\title{
Are computational methods useful for structure elucidation of large and flexible molecules? Belizentrin as a case study
}

\author{
Antonio Hernández Daranas ${ }^{\mathrm{a} *}$ and Ariel M. Sarotti ${ }^{\mathrm{b} *}$ \\ adaranas@ipna.csic.es \\ sarotti@iquir-conicet.gov.ar
}

a) Instituto Universitario de Bio-Orgánica Antonio González, Universidad de La Laguna, 38206, Tenerife, Spain.

b) Instituto de Química Rosario (IQUIR, CONICET-UNR) and Facultad de Ciencias Bioquímicas y Farmacéuticas, Universidad Nacional de Rosario, Suipacha 531 (S2002LRK), Rosario, República Argentina.

\section{Supporting Information}

\section{Table of contents}

- Computational details. Page S2.

- Structures of the diastereoisomers of F1, F2 and F3. Page S3.

- Structures of the eight possible diastereoisomers of belizentrin with the most probable relative configurations of the fragments F1, F2 and F3 found by J-DP4. Page S4.

- B3LYP/6-31G**//MMFF Boltzmann-averaged isotropic shielding values, scaled chemical shifts and coupling constants, absolute scaled errors and DP4/J-DP4 results obtained for all compounds under study. Pages S5-S21.

- J-DP4 probabilities computed using randomly generated ensembles. Page S22.

- DP4 and J-DP4 probabilities recomputed considering only data from the variable regions of the molecule. Page S22.

- Cartesian Coordinates and B3LYP/6-31G**//MMFF energies of all conformations found for the compounds under study at the MMFF level. Pages S21-S3255.

- Fragment F1: Pages S23-S53.

- Fragment F1a: Pages S53-S86.

- Fragment F2: Pages S86-S301.

- Fragment F3: Pages S301-S307.

- Full molecule, isomers 01-08: Pages S307-S3290. 
Computational details. All calculations were done following the general protocols previously described forJ-DP4 methods. ${ }^{1}$ Molecular mechanics conformational searches were undertaken using the Macromodel software (Schrödinger Inc.) and the MMFF94 force field. ${ }^{2} 10^{4}$ (for the fragment approach) or $10^{5}$ (for the full molecule) steps of Mixed Torsional Low-Mode sampling algorithm with a maximum number of 1000 of steps per rotable bond were used. A Polak-Ribier Conjugate Gradient minimization method with a maximum number of 2500 iterations and a convergence threshold of 0.05 were used. A RMSD cutoff of $1.0 \AA$ was used to eliminate redundant conformations. Solvation effects of methanol were simulated using a dielectric constant $\varepsilon=33$ for the media. Extended nonbonded cutoff distances (Van der Waals cutoff of $8.0 \AA$ and an electrostatic cutoff of $20.0 \AA$ ) were used. All local minima within $25 \mathrm{~kJ}$ of the global minimum were saved, and the analysis of the results was undertaken using Maestro software. Quantum mechanical calculations were carried out using the Gaussian 09 package. ${ }^{3}$ Magnetic shielding constants $(\sigma)$ were calculated by means of the gauge including atomic orbitals (GIAO) method, ${ }^{4}$ at the B3LYP/6-31G(d,p)//MMFF level of theory ${ }^{5}$ as recommended for J-DP4. The $J$ coupling calculations were carried out at the same level of theory considering the Fermi contact term (FConly). For $J$ DP4/MIDI! calculations, all conformers found at the MMFF level were first submitted to single point energy calculations at the B3LYP/MIDI! level, and only those with relative energies of $5 \mathrm{kcal} / \mathrm{mol}$ from the corresponding global minima were submitted to NMR calculations at the B3LYP/6-31G** level. Unscaled chemical shifts $\left(\delta_{\mathrm{u}}\right)$ were calculated using TMS as reference standard according to the following expression $\delta_{\mathrm{u}}=\sigma_{0}-\sigma_{\mathrm{x}}$, where $\sigma_{\mathrm{x}}$ is the Boltzmann averaged shielding tensor (over all significantly populated conformations) and $\sigma_{0}$ is the shielding tensor of TMS computed at the same level of theory used to calculate $\sigma_{\mathrm{x}}$. Boltzmann averaging was done according to eq1:

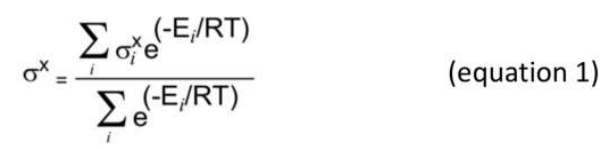

where $\sigma^{\mathrm{x}}$ is the shielding constant for nucleus $\mathrm{x}$ in conformer $i, \mathrm{R}$ is the molar gas constant $\left(8.3145 \mathrm{~J} \mathrm{~K}^{-1} \mathrm{~mol}^{-1}\right), \mathrm{T}$ is the temperature used for the calculation $(298 \mathrm{~K})$, and $E_{\mathrm{i}}$ is the relative energy of conformer $i$ (to the lowest energy conformer) obtained from a single-point NMR calculation at the corresponding level of theory. The scaled chemical shifts $\left(\delta_{\mathrm{s}}\right)$ were computed as $\delta_{\mathrm{s}}=\left(\delta_{\mathrm{u}}-\mathrm{b}\right) / \mathrm{m}$, where $\mathrm{m}$ and $\mathrm{b}$ are the slope and intercept, respectively, resulting from a linear regression calculation on a plot of $\delta_{\mathrm{u}}$ against $\delta_{\mathrm{exp}}$. Proton chemical shifts for each methyl group were averaged due to their conformational freedom. The J-DP4 calculations were carried out using the Excel spreadsheet available for free at sarottinmr.weebly.com. For Random J-DP4 calculations, the isotropic shielding values computed at the J-DP4 level for each isomer were weighted using random conformational amplitudes created as indicated in the original paper. ${ }^{6}$ After 100.000 iterations using a home-made Matlab script, the resulting J-DP4 values were averaged.

1- N. Grimblat, J. A. Gavín, A. Hernández Daranas, A. M. Sarotti, Org. Lett. 2019, 21, 4003-4007.

2- MacroModel Schrodinger release 2018-3; Schrodinger LLC: New York (2018).

3- Frisch, M. J.; Trucks, G. W.; Schlegel, H. B.; Scuseria, G. E.; Robb, M. A.; Cheeseman, J. R.; Scalmani, G.; Barone, V.; Mennucci, B.; Petersson, G. A.; Nakatsuji, H.; Caricato, M.; Li, X.; Hratchian, H. P.; Izmaylov, A. F.; Bloino, J.; Zheng, G.; Sonnenberg, J. L.; Hada, M.; Ehara, M.; Toyota, K.; Fukuda, R.; Hasegawa, J.; Ishida, M.; Nakajima, T.; Honda, Y.; Kitao, O.; Nakai, H.; Vreven, T.; Montgomery, J. A., Jr., Peralta, J. E.; Ogliaro, F.; Bearpark, M.; Heyd, J. J.; Brothers, E.; Kudin, K. N.; Staroverov, V. N.; Kobayashi, R.; Normand, J.; Raghavachari, K.; Rendell, A.; Burant, J. C.; lyengar, S. S.; Tomasi, J.; Cossi, M.; Rega, N.; Millam, J. M.; Klene, M.; Knox, J. E.; Cross, J. B.; Bakken, V.; Adamo, C.; Jaramillo, J.; Gomperts, R.; Stratmann, R. E.; Yazyev, O.; Austin, A. J.; Cammi, R.; Pomelli, C.; Ochterski, J. W.; Martin, R. L.; Morokuma, K.; Zakrzewski, V. G.; Voth, G. A.; Salvador, P.; Dannenberg, J. J.; Dapprich, S.; Daniels, A. D.; Farkas, O.; Foresman, J. B.; Ortiz, J. V.; Cioslowski, J.; Fox, D. J. Gaussian 09, Gaussian, Inc.: Wallingford, CT, 2009.

4- (a) Ditchfield, R. J. Chem. Phys. 1972, 56, 5688-5691. b) Ditchfield, R. Mol. Phys.1974, 27, 789-807. c) Rohlfing, C. M.; Allen, L. C.; Ditchfield, R. Chem. Phys. 1984, 87, 9-15. d) Wolinski, K.; Hinton, J. F.; Pulay, P. J. Am. Chem. Soc. 1990, $112,8251-8260$.

5- (a) Becke, A. D. J. Chem. Phys. 1993, 98, 5648-5652. (b) Lee, C.; Yang, W.; Parr, R. G. Phys. Rev. B: Condens. Matter Mater. Phys. 1988, 37, 785-789. (c) Miehlich, B.; Savin, A.; Stoll, H.; Preuss, H. Chem. Phys. Lett. 1989, 157, $200-206$.

6- M. M. Zanardi, M. O. Marcarino, A. M. Sarotti, Org. Lett.2020, 22, 52-56. 
F1
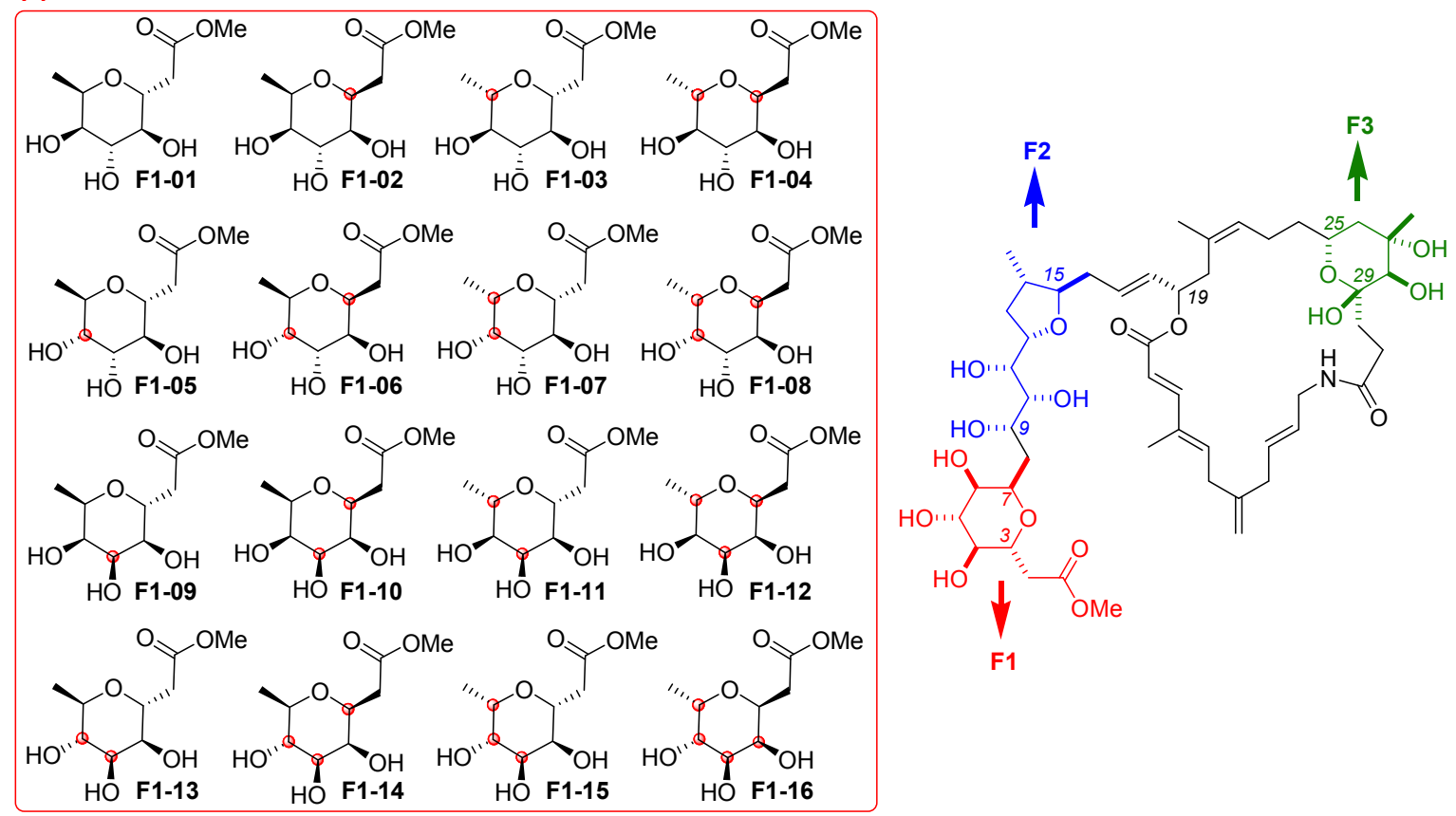

F2

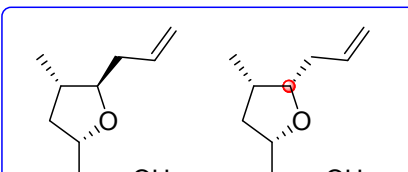

$\mathrm{HO}^{\prime \prime} . \mathrm{OH}$

HO"

"

$\mathrm{HO}_{. .1 \mathrm{OH}} \mathrm{HO}_{. .1 \mathrm{OH}}$

$\mathrm{HO}^{\prime \prime}$

F2-09

1/

HO"

HO"

F2-17

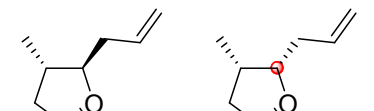

(O)<smiles>C=CCC1OC(C)C[C@@H]1C</smiles><smiles>CCC(C)C(O)O</smiles><smiles>CCCCC1CCCCC1</smiles>

$$
\text { F2-03 }
$$<smiles>C=CCCCC</smiles><smiles>C1CC2CCOC2C1</smiles><smiles>CC(O)C(C)O</smiles><smiles>[CH-]CCCCCC</smiles>

F2-11

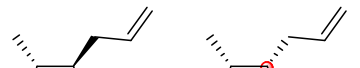<smiles>CCC1OCC[C@@H]1C</smiles><smiles>CC[C@H](O)CO</smiles>
HO"

F2-19

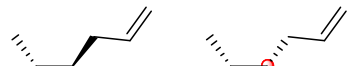<smiles>CCC1OCC[C@@H]1C</smiles>
(O)<smiles>CCCC[C@@H]1OC(CCC)C[C@H]1CC</smiles>
$\mathrm{HO}^{\prime \prime}$ F2-04<smiles>CC[C@H]1OCC[C@@H]1C</smiles>
$\mathrm{HO}$<smiles>[CH]1CCCC1</smiles>
$\mathrm{HO}^{\prime \prime} \overbrace{}^{\mathrm{OH}}$ HO"<smiles>CCC(O)C(O)C(=O)C1C[C@@H](CC)[C@@H](CC)O1</smiles><smiles>C=CCC1O[C]CC1C</smiles>
$\mathrm{HO}^{\prime \prime} \cdot \mathrm{OH}$ F2-05<smiles>C=CCC1OCCC1C</smiles>
. HO'-13<smiles>C=CCC1OCCC1C</smiles>
$\mathrm{HO} " \overbrace{}^{\mathrm{OH}}$ HO" F2-21

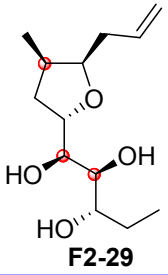<smiles>C=CC[C@H]1C[C@H]([C@@H](O)[C@H](O)[C@H](O)CC)O[C@@H]1CC=C</smiles>
$\mathrm{HO}^{2} \cdot \mathrm{OH}$ F2-14<smiles>C=CC[C]1OCCC1C</smiles>
HOC F2-22<smiles>CC1=C(C)OCC1</smiles>
 F2-30<smiles>C=CCC1OC(C)CC1C</smiles>
$\mathrm{HO}^{\prime \prime \prime} \cdot \mathrm{OH} \mathrm{HO}^{\prime \prime \prime} \cdot{ }^{\prime \prime} \mathrm{OH}$ F2-07 F2-08<smiles>C=CCC1OCCC1C</smiles>
$\mathrm{HO}^{-1} \mathrm{OH}$ HO" F2-15<smiles>C=CCC1OCCC1C</smiles>
HOH F2-23<smiles>C=CCC1OCCC1C</smiles>

$\mathrm{HO}^{2} \mathrm{OH}$ HO" F2-31<smiles>C=CC[C]1OCCC1C</smiles><smiles>CCCCCC(C)O</smiles>
HO" F2-16<smiles>C=CC[C@H]1OCCC1C</smiles><smiles>CC(C)C(O)CO</smiles>
HO" F2-24<smiles>C=CC[C]1OCCC1C</smiles>
$\mathrm{HO}^{\mathrm{OH}}$ $\mathrm{HO}^{\circ}$ F2-32

F3

(n)

Figure $\mathbf{S 1}$. Structures of the diastereoisomers of F1, F2 and F3. 

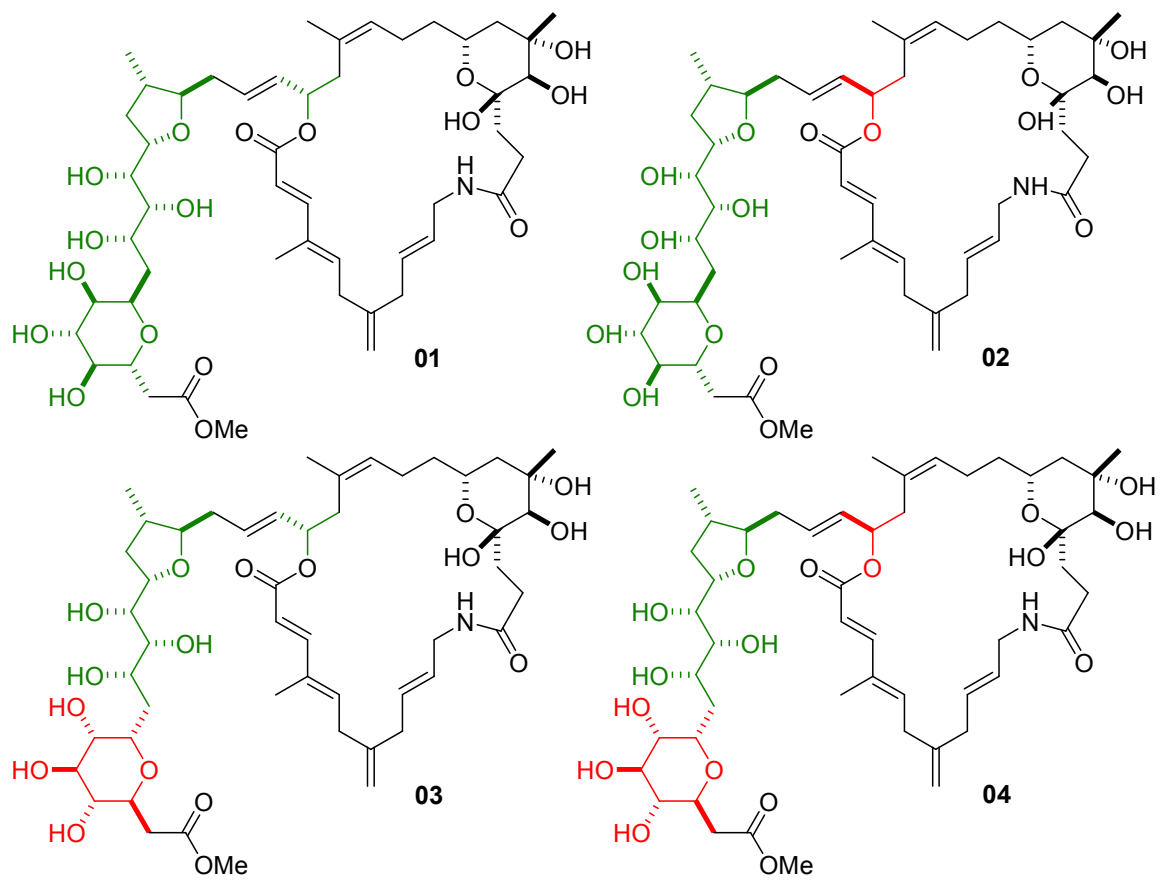

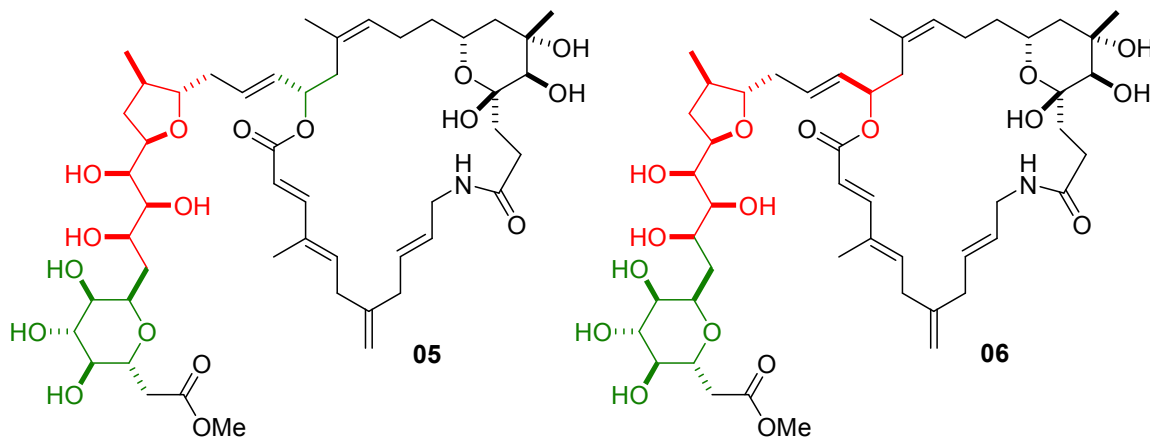

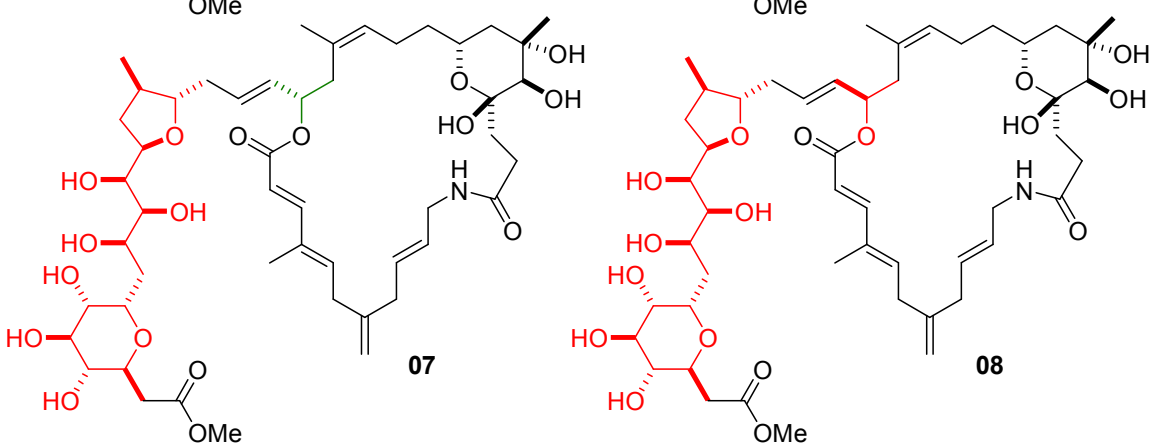

Figure S2. Structures of the eight possible diastereoisomers of belizentrin with the most probable relative configurations of the fragments F1, F2 and F3 found by J-DP4. Green color: right absolute configuration; red color: wrong absolute configuration. 
Table S1. Boltzmann-averaged isotropic shielding values computed for compounds F1-01/F1-16 at the B3LYP/6-31G**//MMFF level of theory following a free conformational sampling procedure.

\begin{tabular}{|c|c|c|c|c|c|c|c|c|c|c|c|c|c|c|c|c|c|}
\hline \multirow{2}{*}{ Atom } & \multirow{2}{*}{ Exp. NMR } & \\
\hline & & F1-01 & $\begin{array}{l}\text { F1-02 } \\
\end{array}$ & $\begin{array}{l}\text { F1-03 } \\
\end{array}$ & F1-04 & F1-05 & F1-06 & $\begin{array}{l}\text { F1-07 } \\
\end{array}$ & $\begin{array}{l}\text { F1-08 } \\
\end{array}$ & F1-09 & F1-10 & F1-11 & F1-12 & F1-13 & F1-14 & F1-15 & F1-16 \\
\hline $\mathrm{C}-1$ & 173.9 & 23.4161 & 23.2603 & 23.0453 & 22.8688 & 23.8120 & 22.8070 & 23.5464 & 24.3733 & 23.2763 & 22.6867 & 23.3103 & 22.9099 & 23.8842 & 22.9464 & 23.4662 & 22.8274 \\
\hline $\mathrm{C}-2$ & & 151.9727 & 154.2531 & 149.9739 & 156.4287 & 151.6653 & 151.8957 & 151.4007 & 156.5572 & 150.5686 & 150.5046 & 150.5793 & 158.0804 & 154.4575 & 154.5613 & 151.6576 & 153.7286 \\
\hline C-3 & & 122.8433 & 117.3311 & 114.9896 & 119.8591 & 121.2028 & 116.3134 & 114.4972 & 119.0428 & 124.4534 & 112.1572 & 118.3876 & 117.6951 & 115.9989 & & 117.9846 & 122.5500 \\
\hline C-4 & & 117.5851 & 120.3200 & 112.6680 & 118.1258 & 118.6391 & 118.2 & 118.4342 & 119.8501 & $\frac{174.4597}{117.4596}$ & 116.3655 & 116.5845 & 121.0571 & 120.5652 & 119.0383 & 120.8902 & 119.0862 \\
\hline & & & & & & & & & & & & & & & & & \\
\hline C-6 & & 7.49 & 120.466 & 115.9 & 116.2 & 117.77 & & 17.0975 & & & & & & & & & \\
\hline C-7 & & 8.4 & 119.3 & 116.9 & 121.4 & 118.3269 & 120.6 & 117.3848 & 122. & & 117.3638 & 121.2836 & 125.1876 & 120.5533 & 117.1377 & 120.3954 & 119.6563 \\
\hline C-36 & & 1.6347 & 141.6852 & 141.5297 & 141.4798 & 141.5827 & 141.4067 & 1.5719 & 141.4341 & 141.5945 & 141.4729 & 141.5838 & 141.6341 & 141.3856 & 141.7420 & 141.6373 & 141.6767 \\
\hline & & & & & & & & & & & & & & & & & \\
\hline$\frac{H-2}{H-2}$ & & & & & & & & $\frac{20.950}{29397}$ & & & & & & & & $\frac{28.99}{29.43}$ & $\frac{28.9589}{29.1308}$ \\
\hline $\mathrm{H}-3$ & & & & & & & & & & & & & & & & & $\frac{29.1308}{27.4481}$ \\
\hline $\mathrm{H}-\mathrm{C}$ & & & & 28.6 & & & & & & & $\frac{20.4}{28.2}$ & & & & & & 28.1335 \\
\hline $\mathrm{H}-5$ & & & & & & 28.0 & & & & & 28.5 & & & & 28.18 & 28.2163 & 27.9281 \\
\hline $\mathrm{H}-6$ & & 27.9994 & 28.1328 & 28.32 & 28.3940 & 28.1267 & 28.5462 & 28.2892 & 28.0 & 28.2205 & 28.1128 & 28.62 & 28.6596 & 27.95 & 27.8093 & 28.1691 & 27.3453 \\
\hline $\mathrm{H}-7$ & & 28.0283 & 27.7448 & 28.31 & & 27.4 & & & 28.0 & & 28.5 & 27.8 & & 28.58 & & 27.7280 & 27.6024 \\
\hline $\mathrm{H}-36$ & 3.6 & 28.1136 & 28.1171 & 28.0963 & 28.0835 & 28.1157 & 28.1164 & 28.1170 & 28.1054 & 28.0974 & 28.1274 & 28.1010 & 28.1127 & 28.1336 & 28.1210 & 28.1148 & 28.1183 \\
\hline
\end{tabular}

Table S2. Scaled chemical shifts computed for compounds F1-01/F1-16 at the B3LYP/6-31G**//MMFF level of theory following a free conformational sampling procedure.

\begin{tabular}{|c|c|c|c|c|c|c|c|c|c|c|c|c|c|c|c|c|c|}
\hline \multirow{2}{*}{ Atom } & \multirow{2}{*}{ Exp. NMR } & \multicolumn{16}{|c|}{ Scaled chemical shifts $(\delta, \mathrm{ppm})$} \\
\hline & & F1-01 & F1-02 & F1-03 & F1-04 & F1-05 & F1-06 & F1-07 & F1-08 & F1-09 & F1-10 & F1-11 & F1-12 & F1-13 & F1-14 & F1-15 & F1-16 \\
\hline C-1 & 173.9 & 173.7 & 173.5 & 172.2 & 172.8 & 173.8 & 174.0 & 173.0 & 173.3 & 174.6 & 173.5 & 173.7 & 173.4 & 173.1 & 172.6 & 173.9 & 174.1 \\
\hline $\mathrm{C}-2$ & 38.4 & 39.2 & 37.8 & 37.3 & 35.7 & 39.4 & 39.3 & 37.8 & 36.4 & 41.5 & 39.0 & 39.6 & 36.0 & 36.7 & 36.0 & 39.4 & 39.3 \\
\hline $\mathrm{C}-3$ & 71.4 & 69.7 & 76.1 & 74.4 & 73.3 & 71.4 & 76.4 & 76.8 & 75.3 & 68.9 & 79.4 & 73.5 & 77.0 & 76.9 & 76.2 & 74.7 & 71.4 \\
\hline C-4 & 75.3 & 75.2 & 73.0 & 76.9 & 75.0 & 74.1 & 74.4 & 72.7 & 74.4 & 76.2 & 74.9 & 75.4 & 73.6 & $\begin{array}{l}72.1 \\
\end{array}$ & 72.9 & 71.6 & 75.0 \\
\hline C-5 & 74.7 & 76.5 & 75.9 & 81.0 & 77.2 & 75.5 & 73.7 & 77.6 & 75.3 & 74.7 & 70.3 & 76.5 & 75.1 & 76.6 & 78.7 & 76.8 & 75.7 \\
\hline $\mathrm{C}-6$ & 73.0 & 75.3 & 72.8 & 73.4 & 77.0 & 75.0 & 73.8 & 74.1 & 75.4 & 71.8 & 74.3 & 75.7 & 76.5 & 76.1 & 73.2 & 75.4 & 72.2 \\
\hline $\mathrm{C}-7$ & 74.8 & 74.2 & 73.9 & 72.4 & 71.6 & 74.5 & 71.9 & 73.8 & 71.5 & 75.1 & 73.9 & 70.4 & 69.4 & 72.1 & 74.9 & 72.2 & $\begin{array}{l}4.4 \\
\end{array}$ \\
\hline C-36 & 52.3 & 50.0 & 50.8 & 46.2 & 51.1 & 50.0 & 50.3 & 48.2 & 52.1 & 50.9 & 48.5 & 49.0 & 52.7 & 50.3 & 49.3 & 49.9 & 51.7 \\
\hline & & & & & & & & & & & & & & & & & \\
\hline $\mathrm{H}-2 \mathrm{a}$ & 2.87 & 2.79 & 2.97 & 2.58 & 2.51 & 2.57 & 2.50 & 2.67 & 2.43 & 2.58 & 2.72 & 2.62 & 3.11 & 2.50 & 2.97 & 2.73 & 2.57 \\
\hline $\mathrm{H}-2 \mathrm{~b}$ & 2.42 & 2.24 & 2.35 & 2.48 & 2.32 & 2.34 & 2.37 & 2.02 & 2.26 & 2.51 & 1.97 & 2.62 & 1.91 & 2.11 & 1.96 & 2.31 & 2.41 \\
\hline $\mathrm{H}-3$ & 3.92 & 4.12 & 4.19 & 4.03 & 4.42 & 3.87 & 4.15 & 3.84 & 4.35 & 4.34 & 3.82 & 4.30 & 4.12 & 4.64 & 4.10 & 4.09 & 3.99 \\
\hline $\mathrm{H}-4$ & 3.10 & 3.32 & 3.08 & 3.22 & 3.74 & 3.70 & 3.60 & 3.96 & 3.93 & 3.05 & 3.76 & 3.13 & 3.64 & 3.94 & 3.48 & 3.56 & 3.35 \\
\hline $\mathrm{H}-5$ & 3.54 & 3.87 & 3.67 & 3.41 & 3.65 & 3.59 & 4.00 & 3.49 & 3.52 & 4.12 & 3.44 & 3.66 & 4.29 & 3.44 & 3.56 & 3.46 & 3.54 \\
\hline $\mathrm{H}-6$ & 3.57 & 3.65 & 3.50 & 3.70 & 3.29 & 3.48 & 3.19 & 3.62 & 3.54 & 3.45 & 4.03 & 3.20 & 2.89 & 3.82 & 4.10 & 3.51 & 4.08 \\
\hline $\mathrm{H}-7$ & 4.05 & 3.62 & 3.86 & 3.72 & 3.55 & 4.09 & 3.75 & 3.67 & 3.57 & 3.54 & 3.39 & 3.93 & 3.61 & 3.08 & 3.33 & 3.92 & 3.84 \\
\hline $\mathrm{H}-36$ & 3.67 & 3.54 & 3.52 & 4.00 & 3.65 & 3.49 & 3.59 & 3.87 & 3.53 & 3.56 & 4.01 & 3.67 & 3.58 & 3.61 & 3.65 & 3.56 & 3.36 \\
\hline
\end{tabular}

Table S3. Absolute scaled errors computed for compounds F1-01/F1-16 at the B3LYP/6-31G**//MMFF level of theory following a free conformational

\begin{tabular}{|c|c|c|c|c|c|c|c|c|c|c|c|c|c|c|c|c|}
\hline \multicolumn{17}{|c|}{ sampling procedure, and DP4 results. } \\
\hline & F1-01 & F1-02 & F1-03 & F1-04 & F1-05 & F1-06 & F1-07 & F1-08 & F1-09 & F1-10 & F1-11 & F1-12 & F1-13 & F1-14 & F1-15 & F1-16 \\
\hline C-1 & 0.2 & 0.4 & 1.7 & 1.1 & 0.1 & 0.1 & 0.9 & 0.6 & 0.7 & 0.4 & 0.2 & 0.5 & 0.8 & 1.3 & 0.0 & 0.2 \\
\hline$c-3$ & 1.7 & 4.7 & 3.0 & 1.9 & 0.0 & 5.0 & 5.4 & 3.9 & 2.5 & 8.0 & 2.1 & 5.6 & 5.5 & 4.8 & 3.3 & 0.0 \\
\hline$c-4$ & 0.1 & 2.3 & 1.6 & 0.3 & 1.2 & 0.9 & 2.6 & $\begin{array}{l}3.9 \\
0.9\end{array}$ & $\begin{array}{l}2.3 \\
0.9\end{array}$ & 0.4 & 0.1 & 1.7 & $\begin{array}{l}3.5 \\
3.2\end{array}$ & 2.8 & $\begin{array}{l}3.3 \\
3.7\end{array}$ & 0.3 \\
\hline$c-5$ & 1.8 & 1.2 & 6.3 & 2.5 & 0.8 & 1.0 & 2.9 & 0.6 & 0.0 & 4.4 & 1.8 & 0.4 & 1.9 & 4.0 & 2.1 & 1.0 \\
\hline$c-6$ & 0.6 & 0.9 & 2.4 & 3.2 & 0.3 & 2.9 & 1.0 & 3.3 & 0.3 & 0.9 & 4.4 & 5.4 & 2.7 & 0.1 & 2.6 & 0.4 \\
\hline$c-36$ & 2.3 & 1.5 & $\begin{array}{l}6.4 \\
6.1\end{array}$ & $\begin{array}{l}1.2 \\
1.2\end{array}$ & 2.3 & 2.0 & $\begin{array}{l}4.1 \\
4.1\end{array}$ & 0.2 & 1.4 & 3.8 & $\begin{array}{l}4.4 \\
3.3\end{array}$ & $\begin{array}{l}0.4 \\
0.4\end{array}$ & 2.0 & 3.0 & $\begin{array}{l}2.0 \\
2.4\end{array}$ & 0.6 \\
\hline Average & 1.2 & 1.5 & 2.8 & 2.1 & 1.0 & 1.7 & 2.3 & 1.7 & 1.3 & 2.5 & 2.0 & 2.5 & 2.6 & 2.3 & 2.2 & 0.5 \\
\hline H-2a & 0.08 & 0.10 & 0.29 & 0.36 & 0.30 & 0.37 & 0.20 & 0.44 & 0.29 & 0.15 & 0.25 & 0.24 & 0.37 & 0.10 & 0.14 & 0.30 \\
\hline $\mathrm{H}-5$ & 0.33 & 0.13 & 0.13 & 0.11 & 0.05 & 0.46 & 0.05 & 0.02 & 0.58 & 0.10 & 0.12 & 0.75 & 0.10 & 0.02 & 0.08 & 0.00 \\
\hline $\mathrm{H}-6$ & 0.08 & 0.07 & 0.13 & 0.28 & 0.09 & 0.38 & 0.05 & 0.03 & 0.12 & 0.46 & 0.37 & 0.68 & 0.25 & 0.53 & 0.06 & 0.51 \\
\hline H-7 & 0.43 & 0.19 & 0.33 & 0.50 & 0.04 & 0.30 & 0.38 & 0.48 & 0.51 & 0.66 & 0.12 & 0.44 & 0.97 & 0.72 & 0.13 & 0.21 \\
\hline $\mathrm{H}-36$ & 0.13 & 0.15 & 0.33 & 0.02 & 0.18 & 0.08 & 0.20 & 0.14 & 0.11 & 0.34 & 0.00 & 0.09 & 0.06 & 0.02 & 0.11 & 0.31 \\
\hline Average & 0.21 & 0.12 & 0.19 & 0.31 & 0.17 & 0.30 & 0.28 & 0.32 & 0.27 & 0.37 & 0.18 & 0.43 & 0.45 & 0.30 & 0.16 & 0.21 \\
\hline
\end{tabular}

\begin{tabular}{|c|c|c|c|c|c|c|c|c|c|c|c|c|c|c|c|c|c|}
\hline \multicolumn{2}{|c|}{ Isomer № } & 1 & 2 & 3 & 4 & 5 & 6 & 7 & 8 & 9 & 10 & 11 & 12 & 13 & 14 & 15 & 16 \\
\hline \multirow{3}{*}{ DP4 (\%) } & C & 4.95 & 1.33 & 0.00 & 0.10 & 13.15 & 0.42 & 0.02 & 0.46 & 3.67 & 0.01 & 0.15 & 0.01 & 0.01 & 0.03 & 0.07 & 75.61 \\
\hline & $\mathrm{H}+\mathrm{C}$ & 1.75 & 73.05 & 0.00 & 0.00 & 13.49 & 0.00 & 0.00 & 0.00 & 0.01 & 0.00 & 0.15 & 0.00 & 0.00 & 0.00 & 0.44 & 11.10 \\
\hline & $\mathrm{J}$ & - & - & - & - & - & - & - & - & - & - & - & - & - & - & - & - \\
\hline
\end{tabular}


Table S4. Boltzmann-averaged isotropic shielding values and coupling constants (FC only) computed for compounds F1-01/F1-16 at the B3LYP/6$31 \mathrm{G}^{* *} / / \mathrm{MMFF}$ level of theory following the iJ-DP4 approach.

\begin{tabular}{|c|c|c|c|c|c|c|c|c|c|c|c|c|c|c|c|c|c|}
\hline \multirow{2}{*}{ Atom } & \multirow{2}{*}{ Exp. NMR } & \multicolumn{16}{|c|}{ Isotropic shielding values $(\sigma, \mathrm{ppm})$} \\
\hline & & F1-01 & F1-02a & F1-03 & F1-04a & F1-05 & F1-06a & F1-07 & F1-08a & F1-09a & F1-10a & F1-11a & F1-12a & F1-13a & F1-14a & F1-15a & F1-16a \\
\hline C-1 & 173.9 & 23.4114 & - & 23.0453 & - & 23.8146 & - & 23.5464 & - & - & - & - & - & - & - & - & - \\
\hline $\mathrm{C}-2$ & 38.4 & 151.9318 & - & 149.9739 & - & 151.6404 & - & 151.4007 & - & - & - & - & - & - & - & - & - \\
\hline $\mathrm{C}-3$ & 71.4 & 122.9131 & - & 114.9896 & - & 121.2453 & - & 114.4972 & - & - & - & - & - & - & - & - & - \\
\hline $\mathrm{C}-4$ & 75.3 & 117.5846 & - & 112.6680 & - & 118.6413 & - & 118.4342 & - & - & - & - & - & - & - & - & - \\
\hline $\mathrm{C}-5$ & 74.7 & 116.2937 & - & 108.8673 & - & 117.3296 & - & 113.7968 & - & - & - & - & - & - & - & - & - \\
\hline C-6 & 73.0 & 117.4656 & - & 115.9766 & - & 117.7663 & - & 117.0975 & - & - & - & - & - & - & - & - & - \\
\hline C-7 & 74.8 & 118.4068 & - & 116.9315 & - & 118.2831 & - & 117.3848 & - & - & - & - & - & - & - & - & - \\
\hline C-36 & 52.3 & 141.6346 & - & 141.5297 & - & 141.5821 & - & 141.5719 & - & - & - & - & - & - & - & - & - \\
\hline & & & & & & & & & & & & & & & & & \\
\hline $\mathrm{H}-2 \mathrm{a}$ & 2.87 & 28.9397 & - & 29.1626 & - & 29.1513 & - & 28.9508 & - & - & - & - & - & - & - & - & - \\
\hline $\mathrm{H}-2 \mathrm{~b}$ & 2.42 & 29.5235 & - & 29.2400 & - & 29.4240 & - & 29.3979 & - & - & - & - & - & - & - & - & - \\
\hline $\mathrm{H}-3$ & 3.92 & 27.4937 & - & 28.0765 & - & 27.6874 & - & 28.1393 & - & - & - & - & - & - & - & - & - \\
\hline $\mathrm{H}-4$ & 3.10 & 28.3439 & - & 28.6857 & - & 27.8794 & - & 28.0527 & - & - & - & - & - & - & - & - & - \\
\hline $\mathrm{H}-5$ & 3.54 & 27.7590 & - & 28.5387 & - & 28.0031 & - & 28.3809 & - & - & - & - & - & - & - & - & - \\
\hline $\mathrm{H}-6$ & 3.57 & 27.9987 & - & 28.3216 & - & 28.1271 & - & 28.2892 & - & - & - & - & - & - & - & - & - \\
\hline $\mathrm{H}-7$ & 4.05 & 28.0314 & - & 28.3117 & - & 27.4393 & - & 28.2570 & - & - & - & - & - & - & - & - & - \\
\hline $\mathrm{H}-36$ & 3.67 & 28.1138 & - & 28.0963 & - & 28.1157 & - & 28.1170 & - & - & - & - & - & - & - & - & - \\
\hline & & & & & & & & & $J$ coupling & only, $\mathrm{H}_{2}$ & & & & & & & \\
\hline$J_{5,6}$ & 8.4 & 8.6 & - & 7.7 & - & 2.5 & - & 2.8 & - & - & - & - & - & - & - & - & - \\
\hline$J_{6.7}$ & 4.6 & 5.8 & - & 9.0 & - & 1.2 & - & 0.6 & - & - & - & - & - & - & - & - & - \\
\hline$J_{2 a, 3}$ & 2.9 & 1.8 & - & 2.0 & - & 2.8 & - & 2.8 & - & - & - & - & - & - & - & - & - \\
\hline$J_{2 \mathrm{~b}, 3}$ & 9.4 & 8.5 & - & 8.9 & - & 8.0 & - & 7.9 & - & - & - & - & - & - & - & - & - \\
\hline
\end{tabular}

${ }^{a}$ No suitable conformation was found within the $10 \mathrm{~kJ} / \mathrm{mol}$ cutoff window

Table S5. Scaled chemical shifts and coupling constants (FC only) computed for compounds F1-01/F1-16 at the B3LYP/6-31G**//MMFF level of theory following the iJ-DP4 approach.

\begin{tabular}{|c|c|c|c|c|c|c|c|c|c|c|c|c|c|c|c|c|c|}
\hline \multirow{2}{*}{ Atom } & \multirow{2}{*}{ Exp. NMR } & \multicolumn{16}{|c|}{ Scaled chemical shifts $(\delta, \mathrm{ppm})$} \\
\hline & & F1-01 & F1-02 & F1-03 & F1-04 & F1-05 & F1-06 & F1-07 & F1-08 & F1-09 & F1-10 & F1-11 & F1-12 & F1-13 & F1-14 & F1-15 & F1-16 \\
\hline C-1 & 173.9 & 173.7 & - & 172.2 & - & 173.8 & - & 173.0 & - & - & - & - & - & - & - & - & - \\
\hline $\mathrm{C}-2$ & 38.4 & 39.2 & - & 37.3 & - & 39.4 & - & 37.8 & - & - & - & - & - & - & - & - & - \\
\hline C-3 & 71.4 & 69.6 & - & 74.4 & - & 71.4 & - & 76.8 & - & - & - & - & - & - & - & - & - \\
\hline $\mathrm{C}-4$ & 75.3 & 75.2 & - & 76.9 & - & 74.1 & - & 72.7 & - & - & - & - & - & - & - & - & - \\
\hline C-5 & 74.7 & 76.5 & - & 81.0 & - & 75.5 & - & 77.6 & - & - & - & - & - & - & - & - & - \\
\hline C-6 & 73.0 & 75.3 & - & 73.4 & - & 75.0 & - & 74.1 & - & - & - & - & - & - & - & - & - \\
\hline C-7 & 74.8 & 74.3 & - & 72.4 & - & 74.5 & - & 73.8 & - & - & - & - & - & - & - & - & - \\
\hline \multirow[t]{2}{*}{$\mathrm{C}-36$} & 52.3 & 50.0 & - & 46.2 & - & 50.0 & - & 48.2 & - & - & - & - & - & - & - & - & - \\
\hline & & - & - & - & - & - & - & - & 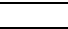 & 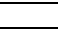 & 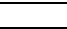 & 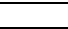 & 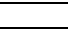 & 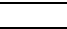 & 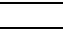 & 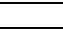 & 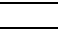 \\
\hline $\mathrm{H}-2 \mathrm{a}$ & 2.87 & 2.78 & - & 2.58 & - & 2.58 & - & 2.67 & - & - & - & - & - & - & - & - & - \\
\hline $\mathrm{H}-2 \mathrm{~b}$ & 2.42 & 2.24 & - & 2.48 & - & 2.34 & - & 2.02 & - & - & - & - & - & - & - & - & - \\
\hline $\mathrm{H}-3$ & 3.92 & 4.11 & - & 4.03 & - & 3.87 & - & 3.84 & - & - & - & - & - & - & - & - & - \\
\hline $\mathrm{H}-4$ & 3.10 & 3.33 & - & 3.22 & - & 3.70 & - & 3.96 & - & - & - & - & - & - & - & - & - \\
\hline $\mathrm{H}-5$ & 3.54 & 3.87 & - & 3.41 & - & 3.59 & - & 3.49 & - & - & - & - & - & - & - & - & - \\
\hline $\mathrm{H}-6$ & 3.57 & 3.65 & - & 3.70 & - & 3.48 & - & 3.62 & - & - & - & - & - & - & - & - & - \\
\hline $\mathrm{H}-7$ & 4.05 & 3.62 & - & 3.72 & - & 4.09 & - & 3.67 & - & - & - & - & - & - & - & - & - \\
\hline \multirow[t]{2}{*}{$\mathrm{H}-36$} & 3.67 & 3.54 & - & 4.00 & - & 3.49 & - & 3.87 & - & - & - & - & - & - & - & - & - \\
\hline & & \multicolumn{16}{|c|}{ Scaled $J(\mathrm{FC}$ only, $\mathrm{Hz})$} \\
\hline$J_{5,6}$ & 8.4 & 9.2 & - & 8.3 & - & 2.8 & - & 3.1 & - & - & - & - & - & - & - & - & - \\
\hline$J_{6.7}$ & 4.6 & 6.3 & - & 9.6 & - & 1.5 & - & 0.8 & - & - & - & - & - & - & - & - & - \\
\hline$J_{2 a, 3}$ & 2.9 & 2.1 & - & 2.3 & - & 3.1 & - & 3.1 & - & - & - & - & - & - & - & - & - \\
\hline$J_{2 b, 3}$ & 9.4 & 9.1 & - & 9.5 & - & 8.6 & - & 8.5 & - & - & - & - & - & - & - & - & - \\
\hline
\end{tabular}

Table S6. Absolute scaled errors computed for compounds F1-01/F1-16 at the B3LYP/6-31G**//MMFF level of theory following the iJ-DP4 approach,

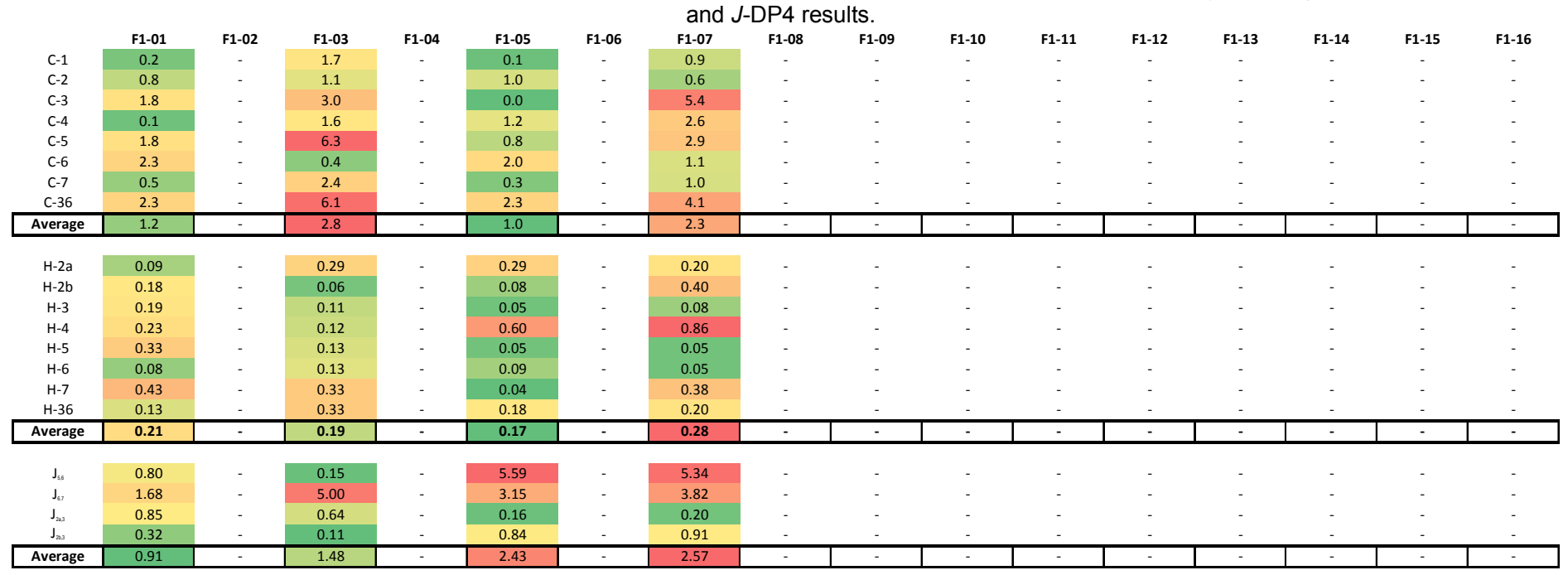

\begin{tabular}{|c|c|c|c|c|c|c|c|c|c|c|c|c|c|c|c|c|c|}
\hline \multicolumn{2}{|c|}{ Isomer № } & 1 & 2 & 3 & 4 & 5 & 6 & 7 & 8 & 9 & 10 & 11 & 12 & 13 & 14 & 15 & 16 \\
\hline \multirow{3}{*}{ J-DP4 (\%) } & $\mathrm{C}$ & 26.19 & - & 0.01 & - & 73.67 & - & 0.13 & - & - & - & - & - & - & - & - & - \\
\hline & $\mathrm{H}+\mathrm{C}$ & 9.64 & - & 0.01 & - & 90.35 & - & 0.00 & - & - & - & - & - & - & - & - & - \\
\hline & $\mathrm{J}$ & 81.33 & - & 17.72 & - & 0.58 & - & 0.36 & - & - & - & - & - & - & - & - & - \\
\hline
\end{tabular}


Calculations of the fragment F1-acid

Given the modest results obtained with DP4 when analyzing fragment F1, we recomputed the full procedure using the free carboxylic acid (F1a) and

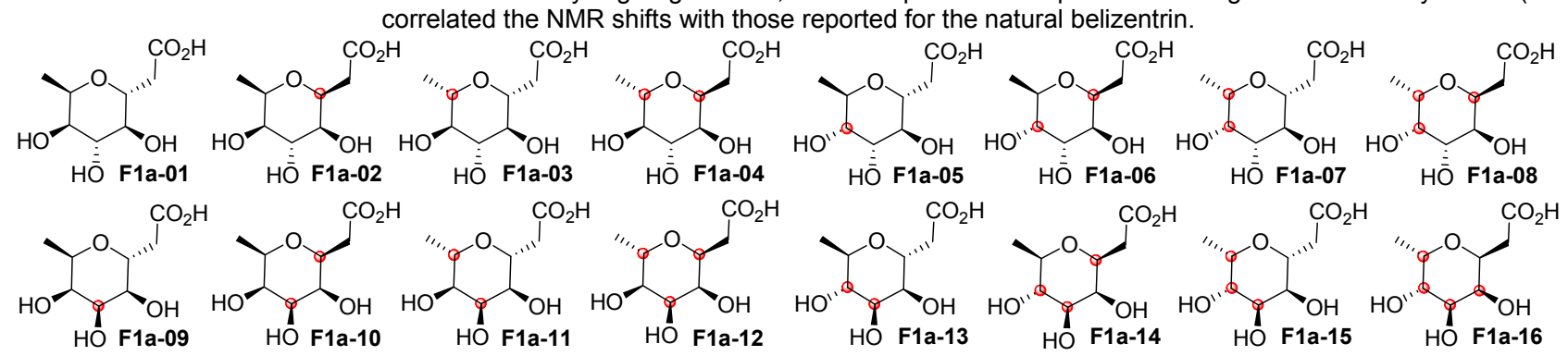

Figure S3. Structures of the F1-acid (F1a)

Table S7. Boltzmann-averaged isotropic shielding values computed for compounds F1a-01/F1a-16 at the B3LYP/6-31G**//MMFF level of theory following a free conformational sampling procedure.

\begin{tabular}{|c|c|c|c|c|c|c|c|c|c|c|c|c|c|c|c|c|c|}
\hline \multirow{2}{*}{ Atom } & \multirow{2}{*}{ Exp. NMR } & \multicolumn{16}{|c|}{ Isotropic shielding values $(\sigma, \mathrm{ppm})$} \\
\hline & & F1a-01 & F1a-02 & F1a-03 & F1a-04 & F1a-05 & F1a-06 & F1a-07 & F1a-08 & F1a-09 & F1a-10 & F1a-11 & F1a-12 & F1a-13 & F1a-14 & F1a-15 & F1a-16 \\
\hline $\mathrm{C}-1$ & 179.4 & 29.2966 & 27.4127 & 27.8782 & 27.1285 & 26.5974 & 26.7157 & 29.2010 & 26.8410 & 28.6612 & 28.0477 & 28.4005 & 26.3769 & 28.5468 & 26.2834 & 29.0230 & 26.4327 \\
\hline $\mathrm{C}-2$ & 41.7 & 151.6284 & 149.6495 & 149.4548 & 151.1442 & 154.1461 & 150.7064 & 151.8204 & 150.3633 & 160.2926 & 150.7288 & 150.8955 & 153.8589 & 160.9172 & 154.0193 & 151.9292 & 153.8253 \\
\hline C-3 & 71.4 & 122.9160 & 116.3203 & 113.5686 & 122.5981 & 119.9103 & 117.6793 & 115.8563 & 121.6965 & 117.2870 & 113.8488 & 118.1206 & 122.6255 & 116.9144 & 115.4676 & 119.5246 & 122.7413 \\
\hline $\mathrm{C}-4$ & 75.6 & 120.1047 & 117.6920 & 114.1931 & 119.9970 & 118.1035 & 114.4341 & 122.7963 & 116.1392 & 116.5609 & 115.5675 & 119.5016 & 119.1440 & 119.0984 & 118.7675 & 123.5140 & 115.6957 \\
\hline C-5 & 74.5 & 116.6689 & 117.6592 & 109.1030 & 117.2801 & 115.5714 & 116.1533 & 113.1218 & 117.0559 & 122.5361 & 120.9493 & 115.8363 & 123.6184 & 115.0516 & 112.4612 & 115.8027 & 117.1130 \\
\hline C-6 & 72.8 & 117.8751 & 120.4204 & 116.3922 & 117.0623 & 119.5901 & 120.4466 & 116.3634 & 123.3264 & 119.0559 & 117.3920 & 117.4664 & 116.2304 & 116.0813 & 116.3401 & 118.2135 & 122.7778 \\
\hline C-7 & 76.2 & 117.9116 & 119.3393 & 117.3300 & 117.3162 & 118.0245 & 119.2000 & 116.6597 & 120.2181 & 123.6500 & 116.0043 & 120.0017 & 114.7693 & 121.4276 & 114.9177 & 119.5951 & 120.3456 \\
\hline & & & & & & & & & & & & & & & & & \\
\hline $\mathrm{H}-2 \mathrm{a}$ & 2.67 & 29.0012 & 29.3626 & 29.1756 & 29.1822 & 28.8866 & 29.2277 & 28.9654 & 29.2724 & 28.9017 & 29.5918 & 29.2337 & 28.5898 & 28.9258 & 28.5536 & 29.2428 & 28.6292 \\
\hline $\mathrm{H}-2 \mathrm{~b}$ & 2.16 & 29.6560 & 29.4897 & 29.6598 & 29.5348 & 29.2546 & 29.6191 & 29.5406 & 29.5635 & 29.9539 & 29.8894 & 29.6255 & 29.4573 & 29.8803 & 29.4890 & 29.4668 & 29.5806 \\
\hline $\mathrm{H}-3$ & 3.85 & 27.8483 & 27.6678 & 28.4700 & 27.3759 & 28.0793 & 27.6102 & 28.3242 & 27.4566 & 26.9717 & 28.3443 & 27.7904 & 27.4935 & 27.0003 & 27.8958 & 27.8992 & 27.4525 \\
\hline $\mathrm{H}-4$ & 2.97 & 27.9454 & 28.7005 & 28.7311 & 27.9258 & 28.0728 & 28.3785 & 27.4248 & 28.0244 & 28.4213 & 27.9896 & 28.4694 & 27.9018 & 27.9027 & 27.9844 & 27.7014 & 28.5793 \\
\hline $\mathrm{H}-5$ & 3.43 & 27.7408 & 27.9276 & 28.5043 & 27.8918 & 28.4719 & 28.0965 & 28.2390 & 27.9856 & 28.3105 & 28.4381 & 27.9974 & 28.0965 & 28.7572 & 28.7731 & 28.2201 & 27.9822 \\
\hline $\mathrm{H}-6$ & 3.48 & 27.9162 & 28.1704 & 28.2639 & 28.6661 & 27.7859 & 28.0587 & 28.4838 & 27.7083 & 28.1499 & 28.2541 & 28.6074 & 28.6067 & 28.0255 & 28.1267 & 28.0896 & 27.3094 \\
\hline $\mathrm{H}-7$ & 3.94 & 27.9873 & 27.7240 & 28.2874 & 27.4921 & 27.4162 & 28.0208 & 28.2075 & 27.7342 & 28.1387 & 28.3046 & 27.9224 & 27.4242 & 28.7136 & 28.8102 & 27.7854 & 27.3636 \\
\hline
\end{tabular}

Table S8. Scaled chemical shifts computed for compounds F1a-01/F1a-16 at the B3LYP/6-31G**//MMFF level of theory following a free conformational sampling procedure.

\begin{tabular}{|c|c|c|c|c|c|c|c|c|c|c|c|c|c|c|c|c|c|}
\hline \multirow{2}{*}{ Atom } & \multirow{2}{*}{ Exp. NMR } & \multicolumn{16}{|c|}{ Scaled chemical shifts $(\delta, \mathrm{ppm})$} \\
\hline & & F1a-01 & F1a-02 & F1a-03 & F1a-04 & F1a-05 & F1a-06 & F1a-07 & F1a-08 & F1a-09 & F1a-10 & F1a-11 & F1a-12 & F1a-13 & F1a-14 & F1a-15 & F1a-16 \\
\hline C-1 & 179.4 & 178.2 & 178.7 & 177.3 & 178.5 & 177.6 & 178.2 & 177.7 & 178.9 & 176.7 & 177.9 & 178.3 & 178.0 & 175.9 & 176.9 & 178.4 & 178.1 \\
\hline $\mathrm{C}-2$ & 41.7 & 37.8 & 38.9 & 34.4 & 38.5 & 35.7 & 37.6 & 35.2 & 40.1 & 32.1 & 36.2 & 37.5 & 37.1 & 29.4 & 33.1 & 37.8 & 37.6 \\
\hline $\mathrm{C}-3$ & 71.4 & 70.7 & 77.0 & 76.6 & 70.7 & 73.8 & 75.0 & 77.0 & 72.3 & 79.3 & 78.8 & 75.2 & 71.6 & 78.1 & 76.5 & 74.9 & 71.9 \\
\hline $\mathrm{C}-4$ & 75.6 & 74.0 & 75.5 & 75.9 & 73.6 & 75.8 & 78.7 & 69.0 & 78.5 & 80.1 & 76.9 & 73.6 & 75.5 & 75.7 & 72.8 & 70.3 & 79.6 \\
\hline $\mathrm{C}-5$ & 74.5 & 77.9 & 75.5 & 81.9 & 76.7 & 78.6 & 76.8 & 80.2 & 77.5 & 73.6 & 70.6 & 77.8 & 70.5 & 80.2 & 79.9 & 79.1 & 78.1 \\
\hline $\mathrm{C}-6$ & 72.8 & 76.5 & 72.3 & 73.3 & 77.0 & 74.2 & 71.9 & 76.4 & 70.4 & 77.4 & 74.7 & 76.0 & 78.7 & 79.1 & 75.5 & 76.4 & 71.8 \\
\hline C-7 & 76.2 & 76.5 & 73.6 & 72.2 & 76.7 & 75.9 & 73.3 & 76.1 & 73.9 & 72.3 & 76.3 & 73.1 & 80.3 & 73.1 & 77.1 & 74.8 & 74.5 \\
\hline $\mathrm{H}-2 \mathrm{a}$ & 2.67 & 2.50 & 2.40 & 2.62 & 2.43 & 251 & 2.46 & 2.34 & 2.35 & 2.81 & 2.20 & 2.51 & 283 & 2.73 & 314 & 223 & 281 \\
\hline $\mathrm{H}-2 \mathrm{~b}$ & 2.16 & 1.83 & 2.29 & 1.97 & $\begin{array}{l}2.70 \\
2.12\end{array}$ & 2.08 & 2.09 & 1.36 & 2.10 & 1.96 & 1.87 & 2.12 & $\begin{array}{l}1.00 \\
\end{array}$ & 1.73 & $\begin{array}{l}1.17 \\
\end{array}$ & $\begin{array}{l}1.209 \\
\end{array}$ & 2.05 \\
\hline $\mathrm{H}-3$ & 3.85 & 3.68 & 3.89 & 3.56 & 4.02 & 3.45 & 3.99 & 3.44 & 3.89 & 4.38 & 3.60 & 3.94 & 3.98 & 4.74 & 4.58 & 3.70 & 3.75 \\
\hline $\mathrm{H}-4$ & 2.97 & 3.58 & 2.98 & 3.21 & 3.54 & 3.46 & 3.26 & 4.97 & 3.40 & 3.20 & 3.99 & 3.27 & 3.55 & 3.79 & 4.39 & 3.92 & 2.85 \\
\hline $\mathrm{H}-5$ & 3.43 & 3.79 & 3.66 & 3.51 & 3.57 & 2.99 & 3.53 & 3.58 & 3.44 & 3.29 & 3.49 & 3.74 & 3.35 & 2.90 & 2.66 & 3.35 & 3.33 \\
\hline $\mathrm{H}-6$ & 3.48 & 3.61 & 3.45 & 3.83 & 2.89 & 3.79 & 3.57 & 3.17 & 3.67 & 3.42 & 3.70 & 3.13 & 2.81 & 3.67 & 4.08 & 3.49 & 3.87 \\
\hline $\mathrm{H}-7$ & 3.94 & 3.53 & 3.84 & 3.80 & 3.92 & 4.23 & 3.60 & 3.64 & 3.65 & 3.43 & 3.64 & 3.81 & 4.06 & 2.95 & 2.57 & 3.83 & 3.83 \\
\hline
\end{tabular}

Table S9. Absolute scaled errors computed for compounds F1a-01/F1a-16 at the B3LYP/6-31G**//MMFF level of theory following a free conformational

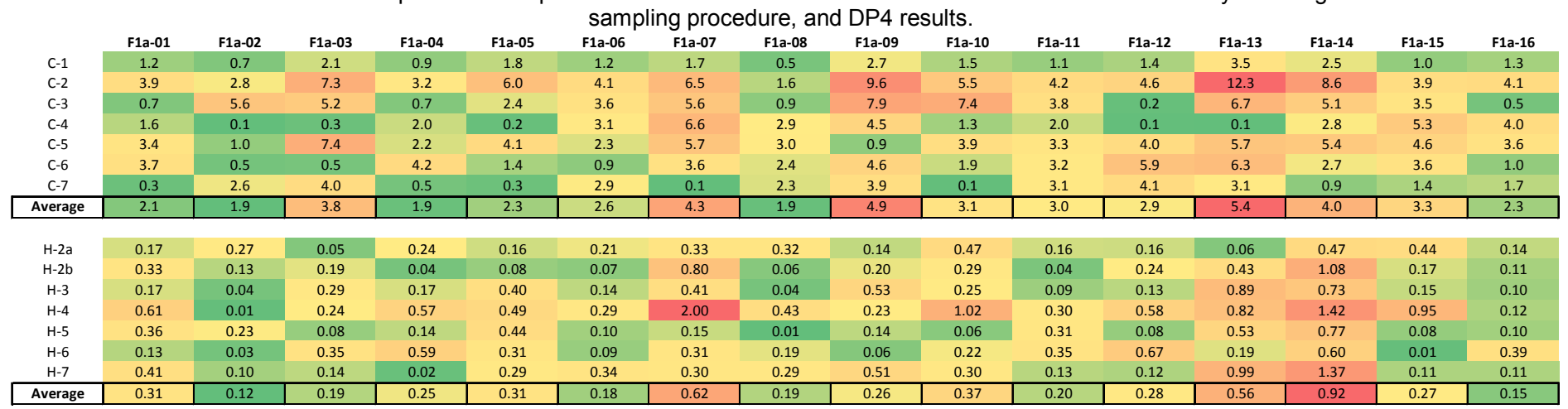

\begin{tabular}{|c|c|c|c|c|c|c|c|c|c|c|c|c|c|c|c|c|c|}
\hline \multicolumn{2}{|c|}{ Isomer № } & 1 & 2 & 3 & 4 & 5 & 6 & 7 & 8 & 9 & 10 & 11 & 12 & 13 & 14 & 15 & 16 \\
\hline \multirow{2}{*}{ DP4 (\%) } & $\mathrm{C}$ & 11.14 & 19.27 & 0.00 & 24.80 & 3.43 & 2.07 & 0.00 & 33.28 & 0.00 & 0.09 & 0.46 & 0.21 & 0.00 & 0.00 & 0.06 & 5.17 \\
\hline & $\mathrm{J}$ & & & & & & & & & & & & & - & & - & \\
\hline
\end{tabular}


Table S10. Boltzmann-averaged isotropic shielding values and coupling constants (FC only) computed for compounds F1a-01/F1a-16 at the B3LYP/6$31 \mathrm{G}^{* *} / / \mathrm{MMFF}$ level of theory following the iJ-DP4 approach

\begin{tabular}{|c|c|c|c|c|c|c|c|c|c|c|c|c|c|c|c|c|c|}
\hline \multirow{2}{*}{ Atom } & \multirow{2}{*}{ Exp. NMR } & \multicolumn{16}{|c|}{ Isotropic shielding values $(\sigma, \mathrm{ppm})$} \\
\hline & & F1a-01 & F1a-02a & F1a-03 & F1a-04a & F1a-05 & F1a-06a & F1a-07 & F1a-08a & F1a-09a & F1a-10a & F1a-11a & F1a-12a & F1a-13a & F1a-14a & F1a-15a & F1a-16a \\
\hline C-1 & 179.4 & 29.2954 & - & 27.8782 & - & 26.5974 & - & 29.2010 & - & - & - & - & - & - & - & - & - \\
\hline $\mathrm{C}-2$ & 41.7 & 151.6197 & - & 149.4548 & - & 154.1461 & - & 151.8204 & - & - & - & - & - & - & - & - & - \\
\hline $\mathrm{C}-3$ & 71.4 & 122.9262 & - & 113.5686 & - & 119.9103 & - & 115.8563 & - & - & - & - & - & - & - & - & - \\
\hline C-4 & 75.6 & 120.1028 & - & 114.1931 & - & 118.1035 & - & 122.7963 & - & - & - & - & - & - & - & - & - \\
\hline C-5 & 74.5 & 116.6669 & - & 109.1030 & - & 115.5714 & - & $\begin{array}{l}113.1218 \\
\end{array}$ & - & - & - & - & - & - & - & - & - \\
\hline C-6 & 72.8 & 117.8764 & - & 116.3922 & - & 119.5901 & - & 116.3634 & - & - & - & - & - & - & - & - & - \\
\hline C-7 & 76.2 & 117.8934 & - & 117.3300 & - & 118.0245 & - & 116.6597 & - & - & - & - & - & - & - & - & - \\
\hline & & & & & & & & & & & & & & & & & \\
\hline $\mathrm{H}-2 \mathrm{a}$ & 2.67 & 29.0021 & - & 29.1756 & - & 28.8866 & - & 28.9654 & - & - & - & - & - & - & - & - & - \\
\hline$H-2 b$ & 2.16 & 29.6550 & - & 29.6598 & - & 29.2546 & - & 29.5406 & - & - & - & - & - & - & - & - & - \\
\hline $\mathrm{H}-3$ & 3.85 & 27.8499 & - & 28.4700 & - & 28.0793 & - & 28.3242 & - & - & - & - & - & - & - & - & - \\
\hline $\mathrm{H}-4$ & 2.97 & 27.9451 & - & 28.7311 & - & 28.0728 & - & 27.4248 & - & - & - & - & - & - & - & - & - \\
\hline $\mathrm{H}-5$ & 3.43 & 27.7403 & - & 28.5043 & - & 28.4719 & - & 28.2390 & - & - & - & - & - & - & - & - & - \\
\hline $\mathrm{H}-6$ & 3.48 & 27.9142 & - & 28.2639 & - & 27.7859 & - & 28.4838 & - & - & - & - & - & - & - & - & - \\
\hline \multirow[t]{2}{*}{$\mathrm{H}-7$} & 3.94 & 27.9877 & - & 28.2874 & - & 27.4162 & - & 28.2075 & - & - & - & - & - & - & - & - & - \\
\hline & & \multicolumn{16}{|c|}{$J$ coupling ( $\mathrm{FC}$ only, $\mathrm{Hz}$ ) } \\
\hline$J_{5,6}$ & 10.5 & 8.7 & - & 7.8 & - & 2.5 & - & 2.4 & - & - & - & - & - & - & - & - & - \\
\hline$J_{6.7}$ & 4.2 & 5.8 & - & 9.1 & - & 1.2 & - & 0.7 & - & - & - & - & - & - & - & - & - \\
\hline
\end{tabular}

${ }^{a}$ No suitable conformation was found within the $10 \mathrm{~kJ} / \mathrm{mol}$ cutoff window

Table S11. Scaled chemical shifts and coupling constants (FC only) computed for compounds F1a-01/F1a-16 at the B3LYP/6-31G**//MMFF level of theory following the iJ-DP4 approach.

\begin{tabular}{|c|c|c|c|c|c|c|c|c|c|c|c|c|c|c|c|c|c|}
\hline \multirow{2}{*}{ Atom } & \multirow{2}{*}{ Exp. NMR } & \multicolumn{16}{|c|}{ Scaled chemical shifts $(\delta, \mathrm{ppm})$} \\
\hline & & F1a-01 & F1a-02 & F1a-03 & F1a-04 & F1a-05 & F1a-06 & F1a-07 & F1a-08 & F1a-09 & F1a-10 & F1a-11 & F1a-12 & F1a-13 & F1a-14 & F1a-15 & F1a-16 \\
\hline C-1 & 179.4 & 178.2 & - & 177.3 & - & 177.6 & - & 177.7 & - & - & - & - & - & - & - & - & - \\
\hline $\mathrm{C}-2$ & 41.7 & 37.8 & - & 34.4 & - & 35.7 & - & 35.2 & - & - & - & - & - & - & - & - & - \\
\hline $\mathrm{C}-3$ & 71.4 & 70.7 & - & 76.6 & - & 73.8 & - & 77.0 & - & - & - & - & - & - & - & - & - \\
\hline $\mathrm{C}-4$ & 75.6 & 74.0 & - & 75.9 & - & 75.8 & - & 69.0 & - & - & - & - & - & - & - & - & - \\
\hline C-5 & 74.5 & 77.9 & - & 81.9 & - & 78.6 & - & 80.2 & - & - & - & - & - & - & - & - & - \\
\hline$C-6$ & 72.8 & 76.5 & - & 73.3 & - & 74.2 & - & 76.4 & - & - & - & - & - & - & - & - & - \\
\hline C-7 & 76.2 & 76.5 & - & 72.2 & - & 75.9 & - & 76.1 & - & - & - & - & - & - & - & - & - \\
\hline & & & & & & & & & & & & & & & & & \\
\hline $\mathrm{H}-2 \mathrm{a}$ & 2.67 & 2.50 & - & 2.62 & - & 2.51 & - & 2.34 & - & - & - & - & - & - & - & - & - \\
\hline $\mathrm{H}-2 \mathrm{~b}$ & 2.16 & 1.83 & - & 1.97 & - & 2.08 & - & 1.36 & - & - & - & - & - & - & - & - & - \\
\hline $\mathrm{H}-3$ & 3.85 & 3.67 & - & 3.56 & - & 3.45 & - & 3.44 & - & - & - & - & - & - & - & - & - \\
\hline $\mathrm{H}-4$ & 2.97 & 3.58 & - & 3.21 & - & 3.46 & - & 4.97 & - & - & - & - & - & - & - & - & - \\
\hline $\mathrm{H}-5$ & 3.43 & 3.79 & - & 3.51 & - & 2.99 & - & 3.58 & - & - & - & - & - & - & - & - & - \\
\hline $\mathrm{H}-6$ & 3.48 & 3.61 & - & 3.83 & - & 3.79 & - & 3.17 & - & - & - & - & - & - & - & - & - \\
\hline $\mathrm{H}-7$ & 3.94 & 3.53 & - & 3.80 & - & 4.23 & - & 3.64 & - & - & - & - & - & - & - & - & - \\
\hline & & \multicolumn{16}{|c|}{ Scaled J (FC only, $\mathrm{Hz})$} \\
\hline$J_{5,6}$ & 10.5 & 9.3 & - & 8.3 & - & 2.8 & - & 2.7 & - & - & - & - & - & - & - & - & - \\
\hline$J_{6.7}$ & 4.2 & 6.3 & - & 9.7 & - & 1.4 & - & 0.9 & - & - & - & - & - & - & - & - & - \\
\hline
\end{tabular}

Table S12. Absolute scaled errors computed for compounds F1a-01/F1a-16 at the B3LYP/6-31G**//MMFF level of theory following the iJ-DP4

\begin{tabular}{|c|c|c|c|c|c|c|c|c|c|c|c|c|c|c|c|c|}
\hline & F1a-01 & F1a-02 & F1a-03 & F1a-04 & F1a-05 & F1a-06 & $\begin{array}{c}\text { proach } \\
\text { F1a-07 }\end{array}$ & $\begin{array}{c}\text { nd } J \text {-[ } \\
\text { F1a-08 }\end{array}$ & $\begin{array}{l}4 \text { results } \\
\text { F1a-09 }\end{array}$ & F1a-10 & F1a-11 & F1a-12 & F1a-13 & F1a-14 & F1a-15 & F1a-16 \\
\hline C-1 & 1.2 & - & 2.1 & - & 1.8 & & 1.7 & & & - & - & & & & & \\
\hline $\mathrm{C}-2$ & 3.9 & - & 7.3 & - & 6.0 & - & 6.5 & - & - & - & - & - & & - & - & - \\
\hline $\mathrm{C}-3$ & 0.7 & - & 5.2 & - & 2.4 & - & 5.6 & - & - & - & - & - & - & - & - & - \\
\hline C-4 & 1.6 & - & 0.3 & - & 0.2 & - & 6.6 & - & - & - & - & - & & - & - & - \\
\hline$c-5$ & 3.4 & - & 7.4 & - & 4.1 & - & 5.7 & - & - & - & - & - & - & - & - & - \\
\hline$c-6$ & 3.7 & - & 0.5 & - & 1.4 & - & 3.6 & - & - & - & - & - & - & - & - & - \\
\hline$c-7$ & 0.3 & - & 4.0 & - & 0.3 & - & 0.1 & - & - & - & - & - & - & - & - & - \\
\hline Average & 2.1 & - & 3.8 & - & 2.3 & - & 4.3 & - & - & - & - & - & - & - & - & - \\
\hline $\mathrm{H}-2 \mathrm{a}$ & 0.17 & - & 0.05 & - & 0.16 & - & 0.33 & - & - & - & - & - & - & - & - & - \\
\hline$H-2 b$ & 0.33 & - & 0.19 & - & 0.08 & - & 0.80 & - & - & - & - & - & - & - & - & - \\
\hline $\mathrm{H}-3$ & 0.18 & - & 0.29 & - & 0.40 & - & 0.41 & - & - & - & - & - & - & - & - & - \\
\hline $\mathrm{H}-4$ & 0.61 & & 0.24 & - & 0.49 & - & 2.00 & - & - & - & - & - & - & - & - & - \\
\hline $\mathrm{H}-5$ & 0.36 & - & 0.08 & - & 0.44 & - & 0.15 & - & - & - & - & - & - & - & - & - \\
\hline $\mathrm{H}-6$ & 0.13 & & 0.35 & - & 0.31 & - & 0.31 & - & - & - & - & - & - & - & - & - \\
\hline $\mathrm{H}-7$ & 0.41 & - & 0.14 & - & 0.29 & - & 0.30 & - & - & - & - & - & - & - & - & - \\
\hline Average & 0.31 & - & 0.19 & - & 0.31 & - & 0.62 & - & - & - & - & - & - & - & - & - \\
\hline$J_{\mathrm{sg}}$ & 1.17 & - & 2.18 & - & 7.68 & - & 7.80 & - & - & - & - & - & - & - & - & - \\
\hline$J_{\mathrm{ata}}$ & 2.09 & - & 5.51 & - & 2.75 & - & 3.32 & - & - & - & - & - & - & - & - & - \\
\hline Average & 1.63 & & 3.85 & & 5.22 & & 5.56 & & - & - & - & - & - & - & - & - \\
\hline
\end{tabular}

\begin{tabular}{|c|c|c|c|c|c|c|c|c|c|c|c|c|c|c|c|c|c|}
\hline \multicolumn{2}{|c|}{ Isomer № } & 1 & 2 & 3 & 4 & 5 & 6 & 7 & 8 & 9 & 10 & 11 & 12 & 13 & 14 & 15 & 16 \\
\hline \multirow{3}{*}{ J-DP4 (\%) } & C & 76.34 & - & 0.02 & - & 23.64 & - & 0.00 & - & - & - & - & - & - & - & - & - \\
\hline & $\mathrm{J}$ & 95.71 & - & 3.11 & - & 0.73 & - & 0.45 & - & - & - & - & - & - & - & - & - \\
\hline & all data & 98.72 & - & 1.01 & - & 0.27 & - & 0.00 & - & - & - & - & - & - & - & - & - \\
\hline
\end{tabular}


Table S13. Boltzmann-averaged isotropic shielding values computed for compounds F2-01/F2-32 at the B3LYP/6-31G**//MMFF level of theory following a free conformational sampling procedure.

\begin{tabular}{|c|c|c|c|c|c|c|c|c|c|c|c|c|c|c|c|c|c|}
\hline \multirow{2}{*}{ Atom } & \multirow{2}{*}{ Exp. NMR } & \multicolumn{16}{|c|}{ Isotropic shielding values $(\sigma, \mathrm{ppm})$} \\
\hline & & F2-01 & F2-02 & F2-03 & F2-04 & F2-05 & F2-06 & F2-07 & F2-08 & F2-09 & F2-10 & F2-11 & F2-12 & F2-13 & F2-14 & F2-15 & F2-16 \\
\hline C-9 & 71.5 & 114.4029 & 114.7797 & 112.7647 & 114.8887 & 115.2156 & 114.7377 & 112.7273 & 115.1287 & 116.2010 & 116.5525 & 114.6265 & 115.0821 & 117.0974 & 116.1476 & 114.3537 & 115.2187 \\
\hline $\mathrm{C}-10$ & 73.5 & 113.5343 & 112.3912 & 118.4183 & 114.0904 & 114.0416 & 111.7394 & 117.0891 & 113.1718 & 119.0931 & 114.4179 & 109.4755 & 111.2171 & 112.6092 & 113.5733 & 109.5815 & 110.1846 \\
\hline C-11 & 75.8 & 114.1909 & 115.8582 & 115.5079 & 113.4398 & 113.3902 & 114.8823 & 116.3460 & 113.6538 & 113.6829 & 117.8714 & 118.0321 & 118.5353 & 117.8511 & 118.9375 & 120.6427 & 118.9548 \\
\hline C-12 & 80.2 & 109.4620 & 108.6220 & 110.5299 & 112.2958 & 110.6420 & 110.7875 & 107.6586 & 111.6161 & 111.3760 & 111.0613 & 114.0118 & 113.2861 & 111.1788 & 110.4219 & 111.3618 & 112.0441 \\
\hline C-13 & 38.3 & 153.3782 & 155.1591 & 157.3529 & 154.6355 & 154.9260 & 151.8814 & 157.1713 & 152.7895 & 151.2646 & 156.2831 & 152.9397 & 154.7148 & 155.6020 & 155.9715 & 154.9417 & 152.8411 \\
\hline C-14 & 40.5 & 151.1861 & 154.6170 & 151.9421 & 153.0660 & 153.7502 & 151.7706 & 153.2098 & 150.6118 & 153.6479 & 153.5517 & 153.3664 & 154.1229 & 154.5567 & 153.0247 & 155.0277 & 151.6816 \\
\hline C-15 & 86.2 & 106.6410 & 1110.4039 & 108.9295 & 110.2080 & 110.4531 & 107.0741 & 1110.4174 & 106.0023 & 106.9797 & 112.8269 & 107.2013 & 109.8634 & 109.4781 & 108.1527 & 110.5505 & 106.2928 \\
\hline C-16 & 37.9 & 149.4978 & 151.7381 & 146.2788 & 152.2587 & 152.4553 & 149.5514 & 151.1571 & 149.7222 & 152.0308 & 150.9841 & 149.8219 & 152.4548 & 152.9021 & 147.8605 & 151.8696 & 149.4464 \\
\hline C-33 & 16.7 & 174.5149 & 176.0598 & 171.0597 & 175.1618 & 175.4700 & 174.5559 & 175.9917 & 174.4364 & 174.7246 & 175.8494 & 173.7980 & 175.3159 & 175.7857 & 172.6438 & 176.2029 & 174.5589 \\
\hline $\mathrm{H}-9$ & 3.99 & 27.9711 & 27.9463 & 28.0800 & 27.9712 & 27.9038 & 27.9592 & 28.0587 & 27.9027 & 27.9450 & 28.2823 & 28.2690 & 28.2451 & 28.1445 & 28.1236 & 28.4007 & 28.3420 \\
\hline $\mathrm{H}-10$ & 3.52 & 28.4798 & 28.4044 & 28.4506 & 28.6658 & 28.4576 & 28.3095 & 28.6301 & 28.6908 & 27.9351 & 27.9812 & 28.2440 & 28.2430 & 28.2427 & 28.2014 & 28.1895 & 28.2407 \\
\hline $\mathrm{H}-11$ & 3.57 & 28.3126 & 28.3369 & 27.8698 & 28.0388 & 28.3304 & 28.5292 & 27.7632 & 28.1400 & 27.9066 & 27.7328 & 28.4669 & 28.4164 & 27.9848 & 27.9686 & 28.3270 & 28.3509 \\
\hline $\mathrm{H}-12$ & 12 & 27.5425 & 27.6277 & 27.4519 & 27.4799 & 27.2595 & 27.6629 & 27.7111 & 27.7149 & 27.7677 & 27.2853 & 27.6531 & 27.4384 & 27.3178 & 27.6066 & 27.7571 & 27.5623 \\
\hline H-13a & 2.10 & 29.9319 & 29.8652 & 29.3407 & 29.4150 & 29.7488 & 29.7283 & 29.6409 & 29.5141 & 29.6381 & 29.8979 & 29.5585 & 29.2464 & 29.3311 & 29.3258 & 29.5772 & 29.6282 \\
\hline $\mathrm{H}-13 \mathrm{~b}$ & 1.56 & 30.0987 & 30.1199 & 29.9733 & 29.8154 & 30.1167 & 30.0739 & 29.8046 & 29.9931 & 30.0926 & 29.9568 & 30.2272 & 30.3679 & 30.0889 & 30.2296 & 30.2875 & 30.1251 \\
\hline $\mathrm{H}-14$ & 1.91 & 29.9436 & 29.4524 & 29.8177 & 29.8006 & 29.8102 & 29.6859 & 29.4587 & 29.9712 & 106 & 985 & 29.6179 & 29.8111 & 29.5077 & 29.7792 & 29.5040 & 29.9952 \\
\hline $\mathrm{H}-15$ & 3.50 & 28.1744 & 27.7167 & 28.0914 & 27.7877 & 27.6863 & 28.2636 & 27.8459 & 28.2872 & 28.0987 & 7.7992 & 28.2447 & 27.6738 & 27.7038 & 28.1512 & 27.7398 & 28.1637 \\
\hline H-16a & 2.35 & 29.3544 & 29.6388 & 29.4705 & 29.5370 & 29.5045 & 29.2768 & 29.8076 & 29.4900 & 29.2474 & 29.5941 & 29.3447 & 29.5090 & 29.6135 & 29.5139 & 29.6333 & 29.3488 \\
\hline H-16b & 2.20 & 29.7764 & 29.8021 & 29.8495 & 29.5521 & 29.5343 & 29.8141 & 29.9100 & 29.6882 & 29.7300 & 29.9633 & 29.7179 & 29.5614 & 29.6822 & 29.6612 & 29.7749 & 29.8144 \\
\hline $\mathrm{H}-33$ & 1.02 & 30.8018 & 30.7791 & 30.7405 & 30.7799 & 30.7602 & 30.8679 & 30.7556 & 30.7892 & 30.8225 & 30.7945 & 30.8632 & 30.7910 & 30.7890 & 30.7816 & 30.7900 & 30.7904 \\
\hline \multirow{2}{*}{ Atom } & \multirow{2}{*}{ Exp. NMR } & \multicolumn{16}{|c|}{ Isotropic shielding values $(\sigma, \mathrm{ppm})$} \\
\hline & & F2-17 & F2-18 & F2-19 & F2-20 & F2-21 & F2-22 & F2-23 & F2-24 & F2-25 & F2-26 & F2-27 & F2-28 & F2-29 & F2-30 & F2-31 & F2-32 \\
\hline C-9 & 71.5 & 115.3242 & 115.3762 & 116.4470 & 115.6365 & 115.2439 & 113.4559 & 115.3551 & 114.1182 & 113.9015 & 113.4266 & 114.6615 & 117.9701 & 113.9087 & 116.2452 & 114.3387 & 115.2715 \\
\hline$C-10$ & 73.5 & 110.4305 & 111.7227 & 110.8739 & 114.3423 & 110.8136 & 116.0011 & 114.9702 & 115.1158 & 115.0312 & 115.5442 & 111.2235 & 110.8474 & 114.6817 & 113.6514 & 110.9987 & 113.4835 \\
\hline C-11 & 75.8 & 120.9494 & 121.6922 & 117.8182 & 116.1836 & 118.5471 & 113.1071 & 118.4804 & & & & 116.9200 & 119.8087 & 115.0127 & 116.0552 & 119.9937 & 116.5221 \\
\hline $\mathrm{C}-12$ & 80.2 & 112.3365 & 111.7220 & 109.5744 & 112.1523 & 114.6326 & 114.6766 & 110.5382 & 111.1832 & 110.9451 & 109.3747 & 109.7981 & 110.4613 & 112.3883 & 112.6758 & 107.2438 & 109.1025 \\
\hline C-13 & 38.3 & 152.9092 & 154.7902 & 154.4637 & 155.2313 & 154.7509 & 154.6192 & 156.7866 & 155.4821 & 151.5124 & 153.9348 & 151.8405 & 153.7599 & 152.2130 & 153.0756 & 155.0055 & 153.2504 \\
\hline C-14 & 40.5 & 151.9037 & 155.1512 & 153.2806 & 153.3645 & 154.0193 & 153.9903 & 153.7669 & 150.5065 & 151.2009 & 154.2978 & 151.9643 & 154.9532 & 153.6254 & 154.6141 & 154.9956 & 151.2016 \\
\hline C-15 & 86.2 & 106.1854 & 110.4942 & 107.4814 & 110.1283 & 109.1076 & 107.7442 & 111.7553 & 106.1620 & 8206 & 4953 & 107.1577 & 108.8016 & 110.3567 & 107.2682 & 110.4889 & 106.7280 \\
\hline C-16 & 37.9 & 149.4526 & 151.9026 & 149.1163 & 152.4004 & 152.4456 & 150.8800 & 151.2218 & 149.3837 & 149.6378 & 151.1544 & 149.6951 & 153.7114 & 152.2064 & 150.5495 & 152.0090 & 149.5753 \\
\hline$C-33$ & 16.7 & 174.5182 & 176.1152 & 173.7050 & 175.2253 & 175.1872 & 173.3683 & 175.8838 & 174.1362 & 174.3963 & 175.9498 & 174.7392 & 175.9536 & 175.1079 & 174.8361 & 176.2569 & 174.4665 \\
\hline & & & & & & & & & & & & & & & & & \\
\hline $\mathrm{H}-9$ & & 28.3105 & 28.5314 & 28.0254 & 28.0580 & 28.4893 & & 28.0278 & 28.2840 & & & 28.3481 & 27.9387 & 28.1765 & 28.0414 & 28.3159 & 28.0175 \\
\hline $\mathrm{H}-10$ & 3.52 & 28.3809 & 28.1000 & 28.2689 & 28.1989 & 28.4464 & 28.3531 & 28.1631 & 28.3788 & 28.6467 & 28.6566 & 27.9099 & 28.4651 & 28.6548 & 28.4588 & 27.9645 & 28.6758 \\
\hline $\mathrm{H}-11$ & 3.57 & 28.2042 & 27.9336 & 28.3889 & 28.0438 & 28.2701 & 28.7186 & 27.9098 & 28.0115 & 27.8882 & 27.8660 & 28.5547 & 28.0416 & 27.9526 & 28.2495 & 28.4421 & 28.0363 \\
\hline $\mathrm{H}-12$ & & 27.1832 & 27.4675 & 27.8573 & 27.1641 & & & & & & & 27.6678 & 27.5162 & 27.3625 & 27.5469 & 27.7313 & 27.5296 \\
\hline H-13a & 2.10 & 29.5269 & 29.4638 & 29.3282 & 29.4211 & 29.1194 & 29.5783 & 29.8829 & 29.8082 & 29.4737 & 29.5726 & 29.6622 & 29.2539 & 29.5166 & 29.2844 & 29.6617 & 29.8739 \\
\hline $\mathrm{H}-13 \mathrm{~b}$ & 1.56 & 30.1088 & 30.2053 & 30.2178 & 30.0765 & 30.3076 & 30.2693 & 29.9814 & 29.8955 & 29.9878 & 29.9267 & 30.1017 & 30.1998 & 29.7429 & 30.1444 & 30.2154 & 30.1382 \\
\hline $\mathrm{H}-14$ & 1.91 & 29.9645 & 29.4945 & 29.8431 & 29.7491 & 29.7838 & 29.6456 & 29.4072 & 29.8898 & 29.9685 & 29.4612 & 29.6883 & 29.5833 & 29.8233 & 29.7900 & 29.4764 & 29.9458 \\
\hline $\mathrm{H}-15$ & & 28.1664 & 27.7654 & 28.2083 & 27.7153 & 27.6860 & 28.1329 & 27.7887 & 28.2310 & 28.2136 & & 28.2861 & 27.5997 & 27.6883 & 28.2476 & 27.7204 & 28.1782 \\
\hline $\mathrm{H}-16 \mathrm{a}$ & 2.35 & 29.3524 & 29.6264 & 29.3959 & 29.5666 & 29.5398 & 29.3482 & 29.6664 & 29.5285 & 29.3460 & 29.5858 & 29.2574 & 29.4309 & 29.5195 & 29.3307 & 29.6309 & 29.3564 \\
\hline $\mathrm{H}-16 \mathrm{~b}$ & 2.20 & 29.7984 & 29.7515 & 29.6599 & 29.5856 & 29.5575 & 29.6158 & 29.8824 & 29.5642 & 29.7908 & 29.9359 & 29.8709 & 29.6315 & 29.5865 & 29.6718 & 29.7726 & 29.7866 \\
\hline $\mathrm{H}-33$ & 1.02 & 30.7920 & 30.7764 & 30.8148 & 30.7788 & 30.7680 & 30.8275 & 30.7778 & 30.7664 & 30.7873 & 30.7768 & 30.8784 & 30.7733 & 30.7708 & 30.8461 & 30.7835 & 30.8065 \\
\hline
\end{tabular}


Table S14. Scaled chemical shifts computed for compounds F2-01/F2-32 at the B3LYP/6-31G**//MMFF level of theory following a free conformational sampling procedure.

\begin{tabular}{|c|c|c|c|c|c|c|c|c|c|c|c|c|c|c|c|c|c|}
\hline \multirow{2}{*}{ Atom } & \multirow{2}{*}{ Exp. NMR } & \multicolumn{16}{|c|}{ Isotropic shielding values $(\sigma, \mathrm{ppm})$} \\
\hline & & F2-01 & F2-02 & F2-03 & F2-04 & F2-05 & F2-06 & F2-07 & F2-08 & F2-09 & F2-10 & F2-11 & F2-12 & F2-13 & F2-14 & F2-15 & F2-16 \\
\hline C-9 & 71.5 & 75.0 & 75.7 & 78.4 & 76.1 & 75.5 & 74.8 & 78.0 & 74.5 & 74.9 & 75.8 & 75.9 & 76.5 & 74.5 & 75.1 & 76.9 & 75.0 \\
\hline C-10 & 73.5 & 75.8 & 78.0 & 72.5 & 76.8 & 76.7 & 77.8 & 73.8 & 76.4 & 72.0 & 78.0 & 81.1 & 80.3 & 78.9 & 77.7 & 81.6 & 80.0 \\
\hline $\mathrm{C}-11$ & 75.8 & 75.2 & 74.6 & 75.6 & 77.5 & 77.3 & 74.7 & 74.5 & 75.9 & 77.4 & 74.5 & 72.5 & 73.1 & 73.8 & 72.2 & 70.8 & 71.3 \\
\hline $\mathrm{C}-12$ & 80.2 & 79.8 & 81.6 & 80.8 & 78.6 & 80.0 & 78.8 & 82.9 & 77.9 & 79.7 & 81.4 & 76.5 & 78.3 & 80.3 & 80.9 & 79.9 & 78.2 \\
\hline C-13 & 38.3 & 36.7 & 36.8 & 32.0 & 36.8 & 36.8 & 38.0 & 34.9 & 37.2 & 39.8 & 35.7 & 37.4 & 37.1 & 36.8 & 34.3 & 37.2 & 37.3 \\
\hline C-14 & 40.5 & 38.9 & 37.3 & 37.6 & 38.4 & 38.0 & 38.1 & 38.8 & 39.3 & 37.4 & 38.5 & 36.9 & 37.7 & 37.8 & 37.3 & 37.1 & 38.5 \\
\hline C-15 & 86.2 & 82.6 & 79.9 & 82.4 & 80.7 & 80.2 & 82.4 & 80.2 & 83.5 & 84.1 & 79.6 & 83.4 & 81.7 & 82.0 & 83.2 & 80.7 & 83.9 \\
\hline C-16 & 37.9 & 40.5 & 40.1 & 43.5 & 39.2 & 39.2 & 40.3 & 40.8 & 40.2 & 39.0 & 41.1 & 40.5 & 39.4 & 39.5 & 42.6 & 40.2 & 40.7 \\
\hline C-33 & 16.7 & 16.0 & 16.7 & 17.7 & 16.6 & 16.8 & 15.5 & 16.7 & 15.7 & 16.30 & 15.95 & 16.37 & 16.63 & 17.06 & 17.23 & 16.31 & 15.61 \\
\hline $\mathrm{H}-9$ & 3.99 & 3.87 & 3.83 & 3.66 & 3.76 & 3.77 & 3.92 & 3.74 & 3.99 & 3.75 & 3.36 & 3.62 & 3.50 & 3.50 & 3.63 & 3.44 & 3.53 \\
\hline $\mathrm{H}-10$ & 3.52 & 3.36 & 3.37 & 3.28 & 3.04 & 3.24 & 3.55 & 3.14 & 3.16 & 3.76 & 3.65 & 3.65 & 3.50 & 3.40 & 3.55 & 3.66 & 3.64 \\
\hline $\mathrm{H}-11$ & 3.57 & 3.53 & 3.44 & 3.88 & 3.69 & 3.36 & 3.32 & 4.05 & 3.74 & 3.78 & 3.88 & 3.41 & 3.33 & 3.66 & 3.79 & 3.52 & 3.52 \\
\hline $\mathrm{H}-12$ & 4.12 & 4.30 & 4.15 & 4.30 & 4.27 & 4.39 & 4.23 & 4.10 & 4.19 & 3.92 & 4.30 & 4.29 & 4.31 & 4.31 & 4.16 & 4.12 & 4.34 \\
\hline $\mathrm{H}-13 \mathrm{a}$ & 2.10 & 1.90 & 1.91 & 2.38 & 2.27 & 2.01 & 2.06 & 2.08 & 2.28 & 2.07 & 1.84 & 2.23 & 2.49 & 2.33 & 2.41 & 2.20 & 2.19 \\
\hline $\mathrm{H}-13 \mathrm{~b}$ & 1.56 & 1.74 & 1.65 & 1.73 & 1.85 & 1.65 & 1.69 & 1.91 & 1.77 & 1.62 & 1.78 & 1.51 & 1.37 & 1.59 & 1.50 & 1.45 & 1.67 \\
\hline $\mathrm{H}-14$ & 1.91 & 1.89 & 2.32 & 1.89 & 1.87 & 1.95 & 2.10 & 2.27 & 1.79 & 2.00 & 2.31 & 2.16 & 1.93 & 2.16 & 1.95 & 2.27 & 1.80 \\
\hline $\mathrm{H}-15$ & 3.50 & 3.67 & 4.06 & 3.65 & 3.95 & 3.98 & 3.60 & 3.96 & 3.58 & 3.59 & 3.82 & 3.65 & 4.07 & 3.93 & 3.61 & 4.14 & 3.72 \\
\hline $\mathrm{H}-16 \mathrm{a}$ & 2.35 & 2.48 & 2.13 & 2.25 & 2.14 & 2.24 & 2.53 & 1.90 & 2.31 & 2.46 & 2.13 & 2.46 & 2.23 & 2.06 & 2.22 & 2.14 & 2.48 \\
\hline $\mathrm{H}-16 \mathrm{~b}$ & 2.20 & 2.06 & 1.97 & 1.86 & 2.13 & 2.21 & 1.97 & 1.80 & 2.10 & 1.98 & 1.78 & 2.06 & 2.18 & 1.99 & 2.07 & 1.99 & 1.99 \\
\hline $\mathrm{H}-33$ & 1.02 & 1.03 & 0.99 & 0.95 & 0.85 & 1.04 & 0.86 & 0.91 & 0.92 & 0.90 & 0.99 & 0.82 & 0.95 & 0.90 & 0.94 & 0.92 & 0.97 \\
\hline \multirow{2}{*}{ Atom } & \multirow{2}{*}{ Exp. NMR } & \multicolumn{16}{|c|}{ Isotropic shielding values $(\sigma, \mathrm{ppm})$} \\
\hline & & F2-17 & F2-18 & F2-19 & F2-20 & F2-21 & F2-22 & F2-23 & F2-24 & F2-25 & F2-26 & F2-27 & F2-28 & F2-29 & F2-30 & F2-31 & F2-32 \\
\hline $\mathrm{C}-9$ & 71.5 & 75.3 & 76.7 & 73.8 & 76.0 & 76.4 & 77.5 & 76.8 & 76.1 & 76.2 & 77.7 & 75.0 & 73.5 & 77.3 & 74.7 & 76.3 & 74.6 \\
\hline $\mathrm{C}-10$ & 73.5 & 80.3 & 80.4 & 79.4 & 77.2 & 80.8 & 75.0 & 77.1 & 75.1 & 75.1 & 75.6 & 78.4 & 80.5 & 76.5 & 77.3 & 79.5 & 76.4 \\
\hline C-11 & 75.8 & 69.7 & 70.4 & 72.5 & 75.4 & 73.1 & 77.9 & 73.6 & 74.2 & 74.1 & 74.6 & 72.8 & 71.7 & 76.1 & 74.9 & 70.8 & 73.4 \\
\hline C-12 & 80.2 & 78.4 & 80.4 & 80.7 & 79.4 & 77.0 & 76.3 & 81.6 & 79.0 & 79.2 & 81.8 & 79.8 & 80.9 & 78.8 & 78.3 & 83.1 & 80.7 \\
\hline C-13 & \begin{tabular}{|l|l}
38.3 \\
\end{tabular} & 37.4 & 37.5 & 36.0 & 36.6 & 37.0 & 36.3 & 35.4 & 34.9 & 38.3 & 37.6 & 38.2 & 38.6 & 38.9 & 38.1 & 37.1 & 36.9 \\
\hline C-14 & 40.5 & 38.4 & 37.1 & 37.2 & 38.4 & 37.8 & 36.9 & 38.4 & 39.9 & 38.7 & 37.3 & 38.1 & 37.4 & 37.5 & 36.6 & 37.1 & 39.0 \\
\hline C-15 & 86.2 & 84.6 & 81.6 & 82.7 & 81.4 & 82.5 & 83.3 & 80.3 & 84.0 & 83.4 & 79.7 & 82.4 & 82.5 & 80.8 & 83.6 & 80.0 & 83.1 \\
\hline C-16 & 37.9 & 40.9 & 40.3 & $\begin{array}{l}41.4 \\
\end{array}$ & 39.4 & 39.3 & 40.0 & 41.0 & 41.0 & 40.2 & 40.4 & 40.3 & 38.6 & 38.9 & 40.6 & 40.0 & 40.6 \\
\hline C-33 & 16.7 & 15.6 & 16.2 & 16.9 & 16.7 & 16.7 & 17.5 & 16.4 & 16.4 & 15.3 & 15.9 & 15.5 & 16.9 & 15.9 & 16.5 & 16.7 & 15.9 \\
\hline $\mathrm{H}-9$ & 3.99 & 3.47 & 3.20 & 3.86 & 3.55 & 3.24 & 3.52 & 3.60 & 3.52 & 3.67 & 3.63 & 3.53 & 3.72 & 3.54 & 3.78 & 3.49 & 3.81 \\
\hline $\mathrm{H}-10$ & 3.52 & 3.40 & 3.64 & 3.60 & 3.41 & 3.28 & 3.47 & 3.47 & 3.43 & 3.17 & 3.11 & 4.00 & 3.19 & 3.05 & 3.34 & 3.86 & 3.16 \\
\hline $\mathrm{H}-11$ & 3.57 & 3.58 & 3.81 & 3.47 & 3.56 & 3.46 & 3.09 & 3.71 & 3.81 & 3.98 & 3.95 & 3.31 & 3.61 & 3.77 & 3.56 & 3.36 & 3.80 \\
\hline $\mathrm{H}-12$ & 4.12 & 4.60 & 4.28 & 4.04 & 4.39 & 4.61 & 4.73 & 4.34 & 4.45 & 4.26 & 4.16 & 4.26 & 4.14 & 4.38 & 4.30 & 4.10 & 4.30 \\
\hline H-13a & 2.10 & 2.26 & 2.25 & 2.46 & 2.26 & 2.60 & 2.20 & 1.86 & 1.95 & 2.30 & 2.15 & 2.12 & 2.41 & 2.16 & 2.47 & 2.09 & 1.96 \\
\hline $\mathrm{H}-13 \mathrm{~b}$ & 1.56 & 1.68 & 1.50 & 1.50 & 1.64 & 1.40 & 1.48 & 1.77 & 1.86 & 1.75 & 1.77 & 1.65 & 1.47 & 1.93 & 1.57 & 1.52 & 1.70 \\
\hline $\mathrm{H}-14$ & 1.91 & 1.82 & 2.22 & 1.90 & 1.95 & 1.93 & 2.13 & 2.31 & 1.86 & 1.77 & 2.26 & 2.09 & 2.08 & 1.84 & 1.94 & 2.29 & 1.89 \\
\hline $\mathrm{H}-15$ & 3.50 & 3.62 & 3.98 & 3.66 & 3.87 & 4.05 & 3.70 & 3.82 & 3.58 & 3.63 & 4.03 & 3.60 & 4.05 & 4.05 & 3.56 & 4.11 & 3.65 \\
\hline $\mathrm{H}-16 \mathrm{a}$ & 2.35 & 2.43 & 2.09 & 2.39 & 2.12 & 2.17 & 2.44 & 2.07 & 2.24 & 2.43 & 2.13 & 2.56 & 2.23 & 2.16 & 2.42 & 2.13 & 2.48 \\
\hline $\mathrm{H}-16 \mathrm{~b}$ & 2.20 & 1.99 & 1.96 & 2.10 & 2.10 & 2.16 & 2.16 & 1.86 & 2.20 & 1.96 & 1.76 & 1.90 & 2.03 & 2.09 & 2.06 & 1.98 & 2.05 \\
\hline $\mathrm{H}-33$ & 1.02 & 0.99 & 0.92 & 0.86 & 0.98 & 0.93 & 0.91 & 1.02 & 0.96 & 0.91 & 0.88 & 0.82 & 0.90 & 0.87 & 0.83 & 0.93 & 1.03 \\
\hline
\end{tabular}

Table S15. Absolute scaled errors computed for compounds F2-01/F2-32 at the B3LYP/6-31G**//MMFF level of theory following a free conformational sampling procedure, and DP4 results.

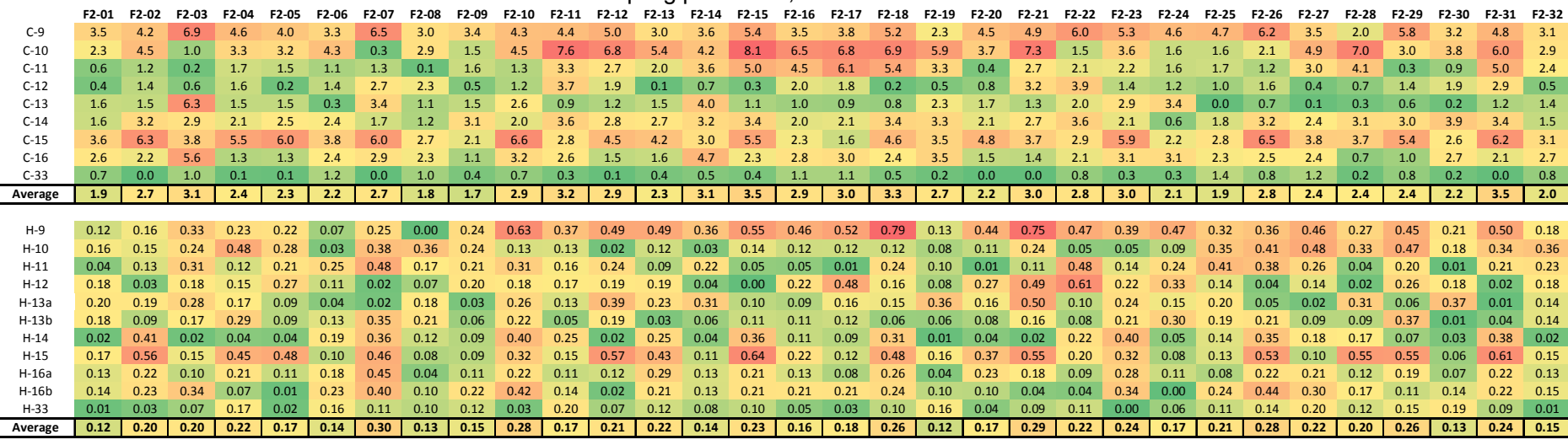

\begin{tabular}{|c|c|c|c|c|c|c|c|c|c|c|c|c|c|c|c|c|c|}
\hline \multicolumn{2}{|c|}{ Isomer № } & 1 & 2 & 3 & 4 & 5 & 6 & 7 & 8 & 9 & 10 & 11 & 12 & 13 & 14 & 15 & 16 \\
\hline \multirow{5}{*}{ DP4 (\%) } & $\mathrm{H}$ & 28.10 & 0.02 & 0.04 & 0.01 & 0.36 & 8.78 & 0.00 & 9.52 & 4.15 & 0.00 & 0.68 & 0.00 & 0.00 & 4.89 & 0.00 & 0.96 \\
\hline & $\mathrm{C}$ & 12.71 & 0.08 & 0.00 & 0.55 & 0.96 & 1.81 & 0.05 & 18.39 & 38.33 & 0.03 & 0.00 & 0.02 & 0.88 & 0.02 & 0.00 & 0.05 \\
\hline & $\mathrm{H}+\mathrm{C}$ & 48.00 & 0.00 & 0.00 & 0.00 & 0.05 & 2.14 & 0.00 & 23.55 & 21.37 & 0.00 & 0.00 & 0.00 & 0.00 & 0.01 & 0.00 & 0.01 \\
\hline & 1 & & & & & & & & & & & & & & & & \\
\hline & all data & 48.00 & 0.00 & 0.00 & 0.00 & 0.05 & 2.14 & 0.00 & 23.55 & 21.37 & 0.00 & 0.00 & 0.00 & 0.00 & 0.01 & 0.00 & 0.01 \\
\hline \multicolumn{2}{|c|}{ Isomer № } & 17 & 18 & 19 & 20 & 21 & 22 & 23 & 24 & 25 & 26 & 27 & 28 & 29 & 30 & 31 & 32 \\
\hline \multirow{5}{*}{ DP4 (\%) } & $\mathrm{H}$ & 0.10 & 0.00 & 32.25 & 0.28 & 0.00 & 0.00 & 0.00 & 0.21 & 0.02 & 0.00 & 0.00 & 0.02 & 0.00 & 7.58 & 0.00 & 2.02 \\
\hline & $\mathrm{C}$ & 0.01 & 0.00 & 0.09 & 1.84 & 0.01 & 0.10 & 0.03 & 4.44 & 9.19 & 0.06 & 0.57 & 0.30 & 0.49 & 2.43 & 0.00 & 6.55 \\
\hline & $H+C$ & 0.00 & 0.00 & 0.40 & 0.07 & 0.00 & 0.00 & 0.00 & 0.13 & 0.02 & 0.00 & 0.00 & 0.00 & 0.00 & 2.48 & 0.00 & 1.78 \\
\hline & 1 & - & - & - & - & - & - & - & - & - & - & - & - & - & - & - & - \\
\hline & all data & 0.00 & 0.00 & 0.40 & 0.07 & 0.00 & 0.00 & 0.00 & 0.13 & 0.02 & 0.00 & 0.00 & 0.00 & 0.00 & 2.48 & 0.00 & 1.78 \\
\hline
\end{tabular}


Table S16. Boltzmann-averaged isotropic shielding values and coupling constants (FC only) computed for compounds F2-01/F2-32 at the B3LYP/6$31 \mathrm{G}^{* * / / M M F F}$ level of theory following the iJ-DP4 approach.

\begin{tabular}{|c|c|c|c|c|c|c|c|c|c|c|c|c|c|c|c|c|c|}
\hline \multirow{2}{*}{ Atom } & \multirow{2}{*}{ Exp. NMR } & \multicolumn{16}{|c|}{ Isotropic shielding values $(\sigma, \mathrm{ppm})$} \\
\hline & & F2-01 & F2-02 & F2-03 & F2-04 & F2-05 & F2-06 & F2-07 & F2-08 & F2-09 & F2-10 & F2-11 & F2-12 & F2-13 & F2-14 & F2-15 & F2-16 \\
\hline C-9 & 71.5 & 113.1659 & 113.0395 & 112.7085 & 112.8468 & 113.3156 & 112.7187 & 112.6016 & 112.6671 & 117.0469 & 117.1247 & 116.1720 & 116.1839 & 116.9618 & 117.4833 & 116.2211 & 116.7161 \\
\hline $\mathrm{C}-10$ & 73.5 & 116.0817 & 115.4371 & 118.6317 & 117.2330 & 117.7404 & 114.7723 & 117.2355 & 117.2517 & 122.1620 & 114.9483 & 119.2965 & 117.8510 & 114.9979 & 116.2323 & 116.7993 & 113.0781 \\
\hline $\mathrm{C}-11$ & 75.8 & 111.6325 & 113.0129 & 115.4190 & 114.3902 & 108.7526 & 113.0193 & 116.2929 & 115.2208 & 109.6159 & 116.4989 & 108.5303 & 108.8611 & 114.1517 & 116.0084 & 110.2234 & 115.2074 \\
\hline $\mathrm{C}-12$ & 80.2 & 108.9436 & 107.8473 & 110.4887 & 109.9537 & 109.9235 & 110.0774 & 107.5487 & 108.9680 & 111.4214 & 111.2084 & 114.2636 & 110.4171 & 113.0111 & 112.8831 & 109.7671 & 111.3726 \\
\hline C-13 & 38.3 & 153.6126 & 155.3938 & 157.3931 & 156.1619 & 155.4647 & 152.3447 & 157.1369 & 154.8342 & 148.4346 & 156.1313 & 151.1981 & 155.7056 & 155.4260 & 156.5010 & 155.3422 & 152.9984 \\
\hline C-14 & 40.5 & 150.9673 & 154.4538 & 151.9300 & 152.5566 & 153.0440 & 151.6406 & 153.2010 & 150.1189 & 155.8976 & 153.4127 & 155.6224 & 153.2307 & 152.4129 & 151.1463 & 153.9085 & 151.4996 \\
\hline C-15 & 86.2 & 106.9349 & 110.6359 & 108.9414 & 109.9801 & 111.2819 & 107.0968 & 110.3305 & 106.9153 & 107.5432 & 113.1555 & 107.6421 & 111.5882 & 109.0182 & 109.6470 & 111.0928 & 106.5321 \\
\hline C-16 & 37.9 & 149.5489 & 151.6396 & 146.2506 & 152.0622 & 152.1431 & 149.2961 & 151.1726 & 149.5833 & 153.8098 & 150.9295 & 153.7553 & 152.2891 & 152.1247 & 145.9788 & 151.7602 & 149.4532 \\
\hline C-33 & 16.7 & 174.4796 & 176.0956 & 171.0269 & 175.0229 & 175.3953 & 174.2995 & 176.0003 & 174.3875 & 174.9822 & 175.8142 & 175.1936 & 175.4707 & 175.3470 & 170.6681 & 175.8244 & 174.4746 \\
\hline $\mathrm{H}-9$ & 3.99 & 28.0493 & 28.0395 & 28.0800 & 28.0682 & 28.0570 & 28.0826 & 28.0494 & 28.0544 & 27.6929 & 28.2917 & 27.3977 & 28.1314 & 28.2333 & 28.1599 & 27.9115 & 28.2619 \\
\hline $\mathrm{H}-10$ & 3.52 & 28.5672 & 28.4933 & 28.4480 & 28.6398 & 28.7070 & 28.3618 & 28.6365 & 28.6592 & 27.8353 & 27.9798 & 28.4070 & 28.7445 & 28.0009 & 27.8602 & 28.6998 & 28.3553 \\
\hline $\mathrm{H}-11$ & 3.57 & 28.2755 & 28.3160 & 27.8695 & 27.7248 & 28.2329 & 28.5344 & 27.7695 & 27.7641 & 28.0225 & 27.6901 & 28.0306 & 27.7505 & 27.6298 & 27.7933 & 27.8287 & 28.2670 \\
\hline $\mathrm{H}-12$ & 4.12 & 27.4696 & 27.5909 & 27.4516 & 27.4452 & 27.1060 & 27.6528 & 27.7169 & 27.6850 & 27.9165 & 27.0104 & 27.7860 & 27.5614 & 26.7271 & 26.8304 & 27.7937 & 27.2043 \\
\hline $\mathrm{H}-13 a$ & 2.10 & 29.8455 & 30.0419 & 29.3371 & 29.2440 & 29.9486 & 29.7377 & 29.6227 & 29.5091 & 29.5153 & 29.9040 & 29.7385 & 30.0793 & 29.4533 & 29.4580 & 30.1443 & 29.7634 \\
\hline$H-13 b$ & 1.56 & 30.3078 & 30.0424 & 29.9707 & 29.8090 & 30.2075 & 30.0708 & 29.8008 & 29.7673 & 30.2255 & 30.0020 & 30.0842 & 30.2948 & 30.1561 & 30.1993 & 30.1640 & 30.1172 \\
\hline $\mathrm{H}-14$ & 1.91 & 29.9324 & 29.4267 & 29.8163 & 29.7795 & 29.8315 & 29.6881 & 29.4613 & 29.9461 & 29.5501 & 29.3768 & 29.3321 & 29.7578 & 29.7349 & 29.8741 & 29.4659 & 29.9618 \\
\hline $\mathrm{H}-15$ & 3.50 & 28.1796 & 27.7040 & 28.0890 & 27.8217 & 27.7239 & 28.2410 & 27.8483 & 28.3191 & 27.9759 & 27.8062 & 28.2030 & 27.6906 & 27.8189 & 28.0116 & 27.7200 & 28.1609 \\
\hline $\mathrm{H}-16 \mathrm{a}$ & 2.35 & 29.3652 & 29.6100 & 29.4706 & 29.5343 & 29.5319 & 29.2826 & 29.8158 & 29.3782 & 29.0883 & 29.5937 & 29.1242 & 29.5486 & 29.3527 & 29.6191 & 29.6203 & 29.3582 \\
\hline$H-16 b$ & 2.20 & 29.7637 & 29.8545 & 29.8508 & 29.5583 & 29.5348 & 29.8029 & 29.9091 & 29.7939 & 29.7468 & 29.9672 & 29.6501 & 29.5849 & 29.7288 & 29.7347 & 29.8531 & 29.8048 \\
\hline \multirow[t]{2}{*}{$\mathrm{H}-33$} & 1.02 & 30.8088 & 30.7774 & 30.7398 & 30.7737 & 30.7466 & 30.8411 & 30.7543 & 30.8024 & 30.8462 & 30.8035 & 30.9193 & 30.7746 & 30.7736 & 30.7058 & 30.7934 & 30.7960 \\
\hline & & \multicolumn{16}{|c|}{$J$ coupling (FC only, $\mathrm{Hz}$ ) } \\
\hline$J_{11,12}$ & 5.8 & 4.9 & 3.6 & 3.4 & 1.6 & 7.2 & 1.4 & 1.5 & 1.5 & 8.9 & 1.8 & 0.8 & 7.9 & 2.6 & 3.8 & 5.0 & 2.4 \\
\hline$J_{12,13 \mathrm{a}}$ & 9.0 & 10.5 & 10.4 & 8.2 & 10.5 & 10.8 & 9.0 & 10.3 & 10.2 & 9.5 & 10.5 & 9.4 & 9.7 & 10.7 & 9.2 & 10.0 & 10.6 \\
\hline$J_{12,13 \mathrm{~b}}$ & 6.1 & 4.4 & 4.6 & 5.7 & 5.3 & 5.2 & 2.4 & 4.5 & 4.6 & 4.4 & 4.5 & 2.0 & 5.4 & 5.3 & 5.4 & 4.9 & 4.3 \\
\hline$J_{14,15}$ & 8.7 & 9.1 & 7.5 & 2.9 & 4.1 & 4.2 & 8.2 & 7.5 & 9.1 & 9.8 & 7.7 & 9.5 & 4.5 & 4.1 & 2.1 & 7.3 & 9.1 \\
\hline \multirow{2}{*}{ Atom } & \multirow{2}{*}{ Exp. NMR } & \multicolumn{16}{|c|}{ Isotropic shielding values $(\sigma, \mathrm{ppm})$} \\
\hline & & F2-17 & F2-18 & F2-19 & F2-20 & F2-21 & F2-22 & F2-23 & F2-24 & F2-25 & F2-26 & F2-27 & F2-28 & F2-29 & F2-30 & F2-31 & F2-32 \\
\hline C-9 & 71.5 & 115.2245 & 116.1485 & 117.2889 & 117.1764 & 117.0881 & 117.2692 & 117.0639 & 118.2038 & 111.8089 & 111.7047 & 113.5537 & 112.9502 & 111.7490 & 112.4216 & 113.4550 & 111.9949 \\
\hline C-10 & 73.5 & 111.5589 & 109.6888 & 114.6033 & 115.0586 & 111.3911 & 110.4008 & 115.3692 & 117.6555 & 117.4600 & 117.5945 & 111.6865 & 115.8148 & 117.6868 & 113.8490 & 111.4682 & 117.8164 \\
\hline C-11 & 75.8 & 121.8231 & 119.0601 & 119.3186 & 118.6683 & 114.1875 & 114.3029 & 120.6969 & 119.9848 & 119.1227 & 120.1883 & 116.4972 & 117.0981 & 118.0542 & 116.0839 & 119.6032 & 112.4432 \\
\hline C-12 & 80.2 & 111.1646 & 112.1725 & 113.1378 & 113.6176 & 115.0701 & 115.0726 & 111.4357 & 112.6087 & 108.8591 & 107.5585 & 109.6270 & 109.5824 & 109.9901 & 113.8613 & 107.0965 & 108.8051 \\
\hline C-13 & 38.3 & 152.9162 & 154.0950 & 156.1645 & 154.7910 & 154.2226 & 150.3995 & 155.5760 & 153.1535 & 154.5986 & 156.5994 & 151.8910 & 155.6717 & 155.7336 & 153.8209 & 155.1224 & 153.8545 \\
\hline C-14 & 40.5 & 152.0941 & 154.3417 & 151.4059 & 152.7203 & 153.3841 & 152.5466 & 153.5694 & 150.5546 & 150.1811 & 153.4543 & 151.9209 & 154.5022 & 152.5542 & 153.4942 & 154.9877 & 150.7813 \\
\hline C-15 & 86.2 & 106.2851 & 110.5240 & 108.1736 & 110.3493 & 110.3142 & 106.3857 & 113.1180 & 107.4418 & 106.9978 & 112.9098 & 107.2040 & 109.9649 & 110.0100 & 107.5600 & 110.5170 & 107.4322 \\
\hline C-16 & 37.9 & 149.3939 & 152.3453 & 146.0792 & 152.1125 & 152.0745 & 150.5807 & 150.9529 & 149.5151 & 149.6627 & 150.6367 & 149.7155 & 152.4823 & 152.1299 & 148.5360 & 152.0099 & 149.5894 \\
\hline C-33 & 16.7 & 174.4929 & 175.7495 & 171.3649 & 175.1425 & 175.2644 & 174.6393 & 175.8230 & 174.2133 & 174.3652 & 175.9027 & 174.8118 & 175.4520 & 175.1927 & 174.4881 & 176.2752 & 174.2761 \\
\hline $\mathrm{H}-9$ & & .9720 & 27.5545 & 27.7314 & 27.7501 & 27.7187 & 27.4696 & 27.7576 & 27.6327 & 28.1256 & 28.1155 & 28.4410 & 28.0677 & 28.1272 & 28.0519 & 28.3971 & 28.1294 \\
\hline $\mathrm{H}-10$ & 3.52 & 28.1498 & 28.5004 & 27.8957 & 27.8898 & 27.9302 & 28.5646 & 27.8520 & 27.6692 & 28.5980 & 28.6080 & 27.7216 & 28.3980 & 28.5642 & 27.9244 & 27.8072 & 28.6188 \\
\hline H-11 & 3.57 & 28.3836 & 27.7588 & 27.7601 & 27.6717 & 28.3830 & 27.8692 & 27.7118 & 27.6547 & 27.6572 & 27.6978 & 28.6691 & 28.2589 & 27.6194 & 28.6867 & 28.5382 & 28.0792 \\
\hline $\mathrm{H}-12$ & 4.12 & 26.9593 & 27.7041 & 27.0612 & 26.7557 & 27.2924 & 27.7075 & 27.0059 & 27.0543 & 27.6987 & 27.7713 & 27.6548 & 27.4094 & 27.4338 & 27.6918 & 27.7126 & 27.3086 \\
\hline $\mathrm{H}-13 \mathrm{a}$ & 2.10 & 29.5121 & 29.5567 & 29.4151 & 29.3697 & 29.2042 & 29.7785 & 29.8799 & 29.8360 & 29.4483 & 29.5816 & 29.6809 & 29.4497 & 29.2382 & 29.3860 & 29.6938 & 29.6745 \\
\hline$H-13 b$ & 1.56 & 30.1717 & 30.3071 & 30.2472 & 30.1514 & 30.4467 & 30.0709 & 30.0207 & 29.9258 & 29.7620 & 29.7807 & 30.1041 & 30.1979 & 29.7500 & 30.2005 & 30.2130 & 30.6871 \\
\hline $\mathrm{H}-14$ & 1.91 & 29.9889 & 29.5012 & 29.9103 & 29.8200 & 29.8203 & 29.5193 & 29.3946 & 29.9279 & 29.9568 & 29.4359 & 29.7066 & 29.7184 & 29.7939 & 30.0604 & 29.4740 & 29.9198 \\
\hline $\mathrm{H}-15$ & 3.50 & 28.1656 & 27.6950 & 28.1672 & 27.7809 & 27.5371 & 28.3065 & 27.8232 & 28.2769 & 28.3425 & 27.8349 & 28.2933 & 27.6915 & 27.8356 & 28.2276 & 27.7223 & 28.2132 \\
\hline $\mathrm{H}-16 \mathrm{a}$ & 2.35 & 29.3623 & 29.5415 & 29.6203 & 29.5579 & 29.5594 & 29.3647 & 29.6083 & 29.3884 & 29.3648 & 29.5615 & 29.2488 & 29.5865 & 29.5442 & 29.3051 & 29.6334 & 29.3793 \\
\hline $\mathrm{H}-16 \mathrm{~b}$ & 2.20 & 29.7925 & 29.7380 & 29.7381 & 29.5784 & 29.5715 & 29.5320 & 29.9554 & 29.7594 & 29.8094 & 30.0494 & 29.8918 & 29.6786 & 29.5855 & 29.9787 & 29.7869 & 29.7541 \\
\hline \multirow[t]{2}{*}{$\mathrm{H}-33$} & 1.02 & 30.8002 & 30.7927 & 30.7297 & 30.7822 & 30.7953 & 30.9298 & 30.8009 & 30.8293 & 30.8026 & 30.7861 & 30.8832 & 30.8078 & 30.7842 & 30.8373 & 30.7865 & 30.8250 \\
\hline & & \multicolumn{16}{|c|}{$J$ coupling ( $\mathrm{FC}$ only, $\mathrm{Hz}$ ) } \\
\hline$J_{11,12}$ & 5.8 & 0.9 & 0.3 & 4.7 & 2.6 & 0.8 & 0.3 & 1.9 & 2.8 & 1.4 & 1.4 & 1.0 & 3.8 & 1.7 & 8.6 & 1.2 & 7.0 \\
\hline$J_{12,13 \mathrm{a}}$ & 9.0 & 10.7 & 10.4 & 7.6 & 10.7 & 10.5 & 9.4 & 10.5 & 10.4 & 10.2 & 10.2 & 9.4 & 6.6 & 10.4 & 7.8 & 10.4 & 10.5 \\
\hline$J_{12,13 \mathrm{~b}}$ & 6.1 & 4.2 & 4.3 & 5.8 & 5.1 & 5.4 & 1.4 & 4.3 & 4.4 & 4.6 & 4.5 & 1.4 & 5.9 & 5.3 & 0.5 & 4.5 & 4.4 \\
\hline$J_{14,15}$ & 8.7 & 9.1 & 7.8 & 3.4 & 4.2 & 4.1 & 9.1 & 7.7 & 9.1 & 9.1 & 7.6 & 9.2 & 5.6 & 4.1 & 8.9 & 7.5 & 9.1 \\
\hline
\end{tabular}


S17. Scaled chemical shifts and coupling constants (FC only) computed for compounds F2-01/F2-32 at the B3LYP/6-31G**//MMFF level of theory following the iJ-DP4 approach.

\begin{tabular}{|c|c|c|c|c|c|c|c|c|c|c|c|c|c|c|c|c|c|}
\hline \multirow{2}{*}{ Atom } & \multirow{2}{*}{ Exp. NMR } & \multicolumn{16}{|c|}{ Scaled chemical shifts $(\delta, \mathrm{ppm})$} \\
\hline & & F2-01 & F2-02 & F2-03 & F2-04 & F2-05 & F2-06 & F2-07 & F2-08 & F2-09 & F2-10 & F2-11 & F2-12 & F2-13 & F2-14 & F2-15 & F2-16 \\
\hline C-9 & 71.5 & 76.0 & 77.0 & 78.5 & 78.0 & 76.9 & 76.6 & 78.1 & 77.2 & 74.2 & 75.3 & 74.9 & 74.8 & 74.5 & 74.5 & 74.6 & 73.5 \\
\hline C-10 & 73.5 & 73.1 & 74.7 & 72.3 & 73.7 & 72.6 & 74.6 & 73.6 & 72.7 & 69.1 & 77.5 & 71.8 & 73.2 & 76.4 & 75.9 & 74.0 & 77.1 \\
\hline C-11 & 75.8 & 77.4 & 77.0 & 75.7 & 76.4 & 81.3 & 76.3 & 74.5 & 74.7 & 81.6 & 75.9 & 82.4 & 82.0 & 77.3 & 76.1 & 80.4 & 75.0 \\
\hline C-12 & 80.2 & 80.1 & 81.9 & 80.8 & 80.8 & 80.2 & 79.2 & 83.0 & 80.9 & 79.8 & 81.3 & 76.8 & 80.4 & 78.4 & 79.5 & 80.9 & 78.8 \\
\hline C-13 & 38.3 & 36.5 & 36.5 & 31.9 & 35.5 & 36.1 & 37.5 & 35.0 & 35.4 & 42.9 & 35.8 & 40.5 & 36.1 & 36.3 & 32.4 & 36.5 & 37.2 \\
\hline C-14 & 40.5 & 39.0 & 37.4 & 37.6 & 39.0 & 38.4 & 38.2 & 38.8 & 40.1 & 35.5 & 38.5 & 36.1 & 38.6 & 39.3 & 38.2 & 37.9 & 38.7 \\
\hline C-15 & 86.2 & 82.0 & 79.3 & 82.4 & 80.8 & 78.9 & 82.1 & 80.3 & 82.9 & 83.6 & 79.3 & \begin{tabular}{|l|l}
83.3 \\
\end{tabular} & 79.3 & 82.4 & 83.0 & 79.6 & $\begin{array}{l}83.7 \\
\end{array}$ \\
\hline C-16 & 37.9 & 40.4 & 40.1 & 43.6 & 39.5 & 39.3 & 40.5 & 40.7 & 40.6 & 37.6 & 41.1 & 38.0 & 39.5 & 39.6 & 43.8 & 40.0 & 40.8 \\
\hline \multirow[t]{2}{*}{ C-33 } & 16.7 & 16.1 & 16.7 & 17.7 & 17.0 & 16.8 & 15.8 & 16.7 & 16.1 & 16.5 & 15.9 & 16.9 & 16.8 & 16.5 & 17.1 & 16.6 & 15.8 \\
\hline & & - & - & - & - & - & - & - & - & - & - & - & - & - & - & - & - \\
\hline $\mathrm{H}-9$ & 3.99 & 3.79 & 3.77 & 3.66 & 3.62 & 3.66 & 3.82 & 3.75 & 3.79 & 3.94 & 3.31 & 4.28 & 3.63 & 3.27 & 3.39 & 3.87 & 3.53 \\
\hline $\mathrm{H}-10$ & 3.52 & 3.28 & 3.31 & 3.29 & 3.03 & 3.04 & 3.52 & 3.13 & 3.14 & 3.80 & 3.59 & 3.27 & 3.04 & 3.48 & 3.66 & 3.10 & 3.44 \\
\hline $\mathrm{H}-11$ & 3.57 & 3.57 & 3.49 & 3.88 & 3.97 & 3.49 & 3.34 & 4.04 & 4.10 & 3.62 & 3.85 & 3.65 & 3.99 & 3.80 & 3.72 & 3.95 & 3.53 \\
\hline $\mathrm{H}-12$ & 4.12 & 4.37 & 4.22 & 4.30 & 4.26 & 4.56 & 4.29 & 4.10 & 4.19 & 3.72 & 4.47 & 3.89 & 4.17 & 4.60 & 4.57 & 3.98 & 4.58 \\
\hline $\mathrm{H}-13 \mathrm{a}$ & 2.10 & 2.02 & 1.74 & 2.38 & 2.40 & 1.87 & 2.05 & 2.10 & 2.23 & 2.16 & 1.84 & 1.95 & 1.76 & 2.20 & 2.25 & 1.68 & 2.05 \\
\hline $\mathrm{H}-13 \mathrm{~b}$ & 1.56 & 1.56 & 1.74 & 1.74 & 1.82 & 1.62 & 1.69 & 1.91 & 1.96 & 1.46 & 1.76 & 1.60 & 1.55 & 1.59 & 1.59 & 1.66 & 1.71 \\
\hline $\mathrm{H}-14$ & 1.91 & 1.93 & 2.36 & 1.89 & 1.85 & 1.98 & 2.10 & 2.26 & 1.77 & 2.12 & 2.32 & 2.35 & 2.07 & 1.96 & 1.88 & 2.34 & 1.86 \\
\hline $\mathrm{H}-15$ & 3.50 & 3.67 & 4.11 & 3.65 & 3.87 & 3.98 & 3.65 & 3.96 & 3.51 & 3.66 & 3.75 & 3.48 & 4.05 & 3.64 & 3.53 & 4.06 & 3.63 \\
\hline $\mathrm{H}-16 \mathrm{a}$ & 2.35 & 2.49 & 2.18 & 2.24 & 2.10 & 2.26 & 2.54 & 1.89 & 2.37 & 2.57 & 2.13 & 2.56 & 2.27 & 2.29 & 2.11 & 2.19 & 2.45 \\
\hline $\mathrm{H}-16 \mathrm{~b}$ & 2.20 & 2.10 & 1.93 & 1.86 & 2.08 & 2.26 & 1.98 & 1.79 & 1.93 & 1.93 & 1.79 & 2.04 & 2.23 & 1.96 & 2.00 & 1.97 & 2.01 \\
\hline \multirow{2}{*}{$\mathrm{H}-33$} & 1.02 & 1.06 & 0.99 & 0.95 & 0.83 & 1.11 & 0.86 & 0.91 & 0.85 & 0.86 & 1.03 & 0.77 & 1.09 & 1.05 & 1.15 & 1.04 & 1.04 \\
\hline & & \multicolumn{16}{|c|}{ Scaled J(FC only, $\mathrm{Hz})$} \\
\hline$J_{11,12}$ & 5.8 & 5.3 & 4.0 & 3.7 & 1.9 & 7.7 & 1.6 & 1.7 & 1.7 & 9.5 & 2.1 & 0.9 & 8.5 & 2.9 & 4.1 & 5.4 & 2.7 \\
\hline$J_{12,13 a}$ & 9.0 & 11.2 & 11.1 & 8.8 & 11.2 & 11.5 & 9.6 & 10.9 & 10.9 & 10.2 & 11.2 & 10.1 & 10.4 & 11.4 & 9.9 & 10.7 & 11.3 \\
\hline$J_{12,13 b}$ & 6.1 & 4.8 & 5.0 & 6.1 & 5.7 & 5.6 & 2.7 & 4.9 & 5.0 & 4.8 & 4.8 & 2.3 & 5.9 & 5.7 & 5.8 & 5.3 & 4.6 \\
\hline$J_{14,15}$ & 8.7 & 9.7 & 8.0 & 3.2 & 4.4 & 4.5 & 8.8 & 8.1 & 9.7 & 10.5 & 8.2 & 10.1 & 4.9 & 4.5 & 2.3 & 7.8 & 9.7 \\
\hline \multirow{2}{*}{ Atom } & \multirow{2}{*}{ Exp. NMR } & \multicolumn{16}{|c|}{ Scaled chemical shifts $(\delta, \mathrm{ppm})$} \\
\hline & & F2-17 & F2-18 & F2-19 & F2-20 & F2-21 & F2-22 & F2-23 & F2-24 & $F 2-25$ & F2-26 & F2-27 & F2-28 & F2-29 & F2-30 & F2-31 & F2-32 \\
\hline C-9 & 71.5 & 75.6 & 75.3 & 74.7 & 75.5 & 74.4 & 73.1 & 76.2 & 74.2 & 78.7 & 80.2 & 75.9 & 78.1 & 79.6 & 78.2 & 77.0 & 77.4 \\
\hline C-10 & 73.5 & 79.3 & 81.7 & 77.6 & 77.7 & 80.1 & 80.0 & 78.0 & 74.8 & 73.0 & 74.3 & 77.8 & 75.3 & 73.7 & 76.7 & 78.9 & 71.7 \\
\hline C-11 & 75.8 & 68.9 & 72.4 & 72.6 & 74.0 & 77.3 & 76.0 & 72.5 & 72.3 & 71.3 & 71.7 & 73.0 & 74.1 & 73.4 & 74.5 & 71.1 & 77.0 \\
\hline C-12 & 80.2 & 79.7 & $\begin{array}{l}79.3 \\
\end{array}$ & 79.2 & 79.2 & 76.5 & 75.3 & 82.0 & 80.1 & 81.6 & 84.3 & 79.8 & 81.4 & 81.4 & 76.7 & 83.1 & 80.6 \\
\hline C-13 & 38.3 & 37.5 & 37.7 & 33.1 & 36.9 & 37.3 & 39.7 & 36.4 & 37.3 & 35.7 & 35.3 & 38.1 & 36.5 & 35.9 & 36.6 & 37.0 & 36.2 \\
\hline C-14 & 40.5 & 38.3 & 37.5 & 38.2 & 39.1 & 38.1 & 37.6 & 38.5 & 40.1 & 40.2 & 38.5 & 38.1 & 37.6 & 39.1 & 37.0 & 37.1 & 39.2 \\
\hline C-15 & 86.2 & 84.6 & 80.9 & 84.5 & 82.5 & 81.2 & 84.0 & 80.3 & 85.5 & 83.5 & 79.0 & 82.2 & 81.0 & 81.3 & 83.1 & 79.8 & 81.9 \\
\hline C-16 & 37.9 & 41.0 & 39.5 & 43.9 & 39.7 & 39.4 & 39.6 & 41.2 & 41.2 & 40.7 & 41.3 & 40.3 & 39.6 & 39.5 & 41.9 & 40.0 & 40.4 \\
\hline C-33 & 16.7 & 15.7 & 16.3 & 16.8 & 16.1 & 16.2 & 15.4 & 15.5 & 15.1 & 15.9 & 16.0 & 15.5 & 17.2 & 16.6 & 15.9 & 16.7 & 16.1 \\
\hline $\mathrm{H}-9$ & 3.99 & 3.69 & 4.03 & 3.76 & 3.61 & 3.80 & 4.22 & 3.68 & 3.79 & 3.71 & 3.67 & 3.44 & 3.65 & 3.54 & 3.81 & 3.41 & 3.67 \\
\hline $\mathrm{H}-10$ & 3.52 & 3.52 & 3.15 & 3.62 & 3.49 & 3.61 & 3.15 & 3.60 & 3.76 & 3.20 & 3.15 & 4.21 & 3.33 & 3.09 & 3.94 & 4.02 & 3.21 \\
\hline $\mathrm{H}-11$ & 3.57 & 3.31 & 3.84 & 3.73 & 3.68 & 3.20 & 3.83 & 3.72 & 3.77 & 4.21 & 4.12 & 3.19 & 3.46 & 4.06 & 3.16 & 3.26 & 3.72 \\
\hline $\mathrm{H}-12$ & 4.12 & 4.63 & 3.89 & 4.34 & 4.44 & 4.18 & 3.99 & 4.32 & 4.28 & 4.16 & 4.04 & 4.28 & 4.30 & 4.25 & 4.17 & 4.12 & 4.44 \\
\hline $\mathrm{H}-13 \mathrm{a}$ & 2.10 & 2.26 & 2.16 & 2.29 & 2.26 & 2.47 & 1.96 & 1.87 & 1.92 & 2.29 & 2.12 & 2.11 & 2.29 & 2.40 & 2.44 & 2.06 & 2.21 \\
\hline $\mathrm{H}-13 \mathrm{~b}$ & 1.56 & 1.65 & 1.46 & 1.57 & 1.61 & 1.36 & 1.67 & 1.75 & 1.85 & 1.95 & 1.91 & 1.66 & 1.56 & 1.88 & 1.61 & 1.52 & 1.26 \\
\hline $\mathrm{H}-14$ & 1.91 & 1.82 & 2.22 & 1.86 & 1.89 & 1.92 & 2.21 & 2.28 & 1.84 & 1.74 & 2.27 & 2.08 & 2.03 & 1.83 & 1.75 & 2.29 & 1.98 \\
\hline $\mathrm{H}-15$ & 3.50 & 3.51 & 3.90 & 3.38 & 3.58 & 3.96 & 3.40 & 3.63 & 3.25 & 3.47 & 3.97 & 3.60 & 4.02 & 3.84 & 3.63 & 4.11 & 3.59 \\
\hline $\mathrm{H}-16 \mathrm{a}$ & 2.35 & 2.40 & 2.18 & 2.12 & 2.10 & 2.15 & 2.36 & 2.10 & 2.30 & 2.38 & 2.14 & 2.57 & 2.16 & 2.09 & 2.53 & 2.13 & 2.49 \\
\hline $\mathrm{H}-16 \mathrm{~b}$ & 2.20 & 2.00 & 1.99 & 2.01 & 2.09 & 2.14 & 2.20 & 1.80 & 1.99 & 1.90 & 1.62 & 1.88 & 2.07 & 2.04 & 1.84 & 1.97 & 2.14 \\
\hline \multirow[t]{2}{*}{$\mathrm{H}-33$} & 1.02 & 1.06 & 1.01 & 1.15 & 1.08 & 1.05 & 0.83 & 1.08 & 1.08 & 0.83 & 0.84 & 0.82 & 0.96 & 0.81 & 0.96 & 0.93 & 1.13 \\
\hline & & \multicolumn{16}{|c|}{ Scaled J (FC only, $\mathrm{Hz})$} \\
\hline$J_{11,12}$ & 5.8 & 1.1 & 0.5 & 5.1 & 2.9 & 1.0 & 0.5 & 2.1 & 3.1 & 1.6 & 1.6 & 1.2 & 4.1 & 1.9 & 9.2 & 1.4 & 7.5 \\
\hline$J_{12,13 a}$ & 9.0 & 11.4 & 11.1 & 8.2 & 11.4 & 11.2 & 10.0 & 11.2 & 11.1 & 10.9 & 10.9 & 10.0 & 7.1 & 11.1 & 8.4 & 11.1 & 11.2 \\
\hline$J_{12,13 b}$ & 6.1 & 4.6 & 4.6 & 6.3 & 5.5 & 5.9 & 1.6 & 4.7 & 4.8 & 5.0 & 4.9 & 1.6 & 6.4 & 5.8 & 0.7 & 4.9 & 4.8 \\
\hline$J_{14,15}$ & 8.7 & 9.7 & 8.3 & 3.7 & 4.6 & 4.5 & 9.7 & 8.2 & 9.7 & 9.7 & 8.2 & 9.8 & 6.0 & 4.4 & 9.5 & 8.1 & 9.7 \\
\hline
\end{tabular}


Table S18. Absolute scaled errors computed for compounds F2-01/F2-32 at the B3LYP/6-31G**//MMFF level of theory following the iJ-DP4 approach, and $J$-DP4 results.

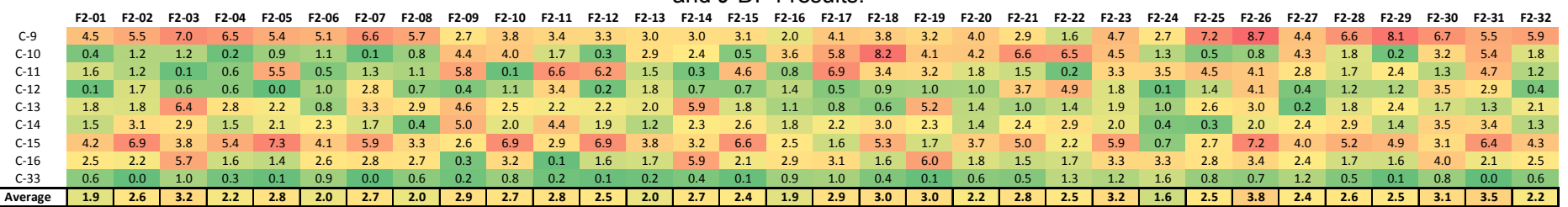

\begin{tabular}{lllllllllllllllllllllllllllllllllllll} 
H-9 & 0.20 & 0.22 & 0.33 & 0.37 & 0.33 & 0.17 & 0.24 & 0.20 & 0.05 & 0.68 & 0.29 & 0.36 & 0.72 & 0.60 & 0.12 & 0.46 & 0.30 & 0.04 & 0.23 & 0.38 & 0.19 & 0.23 & 0.31 & 0.20 & 0.28 & 0.32 & 0.55 & 0.34 & 0.45 & 0.18 & 0.58 & 0.32 \\
\hline
\end{tabular} \begin{tabular}{lllllllllllllllllllllllllllllllllllll}
$\mathrm{H}-10$ & 0.24 & 0.21 & 0.23 & 0.49 & 0.48 & 0.00 & 0.39 & 0.38 & 0.28 & 0.07 & 0.25 & 0.48 & 0.04 & 0.14 & 0.42 & 0.08 & 0.00 & 0.37 & 0.10 & 0.03 & 0.09 & 0.37 & 0.08 & 0.24 & 0.32 & 0.37 & 0.69 & 0.19 & 0.43 & 0.42 & 0.50 & 0.31 \\
\hline
\end{tabular} $\begin{array}{llllllllllllllllllllllllllllllllll}H-11 & 0.00 & 0.08 & 0.31 & 0.40 & 0.08 & 0.23 & 0.47 & 0.53 & 0.05 & 0.28 & 0.08 & 0.42 & 0.23 & 0.15 & 0.38 & 0.04 & 0.26 & 0.27 & 0.16 & 0.11 & 0.37 & 0.26 & 0.15 & 0.20 & 0.64 & 0.55 & 0.38 & 0.11 & 0.49 & 0.41 & 0.31 & 0.15 & 0.35 \\ H\end{array}$

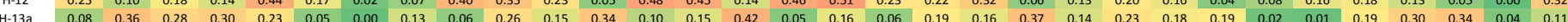
\begin{tabular}{llllllllllllllllllllllllllllllllllllllll}
$H-13 a$ & 0.08 & 0.36 & 0.28 & 0.30 & 0.23 & 0.05 & 0.00 & 0.13 & 0.06 & 0.26 & 0.15 & 0.34 & 0.10 & 0.15 & 0.42 & 0.05 & 0.16 & 0.06 & 0.19 & 0.16 & 0.37 & 0.14 & 0.23 & 0.18 & 0.19 & 0.02 & 0.01 & 0.19 & 0.33 & 0.34 & 0.04 & 0.11 \\
\hline
\end{tabular}

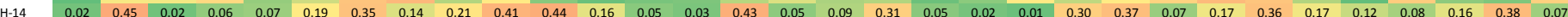

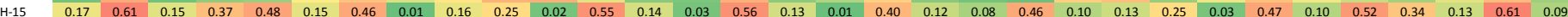
\begin{tabular}{llllllllllllllllllllllllllllllllllllll} 
H-16a & 0.14 & 0.17 & 0.11 & 0.25 & 0.09 & 0.19 & 0.46 & 0.02 & 0.22 & 0.22 & 0.21 & 0.08 & 0.06 & 0.24 & 0.16 & 0.10 & 0.05 & 0.17 & 0.23 & 0.25 & 0.20 & 0.01 & 0.25 & 0.05 & 0.03 & 0.21 & 0.22 & 0.19 & 0.26 & 0.18 & 0.22 & 0.14 \\
\hline
\end{tabular} \begin{tabular}{llllllllllllllllllllllllllllllllllll} 
H-16b & 0.10 & 0.27 & 0.34 & 0.12 & 0.06 & 0.22 & 0.41 & 0.27 & 0.27 & 0.41 & 0.16 & 0.03 & 0.24 & 0.20 & 0.23 & 0.19 & 0.20 & 0.21 & 0.19 & 0.11 & 0.06 & 0.00 & 0.40 & 0.21 & 0.30 & 0.58 & 0.32 & 0.13 & 0.16 & 0.36 & 0.23 & 0.06 \\
\hline
\end{tabular}

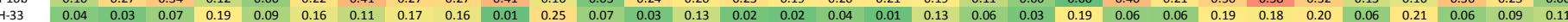

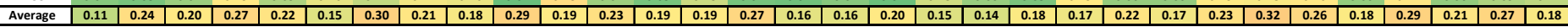

\begin{tabular}{lllllllllllllllllllllllllllllllll} 
J.ux & 0.48 & 1.84 & 2.05 & 3.92 & 1.90 & 4.22 & 4.11 & 4.05 & 3.69 & 3.75 & 4.86 & 2.70 & 2.91 & 1.69 & 0.39 & 3.11 & 4.67 & 5.32 & 0.71 & 2.92 & 4.84 & 5.32 & 3.66 & 2.73 & 4.19 & 4.23 & 4.60 & 1.70 & 3.89 & 3.43 & 4.39 & 1.68 \\
\hline
\end{tabular}

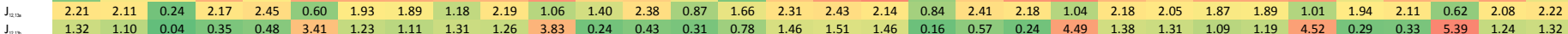

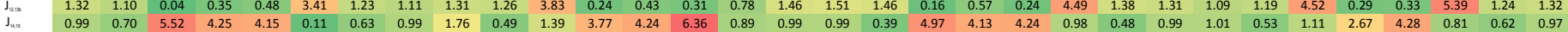

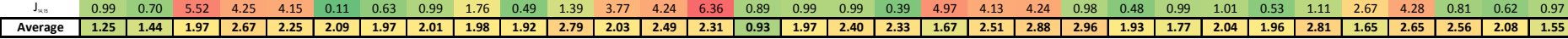

\begin{tabular}{|c|c|c|c|c|c|c|c|c|c|c|c|c|c|c|c|c|c|}
\hline \multicolumn{2}{|c|}{ Isomer № } & 1 & 2 & 3 & 4 & 5 & 6 & 7 & 8 & 9 & 10 & 11 & 12 & 13 & 14 & 15 & 16 \\
\hline \multirow{5}{*}{ J-DP4 (\%) } & $\mathrm{H}$ & 75.58 & 0.00 & 0.08 & 0.00 & 0.00 & 6.43 & 0.00 & 0.01 & 0.41 & 0.00 & 0.11 & 0.00 & 0.02 & 0.04 & 0.00 & 1.12 \\
\hline & $\mathrm{C}$ & 8.61 & 0.13 & 0.00 & 1.13 & 0.03 & 4.51 & 0.06 & 4.14 & 0.02 & 0.09 & 0.06 & 0.14 & 8.26 & 0.09 & 0.34 & 17.14 \\
\hline & $\mathrm{H}+\mathrm{C}$ & 85.68 & 0.00 & 0.00 & 0.00 & 0.00 & 3.82 & 0.00 & 0.01 & 0.00 & 0.00 & 0.00 & 0.00 & 0.02 & 0.00 & 0.00 & 2.52 \\
\hline & $\mathrm{J}$ & 13.57 & 6.21 & 2.02 & 0.10 & 0.38 & 0.90 & 1.11 & 0.93 & 0.89 & 1.20 & 0.08 & 0.82 & 0.16 & 0.84 & 50.67 & 0.84 \\
\hline & all data & 98.59 & 0.00 & 0.00 & 0.00 & 0.00 & 0.29 & 0.00 & 0.00 & 0.00 & 0.00 & 0.00 & 0.00 & 0.00 & 0.00 & 0.00 & 0.18 \\
\hline \multicolumn{2}{|c|}{ Isomer № } & 17 & 18 & 19 & 20 & 21 & 22 & 23 & 24 & 25 & 26 & 27 & 28 & 29 & 30 & 31 & 32 \\
\hline \multirow{5}{*}{ J-DP4 (\%) } & $\mathrm{H}$ & 1.13 & 0.06 & 7.08 & 5.51 & 0.11 & 0.82 & 0.02 & 0.93 & 0.00 & 0.00 & 0.00 & 0.17 & 0.00 & 0.01 & 0.00 & 0.36 \\
\hline & $\mathrm{C}$ & 0.03 & 0.01 & 0.02 & 2.35 & 0.06 & 0.28 & 0.01 & 49.84 & 0.22 & 0.00 & 0.52 & 0.19 & 0.22 & 0.01 & 0.00 & 1.50 \\
\hline & $\mathrm{H}+\mathrm{C}$ & 0.00 & 0.00 & 0.02 & 1.70 & 0.00 & 0.03 & 0.00 & 6.12 & 0.00 & 0.00 & 0.00 & 0.00 & 0.00 & 0.00 & 0.00 & 0.07 \\
\hline & $\mathrm{J}$ & 0.25 & 0.44 & 5.54 & 0.15 & 0.06 & 0.06 & 1.16 & 1.70 & 0.87 & 1.23 & 0.08 & 2.69 & 0.11 & 0.23 & 0.80 & 3.92 \\
\hline & all data & 0.00 & 0.00 & 0.01 & 0.02 & 0.00 & 0.00 & 0.00 & 0.88 & 0.00 & 0.00 & 0.00 & 0.00 & 0.00 & 0.00 & 0.00 & 0.02 \\
\hline
\end{tabular}




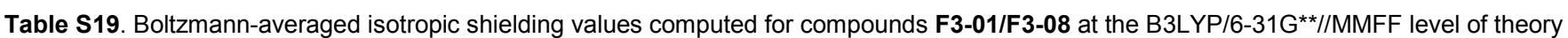
following a free conformational sampling procedure.

\begin{tabular}{|c|c|c|c|c|c|c|c|c|c|}
\hline \multirow{2}{*}{ Atom } & \multirow{2}{*}{ Exp. NMR } & \multicolumn{7}{|c|}{ Isotropic shielding values $(\boldsymbol{\sigma}, \mathbf{p p m})$} \\
\cline { 3 - 10 } & & F3-01 & F3-02 & F3-03 & F3-04 & F3-05 & F3-06 & F3-07 & F3-08 \\
\hline C-25 & 68.0 & 125.2132 & 121.3547 & 125.0934 & 118.6720 & 122.2029 & 120.6412 & 125.8039 & 119.3373 \\
\hline C-26 & 46.5 & 151.1320 & 150.8919 & 142.8621 & 147.1827 & 151.8689 & 146.4655 & 147.0304 & 150.7845 \\
\hline C-27 & 72.8 & 119.6462 & 119.3603 & 118.4826 & 119.0509 & 120.1247 & 120.6704 & 118.5296 & 118.9765 \\
\hline C-28 & 79.4 & 114.6671 & 112.5830 & 115.2674 & 117.0551 & 116.1222 & 108.4717 & 117.5029 & 117.3977 \\
\hline C-29 & 98.5 & 89.0899 & 89.7493 & 89.0675 & 89.0906 & 88.3263 & 87.0318 & 88.3859 & 89.0023 \\
\hline C-35 & 21.8 & 167.6478 & 159.8303 & 161.1712 & 160.7652 & 163.1399 & 161.6525 & 159.7824 & 159.0650 \\
\hline C-24 & 37.0 & 161.9294 & 158.8138 & 159.9719 & 158.6740 & 159.3334 & 159.2112 & 159.6298 & 158.6510 \\
\hline C-30 & 36.6 & 154.0906 & 161.4850 & 156.2197 & 155.9952 & 154.7974 & 165.1439 & 156.4676 & 155.3167 \\
\hline & & & & & & & & & \\
\hline H-25 & 3.90 & 27.4126 & 27.9315 & 27.6452 & 27.9606 & 27.7640 & 27.7859 & 27.6203 & 27.9283 \\
\hline H-26a & 1.73 & 29.6704 & 30.2571 & 30.0976 & 29.6807 & 29.7547 & 30.1479 & 30.2177 & 29.7266 \\
\hline H-26b & 1.45 & 30.3786 & 30.5849 & 30.7560 & 30.3485 & 30.4951 & 30.4290 & 30.9607 & 30.7214 \\
\hline H-28 & 3.28 & 27.9474 & 29.0260 & 28.5217 & 28.3019 & 28.4902 & 28.8108 & 28.5366 & 28.4986 \\
\hline H-35 & 1.38 & 30.4789 & 30.5768 & 30.5769 & 30.5603 & 30.4729 & 30.6133 & 30.5459 & 30.5432 \\
\hline
\end{tabular}

Table S20. Scaled chemical shifts computed for compounds F3-01/F3-08 at the B3LYP/6-31G**//MMFF level of theory following a free conformational sampling procedure.

\begin{tabular}{|c|c|c|c|c|c|c|c|c|c|}
\hline \multirow{2}{*}{ Atom } & \multirow{2}{*}{ Exp. NMR } & \multicolumn{8}{|c|}{ Scaled chemical shifts $(\mathbf{\delta}, \mathbf{p p m})$} \\
\cline { 3 - 10 } & & $\mathbf{F 3 - 0 1}$ & $\mathbf{F 3 - 0 2}$ & $\mathbf{F 3 - 0 3}$ & $\mathbf{F 3 - 0 4}$ & $\mathbf{F 3 - 0 5}$ & $\mathbf{F 3 - 0 6}$ & F3-07 & F3-08 \\
\hline C-25 & 68.0 & 67.4 & 70.3 & 66.2 & 72.5 & 69.8 & 69.7 & 66.2 & 72.2 \\
\hline C-26 & 46.5 & 42.5 & 41.2 & 48.0 & 43.4 & 40.3 & 45.7 & 44.3 & 39.9 \\
\hline C-27 & 72.8 & 72.7 & 72.2 & 73.0 & 72.1 & 71.8 & 69.6 & 73.7 & 72.6 \\
\hline C-28 & 79.4 & 77.5 & 78.9 & 76.3 & 74.1 & 75.8 & 81.0 & 74.7 & 74.2 \\
\hline C-29 & 98.5 & 102.0 & 101.4 & 103.1 & 102.6 & 103.4 & 100.9 & 104.8 & 103.4 \\
\hline C-35 & 21.8 & 26.7 & 32.4 & 29.3 & 29.6 & 29.1 & 31.6 & 31.1 & 31.3 \\
\hline C-24 & 37.0 & 32.2 & 33.4 & 30.5 & 31.7 & 32.9 & 33.8 & 31.3 & 31.8 \\
\hline C-30 & 36.6 & 39.7 & 30.8 & 34.3 & 34.5 & 37.4 & 28.3 & 34.6 & 35.2 \\
\hline & & & & & & & & & \\
\hline H-25 & 3.90 & 3.81 & 4.11 & 3.95 & 3.76 & 3.91 & 4.05 & 3.95 & 3.84 \\
\hline H-26a & 1.73 & 1.94 & 1.76 & 1.85 & 2.04 & 2.00 & 1.78 & 1.82 & 2.11 \\
\hline H-26b & 1.45 & 1.35 & 1.43 & 1.29 & 1.37 & 1.29 & 1.51 & 1.21 & 1.16 \\
\hline H-28 & 3.28 & 3.37 & 3.00 & 3.20 & 3.42 & 3.22 & 3.07 & 3.20 & 3.30 \\
\hline H-35 & 1.38 & 1.27 & 1.44 & 1.45 & 1.16 & 1.31 & 1.33 & 1.55 & 1.33 \\
\hline
\end{tabular}

Table S21. Absolute scaled errors computed for compounds F3-01/F3-08 at the B3LYP/6-31G**//MMFF level of theory following a free conformational sampling procedure, and DP4 results.

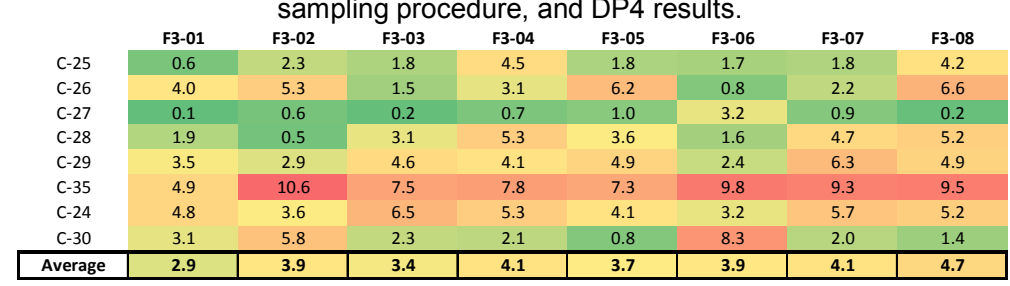

\begin{tabular}{|c|l|l|l|l|l|l|l|l}
\hline H-25 & 0.09 & 0.21 & 0.05 & 0.14 & 0.01 & 0.15 & 0.05 & 0.06 \\
H-26a & 0.21 & 0.03 & 0.12 & 0.31 & 0.27 & 0.05 & 0.09 & 0.38 \\
H-26b & 0.10 & 0.02 & 0.16 & 0.08 & 0.16 & 0.06 & 0.24 & 0.29 \\
H-28 & 0.09 & 0.28 & 0.08 & 0.14 & 0.06 & 0.21 & 0.08 & 0.02 \\
H-35 & 0.11 & 0.06 & 0.07 & 0.22 & 0.07 & 0.05 & 0.17 & 0.05 \\
\hline Average & $\mathbf{0 . 1 2}$ & $\mathbf{0 . 1 2}$ & $\mathbf{0 . 1 0}$ & $\mathbf{0 . 1 8}$ & $\mathbf{0 . 1 1}$ & $\mathbf{0 . 1 0}$ & $\mathbf{0 . 1 3}$ & $\mathbf{0 . 1 6}$ \\
\hline
\end{tabular}

\begin{tabular}{|c|c|c|c|c|c|c|c|c|c|}
\hline \multicolumn{2}{|c|}{ Isomer No } & $\mathbf{1}$ & $\mathbf{2}$ & $\mathbf{3}$ & $\mathbf{4}$ & $\mathbf{5}$ & $\mathbf{6}$ & $\mathbf{7}$ & $\mathbf{8}$ \\
\hline \multirow{4}{*}{$\mathrm{DP4}(\%)$} & $\mathrm{H}$ & 14.40 & 9.88 & 30.31 & 1.60 & 12.35 & 20.00 & 9.85 & 1.62 \\
\cline { 2 - 10 } & $\mathrm{C}$ & 94.99 & 0.17 & 3.45 & 0.12 & 0.87 & 0.31 & 0.08 & 0.00 \\
\cline { 2 - 10 } & $\mathrm{H}+\mathrm{C}$ & 91.68 & 0.11 & 7.00 & 0.01 & 0.72 & 0.42 & 0.06 & 0.00 \\
\cline { 2 - 10 } & $\mathrm{J}$ & - & - & - & - & - & - & - & - \\
\cline { 2 - 10 } & all data & $\mathbf{9 1 . 6 8}$ & $\mathbf{0 . 1 1}$ & $\mathbf{7 . 0 0}$ & $\mathbf{0 . 0 1}$ & $\mathbf{0 . 7 2}$ & $\mathbf{0 . 4 2}$ & $\mathbf{0 . 0 6}$ & $\mathbf{0 . 0 0}$ \\
\hline
\end{tabular}


Table S22. Boltzmann-averaged isotropic shielding values computed for compounds F3-01/F3-08 at the B3LYP/6-31G**//MMFF level of theory following the iJ-DP4 approach.

\begin{tabular}{|c|c|c|c|c|c|c|c|c|c|}
\hline \multirow{2}{*}{ Atom } & \multirow{2}{*}{ Exp. NMR } & \multicolumn{8}{|c|}{ Isotropic shielding values $(\sigma, \mathrm{ppm})$} \\
\hline & & F3-01 & F3-02 & F3-03 & F3-04 & F3-05 & F3-06 & F3-07 & F3-08 \\
\hline $\mathrm{C}-25$ & 68.0 & 125.2132 & - & - & - & 122.2029 & - & - & 120.1098 \\
\hline $\mathrm{C}-26$ & 46.5 & 151.1320 & - & - & - & 151.8689 & - & - & 146.4043 \\
\hline C-27 & 72.8 & 119.6462 & - & - & - & 120.1247 & - & - & 120.2894 \\
\hline C-28 & 79.4 & 114.6671 & - & - & - & 116.1222 & - & - & 103.5284 \\
\hline C-29 & 98.5 & 89.0899 & - & - & - & 88.3263 & - & - & 87.4242 \\
\hline $\mathrm{C}-35$ & 21.8 & 167.6478 & - & - & - & 163.1399 & - & - & 167.9413 \\
\hline $\mathrm{C}-24$ & 37.0 & 161.9294 & - & - & - & 159.3334 & - & - & 158.9769 \\
\hline $\mathrm{C}-30$ & 36.6 & 154.0906 & - & - & - & 154.7974 & - & - & 165.8140 \\
\hline & & & & & & & & & \\
\hline $\mathrm{H}-25$ & 3.90 & 27.4126 & - & - & - & 27.7640 & - & - & 28.3408 \\
\hline $\mathrm{H}-26 \mathrm{a}$ & 1.73 & 29.6704 & - & - & - & 29.7547 & - & - & 30.0768 \\
\hline $\mathrm{H}-26 \mathrm{~b}$ & 1.45 & 30.3786 & - & - & - & 30.4951 & - & - & 30.2330 \\
\hline $\mathrm{H}-28$ & 3.28 & 27.9474 & - & - & - & 28.4902 & - & - & 28.3230 \\
\hline $\mathrm{H}-35$ & 1.38 & 30.4789 & - & - & - & 30.4729 & - & - & 30.4971 \\
\hline
\end{tabular}

Table S23. Scaled chemical shifts computed for compounds F3-01/F3-08 at the B3LYP/6-31G**//MMFF level of theory following the iJ-DP4 approach.

\begin{tabular}{|c|c|c|c|c|c|c|c|c|c|}
\hline \multirow{2}{*}{ Atom } & \multirow{2}{*}{ Exp. NMR } & \multicolumn{7}{|c|}{ Scaled chemical shifts $(\mathbf{\delta}, \mathbf{p p m})$} \\
\cline { 3 - 10 } & & F3-01 & F3-02 & F3-03 & F3-04 & F3-05 & F3-06 & F3-07 & F3-08 \\
\hline C-25 & 68.0 & 67.4 & - & - & - & 69.8 & - & - & 69.5 \\
\hline C-26 & 46.5 & 42.5 & - & - & - & 40.3 & - & - & 46.6 \\
\hline C-27 & 72.8 & 72.7 & - & - & - & 71.8 & - & - & 69.4 \\
\hline C-28 & 79.4 & 77.5 & - & - & - & 75.8 & - & - & 84.0 \\
\hline C-29 & 98.5 & 102.0 & - & - & - & 103.4 & - & - & 98.0 \\
\hline C-35 & 21.8 & 26.7 & - & - & - & 29.1 & - & - & 27.8 \\
\hline C-24 & 37.0 & 32.2 & - & - & - & 32.9 & - & - & 35.6 \\
\hline C-30 & 36.6 & 39.7 & - & - & - & 37.4 & - & - & 29.7 \\
\hline & & - & - & - & - & - & - & - & - \\
\hline H-25 & 3.90 & 3.81 & - & - & - & 3.91 & - & - & 3.63 \\
\hline H-26a & 1.73 & 1.94 & - & - & - & 2.00 & - & - & 1.70 \\
\hline H-26b & 1.45 & 1.35 & - & - & - & 1.29 & - & - & 1.53 \\
\hline H-28 & 3.28 & 3.37 & - & - & - & 3.22 & - & - & 3.65 \\
\hline H-35 & 1.38 & 1.27 & - & - & - & 1.31 & - & - & 1.24 \\
\hline
\end{tabular}

Table S24. Absolute scaled errors computed for compounds F3-01/F3-08 at the B3LYP/6-31G**//MMFF level of theory following the iJ-DP4 approach,

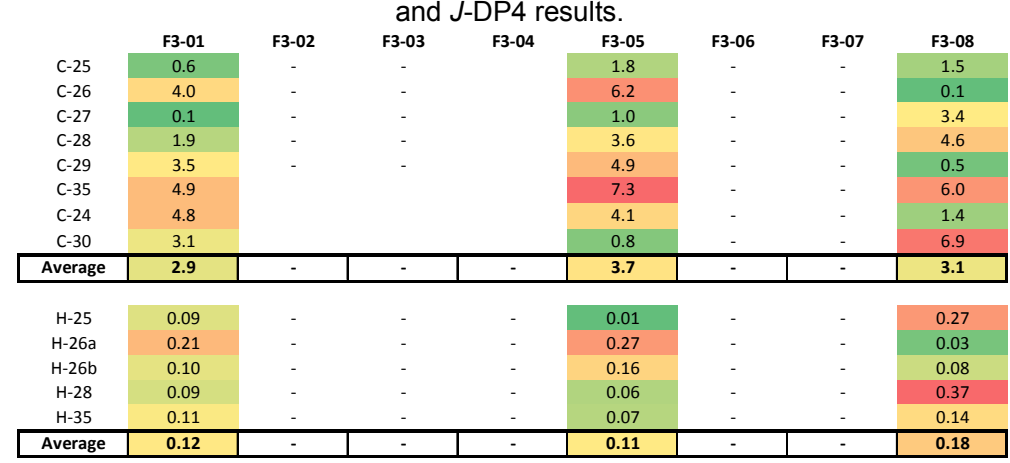

\begin{tabular}{|c|c|c|c|c|c|c|c|c|c|}
\hline \multicolumn{2}{|c|}{ Isomer № } & $\mathbf{1}$ & $\mathbf{2}$ & $\mathbf{3}$ & $\mathbf{4}$ & $\mathbf{5}$ & $\mathbf{6}$ & $\mathbf{7}$ & $\mathbf{8}$ \\
\hline \multirow{4}{*}{ J-DP4 (\%) } & $\mathrm{H}$ & 51.72 & - & - & - & 44.36 & - & - & 3.92 \\
\cline { 2 - 9 } & $\mathrm{C}$ & 82.45 & - & - & - & 0.75 & - & - & 16.80 \\
\cline { 2 - 9 } & $\mathrm{H}+\mathrm{C}$ & 97.73 & - & - & - & 0.77 & - & - & 1.51 \\
\cline { 2 - 10 } & J & - & - & - & - & - & - & - & - \\
\cline { 2 - 10 } & all data & $\mathbf{9 7 . 7 3}$ & - & - & - & $\mathbf{0 . 7 7}$ & - & - & 1.51 \\
\hline
\end{tabular}


Table S25. Boltzmann-averaged isotropic shielding values computed for compounds $01 / 08$ at the B3LYP/6-31G**//MMFF level of theory following a free conformational sampling procedure.

\begin{tabular}{|c|c|c|c|c|c|c|c|c|c|c|c|c|c|c|c|c|c|c|c|}
\hline \multirow{2}{*}{ Atom } & \multirow{2}{*}{ Exp. NMR } & & & & & & \multirow[b]{2}{*}{04} & \multirow[b]{2}{*}{05} & \multirow[b]{2}{*}{06} & \multirow[b]{2}{*}{07} & \\
\hline & & 01 & 02 & 03 & 04 & 05 & 06 & 07 & 08 & Atom & & 01 & 02 & 03 & & & & & 08 \\
\hline $\mathrm{C} 1$ & 173.9 & 22.4342 & 22.2223 & 21.7866 & 21.3169 & 22.8806 & 21.3418 & 22.4920 & 21.7158 & $\mathrm{H} 2 \mathrm{a}$ & 2.87 & 28.9528 & 28.9342 & 28.3735 & 28.0769 & 28.9703 & 28.5466 & 28.1606 & 28.4505 \\
\hline $\mathrm{C} 2$ & 38.4 & 151.2676 & $\frac{151.4402}{151.2 L C}$ & 154.5616 & 155.0446 & 152.7110 & 151.6784 & 154.6722 & 154.9979 & $\mathrm{H} 2 \mathrm{~b}$ & 2.42 & 29.4492 & 29.5730 & 29.8394 & 29.9437 & 29.1256 & 29.3843 & 29.3250 & 29.6511 \\
\hline C3 & 71.4 & 122.3040 & 120.7744 & 112.2321 & 113.5410 & 121.8280 & 120.5722 & 113.8280 & 115.1575 & $\mathrm{H} 3$ & 3.92 & 27.3911 & 27.7943 & 27.0221 & 27.0465 & 27.7709 & 27.9612 & 27.0595 & 27.1160 \\
\hline C4 & 75.3 & 117.4951 & 115.2858 & 121.1583 & 118.3186 & 118.8229 & 115.3001 & 120.8839 & 120.3079 & $\mathrm{H} 4$ & 3.1 & 28.3643 & 28.7904 & 28.3260 & 28.1933 & 28.1092 & 28.7324 & 27.7257 & 28.1245 \\
\hline C5 & 74.7 & 116.0617 & 112.9781 & 117.2736 & 117.8490 & 115.8133 & 112.4413 & 118.5492 & 117.7803 & H5 & 3.54 & 27.7875 & 28.2073 & 27.9923 & 27.8029 & 27.7954 & 28.3754 & 27.7990 & 27.9123 \\
\hline C6 & 73.0 & 117.4194 & 119.9008 & 116.0354 & 119.0705 & 117.6560 & 120.1032 & 116.9168 & 117.8044 & $\mathrm{H} 6$ & 3.57 & 28.0582 & 27.7787 & 28.6179 & 28.5744 & 27.8601 & 27.7562 & 28.8610 & 28.7469 \\
\hline C7 & 74.8 & 115.2821 & 119.8092 & 127.3530 & 125.4789 & 116.9407 & 118.8920 & 122.0745 & 123.4526 & $\mathrm{H} 7$ & 4.05 & 27.6925 & 27.1923 & 26.9173 & 27.2105 & 27.7660 & 27.1900 & 27.3462 & 27.5239 \\
\hline $\mathrm{C} 8$ & 30.4 & 157.2437 & 159.1343 & 155.0068 & 148.1649 & 158.4888 & 158.2771 & 150.8830 & 152.4583 & $\mathrm{H} 8 \mathrm{a}$ & 2.04 & 29.4089 & 29.9574 & 29.2447 & 29.6124 & 29.8785 & 29.8053 & 29.2165 & 29.1838 \\
\hline C9 & 71.5 & 119.0944 & 121.1052 & 119.2498 & 122.6811 & 122.8441 & 123.7945 & 118.5670 & 116.6573 & $\mathrm{H} 8 \mathrm{~b}$ & 1.92 & 29.9617 & 29.9647 & 30.6094 & 30.3094 & 29.9728 & 30.0673 & 30.3061 & 30.4870 \\
\hline $\mathrm{C} 10$ & 73.5 & 110.5259 & 111.6943 & 111.8004 & 109.4737 & 111.0605 & 111.2614 & 111.5286 & 117.1088 & H9 & 3.99 & 27.5698 & 27.4331 & 27.6752 & 27.6044 & 27.3007 & 27.1355 & 27.3358 & 27.5494 \\
\hline C11 & 75.8 & 114.2667 & 114.1885 & 114.0687 & 113.9791 & 113.8159 & 113.5800 & 114.4599 & 111.0406 & $\mathrm{H} 10$ & 3.52 & 28.3029 & 28.2506 & 28.4938 & 28.2455 & 28.1503 & 28.2661 & 27.9978 & 28.4792 \\
\hline $\mathrm{C} 12$ & 80.2 & 113.3652 & 110.3086 & 109.5874 & 113.3467 & 113.1317 & 114.0328 & 113.3548 & 110.5421 & $\mathrm{H} 11$ & 3.57 & 28.1271 & 28.2592 & 27.9256 & 28.2150 & 28.1438 & 28.2462 & 28.0652 & 28.1614 \\
\hline C13 & 38.3 & 153.2975 & 153.8261 & 153.6991 & 153.3559 & 153.5059 & 153.3337 & 153.2080 & 153.3415 & $\mathrm{H} 12$ & 4.12 & 27.1736 & 27.4097 & 27.3876 & 27.2825 & 27.1777 & 27.1505 & 27.3419 & 27.2967 \\
\hline C14 & 4 & 155.3792 & 150.6073 & 150.1688 & 150.5204 & 151.1352 & 151.6003 & 153.7768 & 155.3065 & H13a & 2.1 & 29.6556 & 29.9215 & 29.7348 & 29.7610 & 29.8141 & 29.6735 & 29.6185 & 29.8312 \\
\hline C15 & 3.2 & 106.9209 & 107.2458 & 107.7135 & 106.9894 & 105.1926 & 103.4817 & 107.9678 & 107.1363 & H13b & 1.56 & 30.0831 & 30.0094 & 30.5668 & 30.1869 & 30.0630 & 30.0797 & 30.2704 & 30.4533 \\
\hline C16 & 37.9 & 155.4877 & 151.7970 & 150.0012 & 150.1498 & 151.2463 & 152.7710 & 153.6747 & 156.2545 & $\mathrm{H} 14$ & 1.91 & 29.7303 & 29.9692 & 29.8709 & 30.0519 & 29.8480 & 29.8811 & 29.6774 & 29.3927 \\
\hline C17 & 131.1 & 62.5140 & 63.6566 & 66.2982 & 63.8213 & 63.5042 & 56.4964 & 59.4719 & 63.6519 & & 3.5 & 28.0833 & 27.9989 & 28.1190 & 28.2285 & 28.1913 & 28.0794 & 27.8876 & 28.0781 \\
\hline C18 & 2.1 & 61.6469 & 63.7158 & 60.4463 & 60.1112 & 61.5612 & 64.6599 & 63.3953 & 59.2193 & H16a & 2.35 & 29.0819 & 29.4542 & 29.3700 & 29.5171 & 29.5320 & 29.5251 & 29.2939 & 29.4868 \\
\hline C19 & 3.7 & 117.1632 & 119.3738 & 125.5832 & 116.5299 & 120.1187 & 118.3804 & 115.2653 & 118.3776 & $\mathrm{H} 16 \mathrm{~b}$ & 2.2 & 29.7293 & 29.6306 & 29.8023 & 29.6926 & 29.5695 & 29.5453 & 29.7395 & 29.7090 \\
\hline $\mathrm{C} 20$ & 38.4 & 151.4136 & 152.6335 & 157.1592 & 151.8674 & 152.0161 & 153.2571 & 153.2987 & 152.0670 & H17 & 5.81 & 25.3757 & 25.8355 & 25.9594 & 26.0292 & 25.5705 & 24.9801 & 25.2312 & 25.5519 \\
\hline C21 & 1.9 & 62.4265 & 62.4372 & 59.7194 & 60.4220 & 61.6956 & 64.0206 & 63.3623 & 62.2774 & $\mathrm{H} 18$ & 5.59 & 25.9493 & 25.7348 & 25.6920 & 25.8061 & 26.0062 & 25.9815 & 25.9703 & 25.9658 \\
\hline $\mathrm{C} 22$ & 129.2 & 62.4614 & 63.9096 & 66.1823 & 64.9469 & 63.9956 & 63.4503 & 63.7534 & 63.1520 & $\mathrm{H} 19$ & 5.45 & 26.2484 & 26.4100 & 26.1197 & 26.7057 & 26.2228 & 26.1146 & 26.4343 & 26.5096 \\
\hline $\mathrm{C} 23$ & 24.8 & 166.0407 & 162.8988 & 165.7903 & 163.9755 & 165.7791 & 164.5179 & 163.1966 & 163.1063 & $\mathrm{H} 2 \mathrm{Oa}$ & 2.69 & 29.0657 & 29.2489 & 28.7754 & 28.6785 & 29.0100 & 28.8732 & 28.8026 & 28.8482 \\
\hline $\mathrm{C} 24$ & 37 & 154.2833 & 152.2790 & 153.3672 & 153.4462 & 152.8137 & 152.9755 & 152.2282 & 152.1134 & $\mathrm{H} 2 \mathrm{Ob}$ & 2.09 & 29.9131 & 29.6756 & 29.8302 & 29.8795 & 30.0331 & 30.0153 & 30.0469 & 30.0941 \\
\hline $\mathrm{C} 25$ & .0 & 125.8749 & 123.1454 & 122.8857 & 125.2345 & 124.4257 & 123.1492 & 123.9892 & 122.7732 & $\mathrm{H} 22$ & 5.28 & 26.2201 & 26.2814 & 25.9726 & 26.2880 & 26.1044 & 26.2519 & 26.3112 & 26.3318 \\
\hline $\mathrm{C} 26$ & 5 & 144.8299 & 144.2684 & 148.4400 & 146.3512 & 147.2893 & 146.0720 & 141.6570 & 144.7498 & H23a & 2.13 & 28.5490 & 28.7896 & 29.6122 & 29.0705 & 29.0279 & 28.8522 & 28.3288 & 28.8388 \\
\hline $\mathrm{C} 27$ & 7 & 120.1198 & 119.8615 & 120.3936 & 119.9920 & 119.8963 & 119.9985 & 118.6819 & 120.0339 & $\mathrm{H} 23$ & 2.1 & 30.0773 & 30.1081 & 30.2810 & 29.7463 & 30.0697 & 29.8088 & 30.0385 & 29.9487 \\
\hline $\mathrm{C} 28$ & .4 & 114.0635 & 111.8083 & $\frac{120.050}{114.2816}$ & 109.7260 & $\begin{array}{l}114.0816 \\
11.0816\end{array}$ & 110.2851 & 112.6036 & $\begin{array}{l}114.0457 \\
11.005 \\
\end{array}$ & H24a & 1.52 & 30.0086 & 30.3124 & 29.7802 & 30.1843 & 30.0819 & 30.1696 & 30.5191 & 30.1626 \\
\hline C29 & 3.5 & 88.7351 & 90.2044 & 89.6768 & 90.7830 & 89.2806 & 90.9451 & 89.9499 & 89.5010 & $\mathrm{H} 24 \mathrm{~b}$ & 1.45 & 30.3229 & 30.3230 & 30.1628 & 30.5034 & 30.1197 & 30.4076 & 30.5430 & 30.4438 \\
\hline C30 & 6 & 152.3266 & 152.5722 & 153.2792 & 151.3815 & 152.7413 & 152.2470 & 153.9025 & 153.7528 & $\mathrm{H} 25$ & 3.9 & 27.4284 & 27.7337 & 27.7159 & 27.6266 & 27.4657 & 27.6271 & 27.7042 & 27.6769 \\
\hline C31 & & 160.6055 & 159.4192 & 159.2303 & 158.6529 & 159.3927 & 159.2447 & 158.9896 & 159.6014 & $\mathrm{H} 26 \mathrm{a}$ & & 30.0831 & 30.0765 & 29.9842 & 30.1096 & 29.9572 & 30.1376 & 30.2093 & 30.1184 \\
\hline C32 & 6.3 & 27.4671 & 26.2245 & 29.9537 & 27.5526 & 28.8252 & 28.9222 & 27.1501 & 29.3015 & $\mathrm{H} 26 \mathrm{~b}$ & 1.45 & 30.3364 & 30.3664 & 30.3570 & 30.2453 & 30.2163 & 30.2696 & 30.7272 & 30.2817 \\
\hline C33 & .7 & 175.1277 & $\frac{174.3070}{17.2540}$ & $\frac{174.1318}{1718}$ & 173.8336 & $\frac{174.1597}{17.026}$ & $\frac{174.5181}{1742}$ & 173.9386 & 174.2392 & $\mathrm{H} 28$ & 3.28 & 28.1980 & 28.0817 & 28.0283 & 28.1959 & 28.1154 & 28.3746 & 27.8332 & 28.1952 \\
\hline C34 & 3.9 & 164.0886 & 167.1005 & 168.1076 & 165.4514 & 166.5510 & 164.7584 & 164.0898 & 166.9395 & $\mathrm{H} 30 \mathrm{a}$ & .09 & 29.5081 & 29.3647 & 29.3639 & 29.3655 & 29.4923 & 29.5677 & 28.7091 & 29.5326 \\
\hline C35 & 21.8 & 166.9591 & 167.0300 & 167.4450 & 167.1709 & 167.2761 & 167.9409 & 165.6201 & 167.2193 & H30b & 1.88 & 29.9350 & 29.7794 & 30.3950 & 30.0236 & 30.1706 & 29.8489 & 30.3165 & 29.9710 \\
\hline C36 & & 141.2260 & 141.4633 & 141.6850 & & 141.3430 & 141.1268 & & & & & 29.2205 & & & & 29.3507 & & & 29.3498 \\
\hline $\mathrm{C} 1^{\prime}$ & 8.3 & 30.7106 & 32.2670 & 32.4805 & 31.7004 & 31.7574 & 29.2993 & 30.6209 & 31.0225 & $\mathrm{H} 31 \mathrm{~b}$ & 2.35 & 29.7992 & 29.5407 & 29.7771 & 29.8145 & 29.6286 & 29.8776 & 29.9213 & 29.5739 \\
\hline $\mathrm{C}^{\prime}$ & 117.2 & 78.2470 & 78.5840 & 77.2369 & 76.5503 & 77.6379 & 76.1486 & 78.6373 & 77.3243 & H33 & 1.02 & 30.8989 & 30.8270 & 30.7925 & 30.8281 & 30.7909 & 30.7920 & 30.8052 & 30.8179 \\
\hline C3' & & & 46.8954 & & & 46.9140 & 49.0983 & & & & & & & & & 30.1404 & & & 30.1123 \\
\hline $\mathrm{CH}^{\prime}$ & 5.1 & 8204 & $\frac{7.037}{63.2862}$ & 0751 & 61.6777 & 60.3333 & $\frac{7.07428}{61.7428}$ & 63.1715 & $\frac{17.278}{63.0876}$ & & & 30.4878 & 30.4175 & 30.5282 & 30.5092 & 30.4803 & 30.5420 & 30.3806 & 30.4908 \\
\hline C5' & 140.9 & 54.5962 & 51.7442 & 54.5198 & 54.3435 & 55.3244 & 55.1338 & 53.7404 & 52.9339 & $\mathrm{H} 36$ & 3.67 & 28.0621 & 28.1106 & 28.2043 & 27.9579 & 28.0643 & 28.0341 & 28.1389 & 28.0448 \\
\hline $\mathrm{C}^{\prime}$ & 36.2 & 150.9992 & 152.9046 & 154.1549 & 153.2097 & 153.3279 & 150.9315 & 154.2874 & 153.9034 & $\mathrm{H}^{\prime}$ & 5.79 & 25.9522 & 25.8570 & 25.9216 & 25.8408 & 25.8749 & 25.8316 & 26.0189 & 25.9005 \\
\hline$C 7^{\prime}$ & 147.3 & 48.3026 & 49.4208 & 47.4335 & 49.4709 & 48.0189 & 49.6335 & 49.6792 & & & & 24.4817 & 24.37 & 24.50 & 24.6201 & 24.4697 & 24.6866 & 24.0425 & 24.3262 \\
\hline $\mathrm{C} 8^{\prime}$ & 40.6 & 149.3493 & 146.6719 & 149.9002 & 148.9711 & 149.9396 & 151.5416 & 145.7541 & 146.9433 & $\mathrm{H}^{\prime}$ & 5.93 & 25.5348 & 25.6494 & 25.9443 & 25.9113 & 25.7842 & 25.8972 & 25.5996 & 25.7171 \\
\hline $\mathrm{Cg}^{\prime}$ & 131.3 & 60.1246 & 61.9989 & 65.7356 & 61.0279 & 62.8801 & 63.4022 & 62.7442 & 59.8335 & $\mathrm{H} 6^{\prime} \mathrm{a}$ & 3.04 & 28.5997 & 28.6182 & 28.3794 & 28.8104 & 28.5156 & 28.7075 & 28.3719 & 28.7041 \\
\hline C10' & 129.4 & 66.0291 & & & & 63.2668 & 65.4165 & & & $\mathrm{H}^{\prime} \mathrm{b}$ & 2.8 & 29.0080 & 29.0507 & 29.0891 & 28.8756 & 29.0813 & 28.9432 & 29.4758 & 29.0639 \\
\hline C11' & & & 149.7141 & 148.9513 & 149.6943 & 149.5605 & 149.6645 & 149.6379 & 149.6083 & $\mathrm{H} \varepsilon$ & & 28.9709 & 28.9 & 29.03 & 28.95 & 29.0193 & 29.0 & 29.0 & 28.9276 \\
\hline C12' & 12.8 & 178.5789 & 177.1996 & 177.5978 & 177.2103 & 177.1973 & 177.7697 & 178.6322 & 177.1273 & $\mathrm{H}^{\prime}$ & 5.53 & 25.9634 & 25.8550 & 26.0608 & 25.9748 & 26.0560 & 26.1816 & $\frac{25.07522}{2.752}$ & 25.5936 \\
\hline C13' & $\frac{12.0}{113.1}$ & 80.6760 & 78.0698 & 79.6963 & 78.0879 & 80.0023 & 80.0094 & 78.2086 & 79.0591 & $\mathrm{H} 10^{\prime}$ & 5.46 & 26.2332 & 26.0236 & 26.4049 & 26.1028 & 26.2203 & 26.0276 & $\begin{array}{l}\text { U.IULL } \\
26.1839\end{array}$ & 26.1746 \\
\hline & & & & & & & & & & H11'a & 3.75 & 27.4590 & $\begin{array}{l}27.6954 \\
\end{array}$ & 27.3175 & 27.6951 & 27.4645 & 27.5116 & 27.1410 & 28.0101 \\
\hline & & & & & & & & & & $\mathrm{H} 11^{\prime \prime} \mathrm{b}$ & 3.65 & 28.3498 & 28.3014 & 28.8646 & 28.4599 & 28.6060 & 28.9311 & 28.6480 & 28.0670 \\
\hline & & & & & & & & & & H12' & 1.78 & 29.9879 & 29.9901 & 30.1797 & 30.0686 & 30.0488 & 29.9401 & 30.0286 & 30.0645 \\
\hline & & & & & & & & & & $\mathrm{H} 13^{\prime}$ & 4.84 & 26.7132 & 26.5930 & 26.7932 & 26.7014 & 26.7733 & 26.6893 & 26.6490 & 26.6523 \\
\hline
\end{tabular}


Table S26. Scaled chemical shifts computed for compounds $01 / 08$ at the B3LYP/6-31G**/MMFF level of theory following a free conformational sampling procedure.

\begin{tabular}{|c|c|c|c|c|c|c|c|c|c|c|c|c|c|c|c|c|c|c|c|}
\hline \multirow{2}{*}{ Atom } & \multirow{2}{*}{ Exp. NMR } & & & & & & & & & \multirow{2}{*}{ Atom } & \multirow{2}{*}{ Exp. NMR } & \multirow[b]{2}{*}{01} & \multirow[b]{2}{*}{02} & \multirow[b]{2}{*}{03} & \multirow[b]{2}{*}{04} & \multirow[b]{2}{*}{05} & \multirow[b]{2}{*}{06} & & \\
\hline & & 01 & 02 & 03 & 04 & 05 & 06 & 07 & 08 & & & & & & & & & 07 & 08 \\
\hline $\mathrm{C}-1$ & 173.9 & 174.6 & 175.4 & 176.0 & 176.4 & 174.6 & 176.1 & 175.1 & 175.6 & H-2a & 2.87 & 2.73 & 2.79 & 3.34 & 3.61 & 2.74 & 3.15 & 3.44 & 3.24 \\
\hline $\mathrm{C}-2$ & 38.4 & 39.1 & 38.4 & 35.9 & 34.3 & 37.6 & 38.4 & 34.8 & 35.0 & $\mathrm{H}-2 \mathrm{~b}$ & 2.42 & 2.26 & 2.19 & 1.96 & 1.82 & 2.59 & 2.36 & 2.36 & 2.11 \\
\hline $\mathrm{C}-3$ & $\begin{array}{l}71.4 \\
\end{array}$ & 69.6 & 70.9 & 80.6 & 78.4 & 70.2 & \begin{tabular}{ll|}
71.3 \\
\end{tabular} & \begin{tabular}{ll|}
78.1 \\
\end{tabular} & \begin{tabular}{ll|}
77.0 \\
\end{tabular} & $\mathrm{H}-3$ & 3.92 & 4.21 & 3.86 & 4.61 & 4.60 & 3.88 & 3.70 & 4.45 & 4.50 \\
\hline C-4 & 75.3 & 74.6 & 76.7 & 71.2 & 73.3 & 73.3 & 76.8 & 70.7 & 71.6 & $\mathrm{H}-4$ & 3.1 & 3.29 & 2.93 & 3.38 & 3.50 & 3.56 & 2.98 & 3.84 & 3.55 \\
\hline C-5 & 74.7 & 76.1 & 79.2 & 75.3 & 73.8 & 76.5 & 79.9 & 73.1 & 74.3 & $\mathrm{H}-5$ & 3.54 & 3.83 & 3.48 & 3.70 & 3.88 & 3.85 & 3.31 & 3.77 & 3.75 \\
\hline $\mathrm{C}-6$ & 73.0 & 74.7 & 71.9 & 76.6 & 72.5 & 74.6 & 71.8 & 74.9 & 74.3 & $\mathrm{H}-6$ & 3.57 & 3.58 & 3.88 & 3.11 & 3.13 & 3.79 & 3.90 & 2.79 & 2.96 \\
\hline C-7 & 74.8 & 76.9 & 72.0 & 64.6 & 65.7 & 75.3 & 73.0 & 69.4 & 68.3 & $\mathrm{H}-7$ & 4.05 & 3.93 & 4.43 & 4.71 & 4.45 & 3.88 & 4.43 & 4.19 & 4.11 \\
\hline C-8 & 30.4 & 32.8 & 30.3 & 35.5 & 41.6 & 31.5 & 31.4 & 38.8 & 37.7 & $\mathrm{H}-8 \mathrm{a}$ & 2.04 & 2.30 & 1.83 & 2.52 & 2.14 & 1.87 & 1.96 & 2.46 & 2.55 \\
\hline C-9 & 71.5 & 72.9 & 70.6 & 73.2 & 68.7 & 69.1 & 67.9 & 73.1 & 75.5 & $\mathrm{H}-8 \mathrm{~b}$ & 1.92 & 1.77 & 1.82 & 1.23 & 1.47 & 1.78 & 1.72 & 1.46 & 1.33 \\
\hline C-10 & 73.5 & 81.9 & 80.6 & 81.0 & 82.7 & 81.5 & 81.1 & 80.6 & 75.0 & $\mathrm{H}-9$ & 3.99 & 4.04 & 4.20 & 3.99 & 4.07 & 4.33 & 4.48 & 4.20 & 4.09 \\
\hline C-11 & 75.8 & 78.0 & 77.9 & 78.6 & 78.0 & 78.6 & 78.6 & 77.5 & 81.4 & $\mathrm{H}-10$ & 3.52 & 3.35 & 3.44 & 3.22 & 3.45 & 3.52 & 3.42 & 3.59 & 3.21 \\
\hline C-12 & 80.2 & 79.0 & 82.0 & 83.4 & 78.6 & 79.4 & 78.2 & 78.6 & 81.9 & $\mathrm{H}-11$ & 3.57 & 3.51 & 3.43 & 3.76 & 3.48 & 3.52 & 3.43 & 3.53 & 3.51 \\
\hline $\mathrm{C}-13$ & 38.3 & 37.0 & 35.9 & 36.9 & 36.1 & 36.8 & 36.7 & 36.3 & 36.8 & H-12 & 4.12 & 4.42 & 4.23 & 4.27 & 4.38 & 4.44 & 4.47 & 4.19 & 4.33 \\
\hline $\mathrm{C}-14$ & 40.5 & 34.8 & 39.3 & 40.6 & 39.1 & 39.3 & 38.5 & 35.7 & 34.7 & $\mathrm{H}-13 \mathrm{a}$ & 2.1 & 2.06 & 1.86 & 2.05 & 1.99 & 1.93 & 2.09 & 2.09 & 1.94 \\
\hline C-15 & 86.2 & 85.7 & 85.3 & 85.3 & 85.4 & 87.7 & 89.3 & 84.4 & 85.5 & $\mathrm{H}-13 \mathrm{~b}$ & 1.56 & 1.66 & 1.78 & 1.27 & 1.58 & 1.69 & 1.70 & 1.49 & 1.36 \\
\hline C-16 & 37.9 & 34.6 & 38.1 & 40.8 & 39.5 & 39.1 & 37.2 & 35.8 & 33.7 & $\mathrm{H}-14$ & 1.91 & 1.99 & 1.82 & 1.93 & 1.71 & 1.90 & 1.89 & 2.04 & 2.36 \\
\hline C-17 & 131.1 & 132.4 & 131.5 & 129.0 & 131.2 & 131.7 & 138.9 & 135.8 & 131.4 & $\mathrm{H}-15$ & 3.5 & 3.55 & 3.67 & 3.58 & 3.47 & 3.48 & 3.59 & 3.69 & 3.59 \\
\hline C-18 & 132.1 & 133.4 & 131.4 & 135.2 & 135.2 & 133.8 & 130.3 & 131.7 & 136.1 & H-16a & 2.35 & 2.61 & 2.30 & 2.40 & 2.23 & 2.20 & 2.23 & 2.39 & 2.27 \\
\hline C-19 & 73.7 & 75.0 & 72.4 & 66.5 & 75.2 & 72.0 & 73.6 & 76.6 & 73.6 & $\mathrm{H}-16 \mathrm{~b}$ & 2.2 & 1.99 & 2.14 & 1.99 & 2.06 & 2.16 & 2.21 & 1.98 & 2.06 \\
\hline $\mathrm{C}-20$ & 38.4 & 38.9 & 37.2 & 33.2 & 37.7 & 38.3 & 36.7 & 36.2 & 38.1 & $\mathrm{H}-17$ & 5.81 & 6.12 & 5.71 & 5.61 & 5.58 & 5.97 & 6.52 & 6.14 & 5.97 \\
\hline $\mathrm{C}-21$ & 131.9 & 132.5 & 132.8 & 136.0 & 134.9 & 133.6 & 131.0 & 131.7 & 132.8 & $\mathrm{H}-18$ & 5.59 & 5.58 & 5.80 & 5.86 & 5.80 & 5.56 & 5.57 & 5.46 & 5.58 \\
\hline C-22 & 129.2 & 132.5 & 131.2 & 129.1 & 130.0 & 131.2 & 131.6 & 131.3 & 131.9 & $\mathrm{H}-19$ & 5.45 & 5.30 & 5.17 & 5.46 & 4.93 & 5.35 & 5.45 & 5.03 & 5.07 \\
\hline $\mathrm{C}-23$ & 24.8 & 23.5 & 26.3 & 24.1 & 24.8 & 23.8 & 24.8 & 25.7 & 26.5 & $\mathrm{H}-20 \mathrm{a}$ & 2.69 & 2.62 & 2.50 & 2.96 & 3.03 & 2.70 & 2.84 & 2.84 & 2.87 \\
\hline C-24 & 37.0 & 35.9 & 37.5 & 37.2 & 36.0 & 37.5 & 37.0 & 37.4 & 38.1 & $\mathrm{H}-20 \mathrm{~b}$ & 2.09 & 1.82 & 2.10 & 1.96 & 1.88 & 1.72 & 1.76 & 1.69 & 1.70 \\
\hline $\mathrm{C}-25$ & 68.0 & 65.8 & 68.4 & 69.3 & 66.0 & 67.4 & 68.5 & 67.4 & 69.0 & $\mathrm{H}-22$ & 5.28 & 5.32 & 5.29 & 5.60 & 5.33 & 5.46 & 5.32 & 5.15 & 5.23 \\
\hline $\mathrm{C}-26$ & 46.5 & 45.9 & 46.0 & 42.4 & 43.6 & 43.3 & 44.3 & 48.6 & 45.8 & $\mathrm{H}-23 \mathrm{a}$ & 2.13 & 3.11 & 2.93 & 2.17 & 2.66 & 2.68 & 2.86 & 3.28 & 2.88 \\
\hline C-27 & 72.8 & 71.9 & 71.9 & 72.0 & 71.6 & 72.2 & 71.9 & 73.0 & 71.9 & $\mathrm{H}-23 \mathrm{~b}$ & 2.1 & 1.66 & 1.69 & 1.54 & 2.01 & 1.69 & 1.96 & 1.70 & 1.83 \\
\hline C-21 & 79.4 & 78.2 & 80.4 & 78.4 & 82.5 & 78.4 & 82.1 & 79.4 & 78.2 & $\mathrm{H}-24 \mathrm{a}$ & 1.52 & 1.73 & 1.50 & 2.01 & 1.59 & 1.68 & 1.62 & 1.26 & 1.63 \\
\hline C-29 & 98.5 & 104.9 & 103.3 & 104.4 & 102.6 & 104.5 & 102.6 & 103.5 & 104.1 & $\mathrm{H}-24 \mathrm{~b}$ & 1.45 & 1.43 & 1.49 & 1.65 & 1.28 & 1.64 & $\begin{array}{l}1.39 \\
\end{array}$ & 1.24 & 1.37 \\
\hline $\mathrm{C}-30$ & 36.6 & 38.0 & 37.2 & 37.3 & 38.2 & 37.6 & 37.8 & 35.6 & 36.3 & $\mathrm{H}-25$ & 3.9 & 4.18 & 3.92 & 3.96 & 4.05 & 4.17 & 4.02 & 3.86 & 3.97 \\
\hline $\mathrm{C}-31$ & 31.0 & 29.3 & 30.0 & 31.0 & 30.5 & 30.5 & 30.4 & 30.2 & 30.2 & $\mathrm{H}-26 \mathrm{a}$ & 1.73 & 1.66 & 1.72 & 1.82 & 1.66 & 1.80 & 1.65 & 1.54 & 1.67 \\
\hline C-32 & 176.3 & 169.3 & 171.1 & 167.3 & 169.8 & 168.3 & 168.1 & 170.2 & 167.6 & $\mathrm{H}-26 \mathrm{~b}$ & 1.45 & 1.41 & 1.45 & 1.47 & 1.53 & 1.55 & $\begin{array}{l}1.52 \\
\end{array}$ & 1.07 & 1.52 \\
\hline C-33 & 16.7 & 14.0 & 14.2 & 15.3 & 14.4 & 15.0 & 14.3 & 14.3 & 14.7 & $\mathrm{H}-28$ & 3.28 & 3.45 & 3.59 & 3.66 & 3.50 & 3.55 & 3.31 & 3.74 & 3.48 \\
\hline C-34 & 23.9 & 25.6 & 21.8 & 21.7 & 23.3 & 23.0 & 24.6 & 24.8 & 22.4 & $\mathrm{H}-30 \mathrm{a}$ & 2.09 & 2.20 & 2.39 & 2.40 & 2.37 & 2.24 & 2.19 & 2.93 & 2.22 \\
\hline C-35 & 21.8 & 22.6 & 21.9 & 22.4 & 21.4 & 22.2 & 21.2 & 23.2 & 22.1 & $\mathrm{H}-30 \mathrm{~b}$ & 1.88 & 1.80 & 2.00 & 1.43 & 1.74 & 1.59 & 1.92 & 1.45 & 1.81 \\
\hline C-36 & 52.3 & 49.7 & 49.0 & 49.5 & 49.3 & 49.6 & 49.6 & 49.2 & 49.7 & $\mathrm{H}-31 \mathrm{a}$ & 2.52 & 2.47 & 2.35 & 2.61 & 2.58 & 2.37 & 2.59 & 3.15 & 2.40 \\
\hline$C-1^{\prime}$ & 168.3 & 165.9 & 164.7 & 164.7 & 165.4 & 165.2 & 167.7 & 166.5 & 165.8 & $\mathrm{H}-31 \mathrm{~b}$ & 2.35 & 1.92 & 2.22 & 2.01 & 1.94 & 2.11 & 1.89 & 1.81 & 2.19 \\
\hline C-2' & 117.2 & 115.9 & 115.6 & 117.5 & 117.7 & 116.8 & 118.2 & 115.5 & 117.0 & $\mathrm{H}-33$ & 1.02 & 0.88 & 1.01 & 1.06 & 0.97 & 1.00 & 1.03 & 0.99 & 1.02 \\
\hline $\mathrm{C}-3^{\prime}$ & 150.7 & 148.9 & 149.2 & 148.8 & 147.1 & 149.2 & 146.8 & 150.4 & 148.7 & $\mathrm{H}-34$ & 1.71 & 1.60 & 1.68 & 1.70 & 1.71 & 1.62 & 1.58 & $\begin{array}{l}1.52 \\
\end{array}$ & 1.68 \\
\hline C-4' & 135.1 & 133.2 & 131.9 & 135.6 & 133.5 & 135.1 & 133.4 & $\frac{90.9}{131.9}$ & $\frac{132.0}{132.0}$ & $\mathrm{H}-35$ & 1.38 & 1.27 & 1.40 & 1.31 & 1.27 & $\frac{1.02}{1.30}$ & 1.27 & 1.39 & 1.32 \\
\hline$C-5^{\prime}$ & 140.9 & 140.8 & 144.1 & 141.4 & 141.3 & 140.4 & 140.4 & 141.9 & 142.7 & $\mathrm{H}-36$ & 3.67 & 3.57 & 3.57 & 3.50 & 3.73 & 3.60 & 3.63 & 3.46 & 3.62 \\
\hline C-6' & 36.2 & 39.4 & 36.9 & 36.4 & 36.3 & 36.9 & 39.2 & 35.2 & 36.2 & $\mathrm{H}-2^{\prime}$ & 5.79 & 5.58 & 5.69 & 5.65 & 5.76 & 5.68 & 5.71 & 5.42 & 5.64 \\
\hline C-7' & 147.3 & 147.4 & 146.5 & 148.9 & 146.5 & 148.1 & 146.2 & 146.2 & 147.2 & $\mathrm{H}-3^{\prime}$ & 7.27 & 6.97 & 7.08 & 6.98 & 6.94 & $\begin{array}{l}7.02 \\
\end{array}$ & 6.79 & 7.24 & 7.12 \\
\hline C-8' & 40.6 & 41.1 & 43.5 & 40.9 & 40.8 & 40.5 & 38.5 & 44.3 & 43.5 & $\mathrm{H}-5^{\prime}$ & 5.93 & 5.97 & 5.88 & 5.62 & 5.70 & 5.77 & 5.65 & 5.80 & 5.81 \\
\hline C- $9^{\prime}$ & 131.3 & 135.0 & 133.2 & 129.6 & 134.2 & 132.4 & 131.7 & 132.4 & 135.4 & $\mathrm{H}-6^{\prime} \mathrm{a}$ & 3.04 & 3.06 & 3.09 & 3.33 & 2.91 & 3.17 & 3.00 & 3.24 & 3.00 \\
\hline C-10' & 129.4 & 128.7 & 129.2 & 134.0 & 130.3 & 132.0 & 129.5 & 130.0 & 128.0 & $\mathrm{H}-6^{\prime} \mathrm{b}$ & 2.92 & 2.68 & 2.68 & 2.66 & 2.84 & 2.63 & 2.78 & 2.22 & 2.66 \\
\hline C-11' & 41.9 & 41.1 & 40.3 & 41.9 & 40.0 & 40.9 & 40.5 & 40.1 & 40.7 & $\mathrm{H}-8^{\prime}$ & 2.77 & 2.71 & 2.77 & 2.71 & 2.77 & 2.69 & 2.66 & 2.64 & 2.79 \\
\hline C-12' & 12.8 & 10.4 & 11.1 & 11.7 & 10.8 & $\begin{array}{l}11.8 \\
\end{array}$ & $\begin{array}{l}10.8 \\
\end{array}$ & 9.4 & $\begin{array}{l}11.7 \\
\end{array}$ & $\mathrm{H}-9^{\prime}$ & 5.53 & 5.57 & 5.69 & 5.51 & 5.63 & 5.51 & 5.38 & 5.66 & 5.93 \\
\hline $\begin{array}{ll}-12 \\
C-13^{\prime}\end{array}$ & 113.1 & 113.3 & 116.2 & 114.9 & 116.1 & 114.3 & 114.1 & $\begin{array}{c}0.4 \\
116.0\end{array}$ & 115.1 & $\mathrm{H}-10^{\prime}$ & 5.46 & 5.31 & 5.53 & 5.19 & 5.51 & 5.35 & 5.53 & 5.26 & 5.38 \\
\hline & & & & & & & & & & H-11'a & 3.75 & 4.15 & 3.96 & 4.33 & 3.98 & 4.17 & 4.13 & 4.38 & 3.65 \\
\hline & & & & & & & & & & $\mathrm{H}-11^{\prime \prime b}$ & 3.65 & 3.30 & 3.39 & 2.87 & 3.24 & 3.08 & 2.79 & 2.99 & 3.60 \\
\hline & & & & & & & & & & $\mathrm{H}-12^{\prime}$ & 1.78 & 1.75 & 1.80 & 1.64 & 1.70 & 1.71 & 1.84 & 1.71 & 1.72 \\
\hline & & & & & & & & & & $\mathrm{H}-13^{\prime}$ & 4.84 & 4.85 & 4.99 & 4.82 & 4.94 & 4.83 & 4.90 & 4.83 & 4.93 \\
\hline
\end{tabular}


Table S27. Absolute scaled errors computed for compounds $01 / 08$ at the B3LYP/6-31G*//MMFF level of theory following a free conformational

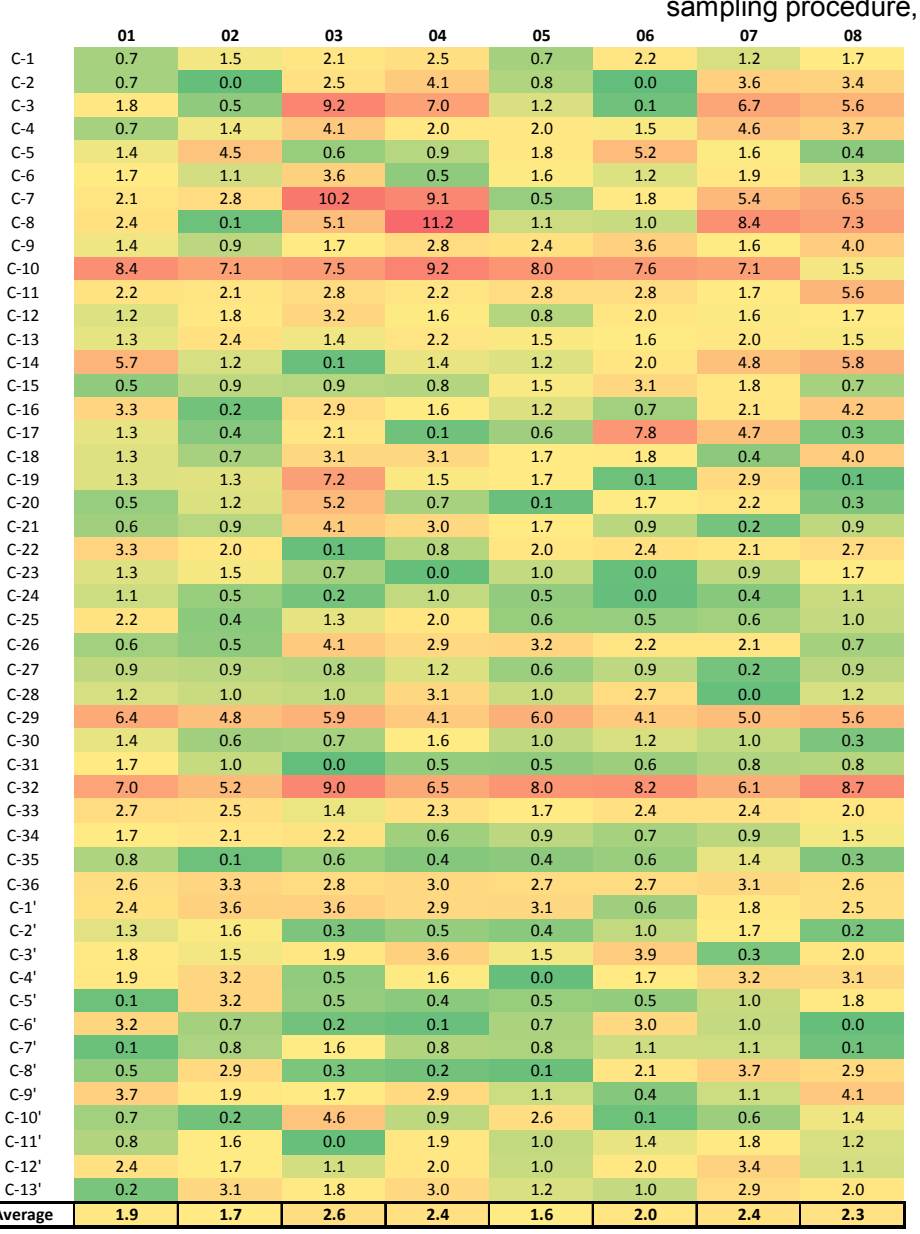

\begin{tabular}{|c|c|c|c|c|c|c|c|c|}
\hline & 01 & 02 & 03 & 04 & 05 & 06 & 07 & 08 \\
\hline H-2a & 0.14 & 0.08 & 0.47 & 0.74 & 0.13 & 0.28 & 0.57 & 0.37 \\
\hline $\mathrm{H}-2 \mathrm{~b}$ & 0.16 & 0.23 & 0.46 & 0.60 & 0.17 & 0.06 & 0.06 & 0.31 \\
\hline $\mathrm{H}-3$ & 0.29 & 0.06 & 0.69 & 0.68 & 0.04 & 0.22 & 0.53 & 0.58 \\
\hline $\mathrm{H}-4$ & 0.19 & 0.17 & 0.28 & 0.40 & 0.46 & 0.12 & 0.74 & 0.45 \\
\hline $\mathrm{H}-5$ & 0.29 & 0.06 & 0.16 & 0.34 & 0.31 & 0.23 & 0.23 & 0.21 \\
\hline $\mathrm{H}-6$ & 0.01 & 0.31 & 0.46 & 0.44 & 0.22 & 0.33 & 0.78 & 0.61 \\
\hline $\mathrm{H}-7$ & 0.12 & 0.38 & 0.66 & 0.40 & 0.17 & 0.38 & 0.14 & 0.06 \\
\hline H-8a & 0.26 & 0.21 & 0.48 & 0.10 & 0.17 & 0.08 & 0.42 & 0.51 \\
\hline H-8b & 0.15 & 0.10 & 0.69 & 0.45 & 0.14 & 0.20 & 0.46 & 0.59 \\
\hline $\mathrm{H}-\mathrm{g}$ & 0.05 & 0.21 & 0.00 & 0.08 & 0.34 & 0.49 & 0.21 & 0.10 \\
\hline $\mathrm{H}-10$ & 0.17 & 0.08 & 0.30 & 0.07 & 0.00 & 0.10 & 0.07 & 0.31 \\
\hline $\mathrm{H}-11$ & 0.06 & 0.14 & 0.19 & 0.09 & 0.05 & 0.14 & 0.04 & 0.06 \\
\hline & 0.30 & 0.11 & 0.15 & 0.26 & 0.32 & 0.35 & 0.07 & 0.21 \\
\hline & 0.04 & 0.24 & 0.05 & 0.11 & 0.17 & 0.01 & 0.01 & 0.16 \\
\hline H-13b & 0.10 & 0.22 & 0.29 & 0.02 & 0.13 & 0.14 & 0.07 & 0.20 \\
\hline H-14 & 0.08 & 0.09 & 0.02 & 0.20 & 0.01 & 0.02 & 0.13 & 0.45 \\
\hline H-15 & 0.05 & 0.17 & 0.08 & 0.03 & 0.02 & 0.09 & 0.19 & 0.09 \\
\hline H-16a & 0.26 & 0.05 & 0.05 & 0.12 & 0.15 & 0.12 & 0.04 & 0.08 \\
\hline H-16b & 0.21 & 0.06 & 0.21 & 0.14 & 0.04 & 0.01 & 0.22 & 0.14 \\
\hline $\mathrm{H}-17$ & 0.31 & 0.10 & 0.20 & 0.23 & 0.16 & 0.71 & 0.33 & 0.16 \\
\hline $\begin{array}{r}H-18 \\
H-18\end{array}$ & 0.01 & 0.21 & 0.27 & 0.21 & 0.03 & 0.02 & 0.13 & 0.01 \\
\hline $\begin{array}{l}\mathrm{H}-19 \\
\mathrm{H}-20 \mathrm{a}\end{array}$ & 0.15 & 0.28 & 0.01 & 0.52 & 0.10 & 0.00 & 0.42 & 0.38 \\
\hline $\begin{array}{l}\begin{array}{l}-20 a \\
H-20 b\end{array} \\
H\end{array}$ & 0.07 & 0.19 & 0.27 & 0.34 & 0.01 & 0.15 & 0.15 & 0.18 \\
\hline $\begin{array}{l}H-200 \\
H-22\end{array}$ & 0.27 & & & 0.21 & 0.37 & 0.33 & 0.40 & 0.39 \\
\hline H-23a & $\begin{array}{l}0.04 \\
0.98\end{array}$ & $\begin{array}{l}0.01 \\
0.80\end{array}$ & $\begin{array}{l}0.32 \\
0.04\end{array}$ & $\begin{array}{l}0.05 \\
0.53\end{array}$ & $\begin{array}{l}0.18 \\
0.55\end{array}$ & $\begin{array}{l}0.04 \\
0.73\end{array}$ & $\begin{array}{l}0.13 \\
1.15\end{array}$ & $\begin{array}{l}0.05 \\
0.75\end{array}$ \\
\hline $\mathrm{H}-23 \mathrm{~b}$ & 0.44 & 0.41 & 0.56 & 0.09 & 0.41 & 0.14 & 0.40 & 0.27 \\
\hline $\mathrm{H}-24 \mathrm{a}$ & 0.21 & 0.02 & 0.49 & 0.07 & 0.16 & 0.10 & 0.26 & 0.11 \\
\hline $\mathrm{H}-24 \mathrm{~b}$ & 0.02 & 0.04 & 0.20 & 0.17 & 0.19 & 0.06 & 0.21 & 0.08 \\
\hline $\mathrm{H}-25$ & 0.28 & 0.02 & 0.06 & 0.15 & 0.27 & 0.12 & 0.04 & 0.07 \\
\hline $\mathrm{H}-26 \mathrm{a}$ & 0.07 & 0.01 & 0.09 & 0.07 & 0.07 & 0.08 & 0.19 & 0.06 \\
\hline $\mathrm{H}-26 \mathrm{~b}$ & 0.04 & 0.00 & 0.02 & 0.08 & 0.10 & 0.07 & 0.38 & 0.07 \\
\hline H-28 & 0.17 & 0.31 & 0.38 & 0.22 & 0.27 & 0.03 & 0.46 & 0.20 \\
\hline $\mathrm{H}-30 \mathrm{a}$ & 0.11 & 0.30 & 0.31 & 0.28 & 0.15 & 0.10 & 0.84 & 0.13 \\
\hline $\mathrm{H}-30 \mathrm{~b}$ & 0.08 & 0.12 & 0.45 & 0.14 & 0.29 & 0.04 & 0.43 & 0.07 \\
\hline $\mathrm{H}-31 \mathrm{a}$ & 0.05 & 0.17 & 0.09 & 0.06 & 0.15 & 0.07 & 0.63 & 0.12 \\
\hline $\mathrm{H}-31 \mathrm{~b}$ & 0.43 & 0.13 & 0.34 & 0.41 & 0.24 & 0.46 & 0.54 & 0.16 \\
\hline $\mathrm{H}-33$ & 0.14 & 0.01 & 0.04 & 0.05 & 0.02 & 0.01 & 0.03 & 0.00 \\
\hline $\mathrm{H}-34$ & 0.11 & 0.03 & 0.01 & 0.00 & 0.09 & 0.13 & 0.19 & 0.03 \\
\hline $\mathrm{H}-35$ & 0.11 & 0.02 & 0.07 & 0.11 & 0.08 & 0.11 & 0.01 & 0.06 \\
\hline $\mathrm{H}-36$ & 0.10 & 0.10 & 0.17 & 0.06 & 0.07 & 0.04 & 0.21 & 0.05 \\
\hline $\mathrm{H}-\mathrm{2}^{\prime}$ & 0.21 & 0.10 & 0.14 & 0.03 & 0.11 & 0.08 & 0.37 & 0.15 \\
\hline H-3' & 0.30 & 0.19 & 0.29 & 0.33 & 0.25 & 0.48 & 0.03 & 0.15 \\
\hline H-5' & 0.04 & 0.05 & 0.31 & 0.23 & 0.16 & 0.28 & 0.13 & 0.12 \\
\hline H-6'a & 0.02 & 0.05 & 0.29 & 0.13 & 0.13 & 0.04 & 0.20 & 0.04 \\
\hline $\mathrm{H}-\mathrm{G}^{\prime} \mathrm{b}$ & 0.24 & 0.24 & 0.26 & 0.08 & 0.29 & 0.14 & 0.70 & 0.26 \\
\hline $\mathrm{H}-\mathrm{-}^{\prime}$ & 0.06 & 0.00 & 0.06 & 0.00 & 0.08 & 0.11 & 0.13 & 0.02 \\
\hline $\begin{array}{l}H-9^{\prime} \\
\end{array}$ & 0.04 & 0.16 & 0.02 & 0.10 & 0.02 & 0.15 & 0.13 & 0.40 \\
\hline $\mathrm{H}-10^{\prime}$ & 0.15 & 0.07 & 0.27 & 0.05 & 0.11 & 0.07 & 0.20 & 0.08 \\
\hline H-11'a & 0.40 & 0.21 & 0.58 & 0.23 & 0.42 & 0.38 & 0.63 & 0.10 \\
\hline H-11'b & 0.35 & 0.26 & 0.78 & 0.41 & 0.57 & 0.86 & 0.66 & 0.05 \\
\hline $\mathrm{H}-122^{\prime}$ & 0.03 & 0.02 & 0.14 & 0.08 & 0.07 & 0.06 & 0.07 & 0.06 \\
\hline $\begin{array}{l}\mathrm{H}-13^{\prime} \\
\mathbf{A}^{2}\end{array}$ & 0.01 & 0.15 & 0.02 & 0.10 & 0.01 & 0.06 & 0.01 & 0.09 \\
\hline Average & 0.17 & 0.15 & & & 0.17 & 0.18 & & 0.20 \\
\hline
\end{tabular}

\begin{tabular}{|c|c|c|c|c|c|c|c|c|c|}
\hline \multicolumn{2}{|c|}{ Isomer № } & $\mathbf{1}$ & $\mathbf{2}$ & $\mathbf{3}$ & $\mathbf{4}$ & $\mathbf{5}$ & $\mathbf{6}$ & $\mathbf{7}$ & $\mathbf{8}$ \\
\hline \multirow{4}{*}{$\mathrm{DP4}$ (\%) } & $\mathrm{H}$ & 0.01 & 99.99 & 0.00 & 0.00 & 0.00 & 0.00 & 0.00 & 0.00 \\
\cline { 2 - 10 } & $\mathrm{C}$ & 0.02 & 6.03 & 0.00 & 0.00 & 93.95 & 0.00 & 0.00 & 0.00 \\
\cline { 2 - 10 } & $\mathrm{H}+\mathrm{C}$ & 0.00 & 99.97 & 0.00 & 0.00 & 0.03 & 0.00 & 0.00 & 0.00 \\
\cline { 2 - 10 } & $\mathrm{J}$ & - & - & - & - & - & - & - & - \\
\cline { 2 - 10 } & all data & $\mathbf{0 . 0 0}$ & 99.97 & $\mathbf{0 . 0 0}$ & $\mathbf{0 . 0 0}$ & $\mathbf{0 . 0 3}$ & $\mathbf{0 . 0 0}$ & $\mathbf{0 . 0 0}$ & $\mathbf{0 . 0 0}$ \\
\hline
\end{tabular}


Table S28. Boltzmann-averaged isotropic shielding values computed for compounds $01 / 08$ at the B3LYP/6-31G**//MMFF level of theory following the iJ-DP4 approach.

\begin{tabular}{|c|c|c|c|c|c|c|c|c|c|c|c|c|c|c|c|c|c|c|c|}
\hline \multirow{2}{*}{ Atom } & \multirow{2}{*}{ Exp. NMR } & & & & & & & & & & & & & & & & & & \\
\hline & & 01 & 02 & 03 & 04 & 05 & 06 & 07 & 08 & & & 01 & 02 & 03 & 04 & 05 & 06 & 07 & 08 \\
\hline $\mathrm{C} 1$ & 173.9 & 23.1953 & 21.5915 & 22.1386 & 22.2162 & 23.5517 & 22.5706 & 23.3445 & 21.7407 & $\mathrm{H} 2 \mathrm{a}$ & 2.87 & 28.9964 & 29.0754 & 29.0285 & 28.2892 & 28.8394 & 28.7205 & 28.3025 & 28.3123 \\
\hline $\mathrm{C} 2$ & 38.4 & 150.7678 & 151.4388 & 153.2851 & 154.9656 & 150.6047 & 149.9256 & 156.1586 & 155.2392 & $\mathrm{H} 2 \mathrm{~b}$ & 2.42 & 29.0577 & 29.5561 & 29.0895 & 30.1578 & 29.5512 & 29.4650 & 29.9090 & 30.0182 \\
\hline C3 & 71.4 & 121.5512 & 121.1905 & 117.3719 & 110.5785 & 120.7226 & 118.8479 & 114.3293 & 115.3384 & H3 & 3.92 & 27.5822 & 27.9599 & 27.4861 & 27.8706 & 27.8854 & 27.4239 & 27.0197 & 27.1168 \\
\hline C4 & 75.3 & 118.1463 & 115.2787 & 118.3179 & 116.1778 & 117.3056 & 115.0385 & 121.0131 & 120.6517 & $\mathrm{H} 4$ & 3.10 & 28.0679 & 28.5776 & 28.3304 & 28.4733 & 28.2582 & 28.8242 & 28.4611 & 28.0223 \\
\hline C5 & 74.7 & 113.7458 & 113.2883 & 117.0563 & 121.1910 & 116.2795 & 114.2362 & 117.6983 & 117.4806 & H5 & 3.54 & 28.0231 & 27.9267 & 27.8721 & 27.5550 & 27.9296 & 28.3204 & 28.0014 & 28.0083 \\
\hline $\mathrm{C} 6$ & 73.0 & 118.5876 & 120.5633 & 119.5068 & 123.1320 & 117.5756 & 120.1416 & 117.4440 & 116.4840 & $\mathrm{H} 6$ & 3.57 & 28.1380 & 27.6776 & 28.1592 & 28.5319 & 27.8531 & 27.9008 & 28.3488 & 28.9608 \\
\hline C7 & 74.8 & 119.0681 & 120.1576 & 122.8192 & 126.0906 & 117.9153 & 116.1057 & 127.1644 & 122.6896 & $\mathrm{H} 7$ & 4.05 & 27.6364 & 26.9677 & 27.4845 & 27.4865 & 27.6599 & 27.7366 & 27.7400 & 27.4440 \\
\hline C8 & 30.4 & 158.2676 & 158.1859 & 153.2060 & 156.2493 & 160.5928 & 159.2667 & 154.5568 & 152.5279 & $\mathrm{H} 8 \mathrm{a}$ & 2.04 & 29.6879 & 29.2716 & 29.7130 & 29.3812 & 29.9478 & 29.6652 & 29.5326 & 28.8563 \\
\hline C9 & 71.5 & 118.2208 & 118.2597 & 122.4481 & 118.7803 & 119.7596 & 113.4035 & 118.1575 & 114.0243 & $\mathrm{H} 8 \mathrm{~b}$ & 1.92 & 29.8741 & 30.0038 & 30.0233 & 29.5740 & 30.2135 & 29.8003 & 30.3538 & 30.6551 \\
\hline C10 & 73.5 & 118.7137 & 115.3533 & 114.7952 & 117.1179 & 116.2007 & 118.3199 & 118.8157 & 118.0069 & H9 & 3.99 & 27.5278 & 27.9604 & 27.4843 & 27.5134 & 27.6208 & 28.2938 & 27.5280 & 27.6867 \\
\hline C11 & 75.8 & 115.0235 & 115.0897 & 115.7020 & 118.0634 & 109.3617 & 118.3843 & 116.9563 & 109.5246 & $\mathrm{H} 10$ & 3.52 & 27.9548 & 28.3244 & 28.5087 & 28.1930 & 28.3253 & 28.0718 & 28.6732 & 28.7192 \\
\hline $\mathrm{C} 12$ & 80.2 & 108.4294 & 110.1546 & 111.4039 & 108.1969 & 108.7927 & 107.7591 & 109.9314 & 109.3434 & H11 & 3.57 & 27.9394 & 28.1959 & 28.2723 & 28.3956 & 27.9307 & 28.4739 & 28.3618 & 28.2142 \\
\hline $\mathrm{C} 13$ & 38.3 & 152.6675 & 154.2789 & 152.6103 & 153.9444 & 152.9841 & 153.4435 & 152.9477 & 153.3380 & $\mathrm{H} 12$ & 4.12 & 27.4360 & 27.6816 & 27.5994 & 27.7450 & 27.0378 & 27.8914 & 27.6719 & 27.3271 \\
\hline C14 & 40.5 & 154.7878 & 151.2865 & 153.7299 & 151.4180 & 155.0003 & 150.6206 & 151.1819 & 155.8653 & H13a & 2.1 & 29.6479 & 29.5122 & 29.9615 & 29.5197 & 29.6367 & 29.5779 & 29.7100 & 29.7331 \\
\hline C15 & 86.2 & 107.7878 & 106.0191 & 107.2713 & 109.4492 & 107.3551 & 110.2375 & 107.2014 & 107.3856 & H13b & 1.56 & 30.1385 & 30.1376 & 30.0017 & 30.1020 & 30.6898 & 30.2370 & 30.2119 & 30.7897 \\
\hline C16 & 37.9 & 154.7159 & 151.1486 & 154.0070 & 153.5777 & 157.0205 & 153.4209 & 151.7040 & 156.9540 & $\mathrm{H} 14$ & 1.91 & 29.7070 & 30.1116 & 29.6872 & 29.9329 & 29.4307 & 30.0752 & 30.0472 & 29.3183 \\
\hline C17 & 131.1 & 62.4417 & 65.6171 & 65.2229 & 63.9965 & 71.2500 & 64.0544 & 61.1505 & 64.1711 & H15 & 3.5 & 27.8798 & 28.0764 & 28.1470 & 27.6523 & 27.9854 & 27.6924 & 28.2454 & 28.0916 \\
\hline C18 & 2.1 & 61.2061 & 59.0207 & 57.5249 & 64.2473 & 56.1333 & 63.6474 & 60.2479 & 58.4131 & H16a & 2.35 & 29.3076 & 29.3282 & 29.4509 & 29.4707 & 29.3720 & 29.5972 & 29.5460 & 29.5169 \\
\hline C19 & & 114.8032 & 122.0960 & 120.0280 & 118.9862 & 126.2524 & 117.2381 & 120.4077 & 119.1519 & & 2.2 & 29.5851 & 29.9288 & 29.7955 & 29.7297 & 29.6306 & 29.6176 & 29.9349 & 29.7250 \\
\hline $\mathrm{C} 20$ & 38.4 & 153.9079 & 151.8158 & 152.1637 & 150.1046 & 157.1698 & 150.9638 & 152.5259 & 152.1205 & $\mathrm{H} 17$ & 5.81 & 25.4174 & 25.6761 & 25.7155 & 25.8597 & 25.7467 & 25.8289 & 25.7216 & 25.5717 \\
\hline $\mathrm{C} 21$ & 131.9 & 61.9490 & 63.4459 & 64.0755 & 61.4023 & 60.7921 & 63.7783 & 61.7762 & 61.7155 & $\mathrm{H} 18$ & 5.59 & 25.9549 & 25.6782 & 25.8321 & 25.5766 & 25.5327 & 25.8641 & 26.0522 & 25.9926 \\
\hline $\mathrm{C} 22$ & 129.2 & 61.9582 & 63.2111 & 63.1647 & 63.3726 & 65.3971 & 63.0799 & 65.0992 & 63.4793 & $\mathrm{H} 19$ & 5.45 & 26.5098 & 26.6059 & 26.3199 & 26.2354 & 26.0021 & 26.5672 & 26.4285 & 26.4907 \\
\hline $\mathrm{C} 23$ & 24.8 & 164.7946 & 163.6936 & 161.8993 & 164.8858 & 164.9212 & 163.4089 & 165.3422 & 163.3122 & $\mathrm{H} 2 \mathrm{Oa}$ & 2.69 & 28.9088 & 28.9118 & 29.0037 & 28.4663 & 28.7998 & 28.7627 & 29.0058 & 28.8644 \\
\hline $\mathrm{C} 24$ & 37.0 & 152.9745 & 151.9132 & 152.2095 & 153.7813 & 153.6538 & 151.7831 & 153.2374 & 152.2998 & $\mathrm{H} 2 \mathrm{Ob}$ & 2.09 & 30.0256 & 30.0057 & 29.8263 & 29.9608 & 29.8011 & 30.1908 & 30.0633 & 30.0879 \\
\hline $\mathrm{C} 25$ & 0 & 124.9062 & 123.8952 & 121.4347 & 127.3951 & 121.9943 & 123.3578 & 122.8841 & 122.6020 & $\mathrm{H} 22$ & 5.28 & 26.3630 & 26.2222 & 25.8320 & 26.2756 & 26.0662 & 26.2676 & 26.1797 & 26.3371 \\
\hline $\mathrm{C} 26$ & 4 & 141.7826 & 145.2587 & 142.0300 & 146.3727 & 148.9028 & 145.5828 & 144.7447 & 145.8601 & $\mathrm{H}_{2}$ & 2.13 & 29.1115 & 28.7475 & 29.5705 & 29.0087 & 29.5988 & 28.8457 & 29.6951 & 28.7928 \\
\hline $\mathrm{C} 27$ & 72.8 & 120.4193 & 120.3802 & 118.8497 & 120.3352 & 120.0054 & 120.0475 & 118.2772 & 120.0081 & $\mathrm{H} 23 \mathrm{~b}$ & 2.1 & 29.8437 & 30.1359 & 29.9452 & 30.1781 & 30.2887 & 30.1884 & 30.1821 & 29.9782 \\
\hline $\mathrm{C} 28$ & 4 & 112.8302 & 115.3177 & 116.7123 & 109.3599 & 112.0682 & 114.7250 & 112.1311 & 113.8335 & H24a & 1.52 & 30.0047 & 30.1764 & 29.9337 & 30.2831 & 29.8562 & 30.2530 & 30.0239 & 30.1541 \\
\hline C29 & 3.5 & 89.4937 & 89.1212 & 89.2677 & 90.8693 & 89.5540 & 89.0829 & 90.3639 & 89.4878 & $\mathrm{H} 24$ & 1.45 & 30.4593 & 30.2726 & 30.3787 & 30.5496 & 30.2687 & 30.2819 & 30.2369 & 30.4035 \\
\hline C30 & 36.6 & 153.3002 & 157.2161 & 154.1308 & 153.8867 & 153.2959 & 155.3345 & 151.8848 & 153.1643 & $\mathrm{H} 25$ & 3.9 & 27.6514 & 27.6978 & 27.5420 & 27.7330 & 27.6868 & 27.5960 & 27.6338 & 27.6823 \\
\hline C31 & & 162.7805 & 159.2151 & 159.6708 & 160.9957 & 159.2555 & 159.0139 & 161.4159 & 159.5527 & & 1.73 & 29.9165 & 30.0514 & & & 29.9808 & 30.1418 & 29.8469 & 30.0874 \\
\hline C32 & 6.3 & 27.9895 & 27.2280 & 30.3281 & 29.0930 & 29.8359 & 28.4780 & 29.0221 & 29.9296 & $\mathrm{H} 2$ & 1.45 & 30.6687 & 30.2173 & 30.58 & 30.3400 & 30.0886 & 30.2221 & 30.1417 & 30.2169 \\
\hline C33 & 16.7 & 173.9201 & 175.1683 & 174.0824 & 174.4866 & 173.6642 & 174.3456 & 174.5773 & 174.1616 & H28 & 3.28 & 28.1002 & 28.0741 & 28.0888 & 28.2703 & 28.5656 & 28.2104 & 27.8574 & 28.1741 \\
\hline C34 & & 164.2554 & 167.3078 & 167.8234 & 166.1125 & 167.7429 & 167.2733 & 167.0496 & 167.5007 & $\mathrm{H} 30 \mathrm{a}$ & 2.09 & 29.2122 & 29.2835 & 28.5856 & 28.6268 & 29.6322 & 28.9286 & 29.0304 & 29.5095 \\
\hline C35 & & 166.7898 & 167.8061 & 165.7014 & 166.8352 & 167.5122 & 166.7546 & 166.0459 & 167.3741 & $\mathrm{H} 30 \mathrm{H}$ & 1.88 & 29.7752 & 29.9031 & 30.6642 & 29.8566 & 30.1609 & 30.6654 & 30.1110 & 30.1116 \\
\hline C36 & & 140.9676 & 141.2368 & 141.1479 & 140.1951 & 141.0418 & 141.2453 & 141.5096 & 140.9368 & $\mathrm{H} 31 \mathrm{a}$ & 2.52 & 29.5808 & 29.2430 & 29.4540 & 28.9042 & 29.2733 & 29.3455 & 29.5597 & 29.3774 \\
\hline $\mathrm{C}^{\prime}$ & 168.3 & 30.3782 & 31.5399 & 30.6680 & 34.2419 & 32.1858 & 32.2845 & 31.7476 & 30.8681 & $\mathrm{H} 31 \mathrm{~b}$ & 2.35 & 29.8966 & 29.4982 & 29.8031 & 29.5437 & 29.6707 & 29.7563 & 30.1388 & 29.6594 \\
\hline $\mathrm{C} 2^{\prime}$ & & 77.6157 & 78.3844 & 77.2450 & 76.5593 & 75.9612 & 75.6688 & 76.9821 & & & 1.02 & 30.8429 & 30.8479 & & 30.7762 & 30.7739 & 30.8204 & 30.8033 & 30.8247 \\
\hline $\mathrm{C}^{\prime \prime}$ & & 46.7555 & 45.9546 & 46.9086 & 49.5551 & 47.9613 & 49.2523 & 47.5152 & 46.5765 & & 1.71 & 30.1796 & 30.1634 & 30.0619 & 30.0040 & 30.0985 & 30.1264 & 30.0805 & 30.0993 \\
\hline C4' & 135.1 & 62.2290 & 61.7407 & 61.9637 & 62.6506 & 59.5641 & 61.7380 & 65.8023 & 63.9600 & $\mathrm{H} 35$ & 1.38 & 30.4457 & 30.4466 & 30.4012 & 30.4727 & 30.5033 & 30.3261 & 30.3296 & 30.4986 \\
\hline$C 5^{\prime}$ & 140.9 & 53.2171 & 52.6606 & 52.6765 & 54.4382 & 55.9312 & 53.3869 & 49.2632 & 51.9426 & $\mathrm{H} 36$ & 3.67 & 28.1106 & 28.1208 & 28.0749 & 28.0519 & 28.1009 & 28.0919 & 28.2161 & 28.0291 \\
\hline$C 6^{\prime}$ & & 150.5404 & 150.8609 & 150.8880 & 152.0579 & & 151.6606 & & & & & & 25.5813 & & & 25.6718 & 25.4611 & 25.7896 & 25.9748 \\
\hline$C 7^{\prime}$ & 147.3 & 45.3096 & 47.9391 & 48.5387 & 47.9112 & 47.6756 & 48.9187 & 49.0872 & 48.8528 & $\mathrm{H}^{\prime}$ & 7.27 & 24.2667 & 24.0949 & 24.4211 & 24.5863 & 24.5454 & 24.7708 & 24.4409 & 24.2727 \\
\hline $\mathrm{C} 8^{\prime}$ & 40.6 & 149.4933 & 147.0415 & 147.8536 & 146.7817 & 150.7741 & 146.6283 & 146.0759 & 146.2166 & $\mathrm{H}^{\prime}$ & 5.93 & 25.3965 & 25.4410 & 25.5839 & 25.4176 & 25.9824 & 26.1139 & 25.5623 & 25.7059 \\
\hline C9' & & 59.0712 & & & & 64.3440 & 62.0987 & & & & & 28.6677 & 28.7553 & & & 28.4938 & 28.7504 & 28.4939 & 28.7940 \\
\hline C10' & 129.4 & 66.6220 & 65.6859 & 64.2566 & 64.5044 & 61.8620 & 65.2105 & 63.6305 & 68.7165 & & 2.92 & 28.8158 & 28.7883 & 28.7366 & 29.1767 & 29.1186 & 28.8192 & 29.0403 & 29.0320 \\
\hline C11' & 41.9 & 148.8712 & 149.6583 & 148.8770 & 148.8535 & 149.9242 & 150.2903 & 148.8528 & 149.6115 & $\mathrm{H} 8^{\prime}$ & 2.77 & 28.9462 & 28.9393 & 28.9446 & 28.9587 & 29.0464 & 28.9196 & 29.0059 & 28.9013 \\
\hline C12' & 12.8 & 177.7699 & 175.2226 & 175.5087 & 179.0216 & 176.9056 & 174.8081 & 174.7909 & 177.2229 & $\mathrm{H}^{\prime}$ & 5.53 & 25.7874 & 25.9754 & & 26.3311 & 26.1453 & 25.9500 & $\begin{array}{l}26.00025 \\
\end{array}$ & 25.3026 \\
\hline & 113.1 & 82.8477 & 80.3761 & 79.8839 & 79.9423 & 79.6246 & 79.5013 & 79.4832 & 78.8159 & & & 26.0784 & 26.0285 & 25.8637 & 25.6412 & 26.1093 & 25.9602 & 25.9275 & 26.2352 \\
\hline & & & & & & & & & & $\mathrm{H} 11$ & 3.75 & 27.7942 & 27.2546 & 27.2806 & 27.2150 & 27.4909 & 27.1963 & 27.4152 & 27.9044 \\
\hline & & & & & & & & & & $\mathrm{H} 11^{\prime} \mathrm{b}$ & 3.65 & 28.2420 & 28.9740 & 29.1168 & 28.9944 & 28.7148 & 28.5155 & 29.0120 & 28.1083 \\
\hline & & & & & & & & & & $\mathrm{H} 12^{\prime}$ & 1.78 & 29.9443 & 29.8803 & 29.9067 & 30.0150 & 30.0442 & 29.8257 & 29.9987 & 30.0891 \\
\hline & & & & & & & & & & $\mathrm{H} 13^{\prime}$ & 4.84 & 26.7570 & 26.5870 & 26.7275 & 26.7847 & 26.8024 & 26.6839 & 26.6076 & 26.6254 \\
\hline
\end{tabular}


Table S29. Scaled chemical shifts computed for compounds $01 / 08$ at the B3LYP/6-31G**//MMFF level of theory following the iJ-DP4 approach.

\begin{tabular}{|c|c|c|c|c|c|c|c|c|c|c|c|c|c|c|c|c|c|c|c|}
\hline \multirow{2}{*}{ Atom } & \multirow{2}{*}{ Exp. NMR } & & & & & & & & & \multirow{2}{*}{ Atom } & \multirow{2}{*}{ Exp. NMR } & \multirow[b]{2}{*}{01} & \multirow[b]{2}{*}{02} & \multirow{2}{*}{03} & & & \multirow[b]{2}{*}{08} \\
\hline & & 01 & 02 & 03 & 04 & 05 & 06 & 07 & 08 & & & & & & 04 & 05 & 06 & 07 & \\
\hline $\mathrm{C} 1$ & 173.9 & 173.7 & 175.8 & 175.9 & 176.2 & 173.9 & 175.5 & 174.5 & 175.5 & $\mathrm{H} 2 \mathrm{a}$ & 2.87 & 2.69 & 2.64 & 2.70 & 3.37 & 2.89 & 3.02 & 3.43 & 3.38 \\
\hline $\mathrm{C} 2$ & 38.4 & 39.5 & 38.8 & 36.5 & 35.3 & 40.4 & 39.9 & 33.8 & 34.7 & $\mathrm{H} 2 \mathrm{~b}$ & 2.42 & 2.64 & 2.19 & 2.65 & 1.57 & 2.22 & 2.29 & 1.91 & 1.79 \\
\hline C3 & 71.4 & 70.2 & 70.7 & 74.7 & 82.4 & 71.8 & 73.0 & 78.1 & 76.8 & $\mathrm{H} 3$ & 3.92 & 4.02 & 3.68 & 4.15 & 3.78 & 3.78 & 4.27 & 4.65 & 4.50 \\
\hline C4 & 75.3 & 73.8 & 76.9 & 73.7 & 76.5 & 75.4 & 77.0 & 71.0 & 71.2 & $\mathrm{H} 4$ & 3.10 & 3.57 & 3.10 & 3.36 & 3.20 & 3.44 & 2.92 & 3.28 & 3.65 \\
\hline $\mathrm{C} 5$ & 74.7 & 78.5 & 79.0 & 75.0 & 71.2 & 76.4 & 77.9 & 74.5 & 74.5 & $\mathrm{H} 5$ & 3.54 & 3.61 & 3.71 & 3.79 & 4.08 & 3.74 & 3.40 & 3.72 & 3.67 \\
\hline $\mathrm{C} 6$ & 73.0 & 73.4 & 71.4 & 72.4 & 69.1 & 75.1 & 71.6 & 74.8 & 75.6 & $\mathrm{H} 6$ & 3.57 & 3.50 & 3.94 & 3.52 & 3.14 & 3.82 & 3.81 & 3.39 & 2.78 \\
\hline $\mathrm{C7}$ & 74.8 & 72.9 & 71.8 & 68.9 & 66.0 & 74.7 & 75.9 & 64.5 & 69.0 & $\mathrm{H} 7$ & 4.05 & 3.97 & 4.61 & 4.15 & 4.15 & 4.00 & 3.97 & 3.96 & 4.19 \\
\hline $\mathrm{C} 8$ & 30.4 & 31.6 & 31.7 & 36.6 & 33.9 & 29.9 & 29.9 & 35.5 & 37.6 & H8a & 2.04 & 2.04 & 2.45 & 2.06 & 2.32 & 1.85 & 2.10 & 2.27 & 2.87 \\
\hline $\mathrm{C9}$ & 71.5 & 73.7 & 73.8 & 69.3 & 73.7 & 72.8 & 78.8 & 74.0 & 78.2 & $\mathrm{H} 8 \mathrm{~b}$ & 1.92 & 1.87 & 1.77 & 1.77 & 2.13 & 1.60 & 1.97 & 1.49 & 1.20 \\
\hline $\mathrm{C} 10$ & 73.5 & 73.2 & 76.9 & 77.4 & 75.5 & 76.5 & 73.5 & 73.3 & 74.0 & $\mathrm{H} 9$ & 3.99 & 4.07 & 3.68 & 4.15 & 4.12 & 4.03 & 3.43 & 4.16 & 3.97 \\
\hline C11 & 75.8 & 77.1 & 77.1 & 76.5 & 74.5 & 83.7 & 73.5 & 75.3 & 82.9 & $\mathrm{H} 10$ & 3.52 & 3.67 & 3.34 & 3.19 & 3.47 & 3.37 & 3.64 & 3.08 & 3.00 \\
\hline $\mathrm{C} 12$ & 80.2 & 84.0 & 82.3 & 81.0 & 85.0 & 84.3 & 84.8 & 82.7 & 83.1 & $\mathrm{H} 11$ & 3.57 & 3.69 & 3.46 & 3.41 & 3.27 & 3.74 & 3.25 & 3.38 & 3.47 \\
\hline $\mathrm{C} 13$ & 38.3 & 37.5 & 35.8 & 37.2 & 36.4 & 37.9 & 36.1 & 37.2 & 36.7 & $\mathrm{H} 12$ & 4.12 & 4.16 & 3.94 & 4.04 & 3.90 & 4.58 & 3.82 & 4.03 & 4.30 \\
\hline C14 & 40.5 & 35.3 & 38.9 & 36.0 & 39.1 & 35.8 & 39.1 & 39.1 & 34.1 & H13a & 2.1 & 2.08 & 2.23 & 1.83 & 2.19 & 2.14 & 2.18 & 2.10 & 2.06 \\
\hline C15 & 86.2 & 84.7 & 86.7 & 85.4 & 83.6 & 85.8 & 82.1 & 85.6 & 85.2 & H13b & 1.56 & 1.62 & 1.64 & 1.79 & 1.62 & 1.15 & 1.54 & 1.63 & 1.07 \\
\hline C16 & 37.9 & 35.4 & 39.1 & 35.7 & 36.8 & 33.6 & 36.1 & 38.5 & 32.9 & H14 & 1.91 & 2.03 & 1.67 & 2.09 & 1.79 & 2.33 & 1.70 & 1.78 & 2.44 \\
\hline C17 & 131.1 & 132.4 & 129.3 & 130.1 & 131.9 & 123.8 & 131.3 & 134.4 & 130.7 & $\mathrm{H} 15$ & 3.5 & 3.74 & 3.57 & 3.53 & 3.99 & 3.69 & 4.01 & 3.49 & 3.59 \\
\hline C18 & 132.1 & 133.7 & 136.3 & 138.3 & 131.6 & 139.6 & 131.8 & 135.4 & 136.8 & H16a & 2.35 & 2.40 & 2.40 & 2.31 & 2.23 & 2.39 & 2.17 & 2.26 & 2.26 \\
\hline C19 & 73.7 & 77.3 & 69.7 & 71.9 & 73.5 & 66.0 & 74.7 & 71.7 & 72.8 & H16b & 2.2 & 2.14 & 1.84 & 1.99 & 1.98 & 2.15 & 2.15 & 1.89 & 2.06 \\
\hline $\mathrm{C} 20$ & 38.4 & 36.2 & 38.4 & 37.7 & 40.5 & 33.5 & 38.7 & 37.6 & 38.0 & $\mathrm{H} 17$ & 5.81 & 6.06 & 5.81 & 5.81 & 5.72 & 5.79 & 5.82 & 5.87 & 5.94 \\
\hline $\mathrm{C} 21$ & 131.9 & 132.9 & 131.6 & 131.3 & 134.6 & 134.7 & 131.6 & 133.7 & 133.3 & $\mathrm{H} 18$ & 5.59 & 5.55 & 5.81 & 5.70 & 5.99 & 5.99 & 5.79 & 5.56 & 5.55 \\
\hline $\mathrm{C} 22$ & 129.2 & 132.9 & 131.9 & 132.3 & 132.5 & 129.9 & 132.4 & 130.2 & 131.5 & $\mathrm{H} 19$ & 5.45 & 5.03 & 4.95 & 5.24 & 5.36 & 5.55 & 5.10 & 5.20 & 5.08 \\
\hline $\mathrm{C} 23$ & 24.8 & 24.8 & 25.8 & 27.3 & 24.8 & 25.3 & 25.5 & 24.1 & 26.2 & $\mathrm{H} 20 \mathrm{a}$ & 2.69 & 2.78 & 2.79 & 2.73 & 3.20 & 2.93 & 2.97 & 2.77 & 2.87 \\
\hline $\mathrm{C} 24$ & 37.0 & 37.2 & 38.3 & 37.6 & 36.6 & 37.2 & 37.9 & 36.9 & 37.8 & $\mathrm{H} 20 \mathrm{~b}$ & 2.09 & 1.73 & 1.77 & 1.96 & 1.76 & 1.99 & 1.59 & 1.77 & 1.72 \\
\hline $\mathrm{C} 25$ & 68.0 & 66.7 & 67.8 & 70.4 & 64.6 & 70.4 & 68.2 & 69.0 & 69.1 & $\mathrm{H} 22$ & 5.28 & 5.17 & 5.30 & 5.70 & 5.32 & 5.49 & 5.40 & 5.44 & 5.23 \\
\hline $\mathrm{C} 26$ & 46.5 & 49.0 & 45.3 & 48.5 & 44.4 & 42.2 & 44.5 & 45.9 & 44.6 & H23a & 2.13 & 2.58 & 2.94 & 2.20 & 2.68 & 2.18 & 2.89 & 2.11 & 2.93 \\
\hline $\mathrm{C} 27$ & 72.8 & 71.4 & 71.6 & 73.1 & 72.1 & 72.5 & 71.7 & 73.9 & 71.9 & H23b & 2.1 & 1.90 & 1.64 & 1.85 & 1.55 & 1.53 & 1.59 & 1.65 & 1.83 \\
\hline C28 & 79.4 & 79.4 & 76.9 & 75.4 & $\begin{array}{l}83.7 \\
\end{array}$ & 80.9 & 77.4 & 80.4 & 78.4 & $\mathrm{H} 24 \mathrm{a}$ & 1.52 & 1.75 & 1.61 & 1.86 & 1.45 & 1.94 & 1.53 & 1.80 & 1.66 \\
\hline $\mathrm{C} 29$ & 98.5 & 103.9 & 104.5 & 104.6 & 103.4 & 104.5 & 104.7 & 103.5 & 104.1 & H24b & 1.45 & 1.32 & 1.52 & 1.44 & 1.19 & 1.55 & 1.50 & 1.60 & 1.43 \\
\hline $\mathrm{C} 30$ & 36.6 & 36.9 & 32.7 & 35.6 & 36.5 & 37.5 & 34.1 & 38.3 & 36.9 & $\mathrm{H} 25$ & 3.9 & 3.96 & 3.92 & 4.10 & 3.91 & 3.97 & 4.11 & 4.06 & 3.97 \\
\hline C31 & 31.0 & 26.9 & 30.6 & 29.7 & 28.9 & 31.3 & 30.2 & 28.2 & 30.2 & H26a & 1.73 & 1.83 & 1.72 & 1.67 & 1.55 & 1.82 & 1.64 & 1.97 & 1.73 \\
\hline C32 & 176.3 & 168.6 & 169.8 & 167.2 & 168.9 & 167.3 & 169.3 & 168.4 & 166.8 & $\mathrm{H} 26 \mathrm{~b}$ & 1.45 & 1.12 & 1.57 & 1.24 & 1.39 & 1.72 & 1.56 & 1.69 & 1.60 \\
\hline C33 & 16.7 & 15.2 & 13.7 & 14.4 & 14.6 & 16.1 & 13.8 & 14.3 & $\begin{array}{ll}14.8 \\
\end{array}$ & $\mathrm{H} 28$ & 3.28 & 3.53 & 3.57 & 3.58 & 3.39 & 3.15 & 3.51 & 3.85 & 3.51 \\
\hline C34 & 23.9 & 25.3 & 22.0 & 21.0 & 23.5 & 22.4 & 21.4 & 22.3 & 21.8 & $\mathrm{H} 30 \mathrm{a}$ & 2.09 & 2.49 & 2.44 & 3.12 & 3.05 & 2.15 & 2.81 & 2.74 & 2.26 \\
\hline C35 & 21.8 & 22.7 & 21.5 & 23.3 & 22.7 & 22.6 & 21.9 & 23.3 & 21.9 & $\mathrm{H} 30 \mathrm{~b}$ & 1.88 & 1.96 & 1.86 & 1.17 & 1.86 & 1.65 & 1.13 & 1.72 & 1.70 \\
\hline C36 & 52.3 & 49.8 & 49.5 & 49.4 & 51.0 & 50.4 & 49.1 & 49.3 & 49.8 & H31a & 2.52 & 2.14 & 2.48 & 2.31 & 2.78 & 2.48 & 2.41 & 2.24 & 2.39 \\
\hline $\mathrm{C} 1^{\prime}$ & 168.3 & 166.1 & 165.3 & 166.9 & 163.5 & 164.8 & 165.2 & 165.6 & 165.9 & $\mathrm{H} 31 \mathrm{~b}$ & 2.35 & 1.85 & 2.24 & 1.98 & 2.16 & 2.11 & 2.01 & 1.70 & 2.13 \\
\hline $\mathrm{C} 2^{\prime}$ & 117.2 & 116.4 & 115.9 & 117.3 & 118.5 & 118.8 & 119.0 & 117.6 & 115.9 & H33 & 1.02 & 0.96 & 0.98 & 1.04 & 0.97 & 1.07 & 0.98 & 1.07 & 1.04 \\
\hline $\mathrm{C} 3^{\prime}$ & 150.7 & 148.9 & 150.1 & 149.6 & 147.2 & 148.2 & 147.1 & 148.9 & 149.3 & H34 & 1.71 & 1.58 & 1.62 & 1.74 & 1.72 & 1.71 & 1.65 & 1.75 & 1.71 \\
\hline C4' & 135.1 & 132.6 & 133.4 & 133.6 & 133.3 & 136.0 & 133.8 & 129.5 & 131.0 & H35 & 1.38 & 1.33 & 1.35 & 1.42 & 1.27 & 1.33 & 1.46 & 1.51 & 1.34 \\
\hline $\mathrm{C} 5^{\prime}$ & 140.9 & 142.1 & 143.0 & 143.5 & 142.0 & 139.8 & 142.7 & 147.0 & 143.6 & $\mathrm{H} 36$ & 3.67 & 3.53 & 3.53 & 3.60 & 3.60 & 3.58 & 3.63 & 3.51 & 3.65 \\
\hline$C 6^{\prime}$ & 36.2 & 39.8 & 39.4 & 39.0 & 38.4 & 36.9 & 38.0 & 35.0 & 35.8 & $\mathrm{H}^{\prime}$ & 5.79 & 5.66 & 5.90 & 5.80 & 5.49 & 5.86 & 6.18 & 5.81 & 5.56 \\
\hline$C 7^{\prime}$ & 147.3 & 150.4 & 148.0 & 147.9 & 149.0 & 148.5 & 147.5 & 147.2 & 146.9 & $\mathrm{H} 3^{\prime}$ & 7.27 & 7.14 & 7.29 & 7.02 & 6.95 & 6.92 & 6.85 & 7.08 & 7.15 \\
\hline $\mathrm{C}^{\prime}$ & 40.6 & 40.9 & 43.4 & 42.3 & 44.0 & 40.2 & 43.4 & 44.5 & 44.2 & $\mathrm{H}^{\prime}$ & 5.93 & 6.08 & 6.03 & 5.93 & 6.14 & 5.57 & 5.54 & 6.02 & 5.82 \\
\hline C9' & 131.3 & 135.9 & 132.7 & 131.6 & 133.4 & 131.0 & 133.4 & 131.8 & 136.9 & H6'a & 3.04 & 3.00 & 2.94 & 2.99 & 3.25 & 3.21 & 2.99 & 3.25 & 2.93 \\
\hline C10' & 129.4 & 128.0 & 129.3 & 131.2 & 131.3 & 133.6 & 130.1 & 131.8 & 125.9 & $\mathrm{H} 6^{\prime} \mathrm{b}$ & 2.92 & 2.86 & 2.90 & 2.98 & 2.52 & 2.63 & 2.92 & 2.73 & 2.71 \\
\hline C11' & 41.9 & 41.5 & 40.7 & 41.2 & 41.8 & 41.1 & 39.5 & 41.5 & 40.7 & $\mathrm{H}^{\prime} \mathrm{8}^{\prime}$ & 2.77 & 2.74 & 2.76 & 2.78 & 2.73 & 2.70 & 2.82 & 2.77 & 2.83 \\
\hline C12' & 12.8 & 11.1 & 13.7 & 12.9 & 9.8 & 12.7 & 13.3 & 14.1 & 11.6 & $\mathrm{H}^{\prime}$ & 5.53 & 5.71 & 5.53 & 5.32 & 5.26 & 5.42 & 5.70 & 5.32 & 6.19 \\
\hline \multirow[t]{5}{*}{ C13' } & 113.1 & 110.9 & 113.8 & 114.5 & 115.0 & 115.0 & 114.9 & 115.0 & 115.3 & $\mathrm{H}^{\prime \prime} \mathrm{O}^{\prime}$ & 5.46 & 5.43 & 5.49 & 5.67 & 5.93 & 5.45 & 5.69 & 5.68 & 5.32 \\
\hline & & & & & & & & & & H11'a & 3.75 & 3.82 & 4.34 & 4.34 & 4.41 & 4.16 & 4.49 & 4.27 & 3.76 \\
\hline & & & & & & & & & & H11'b & 3.65 & 3.40 & 2.73 & 2.62 & 2.69 & 3.01 & 3.21 & 2.76 & 3.57 \\
\hline & & & & & & & & & & $\mathrm{H}^{\prime 2} 2^{\prime}$ & 1.78 & 1.80 & 1.88 & 1.88 & 1.71 & 1.76 & 1.94 & 1.83 & 1.72 \\
\hline & & & & & & & & & & $\mathrm{H}^{\mathrm{H}} 3^{\prime}$ & 4.84 & 4.80 & 4.96 & 4.86 & 4.83 & 4.80 & 4.99 & 5.03 & 4.96 \\
\hline
\end{tabular}


Table S30. Absolute scaled errors computed for compounds $01 / 08$ at the B3LYP/6-31G**//MMFF level of theory following the iJ-DP4 approach, and J-

\begin{tabular}{|c|c|c|c|c|c|c|c|c|c|c|c|c|c|c|c|c|c|}
\hline \multicolumn{18}{|c|}{ DP4 results. } \\
\hline & 01 & 02 & 03 & 04 & 05 & 06 & 07 & 08 & & 01 & 02 & 03 & 04 & 05 & 06 & 07 & 08 \\
\hline$c-1$ & 0.2 & 1.9 & 2.0 & 2.3 & 0.0 & 1.6 & 0.6 & 1.6 & $H-2 a$ & 0.18 & 0.23 & 0.17 & 0.50 & 0.02 & 0.15 & 0.56 & 0.51 \\
\hline c-2 & 1.1 & 0.4 & 1.9 & 3.1 & 2.0 & 1.5 & 4.6 & 3.7 & $H-2 b$ & 0.22 & 0.23 & 0.23 & 0.85 & 0.20 & 0.13 & 0.51 & 0.63 \\
\hline$c-3$ & 1.2 & 0.7 & 3.3 & 11.0 & 0.4 & 1.6 & 6.7 & 5.4 & $\mathrm{H}-3$ & 0.10 & 0.24 & 0.23 & 0.14 & 0.14 & 0.35 & 0.73 & 0.58 \\
\hline c-4 & 1.5 & 1.6 & 1.6 & 1.2 & 0.1 & 1.7 & 4.3 & 4.1 & $H-4$ & 0.47 & 0.00 & 0.26 & 0.10 & 0.34 & 0.18 & 0.18 & 0.55 \\
\hline C-5 & 3.8 & 4.3 & 0.3 & 3.5 & 1.7 & 3.2 & 0.2 & 0.2 & $H-5$ & 0.07 & 0.17 & 0.25 & 0.54 & 0.20 & 0.14 & 0.18 & 0.13 \\
\hline c-6 & 0.4 & 1.6 & 0.6 & 3.9 & 2.1 & 1.4 & 1.8 & 2.6 & $H-6$ & 0.07 & 0.37 & 0.05 & 0.43 & 0.25 & 0.24 & 0.18 & 0.79 \\
\hline$c-7$ & 1.9 & 3.0 & 5.9 & 8.8 & 0.1 & 1.1 & 10.3 & 5.8 & $\mathrm{H}-7$ & 0.08 & 0.56 & 0.10 & 0.10 & 0.05 & 0.08 & 0.09 & 0.14 \\
\hline$c-8$ & 1.2 & 1.3 & 6.2 & 3.5 & 0.5 & 0.5 & 5.1 & 7.2 & $\mathrm{H}-8 \mathrm{a}$ & 0.00 & 0.41 & 0.02 & 0.28 & 0.19 & 0.06 & 0.23 & 0.83 \\
\hline c-9 & 2.2 & 2.3 & 2.2 & 2.2 & 1.3 & 7.3 & 2.5 & 6.7 & $H-8 b$ & 0.05 & 0.15 & 0.15 & 0.21 & 0.32 & 0.05 & 0.43 & 0.72 \\
\hline C-10 & 0.3 & 3.4 & 3.9 & 2.0 & 3.0 & 0.0 & 0.2 & 0.5 & $\mathrm{H}-\mathrm{9}$ & 0.08 & 0.31 & 0.16 & 0.13 & 0.04 & 0.56 & 0.17 & 0.02 \\
\hline C-11 & 1.3 & 1.3 & 0.7 & 1.3 & 7.9 & 2.3 & 0.5 & 7.1 & $\mathrm{H}-10$ & 0.15 & 0.18 & 0.33 & 0.05 & 0.15 & 0.12 & 0.44 & 0.52 \\
\hline C-12 & 3.8 & 2.1 & 0.8 & 4.8 & 4.1 & 4.6 & 2.5 & 2.9 & $\mathrm{H}-11$ & 0.12 & 0.11 & 0.16 & 0.30 & 0.17 & 0.32 & 0.19 & 0.10 \\
\hline C-13 & 0.8 & 2.5 & 1.1 & 1.9 & 0.4 & 2.2 & 1.1 & 1.6 & $\mathrm{H}-12$ & 0.04 & 0.18 & 0.08 & 0.22 & 0.46 & 0.30 & 0.09 & 0.18 \\
\hline C-14 & 5.2 & 1.6 & 4.5 & 1.4 & 4.7 & 1.4 & 1.4 & 6.4 & $\mathrm{H}-13 \mathrm{a}$ & 0.02 & 0.13 & 0.27 & 0.09 & 0.04 & 0.08 & 0.00 & 0.04 \\
\hline C-15 & 1.5 & 0.5 & 0.8 & 2.6 & 0.4 & 4.1 & 0.6 & 1.0 & $H-13 b$ & 0.06 & 0.08 & 0.23 & 0.06 & 0.41 & 0.02 & 0.07 & 0.49 \\
\hline C-16 & 2.5 & 1.2 & 2.2 & 1.1 & 4.3 & 1.8 & 0.6 & 5.0 & $\mathrm{H}-14$ & 0.12 & 0.24 & 0.18 & 0.12 & 0.42 & 0.21 & 0.13 & 0.53 \\
\hline C-17 & 1.3 & 1.8 & 1.0 & 0.8 & 7.3 & 0.2 & 3.3 & 0.4 & $\mathrm{H}-15$ & 0.24 & 0.07 & 0.03 & 0.49 & 0.19 & 0.51 & 0.01 & 0.09 \\
\hline C-18 & 1.6 & 4.2 & 6.2 & 0.5 & 7.5 & 0.3 & 3.3 & 4.7 & $\mathrm{H}-16 \mathrm{a}$ & 0.05 & 0.05 & 0.04 & 0.12 & 0.04 & 0.18 & 0.09 & 0.09 \\
\hline C-19 & 3.6 & 4.0 & 1.8 & 0.2 & 7.7 & 1.0 & 2.0 & 0.9 & $\mathrm{H}-16 \mathrm{~b}$ & 0.06 & 0.36 & 0.21 & 0.22 & 0.05 & 0.05 & 0.31 & 0.14 \\
\hline$c-20$ & 2.2 & 0.0 & 0.7 & 2.1 & 4.9 & 0.3 & 0.8 & 0.4 & $\mathrm{H}-17$ & 0.25 & 0.00 & 0.00 & 0.09 & 0.02 & 0.01 & 0.06 & 0.13 \\
\hline$C-21$ & 1.0 & 0.3 & 0.6 & 2.7 & 2.8 & 0.3 & 1.8 & 1.4 & $\mathrm{H}-18$ & 0.04 & 0.22 & 0.11 & 0.40 & 0.40 & 0.20 & 0.03 & 0.04 \\
\hline$c-22$ & 3.7 & 2.7 & 3.1 & 3.3 & 0.7 & 3.2 & 1.0 & 2.3 & $\mathrm{H}-19$ & 0.42 & 0.50 & 0.21 & 0.09 & 0.10 & 0.35 & 0.25 & 0.37 \\
\hline$c-23$ & 0.0 & 1.0 & 2.5 & 0.0 & 0.5 & 0.7 & 0.7 & 1.4 & $\mathrm{H}-20 \mathrm{a}$ & 0.09 & 0.10 & 0.04 & 0.51 & 0.24 & 0.28 & 0.08 & 0.18 \\
\hline$c-24$ & 0.2 & 1.3 & 0.6 & 0.4 & 0.2 & 0.9 & 0.1 & 0.8 & $\mathrm{H}-20 \mathrm{~b}$ & 0.36 & 0.32 & 0.13 & 0.33 & 0.10 & 0.50 & 0.32 & 0.37 \\
\hline$c-25$ & 1.3 & 0.2 & 2.4 & 3.4 & 2.4 & 0.2 & 1.0 & 1.1 & $\mathrm{H}-22$ & 0.11 & 0.02 & 0.42 & 0.04 & 0.21 & 0.12 & 0.16 & 0.05 \\
\hline$c-26$ & 2.5 & 1.2 & 2.0 & 2.1 & 4.3 & 2.0 & 0.6 & 1.9 & $\mathrm{H}-23 \mathrm{a}$ & 0.45 & 0.81 & 0.07 & 0.55 & 0.05 & 0.76 & 0.02 & 0.80 \\
\hline$C-27$ & 1.4 & 1.2 & 0.3 & 0.7 & 0.3 & 1.1 & 1.1 & 0.9 & $\mathrm{H}-23 \mathrm{~b}$ & 0.20 & 0.46 & 0.25 & 0.55 & 0.57 & 0.51 & 0.45 & 0.27 \\
\hline$c-28$ & 0.0 & 2.5 & 4.0 & 4.3 & 1.5 & 2.0 & 1.0 & 1.0 & $\mathrm{H}-24 \mathrm{a}$ & 0.23 & 0.09 & 0.34 & 0.07 & 0.42 & 0.01 & 0.28 & 0.14 \\
\hline$c-29$ & 5.4 & 6.0 & 6.1 & 4.9 & 6.0 & 6.2 & 5.0 & 5.6 & $\mathrm{H}-24 \mathrm{~b}$ & 0.13 & 0.07 & 0.01 & 0.26 & 0.10 & 0.05 & 0.15 & 0.02 \\
\hline$c-30$ & 0.3 & 3.9 & 1.0 & 0.1 & 0.9 & 2.5 & 1.7 & 0.3 & $\mathrm{H}-25$ & 0.06 & 0.02 & 0.20 & 0.01 & 0.07 & 0.21 & 0.16 & 0.07 \\
\hline C-31 & 4.1 & 0.4 & 1.3 & 2.1 & 0.3 & 0.8 & 2.8 & 0.8 & $\mathrm{H}-26 \mathrm{a}$ & 0.10 & 0.01 & 0.06 & 0.18 & 0.09 & 0.09 & 0.24 & 0.00 \\
\hline C-32 & 7.7 & 6.5 & 9.1 & 7.4 & 9.0 & 7.0 & 7.9 & 9.5 & $H-26 b$ & 0.33 & 0.12 & 0.21 & 0.06 & 0.27 & 0.11 & 0.24 & 0.15 \\
\hline$c-33$ & 1.5 & 3.0 & 2.3 & 2.1 & 0.6 & 2.9 & 2.4 & 1.9 & $\mathrm{H}-28$ & 0.25 & 0.29 & 0.30 & 0.11 & 0.13 & 0.23 & 0.57 & 0.23 \\
\hline C-34 & 1.4 & 1.9 & 2.9 & 0.4 & 1.5 & 2.5 & 1.6 & 2.1 & $\mathrm{H}-30 \mathrm{a}$ & 0.40 & 0.35 & 1.03 & 0.96 & 0.06 & 0.72 & 0.65 & 0.17 \\
\hline C-35 & 0.9 & 0.3 & 1.5 & 0.9 & 0.8 & 0.1 & 1.5 & 0.1 & $\mathrm{H}-30 \mathrm{~b}$ & 0.08 & 0.02 & 0.71 & 0.02 & 0.23 & 0.75 & 0.16 & 0.18 \\
\hline$c-36$ & 2.5 & 2.8 & 2.9 & 1.3 & 1.9 & 3.2 & 3.0 & 2.5 & $\mathrm{H}-31 \mathrm{a}$ & 0.38 & 0.04 & 0.21 & 0.26 & 0.04 & 0.11 & 0.28 & 0.13 \\
\hline$C-1^{\prime}$ & 2.2 & 3.0 & 1.4 & 4.8 & 3.5 & 3.1 & 2.7 & 2.4 & $\mathrm{H}-31 \mathrm{~b}$ & 0.50 & 0.11 & 0.37 & 0.19 & 0.24 & 0.34 & 0.65 & 0.22 \\
\hline$c-2^{\prime}$ & 0.8 & 1.3 & 0.1 & 1.3 & 1.6 & 1.8 & 0.4 & 1.3 & $\mathrm{H}-33$ & 0.06 & 0.04 & 0.02 & 0.05 & 0.05 & 0.04 & 0.05 & 0.02 \\
\hline$c-3^{\prime}$ & 1.8 & 0.6 & 1.1 & 3.5 & 2.5 & 3.6 & 1.8 & 1.4 & $\mathrm{H}-34$ & 0.13 & 0.09 & 0.03 & 0.01 & 0.00 & 0.06 & 0.04 & 0.00 \\
\hline$C-4^{\prime}$ & 2.5 & 1.7 & 1.5 & 1.8 & 0.9 & 1.3 & 5.6 & 4.1 & $\mathrm{H}-35$ & 0.05 & 0.03 & 0.04 & 0.11 & 0.05 & 0.08 & 0.13 & 0.04 \\
\hline$C-5^{\prime}$ & 1.2 & 2.1 & 2.6 & 1.1 & 1.1 & 1.8 & 6.1 & 2.7 & $\mathrm{H}-36$ & 0.14 & 0.14 & 0.07 & 0.07 & 0.09 & 0.04 & 0.16 & 0.02 \\
\hline$c-6^{\prime}$ & 3.6 & 3.2 & 2.8 & 2.2 & 0.7 & 1.8 & 1.2 & 0.4 & H-2' & 0.13 & 0.11 & 0.01 & 0.30 & 0.07 & 0.39 & 0.02 & 0.23 \\
\hline$c-7^{\prime}$ & 3.1 & 0.7 & 0.6 & 1.7 & 1.2 & 0.2 & 0.1 & 0.4 & $\mathrm{H}-3^{\prime}$ & 0.13 & 0.02 & 0.25 & 0.32 & 0.35 & 0.42 & 0.19 & 0.12 \\
\hline$C-8^{\prime}$ & 0.3 & 2.8 & 1.7 & 3.4 & 0.4 & 2.8 & 3.9 & 3.6 & $\mathrm{H}-5^{\prime}$ & 0.15 & 0.10 & 0.00 & 0.21 & 0.36 & 0.39 & 0.09 & 0.11 \\
\hline$C-9^{\prime}$ & 4.6 & 1.4 & 0.3 & 2.1 & 0.3 & 2.1 & 0.5 & 5.6 & H-6'a & 0.04 & 0.10 & 0.05 & 0.21 & 0.17 & 0.05 & 0.21 & 0.11 \\
\hline$c-10^{\prime}$ & 1.4 & 0.1 & 1.8 & 1.9 & 4.2 & 0.7 & 2.4 & 3.5 & $H-6^{\prime} b$ & 0.06 & 0.02 & 0.06 & 0.40 & 0.29 & 0.00 & 0.19 & 0.21 \\
\hline C-11' & 0.4 & 1.2 & 0.7 & 0.1 & 0.8 & 2.4 & 0.4 & 1.2 & H-8' & 0.03 & 0.01 & 0.01 & 0.04 & 0.07 & 0.05 & 0.00 & 0.06 \\
\hline C-12' & 1.7 & 0.9 & 0.1 & 3.0 & 0.1 & 0.5 & 1.3 & 1.2 & H-9' & 0.18 & 0.00 & 0.21 & 0.27 & 0.11 & 0.17 & 0.21 & 0.66 \\
\hline C-13' & 2.2 & 0.7 & 1.4 & 1.9 & 1.9 & 1.8 & 1.9 & 2.2 & $\mathrm{H}-10^{\prime}$ & 0.03 & 0.03 & 0.21 & 0.47 & 0.01 & 0.23 & 0.22 & 0.14 \\
\hline Average & 2.0 & 1.9 & 2.2 & 2.5 & 2.3 & 2.0 & 2.3 & 2.7 & H-11'a & 0.07 & 0.59 & 0.59 & 0.66 & 0.41 & 0.74 & 0.52 & 0.01 \\
\hline & & & & & & & & & $\mathrm{H}-11^{\prime} \mathrm{b}$ & 0.25 & 0.92 & 1.03 & 0.96 & 0.64 & 0.44 & 0.89 & 0.08 \\
\hline & & & & & & & & & $\mathrm{H}-12^{\prime}$ & 0.02 & 0.10 & 0.10 & 0.07 & 0.02 & 0.16 & 0.05 & 0.06 \\
\hline & & & & & & & & & $\mathrm{H}-13^{\prime}$ & 0.04 & 0.12 & 0.02 & 0.01 & 0.04 & 0.15 & 0.19 & 0.12 \\
\hline & & & & & & & & & Average & 0.15 & 0.19 & 0.20 & 0.26 & 0.18 & 0.24 & 0.24 & 0.24 \\
\hline
\end{tabular}

\begin{tabular}{|c|c|c|c|c|c|c|c|c|c|}
\hline \multicolumn{2}{|c|}{ Isomer No } & $\mathbf{1}$ & $\mathbf{2}$ & $\mathbf{3}$ & $\mathbf{4}$ & $\mathbf{5}$ & $\mathbf{6}$ & $\mathbf{7}$ & $\mathbf{8}$ \\
\hline \multirow{4}{*}{ J-DP4 (\%) } & $\mathrm{H}$ & 100.00 & 0.00 & 0.00 & 0.00 & 0.00 & 0.00 & 0.00 & 0.00 \\
\cline { 2 - 10 } & $\mathrm{C}$ & 9.81 & 84.06 & 0.02 & 0.00 & 0.00 & 6.11 & 0.00 & 0.00 \\
\cline { 2 - 10 } & $\mathrm{H}+\mathrm{C}$ & 100.00 & 0.00 & 0.00 & 0.00 & 0.00 & 0.00 & 0.00 & 0.00 \\
\cline { 2 - 10 } & $\mathrm{J}$ & - & - & - & - & - & - & - & - \\
\cline { 2 - 10 } & all data & $\mathbf{1 0 0 . 0 0}$ & $\mathbf{0 . 0 0}$ & $\mathbf{0 . 0 0}$ & $\mathbf{0 . 0 0}$ & $\mathbf{0 . 0 0}$ & $\mathbf{0 . 0 0}$ & $\mathbf{0 . 0 0}$ & $\mathbf{0 . 0 0}$ \\
\hline
\end{tabular}




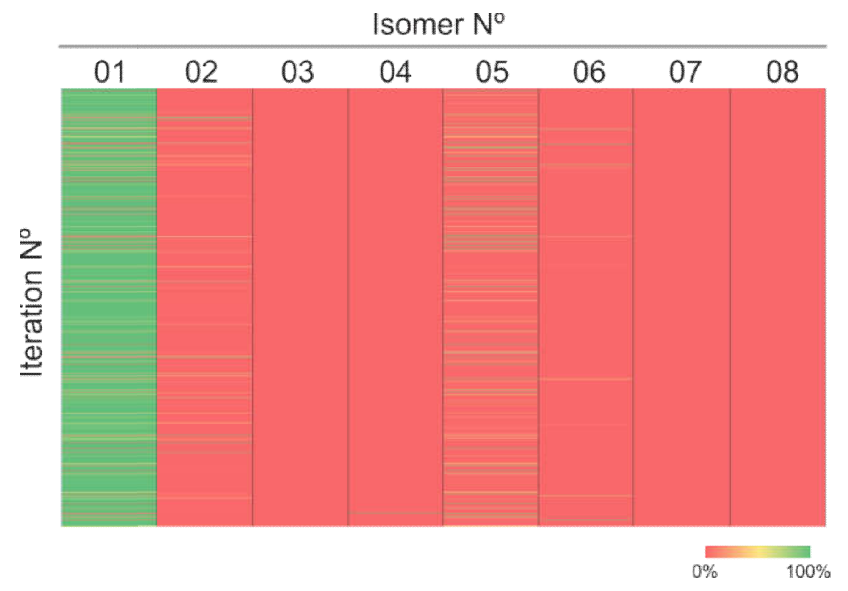

Figure S4. Variations in J-DP4 probabilities computed for compounds $\mathbf{0 1 / 0 8}$ using randomly generated ensembles (showing the first 1000 iterations).

Table S31. Averaged J-DP4 probabilities computed for compounds 01/08 using randomly generated ensembles after 100.000 iterations \begin{tabular}{|c|c|c|c|c|c|c|c|c|}
\hline & 01 & 02 & 03 & 04 & 05 & 06 & 07 & 08 \\
\hline J-DP4 & $91.04 \%$ & $1.81 \%$ & $0.00 \%$ & $0.11 \%$ & $6.46 \%$ & $0.58 \%$ & $0.00 \%$ & $0.00 \%$ \\
\hline
\end{tabular}

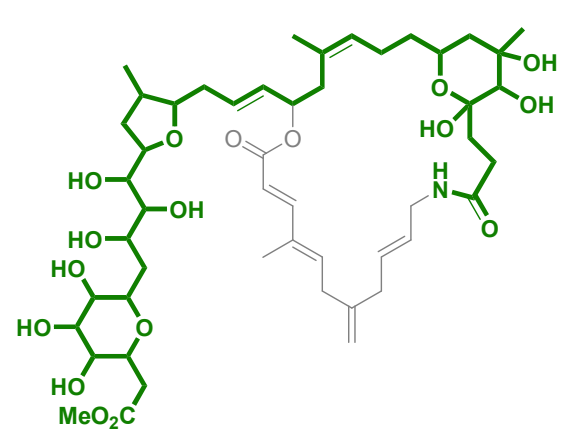

\begin{tabular}{|c|c|c|c|c|c|c|c|c|c|}
\hline \multicolumn{2}{|c|}{ Isomer No } & $\mathbf{1}$ & $\mathbf{2}$ & $\mathbf{3}$ & $\mathbf{4}$ & $\mathbf{5}$ & $\mathbf{6}$ & $\mathbf{7}$ & $\mathbf{8}$ \\
\hline \multirow{4}{*}{ DP4 (\%) } & $\mathrm{H}$ & 1.93 & 82.35 & 0.00 & 0.00 & 10.68 & 5.04 & 0.00 & 0.00 \\
\cline { 2 - 10 } & $\mathrm{C}$ & 0.01 & 98.11 & 0.00 & 0.00 & 1.87 & 0.00 & 0.00 & 0.00 \\
\cline { 2 - 10 } & $\mathrm{H}+\mathrm{C}$ & 0.00 & 99.75 & 0.00 & 0.00 & 0.25 & 0.00 & 0.00 & 0.00 \\
\cline { 2 - 10 } & $\mathrm{J}$ & - & - & - & - & - & - & - & - \\
\cline { 2 - 10 } & all data & $\mathbf{0 . 0 0}$ & $\mathbf{9 9 . 7 5}$ & $\mathbf{0 . 0 0}$ & $\mathbf{0 . 0 0}$ & $\mathbf{0 . 2 5}$ & $\mathbf{0 . 0 0}$ & $\mathbf{0 . 0 0}$ & $\mathbf{0 . 0 0}$ \\
\hline
\end{tabular}

\begin{tabular}{|c|c|c|c|c|c|c|c|c|c|}
\hline \multicolumn{2}{|c|}{ Isomer № } & $\mathbf{1}$ & $\mathbf{2}$ & $\mathbf{3}$ & $\mathbf{4}$ & $\mathbf{5}$ & $\mathbf{6}$ & $\mathbf{7}$ & $\mathbf{8}$ \\
\hline \multirow{4}{*}{ J-DP4 (\%) } & $\mathrm{H}$ & 12.71 & 0.00 & 0.00 & 0.00 & 87.28 & 0.00 & 0.00 & 0.00 \\
\cline { 2 - 10 } & $\mathrm{C}$ & 52.20 & 30.70 & 0.00 & 0.00 & 0.00 & 17.09 & 0.01 & 0.00 \\
\cline { 2 - 10 } & $H+C$ & 100.00 & 0.00 & 0.00 & 0.00 & 0.00 & 0.00 & 0.00 & 0.00 \\
\cline { 2 - 10 } & $\mathrm{J}$ & - & - & - & - & - & - & - & - \\
\cline { 2 - 10 } & all data & $\mathbf{1 0 0 . 0 0}$ & $\mathbf{0 . 0 0}$ & $\mathbf{0 . 0 0}$ & $\mathbf{0 . 0 0}$ & $\mathbf{0 . 0 0}$ & $\mathbf{0 . 0 0}$ & $\mathbf{0 . 0 0}$ & $\mathbf{0 . 0 0}$ \\
\hline
\end{tabular}

Figure S5. DP4 (top) and J-DP4 (bottom) probabilities recomputed considering only the experimental and calculated NMR shifts of the nuclei highlighted in green.

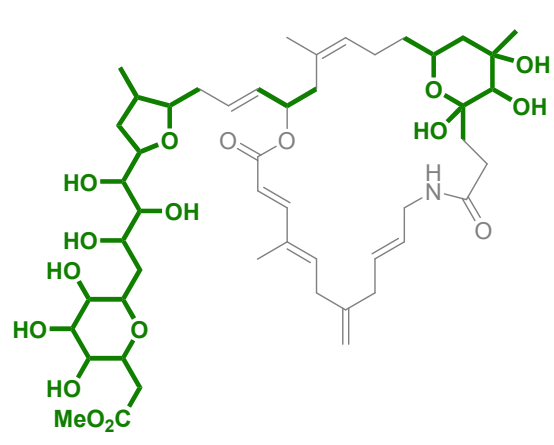

\begin{tabular}{|c|c|c|c|c|c|c|c|c|c|}
\hline \multicolumn{2}{|c|}{ Isomer No } & $\mathbf{1}$ & $\mathbf{2}$ & $\mathbf{3}$ & $\mathbf{4}$ & $\mathbf{5}$ & $\mathbf{6}$ & $\mathbf{7}$ & $\mathbf{8}$ \\
\hline \multirow{4}{*}{ DP4 (\%) } & $\mathrm{H}$ & 39.08 & 34.85 & 0.00 & 0.00 & 0.32 & 25.75 & 0.00 & 0.00 \\
\cline { 2 - 10 } & $\mathrm{C}$ & 0.07 & 90.62 & 0.00 & 0.00 & 6.27 & 3.04 & 0.00 & 0.00 \\
\cline { 2 - 10 } & $\mathrm{H}+\mathrm{C}$ & 0.09 & 97.43 & 0.00 & 0.00 & 0.06 & 2.42 & 0.00 & 0.00 \\
\cline { 2 - 10 } & $\mathrm{J}$ & - & - & - & - & - & - & - & - \\
\cline { 2 - 10 } & all data & $\mathbf{0 . 0 9}$ & $\mathbf{9 7 . 4 3}$ & $\mathbf{0 . 0 0}$ & $\mathbf{0 . 0 0}$ & $\mathbf{0 . 0 6}$ & $\mathbf{2 . 4 2}$ & $\mathbf{0 . 0 0}$ & $\mathbf{0 . 0 0}$ \\
\hline
\end{tabular}

\begin{tabular}{|c|c|c|c|c|c|c|c|c|c|}
\hline \multicolumn{2}{|c|}{ Isomer No } & $\mathbf{1}$ & $\mathbf{2}$ & $\mathbf{3}$ & $\mathbf{4}$ & $\mathbf{5}$ & $\mathbf{6}$ & $\mathbf{7}$ & $\mathbf{8}$ \\
\hline \multirow{4}{*}{ J-DP4 (\%) } & $\mathrm{H}$ & 97.20 & 0.00 & 0.00 & 0.00 & 2.80 & 0.00 & 0.00 & 0.00 \\
\cline { 2 - 11 } & $\mathrm{C}$ & 54.00 & 38.80 & 0.22 & 0.00 & 0.01 & 6.97 & 0.01 & 0.00 \\
\cline { 2 - 11 } & $\mathrm{H}+\mathrm{C}$ & 100.00 & 0.00 & 0.00 & 0.00 & 0.00 & 0.00 & 0.00 & 0.00 \\
\cline { 2 - 10 } & $\mathrm{J}$ & - & - & - & - & - & - & - & - \\
\cline { 2 - 10 } & all data & $\mathbf{1 0 0 . 0 0}$ & $\mathbf{0 . 0 0}$ & $\mathbf{0 . 0 0}$ & $\mathbf{0 . 0 0}$ & $\mathbf{0 . 0 0}$ & $\mathbf{0 . 0 0}$ & $\mathbf{0 . 0 0}$ & $\mathbf{0 . 0 0}$ \\
\hline
\end{tabular}

Figure S6. DP4 (top) and J-DP4 (bottom) probabilities recomputed considering only the experimental and calculated NMR shifts of the nuclei highlighted in green. 
Cartesian Coordinates and B3LYP/6-31G**/MMFF energies of all conformations found for the compounds under study at the MMFF level

Fragment $\mathrm{F} 1$ (cutoff energy $=10 \mathrm{~kJ} / \mathrm{mol}$ )

F1-01_c00001

MMFF Geometry

C $1.211455-2.2222050 .966451$

C $-0.223475 \quad 0.301351 \quad 0.112451$

O $0.033899-0.858226-0.688296$

C $1.262454-1.529274-0.396324$

C $2.448562-0.577419-0.583577$

$\begin{array}{llll}\text { C } 2.269492 & 0.715727 & 0.208725\end{array}$

C $0.898046 \quad 1.332639-0.066561$

C $-1.5779680 .874869-0.330647$

O $3.667132-1.200339-0.191175$

O $3.270306 \quad 1.654345-0.192676$

O 0.6853812 .4187740 .832797

C $-2.736386-0.0220590 .055510$

O $-3.8914190 .510217-0.444395$

O $-2.647818-1.049794 \quad 0.713065$

C $-5.083300-0.228303-0.157941$

H $2.063489-2.8962471 .098419$

H $\quad 0.290305-2.8075541 .059994$

H $1.218997-1.5071781 .794410$

H -0.3027540 .0141391 .168109$

H $1.355157-2.329558-1.141026$

H $2.544440-0.320758-1.646638$

H 2.4158590 .5519651 .282900

H $\quad 0.8783801 .739909-1.085615$

H $-1.753123 \quad 1.8493540 .137969$

H $-1.5934180 .985191-1.421474$

H $3.763531-2.011475-0.718927$

H $4.135408 \quad 1.234538-0.045363$

H 1.4229823 .0416230 .714662

H $-5.244086-0.283407 \quad 0.922876$

H $-5.9270440 .298508-0.611670$

H $-5.021266-1.230828-0.591704$

SCF Energy (B3LYP/6-31G**/MMFF) $=-803.935718475$

F1-01 c00002

MMFF Geometry

C $2.504949-1.9418630 .914156$

C $-0.123400-0.4166330 .874340$

O $0.315783-1.423154-0.041751$

C $1.722901-1.430979-0.297541$

C $2.186302-0.067805-0.824241$

C $1.7612851 .073353 \quad 0.097752$

C 0.2649220 .9892240 .398988

C -1.633904 -0.5876561 .097171$

O $3.601292-0.038966-0.979733$

O $2.0168412 .321572-0.550849$

O $-\begin{array}{llll}0.067116 & 1.934513 & 1.413426\end{array}$

C -2.479096 $-0.362195-0.140513$

$\begin{array}{lllll}0 & -3.747762 & -0.028257 & 0.240897\end{array}$

O $-2.091686-0.495280-1.293296$

C $-4.671908 \quad 0.198571-0.828005$

H $2.062635-2.872448 \quad 1.285709$

H $2.491594-1.2303291 .744913$

H $3.550620-2.1363190 .655911$

H $\quad 0.340648-0.6056361 .850784$

H $1.881696-2.168236-1.094325$

H $1.7483130 .107145-1.815565$

H $2.347831 \quad 1.0829901 .023897$

H $-0.2944971 .267571-0.501451$

H $-1.842177-1.6100491 .434287$

H -1.9626800 .1079231 .878024$

H $3.843538-0.762444-1.582769$

H $2.9637042 .342910-0.772526$

H $\quad 0.2201372 .8092951 .099758$

H $-4.798465-0.713856-1.418542$

H $-5.6366910 .470422-0.391433$

H $-4.3273341 .023533-1.458863$

SCF Energy (B3LYP/6-31G**/MMFF) $=-803.931839342$

F1-01_c00003

MMFF Geometry

C $0.855147-2.2044681 .000998$
C $-0.2828170 .365615-0.120161$

O $0.020708-0.832022-0.844547$

C $1.149963-1.560425-0.354969$

C $2.406707-0.683220-0.384790$

C 2.1880060 .6452690 .337330

C $0.9100041 .328354-0.153284$

C $-1.5179470 .995936-0.772855$

O $3.512043-1.360557 \quad 0.203500$

O $3.302237 \quad 1.507592 \quad 0.092811$

$\begin{array}{lllll}\text { O } & 0.630151 & 2.451250 & 0.679769\end{array}$

C $-2.7451630 .148410-0.536760$

$\begin{array}{llll}\text { O } & -3.222194 & 0.359523 & 0.723329\end{array}$

O $-3.214113-0.617755-1.368323$

C $-4.380020-0.403008 \quad 1.077531$

H $-0.103808-2.7327360 .969062$

H $0.783831-1.4645951 .803705$

H $\quad 1.635310-2.9214001 .274978$

H -0.5343710 .1141310 .917359$

H $1.304013-2.388541-1.057906$

H $2.674817-0.472654-1.428105$

H 2.1587950 .5009881 .423399

H $1.0625931 .704388-1.173000$

H $-1.7209362 .002459-0.389835$

H $-1.384176 \quad 1.085542-1.857874$

H $3.637484-2.193145-0.283139$

H $3.3659021 .652373-0.866641$

H 1.4146853 .0259320 .665691

H $-5.215337-0.1522880 .416729$

H $-4.157349-1.4733091 .031200$

H $-4.655390-0.1461662 .103830$

SCF Energy (B3LYP/6-31G**//MMFF) $=-803.927631501$

F1-01_c00004

MMFF Geometry

C $0.793530-2.1100761 .210935$

$\begin{array}{llll}\text { C } & -0.244508 & 0.494320 & 0.073458\end{array}$

O $-0.181961-0.774744-0.587855$

C $0.934987-1.589564-0.220278$

C $2.247713-0.855633-0.515684$

C $2.283113 \quad 0.534185 \quad 0.119024$

C $1.0152421 .316000-0.225210$

C $-1.5030011 .210924-0.427159$

O $3.368742-1.622499-0.088912$

O $3.4063931 .255150-0.392201$

O $0.9843362 .517552 \quad 0.542119$

C -2.7433220 .5498190 .125605$

O $-3.256734-0.321877-0.788255$

$\begin{array}{lllll}0 & -3.184766 & 0.763995 & 1.247394\end{array}$

C $-4.423265-1.036297-0.368393$

H $-0.212015-2.5142391 .370409$

H $0.940927-1.3202151 .953383$

H $1.519429-2.9030251 .415908$

H -0.3472150 .3411651 .154949$

H $0.893405-2.472562-0.870057$

H $2.347581-0.739102-1.602400$

H 2.4225320 .4779021 .204965

H $1.0381141 .601613-1.284751$

H $-1.5310462 .259225-0.109266$

H $-1.5541151 .207693-1.522763$

H $3.393755-1.6100450 .882786$

H $4.1999840 .724721-0.205627$

H 1.8034423 .0056550 .349854

H -4.729092 -1.696865 -1.183995

H $-4.197593-1.6461340 .511508$

H $-5.239115-0.338457-0.157014$

SCF Energy (B3LYP/6-31G**//MMFF) $=-803.932923103$

F1-01_c00005

MMFF Geometry

C $2.521411-1.9428700 .879366$

C $-0.105876-0.419902 \quad 0.853062$

O $0.327592-1.429592-0.062417$

C $1.733498-1.435242-0.329703$

C $2.187112-0.072735-0.868832$

$\begin{array}{llll}\text { C } & 1.765038 & 1.077949 & 0.044454\end{array}$

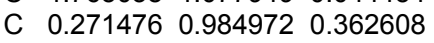

C $-1.612719-0.5945541 .094975$

O $3.596210-0.052956-1.074049$ 
O $2.0233072 .309780-0.632464$

O $\quad-0.061738 \quad 1.9554581 .352716$

C $-2.473149-0.373162-0.132658$

O $-3.731118-0.015950 \quad 0.262492$

O $-2.104456-0.527306-1.288951$

C $-4.6673690 .211172-0.795760$

H $2.069147-2.8614961 .268575$

H $3.560430-2.1583490 .611560$

H $2.528990-1.2195141 .699924$

H $\quad 0.370777-0.6022001 .824698$

H $\quad 1.887639-2.175767-1.124368$

H $1.7284640 .084231-1.853580$

H 2.3678721 .0979610 .959793

H $-0.2908751 .248573-0.539953$

H $-1.932601 \quad 0.102141 \quad 1.878522$

H -1.815207 -1.616325 1.437138

H $4.029592-0.015739-0.204809$

H $1.7044603 .027881-0.059041$

H 0.4336131 .7399932 .161272

H -4.319480 $1.022024-1.442882$

H -4.816683 -0.706920 -1.371839

H $-5.6210600 .504270-0.348690$

SCF Energy (B3LYP/6-31G**//MMFF) $=-803.925558694$

F1-01_c00006

MMFF Geometry

C $2.244215-1.8565611 .289375$

C $-0.193504-0.0629541 .054075$

O $0.049645-1.2957460 .369756$

C $1.419610-1.5400500 .040062$

C $1.980332-0.404173-0.822630$

C $1.7579850 .963910-0.177541$

C $0.291611 \quad 1.1338660 .224656$

C -1.682934 -0.006072 1.416308

O $3.367418-0.621273-1.058206$

O $2.079241 \quad 1.988313-1.121698$

O 0.1703962 .3367850 .979678

C $-2.589591 \quad 0.1570170 .218855$

O $-2.746737-1.036999-0.419424$

O $-3.090271 \quad 1.224277-0.112318$

C $-3.572570-1.006312-1.587434$

H $1.732760-2.6075311 .901045$

H $3.232423-2.2441181 .022588$

H $2.389669-0.9763641 .922566$

H $\quad 0.342273-0.0801122 .011413$

H $1.426641-2.454162-0.566912$

H $1.478969-0.420762-1.799228$

H $2.420007 \quad 1.1167630 .683084$

H $-0.3035951 .252512-0.688921$

H -1.883366 0.8257062 .101601

H $-1.986496-0.9193451 .943214$

H $3.6645570 .041437-1.704049$

H $3.0374391 .950711-1.280530$

H -0.7772252 .5270711 .082589$

H -4.588380 -0.696011 -1.324947

H $-3.143657-0.334121-2.336744$

H -3.610490 -2.016557 -2.003432

SCF Energy (B3LYP/6-31G**//MMFF) $=-803.924950309$

F1-01_c00007

MMFF Geometry

C $2.287533-1.9580261 .049624$

C $-0.148514-0.594790-0.130380$

O $0.788562-1.437557-0.813218$

C $2.136199-1.343728-0.343293$

C $2.6463500 .095981-0.462947$

C 1.7065541 .0896320 .215923

C $0.2665360 .878667-0.253108$

C - $1.522902-0.865751-0.760650$

O $3.947034 \quad 0.2228230 .102154$

O $2.0996212 .419365-0.132432$

$\begin{array}{lllll}\text { O } & -0.601808 & 1.690949 & 0.532077\end{array}$

C $-2.680895-0.4319010 .108869$

O $-3.719516-0.036168-0.683523$

O $-2.685843-0.468014 \quad 1.332226$

C -4.8957420 .3901170 .011330$

H $1.816886-2.9465441 .081343$

H $\quad 1.807030-1.3520431 .823264$
H $3.342511-2.0694401 .318951$

H $-0.190686-0.8874350 .925401$

H $2.736051-1.967732-1.017403$

H $2.7272690 .366219-1.523884$

H $1.774793 \quad 1.0258531 .308337$

H $\quad 0.174081 \quad 1.211208-1.295027$

H -1.582653 -0.368948 - 1.736768

H -1.648366 -1.939194 -0.948769

H $4.527668-0.408132-0.356666$

H 3.0271202 .5269220 .140191

H -0.2937732 .6097960 .449696$

H $-5.303943-0.4334410 .604778$

H $-4.669273 \quad 1.2509480 .647691$

H $-5.6398190 .689571-0.731625$

SCF Energy $\left(B 3 L Y P / 6-31 G^{* *} / / M M F F\right)=-803.933611568$

F1-01_c00008

MMFF Geometry

C 1.2815702 .7597570 .349266

C $-0.073247-0.516215-0.767128$

O $0.3263710 .860049-0.713783$

C 1.0010561 .2654360 .480701

C 2.2842740 .4501870 .689053

C $1.993106-1.0531730 .664134$

C $1.131841-1.446633-0.541605$

C $-1.250759-0.8076350 .176427$

O $3.2228910 .770492-0.336419$

O $1.329005-1.4059921 .873846$

O $1.946821-1.420144-1.717559$

C $-2.513234-0.079323-0.237025$

$\begin{array}{llll}\text { O } & -3.513149 & -0.400378 & 0.637597\end{array}$

O $-2.6167150 .671799-1.196882$

$\begin{array}{llll}\text { C } & -4.778202 & 0.216328 & 0.378607\end{array}$

H 1.8222833 .1410861 .220629

H $1.8597312 .982875-0.553857$

H $\quad 0.3399273 .3123940 .256105$

H -0.421769 -0.668594-1.797029

H $\quad 0.331349 \quad 1.1310391 .336792$

H $2.732546 \quad 0.7344391 .648743$

H $2.941596-1.6030520 .629617$

H $0.780305-2.478883-0.433622$

H -1.029612 -0.5138221 .205706$

H -1.474061 -1.8806470 .155578$

H $4.0365730 .269785-0.155917$

H $1.174882-2.3659101 .854714$

H $2.211331-0.497486-1.875300$

H -5.482946 -0.1187471 .144352$

H $-4.687941 \quad 1.3052310 .437070$

H $-5.154434-0.088704-0.602555$

SCF Energy (B3LYP/6-31G**//MMFF) $=-803.931359412$

F1-01 c00009

MMFF Geometry

C $1.913794-2.1404780 .966346$

C $-0.228384-0.479690-0.386698$

O $0.723670-1.345535-1.017546$

C $1.999648-1.410469-0.375234$

C $2.633529-0.018307-0.304819$

C $1.697796 \quad 0.9988220 .343535$

C $0.3145440 .955363-0.309332$

C $-1.515542-0.572883-1.216156$

O $3.862410-0.0789860 .411485$

O $2.2589502 .301547 \quad 0.164494$

O $-0.570764 \quad 1.7867000 .437098$

C $-2.730663-0.017283-0.516759$

O $-3.040142-0.7830070 .568021$

O $-3.3403580 .973242-0.898915$

C $-4.178550-0.3518801 .320105$

H $1.380962-1.5558011 .722043$

H $1.368341-3.0832280 .850650$

H $2.910809-2.3649451 .358145$

H $-0.442223-0.8544250 .621240$

H $2.632768-2.033083-1.019717$

H $2.8701170 .317005-1.323031$

H $1.6348860 .838841 \quad 1.426172$

H $0.385272 \quad 1.386446-1.315708$

H $-1.730280-1.616453-1.479927$

$\mathrm{H}-1.392646-0.054323-2.175010$ 
H $\quad 4.220821 \quad 0.824336 \quad 0.450233$ H 1.6313182 .9413100 .542550 H $-0.655621 \quad 1.4083381 .329058$ H -4.310732 -1.0387142 .160268$ H -4.0135200 .6557801 .713095$ H $-5.077441-0.3819360 .696916$ SCF Energy (B3LYP/6-31G**//MMFF) $=-803.932490497$

F1-02_c00059

MMFF Geometry

C $1.6177232 .745890-0.310370$

C $-0.152189-0.4427830 .289174$

O $0.226748 \quad 0.812259-0.294353$

C $1.411844 \quad 1.3609230 .292157$

C 2.6080200 .4296420 .043579

C $2.311369-0.9963980 .524474$

C $0.944956-1.4910060 .033426$

C -1.491654 -0.855237 -0.334175

O $2.935958 \quad 0.400318-1.344507$

O $2.309159-1.0241361 .950503$

O $1.013778-1.774473-1.362931$

C $-2.643143-0.0072630 .165760$

O $-3.797359-0.440653-0.423776$

$\begin{array}{lllll}\text { O } & -2.550129 & 0.914033 & 0.965151\end{array}$

C $-4.9825330 .269676-0.051161$

H $\quad 0.763653 \quad 3.390092-0.073924$

H 2.5255223 .2173080 .078140

H $1.6785372 .704164-1.403184$

H $-0.279719-0.2995471 .370194$

H $1.244941 \quad 1.4766661 .370699$

H 3.4828190 .8272570 .570688

H $3.094879-1.6769240 .170485$

H $0.672592-2.4204380 .547939$

H -1.715804 -1.896206 -0.074643

H -1.454466 -0.745040 -1.424087

H $2.1414330 .122326-1.831745$

H $3.189398-0.7399902 .250504$

H $1.689366-2.462335-1.489374$

H $-4.8975841 .323199-0.334648$

H $-5.826249-0.171085-0.588712$

H -5.1609140 .1711091 .023790$

SCF Energy (B3LYP/6-31G**//MMFF) $=-803.937989304$

F1-02 c00060

MMFF Geometry

C $1.6458542 .725100-0.275458$

C $-0.158530-0.4524730 .264345$

O $0.2426670 .802008-0.304081$

C $1.4285361 .330504 \quad 0.299623$

C 2.6186760 .3885950 .055992

C $2.294219-1.0366380 .522703$

C $0.929090-1.5122900 .010140$

C $-1.504152-0.833910-0.364487$

$\begin{array}{lllll}\text { O } & 2.952378 & 0.342257 & -1.329536\end{array}$

O $2.274268-1.0747951 .948453$

O $1.043403-1.794381-1.382998$

C $-2.643660 \quad 0.0182360 .155699$

O $-3.808990-0.407836-0.417548$

$\begin{array}{lllll}0 & -2.533938 & 0.937893 & 0.954705\end{array}$

C $-4.9847280 .307751-0.025603$

H $1.6831502 .708815-1.369902$

H $\quad 0.8056163 .375869-0.008481$

H $2.566627 \quad 3.1749740 .107462$

H $-0.289242-0.3172511 .346220$

H $\quad 1.252716 \quad 1.4330341 .378289$

H 3.4958100 .7646880 .596308

H $3.071699-1.7268350 .172930$

H $0.652034-2.4446390 .517462$

H -1.741052 -1.876731 -0.125366

H -1.469328 $-0.700179-1.451942$

H $3.3488031 .196776-1.568368$

H $3.156696-0.8108962 .260596$

H $0.241163-2.269464-1.657402$

H $-5.146890 \quad 0.2088521 .051985$

H -4.899141 $1.361096-0.309173$

H -5.838795 -0.128531 -0.550306

SCF Energy (B3LYP/6-31G**//MMFF $)=-803.924094486$
F1-02 c00061

MMFF Geometry

C $3.172589-2.018682-0.530687$

C $-0.124290-0.352287-0.379016$

O $0.928886-1.304299-0.168557$

C $2.187851-0.865649-0.691807$

C $2.6545410 .408186 \quad 0.029564$

C $1.5774721 .498000-0.024573$

C $0.1962690 .955995 \quad 0.367615$

C $-1.422442-1.0196000 .089910$

O $2.953241 \quad 0.101944 \quad 1.390198$

O $1.5254562 .007486-1.355650$

O 0.1553390 .7480531 .778183

C $-2.661011-0.239235-0.285196$

O $-3.705835-0.6618390 .486091$

O $-2.7164740 .611684-1.162586$

C $-4.958449-0.0184200 .231105$

H $4.168625-1.743908-0.890197$

H $3.247659-2.3407880 .513590$

H $2.827241-2.890535-1.097401$

H $-0.193939-0.155931-1.457068$

H $2.067588-0.665640-1.764103$

H $3.5789180 .763413-0.441471$

H 1.8560832 .3201490 .645649

H $-0.569261 \quad 1.7037750 .133758$

H $-1.398726-1.1718531 .175126$

H -1.508625 -2.013792 -0.365680

H 3.2781100 .9168371 .809455

H $0.8612532 .717641-1.372075$

H $\quad 0.7837720 .037243 \quad 1.992125$

H -4.8764351 .0573880 .413274$

H $-5.700459-0.4345410 .917813$

H $-5.281881-0.213529-0.795725$

SCF Energy $(B 3 L Y P / 6-31 G * * / / M M F F)=-803.941093508$

F1-02 c00062

MMFF Geometry

C 3.1207452 .0539850 .195004

C -0.2109600 .4609310 .018328$

O $0.919391 \quad 1.286562-0.296779$

C $2.0501841 .025340 \quad 0.541574$

C $2.538999-0.417400 \quad 0.343672$

C $1.400530-1.4230580 .554549$

C $0.134713-1.021752-0.213447$

C $-1.3718160 .956263-0.847050$

O $3.080453-0.584536-0.965448$

O $1.077495-1.4884901 .942244$

O $0.331609-1.251668-1.607153$

C $-2.6735520 .274971-0.512278$

$\begin{array}{lllll}\text { O } & -3.143877 & 0.728268 & 0.684571\end{array}$

O $-3.205925-0.563960-1.227429$

C $-4.375461 \quad 0.1452891 .121390$

H $2.7486583 .065568 \quad 0.391445$

H $4.0281681 .897557 \quad 0.786093$

H $3.3795372 .022250-0.868811$

H $-0.4640050 .617629 \quad 1.075219$

H 1.7513931 .1792831 .586338

H $3.349982-0.6174291 .053412$

H $1.723114-2.4200290 .231251$

H $-0.709363-1.6423020 .109888$

H $-1.1627060 .817952-1.914605$

H -1.506076 $2.038977-0.727841$

H $2.391662-0.336586-1.605791$

H $1.876376-1.7770032 .415648$

H $\quad 0.509205-2.200366-1.725445$

H $-4.258183-0.9348501 .251077$

H -4.637796 0.5877632 .086006

$\mathrm{H}-5.1734410 .3647600 .405616$

SCF Energy $\left(\mathrm{B} 3 L \mathrm{YP} / 6-31 \mathrm{G}^{* *} / / \mathrm{MMFF}\right)=-803.936716022$

F1-02 c00063

MMFF Geometry

C $-2.3998372 .419657-0.033769$

C $-0.045166-0.205941-1.136614$

O $-0.559593 \quad 1.038525-0.649780$

C $-1.9759981 .013082-0.441245$

C $-2.347130-0.0392620 .615442$

C $-1.778177-1.4179010 .254422$ 
C $-0.292544-1.337792-0.122117$

C $1.424315-0.013993-1.528486$

O -1.8617490 .3512941 .898709$

O $-2.494462-1.950552-0.857774$

O $0.473354-1.122151 \quad 1.059328$

C $2.3193150 .459122-0.404610$

O $3.320664-0.443908-0.197539$

$\begin{array}{lllllll}\text { O } & 2.154249 & 1.502979 & 0.213275\end{array}$

C $4.235013-0.117540 \quad 0.853615$

H -2.160820 $3.131009-0.832010$

H -3.4746052 .4692340 .164351$

H -1.8582402 .7609790 .854968$

H $-0.583892-0.445179-2.063685$

H $-2.461923 \quad 0.770942-1.395054$

H -3.439216 -0.0954140 .690475$

H -1.904276 -2.102976 1.101360

H $0.038070-2.285063-0.564728$

H $1.5048250 .744131-2.317405$

H $1.817607-0.948192-1.946441$

H -0.9011050 .4844461 .825337$

H $-3.428050-2.025319-0.596268$

H $\quad 0.320408-1.8780161 .651479$

H $3.705397-0.0483261 .808597$

H $4.975726-0.9187300 .920884$

H 4.7516400 .8203920 .628553

SCF Energy (B3LYP/6-31G**//MMFF) $=-803.932886830$

F1-02_c00064

MMFF Geometry

C -1.743830 $2.597337-0.703562$

C $-0.016952-0.652670-1.025958$

O $-0.248250 \quad 0.751547-0.870546$

C $-1.637667 \quad 1.076852-0.745038$

C $-2.241627 \quad 0.412928 \quad 0.502929$

C $-1.982000-1.0992380 .507837$

C $-0.510442-1.4230940 .212211$

C $1.456587-0.880980-1.382262$

O -1.6735240 .9970691 .673679$

O $-2.810726-1.698816-0.485741$

O $0.266932-1.108441 \quad 1.363681$

C $2.457067-0.462867-0.329959$

O $2.4879990 .894687-0.215756$

O $3.141023-1.2548450 .306331$

C 3.3927091 .4100210 .765485

H -2.782416 $2.920339-0.584968$

H $-1.144475 \quad 3.0202200 .109841$

H $-1.350418 \quad 3.027747-1.631328$

H $-0.597155-0.985970-1.897404$

H $-2.1582950 .722448-1.643966$

H -3.3197390 .6124910 .522418$

H -2.263394 -1.5143321 .483053$

H $-0.394826-2.497446 \quad 0.028859$

H $1.711408-0.334331-2.298945$

H $1.623447-1.943681-1.595744$

H -2.099671 0.5836622 .443779

H - $2.660531-2.658945-0.451754$

H $\quad 0.228749-0.1455801 .495562$

H 3.3093282 .5000360 .765367

H $4.421483 \quad 1.1361410 .512721$

H $3.125806 \quad 1.037848 \quad 1.759227$

SCF Energy (B3LYP/6-31G**/MMFF) $=-803.932394792$

F1-02_c00065

MMFF Geometry

C 1.0941442 .7998080 .195512

C $-0.167020-0.6711710 .140966$

O $0.035904 \quad 0.705980-0.204300$

C 1.0995991 .3137220 .536042

C $2.437296 \quad 0.627980 \quad 0.213193$

C $2.351204-0.8914660 .411123$

C $1.085705-1.490248-0.218075$

C $-1.412807-1.153363-0.604960$

O $2.8114930 .876690-1.141226$

O $2.388158-1.198763 \quad 1.803435$

O $1.268402-1.525461-1.633094$

C -2.662462 -0.6127240 .048321$

O $-3.0189390 .569634-0.529444$

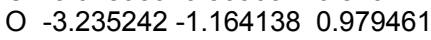

$\begin{array}{llll}C & -4.178244 & 1.199690 & 0.024198\end{array}$

H $\quad 0.149808 \quad 3.2543150 .515559$

H 1.9160273 .3249720 .691040

H $1.1615182 .966571-0.884819$

H $-0.352008-0.7306531 .221330$

H $0.878487 \quad 1.2145531 .606146$

H $3.223725 \quad 1.0397010 .857217$

H $3.231467-1.360462-0.044870$

H $\quad 0.957322-2.5232570 .127442$

H $-1.488667-2.246771-0.586010$

H $-1.406814-0.846133-1.657626$

H $3.079371 \quad 1.808509-1.208625$

H $1.620712-0.7888572 .234307$

H $0.564730-2.079441-2.010639$

H $-5.0527820 .552366-0.090735$

H $-4.010731 \quad 1.4410051 .078228$

H $-4.3548592 .128685-0.524510$

SCF Energy (B3LYP/6-31G**//MMFF)= -803.920829598

F1-03_c00104

MMFF Geometry

C -1.395548 $2.876022-0.406655$

$\begin{array}{lllll}C & 0.238475 & -0.389158 & 0.157167\end{array}$

O $-0.0561510 .903645-0.384706$

C $-1.258000 \quad 1.462845 \quad 0.149317$

$\begin{array}{llll}\text { C }-2.457128 & 0.587763 & -0.227265\end{array}$

C $-2.240730-0.8573350 .223301$

C $-0.873267-1.374577-0.224983$

C $1.595645-0.832179-0.406244$

O -3.6466011 .0952170 .372005$

O $-3.248461-1.687430-0.356629$

O $-0.623410-2.6330690 .396390$

$\begin{array}{lll}\text { C } 2.742389 & -0.008576 & 0.142645\end{array}$

O $3.899525-0.401283-0.469025$

$\begin{array}{lllll}\text { O } & 2.644564 & 0.859879 & 0.998611\end{array}$

$\begin{array}{llll}\text { C } 5.081277 & 0.288821 & -0.050377\end{array}$

H $-0.5113093 .471137-0.153514$

H $-1.4607802 .861921-1.500321$

H $-2.2794653 .382784-0.008260$

H $0.315571-0.3151991 .250560$

H $-1.165518 \quad 1.541857 \quad 1.240558$

H $-2.6125870 .602894-1.313291$

H $-2.349257-0.9523971 .310772$

H $-0.880000-1.536304-1.310481$

H $1.598046-0.717848-1.496865$

H $1.795322-1.877615-0.147644$

H $-3.5161061 .102597 \quad 1.335682$

H $-4.110914-1.322432-0.093765$

H $-1.356098-3.2258120 .155622$

H $4.992587 \quad 1.357731-0.266801$

H 5.2585980 .1232641 .016489

H $5.927504-0.114011-0.613343$

SCF Energy (B3LYP/6-31G**//MMFF) $=-803.940601859$

F1-03_c00105

MMFF Geometry

C $2.210905-2.653709-0.140734$

C $-0.147531-0.072808 \quad 1.051278$

O $0.364792-1.279906 \quad 0.480891$

C $1.792076-1.2912540 .402136$

C $2.287751-0.150046-0.492232$

C $1.7531861 .198251-0.006739$

C $\quad 0.238978 \quad 1.142705 \quad 0.198774$

C $-1.661703-0.2265291 .251740$

O $3.712786-0.114914-0.501737$

O $2.0383842 .198904-0.985644$

$\begin{array}{lllll}\text { O } & -0.193540 & 2.332995 & 0.852481\end{array}$

C $-2.448706-0.387321-0.033254$

$\begin{array}{llll}0 & -3.733645 & 0.027038 & 0.175234\end{array}$

O -2.007936 $-0.849821-1.076576$

C $-4.606846-0.084028-0.953141$

H $1.819662-3.4535430 .497479$

H $1.796358-2.820359-1.141202$

H $3.299352-2.751910-0.190486$

H $\quad 0.2836910 .0404792 .055994$

H $2.207962-1.1942821 .413689$

H $\quad 1.963120-0.310340-1.527872$

H 2.2558121 .5146260 .915587 
H $-0.252356 \quad 1.108630-0.780554$ H $-2.042710 \quad 0.648826 \quad 1.790278$ H $-1.865163-1.1189131 .855352$ H 4.0149240 .0121490 .413863 H $2.9993512 .191278-1.136077$ H $\quad 0.0939713 .0846460 .306126$ H $-4.2324590 .522053-1.783799$ H $-4.706424-1.131703-1.252333$ H $-5.5904880 .292435-0.660207$ SCF Energy (B3LYP/6-31G**//MMFF) $=-803.936897575$

F1-03_c00106

MMFF Geometry

C -1.625467 2.8198990 .269059

C $0.217664-0.2656891 .117306$

$\begin{array}{lllll}\text { O } & -0.062403 & 1.078510 & 0.717457\end{array}$

C $-1.4634991 .341503 \quad 0.608809$

C $-2.0972470 .443540-0.458311$

C -1.809892 -1.030483 -0.171574

C $-0.317086-1.2562410 .075105$

C $1.721908-0.3990141 .376360$

O $\quad-3.506777 \quad 0.654357-0.474532$

O $-2.222114-1.798067-1.303733$

O $-0.106455-2.595151 \quad 0.514434$

C $2.563245-0.2609190 .128760$

O $2.6954181 .053801-0.206438$

O $3.038421-1.211906-0.478681$

C $3.4575541 .313624-1.389159$

H $-1.1412133 .057543-0.684662$

H $-2.6790093 .108644 \quad 0.209787$

H -1.1387113 .4397931 .029928$

H -0.277995 -0.446546 2.081367

H -1.9385821 .1706421 .583964$

H -1.711946 $0.704471-1.452275$

H $-2.409436-1.3824450 .677000$

H $0.211935-1.142378-0.878334$

H $1.950632-1.3720001 .826757$

H 2.0584120 .3617212 .091675

H $-3.8818870 .053762-1.141262$

H $-1.993237-2.726077-1.123297$

H $-0.569719-2.708537 \quad 1.361837$

H $2.9840570 .842638-2.255904$

H $3.4813822 .395159-1.547337$

H $4.4835140 .953944-1.265430$

SCF Energy (B3LYP/6-31G**//MMFF) $=-803.935261298$

F1-03 c00107

MMFF Geometry

C -1.418667 $2.861334-0.353149$

C $0.234299-0.3975870 .162069$

O $-\begin{array}{llll}0.079886 & 0.894936 & -0.367765\end{array}$

C -1.281569 $1.440156 \quad 0.183487$

C $-2.4793150 .561043-0.193396$

C $-2.242220-0.887548 \quad 0.239964$

C $-0.871684-1.390359-0.219982$

C $1.590442-0.823457-0.416033$

$\begin{array}{lllll}\text { O } & -3.662111 & 1.028861 & 0.449294\end{array}$

O $-3.261712-1.721464-0.314863$

$\begin{array}{lllll}\text { O } & -0.616176 & -2.663282 & 0.368847\end{array}$

$\begin{array}{llll}\text { C } & 2.733931 & 0.007493 & 0.128553\end{array}$

O $3.889615-0.371318-0.494517$

$\begin{array}{lllll}\text { O } & 2.634982 & 0.870060 & 0.990325\end{array}$

C $5.0685530 .326956-0.081175$

H -2.276941 3.3802980 .082746

H $-0.5164663 .440786-0.127216$

H -1.524347 2.859288 -1.443639

H $0.320067-0.3298561 .255119$

H -1.181530 $1.505671 \quad 1.274956$

H -2.654196 $0.586958-1.276563$

H -2.340119 -0.9807421 .328303$

H $-0.887971-1.536308-1.307108$

H $1.582319-0.701920-1.505746$

H $1.801960-1.868583-0.165723$

H -3.905518 1.8770860 .042278

H -3.202503 -1.664199 -1.283768

H $-0.593151-2.5472691 .334027$

H $4.9690371 .396026-0.292026$

H 5.2549430 .1581280 .983734
H $5.914100-0.065885-0.651976$

SCF Energy $(B 3 L Y P / 6-31 G * * / / M M F F)=-803.929594461$

F1-03 c00108

MMFF Geometry

C -1.329546 $2.915334-0.237526$

C $0.312770-0.390345-0.106284$

O $-0.0362700 .933496-0.525753$

C - $-1.1347821 .471414 \quad 0.212557$

C $-2.3912040 .629380-0.027376$

C $-2.137178-0.8418370 .305294$

C $-0.858504-1.345298-0.368690$

C $1.560000-0.810587-0.889032$

O -3.4682321 .1165490 .769678$

O $-3.252520-1.602104-0.163188$

O $-0.530137-2.634093 \quad 0.144373$

C $2.763344-0.006668-0.458012$

O $3.263770-0.5061290 .708205$

O $3.1965050 .956627-1.076963$

C $4.4032890 .182471 \quad 1.232420$

H -1.557892 2.964170 -1.307952

H $-2.1348383 .406546 \quad 0.316598$

H $-0.4070273 .487134-0.088053$

H $0.554329-0.3748220 .965215$

H -0.8791231 .4876001 .280256$

H $-2.7060970 .708842-1.075411$

H -2.092200 -0.9899881 .391414$

H - $1.028220-1.448391-1.448178$

H $1.422090-0.644091-1.964314$

H $1.795210-1.872525-0.755835$

H -3.1993241 .0600761 .702448$

H -3.135228 -2.5167710 .144078$

H -1.231032 -3.248550 -0.131258

H 4.1475411 .2226611 .455740

H $4.702150-0.3120652 .160431$

H $5.2356100 .132213 \quad 0.523908$

SCF Energy $(B 3 L Y P / 6-31 G * * / / M M F F)=-803.928323973$

F1-03 c00109

MMFF Geometry

C $3.028908-2.369398-0.107857$

C $-0.168178-0.5290910 .114330$

O $0.823328-1.483168-0.287278$

C $2.102672-1.2268530 .297209$

C $2.6376080 .128574-0.172497$

C 1.6337841 .2417920 .125984

C $0.2358650 .870445-0.373451$

C $-1.504426-0.994005-0.480056$

$\begin{array}{lllll}\text { O } & 3.864991 & 0.408744 & 0.495935\end{array}$

O $2.0705652 .430135-0.536235$

O -0.6935391 .8515510 .076295$

C $-2.712615-0.4023840 .210387$

O $-3.771596-0.412098-0.651461$

O $-2.736129-0.0119111 .369773$

C $-4.9971600 .107874-0.126146$

H $3.135485-2.419671-1.197202$

H $4.022144-2.2623530 .337834$

H $2.608539-3.3298010 .210005$

H $-0.241045-0.5429241 .209822$

H $2.010905-1.2453251 .391211$

H $2.8455010 .102670-1.249815$

H 1.6216251 .4711451 .198569

H $\quad 0.2323500 .904566-1.470014$

H -1.591092 -2.084636 -0.399918

H -1.534840 -0.750252 -1.548994

H 4.1641421 .2849010 .197600

H $1.4023053 .116830-0.368833$

H -0.7209431 .8142231 .047792$

H $-5.7528830 .058679-0.914668$

H $-5.332896-0.4984350 .720579$

$\mathrm{H}-4.8690021 .1526260 .172389$

SCF Energy $(B 3 L Y P / 6-31 G * * / / M M F F)=-803.943069031$

F1-03 c00110

MMFF Geometry

C $2.442832-2.447027-0.306423$

C $-0.149514-0.2353001 .108461$

O $0.478982-1.332157 \quad 0.440032$ 
C $1.895237-1.1688020 .321059$

C $2.2223650 .073315-0.517198$

C $1.540398 \quad 1.314722 \quad 0.063737$

C $0.0519601 .060551 \quad 0.313500$

C $-1.622141-0.5849111 .344807$

$\begin{array}{lllll}\text { O } & 3.628443 & 0.306123 & -0.532136\end{array}$

O $1.6872602 .406773-0.846465$

O -0.5085072 .1628851 .021166$

C -2.406791 -0.7253940 .060469$

O $-3.2716790 .320159-0.074407$

O $-2.256507-1.643670-0.735204$

C $-4.059994 \quad 0.316200-1.268447$

H $2.040926-2.590592-1.315482$

H $3.535058-2.438773-0.360601$

H $2.135377-3.3199010 .280008$

H $0.307189-0.1309232 .102498$

H $2.332006-1.0782911 .324376$

H $1.894684-0.062482-1.555799$

H $2.038758 \quad 1.627756 \quad 0.989036$

H $-0.4601341 .005317-0.654159$

H -2.097662 $0.167597 \quad 1.984718$

H $-1.708790-1.5433451 .870988$

H $4.040442-0.398296-1.059815$

H $1.2552942 .161526-1.682537$

H -0.0560552 .2291451 .879337$

H $-4.7051601 .198586-1.252839$

H -3.412795 $0.369932-2.149084$

H $-4.689190-0.578282-1.304327$

SCF Energy (B3LYP/6-31G**//MMFF) $=-803.924022693$

F1-03 c00111

MMFF Geometry

C $2.894889-2.404753-0.110818$

C $-0.273058-0.504079-0.151084$

O $0.750465-1.442835-0.502483$

C $1.940752-1.2766820 .270042$

C $2.5563850 .099540 \quad 0.003642$

C $1.537303 \quad 1.2075240 .271951$

C $0.2108540 .924195-0.436623$

C $-1.514439-0.881144-0.967151$

$\begin{array}{llll}\text { O } & 3.686616 & 0.275675 & 0.853621\end{array}$

$\begin{array}{lllll}\text { O } & 2.044421 & 2.449722 & -0.219798\end{array}$

$\begin{array}{llll}0 & -0.761964 & 1.861826 & 0.016253\end{array}$

C $-2.787511-0.251494-0.460010$

O $-3.102204-0.7510610 .768806$

O $-3.4334710 .576299-1.090005$

C $-4.295178-0.2257331 .358749$

H $3.819299-2.3674760 .472871$

H $2.418876-3.3772520 .056509$

H $3.148940-2.359765-1.175606$

H $-0.502396-0.615403 \quad 0.917015$

H $1.699776-1.390507 \quad 1.335414$

H $2.9055360 .165534-1.034744$

H 1.3755601 .3411031 .348912

H $\quad 0.3361301 .072217-1.516855$

H $-1.659148-1.969260-0.970304$

H $-1.380214-0.606672-2.020639$

H 4.1256801 .1024560 .592755

H 2.8290992 .6762020 .307059

H $-0.4150792 .751860-0.166672$

H $-5.162277-0.463543 \quad 0.735164$

H $-4.426647-0.6964282 .336629$

H -4.2040190 .8556871 .499152$

SCF Energy (B3LYP/6-31G*//MMFF) $=-803.932937008$

F1-04_c00137

MMFF Geometry

C $-0.9630892 .901122-0.610272$

C $0.137690-0.636332-0.863347$

O $-0.104242 \quad 0.747386-1.145674$

C $-0.808617 \quad 1.460833-0.126975$

C -2.1693100 .8186990 .156654$

C $-2.027474-0.6729780 .456443$

C $-1.195997-1.358981-0.625400$

C $1.165309-0.8282760 .267065$

$\begin{array}{llll}\text { O } & -2.770866 & 1.469760 & 1.273415\end{array}$

O $-3.335394-1.2493710 .480675$

O $-0.982893-2.718112-0.259361$
C $2.509005-0.213888-0.066588$

O $3.302563-0.2567091 .045057$

$\begin{array}{lllll} & 0 & 2.831850 & 0.230928 & -1.159349\end{array}$

$\begin{array}{llll}\text { C } & 4.617101 & 0.285454 & 0.883512\end{array}$

H $-1.462088 \quad 3.5268250 .135458$

H $0.0190153 .336231-0.826399$

H $-1.5355772 .941474-1.543646$

H $0.580833-1.059165-1.774271$

$\begin{array}{llll}H & -0.204281 & 1.496729 & 0.786648\end{array}$

H $-2.8386280 .954656-0.702564$

H $-1.601530-0.8341651 .453672$

H - $1.777392-1.363749-1.556594$

H $1.336196-1.8961110 .442747$

H $0.812679-0.3758611 .198746$

H -3.6192991 .0262921 .445026$

H $-3.227249-2.2041950 .631543$

H $-0.468609-3.134965-0.971753$

H 5.1413390 .1964331 .838675

H $5.169041-0.2792760 .126071$

H 4.5597011 .3438720 .611978

SCF Energy (B3LYP/6-31G**//MMFF) $=-803.934028830$

F1-04_c00138

MMFF Geometry

C $0.931723 \quad 2.898597 \quad 0.597425$

C $-0.136400-0.642963 \quad 0.871974$

$\begin{array}{lllll}\text { O } & 0.100615 & 0.742773 & 1.144870\end{array}$

C $\quad 0.7949021 .4553320 .118094$

C $2.1589850 .817843-0.168890$

C $2.024800-0.679015-0.453108$

C $1.199983-1.360747 \quad 0.638187$

C $-1.166395-0.846357-0.254677$

O $2.760688 \quad 1.433217-1.306130$

O $3.338128-1.243590-0.474754$

O $0.964165-2.7239340 .303258$

C $-2.510495-0.231197 \quad 0.076370$

O $-3.306498-0.285830-1.032884$

$\begin{array}{lllll}\text { O } & -2.831388 & 0.223706 & 1.165436\end{array}$

C $-4.621546 \quad 0.255709-0.873415$

H $1.3871413 .539459-0.162740$

H -0.0526213 .3099160 .847745$

H 1.5345942 .9522261 .510666

H $-0.576454-1.0622281 .786159$

H $0.185798 \quad 1.481191-0.792817$

H 2.8383560 .9572850 .681966

H $1.596443-0.851661-1.447602$

H $1.774848-1.352252 \quad 1.573517$

H -1.335864 -1.915906 -0.421065

H $-0.816638-0.401178-1.191031$

H $3.0087762 .339137-1.056818$

H $3.256879-2.163607-0.777658$

H $\quad 1.823785-3.177140 \quad 0.287497$

H $-5.171425-0.303890-0.110829$

H $-4.5654091 .316277-0.610135$

H -5.1472390.158424 -1.826971

SCF Energy (B3LYP/6-31G**//MMFF) $=-803.925534933$

F1-04_c00139

MMFF Geometry

C $1.2581482 .472703 \quad 0.096255$

C $-0.122566-0.2397310 .336566$

$\begin{array}{lllll}\text { O } & 0.200713 & 0.487553 & -0.854967\end{array}$

C $1.4138231 .246489-0.800170$

C $2.6172340 .360854-0.443723$

C $2.357883-0.5686650 .748192$

C $0.989806-1.2508150 .654203$

C $-1.469189-0.9357380 .095528$

O $2.910856-0.437716-1.594426$

$\begin{array}{llll}\text { O } & 2.412626 & 0.171840 & 1.963863\end{array}$

O $1.033870-2.268216-0.345154$

$\begin{array}{llll}C & -2.627906 & 0.040271 & 0.097791\end{array}$

O $-3.788310-0.653682-0.100230$

$\begin{array}{llllll}0 & -2.535003 & 1.250451 & 0.250421\end{array}$

C $-4.9810880 .136884-0.125158$

$\begin{array}{llll}\text { H } 2.141707 & 3.115636 & 0.032142\end{array}$

H $0.382605 \quad 3.054849-0.211398$

H 1.1048362 .2137091 .146584

H -0.2291300 .4632001 .170681$ 
H $1.5579651 .619166-1.822238$ H $3.493048 \quad 0.993967-0.259949$ H $3.136932-1.3394880 .791596$ H $\quad 0.758660-1.7451681 .605024$ H $-1.656053-1.6659690 .891042$ H -1.468918 -1.440263 -0.877554 H $3.705677-0.960482-1.393085$ H 3.2949550 .5764342 .021833 H $1.169254-1.834470-1.204975$ H $-4.9399050 .858739-0.946440$ H $-5.829554-0.532855-0.289044$ H -5.1169760 .6476230 .832826$

SCF Energy (B3LYP/6-31G**//MMFF) $=-803.933567885$

F1-04_c00140

MMFF Geometry

C $2.931435-2.320186-0.219709$

C $-0.133706-0.360756-0.953568$

O $0.965367-1.275703-1.067535$

C $1.862427-1.2639530 .046294$

C 2.4812980 .1254550 .229509

C 1.4025801 .2030230 .338291

C $0.3934471 .075190-0.802729$

C $-1.111177-0.8209640 .143444$

O $3.2941690 .148476 \quad 1.400791$

O 2.0374062 .4818360 .261089

O $-0.6691781 .995914-0.583586$

C $-2.540584-0.378379-0.087014$

O $-3.263136-0.6100901 .049330$

O $-2.9779450 .089832-1.128989$

C $-4.646233-0.2480960 .983496$

H $2.466661-3.300369-0.373217$

H $3.488834-2.091282-1.134841$

H $3.637979-2.3989510 .611680$

H $-0.657303-0.418219-1.915491$

H $1.327373-1.5680200 .954286$

H $3.1361260 .361960-0.618497$

H $0.908216 \quad 1.166702 \quad 1.316013$

H $\quad 0.8909921 .374634-1.734484$

H $-0.786586-0.454197 \quad 1.123140$

H $-1.121087-1.917056 \quad 0.186975$

H $2.726989-0.0605872 .162602$

H $1.336023 \quad 3.1550110 .293378$

H -1.278919 $1.922543-1.337893$

H $-5.153867-0.831867 \quad 0.209821$

H $-5.103299-0.474367 \quad 1.950439$

H $-4.748516 \quad 0.823947 \quad 0.789190$

SCF Energy (B3LYP/6-31G**//MMFF) $=-803.933890862$

F1-04_c00141

MMFF Geometry

C -1.034461 2.844204-0.660410

C $0.328245-0.609649-0.591441$

O $\quad 0.045645 \quad 0.745179-0.964556$

C $-0.8806191 .426504-0.114756$

C $-2.2257920 .696441-0.069034$

C -2.042272 $-0.774438 \quad 0.307662$

C $-0.972931-1.425085-0.570583$

C $1.150172-0.6879250 .703189$

O

O $-3.283153-1.4619550 .133212$

O $-0.730663-2.764037-0.152073$

C $2.4422130 .082757 \quad 0.573653$

O $3.353294-0.648631-0.129489$

O $2.614011 \quad 1.2049911 .031629$

C $4.620906-0.017367-0.334772$

$\mathrm{H}-0.0593473 .341786-0.704039$

H -1.424564 2.829530-1.684208

H -1.702931 $3.446907-0.038355$

H $0.956244-1.017710-1.393831$

H -0.4616821 .5182060 .893964$

H -2.726189 $0.765238-1.043393$

H $-1.793697-0.8738741 .370460$

H -1.359526 -1.487804 -1.595716

H $1.399241-1.7236520 .960969$

H $0.609491-0.281101 \quad 1.563727$

H $-3.902717 \quad 0.8247850 .919597$

H $-3.530220-1.403591-0.805567$
H $-0.598327-2.7634320 .810876$

H $4.4956260 .906332-0.907989$

H $5.253016-0.701928-0.906715$

H 5.1031270 .1859520 .626050

SCF Energy $\left(B 3 L Y P / 6-31 G^{* *} / / M M F F\right)=-803.930038897$

F1-04 c00142

MMFF Geometry

C $0.7668772 .357767 \quad 0.847518$

C $-0.116677-0.5048530 .251943$

O $0.0112850 .593055-0.660355$

C $1.0798751 .503096-0.378540$

C $2.4318200 .777089-0.305218$

C $2.398306-0.5036200 .536473$

C $1.162016-1.3576880 .234275$

C $-1.352172-1.304295-0.170074$

O $2.8293250 .439713-1.638180$

O $2.409591-0.1494601 .916095$

O $1.340028-1.968241-1.042723$

C $-2.613667-0.5849420 .244495$

$\begin{array}{llll}\text { O } & -3.028816 & 0.245586 & -0.753871\end{array}$

O $-3.148318-0.7258971 .336939$

C $-4.2041291 .010143-0.468773$

H 1.5215833 .1393200 .979068

H -0.2119862 .8362260 .732438$

H $\quad 0.7208681 .774502 \quad 1.770382$

H -0.282049 -0.112529 1.261977

H $1.1056442 .182765-1.239617$

H 3.1937841 .4632020 .081921

H $3.304640-1.087410 \quad 0.332524$

H $1.076083-2.1594400 .977620$

H -1.372030 -2.288678 0.312076

H -1.384252 -1.472290 -1.253255

H $2.139291-0.130347-2.018753$

H $2.431010-0.9739802 .430950$

H $0.650094-2.644572-1.148349$

H $-4.4292591 .626400-1.343204$

H $-5.0524360 .345152-0.279949$

H $-4.029243 \quad 1.6674470 .388339$

SCF Energy (B3LYP/6-31G**//MMFF) $=-803.928955808$

F1-04 c00143

MMFF Geometry

C $0.8415422 .489169-0.160548$

C $-0.187107-0.369470 \quad 0.171751$

O $0.2053760 .299599-1.033152$

C $1.2875331 .226767-0.895093$

C $2.5302760 .558292-0.288693$

C $2.220749-0.2984030 .945452$

C $0.988774-1.1835160 .734237$

C $-1.390360-1.251428-0.171743$

O $3.099816-0.276344-1.301848$

O 1.9901690 .5384952 .075019

O $1.341403-2.241638-0.154787$

C $-2.624451-0.409170-0.391574$

O $-3.185655-0.0832330 .807911$

O $-3.029050-0.068422-1.495592$

C $-4.3569590 .735497 \quad 0.735772$

H $1.6323773 .245719-0.172482$

H $-0.0480892 .909860-0.641894$

H $0.5705412 .300586 \quad 0.880975$

H -0.4986780 .3798850 .908519$

H $1.5310471 .529061-1.921439$

H $3.2755491 .326189-0.050416$

H $3.082018-0.9375981 .175309$

H $0.693811-1.6350991 .689359$

H -1.605292 -1.971698 0.626311

H -1.220645 -1.824328 -1.091261

H $3.910550-0.669648-0.936622$

H $2.790106 \quad 1.0742912 .211344$

H $0.622729-2.895606-0.139654$

H -4.7052630 .9212341 .755219$

H -5.1473990 .2185090 .183322$

H $-4.119813 \quad 1.6945490 .265628$

SCF Energy $\left(B 3 L Y P / 6-31 G^{* *} / / M M F F\right)=-803.917988025$

F1-05 c00196

MMFF Geometry 
C $1.1189662 .340855-1.202171$

C $-0.148371-0.244308-0.211723$

$\begin{array}{llllll}\text { O } & 0.070606 & 0.964169 & 0.524658\end{array}$

C $1.2522891 .697007 \quad 0.175270$

$\begin{array}{llll}\text { C } & 2.497770 & 0.818244 & 0.319559\end{array}$

C $2.345195-0.503866-0.431941$

C $1.040295-1.202015-0.048464$

C $-1.443337-0.877144 \quad 0.319777$

$\begin{array}{lllll}O & 2.668448 & 0.551946 & 1.716600\end{array}$

O $3.441557-1.364616-0.129093$

O $0.849253-2.333566-0.895306$

C $-2.671215-0.068592-0.045577$

O $-3.765700-0.6402730 .539748$

O $-2.678044 \quad 0.927713-0.755532$

C $-5.012523 \quad 0.0134240 .282528$

H $0.1906842 .919154-1.261706$

H $1.9610573 .011458-1.399918$

H $1.0814281 .601733-2.007446$

H $-0.293809-0.007550-1.272950$

H 1.3123012 .5075580 .911881

H $3.3966171 .351268-0.009529$

H $2.367216-0.336767-1.515309$

H $1.101351-1.5689170 .983662$

H -1.401011 -0.9401151 .413749$

H -1.580207-1.880028 -0.099020

H $3.433019-0.0422641 .806609$

H $4.256542-0.900482-0.386639$

H $1.629837-2.905244-0.795217$

H $-5.798951-0.5363920 .806492$

H $-4.988084 \quad 1.039058 \quad 0.662700$

H $-5.2313620 .002910-0.789513$

SCF Energy (B3LYP/6-31G**//MMFF) $=-803.933942328$

F1-05 c00197

MMFF Geometry

C -2.624407-2.063192 -0.835144

C $-0.007539-0.483260-0.926827$

O $-0.422077-1.4594480 .032250$

C $-1.824981-1.481360 \quad 0.327970$

C $-2.316109-0.1000980 .771488$

C $-1.9204730 .988501-0.226183$

C $-0.4205620 .937785-0.516548$

C $1.505014-0.634837-1.149833$

O -1.7125490 .1769302 .040525$

O $-2.2505792 .272740 \quad 0.299535$

O $-0.1136251 .837734-1.579126$

C $2.350379-0.335644 \quad 0.072004$

O $3.613128-0.004118-0.330559$

O $1.968361-0.415361 \quad 1.231387$

C 4.5373180 .2890830 .722035

H $-2.597109-1.424026-1.722219$

H $-2.209465-3.032328-1.132265$

H $-3.672639-2.204648-0.553456$

H $-0.472490-0.726034-1.890989$

H $-1.926131-2.1728221 .173581$

H $-3.399626-0.1016790 .934420$

H $-2.4811040 .879058-1.162029$

H $\quad 0.132753 \quad 1.2761140 .366591$

H $1.8165830 .030301-1.963438$

H $1.732158-1.667510-1.440183$

H -1.9857241 .0729012 .301994$

H $-3.207235 \quad 2.279426 \quad 0.473677$

H $-0.4211192 .720180-1.309158$

H $\begin{array}{llll}5.497637 & 0.548477 & 0.268617\end{array}$

H $4.184822 \quad 1.1422431 .309521$

H $4.675573-0.5893411 .359461$

SCF Energy (B3LYP/6-31G**/MMFF) $=-803.927781938$

\section{F1-05 c00198}

MMFF Geometry

C $-0.690536-2.191457-1.428550$

C $0.202077 \quad 0.326134 \quad 0.037253$

O $-0.036537-0.9749730 .588317$

C $-1.087593-1.728827-0.029205$

C -2.404114 $-0.949466 \quad 0.005234$

C $-2.2389120 .459026-0.561510$

C $-1.069341 \quad 1.1863700 .104346$

C $1.3487650 .949193 \quad 0.841422$
O $-2.809985-0.8643801 .376397$

O $-3.448452 \quad 1.186898-0.354898$

O $-0.8708552 .417599-0.587553$

C $2.6393300 .196362 \quad 0.618714$

$\begin{array}{lllll}\text { O } & 3.168467 & 0.527102 & -0.594072\end{array}$

O $3.113417-0.5958121 .422531$

C $4.390943-0.135291-0.932095$

H $0.277162-2.703591-1.397536$

H $-1.437032-2.880786-1.835365$

H $-0.583617-1.359151-2.129989$

H $\quad 0.529903 \quad 0.225212-1.004532$

H $-1.198525-2.624875 \quad 0.593677$

H -3.197487 -1.490056 -0.522857

H $-2.0831040 .413191-1.645814$

H -1.3323391 .4250551 .142473$

H 1.1315270 .9171521 .916117

H $1.5237341 .997842 \quad 0.579226$

H -3.625739 $-0.335243 \quad 1.402935$

H $-3.3041252 .088717-0.689619$

H $-0.2839732 .971721-0.046698$

H $4.7054620 .214325-1.918975$

H $5.170607 \quad 0.114222-0.206050$

H $4.235395-1.217471-0.976415$

SCF Energy (B3LYP/6-31G**//MMFF) $=-803.933280525$

F1-05 c00199

MMFF Geometry

C $0.690963-2.0273041 .664026$

C $-0.155593 \quad 0.4642330 .142194$

O $-0.137688-0.886211-0.334138$

C $0.911615-1.7123300 .187109$

C $2.280748-1.091521-0.106969$

$\begin{array}{llll}\text { C } 2.352654 & 0.366758 & 0.348017\end{array}$

C $1.1662981 .174006-0.179950$

C $-1.3368031 .171620-0.530331$

O $2.511204-1.164062-1.518947$

O $3.5637620 .964496-0.110212$

O 1.1662882 .4616360 .434215

C -2.6456920 .6567350 .020133$

O $-3.168879-0.283703-0.816790$

O $-3.129151 \quad 1.0280691 .082102$

C $-4.401457-0.870722-0.388264$

H $0.789689-1.1427022 .299738$

H $-0.319902-2.4182711 .821377$

H $1.411874-2.7734772 .012825$

H -0.3297950 .4666831 .225271$

H $\quad 0.832439-2.656276-0.365762$

H $3.083830-1.6724360 .359164$

H $2.370910 \quad 0.423196 \quad 1.443011$

H $1.2685641 .328092-1.261148$

H $-1.3224801 .022835-1.616898$

H $-1.3198942 .253263-0.355577$

H $1.738196-0.786080-1.971453$

H $4.3005220 .422970 \quad 0.220983$

H 2.0258622 .8741180 .242013

H $-4.712492-1.597665-1.143324$

H $-4.261634-1.3915890 .563682$

H $-5.176366-0.103314-0.299520$

SCF Energy (B3LYP/6-31G**//MMFF) = -803.929963062

F1-05_c00200

MMFF Geometry

C $-2.394861-1.797726-1.444780$

C $0.0148590 .055157-1.124031$

O $-0.170911-1.258048-0.586682$

C $-1.514560-1.592871-0.214817$

C $-2.080328-0.568167 \quad 0.774346$

$\begin{array}{llll}C & -1.926752 & 0.859845 & 0.253122\end{array}$

C $-0.478058 \quad 1.138021-0.151703$

C $1.490563 \quad 0.202049-1.516300$

O $-1.350931-0.6849112 .002171$

O $-2.335735 \quad 1.774475 \quad 1.268687$

O $-0.4257532 .419066-0.774562$

C $2.4252190 .263960-0.330930$

$\begin{array}{llll}\text { O } & 2.620685 & -0.983988 & 0.181114\end{array}$

O 2.9152331 .3030770 .092770

C $3.472750-1.0517611 .328413$

H $-1.925717-2.510427-2.131544$ 
H $-3.377575-2.185241-1.158363$

H -2.548660 -0.873111 -2.008157

H $-0.5491040 .129249-2.062393$

H -1.434937 -2.561756 0.292799

H -3.129993 -0.7744941 .011856$

H $-2.5934581 .024882-0.601360$

H $\quad 0.1382701 .1835350 .753682$

H $1.6444521 .110948-2.109491$

H $1.807915-0.636840-2.148455$

H -1.496804 -1.583011 2.345245

H -2.1831732 .6725290 .927843$

H $0.5105442 .654522-0.887223$

H $4.475239-0.6918751 .077871$

H $3.046664-0.4695602 .151071$

H $3.542661-2.0977661 .638719$

SCF Energy $\left(\mathrm{B} 3 L Y P / 6-31 G^{* *} / / \mathrm{MMFF}\right)=-803.926393957$

F1-05_c00201

MMFF Geometry

C $1.4109512 .638803-0.720035$

C $-0.165419-0.730351-0.715189$

O $0.2568620 .591052-1.083118$

C $1.0941421 .263161-0.139045$

C $2.368267 \quad 0.452946 \quad 0.111134$

C $2.029053-0.9627010 .587884$

C $1.041301-1.626457-0.370531$

C $-1.232751-0.6922970 .390266$

O $3.1836151 .107991 \quad 1.076044$

O $1.454895-0.9233231 .892048$

O $1.748392-1.924222-1.578234$

C $-2.534063-0.081057-0.088385$

O $-3.443907-0.1294340 .930430$

O $-2.7327240 .381820-1.202949$

$\begin{array}{lllll}\text { C } & -4.731609 & 0.418766 & 0.631517\end{array}$

H $1.9398392 .548288-1.675358$

H $0.4843913 .186072-0.925760$

H $2.0205953 .236679-0.035977$

H $-0.634481-1.137035-1.620349$

H $\quad 0.5483811 .4302750 .796250$

H $2.9414940 .384472-0.822145$

H $2.950683-1.5523650 .660030$

H $0.692385-2.5788770 .045434$

H $-0.903494-0.1089741 .253963$

H -1.455314 -1.715548 0.714086

H $3.985606 \quad 0.5708421 .194523$

H $2.074244-0.4431782 .467915$

H $1.137972-2.401302-2.165728$

H -5.196788 $-0.133507-0.190733$

H $-5.359023 \quad 0.3175691 .521010$

H $-4.642801 \quad 1.4806490 .383381$

SCF Energy $\left(B 3 L Y P / 6-31 G^{* *} / / M M F F\right)=-803.929439193$

F1-05_c00202

MMFF Geometry

C $-2.848643-1.790437-0.734522$

C $-0.049587-0.597127-0.933339$

O $-0.561292-1.4780190 .070911$

C $-1.947010-1.3052920 .397399$

C $-2.230456 \quad 0.1390410 .823792$

C $-1.710007 \quad 1.149518-0.200847$

C $-0.2455590 .877072-0.548029$

C $1.417834-0.961289-1.190223$

O $-1.593862 \quad 0.3663182 .086784$

$\begin{array}{llll}0 & -1.830770 & 2.474213 & 0.313590\end{array}$

O $0.1544321 .717904-1.628332$

C $2.305879-0.6985740 .004977$

O $3.1543850 .334853-0.262870$

O $2.242204-1.324676 \quad 1.055028$

C $4.0347730 .700021 \quad 0.804391$

H -2.765297 -1.169260-1.630954

H -2.572801 -2.808702 -1.029019

H -3.897624 -1.790091 -0.421812

H - $0.575252-0.800486-1.874821$

H -2.120089 -1.9612831 .259194$

H -3.3012350 .2941190 .994916$

H $-2.3161801 .111976-1.113546$

H $\quad 0.3790831 .1488930 .310036$

H $1.801274-0.414480-2.059618$
H $1.513398-2.028828-1.423027$

H -0.6664490 .0835382 .013930$

H -2.7641302 .6131250 .548332$

H $-0.3986331 .500922-2.398012$

H 4.6550581 .5331590 .463491

H 3.4584521 .0252081 .675816

H $4.686304-0.1401511 .062603$

SCF Energy (B3LYP/6-31G**//MMFF) $=-803.921354776$

F1-05_c00203

MMFF Geometry

C -2.309250 -2.065288 -1.211653

C $0.071102-0.6222800 .034513$

O $-0.898335-1.4504800 .688552$

C $-2.227825-1.3901250 .154808$

C -2.7489790 .0491250 .154069$

C $-1.7681201 .003842-0.525509$

C -0.3637910 .8521290 .060404$

C $1.402985-0.8462490 .766101$

O $-2.9189360 .438772 \quad 1.521936$

O $-2.2033692 .351218-0.352190$

O $0.5469821 .635016-0.706541$

C $2.611195-0.433801-0.043250$

$\begin{array}{lllll}\text { O } & 3.582467 & 0.030981 & 0.795535\end{array}$

O $2.703671-0.537908-1.259102$

C 4.7955290 .4473390 .160801

H -1.885648 -3.074120 -1.161637

H -3.348778 -2.140090 -1.545895

H -1.747326 -1.525549 -1.979183

H $\quad 0.186320-0.961564-1.001684$

H $-2.839427-1.9798230 .848788$

H -3.737852 $0.109907-0.313666$

H $-1.7364340 .820771-1.606052$

H -0.3471351 .2405541 .086200$

H $1.528284-1.9083371 .009944$

H $1.391098-0.3069251 .721296$

H -3.2072741 .3676441 .524725$

H -3.093259 $2.422130-0.737966$

H $\quad 0.2234152 .552136-0.694402$

H $5.261603-0.397138-0.355829$

H $4.5964461 .266132-0.537210$

H 5.4790590 .8053240 .935358

SCF Energy (B3LYP/6-31G**//MMFF) $=-803.931965777$

F1-05_c00204

MMFF Geometry

C $1.8616332 .250276-1.197352$

C -0.1523840 .4988740 .278114$

O $\quad 0.830422 \quad 1.3769380 .840264$

C $2.058861 \quad 1.4863730 .109199$

C $2.7025490 .110561-0.080140$

C $1.720751-0.891843-0.683132$

C $0.411967-0.9214780 .108961$

C -1.3588710 .5370821 .224893$

O $3.113623-0.3464261 .213672$

O $2.320356-2.186094-0.685228$

O $-0.521626-1.752286-0.577174$

C $-2.621295-0.0278090 .623853$

$\begin{array}{llll}\text { O } & -3.045530 & 0.759527 & -0.404952\end{array}$

O $-3.172190-1.0426001 .031122$

C $-4.2390670 .322270-1.061812$

H $1.3640333 .206908-1.005174$

H $2.8237852 .448718-1.679860$

H $1.2338661 .706595-1.909040$

H $-0.4670970 .894038-0.695083$

H $2.7156542 .091186 \quad 0.746563$

H $3.6099270 .179625-0.690431$

H $1.520735-0.638348-1.730800$

H $\quad 0.591229-1.3832291 .087319$

H -1.570975 1.5670551 .539367

H - $-1.136159-0.0056172 .151988$

H $3.484298-1.2385121 .099971$

H $1.658730-2.811533-1.027322$

H $-0.703894-1.347479-1.442429$

H $-5.0768640 .312995-0.358204$

H $-4.4633841 .028906-1.865201$

H -4.089189-0.669616-1.498816

SCF Energy (B3LYP/6-31G**//MMFF) $=-803.932619737$ 
F1-06_c00255

MMFF Geometry

C $1.6315182 .608366-0.715418$

C $-0.229376-0.458196 \quad 0.197619$

O $0.150754 \quad 0.757601-0.464195$

C $1.4044131 .275779-0.008886$

C $2.5221450 .273016-0.315983$

C $2.217703-1.0901270 .316847$

C $0.814624-1.559082-0.073079$

C $-1.617918-0.851421-0.322459$

$\begin{array}{lllll}\text { O } & 3.767537 & 0.765068 & 0.164301\end{array}$

O $2.277449-0.993631 \quad 1.740129$

O $0.799054-1.900519-1.457615$

C $-2.708518 \quad 0.056611 \quad 0.207344$

O $-3.911335-0.366690-0.284506$

$\begin{array}{lllll}\text { O } & -2.536281 & 1.011717 & 0.951874\end{array}$

C $-5.048103 \quad 0.3985770 .128012$

H $0.7986313 .291186-0.514432$

H $2.5583753 .087570-0.386913$

H $1.6706182 .473895-1.802132$

H $-0.284240-0.260671 \quad 1.276210$

H 1.3445031 .4774301 .068469

H $2.6058990 .147067-1.402909$

H $2.975386-1.8190480 .006967$

H $0.540752-2.4547580 .497455$

H -1.858028 -1.871394 -0.001867

H $-1.645732-0.791066-1.416743$

H $4.4481730 .107931-0.059915$

H $3.151305-0.6340581 .969997$

H $1.445295-2.614905-1.590227$

H -4.952089 $1.433491-0.214054$

H $-5.938199-0.041054-0.329744$

H $-5.1569920 .358875 \quad 1.215956$

SCF Energy (B3LYP/6-31G $\left.{ }^{\star *} / / \mathrm{MMFF}\right)=-803.934257656$

F1-06_c00256

MMFF Geometry

C $1.1735482 .730215-0.383376$

C $-0.231655-0.662766 \quad 0.083413$

O $-0.0280460 .670956-0.406588$

C 1.1332681 .2997490 .144269

C $2.3874260 .512830-0.254298$

C $2.278204-0.9518870 .185102$

C $0.960909-1.559458-0.304025$

C $-1.551617-1.169766-0.499963$

$\begin{array}{lllll}\text { O } & 3.555351 & 1.101532 & 0.305829\end{array}$

O $2.325268-1.0160231 .610985$

O $1.041263-1.709575-1.719924$

C $-2.722815-0.5305360 .207202$

O $-3.1046090 .602038-0.448822$

O $-3.219297-0.972740 \quad 1.235245$

C $-4.193368 \quad 1.3178390 .142295$

H $0.2494673 .257296-0.121464$

H 2.0192863 .2889550 .028174

H $1.2416672 .742697-1.476785$

H $-0.315012-0.6144241 .177277$

H $\quad 1.033405 \quad 1.3515841 .236087$

H $2.4989180 .539446-1.345293$

H $3.141756-1.508219-0.197556$

H $0.825622-2.5555360 .133967$

H -1.652793 -2.253771 -0.371240

H $-1.633539-0.961200-1.573322$

H $3.4763281 .047388 \quad 1.273700$

H $2.281832-1.9544001 .862615$

H $\quad 0.282579-2.245320-2.005882$

H -3.924434 1.6523831 .148789

H $-4.3986252 .195869-0.475959$

H -5.0892850 .6904200 .169374$

SCF Energy (B3LYP/6-31G**//MMFF) $=-803.932991472$

F1-06_c00257

MMFF Geometry

C $1.6304802 .638928-0.364269$

C $-0.321198-0.489591-0.086694$

O $0.1562850 .780626-0.553675$

C $1.316678 \quad 1.237836 \quad 0.150345$

C $2.4786010 .262133-0.078393$
C $2.082540-1.1596900 .339225$

C $0.755723-1.569011-0.303358$

C $-1.606700-0.808327-0.852118$

$\begin{array}{lllll}\text { O } & 3.633484 & 0.650497 & 0.656498\end{array}$

O $1.924550-1.2226491 .757766$

O $0.957719-1.802530-1.695734$

C $-2.7400420 .072209-0.383412$

O $-3.283311-0.4366720 .759326$

O $-3.0876261 .097425-0.955062$

C $-4.361193 \quad 0.321217 \quad 1.317477$

H 2.4626613 .0950010 .179269

H $1.8754682 .618240-1.431927$

H $0.7539923 .287611-0.256857$

H $-0.543896-0.3973550 .984419$

H 1.0825001 .3225241 .219323

H $2.7462840 .247009-1.142228$

H $2.880646-1.8600860 .067368$

H $0.406802-2.5109580 .135556$

H -1.907462 -1.854118 - 0.719761

H $-1.486457-0.638955-1.928866$

H 3.9741341 .4711660 .262981

H $2.757565-0.9149682 .154710$

H $1.094234-0.943123-2.129274$

H $-5.193417 \quad 0.3747280 .609089$

H $-4.702794-0.1888532 .222156$

$\mathrm{H}-4.0184031 .3243211 .588276$

SCF Energy $(B 3 L Y P / 6-31 G * * / / M M F F)=-803.937600126$

F1-06 c00258

MMFF Geometry

C $-3.0425842 .191773-0.178934$

C $0.1960100 .398578-0.273320$

O $-0.7999481 .400252-0.013728$

C $-2.1134461 .006826-0.423590$

C $-2.555111-0.2314990 .363640$

C $-1.549396-1.3750960 .186364$

C $-0.131266-0.8945330 .502732$

C 1.5475351 .0128340 .110989

O $-3.846164-0.648903-0.064182$

O $-1.572427-1.843481-1.162122$

O $-\begin{array}{llll}0.020027 & -0.654004 & 1.903891\end{array}$

C $2.7275920 .180958-0.335638$

$\begin{array}{lllll}\text { O } & 3.834749 & 0.563144 & 0.366502\end{array}$

O $2.693749-0.674726-1.209487$

C $5.042062-0.1313900 .037770$

H $-2.6822873 .074388-0.718856$

H $-4.0642841 .979165-0.506872$

H -3.0624002 .4610750 .882971$

H $0.1928960 .193853-1.351960$

H -2.111661 $0.804447-1.502471$

H -2.6185070 .0212951 .429644$

H -1.830402 -2.2172430 .829217$

H $\quad 0.595007-1.6711460 .237410$

H 1.5971011 .1688011 .194904

H $1.6463632 .001253-0.354489$

H $-4.092633-1.4266290 .464927$

H -2.490964 -2.086972 -1.368903

H $-0.203942-1.4925842 .360531$

H $4.928599-1.2020620 .232861$

H $5.307240 \quad 0.045392-1.008988$

H 5.8420380 .2579910 .672993

SCF Energy $\left(B 3 L Y P / 6-31 G^{* *} / / M M F F\right)=-803.935740113$

F1-06 c00259

MMFF Geometry

C -3.070465 2.074157-0.281342

C $0.2626790 .481601-0.021324$

O $-0.8220091 .406346 \quad 0.148852$

C $-2.0387370 .965599-0.461947$

C $-2.499185-0.3467330 .183639$

C $-1.407047-1.4172740 .086548$

C $-0.080180-0.8755200 .625813$

C $1.502431 \quad 1.1438050 .583919$

O $-3.695819-0.819121-0.424041$

O $-1.240041-1.791699-1.281333$

O $-0.176230-0.7205142 .040004$

C $2.7602190 .353104 \quad 0.331911$

O $3.1276330 .464132-0.976347$ 
O $3.343148-0.2910291 .194437$

C $4.307966-0.255217-1.346247$

H $-2.7013143 .010572-0.714003$

H $-4.0213391 .823190-0.760373$

H $-3.252247 \quad 2.270524 \quad 0.781077$

H $\quad 0.4235150 .343598-1.098575$

H $-1.8762530 .836399-1.539839$

H -2.718039 -0.1706501 .243963$

H $-1.728415-2.3109980 .633736$

H $\quad 0.728026-1.5869220 .420516$

H 1.3881391 .2948061 .664043

H 1.6388292 .1529100 .174520

H $-3.496798-1.007635-1.357210$

H $-0.559870-2.485900-1.313189$

H $-0.368052-1.5954392 .418518$

H $4.490191-0.085517-2.410711$

H $5.1688940 .112430-0.779756$

H $4.165172-1.327417-1.181106$

SCF Energy (B3LYP/6-31G**//MMFF) $=-803.933685715$

F1-06 c00260

MMFF Geometry

C $2.2109502 .473807-0.242484$

C $-0.094722-0.0954851 .088633$

$\begin{array}{lllll}\text { O } & 0.403300 & 1.123737 & 0.525137\end{array}$

$\begin{array}{llll}\text { C } & 1.815072 & 1.100372 & 0.290673\end{array}$

C $2.166977-0.015338-0.700315$

C $1.663895-1.373726-0.195492$

C $0.175797-1.2926490 .154280$

C $-1.5693790 .098008 \quad 1.459343$

O $3.574749-0.063995-0.901449$

O $2.382000-1.7582850 .976770$

O $-0.570139-1.162871-1.051988$

C $-2.464912 \quad 0.4727150 .299616$

O $-3.432746-0.4744450 .135435$

O $-2.3254791 .482846-0.377956$

C $-4.343449-0.242121-0.943642$

H $1.6942892 .691998-1.183766$

H $1.914645 \quad 3.255695 \quad 0.465311$

H $3.2897892 .548156-0.408062$

H $\quad 0.442058-0.2617132 .032644$

H $2.3372160 .952552 \quad 1.244738$

H $1.6957050 .199057-1.668016$

H $1.846848-2.141427-0.956148$

H $-0.149307-2.2120950 .655299$

H -1.6680060 .9091942 .191379$

H -1.949559 -0.8101881 .941771$

H $3.754381-0.771482-1.543910$

H $3.328749-1.7421850 .755044$

H $-0.398048-1.954026-1.590704$

H $-5.057472-1.069509-0.969805$

H $-4.8922420 .690464-0.781883$

H -3.803909 -0.213899-1.895153

SCF Energy (B3LYP/6-31G*//MMFF) $=-803.928855651$

F1-06 c00261

MMFF Geometry

C $1.1505072 .718215-0.352886$

C $-0.242749-0.675628 \quad 0.114113$

O $-0.0344330 .656385-0.376216$

$\begin{array}{llll}\text { C } & 1.126532 & 1.286542 & 0.174597\end{array}$

C $2.3822020 .500910-0.225975$

C $2.275957-0.9720670 .191583$

C $0.946586-1.574970-0.271190$

C $-1.556197-1.184912-0.483635$

O 3.5534221 .0619360 .353806

O $2.395867-1.1097301 .607563$

O $0.974914-1.732623-1.689473$

C $-2.735399-0.532230 \quad 0.197460$

O $-3.1148340 .582466-0.489812$

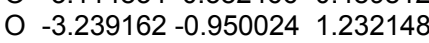

C $-4.210822 \quad 1.3092610 .073897$

H $1.969063 \quad 3.2981740 .082516$

H $1.2492742 .732186-1.443935$

H $0.2079213 .224971-0.117753$

H $-0.337957-0.626813 \quad 1.206486$

H 1.0267511 .3418091 .265651

H $2.4970450 .532595-1.317292$
H $\quad 3.119347-1.522118-0.241767$

H $\quad 0.802428-2.5665290 .174166$

H $-1.663566-2.267065-0.343953$

H -1.619508 -0.989984-1.560913

H $3.706961 \quad 1.926467-0.062618$

H $1.696711-0.5895792 .034982$

H $1.709821-2.332634-1.902689$

H -5.1044130 .6789230 .111871$

H -3.9505071 .6723011 .072752$

H $-4.4152742 .169296-0.569411$

SCF Energy $(B 3 L Y P / 6-31 G * * / / M M F F)=-803.925473273$

F1-06_c00262

MMFF Geometry

C 1.6422572 .6453710 .304616

C $-0.111555-0.5369021 .004785$

$\begin{array}{lllll}\text { O } & 0.136170 & 0.846480 & 0.726884\end{array}$

C 1.5225401 .1414420 .527894

C $2.0653620 .347110-0.667030$

C $1.832304-1.157162-0.481364$

C $0.358355-1.428009-0.162378$

C -1.585053 -0.710784 1.390746

O $3.4525390 .601862-0.856162$

O $2.643914-1.6279990 .594575$

O $-0.413397-1.168443-1.330746$

C $-2.586214-0.3686170 .312128$

$\begin{array}{lllll}0 & -2.619718 & 0.977929 & 0.104972\end{array}$

O $-3.268136-1.204139-0.268408$

C $-3.5275521 .422979-0.907288$

H 2.6845212 .9546620 .184518

H $1.0782672 .955583-0.582088$

H 1.2148583 .1904561 .153469

H $0.471014-0.7978801 .898891$

H $2.0775570 .897452 \quad 1.442973$

H $1.5468750 .667910-1.578988$

H $2.151185-1.685482-1.387249$

H $\quad 0.211649-2.4788830 .113329$

H $-1.823934-0.0832892 .258774$

H -1.767282 -1.747980 1.696962

H $3.9198560 .287802-0.063110$

H $2.491385-2.5848070 .678298$

H $-0.105857-1.773501-2.027083$

H $-3.2617720 .984475-1.874019$

H $-4.5551421 .164751-0.634464$

$\mathrm{H}-3.4468142 .510646-0.981776$

SCF Energy (B3LYP/6-31G**//MMFF $)=-803.929698704$

F1-07_c00295

MMFF Geometry

C $1.3531032 .983468-0.096451$

C $-0.177713-0.378570-0.265302$

$\begin{array}{lllll}\text { O } & 0.097656 & 0.970816 & 0.131062\end{array}$

C $1.2560821 .519644-0.509953$

C $2.5008620 .717253-0.115940$

C $2.302895-0.756624-0.473097$

C $0.996500-1.2938150 .115349$

C -1.471695 -0.814484 0.435554

O $2.7111290 .844725 \quad 1.292152$

O $3.393027-1.530714 \quad 0.021319$

O $0.756126-2.602852-0.396348$

C $-2.688513-0.086711-0.097946$

O $-3.779881-0.4555180 .637050$

O $-2.6902240 .695915-1.038136$

C -5.0165630 .1507430 .248319$

H $2.2346363 .461941-0.533507$

H $0.4621223 .528200-0.427751$

H 1.3933763 .0922470 .992689

H $-0.336732-0.407224-1.351954$

H $1.1088821 .475270-1.596631$

H $3.3938151 .114962-0.610936$

H $2.290093-0.882631-1.562962$

H $1.083770-1.3756761 .205905$

H -1.400576 -0.599076 1.508231

H -1.645291 -1.8858660 .288286$

H 3.4708820 .2835631 .523732

H $4.207192-1.172212-0.371330$

H $1.528239-3.148885-0.168674$

H $-5.801306-0.2219520 .911919$ 
H $-5.266456-0.125182-0.780609$ H -4.9550521 .2381280 .353597$

SCF Energy (B3LYP/6-31G**//MMFF) $=-803.937497425$

F1-07_c00296

MMFF Geometry

C $-2.321631-2.654667-0.168586$

C $0.0524960 .001402-1.157951$

O $-0.474112-1.238060-0.674622$

C $-1.906008-1.256734-0.611691$

C $-2.409870-0.1683450 .342937$

C $-1.8902991 .199154-0.104592$

C $-0.3687811 .178603-0.265112$

C $1.573719-0.137148-1.309305$

O $-1.939690-0.451773 \quad 1.662595$

O $-2.2469522 .194790 \quad 0.851359$

O $\quad 0.0605072 .398073-0.865893$

C $2.312062-0.364223-0.005455$

O $3.6077050 .043710-0.149878$

O $1.829515-0.8666571 .000116$

C $4.438648-0.1273661 .002647$

H -1.977101 -3.398447 -0.895668

H -1.864590 -2.925445 0.789263

H $-3.408879-2.735759-0.078296$

H $-0.3413420 .162661-2.171424$

H $-2.301272-1.081264-1.620504$

H -3.504701 -0.1656360 .386483$

H $-2.3547351 .488399-1.055521$

H $\quad 0.099970 \quad 1.1183110 .723420$

H $1.9707810 .766820-1.785350$

H $1.803503-0.995900-1.951050$

H -2.222725 0.2815452 .235212

H -3.2159092 .1874940 .932791$

H $-0.2544553 .125320-0.301928$

H $4.041846 \quad 0.447093 \quad 1.845153$

H $4.515339-1.1882291 .259478$

H 5.4364650 .2483230 .761183

SCF Energy (B3LYP/6-31G**//MMFF) $=-803.931978196$

F1-07_c00297

MMFF Geometry

C $1.3046132 .944646-0.393521$

C $-0.266089-0.3780200 .004450$

$\begin{array}{llllll}\text { O } & 0.093271 & 0.990655 & 0.230454\end{array}$

C $1.1302001 .452020-0.645078$

C $2.4151970 .650666-0.401855$

C $2.134508-0.840539-0.594723$

C $0.941213-1.2948730 .250018$

C -1.420514 -0.7170170 .951366$

$\begin{array}{lllll}\text { O } & 2.877042 & 0.855133 & 0.935228\end{array}$

O $3.290267-1.602693-0.254610$

O $0.595758-2.631238-0.107326$

C -2.6781040 .0177340 .553364$

O $-3.282307-0.623238-0.487811$

O -3.0673941 .0446401 .094273$

C $-4.483369-0.013538-0.970709$

H $1.497740 \quad 3.1564820 .663499$

H $2.1198703 .357166-0.995384$

H $0.3819123 .477994-0.647924$

H -0.614086 -0.485737 -1.031888

H $0.7981601 .315765-1.682445$

H $3.2194680 .967999-1.075076$

H $1.929622-1.044755-1.652687$

H $1.213051-1.2965361 .312802$

H -1.181063 -0.4244941 .980960$

H $-1.643238-1.7899480 .965828$

H 3.2284251 .7596730 .991090

H $3.518127-1.3958290 .668121$

H $1.388799-3.1796430 .020296$

H -4.864450 -0.620535 -1.796189

H $-4.2732540 .993518-1.343488$

H $-5.2381250 .016325-0.179050$

SCF Energy (B3LYP/6-31G**//MMFF) $=-803.937478688$

F1-07 c00298

MMFF Geometry

C $0.7791002 .983821-0.456281$

C $-0.159379-0.580527-0.163963$
O $-\begin{array}{llll}0.104146 & 0.832745 & 0.067092\end{array}$

C $0.9247981 .484691-0.688471$

C $2.2980430 .956151-0.257365$

C $2.349526-0.563713-0.424571$

C $1.160746-1.2421860 .261983$

C $-1.339997-1.1378020 .634795$

O 2.5472151 .3032751 .106988

O $3.574145-1.0571930 .111537$

O $1.148570-2.626372-0.081840$

C $-2.648307-0.7003110 .020523$

$\begin{array}{lllll}\text { O } & -3.133595 & 0.390634 & 0.678699\end{array}$

O $-3.161067-1.247993-0.947091$

C -4.3618150 .9197590 .169800$

H $1.5541633 .544962-0.987008$

H $-0.2003343 .325892-0.808467$

H $\quad 0.827076 \quad 3.2327880 .609269$

H $-0.342434-0.756696-1.232784$

H $\quad 0.760624 \quad 1.280294-1.754302$

H $3.0966351 .421585-0.845178$

H $2.348639-0.811368-1.493455$

H $1.286817-1.1863891 .349781$

H -1.306177 -0.8046751 .679233$

H -1.343455 -2.233513 0.648380

H 1.7793951 .0253181 .634407

H $3.559316-2.0259060 .025946$

H $1.020871-2.695044-1.043369$

H -4.229896 $1.254995-0.863287$

H -4.6419821 .7793930 .784308$

H -5.1545120 .1683030 .234751$

SCF Energy $\left(B 3 L Y P / 6-31 G^{* *} / / M M F F\right)=-803.934258213$

F1-07 c00299

MMFF Geometry

C $1.7733452 .800224-0.563859$

C $-0.076870-0.339486-1.194009$

O $0.2116701 .028710-0.884663$

C $1.6153661 .300430-0.783901$

C 2.2273630 .4750230 .354021

C $1.956031-1.0131780 .125482$

C $0.465886-1.279495-0.104322$

C $-1.585387-0.469003-1.432274$

O $1.671378 \quad 0.883831 \quad 1.606253$

O $2.419730-1.7541631 .251273$

O $0.310848-2.638844-0.503838$

C $-2.408299-0.296981-0.176989$

O $-2.499872 \quad 1.0233090 .149338$

O $-2.902074-1.2300240 .443312$

C $-3.236789 \quad 1.313121 \quad 1.340919$

H $2.8261583 .076402-0.452662$

H $1.3574753 .351571-1.414393$

H 1.2224123 .1373840 .320719

H $\quad 0.406916-0.579243-2.151114$

H $2.091851 \quad 1.036903-1.736803$

H 3.3067200 .6488430 .423999

H $2.530995-1.361166-0.741921$

H $-0.065983-1.1518490 .845550$

H -1.826902 -1.449869 -1.857725

H $-1.9275450 .276980-2.160330$

H $\quad 0.7029110 .8843391 .521146$

H $2.203427-2.6889491 .092092$

H $-0.639973-2.840640-0.502536$

H $-2.762097 \quad 0.837477 \quad 2.204461$

H -4.2734020 .9791821 .236169$

H -3.2302942 .3961541 .489529$

SCF Energy $\left(B 3 L Y P / 6-31 G^{* *} / / M M F F\right)=-803.934751804$

F1-07 c00300

MMFF Geometry

C -2.645427 -2.357399 0.045712

C $0.041189-0.152526-1.211686$

O $-0.635282-1.264628-0.616747$

C $-2.048402-1.060070-0.486246$

C $-2.329070 \quad 0.1433890 .423481$

C $-1.6320241 .386703-0.134223$

C $-0.1424381 .120884-0.371440$

C $1.509535-0.533101-1.425787$

O $-1.827052-0.1061881 .738238$

O $-1.797712 \quad 2.481596 \quad 0.763446$ 
O $\quad 0.442347 \quad 2.235592-1.039970$

C $2.251678-0.759571-0.128415$

$\begin{array}{lllll}\text { O } & 3.134478 & 0.257021 & 0.087474\end{array}$

O $2.057227-1.7149290 .612118$

C $3.884527 \quad 0.172279 \quad 1.302972$

H -2.161714 -2.6728880 .976277$

H $-3.721396-2.259470 \quad 0.217570$

H -2.482008 -3.168085 -0.673219

H $-0.385066 \quad 0.007240-2.211867$

H -2.466734 - $0.874616-1.484019$

H -3.4045190 .3318560 .517752$

H $-2.1066401 .684597-1.076842$

H $0.361013 \quad 1.0291160 .597525$

H $2.0247770 .235274-2.014003$

H $1.584514-1.466969-1.996276$

H -2.401522 -0.7718952 .152487$

H -1.4196562 .2194901 .620487$

H - $0.0034382 .338221-1.898052$

H $4.493651-0.7366731 .309954$

H 4.5479691 .0396301 .354269

H 3.2113110 .1939302 .165286

SCF Energy (B3LYP/6-31G*//MMFF)= -803.928221326

F1-07 c00301

MMFF Geometry

C $-3.153614-2.277647-0.151903$

C $0.112837-0.542783-0.243122$

O $-0.934719-1.4514440 .122005$

C $-2.176884-1.172898-0.537056$

C $-2.6816280 .217851-0.137930$

C $-1.6208301 .269590-0.465513$

C -0.2685380 .8955620 .146162$

C $1.385549-1.0222660 .467722$

O $-2.951307 \quad 0.230680 \quad 1.265640$

O $-2.024723 \quad 2.542608 \quad 0.033115$

O $0.7235751 .801701-0.327240$

C $2.657572-0.490729-0.152490$

O $3.608629-0.3479700 .816328$

O $2.809068-0.261373-1.345022$

$\begin{array}{llll}\text { C } & 4.877268 & 0.137562 & 0.365927\end{array}$

H $-3.270299-2.3509050 .934697$

H $-4.136487-2.114153-0.603882$

H -2.774459 -3.249328 -0.487188

H $0.265272-0.609992-1.328511$

H $-2.014913-1.216355-1.621770$

H $-3.6231490 .451723-0.647459$

H $-1.512813 \quad 1.371901-1.552579$

H $-0.3152960 .999094 \quad 1.237365$

H $1.447057-2.1170150 .437226$

H $1.341367-0.7406051 .526818$

H -3.2155761 .1362711 .501910$

H $-2.8807282 .757723-0.375219$

H $0.4325212 .699623-0.092359$

H $5.536237 \quad 0.222640 \quad 1.234033$

H $5.321175-0.566512-0.344355$

H $4.7654931 .126816-0.088117$

SCF Energy (B3LYP/6-31G**//MMFF) $=-803.935735642$

F1-07 c00302

MMFF Geometry

C -3.002997-2.345031-0.271241

C $0.193888-0.505578 \quad 0.025100$

O $-0.877509-1.4223790 .285202$

C $-2.005622-1.233903-0.578452$

C $-2.6019990 .160993-0.363428$

C $-1.531516 \quad 1.224932-0.603848$

$\begin{array}{llll}C & -0.278108 & 0.944748 & 0.230576\end{array}$

C $1.339427-0.8889480 .968303$

$\begin{array}{lllll}\text { O } & -3.084510 & 0.259802 & 0.978437\end{array}$

O $-2.062904 \quad 2.506332-0.276119$

O $0.743261 \quad 1.863139-0.150008$

C $2.666823-0.2832380 .587254$

O $3.093667-0.795756-0.601858$

O $3.260545 \quad 0.5383351 .273857$

C $4.347815-0.293187-1.073038$

H -3.288691 -2.348703 0.786137

H -3.905645 -2.248310 -0.882098

H $-2.551233-3.322861-0.472031$
H $\quad 0.525981-0.646495-1.011944$

H $-1.672825-1.344076-1.618354$

H $-3.4596470 .325733-1.025303$

H $-1.272141 \quad 1.251022-1.669527$

H $-0.498923 \quad 1.131047 \quad 1.288435$

H $1.466378-1.9787260 .999840$

H $1.101670-0.5976291 .998843$

H $-3.406673 \quad 1.168972 \quad 1.103222$

H $-1.3485773 .155575-0.394349$

H $0.9625011 .697844-1.082943$

H $5.144787-0.540351-0.365365$

H $4.567826-0.772132-2.030792$

H $4.2885340 .788573-1.227224$

SCF Energy (B3LYP/6-31G**//MMFF) $=-803.936506153$

F1-08 c00322

MMFF Geometry

C $0.9138142 .983861-0.105474$

C $-0.076225-0.485725 \quad 0.789434$

$\begin{array}{lllll}\text { O } & 0.156749 & 0.928826 & 0.827010\end{array}$

C $0.783938 \quad 1.481717-0.337530$

C $2.139087 \quad 0.813187-0.592864$

C $1.970655-0.703463-0.687406$

C $1.242813-1.239708 \quad 0.545443$

C $-1.202116-0.853750-0.194861$

$\begin{array}{lllll}\text { O } & 3.023518 & 1.129371 & 0.485692\end{array}$

O $3.257654-1.307503-0.798062$

O $1.010899-2.6349530 .381998$

C $-2.520648-0.202540 \quad 0.168899$

O $-3.411644-0.412221-0.845735$

$\begin{array}{llll}0 & -2.750188 & 0.397033 & 1.210072\end{array}$

C $-4.7154810 .142019-0.643560$

H $-0.078015 \quad 3.431908 \quad 0.022225$

H 1.4708663 .2030830 .811759

H $1.4097793 .474740-0.948155$

H $-0.426859-0.7614901 .792406$

H $\quad 0.1285301 .334819-1.202841$

H $2.5999841 .204845-1.506623$

H $1.419515-0.964539-1.598426$

H $1.898023-1.1253301 .418315$

H $-1.368768-1.936488-0.189873$

H $-0.947813-0.545920-1.213500$

H 3.8575920 .6563410 .322860

H $3.124828-2.270901-0.811190$

H $\quad 0.562915-2.951812 \quad 1.184910$

H $-5.322807-0.088024-1.522947$

H $-5.186032-0.308247 \quad 0.235709$

H $-4.6526571 .228999-0.534923$

SCF Energy (B3LYP/6-31G**//MMFF) $=-803.932946879$

F1-08 c00323

MMFF Geometry

C $-1.392902-2.2651350 .250058$

C 0.1984880 .3601750 .213265

O $-0.133608-0.502479-0.884745$

C $-1.408426-1.144804-0.789044$

C $-2.527141-0.105082-0.635154$

$\begin{array}{llll}C & -2.259003 & 0.904831 & 0.484657\end{array}$

$\begin{array}{llll}C & -0.848168 & 1.481065 & 0.355955\end{array}$

C $1.5989420 .926133-0.060506$

O $-3.792023-0.721861-0.434356$

$\begin{array}{lllll}0 & -2.385473 & 0.290781 & 1.764334\end{array}$

$\begin{array}{lllll}0 & -0.789062 & 2.333303 & -0.786704\end{array}$

C $2.684326-0.1157420 .118293$

O $3.8970620 .466061-0.123311$

O $2.500442-1.2850840 .426982$

C $5.030323-0.3984330 .004813$

H $-1.242167-1.8965251 .267281$

H $-2.324377-2.8389060 .229691$

H $-0.563048-2.9509120 .046066$

H $0.227703-0.2319881 .135298$

H - $-1.563352-1.643536-1.754440$

H -2.588626 $0.461097-1.573911$

H $-3.009011 \quad 1.7032280 .438353$

H -0.6030142 .0850541 .237662$

H 1.8118091 .7423110 .639164

H $1.6662391 .294790-1.090660$

H -3.939466 -1.335096 -1.174467 
H $-3.265508-0.1214151 .801608$

H $-1.4362023 .046647-0.653598$

H $4.962940-1.220302-0.714398$

H $5.9291270 .184826-0.212089$

H $5.099601-0.7830901 .026828$

SCF Energy (B3LYP/6-31G**//MMFF) $=-803.930409764$

F1-08_c00324

MMFF Geometry

C $0.8595772 .984027-0.159706$

C $-0.066903-0.4861120 .799630$

$\begin{array}{lllll}\text { O } & 0.150658 & 0.931481 & 0.817393\end{array}$

C $0.7563641 .475570-0.362199$

C $2.1221530 .825809-0.611012$

C $1.984522-0.697278-0.674255$

C $1.258713-1.2311590 .562673$

C -1.194132 -0.880239-0.173591

$\begin{array}{llll}\text { O } & 3.031523 & 1.186976 & 0.432371\end{array}$

O $3.283991-1.274528-0.775317$

O $1.012467-2.627175 \quad 0.428531$

C $-2.514450-0.2269030 .179298$

O $-3.404851-0.455486-0.831770$

O $-2.7459450 .388649 \quad 1.210668$

C $-4.7103880 .097860-0.638311$

H $1.418467 \quad 3.2313280 .749230$

H $1.3407573 .467623-1.015074$

H $-0.1395263 .415849-0.033682$

H $-0.410965-0.7519861 .807646$

H $0.0985311 .299368-1.220040$

H $2.5632921 .197090-1.542616$

H $1.439539-0.981838-1.582423$

H $1.902224-1.1057731 .442332$

H -1.356492 -1.963259-0.144858

H $-0.943171-0.594454-1.199564$

H $2.594075 \quad 1.023841 \quad 1.285017$

H $3.171722-2.226880-0.933463$

H $1.873295-3.0782100 .410456$

H $-5.179026-0.3389760 .248598$

H $-4.650878 \quad 1.186725-0.548277$

H $-5.317329-0.149237-1.513451$

SCF Energy $\left(B 3 L Y P / 6-31 G^{* *} / / M M F F\right)=-803.926024996$

F1-08_c00325

MMFF Geometry

C $-2.822847-2.447129-0.203596$

C $0.085545-0.3636580 .792383$

O $-0.983082-1.3220280 .807320$

C $-1.797665-1.330822-0.373064$

C $-2.4564920 .037233-0.576526$

C $-1.3889571 .131078-0.638613$

C -0.4662341 .0585780 .579173$

C $1.179487-0.800269-0.199871$

O $-3.3422050 .293697 \quad 0.516208$

O $-2.0126202 .413594-0.679478$

O 0.5889112 .0004290 .419007

C $2.567757-0.3235260 .171099$

O $3.410955-0.562153-0.877553$

O $2.883396 \quad 0.174890 \quad 1.242627$

C $4.772212-0.170300-0.673895$

H -3.500009 -2.498159-1.061443

H $-2.314771-3.412863-0.104733$

H -3.414577 -2.315406 0.708647

H $\quad 0.515589-0.397948 \quad 1.800667$

H -1.171536 -1.575352 -1.239240

H $-3.0644290 .044234-1.488196$

H -0.800778 1.038369 -1.558877

H -1.035394 1.3641721 .466526

H $\quad 0.946915-0.442774-1.208617$

H $1.220116-1.895843-0.238287$

H $-3.703970 \quad 1.187870 \quad 0.392369$

H -2.586682 2.433968 -1.463956

H 1.1375301 .9622741 .221199

H $5.205944-0.7290860 .160915$

H $5.332944-0.404940-1.582526$

H $4.8334370 .906943-0.492036$

SCF Energy (B3LYP/6-31G**//MMFF) $=-803.931340769$

F1-08_c00326
MMFF Geometry

C 0.9650822 .2372250 .619141

C $-0.211603-0.5634010 .174818$

O $-0.071808 \quad 0.512091-0.765015$

C $1.0975751 .319471-0.595519$

C $2.3633380 .452612-0.655064$

C $2.309938-0.7641810 .274231$

C $0.991987-1.5190270 .086255$

C $-1.525457-1.280267-0.147920$

O $3.5337941 .221749-0.411331$

O $2.431142-0.3428311 .630317$

O $0.993844-2.161512-1.187479$

C $-2.703669-0.4584840 .317795$

O $-3.1419820 .345934-0.691984$

O $-3.159666-0.5072631 .453067$

C $-4.2417701 .200554-0.364011$

H $1.7852952 .960007 \quad 0.662617$

H $\quad 0.0221192 .7930740 .565752$

H $\quad 0.9443011 .6878031 .563091$

H $-0.275461-0.143048 \quad 1.185421$

H $1.1259681 .988943-1.464845$

H $2.4472710 .072769-1.681416$

H $3.169907-1.4099560 .060875$

H $0.885116-2.2962740 .852269$

H -1.587246 -2.247696 0.364072

H $-1.633809-1.475315-1.221581$

H 3.5684641 .4154470 .540951

H $2.413249-1.1393952 .187746$

H $1.747658-2.775535-1.204885$

H $-4.4952841 .784603-1.252664$

H $-5.1126500 .604168-0.075629$

H -3.9600271 .8880520 .439258$

SCF Energy (B3LYP/6-31G**//MMFF $)=-803.927915786$

F1-08_c00327

MMFF Geometry

C -1.063724 -2.296271 0.062741

C 0.2601370 .4707320 .057729

O $-0.138189-0.331514-1.063542$

C $-1.323174-1.108835-0.863752$

C $-2.504514-0.206532-0.478986$

C -2.1827890 .7464530 .676582$

C -0.8540371 .4655610 .429685$

C $1.5524191 .191252-0.335352$

O $-3.666913-0.966148-0.173026$

O $-2.105367 \quad 0.015599 \quad 1.898369$

O $-1.0264582 .432121-0.605451$

C $2.7109580 .223942-0.382239$

O 3.1887710 .0077530 .876693

O $3.128023-0.289990-1.412091$

C $4.281556-0.9113390 .969242$

H -0.820248 -1.991333 1.083176

H -1.929499 -2.964095 0.104217

H - $0.204001-2.871021-0.299635$

H $\quad 0.463415-0.189248 \quad 0.908928$

H -1.558791 -1.547262 -1.842012

H $-2.7489670 .412543-1.351748$

H -3.0043541 .4647010 .782617$

H $-0.5544342 .011767 \quad 1.331656$

H 1.8016551 .9902650 .372697

H $1.4709301 .650709-1.327718$

H -3.851864 -1.539364 -0.936298

H -1.9360390 .6541072 .611950$

H -1.111792 $1.958274-1.449942$

H $5.138352-0.5402440 .398750$

H $4.569024-0.9929792 .020805$

H $3.976694-1.8991680 .611042$

SCF Energy (B3LYP/6-31G**//MMFF) $=-803.924254083$

F1-08_c00328

MMFF Geometry

C $2.692523-2.5117250 .286451$

C $-0.244843-0.371915-0.479635$

O $0.762786-1.392106-0.551266$

C $1.765910-1.3150520 .471839$

C 2.5138690 .0200370 .381393

C 1.5268391 .1866420 .458923

C $0.395504 \quad 1.025315-0.559155$ 
C $-1.158981-0.6099610 .733330$

$\begin{array}{lllll}\text { O } & 3.243775 & 0.089300 & -0.846462\end{array}$

$\begin{array}{llllllll}\text { O } & 2.210803 & 2.417863 & 0.234565\end{array}$

O $-0.588302 \quad 2.033645-0.359048$

C $-2.564680-0.1028130 .519547$

O $-3.216987-0.895083-0.378496$

O $-3.0300780 .874706 \quad 1.090974$

C $-4.565528-0.514346-0.668241$

H $3.124876-2.534396-0.719541$

H $3.503358-2.5035631 .020982$

H $2.129712-3.4447100 .401001$

H $-0.852160-0.496767-1.384615$

H $1.286136-1.4069241 .453292$

H $3.254210 \quad 0.106913 \quad 1.184044$

H $1.107346 \quad 1.2511901 .469188$

H $0.7976241 .174128-1.568998$

H $-0.760117-0.1402101 .639068$

H $-1.227305-1.6805930 .965288$

H $2.640841-0.151118-1.570232$

H $2.6453192 .360067-0.633768$

H $-0.1342832 .893404-0.384158$

H $-4.5910450 .490880-1.099936$

H $-5.175654-0.5617550 .238827$

H $-4.968511-1.220232-1.399278$

SCF Energy (B3LYP/6-31G**//MMFF) $=-803.933113068$

F1-08 c00329

MMFF Geometry

C 2.4288301 .9066900 .753629

C $-0.1594910 .485301-0.104203$

O $0.7680661 .229216-0.909388$

C $2.1405801 .114264-0.521118$

C $2.578561-0.356651-0.507213$

C $1.634267-1.2568690 .294532$

C $0.183551-1.017648-0.127583$

C $-1.5575600 .794796-0.656676$

O $3.907731-0.503092-0.024710$

O $1.750676-1.0007691 .691387$

O $-0.011558-1.531378-1.443986$

C $-2.673623 \quad 0.290871 \quad 0.229210$

O $-\begin{array}{lll}-3.828720 & 0.232673 & -0.497082\end{array}$

O $-2.558351 \quad 0.0121261 .414944$

C $-4.983920-0.215071 \quad 0.219219$

H 1.9054131 .5093881 .626283

H 3.4998561 .9245510 .976917

H 2.0863852 .9407290 .635083

H -0.1040220 .8599640 .924488$

H $2.7156651 .613471-1.311558$

H $2.569139-0.718503-1.543726$

H $1.915041-2.3050370 .137683$

H $-0.494615-1.5555530 .544536$

H $-1.6680780 .373407-1.662703$

H $-1.6805921 .880436-0.754949$

H $4.4814830 .060250-0.571711$

H $2.689283-1.0999831 .926585$

H $0.181878-2.484099-1.418274$

H $-5.829777-0.226960-0.473377$

H $-4.825960-1.2294530 .597549$

H -5.2110070 .4730791 .039069$

SCF Energy (B3LYP/6-31G**/MMFF) $=-803.931632475$

F1-08 c00330

MMFF Geometry

C -3.092103 -2.129645 -0.514181

C $0.060738-0.5826230 .695399$

O $-1.122107-1.3912710 .604846$

C -1.938451-1.132662 -0.546270

C $-2.4250470 .321445-0.535682$

C $-1.2336511 .278265-0.458399$

C -0.3073640 .9127280 .704632$

C $1.078272-1.008717-0.374996$

$\begin{array}{llllll}\text { O } & -3.293607 & 0.536417 & 0.579667\end{array}$

O $-1.7113472 .612533-0.303892$

$\begin{array}{lllll}\text { O } & 0.855921 & 1.733259 & 0.684134\end{array}$

C $2.504122-0.7487990 .052223$

O $3.1246500 .050265-0.862323$

O $3.007665-1.2010061 .072330$

C $4.4848440 .381998-0.566857$
H $-3.775708-1.973019-1.353947$

H -2.706038 -3.153599-0.567215

H $-3.657314-2.0617320 .421558$

H $0.496106-0.8112731 .676226$

H $-1.352008-1.322622-1.452843$

H $-3.0168840 .539058-1.431624$

H $-0.6799711 .251569-1.404232$

H $-0.822271 \quad 1.142473 \quad 1.645290$

H $0.877487-0.512438-1.330775$

H $1.000482-2.084475-0.575796$

H $-2.8504260 .201392 \quad 1.377368$

H $-0.9345563 .190678-0.212824$

H $1.2430711 .691184-0.206553$

H $5.096255-0.524639-0.528638$

H $4.8598651 .024530-1.367992$

H 4.5443490 .9294070 .378699

SCF Energy (B3LYP/6-31G**//MMFF)= -803.930483978

F1-09 c00375

MMFF Geometry

C $-0.9609832 .883120-0.081050$

C $0.166046-0.583527 \quad 0.684164$

$\begin{array}{llll}\text { O } & -0.129013 & 0.820734 & 0.765005\end{array}$

C $-0.7633531 .400369-0.381410$

C $-2.0847490 .689751-0.698521$

C $-1.836607-0.812872-0.849098$

C $-1.100476-1.390068 \quad 0.361627$

C $1.313869-0.870169-0.296229$

$\begin{array}{lllll}0 & -3.021822 & 0.935870 & 0.349575\end{array}$

O $-3.063345-1.508877-1.041561$

O $-1.944303-1.3495901 .516154$

C $2.622056-0.2506310 .149675$

O $3.575283-0.506856-0.795616$

$\begin{array}{lllll}\text { O } & 2.794076 & 0.371292 & 1.188711\end{array}$

C $4.8766460 .017940-0.514382$

H $-1.463883 \quad 3.392667-0.908401$

H $-1.541875 \quad 3.0342540 .835260$

H $0.008413 \quad 3.3659770 .085976$

H $0.497853-0.8620161 .692665$

H $-0.0871831 .322917-1.239720$

H $-2.5268361 .094872-1.615817$

H $-1.228172-0.984188-1.745195$

H $-0.860633-2.446397 \quad 0.196035$

H $1.091266-0.487511-1.296439$

H $1.472850-1.952886-0.361979$

H -3.8193440 .4148720 .154430$

H $-3.501231-1.121018-1.818463$

H $-2.768760-1.8120611 .287369$

H $5.538305-0.252192-1.341752$

H $4.837014 \quad 1.108783-0.438466$

H $5.270512-0.4201280 .407702$

SCF Energy (B3LYP/6-31G**//MMFF) $=-803.922498421$

F1-09 c00376

MMFF Geometry

C $1.438259-2.0638640 .889640$

C $-0.1767450 .384140-0.043123$

O $0.110158-0.831846-0.748409$

C $1.397388-1.400472-0.486477$

C $2.513593-0.383959-0.776444$

C $2.2846480 .963811-0.092099$

C $0.8684521 .453483-0.400156$

C $-1.5883580 .829616-0.452865$

O $3.791757-0.893935-0.422236$

$\begin{array}{lllll}\text { O } & 2.441196 & 0.839764 & 1.321459\end{array}$

$\begin{array}{lllll}0 & 0.616791 & 2.665883 & 0.300842\end{array}$

C -2.661297 -0.0958090 .083433$

O $-3.8742340 .310750-0.396999$

$\begin{array}{lllll}0 & -2.469074 & -1.045341 & 0.830198\end{array}$

C $-4.996621-0.4689690 .027654$

H $0.619833-2.7872010 .977620$

H $2.381674-2.5946611 .048627$

H $1.303147-1.3518611 .707035$

H $-0.176267 \quad 0.1961001 .037234$

H $1.513867-2.218603-1.208848$

H $2.524970-0.197170-1.858480$

H $3.0273521 .701246-0.416688$

H $0.8061461 .673980-1.473165$ 
H $-1.6712970 .835520-1.546108$ H $-1.8020551 .831129-0.064294$ H $\quad 3.911877-1.735927-0.893599$ H 3.3269910 .4714891 .482353 H 0.7075052 .4799721 .251291 H $-5.097668-0.4253881 .116307$ H $-5.896397-0.044642-0.425597$ H $-4.888345-1.504046-0.309906$ SCF Energy $\left(B 3 L Y P / 6-31 G^{* *} / / M M F F\right)=-803.936216387$

\section{F1-09 c00377}

MMFF Geometry

C -2.670479 $2.490705-0.176407$

C $\quad 0.337071 \quad 0.399257 \quad 0.475392$

O

C $-1.689818 \quad 1.356753-0.456382$

C $-2.384625-0.008868-0.526126$

C $-1.336395-1.111507-0.686411$

C $-0.245853-1.0198700 .384806$

C $1.2810550 .762740-0.675305$

O $-3.145809-0.2162210 .663563$

O $-1.969329-2.386214-0.652448$

O $-0.793007-1.3416991 .667419$

C $2.615130 \quad 0.072316-0.544847$

O $3.391003 \quad 0.7239980 .367548$

O $2.924342-0.933682-1.170164$

C $4.6837990 .153877 \quad 0.593636$

H $-2.1459833 .452683-0.184258$

H -3.465212 $2.523306-0.927668$

H -3.1234292 .3950190 .816357$

H $\quad 0.8934780 .4769181 .417966$

H -1.193388 $1.569322-1.410450$

H -3.093584 -0.037232 -1.361594

H $-0.877743-1.011078-1.677080$

H $\quad 0.539463-1.7593340 .195820$

H $1.4525331 .846016-0.709590$

H $\quad 0.8599370 .509529-1.654084$

H $-3.517315-1.1137280 .616444$

H -1.277056 -3.059592 -0.766803

H $-1.217713-2.2132131 .590965$

H $4.585814-0.8639020 .982967$

H $5.2679870 .159811-0.331547$

H 5.1998830 .7659811 .338142

SCF Energy (B3LYP/6-31G**//MMFF) $=-803.924820371$

F1-09 c00378

MMFF Geometry

C $3.000183-2.111752-0.538553$

C $-0.199692-0.6406630 .663434$

O $1.011778-1.4096830 .568149$

C $1.801391-1.171263-0.604402$

C $2.2224250 .302123-0.685365$

C $0.983711 \quad 1.199710-0.618306$

C 0.1000320 .8645590 .585655

C -1.225463 -1.128580 -0.364552

O $3.123391 \quad 0.6188020 .375774$

O $1.3540692 .573388-0.574587$

O $\quad 0.770291 \quad 1.229847 \quad 1.796651$

C $-2.624487-0.7065340 .009453$

O $-3.050056 \quad 0.287329-0.820874$

O $-3.255152-1.1888210 .941425$

C $-4.3621420 .792134-0.553736$

H $2.661281-3.153702-0.531898$

H $3.664826-1.968861-1.395808$

H $3.573633-1.9684550 .383566$

H $-0.587415-0.8575521 .666996$

H $1.212461-1.437797-1.489465$

H $2.7686250 .493436-1.615754$

H $0.3970951 .058515-1.533751$

H $-0.822831 \quad 1.4539110 .559778$

H $-1.218843-2.224029-0.424343$

H $-0.992326-0.785938-1.378418$

H 2.6883260 .3900591 .214912

H $1.9301622 .746213-1.338662$

H 1.0024142 .1715931 .724655

H $-5.104038-0.004609-0.662575$

H -4.4038931 .2254990 .450229$

H $-4.5797961 .576416-1.283541$
SCF Energy $($ B3LYP/6-31G**//MMFF $)=-803.934710385$

F1-09_c00379

MMFF Geometry

C -0.4443892 .8167780 .226646$

C $0.166170-0.8455170 .491841$

$\begin{array}{lllll}\text { O } & 0.090631 & 0.562981 & 0.765373\end{array}$

C $-0.5186601 .370229-0.250520$

C $-1.9551170 .908380-0.531403$

C $-1.950165-0.581769-0.880050$

C $-1.226227-1.4160760 .180914$

C $1.190360-1.157682-0.604023$

O -2.7809101 .1235830 .612810$

O $-3.285338-1.040751-1.060926$

O $-1.986256-1.4419061 .392649$

C $2.584037-0.801888-0.145472$

O $2.902471 \quad 0.459185-0.554318$

O $3.292153-1.5468400 .519572$

C $4.1934540 .928301-0.153715$

H $\quad 0.602375 \quad 3.122950 \quad 0.333055$

H $-0.9327993 .494637-0.479612$

H -0.9006782 .9396151 .214661$

H $\quad 0.518393-1.2955081 .428949$

H $0.0742621 .296434-1.168854$

H $-2.3985451 .478726-1.355863$

H $-1.437978-0.708222-1.841749$

H -1.155924 -2.459425 -0.145584

H $\quad 0.985617-0.640092-1.546303$

H $1.191967-2.228851-0.838813$

H -2.932848 2.0801890 .692866

H $-3.244446-1.991670-1.259540$

H -2.029235 -0.5338791 .737905$

H 4.2626100 .9598540 .937842

H $4.9760090 .288854-0.572964$

H $4.3224401 .941829-0.542573$

SCF Energy $($ B3LYP/6-31G**//MMFF $)=-803.926743378$

F1-09_c00380

MMFF Geometry

C -1.0822942 .8404070 .324764$

C $0.308958-0.6126530 .330597$

$\begin{array}{llll}\text { O } & -0.037543 & 0.734920 & 0.689296\end{array}$

C $-0.8859571 .433475-0.230637$

C $-2.2096010 .684417-0.436769$

C $-1.917412-0.755644-0.865951$

C $-0.952197-1.4575510 .092393$

C $1.277316-0.653241-0.856498$

O $-2.973231 \quad 0.6727650 .768779$

O $-3.127652-1.495859-0.984436$

O $-1.605889-1.667170 \quad 1.347527$

C $2.5773620 .032451-0.512992$

O $3.388969-0.8225350 .172353$

O $2.8308561 .194519-0.803248$

C $4.651689-0.2818320 .573168$

H -1.4711412 .8193251 .348286$

H $-0.1195973 .361706 \quad 0.374336$

H -1.760182 $3.425587-0.303622$

H $0.829609-1.0109911 .210424$

H $-0.3671391 .529016-1.190866$

H -2.825853 $1.176861-1.197799$

H -1.467618 $-0.735102-1.865519$

H $-0.700293-2.452540-0.291714$

H $0.876114-0.163820-1.749367$

H $1.498260-1.686969-1.148253$

H $-3.310748 \quad 1.5724020 .914814$

H $-3.539818-1.520576-0.103613$

H $-0.988923-2.1503641 .923064$

H $4.5032830 .568591 \quad 1.245501$

H $5.200354-1.0609831 .108870$

H $5.2324210 .015768-0.305159$

SCF Energy (B3LYP/6-31G**//MMFF $)=-803.925320339$

F1-09_c00381

MMFF Geometry

C $-2.707666-1.464816-1.002758$

C $0.100002-0.184313-0.880652$

O $-0.389477-1.370373-0.243009$

C $-1.755444-1.3142420 .183481$ 
C $-2.004002-0.098028 \quad 1.091663$

C $-1.496435 \quad 1.2129050 .489511$ C $-0.043074 \quad 1.0314970 .048479$

C $1.544373-0.439915-1.326927$

$\begin{array}{lllll}\text { O } & -3.378132 & 0.029134 & 1.431798\end{array}$

O $-2.2878831 .587124-0.637640$

O $0.4240642 .213962-0.588646$

C $2.491784-0.646777-0.167247$

O $3.3216130 .428496-0.045386$

O $2.483664-1.6362590 .553704$

C $4.255567 \quad 0.3651541 .036699$

H $-3.748603-1.533289-0.672884$

H $-2.627188-0.641917-1.716689$

H $-2.464940-2.376411-1.560303$

H $-0.471880-0.017595-1.801708$

H -1.911618 $-2.209847 \quad 0.798482$

H $-1.450230-0.2581832 .026163$

H - -1.5821112 .0355911 .208277$

H $\quad 0.5622310 .8818190 .950429$

H $1.9050130 .385356-1.952020$

H $1.600419-1.346045-1.942717$

H $-3.658554-0.8097641 .835747$

H -3.211842 $1.635509-0.337791$

H $-0.1437562 .375241-1.361767$

H 4.8549541 .2792751 .022435

H 3.7247410 .3084521 .991834

H $4.921945-0.4936600 .912468$

SCF Energy (B3LYP/6-31G**//MMFF) $=-803.930458974$

F1-09_c00382

MMFF Geometry

C $2.316234-1.5100801 .411567$

C $-0.138422 \quad 0.2887530 .873572$

O $0.062483-1.0967810 .569805$

C $1.397190-1.4519190 .191574$

C $1.890957-0.585663-0.979687$

C $1.6989580 .912087-0.739430$

C $0.2482641 .169584-0.324432$

C $-1.591696 \quad 0.477055 \quad 1.326088$

O $3.250169-0.855898-1.294844$

O $2.588175 \quad 1.357528 \quad 0.283760$

O $0.0688742 .542403 \quad 0.000026$

C -2.6012510 .2475990 .225511$

O $-2.807846-1.0880090 .047231$

O $-3.1344251 .150917-0.406266$

C $-3.733756-1.434745-0.987009$

H $1.889081-2.1798702 .166497$

H $3.309301-1.8847011 .145992$

H $2.434129-0.5396791 .899174$

H 0.4789090 .5526431 .741139

H $1.336717-2.483886-0.177361$

H $1.294481-0.847475-1.863708$

H $1.9590781 .482351-1.638393$

H $-0.3964240 .935229-1.180379$

H -1.7469441 .4905601 .714418$

H -1.830893 -0.2095732 .147645$

H $3.328042-1.810534-1.462681$

H 2.3923722 .2954040 .450511

H $0.3171533 .063369-0.782606$

H -3.804264 -2.524879 -1.028520

H $-4.723723-1.026921-0.761843$

H -3.375309-1.069342 -1.954103

SCF Energy (B3LYP/6-31G**//MMFF) $=-803.924028869$

F1-09_c00383

MMFF Geometry

C $1.138877-2.0422060 .996293$

C $-0.231804 \quad 0.399282-0.284810$

O $0.117170-0.858383-0.879789$

C $1.330139-1.445982-0.397771$

C $2.510418-0.473712-0.557580$

C $2.226877 \quad 0.911765 \quad 0.024236$

C $0.891098 \quad 1.422120-0.522190$

C $-1.5444770 .860147-0.926783$

O $3.704142-1.0017350 .004328$

$\begin{array}{lllllll} & \text { O } & 2.178672 & 0.834291 & 1.448315\end{array}$

$\begin{array}{lllll}\text { O } & 0.575292 & 2.677940 & 0.066855\end{array}$

C $-2.689852-0.033696-0.514637$ $\begin{array}{llll}\text { O } & -3.105391 & 0.291669 & 0.742907\end{array}$

O $-3.149875-0.920951-1.221446$

C $-4.180808-0.4992651 .258056$

H $\quad 0.294798-2.7411100 .988564$

H $2.030043-2.5864501 .322908$

H $0.901532-1.2883801 .750304$

H -0.4033120 .2613030 .789368$

H $1.528712-2.301146-1.056383$

H $2.689360-0.339080-1.632707$

H $3.0421461 .603203-0.217564$

H $1.0053681 .586428-1.600982$

H $-1.4779180 .825713-2.021020$

H $-1.8014801 .889307-0.651908$

H $3.862416-1.869118-0.405505$

H 2.0983951 .7417911 .787398

H 0.3331712 .5211800 .995177

H $-4.415525-0.1402752 .263709$

H $-5.069072-0.3870000 .629044$

H $-3.881527-1.5497311 .321356$

SCF Energy (B3LYP/6-31G**//MMFF)= -803.925718444

F1-09 c00384

MMFF Geometry

C $1.029582-1.9991751 .122118$

C $-0.1976300 .542508-0.099056$

O $-0.109105-0.777067-0.654382$

C $1.081053-1.500900-0.321534$

C $2.332835-0.703860-0.725914$

C $2.3223310 .737300-0.211659$

C $0.9889761 .392262-0.580337$

C $-1.5307521 .142639-0.556265$

O $3.528736-1.372662-0.351285$

$\begin{array}{llll}\text { O } & 2.478712 & 0.767747 & 1.206420\end{array}$

$\begin{array}{lllll}\text { O } & 0.931099 & 2.704230 & -0.032582\end{array}$

C $-2.683702 \quad 0.464734 \quad 0.145275$

O $-3.217333-0.501173-0.655425$

$\begin{array}{llll}\text { O } & -3.046316 & 0.743649 & 1.281001\end{array}$

C $-4.304436-1.238556-0.088021$

H $1.054362-1.1853411 .850552$

H $0.091646-2.5381991 .296786$

H $1.858043-2.6798761 .339221$

H -0.2076580 .4807730 .996060$

H $1.063148-2.405325-0.943161$

H $2.338749-0.647870-1.822369$

H $3.1591691 .310903-0.626000$

H $0.9430981 .488871-1.672312$

H $-1.662172 \quad 1.041618-1.640366$

H $-1.6000882 .211243-0.323770$

H $3.571923-1.4002390 .618763$

H 3.3823690 .4712611 .407614

H 1.0022652 .6225950 .934070

H $-4.640418-1.971345-0.826560$

H $-3.973346-1.7707380 .808882$

H $-5.136980-0.5678380 .144965$

SCF Energy (B3LYP/6-31G**//MMFF) $=-803.931995815$

F1-09 c00385

MMFF Geometry

C $2.448163-1.5393861 .270178$

C $-0.108488-0.510863-0.111384$

O $0.828398-1.502682-0.558666$

C $2.194781-1.254071-0.210057$

C $2.6483830 .125286-0.714945$

C $1.6953581 .251048-0.312907$

C $0.265705 \quad 0.863422-0.695573$

C $-1.497496-0.979083-0.570094$

$\begin{array}{llll}\text { O } & 3.967013 & 0.433558 & -0.284336\end{array}$

$\begin{array}{lllll}\text { O } & 1.758308 & 1.482249 & 1.094237\end{array}$

O $-0.634943 \quad 1.875779-0.265560$

C $-2.635524-0.3416630 .194980$

O $-3.767184-0.382450-0.568089$

O $-2.553594 \quad 0.105824 \quad 1.330759$

$\begin{array}{llll}\text { C } & -4.937675 & 0.175945 & 0.037051\end{array}$

H $2.115499-2.5543021 .515244$

H $3.511540-1.4600701 .515113$

H $1.893331-0.8698421 .931397$

H $-0.098947-0.4788010 .984106$

H $2.781784-2.003503-0.756413$ 
H $2.662687 \quad 0.093174-1.812482$

H $1.9799612 .195989-0.789442$

H $\quad 0.2076260 .816494-1.790229$

H -1.589672 -2.064044 -0.436782

H $-1.616440-0.778447-1.641806$

H $4.547678-0.288680-0.578889$

H 2.6871671 .6679241 .314909

H -0.5673001 .9371730 .702841$

H $-5.7622010 .091447-0.675763$

H $-5.197699-0.3806380 .942400$

H $-4.7782351 .234078 \quad 0.265538$

SCF Energy (B3LYP/6-31G**//MMFF) $=-803.937545646$

F1-09_c00386

MMFF Geometry

C $3.023701-2.084661-0.535520$

C $-0.184351-0.6349970 .664509$

O $1.035481-1.3897450 .572402$

C $1.825156-1.143949-0.598828$

C $2.2324180 .334107-0.682130$

C $0.9791461 .211321-0.625962$

C $0.097071 \quad 0.874737 \quad 0.580172$

C $-1.203863-1.138460-0.362654$

$\begin{array}{lllll}\text { O } & 3.100388 & 0.675046 & 0.398282\end{array}$

O $1.3503122 .585853-0.615747$

O $\quad 0.766912 \quad 1.2796531 .777896$

C $-2.607338-0.7270250 .006872$

O $-3.046030 \quad 0.248119-0.838842$

O $-3.230947-1.2014100 .947606$

C $-4.3636050 .741528-0.578038$

H $2.684598-3.126388-0.559838$

H $3.702269-1.922234-1.378265$

H $3.580251-1.9666200 .400195$

H $-0.571344-0.8555881 .667327$

H $1.238399-1.412978-1.484950$

H $2.7823280 .538669-1.608093$

H $0.4042181 .048334-1.545312$

H $-0.830308 \quad 1.4561090 .539470$

H -1.186832 -2.234020 -0.416213

H $-0.972151-0.799204-1.377769$

H $3.961400 \quad 0.256622 \quad 0.230440$

H $0.5333103 .110087-0.560488$

H $\quad 0.1719331 .0951172 .524430$

H -4.409048 1.1913070 .418519

H $-4.5917381 .510784-1.320524$

$\mathrm{H}-5.096236-0.065722-0.672236$

SCF Energy (B3LYP/6-31G**//MMFF) $=-803.912163988$

F1-09 c00387

MMFF Geometry

C $-2.6779142 .454016-0.205271$

C $0.340291 \quad 0.387870 \quad 0.467919$

O $-0.716518 \quad 1.3562340 .576698$

C - $1.7007121 .312381-0.464903$

C -2.387192 -0.060269-0.509979

C $-1.326670-1.155055-0.659072$

C $-0.229395-1.039316 \quad 0.402319$

C $1.2729230 .742542-0.694854$

O $-3.137263-0.2858840 .682722$

O $-1.919376-2.448491-0.602372$

O $-0.779244-1.3654661 .682910$

C $2.6146190 .067038-0.563300$

$\begin{array}{lllll}\text { O } & 3.388100 & 0.736415 & 0.338251\end{array}$

O $2.931348-0.942134-1.179677$

C 4.6880740 .1825080 .563482

H $-2.1555363 .415512-0.263773$

H -3.490443 $2.457061-0.937816$

H $-3.1040472 .398980 \quad 0.802124$

H $\quad 0.903957 \quad 0.4878461 .403939$

H -1.209060 $1.507531-1.425261$

H -3.094448 $-0.116943-1.345582$

H $-0.866308-1.063902-1.650067$

H $0.554846-1.7796710 .210633$

H $1.4337511 .826853-0.746735$

H $\quad 0.8478420 .470531-1.666852$

H -3.9159040 .2952420 .658225$

H $-2.622579-2.478774-1.273159$

H $-0.054074-1.3362992 .329938$
H $4.602996-0.8330650 .962061$

H $5.2678640 .186768-0.364355$

H 5.2006170 .8066641 .300390

SCF Energy $($ B3LYP/6-31G**//MMFF $)=-803.911012968$

F1-09 c00388

MMFF Geometry

C $-2.716573-1.538530-0.908280$

C $0.087404-0.248160-0.861141$

O $-0.404465-1.391895-0.150407$

C $-1.771151-1.3084400 .270616$

C $-2.014101-0.0366621 .101418$

C $-1.498531 \quad 1.2355610 .424386$

C $-0.0435221 .019514-0.000513$

C $1.523204-0.552900-1.304216$

O $-\begin{array}{llll}-3.378765 & 0.098663 & 1.472588\end{array}$

O $-2.2832581 .548450-0.725424$

O $0.4114312 .170318-0.701051$

C $2.483638-0.673928-0.143636$

O $3.2728980 .436015-0.074551$

O $2.513434-1.6291340 .621453$

C 4.2120410 .4562161 .004837

H -2.662386 -0.741986 -1.654025

H $-2.444653-2.463929-1.428351$

H -3.754990 -1.628627 -0.576001

H $-0.491391-0.130125-1.785518$

H $-1.928525-2.1631600 .940998$

H -1.448502 -0.1491262 .035791$

H -1.5777532 .0988091 .095015$

H $\quad 0.5550200 .919340 \quad 0.913337$

H $1.8858550 .211191-2.001570$

H $1.562756-1.506907-1.844322$

H $-3.900676 \quad 0.2481370 .667175$

H $-3.1655221 .812889-0.414833$

H $1.3728842 .086764-0.816010$

H 4.7776391 .3899790 .945768

H 3.6866720 .4241441 .964125

H $4.908923-0.3827510 .916732$

SCF Energy $\left(B 3 L Y P / 6-31 G^{* *} / / M M F F\right)=-803.924598254$

F1-10 c00431

MMFF Geometry

C $-1.395672 \quad 2.8721350 .334009$

C $0.229692-0.368873-0.423406$

O -0.1013460 .8739660 .217256$

C $-1.2411241 .524415-0.360825$

C $-2.4903930 .644428-0.217436$

C $-2.220049-0.733535-0.829436$

C $-0.936292-1.360716-0.278536$

C $1.513197-0.896848 \quad 0.228880$

O $-\begin{array}{llll}-2.824740 & 0.515277 & 1.164127\end{array}$

O $-3.319039-1.609806-0.609331$

O $-1.099996-1.7008631 .098251$

C $2.733261-0.093498-0.171987$

O $3.830943-0.6205590 .448413$

O $2.731593 \quad 0.868498-0.927647$

C $5.070616 \quad 0.037507 \quad 0.168758$

H -0.5026243 .4852450 .169334$

H -2.265327 $3.417305-0.045153$

H -1.493902 2.7565541 .418755

H $0.410269-0.171708-1.488358$

H -1.033779 $1.705200-1.423393$

H $-3.3501891 .115064-0.707812$

H -2.108381 -0.620097-1.915331

H $-0.715355-2.293672-0.809569$

H $1.687956-1.933025-0.082236$

H $1.433131-0.8468321 .321007$

H -3.572422 -0.1035841 .225274$

H $-4.111499-1.183519-0.977909$

H -1.884165 -2.272310 1.163701

H $5.860231-0.4746450 .724900$

H 5.0289831 .0801980 .497903

H $5.298340-0.022369-0.899807$

SCF Energy (B3LYP/6-31G**//MMFF) $=-803.930027885$

F1-10_c00432

MMFF Geometry

C $-1.3309702 .873208-0.100947$ 
C $0.327458-0.436699-0.134991$

$\begin{array}{lllll}\text { O } & -0.089870 & 0.874960 & 0.277753\end{array}$

C $-1.0992991 .441737-0.569365$

C $-2.3737980 .584960-0.530676$

C $-2.026156-0.859493-0.903653$

C $-0.861557-1.408047-0.072545$

C $1.465824-0.8698460 .789649$

O -2.9492920 .6049170 .775526$

O $-3.168028-1.698706-0.780882$

O $-1.267135-1.6029301 .282708$

C $2.727134-0.099540 \quad 0.481070$

O $3.366424-0.660468-0.585181$

O 3.0893600 .8912841 .102138

C $4.573511-0.007424-0.990442$

H - $0.4131623 .459851-0.219419$

H -2.126789 $3.356164-0.675631$

H -1.5834382 .9132320 .964005$

H $0.693845-0.369170-1.167854$

H $-0.7101071 .476430-1.595061$

H -3.132476 $0.971994-1.220637$

H $-1.732297-0.879460-1.960477$

H $-0.571983-2.395031-0.449834$

H $1.683835-1.9395050 .688787$

H $1.221217-0.6862051 .842624$

H -3.3358651 .4857540 .915066$

H -3.434376 -1.6919080 .154617$

H $-1.475349-0.7309231 .659547$

H $4.984721-0.552646-1.844225$

H $5.305371-0.024855-0.177208$

H $4.3622961 .020680-1.299384$

SCF Energy (B3LYP/6-31G**//MMFF) $=-803.941146728$

F1-10_c00433

MMFF Geometry

C $-0.846808 \quad 2.921872-0.088301$

C $0.228488-0.615606-0.304261$

$\begin{array}{lllll}\text { O } & 0.095215 & 0.744304 & 0.139573\end{array}$

C $-0.903699 \quad 1.480367-0.579472$

C $-2.285998 \quad 0.845315-0.377103$

C $-2.234898-0.633377-0.772516$

C $-1.089463-1.374475-0.074002$

C $1.399408-1.2361330 .458654$

O -2.6744570 .9806070 .989951$

O $-3.486318-1.250628-0.494470$

O $-1.372247-1.4738341 .321471$

C $2.713374-0.733593-0.090299$

O 3.1152150 .3732890 .597065

O $3.296456-1.249209-1.035321$

C 4.3396360 .9637250 .150558

H $0.1405823 .350404-0.292659$

H $-1.6022583 .540196-0.582511$

H -0.9905112 .9834860 .995782$

H $0.459304-0.603289-1.378065$

H $-0.6417831 .468269-1.645192$

H -3.041792 $1.370135-0.972548$

H $-2.080259-0.692756-1.857444$

H $-1.024553-2.398429-0.459954$

H $1.405870-2.3277160 .357268$

H $1.357929-1.0098081 .530740$

H -3.5127700 .5004041 .101268$

H $-3.412929-2.187683-0.743285$

H -0.721781 -2.079259 1.714853

H $4.2415001 .300265-0.885967$

H $5.163090 \quad 0.250006 \quad 0.249563$

H 4.5491991 .8309710 .782438

SCF Energy (B3LYP/6-31G**//MMFF)= -803.923837433

F1-10_c00434

MMFF Geometry

C $3.061133-2.302573-0.021671$

C $-0.182254-0.542640-0.360124$

O $0.848363-1.4258760 .113603$

C $2.109919-1.229885-0.539844$

C $2.6331330 .189919-0.283507$

C $1.5777771 .211101-0.715665$

C $0.2102060 .920144-0.088250$

C -1.480364 $-0.965663 \quad 0.337051$

$\begin{array}{llll}\text { O } & 2.932085 & 0.342786 & 1.103840\end{array}$
O $2.0149142 .524963-0.388932$

$\begin{array}{lllll}\text { O } & 0.254242 & 1.148000 & 1.320057\end{array}$

C $-2.708119-0.294730-0.234345$

O $-3.739587-0.4226560 .651570$

$\begin{array}{llll}\text { O } & -2.767401 & 0.247620 & -1.329357\end{array}$

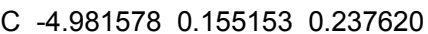

H $2.674491-3.298360-0.265543$

H $4.056130-2.198868-0.464705$

H $\quad 3.152360-2.2663331 .069221$

H $-0.289453-0.699292-1.441693$

H $1.968379-1.384756-1.617103$

H $3.5690380 .359216-0.828149$

H $1.4804361 .164337-1.807839$

H $-0.538907 \quad 1.608292-0.494408$

H $-1.416809-0.7602641 .411856$

H $-1.616668-2.0488860 .230002$

H $3.195876 \quad 1.268517 \quad 1.242374$

H $1.3269613 .144693-0.685945$

H 0.5776722 .0546571 .457633

H $-5.713817-0.0067211 .033188$

H $-5.340355-0.331787-0.674215$

H $-4.865371 \quad 1.2319020 .081475$

SCF Energy (B3LYP/6-31G**//MMFF) $=-803.932596005$

F1-10_c00435

MMFF Geometry

C $2.3843522 .439776-0.522799$

$\begin{array}{llll}\text { C } & -0.032363 & 0.281376 & 1.269273\end{array}$

$\begin{array}{lllll}\text { O } & 0.515673 & 1.300961 & 0.422326\end{array}$

C $1.945901 \quad 1.2530250 .327041$

C $2.404382-0.089686-0.261363$

C $1.825317-1.243651 \quad 0.565158$

C $0.307310-1.1195630 .733091$

C -1.5302830 .5449721 .454721$

O $1.991698-0.199144-1.623623$

O $2.146342-2.500059-0.019797$

O $-0.333967-1.337926-0.521381$

$\begin{array}{llll}\text { C }-2.328157 & 0.564867 & 0.169942\end{array}$

O $-3.279895-0.4119160 .199257$

O $-2.132798 \quad 1.350974-0.748089$

C $-4.098549-0.507750-0.970400$

H $3.4702092 .454226-0.655244$

H $1.9061722 .426653-1.508229$

H $2.0802643 .377895-0.045513$

H $0.430076 \quad 0.393765 \quad 2.259667$

H 2.3622091 .3765541 .334976

H $3.498251-0.150939-0.267574$

H $2.283814-1.2246981 .562117$

H $-0.062500-1.8918901 .417012$

H $-1.682816 \quad 1.5267531 .920218$

H $-1.952874-0.1982732 .141245$

H $1.027948-0.072672-1.654390$

H $3.111967-2.534443-0.129101$

H $-0.047429-2.208946-0.845683$

H -3.482317 $-0.730642-1.846796$

H $-4.807554-1.326832-0.823020$

H $-4.6593190 .420172-1.117206$

SCF Energy (B3LYP/6-31G**//MMFF) $=-803.934968236$

F1-10_c00436

MMFF Geometry

C $-0.8639002 .898913-0.122882$

C $0.229069-0.633556-0.330241$

$\begin{array}{lllll}\text { O } & 0.076582 & 0.720109 & 0.128830\end{array}$

C $-0.9161891 .453367-0.602678$

C $-2.2995090 .815577-0.405330$

C $-2.243716-0.668789-0.779025$

C $-1.080956-1.407347-0.108315$

C $1.398695-1.2555430 .433870$

$\begin{array}{lllll}\text { O } & -2.745707 & 0.969570 & 0.941341\end{array}$

O $-3.480706-1.308610-0.491184$

O $-1.341006-1.579674 \quad 1.284270$

C $2.712375-0.729895-0.092850$

$\begin{array}{llllll}\text { O } & 3.093659 & 0.371132 & 0.615121\end{array}$

O $3.311888-1.224446-1.038978$

C 4.3160310 .9820790 .191164

H $0.1239593 .327012-0.326412$

H -1.617726 $3.511684-0.626185$ 
H -1.0127172 .9707180 .959782$ H $\quad 0.464017-0.607461-1.402697$ H $-0.6470841 .433506-1.666592$ H -3.045491 1.320809-1.028877 H -2.100884 - $0.735801-1.864924$ H -1.002971 -2.416860 -0.527075 H $1.416964-2.3449680 .313567$ H $1.344233-1.0499231 .509540$ H -1.981255 0.8700841 .531398 H -3.618477 -1.2548230 .470206$ H $-1.032147-0.7888891 .755345$ H 5.1467350 .2767840 .289966 H 4.5079121 .8427750 .837284 H $4.2258611 .331864-0.841799$ SCF Energy $\left(B 3 L Y P / 6-31 G^{* *} / / M M F F\right)=-803.936052242$

F1-10_c00437

MMFF Geometry

C 1.6251382 .8191010 .131484

C $-0.096073-0.2358041 .320078$

O $0.143262 \quad 1.0596180 .753800$

C $1.535427 \quad 1.3939710 .665056$

C $2.2821290 .387581-0.222113$

C $2.043596-1.0341350 .295802$

C $0.549295-1.3354650 .460015$

C $-1.600423-0.4054461 .559499$

O $1.8224090 .509240-1.568075$

O $2.657021-1.975365-0.577215$

O $-0.072863-1.392710-0.820937$

C $-2.464466-0.382580 \quad 0.320133$

O $-2.507908 \quad 0.874002-0.205546$

O $-3.043592-1.368183-0.119566$

C $-3.2881111 .016908-1.396244$

H 1.1244923 .5108180 .818099

H $2.6661303 .136106 \quad 0.017917$

H $1.1154052 .920026-0.832709$

H $0.377722-0.2506832 .311480$

H 1.9576791 .3776851 .677945

H $3.3553050 .609708-0.235110$

H $2.533354-1.1319841 .272966$

H $0.409149-2.3173760 .926069$

H $-1.968903 \quad 0.3892942 .220482$

H -1.787460 -1.353557 2.077798

H $2.263555-0.183966-2.088093$

H $2.490387-2.862317-0.215122$

H $0.407381-2.052827-1.349305$

H $-4.3356390 .775105-1.193633$

H $-2.8894590 .377429-2.189740$

H -3.224801 $2.058938-1.721008$

SCF Energy $\left(B 3 L Y P / 6-31 G^{* *} / / M M F F\right)=-803.923999259$

F1-10_c00438

MMFF Geometry

C $2.967469-2.310098-0.077145$

C $-0.288677-0.545931-0.057092$

O $0.811405-1.3830900 .334546$

C $1.945789-1.277150-0.536592$

C $2.4982540 .156137-0.528124$

C $1.3783231 .132659-0.897897$

C $0.1323970 .933264-0.027617$

C $-1.445831-0.8675140 .890466$

$\begin{array}{lllll}\text { O } & 3.008910 & 0.487846 & 0.762651\end{array}$

O $1.8501502 .471728-0.806521$

$\begin{array}{llll}\text { O } & 0.399383 & 1.324028 & 1.318513\end{array}$

C $-2.723099-0.1708050 .498128$

O $-3.249291-0.753219-0.616671$

O $-3.1935590 .781168 \quad 1.107291$

C $-4.464792-0.171709-1.098211$

H $3.219051-2.1853020 .981437$

H $2.551172-3.319000-0.175083$

H $3.884240-2.255159-0.671577$

H $-0.576887-0.821322-1.080175$

H $1.626078-1.541843-1.552903$

H $3.3280680 .262869-1.236567$

H $\quad 1.103890 \quad 0.958750-1.946205$

H $-0.6790381 .575635-0.387464$

H -1.200605 -0.6028881 .926018$

H $-1.633586-1.9485270 .916277$
H $3.831480-0.0129430 .893713$

H $1.115403 \quad 3.059077-1.052560$

H $\quad 0.7434512 .233310 \quad 1.294414$

H $-5.250082-0.253003-0.340593$

H $-4.3005150 .874264-1.374700$

H $-4.777104-0.724727-1.987996$

SCF Energy $(B 3 L Y P / 6-31 G * * / / M M F F)=-803.923493504$

F1-11 c00456

MMFF Geometry

C $-1.5978882 .793201-0.266734$

C $0.197216-0.435544-0.008866$

O $-0.1397820 .910435-0.373744$

C $-1.412803 \quad 1.331410 \quad 0.128216$

C $-2.5247800 .443323-0.446873$

C $-2.256768-1.028742-0.124492$

C $-0.848081-1.402549-0.591391$

C $1.598085-0.730758-0.561785$

$\begin{array}{llll}\text { O } & -3.786594 & 0.835480 & 0.079142\end{array}$

O $-2.342702-1.234408 \quad 1.288840$

O $-0.560129-2.745969-0.222092$

C $2.676697 \quad 0.056208 \quad 0.153899$

O $3.876485-0.188374-0.452739$

$\begin{array}{lllll}\text { O } & 2.499084 & 0.783829 & 1.120977\end{array}$

C $5.002357 \quad 0.4868190 .116976$

H $-1.5871142 .908756-1.356170$

H -2.5359643 .2011590 .120824$

H $-0.770878 \quad 3.398672 \quad 0.120302$

H $0.225523-0.5154821 .085955$

H $-1.401706 \quad 1.2832941 .224805$

H $-2.5587220 .563999-1.537190$

H $-3.007817-1.683431-0.579935$

H $-0.821768-1.347744-1.686805$

H $1.640307-0.461059-1.623657$

H $1.840290-1.792375-0.443518$

H $-4.460657 \quad 0.254393-0.312697$

H $-3.222702-0.9317051 .571886$

H $-0.615924-2.8027350 .747345$

H $\begin{array}{llll}5.890491 & 0.212027 & -0.458361\end{array}$

H $4.865611 \quad 1.5705670 .054965$

H $5.1459160 .173501 \quad 1.155483$

SCF Energy (B3LYP/6-31G**//MMFF) $=-803.940492064$

F1-11 c00457

MMFF Geometry

C $-1.611802 \quad 2.798687-0.100607$

C $0.269531-0.388166-0.258435$

O $-0.1446790 .962936-0.506638$

C -1.3342541 .3294890 .200489$

C $-2.5018530 .432006-0.234247$

C $-2.157257-1.043420-0.023639$

C $-0.830134-1.359253-0.719028$

C $1.573870-0.619740-1.026520$

O

O $-2.034775-1.2917601 .380111$

O $-0.446562-2.700735-0.441375$

C $2.7023990 .186134-0.428949$

O $3.178156-0.4351820 .687998$

$\begin{array}{lllll}\text { O } & 3.103020 & 1.245829 & -0.892862\end{array}$

$\begin{array}{llll}\text { C } & 4.245358 & 0.241185 & 1.359627\end{array}$

H $-2.488433 \quad 3.1636240 .442493$

H -0.7495193 .4131980 .180399$

H $-1.7714752 .954996-1.173179$

H $0.464541-0.5097850 .815243$

H $-1.152617 \quad 1.241121 \quad 1.279343$

H $-2.7034470 .589438-1.301113$

H -2.964555 -1.690342 -0.384333

H $-0.968263-1.262295-1.803476$

H $1.470537-0.313167-2.074416$

H $1.872780-1.673879-1.027536$

H -3.5208500 .5890861 .421758$

H $-1.756722-2.2176741 .486514$

H $-1.162381-3.280491-0.752901$

H $5.110990 \quad 0.3353190 .696986$

H $3.912677 \quad 1.2251011 .703873$

H $4.532460-0.3560022 .229250$

SCF Energy (B3LYP/6-31G**//MMFF) $=-803.938076780$ 
F1-11 c00458

MMFF Geometry

C $2.203157-2.6036520 .235880$

C $-0.099186 \quad 0.2190290 .898791$

O $0.399653-1.1006740 .651756$

C $1.816474-1.1436180 .448993$

C $2.210685-0.272710-0.753515$

C $1.7213951 .165089-0.567684$

C $\quad 0.2170611 .152172-0.282399$

C $-1.5966720 .124793 \quad 1.222641$

O $3.620132-0.282861-0.946251$

O 2.4168931 .7506090 .536623

O $-\begin{array}{llll}0.238128 & 2.469510 & 0.000077\end{array}$

C -2.452905 -0.3781420 .078197$

$\begin{array}{lllll}\text { O } & -3.725442 & 0.094291 & 0.230313\end{array}$

O $-2.068557-1.119592-0.815803$

C $-4.658711-0.316962-0.773642$

H $\quad 1.879287-3.2107721 .088437$

H $1.704660-3.015168-0.648707$

H $3.284158-2.7222700 .117622$

H $\quad 0.3847690 .6010101 .808198$

H $2.318722-0.7983441 .361823$

H $1.750026-0.678674-1.662699$

H $1.959170 \quad 1.777139-1.444598$

H $-0.2978570 .803417-1.184865$

H -1.9600241 .1135651 .526253$

H -1.751482 -0.5702082 .056368$

H $4.0280300 .108134-0.154612$

H 2.0453402 .6390510 .673605

H $-0.0195823 .025700-0.767030$

H $-5.6257840 .139239-0.546183$

H -4.331656 $0.027132-1.759497$

$\mathrm{H}-4.771977-1.405215-0.761398$

SCF Energy (B3LYP/6-31G**//MMFF) $=-803.936957002$

F1-11 c00459

MMFF Geometry

C - 1.5947232 .7294820 .490558

C $0.163680-0.4997390 .918905$

$\begin{array}{lllll}\text { O } & -0.091381 & 0.902972 & 0.779614\end{array}$

C $-1.474743 \quad 1.2108410 .572914$

C $-1.9884820 .532075-0.705138$

C $-1.759223-0.980502-0.643339$

C $-0.283333-1.253251-0.345227$

C $1.645207-0.6964411 .257482$

O $-3.3728550 .806823-0.881905$

O $-2.554978-1.5464950 .401873$

O $-0.064228-2.651116-0.205650$

C $2.576695-0.3175730 .129692$

O $2.729301 \quad 1.0362530 .080139$

O $3.098405-1.130522-0.622696$

C $3.5761111 .522119-0.965823$

H -1.1886773 .1922641 .396814$

H -1.011794 $3.122836-0.349564$

H $-2.635051 \quad 3.0477620 .376397$

H $-0.399999-0.8641941 .788342$

H -2.050207 0.8802911 .447158

H -1.448810 $0.934440-1.572076$

H -2.069603 -1.472744 -1.571381

H $0.299464-0.911033-1.208423$

H $1.845343-1.7422871 .518301$

H $1.922616-0.0987592 .134664$

H $-3.6563610 .367256-1.701640$

H -3.480211 -1.3059850 .222849$

H $-0.616818-2.963606 \quad 0.531197$

H $3.6095572 .612739-0.897016$

H $4.5907181 .131154-0.844268$

H $3.1685831 .244480-1.942497$

SCF Energy $\left(B 3 L Y P / 6-31 G^{* *} / / M M F F\right)=-803.935183159$

F1-11 c00460

MMFF Geometry

C $-1.0756282 .865040-0.080407$

C $0.205366-0.606618-0.061743$

O $\quad 0.065455 \quad 0.793452-0.340033$

C -1.1155921 .3720540 .227774$

C $-2.3636710 .691596-0.355414$

C $-2.308025-0.819224-0.117820$
C $-0.986194-1.371592-0.658680$

C $1.529349-1.070140-0.674827$

O $-3.555163 \quad 1.2069410 .224973$

O $-2.392553-1.0717161 .288190$

O $-0.880906-2.757074-0.353950$

C $2.697355-0.5091190 .100880$

O $3.1673690 .616335-0.508240$

O $3.121410-0.9994361 .139591$

C $4.262131 \quad 1.260413 \quad 0.150432$

H $-1.9056383 .399690 \quad 0.389906$

H -0.1377903 .3022230 .279993$

H -1.108684 $3.042593-1.161019$

H $\quad 0.251762-0.7518401 .025925$

H -1.0824291 .2589601 .318927$

H $-2.4094650 .864577-1.438330$

H $-3.168824-1.319474-0.575110$

H $-0.977991-1.261758-1.750539$

H $1.614012-0.757987-1.722856$

H $1.628887-2.161268-0.657288$

H -3.661157 $2.124089-0.078341$

H $-2.297276-2.0310871 .415807$

H $-1.647728-3.203549-0.751701$

H 5.1210930 .5850970 .206895

H $4.5439352 .138035-0.437505$

H 3.9618521 .5892211 .149818

SCF Energy (B3LYP/6-31G**//MMFF $)=-803.931552595$

F1-11_c00461

MMFF Geometry

C $-1.5607712 .767132-0.147458$

C $0.275331-0.446678-0.232179$

O $-0.1287910 .902036-0.512030$

C -1.3165501 .2981720 .183107$

C $-2.4917840 .404817-0.240477$

C $-2.157436-1.0677490 .009519$

C $-0.833564-1.415885-0.676360$

C $1.585914-0.693491-0.984935$

$\begin{array}{llll}\text { O } & -3.676623 & 0.733475 & 0.474022\end{array}$

O $-2.025464-1.2970581 .416154$

O $-0.500415-2.763722-0.367218$

C $2.6988830 .164945-0.431490$

$\begin{array}{lllll}\text { O } & 3.162101 & -0.367877 & 0.735452\end{array}$

O $3.1000631 .189536-0.967856$

C $4.2144120 .365019 \quad 1.370258$

H -2.4098943 .1735080 .409343$

H -0.6742043 .3634180 .095443$

H -1.744537 2.901941-1.218989

H $0.463864-0.5460040 .844842$

H -1.141355 1.2272071 .264309

H -2.691397 $0.532971-1.312035$

H $-2.963071-1.724657-0.336869$

H $-0.981131-1.339980-1.761343$

H $1.477542-0.444544-2.047597$

H $1.909939-1.738082-0.929422$

H $-3.971691 \quad 1.6091640 .173194$

H $-2.859844-1.0203981 .832351$

H $0.200852-3.045268-0.977925$

H 3.8717451 .3710801 .630440

H $4.488586-0.1605042 .288931$

H 5.0910230 .4113410 .717218

SCF Energy $($ B3LYP/6-31G**//MMFF $)=-803.931775738$

F1-11_c00462

MMFF Geometry

C -3.1167042 .2183520 .177299$

C 0.1353870 .4445920 .114348

O $-0.8709801 .453570-0.061713$

C -2.1755541 .0305970 .351056$

C $-2.627642-0.176664-0.482043$

C $-1.611026-1.315829-0.374653$

C $-0.219668-0.789926-0.735603$

C $1.4743021 .072255-0.295947$

O $-3.905758-0.622006-0.045304$

O $-1.581984-1.8104010 .967425$

O $0.739750-1.826352-0.575242$

C 2.6783230 .3464270 .260515

O $3.7653910 .617368-0.520343$

O $2.676482-0.3442691 .270410$ 
C $4.9902190 .005903-0.103264$

H -4.1306591 .9837380 .514152$

H -2.7520453 .0783850 .749563$

H -3.158116 $2.534847-0.870773$

H $\quad 0.178727 \quad 0.181078 \quad 1.178964$

H -2.1517280 .7806451 .419654$

H -2.712872 $0.120139-1.535205$

H -1.887914 -2.162719-1.012015

H - $0.226233-0.508950-1.796092$

H 1.5283652 .1070970 .063733

H $1.5411831 .110852-1.389925$

H $-4.156679-1.380649-0.599436$

H -2.488011 -2.080804 1.195343

H $0.732113-2.0917790 .360512$

H $5.7710450 .289549-0.813856$

H $\quad \begin{array}{llll}5.272924 & 0.364825 & 0.890982\end{array}$

H $4.890875-1.083769-0.107724$

SCF Energy (B3LYP/6-31G**//MMFF $)=-803.941907208$

F1-11_c00463

MMFF Geometry

C $2.192294-2.6012870 .225212$

C $-0.103614 \quad 0.2219980 .905953$

O $0.394546-1.0955120 .649501$

C $1.810659-1.1407580 .446538$

C $2.206996-0.267012-0.752570$

C $1.7124631 .172711-0.584850$

C $0.213571 \quad 1.166723-0.265721$

C $-1.6029590 .124722 \quad 1.224532$

O $3.616104-0.276724-0.944856$

O $2.443867 \quad 1.8339090 .450203$

O -0.2331262 .4831920 .033368$

C $-2.455781-0.374086 \quad 0.075479$

$\begin{array}{lllll}\text { O } & -3.729310 & 0.096233 & 0.226735\end{array}$

O $-2.068448-1.110961-0.821012$

C $-4.659706-0.311437-0.781341$

H $1.868058-3.2116601 .075432$

H $1.690716-3.007014-0.660308$

H $3.272786-2.7227850 .104334$

H $\quad 0.372856 \quad 0.594614 \quad 1.822325$

H $2.315439-0.806278 \quad 1.361267$

H $1.748026-0.678407-1.660407$

H $1.9081911 .751145-1.494369$

H $-0.316000 \quad 0.829032-1.164150$

H $-1.968151 \quad 1.1114901 .532066$

H $-1.759520-0.5746832 .054239$

H $4.0235770 .123789-0.157769$

H 2.2986501 .3684481 .289095

H $0.0033463 .051521-0.719391$

H -5.628991 $0.138842-0.551510$

H -4.332929 $0.041604-1.764119$

H -4.768654 -1.400194 -0.777628

SCF Energy (B3LYP/6-31G**//MMFF) $=-803.930352139$

F1-11_c00464

MMFF Geometry

C -3.108486 2.1407570 .180397

C $0.1909520 .485095-0.144328$

O $-0.8770141 .435143-0.277764$

C -2.0956051 .0118820 .342782$

C $-2.590367-0.289621-0.303617$

C -1.516499-1.375394 -0.221987

C $-0.207518-0.843228-0.813150$

C $1.4272981 .121700-0.789281$

O $-3.786778-0.742541 \quad 0.317996$

O $-1.316331-1.7229881 .151543$

O $0.818244-1.813697-0.648124$

C $2.722651 \quad 0.450848-0.406287$

$\begin{array}{llll}\text { O } & 2.970872 & 0.630688 & 0.922210\end{array}$

O $3.437220-0.148708-1.199433$

C 4.1772410 .0345551 .408509

H -4.0581991 .9048820 .669299$

H -2.7167773 .0675720 .613639$

H -3.299219 2.346494 -0.878674

H $\quad 0.3894350 .3292220 .923864$

H $-1.922500 \quad 0.8744351 .417895$

H -2.816159-0.107481-1.361668

H $-1.844310-2.289722-0.728823$
H $-0.350223-0.678483-1.888688$

H $1.5062102 .180898-0.512515$

H $1.3365441 .116361-1.882307$

H -3.579363 -0.937565 1.247994

H $-0.589186-2.367991 \quad 1.182947$

H $0.529955-2.625400-1.099333$

H $4.150293-1.0493791 .261422$

H 5.0450660 .4721130 .905821

H 4.2507260 .2413032 .479463

SCF Energy (B3LYP/6-31G**//MMFF) $=-803.937670585$

F1-11 c00465

MMFF Geometry

C -1.095353 $2.849466-0.063664$

C $0.207513-0.613654-0.072396$

O $0.0575410 .787325-0.336921$

C -1.1283291 .3529330 .232661$

C $-2.3727600 .673606-0.359830$

C $-2.316011-0.845855-0.171818$

C $-0.975573-1.381955-0.683308$

C $1.536447-1.061636-0.686819$

$\begin{array}{lllll}\text { O } & -3.566750 & 1.168770 & 0.232381\end{array}$

O $-2.492253-1.1887491 .205646$

O $-0.875404-2.773800-0.408738$

C $2.698440-0.4975380 .095464$

O $3.1650230 .632673-0.507678$

O $3.120989-0.9895381 .133994$

C $4.254244 \quad 1.279720 \quad 0.157456$

H $-0.1613843 .288703 \quad 0.304210$

H -1.124994 $3.035646-1.142922$

H -1.9302373 .3757540 .407684$

H $0.256102-0.7700311 .012920$

H $-1.096143 \quad 1.2358951 .322824$

H -2.418775 $0.874737-1.438168$

H -3.146271 -1.327750 -0.699612

H $-0.954815-1.258681-1.773464$

H $1.620305-0.740702-1.732257$

H $1.644203-2.152082-0.677200$

H $-3.6795352 .091392-0.051589$

H $-1.797778-0.7605041 .730806$

H $-0.934306-2.8887010 .555253$

H $\begin{array}{llll}5.117411 & 0.609427 & 0.211515\end{array}$

H $4.5316142 .162719-0.424323$

H 3.9497081 .6002051 .158366

SCF Energy $\left(B 3 L Y P / 6-31 G^{* *} / / M M F F\right)=-803.931821836$

F1-12 c00494

MMFF Geometry

C -1.036144 $2.612527-0.551380$

C $0.188730-0.180881-0.377630$

O -0.0902280 .7352900 .692106$

C $-1.2568621 .553506 \quad 0.526075$

C -2.5076720 .6986190 .288871$

C $-2.275464-0.335163-0.815992$

C $-0.994361-1.138444-0.582722$

C $1.473349-0.934987-0.009164$

$\begin{array}{llll}\text { O } & -2.818558 & 0.038796 & 1.519329\end{array}$

O $-3.386016-1.219543-0.919012$

O $-1.131634-1.9746300 .566062$

C $2.704864-0.059784-0.121486$

O $3.803619-0.8012100 .211111$

O $2.7100111 .117020-0.455137$

C $5.053826-0.107096 \quad 0.154234$

H $-1.8663003 .325726-0.569789$

H $-0.1084523 .161930-0.358442$

H $-0.9437972 .178974-1.551222$

H $0.3591810 .388529-1.298821$

H -1.372946 2.0796361 .481534

H -3.372139 1.3285750 .050901

H $-2.1889680 .181314-1.779476$

H $-0.806877-1.802789-1.434151$

H $1.612827-1.783355-0.688733$

H $1.420829-1.2940271 .025344$

H $-3.573506-0.5502841 .349638$

H $-4.178087-0.677373-1.075314$

H - $1.926168-2.5195480 .433641$

H $5.0542210 .730700 \quad 0.858061$

H $5.844051-0.8062690 .440604$ 
H $\quad 5.2469730 .242689-0.864294$

SCF Energy (B3LYP/6-31G**//MMFF) $=-803.926035599$

F1-12_c00495

MMFF Geometry

C $-0.6483322 .521028-0.768527$

C $0.257281-0.328044-0.152248$

O -0.0820390 .7131780 .776166$

C -1.123939 1.6069580 .358388

C -2.4091760 .8402810 .016925$

C $-2.127089-0.319561-0.940682$

C $-0.972220-1.198628-0.451794$

C $1.390546-1.1444360 .473811$

O $-2.9606890 .320345 \quad 1.230705$

O $-3.308404-1.091266-1.127862$

O $-1.356799-1.9273350 .714022$

C $2.682282-0.3627300 .461561$

O $3.270335-0.453961-0.765669$

$\begin{array}{lllll}\text { O } & 3.106820 & 0.266886 & 1.421778\end{array}$

C $4.4995410 .263626-0.912630$

H $-0.4616371 .977093-1.698710$

H -1.391153 $3.297517-0.976928$

H $0.2941153 .005447-0.491378$

H $\quad 0.6158370 .131587-1.080597$

H $-1.3178482 .242540 \quad 1.230721$

H $-3.1700431 .503933-0.409273$

H -1.861245 $0.094078-1.920866$

H $-0.731348-1.948523-1.213357$

H $1.554706-2.086193-0.062659$

H $1.173494-1.3990661 .518122$

H $-3.179031 \quad 1.0767791 .801403$

H -3.086248 -1.828791 -1.721218

H -1.531934 -1.285449 1.423019

H $5.246784-0.115959-0.209042$

H $4.8650600 .106919-1.930925$

H $4.3333491 .334343-0.760257$

SCF Energy (B3LYP/6-31G**//MMFF) $=-803.925104029$

F1-12 c00496

MMFF Geometry

C $-0.6071532 .369008-1.131740$

C $0.205701-0.469920-0.360727$

O $0.099571 \quad 0.6821810 .489183$

C -0.9390661 .6130130 .152804$

C $-2.311040 \quad 0.9256330 .115113$

C $-2.273280-0.344857-0.738346$

C $-1.110358-1.261746-0.350298$

C $1.376913-1.3103660 .153118$

$\begin{array}{lllll}\text { O } & -2.680969 & 0.577421 & 1.452574\end{array}$

O $-3.502211-1.056933-0.644841$

O $-1.329747-1.8323050 .940139$

C $2.691306-0.668048-0.220910$

O 3.1189340 .1446530 .786816

O $3.254159-0.846906-1.293459$

C 4.3463060 .8373040 .539294

H $-0.6213571 .722378-2.013573$

H -1.322044 $3.180684-1.299202$

H $0.3989852 .797482-1.070266$

H $0.426232-0.136306-1.381590$

H -0.9375992 .3454210 .969092$

H $-3.087797 \quad 1.604937-0.253629$

H -2.150502 -0.062619-1.790971

H -1.056228 -2.103409-1.049749

H $1.371951-2.313033-0.290423$

H $1.343821-1.4382871 .241628$

H -2.7433331 .4019991 .964182$

H -4.216524 -0.441806 -0.883049

H -1.336499 -1.1085141 .589330$

H 5.1601080 .1228090 .382785

H $4.2397931 .498442-0.326053$

H $4.577117 \quad 1.4448151 .418352$

SCF Energy (B3LYP/6-31G**//MMFF) $=-803.924422205$

F1-12 c00497

MMFF Geometry

C -1.531267 $2.798026-0.363061$

C $0.117158-0.486456-0.933234$

O $-0.3077720 .878049-1.059432$
C $-1.1991201 .343505-0.040308$

C -2.4652310 .4775270 .008165$

C $-2.114579-0.9972430 .208006$

C $-1.106062-1.420276-0.860105$

C $1.146841-0.6587280 .197244$

$\begin{array}{lllll}\text { O } & -3.338612 & 0.913007 & 1.043228\end{array}$

O $-1.565478-1.1785021 .513146$

O $-0.723703-2.772999-0.651617$

C $2.4490050 .058223-0.099912$

O $3.313243-0.1508450 .938127$

O $2.6839840 .715415-1.104331$

C 4.5959870 .4695850 .805160

H $-0.6129013 .392253-0.425012$

H $-2.0238922 .880056-1.338218$

H $-2.180283 \quad 3.2428760 .397019$

H $0.636401-0.725071-1.870929$

H -0.6885081 .3407610 .929251$

H $-3.0062360 .575630-0.941212$

H $-3.016622-1.6180780 .167268$

H $-1.622933-1.374318-1.827977$

H $1.387404-1.7197290 .324947$

H $\quad 0.774640-0.2609391 .144809$

H $-2.8751980 .790829 \quad 1.889670$

H -1.301637 -2.111750 1.587843

H $-0.105694-3.015193-1.362228$

H $4.4863551 .556397 \quad 0.742904$

H 5.1846840 .2267001 .693715

H $5.1147350 .081594-0.076553$

SCF Energy $\left(B 3 L Y P / 6-31 G^{* *} / / M M F F\right)=-803.935154978$

F1-12 c00498

MMFF Geometry

C 2.2654582 .3233230 .889599

C $-0.131306 \quad 0.6348150 .014956$

O $0.8212641 .325574-0.809055$

C $2.1636131 .378045-0.305074$

C $2.706327-0.026240-0.014462$

C $1.726161-0.8387110 .833743$

C $0.312343-0.8191120 .246113$

C $-1.4861380 .750387-0.695784$

O $2.920798-0.673868-1.272032$

O $2.198528-2.1752390 .964894$

O $0.280067-1.530419-0.990779$

C -2.6462310 .2855660 .154190$

O

$\begin{array}{lllll}\text { O } & -2.622799 & 0.168421 & 1.371843\end{array}$

C $-4.911417-0.3809520 .020636$

H 1.7288351 .9490341 .766025

H $3.3109982 .475700 \quad 1.175567$

H 1.8266893 .2959040 .642112

H $-0.198716 \quad 1.1549180 .977786$

H $2.7549791 .816209-1.118528$

H 3.6855110 .0259960 .474667

H $1.695427-0.4112251 .843120$

H $-0.377773-1.3357250 .921946$

H $-1.4699730 .185620-1.635162$

H -1.671721 $1.798462-0.961316$

H $3.211461-1.582495-1.082656$

H $1.558260-2.6594811 .513646$

H $\quad 0.636550-2.419729-0.824035$

H $-5.688051-0.542105-0.731664$

H -4.727773 -1.3246220 .543164$

$\mathrm{H}-5.2536810 .3882080 .719579$

SCF Energy $($ B3LYP/6-31G**//MMFF $)=-803.928337631$

F1-12 c00499

MMFF Geometry

C $-1.4023472 .824292-0.344581$

C $0.109192-0.518211-0.957867$

O $\quad-0.2660040 .862629-1.071368$

C $-1.1303951 .353128-0.040971$

C -2.4312730 .5402870 .003795$

C $-2.142257-0.9503230 .191261$

C $-1.150799-1.402734-0.883054$

C $1.145632-0.7295840 .159453$

O -3.2840931 .0034351 .043979$

O $-1.602838-1.1597051 .495573$

O $-0.826443-2.777868-0.736014$ 
C $2.446084-0.001668-0.118975$

O $3.283658-0.1733130 .947269$

$\begin{array}{llll}\text { O } & 2.703033 & 0.628502 & -1.135381\end{array}$

C 4.5627630 .4583920 .832503

H -1.896877 2.939011 -1.315579

H -2.027158 3.2872150 .424932

H $-0.4598793 .379600-0.405637$

H $0.611596-0.769741-1.901343$

H $-0.614898 \quad 1.3165050 .925423$

H -2.969567 $0.668365-0.943666$

H $-3.070910-1.5304720 .143843$

H $-1.665999-1.310309-1.848150$

H $1.391934-1.7925920 .249756$

H $0.774922-0.3659801 .121344$

H -2.8232590 .8588201 .888177$

H -1.569309 -2.118980 1.648710

H $-0.461080-2.9137210 .153418$

H 4.4429451 .5415310 .733883

H $\quad \begin{array}{lllll}5.128115 & 0.249736 & 1.744723\end{array}$

H $5.1113190 .050093-0.021693$

SCF Energy (B3LYP/6-31G**//MMFF) $=-803.931041612$

F1-12_c00500

MMFF Geometry

C $2.708843-2.136226-0.278338$

C $0.034036-0.767571-0.896694$

O $0.473012-1.367251 \quad 0.328412$

C $1.870853-1.233902 \quad 0.624675$

C $2.318190 \quad 0.233210 \quad 0.594104$

C $1.856406 \quad 0.933457-0.687263$

C $0.3586330 .734208-0.932874$

C $-1.449027-1.099647-1.100566$

O 1.7529880 .8798961 .737952

O $2.1553122 .324472-0.635738$

$\begin{array}{lllll}\text { O } & -0.392415 & 1.428921 & 0.059631\end{array}$

C $-2.359272-0.621443 \quad 0.008545$

O $-3.269904 \quad 0.264833-0.487747$

O $-2.274775-0.988967 \quad 1.173214$

C $-4.185970 \quad 0.805350 \quad 0.469405$

H $3.753924-2.1479250 .046957$

H $2.324262-3.161448-0.251907$

H $2.686026-1.817844-1.324423$

H $0.568857-1.254876-1.721261$

H $1.980140-1.6080631 .650086$

H 3.4056630 .3115560 .703838

H $2.4062690 .521033-1.541587$

H $0.0745791 .163215-1.900481$

H $-1.584775-2.187062-1.154870$

H -1.789653 -0.692101-2.059832

H $\quad 1.984707 \quad 1.8226301 .682086$

H $3.1120342 .412620-0.485177$

H $-0.1090782 .359033 \quad 0.040426$

H -3.6432541 .3535961 .245395$

H $-4.8506831 .500051-0.051030$

H -4.7889050 .0057370 .910023$

SCF Energy (B3LYP/6-31G**//MMFF) $=-803.918403507$

F1-12_c00501

MMFF Geometry

C $-0.5850012 .405421-1.103815$

C $0.193343-0.450491-0.381634$

$\begin{array}{lllll}\text { O } & 0.092567 & 0.682639 & 0.495025\end{array}$

C $-0.9300591 .634963 \quad 0.168620$

C $-2.309806 \quad 0.961962 \quad 0.120067$

C $-2.286001-0.291350-0.758563$

C $-1.130611-1.229959-0.402440$

C $1.353016-1.3145220 .119097$

O -2.7183170 .6029581 .444562$

O $-3.529850-0.978931-0.685854$

$\begin{array}{lllll}\text { O } & -1.363527 & -1.850688 & 0.861535\end{array}$

C $2.676196-0.675857-0.229330$

O $3.101544 \quad 0.1113760 .799359$

O $3.247337-0.836817-1.300333$

C $4.337013 \quad 0.798196 \quad 0.577808$

H $-0.6084371 .773585-1.996112$

H $-1.2872903 .230543-1.259095$

H $0.4275012 .817389-1.034500$

H $0.424822-0.094223-1.392260$
H $-0.9232702 .356107 \quad 0.995115$

H $-3.0713201 .659370-0.245471$

H $-2.1648000 .018374-1.803089$

H $-1.082436-2.044407-1.133967$

H $1.341716-2.305611-0.349400$

H $1.311020-1.4691821 .203923$

H -1.9554240 .6776292 .041737$

H $-3.651806-1.2620650 .236595$

H $-1.262135-1.1754931 .552371$

H $\begin{array}{llll}5.146901 & 0.079794 & 0.418892\end{array}$

H $4.2462221 .474575-0.277494$

H 4.5621551 .3891391 .469539

SCF Energy (B3LYP/6-31G**//MMFF) $=-803.932709616$

F1-12_c00502

MMFF Geometry

C -2.285665 $2.363946-0.525812$

C $0.0214520 .437274-1.128351$

$\begin{array}{lllll}\text { O } & -0.154068 & 1.296642 & 0.004607\end{array}$

C $-1.507581 \quad 1.480048 \quad 0.446551$

$\begin{array}{llll}\text { C } & -2.197459 & 0.138785 & 0.727359\end{array}$

C $-2.017078-0.840986-0.435301$

C $-0.547615-0.963878-0.853329$

C $1.4977390 .460006-1.544173$

O $-1.613789-0.4024791 .916408$

O $-2.551626-2.111337-0.079585$

O $0.190627-1.630745 \quad 0.167480$

C $2.473210-0.086150-0.527813$

$\begin{array}{lllll}\text { O } & 2.558951 & 0.748878 & 0.545779\end{array}$

O $3.096262-1.129444-0.680240$

$\begin{array}{llll}\text { C } 3.446331 & 0.326302 & 1.585438\end{array}$

H -3.263072 $2.629761-0.110618$

H $-1.7302673 .286134-0.727763$

H $-2.4522811 .877744-1.491114$

H $-0.5317410 .873617-1.969179$

H -1.424167 2.031146 1.391401

H -3.2613630 .2836570 .946215$

H $-2.598856-0.479862-1.291966$

H $-0.468187-1.590223-1.748597$

H $1.8107531 .488045-1.766536$

H $1.628226-0.113210-2.469996$

H -2.020132 -1.273503 2.065903

H $-2.400275-2.713131-0.828120$

H $\quad 0.193625-1.0613550 .955736$

H $3.123803-0.6379531 .990013$

H $4.4709960 .263974 \quad 1.206690$

H $3.412077 \quad 1.0709582 .384927$

SCF Energy (B3LYP/6-31G**//MMFF) $=-803.930215257$

F1-13_c00543

MMFF Geometry

C -1.050173 $2.763986 \quad 0.731021$

C $0.207829-0.7383990 .698789$

$\begin{array}{lllll}\text { O } & -0.094779 & 0.613213 & 1.078963\end{array}$

C -0.8172231 .3850990 .117702$

C $-2.1415580 .713019-0.260453$

C $-1.897676-0.726876-0.709904$

C $-1.078481-1.4887030 .331658$

C $1.268263-0.800980-0.410513$

O $-2.7489491 .443280-1.323931$

O $-3.153916-1.372845-0.907172$

O $-1.847376-1.6279521 .532030$

C $2.607158-0.255746 \quad 0.040919$

O $3.481063-0.310309-1.008323$

$\begin{array}{lllll}\text { O } & 2.861536 & 0.159739 & 1.162809\end{array}$

C $4.800006 \quad 0.173679-0.735940$

H $-0.094768 \quad 3.221211 \quad 1.011174$

H -1.5639943 .4347580 .036118$

H -1.642406 2.6881291 .649751

H $0.624617-1.2010131 .602670$

H $-0.1973561 .535381-0.773833$

H -2.8379720 .7265650 .587163$

H $-1.386950-0.728597-1.680197$

H $-0.857041-2.504014-0.015714$

H $0.960026-0.231131-1.291921$

H $1.424180-1.845611-0.703923$

H -3.568469 $0.977450-1.563795$

H $-2.971652-2.283483-1.195410$ 
H $-2.683428-2.0626191 .291536$

H $5.268628-0.4259970 .050225$

H $5.392490 \quad 0.078666-1.649735$

H $4.764597 \quad 1.228829-0.448782$

SCF Energy (B3LYP/6-31G**//MMFF) $=-803.930404007$

F1-13_c00544

MMFF Geometry

C -2.702595 2.4263400 .154652

C $0.404150 \quad 0.4307490 .589707$

O $-0.642762 \quad 1.3997620 .762787$

C $-1.7089031 .319011-0.186653$

C $-2.376490-0.060509-0.154532$

C -1.333413-1.160484 -0.350381

C $-0.172138-0.9910350 .629705$

C $1.2239540 .742039-0.665885$

O $-3.343882-0.136673-1.199165$

O $-1.952679-2.430621-0.155758$

O $-0.643506-1.2171621 .964042$

C $2.5650500 .053694-0.643515$

O $3.431594 \quad 0.7454050 .150065$

O $2.807743-0.982726-1.248163$

C 4.7417230 .1819200 .266340

H -3.1090662 .2897071 .162953$

H -2.202201 3.4009690 .148014

H $-3.5326062 .459168-0.557274$

H 1.0497220 .5595181 .467740

H -1.321812 $1.532098-1.190618$

H $-2.904620-0.2121430 .795054$

H - $0.972130-1.143867-1.385201$

H $0.603234-1.7420770 .444781$

H $1.3884851 .822637-0.763477$

H $0.7048380 .449303-1.584736$

H $-3.726074-1.030707-1.175031$

H -1.271371 -3.111052 -0.291383

H -1.050573 -2.100305 1.981560

H 5.3322290 .8268410 .922606

H $5.2253920 .141595-0.714218$

H $4.686459-0.8159280 .712142$

SCF Energy (B3LYP/6-31G**//MMFF) $=-803.932420017$

F1-13 c00545

MMFF Geometry

C -0.575216 26107051.000233

C $0.233520-0.9826480 .465860$

O $0.1349380 .339066 \quad 1.019833$

C $-0.5792891 .292858 \quad 0.229950$

C $-2.0092620 .822387-0.060801$

C -1.999984 -0.581931-0.665995

C $-1.166299-1.5426930 .181843$

C $1.150453-1.026133-0.759917$

O $-2.6452161 .722678-0.966306$

O $-3.331967-1.080119-0.767234$

O $-1.820455-1.7710751 .435743$

C $2.573699-0.708600-0.369487$

O $2.817848 \quad 0.622342-0.536350$

O $3.363527-1.5406750 .057987$

C $4.1306811 .057729-0.170159$

H -1.074998 2.4989631 .968887

H -1.070546 3.4085430 .439039

H 0.4533392 .9231661 .212371

H $0.689453-1.5874231 .260277$

H $-0.038778 \quad 1.471689-0.706694$

H -2.610533 0.8211250 .855903

H -1.600793 -0.545661 -1.686819

H -1.107519 -2.520991-0.307421

H $0.840956-0.340918-1.555292$

H $1.154219-2.028378-1.204915$

H -2.121280 $1.743975-1.785208$

H -3.848168 -0.428731 -1.272389

H $-1.750657-0.9593341 .966194$

H $4.8822400 .554065-0.785412$

H $4.1940452 .134250-0.349532$

H 4.3098020 .8678810 .892455

SCF Energy (B3LYP/6-31G**//MMFF) $=-803.929365906$

F1-13 c00546

MMFF Geometry
C -1.1609002 .6419010 .985923$

C $0.377339-0.6928790 .382501$

O 0.0252870 .5787250 .950240

C -0.9157821 .3536420 .204248$

C $-2.2212890 .581366-0.020604$

C $-1.933376-0.782777-0.650779$

C $-0.877866-1.5436960 .151988$

C $1.229078-0.538231-0.881238$

O $\quad-3.0886311 .325267-0.873552$

O $-3.130931-1.552290-0.735566$

O $-1.404456-1.8775121 .440910$

C $2.5403810 .139023-0.564854$

O $3.430248-0.771558-0.076887$

O $2.7409691 .337157-0.716204$

C $4.712292-0.2465350 .281147$

H -1.5773812 .4253891 .975875$

H -1.8438893 .3124580 .456097$

H -0.2158663 .1706331 .152333$

H $0.985979-1.1842771 .152301$

H $-0.4727621 .643931-0.755502$

H -2.7514840 .4413660 .929156$

H -1.588711 -0.646316 -1.682292

H $-0.625234-2.490674-0.338325$

H $0.7341140 .054900-1.656568$

H $1.443899-1.514328-1.332722$

H $-2.6322351 .459402-1.721651$

H -3.547336 -1.560399 0.142796

H $-2.123988-2.5168761 .304311$

H $4.607740 \quad 0.500236 \quad 1.074175$

H $5.325257-1.0712040 .654352$

H $5.202748 \quad 0.186559-0.595869$

SCF Energy (B3LYP/6-31G**//MMFF) $=-803.927203291$

F1-13 c00547

MMFF Geometry

C $-1.311948-2.160745-1.179010$

C $0.0814110 .330929-0.060767$

O $-0.177891-0.9206500 .586489$

C $-1.405882-1.5612870 .222046$

C $-2.616379-0.6331490 .428692$

C $-2.4048160 .768318-0.142466$

C $-1.0387391 .322120 \quad 0.277059$

C $1.4419230 .833908 \quad 0.444824$

O $-2.844605-0.4915091 .834154$

O $-2.5007270 .709434-1.563516$

O $-0.8054122 .567631-0.370829$

C $2.591384-0.014272-0.060012$

O 3.7524420 .4384880 .500591

O $2.492288-0.945949-0.846537$

C $4.937057-0.2658330 .115018$

H - $-1.171209-1.405404-1.955675$

H -0.447692 -2.831159-1.241828

H $-2.212573-2.734300-1.419055$

H $\quad 0.1507000 .186934-1.145459$

H -1.500273 -2.404313 0.917766

H $-3.514789-1.088401-0.004296$

H $-3.193428 \quad 1.4472690 .203435$

H -1.0457981 .5016661 .359750$

H 1.4635590 .8032251 .540681

H 1.6230991 .8588190 .103629

H $-3.043310-1.3746132 .189408$

H -2.302631 $1.598128-1.905469$

H $-1.5267063 .168687-0.117790$

H $4.867751-1.3159650 .414141$

H $5.093970-0.180464-0.964502$

H $5.7865060 .190410 \quad 0.630155$

SCF Energy $\left(B 3 L Y P / 6-31 G^{* *} / / M M F F\right)=-803.930810434$

F1-13 c00548

MMFF Geometry

C $3.076442-2.024343-0.284712$

C $-0.265975-0.7506690 .734332$

O $0.958026-1.5011060 .669470$

C $1.852354-1.117061-0.378188$

C $2.2430910 .361240-0.259082$

C $0.9943051 .242091-0.198258$

C $0.0321810 .747145 \quad 0.881116$

C $-1.174963-1.098408-0.448040$ 
O $3.039626 \quad 0.749155-1.376458$

O $1.3607872 .590500 \quad 0.087278$

O $0.630714 \quad 0.932763 \quad 2.170167$

C $-2.604475-0.699380-0.180387$

O $-2.9268940 .402697-0.915460$

O $-3.339511-1.2844730 .604660$

C $-4.2571910 .896711-0.731789$

H $2.775747-3.075977-0.349577$

H $3.793617-1.819581-1.085067$

H $3.581332-1.9020190 .679893$

H $-0.752810-1.0914951 .657010$

H $\quad 1.381126-1.311645-1.349296$

H 2.8500140 .5285830 .638789

H $\quad 0.4934341 .254530-1.173035$

H $-0.887828 \quad 1.3411370 .880493$

H $-1.165211-2.179500-0.634518$

H $-0.831401-0.645324-1.384183$

H $2.5189910 .595415-2.183220$

H $2.0094862 .863139-0.584145$

H 0.8595281 .8748042 .248605

H $-4.387396 \quad 1.770456-1.375933$

H $-4.9879710 .135463-1.021163$

H -4.4072651 .2017660 .308150$

SCF Energy $\left(B 3 L Y P / 6-31 G^{* *} / / M M F F\right)=-803.933504574$

F1-13 c00549

MMFF Geometry

C $-2.719012-1.383593-1.431350$

C $-0.0572770 .065322-0.879931$

O $-0.468281-1.253811-0.510559$

C -1.853534 -1.397353-0.173407

C $-2.295434-0.3898990 .903114$

C -1.8356131 .0407950 .618961$

C $-0.3485991 .060502 \quad 0.249710$

C $1.4235150 .016200-1.286207$

O $-1.722712-0.8137412 .143895$

O $-2.6196961 .579830-0.442100$

O $0.0389432 .372235-0.141751$

C $2.369403-0.358275-0.163177$

O $3.6016270 .172196-0.420434$

O $2.079330-1.0563540 .798722$

C $4.611129-0.1163890 .552023$

H -3.766365 -1.587276-1.187436

H $-2.671493-0.433626-1.968808$

H -2.372001 -2.150729 -2.132367

H $-0.6009940 .366155-1.783966$

H $-1.935557-2.4044650 .254453$

H $-3.385074-0.4323141 .015099$

H -2.0004091 .6808371 .493765$

H $\quad 0.2295920 .793150 \quad 1.141704$

H $\quad 1.7178200 .994922-1.682895$

H $1.565622-0.731491-2.075559$

H -2.044184 -0.2106782 .835656$

H $-2.2660642 .462551-0.645824$

H -0.1542052 .9687730 .601484$

H $\begin{array}{llll}5.536383 & 0.373396 & 0.237217\end{array}$

H 4.3199590 .2791551 .529795

H $4.785817-1.1950490 .607076$

SCF Energy (B3LYP/6-31G**//MMFF) $=-803.925429309$

F1-13_c00550

MMFF Geometry

C $2.713273-2.3852650 .140525$

C $-0.402078-0.4125640 .587520$

O $0.658727-1.3656140 .759662$

C $1.718507-1.277857-0.197752$

C $2.3772560 .108021-0.171365$

C $1.3233491 .203572-0.338114$

C $0.159506 \quad 1.0142760 .636527$

C $-1.214625-0.726990-0.671881$

O $3.3138260 .226852-1.240766$

O $1.9335472 .475993-0.127492$

O $0.609422 \quad 1.270373 \quad 1.972960$

C $-2.565732-0.058578-0.647541$

O $-3.421523-0.7639810 .145654$

O $-2.8244560 .974336-1.251339$

C $-4.739754-0.2199550 .263040$

H $3.148493-2.2293631 .133811$
H $\quad 2.205637-3.3558310 .169931$

H $3.522552-2.444608-0.592624$

H $-1.047749-0.5530381 .463558$

H $1.326029-1.493804-1.198900$

H 2.9249030 .2687770 .765465

H $0.957638 \quad 1.201579-1.371827$

H -0.6207181 .7563610 .438672$

H -1.363162 -1.809143 -0.777554

H $-0.697975-0.419413-1.587388$

H $4.069307-0.350105-1.038864$

H $1.2416073 .151954-0.226777$

H 1.2026430 .5466372 .236159

H $-5.319656-0.8727840 .920773$

H -5.225154 -0.188060 -0.717084

H -4.6989590 .7792090 .707450$

SCF Energy $(B 3 L Y P / 6-31 G * * / / M M F F)=-803.925164992$

F1-13_c00551

MMFF Geometry

C $-2.405660-1.381603-1.608791$

C $-0.0244580 .425402-0.879039$

O $-0.155551-0.993923-0.757944$

C $-1.462866-1.466372-0.410618$

C $-2.005144-0.7863730 .860262$

C -1.8394580 .7343930 .849904$

C $-0.413506 \quad 1.1115710 .436611$

C $1.4057850 .742184-1.333230$

O $-1.280688-1.2876841 .987793$

O $-2.768091 \quad 1.321202-0.058424$

O -0.2975732 .5250350 .324943$

C $2.4560720 .416300-0.296832$

O $2.713504-0.922364-0.292331$

O $2.978178 \quad 1.2539220 .427703$

C $3.680443-1.3594940 .667278$

H -2.576493 -0.355444 -1.942840

H $-1.974163-1.915096-2.463047$

H -3.375666 -1.831174 -1.374938

H $-0.6771090 .774855-1.688162$

H -1.326908 -2.533246 -0.194000

H -3.058342 -1.053559 1.005311

H $-2.045566 \quad 1.159987 \quad 1.839308$

H $\quad 0.2638020 .789312 \quad 1.236022$

H $1.503773 \quad 1.805131-1.583502$

H $1.6524100 .184609-2.245323$

H -1.434312 -2.246808 2.032990

H $-3.6615391 .079557 \quad 0.239771$

H $-0.9319682 .819665-0.350844$

H $3.790435-2.4427770 .569882$

H $4.648622-0.8891920 .471065$

H $3.337554-1.1313831 .681124$

SCF Energy $\left(B 3 L Y P / 6-31 G^{* *} / / M M F F\right)=-803.925461387$

F1-13_c00552

MMFF Geometry

C $0.884627-2.1042071 .316841$

C $-0.1360070 .380955-0.167162$

O $0.153538-0.923611-0.685724$

C $1.257947-1.601302-0.075867$

C $2.540083-0.753019-0.116864$

C 2.3190240 .6926310 .328280

C $1.0857201 .288469-0.359548$

C $-1.3604580 .903549-0.926222$

O $3.003124-0.745629-1.470647$

O $2.160593 \quad 0.721057 \quad 1.745167$

O $\quad 0.8568712 .588307 \quad 0.172675$

C $-2.5860430 .078578-0.611137$

$\begin{array}{llll}\text { O } & -3.066491 & 0.409462 & 0.621598\end{array}$

O $-3.053433-0.763286-1.367260$

C $-4.223387-0.3181101 .045516$

H $0.658904-1.2943552 .014511$

H $-0.017945-2.7229841 .261724$

H $1.691836-2.7077701 .743264$

H $-0.3966540 .309516 \quad 0.895201$

H $1.421430-2.490001-0.698583$

H $3.315128-1.2339690 .491122$

H $3.195847 \quad 1.3080460 .094276$

H $1.3052911 .392445-1.429924$

H -1.198606 $0.850137-2.009699$ 
H $-1.5889311 .946888-0.684379$ H $3.843062-0.256029-1.489556$ H $1.964814 \quad 1.640022 \quad 1.996415$ H $\quad 0.2405493 .048759-0.420901$ H $-3.997253-1.3870911 .105806$ H -4.5030150 .0376792 .040658$ H $-5.057111-0.1369620 .360574$

SCF Energy (B3LYP/6-31G**//MMFF) $=-803.929712046$

F1-13_c00553

MMFF Geometry

C $0.861484-1.9573101 .535179$

C $-0.0955700 .505331-0.014272$

O $-0.055576-0.861915-0.440499$

C $1.045987-1.6312020 .055150$

C $2.393820-0.969516-0.279343$

C 2.4309850 .5271350 .038116

C $1.1722741 .228269-0.484303$

C $-1.352826 \quad 1.135722-0.622180$

O $2.649693-1.142546-1.676608$

$\begin{array}{llll}0 & 2.524073 & 0.718033 & 1.448616\end{array}$

O $1.1543232 .583348-0.048655$

C -2.5926660 .5934580 .048457$

O $-3.124791-0.416839-0.696528$

O -3.0191411 .0022801 .120903$

C $-4.292904-1.035468-0.148545$

H $1.646992-2.6336681 .886220$

H $\quad 0.869610-1.0680232 .169905$

H $-0.108684-2.4408531 .694239$

H $-0.183970 \quad 0.553302 \quad 1.077706$

H $0.992951-2.583023-0.488055$

H $3.197926-1.4910370 .251889$

H $3.3133711 .000374-0.408820$

H $1.1974091 .232158-1.581209$

H $-1.4152000 .940985-1.699750$

H -1.369906 $2.223576-0.492410$

H $1.865095-0.843485-2.166850$

H $3.3409490 .284973 \quad 1.749836$

H $1.9779443 .000683-0.353682$

H $-4.617566-1.819413-0.837829$

H $-4.060899-1.4922840 .818288$

H $-5.098181-0.301668-0.046864$

SCF Energy (B3LYP/6-31G**/MMFF)= -803.925158257

F1-14_c00600

MMFF Geometry

C -1.447541 2.7173090 .751645

C $0.273784-0.380506-0.319274$

$\begin{array}{lllll}\text { O } & -0.063298 & 0.806092 & 0.416274\end{array}$

C -1.259585 $1.434480-0.051928$

C $-2.461096 \quad 0.493503 \quad 0.097235$

C $-2.179417-0.837640-0.601616$

C $-0.840334-1.422503-0.148421$

C $1.617491-0.889480 \quad 0.215894$

O $-3.6090751 .101584-0.489648$

O $-3.235043-1.751047-0.312839$

O $-0.919830-1.7956741 .229116$

C $2.776144-0.015479-0.218001$

$\begin{array}{llll}\text { O } & 3.934314 & -0.538257 & 0.284931\end{array}$

O $2.685175 \quad 0.992380-0.905142$

C $5.1267580 .183654-0.039501$

H -1.5548842 .4973731 .819561$

H $-0.566459 \begin{array}{lll}3.360587 & 0.651607\end{array}$

H $-2.325470 \quad 3.278526 \quad 0.418310$

H $\quad 0.372702-0.118022-1.380839$

H $-1.125548 \quad 1.718949-1.104038$

H $-2.6852250 .318074 \quad 1.156668$

H $-2.176678-0.685580-1.688225$

H $-0.611447-2.332660-0.714107$

H $1.809387-1.898836-0.165383$

H $1.610996-0.9030641 .311991$

H $-4.3439450 .469402-0.410515$

H $-3.034201-2.584070-0.772510$

H $-1.667574-2.4107831 .320091$

H $5.973011-0.3338010 .420283$

H $5.2743830 .203464-1.123516$

H $5.0757851 .199794 \quad 0.362885$

SCF Energy (B3LYP/6-31G**//MMFF) $=-803.936917758$
F1-14 c00601

MMFF Geometry

C -1.463129 2.6825540 .772741

C $0.275464-0.388787-0.333515$

$\begin{array}{lllll}\text { O } & -0.078334 & 0.780542 & 0.420819\end{array}$

C $-1.2736641 .411319-0.049053$

C -2.471857 $0.459594 \quad 0.075015$

C $-2.169602-0.861724-0.638119$

C $-0.828854-1.444565-0.187248$

C $1.621461-0.8932440 .200749$

O $-3.6273291 .036556-0.527879$

O $-3.214922-1.802810-0.405740$

O $-0.909356-1.8430421 .182603$

C $2.7732680 .000283-0.211038$

$\begin{array}{lllllll} & & & \\ \text { O } & 3.935195 & -0.523739 & 0.281832\end{array}$

$\begin{array}{lllll}\text { O } & 2.674467 & 1.023078 & -0.874625\end{array}$

C $5.1217850 .215703-0.024051$

H $-2.316206 \quad 3.270508 \quad 0.422696$

H $-1.6097592 .445017 \quad 1.832182$

H -0.5667343 .3098990 .713826$

H $0.378393-0.106675-1.389651$

H $-1.1314391 .711309-1.095657$

H -2.7082250 .2601891 .127629$

H -2.146331 -0.691691 -1.721612

H $-0.587513-2.340769-0.770161$

H $1.825725-1.893724-0.197136$

H $1.609196-0.9260591 .296389$

H $-3.909183 \quad 1.783616 \quad 0.026065$

H -3.356789 -1.8645260 .553970$

H $-1.532074-2.5878021 .232930$

H 5.0619291 .2213890 .402899

H $5.972055-0.3054130 .423794$

H $5.2699650 .263250-1.107128$

SCF Energy (B3LYP/6-31G**//MMFF)= -803.931929766

F1-14 c00602

MMFF Geometry

C $2.959958-2.3323650 .225977$

C $-0.236685-0.526387-0.260410$

$\begin{array}{lllll}\text { O } & 0.744058 & -1.453178 & 0.233434\end{array}$

C $2.046755-1.237633-0.316365$

C 2.5660590 .1595680 .043876

C $1.557664 \quad 1.225468-0.387473$

C 0.1582490 .9031110 .141244

C $-1.588861-0.9680530 .311115$

$\begin{array}{lrrrr}\text { O } & 3.806146 & 0.385126 & -0.621977\end{array}$

\begin{tabular}{lllll}
\hline & 1.983318 & 2.496316 & 0.098133
\end{tabular}

$\begin{array}{lllll}\text { O } & 0.150776 & 1.006479 & 1.566782\end{array}$

C $-2.761329-0.244415-0.309928$

$\begin{array}{lllll} & \text { O } & -3.863521 & -0.424447 & 0.476310\end{array}$

$\begin{array}{llll}O & -2.726746 & 0.376732 & -1.363462\end{array}$

$\begin{array}{llll}C & -5.063946 & 0.195607 & 0.004547\end{array}$

H $3.022539-2.2834261 .318693$

H $2.556031-3.320489-0.020585$

H $3.970117-2.258642-0.187519$

H $-0.264755-0.609692-1.354986$

H $1.997946-1.356749-1.406793$

H $2.751505 \quad 0.236810 \quad 1.122426$

H $1.5417621 .290942-1.482502$

H $-0.5636521 .635465-0.235267$

H $-1.605470-0.8264361 .397901$

H $-1.729314-2.040708 \quad 0.129199$

H $4.092157 \quad 1.288519-0.402825$

H $1.3301983 .154552-0.194981$

H 0.4598091 .8999991 .794546

H $-5.348994-0.221454-0.966061$

H $-5.860085-0.013708 \quad 0.723878$

H $-4.9292321 .279394-0.064374$

SCF Energy (B3LYP/6-31G**//MMFF) $=-803.939762651$

F1-14 c00603

MMFF Geometry

C $2.2116262 .451985-0.678120$

C $-0.133144 \quad 0.276563 \quad 1.182475$

$\begin{array}{lllll}\text { O } & 0.380138 & 1.313116 & 0.336211\end{array}$

C $1.8036411 .272193 \quad 0.198855$

C $2.263422-0.062545-0.402107$ 
C $1.712394-1.2324810 .416084$

C $0.197649-1.1044430 .597214$

C $-1.625570 \quad 0.5259321 .421635$

O $3.688244-0.112157-0.395870$

O $2.024736-2.455855-0.245617$

O $-0.438043-1.259889-0.671330$

C $-2.465950 \quad 0.5592050 .165133$

O $-3.360323-0.4705860 .176281$

O $-2.3422161 .395322-0.720592$

C $-4.209709-0.559473-0.971812$

H $1.8542523 .391255-0.241778$

H $3.2977012 .514927-0.792566$

H $1.7568212 .375632-1.672042$

H $\quad 0.3608410 .3695252 .159562$

H $2.259761 \quad 1.4206451 .186539$

H $1.934882-0.149779-1.445218$

H $2.210364-1.2617051 .393335$

H $-0.179184-1.9021451 .247172$

H $-1.769371 \quad 1.4991581 .907435$

H -2.021834 -0.2292602 .110976$

H $3.949173-0.979246-0.750867$

H $1.667135-3.1801350 .295772$

H $-0.163539-2.120645-1.031195$

H $-4.824273 \quad 0.341498-1.059088$

H -3.610827 $-0.708638-1.875341$

H $-4.868354-1.422325-0.841510$

SCF Energy (B3LYP/6-31G**/MMFF)= -803.931077571

F1-14 c00604

MMFF Geometry

C $1.5942882 .716094-0.165364$

C $-0.191562-0.208718 \quad 1.232354$

$\begin{array}{lllll}\text { O } & 0.068963 & 1.055748 & 0.609548\end{array}$

C $1.4649591 .331980 \quad 0.461921$

C $2.153340 \quad 0.259478-0.394243$

C $1.876162-1.136822 \quad 0.170295$

C $0.374690-1.3505690 .376024$

C $-1.689590-0.315706 \quad 1.537847$

O $3.5607390 .485803-0.424413$

O $2.374874-2.133277-0.718276$

O $-0.270402-1.367684-0.897398$

C $-2.601737-0.306842 \quad 0.333511$

O $-2.6437260 .937727-0.220367$

O $-3.213217-1.292155-0.060369$

C $-3.468731 \quad 1.067148-1.382055$

H $2.6409613 .017014-0.269083$

H $1.1205152 .743375-1.152964$

H $1.078413 \quad 3.4617190 .449660$

H $\quad 0.323437-0.211613 \quad 2.203101$

H $1.9237631 .375802 \quad 1.458507$

H $1.7997410 .308683-1.431103$

H $2.402744-1.2675591 .123840$

H $0.187299-2.3218540 .847246$

H -2.0029870 .5142912 .183567$

H -1.887962 -1.237559 2.097835

H $3.888540 \quad 0.4562660 .490593$

H $3.316884-1.943138-0.867993$

H $0.145255-2.072264-1.423464$

H $-4.5110540 .847950-1.132356$

H $-3.4014962 .100228-1.733214$

H $-3.1128220 .403106-2.175699$

SCF Energy (B3LYP/6-31G**/MMFF) $=-803.933681733$

F1-14 c00605

MMFF Geometry

C $2.967570-2.296940 \quad 0.194402$

C $-0.234815-0.503012-0.263736$

O $0.756182-1.4198330 .226491$

C $2.054304-1.194313-0.332651$

C $2.570218 \quad 0.204650 \quad 0.033667$

C $1.5450621 .269051-0.364112$

C 0.1459630 .9254750 .154588

C $-1.583276-0.964254 \quad 0.300249$

$\begin{array}{llll}\text { O } & 3.786530 & 0.477588 & -0.658840\end{array}$

$\begin{array}{lllll}0 & 1.959738 & 2.534261 & 0.145978\end{array}$

$\begin{array}{lllll}\text { O } & 0.116927 & 1.043344 & 1.578724\end{array}$

C $-2.761515-0.245974-0.316073$

O $-3.862190-0.4398960 .468987$
O $-2.7317890 .382016-1.365574$

C $-5.0673780 .173891 \quad 0.001189$

H $3.966059-2.245630-0.248957$

H $3.062538-2.2378751 .284279$

H $2.542753-3.281586-0.029967$

H $-0.258216-0.576452-1.359128$

H $1.996849-1.305228-1.423523$

H $2.776928 \quad 0.2820301 .108131$

H $1.524057 \quad 1.358789-1.457535$

H $-0.5749211 .654940-0.228836$

H $-1.603507-0.8340461 .388399$

H -1.712642 -2.0363790 .107323$

H $4.477681-0.090337-0.279147$

H $\quad 1.287313 \quad 3.187977-0.111046$

H 0.6470200 .3189551 .952135

H $-5.348531-0.238203-0.972672$

H $-5.862031-0.0473620 .718587$

H $-4.9413371 .259122-0.059315$

SCF Energy (B3LYP/6-31G**//MMFF) $=-803.933434729$

F1-15 c00621

MMFF Geometry

C $-1.564956 \quad 2.874630 \quad 0.154335$

C $0.112178-0.4217300 .098711$

O $-0.2048320 .948386-0.181722$

C $-1.414577 \quad 1.3860690 .448135$

C $-2.6155610 .566489-0.055071$

C $-2.364438-0.930868 \quad 0.129110$

C $-1.001313-1.322668-0.455565$

C $1.462438-0.730279-0.562505$

O $-2.835450 \quad 0.838984-1.437414$

O $-2.399246-1.2215341 .526680$

O $-0.723279-2.685996-0.157294$

C $2.610986-0.0083990 .111771$

O $3.757316-0.249759-0.591982$

$\begin{array}{llll}\text { O } & 2.523374 & 0.671135 & 1.125071\end{array}$

C $4.9393640 .367822-0.072664$

H $-1.5677213 .073489-0.922758$

H -2.4844093 .2751100 .591686$

H -0.7142443 .4279370 .567493$

H $0.210614-0.557614 \quad 1.183916$

H -1.3033491 .2605091 .532640$

H -3.5121280 .8750600 .495623$

H $-3.154628-1.522641-0.347166$

H $-1.040482-1.217483-1.547209$

H $1.441223-0.411866-1.611540$

H $1.679475-1.802562-0.509706$

H $-3.6166370 .331102-1.715211$

H $-2.176483-2.1623211 .632729$

H $-1.441566-3.220902-0.536108$

H $5.7763450 .101152-0.723551$

H $4.828722 \quad 1.456413-0.071173$

H $5.147483-0.0015110 .936075$

SCF Energy (B3LYP/6-31G**//MMFF) $=-803.934705922$

F1-15 c00622

MMFF Geometry

C $1.6361442 .768014-0.674752$

C $0.009029-0.539120-0.973230$

$\begin{array}{lllll}\text { O } & 0.203924 & 0.875653 & -0.872243\end{array}$

C $1.5759831 .244631-0.687246$

C $2.142890 \quad 0.627400 \quad 0.603911$

C $1.937944-0.8888470 .618027$

C $0.473114-1.2302120 .316531$

C $-1.460202-0.805640-1.318912$

$\begin{array}{lllll}\text { O } & 1.489566 & 1.200701 & 1.734637\end{array}$

O $2.790818-1.467043-0.369782$

$\begin{array}{lllll}\text { O } & 0.313724 & -2.638643 & 0.200044\end{array}$

C $-2.417328-0.431302-0.211120$

O $-2.6262970 .915671-0.205102$

O $-2.911133-1.2417250 .562526$

$\begin{array}{llll}C & -3.499676 & 1.398445 & 0.820102\end{array}$

H $1.2711263 .167396-1.627545$

H 0.9901073 .1890690 .103085

H $2.6586933 .124409-0.518450$

H $0.596397-0.908374-1.825036$

H $2.146797 \quad 0.886588-1.553423$

H 3.2101510 .8690740 .673629 
H $2.224626-1.3142541 .586674$ H $-0.138300-0.8893321 .160501$ H $-1.616007-1.865853-1.550316$ H -1.752476 -0.245670 -2.215927 H $\quad \begin{array}{llll}1.889428 & 0.812821 & 2.531523\end{array}$ H $2.602840-2.421038-0.392318$ H $0.606345-3.0367821 .037553$ H -4.4968810 .9631900 .704305$ H -3.088455 $1.167507 \quad 1.807441$ H -3.5765942 .4841260 .717793$

SCF Energy $\left(\mathrm{B} 3 \mathrm{LYP} / 6-31 \mathrm{G}^{* *} / / \mathrm{MMFF}\right)=-803.931179255$

F1-15_c00623

MMFF Geometry

C -2.519776 -2.389591 -0.294921

C $-0.0034490 .211132-1.055224$

O $-0.604608-1.058098-0.776448$

C -2.005862 -0.967153 -0.490609

C -2.260624 -0.079922 0.741204

C -1.6118921 .2957380 .565208$

C -0.1412681 .1397030 .160707$

C $1.448249-0.021526-1.485527$

O $-1.722542-0.7082501 .902855$

O $-2.3045222 .016927-0.453848$

O $\quad 0.4271512 .415262-0.109609$

C $2.303120-0.603297-0.382814$

$\begin{array}{lllll}\text { O } & 3.166154 & 0.342426 & 0.086426\end{array}$

O $2.202938-1.7534480 .024649$

C $4.017465-0.0788481 .156466$

H -3.587090 -2.396949 -0.054871

H $-2.365174-2.975178-1.208018$

H -1.972814 -2.908336 0.499834

H $-0.5147170 .651724-1.921806$

H $-2.507580-0.539446-1.367896$

H -3.3426060 .0224390 .886633$

H $-1.671789 \begin{array}{lll}1.889147 & 1.484835\end{array}$

H $\quad 0.398658 \quad 0.714577 \quad 1.014758$

H $1.8981660 .911461-1.844669$

H $1.490730-0.730806-2.321175$

H -1.918837 -0.1351722 .663520$

H $-3.2272112 .118341-0.164153$

H $-0.0789772 .814198-0.838190$

H $3.418280-0.3694942 .024669$

H $4.654240-0.9069930 .830940$

H 4.6543680 .7645191 .436450

SCF Energy (B3LYP/6-31G**//MMFF $)=-803.929236398$

F1-15_c00624

MMFF Geometry

C $-1.6152712 .759376 \quad 0.517695$

C $0.207753-0.379843-0.184200$

O $-0.2326340 .982622-0.256621$

C -1.3413531 .2625410 .606357$

C $-2.561591 \quad 0.4075330 .218209$

C -2.194271 -1.0780180 .199855$

C $-0.929584-1.306939-0.636676$

C $1.433575-0.519820-1.090523$

O $-3.036748 \quad 0.760654-1.078769$

O $-1.955545-1.5203451 .536684$

O $-0.536210-2.671921-0.549614$

C $2.6140830 .228412-0.519254$

O $3.199621-0.5088540 .467563$

O $2.9638941 .339336-0.895951$

C 4.3273860 .1009981 .102940

H -0.7455823 .3217610 .875664$

H $-1.7800143 .078325-0.516895$

H -2.482522 3.0421021 .121662

H $\quad 0.502663-0.6067870 .848819$

H - 1.0413321 .0334101 .637100

H -3.3731700 .5826920 .934582$

H -3.010253 -1.686810 -0.206595

H -1.166296 -1.100600 -1.687771

H $1.233844-0.106988-2.086841$

H $1.728882-1.565966-1.228907$

H -3.445681 $1.640056-1.015405$

H $-2.778366-1.3903912 .038320$

H $-0.357669-2.8672310 .386296$

H 4.0294361 .0361161 .586731
H $4.699702-0.5861541 .867327$

H $\quad \begin{array}{llll}5.122983 & 0.279088 & 0.373157\end{array}$

SCF Energy (B3LYP/6-31G**//MMFF) $=-803.934224865$

F1-15_c00625

MMFF Geometry

C $-0.9629562 .899413 \quad 0.390702$

C $0.109110-0.6237060 .028394$

O $0.0298960 .799729-0.134384$

C -1.0715191 .3887550 .565809$

C -2.4057530 .8336430 .039979$

C $-2.416490-0.6953010 .105848$

C $-1.156959-1.269773-0.555927$

C $1.377057-1.093440-0.689461$

O $-2.5985551 .247629-1.310917$

O $-2.457960-1.1038861 .473832$

O $-1.151653-2.680969-0.374659$

C $2.608027-0.5954510 .031119$

O $3.1098470 .502766-0.601876$

O $3.052317-1.1080981 .050410$

C 4.2660911 .0876340 .005170

H $-0.9515263 .181590-0.667668$

H -1.7888513 .4173580 .887375$

H -0.0201843 .2610440 .816099$

H $\quad 0.204283-0.8587531 .096812$

H -0.9642341 .1587531 .633459$

H $-3.224625 \quad 1.2502280 .638309$

H -3.303147 -1.111185 -0.386630

H -1.212072 -1.066363 -1.633105

H $1.400070-0.736150-1.726179$

H $1.452445-2.185278-0.724561$

H -3.457821 $0.899443-1.604159$

H $-3.277271-0.7499271 .859844$

H $-0.493755-3.057383-0.982675$

H 4.0291071 .4317371 .016459

H $\begin{array}{llll}5.089743 & 0.367713 & 0.024285\end{array}$

H $4.5670591 .949162-0.596721$

SCF Energy (B3LYP/6-31G**//MMFF $)=-803.926262515$

F1-15_c00626

MMFF Geometry

C -3.273041-2.040473 -0.422923

C $0.046949-0.418130-0.222648$

O $-1.019024-1.363419-0.051315$

C $-2.268864-0.904054-0.580544$

C -2.7237970 .3804510 .133645$

C -1.6313421 .4492610 .076627$

C -0.2895810 .8709580 .544945$

C $1.326442-1.0911690 .292347$

O -3.0246200 .0892031 .496891$

O $-1.5228681 .907620-1.271137$

$\begin{array}{lllll}\text { O } & 0.736596 & 1.840144 & 0.374198\end{array}$

C $2.594852-0.453378-0.227459$

O $3.607002-0.6749640 .661793$

O $2.6957450 .135777-1.295127$

C $4.880393-0.1373520 .290990$

H -2.937436 -2.920203 -0.983087

H $-3.361545-2.3563540 .622098$

H $-4.261745-1.751385-0.791632$

H $0.161811-0.210106-1.294319$

H $-2.138007-0.709428-1.652693$

H $-3.6415610 .745520-0.342763$

H -1.896873 2.3144020 .694882

H -0.3611170 .6432631 .616077$

H $1.347391-2.144066-0.014506$

H $1.330012-1.0808451 .389192$

H -3.337814 0.9119021 .909739

H $-0.7816772 .536468-1.304858$

H 0.4854892 .6273530 .886657

H 4.8160520 .9496150 .182111

H $5.592107-0.3683751 .087971$

H $5.230186-0.599340-0.637173$

SCF Energy (B3LYP/6-31G**//MMFF) $=-803.933632875$

F1-15 c00627

MMFF Geometry

C -2.544078 -2.378437 -0.232427

C $-0.0104500 .180328-1.071605$ 
O $-0.620409-1.074198-0.750636$

C -2.020852 $-0.965527-0.469558$

C $-2.270190-0.0408270 .735261$

C $-1.601407 \quad 1.3232970 .540326$

C $-0.137744 \quad 1.1510880 .111428$

C $1.439328-0.080206-1.495563$

O $-1.743660-0.6504401 .913533$

O $-2.3277522 .084091-0.424010$

O $\quad 0.4291742 .410641-0.226947$

C $2.290553-0.635902-0.376835$

$\begin{array}{lllll}\text { O } & 3.152967 & 0.320309 & 0.072142\end{array}$

O $2.187629-1.7756550 .058227$

C $4.000275-0.0754691 .155017$

H -2.394622 -2.990799-1.128660

H -1.999811 -2.8785530 .575868$

H $-3.611272-2.3716500 .008624$

H $-0.5144370 .594968-1.954473$

H - $2.519978-0.565183-1.360506$

H -3.3510580 .0798330 .874035$

H -1.630012 1.9015421 .471051

H $\quad 0.4171780 .7527440 .969667$

H $1.8986570 .835905-1.884961$

H $\quad 1.473257-0.815770-2.308708$

H -1.951164 -0.066878 2.663172

H $-2.3027391 .619832-1.276010$

H $\quad 0.317641 \quad 2.9980870 .539884$

H 4.6342280 .7750961 .419079

H $3.397919-0.3480972 .026862$

H $4.640010-0.9091200 .850353$

SCF Energy (B3LYP/6-31G $\left.{ }^{* *} / / \mathrm{MMFF}\right)=-803.922296993$

F1-15 c00628

MMFF Geometry

C -1.613602 27603400.520375

C $0.203165-0.382925-0.178576$

O $-0.2387180 .978568-0.254153$

C $-1.342658 \quad 1.2629390 .612989$

C -2.5669180 .4094980 .233360$

C -2.212279-1.079389 0.191935

C $-0.933980-1.313371-0.624276$

C $1.425240-0.524399-1.090553$

O $-3.0508220 .776749-1.057766$

O $-2.057831-1.5754801 .521735$

O $-0.542328-2.677758-0.527236$

C $2.6072730 .228827-0.528816$

O $3.198845-0.5023480 .458933$

O $2.9532051 .338485-0.913076$

C 4.3287430 .1125951 .085552

H -0.7416493 .3219840 .873974$

H -1.780766 $3.076734-0.514717$

H $-2.478425 \quad 3.046793 \quad 1.126247$

H $0.506801-0.6056040 .852310$

H - $-1.037251 \quad 1.0414481 .643075$

H -3.3724150 .5796690 .957755$

H -3.028194 -1.656123 -0.258726

H -1.161997 -1.112986 -1.678752

H $1.220000-0.116412-2.087636$

H $\quad 1.721740-1.570644-1.225593$

H $-3.4707701 .649834-0.979341$

H -1.327141 -1.1026021 .951544$

H $-0.376760-2.8704720 .411593$

H $4.705043-0.5693531 .852646$

H $\quad 5.1209990 .2868340 .351219$

H 4.0320791 .0504891 .564966

SCF Energy (B3LYP/6-31G**//MMFF) $=-803.931736417$

F1-16 c00661

MMFF Geometry

C -1.165304 $2.413342-0.481608$

C $0.248855-0.277678-0.282281$

$\begin{array}{llll}\mathrm{O} & -0.054568 & 0.641455 & 0.779297\end{array}$

C $-1.280394 \quad 1.3642700 .625973$

C -2.4703980 .4052300 .476970$

C $-2.218099-0.629353-0.619801$

C $-0.870106-1.320422-0.410537$

C $1.598105-0.9291650 .049508$

O

O $-3.250957-1.612810-0.611874$
O $-0.911775-2.1037140 .785130$

C $2.7577980 .028141-0.134620$

O $3.920345-0.6261610 .161974$

O $2.663948 \quad 1.194982-0.489778$

C 5.1141700 .1528930 .035859

H -2.018090 $3.098927-0.466703$

H $-0.2490912 .999831-0.354068$

H -1.116099 $1.960750-1.476162$

H $0.3398650 .277619-1.223268$

H -1.419909 $1.923801 \quad 1.559412$

H -2.639689 -0.1210661 .424820$

H -2.244918 -0.152507-1.606711

H $-0.665103-2.011128-1.236307$

H $1.770595-1.782687-0.615883$

H $1.613572-1.2644371 .092958$

H $-3.807079 \quad 1.7623380 .895637$

H $-4.097850-1.143554-0.704180$

H -1.657823 -2.721782 0.699364

H $5.963986-0.4820600 .299929$

H $5.2373440 .492601-0.996847$

H $5.085373 \quad 1.0042280 .722643$

SCF Energy $\left(B 3 L Y P / 6-31 G^{* *} / / M M F F\right)=-803.934436587$

F1-16 c00662

MMFF Geometry

C $-1.1527572 .419334-0.453020$

C $0.245579-0.275556-0.283641$

O $-0.057050 \quad 0.6263490 .792436$

C -1.2775631 .3596780 .643920$

C -2.4764440 .4126640 .485356$

C $-2.227579-0.622780-0.613478$

C $-0.876146-1.315161-0.419607$

C $1.595372-0.9322350 .034500$

$\begin{array}{lllll}\text { O } & -3.649705 & 1.167016 & 0.198673\end{array}$

O $-3.256800-1.609333-0.607361$

O $-0.915562-2.1424320 .746386$

C $2.7547260 .026840-0.140696$

O $3.918504-0.6337160 .136579$

O $2.659764 \quad 1.199937-0.474293$

C 5.1121770 .1464720 .016172

H $-1.9949593 .117813-0.425556$

H $-0.2270412 .990728-0.324727$

H $-1.1169501 .976933-1.452718$

H $\quad 0.3331500 .292676-1.216967$

H $-1.413926 \quad 1.9114011 .582459$

H -2.653682 -0.1044871 .436550$

H -2.256939 -0.141017-1.598147

H $-0.681435-1.985437-1.264223$

H $1.764641-1.776668-0.643200$

H $1.614792-1.2819011 .073255$

H -4.4061870 .5572220 .224554$

H -4.089320 -1.167903 -0.845348

H $-0.930271-1.5635861 .527219$

H $5.2291850 .503840-1.011278$

H 5.0883610 .9858650 .717688

H $5.963177-0.4935790 .264042$

SCF Energy (B3LYP/6-31G**//MMFF) $=-803.926878797$

F1-16 c00663

MMFF Geometry

C $-2.268414-2.1708190 .812122$

C $0.197683-0.595440-0.048973$

O $-0.712599-1.339083-0.876145$

C $-2.078272-1.322378-0.447033$

C $-2.6042780 .114979-0.334666$

C -1.6773070 .9832370 .515905$

C $-0.233466 \quad 0.8777730 .019903$

C $1.594574-0.792104-0.652376$

O $-\begin{array}{llll}-3.916345 & 0.111332 & 0.218727\end{array}$

O -2.1162362 .3386680 .448967$

O $-0.1343171 .453698-1.285014$

C $2.704362-0.2746030 .233566$

O $3.843187-0.143501-0.508746$

O $2.598450-0.0415051 .429875$

C $4.9917570 .326166 \quad 0.204368$

H $-3.330113-2.3291481 .025799$

H $-1.793043-3.1494560 .684873$

H -1.813684 -1.710042 1.693894 
H $0.189144-1.0289650 .958040$ H $-2.649976-1.823692-1.238036$ H -2.682123 $0.548851-1.339644$ H -1.7440120 .6813201 .567802$ H 0.4307041 .4547810 .672132 H $1.654251-0.309074-1.634494$ H $1.774982-1.861896-0.814878$ H -4.2010571 .0380300 .296958$ H -1.5131572 .8681340 .998256$ H $-0.4633422 .366861-1.224750$ H 4.7992611 .3193580 .621510 H $5.261070-0.3803160 .995196$ H $5.8241390 .397512-0.500866$ SCF Energy (B3LYP/6-31G**//MMFF) $=-803.934823395$

\section{F1-16 c00664}

MMFF Geometry

C $-1.418923 \quad 2.8498750 .253493$

C $0.021458-0.395938-0.840660$

O $-\begin{array}{llll}0.377879 & 0.979967 & -0.784108\end{array}$

C $-1.120031 \quad 1.3589020 .381208$

C $-2.4012910 .520587 \quad 0.525210$

C $-2.085119-0.9749430 .494203$

C $-1.212013-1.306071-0.719688$

C $1.164927-0.7048330 .141512$

O $-3.2969350 .832357-0.540533$

O $-1.424503-1.328408 \quad 1.706222$

O $-0.851099-2.680779-0.690289$

C $2.4389360 .037044-0.210434$

O $3.396370-0.2683420 .715810$

O $2.5835890 .783533-1.168582$

C $4.666327 \quad 0.361326 \quad 0.518173$

H $-1.9595463 .073803-0.672393$

H $-2.004898 \quad 3.212216 \quad 1.103561$

H -0.4835863 .4188510 .209131$

H $0.425603-0.547787-1.850330$

H -0.4877891 .2244391 .265568$

H -2.895839 0.7872391 .466938

H $-3.007983-1.5653380 .448738$

H - $1.829085-1.154813-1.615081$

H $1.400022-1.7745150 .116411$

H $0.903380-0.4235891 .164812$

H $-4.1080240 .314024-0.402595$

H $-1.181974-2.2679571 .640054$

H $-0.323500-2.861880-1.486905$

H $5.3336730 .040613 \quad 1.322461$

H $5.0968570 .052879-0.439313$

H 4.5607211 .4495080 .562682

SCF Energy (B3LYP/6-31G**/MMFF) $=-803.929787394$

F1-16 c00665

MMFF Geometry

C -2.583728 $-2.043060 \quad 0.429530$

C $0.095767-0.6437840 .897719$

O $-0.351479-1.347261-0.268722$

C $-1.748711-1.222718-0.556070$

C $-2.1673490 .250932-0.672782$

C -1.7080651 .0611040 .540373$

C $-0.211644 \quad 0.856493 \quad 0.786076$

C $1.574968-0.9721711 .131870$

O $-\begin{array}{llll}-3.580505 & 0.367725 & -0.804724\end{array}$

$\begin{array}{lllll}O & -1.958977 & 2.448606 & 0.326064\end{array}$

O $0.5210451 .422309-0.301084$

C $2.490489-0.607372-0.015079$

$\begin{array}{llllll}\text { O } & 3.392645 & 0.331774 & 0.390984\end{array}$

O $2.414765-1.094907-1.135564$

C $4.3122580 .774751-0.611791$

H -2.583281 -1.608394 1.433255

H $-3.623790-2.1210600 .097690$

H -2.175063 -3.0551820 .521744$

H $-0.443955-1.0439521 .764884$

H -1.897201-1.688450 -1.538360

H $-1.7243360 .688424-1.576334$

H -2.2784260 .7718081 .430806$

H 0.1020821 .3851341 .693089

H $1.698534-2.0515481 .285253$

H $1.921032-0.4833912 .050492$

H -3.843292 -0.143751-1.589126
H $-2.9065092 .543808 \quad 0.127908$

H $0.2661752 .358671-0.366036$

H $3.7719781 .234686-1.444756$

H $4.9690221 .524958-0.163286$

H $4.923179-0.062777-0.961927$

SCF Energy (B3LYP/6-31G**//MMFF) $=-803.928245913$

Fragment $\mathrm{F} 1 \mathrm{a}$ (cutoff energy $=10 \mathrm{~kJ} / \mathrm{mol}$ )

F1a-01 c00001

MMFF Geometry

C $0.924819-2.2206850 .947455$

C $-0.624006 \quad 0.2370560 .100860$

O $-0.302001-0.900935-0.707260$

C $0.955043-1.513634-0.408363$

C $2.094810-0.503144-0.575056$

$\begin{array}{llll}\text { C } & 1.844271 & 0.772377 & 0.226387\end{array}$

C $0.4468801 .323487-0.057876$

C $-2.0012130 .747389-0.350805$

O $3.338398-1.069067-0.175236$

O $2.8015251 .762735-0.156511$

$\begin{array}{lllll} & 0 & 0.171892 & 2.389970 & 0.848081\end{array}$

C $-3.117715-0.205354 \quad 0.023388$

O $-4.3208420 .241943-0.423034$

O $-2.985364-1.2473620 .649636$

H $\quad 0.032665-2.851161 \quad 1.026474$

H $1.807611-2.8533461 .082094$

H $0.889389-1.5138161 .781672$

H $-0.699352-0.0633961 .153220$

H $1.094398-2.301452-1.159063$

H $2.188659-0.232521-1.634830$

H $1.9875920 .606424 \quad 1.300636$

H $0.4173561 .737990-1.073770$

H $-2.0137470 .861357-1.441193$

H -2.2248581 .7100310 .121640$

H $3.479806-1.869917-0.708527$

H $3.684768 \quad 1.384714-0.003796$

H 0.8800083 .0486630 .743962

H $-4.2904941 .093191-0.909874$

SCF Energy (B3LYP/6-31G**//MMFF) $=-764.619864563$

F1a-01 c00002

MMFF Geometry

C $1.994259-1.9003611 .133896$

C $-0.582715-0.3255370 .808086$

O $-0.105187-1.395224-0.012929$

C $1.316742-1.449573-0.161477$

C $1.853282-0.132478-0.734000$

C $1.385251 \quad 1.0744280 .076914$

C $-0.131346 \quad 1.035480 \quad 0.265714$

C $-2.106077-0.455298 \quad 0.934544$

O $3.276360-0.141129-0.778513$

O $1.7167502 .272230-0.629379$

O $\begin{array}{llll}0.522391 & 2.056563 & 1.179863\end{array}$

C $-2.851057-0.190169-0.353024$

O $-2.711221-1.191350-1.256354$

O $-3.5144920 .816943-0.562789$

H $1.942759-1.136122 \quad 1.914860$

H $1.499183-2.7936111 .529734$

H $3.049501-2.136823 \quad 0.965785$

H $-0.189750-0.4597521 .823671$

H $1.513933-2.238687-0.897713$

H $1.498560-0.013628-1.765984$

H 1.8993521 .1350371 .043299

H $-0.6133651 .250809-0.695557$

H $-2.485748 \quad 0.2460551 .687197$

H $-2.382332-1.4600321 .277155$

H $3.547211-0.906195-1.314302$

H $2.6783242 .263826-0.776502$

H -0.1997622 .9026970 .824736$

H -2.146450 -1.936776 -0.959492

SCF Energy (B3LYP/6-31G**//MMFF) $=-764.622044744$

F1a-01_c00003

MMFF Geometry

C $0.753475-2.1400141 .169746$

C $-0.6369640 .270295-0.020545$

O $-0.337243-0.966005-0.678517$

C $0.875779-1.594138-0.254169$ 
C $2.069007-0.660205-0.479322$

C $1.850510 \quad 0.7072190 .164454$

C $0.493288 \quad 1.283019-0.242698$

C $-1.9593690 .788423-0.595905$

$\begin{array}{lllll}\text { O } & 3.256960 & -1.256784 & 0.030367\end{array}$

O $2.885307 \quad 1.585171-0.284520$

$\begin{array}{lllll}0 & 0.243755 & 2.468514 & 0.509885\end{array}$

C $-3.115323-0.083352-0.167801$

$\begin{array}{llll}0 & -3.530013 & 0.184202 & 1.095634\end{array}$

O $-3.619484-0.947265-0.873247$

H $-0.171121-2.7174901 .276164$

H $1.597546-2.7914661 .416456$

H $\quad 0.717305-1.3415801 .916761$

H $-0.776272 \quad 0.089337 \quad 1.052114$

H $1.008495-2.468027-0.904040$

H $2.214463-0.523616-1.558836$

H 1.9503010 .6478921 .254590

H $0.5339331 .577507-1.298857$

H $-1.9353520 .783978-1.692633$

H -2.173014 $1.817665-0.286398$

H $3.983858-0.627266-0.115501$

H 2.7077902 .4614820 .098509

H 0.2026582 .2227061 .449909

H $-3.037266 \quad 0.898727 \quad 1.552388$

SCF Energy (B3LYP/6-31G**//MMFF) $=-764.614842004$

F1a-01 c00004

MMFF Geometry

C $2.053828-1.9024661 .093243$

C $-0.563782-0.3933620 .783550$

O $-0.051788-1.431678-0.057252$

C $1.372127-1.442763-0.197003$

C $1.875713-0.098916-0.736896$

C $1.3657691 .077450 \quad 0.093758$

C $-0.150503 \quad 0.989848 \quad 0.267444$

C $-2.081538-0.5745390 .901828$

O $3.298778-0.065460-0.768101$

O $1.6679412 .298363-0.585605$

O $-0.5824671 .983831 \quad 1.192664$

C -2.803895 $-0.405954-0.414798$

O $-3.369451 \quad 0.818693-0.553082$

O $-2.860109-1.271589-1.278759$

H $1.977338-1.1563921 .889572$

H $1.580415-2.8168111 .467082$

H $3.116039-2.1074540 .927057$

H $-0.169751-0.5337681 .797992$

H $1.596238-2.209739-0.948768$

H $1.5271400 .030956-1.769647$

H 1.8693621 .1336241 .065927

H $-0.6288051 .204796-0.695654$

H -2.5034300 .1067651 .649083$

H -2.312139-1.588633 1.252461

H $3.596419-0.811898-1.315819$

H $2.6302512 .320149-0.725713$

H $-0.2726602 .843761 \quad 0.859969$

H -3.235961 1.4315820 .200788

SCF Energy (B3LYP/6-31G**//MMFF) $=-764.617617743$

F1a-01 c00005

MMFF Geometry

C $0.746660-2.123397 \quad 1.174732$

C $-0.632063 \quad 0.301156 \quad 0.004487$

O $-0.346721-0.935420-0.659547$

C $0.865577-1.573816-0.247617$

C $2.062728-0.646166-0.485072$

C $1.861125 \quad 0.7260890 .156065$

C $0.501157 \quad 1.307695-0.235243$

C $-1.9625520 .825219-0.547169$

O $3.267861-1.242393-0.016393$

O $2.8938311 .597447-0.309622$

$\begin{array}{llll}0 & 0.269769 & 2.497003 & 0.517311\end{array}$

C $-3.126133 \quad 0.035144 \quad 0.002889$

O $-3.300513-1.150656-0.631309$

O $\quad-3.831100 \quad 0.4128690 .929454$

H $\quad 0.740150-1.327417 \quad 1.925350$

H $-0.190550-2.6785591 .290200$

H $1.576563-2.797647 \quad 1.407884$

H -0.7572710 .1205971 .079359$
H $\quad 0.985801-2.446759-0.901349$

H $2.187101-0.505262-1.566360$

H 1.9724030 .6723551 .245379

H $0.5292531 .598725-1.292769$

H -1.996046 $0.783452-1.642261$

H $-2.128412 \quad 1.870788-0.262684$

H $3.258000-1.2253670 .955408$

H 2.7257002 .4759940 .072265

H $0.2270332 .252408 \quad 1.457475$

H -2.653744 -1.335650 -1.345469

SCF Energy (B3LYP/6-31G**//MMFF) $=-764.616828303$

F1a-01_c00006

MMFF Geometry

C $0.763829-2.1408331 .142695$

C $-0.6323140 .297478 \quad 0.016034$

O $-0.322486-0.918204-0.676714$

C $0.888870-1.555785-0.264915$

C $2.080410-0.613268-0.461413$

$\begin{array}{llll}\text { C } & 1.851316 & 0.737119 & 0.215225\end{array}$

C $0.493013 \quad 1.324263-0.176724$

C $-1.9643580 .807170-0.545798$

O $3.276417-1.1955090 .046015$

O $2.8862301 .643036-0.177599$

$\begin{array}{lllll}\text { O } & 0.260667 & 2.471180 & 0.638357\end{array}$

C -3.098882 $-0.120569-0.180469$

$\begin{array}{lllll}\text { O } & -3.519946 & 0.048727 & 1.097653\end{array}$

O $-3.583087-0.944178-0.945415$

H $-0.159504-2.7236511 .230341$

H $1.609000-2.7967691 .373699$

H $\quad 0.723365-1.3638551 .911646$

H $-0.7690200 .086970 \quad 1.083521$

H $1.025115-2.411205-0.938370$

H $2.233756-0.443634-1.535030$

H 1.9359010 .6474861 .304375

H $\quad 0.5357851 .659099-1.221128$

H $-1.9277200 .862088-1.640810$

H $-2.2180851 .809419-0.185044$

H $3.405520-2.043980-0.411352$

H $2.8449041 .746592-1.143485$

$\begin{array}{llll}\mathrm{H} & -0.468294 & 2.976268 & 0.241485\end{array}$

H -3.044387 0.7412961 .603969

SCF Energy (B3LYP/6-31G**//MMFF) $=-764.603510938$

F1a-01_c00007

MMFF Geometry

C $1.749977-2.0264651 .094345$

C $-0.563136-0.479040-0.106969$

O $0.288335-1.420976-0.772955$

C $1.642948-1.437412-0.313517$

C $2.280739-0.053479-0.474436$

C $1.439013 \quad 1.039753 \quad 0.179835$

C $-0.0170740 .946476-0.278277$

C $-1.965234-0.642713-0.716769$

$\begin{array}{llll}\text { O } & 3.590920 & -0.029713 & 0.082146\end{array}$

O $1.9483742 .318116-0.207953$

$\begin{array}{lllll}\text { O } & -0.804133 & 1.859816 & 0.479997\end{array}$

C -3.085870 -0.1766920 .186651$

O $-4.257051-0.057761-0.491971$

O $-2.9882220 .040702 \quad 1.386206$

H $1.334555-1.3586701 .854812$

H $1.190585-2.9660161 .155896$

H $2.792571-2.2275811 .359414$

H $-0.616634-0.7374890 .956984$

H $2.178181-2.130629-0.974277$

H $2.3794890 .179103-1.542658$

H 1.5077501 .0007381 .273282

H $-0.0831311 .251472-1.330580$

H -2.151439-1.702001 -0.933252

H -2.016385 -0.102309-1.669365

H $4.109245-0.722746-0.361257$

H 2.8832492 .3491500 .058866

H -0.4124822 .7431810 .369424$

H $-4.209519-0.280241-1.446227$

SCF Energy (B3LYP/6-31G**/MMFF)= -764.616056097

F1a-01 c00008

MMFF Geometry 
C $2.034406-1.9341401 .051848$

C $-0.560875-0.3858720 .770404$

O $-0.070215-1.427266-0.079265$

C $1.352974-1.456311-0.231478$

C $1.865608-0.116487-0.773986$

C 1.3786941 .0690840 .060336

C -0.1365110 .9950340 .253805$

C $-2.079405-0.547176 \quad 0.903933$

O $3.287068-0.110419-0.857091$

O $1.6861992 .283755-0.626935$

O -0.5525292 .0064861 .167949$

C $-2.812997-0.373025-0.405623$

O $-3.3544220 .862032-0.546896$

O $-2.897615-1.243894-1.261974$

H $1.537571-2.8322791 .434188$

H $3.087442-2.1733450 .874061$

H $1.990371-1.1830141 .846156$

H $-0.157530-0.5354651 .779861$

H $1.560776-2.223090-0.988112$

H $\quad 1.4950600 .010899-1.799156$

H 1.8956251 .1219831 .025551

H $-0.6170821 .213942-0.706811$

$\mathrm{H}-2.4856610 .1409441 .653603$

H $-2.318838-1.5575531 .259343$

H $3.644218-0.0600890 .045513$

H $2.6455072 .291182-0.787009$

H -0.1184021 .8354122 .021078$

H -3.1982991 .4782780 .199988$

SCF Energy (B3LYP/6-31G**//MMFF) $=-764.610325511$

F1a-01 c00009

MMFF Geometry

C 1.0627742 .7447050 .338332

C $-0.489326-0.461018-0.725924$

O $-0.0249620 .895785-0.686803$

C $0.714374 \quad 1.2659860 .479915$

C 1.9630920 .3880690 .634971

C $1.602533-1.1008000 .617902$

C $0.677741-1.449316-0.553489$

C $-1.641262-0.7009220 .263410$

O $2.8963790 .660368-0.409203$

O $0.954225-1.4528361 .836495$

O $1.436508-1.427680-1.766217$

C -2.886232 $0.079670-0.104484$

O $-3.903681-0.1750840 .759837$

O $-2.9928250 .848393-1.049570$

H 0.1467953 .3432690 .281217

H 1.6544283 .0970301 .188549

H $1.6166502 .940837-0.586025$

H -0.886833 -0.592049-1.740914

H $\quad 0.073822 \quad 1.1609241 .361901$

H 2.4589780 .6409351 .579212

H $2.520216-1.6968830 .542186$

H $\quad 0.281073-2.465277-0.441360$

H -1.368798 $-0.414728 \quad 1.282373$

H $-1.909820-1.763558 \quad 0.252598$

H $2.4437280 .515914-1.257959$

H $1.568640-1.2551622 .563806$

H $2.142634-2.090868-1.681782$

H $-3.688818-0.8101991 .476091$

SCF Energy (B3LYP/6-31G**//MMFF) $=-764.616150966$

F1a-01_c00010

MMFF Geometry

C $2.039177-1.913461 \quad 1.070767$

C $-0.552810-0.3558080 .792417$

O $-0.063886-1.397014-0.059324$

C $1.358549-1.432436-0.211593$

C $1.875251-0.094651-0.754143$

C 1.3900111 .0903850 .082280

C -0.1264431 .0268010 .279143$

C $-2.071496-0.5264850 .926300$

O $3.296493-0.095117-0.837758$

O $1.7299082 .308771-0.584303$

O -0.4996272 .0364571 .213081$

C $-2.833795-0.211072-0.339135$

O $-2.685034-1.162777-1.293102$

O
H $1.995772-1.1642111 .866742$

H $1.541082-2.8117841 .451029$

H $3.091932-2.1536870 .892696$

H $-0.148527-0.5078091 .801138$

H $1.563001-2.199781-0.968567$

H $1.5046730 .031159-1.779389$

H 1.9051931 .1278791 .048809

H $-0.6106371 .261596-0.676460$

H $-2.4603900 .123822 \quad 1.718597$

H -2.322363 -1.553700 1.218370

H $3.654143-0.0286790 .063606$

H $1.2903132 .310660-1.451552$

H $-1.4694022 .101881 \quad 1.206770$

H $-2.104783-1.912346-1.039710$

SCF Energy $\left(B 3 L Y P / 6-31 G^{* *} / / M M F F\right)=-764.615270481$

F1a-02_c00011

MMFF Geometry

C $1.3992462 .698575-0.342991$

C $-0.554402-0.3725160 .298946$

O $-0.1039340 .851008-0.300225$

C $1.113537 \quad 1.335495 \quad 0.276417$

C 2.2519630 .3323460 .035800

C $1.873129-1.0679360 .534650$

C $0.478229-1.4865340 .053430$

C $-1.918160-0.712438-0.315937$

O $2.573047 \quad 0.267525-1.352582$

O $1.873982-1.0788091 .960908$

O $0.524785-1.790177-1.339601$

C $-3.015907 \quad 0.2046750 .181623$

O $-4.216971-0.118673-0.366139$

O -2.8711021 .1256630 .973012$

H $0.5856893 .395171-0.111824$

H 2.3348873 .1195460 .037026

H $1.4533692 .640416-1.435506$

H $-0.669519-0.2094321 .378647$

H $\quad 0.957483 \quad 1.473597 \quad 1.354054$

H 3.1504870 .6838050 .555961

H $2.613860-1.7977610 .186822$

H $\quad 0.153160-2.3920640 .579830$

H $-2.200348-1.736216-0.045704$

H -1.878586 -0.613294 -1.406926

H $1.7617790 .031862-1.834513$

H $2.770461-0.8437272 .255226$

H $1.159867-2.516611-1.460128$

$\mathrm{H}-4.196847-0.877602-0.987573$

SCF Energy (B3LYP/6-31G**//MMFF) $=-764.622190095$

F1a-02 c00012

MMFF Geometry

C 1.2993952 .7100630 .268342

C $-0.598531-0.4581080 .142599$

O $-0.1444760 .866902-0.166733$

C 1.0177321 .2443360 .579694

C 2.2012220 .3312170 .224601

C $1.831349-1.1457180 .406731$

C $0.483401-1.480804-0.244825$

C $-1.902531-0.691700-0.623654$

O $2.5889110 .560080-1.128889$

O $1.761492-1.4182901 .804898$

O $0.638714-1.522060-1.662446$

C -3.0602640 .0049590 .050453$

O $-3.0672321 .342542-0.172167$

$\begin{array}{llll}\text { O } & -3.900713 & -0.568710 & 0.730691\end{array}$

H $1.4253412 .876261-0.806925$

H $0.4508803 .330357 \quad 0.577884$

H 2.1946043 .0622060 .789543

H $-0.793764-0.513196 \quad 1.221799$

H $\quad 0.786863 \quad 1.159726 \quad 1.649296$

H $3.054048 \quad 0.591444 \quad 0.863131$

H $2.618213-1.775384-0.025586$

H $0.156699-2.4784960 .070661$

H -2.148176 -1.760019-0.653402

H $-1.845146-0.342226-1.661175$

H $3.360266-0.003143-1.312052$

H $1.560700-2.363973 \quad 1.909434$

H $0.862232-0.624576-1.963300$

H $-2.3269521 .671848-0.725786$ 
SCF Energy (B3LYP/6-31G**//MMFF) $=-764.626694318$

F1a-02_c00013

MMFF Geometry

C 1.3327292 .7070670 .327975

C $-0.604705-0.4321910 .105260$

O $-0.1341340 .895989-0.163608$

C $1.0303321 .237268 \quad 0.596956$

C $2.203613 \quad 0.319393 \quad 0.220870$

C $1.814204-1.157744 \quad 0.362989$

C $0.466237-1.458400-0.303043$

C - $1.905833-0.626105-0.675997$

O $2.5980280 .578488-1.125207$

O $1.712589-1.4874021 .746841$

O $0.628503-1.467654-1.720254$

C -3.031828 $0.161919-0.051458$

O $-3.562593-0.4564431 .032856$

O $-3.4193791 .247114-0.464465$

H $1.4651922 .901394-0.741881$

H 0.4915053 .3297980 .651839

H 2.2305113 .0323710 .862035

H $-0.805087-0.5136881 .181536$

H $0.794571 \quad 1.1257081 .663013$

H 3.0578360 .5536020 .867045

H $2.587738-1.793494-0.084224$

H $0.126025-2.459186-0.012257$

H $-2.203448-1.680273-0.715860$

H $-1.804430-0.280470-1.711962$

H $3.3593250 .006937-1.323004$

H $2.582250-1.3265452 .151040$

H $0.860261-0.564999-1.998556$

H -3.154871-1.3191391.260630

SCF Energy (B3LYP/6-31G**//MMFF) $=-764.618567222$

F1a-02_c00014

MMFF Geometry

C $1.4377572 .683467-0.341262$

C $-0.542661-0.372805 \quad 0.288089$

O $-0.075294 \quad 0.843577-0.312200$

C $1.137033 \quad 1.3234190 .278737$

C 2.2737490 .3134010 .057649

C $1.883990-1.0939040 .529104$

C $0.482043-1.4982540 .053020$

C $-1.911759-0.691886-0.326344$

O $2.621107 \quad 0.261543-1.326076$

O $1.944455-1.158774 \quad 1.952544$

O $0.562461-1.813018-1.336918$

C $-2.9973890 .235267 \quad 0.179730$

O $-4.205129-0.075162-0.360778$

$\begin{array}{lllll}\text { O } & -2.838337 & 1.154080 & 0.971067\end{array}$

H $0.6258123 .385282-0.120443$

H $2.371498 \quad 3.100006 \quad 0.048197$

H $1.5029002 .622742-1.432835$

H $-0.662221-0.2051591 .366100$

H $\quad 0.966274 \quad 1.469888 \quad 1.352523$

H $3.1638120 .653948 \quad 0.599175$

H $2.614433-1.8145970 .142055$

H $0.160153-2.4027610 .583120$

H $-2.205496-1.713964-0.062048$

H $-1.874325-0.584298-1.416408$

H $1.8240290 .009093-1.822953$

H $1.320366-0.5125552 .321259$

H $-0.260814-2.264672-1.587549$

H $-4.197095-0.833139-0.983646$

SCF Energy (B3LYP/6-31G**//MMFF) $=-764.616865895$

F1a-02 c00015

MMFF Geometry

C $2.7884442 .056156-0.057357$

C $-0.535955 \quad 0.451766 \quad 0.153191$

$\begin{array}{llll}\text { O } & 0.549800 & 1.276377 & -0.294445\end{array}$

C 1.7668811 .0294460 .418150

C $2.237158-0.4144940 .186894$

C $1.132863-1.4203240 .534406$

C $-0.211192-1.032898-0.095579$

C $-1.7865260 .933033-0.585704$

O $2.632949-0.597786-1.171288$

O $0.964006-1.467508 \quad 1.949788$
O $-0.166396-1.280608-1.499286$

C $-3.043727 \quad 0.273600-0.080988$

$\begin{array}{lllll}\text { O } & -3.459190 & 0.782087 & 1.105421\end{array}$

O $-3.625261-0.629383-0.667820$

H 3.7558961 .9101280 .432304

H $2.9286882 .011024-1.142704$

H 2.4358793 .0693600 .165520

H -0.6719270 .6212531 .229374$

H $1.583691 \quad 1.1967211 .487247$

H $3.121617-0.6027120 .806186$

H $1.422061-2.4207300 .190772$

H $-1.012052-1.6515140 .326097$

H -1.706295 $0.760687-1.665700$

H $-1.8979962 .020709-0.489471$

H $1.876929-0.361625-1.735746$

H $1.810571-1.7494702 .336545$

H $-0.000608-2.230487-1.624234$

H -2.901392 $1.503507 \quad 1.466779$

SCF Energy (B3LYP/6-31G**//MMFF) $=-764.620278770$

F1a-02 c00016

MMFF Geometry

C 2.8348102 .0232850 .025622

C $-0.522876 \quad 0.489914 \quad 0.228229$

O $0.574575 \quad 1.306547-0.203630$

C 1.7990490 .9968820 .471411

C $2.232028-0.444200 \quad 0.162024$

C $1.110792-1.4376650 .487519$

C $-0.236930-0.988480-0.092892$

C $-1.7735891 .032934-0.464307$

O $2.576591-0.551727-1.217951$

O $1.004248-1.5400281 .906045$

O $-0.237648-1.198914-1.503684$

C $-3.035196 \quad 0.417646 \quad 0.083611$

O $-3.510140-0.587937-0.691556$

$\begin{array}{llll}0 & -3.565068 & 0.767167 & 1.130502\end{array}$

H $2.510387 \quad 3.0316320 .305489$

H $3.808141 \quad 1.8318510 .486942$

H $2.9525172 .029305-1.063419$

H $-0.6369920 .614694 \quad 1.313333$

H $1.640816 \quad 1.1169831 .550783$

H $3.129114-0.678102 \quad 0.747737$

H $1.372702-2.4264030 .091855$

H -1.038207 -1.6087960 .323909$

H $-1.7239570 .919674-1.553308$

H - $1.8473322 .117530-0.309977$

H $2.881425-1.461887-1.373664$

H $0.314753-2.1973632 .101656$

H $0.428903-0.606834-1.892650$

H -2.981260 -0.785041-1.493803

SCF Energy (B3LYP/6-31G**//MMFF) $=-764.625774884$

F1a-02_c00017

MMFF Geometry

C -1.913652 $2.486733-0.189798$

C $0.411111-0.264530-1.012499$

O $-0.0898601 .006992-0.584850$

C $-1.520608 \quad 1.058766-0.550361$

C -2.0751280 .0289380 .446800$

C -1.538271 $-1.378601 \quad 0.153677$

C $-0.015202-1.376596-0.036514$

C $1.923484-0.152119-1.237192$

O $\begin{array}{lllll} & -1.734793 & 0.396460 & 1.782359\end{array}$

O $-2.138971-1.876912-1.039773$

O $0.607185-1.189422 \quad 1.230634$

C $2.7453580 .193167-0.017431$

$\begin{array}{lllll}O & 2.522128 & 1.452677 & 0.431103\end{array}$

O $3.547491-0.5720520 .502356$

H $-1.5408003 .183109-0.948996$

H $-3.0003432 .595216-0.123552$

H -1.4664512 .7989950 .760152$

H $-0.029321-0.476950-1.996500$

H $-1.8989090 .842869-1.557726$

H $-3.1694450 .030211 \quad 0.384313$

H -1.802647 -2.0536960 .976540$

H $0.319247-2.342188-0.434393$

H $2.1360650 .616144-1.991280$

H $2.306230-1.097904-1.639371$ 
H -0.7663270 .4703951 .832937$

H $-3.100025-1.904886-0.894328$

H $\quad 0.349949-1.9360191 .798070$

H $1.8547831 .958217-0.080593$

SCF Energy (B3LYP/6-31G**//MMFF) = -764.625251975

F1a-02 c00018

MMFF Geometry

C $1.3521872 .715642-0.327228$

C $-0.557679-0.389667 \quad 0.280347$

O $-\begin{array}{llll}0.121110 & 0.844208 & -0.307052\end{array}$

C 1.0835951 .3445830 .283170

C 2.2415520 .3621910 .050890

C $1.878097-1.0433830 .546286$

C $0.497795-1.4844660 .044152$

C $-1.907053-0.750883-0.353341$

O $2.5532550 .311791-1.339919$

O $1.858972-1.0525851 .972528$

O $0.567214-1.784780-1.348342$

C -3.0236350 .1428850 .134972$

O $-4.200418-0.234208-0.428186$

O

H $0.525183 \quad 3.397422-0.099635$

H 2.2772123 .1502880 .063521

H $1.4169242 .664489-1.419416$

H $-0.689770-0.2330681 .358963$

H $\quad 0.9146461 .4760441 .359590$

H 3.1301600 .7333420 .575231

H $2.634158-1.7638020 .212098$

H $\quad 0.181700-2.3967070 .564394$

H $-2.175119-1.780798-0.092202$

H -1.857708 -0.646264 -1.443321

H $3.303360-0.296654-1.450871$

H $2.746366-0.7994482 .279147$

H $1.218899-2.497218-1.462819$

H $-4.9429320 .332864-0.129082$

SCF Energy (B3LYP/6-31G**//MMFF)= -764.619250636

F1a-02 c00019

MMFF Geometry

C 1.3362982 .6920800 .178899

C $-0.595472-0.4557940 .145815$

O $-0.119367 \quad 0.849739-0.209322$

C $1.043797 \quad 1.2372750 .529636$

C 2.2171530 .2952290 .214385

C $1.827660-1.1746140 .421396$

C $0.468005-1.511315-0.206209$

C $-1.912940-0.684973-0.599033$

O $2.634808 \quad 0.455261-1.140605$

O $1.801544-1.4742941 .815613$

O $0.639822-1.589475-1.620973$

C $-3.048824 \quad 0.054146 \quad 0.067432$

O $-3.0312821 .384770-0.193083$

O $-3.893218-0.4826050 .772639$

H $1.4363692 .834412-0.902392$

H $\quad 0.501935 \quad 3.3287170 .494031$

H 2.2464313 .0453240 .672571

H $-0.789506-0.469507 \quad 1.226142$

H 0.8061201 .1919761 .599825

H 3.0694520 .5449230 .857807

H $2.596160-1.813941-0.030111$

H $0.135276-2.4956340 .144731$

H $-2.180087-1.748523-0.594012$

H -1.861673 -0.365619 -1.646429

H $3.0867131 .312708-1.212405$

H $1.132330-0.9153162 .242856$

H -0.161011 -1.992068 -1.996704

H $-2.2910351 .683169-0.763997$

SCF Energy (B3LYP/6-31G**//MMFF $)=-764.611401606$

F1a-03 c00020

MMFF Geometry

C -1.184602 $2.843688-0.420654$

C $0.645346-0.3124090 .163424$

O $0.2728690 .956929-0.386208$

C $-0.961798 \quad 1.4441300 .143202$

C $-2.104438 \quad 0.495352-0.230544$

C $-1.800467-0.9314920 .227902$
C $-0.403018-1.366239-0.215475$

C $2.028532-0.674436-0.395383$

$\begin{array}{lllll}\text { O } & -3.323900 & 0.931833 & 0.364330\end{array}$

O $-2.754284-1.824957-0.349279$

O $-0.077770-2.6039340 .412745$

C $3.1220770 .215404 \quad 0.158113$

O $4.330722-0.090718-0.382579$

O $2.9686591 .100130 \quad 0.988298$

H $-0.3388743 .493239-0.169325$

H -1.246765 2.819819-1.514229

H $-2.0986503 .297463-0.026395$

H $\quad 0.715597-0.2279021 .256514$

H $-0.876271 \quad 1.534576 \quad 1.234022$

H $-2.2588110 .495309-1.316830$

H $-1.904947-1.0274841 .315587$

H $-0.397894-1.533480-1.300247$

H $2.028240-0.560331-1.485829$

H $2.287384-1.706138-0.134100$

H -3.1956250 .9533321 .327993$

H $-3.638005-1.512498-0.089373$

H $-0.773414-3.2410710 .175509$

H $4.317881-0.823099-1.035273$

SCF Energy $($ B3LYP/6-31G**//MMFF $)=-764.624746589$

F1a-03_c00021

MMFF Geometry

C -1.068738 $2.888635-0.117656$

C $0.669636-0.3651050 .068496$

O $\quad 0.315897 \quad 0.961752-0.337635$

C $-0.874193 \quad 1.4338740 .297502$

C $-2.0677800 .564935-0.107027$

C - $-1.795278-0.9071170 .200889$

C $-0.436236-1.342766-0.350578$

C $2.005125-0.716781-0.592842$

O -3.2251170 .9901290 .607600$

O $-2.823456-1.691675-0.406155$

$\begin{array}{llll}\text { O } & -0.140739 & -2.655130 & 0.121955\end{array}$

C 3.1490200 .0051330 .078534

O $3.2562911 .298020-0.316362$

O $3.894638-0.511690 \quad 0.900194$

H $-0.187474 \quad 3.481097 \quad 0.151567$

H -1.183013 $2.973934-1.204037$

H -1.9447493 .3331990 .363804$

H $\quad 0.800542-0.383247 \quad 1.159119$

H $-0.735706 \quad 1.4142651 .386557$

H $-2.2779890 .682033-1.177824$

H -1.861017 -1.092019 1.280092

H $-0.496074-1.400342-1.444531$

H $2.011132-0.466965-1.660385$

H $2.219069-1.788948-0.514593$

H -3.9568170 .4033930 .350102$

H -2.615277 -2.625903 -0.232876

H $-0.064795-2.6141661 .090620$

H $2.5827151 .587568-0.968293$

SCF Energy (B3LYP/6-31G**//MMFF) $=-764.627616912$

F1a-03 c00022

MMFF Geometry

C -1.826412 -2.689697 -0.023552

C $0.598983-0.042518-0.888083$

O $0.048017-1.267298-0.398078$

C $-1.377685-1.309315-0.493358$

C $-2.005425-0.1938310 .348099$

C $-1.4500451 .171743-0.056859$

C $0.0795911 .154502-0.079465$

C $2.129908-0.158850-0.901887$

O $-3.418547-0.2011090 .160795$

O -1.8962522 .1398960 .894331$

O $0.5569662 .373715-0.643353$

C $2.761080-0.3828740 .457812$

O $4.107325-0.1995990 .416208$

O $2.169211-0.7180801 .473844$

H -1.340692 -3.470074-0.619729

H $-1.532780-2.8630541 .017633$

H -2.910015 -2.813903 -0.108488

H $0.2926140 .073626-1.937316$

H -1.671018 -1.205144 -1.546414

H $-1.811278-0.3665241 .414294$ 
H $-1.8571631 .480326-1.027539$ H $\quad 0.4411641 .1193950 .953837$ H $2.5557420 .750721-1.339954$ H $2.418137-1.016211-1.521877$ H -3.7840830 .5310010 .686484$ H -1.5005342 .9930810 .646473$ H $0.2397722 .423222-1.561336$ H $4.4663940 .048378-0.462280$

SCF Energy (B3LYP/6-31G**//MMFF) = -764.621020715

\section{F1a-03 c00023}

MMFF Geometry

C $-1.1790492 .825445-0.383690$

C $0.642264-0.3355640 .179583$

O $0.2619790 .933231-0.366731$

C -0.9709181 .4191310 .169041$

C $-2.1133720 .472485-0.210631$

C $-1.802560-0.9544990 .242763$

C $-0.405425-1.390880-0.201723$

C $2.030620-0.676979-0.379925$

O $-3.328184 \quad 0.8803090 .412924$

O $-2.758462-1.846887-0.335109$

O $-0.112322-2.633947 \quad 0.431912$

C $3.106000 \quad 0.2499980 .148749$

O $4.311288-0.013307-0.421431$

O $2.942704 \quad 1.1255150 .986775$

H -0.311606 $3.454882-0.155853$

H -1.274302 2.807061 -1.474987

H -2.0682183 .3016300 .039163$

H $\quad 0.712686-0.253638 \quad 1.272758$

H $-0.884722 \quad 1.5001741 .260649$

H -2.277347 $0.473346-1.295913$

H $-1.907416-1.0515271 .330623$

H $-0.412243-1.559376-1.286393$

H $2.019807-0.584570-1.472270$

H $2.320804-1.694410-0.099645$

H -3.611857 $1.710575-0.004909$

H -3.640526 -1.542729 -0.060162

H $0.645067-3.031463-0.028903$

H $4.308488-0.744087-1.075888$

SCF Energy $\left(\mathrm{B} 3 \mathrm{LYP} / 6-31 \mathrm{G}^{* *} / / \mathrm{MMFF}\right)=-764.616852752$

F1a-03_c00024

MMFF Geometry

C $-1.1193612 .890093-0.175416$

C $0.669183-0.3350860 .051789$

O $\quad 0.2944290 .980424-0.373374$

C $-0.896444 \quad 1.4470720 .264337$

C -2.081236 $0.551719-0.112415$

C $-1.781966-0.9123490 .215508$

C $-0.421820-1.338100-0.341493$

C $2.004644-0.675569-0.615436$

O -3.2526420 .9643310 .586694$

O $-2.801263-1.743127-0.343596$

O $-0.087478-2.6246630 .173587$

C $3.1237540 .162556-0.045934$

O $3.568159-0.3031561 .147869$

O $3.576408 \quad 1.161128-0.590117$

H -0.2458533 .5021520 .074560$

H -1.245002 2.953413-1.261945

H $-1.998300 \quad 3.3283160 .306293$

H $0.805442-0.3339251 .141805$

H -0.7459801 .4500801 .351975$

H -2.297599 $0.642923-1.183890$

H -1.819851 -1.080535 1.298573

H $-0.480939-1.423360-1.434078$

H $1.963626-0.476589-1.693459$

H $2.272969-1.731244-0.496653$

H $-3.0718220 .896818 \quad 1.539848$

H -2.802397 -1.610017 -1.306942

H $-0.811280-3.229478-0.064017$

H $3.115561-1.1100661 .473777$

SCF Energy (B3LYP/6-31G**//MMFF) $=-764.616347313$

F1a-03 c00025

MMFF Geometry

C 1.10867928518520 .123441

C $-0.673647-0.373247-0.075623$
O $-\begin{array}{llll}0.291963 & 0.942141 & 0.341314\end{array}$

C $0.9007991 .401157-0.299964$

C 2.0841980 .5086050 .090017

C $1.779080-0.957724-0.223994$

C $0.414572-1.3749840 .329852$

C $-2.013262-0.7046700 .587826$

O $3.2516950 .884637-0.635362$

O $2.794190-1.7860240 .347294$

O $0.090566-2.676985-0.152262$

C $-3.1449360 .047734-0.070660$

$\begin{array}{lllll}\text { O } & -3.224931 & 1.337676 & 0.339687\end{array}$

O $-3.903759-0.444478-0.895486$

H $1.9630683 .306222-0.385998$

H $0.2168403 .445703-0.106216$

H 1.2633942 .9254261 .205564

H $-0.809287-0.379189-1.165902$

H $0.7537121 .390363-1.388115$

H 2.3086330 .6071121 .159798

H $1.824351-1.133354-1.305593$

H $\quad 0.474978-1.4427951 .423187$

H $-2.009694-0.4662041 .657973$

H -2.249394 -1.771354 0.499162

H $3.5421051 .750340-0.302772$

H $2.780701-1.6567131 .310862$

H $0.024850-2.629611-1.121382$

H -2.5435391 .6059790 .992585$

SCF Energy $(B 3 L Y P / 6-31 G * * / / M M F F)=-764.615996519$

F1a-03 c00026

MMFF Geometry

C $2.527013-2.464070-0.050958$

C $-0.572966-0.4577250 .102812$

O $0.372197-1.468561-0.271853$

C $1.656343-1.2691540 .324413$

C $2.2666360 .049131-0.158937$

C 1.3175451 .2176490 .106411

C $-0.0904340 .909293-0.407122$

C $-1.927636-0.862682-0.496308$

$\begin{array}{lllll}\text { O } & 3.497584 & 0.277161 & 0.522212\end{array}$

O $1.8241132 .370067-0.569453$

O $-\begin{array}{llll}0.974198 & 1.946437 & 0.006827\end{array}$

C $-3.116707-0.2626820 .220889$

O $-4.265425-0.443872-0.481737$

O $-3.083691 \quad 0.295042 \quad 1.308815$

H $2.053933-3.3963760 .276670$

H $2.643271-2.537378-1.138003$

H $3.519375-2.401120 \quad 0.405213$

H $-0.654533-0.4481801 .197736$

H $1.550940-1.2654531 .417436$

H $2.487801-0.005524-1.232531$

H $1.3024341 .466276 \quad 1.174758$

H -0.075575 $0.920188-1.504067$

H -2.040569 -1.952753 -0.445121

H -1.962019-0.580023 - 1.554984

H $3.8452291 .131880 \quad 0.214407$

H $1.1903233 .093239-0.422901$

H -1.022886 1.9277860 .978029

H -4.161126 -0.921312 -1.332345

SCF Energy $(B 3 L Y P / 6-31 G * * / / M M F F)=-764.627048999$

F1a-03_c00027

MMFF Geometry

C $2.036796-2.546622-0.085185$

C $-0.587528-0.1522690 .931194$

O $0.054725-1.3084160 .384492$

C $1.480410-1.2248810 .434325$

C $1.972338-0.038177-0.399481$

C $1.305395 \quad 1.2618310 .052774$

C -0.2142881 .1025410 .128829$

C $-2.094095-0.4280030 .992054$

O $3.3868780 .093329-0.283085$

O $1.6054492 .294293-0.888670$

O -0.7526922 .2657820 .751875$

C $-2.719608-0.585496-0.375452$

O $-3.5143310 .457360-0.719324$

O $-2.521914-1.544809-1.110323$

H $1.724342-2.722884-1.120387$

H $3.129626-2.571565-0.038771$ 
H $1.645796-3.3818460 .506305$ H -0.252716 -0.032031 1.970917 H $1.798106-1.1183921 .479951$ H $1.751014-0.201661-1.461516$ H 1.7063181 .5965471 .017582 H $-0.6104901 .055577-0.892831$ H -2.609659 0.3549621 .558289 H $-2.285284-1.3664381 .527640$ H 3.5987020 .2261200 .656628 H $2.5740512 .356366-0.951174$ H -1.718070 2.239408 0.646228 H $-3.5931801 .171979-0.052922$

SCF Energy (B3LYP/6-31G**//MMFF) $=-764.608798173$

F1a-03_c00028

MMFF Geometry

C -1.106628 $2.867214-0.155454$

C $0.665715-0.3632690 .071336$

O $\quad 0.2863620 .949708-0.358230$

C $-0.901868 \quad 1.4203350 .282153$

C $-2.088328 \quad 0.527674-0.095328$

C $-1.785488-0.9355810 .233415$

C $-0.425997-1.367915-0.321762$

C $2.007277-0.689754-0.591086$

$\begin{array}{llll}\text { O } & -3.252783 & 0.914318 & 0.629560\end{array}$

O $-2.808017-1.765820-0.322881$

$\begin{array}{llll}\text { O } & -0.127155 & -2.658304 & 0.205423\end{array}$

C $3.1059560 .199647-0.059510$

O $3.556041-0.1981191 .156643$

O $3.5402951 .179240-0.650845$

H -1.264692 $2.930083-1.237666$

H -1.9579713 .3295740 .351892$

H -0.2122693 .4605040 .065196$

H $0.799950-0.3590901 .161293$

H -0.7514361 .4202811 .369800$

H $-2.3156150 .615277-1.165455$

H -1.825206 -1.0997181 .317238$

H $-0.495868-1.458136-1.413313$

H $1.954114-0.529476-1.675012$

H $2.310694-1.730459-0.437215$

H $-3.5412041 .777750 \quad 0.289422$

H -2.802369 -1.643882 -1.287488

H $0.600060-3.033000-0.318947$

H $3.120026-0.9979991 .520300$

SCF Energy (B3LYP/6-31G**//MMFF) $=-764.608762062$

F1a-03_c00029

MMFF Geometry

C $2.554795-2.391264-0.269104$

C $-0.601635-0.4873920 .000409$

O $0.385030-1.421999-0.454004$

C $1.640877-1.2682020 .210681$

C $2.2322060 .110391-0.092530$

C 1.2429331 .2146140 .281052

C $-0.1437510 .945684-0.308456$

C $-1.912701-0.852287-0.704229$

$\begin{array}{lllll}\text { O } & 3.434886 & 0.283201 & 0.651999\end{array}$

O $1.7285442 .467482-0.205895$

$\begin{array}{lllll}\text { O } & -1.068250 & 1.876716 & 0.245979\end{array}$

C $-3.135463-0.289861-0.024753$

O $-3.426215-0.9257701 .137181$

O $-3.801966 \quad 0.634661-0.471081$

H $2.094595-3.365710-0.071597$

H $2.711381-2.333096-1.351997$

H $3.528253-2.3614960 .229003$

H $-0.733087-0.612147 \quad 1.083451$

H $1.496708-1.3956751 .291787$

H $2.4869170 .188633-1.156986$

H $1.1815511 .321221 \quad 1.370831$

H $-0.1183591 .108983-1.393240$

H $-2.032849-1.941716-0.764677$

H -1.902147 $-0.511866-1.746913$

H $3.765428 \quad 1.1788460 .466220$

H $1.7938502 .408196-1.174387$

H -0.7340562 .7694700 .052962$

H -2.817970 -1.658071 1.373692

SCF Energy (B3LYP/6-31G**//MMFF) $=-764.618145472$
F1a-04 c00030

MMFF Geometry

C $-0.7976772 .907283-0.482434$

C $0.606902-0.517918-0.778181$

O $0.278608 \quad 0.854949-1.023893$

C $-0.5681061 .462028-0.045410$

C -1.8910860 .7029630 .088042$

C $-1.654090-0.7858540 .337736$

C $-0.679575-1.351717-0.692872$

C $1.544662-0.6849580 .432031$

O $-2.638016 \quad 1.2505621 .171948$

O $-2.907844-1.4625560 .220928$

O $-0.390369-2.707132-0.367532$

C 2.8676090 .0261950 .237655

O $3.651726-0.0748121 .343023$

O $3.2178730 .615305-0.775138$

H -1.4096923 .4558940 .239425$

H $\quad 0.1604273 .427727-0.590754$

H $-1.2877312 .947150-1.461514$

H $1.160638-0.860680-1.661830$

H $-0.050678 \quad 1.501847 \quad 0.919938$

H $-2.4926430 .827668-0.821641$

H -1.303369 -0.9614101 .361586$

H $-1.175774-1.354950-1.672127$

H $1.770929-1.7461610 .583658$

H $1.085572-0.2919441 .343839$

H $-3.459617 \quad 0.7355371 .247254$

H $-2.735641-2.4123370 .340961$

H $0.213420-3.048400-1.049245$

H $3.256494-0.5785962 .086361$

SCF Energy $(B 3 L Y P / 6-31 G * * / / M M F F)=-764.617558888$

F1a-04_c00031

MMFF Geometry

C $0.8112592 .825400 \quad 0.693787$

C $-0.690474-0.566433 \quad 0.640104$

O $-\begin{array}{llll}0.342144 & 0.773144 & 1.008457\end{array}$

C $0.5875361 .421625 \quad 0.135819$

C 1.8965120 .6299750 .039139

C $1.633090-0.829136-0.334695$

C $0.571716-1.4347350 .580648$

C $-1.544177-0.610405-0.635924$

$\begin{array}{lllll}\text { O } & 2.745434 & 1.194076 & -0.957727\end{array}$

O $2.840816-1.577734-0.176450$

O $\quad 0.240536-2.749303 \quad 0.146061$

C $-2.820505 \quad 0.176643-0.462340$

O $-3.769235-0.5105240 .220828$

$\begin{array}{lllll}0 & -2.982448 & 1.316734 & -0.877537\end{array}$

H $1.454316 \quad 3.4250530 .043513$

H $-0.146193 \quad 3.3468120 .804203$

H $1.2610922 .782374 \quad 1.691745$

H $-1.313544-0.9511221 .457603$

H $0.1409921 .542944-0.857819$

H 2.4394590 .6589630 .992719

H $1.346030-0.927553-1.388262$

H $0.988201-1.5262331 .592247$

H $-1.809135-1.637677-0.911503$

H $-1.019341-0.193298-1.501462$

H $3.0477082 .059602-0.635406$

H $3.516015-1.158103-0.736951$

H $1.068811-3.2571590 .099285$

H $-3.507819-1.4133380 .501808$

SCF Energy (B3LYP/6-31G**//MMFF) = -764.620126314

F1a-04_c00032

MMFF Geometry

C 0.9004652 .8378330 .650599

C $-0.673554-0.5271210 .644530$

$\begin{array}{lllll}\text { O } & -0.310627 & 0.816072 & 0.986632\end{array}$

C 0.6390951 .4328740 .113265

$\begin{array}{llll}\text { C } & 1.932832 & 0.616927 & 0.030286\end{array}$

C $1.646759-0.840197-0.330326$

C $0.578972-1.4133780 .598007$

C $-1.538551-0.584555-0.623694$

O $2.8063921 .184919-0.943387$

O $2.855470-1.586607-0.173793$

O $0.256116-2.735555 \quad 0.180576$

C $-2.908440-0.003999-0.362987$ 
O $\quad-2.9484791 .342460-0.511351$

O $-3.879607-0.672329-0.032714$

H $1.3149632 .798331 \quad 1.664102$

H 1.5913023 .3938080 .009919

H -0.0380363 .3991540 .717494$

H -1.295500 -0.8901871 .473235$

H $0.2027091 .552326-0.884661$

H 2.4624080 .6487760 .990580

H $1.356078-0.937867-1.383073$

H $1.005256-1.4913871 .606759$

H -1.699614 -1.620674 -0.943677

H -1.091706 -0.071175 -1.480075

H $2.3548151 .156126-1.803978$

H $2.647251-2.517298-0.364607$

H $-0.410587-3.0821810 .797920$

H $-2.0989381 .760262-0.765731$

SCF Energy (B3LYP/6-31G**//MMFF) $=-764.612847661$

F1a-04 c00033

MMFF Geometry

C 0.9779942 .4622770 .072912

C $-0.515422-0.189018 \quad 0.322674$

O $-0.1469450 .511101-0.872007$

C $1.0951871 .220828-0.808604$

C $2.2569260 .291388-0.426365$

C $1.944306-0.6137470 .771520$

C $0.550978-1.2409370 .666304$

C $-1.886125-0.8325830 .070625$

O $2.533200-0.531206-1.564498$

O $2.013177 \quad 0.137129 \quad 1.979972$

O $0.566414-2.270270-0.321620$

C -3.0034410 .1895890 .048721$

O $-4.209536-0.400179-0.162615$

O

H $\quad 0.130706 \quad 3.075729-0.252510$

H $\quad 0.8006732 .221092 \quad 1.123807$

H 1.8875393 .0682140 .013783

H $-0.6040580 .526653 \quad 1.147874$

H $1.2678071 .576101-1.832348$

H $3.1550650 .890604-0.237365$

H $2.690752-1.4150990 .833510$

H $0.287328-1.7149721 .619128$

H $-2.111991-1.5464190 .870749$

H $-1.892923-1.345937-0.897918$

H $3.304253-1.082423-1.347246$

H 2.9102660 .5065942 .045462

H $\quad 0.732990-1.852160-1.183819$

$\mathrm{H}-4.179987-1.375066-0.267894$

SCF Energy (B3LYP/6-31G**//MMFF) $=-764.618123441$

F1a-04 c00034

MMFF Geometry

C $2.448611-2.419846-0.132907$

C $-0.636651-0.429959-0.663151$

O $0.404962-1.403768-0.814178$

C $1.464550-1.2853650 .139994$

C $2.1424020 .083167 \quad 0.032301$

C $1.121554 \quad 1.2140880 .153077$

C $-0.0533490 .984848-0.796573$

C $-1.440521-0.6923940 .620077$

O $3.1118840 .210672 \quad 1.069692$

O $1.7715222 .440059-0.191278$

O $-1.0446101 .976479-0.556620$

C $-2.887707-0.2846870 .492780$

O $-3.611398-1.156390-0.253026$

O $-3.3641540 .720401 \quad 1.003396$

H $1.937725-3.387449-0.075454$

H $2.862703-2.343983-1.144505$

H $3.273721-2.4215360 .585443$

H -1.308354 -0.582982 -1.516982

H $1.069707-1.4364461 .152260$

H $2.6712750 .169795-0.925455$

H $\quad 0.785030 \quad 1.327508 \quad 1.190055$

H $0.3044801 .130949-1.824297$

H -1.013146 -0.167755 1.481730

H $-1.416955-1.7562610 .888252$

H 3.5049561 .0969980 .991806

H $1.1020443 .144344-0.145221$
H $-1.7639631 .831802-1.194865$

H -3.108196 -1.923718 -0.600232

SCF Energy $\left(B 3 L Y P / 6-31 G^{* *} / / M M F F\right)=-764.615142136$

F1a-04_c00035

MMFF Geometry

C $2.479652-2.378473-0.340588$

C $-0.604759-0.374973-0.823833$

O $0.464198-1.310521-1.022785$

C $1.462993-1.2918350 .000286$

C 2.1237790 .0867920 .089361

C 1.0771901 .1850430 .280782

C $-0.0430121 .052795-0.751597$

C -1.481647 -0.7985370 .369430$

O $3.0296590 .095704 \quad 1.189976$

O $1.6935332 .462481 \quad 0.100224$

O $-1.0822331 .979381-0.456714$

C $-2.931367-0.3804050 .241834$

O $-3.627316-0.6559781 .376356$

O $-3.4451200 .125164-0.745816$

H $1.981818-3.350708-0.425782$

H $2.951899-2.182554-1.309576$

H $3.260683-2.4557070 .421555$

H -1.217307-0.440135 - 1.731186

H $1.012372-1.5621030 .963253$

H $2.7037180 .288360-0.820335$

H $\quad 0.6742631 .179315 \quad 1.300337$

H $\quad 0.3565491 .322410-1.738137$

H $-1.082255-0.3879281 .302773$

H -1.473942 -1.892333 0.454169

H $3.5207510 .933899 \quad 1.160863$

H 2.3361912 .5830210 .819422

H $-0.6805032 .864155-0.415029$

H -3.102984 -1.071981 2.093694

SCF Energy $\left(B 3 L Y P / 6-31 G^{* *} / / M M F F\right)=-764.612275278$

F1a-04 c00036

MMFF Geometry

C 0.76481324319780 .556761

C $-0.532131-0.3145620 .237413$

O $-0.1848380 .627325-0.785614$

C $0.9984961 .393979-0.538319$

C $2.2127760 .486583-0.289804$

C $1.935776-0.6532100 .697539$

C $0.596694-1.3414880 .414963$

C $-1.843586-0.984130-0.185422$

O $2.610657-0.080679-1.542097$

O $1.912413-0.1504512 .030056$

O $0.705044-2.131882-0.767834$

C $-3.023403-0.0769930 .071169$

O $-3.1293530 .915885-0.846276$

O $-3.800039-0.2138101 .007405$

H $\quad 0.5786691 .981629 \quad 1.534785$

H 1.6275143 .0989850 .650458

H -0.1171823 .0369370 .319633$

H -0.6973820 .2230611 .178089$

H $1.1741141 .949002-1.468349$

H $3.056333 \quad 1.0935340 .057757$

H $2.741602-1.3947140 .634238$

H $\quad 0.336846-2.0144541 .241138$

H $-2.015509-1.8994610 .393493$

H -1.846684 -1.264180 -1.245345

H $1.851600-0.571694-1.900827$

H 2.7767760 .2595162 .204311

H $1.397869-2.796519-0.613518$

$\mathrm{H}-2.4322520 .914314-1.536783$

SCF Energy (B3LYP/6-31G**//MMFF) = -764.621109360

F1a-04 c00037

MMFF Geometry

C $-0.7821052 .829926-0.699335$

C $0.665372-0.590348-0.641882$

O $0.3387250 .753679-1.018468$

C $-0.5726041 .423466-0.143715$

C $-1.8977500 .660827-0.040668$

C $-1.667954-0.8023690 .342938$

C $-0.614437-1.435635-0.567252$

C $1.533443-0.6361430 .623986$ 
O $-2.743717 \quad 1.277514 \quad 0.927142$

O $-2.896326-1.5221880 .218138$

O $-0.324887-2.764567-0.146513$

C 2.8037250 .1595050 .443650

O $3.743506-0.510540-0.268100$

$\begin{array}{lllll}O & 2.968654 & 1.291649 & 0.878931\end{array}$

H -1.442392 $3.423176-0.059872$

H $0.1777623 .351238-0.784346$

H -1.208689 2.792902 -1.707797

H $1.273886-0.992250-1.462060$

H $-0.118423 \quad 1.5379410 .847159$

H -2.429233 $0.699696-0.999649$

H $-1.378612-0.8878341 .396490$

H - $1.035316-1.517365-1.577543$

H $1.809254-1.6627980 .889856$

H $1.013222-0.227878 \quad 1.496749$

H -2.2851001 .2533221 .784144$

H $-3.184517-1.467554-0.708967$

H $-0.167014-2.7533340 .812515$

H $3.481398-1.409012-0.562055$

SCF Energy (B3LYP/6-31G**//MMFF) $=-764.607064833$

F1a-04_c00038

MMFF Geometry

C $0.7871512 .432177 \quad 0.518263$

C $-0.533940-0.3028050 .205468$

$\begin{array}{llll}\text { O } & -0.154788 & 0.620875 & -0.822277\end{array}$

C $1.0319051 .377794-0.559269$

C $2.2333600 .462052-0.274828$

C $1.923066-0.6599430 .721253$

C $0.580344-1.3365390 .424307$

C $-1.840327-0.965913-0.239744$

O $2.635021-0.149244-1.504765$

O $1.903953-0.1160722 .037594$

O $0.712511-2.171708-0.725070$

C $-2.9956990 .001291-0.142625$

O $-3.464696 \quad 0.1282591 .123657$

O $-3.454700 \quad 0.617439-1.095439$

H $1.6561453 .089248 \quad 0.623330$

H $-0.081440 \quad 3.0452040 .253939$

H $0.5727081 .996771 \quad 1.497257$

H -0.7120280 .2507291 .134146$

H $1.2289651 .922342-1.490957$

H $3.081504 \quad 1.0586660 .081257$

H $2.725018-1.4074200 .679054$

H $0.299800-1.9874851 .260500$

H -2.076762 -1.8477270 .366836$

H $-1.783603-1.298663-1.283415$

H $2.9029570 .561380-2.112203$

H $1.750018-0.8501452 .656466$

H $0.906007-1.599873-1.487641$

H $-3.002854-0.4181081 .794885$

SCF Energy (B3LYP/6-31G**//MMFF) $=-764.612601588$

F1a-04_c00039

MMFF Geometry

C $0.8156602 .833447 \quad 0.645360$

C $-0.670018-0.5725250 .650588$

$\begin{array}{lllll}\text { O } & -0.334815 & 0.778530 & 0.993371\end{array}$

C 0.5929021 .4198170 .113846

C $1.9089880 .640580 \quad 0.029474$

C $1.659542-0.823405-0.335189$

C $0.604544-1.4282850 .590941$

C $-1.552885-0.640973-0.603852$

O $2.767094 \quad 1.232497-0.943104$

O $2.879462-1.556608-0.207717$

O $\quad 0.307252-2.766111 \quad 0.205615$

C $-2.911299-0.037530-0.333510$

O $-2.9622331 .291293-0.594146$

O $-3.864486-0.6734720 .098246$

H $-0.138428 \quad 3.367610 \quad 0.714091$

H 1.2348652 .8094021 .657303

H $1.487683 \quad 3.4069540 .000137$

H $-1.273728-0.952720 \quad 1.485077$

H $\quad 0.150054 \quad 1.522133-0.883027$

H 2.4363180 .6860860 .990338

H $1.360874-0.918954-1.385372$

H $1.028093-1.4879841 .601766$
H $-1.735238-1.679307-0.903562$

H $-1.107064-0.150737-1.474214$

H $2.3155651 .194811-1.803490$

H $3.172532-1.4953230 .717335$

H $0.173890-2.784664-0.756913$

H $-2.1282071 .686894-0.923534$

SCF Energy (B3LYP/6-31G**//MMFF)= -764.605573383

F1a-05 c00040

MMFF Geometry

C $0.7606132 .354280-1.175105$

C $-0.541389-0.213703-0.184620$

\begin{tabular}{llll}
\hline & -0.277511 & 0.980570 & 0.560357
\end{tabular}

C 0.9117921 .6909850 .191081

$\begin{array}{lllll}\text { C } & 2.140231 & 0.783197 & 0.295104\end{array}$

C $1.940104-0.526026-0.467713$

C $0.629260-1.199525-0.061123$

C $-1.837386-0.823956 \quad 0.370817$

$\begin{array}{lllll}\text { O } & 2.338058 & 0.496105 & 1.684528\end{array}$

O $3.023877-1.414304-0.201224$

O $0.392940-2.316600-0.915891$

C $-3.055507 \quad 0.0116990 .035888$

O $-4.183255-0.5165220 .579992$

O $-3.056206 \quad 1.031506-0.639183$

H $-0.1553732 .954238-1.205468$

H $1.6128133 .008146-1.385210$

H $\quad 0.6870581 .625928-1.987677$

H $-0.7068190 .039103-1.239196$

H $1.0076572 .491046 \quad 0.935327$

H $3.0427411 .300153-0.049238$

H $1.940007-0.346378-1.549295$

H $\quad 0.706493-1.5801190 .964943$

H $-1.773364-0.8949441 .463041$

H -2.002285 $-1.820492-0.052749$

H $3.091807-0.1151401 .749270$

H $3.842505-0.965222-0.472768$

H $1.163704-2.905272-0.842820$

H $-4.046485-1.3387891 .097228$

SCF Energy (B3LYP/6-31G**//MMFF) $=-764.618201553$

F1a-05_c00041

MMFF Geometry

C $-2.072792-2.021284-1.182352$

C $0.464759-0.342188-0.894161$

O $0.021860-1.426908-0.073807$

C $-1.398051-1.534373 \quad 0.097169$

C $-1.987833-0.227337 \quad 0.635324$

C $-1.560098 \quad 0.975672-0.205898$

C $-0.0404401 .008306-0.368680$

C $1.993059-0.415462-1.006859$

O $-1.505315-0.065681 \quad 1.974284$

$\begin{array}{lllll}0 & -1.988410 & 2.183340 & 0.420535\end{array}$

O $0.316103 \quad 2.031759-1.294524$

C $2.716442-0.102682 \quad 0.282329$

O $2.589299-1.084702 \quad 1.208138$

$\begin{array}{llll}0 & 3.355984 & 0.923515 & 0.474210\end{array}$

H $-1.587880-2.934943-1.542283$

H $-3.131625-2.235201-1.005545$

H $-2.008106-1.290088-1.993257$

H $0.085388-0.499515-1.911812$

H $-1.534004-2.3145340 .856296$

H $-3.079945-0.2862170 .701516$

H -2.035980 $0.947741-1.193131$

H $0.422443 \quad 1.2583560 .593135$

H $2.3509330 .289679-1.766473$

H $2.310966-1.413608-1.331835$

H -1.8416130 .7858962 .302420$

H $-2.955803 \quad 2.1387080 .509504$

H $-0.0449522 .868404-0.954500$

H $2.043751-1.8491770 .924044$

SCF Energy (B3LYP/6-31G**//MMFF) $=-764.620883737$

F1a-05_c00042

MMFF Geometry

C -2.035898 -2.071282 -1.201271

C $0.452863-0.330842-0.847378$

O $-0.001327-1.406970-0.022591$

C $-1.424004-1.5422510 .093471$ 
C $-2.063301-0.2360540 .577853$

C $-1.6321140 .953520-0.279110$

C $-0.108151 \quad 1.024031-0.391133$

C $1.988738-0.368971-0.892157$

O

O $-2.1335742 .164613 \quad 0.284153$

O $0.2496102 .022282-1.344537$

C $2.665941-0.1791090 .450487$

$\begin{array}{lllll}\text { O } & 3.999952 & 0.038718 & 0.306741\end{array}$

O $2.118207-0.2392011 .542211$

H $-1.948890-1.359148-2.026654$

H -1.520545 -2.984304 -1.517917

H -3.097292 -2.300179-1.063502

H $0.125124-0.521587-1.877499$

H -1.573794 -2.310304 0.861851

H -3.156439 -0.3077880 .603929$

H $-2.0713870 .879880-1.280306$

H $0.315493 \quad 1.3287160 .571756$

H $2.3495110 .405710-1.578079$

H $2.311412-1.348273-1.266081$

H $-1.946276-0.7449602 .466065$

H $-1.7973592 .227174 \quad 1.194752$

H $-0.1575652 .857333-1.056364$

H $4.3223720 .045523-0.619630$

SCF Energy $\left(B 3 L Y P / 6-31 G^{* *} / / M M F F\right)=-764.615377989$

F1a-05 c00043

MMFF Geometry

C $0.563382-2.1481581 .544030$

C $-0.555670 \quad 0.2609920 .053040$

O $-0.310671-1.053280-0.459238$

C $0.821141-1.7338980 .097894$

C $2.094608-0.903610-0.080845$

C $1.9178880 .522758 \quad 0.438423$

C $0.6655381 .167933-0.158203$

C $-1.7833430 .814522-0.679220$

O $2.380785-0.865343-1.484151$

O $3.076096 \quad 1.281580 \quad 0.096679$

O $\quad 0.4260402 .418621 \quad 0.483589$

C $-3.0363270 .077065-0.271803$

$\begin{array}{lllll}\text { O } & -3.521089 & 0.494646 & 0.924325\end{array}$

O $-3.556407-0.809040-0.936833$

H $0.477805-1.2895182 .216037$

H $-0.379358-2.7009481 .617152$

H $1.371628-2.7863931 .914603$

H -0.7932550 .1954291 .121687$

H $\quad 0.919987-2.652363-0.493578$

H $2.952030-1.3876920 .399831$

H 1.8542630 .5199011 .532972

H $\quad 0.817867 \quad 1.365935-1.226482$

H $-1.6738580 .698390-1.764548$

H -1.937167 $1.882296-0.487503$

H $3.168275-0.306772-1.602483$

H 2.9951312 .1507960 .523876

H 1.1510753 .0179630 .238852

$\mathrm{H}-3.0057551 .209878 \quad 1.354714$

SCF Energy $\left(B 3 L Y P / 6-31 G^{* *} / / M M F F\right)=-764.612236438$

F1a-05 c00044

MMFF Geometry

C $0.7985772 .297283-1.232618$

C $-0.529544-0.238475-0.198439$

O $-0.259151 \quad 0.967791 \quad 0.526336$

C $\quad 0.934770 \quad 1.6648860 .149756$

C 2.1566400 .7518600 .286152

C $1.954502-0.568956-0.455660$

C $0.635347-1.231182-0.053319$

C $-1.838221-0.8160710 .363258$

$\begin{array}{lllll}\text { O } & 2.348534 & 0.465570 & 1.676470\end{array}$

O $3.032162-1.459068-0.167623$

O $0.432170-2.356445-0.906198$

C $-3.036296 \quad 0.0519840 .036261$

O $-4.167797-0.4256080 .618294$

O $-3.0202741 .053182-0.665933$

H $0.7268491 .551383-2.029141$

H $-0.1132482 .901919-1.284301$

H $1.6567292 .940870-1.450608$

H $-0.688121-0.003397-1.258156$
H 1.0266532 .4829430 .874539

H $3.0698271 .247784-0.061593$

H $1.966387-0.405752-1.539882$

H $\quad 0.713085-1.6019780 .976504$

H -1.768276 -0.8890281 .455090$

H $-2.037421-1.806045-0.058881$

H 2.5172861 .3096012 .128903

H $3.857075-1.010503-0.419961$

H $-0.260317-2.909836-0.508420$

H -4.046316 -1.239306 1.152799

SCF Energy $(\mathrm{B} 3 \mathrm{LYP} / 6-31 \mathrm{G} * * / / \mathrm{MMFF})=-764.604631382$

F1a-05_c00045

MMFF Geometry

C $1.2049962 .647513-0.535122$

C $-0.627532-0.585954-0.690642$

O $-0.1293690 .728870-0.978013$

C $0.8216451 .252223-0.047728$

C 2.0423180 .3327680 .037696

C $1.626415-1.0909990 .419817$

C $0.526210-1.594488-0.513531$

C $-1.610036-0.5693590 .491732$

O 2.9711720 .8391010 .989424

O $1.148165-1.1253161 .762365$

O $1.122879-1.834228-1.791470$

C $-2.8903490 .170676 \quad 0.162992$

$\begin{array}{llll}\text { O } & -3.763303 & 0.136365 & 1.204240\end{array}$

O

H 1.9058503 .1365800 .147851

H $1.6575342 .603722-1.531933$

H $0.3124743 .276670-0.624044$

H -1.188131-0.875967-1.588610

H $\quad 0.356667 \quad 1.3751540 .936884$

H $2.5428180 .305689-0.938661$

H $2.501582-1.7501450 .373567$

H $0.133384-2.552348-0.153187$

H -1.177693 -0.0873861 .372193$

H -1.886843 -1.598988 0.746051

H 3.7352060 .2377061 .001143

H $1.841925-0.7447152 .327692$

H $\quad 0.437872-2.211672-2.369277$

H $-3.444966-0.3491001 .995153$

SCF Energy (B3LYP/6-31G**//MMFF) $=-764.613917314$

F1a-05_c00046

MMFF Geometry

C -2.125623 -1.945835 -1.205952

C $0.435598-0.317097-0.899150$

O $-0.019606-1.403895-0.088161$

C $-1.440788-1.4972110 .082242$

C $-2.008905-0.1921260 .649910$

C $-1.5623251 .025214-0.161652$

C $-0.0443531 .036451-0.354390$

C $1.961558-0.411901-1.022877$

O $-1.554046-0.0567102 .001933$

O -1.9812552 .2224900 .490105$

O $\quad 0.3149162 .079580-1.257559$

C $2.696389-0.1386500 .268703$

O $2.569598-1.1450011 .168356$

O 3.3424710 .8785050 .484663

H -2.058053 -1.195400 -1.998707

H -1.651277 -2.855111-1.590524

H -3.185654 -2.153838 -1.029266

H $0.047720-0.457315-1.915987$

H -1.586482 -2.290746 0.825240

H $-3.102332-0.2281440 .702835$

H -2.055956 $1.023398-1.140192$

H $\quad 0.434815 \quad 1.2693140 .602843$

H $2.3265950 .303408-1.769464$

H $2.261548-1.407840-1.371162$

H $-0.590324-0.1844382 .011807$

H -1.592247 2.2249891 .381518

H $-0.1140621 .898481-2.111223$

H $2.019780-1.8995750 .866575$

SCF Energy (B3LYP/6-31G**//MMFF) = -764.618833337

F1a-05 c00047

MMFF Geometry 
C $-2.197748-1.906845-1.202537$

C $0.413356-0.363189-0.879729$

O $-0.075400-1.443950-0.079744$

C -1.499197-1.493092 0.089820

C $-2.025477-0.1758430 .670646$

C $-1.5409571 .034093-0.129579$

C $-0.0230670 .997314-0.317327$

C $1.934195-0.507725-1.005978$

O $-1.568226-0.0710392 .024581$

O

O $0.3724352 .040990-1.204744$

C $2.652917-0.376400 \quad 0.316981$

O 3.2018960 .8499160 .500026

O $2.719680-1.2714781 .149621$

H -1.752859-2.826924 -1.596225

H -3.263964 -2.082334 -1.027789

H -2.106051 -1.151028 -1.987859

H $0.019923-0.477664-1.897655$

H -1.670638 -2.289079 0.824804

H $-3.119631-0.1781660 .722825$

H $-2.0376281 .058218-1.106732$

H $0.457474 \quad 1.2020450 .645403$

H $2.3403930 .211805-1.725435$

H $2.185980-1.502025-1.396180$

H $-0.609088-0.2302022 .034034$

H -1.5632902 .9778210 .042301$

H $-0.0548301 .881970-2.063763$

H $3.0612751 .488265-0.230941$

SCF Energy (B3LYP/6-31G**//MMFF)= -764.611577565

F1a-05 c00048

MMFF Geometry

C $1.2304282 .597074-0.670147$

C $-0.690347-0.588518-0.582264$

O $-0.183771 \quad 0.703780-0.948335$

C $0.8415941 .233679-0.103931$

C $2.0404560 .282229-0.058699$

C $1.614121-1.1134470 .403551$

C $0.447827-1.618537-0.447339$

C $-1.589511-0.5068140 .657396$

$\begin{array}{llll}\text { O } & 3.056516 & 0.786707 & 0.800567\end{array}$

O $1.226336-1.0608651 .774804$

O $0.929827-1.902478-1.763811$

C $-2.923190 \quad 0.1139230 .315354$

O $-2.861407 \quad 1.464474 \quad 0.212111$

O $-3.950914-0.5278030 .141022$

H $1.6150812 .502008-1.691589$

H $0.3516663 .249170-0.725872$

H $1.9879553 .089897-0.053612$

H -1.314330 -0.893786-1.432594

H $\quad 0.445331 \quad 1.4078420 .902648$

H $2.4741740 .198421-1.062718$

H $2.475499-1.7882390 .337683$

H $0.046595-2.554255-0.041015$

H -1.152978 $0.059072 \quad 1.485015$

H $-1.800874-1.5118341 .042404$

H 2.6878840 .8278051 .699662

H $0.976952-1.9617912 .042938$

H $1.619232-2.583443-1.682766$

$\mathrm{H}-1.9731521 .8510830 .365426$

SCF Energy $\left(B 3 L Y P / 6-31 G^{* *} / / M M F F\right)=-764.615330571$

F1a-05_c00049

MMFF Geometry

C $0.7447082 .324348-1.229158$

C $-0.518548-0.233173-0.165371$

$\begin{array}{lllll}\text { O } & -0.269150 & 0.984048 & 0.546526\end{array}$

C 0.9091351 .7009060 .154314

C $2.151383 \quad 0.814357 \quad 0.279732$

C $1.970163-0.517180-0.446927$

C $0.668401-1.198021-0.020842$

C -1.802099 -0.8492940 .410720$

O $2.355947 \quad 0.5689001 .676245$

O $3.081828-1.361789-0.156068$

O $0.460463-2.355666-0.827834$

C $-3.030169-0.0389570 .064991$

O $-4.123710-0.5716520 .668560$

O $-3.068330 \quad 0.947976-0.654076$
H $-0.1797072 .909994-1.273187$

H $1.5869812 .984310-1.459754$

H $0.6794121 .573083-2.021318$

H $-0.692371-0.009120-1.224981$

H 0.9952362 .5222410 .876245

H $3.0458991 .334311-0.080585$

H $1.973633-0.357209-1.531487$

H $0.759811-1.5435521 .015982$

H -1.731852 -0.8957371 .503881$

H -1.956350 -1.857361 0.011109

H $3.120155-0.0276971 .755009$

H $2.917813-2.216741-0.589780$

H $\quad 0.363816-2.064028-1.750422$

H $-4.936695-0.0649370 .457705$

SCF Energy $\left(B 3 L Y P / 6-31 G^{* *} / / M M F F\right)=-764.631135201$

F1a-05_c00050

MMFF Geometry

C $1.6250432 .226425-1.182710$

C -0.5128230 .4894050 .127489$

O 0.3968301 .3968120 .761570

C 1.6934611 .5017470 .159120

C 2.3663790 .1296830 .076998

C $1.460482-0.901831-0.593679$

C $0.079320-0.9272580 .062449$

C -1.8126180 .5409150 .940720$

O $2.646908-0.2862491 .418560$

O $2.046958-2.198702-0.496934$

O $-0.778998-1.767939-0.704193$

C -3.0135270 .0251530 .188676$

O $-3.430571 \quad 0.879518-0.778492$

O $-3.561812-1.043426 \quad 0.424682$

H $1.1004723 .181420-1.070950$

H $2.6300502 .424078-1.568488$

H $1.0804301 .655002-1.939712$

H $-0.7270910 .848660-0.886088$

H 2.2744832 .1322990 .843391

H $3.3308950 .193832-0.438901$

H $1.354299-0.684886-1.663143$

H $\quad 0.153742-1.3604001 .067674$

H -2.0322291 .5679201 .260399$

H -1.710623 -0.0282351 .872961$

H $3.033457-1.1774431 .370564$

H $2.918391-2.157654-0.926672$

H $-0.365405-2.647377-0.742660$

$\mathrm{H}-2.9096921 .707226-0.854306$

SCF Energy $\left(B 3 L Y P / 6-31 G^{* *} / / M M F F\right)=-764.612000390$

F1a-06 c00051

MMFF Geometry

C $1.4083292 .565933-0.716302$

C $-0.634918-0.372656 \quad 0.227593$

O $-0.1879630 .817684-0.438726$

C $1.1069571 .248646-0.008692$

C $2.1480030 .174415-0.342031$

C $1.766183-1.1677970 .293335$

C $0.326835-1.540077-0.068032$

C $-2.057213-0.669718-0.264641$

O 3.4335350 .5797920 .113109

O $1.861910-1.0813751 .715298$

O $0.259130-1.874233-1.452879$

$\begin{array}{llll}\text { C } & -3.072878 & 0.305801 & 0.292583\end{array}$

O $-4.326256 \quad 0.021500-0.149423$

O $-2.827577 \quad 1.2385111 .044653$

H $2.3719952 .980450-0.406235$

H $1.4153562 .433549-1.803852$

H $0.6276323 .302200-0.495978$

H $-0.654027-0.1762071 .307606$

H $1.0832791 .449627 \quad 1.070090$

H $2.2004390 .047682-1.430827$

H $2.466419-1.944521-0.034667$

H $0.005736-2.4176810 .505761$

H -2.357595 -1.673223 0.057114

H $-2.102707-0.600945-1.357735$

H $4.063581-0.120723-0.127074$

H $2.762477-0.7815521 .927515$

H $0.854451-2.628612-1.601429$

H $-4.387054-0.752368-0.749415$ 
SCF Energy (B3LYP/6-31G**//MMFF) $=-764.618598931$

F1a-06_c00052

MMFF Geometry

C -1.348318 2.6497110 .311735

C $0.658572-0.434346-0.098146$

$\begin{array}{lllll}0 & 0.214290 & 0.849824 & 0.364650\end{array}$

C $-1.0484751 .238996-0.184129$

C $-2.130751 \quad 0.2433760 .249955$

C $-1.757241-1.184880-0.161674$

C $-0.344691-1.525967 \quad 0.320325$

C $2.047102-0.6830950 .496120$

O $-3.3938140 .593019-0.303667$

O $-1.805184-1.288322-1.585015$

O $-0.346832-1.6491251 .741297$

C $3.1034360 .100851-0.245084$

$\begin{array}{llllll}\text { O } & 3.105705 & 1.416520 & 0.083988\end{array}$

O $3.872826-0.390328-1.060684$

H -2.286630 $3.032003-0.100625$

H -1.4074972 .6761281 .405416$

H -0.5404203 .3319820 .025505$

H $0.731681-0.396639-1.193061$

H $-0.9703751 .281481-1.278222$

H $-2.2321420 .273493 \quad 1.341844$

H -2.499588 -1.8826520 .242911$

H $-0.019974-2.485781-0.098755$

H $2.320214-1.7418300 .412318$

H $2.099948-0.4169361 .558306$

H $-3.3167910 .534833-1.271465$

H -1.587055 -2.206749 -1.818826

H $-0.971788-2.357293 \quad 1.972998$

H $2.426703 \quad 1.6773860 .742516$

SCF Energy (B3LYP/6-31G**//MMFF) $=-764.624035221$

F1a-06_c00053

MMFF Geometry

C -1.378033 $2.673333 \quad 0.257345$

C $0.656779-0.403799-0.058957$

$\begin{array}{lllll}\text { O } & 0.199636 & 0.888898 & 0.366366\end{array}$

C $-1.0620561 .253568-0.201634$

$\begin{array}{llll}\text { C }-2.139981 & 0.259714 & 0.247418\end{array}$

C $-1.751945-1.175172-0.126348$

C $-0.341338-1.4931680 .376672$

C $2.040610-0.6250090 .555528$

O $-3.4012370 .585196-0.324994$

O $-1.785690-1.313294-1.547252$

O $-0.356650-1.5837431 .799810$

C $3.0758500 .240731-0.121204$

O $3.491907-0.273178-1.305524$

$\begin{array}{lllll}\text { O } & 3.489070 & 1.298238 & 0.336077\end{array}$

H $-0.5739153 .355926-0.038968$

H -2.316214 $3.037294-0.171566$

H -1.4463912.725959 1.349644

H $0.737386-0.392536-1.153736$

H $-0.9751391 .270164-1.295661$

H -2.2510890 .3152371 .337263$

H -2.492660 $-1.868690 \quad 0.288151$

H $-0.004411-2.459556-0.016885$

H $2.359533-1.6705570 .474576$

H $2.049260-0.3669741 .621610$

H -3.314880 $0.506580-1.290557$

H -1.563058 -2.236483 -1.756339

H $-0.979955-2.2897162 .041999$

H $3.075937-1.124781-1.558323$

SCF Energy (B3LYP/6-31G*//MMFF) $=-764.614360236$

F1a-06 c00054

MMFF Geometry

C $1.4236822 .564817-0.672630$

C $-0.625754-0.3832820 .221801$

O $-0.1711750 .810296-0.432163$

C $1.118987 \quad 1.237524 \quad 0.015214$

C $2.1681910 .172222-0.323455$

C $1.782074-1.1988910 .248013$

C $0.330297-1.550413-0.091826$

C $-2.050573-0.666807-0.271738$

O 3.4439810 .5683160 .165685

O $1.956752-1.2182751 .665056$
O $0.247469-1.898638-1.473515$

C $-3.059714 \quad 0.307313 \quad 0.299342$

O $\begin{array}{llll}\text { O } & -3.317034 & 0.029203 & -0.135347\end{array}$

O $-2.806157 \quad 1.2347531 .055182$

H $1.4383052 .447598-1.761843$

H $0.6404213 .297018-0.447413$

H $2.3846752 .976173-0.350301$

H $-0.644586-0.199604 \quad 1.303455$

H $1.0852461 .428497 \quad 1.095140$

H $2.2430650 .083925-1.414832$

H $2.463103-1.956055-0.157372$

H $\quad 0.018280-2.430340 \quad 0.482465$

H $-2.355133-1.6726260 .038661$

H $-2.098420-0.584415-1.363689$

H $4.074785-0.138119-0.054763$

H $1.405820-0.5225702 .058902$

H $\quad 0.375275-1.088870-1.996304$

H $-4.384664-0.740755-0.739621$

SCF Energy (B3LYP/6-31G**//MMFF)= -764.615992647

F1a-06 c00055

MMFF Geometry

C $2.6355432 .172233-0.222085$

C $-0.616560 \quad 0.436815 \quad 0.126345$

O $0.3945791 .363296-0.297793$

$\begin{array}{llll}\text { C } & 1.690268 & 1.063644 & 0.230292\end{array}$

C $2.149785-0.311207-0.267195$

C $1.131048-1.3930600 .109851$

C $-0.272190-0.989621-0.348050$

C $-1.945936 \quad 0.954615-0.426967$

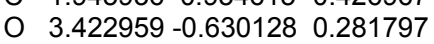

O $1.105603-1.5693881 .526492$

O $-0.336251-1.049184-1.771164$

C -3.1285210 .1831160 .098509$

$\begin{array}{llll}\text { O } & -3.444670 & 0.517941 & 1.373922\end{array}$

O $-3.735510-0.661520-0.546728$

H $2.2630623 .147568 \quad 0.110056$

H 3.6439482 .0325940 .178484

H $2.6939742 .215376-1.315375$

H -0.6554800 .4583391 .223244$

H $1.6498821 .089211 \quad 1.326951$

H $2.250416-0.285578-1.359671$

H $1.427841-2.350759-0.333052$

H $-1.013333-1.6911880 .052155$

H $-1.9631270 .924341-1.523114$

H $-2.0813232 .015342-0.179913$

H $3.682288-1.500202-0.066458$

H $2.014754-1.7665111 .810282$

H $-0.149852-1.966470-2.034607$

H -2.877348 1.2105891 .774607

SCF Energy (B3LYP/6-31G**//MMFF) $=-764.617002119$

F1a-06_c00056

MMFF Geometry

C -1.3744192 .6228780 .367331$

C $0.652957-0.439026-0.099372$

$\begin{array}{lllll}\text { O } & 0.197566 & 0.831322 & 0.389130\end{array}$

C $-1.0649901 .224235-0.156674$

C $-2.144577 \quad 0.215950 \quad 0.253134$

C $-1.764213-1.213450-0.153808$

C $-0.340762-1.5478800 .301979$

C $2.051271-0.6784280 .475739$

O $-3.4047260 .563285-0.307763$

O $-1.868813-1.379538-1.567749$

O $-0.362936-1.705158 \quad 1.720313$

C $3.0895440 .128395-0.267046$

$\begin{array}{llllll}\text { O } & 3.079541 & 1.439339 & 0.079453\end{array}$

O $3.855215-0.342583-1.097919$

H $-2.3142963 .007831-0.039213$

H $-1.4358172 .626811 \quad 1.461202$

H -0.5701373 .3157620 .096843$

H $0.722616-0.379399-1.193135$

H $-0.9829391 .294047-1.248472$

H $-2.2553480 .239691 \quad 1.344469$

H $-2.483591-1.9098200 .292286$

H $-0.025057-2.500785-0.139670$

H $2.336161-1.7323810 .374081$

H $2.114072-0.4243421 .540337$ 
H $-3.3232890 .503033-1.274972$

H $-1.267733-0.754445-2.003893$

H $\quad 0.484111-2.0988391 .989219$

H $2.4056101 .683446 \quad 0.749514$

SCF Energy (B3LYP/6-31G**//MMFF) $=-764.620572274$

F1a-06 c00057

MMFF Geometry

C $1.7334112 .533417-0.084774$

C $-0.536201-0.1996260 .947879$

O $-\begin{array}{llll}0.057949 & 1.060970 & 0.463907\end{array}$

C 1.3693561 .1256550 .377183

C $1.8886880 .058507-0.593639$

C $1.416608-1.339389-0.173534$

C $-0.102218-1.3510290 .018805$

C $-2.048248-0.100177 \quad 1.180069$

O $3.3100710 .095784-0.645040$

O $2.030436-1.715077 \quad 1.059280$

O $-0.722548-1.223341-1.256602$

C $-2.8840090 .171416-0.048734$

O $-2.6868341 .411965-0.558184$

O $-3.674819-0.632358-0.526194$

H 1.3189623 .2778960 .603937

H $2.8169302 .674806-0.135891$

H $1.3034222 .745412-1.069918$

H $-0.084583-0.3601931 .936515$

H 1.7985490 .9840161 .377412

H $1.5098220 .271726-1.601253$

H $1.722091-2.074398-0.927256$

H $-0.424736-2.3017950 .459395$

H $-2.267927 \quad 0.7005521 .897454$

H -2.412754 -1.031296 1.630760

H $3.598004-0.583535-1.278430$

H $2.992665-1.6420510 .937897$

H $-0.456230-1.990463-1.791361$

H $-2.025391 \quad 1.953140-0.076131$

SCF Energy (B3LYP/6-31G**//MMFF)= -764.621932009

F1a-06 c00058

MMFF Geometry

C $1.3880642 .604101-0.644796$

C $-0.617608-0.389998 \quad 0.200483$

O $-0.182760 \quad 0.824407-0.429200$

C 1.0998431 .2653630 .027145

C $2.1627770 .216282-0.318374$

C $1.795322-1.1476640 .277979$

C $0.366418-1.533096-0.114376$

C $-2.028929-0.696874-0.315795$

$\begin{array}{lllll}\text { O } & 3.436096 & 0.633968 & 0.159403\end{array}$

O $1.888891-1.0780161 .701006$

O $0.323557-1.829036-1.508876$

C $-3.063250 \quad 0.245387 \quad 0.255741$

O $-4.296199-0.079134-0.212190$

O $\quad-2.854596 \quad 1.171229 \quad 1.024729$

H $2.3408263 .026703-0.312398$

H $1.4109332 .498249-1.735031$

H $\quad 0.5925173 .322041-0.416748$

H $-0.652023-0.2226561 .285009$

H $1.059317 \quad 1.4398381 .110030$

H $2.2298010 .116900-1.409308$

H $2.519213-1.897400-0.061599$

H $\quad 0.050817-2.431808 \quad 0.428584$

H -2.317149 -1.713283 -0.024902

H -2.065670 -0.599953-1.406984

H $4.076879-0.061186-0.067586$

H $1.670771-1.9582452 .051980$

H $\quad 0.935176-2.567483-1.670678$

H -4.9871520 .5199200 .142609$

SCF Energy $\left(B 3 L Y P / 6-31 G^{* *} / / M M F F\right)=-764.622293421$

F1a-06 c00059

MMFF Geometry

C $-2.598642 \quad 2.230247-0.239103$

C $0.577818 \quad 0.325607-0.275523$

O $-0.382117 \quad 1.371026-0.052516$

C $-1.7105961 .006729-0.439323$

C -2.188494 -0.1835320 .400856$

C $-1.222346-1.3671150 .274036$
C $0.213596-0.9199430 .561049$

C 1.9524240 .9204380 .053965

O $-3.501367-0.5825940 .025483$

O $-1.266436-1.900064-1.049511$

O $0.317834-0.6420201 .955507$

C $3.1051560 .037744-0.364941$

$\begin{array}{lllll}\text { O } & 4.285163 & 0.486981 & 0.136349\end{array}$

O $3.019721-0.946832-1.085387$

H -2.6030632 .5434680 .810865$

H -2.213410 $3.077585-0.816801$

H $-3.6288052 .037697-0.552988$

H $0.5538610 .070237-1.343350$

H -1.721007 $0.760525-1.508903$

H -2.229099 0.1135841 .456073

H -1.524940 -2.1662290.961020

H $\quad 0.900877-1.7413320 .328754$

H 2.0255091 .1396641 .124815

H $2.064597 \quad 1.876897-0.472030$

H -3.511479 -0.711641-0.937960

H -2.152889-2.276338 -1.182172

H $1.262833-0.6143832 .181334$

H $4.221142 \quad 1.2972400 .685839$

SCF Energy (B3LYP/6-31G**//MMFF $)=-764.618220332$

F1a-07_c00060

MMFF Geometry

C $1.1019762 .963395-0.069896$

C $-0.580116-0.325060-0.248316$

$\begin{array}{llll}\text { O } & -0.238251 & 1.006169 & 0.157556\end{array}$

C $0.9329681 .510235-0.496833$

C $2.1467480 .648964-0.131868$

C $1.877549-0.810520-0.501328$

C $0.557908-1.2956750 .102563$

C $-1.881313-0.7106490 .469137$

O $2.384763 \quad 0.750834 \quad 1.273890$

O $2.939609-1.637812-0.032945$

O $0.251393-2.586962-0.418798$

C $-3.0734430 .070435-0.043452$

$\begin{array}{llll}\text { O } & -4.204657 & -0.247886 & 0.639123\end{array}$

O $-3.0528190 .884805-0.955650$

H $0.2309873 .550951-0.380886$

H 1.1645333 .0583871 .019482

H $1.9969403 .407025-0.516556$

H $-0.757663-0.334325-1.332435$

H $0.7664061 .484619-1.581419$

H $3.0486581 .012036-0.637136$

H $1.841949-0.923520-1.592112$

H $\quad 0.658630-1.3936541 .190728$

H - $1.786211-0.5048791 .541848$

H -2.100542 -1.7728580 .317103$

H $3.123043 \quad 0.154766 \quad 1.486968$

H $3.762720-1.311638-0.435256$

H $1.002687-3.168851-0.211646$

H -4.086095 -0.917984 1.345854

SCF Energy $\left(B 3 L Y P / 6-31 G^{* *} / / M M F F\right)=-764.621694610$

F1a-07 c00061

MMFF Geometry

C -2.018284 -2.585246 -0.250159

C $0.552088-0.045310-1.031949$

O $-0.072837-1.248731-0.574464$

C -1.502255 -1.217066 -0.680570

C $-2.072741-0.0816240 .177167$

C $-1.4496811 .251862-0.240578$

C $0.0780391 .169118-0.219818$

C $2.071640-0.243708-1.004215$

O $-1.774223-0.3409471 .550784$

O -1.8749592 .2878090 .641305$

O $0.6262192 .355006-0.789096$

C $2.648229-0.3431170 .388797$

O $2.329893-1.5064671 .007829$

O 3.3356020 .5275490 .906606

H -1.685672 -2.840547 0.761708

H $-3.111131-2.626601-0.285506$

H -1.619743 -3.363054 -0.910855

H $0.2801990 .096351-2.087320$

H -1.770117-1.061141-1.733396

H $-3.164233-0.0380840 .089151$ 
H -1.787246 $1.529631-1.246820$

H 0.4238321 .1103970 .818861

H $2.5716760 .590317-1.510850$

H $2.354566-1.154456-1.545936$

H $-2.095606 \quad 0.4187812 .065840$

H -2.846303 2.3202160 .606701

H $\quad 0.2828713 .107461-0.277170$

H $1.767133-2.1124990 .479834$

SCF Energy (B3LYP/6-31G**//MMFF) $=-764.624670853$

F1a-07 c00062

MMFF Geometry

C -1.947586 $-2.660617-0.378682$

C $0.525050 \quad 0.022265-0.977834$

O $-0.057065-1.226272-0.591941$

C -1.482767-1.254544 -0.739529

C $-2.130599-0.1817820 .143759$

C $-1.5649681 .194813-0.207232$

C $-0.0352191 .187767-0.145759$

C $2.053624-0.102746-0.905118$

O $-1.855497-0.478927 \quad 1.514601$

O -2.0882422 .1616890 .699703$

O $0.4640052 .428015-0.641779$

C $2.598652-0.4009990 .477283$

O $3.946558-0.2322720 .526023$

O $1.942728-0.777708 \quad 1.438060$

H $-3.035744-2.749754-0.449540$

H -1.494394 -3.392671 -1.056217

H $-1.633378-2.9409960 .632428$

H $\quad 0.2839250 .192387-2.036577$

H - $1.727371-1.068661-1.793152$

H $-3.220318-0.1870380 .027651$

H $-1.8988041 .486074-1.210900$

H $\quad 0.2765991 .1180240 .901481$

H $2.5085780 .825656-1.267949$

H $2.376460-0.928729-1.550145$

H -2.2282950 .2442002 .047573$

H -1.6753043 .0154090 .483844$

H $\quad 0.1905692 .510912-1.571254$

H $4.3604650 .054042-0.315921$

SCF Energy (B3LYP/6-31G**//MMFF) $=-764.618186735$

F1a-07_c00063

MMFF Geometry

C $1.0362492 .949203-0.397871$

C $-0.601005-0.354684-0.119559$

O $-0.252899 \quad 1.013607 \quad 0.126158$

C $0.8646411 .456664-0.654140$

C $2.1127330 .650749-0.275769$

C $1.846446-0.844763-0.461934$

C $0.566296-1.2788550 .255635$

C -1.846645 -0.6727760 .712353$

O $2.470704 \quad 0.912640 \quad 1.083074$

O $2.943181-1.6072510 .035323$

O $0.245370-2.615056-0.126340$

C -3.0557650 .0563400 .177926$

O $-3.591631-0.550634-0.910182$

$\begin{array}{lllll}\text { O } & -3.501433 & 1.088920 & 0.660973\end{array}$

H $0.1378323 .491123-0.713319$

H 1.1657563 .1622270 .668782

H $1.8937973 .350248-0.946350$

H -0.844997 -0.471988 -1.184073

H $\quad 0.630617 \quad 1.310794-1.716431$

H $2.9706750 .954655-0.885258$

H $1.756334-1.076768-1.530532$

H $0.726275-1.2775551 .340805$

H -1.709515 -0.3582801 .754321$

H -2.073214 -1.7446410 .730527$

H 1.6785970 .7892321 .633083

H $3.744814-1.297517-0.419662$

H $1.012988-3.1709280 .092552$

H $-3.133998-1.369683-1.196356$

SCF Energy (B3LYP/6-31G**//MMFF) $=-764.618342762$

F1a-07 c00064

MMFF Geometry

C $1.0995632 .954137-0.064457$

C $-0.567709-0.341497-0.266600$
O $\quad-0.2254090 .9858990 .154359$

C $0.9383401 .503079-0.501809$

C $2.1591260 .647064-0.148450$

C $1.893582-0.810324-0.528272$

C $0.575818-1.3136090 .070692$

C $-1.871551-0.7242300 .448218$

$\begin{array}{lllll}\text { O } & 2.402846 & 0.743022 & 1.256238\end{array}$

O $2.978699-1.631512-0.103075$

O $0.309274-2.602240-0.477946$

C $-3.0516440 .095852-0.031440$

O $-4.175411-0.1916870 .676812$

$\begin{array}{llll}\text { O } & -3.029179 & 0.911465 & -0.942349\end{array}$

H $0.2234723 .538071-0.367483$

H 1.1659323 .0413611 .025346

H $1.9896993 .407019-0.511479$

H $-0.747433-0.338629-1.350294$

H $0.7670741 .484945-1.585712$

H $3.0552831 .019433-0.657823$

H $1.852046-0.899606-1.620743$

H $0.687492-1.4271021 .156176$

H $-1.763227-0.5527741 .525595$

H -2.118595 -1.774876 0.266442

H $3.2436850 .289931 \quad 1.437008$

H $2.953054-1.6859560 .867060$

H $-0.387465-3.0159740 .058318$

H -4.060712 -0.8682541 .377995$

SCF Energy $\left(B 3 L Y P / 6-31 G^{* *} / / M M F F\right)=-764.613881013$

F1a-07 c00065

MMFF Geometry

C $1.0155412 .926173-0.412548$

C $-0.598335-0.387939-0.135199$

O -0.2604070 .9831820 .107180$

C $0.8667241 .429693-0.657568$

C $2.1156650 .632088-0.261173$

C $1.856363-0.861052-0.466243$

C $0.576474-1.3007840 .250277$

C $-1.847806-0.7139770 .687473$

O $2.423974 \quad 0.8578051 .116105$

O $2.971383-1.6051370 .018059$

O $0.284181-2.651663-0.101077$

C $-3.078480-0.0885430 .075612$

O $-3.197603 \quad 1.2296990 .370205$

O $-3.878961-0.697253-0.622330$

H $1.8921353 .328624-0.928525$

H $0.1269653 .455279-0.774539$

H 1.0926583 .1549960 .655701

H $-0.837183-0.509909-1.200561$

H $\quad 0.6499491 .276648-1.722609$

H $2.9905120 .941238-0.844512$

H $1.774433-1.075156-1.539330$

H $0.740006-1.2798621 .334515$

H $-1.756341-0.3677101 .723729$

H -2.028860 -1.794397 0.727053

H $2.776716 \quad 1.7600281 .195795$

H $2.756663-2.548318-0.082251$

H $\quad 0.121008-2.683535-1.059159$

$\mathrm{H}-2.4760691 .5950590 .925772$

SCF Energy (B3LYP/6-31G**//MMFF) $=-764.619906348$

F1a-07_c00066

MMFF Geometry

C $-2.205665-2.471058-0.284228$

C $0.519438-0.084412-1.018821$

O $-0.179676-1.252174-0.575887$

C $-1.604811-1.125978-0.674418$

C $-2.0983620 .027706 \quad 0.209515$

C $-1.3932961 .324646-0.193022$

C $0.127298 \quad 1.143331-0.182797$

C $2.021678-0.380970-1.004447$

O $-1.803913-0.245918 \quad 1.581130$

$\begin{array}{llll}\text { O } & -1.770410 & 2.365393 & 0.704656\end{array}$

O $0.7510032 .312079-0.708631$

C $2.556052-0.6619270 .380808$

$\begin{array}{lllll}\text { O } & 3.160231 & 0.414714 & 0.941412\end{array}$

O $2.442407-1.7392500 .951220$

H -3.299065 -2.435253 -0.289825

H $-1.880734-3.245303-0.988317$ 
H $-1.863221-2.7947120 .704319$

H $\quad 0.251090 \quad 0.092365-2.069792$

H -1.864628 $-0.926081-1.722026$

H -3.1840330 .1553500 .130518$

H -1.726645 $1.630953-1.192473$

H $0.462999 \quad 1.0396950 .854943$

H $2.5902640 .433847-1.466099$

H $2.233397-1.275596-1.603702$

H -2.402868 -0.9517591 .877155$

H -1.269047 3.1604600 .454745

H $0.4538022 .422343-1.628017$

H 3.1675651 .2269200 .391830

SCF Energy (B3LYP/6-31G**//MMFF)= -764.612797501

F1a-07_c00067

MMFF Geometry

C $-2.645236-2.369364-0.116681$

C $0.542050-0.490122-0.096868$

O $-0.490580-1.4206290 .253904$

C $-1.691575-1.246768-0.508433$

C $-2.283741 \quad 0.140847-0.242184$

C $-1.2495491 .217262-0.573272$

C 0.0725970 .9536280 .151410

C $1.770844-0.8603300 .741094$

O

O $-1.7425552 .500660-0.196594$

O $1.0541451 .865475-0.333808$

C $3.061363-0.3109340 .188298$

O $3.466267-0.957243-0.933137$

O 3.6846850 .6125760 .694788

H $-2.838079-2.3757720 .961490$

H -3.598380 -2.283948 -0.647035

H $-2.200546-3.341676-0.355847$

H $\quad 0.783915-0.623393-1.159678$

H -1.448772 -1.353777 -1.573478

H $-3.1970230 .294114-0.828020$

H -1.071303 $1.249010-1.655431$

H $-0.0498001 .139438 \quad 1.225710$

H $1.875694-1.9501720 .819814$

H $1.655209-0.5131981 .775172$

H -2.9682431 .1413471 .290063$

H $-2.5766232 .645140-0.675153$

H $0.7156192 .764593-0.181711$

H $2.880426-1.687389-1.226126$

SCF Energy (B3LYP/6-31G**//MMFF) $=-764.615744242$

F1a-07_c00068

MMFF Geometry

C -2.638936 -2.376842 -0.324206

C $0.511746-0.440848-0.213893$

O $-0.491257-1.4235150 .081067$

C -1.721065 -1.196521 -0.619829

C $-2.329060 \quad 0.140797-0.181987$

C -1.325092 $1.273308-0.409123$

C 0.0321920 .9541480 .223235

C $1.791799-0.8744060 .516241$

O

O -1.8230222 .4907270 .140429$

O $\quad 0.979021 \quad 1.938225-0.182113$

C $3.061589-0.326604-0.096792$

O $4.125097-0.4843120 .734120$

O $3.156543 \quad 0.170315-1.210248$

H -2.184638 -3.306510-0.684607

H -2.793652 -2.506963 0.752276

H -3.611466 -2.254853 -0.810374

H $0.696760-0.453281-1.296223$

H $-1.512802-1.179580-1.697255$

H $-3.2528140 .346388-0.733831$

H $-1.1898551 .441893-1.484929$

H -0.0474791 .0090051 .315761$

H $1.875038-1.9680740 .496979$

H $1.734779-0.5709671 .568271$

H $-1.921105-0.2378591 .699810$

H $-2.6911682 .660347-0.263443$

H 0.6250842 .8062850 .077023

H $3.922523-0.9150261 .591914$

SCF Energy (B3LYP/6-31G**//MMFF) $=-764.617359623$
F1a-08 c00069

MMFF Geometry

C $-0.735723 \quad 2.972417 \quad 0.184879$

C $0.508594-0.410468-0.725649$

O $0.1969650 .989241-0.744252$

C $\quad-0.533010 \quad 1.4743740 .390205$

C -1.8600150 .7232660 .543339$

C $-1.609237-0.7834510 .612298$

C $-0.777304-1.243352-0.585035$

C $1.590555-0.7425140 .319456$

O $-2.6932551 .018129-0.580918$

O $-2.863258-1.4623890 .627627$

O $-0.476902-2.627774-0.442413$

C $2.897686-0.0288900 .042385$

O $3.815072-0.2806721 .013035$

O $3.1310200 .680619-0.925805$

H -1.246653 $3.183829-0.760719$

H -1.311206 3.4117441 .005297

H $\quad 0.234121 \quad 3.4794010 .130774$

H $0.936421-0.638341-1.710637$

H $\quad 0.074581 \quad 1.342181 \quad 1.292013$

H $-2.398268 \quad 1.0628681 .435377$

H - $-1.100622-1.0378611 .549560$

H -1.382702 -1.140433 -1.494413

H $1.804478-1.8168500 .306825$

H $1.265640-0.4622061 .325566$

H $-3.5069030 .494485-0.482307$

H -2.675708 -2.4167290 .624448$

H $0.035332-2.896743-1.224096$

H $\quad 3.505073-0.8708761 .732839$

SCF Energy $(B 3 L Y P / 6-31 G * * / / M M F F)=-764.616307573$

F1a-08_c00070

MMFF Geometry

C $-1.170132-2.2366450 .240667$

C $0.6004730 .271595 \quad 0.219904$

O $0.213127-0.561572-0.882637$

C $-1.103651-1.113978-0.794071$

C $-2.1476860 .000664-0.639829$

C -1.8139920 .9850780 .485060$

C $-0.365983 \quad 1.4617780 .363470$

C $2.0379870 .739992-0.047127$

O $-3.453321-0.527164-0.445766$

$\begin{array}{llll}\text { O } & -1.987389 & 0.376273 & 1.761742\end{array}$

O $-0.2431182 .312364-0.775337$

C $3.047083-0.3751990 .132846$

O $4.3132070 .060967-0.099192$

O $2.786687-1.5285200 .445534$

H -2.139149 -2.743985 0.214826

H $-0.389463-2.9779370 .036708$

H $-0.997640-1.8833841 .259985$

H $0.584994-0.3246201 .139536$

H -1.289416 -1.597025 -1.761998

H $-2.1661050 .573434-1.576301$

H $-2.506411 \quad 1.8339450 .439522$

H -0.0824292 .0438541 .248522$

H $2.303713 \quad 1.5373070 .656049$

H $2.1335691 .105368-1.076213$

H $-3.640277-1.126218-1.188560$

H -2.8937990 .0250851 .793993$

H $-0.8413373 .067409-0.642661$

H $4.3856071 .008252-0.343670$

SCF Energy $\left(B 3 L Y P / 6-31 G^{* *} / / M M F F\right)=-764.614803785$

F1a-08_c00071

MMFF Geometry

C 0.6662952 .9647950 .071263

C $-0.569097-0.5018040 .595489$

$\begin{array}{lllll}\text { O } & -0.280101 & 0.892823 & 0.765786\end{array}$

C $0.5109131 .486798-0.272131$

C $1.8612830 .771308-0.394485$

C $1.650708-0.730083-0.605499$

C $0.732221-1.3104040 .470491$

C $-1.569115-0.740325-0.546263$

$\begin{array}{lllll}\text { O } & 2.638945 & 0.988383 & 0.785535\end{array}$

O $2.903151-1.410797-0.566866$

O $\quad 0.435588-2.6725640 .179159$

C $-2.8615430 .000289-0.300668$ 
O $-3.673533-0.642120 \quad 0.575111$

O $-3.145261 \quad 1.071293-0.821125$

H $1.1081873 .103572 \quad 1.063703$

H $1.2853523 .481395-0.668296$

H -0.3161193 .4493240 .100781$

H $-1.060951-0.8207571 .523193$

H $-0.0314921 .420531-1.221619$

H $2.443531 \quad 1.185186-1.225035$

H $1.220382-0.917208-1.596628$

H $1.255287-1.3014181 .434830$

H $-1.801816-1.804495-0.667124$

H $-1.181268-0.405898-1.513899$

H $2.0822870 .786408 \quad 1.556454$

H $3.476400-1.006583-1.240478$

H $1.283085-3.1398340 .081418$

H -3.323154 -1.489806 0.922714

SCF Energy (B3LYP/6-31G*//MMFF) $=-764.615124008$

F1a-08 c00072

MMFF Geometry

C $-0.7023762 .963641 \quad 0.229782$

C $0.511531-0.412805-0.738168$

O $0.2052060 .988094-0.736422$

C $-0.523611 \quad 1.459213 \quad 0.404415$

C $-1.856544 \quad 0.7130950 .540528$

C $-1.608244-0.7958600 .594510$

C $-0.776643-1.244495-0.609101$

C $1.595012-0.7624710 .300184$

O $-2.6934830 .997439-0.583564$

O $-2.864441-1.4701380 .609478$

O $-0.452900-2.626503-0.501415$

C $2.902005-0.0444330 .034085$

O $3.822374-0.3181450 .995794$

O $3.132698 \quad 0.686530-0.918783$

H $0.275248 \quad 3.458510 \quad 0.218262$

H - $-1.1778313 .2054655-0.726735$

H -1.2967813 .3910511 .042703$

H $0.938129-0.628352-1.726459$

H $\quad 0.078902 \quad 1.302706 \quad 1.305982$

H -2.401825 1.0322401 .436212

H $-1.097211-1.0573771 .528729$

H $-1.371578-1.125111-1.523479$

H $1.809106-1.8364980 .269047$

H $1.271333-0.4994021 .311443$

H -3.011852 $1.911177-0.491331$

H $-2.689457-2.4150020 .755084$

H $-1.282950-3.128790-0.560662$

H $3.514356-0.9241391 .703083$

SCF Energy (B3LYP/6-31G**/MMFF) = -764.606341582

F1a-08 c00073

MMFF Geometry

C $2.441973-2.4364350 .304189$

C $-0.540948-0.404126-0.565319$

O $0.505402-1.385351-0.601201$

C $1.457696-1.2830880 .467263$

C $2.151783 \quad 0.082750 \quad 0.435931$

C 1.1120011 .2018210 .499732

C $0.0508131 .017102-0.586285$

C $-1.506444-0.6979260 .593934$

O $2.902513 \quad 0.194424-0.775905$

$\begin{array}{lllll}0 & 1.769034 & 2.456830 & 0.333626\end{array}$

O $-0.9641321 .999700-0.416737$

C $-2.928589-0.3032840 .279063$

O $-3.533554-1.169080-0.571958$

$\begin{array}{lllll} & \mathrm{O} & -3.482512 & 0.687281 & 0.737191\end{array}$

H $2.920053-2.421635-0.681155$

H $3.217698-2.408724 \quad 1.075394$

H $1.914851-3.3942750 .376603$

H $-1.095220-0.532969-1.503301$

H $0.938166-1.4140171 .423954$

H $2.8649180 .176697 \quad 1.262605$

H 0.6445181 .2211641 .490954

H $0.5159481 .203681-1.562791$

H $-1.206105-0.1844371 .513850$

H - $1.506287-1.7660480 .845733$

H $3.2913881 .085837-0.793393$

H 1.0822913 .1455400 .337247
H $-1.6080331 .882089-1.135848$

H -2.977905 -1.924223 -0.861041

SCF Energy (B3LYP/6-31G**//MMFF) = -764.614653721

F1a-08_c00074

MMFF Geometry

C $-0.984385-2.2310700 .531970$

$\begin{array}{llll}\text { C } & 0.615112 & 0.358064 & 0.128364\end{array}$

O $0.256153-0.636894-0.841840$

C -1.020621 -1.251046-0.640065

C $-2.128354-0.188722-0.600776$

$\begin{array}{llll}\text { C }-1.832630 & 0.960597 & 0.367898\end{array}$

C $-0.418601 \quad 1.4985930 .137896$

C $2.0127170 .867386-0.237709$

O $-3.396451-0.768017-0.323268$

$\begin{array}{llll}0 & -1.958587 & 0.504387 & 1.712220\end{array}$

O $-0.3730642 .187844-1.110436$

C $3.076949-0.117190 \quad 0.184692$

O $3.156593-1.188625-0.642604$

$\begin{array}{lllll}\text { O } & 3.789163 & 0.027023 & 1.169768\end{array}$

H $-1.909423-2.8123460 .592752$

H $-0.149283-2.929540 \quad 0.408259$

H $-0.829697-1.7348451 .492902$

H $0.656812-0.1116761 .118161$

H -1.198504 -1.867527 -1.530466

H $-2.1963340 .245085-1.606716$

H $-2.583497 \quad 1.7463490 .224139$

H -0.1505422 .2139330 .924499$

H $2.229568 \quad 1.8099260 .279344$

H $2.1204351 .054117-1.312503$

H $-3.418450-0.9953280 .622037$

H -1.7849851 .2618792 .296545$

H $-1.0151812 .916749-1.066330$

H $2.517510-1.182195-1.386975$

SCF Energy (B3LYP/6-31G**//MMFF)= -764.619010916

F1a-08 c00075

MMFF Geometry

C $-0.989961-2.2545090 .370580$

C $0.625886 \quad 0.3410250 .134654$

O $0.249282-0.574091-0.904832$

C $-1.027801-1.197018-0.732664$

C $-2.133292-0.140049-0.607358$

$\begin{array}{llll}\text { C }-1.819598 & 0.937390 & 0.436766\end{array}$

C $-0.4028801 .481266 \quad 0.245773$

C $2.0206030 .870863-0.209747$

O $-3.386853-0.754167-0.337775$

O $-\begin{array}{llll}-1.925612 & 0.405788 & 1.755178\end{array}$

O $-\begin{array}{llll}0.366656 & 2.301267 & -0.921294\end{array}$

C $3.069459-0.1942030 .002456$

O $3.415756-0.3328521 .306452$

O $3.548951-0.871524-0.897266$

H $-1.922233-2.8263310 .404684$

H $-0.165611-2.9531610 .189221$

H $-0.817381-1.8264131 .360896$

H $0.674031-0.2049421 .083652$

H -1.216561 -1.750472 -1.661526

H $-2.2225600 .356569-1.582103$

H $-2.5554521 .746200 \quad 0.356530$

H $-0.1343192 .119066 \quad 1.096044$

H $2.286601 \quad 1.7427160 .398983$

H $2.081347 \quad 1.180617-1.260143$

H $-4.056551-0.050188-0.299297$

H $-2.8109750 .012398 \quad 1.839629$

H $-0.4244691 .721657-1.699553$

H 2.9506950 .2683301 .926503

SCF Energy (B3LYP/6-31G**//MMFF)= -764.613263824

F1a-08 c00076

MMFF Geometry

C $2.605847-2.3134610 .328974$

C $-0.494180-0.506870-0.627791$

O $0.622964-1.407697-0.631362$

C $1.532404-1.2387090 .465589$

C $2.121594 \quad 0.1760990 .459604$

C 0.9993401 .2141410 .488822

C $-0.0073990 .953329-0.632842$

C $-1.469952-0.8726540 .502140$ 
O $2.9028690 .348516-0.725632$

$\begin{array}{llllll}0 & 1.566049 & 2.515182 & 0.345768\end{array}$

O $-1.0955501 .860282-0.501867$

C $-2.900646-0.541507 \quad 0.150634$

$\begin{array}{llll}\text { O } & -3.411474 & 0.468499 & 0.896142\end{array}$

O $-3.537592-1.115256-0.723870$

H $3.110440-2.257804-0.641578$

H $3.354050-2.2306991 .123087$

H $2.151012-3.3088190 .383249$

H -1.011376 $-0.677387-1.580419$

H $0.996219-1.4140931 .405716$

H 2.7972090 .3202511 .310217

H 0.4980291 .1958201 .463279

H $0.4768391 .172428-1.593058$

H -1.186722 -0.4071001 .451335$

H $-1.442220-1.9527970 .695708$

H $3.224593 \quad 1.266360-0.728213$

H $0.829877 \quad 3.1504950 .321826$

H -1.698335 $1.702127-1.248585$

H -2.799798 0.8628051 .553223

SCF Energy (B3LYP/6-31G**//MMFF) $=-764.618227087$

F1a-08_c00077

MMFF Geometry

C $-0.6877402 .940948-0.047213$

C $0.575661-0.507650-0.609950$

O $\quad 0.2729510 .885811-0.764388$

C $-0.534072 \quad 1.4578620 .273520$

$\begin{array}{llll}C & -1.879432 & 0.728490 & 0.376319\end{array}$

C -1.645771 -0.7691340 .589782$

C $-0.719957-1.328526-0.492700$

C $1.583744-0.743440 \quad 0.524717$

O $-2.627407 \quad 0.905302-0.828860$

O $-2.887638-1.4688210 .552446$

O $-0.432155-2.701376-0.245549$

C $2.854607 \quad 0.0395340 .298099$

O $3.677281-0.549278-0.604960$

O $3.114161 \quad 1.0975390 .856162$

H $-1.336988 \quad 3.4402220 .678223$

H $0.2921393 .431395-0.029978$

H $-1.0895003 .097124-1.053911$

H $1.068304-0.811759-1.542327$

H $-0.001016 \quad 1.380593 \quad 1.227575$

H -2.487613 1.1306161 .194760

H -1.212196 -0.9475551 .580989$

H $-1.246461-1.295726-1.454649$

H $1.848895-1.8025460 .619510$

H $1.185624-0.4430441 .499432$

H -2.939639 $1.825519-0.851149$

H -3.465067 -1.0766321 .229443$

H $-0.183178-2.7973890 .689104$

H $3.346916-1.392729-0.981278$

SCF Energy (B3LYP/6-31G**//MMFF) = -764.604935895

F1a-08_c00078

MMFF Geometry

C 1.9554241 .9946990 .728778

C $-0.5648470 .421357-0.062028$

O $0.288199 \quad 1.228983-0.888473$

C $1.6771771 .197119-0.545293$

C $2.206544-0.242653-0.562297$

C $1.346223-1.2089700 .258692$

C $-0.129429-1.057088-0.115037$

C $-1.9974610 .649817-0.564898$

O $3.560945-0.284184-0.132390$

O $1.491931-0.9600001 .653881$

O $-0.332787-1.567882-1.431442$

C $-3.0552600 .117367 \quad 0.374512$

O $-4.2897340 .157850-0.191376$

O

H $1.488257 \quad 1.555643 \quad 1.613391$

H 3.0298252 .0806390 .917329

H 1.5424663 .0052010 .633229

H -0.4977390 .7889740 .968510$

H $2.194616 \quad 1.739048-1.347487$

H $2.187475-0.585616-1.605080$

H $1.685452-2.2363130 .080366$

H $-0.750498-1.6444150 .570440$
H -2.128723 $0.199471-1.555220$

H $-2.1718121 .727187-0.677689$

H $3.859378-1.207856-0.190087$

H $2.441942-0.9964761 .858112$

H -0.081798 -2.507295 -1.423112

H $-4.3153090 .514009-1.105189$

SCF Energy (B3LYP/6-31G**//MMFF)= -764.615266305

F1a-08 c00079

MMFF Geometry

C $-0.7129422 .932830-0.013080$

C $0.579744-0.500029-0.614762$

O $0.2720960 .894959-0.745730$

C $-0.5492591 .447790 \quad 0.291904$

C -1.8887120 .7070800 .380879$

C $-1.640294-0.789267 \quad 0.581975$

C $-0.712814-1.328307-0.508388$

C $1.599168-0.7474820 .507217$

O $-2.6328600 .888824-0.825579$

O $-2.875572-1.500138 \quad 0.541286$

O $-0.422005-2.704473-0.285549$

C $2.937202-0.1391590 .157758$

O 3.0558491 .1480060 .565150

O $3.818021-0.734648-0.449453$

H $-1.3710003 .418598 \quad 0.713585$

H 0.2626143 .4310450 .016080

H $-1.1095713 .096564-1.020612$

H $1.068311-0.788126-1.554808$

H $-0.0252631 .364193 \quad 1.250091$

H -2.5038641 .0960521 .200333$

H $-1.200927-0.972407 \quad 1.569876$

H $-1.239844-1.281805-1.469598$

H $1.779535-1.8191240 .649579$

H $1.273378-0.3650141 .479028$

H $-2.952223 \quad 1.806620-0.841313$

H $-3.454403-1.120791 \quad 1.224234$

H $-0.197399-2.8213660 .652788$

H 2.2738641 .5145961 .028726

SCF Energy (B3LYP/6-31G**//MMFF) $=-764.602983903$

F1a-09_c00080

MMFF Geometry

C $-0.6582282 .882065-0.148809$

C $0.583652-0.5404090 .638137$

$\begin{array}{llllll}\text { O } & 0.260969 & 0.858670 & 0.701358\end{array}$

C $-0.440814 \quad 1.397737-0.425379$

C $-1.7592650 .651474-0.662870$

C $-1.484445-0.848415-0.791804$

C $-0.679132-1.3813240 .394948$

C $1.689917-0.825957-0.389673$

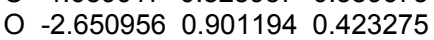

O $-2.702636-1.575294-0.910165$

O $-1.467977-1.331503 \quad 1.587364$

C $3.006450-0.179342-0.013596$

O $3.971760-0.452214-0.930585$

$\begin{array}{lllll}0 & 3.207006 & 0.503986 & 0.980549\end{array}$

H $0.3066023 .389765-0.039711$

H -1.211742 $3.361156-0.961919$

H -1.1979043 .0413910 .790897$

H $0.969314-0.7876331 .635570$

H $0.1948851 .315545-1.313654$

H $-2.253473 \quad 1.025401-1.566835$

H $-0.915197-1.027248-1.711862$

H $-0.424188-2.4360130 .241937$

H $1.416518-0.464144-1.384814$

H $1.861844-1.907418-0.442169$

$\begin{array}{llll}H & -3.444918 & 0.358888 & 0.278051\end{array}$

H $-3.186145-1.214390-1.672745$

H -2.292137 -1.8158761 .408759$

H $3.682403-1.013104-1.681608$

SCF Energy (B3LYP/6-31G**//MMFF) $=-764.605396287$

F1a-09_c00081

MMFF Geometry

C $1.232700-2.0254230 .883167$

C $-0.5816270 .287387-0.028625$

O $-0.204432-0.900149-0.739855$

C $1.126593-1.364340-0.490676$ 
C $2.155270-0.261298-0.787504$

C $1.8252241 .062527-0.097731$

C $0.3716691 .437928-0.392429$

C $-2.0282310 .619252-0.424610$

O $3.473230-0.667741-0.445414$

$\begin{array}{lllll}\text { O } & 2.003446 & 0.948948 & 1.314139\end{array}$

$\begin{array}{lllll}\text { O } & 0.030050 & 2.625012 & 0.313607\end{array}$

C -3.018694 $-0.386200 \quad 0.125268$

O $-4.285790-0.103900-0.276794$

O $-2.744277-1.3363730 .844709$

H $1.048333-1.3282281 .703694$

H $0.475463-2.8121380 .976272$

H $2.217077-2.479061 \quad 1.032397$

H $-0.556521 \quad 0.097627 \quad 1.051242$

H $1.301813-2.169051-1.216254$

H $2.142367-0.072002-1.869088$

H $2.5035881 .857681-0.426868$

H $0.282306 \quad 1.654464-1.464331$

H -2.122752 $0.615431-1.516921$

H -2.314632 $1.601714-0.034473$

H $3.656178-1.496976-0.919393$

H $2.916790 \quad 0.651412 \quad 1.466541$

H 0.1456632 .4459451 .262666

H $-4.3709170 .687813-0.849774$

SCF Energy (B3LYP/6-31G*//MMFF) $=-764.620411810$

F1a-09 c00082

MMFF Geometry

C $-0.689422 \quad 2.869512 \quad 0.107653$

C $0.633624-0.5928160 .479412$

$\begin{array}{lllll}\text { O } & 0.304095 & 0.788839 & 0.696492\end{array}$

C $-0.478527 \quad 1.421943-0.324659$

C $-1.8026340 .682437-0.548183$

C $-1.522557-0.792579-0.838589$

C $-0.635197-1.4248380 .236531$

C $1.674464-0.761391-0.633250$

O -2.6217610 .8120550 .613706$

O $-2.751608-1.499538-0.960479$

O $-1.352482-1.528876 \quad 1.470055$

C $3.035378-0.295588-0.174186$

$\begin{array}{lllll}\text { O } & 3.178718 & 1.050851 & -0.236928\end{array}$

O $3.915602-1.0475130 .223910$

H 0.2762703 .3789390 .200928

H $-1.3007793 .416001-0.616824$

H -1.1656852 .9298971 .092138$

H $1.081959-0.9268751 .423936$

H $0.0949811 .434804-1.257721$

H $-2.3598301 .133531-1.377196$

H $-1.014232-0.863969-1.807970$

H $-0.380725-2.452846-0.043368$

H $1.419754-0.237802-1.559195$

H $1.778798-1.820844-0.897984$

H -3.4256840 .2863730 .460371$

H -2.537637 -2.433629 -1.125564

H $-1.531007-0.6278131 .789077$

H $2.3925261 .537609-0.563040$

SCF Energy (B3LYP/6-31G**/MMFF) $=-764.618221070$

F1a-09 c00083

MMFF Geometry

C -2.306459 $2.478907-0.278133$

C $0.662683 \quad 0.399212 \quad 0.560567$

$\begin{array}{lllll}\text { O } & -0.393614 & 1.373247 & 0.610312\end{array}$

C $-1.306243 \quad 1.348104-0.495245$

C $-1.990627-0.020078-0.605874$

C $-0.930455-1.118865-0.700689$

C $0.092327-1.0224510 .434874$

C $1.6745840 .766043-0.529904$

O $-2.821877-0.229187 \quad 0.535640$

O $-1.559472-2.395844-0.704130$

O $-0.530586-1.3462621 .681825$

C $3.0038450 .088263-0.314178$

$\begin{array}{llllll}\text { O } & 3.752747 & 0.697967 & 0.637737\end{array}$

O $3.367899-0.910531-0.920851$

H $-1.785735 \quad 3.442687-0.255028$

H $-3.0534732 .507781-1.077002$

H $-2.8194142 .382903 \quad 0.684853$

H $1.1588940 .479007 \quad 1.536116$
H $-0.7526171 .561195-1.417180$

H $-2.647122-0.051866-1.482926$

H $-0.412705-1.017447-1.661804$

H $0.891004-1.7586860 .294322$

H $1.8362181 .850728-0.564244$

H $1.3171790 .501448-1.530761$

H $-3.186821-1.1279890 .466891$

H $-0.859019-3.066673-0.775611$

H $-0.945344-2.2199901 .580182$

H $3.340247 \quad 1.4869761 .049409$

SCF Energy (B3LYP/6-31G**//MMFF) = -764.608969545

F1a-09_c00084

MMFF Geometry

C $-0.6405442 .858176 \quad 0.271416$

C $0.636102-0.6345380 .447777$

$\begin{array}{llllll}\text { O } & 0.316844 & 0.733871 & 0.748609\end{array}$

C $-0.460394 \quad 1.430234-0.233464$

C $-1.794866 \quad 0.715803-0.488412$

C - $1.525612-0.743139-0.866904$

C $-0.636405-1.4443670 .162520$

C $1.667873-0.740733-0.680375$

O -2.6192990 .7582430 .675440$

O $-2.745211-1.462542-1.015090$

O $-1.338273-1.594793 \quad 1.400006$

C $2.968529-0.082560-0.289495$

O $3.731264-0.866787 \quad 0.511898$

O $3.2980891 .042244-0.642176$

H $-1.2667573 .444642-0.407371$

$\begin{array}{llll}\mathrm{H} & -1.081768 & 2.878846 & 1.273592\end{array}$

H $\quad 0.3341623 .3515850 .356700$

H $1.089344-1.0246371 .367811$

H $\quad 0.110147 \quad 1.481748-1.167364$

H $-2.3547741 .207877-1.292166$

H $-1.021434-0.768418-1.840514$

H $-0.388886-2.457807-0.172492$

H $1.329322-0.264950-1.606206$

H $1.870637-1.787985-0.934268$

H $-2.930370 \quad 1.6723780 .785811$

H -3.292399 -0.982356-1.659585

H $-2.178679-2.0428261 .202688$

H $3.346044-1.7432240 .725507$

SCF Energy (B3LYP/6-31G**//MMFF)= -764.604293824

F1a-09_c00085

MMFF Geometry

C $2.133197-1.4886261 .271747$

C $-0.534523-0.023570 \quad 0.743730$

$\begin{array}{lllll}\text { O } & -0.073892 & -1.286347 & 0.248193\end{array}$

C $1.330466-1.360251-0.022943$

C $1.772173-0.237235-0.976610$

C $1.308097 \quad 1.148489-0.525539$

C $-0.1944821 .105564-0.242535$

C $-2.034517-0.1398001 .041626$

O $3.180641-0.234937-1.165657$

\begin{tabular}{lllll}
\hline & 1.999408 & 1.548718 & 0.657172
\end{tabular}

$\begin{array}{lllll}\text { O } & -0.632246 & 2.363181 & 0.256594\end{array}$

C $-2.891611-0.299509-0.192352$

O $-2.780516-1.528744-0.753453$

O $-3.613710 \quad 0.581456-0.640838$

H $2.040884-0.6106711 .915183$

H $\quad 1.759922-2.3361421 .857547$

H $3.195092-1.6557511 .068024$

H $-0.0539370 .170861 \quad 1.710385$

H $1.480610-2.308609-0.554526$

H $1.311934-0.427539-1.955277$

H $1.5369041 .906348-1.283217$

H $-0.7078050 .931733-1.195622$

H -2.385261 0.7515021 .575361

H $-2.234433-0.9953891 .698276$

H $3.435580-1.119553-1.478743$

H $2.951515 \quad 1.5096350 .461951$

H -0.1400892 .5421331 .076172$

H $-2.161204-2.135553-0.294333$

SCF Energy (B3LYP/6-31G**//MMFF) $=-764.622155922$

F1a-09 c00086

MMFF Geometry 
C $1.042987-2.0011381 .084442$

C $-0.6065450 .298275-0.130079$

O $-0.254957-0.967162-0.707007$

C $1.037893-1.465013-0.347021$

C $2.131929-0.440890-0.686270$

C $1.8332220 .954716-0.135780$

C $0.416947 \quad 1.366377-0.543846$

C $-2.0103550 .652956-0.632455$

O $3.402101-0.898092-0.242251$

O $1.935270 \quad 0.962640 \quad 1.287645$

O 0.0845052 .6175210 .046957

C $-3.047870-0.269779-0.039226$

O -3.3752780 .0639851 .233835$

O $-3.535869-1.222737-0.632441$

H $1.994937-2.4830421 .326549$

H $\quad 0.852961-1.2239101 .828179$

H $0.244187-2.7412581 .206588$

H $-0.6434630 .206700 \quad 0.961866$

H $1.209505-2.340185-0.986826$

H $2.178253-0.360155-1.780418$

H $2.5665881 .684192-0.497469$

H $0.3881331 .489208-1.634171$

H $-2.0701580 .553648-1.723212$

H $-2.2923071 .683767-0.390611$

H $4.061606-0.232939-0.503004$

H 2.8211010 .6328531 .516760

H $0.7598653 .260754-0.228183$

H -2.9066320 .8481091 .591440$

SCF Energy (B3LYP/6-31G**//MMFF) $=-764.609634074$

F1a-09 c00087

MMFF Geometry

C $2.109780-1.5321301 .342939$

C $-0.509127-0.0215160 .690238$

O $-0.046467-1.294604 \quad 0.225679$

C $1.366405-1.3917550 .014949$

C $1.866499-0.277432-0.919900$

C $1.4099991 .116351-0.488383$

C $-0.1060821 .103317-0.276289$

C -2.025011 $-0.113386 \quad 0.926932$

O $3.280987-0.303439-1.055384$

O 2.0711721 .4909610 .719562

O -0.5388922 .3606320 .229025$

C $-2.844359-0.423801-0.309739$

O $-4.169247-0.210560-0.093809$

O $-2.405619-0.845322-1.370316$

H $1.702111-2.3775051 .908752$

H $3.177783-1.7100451 .186247$

H $1.997406-0.6555821 .985102$

H -0.0697810 .1730561 .676738$

H $1.524007-2.343256-0.509009$

H $1.441546-0.460192-1.915920$

H $\quad 1.6965911 .864020-1.236615$

H $-0.5836970 .950712-1.250535$

H -2.3801370 .8314451 .353753$

H $-2.227187-0.9195421 .642466$

H $3.530505-1.194801-1.353009$

H 1.7106032 .3517720 .992969

H -0.265709 $3.042174-0.408648$

H -4.3981340 .1085150 .805170$

SCF Energy (B3LYP/6-31G**//MMFF) = -764.609749495

F1a-09_c00088

MMFF Geometry

C $-2.3647272 .427733-0.309492$

C 0.6564690 .4622770 .616083

$\begin{array}{lllll}\text { O } & -0.435200 & 1.398438 & 0.632859\end{array}$

C $-1.3197741 .332347-0.493742$

C $-1.951718-0.060451-0.610399$

C $-0.850402-1.122058-0.666380$

C $0.136943-0.9785110 .494212$

C $1.6788650 .855873-0.454810$

O $-2.806379-0.2903470 .509245$

O $-1.410632-2.430673-0.661711$

O $-0.508610-1.3071191 .728674$

C $3.036206 \quad 0.263568-0.170445$

O $3.289174-0.850569-0.900072$

O 3.8221020 .7223360 .648735
H $-1.8797023 .409854-0.282136$

H -3.093066 2.423714 -1.125790

H -2.8965622 .3203560 .641983$

H 1.1297020 .5702851 .600517

H $-0.752227 \quad 1.558877-1.403933$

H -2.582166 -0.121607-1.504833

H $-0.302982-1.014633-1.610247$

H $0.961086-1.6911260 .385706$

H $1.8021291 .946383-0.478045$

H $1.3546070 .591899-1.466512$

H $-3.132943-1.2038770 .443056$

H -2.029478 -2.487222 -1.409767

H $-0.886352-2.1977891 .628826$

H $2.573139-1.126310-1.510836$

SCF Energy $\left(B 3 L Y P / 6-31 G^{* *} / / M M F F\right)=-764.604879598$

F1a-09_c00089

MMFF Geometry

C -0.7121022 .8655010 .136277$

C $0.628844-0.5924850 .469262$

$\begin{array}{llll}\text { O } & 0.287873 & 0.783496 & 0.703376\end{array}$

C $-0.5037451 .421395-0.307383$

C $-1.8230360 .671941-0.535128$

C $-1.523204-0.795511-0.848493$

C $-0.631786-1.4357310 .218386$

C $1.669221-0.738634-0.647411$

O -2.6525380 .7537350 .623131$

O $-2.741757-1.517586-0.991228$

O $-1.362593-1.5430951 .443076$

C $3.026669-0.264760-0.186024$

O $3.1580011 .083391-0.237241$

O $3.913875-1.0124830 .204725$

H 0.2529253 .3807860 .201090

H -1.348606 $3.410663-0.567083$

H -1.155271 2.9200021 .136434

H $1.085847-0.9312961 .407805$

H $\quad 0.064713 \quad 1.444707-1.243445$

H -2.389075 $1.116135-1.362270$

H -1.010213 -0.844199-1.816821

H $-0.373681-2.460044-0.073438$

H $1.407815-0.207245-1.567026$

H $1.783159-1.794074-0.923947$

H $-2.989521 \quad 1.6633640 .683202$

H -2.513739 -2.445790 -1.169587

H $-0.789528-1.9887742 .089864$

H $2.3671131 .565806-0.558414$

SCF Energy (B3LYP/6-31G**//MMFF) = -764.596531895

F1a-09 c00090

MMFF Geometry

C $1.058911-1.9452281 .143643$

C $-0.593600 \quad 0.316528-0.129293$

O $-0.248991-0.967050-0.669165$

C $1.046117-1.456155-0.304149$

C $2.138030-0.445392-0.690642$

C $1.8507960 .971765-0.192416$

C $0.4277851 .368468-0.592917$

C $-2.0043480 .657118-0.621577$

O $3.423284-0.878861-0.266671$

O $1.9944501 .014776 \quad 1.226683$

O $0.1194632 .653619-0.066230$

C -3.044995 -0.154121 0.113031

O $-3.154741-1.419297-0.362101$

O $-3.711664 \quad 0.274514 \quad 1.045900$

H $2.002124-2.4428001 .388236$

H $0.899453-1.1391181 .863433$

H $\quad 0.244504-2.6611831 .301525$

H $-0.6176680 .260748 \quad 0.965824$

H $1.209595-2.352479-0.916018$

H $2.166037-0.400925-1.787183$

H $2.5861801 .676768-0.596641$

H $\quad 0.391167 \quad 1.445917-1.686968$

H -2.116175 $0.479396-1.697683$

H $-2.2455491 .711679-0.445680$

H $3.478532-0.7476820 .695255$

H $1.899771 \quad 1.9425151 .501472$

H 0.0031152 .5638960 .894803

H $-2.547660-1.636249-1.101789$ 
SCF Energy (B3LYP/6-31G**//MMFF) $=-764.622310370$

F1a-09_c00091

MMFF Geometry

C $-0.621868 \quad 2.893416-0.153127$

C $0.546348-0.5590150 .617974$

$\begin{array}{llllll}\text { O } & 0.248820 & 0.845454 & 0.691117\end{array}$

C $-0.432528 \quad 1.406892-0.437364$

C $-1.7647780 .688073-0.686101$

C $-1.522374-0.816470-0.822091$

C $-0.730210-1.375098 \quad 0.362841$

C $1.652048-0.857238-0.406241$

O $-2.676198 \quad 0.952322 \quad 0.380850$

O $-2.764921-1.496159-0.962085$

O $-1.523947-1.318511 \quad 1.551705$

C $2.972613-0.231341-0.022207$

O $3.909131-0.506752-0.966265$

O 3.1988820 .4262640 .981994

H $-1.159616 \quad 3.388399-0.967298$

H -1.1652853 .0576660 .783570$

H $0.352313 \quad 3.380727-0.033642$

H $0.922505-0.8207451 .615335$

H $0.2073621 .316453-1.321696$

H $-2.2425711 .069669-1.595307$

H -0.956662 $-0.993233-1.744965$

H $-0.493275-2.4329880 .203131$

H $1.388678-0.488450-1.401636$

H $1.809848-1.940498-0.463692$

H $-2.245098 \quad 0.6929351 .212859$

H -2.571319 -2.444530 -1.055208

H -2.354852 -1.789309 1.367597

H $4.778322-0.112378-0.739687$

SCF Energy (B3LYP/6-31G**/MMFF) $=-764.625889629$

F1a-09_c00092

MMFF Geometry

C $1.972475-1.6273651 .285965$

C $-0.511303-0.446190-0.105304$

O $0.356985-1.503094-0.542484$

C $1.737427-1.341887-0.197081$

C $2.281496-0.002435-0.717656$

C $1.4046231 .189526-0.329965$

C $-0.0473650 .891588-0.708855$

C $-1.931533-0.825743-0.553918$

$\begin{array}{lllll}\text { O } & 3.621770 & 0.195814 & -0.288835\end{array}$

O 1.4835031 .4335291 .073906

O $-0.8796891 .967319-0.296728$

C $-3.025582-0.165870 \quad 0.256322$

O $-4.244233-0.338833-0.319275$

$\begin{array}{llll}O & -2.867897 & 0.430053 & 1.312608\end{array}$

H $1.569522-2.6134661 .542892$

H $3.039173-1.6204791 .529203$

H $1.467435-0.9126321 .939745$

H $-0.494062-0.4021010 .989673$

H $2.273266-2.134884-0.734647$

H $2.294831-0.057968-1.814270$

H $1.7497352 .107737-0.818619$

H $-0.1071250 .832056-1.802808$

H $-2.070205-1.909498-0.454551$

H -2.063198 $-0.574921-1.613090$

H $3.9286871 .039004-0.663528$

H 2.4228051 .5548891 .294677

H -0.8167272 .0335990 .671666$

H -4.239085 -0.849387 -1.156931

SCF Energy (B3LYP/6-31G**/MMFF) $=-764.619887293$

F1a-09 c00093

MMFF Geometry

C $2.237755-1.4838651 .234634$

C $-0.487471-0.119526 \quad 0.729168$

O $0.029269-1.351293 \quad 0.208924$

C $1.435743-1.360189-0.060636$

C $1.825147-0.200351-0.992119$

C $1.2960321 .154689-0.520897$

C $-0.2041361 .040988-0.239290$

C $-1.977788-0.3244131 .030922$

O $3.230143-0.148179-1.199661$

$\begin{array}{llll}\text { O } & 1.989970 & 1.555475 & 0.660327\end{array}$ $\begin{array}{llll}\text { O } & -0.666561 & 2.278219 & 0.287771\end{array}$

C $-2.806750-0.548530-0.214168$

O $-3.6903760 .451923-0.451279$

O $-2.692521-1.523922-0.945580$

H $3.303686-1.6243011 .032385$

H $2.123067-0.615144 \quad 1.886944$

H $\quad 1.883574-2.346603 \quad 1.810164$

H $-0.0118650 .081866 \quad 1.696974$

H $1.627472-2.290023-0.611430$

H $1.369294-0.401346-1.970365$

H $1.498197 \quad 1.928933-1.269678$

H $-0.7089750 .867784-1.197698$

H -2.3697140 .5233681 .602679$

H $-2.119674-1.2118231 .660381$

H $3.6363390 .212070-0.392826$

H $1.5896852 .389057 \quad 0.961764$

H $-1.6378142 .251618 \quad 0.300419$

$\mathrm{H}-3.693866 \quad \begin{array}{ll}1.183937 & 0.200591\end{array}$

SCF Energy (B3LYP/6-31G**//MMFF) $=-764.610675118$

F1a-10_c00094

MMFF Geometry

C -1.0813942 .8607090 .357789$

C $0.629586-0.332398-0.414373$

$\begin{array}{lllll}\text { O } & 0.262213 & 0.895718 & 0.234819\end{array}$

C $-0.887436 \quad 1.524234-0.348205$

C $-2.1162800 .613063-0.225676$

C $-1.806654-0.751506-0.848477$

C $-0.513476-1.353017-0.291025$

C $1.918646-0.8360320 .246388$

$\begin{array}{llll}\text { O } & -2.460437 & 0.461823 & 1.151333\end{array}$

O $-2.885967-1.656285-0.647855$

O $-0.681865-1.7113161 .080480$

C $3.123413-0.003262-0.138888$

$\begin{array}{llllll}\text { O } & 4.255117 & -0.465538 & 0.454998\end{array}$

$\begin{array}{lllll}0 & 3.111235 & 0.964898 & -0.885884\end{array}$

H -0.2019943 .4967780 .208081$

H $-1.9602413 .388298-0.024745$

H -1.1875002 .7320591 .440421$

H $0.816177-0.120230-1.475501$

H $-0.6743031 .720483-1.406877$

H $-2.982616 \quad 1.067717-0.719385$

H $-1.687355-0.624625-1.931969$

H $-0.264830-2.274894-0.829312$

H $2.118628-1.865891-0.070461$

H $1.827206-0.7943861 .337873$

H $-3.193831-0.1749031 .199027$

H $-3.685112-1.245870-1.020257$

H $-1.453411-2.3010311 .132527$

H $4.128382-1.2486531 .032255$

SCF Energy (B3LYP/6-31G**//MMFF) $=-764.614638777$

F1a-10_c00095

MMFF Geometry

C $-0.9959842 .877837-0.010757$

C $0.642579-0.431046-0.305313$

$\begin{array}{lllll}\text { O } & 0.276997 & 0.871394 & 0.180607\end{array}$

C $-0.8066391 .464227-0.547842$

C $-2.0736950 .608709-0.408206$

C $-1.774945-0.830789-0.839039$

C $-0.539076-1.401234-0.135577$

C $1.881605-0.8820520 .470575$

$\begin{array}{lllllll} & \text { O } & -2.540957 & 0.634531 & 0.940724\end{array}$

O $-2.906467-1.664356-0.620493$

O $-0.832150-1.588412 \quad 1.249228$

C $3.125416-0.202044-0.049931$

$\begin{array}{lllll}\text { O } & 3.226130 & 1.087560 & 0.357239\end{array}$

O $3.953727-0.748185-0.766905$

H -1.1776622 .8765551 .069471$

H $-0.0850773 .465884-0.168892$

H $-1.8272603 .384511-0.510072$

H $0.893295-0.341619-1.370868$

H $-0.5175461 .530955-1.604478$

H $-2.8846161 .018110-1.020512$

H $-1.585480-0.833313-1.919753$

H $-0.303503-2.388466-0.549214$

H $2.038097-1.961348 \quad 0.355847$

H $1.799868-0.6761041 .544148$ 
H $-1.8169250 .328296 \quad 1.513052$

H $-3.087449-1.6644300 .335222$

H $-0.105250-2.1020541 .639834$

H 2.4857021 .4011220 .919554

SCF Energy (B3LYP/6-31G**//MMFF) = -764.629995365

F1a-10 c00096

MMFF Geometry

C -1.035604 2.870237-0.122894

C $0.662817-0.416470-0.269276$

O 0.2667650 .8955680 .162773

C $-0.8277401 .435201-0.591313$

C $-2.0794810 .561751-0.418596$

C $-1.747178-0.883259-0.803612$

C $-0.500510-1.403268-0.080392$

C $1.890043-0.8195650 .549749$

O

O $-2.859222-1.736237-0.560696$

O $-0.771683-1.583607 \quad 1.309874$

C $3.107926-0.0422540 .112209$

O $3.671112-0.545975-1.014128$

$\begin{array}{lllll}\text { O } & 3.536910 & 0.944188 & 0.696441\end{array}$

H $-0.1424463 .467761-0.336887$

H $-1.8901523 .333184-0.625152$

H -1.1855072 .9230260 .960612$

H $0.928006-0.359827-1.333161$

H - $0.5392461 .459924-1.650032$

H -2.906558 $0.927822-1.037766$

H -1.555402 $-0.914924-1.883356$

H $-0.233277-2.391506-0.470383$

H $2.110173-1.8889260 .453699$

H $1.742008-0.6157001 .617323$

H -2.9118561 .4708091 .100595$

H -3.036148 -1.7180230 .395604$

H $-0.956957-0.7093051 .693313$

H $3.224103-1.336308-1.385237$

SCF Energy (B3LYP/6-31G**//MMFF) $=-764.623623496$

F1a-10 c00097

MMFF Geometry

C $2.557004-2.403908-0.115989$

C $-0.594228-0.467125-0.341411$

O $0.397814-1.4144150 .089592$

C $1.653790-1.270174-0.587855$

C $2.2575350 .113399-0.311782$

C $1.2490481 .199114-0.695872$

C $-0.1182840 .965578-0.044162$

C $-1.899263-0.8388510 .373390$

$\begin{array}{lllll}\text { O } & 2.595071 & 0.217067 & 1.071256\end{array}$

O $1.7626532 .479798-0.348858$

O -0.0308381 .1549441 .367815$

C $-3.110128-0.129243-0.187225$

O $-4.209458-0.3347660 .584155$

O $\quad-3.132415 \quad 0.533418-1.215017$

H $2.112338-3.371690-0.372915$

H $3.546275-2.342789-0.579544$

H $2.673904-2.3981620 .973039$

H $-0.730973-0.591955-1.423890$

H $1.480993-1.391740-1.664929$

H $3.1887830 .246182-0.874243$

H $1.1257451 .184171-1.786221$

H $-0.8378611 .702985-0.415516$

H -1.818243 -0.6299961 .445945$

H -2.072431 -1.9171630 .269055$

H $2.911644 \quad 1.1237661 .224338$

H $\quad 1.1026103 .142068-0.615885$

H $\quad 0.3435252 .0394611 .519619$

H -4.061771 $-0.896246 \quad 1.375031$

SCF Energy (B3LYP/6-31G**//MMFF) $=-764.618342386$

F1a-10 c00098

MMFF Geometry

C -2.5975292 .3292760 .128008$

C $0.6223500 .526621-0.203413$

O $-0.418624 \quad 1.3773720 .304379$

C $-1.6364001 .296789-0.448537$

C $-2.205956-0.127881-0.393161$

C $-1.146387-1.126324-0.870398$
C $0.184616-0.946404-0.133872$

C 1.8765820 .8272360 .619092

O $-2.621582-0.4433130 .935674$

O $-1.604071-2.464892-0.721174$

O $0.046281-1.3349041 .232761$

C $3.1008560 .140580 \quad 0.071827$

O $3.5936820 .754776-1.032208$

O $3.595236-0.8673380 .559290$

H $-2.1736383 .334848 \quad 0.029510$

H -3.561553 $2.307102-0.388925$

H $-2.7646062 .169107 \quad 1.198677$

H $0.8056910 .802078-1.250367$

H -1.418276 $1.569667-1.488953$

H -3.100422 -0.207737-1.020596

H $-0.968997-0.962657-1.941029$

H $0.945040-1.601401-0.573524$

H $1.7479500 .536621 \quad 1.668525$

H $2.066315 \quad 1.9076130 .655064$

H -1.858843 -0.3148661 .525155$

H -2.456072 -2.536975 -1.184273

H $-0.281133-2.2508061 .241052$

H $3.1023751 .554443-1.317367$

SCF Energy $($ B3LYP/6-31G**//MMFF $)=-764.621500295$

F1a-10_c00099

MMFF Geometry

C $1.7629162 .664658-0.177287$

C -0.5325870 .0752551 .139309$

$\begin{array}{lllll}\text { O } & -0.031476 & 1.223571 & 0.441732\end{array}$

C 1.3932001 .3660560 .529987

C $2.1012200 .146204-0.076490$

C $1.589958-1.1333920 .592580$

C $0.059466-1.2197560 .558709$

C $-2.0639590 .132201 \quad 1.153813$

O $1.8539720 .104594-1.481697$

O $2.175250-2.270846-0.030081$

O $-0.382350-1.408937-0.782796$

C $-2.7367920 .065516-0.197248$

O $-2.5049091 .163056-0.958180$

O $-3.438541-0.868850-0.563180$

H $2.8444972 .829352-0.166608$

H $1.4123652 .670912-1.214956$

H $\quad 1.2775943 .5138440 .316579$

H -0.2098230 .1631132 .186059$

H 1.6637821 .4612391 .589445

H 3.1866860 .2304990 .049508

H $1.920619-1.1310811 .638976$

H $-0.284199-2.0880321 .132092$

H -2.4026391 .0596991 .632240$

H -2.455660 -0.693288 1.760533

H $2.257313-0.712276-1.822272$

H $1.833161-3.0607620 .422429$

H $0.064858-2.200248-1.128611$

$\mathrm{H}-1.9246621 .835883-0.542035$

SCF Energy (B3LYP/6-31G**//MMFF) $=-764.616324962$

F1a-11 c00100

MMFF Geometry

C -1.455842 $2.728073-0.266672$

C $0.608406-0.3331050 .020551$

O $0.1625990 .979712-0.348409$

C $-1.151857 \quad 1.286610 \quad 0.128858$

C $-2.1708740 .307540-0.470263$

C $-1.782513-1.136911-0.145499$

C $-0.337579-1.385088-0.584822$

C $2.040429-0.503472-0.505000$

O -3.4720460 .5867980 .031351$

O $-1.878333-1.3545411 .265403$

O $0.058765-2.699785-0.212586$

C 3.0327720 .3650900 .239690

O $4.2852110 .236602-0.272209$

O 2.7718841 .0940721 .186396

H $-0.692867 \quad 3.401870 \quad 0.138552$

H -1.433091 $2.848373-1.355410$

H -2.4336003 .0511360 .102430$

H $0.622101-0.4142651 .115462$

H - 1.1582481 .2355881 .225352

H $-2.1936240 .428743-1.560712$ 
H $-2.464680-1.852594-0.617270$

H $-0.294461-1.324221-1.679383$

H $2.081613-0.221300-1.563564$

H $2.367996-1.542094-0.388180$

H -4.085164 -0.049216 -0.375015

H $-2.786467-1.1298101 .531370$

H $-0.012516-2.7651600 .755294$

H $4.360720-0.382343-1.029550$

SCF Energy (B3LYP/6-31G**//MMFF) $=-764.624571532$

F1a-11 c00101

MMFF Geometry

C - $-1.3902462 .750387-0.082938$

C $0.630230-0.354062-0.062199$

O $0.1918670 .984648-0.334863$

C $-1.092137 \quad 1.2860360 .221378$

C $-2.1553770 .355036-0.378543$

C $-1.777901-1.110242-0.155294$

C $-0.360173-1.355374-0.679833$

C $2.031816-0.513344-0.659100$

$\begin{array}{lllll}0 & -3.435605 & 0.617871 & 0.182338\end{array}$

O $-1.825622-1.3891651 .247244$

O $0.039506-2.687741-0.381762$

C $3.062596 \quad 0.209167 \quad 0.175272$

O $3.0767051 .546045-0.051803$

O $3.801033-0.3446440 .979277$

H $-1.4171282 .928138-1.163688$

H $-2.343916 \quad 3.0668740 .349459$

H -0.5976413 .3905880 .319876$

H $0.693892-0.4954421 .025101$

H $-1.046047 \quad 1.1768661 .312461$

H $-2.2252570 .531648-1.458976$

H -2.503896-1.779173 -0.630019

H $-0.362021-1.236471-1.770872$

H $2.084190-0.133714-1.686451$

H $2.334750-1.566130-0.692123$

H -3.3842010 .4278321 .134907$

H -1.523734 -2.305542 1.370000

H $-0.605115-3.286753-0.795537$

H $2.4202011 .859557-0.710036$

SCF Energy $\left(\mathrm{B} 3 \mathrm{LYP} / 6-31 \mathrm{G}^{* *} / / \mathrm{MMFF}\right)=-764.627385551$

F1a-11_c00102

MMFF Geometry

C -1.8698732 .5477440 .209316$

C $0.574088-0.1641220 .822036$

$\begin{array}{llll}\text { O } & 0.011388 & 1.124763 & 0.549635\end{array}$

C -1.4174781 .1114000 .452879$

C $-1.8683860 .176078-0.678457$

C $-1.313459-1.235096-0.466533$

C $0.204975-1.157148-0.293038$

C $2.084828-0.0050291 .024297$

O $-3.288790 \quad 0.135283-0.741787$

O $-1.885351-1.8055920 .713820$

O $0.731038-2.448579-0.015076$

C $2.8315880 .376990-0.232377$

O $2.6095871 .661465-0.605246$

O $3.563230-0.385478-0.850247$

H -1.505492 3.2014961 .009288

H -1.452526 $2.935817-0.726398$

H -2.9601872 .6272040 .167639$

H $0.171803-0.5150631 .782093$

H -1.838876 $0.790357 \quad 1.414284$

H -1.496538 $0.560027-1.636893$

H -1.580011 -1.901213-1.294397

H $0.632804-0.823136-1.245661$

H $2.517978-0.9414111 .395622$

H 2.2967650 .7596151 .781656

H -3.532476 -0.459135 -1.471750

H -2.851600 -1.7873500 .604364$

H $0.315966-2.7653760 .805476$

H $1.9958712 .158384-0.022856$

SCF Energy (B3LYP/6-31G**//MMFF)= -764.627011449

F1a-11 c00103

MMFF Geometry

C -1.426447 $2.765304-0.049010$

C $0.626962-0.317374-0.090301$
O $\quad 0.174628 \quad 1.021588-0.337156$

$\begin{array}{llll}\text { C } & -1.109836 & 1.299727 & 0.230279\end{array}$

C $-2.1665200 .366364-0.378010$

C $-1.772375-1.098183-0.180219$

C $-0.355747-1.319609-0.717535$

C $2.024546-0.452501-0.701347$

O $-3.446317 \quad 0.6061850 .194552$

O $-1.807987-1.3999961 .217933$

O $0.062136-2.651537-0.442916$

C 3.0330370 .3689680 .065224

O $3.436568-0.236851 \quad 1.209762$

O $3.437747 \quad 1.466618-0.294706$

H -0.6389613 .4082800 .359386$

H -1.461810 $2.959857-1.126617$

H $-2.381243 \quad 3.0638800 .393769$

H $0.696791-0.4753890 .994024$

H -1.0564121 .1739871 .319444$

H $-2.2450290 .559227-1.455167$

H $-2.494389-1.767245-0.661022$

H $-0.366269-1.183832-1.806553$

H $2.032381-0.094741-1.738235$

H $2.372836-1.491203-0.720076$

H -3.3862960 .4039611 .144002$

H -1.500026 -2.316398 1.323410

H $-0.578249-3.251437-0.862181$

H $3.030745-1.1131841 .381557$

SCF Energy $\left(B 3 L Y P / 6-31 G^{* *} / / M M F F\right)=-764.621518861$

F1a-11 c00104

MMFF Geometry

C $-1.874686-2.586997-0.303182$

C $0.5439930 .175708-0.771474$

O $-0.007235-1.126974-0.548336$

C $-1.438631-1.139671-0.508735$

C $-1.951129-0.2281580 .615926$

C -1.4156651 .1955110 .444209$

C $0.111397 \quad 1.1502750 .336834$

C $2.0671650 .046062-0.919182$

$\begin{array}{llll}\text { O } & -3.373458 & -0.218546 & 0.626513\end{array}$

O $-1.966787 \quad 1.757923-0.749825$

O 0.6208292 .4509010 .069434

C $2.779662-0.497740 \quad 0.302976$

O $4.124245-0.3190950 .214206$

O $2.247279-1.060267 \quad 1.249217$

H -1.489391 -2.980765 0.643947

H $-2.964082-2.686049-0.307889$

H -1.465959 -3.223186 -1.095852

H $\quad 0.1764590 .539564-1.740953$

H -1.826676 -0.812745 -1.482011

H -1.608161 -0.6177191 .583056$

H $-1.7394011 .839816 \quad 1.268943$

H $0.5107610 .824363 \quad 1.303710$

H $2.4894641 .027296-1.162911$

H $2.289737-0.645937-1.740370$

H -3.6574790 .3770591 .340623$

H $-1.5637142 .634979-0.869215$

H $0.3251283 .034890 \quad 0.788652$

H $4.4316800 .126965-0.603642$

SCF Energy $(B 3 L Y P / 6-31 G * * / / M M F F)=-764.614839513$

F1a-11_c00105

MMFF Geometry

C $1.453713-2.698675-0.311939$

C $-0.603621 \quad 0.358466 \quad 0.045201$

O $-0.153847-0.944761-0.355112$

C $1.162090-1.2626260 .111987$

C $2.176564-0.268141-0.471294$

C $1.7819041 .166290-0.112016$

C $0.3365771 .424474-0.546692$

C $-2.0443010 .518868-0.460322$

O $3.488372-0.5188560 .017246$

O 1.8801531 .3501871 .304017

O $-0.0356632 .734133-0.135046$

C $-3.008769-0.4094480 .249297$

O $-4.261227-0.313546-0.269648$

O $-2.728287-1.1538371 .178113$

H $1.459099-2.791065-1.403623$

H $2.414058-3.0498390 .076135$ 
H $\quad 0.669079-3.3695490 .055260$ H -0.6080650 .4177341 .141591$ H $1.171617-1.2331181 .209049$ H $2.196618-0.353119-1.565415$ H $2.4638621 .893702-0.566290$ H $\quad 0.298014 \quad 1.386381-1.643053$ H $-2.0865420 .286795-1.531047$ H $-2.4016001 .539243-0.290371$ H $3.790297-1.361502-0.361151$ H 2.7920901 .1294061 .560480 H $-0.8446022 .978849-0.614355$ H $-4.3548850 .324274-1.009090$

SCF Energy (B3LYP/6-31G**/MMFF) $=-764.615885595$

F1a-11_c00106

MMFF Geometry

C $-2.011116 \quad 2.490820 \quad 0.202276$

C $0.549886-0.104750 \quad 0.824869$

O $-0.071956 \quad 1.154606 \quad 0.543614$

C -1.499395 1.0736090 .442585

C $-1.8988510 .112819-0.689327$

C $-1.276342-1.269216-0.468954$

C $0.236522-1.114924-0.290893$

C $2.0482240 .130166 \quad 1.038399$

O $-3.311493-0.027198-0.775880$

O $-1.845677-1.852186 \quad 0.706287$

O $0.832293-2.377008-0.019909$

C $2.7478610 .653408-0.194422$

O $3.384497-0.319477-0.892223$

$\begin{array}{lllll}\text { O } & 2.729507 & 1.826578 & -0.544059\end{array}$

H -3.1038852 .5330170 .196132$

H $-1.646197 \quad 3.1643110 .985732$

H - $-1.6371812 .885568-0.748873$

H $0.157514-0.4736341 .782183$

H $-1.9074150 .733763 \quad 1.403070$

H $-1.5358360 .500350-1.649934$

H $-1.516017-1.942428-1.299556$

H $0.644925-0.756141-1.243035$

H $2.545153-0.7803221 .391120$

H $2.201650 \quad 0.882267 \quad 1.822677$

H -3.675341 $0.817328-1.089874$

H $-1.516194-2.7648370 .766961$

H $\quad 0.563949-2.6498360 .873698$

H $3.306786-1.223516-0.520137$

SCF Energy (B3LYP/6-31G**//MMFF) $=-764.614996456$

F1a-11 c00107

MMFF Geometry

C -1.381264 2.744244 -0.029898

C $0.635523-0.361142-0.066731$

$\begin{array}{llll}\text { O } & 0.193945 & 0.980110 & -0.316550\end{array}$

C -1.085922 1.2739860 .252079

C $-2.1571390 .356668-0.353676$

C $-1.783858-1.119443-0.185843$

C $-0.357655-1.353761-0.693843$

C $2.032178-0.511502-0.677849$

$\begin{array}{lllll}0 & -3.420508 & 0.616387 & 0.244860\end{array}$

O $-1.892119-1.5098551 .185565$

O $0.031112-2.697173-0.434370$

C $3.068520 \quad 0.2039100 .155567$

O $3.087064 \quad 1.541444-0.066684$

O $3.806470-0.3556790 .956011$

H -2.331269 3.0579600 .412470

H $-0.584152 \quad 3.3761870 .377042$

H $-1.4136672 .937449-1.107942$

H $0.711956-0.517607 \quad 1.017068$

H $-1.033517 \quad 1.1548311 .341329$

H -2.241437 $0.569679-1.427205$

H -2.490752 -1.757858 -0.726996

H $-0.358540-1.215074-1.782343$

H $2.074854-0.121068-1.701486$

H $2.336153-1.563571-0.724599$

H $-4.0649510 .007173-0.153786$

H -1.302992 -0.9549331 .720978$

H $-0.001985-2.8329080 .528143$

H $2.4302891 .860097-0.722172$

SCF Energy (B3LYP/6-31G**//MMFF) $=-764.620497703$
F1a-11 c00108

MMFF Geometry

C $-1.4216812 .759039-0.064248$

C $0.631616-0.323686-0.084116$

O $0.176848 \quad 1.012160-0.339740$

C $-1.107168 \quad 1.294522 \quad 0.225357$

C $-2.1649490 .360547-0.378807$

C $-1.778216-1.109976-0.198324$

C $-0.348498-1.334287-0.702456$

C $2.028077-0.459824-0.699249$

O $\begin{array}{llll} & -3.444085 & 0.601564 & 0.193489\end{array}$

O $-1.888133-1.4874331 .176470$

O $0.069048-2.662431-0.410572$

C $3.037720 \quad 0.3657690 .061351$

O $3.452306-0.239173 \quad 1.202278$

$\begin{array}{llll}\text { O } & 3.433380 & 1.466399 & -0.299540\end{array}$

H $-0.634407 \quad 3.403758 \quad 0.341531$

H $-1.4545352 .946636-1.143168$

H $-2.376953 \quad 3.0614990 .374852$

H $0.710298-0.472917 \quad 1.000138$

H $-1.054903 \quad 1.1804281 .315155$

H $-2.2428730 .559402-1.455223$

H $-2.480439-1.757214-0.735059$

H $-0.344163-1.210217-1.793213$

H $\quad 2.032710-0.106107-1.737647$

H $2.377404-1.498123-0.714895$

H $-3.3877330 .389561 \quad 1.140925$

H $-1.273564-0.9518451 .702714$

H $-0.580024-3.268020-0.807716$

H $3.052863-1.1181601 .375461$

SCF Energy (B3LYP/6-31G**//MMFF) = -764.614620177

F1a-11_c00109

MMFF Geometry

C $-2.680804 \quad 2.268886 \quad 0.232530$

C $0.5163090 .402297 \quad 0.091594$

O $-0.463523 \quad 1.441386-0.056843$

C $-1.771211 \quad 1.0530110 .379079$

C $-2.274885-0.134759-0.452814$

C $-1.290085-1.304360-0.376296$

C $0.107862-0.812551-0.763165$

C $1.8668550 .993989-0.337708$

$\begin{array}{lllllll}0 & -3.558670 & -0.542699 & 0.004027\end{array}$

$\begin{array}{llll}0 & -1.273819 & -1.806266 & 0.962631\end{array}$

O $1.042618-1.876478-0.648073$

$\begin{array}{llll}\text { C } & 3.061880 & 0.279060 & 0.252842\end{array}$

$\begin{array}{lllll}\text { O } & 4.222526 & 0.694019 & -0.319039\end{array}$

$\begin{array}{lllll}0 & 3.022146 & -0.552400 & 1.148846\end{array}$

H -3.6940742 .0614020 .588690$

H -2.279549 3.1139680 .802766

H $-2.734512 \quad 2.593717-0.812429$

H $\quad 0.569151 \quad 0.127932 \quad 1.153035$

H $-1.7334380 .794772 \quad 1.445302$

H -2.371578 $0.173286-1.501854$

H -1.614779-2.132191-1.016475

H $0.079758-0.516677-1.819345$

H $1.9278462 .040372-0.013685$

H $1.9434380 .984901-1.431292$

H $-3.833424-1.304224-0.534631$

H $-0.711331-2.599070 \quad 0.969426$

H $1.186046-2.0489270 .297899$

H $4.1207001 .376802-1.016174$

SCF Energy (B3LYP/6-31G**//MMFF)= -764.620308816

F1a-12_c00110

MMFF Geometry

C - $-0.6572522 .619597-0.534533$

C $0.580892-0.168139-0.361981$

$\begin{array}{lllll}\text { O } & 0.283402 & 0.740024 & 0.709589\end{array}$

C -0.8855801 .5528150 .533728$

$\begin{array}{llll}\text { C }-2.128614 & 0.692470 & 0.275653\end{array}$

C $-1.876791-0.333278-0.832259$

C $-0.594192-1.130880-0.587985$

C $1.864866-0.9178310 .018784$

$\begin{array}{lllll}0 & -2.450977 & 0.023427 & 1.498270\end{array}$

O $-2.980789-1.223566-0.954436$

O $-0.741131-1.9751940 .553761$

C $3.092474-0.035704-0.075263$ 
O $4.218478-0.716960 \quad 0.264085$

O $3.1011531 .140308-0.410894$

H $0.2650043 .172748-0.326805$

H $-0.5501682 .192717-1.535666$

H -1.490856 $3.328423-0.559014$

H $\quad 0.7598580 .407595-1.277610$

H -1.0163552 .0726301 .490859$

H $-2.993550 \quad 1.3191070 .030724$

H -1.781255 $0.189617-1.791418$

H $-0.392555-1.788787-1.441177$

H $\quad 1.800607-1.2810891 .051048$

H $2.016397-1.762126-0.663274$

H -3.201395 -0.5675491 .316109$

H -3.774026 -0.685116 -1.117538

H -1.531858 -2.5224330 .408717$

H $4.074252-1.6543520 .515185$

SCF Energy (B3LYP/6-31G**//MMFF)= -764.610770197

F1a-12 c00111

MMFF Geometry

C $-0.4820432 .509814-0.885885$

C $0.601472-0.277104-0.269530$

O $0.287058 \quad 0.7755230 .655093$

C $-0.825126 \quad 1.6093520 .298315$

C -2.0961560 .7773570 .079506$

C $-1.834392-0.399086-0.863674$

C $-0.604972-1.210160-0.446501$

C $1.817275-1.0228600 .286364$

O $-2.529518 \quad 0.2698311 .345242$

O $-2.981114-1.238109-0.946448$

O $-0.863027-1.9175990 .766810$

C $3.068988-0.1900620 .149047$

O $3.589706-0.233422-1.102561$

O $3.5576830 .466572 \quad 1.059094$

H $-0.3442911 .947114-1.813438$

H $-1.2739253 .246302-1.054525$

H $0.4559963 .043028-0.697613$

H $\quad 0.863577 \quad 0.170079-1.235214$

H -0.9797032 .2612911 .166550$

H $-2.9174351 .392581-0.304913$

H -1.663043 -0.008009-1.873304

H - $0.392240-1.972637-1.203940$

H $1.693610-1.2486591 .352624$

H $1.979606-1.977116-0.227912$

H $-2.734671 \quad 1.0327151 .912408$

H -3.143186 -1.597490 -0.057222

H $-0.997314-1.2615971 .471927$

H $3.096435-0.793468-1.739113$

SCF Energy (B3LYP/6-31G**//MMFF) $=-764.618706630$

F1a-12 c00112

MMFF Geometry

C $-0.4843742 .439205-1.046077$

C $0.595797-0.311497-0.283869$

O $0.307861 \quad 0.7969280 .581995$

C -0.8060301 .6163080 .199222$

C $-2.0868280 .781896 \quad 0.061649$

C $-1.855863-0.450350-0.815523$

C $-0.621744-1.243775-0.376296$

C $1.819726-1.0329910 .287110$

O -2.4906620 .3569371 .367166$

O $-3.017114-1.273473-0.809694$

O $-0.853925-1.875158 \quad 0.882980$

C $3.089582-0.280830-0.031980$

O $3.266900 \quad 0.805827 \quad 0.759690$

O $3.873648-0.606321-0.913724$

H -1.275341 3.169748 -1.242568

H $\quad 0.4609072 .975929-0.911716$

H $-0.3707681 .819311-1.939948$

H $0.8412810 .073388-1.280711$

H -0.9361662 .3217121 .028813$

H -2.913465 $1.379640-0.338814$

H -1.708316 -0.118988 -1.850395

H - $0.429781-2.051950-1.090831$

H $1.922358-2.031309-0.154885$

H $1.757699-1.1636921 .373822$

H -2.684714 1.1545841 .888380

H $-2.826717-2.053574-1.358104$
H $-0.986905-1.1774101 .546931$

H 2.5546710 .9568971 .417311

SCF Energy $\left(B 3 L Y P / 6-31 G^{* *} / / M M F F\right)=-764.615251115$

F1a-12_c00113

MMFF Geometry

C $1.456388-2.717754-0.271184$

C $-0.5925290 .330928-0.852937$

O $-0.030734-0.985833-0.947226$

C $0.993340-1.2897230 .006025$

C $2.153920-0.292346-0.109251$

C $1.659327 \quad 1.1461100 .045871$

C $0.5204831 .392420-0.943720$

C $-1.532106 \quad 0.4627630 .359065$

O $3.157773-0.5675310 .860462$

O $1.209137 \quad 1.348125 \quad 1.385377$

O $\quad 0.0092132 .707070-0.771027$

C $-2.769400-0.4011630 .219504$

O $-3.596526-0.257291 \quad 1.288423$

O $-3.019807-1.142452-0.720191$

H $2.215267-3.0432140 .446614$

H $0.608410-3.409124-0.215849$

H $1.867582-2.806577-1.282801$

H $-1.2152210 .454480-1.748936$

H $\quad 0.572958-1.2817351 .017950$

H $2.617338-0.390511-1.098926$

H $2.4794211 .855772-0.110985$

H $0.952724 \quad 1.342043-1.951913$

H -1.873752 1.4991340 .454533

H $-1.0372130 .162013 \quad 1.285920$

H $2.759508-0.444227 \quad 1.739172$

H 0.8538662 .2521691 .436683

H $-0.6900112 .838472-1.433886$

H -3.2798490 .3671941 .975657$

SCF Energy $\left(B 3 L Y P / 6-31 G^{* *} / / M M F F\right)=-764.619334137$

F1a-12 c00114

MMFF Geometry

C 1.70944124062600 .916715

C $-0.5498440 .537396 \quad 0.042919$

O $0.330246 \quad 1.318721-0.781143$

C $1.6705581 .475498-0.293075$

C $2.3328740 .117271-0.033729$

C $1.435643-0.7886550 .811589$

C $0.017246-0.8778530 .241438$

C $-1.9206030 .552733-0.647518$

O $2.584116-0.489031-1.304793$

O $2.019880-2.0828670 .913170$

O $0.028855-1.567184-1.008197$

C -3.0365320 .0263820 .225573$

O $-4.189015-0.126743-0.477370$

O $-2.948613-0.2165971 .420965$

H $1.219158 \quad 1.973478 \quad 1.793358$

H 2.7424272 .6418601 .191637

H $1.186553 \quad 3.3420020 .692133$

H -0.6453011 .0339201 .015661$

H $2.2117031 .975256-1.105980$

H 3.3107360 .2435340 .444644

H $1.382916-0.3824851 .828829$

H $-0.617533-1.4630340 .915295$

H $-2.175351 \quad 1.584847-0.918980$

H -1.883663 -0.022932 -1.579196

H $2.952591-1.372986-1.135086$

H $1.430008-2.6281541 .461321$

H $\quad 0.460549-2.426276-0.862060$

$\mathrm{H}-4.1312500 .116394-1.426109$

SCF Energy (B3LYP/6-31G**//MMFF) = -764.614166242

F1a-12 c00115

MMFF Geometry

C $-1.3946982 .679980-0.465283$

C $0.687711-0.388895-0.735138$

O $0.1352640 .919351-0.939243$

C $-0.9542161 .270564-0.078333$

C $-2.1039710 .264941-0.217354$

C $-1.616181-1.1590030 .057139$

C $-0.416669-1.455427-0.843327$

C $1.541145-0.4491310 .540044$ 
$\begin{array}{llll}O & -3.157184 & 0.599674 & 0.678304\end{array}$

O $-1.233279-1.291796 \quad 1.425587$

O $0.106720-2.750704-0.582855$

C $2.843815 \quad 0.291981 \quad 0.347881$

$\begin{array}{lllllll}\text { O } & 2.706453 & 1.635018 & 0.474834\end{array}$

$\begin{array}{lllll}O & 3.909493 & -0.254034 & 0.093679\end{array}$

H $-2.201237 \quad 3.0433540 .178370$

H $-0.5512253 .375169-0.388970$

H $-1.7337652 .711989-1.506604$

H $1.370294-0.557074-1.578844$

H $-0.607509 \quad 1.3189050 .960048$

H -2.501976 $0.309062-1.239298$

H $-2.416464-1.888567-0.109720$

H $-0.774823-1.454360-1.881483$

H $1.805879-1.4832480 .788506$

H $1.043649-0.0353831 .421673$

H -3.866986 -0.0531660 .552849$

H -1.997996 -1.032178 1.967581

H $-0.624186-3.386416-0.667766$

H 1.7949891 .9417810 .667444

SCF Energy (B3LYP/6-31G**//MMFF) = -764.612877686

F1a-12_c00116

MMFF Geometry

C $1.410118-2.675673-0.543268$

C $-0.689823 \quad 0.387793-0.708980$

O $-0.131636-0.910131-0.957197$

C $0.967721-1.277904-0.116664$

C $2.110944-0.263328-0.246404$

C $1.6224821 .151870 \quad 0.070518$

C $0.404317 \quad 1.466410-0.798905$

C $-1.5270330 .404152 \quad 0.577502$

O $3.179249-0.615636 \quad 0.624128$

$\begin{array}{lllll}0 & 1.267974 & 1.252092 & 1.449247\end{array}$

O $-0.1267342 .747334-0.486953$

C $-2.737967-0.4874890 .436346$

O $-3.761747 \quad 0.120296-0.212869$

O $-2.789390-1.6402140 .845305$

H $2.225440-3.0507760 .082338$

H $0.570834-3.376563-0.474739$

H $1.737709-2.680528-1.588734$

H $-1.3829300 .574403-1.539741$

H $0.632152-1.3531040 .923881$

H $2.493258-0.279630-1.275139$

H $2.4169011 .888119-0.094995$

H $0.7451411 .502735-1.842247$

H $-1.871507 \quad 1.4133330 .829395$

H -0.9717820 .0429601 .447924$

H 3.8843490 .0432310 .504584

H 2.0422450 .9763191 .969064

H $0.5975613 .391962-0.559201$

H -3.585668 $1.042622-0.496549$

SCF Energy (B3LYP/6-31G**//MMFF) $=-764.611818692$

F1a-12_c00117

MMFF Geometry

C $1.6291202 .474573 \quad 0.730505$

C $-0.5810540 .523653-0.079132$

O $\quad 0.346547 \quad 1.219137-0.926794$

C $1.6568161 .429097-0.381643$

C $2.2989490 .103670 \quad 0.048099$

C $1.353225-0.7181260 .928169$

$\begin{array}{llll}\text { C }-0.033790 & -0.862571 & 0.297975\end{array}$

C $-1.9063710 .463597-0.843057$

O $2.641189-0.634987-1.128404$

O $1.898315-2.0064891 .191514$

O $0.038951-1.677679-0.871668$

C $-3.033325-0.074897-0.000178$

O -3.5057750 .8383450 .884009$

O $-3.471880-1.213992-0.091328$

H $2.6454422 .740205 \quad 1.038204$

H 1.1208803 .3814180 .385849

H 1.0900722 .1282361 .616755

H -0.7313501 .1139340 .832259$

H $2.245162 \quad 1.846537-1.207949$

H 3.2435780 .2802780 .573824

H $1.240139-0.2167311 .896965$

H $-0.708197-1.371646 \quad 0.995525$
H $-1.820359-0.147298-1.749667$

H $-2.1873001 .459421-1.209309$

H $1.831072-0.752848-1.653356$

H $2.783107-1.877964 \quad 1.573857$

H $0.433411-2.526662-0.607887$

H -3.0658401 .7144660 .851873$

SCF Energy (B3LYP/6-31G**//MMFF) = -764.616339279

F1a-12 c00118

MMFF Geometry

C $-0.6411462 .609891-0.559758$

C $0.559538-0.189846-0.361216$

$\begin{array}{lllll}\text { O } & 0.270149 & 0.728474 & 0.704301\end{array}$

C $-0.886316 \quad 1.556688 \quad 0.517952$

$\begin{array}{llll}\text { C }-2.139800 & 0.708829 & 0.264990\end{array}$

C $-1.900481-0.333665-0.830966$

C $-0.625454-1.142009-0.581786$

C $1.834734-0.9506320 .026182$

O -2.5006680 .0601231 .488159$

O $-3.013321-1.213678-0.944747$

O $-0.783197-1.9747010 .567281$

C $3.068249-0.082779-0.073337$

$\begin{array}{llll}\text { O } & 4.171071 & -0.803202 & 0.256308\end{array}$

O $3.1020711 .094534-0.397524$

H $-0.5414402 .172285-1.557124$

H $-1.4637183 .331395-0.590690$

H $0.2896443 .150545-0.357260$

H $\quad 0.7463750 .377770-1.280214$

H -1.0131382 .0865301 .469989$

H -2.9932971 .3430330 .001460$

H $-1.7998340 .178559-1.795346$

H $-0.430049-1.808725-1.429443$

H $1.767794-1.306774 \quad 1.060753$

H $1.978600-1.801527-0.649300$

H $-1.735466-0.4602621 .787194$

H $-3.805955-0.667469-1.083105$

H $-1.571422-2.5249570 .418737$

H $4.987119-0.2616710 .201754$

SCF Energy (B3LYP/6-31G**//MMFF)= -764.630610476

F1a-12 c00119

MMFF Geometry

C $-1.3523892 .751313-0.228651$

C $0.592160-0.352610-0.875433$

O $0.0695550 .982452-0.947213$

C $-0.937859 \begin{array}{lll}1.303172 & 0.018051\end{array}$

C $-2.1323840 .348700-0.111507$

C $-1.688300-1.1094760 .019285$

C $-0.556821-1.374743-0.976154$

C $1.539170-0.5228820 .325691$

$\begin{array}{lllll}0 & -3.125213 & 0.642455 & 0.864236\end{array}$

O $-1.248405-1.3445031 .356290$

O $-0.088600-2.712201-0.877183$

C 2.7719580 .3518590 .211607

$\begin{array}{lllll}\text { O } & 3.586259 & 0.202373 & 1.289565\end{array}$

O $3.031205 \quad 1.102146-0.718632$

H -2.0956293 .0889230 .499669$

H $-0.4804943 .411666-0.164824$

H $-1.7655522 .874396-1.235868$

H $1.205349-0.482178-1.777239$

H $-0.512773 \quad 1.2603391 .027349$

H -2.593314 $0.479429-1.098574$

H $-2.533918-1.785426-0.151709$

H $-0.988701-1.274198-1.980522$

H $1.890404-1.558347 \quad 0.382717$

H $1.045300-0.2592251 .264311$

H -2.7295700 .4941241 .740259$

H $-1.120408-2.3026751 .458772$

H $0.202158-2.8688800 .035918$

H $3.265528-0.4317241 .965889$

SCF Energy (B3LYP/6-31G**//MMFF) $=-764.614195440$

F1a-12_c00120

MMFF Geometry

C $-1.9821792 .369096-0.604366$

C $0.4419750 .513928-0.908686$

$\begin{array}{lllll}\text { O } & 0.059186 & 1.312800 & 0.217709\end{array}$

$\begin{array}{llll}\text { C } & -1.352751 & 1.447530 & 0.437630\end{array}$ 
C -2.0436750 .0810230 .531150$

C -1.650586 $-0.828066-0.636195$

C $-0.130044-0.908193-0.804262$

C $1.9663870 .580287-1.063605$

O $-1.651701-0.514427 \quad 1.771478$

O $-2.205577-2.124708-0.442951$

O $0.443031-1.5936620 .305719$

C 2.7698930 .0130760 .083202

O $2.657596 \quad 0.743315 \quad 1.219653$

O $3.469755-0.987827-0.005045$

H $-1.4276703 .311818-0.661439$

H -1.970101 $1.934869-1.607991$

H $-3.0225242 .591960-0.347142$

H $0.0293990 .984806-1.809499$

H -1.4412541 .9480511 .409769$

H -3.1325470 .1960320 .577283$

H -2.089356 -0.428162 -1.558079

H $\quad 0.122065-1.488102-1.699329$

H $2.2658270 .046648-1.973844$

H $2.287351 \quad 1.621648-1.192509$

H $-2.044707-1.4035761 .801667$

H -1.940044 -2.672783 -1.201147

H $0.007141-2.4606120 .370162$

H 2.0639861 .5213831 .150401

SCF Energy (B3LYP/6-31G**//MMFF)= -764.611913659

F1a-13 c00121

MMFF Geometry

C $-0.7502832 .787131 \quad 0.620723$

C $0.653211-0.6597550 .635972$

O $\quad 0.321264 \quad 0.6940740 .981844$

C $-0.502637 \quad 1.393741 \quad 0.047127$

C $-1.8204350 .652909-0.204138$

C -1.547705 $-0.794415-0.611147$

C $-0.623444-1.4760670 .397366$

C $1.631478-0.729414-0.546420$

O $-2.5342801 .310786-1.248558$

O $-2.785937-1.498131-0.687934$

O $-1.296239-1.5921951 .656471$

C $2.978746-0.123143-0.214596$

O $3.830109-0.199854-1.271217$

$\begin{array}{lllll}\text { O } & 3.291224 & 0.376451 & 0.856853\end{array}$

H $0.202112 \quad 3.2944860 .810131$

H -1.342381 $3.405796-0.060092$

H $-1.2700172 .727018 \quad 1.583439$

H $1.154412-1.0651351 .524336$

H $0.0434601 .530515-0.893471$

H -2.4537190 .6756670 .691441$

H -1.108908 -0.818498 -1.615622

H $-0.384658-2.4962090 .077004$

H $1.802613-1.779440-0.810641$

H $1.239934-0.204719-1.422529$

H $-3.3485490 .803017-1.406949$

H -2.586001 -2.413062 -0.950011

H -2.128450 -2.069671 1.497125

H $3.457435-0.624741-2.073178$

SCF Energy (B3LYP/6-31G**//MMFF)= -764.613345756

F1a-13 c00122

MMFF Geometry

C -0.8370382 .6924410 .815141$

C $0.723645-0.668901 \quad 0.468129$

O $0.355307 \quad 0.6373330 .937664$

C $-0.5642801 .362097 \quad 0.117052$

C $-1.8594320 .576160-0.111452$

C $-1.542029-0.818784-0.649689$

C $-0.527185-1.5289380 .244026$

C $1.626471-0.600942-0.768459$

O $-2.6690971 .273554-1.055224$

O $-2.738596-1.590520-0.733240$

O $-1.138695-1.7739081 .516184$

C $3.013484-0.133242-0.397987$

O $3.0940221 .212464-0.256675$

O $3.964813-0.885145-0.229818$

H $-1.502787 \quad 3.3298780 .225829$

H -1.2860922 .5314501 .801544$

H $0.101333 \quad 3.2328440 .982421$

H $1.299703-1.1109851 .290932$
H $-0.092527 \quad 1.599223-0.843384$

H -2.4338040 .4980870 .819896$

H -1.156098 $-0.740163-1.672690$

H $-0.274438-2.509565-0.174360$

H $1.2402300 .045324-1.562043$

H $1.739271-1.598433-1.210835$

H $-3.4626480 .733226-1.211648$

H -3.073351 -1.714222 0.171626

H $-0.497927-2.2635192 .059561$

H $2.2553031 .697293-0.408553$

SCF Energy $(\mathrm{B} 3 \mathrm{LYP} / 6-31 \mathrm{G} * * / / \mathrm{MMFF})=-764.618355815$

F1a-13_c00123

MMFF Geometry

C -0.7701122 .6773860 .912808$

C $0.716108-0.6992360 .454412$

$\begin{array}{lllll}\text { O } & 0.367994 & 0.594582 & 0.970249\end{array}$

C $-0.535399 \quad 1.363126 \quad 0.171424$

C -1.846899 0.606291-0.076670

C $-1.558237-0.779189-0.654898$

C $-0.546791-1.5313140 .209386$

C $1.610840-0.599485-0.784909$

O $-2.656036 \quad 1.318137-1.010659$

O $-2.775569-1.519647-0.727693$

O $-1.127704-1.8096791 .488657$

C $2.9228730 .068280-0.452728$

O $3.804715-0.7761690 .137416$

O $3.1651291 .247208-0.675473$

H -1.389616 3.3674450 .333346

H $\quad 0.185725 \quad 3.1699101 .124445$

H -1.2516382 .4994201 .880693$

H $1.288942-1.1770241 .259314$

H $-0.057120 \quad 1.617237-0.781640$

H -2.424654 0.5003520 .850058

H -1.188586 -0.679802 -1.682522

H $-0.298846-2.500438-0.237695$

H $1.148714-0.022265-1.592006$

H $1.820935-1.591168-1.202663$

H -2.976349 $2.123718-0.571753$

H -2.565921 -2.389305 -1.108881

H $-1.955526-2.2953051 .331082$

H $3.481409-1.6933860 .265286$

SCF Energy (B3LYP/6-31G**//MMFF) $=-764.609657811$

F1a-13_c00124

MMFF Geometry

C $2.392614-2.3099030 .565774$

C $-0.685857-0.2380770 .819726$

O $0.388168-1.1474651 .114750$

C $1.356057-1.3035040 .073994$

C $2.0059450 .039350-0.281419$

C $0.9361761 .074776-0.629301$

C -0.1285121 .1481220 .466759$

C $-1.619549-0.833726-0.243989$

O $2.887982-0.123392-1.390280$

O $1.5536682 .350168-0.792692$

O 0.4591541 .6808231 .660468

C $-2.998580-0.212290-0.236822$

O $-3.790606-0.752978-1.199610$

$\begin{array}{lllll}\text { O } & -3.382129 & 0.662479 & 0.526663\end{array}$

H $1.906876-3.2537470 .836986$

H $3.149008-2.516882-0.197026$

H $2.894148-1.9450421 .468923$

H -1.240397 $-0.154851 \quad 1.762926$

H $0.874988-1.741631-0.808948$

H 2.6107880 .4081430 .555913

H $0.4791200 .823451-1.593340$

H $-0.927714 \quad 1.839606 \quad 0.180139$

H -1.739521 -1.907094 -0.051907

H -1.195202 -0.721733 -1.246753

H $2.366824-0.453862-2.141776$

H $0.8535162 .987330-1.015328$

H 0.8524462 .5401341 .430982

H -3.364540 -1.449397 -1.743511

SCF Energy (B3LYP/6-31G**//MMFF)= -764.612077135

F1a-13 c00125

MMFF Geometry 
C $-1.065076-2.151390-1.148810$

C $0.475938 \quad 0.265397-0.060057$

O $0.134981-0.9570850 .604711$

C $-1.128857-1.5253380 .242377$

C $-2.279814-0.5201550 .426881$

C $-1.9777130 .856178-0.164589$

C $-0.581892 \quad 1.3299680 .255020$

C 1.8628330 .6900500 .446615

O $-2.506143-0.3420611 .828499$

O $-2.0694580 .780883-1.585240$

O $-0.2675452 .548122-0.410400$

C $2.959869-0.231348-0.045482$

$\begin{array}{lllll}\text { O } & 4.171217 & 0.130500 & 0.453100\end{array}$

O $2.806773-1.182641-0.798534$

H -0.243717 -2.875049 -1.196209

H $-1.998236-2.671628-1.385576$

H $-0.873440-1.418516-1.936176$

H $0.5421420 .100127-1.142006$

H $-1.279497-2.3496230 .950536$

H -3.202779 $-0.925004-0.004520$

H -2.724171 1.5885210 .165573

H -0.5835291 .5267491 .334733$

H 1.8787130 .6688951 .542696

H 2.1058891 .6983630 .094917

H -2.761686 -1.205139 2.196407

H -1.815151 $1.650100-1.939563$

H $-0.9526143 .196404-0.173404$

H $4.1577450 .913876 \quad 1.043772$

SCF Energy (B3LYP/6-31G**/MMFF) $=-764.614894931$

F1a-13 c00126

MMFF Geometry

C - $-2.3825712 .386842 \quad 0.091704$

C $0.740597 \quad 0.439761 \quad 0.617052$

O $\begin{array}{lllll}0.323383 & 1.394748 & 0.757642\end{array}$

C $-1.365875 \quad 1.290526-0.215771$

C $-2.015030-0.097608-0.186589$

C $-0.952777-1.185497-0.344833$

C $0.182798-0.9908390 .660515$

C $1.5827330 .748543-0.624546$

O $-2.954476-0.196905-1.254525$

O $-1.561496-2.461896-0.156546$

O $-0.334377-1.2271391 .975878$

C $2.9356540 .086673-0.563010$

$\begin{array}{lllll}\text { O } & 3.809687 & 0.757075 & 0.227619\end{array}$

O $3.216234-0.950339-1.149575$

H $-2.8107452 .253658 \quad 1.091338$

H $-1.895526 \quad 3.3682240 .087712$

H $-3.1959232 .401542-0.639786$

H 1.3661710 .5894171 .505809

H $-0.958127 \quad 1.500001-1.212291$

H $-2.565302-0.2471730 .750673$

H -0.566287 -1.173607 - 1.370571

H $0.965961-1.7385690 .496140$

H $1.7255731 .830166-0.739944$

H $1.0899320 .429319-1.549168$

H -3.324522 -1.096179 -1.231789

H $-0.863676-3.133810-0.242193$

H $0.406970-1.1498892 .600534$

H $3.454816 \quad 1.5721380 .642468$

SCF Energy (B3LYP/6-31G**//MMFF) $=-764.610273433$

F1a-13_c00127

MMFF Geometry

C -2.265442 -1.491679-1.382231

C $0.372617-0.001339-0.836969$

O $-0.061823-1.280291-0.365934$

C $-1.462830-1.392089-0.086859$

C $-1.952175-0.2910020 .870730$

C $-1.468198 \quad 1.107257 \quad 0.483420$

C 0.0348381 .0882630 .187022

C $1.869036-0.090963-1.162791$

O $-1.445607-0.6031472 .172048$

O $-2.1956391 .551781-0.658693$

$\begin{array}{llll}O & 0.452936 & 2.359112 & -0.296346\end{array}$

C $2.750163-0.2654180 .052117$

O $2.660609-1.5056090 .592329$

$\begin{array}{llll}\text { O } & 3.472385 & 0.613861 & 0.503429\end{array}$
H -3.323926 -1.674604 -1.172801

H -2.189315 - $0.592802-1.998671$

H $-1.886326-2.317332-1.994529$

H $-0.1263620 .215512-1.789316$

H -1.571077 -2.3563890 .425466$

H $-3.046353-0.3204750 .930415$

H -1.6701841 .8233091 .288619$

H $\quad 0.5672770 .8920801 .125399$

H $2.1986570 .816327-1.682876$

H $2.068570-0.929392-1.841328$

H -1.7983610 .0595872 .790036$

H $-1.8266732 .412209-0.921911$

H $\quad 0.236016 \quad 3.017508 \quad 0.385579$

H $2.037122-2.1093550 .134608$

SCF Energy (B3LYP/6-31G*//MMFF) $=-764.619107575$

F1a-13_c00128

MMFF Geometry

C $-2.182460-1.326776-1.654452$

C $0.3884440 .117000-0.762228$

O $-0.043767-1.219483-0.494070$

C $-1.455136-1.388228-0.313056$

C $-2.021879-0.4316740 .751052$

C $-1.557347 \quad 1.015645 \quad 0.573281$

C $-0.040711 \quad 1.0654360 .364860$

C $1.9058130 .099044-1.005172$

O $-1.574634-0.8996672 .027039$

O $-2.2110901 .603712-0.548830$

$\begin{array}{lllll}\text { O } & 0.366910 & 2.400218 & 0.086625\end{array}$

C $2.733982-0.376697 \quad 0.171753$

O $4.051700-0.091098-0.000730$

O $2.308769-0.974491 \quad 1.150056$

H -1.753989-2.060646 -2.346120

H $-3.247229-1.548238-1.532251$

H $-2.089265-0.354329-2.143552$

H $-0.0561790 .449401-1.708394$

H - $-1.569731-2.4134290 .060973$

H -3.116282 -0.4925730 .747916$

H -1.8144801 .6244431 .447994$

H $\quad 0.4366670 .7706421 .305312$

H $2.236671 \quad 1.106529-1.281367$

H $2.127398-0.582805-1.834939$

H $-1.967666-0.3235892 .704698$

H $-3.1683991 .568572-0.381252$

H $-0.0957472 .686854-0.719439$

H $4.2709050 .373139-0.836552$

SCF Energy (B3LYP/6-31G*//MMFF) $=-764.610693843$

F1a-13 c00129

MMFF Geometry

C $-0.754368 \quad 2.741146 \quad 0.732396$

C $0.641773-0.707164 \quad 0.605070$

O 0.3159330 .6331531 .006207

C $-0.511514 \quad 1.371053 \quad 0.104610$

C $-1.8328010 .642148-0.167250$

C $-1.568096-0.790571-0.631026$

C $-0.638026-1.5113150 .343483$

C $1.611619-0.732815-0.585615$

O $-2.5758191 .333272-1.169264$

O $-2.796091-1.509924-0.720683$

O $-1.301647-1.6750571 .602506$

C $2.957778-0.139964-0.239920$

O $3.775388-0.181472-1.323413$

$\begin{array}{llll}\text { O } & 3.293612 & 0.314866 & 0.842857\end{array}$

H $0.1996103 .240000 \quad 0.936428$

H $-1.349233 \quad 3.3859620 .078636$

H -1.2691792 .6445991 .694792$

H $1.148224-1.1477221 .473356$

H $\quad 0.030644 \quad 1.543098-0.832353$

H -2.4560800 .6231560 .734799$

H $-1.131268-0.787794-1.636644$

H $-0.404493-2.518957-0.018011$

H $1.215355-0.175885-1.439548$

H $1.781592-1.771745-0.891208$

H $-2.0327441 .367069-1.975173$

H $-3.385983-1.004895-1.306280$

H -2.133922 -2.147941 1.430315

H $4.6582690 .197243-1.125269$ 
SCF Energy (B3LYP/6-31G**//MMFF) $=-764.627182809$

F1a-13_c00130

MMFF Geometry

C $2.447083-2.3147670 .028813$

C $-0.736652-0.502870 \quad 0.672282$

O $0.370367-1.414730 \quad 0.770840$

C $1.379518-1.255129-0.230252$

C $1.9764270 .157346-0.196227$

C $0.8714641 .211160-0.286826$

$\begin{array}{llll}\text { C } & -0.233100 & 0.945829 & 0.736888\end{array}$

C $-1.591913-0.822405-0.557194$

O $2.8841610 .331410-1.282529$

O $1.4150062 .510694-0.065552$

\begin{tabular}{llllll}
\hline & 0.270882 & 1.182448 & 2.057848
\end{tabular}

C -2.987381 $-0.267792-0.421055$

O $-3.1543720 .906900-1.076489$

O $-3.873581-0.805017 \quad 0.230826$

H $2.898797-2.1811171 .018081$

H $1.999613-3.3146840 .021326$

H $3.238540-2.284211-0.725838$

H $-1.336255-0.6940271 .571294$

H $0.950596-1.465647-1.217510$

H $2.552306 \quad 0.3114870 .723935$

H $0.4472541 .220083-1.297796$

H -1.0506601 .6606290 .600546$

H -1.687397-1.909101-0.679339

H $-1.136912-0.477288-1.491130$

H $2.3936430 .185843-2.109429$

H $2.1438702 .632465-0.697913$

H 0.8974800 .4715922 .274729

H -2.363250 $1.242100-1.549081$

SCF Energy (B3LYP/6-31G**/MMFF)= -764.609727985

F1a-13_c00131

MMFF Geometry

C $0.874185-1.9540811 .501303$

C $-0.4980640 .290321-0.069194$

O $-0.170825-1.035977-0.501356$

C $1.043544-1.5771030 .031535$

C $2.243458-0.655481-0.246289$

C 1.9697590 .8137090 .082933

C $0.616894 \quad 1.257611-0.483869$

C $-1.8309420 .666931-0.725291$

O $2.579448-0.753704-1.633813$

$\begin{array}{llll}\text { O } & 1.970342 & 0.998693 & 1.497287\end{array}$

O $\quad 0.311903 \quad 2.575267-0.039251$

C $-2.966611-0.143643-0.148472$

O $-3.383572 \quad 0.3253501 .053917$

O $-3.454551-1.124290-0.694752$

H $1.766222-2.463797 \quad 1.878359$

H $0.679912-1.0902032 .141418$

H $0.016103-2.6249071 .619741$

H -0.6332600 .3041441 .018550$

H $1.201642-2.512753-0.519493$

H $3.115488-1.0140810 .312056$

H $2.7560301 .459557-0.325687$

H $0.6815571 .282874-1.578771$

H - $-1.8036370 .469532-1.804039$

H -2.071921 $1.728628-0.601648$

H $1.768995-0.612533-2.151894$

H 2.8452370 .7319191 .827367

H $1.0462903 .152941-0.308568$

H -2.903960 1.1163901 .380397

SCF Energy (B3LYP/6-31G**//MMFF) $=-764.609374692$

F1a-14 c00132

MMFF Geometry

C -1.162544 $2.696543 \quad 0.763645$

C $0.675231-0.323112-0.336945$

$\begin{array}{lllll}\text { O } & 0.296469 & 0.845763 & 0.406848\end{array}$

C $-0.9293371 .425794-0.047190$

C -2.0893270 .4352520 .109546$

C $-1.759599-0.880398-0.597576$

C $-0.392985-1.410998-0.159705$

C $2.044229-0.7779360 .183544$

O $-3.2672880 .997301-0.463407$

O $-2.773545-1.837907-0.302868$
O $-0.443315-1.792673 \quad 1.216877$

C $3.160903 \quad 0.143337-0.261092$

$\begin{array}{lllll}\text { O } & 4.365660 & -0.277976 & 0.205745\end{array}$

O $3.026921 \quad 1.144097-0.951207$

H -2.066092 $3.222204 \quad 0.441314$

H $-1.2503352 .468377 \quad 1.831723$

H -0.3098293 .3762710 .657874$

H $0.752802-0.052665-1.398287$

H $-0.8175461 .719416-1.099384$

H $-2.2954200 .246359 \quad 1.170429$

H $-1.773649-0.723992-1.683480$

H $-0.132223-2.308608-0.731610$

H $2.272682-1.777840-0.202174$

H $2.048558-0.7929181 .279533$

H $-3.9745370 .334901-0.379885$

H $-2.543443-2.659781-0.769265$

H $-1.165369-2.4371811 .312777$

H $4.335755-1.0942750 .749178$

SCF Energy (B3LYP/6-31G**//MMFF)= -764.621116938

F1a-14_c00133

MMFF Geometry

C -1.0599842 .7428450 .503308$

C $0.710840-0.421938-0.244893$

$\begin{array}{lllll}\text { O } & 0.346885 & 0.822533 & 0.373228\end{array}$

C $-0.841251 \quad 1.396138-0.178066$

$\begin{array}{llll}C & -2.039430 & 0.459785 & 0.025351\end{array}$

C $-1.731771-0.927642-0.542555$

C $-0.399604-1.455078-0.007859$

C $2.041892-0.868256 \quad 0.363635$

O $\begin{array}{lllll} & -3.193370 & 0.990440 & -0.621859\end{array}$

O $-2.770106-1.840679-0.197396$

O $-0.507690-1.7029351 .396038$

C $3.193785-0.086570-0.221352$

O 3.2916781 .1643050 .293172

O $3.955009-0.525517-1.073431$

H -1.9334293 .2629750 .099358$

H -0.1816113 .3836800 .368659$

H -1.1931452 .6174631 .583533$

H $0.837238-0.250437-1.322230$

H $-0.6813231 .587140-1.247433$

H -2.282532 $0.370172 \quad 1.090903$

H $-1.696621-0.883875-1.638218$

H $-0.151388-2.410777-0.483511$

H $2.232164-1.9260310 .145826$

H $2.062672-0.7524111 .453498$

H -2.988234 $1.088392-1.567417$

H $-3.608135-1.458865-0.510246$

H $-1.249760-2.3184661 .524117$

H 2.6079851 .3901570 .959737

SCF Energy (B3LYP/6-31G**//MMFF)= -764.627539086

F1a-14_c00134

MMFF Geometry

C -1.1004152 .7572650 .459667$

C $0.718926-0.394001-0.208584$

$\begin{array}{lllll}\text { O } & 0.325007 & 0.854199 & 0.382112\end{array}$

C $-0.863784 \quad 1.400829-0.197066$

C -2.0509920 .4482180 .001939$

C $-1.706790-0.940797-0.540543$

C $-0.377605-1.4403010 .025748$

C $2.045349-0.8065360 .432278$

O $-3.1946920 .927160-0.701149$

O $-2.735791-1.868291-0.204020$

O $-0.508028-1.6692391 .430857$

C $3.1762520 .060490-0.065934$

O $3.624759-0.319061-1.288474$

O $3.633491 \quad 1.012040 \quad 0.553470$

H -1.2797082 .6447311 .534623$

H $-0.212193 \quad 3.3900620 .354936$

H $-1.949772 \quad 3.2828610 .014354$

H $0.858997-0.237461-1.286100$

H $-0.6910351 .576119-1.267129$

H -2.3206400 .3667351 .062259$

H $-1.652376-0.909589-1.636048$

H $-0.105699-2.398676-0.430960$

H $2.292599-1.852733 \quad 0.218334$

H $2.012313-0.7004031 .523431$ 
H $-3.5064601 .728658-0.248391$

H $-3.572849-1.507794-0.543906$

H $-1.243481-2.2935611 .554478$

H $3.166703-1.094416-1.677286$

SCF Energy (B3LYP/6-31G**//MMFF) $=-764.619477532$

F1a-14 c00135

MMFF Geometry

C -2.451022 2.4281710 .200814

C $0.6457240 .450549-0.264554$

O -0.2849331 .4328890 .219329$

C $-1.5970111 .280719-0.329355$

C $-2.189461-0.0831940 .044891$

C -1.238278 -1.205496-0.373511

C $0.175816-0.9512970 .153601$

C 2.0202950 .8274980 .301208

O $-3.439175-0.250172-0.620228$

O $-1.730419-2.446678 \quad 0.125803$

O $0.175938-1.0379291 .580473$

C $3.1588130 .072574-0.345474$

$\begin{array}{llll}\text { O } & 4.335420 & 0.302984 & 0.293723\end{array}$

O $3.066178-0.644169-1.331974$

H -3.463622 2.403667 -0.212559

H -2.516391 2.3941651 .294033

H -1.995349 $3.390844-0.055593$

H $\quad 0.6765360 .520557-1.359987$

H -1.541609 $1.385735-1.421017$

H $-2.379700-0.1388651 .124038$

H -1.225082 -1.284000 -1.467719

H $0.858204-1.725581-0.212564$

H 2.0443450 .6641491 .384497

H 2.1972011 .8970990 .133675

H -3.772514 -1.134873 -0.392312

H $-1.112688-3.141501-0.159480$

H $-0.179540-1.9114091 .818012$

H $4.276890 \quad 0.907470 \quad 1.064260$

SCF Energy (B3LYP/6-31G**//MMFF) $=-764.625246081$

F1a-14 c00136

MMFF Geometry

C $-1.0981402 .738704 \quad 0.475021$

C $0.700465-0.414961-0.230895$

O 0.3149790 .8280720 .375326

C $-0.8712821 .387709-0.196071$

C $-2.0647060 .440960-0.005691$

C $-1.728719-0.948334-0.552873$

C $-0.399625-1.4582680 .009181$

C $2.039853-0.8312750 .380101$

O $-3.2021250 .934451-0.709715$

O $-2.780595-1.851643-0.219606$

O $-0.554881-1.6913121 .410883$

C $3.179747-0.049753-0.228146$

O $3.249464 \quad 1.2219840 .237796$

O $3.956886-0.506816-1.055990$

H -1.9453593 .2740470 .037312$

H $-0.2061993 .367124 \quad 0.374576$

H -1.275292 2.6165721 .549182

H $0.826564-0.251200-1.309572$

H $-0.6989501 .573243-1.264473$

H $-2.3385850 .357242 \quad 1.053473$

H $-1.681363-0.906351-1.648346$

H -0.145570 -2.418933 -0.453199

H $2.241223-1.8911880 .184279$

H $2.066352-0.6889881 .466705$

H $-3.5136721 .730843-0.247883$

H $-2.536983-2.725867-0.568536$

H $\quad 0.235722-2.1631301 .722522$

H $2.556721 \quad 1.4600020 .890641$

SCF Energy (B3LYP/6-31G**//MMFF) = -764.613833544

F1a-14 c00137

MMFF Geometry

C $2.506582-2.2981220 .493121$

C $-0.675520-0.506952-0.127811$

O $0.304970-1.382747 \quad 0.451115$

C $1.592952-1.265721-0.159291$

C $2.144370 \quad 0.1568590 .002817$

C $1.1407091 .179914-0.533692$
C -0.2466380 .9549850 .069924$

C $-2.012209-0.8420280 .536243$

O $3.376530 \quad 0.289516-0.702028$

O $1.5765132 .501912-0.227767$

O $-0.212791 \quad 1.2441401 .469260$

C $-3.170407-0.130623-0.113622$

O $-3.536011-0.696383-1.290476$

$\begin{array}{lllll}\text { O } & -3.718799 & 0.856464 & 0.358456\end{array}$

H $3.503942-2.2968070 .043558$

H $2.080880-3.3021360 .388945$

H $2.604817-2.1097251 .567921$

H -0.741812 -0.727644-1.201359

H $1.506991-1.523959-1.223024$

H 2.3537350 .3718381 .057559

H $1.0819741 .108655-1.626898$

H $-0.9683821 .644001-0.382252$

H -2.002596 -0.5975521 .605098$

H $-2.200743-1.9227150 .503429$

H $3.2090100 .089392-1.638647$

H $2.4762402 .600705-0.583689$

H 0.1075352 .1570721 .567760

H $-3.013915-1.484198-1.553018$

SCF Energy (B3LYP/6-31G**//MMFF) $=-764.620406411$

F1a-14_c00138

MMFF Geometry

C - -1.1571262 .6680600 .802046$

C $0.669470-0.349379-0.325587$

$\begin{array}{lllll}\text { O } & 0.295444 & 0.814181 & 0.429430\end{array}$

C $-0.9258501 .405622-0.021725$

C $-2.091451 \quad 0.4181370 .120844$

C $-1.767256-0.893933-0.597134$

C $-0.404506-1.434381-0.161366$

C $2.035278-0.8162780 .191994$

O $-3.2803020 .977955-0.431684$

O $-2.769297-1.867757-0.316462$

O $-0.459342-1.828614 \quad 1.211707$

C $3.1533120 .103279-0.242107$

O $4.343291-0.3628920 .217315$

O $3.0376161 .122364-0.905723$

H -1.2495422 .4285871 .867152$

H -2.057724 3.2001590 .482029

H -0.3016913 .3457380 .706630$

H $0.750383-0.068384-1.384038$

H -0.807932 $1.710291-1.069998$

H -2.2957290 .2124301 .178533$

H -1.770565 -0.736799-1.682917

H $-0.147582-2.328173-0.740938$

H $2.260213-1.813223-0.203332$

H $2.040464-0.8429681 .287757$

H $-3.109396 \quad 1.180059-1.367473$

H -3.627234 -1.479846 -0.560621

H $-1.181308-2.474749 \quad 1.297847$

H $5.0871320 .218227-0.049087$

SCF Energy $(B 3 L Y P / 6-31 G * * / / M M F F)=-764.634377122$

F1a-14 c00139

MMFF Geometry

C $2.566022-2.2650300 .458328$

C $-0.654162-0.559698-0.200253$

O $0.344618-1.4016620 .397060$

C $1.635610-1.251210-0.200462$

C $2.1518840 .182177-0.027624$

C $1.1239981 .182010-0.561756$

C -0.2718920 .9139540 .006294$

C $-1.989886-0.9377050 .440201$

O $3.3734440 .330996-0.747331$

O $1.5307242 .512211-0.250461$

O -0.2940091 .2327601 .399615$

C $-3.155510-0.294526-0.265553$

$\begin{array}{llll}\text { O } & -3.607815 & 0.814911 & 0.368655\end{array}$

O $-3.631079-0.710629-1.314056$

H $3.568346-2.2382840 .021008$

H $2.165331-3.2782630 .344629$

H $2.646815-2.0794481 .535170$

H $-0.696446-0.782505-1.274782$

H $1.568238-1.506814-1.266273$

H 2.3655270 .3913321 .027536 
H $1.092578 \quad 1.116540-1.656249$

H $-0.9940971 .581431-0.475285$

H -2.010744 -0.7146471 .513059$

H -2.137292 -2.024419 0.388154

H $3.652797 \quad 1.258264-0.656334$

H 1.5097102 .6081900 .717164

H $\quad 0.2329680 .5648001 .870037$

H -3.1271831 .0552991 .189248$

SCF Energy (B3LYP/6-31G**//MMFF) $=-764.628292030$

F1a-15 c00140

MMFF Geometry

C - -1.4347232 .8049520 .130499$

C $0.519214-0.3357730 .106506$

O $0.086517 \quad 0.999529-0.186431$

C $-1.157788 \quad 1.337420 \quad 0.437511$

C $-2.2829170 .413841-0.060555$

C $-1.905027-1.0548980 .137107$

C $-0.511942-1.333562-0.441791$

C $1.892984-0.532916-0.549560$

O $-2.5217840 .654550-1.445749$

O $-1.918489-1.3355701 .536984$

O $-0.119185-2.665640-0.131565$

C 2.9749880 .2818960 .128203

O $4.172107 \quad 0.133634-0.497959$

O $2.822820 \quad 0.9872851 .115624$

H -0.6354793 .4326030 .540159$

H -1.452002 $2.993563-0.948301$

H -2.3861103 .1288440 .562949$

H $\quad 0.626336-0.453636 \quad 1.193095$

H -1.0386491 .2312291 .523245$

H $-3.203950 \quad 0.6492290 .485891$

H $-2.640504-1.716183-0.335344$

H $-0.557208-1.241091-1.534351$

H $1.849835-0.218762-1.599102$

H $2.195986-1.583822-0.492553$

H $-3.2569860 .080308-1.720131$

H -1.616481 -2.252916 1.651950

H $-0.789273-3.263104-0.505169$

H $4.159868-0.462326-1.277089$

SCF Energy $\left(\mathrm{B} 3 \mathrm{LYP} / 6-31 \mathrm{G}^{* *} / / \mathrm{MMFF}\right)=-764.618632886$

F1a-15_c00141

MMFF Geometry

C $-2.129719-2.407484-0.449633$

C $0.4507250 .201803-0.902598$

O $-0.175315-1.065762-0.675873$

C $-1.604727-0.985678-0.616118$

C $-2.056970-0.0586840 .526378$

C -1.3992281 .3172840 .403958$

C $0.118801 \quad 1.1689500 .242313$

C $1.953937-0.025213-1.098284$

O $-1.703746-0.6387351 .780385$

O $\quad-1.9519841 .980063-0.733117$

O $0.7103642 .438449-0.004824$

C $2.671150-0.4786290 .151851$

O $2.378427-1.7582690 .490610$

$\begin{array}{lllll}\text { O } & 3.439237 & 0.227454 & 0.792284\end{array}$

H -1.829134 -3.022806 -1.304961

H -1.708841 -2.8894250 .439342$

H $-3.221404-2.422516-0.378550$

H $\quad 0.0753040 .605963-1.852554$

H -1.966758 $-0.597467-1.576707$

H -3.149034 0.0359350 .495139

H $-1.6185941 .936401 \quad 1.281459$

H 0.5278170 .7830451 .183872

H $2.4349980 .899129-1.439260$

H $2.134836-0.777380-1.875916$

H -2.028070 -0.0454662 .479247$

H -1.481007 $2.825115-0.833214$

H 0.4888203 .0158830 .745542

H $1.741387-2.206113-0.106356$

SCF Energy (B3LYP/6-31G**//MMFF)= -764.623354739

F1a-15 c00142

MMFF Geometry

C -2.068529 -2.456403 -0.633706

C $0.4254980 .266806-0.831606$
O $-0.159065-1.032963-0.696086$

C -1.591569-1.010319-0.703085

C $-2.129245-0.1613670 .462814$

C $-1.524422 \quad 1.244098 \quad 0.443619$

C 0.0043491 .1757130 .332895

C $1.9466720 .108661-0.971974$

O $-1.837825-0.781910 \quad 1.713319$

O $-2.0626231 .945017-0.678347$

O $\quad 0.5395152 .4817910 .151309$

C $2.630278-0.536157 \quad 0.217243$

O $3.979882-0.3875760 .154661$

O $2.072240-1.1477961 .117230$

H -3.160974 -2.514207 -0.617000

H -1.704505 -3.015435 -1.502791

H -1.671972 -2.968302 0.249687

H $\quad 0.077586 \quad 0.703148-1.778139$

H -1.923375 -0.585013-1.658871

H -3.220377 -0.0961320 .380168$

H $-1.805628 \quad 1.801967 \quad 1.344252$

H $\quad 0.399797 \quad 0.784990 \quad 1.276515$

H $2.3962711 .093060-1.143373$

H $2.161608-0.531090-1.836424$

H -0.894086-1.016112 1.721508

H -1.632042 $2.816454-0.709237$

H 0.2540453 .0228120 .907196

H $4.3084680 .105177-0.627379$

SCF Energy $(B 3 L Y P / 6-31 G * * / / M M F F)=-764.623037943$

F1a-15 c00143

MMFF Geometry

C -1.4211202 .7566320 .504070$

C $0.550092-0.326260-0.034207$

O $0.0888421 .027642-0.131199$

C -1.1129481 .2671970 .610670$

C -2.2624430 .3932710 .079441$

C $-1.863812-1.0839780 .073391$

C $-0.509858-1.268789-0.623388$

C $1.869814-0.422662-0.804273$

O $-2.595067 \quad 0.795657-1.247557$

O $-1.786230-1.5288931 .427460$

O $-0.097879-2.627375-0.527675$

C $2.9667920 .335066-0.096190$

O $3.489106-0.3572270 .946758$

O $3.3407071 .456075-0.415096$

H -1.512851 $3.074515-0.540065$

H -2.3434063 .0105881 .035060$

H -0.6008413 .3426650 .933330$

H $\quad 0.735498-0.5663741 .020949$

H -0.9197121 .0319081 .665091$

H -3.1466240 .5479730 .709379$

H $-2.624631-1.692456-0.429165$

H -0.639216 -1.042651-1.689004

H $1.7684980 .010528-1.806905$

H $2.199721-1.459076-0.936807$

H $-3.3408800 .245754-1.542070$

H $-1.639650-2.4898241 .410136$

H $\quad 0.153344-2.8001040 .395379$

H $3.095814-1.2433351 .096084$

SCF Energy (B3LYP/6-31G**//MMFF) = -764.613879965

F1a-15_c00144

MMFF Geometry

C -1.4311602 .7813830 .110280$

C $0.508859-0.3697980 .119351$

O $0.0808010 .964886-0.191026$

C -1.1662151 .3128030 .420962$

C $-2.2883630 .394502-0.091523$

C $-1.919980-1.0779250 .107476$

C $-0.516522-1.369873-0.440868$

C $1.898301-0.559946-0.505011$

O $-2.5431550 .632577-1.474436$

O $-1.945592-1.3774701 .504391$

O $-0.169606-2.710377-0.112366$

C 2.9511050 .2991520 .165125

O $4.1548800 .181236-0.454694$

O $2.773006 \quad 1.010274 \quad 1.143936$

H -0.6326273 .4046640 .527660$

H $-1.4365192 .969757-0.968686$ 
H $-2.385097 \quad 3.1107690 .532849$

H $\quad 0.595220-0.480027 \quad 1.208486$

H -1.059826 1.2050421 .507763

H -3.2123810 .6257490 .450834$

H -2.643414 -1.739458 -0.383331

H $-0.550265-1.289854-1.534626$

H $1.866831-0.280766-1.564701$

H $2.226337-1.599559-0.407403$

H -1.693218 $0.612430-1.945794$

H -2.851743 -1.220182 1.820472

H $0.592354-2.964143-0.659022$

H $4.165516-0.425221-1.225705$

SCF Energy (B3LYP/6-31G**//MMFF) = -764.613520602

F1a-15_c00145

MMFF Geometry

C -1.3713432 .7504640 .436218$

C $0.556834-0.375079-0.002825$

O $\quad 0.112721 \quad 0.980582-0.141736$

C $-1.089757 \quad 1.258706 \quad 0.584430$

C -2.2498150 .3902000 .067169$

C - $1.879208-1.0953250 .078787$

C $-0.509630-1.319658-0.576137$

C $1.882880-0.509467-0.757576$

O $-2.568508 \quad 0.777597-1.268860$

O $-1.880635-1.5809401 .421254$

O $-0.121144-2.679953-0.424358$

C 3.0078650 .1527590 .001348

O $3.0249031 .499383-0.156919$

O $3.812823-0.4535080 .696261$

H -1.448012 $3.043065-0.616487$

H -2.293663 $3.033890 \quad 0.952017$

H $-0.544726 \quad 3.3331120 .857863$

H $\quad 0.737572-0.5885241 .058645$

H $-0.9072491 .052517 \quad 1.646309$

H $-3.134650 \quad 0.5692860 .689156$

H -2.628653 -1.685076 -0.461345

H $-0.610821-1.126325-1.651419$

H $1.829250-0.068894-1.760238$

H $2.164410-1.560940-0.885490$

H $-3.3274810 .241901-1.556177$

H -1.212482 -1.096489 1.932460

H $-0.062979-2.8652820 .528648$

H $2.3112371 .856873-0.727563$

SCF Energy (B3LYP/6-31G**//MMFF) $=-764.620841750$

F1a-15 c00146

MMFF Geometry

C -2.343664 -2.241277 -0.527408

C $0.4324950 .174072-0.894792$

O $-0.292110-1.047007-0.703627$

C $-1.710358-0.858693-0.631742$

C -2.0825260 .0589860 .547254$

C -1.3208501 .3825910 .461864$

C 0.1815501 .1279670 .282879

C $1.909242-0.176968-1.109451$

O $-1.759962-0.557478 \quad 1.791545$

O $-1.8295412 .122869-0.648016$

$\begin{array}{lllll}\text { O } & 0.836695 & 2.374946 & 0.084552\end{array}$

C $2.552845-0.7921640 .112989$

O $3.482476 \quad 0.0115480 .685490$

O $2.262137-1.896268 \quad 0.555809$

H -3.430897 -2.174753 -0.425745

H -2.114857 -2.827712 -1.424358

H -1.938762 -2.807034 0.318427

H $0.0871500 .637686-1.828753$

H -2.043604 -0.404171-1.573476

H -3.1623290 .2508680 .533997$

H $-1.486767 \quad 1.9869921 .361333$

H $\quad 0.5602290 .689733 \quad 1.214171$

H $2.4705370 .706156-1.432381$

H $2.010152-0.915297-1.914767$

H -2.384010 -1.289532 1.930292

H -1.301571 $2.936668-0.718237$

H 1.7941902 .2195730 .138171

H 3.6305690 .8748490 .245744

SCF Energy (B3LYP/6-31G**//MMFF $)=-764.610343893$
F1a-15 c00147

MMFF Geometry

C $-2.135134-2.393219-0.445564$

C $0.4414810 .218719-0.900955$

O $-0.182037-1.048056-0.664343$

C $-1.611034-0.969854-0.604158$

C $-2.058603-0.0501840 .545937$

C $-1.392787 \quad 1.3267640 .458029$

C 0.1209851 .1926240 .241821

C $1.943723-0.009097-1.107556$

O $-1.738171-0.640227 \quad 1.805444$

O $-1.9874522 .079330-0.599219$

O $0.6966752 .465345-0.025948$

C $2.668459-0.4710810 .135202$

O $2.384140-1.7558370 .461944$

O 3.4336850 .2331830 .780851

H -1.837399 -3.002344 -1.306307

H -1.711138 -2.882008 0.438176

H -3.226573 -2.408669 -0.370881

H $0.0627540 .617667-1.851223$

H $-1.974882-0.578626-1.562263$

H -3.1483050 .0613360 .509482$

H -1.5692561 .8955371 .378241$

H 0.5614630 .8174151 .173222

H $2.4245150 .916006-1.447168$

H $2.117981-0.757328-1.890483$

H $-0.799093-0.8910481 .791273$

H -1.827759 $1.621485-1.440132$

H 0.4607713 .0548550 .710522

H $1.749647-2.202208-0.138827$

SCF Energy $($ B3LYP/6-31G**//MMFF $)=-764.618697927$

F1a-15_c00148

MMFF Geometry

C -1.4254862 .7430200 .467362$

C $0.558651-0.338280-0.020570$

O $0.0844251 .008464-0.146015$

C $-1.118848 \quad 1.2544630 .590357$

C $-2.263163 \quad 0.364058 \quad 0.073178$

C $-1.858285-1.1123300 .075786$

C $-0.488689-1.305124-0.589965$

C $1.879851-0.435414-0.789600$

O $-2.605717 \quad 0.718179-1.265963$

O $-1.843237-1.6050161 .415378$

O $-0.052579-2.649722-0.427152$

C $2.9698770 .343286-0.093423$

O $3.501205-0.3298420 .957486$

O $3.3307031 .464260-0.427189$

H -0.6178633 .3307410 .918036$

H -1.483626 $3.057951-0.579969$

H $-2.3622812 .999540 \quad 0.970797$

H $\quad 0.751675-0.5546301 .037939$

H $-0.9258001 .038574 \quad 1.648540$

H -3.1519280 .5070090 .699255$

H -2.600291 -1.712273 -0.464001

H $-0.596363-1.115104-1.665718$

H $1.775135-0.019047-1.799096$

H $2.219356-1.470586-0.905772$

H -3.057841 $1.578180-1.238295$

H $-1.165426-1.1266971 .919403$

H $-0.744210-3.228246-0.791182$

H $3.117607-1.2180681 .118955$

SCF Energy (B3LYP/6-31G**//MMFF) $=-764.610942068$

F1a-15_c00149

MMFF Geometry

C $2.873980-2.0520040 .224501$

C $-0.450500-0.4357300 .045192$

O $0.641853-1.333883-0.195981$

C $1.842374-0.9610730 .490909$

C 2.3251990 .4242630 .028543

C $1.2143591 .463568 \quad 0.183547$

C $-0.0832440 .966016-0.466641$

C $-1.671274-1.023943-0.670541$

O $2.7220820 .360953-1.339650$

O 1.0062501 .6914931 .577794

O $-1.1333331 .886291-0.198456$

C $-2.982716-0.493972-0.148323$ 
O $\quad-3.293440-0.984608 \quad 1.077241$

O

H $2.513792-3.0129640 .608403$

H $3.042307-2.192930-0.848573$

H $3.828807-1.8221880 .706504$

H $-0.651579-0.4059271 .123906$

H $1.631929-0.9443101 .567914$

H 3.2025680 .7107300 .620734

H $1.5080902 .420478-0.263087$

H $\quad 0.0610450 .925908-1.553674$

H -1.689691 -2.117491 -0.576958

H -1.619849 $-0.833851-1.749721$

H $3.0486661 .241725-1.590938$

H $\quad 0.2586262 .307687 \quad 1.662166$

H $-0.8671122 .749187-0.559148$

H -2.639295 -1.612448 1.451507

SCF Energy (B3LYP/6-31G**//MMFF) $=-764.614766553$

F1a-15 c00150

MMFF Geometry

C $-2.823227-2.035875-0.616386$

C $0.446627-0.361137-0.184358$

O $-0.604470-1.335620-0.109095$

C -1.846209-0.866172 -0.649246

C -2.3535380 .3582210 .134434$

C -1.2824251 .4505450 .182227$

$\begin{array}{lllll}\text { C } & 0.055961 & 0.868061 & 0.653047\end{array}$

C $1.725609-1.0384830 .328939$

$\begin{array}{lllll}\text { O } & -2.680420 & 0.001833 & 1.475824\end{array}$

O $-1.1159852 .005409-1.122887$

O $1.059707 \quad 1.8736380 .602670$

C $2.999457-0.392679-0.169570$

O $4.088845-0.8650360 .491073$

O $3.0740110 .431617-1.069963$

H -2.454658 -2.850571 -1.249621

H $-2.919284-2.4526130 .391862$

H -3.812547 -1.738432 -0.976400

H $0.588562-0.083903-1.236841$

H -1.682459 -0.591092 -1.699123

H $-3.2615950 .746602-0.342371$

H -1.5749812 .2678920 .851725$

H $-0.051156 \quad 0.5683331 .702856$

H $1.745724-2.083958-0.003032$

H $1.726240-1.0435181 .425228$

H -3.487390 -0.539402 1.450373

H - $-1.9684872 .391612-1.386798$

H $1.1476242 .155076-0.324370$

H $3.897377-1.5356311 .181003$

SCF Energy (B3LYP/6-31G**//MMFF) $=-764.621597617$

F1a-16 c00151

MMFF Geometry

C $-0.8678822 .411799-0.470354$

C $0.647772-0.224318-0.283688$

$\begin{array}{lllll}\mathrm{O} & 0.309326 & 0.677427 & 0.782341\end{array}$

C -0.9431301 .3537440 .632108$

C $-2.095766 \quad 0.3507270 .477963$

C $-1.803808-0.667911-0.623742$

C $-0.430582-1.308307-0.417354$

C $2.020765-0.8258970 .045861$

O $-3.316563 \quad 1.0216050 .182887$

O $-2.798302-1.690173-0.620851$

O $-0.442612-2.098995 \quad 0.774222$

C $3.1426340 .175195-0.135619$

O $4.350451-0.3771420 .152918$

O $3.0101961 .337039-0.493820$

H -1.745998 $3.064710-0.452172$

H $0.0256143 .032105-0.339949$

H $-0.8015361 .966358-1.467138$

H $0.7178520 .338685-1.221766$

H -1.1040221 .9029141 .568139$

H -2.245048 -0.1862831 .423163$

H -1.848727 -0.187610 -1.608204

H -0.199094 -1.986240 -1.246702

H $2.224580-1.669849-0.622707$

H $2.048102-1.1630031 .088477$

H -3.482802 1.6542430 .902533

H -3.662381 -1.253109 -0.711914
H -1.165599 -2.743475 0.685418

H $4.319075-1.3177190 .430205$

SCF Energy $\left(B 3 L Y P / 6-31 G^{* *} / / M M F F\right)=-764.618996513$

F1a-16_c00152

MMFF Geometry

C -0.852564 $2.418572-0.443799$

C $0.643480-0.224121-0.287300$

$\begin{array}{lllll}\text { O } & 0.309448 & 0.661631 & 0.792896\end{array}$

C $-0.937181 \quad 1.3506120 .649021$

C -2.1008300 .3610030 .488111$

C $-1.815835-0.660146-0.615313$

C $-0.439430-1.303212-0.426552$

C $2.016876-0.8326840 .026436$

O -3.3014531 .0728530 .206066$

O $-2.808250-1.683729-0.611928$

O $\quad-0.446370-2.1364290 .736094$

C $3.1395260 .168890-0.147394$

O $4.348443-0.3912290 .120313$

O $3.0064681 .337332-0.483204$

H $\quad 0.051928 \quad 3.022702-0.314097$

H $-0.8020161 .981821-1.445453$

H -1.719612 $3.085627-0.412552$

H $0.7088290 .350873-1.218303$

H -1.0923491 .8932161 .589952$

H $-2.257624-0.1661161 .437305$

H -1.864590 -0.175844 -1.597965

H $-0.221601-1.962406-1.274109$

H $2.215168-1.667551-0.655074$

H $2.049727-1.1843551 .064070$

H -4.0349120 .4358280 .231230$

H -3.656919-1.271898 -0.845909

H $-0.483891-1.5621101 .519378$

H $4.317320-1.3367200 .380380$

SCF Energy $\left(B 3 L Y P / 6-31 G^{* *} / / M M F F\right)=-764.611119430$

F1a-16 c00153

MMFF Geometry

C 0.6857682 .3906620 .653740

C $-0.665859-0.2994070 .182509$

O $-0.342148 \quad 0.709182-0.787647$

C $0.8630011 .436850-0.529318$

C $2.0660320 .489279-0.411784$

C $1.794263-0.6358880 .586288$

C $0.467691-1.3296980 .269998$

C $-1.984590-0.942686-0.254296$

O $3.2325661 .201382-0.011576$

O $2.860424-1.5817610 .529346$

O $0.569460-2.012914-0.981989$

C $-3.143561-0.002373-0.026060$

O $-\begin{array}{llll}-3.554963 & 0.014481 & 1.266231\end{array}$

O

H $0.609198 \quad 1.8582451 .606256$

H $1.524363 \quad 3.0902200 .726584$

H $-0.2368932 .969586 \quad 0.538734$

H $-0.8040180 .179237 \quad 1.158965$

H $1.0261522 .072491-1.408506$

H $2.2812160 .045924-1.392304$

H $1.782348-0.234667 \quad 1.606569$

H $0.243994-2.0877451 .029156$

H $-2.183259-1.8734030 .289515$

H -1.973917 -1.190183 -1.322753

H $3.3903641 .897494-0.671947$

H $2.665733-2.2804781 .177147$

H $1.327992-2.618454-0.921104$

$\mathrm{H}-3.057841-0.5786341 .868932$

SCF Energy (B3LYP/6-31G**//MMFF) = -764.606387703

F1a-16 c00154

MMFF Geometry

C 0.6652822 .3790210 .657356

C $-0.669613-0.3230710 .218681$

O $-0.3657360 .683296-0.760747$

C $0.8380961 .420952-0.522623$

C $2.0464840 .478419-0.422068$

C $1.797821-0.6498680 .580794$

C $0.469858-1.3490110 .287256$

C $-1.994101-0.973645-0.190420$ 
O $3.226697 \quad 1.196467-0.076698$

O $2.852979-1.6062130 .512489$

O $0.551594-2.031657-0.966624$

C $-3.164974-0.0893240 .166559$

O $-3.3144590 .951562-0.689565$

O $-3.898744-0.2813341 .127358$

H $0.622875 \quad 1.8511121 .614504$

H 1.4886443 .0984870 .706086

H -0.2726962 .9362550 .559437$

H $-0.7943120 .157314 \quad 1.196318$

H $0.9847052 .054007-1.406577$

H $2.2336320 .034786-1.407752$

H $1.793241-0.2572361 .604363$

H $\quad 0.264175-2.1083201 .050238$

H $-2.139748-1.9224560 .339746$

H $-2.038902-1.191045-1.263912$

H 3.1582551 .4648150 .855119

H $3.687778-1.1276530 .654059$

H $1.309194-2.639577-0.915416$

H -2.648613 $0.991703-1.409141$

SCF Energy (B3LYP/6-31G**//MMFF) $=-764.622354427$

F1a-16_c00155

MMFF Geometry

C $-1.358023 \quad 2.7622890 .310305$

C $0.456561-0.286664-0.794152$

O $-0.0799201 .040769-0.719287$

C $-0.919113 \quad 1.3039150 .411450$

C $-2.116173 \quad 0.339470 \quad 0.456479$

C -1.651021 -1.1159110 .398679$

C $-0.685401-1.316268-0.772780$

C $1.569750-0.5179230 .243228$

O $-2.9793160 .599754-0.649283$

O $-1.024256-1.4458811 .635055$

O $-0.191709-2.649366-0.764859$

C $2.7847540 .348963-0.018900$

$\begin{array}{lllll}\text { O } & 3.759577 & 0.126479 & 0.901777\end{array}$

O $2.903796 \quad 1.152993-0.932601$

H $-0.483202 \quad 3.421456 \quad 0.337572$

H $-1.8664472 .964480-0.638540$

H -2.0232173 .0352561 .134963$

H $0.928449-0.362445-1.782789$

H $-0.326026 \quad 1.201148 \quad 1.326648$

H $-2.685240 \quad 0.5226351 .375677$

H -2.506582 -1.7917280 .283010$

H $-1.265144-1.193430-1.697005$

H $1.904783-1.5600520 .202972$

H $1.228485-0.2928051 .256782$

H $-3.740715-0.000199-0.573822$

H -0.689462 -2.355678 1.555514

H $0.391201-2.749931-1.536779$

H $3.539733-0.5439351 .583518$

SCF Energy (B3LYP/6-31G**//MMFF) $=-764.614113131$

F1a-16_c00156

MMFF Geometry

C $1.722605-2.296351-0.604771$

C $-0.637685-0.5094140 .130227$

O $0.246633-1.2173891 .013565$

C $1.602755-1.3188710 .566226$

C 2.2028980 .0691900 .304070

C $1.3088740 .898003-0.618106$

C $-0.1295130 .922346-0.096315$

C -2.027361 -0.5641650 .770116$

O $3.502844-0.062050-0.261975$

O $1.8212122 .226854-0.693665$

O -0.1756001 .6334621 .143019$

C $-3.100860-0.024190-0.138989$

O $-3.445461-0.898975-1.116095$

O $-3.6030181 .085765-0.020614$

H $1.198897-3.230243-0.373522$

H $\quad 1.275023-1.900822-1.521201$

H $2.769877-2.532564-0.817222$

H $-0.674611-1.038268-0.829254$

H $2.161779-1.7706861 .395071$

H 2.3213010 .5958951 .259502

H $1.3405880 .489179-1.634832$

H $-0.7745261 .462360-0.798073$
H $-2.056109-0.0122921 .717291$

H $-2.286130-1.5946691 .045081$

H $3.8360420 .835201-0.434807$

H $1.2363692 .732116-1.283941$

H 0.1943122 .5186210 .983597

H $-2.965960-1.754459-1.096322$

SCF Energy (B3LYP/6-31G**//MMFF)= -764.614290191

F1a-16 c00157

MMFF Geometry

C $1.843146-2.255726-0.630008$

C $-0.613113-0.592577 \quad 0.080238$

O $0.286813-1.2694490 .972313$

C $1.654564-1.3015760 .551445$

C 2.1935840 .1164350 .320376

C $1.2810260 .913992-0.610832$

C $-0.167890 \quad 0.863707-0.121004$

C -2.009218 -0.7213680 .694023$

$\begin{array}{lllll}\text { O } & 3.510068 & 0.054677 & -0.218649\end{array}$

$\begin{array}{lllll}\text { O } & 1.730798 & 2.266660 & -0.659085\end{array}$

$\begin{array}{lllll}0 & -0.277195 & 1.558287 & 1.123732\end{array}$

C $-3.086653-0.250745-0.248525$

O -3.5241791 .0005850 .034536$

O $-3.507621-0.913471-1.187934$

H $1.394249-1.869173-1.549620$

H $2.904336-2.438722-0.825200$

H $1.360661-3.216758-0.421379$

H $-0.610631-1.111959-0.885561$

H $2.217843-1.738477 \quad 1.385521$

H 2.2661170 .6353101 .284521

H $1.3540750 .520610-1.631512$

H $-0.8211811 .380323-0.832649$

H -2.082521 -0.2092861 .660374$

H $-2.220133-1.7726550 .930022$

H $3.8032620 .968779-0.374245$

H $1.1384272 .749855-1.260231$

H 0.0653752 .4579140 .984923

H $-3.092034 \quad 1.4330850 .801454$

SCF Energy (B3LYP/6-31G**//MMFF) $=-764.618967157$

F1a-16 c00158

MMFF Geometry

C 0.6902632 .4056990 .540062

C $-0.659536-0.303188 \quad 0.176598$

O $-0.3348190 .668362-0.831038$

C $0.872663 \quad 1.403193-0.600992$

C $2.071460 \quad 0.455276-0.446262$

C $1.794549-0.638253 \quad 0.587972$

C $0.467959-1.3395710 .290313$

C $-1.993640-0.939577-0.220784$

$\begin{array}{lllll}\text { O } & 3.250486 & 1.175633 & -0.101647\end{array}$

O $2.846945-1.6001590 .577751$

O $0.595513-2.058562-0.939076$

C $-3.136720 \quad 0.0199350 .008352$

$\begin{array}{llll}\text { O } & -3.525357 & 0.067769 & 1.307003\end{array}$

$\begin{array}{lllll}0 & -3.651347 & 0.694812 & -0.873621\end{array}$

H $-0.2415002 .966221 \quad 0.407648$

H 0.6293351 .9140021 .515325

H 1.5185523 .1200090 .574568

H $-0.7806970 .210422 \quad 1.137420$

H $1.0388552 .002112-1.505041$

H $2.274299-0.021224-1.413213$

H $1.770046-0.2104301 .597159$

H $0.254479-2.0757291 .073712$

H -2.193134 -1.855207 0.347582

H $-2.007367-1.209583-1.283741$

H $3.167197 \quad 1.473123 \quad 0.820058$

H $3.681226-1.1194330 .714261$

H $-0.179390-2.638801-1.025317$

H -3.025253 -0.519396 1.913055

SCF Energy (B3LYP/6-31G**//MMFF) $=-764.604483988$

F1a-16_c00159

MMFF Geometry

C $-0.8099382 .438147-0.473002$

C $0.630526-0.238303-0.268987$

$\begin{array}{lllll}\text { O } & 0.320290 & 0.680753 & 0.790552\end{array}$

C $\quad-0.913083 \quad 1.390948 \quad 0.637840$ 
C -2.0931890 .4202030 .494256$

C $-1.834122-0.615178-0.600322$

C $-0.478359-1.292683-0.391791$

C $1.986961-0.8752490 .061742$

$\begin{array}{lllll}\text { O } & -3.286838 & 1.143422 & 0.212037\end{array}$

O $-2.874122-1.590794-0.575429$

O $-0.510577-2.0732280 .805723$

C $3.1320560 .092255-0.130672$

O $4.310349-0.520306 \quad 0.153159$

O $3.0446601 .257696-0.486222$

H $\quad 0.1015263 .033144-0.349178$

H $-0.7588651 .983514-1.466641$

H $-1.6685913 .116526-0.457869$

H $\quad 0.714070 \quad 0.315222-1.211592$

H -1.0566341 .9519581 .569778$

H $-2.250678-0.0975731 .448924$

H -1.875017 -0.134915 -1.584979

H $-0.267098-1.983157-1.216310$

H $2.166432-1.729813-0.600292$

H $2.009688-1.2057091 .106593$

H $-4.001805 \quad 0.4929440 .104098$

H -2.686657 -2.234909-1.279510

H $-1.253086-2.6961850 .725962$

H 5.0715020 .0877780 .038087

SCF Energy (B3LYP/6-31G**//MMFF) $=-764.626957730$

F1a-16 c00160

MMFF Geometry

C -1.931823 -2.182382 0.677415

C $0.555683-0.4117460 .868413$

O $0.147339-1.263450-0.210175$

C -1.267206 -1.345855 -0.417979

C $-1.8867180 .045727-0.620073$

C -1.4752111 .0094860 .494157$

C 0.0462521 .0212910 .657012

C $2.074424-0.5321221 .044099$

O $-3.307688-0.033897-0.667840$

O -1.9208502 .3293790 .188649$

O $\quad 0.6351651 .575808-0.519554$

C $2.910102-0.078785-0.129929$

O $2.789662-0.891204-1.208410$

O $3.6378020 .905678-0.108949$

H -1.939366 -1.668035 1.642933

H -2.967188 -2.4235770 .417502$

H -1.385442 -3.1206190 .821851$

H $\quad 0.115113-0.802098 \quad 1.794238$

H -1.402525 -1.907657 -1.350672

H -1.556893 $0.458360-1.581965$

H -1.952721 0.7265651 .439574

H $\quad 0.336391 \quad 1.6643461 .495509$

H $2.349067-1.5749031 .247190$

H $2.3898220 .048724 \quad 1.919378$

H -3.541687 $-0.638629-1.392615$

H -2.882022 2.2844510 .046256

H $0.2603152 .465180-0.639790$

H $2.172680-1.644304-1.087849$

SCF Energy (B3LYP/6-31G**//MMFF)= -764.620012362

\section{Fragment F2 (cutoff energy $=10 \mathrm{~kJ} / \mathrm{mol}$ )}

F2-01_c00001

MMFF Geometry

C -4.357636 -1.582009 0.426641

C $-4.546395-2.642681-0.366318$

$\begin{array}{llll}C & -2.634924 & 0.239507 & 0.456647\end{array}$

O $-1.593615-0.556506-0.145240$

C $-0.5101870 .311204-0.543961$

C $-0.7873621 .652043 \quad 0.117513$

C -2.3017661 .6887820 .105141$

C $0.820885-0.339173-0.149435$

C -2.8913982 .7100891 .062388$

C $2.0483040 .556971-0.387628$

O $0.974388-1.535092-0.920876$

O $2.0150141 .044796-1.735479$

C $3.411374-0.130962-0.176196$

C $3.566688-0.7696391 .208079$

O $4.4326650 .862227-0.324604$

C -3.997152 -0.211352-0.072817

C $4.939533-1.4014081 .407216$
H $-4.479035-1.7055661 .499650$

H -4.809592 -3.605550 0.057654

H -4.441165 -2.572262 -1.443301

H $-2.5703760 .082027 \quad 1.541275$

H $-0.5853650 .398136-1.635226$

H $-0.3339372 .489513-0.421100$

H -0.4122261 .6638241 .148630$

H $-2.6392941 .915650-0.915196$

H $0.776352-0.6461010 .901664$

H $-3.9851282 .691379 \quad 1.028984$

H -2.5825232 .5172962 .095186$

H -2.562966 3.7201770 .796564

H $1.992103 \quad 1.4432130 .254736$

H $\quad 0.190128-2.087101-0.758497$

H $2.0195580 .272265-2.326512$

H $3.588137-0.880597-0.956653$

H $2.809397-1.5458571 .358358$

H $3.423619-0.0094561 .985513$

H $4.3196721 .264293-1.203081$

H $-4.7784650 .483477 \quad 0.255119$

H $-3.989213-0.202008-1.170049$

H $4.993502-1.8812862 .389648$

H $5.132845-2.1639530 .645951$

H $5.736560-0.6531411 .356659$

SCF Energy $\left(B 3 L Y P / 6-31 G^{* *} / / M M F F\right)=-810.723788275$

F2-01 c00002

MMFF Geometry

C 2.7655432 .5352610 .832480

C 2.0036613 .6321420 .754403

C 2.4038890 .1635320 .105393

$\begin{array}{lllll}\text { O } & 0.967877 & 0.213045 & 0.231633\end{array}$

C $0.400909-0.932465-0.430354$

C $1.561374-1.885426-0.686536$

C $2.692374-0.909800-0.944691$

C $-0.744054-1.5197410 .401393$

C $4.071948-1.534179-0.827742$

C $-1.846810-0.5095660 .778437$

O $-0.215704-2.0498341 .624353$

O $-2.896127-1.2353991 .432126$

C $-2.4536240 .252786-0.412572$

C -3.6275871 .1516440 .001706$

O $-2.898639-0.674712-1.401674$

C $2.9314231 .544800-0.285230$

C $-4.1329492 .003955-1.155807$

H $3.3093992 .342957 \quad 1.753789$

H 1.9293244 .3104381 .597333

H $1.4433273 .871511-0.142621$

H $2.799181-0.1388511 .084011$

H $\quad 0.017024-0.580507-1.395387$

H $\quad 1.370077-2.556307-1.529505$

H $1.785256-2.5008870 .192395$

H $2.572212-0.491640-1.953248$

H $-1.183567-2.372367-0.128978$

H $4.854187-0.790324-1.008606$

H $4.236385-1.9608350 .167440$

H $4.193832-2.337282-1.561892$

H $-1.466256 \quad 0.209544 \quad 1.514133$

H $0.241889-1.3250342 .084579$

H -2.497550 -1.705678 2.184406

H $-1.6822560 .876020-0.877367$

H -3.3185341 .8115670 .820366$

H $-4.4659580 .543471 \quad 0.361362$

H $-3.563737-1.250078-0.986283$

H $3.9981111 .486382-0.529690$

H $2.4083601 .900494-1.181866$

H -4.951036 $2.648991-0.819605$

H $-3.3360602 .643903-1.547704$

$\mathrm{H}-4.5094501 .382041-1.973842$

SCF Energy (B3LYP/6-31G**//MMFF)= -810.725350985

F2-01 c00003

MMFF Geometry

C $4.254786-1.156315-0.182036$

C $5.375520-1.0485820 .540500$

C $2.3872450 .461942-0.793690$

O $1.383098-0.566984-0.884949$

C $0.425451-0.3712070 .180377$ 
C $0.7302520 .999160 \quad 0.771675$

C 2.2335491 .0812340 .593864

C $-0.992288-0.525483-0.383936$

C 2.7880392 .4900590 .706496

C $-2.108162-0.1878210 .620259$

O $-1.146732-1.878485-0.825860$

O $-1.888196-0.9615291 .807539$

C -3.532152 -0.4912810 .118805$

C $-3.906154 \quad 0.146747-1.225486$

O $-4.446730-0.0290831 .119936$

C $3.767440-0.104349-1.137895$

C $-3.8015811 .666747-1.243547$

H $3.659693-2.060928-0.087741$

H $5.676385-1.8470371 .209895$

H $6.007476-0.1694340 .480047$

H $2.1269331 .202407-1.561943$

H $0.634898-1.1586310 .915335$

H $0.4170091 .085417 \quad 1.816421$

H $\quad 0.2326801 .791530 \quad 0.198404$

H 2.7021690 .4504451 .359168

H $-1.0868260 .098883-1.278995$

H $2.3407773 .158775-0.036484$

H 2.5832792 .9057071 .698522

H 3.8722512 .4945190 .555392

H $-2.036543 \quad 0.862623 \quad 0.923932$

H $-1.100002-2.450856-0.040654$

H $-2.596152-0.7353792 .435073$

H -3.673709 -1.5769820 .055826$

H $-4.945611-0.115125-1.460047$

H $-3.289114-0.269999-2.028906$

H $-5.343926-0.2650240 .828170$

H $3.721274-0.567840-2.131320$

H $4.4892340 .718684-1.202411$

H $-2.7710142 .000612-1.091617$

H $-4.1353612 .052327-2.212425$

H $-4.4289872 .118833-0.469291$

SCF Energy (B3LYP/6-31G*//MMFF) $=-810.717181061$

\section{F2-01 c00004}

MMFF Geometry

C $4.545606-1.316841-0.600184$

C $4.734703-2.520357-0.047463$

C $2.7334510 .400720-0.368600$

O $1.696328-0.547518-0.044314$

C $0.5440360 .167274 \quad 0.452686$

$\begin{array}{llll}\text { C } & 0.792824 & 1.631441 & 0.126737\end{array}$

C $2.298745 \quad 1.721270 \quad 0.265867$

C $-0.722126-0.431404-0.171847$

C $2.8963352 .951969-0.393925$

C $-2.007820 \quad 0.343842 \quad 0.175522$

$\begin{array}{llll}\text { O } & -0.837728 & -1.778986 & 0.293051\end{array}$

O $-2.149132 \quad 0.423630 \quad 1.594271$

C -3.292970 $-0.248240-0.440346$

C $-4.5142580 .637009-0.154104$

O $-3.560370-1.546654 \quad 0.084016$

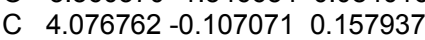

C $-5.7665020 .140369-0.866286$

H $4.750019-1.192558-1.660532$

H $5.079825-3.353502-0.650056$

H $4.549041-2.6966531 .006176$

H $2.7551540 .488110-1.462902$

H $\quad 0.5422120 .011952 \quad 1.539037$

H $\quad 0.259827 \quad 2.3095240 .799754$

H $0.489058 \quad 1.859445-0.902626$

H 2.5530301 .7269971 .334310

H $-0.611009-0.484843-1.261694$

H $2.6688162 .983746-1.464761$

H 2.4967913 .8641930 .061308

H $3.9847092 .966946-0.280221$

H -1.903906 $1.372130-0.188928$

H $-0.011916-2.2379910 .061344$

H -2.258702 -0.4844221 .925597$

H -3.163863 - $0.344871-1.525125$

H $-4.3117131 .665966-0.472941$

H -4.7231350 .6565810 .922241$

H -2.812541 -2.120254 -0.153719

H 4.8408570 .6717390 .055999

H $3.990090-0.3441161 .225713$
H $-6.6068350 .811721-0.662581$

H $-5.6136160 .108179-1.949678$

H -6.047687 -0.861687 -0.527865

SCF Energy (B3LYP/6-31G**//MMFF) $=-810.727411280$

F2-01_c00005

MMFF Geometry

C $-2.7364992 .236802-0.001335$

C $-3.7878002 .771737 \quad 0.629580$

C $-2.168140-0.112509-0.798993$

O $-0.7480650 .069731-0.636609$

C $-0.299308-0.775453 \quad 0.441046$

C $-1.451923-1.7293280 .740490$

C $-2.650619-0.8455640 .452262$

C $1.024373-1.4565350 .079503$

C $-3.946036-1.612925 \quad 0.257369$

C $2.138481-0.483455-0.360208$

O $0.789284-2.362088-1.006605$

O $3.331253-1.254144-0.556531$

C 2.4328430 .6557910 .632220

C 3.6583291 .5083370 .272471

$\begin{array}{llll}\text { O } & 2.655069 & 0.088048 & 1.925293\end{array}$

C $-2.8464411 .225259-1.107138$

C $3.5372502 .215555-1.067069$

H $-1.7384642 .555517 \quad 0.287972$

H -3.642012 3.5028561 .417341

H -4.8042922 .4918600 .376361$

H -2.287391 $-0.760530-1.677691$

H $-0.154067-0.122227 \quad 1.309925$

H -1.427254 -2.099578 1.769848

H $-1.449585-2.5958190 .069042$

H $-2.771247-0.1502811 .292166$

H $1.361509-2.0701090 .923409$

H $-3.869608-2.327176-0.569351$

H $-4.200888-2.173398 \quad 1.162757$

H $-4.772989-0.9302250 .038282$

H $1.885566-0.068119-1.342087$

H $1.639166-2.783741-1.221208$

H $3.606629-1.5937650 .312472$

H 1.5568351 .3095660 .709467

$\begin{array}{lllll}\mathrm{H} & 4.568208 & 0.895946 & 0.289407\end{array}$

H $3.8050112 .261606 \quad 1.057338$

H 2.7778710 .8221232 .551240

H -2.373209 $1.664979-1.994136$

H $-3.8981211 .047084-1.361176$

H $3.5290061 .503515-1.897459$

H $2.6224442 .814816-1.113602$

H $4.3895012 .886453-1.215896$

SCF Energy (B3LYP/6-31G**//MMFF) $=-810.720389797$

F2-01_c00006

MMFF Geometry

C $-4.305489-1.688289-0.201403$

C $-5.397984-2.091523 \quad 0.456852$

C $-2.609328 \quad 0.102957 \quad 0.258012$

O $-1.527151-0.565824-0.421374$

C $-0.438411 \quad 0.365137-0.602491$

C $-0.765538 \quad 1.5615420 .277529$

C $-2.277762 \quad 1.593495 \quad 0.195202$

C $0.884278-0.336196-0.268151$

C $-2.926527 \quad 2.419679 \quad 1.292418$

C $2.1099330 .596198-0.271478$

O $1.082912-1.391099-1.214754$

$\begin{array}{llll}\text { O } & 2.129087 & 1.343913 & -1.493907\end{array}$

C $3.470592-0.115084-0.127409$

C $3.570860-1.0079211 .115334$

O $4.489596 \quad 0.889443-0.061650$

C $-3.938046-0.246841-0.414998$

C $4.943490-1.6549941 .256983$

H -3.643042 - $2.441548-0.620131$

H $-5.610555-3.1487910 .572409$

H $-6.094331-1.3819980 .889727$

H -2.600378 -0.2499941 .297699$

H $-0.4646400 .651793-1.661465$

H $-0.2975442 .485219-0.075823$

H $-0.4419801 .386127 \quad 1.311158$

H $-2.5681352 .002179-0.782113$

H $0.789145-0.8219200 .709274$ 
H -2.6660992 .0414112 .286679$

H -2.597971 3.4625511 .233306

H $-4.0170032 .404017 \quad 1.201161$

H 2.0069251 .3375900 .529248

H $1.098976-0.998009-2.103577$

H $2.297908 \quad 0.722798-2.222394$

H $3.683092-0.703638-1.027345$

H $2.819878-1.8035581 .075651$

H $3.377611-0.4136522 .016462$

H 4.3364721 .4163610 .741261

H $-4.733007 \quad 0.404893-0.034585$

H -3.865352 $-0.078490-1.496563$

H $5.728357-0.9041891 .391091$

H $4.959724-2.3151002 .130118$

H $5.185994-2.2544800 .373831$

SCF Energy (B3LYP/6-31G**//MMFF) $=-810.709006081$

F2-01_c00007

MMFF Geometry

C $2.8281402 .755972 \quad 0.287834$

C 2.3519612 .8182861 .536679

C $2.3855180 .224056-0.053658$

O 0.9524290 .3003090 .082915

C $0.368720-0.922521-0.402724$

C $1.523479-1.900355-0.579136$

C $2.646375-0.964601-0.980531$

C $-0.738859-1.3989890 .543377$

C $4.028106-1.577482-0.830345$

C -1.836284 -0.3508610 .823100$

O $-0.158958-1.7598121 .803940$

O $-2.839767-0.970952 \quad 1.637437$

C $-2.496350 \quad 0.246225-0.433642$

C $-3.6668531 .180669-0.095265$

O $-2.955261-0.801599-1.286416$

C $2.9220391 .547768-0.607472$

C $-4.2262701 .870446-1.333131$

H $3.1982503 .681635-0.149448$

H 2.3450643 .7639012 .068851

H 1.9633411 .9518542 .057617

H $2.798594 \quad 0.0227280 .942897$

H $-0.054479-0.698767-1.389206$

H $1.308962-2.664348-1.332510$

H $1.772339-2.4111250 .358210$

H $2.497566-0.665607-2.026926$

H -1.187846 -2.3167760 .146499$

H $4.220837-1.8846320 .203190$

H $4.127344-2.462423-1.467399$

H $4.806074-0.864450-1.120281$

H -1.423789 0.4620551 .432466

H $0.299230-0.9752512 .151321$

H $-3.186494-1.7387101 .152552$

H $-1.7480970 .813223-0.998027$

H -3.3371151 .9440380 .618707$

H -4.4825710 .6168910 .372548$

H -3.682935 -1.260042 -0.833317

H $3.978234 \quad 1.426117-0.875736$

H $2.3794731 .793022-1.529534$

H -3.453212 2.460320 -1.835703

H $-4.6251211 .144021-2.048082$

H -5.039527 2.547082 -1.052194

SCF Energy $\left(B 3 L Y P / 6-31 G^{* *} / / M M F F\right)=-810.714725239$

F2-01_c00008

MMFF Geometry

C 3.5839792 .0426370 .542014

C 3.2997843 .2394850 .016185

C $2.571008-0.2282060 .227844$

O $1.2593090 .266723-0.108725$

C $0.473054-0.810352-0.647364$

C $1.249984-2.085418-0.343639$

C $2.680701-1.596173-0.444267$

C $-0.955825-0.799871-0.084718$

C $3.688244-2.5274550 .207134$

C $-1.7721110 .454084-0.458834$

O $-0.912628-0.9287851 .336494$

O $-1.112787 \quad 1.6321380 .020566$

C $-3.196857 \quad 0.4898350 .129617$

C $-4.042676-0.740853-0.214699$
O $-3.8606431 .644448-0.398750$

C $3.6309390 .765809-0.248943$

C $-5.463678-0.6425920 .328342$

H 3.8043901 .9790601 .604431

H 3.2860334 .1251230 .641962

H $3.0752853 .357556-1.038131$

H $2.602229-0.3319881 .320483$

H $0.434140-0.659981-1.733817$

H $1.019496-2.890434-1.047982$

H $1.042331-2.4516460 .668818$

H $2.936669-1.475009-1.505532$

H -1.466635 -1.687933 -0.471581

H $3.671331-3.510205-0.275507$

H $4.703089-2.1269440 .121152$

H $3.471962-2.6719401 .270896$

H $-1.8282330 .548721-1.550202$

H $-0.443722-0.1504851 .683935$

H $-0.249281 \quad 1.688689-0.422309$

H $-3.1559830 .622767 \quad 1.217564$

H -3.584970 -1.646701 0.195560

H $-4.099469-0.859105-1.303425$

H -3.317782 $2.418823-0.171178$

H $4.6318680 .334472-0.135508$

H $3.4846910 .979877-1.315176$

H $-6.0067740 .195947-0.118191$

H $-6.017539-1.5587810 .099863$

H -5.458416 -0.511637 1.415168

SCF Energy $(B 3 L Y P / 6-31 G * * / / M M F F)=-810.726912533$

F2-01_c00009

MMFF Geometry

C 3.1112152 .1585200 .740268

C 2.5179973 .3575670 .749583

C 2.311947 -0.1117090 .038999$

O 0.9228630 .1613350 .314904

C $0.113335-0.845193-0.319358$

C $1.068572-1.972183-0.691878$

C $2.319261-1.191317-1.043981$

C $-1.044606-1.2560710 .594887$

C $3.579824-2.038471-1.063143$

C $-1.932348-0.0995491 .103430$

O $-0.508879-1.9141361 .753229$

O $-2.995414-0.6680221 .880385$

$\begin{array}{llll}\text { C }-2.583055 & 0.803447 & 0.039399\end{array}$

C $-3.3531190 .029517-1.039752$

O $-1.6059191 .631676-0.582252$

C $3.0091111 .178860-0.394478$

C $-4.1136830 .957683-1.979397$

H $3.713077 \quad 1.8678291 .597363$

H 2.6342974 .0213501 .599293

H $1.9089003 .695921-0.081345$

H $2.751486-0.4953680 .969050$

H $-0.276742-0.403453-1.243080$

H $\quad 0.692093-2.579683-1.520288$

H $1.264641-2.6385060 .156065$

H $2.180602-0.733553-2.032773$

H -1.663607 -2.006743 0.090796

H $3.499588-2.829940-1.815436$

H $4.456049-1.429466-1.306155$

H $3.758688-2.514009-0.092806$

H $-1.3483080 .525052 \quad 1.791062$

H $0.101062-1.2928162 .187134$

H -2.587620 -1.225103 2.565593

H $-3.293676 \quad 1.4707080 .544320$

H $-4.065191-0.661050-0.574319$

H -2.662709 $-0.564738-1.648467$

H -1.143868 2.1210300 .119605

H $4.0237380 .960316-0.745972$

H $2.4624511 .631381-1.231541$

H -3.433171 $1.623102-2.519815$

H $-4.6686500 .371992-2.719226$

H -4.831056 $1.572323-1.426656$

SCF Energy (B3LYP/6-31G**//MMFF) $=-810.721507779$

F2-01_c00010

MMFF Geometry

C $4.279992-1.1731100 .502542$

C $5.429432-0.7242771 .019307$ 
C $2.468309-0.123171-0.944694$

O $1.415179-0.945885-0.407014$

C $0.506181-0.1023050 .337411$

C $0.895551 \quad 1.3315360 .001809$

C $2.3936751 .204076-0.192966$

C $-0.934609-0.478773-0.028254$

C $3.0165232 .371654-0.937111$

C -2.0098220 .4370880 .584764$

O $-1.169432-1.8186820 .417498$

$\begin{array}{lllll}\text { O } & -1.829800 & 0.494974 & 2.006241\end{array}$

C $-3.462367-0.022940 \quad 0.345000$

C $-3.801114-0.233956-1.136278$

O -4.3132350 .9990590 .878076$

C $3.807681-0.862960-0.889663$

C $-5.251597-0.642016-1.364672$

H $3.648072-1.8108881 .115134$

H $5.717118-0.9886252 .030987$

H $\quad 6.098123-0.0889900 .449057$

H $2.2143290 .033972-2.001710$

H $0.703501-0.3184641 .394752$

H 0.6258942 .0384020 .792038

H $0.4146431 .659290-0.928445$

H $2.859775 \quad 1.1212470 .796519$

H -1.030697 -0.489981-1.119875

H $2.5743502 .496813-1.931125$

H $2.8665513 .304359-0.383400$

H $4.0936292 .222780-1.062914$

H $-1.895018 \quad 1.4618160 .211264$

H $-0.482467-2.3810690 .020320$

H -1.018006 0.9984692 .183698

H $-3.663499-0.9350270 .919991$

H -3.162362 -1.013838 -1.563323

H -3.614404 $0.691455-1.694206$

H -5.2044020 .6224960 .970872$

H $3.704145-1.820300-1.415825$

H $4.563848-0.281169-1.430274$

H -5.943150 $0.156595-1.080013$

H -5.417272 $-0.860312-2.424618$

H $-5.501396-1.539824-0.790485$

SCF Energy (B3LYP/6-31G**//MMFF)= -810.710007095

F2-01 c00011

MMFF Geometry

C 3.2166292 .3796720 .555444

C 2.7536853 .5262500 .045068

C $2.577020-0.0162220 .192640$

O $1.207211 \quad 0.272330-0.152122$

C $0.604426-0.908369-0.710844$

C $1.571116-2.049487-0.420302$

C $2.906906-1.338253-0.499343$

C $-0.811297-1.125542-0.157935$

C $4.044423-2.1094340 .147020$

C $-1.7905760 .015604-0.510468$

O $-0.746236-1.2956091 .257098$

O

C $-3.253954-0.293620-0.129693$

C $-4.2059280 .823222-0.581205$

O $-3.393168-0.448246 \quad 1.280946$

C $3.4718001 .140285-0.255058$

C $-5.667750 \quad 0.467475-0.338738$

H 3.4327022 .3326081 .619712

H 2.5942984 .3875260 .684720

H $2.525043 \quad 3.626563-1.010197$

H $2.613736-0.1333311 .283658$

H $\quad 0.548477-0.749112-1.795286$

H $1.475691-2.868322-1.139799$

H $1.418126-2.4620210 .583941$

H $3.147923-1.159945-1.556035$

H -1.201022 -2.061711 -0.575085

H $4.983795-1.5523490 .076666$

H $3.846534-2.3037821 .206516$

H $4.186270-3.074279-0.350734$

H -1.750032 $0.188839-1.593045$

H $-0.408926-0.4645491 .633795$

H $-0.5054721 .452757-0.178661$

H -3.558562 -1.236494 -0.599750

H -4.063935 $1.024638-1.649140$

H -3.989368 1.749235 -0.035389
H $-2.837227-1.1980101 .552180$

H $4.5269880 .869768-0.135993$

H $3.304391 \quad 1.347357-1.319559$

H -5.8720290 .3224390 .726601$

H $-6.3158301 .273040-0.698496$

H $-5.940537-0.449809-0.870287$

SCF Energy (B3LYP/6-31G**//MMFF) $=-810.726774137$

F2-01 c00012

MMFF Geometry

C $4.561529-1.324652-0.618493$

C $4.754884-2.531167-0.074334$

C $2.7488900 .390757-0.371975$

O $1.711891-0.558857-0.051792$

C 0.5603210 .1532890 .450363

C $0.810096 \quad 1.6194850 .132531$

C 2.3160921 .7073190 .271030

C $-0.709680-0.438778-0.174887$

C $2.9138472 .941746-0.381607$

C -1.9944830 .3439530 .166939$

O $-\begin{array}{lllll}0.816697 & -1.796119 & 0.261578\end{array}$

O $-2.133403 \quad 0.4461131 .584389$

C $-3.279865-0.253074-0.443397$

C $-4.5002830 .635783-0.159804$

O $-3.511088-1.5507010 .099249$

C $4.093228-0.1205430 .148920$

C $-5.7536350 .166174-0.887558$

H $4.761485-1.193228-1.678830$

H $5.098996-3.359869-0.683416$

H $4.573520-2.7144030 .978973$

H $2.7687990 .484968-1.465745$

H $0.561286-0.0056231 .536095$

H 0.2779552 .2941880 .809773

H $\quad 0.506043 \quad 1.853560-0.895491$

H 2.5710171 .7063521 .339335

H $-0.591864-0.481764-1.264191$

H $2.6867602 .979510-1.452339$

H 2.5143113 .8513670 .078811

H $4.0021842 .955919-0.267437$

H -1.886355 $1.367154-0.210661$

H $-0.901874-1.7930911 .229834$

H -2.371603 -0.4346901 .920556$

H $-3.139478-0.358878-1.525916$

H $-4.2855541 .666117-0.466328$

H -4.7174920 .6471880 .915009$

H $-4.190607-1.983626-0.444275$

H 4.8570250 .6592630 .051379

H $4.008259-0.3650341 .215153$

H $-6.575814 \quad 0.866597-0.709237$

H $-5.5829530 .112028-1.967310$

H $-6.075318-0.819235-0.537493$

SCF Energy $\left(B 3 L Y P / 6-31 G^{* *} / / M M F F\right)=-810.707574769$

F2-01_c00013

MMFF Geometry

C $3.171126 \quad 1.913208-0.596731$

C $3.3556952 .329513-1.854533$

C $1.918216-0.0213270 .387305$

O $0.954228-0.107576-0.685148$

C $0.325080-1.395743-0.634270$

C $0.600219-1.9466050 .756517$

C $2.004626-1.4244330 .994996$

C $-1.142158-1.336796-1.067157$

C $2.417693-1.4373482 .456801$

C $-2.137975-0.633064-0.121662$

O $-1.580263-2.692544-1.233031$

O $-3.412243-0.625129-0.784986$

C -1.7890720 .8067210 .293760$

C $-2.906851 \quad 1.4564401 .122315$

O $-1.5670851 .610733-0.863103$

C $3.2551770 .476347-0.164904$

C -2.4996712 .8220951 .662469$

H 2.9548022 .6524290 .170291

H $3.2851153 .383453-2.100233$

H $3.5768871 .633647-2.656126$

H 1.5184400 .6904751 .120656

H $\quad 0.862697-2.026132-1.356792$

H $\quad 0.534921-3.0379460 .798542$ 
H $-0.086746-1.5318871 .501870$ H $2.710042-2.0396390 .420197$ H $-1.197672-0.868966-2.058177$ H $3.429037-1.0373522 .579753$ H $1.739845-0.834523 \quad 3.070646$ H $2.407824-2.4591042 .850149$ H $-2.283740-1.2449830 .775847$ H -2.504091 -2.663478 -1.536407 H $-3.338984-0.030942-1.551868$ H $-0.8726630 .811958 \quad 0.890708$ H -3.1709240 .8069391 .964839$ H -3.8056891 .5930980 .509528$ H $-0.7429281 .306435-1.279446$ H $4.032606 \quad 0.3988050 .603593$ H $3.564239-0.151913-1.009663$ H $-2.284547 \quad 3.5240060 .850833$ H -3.3085803 .2443292 .267064$ H -1.609623 2.7433692 .294913

SCF Energy (B3LYP/6-31G**/MMFF) $=-810.724000259$

F2-01 c00014

MMFF Geometry

C $3.581571-1.7391200 .590991$

C $4.736936-1.7767851 .264358$

C $2.363994 \quad 0.070347-0.721410$

O $1.055205-0.458028-0.434430$

C $\quad 0.354488 \quad 0.476942 \quad 0.407079$

C 1.1892931 .7548210 .402323

C $2.5944361 .198403 \quad 0.281781$

C $-1.0952790 .682565-0.056807$

C $3.6233362 .221530-0.164542$

C -1.970276 -0.5819520 .046520$

O $-1.1047581 .158358-1.402965$

O $-1.382937-1.622345-0.744205$

C $-3.418003-0.392989-0.446177$

$\begin{array}{llll}\text { C } & -4.192068 & 0.765879 & 0.195636\end{array}$

O $-4.121205-1.614452-0.188391$

C $3.403773-1.053222-0.734051$

C -4.3078590 .6727491 .712073$

H $2.715990-2.2422911 .014005$

H $4.801711-2.2942732 .215226$

H $5.631096-1.2951600 .884592$

H $2.3032360 .483512-1.737326$

H $\quad 0.3536580 .051063 \quad 1.418580$

H $1.0386692 .351947 \quad 1.306892$

H $0.9529892 .386253-0.462538$

H $2.8881320 .799419 \quad 1.260538$

H -1.5325811 .4729350 .562503$

H 3.6897693 .0406210 .558979

H $4.6143721 .764680-0.249895$

H $3.3654652 .653016-1.137458$

H -1.967401 -0.9457451 .080908$

H $-0.6992360 .468415-1.956196$

H $-1.945082-2.410543-0.649962$

H $-3.417480-0.266104-1.535613$

H $-5.2107150 .774500-0.212226$

H $-3.7382561 .724493-0.077454$

H $-5.018599-1.517539-0.550127$

H $3.087356-1.818915-1.453386$

H $4.361054-0.655070-1.091291$

H -3.3281170 .7340212 .194516$

H $-4.916474 \quad 1.5000882 .091419$

H $-4.786052-0.2625942 .018599$

SCF Energy (B3LYP/6-31G**/MMFF) $=-810.717071880$

F2-01_c00015

MMFF Geometry

C -2.730896 $2.233203-0.000382$

C $-3.7864932 .765243 \quad 0.625787$

C $-2.152680-0.112978-0.799531$

O $-0.7340570 .073045-0.628956$

C $-0.288468-0.7731390 .449495$

C $-1.440216-1.7310090 .740424$

C $-2.639915-0.850077 \quad 0.447738$

C $1.039645-1.450600 \quad 0.092771$

C $-3.932130-1.6207940 .244245$

C $2.152088-0.469008-0.332705$

O $0.804871-2.346082-1.001591$
O $3.356699-1.220559-0.525803$

C 2.4492770 .6564180 .677286

C 3.6420531 .5448550 .294338

$\begin{array}{lllll}\text { O } & 2.752418 & 0.098401 & 1.958098\end{array}$

C $-2.8328791 .223427-1.108426$

C $3.4548062 .289933-1.016877$

H -1.7349662 .5532870 .294979$

H -3.646216 3.4951951 .415492

H -4.8011252 .4840480 .366636$

H $-2.265160-0.759524-1.680207$

H $-0.152462-0.1211491 .320291$

H -1.420109-2.103395 1.769218

H -1.431907 -2.596151 0.067449

H $-2.767064-0.1568621 .288260$

H $1.373088-2.0703640 .933616$

H $-3.849415-2.332989-0.583511$

H $-4.190154-2.1839421 .146949$

H $-4.759991-0.9399270 .022397$

H $1.897641-0.033377-1.305915$

H $1.585758-2.918718-1.084614$

H $3.233823-1.774438-1.314922$

H 1.5693091 .2981410 .798225

H 4.5649870 .9530840 .256823

H $3.8035702 .276453 \quad 1.096873$

H $1.941978-0.3071622 .308123$

H $-2.3566001 .665931-1.992279$

H $-3.8829551 .042943-1.367844$

H $3.4333181 .604157-1.868727$

H $2.5250912 .867455-1.011575$

H $4.2849612 .986008-1.174705$

SCF Energy (B3LYP/6-31G**//MMFF) $=-810.709279379$

F2-01_c00016

MMFF Geometry

C $4.408918-1.130036-0.205323$

C $4.503408-2.456475-0.060163$

C $2.487993 \quad 0.471826-0.028867$

O $1.481428-0.489387-0.406806$

C $0.1758930 .042325-0.085561$

C $0.396061 \quad 1.522583 \quad 0.183292$

C $1.767108 \quad 1.5109750 .828687$

C $-0.788220-0.289443-1.231806$

C $2.4401372 .872490 \quad 0.847806$

C $-2.2170730 .268550-1.095340$

O $-0.868831-1.710032-1.372363$

O $-2.189783 \quad 1.679192-1.341969$

C $-2.9260390 .057414 \quad 0.258210$

C $-3.049286-1.4069740 .691816$

$\begin{array}{lllll}\text { O } & -4.253482 & 0.588118 & 0.139729\end{array}$

C $3.622834-0.2369100 .712393$

C $-3.812840-1.5649942 .002171$

H $4.937045-0.657168-1.029366$

H $5.091799-3.044704-0.755964$

H $3.999170-2.9765950 .746637$

H $2.8578550 .927622-0.956679$

H $-0.129849-0.4710720 .833580$

H $-0.372961 \quad 1.9581730 .827193$

H $0.4249582 .090469-0.754834$

H $1.667173 \quad 1.145256 \quad 1.859582$

H $-0.3510360 .080331-2.168333$

H 1.8437973 .5879571 .423386

H 3.4312102 .8118601 .308035

H $2.5621603 .274168-0.163741$

H $-2.849042-0.167855-1.879910$

H $\quad 0.040060-2.045835-1.458929$

H $-1.8319091 .813668-2.236063$

H $-2.431760 \quad 0.639507 \quad 1.043967$

H $-2.060685-1.8556070 .824390$

H $-3.575723-1.979699-0.081231$

H $-4.1693401 .519033-0.129822$

H $4.3127490 .499857 \quad 1.138971$

H $3.212321-0.822548 \quad 1.544604$

H -3.837908 -2.6187702 .297631$

H $-3.330841-0.9990002 .805722$

H $-4.847265-1.2201581 .908555$

SCF Energy (B3LYP/6-31G**//MMFF) $=-810.717119021$

F2-01_c00017 
MMFF Geometry

C 3.5962292 .0463820 .558805

C 3.2908533 .2524000 .066895

C $2.587645-0.224607 \quad 0.230309$

O $1.2623640 .267492-0.054002$

C $0.474708-0.798587-0.612118$

C $1.260462-2.076490-0.347544$

C $2.686582-1.578324-0.472689$

C $-0.958036-0.793170-0.056822$

C $3.714078-2.5176540 .134740$

C $-1.7700420 .456862-0.457009$

O $-0.937889-0.8817281 .367185$

O -1.1143831 .6440510 .004859$

C $-3.2001620 .504806 \quad 0.118443$

C $-4.043389-0.732677-0.207380$

O $-3.859371 \quad 1.648344-0.439200$

C $3.6252880 .786622-0.259710$

C $-5.469086-0.623187 \quad 0.321297$

H 3.8483991 .9622001 .612794

H 3.2915114 .1247010 .711279

H $3.0347313 .391251-0.977532$

H $2.654274-0.3501541 .318970$

H $0.435459-0.625513-1.695580$

H $1.017203-2.869396-1.061266$

H $1.084329-2.4629840 .662159$

H $2.916657-1.434460-1.537044$

H -1.470065 -1.683679-0.438681

H $3.690364-3.490087-0.367870$

H $4.724495-2.1096660 .033175$

H $\quad 3.524107-2.6855201 .200195$

H $-1.8186800 .532511-1.550417$

H $-0.671673-1.7843771 .607557$

H $-0.2436381 .688254-0.425056$

H $-3.168822 \quad 0.660278 \quad 1.203614$

H -3.589559 -1.6296080 .225867$

H $-4.090790-0.873665-1.293846$

H -3.317872 $2.427081-0.223579$

H $4.6320640 .359760-0.187675$

H $3.4432911 .021503-1.315909$

H $-6.008361 \quad 0.205557-0.147593$

H $-6.020843-1.5441470 .107368$

H $-5.473141-0.4693511 .405025$

SCF Energy (B3LYP/6-31G**//MMFF) $=-810.714785649$

F2-01_c00018

MMFF Geometry

C $4.264416 \quad 1.7006210 .535292$

C $4.3245812 .892689-0.068691$

C $2.674719-0.2294330 .347377$

O $1.5412910 .572552-0.041888$

C $0.492851-0.296361-0.524208$

C $0.907067-1.704324-0.125971$

C $2.415854-1.618966-0.233217$

C -0.8500040 .1756010 .050705$

C $3.139548-2.7390880 .493868$

C $-2.026868-0.757726-0.295422$

O $-1.1017791 .479374-0.478417$

O $-2.145020-0.864412-1.713112$

C -3.392020 -0.3787110 .317285$

C $-3.9996970 .935524-0.188130$

O $-3.280170-0.317007 \quad 1.737752$

C $3.9615480 .411549-0.175110$

C -5.4495951 .0980660 .256746$

H 4.4539541 .6480601 .604247

H 4.5529673 .7864620 .501528

H $4.1472722 .999198-1.133182$

H $2.678640-0.2650971 .444690$

H $0.497006-0.190897-1.616520$

H $0.475209-2.470518-0.776741$

H $\quad 0.607974-1.9217250 .907048$

H $2.694084-1.641059-1.295461$

H $-0.7660760 .285806 \quad 1.138319$

H $2.861320-3.7118590 .075144$

H $4.224369-2.6292840 .399969$

H $2.893092-2.7499741 .560922$

H $-1.776641-1.7570740 .081248$

H $-0.3440672 .041065-0.241087$

H $-2.861913-1.493541-1.902382$
H $-4.099321-1.1840440 .079948$

H $-3.9621440 .981565-1.281718$

H $-3.443938 \quad 1.7910300 .209972$

H -2.945287 -1.176983 2.044113

H $4.809598-0.265259-0.021748$

H $3.8746580 .589470-1.254398$

H $-5.8570502 .039974-0.124250$

H $-6.0705160 .281687-0.125524$

H -5.5321601 .1122971 .348035$

SCF Energy $\left(B 3 L Y P / 6-31 G^{* *} / / M M F F\right)=-810.711384489$

F2-01 c00019

MMFF Geometry

C $4.269959-1.0577160 .553212$

C $5.310196-0.639871 \quad 1.283113$

C $2.5412150 .125500-0.892998$

O $1.513821-0.854775-0.650406$

C $0.459764-0.2356510 .122159$

C $\quad 0.7496611 .2593750 .102499$

C $2.265374 \quad 1.276160 \quad 0.072643$

C $-0.896827-0.642982-0.467342$

C $2.8548142 .604882-0.365177$

C $-2.0962370 .073750 \quad 0.187302$

O $-1.031007-2.057124-0.301854$

O $-2.069559-0.1774861 .592063$

C $-3.466338-0.331387-0.400147$

C -4.6628410 .4318740 .186266$

O $-3.729584-1.716844-0.173132$

C $3.927701-0.518427-0.806763$

C $-4.6038061 .935316-0.026006$

H $3.634519-1.8429430 .954713$

H $5.507401-1.0739032 .257211$

H 5.9775370 .1371890 .927083

H $2.3919360 .464630-1.927014$

H $\quad 0.566378-0.631587 \quad 1.140076$

H $\quad 0.332956 \quad 1.7800880 .969834$

H $0.343596 \quad 1.724044-0.804839$

H $2.628748 \quad 1.0385791 .080077$

H - $-0.904352-0.452702-1.547484$

H $2.5119072 .886262-1.366528$

H 2.5621343 .4017810 .326110

H $3.9482552 .558031-0.383659$

H -1.9580681 .1507650 .045973$

H -0.258250 -2.473632 -0.720795

H -2.697209 0.4318552 .015099

H $-3.451770-0.176837-1.485906$

H -4.7719690 .2113161 .255104$

H $-5.5804130 .047229-0.278184$

H $-3.027758-2.226803-0.611276$

H $3.969672-1.361394-1.507828$

H $4.6832810 .206372-1.132796$

H -4.457815 2.178184-1.083187

H $\quad-5.5411772 .3970530 .300677$

H -3.7947142 .3912990 .551600$

SCF Energy $\left(B 3 L Y P / 6-31 G^{* *} / / M M F F\right)=-810.715919347$

F2-02 c00196

MMFF Geometry

C -3.5010382 .1659570 .024626$

C $-2.7645153 .276698-0.090393$

C $-2.894310-0.2492320 .368769$

$\begin{array}{lllll}0 & -1.703376 & 0.105783 & 1.104929\end{array}$

C $-0.632856-0.7975070 .755181$

C $-1.109893-1.563856-0.468116$

C $-2.603377-1.631202-0.224003$

C 0.6508640 .0143590 .543367

C -3.404878 -2.035675 -1.448186

C $1.837764-0.8173200 .027968$

O 1.0159950 .6000881 .797249

O $2.007912-1.9616660 .874736$

C $3.185818-0.071315-0.021538$

C $3.1391881 .223352-0.840207$

O $4.148999-0.943605-0.623928$

C $-3.1713860 .864405-0.652874$

C $4.4950631 .916190-0.911408$

H -4.3993472 .1964540 .635920$

H -3.058809 4.1863680 .421498

H $-1.8609973 .299112-0.689803$ 
H $-3.725691-0.3141921 .080891$ H $-0.521983-1.4728841 .612621$ H -0.644861 -2.550895 -0.550418 H -0.894224 -1.009448 -1.389576 H $-2.782184-2.3782360 .562400$ H $0.4450560 .843561-0.142974$ H $-4.478740-1.991200-1.240316$ H -3.193175 -1.393048 -2.308756 H -3.160548 -3.062535-1.740290 H $1.611214-1.211638-0.969120$ H $\quad 0.255122 \quad 1.1220692 .105386$ H $2.167460-1.6365501 .777611$ H 3.5454700 .1391970 .992685 H $2.4201601 .925273-0.405590$ H $2.8131941 .003184-1.863821$ H $4.165547-1.762475-0.099401$ H -2.304908 $0.997998-1.311752$ H $-4.0268110 .598256-1.283376$ H $4.4089392 .854806-1.468141$ H 4.8676162 .1496170 .091022 H $5.2375891 .293292-1.419704$ SCF Energy (B3LYP/6-31G**//MMFF) $=-810.721278780$

F2-02_c00197

MMFF Geometry

C 2.8609152 .2513990 .271067

C $3.8110393 .150907-0.007487$

C $2.2039200 .091604-0.843364$

O $\quad 0.8162590 .284219-0.486916$

C $0.215736-0.990113-0.195330$

C $1.370681-1.9602370 .008255$

C $2.382387-1.425030-0.985553$

C $-0.742754-0.8722990 .994487$

C $3.782666-1.977540-0.788366$

C $-1.830267 \quad 0.2101290 .838435$

O $0.006410-0.5567252 .175567$

O

C $-2.6712150 .094266-0.444912$

C $-3.8164181 .116384-0.491388$

O $-3.212391-1.222128-0.549078$

C 3.0737820 .7627810 .230795

C $-4.5591391 .084990-1.821326$

H 1.8752362 .6089690 .557682

H 3.5950584 .2124190 .046375

H $4.8127252 .849026-0.292823$

H $2.3737460 .579189-1.811037$

H - $0.340290-1.289771-1.091752$

H $1.080075-2.996603-0.189194$

H $1.773679-1.9106071 .026050$

H $2.046108-1.708225-1.993332$

H $-1.209334-1.8452601 .187633$

H 4.487150 -1.504044 -1.479648

H $4.144907-1.8236880 .233142$

H $3.796314-3.055808-0.979716$

H $-1.380379 \quad 1.2091120 .889471$

H $\quad 0.470126 \quad 0.2826502 .013185$

H -2.164784 0.1886162 .766857

H $-2.027610 \quad 0.249308-1.317504$

H -3.419714 2.125200 -0.329731

H -4.5432190 .9120890 .303532$

H $-3.763159-1.3760720 .237629$

H 2.8281610 .3667541 .223118

H 4.1324970 .5501180 .044582

H -5.025108 $0.109906-1.994574$

H $-5.3499451 .841771-1.828912$

$\mathrm{H}-3.8804811 .295767-2.653880$

SCF Energy $\left(B 3 L Y P / 6-31 G^{* *} / / M M F F\right)=-810.723878095$

F2-02 c00198

MMFF Geometry

C 3.8242751 .8999290 .444650

C 3.2114093 .0179780 .849378

C $2.961308-0.297122-0.419898$

$\begin{array}{lllll}\text { O } & 1.795551 & 0.332444 & -0.995428\end{array}$

C $0.648146-0.526856-0.823310$

C $1.071027-1.5991710 .167657$

C $2.544679-1.746280-0.151139$

C $-0.5481150 .326084-0.382512$
C $3.326841-2.5001990 .909265$

C $-1.801670-0.500335-0.036097$

O $-0.8521841 .229000-1.449169$

O $-2.163019-1.311772-1.154084$

C -3.0163070 .3427850 .405801$

C $-4.187721-0.5457200 .847950$

O $\quad-3.4746821 .175617-0.656564$

C 3.3766100 .5133360 .817440

C $\quad-5.3462330 .2662981 .413759$

H $4.7054411 .986832-0.185704$

H 3.5867513 .9898970 .548329

H 2.3304172 .9855451 .480824

H $3.763763-0.270607-1.166968$

H $0.457061-0.970154-1.808724$

H $\quad 0.511236-2.5307190 .039814$

H $0.932164-1.2579411 .200735$

H $2.630482-2.301281-1.096164$

H -0.2678960 .9437180 .479576$

H $2.985859-3.5393950 .966414$

H $4.394992-2.5090920 .669799$

H $3.199893-2.0605891 .903858$

H -1.552820 -1.1850190 .782608$

H $-0.0429011 .733441-1.641872$

H -2.402787 -0.711928 -1.881434

H $-2.722506 \quad 0.992987 \quad 1.238537$

H $-3.849320-1.2563171 .610677$

H $-4.564946-1.125047-0.003292$

H $-2.7497821 .778879-0.891890$

H 2.5443250 .5704861 .529329

H $4.2143100 .025806 \quad 1.328022$

H -5.0239170 .8601632 .274913$

H -5.7624310 .9453270 .663006$

H $-6.148219-0.4016791 .743897$

SCF Energy $\left(B 3 L Y P / 6-31 G^{* *} / / M M F F\right)=-810.724966608$

F2-02 c00199

MMFF Geometry

C 2.9026982 .3341450 .302259

C $2.040393 \quad 3.1268150 .948436$

C $2.7085660 .008154-0.630079$

O $1.3001430 .188605-0.896428$

C $0.600868-1.043630-0.654084$

C $1.565656-1.9252700 .127267$

C $2.898680-1.502369-0.455857$

C $-0.738874-0.8010590 .057366$

C $4.094870-1.9551300 .362109$

C $-1.7455170 .035539-0.758015$

O $-0.507934-0.1653481 .314690$

O $-1.1886671 .321421-1.056438$

C $-3.0838630 .304369-0.041066$

C $-3.802354-0.9642720 .430707$

O $-3.9458200 .984024-0.961943$

C 3.0752710 .8678280 .588872

C $\quad-5.150790-0.6655581 .076147$

H $3.5277882 .767036-0.474554$

H $1.9661884 .178896 \quad 0.695931$

H $1.3963762 .744891 \quad 1.732708$

H $3.2653910 .355629-1.508690$

H $0.409993-1.496220-1.635530$

H $1.355355-2.990427-0.009504$

H $1.531588-1.7109451 .201669$

H $2.984072-1.947688-1.457285$

H -1.177154 -1.781685 0.270735

H $4.025540-1.6309341 .405401$

H $4.162183-3.0483580 .364182$

H $5.024720-1.561182-0.060445$

H -1.941642 -0.452675 -1.720611

H $-0.1151890 .705470 \quad 1.130932$

H $-0.394416 \quad 1.181019-1.599053$

H -2.9319690 .9845320 .805727$

H $-3.189277-1.5021611 .160899$

H -3.971446 -1.633672 -0.421385

H $-3.4820901 .788516-1.251247$

H 2.4612610 .5826121 .451655

$\begin{array}{llll}\text { H } & 4.123712 & 0.709388 & 0.864172\end{array}$

H -5.0347490 .0115831 .928428$

H $-5.844228-0.2084780 .363606$

H $-5.607408-1.5921361 .438830$ 
F2-02_c00200

MMFF Geometry

C -3.511707 2.153158 -0.621840

C -3.723202 2.6953080 .583090

C $-2.845833-0.206946 \quad 0.243077$

O $\begin{array}{llll}-1.678742 & 0.282242 & 0.939207\end{array}$

C $-0.589615-0.6504020 .774616$

C $-1.013882-1.598715-0.334248$

C $-2.513085-1.657517-0.126286$

C 0.6970590 .1345340 .491828

C $-3.267911-2.262482-1.296279$

C $1.908262-0.7522050 .154690$

O $1.008207 \quad 0.9074151 .655642$

O $2.048970-1.7541001 .170092$

C $3.252553-0.004970 \quad 0.043834$

C $3.2317821 .157024-0.956799$

O $4.250708-0.949241-0.361250$

C -3.122285 $0.732397-0.944100$

C $4.588393 \quad 1.839439-1.084023$

H $-3.6358472 .802257-1.487015$

H -4.0026343 .7404460 .667032$

H $-3.6302062 .130287 \quad 1.502324$

H $-3.694110-0.1915250 .937069$

H $-0.509629-1.1860071 .728858$

H $-0.532265-2.577987-0.254592$

H $-0.777117-1.182643-1.320948$

H $-2.704833-2.2803210 .759167$

H $0.5146050 .847714-0.320010$

H $-3.036806-1.757792-2.239808$

H -2.999212 -3.317401-1.417228

H $-4.348621-2.207533-1.130703$

H $1.720868-1.294441-0.779162$

H $\quad 0.2322371 .4600961 .852005$

H $2.183235-1.2954682 .017423$

H $3.5623790 .358028 \quad 1.030584$

H $2.497408 \quad 1.909740-0.652334$

H $2.9389530 .788984-1.947420$

H $4.010571-1.274969-1.245553$

H -2.228102 $0.787489-1.577543$

H $-3.9325590 .320783-1.556315$

H $5.3471691 .153404-1.473030$

H $4.5196942 .687236-1.773146$

H $4.9284912 .217348-0.114545$

SCF Energy (B3LYP/6-31G**//MMFF) $=-810.712726980$

F2-02_c00201

MMFF Geometry

C $2.4587582 .556989-0.220098$

C $1.766084 \quad 3.297508 \quad 0.652567$

C $2.2419310 .157276-0.935674$

$\begin{array}{lllll}\text { O } & 0.817588 & 0.173671 & -0.694556\end{array}$

C $0.409111-1.091942-0.144404$

C $1.687873-1.8096680 .267635$

C $2.662556-1.307817-0.779308$

C $-0.606191-0.8845360 .985302$

C $4.117883-1.571085-0.436312$

C $-1.822929-0.0194160 .595871$

O $0.056092-0.2268532 .074493$

O $-2.700038-0.0144861 .729578$

C -2.595254 -0.504928 -0.646341

C $-3.8615330 .300721-0.970256$

O $-3.010902-1.860653-0.464678$

C 2.9000981 .1431650 .041316

C $-3.597011 \quad 1.769797-1.254910$

H $2.7307782 .995290-1.176783$

H 1.4796704 .3138140 .405123

H 1.4711112 .9063141 .619977

H $2.4136970 .487977-1.967015$

H $-0.055908-1.652202-0.964001$

H $1.570742-2.897750 \quad 0.259235$

H $2.020225-1.5166751 .269651$

H $2.437109-1.824199-1.723344$

H $-0.932971-1.8578791 .370178$

H $4.779508-1.123033-1.184557$

H $4.387422-1.1708020 .546170$

H $4.314500-2.648375-0.414654$
H -1.4876531 .0150330 .456661$

H $-0.600229-0.1079312 .782480$

H $-3.3387890 .707792 \quad 1.606715$

H $-1.943529-0.477421-1.526838$

H -4.597836 $0.202878-0.163388$

H $-4.342921-0.148068-1.849027$

H $-2.215921-2.418976-0.455002$

H 2.6645010 .8659351 .075806

H $3.9900451 .114813-0.066154$

H $-2.848127 \quad 1.888462-2.044089$

H $-4.5184692 .259606-1.586284$

H -3.247123 $2.295602-0.361878$

SCF Energy (B3LYP/6-31G**//MMFF) $=-810.710675963$

F2-02_c00202

MMFF Geometry

C $2.5843092 .522126-0.161904$

$\begin{array}{llll}\text { C } & 1.657686 & 3.279939 & 0.435198\end{array}$

C $2.6611920 .080652-0.753911$

$\begin{array}{llll}\text { O } & 1.228219 & 0.038385 & -0.932679\end{array}$

C $0.721531-1.224154-0.467967$

C $1.843394-1.8532470 .346693$

C $3.067856-1.351761-0.391983$

C $-0.594429-1.047194 \quad 0.303795$

C $4.362197-1.523878 \quad 0.382412$

C $-1.730923-0.440230-0.547761$

O $-0.364395-0.2395411 .457161$

O $-1.424790 \quad 0.905965-0.909620$

C $-3.102278-0.4596030 .160119$

C $-4.2195460 .066642-0.752148$

$\begin{array}{llll}\text { O } & -3.080418 & 0.342882 & 1.338563\end{array}$

C $2.976233 \quad 1.147361 \quad 0.305464$

C $-5.597622-0.079328-0.118456$

H $3.0975082 .916788-1.035081$

H $1.422756 \quad 4.2660150 .049709$

H 1.1188422 .9337451 .310128

H $3.1124470 .366330-1.711778$

H $\quad 0.536005-1.835307-1.360602$

H $1.773899-2.9450520 .371681$

H $1.846059-1.4927811 .381716$

H $3.153442-1.922682-1.327496$

H $-0.913578-2.0328800 .662792$

H $4.311763-1.063521 \quad 1.374327$

H $4.580220-2.5873450 .527209$

H $5.201393-1.079311-0.161885$

H $-1.818693-1.017137-1.476794$

H $-0.1062030 .644623 \quad 1.144531$

H $-0.5922010 .895970-1.411293$

H -3.339206 -1.4871630 .461342$

H -4.209361 -0.475752 -1.704472

H $-4.060352 \quad 1.129159-0.971798$

H -2.415852 -0.0334011 .939851$

H 2.4604210 .9124931 .244360

H $4.0510581 .167414 \quad 0.516857$

H $-5.811817-1.1271420 .115154$

H -5.6762560 .5028930 .804878$

H $-6.3688170 .278564-0.808050$

SCF Energy (B3LYP/6-31G**//MMFF) $=-810.725103239$

F2-02 c00203

MMFF Geometry

C $3.1066491 .790808-1.073951$

C $2.7074582 .975664-0.598341$

C $2.176123-0.530883-0.823827$

O $0.830237-0.020472-0.700427$

$\begin{array}{llll}\text { C } & 0.102410 & -0.811976 & 0.255723\end{array}$

C $1.143081-1.6391021 .000184$

C $2.169775-1.875763-0.089560$

C $-0.7646250 .083315 \quad 1.146465$

C $3.504356-2.3823800 .427320$

$\begin{array}{llll}\text { C } & -1.720252 & 1.035248 & 0.396343\end{array}$

$\begin{array}{lllll}0 & 0.108899 & 0.899823 & 1.941680\end{array}$

$\begin{array}{llll}\text { O } & -2.470349 & 1.741404 & 1.394873\end{array}$

C $-2.7184890 .392741-0.586355$

C -3.542202 $-0.746192 \quad 0.030534$

O $-2.045260-0.087025-1.745331$

C $3.1378110 .526061-0.260648$

C $-4.625125-1.249786-0.916491$ 
H $3.4375121 .727421-2.107325$

H $2.7114053 .853019-1.235814$

H 2.3694383 .0911900 .425427

H $2.381280-0.687741-1.889687$

H $-0.532668-1.495992-0.318173$

H $\quad 0.722069-2.5656301 .402685$

H $1.591280-1.0855191 .832652$

H $1.762743-2.631179-0.776678$

H -1.324997 -0.537835 1.855005

H $3.922614-1.726408 \quad 1.197327$

H $3.386901-3.3755640 .873698$

H $4.230262-2.463246-0.388088$

H $-1.1201511 .787999-0.130470$

H -0.4521511 .4521772 .512883$

H -3.0083542 .4107400 .938477$

H $-3.4186211 .167780-0.923453$

H $-4.015197-0.4099070 .959914$

H -2.894753 -1.5957660 .274650$

H -1.539157 $0.653596-2.120440$

H 2.8860200 .7492840 .783050

H $4.1671110 .151090-0.277326$

H -5.313328 -0.443345 -1.188848

H -4.192928 -1.659516 -1.834662

H $-5.206359-2.043014-0.435527$

SCF Energy (B3LYP/6-31G $\left.{ }^{* *} / / \mathrm{MMFF}\right)=-810.714288886$

F2-02 c00204

MMFF Geometry

C -3.781967-1.840371 0.543619

C $-5.035719-2.2615330 .744044$

C $-2.8490780 .279267-0.442353$

O $-1.690913-0.412141-0.960370$

C $-0.5282950 .435424-0.839903$

C -0.9336771 .5709950 .085264$

C $-2.4004511 .732438-0.256538$

C $0.654562-0.409803-0.349790$

C $-3.1723582 .569727 \quad 0.747321$

C $1.9199000 .416438-0.044188$

O $0.943294-1.376117-1.363862$

O $2.285917 \quad 1.144450-1.216396$

C $3.110686-0.4340020 .447846$

C 4.3133590 .4255450 .863569

O $3.531192-1.349463-0.560837$

C $-3.306812-0.4428420 .834582$

C $5.417246-0.4055541 .506201$

H $-3.051756-2.5477030 .158856$

H -5.313563 -3.2855980 .519431$

H $-5.802339-1.5996931 .131302$

H -3.640885 $0.226556-1.199329$

H $-0.3310740 .816448-1.849850$

H $-0.3517062 .481029-0.089155$

H -0.8123711 .2844451 .136998$

H $-2.4647872 .230697-1.234321$

H $0.362038-0.9733070 .544683$

H -4.2383862 .5872280 .499478$

H -3.0613202 .1923941 .768916$

H -2.808046 3.6025370 .740624

H $1.676822 \quad 1.1503380 .733383$

H $0.127483-1.880690-1.525117$

H $2.9408681 .814301-0.958065$

H 2.783568 -1.029236 1.308406

H 3.9928321 .1971171 .572749

H $4.7411920 .930583-0.010245$

H $3.733818-0.833183-1.359925$

H -2.478079 -0.518112 1.548619

H -4.1106320 .1217801 .320002$

H $\quad 6.246215 \quad 0.2410101 .811376$

H $5.048115-0.9256202 .395697$

H $5.811704-1.1515400 .809179$

SCF Energy (B3LYP/6-31G**//MMFF) $=-810.709190335$

F2-02_c00205

MMFF Geometry

C -2.874945 $2.435708-0.578138$

C $-3.7374263 .406141-0.255916$

C -2.2763860 .2623280 .514872$

$\begin{array}{lllll}\text { O } & -0.936686 & 0.189827 & 0.000732\end{array}$

C $-0.236332-0.7844290 .792035$
C $-1.300452-1.7329931 .351280$

C $-2.629289-1.1910160 .836043$

C $0.860890-1.459066-0.039816$

C $-3.127335-2.004484-0.358845$

C $1.850583-0.484139-0.710598$

O $0.255810-2.239515-1.077799$

O $2.877139-1.267216-1.333871$

C 2.5188700 .5144340 .250729

C $3.5824061 .376942-0.444280$

O $3.121455-0.1906051 .335360$

C $-3.1888790 .968019-0.487888$

C 4.1414072 .4521220 .479390

H -1.888098 $2.715075-0.938216$

$\mathrm{H}-3.4512104 .448206-0.348313$

H $-4.7355853 .182356 \quad 0.103675$

H $-2.2370920 .837377 \quad 1.450947$

H $0.223698-0.249478 \quad 1.631518$

H -1.278684 -1.679873 2.447277

H -1.127457 -2.783292 1.095289

H -3.398909-1.236996 1.615290

H $1.408612-2.1716760 .587753$

H -3.231089-3.061043 -0.089400

H $-4.112187-1.654114-0.684594$

H -2.448449 -1.938866 -1.213567

H $1.3521670 .066830-1.517435$

H $-0.230308-1.628518-1.657732$

H $2.439164-1.888489-1.940784$

H $1.756523 \quad 1.1747270 .677701$

H $3.1496191 .858180-1.328893$

H $4.4201310 .754034-0.779388$

H $3.780725-0.7991640 .959641$

H -3.056391 $0.545812-1.491133$

H $-4.2365270 .823504-0.198299$

H $4.8761203 .062990-0.054843$

H 3.3458373 .1138690 .836194

H 4.6389882 .0112331 .348724

SCF Energy $(B 3 L Y P / 6-31 G * * / / M M F F)=-810.722445097$

F2-02 c00206

MMFF Geometry

C 3.0478842 .0580080 .328945

C 4.1470602 .8185540 .275804

C $2.499387-0.187452-0.668232$

O $1.1087410 .174243-0.818985$

C $0.280264-0.972225-0.563902$

C $1.181779-1.9947550 .114564$

C $2.510461-1.715948-0.558042$

C $-0.965006-0.6009000 .255635$

C $3.696670-2.3487750 .147203$

C $-1.9243180 .364834-0.467547$

O $-\begin{array}{llll}0.566669 & -0.029016 & 1.501899\end{array}$

O $-1.2217211 .572469-0.783374$

C $-3.1655560 .757994 \quad 0.358040$

C $-4.003897-0.4037160 .907304$

O $-4.0073041 .591666-0.447616$

C 3.0641240 .5690590 .544087

C $-4.518994-1.371725-0.150520$

H 2.0783442 .5381570 .223975

H 4.0668593 .8895010 .124155

H 5.1384662 .3920630 .380330

H $3.0294970 .125267-1.576065$

H -0.033974 -1.359433 -1.541568

H $0.830504-3.020135-0.036085$

H $1.253879-1.8182411 .194054$

H $2.464157-2.125628-1.577207$

H $-1.497527-1.5290550 .488428$

H $3.625969-3.4409420 .104136$

H $4.635547-2.053365-0.331975$

H $3.744536-2.0669311 .203915$

H -2.228397 -0.072423 -1.425782

H -0.0851950 .7925001 .302329$

H - $1.8462682 .161146-1.240924$

H -2.854322 1.3884121 .199478

H $-4.876418 \quad 0.011813 \quad 1.427024$

H -3.428772 -0.9608211 .654957$

H $-4.202914 \quad 1.116967-1.272835$

H 2.4660250 .3582621 .438478

H 4.0891400 .2404250 .748783 
H $-5.153770-0.864351-0.882959$

H -3.698723 -1.861139-0.683258

H -5.120169 -2.154662 0.323193

SCF Energy $\left(B 3 L Y P / 6-31 G^{* *} / / M M F F\right)=-810.714193073$

\section{F2-02 c00207}

MMFF Geometry

C $4.157941-0.860377-1.361674$

C $5.287526-0.144365-1.394438$

C $1.998312-0.953187-0.026364$

O $0.600758-0.666309-0.273263$

C $0.0781290 .126858 \quad 0.807161$

C 1.2884140 .6610341 .559318

C $2.262663-0.4916131 .411968$

C $-0.870866 \quad 1.2043180 .271396$

C $3.685517-0.1298891 .793181$

C $-2.0227040 .668761-0.603149$

O $-0.1264792 .134546-0.526677$

O $-2.8807401 .773831-0.916345$

C $-2.871512-0.4317440 .057395$

C $-4.076023-0.840990-0.802687$

O $\quad-3.3343610 .016291 \quad 1.330947$

C $2.793981-0.271825-1.150915$

C $-4.831870-2.021406-0.205469$

H $4.220050-1.934051-1.519584$

H $6.242143-0.631069-1.562318$

H $5.2814890 .931062-1.255358$

H $2.117602-2.040989-0.098534$

H $-0.469969-0.5575911 .465515$

H 1.0551560 .8963802 .602350

H 1.6952831 .5651841 .092684

H $1.930873-1.2959052 .084390$

H $-1.276241 \quad 1.7853581 .107757$

H $4.361515-0.9735941 .624472$

H $4.053976 \quad 0.734651 \quad 1.233140$

H $3.737737 \quad 0.1289512 .856485$

H -1.632673 $0.301931-1.560375$

H $0.2959521 .632666-1.245015$

H $-2.3310302 .456720-1.337573$

H -2.249444 -1.316005 0.232258

H $-3.738205-1.108304-1.810476$

H $-4.779555-0.005386-0.897035$

H $-3.8650690 .818251 \quad 1.184815$

H $2.241129-0.389273-2.092629$

H $2.8433750 .810574-0.980788$

H $-5.241027-1.7754410 .779317$

H $-5.666328-2.302249-0.855989$

$\mathrm{H}-4.177217-2.892194-0.099142$

SCF Energy (B3LYP/6-31G**//MMFF) $=-810.722197805$

F2-02 c00208

MMFF Geometry

C $-3.5445382 .154491-0.581320$

C $-3.7588052 .673797 \quad 0.633144$

C $-2.859292-0.215326 \quad 0.241482$

O $-1.695272 \quad 0.2696150 .945883$

C $-0.601237-0.656156 \quad 0.771802$

C $-1.017387-1.585035-0.356958$

C $-2.516616-1.656646-0.153986$

C 0.6867170 .1360800 .512961

C $-3.264732-2.244822-1.337036$

C $1.896117-0.7494310 .158355$

O $0.9661790 .875163 \quad 1.706511$

O $2.045758-1.7519301 .172873$

C $3.244190-0.0137220 .023183$

C $3.2088341 .132211-0.996004$

O $4.206247-0.986433-0.401140$

C $-3.1432840 .742986-0.928756$

C $4.5498591 .841361-1.141018$

H $-3.6759102 .817423-1.434732$

H $-4.047193 \quad 3.7148230 .735806$

H -3.658853 2.0935201 .542206

H $-3.707338-0.2181200 .936078$

H $-0.526945-1.2101231 .716084$

H $-0.530633-2.563058-0.295174$

H $-0.780946-1.147841-1.334561$

H -2.706808 -2.2971330 .719228$

H $\quad 0.4955170 .868834-0.279195$
H $-4.346027-2.200540-1.172754$

H -3.035073 -1.720699 -2.270267

H -2.988173 -3.295304-1.477335

H $1.696714-1.291810-0.773041$

H 1.6509971 .5318381 .496740

H $2.184071-1.2934012 .019493$

H 3.5731580 .3495861 .004050

H $2.4640521 .879221-0.703725$

H $2.9196970 .743426-1.979761$

H $5.090762-0.609682-0.258046$

H $-2.2496700 .816608-1.561118$

H -3.950249 $0.335732-1.548391$

H $4.4504062 .697134-1.816716$

H $4.9036762 .212152-0.173930$

H $5.3125041 .177367-1.559058$

SCF Energy $(B 3 L Y P / 6-31 G * * / / M M F F)=-810.704203307$

F2-02_c00209

MMFF Geometry

C 3.4683682 .2251170 .003861

C 2.7257553 .2944690 .311629

C $2.876455-0.157726-0.535943$

O $1.6083120 .235777-1.104248$

C $0.598057-0.733559-0.750417$

C $1.215551-1.6092400 .328240$

C $2.676966-1.602993-0.070315$

C $-0.6773840 .011058-0.330596$

C $3.608670-2.1091021 .016150$

C $-1.790563-0.9223260 .184412$

O $-1.1456480 .728120-1.474908$

O $-2.126773-1.863995-0.832998$

C $-3.069295-0.227300 \quad 0.698693$

C $-3.8829800 .524393-0.361731$

$\begin{array}{lllll}\text { O } & -2.734106 & 0.678676 & 1.747932\end{array}$

C 3.2363600 .8498460 .566725

C -5.2501830 .9468880 .166566$

H $4.2976162 .347354-0.688063$

H $2.9471274 .261614-0.126281$

H 1.8887703 .2258370 .997841

H $3.629967-0.120971-1.331945$

H $0.415050-1.318963-1.660294$

H $\quad 0.783100-2.6142580 .348682$

H $1.084494-1.1624691 .321201$

H $2.789240-2.256927-0.946925$

H -0.4317020 .7587980 .432698$

H $3.416746-3.1682921 .218549$

H $4.654256-2.0092600 .707687$

H $3.473692-1.5680531 .958177$

H $-1.379506-1.4931381 .026074$

H $-0.4235951 .309733-1.769039$

H $-2.795396-2.467442-0.466452$

H -3.719086-1.000234 1.129401

H $-4.028791-0.100255-1.249311$

H -3.360699 $1.437161-0.667218$

H -2.2671140 .1733552 .434908$

H 2.4404500 .8866971 .320475

H 4.1578090 .5450891 .074835

H $-5.8380510 .075840 \quad 0.472891$

H -5.1549151 .6184001 .025592$

H $-5.8084051 .474608-0.613350$

SCF Energy $\left(B 3 L Y P / 6-31 G^{* *} / / M M F F\right)=-810.708982615$

F2-02_c00210

MMFF Geometry

C $-4.171946-1.203206-0.172017$

C $-4.099475-2.3240880 .554675$

C $-2.4460740 .481967-0.879652$

O $-1.405289-0.519869-0.895407$

C $-0.1654350 .062180-0.434338$

C -0.5482921 .3806820 .217402$

C $-1.7248271 .810560-0.635303$

C $0.541259-0.948290 \quad 0.479021$

C $-2.5426072 .934924-0.025409$

C $1.855558-0.4716691 .124817$

O $0.808960-2.138321-0.266963$

O $1.5489380 .462816 \quad 2.166069$

C $2.887750 \quad 0.202997 \quad 0.198317$

C $3.339188-0.657195-0.986549$ 
$\begin{array}{lllll}\text { O } & 4.049207 & 0.499677 & 0.986219\end{array}$

C -3.474524 0.0796100 .188122

C $4.4050470 .029913-1.833215$

H $-4.775688-1.205641-1.075930$

H $-4.630245-3.2166650 .242058$

H -3.512875 -2.372922 1.465410

H $-2.9218570 .490956-1.867732$

H $0.4267940 .241434-1.339085$

H $\quad 0.268135 \quad 2.1084150 .211854$

H $-0.8666001 .229971 \quad 1.255803$

H -1.331043 2.161608 -1.599865

H $-0.157442-1.2429481 .272708$

H -3.421230 $3.153165-0.640845$

H $-2.881748 \quad 2.6937190 .987099$

H $-1.942468 \quad 3.8486380 .041109$

H $2.345536-1.3245821 .612510$

H $-0.031107-2.428718-0.662061$

H 0.9732130 .0103102 .806018

H $2.5073501 .165272-0.162075$

H $2.493083-0.891428-1.638792$

H $3.752068-1.604983-0.620815$

H 3.7585061 .0510701 .732818

H $-2.984726-0.0248411 .164020$

H -4.2442140 .8530930 .286316$

H $5.3203340 .207588-1.260349$

H $4.665266-0.597769-2.691637$

H $4.0426980 .990512-2.213110$

SCF Energy (B3LYP/6-31G**//MMFF) $=-810.714623912$

F2-02_c00211

MMFF Geometry

C $-2.702104 \quad 1.979787 \quad 0.044634$

C $-3.1984232 .726930-0.947915$

C $-2.868464-0.460568 \quad 0.843923$

O $-1.560045-0.373696 \quad 1.448834$

C $-0.595222-0.9946950 .572623$

C $-1.236994-0.993035-0.806130$

C $-2.690941-1.240860-0.462134$

C $0.732855-0.232612 \quad 0.665349$

C $-3.662229-0.952739-1.591237$

C $1.795542-0.691970-0.349112$

O $1.242863-0.3962791 .992972$

O $1.929715-2.113897-0.233681$

C $3.189533-0.063898-0.150463$

C $3.1723771 .469103-0.147497$

O $4.027946-0.490693-1.229928$

C $-3.489722 \quad 0.945400 \quad 0.798364$

C 4.5666372 .0670810 .001685

H $-1.6729352 .147966 \quad 0.350322$

H -2.578367 $3.467530-1.441051$

H $-4.2231242 .614912-1.284611$

H -3.488301-1.060202 1.522707

H $-0.474659-2.0227850 .937918$

H $-0.819483-1.762321-1.462921$

H $-1.113504-0.022129-1.298947$

H $-2.789093-2.308678-0.216472$

H $\quad 0.535606 \quad 0.837643 \quad 0.544217$

H -3.494202 -1.648052 -2.420952

H $-4.696721-1.076869-1.255727$

H -3.547530 $0.057407-1.991246$

H $1.444460-0.491227-1.368413$

H $1.459455-1.3371372 .110549$

H $2.436248-2.424041-1.002982$

H $3.649458-0.434950 \quad 0.773409$

H $2.552586 \quad 1.8439760 .673294$

H $2.740598 \quad 1.836885-1.086142$

H $4.202496-1.439184-1.107959$

H -4.5140640 .8792420 .412803$

H -3.5687701 .3115811 .830587$

H 4.5047993 .1592820 .042211

H 5.0440721 .7198860 .923574

H $5.2100751 .800239-0.842280$

SCF Energy (B3LYP/6-31G**//MMFF) $=-810.710587361$

F2-03 c00429

MMFF Geometry

C -2.582096 $2.616886-0.524716$

C $-1.6549843 .571948-0.658992$
C $-2.4157510 .126541-0.269593$

$\begin{array}{llll}\text { O } & -1.174970 & 0.027455 & -0.995627\end{array}$

C $-0.605722-1.276971-0.796415$

C $-1.680165-2.106119-0.100702$

C $-2.436257-1.0598280 .693048$

C $0.689824-1.1370110 .025988$

C $-3.832581-1.4994071 .100019$

C $1.720688-0.192696-0.623955$

O $1.269018-2.428166 \quad 0.209011$

O $1.991773-0.670882-1.948204$

C $3.061908-0.1017340 .126731$

C $2.960560 \quad 0.279698 \quad 1.609609$

O $3.8645360 .875437-0.546522$

C $-2.477594 \quad 1.476665 \quad 0.447848$

C 2.2683481 .6135391 .863162

H $-3.4726762 .662915-1.146085$

H $-1.7908794 .372206-1.378351$

H $-0.7499013 .572342-0.061709$

H $-3.225366 \quad 0.041777-1.006141$

H $-0.383234-1.720820-1.773509$

H $-1.287091-2.9182880 .517402$

H -2.339036 -2.552502 -0.856583

H $-1.864011-0.8207951 .598775$

H $0.441176-0.7478211 .018607$

H $-4.343564-0.7085811 .658071$

H $-4.446349-1.7461420 .227039$

H -3.785044 - 2.3870811 .739222

H $1.2910090 .808701-0.743259$

H $1.527097-2.760277-0.667942$

H $2.635569-0.061565-2.348953$

H $3.598431-1.0536560 .033247$

H $3.9744390 .350378 \quad 2.023277$

H $2.447737-0.5087242 .170738$

H $4.7308120 .897269-0.105329$

H $-3.3507861 .513003 \quad 1.109069$

H -1.5862051 .6019611 .075727$

H $2.285167 \quad 1.8453062 .933119$

H 2.7710912 .4301321 .335776

H 1.2216961 .5904511 .546326

SCF Energy (B3LYP/6-31G**//MMFF) $=-810.719476382$

F2-03 c00430

MMFF Geometry

C $4.485047-1.011486-0.546447$

C $4.691055-2.271650-0.147713$

C $2.4898240 .502900-0.452838$

O $1.518751-0.558031-0.488908$

C $0.1965940 .022815-0.504110$

C $0.375458 \quad 1.523742-0.285314$

C $1.7820851 .643697 \quad 0.269113$

C $-0.656194-0.7087200 .543516$

C $2.405546 \quad 3.0099640 .038488$

C $-2.133987-0.2754890 .602048$

O $-0.101191-0.484787 \quad 1.846660$

O $-2.809907-1.1584941 .507471$

C $-2.869587-0.309922-0.748876$

C $-4.383042-0.064524-0.654869$

O $-2.667606-1.591124-1.350110$

C $3.7502310 .018193 \quad 0.264672$

C $-4.746506 \quad 1.291946-0.074033$

H $4.877063-0.699380-1.511062$

H $5.234140-2.965877-0.779574$

H $4.323996-2.6322640 .806714$

H $2.7152120 .780001-1.491432$

H $-0.211344-0.160941-1.503532$

H $-0.376943 \quad 1.974300 \quad 0.367689$

H $\quad 0.3028772 .034819-1.253952$

H 1.7520241 .4435301 .347888

H $-0.592183-1.791766 \quad 0.380598$

H 1.8060383 .7916810 .516539

H $3.414903 \quad 3.0562960 .458919$

H $2.4744243 .244305-1.029128$

H -2.2100230 .7252001 .041321$

H $0.818001-0.8022901 .826080$

H -2.336543 -1.1201622 .356354$

H -2.431846 $0.437313-1.420455$

H $-4.863894-0.859994-0.072651$

H $-4.814969-0.144549-1.660842$ 
H $-3.090072-1.575249-2.225688$

H 4.4322150 .8574890 .440232

H $3.482529-0.397374 \quad 1.244319$

H $-4.458847 \quad 1.3678320 .978636$

H $-4.2576892 .100023-0.627205$

H $-5.8285851 .447747-0.132496$

SCF Energy (B3LYP/6-31G**//MMFF) $=-810.715738840$

F2-03_c00431

MMFF Geometry

C - $-1.7857332 .267437-0.597808$

C -2.149702 3.2877900 .186950

C $-2.549227-0.153046-0.795113$

O $-1.253893-0.620718-1.213761$

C $-0.735739-1.519033-0.215697$

C $-1.861363-1.7338950 .795796$

C $-2.638552-0.4346450 .703000$

C $0.532978-0.9051390 .407587$

C $-4.060039-0.5333581 .228432$

C $1.611191-0.564940-0.642153$

O $1.052090-1.8353651 .356625$

O $1.910714-1.744455-1.399939$

C $2.939709-0.025617-0.077483$

C $2.769488 \quad 1.214267 \quad 0.807323$

O $3.7743340 .332990-1.185248$

C $-2.7526761 .302649-1.224876$

C 4.1014151 .7551561 .313876

H $-0.7291552 .126469-0.810232$

H -1.402005 3.9527820 .605130

H $-3.190225 \quad 3.4761650 .426813$

H $-3.281107-0.769853-1.333783$

H -0.492942 -2.476177 -0.691698

H $-1.515189-1.958740 \quad 1.808715$

H -2.489015 -2.572158 0.466685

H $-2.1022950 .328753 \quad 1.278973$

H $\quad 0.256858 \quad 0.0094920 .943412$

H -4.637753 -1.2892680 .686131$

H -4.059802 -0.8076142 .288530$

H $-4.579436 \quad 0.424970 \quad 1.127425$

H $\quad 1.212750 \quad 0.161349-1.360222$

H $1.734309-1.3802931 .878049$

H $2.216762-2.424007-0.774888$

H $3.471594-0.8110990 .472053$

H $2.143790 \quad 0.983674 \quad 1.675354$

H 2.2706602 .0092230 .239709

H $3.864790-0.455942-1.746986$

H -2.616996 $1.372001-2.311444$

H $-3.7859291 .601761-1.012200$

H 3.9325692 .6124951 .973291

H 4.6427070 .9918091 .881725

H 4.7386052 .0888420 .489041

SCF Energy (B3LYP/6-31G**//MMFF) $=-810.720339412$

\section{F2-03 c00432}

MMFF Geometry

C 2.9729472 .6753260 .187950

C 2.1082693 .6954280 .150103

C 2.6579750 .1875870 .191058

$\begin{array}{lllll}\text { O } & 1.361222 & 0.232802 & 0.817161\end{array}$

C $0.769902-1.0774290 .787099$

C $1.845145-2.0188320 .250901$

C $2.675437-1.102736-0.625525$

C $-0.501879-1.041182-0.081641$

C $4.069047-1.636701-0.910139$

C -1.5264420 .0061910 .410771$

O $-1.072131-2.348048-0.071410$

O $-1.913445-0.2933751 .751971$

C $-2.7803970 .129425-0.478840$

C $-3.7271351 .234748 \quad 0.011701$

O $-3.490370-1.105745-0.527254$

C $2.8554831 .441732-0.661906$

C $-4.9026391 .446932-0.933772$

H 3.8208142 .7352850 .865356

H $2.251476 \quad 4.5608260 .787848$

H $1.2477823 .685829-0.509575$

H 3.4030610 .1454300 .996461

H $\quad 0.510844-1.3720971 .810785$

H $1.453483-2.889119-0.283311$
H $2.451143-2.3897581 .087637$

H $2.150219-0.953204-1.577980$

H $-0.231535-0.797197-1.116115$

H $4.634359-0.939762-1.536740$

H $4.635122-1.7940270 .014128$

H $4.013127-2.594788-1.437157$

H -1.0400440 .9883290 .437121$

H -1.819689-2.348127 -0.692808

H $-2.231890-1.2119101 .767878$

H $-2.4620770 .356741-1.502878$

H -3.1772882 .1777340 .111104$

H -4.1292370 .9793560 .999324$

H $-3.831382-1.2892090 .364608$

H $3.7709301 .353277-1.257833$

H $2.0174501 .548232-1.362368$

H $-5.5381632 .259739-0.567956$

H $-4.5549391 .713780-1.936790$

H $-5.5200510 .546629-1.011027$

SCF Energy (B3LYP/6-31G**//MMFF) $=-810.723861683$

F2-03 c00433

MMFF Geometry

C -1.528848 $3.085226-0.054699$

C -0.3977263 .7260910 .260414$

C $-2.1196610 .667098-0.350740$

O $-0.8023090 .259666-0.765341$

C $-0.742348-1.173765-0.848625$

C $-2.173352-1.661844-0.624311$

C -2.754704 -0.577785 0.260722

C $0.266194-1.7194940 .180645$

C $-4.273605-0.5568900 .273753$

C $1.747985-1.389057-0.112344$

O $0.154708-3.1489230 .215764$

O $2.532747-1.9626540 .944104$

C $2.1010240 .104983-0.201989$

C $3.6100620 .347819-0.356081$

$\begin{array}{lllll}\text { O } & 1.639156 & 0.778374 & 0.967924\end{array}$

C $-2.009481 \quad 1.8362390 .629211$

C $3.9316031 .820483-0.580008$

H $-2.1523343 .490170-0.847702$

H $-0.1103244 .626578-0.271042$

H $\quad 0.2576323 .3686121 .046675$

H -2.662338 $0.971428-1.255565$

H - $0.416632-1.455245-1.856976$

H -2.251311 -2.661148 -0.187963

H $-2.701265-1.683123-1.586725$

H $-2.389688-0.7238261 .285816$

H $0.014720-1.3629321 .186268$

H $-4.667570-1.5043610 .655715$

H $-4.6482240 .246867 \quad 0.915117$

H $-4.681923-0.404089-0.730931$

H $2.062888-1.890490-1.035821$

H $0.350893-3.483348-0.676322$

H $2.315239-2.9097220 .985376$

H $1.5934540 .551113-1.062707$

H $3.994898-0.233020-1.202097$

H $4.1444190 .021705 \quad 0.544033$

H $2.0686010 .362741 \quad 1.735369$

H -2.9883072 .0506221 .072493$

H -1.3289071 .5705951 .447808$

H $5.0100361 .955203-0.710323$

H $3.4320902 .198218-1.477757$

H 3.6166782 .4311240 .271972

SCF Energy (B3LYP/6-31G**//MMFF) $=-810.719273867$

F2-03 c00434

MMFF Geometry

C $2.173582 \quad 2.837938 \quad 0.437257$

C 1.0846573 .5941390 .258805

C $2.590788 \quad 0.360134 \quad 0.373177$

$\begin{array}{lllll}\text { O } & 1.247690 & 0.019199 & 0.769658\end{array}$

C $0.799212-1.1203270 .021420$

C $1.827704-1.352293-1.079437$

C $3.105598-0.815833-0.462848$

C $-0.620287-0.888516-0.512197$

C $3.790833-1.8672270 .412673$

C $-1.650781-0.5392580 .580868$

O $-1.039532-2.053098-1.222144$ 
O $-1.642398-1.5654271 .582355$

C $-3.100792-0.431751 \quad 0.072201$

C $-3.3185650 .525039-1.107682$

$\begin{array}{llll}\text { O } & -3.912650 & 0.001873 & 1.170408\end{array}$

C $2.5367911 .667331-0.433185$

C $-2.9034031 .963661-0.826501$

H $2.8490093 .081548 \quad 1.253225$

H 0.8808054 .4287350 .920647

H $0.3822063 .397112-0.543077$

H $3.191866 \quad 0.505247 \quad 1.278694$

H $0.812358-1.9856210 .695262$

H $1.580256-0.763022-1.970907$

H $1.885816-2.401527-1.385478$

H $3.820758-0.499021-1.229853$

H $-0.589123-0.062124-1.230837$

H $3.147758-2.2117921 .228821$

H $4.075662-2.739320-0.185115$

H $4.702211-1.4549740 .858900$

H $-1.370804 \quad 0.391127 \quad 1.089110$

H -1.113810 -2.778189 -0.578039

H $-0.827503-1.4614522 .102266$

H -3.466400 -1.429227 -0.200861

H $-4.3866960 .533536-1.358860$

H -2.791345 $0.160444-1.995822$

H -4.8358120 .0144060 .865160$

H $3.518823 \quad 1.870127-0.876560$

H $1.8169621 .578164-1.255903$

H $-3.1333612 .594770-1.691028$

H -3.4371272 .3714750 .037269$

H $-1.8294072 .040975-0.636559$

SCF Energy $\left(\mathrm{B} 3 \mathrm{LYP} / 6-31 \mathrm{G}^{* *} / / \mathrm{MMFF}\right)=-810.711775462$

F2-03_c00435

MMFF Geometry

C -1.9466112 .2968840 .354885$

C -2.4991192 .8910821 .418419$

C $-2.4578250 .154108-0.920751$

O $-1.083121-0.061189-1.288353$

C $-0.623618-1.284790-0.684703$

C -1.847234 -1.926765 -0.030593

C $-2.711002-0.7246440 .300762$

C $0.506252-0.9683820 .314430$

C $-4.173967-1.0655150 .523665$

C $1.679844-0.202959-0.340621$

O $0.951034-2.2046330 .868288$

O $2.243650-0.998609-1.383127$

C $2.788336 \quad 0.2219750 .644256$

C $3.9512190 .980278-0.013226$

O $3.333928-0.9349651 .279756$

C $-2.7342031 .650485-0.749912$

C $3.5417642 .289928-0.666644$

H -0.8631122 .2864420 .269603$

H -1.8744603 .3451702 .179971$

H -3.574599 2.9337551 .549674

H -3.056946 -0.205261 -1.768331

H $-0.246465-1.947161-1.472672$

H -1.616441 -2.5457540 .841178$

H $-2.356440-2.565428-0.763767$

H -2.314598 -0.2571331 .209702$

H $0.110285-0.3539691 .132022$

H $-4.753718-0.1641160 .746684$

H -4.615252 -1.540855 -0.358823

H $-4.283918-1.7551541 .366811$

H $1.277747 \quad 0.699071-0.813111$

H $1.610617-2.0010441 .552460$

H $2.599009-1.805283-0.971622$

H $2.3438020 .844613 \quad 1.430044$

H $4.454494 \quad 0.340288-0.748139$

H 4.7098041 .1920970 .751382

H $3.981043-0.6317541 .939201$

H $-2.4668102 .167200-1.680274$

H -3.808924 $1.804708-0.596611$

H 3.0028612 .9299190 .038995

H $4.4295142 .833134-1.006370$

H $2.9055682 .121567-1.540319$

SCF Energy (B3LYP/6-31G**//MMFF) $=-810.724449835$

F2-03_c00436
MMFF Geometry

C $4.719112-0.3823690 .229765$

C $5.169719-0.5347841 .480031$

C $2.5097200 .309343-0.787311$

O $1.330832-0.284308-1.371933$

C $0.1671270 .133411-0.633174$

C $0.6854140 .700787 \quad 0.680116$

C 2.0248451 .2933520 .283514

C $-0.804896-1.044451-0.506444$

C $1.8708052 .706249-0.284039$

C $-2.104036-0.7234620 .255860$

O $\quad-0.156775-2.1144840 .195728$

O $-2.947455-1.8805060 .188552$

C $-2.8889320 .481442-0.292751$

C $-4.2700870 .684308 \quad 0.347993$

O $-3.0769050 .305974-1.699136$

C $3.368407-0.838358-0.235821$

C $-4.2167410 .932810 \quad 1.846289$

H $5.3676590 .088686-0.504296$

H $6.160278-0.1880351 .753342$

H $4.563792-1.0027232 .248163$

H $3.0574080 .818237-1.589556$

H $-0.2968910 .928194-1.227604$

H $0.850431-0.0953001 .415568$

H $-0.003831 \quad 1.4236101 .127041$

H $2.700561 \quad 1.3542301 .142625$

H -1.037437 -1.445658 -1.500390

H 1.4377803 .3769930 .465373

H $2.8483093 .109523-0.569591$

H $1.2295012 .731181-1.170873$

$\mathrm{H}-1.878115-0.5690421 .317251$

H $0.655371-2.329849-0.294157$

H -2.438863 -2.630321 0.542192

H $-2.2990151 .394630-0.156211$

H $-4.919884-0.1733130 .135345$

H $-4.758718 \quad 1.540694-0.134488$

H $-3.5238381 .100323-2.037704$

H $2.829120-1.3637230 .562307$

H $3.511180-1.586799-1.026148$

H $\quad-5.215809 \quad 1.175027 \quad 2.222537$

H -3.8657440 .0492472 .387339$

H -3.553563 1.7711162 .082055

SCF Energy (B3LYP/6-31G**//MMFF) $=-810.716700328$

F2-03_c00437

MMFF Geometry

C $-3.0430192 .374746-0.076659$

C $-4.2132243 .020033-0.016092$

C $-2.450089-0.063375-0.109611$

O $-1.2356050 .115517-0.863505$

C $-0.517099-1.128355-0.912116$

C -1.417228 -2.171452 -0.254679

C $-2.240501-1.3368280 .705877$

C $0.839187-0.960788-0.200879$

C $-3.529234-2.0132701 .141963$

C $1.6762210 .218501-0.742648$

O $1.559151-2.184492-0.345158$

O $1.8627730 .058956-2.154467$

C $3.0813220 .361736-0.123827$

C $3.0771020 .469673 \quad 1.406164$

O $3.6846301 .543116-0.666963$

C $-2.7033891 .171640 \quad 0.757364$

C $4.4760920 .662347 \quad 1.979120$

H $-2.2813372 .742147-0.759613$

H $-4.3951533 .887157-0.641502$

H $-5.0051572 .698500 \quad 0.651277$

H -3.263297 -0.198497 -0.834896

H - $0.357885-1.407151-1.960142$

H $-0.874413-2.9850190 .235300$

H -2.067705 -2.619839-1.016764

H - $-1.633985-1.1189411 .594600$

H $0.655895-0.7995440 .867279$

H -3.313421 -2.953806 1.659286

H -4.094555 -1.373916 1.827055

H -4.172805 -2.240876 0.285532

H $1.1316101 .160429-0.602719$

H $2.325503-2.1534590 .251127$

H $1.0102390 .236404-2.586304$ 
H $3.717106-0.472909-0.440627$

H $2.646475-0.4315461 .853708$

H 2.4595551 .3210891 .716419

H $3.1492292 .305603-0.388538$

H $-3.5090830 .968782 \quad 1.472152$

H $-1.803657 \quad 1.4123001 .337185$

H 4.4337520 .6923463 .072690

H $5.134690-0.1619981 .687826$

H 4.9239561 .6003121 .636555

SCF Energy (B3LYP/6-31G**//MMFF $)=-810.709209902$

F2-03 c00438

MMFF Geometry

C -3.822899-1.683275 0.001026

C $-3.475284-2.9749070 .018053$

C $-2.488796 \quad 0.432900 \quad 0.151074$

O $-1.426379-0.1476530 .928859$

C $-0.5079470 .899254 \quad 1.290266$

C $-0.8875242 .131008 \quad 0.472586$

C $-1.8198871 .583982-0.592434$

C $0.9500440 .437874 \quad 1.117318$

C $-2.7866652 .621741-1.137106$

C $1.370462-0.005226-0.298656$

O $1.191367-0.6295202 .038447$

O $\quad 0.502972-1.041423-0.769921$

C $2.814986-0.537068-0.408022$

C $3.875124 \quad 0.4408330 .109730$

O $3.085132-0.791527-1.791614$

C $-3.089375-0.625647-0.774327$

C $5.293419-0.089023-0.067717$

H $-4.692749-1.3695680 .572444$

H -4.049703 -3.690759 0.595754

H -2.617969 -3.339083 -0.537009

H $-3.245977 \quad 0.8092270 .852329$

H $-0.676970 \quad 1.1029272 .355271$

H -0.0280592 .6651990 .055847$

H -1.418135 2.8405891 .120408

H -1.214025 $1.192458-1.419434$

H $1.591621 \quad 1.2676831 .433067$

H -3.429884 $3.023966-0.347203$

H $-2.2401113 .458329-1.585040$

H $-3.4312132 .190060-1.908923$

H $1.2474830 .830652-0.996482$

H $0.527267-1.319687 \quad 1.870513$

H $\quad 0.594776-1.803126-0.172963$

H $2.908594-1.4983600 .111504$

H 3.7199640 .6452321 .173927

H $3.7954811 .393020-0.428348$

H $2.403617-1.407544-2.111006$

H $-3.799954-0.162353-1.468022$

H $-2.297455-1.085870-1.377857$

H $5.540834-0.227219-1.124788$

H $\quad \begin{array}{lll}0.014460 & 0.618436 & 0.354147\end{array}$

H $5.419420-1.0478980 .444955$

SCF Energy (B3LYP/6-31G**/MMFF) $=-810.714617358$

F2-03 c00439

MMFF Geometry

C $4.090431-1.579190-0.566173$

C $4.159937-2.7705190 .038266$

C $2.3593480 .233512-0.568304$

O $1.249253-0.646349-0.316526$

C $0.0302480 .129892-0.325388$

C $0.4484601 .597406-0.396543$

C $1.9138221 .578072-0.005361$

C $-0.795112-0.2724660 .905573$

C $26946782767449-0.538486$

C $-2.183327 \quad 0.384842 \quad 1.037242$

$\begin{array}{llll}\text { O } & -0.060422 & 0.072725 & 2.090128\end{array}$

O $-2.832502-0.1841922 .183538$

C $-3.148908 \quad 0.227957-0.150979$

C -3.331471 -1.224989-0.611242

O $-2.7189471 .022047-1.251650$

C $3.614962-0.3216140 .105049$

C $-4.408607-1.356943-1.681547$

H $4.403674-1.502575-1.604238$

H $4.517150-3.640578-0.501675$

H $3.862975-2.9000881 .073132$
H $2.4947280 .292361-1.656638$

H $-0.502799-0.150454-1.238561$

H -0.1479522 .2638380 .232319$

H $0.3342121 .949413-1.429976$

H $1.984041 \quad 1.5678021 .089842$

H $-0.898835-1.3639180 .939668$

H $3.7428452 .719308-0.227807$

H $2.6692642 .805973-1.632713$

H $2.2741293 .704500-0.158881$

H $-2.058515 \quad 1.451876 \quad 1.257226$

H $0.795432-0.3871932 .041862$

H -2.235325 -0.0663542 .942375$

H -4.1327220 .6082180 .153448$

H -3.601066 -1.860085 0.239951

H -2.397264 -1.612690-1.032034

H $-2.6581651 .942495-0.944326$

H 4.4268080 .4126670 .057135

H $3.411492-0.5165431 .165450$

H -4.528293 -2.406884 -1.967704

H -5.373464 -0.994396-1.313090

H $-4.148169-0.791007-2.581325$

SCF Energy $(B 3 L Y P / 6-31 G * * / / M M F F)=-810.719315626$

F2-03 c00440

MMFF Geometry

C $3.6415772 .202494-0.033047$

C $2.9703403 .330972-0.288819$

C $2.910191-0.1649970 .395057$

$\begin{array}{lllll}\text { O } & 1.644030 & 0.234436 & 0.956237\end{array}$

C $0.619406-0.7055630 .571245$

C $1.219058-1.527267-0.561013$

C $2.697168-1.551667-0.217045$

C -0.6690540 .0759370 .268249$

C $3.026087-2.6622040 .783124$

C $-1.917179-0.8088770 .105229$

O $-0.4990170 .863441-0.910659$

O $-1.704012-1.758592-0.944571$

C $-3.215668-0.046238-0.229782$

C -3.5590021 .0615610 .771792$

O $-4.291733-0.991979-0.230807$

C $3.3218470 .874092-0.659248$

C -4.8784501 .7527360 .447554$

H 4.4764282 .2382590 .662161

H 3.2537414 .2600830 .193669

H $2.1322103 .349689-0.976492$

H $3.651967-0.1886651 .202225$

H $0.446146-1.3443101 .445861$

H $1.084208-1.027804-1.527225$

H $\quad 0.777091-2.525103-0.638760$

H $3.313604-1.712264-1.108546$

H $-0.8318610 .780906 \quad 1.091359$

H $2.786411-3.6438300 .360976$

H $4.094656-2.653147 \quad 1.023455$

H $2.473755-2.5535941 .722007$

H -2.068255-1.396160 1.018786

H $\quad 0.266842 \quad 1.443915-0.762096$

H -1.522392 -1.259110 -1.759354

H $-3.1636910 .370047-1.242964$

H -2.7720621 .8224260 .785177$

H $-3.6325240 .639249 \quad 1.781247$

H $-4.061604-1.691250-0.866425$

H $4.2167890 .527920-1.189866$

H $2.5278440 .994636-1.405801$

H $-5.721227 \quad 1.0572640 .508845$

H $-5.0626612 .563872 \quad 1.159237$

$\mathrm{H}-4.8574812 .182651-0.558866$

SCF Energy $\left(B 3 L Y P / 6-31 G^{* *} / / M M F F\right)=-810.722096751$

F2-03 c00441

MMFF Geometry

C $4.492376-0.990416-0.545890$

C $4.707225-2.250946-0.153024$

C $2.4873900 .510731-0.445214$

O $1.523250-0.556397-0.487846$

C $0.1973550 .016148-0.501911$

C $0.3664451 .516703-0.272365$

C 1.7719941 .6423390 .283262

C $-0.653074-0.7290020 .539042$ 
C 2.3867373 .0139510 .061111

C -2.134602 -0.3076540 .597832$

O $-0.098891-0.5060451 .843472$

O $-2.778205-1.2017971 .515786$

C $-2.876694-0.343846-0.752574$

C $-4.375062-0.018257-0.671068$

O $-2.775214-1.646681-1.332297$

C 3.7505490 .0301890 .270300

C $-4.6723891 .369545-0.128124$

H $4.882154-0.671088-1.509048$

H $5.255133-2.938405-0.788113$

H $4.342880-2.6185130 .799789$

H $2.7113670 .795267-1.482102$

H $-0.205292-0.161702-1.504215$

H -0.3889371 .9580810 .383462$

H $0.2903892 .034270-1.237396$

H 1.7428621 .4354801 .360799

H $-0.578536-1.8108030 .372373$

H $1.781866 \quad 3.7889390 .543345$

H 3.3954083 .0643370 .482461

H $2.4546313 .255113-1.005151$

H -2.202270 0.6911391 .042751

H $0.821200-0.8209241 .822754$

H $-3.633753-0.8108171 .759848$

H $-2.4157300 .363240-1.451373$

H $-4.902018-0.773489-0.075507$

H $-4.803685-0.102386-1.678445$

H $-1.844266-1.802234-1.561985$

H 4.4272120 .8729210 .450546

H $3.485114-0.3922371 .247514$

H $-4.1374502 .136796-0.696541$

H $-5.7443091 .579585-0.201995$

H -4.3909891 .4578780 .925057$

SCF Energy (B3LYP/6-31G**//MMFF) $=-810.707334165$

F2-03 c00442

MMFF Geometry

C 2.9810412 .3224290 .558633

C 2.3895343 .4334090 .105257

C $2.290197-0.087920 \quad 0.555796$

$\begin{array}{llll}\text { O } & 0.877803 & 0.194362 & 0.570417\end{array}$

C $0.144188-1.023714 \quad 0.366376$

C $1.174312-2.150816 \quad 0.381074$

C $2.428645-1.455218-0.108655$

C $-0.648296-0.941426-0.953019$

C $3.708351-2.191446 \quad 0.249944$

C $-1.584170 \quad 0.280016-1.080120$

O $-1.392939-2.145902-1.115840$

O $-0.7834601 .442427-1.331436$

C $-2.5098380 .586650 \quad 0.114401$

C $-3.443647-0.564955 \quad 0.500583$

O $-3.3274421 .708082-0.246133$

C $3.0230881 .024065-0.196543$

C $-4.377624-0.1976491 .648267$

H 3.4719662 .3514391 .527912

H 2.3977854 .3412860 .698411

H $1.8885653 .457758-0.856067$

H $2.620894-0.1311241 .601921$

H $-0.543766-1.1638541 .206937$

H $\quad 0.897059-3.018796-0.223840$

H $1.315485-2.4983971 .412676$

H $2.372110-1.351217-1.200054$

H $0.070098-0.889429-1.781361$

H $3.717084-3.189088-0.201100$

H $4.586477-1.649259-0.114252$

H $3.812173-2.3115221 .333680$

H -2.209943 $0.155066-1.972546$

H $-1.838776-2.102491-1.978807$

H $-0.2237701 .591406-0.550337$

H -1.9212370 .9011180 .983938$

H -2.868254 -1.4441020 .803874$

H $-4.058059-0.849344-0.362187$

H -2.729481 $2.435570-0.489750$

H $4.0743840 .752015-0.343320$

H $2.5764111 .154536-1.190396$

$\begin{array}{llll}\mathrm{H} & -3.808676 & 0.104673 & 2.533291\end{array}$

H -5.0496220 .6209391 .372904$

H $-4.995318-1.0594721 .920450$
SCF Energy (B3LYP/6-31G**//MMFF) = -810.721754578

F2-03_c00443

MMFF Geometry

C $-3.9254731 .920089-0.152634$

C $-3.3726553 .137215-0.103243$

C $-2.959317-0.396115-0.310604$

O $-1.7809110 .046728-1.013687$

C $-0.639945-0.723694-0.585731$

C $-1.072800-1.4314810 .689904$

C $-2.557400-1.6531670 .465785$

C $0.5761440 .209340-0.484849$

C $-2.822632-2.922686-0.346489$

C $1.905150-0.536963-0.257337$

O 0.3679651 .1601850 .558648

O $1.890589-1.2008711 .004941$

C $3.1502950 .371396-0.340345$

C $4.449345-0.435610-0.207147$

O $3.133121 \quad 1.3526960 .694175$

C -3.4200270 .7351310 .621580$

C $5.6877240 .425439-0.423899$

H $-4.7994421 .769431-0.780990$

H $-3.7884173 .953319-0.683980$

H -2.502285 3.3408980 .510497

H -3.740582 -0.609918-1.049675

H $-0.454037-1.461146-1.376170$

H $-0.935179-0.7873161 .566002$

H $-0.516586-2.3566810 .867907$

H -3.096710 -1.745911 1.415165

H $0.6505480 .783669-1.416878$

H $-2.450157-3.8046670 .185129$

H -3.898480 -3.055798 -0.503057

H -2.344985 -2.894084 -1.331072

H $1.997194-1.314696-1.024924$

H $-0.458127 \quad 1.6327780 .358692$

H $1.810684-0.5163241 .691490$

H $3.1508610 .899266-1.301534$

H $4.456103-1.253787-0.936514$

H $4.518126-0.8801030 .792967$

H $2.3384601 .898513 \quad 0.570149$

H -4.2361330 .3779361 .260873$

H -2.600399 1.0424211 .282121

H $6.590880-0.188674-0.349454$

H $5.6735220 .888525-1.415613$

H $5.758568 \quad 1.2190810 .326318$

SCF Energy $($ B3LYP/6-31G**//MMFF $)=-810.725843036$

F2-03_c00444

MMFF Geometry

C $3.5102381 .990871-0.347722$

C $2.9991153 .226738-0.318965$

C $2.493859-0.272787 \quad 0.004227$

O 1.4563790 .1941590 .884663

C $0.725113-0.9468481 .368772$

C $1.172391-2.1490890 .541822$

C $1.907524-1.528241-0.631736$

C $-0.788872-0.6720781 .344979$

C $2.939304-2.453848-1.253792$

C $-1.392201-0.315924-0.031251$

O -1.0539290 .3740042 .282948$

O $-0.805990 \quad 0.879777-0.541283$

C $-2.929000-0.164698-0.015758$

C $-3.4875470 .077702-1.425160$

$\begin{array}{lllll}\text { O } & -3.323109 & 0.930144 & 0.808026\end{array}$

C $2.8399940 .819327-1.008075$

C $-5.0111140 .093920-1.446302$

H 4.4728631 .8135130 .125001

H 3.5353614 .0318260 .171354

H $2.0438753 .456684-0.777838$

H $3.367362-0.5205340 .622189$

H $1.030931-1.0896712 .412976$

H $\quad 0.350237-2.8030650 .235083$

H $1.856782-2.7613921 .143004$

H $1.172663-1.248926-1.397406$

H -1.301585 -1.563378 1.725795

H $3.708568-2.741297-0.529176$

H $2.462985-3.369264-1.620070$

H $3.437850-1.972588-2.100876$ 
H $-1.143591-1.116510-0.736189$ H -0.5229421 .1451872 .019736$ H -1.104863 1.6162170 .018804 H -3.379302 -1.0746570 .398650$ H -3.133228 -0.704739-2.105939 H $-3.1343251 .041101-1.811694$ H $-3.0316690 .736286 \quad 1.714922$ H $3.5220110 .426325-1.770138$ H $1.9301741 .150528-1.523986$ H $-5.4104350 .905852-0.830477$ H $-5.371770 \quad 0.239861-2.469423$ H -5.417593 -0.852298-1.075363

SCF Energy (B3LYP/6-31G $\left.{ }^{* *} / / M M F F\right)=-810.720131911$

\section{F2-03_c00445}

\section{MMFF Geometry}

C $-3.605106 \quad 1.731840-0.655711$

C -3.8822582 .6174160 .307954$

C $-2.115771-0.317613-0.827946$

O $-0.798905-0.685320-1.293410$

C $-0.192880-1.591284-0.359882$

C $-1.322595-2.0804240 .535087$

C $-2.249730-0.8802040 .589891$

C $0.926486-0.8611280 .407906$

C $-3.666221-1.2642960 .990746$

C $1.998831-0.249270-0.516929$

O $1.537413-1.7673391 .325290$

O $2.508817-1.267363-1.387910$

C 3.1954720 .3841240 .218549

C 2.8384341 .4401531 .271470

O $4.0483011 .003844-0.750750$

C $-2.2316791 .206016-0.937512$

C 2.0379962 .6182610 .729030

H -4.415729 $1.375251-1.285687$

H -4.8984962 .9647600 .458797$

H -3.108352 3.0075300 .959724

H -2.829560 -0.799073 -1.508379

H $0.225981-2.440821-0.911098$

H $-0.995254-2.4184411 .522504$

H -1.829513 -2.9228170 .046873$

H $-1.858058-0.1618041 .321178$

H $0.486608-0.052281 \quad 1.000241$

H $-4.114540-1.9534720 .267056$

H -3.666663 -1.758186 1.968261

H $-4.313263-0.3865881 .064204$

H $1.5401410 .496184-1.176231$

H $1.856126-2.5349070 .821260$

H $2.976238-1.922025-0.842095$

H $3.797763-0.3980080 .696551$

H 3.7696451 .8407601 .691763

H 2.2905870 .9802092 .100926

H $4.2732490 .328473-1.413343$

H $-1.4840601 .677631-0.286336$

H -1.952275 $1.508819-1.955170$

H 1.8724413 .3539011 .522901

H $2.5684463 .119563-0.086330$

H 1.0576542 .3032050 .360749

SCF Energy $\left(B 3 L Y P / 6-31 G^{* *} / / M M F F\right)=-810.716108591$

F2-03_c00446

MMFF Geometry

C 2.8267192 .7222440 .187959

C 1.9160153 .7020510 .194075

C 2.6219510 .2230790 .170611

O 1.3475090 .2046710 .841589

C $0.796289-1.1226780 .792278$

C $1.896671-2.013768 \quad 0.221910$

C $2.668140-1.055316-0.662933$

C $-0.495113-1.107656-0.050962$

C $4.073885-1.530275-0.989382$

C $-1.494985-0.0108810 .385203$

O $-1.118680-2.3867420 .043176$

O $-1.884705-0.212166 \quad 1.743748$

C $-2.7638600 .052576-0.490985$

C $-3.6853501 .211296-0.082070$

O $-3.497716-1.167045-0.411519$

C $2.7345491 .494274-0.672912$

C $-4.8732601 .361707-1.024035$
H 3.6951232 .8134350 .835191

H 2.0428944 .5661790 .837147

H $1.0332953 .660390-0.434410$

H 3.3956320 .2052140 .949381

H $0.566018-1.4482781 .813256$

H $1.535444-2.895995-0.312321$

H $2.537200-2.3647791 .041336$

H $2.114064-0.914990-1.600318$

H $-0.250894-0.927383-1.105025$

H $4.042228-2.482296-1.529455$

H $4.595938-0.802713-1.618702$

H $4.668626-1.678390-0.081743$

H -1.0033600 .9680160 .328145$

H -0.576517 -3.023045 -0.451592

H $-1.108988-0.0375782 .302651$

H $-2.465140 \quad 0.183005-1.537708$

H $-3.1200232 .150190-0.072119$

H -4.0752321 .0472830 .929564$

H -3.694179 -1.3301400 .527069$

H $3.631717 \quad 1.452967-1.300734$

H $1.8688501 .571267-1.342930$

H -5.490007 2.213259-0.719322

H $-4.5382301 .536189-2.051391$

H $-5.5056190 .468543-1.014459$

SCF Energy (B3LYP/6-31G**//MMFF) $=-810.709715391$

F2-03 c00447

MMFF Geometry

C -4.160236 $1.779555-0.288398$

C -3.9622103 .0544010 .065524$

C $-2.655338-0.223190-0.361430$

O $-1.4456890 .525545-0.567009$

C $-0.353026-0.400460-0.767251$

C $-0.895610-1.785667-0.412901$

C $-2.189671-1.4990280 .326052$

C 0.8433490 .1101810 .056153

C $-3.186028-2.6442950 .261157$

C $2.200313-0.459592-0.399658$

O $0.645624-0.177811 \quad 1.439939$

O $2.144788-1.889505-0.381914$

C $3.396466-0.0311150 .472298$

C $3.562177 \quad 1.480806 \quad 0.674071$

O $4.582921-0.546530-0.143577$

C $-3.6307450 .601248 \quad 0.479286$

C $3.7346002 .266256-0.620146$

H $-4.7468431 .571336-1.179524$

H -4.375982 $3.860593-0.530467$

H $-3.387633 \quad 3.3161320 .947037$

H -3.082108 -0.444800 -1.348861

H $-0.098627-0.357056-1.832947$

H $-0.214126-2.4019730 .178856$

H -1.096990 -2.335921 -1.341013

H -1.952185 -1.291559 1.377481

H $\quad 0.8760301 .202656-0.029317$

H -3.468184 -2.872077 -0.772268

H $-2.758249-3.5512540 .700744$

H -4.098946 -2.401896 0.813711

H $2.377908-0.178729-1.444697$

H $-0.1799280 .258946 \quad 1.710979$

H $3.009855-2.217583-0.681870$

H $3.325464-0.5199921 .451547$

H 4.4555291 .6559391 .286703

H 2.7136491 .8855141 .236116

H $5.332841-0.3248600 .434557$

H $-4.485209-0.0147670 .780906$

H $-3.1330790 .943381 \quad 1.395442$

H $3.9001623 .324891-0.395010$

H $4.5955021 .907406-1.192481$

H $2.8457382 .197480-1.253686$

SCF Energy (B3LYP/6-31G**//MMFF) $=-810.715713301$

F2-03 c00448

MMFF Geometry

C $-4.0108941 .886798-0.616475$

C -4.5477892 .5713560 .399676$

C $-2.434064-0.083693-0.794446$

O $-1.032384-0.360013-0.991630$

C $-0.502024-1.0155960 .171200$ 
C $-1.571022-0.908701 \quad 1.252545$

C $-2.859233-0.8648830 .452808$

C $0.843677-0.3925560 .569535$

C $-3.344115-2.2708520 .091461$

C $1.882912-0.422694-0.568580$

O $1.372660-1.0898391 .695725$

O $2.010296-1.766703-1.051484$

C $3.2929400 .062989-0.177720$

C 3.3116821 .4699310 .428997

O $4.0961340 .080022-1.363620$

C $-2.5802601 .439958-0.662547$

C 4.7224621 .9434180 .758689

H $-4.6374671 .644692-1.470832$

H -5.5895752 .8713050 .370480$

H -3.9641172 .8418091 .272539$

H $-2.970203-0.421840-1.689166$

H $-0.363943-2.074404-0.080332$

H -1.4608590 .0281331 .812362$

H -1.523037 -1.7306731 .973648$

H $-3.659259-0.3710251 .013354$

H 0.6819130 .6519960 .860049

H -4.266864 -2.215099 -0.495997

H -2.607597 -2.826086 -0.498137

H -3.557829 -2.848847 0.996719

H $\quad 1.5208810 .169284-1.417326$

H $\quad 0.771713-0.9434822 .445544$

H $2.284886-2.322267-0.301755$

H $3.765855-0.6477000 .510456$

H 2.7195561 .4975031 .349320

H $2.8667722 .183330-0.275182$

H $4.069942-0.815007-1.743596$

H -2.021646 1.8002420 .210502

H -2.098405 $1.917395-1.525701$

H 4.6873712 .9320981 .227355

H $5.213013 \quad 1.255437 \quad 1.454527$

H $5.3396272 .022605-0.141616$

SCF Energy (B3LYP/6-31G**//MMFF) $=-810.718989733$

\section{F2-03 c00449}

MMFF Geometry

C 2.1493882 .2134910 .293917

C $2.5817503 .085670-0.623438$

C $2.619338-0.2492390 .735164$

O $1.308702-0.4943131 .277424$

C $0.615956-1.4244350 .424364$

C $1.635069-1.895884-0.612825$

C $2.567840-0.704805-0.720755$

C $-0.610883-0.727090-0.198934$

C $3.926007-1.042709-1.310118$

C $-1.579485-0.1626690 .864059$

O $-1.281620-1.665713-1.035940$

O $-2.087233-1.2369601 .653720$

C -2.7492180 .6849170 .321116$

C $-3.750950-0.069319-0.561306$

O $-2.2362081 .795606-0.412237$

C 3.0309251 .2059200 .976853

C $-4.9984380 .761934-0.842424$

H 1.1004982 .2348690 .578038

H $1.8945803 .792345-1.075948$

H $3.6187663 .109805-0.939225$

H $3.305393-0.8927631 .302145$

H $\quad 0.290900-2.2805931 .026849$

H $1.196364-2.189286-1.570757$

H $2.177908-2.764321-0.218022$

H $2.0874890 .051826-1.352312$

H $-0.273580 \quad 0.100284-0.832983$

H $4.445454-1.800891-0.714469$

H $3.817550-1.431592-2.327867$

H $4.562917-0.153578-1.354167$

H $-0.9984150 .483420 \quad 1.533966$

H - $-1.633353-2.369863-0.464449$

H -2.649128 -0.8516592 .347535$

H -3.2997301 .0872971 .181461$

H -4.055214 -1.006484 -0.082946

H -3.299566 -0.310143 -1.529665

H -1.6405472 .2881060 .177843$

H 2.9810911 .4123592 .053305

H 4.0759921 .3416310 .673622
H $-4.749473 \quad 1.687828-1.370314$

H $-5.6958300 .194926-1.467334$

H $-5.513007 \quad 1.0228300 .087910$

SCF Energy $\left(B 3 L Y P / 6-31 G^{* *} / / M M F F\right)=-810.717950165$

F2-03 c00450

MMFF Geometry

C $-2.4747192 .730005-0.212275$

C -1.6343893 .6413240 .290502$

C $-2.3069750 .241993-0.473455$

O $-0.8703300 .234372-0.569908$

C $-0.392754-1.118091-0.525276$

C $-1.633355-2.009631-0.559253$

C $-2.688693-1.1290210 .079363$

C $0.469694-1.3356710 .731636$

C $-4.110402-1.554239-0.246530$

C $1.622684-0.3324940 .966782$

O $1.002416-2.6595060 .680974$

O $1.1074680 .977910 \quad 1.192955$

C $2.692510-0.275966-0.142151$

C 3.8857160 .6032050 .263761

O $2.139610 \quad 0.242885-1.348920$

C $-2.749535 \quad 1.397628 \quad 0.425498$

C $5.0007460 .576200-0.774188$

H -3.002595 2.959925-1.134139

H -1.482073 $4.587358-0.217395$

H -1.084979 3.4616441 .207992

H -2.697801 $0.371343-1.491359$

H $\quad 0.201276-1.320146-1.423041$

H -1.507226 -2.970311 -0.051726

H $-1.899835-2.219329-1.603265$

H $-2.552776-1.1474421 .168561$

H $-0.188707-1.2792421 .608020$

H $-4.295421-1.543168-1.325932$

H $-4.300488-2.5696610 .116528$

H $-4.836516-0.8857440 .226315$

H $2.133464-0.6347641 .890546$

H $1.483781-2.8153771 .511250$

H $\quad 0.4502010 .9183161 .906969$

H $3.051020-1.289340-0.355039$

H 4.2886660 .2629201 .224518

H 3.5646631 .6443690 .387994

H $1.7498411 .110408-1.144892$

H $-3.825351 \quad 1.3321220 .623252$

H -2.2341991 .3321971 .392046$

H $5.8438471 .187713-0.437142$

H $5.363816-0.444327-0.931842$

H $4.6609080 .973797-1.735508$

SCF Energy (B3LYP/6-31G**//MMFF $)=-810.710999704$

F2-04 c00662

MMFF Geometry

C $-4.583350-0.7593230 .513902$

C $-4.826936-1.2248231 .744113$

C $-2.5593580 .266119-0.550740$

O $-1.593535-0.3857830 .298036$

C $-0.2741780 .081445-0.052611$

C $-0.4460340 .972882-1.278189$

C $-1.8575561 .508116-1.108497$

C $0.657207-1.121449-0.237336$

C $-1.8829502 .728766-0.187226$

C $2.109149-0.759697-0.598078$

O $0.151910-1.947610-1.295735$

O $2.869295-1.975271-0.616527$

C $2.786756 \quad 0.219670 \quad 0.376237$

C 4.2669780 .4526740 .040958

O $2.680134-0.2765941 .710124$

C $-3.8501100 .521677 \quad 0.227706$

C 4.8927261 .5246630 .924493

H $-4.942031-1.329679-0.339099$

H $-5.366506-2.1554571 .882465$

H -4.494516 -0.692991 2.628623

H -2.757829 $-0.424987-1.381742$

H $\quad 0.0642200 .6806620 .799894$

H $-0.3953050 .385640-2.203146$

H $\quad 0.321087 \quad 1.750697-1.341595$

H -2.289839 $1.799515-2.072454$

H $0.635777-1.7504050 .660833$ 
H $-1.2466403 .524847-0.588530$

H -2.897468 $3.131945-0.104838$

H -1.531156 2.4946300 .822621

H $2.155381-0.353253-1.615740$

H $-0.763851-2.181954-1.066366$

H $2.433769-2.579116-1.242493$

H 2.2655521 .1823560 .347490

H $4.3664880 .754139-1.008162$

H $4.839929-0.4719410 .178463$

H $3.116641-1.1453551 .735937$

H -3.6276901 .0359381 .170477$

H $-4.5158241 .167396-0.356696$

H $4.869788 \quad 1.2347581 .979548$

H 5.9384341 .6826790 .641837

H 4.3651052 .4776750 .817100

SCF Energy (B3LYP/6-31G**//MMFF $)=-810.721083882$

F2-04_c00663

MMFF Geometry

C $3.0601182 .484808-0.270863$

C 2.8249383 .3628460 .710555

C $2.358807 \quad 0.105426-0.624038$

O $1.151298 \quad 0.1842880 .158015$

C $0.517038-1.1058190 .163641$

C $1.348344-2.005152-0.748255$

C $2.730431-1.379911-0.667173$

C $-0.948641-0.983665-0.273326$

C $3.497676-1.8846270 .555925$

C $-1.772679-0.0342590 .617721$

O $-1.541448-2.281255-0.278490$

O $-1.659655-0.483724 \quad 1.974974$

$\begin{array}{llll}\text { C } & -3.271385 & 0.025478 & 0.269002\end{array}$

C $-3.5951580 .365643-1.191660$

O -3.8698611 .0134081 .116448$

C $3.4177321 .042245-0.042969$

C $-3.0330881 .702669-1.658672$

H $3.0006882 .826955-1.300899$

H 2.5753474 .3923730 .478354

H $2.8744383 .074049 \quad 1.754619$

H $2.0931380 .428981-1.640174$

H $0.575472-1.4955691 .187189$

H $0.986585-1.944820-1.782555$

H $1.312951-3.056767-0.447315$

H $3.322722-1.608046-1.560316$

H $-0.975313-0.606570-1.301638$

H $4.505692-1.4584560 .585402$

H $2.996372-1.6285211 .494909$

H $3.604508-2.9740490 .518134$

H -1.340350 0.9727890 .593204

H -1.550449 -2.602552 0.639569

H $-2.174897 \quad 0.1301792 .526149$

H -3.745167 -0.9280980 .531631$

H $-4.6855770 .407936-1.307779$

H -3.240248 -0.430368 -1.855052

H $-4.824948 \quad 1.0162360 .933789$

H 3.5367080 .8590061 .031749

H $4.3850520 .854665-0.523116$

H $-1.9393261 .707037-1.641881$

H -3.349393 $1.901700-2.687713$

H $-3.3914802 .526080-1.033165$

SCF Energy (B3LYP/6-31G**//MMFF $)=-810.716992249$

F2-04_c00664

MMFF Geometry

C $-4.929504-0.309316-0.203273$

C $-5.844200 \quad 0.6345500 .044965$

C $-2.415478-0.486940-0.139474$

O $-1.322027-0.8910310 .708972$

C $-0.133206-0.182408 \quad 0.297384$

C $-0.5196990 .624115-0.939564$

C $-2.0073250 .863142-0.735225$

C $1.012027-1.1819360 .101037$

C -2.2468252 .0688850 .175330$

C $2.349708-0.547522-0.322542$

O $0.646713-2.119457-0.921547$

O $3.336347-1.586793-0.350946$

C $2.852716 \quad 0.5681670 .610594$

C 4.2645741 .0811130 .292794
O $2.856056 \quad 0.096741 \quad 1.960331$

C $-3.721913-0.5562360 .650691$

C $4.3918861 .695849-1.091178$

H -5.069696 -0.953941-1.067203

H $-6.7003750 .755853-0.609550$

H $\quad-5.757519 \quad 1.297977 \quad 0.898355$

H -2.454765 -1.230086 -0.948444

H $\quad 0.1084450 .4953841 .123545$

H $-0.3741880 .037458-1.854786$

H $0.0695851 .540578-1.042290$

H $-2.5137811 .044294-1.689817$

H $1.146583-1.7792681 .011015$

H -3.3153462 .2797830 .274051$

H -1.8414121 .9177641 .180791$

H -1.777793 $2.965095-0.245050$

H $2.270151-0.169596-1.348027$

H $-0.193833-2.527697-0.651423$

H $3.008432-2.280463-0.948808$

H $2.161266 \quad 1.4168850 .575137$

H 5.0010980 .2769330 .410263

H $4.539353 \quad 1.8337161 .043001$

H $3.440896-0.6795681 .998423$

H -3.812291 -1.558551 1.089531

H -3.6859270 .1240981 .510111$

H $5.3869732 .134366-1.217170$

H $4.2594320 .946159-1.876556$

H $3.6514212 .488357-1.238799$

SCF Energy $($ B3LYP/6-31G**//MMFF $)=-810.721052428$

F2-04_c00665

MMFF Geometry

C $3.9816821 .722923-0.472394$

C $3.6088763 .003311-0.577581$

C $2.694018-0.418959-0.213713$

O $1.5602610 .085045-0.946752$

C $0.369492-0.059981-0.140928$

C $0.757456-0.8969851 .076011$

C $2.097534-1.4942760 .691939$

C $-0.737107-0.647982-1.028660$

C $2.937897-1.9254561 .880810$

C $-2.115611-0.781805-0.354840$

O $-0.350721-1.960517-1.457979$

O $-3.052554-1.186935-1.362424$

C -2.6393110 .5070850 .302034$

C -4.0681160 .3531590 .842945$

O $-2.607604 \quad 1.576416-0.642497$

C 3.3839750 .7502480 .506566

C $-4.5268151 .587276 \quad 1.609696$

H $4.7704351 .354331-1.123056$

H $4.0831823 .655772-1.302623$

H 2.8293263 .4207970 .049885

H $3.387449-0.872315-0.931998$

H 0.0878350 .9495680 .176786

H $\quad 0.020638-1.6586981 .346329$

H $0.862603-0.2377821 .946909$

H $1.903725-2.3823560 .075130$

H $-0.828328-0.054044-1.946562$

H $3.084397-1.1114222 .597772$

H $2.448111-2.7465952 .414947$

H $3.921722-2.2758991 .552717$

H $-2.100999-1.5887520 .386967$

H $\quad 0.500940-1.873171-1.919915$

H $-2.713201-2.007705-1.759308$

H $-1.9795720 .786827 \quad 1.130388$

H $-4.121573-0.5177271 .506372$

H -4.7728210 .1891320 .019186$

H -3.169222 $1.320777-1.394414$

H $2.674143 \quad 1.2705311 .160764$

H $\quad 4.1993120 .3785451 .136965$

H -3.8627331 .7917772 .455593$

H -4.5470732 .4717020 .965421$

H -5.5371671 .4332982 .001651$

SCF Energy (B3LYP/6-31G*//MMFF)= -810.719793822

F2-04 c00666

MMFF Geometry

C $2.1926952 .679056-0.816349$

C $1.4707823 .639238-0.227620$ 
C $2.2222220 .195413-0.504648$

O $0.8702900 .100875-0.019957$

C $0.577002-1.2671330 .304840$

C $1.807403-2.073193-0.109615$

C $2.933309-1.0597700 .005620$

C $-0.718187-1.724917-0.374931$

C $3.452277-0.9649711 .440938$

C $-2.007242-1.0999020 .202282$

O $-0.818723-3.145468-0.215506$

O $-3.102623-1.583436-0.589187$

C -2.0689370 .4376240 .249916$

$\begin{array}{llll}C & -3.438335 & 0.947031 & 0.722807\end{array}$

O $-1.8174970 .977330-1.046385$

C $2.8292711 .532440-0.080510$

C -3.4527292 .4591850 .912714$

H $2.3400412 .723476-1.892399$

H $1.0397364 .441497-0.816449$

H 1.2945203 .6416570 .842255

H $2.1643450 .144630-1.600847$

H $\quad 0.460531-1.3291831 .393974$

H $1.719906-2.394740-1.155510$

H $1.948782-2.9698850 .501847$

H $3.774095-1.323920-0.645595$

H $-0.664003-1.526951-1.452661$

H $3.820776-1.9391181 .779948$

H $4.286780-0.2593301 .506230$

H $2.678081-0.6363442 .141491$

H -2.173157 -1.495822 1.211583

H - $1.640612-3.428566-0.651969$

H -3.016586 -1.193397-1.476099

H -1.3043620 .8218570 .933636$

H -3.705878 $0.468306 \quad 1.671732$

H $-4.2133900 .694335-0.010505$

H $-0.8831050 .811765-1.257743$

H 2.7076831 .6782490 .999733

H $3.903148 \quad 1.542434-0.299879$

H $-4.4338762 .784077 \quad 1.273195$

H -2.702292 $2.768591 \quad 1.647207$

H -3.249922 $2.981357-0.027570$

SCF Energy (B3LYP/6-31G**//MMFF) $=-810.724104087$

F2-04 c00667

MMFF Geometry

C $3.3172032 .489275-0.516318$

C 3.0013873 .4948790 .307532

C $2.6094370 .087298-0.614361$

$\begin{array}{lllll}\text { O } & 1.329980 & 0.279470 & 0.019646\end{array}$

C $0.676606-0.9950710 .143506$

C $1.576266-2.014803-0.550448$

C $2.955107-1.391907-0.416815$

C $-0.741362-0.934099-0.440930$

C $3.588157-1.7277240 .934423$

C -1.6282920 .1291160 .242573$

O $-1.333947-2.226674-0.346050$

O $-1.725645-0.1509991 .639442$

C $-3.041867 \quad 0.254294-0.362710$

C $-3.8370181 .393457 \quad 0.290651$

O $-3.778250-0.953815-0.186311$

C $3.6215511 .089631-0.059282$

C $-5.1833161 .619028-0.386525$

H $3.3688292 .687426-1.583959$

H $2.7969604 .484347-0.086829$

H 2.9393683 .3515451 .380526

H $2.4553460 .270026-1.686950$

H $\quad 0.630933-1.2361191 .212720$

H $1.318701-2.093734-1.614270$

H $1.491727-3.015422-0.115334$

H $3.628390-1.743996-1.206435$

H $-0.679484-0.680356-1.506282$

H $3.002067-1.3419241 .774676$

H $3.676938-2.8125621 .056596$

H $4.596548-1.3075221 .006284$

H -1.132611 1.1028730 .152067

H -1.346004 -2.4804920.592459

H -2.282244 -0.9415111 .742675$

H $-2.9602940 .442590-1.439939$

H -3.259366 2.3239520 .246688

H $-4.0241251 .166746 \quad 1.347015$
H $-3.314791-1.654759-0.675046$

H 3.6268861 .0544971 .036842

H $4.6290000 .833053-0.406944$

H $-5.0527791 .858141-1.446683$

H $-5.8225890 .734145-0.308905$

H -5.7076642 .4549610 .087439$

SCF Energy $(B 3 L Y P / 6-31 G * * / / M M F F)=-810.721243717$

F2-04 c00668

MMFF Geometry

C -4.1886701 .8438230 .355005$

C $-4.3218202 .840170-0.527608$

C $-2.647224-0.1283620 .479382$

O $-1.569847 \quad 0.468973-0.266885$

C $-0.498233-0.491959-0.400008$

C $-0.924491-1.7132820 .409341$

C $-2.441528-1.6401600 .366561$

C 0.8190200 .1899770 .003849

C $-2.993445-2.269153-0.913723$

C $2.079016-0.618548-0.352393$

O 0.8192500 .4625161 .405600

O $2.018078-1.9084030 .265700$

C 3.4110720 .0291220 .078254

C $3.6071901 .453009-0.453096$

O $4.477220-0.783474-0.427076$

C $-3.9890930 .402859-0.024868$

C $4.9580082 .040772-0.061151$

H -4.2358962 .0778371 .415362$

H -4.467916 $3.859505-0.187382$

H -4.286114 2.660571-1.596436

H $-2.5011170 .167115 \quad 1.527784$

H $-0.452882-0.747985-1.465543$

H $-0.593733-1.6333941 .451603$

H $-0.520667-2.6466730 .005632$

H -2.888099 -2.159381 1.221992

H $0.859616 \quad 1.161935-0.500776$

H -2.688307 -3.318617 -0.987321

H $-4.088006-2.246531-0.917218$

H -2.643481-1.753728 -1.813851

H $2.098902-0.802155-1.433229$

H $\quad 0.0219370 .9849201 .599330$

H $1.960836-1.7704701 .226868$

H 3.5033470 .0227221 .170946

H $2.8247522 .115565-0.069457$

H $3.5381331 .453303-1.547520$

H $4.340716-1.684341-0.086845$

H $-4.0521470 .298343-1.114754$

H $-4.808333-0.1780720 .414720$

H 5.0786892 .0516511 .026722

H $5.7849301 .470152-0.494837$

H $5.0384133 .071236-0.421771$

SCF Energy $\left(B 3 L Y P / 6-31 G^{* *} / / M M F F\right)=-810.720769007$

F2-04 c00669

MMFF Geometry

C $3.7812231 .807086-0.813974$

C $3.8608592 .877383-0.015331$

C $2.310465-0.230804-0.806816$

O $1.213123 \quad 0.485962-0.203212$

C $0.633375-0.3061210 .848211$

C $1.485451-1.5666120 .969531$

C $2.088340-1.692579-0.414952$

C $-0.840216-0.5985670 .507408$

C $3.298387-2.607797-0.473225$

C $-1.6752180 .673727 \quad 0.260673$

O $-1.420651-1.354497 \quad 1.569748$

O -1.5198421 .5391741 .392705$

C -3.1841680 .4273350 .064251$

C $-3.510032-0.566693-1.056789$

O $-3.7979141 .686319-0.237255$

C $3.6251630 .395877-0.317534$

C $-5.010275-0.741701-1.260564$

H $3.8406921 .953498-1.889324$

H $3.9772603 .869815-0.436841$

H 3.8098512 .7852561 .063944

H $2.229856-0.118397-1.894758$

H $\quad 0.694717 \quad 0.256739 \quad 1.786870$

H $0.925680-2.4548581 .276958$ 
H $2.273725-1.404126 \quad 1.715085$

H $1.319004-2.106102-1.081812$

H $-0.878337-1.217635-0.395478$

H $4.068894-2.3173690 .248121$

H $3.007284-3.638090-0.242058$

H $3.742550-2.599665-1.473735$

H $-1.2734191 .216992-0.603012$

H $-1.422648-0.7915982 .363023$

H $-2.044758 \quad 2.339448 \quad 1.219571$

H -3.6321310 .0902281 .006123$

H $-3.078578-1.547983-0.834293$

H $-3.073973-0.218343-2.000736$

H -3.438153 $1.996617-1.085923$

H 3.6694490 .3802870 .778160

H $4.481348-0.176952-0.690273$

H $-5.492901-1.081520-0.338748$

H $-5.483740 \quad 0.193807-1.574202$

H $-5.199533-1.488910-2.037886$

SCF Energy (B3LYP/6-31G**//MMFF) $=-810.718718156$

F2-04 c00670

MMFF Geometry

C $3.2439602 .424995-0.618629$

C $3.0505953 .410106 \quad 0.265607$

C $2.4812550 .039273-0.700188$

$\begin{array}{lllll}\text { O } & 1.290345 & 0.233063 & 0.087082\end{array}$

C $0.628596-1.032818 \quad 0.248451$

C $1.420731-2.046468-0.574763$

C $2.818017-1.450817-0.587080$

C $-0.845096-0.927176-0.168204$

C $3.598567-1.838467 \quad 0.669715$

C $-1.618906 \quad 0.140970 \quad 0.631154$

O $-1.443173-2.2100080 .005352$

O $-1.472222-0.1512782 .027375$

C $-3.127783 \quad 0.2292340 .328302$

C $-3.4385350 .464974-1.154866$

O -3.6371401 .3281451 .093346$

C $3.5723141 .007025-0.241725$

C $-4.9312350 .579013-1.439673$

H $3.1705122 .654867-1.678606$

H $2.8203764 .414462-0.072636$

H $3.115666 \quad 3.235048 \quad 1.333804$

H $2.2044980 .257115-1.741116$

H $\quad 0.702510-1.3096141 .307380$

H $1.039200-2.089878-1.602789$

H $1.368118-3.057944-0.160468$

H $3.386438-1.789471-1.460572$

H $-0.884709-0.672300-1.233625$

H $3.123413-1.4686371 .583949$

H $3.679134-2.9279610 .749802$

H $4.616708-1.4376200 .633686$

H $-1.158310 \quad 1.123502 \quad 0.470928$

H $-2.316565-2.192675-0.419964$

H -1.9459740 .5414992 .519071$

H -3.634395 -0.6719650 .693676$

H $-3.042848-0.355536-1.761780$

H $-2.954793 \quad 1.389802-1.491673$

H $-4.605986 \quad 1.251958 \quad 1.111119$

H 3.7075060 .9379080 .844413

H $4.5258580 .744454-0.714676$

H $-5.1018180 .664754-2.517698$

H $-5.467358-0.305042-1.080577$

H $-5.3645301 .464836-0.965493$

SCF Energy (B3LYP/6-31G**//MMFF) $=-810.710285821$

F2-04 c00671

MMFF Geometry

C $-4.297684-0.931374 \quad 0.815034$

C $-4.404265-1.2953832 .097939$

C $-2.4416890 .101325-0.515514$

O $-1.367503-0.4170690 .293466$

C $-0.112943 \quad 0.089621-0.212425$

C $-0.4472680 .852252-1.490973$

C $-1.869718 \quad 1.322968-1.239912$

C $0.868023-1.076718-0.372447$

C $-1.8916842 .619731-0.428987$

C $2.282645-0.696681-0.850122$

O $0.333548-1.997889-1.336753$
O $3.063668-1.898345-0.916245$

C 3.0691390 .2995200 .022009

C $3.110574-0.0796031 .508979$

O $2.5365731 .612699-0.112153$

$\begin{array}{llll}\text { C } & -3.673288 & 0.356188 & 0.353703\end{array}$

C 4.0329120 .8334912 .308379

H $-4.692713-1.5958330 .050683$

H $-4.870851-2.2373382 .364694$

H $-4.030336-0.6676462 .899082$

H -2.668314 $-0.672310-1.262463$

H $\quad 0.251593 \quad 0.7811190 .553367$

H $-0.4409060 .186045-2.361985$

H $0.2569831 .663213-1.696724$

H $-2.400657 \quad 1.500983-2.181821$

H $0.927928-1.6457430 .562932$

H -1.4397552 .4986760 .560696$

H $-1.3432973 .409173-0.954300$

H -2.918446 $2.972898-0.288913$

H $2.223390-0.309600-1.874956$

H $-0.551151-2.258170-1.027073$

H $2.581584-2.525904-1.481851$

H $4.1037540 .334339-0.343403$

H $3.451203-1.114388 \quad 1.626244$

H $2.109894-0.0060581 .948946$

H $2.5964291 .858323-1.051011$

H $-3.4022440 .965287 \quad 1.224498$

H $-4.4260790 .909081-0.220118$

H $3.691605 \quad 1.8727782 .275147$

H 4.0558350 .5204713 .357226

H $5.055746 \quad 0.7934321 .920622$

SCF Energy (B3LYP/6-31G**//MMFF) $=-810.717337595$

F2-04_c00672

MMFF Geometry

C $-4.477639-1.605138-0.493651$

C $-5.711912-1.355311-0.042729$

C $-2.229114-0.469311-0.396325$

O $-1.001920-0.4332540 .357923$

$\begin{array}{llll}\text { C }-0.377471 & 0.848173 & 0.161124\end{array}$

C $-1.2548421 .609812-0.831763$

C $-2.6271460 .992998-0.613509$

C $1.0746070 .672645-0.306560$

$\begin{array}{llll}\text { C } & -3.338634 & 1.649399 & 0.570934\end{array}$

C $1.921072-0.1784100 .660773$

O $1.6842121 .954654-0.446033$

$\begin{array}{lllll}0 & 1.819091 & 0.371681 & 1.980917\end{array}$

C $3.420083-0.2660900 .311759$

C $3.691631-0.772730-1.108635$

O $4.033282-1.175975 \quad 1.232555$

C $-3.228845-1.3864830 .307101$

C $5.181083-0.895004-1.408757$

H $-4.362789-2.007612-1.496842$

H $-6.575259-1.543314-0.671730$

H $-5.883378-0.9624540 .953248$

H -1.969199 -0.912053-1.368377

H $-0.4002311 .375497 \quad 1.122686$

H $-0.9317331 .411937-1.861788$

H -1.220596 $2.692890-0.677812$

H -3.257772 $1.104979-1.502391$

H $1.0780480 .184304-1.287969$

H $-4.348361 \quad 1.2477620 .693818$

H -2.801581 1.4996301 .513012

H -3.437070 2.7282690 .408238

H $1.506206-1.1912290 .723686$

H $1.2207392 .429033-1.156488$

H 2.1373781 .2900581 .940713

H 3.9056610 .7056510 .461642

H $3.232753-1.759522-1.244369$

H $3.250049-0.095680-1.847008$

H $3.847229-0.8492082 .129527$

H $-2.752520-2.3619780 .471808$

H $-3.451662-1.0099331 .312851$

H $5.6862310 .067217-1.277974$

H $5.330597-1.218738-2.443749$

H $5.662706-1.629658-0.756211$

SCF Energy (B3LYP/6-31G**//MMFF) $=-810.718232659$

F2-04_c00673 
MMFF Geometry

C 4.432841-1.616019 0.196081

C $4.678078-2.507915-0.770402$

C $2.698164 \quad 0.172726 \quad 0.466874$

O $1.695281-0.459222-0.352133$

C $0.5382850 .402173-0.427299$

C $0.824886 \quad 1.5787350 .500447$

C 2.3421041 .6609550 .487395

C $-0.716003-0.430290-0.120732$

C $2.8461912 .458735-0.716271$

C $-2.0328740 .316885-0.408620$

O $-0.686845-0.860187 \quad 1.239975$

O $-2.157432 \quad 1.4497730 .448757$

C $-3.291678-0.565798-0.273097$

C $-4.5631990 .197150-0.670183$

O $-3.451579-1.0199031 .069069$

C $4.092813-0.173238-0.055471$

C $-5.795787-0.698776-0.673348$

H $4.490316-1.9415851 .231586$

H $4.923069-3.534655-0.521119$

H $4.637879-2.234301-1.818945$

H $2.570682-0.2311431 .480994$

H $\quad 0.4879630 .751515-1.465759$

H $\quad 0.489112 \quad 1.3682451 .522827$

H $\quad 0.3319992 .4996800 .174232$

H 2.7211962 .1399121 .397157

H -0.686980 -1.340019-0.733251

H $2.5643201 .997001-1.667963$

H $2.4369603 .474600-0.701083$

H $3.9372872 .546151-0.694947$

H $-1.9916280 .698848-1.435827$

H $0.153099-1.3310181 .377656$

H $-2.2006451 .118367 \quad 1.362451$

H $-3.186221-1.449032-0.914431$

H $-4.4401470 .632102-1.668626$

H $-4.745806 \quad 1.0196510 .031728$

H -2.670470 -1.552173 1.295654

H $4.1575550 .037326-1.129818$

H $4.8439150 .443173 \quad 0.452010$

H -6.675296 $-0.128124-0.988287$

H -5.668984 -1.536194 -1.366904

H -5.997509-1.103944 0.323075

SCF Energy (B3LYP/6-31G**//MMFF) $=-810.724567679$

F2-04_c00674

MMFF Geometry

C -3.552531-1.847706 -0.542733

C $-3.903166-2.6638550 .457466$

C $-2.147722 \quad 0.224517-0.634556$

O $-1.329219-0.0960680 .507897$

C -0.4135730 .9881100 .740883$

C $-0.6115001 .979598-0.399580$

C $-2.0584981 .744690-0.799827$

C $1.030624 \quad 0.494276 \quad 0.923661$

C -3.0181962 .5440170 .082851$

C $1.607253-0.384083-0.205490$

O $1.090116-0.2357162 .153606$

O $0.777832-1.540987-0.367549$

C $3.042022-0.8847660 .056270$

C 4.0762820 .2023980 .376358

O $3.468208-1.593579-1.113961$

C -3.552585 -0.347350 -0.445541

C $4.2183531 .264297-0.707085$

H -3.255725 -2.285609 -1.492434

H -3.885336 -3.739512 0.320406

H $-4.209921-2.2786311 .423493$

H -1.668554 -0.248599 -1.503096

H $-0.739928 \quad 1.448545 \quad 1.682275$

H $\quad 0.0357571 .743127-1.250816$

H $-0.3919693 .008879-0.099052$

H $-2.2334482 .033799-1.842327$

H $1.6634761 .377727 \quad 1.057968$

H -2.951213 $2.255476 \quad 1.136713$

H -2.8006373 .6151200 .013068$

H $-4.0542712 .399404-0.239984$

H $1.5728240 .152435-1.159308$

H $0.522848-1.0195622 .051980$

H $1.155974-2.070172-1.090683$
H $3.028399-1.6207110 .869393$

H $5.057125-0.2734650 .502006$

H $3.8388740 .684541 \quad 1.330632$

H $4.350414-1.959035-0.929810$

H $-3.957600-0.0424240 .526947$

H $-4.2207350 .040768-1.222957$

H $5.0097721 .970511-0.435966$

H $4.4836060 .817868-1.670440$

H $3.2941931 .835456-0.833992$

SCF Energy $\left(B 3 L Y P / 6-31 G^{* *} / / M M F F\right)=-810.709374935$

F2-04 c00675

MMFF Geometry

C $3.4494551 .954543-0.103816$

C 3.0427453 .0882950 .478109

C $2.401532-0.312167-0.299789$

O $1.0405560 .147376-0.194770$

C $0.160546-0.986883-0.236093$

C $1.040674-2.211115-0.481434$

C $2.375530-1.7883450 .108223$

C $-0.931265-0.799759-1.298423$

C $2.420967-2.0379851 .616427$

C $-1.7880910 .473463-1.136496$

O $-1.761721-1.957322-1.311130$

O $-0.9991551 .604770-1.528294$

C -2.3686530 .7605480 .262894$

C $-3.242802-0.364040 \quad 0.826976$

O

C 3.3194680 .5932250 .521268

C $-3.839510-0.0121212 .185212$

H $3.9114962 .009976-1.086167$

H $3.1691464 .040405-0.025743$

H 2.5783743 .0885881 .458049

H $2.670253-0.247670-1.363476$

H $-0.292717-1.0831230 .757027$

H $1.163056-2.387944-1.557659$

H $\quad 0.624927-3.123095-0.041935$

H $3.204971-2.335324-0.353965$

H -0.438984 -0.740159-2.278408

H $3.399507-1.7668082 .025706$

H $1.660087-1.4642522 .155185$

H $2.258572-3.0991181 .833926$

H $-2.6210300 .432777-1.849367$

H -2.411666 -1.845834 -2.025629

H $-0.2531121 .675620-0.908333$

H $-1.564951 \quad 1.0015790 .968317$

H -2.661997 -1.283554 0.943413

H $-4.067543-0.5749870 .135720$

H $-2.6225892 .646440-0.196166$

H 2.9356840 .6945601 .543614

H 4.3210660 .1519740 .582539

H -4.5101410 .8499882 .118029$

H $-4.419404-0.8567762 .571046$

$\mathrm{H}-3.0521970 .2178702 .910051$

SCF Energy $\left(B 3 L Y P / 6-31 G^{* *} / / M M F F\right)=-810.718211663$

F2-04 c00676

MMFF Geometry

C -5.055939-0.0551320.295553

C $-5.3440530 .028212-1.008090$

C $-2.484083-0.3642210 .028416$

O $-1.357630-0.8292430 .803195$

C $-0.166898-0.1546270 .346966$

C $-0.5803440 .675520-0.863630$

C $-2.0457390 .969146-0.587600$

C $0.938453-1.1851040 .094075$

C $-2.2034562 .184128 \quad 0.327828$

C $2.278979-0.583109-0.370231$

O $0.484345-2.093263-0.919390$

O $3.222805-1.657563-0.469983$

C 2.8530960 .5065620 .553052

C 4.2682990 .9716190 .179828

O $2.894246-0.0023891 .888354$

C $-3.727055-0.3684540 .923011$

C $4.3626661 .585032-1.207257$

H -5.8717380 .1090010 .997240$

H $-6.354780 \quad 0.258897-1.328151$

H $-4.602986-0.138247-1.780101$ 
H $-2.614319-1.104613-0.772284$

H $\quad 0.129720 \quad 0.507716 \quad 1.167733$

H $-0.5019180 .091418-1.788522$

H $0.0377751 .569811-0.988793$

H -2.581455 $1.174216-1.520445$

H $1.078018-1.7970260 .993543$

H -1.734381 $3.065914-0.121976$

H -3.2606712 .4215210 .482334$

H -1.7466972 .0262331 .310074$

H $2.163683-0.190680-1.386404$

H $\quad 1.194747-2.741263-1.066259$

H $3.379486-1.9940990 .429094$

H 2.1841621 .3743630 .556104

H $4.600213 \quad 1.713816 \quad 0.917339$

H 4.9795120 .1414520 .269096

H 3.2011600 .7159332 .467473

H -3.809613 -1.356755 1.395396

H $-3.5692890 .319846 \quad 1.762904$

H $3.6433722 .401614-1.325405$

H $4.1762090 .842012-1.988135$

H $5.3661201 .991141-1.370791$

SCF Energy (B3LYP/6-31G**//MMFF) $=-810.712947821$

\section{F2-04 c00677}

MMFF Geometry

C $-4.689042-0.5270230 .904786$

C $-4.454301-1.829600 \quad 0.707380$

C $-2.6000450 .332716-0.367626$

$\begin{array}{llll}0 & -1.609384 & -0.279766 & 0.479677\end{array}$

C -0.2991680 .1312940 .036918$

C $-0.5113060 .971393-1.218501$

C $-1.9057001 .538594-1.010816$

C $0.596857-1.103544-0.118778$

C $-1.8703932 .798846-0.144679$

C $2.037486-0.802771-0.577077$

O $-0.002523-2.010722-1.054033$

O $2.762116-2.039061-0.593656$

C 2.7913660 .2193260 .294066

C $4.2576290 .385175-0.130944$

O $2.735326-0.1735321 .664570$

C $-3.863507 \quad 0.6449570 .438729$

C 4.9582381 .4926050 .646297

H -5.581790 -0.2636451 .469306$

H -5.140148 -2.571167 1.103782

H -3.592937 -2.191856 0.159723

H $-2.832465-0.390046-1.161400$

H $\quad 0.0969880 .765380 \quad 0.838162$

H $-0.5128640 .349866-2.120743$

H $0.2640871 .732689-1.348528$

H -2.376114 $1.794775-1.966776$

H $0.610973-1.6525010 .830305$

H -1.477870 2.6029330 .858272

H -1.239786 $3.564899-0.608555$

H $-2.8735313 .223689-0.036005$

H $2.029604-0.451749-1.615631$

H $0.143392-1.668173-1.951244$

H $2.732176-2.4157150 .302008$

H $2.293508 \quad 1.1920620 .220689$

H $4.3096540 .614535-1.201515$

H $4.811574-0.5457980 .037960$

H $3.247924-0.9935131 .762848$

H $-3.5905871 .208627 \quad 1.339516$

H $-4.520978 \quad 1.282685-0.164781$

H 4.4511332 .4522340 .503729

H 4.9841111 .2722521 .717972

H $5.990977 \quad 1.6003860 .299910$

SCF Energy $\left(B 3 L Y P / 6-31 G^{* *} / / M M F F\right)=-810.704528400$

\section{F2-04 c00678}

MMFF Geometry

C $3.1340622 .326240-0.446049$

C $3.3007313 .124673 \quad 0.614219$

C $2.2139890 .010378-0.697581$

$\begin{array}{lllll}\text { O } & 1.351285 & 0.066332 & 0.456781\end{array}$

C $0.703103-1.2079570 .614116$

C $1.097974-2.048168-0.594856$

C $2.454810-1.476987-0.970786$

C $-0.811997-1.0537230 .820866$
C $3.575170-2.108568-0.143160$

C $-1.542610-0.150665-0.198272$

O $-1.016318-0.545602 \quad 2.142596$

O $-1.1600901 .213844-0.024719$

C $-3.079665-0.242524-0.088579$

C $-3.7866250 .623485-1.141631$

O

C $3.4619900 .858971-0.455881$

C $-5.2979290 .431103-1.124104$

H $2.7462652 .756383-1.365919$

H 3.0479854 .1775930 .554060

H 3.6868152 .7467501 .554335

H $1.6386730 .428180-1.535587$

H $1.135681-1.6511191 .520201$

H $0.402873-1.898211-1.428334$

H $1.110848-3.119212-0.370333$

H $2.676842-1.647152-2.030278$

H -1.259446 -2.054450 0.800209

H $4.551391-1.719986-0.450703$

H $3.459221-1.9165440 .928210$

H $3.594452-3.193918-0.289941$

H -1.262451 -0.441002 -1.216446

H -0.6445360 .3528562 .169001$

H $-0.1965561 .269115-0.135895$

H -3.381518 -1.287998 -0.221777

H $-3.4093300 .375935-2.140532$

H -3.580043 $1.685175-0.961491$

H -3.1910121 .0614581 .361344$

H 3.9303210 .5808780 .495887

H $4.1932790 .681980-1.252954$

H $-5.7642031 .040968-1.904362$

H -5.561488 -0.615164 -1.309114

H $-5.7268610 .730016-0.162657$

SCF Energy $\left(B 3 L Y P / 6-31 G^{* *} / / M M F F\right)=-810.715737702$

F2-04 c00679

MMFF Geometry

C 3.1683202 .3797710 .005989

C $4.0215823 .189330-0.631194$

C $2.4459450 .012816-0.397436$

O 1.1398340 .1584940 .192633

C $0.462627-1.1080160 .141643$

C $1.385343-2.065986-0.609045$

C $2.762678-1.484811-0.340268$

C $-0.919287-0.958828-0.509726$

C $3.319035-1.9706490 .999174$

C $-1.821434 \quad 0.082487 \quad 0.192517$

O $-1.542441-2.240299-0.536306$

O $-2.069116-0.3161651 .540448$

C $-3.1742810 .307031-0.515192$

C $-4.077612 \quad 1.3406200 .175555$

O $-3.898922-0.922452-0.575142$

C $3.4459680 .932506 \quad 0.304061$

C $-3.4777552 .735127 \quad 0.246338$

H 2.2180612 .7866710 .342155

H $3.7633494 .227241-0.811229$

H $4.9844702 .835862-0.983089$

H $2.3466590 .310143-1.450783$

H $0.355969-1.465467 \quad 1.172955$

H $1.183648-2.029590-1.687137$

H $\quad 1.265690-3.104796-0.286139$

H $3.472268-1.764106-1.127194$

H $-0.787867-0.635559-1.549743$

H $3.386968-3.0636661 .011886$

H $4.327548-1.5787371 .166186$

H $2.694632-1.6630181 .844071$

H - $1.287712 \quad 1.0387010 .226189$

H -1.753244 -2.482676 0.381823

H $-1.238377-0.2192692 .035497$

H -2.984832 $0.622474-1.548321$

H $-4.3431010 .999544 \quad 1.183817$

H $-5.0289491 .395483-0.369322$

H $-4.708736-0.762998-1.089278$

H $3.3832660 .806651 \quad 1.391508$

H $4.4652940 .672350-0.005140$

H $-3.1695103 .081470-0.745106$

H -4.2169193 .4418950 .637110$

H -2.6101872 .7663860 .911730$ 
SCF Energy (B3LYP/6-31G**//MMFF) $=-810.708119872$

F2-05_c00908

MMFF Geometry

C $-4.239506 \quad 1.772205-0.347545$

C -4.5315562 .5342330 .712516$

C -2.568396 $-0.058091-0.720969$

O $\begin{array}{llll} & -1.619989 & 0.397231 & 0.263757\end{array}$

C $-0.520526-0.537493 \quad 0.322701$

C $-0.765241-1.551289-0.787741$

C $-2.277874-1.547921-0.923314$

C $0.798857 \quad 0.236710 \quad 0.204740$

C -2.930948 -2.474989 0.102894

C $2.044424-0.6622180 .120085$

O $\quad 0.922471 \quad 1.0772301 .356760$

O $2.014850-1.6031021 .201587$

C 3.3929290 .0813140 .187962

C $3.5418291 .177272-0.872533$

O $4.435338-0.879431-0.016577$

C $-3.989160 \quad 0.291259-0.277281$

C $4.9008211 .865002-0.812058$

H $-4.1869462 .240751-1.326969$

H -4.7059183 .5979140 .592839$

H $-4.5999332 .116144 \quad 1.710630$

H -2.328468 $0.474241-1.652022$

H $-0.590222-1.016247 \quad 1.306920$

H $-0.338849-2.533797-0.563330$

H $-0.321383-1.202931-1.729209$

H $-2.587580-1.872988-1.922910$

H $0.7525540 .900801-0.665798$

H $-2.566812-3.500505-0.021214$

H -2.722921 -2.167056 1.132482

H $-4.017568-2.496269-0.028981$

H $2.011783-1.259064-0.798569$

H $\quad 0.1267651 .6354191 .394490$

H $1.999271-1.0951542 .031096$

H 3.5483610 .5034511 .187938

H $2.7675951 .940638-0.745244$

H $3.4216840 .744042-1.872847$

H $4.326980-1.5718320 .657948$

H $-4.716034-0.204531-0.931117$

H $-4.163782-0.0623090 .746070$

H 5.0712012 .3075150 .174614

H $5.7147571 .164044-1.021526$

H $4.9501872 .666321-1.556249$

SCF Energy (B3LYP/6-31G**//MMFF) $=-810.721411338$

F2-05_c00909

MMFF Geometry

C -3.101959 2.179969-0.975150

C $-2.5266373 .376743-0.813352$

C -2.457112 $-0.125226-0.234352$

O $-1.0392710 .127113-0.184001$

C $-0.371593-1.055312 \quad 0.294643$

C -1.443286 -2.1350320 .419437$

C $-2.702430-1.326590 \quad 0.683744$

C $0.799556-1.416814-0.626626$

C $-2.843420-0.9827412 .167512$

C $1.814397-0.277168-0.851594$

O $0.290742-1.793161-1.913416$

O $2.902126-0.809264-1.619197$

C $2.386256 \quad 0.337490 \quad 0.438010$

C $3.480878 \quad 1.3774620 .158140$

O $2.919387-0.6937001 .267918$

C $-3.224846 \quad 1.1501120 .113475$

$\begin{array}{lll}\text { C } 3.944476 & 2.077656 & 1.429424\end{array}$

H -3.517885 $1.929414-1.947671$

H -2.473867 $4.077226-1.639596$

H -2.099262 $3.677410 \quad 0.136845$

H -2.682288 $-0.423856-1.267646$

H $0.008405-0.8194441 .295016$

H $-1.208125-2.8656001 .199617$

H $-1.566118-2.687628-0.519405$

H $-3.598731-1.8760750 .374734$

H $1.313293-2.303131-0.236816$

H $-2.014589-0.3675342 .532104$

H $-3.774190-0.4369222 .352808$

H $-2.874671-1.8961882 .771101$
H $1.3652030 .514700-1.463443$

H $-0.224538-1.042661-2.256026$

H $2.524864-1.186622-2.432475$

H 1.5798370 .8206501 .000084

H $3.1065582 .129748-0.545697$

H $4.3549460 .898554-0.298747$

H $3.617586-1.1452700 .763192$

H -4.2889060 .9211930 .243120$

H $-2.855340 \quad 1.568638 \quad 1.057547$

H 4.7049802 .8278991 .190391

H $3.1101952 .586307 \quad 1.922777$

H 4.3830821 .3679932 .137897

SCF Energy (B3LYP/6-31G**//MMFF) $=-810.722590106$

F2-05_c00910

MMFF Geometry

C $4.467315-1.440316-0.671294$

C $4.767680-2.428967 \quad 0.178445$

C $2.6775640 .314188-0.678291$

$\begin{array}{llll}\text { O } & 1.722444 & -0.438312 & 0.094808\end{array}$

$\begin{array}{lllll}\text { C } & 0.552464 & 0.378353 & 0.318883\end{array}$

C $0.768427 \quad 1.656027-0.482151$

C $2.2812241 .784283-0.515955$

C $-0.697396-0.423464-0.067937$

C $2.8117382 .456863 \quad 0.751153$

C -2.0025660 .3927010 .009589$

O $-0.783251-1.543801 \quad 0.817067$

O -2.1513500 .9324461 .323365$

C $-3.270797-0.399557-0.371296$

C $-4.513706 \quad 0.501563-0.388834$

O $-3.509863-1.457873 \quad 0.553447$

C $4.098861-0.046247-0.245469$

C $-5.750904-0.231649-0.892527$

H $4.497740-1.643889-1.738628$

H $5.030046-3.412984-0.194403$

H $4.755910-2.2778921 .252104$

H $2.5240430 .025036-1.727397$

H $\quad 0.5410800 .594179 \quad 1.394178$

H $\quad 0.2567072 .519059-0.045331$

H $0.3977261 .532276-1.507711$

H $2.6104372 .374188-1.378746$

H $-0.580666-0.831634-1.079028$

H $2.581511 \quad 1.882373 \quad 1.654106$

H 3.8982662 .5807820 .699314

H 2.3742963 .4544240 .866988

H $-1.921923 \quad 1.245586-0.673683$

H $\quad 0.054389-2.0337550 .747501$

H $-2.2403830 .182148 \quad 1.935959$

H -3.134924 $-0.845894-1.363694$

H $-4.3340661 .372212-1.030032$

H -4.7274680 .8703610 .621634$

H $-2.747360-2.0593960 .516697$

H $4.8144880 .646241-0.703719$

H 4.1969190 .0429280 .843193

H $-6.6075300 .449440-0.918533$

H $-5.592673-0.616025-1.905024$

H -6.009839-1.072102-0.241142

SCF Energy (B3LYP/6-31G**//MMFF) $=-810.725039321$

F2-05_c00911

MMFF Geometry

C -2.536619 $2.668478-0.361526$

C -1.8465243 .5690090 .347362$

C $-2.2749380 .188724-0.661481$

O $-0.8475090 .246881-0.473709$

C $-0.469671-0.6618140 .578492$

C -1.707135 -1.490839 0.918902

C $-2.643275-1.234341-0.246269$

C $0.743089-1.489796 \quad 0.127304$

$\begin{array}{llll}\text { C }-4.104823 & -1.491449 & 0.075154\end{array}$

C $1.941664-0.653866-0.369099$

O $0.352504-2.360830-0.941985$

O $3.026985-1.555007-0.622248$

C $2.426427 \quad 0.4199410 .621395$

C 3.7205931 .1349850 .207882

O $2.650293-0.1768331 .901156$

C -2.930465 1.3126200 .156400

C $3.6039371 .895650-1.102448$ 
H $-2.8432732 .924316-1.372398$ H -1.596174 $4.532363-0.083235$ H -1.518745 3.3635301 .360301 H -2.482965 $0.336840-1.727830$ H $-0.194652-0.0608661 .452314$ H $-2.144091-1.1198181 .854573$ H -1.493710 -2.553820 1.070919 H $-2.353478-1.907686-1.064392$ H $1.061204-2.1474460 .944588$ H -4.438736 -0.9273610 .951770$ H $-4.741506-1.217863-0.772286$ H -4.266010 $-2.553310 \quad 0.289471$ H $1.695892-0.189122-1.330663$ H $0.035764-1.803636-1.673531$ H $2.706723-2.224714-1.250794$ H 1.6437201 .1740680 .757638 H 4.5519610 .4218050 .149655 H 4.0003341 .8377491 .003378 H $3.319914-0.8730311 .786862$ H -4.021862 1.2413420 .092269 H -2.6576961 .2261461 .215008$ H $4.5196302 .465943-1.288570$ H $3.4596981 .217314-1.948293$ H $2.7663072 .599756-1.074878$ SCF Energy (B3LYP/6-31G $\left.{ }^{* *} / / M M F F\right)=-810.721367244$

F2-05_c00912

MMFF Geometry

C $2.9715332 .287306 \quad 0.143673$

C 3.9759602 .9653440 .710095

C $2.300626-0.1279020 .208539$

O $0.8955150 .109196-0.003746$

C $0.214011-1.157429-0.073123$

C $1.259960-2.2281660 .227421$

C $2.551115-1.572982-0.233591$

C $-1.002053-1.1653730 .860922$

C $2.762703-1.755477-1.737453$

C -1.9827330 .0060470 .640144$

O $-0.536346-1.0857152 .214959$

O $-3.098361-0.229071 \quad 1.508731$

C $-2.4817350 .156128-0.809231$

C $-3.5749341 .215112-1.012279$

O $-3.000056-1.102108-1.247670$

C $3.1278010 .940709-0.506534$

C $-3.1429642 .619432-0.623983$

H 1.9802922 .7333860 .138971

H 3.7986063 .9367551 .158720

H 4.9845792 .5683240 .737820

H $2.466989-0.0594471 .292715$

H $-0.121080-1.276506-1.109719$

H $1.037293-3.177424-0.269830$

H $1.328775-2.4315731 .302486$

H $3.418237-1.9949340 .286827$

H -1.525185 -2.125041 0.772026

H $1.967213-1.290259-2.328330$

H $3.715133-1.316661-2.051944$

H $2.793286-2.819972-1.993573$

H -1.5113880 .9353080 .981320$

H -1.319684 -1.102688 2.791192

H -3.6045550 .5981161 .571364$

H -1.635157 $0.402442-1.460101$

H -4.484593 $0.937869-0.466212$

H $-3.8680701 .217108-2.070216$

H -3.248998 -1.008114 -2.182953

H $4.1838300 .645645-0.513821$

H $2.8010821 .042936-1.548223$

H -2.9671072 .7030860 .452342$

H $-2.2267042 .907537-1.148762$

H $-3.9245473 .339196-0.887903$

SCF Energy (B3LYP/6-31G $\left.{ }^{\star *} / / M M F F\right)=-810.711661609$

F2-05_c00913

MMFF Geometry

C $4.770567-1.176385-0.136116$

C $5.921244-0.519704-0.320193$

C $2.330762-0.5744120 .066192$

O $1.304953-0.2823651 .035312$

C 0.2850570 .5224000 .402248
C $0.6882730 .642997-1.063631$

C $2.2009660 .501334-1.014687$

C $-1.085492-0.1278570 .635427$

C $2.8643601 .837893-0.677789$

C $-2.2422170 .560794-0.110200$

O $-1.350697-0.1064612 .042289$

$\begin{array}{lllll}\text { O } & -2.218026 & 1.955870 & 0.221192\end{array}$

$\begin{array}{llll}\text { C } & -3.640419 & 0.018667 & 0.240128\end{array}$

C $-3.817824-1.4967930 .077988$

O $-4.5836530 .687264-0.605652$

C $3.675399-0.7152190 .778615$

C $-3.541247-2.008697-1.330246$

H $4.616550-2.112893-0.666024$

H $6.676885-0.912784-0.991486$

H $\quad 6.1297930 .4137040 .190653$

H $2.060504-1.546446-0.370101$

H $\quad 0.3296321 .5005290 .896017$

H $\quad 0.340747 \quad 1.574860-1.520084$

H $0.270344-0.188241-1.645959$

H $2.5970130 .149587-1.973728$

H $-1.028692-1.1847550 .353609$

H $3.9543121 .751551-0.700391$

H $2.5847202 .600079-1.413163$

H 2.5783452 .2059960 .312587

H -2.081267 $0.502769-1.192562$

H -1.4389490 .8244052 .310330$

H -2.949903 2.379711 -0.259287

H -3.8964650 .3049961 .267254$

H $-4.854897-1.7562340 .325451$

H $-3.182080-2.0323980 .791023$

H $-5.4716790 .383658-0.351105$

H $3.9382560 .219741 \quad 1.288250$

H $3.568837-1.4534911 .584210$

H $-3.741543-3.083847-1.382581$

H $-4.180256-1.512952-2.067401$

H $-2.497398-1.852113-1.617055$

SCF Energy $\left(B 3 L Y P / 6-31 G^{* *} / / M M F F\right)=-810.714608352$

F2-05 c00914

MMFF Geometry

C $-4.2243501 .800094-0.355665$

C $-4.5301892 .551100 \quad 0.708241$

C $-2.558790-0.036419-0.724682$

$\begin{array}{llllll}\mathrm{O} & -1.622822 & 0.400234 & 0.279908\end{array}$

C $-0.528762-0.5411330 .343309$

C $-0.764715-1.540534-0.782655$

C $-2.275169-1.525611-0.939999$

C $0.797640 \quad 0.2252620 .248914$

C $-2.948773-2.4602650 .065856$

C $2.050553-0.6664200 .161525$

O $0.901319 \quad 1.0711391 .399352$

O $2.060776-1.5768901 .268582$

C 3.3872290 .1010410 .210240

C 3.514006 1.196817 -0.855186

O $4.446877-0.8464820 .028252$

C $-3.9842350 .316963-0.299277$

C $4.8697731 .892008-0.816639$

H -4.151872 2.280030-1.328279

H -4.6958653 .6173540 .598655$

H -4.618195 2.1215051 .700020

H $-2.3008690 .505137-1.645429$

H -0.616339 -1.030474 1.320646

H $-0.348941-2.528904-0.565142$

H $-0.305227-1.184207-1.713465$

H $-2.572422-1.837003-1.947696$

H $\quad 0.7516810 .893775-0.617618$

H -2.589343 -3.486706 -0.065023

H $-2.753716-2.1657701 .102000$

H $-4.033514-2.472832-0.081672$

H $2.021178-1.277124-0.748569$

H 0.9844270 .4966612 .179874

H $1.348593-2.2231161 .129340$

H 3.5346490 .5310641 .207737

$\begin{array}{llll}\text { H } & 2.737337 & 1.955767 & -0.716808\end{array}$

H $3.3800250 .761836-1.852979$

H $4.354371-1.228720-0.861073$

H $-4.703766-0.166549-0.970042$

H $-4.176885-0.0476180 .716950$ 
H $5.6838811 .195566-1.039545$

H $4.9024982 .693477-1.561585$

H 5.0538042 .3354260 .167060

SCF Energy (B3LYP/6-31G**//MMFF) $=-810.705769327$

F2-05 c00915

MMFF Geometry

C $3.549518 \quad 1.992407 \quad 0.676073$

C $3.397278 \quad 3.104917-0.050966$

C $2.523381-0.2873450 .517011$

O $1.306656 \quad 0.142279-0.123111$

C $0.488215-1.008675-0.397027$

C $1.213595-2.2044760 .215098$

C $2.669422-1.7729690 .177320$

C $-0.944182-0.8151470 .125616$

C $3.300544-2.060789-1.185731$

C $-1.7138360 .335574-0.554210$

O $-0.915502-0.5949541 .536043$

O $-1.0177121 .573261-0.364780$

C $-3.140700 \quad 0.558645-0.014065$

C $-4.026068-0.691272-0.062923$

O $-3.7602381 .570940-0.816955$

C 3.6814750 .6141660 .089685

C $-5.446775-0.4193420 .418475$

H 3.5902512 .0793041 .758728

H 3.3118964 .0709400 .434472

H $3.3535013 .073771-1.134034$

H $2.356712-0.1903341 .598959$

H $\quad 0.456397-1.120684-1.487730$

H $1.008289-3.135173-0.323031$

H $\quad 0.912527-2.357006 \quad 1.258680$

H $3.257675-2.2920240 .942380$

H -1.482933 -1.753822 -0.041363

H $3.243443-3.130192-1.415986$

H $2.805442-1.515947-1.995909$

H $4.359167-1.781711-1.190312$

H $-1.7588740 .163942-1.636764$

H -0.4260530 .2314531 .690416$

H $-0.1488751 .492520-0.793471$

H $-3.103107 \quad 0.9503291 .009615$

H $-3.602857-1.4845770 .561344$

H $-4.079245-1.068220-1.091433$

H -3.192522 2.359710 -0.775894

H $4.6314150 .189827 \quad 0.434630$

H $3.7236680 .683560-1.004104$

H $-5.9572840 .303167-0.225617$

H -6.030476 -1.345395 0.409191

H $-5.444997-0.0298041 .441522$

SCF Energy (B3LYP/6-31G**//MMFF) $=-810.724191943$

F2-05 c00916

MMFF Geometry

C $4.499861-0.720656-0.729626$

C $4.934460-1.869852-0.200557$

C $2.3271040 .533334-0.565816$

O $1.572087-0.686172-0.393705$

C $0.507279-0.476310 \quad 0.562441$

C $0.7941250 .871170 \quad 1.208182$

C 1.4851511 .6289350 .093602

C $-0.822849-0.574285-0.209865$

C 2.2207132 .8707540 .565489

C -2.076552 -0.2664280 .627607$

O $-0.936963-1.916098-0.703268$

O $-2.038670-1.0476111 .829076$

C $-3.413342-0.563073-0.080142$

C $-3.5941790 .103296-1.449290$

$\begin{array}{llll}\text { O } & -4.482245 & -0.122856 & 0.765517\end{array}$

C 3.7248490 .3178730 .034004

C $-3.4832811 .623053-1.424478$

H $4.722762-0.509579-1.772273$

H $5.493060-2.576196-0.804862$

H $4.741843-2.1264760 .835283$

H $2.4129830 .727179-1.641929$

H $0.572277-1.2865611 .297320$

H 1.4846360 .7286862 .049122

H $-0.084137 \quad 1.387527 \quad 1.600684$

H $\quad 0.7187471 .946121-0.627077$

H $\quad-0.790490 \quad 0.075228-1.091334$
H $2.9334142 .647243 \quad 1.365781$

H $2.7655023 .336260-0.261952$

H 1.5108123 .6068350 .957798

H -2.0710220 .7784470 .953054$

H $-0.142228-2.097310-1.234209$

H $-2.001576-1.9841551 .568599$

H $-3.545430-1.645576-0.195342$

H $-4.592527-0.148025-1.829323$

H $-2.872418-0.301249-2.167138$

H $-4.364350-0.5542721 .629304$

H $4.302804 \quad 1.247857-0.003908$

H $3.6428790 .021878 \quad 1.086923$

H $-3.6809662 .027817-2.422313$

H $-4.2084802 .064925-0.734312$

$\mathrm{H}-2.4818031 .948419-1.128172$

SCF Energy $(B 3 L Y P / 6-31 G * * / / M M F F)=-810.718490656$

F2-05_c00917

MMFF Geometry

C 3.4521231 .8363740 .505602

C 3.0902073 .0786010 .165998

C $2.332689-0.3878350 .226683$

O $0.987526 \quad 0.128504 \quad 0.215631$

C $0.071727-0.956626-0.020331$

C $0.906931-2.234407-0.005611$

C $2.267781-1.747904-0.475279$

C -1.069876 -0.923863 1.001992

C $2.342254-1.687498-2.001770$

C -1.842985 0.4097031 .092262

O $-0.530984-1.1852872 .307006$

$\begin{array}{llll}\text { O } & -2.905990 & 0.240024 & 2.040277\end{array}$

C $-2.4770080 .941128-0.206424$

C $-3.351660-0.090014-0.933241$

O $-1.4733201 .417755-1.096729$

C $3.2864880 .637403-0.386501$

C $-4.0870920 .516799-2.122273$

H 3.9028881 .6733691 .481209

H $3.2408273 .902370 \quad 0.855097$

H $2.6392063 .295569-0.796003$

H $2.592475-0.5522501 .281700$

H $-0.331358-0.817639-1.028692$

H $\quad 0.472823-3.021699-0.629657$

H $1.004814-2.6379231 .009016$

H $3.068525-2.407109-0.121368$

H -1.767109-1.745219 0.801946

H $1.615616-0.987261-2.426075$

H $3.339604-1.376924-2.329858$

H $2.149112-2.675244-2.433900$

H -1.1809661 .1805711 .506460$

H $\quad 0.140257-0.5050142 .488676$

H -2.507484 -0.078250 2.868552

H -3.112361 1.7992320 .048391

H $-4.088798-0.514648-0.242523$

H -2.736051 -0.914114-1.310368

H $-0.9454402 .079986-0.619069$

H $4.2747480 .186289-0.533105$

H $2.9167780 .958960-1.367656$

H $-3.3867320 .894242-2.873768$

H $-4.717726-0.239425-2.600599$

H -4.730966 $1.342996-1.804511$

SCF Energy $\left(\mathrm{B} 3 \mathrm{LYP} / 6-31 \mathrm{G}^{* *} / / \mathrm{MMFF}\right)=-810.718536057$

F2-05_c00918

MMFF Geometry

C $4.500190-1.145538-0.813535$

C $4.880522-2.199326-0.082381$

C $2.5834000 .458497-0.623708$

O $1.691543-0.4474760 .054736$

C 0.4657890 .2481380 .369956

C $0.5804201 .622065-0.279006$

C $2.0793601 .866390-0.294349$

C $-0.726902-0.593364-0.106617$

C $2.5658002 .427278 \quad 1.042754$

C -2.0902610 .1109810 .057467$

O $-0.709858-1.8235920 .622182$

$\begin{array}{llll}\text { O } & -2.274798 & 0.485023 & 1.422781\end{array}$

C $-3.299676-0.718703-0.423503$

C $-4.6459760 .006223-0.284176$ 
O $-3.416790-1.925806 \quad 0.331348$

C $4.0302690 .158494-0.231138$

C $-4.7552501 .271864-1.118445$

H $4.538257-1.222633-1.897129$

H $5.213994-3.111335-0.565266$

H $4.864918-2.1735921 .001601$

H $2.4458450 .281016-1.699422$

H $\quad 0.447756 \quad 0.341207 \quad 1.462551$

H $\quad 0.0087102 .3886620 .252737$

H $0.2137031 .589195-1.312903$

H $2.3579722 .574218-1.083103$

H $-0.583212-0.864046-1.159213$

H 2.0554803 .3691061 .271259

H 2.3852091 .7373631 .873148

H 3.6395832 .6378941 .007830

H $-2.060004 \quad 1.039889-0.520206$

H $-0.787309-1.6121031 .567934$

H -2.439770 -0.3274191 .930805$

H -3.154080 -1.002337 -1.472733

H $-4.845900 \quad 0.2389810 .769039$

H -5.446351 -0.682966 -0.583481

H -2.620051 -2.456601 0.162705

H $4.6892610 .950959-0.604479$

H 4.1275610 .1291920 .860845

H $-5.7746451 .668051-1.066727$

H $-4.0808472 .052450-0.755064$

$\mathrm{H}-4.5229721 .071664-2.169007$

SCF Energy (B3LYP/6-31G**//MMFF) $=-810.716440187$

F2-05_c00919

MMFF Geometry

C -2.474504 $2.717430-0.613029$

C $-1.6895703 .656634-0.073137$

C $-2.3516510 .208769-0.625828$

O $-0.9126810 .210648-0.551310$

C $-0.490274-0.6117180 .554026$

C $-1.738974-1.3031541 .098015$

C $-2.753909-1.132882-0.015573$

C $0.626229-1.5604970 .087049$

C $-4.194763-1.2659950 .444601$

C $1.817762-0.852436-0.591680$

O $0.103857-2.486921-0.873942$

O $2.810450-1.859171-0.830686$

C 2.4386040 .2899210 .234586

C $3.6881380 .895619-0.420115$

O $2.778426-0.187824 \quad 1.535918$

C $-2.886476 \quad 1.4561190 .095061$

C 4.1811012 .1308860 .323775

H -2.849700 $2.867961-1.622057$

H -1.431347 $4.546952-0.636175$

H -1.2911913 .5552850 .930222$

H -2.636478 $0.242368-1.684260$

H $-0.095977 \quad 0.054008 \quad 1.329846$

H -2.073451 -0.7814372 .003798$

H -1.576734 -2.347593 1.381418

H $-2.565605-1.911963-0.766464$

H $0.985201-2.1596290 .932974$

H $-4.389023-2.2848120 .796677$

H -4.427667 -0.5856151 .269954$

H -4.885054 -1.056345 -0.378753

H $1.510561-0.478204-1.576163$

H $-0.481416-3.104428-0.404536$

H $3.437052-1.506286-1.484617$

H 1.6968931 .0850500 .366181

H $3.4679851 .170216-1.458068$

H $4.5060310 .165841-0.431530$

H $3.410519-0.9188241 .424401$

H $-3.981596 \quad 1.4383900 .122224$

H -2.532479 1.4809011 .132551

H $5.0581302 .549256-0.180362$

H 3.4071602 .9043370 .354423

H 4.4674891 .8885431 .351969

SCF Energy (B3LYP/6-31G**//MMFF) $=-810.710132638$

F2-05_c00920

MMFF Geometry

C $5.039384-0.565748-0.896526$

C $6.1487610 .001712-0.410267$
C $2.558953-0.391465-0.479950$

O $1.484098-0.9895760 .270671$

C $0.435115-0.0095920 .441042$

C $0.8762661 .222494-0.341690$

C $2.3928081 .120918-0.313575$

C $-0.900029-0.617323-0.009910$

C 2.9549661 .6926040 .989166

C -2.0687310 .3897190 .005383$

O $-1.172108-1.7074190 .875524$

O

C $-3.415337-0.192994-0.470436$

C $-4.5201770 .873114-0.512028$

O $-3.832458-1.2629550 .374102$

C $3.885602-1.017663-0.051920$

C $-5.8185390 .334281-1.099107$

H $4.966561-0.728712-1.968669$

H $6.9511640 .299063-1.076503$

H 6.2768150 .1786120 .651813

H $2.369750-0.652963-1.530798$

H $\quad 0.403267 \quad 0.208445 \quad 1.515581$

H $\quad 0.4831902 .1523890 .080542$

H $\quad 0.5336401 .158382-1.382470$

H $2.8417101 .664014-1.152546$

H $-0.785278-1.045442-1.013163$

H $2.6141641 .136773 \quad 1.868527$

H $4.048414 \quad 1.674710 \quad 0.985781$

H $2.6488032 .737087 \quad 1.112536$

H $-1.8201251 .227670-0.655701$

H -1.956808 -2.1715330 .538147$

H $-2.3123330 .191797 \quad 1.935553$

H $-3.280451-0.611130-1.474847$

H $-4.1883131 .728820-1.111251$

H -4.7327891 .2390510 .499613$

H $-4.011053-0.8948481 .256127$

H $4.067294-0.8370941 .014445$

H $3.802480-2.108825-0.141702$

H $-6.223257-0.480810-0.491208$

H $-6.5711341 .128168-1.140479$

H -5.663006 -0.038605 -2.116414

SCF Energy $\left(B 3 L Y P / 6-31 G^{* *} / / M M F F\right)=-810.718238835$

F2-05 c00921

MMFF Geometry

C $-3.1297432 .302252-0.632245$

C -2.7323043 .4082370 .006933$

C $-2.486902-0.107606-0.401240$

$\begin{array}{lllll}\text { O } & -1.166176 & 0.140265 & 0.117405\end{array}$

C $-0.529612-1.1190610 .398531$

C -1.493474 -2.203343 -0.076664

C $-2.848661-1.5271340 .043678$

C $0.864315-1.191056-0.244776$

C -3.399491-1.632759 1.466417

C $1.840881-0.1209970 .293902$

O $0.737394-1.074691-1.661047$

O $1.4222101 .184276-0.102718$

C $3.295755-0.331786-0.174552$

C 4.2850340 .7285450 .331122

O $3.366741-0.329517-1.601385$

C -3.4390100 .9992080 .051306$

C 4.4110770 .7794711 .844630

H $-3.2480412 .338873-1.712168$

H -2.528784 $4.318800-0.545875$

H -2.6014983 .4261031 .083166$

H $-2.398208-0.096341-1.496597$

H $-0.422328-1.1799691 .488471$

H - $1.400285-3.1258860 .504664$

H -1.314263 -2.460140 -1.127497

H $-3.578516-1.975925-0.639459$

H $1.283413-2.183250-0.039838$

H -2.752693 -1.140490 2.199692

H $-4.392347-1.1763211 .533413$

H -3.502288 -2.682851 1.760393

H $1.818618-0.1547021 .389057$

H $0.382329-0.188790-1.848805$

H $\quad 0.530839 \quad 1.331040 \quad 0.256574$

H $3.635795-1.3207060 .154514$

H $4.0106471 .719721-0.050286$

H $5.2724340 .517759-0.099642$ 
H $2.9935510 .513303-1.911764$

H $-4.4720450 .726008-0.193347$

H $-3.376604 \quad 1.1313101 .138231$

H 5.2099021 .4709662 .131510

H 3.4872891 .1314402 .312746

H $4.654536-0.2064472 .252558$

SCF Energy (B3LYP/6-31G*//MMFF) $=-810.718523979$

F2-05_c00922

MMFF Geometry

C $-4.3868240 .757018-1.166816$

C $-5.365614 \quad 1.463427-0.590509$

C $-2.157349-0.190200-0.468488$

O $-0.746608 \quad 0.103886-0.458876$

C $-0.125872-0.653758 \quad 0.598074$

C $-1.224732-1.5193301 .213698$

C $-2.484275-0.7147860 .932461$

C $1.082066-1.4344080 .064280$

C $-2.690175 \quad 0.367660 \quad 1.993545$

C $2.125946-0.564266-0.666906$

O $0.607998-2.417892-0.864203$

O $3.249302-1.396346-0.983687$

C $2.6341290 .644044 \quad 0.142149$

C $3.766377 \quad 1.388281-0.581967$

O 3.0978820 .1859911 .411241

C $-2.925221 \quad 1.030672-0.973601$

C $4.146342 \quad 2.692843 \quad 0.107530$

H $-4.657614-0.058978-1.831778$

H $-6.4049181 .217444-0.778874$

H $-5.151022 \quad 2.2931110 .073781$

H $-2.286426-1.009544-1.189928$

H $0.2091990 .070901 \quad 1.348511$

H - $1.048811-1.7190112 .275352$

H $-1.301731-2.4895630 .709127$

H $-3.370528-1.3587450 .917941$

H $1.552014-1.9848820 .888354$

H -1.8781951 .1016712 .001618$

H -3.6268750 .9071381 .827003$

H $-2.748006-0.0804242 .991432$

H $1.720461-0.213920-1.624229$

H $1.336451-3.039444-1.030390$

H $2.963608-2.028210-1.664654$

H $1.803197 \begin{array}{llll}1.336266 & 0.318038\end{array}$

H $3.4598321 .613882-1.609970$

H $4.6639650 .760360-0.630559$

H 3.2424230 .9650441 .973721

H -2.756425 $1.889162-0.312184$

H - 2.502968 1.334125 -1.940556

H $3.2790383 .353702 \quad 0.202285$

H 4.5564592 .5142391 .105899

H $4.9095023 .217392-0.476442$

SCF Energy (B3LYP/6-31G**//MMFF) $=-810.709420472$

\section{F2-05 c00923}

MMFF Geometry

C $2.8013792 .321392-0.371855$

C $2.9938102 .738168-1.628428$

C 1.8329620 .2198750 .589043

O $0.972481-0.056884-0.538088$

C $0.445623-1.383231-0.404447$

C $0.716006-1.8092181 .031276$

C $2.035961-1.1122571 .325595$

C $-1.004649-1.483422-0.887235$

C $3.228664-1.9426030 .848459$

C $-2.094508-0.792455-0.041840$

O $-1.322882-2.881000-0.951696$

O $-3.336490-0.956061-0.745684$

C $-1.889738 \quad 0.703175 \quad 0.253489$

C $-3.096800 \quad 1.3226220 .972976$

O $-1.6796341 .419582-0.961574$

$\begin{array}{lllll}\text { C } 3.100396 & 0.928361 & 0.109257\end{array}$

C $-2.8310832 .760273 \quad 1.403243$

H $2.412874 \quad 3.027794 \quad 0.357275$

H $2.7607263 .759086-1.910070$

H $3.3818052 .074843-2.393390$

H $1.2691350 .891818 \quad 1.249327$

H $1.050946-2.018628-1.065006$

H $\quad 0.747690-2.896174 \quad 1.152017$
H $-0.047534-1.417421 \quad 1.712174$

H $2.157987-0.9409102 .401422$

H -1.057907 -1.105724 -1.916000

H $4.172235-1.4546701 .113377$

H $3.225351-2.9274341 .327811$

H $3.222824-2.096980-0.234882$

H $-2.224324-1.3350230 .901550$

H $-2.232750-2.955796-1.287391$

H -3.282916 -0.426753-1.560118

H -1.0079660 .8408430 .884673$

H $-3.3467700 .728291 \quad 1.859262$

H -3.9728641 .3268950 .313745$

H $-0.8114301 .157129-1.311592$

H 3.8206401 .0042360 .932146

H $3.5718050 .354172-0.697484$

H -2.6354313 .4050290 .540723$

H $-3.700623 \quad 3.1616991 .933248$

$\mathrm{H}-1.9694472 .8145052 .076304$

SCF Energy $\left(B 3 L Y P / 6-31 G^{* *} / / M M F F\right)=-810.720698696$

F2-05 c00924

MMFF Geometry

C $-4.626929-1.0496260 .431931$

C $-5.771260-0.761145-0.197720$

C $-2.245188-0.2259350 .370449$

O $-0.962891-0.490851-0.230508$

C $-0.189100 \quad 0.723100-0.226202$

C $-1.037447 \quad 1.7695300 .494749$

C -2.4572431 .2850910 .248153$

C 1.1957530 .5054820 .403544

C $-2.9665511 .756079-1.115210$

C $2.090795-0.482339-0.372213$

O $1.0469370 .051399 \quad 1.749009$

O $1.465299-1.770205-0.430175$

C $3.477980-0.7056560 .260660$

C 4.3022850 .5598590 .524661

O $4.243431-1.548619-0.609225$

C $-3.294090-1.149311-0.247852$

C $4.5614281 .406280-0.715654$

H -4.656948 -1.241649 1.501516

H $-6.704200-0.7121120 .353060$

H -5.799241 -0.570450 -1.264752

H $-2.135320-0.4812651 .434026$

H $-0.0599461 .018074-1.274772$

H -0.8411792 .7837240 .133059$

H -0.8393471 .7639691 .573395$

H -3.141356 1.6538061 .020267

H $1.692461 \quad 1.4802230 .450293$

H $-4.0073871 .458046-1.269472$

H -2.929799 2.848994-1.180835

H $-2.3758481 .349930-1.942411$

H $2.207806-0.149623-1.410337$

H $0.597288-0.8106151 .716031$

H $\quad 0.629109-1.670520-0.915795$

H $3.372409-1.2585241 .202139$

H $\quad 5.2778740 .261270 \quad 0.929106$

H 3.8197911 .1756801 .291242

H $3.722996-2.356362-0.760372$

H $-3.373744-0.969620-1.326896$

H $-2.942062-2.185634-0.161279$

H $5.0692860 .828651-1.494060$

H $3.6318041 .804329-1.132230$

H $5.2007582 .257435-0.459530$

SCF Energy (B3LYP/6-31G**//MMFF $)=-810.723777160$

F2-05 c00925

MMFF Geometry

C -4.9781250 .7975350 .324640$

C $-5.0729951 .509508-0.803870$

C -2.4053010 .4066230 .232020$

O

C $-0.301392-0.6363000 .346452$

C $-0.724885-0.520487-1.111997$

C $-2.240237-0.455715-1.023756$

C 1.0559620 .0024720 .670278

C $-2.849476-1.855114-0.929079$

C $2.217217-0.518153-0.195323$

O $1.344394-0.2496512 .049877$ 
O $2.236849-1.948693-0.102247$

C 3.6062370 .0002610 .224489

C 3.7476821 .5251840 .319298

O $4.580763-0.497586-0.699595$

C $-3.7268160 .311904 \quad 1.000062$

C $3.4076162 .276370-0.962123$

H $-5.9066410 .541647 \quad 0.831586$

H $-6.0470191 .801184-1.182587$

H $-4.2041501 .826839-1.366970$

H -2.212461 $1.457046-0.024704$

H $-0.313733-1.6843030 .669391$

H $-0.348049-1.343409-1.727047$

H $-0.3525530 .416650-1.545435$

H $-2.6584390 .038840-1.906800$

H $0.968351 \quad 1.0898230 .573047$

H -3.942832 -1.804557 - 0.916199

H $-2.562000-2.457450-1.797670$

H $-2.526632-2.388920-0.029529$

H $2.034246-0.283952-1.249842$

H $1.462525-1.2094462 .154684$

H $2.977037-2.264585-0.648401$

H $3.878256-0.4349751 .193338$

H 4.7875441 .7634640 .575729

H $3.125767 \quad 1.9091461 .135335$

H $4.308672-0.238800-1.595916$

H -3.885362 -0.7260211 .318873$

H -3.620869 0.8699591 .940302

H $4.0505811 .966559-1.791117$

H $2.3654432 .122098-1.255813$

H $3.5538943 .351230-0.812777$

SCF Energy (B3LYP/6-31G**//MMFF) $=-810.711693023$

F2-05_c00926

MMFF Geometry

C $-4.5165950 .841141-0.296382$

$\begin{array}{llll}C & -4.757283 & 2.068802 & 0.177568\end{array}$

C $-2.479360-0.619199-0.285455$

O $-1.5226320 .457298-0.234514$

C $-0.192679-0.097282-0.116174$

C $-0.354091-1.602590-0.281153$

C $-1.752776-1.8535220 .255854$

C $0.7221710 .587413-1.141397$

C $-1.750888-1.9843711 .779745$

C $2.1744210 .077156-1.197368$

O $\quad 0.7475291 .991743-0.873392$

O $2.192909-1.203093-1.839099$

C $2.915232-0.0779320 .146675$

C 2.9993621 .2049550 .980013

O $4.256816-0.495366-0.143117$

C $-3.756300-0.217408 \quad 0.453444$

C 3.7919321 .0120982 .268407

H $-4.8982350 .578567-1.279730$

H -5.317342 2.785549-0.412981

H $-4.402202 \quad 2.380228 \quad 1.153711$

H -2.702003 -0.784197-1.348736

H 0.1337990 .1452030 .901152

H $\quad 0.426100-2.1740920 .229813$

H $-0.331215-1.876804-1.343185$

H $-2.179982-2.771071-0.164118$

H $0.2689650 .479622-2.135233$

H $-1.404571-1.0719512 .275430$

H -2.756237 -2.207869 2.151106

H $-1.097020-2.8054652 .092707$

H $2.7659950 .747871-1.834208$

H $-0.1749952 .298627-0.843092$

H $1.814636-1.091070-2.727874$

H $2.465435-0.8811730 .740852$

H $1.999992 \quad 1.556598 \quad 1.251245$

H $3.4851391 .995706 \quad 0.395646$

H $4.198093-1.312904-0.666882$

H $-4.412261-1.0886730 .564198$

H -3.5128260 .1480641 .458492$

H $4.8343190 .749617 \quad 2.063407$

H $3.789874 \quad 1.9378102 .852907$

H $3.350697 \quad 0.2215862 .883673$

SCF Energy (B3LYP/6-31G**//MMFF) $=-810.714462696$

F2-05_c00927
MMFF Geometry

C 4.1911331 .8007430 .474462

C $4.3670322 .758776-0.442421$

C $2.621026-0.1472170 .625316$

O $1.5767010 .435630-0.178163$

C $0.507694-0.524442-0.327673$

C $0.881690-1.7131410 .548955$

C $2.399421-1.6610370 .564069$

C $-0.824320 \quad 0.1515610 .030606$

C $2.992465-2.348307-0.666918$

C $-2.025318-0.8147800 .012719$

O $-1.038021 \quad 1.196782-0.920725$

O $-2.142790-1.399620-1.283178$

C $-3.381285-0.2143310 .441465$

C $-3.9509330 .860997-0.491698$

$\begin{array}{llll}\text { O } & -3.271549 & 0.328675 & 1.755675\end{array}$

C $3.985990 \quad 0.3478210 .146216$

C $-5.397058 \quad 1.203021-0.147332$

H $4.2053282 .077149 \quad 1.525637$

H $4.5147983 .789423-0.138629$

H $4.3653112 .536102-1.503711$

H $2.4442850 .190188 \quad 1.656093$

H $0.509681-0.810302-1.386668$

H $\quad 0.471480-2.656958 \quad 0.176894$

H $\quad 0.509353-1.567107 \quad 1.571093$

H $2.804579-2.1507481 .456710$

H -0.7412740 .6299051 .013542$

H $2.674468-3.395495-0.711174$

H $2.686807-1.864765-1.600261$

H $4.086402-2.340542-0.626795$

H $-1.804044-1.6297650 .712582$

H $-0.2656891 .786883-0.882928$

H $-2.876114-2.037348-1.253075$

H $-4.109903-1.0342440 .486452$

H $-3.9093520 .527453-1.534148$

H $-3.3726441 .786885-0.404770$

H -2.961576 -0.3818342 .342583$

H $4.782502-0.2240410 .636314$

H $4.0841810 .198628-0.935842$

H -5.4821091 .5927710 .871879$

H $-5.7768141 .967051-0.833038$

H $-6.0395990 .320999-0.234205$

SCF Energy (B3LYP/6-31G**//MMFF) $=-810.709082062$

F2-05 c00928

MMFF Geometry

C -2.699264 $2.403928-0.753963$

C $-1.9464023 .459400-0.423981$

C $-2.325407-0.068985-0.525798$

O $-0.896706 \quad 0.068451-0.646942$

C $-0.262261-0.5432490 .493012$

C $-1.349935-1.3016221 .254175$

C $-2.502951-1.3600870 .270397$

C $0.908471-1.4173840 .021721$

C $-3.855898-1.5735890 .925290$

C $1.930747-0.715166-0.897269$

O $0.370397-2.530755-0.707151$

O $2.963882-1.670728-1.175856$

C $2.5954620 .564850-0.353552$

C $3.2326740 .385961 \quad 1.031434$

O $1.6609501 .637885-0.306598$

C $-2.893399 \quad 1.200930 \quad 0.127411$

C 4.0145821 .6195501 .467396

H $-3.2125232 .407991-1.712034$

H -1.849120 $4.298908-1.103615$

H -1.4148653 .5042340 .520034$

H -2.741927 $-0.185476-1.533396$

H $0.115596 \quad 0.259624 \quad 1.134266$

H $-1.632155-0.7287262 .146603$

H -1.033102 -2.289831 1.602641

H $-2.319457-2.201587-0.411607$

H $1.416336-1.8466030 .893591$

H -3.894839 -2.556664 1.406445

H $-4.062500-0.8243521 .696358$

H $-4.657428-1.5303870 .180943$

H $1.444966-0.496444-1.856562$

H $1.120505-3.083876-0.985263$

H $3.566011-1.270374-1.826025$ 
H $3.3885420 .864468-1.050515$

H $3.908388-0.4765691 .028066$

H $2.4594970 .200361 \quad 1.785296$

H $1.2730861 .731366-1.193382$

H $-3.9695801 .089706 \quad 0.299411$

H -2.4221961 .3756841 .101957$

H 4.4736271 .4477092 .446230

H 4.8128151 .8493250 .754530

H 3.3636282 .4954721 .550763

SCF Energy (B3LYP/6-31G**//MMFF) $=-810.713686689$

F2-05_c00929

MMFF Geometry

C 3.5746691 .9783850 .708397

C 3.4102053 .1097950 .013722

C $2.540513-0.2953290 .514043$

O $1.3098580 .152775-0.085253$

C $0.487121-0.990351-0.378404$

C $1.220851-2.2025730 .189363$

C $2.676897-1.7721510 .132422$

C $-0.945990-0.800248 \quad 0.146179$

C $3.280418-2.025836-1.249776$

C $-1.7137650 .338516-0.558187$

O $-0.930204-0.5322811 .547806$

O $-1.0175961 .580456-0.397413$

C $-3.1419300 .575221-0.026205$

C $-4.027690-0.675234-0.044872$

O $-3.759794 \quad 1.567227-0.855110$

C 3.6902930 .6156020 .083925

C $-5.449133-0.3906370 .426613$

H 3.6387042 .0373121 .792008

H 3.3376774 .0630840 .525700

H $3.3431193 .106801-1.068496$

H $2.399150-0.2268901 .601661$

H $\quad 0.450024-1.077578-1.471502$

H $1.003371-3.118530-0.368702$

H $\quad 0.946908-2.384791 \quad 1.234290$

H $3.279508-2.3115120 .871832$

H -1.488565 -1.739684 -0.008257

H $2.771733-1.458611-2.035819$

H $4.339685-1.749358-1.267624$

H $3.215726-3.088633-1.506601$

H $-1.7584080 .143248-1.636744$

H $-0.698101-1.3552062 .008617$

H $-0.1449091 .487669-0.815487$

H -3.1062950 .9928060 .987364$

H -3.606009 -1.452314 0.600651

H -4.078861 -1.078364 -1.063400

H -3.191466 $2.356315-0.832978$

H 4.6470090 .1811160 .395936

H $3.7075470 .713074-1.008299$

H -6.032825 -1.3167550 .440618$

H $-5.449144 \quad 0.025677 \quad 1.438953$

H $-5.9584480 .314754-0.237265$

SCF Energy (B3LYP/6-31G**//MMFF) $=-810.712316201$

F2-06 c01137

MMFF Geometry

C $2.6959992 .717979-0.022070$

C 2.0174373 .6005460 .719779

C $2.3256380 .291416-0.527204$

O $\quad 0.897587 \quad 0.349092-0.363149$

C $0.345099-0.952648-0.632203$

C $1.526176-1.903280-0.820693$

C $2.693244-1.153439-0.207449$

C $-0.611138-1.3465810 .504674$

C $4.042785-1.588053-0.753732$

C $-1.710986-0.3087210 .809295$

O $\quad 0.137045-1.545778 \quad 1.710744$

O $-2.588244-0.8792071 .789326$

C $-2.5522840 .110429-0.408953$

C $-3.7053111 .051229-0.030557$

O $-3.083021-1.049189-1.049659$

C 2.9862641 .3070640 .405564

C $-4.4495671 .567404-1.255711$

H $3.0724783 .032527-0.992066$

H $1.844494 \quad 4.6070160 .354747$

H 1.6232523 .3378701 .695138
H $2.5464580 .525775-1.577241$

H -0.214283 -0.889903-1.572031

H $1.694245-2.055278-1.894748$

H $1.368350-2.892256-0.379164$

H $2.682304-1.3117070 .878423$

H $-1.068783-2.3161480 .275819$

H $4.107512-1.429953-1.835525$

H $4.855495-1.026711-0.282447$

H $4.211647-2.652199-0.558528$

H $-1.2723410 .583706 \quad 1.272518$

H $0.571132-0.7032091 .929354$

H -2.042550 -1.133176 2.553308

H -1.910962 $0.617595-1.137768$

H $-3.3164091 .905740 \quad 0.535131$

H -4.4295790 .5306100 .607003$

H $-3.631830-1.518960-0.398449$

H $2.636761 \quad 1.1477791 .433554$

H 4.0734881 .1710450 .404702

H $-5.2462922 .253427-0.951159$

H -3.773636 2.109081-1.925092

H $-4.9080270 .747910-1.818005$

SCF Energy $(B 3 L Y P / 6-31 G * * / / M M F F)=-810.724905784$

F2-06 c01138

MMFF Geometry

C $4.1338091 .605652-0.052875$

C $3.8989852 .890624-0.341690$

C $2.658476-0.4212890 .013962$

$\begin{array}{llll}\text { O } & 1.731426 & 0.066903 & -0.976364\end{array}$

C $0.556214-0.776301-0.992063$

C $0.907995-1.981770-0.131722$

C $1.862798-1.3911300 .885043$

C $-0.6223960 .079478-0.487650$

C $2.716678-2.4314131 .590006$

C $-1.947467-0.684716-0.321858$

O $-0.8304081 .130133-1.440875$

O $-2.207145-1.433862-1.515763$

C $-3.1822170 .197647-0.047614$

C -3.0208271 .1127551 .170901$

O $-4.297255-0.6689610 .192075$

C 3.2339510 .7576370 .800900

C -4.2706331 .9405531 .446440$

H $5.0326091 .138590-0.447037$

H $4.5915553 .449613-0.961557$

H 3.0189863 .4041840 .029207

H $3.453951-0.951033-0.526184$

H $\quad 0.386089-1.070973-2.033698$

H $1.433381-2.721605-0.749783$

H $\quad 0.053168-2.4889610 .320244$

H $1.283272-0.8384661 .636108$

H -0.3512380 .5668480 .455252$

H $3.399566-1.9584402 .302671$

H $2.086076-3.1353922 .142978$

H $3.318045-3.0068940 .878219$

H -1.857777 -1.420986 0.483813

H $0.0108871 .610464-1.529436$

H -2.255091 -0.801529 -2.253460

H $-3.4337520 .792793-0.933528$

H -2.1815611 .8004131 .024805$

H -2.8064450 .5098492 .061573$

H $-4.385307-1.248402-0.584099$

H 2.4156601 .3678351 .203822

H 3.8204860 .3932531 .651898

H -4.5310482 .5556670 .579279$

H -5.1275141 .3045901 .688922$

H -4.0991492 .6085312 .296726$

SCF Energy (B3LYP/6-31G**//MMFF) $=-810.722210720$

F2-06 c01139

MMFF Geometry

C $2.5104842 .748680-0.178654$

C 1.7184443 .6620920 .393877

C $2.2873590 .269249-0.465307$

O $0.8490700 .275082-0.448426$

C $0.373977-1.073090-0.620885$

C $1.604521-1.978511-0.608778$

C $2.675403-1.1180600 .034274$

C $-0.664620-1.3958910 .466712$ 
C $4.088233-1.539586-0.332884$

C $-1.805164-0.3614630 .579605$

O $0.002692-1.452104 \quad 1.733154$

O $-2.763312-0.8598041 .521311$

C $-2.538149-0.071944-0.743115$

C $-3.7807230 .819406-0.597824$

O $-2.950466-1.312624-1.321691$

C $2.809924 \quad 1.4024570 .418361$

C $-3.4793882 .198951-0.035822$

H $2.9779792 .987000-1.130611$

H $1.5449904 .618204-0.087689$

H 1.2300213 .4744261 .343692

H $2.6049280 .410127-1.507212$

H -0.102621 -1.133752 -1.605525

H $1.881491-2.218649-1.643460$

H $1.444886-2.929640-0.091494$

H $2.561751-1.1689871 .124584$

H -1.073495 -2.398183 0.289444

H $4.828054-0.8963440 .153772$

H $4.276996-2.570020-0.014138$

H $4.254894-1.486088-1.413905$

H -1.4138580 .5728330 .998943$

H $-0.608944-1.8593972 .369239$

H $-2.349514-0.8378302 .400336$

H $-1.8460880 .401308-1.448863$

H $-4.537903 \quad 0.3222310 .020614$

H -4.247531 $0.934690-1.584498$

H $-3.359498-1.114610-2.181368$

H $2.363609 \quad 1.3285831 .418067$

H 3.8958471 .3198940 .537916

H -3.1429052 .1440801 .003594$

H -2.707166 $2.703247-0.625134$

$\mathrm{H}-4.3815762 .818734-0.058445$

SCF Energy (B3LYP/6-31G**//MMFF) $=-810.711042408$

F2-06 c01140

MMFF Geometry

C $3.0609082 .032606-1.112729$

C $2.5516233 .191881-0.680967$

C $2.383310-0.361656-0.755567$

O $0.992290-0.028236-0.571626$

C $0.418374-0.8873200 .427588$

C $1.590265-1.5463361 .142632$

C $2.635988-1.6426740 .046777$

C $-0.528327-0.0947141 .337575$

C $2.438575-2.891713-0.814697$

C -1.636334 0.6774190 .590643

O 0.2284150 .8613702 .090964

O $-2.487978 \quad 1.2839511 .571182$

C $-2.488458-0.176914-0.366066$

C $-3.6544300 .612234-0.978859$

O $-2.996811-1.318327 \quad 0.322782$

C $3.2308300 .815141-0.248082$

C $-4.409713-0.199397-2.023924$

H $3.3820931 .957669-2.148382$

H $2.458546 \quad 4.036177-1.355237$

H 2.2189123 .3192260 .343245

H $2.561826-0.513717-1.826698$

H $-0.150071-1.658410-0.104658$

H $1.319130-2.5093421 .586025$

H $1.976065-0.9114011 .948246$

H $3.649509-1.6799710 .461702$

H - $0.974422-0.7712712 .075466$

H $1.457773-2.911914-1.300258$

H $3.199334-2.934560-1.601520$

H $2.534402-3.797617-0.206915$

H -1.193250 1.5096370 .030625

H $\quad 0.658296 \quad 1.460510 \quad 1.456784$

H -2.852282 0.5768572 .129826

H -1.853817 -0.549655-1.177493

H $-3.2767341 .529287-1.445544$

H $-4.3688340 .904456-0.200049$

H -3.632172 -1.0076230 .989538$

H 2.9676161 .0545610 .789294

H $4.2918690 .539148-0.258841$

H -3.743208 $-0.510368-2.834748$

H -4.859091 -1.095129 -1.584111

H $\quad-5.2144850 .402037-2.458650$
SCF Energy (B3LYP/6-31G**//MMFF) $=-810.713931913$

F2-06 c01141

MMFF Geometry

C 3.4775732 .1406450 .085481

C 2.8677393 .2891860 .399594

C $2.608600-0.180748-0.284734$

O $1.3035880 .248030-0.711451$

C $0.495679-0.906041-0.998978$

C $1.340237-2.128212-0.643623$

C $2.393002-1.5740230 .295960$

C $-0.841345-0.831378-0.235223$

C $3.651584-2.4227840 .360399$

C $-1.7239210 .374404-0.619126$

O $-0.601707-0.8117951 .171071$

O $-1.030087 \begin{array}{llll}1.598601 & -0.349749\end{array}$

C -3.0582700 .4615720 .149181$

C $-3.919918-0.8014860 .045241$

O $-3.8078461 .554134-0.396391$

C 3.1650700 .8194270 .729322

C $-5.257700-0.6556690 .761605$

H $4.2539832 .153499-0.675028$

H $3.1423234 .211528-0.100435$

H 2.0883823 .3305641 .152464

H $3.247012-0.226100-1.177156$

H $0.283571-0.906440-2.074666$

H $1.812221-2.513721-1.556618$

H $\quad 0.762853-2.949792-0.208235$

H $1.963648-1.4995391 .303131$

H -1.388881 -1.755010 -0.451534

H $3.416733-3.4322460 .713438$

H $4.126153-2.511933-0.622607$

H $4.382768-1.9874371 .048552$

H $-1.9265670 .358992-1.697034$

H $-0.1212250 .009918 \quad 1.371729$

H $-0.2321531 .614373-0.904756$

H $-2.875146 \quad 0.703141 \quad 1.203258$

H -3.395706 -1.659209 0.478441

H -4.118879 -1.029090 -1.008970

H $-3.2536332 .350067-0.322334$

H 2.4433000 .9615491 .543654

H 4.0887960 .4335341 .174717

H $\quad \begin{array}{llll}5.870691 & 0.130715 & 0.310679\end{array}$

H $-5.820108-1.5929310 .701568$

H $-5.110322-0.4155531 .819326$

SCF Energy $(B 3 L Y P / 6-31 G * * / / M M F F)=-810.727003525$

F2-06_c01142

MMFF Geometry

C $-3.8210522 .050322-0.289789$

C $-3.1993483 .234699-0.276446$

C $-2.995186-0.3027410 .023612$

$\begin{array}{llll}\text { O } & -1.873212 & 0.093064 & 0.836982\end{array}$

C $-0.715556-0.6918810 .483725$

C $-1.080760-1.435960-0.791584$

C $-2.583447-1.603043-0.671619$

C 0.4972850 .2406390 .362368

C $-2.947088-2.8281860 .169989$

C $1.772509-0.466947-0.135742$

O $0.739408 \quad 0.8059331 .653716$

O $2.080011-1.5615360 .728096$

C $3.0030090 .455749-0.260884$

C $4.201485-0.283646-0.872267$

O $3.3998600 .954702 \quad 1.014332$

C $-3.2837770 .826653-0.977960$

C $5.3821830 .644833-1.128869$

H $-4.777697 \quad 1.9576180 .217741$

H -3.6423044 .0818360 .235695$

H $-2.2444703 .380057-0.769399$

H $-3.861830-0.4512450 .678568$

H $-0.565885-1.3951131 .311996$

H $-0.536655-2.379480-0.898497$

H $-0.855466-0.822642-1.672773$

H $-3.055871-1.717128-1.653525$

H $0.2588521 .077350-0.305491$

H -2.536892 -2.773208 1.183549

H -4.035046 -2.920806 0.256312

H $-2.569224-3.742831-0.298699$ 
H $1.571152-0.893648-1.124872$ H $-0.082159 \quad 1.242470 \quad 1.937973$

H $2.276320-1.1912151 .605860$

H $2.7486151 .315148-0.892745$

H $3.907819-0.750849-1.819208$

H $4.538233-1.080312-0.198134$

H 2.6599351 .4746321 .370656

H $-2.375015 \quad 1.083427-1.535803$

H -4.032149 $0.494370-1.707082$

H $5.1005931 .458006-1.805497$

H $5.754501 \quad 1.083958-0.198137$

H $6.2045680 .089365-1.590701$

SCF Energy $\left(B 3 L Y P / 6-31 G^{* *} / / M M F F\right)=-810.724993981$

F2-06_c01143

MMFF Geometry

C $-4.3270101 .660370-0.038082$

C $-4.5471422 .322093-1.179619$

C $-2.710050-0.1468280 .682589$

O $-1.444447-0.2876941 .360455$

C $-0.479871-0.8779720 .465088$

C $-1.101446-0.820290-0.921469$

C $-2.587418-0.919769-0.633939$

C $0.850859-0.126714 \quad 0.602374$

C $-3.032471-2.374814-0.470873$

C $1.925318-0.571477-0.404799$

O $1.349424-0.3478711 .925681$

O $2.054323-1.998385-0.349347$

C $3.3256380 .030898-0.175735$

C $3.3343041 .562823-0.138599$

O $4.167848-0.392092-1.254274$

C $-2.9626621 .358040 \quad 0.505657$

C 4.7363482 .1330170 .041224

H -5.1818331 .3271330 .544655$

H -5.559754 2.514514 -1.516861

H $-3.7287572 .679631-1.794708$

H -3.485534 $-0.569748 \quad 1.332431$

H $-0.361453-1.9172690 .794952$

H $-0.724726-1.604647-1.584898$

H $-0.8907580 .145842-1.396452$

H -3.179002 $-0.480132-1.443332$

H 0.6698220 .9502800 .510042

H -2.505207 -2.882705 0.343146

H -4.104686 -2.421831 -0.252679

H -2.853339 -2.937793 -1.392941

H $1.599271-0.338306-1.424868$

H $0.657614-0.0625782 .547249$

H $2.301882-2.2347180 .561258$

H $3.769801-0.3689570 .743703$

H 2.7096091 .9292320 .682296

H $2.9211521 .958728-1.074182$

H $4.149213-1.364459-1.271607$

H $-2.8645721 .850473 \quad 1.482014$

H -2.176992 $1.803223-0.117900$

H $5.3861901 .872601-0.799966$

H 4.6929523 .2251480 .104102

H 5.1955491 .7584230 .961530

SCF Energy (B3LYP/6-31G**//MMFF)= -810.723101553

F2-06_c01144

MMFF Geometry

C -4.177031-1.326989 0.178303

C $-4.065980-2.6583360 .112305$

C $-2.5273680 .537258-0.127902$

O $-1.677990-0.195153-1.034265$

C $-0.4260270 .511854-1.199823$

C $-0.6476971 .877363-0.563775$

C -1.6237881 .5642560 .551089$

C $0.675124-0.356094-0.557310$

C -2.3610292 .7861251 .072418$

C $2.0666750 .302231-0.523029$

O $0.739805-1.561502-1.331922$

O $2.3897430 .733516-1.851390$

C $3.207324-0.608247-0.029676$

C $2.980671-1.2589281 .341878$

$\begin{array}{llll}\text { O } & 4.396137 & 0.189746 & 0.031202\end{array}$

C $-3.179678-0.4326960 .858988$

C $2.747679-0.2641732 .472429$
H $-5.042585-0.852818-0.277270$

H -4.823962 -3.248516 -0.391191

H $-3.223663-3.1801500 .552915$

H $-3.2897441 .038077-0.738924$

H $-0.2490920 .604362-2.277381$

H -1.123226 2.540904-1.298219

H $0.2582692 .378702-0.218438$

H -1.0764461 .0986841 .381176$

H $0.362362-0.6501910 .450563$

H -2.926712 3.2835520 .277414

H -3.0646622 .5095981 .863988$

H -1.6549253 .5128081 .487334$

H $2.041593 \quad 1.2069590 .092947$

H $1.257995-2.212154-0.830176$

H $3.2674711 .151130-1.814833$

H $3.404630-1.387439-0.775346$

H $3.868760-1.8494941 .599940$

H $2.140298-1.9595851 .298550$

H $5.128119-0.3948820 .292295$

H -2.406372 -1.038194 1.348313

H -3.7050880 .1237341 .643276$

H 1.8263330 .3060492 .322150

H $2.655856-0.7964053 .424849$

H 3.5799720 .4406762 .561699

SCF Energy $\left(B 3 L Y P / 6-31 G^{* *} / / M M F F\right)=-810.704810830$

F2-06 c01145

MMFF Geometry

C $3.0915842 .307364-0.281992$

C 2.6551463 .3487350 .435302

C $2.225601-0.025565-0.575330$

O $\quad 0.8536810 .319960-0.316185$

C $0.041582-0.860090-0.458091$

C $1.000911-2.034627-0.649847$

C $2.335289-1.492621-0.174086$

C $-0.885354-0.9920760 .759935$

C $3.528555-2.215800-0.775505$

C $-1.7525990 .246073 \quad 1.075744$

O $-0.090950-1.2607251 .924768$

O $-2.593111-0.0764202 .192326$

C $-2.6715680 .760868-0.047543$

C $-3.569119-0.325092-0.657153$

O $-1.9072531 .373669-1.080605$

C 3.1425160 .8955030 .230026

C $-4.5744960 .250294-1.647777$

H $3.4410622 .482747-1.296228$

H 2.6475684 .3455660 .007953

H 2.2984673 .2278841 .452101

H $2.3992220 .097116-1.652757$

H $-0.559108-0.747020-1.366131$

H $1.050723-2.284006-1.717683$

H $0.692058-2.943893-0.124496$

H $2.381431-1.5799450 .918861$

H -1.527768 -1.871317 0.636500

H $3.505596-3.278835-0.513870$

H $3.535898-2.138145-1.867924$

H $4.468742-1.798068-0.402329$

H -1.1018841 .0652791 .406709$

H $0.506688-0.5040192 .052718$

H $-2.014741-0.3856272 .910540$

H $-3.323843 \quad 1.5376810 .372294$

H $-4.115519-0.8519130 .133078$

H -2.962838 -1.064361-1.192376

H -1.361864 2.067301 -0.671963

H $2.8555340 .870648 \quad 1.288842$

H $4.1800180 .549746 \quad 0.163836$

H -5.219989 $0.990841-1.164995$

H -4.071524 $0.729827-2.493352$

H $-5.211164-0.547148-2.043868$

SCF Energy (B3LYP/6-31G**//MMFF) $=-810.720776895$

F2-06 c01146

MMFF Geometry

C $3.0576032 .512923-0.062635$

C 2.2595283 .5478640 .223168

C $2.6035710 .063542-0.338181$

O $1.2391890 .244200-0.756639$

C $0.642302-1.042457-0.992241$ 
C $1.687462-2.086710-0.605964$

C $2.639140-1.3233280 .294269$

C $-0.682367-1.165715-0.214788$

C $4.026617-1.9387010 .365705$

C $-1.736603-0.111855-0.620980$

O $-0.429689-1.089902 \quad 1.186394$

O $-1.3138161 .196758-0.239294$

C $-3.130141-0.374906-0.011679$

C $-4.1682570 .640840-0.509396$

O $-3.087735-0.301036 \quad 1.411675$

$\begin{array}{llll}\text { C } 2.988727 & 1.181828 & 0.631086\end{array}$

C $-5.5757350 .309252-0.028750$

H $3.8109262 .633359-0.836813$

H $2.3625594 .485322-0.312475$

H 1.4936593 .4797640 .987845

H $3.2303610 .096556-1.239219$

H $0.426941-1.118279-2.064605$

H $2.209109-2.422014-1.511724$

H $1.263876-2.976845-0.130626$

H $2.215432-1.2863311 .305788$

H $-1.095896-2.162723-0.409004$

H $4.679064-1.3577761 .024909$

H $3.974361-2.9596280 .758139$

H $4.498010-1.981636-0.621922$

H -1.835889 $-0.118482-1.713558$

H $-0.097236-0.194791 \quad 1.372904$

H - $-0.4696821 .377438-0.686250$

H $-3.458617-1.385630-0.282808$

H $-4.1665990 .668729-1.605140$

H -3.917420 $1.645992-0.149837$

H -2.478891 -0.9907341 .725378$

H 2.2629461 .2254911 .453049

H 3.9710160 .9791991 .072478

H $-5.880851-0.685604-0.368331$

H -5.6414280 .3360601 .063348$

H -6.290425 $1.036943-0.426305$

SCF Energy (B3LYP/6-31G**/MMFF) $=-810.727001998$

\section{F2-06 c01147}

MMFF Geometry

C $2.589652 \quad 2.7533430 .544426$

C $2.006047 \quad 3.334502-0.510169$

C $2.2759780 .313464-0.273582$

$\begin{array}{llll}\text { O } & 0.838260 & 0.335980 & -0.269718\end{array}$

C $0.350041-0.953435-0.684539$

C $1.566382-1.871990-0.789152$

C $2.634182-1.1438670 .004968$

C $-0.728597-1.4366020 .299602$

C $4.048560-1.527456-0.396996$

C $-1.860468-0.416400 \quad 0.546095$

O $-0.102922-1.7103301 .559287$

O $-2.846501-1.0390551 .378706$

C $-2.5643990 .094166-0.725980$

C $-3.7598551 .018920-0.453222$

O $-3.067291-1.008504-1.484252$

C $2.807127 \quad 1.2823310 .787270$

C $-3.3898912 .298030 \quad 0.279442$

H 2.9593163 .4085781 .331232

H $1.9164954 .414995-0.554415$

H $1.6085152 .768048-1.343358$

H $2.615705 \quad 0.605033-1.275949$

H $-0.091352-0.835265-1.679973$

H $1.864073-1.951915-1.842687$

H $1.380411-2.889764-0.432108$

H $2.494287-1.3666101 .070483$

H - $1.141148-2.390074-0.051431$

H $4.787151-0.9873190 .203193$

H $4.213074-2.599532-0.247344$

H $4.241153-1.301412-1.451271$

H -1.4642140 .4325291 .115268$

H $-0.738836-2.2071132 .101139$

H $-2.454730-1.1559632 .260430$

H $-1.8532490 .632987-1.361980$

H -4.5393400 .4842000 .103256$

H $-4.2228101 .282382-1.413131$

H -2.307976 -1.505811 -1.830293

H 2.3367751 .0468821 .750742

H 3.8861661 .1319160 .908537
H -3.058886 2.0936791 .301731

H $-2.5920992 .834332-0.243855$

H -4.2593982 .9604520 .340616$

SCF Energy (B3LYP/6-31G**//MMFF) $=-810.708161083$

F2-06 c01148

MMFF Geometry

C $4.346000-0.3517401 .324974$

C $5.340919-0.9526540 .662999$

C $2.1004110 .604394 \quad 0.664327$

$\begin{array}{lllll}\text { O } & 0.703925 & 0.240182 & 0.665717\end{array}$

C $0.1911150 .300977-0.675719$

C $1.4073300 .345738-1.590832$

C $2.4285711 .091779-0.751843$

C $-0.767009-0.867377-0.938162$

C $2.2613062 .608143-0.873443$

C $-1.929696-0.9810530 .070154$

O $-0.035235-2.099009-0.890986$

O $-2.781366-2.051301-0.358972$

$\begin{array}{lll}\text { C }-2.767103 & 0.300129 & 0.240212\end{array}$

C -3.985892 0.0896331 .150161

O $-3.201520 \quad 0.770382-1.035009$

C $2.892177-0.639007 \quad 1.094808$

C $-4.7308091 .390721 \quad 1.421501$

H 4.5951880 .3825972 .086555

H $6.374143-0.7044540 .880259$

H $5.145582-1.695434-0.102395$

H 2.2373891 .3966941 .409854

H $-0.3550491 .246819-0.765629$

H $1.1878490 .820689-2.552061$

H $1.787291-0.660528-1.800726$

H $3.4498680 .846155-1.060402$

H - $1.161088-0.792499-1.958226$

H $2.4068732 .929729-1.909984$

H $1.2710992 .945121-0.550481$

H $3.0050283 .122703-0.255554$

H -1.541397-1.288384 1.048349

H $0.361001-2.171373-0.005711$

H $-3.096303-1.842411-1.254605$

H -2.1385891 .0858240 .673541$

H $-3.665333-0.3422082 .105248$

H $-4.691276-0.6109620 .687656$

H -3.831779 $0.123539-1.394385$

H $2.465543-1.0260272 .029231$

H $2.756927-1.4451810 .362785$

H -4.0737512 .1264921 .895910$

H $-5.123897 \quad 1.824897 \quad 0.496891$

H -5.5756791 .2080842 .093057$

SCF Energy (B3LYP/6-31G**//MMFF) $=-810.715290472$

F2-06 c01149

MMFF Geometry

C 3.5289302 .1618570 .339378

C $3.3589932 .698755-0.874448$

C $2.511194-0.152507-0.234646$

O $1.1900250 .332196-0.529823$

C $0.336262-0.781215-0.843489$

C $1.164612-2.043903-0.613899$

C $2.293899-1.5722410 .280979$

C $-0.953979-0.724338-0.002636$

C $3.527075-2.4574240 .210901$

C $-1.821127 \quad 0.525936-0.260193$

O $-0.636198-0.8011891 .386406$

$\begin{array}{llll}0 & -1.085139 & 1.709660 & 0.073676\end{array}$

C -3.1180440 .5757550 .570592$

C $-4.022504-0.6598090 .468950$

O $\begin{array}{lllll}\text { O } & -8.861773 & 1.722808 & 0.140576\end{array}$

C 3.1773350 .7686640 .792076

C $-4.481061-0.980298-0.948327$

H 3.9757122 .7946461 .104383

H $3.6610093 .723948-1.062089$

H $2.9250352 .149116-1.700563$

H $3.080607-0.170643-1.172818$

H $0.066212-0.707359-1.903645$

H $1.562405-2.390099-1.576632$

H $0.592871-2.873065-0.185492$

H $1.934575-1.5446701 .317518$

H $-1.537835-1.618998-0.242885$ 
H $3.933212-2.499938-0.805322$

H $4.314996-2.0853060 .872704$

H $3.284023-3.4796650 .519079$

H -2.062499 $0.602962-1.326917$

H $-0.122880-0.0057651 .610337$

H $-0.3109761 .751119-0.512615$

H $-2.868471 \quad 0.7521901 .624154$

H $-4.921350-0.486417 \quad 1.074357$

H -3.522312 -1.5347130 .897685$

H $-4.648911 \quad 1.791177 \quad 0.707581$

H 2.5113360 .8754721 .658218

H $4.102705 \quad 0.303122 \quad 1.150707$

H -5.014280 -0.135588 -1.395131

H -3.637536 -1.237508 -1.595402

H $-5.162477-1.837217-0.935326$

SCF Energy $\left(B 3 L Y P / 6-31 G^{* *} / / M M F F\right)=-810.716786572$

F2-06_c01150

MMFF Geometry

C $-2.9796222 .422055-0.129604$

C $-2.0828573 .348837-0.485031$

C $-2.796964-0.0475680 .303168$

O $-1.4172550 .037497 \quad 0.711582$

C -0.697655 -1.112682 0.240484

C $-1.603301-1.783008-0.784953$

C $-2.993173-1.452276-0.274892$

C $0.686186-0.730195-0.309600$

C $-3.450421-2.4417390 .798930$

C $1.643896-0.1488980 .748934$

O $0.537442 \quad 0.204907-1.378010$

O $1.034150 \quad 0.9999201 .349099$

C $3.017144 \quad 0.2936840 .203391$

C $3.764406-0.812390-0.551676$

O $3.797011 \quad 0.6998041 .334162$

C $-3.0582591 .050460-0.739402$

C $5.140537-0.376644-1.040518$

H -3.7058582 .6673810 .640947$

H $-2.0808174 .322700-0.007824$

H $-1.3389413 .155136-1.249878$

H $-3.4279670 .118975 \quad 1.184309$

H $-0.563963-1.7798961 .100926$

H - $1.405855-2.855573-0.876230$

H -1.470524 -1.339603 -1.778641

H -3.732645 -1.469994-1.083104

H $1.132149-1.635171-0.736000$

H $-4.450032-2.1762061 .159419$

H -3.500503 -3.4567340 .390858$

H $-2.779172-2.4570591 .663544$

H $1.782320-0.8827931 .552591$

H $\quad 0.1402621 .010152-1.003618$

H 1.6480731 .3340322 .025570

H $2.8945521 .178983-0.432366$

H $3.185311-1.133020-1.423578$

H $3.893611-1.6841410 .100925$

H $4.554621 \quad 1.2104391 .002404$

H $-2.3424360 .968937-1.566264$

H -4.061749 $0.927149-1.163803$

H $5.0693690 .521613-1.661983$

H $5.816501-0.169206-0.205596$

H $5.593420-1.170936-1.642668$

SCF Energy $\left(B 3 L Y P / 6-31 G^{* *} / / M M F F\right)=-810.715228204$

F2-06_c01151

MMFF Geometry

C $4.275216-1.332831-0.491538$

C $5.603742-1.278906-0.638339$

C $2.578668 \quad 0.363214 \quad 0.245856$

O $1.656801-0.605787 \quad 0.783823$

C $0.393124 \quad 0.0382931 .071851$

C 0.6556331 .5302210 .922418

C $1.7282681 .569205-0.146485$

C $-0.651447-0.5620880 .108989$

C $2.4955442 .880047-0.185940$

$\begin{array}{llll}C & -2.052626 & 0.075216 & 0.212775\end{array}$

O $-0.748569-1.9579250 .416480$

$\begin{array}{llll}\text { O } & -2.515828 & 0.000278 & 1.560635\end{array}$

C -3.105504 -0.538511 -0.736375

C $-4.497372 \quad 0.104077-0.633344$
O $-3.265992-1.935332-0.487887$

C $3.336574-0.246374-0.935353$

C $-4.5252991 .577663-1.004229$

H $3.837823-2.216851-0.034630$

H $6.227206-2.099826-0.301406$

H $6.090670-0.422742-1.092000$

H $3.2735930 .627137 \quad 1.053821$

H $0.132418-0.2129092 .106224$

H 1.0586961 .9183281 .867266

H -0.2206372 .1326970 .677246$

H $1.2602621 .400941-1.125169$

H $-0.297888-0.496925-0.926792$

H $2.985536 \quad 3.0875590 .771415$

H $3.2676342 .858177-0.961395$

H $1.8206103 .713779-0.405396$

H $-1.9671601 .137185-0.031288$

H $\quad 0.142675-2.337144 \quad 0.325839$

H $-2.484145-0.9332061 .831127$

H $-2.745916-0.444550-1.767754$

H $-4.904959-0.0298300 .376177$

H $-5.182863-0.440275-1.295877$

H -3.603291 -2.040719 0.417708

H $2.627832-0.688967-1.646130$

H $3.8900750 .535430-1.468339$

H $-4.0902581 .742375-1.994956$

H $-5.5584131 .939736-1.023856$

H -3.977917 $2.185378-0.277969$

SCF Energy $\left(B 3 L Y P / 6-31 G^{* *} / / M M F F\right)=-810.712275408$

F2-07_c01330

MMFF Geometry

C -4.260709 -0.9913640 .544977$

C $-4.219127-2.2804440 .189658$

C -2.5140570 .8109720 .701524$

O $-1.489079-0.1494881 .044820$

C $-0.2336580 .265097 \quad 0.469646$

C $-0.5817611 .304157-0.585017$

C -1.7736921 .9876600 .054644$

C $0.524115-0.965469-0.037286$

C $-2.5662322 .856775-0.905437$

C $1.895082-0.661309-0.666933$

O $-0.264698-1.620689-1.040406$

O $2.518183-1.914043-0.980280$

$\begin{array}{llll}\text { C } 2.850297 & 0.141777 & 0.233358\end{array}$

C $4.2423300 .312756-0.391758$

O $2.980029-0.5072321 .497704$

C $-3.5471660 .108188-0.192083$

C $5.137720 \quad 1.2204180 .442379$

H $-4.850990-0.7083871 .412768$

H $-4.760542-3.0255880 .762171$

H -3.648376 $-2.614291-0.669700$

H -2.9927851 .1390661 .632193$

H $\quad 0.3262680 .737871 \quad 1.285159$

H $-0.8708890 .836836-1.533411$

H $\quad 0.247008 \quad 1.989970-0.785209$

H -1.3981412 .6316490 .862543$

H $\quad 0.633370-1.6962680 .773249$

H -3.453696 $3.267888-0.413743$

H -1.954121 $3.696235-1.251977$

H -2.888961 2.301100 -1.791687

H $1.764354-0.136494-1.621107$

H -1.124789-1.830580 -0.637981

H $1.906880-2.404978-1.555985$

H $2.426783 \quad 1.1339150 .421886$

H $4.1472340 .735427-1.398605$

H $4.741910-0.658904-0.483347$

H $3.329840-1.4001331 .334897$

H $-4.3072140 .822529-0.527319$

H $-3.059299-0.297465-1.086489$

H $5.3099120 .803151 \quad 1.439349$

H $6.1106541 .340465-0.044814$

H 4.6912662 .2133050 .556413

SCF Energy $\left(B 3 L Y P / 6-31 G^{* *} / / M M F F\right)=-810.722211140$

F2-07 c01331

MMFF Geometry

C 2.2728682 .6524110 .526470

C 1.2755883 .4428160 .113996 
C 2.5406590 .1591790 .708908

O $1.131966-0.0226350 .972893$

C $0.668957-1.2076290 .305421$

C $1.776372-1.624175-0.653881$

C $3.010775-1.1635260 .093968$

C $-0.671800-0.941632-0.390174$

C $4.262371-1.115576-0.763841$

C $-1.774797-0.4640380 .573703$

O $-1.091365-2.130073-1.058363$

O $-1.898985-1.4306761 .625763$

C $-3.161500-0.301266-0.075796$

C $-3.2072770 .601434-1.315601$

O $-\begin{array}{llll}-4.040991 & 0.236122 & 0.919218\end{array}$

C $2.7015701 .398442-0.184720$

C $-2.7281992 .025950-1.065411$

H 2.8197182 .9283941 .424444

H $1.017234 \quad 4.3371150 .670432$

H $0.7025013 .214581-0.777737$

H $3.045576 \quad 0.3260491 .668055$

H $0.557152-1.9901461 .066639$

H $1.686818-1.100431-1.613268$

H $1.771591-2.699819-0.855027$

H $3.188735-1.8709410 .916375$

H $-0.520663-0.171480-1.154754$

H $4.120883-0.507863-1.663304$

H $5.104297-0.703766-0.198401$

H $4.537541-2.123834-1.091527$

H -1.4719110 .4731531 .054937$

H $-1.259929-2.804539-0.378049$

H $-2.589815-1.1110142 .231335$

H $-3.561503-1.289446-0.333609$

H $-4.2446490 .659632-1.668503$

H $-2.6238670 .158656-2.130010$

H -4.9286460 .2919990 .526143$

H $3.751617 \quad 1.527676-0.469393$

H $2.1210701 .277463-1.107408$

H - $-1.6734132 .049570-0.777991$

H $-2.8369502 .622354-1.977037$

H $-3.3118962 .510404-0.276681$

SCF Energy (B3LYP/6-31G**//MMFF) $=-810.717154941$

F2-07_c01332

MMFF Geometry

C $-4.144600-1.2458850 .350747$

C $-5.463883-1.316600 \quad 0.560986$

$\begin{array}{llll}\text { C }-2.488598 & 0.628225 & 0.639151\end{array}$

$\begin{array}{lllll}0 & -1.431478 & -0.296038 & 0.982651\end{array}$

$\begin{array}{llll}C & -0.183337 & 0.179816 & 0.438917\end{array}$

C $-0.549051 \quad 1.252297-0.576314$

C $-1.780576 \quad 1.8604210 .063973$

C $0.619183-1.004294-0.107640$

C $-2.5833542 .742857-0.875062$

C $1.994813-0.630762-0.691548$

O $-0.149039-1.627692-1.146251$

O $2.648863-1.845383-1.081208$

$\begin{array}{llll}\text { C } 2.910317 & 0.159109 & 0.261675\end{array}$

C $4.3114190 .387332-0.323895$

O $3.021162-0.5258551 .508389$

C $-3.459555-0.083974-0.315509$

C $5.165794 \quad 1.2821750 .565293$

H $-3.523040-2.0801110 .665863$

H -5.897431 -2.186566 1.041959

H $-6.130537-0.5156750 .261323$

H -3.013603 0.8979361 .563595

H $\quad 0.3513370 .635437 \quad 1.280587$

H $-0.7981130 .819276-1.551853$

H $0.257016 \quad 1.977193-0.725557$

H $-1.448468 \quad 2.4801130 .909175$

H $\quad 0.723818-1.7643290 .676038$

H $-3.4998273 .093096-0.389495$

H -1.997837 $3.622470-1.163392$

H -2.858691 2.219071 -1.796044

H $1.858174-0.060054-1.617676$

H $\quad 0.370680-2.379096-1.479773$

H $2.739752-2.403339-0.290384$

H $2.456246 \quad 1.1332180 .472375$

H $4.2282670 .846504-1.315592$

H $4.836053-0.568154-0.441794$
H $3.507993-1.3530581 .354414$

H $-4.2132030 .621801-0.682190$

H -2.920974 $-0.473455-1.187477$

H 4.6925662 .2588120 .708066

H 5.3264310 .8298751 .548823

H $6.1458921 .444477 \quad 0.105713$

SCF Energy (B3LYP/6-31G**//MMFF) $=-810.712340306$

F2-07 c01333

MMFF Geometry

C 2.5456382 .7439190 .020572

C $1.5566353 .448501-0.540806$

$\begin{array}{llll}\text { C } & 2.737293 & 0.323530 & 0.671087\end{array}$

$\begin{array}{lllll}\text { O } & 1.317844 & 0.232241 & 0.920017\end{array}$

C $0.823654-1.0249850 .429404$

C $1.952267-1.647919-0.383656$

C $3.175283-1.102783 \quad 0.325279$

C $-0.466016-0.818786-0.375011$

C $4.459738-1.250990-0.470041$

C $-1.579363-0.1410040 .453386$

O $-0.888546-2.093449-0.852396$

O $-1.899403-0.952877 \quad 1.583579$

C $-2.8612510 .167068-0.346953$

C $-3.9063690 .905045 \quad 0.502454$

O $-3.437833-1.031491-0.859849$

C $2.960837 \quad 1.370532-0.430798$

C $-5.1219521 .322787-0.315410$

H 3.0944173 .1833170 .849501

H $1.3073744 .436786-0.170261$

H $0.9800973 .055267-1.370802$

H 3.2234170 .6512691 .597921

H $\quad 0.629539-1.6624831 .301426$

H $1.923953-1.307250-1.425718$

H $1.909401-2.741475-0.386747$

H $3.291697-1.656314 \quad 1.267887$

H $-0.248513-0.184773-1.243796$

H $4.707176-2.310192-0.598675$

H $4.377491-0.811946-1.469353$

H $5.294759-0.7708120 .049881$

H -1.1956910 .8075380 .847284$

H $-1.652027-1.950804-1.436971$

H -2.120524 -1.8407761 .254782$

H $-2.5944350 .788961-1.209468$

H -3.4568541 .7983430 .950976$

H -4.2538170 .2621801 .319961$

H $-3.736310-1.565998-0.104481$

H $4.0219011 .420571-0.698677$

H $2.405397 \quad 1.093950-1.335208$

H $-4.8304321 .975457-1.144377$

H $-5.6433110 .453387-0.727768$

H $-5.829575 \quad 1.8709050 .314832$

SCF Energy (B3LYP/6-31G**//MMFF) $=-810.721717285$

F2-07_c01334

MMFF Geometry

C $3.5587142 .194379-0.042372$

C $2.8929643 .235684-0.554135$

C $2.846187-0.0964190 .711128$

$\begin{array}{llll}\text { O } & 1.549375 & 0.392947 & 1.118152\end{array}$

C $0.544696-0.6088500 .847920$

C $1.204026-1.607640-0.091745$

C $2.639406-1.5804040 .394973$

$\begin{array}{lllll}\text { C } & -0.723637 & 0.097944 & 0.345934\end{array}$

C $3.622505-2.223623-0.566806$

C $-1.963072-0.810636 \quad 0.273380$

O $-0.487856 \quad 0.667961-0.941850$

O $-1.695551-1.927641-0.580976$

C $-3.239446-0.127917-0.259891$

C -3.6339901 .1362160 .511182$

O $-4.316279-1.066668-0.152558$

C $3.3380520 .766781-0.460329$

C $-4.9314121 .750813-0.001420$

H 4.3142552 .3805060 .716630

H $3.1023884 .243303-0.212105$

H $2.1323513 .104382-1.315669$

H 3.5295930 .0122551 .561950

H $0.330674-1.0912481 .810205$

H $1.146879-1.281288-1.136258$ 
H $\quad 0.760456-2.605137-0.022015$

H $2.682532-2.1340701 .343801$

H $-0.9287180 .935072 \quad 1.022967$

H $3.413763-3.294427-0.664206$

H $3.561942-1.787336-1.568980$

H $4.649229-2.112534-0.203370$

H $-2.165070-1.2301631 .266107$

H $0.276679 \begin{array}{lll}1.263057 & -0.858731\end{array}$

H $-1.467151-1.576969-1.459158$

H $-3.1315260 .104232-1.326189$

H $-2.846607 \quad 1.892794 \quad 0.433822$

H $-3.7643890 .897403 \quad 1.573489$

H $-4.053583-1.865562-0.641336$

H $4.293702 \quad 0.385580-0.837129$

H $2.6192950 .727462-1.287598$

H $-4.8535201 .997598-1.065092$

H $-5.778451 \quad 1.071257 \quad 0.134374$

H -5.1521852 .6730800 .545434$

SCF Energy (B3LYP/6-31G**//MMFF) $=-810.722029693$

F2-07 c01335

MMFF Geometry

C $2.2810162 .643737 \quad 0.519842$

C $1.3027493 .445647 \quad 0.084974$

C 2.5235590 .1491130 .717886

$\begin{array}{llll}\text { O } & 1.109279 & -0.019983 & 0.960094\end{array}$

C $0.650574-1.2143890 .306133$

C $1.765782-1.640937-0.639812$

C $2.993965-1.1802380 .118177$

C $-0.687353-0.961212-0.400488$

C $4.255410-1.145233-0.725899$

C $-1.789666-0.4758550 .563651$

O $-1.082145-2.173699-1.039733$

O $-1.930582-1.453068 \quad 1.602940$

C -3.172122 $-0.271638-0.085804$

C $-3.1923810 .651558-1.310860$

$\begin{array}{llll}\text { O } & -4.060376 & 0.288614 & 0.888160\end{array}$

C $2.7086351 .382482-0.179009$

C $-2.6657322 .056192-1.043263$

H 2.8128742 .9154791 .428056

H 1.0444764 .3448170 .633543

H $0.7454753 .222497-0.817997$

H $3.0141960 .317118 \quad 1.684220$

H $\quad 0.535529-1.9872321 .076838$

H $1.687849-1.122088-1.602866$

H $1.759541-2.717849-0.834573$

H $3.159631-1.8822680 .947736$

H $-0.530574-0.203106-1.176459$

H $4.127118-0.543821-1.631555$

H $5.093121-0.733604-0.154104$

H $4.528983-2.157355-1.042834$

H -1.4702330 .4510061 .054551$

H $-1.828249-1.972360-1.628628$

H -2.453614 -1.049827 2.316115

H $-3.601445-1.240812-0.367114$

H $-4.2297700 .749206-1.655527$

H -2.627985 $0.204273-2.136000$

H $-4.209478-0.385653 \quad 1.571921$

H $3.7638121 .501107-0.448547$

H $2.1404511 .261635-1.109231$

H - $1.6085122 .041463-0.764707$

H -2.762152 $2.669386-1.945085$

H -3.227069 2.547713 -0.242626

SCF Energy (B3LYP/6-31G**/MMFF) $=-810.706770108$

F2-07_c01336

MMFF Geometry

C $-1.761360 \quad 2.789653 \quad 0.506380$

C $-0.892065 \quad 3.1478501 .457797$

C $-2.1558020 .649891-0.753795$

O $-0.761936 \quad 0.280870-0.672097$

C $-0.644192-1.139781-0.499770$

C $-2.037240-1.629697-0.122572$

C $-2.916296-0.671572-0.899278$

C $0.431385-1.4855970 .536985$

C $-4.369193-0.682128-0.459270$

C $1.885537-1.252966 \quad 0.071695$

O $0.308495-2.8726710 .877765$
O $2.747136-1.576771 \quad 1.172614$

C $2.2214270 .174446-0.391114$

C $3.7119150 .351159-0.718419$

O 1.8533541 .1090760 .621890

C -2.5009161 .4801260 .491802$

C $4.0103671 .725689-1.304298$

H $-1.9606353 .483964-0.305895$

H -0.3933334 .1098001 .412419$

H -0.6599492 .4928342 .290263$

H $-2.2917901 .259893-1.654992$

H $-0.370955-1.567048-1.473398$

H -2.211516 -1.526999 0.955515

H -2.200538 -2.676972 -0.395079

H $-2.879882-0.964964-1.958077$

H $\quad 0.256157-0.9211021 .460777$

H $-4.474361-0.5003480 .614995$

H $-4.9423550 .080354-0.996164$

H -4.823269-1.656139 -0.670930

H $2.133864-1.959087-0.730400$

H $\quad 0.436890-3.3902310 .064259$

H $2.535522-2.4850661 .448955$

H $1.6407750 .414262-1.287485$

H $4.028215-0.416327-1.434057$

H 4.3189130 .2340060 .187076

H 2.3437020 .8765231 .429001

H -3.5716341 .7110840 .510299$

H -2.269096 0.9115191 .400722

H $3.7655632 .522625-0.595148$

H $5.0741271 .808096-1.548784$

H $3.4377931 .892857-2.222102$

SCF Energy $($ B3LYP/6-31G**//MMFF $)=-810.714016539$

F2-07_c01337

MMFF Geometry

C $-3.965820-1.1989430 .890058$

C $-3.883581-2.4928400 .560247$

C -2.3346350 .7118090 .780615$

O $-1.214779-0.1736691 .009068$

$\begin{array}{llll}\text { C } & -0.067515 & 0.304420 & 0.275358\end{array}$

C $-0.6153921 .283837-0.752034$

C -1.7603121 .9115860 .017862$

C $0.700210-0.894226-0.289472$

C $-2.7222602 .699252-0.853441$

C $1.991974-0.556150-1.057912$

O $-0.156604-1.599365-1.202028$

O $2.584802-1.789768-1.488151$

C $3.0791760 .216902-0.288508$

C $3.432229-0.4018531 .071282$

O $2.6911221 .573081-0.099589$

C $-3.425984-0.0789000 .044189$

C 4.6225030 .2902221 .725090

H -4.458853 -0.928194 1.820094

H -4.297446 -3.253560 1.213224

H $-3.405081-2.815368-0.357751$

H -2.7104641 .0377581 .758101$

H $\quad 0.5478740 .8367831 .008399$

H $-0.9913370 .768514-1.643159$

H $0.1292832 .015720-1.076580$

H -1.3296532 .6028460 .756357$

H $0.916508-1.6112830 .511375$

H $-3.5657383 .070000-0.262475$

H -2.214722 $3.563766-1.294692$

H -3.116613 $2.096892-1.678072$

H $1.732799-0.003034-1.969240$

H $-0.958865-1.845905-0.710555$

H $1.908945-2.275289-1.991962$

H $3.9916110 .226382-0.898867$

H $3.664299-1.4663130 .953616$

H $2.583269-0.3196231 .759077$

H $2.5543331 .963551-0.979580$

H $-4.2676640 .577155-0.203814$

H $-3.031938-0.479220-0.897735$

H $5.5094850 .230388 \quad 1.086473$

H $4.4105871 .345391 \quad 1.923640$

H $4.859499-0.1902872 .679716$

SCF Energy $\left(\mathrm{B} 3 \mathrm{LYP} / 6-31 \mathrm{G}^{* *} / / \mathrm{MMFF}\right)=-810.718481039$

F2-07_c01338 
MMFF Geometry

C -3.775313 -1.896926 -0.057956

C $-5.035494-2.343091-0.106141$

C $-2.796570 \quad 0.3132640 .647700$

O $-1.600081-0.327147 \quad 1.144290$

C $-0.458403 \quad 0.5198570 .894748$

C $-0.9248231 .550911-0.121680$

$\begin{array}{llll}C & -2.372596 & 1.740412 & 0.285371\end{array}$

C $0.732122-0.362593 \quad 0.493402$

C $-3.2079242 .467414-0.753344$

$\begin{array}{llll}C & 2.067690 & 0.402855 & 0.422481\end{array}$

O $0.463843-0.988712-0.760752$

O $2.020851 \quad 1.385633-0.610268$

C $3.294628-0.5077120 .203661$

$\begin{array}{llll}\text { C } & 4.605559 & 0.290169 & 0.240797\end{array}$

O $3.217497-1.169795-1.056850$

C $-3.349396-0.527831-0.513359$

C $5.831505-0.6114540 .164671$

H $-3.003006-2.5620050 .319908$

H $-5.276703-3.3448920 .232343$

H $-5.843523-1.723659-0.479392$

H $-3.528757 \quad 0.3376391 .463959$

H -0.2337791 .0163001 .847310$

H $-0.8617221 .167963-1.146554$

H - $0.3498712 .480162-0.069153$

H -2.386430 2.3377111 .208140

H $0.828323-1.1709841 .229144$

H -2.850162 $3.495209-0.877143$

H -3.155537 $1.984122-1.734187$

H $-4.2573982 .511427-0.445421$

H 2.2049620 .9409271 .368201

H $-0.369240-1.481448-0.666206$

H $1.8999710 .914901-1.452965$

H $3.319037-1.2774200 .984357$

H 4.6572810 .8792991 .163547

H $4.6474720 .988774-0.603434$

H $2.416241-1.720028-1.052739$

H -4.200664 -0.016298 -0.976451

H $-2.584660-0.659861-1.287711$

H $5.843676-1.3260150 .993769$

H $5.856813-1.172572-0.774593$

H $6.744873-0.0107340 .221788$

SCF Energy (B3LYP/6-31G**//MMFF) $=-810.726070729$

F2-07_c01339

MMFF Geometry

C $-4.212770-1.1117280 .123056$

C $-4.079198-2.341657-0.385742$

C -2.5485690 .6748290 .723351$

O $-1.538141-0.3083351 .042559$

C $-0.246523 \quad 0.1822130 .630403$

C $-0.516573 \quad 1.366381-0.286159$

C $-1.776041 \quad 1.9397850 .332630$

C $0.569187-0.968360 \quad 0.029919$

C $-2.4950492 .937003-0.558165$

C $1.983520-0.575624-0.441527$

O $-0.145801-1.524879-1.082485$

O $2.636057-1.765221-0.902864$

C $2.8674490 .098102 \quad 0.625487$

$\begin{array}{llll}\text { C } & 4.306714 & 0.370579 & 0.165350\end{array}$

O $2.960423-0.7399201 .779846$

C $-3.4542410 .089239-0.370630$

C $4.4019431 .322609-1.015481$

H $-4.915520-0.9575610 .937750$

H -4.658922 -3.1669550 .012936$

H $-3.393273-2.547424-1.199912$

H $-3.136206 \quad 0.8614521 .630326$

H 0.2395290 .5385591 .546298

H $-0.7090301 .052087-1.317490$

H $0.3140292 .078666-0.301738$

H -1.4895902 .4575421 .259212$

H $\quad 0.625797-1.7860210 .757848$

H -2.720731 2.518962 -1.544409

H -3.433686 $3.261324-0.097813$

H - $1.8725913 .824344-0.715118$

H $1.8979780 .077709-1.316467$

H $-0.059653-0.919384-1.837313$

H $2.762850-2.346931-0.133656$
H $2.421591 \quad 1.0471310 .943471$

H $4.817315-0.570243-0.074086$

H $4.8679680 .794921 \quad 1.008021$

H $2.086726-0.7629942 .204031$

H $-4.1926190 .832606-0.690852$

H -2.858059-0.182737-1.250032

H $3.9820100 .879690-1.923038$

H $3.8744472 .259106-0.808959$

H $5.4502361 .562768-1.220402$

SCF Energy (B3LYP/6-31G**//MMFF) $=-810.706510194$

F2-07_c01340

MMFF Geometry

C $-3.5508202 .013522-0.438515$

C $-3.0198773 .229739-0.270126$

C $-2.580063-0.291889-0.307229$

O $-1.2292240 .081762-0.625289$

C $-0.478763-1.119861-0.865870$

C $-1.324797-2.284085-0.341840$

C $-2.445221-1.617240 \quad 0.442916$

C $0.905347-1.013285-0.206601$

C $-2.078852-1.4773501 .920871$

C $1.7001720 .229507-0.652372$

O $1.651295-2.194048-0.501274$

O $1.790790 \quad 0.212772-2.083408$

C $3.1330450 .303145-0.093136$

C 3.2504650 .1996591 .433260

O $3.6944091 .551654-0.515556$

C $-3.267506 \quad 0.8395350 .456862$

C $2.475738 \quad 1.2707772 .191197$

H $-4.2383921 .855310-1.265427$

H $-3.2690014 .036822-0.950343$

H -2.331136 3.4412950 .540308

H $-3.098845-0.470868-1.259632$

H $-0.362574-1.238895-1.950387$

H $-0.758712-3.0221590 .234982$

H $-1.755841-2.818758-1.198409$

H $-3.371853-2.1986140 .376930$

H $0.778114-0.9733010 .879340$

H $-1.211827-0.8278172 .073225$

H $-2.915258-1.0573472 .489051$

H -1.848772 -2.4560892 .355147$

H $1.1493681 .140882-0.392246$

H $1.803040-2.212632-1.461884$

H $2.284853 \quad 1.006905-2.350615$

H $3.746512-0.478705-0.556945$

H $4.307473 \quad 0.298842 \quad 1.710684$

H $2.929263-0.7908451 .772986$

H $4.6159721 .577979-0.206380$

H -4.2221370 .4872530 .864579$

H -2.643339 1.1634851 .298552

H $1.398471 \quad 1.1833892 .022444$

H 2.6518211 .1679053 .266836

H 2.7914272 .2757881 .894910

SCF Energy $\left(B 3 L Y P / 6-31 G^{* * / / M M F F}\right)=-810.716112974$

F2-07_c01341

MMFF Geometry

C $3.0762762 .102315-0.095865$

C $2.3494483 .194085-0.358416$

C $2.605974-0.3191270 .398281$

O $1.382322-0.0132601 .109291$

C $0.490983-1.1354761 .010917$

C $0.944645-1.908332-0.214320$

C $2.449801-1.762174-0.104743$

C $-0.986301-0.7163531 .004956$

C $3.191180-2.140279-1.374995$

C -1.413363 $0.292441-0.078565$

$\begin{array}{lllll}0 & -1.292097 & -0.172897 & 2.293163\end{array}$

$\begin{array}{lllll}0 & -0.634651 & 1.484875 & 0.066001\end{array}$

C $-2.8969790 .705743-0.006365$

C $-3.908702-0.447633-0.021106$

O $-3.1581291 .560085-1.126935$

C $2.8087550 .746035-0.688787$

C $-3.807233-1.348595-1.245577$

H 3.9256632 .1935340 .576450

H $2.601144 \quad 4.1446220 .099043$

H $1.4990223 .162210-1.030167$ 
H $3.431112-0.2719051 .119770$ H $0.674044-1.7389001 .910645$ H $\quad 0.588390-1.448336-1.141958$ H $\quad 0.611625-2.950386-0.197745$ H $2.794677-2.4325420 .695749$ H -1.582805 -1.629230 0.905643 H $2.835062-1.573831-2.241542$ H $4.265762-1.963806-1.264188$ H $3.046627-3.202794-1.597772$ H -1.189186 -0.102727 -1.074754 H -0.7616950 .6359622 .398760$ H $-0.9116752 .099411-0.635247$ H $-3.061441 \quad 1.3188610 .888067$ H -4.921472 -0.025312 -0.005924 H -3.809238 -1.0529330 .886308$ H $-4.0774501 .868083-1.052443$ H $3.6744360 .491918-1.310190$ H $1.9290940 .787333-1.342592$ H -2.844924 -1.867101 -1.284453 H -4.593168 -2.110274-1.214660 H $-3.930061-0.778177-2.171454$

SCF Energy (B3LYP/6-31G**//MMFF) $=-810.708115739$

\section{F2-07 c01342}

MMFF Geometry

C $-3.0815372 .101204-0.473211$

C -2.6380193 .0875510 .314095$

C $-2.238064-0.224369-0.916427$

O $-0.872071 \quad 0.172000-0.666671$

C $-0.194402-0.8669070 .056687$

C $-1.267721-1.8504410 .508503$

C $-2.284056-1.723881-0.607863$

C $0.624247-0.2872021 .217913$

C $-3.641368-2.312903-0.268627$

C 1.6605900 .7834390 .817972

O $1.260533-1.3580641 .909407$

$\begin{array}{lllll}\text { O } & 0.963684 & 1.997380 & 0.507871\end{array}$

C $2.5942220 .455061-0.364510$

C $3.415941-0.825155-0.183272$

O $3.5131771 .546471-0.508395$

C $-3.1532720 .658416-0.054601$

C $4.365195-1.077999-1.349332$

H $-3.4207372 .346696-1.476329$

H -2.614108 4.109984 -0.046737

H $-2.2897402 .895253 \quad 1.322879$

H $-2.451343-0.053621-1.978448$

H $0.462350-1.381922-0.654319$

H - $1.705593-1.5464101 .466931$

H $-0.879609-2.8673530 .623347$

H -1.889539 -2.258788 -1.483472

H -0.0772490 .1832451 .919785$

H -4.049048 -1.8983880 .658920$

H -4.357727 -2.124875 - 1.074620

H -3.562464 -3.397047 -0.134142

H 2.2842881 .0156261 .690290

H $1.726313-0.9793672 .674390$

H $0.4004831 .826806-0.266381$

H $2.0244870 .406878-1.299666$

H $2.760015-1.695273-0.089266$

H $4.012764-0.7560780 .734094$

H $2.9856232 .355699-0.622334$

H $-4.196487 \quad 0.339774-0.157111$

H $-2.8822350 .565193 \quad 1.004087$

H $4.899063-2.022146-1.201210$

H $3.815314-1.143978-2.293486$

H $5.112058-0.283083-1.438860$

SCF Energy (B3LYP/6-31G**//MMFF $)=-810.718621158$

\section{F2-07 c01343}

MMFF Geometry

C $-4.780834-0.2881720 .303567$

C $-5.558316-0.239560-0.783907$

C $-2.3942850 .276761 \quad 0.968717$

O $-1.206060-0.4630571 .340301$

C $-0.075218 \quad 0.0496450 .607614$

C $-0.6541770 .883304-0.524928$

C $-1.894274 \quad 1.459096 \quad 0.130425$

C $0.821752-1.1152980 .178842$
C $-2.8577402 .090350-0.856456$

C $2.081264-0.702455-0.603277$

O $0.070555-1.997649-0.667214$

O $2.862520-1.883705-0.826398$

C 2.9666330 .3351370 .108850

C $4.2672760 .621153-0.655737$

O $3.285012-0.1237621 .422113$

C $-3.345055-0.7239790 .292926$

C $5.0727481 .745281-0.016279$

H $-5.214286-0.0187801 .263315$

H $-6.5934390 .074763-0.705939$

H $-5.180475-0.509436-1.763870$

H -2.8456280 .6419051 .899034$

H 0.4639660 .6984591 .307596

H $-0.9290290 .262122-1.385174$

H $0.041175 \quad 1.652992-0.873268$

H -1.5676412 .2445250 .827227$

H $1.099153-1.7145871 .054554$

H $-3.1521341 .393146-1.646578$

H -3.758332 $2.448536-0.348391$

H $-2.3885072 .951353-1.344938$

H $1.803177-0.326408-1.595358$

H $-0.722435-2.265969-0.171919$

H $2.292692-2.524810-1.285119$

H $2.412518 \quad 1.272986 \quad 0.220292$

H $4.0359300 .894440-1.691598$

H $4.900762-0.273388-0.679939$

H $3.752788-0.9717681 .331469$

H $-2.999790-0.951233-0.723006$

H -3.292016 -1.678253 0.834248

H $5.978177 \quad 1.939078-0.600240$

H 4.4902432 .6711960 .022691

H 5.3782761 .4869311 .002357

SCF Energy $\left(B 3 L Y P / 6-31 G^{* *} / / M M F F\right)=-810.720712909$

F2-08 c01545

MMFF Geometry

C $4.486191-1.114729-0.426523$

C $4.590292-2.214198-1.181356$

C $2.5681410 .402153 \quad 0.127739$

O $1.552269-0.611105-0.020982$

C $0.257195 \quad 0.024792-0.040450$

$\begin{array}{lllll}\text { C } & 0.487161 & 1.461496 & 0.408224\end{array}$

C $1.868897 \quad 1.732588-0.151641$

C $-0.718576-0.787101 \quad 0.816401$

C 2.5518652 .9354730 .475798

C $-2.150067-0.224595 \quad 0.867727$

O $-0.232616-0.8287292 .165650$

O $-2.956740-1.1527831 .604971$

C $-2.802142-0.004630-0.508616$

C $-4.2677940 .440174-0.400110$

O $-2.731865-1.207808-1.272840$

C $3.7243150 .115534-0.831991$

C $-4.8641290 .785636-1.759044$

H $4.987747-1.0952930 .537652$

H $5.159981-3.068360-0.832068$

H $4.112382-2.283602-2.152287$

H $2.9110230 .359324 \quad 1.169843$

H $-0.0640510 .011178-1.088538$

H $0.495415 \quad 1.544821 \quad 1.501422$

H -0.2731732 .1474320 .022946$

H $1.783398 \quad 1.892312-1.235098$

H $-0.733171-1.8301830 .477995$

H 3.5485553 .0858140 .049514

H $1.968283 \quad 3.8448180 .298175$

H 2.6632882 .8140641 .558529

H $-2.166793 \quad 0.714288 \quad 1.434461$

H $0.673633-1.1807292 .134754$

H $-2.537031-1.2738012 .474077$

H $-2.2439430 .760081-1.058956$

H $-4.3419541 .316698 \quad 0.253665$

H $-4.877444-0.3574710 .040553$

H -3.201599 -1.899358 -0.775594

H $3.338289-0.001420-1.852360$

H $4.4249580 .958026-0.841203$

H $-5.8990791 .122180-1.641709$

H $-4.2990991 .589470-2.241477$

H $-4.866093-0.082448-2.425644$ 
SCF Energy (B3LYP/6-31G**//MMFF) $=-810.723842079$

F2-08_c01546

MMFF Geometry

C -3.143636 2.4394410 .350066

C $-2.7899253 .491566-0.396501$

C -2.4169250 .0401010 .360495$

O $-1.097147 \quad 0.304414-0.157449$

C $-0.486546-0.940496-0.535864$

C $-1.372798-2.0412980 .034013$

C $-2.741505-1.396472-0.049494$

C $0.962399-1.001958-0.038079$

C $-3.783553-2.0707680 .825668$

C $1.8389460 .146553-0.573478$

O $1.527990-2.258072-0.408851$

$\begin{array}{lllll}\text { O } & 1.767151 & 0.133948 & -2.005825\end{array}$

C $3.3245770 .050648-0.179786$

C $3.601116-0.0797291 .323643$

O $3.9744881 .230175-0.668328$

C $-3.4029491 .065546-0.200914$

C $3.051266 \quad 1.0694102 .159590$

H $-3.2624142 .577171 \quad 1.421641$

H -2.6211234 .4593590 .062872$

H -2.661027 $3.408328-1.469991$

H $-2.3536190 .123075 \quad 1.453624$

H $-0.518545-1.001522-1.631099$

H $-1.111561-2.2543871 .078138$

H $-1.300330-2.974691-0.532378$

H $-3.078244-1.422022-1.094691$

H $0.956987-0.9551051 .056505$

H $-3.921186-3.1145320 .524604$

H -3.488735 -2.061494 1.880400

H $-4.750755-1.5653580 .741929$

H $1.4271601 .111680-0.256277$

H $1.566411-2.286567-1.380374$

H $2.3153390 .869713-2.328758$

H $3.787630-0.792241-0.706921$

H $4.687128-0.1094901 .478492$

H $3.204822-1.0278541 .702423$

H $4.9220281 .147878-0.465472$

H $-3.3363801 .082060-1.296069$

H -4.4295250 .7853950 .060685$

H 3.4502072 .0320691 .825485

H 1.9593171 .1134822 .113725

H 3.3337610 .9360673 .208981

SCF Energy (B3LYP/6-31G**//MMFF) $=-810.719554101$

F2-08_c01547

MMFF Geometry

C -4.601601 -0.9799780 .732681$

C $-4.462956-1.965123-0.162181$

C $-2.5388660 .389721-0.037525$

O $-1.555858-0.6170390 .277899$

C $-0.245072-0.014603 \quad 0.260819$

C $-0.422707 \quad 1.364827-0.358556$

C $-1.815872 \quad 1.7290250 .113874$

C $0.729533-0.945259-0.467280$

C $-2.4433002 .866180-0.673891$

C $2.177245-0.425729-0.541514$

O $0.277620-1.131968-1.816299$

O $2.978423-1.449340-1.145641$

C $2.798631-0.0632290 .818999$

C $4.2957720 .275090 \quad 0.763346$

O $2.628980-1.1675031 .711097$

C -3.7432490 .2470540 .898417$

C $4.6187421 .470796-0.117260$

H $-5.431700-1.0580801 .432599$

H $-5.160828-2.796017-0.167846$

H $-3.670212-1.976520-0.900106$

H -2.837575 $0.239157-1.082700$

H $0.0484320 .086962 \quad 1.312102$

H $-0.3905191 .321372-1.453722$

H $0.3413172 .074166-0.026652$

H -1.764461 2.0111701 .174173

H $0.706437-1.942161-0.010768$

H - $2.5205012 .622079-1.738747$

H -3.449453 $3.088951-0.305758$

H - $-1.8412593 .776069-0.580768$
H $2.2234060 .438258-1.214441$

H $-0.637936-1.457319-1.771028$

H $2.574398-1.664014-2.004025$

H $2.2603430 .787746 \quad 1.251276$

H $4.872828-0.5972130 .433096$

H $4.647110 \quad 0.482243 \quad 1.782353$

H $2.976319-0.9008212 .579201$

H $-3.3881650 .248351 \quad 1.936993$

H -4.3991301 .1175480 .779452$

H $4.4149401 .259736-1.171079$

H 4.0350312 .3473020 .180981

H $5.6800371 .725221-0.030609$

SCF Energy (B3LYP/6-31G**//MMFF) $=-810.715825097$

F2-08_c01548

MMFF Geometry

C $3.7489041 .295854-1.151014$

C $4.5852302 .236497-0.698035$

C $2.567305-0.682693-0.069694$

O $1.440545-0.636059-0.966594$

C $0.268097-0.263647-0.208886$

C $0.667878-0.345169 \quad 1.260624$

C 2.1309350 .0489701 .198243

C $-0.907001-1.153987-0.624270$

C $2.922072-0.3337672 .436081$

C $-2.232744-0.8435020 .094535$

O $-0.579585-2.521475-0.339923$

O $-3.243944-1.685105-0.476524$

C -2.6899250 .6243510 .005128$

C $-4.075036 \quad 0.834404 \quad 0.634679$

O $-2.7255391 .008283-1.368584$

C $3.825903-0.153542-0.762649$

C -4.4615872 .3053530 .725468$

H $2.9704951 .585422-1.851989$

H $4.4804663 .266504-1.021246$

H $5.3817231 .999915-0.001219$

H $2.728015-1.7463290 .152419$

H $0.0617770 .778697-0.478342$

H $0.563646-1.364783 \quad 1.650004$

H $\quad 0.072000 \quad 0.3196331 .893270$

H 2.1849421 .1360901 .061630

H $-1.042818-1.105379-1.711441$

H $3.971374-0.0399102 .333403$

H $2.5161130 .166013 \quad 3.321645$

H $2.889798-1.4136482 .615737$

H $-2.158877-1.139548 \quad 1.147623$

H $\quad 0.258043-2.718044-0.793669$

H $-3.365576-1.410024-1.401554$

H $-1.9598591 .263357 \quad 0.513848$

H $-4.0888690 .407470 \quad 1.644337$

H -4.8428230 .3210960 .043822$

H -2.834262 $1.973310-1.404610$

H $4.694758-0.331770-0.117779$

H $3.988889-0.726258-1.684213$

H $-4.5611592 .755719-0.266547$

H $-5.4242202 .409601 \quad 1.236191$

H -3.715565 2.8729891 .290420

SCF Energy (B3LYP/6-31G**//MMFF) $=-810.716762295$

F2-08_c01549

MMFF Geometry

C $3.691136-1.7849060 .099508$

C $4.941962-1.904349-0.359703$

C $2.293993 \quad 0.176293 \quad 0.924894$

O $1.044663-0.4025720 .501658$

C $0.5039750 .401758-0.562571$

C $1.3444781 .675180-0.605557$

C $2.7021921 .155640-0.174445$

C $-0.9931630 .653466-0.339854$

C $3.6557292 .242424 \quad 0.288804$

C $-1.809744-0.647282-0.207662$

O $-1.511498 \quad 1.414691-1.428849$

O $-1.543796-1.485127-1.340515$

C $-3.336917-0.453712-0.125176$

C -3.7770920 .4863151 .001870$

O $-3.930288-1.736236 \quad 0.108948$

C $3.304038-0.9222151 .267296$

$\begin{array}{llll}\text { C } & -5.292884 & 0.618844 & 1.091295\end{array}$ 
H $2.901743-2.351425-0.387592$

H $5.157505-2.549171-1.204749$

H $5.766684-1.3650610 .092986$

H $2.0704830 .727148 \quad 1.848520$

H $0.661884-0.147142-1.499854$

H $\quad 0.9705012 .4160120 .112034$

H $1.3565712 .137330-1.597296$

H $3.1497350 .626274-1.024544$

H -1.124858 1.2378010 .578222

H 3.2449672 .8036891 .134676

H 4.6126881 .8127620 .601485

H $3.8524692 .952263-0.521274$

H -1.470515 -1.210573 0.669674

H -1.084847 $2.287753-1.410955$

H - $1.791875-0.986478-2.137980$

H $-3.730131-0.097434-1.084738$

H -3.3560331 .4859050 .852420$

H -3.4089740 .1099341 .963825$

H -3.636454 -2.325985 -0.606460

H $4.193495-0.466671 \quad 1.718825$

H $2.863767-1.5830772 .024442$

H -5.5623501 .3304011 .878342$

H -5.7096940 .9836760 .147200$

H $-5.765629-0.338717 \quad 1.330673$

SCF Energy (B3LYP/6-31G $\left.{ }^{* *} / / \mathrm{MMFF}\right)=-810.721070849$

F2-08_c01550

MMFF Geometry

C $3.3308872 .516791-0.392717$

C 2.8938673 .5917500 .272803

C $2.6410370 .106442-0.373290$

$\begin{array}{lllll}\text { O } & 1.274039 & 0.366852 & 0.005831\end{array}$

C $0.651382-0.8709830 .387415$

C $1.600151-1.978246-0.054358$

C $2.946518-1.3064780 .124112$

C $-0.750716-0.983690-0.224109$

C $4.072791-1.997076-0.625113$

C $-1.685430 \quad 0.1703550 .198660$

O $-1.308196-2.2456730 .132997$

O

C $-3.0865460 .114798-0.444785$

C $-3.9299981 .340762-0.067778$

O $-3.792101-1.050756-0.024569$

C 3.5543691 .1707910 .236923

C $-5.2660761 .368874-0.799946$

H $3.5495952 .613828-1.453057$

H $2.7570134 .537420-0.240359$

H 2.6643173 .5492931 .331778

H $2.6811850 .145290-1.469868$

H $0.589735-0.8811371 .483235$

H $1.436272-2.237433-1.107835$

H $1.490448-2.8890420 .542040$

H $3.188149-1.2839151 .195368$

H $-0.668013-0.961236-1.317656$

H $4.197667-3.025113-0.269347$

H $3.874189-2.036018-1.701452$

H $5.021102-1.471359-0.476661$

H -1.216166 $1.118269-0.090032$

H -1.337298 -2.2943511 .103742$

H $-2.344306-0.576248 \quad 1.881557$

H $-2.9836830 .069182-1.535597$

H -3.379028 $2.258656-0.303016$

H $-4.1354481 .342058 \quad 1.009302$

H $-3.296631-1.824720-0.341535$

H 3.3824011 .2298551 .319053

H 4.6050030 .8982140 .086434

H $-5.8253892 .269736-0.528345$

H $-5.1177751 .376359-1.884528$

H $-5.8812170 .501344-0.542099$

SCF Energy (B3LYP/6-31G**//MMFF) $=-810.723767679$

F2-08_c01551

MMFF Geometry

C -4.2270601 .8589910 .261593$

C $-4.2461523 .007227-0.424432$

C $-2.686693-0.1189190 .235384$

O $-1.5269490 .623383-0.190441$

C $-0.479292-0.302411-0.557579$
C $-0.951216-1.670628-0.082166$

C $-2.453693-1.550327-0.241654$

C 0.8490620 .2182580 .011884

C $-3.231129-2.5981160 .536029$

C $2.092584-0.512283-0.524075$

O $0.835402 \quad 0.132407 \quad 1.437436$

O $1.990618-1.912223-0.242565$

C $3.434570-0.0280770 .062375$

C $3.6741601 .476753-0.099908$

O $4.486646-0.720583-0.620205$

C $-3.9477360 .516254-0.352524$

C 5.0345101 .9116940 .433073

H -4.4345221 .8858641 .328171$

H -4.459402 3.9440890 .078514

H -4.049593 $3.034961-1.490407$

H $-2.708284-0.0774311 .332388$

H $-0.433181-0.287176-1.653804$

H $-0.692227-1.8445150 .968711$

H $-0.532947-2.489001-0.675592$

H $-2.701360-1.636638-1.308281$

H $\quad 0.9222291 .283779-0.233697$

H -3.018508 -2.541426 1.608997

H $-4.308982-2.4670030 .399084$

H -2.967709 -3.604484 0.194184

H $2.122684-0.424655-1.616654$

H $\quad 0.044751 \quad 0.606144 \quad 1.748538$

H $1.918085-2.0127210 .722308$

H $3.511755-0.3057691 .120421$

H 2.9041282 .0460370 .430502

H $3.6191551 .748842-1.160895$

H $4.322808-1.673426-0.513463$

H $-3.8395470 .616264-1.439788$

H $-4.816022-0.126150-0.167073$

H $5.1458512 .996605 \quad 0.338517$

H 5.1415561 .6507061 .490700

H $5.8520531 .442739-0.123178$

SCF Energy $(B 3 L Y P / 6-31 G * * / / M M F F)=-810.723788063$

F2-08 c01552

MMFF Geometry

C -2.3092862 .6196690 .109231$

C -3.1478663 .4677240 .715058$

C -2.1511210 .1166540 .087672$

O $-0.7246290 .092238-0.113394$

C $-0.316967-1.237688-0.472062$

C $-1.521109-2.129118-0.187958$

C $-2.671293-1.193555-0.500411$

C $0.949743-1.6465230 .286034$

C $-3.998669-1.6497820 .079571$

C $2.237542-0.930334-0.175701$

O $1.144473-3.0525780 .090350$

O $3.300209-1.3776480 .678962$

C $2.2109090 .608832-0.172619$

C $3.5773801 .210841-0.531817$

O 1.8394251 .0887201 .118353

C $-2.7444541 .357578-0.581250$

C $3.5159472 .726838-0.675189$

H -1.247871 2.8544550 .101466

H -2.7709804 .3660631 .191819$

H $-4.216057 \quad 3.2838290 .747827$

H -2.3208210 .1468841 .172058$

H $-0.121410-1.241800-1.552295$

H -1.544819 -2.427869 0.867728

H $-1.527520-3.036143-0.800132$

H -2.766101 -1.105217-1.591141

H $0.807019-1.4904231 .362450$

H -4.795171 -0.938109-0.159252

H -4.282866 -2.624205 -0.330964

H $-3.948630-1.746106 \quad 1.169379$

H $2.498315-1.281327-1.181679$

H $1.947619-3.3043140 .577927$

H $3.127893-1.0240531 .568691$

H $1.4732530 .971684-0.896569$

H $3.9376160 .780429-1.473195$

H 4.3133200 .9777690 .246888

H $\quad 0.9059070 .857596 \quad 1.260826$

H -2.409474 $1.416769-1.624175$

H $-3.8384271 .290101-0.591521$ 
H $4.4991223 .118801-0.954652$

H $2.8019703 .017893-1.452294$

H 3.2177663 .2046180 .263291

SCF Energy (B3LYP/6-31G**//MMFF) $=-810.727528058$

\section{F2-08 c01553}

MMFF Geometry

C $-4.146211 \quad 1.5082420 .357980$

C -4.09011428427240 .284126$

C $-2.439941-0.293662-0.000962$

O $-1.309173 \quad 0.5213790 .369580$

C $-0.094233-0.156974-0.018154$

C $-0.506395-1.581630-0.365014$

C -1.888699-1.360858 -0.945735

C $0.935051-0.0172991 .107124$

C $-2.728302-2.624995-1.009645$

C $2.316676-0.6390060 .827767$

O $0.417322-0.6591612 .283713$

O $3.150583-0.3911511 .968614$

C $3.079513-0.117861-0.404340$

C $3.1778651 .412741-0.468494$

O $2.483516-0.600093-1.603516$

C $-3.5160050 .582657-0.644138$

C $4.0775481 .882895-1.605469$

H $-4.6865391 .054498 \quad 1.184728$

H -4.5708153 .4553141 .038976$

H -3.566975 $3.345218-0.521944$

H -2.817103 -0.7541090 .921720$

H $0.2539850 .360673-0.918049$

H $-0.565988-2.2131670 .529124$

H $\quad 0.179237-2.060781-1.069177$

H -1.782189 -0.948652 -1.958269

H $1.046627 \quad 1.0378931 .383195$

H -2.864008 -3.067333 -0.016980

H -3.719666 -2.415213 -1.423310

H -2.247851 -3.373614 -1.648035

H $2.211106-1.7284060 .752328$

H $-0.450808-0.2618442 .470340$

H $2.682919-0.7353162 .748989$

H $4.101293-0.517871-0.370845$

H 3.5691621 .8066450 .476114

H $2.1866831 .851724-0.627901$

H $2.508552-1.571675-1.572500$

H -3.078490 $1.162216-1.466793$

H $-4.308469-0.043443-1.069575$

H $4.1424802 .975667-1.606784$

H $5.0906081 .483572-1.494113$

H $3.687741 \quad 1.568370-2.578636$

SCF Energy (B3LYP/6-31G**//MMFF) $=-810.720154491$

F2-08 c01554

MMFF Geometry

C $4.482075-1.5832890 .274646$

C $4.617071-2.751153-0.363564$

C 2.7407550 .2184680 .187897

O $1.661758-0.660312-0.189328$

C $0.5234220 .133886-0.588995$

C $0.8476191 .563210-0.175286$

C $2.3532311 .596062-0.345858$

C $-0.745532-0.4898620 .009458$

C 3.0201092 .7523020 .379225

C $-2.048428 \quad 0.125887-0.536714$

O $-0.711331-0.374071 \quad 1.431742$

O $-2.1406971 .499714-0.164669$

C $-3.324522-0.612427-0.079690$

C $-4.583295-0.023213-0.731494$

O $-3.480764-0.528547 \quad 1.335140$

C $4.056177-0.304964-0.390638$

C $-5.834905-0.826003-0.398378$

H $4.700485-1.5427551 .338706$

H $4.933141-3.6379660 .174768$

H $4.415327-2.844754-1.424811$

H $2.7773800 .227005 \quad 1.285153$

H $0.4774550 .068027-1.683352$

H 0.5785821 .7514790 .870681

H $\quad 0.3379172 .305481-0.796635$

H $2.5835551 .662839-1.417760$

H $-0.743035-1.564068-0.213978$
H 4.1048542 .7298390 .236688

H $2.6496903 .709767-0.001661$

H $2.8209582 .719221 \quad 1.455658$

H $-2.0086170 .094768-1.632349$

H $0.123305-0.7708261 .735245$

H $-2.178725 \quad 1.5349280 .806649$

H -3.244385 -1.673485 -0.344765

H $-4.4608860 .004376-1.820287$

H -4.741259 $1.005967-0.387446$

H $-2.708707-0.9553841 .743211$

H $3.948942-0.462151-1.471314$

H $4.8531510 .432766-0.244468$

H $-5.733219-1.864498-0.729370$

H $-6.035452-0.8248700 .677490$

H $-6.704855-0.393410-0.902777$

SCF Energy $(B 3 L Y P / 6-31 G * * / / M M F F)=-810.727588607$

F2-08_c01555

MMFF Geometry

C $-3.1109612 .438538 \quad 0.368012$

C $-2.7619153 .489446-0.382455$

C $-2.395906 \quad 0.0357820 .365141$

O $-1.0828910 .294731-0.172065$

C $-0.484470-0.951365-0.563897$

C $-1.366335-2.0497120 .018977$

C $-2.733276-1.398610-0.041634$

C $0.973232-1.022918-0.093798$

C $-3.764470-2.0694610 .849069$

C $1.8374890 .152800-0.597540$

O $1.513882-2.265895-0.538010$

O $1.8047650 .183813-2.030731$

C $3.3216920 .083973-0.191050$

C $3.587893-0.0756541 .312036$

O $3.9477991 .292733-0.639966$

C $-3.3853011 .066683-0.180497$

C 2.9994831 .0376022 .170352

H -3.2141582 .5758991 .441348$

H -2.5815484 .4558880 .075599$

H -2.648338 $3.406536-1.457699$

H $-2.3161520 .117168 \quad 1.457212$

H $-0.537865-1.013816-1.658116$

H -1.089631 -2.265692 1.058513

H -1.307373 -2.982633 -0.549948

H -3.086892 -1.421164 -1.081312

H $0.980426-1.0230711 .002278$

H $-3.452744-2.0634801 .898848$

H $-4.730379-1.5591340 .781867$

H $-3.911970-3.1119190 .548515$

H $1.4170251 .107467-0.259379$

H $2.370099-2.394697-0.097426$

H $0.9265080 .503094-2.298142$

H $3.813424-0.731402-0.735214$

H $4.672863-0.0796561 .476686$

H $3.214776-1.0425621 .665754$

H $4.8962771 .224422-0.436780$

H $-3.3348641 .084109-1.276398$

H -4.4092420 .7910550 .096215$

H $3.2764860 .886546 \quad 3.218807$

H 3.3747362 .0185891 .863202

H 1.9071671 .0527902 .115461

SCF Energy (B3LYP/6-31G**//MMFF) $=-810.706053989$

F2-08_c01556

MMFF Geometry

C $-3.861709-1.484804-0.629743$

C $-4.120416-2.5820880 .090125$

C $-2.2564650 .414630-0.317675$

O $-1.290084-0.3554280 .429294$

C -0.3750120 .5478361 .072743$

C -0.5742351 .8968850 .400440$

C -2.0638721 .8671700 .121063$

C 1.0674380 .0220351 .054712

C $-2.5089742 .888727-0.910954$

C $1.649579-0.355592-0.323012$

O $1.127521-1.1229691 .911436$

O $0.869923-1.401756-0.914878$

C $3.097813-0.882720-0.267003$

C 4.1139460 .0317850 .427376 
O $3.554326-1.097140-1.608312$ C $-3.662768-0.117784-0.038316$ C $4.2103591 .428229-0.175054$ H $-3.798129-1.574160-1.711096$ H $-4.257993-3.540254-0.399108$ H $-4.197215-2.5467951 .171226$ H $-2.0043430 .298488-1.379904$ H -0.7100090 .6112372 .116889$ H $-0.0140631 .969368-0.537982$ H -0.2732802 .7310001 .041326$ H -2.601530 2.0504061 .061302 H 1.6967700 .7881961 .519197 H $-3.5862102 .818236-1.090691$ H -2.289789 $3.904397-0.565732$ H -1.997334 2.741534-1.868044 H $1.6020040 .493272-1.012919$ H $0.566244-1.8114381 .514557$ H $-0.027524-1.057429-1.055042$ H $3.118375-1.8651030 .220307$ H $5.106826-0.4294200 .350008$ H $3.8872820 .111167 \quad 1.495919$ H $2.915855-1.691164-2.039166$ H $-3.838825-0.1480101 .044244$ H $-4.4132160 .551365-0.474029$ H $4.4617871 .387289-1.239291$ H $3.2726971 .980153-0.062949$ H 4.9940302 .0001540 .332457

SCF Energy (B3LYP/6-31G**/MMFF) $=-810.720436087$

F2-08_c01557

MMFF Geometry

C $4.611857-0.983790-0.732594$

C $4.484684-1.9617740 .171777$

$\begin{array}{lllll}C & 2.550139 & 0.384770 & 0.043681\end{array}$

O $1.567911-0.628468-0.253352$

C $0.256801-0.027286-0.243817$

C $0.431618 \quad 1.3553490 .368932$

C $1.8213061 .720938-0.113838$

C $-0.730163-0.963123 \quad 0.461912$

C $2.449416 \quad 2.866188 \quad 0.661755$

C $-2.178731-0.4405150 .533070$

O $-0.272322-1.216551 \quad 1.797771$

O $-2.987620-1.4714651 .113009$

C -2.786842 $-0.053020-0.826777$

C $-4.2826130 .292298-0.777653$

O $-2.616104-1.144110-1.734750$

C $3.7468660 .238076-0.901935$

C -4.6063691 .4760570 .118665$

H $5.436497-1.064283-1.438667$

H $5.186101-2.7896650 .179098$

H $3.697751-1.9703720 .915933$

H $2.858467 \quad 0.244881 \quad 1.087440$

H $-0.0269750 .077138-1.297799$

H $\quad 0.409158 \quad 1.322340 \quad 1.463704$

H -0.3360812 .0608100 .036916$

H $1.763376 \quad 1.995147-1.175881$

H $-0.708382-1.943076-0.029323$

H 3.4528293 .0892960 .286126

H 1.8439213 .7732920 .565095

H 2.5335382 .6304101 .727961

H $-2.229226 \quad 0.413307 \quad 1.218224$

H -0.440034 -0.424878 2.335302

H -2.587299 -1.709262 1.966910

H -2.240323 $0.801528-1.241049$

H -4.866820 $-0.582036-0.465513$

H $-4.6249870 .516924-1.796009$

H -2.953696 -0.861415 -2.601630

H $3.3825520 .228322-1.937370$

H $4.4002241 .112239-0.796783$

H $-4.412495 \quad 1.2476251 .170734$

H $-4.0154232 .353789-0.161157$

H -5.6654051 .7376880 .027236$

SCF Energy (B3LYP/6-31G**//MMFF) $=-810.707856264$

F2-08 c01558

MMFF Geometry

C $3.8403621 .664299-0.135084$

C $3.8342412 .604455-1.086948$
C $2.642076-0.586897-0.092816$

O $1.567247-0.150113-0.947475$

C $0.342472-0.156882-0.181479$

C $0.661078-0.8774241 .124603$

C $2.112314-0.4905541 .336569$

C $-0.780504-0.773987-1.019920$

C $2.844040-1.3774422 .328154$

C $-2.154653-0.815705-0.324855$

O $-0.407320-2.120878-1.345257$

O $-3.084270-1.323642-1.290785$

$\begin{array}{llll}\text { C }-2.646216 & 0.547136 & 0.199640\end{array}$

$\begin{array}{llll}C & -4.068142 & 0.479481 & 0.775769\end{array}$

O $-2.6104881 .490195-0.870596$

C $3.9225050 .189569-0.411725$

C $-4.467401 \quad 1.758909 \quad 1.500978$

H 3.8054891 .9806860 .903745

H $3.7835593 .654705-0.821340$

H $3.8761772 .346816-2.139360$

H $2.818530-1.640531-0.349021$

H 0.1195750 .8964210 .024200

H $0.567338-1.9646151 .018211$

H $0.009978-0.5620331 .945366$

H 2.1370770 .5448361 .696964

H $-0.848856-0.248989-1.980480$

H $3.887358-1.0640362 .433959$

H $2.372588-1.3215913 .314775$

H $2.836639-2.4257702 .011615$

H $-2.127992-1.5451330 .494067$

H $-1.121851-2.494598-1.889245$

H $-3.886994-1.595270-0.814722$

H $-1.9607080 .899400 \quad 0.978452$

H $-4.141208-0.3571311 .480168$

H $-4.7959920 .309595-0.026257$

H $-2.7406892 .375546-0.491911$

H $4.756435-0.222308 \quad 0.168613$

H $4.1681590 .033208-1.470096$

H -4.5032842 .6126830 .817884$

H -5.4623351 .6445761 .943269$

H -3.7633871 .9892842 .306721$

SCF Energy (B3LYP/6-31G**//MMFF) $=-810.709340261$

F2-08 c01559

MMFF Geometry

C $3.1080322 .338262-0.086085$

C $2.489108 \quad 3.472622 \quad 0.259750$

C $2.374886-0.058286-0.076560$

O $\quad 0.9755790 .242139-0.251119$

C $0.206503-0.9430500 .005118$

C $1.204692-2.0942010 .052713$

C $2.422433-1.4086260 .638828$

C $-0.891954-1.128666-1.049151$

C $3.714095-2.1783130 .424477$

C $-1.8708090 .056319-1.186310$

O $-1.604265-2.328817-0.763262$

O $-1.1950101 .128847-1.856207$

C $-2.484960 \quad 0.618706 \quad 0.111615$

C $-3.246753-0.413750 \quad 0.948712$

$\begin{array}{lllll}\text { O } & -3.410618 & 1.649960 & -0.257206\end{array}$

C $3.044144 \quad 1.067468 \quad 0.713357$

$\begin{array}{llll}\text { C }-3.884737 & 0.199382 & 2.190386\end{array}$

H $3.7032232 .325690-0.995567$

H $2.5783684 .358121-0.360155$

H 1.8853313 .5388111 .158080

H $2.811877-0.138868-1.080627$

H $-0.236024-0.834443 \quad 1.002567$

H $1.422036-2.467138-0.955904$

H $\quad 0.850125-2.9339310 .658031$

H $2.261874-1.269098 \quad 1.716434$

H $-0.402349-1.263695-2.022854$

H $3.660489-3.1605590 .905232$

H $3.915088-2.336180-0.640587$

H $4.565538-1.6394960 .851599$

H $-2.691468-0.237803-1.852372$

H $-2.257906-2.459307-1.471279$

H $-0.463424 \quad 1.420983-1.285778$

H $-1.713090 \quad 1.1017860 .721947$

H -2.578157 -1.2152261 .275507$

H $-4.041940-0.8671810 .344701$ 
H $-2.9212202 .301908-0.787865$

H 2.4964261 .2402321 .648239

H 4.0682800 .7842330 .981130

H -3.129187 0.6764552 .822774

H -4.6377940 .9484571 .926749$

H $-4.379665-0.5779872 .781243$

SCF Energy (B3LYP/6-31G**//MMFF) $=-810.720754074$

F2-08 c01560

MMFF Geometry

C $3.4588591 .936117-0.898564$

C $3.5679093 .079147-0.212440$

C $2.329815-0.244792-0.399635$

O $1.321381 \quad 0.3268230 .460099$

C $0.654865-0.7354691 .164778$

C $1.074636-2.0281670 .481125$

C 2.491451-1.694100 0.058413

C $-0.861540-0.5044071 .238958$

C $3.041201-2.623570-1.009724$

C $-1.565460-0.205225-0.103777$

O

O $-1.1459311 .057672-0.620344$

C $-3.104984-0.1975780 .010802$

C $-3.7834280 .042597-1.345926$

O

C $3.6184420 .571148-0.290700$

C $-5.299556-0.081840-1.260905$

H $3.2504931 .985996-1.964239$

H $3.4452824 .033711-0.712351$

H 3.7776803 .0848750 .851360

H $1.934584-0.207692-1.423363$

H $1.065340-0.7173412 .183221$

H $0.456759-2.244145-0.396966$

H $1.018664-2.8903111 .152577$

H $3.141678-1.7387420 .942424$

H -1.317918 -1.3928711 .691112$

H $2.423374-2.605636-1.913843$

H $4.059082-2.336395-1.291464$

H $3.070777-3.654988-0.643514$

H -1.290290 $-0.966646-0.841329$

H $-0.708105 \quad 1.376914 \quad 1.748708$

H $-0.1833371 .020460-0.747735$

H $-3.434449-1.1635200 .411153$

H -3.409416 -0.678591-2.081454

H -3.547852 $1.047576-1.715838$

H -3.1875351 .6578610 .614915$

H 3.9118170 .6614330 .762793

H $4.4341210 .060986-0.815485$

H -5.745599 $0.066603-2.249529$

H -5.591342 -1.074333 -0.903179

H $-5.7240490 .666995-0.585062$

SCF Energy (B3LYP/6-31G**//MMFF) $=-810.717762322$

\section{F2-09 c01756}

MMFF Geometry

C $4.541316-1.275300-0.308371$

C $4.748220-2.4447630 .307249$

C $2.656793 \quad 0.377774-0.245272$

O $1.639497-0.594588 \quad 0.071175$

C 0.4354190 .0977620 .460972

C 0.6502071 .5579040 .086180

C 2.1423501 .7077840 .304082

C $-0.774968-0.585295-0.191364$

C $2.7344582 .929930-0.376006$

C -2.1189980 .0283340 .244028$

O $-0.668455-0.500134-1.612436$

O $-2.232136-0.0481901 .664174$

C $-3.340994-0.648988-0.410337$

C $-4.695098-0.1287870 .093508$

O $-3.285220-2.052681-0.143062$

C $3.989187-0.0534210 .370063$

C $-4.9203301 .350350-0.172215$

H $4.793788-1.189399-1.362149$

H $5.154655-3.288794-0.239159$

H $4.516783-2.5821201 .357619$

H $2.7313040 .417047-1.339936$

H $\quad 0.371476 \quad 0.0031021 .552008$

H 0.0573542 .2392390 .703903
H $\quad 0.397057 \quad 1.745166-0.963925$

H 2.3349551 .7683681 .383765

H $-0.742882-1.6500850 .070136$

H $2.5691032 .906534-1.458500$

H 2.2778633 .8457010 .013748

H $3.8131592 .990488-0.201509$

H $-2.1299241 .089236-0.028897$

H $\quad 0.192726-0.877423-1.861379$

H $-2.262561-0.9902531 .904865$

H $-3.287033-0.524009-1.498253$

H $-4.807751-0.3352901 .164888$

H $-5.494901-0.700460-0.394805$

H $-4.024222-2.471391-0.616475$

H $4.728158 \quad 0.7496420 .271119$

H $3.856412-0.2472101 .441821$

H $-5.940046 \quad 1.6314210 .110210$

H $-4.233861 \quad 1.9717010 .410094$

H $-4.7864791 .583842-1.233109$

SCF Energy (B3LYP/6-31G**//MMFF) $=-810.721237647$

F2-09 c01757

MMFF Geometry

C $2.0091172 .791636-0.172162$

C $1.2778293 .568078-0.979337$

C 2.0530290 .3142680 .192854

O $0.6773750 .114575-0.185335$

C $0.421354-1.291288-0.316596$

C $1.650686-1.9976110 .247243$

C $2.751047-1.013271-0.094839$

C $-0.884198-1.6852930 .385645$

C $4.026702-1.2290200 .700526$

C $-2.164006-1.062227-0.209687$

O $-1.007769-3.1099230 .304141$

O $-2.230377-1.383987-1.606573$

C $-2.3136820 .464317-0.091646$

C -2.1634900 .9937381 .338103$

O

C $2.6322451 .490509-0.593456$

C -2.3399112 .5057921 .417868$

H $2.1747173 .114857 \quad 0.852373$

H $0.8567654 .498123-0.613464$

H $1.0837913 .291794-2.009841$

H $2.061403 \quad 0.5284881 .269823$

H $\quad 0.357139-1.513259-1.389290$

H $1.806495-2.986780-0.194073$

H $1.567886-2.1184541 .334621$

H $2.975819-1.096012-1.166704$

H $-0.818812-1.4337721 .449771$

H $4.786547-0.4897290 .428873$

H $3.846793-1.1470771 .777736$

H $4.438723-2.2241480 .503634$

H -3.042855 -1.540377 0.241428

H $-1.793209-3.3659340 .817078$

H -2.157254 -2.350912 -1.684338

H $-1.6151980 .975462-0.763027$

H $-1.1764220 .747033 \quad 1.741002$

H -2.9139430 .5264081 .987081$

H $-3.7130220 .474566-1.460224$

H $3.7127501 .562373-0.426409$

H $2.4761171 .330723-1.667672$

H -2.1872352 .8482652 .446348$

H -1.613686 3.0180870 .778884

$\mathrm{H}-3.3453422 .8092711 .110100$

SCF Energy (B3LYP/6-31G**//MMFF) = -810.719325949

F2-09_c01758

MMFF Geometry

C $4.212618 \quad 1.455848 \quad 0.421422$

C 4.1711732 .7924620 .455851

C $2.482263-0.293911-0.058965$

O $1.366007 \quad 0.4996150 .391994$

C $0.136523-0.136631-0.022234$

C $0.530188-1.528970-0.495325$

C $1.905366-1.273606-1.079035$

C $-0.869914-0.0570511 .133512$

C $2.730253-2.535843-1.261724$

C $-2.294707-0.5387420 .811026$

O $-0.391993-0.8294382 .241242$ 
O $-2.268562-1.9291490 .466479$

C $-2.9994650 .200464-0.339170$

C $-3.0456591 .721253-0.157488$

O $-4.348178-0.277826-0.408740$

C $3.5597170 .620805-0.643581$

C -3.781392 2.420519-1.294683

H $4.757630 \quad 0.931377 \quad 1.202102$

H 4.6680053 .3373231 .251239

H $3.644395 \quad 3.363797-0.300458$

H $2.865977-0.8322590 .817704$

H $-0.2282660 .450571-0.872985$

H $-0.170857-1.935845-1.230053$

H $\quad 0.597743-2.2349720 .340660$

H $1.787034-0.777716-2.051955$

H -0.9116450 .9759241 .497434$

H $3.716844-2.302126-1.673702$

H $2.877595-3.060150-0.311422$

H $2.231328-3.224602-1.951442$

H $-2.911575-0.4752211 .716534$

H $0.505543-0.5157402 .446592$

H $-1.841967-2.4004531 .202709$

H $-2.531461-0.050763-1.298197$

H $-2.0326982 .132593-0.104008$

H $-3.554936 \quad 1.9668990 .782101$

H $-4.306979-1.243248-0.521007$

H $4.3397460 .023020-1.128559$

H $3.1185961 .269478-1.410708$

H $-3.3125792 .198020-2.258455$

H $-4.8308922 .113869-1.341881$

H -3.756551 3.505019-1.147676

SCF Energy (B3LYP/6-31G**//MMFF) $=-810.722802546$

F2-09_c01759

MMFF Geometry

C 3.2866372 .4409570 .423545

C $2.8530253 .539639-0.204404$

$\begin{array}{llll}C & 2.602631 & 0.033191 & 0.293767\end{array}$

O $1.2425800 .311309-0.095716$

C $0.621240-0.907162-0.534150$

C $1.564203-2.032956-0.126363$

C $2.913901-1.357173-0.258510$

C $-0.794630-1.0501540 .043118$

C $4.029070-2.0807150 .476091$

C $-1.760823 \quad 0.058117-0.432119$

O $-1.310828-2.327338-0.325028$

$\begin{array}{lllll}0 & -1.334045 & 1.325795 & 0.064481\end{array}$

C $-3.217518-0.1777130 .019080$

C $-4.1572230 .954035-0.421219$

O $-3.267920-0.302091 \quad 1.440157$

C $3.5253231 .122093-0.255965$

C $-5.6128910 .653742-0.086238$

H $3.490216 \quad 2.4962121 .489880$

H 2.7041534 .4632210 .344207

H $2.6379043 .538501-1.267150$

H $2.6255240 .025347 \quad 1.391601$

H $0.570831-0.869963-1.630261$

H $1.462232-2.918147-0.761432$

H $1.385429-2.3350840 .913159$

H $3.169872-1.289766-1.324436$

H $-0.726176-1.0118441 .137409$

H $4.979801-1.5506210 .362862$

H $3.815730-2.1648331 .546911$

H $4.157679-3.0928890 .078614$

H $-1.7517040 .107218-1.528282$

H -1.373117 -2.353527 -1.295278

H $-0.448326 \quad 1.502203-0.295457$

H -3.579657-1.123423 -0.399566

H $-4.0665801 .112303-1.501836$

H -3.8844491 .8917280 .077197$

H -2.913020 0.5202801 .819495

H $4.573507 \quad 0.844696-0.097414$

H $3.3731351 .224931-1.337783$

H $-6.2555701 .467738-0.436582$

H -5.943102 -0.270959-0.570012

H -5.7602690 .5498650 .993207$

SCF Energy (B3LYP/6-31G*//MMFF) $=-810.721789159$

F2-09_c01760
MMFF Geometry

C $3.060138 \quad 2.498756 \quad 0.629227$

C $2.3906423 .635507 \quad 0.407237$

C $2.5178710 .093068 \quad 0.177936$

$\begin{array}{lllll}\text { O } & 1.088951 & 0.267831 & 0.265040\end{array}$

C $0.442834-0.908051-0.247870$

C $1.529997-1.968012-0.378779$

C $2.733294-1.117192-0.731365$

C $-0.717036-1.3291740 .656537$

C $4.060136-1.824381-0.516592$

C $-1.754776-0.210998 \quad 0.897262$

O $-1.334448-2.4838670 .092022$

O $-2.739351-0.7050011 .811620$

C $-2.4724380 .316999-0.361042$

C $-3.4971301 .411122-0.024253$

O $-3.136968-0.744200-1.042444$

C $3.1584591 .373029-0.361318$

C $-4.1088142 .030203-1.274827$

H 3.5758722 .3744661 .577869

H $2.361524 \quad 4.4124411 .163323$

H $1.8621053 .810639-0.523301$

H $2.875831-0.1201031 .193823$

H $0.074403-0.669974-1.253457$

H $1.295777-2.719460-1.138871$

H $1.691209-2.4850180 .575340$

H $2.653102-0.815247-1.784295$

H $-0.319449-1.6309201 .634085$

H $4.183214-2.1391340 .525159$

H $4.126453-2.717012-1.147335$

H $4.898295-1.168472-0.771516$

H -1.2618390 .6334581 .394128$

H -2.088769 $-2.716440 \quad 0.659886$

H $-2.279235-0.9565272 .630516$

H -1.731992 $0.731652-1.053332$

H $-3.016817 \quad 2.2015190 .563972$

H -4.3130930 .9958930 .579155$

H -3.763813 -1.147161 -0.417433

H $4.2197651 .203063-0.575181$

H $2.6757301 .659899-1.304052$

H $-4.8114212 .822537-0.997882$

H $-3.3353522 .470697-1.911908$

H $-4.656037 \quad 1.285845-1.861504$

SCF Energy (B3LYP/6-31G**//MMFF) $=-810.713521986$

F2-09 c01761

MMFF Geometry

C $-4.103356-1.912523-0.287183$

C $-5.210330-2.331453 \quad 0.336153$

C -2.5872690 .0504930 .090476$

O $-1.407158-0.596281-0.428418$

C $-0.3717440 .392306-0.608905$

C $-0.863204 \quad 1.649491 \quad 0.095987$

C $-2.3602531 .547677-0.115226$

C $0.963272-0.175563-0.102548$

C $-3.1651852 .431363 \quad 0.822081$

C $2.151327 \quad 0.773572-0.351627$

O $0.861906-0.4590191 .291520$

O $2.2387801 .030806-1.752524$

C 3.5206590 .2680590 .152604

C $3.912405-1.115787-0.384088$

$\begin{array}{llll}\text { O } & 3.547066 & 0.260380 & 1.577538\end{array}$

C $-3.827109-0.476639-0.634072$

C $5.339675-1.493068-0.005352$

H $-3.357286-2.649412-0.573441$

H $-5.352963-3.3842080 .554101$

H $-5.987800-1.6389230 .639248$

H -2.637290 -0.1876191 .161179$

H $-0.3129400 .574832-1.688912$

H $-0.4327742 .561500-0.328563$

H $-0.626097 \quad 1.629242 \quad 1.166353$

H $-2.5879501 .822968-1.153799$

H $1.132225-1.130144-0.614722$

H -4.2386612 .3184110 .640910$

H -2.9727542 .1834201 .871242$

H -2.907081 3.4849130 .673021

H $1.945807 \quad 1.725200 \quad 0.154784$

H $\quad 0.095628-1.0455801 .413621$

H $2.9449641 .685398-1.888075$ 
H $\quad 4.2856450 .984979-0.170583$

H $3.818190-1.139083-1.475381$

H $3.247691-1.8843240 .026404$

H $2.890738-0.3883091 .883812$

H $-4.6962940 .144114-0.387864$

H $-3.679702-0.418560-1.719469$

H $5.591978-2.474683-0.418783$

H $6.055132-0.765046-0.400641$

H $5.463516-1.5437341 .080838$

SCF Energy (B3LYP/6-31G**//MMFF $)=-810.722884613$

F2-09_c01762

MMFF Geometry

C -4.310351-0.950293 -0.743270

C $-4.428224-2.270164-0.926996$

C $-2.629026 \quad 0.3310280 .684116$

O $-1.603856-0.6751650 .569728$

C $-0.495183-0.117226-0.168691$

C $-0.7706821 .378556-0.283920$

C $-2.2858361 .403179-0.347818$

C $0.819310-0.4984810 .526497$

C $-2.8893402 .763993-0.049658$

C $2.066711-0.026684-0.243965$

O $\quad 0.8501690 .0613541 .839385$

O $2.043562-0.568142-1.563506$

C $3.392936-0.4003710 .451871$

C $4.654462-0.051271-0.350517$

O $3.421159-1.8045290 .719963$

C $-4.015770-0.3098370 .583812$

C $4.8117511 .434978-0.625305$

H $-4.456568-0.294680-1.597373$

H -4.652727 -2.670097-1.909854

H $-4.301613-2.970641-0.109199$

H $-2.526791 \quad 0.7482241 .695263$

H -0.534941 -0.575792 -1.164454

H $-0.2981421 .822338-1.165416$

H -0.4163381 .9224240 .599799$

H -2.587097 $1.091201-1.355013$

H $\quad 0.836570-1.5891700 .641778$

H -2.547111 $3.504717-0.779737$

H -3.982192 $2.723402-0.096305$

H -2.606741 3.1193450 .946889

H $2.0331021 .064232-0.340572$

H $0.048800-0.2391612 .301391$

H $2.000829-1.536284-1.488283$

H 3.4435810 .1019321 .424672

H $4.671613-0.605819-1.296758$

H $5.531586-0.4050370 .206465$

H $3.442858-2.271549-0.132327$

H $-4.107493-1.0630331 .377051$

H -4.7851970 .4465940 .777892$

H 4.7748782 .0125250 .303561

H $5.7764661 .627840-1.105636$

H $4.0298561 .803904-1.295328$

SCF Energy (B3LYP/6-31G**//MMFF)= -810.716850291

F2-09 c01763

MMFF Geometry

C $3.5527421 .868784-0.142257$

C $4.5021812 .104128-1.054884$

C $2.421066-0.2409320 .722893$

$\begin{array}{lllll}\text { O } & 1.181417 & 0.424400 & 1.031222\end{array}$

C $0.153009-0.0814750 .148216$

C $0.758251-1.297239-0.545048$

C $2.219085-0.900055-0.639329$

C $-1.123682-0.3150430 .967621$

C $3.154973-2.065143-0.906943$

C $-2.379214-0.685246 \quad 0.158613$

O $-0.898586-1.3619051 .919182$

O $\quad-2.155891-1.915854-0.541034$

C $-2.8176860 .358033-0.882016$

C $-2.9963271 .786470-0.350653$

O $-4.061829-0.083683-1.438969$

C $3.596023 \quad 0.7334200 .841044$

C $-4.028627 \quad 1.9117150 .763049$

H $2.7000282 .540973-0.093343$

H $4.4150732 .945372-1.733752$

H $5.375171 \quad 1.467030-1.142642$
H $2.545712-1.0129941 .494169$

H $-0.0187990 .711987-0.588366$

H $0.309322-1.486823-1.524628$

H $0.649293-2.2037080 .061842$

H $2.320774-0.166795-1.448895$

H $-1.3283350 .580725 \quad 1.564510$

H $4.194809-1.726242-0.952063$

H $3.081931-2.827530-0.124207$

H $2.912553-2.541166-1.862683$

H -3.208189 -0.8943080 .846001$

H $-0.110131-1.1194982 .434852$

H -1.902526 -2.5818480 .121087$

H $-2.1021100 .367904-1.712778$

H $-3.3251432 .428439-1.177722$

H -2.037808 2.188101-0.005114

H $-4.3102910 .545859-2.137185$

H 3.5883011 .1759131 .845030

H $4.5365240 .177966 \quad 0.743778$

H -4.1373222 .9613971 .054870$

H -5.0099531 .5514130 .439517$

H -3.7305351 .3496671 .652669$

SCF Energy $\left(B 3 L Y P / 6-31 G^{* *} / / M M F F\right)=-810.714652026$

F2-09 c01764

MMFF Geometry

C $3.4078232 .022763-0.221499$

C $4.5890422 .264837-0.800778$

C $2.340004-0.0449130 .810029$

$\begin{array}{lllll}\text { O } & 0.996341 & 0.359104 & 0.484308\end{array}$

C $0.462949-0.558460-0.486401$

C $1.448050-1.722542-0.559218$

C $2.764586-1.023123-0.283348$

C $-0.966998-0.986965-0.124559$

C $3.883751-1.9626020 .128325$

C $-1.9758660 .183442-0.154634$

O $-1.389309-2.002315-1.032254$

$\begin{array}{lllll}\mathrm{O} & -1.612822 & 1.161419 & 0.817744\end{array}$

C $-3.433598-0.2611610 .087878$

C -4.4408320 .8946640 .175144$

O $-3.491644-0.9866841 .318791$

C $3.2340281 .181687 \quad 1.011463$

C $-4.5256481 .729203-1.091953$

H $2.5150352 .466549-0.654572$

H $4.6488382 .886192-1.687739$

H $5.5101141 .850444-0.406302$

H $2.269277-0.570814 \quad 1.771750$

H $0.460141-0.040856-1.454627$

H $1.426023-2.231438-1.527738$

H $1.235198-2.4638830 .221138$

H $3.063340-0.488292-1.193233$

H $-0.950783-1.4172420 .884296$

H $3.624805-2.524567 \quad 1.031987$

H $4.091944-2.684887-0.667907$

H $4.804625-1.4059920 .329142$

H $-1.9208480 .665026-1.137511$

H $-1.395687-1.617378-1.925413$

H -1.671974 0.7410031 .692788

H $-3.741920-0.949026-0.708145$

H -4.2073501 .5400391 .030684$

H -5.4337020 .4796830 .391307$

H -4.401294 -1.313659 1.424561

H 2.7844491 .8238871 .779126

H 4.2089960 .8586941 .395708

H $-4.7335151 .100523-1.963311$

H $-5.3325002 .463817-1.004208$

$\mathrm{H}-3.5974872 .279004-1.273205$

SCF Energy (B3LYP/6-31G**//MMFF $)=-810.712789088$

F2-09 c01765

MMFF Geometry

C 2.3637052 .6968610 .060236

C $1.7746923 .585648-0.747578$

C 2.1234840 .2139340 .284712

O $0.7664590 .180199-0.198248$

C $0.380140-1.182664-0.427562$

C $1.484436-2.0425070 .179609$

C $2.702081-1.164663-0.028152$

C $-1.008674-1.4718730 .157979$ 
C $3.885292-1.5531990 .840869$

C $-2.174781-0.684771-0.476402$

O $-1.287926-2.8687250 .011533$

O $-2.143542-0.866678-1.899785$

C $-2.1939910 .831774-0.219322$

C -2.085953 1.2594581 .250303

O $-3.4257021 .339311-0.751379$

C $2.8772931 .358837-0.392455$

C $-3.190214 \quad 0.7050902 .141900$

H 2.4902052 .9501561 .109716

H $1.4281014 .536626-0.358149$

H $1.6258293 .382561-1.802339$

H 2.0733710 .3731551 .370153

H $\quad 0.375747-1.342138-1.513203$

H $1.570671-3.019605-0.305279$

H $1.307871-2.2073281 .249995$

H $2.997242-1.217830-1.084711$

H $-0.996567-1.2775701 .235229$

H $3.633684-1.5064981 .905751$

H $4.207775-2.5752990 .616501$

H $4.734777-0.8855740 .665990$

H -3.128220 -1.119169-0.149999

H -1.177307 -3.100500 -0.926029

H $-2.292677-1.810394-2.077970$

H $-1.4014921 .316527-0.800098$

$\mathrm{H}-2.1406742 .3543301 .300775$

H -1.1112800 .9773201 .661661$

H -3.414334 2.305861 -0.644576

H $3.9444781 .305855-0.149379$

H $2.7841601 .270627-1.482130$

H -3.076497 1.0907013 .160320

H -4.1801391 .0006091 .781052$

H -3.154100 -0.386827 2.194916

SCF Energy (B3LYP/6-31G**//MMFF) $=-810.707563863$

F2-09 c01766

MMFF Geometry

C -2.903187 2.134409 0.364415

C $-3.9659772 .489166 \quad 1.095396$

C $-2.244128 \quad 0.010007-0.872761$

$\begin{array}{llll}\text { O } & -0.830778 & 0.225211 & -0.694589\end{array}$

C $-0.320069-0.797768 \quad 0.177662$

C $-1.427747-1.8379630 .317001$

C $-2.667224-0.969410 \quad 0.221697$

C $0.991466-1.370727-0.364858$

C $-3.934386-1.742546-0.096884$

C $2.078596-0.304627-0.625602$

O $1.468899-2.3803520 .522299$

O $3.215119-0.964394-1.191474$

C 2.5223200 .5266210 .595139

C $3.672074 \quad 1.5032720 .303505$

O $2.952635-0.3498821 .637135$

C $-2.987552 \quad 1.347833-0.913078$

C $3.3356262 .551845-0.743916$

H -1.9154752 .4393080 .700293$

H -3.838965 3.0629932 .006840

H $-4.973237 \quad 2.214747 \quad 0.802433$

H - $2.352313-0.464688-1.857252$

H $-0.156748-0.3382211 .160398$

H -1.363695 -2.396488 1.255735

H $-1.397539-2.557016-0.511056$

H -2.795643 -0.4530491 .180899$

H $0.798003-1.873224-1.321078$

H $-4.793683-1.068286-0.168519$

H -3.848183 -2.281904 -1.046045

H $-4.144861-2.4761470 .688186$

H $1.7048270 .375909-1.398305$

H $1.638470-1.963313 \quad 1.383981$

H $3.558102-1.577515-0.518725$

H 1.6653301 .0909790 .979644

H 4.5722930 .9533350 .003261

H 3.9448942 .0134741 .236354

H 3.1668230 .1964452 .412514

H -2.551223 $1.970587-1.704193$

H $-4.0347921 .170979-1.186103$

H $2.4213613 .092277-0.479375$

H $4.1492783 .280157-0.820728$

H $3.2011022 .103329-1.732417$
SCF Energy (B3LYP/6-31G**//MMFF) $=-810.724107605$

F2-09_c01767

MMFF Geometry

C 2.9761772 .5516030 .608892

C $2.281543 \quad 3.6687890 .366361$

$\begin{array}{llll}C & 2.495568 & 0.127472 & 0.187403\end{array}$

$\begin{array}{llllll}\text { O } & 1.062547 & 0.268854 & 0.263734\end{array}$

C $0.445882-0.929315-0.234772$

C $1.559885-1.963470-0.346130$

C $2.744892-1.089466-0.703914$

C $-0.712747-1.361200 \quad 0.669874$

C $4.086788-1.762418-0.472593$

C $-1.756517-0.2484900 .911141$

O $-1.348610-2.5099820 .112307$

O $-2.702105-0.7574461 .857572$

C $-2.491973 \quad 0.263768-0.346214$

C $-3.4774831 .396260-0.022785$

O $-3.223042-0.787145-0.973247$

C $3.1087721 .415112-0.365230$

C $-4.0596872 .027525-1.282042$

H 3.4877212 .4527291 .562892

H 2.2283394 .4552101 .111389

H $1.7558213 .818336-0.570099$

H $2.852203-0.0634651 .208167$

H $0.077020-0.713988-1.245210$

H $1.349318-2.730044-1.097849$

H $1.727744-2.4638090 .615698$

H $2.663611-0.803816-1.761298$

H $-0.323798-1.6584591 .652595$

H $4.910783-1.090386-0.731659$

H $4.211314-2.0602940 .573833$

H $4.177363-2.661435-1.090954$

H -1.2520960 .5964441 .395739$

H $-0.704275-3.2375910 .114842$

H $-3.211647-0.0053552 .202430$

H $-1.7605040 .638041-1.070929$

H -2.9732872 .1745460 .561401$

H -4.3133791 .0145900 .575112$

H -2.588195 -1.478786 -1.225400

H $4.1752311 .268014-0.569691$

H $2.6256661 .677259-1.314943$

H $-3.2675532 .440287-1.914908$

H $-4.6252371 .297550-1.869409$

H $-4.7390902 .843163-1.014621$

SCF Energy (B3LYP/6-31G**//MMFF) $=-810.711117290$

F2-09_c01768

MMFF Geometry

C $3.3053772 .632871-0.169312$

C 2.4689593 .1297960 .749373

C $2.605465 \quad 0.1538590 .127792$

$\begin{array}{lllll}\text { O } & 1.234581 & 0.332879 & -0.278189\end{array}$

C $0.643148-0.951770-0.532285$

C $1.633517-1.981587-0.001118$

C $2.956514-1.285868-0.248467$

C $-0.757836-1.052293 \quad 0.091327$

C $4.107791-1.8737000 .549074$

C $-1.756183-0.039440-0.513145$

O $-1.269513-2.367205-0.112459$

O $-1.313881 \quad 1.294627-0.270319$

C $-3.195140-0.208077 \quad 0.020325$

C $-4.1592410 .838997-0.555786$

O $-3.198641-0.121346 \quad 1.444983$

C $3.4904161 .192063-0.569283$

C $-5.599906 \quad 0.589767-0.127508$

H $3.9371543 .342115-0.700972$

H 2.4402824 .1982500 .936527

H 1.8017912 .5051571 .330781

H 2.6434330 .2789451 .217479

H $0.569061-1.066346-1.621674$

H $1.553245-2.945668-0.512370$

H $1.483460-2.1498141 .072777$

H $3.191278-1.348238-1.319568$

H $-0.678657-0.8686201 .170346$

H $3.916131-1.8247521 .626262$

H $4.262464-2.924548 \quad 0.282952$

H $5.038725-1.3348160 .347934$ 
H $-1.786635-0.175591-1.601237$ H $-0.737427-2.979957 \quad 0.422340$ H -1.1734921 .3937110 .686473$ H -3.560548 -1.208203 -0.238948 H $-4.1069870 .828437-1.650489$ H $-3.8771841 .843389-0.218543$ H -2.9452960 .7847161 .689620$ H $4.5443040 .942500-0.397961$ H $3.3163391 .138231-1.651755$ H $-6.2611801 .337189-0.577449$ H $-5.938416-0.399980-0.449938$ H -5.7095000 .6545430 .959362$

SCF Energy (B3LYP/6-31G**//MMFF)= -810.709861105

F2-09_c01769

MMFF Geometry

C 3.5200462 .1948130 .414766

C $3.2911733 .288054-0.321179$

C $2.528639-0.1070980 .385453$

O $1.2540520 .312838-0.141133$

C $0.510420-0.845024-0.553319$

C $1.257088-2.0476690 .012572$

C $2.692012-1.564516-0.042981$

C $-0.955573-0.758404-0.100082$

C $3.636817-2.3666130 .834936$

C $-1.7166570 .424237-0.746932$

O $-1.579240-1.995239-0.433750$

O $-1.131801 \quad 1.667618-0.362606$

C $-3.2226770 .504972-0.414896$

C $-3.5188380 .571914 \quad 1.090183$

O $-3.915249-0.607574-0.973784$

C $3.6308130 .807230-0.151584$

C $-4.9956640 .822498 \quad 1.373003$

H 3.6436802 .3061551 .488957

H 3.2261394 .2631920 .149177

H $3.1633553 .231997-1.396560$

H $2.463060-0.0322901 .478937$

H $0.555223-0.887960-1.649564$

H $1.088181-2.959767-0.567791$

H $\quad 0.956373-2.2413221 .049942$

H $3.039011-1.613855-1.083961$

H $-0.964887-0.6398200 .990349$

H $4.656354-1.9736930 .772741$

H $3.328822-2.3394921 .885586$

H $3.658935-3.4145060 .518334$

H $-1.6273640 .333861-1.837356$

H -2.476477 -1.986754 -0.060275

H $-0.2104581 .671431-0.672592$

H -3.630421 $1.411232-0.880904$

H $-2.930811 \quad 1.3691151 .558194$

H $-3.245359-0.3730411 .573160$

H -3.744353 -0.609195 -1.930946

H 4.6160470 .4099900 .117289

H $3.5800580 .844434-1.247078$

H -5.6197430 .0138710 .980117$

H $-5.166811 \quad 0.8852462 .452419$

H -5.3262531 .7636640 .922375$

SCF Energy (B3LYP/6-31G**//MMFF) $=-810.721194309$

F2-09_c01770

MMFF Geometry

C $4.084998-1.614097-0.024161$

C $5.235956-1.5908860 .657356$

C $2.4814260 .186791-0.839594$

O $1.321000-0.663816-0.761665$

C $0.449456-0.1560720 .272210$

C $\quad 0.997141 \quad 1.2150650 .654252$

C 2.4856431 .0162030 .443093

C -1.000859 $-0.174940-0.233591$

C 3.2683952 .3128100 .338020

C -2.0164550 .2490290 .845635$

O $-1.1224930 .683138-1.366015$

O $-1.884788-0.592231 \quad 1.990744$

C -3.4958650 .2633240 .401168$

C $-3.983912-1.059511-0.205581$

O $-3.7141351 .306629-0.545965$

C $3.737102-0.644167-1.117647$

C $-5.484762-1.047472-0.473936$
H $3.355892-2.3847720 .212096$

H $5.429942-2.3237221 .433036$

H $5.998675-0.8464460 .457922$

H $2.3136980 .842252-1.704881$

H $\quad 0.556213-0.843478 \quad 1.120435$

H $\quad 0.744687 \quad 1.4931541 .682078$

H $0.6179651 .999578-0.011388$

H 2.8738950 .4319351 .286479

H -1.219407 -1.193397 -0.575752

H 4.3319112 .1127680 .172834

H $2.9090072 .933489-0.489532$

H 3.1730952 .8953031 .260205

H $-1.769767 \quad 1.268603 \quad 1.166606$

H $-0.4637550 .395038-2.021155$

H $-1.963410-1.5156191 .697890$

H -4.1166890 .4841051 .278857$

H $-3.756218-1.8952290 .464148$

H $-3.479442-1.246378-1.160466$

H -3.436222 2.141951-0.133207

H $3.576857-1.231240-2.030738$

H $4.5794280 .029627-1.314626$

H $-6.046221-0.8728380 .449328$

H $-5.755313-0.269662-1.194717$

H $-5.801070-2.011244-0.885593$

SCF Energy (B3LYP/6-31G**//MMFF) $=-810.713702536$

F2-09 c01771

MMFF Geometry

C -4.233142 -1.772563 -0.115241

C $-4.104405-2.0308421 .191369$

C $-2.4925370 .147808-0.193468$

O $-1.355942-0.6549110 .181834$

C $-0.1438230 .083864-0.087826$

C $-0.5855181 .506926-0.399593$

C $-1.927858 \quad 1.271022-1.062780$

C $0.814335-0.0992451 .097085$

C $-2.8010742 .512692-1.118539$

C 2.2266220 .4802220 .908895

$\begin{array}{lllll}\text { O } & 0.249057 & 0.499588 & 2.269593\end{array}$

O $2.1269221 .900514 \quad 0.745788$

C $3.020640-0.081891-0.284392$

C $3.132590-1.611288-0.278720$

O $4.3339330 .485162-0.208439$

C $-3.520057-0.721270-0.925264$

C $3.943265-2.153608-1.449733$

H $-4.936065-2.386274-0.676067$

H -4.686486 -2.824689 1.648149

H -3.432575 -1.474484 1.833410

H -2.9192170 .5630510 .728499$

H $0.286603-0.372177-0.986945$

H $0.1201162 .031119-1.051020$

H -0.7116762 .0994710 .514107$

H $-1.7552440 .906138-2.084321$

H $0.890536-1.1677611 .328781$

H -2.309150 $3.302737-1.695483$

H $-3.7612752 .294857-1.596089$

H -3.004631 $2.905571-0.116703$

H 2.7952850 .3333251 .836132

H -0.6413720 .1256482 .384724$

H 3.0314002 .2455410 .651832

H $2.5791730 .271401-1.223745$

H $2.137296-2.065109-0.321213$

H $3.609897-1.9439480 .650939$

H $4.7674070 .343161-1.066763$

H -4.290145 -0.077506-1.366675

H -3.020070 -1.242235 -1.752037

H $4.990456-1.841956-1.392128$

H $3.923495-3.248117-1.444502$

H $3.530239-1.811552-2.403849$

SCF Energy (B3LYP/6-31G**//MMFF) $=-810.714229965$

F2-09 c01772

MMFF Geometry

C -3.174709 2.260365 -0.292384

C -3.2484343 .1951990 .661603$

C $-2.199225-0.047864-0.347616$

O

C $-0.569691-1.0305921 .019401$ 
C $-1.077178-2.078780 \quad 0.039481$

C $-2.463111-1.551754-0.277214$

C $0.957311-0.8742831 .107371$

C $-3.058304-2.137597-1.545911$

C $1.612789-0.033054-0.012190$

O $1.248742-0.2483652 .362958$

O $1.374761-0.631597-1.281840$

C $3.1333840 .155790 \quad 0.183330$

C $3.8309870 .898182-0.965968$

$\begin{array}{lllll}\text { O } & 3.742277 & -1.132377 & 0.304883\end{array}$

C $-3.4396140 .799362-0.061299$

C $3.3174992 .311894-1.182610$

H - $2.9166332 .566470-1.302961$

H $-3.049096 \quad 4.235367 \quad 0.427783$

H -3.504951 2.9433481 .684578

H $-1.7632340 .220794-1.318985$

H $-0.959267-1.2711672 .017708$

H $-1.091917-3.0807830 .479847$

H $-0.471372-2.127745-0.869193$

H -3.125850 -1.777583 0.569108

H $1.400475-1.8760641 .142799$

H $-4.053169-1.724453-1.738393$

H $-2.431000-1.925531-2.418253$

H $-3.155705-3.224638-1.458389$

H $1.1503560 .959948-0.027128$

H $\quad 0.824566 \quad 0.6271912 .359737$

H $1.836935-1.487294-1.293224$

H 3.3170000 .6909451 .122239

H $3.7461460 .325083-1.897391$

H $4.9070070 .942596-0.753475$

H $4.686487-0.994267 \quad 0.492367$

H $-4.2630250 .498099-0.718576$

H $-3.7686860 .636594 \quad 0.972658$

H $3.9260902 .820782-1.937087$

H $2.2829042 .313173-1.538205$

H $3.3682682 .894489-0.257442$

SCF Energy (B3LYP/6-31G*//MMFF) $=-810.716989834$

\section{F2-09 c01773}

MMFF Geometry

C $-4.402121-1.4452990 .334495$

C $-4.643586-2.525229-0.416917$

C -2.5747590 .2715510 .319332$

O $-1.572865-0.608018-0.231722$

C $-0.430564 \quad 0.177560-0.631691$

C $-0.641794 \quad 1.562949-0.035618$

C $-2.1514831 .683798-0.084280$

C $0.857757-0.538851-0.197250$

C $-2.7011792 .776626 \quad 0.816063$

C $2.1406200 .193690-0.631919$

O $0.845016-0.681677 \quad 1.222025$

O $2.010078 \quad 0.530337-2.019614$

C $3.435428-0.633181-0.485188$

C $3.783195-1.137996 \quad 0.920240$

O $4.517993 \quad 0.184113-0.951345$

C $-3.953109-0.120796-0.215220$

C $3.977255-0.0320701 .950402$

H $-4.545411-1.5160581 .409684$

H $-4.970410-3.4518620 .042078$

H -4.519595 -2.506675 - 1.494045

H -2.5356870 .1553951 .410471$

H $-0.4759010 .236206-1.725984$

H $-0.1308392 .344873-0.605333$

H -0.2876511 .6131231 .000903$

H $-2.4542801 .885479-1.120572$

H $0.848819-1.550007-0.622823$

H -2.4269392 .6097861 .863164$

H -2.3080513 .7540220 .517814$

H -3.7931122 .8197410 .756602$

H $2.2441451 .137788-0.084146$

H $0.024087-1.145542 \quad 1.460466$

H $2.8197161 .002180-2.280001$

H $3.384795-1.489636-1.168963$

H $4.726156-1.6971180 .864090$

H $3.028856-1.8483921 .272405$

H $5.324945-0.358243-0.930511$

H -4.6968650 .6318570 .069978$

H $-3.928011-0.158540-1.311483$
H $4.254947-0.4669132 .916230$

H $4.7764200 .653391 \quad 1.651758$

H 3.0641330 .5505932 .098782

SCF Energy (B3LYP/6-31G**//MMFF) $=-810.719729834$

F2-09 c01774

MMFF Geometry

C $4.139087 \quad 1.705410 \quad 0.536340$

C 4.1510792 .9559110 .061424

C $2.573367-0.2266910 .216869$

O $1.4093670 .587993-0.031375$

C $0.349317-0.251083-0.535562$

C $0.809235-1.686292-0.317460$

C $2.308109-1.552423-0.495201$

C -0.9709120 .1359450 .148570$

C $3.092318-2.7255480 .066805$

C $-2.182930-0.638234-0.404633$

O $-0.862802-0.0870821 .553272$

O $-2.271174-0.415041-1.809885$

C $-3.536428-0.3097790 .262697$

C $-3.893481 \quad 1.1783220 .395641$

O $-3.548927-0.8920311 .568184$

C $3.8227240 .492996-0.293344$

C $-4.0011951 .923796-0.923460$

H 4.3808571 .5435261 .583571

H 4.3925593 .7884880 .713070

H $3.9206193 .171403-0.975956$

H $2.631070-0.3784461 .302900$

H $0.285674-0.043848-1.610928$

H $0.355004-2.382895-1.028581$

H $0.574077-2.0345120 .695336$

H $2.526949-1.454010-1.567072$

H $-1.113898 \quad 1.2130180 .003984$

H $2.906907-2.8556301 .138329$

H $2.808819-3.654752-0.438400$

H $4.167902-2.580204-0.073573$

H -1.999015 -1.710063 -0.254555

H -0.0836740 .4038821 .866105$

H -2.996938 -0.964909-2.150880

H $-4.332482-0.801880-0.310192$

H $-3.178716 \quad 1.6856891 .054428$

H -4.8573351 .2562870 .915748$

H -4.431165 -0.7439231 .949343$

H $4.688148-0.177961-0.252914$

H $3.6808180 .782749-1.342036$

H $-4.6807831 .411234-1.611320$

H $-4.3920862 .932224-0.752285$

H -3.026779 2.029830-1.407791

SCF Energy (B3LYP/6-31G**//MMFF $)=-810.708334767$

F2-09 c01775

MMFF Geometry

C 3.3130562 .1583020 .526680

C 2.7807543 .3703550 .334265

C $2.424600-0.1565640 .155743$

O $1.0436350 .213250 \quad 0.345017$

C $0.205393-0.851481-0.129257$

C $1.118395-2.057913-0.313180$

C $2.406400-1.389181-0.749387$

C $-0.954176-1.1027340 .834373$

C $3.630089-2.277902-0.608618$

C -1.7946640 .1454791 .182704$

O $-1.773031-2.1396150 .297875$

O $-2.789032-0.2655552 .127793$

C $-2.485016 \quad 0.913414 \quad 0.035364$

C $-3.3985380 .062965-0.854406$

O $-1.5160441 .561392-0.784471$

C $3.1907651 .021439-0.448356$

C $-4.2205880 .918106-1.812679$

H $3.878011 \quad 1.9753871 .437139$

H 2.9088454 .1508131 .076301

H $2.2105003 .604697-0.557822$

H $2.825035-0.4112261 .145785$

H $-0.175213-0.563138-1.116551$

H $0.732165-2.770467-1.048277$

H $1.263808-2.5892050 .635615$

H $2.304489-1.083025-1.799323$

H $-0.550475-1.4900201 .779401$ 
H $4.535560-1.748225-0.920859$

H $3.771221-2.6052580 .426941$

H $3.529694-3.171863-1.232767$

H $-1.133870 \quad 0.8456811 .709557$

H -2.502546 -2.2858200 .924279$

H -3.257010 0.5319932 .428261

H -3.1007291 .7046030 .482670$

H $-4.083258-0.531442-0.239801$

H -2.801083 - $0.624261-1.463591$

H $-0.9629202 .114909-0.207375$

H $4.2018480 .708783-0.732494$

H $2.6846791 .363151-1.360045$

H $-3.5772231 .488924-2.489568$

H $-4.8698440 .281762-2.422516$

H -4.854717 $1.621761-1.264137$

SCF Energy (B3LYP/6-31G**//MMFF) $=-810.716440371$

F2-09_c01776

MMFF Geometry

C $4.209879-1.1649340 .758043$

C $5.238937-0.7422901 .501092$

C $2.578146-0.007554-0.816122$

O $1.506827-0.936198-0.559047$

C $0.449941-0.2355110 .132144$

C $0.794771 \quad 1.2473170 .043056$

C $2.310791 \quad 1.2054800 .073410$

C $-0.908281-0.656496-0.449953$

C $2.9693052 .484520-0.411714$

C $-2.106407-0.0288920 .289943$

O $-0.964170-0.332365-1.838632$

O $-2.047647-0.439066 \quad 1.655041$

C $-3.473830-0.405613-0.321885$

C -4.6899300 .1366780 .441619$

O $-3.618214-1.827176-0.360558$

C $3.935936-0.693718-0.642213$

C $-4.734921 \quad 1.653206 \quad 0.527077$

H $3.531957-1.9026211 .179460$

H $5.385626-1.1256282 .504922$

H $5.946909-0.0113621 .126625$

H $2.4787710 .277752-1.872156$

H $0.512716-0.557257 \quad 1.179334$

H $\quad 0.3669851 .8251800 .867887$

H $0.4478041 .690521-0.896904$

H 2.6255821 .0113381 .106185

H $-0.958116-1.749893-0.384289$

H $4.0599062 .395846-0.382107$

H $2.6777272 .721765-1.440277$

H $2.6814113 .329507 \quad 0.222085$

H -2.002809 1.0618250 .261306

H $-1.107940 \quad 0.624934-1.922951$

H -2.6569750 .1235192 .161612$

H -3.527708 $-0.045729-1.356347$

H $-4.736049-0.295675 \quad 1.448443$

H -5.600629-0.215747 -0.060405

H -3.020649 -2.166149 -1.048162

H $3.971024-1.576786-1.292450$

H $4.728832-0.016022-0.980381$

H $-4.6696752 .104768-0.467793$

H $-5.676011 \quad 1.9756070 .983995$

H -3.919085 2.0455751 .140964

SCF Energy (B3LYP/6-31G**/MMFF) $=-810.710824291$

F2-09_c01777

MMFF Geometry

C $2.7431672 .304105-0.270747$

C $2.7345722 .964994-1.433758$

C $1.9471990 .022340 \quad 0.391081$

O $0.920812-0.052720-0.621852$

C $0.494735-1.419321-0.747666$

C $1.007707-2.1351240 .490994$

C $2.329999-1.4246400 .708094$

C $-1.011879-1.533752-1.007311$

C $2.903694-1.6203202 .100804$

C $-1.961628-0.9809670 .077281$

O $-1.305694-0.895435-2.254360$

O $-3.297789-1.326358-0.316646$

C $-1.907570 \quad 0.5313850 .375904$

C -2.9633460 .9478651 .411623$
O $-2.1268651 .293600-0.807490$

C $3.1192650 .856223-0.128280$

C $-2.8129832 .405305 \quad 1.831315$

H $2.4707482 .839800 \quad 0.634885$

H $2.4561874 .012589-1.468185$

H $3.0006142 .478098-2.365348$

H 1.4947950 .5003631 .269585

H $1.025860-1.806761-1.627846$

H $1.117700-3.2125580 .336487$

H $\quad 0.352319-1.9775621 .353934$

H $3.053136-1.793363-0.031905$

H $-1.257160-2.593958-1.152112$

H $2.213780-1.2632902 .872964$

H $3.099945-2.68071922 .290134$

H $3.846664-1.0766462 .214546$

H $-1.783490-1.5227691 .012023$

H $-0.763067-1.321228-2.939651$

H $-3.490683-0.840860-1.137388$

H -0.9225020 .7985080 .768091$

$\begin{array}{llll}H & -2.882151 & 0.313648 & 2.301786\end{array}$

H -3.9714040 .8196340 .999690$

H $-1.408027 \quad 1.084727-1.427982$

H 3.9645610 .7930470 .566329

H $3.4571940 .459523-1.093827$

H -3.563153 2.6586142 .587256

$\mathrm{H}-1.8231662 .5884242 .261546$

H -2.9500093 .0814800 .981772$

SCF Energy (B3LYP/6-31G**//MMFF) $=-810.717335340$

F2-09_c01778

MMFF Geometry

C $-3.023563-1.914410-0.575695$

C $-4.280442-2.072649-1.005724$

C $-1.852637-0.1541630 .841163$

O $-0.578948-0.338140 \quad 0.195714$

C $-0.248603 \quad 0.857709-0.529397$

C $-1.263409 \quad 1.911295-0.088257$

C -2.4912431 .0615650 .174310$

C $1.2117851 .269016-0.291763$

C -3.5439051 .7507601 .023745$

C $2.2700210 .249808-0.764124$

O $1.4684672 .487105-1.001191$

O $1.995769-0.067096-2.136456$

C $2.351410-1.067898 \quad 0.027958$

C $2.481314-0.9188071 .548857$

O $3.494582-1.803637-0.427389$

C $-2.661071-1.453684 \quad 0.807720$

C $3.697110-0.1160111 .996338$

H -2.207976 -2.141510 -1.257308

H $-4.476171-2.414290-2.016224$

H $-5.129959-1.864742-0.364785$

H -1.6257710 .0739201 .891398$

H $-0.4134710 .649147-1.594107$

H -1.429096 $2.679959-0.849306$

H -0.9356092 .4075640 .833758$

H $-2.9323780 .792026-0.793129$

H 1.3721531 .4816770 .770453

H -3.143385 2.0449651 .999701

H -3.9094842 .6542380 .524860$

H -4.3991781 .0896611 .195171$

H $3.2532300 .737423-0.770302$

H $0.9403103 .189845-0.586709$

H $2.774741-0.525211-2.494118$

H $1.485179-1.700248-0.194931$

H $2.568624-1.9200161 .989589$

H $1.575314-0.4698371 .969114$

H $3.319103-2.083581-1.341407$

H $-2.064960-2.2519461 .267368$

H $-3.562394-1.3341981 .420804$

H $3.748924-0.0961253 .089830$

H $4.626567-0.5593241 .625806$

H 3.6461380 .9195681 .648261

SCF Energy (B3LYP/6-31G**//MMFF) $=-810.709107063$

F2-09 c01779

MMFF Geometry

C $4.043398-1.458947-0.374200$

C $5.355196-1.278422-0.184359$ 
C $2.0818840 .121553-0.739636$

O $1.120741-0.760454-0.128411$

C $0.620624-0.1278871 .066438$

C $1.1478151 .301897 \quad 1.058767$

C $2.471836 \quad 1.132247 \quad 0.337389$

C $-0.904959-0.284437 \quad 1.174549$

C $3.0426332 .428624-0.208986$

C $-1.697408-0.081291-0.136328$

O $-1.162084-1.6113961 .650227$

O $-1.496831 \quad 1.235193-0.639593$

C $-3.211298-0.3472880 .023803$

C $-3.990095-0.095663-1.275633$

O $-3.7435300 .474061 \quad 1.062423$

C $3.224033-0.682884-1.366103$

C $-5.458711-0.480446-1.148509$

H $3.532422-2.2188160 .211524$

H $5.892025-1.8756260 .544546$

H $5.913106-0.537104-0.745509$

H $1.5460710 .632471-1.551159$

H $1.090755-0.6654921 .901100$

H 1.2610821 .7038772 .070455

H $\quad 0.497716 \quad 1.9833040 .504056$

H 3.1894510 .6950191 .042718

H $-1.2650350 .403855 \quad 1.947734$

H $3.9897492 .248698-0.727606$

H $2.3550862 .903761-0.916635$

H $3.231753 \quad 3.1380130 .603299$

H $-1.325542-0.777077-0.897847$

H $-0.811624-2.2331800 .989241$

H $-1.8015301 .862598 \quad 0.037390$

H $-3.362618-1.3879020 .332383$

H $-3.541620-0.670718-2.093934$

H $-3.9427260 .965050-1.549014$

H -3.7053901 .3982110 .763632$

H $2.799605-1.408115-2.071617$

H $3.864068-0.008436-1.947487$

H $-5.975213-0.313972-2.099120$

H $-5.565150-1.537683-0.885696$

H $-5.9625070 .117704-0.382783$

SCF Energy (B3LYP/6-31G**/MMFF) $=-810.715128056$

F2-09 c01780

MMFF Geometry

C -4.323783 -1.528606 -0.392989

C $-5.622357-1.531018-0.071832$

C $-2.5003380 .162306-0.060661$

O $-1.435011-0.8069110 .006314$

C $-0.164179-0.120441-0.077207$

C $-0.482418 \quad 1.3584860 .091882$

C $-1.8500251 .452840-0.554487$

C $0.794260-0.7436640 .946055$

C -2.610521 $2.713286-0.179942$

C $2.248542-0.2317770 .968218$

O $\quad 0.259497-0.5453642 .262952$

O $2.260525 \quad 1.1791151 .177645$

C $3.065109-0.592674-0.287460$

C $4.558516-0.271869-0.124907$

O $2.5723840 .107412-1.426513$

C $-3.607423-0.347670-0.985380$

C $5.384696-0.774757-1.302533$

H $-3.745789-2.434540-0.229360$

H $-6.082392-2.4189080 .348139$

H $-6.245333-0.655839-0.219037$

H -2.8813430 .2895990 .961325$

H $0.196407-0.308319-1.093489$

H $\quad 0.2561772 .004688-0.391580$

H $-0.541667 \quad 1.6386911 .150215$

H - $-1.7259411 .418008-1.645300$

H $\quad 0.809889-1.8303570 .795388$

H $-3.5939212 .734304-0.659725$

H -2.761998 2.7837590 .902339

H -2.059873 $3.603798-0.500566$

H $2.735087-0.6768121 .846182$

H $-0.649261-0.8922092 .261373$

H 3.1683321 .4393841 .406439

H $2.952077-1.664543-0.487946$

H $4.940121-0.7284820 .795353$

H $4.7102210 .811089-0.048476$
H $2.6145731 .058645-1.228203$

H $-4.3200240 .458115-1.195965$

H $-3.178278-0.664798-1.943854$

H $6.444438-0.552804-1.141127$

H $5.279537-1.858020-1.420223$

H $5.079339-0.295115-2.237633$

SCF Energy (B3LYP/6-31G**//MMFF) $=-810.718138458$

F2-10 c02001

MMFF Geometry

C $-3.911374 \quad 1.8688990 .053268$

C $-3.383637 \quad 3.048571-0.292530$

C -2.912628 -0.3849890 .549468$

O $\begin{array}{llll}\text { O } & -1.708095 & 0.214940 & 1.077620\end{array}$

C $-0.575625-0.613250 \quad 0.746226$

C $-1.054100-1.556256-0.347184$

C $-2.496784-1.7773090 .061279$

$\begin{array}{llll}\text { C } & 0.617238 & 0.280796 & 0.380186\end{array}$

C $-3.348762-2.409653-1.024773$

C $1.902805-0.517198 \quad 0.095771$

$\begin{array}{lllll}\text { O } & 0.298143 & 1.064415 & -0.769418\end{array}$

O $2.224175-1.322196 \quad 1.228984$

C $3.1066740 .375865-0.271533$

C $4.386900-0.438771-0.505057$

O 3.3365021 .3340910 .760745

C $-3.4799020 .550990-0.528421$

C $5.5406310 .430846-0.988509$

H -4.7079601 .8507880 .792508$

H -3.7428093 .9653910 .161977$

H -2.589807 $3.121768-1.027501$

H $-3.631057-0.4785261 .372791$

H $-0.343267-1.1872601 .651969$

H $-0.473602-2.483156-0.381138$

H $-1.002658-1.087827-1.336702$

H $-2.500484-2.4514540 .929724$

H $\quad 0.7836100 .979798 \quad 1.208984$

H $-3.311034-1.841268-1.959591$

H $-2.994477-3.422410-1.244472$

H $-4.393503-2.480555-0.705624$

H $1.729579-1.198296-0.746031$

H $-0.5089761 .565660-0.562357$

H $2.312683-0.7334631 .997449$

H $2.8709780 .938946-1.181689$

H $4.197481-1.222748-1.247307$

H $4.701074-0.929004 \quad 0.424071$

H 3.6285150 .8550801 .554509

H $-2.7344870 .716706-1.315458$

H $-4.3598680 .098569-0.998861$

H $5.2831420 .938282-1.923579$

H $5.8060401 .189615-0.245852$

H $6.426471-0.185881-1.170400$

SCF Energy (B3LYP/6-31G**//MMFF) $=-810.717829515$

F2-10 c02002

MMFF Geometry

C $-4.005466-1.468243-0.347358$

C $-3.910967-2.534807 \quad 0.454485$

C $-2.3838370 .337236-1.005977$

O $-1.261884-0.566272-0.889806$

C $-0.1114620 .152962-0.395762$

$\begin{array}{llll}\text { C } & -0.654340 & 1.455647 & 0.170918\end{array}$

C $-1.8064631 .737124-0.773028$

C $0.658865-0.7564850 .570933$

C $-2.763168 \quad 2.802296-0.267697$

C $2.005244-0.2077881 .073182$

O $-0.150660-1.033348 \quad 1.719878$

$\begin{array}{llll}\text { O } & 1.787760 & 0.992246 & 1.825012\end{array}$

C $3.0358640 .121616-0.020009$

C $3.328349-1.049603-0.963747$

$\begin{array}{lllll}\text { O } & 4.261699 & 0.485910 & 0.626160\end{array}$

C $-3.461736-0.108262-0.006442$

C $4.379268-0.703045-2.012058$

H $-4.512254-1.577330-1.302668$

H $-4.328586-3.4886520 .151313$

H -3.418502 -2.478140 1.418949

H -2.773574 $0.260285-2.028198$

H $0.5071960 .364981-1.275764$

H 0.0930002 .2548700 .171469 
H $-1.021615 \quad 1.3302281 .195689$ H $-1.3827602 .087356-1.725128$ H $\quad 0.821711-1.7280050 .090468$ H -2.254074 $3.769793-0.200956$ H -3.612487 2.915288 -0.949061 H -3.1492112 .5677840 .729471$ H $2.443765-0.9202441 .783697$ H $-0.986758-1.4121721 .398649$ H $1.150557 \quad 0.7799422 .528606$ H $2.7160700 .998598-0.594907$ H $2.417027-1.359652-1.485025$ H $3.690731-1.908837-0.386749$ H 4.0640021 .2274321 .223827 H -3.054238 -0.1138421 .011726$ H -4.303909 $0.592390-0.019540$ H $4.0649640 .157835-2.610595$ H $5.343998-0.469713-1.551011$ H $4.528714-1.550381-2.688857$ SCF Energy $\left(B 3 L Y P / 6-31 G^{* *} / / M M F F\right)=-810.721213873$

F2-10_c02003

MMFF" Geometry

C 2.4374412 .6574910 .080885

C 1.4087293 .3098650 .633939

C $2.7004820 .274428-0.672102$

O $1.2920830 .167913-0.969055$

C $0.801214-1.108239-0.530214$

C $1.923073-1.7513190 .277238$

C $3.153558-1.158107-0.377919$

C $-0.508282-0.9573670 .259858$

C $4.421272-1.3200980 .441032$

C $-1.657824-0.345110-0.571237$

O $-0.896110-2.2400820 .747119$

O $-1.3448591 .002441-0.920812$

C $-3.009931-0.3529240 .172339$

C $-4.1321950 .300554-0.646773$

O

C $2.869067 \quad 1.2738660 .482505$

C -5.4885790 .1749740 .035952$

H $3.0074293 .149899-0.702835$

H 1.1488384 .3088210 .301247

H $\quad 0.8090292 .8623801 .418821$

H $3.2082840 .653291-1.567283$

H $\quad 0.623000-1.712935-1.428839$

H $1.896562-2.8444600 .229591$

H $1.867171-1.4596411 .332960$

H $3.300701-1.668500-1.340368$

H $-0.316302-0.3080121 .123055$

H $4.683054-2.3797570 .530781$

H $5.260211-0.805110-0.037630$

H $4.308407-0.9254271 .455855$

H -1.781270 -0.913370 -1.501661

H -1.070694 -2.809104 -0.022092

H $-0.5332390 .989359-1.455495$

H -3.293055 -1.3862200 .402376$

H $-4.188513-0.165617-1.637090$

H -3.926888 1.367767 -0.791682

H -2.5895351 .2409461 .223280$

H 2.2930650 .9455051 .356090

H 3.9203341 .3325300 .785020

H $-6.2669680 .627517-0.586514$

H $-5.750627 \quad-0.875746 \quad 0.195539$

H -5.4953290 .6830901 .005222$

SCF Energy (B3LYP/6-31G**//MMFF) $=-810.719424646$

F2-10_c02004

MMFF Geometry

C $2.8929092 .477448-0.135492$

C 2.2782363 .2656600 .753669

C $2.4178970 .145022-0.942856$

O $1.0000950 .308723-0.722058$

C $0.456044-0.904406-0.178393$

C $1.642850-1.7847410 .194539$

C $2.668989-1.362698-0.837181$

C $-0.468537-0.598796 \quad 1.002363$

C $4.084379-1.800289-0.506259$

C -1.5974590 .3999970 .666953$

O $-1.003077-1.8280121 .488368$
O $-2.335124 \quad 0.6591521 .866189$

C $-2.583099-0.049851-0.429856$

C $-3.6791550 .996291-0.684359$

O $-3.198392-1.286075-0.075593$

C 3.1622721 .0139400 .082693

C $-4.5729200 .618179-1.859041$

H $3.2292852 .911814-1.073323$

H 2.1166774 .3160080 .537316

H 1.9252222 .8811601 .704176

H $2.6447780 .493003-1.957728$

H $-0.101663-1.403385-0.980373$

H $1.401313-2.8511950 .149290$

H $1.998589-1.5627451 .207908$

H $2.386909-1.818037-1.797273$

H $\quad 0.124017-0.168198 \quad 1.820061$

H $4.158402-2.892944-0.523783$

H $4.794056-1.402821-1.238739$

H $4.394965-1.4687540 .489727$

H -1.151665 1.3545720 .361594

H -1.612539 -1.612501 2.214779

H -1.7131781 .0197852 .521081$

H -2.035111 -0.217572 -1.363034

H $-3.2217691 .971547-0.886569$

H -4.3159401 .1038390 .201662$

H $-3.648450-1.1561990 .776775$

H 2.8709670 .7309641 .101533

H $4.2438910 .863542-0.004489$

H $-3.9870110 .506891-2.776905$

H $-5.101766-0.321427-1.671495$

$\mathrm{H}-5.3224181 .397801-2.028130$

SCF Energy (B3LYP/6-31G**//MMFF) $=-810.710998624$

F2-10_c02005

MMFF Geometry

C $1.7939482 .778986-0.216857$

C 1.0163903 .5008530 .597525

C $2.0779520 .344677-0.794613$

O $0.6662170 .063655-0.648810$

C $0.492735-1.294170-0.216850$

C $1.832566-1.7030290 .379552$

C $2.794922-0.989204-0.547239$

C $-0.682470-1.4278890 .759651$

C $4.216620-0.927707-0.017586$

C $-2.076480-1.1972010 .139429$

O $-0.658625-2.758658 \quad 1.290394$

O $-2.235670-2.075086-0.984749$

C $-2.3888310 .225839-0.350539$

C -2.2194321 .2970790 .730775$

O $-3.7572620 .249204-0.778901$

C $2.444248 \quad 1.480270 \quad 0.172448$

C -2.5849432 .6897310 .230362$

H $1.9887403 .155039-1.217961$

H $0.5824804 .434943 \quad 0.257911$

H $\quad 0.7970773 .176113 \quad 1.608648$

H $2.2501140 .668693-1.828327$

H $0.313144-1.904482-1.111273$

H $1.975664-2.7877710 .391038$

H $1.935811-1.3300191 .405902$

H $2.814839-1.538598-1.499316$

H $-0.537847-0.7465541 .604961$

H $4.855956-0.345532-0.688919$

H $4.263881-0.4798340 .980184$

H $4.638292-1.9357940 .057492$

H $-2.848730-1.5016270 .857538$

H - $-1.351634-2.8156971 .970135$

H $-2.061552-2.979524-0.671988$

H $-1.7885580 .473648-1.232552$

$\mathrm{H}-1.186337 \quad 1.3207081 .089323$

H -2.8619001 .0634021 .588318$

H $-3.856377-0.432974-1.465162$

H 2.1572491 .2087151 .195717

H 3.5265091 .6498150 .163129

H -3.641660 2.751116-0.047655

H -2.4031053 .4311491 .014965$

H $-1.9826812 .962738-0.641932$

SCF Energy (B3LYP/6-31G**//MMFF) $=-810.716175063$

F2-10_c02006 
MMFF Geometry

C $3.5060642 .176477-0.290316$

C 2.8337503 .2872380 .032041

C $2.814635-0.215337-0.644393$

O $1.5170500 .186118-1.139523$

C $0.526366-0.774107-0.720833$

C $1.177346-1.5884840 .386897$

C $2.618761-1.626909-0.079563$

C $-0.764922-0.035955-0.335426$

C $3.594614-2.0823980 .990706$

C $-1.915504-0.9931490 .031482$

O $-\begin{array}{lllll}0.505047 & 0.839674 & 0.759530\end{array}$

O $-2.164144-1.850100-1.082134$

C $-3.237843-0.3113090 .445828$

C $-3.7767280 .687259-0.588095$

$\begin{array}{llll}\text { O } & -3.083743 & 0.333408 & 1.707490\end{array}$

C 3.2884930 .8406920 .365241

C $-5.1648601 .197982-0.220492$

H $4.2650942 .233213-1.066346$

H $3.0416224 .222101-0.476842$

H 2.0697393 .2852170 .801449

H $3.504705-0.247684-1.496160$

H $0.340215-1.417140-1.590146$

H $\quad 0.735089-2.5843530 .486535$

H $1.100824-1.0848741 .357441$

H $2.681581-2.334684-0.918353$

H - $1.0552810 .589658-1.187713$

H $3.515285-1.4805561 .901673$

H $3.395175-3.1227821 .269107$

H $4.625067-2.0230950 .626290$

H $-1.590770-1.6179240 .873201$

H $\quad 0.216848 \quad 1.4331420 .490421$

H -2.840405 -2.494647 -0.812333

H $-3.993219-1.0946560 .585121$

H $-3.8227670 .219504-1.577864$

H $-3.1128941 .556518-0.658311$

H -2.433693 1.0483911 .598885

H 2.5603660 .9389981 .179304

H 4.2420780 .5375990 .811501

H $\quad-5.153199 \quad 1.729703 \quad 0.735985$

H $-5.5259511 .891652-0.986523$

H $-5.8794600 .371898-0.147683$

SCF Energy (B3LYP/6-31G**//MMFF) $=-810.720311734$

F2-10_c02007

MMFF Geometry

C -4.001112 $1.615050-0.158845$

C $-3.5773142 .793356-0.629410$

C $-2.810058-0.4658950 .599625$

O -1.6789260 .3003541 .072027$

C $-0.466782-0.4480420 .850422$

C $-0.830441-1.547183-0.136336$

C $-2.255344-1.8578360 .275838$

C $\quad 0.646054 \quad 0.510767 \quad 0.401794$

C $-3.019069-2.684133-0.743420$

C $2.009188-0.1864230 .234444$

O $0.2846261 .123999-0.834880$

O $2.376685-0.746804 \quad 1.493964$

C $3.1240970 .753390-0.275896$

C $4.5138120 .108724-0.370856$

O $3.255727 \quad 1.8770060 .597964$

C -3.437285 $0.287119-0.583240$

C $4.576942-1.078054-1.317829$

H -4.8085011 .6041960 .568724$

H -4.029493 $3.717041-0.285199$

H $-2.7778522 .858923-1.358978$

H -3.535323 -0.5354741 .419454$

H $-0.203320-0.893887 \quad 1.817717$

H - $0.166184-2.413335-0.058659$

H $-0.799359-1.188082-1.171477$

H -2.216564 -2.429292 1.214132

H $\quad 0.719317 \quad 1.3090101 .150400$

H -3.012643 -2.221163-1.735491

H -2.566943 -3.676583 -0.843862

H -4.059906 -2.818510 -0.432020

H $1.896335-1.008796-0.481379$

H $-0.5737221 .561002-0.700631$

H $3.108381-1.3678801 .341384$
H $2.856148 \quad 1.141648-1.265952$

H $4.868916-0.1876490 .623646$

H $5.2274660 .870431-0.711599$

H $2.4794412 .446656 \quad 0.465067$

H $-2.6929270 .433924-1.375017$

H $-4.261064-0.295621-1.010019$

H $4.207671-0.807362-2.311959$

H $5.611616-1.419814-1.422798$

H $3.987485-1.920865-0.945824$

SCF Energy $\left(B 3 L Y P / 6-31 G^{* *} / / M M F F\right)=-810.714510320$

F2-10_c02008

MMFF Geometry

C -3.920442 -1.515673 0.340637

C $-5.198997-1.8440010 .123368$

C $-2.3425020 .015556-0.885845$

O $-1.208989-0.817855-0.554986$

C $-0.047148 \quad 0.010827-0.335303$

C $-0.579812 \quad 1.423307-0.151732$

C $-1.7673121 .420764-1.093796$

C $0.766178-0.5804090 .823980$

C $-2.7110042 .592119-0.888118$

C 2.1263580 .0865131 .093082

O $0.002486-0.5084982 .033798$

O 1.9242591 .4537111 .472160

C $3.1146350 .080774-0.086402$

C $3.370776-1.317643-0.662310$

O $4.349106 \quad 0.616900 \quad 0.404508$

C -3.382092 -0.1147690 .237846$

C $4.370058-1.314184-1.812829$

H $-3.223305-2.3009340 .621590$

H -5.525637 $-2.873590 \quad 0.220283$

H -5.937420 -1.099738 -0.153631

H -2.769434 -0.354931-1.825760

H $\quad 0.536746-0.043677-1.261688$

H $0.1602032 .186222-0.410915$

H -0.9093611 .6017980 .877985$

H -1.380791 $1.475100-2.121682$

H $\quad 0.918302-1.6502550 .641537$

H -3.0562732 .6644190 .148188$

H $-2.2062163 .533707-1.129539$

H $-3.5868582 .503433-1.538797$

H $2.595394-0.3898391 .963610$

H $-0.841908-0.9623001 .869446$

H $1.318463 \quad 1.4551712 .233054$

H $2.7634640 .763789-0.869014$

H $2.436835-1.753944-1.030771$

H $3.757367-1.9766560 .124316$

H $4.8950240 .853382-0.364150$

H $-2.9328790 .138947 \quad 1.205167$

H -4.2099420 .5824210 .067111$

H $5.366983-1.012001-1.478343$

H $4.454940-2.319136-2.238803$

H $4.049225-0.634547-2.608499$

SCF Energy $\left(B 3 L Y P / 6-31 G^{* *} / / M M F F\right)=-810.713554427$

F2-10 c02009

MMFF Geometry

C -2.443454 $2.618610-0.002762$

C $-1.3751093 .340902-0.359004$

C $-2.7083400 .177792 \quad 0.533815$

O

C $-0.744433-1.1206060 .545474$

C $-1.722716-1.737448-0.449169$

C $-3.054023-1.2416200 .076853$

C $0.646498-0.852149-0.052915$

C $-4.194590-1.381987-0.914825$

C $1.648404-0.2751690 .976145$

O $1.127043-2.084704-0.582009$

O 1.2023740 .9929051 .454478

C $3.091790-0.0855410 .460841$

C $3.1881780 .808176-0.784167$

O $3.687152-1.347326 \quad 0.172373$

C $-2.7607371 .257489-0.558097$

C $4.6335881 .112770-1.160339$

H -3.1342883 .0328920 .726944$

H -1.2028434 .3188610 .076955$

H $-0.6562592 .971948-1.082228$ 
H $-3.349230 \quad 0.469577 \quad 1.374442$ H $-0.651362-1.7918331 .409162$ H -1.653151 -2.829342 -0.478453 H -1.542882 -1.362812 -1.464194 H -3.302789 -1.831455 0.970414 H $\quad 0.526157-0.136863-0.875894$ H -3.967241 -0.904345-1.873229 H $-4.394550-2.439723-1.117041$ H $-5.111296-0.935820-0.516410$ H $1.693967-0.9606671 .832405$ H $1.957619-1.903273-1.052984$ H $\quad 0.3384550 .8632081 .880404$ H 3.6843920 .3804291 .258739 H $2.6618401 .754052-0.614971$ H $2.7200250 .314155-1.643104$ H $3.647456-1.8829400 .982872$ H $-2.0597901 .015278-1.366168$ H $-3.7633411 .306821-0.996811$ H $5.1837310 .198466-1.403449$ H $4.6643641 .766875-2.037464$ H $5.1526691 .620250-0.340957$ SCF Energy (B3LYP/6-31G**//MMFF $)=-810.718527286$

F2-10_c02010

MMFF Geometry

C $2.6041272 .434588 \quad 0.342713$

C 3.5242683 .4055570 .341869

C $2.6064950 .109667-0.620883$

O $1.185238 \quad 0.161617-0.870813$

C $0.593773-1.113597-0.575443$

C $1.660963-1.9178130 .156889$

C $2.931128-1.379322-0.468430$

C $-0.714238-0.950446 \quad 0.215813$

C $4.189532-1.7344970 .302669$

C $-1.789831-0.151158-0.554530$

O $-1.234757-2.2428010 .519241$

O $-1.3639841 .199784-0.729204$

C $-3.162904-0.1441310 .152211$

C $-4.1935930 .708759-0.602884$

O

C 2.9099190 .9850680 .605209

C $-5.5984730 .578778-0.027755$

H 1.5670322 .7016750 .155939

H 3.2372304 .4339240 .151582

H 4.5717113 .1954830 .528033

H $3.1199280 .516741-1.500330$

H $0.382480-1.603355-1.535066$

H $1.539633-2.9966780 .018901$

H $1.641621-1.7094981 .233551$

H $3.017563-1.808762-1.476681$

H $-0.496556-0.4272311 .155689$

H $5.066152-1.254001-0.143457$

H $4.127073-1.4321091 .352921$

H $4.353413-2.8173220 .284887$

H -1.927849 -0.590329-1.550706

H $-0.631826-2.6647541 .154079$

H $-0.5459131 .186935-1.253785$

H -3.530853 -1.1734530 .235789$

H -4.219175 $0.407609-1.656596$

H -3.911482 $1.767558-0.564798$

H -3.8190150 .1952111 .964212$

H 2.3004520 .6699011 .460634

H 3.9619060 .8800220 .892659

H $-5.924659-0.466069-0.021026$

H -5.6504200 .9644550 .994795$

H $-6.3071271 .150666-0.635309$

SCF Energy $\left(B 3 L Y P / 6-31 G^{* *} / / M M F F\right)=-810.714319728$

F2-10_c02011

MMFF Geometry

C $-4.224251-1.157098-0.546923$

C $-4.168547-2.420297-0.110270$

C $-2.5011820 .657110-0.797712$

O $-1.459691-0.307645-1.068702$

C $-0.211700 \quad 0.157366-0.506525$

C -0.5907021 .2549320 .475120$

C $-1.7841801 .880928-0.218701$

C $0.549181-1.0481260 .059509$
C $-2.5985142 .797780 \quad 0.676551$

C $1.956450-0.7990090 .636425$

O $-0.231211-1.639668 \quad 1.107909$

O 1.9116620 .1739301 .679086

C $3.012004-0.378934-0.401776$

C $4.423896-0.3450780 .203409$

O $2.713890 \quad 0.909740-0.931400$

C $-3.535415-0.0024520 .126660$

C $5.491265-0.043125-0.840909$

H $-4.807869-0.938616-1.437554$

H $-4.692203-3.208299-0.640343$

H -3.603719 -2.690733 0.774908

H $-2.9735260 .919398-1.752210$

H $0.3406440 .586833-1.348453$

H 0.2212561 .9694210 .638975

H $-0.886321 \quad 0.843707 \quad 1.447087$

H -1.407610 $2.478282-1.061341$

H $\quad 0.624213-1.814213-0.722226$

H -3.485413 3.1653550 .150768

H -2.9239542 .2943191 .592512$

H -2.0008693 .6654170 .976064$

H $2.291768-1.7320201 .108290$

H -1.103213 -1.851653 0.733167

H $1.265245-0.1362882 .336396$

H $2.995878-1.088733-1.236726$

H $4.650643-1.3094080 .672310$

H 4.4845940 .4248420 .981914

H $2.6804551 .533594-0.185857$

H $-3.053146-0.343297 \quad 1.050658$

H -4.3095120 .7204630 .406852$

H $6.483926-0.057758-0.379866$

H $5.478069-0.790033-1.641110$

H $5.3436360 .944590-1.288499$

SCF Energy $\left(B 3 L Y P / 6-31 G^{* *} / / M M F F\right)=-810.721975233$

F2-10_c02012

MMFF Geometry

C 1.5078802 .8716300 .241554

C $0.8266773 .268650-0.839332$

C $2.0569460 .487653-0.634448$

O $0.6741290 .066669-0.653132$

C $0.584581-1.311637-0.269124$

C $1.919332-1.6525450 .381470$

C $2.870854-0.796396-0.427303$

C $-0.615800-1.5669400 .650735$

C $4.245472-0.6529710 .200777$

C $-2.005465-1.2718300 .051405$

O $-0.594684-2.9468151 .038207$

O $-2.145813-1.991576-1.181618$

C $-2.3591190 .204098-0.206715$

C -2.1839291 .1021291 .022304$

O $-3.736451 \quad 0.256493-0.601312$

C $2.212721 \quad 1.556776 \quad 0.460919$

C -2.6557952 .5305290 .777569$

H 1.5768003 .5736941 .070654

H $0.3679014 .251755-0.861059$

H $0.7047542 .643823-1.715344$

H $2.3009160 .915754-1.613663$

H $0.491525-1.904057-1.188061$

H $2.151608-2.7205510 .327597$

H $1.931883-1.3529451 .436483$

H $2.997341-1.272670-1.410188$

H $-0.498574-0.9910671 .574313$

H $4.190404-0.2839451 .229930$

H $4.752869-1.6232200 .229889$

H $4.8678240 .034691-0.380555$

H -2.766543 -1.7034290 .714133$

H $-0.739761-3.4783680 .236494$

H - $1.614297-1.537757-1.857279$

H - $-1.7851650 .603220-1.049899$

H -1.1320121 .1343011 .322914$

H $-2.7567660 .694653 \quad 1.864202$

H $-3.837278-0.319520-1.378482$

H $1.843453 \quad 1.154306 \quad 1.412615$

H 3.2735751 .7959650 .594841

H -3.7349512 .5693850 .599123$

H -2.440009 3.1528421 .651945

H $-2.1490632 .970080-0.086638$ 
F2-10 c02013

MMFF Geometry

C $-2.125546 \quad 2.687994 \quad 0.496751$

C $-0.983798 \quad 3.3802850 .413965$

C $-2.673220 \quad 0.2324360 .502912$

O $-1.432868-0.0880331 .174187$

C $-0.963837-1.3685410 .716061$

C $-1.711159-1.654438-0.575115$

C $-3.061650-1.032928-0.273139$

C $0.571205-1.4023300 .641991$

C $-3.936798-0.848669-1.500210$

C $1.246167-0.159548 \quad 0.020937$

O $1.053782-1.5519751 .982752$

O $0.853556-0.010818-1.339557$

C $2.788320-0.1970990 .106850$

C $3.4401701 .023810-0.558618$

O $3.281184-1.389804-0.501906$

C $-2.4456041 .488154-0.351400$

C $4.9502921 .047987-0.359382$

H -2.8796613 .0096831 .210319$

H -0.8155514 .2408811 .052094$

H $-0.201606 \quad 3.103551-0.284260$

H -3.426675 0.4416301 .272011

H -1.291579 -2.0922231 .475305$

H $-1.771817-2.725965-0.788756$

H $-1.256959-1.165245-1.440082$

H $-3.591058-1.7011490 .421042$

H $\quad 0.866543-2.3059070 .096317$

H $-4.868374-0.336702-1.238633$

H -3.432613 -0.270195 -2.281077

H $-4.196926-1.821339-1.931404$

H $\quad 0.9149520 .7389700 .554020$

H $0.766314-0.7712052 .486836$

H $1.102385-0.822760-1.812440$

H $3.089410-0.2295101 .160182$

H $3.0121301 .944175-0.144921$

H $3.2431901 .020686-1.637174$

H $3.103712-1.335128-1.456002$

$\mathrm{H}-1.6370441 .313584-1.070865$

H $-3.3493511 .725673-0.923384$

H $5.4264420 .174453-0.815616$

H $5.3770561 .943609-0.822095$

H 5.2046041 .0627890 .705162

SCF Energy (B3LYP/6-31G**//MMFF) $=-810.712770035$

F2-10_c02014

MMFF Geometry

C $2.751806 \quad 2.443873 \quad 0.861572$

$\begin{array}{llll}\text { C } 2.088806 & 3.304556 & 0.080439\end{array}$

C $2.3692290 .351922-0.634562$

$\begin{array}{llll}\text { O } & 0.934203 & 0.400463 & -0.480392\end{array}$

C $0.421656-0.930581-0.316526$

C $1.625401-1.828748-0.063897$

C $2.696801-1.127454-0.873536$

C $-0.617851-0.9750810 .805779$

C $4.106970-1.583371-0.544615$

C $-1.772444 \quad 0.034602 \quad 0.621767$

O $-1.117566-2.3065720 .907378$

O $-2.629810-0.052921 \quad 1.764394$

C $-2.626706-0.143138-0.650463$

C $-3.8037650 .838193-0.754474$

O $-3.189925-1.454426-0.703176$

C $3.0056520 .977140 \quad 0.619452$

C $-3.3839872 .297675-0.813560$

H $3.1693612 .834672 \quad 1.787841$

H $1.9837174 .341750 \quad 0.381845$

H $1.6424023 .013225-0.862290$

H $2.6414600 .935758-1.521512$

H $-0.034023-1.227424-1.269232$

H $1.450941-2.860729-0.383384$

H $1.892174-1.8445350 .999745$

H $2.509785-1.343346-1.935184$

H $-0.126404-0.7491201 .760958$

H $4.844453-0.992064-1.096741$

H $4.327181-1.4994280 .524417$

H $4.241225-2.634189-0.822895$
H -1.3489991 .0451690 .624655$

H $-1.797429-2.3087521 .602887$

H -2.0925330 .1436942 .550676$

H $-1.998588-0.023730-1.540276$

H -4.5026240 .6865620 .077267$

H $-4.3781040 .599222-1.659014$

H $-2.455291-2.089567-0.746610$

H 2.6486170 .4452531 .510221

H $4.093786 \quad 0.8535210 .578448$

H $-4.2560962 .931246-1.004872$

H -2.9394152 .6263860 .130211$

H -2.660233 $2.466538-1.617050$

SCF Energy (B3LYP/6-31G**//MMFF) $=-810.713079292$

F2-10_c02015

MMFF Geometry

C $3.6719342 .043584-0.304602$

C $3.0435253 .211473-0.128973$

C $2.870786-0.331104-0.512538$

O $1.6385710 .091959-1.139666$

C $0.567228-0.778535-0.723778$

C $1.085095-1.5247990 .496573$

C $2.552657-1.676567 \quad 0.150211$

C $-0.7075370 .049588-0.502895$

C $3.418546-2.086108 \quad 1.328238$

C $-1.929340-0.796782-0.101369$

O $-0.455749 \quad 1.016482 \quad 0.517352$

O $-1.999835-1.955903-0.940645$

C $-3.292868-0.078478-0.210246$

C $-3.397954 \quad 1.240273 \quad 0.560944$

$\begin{array}{llll}\text { O } & -4.293487 & -0.969490 & 0.299160\end{array}$

C $3.329416 \quad 0.7829990 .440396$

C $-4.796346 \quad 1.845308 \quad 0.502225$

H $4.4880951 .993287-1.020634$

H $3.3422784 .086692-0.695627$

H 2.2260353 .3159410 .575810

H $3.618617-0.472715-1.302124$

H $0.414853-1.483525-1.550193$

H $0.579479-2.4839130 .643977$

H $\quad 0.965729-0.9348501 .412504$

H $2.637365-2.457085-0.619255$

H $-0.9264920 .601618-1.425364$

H $4.474937-2.1111341 .042650$

H $3.306467-1.4039052 .177006$

H $3.139933-3.0869011 .675325$

H $-1.809025-1.1611540 .926224$

H $\quad 0.303543 \quad 1.5511010 .228536$

H $-2.093418-1.651040-1.859477$

H $-3.5380840 .095787-1.265191$

H $-2.698504 \quad 1.975290 \quad 0.150785$

H -3.1400701 .0772581 .614146$

H $-4.224492-1.800127-0.202071$

H $2.5498150 .989024 \quad 1.183718$

H 4.2280660 .4712490 .984069

H $-5.1075892 .010899-0.534151$

H -5.5345001 .1982800 .985936$

H $-4.8098872 .810427 \quad 1.018683$

SCF Energy (B3LYP/6-31G**//MMFF) $=-810.718434013$

F2-10_c02016

MMFF Geometry

C $3.5449722 .053165-0.289619$

$\begin{array}{llll}\text { C } 2.953767 & 3.179816 & 0.123712\end{array}$

C $2.701335-0.277071-0.716227$

O $1.402737 \quad 0.218001-1.114104$

C $0.384414-0.707127-0.683224$

C $1.049928-1.610847 \quad 0.343779$

C $2.458051-1.703115-0.208865$

C $-0.8387380 .079338-0.187034$

C $3.465555-2.264707 \quad 0.778447$

$\begin{array}{llll}C & -2.020558 & -0.830517 & 0.200057\end{array}$

$\begin{array}{lllll}\text { O } & -0.468520 & 0.881701 & 0.932302\end{array}$

O $-2.373220-1.633239-0.924170$

C $-3.271629-0.0969330 .731240$

C $-3.7836901 .083458-0.107851$

O $\begin{array}{llll}-2.989671 & 0.378981 & 2.049374\end{array}$

C $3.2904290 .699172 \quad 0.313147$

C $-4.2060110 .719333-1.520906$ 
H $4.2620472 .109213-1.104489$

H $3.1843594 .127189-0.351333$

H 2.2342683 .1789060 .934767

H $3.339391-0.303098-1.607954$

H $\quad 0.112868-1.293987-1.569501$

H $0.558073-2.5851520 .421767$

H $1.057394-1.1540951 .340151$

H $2.432895-2.369658-1.082803$

H -1.138149 $0.763932-0.988981$

H $3.472606-1.7065391 .720246$

H $3.223324-3.3055281 .018193$

H $4.475429-2.2428790 .356491$

H $-1.681616-1.5092760 .993373$

H $\quad 0.268021 \quad 1.4503870 .649707$

H -3.071802 -2.247100 -0.640744

H $-4.085628-0.8266210 .826254$

H $-3.0363531 .885338-0.135903$

H $-4.647270 \quad 1.5238200 .407818$

H -3.8059540 .7721212 .402483$

H $2.616250 \quad 0.7942641 .172909$

H 4.2490810 .3231100 .686890

H -4.923205 -0.107304 -1.517093

H $-4.6846571 .578910-2.001356$

H -3.348945 $0.436696-2.138045$

SCF Energy (B3LYP/6-31G**//MMFF) $=-810.706665755$

F2-10 c02017

MMFF Geometry

C $2.8541992 .185668-0.785088$

C $2.1365493 .303098-0.622903$

C $2.278390-0.229135-0.453137$

O $0.867016-0.068705-0.663810$

C $0.264977-1.367368-0.742748$

C $1.318082-2.369217-0.255554$

C $2.404379-1.508800 \quad 0.372378$

C $-1.046638-1.4038890 .059993$

C $2.169941-1.3290691 .872367$

C $-2.145468-0.427483-0.408116$

O $-1.587281-2.729044-0.019414$

O $-2.365340-0.647075-1.809579$

C $-1.8732331 .072010-0.198892$

C $-1.459610 \quad 1.4812641 .220252$

O $-3.0757761 .772866-0.545583$

C 2.8514401 .0364010 .184133

C -2.4806121 .1240472 .293242$

H $3.4871842 .095221-1.664009$

H $2.1848004 .098680-1.358335$

H 1.4905963 .4430130 .236802

H $2.735249-0.392721-1.439200$

H $\quad 0.053078-1.581414-1.797486$

H $1.734765-2.890677-1.127241$

H $0.926580-3.1420710 .412799$

H $3.392880-1.9625120 .237370$

H $-0.839612-1.2229651 .118261$

H $2.131725-2.3014132 .375129$

H $2.986886-0.7593492 .327149$

H $1.235452-0.8024382 .085834$

H -3.091702 -0.7012410 .075830$

H -1.812716 -2.897693 -0.950584

H $-3.074905-0.043328-2.089138$

H $-1.1184751 .416089-0.914023$

H -1.323252 2.5698891 .244483

H -0.4919371 .0402971 .479263$

H -2.893130 $2.724604-0.465593$

H 2.2699041 .3107961 .072668

H 3.8846170 .8584470 .503995

H -3.453039 1.5812992 .086368

H $-2.618050 \quad 0.0418642 .373182$

H -2.140220 1.4881323 .268005

SCF Energy (B3LYP/6-31G**//MMFF) $=-810.714346836$

F2-10_c02018

MMFF Geometry

C -4.164782 -1.054461 0.403708

C $-3.934062-2.2545080 .947855$

C $-2.6755050 .670139-0.660498$

O $-1.780720-0.346154-1.161852$

C $-0.4160320 .126997-1.097890$
C $-0.451575 \quad 1.359457-0.208116$

C $-1.8195701 .930899-0.525771$

C $0.472740-1.042236-0.653982$

C -2.2883182 .9795010 .467329$

C $1.986296-0.814606-0.838006$

O $0.187266-1.3942420 .699030$

O $2.621315-2.097458-0.736682$

C 2.6922140 .1542710 .131902

C $4.1934340 .270037-0.176814$

O $2.556253-0.2838891 .481351$

C $-3.3066500 .157148 \quad 0.642512$

C $4.876728 \quad 1.3322080 .676109$

H $-5.028206-0.933705-0.245512$

H $-4.594632-3.0887410 .738469$

H -3.089195 -2.426956 1.605249

H $-3.4589290 .829121-1.411459$

H $-0.1488260 .416611-2.121888$

H $0.3472932 .068185-0.441784$

H -0.3791481 .0976940 .853183$

H -1.763021 $2.404440-1.516348$

H $0.192457-1.925525-1.243663$

H -2.272204 2.6066351 .496445

H -1.635754 3.8582120 .428742

H -3.306768 3.3060990 .234429

H $2.159080-0.473742-1.865756$

H $-0.763890-1.5889630 .753326$

H $2.492085-2.4125560 .174768$

H $2.249773 \quad 1.1501940 .059208$

H $4.3377760 .519776-1.234278$

H $4.695037-0.6864680 .012408$

H $1.607578-0.2964021 .692642$

H -2.525244 -0.077446 1.375098

H -3.9476010 .9292491 .082513$

H 4.4201312 .3144950 .518002

H 4.8136771 .0901951 .741448

H $5.936117 \quad 1.404466 \quad 0.409947$

SCF Energy $(B 3 L Y P / 6-31 G * * / / M M F F)=-810.720464187$

F2-10 c02019

MMFF Geometry

C $4.629490-1.3260640 .258848$

C $4.711727-2.453276-0.456744$

C 2.8174840 .3783720 .551348

$\begin{array}{llll}\text { O } & 1.713655 & -0.493800 & 0.258844\end{array}$

C $0.5260770 .156277 \quad 0.750878$

C 0.8253481 .6574080 .741660

C $2.2696401 .776698 \quad 0.265919$

C $-0.678945-0.298849-0.085646$

C $2.3260232 .195563-1.203716$

C -2.0237750 .2164840 .461448$

O $-0.5268020 .143978-1.432686$

O $-2.186468-0.2180611 .810797$

C $-3.236558-0.229673-0.382463$

$\begin{array}{llll}C & -4.568325 & 0.253722 & 0.209037\end{array}$

O $-3.256567-1.652209-0.494738$

C $4.044581-0.040762-0.257577$

C $-5.751145-0.080053-0.691117$

H $5.015767-1.3252051 .274898$

H $5.150387-3.346637-0.025903$

H $4.345190-2.506950-1.475907$

H $3.029346 \quad 0.289279 \quad 1.626354$

H $\quad 0.396287-0.1809341 .786770$

H $0.7351732 .042041 \quad 1.765268$

H 0.1259842 .2399560 .133650

H 2.8131982 .5293680 .848745

H $-0.681987-1.395758-0.105930$

H $1.8082333 .149453-1.352402$

H $3.3616352 .334447-1.530193$

H $1.8619561 .454361-1.860558$

H $-2.018580 \quad 1.3125850 .475213$

H $0.298045-0.244280-1.771270$

H -2.126162 -1.188233 1.821246

H -3.132341 $0.168086-1.398407$

H $-4.533786 \quad 1.3382810 .363164$

H $-4.744343-0.2153301 .184394$

H $-3.432332-2.0197450 .387763$

H $3.780802-0.157663-1.315644$

H $4.8182160 .732782-0.188487$ 
H $-6.678136 \quad 0.302573-0.252441$

H $-5.6312250 .374531-1.679612$

H -5.861595 -1.161347 -0.819702

SCF Energy (B3LYP/6-31G**//MMFF) $=-810.715678966$

F2-10 c02020

MMFF Geometry

C $3.1084401 .529783-0.679709$

C $4.2425032 .051525-0.198674$

C $2.136216-0.812959-1.109971$

O $0.794006-0.304416-0.950070$

C $\quad 0.331407-0.615048 \quad 0.373629$

C $1.581113-0.8764191 .205609$

C $2.479032-1.5504980 .188762$

C -0.5428670 .5130280 .924706$

C $3.940045-1.6235150 .590504$

C $-1.734617 \quad 0.8781690 .013137$

O $-\begin{array}{llll}0.992219 & 0.141709 & 2.226121\end{array}$

O $-2.420752 \quad 1.9896170 .599122$

C $-2.753066-0.251321-0.239554$

C $-3.9107660 .206687-1.139825$

O $-3.287895-0.7301430 .992004$

C $3.0468030 .331334-1.584159$

C $-4.845270-0.940781-1.502704$

H $2.1701172 .016768-0.428091$

H 4.2167702 .9283280 .439051

H $5.2091441 .617775-0.429268$

H $2.098095-1.547894-1.924172$

H $-0.243772-1.5477920 .314943$

H $1.377352-1.5040122 .078718$

H 2.0267840 .0601461 .559165

H $2.122622-2.5851820 .076422$

H 0.0692891 .4151491 .050124

H $4.544053-2.052394-0.215477$

H $4.351008-0.6441080 .845866$

H $4.057900-2.2619651 .472894$

H $-1.3515971 .222089-0.955359$

H -1.572344 0.8531332 .546890

H -1.7834412 .7199220 .676915$

H -2.246819 -1.094608 -0.721263

H $-3.5128040 .645232-2.062061$

H $-4.5047100 .976986-0.633681$

H $-3.6981310 .025075 \quad 1.447310$

H $4.052000-0.059936-1.781134$

H $2.6597810 .692661-2.546178$

H $-5.317012-1.367730-0.612263$

H -5.639905 -0.583981 -2.165576

$\mathrm{H}-4.303333-1.737712-2.021697$

SCF Energy (B3LYP/6-31G**//MMFF) $=-810.710789951$

F2-11 c02264

MMFF Geometry

C 3.1277732 .5658680 .181514

C $2.3602803 .633674-0.063812$

C 2.6391200 .1090570 .275556

O $1.2626790 .271196 \quad 0.661533$

C $0.665244-1.0290580 .821916$

C $1.710382-2.0508610 .378572$

C $2.684194-1.232921-0.447342$

C $-0.653251-1.091870 \quad 0.026545$

C $4.070494-1.849582-0.529694$

C -1.664450 -0.007475 0.452200

O $-0.388835-0.966195-1.368772$

O $-1.965451-0.1694101 .837868$

C $-2.971135-0.015102-0.369140$

C $-3.957846 \quad 1.0554380 .117564$

O $-3.608071-1.287577-0.266223$

C $3.063814 \quad 1.286057-0.603407$

C $-5.1906501 .148134-0.773431$

H 3.8517722 .6190460 .990432

H 2.4582764 .5305810 .538112

H $1.6248123 .633194-0.860687$

H 3.2371550 .0750001 .196011

H $0.450691-1.1676341 .887843$

H $1.289043-2.900637-0.167582$

H $2.212370-2.4562751 .266475$

H $2.282281-1.126195-1.462794$

H -1.086583 -2.0854360 .189959$
H $4.738924-1.230954-1.136499$

H $4.520769-1.9584840 .462706$

H $4.022563-2.842700-0.988197$

H -1.2072590 .9822500 .333152$

H $\quad 0.014480-0.092887-1.513046$

H $-2.428099-1.0188481 .939667$

H $-2.7460250 .161887-1.427484$

H -3.4638442 .0335120 .140968$

H $-4.2951800 .826475 \quad 1.135343$

H $-3.080822-1.923365-0.778636$

H $4.0563021 .101838-1.029283$

H $2.3628571 .394740-1.440614$

H $-5.7518750 .208600-0.777016$

H $-5.8578881 .937088-0.411885$

$\mathrm{H}-4.9111141 .386925-1.804444$

SCF Energy $(B 3 L Y P / 6-31 G * * / / M M F F)=-810.723215949$

F2-11_c02265

MMFF Geometry

C $-3.0675952 .192442-0.805241$

C $-2.5926343 .348100-0.327424$

C $-2.249515-0.168340-0.612907$

O $-0.8687130 .199905-0.450155$

C $-0.077506-0.990126-0.294515$

C $-1.054779-2.163922-0.258267$

C $-2.380182-1.5150600 .089796$

C $0.812297-0.8788040 .958205$

C $-3.584942-2.330939-0.348126$

C $1.767770 \quad 0.331093 \quad 0.994147$

O $-\begin{array}{llll}-0.003830 & -0.828080 & 2.132322\end{array}$

O 1.0079631 .5457431 .054763

C $2.7509630 .440881-0.184620$

C $3.604221-0.814846-0.391349$

O 3.6340081 .5373290 .079584

C $-3.1385180 .919615-0.009824$

C $4.589711-0.664431-1.544464$

H -3.434692 $2.162310-1.827934$

H $-2.5717614 .233973-0.952845$

H -2.2165513 .4313720 .686150$

H -2.437110 -0.271675 -1.690020

H $0.560528-1.081464-1.180343$

H $-0.764554-2.9556610 .439321$

H -1.105292 -2.617382 -1.256650

H -2.427405 -1.368876 1.176290

H $1.400822-1.7985881 .046279$

H $-3.579437-3.3138080 .134311$

H -4.518028 -1.827911-0.076054

H -3.590853 -2.488004 -1.431989

H 2.3304630 .3065821 .935521

H $-0.521870-0.0056962 .089271$

H $0.496292 \quad 1.618820 \quad 0.231222$

H $2.2153910 .688647-1.108551$

H $2.968777-1.681961-0.597438$

H $\quad 4.174597-1.0295690 .520417$

H 3.0824252 .3263540 .218657

H -4.1830910 .5892840 .007719$

H -2.837555 1.1101171 .028163

H $4.065999-0.440995-2.479317$

H $5.3115860 .135429-1.353118$

H $5.150132-1.594444-1.683651$

SCF Energy (B3LYP/6-31G**//MMFF) $=-810.726475271$

F2-11_c02266

MMFF Geometry

C $-4.393007-0.976415-0.017427$

C $-4.278409-2.301295-0.163271$

C $-2.6948790 .857360 \quad 0.249618$

O $-1.705623-0.0083520 .840601$

$\begin{array}{llll}\text { C } & -0.397882 & 0.333506 & 0.336575\end{array}$

C $-0.6282791 .283880-0.828000$

C $-1.9224821 .978496-0.449945$

C $0.347139-0.955926-0.022201$

C $-1.6723993 .159153 \quad 0.490810$

C $1.703014-0.734596-0.719162$

O $0.550022-1.7068331 .179178$

O $2.239302-2.016926-1.060696$

C 2.7528710 .0638960 .081307

C $4.0501890 .251591-0.718379$ 
O $3.078819-0.602673 \quad 1.298473$

C $-3.5326190 .025898-0.734651$

C $5.0434831 .157264-0.000469$ H $-5.157199-0.5896350 .651726$ H $-4.933806-2.9725200 .380835$ H -3.534098 -2.737706 -0.820135 H -3.3361141 .2463581 .049362$ H 0.1130920 .8455491 .160474 H $-0.7793910 .722551-1.758314$ H $0.2116521 .966822-0.987306$ H $-2.4488152 .357824-1.332795$ H $-0.283745-1.588327-0.659478$ H $-1.045115 \quad 3.913014 \quad 0.003744$ H $-2.620163 \quad 3.6364540 .762122$ H -1.176561 2.854409 1.417980 H $1.526947-0.219748-1.670325$ H $-0.322350-1.8397871 .588604$ H $2.419674-2.485781-0.227524$ H 2.3512101 .0501100 .338968 H $3.8233010 .684371-1.699531$ H $4.536323-0.717067-0.884774$ H $2.264851-0.6683881 .825840$ H $-4.1927340 .685494-1.310216$ H -2.879983 -0.489315 -1.450060 H $5.9420091 .289829-0.611571$ H 4.6101002 .1454810 .183254 H $5.3503200 .731416 \quad 0.959853$

SCF Energy (B3LYP/6-31G**//MMFF) $=-810.721734979$

F2-11_c02267

MMFF Geometry

C 3.5060122 .1653210 .098754

C $2.9257453 .349297-0.127520$

C $2.599369-0.1659800 .260243$

$\begin{array}{lllll}\text { O } & 1.293983 & 0.239575 & 0.709283\end{array}$

C $0.485527-0.933351 \quad 0.918940$

C $1.311644-2.1275180 .445180$

C $2.372007-1.501840-0.439875$

C $-0.861558-0.7681350 .186305$

C $3.622681-2.353919-0.576463$

C -1.645617 0.4799790 .647429

O $-0.641048-0.712463-1.219923$

O -1.8752700 .3790382 .052409$

C -2.994471 $0.713607-0.067756$

C -3.953295 -0.4815770 .017027$

O $-2.779431 \quad 1.037636-1.438664$

C $3.1785680 .909853-0.659130$

C $-5.334834-0.148114-0.534288$

H 4.2687182 .0975290 .870140

H $3.210160 \quad 4.2202340 .452754$

H $2.1616093 .471086-0.887248$

H $3.227994-0.2961831 .151389$

H $0.303081-1.0235121 .995982$

H $0.720973-2.894851-0.065168$

H $1.777816-2.6070031 .315653$

H $1.945776-1.335051-1.437270$

H $-1.452827-1.6682220 .390462$

H $4.095141-2.5317240 .395458$

H $3.378755-3.327027-1.015058$

H $4.358743-1.868105-1.224467$

$\mathrm{H}-1.021314 \quad 1.3655640 .472808$

H - $-0.1155160 .085030-1.403162$

H $-2.308148 \quad 1.2009042 .339672$

H -3.487399 1.5772580 .396373

H -4.059024 -0.8108291 .056620$

H $-3.560419-1.323673-0.564126$

H -2.197549 $1.815874-1.469176$

H $4.1003680 .549906-1.129537$

H $2.4656361 .132757-1.463076$

H $-5.2862070 .126919-1.592449$

H $-5.996390-1.015293-0.442123$

H -5.7865130 .6823890 .017635$

SCF Energy (B3LYP/6-31G**//MMFF) $=-810.712277659$

F2-11_c02268

MMFF Geometry

C $4.410599-1.225802-0.249352$

C $5.730098-1.099960-0.430448$
C $2.5218340 .424135-0.338047$

O $1.529928-0.540156-0.744315$

$\begin{array}{llll}C & 0.212831 & 0.050304 & -0.639436\end{array}$

C $0.4509891 .531497-0.381081$

C 1.7534631 .5238990 .391910

C $-0.539641-0.7095790 .466613$

C $2.4586142 .869611 \quad 0.401633$

C $-1.911584-0.122445 \quad 0.854557$

O $-0.708250-2.0578160 .008153$

O $-2.481644-0.9485451 .875936$

C $-2.9276410 .017088-0.297332$

$\begin{array}{llll}C & -4.254319 & 0.620923 & 0.186394\end{array}$

O $-3.208845-1.250419-0.885891$

C $3.575002-0.2566550 .538455$

C $-5.2127410 .901731-0.964626$

H $3.905105-2.083444-0.685807$

H $6.278225-1.837772-1.006250$

H $6.283617-0.266327-0.012861$

H $2.9804320 .817374-1.254919$

H $-0.283742-0.105044-1.603605$

H $-0.359627 \quad 2.0452480 .139275$

H $0.5920442 .043637-1.342028$

H 1.5497501 .2205631 .427223

H $\quad 0.089620-0.7868221 .361740$

H $2.687706 \quad 3.210273-0.613714$

H 1.8296583 .6294610 .876869

H 3.3993402 .8142710 .958322

H $-1.7651980 .863301 \quad 1.309285$

H $-1.194632-2.5395450 .699036$

H $-1.857369-0.9669422 .621258$

H -2.512065 $0.662269-1.079658$

H -4.0634481 .5572610 .723306$

H $-4.753227-0.0664490 .880038$

H $-2.378067-1.596504-1.253330$

H $4.2158990 .498544 \quad 1.008114$

H $3.085291-0.8176671 .343972$

H $-5.483514-0.018059-1.492242$

H $-6.134007 \quad 1.355235-0.585191$

H $-4.7663301 .594138-1.685347$

SCF Energy (B3LYP/6-31G**//MMFF) $=-810.712276208$

F2-11 c02269

MMFF Geometry

C -2.323869 2.2227750 .196280

C -3.0828522 .7990611 .135046$

C $-2.397618-0.030217-0.986373$

O $-0.972048-0.087815-1.161982$

C $-0.484670-1.310786-0.575339$

C $-1.684237-1.9927120 .084501$

C -2.686179 $-0.866066 \quad 0.255622$

$\begin{array}{llll}\text { C } 0.674626 & -0.998920 & 0.392568\end{array}$

C $-4.125192-1.340073 \quad 0.361983$

C $1.823115-0.220873-0.286471$

O $0.177798-0.247511 \quad 1.497245$

O $2.304739-0.972172-1.400371$

$\begin{array}{llll}\text { C } 2.995929 & 0.114421 & 0.659461\end{array}$

C $4.215100 \quad 0.742445-0.030945$

O $3.433497-1.0710171 .327633$

C $-2.8712011 .425448-0.953852$

C $3.9162412 .069627-0.708263$

$\begin{array}{llll}\text { H }-1.245051 & 2.341798 & 0.254038\end{array}$

H $-2.626076 \quad 3.366517 \quad 1.938469$

H -4.1637582 .7143991 .121567$

H $-2.836630-0.509916-1.871732$

H $-0.112216-1.942684-1.389978$

H $-1.438561-2.4997051 .022868$

H -2.083076 -2.754120 - 0.598236

H $-2.429243-0.3139761 .166762$

H $1.043516-1.9578880 .777183$

H $-4.431917-1.896859-0.529854$

H $-4.250367-1.997707 \quad 1.228386$

H $-4.806582-0.4918480 .480947$

H $1.4306160 .724071-0.678648$

H $0.848465-0.2758942 .200090$

H $2.599481-1.838060-1.071509$

$\begin{array}{llll}H & 2.645732 & 0.799406 & 1.439805\end{array}$

H $4.6448530 .043933-0.759357$

H 5.0013160 .8949670 .719606 
H $3.809344-1.6692820 .659954$ H $-2.5440881 .923398-1.875324$ H $-3.9674331 .449381-0.950010$ H $4.8414592 .512942-1.090401$ H $3.2360001 .942927-1.555515$ H $3.4671742 .777550-0.004620$

SCF Energy (B3LYP/6-31G**//MMFF) $=-810.715907787$

F2-11 c02270

MMFF Geometry

C $-2.6843682 .688039-0.356593$

C - $-1.7255763 .551590-0.003457$

C $-2.7644920 .177599-0.463676$

O $-1.3481090 .057621-0.706821$

C $-0.837090-1.100202-0.024701$

C $-1.918963-1.5217120 .960282$

C $-3.195297-1.1065690 .252163$

C $0.525046-0.7911050 .618151$

C $-3.662814-2.172583-0.740938$

C $1.582654-0.334501-0.407809$

O $\quad 0.370208 \quad 0.218642 \quad 1.613525$

O $1.766316-1.387414-1.353309$

C 2.9348700 .0500800 .232275

C $3.9763330 .478408-0.810706$

O $3.467442-1.0540530 .962353$

C $\quad-3.0003101 .4273890 .398359$

C $5.2430061 .026528-0.163953$

H $-3.2832212 .905216-1.237375$

H -1.549606 $4.446874-0.589666$

H -1.1025393 .3830090 .867938$

H $-3.2709350 .291281-1.429546$

H $-0.711730-1.883557-0.781781$

H -1.836700 -0.9684071 .902897$

H -1.869613 -2.588065 1.201462

H $-4.010375-0.9323210 .963544$

H $0.865430-1.7031381 .122123$

H $-4.578848-1.846526-1.245171$

H $-2.914693-2.378906-1.513022$

H $-3.882381-3.111840-0.222451$

H $\quad 1.2039600 .541576-0.949239$

H $\quad 0.0402141 .0188441 .169983$

H $2.238413-1.019975-2.119147$

H 2.7848840 .8751350 .939056

H $3.5529991 .247481-1.466855$

H $4.264205-0.375850-1.434603$

H $2.919986-1.1747931 .756269$

H -4.0521041 .4725710 .704952$

H -2.3967391 .3790571 .312634$

H 5.0178521 .8947330 .463656

H $5.733190 \quad 0.2701850 .456833$

H $5.9534961 .341287-0.934849$

SCF Energy (B3LYP/6-31G**//MMFF) $=-810.717276034$

\section{F2-11 c02271}

MMFF Geometry

C $3.371979-1.832859-0.401025$

C $2.868704-2.978690 \quad 0.071727$

C $2.4201550 .488126-0.558310$

O $1.0597620 .017693-0.625426$

C 0.2059070 .8860050 .135726

C 1.1301321 .7823300 .951940

C 2.3649181 .8807510 .075861

C -0.8004070 .0879800 .979306$

C $2.2177492 .973834-0.984666$

C $-1.741771-0.837243 \quad 0.181312$

O $-0.080563-0.7183531 .918297$

O $-0.972199-1.814027-0.532234$

C $-2.637944-0.127509-0.846972$

C $-3.4755541 .035728-0.303485$

O $-3.539788-1.090762-1.405305$

C $3.230263-0.5052100 .289318$

C $-4.4162380 .650528 \quad 0.831859$

H $3.924721-1.852271-1.336681$

H $3.007926-3.906078-0.472983$

H $2.310237-3.0124161 .000651$

H $2.8236980 .523055-1.577146$

H $-0.3387971 .503575-0.587393$

H 1.4027381 .3126111 .904035
H $\quad 0.671516 \quad 2.748408 \quad 1.185420$

H $3.2604592 .104456 \quad 0.666217$

H -1.393272 $0.794106 \quad 1.570928$

H $1.3602072 .801964-1.643201$

H $2.0909403 .953649-0.512303$

H $3.1144073 .018062-1.611970$

H -2.363174 -1.416441 0.875111

H $-0.733658-1.1579742 .489053$

H $-0.364792-2.2270450 .105433$

H -2.030396 $0.234977-1.684577$

H $-4.091246 \quad 1.432392-1.120717$

H -2.824965 1.8530560 .025740

H -3.001335 -1.818943 -1.760221

H $4.236458-0.1073190 .466368$

H $2.758453-0.6446221 .269365$

H $-5.104093-0.1444370 .527920$

H $-3.864746 \quad 0.311512 \quad 1.713517$

H -5.0163441 .5166991 .129226$

SCF Energy $\left(B 3 L Y P / 6-31 G^{* *} / / M M F F\right)=-810.710474692$

F2-11 c02272

MMFF Geometry

C $-3.6094122 .022092-0.252110$

C $-3.1404713 .226015-0.598673$

C $-2.513432-0.2166690 .009267$

O $-1.4757180 .039231-0.954560$

C $-0.703441-1.161418-1.113546$

C $-1.128546-2.124970-0.005909$

C $-1.900220-1.2419700 .957860$

C $0.800224-0.835483-1.174715$

C $-2.914238-2.0050941 .793134$

C $1.395793-0.2059930 .103407$

O $1.516552-2.032191-1.475583$

O $\quad 0.7313591 .0188330 .404508$

C 2.9164050 .0471300 .003033

C $3.4821830 .712777 \quad 1.265918$

O $3.2006420 .866028-1.130647$

C -2.9116221 .0894590 .697148$

C $4.9991970 .843697 \quad 1.215135$

H $-4.5569031 .691448-0.669845$

H $-3.6952293 .854293-1.286738$

H -2.202048 $3.603471-0.207760$

H $-3.367971-0.647541-0.530005$

H -0.989662 -1.606061 -2.075593

H $-0.296832-2.6525360 .470286$

H $-1.787070-2.892611-0.432546$

H -1.185261 -0.7487881 .628600$

H $\quad 0.949627-0.133397-2.005064$

H $-2.417510-2.7772452 .389849$

H -3.439163 -1.335084 2.481067

H -3.663796 -2.496273 1.163554

H $1.235022-0.8797410 .952520$

H $1.178414-2.373137-2.321033$

H $0.7706121 .583166-0.386040$

H $3.430375-0.907530-0.156513$

H 3.2053550 .1268052 .149784

H 3.0614731 .7179961 .387781

H $2.808646 \quad 1.741420-0.972511$

H $-3.5929100 .885566 \quad 1.530715$

H -2.0219401 .5768381 .114423$

H 5.3694651 .2963422 .140544

H $5.473148-0.1367251 .104267$

H $5.316955 \quad 1.476676 \quad 0.380821$

SCF Energy (B3LYP/6-31G**//MMFF) $=-810.711961725$

F2-11_c02273

MMFF Geometry

C $3.1785172 .595788-0.536959$

C 2.9716283 .1190100 .676993

C 2.6160930 .1499240 .114225

O 1.2429040 .3941820 .465093

C $0.631149-0.8493940 .856453$

C $1.659134-1.9467510 .590972$

C $2.635040-1.296756-0.371053$

C $-0.705519-1.0247380 .108927$

C $4.010912-1.941975-0.358581$

C $-1.683771 \quad 0.142118 \quad 0.360415$

O $-0.482329-1.122448-1.296105$ 
$\begin{array}{llll}\text { O } & -1.917290 & 0.274925 & 1.761900\end{array}$

C $-3.028407-0.017940-0.380556$

C $-4.0017211 .130093-0.077022$

O $-3.635550-1.260178-0.026833$

C $3.0593771 .153201-0.955162$

C $-5.2771561 .035548-0.904900$

H $3.4705173 .281488-1.330434$

H 3.0951574 .1850440 .837472

H 2.6799172 .5184381 .529826

H 3.2204370 .2545501 .024592

H $0.434787-0.7963851 .933822$

H $1.224527-2.8752960 .208619$

H $2.165447-2.1960491 .532600$

H $2.223684-1.356202-1.386592$

H $-1.162251-1.9639450 .444626$

H $4.681807-1.449170-1.068888$

H $4.471205-1.8863360 .633748$

H $3.942224-2.998420-0.638673$

H $-1.229536 \quad 1.0798120 .017821$

H - $0.051115-1.975672-1.470075$

H $-2.258765-0.5742742 .089366$

H -2.841745 -0.051011 - 1.459936

H $-3.5162042 .091358-0.280638$

H $-4.286057 \quad 1.1185300 .981804$

H $-3.886444-1.2142680 .911105$

H $4.0356370 .852134-1.352842$

H $2.3462001 .122482-1.789189$

H $-5.0509201 .053435-1.975715$

H $-5.8284030 .116240-0.684198$

H $-5.9336541 .882893-0.683167$

SCF Energy (B3LYP/6-31G**//MMFF) $=-810.712973648$

F2-11_c02274

MMFF Geometry

C -1.527291 $2.998022-0.103171$

C -0.3700253 .5800320 .231058$

C $-2.1535620 .589772-0.406753$

O $-0.8230420 .182392-0.768732$

C $-0.785731-1.241503-0.948638$

C $-2.195050-1.750023-0.650149$

$\begin{array}{llll}C & -2.798977 & -0.650611 & 0.201037\end{array}$

C $0.301525-1.866328-0.056629$

C $-4.318203-0.633430 \quad 0.177832$

C $1.741985-1.434600-0.414656$

O $0.016680-1.5859041 .312308$

O $2.636054-2.281480 \quad 0.319463$

C $2.1311440 .039279-0.172522$

C $3.6117340 .301490-0.488642$

$\begin{array}{lllll}\text { O } & 1.898439 & 0.409019 & 1.183753\end{array}$

C -2.072970 1.7676010 .565190

C $3.9626201 .782449-0.404203$

H -2.120659 $3.436486-0.901167$

H $-0.0319424 .469509-0.289204$

H $0.2585883 .182063 \quad 1.020111$

H -2.668411 $0.883084-1.331264$

H $-0.538538-1.442794-1.997567$

H -2.214517 -2.731329 -0.166032

H $-2.747960-1.843581-1.593803$

H $-2.463673-0.7802191 .237676$

H $0.240475-2.957397-0.161535$

H $-4.718997-1.5776240 .561089$

H -4.7095880 .1763770 .801324$

H $-4.702735-0.492283-0.837883$

H $1.912081-1.666375-1.473003$

H $\quad 0.651851-2.0845671 .853304$

H $2.633839-1.9810021 .244271$

H $1.5273740 .694998-0.806989$

H $3.847229-0.060758-1.495883$

H $4.253262-0.2360760 .219566$

H $0.9414990 .345190 \quad 1.343625$

H -3.0687962 .0058860 .955481$

H $-1.442196 \quad 1.497770 \quad 1.421696$

H $5.0160191 .934976-0.659617$

H $3.3563272 .368757-1.102070$

H 3.8012212 .1737890 .605012

SCF Energy (B3LYP/6-31G*//MMFF) $=-810.722438312$

F2-11_c02275
MMFF Geometry

C $3.523596-1.2463470 .730693$

C $4.173947-1.0937601 .889659$

C $2.547847 \quad 0.392728-0.956319$

O $1.493822-0.496904-1.368671$

C $0.216700 \quad 0.100788-1.032854$

C $0.5355951 .505561-0.530789$

C $1.920342 \quad 1.338598 \quad 0.063226$

C $-0.469395-0.828890-0.017609$

C 2.6728332 .6458360 .238520

C $-1.782429-0.2900130 .587958$

O $-0.719582-2.071745-0.686883$

O $-2.311322-1.284528 \quad 1.471687$

C $-2.8735720 .098138-0.429982$

C $-4.1979990 .550462 \quad 0.203185$

O $-3.168813-1.003553-1.289388$

C $3.769494-0.402574-0.487825$

C $-4.0706991 .793544 \quad 1.068072$

H 2.778895-2.034072 0.651570

H $3.954064-1.7405682 .732066$

H $4.929338-0.3268552 .019839$

H $2.837290 \quad 0.953382-1.855414$

H $-0.3685500 .139606-1.958346$

H $-0.189740 \quad 1.9198360 .172115$

H $0.5779692 .190237-1.388255$

H $1.8178840 .852987 \quad 1.041089$

H $0.227114-1.0745690 .793111$

H $2.133473 \quad 3.3113390 .920591$

H 3.6686862 .4684330 .656796

H $2.7957783 .170231-0.715083$

H -1.5520140 .5794711 .211000$

H -1.166792 -2.657341 - -0.052342

H -1.634268 -1.4683952 .145002$

H -2.501702 $0.904753-1.071063$

H $-4.639985-0.2645450 .789360$

H $-4.9186210 .750286-0.600519$

H $-3.440794-1.749267-0.727350$

H $4.078839-1.081297-1.292492$

H $4.6041410 .287120-0.313990$

H -3.603592 2.6128170 .512813

H -5.0611072 .1269351 .394126$

H -3.4767091 .5988971 .965795$

SCF Energy (B3LYP/6-31G**//MMFF) $=-810.705551562$

F2-11_c02276

MMFF Geometry

C $3.8048362 .013395-0.257407$

C $3.1806763 .194365-0.329342$

C $2.977787-0.321578 \quad 0.168707$

$\begin{array}{lllll}\text { O } & 1.833714 & 0.116137 & 0.927834\end{array}$

$\begin{array}{llll}\text { C } & 0.690438 & -0.704816 & 0.606547\end{array}$

C $1.086939-1.510127-0.620595$

C $2.588128-1.660566-0.461249$

C $-0.5393350 .203404 \quad 0.448890$

C $2.943138-2.8352780 .452658$

C $-1.834892-0.555758 \quad 0.099986$

O -0.7365640 .8849381 .691388$

O $-1.741851-1.083993-1.221836$

C $-3.1148920 .301868 \quad 0.211726$

C $-4.370205-0.483252-0.194379$

O $-3.010707 \quad 1.445935-0.634787$

C $3.2891540 .750127-0.887912$

C -5.6478490 .3113210 .047590$

H $4.747551 \quad 1.9537250 .280253$

H 3.6078514 .0711960 .144819

H $2.2392983 .307670-0.855549$

H $3.827300-0.4285350 .853529$

H $\quad 0.544639-1.3670951 .468834$

H $0.882270-0.942532-1.536399$

H $\quad 0.554222-2.463210-0.690599$

H $3.080552-1.825168-1.426011$

H $-0.3244430 .965330-0.310228$

H $2.515041-2.7273111 .454378$

H $2.578696-3.776644 \quad 0.028350$

H $4.029780-2.9160470 .562881$

H - $-1.942328-1.4071670 .783177$

H $0.090241 \quad 1.3534341 .899341$

H $-1.699181-0.331079-1.836043$ 
H $-3.237518 \quad 0.657873 \quad 1.241443$

H $-4.422893-1.419700 \quad 0.372636$

H $-4.330929-0.738754-1.259869$

H $-2.3389502 .035098-0.251893$

H $4.0569920 .379862-1.577420$

H $2.3941950 .970590-1.482582$

H $-5.6662101 .228640-0.548983$

H $-6.521524-0.287330-0.229318$

H -5.7449340 .5843891 .103127$

SCF Energy (B3LYP/6-31G**//MMFF) $=-810.720926531$

F2-11_c02277

MMFF Geometry

C -3.302611 2.073654 -0.076492

C $-4.4684912 .589963-0.480955$

C $-2.242465-0.176438-0.400136$

O $-0.891802 \quad 0.312527-0.331193$

C $0.017451-0.787967-0.504438$

C $-0.835583-2.054761-0.555985$

C $-2.152066-1.6260370 .062509$

C $1.084229-0.7857680 .608169$

C $-3.326828-2.490600-0.363338$

C 1.9479920 .4900200 .688868

O $0.466236-0.949317 \quad 1.888553$

O 1.1137121 .6250120 .957965

C $2.7612670 .812832-0.575486$

C $3.643214-0.326677-1.103102$

O $3.5965241 .938166-0.276390$

C -3.1462470 .6869910 .481294$

C $4.682439-0.822657-0.105113$

H $-2.4100002 .690786-0.141141$

H -4.515754 $3.601172-0.870008$

H $-5.3893192 .019229-0.433396$

H -2.559603 - $0.122052-1.450245$

H $\quad 0.512393-0.657487-1.473008$

H $-0.380056-2.914174-0.054591$

H $-0.985610-2.340643-1.605236$

H $-2.059899-1.6648001 .155144$

H $1.742595-1.6507430 .470582$

H -3.470862 -2.465416 -1.448733

H -3.163232 $-3.532741-0.069736$

H -4.254647 -2.150759 0.106932

H 2.6197190 .4201011 .553335

H $0.116738-1.8547441 .936038$

H $0.571184 \quad 1.4071811 .735245$

H $2.0829451 .143747-1.370902$

H $4.1826680 .030415-1.989534$

H $3.024232-1.167908-1.432065$

H $4.0661822 .179750-1.092858$

H -4.1279770 .2122690 .591118$

H -2.7126190 .7792551 .484828$

H $5.343285-0.0125590 .218179$

H $4.212795-1.2594880 .780772$

H $5.303683-1.596825-0.566965$

SCF Energy (B3LYP/6-31G**//MMFF) $=-810.702739039$

F2-11 c02278

MMFF Geometry

C -4.400016 -0.9523730 .005143$

C $-4.294524-2.280765-0.111854$

C -2.6882660 .8733650 .238110$

$\begin{array}{lllll}\text { O } & -1.707072 & 0.012481 & 0.849241\end{array}$

C $-0.395477 \quad 0.3332140 .341075$

C $-0.6157661 .261190-0.843478$

C $-1.9051291 .973933-0.483037$

C $0.339614-0.9696990 .009871$

C - -1.6476213 .1715810 .433769$

C $1.698060-0.774246-0.693552$

O $0.530845-1.7007251 .224987$

O $2.248396-2.063468-0.983776$

C $2.7434230 .049800 \quad 0.087433$

C $4.0560490 .187531-0.700230$

O $3.011069-0.5731431 .340801$

C $-3.5302670 .028136-0.730688$

C $5.0394221 .147978-0.042825$

H $-5.163498-0.5458270 .663463$

H -4.956402 -2.935602 0.444246

H -3.551282 -2.736489 -0.756702
H $-3.328268 \quad 1.2838581 .028177$

H $\quad 0.1180410 .857524 \quad 1.155626$

H $-0.7694100 .681980-1.762346$

H $\quad 0.2296911 .934189-1.015375$

H -2.426378 $2.339090-1.374833$

H $-0.298975-1.606492-0.615242$

H -2.591886 3.6622390 .692768

H -1.156561 2.8819461 .368413

H -1.013010 $3.910194-0.067195$

H $1.529861-0.286764-1.661221$

H $-0.342329-1.8087421 .639536$

H $1.597684-2.547251-1.520335$

H 2.3358451 .0465420 .289778

H $3.8419110 .548358-1.712951$

H $4.547623-0.788302-0.790765$

H 3.5107180 .0568341 .886402

H $-4.1838090 .680921-1.321320$

H $-2.880071-0.506865-1.433688$

H $\quad 5.3699800 .7786330 .932445$

H $5.9275981 .264581-0.672217$

H 4.5900082 .1364090 .094877

SCF Energy $\left(B 3 L Y P / 6-31 G^{* *} / / M M F F\right)=-810.697824909$

F2-11 c02279

MMFF Geometry

C $4.275329-1.658093-0.541366$

C $4.981758-2.1223930 .495371$

C $2.4508010 .071637-0.813327$

$\begin{array}{llll}\text { O } & 1.014217 & 0.150358 & -0.925420\end{array}$

C 0.4937470 .9696510 .135306

C 1.6192911 .1098001 .150941

C 2.8625131 .0611120 .282303

C $-0.8006020 .363480 \quad 0.700355$

C $3.1974012 .435028-0.302524$

C $-1.9040610 .202132-0.365603$

O $-\begin{array}{llll}0.523600 & -0.909891 & 1.281596\end{array}$

O $-2.1910471 .475995-0.941635$

C $-3.201800-0.4214210 .189844$

C $-4.291113-0.525918-0.887442$

O $-3.673613 \quad 0.3860221 .267692$

C $2.800460-1.390373-0.497938$

C $-5.515194-1.298410-0.412116$

H $4.789350-1.471552-1.480694$

H $6.047090-2.3014020 .398515$

H $4.515335-2.3293441 .452212$

H $2.8750930 .343087-1.787403$

H $0.2748001 .951606-0.300742$

H 1.6361600 .2620641 .845559

H $1.5289982 .021879 \quad 1.748997$

H 3.7327490 .7241620 .854788

H -1.1536181 .0211341 .503315$

H $4.0920182 .372162-0.931324$

H $2.3857322 .834314-0.918895$

H 3.4004613 .1548050 .497433

H -1.540564 -0.445670 -1.172296

H $-0.170963-1.4824020 .578865$

H -2.562522 2.037611-0.239682

H -2.980382 -1.418043 0.589609

H $-3.885358-1.026818-1.774075$

H $-4.6210660 .474706-1.191076$

H $-4.371054-0.1106531 .727349$

H $2.371544-1.6814740 .469202$

H $2.310822-2.039587-1.235312$

H $-5.238815-2.302344-0.074656$

H $-6.020136-0.7830280 .410362$

H $-6.234505-1.402481-1.230730$

SCF Energy (B3LYP/6-31G**//MMFF) $=-810.721831295$

F2-11_c02280

MMFF Geometry

C $3.709122 \quad 1.681954 \quad 0.178574$

C 3.3401802 .7270670 .927614

C $2.561506-0.477035-0.404031$

O $1.764686-0.4922720 .796042$

C $0.423178-0.9352780 .488733$

C $0.328875-0.957925-1.027067$

C $1.756321-1.237448-1.458882$

C $-0.565671-0.0175491 .221602$ 
C $2.069945-2.734837-1.432916$

C $-2.064038-0.3003391 .010960$

O $-0.326815-0.1952972 .631304$

O $-2.292721-1.7121691 .118926$

C $-2.6874830 .163600-0.316263$

C $-2.4606711 .647997-0.621328$

O $-4.103329-0.052749-0.229589$

C $2.8037760 .988093-0.800310$

C $-3.1268472 .082421-1.921821$

H $4.725831 \quad 1.3082340 .269155$

H 4.0431843 .1868201 .613740

H 2.3385513 .1390020 .873105

H $3.517608-0.969569-0.191542$

H $\quad 0.352208-1.9533280 .890614$

H $\quad 0.0303450 .023423-1.411718$

H $-0.390126-1.696769-1.393106$

H $1.949410-0.870948-2.473255$

H $-0.333140 \quad 1.033376 \quad 1.024729$

H $3.108513-2.910998-1.732958$

H $1.933007-3.171642-0.438523$

H $\quad 1.423193-3.275588-2.131876$

H $-2.6343130 .150323 \quad 1.834073$

H $\quad 0.6127190 .0001792 .791297$

H -1.950848 -1.9945401 .984683$

H $-2.341411-0.453038-1.150632$

$\mathrm{H}-1.3917381 .867808-0.699553$

H -2.867571 2.2601760 .192565

H $-4.239740-0.995702-0.033687$

H $3.2780951 .031886-1.787720$

H $1.8511701 .527335-0.870658$

H $-4.2143101 .970628-1.872643$

H -2.907997 $3.136080-2.122511$

H $-2.7563611 .492438-2.766231$

SCF Energy (B3LYP/6-31G**//MMFF) $=-810.715867136$

F2-11 c02281

MMFF Geometry

C $-2.5314582 .690648-0.276481$

C $-1.7939193 .608741 \quad 0.358013$

C $-2.2613200 .214302-0.551753$

O $-0.829147 \quad 0.236879-0.424420$

C $-0.322443-1.103076-0.536017$

C - $-1.540205-2.023108-0.634303$

C $-2.672739-1.177237-0.085085$

C $0.614761-1.4259760 .642536$

C $-4.043959-1.616041-0.570657$

C $1.784561-0.4490400 .900726$

O $-0.165777-1.4869861 .842875$

O $1.299637 \quad 0.8730201 .136560$

C $2.845030-0.405879-0.213891$

C 4.0664690 .4320320 .194171

O $2.2985550 .142835-1.409976$

C $-2.862742 \quad 1.3393750 .291340$

C $5.1722710 .386508-0.852962$

H -2.927052 2.928475-1.260501

H $-1.5930844 .568462-0.105623$

H -1.3775023 .4214301 .341706$

H -2.499444 $0.354567-1.614611$

H $0.239897-1.171786-1.472373$

H -1.416088 -2.974891-0.108342

H -1.723271 -2.261535 - 1.690146

H -2.652061 -1.226857 1.010898

H $1.023498-2.4321380 .486971$

H $-4.118250-1.565884-1.662183$

H -4.246956 -2.648261 -0.266784

H $-4.829969-0.980905-0.150535$

H $2.278499-0.7580191 .830932$

H $0.414753-1.8016822 .556540$

H $\quad 0.5918710 .8095841 .800488$

H $3.169369-1.428112-0.439852$

H $4.466557 \quad 0.066874 \quad 1.147074$

H $3.777177 \quad 1.4802180 .335339$

H $1.9416431 .021681-1.194147$

H -3.953865 1.2456460 .326306

H -2.4943481 .2663691 .322583$

H $4.8370990 .807390-1.805972$

H $6.0357910 .967860-0.514242$

H $5.503752-0.641962-1.027658$
SCF Energy (B3LYP/6-31G**//MMFF) $=-810.715528564$

F2-11_c02282

MMFF Geometry

C 2.6504392 .0556680 .414870

C $3.3335512 .842821-0.423694$

C $2.551520-0.4706660 .726473$

O $1.174797-0.4621491 .139259$

C $0.446566-1.3920540 .312023$

C $1.414552-1.871330-0.771864$

C $2.517284-0.828545-0.754552$

C $-0.826829-0.717250-0.235929$

C $3.841249-1.331786-1.302285$

C $-1.745655-0.1723020 .880232$

O $-0.4724140 .331866-1.131684$

O $-2.110783-1.2325831 .762898$

C -3.0255450 .5463520 .400679$

C $-3.870077-0.272358-0.587134$

O $-2.6560631 .790486-0.190158$

C 3.2316660 .8499421 .097003

C $-5.2195770 .373111-0.880416$

H 1.6151892 .3034120 .633994

H $2.8572923 .704011-0.879163$

H $4.3702892 .643478-0.671405$

H $3.040485-1.2733821 .295422$

H $0.160542-2.2413310 .943523$

H $\quad 0.948233-1.990108-1.754904$

H $1.814369-2.852737-0.485628$

H $2.1878790 .027271-1.354974$

H -1.366229 -1.473003 -0.818442

H $4.214291-2.186962-0.728721$

H $3.730924-1.648486-2.344559$

H $4.601434-0.545118-1.267608$

H -1.1763970 .5560661 .471411$

H $-0.017746 \quad 1.018309-0.614726$

H $-2.503237-1.9460331 .232460$

H $-3.6364720 .769972 \quad 1.284698$

H $-4.049356-1.277120-0.190201$

H -3.339534 -0.377784 -1.540468

H $-3.4624532 .322613-0.293970$

H 3.1265901 .0099202 .177481

H 4.3058330 .7734810 .890001

H $-5.1024411 .338349-1.382063$

H $-5.809372-0.273330-1.538324$

H -5.7892880 .5279370 .041207$

SCF Energy (B3LYP/6-31G**//MMFF $)=-810.713919071$

F2-11_c02283

MMFF Geometry

C $3.2501572 .235741-0.469653$

C $4.4540552 .818374-0.494317$

C $2.493486-0.0681270 .171995$

$\begin{array}{llll}\text { O } & 1.177628 & 0.341326 & 0.585531\end{array}$

C $0.461933-0.8111241 .069074$

C $1.341650-2.0276220 .785517$

C $2.329171-1.524275-0.249773$

C $-0.929123-0.8660120 .405732$

C $3.626283-2.315404-0.278074$

C $-1.763673 \quad 0.4141260 .614943$

O $-0.768763-1.104752-0.991782$

$\begin{array}{llll}\text { O } & -1.673334 & 0.795525 & 1.992882\end{array}$

C -3.2654330 .2742310 .280944$

C $-3.582488-0.230345-1.130403$

$\begin{array}{lllll}\text { O } & -3.867215 & 1.563678 & 0.456923\end{array}$

C $2.9824180 .839763-0.957782$

C $-5.077349-0.233286-1.430052$

H $2.4059722 .802356-0.084834$

H $4.5808423 .832621-0.131570$

H $5.3282182 .299826-0.872012$

H $3.1536470 .011501 \quad 1.046129$

H $0.344298-0.6996832 .153130$

H $0.780536-2.9073630 .455160$

H $1.867343-2.3102791 .706645$

H $1.859343-1.576587-1.240132$

H -1.469087 -1.7232260 .825441$

H $4.142564-2.2737560 .686904$

H $3.429640-3.367502-0.509258$

H $4.307104-1.925467-1.040914$ 
H $-1.339618 \quad 1.236550 \quad 0.025143$ H $-0.281709-0.350136-1.365250$ H $-2.192927 \quad 1.6102262 .103494$ H $-3.739542-0.3834561 .019321$ H -3.213235 -1.252629-1.258074 H $-3.0853850 .404254-1.873696$ H $-3.4853252 .161997-0.208002$ H $3.8872580 .420096-1.411808$ H $2.2201740 .902638-1.744052$ H $-5.260085-0.652042-2.424958$ H $-5.621172-0.841814-0.700602$ H $-5.4919120 .779421-1.413531$

SCF Energy (B3LYP/6-31G**//MMFF) $=-810.719984526$

F2-11_c02284

MMFF Geometry

C $4.425619-0.648037-0.676991$

C $5.351593-0.7265620 .285219$

C $2.0743820 .274135-0.805904$

O $0.707090-0.180715-0.735145$

$\begin{array}{llll}\text { C } & 0.033249 & 0.482000 & 0.346251\end{array}$

C 1.1252201 .1199791 .195383

C 2.1988961 .4456530 .173935

C $-0.874946-0.4882021 .116636$

C $1.9318922 .783849-0.518710$

C $-1.985012-1.1625030 .286398$

O $-0.080798-1.5226281 .707204$

O $-1.395833-1.998767-0.718247$

C $-2.973975-0.208625-0.407072$

C -3.6418170 .7854920 .548623$

O $-4.002436-1.003431-1.009240$

C $2.968023-0.926655-0.461848$

C $-4.6415941 .691611-0.160522$

H $4.739516-0.378254-1.681997$

H $6.392681-0.5181700 .064205$

H $5.090734-0.9963401 .302616$

H $2.2682000 .584996-1.839399$

H $-0.5797461 .272667-0.101050$

H 1.5298510 .4074361 .923388

H $0.763386 \quad 1.988844 \quad 1.753827$

H 3.1865821 .5062870 .642090

H -1.3316950 .0513891 .953239$

H 1.9324313 .6013240 .209973

H $2.7144902 .992441-1.256226$

H $0.9704562 .797608-1.041988$

H $-2.539547-1.8522040 .934636$

H $0.335242-2.0184320 .980961$

H $-0.900493-1.427054-1.329266$

H -2.478944 $0.329346-1.223886$

H -2.890409 1.4191411 .030311

H -4.1735830 .2408161 .337980$

H -3.567347 -1.636765 -1.605671

H $2.773938-1.2603680 .565194$

H $2.682191-1.775089-1.097161$

H $-5.065140 \quad 2.410858 \quad 0.547758$

H -4.157173 $2.253296-0.965503$

H -5.468983 $1.117744-0.589003$

SCF Energy (B3LYP/6-31G**//MMFF) $=-810.725375023$

F2-11_c02285

MMFF Geometry

C -2.1537342 .1386870 .247985$

C -3.0845042 .6430431 .065746$

C $-2.029768-0.018176-1.104405$

O $-0.601326-0.132569-1.000186$

C $-0.284091-1.449310-0.512035$

C $-1.613919-2.102385-0.122460$

C $-2.572402-0.930874-0.012990$

C $0.722053-1.3721930 .647714$

C $-4.030229-1.315965-0.191835$

C $2.122946-0.8514380 .252984$

O $0.180950-0.6077541 .723036$

O $2.984765-1.0561821 .379792$

C $2.2419290 .616621-0.203198$

C $3.686727 \quad 1.084407-0.443923$

O 1.6730421 .4728650 .787098

C -2.460546 $1.449639-1.050893$

C $4.4128570 .302896-1.526265$
H -1.108301 2.2426990 .524205

H $-2.796473 \quad 3.1361201 .987729$

H -4.1415372 .5720270 .834781$

H -2.300760 -0.412021 -2.093753

H $0.168873-2.012241-1.336804$

H $-1.559208-2.6988730 .793585$

H -1.928845 - $2.778999-0.927283$

H -2.442641 -0.4761220 .976179$

H $0.855126-2.3847441 .049632$

H $-4.678625-0.438312-0.105480$

H $-4.208077-1.771575-1.171648$

H $-4.334333-2.0366590 .574276$

H $2.504105-1.500293-0.543561$

H $\quad 0.020256 \quad 0.2916651 .391071$

H $2.656377-0.4912432 .100583$

H $1.6724910 .760061-1.126829$

H $4.260493 \quad 1.0474020 .490214$

H $3.6695702 .144821-0.727458$

H 1.6952912 .3809820 .440125

H $-1.9343122 .001017-1.840138$

H -3.531715 $1.518477-1.275370$

H $4.586332-0.734108-1.224725$

H $3.8431090 .303305-2.460779$

H $5.3894570 .756035-1.725026$

SCF Energy (B3LYP/6-31G**//MMFF)= -810.724724448

F2-11 c02286

MMFF Geometry

C $2.9120962 .652693-0.507426$

C 2.6664953 .1682860 .702680

$\begin{array}{llll}\text { C } & 2.482326 & 0.182038 & 0.150401\end{array}$

$\begin{array}{lllll}\text { O } & 1.094713 & 0.351554 & 0.488781\end{array}$

C $0.546881-0.9226830 .875078$

C $1.637992-1.9622810 .627991$

C $2.586276-1.263794-0.327494$

C $-0.769036-1.1722050 .110362$

C $3.995421-1.831525-0.297989$

C $-1.809373-0.0484260 .310337$

O $-0.514667-1.292802-1.287882$

O $-2.1433650 .050328 \quad 1.693769$

C $-3.108135-0.257179-0.500011$

C $-4.1992150 .785710-0.217720$

O $-3.676373-1.533621-0.198370$

C $2.8781141 .203994-0.920052$

C $-3.790702 \quad 2.209136-0.558992$

H $3.1710503 .350550-1.301636$

H 2.7282384 .2403710 .859142

H 2.4028552 .5557931 .556048

H $3.0719850 .323851 \quad 1.065233$

H $0.335092-0.880337 \quad 1.949586$

H $1.260944-2.9166530 .248146$

H $2.147504-2.1762301 .576678$

H $2.189565-1.350317-1.346906$

H $-1.184268-2.123796 \quad 0.463954$

H $4.441581-1.7461640 .698565$

H $3.988537-2.891118-0.573710$

H $4.645423-1.305026-1.003685$

H $-1.3745230 .908363-0.001647$

H $-0.047447-2.132124-1.434322$

H $-1.3897850 .459552 \quad 2.151199$

H $-2.884191-0.246085-1.573444$

H $-4.5176340 .732300 \quad 0.830586$

H $-5.0911620 .523749-0.801647$

H $-3.115648-2.210904-0.612922$

H $3.8730810 .956694-1.308045$

H $2.1749831 .129678-1.759843$

H $-3.4437792 .280990-1.594459$

H $-4.6450442 .883433-0.440621$

H -2.994665 2.5671730 .100365

SCF Energy (B3LYP/6-31G*//MMFF) $=-810.710765615$

F2-11_c02287

MMFF Geometry

C $-2.5175832 .767663-0.263751$

C $-1.5078243 .640550-0.349563$

C $-2.712040 \quad 0.263428-0.128744$

O $-1.5130290 .015681-0.895089$

C $-0.952429-1.240389-0.495716$ 
C $-1.503000-1.519300 \quad 0.893342$

C -2.903706 -0.9389050 .807847$

C $0.578018-1.264204-0.637662$

C $-3.902258-1.945453 \quad 0.232557$

C $1.348914-0.2316590 .212203$

O $1.048108-2.570649-0.309065$

O $\quad 0.918366 \quad 1.086184-0.115521$

C $2.881419-0.3255960 .037402$

C 3.6168250 .7376790 .866626

O $3.198088-0.154860-1.343503$

C $-2.5265591 .574238 \quad 0.650178$

C $5.129137 \quad 0.552338 \quad 0.851728$

H -3.398173 $2.929889-0.880217$

H $-1.5683114 .485332-1.026929$

H -0.6104503 .5320610 .249135$

H -3.549385 $0.361919-0.829946$

H $-1.356495-1.996723-1.181975$

H -0.946116 -0.9706551 .659799$

H -1.476336 -2.581416 1.154594

H -3.270215 -0.6408361 .796696$

H $0.807626-1.076263-1.694291$

H -3.630461 -2.271432 -0.776301

H $-3.964081-2.8336830 .870107$

H $-4.901952-1.5010140 .179950$

H $1.133569-0.393277 \quad 1.273791$

H $0.606021-3.200806-0.903221$

H $1.1278701 .239802-1.052679$

H $3.218292-1.3249840 .337123$

H 3.2711630 .6980121 .906145

H 3.3964751 .7396450 .479555

H $4.129786-0.402336-1.467134$

H $-3.355092 \quad 1.703317 \quad 1.356863$

H $-1.5989751 .538174 \quad 1.234163$

H $5.5395880 .685719-0.153654$

H 5.6042021 .2907031 .505562

H $5.405702-0.4443411 .209601$

SCF Energy (B3LYP/6-31G*//MMFF) $=-810.709261453$

F2-11 c02288

MMFF Geometry

C $-2.607073 \quad 2.204455 \quad 0.505941$

C $-1.747362 \quad 3.228798 \quad 0.469508$

C -2.572274 $0.050729-0.860007$

O $-1.1454820 .067305-1.035177$

C $-0.615571-1.196416-0.588145$

C $-1.782605-1.974295 \quad 0.022775$

C $-2.820446-0.902758 \quad 0.302798$

C $0.556451-0.9519130 .382360$

C $-4.241380-1.433824 \quad 0.376031$

C $1.678896-0.083360-0.228180$

O $0.079649-0.3336001 .575960$

O $2.130364-0.700823-1.430013$

C 2.8585340 .1346860 .742104

C $3.931316 \quad 1.116540 \quad 0.243095$

O $3.456375-1.1236341 .048889$

C $-3.102574 \quad 1.479585-0.713423$

C $4.756016 \quad 0.652847-0.949489$

H -2.988422 1.8832371 .471390

H $-1.434248 \quad 3.715988 \quad 1.386429$

H -1.340205 $3.595183-0.466277$

H $-2.992251-0.370808-1.783623$

H $-0.246848-1.731525-1.470956$

H $-1.507018-2.5517520 .910765$

H -2.164078 -2.687790 -0.719080

H -2.570801-0.429439 1.258824

H $0.961891-1.9309660 .664501$

H $-4.538600-1.917034-0.560672$

H $-4.335154-2.1718321 .179507$

H $-4.949708-0.623950 \quad 0.577340$

H $1.2744980 .901152-0.491828$

H -0.2802410 .5361381 .331838$

H $2.546255-1.545907-1.187087$

H 2.4649380 .5388391 .682579

H 4.6338231 .2927891 .067937

H 3.4684392 .0827770 .011180

H $4.168777-0.961491 \quad 1.690570$

H -2.821419 $2.046880-1.610247$

H $-4.1982271 .464704-0.682154$
H $5.177069-0.343818-0.787594$

H $4.1636240 .639914-1.867941$

H $5.5890991 .344285-1.114867$

SCF Energy (B3LYP/6-31G**/MMFF) $=-810.721155249$

F2-11_c02289

MMFF Geometry

C $3.6666222 .018843 \quad 0.268039$

C $3.2043923 .272493 \quad 0.334479$

C $2.525193-0.2115780 .209719$

$\begin{array}{lllll}\text { O } & 1.364590 & 0.221436 & 0.942186\end{array}$

C $0.527107-0.9220961 .175000$

C $1.074576-2.0665120 .321876$

C $2.019843-1.372215-0.641524$

C $-0.956449-0.5699340 .952013$

C $3.115065-2.282230-1.170988$

C $-1.329214-0.158837-0.491706$

O $-1.722325-1.6972601 .370232$

O $-0.567190 \quad 0.986140-0.867541$

C $-2.8253390 .151820-0.726035$

C $-3.375565 \quad 1.257896 \quad 0.184880$

O $-3.609588-1.024770-0.551797$

C $3.0798860 .952073-0.612614$

C $-4.796542 \quad 1.658514-0.194757$

H 4.5243191 .7464390 .877728

H 3.6755003 .9978000 .988628

H $2.3534563 .595312-0.255114$

H $3.268036-0.5591520 .940525$

H $0.646795-1.2023902 .229956$

H $0.303471-2.654446-0.184864$

H $1.628047-2.7607890 .967316$

H $1.436230-0.987530-1.487514$

H -1.1829130 .2668751 .624392$

H $3.739045-2.672610-0.359991$

H $2.681780-3.135583-1.702832$

H $3.766373-1.747048-1.868905$

H $-1.058774-0.981027-1.164891$

H -2.660090 -1.442026 1.354876

H $-0.7539891 .171319-1.803484$

H $-2.9527710 .476066-1.766771$

H -2.7347572 .1451530 .135079$

H -3.3955340 .9165901 .226086$

H -3.264933 -1.700605 -1.160091

H $3.8696300 .597924-1.284344$

H $2.2858301 .377911-1.238350$

H $-5.488070 \quad 0.815622-0.097438$

H $-5.1495042 .460544 \quad 0.461394$

H $-4.8385182 .021855-1.226334$

SCF Energy (B3LYP/6-31G**//MMFF) $=-810.709099799$

F2-11_c02290

MMFF Geometry

C $-4.799428-0.623985-0.780182$

C $-5.5119640 .443813-0.403253$

C $-2.636374-0.016742 \quad 0.545234$

O $-1.454244-0.704527 \quad 1.009788$

C $-0.280281-0.088414 \quad 0.443471$

C $-0.7782340 .792953-0.689583$

C $-2.149242 \quad 1.226406-0.206427$

C $0.701644-1.1854960 .021894$

$\begin{array}{llll}C & -2.060106 & 2.441360 & 0.719726\end{array}$

C $1.934345-0.676848-0.750180$

O $1.132993-1.881347 \quad 1.196047$

O $2.720341-1.810383-1.131810$

C $2.8215020 .343693-0.007014$

$\begin{array}{llll}\text { C } 4.028130 & 0.779727 & -0.851902\end{array}$

O $3.287412-0.1913061 .228952$

C $-3.417113-1.010471-0.332246$

C $4.8303291 .887396-0.180408$

H $-5.264533-1.308946-1.486666$

H $-6.5078370 .602030-0.803790$

H $-5.136915 \quad 1.1743130 .302862$

H -3.2309710 .2396531 .429379$

H $0.1560820 .517607 \quad 1.246365$

H $-0.8901120 .206810-1.610088$

H -0.102086 $1.625489-0.906840$

H -2.796700 $1.494413-1.047722$

H $0.184032-1.936513-0.588122$ 
H -3.0583402 .7316551 .064285$ H -1.447265 2.2449851 .605295 H -1.626624 3.2969070 .191372 H $1.597188-0.219458-1.687376$ H $\quad 0.335599-2.1884451 .660803$ H $2.937843-2.302991-0.322124$ H 2.2233291 .2288750 .234945 H $3.6868231 .133100-1.831734$ H $4.701204-0.069364-1.020208$ H $3.855923-0.9541431 .029320$ H -2.823176 -1.247306 -1.224610 H -3.499848 -1.9618970 .210163$ H $5.6632462 .191246-0.822338$ H $4.2053242 .767180 \quad 0.002617$ H 5.2465391 .5549450 .775603 SCF Energy $($ B3LYP/6-31G**//MMFF $)=-810.710950299$

\section{F2-11_c02291}

MMFF Geometry

C $-3.6740681 .823996-0.149623$

C $-3.3072793 .073931-0.454316$

C $-2.407484-0.335599-0.029384$

O $-1.4263010 .040426-1.012546$

C $-0.567494-1.086689-1.247724$

C $-0.885224-2.129741-0.176270$

C $-1.687696-1.3521110 .850884$

C $0.905059-0.642063-1.335198$

C $-2.613090-2.2268421 .679598$

C $1.487170-0.028001-0.040935$

O $1.670617-1.778873-1.730063$

O $\quad 0.7549601 .156921 \quad 0.265455$

C $2.9920710 .306200-0.142424$

C 3.5765041 .0359121 .076034

O $3.2035881 .130321-1.291392$

C $-2.876154 \quad 0.9052210 .731507$

C $3.459707 \quad 0.2516802 .372618$

H $-4.6090091 .442270-0.551519$

H -3.930614 3.688938-1.093960

H $-2.3853213 .502553-0.076935$

H -3.246404 -0.805145 -0.560630

H $-0.845103-1.509590-2.222245$

H $-0.002545-2.6161480 .249316$

H -1.500193 -2.923185 -0.620595

H $-0.989950-0.8357781 .522327$

H $0.961748 \quad 0.105568-2.137071$

H $-3.163745-1.6300632 .413318$

H -3.344222 -2.745620 1.050489

H -2.040021 -2.9842092 .224386$

H $1.349982-0.7364070 .782828$

H $2.518773-1.455915-2.078355$

H $\quad 0.9392691 .3909211 .190269$

H $3.556836-0.620013-0.300674$

H 3.1116732 .0223751 .191596

H 4.6376251 .2423430 .884789

H $4.1608131 .279854-1.374587$

H $-3.510414 \quad 0.6136381 .576049$

H -2.0103731 .4389601 .142549$

H 2.4165730 .1346202 .679779

H $3.905527-0.7427562 .271267$

H $3.984050 \quad 0.777058 \quad 3.177372$

SCF Energy $\left(B 3 L Y P / 6-31 G^{* *} / / M M F F\right)=-810.703577962$

F2-11_c02292

MMFF Geometry

C -1.7600742 .9636660 .467309$

C $-1.3176013 .391457-0.720850$

C $-2.1491810 .502459-0.249396$

O $-0.7744050 .224828-0.561334$

C $-0.642248-1.149454-0.957728$

C $-2.012718-1.792960-0.751578$

C $-2.720315-0.841008 \quad 0.193337$

C $0.494812-1.824742-0.172146$

C $-4.235265-0.9306620 .115419$

C $1.901455-1.271415-0.492328$

O $\quad 0.258145-1.7368101 .232139$

O $2.850840-2.1256220 .159645$

C $2.2027590 .193602-0.112315$

C $3.6637400 .571520-0.401354$
O $1.954183 \quad 0.4228201 .271782$

C -2.218255 1.5776690 .839631

C $3.9274082 .056434-0.180680$

H -1.800781 3.6932721 .274332

H -1.017083 $4.426320-0.848096$

H -1.241948 2.741814 -1.584059

H -2.641623 $0.843499-1.169135$

H $-0.391228-1.168983-2.024930$

H $-1.965333-2.819266-0.374825$

H -2.537295 -1.829706 -1.715235

H $-2.404643-1.0619421 .220658$

H $0.503551-2.895507-0.414574$

H $-4.599920-0.697850-0.890706$

H -4.573997 -1.9400630 .370718$

H $-4.705776-0.2314730 .813607$

H $2.077759-1.389770-1.568274$

H $-0.507746-2.2976551 .439659$

H $2.721135-2.0218651 .118261$

H $1.5571430 .866864-0.684686$

H $3.9160210 .317739-1.437291$

H $\quad 4.338967 \quad 0.0099680 .255183$

H 1.0077280 .2653081 .429197

$\mathrm{H}-3.2503181 .6640021 .198860$

H -1.6037291 .2597941 .691968$

H $4.9689022 .293470-0.420413$

H $3.2845902 .667679-0.822188$

H 3.7484122 .3432360 .860192

SCF Energy $(B 3 L Y P / 6-31 G * * / / M M F F)=-810.718640352$

F2-11_c02293

MMFF Geometry

C $1.6399842 .854505-0.212271$

C $0.6123913 .313431-0.935375$

C $2.2834830 .555707 \quad 0.573933$

O 0.9209620 .0867080 .599085

C $0.869274-1.2865910 .187087$

C $2.220300-1.579776-0.453326$

C $3.161848-0.6716290 .314621$

C $-0.323822-1.551391-0.740367$

C $3.625548-1.3159941 .622666$

C $-1.706142-1.425521-0.061631$

O $-0.246925-0.707275-1.888483$

O $-2.664739-1.934580-0.997740$

C $-2.138344-0.0168350 .396586$

C -3.5443910 .0008531 .016395$

O $-2.110350 \quad 0.897410-0.696508$

C $2.4085211 .605299-0.541126$

C -3.8841621 .3559841 .625538$

H 1.9520123 .4155830 .664759

H $0.1001114 .224441-0.645944$

H $\quad 0.2634202 .792450-1.819968$

H 2.5075281 .0152211 .543965

H $0.756738-1.8912211 .095354$

H $2.226540-1.290965-1.510985$

H $2.483992-2.640685-0.401850$

H $4.053850-0.428168-0.273086$

H -0.236819 -2.576311-1.123090

H $4.294615-0.6383532 .163808$

H $2.789440-1.5574222 .286913$

H $4.176329-2.2409971 .422036$

H $-1.721335-2.1006200 .803284$

H $-0.2548410 .213580-1.575882$

H $-3.476922-2.143582-0.506353$

H -1.4323800 .3523861 .146045$

H $-3.617576-0.7665501 .795327$

H $-4.301018-0.2198640 .254520$

H -2.693451 $0.543600-1.390018$

H $3.4613201 .881973-0.672954$

H $2.0582281 .191845-1.494594$

H -3.1666631 .6248852 .407227$

H -3.8804732 .1456280 .867804$

H -4.8811411 .3267982 .076459$

SCF Energy (B3LYP/6-31G**//MMFF) $=-810.718795942$

F2-12 c02515

MMFF Geometry

C $4.661835-0.839854-0.006549$

C $4.961460-1.664058-1.016674$ 
C 2.5952910 .4498610 .583854

O $1.668588-0.441701-0.066392$

C $0.335343 \quad 0.101450 \quad 0.049568$

C $0.454109 \quad 1.327623 \quad 0.945240$

C 1.8755851 .7973320 .689842

C $-0.603483-0.989998 \quad 0.572494$

C $1.9568302 .670406-0.563237$

C -2.030981 -0.5037710 .888074$

O $-0.665514-2.034850-0.403898$

O $-2.762426-1.6053161 .435984$

C $-2.8182220 .097061-0.295185$

C -4.2118110 .5749760 .137559$

O $-2.985705-0.863997-1.334592$

C $3.9252730 .460616-0.170154$

C $-4.9463361 .298936-0.984326$

H $4.974286-1.1126600 .998308$

H $5.500153-2.586878-0.831329$

H $4.676402-1.437317-2.038072$

H 2.7464460 .0540291 .597782

H $0.0454790 .390611-0.967515$

H $0.356961 \quad 1.0420862 .000482$

H -0.3100902 .0814860 .733190$

H 2.2597722 .3782311 .535853

H $-0.176304-1.453548 \quad 1.470663$

H $2.9771773 .036683-0.715944$

H $1.6545562 .130446-1.466255$

H $1.3070583 .546482-0.462301$

H -1.9763180 .2465121 .684995$

H $\quad 0.246735-2.325917-0.575426$

H -2.837434 -2.278282 0.737396

H $-2.2675450 .947095-0.713223$

H $-4.123746 \quad 1.2509770 .995872$

H $-4.826278-0.2789840 .446580$

H -2.100794 -1.121182 -1.644052

H $3.7539790 .653815-1.236129$

H $4.565914 \quad 1.261755 \quad 0.216097$

H $-5.9221181 .648025-0.631689$

H $-4.3781702 .170092-1.325537$

H $-5.1150280 .639877-1.841587$

SCF Energy (B3LYP/6-31G**/MMFF) $=-810.721645113$

F2-12 c02516

MMFF Geometry

C $3.2687932 .425449-0.503784$

C $2.929483 \quad 3.4572920 .277162$

C $2.5852150 .014810-0.527819$

$\begin{array}{lllll}\text { O } & 1.291829 & 0.217711 & 0.073992\end{array}$

$\begin{array}{lllll}\text { C } & 0.649294 & -1.060356 & 0.240927\end{array}$

C $1.568983-2.092763-0.405525$

C $2.940131-1.452693-0.270495$

C $-0.773533-1.026474-0.339139$

C $3.553504-1.7339801 .102102$

C -1.6638890 .0499280 .315732$

O $-0.715236-0.791195-1.745300$

O $-1.729006-0.1782601 .722661$

C $-3.0893170 .102645-0.273202$

C -3.971229 1.1467130 .426296

O $-3.705711-1.180615-0.175064$

C 3.5775451 .0462560 .009062

C $-5.335217 \quad 1.284144-0.238311$

H $3.3378392 .585177-1.576765$

H $2.7232174 .429735-0.156370$

H 2.8497713 .3524951 .353539

H $2.4502550 .157268-1.609157$

H $0.599834-1.2477291 .320166$

H $1.338006-2.220456-1.469685$

H $1.485571-3.0767920 .066342$

H $3.629855-1.826587-1.035519$

H $-1.218770-2.017244-0.190033$

H $4.557365-1.3029041 .174501$

H $2.951052-1.3225971 .918302$

H $3.648647-2.8128951 .265114$

H $-1.209766 \quad 1.0374710 .169850$

H $-0.275067 \quad 0.065477-1.881345$

H $-2.067385-1.0785681 .863817$

H -3.027553 $0.345213-1.340236$

H $-3.4708332 .121758 \quad 0.415903$

H $-4.1337710 .867372 \quad 1.473980$
H $-3.844422-1.3748890 .767271$

H $3.5624461 .050572 \quad 1.105649$

H $4.5936780 .787258-0.310387$

H $-5.8971720 .346147-0.192708$

H -5.9250802 .0544760 .268443$

H $-5.2313151 .573290-1.289009$

SCF Energy (B3LYP/6-31G**//MMFF) $=-810.716271578$

F2-12 c02517

MMFF Geometry

C $-3.488863 \quad 1.858699-0.264401$

C $-3.1041672 .925339-0.974327$

C $-2.381770-0.3336120 .228186$

$\begin{array}{lllll}\text { O } & -1.031897 & 0.150921 & 0.093608\end{array}$

C -0.119381 $-0.944868 \quad 0.281405$

C $-0.971907-2.1516420 .666529$

C $-2.305514-1.846517 \quad 0.005954$

C $0.969174-0.5915721 .306018$

C $-2.313510-2.281206-1.460454$

C $1.8424440 .629036 \quad 0.953813$

O $0.365665-0.3443442 .580546$

$\begin{array}{lllll}\text { O } & 1.031233 & 1.811138 & 0.923364\end{array}$

C $2.6069650 .541663-0.379232$

C $3.479115-0.711641-0.506589$

O $3.4614821 .687627-0.472094$

C $-3.305756 \quad 0.436148-0.715326$

C $4.250559-0.751704-1.820651$

H -3.9726332 .0189670 .695729$

H $-3.2692783 .927193-0.593319$

H -2.619970 $2.820731-1.938857$

H $-2.676225-0.147648 \quad 1.270441$

H $\quad 0.345803-1.134342-0.692495$

H - $1.115993-2.2062301 .752149$

H $-0.520793-3.0969720 .349418$

H $-3.128528-2.3575840 .518043$

H $1.614502-1.4655141 .445517$

H -3.291393 -2.093738 -1.915632

H $-1.559655-1.754491-2.054107$

H $-2.115577-3.355434-1.542660$

H 2.5546470 .7993891 .770609

H -0.2197320 .4260772 .479084$

H $\quad 0.3862691 .7119390 .202432$

H $1.9116900 .603445-1.224472$

H $2.864345-1.615207-0.445136$

H $4.203238-0.744703 \quad 0.316529$

H $2.8994522 .476285-0.381063$

H $-2.9014380 .423824-1.734808$

H -4.292667 -0.040051 -0.741334

H $4.827950-1.679207-1.889383$

H $3.568151-0.712971-2.675618$

H $4.9516250 .084778-1.900015$

SCF Energy (B3LYP/6-31G**//MMFF) $=-810.723244435$

F2-12 c02518

MMFF Geometry

C $4.450926-1.4833720 .549338$

C $4.722296-2.489900-0.288935$

C $2.6861430 .296503 \quad 0.560603$

O $1.705818-0.456624-0.179442$

C $0.5476340 .376031-0.413465$

$\begin{array}{llll}\text { C } & 0.791402 & 1.658509 & 0.370010\end{array}$

C 2.3065851 .7684330 .380238

C $-0.715982-0.414323-0.038809$

C $2.8283352 .415409-0.903609$

C $-2.0266390 .370243-0.248050$

O $-0.755007-1.583885-0.862090$

O $-2.1061151 .436138 \quad 0.696496$

C $-3.301276-0.495642-0.140155$

C $-4.5789540 .338785-0.307819$

O $-3.349769-1.135268 \quad 1.134544$

C $4.093784-0.0915250 .106932$

C $-5.831033-0.528972-0.343339$

H $4.498305-1.6696421 .619211$

H $4.978488-3.4711550 .095593$

H $4.692950-2.356603-1.364711$

H $2.5494740 .027773 \quad 1.617337$

H $0.5412590 .581244-1.491183$

H 0.4399251 .5491831 .403625 
H $\quad 0.287567 \quad 2.524979-0.068415$

H $2.6551012 .367047 \quad 1.229341$

H $-0.630919-0.7503311 .001889$

H $2.5803161 .830173-1.794751$

H $2.4010723 .416188-1.029268$

H $3.9167852 .527418-0.867312$

H $-2.0135490 .822454-1.247422$

H $0.081243-2.061917-0.726746$

H -2.1714791 .0389301 .581879$

H -3.286909 -1.279072 -0.907047

H -4.524499 $0.920174-1.235309$

H -4.6839541 .0451810 .524353$

H -2.646993 -1.806467 1.154043

H $4.172031-0.022016-0.984863$

H 4.8277160 .5982700 .539579

H $-6.7181180 .095706-0.489023$

H $-5.784994-1.249032-1.166603$

H -5.959570 -1.082453 0.592111

SCF Energy (B3LYP/6-31G**//MMFF) $=-810.720884971$

F2-12 c02519

MMFF Geometry

C $3.5303412 .137027-0.395115$

C 3.3995313 .1231120 .499293

C $2.504538-0.142085-0.572339$

O 1.3006890 .1775660 .152067

C $0.491244-1.0091130 .258577$

C $1.203406-2.090261-0.550028$

C $2.659238-1.662238-0.475031$

C $-0.949424-0.720118-0.197426$

C $3.320372-2.1590830 .811480$

C $-1.627418 \quad 0.390106 \quad 0.634423$

O $-0.947991-0.359780-1.576423$

O $-1.639177-0.0111242 .004013$

C $-3.065956 \quad 0.751788 \quad 0.203990$

C $-4.023105-0.4475160 .180888$

O $-3.0601181 .363444-1.082938$

C $3.6718390 .684519-0.033157$

C $-5.466381-0.023767-0.065854$

H $3.5457582 .391076-1.452001$

H 3.3058084 .1528590 .171887

H 3.3817562 .9243711 .565075

H $2.3150990 .123161-1.621891$

H $0.490208-1.2938221 .317469$

H $0.882096-2.079949-1.598344$

H $1.011207-3.094767-0.160212$

H $3.231567-2.050881-1.324839$

H - $-1.515950-1.653114-0.099627$

H $2.843131-1.7509581 .708164$

H $3.269145-3.2515620 .871044$

H $4.378674-1.8799340 .837173$

H -1.019510 1.3004730 .557186

H $-0.4172570 .450122-1.667698$

H -2.005441 0.7249592 .523238

H -3.4640931 .4896510 .912193$

H $-3.973311-0.9916891 .130485$

H -3.741057 -1.141262 -0.619488

H $-2.4744492 .138120-1.037749$

H 3.7361260 .5827121 .056913

H $4.6142110 .320862-0.459000$

H $-6.122633-0.899919-0.058196$

H -5.8109850 .6642900 .712590$

H $-5.5766710 .470137-1.036330$

SCF Energy (B3LYP/6-31G**//MMFF) $=-810.710091248$

F2-12 c02520

MMFF Geometry

C $4.592996-0.923324-0.497791$

C $5.813636-1.1500430 .000554$

C 2.5893820 .2930990 .394626

O $1.613977-0.482275-0.329157$

C $0.2985570 .052484-0.064596$

C $0.486851 \quad 1.1343860 .991236$

C 1.9021051 .6194360 .730607

C $-0.635574-1.0911450 .346112$

C $1.9363112 .661864-0.388117$

C $-2.032958-0.642131 \quad 0.821113$

O $-0.752474-1.997132-0.756159$
O $-2.737525-1.8187191 .229402$

C $-2.8671260 .138359-0.216266$

C -4.2316020 .5788980 .333459$

O $-3.075829-0.650312-1.385603$

C $3.8831290 .399373-0.413690$

C $-4.9706891 .494257-0.635118$

H $4.079560-1.735445-1.005920$

H $6.274079-2.127102-0.097954$

H $6.371327-0.3735470 .512160$

H $2.782739-0.2482561 .331295$

H $-0.0366760 .492152-1.011382$

H $\quad 0.4386190 .6999991 .997957$

H -0.2723841 .9200560 .931581$

H 2.3397662 .0682571 .629379

H -0.162831 -1.678502 1.142940

H $2.9540293 .035775-0.539866$

H $1.5804302 .259632-1.341903$

H $1.3078753 .521171-0.130124$

H -1.912810 -0.0227831 .717810$

H -1.130879-1.508030-1.506333

H $-3.505057-1.5381021 .755276$

H $-2.3111181 .029975-0.525389$

H $-4.0972291 .104867 \quad 1.285570$

H $-4.868631-0.2937090 .518947$

H -3.522120 -1.469874-1.111217

H $3.6671200 .718528-1.440233$

H 4.5398201 .1513020 .039821

H $-5.9264291 .807096-0.202865$

H $-4.3847262 .393961-0.848026$

H $-5.1797690 .987000-1.582169$

SCF Energy $($ B3LYP/6-31G**//MMFF $)=-810.711340778$

F2-12_c02521

MMFF Geometry

C $3.0635832 .420820-0.624369$

C 2.6720733 .4816530 .090330

C $2.477094-0.013027-0.515034$

$\begin{array}{llll}\text { O } & 1.167687 & 0.173878 & 0.056048\end{array}$

C $0.571292-1.1161180 .287430$

C $1.542079-2.148970-0.280746$

C $2.884655-1.447133-0.164607$

C $-0.845158-1.172756-0.308889$

C $3.485225-1.6209521 .231271$

C $-1.773795-0.0611560 .228701$

O $-0.749514-1.058186-1.728264$

O $-1.883230-0.1788621 .646723$

C $-3.193780-0.102125-0.374918$

C -4.1823400 .8950410 .247002$

O $-3.744987-1.411871-0.217500$

C $3.4195901 .087529-0.027622$

C -3.7692192 .3480850 .082147$

H $3.1415162 .520378-1.703991$

H $2.4333714 .417959-0.402105$

H 2.5810873 .4366061 .169866

H $2.3538830 .059053-1.604825$

H $0.515064-1.2446821 .374612$

H $1.336795-2.351804-1.338381$

H $\quad 1.489068-3.104628 \quad 0.250137$

H $3.601388-1.838117-0.895649$

H - $1.258940-2.161167-0.074329$

H $2.853484-1.1860572 .012384$

H $3.620051-2.6834401 .460670$

H $4.469876-1.1462481 .292140$

H -1.3406720 .9199780 .001425$

H -1.592851 -1.359995 -2.105983

H -1.0502270 .1386442 .034126$

H $-3.1387600 .088403-1.452623$

H $-4.3346620 .671779 \quad 1.310083$

H $-5.1648820 .749382-0.220269$

H $-3.766877-1.6081990 .735031$

H $3.388297 \quad 1.1552641 .066637$

H $4.4497380 .851684-0.318493$

H $-4.561326 \quad 3.007778 \quad 0.450577$

H -2.861435 2.5736160 .649316

H $-3.5904092 .588793-0.970581$

SCF Energy (B3LYP/6-31G**//MMFF) $=-810.716183697$

F2-12_c02522 
MMFF Geometry

C $4.3508720 .176491-1.266959$

C $5.0128091 .321745-1.067106$

C $2.211516-0.740920-0.337952$

O $1.5999920 .535781-0.071820$

C 0.3173950 .3239200 .564598

C $0.089745-1.1796560 .548180$

C $1.501268-1.7412180 .576932$

C $-0.7308951 .181897-0.157972$

C $2.046852-1.8086772 .004115$

C $-2.177998 \quad 1.0921320 .359912$

O -0.3346232 .5556180 .021055$

O $-2.166210 \quad 1.158759 \quad 1.792715$

C $-2.990077-0.153483-0.033048$

C $-3.039574-0.409236-1.542941$

O $-4.336951 \quad 0.046041 \quad 0.419786$

C $3.727415-0.639218-0.168582$

C $-3.885245-1.626943-1.898520$

H $4.260383-0.202115-2.281867$

H $5.443768 \quad 1.860499-1.903860$

H $5.135274 \quad 1.740927-0.074527$

H $1.970136-0.988770-1.380971$

H $0.441856 \quad 0.6796951 .594600$

H $-0.389353-1.486813-0.388717$

H $-0.535060-1.5215951 .378297$

H $1.535236-2.7503940 .151012$

H -0.688909 $1.012140-1.238007$

H $3.047584-2.2524862 .016618$

H $2.111156-0.8210612 .471694$

H $1.403611-2.4360122 .630687$

H -2.7331051 .9812680 .032291$

H $0.5738122 .640139-0.316496$

H -1.704289 1.9784792 .039756

H -2.626663 -1.041785 0.491384

H -2.033259 -0.568811 -1.941315

H -3.465033 $0.463412-2.053059$

H $-4.2982890 .209187 \quad 1.377926$

H $3.970422-0.1930110 .803322$

H $4.173323-1.640198-0.197909$

H -3.502170 -2.525758 -1.405028

H $-4.930225-1.489794-1.604071$

H -3.862361 -1.797728 -2.979574

SCF Energy (B3LYP/6-31G**//MMFF) $=-810.716035398$

F2-12_c02523

MMFF Geometry

C $4.4258261 .426103-0.640822$

C 5.5919751 .4121460 .014217

C $2.1989250 .261681-0.439637$

O $0.881507 \quad 0.4109890 .125318$

C $0.308066-0.8981120 .310996$

C $1.315327-1.894645-0.259086$

C $2.639748-1.164584-0.100512$

C $-1.087673-0.972903-0.329733$

C $3.199464-1.3474301 .311319$

C -2.0716680 .0612060 .255855$

O $-0.984122-0.774775-1.738505$

O $-2.219903-0.178821 \quad 1.654895$

C $-3.4589430 .058079-0.421246$

C $-4.4779951 .001518 \quad 0.232728$

O $-4.023839-1.253774-0.368037$

C 3.0842381 .4159760 .028909

C -4.0723422 .4654320 .192888$

H $4.4409311 .463565-1.727071$

H $6.5289781 .428816-0.531573$

H 5.6340021 .3844091 .097347

H $2.0670270 .339540-1.528315$

H $\quad 0.221318-1.051478 \quad 1.393246$

H $1.134005-2.078156-1.324908$

H $1.273800-2.8630830 .249179$

H $3.382477-1.531402-0.817545$

H -1.471136 -1.986490 -0.165318

H $4.180150-0.8736351 .408868$

H $2.543761-0.9203542 .076843$

H $3.330287-2.4114171 .536526$

H -1.6422891 .0626570 .141527$

H $-0.5972760 .105885-1.883191$

H $-2.649220-1.045214 \quad 1.759778$
H $-3.3589950 .327665-1.479483$

H $-4.6697020 .697794 \quad 1.269265$

H $-5.440510 \quad 0.886708-0.282586$

H -3.530405 -1.812342 -0.992139

H $2.5779822 .361848-0.204363$

H $3.1754411 .408878 \quad 1.121787$

H -4.8864073 .0909360 .572902$

H -3.1928052 .6551760 .815004$

H $-3.8502432 .783932-0.830420$

SCF Energy $\left(B 3 L Y P / 6-31 G^{* *} / / M M F F\right)=-810.721640053$

F2-12_c02524

MMFF Geometry

C -4.459582 -1.120068 0.330332

C $-4.489950-2.1483561 .185401$

C $-2.6090770 .358018-0.512631$

O $-1.631901-0.698133-0.384021$

C $-0.377967-0.1549680 .090914$

C $-0.684631 \quad 1.2706610 .524028$

C $-1.8130381 .661581-0.407581$

C $0.641688-0.303600-1.052076$

C $-2.5738202 .896914 \quad 0.040756$

C $2.0138200 .350142-0.800437$

O $0.836663-1.705327-1.285232$

O $2.8302990 .125306-1.955058$

C $2.765784-0.1223390 .460882$

C $4.1077330 .605694 \quad 0.625834$

O $3.028716-1.5220950 .400873$

C -3.6837410 .1470360 .564500$

C $4.7962480 .254541 \quad 1.939193$

H $-5.034758-1.189669-0.589394$

H -5.073661 -3.033900 0.959052

H -3.936843 -2.127616 2.117906

H -3.062709 $0.277641-1.507874$

H $-0.070188-0.7674470 .945596$

H $\quad 0.165207 \quad 1.954960 \quad 0.484822$

H $-1.040977 \quad 1.263396 \quad 1.562041$

H $-1.3798001 .873384-1.394903$

H $\quad 0.2276420 .089161-1.988706$

H -3.424184 $3.089732-0.621113$

H -2.9484932 .7999201 .064758$

H -1.920629 3.7757450 .014691

H $1.8816601 .435319-0.741194$

H $-0.037986-2.094097-1.458973$

H $2.977628-0.834038-2.022056$

H 2.1515290 .0638401 .349030

H 3.9502901 .6897840 .588479

H $4.7869270 .338533-0.192477$

H $2.173632-1.9815010 .350266$

H -3.2228730 .1228441 .559480$

H -4.4016240 .9745930 .552996$

H $5.033032-0.8125881 .992871$

H 5.7337520 .8118312 .033439

H 4.1624080 .5106362 .794068

SCF Energy $(B 3 L Y P / 6-31 G * * / / M M F F)=-810.719627719$

F2-12 c02525

MMFF Geometry

C $2.7128912 .543611-0.468913$

C $1.9568573 .566329-0.053982$

C $2.3658620 .055841-0.454354$

O $\quad 0.936000 \quad 0.183792-0.574572$

C $0.296609-0.5622300 .476922$

C $1.395213-1.3451911 .195924$

C $2.560995-1.2962730 .228031$

C $-0.824352-1.449185-0.095028$

C $3.909541-1.5404660 .881738$

C $-1.927920-0.701107-0.870402$

O $-0.247061-2.425353-0.968377$

O $-1.365321-0.069403-2.027336$

C $-2.6734530 .385920-0.077359$

C $-3.284230-0.1183301 .234251$

O $-3.738070 \quad 0.878503-0.900079$

C $2.9101001 .272590 \quad 0.310400$

C -4.0450110 .9728321 .978609$

H $3.2272182 .628427-1.422770$

H $1.8579594 .458638-0.662199$

H 1.4250653 .5322270 .890339 
H $2.7905640 .033380-1.465124$ H $-0.1388950 .161196 \quad 1.175013$ H $\quad 1.102759-2.365774 \quad 1.462847$ H $1.649632-0.8333652 .132488$ H $2.404403-2.078433-0.527221$ H -1.277697 -2.009618 0.730436 H $4.090329-0.8590301 .719061$ H $3.962194-2.5614561 .274789$ H $4.719685-1.4163240 .156050$ H -2.652769 -1.427014 -1.260197 H $-0.961742-3.015487-1.262546$ H $-0.857735-0.745198-2.508594$ H $-2.012024 \quad 1.2382380 .117422$ H -2.504154 -0.502652 1.899027 H -3.980015 -0.9404111 .027326$ H -3.339336 $1.196163-1.728481$ H $2.424521 \quad 1.358872 \quad 1.289727$ H 3.9855201 .1597520 .486069 H -4.429683 0.5830552 .926485 H -3.3914291 .8222502 .201515$ $\mathrm{H}-4.8973681 .3362111 .396427$

SCF Energy (B3LYP/6-31G**//MMFF) $=-810.709675612$

F2-12 c02526

MMFF Geometry

C $3.4984422 .223969-0.504337$

C $3.218907 \quad 3.263538 \quad 0.289933$

C $2.481556-0.060348-0.746167$

O $1.1732280 .401738-0.356536$

C $0.682670-0.411250 \quad 0.726854$

C $1.670713-1.5632080 .902744$

C $2.475828-1.544033-0.381890$

C $-0.759740-0.8595780 .423191$

C $3.829660-2.221771-0.265047$

C $-1.725851 \quad 0.3118160 .146513$

O $-0.763948-1.741690-0.697990$

O $-1.7851621 .161101 \quad 1.291358$

C $-3.157364-0.143456-0.212193$

C $-4.0972291 .049165-0.439704$

O $\quad-3.700183-0.943788 \quad 0.836754$

C $3.5425440 .795342-0.036262$

C $-5.4712790 .612503-0.933248$

H $3.7172262 .412349-1.552191$

H $3.2070684 .271959-0.108925$

H $2.9965843 .129705 \quad 1.342724$

H $2.5740300 .060796-1.832125$

H $0.6870350 .205646 \quad 1.632622$

H $1.188998-2.5277011 .092669$

H $2.316438-1.3564731 .765511$

H $1.897544-2.075370-1.149842$

H $-1.111675-1.4305971 .290343$

H $3.703412-3.292466-0.072337$

H $4.399455-2.110950-1.193175$

H 4.426104-1.810746 0.555601

H -1.354876 $0.908997-0.695995$

H $-0.432361-1.242934-1.464435$

H $-0.945987 \quad 1.648813 \quad 1.341471$

H -3.134907 -0.755775 -1.121639

H $-3.6586131 .733754-1.174816$

H -4.2372761 .6062630 .494288$

H $-3.240834-1.8001670 .822522$

H $3.402427 \quad 0.7562621 .050647$

H $4.5456660 .411511-0.251995$

H -6.104033 $1.488313-1.108542$

H $-5.3913990 .058447-1.873881$

$\mathrm{H}-5.974631-0.024646-0.199499$

SCF Energy $\left(B 3 L Y P / 6-31 G^{* *} / / M M F F\right)=-810.715528506$

\section{F2-12 c02527}

MMFF Geometry

C $-2.051517 \quad 2.676976 \quad 0.779838$

C -1.3343313 .6162950 .152915$

C $-2.200970 \quad 0.1995160 .454465$

O $-0.8886630 .053153-0.118259$

C $-0.670498-1.327320-0.454139$

C $-1.908018-2.0822430 .028363$

C $-2.994381-1.021066-0.016259$

C $0.641865-1.8459020 .145939$
C $-3.592863-0.895569-1.417982$

C $1.914153-1.209436-0.457251$

O $0.616268-1.6641221 .561889$

O $3.045034-1.9001610 .089493$

C $2.1195200 .304237-0.240806$

C $3.4657120 .787638-0.802535$

$\begin{array}{llll}\text { O } & 2.076857 & 0.633532 & 1.144866\end{array}$

C $-2.778713 \quad 1.5633780 .078054$

C $3.6025132 .304405-0.740186$

H -2.1252012 .7145941 .863685$

H -0.8348534 .3949760 .718881$

H -1.229639 $3.624579-0.926388$

H $-2.0748370 .137234 \quad 1.544425$

H $-0.613547-1.384556-1.547963$

H -1.783247 -2.423764 1.063142

H $-2.120004-2.965423-0.582542$

H -3.805716 -1.252875 0.682868

H $\quad 0.690598-2.929639-0.020328$

H $-4.398527-0.154409-1.431208$

H -2.847827 -0.596267 -2.162098

H $-4.022385-1.850953-1.738036$

H $1.922359-1.403558-1.537009$

H $1.467464-1.9803511 .910338$

H $2.959052-2.839415-0.147089$

H $1.3220040 .861842-0.742000$

H $3.5711880 .465720-1.844974$

H $4.2945450 .352591-0.231691$

H 1.1842070 .4234161 .467968

H -2.719439 $1.713903-1.006750$

H $-3.835368 \quad 1.6141820 .364992$

H 3.5659922 .6661890 .292040

H $4.5598822 .614012-1.171411$

H $2.8024932 .793300-1.305270$

SCF Energy $\left(B 3 L Y P / 6-31 G^{* *} / / M M F F\right)=-810.716543887$

F2-12 c02528

MMFF Geometry

C $3.472214-1.631554-0.846590$

C $4.676181-2.175540-0.637505$

C $2.300008 \quad 0.341687 \quad 0.162272$

O $0.967363-0.166863-0.039629$

C 0.0167590 .8167690 .405457

C $0.8317131 .947342 \quad 1.029218$

C $2.152324 \quad 1.8610570 .283426$

C $-1.023147 \quad 0.198647 \quad 1.352745$

C $2.0894992 .607744-1.049947$

C $-1.874998-0.9373780 .751190$

O $-0.371404-0.3086952 .522312$

O $-1.029160-2.0303800 .371005$

C $-2.704589-0.557687-0.489088$

C $-3.6062400 .664405-0.274475$

O $-3.522544-1.691554-0.802933$

C $3.224860-0.152186-0.950516$

C $-4.4448611 .008776-1.499505$

H $2.615722-2.291307-0.960307$

H $4.789321-3.252575-0.577906$

H $5.563722-1.563254-0.522177$

H $2.641012-0.0552621 .128683$

H $-0.486321 \quad 1.194780-0.491525$

H 1.0113091 .7655762 .095631

H 0.3311952 .9168640 .942964

$\begin{array}{llll}\text { H } & 2.970926 & 2.286472 & 0.874773\end{array}$

H -1.688861 0.9922311 .708430

H $1.3386792 .187391-1.726660$

H $1.8422383 .662277-0.887147$

H $3.0577252 .574280-1.559853$

H -2.541680 -1.337929 1.525812

H $0.362468-0.8753732 .229275$

H $-0.599687-2.3620561 .177434$

H $-2.040035-0.402019-1.347253$

H -3.002802 $1.542809-0.024638$

H -4.2870030 .4791140 .565024$

H -3.854292 -1.574672 -1.708952

H $2.7742710 .033939-1.932676$

H $4.1752200 .393157-0.908914$

H $-5.0058351 .932104-1.322416$

H $-3.810434 \quad 1.160532-2.378494$

H $-5.1692430 .220369-1.725245$ 
SCF Energy (B3LYP/6-31G**//MMFF) $=-810.705939946$

F2-12_c02529

MMFF Geometry

C $-3.213124 \quad 2.3118170 .213175$

C $-3.4734713 .004590-0.901153$

C $-2.1352260 .087356 \quad 0.622368$

O $-1.3170310 .096548-0.564755$

C -0.592557-1.140693 -0.633659

C $-0.893718-1.903998 \quad 0.652231$

C $-2.271908-1.3840321 .028448$

C $0.894521-0.914697-0.954546$

C $-3.376239-2.1514630 .299838$

C $1.675040-0.0480210 .059473$

O $1.538194-2.182279-1.069663$

$\begin{array}{lllll}\text { O } & 1.073369 & 1.239883 & 0.165991\end{array}$

C $3.1670760 .116560-0.305846$

C $3.9448841 .058750 \quad 0.624731$

O $3.2602600 .639420-1.633563$

$\begin{array}{llll}\text { C } & -3.443393 & 0.833240 & 0.359115\end{array}$

C 3.9774830 .6000832 .073195

H -2.818692 2.8424631 .075965

H $-3.2882594 .072555-0.939926$

H $-3.8706132 .524225-1.788537$

H $-1.5606650 .609973 \quad 1.399757$

H $-1.028490-1.705786-1.468330$

H $-0.189182-1.6428091 .448470$

H $-0.847236-2.9890320 .516320$

H $-2.448963-1.4748932 .106026$

H $0.940580-0.419190-1.932850$

H $-4.364599-1.7980360 .610999$

H $-3.308357-2.045330-0.787487$

H $-3.321582-3.2192500 .537587$

H $1.624770-0.5160001 .047048$

H $1.074138-2.686472-1.759594$

H $1.1285831 .662760-0.708124$

H $3.651129-0.867079-0.310441$

H 3.5366822 .0751580 .565772

H $4.976213 \quad 1.1419130 .258139$

H $4.2029580 .676337-1.869190$

H $-3.9243360 .445218-0.546791$

H -4.1341820 .6804951 .196453$

H 4.6355891 .2506662 .658197

H 2.9841310 .6404522 .529527

H $4.355837-0.4238582 .152713$

SCF Energy (B3LYP/6-31G**//MMFF) $=-810.707933661$

F2-12_c02530

MMFF Geometry

C -3.0996292 .2844210 .405434$

C $-2.510638 \quad 3.417910 \quad 0.008275$

C $-2.435522-0.122851 \quad 0.205354$

$\begin{array}{lllllll}\text { O } & -1.017107 & 0.121552 & 0.146999\end{array}$

C $-0.331263-1.128022-0.041292$

C $-1.400130-2.2173020 .038631$

C $-2.650656-1.496574-0.436037$

C $0.813110-1.2941660 .970155$

C $-2.743246-1.485659-1.962493$

C $1.912572-0.2080510 .980551$

O $0.262166-1.3510242 .292982$

$\begin{array}{lllll}\text { O } & 1.347563 & 1.095935 & 1.108725\end{array}$

C $2.840131-0.231317-0.248677$

C $4.0154090 .746394-0.094935$

O $2.1206910 .105459-1.431127$

C $-3.1895421 .041834-0.436412$

C $5.0037290 .645476-1.250095$

H $-3.552227 \quad 2.2510181 .393163$

H -2.483229 4.2815460 .663653

H -2.046739 $3.504756-0.967922$

H $-2.694890-0.1906661 .271051$

H $0.072813-1.117274-1.057660$

H $-1.553004-2.5520131 .071438$

H $-1.139438-3.099306-0.554699$

H $-3.556176-1.972510-0.043230$

H $1.290332-2.2668120 .801895$

H $-3.667742-1.000295-2.291939$

H $-1.903406-0.958549-2.426146$

H $-2.754652-2.508963-2.352877$
H $2.528368-0.3629291 .876203$

H $-0.298025-0.5656022 .414080$

H $0.819181 \quad 1.1099661 .924207$

H $3.232635-1.245908-0.381945$

H 4.5459980 .5457310 .842959

H $3.647577 \quad 1.778547-0.054056$

H $1.7173140 .979430-1.291104$

H $-2.7890861 .246865-1.436696$

H $-4.2493340 .784398-0.546961$

H $4.5320820 .905715-2.202856$

H $5.8404551 .333124-1.090724$

H $5.408739-0.368197-1.331231$

SCF Energy (B3LYP/6-31G**//MMFF) $=-810.713161871$

F2-12_c02531

MMFF Geometry

C $4.1196381 .789297-0.665391$

C $4.3155212 .802793 \quad 0.185587$

C $2.570369-0.180898-0.640090$

$\begin{array}{lllll}\text { O } & 1.549202 & 0.439262 & 0.165041\end{array}$

C $0.505331-0.5250110 .431216$

C $0.854359-1.754779-0.396107$

C $2.369736-1.689901-0.480288$

C $-0.857420 \quad 0.125645 \quad 0.139142$

C $3.024643-2.2989850 .760348$

C $-2.061052-0.807958 \quad 0.389476$

O $-0.972803 \quad 1.2711970 .986750$

O $-2.042949-1.861822-0.571448$

C $-3.449983-0.1324850 .338904$

C $-3.7078960 .659887-0.950820$

$\begin{array}{llll}\text { O } & -3.631060 & 0.710680 & 1.473450\end{array}$

C $3.9455130 .356623-0.243564$

C $-5.1415321 .170090-1.032377$

H $4.0926602 .001998-1.731036$

H $4.4387393 .814733-0.184460$

H 4.3546132 .6444851 .257556

H $2.3512420 .092103-1.681740$

H $0.576153-0.7522861 .502178$

H $0.441876-1.665155-1.408971$

H $0.475252-2.6825590 .042706$

H $2.741500-2.227198-1.360118$

H $-0.8627640 .483769-0.897424$

H $2.720492-3.344398 \quad 0.879864$

H $4.115722-2.2845980 .670095$

H $2.756283-1.7642791 .676985$

H $-1.941456-1.2552951 .384950$

H $-0.204553 \quad 1.8413740 .810596$

H $-2.750128-2.486865-0.337809$

H $-4.212714-0.917747 \quad 0.411248$

H $-3.5050920 .032927-1.826109$

$\mathrm{H}-3.0431151 .529632-1.001859$

H $-2.9842261 .434074 \quad 1.412832$

H $4.0856450 .273757 \quad 0.841086$

H $4.730619-0.235153-0.728399$

H $-5.3683841 .854337-0.208935$

H -5.295808 1.711220 -1.971390

H $-5.8550330 .340685-0.998413$

SCF Energy (B3LYP/6-31G**//MMFF) $=-810.718891132$

F2-12_c02532

MMFF Geometry

C $4.392605-1.5494740 .579005$

C $4.673751-2.539413-0.275955$

$\begin{array}{llll}\text { C } & 2.665480 & 0.266429 & 0.570529\end{array}$

O $1.700982-0.441105-0.230643$

C $0.5484520 .407438-0.445208$

C $0.806256 \quad 1.672721 \quad 0.362712$

C $2.322991 \quad 1.7487030 .413905$

C $-0.715945-0.387170-0.080678$

C $2.8899752 .409459-0.843714$

C $-2.039200 \quad 0.372177-0.301854$

O $-0.735708-1.558964-0.904255$

$\begin{array}{lllll}0 & -2.138885 & 1.456399 & 0.617777\end{array}$

C $-3.285199-0.523332-0.122223$

C $-4.5965440 .257290-0.293623$

O $-3.264276-1.124987 \quad 1.172122$

C $4.081860-0.1388270 .161843$

C $-5.816913-0.653714-0.244313$ 
H $4.395554-1.765273 \quad 1.644364$ H $4.894019-3.5359620 .090804$ H $4.687585-2.376895-1.347917$ H $2.480765-0.0281281 .612977$ H $\quad 0.5406110 .633192-1.518698$ H $\quad 0.4217971 .5609151 .384249$ H $0.3412692 .558932-0.078418$ H 2.6626182 .3218851 .283901 H $-0.638531-0.7231570 .960624$ H $2.6476961 .848904-1.752256$ H $2.4901073 .422794-0.957467$ H $3.9797042 .493803-0.780740$ H $-2.0640260 .778893-1.321232$ H $0.097868-2.035862-0.749143$ H -1.8180912 .2548200 .166318$ H -3.259293 -1.337016 -0.855874 H -4.588852 $0.789505-1.251696$ H $-4.702947 \quad 1.0040400 .502076$ H -3.250682 -0.407587 1.828854 H $4.205131-0.041702-0.923570$ H 4.8118990 .5232980 .641954 H -6.729692 -0.068944 -0.396061 H -5.768612 -1.415765 -1.028592 H -5.898209 -1.159149 0.722971 SCF Energy (B3LYP/6-31G**//MMFF) $=-810.715919982$

F2-12 c02533

MMFF Geometry

C $3.2766592 .265220-0.788232$

C $3.0618573 .348373-0.033195$

C $2.485785-0.106533-0.621376$

$\begin{array}{lllll}\text { O } & 1.268205 & 0.189865 & 0.090051\end{array}$

C $0.588492-1.0451580 .385300$

C $1.391579-2.152731-0.292302$

C $2.797109-1.576483-0.323910$

C $-0.884152-0.962203-0.051867$

C $3.530532-1.8229280 .995339$

C -1.6531590 .2060110 .599627$

O $-0.935011-0.826546-1.471896$

$\begin{array}{lllll}\text { O } & -1.359760 & 0.242707 & 2.001892\end{array}$

C -3.1906940 .1405950 .463903$

C $-3.7140400 .018431-0.969995$

O $-3.726343 \quad 1.344257 \quad 1.028882$

C $3.5715130 .898257-0.235978$

C $-5.2360740 .071505-1.046920$

H $3.2478192 .370174-1.869769$

H $2.8581254 .308344-0.495044$

H 3.0827663 .2986981 .049765

H $2.252235-0.008957-1.690831$

H $\quad 0.642718-1.181614 \quad 1.471823$

H $1.052182-2.320509-1.321260$

H $\quad 1.310659-3.1072970 .236817$

H $3.389927-2.020385-1.131695$

H -1.371080 -1.909533 0.208054

H $4.555151-1.4402380 .947489$

H $3.031817-1.3434551 .843724$

H $3.592310-2.8964271 .204352$

H $-1.306876 \quad 1.1602350 .183749$

H -0.469843 -0.005046 -1.706207

H -1.647446 -0.6028382 .386913$

H $-3.582334-0.6880701 .066499$

H -3.390609 -0.929321 -1.411434

H $-3.3119780 .833250-1.583778$

H -3.394368 1.4091961 .940763

H 3.6640360 .9554520 .855314

H $4.5387560 .573874-0.637066$

H -5.621853 $1.038168-0.708618$

H $-5.565135-0.075140-2.080643$

H $-5.686155-0.714977-0.432720$

SCF Energy (B3LYP/6-31G**//MMFF) $=-810.715491837$

F2-12 c02534

MMFF Geometry

C -4.550546 -0.9349860 .298989$

C $-4.813079-1.4454301 .507390$

C $-2.536138 \quad 0.199217-0.669145$

O $-1.573423-0.437556 \quad 0.193515$

C $-0.2590050 .092617-0.096095$
C $-0.4312361 .006030-1.302932$

C $-1.8669301 .482423-1.167715$

C $0.706565-1.079741-0.305013$

C $-1.9706632 .674586-0.215163$

C $2.108440-0.712555-0.824906$

O $0.844358-1.7944380 .927050$

O $2.639598-1.900174-1.447299$

C $3.168370-0.2519200 .196545$

C 2.7562510 .9345931 .069132

O $4.3368750 .129118-0.542988$

C -3.8607410 .3813990 .072031$

C 3.8922631 .4421281 .950897

H -4.859364 -1.494195 -0.580615

H $-5.318945-2.4000081 .602364$

H $-4.529787-0.9267782 .416484$

H $-2.679078-0.475123-1.524957$

H $\quad 0.012614 \quad 0.6729910 .789118$

H $-0.3317620 .433480-2.234069$

H $0.3060241 .814153-1.328603$

H $-2.277876 \quad 1.784242-2.137667$

H $\quad 0.243329-1.789672-1.003035$

H $-1.6437172 .425696 \quad 0.799494$

H -1.352990 $3.505364-0.572892$

H -3.002458 $3.036229-0.156697$

H $2.0381520 .016343-1.638758$

H $-0.050819-2.0206851 .233016$

H $2.679230-2.594733-0.767187$

H $3.469897-1.0897750 .836898$

H 1.9312510 .6463501 .726258

H 2.4166891 .7637140 .437538

H $4.600373-0.636256-1.082356$

H $-3.689940 \quad 0.8771501 .035373$

H $-4.5327121 .016852-0.516448$

H 3.5326602 .2475142 .599453

H 4.2806940 .6412742 .588003

H 4.7179641 .8401191 .352958

SCF Energy $(B 3 L Y P / 6-31 G * * / / M M F F)=-810.714680468$

F2-12 c02535

MMFF Geometry

C $4.996315-0.5994930 .556089$

C 6.0625420 .1038820 .159104

C $2.494604-0.4467220 .282123$

O $1.406591-0.920879-0.534941$

C $0.3214340 .033566-0.468529$

C 0.7515631 .0846740 .547407

C $2.268561 \quad 1.0578780 .446305$

C $-0.982202-0.716764-0.153422$

C $2.7552951 .915869-0.722851$

C $-2.2249540 .194083-0.071110$

O $-1.195156-1.670174-1.199638$

O $\quad-2.1228541 .0639081 .053372$

C $-3.555526-0.5867680 .009915$

C $-4.7988150 .287760 \quad 0.227863$

O -3.494002 -1.534835 1.078558

C $3.820063-0.911320-0.320094$

C $-5.0445331 .288091-0.889341$

H $4.978969-0.9879011 .571179$

H 6.8850190 .2875550 .841700

H $6.1351160 .505963-0.845379$

H $2.364475-0.930848 \quad 1.260463$

H $0.2524320 .475324-1.470276$

H $0.4612960 .780621 \quad 1.560996$

H 0.3121862 .0673120 .351380

H 2.7332531 .4302651 .366059

H $-0.855961-1.2798540 .779414$

H $2.3999321 .543299-1.688800$

H $2.4058442 .948079-0.611818$

H $3.8478741 .946905-0.758455$

H $-2.254170 \quad 0.824971-0.966968$

H $-0.405765-2.237066-1.240128$

H -2.0338150 .5136941 .849811$

H -3.692555 -1.165490 -0.910693

H -4.7334320 .8144181 .187835$

H $-5.674907-0.3674040 .318236$

H -3.445746 -1.041235 1.914510

H $3.777650-1.999237-0.461590$

H $3.945716-0.502192-1.329944$ 
H $-5.0944450 .787237-1.861351$ H -5.994786 $1.806865-0.726613$ H -4.256893 $2.046152-0.929134$ SCF Energy (B3LYP/6-31G**//MMFF) $=-810.714062695$

\section{F2-13 c02795}

MMFF Geometry

C $4.449095-1.570527-0.255060$

C $4.696809-2.4350630 .735221$

C $2.6978720 .192751-0.581575$

O $1.696271-0.430141 \quad 0.246938$

C $\quad 0.535867 \quad 0.425340 \quad 0.298987$

C $0.8109181 .580773-0.658180$

C $2.3268561 .677494-0.642570$

C $-0.719732-0.404699-0.007782$

C 2.8194302 .5118990 .540861

C -2.0221480 .4070310 .116653$

O $-0.634132-0.940157-1.328280$

O -2.1110890 .9557331 .431047$

C $-3.288813-0.420199-0.188345$

C $-4.5683750 .410969-0.014408$

O $-3.326786-1.5423870 .692165$

C $4.092818-0.125416-0.042381$

C $-5.817143-0.328057-0.478598$

H $4.516607-1.921409-1.281547$

H $4.953835-3.4652080 .513646$

H $4.646999-2.1355801 .776256$

H $2.581006-0.237567-1.585941$

H $\quad 0.482520 \quad 0.800620 \quad 1.327778$

H $\quad 0.3025862 .502267-0.357916$

H $\quad 0.478479 \quad 1.338484-1.674807$

H $2.7047302 .135758-1.563430$

H $-0.738812-1.2565330 .683177$

H 2.5390802 .0729121 .503701

H 3.9096742 .6095210 .520239

H 2.4000013 .5227910 .497744

H $-1.9957181 .247771-0.586745$

H $\quad 0.197348-1.441837-1.383996$

H -2.1852050 .2123312 .053947$

H -3.232059 -0.801312 -1.214700

H $-4.482417 \quad 1.342845-0.585260$

H -4.7068880 .6772061 .040118$

H -4.013439 $-2.149774 \quad 0.369399$

H $4.8407420 .485638-0.560914$

H $4.1487220 .113044 \quad 1.026620$

H $-6.6920620 .324790-0.395569$

H -5.723675 -0.637847 -1.524171

H $-6.006898-1.2170530 .130461$

SCF Energy (B3LYP/6-31G**//MMFF) $=-810.719468213$

F2-13 c02796

MMFF Geometry

C $-2.433982 \quad 2.3011350 .348721$

C -3.192084 3.260447 -0.193659

C $-1.992264-0.074429-0.314690$

O $-0.667394-0.1290330 .244807$

C $-0.116525-1.4352720 .020375$

C $-1.164782-2.213559-0.775981$

C $-2.464330-1.527926-0.391093$

C $1.241398-1.351780-0.690254$

C $-3.021782-2.0886850 .918052$

C $2.356938-0.6385830 .101288$

O $1.674385-2.692275-0.952000$

O $2.488687-1.2640561 .385841$

C $2.178406 \quad 0.8692360 .349459$

C $1.9156441 .680076-0.923777$

O $3.390364 \quad 1.358787 \quad 0.939979$

C -2.8704820 .8709820 .504739$

C $1.7914423 .173938-0.646745$

H -1.443777 $2.563748 \quad 0.711875$

H $-2.8193154 .276026-0.272498$

H $-4.1887903 .053430-0.567061$

H -1.884573 $0.315310-1.336711$

H $0.002058-1.9131911 .000315$

H -1.143946 -3.286618 -0.561411

H $-0.998847-2.089154-1.853810$

H -3.225450 -1.651858 -1.169486

H $1.117958-0.864142-1.663215$
H $-2.337974-1.9358681 .759136$

H -3.975683 -1.613763 1.169268

H $-3.206330-3.1644100 .826183$

H $3.318611-0.802114-0.402089$

H $2.492986-2.639312-1.474157$

H $2.624765-2.2149281 .232010$

H 1.3886901 .0507541 .086400

H $\quad 0.993061 \quad 1.347555-1.409332$

H $2.7376701 .531066-1.634285$

H 3.5463040 .8418361 .749167

H -3.9176550 .7592660 .199352$

H -2.8051690 .6259991 .571523$

H $1.5583403 .709590-1.572546$

H 0.9900293 .3734460 .071339

H $2.7231063 .586455-0.247590$

SCF Energy $(B 3 L Y P / 6-31 G * * / / M M F F)=-810.716874154$

F2-13_c02797

MMFF Geometry

C $-4.3598580 .981354-0.686751$

C $-4.4747012 .311640-0.769560$

C $-2.485652-0.5276280 .014577$

O $-1.4211920 .377933-0.336245$

C $-0.156671-0.2174220 .030585$

C $-0.478272-1.6286110 .510623$

C $-1.893001-1.4900121 .046911$

C $0.807306-0.094916-1.158323$

C $-1.895812-0.9712992 .485871$

C $2.259903-0.521147-0.884528$

O $0.324012-0.880635-2.254152$

O $2.299731-1.912134-0.543647$

C 2.9728520 .2426610 .244484

C 2.9541521 .7644480 .066277

$\begin{array}{llll}\text { O } & 4.340628 & -0.183068 & 0.268439\end{array}$

C -3.7151780 .2614630 .465054$

C 3.6994212 .4896851 .180634

H $-4.7629360 .372860-1.492118$

H $-4.9560462 .768973-1.627180$

H -4.093192 2.9640190 .007974

H -2.722469-1.095411-0.895984

H $\quad 0.2197650 .3809450 .867468$

H $\quad 0.239997-1.9937051 .250941$

H $-0.484158-2.339070-0.324557$

H -2.419424 -2.451053 1.030320

H $0.7967670 .940236-1.517970$

H $-1.333775-1.6484103 .138035$

H -1.448330 0.0241222 .569810

H -2.917188 -0.914914 2.876278

H $2.843782-0.431419-1.809482$

H $-0.588416-0.595349-2.434222$

H $1.873606-2.398933-1.270000$

H $2.546831-0.0284151 .217571$

H $1.9247302 .136350 \quad 0.047578$

H $3.4222092 .031498-0.888897$

H $4.340551-1.1495040 .379533$

H $-4.458307-0.4201140 .894762$

H -3.4366580 .9851061 .240771$

H 3.2717142 .2473922 .158759

H $4.7610342 .224071 \quad 1.192363$

H 3.6275483 .5726651 .037960

SCF Energy $($ B3LYP/6-31G**//MMFF $)=-810.719763537$

F2-13_c02798

MMFF Geometry

C 3.2592332 .4021850 .546868

C $2.9436893 .431932-0.246539$

C $2.566783-0.0052070 .547834$

O $1.2979370 .204922-0.100504$

C $0.648267-1.063893-0.278187$

C $1.542714-2.105410 \quad 0.390574$

C $2.921368-1.4742680 .300962$

C $-0.783823-1.0385360 .279346$

C $3.575539-1.757743-1.052096$

C $-1.702642-0.029553-0.445688$

O $-1.335674-2.3495120 .177959$

O $-1.2428861 .302067-0.218847$

C $-3.174763-0.1211570 .007431$

C $-4.0669150 .917143-0.687875$ 
$\begin{array}{llll}\text { O } & -3.256310 & 0.062680 & 1.420694\end{array}$

C 3.5823321 .0214480 .046882

C $-5.5380590 .737797-0.334859$

H $3.2955162 .564377 \quad 1.621087$

H 2.7245424 .4055090 .178290

H $2.8961073 .324289-1.324433$

H $2.3939320 .137044 \quad 1.623691$

H $0.615442-1.264074-1.356675$

H $1.468834-3.089631-0.082250$

H $1.269523-2.2239921 .446894$

H $3.583727-1.8532251 .087378$

H $-0.735366-0.7690831 .341635$

H $3.671029-2.837074-1.212808$

H $2.999796-1.343705-1.886169$

H $4.582898-1.331011-1.093500$

H $-1.670902-0.215585-1.526638$

H $-1.379908-2.580405-0.765698$

H $-0.3452411 .372137-0.585318$

H $-3.560621-1.123600-0.209362$

H -3.950291 $0.837952-1.774873$

H $-3.7722581 .931487-0.393975$

H -2.8807670 .9362911 .624954$

H 4.5851070 .7588820 .403471

H $3.6078061 .025181-1.049616$

H $-6.1460681 .475797-0.867821$

H $-5.890031-0.259291-0.618230$

H $-5.7096810 .872046 \quad 0.737631$

SCF Energy (B3LYP/6-31G**//MMFF) $=-810.719052245$

F2-13_c02799

MMFF Geometry

C $-3.3357012 .187873-0.318158$

C $-4.418108 \quad 2.727997-0.889213$

C $-2.470858-0.162718-0.196385$

O $-1.0815040 .201211-0.081691$

C $-0.297634-0.9950730 .056633$

C $-1.264778-2.168942-0.080115$

C $-2.581323-1.5740890 .389484$

C $0.838203-1.019796-0.969634$

C $-2.703143-1.6270951 .913303$

C $1.7495350 .226139-0.918621$

O $1.595362-2.212814-0.777556$

$\begin{array}{llll}\text { O } & 2.714094 & 0.116808 & -1.970833\end{array}$

C $2.495735 \quad 0.4550330 .411018$

C $3.389781 \quad 1.703587 \quad 0.362874$

$\begin{array}{llll}\text { O } & 3.299781 & -0.676059 & 0.736887\end{array}$

C -3.349570 $0.902005 \quad 0.460855$

C 4.0214752 .0106241 .715040

H $-2.3855982 .708464-0.408023$

H $-4.3428153 .662595-1.434386$

H $-5.3906202 .252738-0.825538$

H -2.692038 $-0.211491-1.271895$

H $0.114219-1.003726 \quad 1.072702$

H $-0.942274-3.0482000 .486108$

H -1.360589 -2.469144 -1.131298

H $-3.435038-2.109602-0.040836$

H $\quad 0.409925-1.077570-1.978577$

H $-3.671159-1.2337652 .239809$

H $-2.634703-2.6614462 .267328$

H $-1.919400-1.0485422 .412695$

H $1.148820 \quad 1.117796-1.136057$

H $2.330802-2.195438-1.413470$

H $2.2271210 .056271-2.810329$

H $1.7670170 .576817 \quad 1.219340$

H 2.8005542 .5702060 .041938

H $4.1997531 .564165-0.362950$

H $3.919232-0.8177910 .000715$

H $-4.3760580 .527490 \quad 0.552356$

H -2.984617 1.1290101 .469612

H 3.2534982 .1696852 .478767

H 4.6727601 .1953492 .045321

H 4.6276682 .9196951 .649715

SCF Energy (B3LYP/6-31G*//MMFF) $=-810.711795292$

F2-13 c02800

MMFF Geometry

C $4.1178331 .897088 \quad 0.216886$

C $4.2582342 .785223-0.773675$
C $2.596025-0.0676880 .540605$

$\begin{array}{llll}\text { O } & 1.527790 & 0.426710 & -0.290961\end{array}$

C $0.480005-0.563799-0.343009$

C $0.893446-1.6766410 .615011$

C $2.409728-1.5867010 .602232$

C $-0.8681390 .108867-0.036023$

C $3.003167-2.354902-0.579745$

C $-2.062900-0.854690-0.173083$

O $-0.8411090 .652896 \quad 1.281845$

O $-2.076321-1.372327-1.502927$

C $-3.446083-0.2446350 .142356$

C $-3.7722691 .017436-0.668391$

$\begin{array}{llll}\text { O } & -3.554030 & 0.033402 & 1.535998\end{array}$

C 3.9424670 .4190150 .004609

C $-5.2089831 .478823-0.454964$

H $4.1403792 .254041 \quad 1.243252$

H $4.3860283 .839192-0.552409$

H $4.2467502 .481293-1.814549$

H 2.4247150 .3457331 .544367

H $0.473338-0.943132-1.371569$

H $0.502710-2.6535280 .313799$

H $\quad 0.531619-1.4772181 .630915$

H $2.839031-1.994633 \quad 1.524223$

H $-0.9834600 .949861-0.730101$

H $2.672911-1.954036-1.543387$

H $4.097019-2.318159-0.557231$

H $2.710937-3.409721-0.536621$

H $-1.909989-1.6953520 .515533$

H $-0.074503 \quad 1.2491591 .334164$

H -2.788537 -2.032349 -1.554703

H $-4.208765-0.999556-0.085761$

H -3.615321 $0.831877-1.736696$

H -3.113294 $1.840874-0.370568$

H -2.9007770 .7199291 .753952$

H $4.758525-0.0953140 .525348$

H $4.0297040 .188850-1.064112$

H -5.3931991 .7367730 .592632$

H $-5.4131252 .367191-1.060998$

H $-5.9185200 .698391-0.747969$

SCF Energy (B3LYP/6-31G**//MMFF) = -810.719117182

F2-13 c02801

MMFF Geometry

C $4.983845-0.595921-0.558834$

C $6.0486870 .115378-0.172389$

C $2.482963-0.452399-0.273453$

O $1.400853-0.9248990 .553410$

C $0.3094710 .016204 \quad 0.466930$

C $0.7334491 .074170-0.548528$

C $2.2505641 .050646-0.448367$

C $-0.986555-0.739094 \quad 0.135176$

C $2.7348901 .917523 \quad 0.715220$

$\begin{array}{llll}C & -2.227247 & 0.173751 & 0.120004\end{array}$

O $-0.865152-1.372536-1.138199$

O $-2.356487 \quad 0.821003 \quad 1.384512$

C $-3.533145-0.575323-0.220451$

C $-4.8037320 .281059-0.122104$

O $-3.692755-1.6944110 .655045$

C $3.813573-0.9070050 .325582$

C $-4.8198101 .462480-1.077703$

H $4.963371-0.991693-1.571124$

H $6.8667190 .298165-0.860542$

H 6.1242670 .5249770 .828830

H $2.350142-0.944322-1.247645$

H 0.2223290 .4701131 .461146

H $0.2882362 .052390-0.342172$

H $\quad 0.4373240 .787287-1.564883$

H $2.7139381 .418652-1.370547$

H $-1.112919-1.5352520 .879202$

H 2.3842602 .9484740 .596479

H 2.3782821 .5510641 .683272

H $3.827381 \quad 1.9501740 .752087$

H $-2.0828030 .957346-0.631963$

H $-0.076253-1.940440-1.105419$

H -2.4147300 .1323172 .068104$

H -3.460714 $-0.983549-1.235006$

H -4.9475090 .6355120 .905943$

H $-5.672080-0.357223-0.330705$ 
H $-3.824302-1.3533281 .555787$

H $3.942472-0.4901441 .331853$

H $3.776678-1.9941270 .474539$

H -5.789758 $1.968235-1.032983$

H $-4.0519062 .197565-0.819649$

H $-4.6558151 .135734-2.109233$

SCF Energy (B3LYP/6-31G**//MMFF) $=-810.716231769$

F2-13 c02802

MMFF Geometry

C $-4.424553-1.453244 \quad 0.217357$

C $-4.332053-2.655037-0.362797$

C -2.7614940 .3884090 .610045$

O $-1.653305-0.5272790 .708157$

C $-0.631400-0.142733-0.238341$

C $-1.0368991 .221238-0.795063$

C $-2.116421 \quad 1.6962600 .158543$

C $0.727835-0.1928560 .483216$

C $-3.0424702 .741566-0.437788$

C $1.925542-0.028488-0.471412$

O $\quad 0.786134 \quad 0.823597 \quad 1.481808$

O $1.898255-1.078749-1.437978$

C $3.295313-0.0133870 .240500$

C $4.4560010 .112488-0.756394$

O $3.475646-1.2177950 .983897$

C $-3.813897-0.198712-0.343592$

C $5.800758 \quad 0.266189-0.056414$

H $-4.980419-1.3644451 .147249$

H $-4.799565-3.5218320 .091339$

H -3.791495 -2.796545 -1.292149

H $-3.193414 \quad 0.506027 \quad 1.611007$

H -0.646844 -0.890585 -1.039400

H -1.442723 $1.090743-1.806065$

H $-0.2105331 .933107-0.877286$

H -1.619509 2.1429101 .030738

H $0.796396-1.1581400 .999872$

H $-2.4877803 .660260-0.656886$

H $-3.4957182 .402929-1.374826$

H -3.8459252 .9914970 .262314$

H $1.8306190 .916108-1.018683$

H 0.0388890 .6776692 .087019

H $2.045173-1.915903-0.964973$

H 3.3389310 .8250420 .945799

H $4.2948980 .979164-1.407649$

H $4.508420-0.778890-1.392970$

H $2.884441-1.1797691 .754617$

H -4.626816 $0.519203-0.498834$

H -3.369194 -0.408214 -1.323837

H $6.033416-0.6092460 .557748$

H $6.5996120 .379491-0.796253$

H 5.8077381 .1510590 .587874

SCF Energy (B3LYP/6-31G**//MMFF) $=-810.720479593$

\section{F2-13 c02803}

MMFF Geometry

C 2.9921922 .5524650 .392141

C $2.4484683 .545695-0.319920$

C 2.4130100 .1207380 .649865

$\begin{array}{lllll}\text { O } & 1.009519 & 0.367033 & 0.423543\end{array}$

C $0.484988-0.610620-0.490969$

C $1.659684-1.484617-0.923793$

C $2.627122-1.335620 \quad 0.233057$

C $-0.638150-1.4014700 .197470$

C $4.048942-1.748923-0.102411$

C $-1.745316-0.5108160 .804308$

O $-1.191369-2.317031-0.746851$

O $-2.691306-1.3598181 .462926$

C $-2.5011810 .387751-0.194889$

C $-3.592221 \quad 1.223308 \quad 0.491911$

O $-3.099842-0.397452-1.223187$

C $3.2208581 .163629-0.138109$

C $-4.2480032 .210709-0.465508$

H 3.2997422 .7521291 .415534

H $2.3141864 .527707 \quad 0.120263$

H $2.1276903 .399269-1.345388$

H 2.6075840 .2361651 .722874

H $0.088151-0.093200-1.371760$

H $2.098832-1.077353-1.842985$
H $1.389558-2.525281-1.126538$

H $2.272678-1.9752351 .053327$

H $-0.213809-2.0063151 .008878$

H $4.432568-1.224853-0.983632$

H $4.719916-1.5458270 .738371$

H $4.092307-2.822038-0.316901$

H -1.3063950 .1280801 .580279$

H -1.920766 -2.784371 -0.304890

H -2.211364 -1.860485 2.144648

H -1.791856 $1.067271-0.679216$

H -3.1609191 .7780961 .332985$

H -4.3771700 .5700040 .891110$

H $-3.695655-1.036169-0.795404$

H $4.2932440 .955512-0.055670$

H $2.9579931 .124452-1.202119$

H -4.9985442 .8064960 .063731$

H $-3.5071652 .896929-0.888155$

$\mathrm{H}-4.7491901 .693782-1.289710$

SCF Energy $\left(B 3 L Y P / 6-31 G^{* *} / / M M F F\right)=-810.710690917$

F2-13 c02804

MMFF Geometry

C -2.1101662 .9257110 .466448$

C -1.120287 $3.360565-0.321260$

C $-2.0659370 .461729-0.348400$

$\begin{array}{llll}\text { O } & -0.755659 & 0.123010 & 0.147660\end{array}$

C $-0.534937-1.285713-0.022279$

C $-1.717921-1.802515-0.838060$

C $-2.837989-0.859844-0.436254$

C $0.830957-1.568199-0.665576$

C $-3.504212-1.3086630 .865549$

C $2.052703-1.151714 \quad 0.178744$

O $0.953621-2.979379-0.884374$

O $1.939296-1.7345841 .485123$

C 2.2851970 .3555540 .374459

C $2.3496641 .143615-0.937609$

O $3.541414 \quad 0.5165381 .047462$

C $-2.7005321 .541140 \quad 0.534095$

C $2.6918432 .613165-0.721214$

H -2.5578583 .6491611 .145755$

H $-0.7936364 .393639-0.263972$

H $-0.6150252 .718668-1.031835$

H $-1.9296930 .842168-1.369335$

H -0.575855 -1.744845 0.972956

H -1.933702 -2.858377 -0.648093

H $-1.520997-1.688817-1.911847$

H -3.609858 -0.807892 -1.212473

H $\quad 0.894745-1.087074-1.647366$

H -4.340016 -0.649761 1.121766

H -3.908132 -2.321348 0.759626

H $-2.807774-1.3117361 .710009$

H $2.957747-1.593632-0.258527$

H $0.316351-3.229925-1.574086$

H $1.827874-2.6933061 .364362$

H 1.5273810 .7844041 .038902

H $1.3914591 .087287-1.463536$

H $3.1135670 .709665-1.594032$

H 3.4893950 .0149471 .879235

H -3.7628601 .6331960 .277276$

H -2.6389011 .2304761 .584589$

H $1.9842123 .084327-0.032506$

H $3.7001472 .732443-0.312794$

H $2.6517713 .152667-1.672897$

SCF Energy (B3LYP/6-31G**//MMFF) $=-810.714188972$

F2-13 c02805

MMFF Geometry

C $4.364228 \quad 1.417889-0.430773$

C 3.9739172 .3580130 .437753

C $2.519702-0.3028620 .167387$

O $1.3913840 .503702-0.220225$

C $0.183628-0.278216-0.084098$

C $0.609211-1.6096690 .526106$

C $2.046498-1.7554230 .055429$

C -0.8466630 .5460120 .702119$

C $2.114104-2.348333-1.352853$

C $-2.260294-0.0566370 .785687$

$\begin{array}{llll}\text { O } & -0.381590 & 0.747462 & 2.041749\end{array}$ 
O $-2.205141-1.3235721 .453441$

C $-2.955161-0.284140-0.566832$

C $-3.0113950 .937043-1.494323$

O $-4.293907-0.718043-0.297223$

C $3.7448720 .065343-0.674227$

C $-3.7348622 .138265-0.898232$

H $5.2346701 .640811-1.045512$

H 4.5190583 .2937110 .506544

H 3.1201042 .2312671 .092004

H $2.716330-0.0891321 .226594$

H $-0.180317-0.438948-1.104798$

H $-0.039909-2.4364700 .222268$

H $0.598040-1.5651721 .621646$

H $2.619735-2.4047770 .726634$

H -0.9058491 .5478220 .262061$

H $3.153768-2.488230-1.666085$

H $1.630500-3.330761-1.378272$

H $1.622004-1.712532-2.095648$

H -2.889866 0.5753831 .424122

H $\quad 0.503560 \quad 1.1465691 .983705$

H -1.793976 -1.173579 2.322112

H -2.469158 -1.115118 -1.091797

H $-3.5427930 .654942-2.411985$

H -2.001554 $1.233847-1.796603$

H $-4.715255-0.916233-1.150891$

H $4.528805-0.683220-0.506678$

H $3.4788120 .034273-1.738124$

H $-3.7791592 .949252-1.632346$

H $-4.7618431 .885840-0.616626$

H $-3.2183802 .520353-0.013113$

SCF Energy $\left(B 3 L Y P / 6-31 G^{* *} / / M M F F\right)=-810.709313103$

F2-13_c02806

MMFF Geometry

C $2.6191412 .392564-0.611900$

C $2.4536002 .858998-1.854670$

C 1.8149450 .3175780 .553151

O $\quad 0.622770 \quad 0.257253-0.257250$

C $0.344248-1.100746-0.629205$

C $1.549732-1.921171-0.175614$

C $2.066045-1.1223641 .003603$

C $-0.981440-1.5613920 .007461$

C $3.486325-1.477270 \quad 1.406575$

C $-2.232594-0.766566-0.424385$

O $-1.188191-2.929607-0.367691$

O $-2.309015-0.762078-1.857241$

C $-2.3238720 .698010 \quad 0.037190$

C $-2.1482340 .884588 \quad 1.547507$

O $-3.6262931 .184688-0.315848$

C $2.9311710 .957761-0.285411$

C -2.2647932 .3450061 .968318$

H $2.5329313 .085780 \quad 0.220919$

H $2.2333823 .907466-2.023068$

H $2.5315822 .209746-2.719597$

H $1.5992050 .948847 \quad 1.423741$

H $0.267433-1.157538-1.721235$

H $2.298995-1.940951-0.976902$

H $1.317012-2.9593800 .078081$

H $1.411358-1.3249941 .862618$

H $-0.898757-1.5440491 .098896$

H $3.531031-2.5086841 .772456$

H $4.183773-1.3991120 .566440$

H $3.837222-0.8200952 .208536$

H -3.131879 -1.297958 -0.086960

H -1.977503 -3.246762 0.103437

H $-2.278080-1.688697-2.151463$

H -1.610838 $1.323641-0.510196$

H -1.170144 $0.516770 \quad 1.872044$

H -2.9141170 .3109412 .083328$

H -3.727477 $1.067506-1.276147$

H 3.8767750 .9475110 .267426

H $3.0830740 .389734-1.211166$

H -1.519284 2.9598461 .454180

H -3.257850 2.7482991 .747174

H -2.097655 2.4401053 .045996

SCF Energy (B3LYP/6-31G**//MMFF) $=-810.715054335$

F2-13_c02807
MMFF Geometry

C $-4.8463910 .042640-0.466387$

C -5.7279830 .8301670 .159529$

C $-2.350466-0.310156-0.423509$

O $-1.1308980 .093244-1.076062$

C $-0.030392-0.083815-0.154633$

C $-0.626218-0.7167981 .100168$

C $-2.066384-0.2286871 .077788$

C $1.085120-0.867442-0.861260$

C $-2.178617 \quad 1.1728221 .680670$

C $2.409563-0.983177-0.084249$

O $0.609443-2.186892-1.152042$

O $2.192663-1.7048201 .134046$

C $3.0763150 .351152 \quad 0.291344$

C $3.3114101 .279999-0.904447$

O 4.3492190 .0564190 .879360

C $-3.5159210 .508560-0.977530$

C $4.0035892 .577946-0.504807$

H $-5.108333-0.998974-0.634356$

H -6.6778670 .4311850 .498163$

H -5.5209971 .8784690 .344306$

H -2.500391 -1.364301-0.696933

H $\quad 0.3247520 .9267540 .075502$

H $-0.080515-0.4401202 .007444$

H -0.622228 -1.811132 1.033013

H $-2.721152-0.9018201 .642349$

H $1.269777-0.403651-1.837086$

H -3.2212821 .5003141 .720169$

H -1.798271 1.1791562 .707898

H -1.615341 1.9176511 .109428

H $3.117450-1.591749-0.660967$

H $1.301066-2.642190-1.661868$

H $1.790578-2.5581420 .896738$

H 2.4913590 .8676391 .061370

H $2.3617651 .535211-1.385269$

H $3.9367710 .773931-1.649666$

H $4.188267-0.5324111 .636671$

H $-3.3582431 .576866-0.785622$

H $-3.5184640 .417390-2.071535$

H $4.1170633 .227805-1.378407$

H $3.417763 \quad 3.1180770 .245815$

H $5.0012852 .391158-0.095632$

SCF Energy $\left(B 3 L Y P / 6-31 G^{* *} / / M M F F\right)=-810.714943939$

F2-13_c02808

MMFF Geometry

C 3.4220252 .2486530 .467469

C $3.3101693 .197734-0.468573$

C $2.465400-0.0586610 .661627$

O $1.3011810 .194633-0.147632$

C $0.534589-1.015350-0.257623$

C $1.232196-2.0490480 .624806$

C $2.675197-1.5749110 .625164$

C $-0.937808-0.7798610 .119665$

C $3.429433-2.088805-0.602196$

C $-1.6508800 .215350-0.827639$

O $-1.590951-2.0459350 .096970$

O $-1.0396791 .502582-0.755338$

C $-3.1607510 .411085-0.569104$

C $-3.4870370 .864686 \quad 0.861013$

O $-3.872669-0.791414-0.846995$

C 3.6362640 .7909640 .167872

C $-4.961743 \quad 1.2114851 .029725$

H 3.3644552 .5394331 .513271

H $3.1603024 .233769-0.185180$

H $3.3634722 .961679-1.525626$

H $2.2007560 .233504 \quad 1.687495$

H $0.593282-1.344298-1.302809$

H $1.097310-3.0725430 .261523$

H $0.839633-2.0062321 .648909$

H $3.205818-1.9170501 .520798$

H $-0.964903-0.3851391 .142724$

H $3.417973-3.183743-0.627905$

H $2.993944-1.726245-1.538822$

H $4.477557-1.773798-0.574026$

H -1.540731 $-0.151423-1.856478$

H -2.494932 -1.9208430 .431277$

H $-0.1121651 .407136-1.029913$ 
H $-3.532613 \quad 1.178018-1.260666$

H -2.8866331 .7414891 .128018$

H -3.2510550 .0677121 .575255$

H -3.681592 -1.039591-1.767466

H 4.5610330 .4762030 .665247

H $3.7738700 .654905-0.911620$

H -5.1542551 .5494932 .053090$

H -5.2556652 .0148670 .346836$

H -5.6002370 .3438370 .836796$

SCF Energy (B3LYP/6-31G**//MMFF) $=-810.718523165$

F2-13_c02809

MMFF Geometry

C -4.518012 $1.082024-0.853306$

C $-5.5151871 .461464-0.046559$

C $-2.2509020 .012814-0.584152$

O $-0.8515840 .327082-0.446133$

C $-0.195610-0.770156 \quad 0.213013$

C -1.258877 -1.847763 0.424055

C $-2.547756-1.0435250 .484120$

C $1.012230-1.248095-0.601103$

C $-2.780160-0.4894011 .890860$

C $2.012785-0.121965-0.938799$

O $1.682593-2.2900700 .105866$

O $3.026203-0.673624-1.786396$

C 2.6781110 .5804880 .263050

C $3.6443621 .684831-0.190235$

O $3.413682-0.351279 \quad 1.052042$

C $-3.0649741 .305868-0.558223$

C 4.1997752 .4819590 .983937

H $-4.7667210 .594182-1.792423$

H $-6.5471961 .275810-0.323773$

H $-5.323149 \quad 1.9602230 .896990$

H -2.356717 -0.449447 -1.575906

H $\quad 0.134686-0.413511 \quad 1.195877$

H - $-1.069108-2.4569181 .313228$

H -1.297376 -2.524879 -0.438684

H $-3.411415-1.6577930 .206046$

H $0.673009-1.675929-1.553381$

H -1.9923580 .2054192 .199017$

H $-3.735370 \quad 0.0398601 .951167$

H -2.814850 -1.303803 2.622453

H $1.4949920 .634763-1.539737$

H $1.070733-3.041666 \quad 0.180127$

H $3.510874-1.335822-1.263785$

H $1.909501 \quad 1.0268620 .903619$

H $3.1306812 .372217-0.872194$

H $4.4921401 .248174-0.731654$

H $2.786296-1.0142071 .386543$

H -2.919732 1.8303170 .394052

H $-2.6623081 .988036-1.318559$

H $4.8614423 .274876 \quad 0.621129$

H 3.3922452 .9498611 .556065

H 4.7778441 .8452541 .660808

SCF Energy (B3LYP/6-31G**//MMFF) $=-810.719748118$

F2-13 c02810

MMFF Geometry

C -3.271376 2.5575620 .091418

C $-2.3232273 .124438-0.663860$

C $-2.5409870 .106980-0.331813$

$\begin{array}{lllll}\text { O } & -1.224956 & 0.215386 & 0.240557\end{array}$

C $-0.588675-1.0720350 .198132$

C $-1.567216-2.015751-0.498809$

C $-2.915214-1.374316-0.218054$

C $0.780500-0.991882-0.495407$

C $-3.457024-1.7971111 .148237$

C $1.793758-0.1040040 .262129$

O $1.309074-2.309411-0.629263$

$\begin{array}{lllll}\text { O } & 1.315495 & 1.237414 & 0.333227\end{array}$

C $3.203296-0.114563-0.367482$

C 4.2030580 .8493440 .287356

O $3.1154410 .223463-1.753866$

C $-3.4949261 .089757 \quad 0.352438$

C 4.4724050 .5474461 .752003

H -3.9759813 .2237640 .586148$

H -2.280832 4.204226 -0.762361

H $-1.5757572 .547271-1.194414$
H $-2.4488130 .356419-1.397536$

H $-0.453844-1.4030821 .235473$

H -1.479470 -3.047856 -0.145579

H -1.390246 -2.020678-1.581936

H -3.653078-1.650364 -0.979574

H $\quad 0.631789-0.581030-1.501526$

H -2.800096 -1.489676 1.968309

H $-4.445140-1.3607641 .326603$

H -3.567447 -2.885768 1.196339

H $1.877121-0.4715531 .291397$

H $1.435562-2.6702470 .265024$

H $1.1266911 .536377-0.572285$

H $3.612448-1.130261-0.320907$

H 3.8615631 .8862120 .180235

H $5.1496790 .799819-0.266180$

H $2.804357 \quad 1.142295-1.818272$

H -4.5221980 .8593540 .044873$

H -3.4369830 .9573381 .439874$

H 5.2651871 .2007852 .130464

H $3.583360 \quad 0.7170212 .366381$

H $4.795453-0.4896081 .886497$

SCF Energy (B3LYP/6-31G**//MMFF) $=-810.705228351$

F2-13 c02811

MMFF Geometry

C $-4.449593-0.713686-1.068218$

C $-5.700150-1.055999-0.739023$

C -2.5143800 .1489120 .273399$

O $-1.490028-0.396296-0.580962$

C $-0.1943260 .012246-0.083780$

C $-\begin{array}{llll}-0.459068 & 0.737280 & 1.230883\end{array}$

C -1.8546581 .3034441 .031501$

C $0.717629-1.220696-0.022095$

C $-1.8150042 .630876 \quad 0.272334$

C $2.187832-1.0018720 .386746$

O $0.162811-2.1640090 .905491$

O $2.270319-0.3722881 .664358$

C $3.024355-0.199505-0.625807$

C $4.515377-0.194116-0.254787$

O $2.5701061 .148912-0.699872$

C $-3.7498960 .512523-0.550869$

C $5.3693330 .498429-1.309537$

H -3.901375 -1.348570 -1.759556

H $-6.151080-1.952027-1.151520$

H $-6.292675-0.455404-0.057758$

H $-2.770772-0.6410260 .993164$

H $\quad 0.1876770 .716362-0.828704$

H $\quad 0.296888 \quad 1.4968821 .451807$

H -0.4797740 .0343092 .072141$

H -2.3541301 .4747961 .991794$

H $\quad 0.699047-1.719817-0.998907$

H -1.394610 $2.522881-0.732532$

H -2.8209153 .0512210 .172137$

H -1.2072293 .3637780 .813764$

H $2.649855-1.9906260 .506260$

H $-0.753270-2.3374660 .628268$

H $1.774742-0.9260222 .291840$

H $2.903522-0.641889-1.621363$

H $4.871564-1.223358-0.131391$

H 4.6670110 .3260640 .698488

H 2.6292821 .5287010 .193662

H -4.4378131 .1091640 .060130$

H $-3.4649201 .116107-1.420804$

H $5.2592160 .011357-2.283593$

H $5.0936161 .552006-1.417558$

H $6.4257780 .455003-1.026364$

SCF Energy $\left(B 3 L Y P / 6-31 G^{* *} / / M M F F\right)=-810.721700934$

$\mathrm{F} 2-13$ c02812

MMFF Geometry

C $-3.6459782 .157254-0.288324$

C -3.5891293 .0765710 .681763$

C $-2.3910510 .017982-0.692475$

O $-1.2108900 .514844-0.027411$

C $-0.654243-0.5072080 .815855$

C $-1.636388-1.6755860 .782734$

C $-2.307294-1.503941-0.564838$

C $0.762618-0.8791570 .329492$ 
C $-3.607739-2.275596-0.702420$

C $1.742462 \quad 0.317676 \quad 0.365915$

$\begin{array}{llll}\text { O } & 1.248074 & -1.925196 & 1.167967\end{array}$

O $1.3566841 .287442-0.608280$

C $3.210557-0.0755840 .099081$

C $4.146724 \quad 1.1407830 .049919$

O $3.300571-0.787490-1.134607$

C -3.620311 $0.673268-0.044516$

C $5.6093100 .733636-0.079453$

H -3.724452 2.491707-1.319505

H -3.615959 4.1332910 .439353

H -3.5133452 .7956831 .726303$

H -2.331089 $0.308585-1.748271$

H $-0.590803-0.1191001 .839621$

H -2.369882 -1.557877 1.590187

H $-1.171190-2.6576440 .907442$

H $-1.614543-1.868558-1.335884$

H $0.691456-1.254588-0.699126$

H $-4.312520-2.0404620 .101410$

H -4.090271-2.054173 -1.659762

H $-3.416130-3.353282-0.662914$

H 1.6989920 .7979091 .351478

H $1.993414-2.3479150 .708674$

H $0.4689591 .605980-0.372788$

H $3.554755-0.7529730 .888532$

H $4.023879 \quad 1.7403450 .959121$

H $3.8994281 .778767-0.806769$

H $2.967677-0.201625-1.836098$

H $-4.5408710 .257488-0.468564$

H -3.6333760 .4732931 .033719$

H $5.7891290 .183687-1.008395$

H $6.2483891 .622279-0.085764$

H $\begin{array}{lll}5.915616 & 0.101803 & 0.760256\end{array}$

SCF Energy (B3LYP/6-31G**//MMFF) $=-810.722584631$

F2-13 c02813

MMFF Geometry

C $4.513595-1.337183-0.681203$

C $5.673014-1.311741-0.014665$

C $2.248449-0.253902-0.454129$

$\begin{array}{llll}\text { O } & 0.933822 & -0.466214 & 0.095684\end{array}$

C $0.312824 \quad 0.812030 \quad 0.312823$

C $1.295987 \quad 1.864219-0.199027$

C $2.6409751 .170764-0.053652$

C $-1.0661140 .884931-0.362829$

C 3.1776811 .3142251 .371601

C $-2.078940-0.134998 \quad 0.206692$

O $-1.5792872 .206746-0.209511$

O $-1.653803-1.463826-0.090588$

C $-3.5074320 .057530-0.345059$

C $-4.509860-1.0168940 .101862$

O $-3.4553090 .041576-1.773920$

C $3.166717-1.397431-0.024858$

C $-4.704382-1.0840271 .607566$

H $4.539016-1.330344-1.767884$

H $6.614690-1.275950-0.551420$

H $5.704857-1.3264761 .069167$

H $2.125771-0.292268-1.545989$

H $\quad 0.193736 \quad 0.935198 \quad 1.396665$

H 1.2186852 .8111840 .344306

H $1.1132252 .076246-1.260332$

H $3.3793851 .588832-0.746538$

H $-0.9347110 .690620-1.434456$

H $2.525200 \quad 0.8388822 .110983$

H 4.1707290 .8655851 .464015

H 3.2742592 .3720481 .639563

H -2.115153 -0.0304911 .297499$

H -1.6949032 .3712150 .741982$

H $-0.789161-1.6000320 .332054$

H -3.882050 $1.043804-0.046701$

H $-4.209574-2.000881-0.278649$

H -5.479221 $-0.810599-0.370077$

H $-4.3518020 .224672-2.103067$

H $3.248508-1.4323131 .068231$

H $2.693181-2.348732-0.301254$

H -3.797571 -1.426931 2.114250

H -4.980211 -0.1052752 .012133$

H -5.505355 -1.789392 1.851469
SCF Energy (B3LYP/6-31G**//MMFF) $=-810.712845823$

F2-13_c02814

MMFF Geometry

C $-4.2608741 .794810-0.011761$

C -4.5002332 .4797951 .111928$

C $-2.6110830 .001353-0.598484$

$\begin{array}{lllll}\text { O } & -1.612097 & 0.390268 & 0.365203\end{array}$

C $-0.512391-0.5412710 .297729$

C $-0.814294-1.480077-0.866165$

C $-2.332458-1.469625-0.921266$

C $0.808830 \quad 0.237270 \quad 0.191742$

C $-2.932446-2.4692440 .068602$

C $2.055306-0.6664710 .168585$

O $0.782953 \quad 1.026552-0.998266$

O $1.929472-1.673993 \quad 1.179515$

C 3.3978380 .0563380 .417941

C $3.6893441 .230376-0.520963$

O $4.445457-0.908759 \quad 0.257575$

C $-4.0066540 .313574-0.057859$

C $5.071716 \quad 1.833336-0.295565$

H $-4.2572632 .330889-0.957369$

H -4.6809993 .5486151 .075793$

H $-4.518972 \quad 1.9929382 .080712$

H -2.420502 $0.598575-1.501028$

H $-0.535378-1.0996241 .240908$

H $-0.379712-2.474387-0.724825$

H $-0.419617-1.080448-1.808120$

H $-2.694749-1.723600-1.923882$

H 0.8704560 .9313621 .039000

H $-2.671406-2.2346631 .105519$

H $-4.024443-2.484183-0.008254$

H $-2.576019-3.482325-0.146592$

H $2.116578-1.192877-0.791796$

H $-0.0066581 .592524-0.954153$

H $1.861108-1.2239552 .039130$

H $3.4470780 .400588 \quad 1.458257$

H $2.9505562 .024457-0.374885$

H $3.6276070 .897511-1.563892$

H $4.256070-1.6456240 .863634$

H $-4.766523-0.138500-0.705842$

H $-4.127384-0.1111020 .946046$

H $5.8651261 .113896-0.520062$

H $5.2140002 .700782-0.948161$

H 5.1891672 .1656250 .740851

SCF Energy (B3LYP/6-31G**//MMFF) $=-810.717267732$

F2-13_c02815

MMFF Geometry

C -2.837260 $2.479159-0.191693$

$\begin{array}{llll}\text { C }-2.953722 & 3.220279 & 0.915714\end{array}$

C $-2.086690 \quad 0.122473-0.588177$

\begin{tabular}{lllll}
\hline & -1.233578 & 0.035327 & 0.569627
\end{tabular}

C $-0.640810-1.2764720 .607165$

C -1.120122 -2.014060 -0.637955

C $-2.435900-1.322464-0.955004$

C $0.884431-1.1838150 .787859$

C $-3.588676-1.925922-0.150877$

C $1.595091-0.087099-0.038525$

O $1.132321-0.9257882 .175366$

O $1.408568-0.320403-1.430538$

C $3.106966 \quad 0.013798 \quad 0.261211$

C $3.8596121 .022664-0.618961$

O $3.700048-1.2714470 .058698$

C -3.271006 $1.043133-0.294205$

C $3.3652092 .452130-0.471715$

H $-2.4120002 .935863-1.081717$

H $-2.6245804 .253605 \quad 0.922247$

H $-3.3739502 .814102 \quad 1.829024$

H $-1.4757830 .550344-1.395208$

H $-1.067691-1.767737 \quad 1.491245$

H -1.214061 -3.091478 -0.467859

H $-0.433746-1.883294-1.478973$

H -2.681692 -1.406090 -2.019602

H $1.313836-2.1677890 .567352$

H $-3.689720-2.994511-0.369032$

H -3.443609 -1.8157660 .928562$

H $-4.537849-1.447743-0.413561$ 
H $1.141488 \quad 0.882588 \quad 0.194010$ H $0.719496-0.0723232 .393339$

H $1.867207-1.149035-1.652268$

H $3.253577 \quad 0.2759901 .315552$

H $3.8124160 .719537-1.672088$

H $4.9253030 .989351-0.358350$

H $4.636231-1.2064330 .313827$

H $-4.0088350 .970111-1.101491$

H $-3.7635880 .740932 \quad 0.637883$

H $4.0102463 .132446-1.036927$

H $2.3477852 .566702-0.856864$

H 3.3779352 .7662930 .576651

SCF Energy (B3LYP/6-31G**//MMFF) $=-810.714695190$

F2-13_c02816

MMFF Geometry

C $4.2264881 .929059-0.424278$

C $3.4348832 .948240-0.070522$

C $2.631815 \quad 0.044616 \quad 0.367194$

O $1.5402820 .349976-0.521823$

C $0.508782-0.645402-0.354323$

C $0.971138-1.5569630 .777988$

C $2.484357-1.4424530 .707095$

C $-0.840352 \quad 0.056371-0.129961$

C $3.066510-2.401201-0.332893$

C $-2.027556-0.920703-0.009291$

O $-0.7653300 .858048 \quad 1.048058$

O $-2.136790-1.696936-1.201349$

C $-3.400076-0.2620030 .248081$

C $-3.7772730 .822686-0.771049$

$\begin{array}{llll}\text { O } & -3.447269 & 0.276307 & 1.566972\end{array}$

C $3.9616680 .452827-0.272075$

C $-5.2085591 .311078-0.582718$

H $5.1833172 .178706-0.879476$

H $3.7568283 .970409-0.241345$

H 2.4644762 .8029480 .388070

H $2.466497 \quad 0.622316 \quad 1.286539$

H $0.479335-1.206402-1.295284$

H $0.596033-2.5794420 .670549$

H $\quad 0.628883-1.1825401 .750315$

H $2.945044-1.6683001 .675474$

H $-1.0015180 .737758-0.973791$

H $2.799502-3.435823-0.091720$

H $2.704096-2.187681-1.343553$

H $4.159469-2.341310-0.347784$

H $-1.841833-1.605903 \quad 0.827808$

H -0.0129591 .4647090 .939195$

H - $-1.402214-2.333271-1.209499$

H $-4.163423-1.0489590 .202601$

H -3.665950 $0.439118-1.791256$

H $-3.1156381 .689945-0.664665$

H -2.7915540 .9922941 .619585$

H 4.7838700 .0373610 .323376

H $4.0299050 .021121-1.278247$

H $-5.4501192 .068426-1.335340$

H $-5.9209480 .486942-0.690748$

H $-5.349196 \quad 1.761507 \quad 0.404837$

SCF Energy (B3LYP/6-31G**/MMFF) $=-810.716780439$

F2-13 c02817

MMFF Geometry

C $3.448358 \quad 2.2212810 .166605$

C $2.8415233 .376940-0.126502$

C $2.595903-0.1233880 .458988$

$\begin{array}{lllll}\text { O } & 1.313273 & 0.319009 & 0.940230\end{array}$

$\begin{array}{llll}\text { C } & 0.271957 & -0.231469 & 0.098179\end{array}$

C $0.936718-1.302288-0.766900$

C $2.300401-1.498316-0.130942$

C $-0.871203-0.6886741 .019913$

C $3.340991-2.069800-1.077577$

C $-2.221276-0.9585710 .330613$

O $-0.487464-1.8808541 .713198$

O $-2.084153-2.024031-0.615378$

C $-2.8343050 .235583-0.422191$

C $-2.965627 \quad 1.502605 \quad 0.429125$

O $-4.146273-0.145214-0.854881$

C $3.154378 \quad 0.917847-0.523577$

C $-3.615527 \quad 2.651331-0.333269$ $\begin{array}{llll}\text { H } & 4.208047 & 2.213498 & 0.943884\end{array}$

H 3.1018674 .2848620 .406451

H $2.0787873 .438573-0.894850$

H $3.267654-0.2173431 .320519$

H $-0.0730680 .593205-0.534195$

H $1.034841-0.924879-1.792464$

H $\quad 0.381692-2.240803-0.832135$

H $2.179540-2.2015940 .704614$

H -1.0217700 .0649581 .801593$

H $3.062500-3.084467-1.381477$

H $3.440257-1.471708-1.988940$

H $4.320387-2.120288-0.591160$

H $-2.939487-1.3220841 .076921$

H $0.330980-1.6812592 .199267$

H $-1.766920-2.802816-0.126301$

H -2.260216 $0.450268-1.331029$

H -1.9816751 .8369460 .773252$

H -3.5754171 .2918721 .315972$

H $-4.051026-0.949971-1.392877$

H $4.0929220 .560049-0.961134$

H $2.4512931 .084951-1.348234$

H $-3.651936 \quad 3.5467190 .295479$

H -3.045069 2.893591-1.235630

H $-4.6409942 .407934-0.627662$

SCF Energy (B3LYP/6-31G**//MMFF) $=-810.717539543$

F2-13 c02818

MMFF Geometry

C $-4.591290-0.965680-0.069387$

C $-5.640390-1.2140640 .722532$

$\begin{array}{llll}\text { C }-2.462019 & 0.312000 & 0.278166\end{array}$

O $-1.678268-0.443570-0.663798$

C $-0.3325520 .087414-0.694013$

$\begin{array}{llll}C & -0.298919 & 1.206247 & 0.342291\end{array}$

C -1.7485811 .6565810 .416264$

C $0.644134-1.081168-0.499986$

C $-2.0725142 .668346-0.684503$

C $2.112588-0.760771-0.843245$

O $0.547164-1.5933490 .828502$

O $2.805188-2.012506-0.952007$

$\begin{array}{llll}\text { C } 2.895691 & 0.146949 & 0.126421\end{array}$

$\begin{array}{llll}C & 4.338247 & 0.374571 & -0.352414\end{array}$

$\begin{array}{llll}\text { O } & 2.953727 & -0.427786 & 1.429414\end{array}$

C $-3.9195220 .374913-0.179163$

C $5.080253 \quad 1.384948 \quad 0.514271$

H $-4.199972-1.773158-0.682917$

H $-6.083833-2.2034180 .752422$

H $-6.072764-0.4431811 .350797$

H $-2.398699-0.2206581 .237367$

H $-0.1970680 .504544-1.699089$

H 0.3859792 .0129380 .065985

H $\quad 0.009898 \quad 0.830157 \quad 1.324332$

H $-1.9715272 .120213 \quad 1.383682$

H $0.321335-1.906832-1.148155$

H $-1.9363882 .248638-1.686452$

H $-3.1077253 .014676-0.602279$

H -1.426136 $3.548363-0.598195$

H $2.139878-0.313642-1.844066$

H $-0.386241-1.8160660 .986941$

H $2.804412-2.420716-0.068889$

H $2.404703 \quad 1.1190680 .211593$

H $4.3343730 .733489-1.388076$

H $4.899953-0.566785-0.326960$

H $2.041476-0.5222681 .751067$

H -4.4611341 .1180370 .418110$

H $-3.9775800 .682837-1.229937$

H $6.0921201 .541697 \quad 0.127072$

H 4.5654292 .3509180 .517118

H 5.1662721 .0371111 .548245

SCF Energy (B3LYP/6-31G**//MMFF) $=-810.720117292$

F2-13 c02819

MMFF Geometry

C $-4.993039-0.630898 \quad 0.550992$

C $-6.0628380 .091802 \quad 0.201124$

C $-2.495184-0.469343 \quad 0.249214$

O $-1.419579-0.903908-0.605938$

C $-0.3260820 .031029-0.482273$ 
C -0.7489691 .0513010 .572503$

C -2.2664341 .0268230 .479653$

C $0.970415-0.738118-0.180435$

C $-2.7593701 .935133-0.648164$

C $2.2107200 .178215-0.136831$

O $0.820311-1.417141 \quad 1.065879$

O $2.3205330 .831098-1.401295$

C $3.519958-0.5744840 .183089$

C $4.792751 \quad 0.281914 \quad 0.112692$

O $3.666710-1.655152-0.741390$

C $-3.829915-0.905039-0.354652$

C $4.7958931 .453877 \quad 1.080154$

H -4.962573 -1.064735 1.547198

H $-6.875237 \quad 0.2461430 .902784$

H $-6.1482630 .539023-0.782950$

H $-2.351452-0.9983811 .202178$

H $-0.2398040 .522913-1.458449$

H -0.3079282 .0377380 .398281$

H $-0.446563 \quad 0.730773 \quad 1.576667$

H -2.7258331 .3586691 .417410$

H $1.083537-1.504441-0.957340$

H -2.406146 $1.606601-1.630736$

H $-3.8521321 .965309-0.678535$

H $-2.4115612 .962209-0.492395$

H 2.0552090 .9469640 .628855

H $1.513887-2.096074 \quad 1.116778$

H $2.9442281 .569683-1.301835$

H $3.447133-1.0140751 .184643$

H $4.9574740 .640422-0.910582$

H $5.657623-0.3575090 .332459$

H $4.460939-2.155117-0.486838$

H -3.968886 -0.451092 -1.343494

H $-3.791363-1.985747-0.544885$

H 4.0330852 .1931290 .819333

H $4.616327 \quad 1.1165162 .105870$

H 5.7670651 .9586401 .055130

SCF Energy (B3LYP/6-31G**//MMFF) $=-810.708092964$

$\mathrm{F} 2-13$ c02820

MMFF Geometry

C $-3.6001271 .834866-0.516330$

C $-3.2830953 .053233-0.063804$

C $-2.437598-0.380224-0.371631$

O $-1.1065460 .160406-0.263767$

C $-0.176124-0.921086-0.098358$

C $-0.980897-2.211863-0.235764$

C $-2.366874-1.7907550 .222171$

C $0.970884-0.809183-1.104257$

C -2.492153 -1.853172 1.745354

C $1.7035520 .550808-1.109596$

O $1.881572-1.882233-0.874719$

O $2.6952840 .491344-2.141024$

C $2.371048 \quad 1.0345210 .195341$

C 3.3734220 .0523520 .812085

O 1.3832621 .3579551 .170172

C -3.4391950 .5715640 .282846$

C 4.1607580 .6819961 .956188

H -4.008635 $1.741619-1.519323$

H -3.427653 $3.926720-0.690286$

H $-2.875378 \quad 3.2013650 .929942$

H $-2.652311-0.464263-1.446171$

H $\quad 0.214947-0.8719490 .924318$

H $-0.551110-3.039241 \quad 0.337141$

H $-1.029642-2.526352-1.286110$

H -3.140657 -2.434865 -0.210448

H $0.569542-0.959247-2.115419$

H $-1.793503-1.1763492 .247515$

H -3.505505 -1.587036 2.062964

H $-2.293341-2.8685472 .104756$

H $0.9756321 .311213-1.420119$

H $2.600871-1.795359-1.523573$

H $3.0926381 .375473-2.218401$

H $2.9112811 .963698-0.027652$

H $4.080130-0.2984940 .052091$

H $2.848398-0.8184801 .219665$

H 0.7728602 .0006610 .770540

H -4.4209390 .0889990 .353297$

H $\quad-3.114967 \quad 0.820531 \quad 1.300511$
H 3.4992870 .9973562 .769166

H $4.874558-0.0407852 .364165$

H 4.7233201 .5552071 .610654

SCF Energy $($ B3LYP/6-31G $* * / / M M F F)=-810.713581801$

F2-13 c02821

MMFF Geometry

C $-4.860431-1.1785720 .087511$

C $-6.019881-0.539994-0.105712$

C $-2.411321-0.5817400 .041689$

O $-1.256510-0.570025-0.820645$

C $-0.3284360 .423695-0.334503$

C -0.9360170 .9905150 .946626$

C -2.4275490 .7829870 .735703$

C $1.064858-0.208709-0.184849$

C $-3.0251471 .920804-0.094080$

C 2.1431290 .8166070 .224449

O $0.986639-1.2611820 .774883$

O $2.1641941 .898433-0.705587$

C 3.5755720 .2586720 .373759

C $4.105020-0.459044-0.875681$

O $3.635965-0.642127 \quad 1.477178$

C $-3.642731-0.988986-0.766751$

C $5.570979-0.852836-0.732147$

H $-4.791151-1.8938670 .902928$

H $-6.866350-0.7287350 .545613$

H $-6.1458240 .176554-0.909704$

H -2.213334 -1.357401 0.795236

H $-0.2941971 .203802-1.104006$

H -0.6508822 .0334061 .116933$

H -0.6153910 .4164931 .824253$

H -2.959705 $0.735141 \quad 1.692186$

H $1.317022-0.667426-1.148107$

H -2.596633 $1.970903-1.100069$

H -4.107682 $1.804759-0.197095$

H -2.8482152 .8850040 .394708$

H 1.8616961 .2359671 .198390

H $1.848897-1.7094330 .794039$

H $2.2585441 .527111-1.598897$

H 4.2502241 .0952980 .596039

H $4.0009310 .180727-1.758225$

H $3.533232-1.376264-1.056687$

H $3.344479-0.1578202 .268408$

H -3.822438 -0.272210 -1.577248

H $-3.429343-1.938831-1.274339$

H $5.920573-1.341694-1.647237$

H $6.1975350 .027684-0.558188$

H $5.717430-1.5503580 .098351$

SCF Energy (B3LYP/6-31G**//MMFF) $=-810.713729129$

F2-13 c02822

MMFF Geometry

C $-4.2273681 .646106-0.491015$

C $-4.1450572 .834107 \quad 0.118347$

C $-2.626538-0.276910-0.719071$

O $-1.4689480 .579629-0.668867$

C $-0.578271 \quad 0.1205430 .372961$

C $-1.125880-1.2195290 .861956$

C $-2.108894-1.623962-0.219939$

C $0.8519570 .088254-0.200366$

C $-3.152474-2.6240460 .245153$

C $1.928435-0.1476020 .880784$

O $0.918840-0.922681-1.203440$

O 1.8077750 .8668461 .877253

C $3.386098-0.1747650 .370011$

C $3.8036001 .100852-0.374372$

O $3.589503-1.296349-0.484984$

C $-3.7519530 .354648 \quad 0.115477$

C $5.2922011 .110867-0.702179$

H $-4.6716391 .597927-1.481799$

H $-4.5105823 .729569-0.372244$

H -3.713410 2.9356921 .107825

H -2.942707 $-0.358224-1.765846$

H $-0.633590 \quad 0.8606811 .179268$

H -1.642673 -1.069627 1.818147

H $-0.358092-1.9789681 .034969$

H -1.537423 -2.086294 -1.036390

H $1.021747 \quad 1.051175-0.696838$ 
H -3.694187 -2.272472 1.129071

H -3.880445 -2.821176 -0.548299

H -2.676898 -3.574319 0.510655

H $1.730934-1.1133411 .361276$

H $1.791624-0.862598-1.627252$

H 2.4470500 .6656592 .581682

H $4.053195-0.2975231 .232680$

H $3.569804 \quad 1.9859450 .227533$

H $3.2567001 .183726-1.320431$

H $3.351891-2.0949580 .016146$

H $-4.613323-0.320780 \quad 0.162130$

H -3.4153910 .5291951 .144346$

H $5.894336 \quad 1.0421380 .209337$

H $5.5620740 .276810-1.357502$

H $5.5585052 .040832-1.214637$

SCF Energy (B3LYP/6-31G**//MMFF $)=-810.711487874$

F2-13_c02823

MMFF Geometry

C $-3.7899211 .685430-0.214693$

C $-4.983853 \quad 1.6102130 .383672$

C $-1.7176790 .256548-0.345202$

O -0.4291390 .0609850 .266245$

C $0.003881-1.2859540 .018850$

C $-1.085868-1.936094-0.836657$

C $-2.328855-1.140459-0.471608$

C $1.393410-1.315157-0.635570$

C $-2.967225-1.6819940 .808588$

C $2.529061-0.6519100 .171430$

O $1.774656-2.681271-0.840900$

O $2.592884-1.2508241 .473909$

$\begin{array}{llll}\text { C } & 2.466778 & 0.876698 & 0.350327\end{array}$

C $2.2966441 .649227-0.961647$

O 3.7001701 .2945580 .950501

C -2.4949451 .3110990 .441673$

C $2.2708053 .158186-0.747483$

H -3.743124 $2.055269-1.235770$

H $-5.8834621 .904425-0.145745$

H $-5.0859941 .257097 \quad 1.403868$

H -1.515713 $0.647225-1.352695$

H $0.036680-1.8030020 .985347$

H -1.174842 -3.012045 -0.656817

H $-0.871561-1.796081-1.903988$

H -3.073536 -1.177802 -1.274350

H $\quad 1.350893-0.848607-1.625623$

H $-2.301244-1.5964501 .673180$

H -3.890993 -1.145685 1.043545

H -3.227891 -2.739151 0.688702

H $3.485096-0.910550-0.301444$

H $\quad 1.163738-3.066917-1.491162$

H $1.835611-0.9286641 .991146$

H 1.6781201 .1551231 .057251

H $1.3652411 .362957-1.459895$

H 3.124607 1.413373 -1.641058

H $3.8024980 .795841 \quad 1.779211$

H -2.6442790 .9829091 .477511$

H -1.876350 $2.214873 \quad 0.520013$

H $2.1100693 .670497-1.701458$

H $1.4597613 .442368-0.069385$

H $3.2147073 .520418-0.328499$

SCF Energy $\left(\mathrm{B} 3 \mathrm{LYP} / 6-31 \mathrm{G}^{* *} / / \mathrm{MMFF}\right)=-810.711535138$

F2-13_c02824

MMFF Geometry

C -2.1174392 .6745010 .129933$

C -2.0582563 .2621521 .330185$

C $-1.8388790 .297631-0.605531$

O $-\begin{array}{lll}0.983280 & -0.094428 & 0.487650\end{array}$

C $-0.705591-1.4989820 .378441$

C $-1.298180-1.957436-0.948100$

C $-2.459505-0.995932-1.141851$

C $0.784770-1.8121550 .572314$

C $-3.708422-1.467117-0.395560$

C $1.784045-1.089652-0.358858$

O $1.132333-1.5370421 .932929$

O $3.058033-1.728079-0.179870$

C $1.9735310 .431792-0.177390$

C $3.0241450 .992885-1.148544$
O 2.3935790 .7409561 .148840

C $-2.8197901 .372043-0.137317$

C $3.1072662 .513566-1.089365$

H -1.637358 $3.164116-0.713622$

H -1.5370794 .2050041 .454864$

H -2.521931 2.8172522 .203556

H $-1.1812180 .711753-1.382427$

H -1.263708 -1.969524 1.198087

H -1.589101 -3.012244 -0.932720

H $-0.587601-1.824693-1.770717$

H $-2.715995-0.893415-2.202473$

H $\quad 0.926779-2.8932700 .445074$

H $-4.009182-2.461629-0.742217$

H -3.550868 -1.521645 0.686269

H $-4.548569-0.789528-0.578897$

H $1.503723-1.282505-1.399125$

H $0.559948-2.0805732 .500829$

H $3.343352-1.5510130 .733243$

H $1.0317460 .955296-0.357174$

H $2.7811090 .692489-2.174279$

H $4.0162480 .592468-0.908315$

H $1.6873740 .455831 \quad 1.753118$

H $-3.5766051 .548757-0.910258$

H -3.3388561 .0411250 .770446$

H $3.8444812 .878606-1.811604$

H $2.1413912 .967929-1.332277$

H $3.4104292 .858374-0.095931$

SCF Energy $(\mathrm{B} 3 \mathrm{LYP} / 6-31 \mathrm{G} * * / / \mathrm{MMFF})=-810.714351006$

F2-13_c02825

MMFF Geometry

C -2.372002 2.8430800 .348053

C $-1.2881773 .327084-0.269015$

C $-2.0892190 .389150-0.450694$

O -0.8423020 .1284630 .221180$

C $-0.507831-1.2593180 .079813$

C $-1.545590-1.851413-0.872049$

C $-2.762334-0.976267-0.628129$

C $0.939778-1.447244-0.397784$

C $-3.564191-1.4640860 .579652$

C $2.030194-0.8323020 .505459$

O $1.212605-2.852064-0.475948$

O $1.834853-1.284948 \quad 1.853222$

C 2.1307670 .7039550 .518366

C $2.2346581 .370702-0.859579$

O 3.2939671 .0492261 .282638

C $-2.8979591 .430868 \quad 0.328625$

C $3.4341540 .917525-1.682498$

H -2.9590393 .5425540 .940836$

H -1.026182 $4.374993-0.165638$

H $-0.6435552 .711963-0.883570$

H -1.838399 $0.777173-1.446862$

H $-0.636284-1.7295781 .062322$

H -1.719998 -2.917623 -0.696811

H -1.221754 -1.733633 -1.914050

H $-3.428126-0.971065-1.498583$

H $1.054206-1.048402-1.411123$

H -2.982798 -1.428193 1.506390

H $-4.462399-0.8545140 .721888$

H -3.892426 -2.498232 0.428972

H $3.002361-1.2499480 .215470$

H $\quad 0.649972-3.226083-1.174759$

H $1.072183-0.8095722 .223540$

H $1.283123 \quad 1.131087 \quad 1.064336$

H $2.3239002 .455192-0.717437$

H $1.3173251 .205421-1.433834$

H 3.3321322 .0195031 .336127

H -3.914680 $1.471576-0.080756$

H -2.9753011 .1177841 .377360$

H $3.374787-0.147025-1.926644$

H $3.4707391 .472342-2.625708$

H $4.3737371 .098456-1.151518$

SCF Energy (B3LYP/6-31G**//MMFF) $=-810.701181589$

F2-14 c03089

MMFF Geometry

C $2.8556802 .635791-0.069865$

C $1.9634953 .631449-0.021076$ 
C $2.6006910 .145976-0.225953$

O $1.3347460 .201475-0.911670$

C $0.744637-1.107617-0.938486$

C $1.819433-2.063761-0.428992$

C $2.611503-1.1865940 .519342$

C $-0.544609-1.121140-0.089562$

C $4.003871-1.7173720 .815750$

C $-1.620916-0.133323-0.596950$

O $-1.076556-2.444746-0.089845$

O $-1.170104 \quad 1.210365-0.429959$

C $-2.972273-0.2851040 .133038$

C $-4.0145820 .735582-0.346081$

O $-2.779628-0.132712 \quad 1.539246$

C 2.7280781 .3540560 .703685

C -5.3815580 .4978180 .283363$

H $3.7350102 .753856-0.697712$

H $2.1158224 .534653-0.601729$

H 1.0709723 .5642800 .591144

H $3.3835280 .164966-0.995349$

H $0.495515-1.359350-1.976215$

H $2.453165-2.376339-1.269006$

H $1.429205-2.9690220 .044257$

H $2.058050-1.0960611 .463205$

H $-0.289871-0.8571320 .943744$

H $4.597480-1.820384-0.098843$

H $4.541888-1.0462151 .492520$

H $3.946670-2.7018591 .291710$

H -1.795122 $-0.298524-1.667561$

H -1.308027 -2.671226 -1.006843

H $-0.3645001 .320868-0.962658$

H - $-3.363376-1.295545-0.030743$

H -4.111423 $0.683064-1.436530$

H $-3.6986001 .753163-0.088093$

H -2.3966940 .7488091 .689001$

H $1.855347 \quad 1.4030791 .367179$

H 3.6159281 .2511141 .337643

H -6.104110 $1.225442-0.099561$

H -5.752486 -0.5046390 .047014$

H $-5.342740 \quad 0.6033891 .371968$

SCF Energy (B3LYP/6-31G**//MMFF) $=-810.722782189$

F2-14 c03090

MMFF Geometry

C $4.461443-1.500148 \quad 0.171456$

C $4.357382-2.808515-0.086978$

C 2.7833880 .3597430 .263556

$\begin{array}{lllll}\text { O } & 1.675056 & -0.481261 & 0.628671\end{array}$

C $0.5084490 .346407 \quad 0.827216$

C $0.8902231 .754458 \quad 0.373549$

C $2.1434951 .542184-0.454423$

C $-0.667960-0.3037790 .076426$

C $3.0270722 .775552-0.536106$

C -2.0247720 .3620980 .375980$

O $-0.432268-0.260025-1.329890$

O $-2.2707680 .331364 \quad 1.781078$

C $-3.203708-0.291788-0.376053$

C $-4.5505250 .356872-0.025656$

O $-3.258748-1.686709-0.079776$

C $3.757350-0.428997-0.612761$

C $-5.693315-0.205150-0.861847$

H $5.099488-1.1799550 .991177$

H $4.896318-3.5318890 .515034$

H $3.737263-3.179985-0.895301$

H 3.2712060 .6857111 .192018

H $\quad 0.300020 \quad 0.3462341 .903113$

H $1.1161972 .363817 \quad 1.258175$

H $\quad 0.1041612 .276250-0.179046$

H $\quad 1.8472351 .248587-1.469685$

H $-0.706392-1.3624160 .362570$

H $3.364633 \quad 3.0934770 .456083$

H $3.9140982 .582328-1.147303$

H $2.4816403 .609748-0.989346$

H $-1.991647 \quad 1.4161700 .078299$

H $0.405197-0.725481-1.497086$

H -2.237315 -0.596295 2.069711

H -3.034573 - $0.202249-1.455216$

H -4.493171 $1.439859-0.184670$

H $-4.791343 \quad 0.187961 \quad 1.030548$
H $-3.490874-1.7844550 .859025$

H $3.217514-0.875984-1.457063$

H $4.5195230 .239359-1.028371$

H -5.508089-0.053501-1.930052

H $-5.827759-1.276584-0.683898$

H $-6.6306320 .298727-0.605536$

SCF Energy $(B 3 L Y P / 6-31 G * * / / M M F F)=-810.718535744$

F2-14 c03091

MMFF Geometry

C $2.8396952 .684391-0.060282$

C 2.0815523 .6379660 .492065

C $2.4529290 .241632-0.473073$

$\begin{array}{lllll}\text { O } & 1.021518 & 0.364784 & -0.577641\end{array}$

C $0.433786-0.945167-0.637374$

C $1.593711-1.934675-0.712544$

C $2.707455-1.188969-0.004842$

C $-0.466054-1.1560970 .590700$

C $4.093821-1.712844-0.338777$

C $-1.516922-0.0412480 .791008$

O $-1.098672-2.4295220 .473842$

O $-2.241960-0.3209941 .993314$

C $-2.5246450 .140514-0.361510$

C $-3.5375831 .258290-0.070391$

O $-3.232919-1.072312-0.605339$

C 2.9849441 .2968460 .498046

C $-4.4479791 .533579-1.260793$

H $3.3959422 .918749-0.964292$

H 2.0222334 .6225820 .041101

H 1.5090123 .4561831 .394880

H $2.8643860 .398181-1.478955$

H -0.161941 -1.026477-1.553494

H $1.860602-2.100455-1.764215$

H $1.377466-2.911096-0.269555$

H $2.549737-1.2643521 .078951$

H $0.152765-1.1961561 .495855$

H $4.299523-1.648044-1.412532$

H $4.864964-1.1409480 .186773$

H $4.190314-2.762089-0.040784$

H -1.0005780 .9127370 .953098$

H $-1.689778-2.5313931 .239330$

H -1.599639 -0.351792 2.722856

H -1.982950 $0.388498-1.280495$

H -3.0071612 .1811660 .191120$

H -4.1714370 .9850870 .781608$

H $-3.678353-1.3235870 .221946$

H 2.4525551 .2209201 .454646

H $4.047811 \quad 1.1231890 .700366$

H -3.864575 $1.821876-2.141074$

H $-5.0468510 .653925-1.516704$

H $-5.1360392 .352351-1.027228$

SCF Energy $\left(B 3 L Y P / 6-31 G^{* *} / / M M F F\right)=-810.713452799$

F2-14 c03092

MMFF Geometry

C $2.1502152 .708735-0.671974$

C $1.1845813 .631675-0.600053$

C $2.1664810 .223423-0.345624$

O $0.8060530 .041746-0.778667$

C $0.457609-1.347759-0.671909$

C $1.690974-2.060469-0.113833$

C $2.431354-0.9450310 .597215$

C $-0.792759-1.5154430 .212347$

C $3.902888-1.2450700 .823079$

C $-2.073922-0.825828-0.301065$

O $-1.079577-2.916157 \quad 0.322319$

O $-2.272397-1.217435-1.667466$

C $-2.1024850 .710358-0.234339$

C -1.7849851 .3174291 .137847$

O $-3.4180411 .127469-0.624914$

C 2.3222931 .5919540 .318327

C -2.7193210 .8624832 .252027$

H $2.8654992 .765538-1.488631$

H $1.1173704 .415718-1.346456$

H $0.448578 \quad 3.6222980 .196000$

H $2.8044420 .153844-1.237054$

H $0.254836-1.735091-1.677260$

H $2.293907-2.443039-0.947665$ 
H $1.464980-2.9083160 .539055$ H $1.946656-0.7639951 .565779$ H $-0.578004-1.1677241 .227465$ H $4.021872-2.1426461 .439069$ H 4.427816-1.414533-0.123069 H $4.397250-0.4158261 .338300$ H $-2.937697-1.2322660 .240218$ H -1.303013 -3.239902 -0.567403 H $-3.091727-0.792063-1.974218$ H $-1.4264511 .129331-0.987123$ H $-1.8664302 .409277 \quad 1.065039$ H $-0.7511951 .098677 \quad 1.421925$ H -3.426036 $2.099894-0.633993$ H 1.5943581 .6964541 .131655 H 3.3202051 .6847690 .761424 H -2.634527 -0.2130152 .432948$ H -2.4655321 .3765083 .184859$ H -3.7629971 .0923772 .016693$

SCF Energy (B3LYP/6-31G**//MMFF) $=-810.717245116$

F2-14 c03093

MMFF Geometry

C $4.198247-0.9162450 .795176$

C $4.149085-2253128 \quad 0.789073$

C 2.4636550 .8646830 .418086

O 1.3823480 .0369440 .892416

C $\quad 0.1897630 .309697 \quad 0.128493$

C $0.6547071 .069237-1.104785$

C $1.8554841 .842751-0.591436$

C $-0.555197-1.011574-0.109688$

C 1.4386523 .1537230 .078954

C $-1.936122-0.887243-0.776218$

O $0.242939-1.875924-0.926434$

O $-1.791085-0.331815-2.088885$

C $-2.958299-0.016709-0.025629$

C $-3.176095-0.4399741 .430859$

O $-4.212894-0.122732-0.709279$

C $3.521339-0.045270-0.225563$

C -4.2184780 .4198992 .136230$

H $4.766966-0.4190801 .576672$

H $4.663639-2.8252501 .553446$

H $3.598399-2.7974760 .030036$

H 2.8978731 .3888521 .277617

H -0.4255770 .9632810 .756779$

H $-0.1278591 .707301-1.526116$

H $0.9837750 .381075-1.891921$

H $2.5498132 .089042-1.402489$

H $-0.662793-1.5351040 .847159$

H 2.3201863 .6888520 .448029

H $\quad 0.9265153 .805970-0.636261$

H 0.7685752 .9921290 .929268

H -2.355095 -1.889875 -0.929443

H $1.099043-1.986051-0.478422$

H -1.160460 -0.893744 -2.571267

H -2.668095 $1.039455-0.072232$

H $-2.240518-0.3714511 .994905$

H -3.511419 -1.483410 1.467334

H -4.062713 $0.145050-1.632255$

H $3.064664-0.668030-1.004260$

H $4.2915240 .567456-0.709209$

H $\quad-5.2025590 .3226561 .667593$

H $-4.3146150 .111583 \quad 3.182206$

H -3.9301561 .4759102 .118980$

SCF Energy (B3LYP/6-31G**//MMFF) $=-810.721074591$

F2-14 c03094

MMFF Geometry

C $1.6793692 .974640-0.103672$

C 0.7537963 .6890810 .545786

C $2.1490690 .535428-0.514697$

O $0.764825 \quad 0.127915-0.496261$

C $0.662273-1.2054550 .021392$

C $1.970691-1.4601290 .758703$

C $2.973558-0.676654-0.065855$

C $-0.581950-1.3701800 .904612$

C $3.479892-1.485368-1.262148$

C -1.929492 -1.292398 0.156581

O $-0.505471-2.6579351 .528343$
O $-1.923915-2.250736-0.911627$

C $-2.3029720 .066494-0.457098$

C $-2.306346 \quad 1.2158990 .555004$

O $-3.627704-0.046859-0.994572$

C 2.3154521 .7268850 .441225

C $-2.7269232 .540197-0.071767$

H $2.0115373 .319743-1.079447$

H 0.3387564 .5860930 .099167

H 0.3936893 .3951021 .525380

H $2.4036720 .837913-1.537435$

H $0.604157-1.889917-0.833526$

H $2.206464-2.5248740 .849372$

H $1.927236-1.0381991 .770599$

H $3.845299-0.3898130 .532949$

H $-0.563609-0.6265631 .708454$

H $4.197517-0.895692-1.842647$

H $3.988955-2.394308-0.924616$

H $2.670824-1.781256-1.937431$

H $-2.736883-1.6109800 .828407$

H -1.249559-2.726794 2.150716

H $-1.710613-3.116268-0.522394$

H -1.649571 $0.303551-1.303592$

H -1.312631 1.3441520 .993977

H -3.0021790 .9889511 .371742$

H -3.617095 -0.777858 -1.636190

H 1.8891911 .4852401 .422824

H 3.3820941 .9364070 .585817

H $-2.669813 \quad 3.3425640 .670738$

H -2.071364 $2.803130-0.907851$

H $-3.7564552 .499957-0.440465$

SCF Energy $($ B3LYP/6-31G**//MMFF $)=-810.716130453$

F2-14_c03095

MMFF Geometry

C $-4.150311 \quad 1.8158650 .253116$

C -3.8899103 .1178360 .090317$

C $-2.697171-0.2265340 .258696$

O

C $-0.457637-0.512527 \quad 0.887140$

C $-0.978055-1.8331890 .321630$

C $-2.169435-1.423021-0.523157$

C 0.8144330 .0472270 .221179

C $-3.182856-2.538447-0.716489$

C $2.076637-0.7815430 .534805$

O $0.6264190 .120668-1.189452$

O $2.260739-0.8061991 .950068$

C $3.378378-0.283293-0.130783$

C $3.702673 \quad 1.1899190 .154382$

O $3.325934-0.504528-1.537831$

C $-3.5453070 .725528-0.585479$

C $5.0774701 .585006-0.371370$

H -4.848486 1.5172881 .030916

H $-4.3639083 .856698 \quad 0.727316$

H $-3.204003 \quad 3.468527-0.672881$

H $-3.250341-0.5558251 .148546$

H $-0.294228-0.6087631 .966507$

H -1.301698 -2.472804 1.152950

H $-0.236167-2.401458-0.245994$

H -1.806196 -1.097564 -1.506336

H $0.951413 \quad 1.073607 \quad 0.583076$

H $-2.719054-3.397440-1.212517$

H -3.589016 -2.881660 0.240957

H -4.020257 -2.204325 -1.336662

H $1.912036-1.8123780 .199250$

H $-0.1486780 .686047-1.349210$

H $3.016532-1.3880872 .138620$

H $4.207730-0.8918390 .250631$

H $3.667124 \quad 1.3833441 .232187$

H $2.9647371 .841189-0.327815$

H $2.6105850 .046156-1.899196$

H $-2.9300621 .162929-1.381858$

H $-4.3635140 .178337-1.066809$

H 5.8621610 .9739540 .085822

H $5.1373751 .467101-1.457850$

H $5.2839032 .633760-0.135002$

SCF Energy (B3LYP/6-31G**//MMFF) $=-810.720749357$

F2-14_c03096 
MMFF Geometry

C $4.515388-1.2728990 .160404$

C $4.518131-2.571005-0.163049$

C 2.6919520 .4397620 .321393

O $1.650702-0.504560 \quad 0.627376$

C $\quad 0.413397 \quad 0.212700 \quad 0.828907$

C 0.6937081 .6717390 .474961

C $1.9688111 .604734-0.343760$

C $-0.682579-0.4890280 .004183$

C $2.7524172 .906478-0.345112$

C -2.1029330 .0375130 .296722$

O $-0.399853-0.374718-1.389544$

O $-2.386334-0.1070351 .687397$

C -3.197317 -0.663747 -0.535899

C $-4.632710-0.252095-0.178626$

O $-3.096968-2.080743-0.374367$

C $3.734941-0.223143-0.579206$

C $-4.9178291 .224148-0.399078$

H $5.118705-0.9444261 .002831$

H $5.107733-3.2786020 .409693$

H $3.936533-2.949494-0.996233$

H $3.142557 \quad 0.753823 \quad 1.272489$

H $\quad 0.171680 \quad 0.131526 \quad 1.894834$

H $\quad 0.8675602 .234323 \quad 1.401363$

H $-0.1234262 .172867-0.050632$

H $1.7102961 .343558-1.377909$

H $-0.631311-1.5586010 .242497$

H $3.6595762 .817295-0.950587$

H $2.1479673 .718544-0.762502$

H 3.0509663 .1959050 .668083

H $-2.143279 \quad 1.1078710 .070244$

H $-0.5676500 .544234-1.657797$

H -2.286456 -1.046431 1.916609

H $-3.026532-0.460349-1.599233$

H -4.860546 -0.5212330 .860048$

H -5.327713 $-0.843718-0.788461$

H $-3.306549-2.2922070 .551018$

H $3.241218-0.668309-1.452208$

H $4.4450410 .524383-0.950339$

H $-5.9791221 .431298-0.227805$

H -4.3439021 .8508000 .289943$

H -4.675976 $1.522456-1.423886$

SCF Energy (B3LYP/6-31G**//MMFF) $=-810.710204592$

F2-14_c03097

MMFF Geometry

C -4.674857-1.214964 0.014612

C $-5.034022-1.7959791 .164653$

C -2.766499 $0.284382-0.695996$

O $-1.486037 \quad 0.201565-1.357025$

C $-0.4602250 .708538-0.482624$

C -1.082099 $0.766560 \quad 0.904882$

C -2.5411801 .0587800 .607400$

C $0.795415-0.166406-0.612346$

C -2.7891142 .5569720 .417472$

C $1.979594 \quad 0.3520630 .224067$

O $0.497460-1.502935-0.209379$

O $2.2770791 .695551-0.152780$

C $3.245726-0.5210220 .095974$

C 4.4250330 .0405870 .902806

O $3.622277-0.636447-1.275600$

C -3.263539-1.153943 -0.488935

C $5.632799-0.8874900 .875966$

H -5.446310 -0.766996 -0.606305

H -6.074233 -1.811876 1.471002

H -4.303519 -2.261254 1.817359

H -3.453533 $0.811206-1.368866$

H $-0.2333121 .722768-0.832139$

H -0.6038801 .5119561 .547676$

H -1.007050 -0.202875 1.411277

H $-3.187427 \quad 0.7174451 .422400$

H $1.072482-0.205598-1.672954$

H -2.199650 $2.975514-0.404419$

H -3.845905 2.7418890 .196340

H -2.536565 3.1076351 .329741

H 1.6972220 .3684881 .283457

H -0.246491 -1.810456 -0.755019

H $2.4617121 .703741-1.107150$
H $3.020641-1.5333800 .450494$

H 4.1206590 .1979891 .943967

H $\quad 4.736495 \quad 1.010348 \quad 0.497037$

H $3.9055520 .241056-1.583371$

H $-3.216661-1.687490-1.447098$

H $-2.580542-1.6977690 .175607$

H $6.442618-0.4695701 .482517$

H $5.378971-1.8720361 .281687$

H $6.010290-1.021083-0.142546$

SCF Energy $\left(B 3 L Y P / 6-31 G^{* *} / / M M F F\right)=-810.719388839$

F2-14 c03098

MMFF Geometry

C $2.9377802 .461684-0.029737$

C 4.0651393 .1804100 .014258

C $2.503465-0.001186-0.241257$

O $1.2347480 .158462-0.904514$

C $0.560000-1.108441-0.965988$

C $1.572863-2.146761-0.489739$

C $2.429512-1.3492510 .472687$

C $-0.725340-1.064376-0.112010$

C $3.784418-1.9813880 .743263$

C $-1.737751-0.006509-0.611161$

O $-1.323587-2.357932-0.153475$

O $-1.1789631 .299077-0.465405$

C $-3.101626-0.0557070 .110251$

C $-4.0395971 .068309-0.354607$

O $-2.8788540 .057654 \quad 1.514986$

C 2.7345651 .1716520 .713856

C -5.4500710 .9248590 .203372$

H $2.1086122 .830745-0.627938$

H $4.1470174 .107852-0.542073$

H 4.9201972 .8602650 .599141

H $3.274626-0.019326-1.022516$

H $\quad 0.292311-1.314779-2.009204$

H $2.177583-2.479437-1.343311$

H $1.127765-3.036149-0.035187$

H $1.890165-1.2438931 .423040$

H $-0.448609-0.8376330 .925013$

H $3.663114-2.9716591 .194565$

H $4.363039-2.101102-0.178870$

H $4.372195-1.3668171 .432126$

H -1.919039-0.163604 -1.681509

H -1.956018 -2.4132040 .582700$

H -1.026013 1.4485520 .483391

H -3.574644 -1.026532 -0.077335

H -4.096544 $1.070796-1.449324$

H $-3.6503772 .043038-0.037581$

H $-3.713523-0.1523941 .966209$

H 1.8626721 .2987051 .367529

H $3.5984470 .966091 \quad 1.356419$

H -6.096374 $1.708598-0.204566$

H $-5.881980-0.044329-0.065882$

H -5.4615151 .0183611 .293309$

SCF Energy $\left(B 3 L Y P / 6-31 G^{* *} / / M M F F\right)=-810.712629625$

F2-14 c03099

MMFF Geometry

C -3.605854 -1.631794 0.266694

C $-4.329052-1.8461971 .371559$

C $-2.6796140 .479729-0.817908$

O $-1.523002-0.180922-1.357128$

C $-0.3516220 .577290-0.975131$

C -0.8185421 .6237120 .039472$

C -2.1883221 .1293990 .469112$

C $0.716737-0.410808-0.472806$

C -3.0949112 .2274990 .996478$

C $2.0926310 .236207-0.211634$

O $0.269262-1.0308240 .732523$

O $2.5816540 .826981-1.414204$

C $3.141766-0.771340 \quad 0.306747$

C $4.554660-0.1889610 .462209$

O $3.219867-1.869702-0.605613$

C $-3.849539-0.499420-0.689993$

C 4.6378020 .9623251 .450547

H $-2.800186-2.3216120 .030122$

H -4.106709 -2.687478 2.018990

H $-5.147402-1.1914421 .649987$ 
H $-2.9641751 .252664-1.545202$

H $\quad 0.0048501 .074471-1.883779$

H $-0.9027902 .595275-0.464426$

H $-0.138377 \quad 1.7629120 .884304$

H $-2.046066 \quad 0.377797 \quad 1.254574$

H $0.818797-1.203227-1.224660$

H -2.647485 2.7077371 .872951

H $-3.269536 \quad 3.0012900 .241350$

H $-4.0668661 .821397 \quad 1.294024$

H $1.991316 \quad 1.0287410 .537717$

H $-0.571147-1.4770730 .533155$

H $2.0771211 .642974-1.569737$

H $2.811132-1.1755581 .270905$

H $4.9452590 .128738-0.512310$

H $\quad 5.229410-0.9883700 .794779$

H $3.837665-2.520285-0.230608$

H $-4.7530940 .053380-0.405824$

H -4.045536 $-0.945258-1.673294$

H 4.2273800 .6751982 .423707

H 5.6820851 .2566571 .596939

H 4.0952721 .8409741 .089639

SCF Energy (B3LYP/6-31G**//MMFF) $=-810.713654492$

F2-14 c03100

MMFF Geometry

C 2.5199022 .1211550 .384836

C 3.4696022 .4871431 .253072

C $2.2192170 .105273-1.140750$

O $\quad 0.782391 \quad 0.145030-1.076747$

C $0.306225-1.073180-0.473675$

C $1.536080-1.959585-0.271667$

C $2.653842-0.945002-0.123637$

C $-0.438771-0.7368430 .830901$

C $4.037145-1.517128-0.378561$

C -1.5097280 .3657580 .664328$

O $-1.051426-1.9160891 .351415$

O $-2.091591 \quad 0.6276341 .946088$

C $-2.6402730 .053494-0.337041$

C $-3.6632221 .196404-0.425693$

O $-3.319821-1.145345 \quad 0.027328$

C $2.8005611 .510189-0.958949$

C $-4.7038220 .953667-1.511780$

H 1.4795222 .2826300 .654006

H 3.2014352 .9287022 .206619

H 4.5225982 .3524661 .032238

H $2.467410-0.233404-2.155728$

H - $0.377482-1.569476-1.171695$

H $1.695961-2.565202-1.173238$

H $1.466328-2.6471510 .574855$

H $2.618608-0.5429340 .895879$

H $0.272412-0.3877561 .590127$

H $4.119613-1.935426-1.387258$

H $4.803946-0.743548-0.270212$

H $4.260341-2.316324 \quad 0.335774$

H $-1.020243 \quad 1.2958050 .351360$

H $-0.347989-2.5189061 .642660$

H $-1.372840 \quad 0.8865842 .547510$

H -2.208849 -0.109719-1.330478

H $-3.1475512 .140898-0.633610$

H $-4.192179 \quad 1.3082930 .528130$

H $-3.644247-1.033550 \quad 0.937384$

H 3.880108 1.481663-1.148891

H $2.3608002 .174647-1.713239$

H -4.228442 $0.843353-2.491684$

H $-5.2891340 .051369-1.308805$

H $-5.3971081 .799069-1.564683$

SCF Energy (B3LYP/6-31G**//MMFF) $=-810.709140780$

F2-14 c03101

MMFF Geometry

C $3.1254822 .363663-0.081082$

C $2.3756423 .465091-0.200687$

C $2.554321-0.078182-0.108118$

O $1.3908290 .085558-0.941840$

C $0.623636-1.128560-0.943797$

C $1.497423-2.175017-0.256824$

C $2.302004-1.3381690 .717069$

C $-0.733209-0.897088-0.242073$
C $3.568759-2.0225901 .202013$

C $-1.6016230 .177504-0.943323$

O $-1.418716-2.146492-0.218574$

O $-0.9596221 .451052-0.899128$

C $-3.0210250 .365668-0.364212$

C $-3.034658 \quad 0.708474 \quad 1.132573$

O $-3.805965-0.803648-0.578820$

C $2.742813 \quad 1.173088 \quad 0.751745$

C -4.4337911 .0540671 .628738$

H $4.0754462 .319673-0.607375$

H $2.7103064 .293541-0.815351$

H 1.4211973 .5595220 .305533

H $3.411793-0.219980-0.778789$

H $0.443905-1.429815-1.982668$

H $2.164806-2.632060-0.998886$

H $0.939866-2.9820830 .226413$

H $1.672546-1.1020391 .584934$

H $-0.539720-0.5808330 .789633$

H $4.122779-1.3763971 .890074$

H $3.326123-2.9499511 .731103$

H $4.232876-2.2751840 .368713$

H -1.713432 -0.106602 -1.997812

H -2.225145 -2.034607 0.312038

H $-0.1113341 .372723-1.366954$

H $-3.5074231 .188469-0.903991$

H -2.3704581 .5560241 .334653$

H -2.676521 -0.1442401 .720461$

H -3.813005 -0.981283 -1.534832

H 1.8182301 .3845531 .303756

H 3.5348391 .0076571 .490734

H -4.4028631 .3127922 .691872$

H -4.842076 1.9108821 .083467

H -5.1195790 .2097721 .508025$

SCF Energy $\left(B 3 L Y P / 6-31 G^{* *} / / M M F F\right)=-810.721779760$

F2-14 c03102

MMFF Geometry

C $2.7701002 .670456 \quad 0.497221$

C $2.3935833 .227341-0.659769$

C $2.3687430 .213578-0.230750$

O $0.950797 \quad 0.349743-0.440306$

C $0.371505-0.948878-0.646912$

C $1.532274-1.939466-0.677603$

C $2.578583-1.2456230 .171790$

C $-0.645051-1.2387320 .469696$

C $3.988017-1.760138-0.067683$

C $-1.707625-0.1295920 .639095$

O $-1.262259-2.4956890 .197638$

O $-2.525451-0.464919 \quad 1.764347$

C $-2.6028360 .144852-0.587333$

C $-3.6702961 .223055-0.350533$

O $-3.303249-1.039407-0.970357$

C 2.8262551 .2053550 .843873

C $-3.0978242 .594072-0.030559$

H 3.0778313 .3435251 .295550

H $2.4023694 .306552-0.771360$

H $2.0725962 .641840-1.512566$

H $2.8654290 .417631-1.187804$

H -0.132035 -0.961992-1.620191

H $1.892640-2.043075-1.709169$

H $1.276248-2.939711-0.316248$

H $2.322849-1.3848971 .230368$

H $-0.119663-1.3454161 .427231$

H $4.710547-1.2297800 .560316$

H $4.053373-2.8267880 .171086$

H $4.290567-1.630511-1.112251$

H -1.185962 0.7939500 .911862

H -1.823794 -2.716829 0.959711

H -3.127775 -1.176899 1.489289

H $-1.9858590 .449004-1.440204$

H $-4.3571350 .912590 \quad 0.446370$

H $-4.2926471 .301981-1.251551$

H -2.640972 -1.704103 -1.224801

H $2.2073881 .067782 \quad 1.739989$

H 3.8611670 .9804301 .127526

H -3.9016113 .3363290 .009034$

H -2.594207 2.6025540 .940366

H $-2.3826192 .910335-0.796320$ 
F2-14_c03103

MMFF Geometry

C $2.4951832 .699848-0.124271$

C 1.4760323 .4686490 .275709

C $2.6830650 .222587-0.501157$

$\begin{array}{lllll}\text { O } & 1.279985 & 0.064702 & -0.784070\end{array}$

C $0.777037-1.101750-0.115849$

C $1.904097-1.6123940 .777608$

C $3.152067-1.1160420 .072921$

C $-0.515229-0.7865190 .655643$

C $3.588700-2.077247-1.034618$

C $-1.668977-0.334689-0.269262$

O $-0.895707-1.9595391 .370305$

O $-1.3175740 .891357-0.909304$

C -3.018412 -0.1558720 .457518$

C $-4.1344240 .438949-0.413911$

O $-2.822986 \quad 0.7132801 .575742$

C $2.845534 \quad 1.3778150 .499502$

C $-4.481870-0.409762-1.625586$

H $3.1186923 .045072-0.944886$

H $1.2763264 .415124-0.214642$

H $0.8249293 .169421 \quad 1.089620$

H $3.1999850 .465109-1.437143$

H $0.567360-1.854803-0.886066$

H $1.871541-2.6980470 .913421$

H $1.843841-1.1564231 .773534$

H $3.989962-0.9992640 .769076$

H -0.2948330 .0105391 .377342$

H $2.808999-2.222837-1.789387$

H $4.477852-1.691619-1.544680$

H $3.841145-3.057167-0.615965$

H $-1.800144-1.086834-1.055715$

H -1.540483 -1.6949312.047824

H $-1.2019011 .562511-0.214813$

H $-3.350486-1.1230630 .852387$

H -3.868671 $1.453756-0.734086$

H $-5.0347220 .559548 \quad 0.202420$

H -3.667592 0.7717132 .054061

H 2.2197341 .2088271 .384255

H $3.886133 \quad 1.4298360 .840884$

H $-5.3487050 .012898-2.143798$

H -3.654989 $-0.445585-2.340848$

H -4.730532 -1.433630 -1.329658

SCF Energy (B3LYP/6-31G**//MMFF) $=-810.710083916$

F2-14_c03104

MMFF Geometry

C $4.0151541 .777756-0.370116$

C $3.9444402 .961198 \quad 0.249486$

C $2.450746-0.176084-0.497293$

O $1.2558680 .598622-0.302541$

C $0.107975-0.272987-0.434136$

C $0.656495-1.699521-0.466564$

C $2.085507-1.5590210 .024343$

C -0.8657230 .0785410 .704256$

C $3.000257-2.671671-0.459207$

C $-2.303988-0.449678 \quad 0.548414$

O $-0.354194-0.4099391 .948581$

O $-2.297049-1.8800300 .489038$

C $-3.054036 \quad 0.048739-0.698733$

C $-3.0819201 .569103-0.893166$

O $-4.410880-0.406229-0.624803$

C $3.610487 \quad 0.473830 \quad 0.257680$

C $-3.712138 \quad 2.3351190 .263690$

H $4.3960661 .744143-1.387528$

H $4.2569873 .866833-0.258726$

H 3.5752893 .0490141 .265331

H $2.655959-0.206764-1.575917$

H $-0.349562-0.029619-1.398738$

H $0.641086-2.061332-1.502702$

H $0.089070-2.4199840 .126248$

H $2.078079-1.5548111 .122010$

H $-0.902348 \quad 1.168590 \quad 0.813288$

H $3.054459-2.699278-1.552717$

H $4.016286-2.538803-0.075119$

H $2.635798-3.645449-0.115767$
H $-2.882245-0.2035041 .447661$

H $0.520610-0.0055422 .079465$

H -1.889694 -2.199189 1.312772

H $-2.632768-0.415016-1.598366$

H -3.667506 $1.793567-1.793717$

H -2.070403 $1.948932-1.072737$

H $-4.386223-1.372090-0.513200$

H 3.3270630 .6342041 .305544

H $4.484117-0.1874560 .252113$

H -4.7387802 .0043750 .448960$

H -3.138771 2.2133191 .187051

H -3.7420613 .4045200 .030680$

SCF Energy (B3LYP/6-31G**//MMFF) $=-810.718836083$

F2-14_c03105

MMFF Geometry

C -1.908167 $2.825657 \quad 0.364240$

C $-1.6115992 .916754 \quad 1.665775$

C -2.040910 $0.236973 \quad 0.288227$

O -0.6714990 .0115190 .666602$

C $-0.383635-1.394148 \quad 0.601869$

C $-1.673076-2.0756710 .140181$

C $-2.401216-0.961198-0.585091$

C $0.810214-1.647491-0.337684$

C $-3.893540-1.207661-0.725043$

C $2.141298-0.9903220 .090171$

O $1.022916-3.064900-0.389069$

O $2.409753-1.384553 \quad 1.442953$

C $2.214470 \quad 0.545757 \quad 0.010173$

C $1.8425571 .107266-1.366174$

$\begin{array}{lllll}\text { O } & 3.566428 & 0.937920 & 0.283620\end{array}$

C -2.167618 $1.578613-0.440003$

C $1.9384282 .627686-1.417380$

H -1.974421 $3.756201-0.196590$

H -1.4476953 .8889532 .118755$

$\mathrm{H}-1.5252312 .0483052 .307043$

H $-2.6446830 .235061 \quad 1.205080$

H $-0.141803-1.7491101 .610528$

H $-2.247950-2.392157 \quad 1.020250$

H -1.517851 -2.961516 -0.482204

H $-1.959106-0.845182-1.583542$

H $0.555310-1.333110-1.354815$

H $-4.079604-2.126643-1.290621$

H $-4.376702-1.3107350 .252287$

H $-4.379195-0.382839-1.255420$

H $2.954973-1.419803-0.508698$

H $1.721043-3.233386-1.044655$

H $3.335128-1.1596901 .637135$

H 1.5993201 .0079920 .789387

H $0.8218040 .823607-1.636991$

H $2.5147240 .695834-2.128844$

H $3.7342860 .773348 \quad 1.226766$

H $-1.4672961 .594353-1.284224$

H -3.176746 $1.669091-0.858489$

H $1.2897443 .085976-0.664168$

H $2.9631372 .970956-1.245245$

H $1.6247832 .991316-2.401133$

SCF Energy (B3LYP/6-31G**//MMFF) $=-810.708106626$

F2-14_c03106

MMFF Geometry

C -2.755306 $2.561317-0.070383$

C $-1.964433 \quad 3.383372-0.769086$

C -2.4924750 .1587690 .616893$

O $-1.052896 \quad 0.2120950 .597272$

C $-0.534271-0.979437-0.012125$

C $-1.728722-1.740790-0.577692$

C $-2.869213-1.2947040 .317785$

C $0.517955-0.630449-1.068635$

C $-2.934976-2.1300361 .598179$

C $1.6610480 .262939-0.538261$

O $1.030037-1.844626-1.613893$

O $2.4979890 .575538-1.655848$

C $2.509433-0.3414430 .601026$

C 3.6604610 .5530101 .083889

O $3.102778-1.5737770 .189523$

C $-3.0237741 .131250-0.448202$

C 3.2078841 .8965551 .631201 
H -3.2444822 .9415840 .822620$ H -1.813613 $4.407346-0.445249$ H -1.454561 3.052781-1.667231 H -2.8368090 .4695011 .610358$ H $-0.082073-1.5862640 .781342$ H - $-1.567916-2.823449-0.589492$ H -1.934117 -1.429388-1.609105 H -3.836784 -1.377228 -0.189282 H $0.035874-0.092626-1.895513$ H -3.121963 -3.182696 1.361608 H -2.008750 -2.073206 2.179095 H -3.751590 -1.780547 2.239023 H $1.2180251 .207018-0.201775$ H $1.709706-1.603920-2.266926$ H $3.0707031 .317555-1.399323$ H $1.867429-0.5622601 .461297$ H $4.3927190 .704127 \quad 0.281573$ H 4.2084230 .0185401 .871088 H $2.382909-2.194941-0.012290$ H -2.571529 $0.914847-1.423709$ H $-4.1078411 .007929-0.556292$ H 4.0592632 .4310282 .064883 H 2.7876532 .5300210 .844738 H $2.454387 \quad 1.7682772 .414602$ SCF Energy (B3LYP/6-31G $\left.{ }^{* *} / / M M F F\right)=-810.715669600$

F2-14_c03107

MMFF Geometry

C -3.476823 $2.202224-0.003387$

C $-2.9227293 .410900 \quad 0.143321$

C $-2.531788-0.1184360 .041118$

O $-1.431650 \quad 0.206267 \quad 0.908359$

C $-0.740679-1.0140811 .237087$

C $-1.253918-2.0890920 .283990$

C $-2.032783-1.313301-0.763269$

C $0.779392-0.770270 \quad 1.236527$

C $-3.135548-2.127877-1.418677$

C $1.353408-0.103341-0.032557$

$\begin{array}{lllll}\text { O } & 1.072686 & 0.057778 & 2.367302\end{array}$

O $1.216653-0.981659-1.146582$

C $2.835106 \quad 0.3104330 .110222$

C $3.4033170 .909335-1.184584$

O $3.620416-0.8168370 .496444$

C $-2.8757981 .095186-0.822742$

C $4.8218071 .433685-1.000209$

H -4.4248032 .0001010 .488598$

H -3.410521 4.1693450 .745964

H -1.979985 $3.664188-0.328910$

H $-3.383512-0.401614 \quad 0.674078$

H -1.044728 -1.273397 2.259571

H - $-1.922378-2.7622680 .836603$

H $-0.466493-2.717821-0.141265$

H -1.337544 -0.971478-1.539510

H $1.276678-1.7320921 .408856$

H -3.664284 -1.536670 -2.172749

H $-2.718415-3.010083-1.915183$

H -3.871089-2.471271 -0.683623

H $0.7811870 .802981-0.262813$

H $\quad 0.605161 \quad 0.901597 \quad 2.241115$

H $1.681510-1.808626-0.934882$

H $2.925750 \quad 1.0514160 .912682$

H $2.7622701 .730327-1.525855$

H $3.4250870 .152300-1.977374$

H $3.626397-1.446116-0.244336$

H $-1.9732451 .454505-1.333208$

H $-3.6004190 .816630-1.595833$

H $4.8564952 .204521-0.223835$

H $5.5103300 .630593-0.719688$

H $5.1839371 .876357-1.933628$

SCF Energy (B3LYP/6-31G $*$ //MMFF) $=-810.714890613$

F2-14_c03108

MMFF Geometry

C $4.187950-0.9561850 .791534$

C $4.086848-2.2889280 .846447$

C 2.4986710 .8630780 .394266

$\begin{array}{lllll}\text { O } & 1.401252 & 0.088728 & 0.917969\end{array}$

C $0.215721 \quad 0.3313510 .132453$
C $0.6842361 .057589-1.120367$

C $1.8969901 .827945-0.630986$

C $-0.530700-0.997617-0.058884$

C 1.4990703 .1568380 .014936

C $-1.906571-0.903546-0.741777$

O $0.287169-1.910937-0.799824$

O $-1.724080-0.417469-2.077088$

C $-2.9345690 .000706-0.038658$

C $-3.168393-0.3527341 .434706$

O $-4.175383-0.114561-0.746178$

C $3.508617-0.103920-0.243434$

C -4.2239510 .5345902 .083575$

H $4.802989-0.4487911 .530348$

H $4.605856-2.8481991 .617152$

H $3.487715-2.8424340 .131888$

H $2.970577 \quad 1.3991351 .226156$

H $-0.404941 \quad 1.0054120 .733451$

H $-0.0940531 .694915-1.551151$

H $1.0024110 .353399-1.896617$

H $2.5899972 .048754-1.450464$

H $-0.648636-1.4669760 .924037$

H $0.9904203 .800336-0.710468$

H 0.8321243 .0201940 .872187

H 2.3888673 .6888460 .368363

H -2.313999-1.916202 -0.854018

H $0.234236-1.665584-1.738961$

H $-2.602525-0.371920-2.492304$

H $-2.6329901 .050111-0.131266$

H -2.239596 -0.2517712 .005242$

H -3.497821 -1.395277 1.519042

H $-4.484525-1.032401-0.657002$

H $3.010239-0.742686-0.982588$

H $4.2828430 .464273-0.772624$

H -3.9415261 .5900452 .017239$

H $-5.2013230 .407541 \quad 1.608081$

$\mathrm{H}-4.3315220 .2781923 .142375$

SCF Energy $(B 3 L Y P / 6-31 G * * / / M M F F)=-810.714968223$

F2-14 c03109

MMFF Geometry

C $-4.649560-0.588902-0.317043$

C $-5.299450-0.799436 \quad 0.833070$

C $-2.3485670 .267229-0.922681$

O $-1.049416-0.234655-1.299498$

C $-0.0586750 .230431-0.359700$

C $-0.8381260 .732920 \quad 0.846502$

C $-2.122907 \quad 1.252810 \quad 0.228064$

C $0.951044-0.899970-0.112024$

C $-1.9630132 .685478-0.286063$

C $2.176648-0.5276200 .740530$

O $0.295945-1.9991510 .531283$

O $1.751765-0.1212052 .047355$

C 3.0467960 .6060410 .173738

C $3.5250890 .414072-1.270138$

O $4.2069110 .746797 \quad 1.002563$

C $-3.210944-0.946006-0.544333$

C $4.346468-0.850022-1.493012$

H -5.190792 -0.143295 -1.147618

H $-6.343575-0.5236940 .932608$

H $-4.806431-1.2454231 .689779$

H $-2.7819370 .759319-1.801641$

H $0.4488061 .068729-0.849659$

H $-0.290624 \quad 1.4903591 .415346$

H -1.079404 -0.0883571 .531136$

H $-2.940838 \quad 1.2523970 .955784$

H $1.288330-1.288332-1.079707$

H -1.179448 $2.770277-1.045801$

H -2.899441 $3.034747-0.734165$

H -1.7118613 .3636880 .536117$

H $2.792432-1.4201640 .905952$

H $-0.457701-2.253557-0.028401$

H $1.216561-0.8458442 .414499$

H 2.5101091 .5594490 .244973

H $4.1565291 .268940-1.543583$

H $2.6730540 .419302-1.958232$

H 3.8963580 .8781191 .914953

H -3.163277 -1.682869-1.356557

H $-2.784765-1.4546750 .329563$ 
H $5.226769-0.873692-0.843394$

H $3.755711-1.751428-1.306855$

H $4.694978-0.890416-2.530178$

SCF Energy (B3LYP/6-31G**//MMFF) $=-810.721265241$

\section{F2-14 c03110}

MMFF Geometry

C $-4.378629-0.865623-0.560072$

C $-4.365329-2.203177-0.574671$

C $-2.5688010 .867527-0.349291$

O $-1.5647110 .021513-0.945953$

C $-0.2879740 .262997-0.316292$

C $-0.601144 \quad 1.0110790 .970227$

C -1.8329751 .8169420 .601060$

C $0.452537-1.074151-0.179636$

C $-1.4626953 .129944-0.092293$

C $1.882277-1.0437040 .395542$

O $-0.314841-1.948786 \quad 0.659030$

O $1.894140-0.4488661 .692269$

C $2.919112-0.335343-0.494416$

C $4.347975-0.5197630 .039311$

O $2.642441 \quad 1.059885-0.578872$

C $-3.574030-0.0268120 .392831$

C $5.3933280 .078885-0.893706$

H -5.013564 -0.342621 -1.270633

H -4.973568 -2.750852 -1.286327

H $-3.752358-2.7725620 .115250$

H $-3.0789601 .413736-1.151603$

H $\quad 0.2569840 .913865-1.006385$

H $\quad 0.2349921 .6266991 .315110$

H -0.8589150 .3156961 .777477$

H -2.4296092 .0643021 .486158$

H $0.484085-1.559064-1.163359$

H $-0.8930532 .968466-1.012936$

H $-2.3672183 .688838-0.355271$

H $-0.861355 \quad 3.7596180 .571729$

H $2.204926-2.0835160 .537226$

H $-1.200500-2.0227420 .263949$

H $1.259724-0.9404732 .241623$

H $2.859084-0.744110-1.509671$

H $4.561018-1.5867540 .171067$

H $4.452279-0.0370601 .018418$

H $2.647119 \quad 1.4159740 .326293$

H -3.051942 -0.6722291 .109498$

H -4.2722280 .5963290 .964256$

H $6.397780-0.099040-0.497034$

H $5.336202-0.375300-1.887990$

H $5.2609321 .160015-1.000197$

SCF Energy (B3LYP/6-31G**//MMFF) $=-810.722032879$

F2-14 c03111

MMFF Geometry

C $1.4600913 .051906-0.184224$

C 0.4891213 .7292960 .437652

C $2.1065810 .640324-0.513862$

O $0.755421 \quad 0.133042-0.501772$

C $0.745239-1.1960430 .035746$

C $2.051915-1.3320060 .806621$

C $3.010874-0.493841-0.016390$

C $-0.508695-1.448558 \quad 0.885424$

C $3.606081-1.289154-1.180313$

C -1.849061-1.412532 0.121091

O $-0.411131-2.751301 \quad 1.474834$

O $-1.792392-2.338947-0.973597$

C $-2.309589-0.049626-0.425647$

C -2.3813701 .0467490 .641265$

O $-3.625124-0.221373-0.969388$

C $2.172347 \quad 1.8681920 .407316$

C $-2.9176572 .362216 \quad 0.088605$

H $1.7738223 .382345-1.171114$

H $0.0197924 .581505-0.041525$

H $\quad 0.1452773 .4491051 .427082$

H $2.3544950 .929979-1.541943$

H $\quad 0.768822-1.897750-0.806361$

H $2.367128-2.3726860 .928691$

H $1.953099-0.8901161 .805919$

H $3.843911-0.1269760 .593544$

H $\quad-0.553177 \quad-0.730108 \quad 1.710974$
H $2.838594-1.661943-1.865981$

H $4.290543-0.659724-1.759304$

H $4.174587-2.148154-0.808904$

H -2.633255 -1.808440 0.779136

H $0.308662-2.7289222 .127660$

H $-1.251211-1.942517-1.676982$

H -1.675005 $0.272661-1.258052$

H $-1.390497 \quad 1.2301891 .067380$

H -3.0393080 .7269801 .458343$

H -3.572322 -0.924905-1.638869

H 1.7478381 .6258251 .389385

H 3.2191522 .1567090 .560576

H $-2.3117972 .709427-0.754193$

H -3.953269 $2.261192-0.250855$

H -2.8953403 .1334150 .865074$

SCF Energy $(B 3 L Y P / 6-31 G * * / / M M F F)=-810.710249311$

F2-14_c03112

MMFF Geometry

C $2.6559372 .204873-0.915861$

C $1.8627223 .185905-1.360941$

C $2.168171-0.116386-0.104450$

O $1.006802-0.276185-0.939477$

C $0.567169-1.640395-0.825791$

C $1.266005-2.2348900 .396087$

C $1.884995-1.0304071 .082606$

C $-0.966039-1.738093-0.802088$

C $3.107444-1.3738631 .915918$

C -1.693383 -0.9930510 .338279$

O $-1.473995-1.292361-2.061987$

O $-3.048645-1.466006 \quad 0.353791$

C -1.7183330 .5484010 .287182$

C $-2.472414 \quad 1.1440671 .485704$

O $-2.3502291 .011662-0.903318$

C $2.344031 \quad 1.3526230 .281339$

C -2.3661902 .6638491 .532210$

H $3.5878642 .008676-1.439457$

H $2.1453783 .769232-2.230284$

H $0.9233903 .421659-0.872867$

H $3.038940-0.470954-0.672943$

H $0.929434-2.152605-1.726418$

H $2.044304-2.9317710 .059437$

H $\quad 0.599617-2.8026091 .052848$

H $1.125673-0.5733011 .730409$

H -1.237543 -2.799809-0.733904

H $3.511247-0.4824132 .405788$

H $2.850027-2.0978832 .696018$

H $3.902134-1.8096841 .301108$

H $-1.260690-1.3057741 .294544$

H -1.063488 -1.835866 -2.755995

H $-3.463216-1.180616-0.478923$

H $-0.700724 \quad 0.941977 \quad 0.295426$

H -2.0706780 .7341072 .419501$

H -3.5357280 .8816751 .434652$

H -1.821242 $0.698163-1.656226$

H $1.439103 \quad 1.7224020 .777789$

H 3.1682921 .4578620 .995689

H -1.320236 2.9812601 .594006

H $-2.8136423 .122400 \quad 0.644951$

H -2.8903653 .0511992 .411613$

SCF Energy (B3LYP/6-31G**//MMFF $)=-810.716840178$

F2-14_c03113

MMFF Geometry

C $3.2063622 .286133-0.209624$

C 2.6554143 .3798650 .328564

C $2.373989-0.062732-0.482639$

O $0.9831780 .309076-0.522411$

C $0.171455-0.876241-0.499540$

C $1.134569-2.058773-0.579348$

C $2.398122-1.4965860 .041160$

C $-0.689401-0.8811510 .772304$

C $3.651044-2.271327-0.330230$

C $-1.5050790 .408927 \quad 1.014438$

O $-1.544079-2.0229850 .737478$

$\begin{array}{lllll}\text { O } & -2.192014 & 0.246338 & 2.260700\end{array}$

C $-2.5210260 .851427-0.060154$

C $-3.571950-0.201230-0.430630$ 
O $-1.845645 \quad 1.264122-1.244770$

C 3.1368730 .9184110 .408692

C $-4.6737160 .376807-1.312279$

H $3.7469482 .382238-1.147761$

H $2.7446184 .340421-0.166929$

H 2.1079693 .3381091 .263587

H $2.748807-0.021515-1.513860$

H $-0.472342-0.888253-1.385701$

H $1.312430-2.310153-1.632865$

H $0.773598-2.963193-0.081228$

H $2.287301-1.500597 \quad 1.133445$

H $-0.038456-1.0155181 .645895$

H $3.807821-2.285321-1.414060$

H $4.537521-1.8263660 .132241$

H $3.576886-3.308767 \quad 0.012177$

H -0.7871391 .2261251 .159477$

H -2.077837-2.010205 1.550310

H -2.6287301 .0900232 .468153$

H $-3.053945 \quad 1.7310150 .323601$

H -4.029403 -0.6181150 .473165$

H -3.106536 - $1.021185-0.988468$

H $-1.2019571 .948853-0.994641$

H 2.6539790 .9764951 .392311

H 4.1623330 .5664470 .567849

H -5.191779 $1.196853-0.804832$

H $-4.2711070 .755363-2.257018$

H -5.412420 -0.396344 -1.547102

SCF Energy (B3LYP/6-31G**//MMFF) $=-810.716285365$

F2-14_c03114

MMFF Geometry

C $4.3316961 .539048-0.500906$

C $4.1666682 .859799-0.634410$

C $2.725366-0.351846-0.144857$

O $1.615744 \quad 0.295617-0.792680$

C $0.491255-0.610386-0.803057$

C $0.889833-1.8129190 .050529$

C $2.080343-1.3134460 .846705$

C $-0.751507 \quad 0.165237-0.325547$

C $2.997259-2.4280061 .321674$

C $-2.063366-0.638209-0.412534$

O

O $-2.110756-1.340887-1.659724$

C $-3.3548720 .205707-0.320962$

C -3.4617331 .0972360 .919580$

O $-4.465184-0.700797-0.311308$

C $3.614527 \quad 0.7001170 .518885$

C $-4.798864 \quad 1.8252191 .005847$

H $5.0324881 .032352-1.159478$

H $4.7190213 .408900-1.389229$

H $3.4826823 .413406-0.000686$

H $3.282003-0.902434-0.915099$

H $0.351578-0.923510-1.843837$

H $1.191747-2.634416-0.611829$

H $\quad 0.086568-2.1963860 .685714$

H $1.711285-0.760024 \quad 1.719647$

H $-0.8416541 .075688-0.931369$

H $3.407105-2.9965310 .479957$

H $3.836950-2.0263691 .897085$

H $2.452058-3.1262281 .965310$

H $-2.092263-1.3987110 .376703$

H $\quad 0.253978 \quad 1.123502 \quad 1.046111$

H -2.080818 $-0.680467-2.373096$

H -3.461980 $0.820772-1.222856$

H -2.669611 1.8524990 .912460

H -3.3436390 .4908341 .825352$

H $-4.392772-1.256573-1.106179$

H $3.0073061 .340417 \quad 1.171085$

H $4.370210 \quad 0.215606 \quad 1.147177$

H -4.9712152 .4335980 .112360$

H $-5.6317901 .123542 \quad 1.112496$

H -4.8092422 .4896521 .875910$

SCF Energy (B3LYP/6-31G*//MMFF) $=-810.718937803$

F2-14 c03115

MMFF Geometry

C -4.475089 -1.389947 -0.241894

C $-4.906110-1.9546990 .891560$
C $-2.615600 \quad 0.220907-0.826076$

O $-1.2735650 .210608-1.356795$

C $-0.3772550 .822415-0.409656$

C $-1.136390 \quad 0.882392 \quad 0.907914$

$\begin{array}{llll}\text { C } & -2.575734 & 1.054440 & 0.459371\end{array}$

C $0.9441190 .037060-0.381593$

C $-2.9093412 .522290 \quad 0.183799$

$\begin{array}{llll}\text { C } 2.003591 & 0.679436 & 0.534846\end{array}$

O $0.695684-1.301706 \quad 0.041701$

$\begin{array}{lllll}\text { O } & 2.243690 & 2.012082 & 0.084584\end{array}$

C $3.350097-0.0721450 .619805$

C $4.009944-0.328493-0.742333$

O $3.181831-1.3042701 .316250$

C $-3.028198-1.242333-0.607292$

C $5.411366-0.910064-0.598553$

H $-5.209794-1.022929-0.953833$

H $-5.968143-2.0368381 .095228$

H -4.214088 -2.3408351 .631781$

H $-3.2681140 .671580-1.583328$

H $-0.1890541 .837843-0.778165$

H $-0.778377 \quad 1.6836501 .561600$

H - $-1.043113-0.0602681 .459789$

H -3.2738130 .6943651 .222011$

H $1.320089-0.013953-1.410251$

H $-3.9492122 .619780-0.146451$

H $-2.7890553 .121707 \quad 1.092355$

H $-2.2719752 .955117-0.593746$

H 1.5939000 .7345841 .551408

H $0.030541-1.677362-0.560312$

H 2.8571282 .4292480 .713102

H 4.0390570 .5348901 .220197

H $4.0709050 .603671-1.314779$

H $3.414800-1.041398-1.324385$

H $2.591536-1.8670550 .786919$

H $-2.847713-1.804425-1.532723$

H $-2.378718-1.7100370 .143371$

H $6.060001-0.233416-0.033248$

H $5.390719-1.876779-0.085964$

H $5.858968-1.062407-1.585879$

SCF Energy (B3LYP/6-31G**//MMFF) $=-810.721662668$

F2-14 c03116

MMFF Geometry

C $2.0988092 .177130-0.809102$

C $2.4320813 .226781-0.049758$

C $2.583095-0.318169-0.619794$

O $1.342775-0.709412-1.230710$

C $0.746359-1.722810-0.395866$

C $1.476031-1.685180 \quad 0.947266$

C $2.321044-0.4241360 .877226$

C $-0.782696-1.541848-0.343987$

C $3.580137-0.486552 \quad 1.724485$

C $-1.275393-0.118161-0.010034$

O $-1.302296-1.896007-1.630314$

$\begin{array}{lllll}0 & -0.865373 & 0.214515 & 1.312781\end{array}$

C $-2.8100280 .028370-0.120953$

C $-3.303337 \quad 1.4354010 .245182$

O $-3.447420-0.9248830 .729390$

C $3.0339271 .048994-1.140868$

C $-4.7927251 .608143-0.026576$

H $1.0971222 .135971-1.227925$

H 1.7114394 .0129310 .147183

H 3.4199093 .3196860 .387835

H $3.332208-1.061352-0.926179$

H $0.963903-2.683229-0.881309$

H $2.116480-2.5731031 .028398$

H $0.808622-1.7070411 .813824$

H 1.7083630 .4164561 .220634

H -1.190112 -2.2557590 .382328$

H $4.233112-1.3087121 .412812$

H 4.1498370 .4447701 .645546

H $3.327177-0.6386292 .778852$

H $-0.8167150 .597888-0.701801$

H $-1.078358-2.828341-1.792072$

H $-0.933790 \quad 1.1791191 .410982$

H -3.121648 $-0.197624-1.146808$

H $-2.7478982 .185306-0.329385$

H -3.1342361 .6350101 .309708$ 
H $-3.142508-0.7578831 .637842$

H $4.040758 \quad 1.264207-0.763368$

H $3.1076831 .002863-2.234613$

H $-5.0207661 .434131-1.082922$

H -5.3901180 .9150960 .573857$

H -5.1057662 .6267450 .224070$

SCF Energy (B3LYP/6-31G**//MMFF) $=-810.715015889$

F2-14 c03117

MMFF Geometry

C $4.0958351 .777871-0.099206$

C 3.8759403 .0339490 .304968

C $2.600328-0.216594-0.362096$

O $1.4058950 .546342-0.608148$

C $0.317912-0.365367-0.874760$

C $0.846199-1.769923-0.586803$

C $2.098782-1.526507 \quad 0.233423$

C $-0.8930530 .082018-0.033097$

C $3.097986-2.6687590 .159761$

C $-2.191823-0.667822-0.391417$

O $-0.607479-0.093046 \quad 1.352284$

O $-2.466789-0.481105-1.777937$

C $-3.430992-0.2727780 .444170$

C $-3.730616 \quad 1.2281620 .560914$

O $-3.293601-0.776373 \quad 1.774846$

C 3.5217950 .5641540 .575683

C $-4.0110681 .924475-0.759522$

H $4.7353751 .612773-0.962388$

H $4.3243673 .867760-0.224210$

H $3.2488393 .252977 \quad 1.162111$

H $3.087417-0.390537-1.330987$

H $0.079186-0.268738-1.939969$

H $\quad 1.101117-2.255543-1.537549$

H $0.131341-2.422776-0.078587$

H $1.807863-1.375148 \quad 1.280888$

H $-1.030868 \quad 1.156692-0.200953$

H $2.648789-3.5965670 .529112$

H $3.432898-2.843178-0.868283$

H $3.981267-2.457100 \quad 0.770095$

H -2.024011-1.740509-0.236715

H $\quad 0.1854990 .433043 \quad 1.553198$

H -3.253636 -1.008827 -1.996448

H $-4.316577 \quad-0.7564660 .012810$

H -2.917302 1.7415671 .087665

H -4.606752 1.3521791 .211505

H $-3.168989-1.7387501 .714269$

H $2.968646 \quad 0.8618451 .475391$

H $4.356758-0.0674060 .898648$

H -4.791555 $1.403099-1.322299$

H -4.353722 2.948341-0.577006

H -3.113511 $1.987448-1.380771$

SCF Energy (B3LYP/6-31G**//MMFF) $=-810.708004113$

\section{F2-14 c03118}

MMFF Geometry

C $-4.426335-0.940869-1.043151$

C $-5.340030-1.363649-0.162475$

C $-2.2325540 .306301-0.864880$

O $-0.8148320 .043568-0.821966$

C $-0.2839690 .478506 \quad 0.438515$

C $-1.4805540 .784772 \quad 1.331754$

C $-2.545232 \quad 1.205123 \quad 0.336237$

C $0.656170-0.5787311 .023882$

C $-2.4197862 .686142-0.028415$

C $1.805521-0.9850750 .076352$

O $1.168390-0.0914422 .262522$

O $2.517298-2.047640 \quad 0.718963$

C $2.7829790 .144560-0.312890$

C $3.881449-0.340196-1.270201$

O 3.4168610 .6892330 .841915

C $-2.945810-1.054023-0.834005$

C $4.7288280 .810411-1.800806$

H -4.766432 -0.497415 -1.975371

H $-6.398222-1.258262-0.375231$

H $-5.052523-1.8173140 .779572$

H $-2.4503460 .807082-1.815835$

H 0.2643461 .4116750 .263476

H -1.2560461 .5411302 .090337$
H -1.812662 $-0.119607 \quad 1.856151$

H $-3.550971 \quad 1.0455980 .737993$

H $0.080981-1.4849001 .254966$

H $-3.1893462 .964571-0.756382$

H -2.5551843 .3138090 .858684$

H $-1.4456472 .925706-0.466630$

H $1.366923-1.406335-0.836535$

H $1.772575-0.7690492 .611743$

H $3.073119-2.4802090 .049380$

H $2.2302210 .954480-0.801590$

H $3.431141-0.867498-2.118830$

H $4.552805-1.038549-0.757135$

H $2.724027 \quad 1.076307 \quad 1.403283$

H -2.533567 -1.687848 -1.630062

H -2.714164 -1.582359 0.099413

H $5.246663 \quad 1.331629-0.989702$

H $5.4859970 .431435-2.494666$

H $4.1105131 .536540-2.337997$

SCF Energy $(B 3 L Y P / 6-31 G * * / / M M F F)=-810.719410542$

F2-14 c03119

MMFF Geometry

C $-4.163203-1.812632-0.107922$

C $-4.785186-2.0972321 .041563$

C $-2.457569-0.086806-0.823618$

O $-1.0342720 .043613-1.014883$

C $-0.5105141 .013392-0.095954$

C $-1.629828 \quad 1.3217540 .891894$

C -2.8827281 .0808750 .071589$

C 0.7746670 .5046590 .576945

C $-3.2556442 .312013-0.757481$

C $1.9239650 .248339-0.422811$

O $1.1882101 .458466 \quad 1.552429$

O $1.591499-0.849085-1.272366$

C $3.279772-0.046548 \quad 0.254739$

C $4.382851-0.343694-0.771113$

O $3.162470-1.1731551 .122519$

C $-2.706300-1.473353-0.210505$

C $5.751242-0.490737-0.117008$

H -4.736409-1.837572 -1.031049

H $-5.841994-2.3410801 .048776$

H -4.257367 -2.091066 1.988703

H -2.933077 -0.037588 -1.810421

H $-0.2846891 .920142-0.671171$

H -1.5570802 .3317421 .307301$

H $-1.6062740 .616945 \quad 1.732321$

H -3.737482 0.8388180 .711255

H $0.541454-0.4345821 .093306$

H $-3.4691153 .165236-0.105036$

H $-2.4583672 .605388-1.447878$

H $-4.1536272 .112084-1.351903$

H $2.0560901 .133096-1.058448$

H 1.3920262 .2881431 .087833

H $0.782896-0.613896-1.757783$

H 3.5852930 .8125670 .863746

H $4.4293120 .463019-1.511565$

H $4.164220-1.276007-1.305252$

H $2.610457-0.9081181 .877384$

H $-2.220103-2.230319-0.839790$

H -2.208967-1.551839 0.764466

H $5.768921-1.3279040 .587655$

H $6.514726-0.676609-0.879183$

H $\quad \begin{array}{lll}6.026753 & 0.420522 & 0.423223\end{array}$

SCF Energy $\left(B 3 L Y P / 6-31 G^{* *} / / M M F F\right)=-810.716708199$

F2-14 c03120

MMFF Geometry

C $4.631601-1.275873-0.007650$

C $4.953903-1.887652-1.152765$

C $2.7785450 .290736 \quad 0.706342$

O $1.5012560 .254876 \quad 1.376570$

C 0.4771130 .7357630 .485150

C $1.1023680 .769930-0.902177$

C $2.5637581 .052594-0.605773$

C $-0.783674-0.1294030 .641935$

C $2.8247902 .550542-0.431292$

C $-1.9658710 .354486-0.220364$

O $-0.480839-1.4911120 .338485$ 
O $\quad-2.249727 \quad 1.708846 \quad 0.126509$

C $-3.229631-0.513794-0.043176$

C $-4.415066-0.020958-0.884469$

O $-3.612056-0.535267 \quad 1.331874$

C $3.231489-1.1650380 .517898$

C $-5.597254-0.980713-0.824684$

H $5.426182-0.8395990 .592065$

H $5.987921-1.939371-1.476013$

H $4.199362-2.343040-1.784426$

H $3.486257 \quad 0.807190 \quad 1.365849$

H $\quad 0.249958 \quad 1.7589120 .808131$

H $0.6317591 .512181-1.554329$

H $1.023434-0.202116-1.400752$

H $3.2087940 .696309-1.415449$

H -1.061472 -0.1056101 .702601$

H 2.2375432 .9834700 .384714

H $3.8826732 .728312-0.210014$

H $2.5791333 .093664-1.349941$

H -1.677483 $0.329981-1.278495$

H $-0.415314-1.582163-0.626718$

H $-2.8245302 .078039-0.564785$

H -2.998509 -1.546708 -0.327581

H $-4.106270 \quad 0.097157-1.929309$

H $-4.7599760 .955072-0.523836$

H $-3.7731880 .383730 \quad 1.606525$

H $3.185982-1.6787551 .487016$

H $2.521665-1.702076-0.123654$

H $-6.414868-0.610559-1.451470$

H -5.314314 -1.973596 -1.188384

H $-5.976236-1.0828910 .196904$

SCF Energy $\left(B 3 L Y P / 6-31 G^{* *} / / M M F F\right)=-810.713707467$

F2-14_c03121

MMFF Geometry

C $2.9645782 .311666-0.295624$

C $2.1971563 .398360-0.436576$

C $2.426695-0.137523-0.210560$

O $1.243008-0.020109-1.024112$

C $0.463127-1.221592-0.918370$

C $1.364666-2.239775-0.226563$

C $2.210224-1.3636710 .675102$

C $-0.847637-0.932504-0.152480$

C $3.497796-2.0277811 .133023$

C $-1.7550570 .095815-0.870215$

O $-1.551134-2.158474 \quad 0.029171$

O $-1.0822091 .348810-0.960715$

C $-3.1431720 .308644-0.227075$

C $-3.160658 \quad 0.571420 \quad 1.286687$

O $-3.947022-0.842615-0.494146$

C $2.620421 \quad 1.1501560 .593003$

C -2.4125831 .8205851 .721258$

H $3.8999832 .258511-0.846374$

H $2.5036224 .206126-1.091951$

H 1.2557993 .5004470 .091959

H $3.270082-0.297456-0.894713$

H $0.221035-1.574965-1.927525$

H $1.999693-2.728760-0.976469$

H $\quad 0.827515-3.0242410 .313646$

H $1.619846-1.0920161 .559874$

H $-0.589790-0.5394020 .837128$

H $4.080865-1.3525681 .767269$

H $3.279490-2.9310001 .712301$

H $4.124937-2.3169700 .283135$

H -1.916652 -0.263052 -1.895361

H -1.807250 -2.486029 -0.849959

H -1.638624 $1.946203-1.488632$

H -3.630216 $1.155756-0.726333$

H -2.777980 -0.3001931 .830690$

H $-4.206354 \quad 0.6656191 .608013$

H -4.838033 -0.667855 -0.146124

H $1.707717 \quad 1.3719481 .160706$

H 3.4336141 .0241881 .316775

H -2.750553 2.6973861 .160271

H -2.590266 2.0117112 .784604

H -1.332956 1.7129221 .585838

SCF Energy (B3LYP/6-31G**//MMFF) $=-810.701368379$

F2-14_c03122
MMFF Geometry

C $3.9175191 .766008-0.015309$

C 4.5629951 .9310991 .144668

C $2.0661550 .233508-0.835648$

O $0.6345880 .241825-1.025316$

C $0.089649-1.029474-0.644635$

C $1.278486-1.972177-0.518206$

C $2.392725-1.047427-0.063870$

C $-0.712314-0.8783970 .658768$

C $3.771256-1.626618-0.344125$

C -1.7917940 .2272360 .598321$

O $-1.295741-2.1401010 .979553$

O $-2.426440 \quad 0.303873 \quad 1.878935$

C $-2.8770100 .046669-0.482315$

C $-3.9803371 .116169-0.447579$

O $-3.505140-1.226053-0.324786$

C $2.4417571 .537668-0.125741$

C $-3.4720382 .530649-0.672681$

H $4.4867031 .810638-0.940064$

H 5.6343512 .0989001 .158064

H 4.0403041 .9003832 .094172

H $2.5109560 .209533-1.838575$

H $-0.567877-1.388930-1.444015$

H $1.515654-2.386962-1.506573$

H $1.111256-2.8126780 .161493$

H $2.293089-0.8751331 .015331$

H $-0.033206-0.6310871 .484030$

H $3.886137-2.5974010 .149904$

H $3.932882-1.775728-1.417123$

H $4.563657-0.9719560 .027290$

H $-1.292100 \quad 1.1894380 .439417$

H -1.848597 -2.012291 1.769221

H -1.7369570 .4945122 .537446$

H -2.406422 $0.055279-1.471886$

H -4.5306231 .0653790 .499942$

H $-4.7214020 .881570-1.222647$

H -4.133143 -1.338118 -1.058691

H $2.0072382 .379053-0.681303$

H 1.9588891 .5697340 .859863

H -2.8451702 .8690240 .157473$

H $-2.8907152 .596921-1.597551$

H $-4.3153473 .224100-0.752196$

SCF Energy (B3LYP/6-31G**//MMFF) $=-810.709119867$

F2-14 c03123

MMFF Geometry

C $3.1455502 .364127-0.564349$

C $2.5474793 .494238-0.171157$

C $2.420252-0.035740-0.530625$

O $1.0135060 .261488-0.617078$

C $0.257721-0.950256-0.445438$

C $1.278146-2.084656-0.369692$

C $2.509219-1.3872800 .173603$

C $-0.610478-0.8291190 .819260$

C $3.799979-2.142491-0.094482$

C -1.5418310 .4027430 .843305$

O $-1.369277-2.0246890 .988373$

$\begin{array}{llll}\mathrm{O} & -1.808128 & 0.697716 & 2.229153\end{array}$

C -2.9295240 .2662580 .185176$

C $-2.915922-0.184490-1.276230$

$\begin{array}{lllll}\text { O } & -3.559181 & 1.554279 & 0.241607\end{array}$

C 3.1321341 .0865290 .226455

C $-4.303765-0.177363-1.908262$

H $3.6846252 .360735-1.508139$

H $2.5978484 .385473-0.787210$

H 1.9995523 .5512810 .762775

H $2.798409-0.110237-1.558755$

H $-0.363322-1.100137-1.331171$

H $1.475387-2.461446-1.381655$

H $\quad 0.958197-2.9343410 .240101$

H $2.391787-1.2560651 .257188$

H $0.061642-0.7626661 .684583$

H $4.661430-1.5998820 .307001$

H $3.772876-3.1295640 .378550$

H $3.964176-2.288489-1.167415$

H -1.0304651 .2896760 .452062$

H -1.868614 -1.936910 1.818378

H $-0.962617 \quad 0.9422792 .642978$ 
H $-3.562563-0.4103690 .772411$

H -2.522260 -1.202308-1.351680

H $-2.2690150 .476782-1.864723$

H -3.5813101 .8245451 .175640$

H 2.6399571 .2499271 .193477

H 4.1711540 .8050210 .431315

H $-4.251945-0.561750-2.931946$

H $-4.994386-0.810840-1.342472$

H -4.719664 $0.833937-1.953117$

SCF Energy (B3LYP/6-31G**//MMFF)= -810.714471216

F2-14_c03124

MMFF Geometry

C 2.7514552 .6579280 .548898

C $2.709103 \quad 3.179623-0.682477$

C $2.4731450 .184874-0.183864$

O $1.163047 \quad 0.344960-0.760473$

C $0.566440-0.944751-0.976197$

C $1.679396-1.959872-0.731576$

C $2.532979-1.2609440 .307042$

C $-0.662164-1.117043-0.054727$

C $3.941445-1.8218510 .409462$

C $-1.752566-0.047703-0.304928$

O $-1.235704-2.402735-0.282344$

O $-1.239190 \quad 1.2301810 .067040$

C $-3.056411-0.3220940 .477002$

C $-4.138967 \quad 0.7578020 .341247$

O $-2.757115-0.459366 \quad 1.868884$

C 2.6667051 .2062010 .942022

C $-4.6164040 .967638-1.085954$

H $2.861774 \quad 3.3530501 .379371$

H $2.7823234 .253418-0.820482$

H $2.6029642 .570259-1.571586$

H $3.2058740 .343597-0.984965$

H $0.240979-1.010373-2.021224$

H $2.253664-2.094315-1.657603$

H $1.337052-2.947767-0.414683$

H $2.043507-1.3493201 .285699$

H $-0.343111-1.0501500 .992822$

H $4.524436-1.2799341 .160712$

H $3.914505-2.8770890 .700240$

H $4.472874-1.749989-0.545348$

H - $1.978474-0.025503-1.377777$

H $-0.690141-3.0581650 .183251$

H $-1.8183571 .907599-0.320342$

H -3.480954 -1.278917 0.152240

H -3.7895541 .7087820 .760865$

$\begin{array}{llll}\text { H } & -4.996575 & 0.470431 & 0.963412\end{array}$

H -2.3342350 .3666972 .160324$

H 1.8311621 .1176411 .648712

H 3.5836670 .9668801 .493383

H $-5.4547201 .671604-1.101628$

H $-3.8251621 .382094-1.717271$

H $-4.9567290 .026306-1.528676$

SCF Energy $\left(B 3 L Y P / 6-31 G^{* *} / / M M F F\right)=-810.707112002$

F2-14 c03125

MMFF Geometry

C $2.2363342 .761589-0.060797$

C $2.1319312 .952856-1.380833$

C $2.1132700 .172909-0.142787$

O $\quad 0.792197 \quad 0.107777-0.707028$

C $0.377402-1.264099-0.794839$

C $1.521075-2.095436-0.210210$

C $2.236956-1.1024030 .684703$

C $-0.966331-1.463237-0.068083$

C $3.665867-1.5037441 .007498$

C $-2.114015-0.525157-0.502738$

O $-1.422737-2.798805-0.325043$

O $-2.212229-0.530393-1.934263$

C $-2.0328660 .938602-0.030792$

C -1.8164641 .1389581 .473166$

O $-3.259627 \quad 1.592230-0.383190$

C 2.2646251 .4529610 .684813

C -2.8898220 .5048142 .349368$

H 2.3125373 .6489140 .565219

H $2.1245413 .959893-1.784891$

H $2.0534892 .133917-2.085159$
H $2.8323190 .160201-0.972359$

H $0.262040-1.526614-1.853286$

H $2.184271-2.417003-1.023801$

H $1.204730-2.9951830 .323467$

H $1.669719-1.0010391 .619470$

H $-0.828328-1.3809001 .014656$

H $4.144122-0.7638081 .656542$

H $3.686329-2.4682191 .525565$

H $4.271445-1.5968900 .099719$

H -3.065549 -0.952174 -0.162021

H $-0.843795-3.4126510 .156042$

H -1.455008 -0.034460 -2.289001

H $-1.2503801 .476488-0.575551$

H -1.8136742 .2159581 .683759$

H $-0.8348780 .757497 \quad 1.771340$

H $-3.3898381 .468134-1.339114$

H $1.459476 \quad 1.494791 \quad 1.428570$

H $3.210807 \quad 1.4171771 .237382$

H $-2.901921-0.5839822 .245560$

H -2.6961040 .7351053 .402100$

H -3.8849460 .8867072 .101179$

SCF Energy (B3LYP/6-31G**//MMFF) = -810.710778232

F2-14 c03126

MMFF Geometry

C 3.9369801 .8790340 .082210

C $5.1779342 .159145-0.331327$

C $2.401765-0.057418-0.348295$

O $1.1892810 .698198-0.185559$

C $0.055349-0.167880-0.430981$

C $0.624107-1.575815-0.596781$

C $2.009356-1.4838270 .014454$

C -0.9520590 .0638620 .711060$

C $2.965630-2.545978-0.501842$

C $-2.381612-0.4617950 .472516$

O $-\begin{array}{llll}0.451947 & -0.485135 & 1.934156\end{array}$

O $-2.358825-1.8765620 .256135$

C $-3.1109790 .158647-0.732562$

C $-3.1574581 .690562-0.766685$

O $-4.462647-0.317516-0.738617$

C 3.4943850 .5216620 .551952

C -3.8192422 .3225080 .451903$

H 3.1997922 .6777850 .093170

H $5.4362253 .162054-0.653197$

H $5.952526 \quad 1.400834-0.357376$

H $2.6944580 .009720-1.404849$

H $-0.3839770 .165602-1.376924$

H $\quad 0.698370-1.802289-1.668499$

H $\quad 0.022545-2.370207-0.150830$

H $1.920829-1.5819361 .104070$

$\mathrm{H}-1.006113 \quad 1.1415780 .900074$

H $2.583652-3.547754-0.279214$

H $3.100930-2.470899-1.586056$

H $3.949584-2.449981-0.032683$

H $-2.981860-0.316557 \quad 1.379289$

H $-0.417105-1.4514671 .845672$

H -2.095425 -2.297328 1.091815

H $-2.663593-0.201839-1.666248$

H $-3.7303042 .001273-1.649755$

H $-2.1487602 .100195-0.886736$

H $-4.426944-1.289435-0.731624$

H 3.1173120 .6306181 .576331

H $4.351165-0.1606420 .589209$

H -3.2605872 .1112521 .368120$

H -3.8605273 .4100400 .332004$

$\mathrm{H}-4.844021 \quad 1.9612580 .581591$

SCF Energy (B3LYP/6-31G**//MMFF) $=-810.711748357$

F2-14 c03127

MMFF Geometry

C $-3.687911 \quad 1.950572-0.239028$

C $-3.2348063 .186453-0.477220$

C $-2.530759-0.2240530 .221454$

$\begin{array}{lllll}\text { O } & -1.358882 & 0.373227 & 0.802415\end{array}$

C $-0.520161-0.6818811 .310682$

C $-1.051492-1.9916710 .736315$

C $-2.034269-1.554002-0.335194$

C $0.959179-0.358191 \quad 1.025813$ 
C $-3.133302-2.570784-0.594936$

C $1.300548-0.010619-0.440790$

O $1.314305 \quad 0.739547 \quad 1.870993$

O $1.090762-1.164401-1.251063$

C $2.7413630 .493916-0.684313$

C $3.829613-0.451206-0.154670$

O $2.9098821 .794545-0.126750$

C $-3.1018410 .707928-0.847686$

C $5.2272650 .000273-0.561255$

H $-4.537714 \quad 1.8262590 .427268$

H $-3.7056034 .043859-0.008877$

H -2.392211 3.365059-1.136051

H $-3.258387-0.385391 \quad 1.028305$

H $-0.663795-0.6901762 .399114$

H -1.572306 -2.541283 1.531202

H $-0.271658-2.6600700 .360148$

H -1.485438 -1.381210 -1.268830

H $1.555364-1.2189201 .350074$

H $-2.705149-3.524626-0.920353$

H $-3.727230-2.7580440 .305978$

H $-3.812541-2.222416-1.379145$

H $\quad 0.6147760 .774525-0.782687$

H $\quad 0.7454851 .4906171 .627958$

H $1.219508-0.901613-2.178386$

H $2.883790 \quad 0.599514-1.767093$

H $3.663024-1.467137-0.529468$

H $3.799491-0.4898190 .940135$

H 2.8728771 .7106090 .841012

H $-2.3151210 .976774-1.563829$

H $-3.8953600 .200746-1.407732$

H $5.3223330 .046550-1.650736$

H $5.4617320 .987851-0.152078$

H $5.975028-0.705402-0.185725$

SCF Energy (B3LYP/6-31G**//MMFF) $=-810.720741865$

F2-15 c03345

MMFF Geometry

C $2.6772462 .597500 \quad 0.287621$

C $1.7878643 .412360-0.290690$

C 2.6895490 .1240370 .738769

O $1.251951 \quad 0.1031090 .885313$

C $0.733654-1.1281530 .350445$

C $1.855543-1.728466-0.484887$

C $3.076250-1.2932980 .301146$

C $-0.570118-0.874926-0.420945$

C $4.380423-1.446523-0.460816$

C -1.664364 -0.2201050 .446891$

O $-0.308662-0.041756-1.549319$

O $-1.932357-1.0474251 .578158$

C $-2.9723130 .052792-0.325090$

C -4.0620610 .6594610 .570203$

O $-3.456434-1.156722-0.907601$

C $3.0512131 .243378-0.249237$

C $-5.3069361 .042970-0.219898$

H 3.1723692 .9271941 .197296

H 1.5645174 .3799470 .145486

H $1.2688683 .132096-1.200526$

H $3.1253640 .343126 \quad 1.721131$

H $0.534974-1.7837371 .207396$

H $1.881739-1.307278-1.496169$

H $1.767320-2.815422-0.575637$

H $3.135421-1.9182391 .203592$

H $-0.925647-1.839639-0.801473$

H $4.355867-0.929356-1.425339$

H $5.218320-1.0506440 .121728$

H $4.580766-2.504179-0.663079$

H -1.300566 0.7412230 .829318

H $\quad 0.0412490 .802119-1.215570$

H -2.191083 -1.925545 1.251133

H $-2.7641210 .742682-1.150743$

H -3.6731881 .5508911 .075521$

H -4.362031-0.057143 1.343888

H -3.725096 -1.749960 -0.186024

H $4.1309791 .251819-0.434634$

H $2.5532961 .073812-1.211567$

H $-5.7659520 .167794-0.690093$

H -6.0497831 .4961780 .444126$

H $-5.0652731 .768451-1.003253$
SCF Energy $(B 3 L Y P / 6-31 G * * / / M M F F)=-810.717182796$

F2-15_c03346

MMFF Geometry

C $4.285928-1.111075-0.039382$

C $4.136800-2.3277740 .496449$

C $2.6414460 .678080-0.682650$

O $1.616242-0.298439-0.969921$

C $0.3336550 .208247-0.542373$

C $0.629291 \quad 1.4218370 .323592$

C $1.8861671 .962071-0.327048$

C $-0.432429-0.9151510 .161225$

C 2.6249342 .9801060 .522926

C -1.755663 -0.472335 0.813757

O $-0.698218-1.945867-0.795496$

O $-2.310780-1.6004531 .497728$

C $-2.8142810 .121512-0.138720$

C -4.0755240 .5583760 .620546$

O $-3.202208-0.831843-1.124964$

C 3.5409750 .1099550 .425534

C $-5.0734301 .273101-0.282402$

H $4.991996-0.983635-0.855824$

H $4.707638-3.1685010 .117686$

H $3.447040-2.5071771 .313743$

H $3.229100 \quad 0.830177-1.595919$

H $-0.1861920 .508296-1.460600$

H $\quad 0.8287901 .126683 \quad 1.360816$

H -0.1933992 .1432440 .328700$

H $1.5944012 .451501-1.267149$

H $\quad 0.203854-1.3811930 .924269$

H $2.8548372 .593031 \quad 1.520656$

H 3.5630693 .2774360 .043482

H 2.0142943 .8797190 .655202

H - $-1.531478 \quad 0.268771 \quad 1.589321$

H $0.154994-2.211816-1.179736$

H $-2.533261-2.2660400 .824055$

H -2.397677 $0.990042-0.660885$

H $-3.8013391 .228173 \quad 1.443729$

H $-4.578347-0.3145721 .053746$

H -2.410412 -1.063157-1.639290

H 4.2876790 .8523840 .727779

H $2.942013-0.1350251 .311085$

H $-5.4268380 .617736-1.084468$

H $-5.944451 \quad 1.5931240 .298353$

H $-4.6237992 .161916-0.736264$

SCF Energy $($ B3LYP/6-31G**//MMFF $)=-810.721732898$

F2-15_c03347

MMFF Geometry

C $-3.1602591 .884279-0.738794$

C $-2.8027382 .965275-0.036607$

C $-2.184516-0.424794-0.919854$

O $-0.8516970 .076482-0.675979$

C $-0.114337-0.8764200 .107672$

C $-1.145640-1.8477740 .666342$

C $-2.160391-1.885837-0.458634$

C $0.738736-0.1790221 .176807$

C $-3.489271-2.503853-0.062701$

C $1.769140 \quad 0.8382120 .647919$

$\begin{array}{lllll}\text { O } & -0.114585 & 0.504460 & 2.101178\end{array}$

O 1.0884301 .9339240 .021726

C $2.8020280 .285207-0.350004$

C $3.573846-0.9299390 .175032$

O $3.7476551 .325163-0.625776$

C $-3.1767230 .487047-0.182747$

C $4.612631-1.433355-0.820451$

H -3.466773 $2.012404-1.773783$

H -2.815443 $3.947596-0.495981$

H -2.4911402 .8917680 .999351$

H -2.370557 -0.377292 -1.999615

H $0.538184-1.415667-0.589435$

H -1.607577 -1.468710 1.584994

H $-0.712451-2.8279490 .887919$

H -1.735653 -2.488077 -1.274334

H $1.258976-0.9449421 .761955$

H -3.924682 -2.014696 0.814516

H -4.207774 -2.438629 -0.886028

H $-3.357227-3.5622380 .185995$ 
H 2.2924001 .2852991 .502156 H -0.5850561 .1954971 .603721$ H $\quad 0.6111551 .586541-0.750868$ H $2.3208100 .041683-1.304351$ H $2.888234-1.753817 \quad 0.397581$ H $4.091108-0.6668561 .105611$ H $3.2467972 .093728-0.948886$ H $-4.1974670 .103831-0.290291$ H -2.945084 0.5103810 .888958 H $5.379954-0.678019-1.015838$ H $5.112624-2.323093-0.424544$ H $4.143178-1.703130-1.771873$

SCF Energy (B3LYP/6-31G**//MMFF) $=-810.724270190$

\section{F2-15_c03348}

MMFF Geometry

C 2.8896132 .3763590 .342364

C $2.0258183 .312851-0.065413$

C $2.701041-0.115000 \quad 0.623026$

O $1.295929-0.0118330 .944927$

C $0.593664-1.1413980 .395197$

C $1.543430-1.777581-0.610094$

C $2.885392-1.5236230 .046935$

C $-0.757672-0.703081-0.192745$

C $4.069516-1.738809-0.878526$

C $-1.674482-0.0299980 .851160$

O $-0.535647 \quad 0.186558-1.284080$

O $-1.895326-0.9501151 .919443$

$\begin{array}{llll}C & -3.039416 & 0.456554 & 0.317517\end{array}$

C $-3.864037-0.636548-0.376943$

O $-2.8563181 .561091-0.564499$

C $3.0583471 .039397-0.325302$

C $-5.266990-0.157095-0.730113$

H 3.5190012 .5866351 .203055

H 1.9546584 .2598540 .458287

H $\quad 1.377541 \quad 3.153942-0.920052$

H $3.268723-0.0159631 .556128$

H $0.428031-1.8413861 .223576$

H $1.496016-1.282541-1.586550$

H $1.333136-2.840752-0.761541$

H $2.983880-2.2207460 .891328$

H -1.246381-1.599282 -0.591198

H $4.134222-2.791230-1.174645$

H $3.987059-1.146730-1.795592$

H $5.005932-1.471474-0.378500$

H $-1.1508950 .843571 \quad 1.260378$

H $-0.0640590 .963274-0.937004$

H -2.410835 -0.4890512 .602987$

H -3.621565 0.8294501 .169257

H -3.943799 -1.5181290 .268651$

H $-3.376406-0.945944-1.308333$

H -2.390677 $1.237350-1.354393$

H $4.1044010 .961049-0.641139$

H $2.4375970 .993257-1.228115$

H -5.832743 -0.965179-1.204762

H -5.8112660 .1559740 .166491$

H $-5.2360170 .686282-1.427025$

SCF Energy (B3LYP/6-31G**//MMFF) $=-810.721431219$

F2-15_c03349

MMFF Geometry

C -2.625149 $2.628277-0.251244$

C -1.7062653 .4072420 .330856$

C $-2.6811930 .165659-0.752802$

O $-1.244280 \quad 0.120777-0.897726$

C $-0.745922-1.117392-0.358792$

C $-1.887130-1.7149020 .452770$

C $-3.091562-1.253572-0.343958$

C $0.559872-0.8825120 .418151$

C $-4.408436-1.4010880 .396997$

C $1.663795-0.262499-0.463246$

O $0.328353-0.0074231 .519752$

O $1.942168-1.143452-1.551449$

$\begin{array}{llll}\text { C } & 2.970102 & 0.050898 & 0.297657\end{array}$

C $4.0480990 .627276-0.630749$

O $3.486448-1.1370740 .895878$

C $-3.0213881 .270261 \quad 0.259151$

C 5.2900831 .0675270 .134710
H $-3.1277392 .991670-1.143826$

H -1.467094 $4.379757-0.085258$

H -1.177572 3.0911931 .223336

H -3.112124 $0.413070-1.730471$

H $-0.546480-1.771529-1.217054$

H $-1.929594-1.3101491 .469008$

H -1.813397 -2.8040810 .528564$

H -3.145311 -1.863707 -1.256814

H $0.899878-1.8483640 .811212$

H -5.232414 -0.983374 -0.190322

H $-4.626290-2.4590130 .578097$

H $-4.390537-0.9007251 .370488$

H $1.2991970 .678769-0.892260$

H $-0.130165-0.5129562 .211276$

H $2.324567-1.956650-1.179385$

H 2.7699950 .7700621 .100821

H $3.6464611 .487532-1.178346$

H $4.356247-0.123914-1.367682$

H $2.906834-1.3673811 .641261$

H -4.1001491 .2935930 .448496$

H $-2.5232201 .073376 \quad 1.216134$

H 5.0407341 .8290710 .880527

H 5.7622940 .2241010 .647896

H $6.0249311 .495967-0.554351$

SCF Energy $\left(B 3 L Y P / 6-31 G^{* *} / / M M F F\right)=-810.714613148$

F2-15 c03350

MMFF Geometry

C $4.239257-1.1052420 .270083$

C $4.062259-2.2474220 .943734$

C $2.6342950 .569266-0.699356$

O $1.635576-0.448198-0.932155$

C $0.3232390 .092728-0.666459$

C $\quad 0.553273 \quad 1.405474 \quad 0.065044$

C $1.8510201 .878108-0.557121$

C $-0.495870-0.9438570 .107968$

C $2.5232393 .001450 \quad 0.211548$

C $-1.858287-0.4254320 .614143$

O $-0.674458-2.074304-0.750077$

O $-2.471550-1.4619431 .386819$

C $-2.8396270 .061067-0.472090$

$\begin{array}{llll}C & -4.197066 & 0.526847 & 0.077045\end{array}$

O $-3.089759-0.998504-1.396328$

C 3.4581710 .1536120 .528744

C $-4.102303 \quad 1.7140831 .021122$

H $4.996979-1.070844-0.508393$

H $4.661641-3.1208120 .711199$

H $3.321221-2.333167 \quad 1.730775$

H $3.2827550 .615382-1.582602$

H $\quad-0.1255990 .275856-1.650653$

H $\quad 0.680672 \quad 1.239459 \quad 1.141593$

H $-0.2695382 .113196-0.074184$

H $\quad 1.6250712 .245745-1.568294$

H $\quad 0.091837-1.3113930 .958864$

H 2.6831752 .7417421 .262795

H $3.4918963 .250287-0.233594$

H 1.9010283 .9025900 .189590

H $-1.666848 \quad 0.394297 \quad 1.313818$

H -1.102821 -2.773084 -0.227774

H $-2.808374-2.1314640 .767104$

H $-2.3818020 .881743-1.035513$

H -4.711995 -0.3037520 .574965$

H $-4.8428180 .800350-0.767435$

H -3.651478 $-0.644167-2.106594$

H $\quad 4.1788700 .9374440 .786699$

H 2.8004890 .0116251 .394921

H -3.559933 2.5434860 .556518

H -5.1053472 .0682451 .280079$

H $-3.597172 \quad 1.4467411 .953619$

SCF Energy $\left(B 3 L Y P / 6-31 G^{* *} / / M M F F\right)=-810.702562360$

F2-15 c03351

MMFF Geometry

C $3.7710231 .933348-0.374487$

C $3.1380863 .027720-0.812038$

C $2.944455-0.2583390 .537918$

O $1.760404 \quad 0.3613751 .085095$

C $0.632202-0.5294040 .935838$ 
C $1.085375-1.612043-0.029098$

C $2.559899-1.7212940 .302530$

C $-0.5902310 .294614 \quad 0.504264$

C $3.364345-2.484865-0.734175$

C $-1.870628-0.5401790 .301390$

O $-0.841534 \quad 1.2619501 .528687$

O $-1.709693-1.411140-0.816453$

C -3.1373410 .3185680 .092886$

C $-4.381200-0.545816-0.161364$

O $-2.9224101 .184775-1.020841$

C $3.3558550 .528754-0.715757$

C $-5.6675490 .270184-0.189787$

H 4.6449312 .0547200 .260432

H $3.4904174 .014629-0.532729$

H $2.2632092 .960586-1.449224$

H $3.738484-0.1963031 .291854$

H $0.456841-0.9577301 .931082$

H $0.951293-1.294458-1.069893$

H $\quad 0.548829-2.5546400 .115486$

H $2.650892-2.2511381 .261407$

H $-0.3444820 .847345-0.411009$

H $4.430870-2.466094-0.488128$

H $3.044674-3.531939-0.767771$

H $3.235140-2.072520-1.739981$

H -2.030614 -1.169570 1.185393

H -0.0270101 .7815651 .642223$

H -1.612383 $-0.855556-1.608919$

H -3.300710 0.9447810 .977783

H $-4.467544-1.3060770 .623483$

H -4.291902 -1.066309-1.122200

H -3.636431 $1.843974-1.026009$

H $4.2085630 .045930-1.205543$

H $2.5297700 .550544-1.436923$

H $-5.6747750 .978390-1.023590$

H -5.7978010 .8299930 .741651$

H $-6.530136-0.393291-0.309504$

SCF Energy (B3LYP/6-31G*//MMFF) $=-810.718095070$

\section{F2-15 c03352}

MMFF Geometry

C $3.764031-0.3100521 .414873$

C $3.386956-0.8522912 .578158$

C $2.5436840 .100233-0.744320$

O $1.689211-1.044524-0.534641$

C $0.343793-0.734372-0.967280$

C $0.3159180 .767259-1.187156$

C $1.7357121 .041236-1.640876$

C $-0.635860-1.2929570 .073370$

C $2.1135972 .511239-1.610512$

C $-2.133325-1.035128-0.171483$

O $-0.475654-2.7246920 .064017$

O $-2.434847-1.303229-1.547759$

C -2.6655800 .3721910 .147753$

C $-2.346837 \quad 0.847457 \quad 1.569184$

$\begin{array}{llll}\text { O } & -4.093299 & 0.337993 & 0.008519\end{array}$

$\begin{array}{llll}C & 2.928586 & 0.664955 & 0.631669\end{array}$

C $-2.9249442 .226808 \quad 1.864101$

H $4.734810-0.5767551 .004962$

H $4.037860-1.5475413 .096899$

H $2.429583-0.6173513 .030002$

H $3.443434-0.240152-1.270741$

H $\quad 0.217818-1.258324-1.923371$

H $0.107711 \quad 1.301834-0.254117$

H $-0.4271321 .067207-1.931674$

H $1.8330620 .685616-2.676501$

H $-0.344342-0.9815491 .081108$

H $1.5158093 .072487-2.336537$

H $1.9381862 .960629-0.627838$

H $3.1691732 .645546-1.867592$

H - $2.721939-1.7634170 .402443$

H $0.462739-2.9109920 .240780$

H $-2.150220-2.214760-1.733334$

H $-2.3114871 .102783-0.584924$

H $-1.2652690 .893852 \quad 1.727656$

H -2.7602240 .1399892 .298123$

H $-4.2881500 .034979-0.895008$

H $3.517924 \quad 1.581006 \quad 0.515774$

H $2.0272920 .922217 \quad 1.201280$
H $-4.0178852 .224208 \quad 1.809651$

H -2.641292 2.5455272 .872341

H -2.5451542 .9691031 .154633$

SCF Energy (B3LYP/6-31G**//MMFF) $=-810.715751265$

F2-15 C03353

MMFF Geometry

C $-1.619496 \quad 2.732706 \quad 0.249301$

C $-0.694167 \quad 3.108618 \quad 1.139188$

C $-2.1794500 .530165-0.825387$

O $-0.8006920 .102843-0.770588$

C $-0.741195-1.311475-0.527437$

C $-2.128809-1.704213-0.038038$

C $-3.006080-0.759771-0.834060$

C $0.382068-1.6663750 .453277$

C $-4.431091-0.668347-0.318388$

C $1.808285-1.426945-0.088142$

O $0.193028-0.970753 \quad 1.685285$

O $2.725818-2.010768 \quad 0.845471$

C $2.2307330 .030210-0.365495$

C $3.6937390 .132384-0.825765$

$\begin{array}{lllll}\text { O } & 2.051188 & 0.839626 & 0.793362\end{array}$

C $-2.432266 \quad 1.4721760 .361036$

C $4.0690191 .551118-1.235670$

H $-1.8102653 .374616-0.606928$

H -0.1434024 .0327701 .002288$

H -0.4676772 .5056432 .011568$

H -2.326958 $1.073477-1.766675$

H $-0.547713-1.794534-1.493618$

H $-2.245200-1.5220371 .036634$

H $-2.350650-2.759129-0.226846$

H -3.043131-1.127451-1.869385

H $0.297942-2.7320120 .701081$

H $-4.9994690 .079472-0.880404$

H $-4.938930-1.632177-0.431588$

H $-4.467610-0.4033170 .743014$

H $1.921068-1.998666-1.017209$

H $0.186445-0.0188451 .485843$

H $2.567684-1.5961711 .710838$

H $1.5942630 .447058-1.151751$

H $3.863285-0.539193-1.675279$

H $4.369635-0.174069-0.018706$

H $2.6553050 .514376 \quad 1.481867$

H $-3.487241 \quad 1.7658080 .394928$

H -2.201196 0.9622101 .304175

H $3.4289801 .905735-2.049896$

H $3.9756062 .246638-0.395755$

H $5.1067501 .580705-1.582956$

SCF Energy (B3LYP/6-31G**//MMFF) $=-810.721404545$

F2-15 c03354

MMFF Geometry

C $-2.7294752 .525017-0.375272$

C $-1.8063893 .486640-0.262861$

C $-2.7232390 .008798-0.418598$

O $-1.444271-0.050940-1.097887$

C $-0.811153-1.298391-0.784007$

C $-1.421320-1.7448910 .534257$

C $-2.864942-1.3243290 .334086$

C $0.722833-1.208343-0.822166$

C $-3.678461-1.3178161 .616503$

C $1.354704-0.1493010 .106868$

O $1.257950-2.491704-0.503962$

$\begin{array}{lllll}\text { O } & 0.978716 & 1.157076 & -0.324146\end{array}$

C $2.897040-0.2203020 .143343$

C 3.5122590 .8742141 .027603

O $3.417786-0.100451-1.180262$

C -2.7489041 .2710410 .455260$

C $5.0217850 .719611 \quad 1.164657$

H $-3.5294992 .655431-1.099511$

H $-1.8550864 .370695-0.889131$

H -0.9926183 .4125200 .449873$

H $-3.5035950 .076943-1.186732$

H $-1.122502-2.013425-1.558907$

H $-0.988629-1.2097411 .385715$

H $-1.309529-2.8192350 .708448$

H $-3.332052-2.044867-0.352758$

H $1.009796-0.964841-1.853332$ 
H -3.225384 -0.684759 2.386132

H -4.695992 -0.9601961 .429139$

H -3.749334 -2.330757 2.027125

H $0.996496-0.2809001 .133538$

H $0.906319-3.126213-1.151508$

H $0.0088221 .198986-0.325492$

H $3.207231-1.1999510 .523930$

H 3.0600140 .8429032 .025456

H 3.3133311 .8649410 .602186

H $3.1164650 .752458-1.537888$

H -3.6662431 .2980131 .053675$

H -1.8992881 .2644741 .148858$

H 5.4220231 .4987481 .821090

H $5.278134-0.2526091 .597348$

H $5.521244 \quad 0.8078500 .194767$

SCF Energy (B3LYP/6-31G**//MMFF $)=-810.717687323$

F2-15_c03355

MMFF Geometry

C $-3.179618 \quad 1.888862-0.735859$

C $-2.7940042 .968655-0.046810$

C $-2.212462-0.423379-0.926481$

O $-0.8744360 .074067-0.706859$

C $-0.135769-0.8640700 .093384$

C $-1.162913-1.8368210 .659687$

C $-2.183443-1.882104-0.459645$

C $0.710386-0.1526361 .159770$

C $-3.509656-2.499777-0.053971$

C $1.745477 \quad 0.8529210 .617010$

O -0.1638390 .5431182 .055790$

O $1.0522401 .917379-0.047276$

C $2.7843830 .274206-0.360967$

C $3.546701-0.9318930 .201011$

O $3.7144921 .326465-0.643287$

C $-3.1894170 .493470-0.175055$

C $4.590444-1.479461-0.765088$

H -3.514499 2.016426 -1.761985

H $-2.8122533 .950082-0.507671$

H -2.4517352 .8940620 .979556$

H -2.417105 -0.379023 -2.003108

H $0.521716-1.409576-0.593965$

H -1.620980 -1.457573 1.580080

H $-0.725537-2.8149560 .882053$

H -1.761699-2.487181-1.274933

H $1.220986-0.9127041 .761735$

H $-4.232823-2.437498-0.873299$

H -3.375444 -3.557393 0.197262

H $-3.940385-2.0082870 .824253$

H 2.2594001 .3252821 .463925

H $\quad 0.3857420 .9473582 .748551$

H $1.7226282 .544609-0.368388$

H $2.2994450 .019490-1.310613$

H $2.851741-1.7434290 .439358$

H $4.056201-0.6476701 .129654$

H $4.2223201 .068572-1.430932$

$\mathrm{H}-4.2128120 .112285-0.262802$

H -2.938875 0.5202930 .892203

H $5.392471-0.756668-0.942223$

H $5.046550-2.384354-0.350691$

H $4.136645-1.738210-1.726881$

SCF Energy $\left(B 3 L Y P / 6-31 G^{* *} / / M M F F\right)=-810.701045977$

F2-15_c03356

MMFF Geometry

C $-2.8274702 .216795-0.755850$

C $-2.3383073 .214387-0.010707$

C $-2.230300-0.218144-0.940657$

O $-0.8481520 .060721-0.625460$

C $-0.303317-1.017700 \quad 0.152072$

C $-1.502306-1.8119610 .656507$

C $-2.463863-1.669003-0.506515$

C $0.621216-0.4971691 .261909$

C $-3.890498-2.066401-0.172725$

C $1.8243390 .372691 \quad 0.833202$

O -0.1517690 .2771782 .188860$

O 1.4055081 .4643190 .015295

C $2.937940-0.4001920 .102350$

C $4.1779150 .475197-0.136372$
O $2.474972-0.885310-1.154554$

C $-3.0998880 .832673-0.234591$

C $5.329119-0.311607-0.750395$

H -3.052336 $2.408584-1.801797$

H $-2.1667844 .192494-0.446627$

H -2.0994273 .0751691 .037750$

H -2.352844 -0.129598 -2.026886

H $\quad 0.261891-1.652426-0.538196$

H -1.932999-1.372324 1.563149

H -1.245461 -2.8528160 .876426$

H $-2.110769-2.322502-1.317035$

H $1.000055-1.3541241 .831297$

H -3.942704 -3.1349420.061708

H $-4.275825-1.5228200 .695843$

H -4.554709-1.875178-1.021622

H $2.255503 \quad 0.8183381 .739105$

H -0.6145010 .9669181 .683769$

H $\quad 0.753197 \quad 1.9784840 .519601$

H $3.223752-1.2707710 .703880$

H $4.513408 \quad 0.911160 \quad 0.811687$

H $3.9338561 .301908-0.814087$

H $2.161250-0.120413-1.667243$

H $-4.1618140 .622257-0.403044$

H -2.9263600 .8017420 .847750$

H $5.618135-1.148693-0.107039$

H $5.060946-0.709175-1.734242$

H $6.2025840 .336072-0.876641$

SCF Energy (B3LYP/6-31G**//MMFF) $=-810.714684299$

F2-15_c03357

MMFF Geometry

C 2.8333752 .4090250 .333836

C $1.9293553 .314445-0.056896$

C $2.703101-0.0840520 .628430$

O $1.298715-0.0112610 .958990$

C $0.610808-1.1378860 .384578$

C $1.578562-1.762000-0.611842$

C $2.911628-1.4909570 .057508$

C $-0.744590-0.702753-0.199242$

C $4.107171-1.690245-0.856764$

C $-1.667494-0.0736300 .867643$

O $-0.5425990 .242440-1.246213$

O $-1.891854-1.0382221 .895738$

$\begin{array}{llll}\text { C } & -3.032686 & 0.431154 & 0.350398\end{array}$

C $-3.839969-0.625950-0.415794$

O $-2.852899 \begin{array}{lll}1.587525 & -0.463389\end{array}$

C $3.0256801 .073054-0.329199$

C $-5.245346-0.140483-0.750639$

H 3.4783952 .6451071 .176194

H $1.841676 \quad 4.2628170 .461797$

H $1.2629403 .127224-0.891749$

H 3.2757530 .0323501 .556477

H $0.444725-1.8498841 .202968$

H $1.541094-1.271466-1.589769$

H $1.382972-2.827842-0.764212$

H $3.010806-2.1856430 .903697$

H -1.228410 -1.590305 -0.624329

H $4.190895-2.742354-1.149199$

H $4.023945-1.102030-1.776245$

H $5.034612-1.406846-0.348918$

H $-1.147807 \quad 0.783278 \quad 1.315013$

H -0.239681 -0.242435 -2.031674

H -2.405833 -0.6049392 .598343$

H $-3.6267110 .748414 \quad 1.216486$

H -3.914762 -1.545951 0.174412

H $-3.341909-0.872771-1.360374$

H -2.340884 $1.323485-1.246645$

H $4.0698601 .016587-0.656063$

H $2.3966691 .009525-1.225457$

H $-5.798399-0.923691-1.278924$

H -5.7999420 .1107110 .159017$

H $-5.2184680 .744472-1.393946$

SCF Energy $\left(B 3 L Y P / 6-31 G^{* *} / / M M F F\right)=-810.713028868$

F2-15 c03358

MMFF Geometry

C $3.3463112 .287857-0.225105$

C $2.5565673 .304394-0.589335$ 
C $2.856336-0.0643320 .513351$

$\begin{array}{lllll}\text { O } & 1.591716 & 0.329618 & 1.088645\end{array}$

C $0.610100-0.7064620 .860920$

C $1.222173-1.634377-0.175045$

C $2.695881-1.5469230 .166968$

C $-0.723582-0.044551 \quad 0.478220$

C $3.607377-2.102705-0.912482$

C $-1.860229-1.0484290 .191261$

O

O $-1.556759-1.767203-1.002844$

C $-3.269723-0.4333230 .038841$

C $-3.3442640 .697892-0.996803$

O $\begin{array}{llll}-3.746237 & 0.028408 & 1.300163\end{array}$

C $3.1478630 .866203-0.673699$

C $-4.775977 \quad 1.162346-1.235026$

H $4.190782 \quad 2.4913170 .428214$

H $2.7556234 .309479-0.233918$

H $1.7028983 .154409-1.241008$

H $3.6303210 .059381 \quad 1.280276$

H $0.500993-1.2289811 .819980$

H $1.044547-1.263266-1.191322$

H $0.832528-2.654506-0.107456$

H $2.863219-2.1270011 .085525$

H $-0.559123 \quad 0.601775-0.392645$

H $3.447993-3.180284-1.026952$

H $3.419890-1.640335-1.886869$

H $4.658500-1.943658-0.651726$

H $-1.895201-1.7671151 .020376$

H -0.3789611 .4222191 .723092$

H -2.234132 -2.455947 -1.113144

H $-3.954966-1.228801-0.279413$

H -2.916616 $0.366194-1.949511$

H $-2.7670311 .564720-0.655295$

H $-3.1710790 .758037 \quad 1.586532$

H $4.0647490 .553971-1.185725$

H $2.3297950 .817582-1.402650$

H $-5.4011880 .337602-1.591172$

H $-5.2223151 .563372-0.319663$

H $-4.7944061 .952664-1.992274$

SCF Energy (B3LYP/6-31G**//MMFF) $=-810.719001882$

F2-15 c03359

MMFF Geometry

C 3.5201192 .3241310 .222475

C $2.9804143 .403619-0.354702$

C $2.734181-0.040923 \quad 0.469133$

$\begin{array}{lllll}\text { O } & 1.327892 & 0.241907 & 0.386945\end{array}$

C $0.634877-0.9163670 .881744$

C $1.582815-2.1030590 .688242$

C $2.842568-1.5119300 .065350$

C $-0.729017-1.062722 \quad 0.188389$

C $2.876936-1.758934-1.442973$

C -1.6338200 .1735260 .372439$

O $-0.540083-1.303199-1.204023$

O -1.8309340 .4084441 .766304$

$\begin{array}{llll}\text { C } & -3.000848 & 0.038639 & -0.330771\end{array}$

C $-3.8897951 .267485-0.090399$

O $-3.657217-1.1280220 .163033$

C $3.5173530 .951106-0.390149$

C $-5.169801 \quad 1.237112-0.916058$

H 4.0014282 .4371061 .190539

H 3.0198204 .3682220 .139390

H $2.4915803 .346155-1.320852$

H 3.0274940 .0634991 .523421

H $0.477464-0.7633741 .956762$

H $1.146291-2.9189630 .102773$

H $1.820815-2.5273541 .672028$

H $3.742170-1.9644710 .498199$

H -1.221601 -1.947259 0.609160

H $3.812696-1.390681-1.876041$

H $2.818596-2.831028-1.659520$

H $2.050251-1.264459-1.960705$

H $-1.1321791 .057728-0.039014$

H $-0.112025-0.515532-1.581246$

H $-2.322249-0.3509682 .124140$

H - $2.840736-0.097185-1.406777$

H $-3.3344042 .178581-0.341592$

H -4.1724891 .3299170 .967192$
H $-4.417799-1.307535-0.414622$

H $4.5586270 .621618-0.484168$

H $3.0894151 .001276-1.398664$

H $-5.8120210 .398851-0.629829$

H $-5.7383972 .159700-0.760758$

H $-4.945904 \quad 1.153026-1.984210$

SCF Energy (B3LYP/6-31G**//MMFF) $=-810.718046898$

F2-15 c03360

MMFF Geometry

C $3.790300-1.7708891 .036229$

C $3.971529-2.630482 \quad 0.027022$

C $2.9032720 .192169-0.420365$

O $1.737723-0.542014-0.853833$

C $0.5955980 .341306-0.906200$

C $1.0068481 .589194-0.144359$

C $2.4862541 .666388-0.462526$

C $-0.632233-0.410043-0.369428$

C $3.2526872 .627000 \quad 0.429125$

C $-1.9266870 .427829-0.358290$

O $-0.838965-1.549481-1.210766$

$\begin{array}{llll}O & -1.794186 & 1.469676 & 0.606025\end{array}$

C $-3.185033-0.409045-0.036994$

C $-4.467734 \quad 0.432562 \quad 0.016934$

O $-3.012079-1.075227 \quad 1.213967$

C $3.315613-0.3413200 .962946$

C $-5.713320-0.432091 \quad 0.169124$

H $4.012127-2.1298422 .039721$

H $4.322381-3.6375160 .227590$

H $3.783861-2.366088-1.006305$

H $3.7093980 .019130-1.142772$

H $0.4488800 .576862-1.968138$

H $\quad 0.8550301 .4615050 .934047$

H $0.4582612 .478896-0.467680$

H $2.5887312 .013668-1.500695$

H $-0.411264-0.7844580 .637853$

H 2.9136423 .6547440 .260385

H 3.1068712 .4068001 .491489

H 4.3247152 .5864050 .211738

H -2.059669 $0.891676-1.343910$

H $-0.017232-2.070039-1.200130$

H -2.482961 2.1309580 .425220

H $-3.306910-1.186119-0.800079$

H $-4.559397 \quad 1.029551-0.897505$

H $-4.436567 \quad 1.1231690 .867665$

H -2.860047 $-0.393701 \quad 1.890989$

H 4.1268110 .2749251 .366651

H $2.465395-0.2567041 .651290$

H $-5.805644-1.135930-0.664216$

H $-5.689762-1.0037651 .102164$

H -6.6091480 .1967750 .182175$

SCF Energy (B3LYP/6-31G**//MMFF) $=-810.715262247$

F2-15 c03361

MMFF Geometry

C $2.942502 \quad 1.9000610 .090447$

C $3.9245762 .489588-0.600904$

C $2.473844-0.4005851 .141215$

O $1.044598-0.2017891 .037898$

C $0.543523-1.049817-0.010598$

C $1.714645-1.216581-0.967640$

C $2.866052-1.3601150 .009109$

C $-0.726714-0.463532-0.642499$

C $4.240268-1.203604-0.614289$

C $-1.861733-0.2483450 .380226$

O $-0.4265690 .775666-1.279956$

O $-2.170256-1.4878891 .016141$

C $-3.1384960 .353209-0.243605$

C $-4.2695720 .512737 \quad 0.782242$

O $-3.588266-0.467347-1.321107$

C $3.1665380 .966464 \quad 1.245656$

C $-5.483118 \quad 1.220741 \quad 0.193359$

H $1.9161712 .135708-0.173450$

H $3.6929733 .167846-1.414824$

H $4.9685022 .307692-0.371035$

H $2.644708-0.9147652 .095987$

H $0.308942-2.0196130 .447259$

H $1.855590-0.330428-1.596120$ 
H $\quad 1.596745-2.085730-1.621655$

H $2.818591-2.3791480 .421395$

H -1.058874 -1.158498 -1.422746

H $4.337184-0.274224-1.179764$

H $5.021946-1.2290320 .151388$

H $4.432229-2.024551-1.313959$

H -1.5211990 .4394581 .163486$

H $-0.169574 \quad 1.406586-0.586507$

H -2.409206 -2.127137 0.323866

H $-2.901547 \quad 1.335222-0.668717$

H $-3.9092601 .083813 \quad 1.645528$

H $-4.595670-0.4690121 .145453$

H -3.882677 -1.315645 -0.948955

H 2.7724041 .4767572 .134522

H $4.2385770 .818111 \quad 1.421828$

H $-5.2146522 .215034-0.177392$

H $-5.9151630 .649313-0.634130$

H $-6.256676 \quad 1.3418050 .958421$

SCF Energy (B3LYP/6-31G**//MMFF) $=-810.714032762$

F2-15 c03362

MMFF Geometry

C $4.174733-1.153568-0.411936$

C $4.009760-2.452077-0.136074$

C $2.5608630 .764804-0.596219$

O $1.497788-0.108164-1.037432$

C $0.2452060 .323143-0.457839$

C 0.6109021 .3216970 .628375

C $1.8572211 .960650 \quad 0.051273$

C $-0.516036-0.9143130 .029775$

C 2.6565722 .7627141 .062541

C -1.775721 -0.6424410 .871633$

O $-0.879281-1.712845-1.100530$

O $-2.014728-1.8330321 .649651$

C $-3.095541-0.3396690 .133329$

C $-3.032730 \quad 0.824144-0.856952$

O $-4.077807-0.019368 \quad 1.128493$

C $3.484496-0.0417630 .329332$

C $-4.3957921 .173329-1.444963$

H $4.853048-0.872438-1.213449$

H $4.540573-3.206597-0.706421$

H $3.346409-2.784490 \quad 0.654742$

H $3.1182891 .093270-1.481735$

H $-0.2855560 .823823-1.272304$

H $\quad 0.8409340 .8115851 .571689$

H -0.1890072 .0434320 .818898$

H $1.5443112 .642068-0.752604$

H $\quad 0.174192-1.5363970 .615317$

H 3.5838253 .1354100 .615944

H 2.0771463 .6264141 .406008

H 2.9130452 .1703051 .946646

H - 1.5805580 .1370811 .615315

H $-0.067950-1.880484-1.610220$

H -2.149964 -2.567775 1.026591

H -3.459771 -1.237921 -0.379945

H $-2.3688660 .571931-1.688203$

H -2.635322 $1.717066-0.360552$

H $-4.121433-0.7708831 .744503$

H $4.263757 \quad 0.6049720 .747452$

H $2.912292-0.4543531 .169166$

H $-4.8442330 .303769-1.936017$

H -5.086183 $1.530264-0.674606$

$\mathrm{H}-4.290351 \quad 1.967310-2.191330$

SCF Energy (B3LYP/6-31G**//MMFF) $=-810.714685799$

F2-15 c03363

MMFF Geometry

C $4.587968-0.902041-0.755521$

C $4.910239-2.032931-0.117604$

C $2.5604750 .567668-0.694282$

O $1.628173-0.448306-0.290543$

C $0.2919630 .086764-0.426855$

C $0.4478741 .598060-0.595060$

C $1.9058221 .877855-0.261799$

C $-0.530343-0.3807390 .781808$

C 2.0759682 .2178801 .219275

$\begin{array}{llll}C & -1.966148 & 0.174881 & 0.852847\end{array}$

$\begin{array}{llll}\text { O } & -0.593277 & -1.812770 & 0.744427\end{array}$
O $-2.582407-0.3422222 .037642$

C $-2.866672-0.119317-0.364702$

C $-4.2652130 .492132-0.195623$

O $-3.020576-1.523429-0.555884$

C $3.9372060 .281509-0.095272$

C $-5.1183450 .333848-1.448252$

H $4.815235-0.819331-1.815377$

H $5.383271-2.849428-0.652127$

H $4.708940-2.1654990 .939567$

H $2.6152290 .539337-1.791752$

H $-0.119617-0.353267-1.343101$

H $-0.2528692 .199349-0.011697$

H $0.2672551 .850010-1.648389$

H $2.2905532 .718832-0.849794$

H $-0.008702-0.1391881 .715070$

H 1.7836801 .3889551 .870649

H 3.1179252 .4692831 .442719

H 1.4664953 .0877671 .486712

H -1.9200831 .2592840 .994614$

H $\quad 0.321585-2.1420630 .717235$

H $-2.652385-1.3064131 .929112$

H -2.408821 $0.292812-1.270958$

H -4.1791131 .5589430 .040420$

H -4.792729 0.0077950 .634712

H $-2.138139-1.898346-0.716262$

H $4.5920221 .149226-0.236476$

H 3.8519790 .0985340 .982794

H $-5.285587-0.720963-1.686782$

H $-6.0957490 .803978-1.299838$

$\mathrm{H}-4.6404460 .810800-2.309935$

SCF Energy (B3LYP/6-31G**//MMFF) $=-810.719604785$

F2-15_c03364

MMFF Geometry

C -3.699317 -0.082919-1.498977

C $-4.9755130 .192146-1.791158$

C $-2.452684-0.1944110 .684774$

O $-1.567734-1.2211630 .186418$

C $-0.230339-1.0003490 .692802$

C -0.2381310 .4009221 .274936$

C $-\begin{array}{llll}-1.660258 & 0.515067 & 1.785484\end{array}$

C $0.765168-1.264224-0.444616$

C $-2.073496 \quad 1.9354892 .126459$

C $2.255228-1.039160-0.131735$

O $0.638009-2.657329-0.789990$

O $2.539896-1.6389451 .140109$

C $2.7563920 .416346-0.099885$

C $2.4286371 .208034-1.371667$

$\begin{array}{lllll}\text { O } & 4.181283 & 0.350839 & 0.052384\end{array}$

C $-2.8797940 .687136-0.499517$

C $2.9595822 .636253-1.337491$

H -3.212624 -0.903390 -2.020058

H $-5.509797-0.398190-2.527490$

H -5.507422 $1.002834-1.305585$

H -3.333493 -0.6861101 .115073$

H $-0.095271-1.7410201 .491344$

H $-0.052802 \quad 1.1535290 .500755$

H 0.5032830 .5288682 .068872

H $-1.739282-0.0902412 .699714$

H $0.467933-0.721898-1.347283$

H -1.9143262 .6222541 .288970$

H -3.130142 1.9746112 .409511

H -1.484593 2.310132 2.970696

H $2.855891-1.601617-0.859134$

H $-0.296864-2.816743-1.007096$

H $3.490480-1.5217501 .309241$

H 2.3794210 .9317390 .788182

H $1.3459251 .259291-1.521274$

H $2.8608790 .703194-2.243925$

H $4.491307 \quad 1.2333960 .316510$

H $-3.4559811 .547347-0.141209$

H -1.998703 $1.074964-1.024684$

H $2.6291843 .180358-2.228206$

H $2.5892423 .170700-0.456943$

H $4.0532692 .658540-1.323812$

SCF Energy (B3LYP/6-31G**//MMFF) $=-810.709704438$

F2-15_c03365 
MMFF Geometry

C $3.345962-0.6098111 .287959$

C 4.0302400 .0620982 .220702

C $2.597065-0.244259-1.146137$

O $1.470375-1.127059-0.953914$

C $0.282651-0.333373-0.744316$

C $0.779398 \quad 1.026468-0.279891$

C $2.0415371 .182983-1.102216$

C $-0.635504-1.0586800 .242581$

C $2.9692902 .284977-0.626320$

C $-1.861646-0.2387760 .691104$

O $-1.070810-2.281145-0.360618$

O $-2.588426-1.0164541 .647390$

C $-2.8166130 .218261-0.431120$

$\begin{array}{llll}C & -4.053742 & 0.977556 & 0.072986\end{array}$

O $-3.277833-0.919373-1.160870$

C $3.714179-0.644208-0.168523$

C -3.7259642 .2666360 .808052$

H $2.485429-1.1943791 .601747$

H 3.7177050 .0326143 .258878

H 4.9064610 .6491131 .968993

H $2.976039-0.435780-2.158167$

H $-0.200857-0.253406-1.726208$

H 1.0114691 .0180120 .791121

H $0.0527611 .823901-0.463172$

H $1.7322551 .441114-2.125902$

H - $0.067337-1.3600551 .131393$

H $3.8939392 .295083-1.212043$

H $2.4876133 .261631-0.744790$

H 3.2304242 .1822960 .429428

H -1.5002920 .6422701 .231261$

H - $0.275008-2.773483-0.626037$

H -2.918642 -1.806446 1.185432

H -2.270382 $0.854897-1.136019$

H -4.6634640 .3288420 .713766$

H $-4.6938511 .216312-0.786329$

H -3.820079 $-0.595883-1.900387$

H $3.998498-1.681697-0.388984$

H $4.602942-0.031121-0.359673$

H -3.218059 $2.070403 \quad 1.756695$

H -3.0882802 .9152330 .199092$

H -4.646859 2.8135421 .034841

SCF Energy (B3LYP/6-31G**//MMFF) $=-810.709035788$

F2-16_c03589

MMFF Geometry

C $4.597492-0.987590-0.164370$

C $4.760621-2.125568-0.848356$

C $2.6139110 .494657 \quad 0.231111$

O $1.637311-0.5592830 .107002$

C $0.3241740 .028995-0.016744$

C $0.489416 \quad 1.492296 \quad 0.361090$

C $1.8847151 .781556-0.153100$

C $-0.658492-0.757746 \quad 0.854322$

C $2.498573 \quad 3.0404260 .434419$

C $-2.064932-0.136034 \quad 0.946736$

O $-0.761547-2.085053 \quad 0.328994$

O $-2.840861-0.929211 \quad 1.850851$

C $-2.8265870 .015046-0.386530$

C -4.198446 $0.674026-0.182042$

O $-3.035474-1.254953-0.999327$

C $3.8141770 .191922-0.667635$

C $-4.9028420 .959789-1.502813$

H $5.063920-0.8958310 .813064$

H $5.342810-2.939283-0.429949$

H $4.319529-2.266827-1.828759$

H $2.9184770 .524677 \quad 1.285493$

H $0.056372-0.066970-1.076217$

H 0.4484891 .6215131 .449886

H - $0.2741012 .133562-0.089081$

H $1.8430851 .878427-1.246428$

H $-0.251853-0.8741631 .866823$

H 1.8949753 .9175520 .179221

H 2.5633542 .9835601 .526221

H 3.5087613 .2012530 .045081

H -1.9794220 .8526021 .411801$

H $0.138316-2.453068 \quad 0.292323$

H $-2.943720-1.8089311 .448301$
H -2.240635 $0.627388-1.080976$

H $-4.081166 \quad 1.6156080 .366488$

H -4.8482770 .0201330 .411823$

H -2.162594 -1.643333 -1.178285

H $3.4707740 .004360-1.692799$

H $4.4882961 .055327-0.700676$

H $-4.2989631 .619969-2.133402$

H $-5.0995910 .037515-2.058161$

H -5.862952 $1.452309-1.318472$

SCF Energy (B3LYP/6-31G**//MMFF) $=-810.724087526$

F2-16_c03590

MMFF Geometry

C $3.2656552 .457053-0.507337$

C 2.8020553 .5802540 .052069

C $2.610607 \quad 0.047828-0.281296$

$\begin{array}{lllll}\text { O } & 1.234190 & 0.320001 & 0.052809\end{array}$

C $0.624268-0.888714 \quad 0.543615$

C $1.593247-2.0145700 .206925$

C $2.927837-1.3096980 .345008$

C $-0.782903-1.060951-0.046792$

C $4.075534-2.046963-0.323042$

C -1.7195550 .1206580 .278931$

O $-0.695492-1.211007-1.463086$

O

C $-3.131095-0.036041-0.324524$

C $-4.059136 \quad 1.1270110 .053891$

O $-3.712285-1.265998 \quad 0.106798$

C $3.498823 \quad 1.1767490 .244035$

C $-5.4101291 .034046-0.644126$

H $3.5000722 .461946-1.568646$

H $2.6597034 .473709-0.545939$

H 2.5560443 .6292331 .107059

H $2.667334-0.011125-1.376144$

H $\quad 0.559150-0.7863091 .634083$

H $1.457035-2.373040-0.820120$

H $1.485876-2.8703010 .880326$

H $3.151954-1.1881251 .413303$

H $-1.203289-1.9898880 .356251$

H $3.894779-2.184474-1.394435$

H $5.013894-1.495676-0.207832$

H $4.208802-3.0372380 .124864$

H $-1.2909421 .047459-0.121171$

H $-0.270221-0.410960-1.816943$

H -2.127492 -0.5623042 .067553$

H $-3.050460-0.087719-1.416231$

H $-3.5868232 .079873-0.211456$

H -4.2388581 .1345441 .135435$

H -3.868262 -1.2039391 .064294$

H 3.3090191 .3299731 .313878

H 4.5556550 .9085240 .133770

H $-5.2893251 .033294-1.732140$

H $-5.9458990 .124295-0.356003$

H $-6.0339851 .891656-0.373097$

SCF Energy (B3LYP/6-31G**//MMFF) $=-810.718947473$

F2-16 c03591

MMFF Geometry

C $-3.1445152 .284128-0.093291$

C $-2.544078 \quad 3.426317-0.445884$

C $-2.353190-0.092981-0.032496$

O $-0.9664650 .244837 \quad 0.172313$

C $-0.159483-0.923414-0.055315$

C $-1.128545-2.098701-0.091110$

C $-2.349807-1.458250-0.719665$

C $0.948245-1.0500750 .999585$

C $-3.626146-2.256084-0.517505$

C $1.9305190 .135501 \quad 1.077545$

$\begin{array}{llll}\text { O } & 0.359674 & -1.217429 & 2.293935\end{array}$

$\begin{array}{llll}\text { O } & 1.229748 & 1.317743 & 1.486910\end{array}$

C $2.6917010 .459081-0.220544$

C $3.449006-0.738183-0.803965$

$\begin{array}{lllllll}\text { O } & 3.645961 & 1.486191 & 0.073548\end{array}$

C $-3.0276440 .999218-0.863651$

C $4.219692-0.377994-2.068676$

H $-3.764503 \quad 2.2752780 .799517$

H $-2.672068 \quad 4.321647 \quad 0.152567$

H $-1.9174253 .489097-1.328657$ 
H -2.814381 -0.1631350 .961368$

H $\quad 0.288897-0.804562-1.048865$

H -1.364800 -2.459478 0.916916

H $-0.738191-2.941742-0.669382$

H -2.168294 -1.337509-1.796213

H $1.511075-1.9695760 .806703$

H -3.536762 -3.246667 -0.975283

H $-3.847825-2.3960740 .545850$

H $-4.480805-1.748476-0.975568$

H $2.650266-0.0562161 .882914$

H $-0.157164-0.4151742 .482629$

H $\quad 0.584387 \quad 1.5371780 .793566$

H $2.0094250 .874680-0.971164$

H $2.755695-1.549642-1.047321$

H $4.163301-1.120738-0.064922$

H 3.1562772 .2372420 .450743

H -2.458710 $1.166245-1.786950$

H $-4.0368900 .685061-1.153003$

H $4.712794-1.267074-2.474576$

H $3.5472360 .017286-2.836741$

H $4.9925830 .370231-1.867951$

SCF Energy (B3LYP/6-31G**//MMFF) $=-810.725990231$

\section{F2-16 c03592}

MMFF Geometry

C $4.516967-1.4233030 .463531$

C $4.678423-2.621602-0.108469$

C 2.7357300 .3312390 .274240

O $1.678062-0.591956-0.054585$

C $0.5378820 .149543-0.544115$

C $0.813687 \quad 1.601674-0.190077$

C $2.3205431 .668909-0.336033$

C $-0.741357-0.462251 \quad 0.045746$

C 2.9421802 .8781260 .340809

C $-2.0334750 .261740-0.381442$

O $-0.815659-1.820243-0.398695$

O

C $-3.328452-0.503139-0.029611$

C $-4.585990 \quad 0.281181-0.429577$

O $-3.383334-0.748768 \quad 1.375018$

C $4.064428-0.193651-0.272181$

C $-5.860044-0.526635-0.213259$

H $4.732674-1.3187681 .523773$

H $5.012872-3.4699970 .478665$

H $4.480530-2.778588-1.162936$

H 2.7689400 .4012661 .369401

H $0.5419880 .017647-1.633753$

H $0.523948 \quad 1.8133820 .846571$

H $0.2927352 .303783-0.847383$

H $2.5694941 .688392-1.405672$

H $-0.655790-0.481178 \quad 1.139139$

H $4.0301192 .876670 \quad 0.221156$

H $2.5558313 .804510-0.096880$

H 2.7211652 .8957861 .413433

H -2.015965 $0.407739-1.468542$

H $0.009176-2.259483-0.128406$

H -2.146838 1.4302281 .180491

H $-3.339393-1.473791-0.539326$

H $-4.5243870 .569315-1.485228$

H -4.6664291 .1995290 .164123$

H $-2.696706-1.4034661 .586435$

H $3.963876-0.412907-1.342538$

H $4.8438050 .569226-0.165558$

H $-6.7319790 .053106-0.532288$

H $-5.838698-1.454108-0.794007$

H $-5.994977-0.7836420 .842047$

SCF Energy (B3LYP/6-31G**//MMFF) $=-810.723382534$

\section{F2-16 c03593}

MMFF Geometry

C $3.5855502 .089693-0.542076$

C 3.3310313 .2762300 .020765

C $2.554732-0.179194-0.268910$

$\begin{array}{lllll}\text { O } & 1.253199 & 0.316197 & 0.106361\end{array}$

C $0.470825-0.773674 \quad 0.630050$

C $1.231630-2.0448880 .275172$

C $2.667120-1.5671640 .362253$

C $-0.965929-0.7175590 .086385$
C $3.655852-2.486131-0.334142$

C -1.6894480 .5982070 .445422$

O $-0.944545-0.888947-1.328512$

$\begin{array}{llll}\text { O } & -1.716315 & 0.729012 & 1.866361\end{array}$

C $-3.1290300 .729372-0.098810$

C $-4.052621-0.4244420 .314707$

$\begin{array}{llll}\text { O } & -3.115618 & 0.823756 & -1.520517\end{array}$

C 3.6324200 .7922430 .214585

C $-5.502265-0.167810-0.080467$

H $3.7807262 .050903-1.610706$

H $3.3156994 .178017-0.581648$

H 3.1332063 .3698511 .082748

H $2.564067-0.252679-1.364408$

H $0.460434-0.6560491 .720777$

H $1.004479-2.382863-0.742586$

H $1.008816-2.8674130 .961524$

H $2.944057-1.4776701 .421290$

H -1.510050 -1.567050 0.514260

H $4.674972-2.095065-0.255569$

H $3.640558-3.4820610 .120496$

H $3.419034-2.598542-1.397507$

H -1.1050121 .4354560 .042986$

H -0.427819 -0.155977 -1.704941

H -2.112715 1.5921132 .074895

H -3.5603831 .6629210 .284501$

H $-4.005253-0.5793611 .398264$

H -3.737432 -1.354383 -0.172203

H $-2.5517001 .578835-1.759724$

H 3.5066620 .9796781 .288317

H 4.6269360 .3543520 .072509

H -5.8799160 .7462970 .388740$

H $-5.608246-0.068055-1.165145$

H $-6.134075-1.0006990 .243974$

SCF Energy $\left(B 3 L Y P / 6-31 G^{* *} / / M M F F\right)=-810.712681335$

F2-16 c03594

MMFF Geometry

C $3.2570612 .440289-0.004166$

C $4.2423413 .122817-0.598379$

C $2.5475520 .036640-0.123114$

O $1.157340 \quad 0.299504 \quad 0.157988$

C $0.504195-0.9409180 .487598$

C $1.463411-2.0429460 .058228$

C $2.803083-1.3954490 .348545$

C $-0.895691-1.003676-0.142896$

C $3.969705-2.076952-0.345650$

C -1.8013210 .1583680 .316067$

O $-0.797754-0.954337-1.564974$

$\begin{array}{lllll}\text { O } & -1.915806 & 0.132827 & 1.738639\end{array}$

C $-3.2086520 .123569-0.317352$

C $-4.0948201 .267206 \quad 0.197599$

O $-3.819804-1.128425-0.008591$

C 3.4237491 .0693230 .588604

C $-5.4229971 .360740-0.543045$

H 2.2754462 .9014800 .068658

H $4.0588234 .112437-1.002252$

H $5.2410302 .711369-0.693440$

H $2.6721790 .111297-1.211461$

H $0.412505-0.9626891 .581044$

H $1.380046-2.266730-1.010722$

H $1.304398-2.9725030 .613126$

H $2.973063-1.4095541 .433621$

H -1.354646 -1.960392 0.135426

H $3.843290-2.079879-1.433526$

H $4.911617-1.568291-0.117621$

H $4.059234-3.116948-0.015198$

H -1.336263 1.1134110 .042968

H $-0.502271-1.827114-1.873124$

H $-2.376009-0.6893311 .980332$

H $-3.1154540 .190637-1.407723$

H -3.5662332 .2211440 .086909$

H -4.312546 1.1276021 .263159

H -4.614871 -1.214530 -0.560722

H $3.146578 \quad 1.1287951 .648240$

H 4.4753690 .7634840 .541579

H -5.991133 2.226232 -0.187047

H -5.264523 $1.479309-1.619501$

H $-6.0371270 .470156-0.379464$ 
F2-16_c03595

MMFF Geometry

C $4.620058-0.995597-0.146620$

C $4.792926-2.135718-0.824619$

C $2.630147 \quad 0.4825530 .232042$

O $1.655651-0.5735790 .107703$

C $0.341806 \quad 0.011885-0.022854$

C $0.503688 \quad 1.477245 \quad 0.350030$

C $1.9004761 .766538-0.160378$

C $-0.645544-0.7714360 .847579$

C $2.510067 \quad 3.028830 \quad 0.424234$

C $-2.051483-0.145174 \quad 0.949752$

O $-0.731521-2.108068 \quad 0.342673$

O $-2.810090-0.956713 \quad 1.851635$

C $-2.8085490 .006578-0.386395$

C $-4.1845840 .666186-0.213714$

O $-2.982965-1.263913-1.009335$

C $3.8346810 .178126-0.660102$

C $-4.8464250 .969203-1.552640$

H $5.079495-0.8975680 .833623$

H $5.375931-2.945003-0.398822$

H $4.358977-2.283196-1.807291$

H $2.9298660 .517456 \quad 1.287767$

H $0.079127-0.085889-1.083443$

H $\quad 0.458905 \quad 1.610574 \quad 1.438279$

H $-0.2590972 .115759-0.105028$

H $1.8626341 .858794-1.254242$

H $-0.232659-0.8792051 .858291$

H 2.5704192 .9767641 .516430

H 3.5215373 .1894060 .038133

H 1.9061943 .9040380 .162749

H -1.9586720 .8412591 .419484$

H -1.056165 -2.061801-0.572659

H $-3.595227-0.4501092 .118670$

H $-2.2106700 .619441-1.069491$

H $-4.081782 \quad 1.6002850 .350296$

H $-4.856420 \quad 0.0071140 .348349$

H $-3.467279-1.832470-0.386175$

H $3.496199-0.016716-1.685545$

H $4.5061661 .043495-0.695345$

H -5.812632 $1.458394-1.392940$

H $-4.223412 \quad 1.638203-2.154673$

H -5.023605 $0.053838-2.125724$

SCF Energy (B3LYP/6-31G**//MMFF) $=-810.712416749$

F2-16_c03596

MMFF Geometry

C $3.611768 \quad 2.113103-0.365676$

C 3.7194763 .0767040 .555762

C $2.385713-0.072851-0.409690$

$\begin{array}{lllll}\text { O } & 1.344317 & 0.345436 & 0.499290\end{array}$

C $0.642493-0.8176890 .962302$

C $1.036700-1.9547870 .030097$

C $2.478710-1.593948-0.270700$

C $-0.864528-0.559176 \quad 1.115618$

C $3.036591-2.293664-1.498106$

C $-1.605069-0.119320-0.166655$

O $-1.478116-1.7425501 .623591$

O $-\begin{array}{llll}-1.087887 & 1.126618 & -0.628863\end{array}$

C $-3.127578 \quad 0.036019 \quad 0.044417$

C $-3.8479130 .513139-1.225048$

$\begin{array}{lllll}\text { O } & -3.377679 & 0.963417 & 1.100283\end{array}$

C $3.6907140 .644055-0.059947$

C $-5.3611520 .540506-1.049787$

H $3.4671362 .399244-1.404316$

H 3.6579114 .1207260 .268764

H 3.8678262 .8451461 .604712

H $2.0551600 .199881-1.420391$

H $1.047577-1.0471161 .957717$

H $\quad 0.447214-1.948345-0.892427$

H $0.929118-2.9381560 .497500$

H $3.094680-1.8543930 .600640$

H $-0.9832780 .235647 \quad 1.863201$

H $2.452548-2.058403-2.394213$

H $4.072366-1.992131-1.682387$

H $\quad 3.020219-3.380039-1.361917$
H $-1.461691-0.859855-0.961119$

H $-1.049619-1.9564742 .469871$

H $-0.1517330 .994255-0.851832$

H $-3.551982-0.9257550 .353690$

H $-3.599250-0.147627-2.063363$

H $-3.5221811 .525766-1.490716$

H $-2.970824 \quad 1.810447 \quad 0.848977$

H 3.9226650 .4936601 .001858

H $4.5197760 .222525-0.639572$

H $-5.8424520 .858946-1.980009$

H $-5.743474-0.452592-0.793395$

H $-5.6583521 .238731-0.261202$

SCF Energy (B3LYP/6-31G**//MMFF) $=-810.716993209$

F2-16_c03597

MMFF Geometry

C $4.484667-0.2059580 .915191$

C $5.067965-1.4071290 .835239$

C $2.277876 \quad 0.674352 \quad 0.114141$

$\begin{array}{llllllll}\text { O } & 1.598908 & -0.595682 & 0.179175\end{array}$

C $0.334349-0.494497-0.517481$

C $0.115398 \quad 0.989886-0.748337$

C $1.5349381 .482790-0.946569$

C $-0.732205-1.226773 \quad 0.307144$

C $1.6808442 .986983-0.794351$

C $-2.171489-1.202513-0.238408$

O $-0.346992-2.6147340 .340003$

O $-2.142826-1.479897-1.645338$

C $-2.9753390 .094438-0.043984$

C $-3.031313 \quad 0.576971 \quad 1.409174$

O $-4.320922-0.161222-0.471413$

C $3.7535110 .451872-0.220933$

C $-3.872321 \quad 1.838197 \quad 1.570780$

H 4.5450450 .3411491 .852410

H $5.584961-1.8248921 .692296$

H $5.039899-1.992274-0.077307$

H $2.172143 \quad 1.144112 \quad 1.100929$

H $0.487944-1.007441-1.475501$

H -0.3280251 .4642220 .134612$

H $-0.5223791 .192525-1.613160$

H $1.872297 \quad 1.188500-1.949597$

H $-0.703410-0.8992881 .350790$

H 1.3583613 .3230950 .196858

H $2.7220443 .294537-0.932287$

H $1.0728613 .509348-1.540204$

H $-2.739410-2.0288860 .209247$

H $0.555496-2.6551970 .701130$

H $-1.685201-2.330266-1.762779$

H $-2.6015380 .890846-0.693574$

H -2.0258950 .7909911 .783853$

H $-3.463458-0.2061992 .043717$

H $-4.277530-0.467523-1.393608$

H $3.839138-0.161222-1.126942$

H $4.2427681 .410680-0.426628$

H -3.853162 2.1716952 .613267

H -3.482766 2.6498040 .947956

H -4.916812 1.6622421 .295482

SCF Energy (B3LYP/6-31G**//MMFF) $=-810.718327853$

F2-16_c03598

MMFF Geometry

C $2.7944462 .123722-0.575929$

C 3.7320822 .8810690 .004565

C $2.268496-0.366444-0.544244$

O $0.855431-0.172755-0.746164$

C $0.160263-0.5772710 .447923$

C $1.186505-1.3154591 .303912$

C $2.459501-0.5670970 .958207$

C $-1.092368-1.4015100 .120104$

C $3.729303-1.3139811 .325221$

C $-2.146035-0.679682-0.744441$

O $-0.692738-2.592875-0.566814$

O $-1.580241-0.344589-2.017656$

C $-2.7177750 .617046-0.144331$

C $-3.2876910 .449712 \quad 1.269356$

O $-3.7596831 .083159-1.010900$

C $3.061507 \quad 0.776084-1.184500$

$\begin{array}{llll}\text { C } & -3.887319 & 1.741953 & 1.810777\end{array}$ 
H $\quad 1.7769002 .502472-0.625931$

H 3.4760123 .8490820 .421123

H 4.7628902 .5521250 .076358

H 2.522374 -1.292222 -1.077859

H -0.1296750 .3443590 .966638$

H $1.273449-2.3690011 .013457$

H $0.941039-1.2782342 .369574$

H $2.444780 \quad 0.393481 \quad 1.487604$

H -1.551562 -1.7287841 .059567$

H $3.783275-2.2858800 .823264$

H $4.615328-0.7377001 .040983$

H $3.772946-1.4908662 .404857$

H -2.967769-1.372722 -0.964702

H -1.489239 $-3.133189-0.705408$

H -1.182767 -1.154481 -2.380763

H $-1.9513241 .400323-0.149544$

H -2.505963 0.1202891 .961115

H $-4.071978-0.3168581 .265393$

H -4.466319 $0.415013-1.012850$

H $4.1318340 .541326-1.141835$

H $2.7905640 .842296-2.245760$

H -4.2441611 .5908762 .834597$

H $-3.1409062 .542576 \quad 1.826287$

H $-4.7373802 .073348 \quad 1.206388$

SCF Energy (B3LYP/6-31G $\left.{ }^{* *} / / \mathrm{MMFF}\right)=-810.706454867$

F2-16 c03599

MMFF Geometry

C -1.972889 2.8279090 .489827

C $-1.1273203 .715026-0.046341$

C $-2.209013 \quad 0.3516310 .193486$

O $-0.825566 \quad 0.142350-0.151963$

C $-0.664993-1.182070-0.686175$

C -1.962239-1.915631-0.366922

C $-2.975414-0.793040-0.466946$

C $0.586968-1.861876-0.121363$

C $-4.303978-1.1125710 .195827$

C $1.920767-1.230371-0.576722$

O $0.517133-1.9038471 .304010$

O $2.978435-2.085028-0.124628$

C $2.2133290 .216956-0.130674$

C $3.6064740 .691681-0.573218$

$\begin{array}{llll}\text { O } & 2.106838 & 0.342798 & 1.284657\end{array}$

C -2.650263 $1.734377-0.287580$

C $3.8350032 .166010-0.263226$

H -2.1963702 .8963971 .551399$

H -0.672882 4.4827960 .570227

H $-0.8719553 .690884-1.099865$

H $-2.2791830 .282303 \quad 1.286946$

H - $0.566111-1.078581-1.774100$

H $-1.950193-2.3296660 .648333$

H -2.158276 -2.735796 -1.064182

H -3.147452 -0.568438 -1.528284

H $0.589267-2.909446-0.447616$

H -4.995762 -0.2688090 .110913$

H $-4.771277-1.981412-0.279096$

H -4.177526 -1.339100 1.259857

H $1.956574-1.262336-1.672371$

H $0.426041-0.9883961 .619609$

H $2.902702-2.1593490 .841965$

H $1.4682230 .886541-0.570841$

H $3.7279270 .532668-1.650901$

H $4.3861130 .115497-0.061098$

H $2.802385-0.2048311 .686515$

H -2.428714 $1.842911-1.356787$

H $-3.7328151 .850900-0.163959$

H $3.0862962 .791264-0.760079$

H 3.7860152 .3593490 .812948

H $4.8236612 .476281-0.615981$

SCF Energy (B3LYP/6-31G**//MMFF) $=-810.723767230$

F2-16_c03600

MMFF Geometry

C -3.205815 2.217636 -0.962209

C -2.876587 2.8802390 .152726

C $-2.340560-0.096757-0.176709$

O $-0.9603130 .294921-0.042587$

C $-0.142958-0.8827730 .068029$
C $-1.106927-2.0429110 .286489$

C $-2.310232-1.588092-0.514532$

C $0.914731-0.7294331 .170405$

C $-3.587681-2.330855-0.162697$

C 1.8992400 .4417150 .988145

O $0.264562-0.5624372 .435548$

O 1.1645211 .6724021 .028964

C $2.7239650 .420619-0.311523$

C $3.500193-0.884942-0.521990$

O $3.6511961 .509487-0.228133$

C $-3.0151420 .752648-1.258176$

C $4.332160-0.883816-1.798744$

H -3.669965 2.791512 -1.762385

H -3.0734823 .9445010 .230313$

H -2.4116242 .3974061 .003483$

H -2.8212860 .0597160 .797419$

H $0.350860-1.012781-0.902421$

H $-1.373846-2.1538411 .344101$

H $-0.694540-2.995879-0.058881$

H -2.094859-1.728112 -1.582367

H $1.479235-1.6646621 .247390$

H $-4.430241-1.963346-0.756617$

H -3.476636 -3.401857 -0.361815

H -3.843482 -2.210582 0.895398

H $2.5714650 .475716 \quad 1.855141$

H -0.2575900 .2569552 .385653$

H 1.8059592 .3964960 .927029

H $2.0720780 .627545-1.168446$

H $2.810244-1.733377-0.571969$

H $4.175065-1.055168 \quad 0.325548$

H $4.0046101 .665651-1.120038$

H -2.422985 $0.689521-2.180299$

H $-4.0048770 .336511-1.479827$

H $5.132368-0.138884-1.756319$

H $\quad 4.799605-1.863303-1.942577$

H $3.707877-0.674395-2.673136$

SCF Energy $(B 3 L Y P / 6-31 G * * / / M M F F)=-810.714405059$

F2-16 c03601

MMFF Geometry

C -2.6090302 .6039020 .569221$

C -1.790886 3.6501560 .409736

C -2.3472310 .1648150 .056042$

$\begin{array}{lllll}\text { O } & -0.912649 & 0.166060 & 0.200123\end{array}$

C $-0.382729-1.048297-0.356969$

C $-1.579019-1.974889-0.544940$

C $-2.668061-0.982713-0.901627$

C $0.737855-1.6230220 .519771$

C $-4.072360-1.541009-0.749956$

C $1.934478-0.6994460 .838857$

O $0.181571-2.0375501 .774218$

O $1.499592 \quad 0.4784791 .518339$

C $2.804281-0.320027-0.373897$

C 4.0762890 .4304860 .050210

O $2.0752110 .491983-1.289483$

C $-2.8095561 .527982-0.460484$

C $5.0007500 .709927-1.127937$

H -3.1747912 .5207081 .493529$

H -1.693516 4.3954751 .191502

H -1.206134 $3.780997-0.494072$

H $-2.767073-0.0344411 .050846$

H $0.013183-0.796431-1.346555$

H -1.835941 -2.501877 0.381305

H -1.406482 -2.723763 -1.323844

H -2.521196 -0.658443 -1.940743

H $1.119447-2.5321630 .040475$

H -4.822370 -0.785587 -1.003992

H -4.218696 -2.398958 -1.414271

H $-4.263486-1.8740620 .275719$

H $2.568547-1.2316461 .559430$

H $-0.172119-1.2430482 .210342$

H $0.872657 \quad 0.944030 \quad 0.939716$

H $3.088531-1.235059-0.906206$

H $4.622512-0.1568800 .797255$

H 3.8143301 .3906850 .510566

H $1.8770901 .336373-0.850470$

H $-2.2628431 .780943-1.377645$

H $-3.8754301 .494909-0.713099$ 
H $5.298199-0.220860-1.621423$

H $4.5187621 .354719-1.869315$

H $5.9083401 .215421-0.782793$

SCF Energy (B3LYP/6-31G**//MMFF) $=-810.725002362$

F2-16 c03602

MMFF Geometry

C -3.377943 $2.039166-0.389850$

C $-2.8012873 .225990-0.610964$

C $-2.403194-0.0221870 .751499$

$\begin{array}{lllll}\text { O } & -1.045154 & 0.298219 & 0.391572\end{array}$

C $-0.572918-0.693587-0.541210$

C $-1.600256-1.821966-0.516207$

C $-2.883480-1.050963-0.270891$

C $0.862252-1.123071-0.200101$

C $-4.034200-1.9138980 .215030$

C $1.8523980 .061395-0.144794$

O $0.852806-1.7874981 .062856$

O $1.8715990 .723520-1.408849$

C $3.295059-0.3604920 .206550$

C 4.3305830 .7705900 .124589

O $3.720204-1.399966-0.678204$

C $-3.231141 \quad 1.2587420 .885883$

C 4.0556521 .9210481 .078552

H -4.008699 $1.617965-1.167808$

H $-2.9547383 .744623-1.550971$

H -2.1687403 .6972750 .133145$

H $-2.346846-0.4893021 .744137$

H $-0.592745-0.223887-1.532260$

H -1.415569 -2.522994 0.306048

H - $-1.612348-2.392511-1.449806$

H $-3.172990-0.566711-1.210985$

H $1.176205-1.846803-0.962226$

H $-4.928259-1.3070730 .389878$

H -4.283579 -2.676961 -0.529370

H $-3.785512-2.4261681 .150549$

H 1.5197620 .7821780 .611459

H $1.686512-2.2807521 .142976$

H $1.0474141 .232949-1.487467$

H $3.317204-0.7803361 .218379$

H $4.4032201 .150543-0.901836$

H $\begin{array}{llll}5.321199 & 0.353163 & 0.346804\end{array}$

H $3.670535-1.051455-1.584966$

H -4.2334031 .0124651 .255602$

H $-2.762516 \quad 1.8957381 .647324$

H 3.9577131 .5630742 .108315

H 4.8806432 .6400771 .047360

H 3.1407552 .4560920 .808203

SCF Energy (B3LYP/6-31G**//MMFF) $=-810.717916321$

F2-16_c03603

MMFF Geometry

C $4.216078-1.6816440 .645711$

C $4.300918-2.8826470 .062758$

C $2.631490 \quad 0.241303 \quad 0.365691$

O $1.515967-0.568477-0.057322$

C $0.4912990 .288658-0.610233$

C $0.8811541 .701141-0.206499$

C $2.3939851 .621422-0.244223$

C $-0.881705-0.206136-0.127508$

C 3.0798842 .7538060 .500295

C $-2.0650100 .648852-0.628066$

O $-1.040436-1.546197-0.599524$

O $-2.0068091 .932019-0.007355$

C $-3.4698330 .055317-0.378167$

C $-3.730452-0.3272871 .085781$

O $-3.687400-1.071549-1.223004$

C $3.939293-0.403976-0.095480$

C $-5.175181-0.7533041 .317819$

H $4.364184-1.6124941 .720351$

H $4.508056-3.7673450 .655010$

H 4.164821 -3.005839-1.005889

H $2.591070 \quad 0.292176 \quad 1.461767$

H $0.5623920 .173240-1.699632$

H $\quad 0.5392081 .9254850 .811524$

H $0.4807382 .460894-0.884039$

H $2.7203881 .631335-1.292958$

H $-0.877963-0.2441080 .968628$
H $4.1683002 .646700 \quad 0.457767$

H 2.8180663 .7197080 .056122

H $2.7846892 .777787 \quad 1.554585$

H -1.947837 $0.790020-1.710327$

H $-0.283968-2.061729-0.270399$

H $-2.7014872 .484100-0.404842$

H $-4.2133280 .808360-0.667974$

H $-3.5008130 .518267 \quad 1.743705$

H -3.086796 -1.163986 1.380496

H $-3.058825-1.765463-0.961183$

H $3.896089-0.598130-1.174602$

H 4.7791420 .2772580 .081588

H $-5.330927-0.9971772 .373583$

H -5.8683200 .0505311 .049911$

H $-5.428983-1.6389860 .727172$

SCF Energy $(B 3 L Y P / 6-31 G * * / / M M F F)=-810.721295295$

F2-16_c03604

MMFF Geometry

C $4.656776-1.390853-0.270354$

C $4.250103-2.2951150 .628222$

C 2.7108710 .2804410 .113713

O $1.655790-0.603924-0.313633$

C $0.4582300 .167331-0.557808$

C $0.725966 \quad 1.537440 \quad 0.043455$

C $2.2136651 .691269-0.200523$

C $-0.749858-0.5966770 .005521$

C 2.8559332 .7785100 .643728

C $-2.0979300 .135494-0.166624$

O $-0.814361-1.859251-0.666426$

$\begin{array}{llll}\text { O } & -2.116877 & 1.310657 & 0.640557\end{array}$

C -3.320509 -0.7378300 .188236$

C -4.6637130 .0050330 .143241$

O $-3.146309-1.2491331 .512292$

C $4.010887-0.074231-0.615572$

C $-5.0061760 .566959-1.226482$

H $5.564063-1.617439-0.827419$

H $4.817809-3.2080870 .775310$

H $3.361137-2.1598811 .231829$

H 2.8249180 .1534351 .197655

H $\quad 0.3714700 .245738-1.649262$

H $0.516636 \quad 1.5399411 .120193$

H $0.1357152 .328710-0.427833$

H $2.3738321 .919412-1.263067$

H $-0.564080-0.8117951 .064645$

H 3.9299042 .8454690 .444196

H 2.4098053 .7529200 .419447

H 2.7233012 .5867201 .713816

H -2.190683 $0.454229-1.211038$

H $-0.973948-1.685696-1.610015$

H $-2.067103 \quad 1.028787 \quad 1.570189$

H -3.363417 -1.598884 -0.488978

H $-4.6806110 .808500 \quad 0.889650$

H $-5.458466-0.6874480 .449482$

H -3.889748 -1.848429 1.695878

H $3.819413-0.088282-1.696352$

H $4.755510 \quad 0.708532-0.428725$

H $-6.0167960 .987856-1.217542$

H -4.318295 $1.366995-1.515236$

H $-4.971777-0.214717-1.991668$

SCF Energy (B3LYP/6-31G**//MMFF $)=-810.710653663$

F2-16_c03605

MMFF Geometry

C $-4.925440-0.2549290 .190925$

C $-5.6093980 .362290 \quad 1.160952$

C $-2.479744-0.376407-0.477492$

O $-1.329443-1.234954-0.308380$

C $-0.175186-0.423945-0.010001$

C $-0.5410290 .974068-0.473168$

C $-2.0155561 .039501-0.122341$

C $1.053878-1.030798-0.690405$

C $-2.7370962 .152439-0.866168$

C $2.321354-0.154583-0.613049$

O $1.296977-2.304604-0.085593$

O $3.370156-0.816641-1.327010$

C 2.8228600 .1748560 .808612

C 4.1095731 .0128590 .836951 
O $3.106060-1.0211121 .536083$

C $-3.606243-0.9325130 .398540$

C 3.9648792 .3837840 .197093

H $-5.349299-0.282961-0.809421$

H $-6.564180 \quad 0.8302450 .947525$

H $-5.232743 \quad 0.4138142 .176423$

H $-2.759887-0.442123-1.536391$

H $-0.062219-0.4523521 .080999$

H $-0.4012811 .072356-1.557085$

H $0.048150 \quad 1.7524930 .020773$

H -2.1118481 .2080520 .958465$

H $\quad 0.821271-1.245869-1.740875$

H $-2.2774703 .121573-0.644346$

H -2.695136 $2.002359-1.950224$

H -3.788906 $2.211754-0.575292$

H $2.1260280 .781759-1.146298$

H $2.088475-2.679226-0.508841$

H $3.063893-0.952974-2.239903$

H 2.0459860 .7138851 .362408

H 4.9324090 .4660200 .360173

H $4.418721 \quad 1.1426061 .882407$

H $2.275647-1.5192821 .619903$

H $-3.732260-2.0000760 .175350$

H -3.301748 -0.8973251 .452887$

H 3.1263032 .9351310 .633829

H 4.8755382 .9701250 .356759

H $3.8074572 .308778-0.882717$

SCF Energy $($ B3LYP/6-31G**//MMFF $)=-810.713694360$

F2-16_c03606

MMFF Geometry

C $-4.480422-1.1365840 .001863$

C $-4.610014-2.3421490 .566657$

C $-2.5515510 .451459-0.210584$

O $-1.536608-0.572475-0.177389$

C $-0.245306 \quad 0.0492360 .018510$

C $-0.4664891 .536210-0.209989$

C $-1.876768 \quad 1.7185260 .312599$

C $0.763353-0.610648-0.927426$

C $-2.5332513 .007109-0.151444$

C $2.1421120 .067438-1.022172$

O $0.945577-1.974586-0.535070$

O $2.712272-0.335562-2.284095$

C $3.195974-0.2690550 .052462$

C $2.748025-0.0353411 .496100$

O $4.3387330 .561977-0.195080$

C $-3.749845 \quad 0.014527 \quad 0.633532$

C $3.873732-0.2402712 .504122$

H $-4.935936-0.964879-0.970039$

H -5.154657 -3.1307960 .059172$

H $-4.178019-2.564277 \quad 1.536062$

H -2.844166 $0.576765-1.261431$

H $\quad 0.004748-0.142423 \quad 1.065745$

H $-0.4201681 .778339-1.279136$

H $\quad 0.2665832 .1565070 .314312$

H $-1.8488841 .704374 \quad 1.410554$

H $\quad 0.319867-0.653713-1.931174$

H -3.5527003 .0889570 .238370$

H -1.966809 3.8754600 .200573

H -2.585045 $3.060332-1.244123$

H $2.0317581 .153509-1.104984$

H $0.064324-2.383772-0.489189$

H $2.787939-1.305471-2.274884$

H $3.538944-1.304627-0.061252$

H $\quad 1.942847-0.728414 \quad 1.754254$

H $2.3690120 .986861 \quad 1.609623$

H $4.6254490 .394917-1.109321$

H $-3.412080-0.262517 \quad 1.639907$

$\begin{array}{llll}\mathrm{H} & -4.457517 & 0.844228 & 0.742135\end{array}$

H $3.490659-0.1239503 .523097$

H $4.300939-1.2440932 .414756$

H 4.6758150 .4911142 .364662

SCF Energy (B3LYP/6-31G**//MMFF) $=-810.717110438$

F2-16_c03607

MMFF Geometry

C 3.5730582 .1331330 .081484

C $4.6081642 .704033-0.544884$
C $2.506242-0.129034-0.118738$

$\begin{array}{lllll}\text { O } & 1.192987 & 0.314464 & 0.279887\end{array}$

C $0.374509-0.8369580 .559031$

C $1.138176-2.0373880 .014207$

C $2.572734-1.6108840 .252700$

C $-1.036153-0.657247-0.024944$

C $3.583716-2.407348-0.553907$

C -1.7500530 .5975820 .527268$

O $-0.935618-0.578149-1.444894$

O

C -3.1841570 .8389910 .006531$

C $-4.130753-0.3547450 .203659$

O $-3.1171191 .182163-1.375523$

C 3.5718510 .7159750 .581853

C $-5.577603-0.018528-0.138946$

H 2.6779022 .7253240 .252823

H $4.5481833 .734583-0.877638$

H $5.5260672 .159710-0.736908$

H $2.569154-0.001782-1.207523$

H $\quad 0.315133-0.9203241 .651447$

H $0.958011-2.181371-1.057457$

H $0.873598-2.9661440 .528682$

H $2.799069-1.7206491 .321909$

H -1.608141 -1.5586060 .224127$

H $3.397501-2.319486-1.629578$

H $4.602369-2.057961-0.359004$

H $3.535198-3.469013-0.290204$

H -1.158682 1.4781030 .245752

H -1.837219 -0.533329-1.805027

H -2.165819 -0.3007222 .219796$

H -3.5943541 .7044550 .542661$

H $-4.095426-0.6991981 .242576$

H -3.824866 -1.189859 -0.436836

H $-3.9795281 .547901-1.634141$

H 3.3772900 .7440731 .660995

H 4.5605230 .2649090 .438663

H $-6.222586-0.8769590 .074785$

H -5.9345580 .8291290 .454344$

H $-5.6924030 .226922-1.198857$

SCF Energy (B3LYP/6-31G**//MMFF) $=-810.713689006$

F2-16 c03608

MMFF Geometry

C -3.087263 2.3630410 .704211

C -2.6894713 .5162430 .155279$

C $-2.430157-0.0240260 .310293$

O $-1.093873 \quad 0.283299-0.137383$

C $-0.520073-0.893344-0.737751$

C $-1.440870-2.048141-0.363268$

C $-2.789872-1.357572-0.344515$

C $0.941333-1.064106-0.290925$

C -3.862347 -2.1401990.393286

C $1.8291750 .152684-0.626521$

O $0.966431-1.3070121 .113807$

O $1.5970130 .505209-1.997094$

C $3.344954-0.095983-0.473948$

C $3.846493-0.5123330 .913678$

O $4.015606 \quad 1.114457-0.851144$

C $-3.3757671 .113391-0.079083$

C 3.5783330 .5131452 .008416

H -3.2221772 .3179981 .781865$

H -2.501786 4.3846810 .777155

H $-2.5430573 .614808-0.914661$

H $-2.383747-0.1314511 .401938$

H $-0.564454-0.745108-1.823884$

H -1.206907 -2.450452 0.629235

H -1.387872 -2.871358 -1.082142

H -3.114465 -1.192611-1.380811

H $1.344647-1.955259-0.786402$

H -4.025738 -3.112022 -0.083861

H $-3.581320-2.3210941 .436245$

H -4.813362 -1.598655 0.389801

H $1.5353941 .018659-0.021465$

H $0.534025-0.5527641 .549795$

H $2.1493521 .281457-2.193321$

H $3.651296-0.853944-1.205425$

H $4.934177-0.6514070 .861065$

H $3.429755-1.4827931 .200085$ 
H $4.9719140 .939217-0.828266$

H $-3.2883581 .315867-1.153993$

H $-4.4145930 .824808 \quad 0.116715$

H 2.5084230 .6846302 .152470

H 3.9896210 .1594372 .959489

H 4.0510101 .4729321 .777990

SCF Energy (B3LYP/6-31G**//MMFF) $=-810.718937315$

\section{F2-16 c03609}

MMFF Geometry

C $-4.623196-1.3617880 .189121$

C $-5.302353-2.182860-0.619433$

C $-2.407173-0.196596-0.275339$

O $-0.987920-0.433571-0.128253$

C -0.3909720 .6982030 .524204$

C $-1.343448 \quad 1.8525750 .264835$

C $-2.691808 \quad 1.1632070 .375453$

C 1.0421450 .9208980 .019880

C $-3.8003431 .964533-0.291328$

C $1.957444-0.3079610 .203179$

O $1.012841 \quad 1.271979-1.363670$

O $2.020361-0.6589451 .584394$

C $3.392261-0.066414-0.311341$

C $4.302786-1.283022-0.091655$

O $3.9585651 .066300 \quad 0.347241$

C $-3.134780-1.3908620 .351225$

C $5.680419-1.088371-0.712372$

H $-5.179319-0.6448190 .786573$

H $-6.383270-2.120621-0.683474$

H -4.797955 -2.924526 -1.228890

H $-2.604073-0.163867-1.354129$

H -0.3774380 .4793351 .599267$

H -1.212202 $2.264596-0.742581$

H -1.2185952 .6673140 .984477$

H -2.9339491 .0300041 .438001$

H 1.4565351 .7764450 .565950

H -3.606210 2.103160 -1.360438

H $-4.7702591 .471601-0.188899$

H -3.8826132 .9567340 .164995$

H $\quad 1.548990-1.165579-0.345914$

H $0.5948050 .536712-1.844059$

H $1.170934-1.0656001 .824594$

H $3.3599600 .166720-1.381669$

H $3.839893-2.176177-0.526903$

H $4.440109-1.4676620 .980428$

H $3.9568920 .881706 \quad 1.302161$

H -2.723487 -2.314835 -0.077255

H -2.895814 -1.450973 1.420911

H $6.205516-0.241740-0.259381$

H $6.292001-1.983491-0.560400$

H $5.602869-0.909798-1.789621$

SCF Energy (B3LYP/6-31G**//MMFF) $=-810.722008983$

\section{F2-16 c03610}

MMFF Geometry

C $-4.280266-0.7066050 .097494$

C $-5.272223-0.3606480 .925772$

C $-2.2226960 .500747-0.791213$

O $-1.446008-0.704131-0.927683$

C $-0.357769-0.6669410 .028175$

C -0.3304360 .7562460 .563548$

C $-1.801337 \quad 1.125526 \quad 0.535881$

C $0.918487-1.162624-0.665426$

C -2.0596392 .6188880 .625773$

C $2.215124-1.1584550 .164870$

O $0.675341-2.531481-1.045121$

O $1.919588-1.7117671 .454501$

C 2.9142130 .1975770 .368798

C $3.2059840 .953201-0.932854$

O $4.155899-0.0535891 .042858$

C $-3.714496 \quad 0.199320-0.959393$

C $3.9471522 .262678-0.690323$

H $-3.859250-1.7053910 .178264$

H -5.640874 -1.063424 1.665069

H -5.7300420 .6213690 .883934$

H -1.915288 $1.149952-1.622054$

H $-0.649150-1.3615440 .826516$

H $\quad 0.235691 \quad 1.416805-0.103069$
H $\quad 0.0985520 .8201991 .567460$

H -2.294163 $0.626326 \quad 1.379285$

H $1.058933-0.636173-1.613742$

H -1.6701873 .0204781 .566857$

H -1.577840 $3.160435-0.195239$

H -3.1327522 .8310140 .586029$

H $2.926671-1.857058-0.294997$

H $\quad 0.574871-3.049414-0.227695$

H $2.748676-1.7218691 .963084$

H 2.3382150 .8280561 .051140

H 2.275787 1.182606 -1.461419

H $3.8188610 .329991-1.595230$

H $4.716452-0.5807780 .447881$

H $-4.2718051 .143131-0.993450$

H -3.867645 -0.297656-1.925524

H $4.0992902 .789685-1.637680$

H $3.3751082 .917855-0.025749$

H $4.9307342 .089697-0.242712$

SCF Energy $($ B3LYP/6-31G**//MMFF $)=-810.712867187$

F2-16 c03611

MMFF Geometry

C $-3.6128432 .024067-0.547063$

C -4.4302762 .8570580 .106468$

C $-2.368728-0.0339190 .166336$

O $-1.2893370 .136789-0.777545$

C $-0.542441-1.086558-0.850802$

C $-0.978342-1.9278440 .341190$

C $-2.438549-1.5345630 .454751$

C $0.967180-0.833527-0.987819$

C $-3.056211-1.8777381 .799402$

C $1.610840-0.0093020 .151215$

O $1.602111-2.104809-1.103257$

O $1.067223 \quad 1.310407 \quad 0.157249$

C 3.1483870 .0981100 .041422

C 3.7419821 .0070371 .128771

O $3.4844770 .611664-1.246402$

C $-3.6642890 .527105-0.422699$

C $5.2648500 .970217 \quad 1.158344$

H -2.869695 2.445395-1.219211

H $-4.3455403 .929617-0.030625$

H -5.1918282 .4893450 .785254$

H $-2.0895170 .517958 \quad 1.073450$

H $-0.879206-1.601472-1.761556$

H $-0.441660-1.6509471 .254245$

H $-0.835279-2.9996840 .173956$

H -3.002280 -2.043328 -0.338879

H $1.110016-0.290445-1.931240$

H $-4.104184-1.5646111 .839815$

H $-3.019888-2.9579231 .974578$

H -2.525882 -1.386010 2.621982

H $1.389154-0.465717 \quad 1.121897$

H $2.481545-1.960683-1.491589$

H $\quad 0.112997 \quad 1.2373610 .323202$

H $3.585313-0.9036130 .126876$

H 3.3669440 .6985172 .111531

H 3.4343952 .0465100 .964522

H $4.4429150 .506684-1.367941$

H $-3.8292280 .114969-1.425759$

H $-4.517037 \quad 0.229417 \quad 0.198475$

H $\begin{array}{llll}5.693902 & 1.366889 & 0.233464\end{array}$

H 5.6388611 .5805041 .986747

H $5.630034-0.0519911 .299349$

SCF Energy $($ B3LYP/6-31G**//MMFF $)=-810.714064758$

F2-16 c03612

MMFF Geometry

C $4.571914-0.992194 \quad 0.281107$

C $4.144887-1.8793331 .187277$

C $2.3869590 .402677 \quad 0.203447$

O $1.542997-0.653959-0.294856$

C $0.307258-0.084669-0.787468$

C $0.2915751 .347718-0.285732$

C $1.7657821 .698664-0.315664$

C $-0.853003-0.982835-0.338951$

C 2.1119092 .9269170 .508505

C $-2.273190-0.527569-0.722077$

O $-0.660279-2.257047-0.982597$ 
O $-2.266242-0.067221-2.080793$

$\begin{array}{llll}\text { C }-2.897919 & 0.582932 & 0.138572\end{array}$

C -2.8848590 .3353411 .653011$

O $\quad-4.2608120 .732907-0.284473$

C $3.8244230 .188874-0.281191$

C $-3.589818-0.9460512 .080835$

H $5.580659-1.124369-0.106209$

H $4.795535-2.6874601 .504789$

H $3.158416-1.8312521 .631876$

H $2.336411 \quad 0.3724001 .299265$

H $\quad 0.395500-0.101177-1.881290$

H $-0.0863951 .400650 \quad 0.741563$

H $-0.3101742 .008992-0.915301$

H $2.0655321 .869163-1.358526$

H $-0.783636-1.1911530 .733198$

H 1.8331422 .7966291 .559589

H 3.1855343 .1353050 .468306

H 1.5829683 .8067620 .127559

H $-2.947023-1.394229-0.718211$

H $\quad 0.226499-2.573062-0.737444$

H $-1.928834-0.795416-2.630545$

H -2.418265 $1.542016-0.077547$

H $-3.393512 \quad 1.1719302 .148670$

H -1.8575020 .3273152 .030953$

H $-4.644042 \quad 1.4720420 .218091$

H $3.817326 \quad 0.070043-1.372417$

H $4.4183291 .082697-0.056585$

H $-3.588688-1.0259203 .172918$

H $-4.631325-0.9594851 .745313$

H $-3.087271-1.8326851 .683687$

SCF Energy $\left(\mathrm{B} 3 \mathrm{LYP} / 6-31 \mathrm{G}^{* *} / / \mathrm{MMFF}\right)=-810.708233472$

F2-16_c03613

MMFF Geometry

C $-4.211786-1.1467730 .797892$

C $-5.240661-0.7104121 .533224$

C $-2.590871-0.023174-0.812005$

O $-1.514478-0.938046-0.531200$

C $-0.463292-0.2203130 .158301$

C $-0.812074 \quad 1.2548230 .015711$

C $-2.327828 \quad 1.2150150 .041727$

C $0.895390-0.656572-0.410533$

C $-2.9835092 .479139-0.484585$

C $2.098323 \quad 0.0723750 .226760$

O $1.006398-2.061890-0.165689$

O $2.091119 \quad 1.445329-0.160341$

C $3.468343-0.544934-0.129384$

C $4.682318 \quad 0.2643250 .349287$

O $3.573316-0.697952-1.546789$

C -3.944742 -0.710790 -0.615159

C 4.7467010 .4313261 .858209

H -3.528685 -1.869718 1.235908

H $-5.382087-1.0684242 .547111$

H $-5.9538430 .006848 \quad 1.142285$

H -2.495489 $0.231936-1.876000$

H $-0.537756-0.5244101 .210511$

H $-0.4607241 .649070-0.945929$

H $-0.390984 \quad 1.8679770 .817798$

H -2.646920 1.0523191 .078607

H $0.880136-0.512013-1.497960$

H -2.686259 $2.686133-1.518151$

H -4.074268 $2.391365-0.458293$

H $-2.698777 \quad 3.342550 \quad 0.125721$

H $1.986857 \quad 0.0435321 .316997$

H $\quad 1.708047-2.405778-0.744305$

H $2.1255181 .482571-1.131118$

H $3.532476-1.5520710 .297884$

H $4.701096 \quad 1.249064-0.133318$

H $5.595038-0.2404300 .007396$

H $3.5844050 .189409-1.943584$

H $-4.742089-0.045818-0.968123$

H -3.977974 -1.610805-1.241830

H 5.6806030 .9270862 .141791

H $3.920667 \quad 1.0451742 .229279$

H $4.712289-0.5393072 .362804$

SCF Energy (B3LYP/6-31G**//MMFF) $=-810.712957143$

F2-16_c03614
MMFF Geometry

C $4.506845-1.293653-0.223243$

C $5.763035-1.3186540 .235890$

C $2.6030160 .302247 \quad 0.129780$

O $1.569303-0.686225-0.056198$

C $0.299809-0.015360-0.214921$

C $\quad 0.5398531 .427272 \quad 0.200048$

C $1.9694091 .638923-0.254369$

C $-0.760742-0.7574490 .603070$

C 2.6347032 .8462540 .383435

C $-2.128087-0.0472900 .680322$

O $-0.934689-2.0599350 .035462$

O $-2.952854-0.808101 \quad 1.566306$

C $-2.8447840 .155207-0.670245$

C $-4.0899151 .056078-0.599098$

O $-3.198906-1.105390-1.232742$

C $3.820563-0.054530-0.725238$

C $-5.2698580 .495803 \quad 0.182710$

H $3.943889-2.223284-0.248804$

H $\quad 6.203818-2.2479940 .579658$

H $6.370121-0.4211190 .278333$

H $2.865671 \quad 0.292717 \quad 1.195859$

H $0.068394-0.074945-1.285475$

H $0.464257 \quad 1.5381291 .289013$

H $-0.1649512 .122831-0.265015$

H $1.9772081 .758834-1.346176$

H $-0.391080-0.9293111 .621941$

H 3.6674742 .9525040 .036987

H 2.0959393 .7636280 .124241

H $2.6525532 .764517 \quad 1.475435$

H -1.9890630 .9302181 .155178$

H $-0.059751-2.4838040 .007745$

H $-3.109125-1.6714111 .146049$

H -2.147052 $0.632548-1.367449$

H $-4.4384911 .230895-1.625197$

H $-3.8144922 .034520-0.188408$

H -3.610149 -0.937411-2.097517

H $3.509203-0.242425-1.760100$

H $4.5246870 .785456-0.742202$

H $-6.147407 \quad 1.134870 \quad 0.037917$

H $-5.532763-0.512696-0.149597$

H -5.0648030 .4708581 .256114$

SCF Energy (B3LYP/6-31G**//MMFF) $=-810.711891930$

F2-16 c03615

MMFF Geometry

C -3.664762 1.7773530 .242851

C $-4.9026441 .988657-0.218353$

C $-2.317010-0.3128030 .784485$

$\begin{array}{lllll}\text { O } & -1.049130 & 0.301329 & 0.482447\end{array}$

C $-0.501636-0.333642-0.690764$

C $-1.353917-1.574729-0.939750$

C $-2.714153-1.104343-0.461024$

C $0.997976-0.612416-0.499892$

C $-3.693166-2.232601-0.189920$

C $1.8223470 .650066-0.176910$

O $1.157445-1.5617910 .554154$

O $1.4303691 .692383-1.078172$

C $3.3517470 .482161-0.316409$

C $3.965725-0.6637850 .494439$

O $3.958941 \quad 1.7150470 .092360$

C -3.3179840 .7383221 .271426$

C $5.487132-0.6983610 .402075$

H -2.855806 $2.397370-0.134667$

H $-5.0889152 .759614-0.958140$

H $\quad-5.745458 \quad 1.4012180 .128471$

H -2.123265 -1.003294 1.616318

H $-0.6487060 .366294-1.522814$

H -1.015069 -2.428277 -0.341174

H -1.348252 -1.877314 -1.991266

H $-3.135344-0.442743-1.228025$

H $1.378593-1.071986-1.419685$

H -4.650861 -1.8394640 .165318$

H -3.881033 -2.806459-1.103265

H -3.309849 -2.923903 0.568126

H 1.5898710 .9978800 .837509

H $\quad 0.773149-1.1746721 .359512$

H $1.9496352 .482923-0.851545$ 
H $3.605890 \quad 0.357386-1.375831$ H $3.586342-1.6258820 .136154$ H $3.688968-0.563248 \quad 1.550664$ H 3.7741881 .8362671 .039525 H $-4.2244860 .235386 \quad 1.628975$ H -2.885412 1.2665622 .130188 H $\quad \begin{array}{llll}5.935800 & 0.200538 & 0.835859\end{array}$ H $5.877019-1.5627240 .949298$ H $5.814063-0.780483-0.639329$ SCF Energy (B3LYP/6-31G**//MMFF) $=-810.720894430$

F2-17_c03842

MMFF Geometry

C -3.540891 $2.155284-0.598522$

C $-3.2120443 .349192-0.092935$

C $-2.607771-0.147963-0.268541$

$\begin{array}{lllll}\text { O } & -1.272359 & 0.298980 & 0.043142\end{array}$

C $-0.523747-0.8056220 .584999$

C -1.354225 -2.051878 0.306441

C $-2.762145-1.5032140 .421179$

C $0.893825-0.839853-0.006476$

C $-3.815961-2.400338-0.204678$

C 1.6868930 .4519260 .265462

O $0.821334-1.058950-1.414185$

$\begin{array}{lllll}\text { O } & 1.794716 & 0.635390 & 1.677416\end{array}$

C $3.0931410 .467493-0.370147$

C $4.066284-0.5473690 .243958$

O $3.6483231 .774859-0.193545$

C -3.6214920 .8918660 .211222$

C $5.424524-0.533148-0.448200$

H $-3.7750582 .084390-1.657630$

H -3.176074 $4.225148-0.731500$

H $-2.972923 \quad 3.4744290 .957228$

H $-2.658937-0.263451-1.359251$

H $-0.469818-0.6457041 .669142$

H -1.146116 -2.857183 1.017391

H - $1.177930-2.438739-0.704070$

H -2.997838 $-1.359698 \quad 1.484351$

H $1.413562-1.6972390 .434979$

H -3.621717 -2.564996 -1.269863

H $-3.831235-3.3775070 .288995$

H $-4.812499-1.958273-0.108748$

H $\quad 1.129093 \quad 1.311743-0.126228$

H $0.306062-0.328730-1.798390$

H 2.3037521 .4507621 .825746

H $3.0182380 .296999-1.450210$

H $3.655779-1.5597960 .175952$

H $4.227507-0.3233251 .305044$

H $3.0622202 .405773-0.645200$

H -4.6396300 .4968170 .119972$

H -3.4498801 .1146971 .271762$

H $6.080441-1.289392-0.005215$

H $5.322151-0.756301-1.514910$

H $5.9164030 .439088-0.344554$

SCF Energy (B3LYP/6-31G**/MMFF) $=-810.721914267$

F2-17 c03843

MMFF Geometry

C 2.8456412 .5488430 .365568

C 2.1029243 .6441050 .170080

C $2.3931170 .111391-0.001296$

$\begin{array}{lllll}\text { O } & 0.967142 & 0.223109 & 0.180884\end{array}$

C $0.338727-0.988117-0.272844$

C $1.463745-2.001303-0.447095$

C $2.601387-1.107804-0.899284$

C $-0.771191-1.4338530 .688932$

C $3.970340-1.751112-0.761893$

C $-1.906873-0.4124970 .919489$

O $-0.201006-1.7467951 .964025$

O

C $-2.688180-0.042927-0.354013$

C $-3.8824390 .874725-0.048376$

O $-3.152197-1.228057-0.994934$

C $2.9338301 .407087-0.607429$

C $-4.6375911 .279565-1.307978$

H 3.4321662 .4728251 .277586

H 2.0857224 .4354330 .911460

H $1.5019433 .770399-0.723552$
H $2.828695-0.0623690 .991409$

H -0.092695 -0.768309-1.256649

H $1.212683-2.780226-1.173401$

H $1.718614-2.4906790 .500231$

H $2.436884-0.834106-1.950241$

H -1.203153 -2.367647 0.310945

H $4.177515-2.0364530 .274988$

H $4.034176-2.654174-1.377693$

H $4.758861-1.064993-1.086217$

H -2.597109-0.853756 1.649662

H $\quad 0.200038-0.9307802 .309891$

H $-0.790887 \quad 1.1962880 .893407$

H -2.029254 $0.480831-1.055790$

H -3.5424991 .7815200 .464403$

H -4.5840090 .3607090 .619874$

H $-3.747961-1.685804-0.377448$

H $3.9856271 .282796-0.888983$

H $2.3762181 .649459-1.520880$

H $-5.4601771 .955308-1.052848$

H $-3.9774851 .798828-2.009957$

H $-5.0642360 .408643-1.815324$

SCF Energy $\left(B 3 L Y P / 6-31 G^{* *} / / M M F F\right)=-810.725161088$

$\mathrm{F} 2-17 \mathrm{c} 03844$

MMFF- Geometry

C $-4.593465-1.044109-0.165370$

C -4.762016 -2.150797 -0.897677

C $-2.6134810 .434716 \quad 0.259834$

$\begin{array}{lllll}0 & -1.631472 & -0.603921 & 0.068674\end{array}$

C $-0.3228900 .000172-0.050404$

C -0.4947321 .4427120 .398110$

C $-1.9012271 .743645-0.077743$

C $0.672562-0.8200180 .773856$

C -2.5138352 .9692250 .577559$

C $2.087283-0.2217980 .916377$

O $0.787059-2.1195300 .181098$

O $2.762016-0.9694761 .935790$

C $2.952914-0.234774-0.360871$

C $4.3717720 .300558-0.101555$

O $2.3418670 .549808-1.379262$

C $-3.8283620 .163236-0.629096$

C $5.2419690 .267283-1.352001$

H $-5.041324-1.0009070 .824138$

H $-5.330201-2.987388-0.505988$

H -4.339336 -2.243451-1.892001

H $-2.8978790 .413543 \quad 1.320049$

H -0.079905 -0.050127-1.117637

H $0.2535992 .113526-0.033131$

H $-0.435857 \quad 1.5209821 .491031$

H -1.879692 $1.890863-1.165918$

H $\quad 0.257588-0.991349 \quad 1.775658$

H -2.558465 2.8620521 .666588

H -1.921606 3.8621990 .351978

H -3.5320403 .1391010 .214088$

H 2.0039650 .8043131 .290910

H $-0.111205-2.4862960 .110785$

H $2.823530-1.8910741 .630161$

H $3.041189-1.260061-0.738560$

H $4.864067-0.2873140 .680999$

H $\quad 4.317574 \quad 1.3381910 .249659$

H $2.314327 \quad 1.470872-1.069022$

H -4.509286 $1.021711-0.610133$

H $-3.5033870 .024924-1.667898$

H $5.323103-0.751223-1.744979$

H $4.8357130 .909801-2.139298$

H $6.2508000 .621759-1.118000$

SCF Energy $\left(B 3 L Y P / 6-31 G^{* *} / / M M F F\right)=-810.718307771$

$\mathrm{F} 2-17 \mathrm{c} 03845$

MMFF Geometry

C $-4.380546-1.5762210 .497031$

C -4.541385 -2.719134-0.179226

C $-2.6745680 .257093 \quad 0.369939$

O $-1.611052-0.587713-0.114120$

C $-0.5308530 .242128-0.598157$

C $-0.8309171 .641296-0.085918$

C $-2.3456171 .662365-0.130410$

C $0.804638-0.372962-0.153144$ 
C -2.9612262 .7754810 .699682$

C $2.0389640 .438872-0.585716$

O $0.892278-1.677370-0.734054$

$\begin{array}{lllll}\text { O } & 2.078875 & 1.663110 & 0.146972\end{array}$

C $3.379792-0.303968-0.397192$

C $3.734734-0.5761031 .070576$

O $4.4204820 .497975-0.965058$

C $-4.018696-0.264740-0.140848$

C $5.033948-1.3608571 .211993$

H -4.527529 -1.5829941 .574127$

H $-4.807671-3.6325380 .341278$

H -4.410053 -2.765592 -1.254540

H $-2.6372630 .220074 \quad 1.466681$

H $-0.6027490 .216035-1.693290$

H $-0.3781782 .423656-0.702001$

H $-0.480544 \quad 1.7634080 .946427$

H -2.664952 $1.774786-1.175231$

H $\quad 0.790930-0.4995830 .935934$

H -2.670162 2.6988871 .752715

H $-2.635993 \quad 3.7540310 .331620$

H -4.0539452 .7421660 .649012$

H $1.9395680 .703505-1.646185$

H $\quad 0.105493-2.174395-0.450723$

H $2.8668372 .152038-0.146313$

H $\quad 3.369480-1.248219-0.953322$

H $2.934142-1.1427581 .557157$

H 3.8520220 .3691321 .613390

H $4.2203720 .610751-1.909939$

H -4.8142390 .4530910 .088992$

H $-3.982131-0.375913-1.231889$

H $5.238237-1.5630792 .268279$

H $4.969934-2.3205620 .689481$

H $5.884115-0.8034450 .806754$

SCF Energy (B3LYP/6-31G**//MMFF) $=-810.720224352$

F2-17 c03846

MMFF Geometry

C $-3.167726 \quad 2.451872-0.670891$

C -2.684905 $3.598569-0.179554$

C $-2.5783330 .048721-0.268358$

O $-1.202176 \quad 0.3102350 .074782$

C $-0.633337-0.8750630 .662225$

C $-1.622807-1.9988600 .382097$

C -2.941838-1.255044 0.442393

C $0.782760-1.1179540 .117983$

C $-4.094024-2.009326-0.198375$

C 1.7428880 .0654150 .377976

O $0.711424-1.382293-1.281690$

$\begin{array}{lllll}\text { O } & 1.719730 & 0.385875 & 1.767925\end{array}$

C $3.199231-0.216619-0.055364$

C $4.139794 \quad 0.9441360 .305690$

O $3.270765-0.419923-1.463769$

C -3.4474641 .2318430 .160604$

C $5.5865700 .661805-0.081225$

H $-3.3830072 .390317-1.734478$

H $-2.5085754 .445480-0.833776$

H -2.456553 3.7131130 .874118

H -2.615726 $-0.083769-1.357694$

H $-0.591377-0.6993921 .744356$

H -1.550323 -2.808227 1.115011

H -1.475602 $-2.430880-0.614569$

H -3.184607 -1.054824 1.494751

H $1.181081-2.0184400 .600681$

H $-4.261252-2.9626540 .313489$

H $-5.020362-1.429100-0.142093$

H $-3.895240-2.224837-1.253602$

H $\quad 1.3840470 .940799-0.177707$

H $\quad 0.317280-0.601621-1.707768$

H $2.049797-0.3881252 .255706$

H $3.563461-1.1246200 .440901$

H $4.102177 \quad 1.1467671 .381630$

H $3.8186531 .854926-0.214213$

H $2.751575-1.214313-1.673938$

H -4.5084040 .9818710 .046945$

H -3.274356 $1.453248 \quad 1.221303$

H $5.6944930 .534953-1.162878$

H 6.2268951 .4957830 .223461

H $5.953122-0.2445830 .411184$
SCF Energy $($ B3LYP/6-31G**//MMFF $)=-810.722255349$

F2-17_c03847

MMFF Geometry

C $-3.673460-1.826138-0.098756$

C $-4.864043-2.000727-0.683111$

C -2.4217010 .2072460 .783009$

O $-1.119234-0.3523060 .525358$

C $-0.4880470 .417815-0.517380$

C $-1.3461101 .666260-0.702029$

C $-2.7303321 .126277-0.398017$

C $0.9829030 .701570-0.173191$

C $-3.7554262 .203315-0.091256$

C $1.798039-0.5891050 .036039$

O 1.0329421 .4981141 .008420

O $1.751924-1.355166-1.169274$

C $3.269168-0.3596560 .442129$

C $4.1149790 .320246-0.642214$

O $3.857180-1.6327680 .729567$

C $-3.436032-0.9047431 .064071$

C $5.5465440 .571236-0.182076$

H -2.822421 -2.389155 -0.473052

H $-4.971523-2.685968-1.516928$

H -5.745591-1.467207-0.345564

H $-2.3143040 .803603 \quad 1.699146$

H $-0.543955-0.189441-1.429620$

H - $1.2583902 .084228-1.709443$

H -1.0786222 .4521640 .013987$

H -3.068928 $0.548157-1.266532$

H $1.4013851 .288364-0.999277$

H -4.7302961 .7594550 .134055$

H -3.454547 2.8116740 .768299

H $-3.8787512 .872309-0.949186$

H $1.324267-1.1957830 .817915$

H $1.9174051 .897646 \quad 1.058371$

H $2.269903-2.164562-1.018538$

H 3.3175550 .2254781 .367124

H $3.6715401 .279980-0.926188$

H $4.155214-0.307626-1.540128$

H $3.354082-2.0297091 .461063$

H $-3.064521-1.5204841 .892739$

H $-4.379857-0.4564151 .396829$

H $6.1095561 .084772-0.967993$

H $5.564778 \quad 1.2000020 .713731$

H $6.064625-0.3660520 .043301$

SCF Energy (B3LYP/6-31G**//MMFF $)=-810.717055077$

F2-17_c03848

MMFF Geometry

C -3.6155622 .3679870 .026749$

C $-2.8641302 .899869-0.944176$

C $-2.623506-0.022431-0.130917$

O -1.2776730 .3446010 .232720$

C $-0.536770-0.846610 \quad 0.556917$

C $-1.401410-2.0168170 .105854$

C $-2.794303-1.4675120 .340060$

C $0.859174-0.803568-0.082684$

C $-3.879170-2.236181-0.394497$

C 1.6878020 .4139290 .371599

O $0.736337-0.783577-1.503745$

O $1.837897 \quad 0.394870 \quad 1.791662$

C $3.0742020 .517917-0.298138$

C $4.058159-0.5946230 .088764$

$\begin{array}{llll}\text { O } & 3.643671 & 1.782015 & 0.057769\end{array}$

C -3.6169320 .9447250 .521188$

C $5.379387-0.476953-0.662642$

H -4.3180773 .0298590 .530185$

H -2.968984 3.949510-1.198966

H $-2.1381122 .322820-1.503657$

H -2.692021 $0.027953-1.225116$

H $-0.444094-0.8699121 .649847$

H -1.196030 -2.928314 0.675298

H -1.255192 -2.242884 -0.956872

H -3.004213 -1.489792 1.417986

H $1.376185-1.7302430 .189921$

H -3.711435 -2.231881-1.476768

H -3.902752 -3.279278 -0.062536

H $-4.864784-1.799892-0.204949$ 
H 1.1436291 .3359320 .132444 H $\quad 0.228170 \quad 0.010596-1.742913$ H $2.182373-0.4775692 .046937$ H $2.9511090 .532441-1.386805$ H $3.625542-1.577347-0.123429$ H $4.279392-0.5510311 .161420$ H 3.7254291 .8044861 .026766 H $-4.6352550 .559506 \quad 0.391902$ H -3.4194260 .9831561 .600289$ H $6.045101-1.300311-0.384660$ H $5.219375-0.521156-1.744682$ H $5.8905660 .461688-0.427353$

SCF Energy (B3LYP/6-31G**/MMFF) $=-810.720258283$

F2-17 c03849

MMFF Geometry

C $4.491797-1.237818-0.063996$

C $4.565469-2.5040300 .361120$

C $2.6195810 .428577-0.141409$

O $1.571462-0.556483-0.247874$

C $0.2988450 .081992-0.004204$

C $0.570258 \quad 1.577205-0.063412$

C 1.9724871 .6526290 .504908

C $-0.722776-0.429940-1.021611$

C $2.680786 \quad 2.961220 \quad 0.200014$

C $-2.0753770 .304192-0.995994$

O $-0.906980-1.827625-0.786144$

O $-2.902548-0.275146-2.011127$

C $-2.834620 \quad 0.318962 \quad 0.347664$

C $-3.420609-1.0290290 .786281$

$\begin{array}{llll}\text { O } & -3.923798 & 1.242422 & 0.217345\end{array}$

C $3.779651-0.1414920 .676763$

C $-4.183068-0.9278842 .103694$

H $4.981704-0.972248-0.997170$

H $5.099531-3.248314-0.219362$

H $4.096957-2.8201891 .286573$

H $2.9450020 .659926-1.164132$

H $0.008822-0.2164281 .010912$

H -0.1552372 .1623710 .509173$

H $0.558243 \quad 1.935340-1.100448$

H 1.9176621 .5188061 .593746

H $-0.296629-0.361737-2.030696$

H $2.761338 \quad 3.132173-0.878758$

H 2.1336953 .8044820 .633820

H 3.6923342 .9644900 .617973

H $-1.9073701 .341151-1.311713$

H - $-1.556148-2.147066-1.436286$

H -3.748385 $0.205129-2.004698$

H -2.1886230 .7095491 .141133$

H -2.626264 -1.7688220 .915922$

H $-4.112677-1.4087490 .025828$

H $-3.544708 \quad 2.1197490 .037503$

H 4.5092990 .6460900 .896592

H $3.405211-0.5190931 .636481$

H -4.545364 -1.916170 2.404724

H -3.537254 -0.546292 2.900990

H $-5.050779-0.2669982 .015379$

SCF Energy (B3LYP/6-31G**/MMFF) $=-810.713508204$

F2-17_c03850

MMFF Geometry

C -3.380200 $2.270416-0.579557$

C $-3.086012 \quad 3.4107840 .054627$

C $-2.515010-0.072156-0.358532$

O

C $-0.534222-0.8444450 .633409$

C $-1.350074-2.0451270 .170695$

C $-2.754882-1.4768270 .195108$

C $0.936226-0.856166 \quad 0.183439$

C $-3.754726-2.294951-0.603667$

C 1.7109030 .4054830 .621326

O $0.986318-0.981438-1.235462$

$\begin{array}{lllll}\text { O } & 1.531995 & 0.568127 & 2.032563\end{array}$

$\begin{array}{lllll}\text { C } 3.230798 & 0.383239 & 0.347076\end{array}$

C $3.6017820 .483874-1.135400$

O $3.804726-0.810806 \quad 0.880303$

$\begin{array}{lllll}\text { C } & -3.556280 & 0.946004 & 0.108437\end{array}$

C $5.0935780 .731631-1.331567$
H -3.5112912.293080 -1.658385

H $-2.9762494 .335799-0.500746$

H $-2.9486593 .443148 \quad 1.129806$

H $-2.460959-0.094960-1.454864$

H $-0.589250-0.7788141 .727161$

H $-1.223798-2.9109820 .827674$

H $-1.082072-2.351065-0.847260$

H -3.091787 $-1.417237 \quad 1.238864$

H $1.407111-1.749008 \quad 0.610426$

H $-3.459153-2.374679-1.655314$

H $-3.832204-3.309236-0.198662$

H $-4.749718-1.840427-0.567219$

H 1.2824531 .2897250 .133520

H $\quad 0.523735-0.214524-1.614943$

H $1.932398 \quad 1.4179342 .283693$

H $3.695744 \quad 1.2184450 .885892$

H $3.0437221 .298969-1.609243$

H $3.357731-0.447189-1.657499$

H $3.561231-0.8542801 .821029$

H $-4.5660480 .582323-0.113152$

H -3.4853361 .0759231 .195606$

H $5.3254340 .808351-2.398677$

H $5.4022851 .665156-0.850320$

H $5.690843-0.085305-0.914648$

SCF Energy (B3LYP/6-31G**//MMFF) $=-810.719668376$

F2-17 c03851

MMFF Geometry

C $-4.623525-0.3347150 .727881$

C $-5.192570-1.498587 \quad 0.394667$

$\begin{array}{llll}\text { C }-2.384808 & 0.676803 & 0.225355\end{array}$

O $-1.698818-0.5840570 .092798$

C $-0.400977-0.358544-0.506969$

C $-0.182337 \quad 1.142518-0.446652$

C $-1.5930611 .666292-0.626263$

C $0.629301-1.2281350 .224595$

C $-1.7590863 .116610-0.207018$

C $2.092416-1.122858-0.251746$

O $0.241055-2.5980430 .009616$

O $2.139853-1.125961-1.678654$

$\begin{array}{llll}\text { C } & 2.870779 & 0.096828 & 0.270639\end{array}$

C $4.3607640 .024274-0.104510$

$\begin{array}{lllll}\text { O } & 2.746237 & 0.180838 & 1.687497\end{array}$

C $-3.8381190 .521318-0.225404$

C $5.135802 \quad 1.248763 \quad 0.366238$

H $-4.7378960 .028541 \quad 1.745983$

H $-5.751132-2.0674131 .130059$

H $-5.111775-1.903504-0.607941$

H -2.3368020 .9564501 .285957$

H $-0.500317-0.686621-1.549459$

H $0.4995051 .500858-1.222955$

H $\quad 0.2098231 .4416810 .532758$

H $-1.8742891 .563299-1.683044$

H $\quad 0.563116-1.0718091 .307064$

H -1.492638 3.2633210 .845096

H $-1.116583 \quad 3.766777-0.809925$

H $-2.7939763 .446491-0.341599$

H $2.617720-2.0283070 .080971$

H $-0.675156-2.6907280 .323464$

H $1.681250-1.928146-1.982191$

H $2.4685521 .018769-0.157014$

H $4.474198-0.067716-1.190434$

H $4.816003-0.8631240 .351839$

H $3.122247-0.6314682 .067668$

H $-4.3241711 .502199-0.276616$

H $-3.8689110 .086678-1.232373$

H $\quad 5.1152941 .3396541 .456664$

H 6.1827391 .1738170 .055665

H $4.7191052 .164695-0.064553$

SCF Energy (B3LYP/6-31G**//MMFF) $=-810.718512853$

F2-17 c03852

MMFF Geometry

C $2.6262072 .335008 \quad 0.264879$

C $3.5593403 .051390-0.372119$

C $2.276857-0.1558500 .654273$

O $0.841253-0.047548 \quad 0.702764$

C $0.290352-0.709982-0.450676$ 
C $1.438517-1.504993-1.067760$

C $2.622074-0.606666-0.763992$

C $-0.936080-1.556585-0.083607$

C $3.966548-1.302115-0.880923$

C $-2.104710-0.7806380 .565306$

O $-0.529227-2.5862690 .824131$

O

C $-2.7075950 .311258-0.335914$

C $-3.950757 \quad 0.9491660 .305592$

O $-3.059448-0.271268-1.587338$

C 2.9274291 .1452041 .131909

C $-4.5250712 .090919-0.523634$

H 1.5850322 .6305560 .165096

H $3.2771473 .904305-0.979430$

H $4.6121922 .801790-0.302043$

H $2.541014-0.9462891 .369598$

H $-0.0039900 .078996-1.153106$

H $1.293887-1.679318-2.138259$

H $1.565416-2.478890-0.580406$

H $2.5961910 .239387-1.461797$

H -1.292206 -2.061543 -0.989424

H $4.123939-1.665633-1.901653$

H $4.782461-0.614237-0.637489$

H $4.035399-2.160492-0.204394$

H -2.887764 -1.506048 0.821338

H -1.299947 -3.1568620 .985300$

H -1.261822 -0.8787232 .323891$

H $-1.9633751 .096687-0.512922$

H $-3.705215 \quad 1.335343 \quad 1.301259$

H -4.7332730 .1906320 .429140$

H $-3.239673 \quad 0.451915-2.211129$

H 2.5553131 .3771852 .137783

H 4.0106320 .9975311 .217912

H -5.3632302 .5549400 .006443$

H -3.769964 $2.862193-0.704961$

H -4.898763 $1.737222-1.489194$

SCF Energy (B3LYP/6-31G**//MMFF) $=-810.713255018$

\section{F2-17 c03853}

MMFF Geometry

C 3.4734492 .2202120 .339072

C $3.4801343 .250555-0.514034$

C $2.384110-0.036506 \quad 0.278997$

O $1.278864 \quad 0.375277-0.551988$

C $0.577326-0.794678-0.999520$

C $1.117813-1.964945-0.188236$

C $2.556544-1.5346630 .024576$

C $-0.946692-0.593845-0.968858$

C $3.251555-2.2786601 .151599$

C $-1.547590-0.2693310 .413778$

O $-1.574515-1.765177-1.485313$

O

C $-3.088301-0.1478660 .408529$

C $-3.6168061 .062226-0.373169$

O $-3.531054-0.0250671 .764393$

C $3.6252660 .783624-0.074780$

C $-5.1403901 .115052-0.390664$

H 3.3603582 .4236621 .400857

H $3.3695444 .265972-0.149565$

H $3.5928453 .102388-1.582233$

H 2.0854650 .1439821 .320178

H $0.875978-0.949831-2.045510$

H $1.028820-2.918137-0.718122$

H $\quad 0.609217-2.064707 \quad 0.775771$

H $3.112650-1.695402-0.909026$

H -1.171134 $0.240891-1.643672$

H $4.281463-1.9293941 .274133$

H $2.732301-2.1378172 .105424$

H $3.282412-3.3528170 .941220$

H -1.282659 -1.059022 1.125340

H -1.230106 -1.912624 -2.382644

H -1.3819291 .1214311 .774016$

H -3.536004 -1.065616 0.010667

H $-3.2619201 .033104-1.408232$

H -3.2534551 .9928270 .078308$

H $-3.254693-0.8287882 .236853$

H $4.505090 \quad 0.376638 \quad 0.436365$

H $3.8147300 .721938-1.153736$
H $-5.4803811 .970537-0.983050$

H $-5.5580450 .206775-0.836475$

H $-5.548708 \quad 1.2232660 .618928$

SCF Energy $($ B3LYP/6-31G**//MMFF $)=-810.708000163$

F2-17 c03854

MMFF Geometry

C 2.9755762 .5011280 .252450

C $2.3191983 .600485-0.134880$

C 2.4323430 .0591110 .095948

$\begin{array}{llll}\text { O } & 1.003042 & 0.248479 & 0.113644\end{array}$

C $0.360679-0.979798-0.268799$

C $1.445007-2.050226-0.218643$

C $2.665704-1.258300-0.642309$

C $-0.857513-1.2760100 .617610$

C $3.979779-1.937422-0.297263$

C $-1.968209-0.2003330 .620510$

O $-0.425705-1.457761 \quad 1.971121$

O -1.4354181 .0290961 .109065$

C $-2.6163100 .019182-0.758115$

C $-3.7468011 .061973-0.776252$

O $-3.116512-1.226485-1.239648$

C $3.099078 \quad 1.257225-0.581552$

C $-4.9244580 .727306 \quad 0.129814$

H 3.4603432 .4992001 .225255

H 2.2701394 .4682230 .513951

H $1.8207753 .654940-1.096405$

H $2.756147-0.0229331 .141903$

H $0.039409-0.855862-1.309615$

H $1.227284-2.893399-0.881161$

H $1.580336-2.4429050 .795932$

H $2.619562-1.095227-1.727556$

H $-1.289668-2.2338300 .305750$

H $4.057356-2.904628-0.804511$

H $4.830531-1.323697-0.609152$

H $4.069236-2.1148540 .779760$

H $-2.722532-0.5328271 .344135$

H $-0.022852-0.6207302 .260297$

H -2.1821681 .6104821 .329417$

H $-1.8535110 .366945-1.464411$

H $-4.1317011 .139680-1.800996$

H -3.354841 $2.050290-0.511796$

H -3.480592 -1.073934 -2.128209

H $4.1656231 .058540-0.736475$

H $2.6493821 .421805-1.568849$

H -4.6356480 .7440601 .184663$

H $-5.7208251 .466488-0.005153$

H $-5.339474-0.258391-0.101348$

SCF Energy (B3LYP/6-31G**//MMFF) $=-810.712199897$

F2-17 c03855

MMFF Geometry

C $3.999229-0.5223841 .212725$

C 4.8825920 .2768661 .821476

C $2.500147-0.263349-0.828288$

O $1.484730-1.152751-0.325078$

C $0.290686-0.381389-0.050516$

C $0.5226980 .983035-0.685683$

C $2.0193391 .150790-0.511953$

C $-0.922890-1.146432-0.585215$

C $2.6233952 .222193-1.401978$

C $-2.266128-0.385255-0.550820$

O $-1.062192-2.3554330 .171168$

O $-3.221446-1.153878-1.290558$

C $-2.845168-0.1338410 .855825$

C -4.2661930 .4586440 .851619$

O $-1.984596 \quad 0.7385561 .581359$

C $3.874796-0.654068-0.278913$

C -4.3766951 .8122850 .161110$

H $3.334705-1.1258531 .825573$

H 4.9263580 .3233132 .904097

H $5.5710400 .895821 \quad 1.256993$

H $2.517500-0.415123-1.916013$

H $0.248034-0.2999161 .041619$

H $-0.0546331 .777928-0.204817$

H $0.2609380 .967022-1.751184$

H 2.2139881 .4061690 .537000

H $-0.720553-1.468319-1.614756$ 
H $\quad 2.4453172 .011677-2.461796$

H $2.1868033 .200611-1.176386$

H $3.7049812 .291198-1.248493$

H $-2.1437390 .567683-1.076758$

H $-0.211422-2.8249090 .127255$

H -2.890146 -1.237568 -2.200941

H -2.893808 -1.082773 1.403269

H -4.5925190 .5904751 .890946$

H $-4.967782-0.2431050 .386520$

H -2.3502360 .8361072 .477100$

H $4.068404-1.705295-0.526913$

H $4.646798-0.059361-0.781772$

H $-5.393862 \quad 2.2033060 .267316$

H -3.689331 2.5421350 .599679

H $-4.1636591 .734251-0.909003$

SCF Energy (B3LYP/6-31G**//MMFF) $=-810.712892574$

F2-17_c03856

MMFF Geometry

C 3.1121792 .2115590 .171383

C $2.5203483 .393282-0.035272$

C $2.283140-0.144477-0.034350$

O 0.9063120 .1914530 .230754

C $0.071424-0.916776-0.143475$

C $1.012370-2.102615-0.322922$

C $2.245873-1.418626-0.878422$

C $-1.050668-1.1395840 .878195$

C $3.504981-2.262338-0.780708$

C $-1.968698 \quad 0.064314 \quad 1.186212$

O $-0.476633-1.5616192 .122327$

O $\quad-1.217058 \quad 1.1292431 .776500$

C -2.7969190 .6358350 .020577$

C $-3.706945-0.411552-0.628222$

O $-1.953897 \quad 1.195787-0.982899$

C $2.9665811 .026886-0.741392$

C $-4.6577460 .205594-1.646740$

H 3.7459022 .0918071 .046383

H 2.6690174 .2113660 .661143

H $1.8803033 .565111-0.893609$

H $2.756213-0.3389280 .937131$

H $-0.362050-0.671995-1.119447$

H $0.601104-2.861602-0.995124$

H $\quad 1.245917-2.5858760 .632771$

H $2.063578-1.167040-1.931967$

H -1.666691 -1.9780200 .535177$

H $3.726501-2.5341530 .256841$

H $3.392528-3.188403-1.353914$

H $4.369212-1.721603-1.179007$

H -2.669195 -0.2532021 .969151$

H $\quad 0.088198-0.8363742 .440948$

$\mathrm{H}-0.5022051 .3681371 .162900$

H -3.410101 1.4615690 .403273

H $-4.295771-0.923657 \quad 0.141212$

H -3.105774 -1.165718 -1.149262

H $-1.4579381 .926293-0.576490$

H $3.9672150 .733282-1.078326$

H $2.3902251 .308770-1.631696$

H $-5.306311-0.566645-2.072625$

H $-5.2948710 .962851-1.178764$

H $-4.1103240 .676122-2.469325$

SCF Energy (B3LYP/6-31G**//MMFF) $=-810.725123563$

F2-17_c03857

MMFF Geometry

C $-4.507225-1.719248-0.087666$

C $-3.920291-2.6195250 .709362$

C -2.7210880 .1358650 .221446$

O $-1.626098-0.601544-0.357223$

C $-0.547766 \quad 0.310489-0.663339$

C $-0.897092 \quad 1.608276 \quad 0.046675$

C $-2.409577 \quad 1.608228-0.047265$

C $0.785720-0.342089-0.266208$

C -3.0759222 .5705910 .920985$

C $2.0190320 .546736-0.514730$

O $0.916077-1.551217-1.020191$

O 2.0006341 .6455890 .395810

C $3.366415-0.195782-0.381381$

C $3.666428-0.681826 \quad 1.042745$
O $4.4120250 .698244-0.775670$

C $-4.041917-0.321953-0.405308$

C $4.973877-1.4617921 .120368$

H $-5.435922-2.015618-0.572164$

H $-4.370491-3.5968160 .848815$

H -2.995505 -2.418313 1.236040

H -2.713236 -0.060678 1.300972

H $-0.5803330 .456093-1.750852$

H $-0.4429692 .484389-0.425592$

H $-0.5831231 .576343 \quad 1.097300$

H $-2.6979671 .873656-1.073356$

H $0.727902-0.6307760 .789610$

H -4.1654462 .5283090 .826850$

H -2.8182662 .3375941 .959632$

H -2.7595913 .5991680 .719069$

H $1.957900 \quad 0.968638-1.525941$

H $0.957522-1.311240-1.961808$

H 2.7902712 .1846450 .217358

H $3.398717-1.045039-1.073275$

H $2.857943-1.3259531 .403660$

H $3.7430390 .172104 \quad 1.726139$

H $4.2484960 .950496-1.700435$

H $-4.8430590 .361657-0.100084$

H $-3.958283-0.255873-1.497821$

H $5.828759-0.8385990 .839769$

H $5.139504-1.8177252 .142253$

H $4.949474-2.3328520 .457942$

SCF Energy (B3LYP/6-31G**//MMFF $)=-810.712158900$

F2-17 c03858

MMFF Geometry

C -4.507962 -1.154108 -0.578121

C $-5.785391-1.212610-0.185446$

C -2.5746640 .2702760 .149595$

O $-1.564379-0.707228-0.172957$

C $-0.266638-0.070096-0.149328$

C -0.4834831 .2782050 .519239$

C -1.8809001 .6284160 .050731$

C $0.725569-0.9942870 .563494$

C $-2.5390912 .722147 \quad 0.873892$

C $2.112641-0.3941330 .878891$

O $0.889285-2.182680-0.219982$

O $2.765755-1.2908501 .786464$

C $3.043162-0.152498-0.327491$

C 4.3945830 .4366330 .114217

O $2.4154910 .745362-1.234739$

C $-3.7581540 .127688-0.809410$

C $5.3675740 .624531-1.043213$

H $-3.974388-2.084931-0.753203$

H $-6.272087-2.171011-0.040702$

H $-6.364528-0.314620-0.001270$

H -2.8909080 .0736001 .182509$

H $\quad 0.0070770 .061417-1.202315$

H $\quad 0.2641852 .021000 \quad 0.226210$

H $-0.460197 \quad 1.180376 \quad 1.611821$

H $-1.8296861 .949319-0.998498$

H $\quad 0.272821-1.3380441 .502183$

H -3.5491382 .9323160 .508727$

H -2.614531 2.4395921 .929323

H -1.959382 3.6491140 .814944

H $1.9736690 .546675 \quad 1.422864$

H $1.130449-1.917472-1.123577$

H $2.865872-2.145240 \quad 1.332225$

H $3.228492-1.100252-0.847055$

H $4.866960-0.2156380 .857158$

H 4.2341751 .4133590 .586707

H $2.9050450 .712590-2.073698$

H $-4.4334360 .984110-0.700000$

H $-3.4011570 .122105-1.846681$

H $5.0016401 .366759-1.758925$

H $\quad 6.3341100 .975444-0.667375$

H $5.532250-0.318393-1.573974$

SCF Energy (B3LYP/6-31G**//MMFF) $=-810.715421178$

F2-17 c03859

MMFF Geometry

C -2.916217 $2.536807-0.680056$

C $-2.3690613 .649939-0.179060$ 
C $-2.4850210 .099472-0.280657$

O $\begin{array}{lllll} & -1.098013 & 0.273011 & 0.074760\end{array}$

C $-0.609699-0.947477 \quad 0.662847$

C -1.666542 -2.0061250 .372477$

C $-2.936200-1.1798970 .424062$

C $0.795363-1.2789590 .135025$

C $-4.128790-1.858100-0.227727$

C $1.817206-0.1461540 .396323$

O $0.701677-1.540431-1.264174$

$\begin{array}{lllll}\text { O } & 1.768560 & 0.188967 & 1.781223\end{array}$

C $3.261499-0.5410930 .017483$

C $4.3321710 .493304 \quad 0.404451$

O $3.339279-0.783182-1.385460$

C $-3.281377 \quad 1.334432 \quad 0.143812$

C $4.1475861 .855630-0.251861$

H $-3.1216302 .491450-1.746507$

H -2.132314 $4.485828-0.828199$

H $-2.146863 \quad 3.7472850 .877858$

H -2.521432 $-0.028224-1.370498$

H $-0.569051-0.7774171 .746025$

H $-1.651617-2.8193251 .104281$

H - $1.539965-2.445432-0.623837$

H $-3.173951-0.9668831 .474949$

H $1.129566-2.2002340 .628167$

H $-5.016944-1.220512-0.176800$

H $-3.935698-2.083447-1.281851$

H $-4.360254-2.8000160 .280233$

H $1.5144220 .728283-0.192820$

H $1.575861-1.835953-1.569231$

H 2.1878901 .0587861 .890633

H $3.512166-1.4794250 .528137$

H 5.3142810 .1104620 .099559

H 4.3667930 .6153391 .492783

H $4.244153-1.078529-1.584295$

H $-4.354797 \quad 1.152658 \quad 0.018412$

H -3.1054531 .5409471 .206944$

H 4.9804572 .5142780 .015188

H $4.1222991 .773150-1.342755$

H 3.2245512 .3393350 .080126

SCF Energy (B3LYP/6-31G**//MMFF) $=-810.714960750$

F2-17_c03860

MMFF Geometry

C -3.614328 2.3753870 .037386

C -2.900962 $2.896631-0.967565$

C $-2.633494-0.019007-0.135960$

O $-1.273195 \quad 0.3478530 .171228$

C -0.534542 -0.8375490 .520853$

C -1.404873 $-2.012003 \quad 0.094476$

C $-2.792339-1.4596880 .355521$

C $0.873262-0.803485-0.095266$

C $-3.895189-2.235490-0.344135$

$\begin{array}{llll}\text { C } & 1.686320 & 0.423099 & 0.366047\end{array}$

O $0.787321-0.771047-1.518104$

$\begin{array}{lllll}\text { O } & 1.804693 & 0.419378 & 1.789228\end{array}$

C $3.0880900 .527833-0.274398$

C $4.063505-0.5774970 .150554$

O 3.6604001 .7863010 .096578

C $-3.598436 \quad 0.9570490 .544938$

C $5.398642-0.475982-0.578910$

H -4.2950883 .0436570 .562028$

H -3.013734 $3.943891-1.227862$

H -2.198759 2.312547 -1.549683

H -2.743953 $0.021366-1.226940$

H -0.453022 -0.8409891 .615316$

H $-1.187592-2.9196260 .665687$

H -1.288814 -2.244619-0.969527

H -2.977259-1.470315 1.438191

H $1.392771-1.7236890 .195885$

H -3.752411 -2.245189-1.429945

H $-3.912869-3.2742630 .001472$

H $-4.875263-1.794533-0.137438$

H 1.1399661 .3378010 .105264

H $0.556266-1.664334-1.822823$

H $2.153362-0.4462292 .061147$

H $2.9966910 .531951-1.366773$

H $3.635160-1.564389-0.050680$

H $4.265766-0.5124261 .225864$
H $3.0678842 .485570-0.228244$

H -4.6217290 .5724420 .459729$

H -3.358349 1.0054621 .614965

H $5.258429-0.542270-1.662568$

H $5.9068260 .466767-0.353775$

H $6.058039-1.294254-0.271967$

SCF Energy (B3LYP/6-31G**//MMFF) $=-810.707919308$

F2-17 c03861

MMFF Geometry

C 3.6822832 .1063700 .104239

C $3.8476662 .943102-0.926149$

C $2.390732-0.0239950 .383505$

$\begin{array}{llll}\text { O } & 1.388329 & 0.302580 & -0.602512\end{array}$

C $0.641666-0.884828-0.911567$

C $0.991594-1.9061110 .161996$

C $2.440491-1.5525240 .437226$

C $-0.852567-0.584221-1.100205$

C $2.961890-2.108668 \quad 1.750962$

C $-1.605159-0.0897930 .159188$

O $-1.460033-1.771838-1.605064$

$\begin{array}{llll}\text { O } & -0.879222 & 0.964133 & 0.784397\end{array}$

C $-3.0375400 .406338-0.146067$

C $-3.7484590 .939967 \quad 1.108299$

O $-3.815115-0.646202-0.709872$

C $3.7256740 .608808-0.012090$

$\begin{array}{llll}\text { C } & -5.148769 & 1.458617 & 0.804817\end{array}$

H 3.5143432 .5247621 .093446

H $3.8091354 .016183-0.773985$

H $4.0214332 .577305-1.932092$

H 2.0401660 .3821691 .341376

H $1.036091-1.254454-1.868440$

H $0.857281-2.936969-0.179100$

H $\quad 0.393675-1.765827 \quad 1.068548$

H $3.058217-1.937374-0.385579$

H $-0.9295310 .191895-1.872646$

H $4.004609-1.8179501 .911991$

H $2.375529-1.7451202 .601408$

H $2.912359-3.202547 \quad 1.751740$

H -1.682796 -0.9191550 .872086$

H $-2.370181-1.550312-1.864566$

H -0.7725421 .6777830 .132266$

H -2.988372 $1.214277-0.885898$

H -3.1669041 .7509001 .560540$

H $-3.8341350 .140013 \quad 1.853607$

H $-3.875012-1.357328-0.049067$

H $4.525516 \quad 0.2425010 .641415$

H $3.9823810 .319635-1.038895$

H $-5.606753 \quad 1.859743 \quad 1.714606$

H -5.1160192 .2599930 .059920$

H -5.797162 0.6621230 .426804

SCF Energy (B3LYP/6-31G**//MMFF) $=-810.716965881$

F2-17_c03862

MMFF Geometry

C $3.780253 \quad 1.6360650 .299350$

C $4.6564732 .131265-0.582122$

C $2.584383-0.5991180 .531585$

O $1.428146-0.0321311 .177540$

C $0.295105-0.1580150 .287671$

C $0.742104-1.095371-0.826642$

C $2.214090-0.751672-0.942327$

C $-0.918855-0.6379101 .085924$

C $3.031379-1.796957-1.679923$

C $-2.150268-0.9936890 .231055$

$\begin{array}{llll}\text { O } & -1.245424 & 0.380715 & 2.033597\end{array}$

O $-3.198434-1.434394 \quad 1.101874$

C $-2.7003730 .114662-0.690786$

C $-3.4159601 .268020 \quad 0.026741$

O $-3.628835-0.522476-1.578365$

$\begin{array}{llll}\text { C } 3.831716 & 0.234109 & 0.837567\end{array}$

C $-3.9340542 .332831-0.934344$

H $2.9860172 .282863 \quad 0.663232$

H $4.5680883 .155268-0.928164$

H $5.4697251 .529925-0.972914$

H $2.723096-1.5903260 .984075$

H $0.1185910 .850327-0.106663$

H $0.191487-0.936768-1.758460$ 
H $0.616511-2.143981-0.528801$

H $2.2999450 .206366-1.469776$

H $-0.633522-1.5102071 .687562$

H $2.671956-1.911120-2.707721$

H $4.086331-1.507926-1.720970$

H $2.967968-2.775167-1.191510$

H $-1.903476-1.860588-0.394763$

H -2.0192790 .0722082 .535710$

H -2.857040 -2.186057 1.615696

H -1.890514 $0.506965-1.314862$

H $-2.736917 \quad 1.7592240 .728515$

H -4.2705640 .8879620 .598268$

H $-3.7867820 .080079-2.324271$

H $3.9471350 .310613 \quad 1.925994$

H $4.717426-0.2896930 .458225$

H $-4.7216401 .939244-1.583846$

H $-4.3580673 .170962-0.371711$

H -3.126067 2.720787-1.562498

SCF Energy (B3LYP/6-31G**//MMFF) $=-810.708772230$

F2-17 c03863

MMFF Geometry

C $-4.504646-1.4159450 .554833$

C -4.644462 -2.643124 0.041097

C $-2.746368 \quad 0.352317 \quad 0.292608$

O $-1.673423-0.5702250 .016555$

C $-0.5356570 .162728-0.493684$

C $-0.8382581 .626101-0.214983$

C $-2.3444081 .663487-0.378853$

C $0.742577-0.4094690 .140351$

C -2.9903922 .8952520 .231488$

C $2.0445220 .292308-0.309720$

O $0.806507-1.792662-0.217247$

$\begin{array}{lllll}\text { O } & 2.022138 & 1.651864 & 0.117579\end{array}$

C $3.334035-0.3761100 .224546$

C $4.5921410 .443314-0.107469$

O $3.497863-1.672635-0.344367$

C $-4.063380-0.217293-0.236953$

C $5.863635-0.1947270 .439976$

H -4.730512 -1.262578 1.606886

H $-4.971614-3.4665360 .666654$

H -4.435696 -2.848861 -1.002835

H -2.7891790 .4750841 .382883$

H - $0.523655-0.021540-1.575741$

H $-0.3237322 .303612-0.902630$

H $-0.562074 \quad 1.8938620 .812254$

H -2.582319 $1.626596-1.450441$

H $0.654845-0.363121 \quad 1.233056$

H -2.612576 $3.804524-0.247579$

H $-4.0768742 .871861 \quad 0.101331$

H -2.7808982 .9689031 .303837$

H $2.0815230 .281391-1.406342$

H $-0.021617-2.2084320 .078873$

H 2.0027241 .6566081 .089973

H $3.268468-0.4849371 .314164$

H $4.510204 \quad 1.4555380 .303396$

H $4.6963120 .534653-1.195484$

H $2.745492-2.217539-0.058697$

H $-4.8543870 .538542-0.173553$

H $-3.951231-0.487138-1.294523$

H $\quad \begin{array}{llll}6.728503 & 0.437314 & 0.214373\end{array}$

H $5.804714-0.3136321 .526502$

H $\quad 6.042867-1.177961-0.005937$

SCF Energy (B3LYP/6-31G**//MMFF) $=-810.721827377$

F2-17 c03864

MMFF Geometry

C 2.1044762 .7132160 .230981

C $1.3734483 .612978-0.436452$

C $2.150694 \quad 0.208757 \quad 0.185413$

O $0.773267 \quad 0.071510-0.214289$

C $0.525971-1.288919-0.607698$

C $1.749321-2.078605-0.155458$

C $2.850948-1.051129-0.319298$

C $-0.793169-1.808088-0.027403$

C $4.122285-1.3982580 .435456$

C $-2.058415-1.154422-0.621309$

O $-0.770888-1.6735121 .392460$
O $-3.203899-1.786664-0.033398$

C $-2.2305570 .370648-0.483829$

C $-2.3787070 .894300 \quad 0.950287$

O $-3.3997360 .739625-1.229166$

C $2.7257871 .498896-0.399978$

C -2.5906762 .4035910 .996404$

H $2.2716722 .863147 \quad 1.294530$

H 0.9537904 .4704040 .078183

H $1.1779303 .510617-1.498055$

H $2.163350 \quad 0.2432531 .282798$

H $0.473866-1.300751-1.703696$

H $1.907705-2.979115-0.756516$

H $1.664518-2.3836920 .894533$

H $3.082455-0.951291-1.388269$

H $-0.851590-2.886412-0.224248$

H $4.882251-0.6226220 .298446$

H $3.935846-1.5014221 .509728$

H $4.537377-2.3448510 .074254$

H -2.100189-1.396356-1.691304

H -1.622615 -2.000560 1.729143

H $-3.140666-2.739400-0.217402$

H $-1.3985430 .882118-0.975878$

H -1.4842370 .6630251 .535432$

H -3.2337740 .4171961 .442900$

H $-4.1587730 .284451-0.825650$

H $3.8070891 .543467-0.227993$

H $2.5651541 .517046-1.485305$

H -2.6425912 .7442902 .035546$

H -1.7644292 .9297340 .508122$

H -3.5239932 .6922660 .503153$

SCF Energy $($ B3LYP/6-31G**//MMFF $)=-810.717112504$

F2-17_c03865

MMFF Geometry

C $-4.591681-0.7618620 .454201$

C $-5.698049-0.4142951 .121177$

C -2.4064630 .4084430 .065882$

O $-1.631086-0.800451-0.056599$

C $-0.325633-0.478458-0.593745$

C $-0.2146391 .033342-0.502714$

C $-1.6489961 .463241-0.736264$

C $0.729310-1.287360 \quad 0.171315$

C $-1.9306732 .891883-0.303828$

C $2.206090-1.076018-0.228989$

O $0.418082-2.670636-0.080818$

O $2.336044-1.112549-1.650293$

C $2.871473 \quad 0.195627 \quad 0.327732$

C 4.3798100 .2254870 .029130

O $2.6677190 .270864 \quad 1.735880$

C $-3.8274810 .166882-0.446821$

C 5.0441701 .4999350 .534863

H $-4.221123-1.7787250 .555428$

H $-6.207496-1.1339881 .752561$

H $-6.112596 \quad 0.585326 \quad 1.051674$

H $-2.4218980 .671795 \quad 1.131617$

H $-0.356562-0.794861-1.644192$

H $0.4719321 .452951-1.243033$

H $\quad 0.114233 \quad 1.3418450 .497106$

H $-1.8775751 .359204-1.805568$

H $0.593143-1.1472081 .249755$

H $-2.9786873 .154219-0.478633$

H -1.719744 3.0379690 .760893

H -1.308556 $3.594001-0.868653$

H $2.770897-1.9399770 .145113$

H $0.988759-3.2091530 .493541$

H $1.973805-0.284509-2.007677$

H $2.4291031 .087869-0.122669$

H $4.5538910 .142243-1.049547$

H $4.870953-0.6288750 .510373$

H $3.081178-0.5118932 .138496$

H $-4.3581881 .121376-0.541401$

H -3.793330 -0.289599-1.443750

H $6.108205 \quad 1.497110 \quad 0.278015$

H 4.5881262 .3854600 .080973

H $4.9620161 .588517 \quad 1.622574$

SCF Energy $\left(\mathrm{B} 3 \mathrm{LYP} / 6-31 \mathrm{G}^{* *} / / \mathrm{MMFF}\right)=-810.703946869$

F2-17_c03866 
MMFF Geometry

C 3.7938081 .6683410 .072960

C 3.4600482 .9638130 .081110

C $2.390003-0.3476120 .756520$

O $1.138122 \quad 0.2906730 .437354$

C $0.548358-0.395201-0.684615$

C $1.347542-1.683009-0.863049$

C $2.733427-1.233531-0.440101$

C $-0.958810-0.596052-0.460642$

C $3.675402-2.377924-0.111038$

C $-1.7226380 .717002-0.178719$

O $-1.148747-1.4948550 .630548$

O $-1.410701 \quad 1.677220-1.192979$

C $-3.2619160 .594146-0.158853$

C $-3.814563-0.1811911 .040444$

O $-3.717422-0.035987-1.356971$

C $3.4295490 .700273 \quad 1.163175$

C $-5.328521-0.0425831 .157636$

H $4.3815861 .291359-0.759436$

H $3.7624853 .614132-0.732507$

H 2.8819603 .3960090 .890262

H $2.188971-0.9769021 .634178$

H $\quad 0.711646 \quad 0.242047-1.562613$

H $1.310246-2.055072-1.891374$

H $\quad 0.989210-2.480422-0.201864$

H $3.162399-0.642370-1.257917$

H -1.364130 -1.078725 -1.357157

H $3.824631-3.017206-0.987312$

H $4.653565-1.9986820 .201246$

H $3.281629-3.0030620 .697423$

H -1.4072621 .1401330 .783230$

H $-0.745841-1.0907681 .418160$

H $-0.5130682 .007254-1.018326$

H $-3.6899741 .604834-0.157843$

H $-3.354613 \quad 0.179879 \quad 1.967069$

H $-3.593979-1.249250 \quad 0.942376$

H $-3.3624890 .474251-2.105056$

H $3.040607 \quad 1.2571392 .025607$

H $4.3453380 .199979 \quad 1.498871$

H $-5.834307 \quad-0.438088 \quad 0.271247$

H -5.691192 -0.5977922 .028601$

H $-5.6175731 .006087 \quad 1.280549$

SCF Energy (B3LYP/6-31G**//MMFF) $=-810.720464829$

F2-18_c04096

MMFF Geometry

C -2.911786 2.4364800 .069303

C $-2.0662713 .302985-0.500125$

C -2.724015 -0.0011790 .655188$

O $-1.302517 \quad 0.1220550 .885509$

C $-0.640357-1.0791520 .450014$

C -1.648016 -1.824431 -0.413681

C $-2.950237-1.4688260 .275384$

C $0.680095-0.741643-0.259875$

C $-4.184358-1.783468-0.550796$

C 1.6555410 .0467230 .633760

O $\quad 0.4118580 .011621-1.440966$

O $1.957504-0.7387651 .787207$

C $2.9640070 .453730-0.076411$

C $3.869810-0.729919-0.440516$

O $3.696427 \quad 1.3183250 .798035$

C -3.124176 $1.027834-0.412695$

C $5.124138-0.286435-1.184883$

H -3.492112 $2.762110 \quad 0.928812$

H -1.961587 4.307819-0.105803

H -1.466943 3.028849 -1.361149

H -3.238844 0.2240121 .596991

H $-0.435266-1.669357 \quad 1.352047$

H -1.454592 -2.901112 -0.438570

H -1.649477 -1.458667 -1.446635

H $-3.007903-2.0507331 .206254$

H $1.137811-1.687824-0.568676$

H -4.273388 -2.864229 -0.704783

H -5.090192 -1.440696 -0.040561

H -4.147257 -1.315329 -1.539652

H 1.1657430 .9616520 .989920

$\mathrm{H}-0.0309200 .834000-1.169690$

H $2.578239-0.2261372 .332786$
H $2.736347 \quad 1.032922-0.978588$

H $3.330149-1.444189-1.070384$

H $4.186318-1.2577890 .466826$

H 3.1432182 .0995600 .968896

H $-2.5528450 .859389-1.333422$

H $-4.1860680 .923748-0.661034$

H $5.728949-1.157957-1.455515$

H $4.8648450 .245261-2.105869$

H $5.7433340 .372618-0.568477$

SCF Energy $\left(B 3 L Y P / 6-31 G^{* *} / / M M F F\right)=-810.720186780$

F2-18 c04097

MMFF Geometry

C $2.8701172 .235409-0.627999$

C 2.3985423 .1841770 .188638

C $2.218798-0.163516-0.998291$

O $0.843017 \quad 0.116890-0.658484$

C $0.286067-0.9989340 .056060$

C $1.469592-1.8593350 .478921$

C $2.421754-1.648612-0.681132$

C $-0.601936-0.5307881 .217844$

C $3.842153-2.102980-0.396618$

C -1.7803490 .3906230 .832161$

O $\quad 0.196243 \quad 0.1643992 .181447$

O

C $-2.785468-0.245491-0.144686$

C $-3.9978220 .669866-0.379853$

O $-3.220111-1.4905130 .394021$

C $3.1093090 .810066-0.212185$

C $-4.9772940 .105369-1.401141$

H $3.1051102 .501095-1.655501$

H $2.2505164 .196046-0.172404$

H 2.1507652 .9712401 .222653

H $2.3424310 .008723-2.074404$

H $-0.319225-1.561827-0.664569$

H $1.188863-2.9075980 .619731$

H $1.920435-1.503707 \quad 1.412338$

H $2.044530-2.228745-1.535432$

H $-0.994589-1.4162071 .730751$

H $3.871623-3.187651-0.247283$

H $4.501896-1.860605-1.235750$

H $4.248824-1.6382760 .507283$

H $-2.3009560 .656679 \quad 1.760854$

H $\quad 0.548557 \quad 0.9592821 .745349$

H $-0.8334541 .412044-0.544605$

H $-2.296587-0.434232-1.107736$

H -3.664536 $1.653880-0.728299$

H $-4.5372020 .818440 \quad 0.563527$

H -3.685817 -1.972433 -0.309869

H 2.9305880 .7016150 .864304

H $4.1668950 .589051-0.393855$

H -5.434808 -0.823408 -1.047434

H $-5.7834160 .823124-1.584396$

H -4.478151-0.094539-2.354464

SCF Energy (B3LYP/6-31G**//MMFF $)=-810.722918035$

F2-18 c04098

MMFF Geometry

C -3.539718 2.147671 -0.226099

C $-2.8123073 .227294-0.534313$

C $-2.910521-0.1824230 .477085$

$\begin{array}{lllll}0 & -1.701567 & 0.285601 & 1.113784\end{array}$

C $-0.639498-0.6708710 .901870$

C -1.140601-1.612545 -0.180269

C $-2.628994-1.640240 \quad 0.102672$

C 0.6558920 .0933590 .587623

C -3.454514 -2.231300 -1.025951

C $1.868136-0.8173880 .321623$

O $0.950303 \quad 0.923674 \quad 1.714794$

O $1.680609-1.504235-0.915298$

C $3.219768-0.0712270 .282808$

C $3.3521020 .907642-0.891497$

O $4.265167-1.0424740 .172564$

C $-3.2212090 .756013-0.698917$

C $4.6741141 .666056-0.861689$

H -4.4218102 .2731330 .396590$

H -3.097627 $4.205993-0.164338$

H -1.925254 3.155810-1.153776 
H $-3.721401-0.134677 \quad 1.213783$

H $-0.529727-1.2095961 .851960$

H $-0.679863-2.603133-0.121015$

H -0.950452 -1.202804 -1.179402

H $-2.789704-2.2549390 .999766$

H $0.477427 \quad 0.755112-0.268393$

H -3.212770 -3.291267-1.159041

H $-4.523942-2.155025-0.804877$

H -3.263080 -1.731128 -1.980702

H $1.920714-1.5817001 .107339$

H $\quad 0.175926 \quad 1.492637 \quad 1.865679$

H $2.460283-2.068379-1.056016$

H $3.3829750 .462819 \quad 1.225759$

H $2.5352931 .636400-0.873571$

H $3.2960300 .366815-1.843577$

H $4.212936-1.6187570 .954057$

H -2.373302 $0.785134-1.394026$

H $-4.0918510 .392719-1.255802$

H $5.5270930 .986494-0.952972$

H $4.7197852 .375263-1.694208$

H 4.7803222 .2303050 .070358

SCF Energy (B3LYP/6-31G**//MMFF) $=-810.717945113$

\section{F2-18 c04099}

MMFF Geometry

C $2.4878682 .585854-0.531641$

C $1.5246913 .376664-0.045206$

C $2.6628720 .089285-0.769787$

O $1.232714-0.034084-0.931951$

C $0.773352-1.220591-0.258638$

C $1.927167-1.6842940 .621036$

C $3.124077-1.258583-0.205719$

C $-0.536093-0.9390510 .495527$

C $4.433213-1.2693250 .563033$

C $-1.656518-0.408180-0.428689$

O $-\begin{array}{lll}0.269230 & 0.005114 & 1.530741\end{array}$

O $-1.804236-1.288609-1.541567$

C $-3.021053-0.2586840 .279904$

C $-4.1282530 .201120-0.682374$

O $-2.9212040 .673813 \quad 1.352666$

C $2.937114 \quad 1.3086960 .123157$

C -5.4823630 .3199910 .006043$

H $2.9879342 .877496-1.451549$

H $1.2479634 .288177-0.563784$

H $\quad 0.996523 \quad 3.1304310 .869187$

H $3.1027170 .250550-1.761392$

H $0.598151-1.973188-1.037438$

H $1.895424-2.7620260 .808062$

H $1.932809-1.1765821 .591992$

H $3.218146-1.959904-1.047134$

H $-0.858971-1.8748780 .968631$

H $5.249011-0.881798-0.055564$

H $4.691564-2.2914820 .859535$

H $4.378175-0.6690311 .476689$

H -1.358912 $0.575756-0.812288$

H $-1.072411 \quad 0.084128 \quad 2.072443$

H -2.070198 -2.158479-1.197550

H $-3.312603-1.2254900 .707476$

H $-4.222778-0.500590-1.518441$

H $-3.8705191 .181261-1.101478$

H -2.6644131 .5329620 .976007$

H 2.4343601 .1906311 .090541

H $4.010807 \quad 1.4014970 .320415$

H $-5.462996 \quad 1.0740810 .798939$

H $-6.2479660 .615457-0.718319$

H $-5.782616-0.6362580 .446027$

SCF Energy (B3LYP/6-31G**//MMFF) $=-810.716701234$

\section{F2-18 c04100}

MMFF Geometry

C $-4.262900-1.164571-0.135779$

C $-4.096540-2.427206 \quad 0.273840$

C $-2.644690 \quad 0.697857-0.617913$

O $-1.616002-0.233895-1.018188$

C $-0.3283110 .242586-0.565566$

C -0.6223221 .3560830 .426135$

C $-1.8951541 .946585-0.144675$

C $0.455591-0.9417630 .007147$
C -2.6277942 .8641310 .817602$

C $1.784377-0.5948520 .711903$

O $0.729659-1.859449-1.058390$

O $2.223494-1.7677701 .408271$

C $2.930001-0.140529-0.216030$

C 4.2350760 .1132000 .558961

O $2.5654041 .050108-0.905817$

C $-3.5220340 .010948 \quad 0.439538$

C $5.3790480 .537557-0.353846$

H $-4.980822-0.963025-0.926549$

H $-4.665307-3.231445-0.180099$

H -3.394645 -2.680856 1.060559

H $-3.2480080 .936050-1.502233$

H $0.1618920 .638121-1.462000$

H 0.1907992 .0844090 .495230

H $-0.8017020 .951981 \quad 1.429876$

H -1.624339 $2.533026-1.034173$

H $-0.187756-1.4912620 .706505$

H -2.8369422 .3739571 .773821$

H -2.0226393 .7515591 .031939$

H -3.5765813 .1995300 .386952$

H 1.6035370 .1754931 .470244

H - $0.119564-2.076508-1.480115$

H $1.537219-1.9988602 .057110$

H $3.127039-0.914271-0.967132$

H $4.538889-0.7890991 .101082$

H 4.0773770 .9061161 .300166

H $2.4456401 .752470-0.244220$

H -2.907045 $-0.315804 \quad 1.286881$

H $-4.270704 \quad 0.7111220 .826022$

H 6.2951640 .6739660 .229670

H $5.572960-0.222757-1.117043$

H $5.1590241 .483991-0.857396$

SCF Energy $\left(B 3 L Y P / 6-31 G^{* *} / / M M F F\right)=-810.709137531$

F2-18 c04101

MMFF Geometry

C -2.8823672 .4593220 .082918$

C $-2.0054363 .312575-0.458064$

C -2.7342840 .0163960 .656829$

O $-1.315290 \quad 0.1194770 .908382$

C $-0.656755-1.0724850 .442706$

C $-1.670765-1.805208-0.425697$

C $-2.971239-1.4458330 .264710$

C $0.659340-0.722744-0.270859$

C $-4.206861-1.742548-0.566030$

C $1.630874 \quad 0.059136 \quad 0.636086$

O $0.3632970 .048728-1.432795$

O $1.922463-0.711351 \quad 1.803841$

C $2.9503360 .482046-0.043124$

C $3.857791-0.683525-0.459015$

$\begin{array}{lllll}\text { O } & 3.667009 & 1.324426 & 0.865861\end{array}$

C $-3.1046791 .056483-0.411083$

C $5.107871-0.204023-1.187831$

H -3.4813832 .7914160 .926863$

H $-1.8940074 .313930-0.056801$

H -1.385220 $3.030004-1.301386$

H -3.2604710 .2428041 .592039$

H $-0.444561-1.6795631 .331805$

H -1.484341 -2.883052 -0.457173

H - $1.669630-1.434758-1.456980$

H -3.034636 -2.035520 1.190416

H $1.113592-1.666133-0.595402$

H $-5.110250-1.395210-0.054549$

H $-4.305405-2.821068-0.728966$

H -4.163799 -1.266862 -1.551035

H 1.1428300 .9767880 .986994

H $1.147506 \quad 0.033093-2.006526$

H $2.220352-1.5913231 .518017$

H $2.727501 \quad 1.101028-0.918720$

H $3.317099-1.372199-1.115924$

H $4.181325-1.2478140 .423220$

H 3.8397710 .8069851 .671068

H $-2.5203630 .886973-1.323547$

H $-4.1635650 .967192-0.677781$

H $4.8436990 .362933-2.086243$

H $5.7245600 .433179-0.546442$

H $5.716607-1.060611-1.494531$ 
H $\quad \begin{array}{lll}1.550327 & 0.524986 & 1.767055\end{array}$

F2-18_c04102

MMFF Geometry

C -2.3871642 .5136890 .535203$

C $-1.416636 \quad 3.344467 \quad 0.138224$

C $-2.5307250 .007697 \quad 0.680467$

O $-1.114260-0.0941830 .946893$

C $-0.587679-1.2685410 .302550$

C $-1.651879-1.717689-0.689445$

C $-2.922070-1.3292260 .040663$

C $0.785309-0.967924-0.321932$

C $-4.159089-1.334010-0.839503$

C $1.813866-0.4568300 .710403$

O $0.625767-0.006111-1.360657$

O $1.889803-1.4005641 .786616$

C $3.249696-0.2512740 .183227$

C $3.4620710 .907935-0.797347$

O $3.680253-1.460402-0.449765$

C $-2.7633921 .251439-0.190672$

C $3.0441692 .263744-0.253546$

H -2.949901 2.7596321 .431969

H $-1.195903 \quad 4.2420160 .705326$

H $-0.8275753 .146175-0.750377$

H $-3.046630 \quad 0.127482 \quad 1.640803$

H $-0.482156-2.0334391 .081924$

H -1.589901 -2.788862 -0.904483

H -1.578058 -1.179135 -1.640911

H $-3.079516-2.0551720 .851017$

H $1.149462-1.892897-0.783725$

H $-4.034040-0.705712-1.727305$

H -4.374646 -2.350093 -1.186738

H $-5.031465-0.975986-0.283690$

H 1.4631330 .4823821 .150486

H $\quad 0.254820 \quad 0.798859-0.960678$

H $2.229680-2.2351171 .419819$

H $3.912864-0.1069731 .045846$

H $2.9590820 .710910-1.750052$

H $4.529240 \quad 0.950488-1.053047$

H $4.608926-1.340637-0.712404$

H $-2.1910421 .173445-1.122800$

H $-3.8219101 .332138-0.461536$

H 3.5284732 .4681330 .706456

H $3.3344843 .053972-0.953434$

H $1.9612222 .327609-0.116913$

SCF Energy $\left(\mathrm{B} 3 \mathrm{LYP} / 6-31 \mathrm{G}^{* *} / / \mathrm{MMFF}\right)=-810.716478913$

F2-18_c04103

MMFF Geometry

C $-4.096682-1.4269200 .089712$

C $-5.413647-1.607686-0.059849$

C $-2.5443120 .389767-0.700593$

O $-1.443088-0.537366-0.826104$

C $-0.2162840 .114866-0.433911$

C -0.6322921 .3911020 .278369$

C $-1.8950591 .759069-0.473055$

C $0.613408-0.8548450 .411623$

C -2.7347362 .8137880 .224899$

C $1.871617-0.2352941 .044603$

O $0.980331-1.968301-0.405984$

O $2.515322-1.2506331 .822280$

C 2.8919530 .4080890 .081445

C $3.677859-0.574639-0.795736$

$\begin{array}{llll}\text { O } & 3.840511 & 1.130569 & 0.877894\end{array}$

C $-3.461487-0.105808 \quad 0.428399$

C $4.6873580 .131918-1.695091$

H -3.438291 -2.284167 -0.026192

H $-5.809713-2.588817-0.298325$

H $-6.115587-0.7884920 .050267$

H -3.094963 $0.390705-1.649030$

H $\quad 0.3061600 .348001-1.369919$

H $\quad 0.1357202 .1688410 .230045$

H $-0.855565 \quad 1.197670 \quad 1.334637$

H -1.598733 2.156487 -1.454233

H -0.010365 -1.277292 1.209716

H $-2.192612 \quad 3.7645910 .266814$

H -3.671352 $2.984040-0.315594$

H -2.9763052 .5315681 .254639$

H $\quad 0.161417-2.338530-0.778080$

H $3.299670-0.8477002 .232874$

H $2.3943981 .146661-0.556060$

H $2.999924-1.143791-1.437092$

H $4.226664-1.287995-0.170138$

H 3.3537501 .8204261 .360308

H $-2.888764-0.2426021 .353498$

H -4.2442840 .6335410 .630715$

H $4.1909180 .863568-2.340539$

H $5.4525650 .650432-1.109161$

H $5.194237-0.596737-2.335796$

SCF Energy (B3LYP/6-31G**//MMFF $)=-810.710404967$

F2-18_c04104

MMFF Geometry

C $3.1666871 .821441-0.711066$

C $2.8309102 .917873-0.022010$

C $2.130991-0.462729-0.880161$

O $\quad 0.810000 \quad 0.076170-0.653355$

C $0.039013-0.8498540 .128872$

C $1.040019-1.8415450 .709748$

C $2.064077-1.917991-0.404929$

C $-0.815919-0.1247861 .177194$

C $3.372414-2.5667460 .010165$

C -1.7803670 .9670340 .665622$

$\begin{array}{lllll}\text { O } & 0.048417 & 0.495140 & 2.139395\end{array}$

O -1.0277022 .0077360 .034595$

C $-2.8979990 .517303-0.293830$

C $-3.790944-0.5756950 .300210$

O $-2.353006 \quad 0.051422-1.525781$

C $3.1405900 .429442-0.142406$

C $-5.001202-0.868671-0.578262$

H $3.486727 \quad 1.931879-1.743892$

H $2.8739903 .895217-0.489843$

H 2.5069012 .8617511 .011330

H $2.327896-0.430687-1.958564$

H $-0.611525-1.389368-0.568154$

H $0.579414-2.8078520 .937351$

H $1.504279-1.4657091 .628197$

H $1.631449-2.517204-1.218709$

H -1.388972 -0.8744631 .733518$

H $3.812531-2.0802040 .886488$

H $3.210026-3.6186060 .268401$

H $4.099965-2.529078-0.806914$

H $-2.2463001 .430528 \quad 1.545089$

H $\quad 0.571273 \quad 1.1658901 .666901$

H $-1.6479142 .717885-0.202880$

H $-3.5132201 .390830-0.543434$

H $-4.139203-0.2745431 .294704$

H $-3.226381-1.5088550 .409378$

H -1.755761 $0.742631-1.859666$

H 2.9000870 .4688260 .926771

H $4.1516260 .018094-0.236949$

H $-4.698712-1.232743-1.565117$

H $-5.626759-1.637351-0.113312$

H $-5.6127030 .028927-0.714400$

SCF Energy (B3LYP/6-31G**//MMFF) $=-810.713958340$

F2-18 c04105

MMFF Geometry

C $2.6661402 .523546-0.408388$

C $1.7552003 .470594-0.157930$

C $2.6740450 .015147-0.601061$

O $1.342518-0.019930-1.168706$

C $0.719867-1.264296-0.821574$

C $1.470850-1.7816220 .394735$

C $2.887025-1.3575910 .055582$

C $-0.804797-1.134027-0.673150$

C $3.840412-1.4231111 .235797$

C $-1.286728-0.1268350 .388772$

O $-1.346035-2.419472-0.376699$

$\begin{array}{lllll}\text { O } & -0.851717 & 1.181766 & 0.024937\end{array}$

C $-2.818847-0.1168520 .589929$

C $-3.6039740 .399245-0.623167$

O $-3.1175720 .731451 \quad 1.703634$

C 2.7621231 .2235840 .342483

C $-5.1109230 .346797-0.397865$ 
H $3.3974942 .701333-1.192659$

H $1.7457704 .389469-0.733882$

H 1.0099823 .3503790 .620190

H $3.3853330 .139160-1.426821$

H $\quad 0.916008-1.954002-1.654685$

H $1.366883-2.8630420 .524260$

H $1.144077-1.2918721 .317510$

H $3.265432-2.040784-0.718340$

H -1.194080 -0.814869-1.647607

H $3.953220-2.4579001 .576634$

H $4.831274-1.0521620 .955191$

H $3.479942-0.8368612 .087241$

H $-0.828134-0.3601401 .355810$

H -1.087277 -3.021066 -1.095511

H $-1.170368 \quad 1.7953880 .708955$

H $-3.169525-1.1210600 .853721$

H -3.367823 -0.195202 -1.511555

H -3.331833 $1.439752-0.835671$

H -2.673005 0.3583442 .483710

H 1.9713471 .1669671 .100255

H 3.7249371 .2262250 .864866

H -5.4133550 .9883140 .435623$

H $-5.6372940 .690928-1.293898$

H $-5.438924-0.675297-0.183647$

SCF Energy (B3LYP/6-31G $\left.{ }^{* *} / / \mathrm{MMFF}\right)=-810.704821143$

F2-18 c04106

MMFF Geometry

C $2.7368252 .382960-0.420548$

C 2.0757423 .2644300 .338194

C $2.339064-0.051373-0.901676$

O $\quad 0.913728 \quad 0.107414-0.730778$

C $0.373822-1.052280-0.075240$

C $1.569169-1.8286520 .464176$

C $2.623097-1.522045-0.580797$

C $-0.661298-0.6618550 .990019$

C $4.035483-1.868891-0.145491$

C -1.8535010 .1848930 .486696$

O -0.0018340 .0735272 .026538$

O $-1.4018641 .446925-0.001363$

C $-2.704557-0.511463-0.589875$

C $-3.8973800 .319032-1.093068$

O $-3.167150-1.772347-0.113637$

C 3.0418490 .9723490 .002362

C $-4.9094740 .703256-0.021538$

H $3.0853082 .695874-1.401251$

H $1.8891714 .269761-0.023247$

H 1.7077273 .0010611 .323760

H $2.5789060 .155863-1.951571$

H $-0.110183-1.653683-0.853794$

H $1.356956-2.8979260 .559627$

H $1.887003-1.4615301 .446398$

H $2.388897-2.112623-1.477919$

H -1.040639 -1.5812501 .451911$

H $4.135945-2.950832-0.007679$

H $4.301327-1.3934180 .804161$

H $4.761884-1.556888-0.902555$

H $-2.4780630 .401913 \quad 1.361040$

H $-0.657594 \quad 0.2570292 .720565$

H $-0.838306 \quad 1.282562-0.776446$

H $-2.081599-0.711827-1.468951$

H -4.429059 -0.264997-1.854695

H $-3.5352791 .229605-1.583940$

H -3.618093 -1.6274270 .735032$

H $2.7416380 .824688 \quad 1.046786$

H $4.1284350 .842444-0.048371$

H $-4.467103 \quad 1.362488 \quad 0.730726$

H $-5.7473131 .241050-0.477661$

H -5.316049 -0.1782490 .482706$

SCF Energy (B3LYP/6-31G**//MMFF) $=-810.711342532$

F2-18_c04107

MMFF Geometry

C -3.971928 -0.7093981 .133130$

C $-3.673362-1.7108911 .968343$

C $-2.627428 \quad 0.517511-0.600710$

O $-1.816826-0.647244-0.866841$

C $-0.445215-0.246041-1.093842$
C $-0.361686 \quad 1.202890-0.649614$

C $-1.7496101 .711014-0.983693$

C $0.472807-1.234802-0.363530$

C $-2.0834253 .044366-0.338966$

C $1.991404-0.985293-0.470903$

O $0.241997-2.527623-0.953999$

O $2.334990-0.671136-1.820774$

C 2.5511780 .1097460 .453503

C 4.0891340 .1412850 .413470

O $2.114136-0.1437641 .785070$

C -3.0685320 .4639030 .869970$

C $4.680417 \quad 1.2695311 .249232$

H $-4.926429-0.7299520 .613652$

H $-4.370800-2.5278042 .117921$

H -2.735376-1.736094 2.511914

H $-3.5075100 .474145-1.253647$

H $-0.295358-0.320375-2.178215$

H $0.4235851 .756258-1.172618$

H -0.1798551 .2752960 .428973$

H -1.808829 $1.836029-2.074255$

H $\quad 0.170423-1.3343130 .685012$

H -1.942071 3.0229010 .746357

H -1.434436 $3.831916-0.736508$

H -3.120743 $3.326098-0.546248$

H $2.501350-1.927529-0.228315$

H $-0.708910-2.715902-0.872344$

H $2.012013-1.399446-2.378803$

H $2.182895 \quad 1.0922180 .144801$

H $4.4364730 .257699-0.619284$

H $4.488585-0.8076590 .791800$

H 2.2964810 .6508652 .313946

H -2.191578 0.4117481 .526630

H $-3.624721 \quad 1.3701601 .133961$

H 4.4619551 .1378092 .313280

H 5.7689611 .2907771 .134307

H 4.2870022 .2403780 .931697

SCF Energy $(B 3 L Y P / 6-31 G * * / / M M F F)=-810.714749610$

F2-18 c04108

MMFF Geometry

C $-3.5513302 .158999-0.192644$

C $-2.8307153 .243206-0.500734$

C $-2.910612-0.1749350 .486637$

$\begin{array}{lllll}\text { O } & -1.694611 & 0.288447 & 1.113694\end{array}$

C $-0.638177-0.6722680 .893646$

C $-1.147638-1.603316-0.193772$

C $-2.634227-1.6302610 .098562$

C 0.6627660 .0841210 .581915

C $-3.468224-2.211312-1.029162$

C $1.870369-0.8309920 .296048$

O $0.9593360 .916924 \quad 1.707192$

O $1.683996-1.532401-0.932617$

C $3.228490-0.0950450 .261064$

C $3.3717490 .908331-0.890800$

O $4.268772-1.0694800 .132141$

C $-3.2339020 .771742-0.679237$

C $4.6860821 .677712-0.820182$

H -4.4267772 .2771640 .440759$

H -3.114951 $4.218078-0.119980$

H -1.950196 $3.179335-1.130268$

H $-3.713386-0.1322391 .232614$

H $-0.531594-1.2202041 .838819$

H $-0.688787-2.595416-0.146342$

H $-0.963713-1.184548-1.190325$

H $-2.790401-2.2516380 .991849$

H $0.4787980 .753546-0.266296$

H -4.535996 -2.134724 -0.800808

H -3.281705 -1.704383 -1.981318

H $-3.229457-3.270620-1.171708$

H $1.928882-1.5957621 .080479$

H 1.1153820 .3376722 .472615

H $1.489691-0.880152-1.626592$

H 3.3962530 .4197291 .213996

H $2.547761 \quad 1.628997-0.874314$

H $3.3421130 .387051-1.854669$

H $4.204437-1.6702760 .893898$

H $-2.3923230 .807900-1.381667$

H $-4.1089310 .411300-1.231075$ 
H $4.7430942 .401040-1.639886$

H 4.7672642 .2273070 .122954

H $5.5468111 .007026-0.904066$

SCF Energy (B3LYP/6-31G**//MMFF) $=-810.703351577$

\section{F2-18 c04109}

MMFF Geometry

C -3.7931072 .0087910 .085653$

C $-3.3993773 .094909-0.588910$

C $-2.716144-0.2130620 .505223$

$\begin{array}{lllll}\text { O } & -1.356868 & 0.232398 & 0.369841\end{array}$

C $-0.520040-0.7867980 .942250$

C $-1.315497-2.0927930 .865485$

C $-2.645621-1.714430 \quad 0.223837$

C $0.844460-0.8226520 .234171$

C $-2.665498-2.087749-1.258652$

C 1.5926260 .5218970 .304609

O $0.670072-1.194916-1.130350$

O $1.806208 \quad 0.857007 \quad 1.676098$

C $2.9398360 .527750-0.448434$

C $4.008491-0.3775750 .178262$

O $3.4429531 .867703-0.452714$

C $-3.6266920 .600505-0.414197$

C $5.303766-0.379750-0.625703$

H $-4.2727962 .141531 \quad 1.051898$

H $-3.5529514 .084892-0.173592$

H $-2.9193223 .018146-1.558228$

H $-3.008000-0.0586551 .553765$

H $-0.371057-0.5267531 .997725$

H -1.487907 -2.460240 1.885173

H $-0.787714-2.894557 \quad 0.338564$

H $-3.477417-2.2367910 .710571$

H $1.441148-1.6054610 .715329$

H $-1.912146-1.540300-1.831803$

H -3.644356 -1.874195 -1.700283

H $-2.477020-3.158797-1.389090$

H $0.9632051 .312636-0.122485$

H $0.144642-0.497524-1.558741$

H 2.2848681 .7034221 .696541

H $2.785340 \quad 0.242519-1.495330$

H $3.644070-1.4074400 .246993$

H $4.243868-0.0383031 .193931$

H $2.7915522 .423880-0.913257$

H -3.220090 $0.619142-1.432460$

H $-4.6199030 .138741-0.458481$

H $5.126285-0.715842-1.652199$

H $5.7538980 .617141-0.661740$

H $\quad 6.029678-1.058978-0.167370$

SCF Energy $($ B3LYP/6-31G**//MMFF $)=-810.717928643$

F2-18 c04110

MMFF Geometry

C $1.6986052 .696226-0.001654$

C 0.8023023 .2450040 .825625

C $2.1691940 .358520-0.802854$

O $0.771485-0.005650-0.745760$

C $0.649027-1.393879-0.396574$

C $1.995776-1.7839250 .197094$

C $2.942723-0.955949-0.647105$

C $-0.533145-1.6396890 .546006$

C $4.348206-0.868404-0.079756$

C $-1.918886-1.453998-0.103433$

O $-0.393936-0.8207051 .704426$

O $-2.895322-1.854014 \quad 0.867605$

C $-2.293986-0.063013-0.652904$

C $-2.469740 \quad 1.0330780 .404205$

O $-3.542383-0.195887-1.347940$

C $2.431301 \quad 1.4138370 .281808$

C $-2.8519332 .374648-0.212274$

H $1.9284483 .206991-0.933244$

H $\quad 0.310781 \quad 4.1757890 .564499$

H $\quad 0.5408092 .7786201 .769040$

H $2.3646250 .788775-1.792631$

H $0.492158-1.948225-1.330704$

H $2.184561-2.8594290 .125377$

H $2.070623-1.4957121 .252179$

H $3.007542-1.425551-1.639049$

H $-0.473577-2.675791 \quad 0.904237$
H $4.818614-1.857533-0.075122$

H $4.352630-0.5006780 .951324$

H $4.970515-0.203026-0.686693$

H -2.001177 -2.180544 -0.922154

H -1.154971 -1.003480 2.281807

H $-3.770030-1.7701110 .450482$

H - $1.5639910 .259875-1.400733$

H -1.544442 1.1744190 .967591

H -3.2553880 .7529691 .115506$

H -3.405724 -0.822801-2.078600

H $2.137348 \quad 1.0248151 .264267$

H 3.4990831 .6546630 .326120

H -3.821703 2.322372 -0.716560

H -2.921042 $3.140190 \quad 0.567173$

H -2.101105 2.698719-0.939863

SCF Energy (B3LYP/6-31G**//MMFF) $=-810.716042681$

F2-18 c04111

MMFF Geometry

C $2.2725042 .664266-0.361367$

C 1.3234043 .3719400 .261623

C $2.5048310 .205913-0.831694$

O $1.0706770 .041969-0.890714$

C $0.706495-1.218274-0.297749$

C $1.939920-1.7068890 .449600$

C $3.052449-1.167274-0.427872$

C $-0.564684-1.0639810 .553975$

C $4.419562-1.1934700 .231630$

C $-1.764347-0.528239-0.263104$

O $-0.317038-0.172731 \quad 1.638724$

O $-1.963645-1.364397-1.401032$

C $-3.075118-0.4572030 .552052$

C $-4.323184-0.147677-0.290818$

$\begin{array}{llll}\text { O } & -2.983809 & 0.530536 & 1.575951\end{array}$

C $2.810673 \quad 1.3535860 .142522$

C $-4.2610471 .181184-1.033737$

H $2.6922543 .053546-1.285284$

H $0.9791194 .313334-0.152365$

H $0.8732593 .026901 \quad 1.185875$

H $2.8568830 .468779-1.836429$

H $0.508244-1.906172-1.129351$

H $1.958845-2.7968630 .546095$

H $2.013171-1.2802431 .455073$

H $3.099414-1.789692-1.332654$

H $-0.824820-2.0447740 .971373$

H $5.169448-0.723172-0.412296$

H $4.420569-0.6763561 .196493$

H $4.733389-2.2266720 .415172$

H $-1.5190090 .480359-0.616758$

H $\quad 0.196965-0.6536512 .308589$

H $-2.186556-2.254659-1.079016$

H -3.249154 -1.424001 1.040785

H $-5.194034-0.1139880 .375930$

H -4.503915 -0.954948 -1.009601

H -2.2397420 .2907132 .153225$

H 2.3884531 .1345121 .130696

H 3.8931051 .4694210 .266069

H $-3.4685001 .181484-1.787718$

H $-5.2087431 .364526-1.550520$

H $-4.0868782 .015317-0.347167$

SCF Energy (B3LYP/6-31G**//MMFF) $=-810.712013377$

F2-18_c04112

MMFF Geometry

C $-4.272919-1.192453-0.094784$

C $-4.090574-2.4491840 .325716$

C $-2.6791320 .681981-0.612029$

O $-1.644592-0.242518-1.014753$

C $-0.3562880 .255322-0.585891$

C -0.6518051 .3729290 .400882$

C $-1.9385361 .943775-0.158652$

C $0.457055-0.914294-0.021609$

C -2.6696892 .8610440 .805176$

C $1.765489-0.5380990 .705626$

O $0.747945-1.798240-1.109762$

O $2.147081-1.6636381 .508599$

C $2.960757-0.150487-0.188161$

C 4.1839740 .2470350 .654960 
O $2.624336 \quad 0.949693-1.023589$

C $-3.537672-0.0041930 .461513$

C $5.3969300 .581455-0.204968$

H $-5.000468-1.005446-0.880310$

H $-4.656109-3.263181-0.114419$

H $-3.378510-2.6881131 .107893$

H $-3.2944260 .905356-1.491954$

H $0.1077080 .657475-1.492295$

H 0.1552002 .1101020 .451796

H -0.8145260 .9750311 .409817$

H -1.686002 2.525724 -1.056439

H $-0.184959-1.486750 \quad 0.660825$

H -3.627606 3.1820710 .383626

H -2.8614272 .3767181 .768001$

H -2.071982 3.7569781 .004502

H $1.559014 \quad 0.274513 \quad 1.409936$

H $1.148872-2.601438-0.737051$

H $2.414815-2.381600 \quad 0.910412$

H $3.249682-0.996800-0.824041$

H $4.455510-0.5636611 .340233$

H $3.943713 \quad 1.1280831 .262594$

H $2.2047880 .588368-1.822347$

H $-2.910016-0.3169051 .304857$

H -4.2893040 .6918730 .849948$

H $5.677071-0.269591-0.833769$

H $5.202708 \quad 1.440913-0.853891$

H $\quad \begin{array}{llll}6.252563 & 0.828924 & 0.431444\end{array}$

SCF Energy (B3LYP/6-31G**//MMFF) $=-810.707228906$

F2-19_c04353

MMFF Geometry

C - $1.1197503 .124806-0.459742$

C $0.1053843 .634056-0.288224$

C $-2.0197700 .784371-0.397287$

O $-0.7870870 .174156-0.822909$

C $-0.881597-1.251724-0.689743$

C $-2.336060-1.542345-0.321677$

C $-2.745402-0.283667 \quad 0.416502$

C $0.124806-1.751480 \quad 0.364955$

C $-4.250067-0.0884530 .492941$

C $1.614414-1.4862350 .041148$

O $-0.045815-3.169766 \quad 0.482007$

O $1.914014-1.956122-1.273734$

C $2.060970-0.0183670 .154882$

C 3.5836770 .1297000 .000098

$\begin{array}{lllll}\text { O } & 1.663014 & 0.512748 & 1.415337\end{array}$

C $-1.713558 \quad 2.049570 \quad 0.406092$

C 4.0293531 .5857370 .057524

H -1.736315 $3.511388-1.267188$

H $0.4739404 .412553-0.947036$

H $\quad 0.759458 \quad 3.2859980 .503451$

H -2.586861 $1.030794-1.304518$

H $-0.660682-1.712957-1.659112$

H -2.479487 -2.4527590 .266996$

H -2.926971 -1.647601-1.240867

H $-2.338992-0.3231631 .435539$

H $-0.113440-1.3176351 .342602$

H -4.4978880 .8361111 .023486$

H $-4.699259-0.035059-0.504475$

H $-4.718920-0.920644 \quad 1.028348$

H $2.219907-2.0858180 .733435$

H $\quad 0.514961-3.4732851 .216152$

H $1.639128-2.887878-1.318726$

H $1.5916950 .582040-0.630475$

H $3.911786-0.301576-0.952217$

H $4.093855-0.4153340 .803471$

H $2.099517-0.0099872 .109578$

H -2.632939 2.4445040 .852795

H $-1.027594 \quad 1.8093151 .227894$

H 3.7877052 .0397271 .023666

H $5.1127271 .653551-0.083370$

H $3.5472502 .173762-0.729846$

SCF Energy (B3LYP/6-31G**//MMFF) $=-810.716972538$

F2-19 c04354

MMFF Geometry

C -2.934191 2.5276310 .057552

$\begin{array}{llll}\text { C } & -2.092118 & 3.566761 & 0.033777\end{array}$
C $-2.5683020 .053212-0.125304$

O $-1.3697010 .172011-0.915965$

C $-0.716368-1.104627-0.993570$

C $-1.692834-2.114103-0.396788$

C $-2.447655-1.2768300 .615809$

C $0.638418-1.047184-0.254990$

C $-3.781027-1.8784331 .026254$

C $1.608426-0.000858-0.846661$

O $1.239998-2.339031-0.304215$

O $1.1168311 .319401-0.610915$

C $3.041688-0.087933-0.282109$

$\begin{array}{llll}\text { C } & 3.150706 & 0.259570 & 1.209264\end{array}$

O $3.8689770 .808808-1.030678$

C $-2.677826 \quad 1.255256 \quad 0.814616$

C $4.5759870 .116212 \quad 1.729457$

H -3.867892 $2.600149-0.494076$

H $-2.337715 \quad 4.460271-0.529854$

H -1.1491923 .5455340 .568905$

H $-3.4136680 .030624-0.825469$

H $-0.542125-1.346571-2.048651$

H $-1.216855-2.9959770 .041053$

H -2.377295 -2.462477 -1.181067

H $-1.823221-1.155328 \quad 1.510507$

H $\quad 0.452976-0.7969750 .795726$

H -3.633070 -2.856982 1.494609

H $-4.295260-1.2340481 .746158$

H $-4.442339-2.0151180 .164020$

H $1.671220-0.130312-1.934689$

H $1.407273-2.552827-1.238165$

H $\quad 0.249516 \quad 1.394093-1.043313$

H $3.445200-1.091584-0.454631$

H $2.498816-0.392528 \quad 1.799699$

H 2.8315191 .2939301 .382735

H $3.510567 \quad 1.705441-0.912650$

H -3.5031581 .1088751 .520588$

H $-1.7560861 .349527 \quad 1.402660$

H $4.944474-0.9042821 .584692$

H $\begin{array}{llll}5.257958 & 0.804834 & 1.221099\end{array}$

H 4.6100780 .3401822 .800458

SCF Energy (B3LYP/6-31G**//MMFF) $=-810.723663767$

F2-19 c04355

MMFF Geometry

C 3.3708831 .9347950 .100408

C $4.1070302 .409438-0.910653$

C $2.721773-0.4226110 .816709$

O $1.453224-0.0179951 .357115$

C $0.428612-0.865247 \quad 0.788056$

C $1.099176-1.679230-0.321367$

C $2.400687-0.941092-0.579155$

$\begin{array}{llll}\text { C } & -0.742852 & 0.031082 & 0.344794\end{array}$

C $3.486931-1.813416-1.183281$

C $-1.995744-0.767460-0.054290$

O $-0.3427280 .847063-0.753963$

O $-2.426033-1.5375301 .068791$

C $-3.1616930 .108839-0.560235$

C $-3.7795341 .008827 \quad 0.518040$

O $-4.188658-0.757021-1.054451$

C $3.735436 \quad 0.7211310 .907269$

C $-4.892171 \quad 1.890862-0.037350$

H 2.4589142 .4600880 .371543

H $3.7919923 .295700-1.450496$

H $5.0278511 .926206-1.217705$

H $3.082337-1.2446531 .450430$

H $0.094450-1.5347331 .588893$

H $0.492722-1.789400-1.224594$

H $1.301013-2.6913730 .052200$

H $2.189867-0.111277-1.264031$

H $-0.9811680 .703057 \quad 1.177709$

H $3.162390-2.219990-2.146700$

H $4.401484-1.236266-1.351868$

H $3.735699-2.655930-0.529256$

H $-1.740930-1.479366-0.848237$

H $0.4192091 .374591-0.460129$

H -3.223534 -2.024092 0.798595

H $-2.8299120 .723255-1.405002$

H $-3.015177 \quad 1.6562550 .959340$

H -4.2049300 .3980441 .323072$ 
H $-3.809748-1.273130-1.786312$

H 3.8132021 .0417591 .953796

H 4.7248990 .3501530 .614234

H $-5.7222351 .291808-0.424442$

H -5.2865462 .5398180 .751124$

H $-4.5194892 .527825-0.845766$

SCF Energy (B3LYP/6-31G**//MMFF) $=-810.721284800$

F2-19 c04356

MMFF Geometry

C $4.346420-0.879750-0.559772$

C $4.321307-2.214812-0.479648$

C $2.5578820 .881516-0.410479$

O $1.528900 \quad 0.005258-0.913203$

C $0.2787820 .294573-0.254183$

C $0.638997 \quad 1.1316900 .963695$

C 1.8607481 .9005570 .495099

C $-0.458017-1.0268530 .006198$

C $1.4722013 .163431-0.276712$

C $-1.882497-0.9000820 .581263$

O $0.302718-1.8318850 .913692$

O $-1.850414-0.2617841 .856474$

C $-2.858669-0.136517-0.330013$

C $-4.293136-0.1555870 .221629$

O $-2.846201-0.707275-1.635656$

C 3.5817730 .0320780 .358321

C $-5.2592960 .633778-0.652723$

H $4.960805-0.414831-1.326408$

H $4.900037-2.817458-1.171202$

H $\quad 3.727585-2.7277300 .268906$

H $3.0444031 .366624-1.265171$

H - $0.3000160 .897292-0.962901$

H 0.9209250 .4955541 .810620

H $-0.183168 \quad 1.775323 \quad 1.290217$

H 2.4896742 .2059331 .338756

H $-0.499765-1.595794-0.930452$

H $0.8686702 .940804-1.162463$

H $0.898473 \quad 3.8436420 .361562$

H $2.3692823 .695352-0.611357$

H $-2.266169-1.9150190 .748840$

H $1.186617-1.9470610 .524831$

H -1.236418 -0.7662652 .417240$

H $-2.5432680 .909437-0.417867$

H -4.315969 0.2609821 .234904

H $-4.653955-1.1896180 .281280$

H $-3.138920-1.631480-1.558728$

H $4.3038990 .687347 \quad 0.859787$

H $3.080654-0.5559071 .136579$

H $-4.9412141 .677170-0.743868$

H $-5.3297890 .205387-1.657433$

H $-6.2613670 .621677-0.212184$

SCF Energy (B3LYP/6-31G**//MMFF) $=-810.719692530$

\section{F2-19 c04357}

MMFF Geometry

C -4.477948 -1.459959 0.303154

C $-4.392419-2.7873650 .161306$

C $-2.769497 \quad 0.373758 \quad 0.261694$

O $-1.681817-0.450394 \quad 0.717309$

C -0.5040370 .3726550 .861702$

C -0.8539861 .7418200 .281630$

C $-2.0979721 .478563-0.545522$

C $0.672520-0.3580550 .187684$

C $-2.9584292 .714426-0.748346$

C 2.0388920 .3198020 .428060

O $0.431396-0.460143-1.214172$

O $2.214438 \quad 0.5322901 .827476$

C $3.233605-0.496560-0.116586$

$\begin{array}{llll}\text { C } & 4.581000 & 0.157352 & 0.229248\end{array}$

O $3.152608-0.615254-1.534222$

C $-3.743342-0.472570-0.559125$

C $5.767970-0.658309-0.269431$

H -5.123178 -1.0594631 .080736$

H -4.953075 -3.4468520 .814668$

H -3.766117 -3.237695 -0.600844

H $-3.265870 \quad 0.7871551 .149939$

H -0.3127620 .4624831 .936933$

H $-0.0500862 .200851-0.300229$
H $-1.0829772 .429177 \quad 1.106281$

H $-1.7917101 .093123-1.526412$

H $\quad 0.707169-1.3822250 .580878$

H -3.305466 3.1226430 .206843

H -2.391342 $3.497169-1.262674$

H -3.839066 2.483588 -1.355539

H $2.0443671 .297992-0.066681$

H $-0.413181-0.928474-1.329520$

H $2.223545-0.3386512 .260291$

H $3.218107-1.5056130 .313252$

H 4.6781410 .2832461 .313145

H $4.6355911 .153591-0.226271$

H $2.336340-1.099309-1.744415$

H $-4.4873440 .169344-1.043857$

H -3.198660 -1.000115-1.352273

H $5.765768-0.742890-1.360456$

H $6.705611-0.1773490 .027004$

H $5.755048-1.6670750 .155272$

SCF Energy (B3LYP/6-31G**//MMFF) $=-810.721615051$

F2-19 c04358

MMFF Geometry

C -3.1838202 .2770950 .012699$

C $-4.3354282 .893595-0.275690$

C $-2.363182-0.058846-0.394750$

O $-0.9730260 .310757-0.471559$

C $-0.167812-0.877840-0.540118$

C $-1.138398-2.052527-0.631092$

C $-2.371617-1.5146140 .066881$

C $0.750598-0.9434170 .689820$

C $-3.642090-2.270201-0.284635$

C 1.5865990 .3324850 .915523

O $1.590739-2.0887140 .569380$

O 2.3732370 .1311432 .095172

C $2.4972880 .786536-0.244096$

C $3.688609-0.133066-0.538556$

O 3.0179092 .0786420 .094890

C $-3.0933840 .882536 \quad 0.565087$

C $4.5634230 .396257-1.670395$

H -2.252241 $2.813476-0.148627$

H $-4.3339963 .904956-0.667486$

H -5.292855 $2.406062-0.128831$

H $-2.7767830 .027386-1.408319$

H $0.436664-0.846213-1.453686$

H $-0.761384-2.979396-0.189059$

H -1.361594 -2.256645 -1.686196

H -2.214220 -1.566075 1.152119

H $0.144566-1.0992731 .591452$

H $-3.845341-2.235204-1.360241$

H $-4.506668-1.8458610 .235126$

H $-3.556616-3.3221760 .007029$

H 0.8984501 .1528941 .156121

H $2.173645-2.1036221 .347932$

H 2.8844460 .9450822 .244555

H $1.9060460 .919622-1.156337$

H $3.341013-1.130140-0.822360$

H $4.317804-0.2375340 .352868$

H 2.2625142 .6837260 .188772

H -4.0965920 .4959010 .778444$

H $-2.553407 \quad 0.936777 \quad 1.518446$

H $5.373671-0.309467-1.880250$

H $3.9802920 .525308-2.587702$

H $5.0170041 .357835-1.410891$

SCF Energy $($ B3LYP/6-31G**//MMFF $)=-810.721150841$

F2-19 c04359

MMFF Geometry

C $4.267169 \quad 1.742512 \quad 0.302304$

C 4.0453293 .0527080 .148630

C $2.760269-0.2600500 .257106$

O 1.5861890 .4482140 .692386

C $0.492463-0.4877590 .810750$

C $1.001091-1.8217850 .267958$

C $2.221186-1.437199-0.546993$

C $-0.730048 \quad 0.1172280 .093714$

C $3.210342-2.576990-0.723979$

C $-2.031151-0.6718100 .334542$

O $-0.4763720 .225783-1.305641$ 
O $-2.304897-0.7403631 .734381$

C $-3.258660-0.104223-0.409199$

C $-3.725555 \quad 1.272910 \quad 0.081533$

O $-4.335016-1.036159-0.261349$

C $3.6498280 .677807-0.559902$

C $-4.8903421 .809532-0.742730$

H 4.9413651 .4171671 .090453

H $4.526233 \quad 3.7716770 .802812$

H $3.3844433 .429696-0.624189$

H $3.285968-0.6109051 .155329$

H $0.273545-0.5838391 .880357$

H $0.262981-2.383396-0.310913$

H $1.294642-2.4593311 .111941$

H $1.890845-1.095211-1.536036$

H -0.8454651 .1414300 .466708$

H $4.070893-2.260028-1.321039$

H $3.583958-2.9373730 .240386$

H $2.736996-3.420178-1.237791$

H -1.901712 -1.706922 -0.001944

H $-0.499855-0.667826-1.687341$

H $-2.278733 \quad 0.1633812 .091511$

H -3.041523 -0.054150 -1.482000

H -2.9044121 .9949320 .030949$

H $-4.054752 \quad 1.213533 \quad 1.125343$

H $-4.520718-1.1213060 .689656$

H $4.462545 \quad 0.113801-1.031014$

H $3.0622791 .139683-1.363347$

H $-5.1786602 .802910-0.384648$

H -4.614981 $1.896874-1.798522$

H $-5.7666411 .158289-0.667275$

SCF Energy $\left(\mathrm{B} 3 \mathrm{LYP} / 6-31 \mathrm{G}^{* *} / / \mathrm{MMFF}\right)=-810.715181129$

F2-19_c04360

MMFF Geometry

C $-1.8061932 .750295-0.326866$

C $-2.6643383 .739012-0.601583$

C $-2.0537320 .252673-0.312602$

O $-0.6848980 .009705-0.685223$

C $-0.424141-1.402128-0.656458$

C -1.749764 -2.074042 -0.297155

C $-2.477687-0.9873120 .468474$

C $0.704814-1.7066760 .346003$

C $-3.980393-1.1989130 .535278$

C $2.078092-1.0653170 .037206$

O $0.897731-3.126440 \quad 0.390432$

O $2.488312-1.433527-1.281846$

C 2.1681990 .4640240 .195995

C 3.6164130 .9569890 .041483

O $1.699105 \quad 0.8533821 .482458$

C $-2.151231 \quad 1.5444970 .500845$

C 3.7232562 .4745280 .132236

H -0.791746 $2.820262-0.711016$

H -2.351413 4.587098 -1.200903

H -3.685930 $3.721602-0.238309$

H -2.631752 $0.341437-1.242266$

H $-0.127118-1.726752-1.660148$

H -1.645535 $-3.003160 \quad 0.270344$

H -2.294047 -2.313587 -1.219944

H -2.074735 -0.945172 1.488993

H $0.395094-1.4068231 .352901$

H $-4.465278-0.3919521 .093126$

H -4.425862 -1.232198 -0.464568

H -4.213431 -2.1430341 .038620$

H $2.804144-1.5272350 .719015$

H $1.212533-3.406623-0.486345$

H $1.968049-0.910321-1.914744$

H $1.5654640 .970613-0.565282$

H $4.0273750 .634145-0.921667$

H $4.2419350 .523477 \quad 0.831268$

H $\quad 0.732636 \quad 0.753707 \quad 1.485314$

H -3.1599051 .6484030 .917301$

H -1.453118 1.5104861 .345449

H $4.7621932 .787906-0.011121$

H $3.1142972 .956228-0.639310$

H 3.3943852 .8407541 .109587

SCF Energy (B3LYP/6-31G**//MMFF) $=-810.720899085$

F2-19_c04361
MMFF Geometry

C $-2.6844132 .744626-0.023984$

C -1.8670813 .6660310 .498213$

C $-2.4204140 .283843-0.424486$

O $-0.9881690 .339533-0.566037$

C $-0.463219-0.995326-0.638495$

C $-1.670081-1.931438-0.670843$

C $-2.727687-1.1303730 .062243$

C $0.463277-1.2518290 .561388$

C $-4.145688-1.591703-0.228303$

C $1.544701-0.1726190 .801803$

O $1.076436-2.5292230 .380763$

O $2.106819-0.4106652 .097836$

C $2.681560-0.117512-0.239709$

C 3.7602230 .9127330 .136386

O $2.1598370 .213675-1.522301$

C -2.8778541 .3697260 .550504$

C $4.8989590 .956431-0.875215$

$\mathrm{H}-3.2548612 .998051-0.913891$

H -1.7748274 .6432760 .037102$

H -1.2779503 .4643521 .386004$

H -2.850094 $0.451636-1.420892$

H $\quad 0.090333-1.113338-1.575523$

H $-1.484674-2.913115-0.225251$

H -1.975269-2.093176 -1.712786

H $-2.542779-1.2054291 .141854$

H $-0.143935-1.3194271 .473226$

H -4.281956 -2.633295 0.080797

H $-4.874421-0.9813900 .314077$

H -4.378667 -1.524757 -1.296340

H 1.0552590 .8065910 .856837

H $1.556064-2.7438161 .198499$

H $2.651876-1.2138382 .044890$

H $3.160270-1.100321-0.318055$

H 4.1794020 .6838581 .122411

H $3.313311 \quad 1.9128310 .191555$

H $1.7254121 .080935-1.452361$

$\mathrm{H}-3.941824 \quad 1.2466890 .782037$

H -2.3242121 .2768161 .493444$

H $5.6602921 .674282-0.553607$

H $5.375718-0.024351-0.969641$

H $4.5444361 .264828-1.863495$

SCF Energy (B3LYP/6-31G**//MMFF $)=-810.714683056$

F2-19_c04362

MMFF Geometry

C -2.9373162 .5362670 .086006$

C -2.0925313 .5735900 .082880$

C $-2.5791770 .063841-0.134016$

O $-1.3755790 .188278-0.915806$

C $-0.729726-1.091046-1.013007$

C $-1.717063-2.103440-0.437383$

C $-2.469904-1.2779230 .586280$

C $0.624521-1.060198-0.270187$

C $-3.808470-1.8772190 .982578$

C $1.599907-0.015532-0.856974$

O $1.196843-2.362569-0.358265$

O $1.0774911 .291710-0.615053$

C $3.035829-0.098338-0.297469$

C 3.1449880 .2395251 .196211

O $3.8634640 .797106-1.046825$

C $-2.6878651 .251500 \quad 0.824013$

C $4.5709730 .096788 \quad 1.714641$

H -3.868035 $2.619995-0.469260$

H $-2.3328584 .476185-0.468017$

H -1.1523093 .5412700 .622195$

H -3.420495 $0.056796-0.839211$

H $-0.554330-1.317436-2.071551$

H -1.250897 -2.996681-0.012469

H -2.400937 -2.432631 -1.230412

H $-1.847540-1.1748021 .484842$

H $\quad 0.435977-0.8262550 .784551$

H -3.668768 -2.864607 1.434633

H -4.320291 -1.241543 1.711884

H $-4.468198-1.9945010 .116283$

H $1.650764-0.146267-1.945674$

H $1.872750-2.4359360 .336394$

H $1.6068871 .920617-1.133330$ 
H $3.440797-1.099933-0.477110$

H $2.495029-0.4178961 .782860$

H 2.8220791 .2716521 .376588

H $3.6240661 .706104-0.799056$

H -3.5162511 .0970371 .524510$

H -1.768044 1.3332691 .417098

H 4.6050140 .3135572 .787124

H $4.942156-0.9218061 .562794$

H $\quad 5.250680 \quad 0.7907201 .210490$

SCF Energy (B3LYP/6-31G**//MMFF) $=-810.706223233$

F2-19 c04363

MMFF Geometry

C $3.6865081 .955456-0.072957$

C $3.2367983 .214845-0.110510$

C $2.531682-0.2548840 .174655$

$\begin{array}{lllll}\text { O } & 1.416137 & 0.267032 & 0.917147\end{array}$

C $0.587010-0.8379531 .325659$

C $1.065409-2.0645110 .555246$

C $1.974112-1.493218-0.518163$

C $-0.898305-0.465347 \quad 1.159737$

C $3.034328-2.472947-0.992776$

C $-1.3050940 .060595-0.231325$

O $-1.193631 \quad 0.5330012 .141493$

O $-1.219922-1.004572-1.176649$

C $-2.7256280 .665465-0.282545$

C -3.844095 -0.356207 -0.039037

O $-2.9218951 .238329-1.579546$

C $3.0397960 .809413-0.798324$

C $-5.2242810 .290998-0.041601$

H $4.579096 \quad 1.7378910 .508008$

H 3.7525263 .9987020 .433302

H $2.3529253 .485169-0.677432$

H $3.313164-0.5291190 .896155$

H $0.783105-0.9910162 .394801$

H $\quad 0.255338-2.680507 \quad 0.154057$

H $1.637190-2.7090201 .235569$

H $1.361694-1.192034-1.376640$

H -1.493611 -1.350169 1.412122

H $3.689255-2.783799-0.171992$

H $2.567881-3.371295-1.410416$

H $3.660115-2.026446-1.771711$

H $-0.5978820 .833917-0.554707$

H $-0.6362681 .306727 \quad 1.948545$

H -1.480445 $-0.646580-2.042740$

H $-2.812520 \quad 1.4840580 .440885$

H $-3.699478-0.8573990 .923501$

H $-3.831812-1.124800-0.820834$

H $-2.2489231 .929972-1.699136$

H $3.7834620 .377844-1.477490$

H $2.208317 \quad 1.176583-1.413057$

H -5.451609 $0.744593-1.011284$

H $-5.992704-0.4607740 .165120$

H $-5.293766 \quad 1.0669440 .727455$

SCF Energy (B3LYP/6-31G**//MMFF) $=-810.719382241$

F2-19 c04364

MMFF Geometry

C $3.373001-1.8702990 .235802$

C $4.159585-2.1925121 .268843$

C $2.8185330 .347626-0.911466$

O $1.496264-0.083179-1.291989$

C $0.5415850 .627648-0.484868$

C $1.261688 \quad 0.871120 \quad 0.831520$

C $2.686574 \quad 1.158245 \quad 0.386931$

C $-0.760576-0.182128-0.397524$

C 2.9063442 .6521820 .138592

C $-1.867110 \quad 0.5450090 .383594$

O $-0.508075-1.441166 \quad 0.223509$

O $-2.1361281 .794103-0.253368$

C $-3.174868-0.2643240 .515696$

C -3.918037 -0.469849 -0.810895

O $-4.047007 \quad 0.439800 \quad 1.405913$

C $3.744549-0.874362-0.826351$

C $-5.174769-1.315912-0.640894$

H $2.412151-2.3680230 .141631$

H $3.835469-2.9272531 .997856$

H $5.131761-1.7326231 .406981$
H $3.1878560 .981369-1.727444$

H $0.3464081 .580485-0.992232$

H $1.258512-0.033047 \quad 1.450944$

H $\quad 0.8111311 .6779961 .417372$

H 3.4092520 .8482081 .149645

H -1.092892 $-0.387791-1.421479$

H 2.7559853 .2217101 .061779

H $3.9296782 .835828-0.205683$

H $22211303.048645-0.617639$

H -1.5081730 .7724961 .394941$

H $\quad 0.171489-1.892054-0.306071$

H -2.842865 2.2310530 .251958

H -2.968976 -1.237544 0.975771

H $-3.267825-0.962240-1.540762$

H $-4.2184160 .497481-1.230582$

H -3.5986190 .5029572 .266449$

H $3.720411-1.403396-1.787282$

H $4.774759-0.527547-0.678719$

H $-5.897166-0.8306320 .022536$

H $-5.659596-1.468131-1.610502$

H $-4.930889-2.299088-0.225856$

SCF Energy $\left(B 3 L Y P / 6-31 G^{* *} / / M M F F\right)=-810.720067595$

F2-19 c04365

MMFF Geometry

C 4.2640861 .6335930 .189396

C 4.0735542 .9557580 .118566

C $2.694279-0.3205050 .161988$

$\begin{array}{lllll}\text { O } & 1.581141 & 0.397948 & 0.722886\end{array}$

C $0.477973-0.5176050 .898345$

C $0.888871-1.8293550 .231755$

C $2.054355-1.436129-0.655771$

C $-0.791100 \quad 0.1519010 .334382$

C $2.987901-2.592804-0.971168$

C $-2.082296-0.6451290 .609391$

O $-0.6399420 .333784-1.071668$

O $-2.125378-0.9611082 .004609$

C $-3.3993380 .083173 \quad 0.258775$

C $-3.6292430 .274217-1.243065$

O $-3.431206 \quad 1.3637320 .890592$

C $3.5489630 .633012-0.674053$

C $-5.0474580 .746197-1.545093$

H $4.985372 \quad 1.248746 \quad 0.905933$

H 4.6257913 .6259380 .768374

H $3.3685553 .390869-0.580977$

H $3.275400-0.7341630 .997122$

H $\quad 0.364766-0.6690701 .977706$

H $0.084843-2.322256-0.321518$

H $1.220054-2.5329761 .006468$

H $1.658207-1.031922-1.596085$

H $-0.869721 \quad 1.1501880 .781834$

H $3.808508-2.268896-1.618610$

H $3.424456-3.015720-0.060111$

H $2.447657-3.392856-1.487964$

H -2.058058 -1.593213 0.059661

H $\quad 0.1521950 .880982-1.209671$

H -2.890380 -1.543195 2.151265

H $-4.237131-0.4894270 .676267$

H -3.450347 -0.667040 -1.774451

H $-2.9450571 .029505-1.643636$

H $-3.283157 \quad 1.2216801 .841201$

H $4.3047560 .071332-1.234276$

H $2.9172681 .152613-1.405520$

H $-5.2548671 .711238-1.072221$

H $-5.1833190 .863498-2.624876$

H $-5.7859830 .021349-1.187961$

SCF Energy (B3LYP/6-31G**//MMFF) $=-810.717890698$

F2-19 c04366

MMFF Geometry

C $-2.9749312 .598480-0.024893$

C $-2.111763 \quad 3.6182730 .041479$

C $-2.6418350 .119401-0.200301$

O $-1.369727 \quad 0.220502-0.868553$

C $-0.734651-1.067581-0.896203$

C $-1.788904-2.061644-0.414416$

C $-2.621017-1.2202950 .531920$

C $0.542625-1.048861-0.027157$ 
C $-3.999869-1.797300 \quad 0.803583$

C $1.605694-0.032718-0.520674$

O $1.077832-2.370055-0.034222$

O $1.0716591 .289107-0.483965$

C $2.901305-0.0381810 .322818$

C $3.8986861 .042661-0.127815$

O $3.538305-1.3107370 .247036$

C -2.8184641 .3138820 .738738$

C $5.164247 \quad 1.0546810 .720818$

H $-3.8510252 .697159-0.660560$

H $-2.2838114 .522003-0.532813$

H -1.2236153 .5704430 .661825$

H $-3.4148370 .122046-0.979946$

H $-0.459819-1.302089-1.931473$

H -1.381429 -2.9616530 .054060$

H -2.400371 -2.381535-1.268198

H -2.083385 -1.122356 1.484036

H $\quad 0.266131-0.7885401 .002227$

H $-4.577118-1.910128-0.120277$

H $-3.918122-2.7838411 .271407$

H $-4.568165-1.1498791 .478724$

H $\quad 1.868738-0.274231-1.558664$

H $\quad 1.798270-2.4004050 .617719$

H $\quad 0.3266241 .325985-1.107139$

H $2.645614 \quad 0.143139 \quad 1.373872$

H $3.4370862 .034825-0.076277$

H $4.1878080 .866803-1.171000$

H $3.759791-1.476733-0.685244$

H $-3.711178 \quad 1.1778561 .359492$

H -1.9567171 .3834971 .414633$

H $5.712003 \quad 0.1111280 .636187$

H $5.8302901 .858843 \quad 0.391733$

H 4.9259491 .2223301 .775928

SCF Energy (B3LYP/6-31G**//MMFF) $=-810.722432919$

\section{F2-19_c04367}

MMFF Geometry

C -2.2037172 .2259530 .304547$

C $-3.0719262 .740523 \quad 1.182541$

C $-2.1596400 .085416-1.072493$

O $-0.723207-0.000358-1.067629$

C $-0.330302-1.209894-0.396013$

C -1.620913 -1.964374 -0.070930

C $-2.638996-0.8452970 .037958$

C $0.490310-0.8587160 .854750$

C $-4.076400-1.308466-0.121700$

C 1.6728550 .1115450 .619051

O $0.968839-2.0744851 .433012$

O $2.185803 \quad 0.4831851 .903114$

C $2.835417-0.459578-0.216153$

C $4.0938260 .427309-0.230598$

O $2.403014-0.662847-1.558345$

C $-2.6054851 .547057-0.974551$

C $3.8679731 .821408-0.802291$

H -1.140556 2.3094570 .514466

H $-2.717503 \quad 3.2239232 .086338$

H -4.1425662 .6882441 .019128$

H -2.482650 -0.301482 -2.048432

H $\quad 0.266374-1.823275-1.079056$

H -1.560460 -2.583336 0.829107

H - $-1.875562-2.625116-0.909690$

H -2.526150 -0.373639 1.021379

H $-0.172504-0.3992911 .598842$

H -4.767925 -0.463522 -0.045587

H -4.238076 -1.790672 -1.091536

H -4.336936 -2.0299500 .659574$

H $1.290627 \quad 1.0227330 .145093$

H $1.480909-1.8368922 .225025$

H $1.471673 \quad 0.9338632 .385264$

H $3.130408-1.4346470 .189472$

H $4.859609-0.062333-0.845329$

H 4.5078590 .5134590 .780309

H $3.138600-1.068939-2.047401$

H $-2.1476692 .111745-1.796500$

H $-3.6916421 .603760-1.114474$

H $3.1839552 .403889-0.178229$

H $4.8175362 .364642-0.847382$

H $3.4590431 .776097-1.816301$
SCF Energy (B3LYP/6-31G**//MMFF)= -810.714527335

F2-19_c04368

MMFF Geometry

C $1.5413823 .069028-0.078617$

C $0.5742053 .625936-0.815906$

C 2.1609580 .7184700 .566929

O 0.8093280 .2196120 .511936

C $0.810119-1.1341990 .039732$

C $2.186632-1.361133-0.573444$

C $3.079315-0.4704780 .268588$

C $-0.342810-1.380722-0.943069$

C $3.524438-1.1709161 .554187$

C $-1.762265-1.296696-0.336781$

O $-0.188766-2.682265-1.520770$

O $-1.864925-2.1903550 .773955$

C $-2.205578 \quad 0.1012340 .130224$

C -3.6882530 .1112530 .537772$

O $\quad-2.0248551 .049932-0.915696$

C $2.3026701 .835950-0.478457$

C -4.1351651 .4709391 .061318$

H 1.8106023 .5375090 .864577

H $0.0670374 .520589-0.471644$

H $\quad 0.2720653 .204408-1.768131$

H $2.338241 \quad 1.121815 \quad 1.571128$

H $\quad 0.701022-1.7892040 .912679$

H $2.205151-1.011245-1.613195$

H $2.481278-2.415122-0.571970$

H $3.980865-0.173549-0.278768$

H $-0.273023-0.668363-1.772535$

H $4.159608-0.5058222 .148890$

H $2.677419-1.4685972 .180536$

H $4.105814-2.0692131 .321612$

H -2.469588 -1.653312 -1.097269

H $-0.108402-3.324788-0.795730$

H $-1.738546-3.0933520 .437481$

H $-1.6214500 .420577 \quad 1.000622$

H $-3.875487-0.641047 \quad 1.312218$

H $-4.312214-0.141781-0.328159$

H $-1.0710081 .218361-0.995679$

H $3.3580592 .114917-0.580555$

H $1.9574981 .484091-1.458488$

H -5.1857381 .4301721 .365922$

H -3.5411921 .7693811 .931086$

H -4.0381482 .2461880 .295113$

SCF Energy $($ B3LYP/6-31G**//MMFF $)=-810.715251074$

F2-19_c04369

MMFF Geometry

C $4.219343-1.656877-0.445307$

C $4.292755-2.7767770 .282618$

C $2.5056080 .164994-0.601624$

O $1.394645-0.665779-0.225200$

C $0.1767420 .109935-0.326372$

C $0.6108571 .559769-0.541180$

C $2.0837501 .564253-0.175980$

C $-0.663074-0.1913420 .927116$

C $2.8658352 .683169-0.842794$

C -2.1432270 .2429920 .873330$

$\begin{array}{lllll}\text { O } & -0.067650 & 0.432602 & 2.069387\end{array}$

O $-2.247807 \quad 1.6535430 .698386$

C $-2.962896-0.452136-0.228801$

C $-4.455667-0.095768-0.129942$

O $-2.798385-1.861467-0.103752$

C $3.772815-0.3285430 .097140$

C $-5.285470-0.702463-1.254398$

H $4.506756-1.696601-1.492765$

H $4.627572-3.704395-0.168562$

H $4.020864-2.7904581 .332393$

H $2.6126060 .103733-1.692982$

H $-0.343758-0.257899-1.216398$

H 0.0445282 .2954720 .033311

H $\quad 0.4779301 .815813-1.600292$

H 2.1723881 .6666810 .913331

H -0.624232 -1.269486 1.126424

H $2.4602893 .659172-0.556480$

H $3.9187282 .656722-0.545790$

H $2.8217692 .607903-1.934516$ 
H -2.591997 0.0130921 .848816

H 0.8394030 .0895502 .144985

H -1.784021 2.0712421 .444426

H -2.595190 -0.143265 -1.214217

H $-4.5860570 .991995-0.153335$

H $-4.859149-0.4564300 .824162$

H -3.140368 -2.273475 -0.914854

H $4.5897020 .386650-0.050354$

H $3.594908-0.4052531 .177201$

H -6.324064 -0.364592 -1.178551

H $-4.900797-0.399100-2.233136$

H -5.288466 -1.795497-1.206219

SCF Energy (B3LYP/6-31G $\left.{ }^{* *} / / \mathrm{MMFF}\right)=-810.717684716$

F2-19_c04370

MMFF Geometry

C $4.130989-1.013282-0.775574$

C $4.092170-2.341824-0.623328$

C $2.3942960 .791202-0.549859$

O $1.306284-0.087320-0.902930$

C $0.1276800 .271229-0.150199$

C 0.6200081 .1574060 .983439

C 1.8061461 .8717670 .362006

C $-0.612071-1.0134570 .244920$

C $1.3702753 .103132-0.435031$

C $-1.957536-0.8567520 .980293$

O $0.236922-1.8018551 .091260$

O $-1.775148-0.1533832 .211572$

C -3.097664 -0.1643920 .212655$

C -3.431309-0.854972 -1.112735

O $-2.7822831 .201795-0.044476$

C $3.473203-0.0399390 .161779$

C $-4.683573-0.277740-1.761512$

H $4.676470-0.601126-1.620518$

H $4.591883-2.990974-1.333983$

H $3.564743-2.8032630 .204209$

H $2.8033531 .219793-1.472396$

H $-0.4925050 .851439-0.840285$

H $\quad 0.9704210 .555683 \quad 1.830077$

H $-0.1523321 .836931 \quad 1.355398$

H 2.5182822 .2040651 .125550

H - $0.765915-1.620090-0.655071$

H $2.2418093 .596282-0.879027$

H $0.6819432 .851007-1.247915$

H 0.8722563 .8277530 .217767

H -2.303963 -1.859725 1.262104

H $1.066584-1.9524530 .606562$

H -1.086077 -0.6224002 .712921$

H -3.989281 -0.1564820 .852205$

H -3.579760 -1.928227 -0.948170

H $-2.604261-0.734374-1.821878$

H -2.576229 1.6167420 .810579

H $\quad 4.2508370 .6243270 .557188$

H $3.039436-0.5766891 .014127$

H -4.903671 -0.808393 -2.693238

H -5.549821 -0.380616-1.100316

H $-4.5560920 .782627-2.000428$

SCF Energy (B3LYP/6-31G**//MMFF) $=-810.721783585$

F2-19_c04371

MMFF Geometry

C $3.891429-0.8193620 .381437$

C $4.063156-2.1056930 .056522$

C $2.4865361 .026550-0.705153$

O $1.4848500 .098633-1.165356$

C $0.3027250 .261037-0.358358$

C $\quad 0.822142 \quad 0.734166 \quad 0.989287$

C 1.9827311 .6387920 .608785

C $-0.490129-1.052905-0.373864$

C 1.5225723 .0851220 .410998

C $-1.854748-1.022351 \quad 0.342234$

O $0.287784-2.0933910 .227804$

O $-1.683040-0.7419531 .730221$

C $-2.848408-0.007665-0.248884$

C $-4.233305-0.109006 \quad 0.410023$

O $-2.975323-0.218868-1.652420$

C $3.8372060 .300195-0.619892$

C $-5.2116420 .921518-0.139733$
H $3.804392-0.5600041 .432932$

H $4.102128-2.8665410 .828359$

H $4.163679-2.422280-0.975648$

H $2.5805191 .798498-1.479034$

H $-0.2884941 .051283-0.835001$

H $1.204640-0.107141 \quad 1.578078$

H $\quad 0.055859 \quad 1.238258 \quad 1.585557$

H 2.7523041 .6487591 .388246

H - $0.639529-1.359700-1.416231$

H $1.1134503 .489618 \quad 1.342913$

H 2.3659753 .7170970 .113102

H $0.7505963 .169707-0.360820$

H $-2.287902-2.0290230 .277795$

H $1.135429-2.130043-0.247292$

H $-1.054062-1.3952112 .081778$

H $-2.4742491 .010841-0.095105$

H -4.1493380 .0294601 .493691$

H $-4.654967-1.1064740 .236160$

H -3.320726 -1.117991-1.787425

H $4.082545-0.091443-1.615680$

H $4.6194091 .025052-0.363549$

H $-5.3885520 .773498-1.209548$

H -6.1746090 .8380420 .373953$

$\begin{array}{llll}H & -4.834250 & 1.937981 & 0.010361\end{array}$

SCF Energy $\left(B 3 L Y P / 6-31 G^{* *} / / M M F F\right)=-810.721373933$

F2-19 c04372

MMFF Geometry

C -3.354858 $2.030827-0.617921$

C $-2.7962143 .164381-0.178663$

C $-2.550426-0.349901-0.631765$

O $-1.1577940 .019168-0.648130$

C $-0.424042-0.8273800 .249117$

C $-1.452583-1.6485901 .019800$

C $-2.621950-1.7164170 .055657$

C $0.4874880 .006622 \quad 1.152942$

C $-2.453153-2.857515-0.949634$

C 1.4296570 .9570520 .388347

O $1.218778-0.8759341 .998989$

O 2.1932101 .6809881 .359996

C $2.3778070 .322408-0.649553$

C $3.488744-0.558507-0.062350$

O $2.9767221 .410156-1.365812$

C $-3.3253250 .731727 \quad 0.137577$

C $4.407582-1.144636-1.128748$

H $-3.8705402 .039868-1.574542$

H -2.855358 $4.071192-0.770556$

H -2.271572 3.2081490 .769365

H -2.907774 -0.399008-1.667237

H $0.177934-1.512553-0.359549$

H - $-1.763809-1.123777 \quad 1.931337$

H -1.066262 -2.627021 1.322057

H $-3.570837-1.8678550 .581985$

H -0.1319450 .6271191 .813919$

H -2.420954 -3.823035 -0.433904

H $-3.297478-2.878368-1.646875$

H -1.536554 -2.758761-1.540126

H $\quad 0.814723 \quad 1.708093-0.124447$

H $1.800044-0.3287252 .554721$

H 2.7661902 .2991880 .874539

H $1.792040-0.247827-1.378520$

H $3.054710-1.3928510 .495134$

H 4.1069110 .0227140 .631542

H $3.3597901 .050457-2.183528$

H -4.3611680 .4073830 .292251$

H -2.8809230 .8889361 .127911$

H $4.978055-0.364039-1.640884$

H $5.125964-1.830624-0.668385$

H $3.835133-1.704092-1.875380$

SCF Energy (B3LYP/6-31G**//MMFF) $=-810.716511517$

F2-19_c04373

MMFF Geometry

C -4.005924 $1.911189-0.301837$

C $-4.5310782 .446870 \quad 0.805728$

C $-2.444338-0.023598-0.769217$

O $-1.046490-0.277718-1.015226$

C $-0.508651-1.0836360 .043423$ 
C $-1.571262-1.1287531 .135493$

C $-2.864185-0.9710240 .358414$

C $0.842442-0.5335480 .528757$

C $-3.354119-2.311801-0.193318$

C $1.930514-0.544243-0.565525$

O $1.273972-1.3052641 .646791$

O $1.5950750 .375178-1.605395$

C $3.340693-0.194612-0.046503$

C 3.4855601 .2493210 .454100

O $4.272389-0.413554-1.110753$

C $-2.5788911 .467036-0.422275$

C $4.881277 \quad 1.5315850 .997408$

H -4.640046 1.797231-1.177197

H -5.570960 2.7539530 .827756

H $-3.9394392 .586413 \quad 1.703613$

H -2.989688 $-0.228865-1.698072$

H $-0.366495-2.095668-0.356141$

H $-1.454868-0.280031 \quad 1.820877$

H -1.520069-2.043923 1.733635

H -3.659724 -0.5592690 .987480$

H $\quad 0.6885010 .4990440 .863643$

H -4.279659-2.173353 -0.762432

H -2.621848 -2.780360 -0.858599

H -3.564459 -3.010241 0.623494

H $1.982899-1.540497-1.023193$

H $1.410014-2.2186341 .341823$

H $\quad 0.7437380 .096967-1.983288$

H $3.621291-0.8891020 .752914$

H 2.7577601 .4515881 .246691

H $3.2931141 .955619-0.362111$

H $4.0240180 .176280-1.843383$

H -2.011347 1.6974350 .488152

H $-2.1000582 .058725-1.213480$

H 5.1241020 .8543221 .822465

H $5.643211 \quad 1.4165130 .220422$

H 4.9382242 .5579991 .373704

SCF Energy (B3LYP/6-31G**//MMFF) $=-810.721406126$

\section{F2-19 c04374}

MMFF Geometry

C $4.053951-1.662592-0.055508$

C $3.630302-2.928700-0.138777$

C $2.8664060 .552982-0.120174$

$\begin{array}{llll}\text { O } & 1.791145 & 0.055709 & -0.943355\end{array}$

C $0.5561410 .687968-0.556731$

C 0.8185551 .3269310 .798821

C 2.2865341 .7009340 .713024

C $-0.580287-0.345346-0.593063$

C 2.4857983 .0516270 .022059

C $-1.9654430 .243836-0.246659$

O $-0.276825-1.4074490 .310993$

O $-2.2344381 .358729-1.092907$

C $-3.108067-0.780879-0.417629$

C $-4.518381-0.180443-0.281992$

O $-2.958123-1.8325460 .532781$

C $3.373081-0.5998020 .760335$

C -4.7944530 .4607591 .072148$

H $4.949890-1.375747-0.600103$

H $4.170122-3.649776-0.742750$

H $2.744661-3.2667500 .387598$

H $3.6717270 .899191-0.778892$

H $\quad 0.3668151 .464937-1.306483$

H $\quad 0.6796780 .601815 \quad 1.609250$

H $\quad 0.156430 \quad 2.1749570 .998659$

H $2.742591 \quad 1.764522 \quad 1.707271$

H $-0.611220-0.781466-1.599497$

H $2.0881783 .060141-0.997840$

H 1.9899183 .8490910 .585440

H $3.5521063 .295334-0.035179$

H -1.955564 0.5828690 .796590

H $\quad 0.582210-1.7773390 .044908$

H -1.699777 2.106184 -0.776618

H $-3.035278-1.226170-1.417871$

H $-5.254860-0.980893-0.425959$

H $-4.693636 \quad 0.556874-1.073463$

H $-3.659855-2.4842390 .364748$

H $4.098868-0.2180251 .488203$

H $2.543095-1.0384671 .327493$
H $-5.834708 \quad 0.798967 \quad 1.120023$

H $-4.634467-0.2482431 .890068$

$\mathrm{H}-4.155821 \quad 1.333038 \quad 1.239171$

SCF Energy (B3LYP/6-31G**//MMFF) $=-810.706747967$

F2-19 c04375

MMFF Geometry

C -3.1963142 .2803420 .031050$

C $-4.3494632 .896180-0.252552$

C $-2.368086-0.049643-0.395327$

O $-0.9790640 .324775-0.467309$

C $-0.169827-0.860383-0.545279$

C $-1.137088-2.037137-0.647597$

C $-2.372726-1.5090660 .053606$

C $0.748236-0.9346880 .685671$

C $-3.640354-2.265590-0.305645$

C 1.5821850 .3433170 .917243

O $1.587350-2.0786030 .550550$

$\begin{array}{lllll}\text { O } & 2.350776 & 0.184582 & 2.114433\end{array}$

C $2.4954670 .808131-0.236188$

C $3.691982-0.096894-0.556245$

O 2.9896542 .1099350 .105254

C -3.1020690 .8812220 .571689$

C $4.5239520 .439497-1.716699$

H $-2.2662102 .820576-0.125173$

H $-4.3504083 .910742-0.635702$

H -5.305620 $2.404740-0.110520$

H $-2.7809230 .043909-1.408558$

H $0.435203-0.819188-1.457978$

H $-0.758050-2.966799-0.213481$

H - $-1.358585-2.232435-1.704831$

H $-2.216279-1.5692581 .138634$

H $0.140093-1.0970021 .584739$

H -4.506836 -1.848299 0.216645

H -3.842503 -2.222242 -1.381154

H $-3.551934-3.319597-0.022703$

H $\quad 0.888721 \quad 1.1644591 .137882$

H $2.023172-2.2279161 .406327$

H $2.972058-0.5500311 .982574$

H $1.8945000 .944300-1.141230$

H $3.352966-1.101933-0.820916$

H $4.351737-0.181846 \quad 0.314550$

H 3.5019652 .0221230 .927547

H -4.1042770 .4896790 .780752$

H $-2.5632700 .929217 \quad 1.526163$

H $4.9618521 .413815-1.478668$

H $5.343910-0.250044-1.941577$

H $3.9136090 .545784-2.619104$

SCF Energy (B3LYP/6-31G**//MMFF $)=-810.716110993$

F2-19 c04376

MMFF Geometry

C $-4.485690-1.4829350 .264923$

C $-4.391495-2.8091050 .117132$

C $-2.7854600 .359001 \quad 0.259340$

O $-1.699593-0.4620810 .724716$

C $-0.522700 \quad 0.361496 \quad 0.874241$

C $-0.879702 \quad 1.7376550 .316225$

C $-2.1107491 .474717-0.529983$

C $0.655349-0.3605980 .191541$

C $-2.9761982 .706960-0.732837$

C $2.024638 \quad 0.3098320 .448441$

O $0.402391-0.474269-1.207335$

O 2.2011900 .4869601 .852746

C $3.215066-0.495547-0.119185$

C 4.5642250 .1530210 .233766

O $3.090995-0.579385-1.535793$

C $-3.742977-0.487243-0.580703$

C $5.758885-0.651348-0.263668$

H $-5.144580-1.0898781 .034806$

H $-4.958584-3.4751200 .758375$

H -3.751403 -3.251962 -0.637763

H -3.2965850 .7625541 .143737$

H -0.3283910 .4387901 .949902$

H $-0.0732722 .218748-0.243707$

H -1.128972 2.4053991 .151178

H $-1.7907031 .099227-1.510220$

H $0.682046-1.3855210 .582549$ 
H $-3.337137 \quad 3.1052580 .221387$

H $-2.4077723 .497420-1.233927$

H $-3.8481342 .475908-1.352381$

H $2.0307091 .301157-0.019653$

H $0.5444620 .398084-1.611570$

H $2.204986-0.3943352 .263917$

H $3.188625-1.5127530 .291230$

H 4.6569050 .2673991 .319531

H $4.6186921 .154748-0.209569$

H $3.697762-1.271882-1.847037$

H $-4.4826850 .154088-1.072725$

H -3.183915 -1.007383 -1.368720

H $5.788510-0.692051-1.356609$

H $6.690820-0.1877250 .075651$

H $5.729595-1.6753990 .121548$

SCF Energy $\left(B 3 L Y P / 6-31 G^{* *} / / M M F F\right)=-810.706846209$

F2-19_c04377

MMFF Geometry

C $1.8026142 .254513 \quad 0.131075$

C $2.2628433 .066544-0.827031$

C $2.422953-0.1010850 .871652$

O $1.089815-0.4251691 .306072$

C $0.594523-1.5224210 .517389$

C $1.755944-1.968237-0.372914$

C $2.560347-0.692149-0.527838$

C $-0.652939-1.080521-0.277520$

C $3.996533-0.923059-0.963605$

C -1.829379 -0.6531340 .624798$

O $-1.069609-2.154572-1.118432$

$\begin{array}{llll}\text { O } & -1.466587 & 0.505240 & 1.371431\end{array}$

C $-3.145353-0.403162-0.141487$

C $-3.1051160 .644401-1.265344$

O $-4.147813-0.0421770 .812576$

C 2.6792521 .4026561 .004604

C $-2.7662902 .062575-0.829273$

H $\quad 0.7309712 .210503 \quad 0.304647$

H $1.5759243 .660700-1.419887$

H $3.3229583 .151470-1.037824$

H $3.100561-0.6200501 .563190$

H $\quad 0.321210-2.342717 \quad 1.191723$

H $1.450683-2.403613-1.328427$

H $2.348530-2.7245170 .158167$

H $2.060453-0.060606-1.271861$

H $-0.375557-0.234436-0.916936$

H $4.027324-1.417992-1.939771$

H $4.5345080 .026348-1.049383$

H $4.536769-1.554128-0.249909$

H -2.021482 -1.446579 1.358914

H -1.306753 -2.904171 -0.545881

H $-2.2314640 .748573 \quad 1.920707$

H -3.471012 -1.353177 -0.582383

H $-4.0986960 .679416-1.730658$

H -2.406688 $0.331098-2.048888$

H $-4.987303 \quad 0.0591810 .332426$

H 2.4888571 .7030562 .042562

H 3.7370961 .6080560 .801901

H -2.913051 2.753660 -1.666128

H $-3.4048772 .394556-0.005228$

H -1.721005 2.147259-0.521283

SCF Energy $($ B3LYP/6-31G**//MMFF $)=-810.710722805$

F2-19_c04378

MMFF Geometry

C $2.2645072 .142750-0.189371$

C $2.7393482 .782703-1.263814$

C $2.606541-0.1998430 .748405$

O $1.294292-0.266197 \quad 1.335193$

C $0.547090-1.3188610 .698887$

C $1.530836-2.036993-0.226592$

C $2.508687-0.934783-0.584933$

C $-0.675833-0.726411-0.038181$

C $3.839783-1.444034-1.109042$

C $-1.688627-0.0319370 .904951$

O $-1.320049-1.779483-0.748171$

O

C $-2.9314620 .578900 \quad 0.220373$

C $-3.956820-0.449951-0.265639$
O $\quad-2.531317 \quad 1.385004-0.888478$

C 3.0931551 .2508830 .691551

C $-5.2745370 .207551-0.662565$

H 1.2196852 .2792050 .076827

H $2.089703 \quad 3.417271-1.856625$

H $3.7745872 .684924-1.571101$

H $3.269279-0.7506931 .429614$

H $\quad 0.199023-2.0167501 .469815$

H $1.070392-2.504803-1.101045$

H $2.044907-2.8269430 .336130$

H $2.045485-0.301085-1.350559$

H $-0.3180910 .008517-0.768630$

H $3.695084-2.024507-2.026059$

H $4.511601-0.610990-1.338929$

H $4.339479-2.089056-0.378635$

H $-2.031778-0.7402801 .668945$

H $-1.635118-2.428418-0.095817$

H -0.3391080 .6792202 .135318$

H $-3.4222831 .253846 \quad 0.933457$

H $-4.153863-1.1867340 .520914$

H $-3.585221-0.981695-1.147167$

H -1.891698 $2.037937-0.555743$

H 3.0583961 .6748851 .702917

H 4.1427451 .2672000 .374335

H $-5.7178830 .739790 \quad 0.184970$

H $-5.1328050 .920074-1.481193$

H -5.988886 -0.551939-0.996104

SCF Energy $\left(B 3 L Y P / 6-31 G^{* *} / / M M F F\right)=-810.719834765$

F2-19_c04379

MMFF Geometry

C $2.7166412 .631938-0.205308$

C $1.7506783 .417036-0.695457$

C $2.7294530 .206210 \quad 0.448013$

O 1.3166290 .1754910 .725791

C $0.742975-1.0279170 .195066$

C $1.836596-1.708753-0.622379$

C $3.111750-1.2170050 .035300$

C $-0.528771-0.729629-0.617559$

C $3.480142-2.0716531 .249950$

C $-1.655895-0.0745200 .220292$

O $-0.979689-1.948064-1.202489$

O $-1.225163 \quad 1.199108 \quad 0.693991$

C $-2.9625010 .125188-0.577728$

C -4.0161360 .9882040 .138271$

O $-3.536649-1.144309-0.881854$

C $2.9762221 .227339-0.673632$

C -4.4767560 .4239721 .476376$

H $3.3633143 .025190 \quad 0.574629$

H $1.6160284 .424209-0.316497$

H $1.0793093 .071838-1.473934$

H $3.254727 \quad 0.5185631 .358417$

H $0.485926-1.6693041 .047484$

H $1.809791-1.365410-1.663919$

H $1.733578-2.798324-0.634793$

H $3.958119-1.235226-0.660004$

H -0.266148 -0.040740 -1.430423

H $4.390930-1.6888821 .722618$

H $2.689905-2.0789462 .007618$

H $3.669150-3.1073150 .948413$

H $-1.866143-0.7181151 .083547$

H -1.264627 -2.534826 -0.481187

H $-0.458743 \quad 1.056382 \quad 1.274519$

H -2.722802 $0.617238-1.528779$

H $-4.8990341 .071437-0.507973$

H -3.6358032 .0052020 .286050$

H -4.323209-0.987695 -1.431547

H 4.019108 1.169121-1.006609

H $2.3435311 .003601-1.541166$

H -4.874065 -0.589746 1.366766

H -3.6594170 .3993182 .202989$

H -5.2708431 .0521581 .893045$

SCF Energy (B3LYP/6-31G**//MMFF) $=-810.710774127$

F2-19_c04380

MMFF Geometry

C $4.2531691 .830267-0.428567$

C 4.1233292 .4943870 .725699 
C $2.685844-0.1356220 .203754$

O 1.4887920 .6503940 .334492

C $0.400680-0.2188400 .715664$

C $0.926752-1.6489820 .605603$

C $2.186139-1.510032-0.228983$

C $-0.8106050 .119455-0.172950$

C $3.181182-2.636680-0.005962$

C $-2.106014-0.5769780 .276190$

O $-0.535307-0.235339-1.526499$

O $-2.399549-0.1890421 .616645$

C $-3.313735-0.305961-0.644951$

C $-3.7096001 .162657-0.863107$

O $-4.432662-1.027724-0.122631$

C $3.6221670 .518401-0.817138$

C $\quad-4.127725 \quad 1.9222700 .387629$

H $4.8904812 .276874-1.189910$

H 4.6407623 .4371190 .871223

H 3.5132642 .1304161 .543288

H $3.161646-0.1958551 .191139$

H $0.163496 \quad 0.011042 \quad 1.760707$

H $0.213606-2.3572020 .174802$

H $1.172576-2.0157011 .610498$

H $1.902959-1.488905-1.289214$

H $-0.9497821 .207042-0.145478$

H $2.730777-3.602493-0.257685$

H $4.068675-2.506687-0.632995$

H $3.510014-2.6805871 .037888$

H -1.953539 -1.6627410 .296194$

H $\quad 0.2626910 .250965-1.796053$

H -3.229782 -0.6277581 .870008$

H -3.098372 $-0.742703-1.627837$

H -4.562956 $1.184470-1.553376$

H -2.896374 $1.703564-1.359296$

H $-5.174621-0.906084-0.739285$

H $4.438965-0.172469-1.055833$

H $3.0662030 .696554-1.746714$

H -3.2760172 .1032821 .048955$

H -4.5342502 .9004010 .109708$

H $-4.900114 \quad 1.3850900 .945920$

SCF Energy (B3LYP/6-31G**//MMFF) $=-810.717368606$

F2-19 c04381

MMFF Geometry

C -4.819324 -0.971735 0.229520

C $-5.420119-1.479224-0.852704$

C $-2.5650990 .076672 \quad 0.750015$

O $-1.285974-0.2939631 .301469$

C $-0.286618 \quad 0.6463710 .858594$

C $-0.9777921 .582991-0.126774$

C $-2.2354080 .834597-0.533052$

C $0.905872-0.1539600 .301022$

C -3.323808 $1.771657-1.032273$

C 2.1359370 .7219200 .000784

O $0.521508-0.839114-0.888943$

O $2.550791 \quad 1.398906 \quad 1.187700$

C $3.328804-0.051222-0.601154$

C $3.986324-1.059400 \quad 0.351298$

O $4.3126870 .903938-1.012049$

C $-3.381200-1.2064880 .574772$

C $5.105356-1.841637-0.326914$

H $-5.405272-0.363750 \quad 0.913895$

H -6.468653 -1.278153-1.043762

H -4.882379 -2.097482 -1.562844

H $-3.0477380 .736640 \quad 1.482891$

H $0.034586 \quad 1.207383 \quad 1.743394$

H $-0.3612471 .865914-0.984299$

H -1.241825 2.5121340 .395112

H -1.979415 $0.127618-1.332111$

H $1.168075-0.9187651 .041573$

H -2.950509 2.387356-1.857648

H $-4.1891111 .217685-1.404239$

H -3.667728 $2.445030-0.239965$

H $1.8652281 .503429-0.718517$

H $-0.225507-1.418549-0.660953$

H 2.6571590 .7365841 .891258

H $3.004610-0.568070-1.511278$

H $3.244070-1.7714940 .725345$

H $4.417863-0.5411001 .215470$
H $4.5869621 .400897-0.222021$

H -2.893397 -1.853717 -0.165855

H $-3.348330-1.7741741 .514057$

H $5.912159-1.179919-0.656927$

H $5.532098-2.5691660 .370755$

H $4.728653-2.387720-1.197568$

SCF Energy $(B 3 L Y P / 6-31 G * * / / M M F F)=-810.721156365$

F2-19 c04382

MMFF Geometry

C -2.9460282 .3261110 .006407$

C $-3.9802673 .117327-0.299808$

C $-2.404505-0.060227-0.575294$

O $-0.979457 \quad 0.136959-0.494372$

C $-0.370098-0.9879730 .156239$

C $-1.509831-1.8363400 .712978$

C $-2.652151-1.532770-0.237617$

C $0.624182-0.5219951 .226274$

C $-2.590267-2.411666-1.488707$

C 1.6582550 .5259930 .752580

O $1.316560-1.6523591 .757112$

O 2.3246631 .0315851 .915972

C $2.7342650 .006262-0.222970$

C $3.7835011 .081922-0.555043$

O $2.131323-0.431490-1.436246$

$\begin{array}{llll}\text { C } & -3.082408 & 0.888974 & 0.426228\end{array}$

C $4.8625930 .567947-1.500379$

H -1.942503 $2.741862-0.036898$

H -3.815159 4.149112 -0.590523

H $-5.0017102 .754614-0.267907$

H $-2.7301390 .181160-1.594120$

H $0.145704-1.578781-0.608193$

H -1.781766 -1.505957 1.722863

H -1.251753 -2.8982490 .773612$

H -3.625869 -1.693116 0.238305

H $0.066932-0.0729252 .058855$

H -1.654779 -2.281977 -2.042251

H $-2.681000-3.469608-1.220808$

H -3.414381 -2.166189-2.167132

H $1.130719 \quad 1.3708580 .293842$

H $\quad 0.659319-2.2393642 .167051$

H 1.6528291 .4475112 .482551

H $3.254172-0.8507420 .220513$

H $4.266108 \quad 1.4409130 .360564$

H $3.2944991 .941353-1.029622$

H $1.6638110 .326634-1.826925$

H -4.1423210 .6246590 .523557$

H -2.6270140 .7913891 .419015$

H $5.378817-0.296689-1.071349$

H $4.4412630 .274224-2.466774$

H $5.6060521 .350367-1.683286$

SCF Energy $\left(B 3 L Y P / 6-31 G^{* *} / / M M F F\right)=-810.709956004$

F2-19 c04383

MMFF Geometry

C -2.5414222 .7728510 .625056$

C $-2.1473553 .335270-0.523381$

C $-2.3302690 .316165-0.179225$

$\begin{array}{llll}0 & -0.910518 & 0.356712 & -0.413985\end{array}$

C $-0.432189-0.970272-0.684673$

C $-1.662483-1.875230-0.710770$

C $-2.634902-1.1354810 .186349$

C $0.590843-1.3780640 .387609$

C $-4.083077-1.540673-0.030175$

C $1.728765-0.3578260 .631785$

O $1.137923-2.6447560 .017734$

O $2.422942-0.7639061 .816261$

C $2.740007-0.200136-0.521462$

C $3.9746040 .645541-0.161269$

O $2.0930050 .387265-1.646428$

C -2.6950581 .3058740 .932005$

C 3.6520072 .0765140 .250559

H $-2.785948 \quad 3.442549 \quad 1.447712$

H $-2.0813794 .415288-0.604937$

H -1.884181 2.753681-1.398366

H $-2.8293400 .583132-1.119524$

H $0.033015-0.989604-1.675752$

H $-1.468541-2.901426-0.384973$ 
H -2.055560 -1.922482 -1.734588

H $-2.363490-1.3250541 .233145$

H $0.069220-1.5258181 .342200$

H -4.218924 $-2.606940 \quad 0.178214$

H $-4.749790-0.9806940 .632870$

H -4.401455 -1.356569-1.061778

H 1.2721690 .6121240 .857262

H $1.675936-2.9612580 .762559$

H $2.933109-1.5638111 .604921$

H $3.100958-1.189860-0.825555$

H $4.6329470 .694228-1.037911$

H 4.5467390 .1595980 .637041

H $2.7405080 .430720-2.370457$

H $-3.7376841 .146578 \quad 1.231053$

H -2.0704861 .0988401 .810761$

H 3.0754292 .1052311 .179726

H 4.5789412 .6342190 .419571

H $3.0841732 .597593-0.526315$

SCF Energy (B3LYP/6-31G**//MMFF $)=-810.709759975$

F2-19 c04384

MMFF Geometry

C $-4.427152-1.4698590 .224201$

C -4.767214 -2.179348 -0.857661

C -2.6544150 .2624020 .725200$

O $-1.3504160 .361302 \quad 1.336108$

C $-0.411330 \quad 0.889604 \quad 0.380348$

C $-1.0823860 .767189-0.979899$

C $-2.552640 \quad 0.939862-0.646217$

C 0.9319960 .1553260 .525752

C $-2.9443752 .417224-0.570318$

C $2.0234780 .696942-0.417809$

O $0.733242-1.2333920 .269394$

O $2.0889572 .117657-0.255983$

C $3.4442030 .137564-0.182532$

C $3.608643-1.336518-0.563217$

O 3.8092230 .2969821 .189000

C $-3.011868-1.2297690 .658286$

C $5.073118-1.761491-0.568940$

H $-5.216837-1.0472800 .839798$

H $-5.810936-2.322056-1.115378$

H $-4.017715-2.626022-1.501768$

H $-3.3675420 .776302 \quad 1.380859$

H $-0.278761 \quad 1.9472560 .637036$

H $-0.927143-0.230216-1.407634$

H $-0.7032841 .497581-1.701370$

H -3.188094 $0.467763-1.402318$

H $1.2552490 .247522 \quad 1.569562$

H -2.372927 2.9611320 .188616

H -2.780005 2.909057-1.534739

H $-4.0062842 .516075-0.320800$

H $1.7408330 .498292-1.458885$

H $0.053892-1.5486620 .889753$

H $2.7077912 .461511-0.922740$

H $4.1563740 .735893-0.764667$

H $3.184844-1.518851-1.556941$

H $3.088414-1.9795320 .154362$

H $3.6986551 .237437 \quad 1.410967$

H $-2.302019-1.7597310 .010904$

H $-2.877629-1.6724801 .653851$

H $5.158583-2.814973-0.853965$

H $5.650173-1.169036-1.286137$

H $5.525971-1.6421410 .420307$

SCF Energy (B3LYP/6-31G**//MMFF) $=-810.718792941$

F2-19_c04385

MMFF Geometry

C $-2.0591332 .640638-0.292447$

C $-3.0004273 .558477-0.539388$

C $-2.0826490 .131833-0.366232$

O $-0.692227 \quad 0.020543-0.723519$

C $-0.291953-1.356859-0.675113$

C $-1.561901-2.158601-0.393641$

C $-2.417677-1.1649980 .365658$

C $0.798891-1.5511590 .396006$

C $-3.895999-1.5150410 .364059$

C $2.103636-0.7435340 .189577$

O $1.154046-2.9396050 .427661$
O $2.613047-1.013231-1.116925$

C 1.9909440 .7771150 .413690

C $3.347476 \quad 1.4870660 .567616$

O 1.2499991 .0142721 .611044

C -2.2995311 .3806420 .490322$

C $4.2289531 .464872-0.669139$

H $-1.0530732 .813380-0.666332$

H -2.761399 4.451274 -1.106769

H $-4.0178703 .437705-0.184829$

H $-2.6512700 .205340-1.302645$

H $0.096744-1.646846-1.657986$

H -1.394110 -3.090042 0.154287

H -2.042863 -2.419006 -1.345497

H -2.063258 -1.114112 1.403531

H $0.392286-1.3155161 .385363$

H $-4.475611-0.7687700 .916183$

H -4.295413 - $1.565620-0.654457$

H $-4.060896-2.4880680 .838226$

H $2.843715-1.1450190 .893721$

H $1.558669-3.157837-0.429658$

H $2.067759-0.527477-1.758701$

H $1.4529371 .245193-0.417918$

H 3.8949741 .0611181 .417970

H 3.1623792 .5343480 .840240

H $\quad 1.111148 \quad 1.9737461 .686489$

H -3.3153861 .3795560 .901726$

H - 1.6045111 .3786721 .338540

H $5.0849272 .133367-0.529681$

H $4.6283940 .465237-0.860139$

H $3.6790251 .800831-1.553562$

SCF Energy (B3LYP/6-31G**//MMFF) $=-810.717296675$

F2-19_c04386

MMFF Geometry

C $-1.7155602 .871713-0.560652$

C $-0.6994893 .716594-0.354054$

C $-2.0180330 .390053-0.368485$

O $-0.6562620 .061146-0.701217$

C $-0.453322-1.355889-0.557391$

C $-1.823480-1.937264-0.202602$

C $-2.511423-0.7747970 .482760$

C $0.631785-1.6115700 .507652$

C $-4.023297-0.9123620 .534476$

C $2.090360-1.3011920 .099048$

O $0.603321-3.0007980 .866864$

O $2.388151-2.012535-1.108048$

C $2.5083940 .169101-0.080718$

C 2.2367861 .0259511 .158265

O $1.8656590 .756171-1.207163$

C -2.0648721 .7349820 .357842$

C 2.8731402 .4063101 .049025

H -2.340542 $3.014264-1.438516$

H $-0.5023844 .521066-1.054136$

H -0.0518413 .6237710 .510232$

H -2.577777 $0.442135-1.311893$

H $-0.133767-1.765402-1.522028$

H -1.794137 -2.841367 0.411079

H -2.354328 -2.193041-1.129210

H $-2.122384-0.6871191 .505777$

H $\quad 0.393683-1.0526901 .418808$

H -4.478266 -0.0500291 .031563$

H -4.454334 -0.988684 -0.469439

H -4.308302 -1.810967 1.091527

H $2.740801-1.7387810 .868414$

H $\quad 0.826245-3.5125710 .070162$

H $3.332993-1.884720-1.299601$

H $3.5828910 .192032-0.302569$

H 2.6276170 .5271762 .052397

H 1.1587261 .1646531 .294901

H $2.0080160 .165036-1.966015$

H $-3.072598 \begin{array}{lll}1.916835 & 0.747963\end{array}$

H -1.379061 1.7183011 .213876

H 2.4882302 .9573710 .185631

H 2.6572762 .9930111 .947543

H 3.9603552 .3280800 .949061

SCF Energy (B3LYP/6-31G**//MMFF) $=-810.707925001$

F2-19_c04387 
MMFF Geometry

C $3.5436202 .103441-0.106931$

C $4.4133572 .109382-1.123148$

C $1.883244 \quad 0.4231330 .798110$

$\begin{array}{lllll}\text { O } & 0.479677 & 0.106646 & 0.715095\end{array}$

C $0.304227-1.1840620 .114910$

C $1.669497-1.584179-0.436737$

C $2.636395-0.8731420 .490414$

C $-0.803705-1.153616-0.948796$

C $2.894317-1.6842901 .762158$

C $-2.218644-0.825221-0.421192$

O $-0.888207-2.444158-1.564766$

O $-2.551158-1.7073520 .652203$

C $-2.427410 \quad 0.6235990 .052108$

C $-3.904911 \quad 0.9112360 .367373$

O $-1.973248 \quad 1.532336-0.946457$

C $2.1494361 .559673-0.200572$

C -4.1199492 .3274190 .886546$

H 3.8518072 .5263100 .845611

H $5.4074502 .522991-0.993353$

H $4.1531951 .704285-2.094814$

H $2.0859720 .780757 \quad 1.814740$

H $\quad 0.032315-1.8846550 .913786$

H $1.799742-1.200198-1.456159$

H $1.806414-2.669470-0.473172$

H $3.601652-0.7008990 .003833$

H -0.548204 -0.434389-1.735894

H $1.975885-1.8778032 .325738$

H $3.350606-2.6492351 .517360$

H $3.582638-1.1462522 .422773$

H -2.930832 -1.034111 -1.230634

H - $0.072786-2.584445-2.074856$

H $-2.429917-2.616474 \quad 0.328254$

H -1.8532590 .8085080 .965632$

H -4.2781630 .2018851 .114410$

H $-4.509407 \quad 0.785122-0.539077$

H -2.498613 $1.377398-1.750037$

H $1.9176281 .230190-1.221245$

H 1.4462162 .3785040 .000381

H $-3.830951 \quad 3.0742730 .140771$

H -5.1765282 .4840201 .126310$

H -3.536220 2.5036731 .795581

SCF Energy (B3LYP/6-31G**//MMFF) $=-810.715544356$

F2-19_c04388

MMFF Geometry

C $3.570824-2.1961580 .143746$

C $3.822690-2.341594-1.162261$

C $2.7390460 .263440-0.005119$

O $1.603132-0.042009-0.839416$

C $0.4786220 .764191-0.441242$

C $0.829997 \quad 1.327056 \quad 0.927836$

C 2.3384411 .4708880 .851646

C $-0.803289-0.082267-0.502977$

C 2.7469822 .7886350 .189255

C $-2.0719490 .720592-0.153929$

O $-0.678253-1.1857190 .390785$

O $-2.1130041 .897582-0.970147$

C $-3.410571-0.020774-0.356065$

C $-3.698024-1.1903080 .592272$

O $-3.461992-0.509906-1.700104$

C $3.056698-0.9721030 .856353$

C $-3.667253-0.8157442 .064354$

H $3.756435-3.056576 \quad 0.784277$

H $4.194267-3.287930-1.541783$

H $3.672180-1.543206-1.878522$

H $3.5938280 .500605-0.648793$

H $0.4127351 .578088-1.173051$

H $\quad 0.5765600 .6166451 .723286$

H $\quad 0.3060192 .2632641 .143366$

H $2.7939301 .444021 \quad 1.847712$

H $-0.885510-0.496096-1.515161$

H 2.3768103 .6414050 .768046

H 3.8382712 .8665410 .139435

H $2.3608202 .878913-0.831087$

H -2.0163901 .0671260 .883198$

H $0.114591-1.6836910 .127398$

H $\quad-2.1921141 .608358-1.895687$
H $-4.2230610 .711898-0.267870$

H -3.015300 -2.024954 0.399513

H $-4.693729-1.5882530 .355556$

H $-4.337980-0.910392-1.834311$

H $3.809255-0.6976231 .606131$

H $2.155659-1.2788741 .401723$

H -3.998036 -1.662803 2.673994

H $-2.658202-0.5494482 .391586$

H -4.3333880 .0282702 .268890$

SCF Energy $(B 3 L Y P / 6-31 G * * / / M M F F)=-810.716621364$

F2-19 c04389

MMFF Geometry

C - 2.7834132 .5282600 .239439

C -2.0902673 .4307920 .942783$

C $-2.2694210 .172181-0.444485$

O $-0.842108 \quad 0.362853-0.449170$

C $-0.192031-0.901657-0.647568$

C $-1.300897-1.921134-0.902421$

C $-2.480977-1.314416-0.169443$

C $0.663113-1.2377110 .580577$

C $-3.823910-1.847085-0.639297$

C $1.595625-0.1071831 .072336$

$\begin{array}{lllll}\text { O } & 1.409548 & -2.421241 & 0.295491\end{array}$

O $2.235355-0.5875072 .268959$

C 2.7310780 .3825940 .149286

C $2.2757711 .397743-0.904914$

O $3.372499-0.707383-0.506252$

C -2.8987951 .0779700 .615144$

C $3.4574342 .049514-1.615404$

H $-3.3052782 .854314-0.656495$

H $-2.049007 \quad 4.4652780 .619531$

H $-1.553645 \quad 3.1572301 .844517$

H -2.633891 $0.435993-1.446068$

H $0.441496-0.850943-1.538635$

H $-1.059233-2.934811-0.569414$

H -1.509798 -1.967333 -1.978968

H $-2.372753-1.5176460 .903940$

H $-0.002056-1.4920891 .415913$

H -3.886333 -2.928250 -0.477404

H $-4.645226-1.376286-0.090208$

H -3.979991 -1.657426 -1.706496

H $\quad 0.996573 \quad 0.756934 \quad 1.383736$

H $1.935655-2.6294761 .086443$

H $1.536521-0.7855572 .915614$

H $3.4981810 .860793 \quad 0.772218$

H $1.6727422 .182883-0.435139$

H $1.6645900 .917060-1.672635$

H $3.663615-1.3293230 .182228$

H -3.9624490 .8418560 .732056$

H $-2.4158760 .903221 \quad 1.584958$

H $4.0611371 .307208-2.146870$

H $3.0999392 .780042-2.348135$

H $4.1028722 .573354-0.903106$

SCF Energy (B3LYP/6-31G**//MMFF $)=-810.709440391$

F2-19 c04390

MMFF Geometry

C -3.655778 -1.955036 -0.876500

C $-3.923684-2.5099310 .311283$

C -2.5018820 .1673290 .062945$

O $-1.401817-0.5143580 .689158$

C $-0.5964370 .461672 \quad 1.377468$

C -1.0555541 .8355600 .899785$

C $-1.9290571 .532968-0.304651$

C 0.8964500 .1364701 .185576

C -2.978225 $2.600404-0.568503$

C $1.358717-0.053095-0.274295$

O $1.163473-1.0630621 .918972$

O $1.2878991 .185235-0.979478$

C $2.783079-0.634363-0.416929$

C 3.9012290 .2792770 .102981

O $3.014882-0.907665-1.802743$

C $-2.980810-0.636155-1.150022$

C $5.270341-0.3850870 .013818$

H $-3.957154-2.509749-1.763480$

H -4.419869 -3.473644 0.361285

H $-3.664460-2.0348181 .249460$ 
$\begin{array}{llll}H & -3.303698 & 0.275530 & 0.804936\end{array}$ H -0.8339740 .3589962 .444230$ H $-0.2367362 .525740 \quad 0.678087$ H -1.6502222 .3058581 .693734$ H $-1.2894461 .439170-1.190445$ H 1.4762290 .9362251 .659946 H -3.660044 2.7109920 .281284 H $-2.5028243 .570478-0.747319$ H -3.577226 2.352591-1.450138 H $0.678371-0.741123-0.790105$ H $0.622349-1.7710731 .528537$ H $1.7842961 .850608-0.473856$ H $2.834736-1.6005570 .096803$ H 3.7154930 .5528001 .146664 H $3.9410171 .204517-0.483349$ H $2.935238-0.066630-2.284685$ H -3.683942 -0.030055 -1.732846 H $-2.120776-0.848120-1.798373$ H $5.535671-0.615100-1.022823$ H 6.0391320 .2815510 .417182 H $5.292022-1.3154680 .590262$ SCF Energy (B3LYP/6-31G**//MMFF)= -810.717805505

\section{F2-19 c04391}

MMFF Geometry

C $2.3901762 .199105-0.212319$

C $1.762622 \quad 3.203690 \quad 0.409743$

C $2.671639-0.2343930 .509319$

O $1.451085-0.265227 \quad 1.267122$

C $0.719463-1.4447080 .874605$

C $1.375600-1.976776-0.399487$

C $2.305322-0.855905-0.832894$

C $-0.787215-1.1346570 .785970$

C $3.498997-1.332092-1.642112$

C $-1.1587180 .096443-0.064995$

O $-1.234630-0.935358 \quad 2.130005$

O $-0.860118-0.176527-1.433341$

C -2.6368200 .5308300 .044559$

C -3.627832 -0.499591-0.512126

O $-2.8018651 .748043-0.690911$

C 3.2492241 .1833760 .486781

C $-5.076273-0.060639-0.330089$

H $2.2937382 .119156-1.291573$

H $1.1637333 .910226-0.154419$

H 1.8302703 .3369601 .483693

H $3.385691-0.8803961 .038839$

H $0.869419-2.1780291 .677810$

H $0.662396-2.269817-1.175568$

H $1.951831-2.877876-0.152220$

H $1.727565-0.151683-1.440228$

H -1.285188 -2.0318410 .399170$

H $4.133769-0.489205-1.932865$

H $4.114371-2.037116-1.073149$

H $3.167831-1.835982-2.556141$

H $-0.5392900 .949154 \quad 0.235323$

H -2.198137-1.062955 2.142543

H $-1.0866710 .618925-1.944894$

H $-2.8872760 .758447 \quad 1.086682$

H -3.494424 -1.465269-0.013875

H -3.454529 $-0.650792-1.584132$

H -2.212581 2.412108 -0.294270

H 3.4183961 .5041331 .522957

H $4.2302171 .171566-0.002491$

H -5.753654 -0.831573 -0.710831

H -5.3059910 .0998540 .728033$

H $-5.2843870 .866458-0.873129$

SCF Energy (B3LYP/6-31G**//MMFF) $=-810.713551431$

\section{F2-19 c04392}

MMFF Geometry

C $-1.8014092 .955007-0.079522$

C $-0.770972 \quad 3.5833670 .497139$

C $-2.3411540 .540867-0.527068$

O $-0.9604880 .137538-0.610674$

C $-0.789761-1.153615-0.011760$

C $-2.080204-1.4367230 .750970$

C -3.123039-0.688365 -0.056449

C $0.469200-1.1994590 .868569$
C $-3.624389-1.520245-1.238748$

C $1.811506-0.988507 \quad 0.127145$

O $0.544395-2.489863 \quad 1.488347$

O $1.857985-1.864003-0.999019$

C $2.0828910 .459238-0.320221$

C $3.442897 \quad 0.671163-1.009139$

$\begin{array}{lllll}\text { O } & 1.992648 & 1.319975 & 0.812618\end{array}$

$\begin{array}{lllll}\text { C } & -2.439866 & 1.708601 & 0.467064\end{array}$

C $4.6483020 .328934-0.143281$

H $-2.2173943 .368385-0.994750$

H $-0.357802 \quad 4.482593 \quad 0.053735$

H $-0.323093 \quad 3.2153451 .413593$

H -2.665670 $0.869293-1.521622$

H $-0.700065-1.885125-0.824051$

H $-2.030740-1.0099341 .760441$

H -2.283152 -2.507336 0.853581

H $-3.990550-0.4205440 .556769$

H $0.391943-0.4591621 .673470$

H $-4.102927-2.440202-0.886716$

H $-4.366575-0.955530-1.813151$

H $-2.816773-1.799283-1.923204$

H $2.601641-1.3104650 .816984$

H $-0.188574-2.5580152 .122935$

H $2.780542-1.911141-1.300783$

H $1.3206080 .771814-1.040438$

H $3.5287961 .729137-1.288101$

H $3.4896850 .095662-1.940240$

H 2.1189402 .2319820 .500110

H -3.4933811 .9333340 .671258$

H -1.9705851 .4369041 .420555$

H $5.5717920 .594473-0.668334$

H 4.6307130 .8786340 .802654

H $4.692281-0.7415670 .076926$

SCF Energy (B3LYP/6-31G*//MMFF) $=-810.707340564$

F2-19 c04393

MMFF Geometry

C $3.398111-2.006129-0.187043$

C $4.659458-2.450191-0.222471$

C $2.406258 \quad 0.297207-0.286863$

O $1.171084-0.129863-0.892902$

C $0.2218130 .948491-0.866111$

C $1.0022922 .178861-0.409530$

C 2.0712741 .5753360 .478310

C $-0.965963 \quad 0.5893670 .057699$

C 3.2617092 .4927150 .701601

C $-1.776431-0.632921-0.436489$

O $-1.816147 \quad 1.7270170 .157013$

O $-0.900047-1.764529-0.495386$

C $-2.975492-1.041320 \quad 0.449317$

C $-4.189866-0.107668 \quad 0.449023$

O $-2.544061-1.1778161 .806676$

C $2.949005-0.8155550 .612296$

C $-4.7812580 .137933-0.928401$

H $2.642782-2.539022-0.758945$

H $4.918847-3.320259-0.815632$

H $5.450184-1.958557 \quad 0.333186$

H $3.1085310 .508902-1.103798$

H $-0.151873 \quad 1.112778-1.883795$

H 0.3981752 .9362600 .097335

H $1.4654312 .656790-1.282474$

H 1.6259551 .3276581 .450797

H -0.5741010 .3677911 .057310$

H $3.7435432 .762666-0.244023$

H 2.9463203 .4183871 .194233

H 4.0122232 .0117751 .336531

H -2.123896 $-0.447861-1.458810$

H $-2.1390261 .935894-0.736117$

H $-1.387385-2.493285-0.916075$

H $-3.317890-2.0373320 .140369$

H -3.9554190 .8449820 .935129$

H $-4.964012-0.5560541 .086135$

H $-1.790939-1.7932351 .810801$

H $3.777843-0.4347011 .220271$

H $2.165529-1.1544151 .301475$

H $-4.1013780 .714540-1.561843$

H -5.009491 $-0.806554-1.432388$

H $-5.7120550 .707626-0.840910$ 
F2-20_c04619

MMFF Geometry

C $4.279606 \quad 1.778502-0.241025$

C 4.4357942 .6945490 .721353

C $2.676938-0.124878-0.541254$

O 1.6096360 .4468690 .240429

C $0.515255-0.4920110 .293298$

C $0.904459-1.656249-0.612363$

C $2.422352-1.634883-0.555721$

C $-0.7894420 .230655-0.078032$

C $2.944428-2.3859450 .670006$

C -2.029292 -0.6695560 .047203$

O $-0.7046920 .721655-1.415217$

O $-2.125256-1.135047 \quad 1.393576$

C $-3.3485690 .018516-0.362482$

C -3.7864751 .1387120 .592065$

O $-4.356091-0.997481-0.388896$

C 4.0288810 .3187990 .017655

C $-5.044006 \quad 1.857741 \quad 0.117935$

H $4.3460902 .096727-1.278272$

H $4.619173 \quad 3.7328170 .466937$

H $4.3826842 .429447 \quad 1.771507$

H $2.553491 \quad 0.259619-1.563372$

H $0.459589-0.8338391 .333561$

H $0.582311-1.477309$-1.645190

H $0.460134-2.602990-0.289922$

H $2.859286-2.094787-1.449229$

H $-0.885377 \quad 1.1022380 .579673$

H $2.604967-1.9361341 .608517$

H $2.605180-3.4272660 .654379$

H $4.039195-2.3996230 .680240$

H $-1.902720-1.554780-0.588741$

H $0.089822 \quad 1.279873-1.469030$

H $-2.914752-1.7003821 .448737$

H $-3.2563600 .405378-1.383781$

H $-3.9893440 .728586 \quad 1.588426$

H -2.9890821 .8816930 .694591$

H -5.128596 $-0.636423-0.855225$

H $4.074758 \quad 0.1238151 .095907$

H $4.834455-0.251034-0.459834$

H -5.9100581 .1892040 .109749$

H -5.2751402 .6910810 .789284$

H $-4.9080512 .262421-0.889874$

SCF Energy $($ B3LYP/6-31G**//MMFF $)=-810.717916108$

F2-20_c04620

MMFF Geometry

C $4.520193-1.045963-0.165287$

C $4.698832-2.076264-0.999559$

C $2.5611790 .444322 \quad 0.306785$

O $1.548886-0.512391-0.062209$

C $\quad 0.249408 \quad 0.109720 \quad 0.051525$

C 0.4934281 .4893840 .653441

C 1.9006431 .8198960 .185377

C $-0.678699-0.8302170 .834205$

C $1.8948972 .408324-1.226499$

C -2.155786 -0.3977620 .918536$

O $-0.194693-0.9780842 .173901$

O -2.2710640 .8556421 .589583$

C -2.854841-0.293095 -0.447862

C $-4.3554480 .007057-0.303763$

O $-2.683231-1.508361-1.171950$

C $3.8160630 .228810-0.539406$

C $-5.0478290 .153466-1.652807$

H $4.913049-1.1203070 .845508$

H $5.220664-2.967323-0.668129$

H $4.330036-2.051839-2.018921$

H $2.7912390 .259674 \quad 1.365349$

H $-0.118596 \quad 0.212673-0.975166$

H $\quad 0.4848251 .4499311 .749197$

H $\quad-0.257815 \quad 2.2213880 .342221$

H 2.3812242 .5430030 .853910

H $-0.623055-1.8304790 .387720$

H $1.4927361 .709039-1.966522$

H $1.2871423 .318944-1.259490$

H $2.9085352 .680990-1.537592$
H $-2.684669-1.1344711 .537348$

H $0.731432-1.2694402 .115981$

H -1.8385720 .7607602 .455550$

H -2.402878 $0.514223-1.035199$

H $-4.845524-0.8065450 .244732$

H -4.5048710 .9286180 .269785$

H -3.096431-2.222145 -0.656650

H $3.5547440 .204824-1.604248$

H $4.5160801 .058065-0.385297$

H $-6.1055930 .395905-1.508842$

H $-4.5928630 .956897-2.240918$

H $-4.992260-0.772910-2.233038$

SCF Energy $($ B3LYP/6-31G**//MMFF $)=-810.718286836$

F2-20_c04621

MMFF Geometry

C -3.2731062 .3304950 .631716$

C $-3.0726333 .341500-0.220997$

C -2.485104 -0.047268 0.649677

O $-1.2890860 .187315-0.117960$

C $-0.605708-1.061218-0.311365$

C -1.392894 -2.111165 0.469649

C $-2.798913-1.5367590 .484706$

C $0.867995-0.9594230 .113865$

C $-3.559109-1.894334-0.793302$

C $1.6692300 .063647-0.718862$

O $1.465330-2.2488150 .001132$

O $1.1967961 .387274-0.464747$

C $3.1883780 .032401-0.452144$

C 3.5868610 .4750190 .962717

O $3.8239040 .891353-1.404621$

C -3.5830430 .9210610 .209522$

C $5.0910850 .384276 \quad 1.190655$

H -3.219082 2.5306261 .698767

H $-2.856054 \quad 4.3376770 .149249$

H $-3.1180973 .196336-1.294675$

H -2.222455 $0.140977 \quad 1.700201$

H $-0.660579-1.299700-1.381030$

H -1.021814 -2.180770 1.500270

H -1.320851 -3.108500 0.024607

H $-3.372351-1.9116771 .339915$

H $0.898240-0.6582751 .167766$

H -3.623533 -2.981605 -0.908950

H $-4.582979-1.508354-0.757299$

H -3.078153 -1.489284-1.689395

H $1.516200-0.131123-1.788148$

H $1.437688-2.507494-0.936030$

H $\quad 0.2582941 .420298-0.715945$

H $3.574834-0.974679-0.642785$

H $3.282646 \quad 1.5140751 .135661$

H $3.084700-0.1468991 .710808$

H $3.4726541 .788387-1.270090$

H $-3.7069700 .883407-0.879503$

H -4.5379870 .6339750 .664603$

H 5.6381721 .0489820 .514953

H 5.3343800 .6753512 .217414

H $5.449306-0.6381441 .034181$

SCF Energy (B3LYP/6-31G**//MMFF) $=-810.719781517$

F2-20_c04622

MMFF Geometry

C $1.8631132 .951820-0.452378$

C $1.111183 \quad 3.7513670 .312498$

C $2.1327370 .463374-0.515227$

$\begin{array}{lllll}\text { O } & 0.832297 & 0.165235 & 0.024995\end{array}$

C $0.684121-1.2577000 .140693$

C $1.955491-1.869004-0.448505$

C $2.988509-0.775044-0.238514$

C $-0.598656-1.736510-0.552510$

C $3.588547-0.8389271 .166593$

C $-1.917527-1.2388840 .082605$

O $-0.604227-3.167886-0.514177$

O $-1.942777-1.5798841 .469681$

C $-2.1856670 .269590-0.056258$

C $-3.5960310 .645140 \quad 0.428163$

O $-2.0331710 .667244-1.415602$

C 2.6462301 .7789600 .069389

C $-3.8536912 .144556 \quad 0.346191$ 
H $1.9245963 .157552-1.517959$ H $0.5721314 .583746-0.126733$ H 1.0169303 .5906981 .380678 H $2.0034810 .564919-1.601865$ H $0.644423-1.4977941 .210052$ H $1.833206-2.045352-1.524922$ H $2.216045-2.8255500 .015243$ H $3.805781-0.855313-0.963898$ H $-0.574200-1.440662-1.608061$ H $2.834161-0.6958271 .946985$ H $4.066264-1.8100101 .335312$ H $4.356921-0.0698991 .296146$ H $-2.744117-1.785741-0.389710$ H $-1.370061-3.472373-1.030265$ H $-1.778578-2.536195 \quad 1.536456$ H -1.4684470 .8364670 .546279$ H $-4.3478960 .135704-0.186593$ H -3.740455 0.3192241 .464252 H $-2.685376 \quad 0.175980-1.943849$ H $2.585811 \quad 1.756074 \quad 1.164184$ H $3.6976341 .924305-0.204215$ H $-3.7954932 .504080-0.685829$ H -4.8539152 .3760820 .726082$ H -3.1259752 .6997960 .946612$ SCF Energy (B3LYP/6-31G*//MMFF) $=-810.712629823$

F2-20 c04623

MMFF Geometry

C $-4.450037-1.579648-0.010556$

C $-4.721097-2.2831791 .094438$

C $-2.6785440 .102947-0.569330$

$\begin{array}{llll}\text { O } & -1.695796 & -0.394354 & 0.360627\end{array}$

C -0.5293620 .4527290 .303486$

C $-0.778488 \quad 1.452085-0.821711$

C $-2.2936111 .558922-0.847271$

$\begin{array}{llll}C & 0.725426 & -0.419961 & 0.145177\end{array}$

C $-2.798147 \quad 2.565486 \quad 0.187930$

$\begin{array}{lll}\text { C } 2.036203 & 0.394150 & 0.160824\end{array}$

O $0.637135-1.159868-1.071699$

O 2.0562791 .2202091 .323633

C $3.307565-0.4842760 .136819$

C $4.5884650 .356707 \quad 0.258138$

O $3.380270-1.224614-1.078481$

C $-4.084511-0.121258-0.012268$

C $5.847982-0.5011610 .274162$

H $-4.504100-2.081150-0.973438$

H $-4.983577-3.3332381 .026455$

H $-4.685265-1.8303072 .078983$

H -2.549597 $-0.474700-1.495222$

H -0.4915460 .9782821 .264859$

H $-0.4316971 .056948-1.784184$

H $-0.2667942 .405053-0.655186$

H -2.652537 $1.874978-1.833201$

H $\quad 0.739586-1.1514160 .963003$

H -2.369486 $3.555735-0.000060$

H -3.887107 2.6656510 .133730

H -2.537569 2.2753621 .210709

H $2.0560641 .047473-0.720189$

H $-0.198875-1.656414-1.049788$

H 2.0502600 .6363522 .101466

H $3.280565-1.1964400 .970787$

H $4.5665970 .957171 \quad 1.174273$

H $4.657257 \quad 1.048729-0.590004$

H $2.606887-1.812359-1.113383$

H $-4.1551810 .276176 \quad 1.007439$

H $-4.8185690 .409157-0.629903$

H $6.732626 \quad 0.1319450 .396436$

H $5.963512-1.060514-0.659256$

H $5.824376-1.2147111 .104016$

SCF Energy (B3LYP/6-31G*//MMFF) $=-810.720105141$

F2-20_c04624

MMFF Geometry

C $-4.171764-1.847087-0.140288$

C $-4.409426-2.5783490 .954341$

C $-2.5630760 .010837-0.632511$

O $-1.570382-0.386292 \quad 0.334175$

$\begin{array}{llll}\text { C } & -0.494076 & 0.574588 & 0.318777\end{array}$
C $-0.8030761 .548175-0.814270$

C $-2.319198 \quad 1.499613-0.896002$

C $0.848550-0.1670410 .205701$

C -2.9619332 .4452490 .119889$

$\begin{array}{llll}\text { C } & 2.066446 & 0.775994 & 0.251469\end{array}$

O $0.869074-0.907528-1.013146$

O $1.936314 \quad 1.617214 \quad 1.402260$

C $3.443495 \quad 0.0813110 .338413$

C $3.853738-0.658911-0.937773$

O $3.453843-0.8453421 .425265$

C $-3.958692-0.358700-0.129351$

C $5.315877-1.090141-0.898911$

H $-4.138294-2.348394-1.104207$

H $-4.559432-3.649762 \quad 0.877337$

H $-4.456803-2.1273611 .939263$

H -2.340633 $-0.547042-1.552768$

H $-0.544742 \quad 1.0972151 .281153$

H $-0.3822741 .195104-1.763489$

H $-0.3980702 .547722-0.628292$

H -2.671970 $1.781163-1.894531$

H $0.902160-0.8935541 .025358$

H -2.630190 $3.474963-0.051971$

H $-4.0526922 .433608 \quad 0.025462$

H $-2.710798 \quad 2.179398 \quad 1.151588$

H $2.0613611 .426062-0.631909$

H $0.094510-1.495732-1.008998$

H $2.6535422 .273033 \quad 1.369886$

H 4.2033660 .8385620 .569140

H $3.252808-1.565156-1.066726$

H $3.698368-0.017184-1.812190$

H $3.191125-0.3575302 .224620$

H -4.1089270 .0262970 .886610$

H $-4.7195610 .095366-0.774933$

H $5.976911-0.224010-0.792989$

H $5.507138-1.773731-0.065812$

H $5.581117-1.606668-1.826928$

SCF Energy (B3LYP/6-31G**//MMFF) $=-810.716264768$

F2-20 c04625

MMFF Geometry

C $3.525617 \quad 1.9874820 .199618$

C $4.6672522 .541865-0.222880$

C $2.455498-0.239415-0.229137$

$\begin{array}{llll}\text { O } & 1.100442 & 0.214357 & -0.045239\end{array}$

C $0.211820-0.905138-0.191786$

C $1.076422-2.100304-0.586848$

C $2.425589-1.7510590 .017417$

C $-0.892882-0.592043-1.203733$

C $2.498215-2.1613891 .489161$

C $-1.6824790 .694005-0.889840$

O $-1.758686-1.719960-1.294517$

O $-2.657224 \quad 0.865467-1.924916$

$\begin{array}{llll}C & -2.370798 & 0.775591 & 0.488378\end{array}$

C -3.541169 -0.1940090 .692659$

$\begin{array}{lllll}\text { O } & -2.876567 & 2.108307 & 0.641869\end{array}$

$\begin{array}{llll}\text { C } 3.404256 & 0.562172 & 0.662517\end{array}$

C $-4.196330-0.0319152 .060403$

H 2.6276422 .5997350 .223543

H $4.6899083 .578906-0.539380$

H $5.592443 \quad 1.977567-0.261764$

H $2.702466-0.061527-1.285254$

H $-0.228208-1.1084870 .791747$

H $1.175736-2.160408-1.678091$

H $0.662245-3.052130-0.240153$

H $3.241610-2.249932-0.517386$

H $-0.444730-0.464026-2.197794$

H $1.753441-1.6432682 .101721$

H $2.330191-3.2385131 .595247$

H $3.486969-1.9426511 .905223$

H $-0.9970811 .545832-0.984220$

H $-2.460583-1.495839-1.929389$

H -3.140522 $1.687660-1.734205$

H -1.6340720 .6298701 .285675$

H $-4.309733-0.028364-0.071087$

H $-3.199455-1.2289960 .605729$

H -2.118132 2.7158230 .603697

H $3.0312870 .585727 \quad 1.693473$

H 4.3909480 .0840330 .674061 
H -4.6403940 .9613672 .177408$

H $-4.994222-0.7711632 .184767$

$\mathrm{H}-3.467547-0.1816482 .863276$

SCF Energy (B3LYP/6-31G**//MMFF) $=-810.718334260$

\section{F2-20 c04626}

MMFF Geometry

C $-4.226696 \quad 1.765742-0.414540$

C -5.2657442 .3590840 .183595$

C $-2.651120-0.0421310 .318409$

O $-1.5399340 .396124-0.487619$

C $-0.443585-0.524613-0.309725$

C $-0.886333-1.5204280 .757595$

C $-2.398174-1.5248740 .606287$

C $0.8417670 .264398-0.013065$

C $-2.844487-2.478556-0.503204$

C $2.088251-0.6317460 .104749$

O $0.677349 \begin{array}{llll}1.037804 & 1.174969\end{array}$

O $2.260706-1.383691-1.096807$

C 3.3864340 .1353160 .432140

C $3.877991 \quad 1.068581-0.682484$

O $4.409098-0.8260920 .713288$

C $-3.9711260 .284502-0.381010$

C $5.1181081 .852148-0.267473$

H $-3.5245712 .382397-0.970007$

H -5.3970643 .4336850 .117676$

H -5.9977841 .7903440 .746053$

H $-2.5872690 .513911 \quad 1.264211$

H - 0.330035 -1.045367 -1.268199

H $-0.632489-1.1678561 .763485$

H $-0.423000-2.5035150 .628856$

H -2.889220 -1.8302631 .537088$

H $0.9711930 .982379-0.830836$

H -2.449872 -2.190238-1.482643

H -2.505562 -3.498232 -0.290901

H $-3.936633-2.506056-0.575766$

H $1.934922-1.3650640 .905950$

H $\quad 0.755518 \quad 0.443777 \quad 1.939967$

H $2.248251-0.763479-1.845245$

H $3.2450550 .711270 \quad 1.353350$

H $4.1329840 .490580-1.578334$

H $3.0940131 .781352-0.957229$

H $4.525972-1.371523-0.083574$

H -3.949873 -0.065762 -1.419844

H $-4.795327-0.2284680 .128763$

H $5.9583641 .185094-0.051326$

H $5.4240142 .526970-1.073194$

H 4.9189432 .4563530 .623391

SCF Energy (B3LYP/6-31G**//MMFF) $=-810.713086378$

F2-20 c04627

MMFF Geometry

C $3.1365462 .284292-0.867600$

C $2.5913913 .452837-0.511203$

C $2.501546-0.094262-0.399850$

O $1.094248 \quad 0.161154-0.226127$

C $0.437418-1.0667960 .123493$

C $1.497040-2.1648900 .044907$

C $2.782648-1.4090870 .334635$

C $-0.765299-1.320592-0.788871$

C $3.017057-1.2663811 .839212$

C $-1.769920-0.150562-0.902039$

O $-1.432828-2.497926-0.334006$

O $-2.680928-0.469953-1.960392$

C $-2.596904 \quad 0.1418860 .366650$

C -3.6565851 .2321440 .129331$

$\begin{array}{lllll}\text { O } & -1.743228 & 0.560878 & 1.426583\end{array}$

C 3.3106741 .1171250 .064018

C $-4.4903451 .512601 \quad 1.373702$

H $3.4868942 .163111-1.889432$

H $2.4984994 .258083-1.231681$

H $2.228923 \quad 3.6265180 .495959$

H $2.657360-0.254996-1.475809$

H $0.114093-0.9878161 .166894$

H $1.546936-2.582731-0.968605$

H $1.297904-2.9920930 .733391$

H $3.649756-1.919851-0.098978$

H $-0.396544-1.538685-1.799732$
H $\quad 3.070840-2.2521092 .313691$

H $3.965236-0.7564302 .037943$

H $2.220475-0.6994902 .331614$

H -1.232727 $0.757060-1.202336$

H -2.189446 -2.649417 -0.926061

H $-2.163392-0.549418-2.779903$

H $-3.111818-0.7694660 .691822$

H $-3.1657702 .165958-0.170773$

H $-4.3318000 .938765-0.682058$

H $-1.2768511 .362706 \quad 1.134485$

H 3.0082331 .4084351 .077250

H 4.3769390 .8643510 .092962

H $-3.871688 \quad 1.8921912 .192778$

H -5.2530052 .2664161 .153517$

H -4.998763 0.6057951 .716284

SCF Energy $(B 3 L Y P / 6-31 G * * / / M M F F)=-810.715618762$

F2-20_c04628

MMFF Geometry

C $4.501683-1.1899210 .156056$

C $4.709419-2.263054-0.615094$

C 2.5797440 .4034800 .380261

O $1.561226-0.523508-0.042741$

C $\quad 0.286150 \quad 0.157237-0.065718$

C 0.5421551 .5532990 .492863

C $1.999457 \quad 1.799128 \quad 0.139707$

C $-0.747108-0.7040740 .674327$

C $2.1464042 .318696-1.291315$

C -2.207944 -0.2070800 .612555$

O $-0.358887-0.8019932 .049946$

O -2.3211071 .0844491 .205176$

C $-2.785640-0.151885-0.811217$

C $-4.2462370 .326615-0.877324$

O $-2.677014-1.440478-1.410082$

C $3.8956680 .095567-0.334635$

C $-5.236010-0.569326-0.143784$

H $4.794256-1.2356141 .201945$

H $5.155863-3.159776-0.199500$

H $4.439188-2.268413-1.665167$

H 2.7023850 .2574561 .462590

H $0.0118960 .226667-1.123852$

H $\quad 0.4349081 .5692321 .583889$

H -0.1432322 .3010240 .082199$

H 2.4533242 .5317560 .816655

H $-0.691163-1.7247280 .276429$

H $3.1955462 .531214-1.521412$

H $1.7778081 .603122-2.033097$

H $1.5883773 .252381-1.418987$

H $-2.809039-0.8902301 .224538$

H $-0.971725-1.4193672 .484504$

H $-1.961974 \quad 1.0221062 .106890$

H -2.195887 $0.547580-1.415034$

H -4.326199 $1.348206-0.488795$

H $-4.5524810 .362236-1.930421$

H -3.015157-1.372013-2.319114

H $3.7323510 .034499-1.417487$

H $4.6168910 .900056-0.150059$

H $-5.058018-0.5633750 .935319$

H $-6.257311-0.211378-0.310645$

H $-5.180801-1.602488-0.499877$

SCF Energy $\left(B 3 L Y P / 6-31 G^{* *} / / M M F F\right)=-810.709865647$

F2-20_c04629

MMFF Geometry

C $-4.2789391 .207948 \quad 0.430793$

C -4.3632212 .5232850 .202258$

C $-2.436479-0.4615450 .114285$

O -1.3533460 .4785410 .254080$

C $-0.100298-0.2097770 .035287$

C $-0.451339-1.687236-0.099949$

C $-1.862864-1.650266-0.661027$

C $0.8700600 .168838 \quad 1.161709$

C $-1.854614-1.483763-2.181515$

C $2.311007-0.3697121 .063988$

O $0.336345-0.2884832 .412802$

O $2.310375-1.7993491 .050637$

C $3.1560390 .106825-0.130860$

C $3.2795781 .631304-0.205567$ 
O $2.616931-0.374200-1.359576$ C $-3.6468050 .226945-0.517126$ C $4.2744572 .074877-1.271416$ H $-4.6990660 .811491 \quad 1.351599$ H $-4.837013 \quad 3.1773760 .926166$ H -3.963783 $2.969302-0.701747$ H $-2.688488-0.796768 \quad 1.130118$ H $0.2749540 .175402-0.917540$ H $-0.473548-2.1789750 .879853$ H $\quad 0.258275-2.233026-0.728755$ H -2.408414 -2.570277-0.422381 H $0.902372 \quad 1.2613621 .244062$ H -2.874380 -1.500811 -2.579477 H $-1.387484-0.544739-2.494489$ H $-1.305941-2.305755-2.653712$ H $2.838796-0.0833661 .983133$ H $-0.561417 \quad 0.0767902 .494929$ H $1.821221-2.0950731 .837547$ H $4.156779-0.334430-0.043331$ H $2.3093742 .078832-0.450227$ H 3.5987362 .0287560 .764570 H $2.546809-1.341450-1.285670$ H $-3.3501650 .744563-1.437359$ H $-4.403818-0.520146-0.782943$ H $5.2699311 .665643-1.072146$ H $3.9614151 .750287-2.268701$ H $4.3529323 .166607-1.282200$

SCF Energy (B3LYP/6-31G**//MMFF) $=-810.720659630$

F2-20_c04630

MMFF Geometry

C $4.505376-1.4596310 .657596$

C $5.734956-1.268958 \quad 0.166728$

C $2.249750-0.358805 \quad 0.421428$

O $1.018122-0.431665-0.322287$

C $0.3861780 .859331-0.296418$

C $1.267218 \quad 1.7551790 .574124$

C 2.6414611 .1209600 .431896

$\begin{array}{llll}C & -1.065695 & 0.763249 & 0.201547\end{array}$

C $3.3398301 .610922-0.837828$

C $-1.967368-0.075627-0.726706$

$\begin{array}{lllll}0 & -1.596845 & 2.080346 & 0.325921\end{array}$

O $-1.517810-1.430379-0.722892$

C $-3.464349-0.031975-0.352335$

C $-3.787384-0.6977520 .991713$

O $-4.201431-0.712338-1.373193$

C $3.249551-1.360222-0.155744$

C $-5.265176-0.5858261 .348643$

H $4.399960-1.7193431 .707837$

H $6.603653-1.3637200 .808840$

H $5.896905-1.017500-0.875504$

H $1.998350-0.6644501 .447020$

H $0.394098 \quad 1.245559-1.323575$

H $\quad 0.952326 \quad 1.699508 \quad 1.624110$

H 1.2251572 .8064530 .272488

H $3.278167 \quad 1.357275 \quad 1.291606$

H -1.0535920 .3032121 .196582$

H $2.795944 \quad 1.330333-1.745477$

H $3.4338882 .702215-0.826286$

H $4.3506921 .200235-0.913156$

$\mathrm{H}-1.8611260 .292366-1.755200$

H -1.596652 $2.484674-0.558542$

H $-2.105223-1.933547-1.312404$

H $-3.819293 \quad 1.004806-0.342527$

H $-3.528986-1.7628510 .958432$

H -3.202651 -0.2379921 .795015$

H $-4.041780-0.245126-2.210919$

H $2.777556-2.351081-0.179885$

H $3.463041-1.125690-1.205690$

H $-5.894095-1.0978230 .613776$

H $-5.451806-1.0425202 .325868$

H $-5.5769090 .462221 \quad 1.399328$

SCF Energy (B3LYP/6-31G**//MMFF) $=-810.709425620$

F2-20 c04631

MMFF Geometry

C $3.3725442 .355829-0.576301$

C 3.0766623 .4038430 .200592
C $2.601421-0.027838-0.571028$

$\begin{array}{lllll}\text { O } & 1.325656 & 0.229845 & 0.045810\end{array}$

C $0.632631-1.0152560 .227395$

C $1.505920-2.091341-0.414755$

C $2.902474-1.504717-0.302659$

C $-0.790876-0.953456-0.351880$

C $3.519524-1.7955261 .066189$

C -1.7047150 .0756330 .359383$

O $-1.361119-2.257360-0.275379$

$\begin{array}{lllll}O & -1.141603 & 1.380780 & 0.248018\end{array}$

C $-3.1386800 .124021-0.216663$

C $-3.9873301 .225042 \quad 0.440888$

O $-3.803558-1.119453-0.011607$

C $3.6385430 .970309-0.056598$

C $-5.3923831 .303981-0.144588$

H $3.4357982 .505611-1.651073$

H $2.8993264 .379830-0.237747$

H $3.0040213 .309208 \quad 1.278409$

H $2.4575580 .109349-1.651900$

H $\quad 0.576277-1.201386 \quad 1.307324$

H $1.249887-2.209695-1.475493$

H $1.390710-3.0684960 .064417$

H $3.567925-1.913222-1.071474$

H $-0.722469-0.674186-1.410736$

H $3.575452-2.8755811 .239494$

H $4.539621-1.4022551 .123869$

H $2.941651-1.3536011 .884328$

H $-1.771131-0.1861641 .423336$

H $-1.427195-2.4967050 .665096$

H -0.2893541 .3777340 .715400$

H -3.096462 $0.317976-1.295657$

H $-4.0759421 .029734 \quad 1.516616$

H -3.5077372 .2026170 .320642$

H $-3.339919-1.791238-0.539549$

H 3.6393760 .9837651 .040119

H $4.6398460 .671482-0.387882$

H $-5.356737 \quad 1.493170-1.222125$

H -5.9499080 .3776750 .025220$

H -5.9499962 .1207510 .324797$

SCF Energy (B3LYP/6-31G**//MMFF) $=-810.718926040$

F2-20 c04632

MMFF Geometry

C 3.3398012 .5460720 .032793

C $2.2909003 .177143-0.507641$

C $2.5222980 .142575-0.488551$

$\begin{array}{lllll}\text { O } & 1.315857 & 0.193943 & 0.294494\end{array}$

C $0.666444-1.0863440 .237619$

C $1.509683-1.958848-0.690415$

C $2.892686-1.341881-0.572035$

C $-0.795320-0.947073-0.217171$

C $3.640997-1.8868630 .645502$

C $-1.659081-0.131086 \quad 0.768426$

O $-1.354607-2.247905-0.380876$

$\begin{array}{llllll}\text { O } & -1.172777 & 1.206892 & 0.870930\end{array}$

C $-3.162172-0.107343 \quad 0.415288$

C $-3.4930950 .643403-0.881108$

$\begin{array}{lllll}\text { O } & -3.868345 & 0.527750 & 1.486081\end{array}$

C 3.5845001 .0612750 .120850

C $-4.9752000 .561469-1.229036$

H $4.121808 \quad 3.1661100 .467861$

H $2.2462914 .261296-0.499641$

H $1.4621792 .649040-0.963092$

H $2.2640990 .488349-1.498629$

H $0.695826-1.5117221 .248686$

H $1.161580-1.864967-1.727110$

H $1.467421-3.019448-0.423447$

H $3.496003-1.544420-1.464091$

H $-0.800690-0.448566-1.193448$

H $3.127162-1.6584021 .584729$

H $3.745081-2.9749540 .575456$

H $4.649831-1.4654160 .702266$

H $-1.561961-0.5695671 .769610$

H $-1.339078-2.6884160 .486107$

H $-1.0678601 .559980-0.028572$

H $-3.544172-1.1326150 .349499$

H $-3.2314441 .703394-0.783066$

H -2.919022 $0.232562-1.717779$ 
H -3.6984430 .0154502 .294694$

H $3.698970 \quad 0.829837 \quad 1.187134$

H $4.5469450 .862801-0.366488$

H -5.593894 $1.026169-0.455096$

H $-5.1696701 .082638-2.171868$

H $-5.292639-0.479556-1.345868$

SCF Energy (B3LYP/6-31G**//MMFF) $=-810.702418675$

F2-20_c04633

MMFF Geometry

C 2.1701812 .6728510 .075657

C $2.8457723 .666004-0.513125$

C $2.1124980 .196770-0.305436$

$\begin{array}{lllll}\text { O } & 0.762349 & 0.016541 & 0.159536\end{array}$

C $0.417699-1.3747300 .075265$

C $1.625120-2.078877-0.544042$

C $2.782031-1.171713-0.161579$

C $-0.877206-1.574633-0.725373$

C $3.295668-1.4881291 .243811$

C $-2.156552-0.988343-0.084848$

O $-1.086943-2.979850-0.907432$

O $-2.295703-1.539227 \quad 1.226228$

C $-2.2057580 .549501-0.011041$

C -3.5918311 .0600020 .418559$

O $-1.8845651 .081208-1.293185$

C 2.7769371 .3485510 .448337

C -3.6539782 .5780520 .536591$

H 1.1202252 .8265720 .312230

H $2.3506774 .600896-0.752322$

H $3.8954633 .566465-0.766434$

H $2.0447740 .445212-1.373842$

H $0.286149-1.7422971 .100126$

H $1.535423-2.104603-1.637668$

H $1.732438-3.111459-0.197680$

H $3.616910-1.274340-0.863565$

H $-0.755901-1.145003-1.726332$

H $4.151598-0.8540521 .496629$

H $2.528103-1.3381002 .009776$

H $3.631135-2.529071 \quad 1.303688$

H -3.007730 -1.351366 -0.676247

H -1.224505 -3.371021 -0.027431

H -3.214345 -1.401867 1.511589

H -1.4694470 .9106530 .715426$

H $-3.8705630 .630671 \quad 1.386664$

H $-4.3452290 .744191-0.313156$

H -1.675615 2.023626 -1.179293

H 2.6458401 .2240651 .529932

H 3.8533831 .3533410 .239646

H -4.6350112 .8850260 .913325$

H $-3.5054813 .062422-0.433145$

H -2.892875 2.9488431 .230254

SCF Energy (B3LYP/6-31G**//MMFF) $=-810.714763692$

\section{F2-20 c04634}

MMFF Geometry

C $3.319776 \quad 2.210032-0.359821$

C 3.5209713 .0215220 .684423

C $2.247287-0.043180-0.581935$

$\begin{array}{lllll}\text { O } & 1.366576 & 0.093358 & 0.550147\end{array}$

C $0.594652-1.1151190 .681745$

C $0.987674-2.019953-0.480672$

C $2.394118-1.550105-0.814220$

C $-0.906119-0.797976 \quad 0.806759$

C $3.434146-2.235848 \quad 0.073347$

C $-1.4406920 .304660-0.131102$

O $-1.142081-0.3873582 .158604$

O $-1.265826-0.086861-1.489742$

C -2.9257280 .6570930 .110584$

C $-3.901041-0.466012-0.266363$

O $-3.2469351 .805407-0.681678$

C $3.5448830 .723824-0.327771$

C $-5.348477-0.096418 \quad 0.037661$

H $2.9814692 .642699-1.297860$

H $3.3438284 .087607 \quad 0.593734$

H 3.8613222 .6415921 .641364

H $\quad 1.7206270 .393454-1.441913$

H $0.932093-1.5801351 .617191$

H $\quad 0.342219-1.869399-1.350673$
H $\quad 0.924493-3.080565-0.216672$

H $2.645390-1.760944-1.859672$

H $-1.459462-1.7312740 .657665$

H $3.386491-3.323059-0.050676$

H $4.446421-1.920475-0.199465$

H $3.285989-2.0119251 .134608$

H -0.8542871 .2204760 .014703$

H -0.6182010 .4169332 .316733$

H -1.613475 $0.630439-2.047031$

H $-3.0799940 .940987 \quad 1.157667$

H $-3.657705-1.3828320 .279677$

H -3.828610 -0.686794 -1.337858

H $-2.6726382 .533422-0.388865$

H 3.9698280 .4376590 .641910

H $4.2810230 .477420-1.101745$

H $-5.6698380 .773321-0.543711$

H -5.4790420 .1310101 .100486$

$\mathrm{H}-6.010523-0.931109-0.214039$

SCF Energy (B3LYP/6-31G**//MMFF) $=-810.715651580$

\section{F2-20 c04635}

MMFF Geometry

C $4.581495-1.511626-0.509189$

C $3.920120-2.657952-0.312303$

C 2.7216490 .0478040 .401684

O $1.714973-0.288831-0.572134$

C $0.5558480 .541904-0.351778$

C $0.8483321 .365626 \quad 0.898334$

C $2.365074 \quad 1.4537210 .896457$

C $-0.697323-0.344301-0.278683$

C 2.8547612 .5941020 .002529

C $-2.0107370 .448337-0.104620$

O $-0.554364-1.2674840 .801089$

O $-2.1063951 .434753-1.129814$

C $-3.270645-0.444806-0.173431$

C $-4.5657900 .380481-0.099894$

O $-3.283319-1.3765850 .904647$

C $4.117497-0.109427-0.207860$

C $-5.814709-0.486796-0.204354$

H $5.579103-1.581822-0.939442$

H $4.381036-3.603478-0.578720$

H $2.922971-2.6926800 .109115$

H $2.600218-0.6498411 .241214$

H $\quad 0.4905691 .200575-1.225338$

H 0.5216010 .8387931 .803014

H $0.3451832 .337430 \quad 0.886103$

H $2.7539251 .621247 \quad 1.907262$

H $-0.749605-0.937190-1.200538$

H 3.9458762 .6758290 .039615

H $2.5630832 .456451-1.043580$

H $2.443857 \begin{array}{llll}3.550719 & 0.342714\end{array}$

H -2.0046150 .9515940 .870788$

H $0.274170-1.7562170 .657485$

H -1.497257 $2.157388-0.903232$

H -3.272996-1.008466-1.114659

H $-4.592727 \quad 1.124005-0.904123$

H -4.6044990 .9219270 .853158$

H -2.507480 -1.953535 0.805690

H $4.1657430 .442267-1.154926$

H 4.8542300 .3348660 .472595

H $-5.881992-1.1940960 .628059$

H -5.819720 -1.053977 -1.140532

H $-6.7112760 .140975-0.183393$

SCF Energy $($ B3LYP/6-31G**//MMFF $)=-810.716730318$

F2-20 c04636

MMFF Geometry

C $3.782873 \quad 1.895486-0.501241$

C 3.8680192 .8557380 .426179

C $2.314226-0.130067-0.736568$

O $1.2254920 .503901-0.033217$

C $0.625237-0.425170 \quad 0.884424$

C $1.492123-1.6816360 .854112$

C $2.098138-1.630386-0.533455$

C $-0.845118-0.6811670 .489267$

C $3.311857-2.526797-0.703375$

C -1.7034650 .6035780 .519760$

O $-1.376862-1.6399631 .400315$ 
O $-1.2918921 .494726-0.518613$

C -3.2197020 .3635150 .366050$

C $-3.627770-0.208811-0.998556$

O -3.8907291 .6121720 .566193$

C $3.6342000 .432141-0.186863$

C $-5.125420-0.478682-1.082797$

H $3.8318732 .177444-1.549891$

H 3.9787123 .8941370 .133209

H 3.8274022 .6270161 .485302

H $2.2202790 .120735-1.800167$

H 0.6553850 .0090421 .891053

H $\quad 0.946145-2.6091451 .049137$

H $2.278334-1.6001961 .615090$

H $1.332082-1.959773-1.249166$

H $-0.861926-1.104131-0.522558$

H $4.079846-2.3261900 .050348$

H $3.024160-3.578915-0.603579$

H $3.757570-2.391866-1.694083$

H - $-1.547231 \quad 1.1269901 .471903$

H $-2.174750-2.0209640 .996344$

H $-0.3617581 .726810-0.357470$

H -3.571332 -0.2998931 .163364$

H $-3.3669210 .494178-1.798467$

H $-3.095205-1.145359-1.193077$

H $-3.5694022 .229549-0.113464$

H $3.690997 \quad 0.2770830 .897333$

H $4.486475-0.086357-0.639530$

H $-5.375283-0.914022-2.055636$

H $-5.440258-1.182354-0.305704$

H $-5.7044470 .443035-0.969324$

SCF Energy $\left(\mathrm{B} 3 \mathrm{LYP} / 6-31 \mathrm{G}^{* *} / / \mathrm{MMFF}\right)=-810.718445361$

F2-20_c04637

MMFF Geometry

C $-4.970070-0.765381-0.382209$

C $-6.0417760 .031999-0.314849$

C $-2.480164-0.517220-0.075246$

O $-1.432271-0.6589620 .904570$

$\begin{array}{lllll}C & -0.330524 & 0.194446 & 0.527588\end{array}$

C $-0.7087880 .819365-0.812784$

C $-2.2286470 .826203-0.765821$

C $0.971141-0.6226940 .530786$

C $-2.7464152 .048726-0.005793$

C $2.221256 \quad 0.230073 \quad 0.224794$

O $0.869000-1.680943-0.420758$

O $2.252648 \quad 1.349803 \quad 1.106745$

C $3.540861-0.5604060 .356753$

C 4.8064750 .3079860 .254366

O $3.592634-1.579424-0.638437$

C -3.835124 -0.7346650 .597309$

C $4.9507241 .044736-1.071320$

H -4.916432 -1.496318 -1.184968

H $-6.832738-0.046859-1.052617$

H -6.1500530 .7718720 .470509$

H -2.317078 $-1.323147-0.804866$

H -0.2759490 .9728561 .297635$

H -0.379349 $0.190406-1.648626$

H $-0.2621791 .808669-0.952535$

H -2.655831 $0.839459-1.774729$

H $1.078899-1.0897321 .517868$

H $-3.8391942 .087447-0.022967$

H -2.427765 $2.052613 \quad 1.041497$

H $-2.3825792 .971233-0.471094$

H $2.1390600 .605370-0.802089$

H $\quad 0.076966-2.198528-0.195937$

H 2.3244051 .0093772 .014884

H $3.558925-1.0545111 .336233$

H $5.685170-0.3386060 .371255$

H 4.8351611 .0327361 .075896

H $4.409804-2.087325-0.498308$

H $-3.812892-1.6968721 .125557$

H $-3.996541 \quad 0.0129491 .383292$

H $5.904551 \quad 1.581678-1.098039$

H $4.1534201 .780992-1.208619$

H $4.9324270 .351429-1.917643$

SCF Energy (B3LYP/6-31G**//MMFF) $=-810.709118290$

F2-20_c04638
MMFF Geometry

C -3.6608572 .1444050 .152896$

C $-3.1030753 .344012-0.045926$

C $-2.706194-0.1693200 .366062$

O

C $-0.362864-0.1533790 .132101$

C $-0.929062-1.201572-0.825672$

C $-2.313729-1.494339-0.278695$

C $0.746342-0.6128861 .093489$

C $-3.273037-2.058706-1.311533$

C $2.144975-0.8066600 .471294$

O $\quad 0.369063-1.8483791 .709797$

O $2.109683-1.797846-0.552041$

C $2.7550940 .480513-0.111867$

C $4.2026090 .266661-0.583637$

O 2.7254681 .5163350 .866305

C $-3.2631050 .898243-0.588917$

C $4.8015341 .524244-1.200748$

H -4.4574362 .0549230 .886931$

H -3.4383774 .2063420 .519655$

H $-2.3071333 .486948-0.768400$

H $-3.416876-0.3422141 .182977$

H $-0.0211970 .719730-0.433364$

H $-0.327596-2.109364-0.910955$

H $-0.990568-0.770982-1.832974$

H $-2.204029-2.2370480 .523621$

H $\quad 0.8269780 .112924 \quad 1.912090$

H -3.351552 -1.416215 -2.194374

H -2.929861 -3.041387 -1.652490

H $-4.273777-2.180207-0.884772$

H $2.808410-1.1905381 .257484$

H -0.481031 -1.699244 2.158182

H $1.790936-2.621354-0.144383$

H $2.1657930 .815087-0.972880$

H $4.245710-0.541784-1.321957$

H $4.830043-0.0283120 .266238$

H $3.260693 \quad 1.2247131 .624224$

H $-2.5257171 .145501-1.361857$

H $-4.1570410 .521185-1.097881$

H $5.8191271 .323720-1.550982$

H $4.8523152 .340753-0.473805$

H $4.2082141 .860615-2.056887$

SCF Energy (B3LYP/6-31G**//MMFF) $=-810.715627212$

F2-20_c04639

MMFF Geometry

C 4.2960781 .8331910 .447291

C 3.5045462 .8988050 .278608

C $2.6091790 .083120-0.453907$

O 1.5890460 .2910370 .541798

C $0.527124-0.6625840 .328571$

C $0.888723-1.433228-0.937450$

C $2.405341-1.348232-0.962225$

C $-0.819727 \quad 0.0778970 .288753$

C $3.037307-2.436164-0.092517$

C $-2.035015-0.8558250 .118450$

O $-0.7965951 .018614-0.784021$

O $-1.990430-1.8799381 .117220$

C $-3.415816-0.1780100 .258962$

C $-3.7774860 .757708-0.897977$

O $-\begin{array}{llll}-3.475210 & 0.562368 & 1.478942\end{array}$

C 3.9892120 .3910450 .133759

C $-5.241616 \quad 1.181118-0.849331$

H 5.2872272 .0116810 .860852

H 3.8594663 .8875090 .550920

H $2.5020532 .824488-0.124618$

H $2.3934780 .772453-1.281286$

H $0.552284-1.3343541 .194197$

H $0.487574-0.937006-1.829411$

H $0.499676-2.456223-0.929946$

H $2.792162-1.458371-1.981547$

H -0.9140380 .6523731 .218061$

H $4.129697-2.393057-0.149038$

H $2.750939-2.3450330 .960022$

H $2.731545-3.429246-0.439216$

H $-2.000815-1.347762-0.861467$

H $-0.0320811 .602950-0.641838$

H $-1.302740-2.5160590 .857960$ 
H $-4.180028-0.9622240 .334555$

H $-3.5851250 .263656-1.856752$

H $-3.1754581 .671424-0.856631$

H $-3.250845-0.0502532 .200265$

H $4.117803-0.1634211 .071661$

H $4.7586280 .042099-0.565674$

H -5.4682981 .7244020 .073376$

H $-5.9031840 .311083-0.908623$

H $-5.4715011 .839208-1.693262$

SCF Energy (B3LYP/6-31G**//MMFF) $=-810.713918179$

F2-20_c04640

MMFF Geometry

C 4.468041-1.260918 0.228516

C $4.441695-2.523253-0.213362$

C 2.6898140 .4911570 .512763

O $1.661773-0.4844450 .772618$

C $0.566829-0.279686-0.147658$

C $0.8399391 .036478-0.873387$

C $1.9253921 .691012-0.040467$

C $-0.746538-0.3388810 .653933$

C $2.7386582 .724883-0.799118$

C $-2.009435-0.340692-0.235206$

O $-0.803298 \quad 0.760847 \quad 1.560257$

O $-1.898846-1.383815-1.201049$

C $-3.316634-0.5254480 .567557$

C $-4.562687-0.754591-0.303506$

O -3.5649980 .6060951 .398376$

C $3.736765-0.123571-0.429810$

C $-4.8662710 .382198-1.271602$

H $5.058230-1.0295231 .111400$

H $4.995604-3.2983950 .304998$

H $3.871112-2.805668-1.091077$

H $3.159696 \quad 0.7512291 .468736$

H $\quad 0.598513-1.110595-0.861509$

H $-0.0413561 .674073-0.987283$

H $1.2053420 .820327-1.885122$

H 1.4393042 .1972140 .804754

H $-0.734330-1.2530841 .261049$

H $3.1702452 .315258-1.717980$

H $2.1056083 .571595-1.085107$

H $3.5541483 .108157-0.177471$

H $-2.0630580 .614772-0.768641$

H $-0.013945 \quad 0.7110322 .126288$

H -1.858476 -2.228790 -0.721113

H $-3.217834-1.4003301 .222285$

H -4.464563 -1.690404 -0.865309

H $-5.432266-0.8686380 .356125$

H -2.811216 0.6990962 .004961

H $3.262697-0.472703-1.354957$

H $4.4838590 .627954-0.707624$

H $-4.9766671 .335866-0.746415$

H $-4.078250 \quad 0.489151-2.022735$

H $-5.802840 \quad 0.180825-1.801785$

SCF Energy (B3LYP/6-31G**/MMFF) $=-810.718598010$

F2-20 c04641

MMFF Geometry

C 3.2041442 .2743680 .104685

C $4.2886132 .951791-0.288335$

C $2.367022-0.034820-0.439954$

O $\quad 0.987141 \quad 0.366588-0.309388$

C $0.265195-0.6133160 .456023$

C $1.282552-1.660598 \quad 0.901773$

C $2.365557-1.537178-0.150700$

C $-0.868676-1.195631-0.401389$

C $3.688872-2.1585070 .259205$

C $-1.794362-0.129968-1.024540$

O $-1.615369-2.1139960 .393032$

O $-2.802825-0.795657-1.793901$

C $-2.4831290 .849874-0.053953$

C -3.5409740 .2326990 .870242$

O $-3.1072851 .869468-0.845559$

C 3.2177520 .8250570 .508583

C -4.2066791 .2734021 .764334$

H 2.2526072 .7979300 .149729

H $4.2131313 .998812-0.561112$

H $5.2631392 .479894-0.345940$
H $2.6751840 .147145-1.476514$

H $-0.154147-0.1292281 .345420$

H $0.874157-2.6723420 .981921$

H $1.677603-1.3893021 .888779$

H $2.016350-2.052632-1.056207$

H $-0.437684-1.778966-1.225084$

H $4.045450-1.7713681 .219072$

H $3.582425-3.2433570 .365186$

H $4.457371-1.967739-0.496696$

H $-1.2150960 .463212-1.743786$

H $-2.338642-2.455699-0.160200$

H -2.351030 -1.344266 -2.457728

H -1.7266631 .3611610 .549962$

H $-4.324960-0.2551210 .279622$

H $-3.088165-0.5248951 .515640$

H $-3.772511 \quad 1.436422-1.408124$

H $2.8310180 .764951 \quad 1.532750$

H $\quad 4.2507740 .460707 \quad 0.523931$

H -4.9192790 .7884642 .439333$

H $-3.464036 \quad 1.7970002 .374821$

$\mathrm{H}-4.7556232 .0149641 .175913$

SCF Energy $\left(B 3 L Y P / 6-31 G^{* *} / / M M F F\right)=-810.715606731$

F2-20 c04642

MMFF Geometry

C $-3.6564902 .053010 \quad 0.474087$

C $-3.5476603 .074921-0.382240$

C $-2.550062-0.1925180 .583422$

O $-1.3503240 .197057-0.111793$

C $-0.493486-0.948066-0.248287$

C $-1.177053-2.0871530 .506291$

C $-2.646614-1.7122730 .426888$

C $0.931687-0.6493910 .252372$

C $-3.270877-2.184091-0.887170$

C $1.6646280 .417693-0.593308$

O $1.668479-1.8676090 .250827$

O $\quad 0.8913341 .621863-0.575307$

C $3.0894440 .785430-0.121153$

C $4.140083-0.297862-0.383921$

O $3.078406 \quad 1.0824421 .275472$

C -3.7400890 .6105920 .058210$

C $5.5570160 .222043-0.164995$

H -3.6967842 .2677691 .538797$

H -3.495732 $4.094664-0.016771$

H -3.506214 $2.915522-1.454060$

H $-2.384071 \quad 0.0401441 .644608$

H $-0.456930-1.201340-1.315434$

H $-0.863697-2.0915221 .558201$

H -0.942604 -3.070365 0.086981

H -3.214612 -2.154162 1.253259

H $\quad 0.860657-0.2932321 .287194$

H -2.796467 -1.725127 -1.760346

H -3.178265 -3.270916 -0.986754

H $-4.338383-1.943196-0.918595$

H $1.7161570 .080392-1.635892$

H $1.724754-2.179688-0.668625$

H $1.2981982 .243479-1.202541$

H $3.4002031 .706076-0.631394$

H $4.000642-1.1457060 .294213$

H $4.054570-0.664400-1.412948$

H 2.4110611 .7752141 .419236

H -3.786766 $0.546517-1.035621$

H -4.6738350 .1978350 .457465$

H 5.7029590 .5565800 .866856

H $6.284590-0.569862-0.369132$

H $5.7742291 .061291-0.833462$

SCF Energy $\left(B 3 L Y P / 6-31 G^{* *} / / M M F F\right)=-810.705029331$

F2-20 c04643

MMFF Geometry

C $-4.351873-1.306388-0.659843$

C $-4.371263-2.560599-0.194774$

C $-2.5915790 .486306-0.676299$

O $-1.512429-0.466472-0.740560$

C $-0.591696-0.2181990 .345356$

C -1.0134041 .1014650 .989187$

C -1.958532 $1.714502-0.026192$

C $0.839841-0.255535-0.224656$ 
C $-2.9113742 .738230 \quad 0.565344$

C $1.935207-0.1843310 .858335$

O $1.0076180 .826649-1.137536$

O $1.649995-1.1767481 .849631$

C $3.377858-0.4281450 .361076$

C $3.9355330 .694113-0.518934$

O $3.441094-1.653601-0.369686$

C $-3.767852-0.1402570 .089005$

C $5.4337460 .538406-0.756486$

H -4.790348 -1.104500 -1.633667

H -4.812112 $-3.357848-0.783150$

H $-3.949884-2.8141280 .771707$

H $-2.8996190 .719018-1.702695$

H $-0.724151-1.0384711 .059923$

H $-0.178723 \quad 1.763540 \quad 1.236458$

H -1.5382770 .8912561 .929372$

H -1.349597 $2.220106-0.787944$

H $\quad 0.947297-1.183017-0.800155$

H $-3.4812282 .331657 \quad 1.406839$

H -2.3549253 .6054940 .936691$

H $-3.6196573 .090471-0.191379$

H 1.9040820 .7924561 .355116

H $\quad 0.3324120 .728983-1.830584$

H $2.288038-1.0608872 .574227$

H $4.031854-0.5532331 .233262$

H $3.4505760 .689983-1.500680$

H $3.7520851 .667347-0.049956$

H $3.082747-2.3522130 .204316$

H -3.448312 -0.4623151 .087246$

H -4.5680430 .5959850 .222279$

H $5.8037321 .359670-1.378582$

H 5.9844500 .5561600 .189325

H $5.659707-0.401542-1.269468$

SCF Energy (B3LYP/6-31G**//MMFF) $=-810.715114905$

F2-20 c04644

MMFF Geometry

C $3.3815692 .265171-0.559729$

C $2.9113333 .427057-0.092174$

C $2.572675-0.098233-0.358368$

O $1.1815110 .232461-0.181714$

C $0.435101-0.9805850 .015637$

C $1.424731-2.131312-0.160258$

C $2.748252-1.499300 \quad 0.234983$

C $-0.749557-1.046416-0.954789$

C $2.950595-1.5337841 .750546$

C - $1.7305870 .144708-0.854004$

O $-1.443503-2.276696-0.758289$

$\begin{array}{lllll}0 & -2.438262 & 0.226498 & -2.099573\end{array}$

C -2.7949880 .0585540 .258793$

C $-3.6375291 .343927 \quad 0.324445$

O $-2.189864-0.1380011 .529508$

C 3.4496890 .9981400 .246808

C $-4.7429791 .260349 \quad 1.370184$

H $3.7486222 .225459-1.582005$

H $2.893674 \quad 4.307225-0.725525$

H 2.5365423 .5213340 .921022

H $2.743290-0.154327-1.442594$

H $0.090736-0.9831371 .053495$

H $1.474303-2.440114-1.212251$

H $\quad 1.154217-3.0124200 .429998$

H $3.591865-2.018141-0.233949$

H $-0.347425-1.069898-1.976338$

H $2.927672-2.5658272 .116666$

H $3.923962-1.1126142 .022134$

H $2.179300-0.9703482 .285347$

H -1.163363 $1.077017-0.750514$

H -2.160439 -2.313316 -1.414499

H -1.785775 $0.415786-2.795269$

H -3.475990 -0.7799200 .065186$

H -2.9935652 .1952210 .577030$

H $-4.0938601 .552079-0.649717$

H -1.983133 $-1.084870 \quad 1.607411$

H 3.1403421 .2031921 .278907

H 4.4949240 .6689300 .270052

H -4.3328921 .1326852 .376675$

H -5.3365392 .1802631 .364563$

H $\quad-5.4153450 .4219891 .162274$
SCF Energy $($ B3LYP/6-31G**//MMFF $)=-810.714005004$

F2-20_c04645

MMFF Geometry

C -2.8756692 .5608550 .493674$

C $-2.3206263 .548470-0.217652$

C -2.3580820 .1103690 .692109$

O $-0.9566350 .322326 \quad 0.425182$

C $-0.494579-0.637830-0.539111$

C $-1.704047-1.481190-0.938861$

C $-2.623602-1.3315180 .256318$

C $0.643266-1.4723970 .071138$

C $-4.067002-1.702974-0.032730$

C $1.788953-0.6654480 .724802$

O $1.175391-2.348967-0.923674$

O $2.595515-1.5936701 .460568$

C $2.6996020 .127995-0.234290$

C 3.8402890 .8400680 .513422

O $1.9417231 .099702-0.946262$

C $-3.1614381 .188646-0.051666$

C $4.7634821 .603725-0.428287$

H -3.1485372 .7527481 .528222$

H $-2.143737 \quad 4.518410 \quad 0.234084$

H -2.032398 $3.409029-1.253600$

H $-2.515798 \quad 0.211255 \quad 1.772710$

H $-0.139740-0.105608-1.427478$

H -1.462037 -2.523480 -1.167632

H -2.169166 -1.051566 -1.834928

H $-2.254576-1.9952871 .050417$

H $0.225784-2.1207950 .852033$

H $-4.469851-1.152489-0.888912$

H -4.145624 -2.770433 -0.265179

H $-4.700718-1.4998360 .836468$

H $1.356597 \quad 0.020671 \quad 1.462193$

H $1.419237-1.813149-1.697248$

H $2.962407-2.2309090 .823809$

H $3.148151-0.551036-0.968264$

H 4.4387900 .1150241 .075959

H 3.4219051 .5530311 .234260

H $1.5372601 .697847-0.294662$

H $-2.9346871 .161133-1.124315$

H -4.2360331 .0089380 .062660$

H $5.2047930 .933027-1.172302$

H $5.5786962 .066307 \quad 0.137234$

H $4.2280452 .399420-0.955231$

SCF Energy (B3LYP/6-31G**//MMFF $)=-810.717925367$

F2-20_c04646

MMFF Geometry

C $4.164685-1.3246690 .750459$

C $4.381138-2.4783200 .108838$

C $2.3147680 .371195 \quad 0.640912$

O $1.322721-0.6177520 .302816$

C $0.688769-0.238654-0.935399$

C $1.0992041 .200545-1.229482$

C 1.7491231 .6660140 .060618

C $-0.824847-0.511870-0.854542$

C $2.7275692 .812019-0.126883$

C -1.5513550 .0956590 .362109$

O $-0.996207-1.932481-0.838452$

$\begin{array}{llll}\text { O } & -1.561809 & 1.516868 & 0.239802\end{array}$

C -3.002242 -0.4045800 .539322$

C $-3.9502390 .029329-0.586470$

$\begin{array}{llll}\text { O } & -3.508574 & 0.117776 & 1.772074\end{array}$

C $3.677091-0.0688790 .082133$

C $-5.355911-0.531050-0.402469$

H $4.358185-1.2779901 .819079$

H $4.737703-3.3486370 .648792$

H $4.206506-2.576468-0.956796$

H $2.3669320 .438347 \quad 1.734110$

H $1.111015-0.893245-1.709093$

H $0.2718321 .839961-1.550761$

H $1.8273201 .204146-2.050664$

H 0.9544752 .0102000 .735167

H -1.279016 -0.144344 -1.781717

H $2.2023303 .707201-0.476506$

H 3.2210533 .0581940 .818875

H $3.4997422 .576755-0.866173$ 
H $-0.998168-0.143297 \quad 1.278425$

H $-0.555531-2.271126-0.039917$

H -2.0250891 .8726401 .017457$

H -3.015688 -1.4966600 .629749$

H $-3.569059-0.306483-1.556274$

H $-4.0255021 .122590-0.617913$

H $-2.940091-0.2159052 .487085$

H $3.616588-0.223949-1.001659$

H 4.4277690 .7090190 .260169

H $-5.815150-0.1641710 .520683$

H $-5.339431-1.625107-0.368746$

H -5.994302 -0.227388 -1.238169

SCF Energy (B3LYP/6-31G**/MMFF) $=-810.716530407$

F2-20_c04647

MMFF Geometry

C $2.3597132 .741541 \quad 0.291174$

C 2.0754323 .3256881 .460646

C $1.8860170 .455721-0.637964$

$\begin{array}{lllll}\text { O } & 0.669656 & 0.334611 & 0.128313\end{array}$

C $0.555053-0.9899830 .669322$

C $1.874898-1.6931580 .359160$

C $2.340265-0.982042-0.895142$

C $-0.669706-1.6994850 .061282$

C $3.811713-1.191410-1.205371$

C $-2.041500-1.0420380 .338191$

O $-0.722906-3.0347750 .579193$

O $-2.232898-0.9150891 .749120$

C $-2.2952030 .318640-0.337387$

C $-3.7600280 .756017-0.173384$

O $-2.002606 \quad 0.241319-1.728548$

C $2.8670491 .332114 \quad 0.155365$

C $-4.0247672 .130089-0.776800$

H $2.2277883 .314171-0.623298$

H 1.7161474 .3485021 .490761

H 2.1926742 .7992522 .401297

H $1.6389060 .944993-1.587859$

H $0.440043-0.9185791 .756728$

H $1.787684-2.7758870 .231604$

H $2.582492-1.517478 \quad 1.179096$

H $1.758124-1.372287-1.741418$

H $-0.541605-1.794817-1.022316$

H $4.113734-0.594655-2.071993$

H $4.452647-0.920787-0.360187$

H $4.005294-2.244222-1.437311$

H $-2.806510-1.749512-0.007360$

H - $0.879146-2.9709341 .537240$

H - $-1.676108-0.1825142 .062954$

H -1.667618 1.0973350 .109950

H -4.0326290 .7803220 .887689$

H $-4.4202330 .033722-0.668972$

H -1.035414 $0.217334-1.824133$

H 3.0418420 .9022591 .149057

H $3.8336851 .381654-0.357743$

H -3.838672 2.135808-1.855145

H -3.388181 2.890216 -0.312760

H -5.068526 $2.417409-0.615049$

SCF Energy (B3LYP/6-31G**//MMFF) $=-810.717994964$

F2-20_c04648

MMFF Geometry

C $4.2947091 .628525-0.326353$

C 4.4288812 .6540880 .522132

C $2.639850-0.247175-0.488319$

O $1.550760 \quad 0.4526640 .145059$

C $0.420871-0.4384490 .246854$

C $0.816479-1.719836-0.479839$

C $2.328380-1.738294-0.329195$

C $-0.8391820 .275021-0.268802$

C $2.747779-2.3428421 .011699$

C $-2.117556-0.566989-0.119728$

O $-0.6597970 .660896-1.630983$

O $-2.291142-0.908127 \quad 1.253631$

C $-3.3835280 .115006-0.677299$

C $-3.7501121 .488678-0.094393$

O $-4.479935-0.785969-0.498706$

C 3.9723790 .2220960 .096225

C -4.0476181 .5102461 .397888$
H $4.4349611 .810046-1.388873$

H 4.6672083 .6453820 .152142

H 4.3029242 .5263541 .591490

H $2.5926730 .007271-1.556416$

H $\quad 0.299926-0.6493251 .316272$

H $\quad 0.567907-1.668210-1.545721$

H $0.317484-2.603315-0.069739$

H $2.799493-2.321676-1.128253$

H $-0.936136 \quad 1.2041180 .304813$

H $2.371764-1.7666791 .863050$

H $2.369413-3.3665551 .105255$

H $3.838511-2.3892971 .092403$

H -1.994656-1.514352 -0.659807

H $-0.760440-0.128661-2.188188$

H $-3.112741-1.4240241 .322728$

H $-3.2580240 .235243-1.760342$

H $-2.9613172 .215824-0.316163$

H $-4.6529261 .844288-0.607845$

H -5.263992 -0.378891-0.905035

H 3.9464000 .1645091 .191104

H $4.782379-0.428517-0.253743$

H -4.7901870 .7550061 .671819$

H -3.1423471 .3453711 .988502$

H -4.4453582 .4899401 .683308$

SCF Energy $\left(B 3 L Y P / 6-31 G^{* *} / / M M F F\right)=-810.709758193$

F2-20 c04649

MMFF Geometry

C $4.559552-0.9205160 .120050$

C $4.850330-1.963296-0.665762$

C 2.5102490 .4981810 .385946

O $1.569664-0.508289-0.035877$

C $0.2412610 .060871-0.037982$

C 0.3829101 .4669930 .535861

C 1.8092201 .8414830 .169836

C $-0.707163-0.8947100 .700546$

C $1.8933842 .388578-1.255988$

C $-2.205896-0.5256690 .637202$

O $-0.311322-0.9657642 .075226$

O $-2.415116 \quad 0.736547 \quad 1.266216$

C $-2.781148-0.481291-0.790310$

C $-4.317866-0.443115-0.848686$

O $-2.357444-1.651107-1.492787$

C $3.8380860 .312511-0.348610$

C $-4.9547050 .811660-0.276286$

H $4.868035-0.9516741 .161893$

H $5.377981-2.822212-0.265776$

H $4.568545-1.981174-1.712758$

H $2.6589510 .351321 \quad 1.464890$

H $-0.0510320 .120145-1.092312$

H $\quad 0.287915 \quad 1.460896 \quad 1.628221$

H $-0.3690762 .157780 \quad 0.142387$

H $2.2066022 .602428 \quad 0.851047$

H $-0.559871-1.9045730 .298734$

H $2.9172772 .693565-1.495181$

H $1.5786261 .652688-2.002675$

H $1.2554883 .272320-1.365481$

H -2.761210 -1.268030 1.224597

H $-0.872823-1.6314382 .507841$

H -2.0596350 .6737592 .169237$

H $-2.3888520 .393732-1.322466$

H -4.627871 -0.538422 -1.897694

H $-4.730098-1.327150-0.346078$

H -2.660019-1.567922 -2.413162

H $3.6665360 .248631-1.430026$

H $4.489516 \quad 1.174552-0.163735$

H $-4.4953191 .713825-0.691682$

H $-6.0220170 .833066-0.520478$

H $-4.873178 \quad 0.8427120 .813528$

SCF Energy (B3LYP/6-31G**//MMFF) $=-810.708198411$

F2-20_c04650

MMFF Geometry

C $-4.2089721 .393870 \quad 0.182704$

C $-4.3092452 .520209-0.531962$

C $-2.416462-0.3469740 .377403$

$\begin{array}{lllll}0 & -1.319360 & 0.524095 & 0.039634\end{array}$

C $-0.097828-0.2495490 .002135$ 
C $-0.470680-1.647206 \quad 0.483421$

C $-1.930327-1.7672200 .079315$

$\begin{array}{lllll}\text { C } & 0.977707 & 0.493127 & 0.806284\end{array}$

C $-2.070849-2.202584-1.380270$

C $2.397823-0.1067020 .808820$

$\begin{array}{llllllllll}\text { O } & 0.557284 & 0.587674 & 2.175158\end{array}$

O $2.357926-1.4270141 .358965$

C $3.129612-0.159062-0.545986$

C $3.2489701 .185003-1.273538$

O $2.473632-1.086811-1.411112$

C $-3.680307 \quad 0.095428-0.360504$

C $3.9882982 .248356-0.478833$

H -4.5363761 .4040011 .219280$

H $-4.7045573 .423595-0.080563$

H -4.000469 2.562949-1.570489

H -2.566641 -0.2498851 .461654$

H $0.189038-0.280431-1.053340$

H $-0.397979-1.7208811 .575125$

H $0.169932-2.4240810 .055478$

H -2.456065 -2.497703 0.704606

H $1.027175 \quad 1.5282580 .449224$

H -1.630038 -1.479589-2.074051

H - $1.577227-3.166839-1.541559$

H $-3.124980-2.326446-1.648696$

H 3.0019370 .4799891 .512159

H -0.3272900 .9919822 .178943$

H $3.272432-1.7532911 .409851$

H $4.134042-0.566908-0.376782$

H $3.7769921 .024631-2.222442$

H $2.2568021 .553422-1.559829$

H $2.994067-1.146167-2.230372$

H $-3.4747220 .202429-1.432537$

H $-4.463853-0.662338-0.244312$

H $4.9776761 .893299-0.173883$

H $4.1248093 .146457-1.089870$

H 3.4330012 .5398230 .417320

SCF Energy (B3LYP/6-31G*//MMFF) $=-810.705171960$

\section{F2-20 c04651}

MMFF Geometry

C $-4.430468-1.081138-0.985315$

C $-5.447088-1.510901-0.229871$

C $-2.1970180 .020218-0.593336$

O $-0.797084-0.272057-0.418218$

C $-0.186623 \quad 0.809820 \quad 0.305364$

C $-1.279795 \quad 1.8576340 .521552$

C $-2.552724 \quad 1.0263880 .504333$

C $1.0355471 .348251-0.446878$

C $-2.821810 \quad 0.4114001 .878874$

C $2.0656310 .280610-0.880410$

$\begin{array}{lllll}\text { O } & 1.695513 & 2.327810 & 0.356032\end{array}$

O $2.977935 \quad 0.916707-1.783895$

C $2.871082-0.3789330 .258358$

C $3.953326-1.333070-0.275825$

O $2.002154-1.1089601 .118144$

C $-2.984409-1.288441-0.647474$

C $4.768267-1.9712450 .842601$

H $-4.656413-0.561534-1.912963$

H $-6.472460-1.334451-0.536196$

H $-5.277864-2.0425890 .699964$

H $-2.2778340 .520012-1.569223$

H $\quad 0.1112910 .428155 \quad 1.287618$

H $-1.3037062 .569163-0.313578$

H -1.1336992 .4336551 .440674$

H -3.418949 1.6340980 .220285

H $0.7008191 .858920-1.359566$

H $-2.898302 \quad 1.194827 \quad 2.640499$

H -3.767175 -0.1383421 .884392$

H $-2.030755-0.2800182 .186707$

H $1.547941-0.495013-1.456465$

H 1.0712573 .0566660 .509859

H $3.4304721 .623745-1.292321$

H $3.3642620 .393408 \quad 0.859954$

H $4.638137-0.798213-0.943133$

H $3.484232-2.135803-0.857856$

H $1.557262-1.7892440 .584069$

H $-2.541316-1.931522-1.419160$

H $-2.861722-1.8478100 .287953$
H $5.254182-1.2068001 .457319$

H $5.547884-2.6135200 .420644$

H $4.140798-2.5893391 .492181$

SCF Energy (B3LYP/6-31G**//MMFF) $=-810.716284706$

F2-20 c04652

MMFF Geometry

C $2.1935032 .635819-0.329484$

C 1.6034493 .4890700 .515211

C $2.0114310 .152027-0.576730$

O $0.6930920 .051181-0.009064$

C $0.290247-1.3266760 .018276$

C $1.413082-2.106157-0.667142$

C $2.633891-1.236439-0.420396$

C $-1.079814-1.502925-0.650893$

C $3.247274-1.5137080 .952679$

C $-2.305654-0.9858180 .134365$

O $-1.294081-2.901105-0.887773$

O $-2.342611-1.6436091 .406485$

C -2.4421840 .5311650 .362463$

C $-2.3820961 .344571-0.933428$

O $\quad-1.4474281 .0074081 .264047$

C 2.7674181 .3070910 .078950

C $-2.6944442 .818067-0.702745$

H $2.2734142 .911985-1.377776$

H 1.2104614 .4340200 .156366

H 1.4978713 .2615441 .570100

H $1.8752780 .357241-1.647782$

H $0.238766-1.6337821 .069631$

H $1.231566-2.169307-1.747887$

H $1.508010-3.128218-0.287261$

H $3.404220-1.407159-1.180664$

H -1.065723 -1.021646-1.635023

H $2.551853-1.2962701 .769673$

H $3.543681-2.5649691 .034382$

H $4.146205-0.9078091 .105638$

H -3.200220 -1.326905 -0.403645

H -1.329941 -3.341398 -0.020945

H -3.167047 -1.379966 1.849611

H -3.4051200 .7157770 .855372$

H $-1.3815571 .285074-1.375832$

H -3.094486 $0.939507-1.661041$

H -1.474444 0.4419102 .054521

H 2.7311681 .2125451 .171065

H $3.8207651 .284736-0.223369$

H $-1.9646763 .279736-0.030264$

H -2.666940 $3.361934-1.652271$

$\mathrm{H}-3.6914932 .943904-0.268915$

SCF Energy (B3LYP/6-31G**//MMFF) $=-810.707902683$

F2-20 c04653

MMFF Geometry

C $-4.665795-0.8272620 .304269$

C $-5.464314-1.745402-0.251695$

C $-2.315005-0.099328-0.249262$

O $-0.933707-0.457340-0.046310$

C $-0.1286420 .726929-0.171936$

C $-1.0779591 .863409-0.549163$

C -2.4029411 .4029600 .036857$

C $0.9964630 .512635-1.187445$

C -2.5033291 .7700911 .518760$

C $1.882515-0.715223-0.891108$

O $1.7748771 .703415-1.262280$

O $2.884087-0.810188-1.910577$

C $2.574115-0.7614830 .487106$

C $3.6711680 .287847 \quad 0.704619$

O $3.174634-2.0555290 .631643$

C -3.204052 -1.038394 0.565285

C 4.3304600 .1619872 .074302

H -5.0986990 .1256710 .595809$

H $-6.516476-1.534954-0.409885$

H -5.087866 -2.715117 -0.557709

H -2.513581 -0.266952-1.317405

H $\quad 0.294416 \quad 0.9451830 .815728$

H -1.174183 $1.939991-1.639650$

H $-0.7374002 .835666-0.179479$

H $-3.2483691 .858246-0.490896$

H $\quad 0.5568140 .366289-2.182662$ 
H -1.729638 1.2827022 .120609

H -2.400669 2.8524341 .652530

H -3.476340 1.4834521 .929079

H $1.268672-1.619314-0.991619$

H $2.4957541 .540376-1.894349$

H $2.427184-0.865635-2.767156$

H $1.827893-0.677117 \quad 1.284287$

H $4.4533130 .183693-0.056339$

H 3.2569031 .2965140 .624004

H $2.463995-2.7169270 .577670$

H -2.918984 -2.074384 0.337855

H $-3.004210-0.9253981 .637380$

H 3.5894090 .2531352 .874867

H $4.843674-0.7981522 .185910$

H 5.0731550 .9551442 .208077

SCF Energy $\left(B 3 L Y P / 6-31 G^{* *} / / M M F F\right)=-810.710181061$

F2-20_c04654

MMFF Geometry

C $-4.7734810 .024167-0.562184$

C $-5.657291-0.870219-0.105975$

C $-2.2825550 .381027-0.407638$

O $-1.0474620 .113532-1.100078$

C $0.0353190 .119837-0.139453$

C -0.5886840 .5139331 .196320$

C -2.0238450 .0296561 .058754$

C $1.1597701 .019746-0.668173$

C $-2.133848-1.4584831 .395390$

C $2.4653101 .071283 \quad 0.152843$

O $0.6489492 .356900-0.772478$

O 2.2061381 .5576451 .471839

C $3.259491-0.2403600 .287020$

C $3.644037-0.847129-1.065324$

O $2.526073-1.2061291 .035933$

C $-3.429289-0.331563-1.123526$

C $4.589785-2.032501-0.914579$

H $-5.0437341 .076983-0.544072$

H $-6.617296-0.5462580 .280781$

H -5.442437 -1.933114 -0.109894

H -2.439304 $1.466189-0.487098$

H $0.383871-0.916441-0.098276$

H $-0.5940361 .602971 \quad 1.324763$

H -0.0582300 .0839022 .051158$

H -2.694209 $0.585370 \quad 1.723686$

H $1.3871350 .714984-1.696317$

H -1.555469 -2.0843220 .708401$

H -1.769828 -1.649296 2.410762

H $-3.174223-1.793306 \quad 1.356987$

H $3.1266421 .814529-0.311259$

H $1.3308772 .898939-1.204600$

H 1.7470672 .4102661 .381053

H $4.169908-0.0344380 .864102$

H $2.749198-1.201014-1.590171$

H $4.124355-0.087831-1.692826$

H $2.292672-0.7962061 .886370$

H -3.413540 -0.043044 -2.182721

H -3.263934 -1.415825 -1.125661

H $4.125305-2.843060-0.344331$

H $4.859985-2.426239-1.899521$

H $5.511332-1.735681-0.403896$

SCF Energy $(B 3 L Y P / 6-31 G * * / / M M F F)=-810.714976391$

F2-21_c04931

MMFF Geometry

C $-3.5264442 .133060-0.617068$

C -3.3289073 .2196060 .137808$

C $-2.569074-0.181779-0.550002$

O $-1.3229740 .187478 \quad 0.072153$

C $-0.541453-1.0018750 .293804$

C $-1.319594-2.152426-0.339145$

C $-2.758442-1.672420-0.253832$

C $0.882699-0.821208-0.257262$

C $-3.369115-1.9829421 .113545$

C $1.627826 \quad 0.362508 \quad 0.387576$

O $0.830739-0.629535-1.669770$

O $1.719371 \quad 0.135444 \quad 1.794331$

C $3.0370850 .604888-0.192954$

C $4.040525-0.5132600 .118381$
O $\quad 3.545477 \quad 1.8222190 .362262$

C $-3.6867500 .742940-0.067175$

C $5.402715-0.257056-0.516602$

H $-3.5858752 .252346-1.695968$

H $-3.2256804 .196436-0.321851$

H $-3.2653593 .156023 \quad 1.218375$

H -2.424808 -0.056400 -1.632254

H $-0.497444-1.1504591 .379299$

H -1.134959 -3.1060320 .165311$

H -1.048782 -2.283209-1.393597

H $-3.381164-2.146142-1.021165$

H $1.428153-1.752548-0.069634$

H -2.838454 -1.481378 1.929010

H $-4.417262-1.6690011 .151172$

H -3.343720 -3.060430 1.308629

H $1.042700 \quad 1.2804210 .250057$

H $0.2996980 .167812-1.838229$

H $2.197820 \quad 0.8897202 .179176$

H $2.9756840 .751298-1.277495$

H $3.666255-1.475009-0.246462$

H $4.185685-0.6008701 .201592$

H 2.9407852 .5380280 .102403

H $-4.6580480 .358632-0.399652$

H -3.6993600 .7810091 .028791$

H $5.8597760 .658010-0.127705$

H $6.080868-1.088708-0.300197$

H $5.316149-0.164615-1.603851$

SCF Energy $\left(B 3 L Y P / 6-31 G^{* *} / / M M F F\right)=-810.719336922$

F2-21_c04932

MMFF Geometry

C $3.1926552 .226348 \quad 0.365134$

C $2.6371483 .381233-0.018742$

C $2.445546-0.1558160 .162297$

O $1.0356800 .136107 \quad 0.123269$

C $0.306984-1.092142-0.047354$

C $1.340723-2.2149350 .005134$

C $2.604677-1.532150-0.489333$

C $-0.815516-1.2206000 .993218$

C $2.668194-1.515667-2.017325$

C $-1.865131-0.0871570 .988322$

O $-0.246918-1.2812732 .305438$

O $-1.251412 \quad 1.1604741 .312061$

C $-2.6408930 .046625-0.334223$

C $-3.7562921 .099551-0.241057$

O $-3.205344-1.212351-0.690592$

C $3.2297950 .986077-0.483901$

C $-4.5030981 .268688-1.558147$

H 3.6562062 .1724831 .346972

H 2.6471534 .2417110 .641272

H $2.1640583 .488966-0.988537$

H $2.716579-0.2383571 .224148$

H $-0.130790-1.060376-1.051298$

H $1.040259-3.084341-0.587796$

H $1.501176-2.5603861 .033351$

H $3.501773-2.038739-0.116017$

H -1.324882 -2.178384 0.836976

H $2.640066-2.536744-2.412942$

H $1.835996-0.960380-2.461702$

H $3.600699-1.057462-2.362391$

H $-2.572783-0.2966821 .800488$

H $0.218062-0.4405182 .458601$

H $-0.629188 \quad 1.380680 \quad 0.598118$

H -1.958107 $0.346606-1.137224$

H -3.3390262 .0690520 .053451$

H -4.4815700 .8049330 .527007$

H -3.821672 -1.472070 0.015550

H $4.2786660 .693790-0.609886$

H $2.8231911 .209729-1.477694$

H $-3.8194751 .564165-2.360333$

H $-5.0038030 .342039-1.855346$

H -5.267375 $2.046297-1.460304$

SCF Energy (B3LYP/6-31G**//MMFF) $=-810.722419026$

F2-21_c04933

MMFF Geometry

C -4.650887 -0.919762 -0.073474

C $-4.955032-1.645800-1.155174$ 
C -2.5936640 .3384690 .609702$

O $-1.664620-0.468562-0.139812$

C -0.3352850 .0806860 .011780$

C $-0.460617 \quad 1.2094371 .026841$

C -1.8922031 .6800250 .838276$

C $0.618312-1.0473420 .416757$

C $-2.0042142 .670657-0.321537$

C $2.051501-0.6186540 .793742$

O $0.695219-1.983668-0.665309$

O $2.684916-1.7505291 .402950$

C $2.933917-0.126299-0.372485$

C 4.3674340 .1949210 .084829

O $2.3662281 .037347-0.963861$

C $-3.9358580 .402028-0.119488$

C $5.2533950 .658374-1.065063$

H $-4.942912-1.2947940 .904095$

H -5.477081 -2.591006 -1.053155

H $-4.689927-1.315650-2.153386$

H -2.722109 -0.1584681 .581369$

H $-0.0715160 .468996-0.977607$

H $\quad 0.287464 \quad 1.9945020 .883849$

H -0.3444840 .8215002 .046902$

H $-2.2717212 .168974 \quad 1.742529$

H $0.182608-1.6053971 .255615$

H -1.708340 2.226828 -1.277374

H $-3.0316753 .034657-0.423590$

H -1.365169 $3.542246-0.143674$

H 2.0001370 .1578641 .564782

H $-0.214416-2.252383-0.881404$

H $2.717291-2.4602310 .738409$

H $2.990230-0.899746-1.147252$

H $4.827138-0.6864990 .545384$

H 4.3456460 .9889800 .841112

H $2.3647001 .741579-0.293327$

H $-4.581416 \quad 1.1509230 .353635$

H $-3.7851990 .701618-1.163662$

H $\quad 6.2706690 .842862-0.705221$

H $5.304161-0.102104-1.850573$

H $4.8811781 .587605-1.507438$

SCF Energy (B3LYP/6-31G**//MMFF) $=-810.715946145$

F2-21_c04934

MMFF Geometry

C $4.280787 \quad 1.699798 \quad 0.524847$

C $4.5449772 .625011-0.404611$

C $2.614056-0.1684720 .641549$

O $1.640066 \quad 0.442839-0.226521$

C $0.541852-0.475808-0.425953$

C $0.807283-1.6508730 .505218$

C $2.322983-1.6706610 .604850$

C $-0.7807540 .275907-0.205450$

C $2.950583-2.419651-0.571669$

C $-2.032951-0.600189-0.392893$

O $-0.8310941 .346263-1.153602$

O $-2.107207-1.5534010 .666582$

C $-3.355627 \quad 0.195417-0.447830$

C -3.710896 0.8959490 .870283

O $-4.413131-0.714612-0.766996$

$\begin{array}{llll}\text { C } 4.023180 & 0.250058 & 0.221357\end{array}$

C $-4.990800 \quad 1.716203 \quad 0.758389$

H 4.2578252 .0023541 .568743

H $4.7265723 .655121-0.118191$

H $4.5832652 .375543-1.459129$

H $2.402832 \quad 0.203247 \quad 1.653977$

H $0.607356-0.795310-1.473349$

H $\quad 0.375157-2.587157 \quad 0.139574$

H $\quad 0.392078-1.4523071 .501332$

H $2.653322-2.1538001 .531376$

H $-0.771017 \quad 0.7247940 .794909$

H $2.723693-1.948228-1.533257$

H $4.039540-2.463471-0.467563$

H $2.584153-3.451185-0.608381$

H -1.935479 -1.167544 -1.327299

H -0.033176 $1.887016-1.022995$

H -2.905921 -2.0888960 .520802$

H $-3.3187410 .933306-1.257112$

H -2.898927 1.5627651 .177935

H -3.8543490 .1555031 .666055$
H $-4.211115-1.105634-1.634090$

H $4.766135-0.3451360 .764879$

H $4.1678500 .068149-0.850431$

H $\quad-5.8524791 .0831980 .524884$

H $-5.1955302 .225091 \quad 1.705747$

H $-4.9001582 .477741-0.022632$

SCF Energy $\left(B 3 L Y P / 6-31 G^{* *} / / M M F F\right)=-810.717783760$

F2-21 c04935

MMFF Geometry

C $3.1598802 .392710-0.312734$

C 3.9890653 .2784950 .250540

C 2.4980540 .0551140 .307093

O $1.1897310 .111853-0.293960$

C $0.548309-1.168205-0.136537$

C $1.490679-2.0221320 .707825$

C $2.854076-1.4321900 .389884$

C $-0.858408-0.9926230 .458890$

C $3.431795-2.025215-0.896170$

C $-1.773593-0.093000-0.403397$

O $-0.751004-0.4504611 .773750$

O $-1.786781-0.589638-1.740801$

C $-3.224337-0.0069110 .122141$

C $-4.1250870 .815566-0.813198$

O -3.2534720 .6019701 .409846$

C $3.4755950 .931867-0.476363$

C $-5.5679670 .875752-0.326008$

H $2.2014442 .742761-0.687768$

H 3.7039984 .3215220 .334040

H 4.9585022 .9832180 .636450

H $2.3884750 .446313 \quad 1.328247$

H $\quad 0.470871-1.605360-1.139030$

H $1.400192-3.0888280 .480486$

H $1.289506-1.8986711 .778517$

H $3.566162-1.6180131 .201783$

H $-1.313253-1.9862890 .551064$

H $2.805971-1.813085-1.768931$

H $4.431260-1.624602-1.094074$

H $3.527495-3.112699-0.807273$

H $-1.3558870 .921285-0.422787$

H -0.3140310 .4153591 .699214$

H -2.168397 -1.484018 -1.720706

H -3.646108 -1.015870 0.210110

H $-4.1179640 .390457-1.822936$

H $-3.7449421 .842003-0.883112$

H $-2.763606 \quad 0.0253302 .020012$

H $4.5006980 .728006-0.144494$

H $3.4187860 .704755-1.547478$

H $-6.1814541 .439643-1.035804$

H $-5.992050-0.129356-0.235017$

H -5.6418401 .3709770 .647022$

SCF Energy $\left(B 3 L Y P / 6-31 G^{* *} / / M M F F\right)=-810.720610697$

F2-21_c04936

MMFF Geometry

C $4.482237-1.0539440 .631013$

C $5.713981-1.3344750 .191069$

C $2.5732170 .233936-0.362501$

$\begin{array}{llll}\text { O } & 1.534429 & -0.494085 & 0.320945\end{array}$

C $0.2552870 .090666-0.009675$

C $0.5374251 .153816-1.063937$

C $1.9574641 .583946-0.740485$

C $-0.703496-1.017083-0.454182$

C 1.9815842 .6346100 .370773

C $-2.052450-0.515843-1.000840$

O $-0.908262-1.8871810 .661377$

O $-2.819635-1.663477-1.381236$

C $-2.8908190 .384495-0.068847$

C $-3.502617-0.3195331 .148896$

O $-3.969630 \quad 0.922759-0.844875$

C $3.8314570 .295473 \quad 0.504241$

C -4.3421280 .6253062 .002735$

H $3.913305-1.8399601 .120977$

H $\quad 6.129275-2.3283770 .317745$

H $6.325735-0.585950-0.300027$

H $2.787427-0.323642-1.285009$

H -0.1066310 .5504740 .917635$

H $\quad-0.1905301 .970434-1.047468$ 
H $0.5189440 .711545-2.068318$

H $2.4546072 .006333-1.620949$

H $-0.214747-1.636991-1.216641$

H 1.5655412 .2559421 .309745

H 3.0055172 .9684240 .567538

H 1.4013053 .5159800 .077267

H $-1.8624450 .031054-1.932500$

H - $-1.518102-2.5879840 .373079$

H $-3.662767-1.341046-1.743607$

H $-2.293262 \quad 1.238227 \quad 0.267771$

H $-2.717664-0.7366751 .785236$

H $-4.148033-1.1449360 .826712$

H -3.579936 $1.452910-1.561000$

H 4.5387451 .0158190 .076272

H $3.5813980 .632534 \quad 1.517243$

H -3.7448281 .4750002 .348281$

H $-5.202590 \quad 1.0105751 .447159$

H -4.7223280 .0979622 .883612$

SCF Energy (B3LYP/6-31G*//MMFF) $=-810.712396844$

F2-21 c04937

MMFF Geometry

C $-3.7422311 .979327-0.418085$

C -3.5120413 .0650740 .328646$

C $-2.530591-0.206791-0.671986$

O $-1.2490640 .386592-0.384511$

C $-0.610320-0.346267 \quad 0.679163$

C $-1.473254-1.5765570 .953002$

C $-2.360797-1.671650-0.272472$

C $0.841542-0.6747770 .280590$

C $-3.633908-2.467440-0.044984$

C $1.6746620 .579238-0.045275$

O $0.848602-1.552542-0.842968$

$\begin{array}{lllll}\text { O } & 1.697699 & 1.425703 & 1.104572\end{array}$

C $3.1181110 .272975-0.497076$

C $4.004883-0.310368 \quad 0.612477$

O $3.6826991 .507437-0.950277$

C $-3.6185010 .568492 \quad 0.087829$

C $5.397402-0.6835530 .117696$

H -4.046052 2.113096 -1.453154

H -3.622881 $4.057024-0.095490$

H -3.2092862 .9860751 .366924$

H -2.705752 $-0.128140-1.751543$

H $-0.6043150 .296847 \quad 1.566993$

H -2.076095 -1.401644 1.852911

H $-0.892552-2.4867541 .133212$

H - $1.787720-2.171146-1.065295$

H $1.295391-1.2131111 .119970$

H -4.209985 -2.0880100 .804908$

H $-4.272587-2.438397-0.933586$

H $-3.394755-3.515548 \quad 0.164674$

H $1.1858221 .145303-0.848327$

H $0.422520-1.085430-1.582161$

H $2.2262352 .209553 \quad 0.876141$

H $3.094390-0.404281-1.358584$

H $3.542481-1.2090121 .033181$

H 4.1205660 .4161001 .425334

H $4.4960681 .299240-1.439643$

H $-4.5927490 .086073-0.048006$

H -3.4030210 .5769791 .163017$

H $5.340930-1.386291-0.719502$

H $5.9582590 .198205-0.206336$

H $5.965857-1.1604720 .922677$

SCF Energy (B3LYP/6-31G**//MMFF) $=-810.718001127$

F2-21_c04938

MMFF Geometry

C $-3.285188 \quad 2.374627-0.319218$

C $-3.107960 \quad 3.2781840 .651151$

C $-2.4563790 .030697-0.632429$

$\begin{array}{lllll}\text { O } & -1.259971 & 0.186943 & 0.155218\end{array}$

C $-0.563887-1.073377 \quad 0.201058$

C $-1.333508-2.024620-0.711977$

C $-2.747964-1.472573-0.655985$

C $0.915820-0.882481-0.176901$

C $-3.503722-1.9988840 .565161$

C $1.647500 \quad 0.1214350 .740014$

O $0.996799-0.442454-1.530367$
O $1.440310-0.2854702 .097158$

C 3.1718240 .2377250 .519330

C $3.5649590 .920736-0.793613$

O $3.769693-1.058703 \quad 0.555354$

C $-3.5681590 .918534-0.073183$

C $5.052391 \quad 1.254348-0.836934$

H $-3.2329042 .704235-1.353615$

H -2.911328 4.3161980 .405752

H $-3.153523 \quad 3.002427 \quad 1.698809$

H $-2.2013730 .352734-1.651774$

H $-0.635931-1.433998 \quad 1.233838$

H $-1.246935-3.067707-0.392310$

H $-0.969673-1.968674-1.744628$

H $-3.316162-1.745371-1.552372$

H $1.402292-1.862865-0.118507$

H $-4.533685-1.6278230 .573920$

H $-3.550978-3.0930630 .544138$

H -3.030939-1.701008 1.506381

H $1.202740 \quad 1.117746 \quad 0.626169$

H $0.5272930 .407187-1.592168$

H 1.8133250 .4049912 .671341

H 3.6040790 .8020201 .355270

H $2.9922991 .845780-0.923665$

H $3.3560200 .266103-1.646025$

H $3.510879-1.4740501 .395977$

H $-4.5203160 .673204-0.557813$

H $-3.6860240 .744347 \quad 1.003161$

H $5.6652810 .350932-0.758602$

H $5.2999781 .749144-1.781522$

H $5.326577 \quad 1.928872-0.019495$

SCF Energy (B3LYP/6-31G**//MMFF) $=-810.716983780$

F2-21_c04939

MMFF Geometry

C $4.512804-0.498909-1.023080$

C $5.161349-1.503562-0.423494$

C $2.3238920 .630742-0.560380$

O $1.700228-0.499675 \quad 0.078106$

C $0.383651-0.1167180 .541719$

C $0.155648 \quad 1.293740 \quad 0.020414$

C $1.5625621 .861362-0.063106$

C $-0.622377-1.180580 \quad 0.081592$

C 2.0271212 .4034521 .289555

C $-2.094500-0.9685690 .490884$

O $-0.219814-2.4224540 .689285$

O $-2.164968-0.5742341 .861443$

C $-2.879715 \quad 0.044631-0.359002$

C $-4.3757720 .048893-0.001003$

O $-2.728308-0.261699-1.742015$

C $3.828315 \quad 0.619928-0.288104$

C $-5.1592751 .081140-0.802218$

H $4.480687-0.474652-2.109335$

H $5.639018-2.278425-1.013037$

H $5.226258-1.5728890 .656638$

H $2.1408470 .518124-1.638060$

H $0.449184-0.1153191 .636559$

H -0.5150641 .8767360 .658113$

H $-0.271414 \quad 1.264689-0.989518$

H $1.6187552 .675712-0.794169$

H $-0.540792-1.343221-0.998894$

H 3.0250442 .8465311 .208089

H $1.349015 \quad 3.188557 \quad 1.640929$

H 2.0668031 .6249292 .057980

H $-2.603473-1.9392120 .418216$

H $0.702927-2.5855310 .427952$

H $-1.701131-1.2520892 .382326$

H $-2.4996981 .056324-0.194235$

H $-4.509516 \quad 0.256744 \quad 1.066559$

H $-4.808759-0.938526-0.202241$

H -3.084991 -1.154419-1.889590

H $4.2740821 .564410-0.621121$

H 4.0171900 .5218770 .787853

H $-5.1181710 .869003-1.875074$

H $-6.2111251 .073629-0.499031$

H $-4.7649972 .088227-0.634138$

SCF Energy (B3LYP/6-31G**//MMFF) $=-810.716286837$

F2-21_c04940 
MMFF Geometry

C $4.644961-1.186065-0.689948$

C $5.827727-1.015915-0.088877$

C $2.316660-0.250300-0.447458$

O $1.045940-0.504233 \quad 0.183292$

C $0.344183 \quad 0.746185 \quad 0.326377$

C $1.2179021 .810045-0.336186$

C $2.6170931 .230530-0.199610$

C $-1.075903 \quad 0.637289-0.253294$

C $3.208258 \quad 1.5467391 .175351$

C $-1.916389-0.4846280 .388363$

O $-1.0008140 .413067-1.660770$

O $-2.060747-0.2445651 .788312$

C $-3.321675-0.637364-0.231256$

$\begin{array}{llll}\text { C } & -4.259569 & 0.550267 & 0.027054\end{array}$

O $-3.915907-1.8224710 .309010$

C $3.337044-1.2795670 .037452$

C $-5.6174310 .365277-0.640161$

H $4.621536-1.279886-1.772638$

H $6.739236-0.963993-0.674380$

H $5.908853-0.9261330 .988750$

H $2.150674-0.400389-1.523842$

H 0.2862670 .9523891 .401347

H 1.0937292 .7947410 .125446

H $0.9784831 .917419-1.400827$

H $3.2887881 .632513-0.966144$

H - $-1.5665311 .604660-0.099682$

H 2.6313391 .0964851 .989478

H 4.2365291 .1826911 .253381

H 3.2347782 .6292181 .340996

H - $1.405205-1.4494510 .276415$

H $-0.526151-0.424557-1.799423$

H $-1.203051-0.4228762 .208977$

H -3.231020 -0.804526 -1.310126

H -3.813445 $1.476563-0.348577$

H $-4.4277880 .674548 \quad 1.103219$

H -3.980163 -1.704776 1.272423

H $3.469765-1.2044801 .123499$

H $2.925269-2.284063-0.126297$

H -6.246446 $1.243515-0.462946$

H $-5.5078670 .239813-1.722013$

H -6.142528 -0.508765 - 0.242627

SCF Energy (B3LYP/6-31G**//MMFF) $=-810.720508225$

F2-21_c04941

MMFF Geometry

C $3.2551742 .230176-0.545523$

C 3.9009103 .2262230 .071208

$\begin{array}{llll}C & 2.283897 & 0.069617 & 0.274323\end{array}$

O $1.260760-0.023712-0.736816$

C $0.526197-1.240448-0.535961$

C $1.077421-1.8903290 .730173$

C $2.501056-1.3596140 .781273$

C $-0.994104-1.003742-0.565407$

C $3.442745-2.206414-0.076709$

C -1.5276540 .0157610 .461692$

O $-1.656384-2.251892-0.376836$

O -0.9625051 .2981950 .191750$

C -3.068352 0.1319190 .481702

C $-3.665490 \quad 0.727946-0.799923$

O -3.4386430 .9763491 .576698$

C $3.5188350 .772099-0.289970$

C $-5.188520 \quad 0.780330-0.754734$

H $2.4988232 .479733-1.285269$

H $3.6672034 .259342-0.161574$

H 4.6668893 .0324540 .813796

H 1.8602990 .6690691 .092127

H $0.775570-1.893946-1.382599$

H $1.009092-2.9823720 .703638$

H $\quad 0.540362-1.5512541 .621903$

H $2.885807-1.3548961 .807296$

H -1.238992 -0.634289-1.568764

H $4.472126-1.8416760 .000293$

H $3.438786-3.2475150 .263546$

H $3.161446-2.197459-1.134509$

H $-1.204145-0.2689531 .468758$

H -1.350438 -2.855854 -1.074826

H -1.3197701 .9183090 .850364$
H -3.516555 -0.8498030 .672886$

H -3.367071 $0.136272-1.671139$

H $-3.298193 \quad 1.750160-0.949137$

H -3.1176220 .5557252 .392530$

H 4.3583260 .6571070 .405978

H $3.8116210 .321637-1.245832$

H $-5.542924 \quad 1.4255080 .055114$

H $-5.5791431 .179392-1.696292$

H $-5.610019-0.219369-0.609512$

SCF Energy (B3LYP/6-31G**//MMFF) $=-810.706841465$

F2-21_c04942

MMFF Geometry

C $3.4810911 .788786-0.073028$

C $3.1246942 .923097-0.686239$

C $2.310483-0.405156 \quad 0.230821$

$\begin{array}{lllll}\text { O } & 0.975762 & 0.129080 & 0.142142\end{array}$

C $0.030813-0.951494 \quad 0.218543$

C $0.844813-2.212108 \quad 0.505645$

C $2.191359-1.891042-0.120701$

C $-1.070916-0.6602421 .247532$

C $2.197595-2.199698-1.618618$

C $-1.8735740 .646212 \quad 1.065424$

O $-0.489972-0.6179482 .558231$

O $\begin{array}{llll}0.988690 & 1.766059 & 1.168790\end{array}$

$\begin{array}{lll}\text { C }-2.694550 & 0.786562 & -0.230270\end{array}$

C $-3.700026-0.351578-0.426577$

O $-1.8394940 .851362-1.369011$

C $3.2571840 .416209-0.644351$

C $-4.632705-0.096821-1.604490$

H 3.9712961 .8519100 .895106

H $3.3184353 .883258-0.220968$

H $2.6352782 .915595-1.653886$

H $2.609639-0.3186021 .284773$

H $-0.417871-1.051260-0.774985$

H $0.369159-3.1136090 .106923$

H $\quad 0.981472-2.3635801 .582898$

H $2.995451-2.4681520 .349573$

H $-1.765920-1.5069861 .258227$

H $1.968102-3.256406-1.792927$

H $1.464825-1.601484-2.169378$

H $3.184313-2.004232-2.051036$

H $-2.5589770 .730612 \quad 1.918937$

H $\quad 0.1446660 .1193872 .568503$

H $-1.5294862 .573423 \quad 1.133226$

H -3.232176 $1.742480-0.198218$

H $-4.301362-0.479890 \quad 0.480577$

H $-3.173649-1.293813-0.617769$

H $-1.1815451 .547380-1.199858$

H $4.230229-0.083869-0.713333$

H $2.8533010 .503352-1.660243$

H $-5.348942-0.918766-1.702264$

H $-5.1979370 .829944-1.463111$

H $-4.076713-0.021451-2.544120$

SCF Energy (B3LYP/6-31G**//MMFF) $=-810.713350577$

F2-21_c04943

MMFF Geometry

C $3.2602122 .347678-0.545062$

C $2.544066 \quad 3.021256 \quad 0.362797$

$\begin{array}{llll}\text { C } 2.461453 & -0.014217 & 0.162654\end{array}$

O $1.0642450 .181817-0.121385$

C $0.358792-1.0478470 .121450$

C $1.393244-2.0392050 .653258$

C $2.692759-1.5249570 .057370$

C $-0.840176-0.8292161 .056973$

C $2.896808-2.046258-1.365892$

C -1.8952110 .1873450 .560894$

$\begin{array}{llll}\text { O } & -0.354468 & -0.374882 & 2.325496\end{array}$

O $-1.305742 \quad 1.4736610 .381319$

C $-2.588949-0.226903-0.747170$

C $-3.6494590 .773180-1.238931$

O $-3.185021-1.509904-0.574537$

C $3.3134960 .857105-0.763940$

C $-4.8182860 .972239-0.282711$

H $3.8862312 .932569-1.216667$

H $2.6030604 .103767 \quad 0.406553$

H 1.8907932 .5302211 .073588 
H $2.621231 \quad 0.284574 \quad 1.207487$

H $\quad 0.001739-1.401926-0.851824$

H $\quad 1.154737-3.072510 \quad 0.382470$

H $1.460726-1.9969941 .746965$

H $3.555010-1.8307800 .660688$

H -1.322744 -1.797539 1.234658

H $3.853423-1.702489-1.772190$

H $2.913262-3.141505-1.373829$

H $2.104642-1.717381-2.046098$

H $-2.643960 \quad 0.290714 \quad 1.355251$

H -1.114592 -0.3230372 .929559$

H -0.8220051 .6886951 .197110$

H -1.840477 $-0.311632-1.543577$

H -4.059492 $0.405590-2.188045$

H $-3.1845681 .743151-1.448761$

H $-3.586989-1.763896-1.422754$

H $4.3633670 .553933-0.669984$

H $3.0112210 .686785-1.804733$

H $-5.3057900 .022469-0.043271$

H $-4.496506 \quad 1.4432300 .650576$

H $-5.5669231 .627256-0.740445$

SCF Energy (B3LYP/6-31G**//MMFF) $=-810.707361192$

\section{F2-21 c04944}

MMFF Geometry

C 2.4812272 .7321880 .446632

C $1.6779593 .659964-0.086003$

C 2.3572270 .2249230 .532549

$\begin{array}{lllll}\text { O } & 0.916588 & 0.226220 & 0.519346\end{array}$

C $0.444716-0.622252-0.542799$

C $1.669453-1.331957-1.120352$

C $2.732279-1.132810-0.057666$

C $-0.642411-1.581226-0.024549$

C $4.151455-1.281061-0.576506$

C $-1.868511-0.9046560 .630231$

O $-0.060356-2.4717990 .932454$

O $-1.473643-0.172626 \quad 1.789897$

C $-2.6400820 .036717-0.310931$

C $-3.922571 \quad 0.5684860 .349870$

O $-2.972985-0.675957-1.498684$

C $2.8612271 .451336-0.244044$

C $-4.6692951 .567205-0.525353$

H 2.8967022 .9075051 .435569

H 1.4446894 .5654420 .463392

H $1.2395133 .533958-1.069667$

H 2.6866860 .2867641 .576553

H $0.0131560 .024338-1.314961$

H $1.963514-0.840803-2.056495$

H $1.493550-2.385889-1.358373$

H $2.575803-1.8916260 .721202$

H $-0.978689-2.203240-0.862861$

H $4.329415-2.309324-0.908873$

H $4.348516-0.623822-1.429356$

H $4.877221-1.0506240 .209961$

H -2.543681 -1.6983290 .975766$

H $-0.743117-3.115269 \quad 1.188079$

H $-0.941963-0.7695892 .343534$

H $-2.0060350 .889545-0.581081$

H -3.6823631 .0556511 .301463$

H $-4.599355-0.2666240 .567870$

H $-3.267127-0.028082-2.160747$

H $3.9541541 .430819-0.317723$

H $2.4630901 .448647-1.265813$

H -5.039078 $1.099171-1.442555$

H $-5.5338701 .966406 \quad 0.014772$

H $-4.0236192 .407126-0.800247$

SCF Energy (B3LYP/6-31G**//MMFF) $=-810.708396920$

\section{F2-21 c04945}

MMFF Geometry

C $4.939726 \quad 0.261501 \quad 0.508093$

C $5.870550-0.5990000 .081151$

C $2.4319190 .472290 \quad 0.356706$

O $1.3840231 .081840-0.422481$

C $0.1749930 .302225-0.265809$

C $0.502846-0.7832990 .752986$

C $1.999539-0.9797570 .574055$

C $-\begin{array}{llll}0.967317 & 1.239821 & 0.136864\end{array}$
C $2.291037-1.922277-0.594860$

C -2.2978470 .5558680 .516719$

O $-1.2139502 .141677-0.949231$

O -3.1455621 .5602881 .085429$

C $-3.047644-0.130930-0.642671$

C $-4.456003-0.627169-0.269300$

O $-2.282349-1.231581-1.123542$

C $3.7788070 .724816-0.320223$

C $-4.477456-1.6627540 .847773$

H $5.0306470 .668906 \quad 1.511748$

H $6.690685-0.8876410 .729216$

H $5.832741-1.027793-0.914044$

H $2.422657 \quad 0.991440 \quad 1.325653$

H $-0.016766-0.130631-1.253617$

H $-0.086237-1.6935290 .607042$

H $\quad 0.309893-0.4232301 .771733$

H $2.455177-1.3978321 .478459$

H $-0.641886 \quad 1.8718550 .973270$

H $1.802968-2.890176-0.437187$

H $1.939434-1.520871-1.550693$

H $3.363993-2.112741-0.686791$

H -2.097719 -0.1705721 .310961$

H $-0.3689412 .571970-1.165919$

H -3.305909 2.2304020 .398716

H -3.164379 $0.578485-1.470703$

H $-4.910229-1.087720-1.155663$

H -5.0938980 .2192240 .009459$

H -2.757689-1.613227 -1.881143

H $3.7922000 .280867-1.323069$

H $3.8882261 .803976-0.489354$

H $-3.851077-2.5263020 .604447$

H -4.130375 -1.237839 1.794134

H -5.499725 -2.022836 1.003057

SCF Energy $\left(B 3 L Y P / 6-31 G^{* *} / / M M F F\right)=-810.717594909$

F2-21 c04946

MMFF Geometry

C $-4.671149-0.910214-0.060393$

C $-4.986609-1.626400-1.145415$

C $-2.6035510 .332300 \quad 0.620957$

O $-1.682555-0.476213-0.137036$

C -0.3506290 .0678280 .006556$

C $-0.463393 \quad 1.1903271 .030140$

C -1.8938371 .6690240 .853711$

C $0.607728-1.0638790 .394198$

C $-2.0087982 .668188-0.298602$

C $2.039739-0.6412740 .788280$

O $0.670935-2.001986-0.687081$

O $2.669099-1.7830641 .383738$

C $2.938489-0.124426-0.354239$

C 4.3411850 .2475930 .158173

O $2.3373151 .021408-0.944895$

C $-3.9488270 .407764-0.101593$

C $5.2799080 .698418-0.954267$

H $-4.958645-1.2907940 .916374$

H $-5.513216-2.569357-1.047145$

H $-4.726181-1.290290-2.142977$

H -2.730280 -0.169322 1.590439

H $-0.0968320 .466835-0.981181$

H $\quad 0.288903 \quad 1.971675 \quad 0.886608$

H $-0.343270 \quad 0.7948712 .046954$

H -2.265150 $2.153717 \quad 1.763644$

H $\quad 0.168071-1.626566 \quad 1.227488$

H -1.363596 $3.535029-0.119765$

H -1.722432 2.229004-1.259581

H $-3.0350373 .038252-0.390655$

H $1.9790260 .120744 \quad 1.572986$

H $0.893221-1.513553-1.497637$

H $2.701038-2.4839700 .710050$

H $3.041834-0.899500-1.122935$

H $4.797966-0.6091110 .666078$

H 4.2629551 .0616250 .889065

H $2.7914741 .196263-1.786062$

H $-4.588014 \quad 1.1574500 .379099$

H $-3.8015170 .713108-1.144682$

H $6.2808370 .880115-0.549651$

H $5.362548-0.067171-1.732067$

H $4.936398 \quad 1.628290-1.417294$ 


\section{F2-21_c04947}

MMFF Geometry

C $4.271243 \quad 1.712295 \quad 0.533886$

C $4.5265662 .650602-0.384863$

C $2.618464-0.1693090 .633536$

$\begin{array}{lllll}\text { O } & 1.636704 & 0.445054 & -0.223558\end{array}$

C $0.545746-0.480029-0.432130$

C $0.822107-1.6633160 .485182$

C $2.338215-1.6730890 .579982$

C $-0.782526 \quad 0.258600-0.202351$

C $2.967649-2.404073-0.606966$

C $-2.028740-0.627213-0.397799$

O $-0.843346 \quad 1.338458-1.139044$

$\begin{array}{llll}0 & -2.104683 & -1.612927 & 0.631799\end{array}$

C $-3.361456 \quad 0.152314-0.457297$

C -3.7438230 .8400600 .861247$

O $-4.382517-0.784642-0.814500$

$\begin{array}{llll}\text { C } 4.022944 & 0.264558 & 0.213080\end{array}$

C -4.9854291 .7143820 .727934$

H 4.2491922 .0016541 .581433

H $4.7019203 .678447-0.086296$

H $4.5636802 .414301-1.442553$

H $2.4083090 .189108 \quad 1.650862$

H $0.612640-0.787265-1.483206$

H $0.395831-2.5986650 .110687$

H $0.408762-1.4785511 .484609$

H $2.674791-2.1644721 .499904$

H -0.7750490 .6986830 .801714$

H $2.734441-1.923612-1.562434$

H $4.057204-2.441219-0.506581$

H $2.608525-3.437854-0.654158$

H $-1.929368-1.176819-1.342439$

H -0.047542 $1.882010-1.007166$

H $-2.059613-1.1580151 .489478$

H $-3.3127080 .891274-1.265244$

H $-2.9199261 .470053 \quad 1.211805$

H -3.9449890 .0904201 .635344$

H -5.174364 - $0.279858-1.064633$

H $4.772278-0.3320680 .746379$

H $4.1650380 .096956-0.861485$

H $-5.871776 \begin{array}{lll}1.119721 & 0.487925\end{array}$

H $-5.1806102 .234528 \quad 1.671300$

H -4.851698 $2.469015-0.053460$

SCF Energy (B3LYP/6-31G**//MMFF) $=-810.710970112$

F2-21_c04948

MMFF Geometry

C $3.1708252 .402938-0.256988$

$\begin{array}{llll}C & 4.020804 & 3.268693 & 0.306672\end{array}$

C $2.511462 \quad 0.051582 \quad 0.312157$

$\begin{array}{lllll}\text { O } & 1.188437 & 0.132328 & -0.253145\end{array}$

C $0.549389-1.153005-0.131214$

C $1.502256-2.0321730 .673825$

C $2.862126-1.4392760 .344105$

C $-0.862976-0.9978740 .459082$

C $3.411897-1.997667-0.969526$

C $-1.765740-0.075305-0.392888$

O $-0.776459-0.4611011 .777255$

O $-1.746210-0.515923-1.749703$

C $-3.230311-0.0264500 .098876$

C $-4.1135000 .853670-0.800719$

O

C $3.4735780 .945766-0.470305$

C $-5.5664940 .878189-0.342603$

H $2.2040182 .767858-0.594161$

H 3.7439994 .3102830 .427542

H 4.9992212 .9581630 .655927

H 2.4294770 .4140151 .346430

H $0.473051-1.559285-1.147019$

H $\quad 1.403063-3.0924370 .420979$

H $1.329776-1.9355951 .751241$

H $3.589739-1.6513791 .135540$

H $-1.324862-1.9911740 .522992$

H $2.771764-1.756417-1.824030$

H $4.410043-1.597122-1.174114$

H $3.502544-3.087807-0.914218$
H -1.358182 $0.943032-0.361986$

H $-0.532193-1.1843812 .378260$

H $-2.112256-1.416584-1.773728$

H $-3.643207-1.0424530 .101495$

H $-4.0802900 .495449-1.835569$

H $-3.7361001 .883482-0.795619$

H -2.9004881 .3556181 .441262$

H $4.5055500 .727357-0.170900$

H $3.3879940 .749735-1.545739$

H $-5.662901 \quad 1.3082970 .658998$

H $-6.166517 \quad 1.486156-1.027072$

H $-5.988727-0.131686-0.327080$

SCF Energy (B3LYP/6-31G**//MMFF) $=-810.705241572$

F2-21_c04949

MMFF Geometry

C $-3.227628 \quad 2.294998 \quad 0.029404$

C $-4.210077 \quad 3.024081-0.511836$

C $-2.297987 \quad 0.028826-0.540205$

O $-0.9696210 .385015-0.109022$

C $-0.500320-0.586528 \quad 0.845994$

C $-1.530801-1.713493 \quad 0.877758$

C $-2.343788-1.490189-0.382300$

C $0.920061-1.0422060 .460069$

C $-3.719515-2.131848-0.343655$

$\begin{array}{llll}\text { C } & 1.928935 & 0.125847 & 0.362661\end{array}$

O $0.876894-1.747598-0.778147$

$\begin{array}{lllll}\text { O } & 1.890989 & 0.877378 & 1.573839\end{array}$

C $3.376827-0.342202 \quad 0.098049$

C $4.427276 \quad 0.775410 \quad 0.203389$

O $3.490596-0.931033-1.195048$

C $-3.323006 \quad 0.816693 \quad 0.291585$

C $4.2149981 .926961-0.771405$

H -2.3045562 .7949670 .311427$

H $-4.0802974 .089280-0.669521$

H $-5.1529832 .577033-0.806427$

H $-2.3950290 .300988-1.598053$

H $-0.469943-0.0983101 .827001$

H -2.161903 -1.600412 1.768258

H - $1.085593-2.7120920 .930080$

H $-1.790520-1.930808-1.222676$

H $1.264018-1.7496491 .224247$

H -3.629081 -3.222776 -0.306509

H $-4.293477-1.8225420 .535701$

H - $-4.293646-1.871371-1.238581$

H $1.6189730 .783581-0.457950$

H $0.556932-1.128496-1.456878$

H 2.1715150 .2896352 .296207

H $3.649189-1.1082690 .834953$

H $5.4153530 .344936-0.003208$

H $4.459223 \quad 1.169303 \quad 1.225449$

H $2.904616-1.706163-1.219215$

H -4.3373580 .4700020 .065235$

H $-3.1534310 .660908 \quad 1.363531$

H $5.0389982 .643132-0.688156$

H $4.1812631 .573518-1.806505$

H $3.2870292 .465400-0.558436$

SCF Energy (B3LYP/6-31G**//MMFF) $=-810.719773235$

F2-21_c04950

MMFF Geometry

C $4.061773-1.543873-0.736477$

C $4.295474-2.6239780 .017313$

C $2.261076 \quad 0.208132-0.711465$

O $1.270790-0.708364-0.203943$

C $0.690275-0.1572360 .989588$

C $1.182771 \quad 1.2832751 .115833$

C $1.7651851 .579446-0.253352$

C $-0.839590-0.3428820 .983480$

C $2.7831352 .706221-0.253712$

C $-1.5912220 .396921-0.140918$

$\begin{array}{llll}0 & -1.356940 & 0.080706 & 2.243456\end{array}$

O $-1.149250-0.091286-1.406761$

C $-3.1284540 .269110-0.051944$

C $-3.651571-1.151616-0.301479$

O $-3.705927 \quad 1.132343-1.037107$

C $3.644065-0.207985-0.186351$

C $-5.166612-1.240650-0.156122$ 
H $4.186890-1.622484-1.813387$ H $4.598582-3.558706-0.441545$ H $4.187935-2.5973491 .095938$ H $2.247424 \quad 0.148814-1.806374$ H $\quad 1.092128-0.726879 \quad 1.837637$ H 1.9631551 .3322801 .885825 H 0.4058951 .9944691 .412011 H $\quad 0.938367 \quad 1.872525-0.914048$ H -1.032519 -1.417390 0.878780 H 3.5910752 .5308090 .463654 H $3.2259782 .827544-1.247461$ H 2.3033923 .6520170 .019745 H -1.345191 $1.464539-0.104631$ H $-0.922025-0.4485902 .933569$ H -1.640912 $0.391922-2.092574$ H $-3.479180 \quad 0.6227640 .924231$ H -3.196467 -1.8553250 .402669$ H $-3.391428-1.478425-1.315192$ H $-3.4269532 .040728-0.830309$ H $4.4008130 .522622-0.492756$ H $3.644330-0.2407040 .909761$ H -5.482532 -0.9276720 .844072$ H $-5.676922-0.611616-0.892064$ H $-5.499054-2.272375-0.309080$ SCF Energy (B3LYP/6-31G**//MMFF) $=-810.706601612$

F2-21 c04951

MMFF Geometry

C $5.015586-0.4051980 .009566$

C 5.9230210 .5557740 .215159

C $2.501548-0.558272-0.105025$

O $1.339598-0.8481750 .696598$

C $0.195449-0.1843870 .113196$

C $0.692197 \quad 0.457902-1.177432$

C $2.1626190 .712584-0.888120$

C $-0.936017-1.199804-0.068404$

C $2.3488742 .017998-0.112759$

C $-2.158209-0.670074-0.841412$

O $-1.324716-1.6515931 .231040$

O $-3.096749-1.746324-0.945719$

C $-2.8529540 .580589-0.262665$

C $-3.6530820 .358903 \quad 1.028173$

O $-3.737157 \quad 1.088480-1.270641$

C $3.741406-0.5400250 .788530$

C -4.3301241 .6359331 .514732$

H $5.212465-1.149902-0.757392$

H $6.8293750 .592102-0.379267$

H $5.780770 \quad 1.3176110 .973639$

H $2.590890-1.394484-0.813001$

H -0.1004310 .5821610 .839111$

H $0.128172 \quad 1.355930-1.446645$

H $0.610701-0.248594-2.013462$

H $2.7437080 .774089-1.814884$

H - $0.546501-2.090129-0.578409$

H 3.4097232 .2333560 .043445

H $1.9268932 .859425-0.672969$

H 1.8647051 .9917440 .868588

H -1.846387 $-0.448458-1.869547$

H -2.039184 -2.300391 1.109623

H -3.804952 -1.461807-1.547456

H $-2.1102751 .367681-0.098160$

H -2.999577 -0.000809 1.827313

H $-4.432125-0.395486 \quad 0.868725$

H -4.485476 $0.473346-1.351918$

H 3.6492240 .2391051 .554969

H $3.781671-1.4826741 .349936$

H -3.593866 2.4276261 .686529

H -5.0666341 .9995930 .791534$

H -4.8525811 .4483132 .458239$

SCF Energy (B3LYP/6-31G**//MMFF) $=-810.708856170$

F2-21_c04952

MMFF Geometry

C 2.1563552 .6070000 .504095

C $1.5790023 .526275-0.277595$

C 2.1301340 .1026070 .485483

O $0.845547-0.027195-0.151030$

C $0.546166-1.422054-0.330740$
C $1.695755-2.190030 \quad 0.320220$

C $2.857647-1.2169870 .218965$

C $-0.830543-1.7770540 .242615$

C $3.542477-1.307997-1.145396$

C $-2.023435-1.174239-0.528005$

O $-0.879405-1.4105661 .620264$

O $-3.232026-1.6564990 .075753$

C $-2.1255230 .359074-0.641597$

C -2.3280271 .1096580 .680815$

O $-3.2299410 .651939-1.509269$

C $2.8311811 .366464-0.011843$

C -2.4863222 .6124330 .476788$

H 2.1503782 .7606051 .580170

H 1.1106994 .4021230 .158226

H $1.5587993 .422278-1.356707$

H $1.936891 \quad 0.1851491 .564309$

H $0.547423-1.612794-1.410827$

H $1.876684-3.154874-0.163568$

H $1.486893-2.3896971 .378378$

H $3.608511-1.4123330 .992659$

H $-0.937294-2.8694150 .219901$

H 2.865079-1.055341-1.967518

H $4.400165-0.629434-1.195520$

H $3.917602-2.322342-1.318795$

H -2.015755 -1.581774 -1.547328

H $-1.764423-1.6408241 .951436$

H -3.207826 -2.628262 0.046647

H $-1.2418770 .751611-1.152522$

H -1.473929 0.9486531 .344557

H $-3.224440 \quad 0.742175 \quad 1.193702$

H $-4.0328600 .298377-1.088965$

H $3.870773 \quad 1.3773300 .335697$

H $2.8446221 .385447-1.108396$

H -2.570160 3.1164011 .444959

H $-1.6219163 .029276-0.049080$

$\mathrm{H}-3.3866402 .845328-0.099996$

SCF Energy $(B 3 L Y P / 6-31 G * * / / M M F F)=-810.714936087$

F2-21 c04953

MMFF Geometry

C $4.443823-1.439218-0.713603$

C $4.697354-2.5244590 .026452$

C $2.6970510 .352515-0.568606$

O $1.702475-0.4567840 .087601$

C $0.5458910 .359850 \quad 0.383884$

C $0.8141701 .710099-0.267328$

C $2.3303741 .803858-0.249907$

C $-0.716640-0.385334-0.081707$

C 2.8425872 .3165921 .096869

C -2.0377820 .3674550 .198701$

O $-0.740708-1.6415170 .601591$

O $-2.0517991 .592953-0.528140$

C $-3.307482-0.440180-0.163187$

C $-4.5883390 .398064-0.017342$

O $-3.435328-1.5790270 .684258$

C $4.096261-0.092488-0.142610$

C $-5.841112-0.381318-0.400247$

H $4.499452-1.522473-1.795881$

H $4.947455-3.466898-0.448310$

H $4.659287-2.4944621 .109669$

H $2.5675570 .188148-1.647298$

H $\quad 0.5242410 .4600371 .476085$

H $\quad 0.3165622 .536408 \quad 0.249058$

H $0.4737241 .707061-1.310370$

H $2.6950872 .478609-1.032654$

H $-0.632675-0.602923-1.153730$

H 2.5773611 .6499361 .923665

H 3.9324932 .4192161 .084160

H $2.4239183 .304978 \quad 1.314795$

H $-2.0775410 .607141 \quad 1.268765$

H $0.098595-2.093720 \quad 0.408648$

H -2.035139 $1.375405-1.475849$

H -3.238279 -0.790416-1.200470

H $-4.5344721 .293816-0.645712$

H $-4.695996 \quad 0.728882 \quad 1.022926$

H -2.667342 -2.1530300 .525173$

H $4.8411110 .627691-0.500654$

H $4.164884-0.1288910 .951436$ 
H -5.992598 -1.244088 0.255627

H $-6.7234740 .261058-0.316952$

H -5.778259 -0.739108-1.432809

SCF Energy (B3LYP/6-31G**//MMFF) $=-810.719340717$

\section{F2-21 c04954}

MMFF Geometry

C $-3.6078512 .077280-0.291417$

C -3.2845863 .1509160 .438136$

C $-2.502268-0.153801-0.624752$

O $-1.1825540 .388756-0.422867$

C $-0.505877-0.3682250 .599664$

C $-1.394815-1.5660290 .928769$

C $-2.362805-1.624761-0.236609$

C $0.904673-0.7493680 .111272$

C $-3.648069-2.3717240 .072719$

C $1.7610470 .471427-0.272080$

O $0.810649-1.629767-1.006425$

O 1.8737761 .3330640 .858309

C $3.1569540 .106568-0.820171$

C $4.060752-0.7458890 .082515$

O $3.8569851 .310374-1.146613$

C -3.5067840 .6611470 .204938$

C $4.417571-0.1290271 .427487$

H -3.975345 2.225725 -1.303476

H -3.3840384 .1477560 .022472$

H -2.9157363 .0570301 .453547$

H -2.745081 $-0.066217-1.690436$

H -0.4206880 .2734651 .484453$

H -1.930848 -1.3710741 .866057$

H $-0.838022-2.4978031 .070705$

H $-1.861461-2.143874-1.064653$

H $1.393150-1.3005360 .922949$

H $-4.341103-2.316231-0.772724$

H -3.436445 $-3.428630 \quad 0.266701$

H -4.153099 -1.9725560 .958144$

H $1.2457161 .047901-1.050796$

H $\quad 0.351980-1.151399-1.718417$

H 2.4342332 .0836690 .597088

H $3.030609-0.440427-1.762521$

H $5.003588-0.919272-0.452427$

H $3.609624-1.7306740 .246226$

H $3.3118661 .803759-1.782992$

H -4.5059720 .2175080 .133191$

H $-3.2211850 .658306 \quad 1.263690$

H $3.550607-0.0912612 .092673$

H $5.179928-0.7379501 .924967$

H $4.8192280 .882277 \quad 1.313511$

SCF Energy (B3LYP/6-31G**//MMFF) $=-810.715756503$

F2-21 c04955

MMFF Geometry

C $-3.726906 \quad 1.816552-0.824372$

C $-3.6169252 .984723-0.181937$

C $-2.398571-0.313893-0.732841$

O $-1.177756 \quad 0.375891-0.400311$

C $-0.613460-0.2012660 .793519$

C -1.436917 -1.446285 1.119124

C $-2.199428-1.717308-0.163057$

C $0.887226-0.4774920 .571941$

C $-3.449998-2.5555870 .034941$

C 1.6811650 .7835090 .164959

O $1.038063-1.485147-0.424018$

O $1.409319 \quad 1.826494 \quad 1.107625$

C 3.2149590 .6194880 .093375

C $3.690992-0.252667-1.073896$

O $3.675803 \quad 0.068184 \quad 1.327653$

C $-3.5869510 .474479-0.160092$

C $5.190790-0.126801-1.318390$

H -3.938755 $1.825621-1.890387$

H $-3.7324643 .919971-0.718689$

H -3.409068 3.0303170 .881379

H -2.475939 $-0.358096-1.825815$

H $-0.7322630 .529307 \quad 1.602100$

H -2.129502 -1.2167011 .938687$

H $-0.831735-2.2976661 .446315$

H -1.531520 -2.263258 -0.842998

H $1.289303-0.8810501 .508376$
H $-4.120702-2.1231100 .784266$

H -4.002557 -2.654506 -0.904879

H $-3.183498-3.5612920 .377312$

H $1.3326701 .141329-0.811171$

H $\quad 0.666125-1.137171-1.252703$

H $1.7449201 .533341 \quad 1.972363$

H 3.6582081 .6199550 .010413

H $3.1658630 .033010-1.992268$

H $3.480859-1.308700-0.874192$

H 4.6458250 .1230461 .334966

H $-4.521140-0.072386-0.328471$

H -3.4736930 .6061300 .922568$

H $5.481138-0.727612-2.186326$

H $5.4699550 .912535-1.518382$

H $5.768683-0.481100-0.459701$

SCF Energy $(B 3 L Y P / 6-31 G * * / / M M F F)=-810.715170651$

F2-21_c04956

MMFF Geometry

C $-3.3927282 .334939-0.050934$

C -3.6063562 .9839691 .099210$

C $-2.2874040 .153552-0.598058$

O $-1.399410 \quad 0.1493990 .538969$

C $-0.672443-1.0885770 .551957$

C $-1.008278-1.810430-0.748136$

C $-2.411419-1.307157-1.047345$

C $0.823077-0.8731790 .838596$

C $-3.469250-2.122711-0.301999$

C $1.603774-0.047595-0.212260$

O $1.440638-2.1502960 .986651$

O $0.922118 \quad 1.175294-0.474018$

C 3.0554490 .2711910 .219111

C $3.7752271 .166794-0.802364$

O $3.810874-0.9285780 .361332$

C $-3.5955580 .856541-0.234540$

C $5.1941211 .517440-0.370666$

H $-3.0605242 .903565-0.915731$

H -3.4448664 .0544161 .164052$

H -3.9413642 .4648051 .990247$

H $-1.7736810 .716107-1.389880$

H $-1.087519-1.6768061 .381258$

H $-0.932893-2.898212-0.656922$

H $-0.343438-1.499690-1.560954$

H -2.632224 -1.365406 -2.119291

H $0.901732-0.3457351 .798284$

H $-3.401675-3.180548-0.577856$

H -3.360193 -2.051252 0.784808

H $-4.476527-1.781235-0.561564$

H $1.653268-0.617771-1.147196$

H $\quad 0.983017-2.6184021 .705692$

H $\quad 0.851529 \quad 1.661136 \quad 0.365464$

H 3.0478990 .7878881 .186890

H $3.2190952 .098464-0.953455$

H $3.8294950 .652900-1.769803$

H $3.398732-1.4558361 .065983$

H $-4.3302330 .714186-1.035430$

H -4.0133990 .4262980 .683518$

H $5.6571282 .183351-1.105891$

H 5.1932742 .0288770 .597104

H $5.8203320 .623698-0.288217$

SCF Energy (B3LYP/6-31G**//MMFF $)=-810.710719115$

F2-21_c04957

MMFF Geometry

C 4.3490041 .3634990 .101848

C $4.3172362 .587927-0.436181$

C $2.595143-0.4018150 .460089$

O 1.5494900 .5845160 .602720

C $0.3405690 .118912-0.041224$

C $0.746568-1.111928-0.838574$

C $1.889461-1.672760-0.018408$

C $-0.704884-0.1157811 .061991$

C $2.735704-2.688236-0.765548$

C $-2.014341-0.7814440 .601928$

O $-0.9862931 .157191 \quad 1.655631$

O $-2.885655-0.858608 \quad 1.736350$

C $-2.753396-0.117185-0.578237$

C $-3.3760341 .250685-0.272980$ 
O $-3.811254-0.998144-0.977948$

C $3.6601810 .165118-0.491214$

C $-4.115687 \quad 1.831212-1.473998$

H 4.9120951 .2115681 .019016

H 4.8396113 .4106770 .039578

H $3.7727642 .791673-1.351465$

H $3.036241-0.5705141 .449898$

H $0.0050340 .923153-0.705596$

H $1.111000-0.798578-1.825128$

H $-0.056139-1.832047-1.010490$

H $1.461922-2.1732760 .861395$

H $-0.265762-0.7137371 .870229$

H $2.142782-3.579575-0.997624$

H $3.112199-2.291748-1.713924$

H $3.591053-3.001713-0.158524$

H $-1.797216-1.8223200 .335301$

H $-1.649706 \quad 1.0108822 .351639$

H $-3.705725-1.2916431 .442900$

H -2.081441 $-0.026703-1.438581$

H -2.602887 1.9655620 .021870

H -4.090132 1.1673470 .554398

H -3.404862 -1.834617 -1.262061

H $4.431274-0.586951-0.691033$

H $3.2047890 .435044-1.451802$

H -4.505506 $2.824920-1.230746$

H -3.446322 $1.931148-2.334296$

H $-4.9625071 .202584-1.765912$

SCF Energy (B3LYP/6-31G**//MMFF) $=-810.708732960$

F2-22_c05199

MMFF Geometry

C -3.429447 2.175226 -0.219481

C $-4.6546242 .704198-0.126901$

C $-2.537726-0.1269620 .224744$

O $\begin{array}{lllll}\text { O } & -1.214309 & 0.315998 & 0.574743\end{array}$

C $-0.414484-0.829948 \quad 0.922145$

C $-1.265717-2.064498 \quad 0.632955$

C $-2.344832-1.546626-0.298061$

C $0.915143-0.7867350 .143597$

C $-3.607021-2.392738-0.285529$

$\begin{array}{llll}\text { C } & 1.723991 & 0.497901 & 0.405547\end{array}$

O $0.664904-0.909436-1.254597$

$\begin{array}{lllll}\text { O } & 2.011476 & 0.582309 & 1.801556\end{array}$

C $3.0357590 .586643-0.402828$

C $4.090829-0.4502070 .004414$

O $3.5957361 .888715-0.202975$

C $-3.1403770 .824018-0.810567$

C $5.347981-0.359056-0.853117$

H $-2.584022 \quad 2.7550830 .141894$

H $-4.796545 \quad 3.6885150 .305921$

H $-5.5314902 .170553-0.476546$

H $-3.138243-0.1295061 .144328$

H $-0.205598-0.7764341 .996825$

H -1.711497 -2.418825 1.571399

H $-0.697971-2.9003670 .212600$

H $-1.945841-1.518888-1.319875$

H $1.499768-1.6612230 .449903$

H $-4.356596-1.989123-0.973238$

H $-3.384603-3.419059-0.595370$

H $-4.053552-2.4320780 .713733$

H 1.1118871 .3726020 .152416

H $\quad 0.126240-0.145613-1.523967$

H $2.527177 \quad 1.394617 \quad 1.942852$

H $2.8231140 .492755-1.473894$

H $3.685571-1.463038-0.085511$

H $4.385490-0.3000151 .049742$

H $2.9494982 .538734-0.527351$

H -2.438094 $0.967364-1.640892$

H -4.057183 $0.392078-1.227832$

H $6.063520-1.132076-0.555103$

H $5.110944-0.506850-1.911474$

H $5.8387110 .612810-0.741883$

SCF Energy (B3LYP/6-31G*//MMFF)= -810.722246986

F2-22 c05200

MMFF Geometry

C $2.7655582 .593142-0.409084$

$\begin{array}{llll}\text { C } & 2.113189 & 3.583123 & 0.210427\end{array}$
C $2.3050680 .136999-0.602346$

$\begin{array}{lllll}\text { O } & 0.881214 & 0.270967 & -0.450316\end{array}$

C $0.274670-1.031264-0.502331$

C $1.417272-2.041533-0.599880$

C $2.621788-1.267603-0.100540$

C $-0.646364-1.2449940 .713875$

C $3.945098-1.822921-0.599588$

C $-1.761893-0.190613 \quad 0.895711$

O $\quad 0.145392-1.2624671 .905515$

O $-1.198274 \quad 1.102994 \quad 1.108415$

C $-2.745997-0.114312-0.284155$

C $-3.9074310 .852220-0.002632$

O $-3.266754-1.411628-0.561603$

C $3.0060331 .238136 \quad 0.194187$

C $-4.8636070 .966405-1.183200$

H $3.1569722 .771135-1.407315$

H $1.9755894 .541110-0.278927$

H 1.7048643 .4570641 .207073

H $2.5310510 .227373-1.673134$

H $-0.325054-1.082055-1.417805$

H $1.556887-2.322411-1.651811$

H $1.235539-2.965633-0.042257$

H $2.622006-1.283026 \quad 0.996651$

H $-1.100177-2.2398750 .628818$

H $4.077259-2.857986-0.267625$

H $3.998478-1.810968-1.693421$

H $4.785655-1.236746-0.215435$

H $-2.316284-0.4435161 .808627$

H $-0.444830-1.4769092 .647952$

H $-0.530714 \quad 1.0182181 .810508$

H $-2.2232250 .236453-1.181312$

H $-3.522248 \quad 1.8500850 .234976$

H -4.4784850 .5029020 .866186$

H $-3.746158-1.7144020 .228495$

H $2.654515 \quad 1.2235251 .233663$

H $4.087638 \quad 1.063261 \quad 0.212317$

H $-4.3391421 .314009-2.079010$

H $-5.3346210 .004996-1.410246$

H $-5.6584611 .683765-0.955712$

SCF Energy (B3LYP/6-31G**//MMFF) $=-810.711658958$

F2-22 c05201

MMFF Geometry

C -2.432450 2.1933590 .494695

C $-3.163336 \quad 3.016404-0.265465$

C $-2.505287-0.322279 \quad 0.888394$

O $-1.086594-0.4277291 .093383$

C $-0.574951-1.4453240 .208864$

C $-1.730172-1.859564-0.705128$

C $-2.724009-0.720653-0.566604$

C $0.660733-0.908659-0.539509$

C $-4.153586-1.115441-0.893564$

C $1.774140-0.4029420 .406372$

$\begin{array}{llll}\text { O } & 0.268688 & 0.134135 & -1.428372\end{array}$

O $2.077979-1.424606 \quad 1.354417$

C $3.0680790 .009473-0.330913$

C 4.1808250 .4162950 .648043

O $2.8185251 .112458-1.197796$

C $-3.006448 \quad 1.0639551 .302085$

C $5.4753870 .787646-0.065248$

H -1.3587262 .3520860 .549973$

H -2.688558 $3.818615-0.819706$

H $-4.2385652 .905196-0.350455$

H -2.970448 -1.0620531 .554352$

H $-0.279845-2.3015050 .826538$

H $-2.166867-2.793578-0.328483$

H -1.426044 -2.043630 -1.740288

H $-2.4160600 .085774-1.242087$

H $1.058077-1.724553-1.155646$

H $-4.508590-1.918030-0.238365$

H $-4.829472-0.262196-0.778241$

H $-4.228320-1.467714-1.927654$

H 1.4016450 .4700970 .956535

H $-0.0759120 .865783-0.888641$

H $2.419812-2.191526 \quad 0.863765$

H $3.430804-0.828952-0.938450$

H $4.392270-0.4001361 .347379$

H 3.8551461 .2817261 .237901 
H $2.1884740 .817517-1.876641$

H $-4.101948 \quad 1.079907 \quad 1.258082$

H -2.727632 1.2422062 .348296

H $6.249967 \quad 1.0370670 .666996$

H $5.839650-0.046178-0.673689$

H $5.3388191 .656393-0.716661$

SCF Energy (B3LYP/6-31G*//MMFF) $=-810.722961037$

F2-22 c05202

MMFF Geometry

C $2.9848962 .488796-0.234330$

C 2.1009963 .4535870 .044248

C $2.799894-0.016108-0.341218$

O $1.4027650 .016918-0.696609$

C $0.716377-1.074201-0.060193$

C $1.664112-1.6046891 .006760$

C $3.030290-1.3351580 .403671$

C $-0.650473-0.6153650 .472887$

C $3.456140-2.451888-0.551880$

C $-1.566399-0.054518-0.633313$

O $-\begin{array}{llll}0.467907 & 0.378617 & 1.479225\end{array}$

O $-1.779848-1.049085-1.635256$

C $-2.9236680 .472840-0.119543$

C $-3.856445-0.6116300 .434818$

O $-3.5963631 .119279-1.205131$

C 3.1011431 .2060480 .540106

C -5.140581-0.024209 1.010096

H $3.6713232 .634286-1.064392$

H $2.0702024 .359115-0.551749$

H $1.396638 \quad 3.3589740 .863171$

H $3.3909830 .035845-1.263414$

H $0.566596-1.844028-0.826512$

H $1.479835-2.6577951 .240687$

H $1.567881-1.0396731 .941068$

H $3.800429-1.2463071 .177977$

H $-1.125264-1.480710 \quad 0.948146$

H $3.530269-3.406246-0.019950$

H $2.753623-2.580327-1.381412$

H $4.439314-2.230557-0.980905$

H -1.060177 $0.780511-1.133188$

H -0.0186791 .1354791 .065336$

H -2.093750 -1.857588 -1.196204

H $-2.756663 \quad 1.2364360 .648874$

H -3.355137 -1.181811 1.223041

H -4.137513 -1.312881 -0.359475

H $-3.0268391 .844404-1.513682$

H 2.4252451 .2290561 .403335

H $4.123921 \quad 1.1384370 .929268$

H -5.768400 -0.8217311 .420182$

H $-4.9203780 .682276 \quad 1.816656$

H -5.7203130 .4979610 .242658$

SCF Energy (B3LYP/6-31G**//MMFF) $=-810.713316341$

\section{F2-22 c05203}

MMFF Geometry

C $-3.284222 \quad 2.3522660 .230670$

C -2.5992643 .4983430 .148320$

C $-2.581516-0.053348 \quad 0.183040$

O $-1.268890 \quad 0.190904 \quad 0.718962$

C $-0.573224-1.0639200 .841705$

C $-1.469766-2.1281190 .211423$

C $-2.432898-1.326680-0.642887$

C $0.820835-0.9435890 .191820$

C -3.741802 -2.048847 -0.914584

C 1.6706660 .1982830 .790801

O $0.670690-0.748387-1.211333$

$\begin{array}{lllll}\text { O } & 1.697511 & 0.031207 & 2.212566\end{array}$

C 3.1351530 .2657390 .304470

C $3.2914880 .712703-1.151885$

O $3.759756-1.0088880 .459692$

C $-3.028451 \quad 1.155054-0.640577$

C $4.7434441 .026311-1.496553$

H $-4.083215 \quad 2.2741370 .963524$

H -2.836308 4.3284890 .804679

H -1.794990 $3.628709-0.567272$

H $-3.258362-0.2175631 .032002$

H -0.458583 -1.277604 1.910645

H $-2.014393-2.6529381 .006968$
H $-0.919400-2.886723-0.353863$

H -1.948577 -1.098241-1.600767

H $1.334517-1.9010550 .336130$

H -3.558770 -2.990873 -1.442154

H $-4.272325-2.2814830 .014872$

H -4.404126-1.438799-1.536405

H $1.191684 \quad 1.1633030 .584111$

H $\quad 0.2060400 .095990-1.342757$

H 2.1437140 .8075882 .591639

H 3.6860230 .9618990 .949427

H $2.6839461 .605017-1.339576$

H $2.960791-0.076699-1.834739$

H $3.651583-1.2733931 .389258$

H $-2.2645941 .394374-1.391267$

H $-3.9550460 .925319-1.178351$

H $5.3813510 .145964-1.370082$

H $4.8212981 .352457-2.538635$

H $5.1325421 .828250-0.861050$

SCF Energy (B3LYP/6-31G**//MMFF) $=-810.719049982$

F2-22 c05204

MMFF Geometry

C $3.5391682 .124382-0.292880$

C $3.0139803 .345182-0.445416$

C $2.516397-0.160971-0.178238$

O $1.3729350 .172138-0.986039$

C $0.605499-1.023714-1.196332$

C $1.170447-2.095247-0.264150$

C $2.031383-1.3059760 .705316$

C $-0.901220-0.736192-1.049695$

C $3.145685-2.129627 \quad 1.328376$

C $-1.351628-0.2685850 .348410$

O $-1.627357-1.912043-1.402446$

$\begin{array}{llll}\text { O } & -0.712579 & 0.968113 & 0.661382\end{array}$

C $-2.881257-0.1020810 .488182$

C $-3.4638411 .040001-0.354806$

$\begin{array}{llll}\text { O } & -3.177223 & 0.167331 & 1.862596\end{array}$

C 2.9658091 .0694220 .610517

C $-4.9796021 .136604-0.223513$

H $4.4409861 .872020-0.844594$

H $3.4789054 .064072-1.111135$

H 2.1171553 .6472650 .084087

H $3.312456-0.500709-0.854353$

H $\quad 0.785842-1.346972-2.229844$

H $1.789382-2.787974-0.848953$

H $0.407332-2.6994460 .235502$

H $1.389322-0.9136831 .504159$

H -1.150764 $0.049515-1.773132$

H $2.732348-2.9792761 .881599$

H $3.827230-2.5231500 .566734$

H $3.734222-1.5278272 .027628$

H -1.027498 -0.9973391 .100415$

H -1.381744 -2.147698 -2.313326

H -1.0139211 .2357411 .546438$

H -3.386262 -1.040802 0.233255

H $-3.2183760 .897738-1.411999$

H -3.037336 $1.999446-0.039128$

H -2.867246 -0.5931462 .383503$

H $2.1190021 .479377 \quad 1.174665$

H $3.7380290 .792197 \quad 1.336727$

H $-5.3621181 .939350-0.861987$

H $-5.4591170 .201740-0.530862$

H -5.2789571 .3560450 .806023$

SCF Energy (B3LYP/6-31G**//MMFF) $=-810.709969015$

F2-22_c05205

MMFF Geometry

C $-4.401803-1.244148-0.306510$

C -5.723761-1.107299-0.459505

C $-2.5165600 .412039-0.284323$

O $-1.529289-0.513602-0.781007$

C $-0.2102910 .071084-0.649070$

C $-0.4481351 .524091-0.264265$

C -1.7395591 .4490270 .523519$

C $0.553672-0.7796530 .379995$

C -2.4465682 .7874690 .655004$

C $1.933204-0.2594220 .833038$

O $0.741028-2.086007-0.186073$ 
O $2.375464-1.1069921 .901058$

C $3.023835-0.218537-0.257272$

$\begin{array}{llll}\text { C } 4.392406 & 0.198696 & 0.308065\end{array}$

O $2.6543110 .693338-1.286833$

C $-3.557346-0.3405920 .547062$

C $5.4791050 .221381-0.759895$

H -3.901039 -2.062136 -0.818075

H $-6.278299-1.796945-1.086415$

H $-6.272977-0.311806 \quad 0.031897$

H -2.987620 $0.878413-1.159652$

H $0.262966-0.008284-1.632877$

H $-0.6029282 .114492-1.176991$

H $0.367714 \begin{array}{lll}1.995371 & 0.286787\end{array}$

H $-1.520612 \quad 1.0613831 .527211$

H $-0.070603-0.9387751 .268392$

H -2.690836 $3.210592-0.325181$

H -3.3788842 .6836251 .218665$

H -1.8118123 .5068251 .182657$

H 1.8156970 .7379411 .267723

H $-0.136221-2.417666-0.444482$

H $2.465409-2.0065951 .541670$

H $3.130973-1.209807-0.712620$

H $4.701381-0.4875461 .104410$

H $4.322026 \quad 1.2020020 .745550$

H $2.589427 \quad 1.579168-0.891036$

H $-3.056539-0.9645581 .297720$

H $-4.1930980 .372211 \quad 1.085008$

H $6.4416420 .487681-0.311593$

H $5.584695-0.760879-1.231239$

H $5.2582350 .956592-1.539750$

SCF Energy (B3LYP/6-31G**//MMFF) $=-810.718279456$

F2-22_c05206

MMFF Geometry

C -3.524172 2.272301-0.192382

C $-2.783902 \quad 3.386571-0.181740$

C -2.944252 $-0.153440 \quad 0.118507$

O $\begin{array}{llll}-1.785392 & 0.125823 & 0.928159\end{array}$

C $-0.717957-0.783568 \quad 0.584432$

C $-1.157314-1.478371-0.694564$

C $-2.669940-1.488048-0.577247$

C $0.597147 \quad 0.0085350 .504638$

C $-3.162593-2.6695510 .260936$

C $1.821657-0.851800 \quad 0.143448$

O $\quad 0.822406 \quad 0.6005831 .787463$

O $1.710218-1.289951-1.210147$

C $3.174491-0.1300260 .328818$

C $3.3812701 .051169-0.628473$

O $4.223079-1.0769750 .099974$

C $-3.1183970 .999911-0.882078$

C $4.700772 \quad 1.771612-0.376140$

H -4.4833542 .2783920 .318778$

H -3.1354834 .2747130 .331810$

H - $-1.8211713 .433080-0.678421$

H -3.819723 -0.2122700 .776080$

H $-0.659203-1.5029251 .410690$

H $-0.718276-2.474385-0.806263$

H $-0.873060-0.885409-1.572132$

H $-3.149476-1.550733-1.560443$

H $\quad 0.4750560 .825847-0.216061$

H - $2.752611-2.6593211 .275913$

H $-4.254561-2.647790 \quad 0.343479$

H $-2.881150-3.618133-0.208114$

H $1.824314-1.7515570 .771888$

H $0.041114 \quad 1.1402571 .998366$

H $2.496017-1.828030-1.407480$

H $3.279890 \quad 0.2140191 .363961$

H $2.566498 \quad 1.774807-0.522159$

H $3.3828150 .700915-1.667420$

H $4.121578-1.7895820 .753771$

H $-2.1895151 .161496-1.442665$

H $-3.8993450 .746677-1.608773$

H $4.7995192 .624501-1.055094$

H 4.7505842 .1481950 .650433

H $5.5562051 .109635-0.542669$

SCF Energy (B3LYP/6-31G**//MMFF) $=-810.717963534$

F2-22_c05207
MMFF Geometry

C $2.9896982 .237786-0.616360$

C $2.3522203 .292806-0.096749$

C $2.449277-0.216123-0.654357$

O $1.0278050 .010224-0.576220$

C $0.420224-0.9957600 .248622$

C $1.563006-1.6894810 .979900$

C $2.708830-1.581065-0.008956$

C $-0.647394-0.3902141 .172594$

C $2.669503-2.706998-1.044151$

C -1.7941360 .3729270 .470656$

$\begin{array}{lllll}\text { O } & -0.026928 & 0.513690 & 2.093324\end{array}$

O $-1.2823541 .505994-0.229546$

C $-2.629544-0.493426-0.486929$

C $-3.7786470 .263315-1.174205$

O $-3.150520-1.6107990 .227695$

C 3.1579880 .9235870 .093477

C $-4.8254510 .820553-0.218103$

H $3.4229172 .327143-1.609178$

H $2.2675144 .215693-0.659993$

H 1.9042903 .2559450 .890127

H $2.742019-0.206051-1.711009$

H $-0.055317-1.714347-0.428335$

H $1.313360-2.7171131 .261609$

H $1.832227-1.1546941 .898028$

H $3.680449-1.6242140 .495674$

H -1.075759 -1.196355 1.779467

H $2.767497-3.682353-0.555967$

H $1.739403-2.709399-1.621452$

H $\quad 3.499065-2.601917-1.751589$

H $-2.437741 \quad 0.768971 \quad 1.264882$

H $\quad 0.344175 \quad 1.246817 \quad 1.572793$

H $-0.7089641 .180680-0.944222$

H -1.983244 -0.880068 -1.283458

H $-4.290314-0.427147-1.856499$

H $-3.3788391 .078547-1.787840$

H $-3.648376-2.157924-0.403459$

H $2.7763411 .002273 \quad 1.118506$

H 4.2315260 .7114260 .161282

H -5.2447150 .0352150 .418085$

H $-4.406713 \quad 1.6011740 .423526$

H -5.648269 $1.267403-0.785642$

SCF Energy $\left(B 3 L Y P / 6-31 G^{* *} / / M M F F\right)=-810.721800200$

F2-22_c05208

MMFF Geometry

C -2.7135342 .7119050 .087961$

C $-1.8328983 .674102-0.209307$

$\begin{array}{llll}\text { C } & -2.493470 & 0.224444 & 0.305108\end{array}$

O

C $-0.648750-1.0887020 .905799$

C -1.797969-2.015076 0.512878

C $-2.685706-1.139189-0.349976$

C $0.655696-1.3239520 .119235$

C $-4.130255-1.607971-0.398963$

C $1.767761-0.2960710 .439808$

O $0.376993-1.310666-1.279304$

O $1.987885-0.2561061 .847136$

C $3.105630-0.632167-0.253125$

C $4.2867290 .244557 \quad 0.198492$

O $2.956631-0.517832-1.666400$

C $-2.7948201 .395115-0.631459$

C $4.0946641 .732592-0.065924$

H -3.424237 2.8856780 .891736

H -1.8284524 .6054110 .346377$

H $-1.1049303 .551553-1.003641$

H -3.0863230 .3003221 .226537$

H $-0.450007-1.1993951 .977929$

H -2.334545 -2.322481 1.419814

H -1.471415 -2.930031 0.008746

H $-2.282810-1.125268-1.370646$

H $1.015042-2.3300830 .367310$

H $-4.733677-0.951727-1.033737$

H $-4.191733-2.621623-0.808543$

H -4.582139-1.620225 0.598591

H 1.4422330 .6963890 .103799

H $0.077935-0.414507-1.510415$

H $\quad 1.2433580 .2236472 .247754$ 
H $3.365874-1.674710-0.030766$

H $5.186540-0.074615-0.342172$

H 4.4866090 .0898081 .264840

H $3.797693-0.786166-2.074043$

H $-2.0924241 .385052-1.474433$

H $-3.8045981 .298619-1.045698$

H 5.0002882 .2811450 .212896

H $3.8965991 .927947-1.124271$

H 3.2675492 .1400600 .522878

SCF Energy (B3LYP/6-31G**//MMFF) $=-810.713190712$

F2-22_c05209

MMFF Geometry

C -4.406463 -1.221775 -0.305361

C $-4.428265-2.5075880 .062949$

C $-2.5616120 .467936-0.481324$

O $-1.525406-0.501303-0.736962$

C $-0.2317430 .138380-0.631837$

C $-0.5218011 .630332-0.544388$

C -1.8612301 .6627730 .161934$

C $0.482869-0.4744730 .584253$

C $-2.6029792 .977896-0.006076$

C 1.8087660 .1992290 .980424

O $\quad 0.705714-1.8585650 .290803$

O $2.359378-0.5358872 .079737$

C $2.8699040 .349658-0.129207$

C $3.502232-0.961769-0.611130$

O 3.9211801 .1768910 .385989

C $-3.633920-0.1533660 .415394$

C $4.563634-0.733691-1.682410$

H $-4.986896-0.918228-1.172717$

H -5.010600 -3.229974 -0.498463

H -3.869284 -2.8614170 .922174$

H $-2.9857760 .747237-1.454699$

H $\quad 0.318545-0.102557-1.548184$

H $-0.6268172 .034252-1.560086$

H $0.2477422 .220307-0.042630$

H $-1.7019371 .477904 \quad 1.232371$

H $-0.189853-0.4743211 .451025$

H -3.5687852 .9502380 .507894$

H -2.0202643 .8039290 .414802$

H -2.789347 $3.202003-1.061778$

H 1.5835281 .1933431 .383734

H $1.160166-2.2470641 .058063$

H $3.189826-0.0979842 .334179$

H $2.4442010 .883773-0.985467$

H $2.738847-1.622476-1.031192$

H $3.976484-1.4856980 .226736$

H 3.5341092 .0422020 .602725

H -3.166609 -0.5716331 .315805$

H $-4.3443350 .614576 \quad 0.741849$

H $4.956515-1.693659-2.032372$

H $4.142666-0.205428-2.543730$

H $5.404602-0.149655-1.295905$

SCF Energy (B3LYP/6-31G**//MMFF)= -810.711322299

F2-22 c05210

MMFF Geometry

C -3.447861 $2.139119-0.020285$

C -4.6738222 .6337950 .184057$

C $-2.470124-0.1498250 .290901$

$\begin{array}{lllll}0 & -1.132692 & 0.316068 & 0.544600\end{array}$

C $-0.283648-0.8183290 .801247$

C -1.128103 $-2.065848 \quad 0.549171$

C $-2.288453-1.552064-0.280939$

C $0.979502-0.726767-0.076676$

C -3.527002 -2.427862 -0.190618

C 1.7796430 .5691620 .150551

O $0.624904-0.826340-1.453980$

$\begin{array}{lllll}\text { O } & 2.157787 & 0.646848 & 1.522887\end{array}$

C $3.0154270 .707496-0.763832$

C $4.056813-0.419658-0.705417$

O $3.674571 \quad 1.942405-0.470574$

C $-3.1755960 .810566-0.667994$

C $4.710190-0.6420760 .651203$

H -2.5900922 .7314970 .287754$

H -4.803950 3.6031420 .652876

H $-5.5630842 .086355-0.108254$
H $-2.994223-0.1883841 .255430$

H $0.007115-0.7840811 .857627$

H -1.490284 -2.452089 1.510726

H $-0.577279-2.8781670 .064754$

H -1.972284 -1.490812 -1.330021

H $1.606865-1.5926510 .163898$

H $-4.337201-2.026154-0.806994$

H $-3.307537-3.441168-0.542857$

H -3.892176 -2.501316 0.839186

H $1.1332891 .434698-0.042170$

H $\quad 0.049657-0.070553-1.663795$

H 2.6784431 .4604581 .635637

H $2.6744720 .780839-1.803812$

H $4.859518-0.173458-1.412831$

H $3.614639-1.360501-1.050986$

H $3.0265832 .657317-0.592103$

H -2.545167 $0.992163-1.547318$

H $-4.1125700 .366259-1.022798$

H $5.537261-1.3532690 .553112$

H 5.1155350 .2878121 .060886

H $4.004099-1.0660351 .370381$

SCF Energy $(B 3 L Y P / 6-31 G * * / / M M F F)=-810.719447221$

F2-22 c05211

MMFF Geometry

C $2.7787592 .591826-0.419102$

C $2.1324763 .587447 \quad 0.197744$

C $2.3121320 .135828-0.597633$

O $\quad 0.889018 \quad 0.271099-0.439024$

C $0.281676-1.030883-0.490723$

C $1.423703-2.042231-0.586963$

C $2.629320-1.267185-0.092031$

C $-0.641728-1.2453750 .724184$

C $3.951469-1.824673-0.591735$

C $-1.759238-0.1920780 .907781$

O $0.148844-1.272587 \quad 1.916547$

O -1.1709931 .0845331 .152711$

C $-2.729058-0.105351-0.285723$

C $-3.9104150 .841101-0.018700$

O $-3.229432-1.405788-0.588510$

C 3.0181501 .2394770 .190776

C $-4.8689410 .920478-1.200731$

H $3.1657242 .762704-1.420415$

H $1.9953064 .542828-0.296579$

H 1.7288473 .4683831 .197159

H $2.5329140 .221499-1.669995$

H -0.316248 -1.081552 -1.407379

H $1.561306-2.326659-1.638206$

H $1.242488-2.964510-0.026361$

H $2.631026-1.2790451 .005304$

H $-1.096567-2.2393000 .633595$

H $4.792926-1.238247-0.209941$

H $4.083078-2.859119-0.257629$

H $4.003244-1.815295-1.685570$

H -2.316710 -0.463654 1.813914

H $-0.445254-1.4727202 .659627$

H -1.855448 1.6618691 .529821

H $-2.1958570 .262603-1.169761$

H -3.5479601 .8507320 .201561$

H -4.4741300 .4933200 .855386$

H $-3.717026-1.7248930 .190065$

H 2.6702691 .2313971 .231545

H $4.099441 \quad 1.0623740 .205734$

H $-4.349971 \quad 1.259400-2.102924$

H $-5.327064-0.050994-1.410153$

H $-5.6736791 .630545-0.985440$

SCF Energy (B3LYP/6-31G**//MMFF) $=-810.702942040$

F2-22 c05212

MMFF Geometry

C $3.0771932 .192498-0.565031$

C 2.6344673 .3097750 .022672

C $2.188877-0.150483-0.608258$

O $0.8184350 .238915-0.412326$

C $-0.007556-0.936473-0.378111$

C $0.936804-2.136495-0.457357$

C $2.278104-1.563640-0.042729$

C $-0.907350-0.9263600 .871143$ 
C $3.461642-2.365974-0.556906$

C $-1.7769990 .331841 \quad 1.094367$

O $-0.082946-1.0900812 .032382$

O -0.9512831 .4898541 .250535$

C -2.8297080 .6535210 .019085$

C $-3.825263-0.488714-0.201692$

O $-2.2076720 .971685-1.223450$

C 3.1055230 .8462190 .101930

C $-4.969340-0.083340-1.123122$

H $3.4488032 .251545-1.584723$

H $2.6434704 .252847-0.512950$

H 2.2549243 .3044461 .038362

H $2.378822-0.151715-1.689906$

H $-0.637297-0.930011-1.273518$

H $0.980346-2.488582-1.496252$

H $\quad 0.620606-2.9863740 .155549$

H $2.323738-1.5270891 .053013$

H - $1.554216-1.8103590 .831837$

H $4.407317-1.919343-0.234522$

H $3.426131-3.391305-0.174490$

H $3.468284-2.414868-1.650994$

H $-2.300896 \quad 0.2141282 .051770$

H $-0.671633-1.1845882 .800547$

H $-0.261606 \quad 1.2700241 .899977$

H $-3.373609 \quad 1.5552570 .327485$

H $-4.241564-0.812850 \quad 0.758957$

H -3.320827 -1.348664 -0.656934

H $-1.5515291 .668332-1.049369$

H $2.803674 \quad 0.942842 \quad 1.152671$

H $\quad 4.1399220 .4850400 .090574$

H $-5.5199090 .768694-0.711951$

H $-4.6025820 .190526-2.117313$

$\mathrm{H}-5.671004-0.915174-1.240887$

SCF Energy (B3LYP/6-31G**//MMFF) $=-810.715625961$

F2-22 c05213

MMFF Geometry

C $-3.534023-1.3689950 .443514$

C $-4.162833-1.5030881 .616569$

C $-2.5415620 .625027-0.790605$

O $-1.516475-0.152603-1.436090$

C $-0.2186390 .315769-0.987358$

C $-0.496121 \quad 1.551440-0.137801$

C - $-1.875885 \quad 1.267086 \quad 0.422513$

C $0.453660-0.859176-0.257316$

C -2.5928172 .5016550 .939607$

C $1.792693-0.5656250 .451404$

O $0.683834-1.898360-1.221422$

O $2.164917-1.749907 \quad 1.166233$

C $2.953973-0.149437-0.473121$

C $4.323059-0.0898400 .227508$

O $2.6728781 .124818-1.045237$

C $-3.776102-0.237670-0.514978$

C $4.3919190 .904478 \quad 1.379741$

H -2.810835 -2.126020 0.151513

H $-3.947035-2.3473902 .262112$

H -4.896341 -0.7788211 .953210$

H -2.829295 $1.401797-1.511938$

H $\quad 0.3456300 .573006-1.889587$

H $-0.5335252 .433114-0.791311$

H $0.248526 \quad 1.7579160 .633210$

H -1.771324 0.5464971 .242616

H $-0.241032-1.2928240 .472740$

H -2.715899 3.2542080 .153492

H -3.5867902 .2440951 .318858$

H -2.0274352 .9600981 .757519$

H $1.6252550 .208047 \quad 1.206107$

H $-0.170723-2.096648-1.641613$

H $2.287024-2.4593360 .511909$

H $3.040902-0.870019-1.295019$

H $5.0802890 .205553-0.509658$

H $4.605920-1.0845240 .590261$

H $3.3967501 .339176-1.658118$

H $-4.5903480 .402225-0.154327$

H -4.114107 -0.682424 -1.459443

H $5.4108230 .943521 \quad 1.778634$

H 4.1206541 .9131831 .053854

H 3.7277560 .6142412 .199050
SCF Energy (B3LYP/6-31G**//MMFF) $=-810.718993764$

F2-22_c05214

MMFF Geometry

C -2.2733622 .1000920 .422284$

C -2.942938 $2.909140-0.406139$

C $-2.353296-0.414470 \quad 0.822073$

O $-0.954158-0.4985331 .140158$

C $-0.356432-1.5077020 .301138$

C $-1.431740-1.949155-0.694176$

C $-2.451831-0.826338-0.642040$

C $0.921324-0.946840-0.357129$

C $-3.845178-1.247790-1.075300$

C $1.942649-0.4138950 .670729$

O $0.5721850 .082442-1.275611$

O $2.236284-1.4612011 .603998$

C $3.2893420 .082305 \quad 0.101125$

C $3.2627571 .382886-0.710723$

O $3.876386-0.942131-0.707021$

C $-2.9030560 .967878 \quad 1.183061$

C $2.7005802 .573710 \quad 0.047217$

H -1.211078 2.2734520 .572726

H -2.430335 $3.714296-0.921019$

H $-4.0047802 .783488-0.586764$

H $-2.860307-1.155617 \quad 1.455320$

H $-0.089552-2.3549630 .943461$

H - $1.880443-2.887308-0.342778$

H -1.046662 -2.136418 -1.701330

H -2.106718 -0.019306 -1.298403

H $1.371013-1.761517-0.936368$

H $-4.236578-2.051727-0.442880$

H -4.541919-0.405549-1.019495

H -3.833814 -1.608308 -2.109081

H $1.492586 \quad 0.396874 \quad 1.252694$

H $0.1541950 .799203-0.769136$

H $2.676261-2.1761591 .112475$

H 3.9803090 .2204190 .942289

H $2.7199001 .247653-1.652254$

H $4.2915811 .615574-1.016011$

H $3.388064-0.979957-1.546832$

H -3.9909570 .9716851 .045262$

H -2.7170531 .1540302 .248339$

H 3.2216052 .7150590 .999311

H $2.8237113 .486653-0.544172$

H 1.6324792 .4549160 .249729

SCF Energy (B3LYP/6-31G**//MMFF $)=-810.720111063$

F2-22_c05215

MMFF Geometry

C $1.9839532 .787640-0.402821$

C $1.0245443 .667648-0.096620$

C $2.1734800 .286828-0.412056$

O $0.7897220 .036513-0.716523$

C $0.578642-1.383121-0.813302$

C $1.901830-2.045146-0.427637$

C $2.637203-0.9597900 .332206$

C $-0.599821-1.8160120 .073203$

C $4.144218-1.1525750 .350038$

C $-1.981024-1.337541-0.415622$

O $-0.364833-1.4129341 .419656$

O $-2.959428-1.9469590 .438769$

C -2.255336 $0.177504-0.492586$

C -2.2454480 .9132030 .852764$

O $-3.5527320 .350504-1.079877$

C 2.2972591 .5669950 .415330

C -2.6550512 .3762820 .721422$

H $2.5890792 .964676-1.288287$

H $0.8515854 .533718-0.725851$

H $0.399778 \quad 3.5427320 .780745$

H $2.7067870 .388423-1.366910$

H $0.344334-1.621138-1.857592$

H $2.449959-2.306452-1.342088$

H $1.781576-2.9678250 .148862$

H $2.268037-0.9398161 .365561$

H $-0.625460-2.9136560 .091730$

H $4.560231-1.158079-0.663167$

H $4.635482-0.351480 \quad 0.910945$

H $4.404201-2.1039540 .825529$ 
H $-2.149574-1.767545-1.411319$ H $-1.126989-1.7063861 .947818$ H $-3.835551-1.6726000 .117696$ H -1.546932 $0.657202-1.174735$ H -1.2446720 .8798121 .293409$ H $-2.9362210 .433192 \quad 1.555358$ H $-3.525233-0.037977-1.970989$ H $1.627517 \quad 1.5134691 .282756$ H 3.3197901 .6724990 .794615 H $-2.5453602 .885548 \quad 1.684222$ H $-2.0305202 .895285-0.011825$ $\mathrm{H}-3.7003672 .4718800 .411414$

SCF Energy (B3LYP/6-31G**/MMFF) $=-810.718801227$

F2-22_c05216

MMFF Geometry

C -3.441756 -1.475742 -0.820548

C $-3.147025-2.720851-0.430318$

C $-2.2225880 .708742-0.575925$

O $-0.9272820 .077908-0.532719$

C -0.0803420 .7702930 .396310$

C $-0.999146 \quad 1.672026 \quad 1.213060$

C -2.1014032 .0082730 .225600$

C $0.755908-0.209452 \quad 1.232467$

C $-1.7162843 .186510-0.671421$

C $1.636545-1.2168790 .461874$

O $-0.117695-0.9736662 .074748$

O $0.803413-2.056687-0.343344$

C $2.751652-0.633536-0.426081$

C $3.7259190 .258907 \quad 0.348018$

O $2.2014620 .111822-1.509362$

C $-3.247441-0.2574730 .037981$

C $4.9259180 .667510-0.497747$

H $-3.854288-1.324536-1.814793$

H -3.313739 -3.559513-1.097396

H $-2.734820-2.9252990 .551625$

H -2.476552 $0.902790-1.624915$

H $0.590013 \quad 1.404059-0.193225$

H -0.4760902 .5468281 .611891$

H -1.434676 1.1352662 .063471

H -3.0339862 .2691110 .738250$

H 1.3894350 .3709321 .911952

H -1.545399 $4.086829-0.072048$

H $-0.8095122 .988994-1.251853$

H -2.522802 $3.404013-1.379873$

H $2.098486-1.8818121 .203399$

H -0.686894 -1.505558 1.492130

H $1.371673-2.732144-0.751029$

H $3.305312-1.465623-0.878783$

H $4.083906-0.2652231 .241399$

H $3.222271 \quad 1.1759790 .674747$

H $1.552697-0.458977-1.955729$

H -2.932408 -0.5574871 .044598$

H -4.2173550 .2443630 .136670$

H $4.6179151 .248261-1.372717$

H 5.6098221 .2856280 .092469

H $5.478061-0.211870-0.844441$

SCF Energy (B3LYP/6-31G**//MMFF) $=-810.714071154$

F2-22 c05217

MMFF Geometry

C $4.339937-1.4892670 .563527$

C $4.970379-2.099123-0.446322$

C $2.4955390 .234170 \quad 0.721043$

O 1.0709990 .3075440 .937783

C $0.4420150 .920881-0.200655$

C $1.4856330 .926299-1.310146$

C $2.7899461 .047211-0.544215$

C $-0.8644120 .192407-0.559740$

C $3.1142802 .504597-0.208580$

C -1.8738380 .1862330 .604473$

O $-0.556737-1.148419-0.934476$

O $-2.185876 \quad 1.539302 \quad 0.941507$

C -3.179430 -0.584993 0.317390

C $-4.0430790 .042463-0.784370$

O $-3.952745-0.618451 \quad 1.521692$

C $2.859459-1.2539210 .607085$

C $-5.297623-0.775818-1.068336$
H $4.924653-1.1501291 .414670$

H $6.044726-2.244681-0.415549$

H $4.431904-2.460155-1.315588$

H 2.9884560 .6605781 .602939

H $0.214006 \quad 1.955160 \quad 0.084003$

H $1.3180391 .729616-2.034236$

H $1.483339-0.019551-1.864039$

H $3.6283460 .646184-1.122878$

H -1.290172 $0.702407-1.431934$

H $4.0538492 .565247 \quad 0.351075$

H $3.2315273 .093544-1.124233$

H 2.3349352 .9753570 .399002

H -1.405879 -0.2632631 .489241$

H -1.322520 -1.503988 -1.415867

H $-2.822297 \quad 1.5155531 .676595$

H $-2.953361-1.6255910 .059733$

H $-3.4713680 .129857-1.713820$

H $-4.3582301 .050484-0.490354$

H -3.422624 -1.074028 2.197840

H $2.452013-1.7862021 .476344$

H $2.359535-1.700532-0.261513$

H -5.870757 -0.316064 -1.879823

H $-5.039172-1.795356-1.371476$

H $-5.946918-0.831088-0.189091$

SCF Energy $\left(B 3 L Y P / 6-31 G^{* *} / / M M F F\right)=-810.715738669$

F2-22 c05218

MMFF Geometry

C $3.7640961 .874542-0.405168$

C $3.3695513 .076360-0.840687$

C $2.534735-0.2692980 .012755$

O $1.516465-0.023586-0.974329$

C $0.681873-1.189498-1.056637$

C $1.038760-2.0883530 .127649$

C $1.857596-1.1830001 .029456$

C $-0.802318-0.796694-1.173087$

C $2.820988-1.9400831 .927774$

C $-1.393478-0.0504360 .048175$

O $-1.530638-1.994186-1.436385$

O $-0.609434 \quad 1.104536 \quad 0.334920$

C $-2.8626380 .388578-0.150750$

C $-3.3966181 .196878 \quad 1.043418$

O $-3.696644-0.749584-0.349142$

C 3.0099161 .0577090 .605674

C -4.8353201 .6547130 .837284$

H $4.6901081 .457581-0.792744$

H $3.9621643 .618182-1.569793$

H $2.4556313 .537451-0.483123$

H $3.363137-0.789367-0.487228$

H $0.958301-1.720814-1.977026$

H $1.649519-2.926926-0.230811$

H $\quad 0.172342-2.523787 \quad 0.634375$

H $1.169816-0.6006401 .655635$

H $-0.895492-0.143487-2.050596$

H $3.381178-1.2527762 .569262$

H $2.276865-2.6356632 .575171$

H $3.543306-2.5193931 .342863$

H -1.353691 -0.7129830 .920713$

H -2.442772 -1.742625 -1.658899

H $-0.6105861 .668785-0.457152$

H -2.933205 $1.011891-1.050335$

H -2.771909 2.0800831 .216701

H -3.3598280 .5831451 .951564$

H -3.636655 -1.304051 0.447676

H $2.150868 \quad 1.6271040 .981194$

H 3.6765040 .8748311 .455835

H -5.165178 2.2548121 .691358

H $-4.9247262 .269395-0.064004$

H -5.5165470 .8033110 .743712$

SCF Energy $(B 3 L Y P / 6-31 G * * / / M M F F)=-810.715004146$

F2-22 c05219

MMFF Geometry

C $-3.2639842 .277230 \quad 0.154494$

C -4.4793922 .8246800 .266401$

C $-2.469349-0.1019770 .155164$

$\begin{array}{lllll}\text { O } & -1.168781 & 0.198771 & 0.691755\end{array}$

C $-0.427344-1.0275260 .837429$ 
C $-1.286637-2.1349330 .230873$ C $-2.270545-1.384510-0.645805$ C $0.967144-0.8654930 .195915$ C -3.551080 -2.158424 -0.911270 C $1.7626790 .316516 \quad 0.793414$ O $0.833591-0.663825-1.207493$ $\begin{array}{lllll}\text { O } & 1.784757 & 0.193948 & 2.219787\end{array}$ C 3.2268130 .4529330 .319686 C $3.3700440 .944050-1.124587$ O $3.903915-0.798366 \quad 0.439673$ C $-2.9596861 .073258-0.692185$ C $4.8102411 .320480-1.455883$ H -2.4376342 .7300040 .696444$ H -4.632853 3.6981620 .890596 H $-5.3367232 .415700-0.256501$ H $-3.143997-0.2751431 .004356$ H $-0.309778-1.2174271 .910634$ H -1.819700 -2.654264 1.037907 H -0.712053 -2.893329 -0.309462 H -1.788358 -1.155474 -1.604505 H $1.522651-1.7980210 .353310$ H -3.330646 -3.103639 -1.418224 H -4.079582 -2.390968 0.019360 H -4.230481-1.585341-1.549785 H $\quad 1.248471 \quad 1.2585640 .568805$ H $0.552604-1.505020-1.604530$ H $2.155391-0.6774312 .439576$ H 3.7442681 .1550860 .985980 H $2.7306381 .817970-1.291214$ H $3.0700860 .161723-1.829424$ H $3.905863-1.0426181 .380378$ H -2.186927 $1.361892-1.415332$ H $-3.8485990 .780002-1.262317$ H $4.8781711 .677241-2.488586$ H $5.1675292 .118920-0.797864$ H $5.4803860 .461477-1.351248$ SCF Energy (B3LYP/6-31G**//MMFF) $=-810.710106657$

F2-22 c05220

MMFF Geometry

C $-4.374687-0.749379-0.954439$

C $-5.340357-1.011097-0.066505$

C $-2.0975580 .343827-0.828212$

O $-0.705286-0.018961-0.727259$

C $-0.146391 \quad 0.5361880 .473743$

C -1.3301350 .9924251 .318600$

C -2.3629841 .3736870 .274394$

C $0.779119-0.4664851 .179208$

C -2.152134 $2.802046-0.232707$

C $1.977480-0.9640450 .339972$

O $0.009167-1.6085111 .570359$

O $1.520805-1.634093-0.834135$

C $2.9545860 .149672-0.074612$

C $4.194137-0.410570-0.790377$

O 3.3639820 .8840621 .076030

C $-2.912932-0.950169-0.686565$

C $5.1475600 .688856-1.241263$

H -4.656012 -0.379672 -1.936939

H $-6.381549-0.849978-0.323602$

H -5.112456 -1.3860730 .925150$

H $-2.2599120 .765910-1.827111$

H $\quad 0.4348551 .415848 \quad 0.175197$

H -1.064813 1.8065882 .000269

H -1.724978 0.1712081 .927809

H -3.3782861 .3121390 .679313$

H $1.147910-0.0064262 .103682$

H -2.2535533 .5205390 .587358$

H -1.165101 $2.941944-0.684993$

H $-2.9028443 .050551-0.990429$

H $2.518230-1.7106040 .936129$

H $0.591971-2.1946742 .082335$

H $\quad 0.863213-2.294029-0.555534$

H $2.455608 \quad 0.846527-0.757555$

H $3.894216-0.996650-1.666228$

H $4.740878-1.080614-0.115776$

H $3.8229180 .268754 \quad 1.673294$

H -2.530321 -1.690210 -1.401487

H $-2.746835-1.395900 \quad 0.302163$
H $6.0004250 .253307-1.771628$

H $4.6467041 .386847-1.919732$

H $5.5361441 .254965-0.389069$

SCF Energy (B3LYP/6-31G**//MMFF) $=-810.712169082$

F2-22 c05221

MMFF Geometry

C $3.934216-1.420975-0.652148$

C $3.650922-2.713491-0.455707$

C $2.6383140 .686476-0.208903$

O 1.8710320 .1076190 .864191

C $\quad 0.4999630 .5596870 .787686$

C $0.3658151 .251650-0.557543$

C $1.7630331 .784515-0.814517$

C $-0.419346-0.647027 \quad 1.023383$

C $1.9893953 .129395-0.120784$

C $-1.938750-0.380798 \quad 0.995697$

O $-0.128841-1.1370972 .346004$

$\begin{array}{llll}\text { O } & -2.235653 & 0.801392 & 1.739838\end{array}$

C $-2.567091-0.259075-0.403138$

C $-4.102273-0.194353-0.321755$

O $-2.178167-1.386090-1.182190$

C $2.962778-0.424474-1.220130$

C $-4.7593440 .012253-1.680772$

H $4.927152-1.059876-0.396701$

H $4.398286-3.383543-0.045074$

H $2.676289-3.123646-0.696095$

H $3.566598 \quad 1.0931200 .209453$

H 0.3831141 .2819911 .604616

H $-0.4033272 .029632-0.556091$

H $\quad 0.1136170 .526735-1.340337$

H $1.950617 \quad 1.922846-1.885140$

H $-0.157312-1.4659000 .344161$

H $3.0077873 .486617-0.307152$

H $1.2944863 .882922-0.506196$

H $1.8525953 .066508 \quad 0.963513$

H -2.431395 -1.212177 1.518010

H $\quad 0.823229-1.3349242 .377011$

H -1.8702700 .6808302 .633205$

H -2.214613 $0.649211-0.899864$

H -4.4098370 .6241590 .338715$

H -4.489918 -1.127380 0.105049

H -2.404249-1.199105 -2.108646

H $2.044494-0.937541-1.531104$

H $3.4143540 .011734-2.118960$

H $-5.8423260 .119493-1.561454$

H $-4.3804600 .917242-2.166152$

$\mathrm{H}-4.581674-0.838237-2.345720$

SCF Energy (B3LYP/6-31G**//MMFF $)=-810.714997063$

F2-22 c05222

MMFF Geometry

C $-3.613380-2.019863-0.325726$

C $-4.908523-2.353564-0.351999$

C $-2.4405340 .191767-0.187244$

O $-1.272514-0.235152-0.912643$

C $-0.4620580 .924738-1.161488$

C $-0.9703222 .036185-0.241024$

C - -1.9211351 .3176780 .700816$

C $1.0362200 .589816-1.043264$

C -3.0034802 .2212981 .266205$

C 1.4892680 .0956810 .348047

O $1.7602821 .758634-1.420385$

O $0.911875-1.1817620 .622064$

C $3.019695-0.0265980 .513560$

C $3.657631-1.097863-0.380934$

O $3.302483-0.3613511 .876548$

C $-3.031659-0.9830110 .593483$

C $5.174515-1.139723-0.234609$

H -2.930496-2.532050 -0.998706

H $-5.265536-3.115511-1.036350$

H -5.630980 -1.877315 0.301414

H $-3.1670090 .573639-0.917538$

H $-0.6517421 .236148-2.197387$

H -1.508639 2.780854 -0.841365

H -0.1779892 .5737350 .288620$

H -1.3367260 .8967341 .529463$

H $1.241483-0.196869-1.780340$ 
H $-3.6293612 .641997 \quad 0.472146$ H -3.654902 1.6719381 .952751 H -2.558168 3.0539661 .820629 H 1.1322890 .7812501 .124796 H $2.661731 \quad 1.484579-1.659275$ H $-0.052997-1.0901610 .564815$ H $3.496108 \quad 0.941783 \quad 0.324860$ H $3.417494-0.909836-1.432289$ H $3.267375-2.089155-0.121832$ H $2.940218 \quad 0.3479472 .434422$ H -2.253203 -1.469380 1.193286 H $-3.799723-0.6228891 .287304$ H $5.618632-0.171834-0.487791$ H $5.470928-1.3991350 .786554$ H $5.596769-1.892637-0.907803$ SCF Energy (B3LYP/6-31G**//MMFF) $=-810.711045850$

\section{F2-22_c05223}

MMFF Geometry

C $2.7014072 .633032-0.277049$

C $1.7704173 .592511-0.234258$

C $2.7051640 .115889-0.260495$

O $1.428551-0.017821-0.923909$

C $0.807423-1.238866-0.505899$

C $1.466561-1.6140160 .812179$

C $2.892595-1.1356910 .608834$

C $-0.727891-1.139036-0.484814$

C $3.749219-2.187408-0.099619$

C -1.3029430 .0298620 .341386$

O $-1.256000-2.372694-0.003939$

O $-1.006358 \quad 1.270373-0.301652$

C -2.829907 -0.0440680 .554963$

C $-3.6525650 .094959-0.733736$

O $-3.2030571 .000707 \quad 1.459615$

C 2.6835641 .4000010 .582736

C $-5.149431-0.031897-0.475887$

H $3.5363272 .750220-0.963157$

H $1.8473694 .461652-0.878316$

H 0.9221563 .5315020 .438378

H $3.4826360 .188489-1.030572$

H $1.074699-2.001734-1.249443$

H $1.385031-2.6817631 .037483$

H $1.024788-1.0617541 .648162$

H $3.375376-0.9088061 .566144$

H -1.056541 -1.008952 -1.523733

H $3.803396-3.1048180 .495921$

H $3.355991-2.447982-1.087355$

H $4.770600-1.816171-0.236163$

H $-0.837244 \quad 0.065566 \quad 1.332330$

H $-0.928778-3.079969-0.585531$

H $-0.0454941 .311450-0.436149$

H $-3.086296-0.9853201 .053612$

H $-3.359111-0.673099-1.456617$

H -3.472451 $1.072729-1.195686$

H -2.9545861 .8463991 .048260$

H 1.8022051 .4072271 .235854

H 3.5707761 .4331931 .226216

H $-5.7011570 .040452-1.418496$

H $-5.386805-0.997051-0.017324$

H -5.5096600 .7623940 .185208$

SCF Energy $\left(B 3 L Y P / 6-31 G^{* *} / / M M F F\right)=-810.718456873$

F2-22_c05224

MMFF Geometry

C $-4.208710-1.565572-0.801356$

C $-4.920485-2.1833880 .147918$

C $-2.4109020 .212825-0.796731$

O $-0.974530 \quad 0.328304-0.874435$

C $-0.482448 \quad 0.997486 \quad 0.299035$

C -1.6230390 .9743381 .307565$

C -2.8541121 .0300310 .421865$

$\begin{array}{lllll}\text { C } & 0.817129 & 0.338246 & 0.789035\end{array}$

C -3.2058392 .4677670 .032855$

C $1.9380330 .342363-0.275850$

O $0.544311-1.0009271 .196400$

O $2.1263771 .675514-0.747468$

C $3.284794-0.208000 \quad 0.245595$

C $4.396785-0.097561-0.809846$
O $3.156525-1.5797980 .608599$

C $-2.739273-1.284321-0.697670$

C $5.739596-0.601173-0.293980$

H -4.713403 -1.254907 -1.712348

H $-5.981136-2.3626590 .009997$

H -4.463256 -2.517460 1.072603

H $-2.8271700 .613475-1.728671$

H -0.2786482 .0354370 .010182$

H -1.556249 1.7937192 .029968

H $-1.6346200 .036642 \quad 1.874847$

H -3.7255040 .6018230 .927515$

H 1.1602630 .8855911 .675153

H $-4.0919202 .480944-0.610690$

H -3.430378 3.0638110 .923673

H $-2.3941402 .962813-0.509772$

H $1.621490-0.275850-1.124980$

H $0.213074-1.4822470 .418613$

H 2.4185182 .2175290 .005409

H $3.5943790 .354421 \quad 1.135117$

H $4.5172130 .942867-1.131318$

H $4.126674-0.688250-1.693642$

H $2.523686-1.6324991 .344481$

H -2.228886 -1.814507 -1.512270

H -2.317911 -1.7033140 .224808$

H $5.696852-1.664001-0.036954$

H $6.509330-0.473238-1.061775$

H $6.051102-0.0431310 .594672$

SCF Energy (B3LYP/6-31G**//MMFF) $=-810.722187820$

F2-23_c05457

MMFF Geometry

C 3.7121142 .0725790 .112173

C $3.1121973 .193746-0.303512$

C $2.870073-0.2368540 .642932$

O 1.5970320 .2820521 .090315

C $0.550556-0.6440220 .735667$

C $1.156253-1.581373-0.298188$

C $2.589218-1.6731790 .186811$

C -0.6883480 .1409390 .280334$

C $3.541678-2.270928-0.833362$

C $-1.891462-0.763960-0.030073$

O $-0.3731940 .906684-0.881714$

O $-2.220043-1.5109011 .141440$

C $-3.1370580 .000311-0.526834$

C -3.7843610 .8966320 .536972$

O $-4.111325-0.960353-0.947350$

C $3.4164760 .705457-0.440085$

C $-4.9786291 .669497-0.011490$

H 4.4678952 .1433870 .890076

H 3.3740024 .1512190 .133344

H $2.3546103 .178180-1.079094$

H $3.550904-0.2456191 .502666$

H $0.317881-1.2047761 .649596$

H $1.118345-1.151313-1.305574$

H $\quad 0.652685-2.552539-0.325532$

H $2.601466-2.3159501 .078526$

H $-0.943110 \quad 0.851017 \quad 1.075520$

H $3.506917-1.738432-1.789190$

H $4.571386-2.247797-0.462504$

H $3.279051-3.315496-1.032325$

H $-1.610378-1.489537-0.803337$

H $0.383036 \quad 1.475222-0.656688$

H -2.987137 -2.0682350 .924438$

H $-2.8821710 .601216-1.407391$

H -3.056782 1.6167810 .924197

H -4.1352410 .2904561 .380492$

H -3.719517 -1.477226 -1.671844

H $4.3524530 .310001-0.849842$

H $2.7018670 .784110-1.268143$

H $-4.6824892 .297663-0.857704$

H $-5.7734440 .994578-0.343854$

H -5.3942182 .3201680 .764578$

SCF Energy (B3LYP/6-31G**//MMFF) $=-810.719515539$

F2-23_c05458

MMFF Geometry

C $4.187996-1.212468-0.465765$

C $4.124773-2.4131270 .120860$ 
C $2.4864690 .590758-0.886356$

O $1.422829-0.379689-1.010884$

C $0.2022840 .169143-0.468226$

C $0.625931 \quad 1.3712820 .361179$

C $1.8050371 .888014-0.438849$

C $-0.555531-0.945780 \quad 0.264818$

C 2.6602072 .8917310 .313996

C $-1.954059-0.572377 \quad 0.794248$

O $0.219658-1.4004301 .379845$

$\begin{array}{lllll}0 & -1.859039 & 0.476214 & 1.756561\end{array}$

C $-2.944944-0.148626-0.303454$

C -4.3572550 .0845690 .256612$

O $-2.997042-1.148180-1.317753$

C 3.5370880 .0272650 .082407

C $-5.3358630 .545199-0.816327$

H $4.749356-1.113343-1.391184$

H $4.620846-3.268700-0.324409$

H $3.581117-2.5646621 .046702$

H $2.9345800 .728160-1.877854$

H $-0.3818030 .505625-1.332804$

H 0.9458201 .0768241 .367251

H -0.1722552 .1128820 .459986$

H $1.4099762 .385800-1.335965$

H $-0.644787-1.809314-0.405490$

H $2.084883 \quad 3.7997690 .523596$

H 3.0075242 .4968461 .274103

H $3.5351713 .177258-0.278692$

H $-2.355341-1.4469321 .322729$

H $1.088977-1.6685631 .036123$

H -1.2349370 .1833832 .442776$

H $-2.6067120 .784221 \quad-0.768633$

H -4.3304590 .8359801 .053620$

H $-4.741772-0.8455770 .692439$

H $-3.310158-1.972217-0.907050$

H 4.3299620 .7640150 .252379

H $3.076945-0.1912091 .053525$

H $-5.456081-0.210766-1.598488$

H $-6.3201020 .729454-0.373956$

H $-4.9947971 .474256-1.283720$

SCF Energy (B3LYP/6-31G**//MMFF) $=-810.719759723$

F2-23 c05459

MMFF Geometry

C -2324724 26743690.051288

C $-1.235678 \quad 3.326647 \quad 0.473452$

C $-2.697750 \quad 0.267794-0.568723$

O $-1.3262210 .120031-0.992870$

C $-0.812744-1.140922-0.535695$

C $-1.866682-1.7289690 .396201$

C $-3.142947-1.141829-0.170664$

C $0.561574-0.970075 \quad 0.130962$

C $-4.332667-1.243997 \quad 0.766433$

C $1.635275-0.406221-0.823183$

O $\quad 0.980798-2.233610 \quad 0.640369$

O $1.3200890 .939431-1.183231$

C $3.062569-0.427048-0.237837$

C $3.261248 \quad 0.506702 \quad 0.964571$

O $3.973495-0.049988-1.275459$

C $-2.738837 \quad 1.3179170 .552150$

C 4.6722010 .4173951 .533883

H $-2.9597703 .145925-0.694021$

H $-0.992134 \quad 4.3052030 .074385$

H $-0.5699912 .898997 \quad 1.215004$

H $-3.2825050 .619040-1.427466$

H $-0.719147-1.790436-1.415522$

H $-1.711452-1.3948151 .429262$

H $-1.859918-2.8233460 .393921$

H -3.384864 -1.690564 -1.091863

H $\quad 0.445639-0.2814920 .976376$

H $-4.121409-0.8081051 .748133$

H $-5.203966-0.7340360 .343435$

H $-4.601273-2.2936740 .926782$

H $1.647746-0.991911-1.751255$

H $1.085562-2.837592-0.114735$

H $\quad 0.451708 \quad 0.933852-1.620107$

H $3.329147-1.4510840 .045722$

H $2.5503300 .259147 \quad 1.759492$

H 3.0821651 .5476650 .670973
H $3.7358570 .848860-1.561368$

H -3.7554101 .4108660 .949993$

H -2.0877041 .0150611 .381038$

H $4.902989-0.6052161 .848770$

H $\begin{array}{llll}5.420288 & 0.729430 & 0.798404\end{array}$

H $4.767801 \quad 1.0707402 .407146$

SCF Energy (B3LYP/6-31G**//MMFF) $=-810.720111883$

F2-23 c05460

MMFF Geometry

C $-1.4814562 .923208 \quad 0.028086$

C $-0.548772 \quad 3.3797760 .871188$

C -2.104023 $0.634984-0.805959$

O $-0.7405690 .167549-0.709181$

C $-0.728243-1.211093-0.312967$

C $-2.130476-1.508387 \quad 0.205245$

C $-2.974854-0.619942-0.684704$

C $0.372139-1.4860490 .719851$

C $-4.395291-0.428146-0.184503$

C $1.820053-1.307240 \quad 0.208297$

$\begin{array}{llll}\text { O } & 0.225483 & -2.842976 & 1.154607\end{array}$

O $2.007995-2.070259-0.984279$

C $2.242193 \quad 0.146614-0.063471$

C $3.7363780 .252159-0.411464$

$\begin{array}{lllll}\text { O } & 1.968213 & 0.950174 & 1.080269\end{array}$

$\begin{array}{llll}\text { C }-2.318507 & 1.699282 & 0.280407\end{array}$

C $4.1562791 .682779-0.725102$

H $-1.6609203 .468470-0.894980$

H $\quad 0.019314 \quad 4.271963 \quad 0.631560$

H -0.3338672 .8754291 .806685$

H $-2.2367641 .085511-1.796997$

H $-0.559312-1.811172-1.216102$

H $-2.232808-1.2117391 .256405$

H $-2.391452-2.5681040 .123984$

H -3.025454 -1.089728 -1.677388

H $\quad 0.217025-0.8470691 .597197$

H $-4.9379230 .278798-0.820059$

H $-4.936312-1.380340-0.201918$

H $-4.421670-0.0573110 .845135$

H $2.497241-1.7307660 .961574$

H $0.859968-2.9898921 .876691$

H $1.746714-2.986142-0.787196$

H $1.6762500 .552996-0.907693$

H $3.970487-0.380532-1.274988$

H $4.340357-0.1032730 .432246$

H $2.4884240 .598521 \quad 1.822752$

H -3.3659722 .0200330 .297001$

H -2.083158 $1.283514 \quad 1.267685$

H $5.2163591 .713023-0.996146$

H $3.5799242 .084552-1.564472$

H 4.0120472 .3396650 .138268

SCF Energy (B3LYP/6-31G**//MMFF) $=-810.712488190$

F2-23 c05461

MMFF Geometry

C $3.6304392 .031408-0.075569$

C $4.8622102 .553361-0.071788$

C $2.757514-0.232357 \quad 0.597248$

$\begin{array}{llll}\text { O } & 1.502587 & 0.335335 & 1.036819\end{array}$

C $0.432139-0.587027 \quad 0.749650$

C $1.001467-1.589463-0.242381$

C $2.440008-1.6852310 .224704$

$\begin{array}{llll}\text { C } & -0.799339 & 0.196463 & 0.272824\end{array}$

C $3.361896-2.359854-0.775265$

C $-2.024667-0.692621-0.004537$

O $-0.4745120 .914013-0.918188$

O $-2.403121-1.385096 \quad 1.184764$

C $-3.2484430 .087838-0.532490$

C $-3.876117 \quad 1.038187 \quad 0.496182$

O $-4.248600-0.857365-0.927150$

C $3.3083800 .638234-0.542754$

C $-5.043276 \quad 1.827027-0.086809$

H 2.8036762 .6489370 .266031

H 5.0273943 .5684780 .272292

H $5.7215491 .983628-0.407421$

H $3.452469-0.2090701 .445461$

H $0.207405-1.0914731 .697163$

H $0.955686-1.211574-1.270156$ 
H $\quad 0.477955-2.549895-0.210826$

H $2.453834-2.2781641 .150404$

H -1.037842 0.9398981 .042003

H $4.398423-2.336902-0.424217$

H $3.075176-3.408454-0.909099$

H $3.319865-1.882699-1.759605$

H $-1.771427-1.451829-0.755231$

H $\quad 0.290342 \quad 1.479668-0.715744$

H -1.733177 -2.067812 1.356997

H -2.974019 $0.651446-1.431519$

H $-3.1302091 .749457 \quad 0.864780$

H $-4.250598 \quad 0.4713851 .356631$

H -3.866237 -1.414278 -1.626444

H $4.2083550 .178428-0.966311$

H $2.5709210 .721957-1.349748$

H $-5.8557681 .165119-0.402506$

H -5.4444532 .5152710 .664166$

H -4.723409 2.417591-0.951140

SCF Energy $\left(B 3 L Y P / 6-31 G^{* *} / / M M F F\right)=-810.711750479$

F2-23 c05462

MMFF Geometry

C $-3.821608-1.8379860 .158729$

C $-5.092175-2.256371 \quad 0.166214$

C -2.7934490 .4337690 .507782$

O $-1.616803-0.1453371 .116688$

C $-0.4582490 .632010 \quad 0.754185$

C $-0.8853501 .492195-0.425267$

C $-2.3328101 .774670-0.076691$

C $0.726733-0.3095160 .493955$

C $-3.1390532 .338640-1.232909$

C 2.0372420 .4388290 .172883

O $0.404163-1.189177-0.582104$

O $2.2844831 .403087 \quad 1.194497$

C $3.263247-0.4934290 .043698$

C $4.5639910 .295733-0.174610$

O $3.099068-1.385103-1.055530$

C $-3.356185-0.572393-0.508033$

C $5.785890-0.610716-0.265135$

H -3.070481 -2.4502810 .651145$

H $-5.362188-3.1834070 .660233$

H -5.880305 -1.687601 -0.314470

H $-3.5316150 .607052 \quad 1.300280$

H -0.2408721 .2725241 .617839$

H $-0.8183200 .943238-1.371622$

H $-0.2829332 .400945-0.517683$

H -2.344284 2.5148830 .736103

H $0.870981-0.9337161 .384693$

H -2.756516 $3.324674-1.517663$

H -3.086067 $1.700276-2.120631$

H $-4.1907872 .454104-0.952422$

H $1.9141010 .974085-0.776836$

H $-0.417485-1.651079-0.342736$

H 2.4049210 .9230472 .031758

H $3.370284-1.0917690 .957022$

H $4.721134 \quad 1.0082440 .642552$

H $4.4950750 .870263-1.106348$

H $2.318777-1.935899-0.875645$

H $-4.189394-0.123430-1.060277$

H $-2.587110-0.847207-1.239535$

H $5.895552-1.2108170 .643711$

H $5.718593-1.289472-1.120981$

H $6.692298-0.009020-0.385702$

SCF Energy $\left(B 3 L Y P / 6-31 G^{* *} / / M M F F\right)=-810.721286024$

F2-23 c05463

MMFF Geometry

C $-3.5619832 .102425-0.358606$

C $-2.8918073 .252449-0.224370$

C $-2.844512-0.304338-0.486818$

O $-1.5993480 .053843-1.128386$

C $-0.557880-0.838508-0.683917$

C $-1.099838-1.5252970 .560654$

C $-2.572471-1.637108 \quad 0.220669$

C $0.745135-0.046464-0.491877$

C $-3.449623-1.9763311 .412501$

C $1.936072-0.931793-0.077237$

O $\quad 0.536251 \quad 0.957910 \quad 0.498760$
O $2.023061-2.023177-0.999465$

C $3.312270-0.230281-0.058082$

C 3.4740140 .8019941 .061350

O $3.5472160 .415569-1.310054$

C -3.2619950 .8564550 .428338$

C 4.9208451 .2617231 .205049

H -4.381823 $2.056867-1.070896$

H $-3.1610184 .117828-0.820009$

H -2.0686693 .3517530 .474407$

H -3.598325 -0.446298 -1.270772

H $-0.430094-1.576110-1.485805$

H $-0.958225-0.9098551 .456417$

H $-0.628027-2.4962450 .739343$

H $-2.685719-2.439643-0.522158$

H $0.9678740 .469982-1.433144$

H $-3.311869-1.2704152 .237906$

H $-4.506858-1.9736041 .128960$

H $-3.205430-2.9740511 .792486$

H $1.749576-1.3565880 .916723$

H -0.2062331 .5100590 .199418$

H $2.711820-2.628167-0.675181$

H $4.092700-0.9942780 .048235$

H 3.1422920 .3781192 .015734

H 2.8709541 .6921850 .853687

H $3.439478-0.254574-2.006654$

H -4.1698780 .5946610 .982911$

H -2.474149 1.0601521 .163564

H 5.2804491 .7372620 .287302

H 5.0055661 .9901672 .017932

H 5.5787990 .4180791 .436846

SCF Energy $($ B3LYP/6-31G**//MMFF $)=-810.717343753$

F2-23_c05464

MMFF Geometry

C $3.924108-1.488147-0.547989$

C $3.835951-2.6266310 .148807$

C $2.3131650 .391651-0.989507$

O $1.181612-0.505956-0.936763$

C $0.0481650 .178586-0.357461$

C 0.6215921 .4100340 .325648

C $1.7604181 .768931-0.608117$

C $-0.721112-0.8096820 .527330$

C $2.7412932 .766884-0.018615$

C $-2.028698-0.3116421 .173451$

O $0.133136-1.2427271 .596089$

$\begin{array}{llll}\text { O } & -1.770776 & 0.809717 & 2.022021\end{array}$

$\begin{array}{llll}\text { C } & -3.174604 & 0.076881 & 0.222577\end{array}$

C $-3.587369-1.062782-0.713197$

O $-2.8219731 .213913-0.561554$

C $3.405798-0.162299-0.063019$

C $-4.844388-0.726991-1.506748$

H $4.406167-1.508856-1.521920$

H $4.234232-3.550601-0.256173$

H $3.368292-2.6592131 .126529$

H $2.6798640 .410701-2.022910$

H $-0.5726490 .479229-1.207890$

H 1.0064961 .1760501 .324696

H -0.1113642 .2161180 .422618$

H $1.3247072 .217200-1.512490$

H $-0.932756-1.712634-0.057082$

H 2.2472843 .7291650 .153583

H 3.1422882 .4297190 .942577

H $3.5795172 .936507-0.701972$

H $-2.397907-1.1047711 .836954$

H $0.939984-1.6046431 .191164$

$\mathrm{H}-1.0772000 .5434512 .649875$

H -4.0380220 .3796510 .828305$

H $-3.766819-1.975724-0.134308$

H $-2.786822-1.271637-1.432182$

H $-2.567014 \quad 1.9219490 .054587$

H $4.2569530 .526559-0.026277$

H $3.019824-0.2636190 .958504$

H $-5.121475-1.569970-2.147653$

H $-5.685707-0.519269-0.837867$

$\mathrm{H}-4.6897860 .146300-2.147975$

SCF Energy (B3LYP/6-31G**//MMFF) $=-810.721835787$

F2-23 c05465 
MMFF Geometry

C $-2.7059892 .530046-0.319651$

C -2.0311323 .3534040 .490509$

C $-2.3805760 .111754-0.921850$

O $-0.9530150 .210629-0.728382$

C $-0.473101-0.975655-0.077500$

C $-1.705228-1.7502260 .379393$

C $-2.716823-1.362967-0.680105$

C $0.473790-0.6188441 .070632$

C $-4.151319-1.685693-0.302752$

C 1.6299640 .3397500 .703505

O $0.994918-1.8305251 .616798$

O 2.2462490 .7550321 .928398

C $2.726078-0.256880-0.202556$

C $3.8937820 .721358-0.420323$

O $2.181865-0.605833-1.471126$

C -3.0590021 .1100560 .028667$

C $4.9907520 .130144-1.297474$

H -3.030232 $2.901016-1.288465$

H -1.810305 4.3691570 .181281

H $-1.6867303 .032171 \quad 1.467299$

H $-2.6010150 .381583-1.961624$

H $0.041541-1.583412-0.829905$

H -2.037953 -1.420259 1.370830

H -1.524793 -2.8285600 .427878$

H $-2.472030-1.917765-1.596901$

H $-0.111025-0.1408811 .867593$

H -4.431072 -1.248367 0.661017

H -4.844929 -1.314499-1.063993

H -4.288379 -2.769221 -0.221233

H 1.2165501 .2386350 .230613

H $1.587212-1.5876492 .349021$

H 1.5747951 .2269292 .449958

H $3.126573-1.1701960 .252301$

H 4.3350391 .0097440 .540128

H $3.5257971 .635129-0.902361$

H $1.8192790 .202460-1.872311$

H $-4.148742 \quad 1.017698-0.036730$

H -2.7720750 .9006881 .066361$

H $5.388067-0.790824-0.859173$

H $4.620708-0.097193-2.302012$

H $5.8164040 .841714-1.398050$

SCF Energy (B3LYP/6-31G**//MMFF) $=-810.715627844$

\section{F2-23_c05466}

MMFF Geometry

C $-3.1490202 .199709-0.493052$

C -2.6161493 .1913110 .229593$

C $-2.427562-0.172545-0.904863$

O $-1.0341340 .178434-0.759343$

C $-0.352045-0.857753-0.035643$

C $-1.428842-1.7817030 .520321$

C $-2.508506-1.656933-0.535420$

C $0.550365-0.2569191 .043825$

C -3.862232 -2.179615 -0.090170

C 1.5489560 .7921750 .513818

O $1.224943-1.3169381 .715232$

O $2.322140 \quad 1.2823571 .615489$

C $2.5133770 .338593-0.599904$

C $3.564417-0.698728-0.183545$

O $3.1907021 .506547-1.082499$

C $-3.2514190 .775722-0.020306$

C $4.505928-1.060183-1.327456$

H $-3.5419192 .426706-1.480703$

H -2.574936 4.199696-0.167396

H -2.2119323 .0172701 .220675$

H -2.704081-0.032081-1.956690

H $\quad 0.245983-1.426150-0.758771$

H -1.793909 -1.4262881 .491371$

H -1.071142 -2.807765 0.650551

H $-2.189859-2.238657-1.412074$

H $-0.0756320 .239101 \quad 1.797136$

H -3.814956 -3.260331 0.081584

H -4.195049 -1.7155310 .843761$

H $-4.620862-1.993377-0.857088$

H $0.985757 \quad 1.6557440 .137572$

H $1.807004-0.9145872 .382371$

H 1.7019061 .6437342 .271445
H $1.939223-0.046307-1.448895$

H $3.079126-1.6180120 .155795$

H $4.171508-0.3148170 .644397$

H $3.6934801 .881131-0.338828$

H $-4.3106500 .497044-0.046907$

H $-2.9185350 .706351 \quad 1.022347$

H $5.089758-0.194320-1.654773$

H $5.209238-1.834646-1.004735$

H $3.947658-1.445886-2.186361$

SCF Energy $\left(B 3 L Y P / 6-31 G^{* *} / / M M F F\right)=-810.714726035$

F2-23_c05467

MMFF Geometry

C $4.260054-1.2742290 .272038$

C $4.216658-2.196775-0.696089$

C $2.5180680 .336885-0.792590$

O $1.450900-0.634164-0.715439$

C $0.2124790 .030036-0.384649$

C 0.6097921 .4023230 .134947

C $1.8274401 .703791-0.715815$

C $-0.587646-0.8673290 .568723$

C $2.6547232 .869753-0.204572$

C $-2.005582-0.3764010 .922678$

O $0.132579-1.0258251 .796466$

O

C $-2.942144-0.240033-0.290395$

C -4.3748920 .1142080 .140835$

O $-2.947411-1.472050-1.005253$

C $3.521527 \quad 0.0389190 .335134$

C $-5.3111990 .335329-1.040472$

H $4.906359-1.4786201 .123966$

H $4.807644-3.102737-0.610010$

H $3.609218-2.081736-1.585408$

H $3.0103040 .230783-1.766519$

H $-0.3288800 .137236-1.331915$

H $\quad 0.882653 \quad 1.370892 \quad 1.195767$

H -0.1851452 .1433740 .008562$

H $1.4759101 .957296-1.726292$

H $-0.653978-1.8716090 .133094$

H $3.5583192 .998555-0.808785$

H $2.0773353 .798712-0.260451$

H $2.9535542 .734697 \quad 0.839855$

H -2.438867 -1.093137 1.632563

H $1.012868-1.3728731 .571522$

H -1.3545750 .7654962 .364420$

H $-2.5717080 .548594-0.956096$

H $-4.370671 \quad 1.0231600 .752854$

H -4.786655 -0.6957590 .755143$

H -3.366177 -1.313326 -1.867599

H 4.2865850 .8232800 .361243

H 2.9988280 .0609991 .299233

H -4.925642 $1.114082-1.705919$

H $-5.447420-0.581536-1.621728$

H $-6.2968060 .651278-0.683929$

SCF Energy $\left(B 3 L Y P / 6-31 G^{* *} / / M M F F\right)=-810.717116449$

F2-23 c05468

MMFF Geometry

C -2.525565 2.5624240 .755755

C $-2.2135373 .259359-0.342833$

C $-2.6777670 .244963-0.413984$

O $-1.289108 \quad 0.256817-0.807910$

C $-0.717611-1.045449-0.611844$

C $-1.731162-1.8405660 .201330$

C $-3.038364-1.238303-0.271346$

C $0.663930-0.9536210 .055220$

C $-4.223024-1.5776940 .615234$

C $1.701364-0.203964-0.804905$

O $1.129476-2.2722300 .331451$

O $1.3010571 .159114-0.945675$

C $3.137803-0.259194-0.242230$

C 3.3130260 .4908181 .084858

O $4.0187260 .329124-1.204469$

C $-2.819778 \quad 1.0874490 .866457$

C 4.7302480 .3667001 .632742

H -2.5817073 .1126721 .693488$

H -2.026289 4.326008 -0.274712

H $-2.1378052 .802910-1.322131$ 
H $-3.2702080 .690604-1.221494$ H - $0.619961-1.509760-1.601927$ H -1.593469 -1.673022 1.276476 H -1.662826 -2.917031 0.016224 H -3.243805 -1.630829 -1.277526 H $0.545303-0.4257721 .008843$ H $-5.122723-1.0537350 .277361$ H -4.427061 -2.653293 0.581522 H -4.042091-1.313700 1.662000 H $1.711009-0.639003-1.812345$ H $\quad 1.225517-2.736670-0.517565$ H $1.9736821 .605575-1.487896$ H $3.453437-1.301495-0.118942$ H $2.617456 \quad 0.103548 \quad 1.836384$ H 3.0974691 .5571500 .948608 H $3.948291-0.191001-2.023047$ H -3.8435521 .0033911 .248317$ H -2.1486210 .6902701 .638095$ H 5.4629300 .8093640 .950880 H 4.8092440 .8864512 .592944 H $4.997179-0.6827571 .792545$ SCF Energy (B3LYP/6-31G**//MMFF) $=-810.705756645$

\section{F2-23 c05469}

MMFF Geometry

C 2.5340002 .4745820 .328291

C 1.5131183 .3300160 .202820

C $2.701509-0.0311750 .484456$

O $1.435323-0.1184801 .177743$

C $0.769042-1.3348540 .795169$

C $1.451627-1.809203-0.476460$

C $2.884098-1.385423-0.213557$

C $-0.753882-1.1344560 .719824$

C $3.764927-1.411250-1.450090$

C -1.2302860 .1562080 .023190$

O $-1.244613-1.1276502 .065417$

O $-0.846836 \quad 0.131916-1.348723$

C -2.7546490 .3898780 .118326$

C -3.590194 -0.635062 -0.659972

O $-3.0413051 .688650-0.411023$

C $2.6563831 .190739-0.445133$

C $-5.087431-0.399670-0.496673$

H 3.3353192 .7174991 .021434

H 1.4864904 .2432210 .787190

H $0.6914953 .137214-0.478125$

H 3.4873280 .1070801 .237015

H $0.985994-2.0551651 .596210$

H $1.068193-1.309500-1.369604$

H $1.345400-2.888254-0.624303$

H $3.311743-2.0841930 .519601$

H -1.183229 $-2.013990 \quad 0.228128$

H $3.869080-2.436442-1.821325$

H $3.347323-0.810422-2.264326$

H $4.766302-1.033235-1.220480$

H $-0.733455 \quad 1.0202080 .480327$

H $-0.835060-0.3731982 .523028$

H -1.153779 $0.962076-1.751924$

H $-3.069808 \quad 0.399283 \quad 1.167889$

H -3.361844 -1.650379 -0.320329

H -3.356260 -0.580471 -1.729732

H -2.5584042 .3379940 .128162$

H $3.5783211 .252373-1.034024$

H $1.8214151 .098634-1.149561$

H $-5.3844420 .576421-0.892581$

H -5.650128 -1.166477-1.038656

H $-5.378125-0.4477370 .557526$

SCF Energy (B3LYP/6-31G**//MMFF) $=-810.716759654$

\section{F2-23 c05470}

MMFF Geometry

C $2.5723582 .651159-0.251415$

C $1.5851533 .312415-0.866130$

C 2.7311610 .3070470 .641108

$\begin{array}{lllll}\text { O } & 1.314926 & 0.270675 & 0.917437\end{array}$

C $0.784541-1.0162080 .562659$

C $1.890288-1.742243-0.195768$

C $3.134902-1.1554510 .437900$

C $-0.518863-0.879103-0.241237$
C $4.403961-1.411717-0.354654$

C $-1.675740-0.2368680 .562754$

O $-0.902426-2.170224-0.707156$

O $\quad-1.268808 \quad 1.0347591 .061890$

C $-2.974156-0.060391-0.257854$

C -4.0668850 .6654720 .543618$

O $-3.490643-1.326899-0.656566$

C $2.9563101 .231322-0.565514$

C $-5.3391240 .878423-0.268320$

H 3.1430773 .1632970 .518875

H $1.3589544 .337837-0.595453$

H $0.9869532 .846544-1.641541$

H $3.238158 \quad 0.717970 \quad 1.522374$

H $0.586223-1.5552821 .498081$

H $1.853520-1.508077-1.266648$

H $1.825660-2.829315-0.087094$

H $3.254055-1.6125181 .430438$

H $-0.319753-0.248984-1.117360$

H $4.315743-1.074571-1.392400$

H $5.256450-0.8996730 .102993$

H $4.627550-2.483695-0.378186$

H -1.897265 -0.8814121 .422729$

H -1.082146 -2.724657 0.071593

H $-1.034244 \quad 1.591378 \quad 0.299715$

H $-2.7623810 .523839-1.162108$

H -3.7068041 .6422350 .884786$

H $-4.3236790 .077673 \quad 1.433292$

H -2.844388 -1.732111-1.259174

H $4.0137871 .232981-0.851626$

H $2.3819250 .874642-1.429129$

H $-5.789924-0.073879-0.563941$

H $-6.076628 \quad 1.4278250 .325494$

H -5.132665 $1.458330-1.173433$

SCF Energy (B3LYP/6-31G**//MMFF) $=-810.712707522$

F2-23 c05471

MMFF Geometry

C - -1.4475992 .6938340 .259232$

C $-2.0015803 .904641 \quad 0.130805$

C $-2.0508450 .447439-0.701016$

O $-\begin{array}{llll}0.669853 & 0.027289 & -0.725641\end{array}$

C $-0.574253-1.356482-0.360992$

C $-1.941891-1.7429310 .192595$

C $-2.861434-0.848861-0.613069$

C $0.575914-1.596147 \quad 0.627505$

C $-4.265528-0.746818-0.045239$

C $1.986460-1.2078350 .127816$

O $0.620303-2.992908 \quad 0.943741$

O $2.238418-1.815871-1.139574$

$\begin{array}{llll}\text { C } & 2.265713 & 0.304736 & 0.044277\end{array}$

C $3.7389500 .593891-0.287186$

$\begin{array}{lllll}\text { O } & 1.929084 & 0.928989 & 1.279897\end{array}$

C -2.2320991 .4270670 .468952$

C $4.0187652 .085634-0.420463$

H -0.3642492 .6126110 .222848$

H $-1.3788094 .780873-0.012421$

H -3.076342 4.0440410 .164870

H $-2.2657260 .959373-1.646797$

H $-0.404836-1.928182-1.282146$

H -2.015213 -1.5089681 .261639$

H $-2.159600-2.8076490 .063407$

H -2.934103 -1.268036 -1.626712

H $0.380085-1.0614691 .564332$

H $-4.768653-1.718362-0.098786$

H $-4.262598-0.439508 \quad 1.005333$

H $-4.862427-0.026873-0.614238$

H $2.707826-1.6533960 .825306$

H $-0.190009-3.2129471 .433196$

H $1.728236-1.332282-1.811160$

H $1.6548690 .761163-0.740603$

H $4.0212740 .097881-1.222508$

H 4.3833150 .1955890 .505985

H 2.4864540 .5357151 .973028

H $-3.292413 \quad 1.6720070 .595756$

H $-1.8827980 .973937 \quad 1.404470$

H $5.0681212 .249719-0.685182$

H $3.3976642 .531551-1.203891$

H 3.8228682 .6142180 .517586 
H $-1.871686-0.4858541 .721116$

H $-1.494024-2.4120850 .190063$

H -0.2783161 .1166681 .761502$

H -3.5043921 .2430190 .825128$

H $-4.043816-1.2780360 .902744$

H $-3.651230-1.327394-0.826617$

H $-2.2017291 .872595-0.929401$

H $4.0089640 .876602-0.968175$

H $2.3015650 .769791-1.411805$

H $\quad \begin{array}{llll}-5.802739 & 0.437023 & 0.415998\end{array}$

H $-5.3919870 .363304-1.309332$

H -6.060649-1.059545-0.495337

SCF Energy (B3LYP/6-31G**//MMFF) $=-810.715483376$

F2-23_c05474

MMFF Geometry

C $-4.4812971 .477746-0.485988$

C -4.4705702 .7171150 .017291$

C $-2.799070-0.377947-0.479617$

O $-1.6320640 .451096-0.355244$

C $-0.502272-0.359547-0.731367$

C $-0.903765-1.809465-0.448180$

C $-2.343918-1.7384820 .049275$

C $0.7485120 .152261-0.000115$

C $-2.402010-1.8827381 .570547$

C $2.040725-0.542760-0.461175$

O $0.595043-0.0332931 .405103$

O $2.213624-0.309531-1.859350$

C $3.302761-0.0869160 .301828$

C 3.7028961 .3695940 .031911

O $4.392222-0.925484-0.096652$

C -3.9789750 .2656830 .248239$

C 4.9229831 .7905500 .843257

H -4.885182 $1.324492-1.483464$

H -4.852952 $3.549211-0.563692$

H -4.0822502 .9240561 .008404$

H -3.024421 -0.467682 -1.551925

H $-0.371011-0.224272-1.812154$

H $-0.235172-2.3203850 .251886$

H $-0.858399-2.378218-1.385362$

H $-2.948760-2.542353-0.385919$

H $0.8201201 .231594-0.178398$

H -1.875313 -1.071248 2.080913

H -3.438633 -1.886477 1.922661

H -1.949075 -2.829192 1.885151

H $1.940864-1.626924-0.330225$

H -0.1967390 .4623141 .675676$

H $3.040115-0.747206-2.126378$

H $3.164399-0.2359421 .378797$

H 2.8767132 .0443760 .277351

H $3.9417851 .506310-1.029377$

H $4.162198-1.8389500 .144655$

H $-4.805978-0.4500010 .322512$

H -3.6883850 .5493491 .266984$

H $5.803011 \quad 1.1954820 .580281$

H 5.1591192 .8417360 .649309

H 4.7373461 .6770651 .916015

SCF Energy (B3LYP/6-31G**//MMFF) $=-810.717293585$

F2-23_c05475

MMFF Geometry

C -3.857929 $1.879312-0.235902$

C $-3.2850353 .075785-0.062827$

C $-2.918366-0.434907-0.525417$

O $-1.7319620 .078070-1.172094$

C $-0.590312-0.703606-0.767338$

C - $-1.041085-1.5220850 .433689$

C $-2.497140-1.7721620 .094103$

C $0.6128910 .225574-0.542212$

C $-3.319984-2.2762841 .266161$

C $1.908402-0.526001-0.165032$

$\begin{array}{lllll}\text { O } & 0.285534 & 1.196292 & 0.451634\end{array}$

O $2.129704-1.542535-1.139868$

C $3.1439610 .399725-0.090102$

C $4.442412-0.3731390 .191098$

$\begin{array}{lllll}\text { O } & 2.975238 & 1.364514 & 0.945642\end{array}$

C -3.4162070 .6259860 .467995$

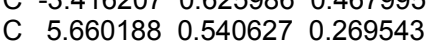


H $-4.700063 \quad 1.797846-0.918037$

H $-3.6535443 .943794-0.598257$

H -2.443456 3.2102540 .607684

H -3.677821 -0.603924 -1.298341

H $-0.377897-1.379503-1.605128$

H $-0.959839-0.9578241 .368704$

H $-0.464320-2.4451190 .547015$

H -2.531510 -2.534201 -0.697601

H $0.7749840 .783143-1.472939$

H -3.247490 -1.613561 2.134442

H $-4.374991-2.3668680 .988451$

H -2.968109 -3.2648331 .580227$

H $1.764813-1.0023180 .813350$

H $\quad 0.315774 \quad 0.764126 \quad 1.321415$

H $2.729936-2.200262-0.750373$

H $3.2622290 .934517-1.040701$

H $4.622318-1.116673-0.592646$

H $4.354390-0.9088001 .144026$

H 2.2349301 .9416820 .692670

H $-4.276866 \quad 0.246293 \quad 1.029633$

H -2.6290310 .8644491 .193419$

H $5.7847231 .106455-0.659154$

H 5.5760951 .2507041 .097922

H $6.565847-0.0530550 .429149$

SCF Energy (B3LYP/6-31G $\left.{ }^{* *} / / \mathrm{MMFF}\right)=-810.714233052$

F2-23 c05476

MMFF Geometry

C -3.544055 2.122117 -0.337558

C $-2.8641603 .267009-0.209468$

C $-2.848540-0.290542-0.475575$

O $-1.6083980 .057459-1.132295$

C $-0.569414-0.845519-0.702752$

C -1.101291-1.527811 0.548501

C $-2.579252-1.6260830 .228007$

C $0.743301-0.066449-0.526775$

C $-3.443769-1.958270 \quad 1.431001$

C $1.937293-0.951218-0.114530$

O $\quad 0.5485650 .9475090 .457826$

O $2.064852-2.036532-1.039044$

C $3.310034-0.242441-0.085191$

C $3.465711 \quad 0.7600741 .064478$

O $3.5168790 .420174-1.333239$

C -3.244895 0.8732790 .445144

C $4.915921 \quad 1.182127 \quad 1.273708$

H $-4.3716032 .083193-1.041122$

H -3.133206 4.134820 -0.801633

H $-2.032935 \quad 3.3594970 .480774$

H -3.613173 -0.425562 -1.250221

H $-0.460857-1.581691-1.508505$

H $-0.942396-0.913817 \quad 1.442480$

H $-0.636408-2.5031350 .721419$

H -2.709577 -2.427006 -0.513759

H $\quad 0.960747 \quad 0.441817-1.473715$

H -3.289107 -1.254123 2.254787

H $-4.504627-1.9462761 .161178$

H -3.203557 -2.958290 1.807460

H $1.762433-1.3839410 .878236$

H $-0.190331 \quad 1.5041190 .158192$

H $\quad 1.355450-2.672855-0.848175$

H $4.086798-1.013873-0.006094$

H 3.0962530 .3197531 .997310

H 2.8882681 .6685450 .862727

H $4.4427360 .712994-1.365669$

H $-4.1481180 .618740 \quad 1.010597$

H -2.4464691 .0697861 .170700$

H $5.551973 \quad 0.314477 \quad 1.475084$

H 5.3118361 .7055100 .398404

H 4.9893761 .8608072 .129620

SCF Energy (B3LYP/6-31G**//MMFF) $=-810.710192162$

F2-24_c05724

MMFF Geometry

C $4.3155181 .754623-0.259915$

C 4.3533052 .9008130 .428753

C $2.713023-0.173656-0.277330$

O $1.566038 \quad 0.6053340 .119946$

C $0.492340-0.2901860 .476162$
C $0.923158-1.6720910 .002918$

C $2.425223-1.5996820 .189988$

C $-0.8228480 .236778-0.116518$

C $3.183591-2.668504-0.577638$

C $-2.044028-0.6019900 .293194$

O $-0.7332290 .260119-1.540610$

O $-2.145403-0.6023641 .717290$

C $-3.372556-0.114903-0.323370$

C $-3.839326 \quad 1.2502130 .199092$

O $-4.386751-1.076435-0.013903$

C 3.9779510 .4207630 .343966

C $-5.1261131 .714269-0.474135$

H $4.5509351 .776278-1.320895$

H $4.6089833 .831163-0.066659$

H 4.1305122 .9332081 .489423

H $2.763931-0.131103-1.373223$

H $0.437710-0.2766591 .571718$

H $0.677004-1.830196-1.053730$

H $0.458616-2.4755130 .582562$

H $2.650787-1.6972861 .260538$

H -0.9459351 .2738990 .216144$

H $2.991154-2.602542-1.653779$

H $4.262532-2.570593-0.421981$

H $2.883803-3.667297-0.243623$

H -1.890170 -1.643052 -0.016529

H $\quad 0.0543900 .781526-1.772854$

H -2.927973 -1.129948 1.952215

H -3.289291 -0.080917 -1.415775

H -3.0688072 .0086940 .028334$

H -4.024242 1.1995661 .278598

H $-4.119148-1.922616-0.411306$

H $3.8452300 .522546 \quad 1.428343$

H $4.829913-0.2486820 .179649$

H -4.995742 $1.786632-1.558632$

H $-5.9540751 .028050-0.270925$

$\mathrm{H}-5.4107872 .702927-0.100415$

SCF Energy $(B 3 L Y P / 6-31 G * * / / M M F F)=-810.721213595$

F2-24 c05725

MMFF Geometry

C $4.401399-1.3582650 .066638$

C $4.439448-2.678362-0.146292$

C $2.5596340 .338414-0.058798$

O $1.502717-0.592097 \quad 0.251287$

C $0.2283600 .034151-0.018376$

C $0.5299181 .511055-0.231016$

C $1.9022791 .451939-0.871701$

C $-0.740857-0.3168691 .118192$

C $2.6486262 .774016-0.828893$

C -2.1982530 .1464860 .926081$

O $-\begin{array}{llll}0.266237 & 0.249833 & 2.345005\end{array}$

O -2.2610851 .5686330 .834153$

C $-2.888915-0.460082-0.307381$

C $-4.374946-0.074533-0.381098$

O $-2.769265-1.879739-0.277659$

C $3.674545-0.383897-0.816763$

C $-5.057727-0.637579-1.621318$

H $4.933537-0.9532270 .923449$

H $4.986959-3.3279240 .527830$

H $3.928561-3.132428-0.988160$

H $2.9323030 .728450 \quad 0.897546$

H - $0.125053-0.408396-0.956941$

H $0.5780062 .053476 \quad 0.720478$

H $-0.2117602 .002757-0.867396$

H $1.7878821 .137257-1.917823$

H $-0.726844-1.4030411 .269134$

H $3.6360752 .680929-1.291722$

H $2.0934043 .546332-1.371168$

H $2.790258 \quad 3.1218680 .199893$

H $-2.759457-0.134371 \quad 1.826822$

H $0.649891-0.0530972 .467840$

H -1.829400 1.9292681 .627589

H -2.400120 -0.101203 -1.220343

H $-4.4847651 .015646-0.383914$

H -4.901689 -0.4572190 .501423$

H $-3.215097-2.1985790 .525667$

H $3.253695-0.906461-1.684899$

H $4.4059460 .340286-1.193072$ 
H $-5.042385-1.731869-1.623532$

H $-6.103765-0.316764-1.653428$

H $-4.566299-0.283572-2.533209$

SCF Energy $\left(B 3 L Y P / 6-31 G^{* *} / / M M F F\right)=-810.721338900$

\section{F2-24 c05726}

MMFF Geometry

C $-3.3636392 .296690 \quad 0.569417$

C $-3.051253 \quad 3.419942-0.086654$

C $-2.542893-0.063500 \quad 0.375982$

O $-1.2383890 .298401-0.119796$

C $-0.577326-0.880336-0.606141$

C $-1.413903-2.062021-0.130393$

C $-2.809276-1.471968-0.154946$

C $0.884423-0.932546-0.136922$

C $-3.816292-2.2624420 .662073$

C $1.7348140 .237573-0.675198$

O $1.452600-2.172950-0.549641$

O $1.2854071 .473506-0.118447$

C $3.2437010 .096509-0.386610$

C 3.6083520 .1724901 .102614

O $3.9289161 .139936-1.087072$

C $-3.5724560 .964764-0.094267$

C $5.102173-0.0167741 .338324$

H -3.484482 2.340031 1.648708

H $-2.917404 \quad 4.3515920 .452098$

H -2.922178 $3.431368-1.163183$

H -2.477458 -0.0713761 .471902$

H $-0.612323-0.843501-1.702865$

H -1.136506 -2.351303 0.890986

H -1.306618 $-2.939897-0.774673$

H -3.151091-1.422248 -1.197516

H $0.891366-0.9001720 .958978$

H -3.911912 -3.281343 0.272836

H -3.515282 -2.331012 1.712860

H $-4.804336-1.7930700 .624826$

H $1.6111070 .312617-1.763220$

H $1.445883-2.193037-1.521910$

H $\quad 0.3563511 .594669-0.377585$

H $3.612016-0.846222-0.805803$

H $3.068321-0.5957651 .665604$

H 3.3238041 .1480141 .514024

H $3.595691 \quad 1.988373-0.747571$

H -3.513546 $1.075888-1.184335$

H $-4.585340 \quad 0.6224700 .145896$

H 5.3205060 .0110542 .410669

H $5.440518-0.9821770 .948596$

H $5.686096 \quad 0.7736310 .856655$

SCF Energy $\left(B 3 L Y P / 6-31 G^{* *} / / M M F F\right)=-810.722490260$

\section{F2-24 c05727}

MMFF" Geometry

C -1.8274213 .0117860 .143599$

C $-0.9558943 .778151-0.521659$

C -2.1398650 .5280440 .243251$

O $-0.763247 \quad 0.223571-0.054578$

C $-0.640990-1.174499-0.355476$

C $-1.966367-1.8068030 .057705$

C $-2.940524-0.682768-0.231605$

C $0.576230-1.7859740 .349083$

C $-4.282067-0.8489340 .460567$

C $1.948745-1.268186-0.139306$

O $0.538342-3.2011570 .134902$

O $2.054870-1.446166-1.552798$

C 2.2519390 .2027780 .193418

C $3.6999860 .580865-0.159753$

O $2.026836 \quad 0.4435041 .579268$

C $-2.5364441 .829326-0.454802$

C 3.9963202 .0498410 .115851

H -2.049286 3.2536461 .179865

H $-0.4792514 .620146-0.031765$

H $-0.7009773 .580261-1.556822$

H $-2.2148760 .636283 \quad 1.333325$

H - $0.537037-1.268227-1.443712$

H -1.966612 $-2.049710 \quad 1.127679$

H $-2.188198-2.721755-0.500099$

H -3.099730 -0.626578 -1.316952

H $0.492645-1.6194251 .429632$
H $-4.945407-0.0083040 .234181$

H $-4.775512-1.7676280 .126920$

H $-4.168271-0.9048191 .548404$

H $2.725271-1.8973430 .315176$

H $1.258939-3.5961210 .654811$

H $1.866216-2.381207-1.742691$

H $1.5901420 .861759-0.378048$

H $3.8989590 .374151-1.217221$

H $4.397154-0.024506 \quad 0.432237$

H $2.630480-0.1293972 .082412$

H $-2.3089651 .759113-1.525965$

H $-3.6151331 .996955-0.359369$

H 3.8848232 .2871841 .178371

H $5.0244432 .287565-0.175009$

H $3.3238782 .698207-0.454947$

SCF Energy (B3LYP/6-31G**//MMFF $)=-810.715129567$

F2-24_c05728

MMFF Geometry

C $4.424465-1.576369-0.448493$

C $5.577385-1.9134380 .140463$

C 2.6761920 .1355950 .106305

O $1.584188-0.598669-0.484617$

C $0.4347400 .267980-0.579859$

C $0.766057 \quad 1.499947 \quad 0.251283$

C 2.2638001 .6062170 .047304

C $-0.820596-0.503667-0.147349$

C 2.9536242 .4856051 .076130

C $-2.1184380 .317638-0.294331$

O $-0.672725-0.923400 \quad 1.208591$

O $-2.1924780 .836556-1.621035$

C $-3.398660-0.4934910 .008444$

C $-4.6722740 .328303-0.246371$

O $-3.411983-0.9162411 .369122$

C $3.970048-0.158797-0.655133$

C $-5.942756-0.4728610 .012271$

H $3.775393-2.368473-0.812673$

H $5.851216-2.9566550 .254802$

H $6.263209-1.1628760 .517304$

H $2.759001-0.1990871 .148771$

H $0.3551090 .547570-1.637431$

H $\quad 0.5352391 .343708 \quad 1.311663$

H $0.2238382 .388706-0.085437$

H $2.4540422 .009800-0.956504$

H $-0.891735-1.412920-0.757305$

H 4.0325392 .5251230 .896985

H 2.5662893 .5087081 .028616

H 2.7944952 .1130722 .093656

H $-2.079367 \quad 1.1657820 .400316$

H $\quad 0.159446-1.4234041 .268864$

H $-2.2387840 .081305-2.231942$

H -3.429427 -1.386510 -0.628109

H $-4.6945150 .688699-1.280759$

H -4.6824501 .2065080 .410775$

H $-2.646727-1.4997921 .504917$

H $3.813041-0.012757-1.730870$

H $4.7557960 .538213-0.341393$

H $-6.8238950 .136215-0.213535$

H -5.976940 -1.366115 -0.619455

$\mathrm{H}-6.012352-0.7867631 .058270$

SCF Energy $\left(B 3 L Y P / 6-31 G^{* *} / / M M F F\right)=-810.723788369$

F2-24_c05729

MMFF Geometry

C $-4.225310-1.8425930 .278268$

C $-5.364198-2.211697-0.318281$

C $-2.6569350 .077927-0.104988$

O $-1.483799-0.6189590 .362582$

C -0.4111370 .3306950 .532693$

C $-0.8808081 .618914-0.129282$

C $-2.373379 \quad 1.5620310 .125927$

C $0.887858-0.269089-0.024926$

C $-3.1758952 .493038-0.766571$

C 2.1118880 .6349640 .203188

O $0.744752-0.511833-1.423929$

$\begin{array}{lllll}\text { O } & 2.271415 & 0.890417 & 1.598965\end{array}$

C $3.4265530 .077454-0.383988$

C $3.953747-1.1760370 .328950$ 
O $4.403878 \quad 1.118464-0.285224$

C $-3.891231-0.4242730 .646328$

C $5.174931-1.773159-0.361163$

H -3.495502 $-2.609428 \quad 0.525515$

H $-5.548235-3.254358-0.553535$

H $-6.127472-1.488031-0.582183$

H $-2.747614-0.134073-1.178504$

H $-0.3135310 .486647 \quad 1.614155$

H $-0.6771321 .614931-1.206693$

H -0.4071152 .5064290 .301072$

H -2.560354 1.8211841 .176814

H $1.039384-1.2423330 .455753$

H - $-2.8782353 .533923-0.602309$

H -3.023201 $2.263230-1.826447$

H -4.246595 $2.411411-0.555261$

H $1.9350661 .609001-0.269689$

H $-0.045841-1.065885-1.542816$

H 2.2857050 .0359822 .062339

H $3.285920-0.128269-1.451487$

H $3.176276-1.9453850 .368583$

H $4.237870-0.933948 \quad 1.359747$

H $5.1604080 .869034-0.841858$

H $-3.709124-0.3947681 .727603$

H $-4.746444 \quad 0.2296120 .440223$

H $6.028265-1.089258-0.330589$

H $5.473784-2.6991560 .140856$

H $4.957250-2.010244-1.407373$

SCF Energy (B3LYP/6-31G**//MMFF) $=-810.716219970$

F2-24_c05730

MMFF Geometry

C $3.2909302 .321115-0.370871$

C $2.7196283 .494679-0.077950$

C $2.514408-0.051579-0.140898$

O $1.112246 \quad 0.263273-0.264699$

C $0.340506-0.8825640 .127915$

C $1.319113-2.047640 \quad 0.209089$

C $2.579244-1.3449060 .672089$

C $-0.815007-1.120202-0.845577$

C $3.843649-2.1555150 .446378$

C $-1.7237650 .107918-1.048502$

O $-1.562481-2.247018-0.396869$

O $-2.737599-0.256006-1.992071$

C -2.3801750 .7097810 .211062$

C $-3.441525-0.1723160 .879691$

O $-3.0133321 .936910-0.174637$

C $3.244367 \quad 1.115722 \quad 0.525213$

C -4.0726640 .5000682 .094516$

H $3.8324832 .224696-1.308418$

H $2.7936154 .328883-0.767143$

H 2.1709363 .6442430 .845354

H $2.895333-0.216474-1.157309$

H $-0.046340-0.6904251 .136628$

H $1.472882-2.500977-0.778063$

H $0.984931-2.8311020 .895954$

H $2.482703-1.1207691 .743035$

H $-0.407512-1.390281-1.828628$

H $4.726845-1.6037530 .782880$

H $3.801221-3.097078 \quad 1.003768$

H $3.981240-2.398063-0.612727$

H $-1.1319400 .892256-1.537218$

H -2.299985 -2.371470 -1.018601

H $-3.2971140 .527225-2.132208$

H $-1.611903 \quad 0.9747260 .945394$

H -3.000431 -1.116333 1.211036

H $-4.243399-0.406640 \quad 0.169941$

H -2.321077 $2.530172-0.512847$

H 2.7495111 .3706211 .470840

H 4.2757750 .8305590 .761594

H -4.6104791 .4111201 .814429$

H $-4.789088-0.1779552 .569498$

H -3.311123 0.7621892 .836092

SCF Energy (B3LYP/6-31G*//MMFF) $=-810.720313663$

F2-24_c05731

MMFF Geometry

C $4.203822-1.765458-0.345606$

C $5.294982-2.2109650 .287326$
C $2.5795490 .076467 \quad 0.168330$

O $1.460373-0.550877-0.490762$

C $0.3925120 .411051-0.617404$

C $0.785066 \quad 1.593852 \quad 0.257523$

C $2.294548 \quad 1.577503 \quad 0.125880$

C $-0.942992-0.261653-0.262060$

C $3.0049462 .374718 \quad 1.206224$

C $-2.1562140 .672680-0.431974$

O $-0.885851-0.7207201 .087346$

O $-2.0975341 .246736-1.742015$

C $-3.539436 \quad 0.001718-0.281615$

C $-3.868144-0.4441711 .145975$

O $-3.626335-1.133236-1.144217$

C $3.880084-0.310652-0.538325$

C $-5.331644-0.846657 \quad 1.292581$

H $3.508940-2.492319-0.758378$

H $5.474729-3.2756520 .388687$

H $6.022488-1.5290680 .713304$

H $2.583067-0.2849101 .205257$

H $0.3876050 .717393-1.670647$

H $\quad 0.491226 \quad 1.436506 \quad 1.302030$

H $0.3358102 .531876-0.082409$

H $2.5657401 .983427-0.858073$

H $-1.055002-1.146909-0.899480$

H 4.0907762 .3262901 .078396

H 2.7073043 .4275841 .165144

H $2.7662551 .996906 \quad 2.206098$

H -2.089982 1.4958370 .289978

H $-0.109821-1.3021361 .164011$

H -2.807474 $1.908343-1.805396$

H $-4.3091180 .707422-0.619042$

H $-3.652436 \quad 0.3649331 .852644$

H $-3.264252-1.3137541 .425946$

H $-3.414672-0.830417-2.043911$

H $3.788386-0.129740-1.616310$

H $4.7048970 .310688-0.170828$

H $-5.537277-1.1505392 .323852$

H $-5.994133-0.0103761 .047857$

H $-5.580755-1.6871890 .637310$

SCF Energy (B3LYP/6-31G**//MMFF) $=-810.719964825$

F2-24 c05732

MMFF Geometry

C $4.431515-1.3535040 .234146$

C $4.505925-2.652547-0.076324$

$\begin{array}{lllll}\text { C } & 2.590895 & 0.341020 & 0.064548\end{array}$

$\begin{array}{llll}\text { O } & 1.519682 & -0.614613 & 0.198914\end{array}$

C $0.2687830 .028376-0.135116$

C $0.5747621 .518222-0.206163$

C $2.0010991 .512039-0.718576$

C $-0.798178-0.411627 \quad 0.875857$

C $2.7270932 .829115-0.505735$

$\begin{array}{lllll}\text { C }-2.238780 & 0.063881 & 0.587566\end{array}$

$\begin{array}{llll}0 & -0.417183 & 0.059657 & 2.174073\end{array}$

O -2.3045721 .4878490 .593507$

C $-2.809480-0.454397-0.742580$

C $-4.2502820 .003308-1.027663$

O $-2.748677-1.878151-0.754251$

C $3.781137-0.315774-0.636620$

C $-5.276274-0.475860-0.008752$

H $4.873901-1.0147421 .167359$

H $4.993869-3.3506710 .594998$

H $4.084088-3.041376-0.996418$

H $2.8666340 .656781 \quad 1.079371$

H $0.008167-0.340911-1.133576$

H 0.5289061 .9871110 .783743

H $-0.1093602 .055111-0.869912$

H $1.9875911 .278353-1.791745$

H $-0.780206-1.5065250 .939621$

H $3.7539372 .774704-0.880356$

H $2.2169133 .639381-1.036765$

H 2.7693863 .0972390 .555295

H $-2.866977-0.2855131 .415579$

H $-1.053858-0.2997812 .815175$

H $-1.943688 \quad 1.7914861 .444105$

H $-2.191219-0.087218-1.569845$

H $-4.551544-0.388604-2.007312$

H $-4.2919631 .096245-1.096562$ 
H $-3.081332-2.179751-1.616770$

H $3.450506-0.770504-1.578943$

H $4.5368350 .438201-0.884048$

H -5.260122 -1.5651300 .093739$

H $-5.101417-0.0312630 .975198$

H $-6.282320-0.184065-0.327396$

SCF Energy (B3LYP/6-31G**//MMFF) $=-810.712330971$

F2-24 c05733

MMFF Geometry

C -2.9483822 .4595730 .373000$

C $-4.0109193 .080850 \quad 0.897155$

C $-2.376150 \quad 0.0338210 .083413$

$\begin{array}{lllll}\text { O } & -0.949452 & 0.232041 & 0.157389\end{array}$

C $-0.287646-0.964145-0.281883$

C $-1.360697-2.045900-0.340032$

C $-2.576151-1.236293-0.744186$

C $0.878931-1.3158170 .642999$

C $-3.892473-1.946802-0.480500$

C $1.893140-0.1757990 .892943$

O $1.547630-2.4580830 .107837$

O $2.760069-0.5994561 .951989$

C $2.7702560 .210663-0.315363$

C 3.8357031 .2583640 .052548

O $1.9619050 .733055-1.364728$

C $-3.0373671 .266356-0.536243$

C $4.7184891 .629833-1.132766$

H -1.9568972 .8400580 .604384$

H -3.8802243 .9420601 .543371$

H -5.0220182 .7452760 .694689$

H $-2.731134-0.1169611 .111341$

H $0.067367-0.793250-1.304399$

H -1.514271 -2.5017630 .645987$

H -1.117457 -2.842235 -1.049932

H -2.502134 -1.002195-1.814795

H $\quad 0.472411-1.6174971 .617224$

H -3.946857 -2.879526 -1.051354

H $-4.009205-2.1947590 .579959$

H -4.740711 -1.321169-0.775095

H 1.3568520 .7112191 .250892

H $2.280886-2.6733960 .709440$

H $2.211521-0.7403092 .742443$

H $3.284368-0.677786-0.699787$

H 4.4764780 .8850110 .858994

H 3.3475062 .1712590 .414538

H $1.4989491 .516841-1.022234$

H -2.536513 $1.526643-1.476884$

H $-4.0856541 .049485-0.772285$

H $5.2255300 .746875-1.534566$

H $4.1359402 .089383-1.937094$

H $5.4836622 .348047-0.821241$

SCF Energy (B3LYP/6-31G**//MMFF) $=-810.718908400$

\section{F2-24 c05734}

MMFF Geometry

C -4.105567 -1.565393 0.499935

C $-5.210148-1.368177 \quad 1.228436$

C $-2.592515-0.034318-0.858760$

O $-1.413356-0.833709-0.643206$

C $-0.490763-0.0775220 .170868$

C $-1.034061 \quad 1.3466670 .217097$

C -2.5296851 .0991760 .163784$

C $0.933565-0.246074-0.379675$

C $-3.3407862 .323782-0.220522$

C 2.0023280 .4520610 .480260

O $0.9941520 .231192-1.723344$

O $1.939479-0.0791031 .803660$

C $3.4345230 .312199-0.076888$

C $3.998866-1.114258-0.014582$

O $4.289597 \quad 1.1894250 .662958$

C $-3.847189-0.910845-0.827700$

C $5.395622-1.206267-0.617736$

H -3.348261 -2.2477040 .877150$

H -5.339575 -1.875844 2.178109

H -5.997934 -0.7015690 .895669$

H -2.493842 $0.375585-1.873021$

H $-0.547752 \quad-0.517480 \quad 1.174490$

H $-0.716811 \quad 1.932315-0.652668$
H $-0.7215621 .882921 \quad 1.118235$

H $-2.853127 \quad 0.752897 \quad 1.153170$

H $1.132725-1.322758-0.425326$

H -3.1964513 .1255520 .511158$

H -3.047046 2.708565 -1.202817

H $-4.4089072 .087191-0.256459$

H 1.7709191 .5223010 .551702

H $1.0099691 .202622-1.701690$

H 2.5317350 .4518082 .362191

H $3.4580310 .669064-1.112366$

H $3.343336-1.806818-0.552178$

H $4.058303-1.4538821 .026089$

H 4.3958380 .8229561 .556937

H $-4.713143-0.312179-1.134709$

H -3.735394 -1.713982-1.566835

H $5.392379-0.881230-1.662958$

H $6.110559-0.588099-0.065971$

H $5.751900-2.240868-0.585544$

SCF Energy (B3LYP/6-31G**//MMFF) $=-810.712518273$

\section{F2-24 c05735}

MMFF Geometry

C $-4.066017 \quad 1.656090 \quad 0.210508$

C $-4.0072322 .938225-0.166473$

C $-2.410120-0.2248860 .126910$

O -1.2464090 .6222720 .211625$

C $-0.070203-0.157220-0.106642$

C $-0.523842-1.610086-0.093294$

C $-1.948325-1.488393-0.596365$

C 1.0484750 .2275490 .869132

C $-2.799520-2.713164-0.308672$

C $2.433715-0.4090390 .643619$

O $0.643087-0.1156442 .202428$

O $2.346257-1.8346470 .723140$

C $3.152381-0.060331-0.672078$

C $3.3436391 .436225-0.934617$

O $2.449210-0.604804-1.789344$

C $-3.5311380 .513624-0.606062$

C 4.1552332 .1474510 .135270

H -4.5360691 .4132501 .160060$

H -4.4165293 .7167310 .468163$

H -3.552407 $3.233575-1.105413$

H $-2.710229-0.4587711 .156772$

H $0.2029770 .138365-1.124880$

H $-0.514294-2.0252850 .921404$

H $0.094158-2.249965-0.729542$

H -1.924066 -1.314221 -1.680597

H $\quad \begin{array}{llll}1.151634 & 1.318683 & 0.870567\end{array}$

H -2.379036 -3.597079 -0.799277

H -2.855372 -2.919873 0.765393

H $-3.820269-2.575519-0.678584$

H $3.081438-0.1133301 .478268$

H -0.2200380 .3045822 .359178$

H $1.931439-2.0513961 .575836$

H $4.135128-0.548466-0.671976$

H $2.372361 \quad 1.930259-1.056527$

H $3.8481031 .559265-1.901851$

H $2.343176-1.558010-1.627170$

H $-3.1620800 .882824-1.571118$

H $-4.361723-0.170771-0.812774$

H 5.1257651 .6621310 .279078

H $4.3366983 .186034-0.159691$

H 3.6297992 .1632901 .094471

SCF Energy $(\mathrm{B} 3 \mathrm{LYP} / 6-31 \mathrm{G} * * / / \mathrm{MMFF})=-810.722230459$

F2-24 c05736

MMFF Geometry

C $2.6592312 .144738 \quad 0.823230$

C 3.8387812 .4639031 .367505

C $1.9017170 .351325-0.815937$

O $0.5731970 .269480-0.266449$

C $0.402677-1.020975 \quad 0.340917$

C $1.605233-1.856504-0.095237$

C $2.688228-0.800095-0.193631$

C $-0.943183-1.646299-0.046210$

C $3.895920-1.233504-1.004966$

C -2.204555 -0.8953710 .437130$

O $-0.994951-2.9729450 .491202$ 
O $-2.147865-0.719876 \quad 1.853776$

C $-2.470657 \quad 0.459260-0.244210$

C $-3.830540 \quad 1.0458800 .168708$

O $-2.4325810 .305730-1.659900$

C $2.4766601 .756072-0.616705$

C $-4.0875952 .408841-0.461619$

H 1.7712732 .1770691 .449372

H 3.9036952 .7420822 .413709

H 4.7538962 .4520900 .786013

H $1.7898990 .183767-1.895605$

H $0.460029-0.881848 \quad 1.427647$

H $1.427136-2.316728-1.075310$

H $1.844174-2.6523510 .616821$

H $3.012367-0.5487740 .823807$

H -0.994323 -1.749019-1.136187

H $4.638324-0.430790-1.056319$

H $4.373100-2.106627-0.548064$

H $3.616927-1.501167-2.029653$

H $-3.061295-1.5551970 .248523$

H $-0.986969-2.8964171 .460954$

H -1.512014 -0.0093592 .043598$

H -1.6981291 .1820830 .037416$

H $-3.884493 \quad 1.1463161 .258561$

H $-4.636322 \quad 0.369417-0.141268$

H -3.130184 - $0.324268-1.909317$

H $3.4258501 .838399-1.159652$

H $1.7888422 .485489-1.062468$

H $-4.1194792 .344158-1.553640$

H $-5.0491412 .805725-0.120940$

H -3.307058 $3.123203-0.180875$

SCF Energy (B3LYP/6-31G**//MMFF) $=-810.718992328$

F2-24_c05737

MMFF Geometry

C $-3.374300 \quad 2.4233360 .402579$

C $-2.9509263 .524508-0.228202$

C -2.633810 0.0299200 .317266

O $-1.2716610 .331391-0.046280$

C $-0.615913-0.877897-0.461369$

C $-1.543278-2.017676-0.055169$

C $-2.904193-1.373482-0.222951$

C $0.793707-0.984782 \quad 0.141120$

C $-4.017979-2.1118870 .498846$

C $1.7686920 .099536-0.380245$

O $1.311202-2.282775-0.138744$

$\begin{array}{lllll}\text { O } & 1.218760 & 1.394456 & -0.151069\end{array}$

C $3.171925 \quad 0.028888 \quad 0.265024$

C $4.0809481 .174474-0.208895$

O $3.811046-1.202170-0.060230$

C $-3.5669851 .091812-0.266765$

$\begin{array}{llll}\text { C } & 5.455065 & 1.136784 & 0.449332\end{array}$

H -3.604393 2.4863221 .463078

H -2.836226 4.4575180 .312610

H -2.710568 $3.516112-1.285493$

H -2.680579 0.0357321 .414154

H $-0.547833-0.852456-1.556760$

H -1.378356 -2.3005180 .992146$

H $-1.410620-2.909547-0.674863$

H $-3.139978-1.325132-1.294552$

H $0.713425-0.8802711 .230376$

H $-4.977444-1.6040640 .360086$

H $-4.116820-3.1314130 .112062$

H $-3.824190-2.1781191 .574728$

H $1.881759-0.031333-1.463831$

H $1.384209-2.370437-1.104731$

H 1.0858361 .4946070 .807083

H $3.079536 \quad 0.089534 \quad 1.356543$

H 3.6197522 .1442340 .008368

H $4.2203401 .109790-1.294978$

H $3.291815-1.9190190 .341647$

H $-3.3917431 .185770-1.345797$

H $-4.6122600 .792933-0.129475$

H $\begin{array}{llll}5.996846 & 0.220997 & 0.193365\end{array}$

H $\quad 6.056855 \quad 1.986728 \quad 0.112294$

H 5.3681491 .1936181 .539095

SCF Energy (B3LYP/6-31G*//MMFF) $=-810.714644342$

F2-24_c05738
MMFF Geometry

C -3.789208 $1.707524-0.284281$

C $-3.4204772 .963039-0.562539$

C $-2.432552-0.1510790 .815314$

$\begin{array}{llll}\mathrm{O} & -1.167283 & 0.362813 & 0.357631\end{array}$

C $-0.599410-0.572778-0.575742$

C -1.446342 -1.838960 -0.472751

C $-2.813748-1.264463-0.157603$

C $0.897063-0.798065-0.303663$

C $-3.787716-2.2788560 .414520$

C $1.7304770 .489758-0.465641$

O $1.393063-1.780923-1.208743$

O $1.349884 \quad 1.4281210 .540814$

C $3.2564620 .263349-0.399658$

C $3.755403-0.2008550 .974987$

O $3.9068871 .498783-0.714278$

C -3.4367470 .9914890 .989089$

C $5.256848-0.4648600 .982964$

H $-4.3981871 .174843-1.009457$

H $-3.7159503 .428103-1.496760$

H -2.8198163 .5444730 .128159$

H $-2.240028-0.5798381 .808190$

H $-0.729078-0.147825-1.579836$

H -1.097851 -2.475827 0.349930

H -1.431479 -2.430797 -1.392958

H $-3.229922-0.852632-1.084698$

H $1.013833-1.1690640 .721859$

H $-3.963385-3.088972-0.300629$

H -3.406990 -2.724420 1.339595

H -4.751229 -1.809733 0.637493

H $1.4947640 .947984-1.434762$

H $0.986002-2.632274-0.976167$

H 1.8895552 .2270060 .412406

H $3.563040-0.459480-1.164344$

H $3.242078-1.1196901 .276361$

H 3.5442170 .5631351 .732347

H $3.6338421 .753164-1.612219$

H $-4.3614350 .604574 \quad 1.432826$

H -3.021046 1.7120091 .705476

H $5.570519-0.8235681 .968514$

H $5.523758-1.2273720 .244247$

H 5.8240770 .4443800 .761056

SCF Energy (B3LYP/6-31G**//MMFF) $=-810.709083668$

F2-24 c05739

MMFF Geometry

C $4.7980350 .744667-0.389640$

C $5.398024 \quad 1.7071790 .319602$

C $2.453914-0.226973-0.473472$

O $1.233306-0.100553-1.236275$

C $0.107672-0.102386-0.334033$

C $0.636396-0.6876730 .964022$

C $2.049474-0.1343001 .000289$

C $-1.056884-0.843298-1.004288$

C $2.945696-0.9033201 .958530$

C $-2.394225-0.826772-0.238427$

O $-0.696762-2.213724-1.213403$

O $-2.252487-1.471755 \quad 1.025900$

C $-2.9679780 .582704-0.013591$

C -4.3623290 .5389590 .631575$

O $-3.0429481 .276671-1.255934$

C $3.4170530 .861484-0.956869$

C $-4.920306 \quad 1.931474 \quad 0.897310$

H $5.341169-0.176732-0.582418$

H $6.403783 \quad 1.5639380 .699167$

H 4.9012762 .6467580 .534907

H $2.860713-1.219385-0.705679$

H $-0.1585220 .950508-0.185000$

H $0.659103-1.7832880 .929296$

H $0.046060-0.3844921 .833747$

H 2.0061400 .9169941 .314146

H -1.211525 -0.422013 -2.005075

H $3.946927-0.4676132 .006966$

H $2.528320-0.8853182 .970922$

H $3.048352-1.9512261 .657169$

H $-3.115828-1.421305-0.813930$

H $0.143728-2.220134-1.703173$

H $-1.895570-2.3610650 .858918$ 
H -2.3058381 .1515000 .649009$ H $-4.325632-0.011377 \quad 1.578329$ H $-5.0607850 .012047-0.029858$ H $-3.6417630 .778780-1.838580$ H $3.4920800 .804799-2.050735$ H $2.9850961 .850056-0.753096$ H $-4.2560812 .499701 \quad 1.556152$ H $-5.0486582 .494523-0.032321$ H -5.8986631 .8587541 .382777$ SCF Energy (B3LYP/6-31G**//MMFF) $=-810.722463849$

F2-24_c05740

MMFF Geometry

C -4.688444 -1.281466 0.415964

C $-4.269126-2.325613-0.308403$

C $-2.6833780 .266636-0.138341$

O $-1.673203-0.5607240 .473059$

C $-0.457931 \quad 0.2044130 .607956$

C $-0.6689951 .478348-0.198799$

C $-2.1587721 .698913-0.029521$

C $0.736169-0.6606190 .178749$

C -2.747912 $2.655795-1.051603$

C 2.0953710 .0460470 .368209

O $0.578846-1.037040-1.188793$

O $2.193178 \quad 0.523379 \quad 1.708063$

C $3.301201-0.8684970 .063411$

C $4.663290-0.2564260 .432803$

O $3.305144-1.214741-1.319187$

C -4.0226340 .0604750 .576387$

C $4.9867281 .035635-0.306391$

H $-5.623849-1.3983750 .960391$

H -4.854523 -3.238902 -0.334024

H $-3.352841-2.305785-0.885392$

H $-2.754749-0.029045-1.192744$

H $-0.3749050 .452661 \quad 1.673267$

H -0.422688 1.329818 -1.256903

H -0.0672592 .3128170 .174120$

H -2.3431232 .0914300 .979651$

H $\quad 0.718051-1.5855690 .768838$

H -2.281414 $3.642760-0.967422$

H $-2.5916482 .296465-2.074415$

H -3.824658 $2.777989-0.898637$

H $2.1311860 .909693-0.306436$

H - $0.288417-1.469091-1.273275$

H $2.159595-0.2475082 .300141$

H $3.191770-1.7980370 .635975$

H $5.450209-0.9819040 .191369$

H $4.717351-0.0783651 .512788$

H $4.045156-1.827691-1.467651$

H $-3.8779800 .216560 \quad 1.653159$

H -4.7371850 .8176380 .232564$

H $4.9484650 .895643-1.391025$

H $4.2924021 .836236-0.035160$

H $5.9959231 .371730-0.046831$

SCF Energy (B3LYP/6-31G**//MMFF)= -810.709986531

F2-24 c05741

MMFF Geometry

C $2.3776632 .792224-0.174050$

C 1.6505103 .7024890 .483095

C $2.2660350 .290632-0.255327$

$\begin{array}{lllll}\text { O } & 0.857190 & 0.223147 & 0.039770\end{array}$

C $0.502016-1.1331860 .350566$

C $1.703632-1.980447-0.055773$

C $2.851773-1.0338120 .229683$

C $-0.799050-1.533673-0.354582$

C $4.147861-1.426981-0.457357$

C $-2.072529-0.8182840 .154701$

O $-0.982738-2.941931-0.170245$

O $-2.225735-1.0557541 .555479$

C $-2.1278960 .700071-0.085920$

C -3.4531891 .3495840 .350258$

O $-1.9116530 .998748-1.461246$

C $2.874167 \quad 1.5116830 .435955$

C $-4.6825230 .851448-0.399379$

H $2.6377002 .983450-1.212021$

H $1.3253544 .609481-0.014896$

H 1.3658753 .5606871 .519820
H $2.3603200 .377299-1.345833$

H $0.381308-1.1999551 .439161$

H $1.664894-2.225961-1.124552$

H $1.768518-2.9162680 .507525$

H $3.016218-0.9980791 .315014$

H $-0.696538-1.360461-1.432247$

H $4.942393-0.708431-0.233488$

H $4.479277-2.413511-0.117166$

H $4.028625-1.469544-1.545214$

H -2.926512 -1.293706 -0.341627

H $-1.760850-3.206997-0.689897$

H -2.195383 -2.018170 1.691824

H $-1.346322 \quad 1.1998010 .497339$

H -3.3794462 .4306380 .175933$

H -3.6052801 .2101251 .426555$

H $-0.9717690 .840062-1.652077$

H 2.6344141 .4891031 .506504

H $3.965974 \quad 1.4937120 .344241$

H $-4.890895-0.198478-0.175329$

H $-5.5615521 .431321-0.099246$

$\mathrm{H}-4.5636640 .959728-1.481600$

SCF Energy $\left(B 3 L Y P / 6-31 G^{* *} / / M M F F\right)=-810.719314465$

F2-24 c05742

MMFF Geometry

C $3.583993 \quad 1.936151-0.646954$

C 3.7293583 .0363600 .099940

C $2.342414-0.212693-0.294820$

$\begin{array}{lllll}\text { O } & 1.335859 & 0.359319 & 0.564503\end{array}$

C $0.553001-0.7062561 .134586$

C $0.971896-1.9846380 .422507$

C $2.422142-1.6880310 .094836$

C $-0.947618-0.3724621 .088028$

C $2.994036-2.586270-0.988290$

C $-1.4488860 .282118-0.216354$

$\begin{array}{llll}\text { O } & -1.217319 & 0.525470 & 2.170963\end{array}$

O $-1.231768-0.597459-1.315629$

C $-2.9398900 .686189-0.172813$

C $-3.906339-0.504724-0.123916$

O $-3.2320881 .443365-1.352128$

C $3.6627850 .535985-0.107106$

C $-5.361531-0.060491-0.028139$

H $3.4067562 .050035-1.713343$

H $3.6658294 .019856-0.352654$

H 3.9113432 .9777711 .167252

H $1.981368-0.106769-1.326445$

H $\quad 0.868513-0.7739952 .184512$

H $0.400956-2.156926-0.493936$

H $\quad 0.849900-2.8679751 .057143$

H $3.018624-1.8027001 .010009$

H $-1.502656-1.2938311 .294125$

H $4.037869-2.332104-1.196915$

H $2.958392-3.635094-0.676034$

H $2.431638-2.494670-1.923588$

H $-0.864540 \quad 1.189453-0.414167$

H $-0.692573 \quad 1.3310542 .021937$

H -1.558972 -0.151271-2.115385

H $-3.126670 \quad 1.3459450 .682074$

H -3.683572 -1.142697 0.737196

H -3.800423 -1.115840 -1.028025

H $-2.6649042 .233138-1.341137$

H 3.9274700 .5587800 .957549

H $4.4704560 .016418-0.634625$

H $-6.018030-0.9342730 .035897$

H -5.5251310 .5525730 .863789$

H $-5.6612840 .520147-0.905921$

SCF Energy (B3LYP/6-31G**//MMFF) $=-810.717860143$

F2-24 c05743

MMFF Geometry

C $-4.260785-1.388502-0.472696$

C $-5.328827-1.656200 \quad 0.287129$

C $-2.6287540 .561141-0.594918$

O $-1.528851-0.250094-1.051638$

C $-0.524780-0.283317-0.014255$

C -0.9269130 .7801261 .001963$

C -2.4400940 .7229410 .912536$

C $0.868254-0.127895-0.642424$ 
C $-3.1352311 .940606 \quad 1.495041$

C $2.017758-0.2281140 .383657$

O $0.9350991 .106505-1.355247$

O $1.857161-1.4215621 .148374$

C $3.421567-0.221315-0.261317$

C $4.532845-0.4000250 .785341$

O $3.6511681 .008060-0.943732$

C $-3.961897-0.035587-1.054155$

C $5.921527-0.4362460 .158339$

H -3.567529 -2.194534 -0.698179

H $-5.491472-2.6565250 .673183$

H $-6.053279-0.8893750 .538267$

H -2.507687 $1.534160-1.090173$

H $-0.608420-1.2743570 .449330$

H $-0.568848 \quad 1.774657 \quad 0.714184$

H $-0.548860 \quad 0.5619242 .005326$

H $-2.779224-0.1721571 .448445$

H $0.981171-0.920351-1.392392$

H -4.2216381 .8540051 .393213$

H -2.902534 2.0434622 .559870

H -2.821285 2.8615220 .992234

H $1.9526390 .624327 \quad 1.071053$

H $\quad 1.035957 \quad 1.826147-0.709853$

H $1.924167-2.1756500 .537791$

H $3.497914-1.037881-0.990028$

H $4.382289-1.3276401 .348353$

H 4.5029090 .4305921 .501140

H $3.0233141 .055358-1.684499$

H $-4.7707010 .667843-0.823211$

H $-3.940064-0.150945-2.145055$

H $6.1571240 .504981-0.347919$

H $6.679036-0.5990680 .931526$

H $6.000371-1.249445-0.570112$

SCF Energy (B3LYP/6-31G**//MMFF) $=-810.718102323$

F2-24 c05744

MMFF Geometry

C -3.6888042 .0694800 .341720$

C $-3.4490213 .206405-0.321314$

C $-2.592137-0.183878 \quad 0.294371$

O $\begin{array}{lllll}-1.298377 & 0.316487 & -0.098464\end{array}$

C $-0.468820-0.785716-0.498749$

C -1.203009-2.046492 -0.056582

C -2.649313 -1.623463 -0.213991

C $0.949615-0.6605690 .082476$

C $-3.625760-2.5086900 .540578$

C $1.7206250 .573128-0.443588$

O $1.660195-1.855164-0.224260$

O $1.0175391 .767457-0.083609$

C 3.1561230 .7488910 .098567

C $4.175409-0.236733-0.481363$

O $3.159991 \quad 0.626683 \quad 1.521223$

C $-3.6861500 .708083-0.294203$

C $5.6067770 .159911-0.135700$

H -3.9084002 .1226151 .404981$

H -3.4696904 .1589830 .196670$

H -3.227601 3.208471-1.382764

H -2.623851 -0.1612211 .391578$

H $-0.419279-0.773473-1.595463$

H $-0.982185-2.2788460 .992749$

H $-0.942250-2.919788-0.662085$

H -2.904176 -1.635626 -1.282345

H $\quad 0.864336-0.5831891 .173113$

H -3.571250 -3.539552 0.175370

H -3.409444 -2.520802 1.614236

H $-4.654016-2.1578790 .408089$

H $1.7697830 .543048-1.538973$

H $1.733348-1.917196-1.192019$

H $0.1507401 .745148-0.523086$

H $3.4884011 .770734-0.126364$

H $4.076456-0.283686-1.571475$

H $4.014798-1.242236-0.079994$

H $2.519598 \quad 1.270938 \quad 1.868762$

H -3.544404 $0.801126-1.378223$

H $-4.6708910 .256314-0.129983$

H $6.311605-0.555553-0.571243$

H $5.8448151 .152523-0.531208$

H 5.7655490 .1733020 .947241
SCF Energy (B3LYP/6-31G**//MMFF) $=-810.718110544$

F2-24_c05745

MMFF Geometry

C $3.3075742 .314691-0.397283$

C $2.7410703 .494048-0.118657$

C $2.518816-0.051401-0.141319$

O $1.1185540 .268740-0.270616$

C $0.340285-0.8688600 .133265$

C $1.313698-2.0375470 .229253$

C $2.576500-1.3357310 .686330$

C $-0.814958-1.113064-0.840180$

C $3.837487-2.1546130 .471632$

C $-1.7192370 .118737-1.052002$

O $-1.561952-2.233696-0.376392$

O $-2.720987-0.203436-2.022367$

C -2.3789830 .7343790 .199501$

C $-3.442617-0.1257480 .893558$

O $-2.9839081 .971898-0.198123$

C 3.2540661 .1197450 .512303

C $-4.023703 \quad 0.5617372 .124916$

H $3.8501312 .205184-1.332809$

H $2.8195964 .319989-0.817081$

H 2.1912623 .6562450 .801846

H $2.900304-0.229812-1.155341$

H $-0.047432-0.663591 \quad 1.139052$

H $1.466599-2.502826-0.752463$

H $\quad 0.974986-2.8115860 .924531$

H $2.479218-1.0991631 .754635$

H - $0.405201-1.391615-1.819922$

H $3.975689-2.409008-0.584610$

H $4.722657-1.6034290 .803867$

H $3.789631-3.0898751 .038907$

H $-1.1153110 .904326-1.523375$

H -2.170640 -2.497241-1.086630

H $-3.275558-0.915116-1.662933$

H $-1.602297 \quad 1.0009320 .923735$

H $-3.016396-1.0811291 .210842$

H $-4.271279-0.3370010 .208380$

H $-3.6572451 .766166-0.869269$

H $2.759403 \quad 1.387740 \quad 1.454404$

H 4.2836310 .8320950 .753200

H -3.2371850 .7977082 .848696$

H -4.5390131 .4901211 .859419$

H $-4.749206-0.0954012 .615001$

SCF Energy (B3LYP/6-31G**//MMFF $)=-810.714829071$

F2-24_c05746

MMFF Geometry

C $-4.271689-1.669023-0.488633$

C $-4.418824-2.8014650 .208051$

C $-2.6169430 .210626-0.362984$

O $-1.542923-0.5947630 .164603$

C -0.4860920 .2757620 .620806$

C $-0.821041 \quad 1.6609670 .084495$

C -2.3358891 .6358680 .111704$

C $0.869995-0.2952270 .177928$

C $-2.9752082 .712835-0.747464$

C 2.0777790 .5337200 .660096

O $0.892587-0.373487-1.246745$

O 1.9946590 .7125312 .077849

C $3.464240-0.0889840 .381513$

C $3.861194-0.081639-1.099494$

O $3.479624-1.4280600 .877605$

C $-3.958223-0.3355380 .129156$

C $5.342564-0.379026-1.304726$

H -4.393575 -1.701946-1.568175

H -4.649792 -3.731964 -0.298867

H $-4.311193-2.8223691 .286793$

H $-2.5529980 .150327-1.457460$

H $-0.552490 \quad 0.276216 \quad 1.715494$

H $-0.4597791 .792499-0.942394$

H -0.3951782 .4610140 .697553$

H -2.6702611 .7589621 .150706$

H $0.941778-1.3213540 .557696$

H -4.066971 $2.649605-0.705428$

H -2.681157 $3.708026-0.397960$

H $-2.6725652 .622000-1.796091$ 
H $2.059708 \quad 1.530223 \quad 0.201531$

H $\quad 0.123963-0.902577-1.521030$

H $1.302454 \quad 1.3718172 .252745$

H $4.2084540 .470343 \quad 0.962911$

H $3.6411490 .896989-1.540929$

H $3.297218-0.840640-1.651845$

H $4.397646-1.745200 \quad 0.851828$

H $-3.943719-0.4227391 .222890$

H $-4.766616 \quad 0.355992-0.133647$

H $5.602040-1.383274-0.956886$

H $5.593257-0.319513-2.368774$

H $5.9658760 .344537-0.769853$

SCF Energy (B3LYP/6-31G**/MMFF) $=-810.711787289$

F2-25_c05991

MMFF Geometry

C $4.319677 \quad 1.348273 \quad 0.370865$

C $4.3302482 .685757 \quad 0.343779$

C $2.526529-0.353227-0.048744$

$\begin{array}{lllll}\text { O } & 1.438055 & 0.503347 & 0.354134\end{array}$

C $0.192408-0.103532-0.048385$

C $0.531876-1.531454-0.453117$

C $1.922095-1.356452-1.030269$

C $-0.8286810 .038101 \quad 1.083821$

C $2.699094-2.656568-1.144410$

C $-2.214373-0.5536510 .778894$

O $-0.330700-0.6357192 .249183$

O $-3.085356-0.2287941 .872091$

C $-2.873116-0.057387-0.519623$

C $-3.1771261 .446947-0.532618$

O $-4.093835-0.781718-0.704272$

C $3.644750 \quad 0.490940-0.662217$

C $-3.7725341 .900131-1.860282$

H 4.8362490 .8394641 .180702

H 4.8404423 .2468381 .119040

H $3.8333913 .241434-0.443800$

H $2.880773-0.8654260 .855440$

H $-0.1416740 .461133-0.926563$

H $-0.181721-1.943747-1.172734$

H $0.560862-2.1997940 .415545$

H $1.833855-0.901934-2.026316$

H -0.9199521 .0910511 .375469$

H $3.698492-2.480087-1.554273$

H $2.815591-3.141437-0.169435$

H $2.181239-3.356818-1.807947$

H $-2.162853-1.649500 \quad 0.761368$

H $0.548436-0.2665822 .442046$

H -2.664839 -0.5591982 .684584$

H $-2.240363-0.311326-1.375818$

H -2.263099 $2.021027-0.348195$

H -3.892201 1.6970020 .259994

H $-4.664137-0.5951380 .061419$

H $4.405263-0.158203-1.110805$

H $3.2363791 .119868-1.463173$

H -3.092847 $1.679196-2.689355$

H $-4.7296191 .407426-2.057618$

H -3.948056 $2.980557-1.844819$

SCF Energy (B3LYP/6-31G**//MMFF) $=-810.722423967$

F2-25_c05992

MMFF Geometry

C -3.632709 $1.627954-0.697489$

C -4.5135802 .3186550 .035114$

C -2.497653 $-0.633550-0.421246$

O $-1.311473-0.242822-1.140127$

C $-0.203277-0.217551-0.213773$

C $-0.702422-0.881277 \quad 1.065387$

C $-2.164959-0.4803481 .061953$

C $1.019855-0.869664-0.863188$

C -3.033292 -1.321915 1.979771

C $2.283194-0.8804530 .013209$

O $0.688151-2.230085-1.177873$

O $3.348198-1.407302-0.790880$

C $2.709811 \quad 0.487612 \quad 0.575753$

C $3.0977831 .506639-0.503236$

O $3.8416400 .288531 \quad 1.428784$

C $-3.713370 \quad 0.145767-0.930132$

C 3.4450342 .8668930 .090324
H -2.8120112.164259-1.166726

H -4.4030613 .3901600 .160822$

H $-5.352631 \quad 1.8328520 .520598$

H -2.654962 -1.694365 - -0.658746

H $-0.0042460 .843643-0.024277$

H $-0.165530-0.5340361 .953208$

H $-0.605600-1.9724391 .017398$

H -2.230424 0.5699731 .372065

H $1.220486-0.390198-1.828565$

H -2.699895 -1.226878 3.018337

H $-4.078358-1.0002651 .929492$

H -2.993167 -2.382663 1.710202

H $2.155385-1.5960170 .834952$

H $1.469205-2.627513-1.600182$

H $4.149905-1.414550-0.240101$

H $1.915037 \quad 0.902357 \quad 1.204694$

H $2.2764601 .639409-1.214998$

H $3.9704901 .150973-1.063572$

H $3.568522-0.3133492 .142159$

H -3.802199 -0.006287-2.013209

H $-4.622122-0.264940-0.474038$

H 4.3103042 .8029850 .757358

H $3.6885263 .574950-0.708259$

H $2.601263 \quad 3.2728020 .657472$

SCF Energy (B3LYP/6-31G**//MMFF) = -810.716557606

F2-25 c05993

MMFF Geometry

C -3.195462 $2.550227-0.476281$

C $-2.743163 \quad 3.631202 \quad 0.168979$

C $-2.5879020 .120637-0.360048$

$\begin{array}{lllll}\text { O } & -1.225231 & 0.348876 & 0.053094\end{array}$

C $-0.653563-0.8953860 .491257$

C $-1.626348-1.9842540 .054991$

C $-2.954503-1.2644260 .171958$

C $0.762992-1.064840-0.072220$

C $-4.080724-1.942481-0.588712$

C $1.7057130 .095160 \quad 0.317362$

O $1.316504-2.2902220 .404789$

$\begin{array}{lllll}\text { O } & 1.726992 & 0.239226 & 1.737314\end{array}$

C $3.147723-0.092171-0.194542$

C $4.0429341 .106510 \quad 0.156470$

O $3.133649-0.274357-1.608383$

C $-3.4831991 .233162 \quad 0.187536$

C $5.4782890 .914921-0.316925$

H $-3.3782592 .620024-1.545423$

H $-2.558994 \quad 4.554840-0.368715$

H $-2.547698 \quad 3.615343 \quad 1.235499$

H $-2.5935940 .124996-1.457925$

H $-0.625251-0.8748901 .588300$

H $-1.565962-2.8787640 .682271$

H $-1.440660-2.281913-0.984608$

H $-3.226062-1.1994931 .234277$

H $0.716994-1.117149-1.166906$

H -3.851964 -2.022960 -1.656829

H $-4.250004-2.953569-0.204198$

H $-5.015101-1.381899-0.485671$

H $1.2996421 .025398-0.097987$

H $\quad 0.793877-3.0183450 .028906$

H $2.051882-0.5976542 .111517$

H $3.587329-0.991450 \quad 0.252824$

H $4.049513 \quad 1.2710111 .239630$

H $3.6469772 .015131-0.313207$

H $2.7547380 .527509-2.007374$

H -4.5372750 .9901250 .012508$

H -3.342395 1.3212841 .272090

H $5.5310110 .824961-1.406346$

H $6.0898131 .773491-0.021522$

$\begin{array}{lllllll}H & 5.919411 & 0.016221 & 0.125433\end{array}$

SCF Energy (B3LYP/6-31G**//MMFF) $=-810.717966870$

F2-25 c05994

MMFF Geometry

C $-4.439124-1.6127520 .333959$

C $-4.538151-2.802856-0.268774$

C $-2.7432490 .229944 \quad 0.209315$

O $-1.638015-0.631605-0.128065$

C $-0.5131440 .178467-0.535722$ 
C $-0.8807951 .611297-0.172864$

C $-2.3844201 .599368-0.363429$

C $0.759462-0.3977030 .101424$

C $-3.0904882 .760338 \quad 0.315525$

C $2.0639330 .214480-0.446969$

O $0.717565-0.2171301 .517947$

O $2.0909631 .618961-0.205163$

C $3.331465-0.4221290 .158909$

C $4.6170160 .167120-0.442673$

O $3.311593-1.831155-0.058247$

C $-4.037979-0.345332-0.366639$

C $5.873503-0.4342180 .174569$

H $-4.669947-1.5447841 .393992$

H -4.837898 -3.680325 0.293860

H -4.322834 -2.924162 -1.324637

H $-2.792956 \quad 0.272157 \quad 1.305376$

H $-0.4481640 .078460-1.626522$

H $-0.3835762 .346895-0.812008$

H -0.6307611 .8408140 .869364$

H -2.601929 $1.625886-1.439751$

H $0.768528-1.480780-0.070658$

H -4.1724112 .7048250 .160375$

H -2.904762 2.7670221 .394943

H -2.739823 $3.714508-0.091438$

H $2.0755300 .068715-1.533978$

H $-0.110704-0.6157101 .836144$

H 2.0416791 .7515690 .757194

H $3.349686-0.2530791 .242045$

H $4.6408321 .253064-0.298736$

H $4.642132-0.023477-1.522493$

H $3.306864-1.983731-1.018692$

H $-4.8552040 .375712-0.252554$

H -3.913823 -0.533494 -1.440533

H $\quad 6.765006 \quad 0.032280-0.256674$

H $5.894513-0.2703611 .256631$

H $5.934999-1.510704-0.012802$

SCF Energy (B3LYP/6-31G**//MMFF) $=-810.721554213$

\section{F2-25 c05995}

MMFF Geometry

C $3.4601612 .310866 \quad 0.388927$

C $3.2114593 .372155-0.386479$

C $2.526482-0.0152720 .437663$

O $1.243800 \quad 0.350181-0.111074$

C $0.535995-0.845283-0.479460$

C $1.311366-2.0052730 .133315$

C $2.732292-1.4835900 .065125$

C $-0.925973-0.772667-0.018621$

C $3.694841-2.2250950 .976400$

C $-1.6814630 .439006-0.606267$

O $-1.573670-1.992205-0.370124$

O

C $-3.1635020 .568859-0.198075$

C $-3.346535 \quad 0.764758 \quad 1.310081$

O $-3.903995-0.579997-0.605318$

C $3.6076820 .907485-0.127373$

C -4.7909501 .0843851 .675833$

H 3.5732092 .4627261 .459416

H 3.1203734 .3613370 .048927

H $3.0932663 .275646-1.459989$

H $2.4519780 .098561 \quad 1.527101$

H $0.587203-0.932109-1.572421$

H $1.169238-2.942040-0.413988$

H $1.011740-2.1697041 .175820$

H $3.085627-1.562393-0.971903$

H $-0.944069-0.6933801 .074057$

H $4.703514-1.8069100 .902837$

H $3.381292-2.1666632 .024129$

H $3.747476-3.2834020 .700282$

H $-1.1593701 .357356-0.311645$

H $-1.607562-2.036814-1.341109$

H -2.022986 $1.176078-2.387995$

H $-3.6005791 .423002-0.730082$

H -2.7034201 .5790871 .662286$

H $-3.061247-0.1461421 .848680$

H $-3.744506-0.705682-1.556382$

H $4.6013370 .545000 \quad 0.159422$

H $3.5611800 .904287-1.223583$
H -4.8806691 .2383242 .755936$

H -5.1289411 .9967791 .174387$

H -5.4634310 .2675881 .395579$

SCF Energy (B3LYP/6-31G**//MMFF) $=-810.713652954$

F2-25 c05996

MMFF Geometry

C $2.3223262 .657198 \quad 0.165148$

C $1.6749253 .604805-0.521937$

C 2.1549650 .1556130 .240209

O $0.7526290 .110294-0.089995$

C $0.387249-1.238038-0.424702$

C $1.562824-2.1056480 .012570$

C $2.733451-1.176915-0.233386$

C $-0.943648-1.6333480 .225323$

C $4.001662-1.5946390 .490425$

C $-2.193460-0.966179-0.384727$

O $-1.111067-3.0517610 .093817$

O $-3.335958-1.4033630 .368143$

C $-2.212170 \quad 0.570941-0.413312$

C -2.2012791 .2244200 .975820$

O $-3.3935030 .985521-1.110084$

C $2.8039131 .365830-0.433139$

C $-2.1744452 .745787 \quad 0.894041$

H 2.5271372 .8259981 .219249

H $1.3567104 .518283-0.031371$

H $1.4486773 .487796-1.575895$

H 2.2230010 .2389651 .332824

H $0.296152-1.291261-1.517320$

H $1.630118-3.039836-0.553342$

H $1.487934-2.3551621 .078497$

H $2.931087-1.139237-1.313202$

H $-0.912765-1.4256351 .301119$

H $4.814415-0.8882690 .294452$

H $3.848917-1.6403431 .573856$

H $4.327162-2.5848070 .155131$

H -2.353421 -1.341934-1.403308

H -1.115908 -3.262835 -0.855591

H $-3.328100-2.3760310 .368051$

H $-1.3732530 .942629-1.008497$

H -1.3299550 .8908161 .548420$

H $-3.0966320 .935017 \quad 1.538565$

H $-4.1593250 .644840-0.616526$

H $3.8925031 .324373-0.313761$

H $2.5905641 .348637-1.509361$

H -3.0730903 .1349800 .405496$

H -2.1258803 .1762751 .899263$

$\mathrm{H}-1.3007343 .0922500 .334195$

SCF Energy $(B 3 L Y P / 6-31 G * * / / M M F F)=-810.720419112$

F2-25 c05997

MMFF Geometry

C $2.765212 \quad 2.6293290 .336237$

C 1.9979393 .6847180 .041472

C 2.3973600 .1597710 .118275

O $0.9633830 .240266 \quad 0.244828$

C $0.388179-1.018020-0.137575$

C $1.545289-2.006799-0.218798$

C $2.671588-1.108995-0.689794$

C $-0.712641-1.4454570 .841531$

C $4.052736-1.699464-0.465684$

C -1.852756 -0.421004 1.041089

O $-1.252421-2.6978380 .425496$

O $-1.3694850 .676796 \quad 1.814144$

C $-2.5059630 .106933-0.252469$

C -3.6781791 .0566520 .043922$

O $-2.970002-0.981219-1.045885$

C $2.9228141 .429070-0.554071$

C $-4.3038581 .618011-1.226690$

H 3.3196212 .6327591 .271101

H $1.929764 \begin{array}{lll}4.522697 & 0.726514\end{array}$

H $1.4266303 .731874-0.879020$

H 2.8026350 .0643651 .134166

H $-0.027240-0.896020-1.145628$

H $1.341766-2.837358-0.901459$

H $1.774806-2.4243490 .769434$

H $2.535767-0.908722-1.761207$

H $-0.250079-1.6056221 .823930$ 
H $4.232284-1.9097190 .594145$

H $4.163737-2.638488-1.017895$

H $4.832090-1.012021-0.809139$

H -2.631456 -0.9147311 .636560$

H -1.625233 -2.579467 -0.464764

H $-0.684741 \quad 1.1288081 .292064$

H $-1.7661240 .659752-0.842407$

H -3.3415791 .8923000 .667582$

H -4.4572570 .5218790 .600721$

H -3.652559-1.450175 -0.535840

H $3.9874661 .319246-0.789087$

H 2.393109 $1.592731-1.501010$

H $-5.1120192 .311527-0.973590$

H -3.562651 2.163062 -1.819718

H -4.726859 $0.823164-1.848783$

SCF Energy (B3LYP/6-31G**//MMFF) $=-810.725558600$

F2-25_c05998

MMFF Geometry

C -3.379182 2.0074410 .353855

C -4.6009152 .1829250 .869881$

C -2.251839 $0.101486-0.901331$

O $-0.9349210 .420528-0.411886$

C $-0.493794-0.6500810 .442302$

C -1.491620 -1.7895330 .255289$

C $-2.773953-1.025592-0.011510$

C $0.951792-1.0429090 .114758$

C $-3.864865-1.864272-0.652724$

C $1.948578 \quad 0.1293880 .261845$

O $1.341354-2.128400 \quad 0.954144$

$\begin{array}{llllllll} & & 1.851024 & 0.685407 & 1.571785\end{array}$

C $3.407968-0.278278-0.016430$

C 4.4248960 .8586120 .178937

O $3.503333-0.780807-1.347216$

C $-3.1145461 .363940-0.977828$

C $4.2093612 .057152-0.736041$

H -2.5199252 .3589500 .919266$

H -4.7257562 .6605831 .835600$

H $-5.491161 \quad 1.855000 \quad 0.344666$

H -2.108462 -0.270203 -1.924939

H $-0.566257-0.2917231 .477113$

H $-1.552840-2.4417661 .131713$

H $-1.224230-2.406831-0.611355$

H $-3.140890-0.6320270 .944342$

H $0.996875-1.400789-0.920819$

H $-4.144545-2.6965840 .001373$

H $-4.760866-1.262848-0.836082$

H $-3.538400-2.284647-1.609891$

H $1.6480930 .909479-0.446635$

H $1.235263-1.8446161 .877826$

H 2.1797490 .0227332 .202330

H $3.694047-1.0891320 .664645$

H $5.4294940 .465839-0.022506$

H 4.4183921 .1946041 .222040

H $4.422462-1.064645-1.489337$

H $-2.5983752 .107373-1.598089$

H $-4.0583781 .125921-1.482862$

H $4.2052281 .760618-1.789389$

H $3.2666022 .565497-0.514251$

H $5.0162802 .783723-0.595394$

SCF Energy (B3LYP/6-31G**//MMFF) $=-810.715674981$

F2-25_c05999

MMFF Geometry

C -3.629119 $1.928245-0.646993$

$\begin{array}{llll}C & -3.777824 & 2.986521 & 0.157607\end{array}$

C -2.355863 -0.218246 -0.416918

O $\begin{array}{llll}\text { O } & -1.347565 & 0.324002 & 0.462939\end{array}$

C $-0.625336-0.763217 \quad 1.066654$

$\begin{array}{llll}\text { C }-0.974398 & -2.001307 & 0.255971\end{array}$

C $-2.417497-1.714796-0.109589$

C $0.876075-0.4641521 .181380$

C $-2.931987-2.561450-1.260908$

C $1.598237-0.043688-0.119196$

O $1.040673 \quad 0.5711102 .156524$

$\begin{array}{lllll}\text { O } & 1.044957 & 1.166397 & -0.631804\end{array}$

C 3.1110090 .1784120 .089754

C $3.8278700 .546111-1.219521$
O $3.699426-1.001687 \quad 0.632270$

C $-3.6843760 .501198-0.179457$

C $5.3162720 .797511-1.013342$

H $-3.4665322 .099254-1.708073$

H -3.731288 $3.992892-0.244151$

H -3.9453132 .8708201 .222626$

H $-2.005445-0.051819-1.443998$

H $-1.039695-0.8654992 .078637$

H $-0.851163-2.924358 \quad 0.830293$

H $-0.363071-2.079717-0.649344$

H $-3.045555-1.891597 \quad 0.773897$

H $1.361622-1.3559921 .593790$

H $-3.970152-2.308850-1.497801$

H -2.334012 -2.413524 -2.166451

H -2.893638 -3.624819-1.002875

H $1.462256-0.824822-0.875290$

H $\quad 0.6170251 .372282 \quad 1.802716$

H $0.1105561 .000631-0.839251$

H 3.2702210 .9911430 .808386

H $3.3788821 .442975-1.660410$

H $3.716500-0.269292-1.944714$

H $3.568909-1.720746-0.009350$

H $-4.489489-0.002605-0.726240$

H $-3.9393580 .465344 \quad 0.887101$

H $5.826185-0.099172-0.647748$

H $5.7839541 .086252-1.960173$

H $5.4802981 .605774-0.293650$

SCF Energy (B3LYP/6-31G**//MMFF) $=-810.719811440$

F2-25 c06000

MMFF Geometry

C $-4.762600 \quad 0.572883-0.544200$

C $-5.403026 \quad 1.5823690 .055996$

C $-2.400792-0.354511-0.461723$

O $-1.155740-0.271848-1.191832$

C $-0.068660-0.160027-0.253479$

C $-0.627476-0.6417991 .074523$

C $-2.051905-0.121964 \quad 1.012214$

C $1.141254-0.934573-0.783634$

C $-2.966518-0.8247612 .003217$

C $2.382944-0.885766 \quad 0.120907$

O $0.780757-2.315881-0.933312$

O $3.447573-1.538328-0.585963$

$\begin{array}{llll}\text { C } 2.846861 & 0.523898 & 0.531531\end{array}$

C $3.2978421 .394803-0.648132$

$\begin{array}{lllll}\text { O } & 3.950198 & 0.388305 & 1.432972\end{array}$

C $-3.364848 \quad 0.666428-1.073676$

C $3.6819302 .802784-0.208528$

H $-5.283165-0.372663-0.671895$

H -6.4189341 .4533840 .413277$

H -4.9301512 .5469840 .203012$

H $-2.781661-1.371868-0.617321$

H $0.1670580 .909050-0.193446$

H $-0.069257-0.2532881 .931557$

H $-0.625152-1.7366811 .135828$

H $-2.0422980 .953796 \quad 1.232377$

H $1.391387-0.589800-1.793802$

H -3.977993 -0.411644 1.977057

H $-3.035339-1.8975451 .793264$

H -2.587695 -0.7070473 .024050$

H $2.210269-1.4946311 .017063$

H $-0.006140-2.348228-1.504180$

H $4.233557-1.511770-0.013539$

H $2.053510 \quad 1.0366831 .085311$

H $2.4988431 .470644-1.393030$

H $4.1695580 .945973-1.138800$

H $3.639350-0.1184192 .202495$

H $-2.9573191 .677823-0.945673$

H $-3.4005670 .510208-2.159818$

H $2.8414543 .301126 \quad 0.284831$

H 4.5292292 .7880940 .483989

H $3.9691253 .403243-1.077629$

SCF Energy (B3LYP/6-31G**//MMFF) $=-810.715191315$

F2-25 c06001

MMFF Geometry

C -3.257516 $2.703347 \quad 0.157728$

C $-2.3942413 .212287-0.728978$ 
C $-2.593418 \quad 0.221892-0.198021$

$\begin{array}{lllll}\text { O } & -1.230160 & 0.361983 & 0.249320\end{array}$

C $-0.675779-0.9430850 .479520$

C $-1.668406-1.938800-0.108802$

C $-2.983263-1.2220920 .122751$

C $0.728816-1.050019-0.125013$

C $-4.123130-1.760569-0.724481$

C 1.7029730 .0359190 .387326

O $1.232416-2.3513830 .169752$

O $1.778709-0.0044651 .811843$

C $3.132334-0.105748-0.171660$

C 4.0362521 .0596720 .261951

O $3.089104-0.165616-1.594946$

C $-3.477228 \quad 1.254667 \quad 0.508724$

C $5.4624580 .907123-0.251582$

H $-3.886856 \quad 3.4076870 .698917$

H $-2.3423914 .285332-0.882417$

H $-1.7277602 .593274-1.316968$

H -2.599819 $0.381646-1.283813$

H -0.637395 -1.098525 1.565278

H -1.619595 -2.9180760 .376902$

H -1.492443 -2.081485 -1.182360

H $-3.249993-1.3125661 .184425$

H $0.647985-0.958811-1.215424$

H -4.306608 -2.815497 -0.495857

H -5.048300 -1.208352 -0.532575

H -3.899825 -1.682491 -1.793828

H 1.3111841 .0200390 .100070

H $2.007420-2.503665-0.396784$

H $\quad 0.9390280 .3372602 .162199$

H $3.582560-1.0374990 .190293$

H 4.0622551 .1338461 .354829

H $3.6341422 .004415-0.123716$

H $2.7021950 .667165-1.915458$

H $-4.530757 \quad 1.029746 \quad 0.304813$

H $-3.330616 \quad 1.1649411 .592882$

H $5.910078-0.0248610 .107848$

H $5.4952320 .906237-1.345491$

H $\quad \begin{array}{llll}6.080616 & 1.738519 & 0.101703\end{array}$

SCF Energy (B3LYP/6-31G**/MMFF) $=-810.715942210$

F2-25_c06002

MMFF Geometry

C $4.092875-1.5161320 .651128$

C $5.131091-1.3011001 .466957$

C $2.6041590 .005591-0.744638$

O $1.455025-0.857038-0.649967$

C $0.427918-0.1787800 .109889$

C 0.9057761 .2610790 .268005

C 2.4109811 .0819690 .319534

C $-0.920738-0.391362-0.592527$

C 3.1893052 .3586960 .056069

C $-2.135476 \quad 0.0665310 .240940$

O $\quad-0.927018 \quad 0.293181-1.846538$

O $-2.0504661 .462273 \quad 0.513787$

C $-3.481350-0.229313-0.449723$

$\begin{array}{llll}\text { C } & -4.709730 & 0.278162 & 0.324027\end{array}$

O $-3.597603-1.635104-0.658116$

C $3.896199-0.811589-0.661405$

C $-4.874035-0.3331461 .709577$

H $3.347016-2.2517710 .940750$

H $5.218722-1.8472322 .399919$

H $5.904873-0.5817581 .222996$

H $2.5568950 .458516-1.744192$

H $0.405567-0.6695961 .091157$

H $0.5092871 .737546 \quad 1.169455$

H $0.6246261 .876751-0.594293$

H $2.674376 \quad 0.701845 \quad 1.314289$

H - $1.023080-1.458598-0.823837$

H $2.9520482 .778356-0.927211$

H 2.9521913 .1161560 .810282

H 4.2672102 .1716850 .092091

H -2.093522 -0.4620501 .200137$

H $-0.154277-0.016729-2.349503$

H $-2.0392911 .927765-0.340225$

H $-3.4964960 .253080-1.434739$

H -5.610446 $0.034647-0.253448$

H $-4.674813 \quad 1.3699110 .412695$
H $-4.434054-1.794730-1.127617$

H $3.877194-1.582768-1.441642$

H $4.750516-0.159001-0.877747$

H -5.8083530 .0145452 .162311$

H $-4.910695-1.4257261 .662708$

H $-4.056853-0.0409712 .375344$

SCF Energy $(B 3 L Y P / 6-31 G * * / / M M F F)=-810.722058375$

F2-25 c06003

MMFF Geometry

C $4.123741 \quad 1.904398 \quad 0.448212$

C $4.1169713 .113108-0.124863$

C $2.642291-0.1046380 .212354$

O $1.465710 \quad 0.642010-0.156950$

C $0.452738-0.273945-0.629114$

C $0.957195-1.667378-0.277402$

C $2.457379-1.490126-0.401408$

C $-0.9012150 .146838-0.037272$

C $3.255653-2.5844930 .285743$

C $-2.101953-0.592513-0.659772$

O $-0.888372-0.0508411 .376012$

O $-1.993662-1.996449-0.414545$

C $-3.497085-0.143140-0.176931$

C $-3.8098721 .317815-0.519799$

O $-3.634942-0.3455001 .228702$

C $3.8904080 .619333-0.294936$

C $-5.2556501 .682293-0.205397$

H 4.3183931 .8348551 .515276

H 4.2971574 .0032120 .468101

H $3.9315993 .237244-1.186066$

H $2.650274-0.1672601 .308538$

H $0.423122-0.155907-1.719544$

H $\quad 0.569125-2.436764-0.951408$

H $0.689955-1.9464830 .748433$

H $2.721144-1.467821-1.467435$

H $-1.0193311 .224523-0.197395$

H $4.330885-2.4100540 .179083$

H $3.027296-2.6363851 .355716$

H $3.027300-3.560753-0.154232$

H -2.061223 -0.464699-1.748136

H $-0.1185850 .427279 \quad 1.729255$

H -2.025106 -2.125217 0.549182

H $-4.244858-0.793294-0.647383$

H $-3.6207471 .500193-1.583738$

H $-3.165013 \quad 1.9914190 .055884$

H -2.9996210 .2372701 .678057$

H $4.775076-0.013234-0.159957$

H $3.7913200 .819722-1.369223$

H -5.4713461 .5718980 .861944$

H $-5.4501962 .723465-0.481949$

H $-5.9500951 .047722-0.765250$

SCF Energy $(B 3 L Y P / 6-31 G * * / / M M F F)=-810.725445337$

F2-25 c06004

MMFF Geometry

C $-3.5990682 .235029-0.486265$

C $-3.281307 \quad 3.3775450 .132923$

C $-2.657518-0.085677-0.368544$

$\begin{array}{llll}\text { O } & -1.326576 & 0.333479 & -0.004070\end{array}$

C $-0.575830-0.8150920 .424384$

C $-1.402457-2.0334010 .031477$

C $-2.812339-1.5010920 .187711$

C $0.831456-0.803471-0.187756$

C $-3.858937-2.337216-0.528028$

C 1.6381670 .4532230 .197363

O $1.507361-1.9887510 .217271$

O $1.801277 \quad 0.489673 \quad 1.615851$

C $3.0124010 .571321-0.496071$

C $4.115917-0.3370350 .062624$

O $3.4592381 .925094-0.343044$

C -3.6794090 .9003090 .199427$

C $5.435331-0.166388-0.684023$

H -3.824072 2.263686-1.549234

H $-3.2451104 .309966-0.419727$

H -3.051699 3.4040691 .192302

H -2.699584 -0.096729 -1.465682

H $-0.513679-0.7742461 .519462$

H $-1.197523-2.9025410 .663831$ 
H -1.211501-2.316471-1.011239 H $-3.055446-1.4598961 .258078$ H $\quad 0.745000-0.827662-1.280775$ H -3.874250 -3.357034 -0.129853 H -4.857621 -1.909106 -0.397402 H $-3.656893-2.399354-1.602635$ H $1.0450421 .337941-0.065516$ H $1.627896-1.9429641 .181387$ H 2.2912831 .3019761 .830350 H $2.8970260 .394768-1.571994$ H $3.826258-1.388371-0.004941$ H $4.297752-0.1079381 .119186$ H $2.8155152 .499258-0.792169$ H -4.694896 0.5116870 .063279 H $-3.516548 \quad 1.0221301 .277714$ H $6.185778-0.855543-0.283423$ H $5.311945-0.382360-1.750090$ H $5.8264930 .850444-0.580311$ SCF Energy (B3LYP/6-31G**//MMFF)= -810.719957834

\section{F2-25 c06005}

MMFF Geometry

C 3.3224942 .2598450 .122831

C $3.0683093 .258345-0.730299$

C $2.389264-0.0549760 .357837$

O $1.1025790 .268926-0.207126$

C $0.389103-0.949514-0.473657$

C $1.170183-2.0605590 .218870$

C $2.590841-1.5475110 .097842$

C $-1.066769-0.839975-0.003899$

C $3.561010-2.2180481 .054872$

C $-1.820607 \quad 0.356434-0.629005$

O $-1.712593-2.072882-0.309638$

O $-1.8411990 .227701-2.054456$

C $-3.2816200 .538011-0.169146$

C $-3.463056 \quad 0.7732191 .335409$

O $-4.046187-0.612684-0.536306$

C $3.466168 \quad 0.820543-0.284815$

C $-2.7233521 .991067 \quad 1.863203$

H 3.4432672 .4937041 .177484

H $2.9803034 .278194-0.371802$

H $2.9423653 .079422-1.792293$

H $2.323660 \quad 0.142127 \quad 1.436007$

H $\quad 0.429333-1.125223-1.556152$

H $1.022546-3.036248-0.253896$

H $\quad 0.880921-2.1451241 .273766$

H $2.934654-1.706407-0.933190$

H $-1.068399-0.7205391 .085673$

H $3.256516-2.0790382 .097667$

H $3.610898-3.2943370 .860143$

H $4.569200-1.8075570 .940939$

H -1.277062 $1.282597-0.410423$

H -2.617373 -2.0279570 .042420$

H $-0.9367390 .375101-2.378205$

H $-3.7146161 .380323-0.723503$

H -3.164451 $-0.118077 \quad 1.899977$

H -4.5340380 .8946941 .543223$

H -4.973590 $-0.436672-0.303258$

H $4.461874 \quad 0.4807370 .021582$

H $3.4113770 .733004-1.377144$

H -2.9865342 .1646372 .911629$

H -1.639102 1.8545791 .814316

H -2.9876172 .8882881 .294987$

SCF Energy $\left(B 3 L Y P / 6-31 G^{* *} / / M M F F\right)=-810.711385249$

F2-25_c06006

MMFF Geometry

C $-3.753822-1.735911-0.020325$

C $-5.004882-1.779630-0.492179$

C -2.2934410 .1158490 .937451$

O $-1.062237-0.4790330 .483403$

C $-0.4861090 .374586-0.521650$

C $-1.2806791 .677294-0.486967$

C $-2.6588191 .178723-0.097964$

C $1.0159990 .566429-0.273540$

C -3.5775242 .2660390 .429916$

C $1.798477-0.765244-0.260788$

O $1.5300331 .447771-1.268055$
O $1.575470-1.436619-1.505032$

C $3.321227-0.649226-0.036816$

C $3.665623-0.0303231 .323434$

O $3.9046500 .123160-1.084097$

C $-3.344342-0.9654761 .203016$

C $5.148881-0.1340491 .657287$

H $-2.981730-2.297844-0.539627$

H $-5.237532-2.359748-1.378450$

H $-5.812682-1.241113-0.009308$

H -2.0571190 .5974001 .895962$

H $-0.653529-0.107288-1.493388$

H -1.267482 2.202736-1.446660

H $-0.886921 \quad 2.3567020 .279327$

H -3.117872 $0.721664-0.983151$

H 1.1496411 .0540010 .698670

H -4.5513221 .8509900 .708487$

H -3.1541092 .7562611 .313016$

H $-3.7429873 .033593-0.333162$

H $1.382119-1.4092430 .523496$

H $1.4872500 .983280-2.121501$

H $2.016113-2.301910-1.455303$

H $3.755285-1.654302-0.109367$

H $3.095120-0.5320222 .113536$

H 3.3947771 .0316081 .336122

H $4.8699240 .030671-1.021699$

H $-2.933076-1.6897181 .917287$

H -4.220016 -0.5085521 .679406$

H $5.483189-1.1760901 .639453$

H $5.759694 \quad 0.4378420 .952374$

H 5.3364020 .2644962 .659467

SCF Energy $($ B3LYP/6-31G**//MMFF $)=-810.710722893$

F2-25_c06007

MMFF- Geometry

C $-4.487731-1.079270-0.611167$

C $-4.593342-2.034481-1.541683$

C $-2.5672570 .321638 \quad 0.186513$

O $-1.552211-0.650709-0.137121$

C $-0.254977-0.018751-0.060707$

C -0.4846691 .3176810 .632634$

C -1.8695621 .6801070 .135487$

C $0.721952-0.9688220 .636882$

C $-2.5486022 .756707 \quad 0.964277$

C $2.176239-0.4741410 .778145$

O $0.237111-1.226741 \quad 1.964587$

O $2.912516-1.5145361 .434627$

C $2.882174-0.153336-0.554248$

C $4.3993560 .045162-0.390018$

O $2.3320231 .028091-1.129616$

C $-3.7272220 .202449-0.803337$

C $5.0914080 .360255-1.710759$

H -4.986974 -1.224371 0.343438

H -5.162007 -2.936060 -1.341351

H $-4.117792-1.937497-2.511297$

H -2.906166 0.1010951 .207348

H $0.0491220 .141301-1.100386$

H 0.2695862 .0653200 .372523

H $-0.488374 \quad 1.2093521 .723663$

H $-1.7903472 .023832-0.904735$

H $\quad 0.714510-1.9416120 .129681$

H -2.6533792 .4512852 .010796$

H -1.9662673 .6836280 .941682$

H -3.548055 2.9767080 .576177

H 2.2039260 .4019351 .437792

H $-0.673097-1.5591191 .879784$

H $2.464093-1.6988382 .277892$

H $2.715541-0.976721-1.259090$

H $4.859316-0.854048 \quad 0.034219$

H 4.5909980 .8730530 .303408

H $2.5121791 .766258-0.522649$

H $-4.4278321 .033874-0.665950$

H $-3.3450240 .261501-1.830228$

H $6.1711860 .453481-1.556622$

H $4.920611-0.436562-2.441552$

H $4.7321371 .302162-2.136469$

SCF Energy (B3LYP/6-31G**//MMFF) $=-810.721292810$

F2-25_c06008 
MMFF Geometry

C $3.820909-1.587159-0.747792$

C $3.988587-2.753920-0.115342$

C $2.3582730 .396767-0.294176$

O $1.329885-0.3553630 .384146$

C $0.4525860 .562257 \quad 1.059758$

C $0.776698 \quad 1.9424490 .508331$

C 2.2669021 .8187340 .259452

C $-1.020577 \quad 0.1436050 .935852$

C $2.8168592 .882878-0.674465$

C $-1.530393-0.125846-0.498367$

O $-1.201228-1.0275441 .736810$

O $-0.861715-1.260175-1.057638$

C -3.043726 -0.406045 -0.618036

C $-3.9039340 .803373-0.237262$

O $-3.422294-1.5122490 .198867$

C $3.718088-0.259755-0.051206$

C $-5.3782000 .579762-0.553049$

H $3.762838-1.586545-1.833140$

H $4.059674-3.678474-0.677929$

H $4.055622-2.8092690 .965468$

H $2.109437 \quad 0.384728-1.363438$

H $\quad 0.741900 \quad 0.5210572 .118570$

H $\quad 0.5233472 .741379 \quad 1.211749$

H $0.2499042 .139568-0.431400$

H 2.7905301 .8815861 .222959

H -1.625221 0.9311621 .397096

H $3.8905652 .744784-0.835081$

H $2.3234142 .852839-1.651855$

H $2.6644963 .881192-0.251686$

H $-1.299007 \quad 0.728709-1.142913$

H - $0.677994-1.7401471 .330901$

H $0.089593-1.063908-1.075089$

H $-3.264386-0.695373-1.653249$

H $-3.557977 \quad 1.693158-0.775197$

H $-3.822422 \quad 1.0065270 .836452$

H -2.838717 $-2.255324-0.031423$

H $4.5209950 .383181-0.429319$

H $3.880319-0.388072 \quad 1.026423$

H $-5.5246860 .384738-1.620128$

H $-5.782764-0.2664090 .010896$

H -5.959055 $1.468966-0.288334$

SCF Energy (B3LYP/6-31G**//MMFF) $=-810.716831241$

F2-25_c06009

MMFF Geometry

C -2.509915 2.3720410 .182978

C $-3.4242203 .185112-0.357767$

C $-2.067561-0.082832-0.086277$

O -0.6525630 .0118200 .172070$

C $-0.121999-1.3029500 .407495$

C $-1.210756-2.268653-0.047521$

C $-2.465978-1.5021450 .313853$

C $1.224776-1.493184-0.301197$

C $-3.712948-2.016281-0.384566$

C $2.400189-0.7153000 .327109$

O $1.587955-2.879215-0.240684$

O $3.563395-0.953960-0.482188$

C 2.2315320 .8069480 .470927

C $2.1104301 .543321-0.871144$

O $3.3825691 .291932 \quad 1.172327$

C $-2.8123450 .995350 \quad 0.703326$

C $1.8513463 .035532-0.702459$

H -1.485352 2.7240480 .265704

H -3.142228 $4.171317-0.710240$

H $-4.4614192 .885448-0.459469$

H -2.205461 $0.066625-1.165078$

H $0.014057-1.412243 \quad 1.491436$

H -1.145454 -3.242061 0.448166

H -1.160501 -2.429995 -1.131730

H -2.612750 -1.559893 1.400769

H $1.143888-1.228145-1.362207$

H -3.610244 -1.971760 -1.474030

H -3.906539-3.057950 -0.108029

H -4.590074 -1.424662 -0.104657

H $2.637007-1.1408201 .310875$

H $0.991903-3.368343-0.832512$

H $3.682595-1.917614-0.539715$
H $\quad 1.369931 \quad 1.024453 \quad 1.109369$

H $1.2933371 .119117-1.463778$

H $3.0338531 .427516-1.450777$

H 3.1958292 .2031181 .454208

H -3.8915990 .8074650 .666643$

H $-2.5098640 .964071 \quad 1.757231$

H $2.7014043 .539348-0.232752$

H $1.6891673 .500964-1.679925$

H $0.9623553 .213628-0.090241$

SCF Energy $\left(B 3 L Y P / 6-31 G^{* *} / / M M F F\right)=-810.712043572$

F2-25_c06010

MMFF Geometry

C -4.395573 -1.669242 0.317193

C $-4.484700-2.837911-0.327355$

C $-2.7373130 .208726 \quad 0.222537$

O $-1.626135-0.618120-0.173978$

C $-0.5116030 .225908-0.545189$

C $-0.9086671 .639540-0.136326$

C $-2.4145871 .602672-0.306921$

C $0.760118-0.3580350 .088569$

C -3.1339742 .7255850 .420340$

C $2.0772140 .219983-0.467510$

O $0.724226-0.1729371 .504874$

O $2.1329721 .628287-0.261389$

C $3.326979-0.4079830 .183390$

C $4.6294770 .130375-0.430331$

O $3.287158-1.8253990 .033263$

C $-4.035317-0.368798-0.343996$

C $5.868203-0.4608830 .230747$

H $-4.603768-1.645307 \quad 1.383800$

H -4.754199 -3.7415120 .208383$

H $-4.290514-2.915480-1.391403$

H -2.7611880 .2094441 .320278$

H $-0.4361290 .161136-1.637771$

H $-0.4433302 .406968-0.760857$

H $-0.647350 \quad 1.848190 \quad 0.907414$

H -2.647089 $1.660939-1.378849$

H $0.754419-1.442508-0.076643$

H -2.9343582 .6989851 .496866$

H -2.8069843 .6997620 .042078$

H -4.2165712 .6543630 .276738$

H $2.1083120 .031209-1.548511$

H $-0.104958-0.5654281 .827915$

H $1.6666252 .050317-1.001941$

H $3.336462-0.186328 \quad 1.257211$

H $4.6705521 .221393-0.339062$

H $4.663810-0.112967-1.499244$

H $3.290742-2.023564-0.918826$

H $-4.8625480 .331653-0.182582$

H $-3.934843-0.513806-1.426920$

H $6.772489-0.031425-0.212199$

H $5.880265-0.2440841 .303594$

H $5.912840-1.5462210 .097479$

SCF Energy (B3LYP/6-31G**//MMFF) $=-810.713692867$

F2-26 c06208

MMFF Geometry

C $4.109424-1.1170860 .759561$

C $4.032717-2.4147800 .443627$

C 2.4412280 .7644640 .734905

O $1.351942-0.1376701 .035782$

C 0.1599630 .3104970 .359889

C $0.6273281 .295862-0.700331$

C $1.8022081 .948839-0.000102$

C $-0.616210-0.902861-0.159622$

C $2.6982442 .748466-0.928890$

C $-1.923339-0.564134-0.895285$

O $0.210042-1.626445-1.083180$

O $-2.592183-1.796472-1.200124$

C $-2.9032200 .334482-0.119549$

C $-3.440175-0.3020031 .168992$

O $-4.0170330 .629490-0.968841$

C $3.499858-0.013810-0.060577$

C -4.3468420 .6471201 .943982$

H $4.651022-0.8302351 .657191$

H $4.498350-3.1624941 .076109$

H $3.507633-2.753192-0.442641$ 
H $2.869214 \quad 1.1039451 .685984$ H -0.4326080 .8351751 .118471$ H $-0.153421 \quad 2.009457-0.980485$ H $0.9597620 .783414-1.610452$ H 1.4013442 .6365870 .758180 H $-0.811614-1.6017420 .662118$ H $2.1507633 .600963-1.344796$ H $3.5673693 .137693-0.388991$ H $3.0559102 .148351-1.771687$ H -1.699556 -0.104633 -1.865950 H $1.029334-1.859709-0.614027$ H -1.966578 -2.349382 -1.698983 H $-2.429676 \quad 1.2936380 .115016$ H -2.612283 -0.598902 1.821105 H -4.019092 -1.202889 0.933723 H -3.671618 $1.071561-1.762963$ H $3.056805-0.428948-0.973876$ H $4.3134390 .654398-0.363767$ H -4.6868910 .1709942 .869206$ H -3.814243 1.5654222 .211242 H $-5.2326210 .918976 \quad 1.361572$

SCF Energy (B3LYP/6-31G**//MMFF) $=-810.713614362$

F2-26_c06209

MMFF Geometry

C $-2.300212 \quad 2.7644290 .217677$

C -1.246699 $3.425846-0.274884$

C -2.6753930 .3279940 .708291$

$\begin{array}{lllll}\text { O } & -1.272331 & 0.122667 & 0.978379\end{array}$

C $-0.844835-1.1183340 .391740$

C $-2.001241-1.613594-0.469389$

C $-3.197836-1.0401660 .261958$

C $0.461299-0.916208-0.388301$

C -4.476885 -1.050630 -0.555507

C $1.592176-0.3196790 .477978$

O $0.894188-2.168780-0.916101$

O $1.803754-1.1441841 .623422$

C $2.922378-0.157161-0.283844$

C $4.007896 \quad 0.494516 \quad 0.586850$

O $2.7117340 .636082-1.449734$

C $-2.8010471 .455459-0.328012$

C $5.3357670 .628117-0.147971$

H -2.8387983 .1924651 .059141$

H -0.9367174 .3687040 .162232$

H $-0.6766663 .041207-1.113357$

H -3.1605340 .6293081 .644442$

H - $0.691262-1.8315511 .211377$

H -2.027096 -2.705167 -0.543623

H -1.938954 -1.209436 -1.486890

H -3.363274 -1.647149 1.163314

H $0.281115-0.237527-1.230986$

H -4.789813 -2.080267 -0.758948

H -5.287229 $-0.553043-0.013182$

H -4.351466 -0.551438 -1.521544

H 1.2692170 .6639930 .839695

H $0.233051-2.462491-1.565267$

H $2.054493-2.0283961 .305223$

H $3.286727-1.138402-0.609865$

H $4.168003-0.0936241 .497353$

H 3.6850541 .4967380 .893565

H $2.3960381 .509650-1.161349$

H -2.246802 $1.197832-1.238732$

H $-3.8499161 .597165-0.610702$

H $6.089467 \quad 1.0671760 .513491$

H $5.701305-0.350082-0.476416$

H $5.2430851 .274506-1.026210$

SCF Energy (B3LYP/6-31G**//MMFF) $=-810.715609061$

F2-26 c06210

MMFF Geometry

C -2.3086362 .6897360 .436489$

C $-1.2121883 .409308 \quad 0.171419$

C -2.6882070 .2097090 .593274$

O $-1.331473-0.0099301 .033568$

C $-0.784151-1.1513370 .352155$

C $-1.803923-1.564646-0.703283$

C -3.103317 -1.103834-0.074747

C $0.593640-0.811582-0.234113$
C $-4.264786-1.036642-1.049682$

C $1.600165-0.3335810 .834410$

O $1.090272-1.956071-0.922268$

O $1.742457-1.3643101 .816812$

C 3.0047340 .0360270 .314614

C $2.9811431 .219973-0.657521$

O $3.615260-1.078275-0.332959$

C $-2.7010841 .448329-0.316244$

C $4.3837011 .700717-1.009642$

H -2.966452 3.0161061 .237808

H $-0.985247 \quad 4.2982150 .749764$

H $-0.5244113 .125727-0.617547$

H -3.3070290 .3941921 .479467$

H $-0.697404-1.9600761 .088541$

H -1.781556-2.640480 -0.903312

H $-1.625862-1.044743-1.652360$

H -3.363723 -1.817468 0.719706

H $0.469609-0.011528-0.972562$

H $-5.153914-0.621360-0.564768$

H $-4.031530-0.422524-1.925363$

H -4.515382 -2.039299-1.412097

H 1.1822400 .5398491 .349609

H $1.253371-2.648680-0.259213$

H $2.313847-1.0192942 .523810$

H $3.6427420 .283847 \quad 1.172028$

H $2.4208122 .053472-0.218745$

H $2.4835200 .934975-1.591524$

H $3.584770-1.8266300 .287356$

H -2.024412 $1.302568-1.166903$

H -3.705962 $1.612395-0.720770$

H $4.3286462 .558223-1.687729$

H $4.9274582 .012886-0.112287$

H $4.9611810 .915253-1.506910$

SCF Energy $\left(B 3 L Y P / 6-31 G^{* *} / / M M F F\right)=-810.711389339$

F2-26 c06211

MMFF Geometry

C $-3.8293251 .956574-0.102892$

C $-3.2626953 .060291-0.603072$

C $-2.904665-0.2498410 .671997$

O -1.6728110 .3677551 .106230$

C $-0.568271-0.5300120 .863632$

C $-1.101742-1.589959-0.088512$

C $-2.543452-1.7058840 .365012$

C 0.6371260 .2940620 .388898

C $-3.434770-2.444463-0.617479$

C $1.954175-0.5049690 .327477$

O $0.3686170 .850868-0.898812$

O $1.829526-1.594545-0.582890$

C $3.1646080 .356756-0.086753$

C $4.472792-0.449720-0.089255$

$\begin{array}{lllll}\text { O } & 3.293620 & 1.462627 & 0.803767\end{array}$

C $-3.4548570 .558851-0.512232$

C $5.6674150 .386890-0.529723$

H -4.6161172 .0647750 .639279$

H $-3.5815234 .041274-0.268167$

H -2.476401 $3.007334-1.347816$

H $-3.614217-0.2099591 .507410$

H $-0.331302-0.9887051 .831998$

H $-0.561041-2.537411-0.005973$

H $-1.053257-1.259644-1.132217$

H -2.553263 -2.260813 1.314032

H $\quad 0.761502 \quad 1.1431001 .071844$

H -3.118062 -3.489149-0.706975

H $-4.475617-2.436277-0.278452$

H $-3.394627-2.004820-1.619212$

H $2.140656-0.9283371 .321939$

H $-0.4472721 .374384-0.822538$

H $1.623595-1.223094-1.457968$

H $3.0060380 .758211-1.094722$

H $4.385134-1.314152-0.756648$

H $4.675730-0.8301990 .919111$

H $3.446093 \quad 1.1088431 .696709$

H -2.716855 $0.592703-1.322625$

H $-4.3577650 .082510-0.909969$

H $6.572110-0.229194-0.548051$

H $5.5121420 .791462-1.534890$

H $5.844683 \quad 1.2220630 .154947$ 
F2-26_c06212

MMFF Geometry

C $1.9127092 .690281 \quad 0.288724$

C 1.1145403 .2426241 .208914

C $2.1388920 .403909-0.738224$

$\begin{array}{llllll}\text { O } & 0.719090 & 0.149662 & -0.644943\end{array}$

C $0.492812-1.250349-0.416252$

C $1.826372-1.8024670 .070493$

C $2.804140-0.977128-0.740125$

C $-0.666641-1.4727160 .560562$

C $4.234065-1.057800-0.236399$

C $-2.065843-1.214111-0.033679$

O $-0.628529-2.842596 \quad 0.981836$

O $-3.013766-1.416117 \quad 1.025911$

C $-2.305980 \quad 0.172953-0.653715$

C $-2.244243 \quad 1.321674 \quad 0.360464$

O $-3.6114490 .179429-1.243673$

C $2.535615 \quad 1.3280290 .422787$

C $-2.3634422 .684040-0.312087$

H $2.1430943 .258354-0.609052$

H 0.7006374 .2328881 .054040

H 0.8576632 .7209242 .124209

H $2.3284550 .907663-1.693760$

H $0.253587-1.708694-1.384909$

H $1.926080-2.877763-0.106394$

H $1.960033-1.6164351 .143207$

H $2.788145-1.354670-1.772436$

H $-0.524512-0.8599361 .458800$

H $4.311942-0.7919710 .822779$

H $4.618575-2.077581-0.345391$

H $4.885263-0.389949-0.809168$

H $-2.287853-1.991096-0.776170$

H $-1.359285-2.9717431 .610849$

H -3.899954 -1.2695730 .652627$

H $-1.6002050 .351572-1.470570$

H -1.3038281 .2904770 .918766$

H $-3.0626001 .231507 \quad 1.084669$

H -3.626921 -0.511761-1.927470

H $2.2449040 .876468 \quad 1.379108$

H $3.621676 \quad 1.470616 \quad 0.438933$

H $-2.294428 \quad 3.4805570 .435542$

H -1.559538 2.831999 -1.040094

H -3.321671 2.791488-0.829950

SCF Energy (B3LYP/6-31G**//MMFF) $=-810.717272371$

F2-26_c06213

MMFF Geometry

C $4.097825-1.1326280 .746379$

C $3.978935-2.4348150 .463743$

C $2.456711 \quad 0.772163 \quad 0.731512$

O $1.361483-0.1085581 .069131$

$\begin{array}{llll}\text { C } & 0.172111 & 0.319779 & 0.375591\end{array}$

C $0.635408 \quad 1.296357-0.695067$

C $1.816088 \quad 1.952705-0.007205$

C $-0.603212-0.907858-0.113710$

C $2.7094492 .741876-0.947282$

C $-1.905556-0.594130-0.872147$

O $0.239557-1.693477-0.969145$

O $-2.571871-1.835559-1.141182$

C $-2.890508 \quad 0.330815-0.134515$

C $-3.443547-0.263782 \quad 1.167401$

O $-3.9943370 .602136-1.004322$

C $3.484363-0.034056-0.076979$

C $-4.356302 \quad 0.711252 \quad 1.902028$

H $4.677975-0.8385421 .617178$

H $4.449515-3.1795391 .096262$

H $3.412627-2.779801-0.394280$

H 2.9116371 .1193791 .667051

H -0.4265280 .8564601 .121336$

H $-0.1459252 .009531-0.974666$

H $0.9631490 .783395-1.605276$

H $1.4216522 .646813 \quad 0.748503$

H $-0.812734-1.5627630 .739635$

H $3.0579032 .135105-1.789171$

H $2.1630513 .594660-1.364284$

H $3.5843993 .129967-0.416043$
H $-1.675357-0.165666-1.855314$

H $0.326321-1.234476-1.820968$

H $-1.939941-2.409133-1.607689$

H -2.4166381 .2954810 .075509$

H -2.623769 -0.5418001 .837831$

H $-4.022173-1.1702840 .954000$

H -3.639771 $1.022596-1.806110$

H $3.012910-0.458563-0.971561$

H $4.2995510 .616752-0.412024$

$\begin{array}{llll}\text { H } & -4.707759 & 0.265233 & 2.837929\end{array}$

H -3.8243521 .6361862 .146313$

H $-5.2349800 .966607 \quad 1.301487$

SCF Energy (B3LYP/6-31G**//MMFF) $=-810.704763570$

F2-26_c06214

MMFF Geometry

C $2.7669572 .449367-0.161187$

$\begin{array}{llll}\text { C } 2.203227 & 3.232034 & 0.765824\end{array}$

C $2.2655970 .114108-0.943416$

$\begin{array}{lllll}\text { O } & 0.862525 & 0.260137 & -0.633935\end{array}$

C $0.364807-0.963239-0.071540$

C $1.582065-1.8307110 .226210$

C $2.540712-1.390738-0.861146$

C $-0.501469-0.6909281 .165558$

C $3.978238-1.812693-0.616608$

C -1.6867440 .2759320 .944481$

O $-0.976663-1.9289811 .689316$

O -1.1903551 .6068760 .808410$

C $-2.602900-0.058339-0.250087$

C $-3.7966470 .905896-0.343992$

O $-3.083880-1.394485-0.139238$

C 3.0615220 .9880770 .037752

C $-4.6857660 .610665-1.545391$

H $3.0403532 .887308-1.117760$

H $2.019875 \quad 4.2812020 .561446$

H 1.9133312 .8439891 .735920

H $2.4246200 .468750-1.968829$

H $-0.228095-1.462686-0.847702$

H $1.351159-2.8999340 .189117$

H $1.993873-1.6088601 .218151$

H $2.208349-1.844445-1.805680$

H $0.138684-0.241074 \quad 1.935622$

H $4.638971-1.402952-1.387265$

H $4.342826-1.4823410 .361304$

H $4.064047-2.904268-0.644569$

H -2.297455 $0.253376 \quad 1.856210$

H $-1.508521-2.3589540 .998217$

H -0.6482981 .6404380 .001806$

H -2.035296 $0.016462-1.184670$

H -3.444161 $1.940965-0.414303$

H -4.4096200 .8264830 .562058$

H $-3.617844-1.451090 \quad 0.671705$

H 2.8336310 .7008761 .071459

H $4.1373770 .847897-0.113924$

H -5.504719 $1.335263-1.594622$

H $-4.1172980 .677609-2.478403$

H -5.126415 -0.388996-1.480457

SCF Energy (B3LYP/6-31G**//MMFF) $=-810.723462758$

F2-26_c06215

MMFF Geometry

C $-2.5552972 .548844-0.068436$

$\begin{array}{lllll}C & -3.454215 & 3.533617 & 0.040541\end{array}$

$\begin{array}{llll}\text { C } & -2.616529 & 0.125657 & 0.609207\end{array}$

$\begin{array}{lllll} & \mathrm{O} & -1.196000 & 0.112576 & 0.871496\end{array}$

C $-0.637077-1.1334200 .424208$

C $-1.715923-1.819259-0.404140$

C $-2.976551-1.3267250 .276411$

C $0.664198-0.891564-0.350663$

C $-4.238133-1.554762-0.536630$

$\begin{array}{llllll}C & 1.735839 & -0.144261 & 0.474798\end{array}$

O $1.178061-2.146847-0.793345$

O $1.976596-0.8791801 .673835$

C $3.0603670 .044917-0.292759$

C $4.089245 \quad 0.8523370 .514995$

O $2.7783540 .723521-1.514823$

C $-2.889708 \quad 1.148323-0.504935$

C $5.4114691 .035574-0.220138$ 


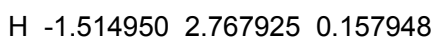
H -3.146953 4.5251100 .355008 H $-4.5036893 .371507-0.178620$ H -3.1274740 .4369441 .528330$ H $-0.443330-1.7435581 .315667$ H -1.619532-2.909265 -0.394704 H -1.684639-1.486267 -1.448596 H -3.080672 -1.871567 1.225498 H $0.438565-0.296607-1.243651$ H $-4.160531-1.131364-1.543149$ H $-4.429330-2.627316-0.648594$ H -5.105556 -1.108241 -0.040077 H 1.3302290 .8367810 .752475 H $1.418530-2.660030-0.002799$ H $2.403928-0.2793882 .308047$ H $3.490399-0.935634-0.531510$ H 4.2961120 .3550461 .468605 H 3.6839751 .8460950 .740721 H $3.5546030 .629571-2.092058$ H $-2.2794420 .923956-1.387979$ H $-3.9411491 .102425-0.809771$ H $5.2878751 .640024-1.123818$ H $6.131591 \quad 1.5485680 .425331$ H $5.8392450 .069056-0.504358$ SCF Energy (B3LYP/6-31G**//MMFF) $=-810.716881065$

F2-26_c06216

MMFF Geometry

C 3.4249742 .2711910 .005021

C 2.7263413 .2836500 .530646

C $2.794200-0.042241-0.752522$

O $1.474838 \quad 0.398095-1.143464$

C $0.514974-0.645502-0.871205$

C $1.221194-1.6215250 .057928$

C $2.649702-1.534779-0.441866$

C $-0.7788530 .001029-0.353001$

C $3.666754-2.1426040 .507709$

C $-1.963489-0.980684-0.259239$

$\begin{array}{lllll}\text { O } & -0.540807 & 0.596568 & 0.921641\end{array}$

O $-1.661661-2.0252790 .668303$

C $-3.317580-0.3680530 .156195$

C -3.841582 $0.666810-0.845781$

$\begin{array}{llll}\text { O } & -3.229161 & 0.221799 & 1.452459\end{array}$

C 3.2644250 .8341310 .417932

C $-5.259603 \quad 1.116317-0.515439$

H $4.1632062 .489914-0.762274$

H 2.8927014 .3002400 .191516

H 1.9810873 .1195241 .300948

H $3.4636600 .096779-1.609988$

H $0.313822-1.132021-1.834192$

H $0.815265-2.634994-0.012334$

H $1.160857-1.3019721 .104362$

H $2.705995-2.082136-1.393660$

H - $1.0423230 .819700-1.032395$

H $3.501653-3.2211470 .602311$

H $4.684619-1.9895360 .135323$

H $3.598616-1.7132471 .512387$

H $-2.093043-1.462035-1.236105$

H $0.192746 \quad 1.2260490 .814826$

H -1.538548 -1.611564 1.540208

H $-4.045375-1.183597 \quad 0.247797$

H -3.828960 $0.246418-1.857763$

H -3.202671 $1.557196-0.844402$

H -2.607083 0.9670531 .399655

H $2.5566420 .762777 \quad 1.252357$

H 4.2381590 .4885950 .782631

H -5.3060021 .5989790 .465682$

H $-5.6098201 .836525-1.261775$

H -5.949605 $0.266584-0.514902$

SCF Energy (B3LYP/6-31G**//MMFF) $=-810.723729108$

F2-26_c06217

MMFF Geometry

C $-2.770203 \quad 2.450395 \quad 0.234741$

C $-1.863773 \quad 3.428242 \quad 0.128087$

C $-2.717138-0.063927 \quad 0.330399$

O $-1.455242-0.0873791 .042613$

C $-0.796978-1.3371880 .781466$
C $-1.360460-1.813948-0.544657$

C $-2.815626-1.413985-0.397390$

C $0.732957-1.2194460 .829153$

C $-3.589138-1.447851-1.703789$

C $1.368569-0.111595-0.039617$

$\begin{array}{lllll}\text { O } & 1.113531 & -1.010987 & 2.193407\end{array}$

$\begin{array}{lllll}\text { O } & 0.945250 & 1.174587 & 0.405372\end{array}$

C $2.910696-0.141401-0.000964$

C $3.5327470 .938439-0.900954$

O $3.376757-1.423903-0.415031$

C $-2.7411211 .179659-0.569809$

C $5.0552260 .934028-0.842681$

H -3.5929672 .5790430 .933523$

H $-1.947692 \quad 4.323524 \quad 0.734360$

H $-1.0287703 .356153-0.559866$

H -3.518096 $0.005807 \quad 1.076880$

H $-1.117930-2.0187281 .581375$

H $-1.223939-2.889105-0.694505$

H $-0.908267-1.287420-1.391731$

H -3.292042 $-2.127560 \quad 0.290329$

H $1.147525-2.1917450 .539354$

H $-4.617432-1.103397-1.555086$

H $-3.122448-0.823604-2.472621$

H -3.631082 -2.470200 -2.094591

H $1.042540-0.235574-1.078104$

H $\quad 0.756222-0.1479992 .465294$

H $-0.024740 \quad 1.193723 \quad 0.370625$

H 3.2583350 .0242571 .025690

H $3.1740031 .931431-0.607989$

H $3.2304910 .772397-1.942127$

H $3.078633-1.567361-1.329524$

H $-1.8709721 .175674-1.237488$

H $-3.6406081 .176694-1.195137$

H $5.4582371 .735167-1.470476$

H 5.4078551 .0953430 .181037

H $5.466245-0.013592-1.204028$

SCF Energy (B3LYP/6-31G*//MMFF) $=-810.720182400$

F2-26 c06218

MMFF Geometry

C $-2.8072482 .572010-0.107061$

C $-1.8657393 .362538-0.634634$

C $-2.789195 \quad 0.157087 \quad 0.589360$

$\begin{array}{lllll}0 & -1.381983 & 0.213046 & 0.908812\end{array}$

C $-0.742171-0.9979820 .473387$

C $-1.760556-1.745267-0.379097$

C $-3.065844-1.3119320 .256252$

C $0.561642-0.680552-0.272365$

C $-4.287247-1.602588-0.596989$

C $1.564305 \quad 0.1134850 .588993$

O $1.131015-1.904670-0.722752$

O $1.919800-0.6647341 .732564$

C $2.8363550 .565181-0.159918$

C $3.881326-0.530359-0.409959$

O $3.466821 \quad 1.5805160 .632161$

C $-3.0591061 .155949-0.546439$

C $5.100032-0.003466-1.161354$

H -3.4398182 .9690890 .682720$

H $-1.7361174 .377722 \quad-0.275529$

H $-1.2102243 .014421-1.425228$

H $-3.3515700 .451573 \quad 1.483532$

H $-0.531470-1.5953611 .369629$

H $-1.609461-2.829009-0.354535$

H $-1.712975-1.422571-1.426167$

H $-3.174827-1.8567361 .204815$

H $0.327568-0.077687-1.158217$

H $-4.421935-2.683301-0.712670$

H $-5.191162-1.198487-0.130077$

H $-4.197991-1.175990-1.601297$

H 1.0577791 .0077980 .972457

H $1.385404-2.4168510 .064079$

H $2.533138-0.1311592 .266662$

H $2.5555501 .033091-1.110981$

H $3.454883-1.348848-0.994950$

H $4.229945-0.9468430 .542228$

H 2.8463122 .3259180 .702212

H $-2.4329430 .920452-1.415468$

H $-4.1046421 .093609-0.867514$ 
H $5.803917-0.819409-1.354666$

H $4.8090150 .428167-2.124241$

H $5.627080 \quad 0.762525-0.584128$

SCF Energy (B3LYP/6-31G**//MMFF) $=-810.717529727$

\section{F2-26 c06219}

MMFF Geometry

C $-2.637215 \quad 2.4490920 .211482$

C $-3.607168 \quad 3.3588380 .357303$

C $-2.606321-0.0491110 .516132$

O $-1.224549-0.0299970 .936751$

C $-0.536919-1.1610240 .377366$

C $-1.473224-1.766643-0.660568$

C $-2.829310-1.449846-0.064253$

C $0.820337-0.735286-0.199016$

C $-3.979514-1.615397-1.040800$

C $1.736917-0.0663390 .849366$

O $1.450043-1.874314-0.778054$

O $1.912659-0.9473401 .963111$

C 3.1330690 .3651700 .355536

C $3.0601781 .411553-0.762708$

O $3.856353-0.778824-0.094430$

C $-2.8273591 .116643-0.460786$

C $4.4196492 .017449-1.088882$

H -1.6446032 .6834290 .587483$

H -3.4036094 .3048850 .846893$

H -4.613543 $3.178912-0.004301$

H -3.2325140 .0916921 .405405$

H $-0.395583-1.8899461 .185575$

H -1.304153 -2.838903 -0.799800

H -1.354383 -1.276614 -1.634477

H $-2.995618-2.1382940 .776617$

H $0.645445-0.014581-1.005993$

H $-4.922653-1.295324-0.586717$

H -3.825470 -1.039488 -1.958933

H $-4.084276-2.666663-1.329844$

H $1.2278770 .821976 \quad 1.241906$

H $1.582270-2.531806-0.074351$

H $2.466125-1.6895971 .665969$

H 3.6865530 .7690501 .212497

H $2.3799992 .219637-0.469805$

H $2.6678560 .958699-1.680554$

H $4.781778-0.513676-0.226659$

H $-2.1123341 .061238-1.290365$

H $-3.8344231 .064307-0.889064$

H $4.3095822 .799952-1.846616$

H $4.8718182 .468284-0.199937$

H $5.1090911 .266044-1.484984$

SCF Energy $\left(B 3 L Y P / 6-31 G^{* *} / / M M F F\right)=-810.710637020$

F2-26 c06220

MMFF Geometry

C $-2.0911092 .360845-0.553700$

C $-2.9177933 .410066-0.481817$

C $-2.0810970 .145648 \quad 0.641749$

O $-\begin{array}{llll}0.639124 & 0.057185 & 0.599558\end{array}$

C $-0.241847-1.3140520 .440146$

C $-1.493613-2.053861-0.014288$

C $-2.574237-1.3024280 .734717$

C $0.938957-1.441175-0.530148$

C $-3.977463-1.5908170 .232307$

C $2.296496-0.9701430 .030951$

O $1.082292-2.820457-0.896160$

O $3.255718-1.098267-1.029980$

C 2.3567110 .4651470 .581397

C $2.1415661 .544214-0.489051$

O $3.655622 \quad 0.634647 \quad 1.161185$

C $-2.5494450 .928648-0.594448$

C 2.0534022 .9456250 .103534

H -1.021806 $2.546287-0.598793$

H -2.522553 $4.419904-0.462560$

H -3.993903 $3.283717-0.440217$

H $-2.3589200 .691995 \quad 1.551303$

H $\quad 0.050407-1.6890901 .429640$

H -1.463909 -3.119634 0.232606

H -1.635778 -1.956959-1.097611

H -2.524438 -1.605376 1.790380

H $\quad 0.721773-0.898279-1.457256$
H $-4.072572-1.415336-0.844042$

H $-4.238758-2.6385160 .415378$

H $-4.711255-0.9656030 .750984$

H $2.626983-1.6693720 .809644$

H $1.259710-3.326011-0.084560$

H $4.118293-0.823711-0.673715$

H 1.6373050 .5782501 .398059

H $1.2195901 .349838-1.045728$

H $2.9696261 .534319-1.207600$

H 3.6426841 .4544251 .682906

H $-2.1442370 .476878-1.507816$

H $-3.6421920 .900491-0.668274$

H 1.2587103 .0047590 .853799

H 2.9958803 .2433960 .572704

H $1.8301323 .672461-0.684151$

SCF Energy $(B 3 L Y P / 6-31 G * * / / M M F F)=-810.709916115$

F2-26_c06221

MMFF Geometry

C $-3.8247661 .970323-0.089328$

C $-3.2500973 .075022-0.578038$

C $-2.915090-0.2495320 .664961$

O -1.6809150 .3571901 .107521$

C $-0.580034-0.5429540 .856647$

C $-1.117548-1.590625-0.106902$

C $-2.560501-1.7043010 .343225$

C 0.6295300 .2816350 .391892

C $-3.454030-2.428331-0.648048$

C $1.947036-0.5197110 .330274$

O $0.3650660 .852366-0.890659$

O $1.800609-1.597583-0.590101$

C $3.1535340 .349006-0.085673$

C $4.471162-0.443092-0.079609$

$\begin{array}{lllll}\text { O } & 3.255354 & 1.443713 & 0.822628\end{array}$

C $-3.459000 \quad 0.574262-0.511991$

C $5.6722120 .395989-0.498399$

H -4.6117012 .0763840 .652855$

H -3.562653 $4.054991-0.234051$

H -2.463059 $3.023832-1.322265$

H -3.625849 -0.2143241 .499426$

H $-0.346083-1.0128141 .820495$

H - $0.582192-2.541925-0.032895$

H $-1.065720-1.250170-1.147181$

H -2.574416 -2.268612 1.286528

H $\quad 0.752008 \quad 1.1242751 .083215$

H $-4.495191-2.419166-0.310015$

H -3.410873 -1.978144 -1.644962

H $-3.141963-3.473436-0.748300$

H $2.126558-0.9435051 .326715$

H $-0.4522421 .373287-0.811853$

H $2.491918-2.251718-0.394139$

H $2.9873590 .749314-1.093417$

H $4.401229-1.299386-0.758985$

H $4.662394-0.8337700 .927145$

H 3.8470002 .1044520 .425175

H $-2.7202240 .611232-1.321407$

H $-4.3645930 .107435-0.914872$

H $6.567950-0.231400-0.551715$

H $5.5129460 .844322-1.484189$

H $5.872030 \quad 1.197207 \quad 0.219511$

SCF Energy (B3LYP/6-31G**//MMFF) $=-810.713629265$

F2-26_c06222

MMFF Geometry

C $-4.259679-1.052462-0.312723$

C $-4.176829-2.3458510 .018521$

C $-2.5445120 .761775-0.613494$

O $-1.553544-0.188349-1.066905$

C $-0.2468250 .231616-0.619907$

C -0.4972251 .2504880 .481845$

C $-1.7508811 .934217-0.025998$

C $0.564525-1.001069-0.212655$

C -2.4489932 .7839571 .020814$

C $2.007604-0.7382420 .265869$

O $-0.118646-1.664810 \quad 0.862373$

O $2.591018-2.0117530 .568478$

C $2.907269-0.032926-0.766438$

C $4.411016-0.091221-0.442724$ 
O $\quad 2.523517 \quad 1.334437-0.883385$

$\begin{array}{lllll}\text { C } & -3.483157 & 0.041796 & 0.366268\end{array}$

C 4.7847420 .5529710 .886679

H $-4.933587-0.761800-1.114418$

H $-4.767975-3.086859-0.508557$

H -3.522909 -2.687599 0.813067

H -3.112516 $1.098956-1.489080$

H $0.2148120 .716828-1.486592$

H $\quad 0.3394491 .9418370 .615917$

H $-0.6900640 .765006 \quad 1.445347$

H -1.461116 2.592495 -0.857495

H $0.578601-1.722376-1.039181$

H $-1.810124 \quad 3.6224551 .317850$

H $-3.382938 \quad 3.1961880 .625616$

H -2.679204 2.213210 1.926142

H $1.978549-0.1649971 .200373$

H $-1.016620-1.8688840 .549720$

H $2.021225-2.4412001 .229433$

H $2.760053-0.505179-1.745802$

H $4.9606940 .436027-1.232616$

H $4.762005-1.129334-0.453893$

H $3.0688741 .735537-1.581290$

H $-2.909310-0.3724651 .203895$

H -4.2105730 .7473560 .782670$

H $5.8711230 .528718 \quad 1.020821$

H $4.465247 \quad \begin{array}{lll}1.598808 & 0.927181\end{array}$

H 4.3352220 .0207701 .730073

SCF Energy (B3LYP/6-31G**//MMFF)= -810.718710475

F2-26_c06223

MMFF Geometry

C $-4.1698161 .484306-0.735149$

C $-5.2390812 .185291-0.341450$

C $-2.414116 \quad 0.210433 \quad 0.519363$

O $-1.3759940 .355669-0.463580$

$\begin{array}{llll}\text { C } & -0.132901 & 0.041779 & 0.193761\end{array}$

C $-0.484105-0.8990281 .348339$

C $-2.002970-1.0324881 .309946$

C $0.859384-0.499862-0.840321$

C $-2.417103-2.3590970 .671697$

C $2.257287-0.830590-0.290280$

O $0.334490-1.708056-1.406229$

O $3.100193-1.169003-1.401618$

C $2.941637 \quad 0.296654 \quad 0.501386$

C $3.2318761 .556419-0.326064$

$\begin{array}{lllll}\text { O } & 4.173405 & -0.211266 & 1.024794\end{array}$

C $-3.7816440 .149969-0.161233$

C 3.8526962 .6647680 .515610

H -3.543128 $1.890719-1.525008$

H -5.468211 $3.141238-0.799732$

H -5.8996691 .8233160 .438627$

H -2.3654701 .0882301 .179504$

H 0.2420650 .9891660 .598126

H $-0.172755-0.4326242 .291523$

H $\quad 0.031547-1.8632251 .299790$

H -2.424388 -1.002182 2.321342

H $0.9416650 .201642-1.679162$

H $-2.092330-2.433433-0.370126$

H -1.984838 -3.2022441 .221182$

H -3.504918 -2.480346 0.695027

H $2.219165-1.7339490 .330573$

H $-0.524039-1.487361-1.806445$

H $2.664706-1.892345-1.884602$

H $2.3314920 .559614 \quad 1.371285$

H $2.3087191 .937015-0.775456$

H $3.9268781 .323537-1.141435$

H $4.723664-0.4811360 .269330$

H $-3.766625-0.567572-0.990222$

H $-4.537307-0.1847180 .559371$

H 4.8183702 .3586710 .929723

H $4.0180963 .555866-0.098357$

H 3.1936852 .9420651 .344572

SCF Energy (B3LYP/6-31G**//MMFF) $=-810.719489276$

F2-26_c06224

MMFF Geometry

C $4.101608-1.1373830 .747218$

C $3.985596-2.4374150 .454132$
C $2.4586150 .765806 \quad 0.735085$

$\begin{array}{lllll}\text { O } & 1.362392 & -0.118835 & 1.058944\end{array}$

$\begin{array}{llll}\text { C } & 0.177052 & 0.312842 & 0.360383\end{array}$

C $0.6464161 .298133-0.699741$

C 1.8216181 .9508120 .000873

C $-0.591916-0.912586-0.144855$

C $2.7202482 .748174-0.927356$

C $-1.894739-0.593761-0.902374$

O $0.257805-1.685041-1.005052$

O $-2.542869-1.832200-1.224843$

C $-2.8880020 .322704-0.162593$

C $-3.455410-0.281774 \quad 1.129377$

O $-3.961728 \quad 0.597927-1.067807$

C $3.492914-0.033061-0.072159$

C $-4.3122800 .706841 \quad 1.911621$

H $4.675805-0.8493541 .624091$

H $4.452546-3.1864961 .084193$

H $3.425382-2.776394-0.410262$

H $2.906497 \quad 1.1066291 .676356$

H $-0.4277140 .842297 \quad 1.106203$

H $-0.1339482 .012359-0.979361$

H $0.9808820 .791929-1.611407$

H 1.4212012 .6389470 .759040

H $-0.798758-1.5758860 .702638$

H $3.5911253 .133389-0.387403$

H $3.0755302 .148010-1.771125$

H $2.1755703 .603210-1.341991$

H $-1.655232-0.141832-1.872615$

H $0.325242-1.228993-1.860318$

H -2.645348 - $2.343282-0.404430$

H -2.4057531 .2823580 .051799$

H -2.640798 $-0.615127 \quad 1.780598$

H $-4.077949-1.1538830 .898493$

H $-4.482773 \quad 1.329848-0.697403$

H $3.028336-0.451378-0.973351$

H $4.3096250 .621127-0.396417$

H $-5.2078130 .992917 \quad 1.352142$

H $-4.6398560 .255372 \quad 2.853786$

H -3.7469131 .6134122 .149149$

SCF Energy (B3LYP/6-31G**//MMFF) $=-810.700072778$

F2-26 c06225

MMFF Geometry

C $-3.104298 \quad 2.138953-0.321753$

C $-2.360570 \quad 3.176214-0.722254$

C $-2.675136-0.2316130 .407994$

O $-1.412627 \quad 0.1024391 .034831$

C $-0.563976-1.0569041 .024416$

C $-1.091880-1.933932-0.097046$

C $-2.585528-1.7237240 .052880$

C $0.924525-0.6934210 .911412$

C $-3.389677-2.205338-1.141790$

C $1.297290 \quad 0.283582-0.225381$

O $1.329834-0.142513 \quad 2.167704$

$\begin{array}{llllll}\text { O } & 0.752496 & 1.578572 & 0.041802\end{array}$

C $2.8109030 .483572-0.450669$

C $3.507889-0.783102-0.958603$

O 3.4513850 .9074790 .751170

C $-2.8916830 .721042-0.776661$

C $4.952494-0.517817-1.365567$

H -3.9274982 .3242370 .363665$

H -2.572640 $4.176450-0.360736$

H $-1.5341673 .049399-1.412676$

H $-3.466580-0.0808841 .152511$

H $-0.729618-1.5593841 .987338$

H $-0.792618-2.9803150 .016083$

H $-0.756522-1.585877-1.079603$

H -2.918432 -2.2947920 .931491$

H $1.480310-1.6287370 .790357$

H $-4.452820-1.979808-1.010519$

H $-3.051085-1.745810-2.076015$

H $-3.288113-3.289906-1.256156$

H $0.860828-0.056695-1.170345$

$\begin{array}{llll}\text { H } & 0.851159 & 0.696206 & 2.285011\end{array}$

H $-0.204271 \quad 1.4742270 .171955$

H $2.9525301 .291337-1.179877$

H $2.966160-1.188234-1.820933$

H $3.519779-1.551487-0.177716$ 
H 2.9699001 .6874671 .076050

H $-2.0355890 .668893-1.460454$

H $-3.7855720 .429038-1.338442$

H $5.0038620 .236023-2.157687$

H $5.547784-0.167276-0.516767$

H $5.412391-1.436954-1.742465$

SCF Energy (B3LYP/6-31G**//MMFF) $=-810.717482320$

F2-26_c06226

MMFF Geometry

C $2.8031862 .293178 \quad 0.249155$

C 3.7896023 .1678520 .022503

C $2.3033830 .025458-0.721114$

O $\quad 0.880092 \quad 0.227193-0.582338$

C $0.259749-0.995817-0.158993$

C $1.389300-1.9357780 .245692$

C $2.497772-1.494039-0.687624$

C $-0.750489-0.7438250 .967948$

C $3.870967-2.003831-0.289163$

C $-1.857946 \quad 0.2893880 .650515$

O $-1.338904-1.982372 \quad 1.359051$

$\begin{array}{llll}O & -1.292661 & 1.597423 & 0.593433\end{array}$

C $-2.6385600 .033756-0.653435$

C $-3.7240851 .084183-0.948503$

O $-3.230681-1.261077-0.612046$

C 3.0102840 .8100870 .395351

C -4.8278431 .1590210 .098666$

H 1.7880862 .6692420 .347722

H $3.5745034 .227138-0.066947$

H $4.8206932 .847602-0.078570$

H $2.6072030 .420274-1.698132$

H -0.244912 -1.427956-1.031784

H $1.119097-2.9893200 .124231$

H $1.679343-1.7796631 .291653$

H $2.268164-1.883383-1.689808$

H $-0.197309-0.366017 \quad 1.837419$

H $3.909203-3.095480-0.369927$

H $4.643457-1.590242-0.945342$

H $4.121314-1.7451670 .744659$

H $-2.565131 \quad 0.266493 \quad 1.488701$

H -1.830766 -2.3309210 .596066$

H $-0.846847 \quad 1.7640141 .441177$

H -1.942425 $0.054733-1.500223$

H $-4.1962710 .835719-1.907457$

H -3.269314 2.074194-1.066145

H -3.684266 -1.404919 -1.460063

H $2.6168600 .517701 \quad 1.376187$

H 4.0833370 .5890100 .390856

H -4.444370 1.5127921 .059902

H $-5.6021391 .862144-0.225539$

H $-5.302193 \quad 0.1848710 .250580$

SCF Energy (B3LYP/6-31G**//MMFF) $=-810.711641398$

\section{F2-27 c06457}

MMFF Geometry

C $-2.9904062 .449374-0.427803$

C -2.447230 3.5035490 .191161

C $-2.3153660 .040379-0.578156$

O $-0.9104360 .293842-0.400679$

C $-0.199085-0.956457-0.461989$

C $-1.252554-2.059509-0.547708$

C $-2.520426-1.381120-0.065351$

C $0.743984-1.0712500 .745729$

C -3.789537 -2.048011 -0.568832

C 1.6789690 .1363860 .943261

O $-0.043948-1.205804 \quad 1.935679$

O $2.574657-0.1957172 .013822$

C $2.5026610 .544593-0.291630$

C $3.497166-0.528069-0.754035$

$\begin{array}{lllll}\text { O } & 3.241589 & 1.726587 & 0.033172\end{array}$

C -3.1225441 .0865340 .191319$

C $4.239536-0.115082-2.019665$

H -3.370778 2.576788 -1.438076

H -2.385269 $4.463115-0.310370$

H -2.055903 3.4298941 .199697

H $-2.5272040 .099813-1.654193$

H $0.392741-0.957695-1.383987$

H $\quad-0.996805-2.9565790 .024818$
H $-1.362193-2.368086-1.595422$

H -2.527995 -1.386064 1.032012

H $1.326821-1.9963560 .664655$

H -4.678696-1.530748 -0.195273

H $-3.835347-2.048878-1.663073$

H $-3.837810-3.087902-0.229241$

H 1.0993070 .9956751 .303070

H $\quad 0.572617-1.2869192 .683618$

H 3.1657020 .5660022 .142638

H $1.8365190 .810974-1.119132$

H $2.976626-1.471083-0.950425$

H $4.242977-0.7152170 .027524$

H 2.5992012 .4181250 .267412

H -4.1849300 .8188220 .193918$

H -2.7892571 .1159521 .236365$

H $4.917522-0.914283-2.335864$

H $3.5389830 .078492-2.838141$

H $4.8372630 .786993-1.856283$

SCF Energy $\left(B 3 L Y P / 6-31 G^{* *} / / M M F F\right)=-810.719677325$

F2-27 c06458

MMFF Geometry

C $3.7530322 .084589-0.114647$

C 3.1136083 .2516090 .022860

C $2.972393-0.3012880 .009344$

O 1.8687870 .0007590 .885796

C $0.711721-0.7654480 .491818$

C $1.052060-1.388635-0.853377$

C $2.559618-1.541454-0.787836$

C -0.5131410 .1578170 .479792$

C $2.962914-2.830070-0.067882$

C $-1.795023-0.515452-0.048794$

O $-0.7483130 .594843 \quad 1.822831$

$\begin{array}{llll}\text { O } & -2.032090 & -1.723731 & 0.673040\end{array}$

C -3.0421280 .3859580 .042920$

C $-4.285750-0.291229-0.553705$

O $-2.796021 \quad 1.612316-0.641256$

C $3.2145140 .917839-0.894586$

C $-5.5306250 .579244-0.437021$

H 4.7256851 .9641940 .355251

H 3.5582304 .0574890 .596454

H $2.1422663 .423110-0.427700$

H $3.860133-0.4923100 .623950$

H $\quad 0.590787-1.5405141 .258238$

H $0.794406-0.704068-1.670929$

H $0.517866-2.327531-1.028939$

H $3.006557-1.560660-1.787944$

H $\quad-0.2923391 .057496-0.106839$

H $2.579492-2.8713340 .956751$

H $2.585231-3.705845-0.606044$

H $4.053976-2.912421-0.018040$

H -1.635781 -0.789947-1.098056

H $\quad 0.0693121 .0185532 .136357$

H -2.140731 -1.488084 1.610491

H -3.2523040 .6256681 .091843$

H $-4.476219-1.245724-0.050497$

H $-4.117737-0.505534-1.616059$

H $-2.6219831 .402069-1.574670$

H $3.9463130 .665101-1.670914$

H $2.2860861 .207452-1.401868$

H $-5.4168661 .515542-0.992102$

H -6.398639 $0.051272-0.844656$

H -5.7418020 .8216580 .609300$

SCF Energy $\left(B 3 L Y P / 6-31 G^{* *} / / M M F F\right)=-810.718387419$

F2-27 c06459

MMFF Geometry

C $-3.1833992 .452412-0.121630$

C -2.4305493 .5273940 .137350$

C $-2.6286770 .015656-0.310156$

O $-1.2673330 .236226-0.718917$

C $-0.616826-1.032438-0.904161$

C $-1.626397-2.103995-0.497366$

C $-2.617951-1.3501780 .367007$

C $0.699764-1.081789-0.105274$

C $-3.981122-2.0171200 .443374$

C $1.732358-0.012113-0.529503$

O $0.430608-0.9505361 .290054$ 
O $\quad 1.225461 \quad 1.299014-0.287982$

C $3.072975-0.1502880 .219981$

C $4.1073750 .885423-0.249382$

O $3.599234-1.461194 \quad 0.026374$

$\begin{array}{llll}\text { C } & -3.069878 & 1.148826 & 0.617007\end{array}$

C $5.4235650 .773646 \quad 0.509697$

H $-3.9318792 .516734-0.907118$

H $-2.5644144 .441654-0.430408$

H $-1.671504 \quad 3.5155250 .911711$

H -3.244827 $-0.005619-1.218974$

H $-0.382558-1.134828-1.970438$

H $-1.175486-2.9618260 .011079$

H $-2.123146-2.487013-1.398067$

H $-2.208298-1.2638391 .381196$

H $1.138491-2.075856-0.249443$

H $-3.893793-3.0221740 .869128$

H $-4.663540-1.4416801 .076356$

H $-4.438479-2.109957-0.547528$

H $1.913030-0.111995-1.607208$

H $0.057360-0.0633101 .430968$

H $0.420415 \quad 1.408424-0.821569$

H $2.910931-0.0116461 .295542$

H $3.7147301 .900276-0.121931$

H $4.3156970 .743692-1.316746$

H $3.755192-1.581095-0.926021$

H -4.0484420 .9216011 .054432$

H -2.356299 1.2487811 .444715

H 6.1208311 .5461050 .169852

H $5.267603 \quad 0.9069201 .584901$

H $5.897197-0.1995610 .347667$

SCF Energy (B3LYP/6-31G**//MMFF) $=-810.725846234$

F2-27_c06460

MMFF Geometry

C $-4.267658-1.328341-0.392386$

C $-5.588078-1.258108-0.595534$

$\begin{array}{llll}\text { C } & -2.551248 & 0.396568 & 0.221794\end{array}$

O $-1.677006-0.5296680 .897498$

C $-0.405791 \quad 0.110346 \quad 1.157545$

C $-0.619667 \quad 1.5832850 .839442$

C $-1.653888 \quad 1.532062-0.266035$

C $0.649839-0.6127250 .298230$

C $-2.381965 \quad 2.849177-0.475052$

$\begin{array}{llll}C & 2.061311 & 0.006992 & 0.358393\end{array}$

O $0.732585-1.9640230 .771900$

$\begin{array}{llll}\text { O } & 2.453855 & 0.172267 & 1.719405\end{array}$

C $3.126018-0.824763-0.383147$

C $4.547661-0.244514-0.295485$

O $2.744238-0.950992-1.751071$

C -3.282924 -0.320465 -0.914776

C $4.698414 \quad 1.143552-0.904574$

H -3.872626 -2.1676690 .174223$

H $-6.246500-2.021678-0.196056$

H -6.033643 $-0.445031-1.157746$

H $-3.2670280 .763957 \quad 0.968835$

H $-0.184000-0.0346322 .220930$

H $\quad 0.283173 \quad 2.1313050 .563498$

H -1.043001 2.0818521 .721385

H -1.157742 $1.246969-1.203023$

H $\quad 0.314572-0.673082-0.743695$

H -3.127448 2.761710 -1.271630

H -2.898335 3.1716020 .435249

H $-1.6766193 .637316-0.758126$

H $2.0130351 .001757-0.094802$

H $-0.161167-2.3440550 .717789$

H $2.463715-0.7093582 .130477$

H $3.163901-1.8337920 .045100$

H $5.230816-0.915535-0.831095$

H $4.880259-0.2193290 .748422$

H $3.409121-1.507108-2.191783$

H $-3.794220 \quad 0.411355-1.550914$

H -2.561857 $-0.855634-1.544908$

H $5.7495001 .449534-0.879708$

H $4.3691791 .160602-1.947928$

H $4.1253961 .889869-0.346846$

SCF Energy (B3LYP/6-31G**//MMFF) $=-810.719459718$

F2-27_c06461
MMFF Geometry

C $2.929054-1.767099-0.586375$

C $4.034760-2.260123-0.017357$

C $2.0693270 .591089-1.009918$

O $0.6883110 .204137-0.914098$

C 0.0518891 .0191640 .089772

C $1.146656 \quad 1.881573 \quad 0.720179$

C 2.4278601 .1512360 .362078

C -0.6989500 .1198141 .084958$

C 3.6588322 .0410430 .365187

C $-1.668558-0.8849990 .431703$

O $0.256258-0.6330941 .842526$

O $-2.373636-1.5711411 .476081$

C $-2.684789-0.283842-0.557598$

C -3.6694790 .7077990 .074766$

O $-3.440819-1.352713-1.135755$

C $2.916560-0.581981-1.510197$

C $-4.5753221 .356793-0.965744$

H $1.978250-2.252738-0.382714$

H $3.977468-3.1226260 .637582$

H $5.008562-1.815765-0.190458$

H $2.1148021 .388393-1.764345$

H $-0.6659481 .672394-0.418715$

H $1.0202592 .032847 \quad 1.796678$

H $1.1334072 .874608 \quad 0.252343$

H 2.5759850 .3430991 .087316

H -1.2293310 .7487631 .809521$

H $3.5603242 .865838-0.348433$

H 3.8185002 .4730541 .358504

H $4.553158 \quad 1.4691770 .098169$

H -1.093204 -1.667872 -0.077480

H -0.240977 -1.192589 2.463552

H -2.795921 -0.9056082 .044873$

H $-2.1558090 .204136-1.383274$

H -3.1284541 .4988790 .604191$

$\mathrm{H}-4.3104520 .1955380 .801523$

H $-2.813408-1.951049-1.576331$

H $2.511104-0.926011-2.469935$

H $3.938699-0.234035-1.701757$

H $-5.2471142 .074535-0.484403$

H $-3.9864641 .894450-1.715770$

H -5.191406 $0.612272-1.479398$

SCF Energy (B3LYP/6-31G**//MMFF) $=-810.713719635$

F2-27_c06462

MMFF Geometry

C $-3.991023 \quad 1.749150 \quad 0.059354$

C $-3.6810683 .036282-0.132115$

C $-2.633287-0.357769-0.018127$

O $-1.6706530 .149287-0.964181$

C $-0.541272-0.752589-1.032285$

C $-0.970983-1.998752-0.271025$

C $-1.902090-1.4340390 .781808$

C $0.6749110 .000380-0.455012$

C $-2.820058-2.4748291 .400164$

C $1.956177-0.849571-0.341965$

O $0.929244 \quad 1.115049-1.317581$

O $2.220375-1.480809-1.597722$

C $3.236222-0.1086420 .098809$

C 3.1059360 .5326791 .484775

O $3.6064820 .883181-0.857573$

C -3.1486470 .7900930 .851894$

C 4.4291641 .1036841 .979121

H $-4.9114681 .364883-0.372720$

H $-4.3351063 .678442-0.711962$

H -2.7765383 .4696490 .280126$

H -3.452378 -0.799202 -0.600889

H $-0.372354-0.976828-2.091511$

H $-0.153430-2.5897060 .145812$

H -1.531454 -2.656151 -0.948744

H $-1.300802-0.973514 \quad 1.576637$

H $0.4232460 .421670 \quad 0.524538$

H -3.445452 -2.959568 0.642949

H $-2.235622-3.253121 \quad 1.901664$

H $-3.482708-2.0194172 .142743$

H $1.783908-1.6557820 .378709$

H $0.1095591 .636452-1.366564$

H $2.391426-0.775450-2.245599$ 
H $4.059621-0.8331210 .109575$

H $2.746947-0.2093762 .206910$

H 2.3786811 .3523141 .457681

H $2.9073941 .558626-0.872542$

H $-3.7628570 .397271 \quad 1.669949$

H -2.301166 1.3209301 .303619

H 4.3047681 .5315362 .978930

H 5.1949290 .3235702 .037373

H 4.7933831 .8957661 .317395

SCF Energy (B3LYP/6-31G*//MMFF) $=-810.724278873$

F2-27 c06463

MMFF Geometry

C $3.7635952 .075816-0.158026$

C $3.1277363 .248269-0.055656$

C $2.974854-0.3038590 .021458$

$\begin{array}{lllll}\text { O } & 1.867602 & 0.023853 & 0.883813\end{array}$

C $0.711486-0.7516840 .505618$

C $1.055977-1.407769-0.822773$

C $2.562907-1.561941-0.746811$

C $-0.513797 \quad 0.171454 \quad 0.466953$

C $2.960040-2.8334790 .005972$

C $-1.794581-0.509304-0.057310$

O -0.7548840 .6413661 .797462$

O $-1.995012-1.7190230 .673945$

$\begin{array}{llll}\text { C } & -3.054456 & 0.374633 & 0.055991\end{array}$

C $-4.288983-0.293135-0.569935$

O $-2.833787 \quad 1.615039-0.611543$

C $3.2264030 .891218-0.911527$

C $-5.5380560 .573358-0.458152$

H 4.7322351 .9640720 .322184

H $3.571296 \quad 4.0670280 .500442$

H $2.1605333 .411649-0.517984$

H $3.858399-0.4823480 .645799$

H $0.588917-1.5074471 .290965$

H $0.803374-0.742449-1.657609$

H $0.520380-2.349414-0.978181$

H $3.014200-1.606473-1.744153$

H $-0.2842281 .055887-0.138991$

H $2.572294-2.849057 \quad 1.029689$

H $2.582368-3.721222-0.512217$

H $4.050683-2.9171950 .062303$

H -1.632352 - $0.774397-1.109260$

H $\quad 0.065708 \quad 1.0601662 .109832$

H -2.605804 -2.276675 0.163698

H -3.279291 $0.582978 \quad 1.109295$

H $-4.491737-1.253692-0.084688$

H $-4.104398-0.490052-1.633008$

H -2.233312 2.143527 -0.059177

H $3.9620070 .616739-1.676655$

H $2.3019341 .170255-1.431589$

H $-6.3998520 .047079-0.880888$

H -5.7616340 .8039370 .588268$

H -5.421834 $1.515753-1.002298$

SCF Energy (B3LYP/6-31G**/MMFF) $=-810.714670695$

F2-27 c06464

MMFF Geometry

C $-3.5369682 .049303-0.160348$

C $-2.9769213 .244596 \quad 0.057210$

C $-2.570999-0.257775-0.319514$

O $-1.2795340 .186361-0.771337$

C $-0.422321-0.953875-0.950304$

C $-1.218834-2.174710-0.494483$

C $-2.305662-1.5848010 .382482$

C $0.903146-0.752218-0.188370$

C $-3.529319-2.476506 \quad 0.509379$

C $1.7122620 .472428-0.672255$

O $0.643882-0.647962 \quad 1.209575$

O $1.003656 \quad 1.681677-0.385030$

C $3.1160820 .626418-0.051121$

C $4.063572-0.516320-0.430257$

$\begin{array}{llllll}\text { O } & 3.035327 & 0.700974 & 1.371038\end{array}$

C $-3.179396 \quad 0.802647 \quad 0.598662$

C $5.494218-0.247754 \quad 0.021413$

H $-4.3045321 .964339-0.925187$

H -3.282331 $4.108149-0.523429$

H $-2.208500 \quad 3.382814 \quad 0.809770$
H -3.196568 -0.407081-1.209690

H $-0.200631-1.039543-2.020737$

H $-0.612668-2.9284920 .017461$

H - $-1.662752-2.660138-1.373381$

H $-1.893368-1.4047461 .383312$

H $1.504033-1.656363-0.333003$

H $-4.286288-2.013598 \quad 1.149853$

H -3.987195 -2.671048 -0.466420

H $-3.257870-3.4406540 .951578$

H $1.8252890 .419660-1.762039$

H $\quad 0.1323470 .167301 \quad 1.349679$

H $0.1508111 .642109-0.849652$

H $3.5443161 .578893-0.387656$

H $4.057052-0.663226-1.516246$

H $3.738717-1.452210 \quad 0.038098$

H $2.401877 \quad 1.4062101 .588156$

H -4.0921410 .4192411 .068189$

H -2.473581 1.0441691 .403373

H $6.146290-1.072535-0.283034$

H $5.8804420 .672746-0.427896$

H $5.557448-0.1529941 .109968$

SCF Energy (B3LYP/6-31G**//MMFF) $=-810.722077947$

F2-27 c06465

MMFF Geometry

C $-4.425470-0.5327820 .984425$

C $-5.375185-0.9212310 .126178$

$\begin{array}{llll}C & -2.110957 & 0.456550 & 0.742396\end{array}$

$\begin{array}{llll}\text { O } & -0.728303 & 0.043396 & 0.762365\end{array}$

C $-0.104176 \quad 0.397738-0.482806$

C $-1.238466 \quad 0.707590-1.450274$

C $-2.308887 \quad 1.276867-0.537291$

C $0.846051-0.713932-0.942531$

C $-2.097796 \quad 2.770727-0.281086$

C $1.914232-1.1168780 .092060$

O $0.085241-1.890817-1.247552$

O $2.803387-2.053893-0.532716$

$\begin{array}{llll}\text { C } 2.758301 & 0.038850 & 0.656704\end{array}$

C $3.6105530 .764015-0.393877$

O $3.619047-0.4923221 .669559$

C $-2.964553-0.8188540 .803636$

C $4.379937 \quad 1.9367890 .202388$

H $-4.7207620 .013645 \quad 1.876374$

H $-6.417019-0.6880920 .316881$

H $-5.133425-1.473074-0.775456$

H -2.2909891 .0563491 .642474$

H $0.4681541 .314910-0.303275$

H $-1.622366-0.204225-1.921897$

H $-0.9286491 .385938-2.251254$

H -3.306171 $1.153524-0.971802$

H $1.326327-0.421157-1.882837$

H $-2.878182 \quad 3.155617 \quad 0.383986$

H $-1.130392 \quad 2.9806650 .186037$

H -2.149941 $3.333317-1.219179$

H $1.451123-1.6620540 .923891$

H $-0.396229-2.145842-0.441764$

H $2.259844-2.784184-0.875326$

H $2.1071620 .758511 \quad 1.162921$

H $2.9768921 .140565-1.203501$

H $4.3383440 .073105-0.835279$

H $4.189933-1.1584341 .249173$

H $-2.787033-1.435461-0.086504$

H -2.626573 -1.432008 1.649069

H $4.9520062 .447731-0.578664$

H 3.6968252 .6638650 .652795

H 5.0846581 .6031840 .970364

SCF Energy (B3LYP/6-31G**//MMFF) $=-810.722282166$

F2-27 c06466

MMFF Geometry

C $-2.861385 \quad 2.462203-0.217510$

C $-2.005747 \quad 3.379797 \quad 0.246830$

C $-2.677772-0.024776-0.527985$

O $-1.247400 \quad 0.016073-0.701191$

C $-0.648810-1.128606-0.073899$

C $-1.731083-1.7427670 .804525$

C $-3.005860-1.4018840 .055969$

C $0.632561-0.7469350 .683233$ 
C $-3.302556-2.421488-1.045660$

C $1.743587-0.166198-0.222322$

O $\quad 0.3219490 .194087 \quad 1.711426$

O $1.3144911 .053366-0.822814$

C 3.0475770 .1116250 .550005

C $4.1564640 .758141-0.297425$

O $3.527547-1.1106291 .106573$

C $-3.0792801 .117603 \quad 0.417772$

C $4.610071-0.083816-1.483087$

H -3.445545 $2.695242-1.104088$

H -1.897084 $4.334126-0.256747$

H -1.401431 $3.197752 \quad 1.128515$

H $-3.1477230 .121418-1.507825$

H $-0.388298-1.829223-0.876817$

H -1.757133 -1.2673321 .791914$

H -1.579995 -2.815257 0.962218

H $-3.871141-1.3733600 .727560$

H $1.009708-1.6477811 .180901$

H -2.495676 -2.484275 -1.782796

H $-3.448551-3.418760-0.617690$

H $-4.219405-2.148568-1.579125$

H $1.939832-0.890783-1.021420$

H $\quad 0.002415 \quad 1.003070 \quad 1.276007$

H $\quad 0.5310670 .857683-1.363869$

H $2.8360450 .793970 \quad 1.382513$

H $\begin{array}{llll}5.031612 & 0.925716 & 0.342967\end{array}$

H $3.8324901 .741825-0.655759$

H $4.325619-0.9051421 .622541$

H -4.1425451 .0323820 .671774$

H $-2.5143791 .052646 \quad 1.355427$

H $3.809910-0.201581-2.219479$

H $5.4517110 .403419-1.986128$

H $4.939204-1.077749-1.165216$

SCF Energy (B3LYP/6-31G**//MMFF) $=-810.723045729$

F2-27 c06467

MMFF Geometry

C $-3.0727192 .495290 \quad 0.578243$

C -2.434760 $3250160-0.323806$

C $-2.3176840 .210693-0.386948$

O $-0.9108170 .405500-0.160050$

C $-0.200298-0.771245-0.588371$

C $-1.255338-1.806802-0.971184$

C $-2.514434-1.300242-0.294092$

C $0.771683-1.2126790 .518247$

C $-3.791365-1.804923-0.945190$

C $1.726128-0.1043041 .004825$

O $0.036128-1.6431061 .670903$

O $2.663013-0.6986461 .915684$

C $2.5201120 .621295-0.096189$

C $3.497113-0.289757-0.853095$

O $3.249444 \quad 1.677937 \quad 0.536425$

C -3.1190650 .9915720 .659032$

C $4.1887160 .422304-2.009481$

H $-3.6323103 .014606 \quad 1.354130$

H $-2.4877084 .332279-0.261559$

H -1.853424 2.826500-1.133538

H -2.547689 $0.563391-1.400677$

H $0.371504-0.506840-1.484736$

H $-0.991426-2.831138-0.691648$

H -1.383428 -1.798195 -2.061552

H -2.502654 -1.615375 0.756917

H $\quad 1.351007-2.0811580 .183252$

H -3.831209-2.898792 -0.915417

H -4.674772 -1.422418 -0.424828

H $-3.857170-1.494268-1.993239$

H $1.1728960 .636157 \quad 1.596382$

H $-0.391799-2.4876831 .451900$

H $2.147848-1.1305192 .618565$

H $1.8255751 .097026-0.796577$

H $2.966983-1.158799-1.256522$

H $4.273509-0.659603-0.173009$

H $3.5817892 .267923-0.160471$

H -4.173905 0.6985590 .601342

H -2.7605050 .7163871 .659359$

H $4.819553-0.282690-2.560494$

H $3.4560380 .838600-2.708072$

H $4.8306151 .234386-1.655161$
SCF Energy (B3LYP/6-31G**//MMFF) $=-810.708115851$

F2-27_c06468

MMFF Geometry

C $-3.1966042 .470365-0.096548$

C -2.4520633 .5417380 .199307$

C $-2.6383290 .036980-0.316784$

O $-1.2675180 .259994-0.689592$

C $-0.624590-1.007831-0.907295$

C $-1.641366-2.083138-0.527751$

C $-2.643590-1.3408780 .335018$

C $0.699999-1.084289-0.122736$

C $-4.010074-2.0040160 .378037$

C $1.738354-0.028055-0.566844$

O $0.461648-0.9248291 .275356$

O $1.2188591 .287734-0.384757$

C $3.079535-0.1426920 .187029$

C $4.1076820 .877900-0.327659$

O $3.588280-1.4626860 .013031$

C -3.0981651 .1537290 .621007$

C 5.4205090 .8373040 .444280

H -3.926589 $2.547872-0.898088$

H $-2.5741804 .465782-0.355104$

H -1.711353 3.5168930 .990922

H $-3.2341150 .033323-1.239318$

H $-0.399127-1.086462-1.977685$

H -1.199350 -2.950808 -0.028488

H -2.127261 -2.452089-1.440409

H -2.249854 -1.273130 1.356894

H $1.130252-2.082501-0.269101$

H $-3.932873-3.0173260 .785526$

H $-4.699910-1.4384271 .012030$

H -4.452831 -2.075714 -0.620997

H $1.919628-0.163200-1.640188$

H $-0.007520-1.7163521 .587723$

H $\quad 0.967220 \quad 1.3763310 .550539$

H 2.9158510 .0274021 .258227

H $3.6993381 .892360-0.259215$

H $4.3268260 .680520-1.384062$

H $4.308805-1.5895520 .652675$

H $-4.0857220 .919771 \quad 1.033986$

H -2.4017831 .2385631 .464893$

H 6.0900091 .6266960 .087603

H 5.2512560 .9943561 .514148

H $5.935332-0.1187210 .310822$

SCF Energy $(B 3 L Y P / 6-31 G * * / / M M F F)=-810.710980812$

F2-27_c06469

MMFF Geometry

C $-1.4364722 .995746-0.435771$

C $-0.3056343 .618772-0.085030$

C $-2.0740280 .572286-0.465736$

O $-0.726795 \quad 0.109036-0.658984$

C $-0.713682-1.327213-0.692538$

C $-2.150360-1.776760-0.426481$

C $-2.783745-0.5738200 .244577$

C $0.303728-1.8779390 .325127$

C $-4.297628-0.5395910 .119824$

C $1.783415-1.5289970 .038274$

O $-0.054072-1.4653401 .641105$

O $2.159748-2.096507-1.216798$

C $2.163024-0.034260 \quad 0.032110$

C $3.6831030 .158470-0.103077$

O 1.7421930 .5984981 .235327

C $-2.052670 \quad 1.869020 \quad 0.344547$

C $4.0751491 .629453-0.178536$

H -1.953411 $3.320465-1.335160$

H $0.0864554 .428173-0.691063$

H $\quad 0.247517 \quad 3.3338790 .802984$

H $-2.5078990 .742708-1.459988$

H $-0.416902-1.637733-1.700861$

H -2.221364 -2.691105 0.170899

H -2.644448 -1.978824 -1.385644

H $-2.515963-0.5807681 .308781$

H $\quad 0.213158-2.9720570 .302169$

H -4.7113260 .3434920 .616543$

H -4.613538 -0.515053 -0.928537

H $-4.741550-1.4262420 .584376$ 
H $\quad 2.386769-2.0164850 .815823$

H $0.581129-1.8653122 .259093$

H $\quad 1.994847-3.053616-1.168713$

H $1.6926710 .475312-0.815961$

H $4.053307-0.348833-1.000814$

H $4.190979-0.2854020 .761798$

H 0.7708750 .5649421 .259596

H $-3.0726402 .162671 \quad 0.616556$

H -1.497217 1.7136031 .278027

H $5.158337 \quad 1.724073-0.304627$

H $3.5904362 .120397-1.028346$

H $3.7959862 .165216 \quad 0.734128$

SCF Energy (B3LYP/6-31G**/MMFF)= -810.718715395

F2-27_c06470

MMFF Geometry

C - -2.5153112 .7504770 .207539$

C -1.7748553 .5439240 .989763$

C $-2.2789410 .349330-0.468596$

O $-0.8473520 .324890-0.331988$

C $-0.365312-0.977017-0.711046$

C $-1.596900-1.855375-0.932192$

C $-2.711841-1.094697-0.240123$

C $0.600667-1.4964570 .363869$

C $-4.092698-1.424179-0.781650$

C $1.765149-0.5559310 .750930$

O $-0.138214-1.7696821 .563297$

O $2.576966-1.2610321 .698781$

C $2.663539-0.114937-0.421390$

C 3.9496040 .5856680 .050287

O $1.953848 \quad 0.776794-1.277165$

C -2.8694441 .3295970 .545322$

C $4.8380901 .014767-1.111196$

H $-2.8962753 .150093-0.728807$

H $-1.557764 \quad 4.5633370 .689724$

H -1.3730183 .1947831 .934398$

H -2.507242 $0.666296-1.495083$

H $\quad 0.167818-0.879051-1.662467$

H -1.478346 -2.878982 -0.563269

H -1.796483 -1.925125 -2.009442

H $-2.685684-1.3288400 .831688$

H $1.009490-2.4616440 .041674$

H $-4.866191-0.858831-0.252687$

H -4.172483 -1.187420 -1.847972

H $-4.310381-2.490007-0.656548$

H $\quad \begin{array}{lll}1.368063 & 0.327230 & 1.266872\end{array}$

H $-0.523429-0.9297271 .867312$

H $1.999976-1.5232032 .436547$

H $2.944708-0.994247-1.013242$

H $4.529808-0.0770120 .701868$

H 3.6916751 .4789230 .632316

H $1.6827391 .546131-0.747660$

H -3.9619351 .2483810 .559715$

H -2.5088461 .0830251 .552029$

H $5.1166290 .154912-1.728622$

H $4.3371461 .749922-1.748623$

H $5.7575531 .472286-0.732207$

SCF Energy $($ B3LYP/6-31G**//MMFF $)=-810.721151048$

F2-27_c06471

MMFF Geometry

C $4.396153-1.2099130 .039515$

C $4.330745-2.5460490 .052633$

C $2.6504180 .577522-0.176234$

O $1.736674-0.231904-0.943307$

C $0.4554770 .434898-1.024299$

C $0.6894081 .835664-0.476371$

C $1.785347 \quad 1.6090690 .544358$

C $-0.554573-0.426885-0.241329$

C 2.5274602 .8779910 .928256

C $-1.9661140 .185199-0.118897$

O $-0.671368-1.680918-0.927816$

O $-2.4053620 .592357-1.413132$

C -3.003213 -0.7716250 .506403$

C $-4.332014-0.0981090 .888643$

O $-2.447347-1.3257581 .701150$

C $3.444361-0.3138860 .780483$

C $-5.1517710 .421802-0.279711$
H $\quad 5.188225-0.732307-0.531522$

H $5.052227-3.136772-0.501129$

H $3.562362-3.0711930 .608975$

H $3.3213481 .069441-0.892805$

H $0.1769290 .467720-2.083700$

H $-0.1960532 .321037-0.061163$

H $1.0612172 .478656-1.284890$

H $\quad 1.343067 \quad 1.1686331 .447569$

H $-0.159616-0.6575660 .754778$

H 1.8414523 .6029191 .378696

H 3.3172322 .6640951 .655194

H $2.991346 \quad 3.3514740 .056426$

H -1.903982 1.0831500 .505031

H $0.222088-2.060999-0.987939$

H -2.440343 -0.201151-1.974618

H $-3.212540-1.598775-0.182780$

H -4.1439320 .7172281 .598517$

H $-4.942921-0.8244551 .440745$

H -3.075634 -1.986465 2.039207

H $4.0297620 .302021 \quad 1.472553$

H $2.753434-0.9173001 .382628$

H -5.271974 -0.344763 -1.051489

H -6.1493910 .7133360 .064942$

H -4.695194 $1.307754-0.729078$

SCF Energy $\left(B 3 L Y P / 6-31 G^{* *} / / M M F F\right)=-810.716127492$

F2-27 c06472

MMFF Geometry

C -3.130398 2.4160090 .082041

C -2.3592043 .4845610 .312038$

C $-2.593810-0.011428-0.237591$

O $-1.2747370 .228876-0.758726$

C $-0.642968-1.030748-1.045185$

C $-1.608043-2.120086-0.582575$

C $-2.520699-1.3990150 .390039$

C $0.743770-1.094317-0.374547$

C -3.870749-2.074155 0.564486

C $1.7025850 .014730-0.856690$

O $0.592643-1.0378301 .040586$

O $1.2806991 .294042-0.382815$

C $3.173976-0.202641-0.446446$

C 3.4932910 .0338721 .036228

O $3.9749710 .690839-1.230314$

C -2.9518541 .0884520 .762864$

C $4.969587-0.1843631 .350152$

H $-3.9440562 .504151-0.633334$

H -2.542782 $4.417188-0.210324$

H -1.5362153 .4493201 .017284$

H -3.288819-0.005219-1.087866

H -0.509756-1.096635 -2.131668

H $-1.110145-2.990227-0.143641$

H -2.182443 -2.478819-1.446474

H $-2.023378-1.3440181 .366622$

H $1.186976-2.068820-0.611205$

H $-4.413577-2.136918-0.384651$

H $-3.744776-3.0920810 .947868$

H -4.495677 -1.5223981 .273528$

H $\quad 1.6668970 .060547-1.952753$

H $\quad 0.233889-0.1606351 .260050$

H $\quad 0.376793 \quad 1.446131-0.706545$

H $3.478656-1.216611-0.729733$

H $2.910468-0.6424061 .667315$

H 3.2376021 .0607271 .321932

H $3.7004881 .598108-1.011541$

H -3.8879450 .8430701 .276751$

H -2.1681371 .1625311 .527302$

H $5.150128-0.0486172 .421294$

H $5.281228-1.1982511 .079451$

H 5.6030770 .5272420 .811617

SCF Energy (B3LYP/6-31G**//MMFF) $=-810.727216787$

F2-27_c06473

MMFF Geometry

C $4.414555-0.838989-0.048264$

C $4.392944-2.1618980 .148933$

C $2.5916260 .868498-0.330404$

O $1.647433-0.083106-0.860107$

C $0.3257800 .208896-0.356725$ 
C $\quad 0.524077 \quad 1.2008880 .778284$

C 1.7654251 .9618140 .352450

C $-0.355856-1.1110900 .028790$

C $1.4266353 .096986-0.615957$

C $-1.758603-0.9875470 .659817$

O $-0.463269-1.924552-1.143968$

O $-1.647691-0.4956671 .993160$

C $-2.753386-0.099269-0.116407$

C $-4.151101-0.1104670 .524761$

O $-2.856790-0.533337-1.468901$

C 3.5091010 .1311630 .657617

C $-5.1298630 .801380-0.204566$

H $5.132378-0.428557-0.753844$

H $5.076372-2.808975-0.389916$

H $3.697887-2.6209570 .843256$

H $3.1833921 .268207-1.162353$

H $-0.2096960 .675560-1.191808$

H $\quad \begin{array}{llll}0.735791 & 0.674537 & 1.716721\end{array}$

H $-0.348906 \quad 1.8390250 .943505$

H $2.2842072 .398728 \quad 1.212990$

H $0.300140-1.6559350 .719673$

H $2.338033 \quad 3.624124-0.917522$

H $0.9358512 .735970-1.525445$

H $0.761556 \quad 3.824344-0.138606$

H -2.186111 -1.997581 0.717534

H $0.427344-1.999010-1.527907$

H $-1.074664-1.1076542 .485780$

H -2.396252 $0.936552-0.125875$

H $-4.091804 \quad 0.2057401 .572060$

H $-4.557503-1.1289730 .509448$

H $-3.151135-1.460194-1.461642$

H 4.1390370 .8539651 .189435

H $2.911457-0.3982611 .409908$

H -6.1017450 .7896910 .298881$

H $-4.7669441 .833984-0.217445$

H $-5.2841460 .476797-1.238275$

SCF Energy (B3LYP/6-31G*//MMFF) $=-810.710514292$

\section{F2-27 c06474}

MMFF Geometry

C $-3.3776001 .429488-0.689801$

C $-3.0666392 .692426-1.001567$

C $-2.028874-0.609125-0.152211$

O $-0.857163-0.233024-0.898879$

C $-0.027043-1.398633-0.990131$

C $-0.431592-2.332366 \quad 0.153071$

C $-1.480481-1.5437520 .921675$

C $1.460566-1.040331-1.080212$

C $-2.530737-2.4224121 .580078$

C $2.127041-0.4312370 .167968$

O $2.153452-2.256819-1.398941$

O $3.501453-0.186655-0.178682$

$\begin{array}{llll}\text { C } & 1.524847 & 0.878751 & 0.703449\end{array}$

C $1.6126672 .053552-0.279040$

O $2.234731 \quad 1.2400781 .893944$

C $-2.713876 \quad 0.6395380 .403249$

C $0.996867 \quad 3.326330 \quad 0.290183$

H $-4.1676730 .935988-1.250184$

H -3.590428 $3.205317-1.800907$

H -2.292611 $3.234920-0.471486$

H $-2.701613-1.150744-0.831499$

H $-0.298924-1.899476-1.929715$

H $0.401175-2.6374880 .793227$

H $-0.854310-3.254165-0.266052$

H $-0.969903-0.9598741 .697860$

H $1.612508-0.367474-1.932684$

H -3.253433 -1.819213 2.138070

H -3.083154 -3.009661 0.838690

H -2.062970 -3.120454 2.282030

H $2.164976-1.1776520 .969754$

H $3.096325-2.036240-1.492085$

H 3.9352820 .1877360 .607301

H 0.4856550 .7284851 .001928

H $1.0990501 .806233-1.213715$

H $2.6597562 .268654-0.522369$

H 2.1172140 .5182442 .534961

H -3.486329 0.3565201 .126822

H -1.9834841 .2655400 .929938$
H $1.0191744 .123546-0.459549$

H -0.0445463 .1618580 .581397$

H 1.5476013 .6773891 .168465

SCF Energy $($ B3LYP/6-31G**//MMFF $)=-810.714979974$

F2-27 c06475

MMFF Geometry

C $-2.6183382 .565055 \quad 0.367041$

C -2.0047063 .3402651 .268122$

C $-2.1350620 .255163-0.473415$

O $-0.7220950 .326926-0.216081$

C $-0.105104-0.893699-0.662137$

C $-1.238616-1.843104-1.051522$

C $-2.463198-1.231880-0.397002$

C $0.820479-1.4277190 .437606$

C $-3.766079-1.618376-1.076262$

C $1.867529-0.4350770 .987128$

O $0.021072-1.8538101 .551699$

O $2.685278-1.1795541 .911926$

C $2.8491610 .202929-0.013962$

C $2.3152461 .472619-0.686493$

O $3.221104-0.740773-1.016240$

C $-2.882477 \quad 1.0983850 .559955$

C $3.4004402 .196052-1.477018$

H -2.957601 $3.014608-0.562558$

H $-1.8470154 .394721 \quad 1.069088$

H -1.6489012 .9413262 .211612$

H $-2.3063850 .640289-1.487609$

H $\quad 0.485619-0.672436-1.556453$

H -1.063472 -2.881795 -0.754239

H $-1.349024-1.837761-2.143743$

H $-2.499870-1.5541950 .651252$

H $1.323897-2.3326880 .077474$

H -4.622295 -1.161709-0.570237

H -3.783376 -1.297270 -2.123189

H $-3.903100-2.704564-1.055131$

H $1.3871370 .345528 \quad 1.588770$

H $-0.454922-1.0738371 .885483$

H $2.089982-1.5656922 .577403$

H 3.7716790 .4664890 .519986

H 1.9164232 .1574610 .070427

H $1.5093371 .240688-1.386533$

H $3.601590-1.511675-0.561387$

H -3.9624910 .9351600 .473428$

H -2.5848990 .7940411 .571335$

H $2.993523 \quad 3.106476-1.928587$

H $4.2329792 .483995-0.827144$

H $3.7926491 .566843-2.282125$

SCF Energy (B3LYP/6-31G**//MMFF) $=-810.719953050$

F2-27 c06476

MMFF Geometry

C $4.281587 \quad 1.661760 \quad 0.276823$

C $4.5339262 .476998-0.753300$

C $2.662909-0.2398790 .681221$

O $1.376388-0.4825891 .286831$

C $0.449738-0.9485890 .284205$

C $1.119401-0.695808-1.057503$

C $2.595374-0.824355-0.732913$

C $-0.895153-0.233400 \quad 0.478734$

C $3.051344-2.285072-0.755789$

C $-1.925815-0.545249-0.624451$

O $-1.417168-0.6326331 .748666$

O $-2.085411-1.964586-0.712610$

C $-3.3208810 .090147-0.445156$

C $-3.2758471 .621485-0.445119$

O $-3.930901-0.3512950 .765828$

C $2.902437 \quad 1.277027 \quad 0.722611$

C $-4.6698102 .236757-0.461603$

H 5.1190241 .2573110 .839188

H $5.5555412 .721693-1.022685$

H $3.7336732 .910432-1.342922$

H $3.420556-0.7424811 .294143$

H $0.331326-2.0243490 .461605$

H $0.9146260 .325418-1.402166$

H $\quad 0.775400-1.383610-1.836017$

H $3.209887-0.273444-1.451955$

H -0.7254650 .8478610 .536143$ 
H $2.910970-2.716401-1.752531$

H $4.115903-2.354589-0.507670$

H $2.502043-2.903937-0.038948$

H -1.522887 -0.213126 -1.588524

H $-0.744978-0.4205492 .418984$

H -2.681649 -2.147954 -1.458427

H -3.969539 -0.253536 -1.260480

H $-2.7203751 .979287-1.319559$

H $-2.762011 \quad 1.9858600 .451775$

H -3.889318 -1.3228670 .774487$

H $2.134303 \quad 1.7981730 .137424$

H 2.7634201 .6292831 .753132

H $-5.2288151 .916306-1.346462$

H -5.2410451 .9541450 .428201$

H -4.599113 $3.328962-0.483013$

SCF Energy $\left(\mathrm{B} 3 L \mathrm{LP} / 6-31 \mathrm{G}^{* *} / / \mathrm{MMFF}\right)=-810.710557195$

F2-27_c06477

MMFF Geometry

C $3.355297-2.3129880 .159875$

C $3.667866-2.426101-1.136177$

C $2.706100 \quad 0.203360 \quad 0.069887$

O $1.593890 \quad 0.020811-0.827487$

C $\quad 0.478838 \quad 0.826477-0.393474$

C 0.8379951 .3342510 .994221

C 2.3519591 .4072590 .948914

C $-0.799435-0.021076-0.458322$

C 2.8325372 .7185220 .323008

C -2.0423940 .6934290 .107760$

O $-1.029163-0.355761-1.829285$

O $-2.219598 \quad 1.947048-0.560032$

C -3.369126 -0.0893990 .022954$

C $-3.369538-1.4366890 .753483$

O $-3.720583-0.349838-1.336922$

C $2.880213-1.0855960 .894146$

C $-3.068429-1.3333682 .239364$

H $3.451548-3.2068230 .773752$

H $3.999493-3.379723-1.533758$

H $3.605861-1.594160-1.826770$

H $3.6088240 .395957-0.520868$

H $0.414423 \quad 1.658233-1.105516$

H 0.5344770 .6095911 .759685

H $0.3546662 .287021 \quad 1.231568$

H 2.7887351 .3339981 .951012

H $-0.636090-0.9660210 .072144$

H $3.9269702 .744390 \quad 0.287948$

H $2.4641202 .850784-0.699315$

H 2.4962703 .5745710 .917439

H -1.862969 0.9417651 .158818

H $-0.232274-0.808819-2.154931$

H $-2.2073601 .775423-1.517088$

H $-4.173327 \quad 0.5361630 .430116$

H -2.664626 -2.1293820 .278291$

H $-4.355428-1.9021080 .623899$

H -3.825532 $0.507981-1.781800$

H $3.598539-0.896426 \quad 1.701382$

H $1.925534-1.3514851 .365037$

H -2.038691 -1.010465 2.418834

H $-3.745357-0.6267342 .729414$

H $-3.195479-2.3102992 .716681$

SCF Energy (B3LYP/6-31G**//MMFF) $=-810.709712941$

F2-27_c06478

MMFF Geometry

C -3.715206 2.1813790 .217635

C -3.0360753 .3289160 .325572$

C $-2.987119-0.191480-0.178101$

O $-1.7858310 .188425-0.878047$

C $-0.701177-0.680218-0.489531$

C $-1.202999-1.475218 \quad 0.706377$

C $-2.698179-1.5505750 .463822$

C $0.5466940 .173836-0.222173$

C $-3.057308-2.702485-0.477102$

C $1.738897-0.6321150 .326183$

O $0.908514 \quad 0.800794-1.452989$

O $2.051318-1.690859-0.579708$

C 3.0015920 .2073520 .619557

C $3.8605710 .561742-0.602366$
O $3.830560-0.5551121 .507632$

C $-3.296190 \quad 0.892107 \quad 0.867106$

C $5.0632661 .422767-0.228305$

H $-4.6319442 .170725-0.366172$

H $-3.3941234 .227258-0.165045$

H -2.1172293 .3929640 .897886$

H -3.805877 -0.253264 -0.904677

H $-0.525214-1.341781-1.346597$

H -1.016225 -0.9272301 .638167$

H $-0.718464-2.4525020 .795000$

H $-3.251278-1.6947301 .398510$

H 0.2994030 .9805930 .478668

H -2.566495 -2.612115 -1.451424

H $-2.765139-3.662614-0.038886$

H $-4.138443-2.728863-0.650690$

H $1.426060-1.1137281 .260374$

H $\quad 0.1339711 .300058-1.764380$

H $2.787891-2.195121-0.193533$

H 2.7195501 .1212101 .155457

H $3.2767851 .105879-1.348319$

H $4.239322-0.350985-1.077078$

H $3.334098-0.6850352 .333591$

H -4.1153910 .5598891 .515669$

H -2.4221001 .0655831 .506686$

H $5.6333741 .682137-1.126121$

H 4.7436402 .3536440 .250939

H 5.7367410 .8960010 .454821

SCF Energy (B3LYP/6-31G**//MMFF $)=-810.712022749$

F2-27 c06479

MMFF Geometry

C $3.0085232 .011744-0.113881$

C $3.4768292 .682314-1.172402$

C $2.922067-0.4721410 .497577$

O $1.714570-0.3081281 .265563$

C $0.602462-0.8007770 .495149$

C $1.034040-0.664097-0.954718$

C $2.521329-0.964772-0.898986$

C $-0.653738-0.0054280 .871879$

C $2.792789-2.462205-1.061573$

C $-1.882437-0.328243-0.004224$

O $-0.967856-0.3034502 .236742$

O $-2.098082-1.7379500 .012209$

C $-3.158414 \quad 0.400318 \quad 0.461272$

C $-4.4150820 .086003-0.367660$

O -2.9192051 .8066330 .438269$

C 3.7119110 .8459220 .522132

C $-4.2993200 .446948-1.842942$

H 2.0687482 .3310470 .329086

H $2.9227283 .520650-1.580473$

H $4.4117622 .409297-1.648920$

H $3.528032-1.2249431 .016292$

H $\quad 0.480202-1.8556190 .770413$

H $\quad 0.8867210 .366247-1.300003$

H $\quad 0.475153-1.322137-1.626954$

H $3.064585-0.446738-1.696879$

H -0.4369531 .0686090 .829918$

H $2.291622-3.057696-0.291490$

H $2.444832-2.812435-2.039186$

H $3.867083-2.664913-0.996266$

H $-1.643231-0.032560-1.032012$

H $-0.180314-0.0932342 .767527$

H $-2.710706-1.952726-0.710950$

$\mathrm{H}-3.3786400 .118324 \quad 1.498176$

H -5.2566120 .6558510 .046064$

H $-4.675738-0.974008-0.272833$

H -3.7152682 .2459310 .782752$

H 3.9071321 .1195551 .566785

H 4.6873710 .6832250 .047352

H $-3.547499-0.166633-2.347601$

H $-5.2567160 .274220-2.345519$

H -4.035471 $1.500328-1.977283$

SCF Energy (B3LYP/6-31G**//MMFF) $=-810.712440654$

F2-28_c06677

MMFF Geometry

C -4.438088 -1.530430 -0.472787

C $-4.763704-2.3874310 .501499$ 
C $-2.6459810 .209124-0.683361$ $\begin{array}{llll}\text { O } & -1.712918 & -0.423609 & 0.213988\end{array}$ C -0.5472310 .4184750 .348419$ C $-0.740186 \quad 1.569531-0.630656$ C $-2.251503 \quad 1.688505-0.721287$ C $0.709611-0.4266650 .106284$ C -2.8131842 .5330530 .423404$ $\begin{array}{llll}\text { C } 2.016884 & 0.390159 & 0.098868\end{array}$ O $\quad 0.789532-1.404678 \quad 1.148814$ $\begin{array}{lllll}\text { O } & 2.102848 & 1.164412 & 1.294951\end{array}$ C $3.281993-0.480372-0.036064$

C $4.5599180 .369869-0.107753$

O $3.183542-1.287576-1.206988$

C $-4.079025-0.088859-0.241576$

C $5.817894-0.483632-0.206656$ H $-4.439290-1.885228-1.500147$ H $-5.017595-3.4153210 .266720$ H $-4.781424-2.0836581 .542331$ H -2.465236 -0.226157 -1.676056 H $-0.561540 \quad 0.7834651 .382502$ H $-0.3432241 .302663-1.618436$ H $-0.2382142 .486603-0.307400$ H $-2.5574252 .149224-1.667293$ H $\quad 0.607219-0.980542-0.834487$ H -2.377582 3.5379910 .407533 H -3.897967 $2.645887 \quad 0.326871$ H -2.606886 2.0929941 .404300 H $1.9824281 .093351-0.741233$ H $-0.041917-1.909344 \quad 1.136575$ H 2.1000350 .5452992 .045176 H $3.365309-1.1524590 .826045$ H 4.6392101 .0110180 .777232 H $4.5213791 .024108-0.987216$ H $3.122699-0.692698-1.973914$ $\begin{array}{llll}\text { H } & -4.206032 & 0.155258 & 0.820039\end{array}$ H $-4.7813030 .529116-0.813037$ H $5.902233-1.1568640 .652421$ H $5.820553-1.086967-1.119723$ H $6.7061640 .155907-0.224924$ SCF Energy (B3LYP/6-31G**//MMFF) $=-810.718399449$

F2-28_c06678

MMFF Geometry

C $3.3761452 .078214-0.325521$

C $2.938553 \quad 3.2485540 .152524$

C $2.434066-0.242735-0.256830$

O $1.0542250 .160599-0.159089$

C $0.232081-1.015397-0.041407$

C $1.165658-2.213016-0.198417$

C $2.494003-1.6686480 .299623$

C $-0.924823-0.965126-1.045506$

C $2.597762-1.7597531 .822977$

C $-1.7771550 .315491-0.971628$

O $-0.381788-1.041908-2.370812$

O $-2.881986 \quad 0.136777-1.869596$

C $-2.318675 \quad 0.6727640 .424500$

$\begin{array}{llll}C & -3.288357 & -0.369042 & 0.996787\end{array}$

$\begin{array}{llllll}\text { O } & -3.011002 & 1.921974 & 0.330812\end{array}$

C $3.326856 \quad 0.7846450 .439176$

C $-3.743448-0.0154272 .407827$

H $3.8037442 .050795-1.324400$

H $3.0065384 .147833-0.449998$

H 2.5065323 .3310961 .143610

H $2.672218-0.275986-1.329098$

H -0.169761-1.016405 0.978160

H $1.272575-2.503270-1.250175$

H $\quad 0.808916-3.0920660 .347418$

H $3.336191-2.220292-0.133028$ H $-1.554879-1.853655-0.923107$ H $3.574045-1.4038082 .167595$ H $1.827059-1.1689242 .328039$ H $2.494215-2.7993242 .152328$ H $-1.2062361 .161407-1.374796$ H $-1.130111-1.018457-2.991628$ H $-3.4217200 .944776-1.827464$ H -1.4896050 .8280431 .122909$ H $-2.815457-1.3563141 .022952$ H $-4.180717-0.443436 \quad 0.364132$
H $-2.3725792 .587975 \quad 0.023217$

H 2.9638450 .9738151 .456619

H 4.3497990 .3983890 .515527

H $-2.8876950 .064593 \quad 3.085804$

H -4.2882700 .9335422 .426214$

H $-4.410238-0.7930002 .794012$

SCF Energy (B3LYP/6-31G**//MMFF) $=-810.716093143$

F2-28 c06679

MMFF Geometry

C $2.6851352 .505961-0.781131$

C $1.9275693 .543381-0.407908$

C $2.3847950 .021733-0.563077$

$\begin{array}{lllll}\text { O } & 0.948567 & 0.119786 & -0.624959\end{array}$

C $0.378684-0.5272790 .528981$

C $1.516910-1.2589511 .238827$

C $2.632155-1.271514 \quad 0.211284$

C $-0.778915-1.4347160 .087159$

C $4.015278-1.4502360 .811758$

C $-1.855068-0.738204-0.768087$

O $-0.258964-2.526729-0.682867$

O $-2.931738-1.665813-0.965502$

C $-2.4395160 .553783-0.171284$

C $-3.191866 \quad 0.349341 \quad 1.150637$

O $-3.3336951 .123454-1.133112$

C $2.944046 \quad 1.3005390 .080053$

C -3.6959451 .6647101 .732260$

H $3.1536992 .528017-1.761708$

H $1.7826324 .385975-1.075280$

H 1.4399403 .5707970 .560169

H $2.762668-0.073295-1.588120$

H $-0.0063150 .255398 \quad 1.191953$

H $1.243791-2.2602401 .586955$

H $1.815664-0.6868602 .126253$

H $2.449290-2.111560-0.472517$

H $-1.241940-1.8919090 .968964$

H $4.102330-2.4367801 .279322$

H $4.785964-1.3745570 .038012$

H $4.228272-0.7036401 .583364$

H $-1.464747-0.524929-1.771088$

H $0.187697-2.150806-1.460765$

H $-2.550989-2.473174-1.351700$

H $-1.641103 \quad 1.289455-0.030174$

H $-2.540432-0.1295351 .888957$

H $-4.058007-0.305200 \quad 0.997441$

H $-4.0386960 .473995-1.298406$

H $2.506516 \quad 1.453786 \quad 1.073841$

H 4.0286681 .2165520 .209135

H $-2.8664072 .356071 \quad 1.911856$

H -4.4111102 .1502811 .061089$

$\mathrm{H}-4.2003241 .4852842 .687130$

SCF Energy (B3LYP/6-31G**//MMFF) $=-810.719304831$

F2-28_c06680

MMFF Geometry

C $4.143441-1.8398290 .400414$

C $4.406258-2.640581-0.638543$

C $2.5359570 .054894 \quad 0.729143$

O $1.567096-0.396205-0.237300$

C $0.4993730 .573282-0.318808$

$\begin{array}{llll}\text { C } & 0.784099 & 1.608729 & 0.761855\end{array}$

C $2.2964501 .558928 \quad 0.890156$

C $-0.846423-0.152626-0.178629$

C $2.9739022 .434513-0.165057$

C $-2.0574390 .799442-0.116616$

O $-0.983107-1.029312-1.299947$

O $-2.026611 \quad 1.653355-1.264627$

$\begin{array}{llll}\text { C } & -3.444240 & 0.125788 & -0.049987\end{array}$

C $-3.622524-0.734573 \quad 1.205011$

O $-3.672946-0.686267-1.199779$

C $3.941706-0.3540890 .289100$

C $-5.053066-1.2370431 .357796$

H $4.079190-2.2786631 .392818$

H $4.546008-3.705672-0.489576$

H $4.484587-2.253202-1.648251$

H $2.283723-0.4422601 .676137$

H $0.5812831 .022695-1.315948$

H 0.3345991 .3012731 .714707 
H $0.3895412 .598306 \quad 0.512185$

H 2.6225371 .9017501 .878658

H $-0.824551-0.7877510 .714318$

H $4.0613622 .423525-0.038977$

H $2.7501552 .104572-1.184481$

H $2.6443373 .474586-0.068534$

H $-1.952298 \quad 1.4481730 .760987$

H $-0.205808-1.614019-1.308271$

H $-2.7527832 .293641-1.174764$

H $-4.2141860 .907159-0.066418$

H $-3.353083-0.1565372 .096231$

H $-2.965270-1.6105241 .160946$

H -3.489586 $-0.139413-1.982776$

H $4.122049-0.035498-0.744693$

H $4.688354 \quad 0.135913 \quad 0.924937$

H $-5.148409-1.8291062 .273504$

H $-5.756939-0.4010931 .420243$

H $-5.345234-1.8714210 .515232$

SCF Energy (B3LYP/6-31G**//MMFF) $=-810.708695902$

F2-28 c06681

MMFF Geometry

C $-4.442745-1.544746-0.454422$

C $-4.765711-2.392467 \quad 0.528824$

C -2.654651 $0.196678-0.685090$

O $-1.718692-0.4243940 .217535$

$\begin{array}{llll}\text { C } & -0.556260 & 0.423414 & 0.343927\end{array}$

C $-0.7524331 .562550-0.648207$

C $-2.2641021 .676428-0.739523$

C $0.704641-0.4200290 .113458$

$\begin{array}{llll}\text { C }-2.827588 & 2.532392 & 0.395762\end{array}$

C $2.0102690 .402014 \quad 0.092749$

$\begin{array}{lllll}\text { O } & 0.786162 & -1.385991 & 1.167449\end{array}$

$\begin{array}{lllll}\text { O } & 2.070798 & 1.187967 & 1.282262\end{array}$

C $3.274495-0.475335-0.017820$

C $4.5572140 .365350-0.123511$

O $3.149259-1.298055-1.175803$

C $-4.086325-0.100197-0.238192$

C $5.818811-0.484034-0.217779$

H $-4.444397-1.909934-1.478240$

H $-5.017914-3.4231100 .304528$

H - $-4.782868-2.0782621 .566460$

H -2.474137 $-0.248662-1.673466$

H -0.5738650 .7999241 .373927$

H $-0.355306 \quad 1.285440-1.633096$

H $-0.2528092 .484562-0.335887$

H $-2.5716452 .125566-1.690559$

H $0.602358-0.986008-0.820093$

H $-3.9125642 .641494 \quad 0.298239$

H -2.619861 2.103951 1.381488

H -2.3943693 .5380030 .368370$

H $1.9697941 .086783-0.763196$

H $-0.047006-1.887764 \quad 1.165202$

H $2.716787 \quad 1.899007 \quad 1.135580$

H $3.351148-1.1245200 .863016$

H $4.652548 \quad 1.0211270 .748585$

H $4.5085031 .003401-1.014168$

H $3.815805-2.002501-1.112074$

H -4.2122290 .1544820 .821175$

H $-4.7906080 .510574-0.814905$

H $6.7049830 .158999-0.213967$

H $5.894076-1.1702520 .631685$

H $5.839677-1.070634-1.141217$

SCF Energy (B3LYP/6-31G**//MMFF) $=-810.712171667$

F2-28_c06682

MMFF Geometry

C $-3.3182652 .294208 \quad 0.536245$

C $-3.0022553 .356994-0.212309$

C $-2.572121-0.096396 \quad 0.478753$

O $-1.288611 \quad 0.164523-0.120644$

C $-0.605842-1.084110-0.329778$

C $-1.493950-2.1655220 .280402$

C $-2.884139-1.563237 \quad 0.170914$

C $0.817608-1.0456700 .247409$

C $-3.492499-1.814136-1.209670$

C $1.7349520 .003926-0.419972$

O $\quad 0.759311-0.796210 \quad 1.652344$
O $1.2187421 .318974-0.224462$

C $3.175509-0.0304310 .128728$

C $4.0897440 .979521-0.583203$

O $3.715816-1.341338-0.021260$

C $-3.5949780 .924757-0.019224$

C $5.5065170 .972464-0.023063$

H $-3.3907632 .419178 \quad 1.613601$

H -2.818146 4.3199780 .251269

H $-2.919707 \quad 3.287119-1.291202$

H $-2.4365280 .012378 \quad 1.563817$

H $-0.544933-1.231730-1.414991$

H $-1.254733-2.3227111 .339192$

H $-1.382357-3.129448-0.225666$

H $-3.559977-1.9843600 .923548$

H $1.259199-2.040574 \quad 0.119430$

H $-2.904033-1.357560-2.011926$

H $-3.556984-2.889019-1.410047$

H $-4.508420-1.409947-1.265539$

H $1.758719-0.192572-1.499273$

H $\quad 0.3642870 .084393 \quad 1.774143$

H $0.341041 \quad 1.358052-0.640505$

H 3.1688530 .2068201 .199233

H $3.6818961 .992356-0.491642$

H $4.1439130 .740898-1.652359$

H $3.734283-1.548193-0.971326$

H -3.586833 $0.965120-1.115151$

H -4.6018810 .6287150 .296909$

H $5.987289-0.000484-0.164283$

H $6.1166161 .724862-0.533009$

H 5.5054521 .2049251 .046567

SCF Energy (B3LYP/6-31G**//MMFF) $=-810.722731137$

F2-28_c06683

MMFF Geometry

C $4.472151-0.026424-1.155605$

C $5.4365820 .886092-0.993250$

C $2.161724-0.436160-0.233928$

O $0.767028-0.194520-0.505552$

C $0.042792-0.2282950 .739536$

C $1.055732-0.6055531 .819117$

C $2.366482-0.0915891 .243867$

C $-1.161899-1.1713840 .633554$

C $2.546694 \quad 1.398494 \quad 1.539089$

C $-2.118496-0.863430-0.534019$

O $-0.693798-2.5162010 .459946$

O $-3.247755-1.737829-0.396714$

C $-2.618797 \quad 0.590699-0.614636$

C -3.4639331 .0222970 .590612$

O $-3.4215590 .724174-1.792203$

C $3.013190 \quad 0.301686-1.266404$

C -3.8794442 .4863810 .505182$

H $4.755089-1.073363-1.229333$

H $6.4755400 .582047-0.927957$

H $5.2102831 .944308-0.923319$

H $2.307903-1.517649-0.366116$

H -0.3070650 .7928260 .927874$

H $1.123319-1.6928181 .943126$

H $0.798367-0.1825052 .795081$

H $3.221881-0.6333341 .662372$

H - $-1.712705-1.1704661 .580745$

H $3.515928 \quad 1.7552131 .179510$

H $2.5148751 .581542 \quad 2.618618$

H 1.7699692 .0120471 .071522

H $-1.645693-1.142867-1.483756$

H $-0.153120-2.537384-0.348364$

H $-3.853001-1.546198-1.133535$

H $-1.7724801 .275032-0.735796$

H -2.905065 $0.874993 \quad 1.520545$

H -4.3758500 .4165080 .649946$

H $-2.8603870 .504626-2.555513$

H $2.673000 \quad 0.013982-2.269839$

H $2.8339031 .382194-1.208695$

H -4.4554692 .7664251 .393077$

H -3.0023913 .1390130 .450419$

H $-4.5048442 .673942-0.373116$

SCF Energy (B3LYP/6-31G**//MMFF) $=-810.714001622$

F2-28_c06684 
MMFF Geometry

C -4.643148 $-0.987502-0.460913$

C $-4.981158-2.0268290 .310443$

C $-2.5484980 .386749-0.669642$

O $-1.711241-0.729409-0.296167$

C $-0.631526-0.2740820 .552091$

C $-0.973456 \quad 1.162044 \quad 0.920317$

C $-1.749972 \quad 1.636297-0.290517$

C $0.678580-0.467284-0.236320$

C $-2.5410692 .909442-0.047396$

$\begin{array}{llll}\text { C } & 1.940187 & 0.064400 & 0.472769\end{array}$

O $0.846822-1.875842-0.448161$

O $1.988533-0.4412941 .806178$

C $3.251936-0.293696-0.255296$

C 4.4792650 .3179530 .438243

O $3.1908810 .166464-1.603312$

C $-3.903510 \quad 0.2243020 .035859$

C $5.782740-0.060753-0.253555$

H $-4.923427-1.004683-1.510913$

H $-5.519284-2.870030-0.108831$

H $-4.728511-2.0571731 .364461$

H -2.691521 $0.351823-1.756415$

H -0.629167 -0.9166211 .439737$

H $-0.113683 \quad 1.7925921 .155321$

H $-1.622104 \quad 1.1626171 .805620$

H $-1.0311901 .835640-1.097484$

H $0.592239-0.015250-1.231130$

H -3.143919 3.164559-0.924809

H -3.2083672 .8203390 .815820$

H $-1.862815 \quad 3.7463870 .149984$

H 1.8701241 .1553000 .539788

H $0.049668-2.196537-0.904275$

H $2.023710-1.4117451 .750210$

H $3.379977-1.382218-0.279752$

H $4.527584-0.0099471 .482611$

H 4.3985501 .4117790 .438818

H $3.0918641 .133695-1.582903$

H -3.759439 0.1536161 .120754

H $-4.5397291 .095061-0.156822$

H 6.6334610 .3677950 .285878

H $5.910229-1.147594-0.279500$

H $5.8135610 .314996-1.280966$

SCF Energy (B3LYP/6-31G**//MMFF) $=-810.716300388$

F2-28_c06685

MMFF Geometry

C $-4.988994-0.892858-0.623014$

C $-6.110055-0.188813-0.432094$

C $-2.520165-0.542297-0.251617$

O $-1.465318-0.7798640 .701230$

$\begin{array}{lllll}\text { C } & -0.419968 & 0.194641 & 0.487673\end{array}$

C $-0.8402201 .008325-0.731600$

C $-2.3569400 .912043-0.700095$

C $0.923608-0.5321460 .339725$

C $-2.950828 \quad 1.951677 \quad 0.251976$

$\begin{array}{llll}C & 2.101869 & 0.394333 & -0.025748\end{array}$

O $1.206157-1.2017471 .573358$

O 2.1598571 .4853910 .892319

C $3.463328-0.328278-0.053879$

C $4.6020130 .607098-0.489318$

O $3.396056-1.439581-0.944282$

C $-3.858041-0.9575750 .359461$

C $5.956331-0.090444-0.486913$

H $-4.888582-1.471230-1.537995$

H -6.894604 -0.190907 -1.180839

H $-6.2654170 .397768 \quad 0.466753$

H -2.304177 -1.200473 -1.105207

H $-0.4121070 .824379 \begin{array}{rr}1.385513 \\ \text { H }\end{array}$

H $-0.4718130 .537945-1.652331$

H $-0.4564292 .032810-0.706579$

H $-2.7847791 .070035-1.696363$

H $0.824891-1.322104-0.413851$

H $-2.6475242 .961196-0.045983$

H $-4.043967 \quad 1.9234180 .231268$

H -2.631691 $1.797273 \quad 1.287573$

H $1.9090190 .819386-1.017722$

H $1.231564-0.5334512 .278708$

H 2.4124701 .1312631 .761662
H $3.699899-0.7151970 .944220$

H $4.6536451 .476756 \quad 0.175037$

H $4.4106260 .977223-1.503886$

H $3.198013-1.097107-1.832787$

H -3.773312 -1.9924960 .716339$

H $-4.067338-0.3668921 .259605$

H $5.978449-0.922439-1.197551$

H $6.7434870 .614876-0.772005$

H $6.194042-0.4798440 .508080$

SCF Energy (B3LYP/6-31G**//MMFF) $=-810.709546965$

F2-28_c06686

MMFF Geometry

C $4.642710-0.735808 \quad 0.039236$

C $4.956261-1.708988-0.823345$

C 2.5283410 .5384690 .473058

$\begin{array}{llllll}\text { O } & 1.633246 & -0.497142 & 0.022731\end{array}$

$\begin{array}{lllll}\text { C } & 0.276278 & 0.000411 & 0.064036\end{array}$

C $0.352670 \quad 1.359923 \quad 0.745360$

C 1.7417821 .8478150 .371608

C $-0.611848-1.0406260 .759079$

C $1.7512322 .500236-1.011620$

C $-2.090303-0.6435850 .954791$

O $-0.571410-2.251192-0.003679$

O -2.1961370 .3359861 .984980$

C $-2.802237-0.115337-0.308246$

C $-4.2925760 .163110-0.050977$

O $-2.679526-1.050380-1.375611$

C $3.8344380 .480124-0.319537$

C $-4.9959220 .730374-1.277709$

H $4.997372-0.8139711 .063878$

H $5.547653-2.559042-0.501160$

H $4.630747-1.677824-1.857266$

H $2.728750 \quad 0.333482 \quad 1.533905$

H $-0.0259160 .108224-0.984263$

H 0.3020051 .2460041 .835453

H -0.4517862 .0356850 .441065$

H 2.1127302 .5813201 .096429

H $-0.173285-1.2797871 .736192$

H $1.0570093 .347073-1.040303$

H $2.7471952 .886150-1.251858$

H $1.4616451 .801893-1.803204$

H -2.630860 -1.536958 1.294975

H $0.364276-2.491259-0.117713$

H $-1.807561-0.0425592 .791876$

H -2.336308 $0.821968-0.631834$

H $-4.4098500 .869746 \quad 0.778162$

H $-4.799511-0.7670120 .233383$

H -3.071961-1.890122 -1.081361

H $3.623600 \quad 0.476211-1.395875$

H $4.4427171 .365526-0.101105$

H $-6.0439880 .944191-1.045354$

H $-4.5235591 .662780-1.603068$

H $-4.9754460 .023391-2.112723$

SCF Energy (B3LYP/6-31G**/MMFF) $=-810.710373614$

F2-28 c06687

MMFF Geometry

C $3.3813392 .046662-0.465070$

C $2.9263953 .253765-0.110620$

C $2.434721-0.260840-0.222407$

O $1.0529400 .147883-0.211973$

C $0.225141-1.0088550 .010382$

C $1.157237-2.216492-0.024013$

C $2.474086-1.6318770 .460274$

C $-0.937803-1.037867-0.989095$

C $2.539366-1.5887521 .987772$

C $-1.7813940 .252278-1.015882$

O $-0.426998-1.207146-2.317974$

O $-2.9065870 .023898-1.877535$

$\begin{array}{lll}\text { C }-2.310152 & 0.732536 & 0.347647\end{array}$

C $-3.291828-0.2466921 .006866$

$\begin{array}{lllll}\text { O } & -2.965017 & 1.986131 & 0.128589\end{array}$

C $3.303510 \quad 0.826632 \quad 0.410135$

C $-3.713357 \quad 0.194992 \quad 2.403330$

H $3.8460691 .931123-1.440901$

H $3.0169224 .095568-0.788507$

H $2.457305 \quad 3.4234110 .852127$ 
H $2.710969-0.389284-1.278234$ H $-0.177415-0.914457 \quad 1.025396$ H $\quad 1.296846-2.592953-1.042959$ H $\quad 0.785025-3.0467140 .584182$ H $3.325296-2.2209950 .100279$ H -1.583640 -1.901826 -0.792166 H $2.421291-2.5945702 .404727$ H $3.509154-1.2076002 .323987$ H $1.760522-0.9523612419695$ H -1.212788 $1.064546-1.486194$ H $-0.212622-2.147705-2.435394$ H $-2.556490-0.251293-2.742317$ H $-1.4675950 .933614 \quad 1.017654$ H -2.837689 -1.239867 1.086765 H -4.197524 -0.3429220 .396499$ H $-3.1144572 .398403 \quad 0.995823$ H $2.904517 \quad 1.105147 \quad 1.393092$ H 4.3228610 .4511720 .557129 H -2.841385 0.3346563 .050097 H -4.2782961 .1315262 .375001$ H -4.355146 -0.565694 2.859454 SCF Energy (B3LYP/6-31G**//MMFF) $=-810.705858977$

F2-28_c06688

MMFF Geometry

C $-2.9230562 .084968-0.055762$

C $-3.0692772 .705625-1.231706$

C $-1.822362-0.0699020 .590400$

O $-0.874949-0.066026-0.500134$

C $-0.231122-1.344934-0.560583$

C $-0.545044-2.044606 \quad 0.753986$

C -1.941141-1.525430 1.064357

C $1.248612-1.236753-0.943102$

C $-3.018137-2.3513490 .359156$

C $2.218846-0.6308750 .088591$

O $1.696498-2.570017-1.230267$

O $3.505693-0.561469-0.548739$

C 1.8670110 .7650530 .628217

C $1.8539711 .863267-0.442436$

O $2.841646 \quad 1.1229971 .615141$

C -3.1198360 .6081300 .148298$

C 1.3857063 .2011130 .118535

H -2.651643 2.6764570 .815020

H -2.914827 $3.776172-1.310536$

H -3.340701 2.162611 -2.130133

H $-1.3646350 .515147 \quad 1.398984$

H - $0.732585-1.899006-1.365581$

H $0.135510-1.7193661 .548458$

H $-0.479527-3.1342680 .680516$

H -2.143630 -1.562892 2.140939

H $1.329306-0.676349-1.882583$

H -2.932432 -2.305018 -0.730899

H $-2.949960-3.4032820 .656962$

H -4.018304 -1.999595 0.632547

H $2.351839-1.3297540 .923236$

H $2.624575-2.508209-1.514870$

H $4.128685-0.1882980 .098385$

H $\quad 0.906397 \quad 0.738021 \quad 1.146403$

H $1.1937441 .587349-1.270627$

H $2.8610552 .003370-0.852881$

H 2.8032710 .4545922 .320352

H -3.486440 $0.154291-0.780230$

H -3.8918550 .4740410 .914746$

H $\quad 0.3763403 .1185290 .534143$

H 2.0549443 .5604030 .906408

H $1.3647403 .955326-0.674479$

SCF Energy (B3LYP/6-31G**//MMFF) $=-810.713006269$

F2-28 c06689

MMFF Geometry

C $-3.601427 \quad 1.9850700 .399366$

C -3.492266 $3.004791-0.459680$

C $-2.492678-0.2583230 .510666$

O $-1.2958210 .132896-0.188785$

C $-0.436002-1.012566-0.323691$

C $-1.114769-2.1499580 .435537$

C $-2.585728-1.778793 \quad 0.356907$

C $0.989427-0.6997620 .160262$
C $-3.210570-2.254273-0.955554$

C $1.6939640 .399784-0.665734$

O $0.951305-0.3282421 .537108$

O $1.0020451 .644414-0.533002$

C $3.1681080 .668200-0.297358$

C $4.079645-0.538084-0.551682$

O 3.2855701 .0775711 .064501

C $-3.6853010 .541492-0.012776$

C $5.551934-0.192586-0.363884$

H -3.6413012 .2026071 .463630$

H $-3.4399934 .025463-0.096864$

H $-3.4512152 .842615-1.531098$

H $-2.324248-0.0234371 .570874$

H $-0.403012-1.257107-1.392476$

H $-0.805191-2.1636151 .487547$

H $-0.877498-3.1312370 .013037$

H $-3.151890-2.2208331 .184590$

H $1.571885-1.6248290 .095166$

H $-2.738440-1.795712-1.830301$

H -3.115532 -3.341043-1.053443

H $-4.278740-2.015759-0.986081$

H $1.6540470 .125550-1.727257$

H $\quad 0.4424980 .4984851 .600185$

H $\quad 0.0949301 .515497-0.857811$

H $3.5190591 .517518-0.896259$

H $3.929881-0.910859-1.571293$

H $3.838900-1.3516250 .141680$

H 3.0097580 .3329161 .625384

H $-3.7352970 .474536-1.106288$

H -4.6172330 .1285430 .390225$

H 5.7581480 .1275610 .662124

H $\quad 6.174077-1.068328-0.574554$

H $5.8556020 .610027-1.043565$

SCF Energy $(B 3 L Y P / 6-31 G * * / / M M F F)=-810.721949645$

F2-28 c06690

MMFF Geometry

C $-4.919051-0.870745-0.153108$

C $-5.597986-2.023245-0.130768$

C $-2.447043-0.381191-0.041210$

O $-1.354443-0.2389710 .887798$

$\begin{array}{llll}C & -0.351618 & 0.618961 & 0.298817\end{array}$

C $-0.8350800 .927780-1.113950$

C $-2.345800 \quad 0.815602-0.990505$

C $1.014814-0.0786670 .372805$

C $-2.9504692 .115267-0.455867$

C $2.1424980 .684592-0.351387$

O $1.339574-0.2442591 .755559$

O 2.1973472 .0158620 .170601

C $3.5515640 .064706-0.247398$

C $3.628314-1.333323-0.868717$

O $3.973945-0.0127341 .112513$

C $-3.748921-0.5725800 .736438$

C $5.058471-1.855857-0.931001$

H $-5.236683-0.097475-0.846958$

H $-6.440487-2.177484-0.796170$

H $-5.328787-2.8281260 .544229$

H -2.238883 -1.299752 -0.608109

H $-0.343568 \quad 1.5296820 .909958$

H $-0.4770070 .164730-1.816989$

H $-0.4872371 .900629-1.474592$

H -2.806129 $0.592740-1.959406$

H $0.920429-1.088613-0.040717$

H $-2.6983742 .953682-1.114204$

H $-4.0420742 .052235-0.414629$

H -2.5913562 .3580130 .549251$

H $1.8857610 .772172-1.413597$

H 1.4842720 .6410442 .131393

H $2.8597632 .506764-0.344939$

H $4.2641470 .726655-0.754802$

H $3.213146-1.315144-1.882770$

H $3.040123-2.044370-0.277212$

H 3.8823130 .8763001 .495892

H $-3.604930-1.3810861 .465566$

H $-3.9686540 .312877 \quad 1.344531$

H $5.693769-1.188621-1.522238$

H $5.493023-1.9488420 .069223$

H $5.077882-2.844995-1.399379$ 
F2-28_c06691

MMFF Geometry

C $4.360567 \quad 1.658950-0.593941$

C $4.5629532 .676403 \quad 0.250612$

C $2.700784-0.218874-0.631882$

$\begin{array}{lllll}\text { O } & 1.669956 & 0.473108 & 0.099384\end{array}$

C $0.553781-0.421693 \quad 0.298822$

C $0.886447-1.691029-0.475830$

C $2.404929-1.711142-0.459748$

C $-0.7394620 .282753-0.138081$

C $2.940190-2.3282520 .833208$

C $-1.984330-0.623148-0.091501$

O $-0.915428 \quad 1.4166550 .712583$

O $-2.152232-1.121451 \quad 1.236162$

C $-3.2878710 .048077-0.576374$

C -3.9582940 .9977640 .425589$

O $-4.229395-0.995161-0.862936$

C $4.078253 \quad 0.247447-0.160154$

C $-5.226958 \quad 1.628740-0.139933$

H $4.4104101 .851506-1.662486$

H $4.7666713 .672130-0.127765$

H 4.5275012 .5378301 .325537

H $2.5611150 .045378-1.689457$

H $0.524752-0.626917 \quad 1.375961$

H $0.541522-1.607318-1.514351$

H $0.423881-2.582968-0.042528$

H $2.803308-2.286830-1.302882$

H - $0.6111110 .678168-1.152500$

H $2.640828-1.7600941 .719621$

H $2.570899-3.3528700 .950186$

H $4.033767-2.3752400 .817461$

H $-1.798206-1.498731-0.724766$

H $-1.071021 \quad 1.0865801 .614241$

H -2.928397 -1.7073581 .230025$

H $-3.1009390 .574563-1.519868$

H $-3.281231 \quad 1.8060390 .711653$

H -4.2333050 .4547941 .337415$

H -3.859898 -1.534721 -1.582594

H 4.1472200 .1784730 .932300

H $4.855538-0.397715-0.585887$

H -5.6564662 .3255670 .587077$

H -5.010979 2.186174 -1.056867

H -5.985002 $0.872386-0.365788$

SCF Energy (B3LYP/6-31G**//MMFF) $=-810.711415753$

F2-28_c06692

MMFF Geometry

C $3.543676 \quad 2.1163730 .335111$

C $4.6100622 .797257-0.099237$

C $2.4429830 .006430-0.477497$

O $1.134656 \quad 0.444210-0.061433$

C $0.526329-0.5672960 .762031$

C $1.459184-1.7767460 .738100$

C $2.347386-1.518072-0.462539$

C $-0.899984-0.875595 \quad 0.269284$

C $3.657212-2.285238-0.430186$

C -1.8615620 .3332190 .329556$

O $-0.854378-1.379292-1.065298$

O $-1.406240 \quad 1.380766-0.524145$

C -3.300857 -0.030851-0.087522

C $-4.257324 \quad 1.166698 \quad 0.029623$

$\begin{array}{llll}\text { O } & -3.781916 & -1.098177 & 0.726298\end{array}$

C 3.4960270 .6178910 .460820

C $-5.6741130 .821220-0.411291$

H 2.6567472 .6720510 .628621

H $4.5825403 .879733-0.161245$

H $5.5219762 .293359-0.399580$

H $2.6101300 .363704-1.500702$

H $0.465711-0.1723061 .783226$

H $\quad 0.932670-2.7348140 .682987$

H $2.053654-1.7924961 .660285$

H $1.797439-1.828152-1.361489$

H $-1.302329-1.6795150 .896394$

H $4.213498-2.1111310 .496422$

H $3.468758-3.361976-0.499482$

H $4.294483-1.999612-1.273199$
H -1.873293 0.7159991 .357860

H $-0.509692-0.667758-1.631916$

H $-0.5314531 .661857-0.207099$

H $-3.307801-0.375959-1.128169$

H -3.895651 $2.003620-0.578088$

H -4.2976511 .5083281 .071080$

H $-3.788259-0.7891671 .648377$

H $3.2653470 .382481 \quad 1.506507$

H 4.4847420 .2008950 .239695

H $-5.6865700 .471686-1.448614$

$\begin{array}{llll}H & -6.110558 & 0.041744 & 0.220884\end{array}$

H $-6.315517 \quad 1.705627-0.343654$

SCF Energy (B3LYP/6-31G**//MMFF) $=-810.723118172$

F2-28_c06693

MMFF Geometry

C $-4.652172-1.327063-0.102648$

C $-4.050363-2.466331-0.463398$

C $-2.6197710 .220628-0.547807$

O $-1.729041-0.3728820 .415642$

$\begin{array}{llll}\text { C }-0.504813 & 0.391938 & 0.457362\end{array}$

C $-0.629521 \quad 1.452131-0.630022$

C $-2.1305391 .661246-0.729871$

C $0.687919-0.5601110 .290526$

C $-2.625995 \quad 2.648550 \quad 0.327871$

C $2.0524640 .153434 \quad 0.180114$

O $0.703857-1.445076 \quad 1.415975$

$\begin{array}{llll}\text { O } & 2.186345 & 1.041572 & 1.289122\end{array}$

C $3.250474-0.8177640 .150922$

C $4.624935-0.133044 \quad 0.071993$

O $3.135746-1.704655-0.959944$

C $-4.0724010 .064323-0.089769$

C $4.8187990 .751421-1.153114$

H $-5.688157-1.395156 \quad 0.224687$

H $-4.593918-3.404557-0.421916$

H $-3.022211-2.502431-0.802080$

H $-2.467146-0.313899-1.495039$

H $-0.4802430 .859196 \quad 1.449237$

H $-0.258947 \quad 1.064667-1.587651$

H $-0.0656132 .361059-0.399045$

H $-2.4127242 .046852-1.716003$

H $\quad 0.520640-1.190969-0.589584$

H $-3.701376 \quad 2.8243770 .221905$

H -2.442068 2.2928141 .346689

H -2.124153 $3.615547 \quad 0.213976$

H $2.0396260 .753264-0.736608$

H $\quad 0.887732-0.910325 \quad 2.207414$

H 2.9156661 .6532731 .092902

H $3.258227-1.4258851 .064025$

H $5.397795-0.9116670 .041347$

H 4.8066240 .4573750 .976954

H $2.409129-2.322148-0.771495$

H -4.1712200 .4305590 .939665$

H $-4.7137660 .686685-0.725777$

H $4.1515181 .617831-1.131468$

H $5.846348 \quad 1.128402-1.181997$

H $4.6388880 .197317-2.079448$

SCF Energy (B3LYP/6-31G**//MMFF) $=-810.710439437$

F2-28_c06694

MMFF Geometry

C -4.434477 -1.021810 -0.833311

C $-4.802491-2.135736-0.190732$

C $-2.3918490 .437802-0.705265$

O $-1.549327-0.676627-0.338530$

C $-0.580653-0.2588890 .651619$

C $-1.022511 \quad 1.126254 \quad 1.100402$

C $-1.6917541 .676769-0.141798$

C $0.809844-0.338008-0.011144$

C $-2.558765 \quad 2.895375 \quad 0.121708$

C $1.9667160 .176595 \quad 0.868792$

O $1.046383-1.713525-0.332392$

O $1.907582-0.4559592 .150139$

C $3.392779-0.0111090 .309153$

C $3.6079840 .708851-1.026819$

O $3.706753-1.3952630 .166182$

C $-3.8036190 .167729-0.162986$

C $5.0634520 .663500-1.474711$ 
H $-4.605593-0.957369-1.904923$

H -5.257301 -2.957773 -0.732729

H -4.656868 -2.248943 0.877765

H -2.420919 $0.492152-1.800132$

H $-0.638914-0.9759981 .478273$

H -0.2213071 .7648041 .477409$

H -1.7578301 .0256261 .908911$

H $-0.9043151 .971107-0.849376$

H $0.8008040 .202980-0.963818$

H $-3.0800973 .204667-0.789840$

H $-3.305607 \quad 2.7081070 .899864$

H -1.941524 3.7353330 .458109

H $1.827197 \quad 1.2478701 .046863$

H $0.306832-2.012757-0.888872$

H $2.047013-1.4083332 .009134$

H $4.1009790 .379176 \quad 1.050166$

H $3.2948681 .755552-0.940959$

H $3.0026790 .241949-1.812201$

H $3.112519-1.767802-0.507074$

H -3.7684490 .0085740 .921534$

H $-4.4542581 .029021-0.349392$

H $5.7169991 .128583-0.729914$

H $5.399463-0.365812-1.633849$

H $5.1845661 .206345-2.417589$

SCF Energy (B3LYP/6-31G**//MMFF) $=-810.721819030$

F2-28 c06695

MMFF Geometry

C -1.856559 2.8386520 .524667

C -1.041706 $3.667830-0.137289$

C -2.1435650 .3543630 .422468$

O $-0.8110630 .077687-0.046011$

C $-0.661321-1.339330-0.238127$

C -1.970897 -1.970939 0.232163

C $-2.983091-0.858476 \quad 0.017311$

C $0.579053-1.8712290 .495884$

C -3.487762 -0.835409-1.426029

C $1.934628-1.380590-0.063343$

O $0.478331-1.5611761 .884119$

O $2.090681-1.872910-1.394785$

C $2.1733250 .142866-0.076653$

C $3.6089580 .482245-0.512358$

O $1.959551 \quad 0.6980541 .216238$

C -2.609354 $1.706215-0.117205$

C $3.8475521 .985105-0.598177$

H -1.9954762 .9870521 .592492$

H -0.5288024 .4676490 .385758$

H $-0.8690913 .563562-1.202671$

H -2.087447 0.3915531 .519404

H -0.547848 -1.504219-1.316101

H -1.929456 -2.215822 1.300472

H -2.201874 -2.896067 -0.305161

H $-3.847789-0.9742850 .680382$

H $0.558186-2.9656220 .410715$

H -4.242677 -0.053932 -1.560947

H $-2.682381-0.652813-2.144630$

H -3.957129 -1.791094-1.683017

H $2.723920-1.8328060 .551753$

H $1.252823-1.9454292 .328955$

H $2.025595-2.842404-1.359960$

H $1.4925060 .628505-0.784607$

H $3.8258980 .037360-1.489780$

H 4.3229880 .0645890 .207848

H 1.0198140 .5781641 .435073

H $-2.4747541 .746892-1.204954$

H $-3.676028 \quad 1.8454100 .092670$

H $3.1551542 .451136-1.306379$

H 3.7201432 .4666650 .376286

H $4.8681592 .184896-0.939957$

SCF Energy (B3LYP/6-31G**//MMFF) $=-810.716022720$

F2-28_c06696

MMFF Geometry

C $4.578099-1.314490 \quad 0.615683$

C $5.758504-1.226348-0.007039$

C $2.306184-0.2444250 .395910$

O $1.012961-0.430330-0.211476$

C $\quad 0.3817690 .855027-0.360439$
C $\quad 1.329561 \quad 1.874088 \quad 0.269939$

C 2.6898621 .2115850 .119640

C -1.0302640 .8601360 .245595$

C $3.2739121 .471365-1.270102$

C $-2.011189-0.115374-0.444314$

O $-0.956763 \quad 0.5500641 .637688$

O $-1.550987-1.459316-0.317514$

C $-3.436816-0.039870 \quad 0.139012$

C $-4.405287-0.973276-0.605992$

O $-3.899791 \quad 1.304503 \quad 0.038615$

C $3.254729-1.340098-0.088969$

C $-5.807221-0.971085-0.009392$

H $4.567270-1.3888011 .700086$

H $6.681233-1.2204410 .562765$

H $5.826560-1.159336-1.087124$

H $2.149226-0.368317 \quad 1.476870$

H $0.3064571 .045389-1.438066$

H 1.1129952 .0086701 .336587

H $1.2557442 .857952-0.203868$

H $3.397248 \quad 1.5848790 .868369$

H $-1.425161 \quad 1.8795570 .167794$

H $4.2767691 .044606-1.360188$

H $2.6560561 .045017-2.066876$

H $3.3626432 .547725-1.453073$

H -2.049297 $0.130225-1.513096$

H $-0.604288-0.3532141 .715120$

H -0.688929 -1.520274 -0.762619

H $-3.415968-0.3219821 .198808$

H -4.026582 -2.001337 -0.590730

H $-4.480833-0.664832-1.655813$

H -4.6914201 .3878540 .596259$

H $2.786258-2.3153970 .097342$

H $3.373197-1.289434-1.178175$

H $-6.2839780 .008693-0.108355$

H $-6.437302-1.700233-0.529149$

H $-5.782321-1.2397481 .051432$

SCF Energy $(B 3 L Y P / 6-31 G * * / / M M F F)=-810.723608018$

F2-29 c06930

MMFF Geometry

C $4.452816-0.7980560 .752486$

C $4.623842-1.0558942 .054015$

C $2.5203620 .096698-0.568905$

O $1.492915-0.3704110 .327802$

C $0.2100440 .072811-0.162140$

C $0.4713750 .737190-1.509932$

C $1.8943931 .248061-1.361603$

C $-0.760055-1.113267-0.188291$

C $1.9278362 .605846-0.658275$

C $-2.178403-0.773390-0.673837$

O $-0.240964-2.121555-1.067892$

O $-2.978084-1.952417-0.503376$

C -2.8612080 .4018590 .049943$

C $-3.106418 \quad 0.150247 \quad 1.543271$

O $-4.1263170 .640055-0.575661$

C 3.7847440 .4388560 .219346

C $-3.716097 \quad 1.362740 \quad 2.237057$

H $4.825022-1.5160300 .026163$

H $5.119177-1.9663532 .373152$

H $4.274869-0.3712552 .818995$

H $2.727276-0.731260-1.261346$

H -0.1402920 .8184850 .559910$

H $-0.260208 \quad 1.517212-1.741971$

H $0.4383770 .003620-2.324509$

H $2.3801951 .357736-2.337690$

H $-0.798843-1.5846590 .800949$

H $1.5223122 .557850 \quad 0.357301$

H $2.9530812 .984336-0.593426$

H $1.3426403 .342281-1.219232$

H $-2.162727-0.583765-1.754312$

H $0.652069-2.345899-0.754352$

H $-3.875278-1.740568-0.813712$

H $-2.2738591 .317188-0.077416$

H $-2.166955-0.1004342 .046852$

H $-3.792368-0.6945141 .677222$

H $-3.9559850 .853567-1.508914$

H $4.5007340 .954862-0.430848$

H 3.5419021 .1122231 .050354 
H $-3.849994 \quad 1.1578213 .303935$

H -3.0652442 .2373552 .139143$

H -4.695706 1.6126151 .817964

SCF Energy (B3LYP/6-31G**//MMFF) $=-810.711639544$

F2-29 c06931

MMFF Geometry

C -3.164655 2.536201-0.518475

C -2.8451693 .5203830 .329477$

C $-2.5435960 .110528-0.615570$

$\begin{array}{llll}\mathrm{O} & -1.285382 & 0.248212 & 0.073212\end{array}$

C $-0.684414-1.0502330 .205724$

C $-1.591959-2.029762-0.534960$

C $-2.951276-1.356951-0.449832$

C $0.754755-1.035163-0.324473$

C $-3.650938-1.6826640 .870565$

C $1.656264 \quad 0.024348 \quad 0.348105$

O $1.319441-2.334452-0.154111$

O $1.668512-0.1733731 .760985$

C $3.111643-0.017923-0.158468$

C $3.974361 \quad 1.0855460 .474339$

O $3.130366 \quad 0.124189-1.576944$

C $-3.5400801 .144342-0.091160$

C 5.4225361 .0285850 .004663

H -3.165313 2.747362 -1.584767

H -2.587754 $4.505399-0.044181$

H -2.8326073 .3635991 .402410$

H $-2.3380260 .298151-1.678745$

H $-0.691559-1.3093571 .271451$

H -1.561907 -3.036669 -0.107238

H -1.295431 -2.109166 -1.588551

H $-3.604063-1.675371-1.270219$

H $0.731240-0.828120-1.400991$

H $-3.085671-1.3275041 .738276$

H -4.645145 -1.225655 0.906376

H $-3.784667-2.7645970 .976491$

H 1.2409861 .0203600 .149734

H $1.392573-2.4992740 .801730$

H $\quad 0.802616 \quad 0.1035092 .104997$

H $3.561389-0.9876300 .085885$

H 3.9564771 .0027991 .566663

H 3.5687332 .0699570 .210957

H $2.7408470 .988397-1.794144$

H $-4.5409860 .929681-0.483006$

H -3.5923401 .0981991 .003321$

H 6.0097701 .8085410 .499833

H $\begin{array}{llll}5.873805 & 0.060613 & 0.244531\end{array}$

H $5.4986931 .186749-1.075567$

SCF Energy (B3LYP/6-31G**//MMFF) $=-810.717400581$

F2-29 c06932

MMFF Geometry

C $-2.5067442 .438196-0.535007$

C $-2.0626783 .450588 \quad 0.218013$

C $-2.143131-0.043380-0.523545$

O $-0.817543-0.009573 \quad 0.038715$

C $-0.342830-1.3553330 .209370$

C $-1.418223-2.262884-0.387960$

C $-2.685299-1.443190-0.224735$

C $1.039327-1.544491-0.427405$

C $-3.283790-1.6158621 .172067$

C $2.199113-0.8650100 .326994$

O $1.315635-2.951412-0.456879$

O $3.392981-1.120120-0.430249$

C 2.0808620 .6507670 .561757

C $2.0659511 .478114-0.730276$

O $3.2069661 .069216 \quad 1.342227$

C $-2.973957 \quad 1.1205380 .017353$

C $1.9147252 .969600-0.456097$

H -2.548375 2.571047-1.613055

H -1.744309 4.379326 -0.242669

H -2.005142 3.3725251 .298068

H -2.021919 $0.060564-1.610883$

H - $0.276861-1.5452951 .287985$

H -1.455874 -3.2448470 .093974$

H -1.231639 -2.424831 -1.457598

H -3.444101 -1.733247 -0.960168

H $1.023552-1.201599-1.468898$
H $-2.603937-1.276217 \quad 1.959982$

H $-4.217321-1.0520921 .267211$

H $-3.517672-2.6692121 .360890$

H $2.348611-1.3743991 .287442$

H $2.186836-3.066091-0.874015$

H $4.132177-0.7066040 .048017$

H 1.1930070 .8753221 .160740

H $1.2446521 .158131-1.379466$

H $3.0010301 .333347-1.283940$

H 3.1747000 .5854372 .185242

H $-4.0242000 .990225-0.268413$

H -2.9246291 .1431441 .112710$

H $2.7692103 .360598 \quad 0.105068$

H $1.8524133 .521586-1.399272$

H 1.0055243 .1723780 .117358

SCF Energy $(B 3 L Y P / 6-31 G * * / / M M F F)=-810.717768850$

F2-29_c06933

MMFF Geometry

C $-4.399990-1.6171490 .376417$

C $-4.607729-2.615296-0.489756$

C $-2.703607 \quad 0.2248690 .477871$

O $-1.674227-0.472410-0.249604$

C $-0.5290290 .396505-0.395364$

C -0.8567341 .6625820 .390590$

C $-2.374470 \quad 1.7126570 .340132$

C $0.729066-0.378136 \quad 0.026126$

C $-2.8677552 .362051-0.953944$

C $2.0483700 .339496-0.323012$

O $0.694127-0.636958 \quad 1.430549$

$\begin{array}{llll}\text { O } & 2.110657 & 1.605525 & 0.328734\end{array}$

C $3.299910-0.479746 \quad 0.053134$

C $4.5992950 .232278-0.354756$

O $3.244078-1.757733-0.576580$

C $-4.081525-0.204216-0.026959$

C $5.840598-0.5568930 .042018$

H $-4.473353-1.8275111 .440342$

H $-4.838567-3.613166-0.132928$

H -4.550221 -2.458397 -1.561204

H -2.588494 -0.0625231 .532365$

H $-0.4605260 .629491-1.464838$

H $-0.3748202 .551972-0.026564$

H $-0.538567 \quad 1.5737601 .436044$

H -2.780846 2.2816891 .183968

H $0.709451-1.357253-0.467530$

H $-3.9603532 .429243-0.963017$

H -2.477309 $3.381535-1.043979$

H $-2.5573971 .803963-1.843092$

H $2.0558050 .527148-1.403614$

H $-0.139870-1.1005651 .618877$

H $2.069824 \quad 1.4440301 .287064$

H $3.323442-0.6440721 .136894$

H 4.6509291 .2236250 .108821

H $4.6182840 .374652-1.442009$

H $3.235052-1.614863-1.538485$

H -4.8532310 .4491200 .396450$

H -4.129304 -0.114491-1.118938

H $5.874324-1.528293-0.460929$

H $6.743259-0.003780-0.236618$

H $5.867010-0.7267271 .123141$

SCF Energy $\left(B 3 L Y P / 6-31 G^{* *} / / M M F F\right)=-810.718563883$

F2-29_c06934

MMFF Geometry

C $-3.3594362 .400214-0.290819$

C $-3.228432 \quad 3.260307 \quad 0.725312$

C $-2.4640160 .094464-0.693654$

O $-1.291744 \quad 0.2399910 .131274$

C $-0.563218-0.9993290 .125869$

C $-1.289843-1.927655-0.844519$

C $-2.717982-1.412471-0.795887$

C $0.907664-0.759253-0.244593$

C $-3.486297-2.0146790 .381441$

C 1.6169500 .2209910 .714881

O $1.579356-2.015605-0.271980$

O $1.518983-0.2948572 .046393$

C 3.1047800 .4969530 .414153

C $3.319851 \quad 1.156897-0.951362$ 
$\begin{array}{lrr}\text { O } & 3.864576 & -0.708893 \quad 0.466041\end{array}$

C -3.610898 $0.927644-0.121062$

C $4.7643961 .598265-1.153997$

H $-3.2908242 .780348-1.306870$

H -3.0522584 .3133450 .534445$

H -3.291978 2.9336851 .757293

H $-2.1920800 .471483-1.689390$

H $-0.632244-1.4239571 .134886$

H $-1.183933-2.983242-0.575761$

H $-0.897484-1.804218-1.862027$

H $-3.259275-1.655002-1.717277$

H $0.949257-0.341225-1.256671$

H $-3.506729-3.1072450 .306847$

H $-3.040061-1.752214 \quad 1.346103$

H $-4.524784-1.6681030 .385806$

H $1.078472 \quad 1.176457 \quad 0.705084$

H $1.591720-2.3612110 .636953$

H 1.8960160 .3701932 .647322

H 3.5063841 .1496611 .199210

H $2.6653212 .030074-1.052107$

H $3.0710140 .455310-1.755769$

H $3.684030-1.1287181 .324473$

H $-4.5447180 .682865-0.640404$

$\begin{array}{lllll}H & -3.749981 & 0.699477 & 0.942689\end{array}$

H $5.0669972 .313875-0.382969$

H $5.4499640 .745833-1.121345$

H $4.8767032 .083343-2.128910$

SCF Energy (B3LYP/6-31G**/MMFF) $=-810.711089402$

F2-29_c06935

MMFF Geometry

C $4.526147-0.4798241 .142901$

C $4.260789-1.7913121 .133149$

C $2.545128 \quad 0.221174-0.375395$

O $1.487652-0.2295840 .493039$

$\begin{array}{llll}\text { C } & 0.218870 & 0.145645 & -0.082595\end{array}$

C $0.5298430 .770948-1.438631$

C $1.9290171 .327732-1.238709$

C $-0.703125-1.077513-0.116377$

C $1.8891582 .709069-0.582969$

C $-2.103454-0.813694-0.694836$

O $-0.098823-2.097479-0.925435$

O $-2.881111-2.008590-0.530565$

C $-2.874393 \quad 0.354834-0.055286$

C $-3.210827 \quad 0.1332201 .426319$

O $-4.086400 \quad 0.517574-0.798659$

C $3.7693210 .624846 \quad 0.450896$

C -3.8700151 .3487912 .067208$

H $5.391889-0.1530691 .716478$

H $4.896849-2.4782011 .682031$

H $3.422513-2.2141520 .593346$

H $2.799437-0.621395-1.032408$

H -0.1987410 .9023140 .590748$

H $-0.2103221 .520888-1.733992$

H $0.5571730 .009463-2.227393$

H $2.4600641 .418143-2.193074$

H $-0.781283-1.5134800 .886468$

H $1.3124413 .408306-1.197905$

H 1.4342632 .6833990 .412410

H $2.8986443 .120055-0.481003$

H $-2.039500-0.652877-1.778301$

H $0.784326-2.272275-0.557143$

H $-2.390721-2.733186-0.955563$

H $-2.304887 \quad 1.281860-0.179025$

H -2.300358 -0.0965421 .989596$

H -3.894014 -0.7172461 .536119$

H -4.479692 $1.368757-0.543309$

H 4.481799 1.144482 -0.201273

H 3.4636121 .3283941 .235237

H -4.8462001 .5588931 .620268$

H -4.0261001 .1707423 .136199$

H -3.240500 2.2378651 .960403

SCF Energy (B3LYP/6-31G*//MMFF) $=-810.710411272$

F2-29 c06936

MMFF Geometry

C -3.808335 $1.829962-0.475542$

C $-3.3821893 .097353-0.432765$
C $-2.618177-0.379140-0.348861$

O $-1.422183 \quad 0.144738-0.958714$

C $-0.293260-0.121422-0.096904$

C $-0.787734-1.0554451 .005426$

C $-2.119331-1.5588260 .482712$

C $0.851379-0.663167-0.965101$

C $-3.049980-2.0670831 .569499$

C $2.175216-0.914984-0.222168$

O $0.452944-1.913100-1.545119$

O $3.173827-1.241535-1.200036$

$\begin{array}{llll}\text { C } 2.695993 & 0.262595 & 0.620815\end{array}$

C $3.0411281 .509433-0.203834$

O $3.879100-0.1590421 .307181$

C -3.3115320 .7439190 .438466$

C 3.4754362 .6767130 .674986

H $-4.5664041 .559078-1.205819$

H $-3.7840443 .835408-1.118278$

H -2.6299793 .4194590 .278666$

H $-3.279292-0.733525-1.148642$

H -0.0027160 .8420560 .334744$

H $-0.926504-0.4797501 .929095$

H $-0.096043-1.8683991 .243858$

H $-1.916001-2.391251-0.204781$

H $1.0215990 .009174-1.814650$

H $-4.021554-2.3448501 .148481$

H $-2.624298-2.9544442 .050196$

H -3.215339 -1.319772 2.352016

H $2.090884-1.8052870 .412482$

H $-0.362850-1.749470-2.049147$

H $2.838432-1.995208-1.715430$

H $1.9655970 .522637 \quad 1.394213$

H $2.1769461 .825967-0.796963$

H $3.859793 \quad 1.288838-0.899130$

H $3.641352-0.9229971 .859820$

H -4.1791280 .3483320 .977825$

H -2.628071 1.1691681 .183166

H $2.6878012 .944167 \quad 1.386652$

H 4.3827342 .4375221 .238094

H 3.6858853 .5552060 .056658

SCF Energy (B3LYP/6-31G**//MMFF) $=-810.711187832$

F2-29 c06937

MMFF Geometry

C -3.123494 $2.298234-0.487030$

C $-2.551007 \quad 3.431204-0.065038$

C $-2.448688-0.106579-0.297203$

O $-1.0337170 .146430-0.204495$

C $-0.346643-1.103446-0.039382$

C $-1.406424-2.199040-0.135209$

C $-2.666716-1.4878480 .327548$

C $0.779167-1.255735-1.071654$

C $-2.778846-1.4911351 .852828$

C $1.820477-0.113395-1.086849$

O $1.433875-2.506674-0.873123$

O $1.2325341 .052151-1.663177$

$\begin{array}{llll}\text { C } & 2.437254 & 0.242376 & 0.281253\end{array}$

C 3.5162991 .3306340 .157718

O $3.005432-0.9190200 .878957$

C $-3.224090 \quad 1.046348 \quad 0.339695$

C 4.1026421 .7201381 .508957

H $-3.5533222 .272216-1.485098$

H -2.514442 $4.301849-0.710793$

H -2.1100653 .5109070 .922454$

H $-2.684492-0.166138-1.368954$

H $0.070876-1.1168860 .974428$

H $-1.149497-3.0853270 .453174$

H - $-1.536365-2.519505-1.176700$

H $-3.564606-1.963857-0.082314$

H $0.323382-1.286271-2.069678$

H -3.708395 -1.0107852 .174991$

H $-2.792851-2.5180932 .233700$

H $-1.945497-0.9668392 .331379$

H $2.634119-0.425740-1.754047$

H $1.803424-2.5087740 .026316$

H $0.515357 \quad 1.344737-1.075124$

$\begin{array}{llll}H & 1.657109 & 0.617109 & 0.953358\end{array}$

H $3.0994472 .226831-0.315172$

H $4.3343320 .971680-0.478634$ 
H $\quad 3.721915-1.2303350 .299574$

H $-4.284327 \quad 0.7810580 .424393$

H $-2.847006 \quad 1.2434351 .350494$

H 4.8423392 .5170301 .382627

H 3.3217422 .0859552 .183302

H 4.6022490 .8721151 .987228

SCF Energy (B3LYP/6-31G**//MMFF) $=-810.722925183$

F2-29 c06938

MMFF Geometry

C $-3.3170472 .242774-0.414735$

C -3.5274793 .0227320 .651485$

C $-2.241517-0.002952-0.690377$

O $-1.368727 \quad 0.1111350 .453322$

C $-0.663861-1.129710 \quad 0.626163$

C $-0.991126-1.984376-0.591576$

C $-2.381335-1.502380-0.970890$

C $0.836787-0.9123990 .873864$

C -3.462192 -2.212161-0.153988

C $1.577063-0.000383-0.131137$

O $0.988941-0.3649022 .188074$

O $1.0681561 .330499-0.069399$

C 3.0952700 .0621390 .138889

C $3.8305350 .939403-0.887161$

O $3.642746-1.2542360 .108882$

C $-3.5423870 .756225-0.429115$

C $5.3253691 .028833-0.606394$

H $-2.9698302 .703162-1.336293$

H $-3.3491034 .091040 \quad 0.594409$

H -3.876552 2.6144891 .593509

H $-1.7082440 .457269-1.533821$

H -1.097103 -1.593217 1.521812

H $-0.932595-3.055903-0.377343$

H $-0.303597-1.776362-1.418845$

H -2.586472 -1.681237 -2.032458

H $1.314647-1.8984070 .894707$

H -3.367455 -2.017977 0.919076

H $-4.460316-1.888844-0.467072$

H $-3.407420-3.295528-0.305640$

H $\quad 1.418150-0.377516-1.147433$

H $\quad 0.5713690 .5137162 .186089$

H $0.123527 \quad 1.299312-0.292421$

H 3.2764450 .4759951 .137994

H $3.4137891 .952643-0.890023$

H $3.6963380 .524326-1.893477$

H $3.492293-1.618413-0.780216$

H -4.269434 $0.533128-1.218460$

H -3.9787180 .4415250 .526683$

H $5.8070121 .685948-1.337549$

H 5.5120061 .4376200 .391819

H $5.8038580 .046782-0.672245$

SCF Energy (B3LYP/6-31G**//MMFF) $=-810.718188938$

\section{F2-29 c06939}

MMFF Geometry

C $-3.268514 \quad 2.6971470 .121312$

C $-2.2511913 .281222-0.522444$

C $-2.5897190 .257454-0.432899$

$\begin{array}{lllll}0 & -1.316974 & 0.271180 & 0.240121\end{array}$

C $-0.730246-1.0378960 .151222$

C $-1.681594-1.887386-0.688622$

C $-3.023503-1.212140-0.462144$

C $0.686547-0.952825-0.432790$

C $-3.686549-1.7122280 .822362$

C $1.630816-0.0416030 .382720$

O $1.231866-2.268322-0.516439$

O $1.622225-0.4838411 .740068$

C $3.081672-0.033129-0.142439$

C $3.9714960 .953040 \quad 0.630701$

O $3.0947110 .341621-1.517944$

C $-3.558796 \quad 1.2238920 .253640$

C 5.4074690 .9642550 .119779

H $-3.983805 \quad 3.3517370 .616427$

H -2.166084 $4.362903-0.535307$

H -1.487989 2.716640 -1.043860

H $-2.4087800 .581155-1.466805$

H -0.696077 -1.4512861 .166668$

H $-1.658340-2.945224-0.409068$
H $-1.420463-1.821217-1.752445$

H $-3.708548-1.400132-1.296500$

H $0.622476-0.557845-1.453457$

H -4.669719-1.2499690.957569

H -3.838632 -2.7959110 .778048$

H $-3.086446-1.4933351 .711367$

H 1.2255720 .9776600 .355747

H $1.324805-2.6050880 .391466$

H $1.955270 \quad 0.2442482 .291028$

H $3.523865-1.032928-0.051463$

H 3.9870310 .7000811 .696072

H 3.5676341 .9683800 .536095

H $2.761313-0.413146-2.031900$

H $-4.5675661 .056436-0.143263$

H -3.5862231 .0099181 .329321$

H $\quad 6.0145821 .6500090 .719381$

H $5.854579-0.0325330 .188017$

H $5.4595881 .295521-0.921978$

SCF Energy $\left(B 3 L Y P / 6-31 G^{* *} / / M M F F\right)=-810.716734073$

F2-29 c06940

MMFF Geometry

C $4.530076-1.616257-0.487616$

C $5.730597-1.4580410 .080383$

C $2.299526-0.443633-0.413136$

O $1.019832-0.4687830 .249073$

C $0.434107 \quad 0.8422590 .164875$

C $1.3964641 .699251-0.656233$

C $2.7374331 .023933-0.416729$

C $-0.9757210 .765601-0.436123$

C 3.3676781 .5105960 .889385

C $-1.933837-0.1399290 .370092$

O $-1.5137052 .083195-0.534657$

$\begin{array}{llll}\text { O } & -1.964827 & 0.284359 & 1.732413\end{array}$

C $-3.369239-0.165318-0.192431$

C $-4.268976-1.1288510 .597982$

O $-3.314416-0.571469-1.557433$

C $3.228015-1.4663190 .240640$

C $-5.712412-1.1258110 .110920$

H $4.484961-1.886386-1.539653$

H $6.635846-1.588624-0.502378$

H $5.832541-1.1978961 .128169$

H $2.106098-0.756076-1.449198$

H $\quad 0.387449 \quad 1.245537 \quad 1.183885$

H $1.3675662 .754960-0.369260$

H $1.1495801 .640646-1.723997$

H $3.4356821 .227891-1.235990$

H $-0.9056610 .366598-1.455044$

H $2.757726 \quad 1.260597 \quad 1.763357$

H 4.3578701 .0699571 .035394

H 3.4970482 .5982610 .870180

H -1.529772 -1.159411 0.358286

H -1.5118672 .4738390 .355496$

H -2.3898431 .1580791 .763301$

H $-3.801600 \quad 0.841781-0.147653$

H $-4.263208-0.8634531 .661038$

H -3.881823 -2.151320 0.510565

H $-4.179539-0.383374-1.958154$

H $3.380096-1.2230791 .299259$

H $2.724631-2.4420020 .246408$

H $-6.324520-1.7725950 .747600$

H -6.135701 -0.1170650 .145413$

H $-5.790892-1.500168-0.914249$

SCF Energy $\left(B 3 L Y P / 6-31 G^{* *} / / M M F F\right)=-810.713736746$

F2-29 c06941

MMFF Geometry

C $4.078542 \quad 1.895697 \quad 0.484338$

C $4.1858922 .955108-0.325497$

C $2.601317-0.1285410 .455940$

O $1.5105690 .485467-0.257013$

C $0.474102-0.498462-0.470665$

C $0.931752-1.7591690 .256822$

C $2.446018-1.634900 \quad 0.235940$

C $-0.8715700 .104973-0.036349$

C $3.029395-2.155333-1.078783$

C $-2.089349-0.742362-0.455358$

O $-0.877193 \quad 0.299964 \quad 1.377255$ 
O $-2.022968-2.032445 \quad 0.156109$

C $-3.474159-0.144190-0.129375$

C $-3.7409901 .181581-0.850907$

O

C 3.9291200 .4790520 .003399

C $-5.1769971 .656211-0.664142$

H 4.1100482 .0564631 .559023

H 4.2962743 .9523270 .086481

H $4.1641782 .849408-1.404415$

H $2.4378300 .088023 \quad 1.520915$

H $0.449077-0.681041-1.551872$

H $0.560607-2.673987-0.215250$

H $0.589281-1.7621141 .298359$

H $2.901747-2.1980621 .058094$

H $-0.955307 \quad 1.101178-0.485549$

H $4.122650-2.098124-1.068427$

H $2.758993-3.206124-1.228461$

H $2.671017-1.589683-1.944793$

H $-2.037648-0.910507-1.537756$

H $-0.1012450 .842927 \quad 1.598722$

H -2.068187-1.899199 1.118679

H $-4.237377-0.875619-0.422120$

H $-3.5403171 .070442-1.922529$

H -3.079744 $1.965809-0.465526$

H -2.979019 0.7002201 .566300

H $4.763614-0.1043950 .409434$

H $4.003800 \quad 0.452845-1.090442$

H $-5.401879 \begin{array}{lll}1.839332 & 0.391237\end{array}$

H $-5.3386452 .591421-1.209688$

H -5.886972 $0.915057-1.044637$

SCF Energy (B3LYP/6-31G**//MMFF) $=-810.722474815$

F2-29_c06942

MMFF Geometry

C $-3.5745412 .218582-0.587352$

$\begin{array}{llll}C & -3.381949 & 3.263762 & 0.225185\end{array}$

C $-2.617256-0.096893-0.634434$

O -1.3764870 .2379410 .016934$

C $-0.594699-0.9586300 .171878$

C -1.370482 -2.077313 -0.519595

C $-2.809266-1.600907-0.418109$

C $0.820103-0.757576-0.390789$

C $-3.427173-1.9816530 .928103$

C $1.5775280 .395117 \quad 0.299129$

O $1.531721-1.983927-0.265368$

$\begin{array}{llll}\text { O } & 1.722429 & 0.096568 & 1.688240\end{array}$

C $2.955050 \quad 0.718360-0.318337$

C $4.082289-0.2638880 .026928$

$\begin{array}{lllll}\text { O } & 3.353247 & 2.008515 & 0.164373\end{array}$

C $-3.7390130 .801498-0.112658$

C $5.4046800 .123403-0.627857$

H -3.626456 $2.394620-1.658746$

H $-3.2751254 .263387-0.181604$

H -3.325884 3.1434301 .301328

H -2.464004 $0.085122-1.707367$

H $-0.542716-1.178047 \quad 1.245453$

H -1.189252 -3.056996 -0.066971

H $-1.087519-2.144000-1.577891$

H $-3.427808-2.033391-1.212553$

H $0.748820-0.522608-1.459648$

H -2.899709-1.524029 1.771151

H -4.475161 -1.6687120 .976856$

H -3.403755 -3.0678831 .066804$

H $0.957146 \quad 1.2981110 .243108$

H $1.638341-2.165561 \quad 0.684256$

H 2.1814930 .8488432 .099929

H $2.8583480 .802733-1.407201$

H $3.830270-1.275745-0.299609$

H $4.242015-0.2928431 .111245$

H $2.6952802 .653863-0.145733$

H $-4.707527 \quad 0.435440-0.472710$

H -3.7606450 .7818820 .983653$

H $6.173126-0.619387-0.390956$

H $5.3027630 .170065-1.716803$

H $5.7590581 .095760-0.271977$

SCF Energy (B3LYP/6-31G*//MMFF)= -810.717396331

F2-29_c06943
MMFF Geometry

C -2.590840 $2.635798-0.067521$

C -1.700980 $3.041044-0.980557$

C $-2.1797490 .092935-0.374453$

$\begin{array}{lllllllllll}\text { O } & -0.819988 & 0.058521 & 0.100398\end{array}$

C $-0.371473-1.306697 \quad 0.151104$

C $-1.494798-2.142380-0.459408$

C $-2.732459-1.318577-0.152531$

C $0.979309-1.471954-0.557091$

C $-3.249650-1.5943231 .260322$

C $2.192910-0.9261990 .224439$

O $1.214155-2.869258-0.779168$

O $3.357727-1.094077-0.598082$

C $2.1279330 .547796 \quad 0.658675$

C $2.1146261 .540047-0.511119$

O 3.2798330 .8310001 .462315

C $-2.950034 \quad 1.222955 \quad 0.314452$

C $1.9814142 .982362-0.036280$

H $-3.134034 \quad 3.409268 \quad 0.472805$

H $-1.5436524 .100690-1.153302$

H $-1.1125482 .349271-1.570362$

H -2.135371 $0.273163-1.456752$

H $-0.263048-1.5763931 .209101$

H $-1.526326-3.161367-0.061435$

H - $1.371247-2.212960-1.547847$

H $-3.540053-1.536658-0.860351$

H $\quad 0.945372-1.003559-1.547489$

H $-2.518792-1.3270142 .030089$

H $-4.166029-1.0281151 .455886$

H -3.490386 -2.656177 1.379459

H $2.368313-1.5508781 .109480$

H $1.218120-3.3108710 .087497$

H $3.399001-2.031241-0.854929$

H 1.2582880 .7152891 .301618

H $1.2869151 .318136-1.191824$

H $3.0451921 .461679-1.085574$

H $3.255610 \quad 0.2349342 .230095$

H -4.0211291 .0991010 .112945$

$\begin{array}{llll}\text { H } & -2.805960 & 1.149688 & 1.399681\end{array}$

H 2.8416623 .2836410 .569637

H $1.9234233 .658006-0.895519$

H 1.0756103 .1160240 .563190

SCF Energy (B3LYP/6-31G**//MMFF) $=-810.707449534$

F2-29_c06944

MMFF Geometry

C $-4.571484-1.462795-0.412080$

C $-3.991232-2.555557 \quad 0.097782$

$\begin{array}{llll}C & -2.706257 & 0.197717 & 0.283368\end{array}$

O $-1.645274-0.388642-0.493313$

C $-0.456623 \quad 0.420702-0.354652$

C $-0.808651 \quad 1.523090 \quad 0.639774$

$\begin{array}{llll}C & -2.312392 & 1.665138 & 0.474042\end{array}$

C $0.719540-0.4971020 .012502$

C -2.658555 $2.581930-0.700412$

C $2.0953260 .198239-0.055057$

O $\quad 0.526971-1.0334391 .322524$

$\begin{array}{llll}\text { O } & 2.143611 & 1.289908 & 0.859107\end{array}$

C $3.265745-0.7593330 .242903$

C $4.646051-0.0812290 .251105$

O $3.259230-1.813688-0.717185$

C $-4.055533-0.046620-0.398035$

C $5.0357790 .562522-1.073220$

H $-5.535263-1.594876-0.900906$

H $-4.481627-3.5199880 .013849$

H $-3.032930-2.5273800 .601519$

H $-2.690793-0.2905691 .267034$

H $-0.2709650 .857358-1.343395$

H $-0.2569832 .449256 \quad 0.451702$

H -0.5919051 .2129991 .668937$

H -2.7739302 .0787651 .377863$

H $0.714128-1.352644-0.673784$

H -2.223524 $3.576040-0.551496$

H $-2.2881842 .192695-1.654188$

H $-3.7423872 .709828-0.787220$

H $2.2067300 .607318-1.065730$

H $-0.339467-1.4752331 .329868$

H 2.0028310 .9309491 .752012 
H $3.120632-1.2117361 .231570$

H $5.404768-0.8380730 .486441$

H 4.6914510 .6717021 .046108

H $3.980533-2.424182-0.488307$

H -4.8180730 .5695720 .093707$

H $-3.9976440 .271624-1.446470$

H $4.3786931 .401799-1.318677$

H $\quad 6.0577260 .950824-1.012785$

H $4.999600-0.159368-1.894839$

SCF Energy $\left(B 3 L Y P / 6-31 G^{* *} / / M M F F\right)=-810.716775320$

F2-29_c06945

MMFF Geometry

C $3.695616 \quad 1.8489130 .427360$

C $3.7748592 .801999-0.507940$

C $2.182661-0.1358460 .722946$

O 1.0873380 .5206430 .051193

C $0.451410-0.395537-0.857531$

C $1.279398-1.677766-0.834606$

C $1.921596-1.6317640 .537109$

C $-1.012967-0.605334-0.425588$

C $3.115111-2.5586650 .684118$

C $-1.8035550 .720023-0.340273$

O $-1.619072-1.497514-1.357936$

O $-1.7538851 .364469-1.617878$

C $-3.2830850 .593000 \quad 0.077793$

C -3.515732 -0.0421971 .452245$

O $-4.005321-0.186309-0.877767$

C 3.4980580 .3883890 .126595

C -2.8492510 .7040082 .595730$

H 3.7871112 .1346051 .472042

H $3.9223933 .838459-0.224876$

H $3.6924532 .569244-1.563856$

H $2.1302340 .123671 \quad 1.787208$

H $\quad 0.4822690 .031362-1.866828$

H $2.047691-1.630106-1.616324$

H $0.697718-2.588210-1.005947$

H $1.165551-1.9350631 .274515$

H -1.026064 -1.086690 0.558852

H $3.868631-2.384761-0.090693$

H $3.589318-2.4276311 .661925$

H $2.797492-3.6035920 .600883$

H -1.2969081 .3904540 .362855$

H -2.512701 -1.700298 -1.034060

H -2.186831 $2.230402-1.526885$

H -3.7419891 .5893190 .065006$

H -3.189239 -1.088959 1.450545

H $-4.597020-0.082799 \quad 1.638124$

H -3.846173 $0.208124-1.752547$

H $4.351075-0.1501080 .553798$

H $3.5149360 .226473-0.957955$

H -3.143630 1.7581592 .601764

H $-3.1449440 .264572 \quad 3.553764$

H - 1.7585760 .6477242 .530043

SCF Energy (B3LYP/6-31G**//MMFF) $=-810.715456433$

F2-29 c06946

MMFF Geometry

C -4.394322 $1.506181-0.149307$

C -5.6207101 .1326640 .232310$

C $-2.1090390 .447408-0.256172$

$\begin{array}{llllll}\text { O } & -0.883796 & 0.302047 & 0.488034\end{array}$

C $-0.213527-0.8897820 .040665$

C -1.065081 -1.471388 -1.087566

C $-2.457472-0.955367-0.761092$

C $1.226747-0.571539-0.384618$

C $-3.146048-1.8560010 .265896$

C $2.057720 \quad 0.0530870 .758339$

O $1.821635-1.781433-0.846017$

O $2.082065-0.8470521 .871665$

C 3.5150110 .4194500 .413489

C $3.6743141 .438890-0.720560$

O $4.230679-0.7648590 .054741$

C -3.1410611 .1750730 .604486$

C $2.9842562 .766664-0.456525$

H $-4.2902992 .101122-1.053193$

H -6.488085 $1.412330-0.355557$

H $\quad-5.7814390 .546716 \quad 1.130505$
H $-1.8609681 .081402-1.119418$

H $-0.209909-1.5979890 .878423$

H $-0.993509-2.561854-1.148326$

H $-0.749062-1.065648-2.057222$

H -3.083895 -0.910343 -1.658814

H $1.1957360 .133117-1.223755$

H $-3.209328-2.883751-0.107770$

H -2.613311 -1.878646 1.221953

H $-4.168245-1.5194050 .460263$

H $1.5498770 .955423 \quad 1.115197$

H $2.709889-1.566198-1.176869$

H $\quad 2.521207-1.6626871 .574297$

H $3.9976210 .803617 \quad 1.320877$

H $3.3182541 .015431-1.667247$

H $4.7448981 .623610-0.876939$

H $5.159846-0.515577-0.087603$

H -3.3537840 .6008611 .514432$

H -2.6983372 .1139840 .962320$

H $3.2301403 .481474-1.248470$

H $1.8960322 .656887-0.438258$

H 3.3072693 .1944690 .497716

SCF Energy $(B 3 L Y P / 6-31 G * * / / M M F F)=-810.719263989$

F2-29 c06947

MMFF Geometry

C $4.600765-0.8780720 .216733$

C $4.950025-1.4176981 .389989$

C $2.5056190 .235439-0.591041$

O $1.611616-0.4503920 .307863$

C 0.2727760 .0568120 .114448

C $0.3476791 .011993-1.072893$

C $1.7791271 .516569-1.009469$

C $-0.690859-1.123167-0.046102$

C $1.9153682 .682609-0.028926$

C $-2.180796-0.765186-0.228025$

O $-0.298319-1.882719-1.200328$

O $-2.902530-1.999251-0.327815$

C $-2.789310 \quad 0.053417 \quad 0.926018$

C -4.3278240 .1026090 .926919$

$\begin{array}{lllll}\text { O } & -2.295141 & 1.389297 & 0.879829\end{array}$

C $3.868640 \quad 0.427403 \quad 0.074472$

C $-4.9296730 .718059-0.330324$

H $4.866398-1.402776-0.697311$

H $5.482302-2.3619941 .423539$

H $4.712584-0.9336782 .330762$

H $2.611703-0.409390-1.474513$

H $0.0371450 .606591 \quad 1.031165$

H $-0.4039861 .805127-1.026863$

H $\quad 0.1997660 .477185-2.018861$

H $2.1239191 .856096-1.992637$

H $-0.580115-1.8069180 .804691$

H 1.6554442 .3970140 .995293

H $2.9408643 .065866-0.021267$

H $1.2600273 .509258-0.323651$

H -2.310798 -0.236038-1.179788

H $0.633158-2.134195-1.076816$

H -2.517502 -2.500533 -1.067017

H $-2.470729-0.3868571 .879177$

H $-4.6589850 .705257 \quad 1.782158$

H $-4.739741-0.902576 \quad 1.071577$

H -2.6498191 .8622001 .651796$

H $4.4886461 .096711-0.533207$

H 3.7459800 .8891041 .061770

H $-6.018607 \quad 0.776072-0.233143$

H $-4.5538001 .732082-0.497561$

$\mathrm{H}-4.7080830 .116194-1.216481$

SCF Energy (B3LYP/6-31G**//MMFF $)=-810.717590708$

F2-29 c06948

MMFF Geometry

C $-3.5858331 .825632-0.744585$

C -3.8490292 .7778640 .157314$

C $-2.250139-0.290518-0.635708$

$\begin{array}{lllll}O & -1.356488 & 0.152545 & 0.406214\end{array}$

C $-0.474646-0.9289880 .752909$

C $-0.755064-2.051988-0.238614$

C $-2.212447-1.821344-0.601126$

C $0.993719-0.4760150 .809841$ 
C $-3.151014-2.4639340 .421241$

C $1.5058730 .309371-0.419002$

O 1.1525590 .3223951 .986245

O $0.861027 \quad 1.584686-0.495130$

C $3.0245200 .585917-0.434537$

C $3.859138-0.691746-0.573253$

O 3.4281391 .2695620 .750270

C $-3.6247490 .351883-0.449523$

C $5.337201-0.389294-0.789429$

H $-3.3359032 .124432-1.759387$

H -3.807505 $3.825177-0.121556$

H -4.106496 2.5339111 .181962

H -1.810214 $0.045350-1.585160$

H $-0.778930-1.2488571 .757808$

H $-0.550814-3.0406410 .183948$

H -0.141281 -1.949299-1.140194

H -2.446378 -2.238576 -1.587012

H $1.606674-1.3708880 .958423$

H -3.025428 -2.0421811 .423489$

H -4.196952 -2.3274020 .127679$

H $-2.967768-3.5419330 .484654$

H $1.255178-0.228579-1.339331$

H $\quad 0.620048 \quad 1.127277 \quad 1.864824$

H $-0.0963801 .430963-0.551385$

H $3.2492411 .257459-1.272749$

H $3.493291-1.288285-1.416636$

H $3.775314-1.3028970 .332301$

H 2.8601822 .0540510 .839442

H -4.347966 -0.109885 -1.131712

H $-3.9814970 .191676 \quad 0.575082$

H $5.899335-1.321422-0.904717$

H $5.4861540 .209849-1.693304$

H 5.7610050 .1561540 .059575

SCF Energy (B3LYP/6-31G**//MMFF) $=-810.715225389$

F2-29 c06949

MMFF Geometry

C $-3.6084792 .155449-0.553436$

C -3.5590593 .1345170 .356889$

C $-2.369020-0.016863-0.798318$

O $-1.193737 \quad 0.508625-0.146757$

C $-0.691411-0.4499530 .799851$

C $-1.673851-1.6186700 .805263$

C $-2.303197-1.527862-0.570279$

C $0.737713-0.8607110 .400403$

C $-3.606365-2.296474-0.697648$

C $1.697166 \quad 0.339998 \quad 0.220968$

O $1.235434-1.7418241 .407073$

O 1.7143641 .1332211 .407308

C $3.147661-0.070077-0.102669$

C $4.0258041 .156588-0.401490$

O $3.136048-0.937186-1.232922$

C $-3.6021710 .689949-0.214932$

C $5.4847660 .794675-0.649481$

H -3.665676 2.423698-1.605160

H -3.5709124 .1737460 .047045$

H -3.504081 2.9202301 .418381

H -2.288665 $0.204192-1.869499$

H $-0.682240 \quad 0.007981 \quad 1.795886$

H -2.431122 -1.4555101 .582146$

H -1.209468 -2.5894291 .002272$

H -1.591406 -1.946072 -1.295410

H $0.696073-1.418425-0.543096$

H -4.058404 -2.132509-1.681083

H -3.426672 -3.370862 -0.584233

H -4.332334 $-2.005018 \quad 0.068113$

H $1.3219360 .967451-0.597440$

H $2.022792-2.1846371 .048501$

H $\quad 0.8592591 .5915201 .468438$

H $3.578289-0.6110730 .748843$

H $3.982764 \begin{array}{lll}1.863647 & 0.434568\end{array}$

H $3.6471331 .673988-1.291487$

H $4.014666-1.346591-1.302302$

H $-4.5197890 .256302-0.627568$

$\begin{array}{llll}\mathrm{H} & -3.636124 & 0.559743 & 0.873482\end{array}$

H $\quad 6.078578 \quad 1.703985-0.787797$

H $5.9016690 .243406 \quad 0.199179$

H $5.5995150 .184159-1.550124$
SCF Energy (B3LYP/6-31G**//MMFF) $=-810.712796330$

F2-29_c06950

MMFF Geometry

C $-3.0633272 .342127-0.497817$

C $-2.4674853 .468341-0.090316$

C $-2.433908-0.073653-0.287185$

O $-1.013791 \quad 0.149741-0.202029$

C $-0.350464-1.112033-0.025480$

C $-1.435053-2.184865-0.110183$

C $-2.678626-1.4441060 .350757$

C $0.776982-1.292623-1.053028$

C $-2.785381-1.4318181 .876377$

C $1.821224-0.151448-1.081226$

O $1.449353-2.527783-0.817466$

O $1.2358541 .017181-1.654417$

C $2.4289510 .222800 \quad 0.286673$

C 3.5280921 .2893110 .140303

O $2.976763-0.942587 \quad 0.893643$

C -3.1830391 .1008630 .342091$

C 4.0945921 .7448441 .479304

H -3.499088 $2.314213-1.493258$

H -2.418133 $4.331541-0.744983$

H -2.019390 3.5495090 .893831

H -2.674953 -0.137778 -1.357506

H $0.065989-1.1259180 .988762$

H -1.196881 -3.072307 0.484319

H -1.574762 -2.509181-1.149215

H -3.587507 -1.905062 -0.051752

H $\quad 0.329549-1.346635-2.054163$

H $-1.940109-0.9201662 .347646$

H -3.703829 -0.9298642 .197442$

H -2.818714 -2.454827 2.266278

H $2.641108-0.468273-1.739600$

H $\quad 0.808169-3.246759-0.946099$

H $0.9154710 .782094-2.541694$

H 1.6441460 .6210560 .940894

H $3.1347652 .167521-0.383928$

H $4.3537030 .888579-0.460322$

H $3.152508-0.7371221 .826970$

H -4.2477800 .8572990 .435131$

H -2.7964081 .3006501 .348745$

H 4.8194962 .5506971 .325343

H 3.3015632 .1224642 .132495

H 4.6103160 .9294101 .995202

SCF Energy $\left(B 3 L Y P / 6-31 G^{* *} / / M M F F\right)=-810.698783108$

F2-29_c06951

MMFF Geometry

C $2.7249822 .376292-0.214485$

C $2.5588533 .058116-1.353444$

C 1.8881550 .1428650 .572699

O $0.690458 \quad 0.255241-0.224984$

C $0.322065-1.027794-0.755682$

C $1.526811-1.932191-0.512107$

C $2.113514-1.3591120 .761582$

C $-0.976185-1.513975-0.082508$

C $3.536714-1.8123451 .035305$

C $-2.251543-0.742764-0.488839$

O $-1.191772-2.888256-0.430691$

O $-3.345972-1.2723060 .276238$

C $-2.2361830 .781357-0.288563$

C $-2.1092931 .221012 \quad 1.176747$

O $-3.4549781 .312263-0.823507$

C $3.0108870 .900904-0.150403$

C -1.9777572 .7336831 .307515$

H 2.6586362 .9126600 .728635

H $2.3577814 .123688-1.331461$

H $2.6174152 .570806-2.320298$

H $1.690951 \quad 0.613587 \quad 1.543524$

H $\quad 0.153358-0.928737-1.834283$

H $2.239232-1.817452-1.338762$

H $1.287633-2.996222-0.435060$

H $1.488387-1.6907541 .602171$

H -0.875335 -1.485404 1.008367

H $4.203390-1.6050220 .192140$

H $3.938192-1.3140301 .923363$

H $3.563900-2.8925651 .214381$ 
H $-2.495929-0.959344-1.536635$ H $-1.252911-2.942518-1.399850$ H $-3.362878-2.2338390 .130250$ H -1.436181 $1.226453-0.886766$ H -1.2364290 .7558891 .645235$ H -2.9940350 .9092051 .744286$ H $-4.1911030 .907532-0.332943$ H 3.9601240 .7754620 .381888 H $3.1440810 .502458-1.163552$ H -1.8694033 .0125872 .360426$ H -1.0967463 .0969140 .768765$ H -2.860143 3.2468430 .912836

SCF Energy (B3LYP/6-31G**//MMFF) $=-810.716299704$

F2-29_c06952

MMFF Geometry

C $4.3162211 .700627-0.003947$

C $4.1339442 .810460-0.728347$

C $2.790818-0.1856010 .648688$

O $1.621687 \quad 0.6548750 .655553$

C $0.6295820 .102776-0.241672$

C $1.113765-1.299303-0.612179$

C $2.230458-1.5774920 .376663$

C $-0.737005 \quad 0.205656 \quad 0.458159$

C $3.219202-2.628099-0.096458$

C $-1.9372970 .098149-0.506276$

O $-0.847889-0.798197 \quad 1.466707$

O $-1.895428-1.132347-1.222544$

C -3.3004350 .2219330 .205381$

C $-4.4744850 .177336-0.785106$

O $-3.349058 \quad 1.4423680 .940768$

C $3.7889930 .346553-0.391539$

C $-5.8252270 .265077-0.085710$

H $4.886617 \quad 1.768046 \quad 0.918914$

H $4.5444313 .757399-0.395258$

H $3.5753282 .796285-1.657569$

H $3.241521-0.1481581 .647576$

H $\quad 0.6237760 .744483-1.131014$

H $1.497807-1.286592-1.639787$

H $\quad 0.339013-2.069193-0.576943$

H $1.769663-1.9399681 .305985$

H -0.7897941 .1712410 .976104$

H $3.642936-2.382591-1.075510$

H $4.042142-2.7345020 .617404$

H $2.724944-3.600991-0.189483$

H $-1.8466490 .904988-1.243977$

H -0.116470 -0.660959 2.092764

H -1.995033 -1.851696 -0.575276

H -3.421751 -0.601265 0.919328

H -4.442507 -0.747762 -1.371017

H $-4.3986591 .016082-1.487762$

H -3.252872 2.1745390 .307819

H $4.648357-0.328362-0.471055$

H $3.3211810 .399636-1.381815$

H $-6.6343340 .198934-0.820106$

H $-5.948935-0.5538860 .629933$

H -5.9364221 .2122600 .450921$

SCF Energy (B3LYP/6-31G**//MMFF) $=-810.716080362$

F2-29_c06953

MMFF Geometry

C $4.441874-0.6394690 .572760$

C $4.688824-0.991076 \quad 1.839685$

C $2.3909150 .206352-0.593727$

O $1.442629-0.4160290 .295834$

C $0.107121-0.023665-0.092474$

C $0.2584550 .760245-1.392371$

C $1.6451541 .366076-1.259334$

C $-0.776410-1.269982-0.170240$

C $1.6108312 .661163-0.446163$

C $-2.255540-1.032007-0.528533$

O $-0.240341-2.146255-1.175959$

O $-2.893594-2.324992-0.496631$

C $-3.088004-0.1416610 .414483$

C $-3.0084531 .355306 \quad 0.098113$

O $-2.700848-0.3550561 .770650$

C $3.658928 \quad 0.5805830 .174413$

C -4.0327282 .1593020 .892270$
H $4.835500-1.262372-0.226512$

H $5.265585-1.8828362 .059839$

H $4.322647-0.4027982 .673811$

H $2.630058-0.541250-1.362806$

H $-0.244490 \quad 0.6281030 .711947$

H $-0.5342221 .498537-1.540338$

H $0.2448980 .090837-2.260968$

H $2.0752081 .592044-2.241672$

H $-0.704532-1.8341810 .767685$

H $0.9467123 .392641-0.918993$

H 1.2581142 .5000590 .577557

H $2.6071043 .111756-0.391460$

H $-2.355426-0.683989-1.563212$

H $0.687084-2.321656-0.940878$

H $-2.408317-2.900881-1.112422$

H $-4.140795-0.4451290 .342777$

H $-3.1837041 .521942-0.970568$

H -2.0240091 .7591760 .345292$

H -2.815106 -1.302360 1.959096

H $4.3045531 .204506-0.454510$

H 3.4019881 .1602901 .069216

H -3.962710 3.2200950 .631152

H $-5.051051 \quad 1.8236190 .672081$

H -3.8636742 .0654901 .969457$

SCF Energy (B3LYP/6-31G**//MMFF) $=-810.715758760$

F2-30 c07190

MMFF Geometry

C -4.235491-1.536231 -0.368085

C $-4.316417-2.6578100 .356636$

C $-2.4611770 .219066-0.598924$

O $-1.366565-0.660321-0.285300$

C $-0.1309480 .079313-0.394411$

C $-0.5137391 .541250-0.614057$

C $-1.9685531 .602538-0.189630$

C $0.708744-0.2105510 .858294$

C $-2.7333782 .750909-0.825917$

C $2.106346 \quad 0.432197 \quad 0.874387$

$\begin{array}{lllll}\text { O } & 0.013878 & 0.271351 & 2.016681\end{array}$

O $2.813087-0.0775892 .015256$

C $2.9683800 .176697-0.373436$

C $3.316541-1.300468-0.602298$

O $4.1807320 .925941-0.238097$

C $-3.711182-0.2301580 .158586$

C $4.116524-1.509040-1.882571$

H $-4.575917-1.557129-1.400190$

H -4.709416 -3.568291-0.082360

H $-3.993617-2.6894651 .391392$

H $-2.6244350 .169444-1.683888$

H $\quad 0.384169-0.307808-1.279746$

H $-0.4179381 .776957-1.681966$

H $0.1152982 .254635-0.074687$

H -2.0111721 .7079220 .902068$

H $0.794878-1.294403 \quad 1.002574$

H -2.280615 $3.710556-0.555937$

H $-2.7349622 .675093-1.918488$

H -3.774235 $2.763583-0.488029$

H 2.0284901 .5130271 .042539

H $-0.853667-0.1681562 .035240$

H 2.2658860 .1038902 .798697

H $2.4644740 .578312-1.258394$

H $2.403060-1.901556-0.661385$

H $3.913110-1.6840090 .233690$

H $4.6340960 .607472 \quad 0.561514$

H -3.483716 -0.3215921 .228149$

H -4.5056700 .5173390 .055656$

H $5.076696-0.985365-1.843258$

H $4.321092-2.574311-2.029197$

H $3.561142-1.147465-2.753756$

SCF Energy $\left(B 3 L Y P / 6-31 G^{* *} / / M M F F\right)=-810.721257850$

F2-30 c07191

MMFF Geometry

C -2.8438312 .7108770 .236059$

C -1.9465243 .7031090 .231108$

C -2.6079050 .2144670 .207109$

$\begin{array}{lllll}0 & -1.323037 & 0.209000 & 0.858802\end{array}$

C $-0.773486-1.1187960 .818250$ 
C $-1.865545-2.017793 \quad 0.246168$

C $-2.650720-1.062332-0.629981$

C $0.513512-1.106866-0.028293$

C $-4.054882-1.547609-0.947956$

C $1.567864-0.0979470 .481229$

O $1.070681-2.420384-0.052025$

O $1.841729-0.3405241 .860431$

C $2.885232-0.135398-0.318565$

C 3.8855650 .9232800 .170998

O $2.6114350 .075694-1.701712$

C $-2.7484331 .486819-0.630190$

C $5.2030230 .870674-0.592079$

H -3.7030512 .7881320 .897212$

H -2.075232 4.5633900 .878956

H -1.073396 $3.675686-0.411233$

H $-3.369714 \quad 0.1844930 .997130$

H $-0.543893-1.4377031 .841597$

H -2.498418 -2.384074 1.064725

H -1.488710 -2.890277 -0.295079

H -2.104076 -0.914975 -1.570530

H $0.264353-0.843421-1.062571$

H -4.019284 -2.498887 -1.488890

H $-4.642512-1.701126-0.036591$

H $-4.586438-0.823719-1.573369$

H 1.1394490 .9092010 .409932

H $1.233714-2.6926010 .866831$

H $2.291527-1.1995841 .928803$

H $3.352674-1.122111-0.218625$

H 4.0920960 .7855821 .238108

H 3.4572621 .9249630 .043883

H $2.2042880 .954226-1.793170$

H -1.893316 $1.576824-1.312070$

H $-3.6538541 .436380-1.245370$

H $5.671439-0.114088-0.496167$

H $5.0571461 .081496-1.656027$

H $5.8982651 .617009-0.194599$

SCF Energy (B3LYP/6-31G**//MMFF) $=-810.714828319$

\section{F2-30 c07192}

MMFF Geometry

C -2.9296472 .3042210 .577758$

C $-2.150794 \quad 3.3764990 .761017$

C $-2.405633-0.126458 \quad 0.243411$

O $-1.173481-0.0740610 .988562$

C $-0.424930-1.2802050 .763354$

C $-1.361576-2.2266420 .019384$

C $-2.242827-1.269189-0.757418$

C $0.855932-0.937730-0.023962$

C -3.556256-1.885004-1.209360

C 1.7297680 .1199490 .685844

O $1.596639-2.136541-0.237704$

O $2.082428-0.3767551 .981484$

C $3.0268270 .513904-0.050188$

C $2.8233151 .108860-1.447416$

O $3.886893-0.618300-0.191150$

C $-2.6499441 .225580-0.429965$

C $1.9555052 .356283-1.465550$

H -3.8260492 .2008181 .183485$

H -2.409862 4.1230891 .503734

H -1.246985 3.5272890 .181091

H $-3.204975-0.3514840 .961450$

H -0.160898 -1.7200211 .731892$

H -1.964251 -2.785332 0.746966

H $-0.849683-2.955350-0.615370$

H -1.693459 -0.921038-1.641687

H $0.576099-0.546623-1.007595$

H -4.144901 -2.244618 -0.358691

H -4.162839 -1.154582 -1.753853

H $-3.373348-2.735084-1.874728$

H $1.131643 \quad 1.0224960 .853159$

H $1.897921-2.4536280 .631106$

H 2.5673810 .3281422 .443546

H 3.5766181 .2364940 .565622

H $2.4069860 .355880-2.127124$

H $3.8073621 .355557-1.866889$

H $3.998313-1.0094670 .692323$

H -1.777263 $1.498866-1.036620$

H $-3.5102341 .160790-1.105599$
H $1.9433992 .790480-2.470385$

H $0.9207952 .129497-1.192493$

H $2.3399523 .112674-0.774330$

SCF Energy (B3LYP/6-31G**//MMFF) = -810.712565555

F2-30 c07193

MMFF Geometry

C $2.5953352 .691573-0.295836$

C 1.8120423 .6460700 .219039

C $2.3206720 .208850-0.514244$

O $0.8820110 .255045-0.567848$

C $0.357546-1.079535-0.490860$

C $1.560958-2.016935-0.550766$

C $2.666630-1.1689850 .045482$

C $-0.473282-1.2411040 .797489$

C $4.061157-1.651735-0.315374$

C $-1.596181-0.1966470 .997155$

O $-1.029214-2.5544290 .837354$

O

C $-2.5402860 .001087-0.206152$

C -3.6661081 .0002660 .107010$

O $-3.110385-1.246443-0.590674$

C $2.834109 \quad 1.358320 \quad 0.354724$

C $-4.5811311 .234253-1.088413$

H $3.1018972 .884587-1.237904$

H $1.6838164 .590276-0.298816$

H 1.2867563 .5044811 .157096

H $2.6847750 .309952-1.545172$

H $-0.276221-1.261566-1.366189$

H $1.788067-2.248020-1.599559$

H $1.414523-2.966540-0.028074$

H $2.561898-1.1688171 .138275$

H $0.200851-1.1537011 .658613$

H $4.825367-1.0060710 .128260$

H $4.223145-2.6693780 .054720$

H $4.215192-1.659999-1.399656$

H -2.200684 -0.5301521 .850558$

H -1.597350 -2.660032 0.055409

H $-0.488744 \quad 1.368507 \quad 0.608532$

H -1.974912 $0.388159-1.061444$

H $-3.2440951 .962000 \quad 0.419312$

H -4.2770320 .6226830 .935969$

H $-3.643409-1.5713900 .155275$

H $2.343730 \quad 1.327701 \quad 1.335876$

H $3.911601 \quad 1.2523160 .523703$

H $-4.0153021 .612293-1.945905$

H $-5.0892110 .312802-1.389235$

H $-5.3485601 .972999-0.836075$

SCF Energy (B3LYP/6-31G**//MMFF $)=-810.726765549$

F2-30 c07194

MMFF Geometry

C -2.4190902 .3437190 .142651$

C $-3.502448 \quad 3.121422 \quad 0.247315$

C $-2.045510-0.1294180 .316085$

O $-0.667075-0.0418400 .725796$

C $-0.100828-1.3629090 .795514$

C $-1.253517-2.3254700 .507006$

C $-2.173359-1.490369-0.359663$

C $1.073696-1.479766-0.193179$

C $-3.594137-2.024804-0.420813$

C $2.343612-0.7047860 .216926$

O $1.441047-2.863815-0.278335$

O $3.316359-0.923634-0.817887$

C 2.1980030 .8108630 .437354

C $1.8147851 .585724-0.829883$

O $3.457104 \quad 1.315890 \quad 0.898671$

C $-2.3893581 .041220-0.606360$

C $1.6381003 .075644-0.561926$

H -1.4985572 .6696280 .618976$

H $-3.4578094 .053470 \quad 0.799976$

H -4.445736 $2.846079-0.211053$

H -2.656814 -0.0927741 .227681$

H $0.266482-1.5356361 .814122$

H $-1.754531-2.5778371 .450997$

H $-0.958508-3.2669190 .036125$

H -1.760173 -1.447903-1.376067

H $0.759505-1.171249-1.197159$ 
H $-4.049280-2.0696950 .574321$

H $-4.224925-1.390143-1.050911$

H $-3.606450-3.035389-0.841968$

H $2.768027-1.1667401 .117468$

H $2.172286-2.930132-0.916482$

H $4.127536-0.455009-0.555739$

H 1.4771401 .0139911 .234808

H $\quad 0.8838591 .193329-1.250213$

H $2.5942521 .474345-1.592851$

H $3.664551 \quad 0.864978 \quad 1.734857$

H $-1.6367481 .127769-1.399215$

H -3.356612 $0.865468-1.090868$

H $1.3122793 .585989-1.473814$

H 0.8847283 .2490490 .212153

H $2.5763423 .536092-0.237273$

SCF Energy $($ B3LYP/6-31G**//MMFF $)=-810.719607292$

F2-30_c07195

MMFF Geometry

C -4.748752 -0.233904 -0.249141

C $-5.366868-0.7205360 .832785$

C $-2.4066450 .585638-0.736182$

O $-1.1746310 .118065-1.325672$

C $-0.1116130 .220820-0.358990$

C -0.7884470 .4069790 .990788$

C -2.0461801 .1762120 .631775$

C $0.802716-1.002588-0.477277$

C -1.7804702 .6804890 .538655$

C $1.991310-1.0145670 .497919$

O $0.036270-2.187944-0.219536$

O $2.817830-2.1424380 .175434$

C $2.874580 \quad 0.244854 \quad 0.478727$

C $3.5850810 .491700-0.859132$

O 3.8571370 .1149951 .511502

C $-3.359529-0.616958-0.664026$

C $4.3971881 .781243-0.847398$

H $-5.2810140 .473714-0.879478$

H $-6.376377-0.4076341 .076383$

H $-4.881327-1.4325511 .491135$

H $-2.8291251 .342652-1.407563$

H $0.449415 \quad 1.123451-0.625155$

H $-0.148060 \quad 0.919047 \quad 1.715484$

H -1.071609 -0.5585661 .426006$

H -2.830103 1.0246781 .380835

H $1.156737-1.105922-1.509791$

H $-2.699743 \quad 3.2125420 .271189$

H -1.435877 3.0708451 .501933

H -1.024793 $2.924221-0.214947$

H $1.639357-1.1925261 .521680$

H $-0.707446-2.191690-0.846292$

H $2.251726-2.9329320 .203532$

H $2.274802 \quad 1.121560 \quad 0.742927$

H $2.8542130 .549162-1.672492$

H $4.268892-0.334932-1.084383$

H $4.386237-0.6770541 .314381$

H $-3.413140-1.087361-1.654264$

H -2.945436 -1.388132 -0.002297

H $4.864274 \quad 1.940796-1.824507$

H $3.7583972 .643686-0.632255$

H $5.1929081 .745222-0.096903$

SCF Energy (B3LYP/6-31G**//MMFF) $=-810.722292460$

F2-30_c07196

MMFF Geometry

C $4.167366 \quad 1.5982560 .473307$

C 5.3916561 .9250890 .044663

C $2.4286810 .020065-0.408655$

O 1.3083920 .7504650 .122016

C $0.089417 \quad 0.089613-0.281826$

C $0.501885-1.218578-0.952367$

C $1.935741-1.419479-0.499950$

C $-0.817405-0.0385930 .952269$

C $2.744446-2.298079-1.439599$

C $-2.209687-0.6407850 .688471$

O $-0.162072-0.8409821 .944779$

O $-2.960360-0.514496 \quad 1.904927$

C -3.004804 $0.006540-0.460197$

C $-3.3559911 .479854-0.216323$
O $-4.226485-0.720580-0.625759$

C 3.6383360 .1920630 .511473

C $-4.0774412 .102463-1.406012$

H 3.5118942 .3896440 .828054

H 5.7164382 .9599150 .047500

H $6.0866391 .174421-0.314787$

H $2.6420820 .422179-1.408279$

H $-0.3872890 .746240-1.017242$

H $0.461781-1.086923-2.041506$

H $-0.143633-2.068018-0.713487$

H $1.929704-1.8760930 .497924$

H $-0.9220370 .947358 \quad 1.420292$

H $2.796673-1.867841-2.445507$

H $3.768233-2.424977-1.074567$

H $2.291564-3.291656-1.521622$

H $-2.123661-1.7196620 .512579$

H $-0.150656-1.7610281 .631771$

H -3.842241 -0.8908291 .741205$

H -2.454161 -0.091732 -1.401674

H -2.449097 $2.060795-0.019202$

H -4.0086321 .5734740 .659558$

H -3.993681 -1.640791-0.837350

H $3.355234-0.0291751 .547965$

H $4.427680-0.5140990 .229923$

H $-3.4640862 .041609-2.310579$

H $-5.0313551 .602685-1.601457$

H -4.286408 $3.158667-1.208740$

SCF Energy (B3LYP/6-31G**//MMFF) $=-810.708266117$

F2-30_c07197

MMFF Geometry

C -3.644865 $1.906102-0.054889$

C -3.2071103 .1525250 .155449$

C $-2.494527-0.320225-0.027973$

O -1.4860020 .0753910 .918869$

C $-0.687577-1.0784121 .238206$

C -1.065388 -2.173639 0.244561

C $-1.833545-1.433074-0.834468$

C $0.808346-0.719731 \quad 1.261518$

C $-2.807393-2.317132-1.594751$

C $1.395197-0.137700-0.043925$

$\begin{array}{lllll}\text { O } & 1.024590 & 0.205614 & 2.331442\end{array}$

O $0.6935301 .041497-0.428722$

C 2.8999380 .1871310 .064259

C $3.4742960 .707062-1.262971$

O $3.615138-0.9799570 .463383$

C $-2.9087110 .884047-0.874211$

C $4.9524601 .059817-1.155062$

H $-4.594121 \quad 1.6061400 .381579$

H $-3.788283 \quad 3.8452860 .754166$

H $-2.2682483 .501823-0.259824$

H -3.350614 -0.7071340 .541340$

H $-0.981107-1.3868852 .249627$

H -1.714521 -2.9028210 .746204$

H $-0.207322-2.729826-0.145427$

H -1.114432 -1.003024 -1.543299

H $1.364313-1.6268441 .525526$

H -2.277276 -3.141073 -2.083752

H $-3.560477-2.750238-0.927858$

H -3.330932 -1.748248 -2.369340

H $1.254209-0.875570-0.841839$

H $\quad 0.4340130 .9646572 .187798$

H $0.842458 \quad 1.7147910 .256460$

H $3.0595940 .953657 \quad 0.831606$

H $2.9250301 .595966-1.592358$

H $3.361665-0.057993-2.040773$

H $3.484398-1.657440-0.221949$

H -2.022728 1.339929 -1.332899

H $-3.5693140 .565020-1.688080$

H $5.1176351 .822362-0.387343$

H $5.5555200 .181568-0.904645$

H $5.3157551 .454900-2.109156$

SCF Energy (B3LYP/6-31G**//MMFF) $=-810.712150292$

F2-30 c07198

MMFF Geometry

C $1.9595182 .953826-0.043184$

C $0.800643 \quad 3.5199710 .312437$ 
C $2.5978620 .553541-0.424380$

O $1.257052 \quad 0.157850-0.770966$

C $0.933684-1.077225-0.114265$

C $2.096598-1.4008400 .818829$

C $3.266735-0.691160 \quad 0.163825$

C $-0.411836-0.9597420 .614066$

C $3.902024-1.553241-0.929075$

C $-1.577558-0.510655-0.296889$

O $-0.716817-2.216347 \quad 1.217131$

O $-1.718698-1.416524-1.388984$

C $-2.922121-0.4366690 .450909$

C $-4.114382-0.044567-0.438376$

O -2.8051410 .4926971 .525983$

C 2.5136151 .7071670 .588027

C $-3.9900481 .329438-1.084440$

H $2.5475093 .418161-0.830592$

H $0.456053 \quad 4.422542-0.180272$

H $0.177526 \quad 3.0957291 .092106$

H $3.1049920 .893508-1.335058$

H $0.879626-1.855858-0.884693$

H $2.242796-2.4780320 .946156$

H $1.925441-0.9755941 .815353$

H $4.045653-0.4392800 .891962$

H $-0.312200-0.2259621 .422738$

H $3.186696-1.823893-1.712568$

H $4.729701-1.016368-1.404661$

H $4.303817-2.478938-0.503762$

H $-1.3348890 .478580-0.702082$

H $-0.860869-2.8580080 .500426$

H - $0.966152-1.275788-1.988022$

H $-3.150720-1.4182870 .883757$

H -5.021709 -0.0380520 .178701$

H $-4.266669-0.798510-1.219001$

H -3.6502580 .4933642 .007163$

H 1.8933811 .4202111 .445639

H 3.5141271 .9378150 .972567

H $-4.9088171 .570019-1.629224$

H $-3.8300102 .110890-0.335298$

H -3.164606 $1.361273-1.801552$

SCF Energy (B3LYP/6-31G**/MMFF) $=-810.716365439$

F2-30 c07199

MMFF Geometry

C $3.900055-1.1523050 .057335$

C $3.892830-2.4373630 .429198$

C $2.3829700 .752403 \quad 0.821039$

O $1.270045-0.107107 \quad 1.119928$

$\begin{array}{lllll}\text { C } & 0.103294 & 0.410681 & 0.440374\end{array}$

C $0.5897441 .537287-0.472920$

C $2.0872411 .306461-0.566994$

C $-0.601113-0.759972-0.260661$

C $2.8762752 .555616-0.917539$

C $-1.923232-0.404525-0.963858$

O $0.268978-1.306409-1.261300$

O $-2.529427-1.629345-1.403831$

C $-2.9482820 .358994-0.106993$

C $-3.464148-0.4437061 .095924$

O $-4.046792 \quad 0.693945-0.960883$

C $3.702169-0.0024691 .004185$

C -4.3873720 .3709721 .993996$

H $4.078998-0.917549-0.988404$

H $4.051323-3.221930-0.302712$

H $3.727093-2.7290301 .460298$

H 2.3553581 .5644401 .560914

H $-0.5495950 .819307 \quad 1.218696$

H 0.3819402 .5012340 .009335

H $0.1026091 .561203-1.451628$

H $2.2633050 .547499-1.337692$

H $-0.766012-1.5741990 .455296$

H $2.5606662 .950011-1.888955$

H $2.7323103 .344241-0.171491$

H $3.9472392 .336600-0.974260$

H $-1.7257220 .164828-1.880263$

H $1.084585-1.585712-0.811481$

H -1.878203 -2.090536-1.960029

H $-2.512422 \quad 1.3066360 .226870$

H -2.623555 $-0.796771 \quad 1.702547$

H $-4.019584-1.3243940 .752771$
H $-4.5972131 .345466-0.495102$

H 4.5422930 .6908290 .880075

H $3.749234-0.3717802 .036822$

H $-4.672490-0.2181382 .871632$

H -3.890736 1.2813412 .344012

H -5.3064500 .6552941 .472929$

SCF Energy (B3LYP/6-31G**//MMFF)= -810.714928664

F2-30 c07200

MMFF Geometry

C $2.646535 \quad 2.794617 \quad 0.359215$

C $2.6212463 .168780-0.925155$

C $2.4546240 .246777-0.087418$

$\begin{array}{lllll}\text { O } & 1.153792 & 0.297670 & -0.703158\end{array}$

C $0.647412-1.038580-0.861258$

C $1.769356-1.977928-0.428548$

C $2.539869-1.1321670 .565607$

C $-0.636378-1.195956-0.024261$

C $3.958916-1.6211520 .801772$

C $-1.725263-0.155926-0.378176$

O $-1.149347-2.514896-0.210618$

O $-2.003125-0.245680-1.774164$

C $-3.025592-0.357518 \quad 0.424197$

$\begin{array}{llll}C & -4.150910 & 0.632733 & 0.080398\end{array}$

O $-2.727030-0.265144 \quad 1.816144$

C $2.592553 \quad 1.396107 \quad 0.916710$

C $-3.7933332 .094216 \quad 0.317768$

H $2.7161783 .584343 \quad 1.105225$

H $2.668092 \quad 4.221127-1.185566$

H $2.5552182 .457650-1.739428$

H $3.2020820 .333837-0.886313$

H $\quad 0.424988-1.208990-1.921043$

H $2.401617-2.209843-1.295259$

H $1.422212-2.927362-0.010858$

H $1.999749-1.1304851 .521344$

H $-0.391079-1.0886611 .038637$

H $4.479450-0.9800901 .519809$

H $3.952439-2.6399641 .202754$

H $4.540429-1.629024-0.126260$

H $-1.3127670 .839159-0.174934$

H $-1.412432-2.597680-1.143398$

H $-2.494600 \quad 0.552632-2.030181$

H $-3.414252-1.365651 \quad 0.234985$

H -5.0220770 .4007730 .706138$

H $-4.4665720 .500767-0.960515$

H $-3.547789-0.4449202 .305631$

H $1.7429821 .364194 \quad 1.611400$

H 3.5022201 .2508851 .511092

H $-3.4707212 .264416 \quad 1.349488$

H -2.996876 $2.426151-0.354448$

H -4.6670292 .7271170 .130958$

SCF Energy (B3LYP/6-31G**//MMFF) $=-810.716364382$

F2-30 c07201

MMFF Geometry

C $-3.9044551 .970221-0.025868$

C $-3.3364693 .168769-0.199986$

C $-2.966800-0.3367750 .323108$

$\begin{array}{lllll}O & -1.766021 & 0.149885 & 0.955397\end{array}$

C $-0.644636-0.6724770 .572716$

C $-1.119241-1.488118-0.621166$

C $-2.600709-1.665650-0.342869$

$\begin{array}{llll}\text { C } & 0.577752 & 0.233727 & 0.360379\end{array}$

C $-2.862216-2.8504180 .589575$

C $1.902473-0.5361990 .185140$

O $0.3714541 .072504-0.777133$

O $1.829821-1.387983-0.955577$

C 3.1270240 .3911180 .040952

C $4.433926-0.409991-0.075328$

$\begin{array}{lllll}\text { O } & 3.189129 & 1.243237 & 1.181792\end{array}$

C $-3.4344560 .711338-0.698714$

C $5.655520 \quad 0.475768-0.285547$

H -4.7649291 .8919790 .633536$

H $-3.7268094 .041244 \quad 0.312423$

H -2.478231 $3.301348-0.849427$

H -3.732961 -0.4692961 .096250$

H $-0.447096-1.3360581 .423497$

H - $0.581277-2.435058-0.725828$ 
H - $-0.994882-0.929952-1.556270$

H -3.164358 -1.837382 -1.266647

H $\quad 0.6605430 .902981 \quad 1.225246$

H -2.359785 -2.738165 1.555665

H $-3.935447-2.9521200 .783259$

H -2.514558 -3.783236 0.133493

H $2.041050-1.1762711 .065080$

H $-0.4460601 .575253-0.620059$

H $1.666823-0.824949-1.731706$

H $3.0126481 .016552-0.852883$

H $4.371520-1.114655-0.912056$

H $4.590605-0.9955240 .838769$

H 3.8211681 .9550570 .986253

H -2.626804 $0.943601-1.403189$

H $-4.2705850 .309871-1.283544$

H 5.8384951 .1192830 .580294

H $6.546661-0.142919-0.432796$

H $5.5329221 .109383-1.169534$

SCF Energy (B3LYP/6-31G**//MMFF) $=-810.720251465$

F2-30 c07202

MMFF Geometry

C -3.3119382 .3903400 .010004$

C -2.585493 $3.510392-0.074865$

C $-2.680645-0.0320090 .157490$

O $-1.4130630 .213491 \quad 0.796468$

C $-0.660167-1.0103520 .850441$

C $-1.595401-2.1094220 .353479$

C $-2.516663-1.356319-0.584898$

C $0.616846-0.859627-0.000789$

C $-3.824798-2.079170-0.857922$

C $1.497114 \quad 0.325737 \quad 0.448105$

O $1.345514-2.081321 \quad 0.060148$

$\begin{array}{lllll}\text { O } & 1.906794 & 0.120412 & 1.800685\end{array}$

C $2.7296470 .584689-0.444888$

C $3.905442-0.381568-0.247786$

O $3.2096921 .902770-0.147273$

C $-3.0202871 .137978-0.767386$

C $5.075082-0.061846-1.173624$

H -4.1736602 .3767880 .672440$

H $-2.851364 \quad 4.3834390 .511004$

H - $1.7182523 .576698-0.722491$

H -3.430084 $-0.126050 \quad 0.954423$

H $-0.389137-1.2118291 .893324$

H -2.166767 -2.5087261 .201443$

H - $1.085246-2.950374-0.124770$

H -1.994841 -1.192947 -1.536831

H $0.338634-0.693097-1.048043$

H -3.636647 -3.049781-1.328368

H $-4.385749-2.2565810 .065832$

H -4.460687 -1.495865 - 1.531058

H $\quad 0.880542 \quad 1.2330670 .449155$

H $1.636665-2.2037950 .980135$

H 2.4369910 .8924942 .063220

H $2.4254910 .588211-1.498353$

H $3.598680-1.412707-0.439211$

H $4.271683-0.3283250 .784157$

H $2.5032162 .530987-0.374861$

H -2.188867 $1.313604-1.461780$

H $-3.9045810 .899974-1.369180$

H $5.877312-0.793039-1.031670$

H $4.764622-0.098896-2.222723$

H $5.4881140 .930994-0.970193$

SCF Energy $\left(B 3 L Y P / 6-31 G^{* *} / / M M F F\right)=-810.719689043$

F2-30_c07203

MMFF Geometry

C $-2.259606 \quad 2.243571-0.111192$

C - $-1.5258763 .243847 \quad 0.389603$

C $-2.508218-0.183160 \quad 0.640770$

O $-1.161866-0.2745371 .139325$

C $-0.508064-1.3795530 .488003$

C $-1.524018-1.981624-0.486166$

C $-2.466918-0.820729-0.743795$

C $0.774671-0.887456-0.207942$

C $-3.825153-1.243565-1.274209$

C $1.723755-0.0938010 .718465$

O $1.443119-2.018970-0.761670$
O $2.027115-0.8681101 .883507$

C 3.0718660 .3217510 .093601

C $2.9698101 .180802-1.170167$

O $3.846541-0.832225-0.241023$

C $-3.017088 \quad 1.257324 \quad 0.731508$

C $2.2235732 .487599-0.966313$

H $-2.3362742 .149452-1.190889$

H -1.011709 $3.932347-0.272017$

H -1.421895 $3.392447 \quad 1.458627$

H -3.125476 -0.7965901 .311797$

H $-0.256499-2.1332721 .243459$

H -2.058908 -2.801736 0.009852

H -1.080222 -2.386667 -1.400399

H -1.990421 -0.151750-1.469942

H $\quad 0.495529-0.236063-1.043733$

H $-4.467445-0.372660-1.438272$

H -3.718579 -1.768919-2.228909

H $-4.337817-1.914657-0.576876$

H 1.2126290 .8100761 .069453

H $1.761782-2.562896-0.021160$

H $1.221074-0.9135092 .425181$

H 3.6539440 .8657730 .847970

H $2.5060250 .610271-1.983758$

H $3.9852851 .404151-1.522741$

H $3.903562-1.3833670 .558194$

H -4.0720021 .2924590 .434834$

H -2.9753001 .5721361 .782371$

H $1.1659702 .311683-0.753759$

H $2.6541093 .062917-0.141000$

H $2.2809633 .099061-1.872387$

SCF Energy $($ B3LYP/6-31G**//MMFF $)=-810.714238082$

F2-30_c07204

MMFF Geometry

C $3.0593632 .278608-0.365166$

C 2.4889093 .2671220 .332591

C $2.413227-0.117439-0.762836$

O $1.0046820 .129359-0.588424$

C $0.418968-0.9296470 .181794$

C $1.572695-1.7868830 .693776$

C $2.644518-1.575661-0.358633$

C $-0.466283-0.3736621 .306094$

C $2.457742-2.521744-1.546721$

C -1.5998150 .5711870 .841461$

O $-1.010137-1.4710692 .035653$

O $-1.036877 \begin{array}{lll}1.787175 & 0.352200\end{array}$

C $-2.544653-0.007823-0.231974$

C $-3.6653250 .981144-0.593302$

O $-3.148789-1.2108740 .233432$

C 3.1876160 .8651720 .129869

C $-4.5814310 .445929-1.687318$

H $3.4655822 .501388-1.348378$

H $2.4305284 .268308-0.080153$

H 2.0703963 .0975321 .318387

H $2.6672590 .055533-1.815282$

H $-0.183681-1.539618-0.501466$

H $1.286563-2.8339730 .834774$

H $1.934110-1.4130961 .659619$

H $3.648117-1.7401530 .048884$

H $\quad 0.1763230 .193403 \quad 1.992461$

H $1.483736-2.394166-2.030293$

H $3.230150-2.341309-2.301899$

H $2.541464-3.564961-1.224675$

H -2.2072330 .8197511 .721599$

H -1.527674 -1.106361 2.773562

H -0.4850892 .1630191 .058914$

H -1.985998 -0.231715 -1.148299

H $-3.2403321 .933253-0.930094$

H -4.2771351 .1864660 .293547$

H $-2.441525-1.8607140 .382013$

H $2.8325270 .801579 \quad 1.165648$

H 4.2524800 .6041160 .132371

H $-4.015071 \quad 0.222481-2.596965$

H $-5.095663-0.465883-1.368140$

H $-5.3440591 .190585-1.936826$

SCF Energy $\left(B 3 L Y P / 6-31 G^{* *} / / M M F F\right)=-810.707999303$

F2-30_c07205 
MMFF Geometry

C $3.851906-1.606776-0.205812$

C $3.527808-2.896788-0.348185$

C 2.4780940 .4536020 .180225

O $1.415885-0.233257 \quad 0.865430$

C $0.469478 \quad 0.745272 \quad 1.331200$

C 0.8387732 .0730140 .674753

C $1.8006611 .675588-0.429753$

C $-0.975722 \quad 0.279128 \quad 1.074264$

C $2.7569482 .788273-0.824365$

C $-1.339650-0.019239-0.396480$

O $-1.201724-0.8806951 .878754$

O $-0.511948-1.079090-0.881641$

C -2.810296 -0.416855 -0.650002

C $-3.796316 \quad 0.703732-0.304492$

O $-3.158002-1.580177 \quad 0.098024$

C $3.107166-0.476194-0.857697$

C $-5.2166040 .374171-0.748400$

H $4.709894-1.3495180 .410016$

H $4.109048-3.6671800 .146583$

H $2.682786-3.206116-0.953490$

H 3.2195130 .7537650 .933009

H 0.6153550 .8236822 .416031

H 1.3435932 .7086981 .413528

H -0.0239632 .6380250 .308473$

H $1.2184761 .377394-1.310904$

H -1.643975 1.0574201 .457882

H $3.4240892 .464708-1.629177$

H $2.2027963 .663680-1.178772$

H 3.3774963 .1024650 .021476

H $-1.1171910 .864867-1.004286$

H $-0.617870-1.5829101 .543569$

H $-0.691539-1.182578-1.831591$

H -2.924075 -0.684190 -1.707932

H $-3.483707 \quad 1.638406-0.783947$

H -3.8181140 .8714990 .778170$

H -2.493130 -2.261784 -0.099747

H $2.329322-0.873731-1.521272$

H $3.8161950 .078571-1.482255$

H $\quad-5.2603080 .208785-1.829651$

H -5.592987 $-0.522761-0.246842$

H -5.888872 $1.202983-0.504935$

SCF Energy (B3LYP/6-31G**//MMFF) $=-810.706471655$

F2-30_c07206

MMFF Geometry

C 2.6157152 .0885020 .296708

C 3.5857132 .4904861 .125696

C $2.216296-0.053803-1.023053$

O $0.7817950 .053896-1.001443$

C $0.240997-1.110304-0.351138$

C $1.431378-2.0006030 .013685$

C $2.572858-1.0068680 .113153$

C $-0.595175-0.6842320 .870255$

C $3.947364-1.638775-0.015931$

C -1.7369210 .3198320 .579965$

O $-1.133457-1.8458241 .500561$

O $-1.183773 \quad 1.6090630 .325937$

C -2.684917 -0.069784 -0.571704

C $-3.7979800 .958634-0.838247$

O $-3.266748-1.341750-0.302990$

C $2.8609731 .334243-0.979163$

C $-4.752414 \quad 1.169170 \quad 0.330028$

H $1.5862462 .328240 \quad 0.547675$

H 3.3433043 .0372522 .030364

H 4.6299552 .2817490 .920970

H $2.471065-0.512075-1.988455$

H $-0.391423-1.651078-1.064150$

H $1.617552-2.708541-0.804440$

H $1.289481-2.5861990 .926559$

H $2.501421-0.5028481 .084549$

H $0.075465-0.2074211 .596176$

H $4.732882-0.8797090 .054282$

H $4.111207-2.3693560 .782814$

H $4.063304-2.156594-0.973994$

H $-2.3250490 .396658 \quad 1.502199$

H -1.731405 -2.277005 0.866445

H $-0.663276 \quad 1.551779-0.493549$
H $-2.108185-0.157429-1.499913$

H $-4.3939710 .612189-1.692095$

H $-3.3608311 .921370-1.126409$

H -3.832936 -1.572523 -1.059251

H $3.9377601 .236368-1.162744$

H $2.4513541 .940383-1.797010$

H -4.2436231 .6208131 .186503$

H -5.5610631 .8454150 .033709$

H -5.2050950 .2264940 .651738$

SCF Energy $\left(B 3 L Y P / 6-31 G^{* *} / / M M F F\right)=-810.725246948$

F2-30_c07207

MMFF Geometry

C -2.014419 2.8480770 .079821

C $-1.1105263 .744470-0.329847$

C $-2.1333010 .357310 \quad 0.374897$

$\begin{array}{lllll}\text { O } & -0.751459 & 0.161549 & 0.738795\end{array}$

C $-0.466232-1.2525050 .791611$

C -1.817861 -1.946483 0.637230

C $-2.587549-0.970479-0.225595$

C $0.576989-1.585962-0.293725$

C $-4.090774-1.187950-0.189618$

C $2.027360-1.2458640 .112097$

O $0.569562-2.994382-0.554070$

O $2.881946-1.551237-1.001181$

C 2.3082970 .2042600 .536324

C $2.1468891 .232459-0.591123$

O $3.654134 \quad 0.264837 \quad 1.025024$

C $-2.2602021 .530434-0.598864$

C $2.4174962 .653336-0.110749$

H -2.6269223 .0867920 .945639$

H -0.9869684 .6825340 .200162$

H $-0.4828223 .562292-1.195009$

H -2.6822450 .5654641 .302882$

H $-0.053005-1.4903771 .778907$

H $-2.293547-2.0237501 .624263$

H -1.778477 -2.9572270 .224961$

H -2.235796 -1.058441-1.261895

H $0.340740-1.079363-1.237396$

H $-4.343050-2.186998-0.560137$

H $-4.485542-1.0975540 .827953$

H $-4.607720-0.455767-0.817446$

H $2.348037-1.9187980 .918007$

H -0.165583 -3.174704-1.164167

H $2.718566-2.478503-1.244976$

H $1.6722140 .475657 \quad 1.384014$

H $1.1355291 .186224-1.006988$

H $2.8480381 .017532-1.406164$

H $4.2424150 .000497 \quad 0.296880$

H -1.558661 $1.397923-1.431877$

H $-3.2715161 .558738-1.019839$

H $3.4524802 .770428 \quad 0.225124$

H $2.2482523 .365099-0.924935$

H $1.7550992 .919548 \quad 0.718753$

SCF Energy $\left(B 3 L Y P / 6-31 G^{* *} / / M M F F\right)=-810.716344008$

F2-30 c07208

MMFF Geometry

C $3.5374742 .254750-0.101986$

C 2.8422953 .3725980 .136451

C $2.861534-0.142818-0.445475$

O $1.5719520 .205605-0.987764$

C $0.585556-0.753205-0.554914$

C $1.231765-1.5242170 .586806$

C $2.702046-1.5169280 .210460$

C $-0.722258-0.007658-0.243345$

C $3.041070-2.646463-0.764748$

C $-1.920271-0.947066-0.000845$

$\begin{array}{lllll}0 & -0.533789 & 0.839493 & 0.889288\end{array}$

O $-1.688094-1.752278 \quad 1.157133$

C $-3.290378-0.2577250 .173273$

C $-3.7539020 .460581-1.098751$

$\begin{array}{lllll}\text { O } & -3.256978 & 0.687611 & 1.240372\end{array}$

C 3.2675190 .9374140 .569176

C $-5.1866260 .966931-0.981304$

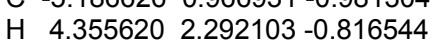

H $3.0902274 .294067-0.378879$

H $2.0192793 .389640 \quad 0.842338$ 
H $3.585296-0.169333-1.268748$

H $0.414823-1.422308-1.407170$

H $\quad 0.817752-2.5302340 .703332$

H $1.104063-0.9998061 .540808$

H $3.342080-1.6339741 .092057$

H $-0.9442760 .651293-1.090262$

H $2.466164-2.580693-1.694009$

H $4.103597-2.614448-1.028745$

H $2.838566-3.621617-0.309674$

H -1.996859-1.641805 -0.845832

H $\quad 0.198407 \quad 1.4440370 .679579$

H -1.464644 -1.155440 1.891450

H -4.032150 -1.016947 0.451126

H -3.688037 -0.217743 -1.956968

H -3.112292 $1.325161-1.303287$

H -3.0296080 .2055592 .052975$

H $2.4864381 .058687 \quad 1.329267$

H 4.1827370 .6308791 .089488

H $-5.2863521 .696936-0.171936$

H $-5.4914281 .453938-1.913006$

H $-5.8790830 .141440-0.788385$

SCF Energy (B3LYP/6-31G**//MMFF) $=-810.713620417$

F2-30_c07209

MMFF Geometry

C $-4.530418-0.837198-0.724046$

C $-4.768672-2.149462-0.620895$

C $-2.4915650 .564123-0.320411$

O $-1.551794-0.484363-0.615992$

C $-0.2129030 .052576-0.525171$

C $-0.347587 \quad 1.4654840 .038649$

C $-1.743101 \quad 1.4889270 .632002$

C $0.632232-0.9214550 .307620$

C -2.3302752 .8865370 .734416$

C $2.128338-0.5764880 .470722$

O $0.080458-1.0069071 .629864$

O $2.740441-1.6782211 .153474$

C $2.884930-0.343757-0.849448$

C $4.418129-0.374952-0.718468$

O $2.5034710 .916421-1.397095$

C $-3.757453-0.040240 \quad 0.288619$

C $4.9818250 .675607 \quad 0.230499$

H $-4.923787-0.302328-1.584711$

H -5.338495 -2.666036 -1.385481

H $-4.402120-2.728648 \quad 0.219278$

H $-2.7206131 .078478-1.263288$

H $\quad 0.170324 \quad 0.088722-1.549642$

H $-0.2723332 .185770-0.786258$

H 0.4253041 .7329030 .764355

H $-1.7052981 .044077 \quad 1.634701$

H $0.539475-1.930921-0.113213$

H - 1.7022523 .5216411 .367863

H $-2.4066083 .362466-0.248899$

H -3.3321472 .8595921 .174004$

H $2.2304730 .298888 \quad 1.122764$

H $-0.846880-1.2855091 .537284$

H $2.263249-1.8000751 .992274$

H $2.596323-1.121690-1.567191$

H $4.858151-0.197849-1.707954$

H $4.751321-1.368661-0.398617$

H $2.9506671 .012502-2.255201$

H -3.490923 -0.676295 1.142120

H -4.4133790 .7532260 .663762$

H 6.0758850 .6328590 .227238

H $4.6851961 .685147-0.070053$

H 4.6469850 .5080891 .258331

SCF Energy (B3LYP/6-31G**//MMFF) $=-810.719030986$

F2-30 c07210

MMFF Geometry

C $4.031697 \quad 1.900047-0.379513$

C 4.5370432 .4865550 .711392

C $2.446992-0.030520-0.781397$

O $1.052183-0.273053-1.057968$

C $0.470010-1.0158200 .024375$

C $1.493901-1.0135781 .153323$

C $2.813933-0.9184170 .412076$

C $-0.882770-0.410600 \quad 0.426954$
C $3.300758-2.295100-0.046041$

C $-1.895119-0.370729-0.738163$

O $-1.403986-1.1544701 .524830$

O $-2.077567-1.703068-1.227976$

C $-3.2831410 .212603-0.401946$

C -3.2184581 .6808560 .030218$

O $-3.914128-0.542566 \quad 0.630345$

C $2.6001671 .474917-0.515486$

C -4.6042582 .2989880 .170729$

H $4.6867541 .727153-1.229430$

H 5.5815782 .7762490 .745524

H 3.9243462 .6852201 .583778

H $3.016734-0.298797-1.679023$

H $0.331184-2.047595-0.321426$

H $1.407934-1.8926471 .799679$

H $1.369133-0.1259771 .785784$

H $3.594732-0.4826861 .043629$

H $-0.7166590 .615536 \quad 0.773693$

H $3.472984-2.9485900 .815535$

H $2.583704-2.790633-0.708346$

H $4.246747-2.201739-0.590164$

H $-1.4642340 .212811-1.560702$

H -1.604928 -2.050036 1.203063

H $-2.651963-1.652062-2.010938$

H $-3.9253580 .123350-1.286943$

H $-2.6443602 .261114-0.701020$

H -2.7153531 .7696360 .999868$

H -3.910893 -1.474211 0.351450

H $2.1549002 .029129-1.352128$

H $2.012301 \quad 1.766610 \quad 0.363944$

H $-5.1525242 .250205-0.775407$

H -5.1937251 .7864180 .937365$

H -4.519586 3.3515870 .459232

SCF Energy (B3LYP/6-31G**//MMFF) $=-810.712996487$

F2-30 c07211

MMFF Geometry

C $-2.5310652 .200136-0.300678$

C -2.0404243 .2521810 .364491$

C $-2.668372-0.2842020 .313036$

O $-1.492574-0.3179861 .141244$

C $-0.760779-1.507840 \quad 0.781341$

C $-1.281550-1.952937-0.588172$

C $-2.176044-0.806102-1.030849$

C $0.758963-1.2843580 .842414$

C $-3.282221-1.226668-1.981383$

C $1.313678-0.121017-0.008164$

O $1.122522-1.0779372 .210668$

$\begin{array}{lllll}\text { O } & 0.817231 & 1.125887 & 0.470491\end{array}$

C $2.854773-0.0561160 .012570$

C $3.3978351 .081671-0.866924$

O $3.393792-1.296590-0.439270$

C $-3.325776 \quad 1.098413 \quad 0.339434$

C $4.9183791 .169249-0.826510$

H -2.370020 2.147246-1.373975

H $-1.4835604 .021794-0.158915$

H -2.179654 3.3634091 .434148

H -3.382019 -0.9955350 .753051$

H $-1.025669-2.2657701 .529887$

H -1.860161 -2.877676 -0.469281

H $-0.488108-2.170022-1.310302$

H -1.540053 -0.061961-1.524896

H $1.240052-2.2196370 .534157$

H -2.861208 -1.669225 -2.890182

H -3.945355 -1.967421 -1.522272

H $-3.890735-0.366072-2.276368$

H $\quad 0.980838-0.244635-1.045059$

H $0.704176-0.2484362 .499544$

H -0.1521581 .0777960 .464483$

H $3.2043050 .105071 \quad 1.039185$

H $2.9831702 .043294-0.544599$

H $3.0924390 .923156-1.908355$

H $3.092604-1.436069-1.353374$

H $-4.2946621 .045381-0.171643$

H -3.5354401 .3606181 .384534$

H $\quad \begin{array}{llll}5.273924 & 1.326647 & 0.196702\end{array}$

H $5.3817270 .257797-1.216734$

H $5.2634272 .008808-1.438434$ 
F2-30_c07212

MMFF Geometry

C $3.690562-1.5358380 .125055$

C $4.680027-1.651074-0.767911$

C $2.3779520 .462587 \quad 1.006636$

$\begin{array}{lllll}\text { O } & 1.170029 & -0.288914 & 1.210874\end{array}$

C $0.085205 \quad 0.421814 \quad 0.570487$

C $0.720597 \quad 1.541135-0.257477$

C $2.1842351 .146139-0.341006$

C $-0.756856-0.594020-0.216621$

C $3.1188172 .318113-0.583649$

C $-2.031945-0.023193-0.859136$

O $0.039345-1.144126-1.274828$

O $-2.737310-1.117140-1.458493$

C $-2.967117 \quad 0.7415880 .094485$

C $-3.457595-0.0154241 .339213$

O $-4.092767 \quad 1.193833-0.663616$

C $3.606155-0.4398941 .148816$

C $-4.323921-1.2375201 .068930$

H $2.902659-2.2843940 .127761$

H $4.686716-2.471036-1.477518$

H $5.493143-0.934950-0.809320$

H $2.4165521 .213747 \quad 1.807791$

H -0.5150800 .8554161 .376722$

H $0.6104462 .490063 \quad 0.283012$

H $0.2649121 .682992-1.241443$

H $2.2948660 .427248-1.161403$

H -1.013238 -1.4431330 .428380$

H $4.159172 \quad 1.982208-0.638224$

H $2.8750632 .815381-1.528167$

H 3.0461833 .0620630 .216804

H $-1.7641330 .630605-1.698061$

H $0.817282-1.558357-0.864227$

H $-3.541980-0.753268-1.865990$

H $-2.448781 \quad 1.646496 \quad 0.432307$

H -4.0604950 .6785531 .939274$

H -2.607879 -0.3091301 .964658$

H $-4.6536951 .721871-0.070238$

H $4.5119420 .177691 \quad 1.122947$

H $3.577903-0.9213232 .134460$

H $-5.154397-1.003247 \quad 0.396427$

H $-3.739729-2.0545780 .637141$

H -4.748495-1.606609 2.008480

SCF Energy (B3LYP/6-31G**//MMFF) $=-810.712203367$

F2-30_c07213

MMFF Geometry

C $1.8297452 .402090 \quad 0.405991$

C $0.765154 \quad 3.179406 \quad 0.177663$

C $2.535527 \quad 0.284029-0.826571$

O $1.191208-0.044801-1.224050$

C $0.805596-1.278431-0.593044$

C $2.059594-1.8236090 .089128$

C $2.833930-0.5593120 .410448$

C $-0.349193-1.003507 \quad 0.388957$

C $4.314595-0.7939950 .653359$

C $-1.558996-0.284818-0.254232$

O $-0.773916-2.2415380 .958984$

O $-2.028302-1.054774-1.358560$

C -2.725513 -0.0677010 .731836$

$\begin{array}{llll}\text { C }-3.833021 & 0.864530 & 0.212697\end{array}$

O $-2.227372 \quad 0.508967 \quad 1.941645$

C $2.6810191 .801377-0.677700$

C $-4.6289020 .328924-0.964909$

H $2.1127212 .203647 \quad 1.436159$

H 0.1961253 .5885301 .005288

H $0.4406523 .415832-0.829565$

H $3.181080-0.035716-1.655599$

H $0.482229-1.986888-1.364095$

H $2.622646-2.438293-0.624918$

H $1.855473-2.441408 \quad 0.968364$

H $2.397041-0.1104451 .309694$

H $0.021932-0.3798531 .209860$

H $4.462914-1.4615591 .508569$

H $4.797533-1.251065-0.216882$

H $\quad 4.8276150 .1489450 .867425$
H -1.233390 $0.691029-0.634302$

H $-1.157497-2.7772530 .243429$

H $-1.380257-0.965538-2.077488$

H -3.178851 -1.0315100 .991931$

H -3.405813 $1.841301-0.047495$

H -4.5286011 .0648861 .038305$

H -1.8145041 .3602651 .716294$

H $3.7280322 .053018-0.471993$

H $2.4271092 .270117-1.637261$

H $-5.5011640 .965639-1.145957$

H $-4.0362090 .326607-1.883493$

H $-4.986973-0.687013-0.772167$

SCF Energy (B3LYP/6-31G**//MMFF)= -810.716638496

F2-30_c07214

MMFF Geometry

C 2.3484821 .8848451 .230258

C 1.4453802 .6284461 .879237

C $2.1039720 .479626-0.896179$

$\begin{array}{lllll}\text { O } & 0.710933 & 0.220208 & -0.642592\end{array}$

C $0.561028-1.123706-0.171983$

C $1.893678-1.4575230 .486316$

C $2.900144-0.727665-0.385738$

C $-0.638787-1.2487940 .773191$

C $3.378160-1.609618-1.541631$

C $-2.017032-1.143207 \quad 0.089551$

O $-0.567256-2.5366441 .399148$

O $-3.004319-1.2244471 .129423$

C $-2.2806530 .124508-0.741528$

C $-2.2820591 .416643 \quad 0.085237$

O $-3.567147-0.003309-1.358498$

C $2.4733581 .831201-0.266585$

C $-2.4159592 .655901-0.791625$

H 3.0481821 .2939091 .814993

H 1.4112442 .6291632 .963200

H 0.7270053 .2419951 .346868

H $2.2150200 .585237-1.982523$

H $0.413940-1.774844-1.043126$

H $2.071210-2.5349540 .560859$

H $1.928797-1.0442321 .501554$

H $3.786603-0.4328250 .186243$

H $-0.565541-0.5030721 .573856$

H $4.083578-1.060662-2.174479$

H $3.891745-2.498614-1.160630$

H $2.549441-1.943609-2.174467$

H $-2.176382-2.031274-0.535134$

H $-1.323014-2.6044752 .007732$

H $-3.877334-1.1718460 .703719$

H $-1.5569970 .199773-1.558665$

H -1.3589951 .5038370 .666104$

H $-3.119847 \quad 1.4119620 .792628$

H $-3.542450-0.792811-1.925801$

H $3.5068622 .083104-0.532942$

H $1.8345452 .605718-0.710911$

H -1.591304 $2.717262-1.508944$

H $-3.3582122 .652047-1.348075$

H -2.394056 $3.558816-0.173052$

SCF Energy (B3LYP/6-31G**//MMFF) $=-810.717627393$

F2-30_c07215

MMFF Geometry

C $-4.276610-0.662358-1.150599$

C $-4.530691-1.972704-1.239347$

C $-2.301703 \quad 0.639817-0.321667$

O $-1.336128-0.384146-0.620032$

C $-0.0176750 .109065-0.291120$

C $-0.2184331 .447717 \quad 0.416020$

C $-1.679161 \quad 1.4289050 .823820$

C $0.702072-0.9735890 .521986$

C -2.2675812 .8162091 .018053$

C $2.160823-0.6817990 .924728$

O $-0.023129-1.1950151 .742180$

O $2.656009-1.885298 \quad 1.546738$

C $3.165505-0.351541-0.194849$

C $3.2134001 .134967-0.565214$

O $2.882337-1.121193-1.361314$

C $-3.638102-0.0079870 .041967$

C $4.3664481 .448750-1.512656$ 
H $-4.553230-0.020296-1.982950$

H -4.998525 -2.382568 -2.127763

H $-4.277799-2.655672-0.436037$

H -2.404273 $1.268096-1.216658$

H $0.497257 \quad 0.254621-1.244745$

H $-0.0353682 .259293-0.300065$

H $0.456106 \quad 1.611772 \quad 1.260902$

H -1.7746330 .8673561 .761931$

H $0.653188-1.927588-0.017980$

H -2.2125613 .4077240 .098094$

H -3.3182942 .7578361 .318202$

H -1.7244133 .3579741 .799388$

H $2.2061090 .083758 \quad 1.707587$

H $-0.930334-1.4452841 .496231$

H $2.059052-2.0940732 .285792$

H $4.169599-0.6487580 .135061$

H 3.3273091 .7418010 .340078

H $2.2932281 .448331-1.063197$

H $2.908198-2.058665-1.103578$

H $-3.487142 \quad-0.7468880 .839027$

H -4.3338460 .7477640 .423487$

H $5.3277041 .181505-1.062414$

H $4.2645380 .905254-2.457196$

H $4.3856742 .519200-1.740506$

SCF Energy (B3LYP/6-31G $\left.{ }^{* *} / / M M F F\right)=-810.717794764$

F2-30 c07216

MMFF Geometry

C $4.1189711 .887657-0.456717$

C 4.7448692 .3728630 .621244

C $2.4507190 .027488-0.853074$

O $1.029038-0.165987-1.002058$

C $0.540979-0.9850150 .071494$

C $1.674870-1.0977631 .083649$

C $2.913926-0.9646320 .218778$

C $-0.743798-0.3880550 .661657$

C $3.313927-2.306306-0.399311$

C $-1.874715-0.218936-0.377161$

O $-1.188730-1.2167651 .734120$

O $-2.097238-1.479257-1.009935$

C $-3.1984410 .287156 \quad 0.233141$

C $-4.2728070 .534338-0.837701$

O $\quad-2.972749 \begin{array}{lll}1.512374 & 0.926545\end{array}$

C $2.6701281 .501994-0.481547$

C $-5.5847551 .030852-0.241503$

H $4.682031 \quad 1.773021-1.379308$

H 5.7950562 .6392370 .573361

H 4.2267062 .5106091 .563709

H $2.918525-0.173960-1.824099$

H $\quad 0.338656-1.981632-0.339881$

H $1.631536-2.0265741 .661024$

H $1.640187-0.2648751 .796745$

H $3.766857-0.5982670 .798808$

H $-0.511530 \quad 0.597374 \quad 1.082199$

H $2.519862-2.730523-1.022279$

H $4.201766-2.184907-1.029141$

H $3.555353-3.0336090 .382986$

H -1.529853 $0.488141-1.141988$

H -1.430483 -2.079966 1.356514

H -2.570107 -1.313341 -1.842558

H $-3.592650-0.4471010 .946496$

H -4.474695 -0.385499-1.396615

H -3.916768 $1.286156-1.552527$

H -2.506532 1.3013631 .753040

H $2.1589082 .132694-1.220490$

H $2.180679 \quad 1.7294410 .473846$

H -5.9794950 .3143050 .485895$

H -5.4578581 .9961110 .258381$

H -6.331963 1.159047-1.031195

SCF Energy (B3LYP/6-31G**//MMFF) $=-810.719479693$

F2-30_c07217

MMFF Geometry

C $4.1354371 .906673-0.213621$

C 3.8697283 .1897010 .055970

C $2.705404-0.149679-0.305614$

O $1.4951240 .544398-0.651443$

C $0.452620-0.428556-0.891761$
C $1.005729-1.774172-0.420112$

C $2.222449-1.4045450 .408003$

C $-0.8259620 .079915-0.199066$

C $3.255948-2.5154270 .487998$

C $-2.115924-0.576707-0.735299$

O $-0.719047-0.1108661 .209660$

O $-2.045950-1.992117-0.557091$

C $-3.433323-0.081400-0.100668$

C $-3.6876751 .409542-0.344434$

O $-3.441050-0.3240501 .304920$

C 3.5797660 .7500410 .568389

C -5.0773961 .8363720 .112808$

H $4.8007421 .675201-1.041485$

H $4.3070843 .979285-0.545209$

H 3.2156223 .4743800 .872687

H $3.221659-0.405674-1.240605$

H $0.288891-0.449042-1.975673$

H $1.302826-2.362866-1.297550$

H $\quad 0.295904-2.3837140 .144335$

H $1.889573-1.152813 \quad 1.423379$

H $-0.894258 \quad 1.162119-0.360442$

H $3.632827-2.784484-0.504381$

H $4.110140-2.2132081 .101783$

H $2.820447-3.4139830 .937641$

H -2.164464 -0.399325 -1.816977

H $\quad 0.0620330 .384042 \quad 1.510885$

H -2.830466 -2.382423 -0.978475

H $-4.264489-0.662698-0.518860$

H -3.580095 $1.637959-1.410955$

H -2.9575302 .0145990 .204921$

H $-3.216395-1.2606851 .439091$

H 2.9958551 .1197091 .420716

H 4.4243690 .1807300 .972232

H $-5.8535001 .272291-0.414301$

H -5.2083991 .6810801 .188184$

$\mathrm{H}-5.2317842 .900047-0.094864$

SCF Energy $(B 3 L Y P / 6-31 G * * / / M M F F)=-810.711259939$

F2-31 c07418

MMFF Geometry

C $3.7727341 .943470-0.185921$

C $3.1445633 .102721-0.412294$

C $2.941150-0.3582110 .388471$

$\begin{array}{lllll}\text { O } & 1.791616 & 0.177661 & 1.080052\end{array}$

C $0.640785-0.6540740 .819695$

C $1.036992-1.576107-0.322182$

C $2.519268-1.758051-0.067866$

C -0.5661730 .2459520 .530029$

C $3.272291-2.352461-1.244412$

C $-1.830305-0.5257460 .103708$

O $-\begin{array}{llll}0.861444 & 0.983025 & 1.721374\end{array}$

O $-2.124047-1.5329111 .071301$

C $-3.0653800 .377791-0.081854$

C $-4.287875-0.413606-0.572092$

O $-2.7647991 .411896-1.015992$

C $3.3181090 .619354-0.735238$

C $-5.5226870 .465566-0.724897$

H $4.672206 \quad 1.9480570 .424228$

H $3.525746 \quad 4.025878 \quad 0.010342$

H $2.2447903 .152370-1.015689$

H $3.765413-0.431301 \quad 1.108040$

H $0.475871-1.2305311 .738698$

H $0.868456-1.095412-1.293521$

H $\quad 0.481917-2.518984-0.309961$

H $2.632529-2.4387370 .787879$

H $-0.3008390 .984297-0.236115$

H $2.931317-3.375378-1.436351$

H $3.116610-1.779281-2.163856$

H $4.346898-2.388804-1.038290$

H -1.622105 -1.036229 -0.843770

H -0.0540921 .4646441 .971116$

H -2.276586 -1.086109 1.921739

H $-3.3248190 .854214 \quad 0.870819$

H $-4.519314-1.2255750 .126106$

H $-4.069532-0.867734-1.546179$

H $-2.5472270 .991219-1.865374$

H $4.1418020 .213296-1.332538$

H $2.4651030 .769609-1.408123$ 
H $-5.3656991 .248217-1.473579$

H $-6.377525-0.138301-1.045436$

H $-5.782357 \quad 0.9436160 .225054$

SCF Energy $\left(B 3 L Y P / 6-31 G^{* *} / / M M F F\right)=-810.718263788$

\section{F2-31 c07419}

MMFF Geometry

C -3.078335 $1.935958-1.040414$

C $-2.6693903 .075285-0.470847$

C $-2.245736-0.432497-0.906634$

O $-\begin{array}{llll}0.886699 & 0.016034 & -0.703382\end{array}$

C $-0.215512-0.8843620 .195549$

C - $-1.310242-1.7040370 .864199$

C $-2.319720-1.820727-0.260652$

C $0.686049-0.1065021 .159790$

C $-3.688108-2.296447 \quad 0.193369$

C 1.6997740 .8321160 .477798

O $-\begin{array}{llll}0.128377 & 0.701791 & 2.020199\end{array}$

O 2.5551081 .3732461 .494911

C $2.5876400 .179242-0.595573$

C $3.509338-0.924864-0.059818$

O $3.3874541 .203999-1.195042$

C $-3.1891480 .624314-0.313280$

C $4.323876-1.580135-1.168885$

H $-3.3579421 .948861-2.090654$

H $-2.6148443 .990366-1.050323$

H -2.3817083 .1163720 .573890$

H -2.415157 -0.514485-1.987062

H $0.393623-1.553436-0.423988$

H -1.751433 -1.179668 1.719153

H $-0.944142-2.6734401 .216252$

H $-1.931411-2.549229-0.986725$

H $1.208517-0.8070251 .820739$

H $-3.626466-3.3198820 .578680$

H -4.095774 -1.670827 0.993854

H $-4.396301-2.293223-0.641388$

H 1.1835631 .6953900 .039468

H -0.6430841 .3041951 .455990$

H $1.983267 \quad 1.7920682 .161043$

H $1.961322-0.221913-1.398796$

H $2.921615-1.6996880 .443209$

H $4.211161-0.5110010 .673840$

H $3.9338431 .597471-0.493056$

H $-4.2299500 .292199-0.394223$

H $-2.971913 \quad 0.769480 \quad 0.751842$

H $4.986308-0.858914-1.657490$

H $4.945539-2.381044-0.756297$

H $3.668565-2.017256-1.929056$

SCF Energy $($ B3LYP/6-31G**//MMFF $)=-810.720596043$

F2-31 c07420

MMFF Geometry

C $-3.3553312 .257851-0.178615$

C $-2.5667383 .335751-0.099694$

C $-2.860196-0.197917-0.385335$

O $-1.6343090 .061231-1.103899$

C $-0.618511-0.872577-0.678454$

C $-1.165787-1.5522100 .566496$

C $-2.653798-1.560326 \quad 0.282455$

C $0.698089-0.111949-0.472557$

C $-3.506743-1.8634451 .501156$

C $1.827306-0.9801630 .116239$

O $1.1062490 .401002-1.743232$

O $2.005947-2.142017-0.699289$

C $3.200667-0.297156 \quad 0.280542$

C 3.1581040 .9054101 .229853

O $3.7222920 .107129-0.984230$

C -3.1079680 .9781310 .571614$

C $4.548931 \quad 1.455367 \quad 1.521077$

H $-4.2359082 .300975-0.814284$

H $-2.8023854 .231189-0.664127$

H -1.677955 3.3442480 .521353

H -3.675024 -0.258708 -1.116848

H $-0.517303-1.595866-1.497402$

H $-0.947895-0.9629751 .465427$

H $-0.752846-2.5552870 .710746$

H -2.847998 -2.336648 -0.471105

H $\quad 0.5194530 .7559530 .172463$
H $-4.571152-1.7779001 .260152$

H -3.321422 -2.884908 1.850373

H $-3.286558-1.1891842 .334846$

H $1.516094-1.3422831 .102765$

H $\quad 0.373840 \quad 0.941082-2.087078$

H $2.291362-1.837360-1.578071$

H $3.905402-1.0422480 .669048$

H 2.6845550 .6176942 .175352

H $2.564615 \quad 1.715726 \quad 0.791248$

H $3.1320580 .789764-1.346169$

H $-3.9914630 .785921 \quad 1.190348$

H -2.2529971 .1034221 .247111$

H 5.0349821 .8130160 .608086

H 4.4828572 .2962272 .218743

H 5.1861080 .6887961 .973562

SCF Energy $(B 3 L Y P / 6-31 G * * / / M M F F)=-810.723204148$

F2-31 c07421

MMFF Geometry

C -2.7049422 .5145590 .021629$

C -1.8084773 .2708960 .665067$

C $-2.6879920 .112758-0.718469$

O $-1.2549440 .137135-0.899163$

C $-0.698361-1.131164-0.514781$

C $-1.792999-1.8516530 .260445$

C $-3.038128-1.353688-0.445116$

C $0.611746-0.9554910 .266969$

C $-4.322582-1.6228860 .317996$

C $1.734012-0.262985-0.538579$

O $0.363158-0.2136631 .461573$

O $1.3472281 .061051-0.901426$

C $3.060056-0.1754110 .243534$

C $4.1813680 .466244-0.589302$

O $3.464699-1.4804790 .651276$

C $-3.041678 \quad 1.099630 \quad 0.404123$

C 5.4855250 .5847200 .189477

H -3.234035 $2.941003-0.826767$

H -1.6131234 .2870680 .340458$

H -1.255699 $2.893917 \quad 1.518314$

H $-3.1520490 .438600-1.657207$

H $-0.492020-1.678272-1.443544$

H -1.805810 -1.555228 1.315521

H -1.681511 -2.9394550 .218614$

H -3.104399 -1.868973 -1.414018

H $0.953158-1.9499160 .576443$

H $-4.499145-2.7010830 .396837$

H -4.286822 -1.223055 1.336439

H $-5.179946-1.178193-0.197100$

H $1.900399-0.831846-1.461922$

H 0.0663360 .6734281 .194902

H $0.5509830 .996105-1.455104$

H 2.9161530 .4268251 .148474

H $3.8820401 .465208-0.925180$

H $4.371670-0.139375-1.483718$

H $3.604838-2.012199-0.150719$

H $-4.115972 \quad 1.0646110 .616267$

H $-2.514757 \quad 0.8282451 .326867$

H $\quad 5.868717-0.3985810 .479291$

H $6.247918 \quad 1.073529-0.425317$

H 5.3466651 .1820911 .096127

SCF Energy (B3LYP/6-31G**//MMFF) $=-810.723888258$

F2-31_c07422

MMFF Geometry

C $3.8524711 .692385-0.445820$

C $3.3152612 .870025-0.783476$

C $2.853031-0.4506100 .407341$

O $1.7780350 .255677 \quad 1.064700$

C $0.553573-0.4978350 .932520$

C $0.829857-1.570455-0.108711$

C $2.300133-1.8508200 .123667$

C -0.5858050 .4658260 .578385$

C $2.957439-2.634296-0.998269$

C $-1.925079-0.2371440 .271867$

O -0.7716851 .3521151 .687434$

O $-2.251950-1.0940031 .364110$

C -3.0785820 .7544020 .023120$

C $-4.4398340 .097233-0.259637$ 
O $-2.728858 \quad 1.601237-1.070645$

C $3.2683110 .360686-0.829289$

C $-4.457516-0.805739-1.486204$

H 4.7718351 .6852850 .134083

H $3.7882553 .797211-0.478580$

H $2.4005552 .931397-1.362673$

H $3.694024-0.5148931 .108148$

H $0.377073-0.9533841 .915062$

H $0.666107-1.186643-1.122922$

H $0.199330-2.4548090 .024880$

H $2.388326-2.4402101 .047411$

H $-0.2882391 .090465-0.272515$

H $4.032081-2.740245-0.819187$

H $\quad 2.526030-3.638822-1.063665$

H $2.815564-2.154992-1.972182$

H $-1.776744-0.860877-0.616802$

H $\quad 0.083519 \quad 1.7819341 .860717$

H $-2.956333-1.6949541 .069314$

H $-3.206394 \quad 1.3907340 .907267$

H -5.181483 $0.889401-0.423081$

H $-4.774551-0.4718590 .614810$

H -3.443614 2.251415 - 1.179090

H $4.032272-0.178918-1.399650$

H $2.4064610 .509921-1.490874$

H $-5.474957-1.166456-1.669241$

H $-4.125373-0.270887-2.381249$

H -3.817641-1.681975 -1.347748

SCF Energy (B3LYP/6-31G**//MMFF) $=-810.710416504$

F2-31_c07423

MMFF Geometry

C -3.177886 2.205451 -0.193663

C -2.524333 2.869620-1.154006

C $-2.269017-0.151645-0.810213$

O $-0.8994760 .218140-0.534658$

C $-0.214351-0.8918240 .071148$

C $-1.300727-1.8488130 .539690$

C $-2.343565-1.661375-0.544286$

C $0.731413-0.4016451 .172183$

C $-3.703490-2.235007-0.188216$

C 1.7378730 .6755540 .723107

$\begin{array}{lllll}\text { O } & -0.040673 & 0.158491 & 2.243325\end{array}$

$\begin{array}{lllll}\text { O } & 2.636586 & 0.922781 & 1.813776\end{array}$

C $2.5749440 .320902-0.519060$

C $3.497130-0.888862-0.320294$

O $3.387506 \quad 1.450211-0.855249$

C $-3.185308 \quad 0.720058 \quad 0.065791$

C $4.252069-1.251067-1.593895$

H -3.7872492 .7938030 .490287$

H -2.612264 $3.948963-1.224326$

H $-1.8985462 .373154-1.885456$

H -2.471759 $0.042928-1.869888$

H $0.362720-1.379328-0.723610$

H -1.708977 -1.5611101 .514942$

H $-0.939652-2.8791050 .616078$

H $-1.985745-2.177172-1.446959$

H $1.263356-1.2551131 .607141$

H $-3.642520-3.324158-0.089653$

H $-4.078710-1.8426560 .762268$

H -4.436371 -2.008387 -0.969176

H 1.2193701 .6267770 .549391

H $-0.5649990 .890610 \quad 1.875326$

H 2.0954801 .1547312 .587956

H $1.9181520 .145129-1.377658$

H $2.917817-1.761653-0.001801$

H $4.237094-0.6768480 .460613$

H $2.7919062 .199992-1.024517$

H $-4.2241110 .390342-0.049930$

H -2.914675 0.5828331 .119991

H $4.875836-2.134198-1.423180$

H $3.557376-1.479271-2.408569$

H $4.906426-0.435330-1.916542$

SCF Energy (B3LYP/6-31G**//MMFF) $=-810.712595501$

F2-31_c07424

MMFF Geometry

C 2.9514452 .2712690 .023536

C $2.1124993 .171382-0.501512$
C $2.679578-0.1484180 .647140$

$\begin{array}{lllll}\text { O } & 1.282081 & 0.047525 & 0.955667\end{array}$

C $0.532863-1.1132280 .558151$

C $1.456482-1.920635-0.343698$

C $2.809282-1.6287200 .273433$

C $-0.801261-0.721733-0.096757$

C $3.981008-2.014207-0.611488$

C -1.7450500 .0560390 .847362$

O $-0.544636 \quad 0.044019-1.272627$

O -1.1715251 .3206541 .191922$

$\begin{array}{llll}\text { C } & -3.151562 & 0.347798 & 0.285307\end{array}$

C $-3.968879-0.924066 \quad 0.036942$

O $-\begin{array}{llll}-3.067503 & 1.082792 & -0.934257\end{array}$

C $3.0672930 .849782-0.453926$

C $-5.416482-0.613335-0.324682$

H 3.5993552 .5767280 .841008

H $2.0794154 .183436-0.113192$

H $1.4473422 .918207-1.319543$

H $3.2580320 .059807 \quad 1.555317$

H $\quad 0.330382-1.6848151 .472917$

H $1.423931-1.566468-1.380387$

H $1.205354-2.985729-0.344801$

H $2.884313-2.204887 \quad 1.206633$

H $-1.294292-1.645056-0.418348$

H $4.005928-3.099382-0.757974$

H $3.916721-1.553037-1.602224$

H $4.928471-1.714692-0.152409$

H $-1.860865-0.506823 \quad 1.781804$

H $-0.1213200 .873352-0.991625$

H $-0.321573 \quad 1.1502651 .631618$

H -3.6862780 .9887400 .997584$

H $-3.955800-1.5578940 .930859$

H $-3.536935-1.499001-0.789891$

H $-2.513648 \quad 1.863753-0.763665$

H $4.1068730 .691306-0.761330$

H $2.4362940 .701888-1.338516$

H $-5.480336-0.029532-1.248158$

H $-5.974877-1.542730-0.474908$

H $-5.907130-0.0489270 .474741$

SCF Energy (B3LYP/6-31G**//MMFF) $=-810.720143380$

F2-31 c07425

MMFF Geometry

C $4.301868-1.023080-0.029583$

C $4.245122-2.171300 \quad 0.654580$

C $2.5343810 .589620-0.800542$

O $1.555451-0.466116-0.920022$

C $0.2565150 .031136-0.527454$

C $0.519347 \quad 1.344073 \quad 0.191086$

C $1.7239371 .867749-0.564267$

C $-0.452632-1.048354 \quad 0.300545$

C $2.433733 \quad 3.0124890 .136226$

C $-1.828852-0.6597240 .880059$

O $-0.622889-2.209063-0.519273$

O

C -2.822702 $-0.055710-0.133981$

C -4.1970870 .2118160 .501886$

O $-2.985882-0.926672-1.248944$

$\begin{array}{llll}\text { C } 3.509823 & 0.206019 & 0.322548\end{array}$

C $-5.1729160 .853592-0.476656$

H $4.963883-0.960323-0.889321$

H $4.845703-3.0217910 .351086$

H $3.602598-2.2845231 .520759$

H $3.0741000 .659757-1.752663$

H $-0.2842810 .207466-1.465226$

H $0.775248 \quad 1.1735201 .243474$

H $-0.335122 \quad 2.025847 \quad 0.152802$

H $1.3764032 .230918-1.541911$

H $0.208778-1.349497 \quad 1.123087$

H 1.7793553 .8891630 .190224

H 2.7174342 .7540301 .161448

H $3.3376043 .300347-0.409946$

H -2.283286 -1.5701631 .293137$

H $0.252165-2.446152-0.871713$

H $-1.085130-0.1684132 .618631$

H -2.435010 $0.897348-0.510428$

H $-4.0920810 .868266 \quad 1.372866$

H -4.634277 -0.7317490 .850167$ 
H $-3.308166-1.780906-0.914021$ H $4.225783 \quad 1.0163470 .498301$ H 2.9622980 .0399551 .258406 H $-5.3729540 .197761-1.329640$ H $-6.126657 \quad 1.055998 \quad 0.021117$ H $-4.7798191 .802437-0.855227$

SCF Energy (B3LYP/6-31G*//MMFF) $=-810.710413999$

F2-31_c07426

MMFF Geometry

C $3.398373-1.968902-0.137300$

C $4.622683-2.503365-0.067615$

C $2.6050760 .396616-0.470158$

O $1.418550-0.045523-1.165735$

C $0.3004720 .788459-0.792222$

C 0.7589891 .5875880 .416726

C $2.235936 \quad 1.7579150 .127539$

C $-0.924667-0.103372-0.549371$

C 3.0420112 .2263271 .325646

C $-2.1458710 .660465-0.000620$

O $-1.264195-0.722641-1.792459$

O $-2.442715 \quad 1.746291-0.885284$

C -3.427852 -0.1756040 .196809$

C $-3.277402-1.3734961 .142672$

O $-3.889584-0.655632-1.067364$

C $2.998796-0.688570 \quad 0.544322$

C -2.823287 -0.9983472 .543359$

H $2.635501-2.491136-0.709022$

H $4.845455-3.434107-0.577957$

H $5.419270-2.0272240 .493333$

H $3.4056080 .517405-1.209962$

H $\quad 0.125472 \quad 1.452777-1.647865$

H 0.6105951 .0200511 .343468

H $\quad 0.2296742 .5403380 .513503$

H $2.3384172 .511481-0.666386$

H $-0.651633-0.9125890 .137533$

H $4.1100902 .253722 \quad 1.087725$

H 2.7361823 .2366421 .617903

H 2.8983621 .5784882 .196300

H -1.8763611 .1134930 .959719$

H - $0.473534-1.192668-2.108805$

H $-3.1835142 .245172-0.500964$

H -4.2165130 .4867830 .575102$

H -2.592128 -2.1141810 .713862$

H $-4.244615-1.8881021 .212267$

H $-4.727832-1.125027-0.917127$

H $3.824940-0.3363241 .171815$

H $2.155091-0.9170821 .206332$

H -1.800329 -0.6105422 .543959$

H -3.482321 -0.2419492 .980676$

H -2.841870 -1.879663 3.192607

SCF Energy (B3LYP/6-31G*//MMFF) $=-810.698542811$

\section{F2-31 c07427}

MMFF Geometry

C $-2.6577512 .533064 \quad 0.062972$

C $-1.725468 \quad 3.2502050 .700573$

C $-2.7009410 .146850-0.726207$

O $-1.2709840 .147826-0.927824$

C $-0.726849-1.125962-0.542646$

C $-1.832586-1.8432730 .221263$

C $-3.071623-1.317655-0.474686$

C $0.578499-0.966712 \quad 0.252242$

C $-4.358519-1.578576 \quad 0.287185$

C $1.712126-0.299674-0.560013$

O $0.311776-0.201497 \quad 1.427791$

O $1.3142090 .999809-0.993164$

C $3.041616-0.178153 \quad 0.211843$

C $4.1628310 .377063-0.681928$

O $3.408614-1.4669430 .695684$

C $-3.019943 \quad 1.117797 \quad 0.420538$

C $5.472460 \quad 0.5826910 .068832$

H $-3.1974152 .992965-0.760803$

H $-1.512276 \quad 4.268787 \quad 0.395675$

H $-1.1599482 .837158 \quad 1.528524$

H -3.173001 $0.499017-1.651230$

H $-0.518356-1.672256-1.471409$

H $-1.840256-1.5653391 .281409$
H $-1.735800-2.9317610 .161779$

H $-3.146629-1.817622-1.450722$

H $0.897869-1.9647390 .575811$

H $-5.209962-1.113068-0.219485$

H $-4.551288-2.6548190 .350459$

H $-4.314780-1.1946801 .311431$

H $1.879036-0.905903-1.459043$

H $1.070154-0.305346 \quad 2.026517$

H $1.0622751 .507863-0.202875$

H $2.9114860 .491987 \quad 1.070172$

H $3.8593421 .336672-1.115314$

H $4.350825-0.315239-1.511594$

H $4.124549-1.3489361 .342291$

H $-4.091958 \quad 1.100546 \quad 0.645983$

H -2.4877260 .8172551 .331005$

H $5.885863-0.3666850 .422157$

H $6.215097 \quad 1.042449-0.591244$

H $5.330861 \quad 1.242144 \quad 0.930807$

SCF Energy (B3LYP/6-31G**//MMFF) $=-810.712103972$

F2-31 c07428

MMFF Geometry

C -3.688542 2.0969800 .298693

C $-3.020006 \quad 3.163878 \quad 0.750905$

C $-2.939552-0.154555-0.531245$

O $-1.6973420 .389449-1.028791$

C $-0.628654-0.553000-0.794628$

C $-1.189786-1.5950430 .159619$

C $-2.647741-1.631549-0.250205$

C $0.5988610 .208425-0.275928$

C $-3.547210-2.3300530 .753625$

C $1.762848-0.7096710 .140637$

O $1.0239121 .093106-1.312887$

O $2.130212-1.538424-0.962591$

C 3.0031390 .0320390 .685353

C $3.9249600 .649936-0.375154$

O $3.787004-0.9185351 .419837$

C $-3.369902 \quad 0.6788750 .685297$

C $5.1019881 .392727 \quad 0.249603$

H $-4.5197812 .252934-0.384002$

H -3.3020734 .1627890 .436306$

H -2.184906 3.0622461 .434999

H $-3.690139-0.065660-1.325756$

H $-0.411104-1.005390-1.770376$

H $-1.090232-1.268854 \quad 1.201944$

H $-0.693265-2.5648500 .058554$

H -2.715512 - $2.174580-1.203612$

H 0.3103260 .8386930 .574189

H $-4.596239-2.2591150 .449232$

H -3.287531 -3.3919010 .822378$

H $-3.449884-1.9053411 .757873$

H $1.401311-1.3874240 .923244$

H $0.2652661 .656195-1.544679$

H $2.846960-2.122351-0.660834$

H 2.6874040 .8017971 .399409

H $3.3794221 .355063-1.006439$

H $4.332485-0.132840-1.025753$

H $3.247104-1.2296362 .166353$

H $-4.2720120 .253207 \quad 1.138445$

H $-2.5817360 .670051 \quad 1.447838$

H 4.7530592 .1911560 .912105

H 5.7406820 .7180350 .827836

H $5.7176981 .847082-0.533233$

SCF Energy (B3LYP/6-31G**//MMFF) $=-810.711964449$

F2-31_c07429

MMFF Geometry

C $3.863972-1.5078521 .130799$

C $4.185925-2.3195470 .117023$

C $2.813795 \quad 0.368291-0.331835$

O $1.759068-0.485757-0.826831$

C $0.5269220 .261797-0.908521$

C $0.760387 \quad 1.535903-0.113765$

C $2.232213 \quad 1.786546-0.368557$

C $-0.624453-0.614406-0.400363$

C 2.8475852 .8102650 .568591

C $-1.971788 \quad 0.132629-0.300631$

O $-0.746360-1.709382-1.314029$ 
O $-2.2698450 .750690-1.551599$

C $-3.152795-0.7644110 .114642$

C $-4.497323-0.0233820 .212046$

O $-2.852124-1.3788901 .364811$

C $3.228243-0.1422511 .059763$

C $-4.528708 \quad 1.0898191 .251245$

H $4.085490-1.8588812 .137158$

H $4.644341-3.282608 \quad 0.316546$

H $4.010401-2.057685-0.919184$

H $3.6632950 .303648-1.021636$

H $0.3890840 .494328-1.972211$

H $\quad 0.576525 \quad 1.3729250 .955161$

H $0.1225352 .360304-0.446930$

H $2.3354112 .163554-1.396125$

H $-0.349585-1.0419480 .571556$

H 2.4004583 .7960000 .401005

H 2.6854212 .5542571 .620509

H 3.9251802 .8958200 .395851

H $-1.853350 \quad 0.9313560 .438984$

H -1.261116 -2.405256 -0.871795

H $-2.3248290 .046642-2.220508$

H $-3.282405-1.562428-0.626236$

H $-5.274805-0.7487670 .482983$

H $-4.772593 \quad 0.387175-0.766047$

H -3.597102 -1.960430 1.593679

H 3.9443830 .5555031 .508162

H $2.346313-0.1713341 .711839$

H $-3.853747 \quad 1.907457 \quad 0.982778$

H -5.5388781 .5067001 .320475$

H -4.250187 0.7199662 .242552

SCF Energy (B3LYP/6-31G**//MMFF) $=-810.711094732$

F2-31_c07430

MMFF Geometry

C $4.736023-0.999481-0.034851$

C $4.821198-2.2190550 .508374$

C $2.8170620 .568465-0.400440$

O $1.786675-0.431843-0.446049$

C $0.5730410 .194517-0.919280$

C $0.798706 \quad 1.703371-0.817907$

C $2.0832591 .851254-0.015644$

C $-0.602339-0.367392-0.103926$

C $1.785616 \quad 1.9926391 .477631$

C $-1.9745350 .239451-0.461008$

O $-0.662565-1.778917-0.348956$

O $-2.179301 \quad 0.162694-1.871254$

C -3.153781 $-0.442898 \quad 0.262025$

C $-4.4951640 .233437-0.061561$

O $-2.933799-0.4098101 .670263$

C 3.9315210 .1285740 .548655

C $-5.669727-0.455165 \quad 0.621797$

H $5.286621-0.797542-0.950074$

H $5.423990-2.9886490 .038792$

H $4.294919-2.4714971 .422281$

H $3.2124450 .673339-1.420692$

H $0.465082-0.100056-1.970460$

H $-0.0396932 .264211-0.398521$

H $0.9603702 .099051-1.829186$

H $2.6482542 .734393-0.334774$

H -0.408584 -0.2484350 .967619$

H 1.2888831 .1077791 .886427

H 2.7089602 .1512872 .044264

H $1.1386082 .857366 \quad 1.660741$

H $-1.9715961 .300718-0.191325$

H $\quad 0.205479-2.150074-0.114260$

H $-2.157278-0.777651-2.119457$

H -3.219055 -1.494509-0.040839

H $-4.6689870 .233183-1.143385$

H $-4.473027 \quad 1.2789330 .269225$

H $-2.888490 \quad 0.5228301 .942083$

H 4.6125170 .9665620 .736953

H $3.509312-0.1823991 .511872$

H -6.6078970 .0350560 .342710$

H $-5.734594-1.5063590 .323216$

H -5.579598 -0.410840 1.711578

SCF Energy (B3LYP/6-31G**//MMFF) $=-810.716416306$

F2-31_c07431
MMFF Geometry

C -2.493788 $2.545696-0.173220$

C -1.501785 $3.301796 \quad 0.310190$

C $-2.6803220 .083940-0.644006$

O $-1.269161-0.005366-0.939353$

C $-0.753663-1.257336-0.455510$

C $-1.818726-1.819598 \quad 0.476054$

C $-3.088275-1.319458-0.183146$

C $0.625695-1.0759380 .197111$

C $-4.317885-1.4216860 .701295$

C $1.688834-0.527015-0.776718$

O $0.500811-0.2172821 .327608$

O $1.4072090 .828806-1.125459$

C $3.134275-0.609677-0.244000$

C 3.4946310 .3931680 .860979

O $4.014180-0.394756-1.354822$

C $-2.8796791 .204586 \quad 0.387339$

C $4.9440350 .255006 \quad 1.314928$

H $-3.0676892 .916511-1.018493$

H -1.275047 $4.262962-0.138018$

H -0.9007542 .9788101 .153021$

H -3.204172 $0.339027-1.573125$

H $-0.655104-1.913930-1.329396$

H $-1.728339-1.4138621 .490058$

H -1.771939 -2.910661 0.545949

H $-3.263754-1.929597-1.080708$

H $0.955971-2.0530090 .568760$

H $-4.547670-2.4714670 .913052$

H $-4.175053-0.9187911 .663098$

H $-5.188740-0.9795860 .207019$

H $1.645087-1.104420-1.709143$

H $\quad 0.2339040 .658490 \quad 0.999377$

H $0.5180050 .855090-1.517383$

H $3.328191-1.6291150 .108645$

H 2.8526450 .2499791 .734155

H 3.3506841 .4200280 .505323

H $3.8382550 .497994-1.698896$

H -3.9323881 .2641790 .684615$

H -2.2939010 .9966951 .290764$

H $5.145005-0.7576861 .678841$

H 5.6414840 .4746600 .500663

H $5.1509280 .955572 \quad 2.130263$

SCF Energy (B3LYP/6-31G**//MMFF) $=-810.725354722$

F2-31_c07432

MMFF Geometry

C $2.868746 \quad 1.872642 \quad 0.285067$

C $3.3564882 .752912-0.596224$

C $2.927484-0.6737720 .649989$

$\begin{array}{llll}\text { O } & 1.661334 & -0.624840 & 1.342733\end{array}$

C $0.614399-1.0381320 .437999$

C $1.179330-0.831898-0.958511$

C $2.632039-1.208439-0.754641$

C $-0.655779-0.2371010 .745363$

C $3.556106-0.780475-1.879023$

C $-1.810810-0.467044-0.250797$

O $-1.101934-0.6042472 .055291$

O $-2.127116-1.856628-0.321873$

C $-3.092676 \quad 0.301484 \quad 0.128610$

C $-4.2068690 .113319-0.913428$

$\begin{array}{llll}\text { O } & -2.798019 & 1.690574 & 0.258210\end{array}$

C 3.6304890 .6850850 .802622

C $-5.4887010 .840797-0.527371$

H 1.8693752 .0379410 .678577

H $2.7573503 .597981-0.917548$

H $4.3531152 .648276-1.010341$

H $3.544669-1.412377 \quad 1.177294$

H $0.451321-2.1051290 .636015$

H $1.0884130 .214638-1.271103$

H $0.682006-1.454070-1.708595$

H $2.678084-2.306167-0.699029$

H -0.4112690 .8306950 .788957$

H $3.301106-1.313588-2.801339$

H $3.4817870 .287749-2.095153$

H $4.597803-1.012749-1.635988$

H - $1.490453-0.142183-1.248196$

H $-0.363756-0.4444332 .668391$

H $-1.379224-2.312348-0.742798$ 
H $-3.465450-0.0527961 .096804$

H $-4.432485-0.951225-1.040647$

H $-3.8749200 .498993-1.884930$

H -2.478670 $2.008247-0.603681$

H 3.7872970 .8642361 .874602

H $\quad 4.6267420 .6334890 .347507$

H $-5.3312931 .922008-0.464787$

H $-6.2654700 .658093-1.276782$

H $-5.861670 \quad 0.4895950 .439946$

SCF Energy (B3LYP/6-31G**//MMFF) $=-810.710194533$

F2-31_c07433

MMFF Geometry

C -1.680555 $2.772200-0.120480$

C $-0.870783 \quad 3.3385570 .781156$

C $-2.0712840 .410917-0.893398$

O $-0.692717 \quad 0.044676-0.660972$

C $-0.621423-1.328246-0.242334$

C -2.033566 -1.694538 0.194942

C $-2.866031-0.895496-0.786616$

C $0.434281-1.5310580 .853517$

C $-4.329279-0.784411-0.397390$

C $1.899940-1.3394080 .400493$

O $\quad 0.141052-0.6793961 .958645$

O $2.211184-2.322751-0.586958$

C $2.2812070 .048094-0.152684$

C $3.7880400 .142078-0.446577$

O $1.948164 \quad 1.0690740 .781223$

C -2.4503141 .5023940 .118437$

C $4.1775191 .485464-1.051716$

H -1.806560 $3.257763-1.084804$

H -0.3469364 .2590550 .547898$

H $-0.7138802 .897318 \quad 1.759222$

H $-2.1466400 .808806-1.912699$

H - $0.355532-1.919613-1.127577$

H $-2.235896-1.3697751 .222300$

H -2.218268 -2.771642 0.138146

H -2.813008 -1.400286 -1.761724

H $\quad 0.329809-2.5640011 .210895$

H -4.456298 -0.3807120 .612195$

H -4.869032 $-0.139157-1.097878$

H -4.803549 -1.771649 -0.415550

H $2.538262-1.532373 \quad 1.273174$

H $0.799842-0.8579842 .651222$

H $2.048279-3.196965-0.193793$

H $1.7499010 .243425-1.090713$

H $4.092440-0.653112-1.136194$

H 4.3553120 .0098900 .482777

H 0.9829191 .0575420 .895033

H $-3.514409 \quad 1.747770 \quad 0.031179$

H $-2.2746491 .145826 \quad 1.140782$

H $3.9654962 .310468-0.364602$

H $5.2492671 .501518-1.273638$

H $3.6351561 .665785-1.985245$

SCF Energy (B3LYP/6-31G**//MMFF)= -810.716610969

F2-31 c07434

MMFF Geometry

C -3.126790 2.0620740 .018868

C $-4.2143982 .774738-0.295233$

C $-2.304242-0.187090-0.756357$

O $-0.9247460 .220985-0.618268$

C $-0.140231-0.891288-0.150941$

C -1.135499-1.927475 0.353111

C $-2.294515-1.712186-0.599611$

C $0.887427-0.4287230 .887572$

C $-3.586918-2.367846-0.147132$

C 1.8016070 .7142480 .410776

$\begin{array}{lllll}\text { O } & 0.182561 & 0.036821 & 2.046360\end{array}$

O $2.7372740 .981261 \quad 1.461966$

C $2.5525310 .451773-0.906699$

C $3.430190-0.808771-0.965179$

O $3.3512691 .603369-1.193360$

C -3.1379640 .5807650 .281202$

C $4.602744-0.8410970 .004801$

H -2.1740782 .5785800 .104584$

H -4.140343 $3.843213-0.466215$

H $\quad-5.190220 \quad 2.310937-0.387885$
H $-2.6358510 .081801-1.766547$

H $0.374994-1.300190-1.027991$

H $-1.448258-1.7295711 .384408$

H $-0.729649-2.9429890 .314248$

H -2.016542 -2.146480 -1.570590

H $1.487099-1.2846751 .218320$

H -3.870925 -2.061365 0.864687

H $-4.407458-2.117322-0.827073$

H -3.477451 -3.457629-0.138598

H 1.2147891 .6363220 .312234

H $\quad 0.8488600 .3235242 .694428$

H 3.3136841 .7036091 .158469

H $1.8158920 .386711-1.715886$

H $3.848999-0.880736-1.977288$

H $2.817637-1.704795-0.818198$

H $3.777708 \quad 1.457923-2.055078$

H -4.1716450 .2173790 .277559$

H -2.7357190 .4207331 .288516$

H $5.249827-1.694711-0.223432$

H $5.2096160 .066346-0.064994$

H $4.264527-0.9608321 .037659$

SCF Energy $\left(B 3 L Y P / 6-31 G^{* *} / / M M F F\right)=-810.714998803$

F2-31 c07435

MMFF Geometry

C 3.0288692 .4867080 .165925

C $2.7181333 .289584-0.858058$

C 2.2700910 .1870470 .802187

$\begin{array}{lllll}\text { O } & 0.996423 & 0.245236 & 0.139566\end{array}$

C $0.195228-0.8207160 .675373$

C $1.173059-1.8819191 .188090$

C $2.560849-1.3099020 .919472$

C $-0.807096-1.314101-0.374830$

C $3.182161-1.939084-0.327657$

C $-1.692556-0.211686-0.986963$

O $-0.094484-1.942206-1.448017$

O $-2.656522-0.861930-1.827219$

C -2.4324630 .6813960 .026069$

C $-3.449570-0.0746800 .890359$

O $-3.1324141 .697650-0.699329$

C 3.2979601 .0136380 .030029

C -4.1073280 .8297561 .926286$

H $3.1029582 .917261 \quad 1.161266$

H $2.5398964 .346580-0.692684$

H $2.6346122 .911446-1.870848$

H 2.1338510 .6050861 .809730

H -0.352078 -0.4142791 .534324$

H $1.014594-2.8716270 .747438$

H $1.025970-2.0035842 .268863$

H $3.234139-1.5056921 .762088$

H $-1.434614-2.0998970 .060514$

H $3.238193-3.027836-0.223049$

H $2.608301-1.715912-1.231327$

H $4.203302-1.574382-0.479545$

H $-1.0929230 .414504-1.659402$

H $\quad 0.462535-1.263778-1.866614$

H -3.216427 -0.167811 -2.215615

H $-1.713014 \quad 1.1980960 .670010$

H $-2.963025-0.9038731 .414304$

H $-4.241722-0.496840 \quad 0.260780$

H $-2.4721412 .208433-1.198225$

H 4.3034340 .8167610 .419511

H $3.2892740 .736449-1.031028$

H -3.3580111 .2746562 .588824$

H -4.6713681 .6381301 .450784$

$\mathrm{H}-4.8037880 .2518672 .542206$

SCF Energy (B3LYP/6-31G**//MMFF) $=-810.712558892$

F2-31_c07436

MMFF Geometry

C $-2.3693822 .575963-0.377275$

C -1.697099 $3.364606 \quad 0.468767$

C $-2.2108340 .125139-0.913948$

O $-0.7931160 .117682-0.633550$

C $-0.429583-1.138157-0.034276$

C $-1.735165-1.7785280 .419067$

C $-2.684894-1.311743-0.666466$

C $0.601469-0.9231021 .077422$ 
C $-4.150621-1.503209-0.319738$

C $1.878467-0.1471180 .679720$

O $-0.016173-0.1952182 .149238$

O $2.733640-0.127117 \quad 1.829277$

C $2.663143-0.758604-0.495721$

C $4.096911-0.220650-0.650812$

O $1.958363-0.523050-1.712564$

C $-2.863951 \quad 1.198717-0.030852$

C $4.1734271 .282752-0.886581$

H $-2.5835202 .945793-1.376745$

H -1.3700424 .3508430 .158105$

H $-1.4602673 .043913 \quad 1.477226$

H $-2.3438910 .384926-1.971094$

H $\quad 0.002181-1.760535-0.826527$

H -2.059996 -1.403507 1.396002

H -1.659703 -2.8682180 .485190$

H -2.472268 -1.895610 -1.573422

H $0.884483-1.8943631 .500609$

H -4.383448 -2.569542 -0.228037

H -4.413036 -1.029240 0.631503

H $-4.791758-1.084643-1.102114$

H 1.6137220 .8935410 .455338

H $-0.3140540 .657907 \quad 1.789254$

H $2.229323 \quad 0.2724842 .558614$

H $2.728826-1.844344-0.352609$

H $4.565992-0.715270-1.510685$

H $4.696986-0.4808120 .228395$

H $2.446571-0.966257-2.427118$

H -3.950662 $1.196163-0.170890$

H -2.668279 $0.988057 \quad 1.027385$

H $5.2140741 .583221-1.045829$

H $3.6005171 .577702-1.771042$

H $3.7951861 .843024-0.026554$

SCF Energy $\left(B 3 L Y P / 6-31 G^{* *} / / M M F F\right)=-810.715070361$

\section{F2-32 c07637}

MMFF Geometry

C $-4.533434-1.344968-0.532542$

C $-4.751213-2.5088560 .090070$

C $-2.7060950 .364101-0.366801$

O $-1.688208-0.572722 \quad 0.040887$

C -0.5392150 .1600950 .518309$

C $-0.762703 \quad 1.6032160 .094782$

C $-2.269979 \quad 1.717630 \quad 0.192991$

C $0.733307-0.486872-0.040174$

C $-2.8377282 .910251-0.556925$

C $2.022506 \quad 0.2917250 .287481$

O $0.839916-1.8010980 .517945$

O $2.099130 \quad 0.517674 \quad 1.694699$

C $3.305051-0.421351-0.184560$

C 4.5641430 .4127710 .098940

O $3.217157-0.686267-1.582671$

C $-4.067215-0.0949360 .158954$

C $5.839306-0.299553-0.333846$

H -4.710544 -1.285811 -1.603383

H $-5.092413-3.374445-0.467137$

H -4.593296 -2.619951 1.157040

H $-2.7017120 .382132-1.464644$

H $-0.563587 \quad 0.0727831 .611858$

H $-0.433001 \quad 1.761981-0.939702$

H -0.2361362 .3168980 .735438$

H -2.548144 1.7938351 .252850

H $0.637499-0.615128-1.124924$

H -2.586942 2.870718-1.622291

H -3.928133 $2.944547-0.468768$

H $-2.4368783 .845200-0.151961$

H $1.9671291 .273691-0.196823$

H $\quad 0.019262-2.2755770 .299902$

H $2.114841-0.3518952 .130300$

H $3.407894-1.3831920 .331134$

H 4.6349550 .6414811 .168162

H $4.5064181 .366448-0.439705$

H $3.1395450 .167059-2.042636$

H -4.007871 -0.2650951 .241254$

H $-4.8191470 .684234-0.009835$

H $5.849405-0.480068-1.413209$

H $6.7136370 .311404-0.088031$

H $5.942882-1.2614640 .178157$
SCF Energy (B3LYP/6-31G**//MMFF) $=-810.720716954$

F2-32_c07638

MMFF Geometry

C $3.0675562 .355409-0.589782$

C $2.4286743 .525113-0.475490$

C $2.399606-0.000132-0.050967$

O $0.9882240 .223191-0.247555$

C $0.258415-0.8882340 .303533$

C $1.287177-1.9840040 .551277$

C $2.503491-1.1646910 .934626$

C $-0.895179-1.284283-0.623170$

C $3.808617-1.9363100 .845154$

C $-1.852137-0.133028-0.987310$

O $-0.359529-1.795572-1.851534$

O $-2.948291-0.687317-1.729223$

C -2.4288230 .6502060 .204473$

C $-3.308861-0.1901641 .139846$

O $\quad-3.2028631 .738389-0.310914$

C 3.0540351 .2831820 .462955

C -3.8051230 .6140572 .335475$

H $3.6420602 .161128-1.491873$

H $2.4814974 .259903-1.271376$

H 1.8443663 .7691720 .404695

H $2.816223-0.282931-1.026590$

H $-0.138607-0.5562401 .270325$

H $1.491567-2.564172-0.356073$

H $\quad 0.972189-2.6803861 .334290$

H $2.371804-0.8006311 .962537$

H -1.456958-2.113189-0.178335

H $4.657276-1.3012821 .117603$

H $3.795787-2.7934861 .526249$

H $3.982561-2.314719-0.167769$

H $-1.3596170 .568393-1.672511$

H $\quad 0.204379-1.102759-2.236708$

H -2.571406 -1.169733 -2.485118

H -1.613932 1.1054310 .776391

H $-2.750611-1.0557161 .511309$

H $-4.185498-0.5667860 .599904$

H $-3.9184301 .359459-0.850095$

H 2.5212301 .6394661 .353723

H $4.0908341 .086376 \quad 0.758441$

H $-4.403325-0.0225932 .995171$

H -2.9657301 .0098042 .916140$

H -4.4326751 .4532322 .019762$

SCF Energy (B3LYP/6-31G**//MMFF $)=-810.722476917$

F2-32_c07639

MMFF Geometry

C $-4.317507-0.9943140 .531752$

C $-5.368318-0.5319841 .218597$

C $-2.5164780 .106873-0.890837$

O $-1.522830-0.892847-0.593264$

C $-0.474556-0.2798350 .191387$

C $-0.725864 \quad 1.2209360 .127691$

C -2.2392421 .2740230 .054017$

C $0.885599-0.730571-0.353504$

C $-2.7828562 .605409-0.432632$

C $2.086634-0.0336350 .318136$

O $0.997132-2.142098-0.140897$

O $1.997654-0.1873321 .733639$

C $3.448189-0.564847-0.168815$

C 4.6580650 .0795070 .528425

O $3.545634-0.365394-1.577229$

C $-3.921209-0.499270-0.830374$

C 4.7718191 .5848300 .324691

H -3.714500 -1.783957 0.972431

H -5.605624 -0.9353532 .196922$

H -6.0047790 .2515820 .822807$

H $-2.3281200 .415721-1.927957$

H $-0.616488-0.6481851 .215223$

H $-0.2829761 .653129-0.778349$

H $-0.320095 \quad 1.7523250 .993735$

H -2.6363751 .0704441 .056030$

H $0.920198-0.574380-1.438272$

H $-3.8761892 .584735-0.481064$

H -2.489741 3.4122020 .247055

H $-2.4053072 .853183-1.430283$ 
H 2.0191591 .0372140 .098608

H $0.231898-2.560803-0.571302$

H $2.016138-1.1405921 .926247$

H $3.505575-1.644154 \quad 0.017660$

H $5.572904-0.3773080 .130635$

H $4.634423-0.1393301 .601993$

H $4.394710-0.738988-1.868880$

H $-4.6475420 .235767-1.197379$

H $-3.964229-1.358895-1.511007$

H $4.7927461 .844139-0.738132$

H 3.9401892 .1165760 .795897

H 5.6976571 .9535590 .778199

SCF Energy (B3LYP/6-31G**/MMFF) $=-810.721602248$

F2-32_c07640

MMFF Geometry

C $4.251028-1.5910790 .680068$

C $4.402056-2.759562 \quad 0.046814$

C $2.601490 \quad 0.281808 \quad 0.436386$

O $1.531534-0.549743-0.056938$

C $0.4863570 .294255-0.587290$

C $0.813427 \quad 1.702998-0.117511$

C $2.3282331 .678758-0.118444$

C $-0.873429-0.244148-0.124408$

C $2.9529112 .800790 \quad 0.692653$

C $-2.0594980 .661649-0.510006$

O $-1.050878-1.535647-0.711907$

O $-2.0312300 .905317-1.919520$

C $-3.4653340 .142374-0.144502$

C $-3.655176-0.0521751 .363800$

O $-3.749607-1.078103-0.826063$

C $3.946636-0.292280-0.011506$

C $-5.097097-0.3909341 .720817$

H $4.362560-1.5658891 .761038$

H $4.626008-3.6620410 .605145$

H $4.304608-2.838301-1.030325$

H 2.5267590 .2813231 .531840

H $0.5724310 .224211-1.679165$

H $\quad 0.433715 \quad 1.8739800 .897591$

H $0.3972092 .473306-0.773427$

H $2.6799801 .746779-1.156775$

H $-0.855714-0.3938310 .961077$

H 2.6309612 .7670701 .738920

H 4.0451942 .7353450 .674060

H 2.6662953 .7755710 .284390

H $-1.9296381 .637005-0.027271$

H $-0.286591-2.079564-0.454305$

H -2.154289 $0.048490-2.363416$

H $-4.2009380 .867745-0.512869$

H -3.361555 0.8588521 .897479

H $-3.021656-0.8704691 .724721$

H -3.128815 -1.752505 -0.502068

H $3.943047-0.438228-1.099050$

H $4.7537640 .411306 \quad 0.221965$

H $-5.412307-1.3306491 .256588$

H $-5.201112-0.4998532 .805006$

H $-5.7780840 .401021 \quad 1.393048$

SCF Energy (B3LYP/6-31G**/MMFF) $=-810.725668359$

F2-32_c07641

MMFF Geometry

C -3.320345 2.3601890 .435455

C $-2.8795103 .491257-0.126736$

C -2.601679 -0.0310120 .218160$

O $-1.233256 \quad 0.280907-0.111857$

C $-0.583482-0.907161-0.596620$

C $-1.520926-2.061014-0.263642$

C $-2.875680-1.397549-0.408190$

C $0.826593-1.054548-0.007979$

C $-4.003214-2.1686390 .255768$

C $1.7902890 .088391-0.399440$

O $0.745503-1.137724 \quad 1.415289$

$\begin{array}{lllll}\text { O } & 1.310811 & 1.339259 & 0.089632\end{array}$

C $3.217041-0.1230190 .145665$

C $4.1768220 .992297-0.298822$

O $3.719801-1.381083-0.298646$

C $-3.5197411 .071536-0.311352$

C 5.5809770 .8047610 .261740
H -3.559996 2.3643511 .495700

H $-2.760522 \quad 4.3902240 .468108$

H $-2.6291893 .541340-1.180660$

H $-2.660471-0.0903431 .312878$

H $-0.510126-0.803168-1.686717$

H $-1.376824-2.4133640 .764655$

H $-1.385173-2.914055-0.935331$

H $-3.098887-1.283618-1.477621$

H $1.239582-2.007236-0.358752$

H $-4.104550-3.162873-0.191728$

H $-3.823010-2.2998041 .328049$

H $-4.957415-1.6459990 .136279$

H $1.8312790 .147126-1.494400$

H $0.371463-0.2980851 .733783$

H $0.4449521 .505659-0.319729$

H $3.195022-0.1411681 .241793$

H 3.7991191 .9694640 .021841

H $4.2453661 .007885-1.393267$

H $3.752648-1.361508-1.270294$

H $-3.333848 \quad 1.226057-1.381696$

H $-4.5689160 .775377-0.200451$

H $6.2244471 .633559-0.050365$

H $5.565406 \quad 0.7809081 .355969$

H $6.033894-0.124528-0.097424$

SCF Energy (B3LYP/6-31G**//MMFF) $=-810.725517531$

F2-32 c07642

MMFF Geometry

C $-4.530332-1.374296-0.537254$

C $-4.753614-2.5252040 .106948$

C $-2.7098420 .343330-0.383521$

O $-1.694554-0.5807330 .058011$

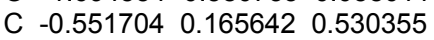

C $-0.776426 \quad 1.599127 \quad 0.073886$

$\begin{array}{llll}C & -2.285211 & 1.709863 & 0.152807\end{array}$

C $0.729027-0.485865-0.004693$

C $-2.8484642 .884455-0.628305$

C 2.0227520 .2998790 .296908

$\begin{array}{lllll}\text { O } & 0.828090 & -1.795060 & 0.567239\end{array}$

$\begin{array}{lllll}\text { O } & 2.146466 & 0.505306 & 1.703818\end{array}$

C $3.292453-0.419090-0.201179$

C $4.5550710 .420417 \quad 0.053640$

O $3.156352-0.664191-1.598634$

C $-4.076528-0.1086850 .133640$

C $5.834720-0.291999-0.365899$

H $-4.693012-1.338415-1.611412$

H $-5.084876-3.403538-0.436131$

H $-4.609776-2.613198 \quad 1.178158$

H $-2.6909490 .337669-1.481244$

H $-0.5882580 .101620 \quad 1.624975$

H $-0.4359061 .737514-0.960021$

H $-0.2604142 .329100 \quad 0.704352$

H -2.5753991 .8069601 .207722$

H $0.626214-0.635381-1.085771$

H $-2.5851532 .823744-1.689646$

H $-3.9398342 .916244-0.553394$

H -2.455573 $3.829138-0.238411$

H $1.9642811 .282912-0.185998$

H $0.966870-1.6877891 .523993$

H 1.4515501 .1273921 .976402

H $3.397724-1.3825630 .312674$

H 4.6331210 .6714601 .117437

H $4.4909441 .362550-0.504054$

H $3.845879-1.298579-1.856889$

H $-4.031099-0.2555371 .220018$

H $-4.8283860 .664407-0.061384$

H $5.929208-1.2584420 .139065$

H $5.864317-0.460746-1.446503$

H $6.7062810 .315458-0.101392$

SCF Energy (B3LYP/6-31G**//MMFF) $=-810.712013553$

F2-32 c07643

MMFF Geometry

C $-3.1040752 .552013-0.243128$

C -2.6982282 .8882630 .986696$

C $-2.3881940 .063543-0.102710$

$\begin{array}{llll}\text { O } & -0.978431 & 0.318761 & 0.057423\end{array}$

C $-0.250121-0.898218-0.187751$ 
C $-1.288048-2.013485-0.211167$

C $-2.483048-1.293307-0.803847$

C $0.865946-1.0765940 .846168$

C $-3.799289-2.017412-0.579032$

$\begin{array}{llll}\text { C } & 1.822826 & 0.124602 & 0.968472\end{array}$

O $0.264819-1.2780382 .132857$

O $2.865549-0.2669091 .872643$

C $2.4514420 .604566-0.352220$

C $3.358362-0.434082-1.026934$

O $3.2048371 .791913-0.086957$

C $-3.0307021 .200202-0.904468$

C $3.9101290 .063849-2.357420$

H -3.544741 $3.338649-0.853279$

H -2.813598 3.9088621 .336710

H -2.249322 $2.176601 \quad 1.668697$

H -2.825046 -0.0000490 .901981$

H $0.184531-0.807054-1.190397$

H -1.524249 -2.3730890.796856

H $-0.962369-2.871070-0.807619$

H -2.319646 -1.171443 -1.883167

H $1.425972-1.992920 \quad 0.625734$

H $-4.005099-2.1540610 .487942$

H $-4.633129-1.457528-1.013718$

H -3.777897 -3.007382 -1.046502

H 1.3055690 .9602281 .456796

H $0.986719-1.3988912 .773482$

H 3.3859800 .5275892 .079773

H $1.6596290 .905164-1.045792$

H $2.805338-1.362133-1.205574$

H $4.208627-0.674675-0.377890$

H 4.0045581 .5378390 .403829

H -2.472616 $1.334418-1.840134$

H -4.054769 $0.917880-1.175897$

H $4.535466 \quad 0.951928-2.223329$

H $4.525275-0.712270-2.823900$

H $3.0986380 .313949-3.048289$

SCF Energy (B3LYP/6-31G*//MMFF) $=-810.713845568$

\section{F2-32 c07644}

MMFF Geometry

C $2.803242 \quad 2.104523-0.605959$

C $3.7800342 .830741-0.050809$

C $2.263822-0.382328-0.657372$

O $0.842138-0.172619-0.761016$

C $0.224140-0.6178850 .462217$

C $1.291895-1.406523 \quad 1.214556$

C $2.549039-0.6550740 .819330$

C $-1.062850-1.3941550 .167495$

C $3.830764-1.4299501 .067678$

C $-2.074761-0.637954-0.715393$

O $-0.717490-2.606162-0.516280$

O $-3.265250-1.433140-0.813575$

C -2.451104 $0.777113-0.239987$

C -3.1508690 .8255901 .124809$

O $-3.3098591 .364162-1.223257$

C $3.0232990 .785753-1.291910$

C $-3.403988 \quad 2.256150 \quad 1.585912$

H $1.7870402 .488834-0.574471$

H 3.5565813 .7791350 .425383

H $4.8111132 .495305-0.058999$

H $2.476524-1.282972-1.248863$

H -0.0121590 .2839991 .039538$

H $1.348496-2.4462940 .872349$

H $1.117008-1.4155412 .294652$

H $2.5814360 .280179 \quad 1.391747$

H $-1.527772-1.6992441 .112060$

H $4.703378-0.8495850 .752000$

H $3.942675-1.656828 \quad 2.133055$

H $3.839808-2.3783470 .520025$

H -1.693261 $-0.577242-1.742019$

H $-1.545208-3.084927-0.694639$

H -3.570980 -1.633265 0.087019

H - $-1.5536911 .403604-0.212663$

H -2.544337 0.3185191 .882308

H $-4.1192340 .314727 \quad 1.074779$

H -4.099826 $0.801533-1.296410$

H $4.0924880 .545030-1.326548$

H $2.6877820 .902724-2.329983$
H $-4.0645562 .787602 \quad 0.893871$

H -3.8804242 .2553862 .571385$

$\mathrm{H}-2.4651772 .8137331 .664036$

SCF Energy $($ B3LYP/6-31G**//MMFF $)=-810.717893912$

F2-32 c07645

MMFF Geometry

C $-3.207538 \quad 1.759497-0.247684$

C $-3.3377872 .471381-1.372716$

C $-1.874702-0.2844410 .317460$

O $-0.843635-0.037916-0.663569$

C $-0.110961-1.252665-0.875887$

C $-0.430180-2.1521700 .308612$

C $-1.886048-1.7978480 .548841$

C $1.371856-0.992049-1.155551$

C $-2.392537-2.2069881 .921223$

C $2.243769-0.4909400 .011674$

O $1.928811-2.232657-1.614031$

O $3.548905-0.229880-0.531351$

C 1.7564110 .7720880 .741105

C $1.7137632 .025148-0.142390$

O 2.6481551 .0323181 .831577

C $-3.2092920 .257467-0.196573$

C 1.1041813 .2163060 .587709

H -3.099733 2.2899290 .694966

H $-3.3310863 .555481-1.340490$

H -3.450082 $1.991380-2.338365$

H $-1.5772960 .241023 \quad 1.233944$

H $-0.544334-1.715789-1.773754$

H $\quad 0.169986-1.8945841 .187892$

H $-0.277129-3.2123890 .086468$

H $-2.498511-2.290302-0.218676$

H $1.454934-0.288912-1.993207$

H -1.809023 -1.737615 2.720386

H -3.439383 -1.916072 2.053175

H -2.324385 -3.292240 2.048392

H $2.393019-1.3028690 .733648$

H $2.862499-2.067413-1.831427$

H 4.1099450 .0790380 .200734

H $0.7749420 .595875 \quad 1.187206$

H $1.1277731 .840009-1.047957$

H $2.7277822 .303022-0.453972$

H 2.6241840 .2585592 .420201

H $-3.416447-0.151868-1.193257$

H $-4.024681-0.0581280 .464136$

H 0.0816042 .9949590 .910087

H 1.6928043 .4886961 .469207

H $1.0691314 .086573-0.075281$

SCF Energy (B3LYP/6-31G**//MMFF $)=-810.716090786$

F2-32 c07646

MMFF Geometry

C $-3.6332612 .012043 \quad 0.341934$

C $-3.3864693 .176360-0.268943$

C $-2.541589-0.2392270 .198512$

O $-1.2458860 .274630-0.170123$

C $-0.419283-0.813207-0.620259$

C $-1.155102-2.087988-0.226350$

C $-2.600510-1.656441-0.370219$

C $1.003912-0.706509-0.051353$

C $-3.580935-2.5705150 .343915$

C $1.7573350 .560350-0.515105$

O $0.946993-0.7510081 .373216$

O $1.1090911 .734861-0.018194$

C $3.2337130 .651508-0.077723$

C $4.104427-0.451637-0.687852$

O $3.346124 \quad 0.589628 \quad 1.342894$

C $-3.6322300 .679861-0.353550$

C $5.586546-0.231225-0.409788$

H -3.8575942 .0187911 .405512$

H -3.4061914 .1052010 .290526$

H -3.159956 $3.224844-1.328211$

H $-2.575145-0.263347 \quad 1.295734$

H $-0.376831-0.743889-1.714745$

H $-0.943409-2.3712840 .811447$

H $-0.892289-2.934594-0.867803$

H -2.852610 -1.623049-1.438776

H $1.558037-1.593141-0.377040$ 
H $-3.367838-2.6279341 .416648$

H $-4.608029-2.2124200 .223265$

H -3.527185 -3.585359-0.063796

H $1.7222460 .616435-1.610108$

H $\quad 0.4559420 .034915 \quad 1.668748$

H $\quad 0.2023831 .743065-0.368612$

H $3.6267521 .631029-0.377569$

H $3.950172-0.493826-1.772021$

H $3.830606-1.427316-0.270636$

H 2.7580581 .2718321 .709680

H $-3.4876650 .820330-1.432178$

H $-4.6184600 .223741-0.211838$

H $5.796668-0.2417110 .664200$

H $6.179263-1.024549-0.876360$

H $5.923180 \quad 0.727116-0.817721$

SCF Energy $\left(B 3 L Y P / 6-31 G^{* *} / / M M F F\right)=-810.721738729$

F2-32_c07647

MMFF Geometry

C $4.562409-0.954161-0.075713$

C $4.741947-2.199881-0.528911$

C 2.5412420 .5180350 .110242

O $1.600951-0.5676380 .241038$

C $0.262083-0.0592620 .042008$

C $0.385016 \quad 1.4533430 .131014$

C $1.7501131 .681536-0.485892$

C $-0.672033-0.7132031 .067989$

C $2.3340343 .049334-0.177804$

C $-2.134014-0.2198431 .060465$

O $-0.674699-2.1262380 .841338$

O -2.2043151 .0906291 .617292$

C $-2.818016-0.210084-0.322610$

C $-4.2970240 .199889-0.226230$

O $-2.728514-1.494985-0.930598$

C $3.7158750 .078244-0.765369$

C $-4.9721300 .258566-1.590946$

H $5.062612-0.6565960 .842222$

H $5.370471-2.8969330 .014628$

H $4.268679-2.545831-1.441075$

H $2.8854580 .765136 \quad 1.123080$

H $-0.017452-0.363246-0.974183$

H $0.3825821 .784927 \quad 1.176593$

H $-0.4142251 .978054-0.399679$

H $1.6679601 .562939-1.574776$

H $-0.250598-0.5649102 .070331$

H $1.6893783 .840458-0.574477$

H 2.4361683 .2084550 .900951

H $3.3241293 .162458-0.630160$

H -2.711741 -0.8860691 .715169$

H $\quad 0.251351-2.4236890 .851314$

H -1.8336881 .0491362 .515332$

H -2.313003 $0.505076-0.981115$

H $-4.389273 \quad 1.1810730 .252650$

H -4.842268 -0.5226090 .393008$

H $-3.156281-2.135150-0.336658$

H $3.340094-0.319305-1.716585$

H $4.3549530 .937341-0.998883$

H $-6.0117480 .583629-1.482877$

H $-4.4615670 .968273-2.249574$

H $-4.976374-0.721849-2.077238$

SCF Energy (B3LYP/6-31G**//MMFF) $=-810.712901265$

F2-32_c07648

MMFF Geometry

C $-4.156254-1.236583-0.240305$

C $-5.303109-1.1607540 .444299$

C $-2.3316620 .442602-0.816757$

O $-1.286877-0.548128-0.860571$

C $-0.371582-0.3006240 .230902$

C $-0.747834 \quad 1.064893 \quad 0.792017$

C -2.2466391 .0863180 .565341$

C $1.069197-0.410259-0.286245$

C -2.8576492 .4740540 .639378$

C $2.136231-0.0189960 .756071$

O $1.276131-1.754681-0.726741$

O $1.940065-0.7834761 .949982$

C $3.605681-0.1815870 .316085$

C $4.0061480 .616030-0.930196$
O $3.906367-1.5591650 .080851$

C -3.677770 -0.180731-1.196357

C $3.8126272 .116338-0.786991$

H -3.531393 -2.116760 -0.113076

H $-5.595619-1.9605001 .116014$

H $-5.964687-0.3068500 .350112$

H $-2.0749401 .182047-1.587340$

H $-0.573849-1.0843430 .971806$

H $-0.263038 \quad 1.868210 \quad 0.223052$

H $-0.472323 \quad 1.1772691 .844776$

H $-2.7151590 .448274 \quad 1.324813$

H $1.1747300 .218055-1.177427$

H -2.412618 $3.149093-0.099360$

H -3.9358002 .4348240 .454444$

H -2.7003782 .9110381 .630900$

H $1.979779 \begin{array}{lll}1.025727 & 1.043962\end{array}$

H $1.130608-2.3440230 .032648$

H $2.195838-1.7015331 .756816$

H $4.2483360 .118014 \quad 1.152736$

H $3.4592780 .253904-1.808894$

H $5.0625750 .413042-1.148608$

H $3.386369-1.850601-0.687286$

H $-4.4274550 .613247-1.296871$

H -3.581799 -0.656443 -2.180509

H $2.7536922 .378399-0.705762$

H 4.3340252 .4972120 .096756

H $4.2140362 .632094-1.665315$

SCF Energy (B3LYP/6-31G**//MMFF) $=-810.719561268$

F2-32_c07649

MMFF Geometry

C $4.390956-1.769311-0.052031$

C $3.753671-2.6882050 .682825$

C $2.653617 \quad 0.1241620 .298934$

O $1.554657-0.549572-0.346496$

C $0.5084890 .407737-0.620001$

C $\quad 0.880741 \quad 1.661726 \quad 0.155151$

C 2.3938201 .6175600 .095453

C $-0.844332-0.210091-0.242852$

C $3.0656902 .510631 \quad 1.124227$

C $-2.0245770 .775455-0.357638$

O $-1.066639-1.325053-1.109897$

O $-2.047911 \quad 1.334829-1.674573$

C $-3.4256790 .203626-0.056861$

C $-3.544905-0.3216791 .378704$

O $-3.731768-0.836871-0.982840$

C $3.978010-0.343957-0.312773$

C $-4.979223-0.6655361 .761008$

H $5.323973-2.071067-0.524760$

H $4.169660-3.6850530 .786205$

H $2.820519-2.4834031 .193108$

H $2.608010-0.1245211 .366709$

H $\quad 0.5533030 .597249-1.699882$

H $0.536398 \quad 1.5948251 .194766$

H $0.4611472 .570379-0.286828$

H $2.7147191 .922078-0.909920$

H $-0.789931-0.6110530 .775718$

H 2.7758022 .2365102 .144171

H 4.1554012 .4387981 .052951

H 2.7858993 .5572290 .965622

H -1.8535291 .6132980 .327912$

H -0.307297 -1.925085 -1.012391

H $-2.2017790 .602416-2.296052$

H $-4.1603871 .000570-0.227600$

H -3.1683720 .4309532 .080988$

H $-2.940310-1.2276241 .501852$

H -4.675800 -1.048391-0.893020

H $3.928807-0.221997-1.402566$

H $\quad 4.7901440 .2979890 .048388$

H $-5.370323-1.4871781 .153554$

H $-5.023600-0.9769662 .809639$

H $-5.6368380 .200672 \quad 1.637010$

SCF Energy (B3LYP/6-31G**//MMFF) $=-810.713340724$

F2-32_c07650

MMFF Geometry

C -4.412753 -1.599144 -0.502986

C $-4.493954-2.7964310 .088075$ 
C $-2.7445190 .266780-0.353399$

O $-1.628378-0.582677-0.018279$

C $-0.531196 \quad 0.239307 \quad 0.436820$

C $-0.904863 \quad 1.667066 \quad 0.070624$

C $-2.410738 \quad 1.6376680 .233097$

C $0.771400-0.275139-0.190299$

C -3.117093 $2.793888-0.453177$

C $1.9922590 .614566 \quad 0.108402$

O $\quad 0.995930-1.593413 \quad 0.310339$

$\begin{array}{llll}\text { O } & 2.144629 & 0.750290 & 1.521647\end{array}$

C $3.3138830 .126834-0.524769$

C $4.021059-1.0170920 .215206$

O $4.220384 \quad 1.238337-0.542713$

C $-4.033899-0.3321910 .210742$

C $5.294928-1.460768-0.497441$

H $-4.642066-1.524737-1.562914$

H -4.778076 -3.673187 -0.483703

H $-4.278925-2.9242481 .143228$

H -2.789028 $0.317468-1.449325$

H $-0.5011740 .119316 \quad 1.527227$

H $-0.6362211 .886093-0.970388$

H -0.4195992 .4076540 .713356$

H -2.648784 1.6562901 .305230

H $0.646931-0.369761-1.275898$

H -2.909469 2.809796 -1.528399

H $-4.2011722 .723847-0.320595$

H -2.786956 $3.749748-0.033219$

H $1.7836681 .621163-0.273413$

H $\quad 0.206719-2.1225720 .102766$

H 2.9041901 .3376761 .676552

H $3.138977-0.156110-1.569491$

H $3.367574-1.8874910 .308478$

H $4.298726-0.6995341 .227209$

H $3.8299311 .926676-1.107777$

H -3.912689 -0.5280931 .283486$

H -4.8608720 .3781370 .098731$

H $6.030121-0.651527-0.545736$

H $5.753615-2.2975750 .039165$

H $5.076973-1.791387-1.517900$

SCF Energy (B3LYP/6-31G**/MMFF) $=-810.714437305$

F2-32 c07651

MMFF Geometry

C $-4.334225-0.9811520 .549619$

C $-5.387080-0.493878 \quad 1.215781$

C $-2.5220040 .068113-0.897959$

O $-1.533193-0.925111-0.564660$

C $-0.486489-0.2924180 .207282$

C $-0.731961 \quad 1.2068840 .093751$

C $-2.244658 \quad 1.2635210 .011320$

C $0.877098-0.765897-0.311362$

C $-2.7806152 .581000-0.519366$

C $2.073957-0.0352880 .337062$

O $0.958400-2.169020-0.040177$

O $1.953946-0.1293811 .755388$

C $3.443416-0.587683-0.103182$

$\begin{array}{llll}C & 4.653610 & 0.139760 & 0.507247\end{array}$

O $3.527882-0.531843-1.525683$

C $-3.929247-0.531092-0.825497$

C $4.732421 \quad 1.6225690 .167829$

H $-3.736170-1.7584121 .018179$

H $-5.630799-0.8650952 .205304$

H -6.0189220 .2786070 .791813$

H $-2.3277670 .343757-1.943213$

H $-0.638671-0.6262421 .241539$

H $-0.283247 \quad 1.607722-0.823883$

H -0.3283531 .7647700 .943998$

H -2.647415 1.0932141 .017397

H $0.904415-0.652839-1.401977$

H -2.397278 $2.796228-1.522458$

H -3.873788 $2.563252-0.572348$

H -2.487424 3.4074320 .136268

H 1.9996591 .0223460 .060569

H $1.670644-2.534403-0.591352$

H 2.5591210 .5218542 .148128

H $3.522506-1.6400710 .194738$

H $5.569501-0.3330830 .130617$

H $4.6603910 .016127 \quad 1.595929$
H $4.378697-0.924276-1.785822$

H $-4.6510170 .194408-1.219209$

H $-3.972048-1.411687-1.478618$

H 3.9041872 .1802440 .614401

H 5.6629902 .0458590 .559839

H $4.7191611 .786508-0.913939$

SCF Energy (B3LYP/6-31G**//MMFF)= -810.709447625

F2-32 c07652

MMFF Geometry

C $3.549490-1.953481-0.324450$

C $4.746947-2.139565-0.891175$

C $2.397497 \quad 0.046946 \quad 0.747817$

O $1.073659-0.3913840 .387164$

C $0.5292700 .529996-0.576767$

C $1.4710031 .731073-0.588796$

C $2.8070321 .071121-0.308882$

C $-0.928493 \quad 0.887877-0.252385$

C 3.8842962 .0368390 .151361

C $-1.903483-0.306669-0.351332$

$\begin{array}{llll}\text { O } & -1.004492 & 1.461463 & 1.052816\end{array}$

O $-1.470219-1.3750390 .488429$

C $-3.3609560 .050300 \quad 0.008105$

C $-4.301796-1.148631-0.189734$

O $-3.818798 \quad 1.119442-0.815093$

C $3.328569-1.1546530 .928896$

C $-5.738414-0.8293240 .205793$

H $2.678756-2.413677-0.784462$

H $4.840842-2.732600-1.794420$

H $5.647497-1.706548-0.470208$

H 2.2929680 .5410901 .723203

H $0.5714530 .028500-1.551964$

H 1.2231342 .4443420 .206402

H $1.4470092 .267290-1.542299$

H $3.1412570 .574533-1.228058$

H $-1.2492441 .663289-0.957262$

H $4.0811952 .789061-0.619482$

H 3.5889702 .5623741 .065723

H $4.820631 \quad 1.5072510 .353751$

H -1.870752 -0.675505 -1.384055

H -0.5983820 .8335401 .674385$

H $-1.518925-1.0683281 .409588$

H $-3.4269320 .367647 \quad 1.056126$

H -3.958916 -2.0043080 .402556$

H $-4.297698-1.452075-1.243715$

H -3.394702 $1.935617-0.500790$

H $4.286190-0.810757 \quad 1.338100$

H $2.889045-1.8334811 .670421$

H $-6.155730-0.029807-0.414152$

H $-6.369144-1.7150850 .079402$

H -5.795485-0.519193 1.253987

SCF Energy (B3LYP/6-31G*//MMFF) $=-810.717651630$

F2-32 c07653

MMFF Geometry

C $-2.5513502 .528755 \quad 1.012588$

C $-1.718979 \quad 3.575240 \quad 0.974282$

C -2.351185 0.1829920 .151262

O $-0.912691 \quad 0.144506 \quad 0.247162$

C $-0.422043-0.969789-0.519303$

C $-1.634395-1.852533-0.789924$

C $-2.724275-0.810975-0.949840$

C $0.730569-1.658794 \quad 0.215407$

C $-4.128528-1.375479-0.822703$

C $1.902812-0.7497090 .650399$

O $0.217596-2.2783401 .404512$

O $2.898859-1.5999051 .233230$

C $2.5595270 .056909-0.487281$

C $3.8825610 .715400-0.059195$

O $1.674801 \quad 1.078849-0.939680$

C $-2.8041791 .612361-0.151073$

C $4.5303861 .507333-1.188682$

H $-3.0892802 .323803 \quad 1.934530$

H -1.5830854 .2014541 .849104$

H $-1.160513 \quad 3.8238220 .078479$

H -2.743762 $-0.150251 \quad 1.120910$

H $-0.066945-0.571464-1.476736$

H $-1.860457-2.5085710 .058572$ 
H $-1.503249-2.478800-1.677418$

H -2.616434 -0.338450 -1.935636

H $1.116368-2.476801-0.404542$

H -4.281625 -1.855585 0.149677

H -4.878198 $-0.585283-0.930009$

H $-4.313561-2.124374-1.599842$

H $1.564662-0.0677411 .440604$

H $-0.189029-1.5798981 .945968$

H $2.473037-2.0924051 .955909$

H $2.762009-0.609549-1.334256$

H $4.594749-0.0435170 .282791$

H 3.7007041 .3997020 .778438

H $1.4748881 .657006-0.183559$

H -2.282252 $1.985331-1.041451$

H $-3.8778511 .630065-0.369459$

H $5.4855331 .925400-0.855091$

H $4.7244810 .866107-2.054306$

H $3.8957602 .338864-1.510081$

SCF Energy $\left(B 3 L Y P / 6-31 G^{* *} / / M M F F\right)=-810.721865823$

\section{F2-32 c07654}

MMFF Geometry

C $-4.669280-1.4866680 .095266$

C -4.152637-2.440971-0.687441

C $-2.753396 \quad 0.227086-0.250732$

O $-1.712865-0.577674 \quad 0.339069$

C $-0.564650 \quad 0.2566730 .609098$

C $-0.8268311 .567260-0.116683$

C $-2.3340311 .677723-0.010036$

C $0.705209-0.4819520 .169545$

C $-2.9382122 .669675-0.988994$

C $1.993273 \quad 0.365528 \quad 0.258086$

O $0.828145-1.634191 \quad 1.010036$

$\begin{array}{lllll}\text { O } & 2.160537 & 0.862318 & 1.585858\end{array}$

C $3.269568-0.409094-0.127255$

C $4.5019420 .510672-0.147778$

O $3.081484-0.985463-1.416627$

C $-4.101955-0.1217790 .387439$

C $5.794600-0.237656-0.447428$

H $-5.615318-1.7057660 .587038$

H $-4.673560-3.385170-0.809039$

H -3.217442 -2.317064 -1.219571

H $-2.7639470 .011226-1.326641$

H -0.5616210 .4122521 .695207$

H $-0.521527 \quad 1.498727-1.168320$

H $-0.303016 \quad 2.4137270 .336889$

H -2.592496 $1.979627 \quad 1.013835$

H $0.561131-0.852026-0.852940$

H -2.707945 $2.401560-2.025601$

H $-4.0269752 .708351-0.885024$

H $-2.5461683 .675857-0.807546$

H $1.8949411 .226683-0.414009$

H $1.467582-2.2359690 .593417$

H 1.4562811 .5104861 .753498

H $3.445653-1.2160580 .594391$

H $4.611627 \quad 1.0158340 .818430$

H $4.3717061 .285701-0.912853$

H $3.793763-1.630953-1.560118$

H -4.009408 -0.0431541 .478301$

H -4.8522940 .6129980 .072500$

H $5.954244-1.0479660 .270793$

H $5.787392-0.663609-1.455181$

H $6.6474020 .445806-0.382661$

SCF Energy (B3LYP/6-31G**//MMFF) $=-810.707144266$

F2-32 c07655

MMFF Geometry

C $-2.6453942 .266791 \quad 1.090806$

C - -1.8872253 .3682261 .132406$

C $-2.214592-0.0286240 .178923$

O -0.7864840 .0440040 .367242$

C $-0.155970-0.962891-0.442624$

C $-1.260895-1.946711-0.805085$

C $-2.439146-1.007786-0.975395$

C $1.039742-1.5680470 .291853$

C $-3.786531-1.707614-0.936018$

C $2.086193-0.5647990 .822368$

O $0.559444-2.3073361 .425750$
O $3.168093-1.3532021 .357272$

C $2.7349950 .401457-0.187015$

C $1.9370231 .690384-0.416381$

O $2.945390-0.252141-1.436822$

C $-2.7604571 .370787-0.109920$

C $2.7275022 .711784-1.227251$

H -3.2235331 .9977441 .970988$

H -1.849535 3.9757382 .029986

H -1.292556 3.6804740 .280931

H -2.638054 -0.424983 1.110870

H $\quad 0.184780-0.475088-1.362557$

H -1.458263 -2.657109 0.005974

H -1.030639 -2.517577 -1.709601

H $-2.335947-0.486623-1.936759$

H $1.527729-2.306775-0.354694$

H $-3.866074-2.434802-1.750681$

H -3.933360 -2.243319 0.007870

H -4.603359 -0.987312 -1.045049

H $1.688880-0.0016781 .675215$

H $\quad 0.061443-1.6893501 .988268$

H $2.787839-1.9490002 .025681$

H 3.7287600 .6821180 .186167

H 1.6666322 .1394030 .545934

H $1.0131801 .492453-0.964720$

H $3.493924-1.036571-1.264963$

H $-2.2196851 .810707-0.957459$

H $-3.8180891 .312686-0.391053$

H $3.6595292 .979315-0.719411$

H $2.9743602 .325570-2.221148$

H $2.1390203 .625642-1.357332$

SCF Energy (B3LYP/6-31G**//MMFF) $=-810.720255405$

F2-32_c07656

MMFF Geometry

C -1.7413892 .9398570 .281135$

C $-0.8082503 .723221-0.271164$

C $-2.1243330 .467016 \quad 0.169638$

O $-0.7403740 .147035-0.071557$

C $-0.635002-1.224787-0.487979$

C $-1.993992-1.849646-0.192333$

C $-2.924559-0.680320-0.444248$

C $0.537070-1.9306840 .207632$

C $-4.306843-0.8629790 .157730$

C $1.941328-1.425994-0.197437$

O $0.370446-1.8443051 .621126$

O $2.150811-1.705238-1.582318$

C $2.2438810 .065700 \quad 0.049696$

C $3.7135890 .399194-0.257697$

O 1.9844640 .4145721 .404972

C $-2.4459041 .829119-0.445933$

C $4.0183551 .882628-0.086172$

H -2.019502 3.1117811 .317691

H -0.3383474 .5103040 .308346$

H $-0.4955353 .593579-1.301370$

H $-2.2610890 .493222 \quad 1.258773$

H $-0.472791-1.216974-1.572993$

H -2.066984 -2.177647 0.851410

H -2.202729 -2.711962 -0.832905

H $-3.020599-0.534933-1.528638$

H $0.474374-2.994440-0.056710$

H $-4.256583-1.0060841 .242275$

H $-4.9365810 .010453-0.037864$

H -4.801465 -1.739223 -0.274209

H $2.675378-2.0105530 .373093$

H $1.102054-2.3333072 .034642$

H $2.042954-2.663152-1.709339$

H $1.6223880 .692565-0.599754$

H $3.9641690 .107348-1.283550$

H $4.369793-0.1638410 .417106$

H $1.0305600 .305471 \quad 1.558368$

H $-2.1591951 .833994-1.505103$

H $-3.5235812 .021610-0.397583$

H $5.0631912 .084128-0.342813$

H $3.3851272 .490169-0.740606$

H 3.8585062 .2070340 .946747

SCF Energy (B3LYP/6-31G**//MMFF $)=-810.718294334$

F2-32 c07657 
MMFF Geometry

C $4.2120181 .271737-0.910494$

C $4.9436582 .097077-0.153378$

C $2.146024-0.151125-0.523722$

O $0.714321-0.095353-0.716503$

C $0.064228-0.5762420 .471271$

C $1.115516-1.3939941 .203922$

C $2.370211-0.5849670 .929076$

C -1.214424 -1.340606 0.113747

C $3.639668-1.3885031 .164962$

C $-2.213303-0.555018-0.758078$

O $-0.864817-2.532414-0.603999$

O $-3.393972-1.362131-0.869341$

C $-2.6002280 .836470-0.224480$

C -3.3363020 .8081051 .122340$

O $-3.4239241 .480494-1.201768$

C $2.7154691 .223068-0.888591$

C -3.6440662 .2088121 .637677$

H $4.7238840 .600448-1.594962$

H $6.0261192 .088291-0.219272$

H 4.4814082 .7892440 .541558

H $2.521000-0.911084-1.220960$

H $-0.184197 \quad 0.3024591 .078478$

H $1.215196-2.3996940 .779322$

H $0.895514-1.4959622 .270915$

H 2.3734220 .2930001 .588612

H -1.705646 -1.682866 1.031656

H $4.533592-0.7819770 .999256$

H $3.674855-1.7546402 .196547$

H $3.693393-2.2567420 .499412$

H - $1.821371-0.461168-1.778583$

H $-0.368533-2.263077-1.396025$

H -3.966150 -0.952444 -1.539683

H -1.702761 $1.457228-0.138682$

H $-2.734290 \quad 0.286179 \quad 1.873327$

H -4.2855650 .2681991 .024909$

H -4.293034 $1.045059-1.197059$

H $2.3501171 .502546-1.885484$

H $2.3019721 .982301-0.211857$

H -4.140606 2.1508972 .611455

H $-2.7251232 .791046 \quad 1.759806$

H -4.306885 2.7490930 .954685

SCF Energy (B3LYP/6-31G**//MMFF) $=-810.713730521$

Fragment F3 (cutoff energy $=10 \mathrm{~kJ} / \mathrm{mol}$ )

F3-01_c00001

MMFF Geometry

C $3.855074-0.1576130 .265248$

C $2.801865-0.958612-0.489467$

C $1.390071-0.7304240 .065997$

C $0.377315-1.613538-0.667340$

C $-1.064125-1.338477-0.213251$

O $1.059716 \quad 0.651614-0.108930$

O $-1.950380-1.956685-1.159013$

C $-1.3221680 .181624-0.280201$

C $-1.368870-1.9773871 .146533$

$\begin{array}{lllll}\text { O } & -2.599102 & 0.508316 & 0.262457\end{array}$

C $-0.225672 \quad 1.0218670 .409774$

C -0.4085322 .5387730 .176455$

O

C $-0.3551072 .981460-1.279808$

H $3.859299-0.4203891 .327830$

H $4.850013-0.367795-0.140077$

H 3.6772130 .9187390 .177148

H $3.056889-2.023166-0.433593$

H $2.839567-0.662761-1.544939$

H $1.397389-0.9660841 .136951$

H $0.623391-2.675233-0.540463$

H $0.441236-1.419421-1.747374$

H -1.745022 -2.906992 -1.185386

H $-1.3591680 .447826-1.343872$

H -1.243162 -3.065719 1.094557

H $-0.724178-1.6108831 .947964$

H -2.412037 -1.810397 1.436425

H -3.258767 -0.035992 -0.200708

H $\quad 0.3909913 .0718090 .708750$

H -1.346731 2.8805880 .630716

H 0.1392531 .5561192 .270264
H $-0.3751564 .074899-1.336667$

H $0.5604032 .638080-1.770713$

$\mathrm{H}-1.2148132 .610793-1.844833$

SCF Energy (B3LYP/6-31G**//MMFF $)=-694.025705043$

F3-01 c00002

MMFF Geometry

C $3.5381590 .201031-0.755143$

C $2.979610-0.2901960 .574661$

C $1.448399-0.3989350 .628172$

C $0.894240-1.438976-0.349007$

C $-0.640425-1.497419-0.312993$

O $0.895067 \quad 0.892921 \quad 0.355917$

O $-1.056417-2.263324-1.454362$

C $-1.188809-0.063288-0.476667$

C $-1.150442-2.2544960 .918096$

O $-2.607471-0.043726-0.338594$

C -0.5329090 .9625220 .474728$

C -0.96189124140290 .163194$

O $-0.950631 \quad 0.682046 \quad 1.816492$

C $-0.5891202 .914321-1.226151$

H $3.1067921 .165428-1.039987$

H $4.6229120 .329272-0.678386$

H $3.348943-0.515559-1.559428$

H $3.301840 \quad 0.418800 \quad 1.347681$

H $3.428171-1.2599770 .819556$

H $1.188124-0.6797881 .655854$

H $1.323022-2.428811-0.149006$

H $1.193143-1.184058-1.374111$

H -2.028390 -2.239481-1.486617

H $-0.9828510 .226679-1.513986$

H -2.242233 -2.344749 0.904402

H $-0.766984-3.2819740 .917869$

H $-0.854673-1.7909811 .861336$

H -2.813395 -0.1123390 .609272$

H $-0.482053 \quad 3.0829590 .890360$

H -2.041352 2.5309190 .319043

H -0.8305121 .4849402 .350289$

H $-0.8267303 .979640-1.313679$

H $0.4803182 .793214-1.423401$

$\mathrm{H}-1.1493492 .390974-2.006032$

SCF Energy $(B 3 L Y P / 6-31 G * * / / M M F F)=-694.031846217$

F3-01 c00003

MMFF Geometry

C $-3.442804-1.314501-0.867151$

C $-2.981559-0.9006810 .524741$

C $-1.580007-0.2748390 .579159$

C $-1.4863191 .035819-0.200897$

C $-0.0694241 .621932-0.146521$

O $-0.642036-1.2208410 .061291$

O $-0.0138752 .670573-1.125846$

C $0.9438360 .529895-0.558391$

C 0.2129622 .2845421 .207639

O $2.271279 \quad 1.019222-0.382922$

C $0.737404-0.8222430 .166181$

C $1.537988-1.975797-0.481687$

O $1.130396-0.6963831 .533885$

C $3.054244-1.860075-0.428374$

H $-4.409308-1.824887-0.802486$

H -3.572909-0.447577-1.521135

H -2.732809 -2.001620 -1.337310

H $-2.981486-1.8011481 .151820$

H $-3.712771-0.2074900 .956564$

H -1.353335 -0.0998751 .637611$

H -2.2202021 .7633850 .166871$

H -1.735779 $0.863458-1.255992$

H $0.9017272 .998241-1.154850$

H $0.8202870 .364513-1.637116$

H $\quad \begin{array}{llll}1.186647 & 2.787028 & 1.209332\end{array}$

H -0.5260543 .0694221 .409060$

H $\quad 0.188151 \quad 1.5809262 .042368$

H 2.4842190 .9641640 .564277

H $1.219692-2.106092-1.524010$

H $1.261615-2.9109930 .024877$

H $1.318360-1.5847551 .879813$

H $3.509191-2.798700-0.762245$

H $3.420137-1.073267-1.093146$ 
H $3.410584-1.6595970 .586243$

SCF Energy (B3LYP/6-31G**//MMFF) $=-694.030508478$

F3-02 c00014

MMFF Geometry

C -3.263857 -1.704276 -0.183203

C $-1.820124-2.166394-0.334299$

C $-0.818112-1.1130660 .158345$

C $0.613768-1.6572630 .072687$

C $1.651030-0.5838680 .436182$

O $-0.964658 \quad 0.053933-0.656587$

O $1.548493-0.3300661 .839026$

C $1.3710780 .713775-0.349739$

C $3.066211-1.1184130 .185500$

O $1.7421640 .512530-1.721870$

C $-0.1143561 .153746-0.313043$

C -0.5314231 .7867851 .033649$

O $-0.2921572 .196589-1.287946$

C -1.988780 2.2444551 .056393

H -3.947921 -2.491396 -0.515840

H -3.493631 -1.4742610 .861999$

H -3.462231 -0.811236 -0.783901

H -1.640757 -2.384679-1.394190

H -1.685174 -3.0996910 .224431$

H -1.065438 -0.8693501 .197465$

H $0.802757-2.014451-0.947815$

H $\quad 0.709024-2.5186870 .745697$

H 2.1956340 .3591302 .067002

H 1.9996861 .5328760 .019662

H $3.816688-0.3602350 .436828$

H $3.272523-1.9830360 .826995$

H $3.217378-1.422080-0.855108$

H $1.109950-0.113152-2.114794$

H $\quad 0.1000222 .661387 \quad 1.234684$

H -0.3980091 .0957921 .868892$

H $0.2878322 .003138-2.043316$

H $-2.672027 \quad 1.404606 \quad 0.897958$

H -2.225021 2.6890842 .028659

H -2.1846332 .9994640 .288868$

SCF Energy (B3LYP/6-31G**//MMFF) $=-694.027177398$

F3-02 c00015

MMFF Geometry

C $3.813747-0.7421950 .010535$

C $2.520347-1.097971-0.704052$

C $1.262461-0.518805-0.040457$

C $1.2425411 .015293-0.063493$

C -0.0874361 .5765620 .463952$

O $0.145403-1.047192-0.766045$

O -0.1531501 .3167641 .868008$

C $-1.2676950 .886716-0.249396$

C -0.1075603 .1013880 .301378$

O $-1.3114961 .333688-1.612621$

C $-1.145168-0.657952-0.284563$

C $-1.461884-1.3300471 .070516$

O $-2.143593-1.157960-1.192547$

C -1.378453 -2.854782 1.018538

H $3.771699-1.0299151 .065599$

H $4.654582-1.269958-0.451039$

H $4.0245460 .329413-0.049617$

H $2.425918-2.190910-0.742946$

H $2.574640-0.772238-1.750483$

H $1.226518-0.8843060 .991752$

H $1.3962421 .359617-1.094397$

H 2.0692191 .4008220 .544654

H -1.0033401 .6565212 .195876$

H -2.226136 $1.159640 \quad 0.209442$

H $0.671358 \quad 3.5663780 .917140$

H $0.0505993 .412322-0.735934$

H -1.062290 3.5145910 .645982

H -1.665895 2.238776 -1.614088

H -2.477935 -1.057884 1.382631

H $-0.782982-0.9990521 .859323$

H -2.212392 -0.530070 -1.931332

H -1.614655 -3.275034 2.001596

H $-2.089585-3.2717360 .299015$

H $-0.372822-3.188610 \quad 0.744718$

SCF Energy (B3LYP/6-31G**/MMFF) $=-694.022708487$
F3-02_c00016

MMFF Geometry

C -3.543408 -0.8516640 .480728$

C $-2.793841-0.860226-0.845938$

C $-1.336030-0.379962-0.765310$

C $-1.2226181 .096721-0.366839$

C $0.2466541 .525224-0.214958$

O $-0.637016-1.2219300 .156148$

O $0.883818 \quad 1.499639-1.493565$

C 0.9735090 .5383440 .719577

C $0.3244172 .971148 \quad 0.289291$

O 0.4553930 .6873622 .050641

C $0.762792-0.943240 \quad 0.317751$

C $1.529559-1.412516-0.940379$

O $1.258613-1.7722871 .384722$

C $3.042710-1.242836-0.867050$

H -3.020627 -1.4395601 .241079$

H $-3.6782760 .165156 \quad 0.859714$

H -4.539265 -1.286901 0.347905

H $-3.343302-0.255032-1.576190$

H -2.795774 -1.893107-1.216665

H -0.911636 -0.517978 -1.765791

H -1.7337451 .2654470 .588115$

H -1.724149 $1.710551-1.125040$

H $0.3839842 .080088-2.092570$

H 2.0451470 .7583330 .775239

H 1.3681623 .2775380 .421577

H $-0.1105883 .662045-0.442504$

H -0.2028043 .1112581 .238037$

H 0.8190191 .5109712 .417278

H $1.169003-0.924114-1.849165$

H $1.320924-2.481071-1.088041$

H $1.066491-1.3206562 .223827$

H $3.462886-1.7365930 .014138$

H $3.509568-1.687783-1.752098$

H $3.326051-0.186837-0.843658$

SCF Energy $\left(B 3 L Y P / 6-31 G^{* *} / / M M F F\right)=-694.020635618$

F3-03_c00028

MMFF Geometry

C $3.400658-1.6378110 .234554$

C $2.070715-2.101075-0.346030$

C $0.900683-1.2185180 .106703$

C $-0.427571-1.767627-0.433650$

C $-1.600749-0.843691-0.073650$

O $1.126797 \quad 0.107907-0.380945$

O $-1.788901-0.867433 \quad 1.344224$

C $-1.2546310 .592673-0.511886$

C $-2.901003-1.331646-0.717060$

O $-2.276508 \quad 1.500886-0.111384$

C $\quad 0.1246681 .0569390 .011501$

C $0.5059082 .411230-0.621818$

O $\quad 0.109876 \quad 1.2584581 .426850$

C $1.8824812 .914331-0.193968$

H $3.372585-1.6422231 .328785$

H $4.205234-2.306815-0.086756$

H $3.651688-0.626186-0.099407$

H $1.892986-3.139779-0.044101$

H $2.150560-2.081817-1.439850$

H $0.883686-1.1992891 .203503$

H -0.593850 -2.772244 -0.025601

H $-0.365323-1.855180-1.526281$

H -1.981640 -1.7839481 .606527$

H $-1.2268630 .606154-1.609752$

H $-3.752509-0.719423-0.400107$

H -2.843307 -1.309422 -1.810013

H $-3.127414-2.357955-0.405833$

H -2.3229431 .4790910 .859662$

H $-0.2308923 .175765-0.347696$

H $0.5062432 .321763-1.715063$

H -0.4959550 .6117281 .824022$

H $2.0949053 .874740-0.674900$

H 1.9341323 .0639070 .888830

H $2.6716032 .213748-0.484110$

SCF Energy (B3LYP/6-31G**//MMFF) $=-694.035086577$

F3-03_c00029 
MMFF Geometry

C $3.340300-1.164111-0.835213$

C $2.730292-1.1010920 .559519$

C $1.244700-0.7135150 .593802$

C $0.344414-1.748752-0.095561$

C $-1.125966-1.302268-0.075786$

O $1.0986510 .572456-0.016932$

O $-1.592081-1.2983401 .276533$

C $-1.2144240 .127495-0.645150$

C $-2.006493-2.277431-0.861134$

O $-2.5495700 .618171-0.567003$

C $-0.2331151 .104756 \quad 0.044025$

C $-0.2183912 .456072-0.700881$

O $-\begin{array}{llll}0.629937 & 1.384980 & 1.388874\end{array}$

C $0.764293 \quad 3.464175-0.110227$

H $4.414497-1.363865-0.762934$

H $2.896902-1.966592-1.431536$

H $3.211401-0.219683-1.372535$

H $3.294776-0.353606 \quad 1.131087$

H $2.873037-2.0653031 .060873$

H $0.963511-0.6294201 .651331$

H $0.458409-2.7163730 .408110$

H $0.651338-1.873466-1.140710$

H $-1.502566-2.2008871 .627741$

H $-0.9547470 .075768-1.710953$

H $-1.729779-2.308033-1.919944$

H $-1.921385-3.291610-0.454154$

H $-3.064528-2.003403-0.784911$

H -2.7960580 .6466840 .373206$

H - $1.2169752 .908715-0.679046$

H $0.0553902 .296625-1.751133$

H $-1.043030 \quad 0.5887531 .760651$

H $0.519801 \quad 3.697357 \quad 0.930611$

H $1.7918713 .089545-0.148278$

H $0.7279834 .398670-0.679620$

SCF Energy (B3LYP/6-31G**//MMFF) $=-694.033768103$

F3-03_c00030

MMFF Geometry

C $3.418494-1.6056450 .184990$

C $2.080527-2.088709-0.360210$

C $0.910378-1.2202640 .120029$

C $-0.422608-1.789336-0.383648$

C $-1.606229-0.872082-0.035832$

$\begin{array}{lllll}\text { O } & 1.105736 & 0.106879 & -0.377681\end{array}$

O $-1.842598-0.930714 \quad 1.373481$

C $-1.2733170 .570532-0.475033$

C $-2.884644-1.391739-0.699759$

O $-2.284298 \quad 1.480945-0.050798$

C $0.106352 \quad 1.046848 \quad 0.040880$

C $0.453622 \quad 2.421895-0.574560$

$\begin{array}{lllll}\text { O } & 0.052113 & 1.164729 & 1.464980\end{array}$

C $1.8788242 .893855-0.295118$

H $3.646799-0.591725-0.157870$

H $3.418430-1.6071721 .279689$

H $4.223670-2.264607-0.155022$

H $1.924694-3.128770-0.050946$

H $2.132566-2.071676-1.455642$

H $\quad 0.924393-1.1993001 .216539$

H $-0.573949-2.7859750 .049290$

H $-0.371964-1.901751-1.474804$

H $-1.103921-0.4954011 .830074$

H -1.249116 $0.591307-1.573370$

H -3.762657 -0.822118 -0.376455

H $-2.820792-1.346092-1.791642$

H - $3.075378-2.431781-0.409886$

H $-3.098563 \quad 1.270532-0.537522$

H $-0.2383323 .186193-0.201250$

H $0.3392972 .371230-1.664510$

H $\quad 0.7373081 .7849401 .758517$

H 2.0593703 .0370450 .773621

H $2.6176482 .183558-0.678667$

H $2.0544873 .854722-0.790436$

SCF Energy (B3LYP/6-31G**//MMFF) $=-694.026697231$

F3-04_c00034

MMFF Geometry

C $-2.237688 \quad 2.5524990 .430135$
C $-0.731893 \quad 2.331702 \quad 0.358123$

C $-0.327557 \quad 1.463637-0.844102$

C $1.1903301 .275479-0.996719$

C $1.7699070 .161502-0.114248$

O $-1.0393530 .226387-0.902897$

$\begin{array}{llll}\text { O } & 1.738569 & 0.569945 & 1.253943\end{array}$

C $0.910304-1.102914-0.289029$

C $3.236337-0.104274-0.470034$

O $1.377011-2.1497290 .557723$

C $-0.591414-0.834013-0.048263$

C $-1.416689-2.085037-0.423197$

O $-0.864465-0.5799861 .330384$

C $-2.922400-1.891121-0.257151$

H $-2.610095 \quad 3.017558-0.487995$

H $-2.772515 \quad 1.6098260 .581198$

H -2.4812593 .2142821 .267443$

H -0.4099491 .8918991 .304143$

H -0.2344503 .3061850 .282734$

H $-0.6557382 .018283-1.733197$

H $1.6991862 .228418-0.807132$

H $1.3838121 .011872-2.045736$

H $2.2561301 .389243 \quad 1.335420$

H $1.036154-1.450122-1.323375$

H $3.343985-0.464445-1.498273$

H $3.8336990 .808429-0.361478$

H $3.679703-0.8435560 .206314$

H $\quad 1.305690-1.8391371 .476316$

H -1.117892 -2.935305 0.201716

H $-1.226535-2.354222-1.469519$

H $-0.072085-0.1955591 .738560$

H $-3.294181-1.084313-0.896331$

H $-3.449941-2.808735-0.536920$

H -3.183227 -1.658299 0.779794

SCF Energy (B3LYP/6-31G**//MMFF)= -694.027229173

F3-04 c00035

MMFF Geometry

C 3.5203840 .5142010 .315396

C $2.515098-0.616866 \quad 0.138927$

C $1.455122-0.293273-0.926452$

C $0.467844-1.440333-1.192299$

C $-0.692646-1.507718-0.191821$

O $0.7961420 .953139-0.696643$

O $-0.187053-1.9535201 .066621$

C -1.312537-0.104002 -0.054829

C $-1.727952-2.541931-0.649256$

$\begin{array}{llll}\text { O } & -2.341175 & -0.129968 & 0.930130\end{array}$

$\begin{array}{llll}\text { C } & -0.256706 & 0.974746 & 0.277291\end{array}$

$\begin{array}{llll}\text { C } & -0.834873 & 2.407485 & 0.259562\end{array}$

$\begin{array}{lllll}\text { O } & 0.244364 & 0.818046 & 1.609259\end{array}$

C $-1.4411542 .846985-1.066517$

H $3.0358831 .425757 \quad 0.678461$

H $4.2851670 .227683 \quad 1.044205$

H $4.0227640 .743112-0.629899$

H $2.064215-0.8219411 .112012$

H $3.053961-1.526310-0.152741$

H $2.011201-0.147885-1.862230$

H $1.011403-2.392637-1.221477$

H $0.044232-1.281143-2.193585$

H $-0.933944-1.9923471 .688860$

H $-1.780814 \quad 0.129278-1.019042$

H $-1.268647-3.532646-0.743900$

H $-2.535942-2.6471480 .083301$

H $-2.169574-2.272412-1.614210$

H -2.8326080 .7061530 .868561$

H $-0.024435 \quad 3.1092680 .501417$

H $-1.5754212 .531261 \quad 1.059224$

H $0.139886-0.1098791 .873297$

H $-0.7339652 .725664-1.892350$

H $-2.3492282 .283586-1.298776$

$\mathrm{H}-1.7178303 .905253-1.016078$

SCF Energy (B3LYP/6-31G**//MMFF) $=-694.026352369$

F3-04 c00036

MMFF Geometry

C -3.606792 -1.2106190 .069289$

C $-2.122623-1.3696430 .365648$

C $-1.214474-0.915664-0.789607$ 
C $-1.2553420 .581620-1.133444$ C $-0.3993741 .450975-0.203883$

O $0.126750-1.386909-0.607647$

O $-1.019503 \quad 1.4978811 .080583$

C $1.0076330 .830253-0.108491$

C $-0.3472312 .892515-0.722560$

O $1.802044 \quad 1.5875580 .800368$

C $0.966771-0.6585290 .300050$

C $2.360256-1.3293620 .262744$

O $0.501994-0.7564321 .650453$

C $3.058276-1.295676-1.090461$

H -3.880584-1.720972 -0.859424

H $-4.199076-1.6465050 .880467$

H $-3.889786-0.157696-0.015732$

H $-1.916344-2.4266740 .576928$

H $-1.896036-0.8370231 .292453$

H $-1.572528-1.448025-1.680424$

H $-2.2899370 .939969-1.154516$

H $-0.8631140 .691479-2.154066$

H $-0.411898 \quad 1.9572761 .685333$

H $1.4624150 .923512-1.102525$

H $-1.3563923 .314298-0.794174$

H $0.208293 \quad 3.541777-0.036536$

H $\quad 0.1218482 .950304-1.710088$

H $2.723807 \quad 1.295560 \quad 0.703974$

H $2.244428-2.384898 \quad 0.544434$

H $3.015396-0.892067 \quad 1.025839$

H $0.783094-1.6130552 .012382$

H $2.423881-1.708919-1.880289$

H $3.344368-0.277587-1.369040$

H $3.975299-1.892768-1.050770$

SCF Energy (B3LYP/6-31G**//MMFF) $=-694.017706416$

F3-05 c00053

MMFF Geometry

C $-3.616175-1.4350090 .005897$

C $-2.991291-0.137248-0.489864$

C $-1.600105 \quad 0.1046490 .110272$

C $-1.0479841 .456571-0.350544$

C $0.386476 \quad 1.678727 \quad 0.143398$

O $-0.739562-0.955396-0.315789$

$\begin{array}{llll}\text { O } & 0.906888 & 2.828857 & -0.537475\end{array}$

C $1.253616 \quad 0.451006-0.215811$

C $0.416798 \quad 2.019598 \quad 1.638636$

O $1.4441270 .429783-1.637143$

C $0.603817-0.8912840 .196295$

C $1.332552-2.118084-0.399743$

O $0.621284-1.0039521 .620438$

C $2.794211-2.246587 \quad 0.007264$

H $-4.616621-1.558253-0.420798$

H $-3.017447-2.303537-0.285472$

H $-3.710503-1.4319731 .096420$

H $-3.6563230 .696732-0.237583$

H -2.921862 $-0.187987-1.583488$

H -1.6868490 .0765101 .202672$

H -1.698407 2.275767 -0.018979

H -1.052650 $1.500519-1.448555$

H $1.8250672 .958673-0.243805$

H $2.2450350 .560810 \quad 0.239298$

H -0.0599861 .2662802 .268540$

H 1.4490782 .1502091 .983589

H $\quad-0.0890372 .9749281 .823050$

H $1.827098 \quad 1.286625-1.891535$

H $1.254573-2.115228-1.494065$

H $0.810468-3.028842-0.075906$

H $0.532495-1.942764 \quad 1.854437$

H $2.906462-2.2733191 .095363$

H $3.213048-3.174797-0.394989$

H $3.392983-1.418715-0.383245$

SCF Energy (B3LYP/6-31G**//MMFF) $=-694.026539315$

F3-05_c00054

MMFF Geometry

C -3.922231 -1.101720 0.022063

C $-2.540768-1.538487-0.436837$

C $-1.398406-0.6889220 .137160$

C $-1.455500 \quad 0.765524-0.340702$

C $-0.223772 \quad 1.562468 \quad 0.109205$
O $-0.178058-1.306468-0.291484$

$\begin{array}{lllll}\text { O } & -0.226808 & 2.805450 & -0.606076\end{array}$

C $1.0678970 .786601-0.235037$

C -0.3185011 .9292491 .596521$

O $1.2708150 .841667-1.653640$

C $1.023601-0.6940790 .209081$

C $2.189066-1.528717-0.370405$

O $1.085521-0.760777 \quad 1.634779$

C $3.575864-1.0422270 .028012$

H $-4.677623-1.807852-0.337890$

H $-3.981913-1.0703261 .114483$

H $-4.182104-0.113752-0.368811$

H $-2.496374-1.533233-1.533226$

H $-2.383636-2.584506-0.143409$

H $-1.451051-0.7398081 .230912$

H $-2.367222 \quad \begin{array}{lll}\text { H } & 1.262035 & 0.012216\end{array}$

H $-1.5132790 .789928-1.437310$

H $\quad 0.586150 \quad 3.285242-0.371877$

H 1.9219601 .3022650 .218948

H $-0.442557 \quad 1.0644502 .251318$

H $0.5754662 .476767 \quad 1.916994$

H $-1.1662652 .602557 \quad 1.771116$

H $0.5835210 .300040-2.076784$

H $2.117334-1.579050-1.463899$

H $2.083337-2.566607-0.026446$

H $1.388170-1.6488981 .887072$

H $3.689120-1.0016691 .115579$

H $4.337105-1.725680-0.362127$

H $3.784596-0.049018-0.379868$

SCF Energy (B3LYP/6-31G**//MMFF) $=-694.024617844$

F3-05 c00055

MMFF Geometry

C -2.686276 2.5522800 .021509

C $-1.2491692 .538336-0.483741$

$\begin{array}{llll}\text { C } & -0.436650 & 1.379533 & 0.108508\end{array}$

C $1.0169751 .448178-0.368084$

C $1.8299910 .247000 \quad 0.133700$

$\begin{array}{lllll}0 & -1.035703 & 0.153207 & -0.315269\end{array}$

$\begin{array}{llllll}\text { O } & 3.086374 & 0.221760 & -0.559391\end{array}$

C $1.078866-1.062908-0.201773$

C 2.1720380 .3868851 .622111

O $1.144704-1.260781-1.622449$

C $-0.418867-1.0352680 .206278$

C $-1.183653-2.234520-0.397238$

$\begin{array}{lllll} & -1.572697 & -1.132534 & 1.623943\end{array}$

C $-2.655460-2.282107 \quad 0.008689$

H $-3.2250833 .407098-0.399349$

H $-3.2205651 .642441-0.269075$

H $-2.7165952 .636023 \quad 1.112480$

H $-0.7742373 .493708-0.232029$

H $-1.2728182 .456725-1.577446$

H $-0.4892001 .444166 \quad 1.201438$

H $1.4860562 .387777-0.049989$

H $1.0456311 .458034-1.466466$

H $3.5216881 .080882-0.423886$

H $\quad 1.589197-1.917388 \quad 0.259091$

H 2.8412751 .2403231 .784948

H $1.297157 \quad 0.5342342 .258428$

H $2.714581-0.4968531 .976891$

H $2.085105-1.257845-1.870620$

H $-0.712238-3.171196-0.075375$

H $-1.149796-2.198238-1.492414$

H $0.291010-1.3107572 .027860$

H $-3.185346-1.378427-0.308002$

H $-3.144874-3.140438-0.462827$

H -2.769153 -2.387946 1.091909

SCF Energy (B3LYP/6-31G**//MMFF) $=-694.020588121$

F3-06_c00069

MMFF Geometry

C -3.642591-0.972965 -0.000471

C $-2.369709-1.758622-0.288496$

C $-1.105743-0.9824300 .105483$

C $0.142790-1.843042-0.124856$

C $1.422910-1.0576340 .185066$

O $-1.0454780 .202979-0.691880$

O $1.465386-0.8195351 .593247$ 
C $1.3972590 .275292-0.585212$

C $2.660954-1.889101-0.164677$

O $2.510407 \quad 1.079230-0.200795$

C $0.0714761 .061772-0.434809$

C $-0.087874 \quad 1.799456 \quad 0.913894$

O $0.0732402 .099523-1.432998$

C -1.408466 $2.559013 \quad 1.030465$

H $-4.521447-1.569611-0.264622$

H $-3.712686-0.7173481 .061656$

H -3.679591-0.045802 -0.580928

H - $2.346533-1.989844-1.360395$

H $-2.408239-2.709300 \quad 0.255712$

H $-1.187577-0.7086921 .163484$

H $\quad 0.168360-2.170677-1.172417$

H $\quad 0.084269-2.738340 \quad 0.506527$

H $2.237391-0.2561141 .774103$

H $1.5377390 .039925-1.649171$

H $2.700733-2.124569-1.233115$

H $3.582302-1.3628280 .108658$

H $2.666595-2.830467 \quad 0.396974$

H $2.466812 \quad 1.901920-0.717551$

H 0.7245872 .5253021 .039612

H -0.0366831 .1069181 .757685$

H $0.4405941 .733199-2.254381$

H $-1.460032 \quad 3.0710791 .996869$

H $-1.506148 \quad 3.3158610 .246301$

H -2.264915 1.8813800 .966088

SCF Energy (B3LYP/6-31G**/MMFF) $=-694.027244197$

F3-06_c00070

MMFF Geometry

C -3.605611-1.079905 -0.021393

C $-2.305627-1.817595-0.315675$

C $-1.070010-1.0017170 .087931$

C $0.207703-1.814030-0.150972$

C $1.461195-0.9892950 .176051$

O $-1.0498370 .192197-0.697783$

O $1.499881-0.7423411 .582917$

C $1.3908850 .352585-0.580780$

C $2.726166-1.779103-0.176949$

O $2.452598 \quad 1.221156-0.190762$

C $0.0345611 .088178-0.428534$

C $-0.157783 \quad 1.8070260 .926710$

O $-0.0022312 .136022-1.416169$

C -1.5074202 .5132091 .046139$

H $-3.674198-0.148748-0.592316$

H $-4.462652-1.704346-0.292876$

H $-3.685693-0.8377771 .043186$

H -2.273277 -2.036886 -1.389842

H $-2.311407-2.7746450 .218813$

H $-1.161861-0.7421251 .148673$

H $0.248890-2.123777-1.203571$

H $0.177229-2.7214150 .464766$

H $1.511985-1.6003432 .040354$

H $1.5368640 .140591-1.649214$

H $2.803048-1.958538-1.254103$

H $3.628942-1.2567070 .157388$

H $2.731844-2.7497130 .332716$

H $3.2892950 .817888-0.475584$

H 0.6250052 .5633601 .061511

H $-0.081806 \quad 1.1095651 .764558$

H $0.4008531 .801382-2.234070$

H -2.336552 1.8030210 .973830

H -1.581339 3.0145192 .016721

H -1.632819 3.2723020 .268252

SCF Energy (B3LYP/6-31G**//MMFF) $=-694.014134802$

F3-06_c00071

MMFF Geometry

C -3.545252 -0.426511-0.868264

C $-2.918257-0.1232890 .486834$

C $-1.382645-0.1519720 .506032$

C $-0.811848-1.5376120 .184058$

C $0.722870-1.510516 \quad 0.204993$

O $-0.902457 \quad 0.813682-0.432418$

O $1.165005-1.2861751 .545121$

C $1.215975-0.367687-0.702712$

C $1.296260-2.860934-0.237204$
O $2.632634-0.237438-0.593751$

C $0.5181170 .994285-0.455875$

C 1.0286091 .7542420 .791895

O $0.8474241 .778568-1.618043$

C 0.2446693 .0262061 .111786

H $-3.1725860 .249968-1.643357$

H -3.347409 -1.455769-1.180778

H $-4.631869-0.303809-0.811405$

H -3.310403 -0.8255991 .231573$

H $-3.2480240 .880077 \quad 0.784455$

H $-1.0754180 .148498 \quad 1.514706$

H -1.137902 -1.853889-0.813925

H -1.193452 -2.263832 0.911735

H $0.820328-2.0054522 .101359$

H $1.013275-0.662731-1.742309$

H $1.023714-3.096869-1.270955$

H $2.388722-2.872364-0.153801$

H $\quad 0.930040-3.6686880 .407057$

H $2.9054040 .472755-1.199700$

H 2.0804572 .0319550 .653179

H $\quad 0.976664 \quad 1.1222891 .682045$

H $0.6774022 .713365-1.424228$

H 0.3186523 .7653360 .309590

H $-0.8135352 .810547 \quad 1.287145$

H 0.6440973 .4878522 .021073

SCF Energy $($ B3LYP/6-31G**//MMFF $)=-694.020358194$

F3-07_c00084

MMFF Geometry

C -2.713394 $2.508788-0.016105$

C $-1.2672232 .517263-0.494922$

C -0.4505691 .3656890 .104914$

C $1.0151031 .458611-0.337826$

C $1.8263200 .253368 \quad 0.163858$

O $-1.0250450 .132843-0.336962$

$\begin{array}{lllll}\text { O } & 1.924719 & 0.329434 & 1.590647\end{array}$

C $1.104268-1.064051-0.196797$

C $3.2525480 .297984-0.393733$

O $1.229973-1.309550-1.598315$

C $-0.394786-1.038180 \quad 0.202472$

C $-1.147005-2.255403-0.378390$

O $-0.538849-1.112026 \quad 1.624285$

C $-2.619607-2.3068840 .023782$

H -2.764606 2.5850241 .074636

H -3.255838 $3.359079-0.441437$

H $-3.2300571 .593744-0.322185$

H $-0.8096673 .477151-0.229042$

H $-1.2697372 .441926-1.589229$

H $-0.5197631 .423672 \quad 1.198394$

H 1.4457392 .3926110 .044332

H $1.0622041 .497291-1.433533$

H $2.359126 \quad 1.169096 \quad 1.818716$

H $1.594154-1.8945630 .327176$

H $3.7742531 .201194-0.056552$

H $3.837335-0.552736-0.025906$

H $3.2697420 .284768-1.488018$

H $0.694162-0.648292-2.068228$

H $-0.669020-3.181348-0.036153$

H $-1.108351-2.240873-1.473912$

H $0.222392-0.6717062 .036190$

H $-3.157069-1.416467-0.316490$

H $-3.099442-3.181046-0.428330$

H $-2.735868-2.3872381 .108816$

SCF Energy (B3LYP/6-31G**//MMFF) $=-694.033162398$

F3-07_c00085

MMFF Geometry

C -0.1189913 .3822700 .845640$

C $-0.2082372 .831974-0.572304$

C $-0.2887201 .301094-0.663433$

C $-1.567168 \quad 0.735154-0.032212$

C $-1.580358-0.801117-0.094770$

O $0.8719740 .749462-0.036223$

O $-1.692993-1.205066-1.463885$

C $-0.256018-1.366624 \quad 0.466111$

C $-2.800836-1.3572670 .645603$

O $-0.236010-1.2165931 .886670$

C $0.986640-0.676354-0.155695$ 
C $2.277752-1.0821630 .588294$

O $1.157866-1.082153-1.517438$

C $3.542496-0.472818-0.013430$

H 0.7270042 .9524841 .390479

H $0.019781 \quad 4.4680160 .815249$

H -1.033373 3.1843971 .411761

H $0.6898553 .162196-1.109380$

H -1.070257 $3.279102-1.080883$

H -0.262762 $1.045610-1.730363$

H $-2.4374491 .146815-0.557888$

H -1.6365521 .0437601 .016943$

H -2.514123 -0.827424 -1.822897

H $-0.207072-2.4411190 .249009$

H $-3.730158-1.0080130 .181041$

H $-2.819597-2.4516370 .590841$

H -2.816371 -1.061558 1.699389

H $-0.147019-0.2695042 .087355$

H $2.385624-2.1735040 .570692$

H $2.229806-0.7649861 .636718$

H $0.282500-1.250791-1.902826$

H 3.5043440 .6207010 .002472

H $4.418552-0.7860210 .563837$

H $3.691216-0.798613-1.047361$

SCF Energy (B3LYP/6-31G**//MMFF) $=-694.031898096$

F3-07 c00086

MMFF Geometry

C $-3.126286-2.368197-0.028942$

C -1.685652 -2.217142 -0.488884

C $-0.964711-1.0038490 .113999$

C $-1.5893640 .327193-0.323370$

C -0.7591591 .5241280 .167953$

O $0.397462-1.081022-0.325180$

O $-0.8667191 .602842 \quad 1.593837$

C $0.7293421 .328618-0.195695$

C $-1.3204572 .831925-0.400526$

O $0.9068661 .522419-1.599911$

C $1.258044-0.0701600 .218277$

C $2.679216-0.308386-0.344784$

O $1.301072-0.1195221 .646978$

C $3.257498-1.689018-0.041321$

H $-3.189871-2.3907811 .063376$

H -3.753479 -1.551126 -0.396940

H -3.546022 $-3.304151-0.411849$

H -1.650909 -2.166408 -1.584460

H -1.130609 -3.125395 -0.221061

H -0.988831 -1.0959411 .206860$

H -2.6097630 .3999590 .070237$

H -1.653641 $0.353921-1.418559$

H -1.8071291 .7005591 .821085$

H 1.3235982 .0950180 .318325

H -2.350978 $2.992660-0.063117$

H $-0.7399963 .689253-0.041290$

H -1.316237 2.843435 -1.494957

H $\quad 0.485754 \quad 0.777179-2.060629$

H 3.3656070 .4469040 .056384

H $2.670548-0.206982-1.436450$

H $1.859524-0.8632341 .920753$

H $3.428969-1.8329111 .028920$

H $2.599323-2.486495-0.398939$

H $4.223867-1.803880-0.543716$

SCF Energy (B3LYP/6-31G**//MMFF) $=-694.023192953$

F3-08 c00092

MMFF Geometry

C -2.066900 $2.694445-0.087650$

C -0.5793352 .3851520 .024336$

C $-0.1361821 .289078-0.957965$

C $1.3706720 .994182-0.924673$

C $1.7868830 .023527 \quad 0.189937$

O $-\begin{array}{llll}0.924275 & 0.102378 & -0.864722\end{array}$

O $1.680357 \quad 0.680873 \quad 1.455081$

C $0.854029-1.2050030 .200469$

C $3.256467-0.380187 \quad 0.019758$

O $1.128784-2.026790-0.935094$

C $-0.640855-0.7997450 .213067$

C -1.546267 -2.033785 -0.004855

O $-1.004230-0.2657241 .488200$
C $-3.038164-1.7100960 .052897$

H $-2.676698 \quad 1.8259550 .179492$

H $-2.333307 \quad 3.5122880 .589496$

H $-2.3281742 .999787-1.105919$

H $-0.3676502 .112637 \quad 1.060380$

H $-0.0114153 .301402-0.177961$

H $-0.3459881 .687459-1.959513$

H $1.9263451 .936954-0.846975$

H $1.6409700 .548132-1.891235$

H 2.2440821 .4729901 .432226

H $1.064289-1.8038201 .095855$

H $3.564259-1.0626400 .820219$

H $3.444667-0.866330-0.942623$

H 3.9113570 .4960850 .091466

H $\quad 0.819212-1.557244-1.728104$

H $-1.333240-2.7877540 .762830$

H $-1.348116-2.484754-0.984374$

H -0.2121420 .1075821 .907821$

H $-3.624000-2.618544-0.121239$

H $-3.324174-1.3118321 .031088$

$\mathrm{H}-3.317458-0.979670-0.712587$

SCF Energy (B3LYP/6-31G**//MMFF) $=-694.025944671$

F3-08 c00093

MMFF Geometry

C -3.623206-1.010463 0.036960

C $-2.348398-1.777680-0.289666$

C $-1.084870-0.9970270 .096832$

C $0.164062-1.833924-0.182310$

C $1.450501-1.0627620 .135630$

O $-1.0484410 .201752-0.681033$

O $2.533911-1.801251-0.455608$

C $1.3999170 .317918-0.548703$

C $1.737940-1.0407551 .640568$

O $2.4862321 .128649-0.103290$

C $0.0542671 .074385-0.414800$

C -0.1399521 .8225870 .922575$

O $0.0499552 .110033-1.417465$

C $-1.4733442 .564117 \quad 1.009714$

H -3.675711 -0.7751621 .104783$

H $-3.680026-0.073230-0.525442$

H $-4.501041-1.610956-0.221813$

H $-2.342873-1.987877-1.366224$

H $-2.367710-2.7391430 .236518$

H -1.150416 -0.7443441 .160466$

H $0.182034-2.116340-1.244665$

H $\quad 0.135395-2.777638 \quad 0.376643$

H $3.350226-1.291575-0.312798$

H $1.5903860 .144279-1.617362$

H $0.929094-0.6077852 .229131$

H $1.900796-2.0607662 .010276$

H $2.660452-0.4935291 .863359$

H $2.4397801 .969713-0.589306$

H $0.6590562 .561578 \quad 1.057588$

H -0.0974321 .1406021 .772573$

H $\quad 0.409696 \quad 1.740457-2.240641$

H -2.318737 1.8734600 .936503

H -1.5490473 .0830981 .970825$

H -1.5686613 .3133250 .217958$

SCF Energy $\left(B 3 L Y P / 6-31 G^{* *} / / M M F F\right)=-694.021249544$

F3-08 c00094

MMFF Geometry

C $3.926648-0.2668330 .259641$

C $2.750334-0.861606-0.497282$

C $1.383386-0.348752-0.023299$

C $1.193387 \quad 1.144235-0.295247$

C $-0.2100851 .623790 \quad 0.100767$

O $0.399527-1.098308-0.744971$

O $-0.4135512 .919410-0.490208$

C $-1.2665840 .687503-0.519420$

C -0.3346761 .8314461 .614316$

O $-2.5670350 .967616-0.002996$

C $-0.962286-0.825914-0.400380$

C $-1.327407-1.4586070 .960566$

O $-1.802027-1.506187-1.354916$

C -1.016432 -2.953335 1.031904

H $3.815055-0.414707 \quad 1.338349$ 
H $4.857329-0.750668-0.053575$ H 4.0305530 .8044150 .064628 H $2.773843-1.953459-0.386606$ H $2.868962-0.674367-1.571859$ H $1.290533-0.5657121 .046070$ H $1.3382581 .339529-1.366907$ H $1.949782 \quad 1.7441800 .223758$ H $0.2976943 .506066-0.180504$ H -1.322950 $0.934067-1.589228$ H $-1.332112 \quad 2.193508 \quad 1.886223$ H $-0.122146 \quad 0.932497 \quad 2.192577$ H 0.3644422 .6064101 .952062 H -2.736162 $1.915452-0.140666$ H $-2.399254-1.3332601 .155968$ H $-0.790208-0.9817611 .780936$ H - $1.816815-0.987252-2.175838$ H $-1.303254-3.3472902 .012382$ H $-1.568882-3.5149370 .272512$ H $\quad 0.052312-3.1445230 .895059$ SCF Energy (B3LYP/6-31G**//MMFF) $=-694.015628373$

F3-08 c00095

MMFF Geometry

C -2.091480 $2.678148-0.067475$

C -0.6000512 .3864310 .041595$

C $-0.1441421 .303389-0.949905$

C $1.367004 \quad 1.031343-0.921547$

C 1.7985730 .0608900 .186000

O $-0.9139230 .103718-0.860556$

O $1.703774 \quad 0.7402121 .439968$

C $0.878074-1.1783170 .193533$

C $3.272708-0.3200910 .000745$

O $1.160023-1.990205-0.947625$

C $-0.622846-0.7943240 .217935$

C -1.507602 -2.050452 0.021874

O $-0.904497-0.2278371 .498485$

C $-3.008043-1.767794-0.026518$

H -2.3680423 .4852730 .618244$

H -2.356260 $2.990921-1.082470$

H $-2.690634 \quad 1.7994040 .190047$

H $-0.3839722 .106992 \quad 1.075307$

H $-0.0438393 .311268-0.153481$

H $-0.3632921 .704435-1.948383$

H $1.9088311 .981547-0.836906$

H $1.6411860 .596081-1.891925$

H 1.9503100 .1129542 .140918

H $1.100957-1.7806411 .083496$

H 3.9144530 .5645370 .085899

H $3.595102-1.0134220 .785965$

H $3.462029-0.787265-0.970880$

H $0.841877-1.519925-1.736675$

H -1.319233 -2.7644390 .832733$

H $-1.252732-2.542987-0.924045$

H $-1.863686-0.2214431 .638669$

H -3.383675 -1.393085 0.929524

H $-3.254168-1.040465-0.806062$

H -3.551405-2.692169-0.249570

SCF Energy (B3LYP/6-31G**//MMFF) $=-694.019916902$

F3-08_c00096

MMFF Geometry

C -2.601282 -2.605641 -0.153238

C $-1.195763-2.082862 \quad 0.106374$

C $-0.737896-1.026212-0.912782$

C $-1.5059450 .303077-0.897358$

C $-1.045760 \quad 1.265064 \quad 0.207575$

O $0.678094-0.821512-0.849699$

$\begin{array}{lllll}\text { O } & -1.464259 & 0.764901 & 1.479422\end{array}$

C $0.493488 \quad 1.3767490 .203548$

C -1.7141942 .6342080 .028393$

$\begin{array}{lllll}\text { O } & 0.915821 & 2.125021 & -0.938027\end{array}$

C $1.185998-0.0099990 .217798$

C $2.7112040 .143377-0.006350$

O $0.967101-0.592607 \quad 1.503369$

C $3.481172-1.174883-0.057280$

H - $2.829929-3.4230070 .538455$

H -3.354386 -1.827059 - 0.003617

H -2.696773 -2.991006 -1.173192
H $-0.494086-2.9260600 .075501$

H $-1.153041-1.7030421 .130046$

H $-0.905947-1.470210-1.902736$

H $-2.5832170 .119077-0.825993$

H - $1.3397640 .788888-1.868365$

H -2.430156 0.6547381 .457774

H $0.809401 \quad 1.9356651 .093917$

H -2.8039622 .5484510 .110278$

H $-1.397814 \quad 3.3242110 .818972$

H $-1.4817443 .086398-0.940844$

H $0.7639021 .579158-1.728044$

H $3.1409240 .760258 \quad 0.792118$

H $2.8994930 .650864-0.959930$

H $1.583783-1.3305981 .625849$

H $3.465344-1.6936600 .905084$

H $3.077437-1.845810-0.821421$

H $4.530344-0.980946-0.304444$

SCF Energy (B3LYP/6-31G**//MMFF) $=-694.016882174$

F3-08_c00097

MMFF Geometry

C -3.559456-1.128985 0.054236

C $-2.258069-1.858126-0.252804$

C $-1.022221-1.0214330 .104866$

C $0.253215-1.820942-0.159815$

C $1.510391-0.9949140 .143804$

O $-1.027007 \quad 0.151662-0.711492$

O $2.639749-1.689989-0.413087$

C $1.404026 \quad 0.379172-0.555584$

C $1.792095-0.9490841 .648882$

$\begin{array}{lllll}\text { O } & 2.464049 & 1.247794 & -0.156081\end{array}$

C $0.025768 \quad 1.086793-0.453281$

$\begin{array}{llll}\text { C } & -0.209773 & 1.873558 & 0.857015\end{array}$

O $0.039268 \quad 2.042009-1.533841$

C -1.6255932 .4273491 .012463$

H $-3.619179-0.8650561 .114964$

H $-3.651449-0.211473-0.535373$

H $-4.415027-1.768559-0.184702$

H -2.247697-2.101007-1.322426

H -2.241013 -2.803161 0.302419

H $-1.090325-0.7376121 .160767$

H $0.277992-2.122995-1.216305$

H $0.258963-2.7540460 .417170$

H $2.496565-1.787847-1.369896$

H $1.5893750 .201637-1.624606$

H $1.943596-1.9641082 .037265$

H $2.721781-0.4119611 .865871$

H $0.985392-0.4987512 .227080$

H $2.3485801 .463977 \quad 0.783582$

H 0.4865132 .7191520 .912919

H -0.0232811 .2438041 .727920$

H $-0.6502612 .705681-1.376991$

H $-1.7110952 .960512 \quad 1.965181$

H -1.8799553 .1332300 .217405$

H -2.3702401 .6264401 .015063$

SCF Energy (B3LYP/6-31G**//MMFF) $=-694.011445749$

Belizentrin (cutoff energy $=25 \mathrm{~kJ} / \mathrm{mol}$ )

011

MM̄FF Geometry

C $0.546873-3.704405-0.625039$

C $0.439328-2.910198-1.701437$

C $-0.789167-2.089652-1.990801$

O $-0.379535-0.717703-2.194399$

C $-1.482890-2.566728-3.276094$

C $-2.911141-2.061230-3.365450$

C $-3.987018-2.759209-2.950217$

C $-3.085311-0.707934-4.003094$

C $-3.993605-4.123985-2.321847$

C $-4.914899-4.256929-1.100405$

C $-4.403936-3.534541 \quad 0.158554$

C $-5.151403-4.0618481 .388728$

C $-4.754465-3.3199092 .670514$

O $-4.633708-2.1285190 .012898$

O $-5.706603-3.657532 \quad 3.690651$

C $-4.880924-1.8062802 .417690$

C $-3.383219-3.7667113 .190265$

O $-4.394234-1.063582 \quad 3.534129$ 
C $-4.181617-1.3343891 .120888$

$\begin{array}{llll}\text { C } & -4.559540 & 0.142745 & 0.849372\end{array}$

O $-2.769108-1.4352851 .300074$

C $-0.230417 \quad 0.055515-1.087412$

O $-0.335290-0.2961170 .075993$

C $0.0419821 .434982-1.539842$

C $-0.2144542 .451357-0.703639$

C $-0.0155503 .873590-0.981979$

C $0.5845034 .244634-2.313071$

C $-4.1037600 .685048-0.510812$

C $-4.5483472 .123476-0.724097$

C $-4.5652743 .907843-2.464156$

O $\quad \begin{array}{llll}-5.166968 & 2.767724 & 0.119095\end{array}$

C $-3.4002524 .841263-2.373206$

C $-3.3733485 .914655-1.568512$

C $-2.2560696 .918814-1.534478$

C $-1.5548576 .975833-0.196123$

C -2.0545017 .7021930 .817922$

C $-0.2271956 .264851-0.069045$

C $-0.3730124 .769038-0.037619$

N $-4.1819262 .597156-1.976259$

H $-0.307485-3.7721100 .046669$

H $1.261655-2.815821-2.404015$

H -1.497765 -2.140746 -1.154138

H -1.473338 -3.661539-3.334318

H $-0.930330-2.221786-4.160501$

H -4.972175 -2.317734 -3.093347

H -2.679513 -0.712703 -5.020007

H $-4.137577-0.411722-4.069854$

H $-2.5653600 .061158-3.425639$

H $-4.347031-4.828485-3.085428$

H -2.989763 -4.451920 -2.035435

H -5.917903 -3.893688-1.358794

H $-5.004749-5.329264-0.884185$

H -3.327840 -3.7177820 .260111$

H -4.990116 -5.1405151 .509274$

H $-6.232995-3.9370891 .236890$

H -5.692407 -4.623114 3.806406

H -5.952642 -1.575934 2.336429

H -3.392301 -4.838402 3.422491

H -3.131767 -3.259529 4.128283

H -2.575937 -3.593036 2.475858

H -4.867123 -1.382056 4.322102

H $-5.652188 \quad 0.2444770 .894472$

H $-4.141031 \quad 0.781521 \quad 1.637751$

H $-2.328019-0.7721000 .747478$

H $\quad 0.4188691 .573945-2.546132$

H -0.6161012 .2224420 .282915$

H $-0.0362783 .868508-3.133875$

H $\quad 1.5902313 .821497-2.412092$

H $0.6755875 .321445-2.467248$

H -3.014922 $0.654950-0.594970$

H -4.531408 $0.079855-1.317372$

H -5.430623 4.274259-1.901880

H -4.864920 $3.793256-3.510689$

H -2.561658 4.649136 -3.037434

H -4.225862 $6.111472-0.921976$

H $-2.6817277 .904511-1.765259$

$\mathrm{H}-1.5268416 .728816-2.331547$

H -1.541129 $7.772787 \quad 1.770947$

H $-2.991694 \quad 8.2396470 .716658$

H $\quad 0.275772 \quad 6.561060 \quad 0.861421$

H $0.4412956 .607950-0.863905$

H -0.8070284 .3983140 .892153$

H -3.631407 $2.004652-2.590350$

C $3.030763-4.334473-0.993426$

O $3.536650-3.002370-0.772193$

C $4.967511-3.065250-0.627816$

C $5.372362-4.488566-0.995625$

C $4.153851-5.273226-0.552932$

H $5.184062-2.9049500 .434758$

C $5.640298-1.979272-1.472506$

H $5.526732-4.601868-2.075073$

H $6.292094-4.800746-0.491513$

C $4.076767-6.664001-1.158622$

H $4.167986-5.356370 \quad 0.542125$

C $5.190593-0.540779-1.139957$

O $5.333597-2.223126-2.851727$
H $\quad 6.729421-2.067905-1.380379$

H $4.149680-0.402397-1.452858$

O $5.9670910 .345825-1.957568$

C $5.353064-0.1358920 .337476$

C 5.1860491 .3699950 .609806

H $4.646642-0.7021180 .950745$

O $6.668329-0.5043210 .766214$

H 5.3972101 .5417821 .672289

H $5.976707 \quad 1.9172530 .080981$

H $3.178725-7.189509-0.819398$

H $4.050901-6.624417-2.252801$

H $4.947142-7.259649-0.864587$

C $1.734375-4.530037-0.203835$

H $5.766795-1.525185-3.372467$

H $6.8936490 .273061-1.670277$

H $6.728584-0.3197651 .719139$

H $1.927330-4.2892720 .849714$

H $1.432583-5.582980-0.244668$

H $2.852355-4.444110-2.070763$

C $2.656631 \quad 1.4971612 .321595$

O $2.730771 \quad 1.2749690 .908173$

C 3.8051301 .9465870 .241504

C 3.6944583 .4621320 .460645

C 3.6255073 .8145751 .941933

C 2.5386822 .9968472 .634335

H 3.5444491 .0743882 .806954

C 1.4211200 .7544782 .857673

H $3.6567891 .772781-0.830616$

O $4.7912684 .148497-0.133352$

H $2.7864253 .835726-0.024951$

H 4.5949263 .6791322 .435427

O 3.2959245 .1992512 .079583

H 1.5535633 .3677932 .322720

O $2.637803 \quad 3.1907574 .044316$

C $1.543876-0.7526972 .783184$

H $1.262767 \quad 1.0127293 .910976$

H 0.5337701 .0398562 .282286

H $4.8030423 .917186-1.077869$

H 3.9711005 .7061941 .596581

H 2.5707864 .1467414 .210896

O $0.474345-1.3080583 .428236$

O $2.442841-1.3666312 .227117$

C $0.437642-2.738053 \quad 3.459149$

H $0.159714-3.1194822 .473734$

H $\quad 1.400799-3.1493693 .776389$

$\mathrm{H}-0.324273-3.0419454 .181647$

SCF Energy (B3LYP/6-31G**//MMFF) $=-3245.91425398$

01_10

MM̄FF Geometry

C $-2.579483-3.7152001 .419155$

C -1.855487 -2.626744 1.121097

C $-0.885793-1.9673472 .063332$

O $0.378456-1.8706701 .362988$

C $-1.361699-0.5443872 .405703$

C $-0.947083-0.1085683 .797362$

C 0.0246840 .7839414 .064979

C $-1.761503-0.6936004 .924185$

C $0.927392 \quad 1.4749103 .085141$

C 0.3879242 .8618502 .717508

C 1.4143063 .6958391 .938655

C $0.8062645 .028667 \quad 1.497345$

C $1.7662965 .839826 \quad 0.613147$

O 1.8294052 .9451230 .796656

O $1.0070016 .874052-0.031567$

C $2.3228484 .927622-0.502304$

C 2.8402446 .5487881 .447193

O $3.3362515 .597978-1.248705$

C 2.8570383 .5739820 .019851

C $3.1917202 .635189-1.166052$

O 4.0346523 .8102890 .789637

C $1.228184-2.9288691 .465444$

O $1.133678-3.8751272 .230962$

C $2.266194-2.7775950 .419123$

C $3.212966-3.7198820 .294188$

C $4.252677-3.782745-0.736420$

C $4.142950-2.828111-1.895370$

C $3.5952771 .208803-0.760310$ 
C $5.055424 \quad 1.071397-0.361327$

C $6.601871-0.4377370 .883283$

O $5.9219591 .866076-0.717093$

C $7.227038-1.364050-0.111716$

C $7.699605-2.5807430 .198492$

C $8.352041-3.496191-0.797342$

C $7.715012-4.867099-0.843814$

C $8.353867-5.943890-0.355498$

C $6.375281-5.003565-1.535033$

C $5.221803-4.714106-0.612643$

N $5.282739-0.0569090 .413891$

H -2.495385 -4.168530 2.403085

H -1.965005 -2.176306 0.139003

H $-0.764951-2.5658102 .973889$

H -2.458150 -0.4822742 .364017$

H -0.9979980 .1650611 .653480$

H 0.2058791 .0522345 .104952

H -1.460350 -0.301937 5.901839

H -2.821799 -0.4561614 .788462$

H -1.651413 -1.7815684 .959699$

H 1.9182431 .5685863 .546732

H 1.0714380 .8671522 .185261

H -0.5157942 .7277632 .108837$

H 0.0916053 .4089193 .621376

H 2.2790523 .8664052 .591244

H 0.4908505 .6188982 .366821

H $-0.1089304 .835370 \quad 0.923078$

H $0.584007 \quad 7.4111310 .660222$

H $1.5030114 .728518-1.205345$

H 2.3762107 .2440532 .157298

H $3.493257 \quad 7.1601200 .814764$

H 3.4613905 .8590772 .022277

H $2.956116 \quad 6.435177-1.565795$

H $2.2942882 .540661-1.790877$

H $3.9778453 .079271-1.787882$

H 4.4416792 .9566041 .001993

H $2.196091-1.924429-0.245733$

H $3.224424-4.5408151 .010697$

H $4.229598-1.793383-1.555686$

H $3.177658-2.947675-2.400041$

H $4.911369-2.972067-2.657066$

H 2.9483940 .8317920 .038983

H $3.4543370 .552001-1.627706$

H $6.476335-0.9059611 .864577$

H 7.2220360 .4570990 .997435

H $7.311436-1.007236-1.135753$

H $7.638654-2.9299121 .226820$

H $9.411810-3.580767-0.523660$

H $8.339171-3.062803-1.805239$

H $7.914333-6.934384-0.411828$

H $9.327396-5.8588820 .115695$

H $6.249719-6.032117-1.899447$

H $6.375517-4.383640-2.435102$

H $5.190673-5.3743950 .255806$

H $4.516008-0.6975370 .596001$

C $-4.962310-3.9230460 .669502$

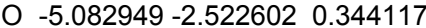

C $-6.228582-2.335139-0.503697$

C -7.052525 -3.609112 -0.372040

C $-5.960169-4.650967-0.232208$

H $-5.852594-2.263036-1.531406$

C $-6.963777-1.046414-0.128874$

H -7.681131 -3.5974270 .525820$

H -7.700066 -3.777181-1.237902

C $-6.448350-5.9740230 .331668$

H $-5.515328-4.826853-1.221087$

C $-6.071125 \quad 0.213017-0.124647$

O $-7.502933-1.1976601 .191057$

H -7.822223 -0.907472 -0.796764

H $\quad-5.367300 \quad 0.1529050 .712562$

$\begin{array}{llll}\text { O } & -6.920736 & 1.333268 & 0.159434\end{array}$

C $-5.3059190 .477365-1.436910$

C $-4.6275421 .859691-1.502317$

H -4.560049 -0.308473 -1.593453

O $-6.2359750 .399873-2.521691$

H $-4.2531812 .005040-2.522524$

H -5.390396 2.637340 -1.375566

H $\quad-5.621820-6.6849640 .429450$
H $-6.901581-5.8461551 .320453$

H -7.200613 -6.418851 -0.327737

C $-3.518080-4.3653850 .441604$

H $-7.964647-0.3705351 .411591$

H $-7.5304591 .435859-0.591600$

H $-5.7301770 .484331-3.348003$

H $-3.211651-4.116893-0.582641$

H -3.426339 -5.4521560 .547854$

H -5.239930 -4.030886 1.725922

C $-1.6220501 .343961-1.855945$

O $-2.4185081 .120189-0.684403$

C $-3.4877262 .052221-0.482620$

C $-2.9390623 .485305-0.422521$

C $-2.1045213 .819351-1.653060$

C $-1.0267572 .758388-1.855167$

H $-2.2378571 .202887-2.752155$

C $-0.5012710 .290332-1.865288$

H $-3.897995 \quad 1.8365370 .510324$

O $-3.9933364 .433949-0.299161$

H -2.3056993 .5943560 .466876$

H $-2.7279563 .923844-2.548519$

O $-1.4623895 .081149-1.462943$

H $-0.2743402 .847688-1.062572$

O $-0.3700072 .992417-3.099336$

C $-0.995320-1.081773-2.272029$

H $\quad 0.2790210 .568050-2.582628$

H $-0.0604380 .205267-0.865027$

H $-4.503214 \quad 4.2022740 .495891$

H $-2.1586975 .736118-1.283715$

H $-0.0212243 .900051-3.076972$

O $0.054774-1.955443-2.232032$

O $-2.144351-1.358466-2.586509$

C $-0.250464-3.305655-2.594294$

H $-0.987454-3.726222-1.904417$

H $-0.616622-3.347807-3.624442$

H $\quad 0.668949-3.892732-2.524382$

SCF Energy $\left(B 3 L Y P / 6-31 G^{* *} / / M M F F\right)=-3245.92433792$

01100

MM̄FF Geometry

C $4.435958-0.4963122 .953196$

C $3.424681-1.2413732 .481358$

C $2.171209-1.5535653 .257886$

O $1.032531-1.0712292 .508818$

C $2.043041-3.0813953 .405644$

C $0.793364-3.5224714 .140419$

C $-0.299982-4.0466313 .550476$

C $0.846616-3.3751625 .639055$

C $-0.548962-4.2522062 .079188$

C $-1.803976-3.4985161 .637211$

C $-2.109824-3.5888900 .133764$

C -1.019057 -2.960743 -0.739903

C $-1.421893-2.900957-2.221553$

O $-3.345949-2.886988-0.049749$

O $-0.507530-2.022471-2.894504$

C $-2.822579-2.266246-2.335499$

C -1.286241 -4.267999-2.902586

O $-3.293954-2.314315-3.680479$

C $-3.862362-2.902180-1.386592$

C $-5.162392-2.062746-1.381210$

O $-4.152815-4.228066-1.828654$

C $0.660207 \quad 0.2246522 .700755$

O $1.223658 \quad 1.059782 \quad 3.389563$

C $-0.5855950 .446812 \quad 1.933277$

C -1.1977481 .6394961 .956021$

C -2.4349861 .9793361 .247275$

$\begin{array}{llll}C & -3.232611 & 0.847271 & 0.655192\end{array}$

C $-6.241471-2.606962-0.437586$

C $-7.250886-1.538970-0.071689$

C $-7.623590 \quad 0.334636 \quad 1.532284$

O $-8.329263-1.442360-0.652612$

C -7.2341561 .6463190 .928920$

C -6.7771532 .6830361 .647618$

C -6.4576904 .0288421 .064222$

C $-5.0324594 .455705 \quad 1.330935$

C -4.7584205 .3709522 .276929$

C -3.9619503 .8802980 .429105$

C -2.7972803 .2767751 .168052$ 
N $-6.812545-0.7211700 .958306$ H $4.336360-0.0668363 .948525$ H $3.485294-1.6788801 .489125$ H $2.186874-1.0886434 .251701$ H $2.917425-3.4732563 .943676$ H $2.088175-3.5526642 .417723$ H -1.125601 -4.356414 4.190490 H $-0.059206-3.7504146 .126823$ H $1.695130-3.9349326 .045856$ H $0.957431-2.3226075 .917551$ H $\quad 0.304358-3.929176 \quad 1.482138$ H $-0.680217-5.3244551 .892884$ H $-2.672819-3.8834802 .189620$ H -1.733612 -2.444096 1.929340 H -2.257134 -4.643303 -0.127746 H $-0.068422-3.496235-0.638319$ H $-0.822594-1.936257-0.397857$ H $0.392746-2.365006-2.759881$ H -2.724141 -1.202074 -2.081003 H - $0.246866-4.615532-2.859109$ H - $1.536180-4.205870-3.967485$ H -1.909364 -5.038972 -2.444805 H -2.616863 -1.896897 -4.240003 H $-4.918412-1.033017-1.092177$ H -5.589670 -2.019565 -2.390872 H $-4.994336-4.514706-1.440858$ H $-0.972688-0.3848481 .359873$ H -0.7449692 .4483352 .527590$ H -3.448394 0.0892521 .416953 H -2.683485 $0.371201-0.162521$ H $-4.198733 \quad 1.1603530 .255517$ H -6.781929 -3.437960 -0.905095 H $-5.807960-2.9989830 .489798$ H -7.4626750 .3136862 .614788$ H $-8.682306 \quad 0.136868 \quad 1.336385$ H -7.370588 1.758798 -0.144008 H -6.6644042 .5751252 .724164$ H $-7.1579024 .753807 \quad 1.499805$ H -6.646120 $4.053084-0.016507$ H $-3.747725 \quad 5.7228952 .452968$ H -5.5427255 .7955042 .895042$ H -3.577947 $4.687840-0.208782$ H -4.402056 $3.165002-0.272118$ H $-2.149017 \quad 4.0182801 .637314$ H -5.877311 -0.8526751 .332330$ C $6.020530-0.9249990 .951126$

O $5.130293-0.451087-0.074906$ C $5.816273-0.486688-1.340628$

C $7.202691-1.063555-1.067386$

C $7.418764-0.6856560 .386337$

H $5.9307440 .553892-1.664461$

C $4.981427-1.268683-2.363320$

H $7.223256-2.152924-1.186921$

H $7.961238-0.644900-1.736164$

C $8.514216-1.4924011 .061317$

H 7.6678630 .3828100 .439363

C $3.537739-0.743366-2.518416$

O $4.904636-2.633209-1.931107$

H $5.496923-1.275843-3.331062$

H $2.973698-0.932846-1.597759$

O $2.895926-1.499333-3.552309$

C $3.4493560 .746928-2.892636$

C $2.0292791 .248136-3.202597$

H $3.8680801 .356579-2.088958$

O $4.2561550 .993472-4.048513$

H $2.1160862 .284436-3.551820$

H $1.6280710 .697904-4.063030$

H $8.638648-1.1886222 .105217$

H $8.289947-2.5642961 .046880$

H $9.471463-1.3422420 .551262$

C $5.741011-0.1852782 .261763$

H $4.575383-3.159200-2.679244$

H $2.784655-2.407586-3.224766$

H $3.9178580 .432267-4.767502$

H 5.7640190 .8951462 .080291

H $6.540727-0.4120002 .976399$

H $5.843599-2.0019871 .072927$

C $1.6337983 .237412-0.920096$
O $1.5386451 .810386-0.838874$

C $1.0469221 .164059-2.017592$

C $-0.3578051 .684308-2.347175$

C $-0.3734973 .202189-2.489290$

C $0.2764323 .862892-1.276459$

H $2.387703 \quad 3.513417-1.667225$

C 2.0997913 .7530420 .453038

H $0.9416060 .104003-1.760752$

O $-0.8549131 .098474-3.545403$

H $-1.0471601 .403347-1.543451$

H $\quad 0.1088783 .525975-3.418759$

O $\quad-1.7290203 .651937-2.567019$

H $-0.4008053 .787901-0.418899$

O $0.4582955 .252637-1.545067$

C 3.5525083 .4472380 .748650

H 1.9851014 .8424170 .495026

H 1.5042163 .2981161 .252482

H $-0.8406400 .133324-3.426104$

H $-2.1441313 .189867-3.315694$

H $-0.4132465 .620377-1.772053$

O $3.9487134 .202728 \quad 1.816289$

O 4.2444922 .6411530 .144570

C 5.3088114 .0459862 .234328

H 5.9877023 .9866471 .378000

H 5.5817344 .9201262 .831753

H 5.3956403 .1535032 .858946

SCF Energy $(B 3 L Y P / 6-31 G * * / / M M F F)=-3245.91998506$

01101

MM̄̄F Geometry

C $-4.4386571 .044276-1.857744$

C $-3.6236992 .017836-2.292469$

C $-2.7153902 .823406-1.403731$

O $-1.4353652 .862748-2.084436$

C $-3.2332454 .258278-1.189721$

C $-4.4944324 .347100-0.358101$

C $-4.520004 \quad 4.357310 \quad 0.989118$

C $-5.7762614 .506769-1.135454$

C -3.3599724 .2751351 .946621$

C -3.6026423 .2501233 .067293$

C -3.2101331 .8151872 .677381$

C $-3.8416950 .813936 \quad 3.647146$

C $-3.390073-0.624373 \quad 3.361335$

O

O $-3.788920-1.4404214 .472931$

C $-1.851431-0.6526103 .303149$

C $-4.082306-1.2047372 .125480$

O $-1.379559-1.9420212 .914085$

C $-1.2322780 .447828 \quad 2.406075$

C 0.2947010 .4906212 .660977

O $\begin{array}{llll}-1.474907 & 0.126213 & 1.036500\end{array}$

C $-0.3409783 .199794-1.353706$

O $-0.3183493 .526466-0.178680$

C $0.843443 \quad 3.096823-2.235655$

C $2.0671883 .266547-1.712651$

C $3.3405943 .206823-2.434114$

C $3.2978502 .928054-3.913573$

C 1.0287551 .6411141 .971506

C 2.4510781 .7584262 .480195

C 4.7047170 .7141912 .280815

O 2.7742462 .6153603 .299749

C 5.4871561 .6538331 .419779

C $6.333916 \quad 1.2537120 .459857$

C $7.1582912 .188166-0.378537$

C $6.7022642 .242289-1.819636$

C $7.0761651 .301141-2.702490$

C $5.8922783 .442102-2.256464$

C $4.4803493 .408839-1.739969$

N $3.2982140 .805781 \quad 1.940388$

H $-4.4767200 .821776-0.795565$

H $-3.6092612 .261225-3.352901$

H $-2.5792002 .311628-0.444059$

H $-3.3908744 .744099-2.162938$

H $-2.4547024 .874630-0.723704$

H $-5.4894354 .473107 \quad 1.473327$

H $-6.6506034 .598137-0.482276$

H $-5.7326085 .410228-1.752787$

H $-5.9479923 .649034-1.790944$ 
H -2.4131984 .0680751 .441688$ H -3.260396 5.2652382 .409057 H -3.0013493 .5548403 .934137$ H -4.6540943 .2875963 .378217$ H -3.5392481 .6109481 .653177$ H -4.9365680 .8824683 .618110$ H $-3.553357 \quad 1.0706214 .676434$ H $-4.755290-1.3753224 .561484$ H -1.494882 -0.501875 4.331397 H -5.171966 -1.174801 2.244967 H -3.826855 -2.261067 1.987526 H $-3.834029-0.6651341 .210109$ H - $-1.523515-2.0315641 .956680$ H $\quad 0.473003 \quad 0.5839603 .740477$ H $0.747452-0.4537222 .337793$ H $-0.829840 \quad 0.5976450 .486110$ H $\quad 0.6764302 .884138-3.285279$ H $2.1502423 .473980-0.646208$ H $2.8224903 .760018-4.444392$ H $2.7336972 .013884-4.124789$ H $4.2848572 .780002-4.357193$ H $1.055606 \quad 1.4945290 .887400$ H 0.5338222 .5986092 .164557 H $5.010208-0.3263052 .137843$ H 4.8429840 .9739243 .335333 H 5.3653272 .7167831 .613467 H $6.4663430 .189797 \quad 0.280646$ H $8.2029161 .852716-0.335700$ H 7.1650813 .1983350 .049925 H $6.7764671 .351476-3.744062$ H $7.6859010 .456170-2.400050$ H $5.9229573 .549951-3.345305$ H 6.374854 4.353829-1.880620 H $4.4033833 .611627-0.671462$ H $2.9371320 .158501 \quad 1.246644$ C $-5.277232-1.257904-2.572227$ O $-3.912378-1.718730-2.584197$ C $-3.753752-2.698814-1.539667$ C $-5.163178-3.095561-1.117317$ C $-5.901488-1.780912-1.278762$ H $-3.268029-2.190875-0.699185$ C -2.880603 -3.858786 -2.024241 H $-5.587380-3.854616-1.784808$ H -5.197201-3.485976 -0.095812 C -7.411375 -1.935257-1.337705 H $-5.647697-1.130279-0.434862$ C $-1.476360-3.433768-2.501091$ O $-3.536370-4.511462-3.118924$ H $-2.795514-4.611743-1.232526$ H -1.559841 -2.831686 -3.413451 O $-0.770354-4.623321-2.879837$ C $-0.644575-2.669025-1.451187$ C $0.777992-2.364780-1.961654$ H -1.141845 -1.723692 -1.209253 O $-0.595398-3.452861-0.262271$ H $1.294603-3.307127-2.169078$ H $0.688094-1.832075-2.915833$ H -7.897759 -0.963746-1.470097 H -7.717606 -2.581633 -2.167092 H -7.788628 -2.378092 -0.410018 C $-5.3349280 .258580-2.776659$ H -3.667708 -3.849334 -3.819269 H $-0.641546-5.155821-2.076097$ H $-0.146629-2.9256490 .419959$ H $-6.3659930 .608291-2.650864$ H $-5.0527810 .468688-3.816569$ H -5.767321 -1.734602 -3.431782 C $2.676791-3.2973890 .261600$

O $1.811090-2.157613 \quad 0.287391$

C $1.625804-1.517471-0.982011$

C $2.984284-1.078338-1.556119$

C $3.985486-2.231981-1.598461$

C $4.074753-2.911619-0.230272$

H $2.259262-4.046314-0.422484$

C $2.654561-3.9719701 .636573$

H $1.048101-0.605919-0.782965$

O $2.836259-0.539464-2.864686$

H $3.401095-0.279769-0.930574$
H $3.717332-2.956181-2.376178$

O $5.274251-1.735175-1.962992$

H $4.564310-2.2320410 .476174$

O $4.879442-4.082253-0.333815$

C $3.169738-3.1099532 .765171$

H $1.625792-4.2684371 .879730$

H $3.241484-4.8978011 .633037$

H $2.1847320 .180055-2.810109$

H $5.530070-1.060131-1.313001$

H $5.743194-3.810584-0.688890$

O $2.124804-2.5928013 .471943$

O $4.357323-2.9301323 .000719$

C $2.478493-1.8225894 .624832$

H $3.075575-0.9538684 .335926$

H $1.555776-1.4734825 .096025$

H $3.024261-2.4440705 .341040$

SCF Energy (B3LYP/6-31G**//MMFF) $=-3245.91878558$

01102

MM̄FF Geometry

C $-5.8349612 .251970 \quad 0.507023$

C -4.5954812 .7335370 .330564$

C -3.7217103 .1947521 .468612$

O -2.5425152 .3591241 .508163$

C $-3.2650524 .647291 \quad 1.244745$

C -2.4352735 .1926072 .392441$

C -1.1265445 .5086182 .325588$

C $-3.1859245 .410913 \quad 3.683274$

C $-0.1931885 .426317 \quad 1.149243$

C 0.8881184 .3636951 .362001

C 2.0402664 .4498340 .347736

C $1.5829554 .208343-1.092742$

C $2.7626774 .181056-2.078347$

O 3.0034283 .4559800 .729208

O $2.2908913 .635344-3.319657$

C $3.8403373 .216569-1.541952$

C $3.2733825 .591589-2.395985$

O $5.0166683 .263432-2.345045$

C $4.2009023 .462298-0.062420$

C 5.1509812 .3827510 .505342

O $4.8843374 .716792 \quad 0.052585$

C -2.6563181 .1590122 .139907$

O $-3.656420 \quad 0.6894912 .658761$

C -1.3339070 .5013332 .083583$

C $-1.222011-0.7871252 .436899$

C $0.007555-1.5778612 .411637$

C $1.302397-0.8557462 .142040$

C 4.6270760 .9451040 .440640

C $5.518857-0.0178421 .208550$

C $5.716332-2.4143011 .828044$

O $6.5469430 .329799 \quad 1.783793$

C $4.733073-3.4922532 .149379$

C $4.205547-3.6720173 .369964$

C $3.195616-4.7343293 .700175$

C $1.804999-4.1560433 .868118$

C $1.305503-3.9288185 .094769$

C $1.054639-3.9023602 .577025$

C $-0.069143-2.9046932 .645121$

N $5.021736-1.3138741 .185144$

H -6.2486742 .2049821 .511440$

H $-4.1734202 .772258-0.670837$

H -4.255715 3.1191462 .423992

H $-4.1406715 .297306 \quad 1.115071$

H -2.7104254 .7051270 .302952$

H -0.6538375 .8975373 .227590$

H -2.5828595 .9367804 .431390$

H -4.0809246 .0157763 .504605$

H -3.4903334 .4557834 .121334$

H $-0.7277305 .230616 \quad 0.217463$

H 0.2771696 .4104391 .031356

H 1.3131934 .4560962 .370919

H 0.4404683 .3622051 .328332

H 2.5003085 .4405320 .443614

H $0.8525244 .960530-1.411621$

H $1.0623693 .243355-1.145019$

H $1.5518344 .187015-3.628534$

H $3.4365372 .203146-1.644314$

H $2.4776636 .192701-2.852325$ 
H $4.0845225 .561732-3.131703$ H $3.6257096 .130363-1.514160$ H $4.7474763 .089429-3.263310$ H 6.1267842 .4427250 .005306 H 5.3499052 .6282111 .558366 H 5.3961824 .7148130 .878428 H -0.4924091 .0878411 .733876$ H -2.117072 -1.323065 2.750282 H $1.439786-0.0346362 .854864$ H $1.318166-0.442498 \quad 1.129108$ H $2.180467-1.4966122 .243581$ H 3.6199490 .8891510 .868057 H $4.5847740 .602075-0.598803$ H $6.231747-2.0477862 .722546$ H $\quad 6.476294-2.7870541 .133867$ H $4.436096-4.1490351 .335738$ H $4.498014-3.0067264 .179383$ H $3.520127-5.2404524 .618226$ H $3.172839-5.5146542 .928618$ H $\quad 0.307038-3.5357305 .249115$ H $1.889617-4.1387875 .985172$ H $0.642892-4.8571082 .227897$ H $1.754265-3.5914881 .794561$ H -1.041960 -3.347351 2.859598 H $4.260435-1.5304050 .547330$ C $-6.7762370 .228729-0.718165$ O $-5.507233-0.287253-1.162650$ C $-5.282683-1.564468-0.531130$ C $-6.583373-1.9250930 .183358$ C $-7.121767-0.5534640 .547337$ H $-4.502959-1.4052790 .223192$ C - $4.803762-2.593856-1.563005$ H -7.285220 -2.443767 -0.479669 H $-6.411917-2.5649511 .054504$ C $-8.599491-0.5455020 .893727$ H $-6.549521-0.1852871 .407413$ C -3.600162 -2.126194 -2.406591 O $-5.881437-2.863267-2.469388$ H $-4.578714-3.541183-1.059221$ H -3.911857 -1.299446 -3.055934 O $-3.234321-3.194831-3.289094$ C $-2.367037-1.700812-1.590849$ C - $-1.215590-1.212326-2.493885$ H $-2.642623-0.876201-0.925032$ O $-1.939715-2.777197-0.762943$ H $-0.935020-2.007344-3.190439$ H -1.598721 -0.378546 -3.092702 H -8.9366920 .4676891 .134328$ H -9.209216 -0.9158530 .062872$ H -8.794307 -1.182199 1.762904 C $-6.7130161 .754662-0.605365$ H -5.560807 -3.516277 -3.115080 H $-3.006267-3.966471-2.743621$ H -1.530164 -3.449943 -1.332732 H $-7.7215882 .154350-0.446858$ H $-6.3580852 .164991-1.559503$ H -7.493353 -0.017411-1.513063 C $1.525663-2.604427-2.126840$ O $0.758695-1.874759-1.160562$ C $0.013992-0.763849-1.674300$ C $0.9472840 .216334-2.395717$ C $1.795247-0.471627-3.457269$ C $2.509441-1.679106-2.856010$ H $0.855138-3.076001-2.854322$ C $2.281211-3.713657-1.378373$ H $-0.373425-0.226186-0.800346$ O $\quad 0.212331 \quad 1.275857-2.998952$ H $1.6198790 .672547-1.658605$ H $1.192401-0.758832-4.326858$ O $2.7856530 .442069-3.933576$ H $3.283709-1.328998-2.161432$ O $3.175731-2.394145-3.895361$ C $1.384076-4.868661-0.985667$ H $3.070969-4.131532-2.012583$ H $2.726429-3.307315-0.463852$ H $\quad-0.3287691 .689595-2.304982$ H $2.3204891 .236762-4.247304$ H $2.501752-2.707933-4.522294$
O $2.135865-5.804795-0.332725$

O $0.188161-4.951982-1.226673$

C $1.438697-6.9877270 .071316$

H $1.167143-7.576693-0.809589$

H $2.110379-7.5798610 .698632$

H $\quad 0.548025-6.7358300 .654485$

SCF Energy $\left(B 3 L Y P / 6-31 G^{* *} / / M M F F\right)=-3245.91819437$

01103

MM̄FF Geometry

C $1.092396-4.0647900 .042287$

C $0.972327-3.563256-1.196344$

C $-0.193374-2.741910-1.679733$

O $0.408269-1.523961-2.190798$

C $-0.952218-3.412757-2.842305$

C $-2.113628-4.279984-2.402234$

C $-3.405341-4.018742-2.683281$

C $-1.763971-5.527040-1.633318$

C $-3.969611-2.873817-3.476473$

C $-3.990990-1.519519-2.761908$

C $-4.521499-1.518594-1.321070$

C $-5.967618-2.008927-1.226690$

C $-6.509568-1.9080940 .206876$

O $-4.437212-0.164431-0.849802$

O $-7.933883-2.0814260 .153931$

C $-6.247059-0.4856270 .740277$

C $-5.968449-3.0300601 .101649$

O $-6.600785-0.3866912 .118472$

C -4.793018 -0.0005820 .529451$

C $-4.6926751 .499690 \quad 0.890654$

O $-3.926391-0.7409981 .388976$

C $-0.139776-0.326566-1.863999$

O $-1.142679-0.122156-1.201723$

C $0.7309200 .715696-2.451945$

C $0.3795432 .007492-2.379314$

C $1.1709643 .138070-2.870019$

C $2.5585072 .848924-3.382633$

C -3.3628102 .1672760 .513399$

C -3.3298273 .6239910 .949323$

C -1.8729255 .6337550 .898512$

O -4.2158964 .1428601 .623762$

C -1.189763 $6.328853-0.232363$

C $0.1173986 .629293-0.244605$

C $0.7907017 .366452-1.365436$

C $1.7782206 .507174-2.121534$

C $3.0913986 .552872-1.841412$

C $1.2538705 .671503-3.267860$

C $0.6375294 .375988-2.812170$

N $-2.188784 \quad 4.2746500 .501072$

H $\quad 0.268211-3.9275950 .737292$

H $1.776231-3.703883-1.913895$

H $-0.874265-2.505309-0.852933$

H $-0.267457-4.021446-3.448541$

H -1.299741-2.633927 -3.530685

H -4.158336 -4.714204 -2.314038

H $-2.607777-6.222840-1.568572$

H -0.943448 -6.062397 -2.121910

H -1.468021 -5.277982 -0.610943

H -3.427254 -2.775812 -4.424742

H $-4.994701-3.139098-3.765508$

H $-2.978831-1.099898-2.758543$

H -4.577005 -0.812467 -3.364706

H $-3.858985-2.138596-0.707057$

H $-6.068640-3.037606-1.590847$

H -6.601965 -1.395872 -1.882104

H $-8.115636-2.947717-0.249129$

H -6.9191120 .1951130 .198818$

H $-6.251319-4.0103340 .699406$

H $-6.405737-2.9809182 .104994$

H -4.880984 -3.016096 1.197837

H $-7.522557-0.6849052 .203092$

H -5.4912332 .0488660 .373958$

H -4.8572961 .6254941 .968522$

H $-3.102499-0.2447261 .513963$

H $1.6561430 .389250-2.914280$

H $-0.5696542 .264588-1.911269$

H $3.1425112 .306598-2.630278$

H $3.1321573 .747408-3.619519$ 
H $2.5125482 .244999-4.295355$ H -2.5195491 .6603500 .993756$ H -3.216783 2.129033 -0.572156 H -1.242442 5.5765861 .790856 H -2.786771 $6.171368 \quad 1.170957$ H -1.811006 $6.615407-1.077703$ H $\quad 0.737779 \quad 6.347824 \quad 0.602945$ H $1.3014888 .237064-0.933307$ H $0.0567197 .783497-2.066288$ H $3.8099165 .964705-2.402288$ H $3.4761017 .175807-1.040676$ H $2.0433415 .499309-4.007445$ H $0.4944946 .243162-3.817150$ H $-0.373176 \quad 4.484253-2.419488$ H $-1.447903 \quad 3.7188740 .082654$ C $3.633562-4.450203-0.047640$

O $3.893083-3.0434390 .120933$ C $5.288936-2.8538730 .416303$

C $5.954969-4.2063570 .190514$ C $4.834950-5.1578670 .576212$ H $5.354192-2.6127181 .483655$ C $5.840310-1.689127-0.414577$ H $6.234571-4.353238-0.859097$ H $6.855351-4.332000 \quad 0.799448$ C $5.044611-6.5776650 .080784$ H $4.741461-5.1668211 .670389$ C $5.014571-0.389274-0.281431$ O $5.824021-2.072062-1.795887$ H $6.890181-1.510514-0.154729$ H $4.028553-0.546327-0.733449$ O $5.671617 \quad 0.608742-1.071383$ C 4.8783880 .1168591 .166418 C 4.0874491 .4270791 .331287 H $4.398687-0.6498811 .781201$ O $\quad 6.181203 \quad 0.317885 \quad 1.721157$ H 4.1370191 .7087582 .390554 H 4.5992392 .2435060 .806744 H $4.204992-7.2187210 .367556$ H $5.138218-6.612893-1.009739$ H $5.957139-7.003800 \quad 0.510327$

C $2.290466-4.7891130 .605372$

H $6.173645-1.322316-2.307540$ H $5.055598 \quad 1.352733-1.179027$ H $\quad \begin{array}{llll}6.639667 & 0.969827 & 1.163547\end{array}$ H $2.347613-4.5156721 .667449$ H $2.099747-5.8670150 .559636$ H $3.606828-4.652135-1.125562$ C 1.7062630 .1863072 .776308 $\begin{array}{lllll}\text { O } & 1.957421 & 0.171226 & 1.367906\end{array}$ C 2.6177631 .3406190 .870824 C 1.7826592 .5906041 .187613 C 1.4484192 .6901762 .675485 C 0.8339691 .3818853 .175681 H $2.665767 \quad 0.2625563 .303816$

C $1.132171-1.1771283 .188048$ H $2.6418531 .238248-0.219215$ $\begin{array}{lllll}\text { O } & 2.461561 & 3.773001 & 0.778837\end{array}$ H 0.8390852 .5504310 .628935 H 2.3290852 .9617103 .269077 O 0.4956553 .7353982 .875682 H -0.1788101 .2875782 .768859$ O $\quad 0.7189161 .4253424 .595665$ C $-0.212874-1.5219402 .583090$ H $1.826927-1.9643162 .869462$ H $1.036009-1.2147184 .279274$ H $2.6277273 .699159-0.176512$ H 0.8877064 .5532002 .524055 H 0.1910982 .2110244 .819844 O $-0.690587-2.645857 \quad 3.197107$ O $-0.765100-0.9102181 .680665$ C -1.955099 -3.1317892 .733192$ H -2.757454 -2.541788 3.183738 H $-2.020308-3.1030081 .641015$ H -2.057988 -4.170801 3.057497

SCF Energy (B3LYP/6-31G**//MMFF) $=-3245.90807146$

01104

MM̄FF Geometry
C $-3.8455832 .709618-0.118269$ C $-3.751533 \quad 3.7021720 .779589$ C -2.6741333 .8579031 .830795$ O -1.6979792 .7942741 .766525$ C -1.9131355 .1821201 .625077$ C -0.8927205 .4564172 .715514$ C 0.4411575 .5141582 .529666 C -1.4616585 .6929384 .092336$ C 1.2157535 .3749121 .248719 C 1.8037093 .9719951 .087119 C $2.8728623 .871878-0.013382$ C $2.3295964 .237808-1.395860$ C $3.3610414 .007799-2.509024$ O $3.3393302 .514282-0.012705$ O $2.6501994 .064083-3.755126$ C $3.9507782 .590560-2.361661$ C $4.3952985 .138750-2.553346$ O $5.0064922 .382824-3.297497$ C $4.4154772 .258212-0.925068$ C $4.7962650 .760520-0.834379$ O $5.5678863 .046508-0.618452$ C -1.9458191 .6525202 .462443$ O -2.9383201 .3849593 .118965$ C -0.7796020 .7659402 .261277$ C $-0.860824-0.5384452 .559923$ C $0.210461-1.5181282 .372876$ C $1.570903-0.9996581 .983342$ C $5.0232600 .253280 \quad 0.594887$ C $5.433708-1.2103530 .625309$ C $5.885840-3.0539472 .248806$ O $5.692997-1.860390-0.383630$ C $4.678203-3.9363732 .223234$ C $4.041890-4.3544863 .327850$ C $2.852614-5.2720093 .318808$ C $1.558114-4.5301483 .574151$ C $1.075308-4.4177844 .823395$ C $0.868678-3.9902632 .338101$ C $-0.059772-2.8264802 .562088$ N $5.498450-1.694419 \quad 1.924125$ H $-3.0898711 .932733-0.143218$ H -4.5288604 .4639040 .791515$ H -3.159948 3.8557892 .814019 H -2.619292 6.0224381 .596597 H -1.430766 5.1574840 .642135 H 1.0685795 .7038943 .400302 H -0.7022026 .0387504 .801942$ H -2.242462 6.4595404 .053961 H $-1.8931254 .772628 \quad 4.497206$ H $\quad 0.600121 \quad 5.633793 \quad 0.382982$ H $2.0258206 .114775 \quad 1.270234$ H 2.2516823 .6371902 .032713 H $0.9991043 .255726 \quad 0.878223$ H 3.7051124 .5313870 .259966 H $1.9766175 .275187-1.425956$ H $1.4503323 .617652-1.606039$ H $3.280553 \quad 3.847390-4.463809$ H $3.1573441 .884712-2.642625$ H $3.8997526 .100482-2.733552$ H $5.0953355 .005434-3.385388$ H $4.9697965 .230164-1.629192$ H $5.7960822 .838263-2.958844$ H $3.9949580 .153743-1.273855$ H $5.7021950 .576194-1.425835$ H 6.0822802 .6030340 .073960 H 0.1154011 .2100061 .841020 H -1.800124 -0.9302752 .947358$ H $1.908601-0.2345202 .691833$ H $1.547128-0.5611480 .980371$ H $2.343802-1.7706541 .983084$ H 5.8152750 .8253551 .089326 H 4.1046790 .3651501 .180870 H $6.356992-3.0322783 .236690$ H $6.624141-3.4115811 .523675$ H $4.323600-4.2591021 .247252$ H $4.397363-4.0269424 .302356$ H $3.010147-6.0427014 .084564$ H $2.781998-5.8200012 .370534$

H $0.139154-3.9152405 .039162$ 
H $1.610051-4.8372655 .669797$ H $0.292794-4.8060871 .881839$ H $1.616077-3.7219511 .583724$ H -1.066989 -3.1161522 .862685$ H $5.192628-1.0986152 .688158$ C $-5.7908411 .297473-0.919812$ O $-4.9868580 .114252-1.121695$ C $-5.365646-0.863605-0.134093$ C $-6.657624-0.3515710 .500562$ C $-6.444332 \quad 1.145803 \quad 0.454184$ H $-4.577522-0.8624520 .627677$ C $-5.510544-2.258602-0.750792$ H $-7.538349-0.628262-0.090741$ H $-6.800065-0.7352181 .515460$ C $-7.718697 \quad 1.953545 \quad 0.624730$ H -5.7448501 .4120381 .255940$ C $-4.242152-2.864829-1.387781$ O $-6.524503-2.216543-1.765499$ H $-5.889948-2.9458940 .015570$ H -4.031359-2.373577 -2.345032 O $-4.584993-4.225979-1.690807$ C $-2.988532-2.840539-0.491253$ C $-1.841880-3.775064-0.917041$ H $-2.614070-1.814162-0.425993$ O $-3.350073-3.223003 \quad 0.842030$ H -1.048955 -3.673011 -0.167766 H -2.176782 -4.815597 -0.816657 H -7.509090 3.0266190 .572505 H $-8.453014 \quad 1.714431-0.151703$ H -8.1782051 .7459051 .596719$ C $-4.9571472 .567144-1.118785$ H -6.246946 -1.560857 -2.428208 H -3.936351 -4.565820 -2.328773 H $-3.731872-4.1166910 .798543$ H -5.614976 $3.443711-1.091134$ H -4.500422 $2.540308-2.115276$ H $-6.5597271 .271338-1.703505$ C $0.350032-1.833878-1.789244$ O $-0.808200-2.211199-2.540930$ C -1.264725 -3.547734 -2.328089 C $-0.146955-4.534905-2.693514$ C $1.126531-4.256938-1.898859$ C $1.527983-2.789856-2.038349$ H $0.095769-1.810984-0.724008$ C $0.738237-0.412621-2.226719$ H $-2.078217-3.708148-3.044063$ O $-0.581821-5.872111-2.469325$ H $0.074493-4.444308-3.765026$ H $1.009325-4.546363-0.848594$ O $2.167159-5.082849-2.427070$ H $1.937094-2.624715-3.043596$ O $2.562804-2.490796-1.103142$ C $-0.3394760 .603454-1.932048$ H $1.647573-0.080558-1.716703$ H $0.912280-0.408108-3.310119$ H $0.161733-6.459746-2.687873$ H $2.949256-4.967781-1.861329$ H $3.344731-3.013385-1.347860$ O $-0.152413 \quad 1.692911-2.733382$ O $-1.2197990 .467707-1.095017$ C $-1.1053362 .748108-2.577644$ H -2.103124 2.395057 -2.851649 H $-1.0907623 .124639-1.550586$ H $-0.8241263 .561909-3.251405$ SCF Energy (B3LYP/6-31G**/MMFF) $=-3245.92253741$

01105

MM̄FF Geometry

C $-0.979044-2.7195530 .844392$

C $-1.445436-2.052796 \quad 1.912453$

C $-0.637178-1.2295212 .892982$

O $0.773854-1.4720382 .695330$

C $-0.952673 \quad 0.2642532 .680816$

C $-0.611821 \quad 1.1471503 .864478$

C $0.4296592 .000292 \quad 3.913612$

C -1.5737571 .0673435 .022566$

C 1.4861892 .2168082 .868783

C 1.2905523 .5633252 .162591
C $2.3583023 .806656 \quad 1.087843$

$\begin{array}{llll}\text { C } 2.163501 & 5.172680 & 0.427297\end{array}$

C $3.1488415 .397919-0.729942$

$\begin{array}{lllll}\text { O } & 2.238091 & 2.772469 & 0.107877\end{array}$

O $2.6883276 .526569-1.488623$

C $3.1062754 .174562-1.670143$

C $4.5502025 .758595-0.223186$

O $4.1114414 .268910-2.676802$

C $3.2238842 .823279-0.929964$

C $2.9446981 .657302-1.906803$

O $4.5479792 .687252-0.409501$

C $1.582566-1.4519223 .785835$

O $1.265796-1.1692454 .932885$

C $2.975466-1.7941723 .426737$

C $3.269651-2.6755552 .458448$

C $4.611319-3.1396292 .096398$

C $5.789169-2.4313062 .712504$

C $2.7603560 .300086-1.214277$

C $2.499891-0.819168-2.206732$

C $2.048970-3.275426-2.256439$

O $2.544196-0.666635-3.423912$

C $3.255390-4.156073-2.119651$

C $4.528858-3.742418-2.019589$

C $5.696598-4.664743-1.819832$

C $6.488332-4.320627-0.575952$

C $7.639607-3.633211-0.662886$

C $5.992437-4.8399560 .755456$

C $4.731336-4.1670631 .229568$

N $2.232598-2.016564-1.558694$

H $\quad 0.086720-2.682606 \quad 0.631232$

H -2.510291 -2.0769782 .129907$

H $-0.930663-1.5636723 .895679$

H -2.0258780 .3927252 .488749$

H $-0.450517 \quad 0.6197821 .773572$

H $0.5563362 .606828 \quad 4.809393$

H $-1.317424 \quad 1.7670535 .825164$

H -2.5888241 .3077654 .689609$

H -1.5778160 .0618305 .453821$

H 2.4650612 .1996523 .363903

H 1.5053891 .3991852 .142325

H $\quad 0.2961353 .5755461 .698853$

H 1.3178494 .3761732 .899205

H 3.3454923 .7454931 .560927

H 2.2470855 .9787141 .166858

H $1.143247 \quad 5.2401190 .026027$

H $2.6389267 .290771-0.889035$

H $2.1401004 .198299-2.193558$

H 4.5163946 .6781400 .373509

H $5.2300515 .967936-1.056314$

H 4.9950184 .9793610 .399051

H $3.9931295 .124001-3.124878$

H $2.026797 \quad 1.868157-2.470082$

H $3.7619431 .580181-2.635123$

H $4.7637061 .745698-0.324310$

H $3.737980-1.3561254 .061765$

H $2.452727-3.1341701 .903068$

H $5.828496-2.6163883 .791438$

H $5.721195-1.3505652 .542762$

H $6.748619-2.7453802 .294928$

H $3.6553620 .031636-0.642996$

H $1.9131620 .342105-0.520380$

H $1.819620-3.108451-3.312601$

H $1.189425-3.763435-1.785910$

H $3.050578-5.223891-2.080122$

H $4.759183-2.681585-2.079485$

H $6.337251-4.591971-2.708209$

H $5.378201-5.713268-1.768587$

H $8.230502-3.4047880 .217715$

H $8.015534-3.279697-1.617427$

H $6.775226-4.7688701 .519695$

H $5.797928-5.9168650 .665804$

H $3.821807-4.618440 \quad 0.832638$

H $2.262961-2.043609-0.543408$

C $-3.284478-3.646784 \quad 0.079397$

O $-3.902270-2.355303-0.057827$

C $-5.191073-2.508909-0.685665$

C $-5.421901-4.010101-0.821537$

C $-3.999672-4.529388-0.942627$ 
H $-5.094906-2.084529-1.691691$ C $-6.254699-1.7213170 .096834$ H $-5.899672-4.4389400 .065389$ H $-6.044596-4.255640-1.687247$ C $-3.875135-6.019345-0.676803$ H $-3.630293-4.309436-1.953401$ C $-5.820620-0.2789110 .435461$ O $-6.500005-2.365764 \quad 1.353704$ H $-7.206659-1.714798-0.446860$ H $-4.982093-0.3208991 .138408$ $\begin{array}{lllll}\text { O } & -6.892542 & 0.370352 & 1.129154\end{array}$ C $-5.4557110 .578448-0.787898$ C $-4.9323251 .985027-0.442637$ H $-4.6968050 .072300-1.383802$ O $-6.6007610 .715855-1.631542$ H $-4.8107112 .539015-1.381634$ H $-5.6985882 .536247 \quad 0.116297$ H -2.835904 -6.348662 -0.773041 H $-4.218437-6.2774040 .330675$ H -4.476335 -6.588918-1.393009 C $-1.774181-3.530307-0.150562$ H -7.101431 -3.1114281 .189220$ H -7.115828 -0.1825681 .897512$ H -7.301058 $1.140499-1.106750$ H -1.598142 -3.079296 -1.133941 H $-1.335262-4.534646-0.173481$ H -3.489465 -4.0037841 .097304$ C -2.057060 $1.663200-1.489825$ O $-2.6184161 .137742-0.280466$ C -3.6045001 .9689510 .343061$ C $-3.021984 \quad 3.3532620 .656804$ C -2.372065 $3.992383-0.567045$ C -1.391695 $3.018093-1.218753$ H $-2.8454211 .785499-2.242632$ C $-1.0324270 .638857-2.008239$ H -3.8233461 .5078811 .312638$ O $-4.040842 \quad 4.199976 \quad 1.177175$ H -2.262243 3.2437831 .441783 H $-3.1225854 .333180-1.290004$ O $-1.641115 \quad 5.152149-0.164398$ H $-0.5174672 .884807-0.570300$ O $-0.9323973 .575103-2.448725$ C -1.669776 $-0.487481-2.795877$ H $-0.3248911 .127256-2.686709$ H $-0.4859730 .189999-1.171558$ H -3.616071 5.0169291 .488218 H -2.2842485 .8120240 .144688$ H - $0.5258694 .434567-2.243291$ O $-0.686743-1.218434-3.400737$ O $-2.869145-0.707980-2.877535$ C $-1.140952-2.297684-4.224034$ H $-1.819410-1.927847-4.999036$ H $-0.270142-2.745672-4.709467$ H -1.632966 -3.058439-3.611551 SCF Energy (B3LYP/6-31G**/MMFF) $=-3245.90776475$

01_106

MM̄FF Geometry

C $3.189574-3.1360960 .511331$

C $2.265636-4.0634920 .809855$

C $0.771787-3.8298470 .767626$

O $0.492281-2.728907-0.126235$

C $0.007235-5.0655800 .254686$

C $-1.461471-5.0646960 .645832$

C $-2.501255-4.942311-0.202794$

C $-1.735969-5.2671202 .117118$

C $-2.487707-4.791008-1.696088$

C $-2.742693-3.363979-2.203287$

C $-4.115046-2.771680-1.834656$

C $-4.354398-1.475110-2.615205$

C $-5.663691-0.779678-2.214579$

O $-4.144397-2.495387-0.429564$

O $-5.6043290 .549223-2.756296$

C $-5.726031-0.668259-0.675165$

C $-6.878462-1.458224-2.858480$

O $-6.997889-0.187842-0.248315$

C $-5.404177-1.9941770 .045671$

C $-5.307871-1.8418491 .585863$
O $-6.456864-2.934245-0.207692$

C $-0.267853-1.7022090 .330031$

O $-0.819989-1.6157541 .414275$

C $-0.309453-0.682633-0.743740$

C $-1.2001090 .314167-0.659395$

C $-1.408193 \quad 1.387262-1.630427$

C $-0.4294651 .514073-2.765207$

C $-4.258549-0.8475392 .090989$

C $-4.791170 \quad 0.555622 \quad 2.340817$

C -3.995390 2.8948512 .721925

$\begin{array}{llll}\text { O } & -5.986735 & 0.813254 & 2.449186\end{array}$

C $-4.238988 \quad 3.591497 \quad 1.420403$

C $-3.375691 \quad 4.4478190 .852131$

C $-3.624963 \quad 5.127289-0.465365$

C $-2.6443094 .707182-1.540606$

C $-1.630628 \quad 5.510367-1.904387$

C $-2.889928 \quad 3.395715-2.257574$

C $-2.4589752 .210823-1.441969$

N -3.7647951 .4822462 .473185$

H $2.868193-2.1438900 .204691$

H $2.598737-5.0307581 .179062$

H $0.473382-3.5904061 .794680$

H $0.450170-5.9835630 .663536$

H $\quad 0.124870-5.133028-0.831684$

H $-3.504334-4.9804710 .221773$

H $-2.801085-5.4156492 .325369$

H $-1.208056-6.1536202 .483796$

H $-1.409254-4.4000002 .698287$

H $-1.541104-5.143716-2.117100$

H -3.253562 $-5.459540-2.109654$

H $-1.949162-2.713368-1.825166$

H -2.646287 -3.384203-3.296257

H $-4.886618-3.511267-2.078259$

H $-4.338160-1.663237-3.696237$

H $-3.524207-0.782578-2.424555$

H $-6.4433070 .995955-2.552999$

H $-4.9862420 .086427-0.380937$

H -6.808569 -1.405679 -3.951689

H $-7.808227-0.942808-2.593485$

H $-6.977803-2.510316-2.583345$

H $-7.1164120 .703000-0.617846$

H $-6.298447-1.6267892 .004764$

H -5.029055 -2.826070 1.989485

H $-6.429063-3.6200630 .480118$

H $0.367170-0.796919-1.582763$

H -1.8582950 .3435550 .205687$

H $-0.5527970 .683481-3.468477$

H $0.6005641 .504615-2.395526$

H $-0.5405962 .442847-3.329447$

H -3.881760 -1.201050 3.059382

H -3.408483 -0.8077631 .406686$

H -3.1130563 .2840083 .240009$

H $-4.8647293 .013742 \quad 3.376511$

H -5.1775543 .3720490 .916392$

H -2.436884 4.6705751 .353588

H $-3.5638406 .210509-0.297844$

H $-4.646953 \quad 4.944545-0.820621$

H $-0.9408835 .227329-2.692655$

H $-1.4623606 .464553-1.416306$

H -2.409648 $3.402230-3.241381$

H $-3.961433 \quad 3.298660-2.474790$

H $-3.1212851 .998722-0.604467$

H -2.8066811 .1854352 .312655$

C $5.435339-2.841849-0.578459$

O $5.205335-1.428952-0.756297$

C $6.464585-0.765115-0.951247$

C $7.460267-1.864527-1.297289$

C $6.950556-3.006942-0.440760$

H $6.740281-0.3240340 .014252$

C $6.3269860 .339228-2.003963$

H $7.413501-2.139347-2.357244$

H $8.491118-1.579735-1.066434$

C $7.462741-4.369727-0.873819$

H $7.256015-2.8257450 .598636$

C $5.1753791 .335156-1.749134$

O $6.090623-0.257766-3.286966$

H $7.2767730 .878258-2.097700$

H $4.2153230 .834286-1.918363$ 
O $5.2704732 .365937-2.741506$

C $5.1755841 .996395-0.357877$

C $4.1748923 .160747-0.208861$

H 4.9727321 .2390830 .405263

O $6.4846702 .512197-0.098754$

H 4.3144943 .6001390 .786115

H $4.4547093 .960020-0.906195$

H $7.056109-5.159948-0.234968$

H $7.180991-4.594129-1.908093$

H $8.554887-4.409082-0.806915$

C $4.672324-3.3332770 .654085$

H $5.287686-0.802055-3.213619$

H $5.259594 \quad 1.932188-3.611962$

H $6.487213 \quad 2.8646620 .807515$

H $4.992322-2.7572041 .531223$

H $4.906578-4.3866390 .845267$

H $5.065173-3.340953-1.483101$

C $2.1307672 .176851 \quad 1.860376$

O $2.2750201 .747343 \quad 0.500257$

C $2.6995102 .763020-0.415776$

C $1.7170893 .943200-0.395814$

C $1.509976 \quad 4.4829891 .014800$

C 1.1380063 .3442291 .961040

H 3.1076372 .4786382 .257114

C 1.6260790 .9788782 .681199

H $2.6267892 .323278-1.416176$

O $2.1782644 .974136-1.261502$

H $0.7499293 .604225-0.786825$

H $2.393248 \quad 5.022606 \quad 1.376073$

$\begin{array}{lllll}\text { O } & 0.432812 & 5.422008 & 1.013348\end{array}$

H $\quad 0.1223152 .9958091 .731737$

O $1.128163 \quad 3.8386603 .299291$

C $2.700878-0.0550242 .941475$

H $1.272520 \quad 1.3225203 .660037$

H $0.8045870 .479262 \quad 2.158679$

H $1.4747525 .641221-1.328326$

H $0.715257 \quad 6.192170 \quad 0.492132$

H $0.503274 \quad 4.583518 \quad 3.328392$

O $2.255871-0.9155603 .905344$

O $3.782541-0.1109982 .374572$

C $3.178388-1.9296434 .316008$

H $4.166156-1.5039744 .518074$

H $2.799234-2.3788025 .237737$

H $3.241815-2.7048283 .549218$

SCF Energy (B3LYP/6-31G**/MMFF) $=-3245.90809561$

01_107

MM̄FF Geometry

C -3.609143 2.4379070 .983240

C -3.194453 2.0183682 .187302

C -2.923173 0.5754102 .509617

$\begin{array}{llll}\text { O } & -1.498458 & 0.498667 & 2.758355\end{array}$

C -3.6720220 .0852093 .759751$

C $-5.168205-0.0396403 .585157$

C $-5.784710-1.133123 \quad 3.095839$

C $-5.981358 \quad 1.1330684 .068565$

C $-5.153117-2.4005152 .579641$

C $-5.770599-2.8414201 .242741$

C $-5.293843-1.995843 \quad 0.049011$

C $-6.341220-2.016684-1.066373$

C $-5.868479-1.246929-2.305077$

O $-4.061241-2.560201-0.424579$

O $-6.762763-1.556000-3.384877$

C $-4.478301-1.772665-2.702942$

C $-5.9367720 .270992-2.106325$

O $-3.925813-0.994124-3.763718$

C $-3.471249-1.857964-1.527037$

C $-2.226393-2.640006-2.011534$

O $-3.079426-0.538894-1.149825$

C -0.888629 -0.6985012 .557985$

O $-1.416519-1.7474712 .226502$

C $0.555787-0.5075132 .813942$

C $1.373252-1.5689592 .768948$

C $2.819844-1.5600262 .987262$

C $3.495863-0.2312803 .195491$

C $-1.202132-3.011735-0.931923$

C $-0.000134-3.712500-1.548860$

C $2.097965-4.882526-0.997710$
O $0.127125-3.879799-2.760360$

C $3.068264-4.9852320 .132739$

C $4.299492-4.4519210 .102913$

C $5.317919-4.6185761 .195773$

C $5.658137-3.3170081 .887024$

C $6.622911-2.5118981 .411911$

C $4.939782-2.9948213 .178230$

C $3.473984-2.7392882 .974483$

N $0.930723-4.117122-0.600061$

H $-3.7982521 .707517 \quad 0.203223$

H -2.9852812 .7505902 .963535$

H -3.166897 -0.0561951 .647071$

H -3.4489480 .7439974 .610655$

H -3.262909 -0.882647 4.077282

H $-6.873907-1.1289213 .066490$

H -7.0585320 .9621913 .967670$

H -5.7777821 .3250015 .127114$

H -5.7396792 .0349673 .499240$

H $-4.068503-2.3145702 .474315$

H -5.342280 -3.186506 3.320860

H $-5.501890-3.8919991 .073020$

H -6.863889 -2.800476 1.329610

H $-5.116634-0.9652100 .374031$

H -7.296918 -1.611550 -0.710599

H -6.545121 -3.056872 -1.357359

H -7.659737 -1.293772 -3.115388

H -4.620837 -2.785135 -3.106732

H $-6.9625060 .581594-1.874102$

H $-5.6631990 .802755-3.024326$

H $-5.2939620 .625214-1.299512$

H $-4.577153-0.985073-4.485819$

H -2.555175 -3.578857 -2.477778

H -1.717243 -2.056436 -2.789170

H $-2.212970-0.578234-0.715090$

H 0.9046450 .4952913 .032431

H $0.942579-2.5461152 .552852$

H 3.2235810 .1858884 .170948

H 3.2022340 .4824732 .419855

H $4.586281-0.2894903 .152918$

H $-0.838946-2.119905-0.414620$

H $-1.655968-3.683036-0.194842$

H $2.541158-4.412090-1.882857$

H $1.758561-5.882347-1.289745$

H $2.756651-5.5624270 .999773$

H $4.612871-3.891187-0.774847$

H $6.224417-5.0471530 .748106$

H $4.987981-5.3569641 .937387$

H $6.892330-1.5910561 .918128$

H $7.160413-2.7540330 .500920$

H $5.421187-2.1582253 .695742$

H $5.050185-3.8437223 .865661$

H $2.893032-3.6494502 .824028$

H $0.668066-4.0944850 .381657$

C $-2.5611184 .488247-0.084091$

O -1.4689584 .5634860 .853236$

C $-0.2812914 .003687 \quad 0.245532$

C $-0.5528464 .080866-1.250056$

C $-2.0312763 .742353-1.308087$

H -0.2325212 .9602340 .581529$

C 0.9452684 .7732210 .752016

H $-0.3768725 .096094-1.625977$

H $0.0413813 .385339-1.843628$

C $-2.7050224 .143055-2.608489$

H $-2.1311362 .657647-1.186114$

C 2.3055074 .0714360 .564900

O 0.7965454 .9321882 .176782

H 0.9670105 .7893970 .341447

H $2.3245653 .187344 \quad 1.212721$

O 3.3157924 .9531421 .079001

C $2.6984383 .667163-0.863038$

C $4.1355263 .117944-0.992516$

H $2.0031782 .909258-1.230591$

O $2.5810904 .809645-1.711485$

H $4.3073692 .873687-2.048185$

H $4.8559083 .915246-0.770196$

H $-3.7701393 .891263-2.586257$

H -2.614988 $5.218749-2.792935$

H $-2.2505573 .618366-3.455229$ 
C $-3.788476 \quad 3.8817350 .608131$ H $-0.056603 \quad 5.3737552 .329433$ H 3.3375245 .7380010 .504663 H $2.7725264 .521491-2.620265$ H $-4.6636453 .962524-0.047008$ H $-4.013014 \quad 4.4639391 .510853$ H -2.797773 $5.526841-0.348330$ C $3.3972130 .191009-1.486067$ O $3.3985430 .892143-0.237913$ C $4.4171331 .887417-0.108288$ C $5.8047651 .258510-0.295025$ C $5.9001320 .434611-1.576428$ C $4.727396-0.539728-1.680105$ H $3.241270 \quad 0.899870-2.308895$ C $2.215125-0.781506-1.456993$ H $4.3754682 .233700 \quad 0.931367$ O $\quad 6.8206132 .255673-0.303470$ H 6.0139500 .5956020 .554528 H $5.9531241 .077631-2.462887$ O $7.111397-0.324307-1.557759$ H $4.837397-1.331513-0.929434$ O $4.744326-1.157132-2.964630$ C $0.925374-0.036983-1.712624$ H $2.308088-1.569524-2.212892$ H $2.126917-1.278460-0.484012$ H $\quad 6.7505952 .7510720 .530457$ H $7.8450170 .305295-1.450297$ H $5.607759-1.593674-3.064923$ O $\quad 0.683200 \quad 0.008908-3.053253$ O $0.2443490 .468859-0.830572$ C $-0.483604 \quad 0.717714-3.486104$ H $-0.1949961 .732658-3.771135$ H $-0.884204 \quad 0.205617-4.365070$ H -1.262188 $0.750006-2.720245$ SCF Energy (B3LYP/6-31G**//MMFF) $=-3245.91891467$

01108

MM̄FF Geometry

C -3.547871 -1.010057 -3.160201

C $-2.769770-0.623689-2.137734$

C $-1.406740-1.190012-1.843192$

O $-0.605115-0.072860-1.393085$

C $-1.510967-2.236628-0.714902$

C $-0.330711-3.185720-0.618317$

C $0.470257-3.3245540 .456988$

C $-0.111543-4.074519-1.818690$

C $0.395142-2.6195621 .781469$

C $1.411762-1.4827601 .960149$

C $2.880093-1.9383062 .013514$

C $3.783144-0.7707932 .419047$

C $5.270881-1.1526302 .398029$

O $3.267669-2.413850 \quad 0.722015$

O $6.0357790 .062232 \quad 2.456630$

C $5.603777-1.8316091 .050738$

C $5.663424-1.9696033 .635244$

O $6.937561-2.3338391 .084197$

C $4.602193-2.9418220 .660188$

C $4.810810-3.497631-0.771938$

O $4.758690-4.0483601 .557806$

C $0.720340-0.058602-1.682692$

O $1.357154-0.904978-2.287090$

C $1.2741181 .190880-1.112697$

C $2.5989741 .316490-0.952538$

C $3.2966692 .471984-0.386177$

C $2.4865593 .707858-0.096225$

C $4.707728-2.469750-1.902433$

C $6.033210-1.825831-2.273446$

C $6.9657110 .188200-3.411714$

O $7.121316-2.329858-2.008005$

C $7.3494701 .114873-2.301345$

C $7.1837012 .445430-2.346402$

C $7.5672073 .370433-1.226626$

C $6.3803114 .109304-0.646945$

C $6.1186305 .379193-0.999315$

C 5.5511923 .4088020 .407251

C $4.6250382 .376664-0.170094$

N $5.857413-0.640164-2.973327$

H $-3.202223-1.782910-3.841321$
H $-3.1485920 .129365-1.451777$

H $-0.985117-1.608802-2.763740$

H $-2.403070-2.860169-0.866328$

H -1.666639 -1.710495 0.232020

H $1.266481-4.0661410 .399499$

H $0.631773-4.856102-1.627042$

H -1.044795 -4.576532 -2.094460

H $\quad 0.240417-3.494976-2.676592$

H $-0.609086-2.2214361 .954258$

H $\quad 0.544987-3.3653742 .572416$

H $1.276453-0.7576091 .150773$

H $1.169784-0.9679372 .898366$

H $2.960878-2.7531822 .742585$

H $3.501261-0.3852843 .406858$

H $3.6310810 .062788 \quad 1.721533$

H $5.7805770 .538928 \quad 3.264835$

H $5.581956-1.0388270 .293261$

H $5.467950-1.3967584 .549907$

H $6.737532-2.1854123 .642866$

H $5.119862-2.9131083 .716383$

H $7.171765-2.6228890 .186750$

H $5.748741-4.063194-0.826297$

H $4.017522-4.238345-0.949069$

H $4.386916-4.841507 \quad 1.137440$

H $0.5744121 .963150-0.815348$

H $3.2372000 .485708-1.245788$

H $1.7724933 .517740 \quad 0.712530$

H $1.9320044 .024308-0.987036$

H 3.0948304 .5642550 .203288

H $4.359188-2.980729-2.808913$

H $3.969073-1.701547-1.659287$

H $\quad 6.6382750 .726225-4.306837$

H $7.816837-0.446773-3.677726$

H $7.7874220 .663604-1.413851$

H $6.7520282 .899381-3.235480$

H $8.3011454 .085296-1.621203$

H $8.0867442 .832779-0.423596$

H $5.2879715 .926291-0.566075$

H $6.7266005 .898359-1.732844$

H 5.0020754 .1369941 .013970

H $6.2211642 .912741 \quad 1.121398$

H $5.1173231 .433703-0.406583$

H $4.919618-0.266701-3.086974$

C $-5.994470-1.211420-2.638473$

O $-5.804986-1.003149-1.224332$

C $-7.069573-0.686547-0.617114$

C $-8.125145-1.082562-1.640019$

C $-7.416923-0.738060-2.938085$

$\mathrm{H}-7.0963150 .402113-0.488984$

C $-7.174455-1.3732790 .749222$

H $-8.346351-2.155366-1.602940$

H -9.063283 -0.536665 -1.503161

C $-8.037560-1.388621-4.161901$

H $-7.4307240 .352814-3.066561$

C $-5.973104-1.0940101 .679534$

O $-7.244834-2.7923750 .556135$

H -8.111233 -1.089037 1.241150

H -5.071642 -1.549475 1.255482

O $-6.211444-1.7584622 .926375$

C -5.7288620 .3985551 .969696$

C -4.5448920 .6737152 .917056$

H -5.5608330 .9370231 .034782$

O

H -4.5927491 .7255393 .223500$

H $-4.677751 \quad 0.103078 \quad 3.844015$

H -7.482172 -1.124851 -5.067385

H -8.043914 -2.480373 -4.076572

H -9.072612 -1.055769 -4.291066

C $-4.918311-0.438975-3.403056$

H $-6.449704-3.0616530 .064610$

H $-6.372412-2.6970212 .728023$

H $-7.0852050 .472632 \quad 3.377663$

H $-4.9277880 .613783-3.096588$

H $-5.117368-0.461279-4.480292$

H $-5.897353-2.289883-2.818562$

C -2.7677042 .4840951 .196590$

O -2.9700201 .0722561 .058818$

C -3.1716930 .3626922 .286435$ 
C $-1.974148 \quad 0.574177 \quad 3.221647$ C -1.661870 $2.053123 \quad 3.428917$ C -1.5461952 .7636392 .081228$ H -3.6585832 .9461791 .638322$ C $-2.5660093 .066394-0.212337$ H -3.175104 -0.701946 2.026569 O -2.214002 -0.0653304 .469676$ H -1.0952670 .0929002 .776391$ H -2.410088 2.5390874 .066179 O $-0.407624 \quad 2.1830234 .102312$ H -0.6294372 .4378201 .573171$ O $-1.437156 \quad 4.1681332 .303836$ C -3.857392 $3.177693-0.996470$ H -2.149813 $4.078154-0.151665$ H -1.884804 2.427881-0.784809 H $-1.389640-0.030548 \quad 4.983110$ H -0.5193551 .8300535 .001041$ H -0.6671324 .3113112 .880475$ O $-3.570974 \quad 3.645699-2.248409$ O $-4.9725092 .912994-0.571080$ C $-4.6999523 .866201-3.100286$ H -5.286795 $4.710520-2.726687$ H $-4.3291034 .109522-4.099462$ H -5.320066 $2.967873-3.166814$ SCF Energy (B3LYP/6-31G*//MMFF)= -3245.91991637

01_109

MM̄FF Geometry

C $4.188314-1.716339-1.941603$

C $3.269248-2.674347-2.132977$

C $2.359245-3.200496-1.058000$

O $1.029810-3.161370-1.635305$

C $2.690611-4.646316-0.650362$

C $3.965379-4.7875480 .151121$

C $4.042186-4.6396951 .488382$

C $5.187109-5.190732-0.633843$

C $2.938938-4.285522 \quad 2.451945$

C $3.359436-3.1739213 .427043$

C $3.267363-1.7643642 .817662$

C $4.183597-0.802117 \quad 3.575873$

C 4.0621020 .6327583 .049495

O $1.903895-1.3298522 .928294$

O 4.7248311 .5008093 .981126

C 2.5738251 .0233013 .039214

C $4.7726220 .814597 \quad 1.705940$

$\begin{array}{llll}\text { O } & 2.390826 & 2.301298 & 2.432234\end{array}$

C $1.639436-0.0286972 .386354$

C $0.175936 \quad 0.3567192 .717014$

O $1.828670-0.0071810 .972221$

C $-0.033376-3.161909-0.791564$

$\begin{array}{lllll}\text { O } & -0.006700 & -3.208927 & 0.426937\end{array}$

C -1.256165 -3.107272 -1.622306

C $-2.449160-3.309029-1.045478$

C $-3.749710-3.296087-1.716474$

C $-3.790068-2.907090-3.170497$

C $-0.892731-0.6497322 .276359$

C -2.273912 -0.2379292 .763301$

C -4.614866 -1.019325 2.901686

O $-2.489604 \quad 0.8140243 .360440$

C -5.507317 -1.963049 2.164691

C $-6.456075-1.5647621 .304122$

C -7.380137-2.494072 0.569889

C $-7.101570-2.532796-0.916945$

C $-7.628114-1.615648-1.745547$

C $-6.274715-3.682175-1.449109$

C $-4.842675-3.618589-0.994892$

N -3.246974 -1.175704 2.442288

H $4.338444-1.317164-0.943389$

H $3.128953-3.080701-3.132445$

H $2.379243-2.535157-0.187414$

H $2.736527-5.285040-1.543516$

H $1.857359-5.072868-0.077814$

H $5.008694-4.8150381 .960380$

H $6.067129-5.3232420 .004833$

H $5.009618-6.141156-1.147863$

H $5.438803-4.434467-1.382040$

H $2.012702-4.0059971 .944493$

H $2.720839-5.1875903 .036850$
H $2.701868-3.2272394 .304498$

H $4.379459-3.3735673 .778842$

H $3.553120-1.8003321 .761425$

H $5.227282-1.1375463 .525903$

H $3.917736-0.8071804 .642425$

H 5.6533421 .2188624 .045381

H 2.2755191 .1458924 .089617

H $5.827706 \quad 0.5271541 .785103$

H 4.7719981 .8657681 .397105

H 4.3247350 .2196610 .909025

H 2.4455392 .1824171 .468680

H $\quad 0.0840170 .478198 \quad 3.804799$

H $-0.058576 \quad \begin{array}{lll}1.327580 & 2.262592\end{array}$

H $1.041198-0.3788330 .544605$

H -1.140313 -2.917748 -2.683303

H -2.479547 -3.5139020 .024052$

H -3.332222 $-3.688589-3.786563$

H -3.249475 -1.970037 -3.341245

H $-4.802779-2.742547-3.545860$

H $-0.931280-0.7158421 .184638$

H $-0.670147-1.6416452 .684092$

H -4.9151340 .0263412 .770539$

H -4.636434 -1.240326 3.974037

H $-5.374831-3.0240702 .361374$

H $-6.593165-0.5019651 .123607$

H $-8.410678-2.1582000 .745329$

H -7.333946 -3.5087990 .984671$

H -7.451734 -1.651994 -2.815396

H $-8.243006-0.804528-1.369469$

H -6.349598 -3.743228 -2.539637

H $-6.707124-4.625350-1.090489$

H -4.703879 -3.8997220 .049043$

H -2.947985 -2.087381 2.106847

C $4.8453930 .393285-3.192103$

O $3.4492830 .754174-3.120040$

C $3.2957331 .759322-2.102361$

C $4.6602362 .422672-1.999274$

C $5.5719231 .216696-2.124715$

H $3.0802891 .236626-1.162030$

C $2.1383812 .711716-2.418420$

H $4.8301853 .119558-2.828598$

H $4.7932192 .965483-1.058907$

C $7.0027551 .570928-2.492834$

H $5.5840700 .681526-1.168565$

C $0.7615142 .007806-2.374175$

$\begin{array}{lllll}\text { O } & 2.372398 & 3.307682 & -3.691481\end{array}$

H $2.1460283 .515163-1.671250$

H $0.6841631 .478691-1.417821$

$\begin{array}{llll}\text { O } & 0.652018 & 1.033374 & -3.409396\end{array}$

C $-0.4238482 .984418-2.491087$

C - $-1.8119242 .327811-2.387956$

H $-0.3296673 .748180-1.714631$

O $-0.373123 \quad 3.675952-3.741158$

H $-2.5581293 .122258-2.514843$

H $-1.9655831 .661480-3.246639$

H $7.6120910 .667539-2.596502$

H $7.0504122 .120730-3.438831$

H $7.4550262 .197675-1.717306$

C $5.004970-1.127195-3.060491$

H $1.6810093 .975303-3.835888$

H $1.3724850 .391693-3.289620$

H $-0.3938553 .006553-4.446856$

H $6.060335-1.385258-2.917707$

H $4.694556-1.582566-4.009820$

H $5.1893570 .692566-4.190304$

C $-2.529284 \quad 3.427960 \quad 0.380520$

O $-1.697092 \quad 2.3006160 .082848$

C $-2.0761041 .556771-1.078778$

C $-3.5174001 .044223-0.934591$

C $-4.4900672 .175468-0.608179$

C $-3.9836782 .985404 \quad 0.583807$

H -2.474698 $4.153999-0.439625$

C -1.977408 4.0836291 .656050

H $-1.4284940 .671580-1.099914$

O $-3.918753 \quad 0.383354-2.128607$

H -3.551164 $0.302106-0.127417$

H $-4.6612602 .823370-1.475688$

O $-5.7624221 .627980-0.260971$ 
H -4.0741002 .3856251 .498222$

O $\quad-4.805917 \quad 4.139573 \quad 0.746394$

C $-0.663586 \quad 4.796950 \quad 1.417429$

H -2.6726894 .8299202 .054644$

H -1.814794 3.3118042 .417153

H $-4.763647-0.062826-1.949567$

H $-6.1203771 .191157-1.052070$

H -5.7219113 .8301690 .852096$

O $\quad 0.0625344 .8051952 .573834$

O $-0.333095 \quad 5.321767 \quad 0.362891$

C 1.3186955 .4879932 .507016

H 1.9340695 .0855851 .696403

H 1.1532486 .5606092 .369330

H 1.8418275 .3309473 .453988

SCF Energy (B3LYP/6-31G**//MMFF) $=-3245.93364215$

01 11

MM̄FF Geometry

C $0.508840-2.336949-2.440314$

C $-0.502077-3.216787-2.382982$

C $-1.357415-3.421520-1.162818$

O $-2.674335-2.923622-1.504212$

C $-1.484773-4.905312-0.772687$

C $-0.243527-5.502396-0.148791$

C $0.005559-5.490276 \quad 1.175228$

C $0.689719-6.214808-1.090837$

C $-0.834297-4.8388502 .241585$

C $-0.001283-4.3321163 .426748$

C $0.925624-3.1547393 .082014$

C $1.932014-2.9509754 .217763$

C $2.839664-1.7428673 .967873$

O $0.124846-1.9752592 .911419$

O $3.529349-1.4491685 .192453$

C $1.951143-0.5284443 .645504$

C $3.919832-2.0403692 .922417$

O $2.744191 \quad 0.597903 \quad 3.278418$

C $0.875975-0.8023262 .562359$

C -0.0816490 .4136012 .502622$

O $1.516383-0.9510951 .293135$

C $-3.458723-2.500378-0.477247$

O $-3.186392-2.523160 \quad 0.712264$

C $-4.718490-1.978892-1.049088$

C $-5.611388-1.417640-0.221154$

C $-6.884891-0.807690-0.595825$

C -7.293223 -0.848066 -2.043602

C -1.3548660 .2198381 .667765$

C -2.1011731 .5374681 .502475$

C -3.9178712 .6048470 .174920$

O -1.7853222 .5613212 .104882$

C $-5.2996572 .557732 \quad 0.747075$

C -6.3935632 .8447170 .023300$

C -7.796840 2.8577470 .565392

C $-8.7297591 .967523-0.228693$

C -9.420595 2.454558 -1.273494

C -8.9104550 .5326320 .215836$

C $-7.612244-0.2079710 .368969$

N -3.155924 1.4443720 .605201

H $0.728623-1.722954-1.571298$

H -0.738534 -3.814099-3.260333

H $-0.957512-2.835939-0.324370$

H -1.784080 -5.499907 -1.647143

H -2.330293 -5.030695 -0.083659

H $0.894807-6.007387 \quad 1.532960$

H $1.549146-6.653212-0.572523$

H $\quad 0.163872-7.027252-1.602917$

H $\quad 1.079917-5.526534-1.844071$

H -1.421897 -4.010338 1.840095

H -1.539708 -5.5890262 .618919$

H -0.697342 -4.0215134 .216806$

H $0.587106-5.1681013 .826040$

H $1.454264-3.3677542 .146447$

H $2.535525-3.8547124 .370475$

H $\quad 1.390289-2.7905885 .160774$

H $4.039403-2.2376705 .445556$

H $1.435582-0.2502844 .575711$

H $4.522244-2.9035933 .230143$

H $4.622849-1.2053142 .835960$

H $3.514438-2.2583171 .932266$
H $3.385780 \quad 0.746017 \quad 3.994359$

H -0.4029290 .6707053 .520924$

H $0.469068 \quad 1.2788112 .110542$

H $0.911322-0.6581020 .593880$

H -4.864482 -2.046840 -2.120555

H $-5.378493-1.3773780 .842606$

H -7.367662 -1.883791-2.393303

H $-6.562297-0.318518-2.664622$

H $-8.264235-0.382440-2.230037$

H -1.114877 -0.1679630 .673245$

H $-2.023778-0.4907402 .164857$

H $-3.9287302 .591691-0.919647$

H -3.4165313 .5204140 .504380$

H -5.4041842 .3114831 .800849$

H $-6.273953 \quad 3.120131-1.022758$

H $-8.1588793 .893906 \quad 0.531843$

H -7.8232932 .5713281 .624011$

H $-10.107100 \quad 1.832347-1.837916$

H -9.311919 $3.486919-1.589160$

H -9.580614 -0.017258 -0.455759

H $-9.4197890 .533628 \quad 1.188442$

H -7.232303 -0.2129941 .391235$

H -3.3495770 .5544590 .155987$

C $2.866784-2.116778-3.371234$

O $3.227737-0.928300-2.639691$

C $4.249378-1.272823-1.685901$

C $4.692525-2.695932-2.020933$

C $3.413215-3.295612-2.567996$

H $3.765774-1.279137-0.702273$

C $5.386173-0.245994-1.704434$

H $5.475567-2.710441-2.787791$

H $5.075062-3.226017-1.143216$

C $3.627892-4.553651-3.390669$

H $2.761394-3.530201-1.717662$

C $4.9574031 .218467-1.475615$

O $6.034860-0.305678-2.982380$

H $6.142658-0.538387-0.965755$

H $4.4516631 .597078-2.371092$

O $\quad 6.1625391 .988493-1.352821$

C $4.0726221 .463440-0.240683$

C 3.9627542 .9350520 .198941

H $3.0735241 .054494-0.423961$

$\begin{array}{lllll}\text { O } & 4.629588 & 0.740360 & 0.860934\end{array}$

H 3.2929482 .9687631 .067062

H 4.9367123 .2577490 .589092

H $2.678286-4.926841-3.786785$

H $4.297120-4.370438-4.238035$

H $4.072293-5.343474-2.776265$

C $1.362867-2.125203-3.658842$

H $6.7613230 .340869-2.969003$

H $6.6074601 .707874-0.534507$

H $4.0157150 .832517 \quad 1.609419$

H $1.147779-2.894445-4.410052$

H $1.082451-1.156925-4.091523$

H $3.393174-2.043157-4.332357$

C $1.0938373 .740193-0.540401$

O $2.2066103 .568551-1.426026$

C $3.4823953 .918742-0.884804$

C $3.4750795 .383916-0.425154$

C 2.3531765 .6488980 .571841

C $1.0203325 .176708-0.000354$

H $1.1717103 .025208 \quad 0.287538$

C $-0.1803053 .429388-1.342049$

H $4.1935543 .857672-1.716512$

O 4.7197965 .7424780 .165396

H $3.3306846 .039011-1.294283$

H 2.5537625 .1827451 .543501

O 2.2609537 .0548810 .814224

H $0.7094935 .861877-0.799922$

O $0.0251115 .238975 \quad 1.020424$

C $-0.3333841 .964008-1.680373$

H -1.066658 $3.720063-0.767778$

H $-0.1652533 .982913-2.288808$

H $5.4152765 .569900-0.492233$

H 3.1296617 .3493751 .137898

H 0.0105586 .1516501 .356515

O $-1.5040381 .802378-2.367493$

O $0.4561011 .079436-1.383692$ 
C $-1.8341430 .460571-2.734016$

H $-2.8640710 .451456-3.100718$

H $-1.1713840 .122543-3.534863$

H -1.769095 -0.201576 -1.866975

SCF Energy $\left(B 3 L Y P / 6-31 G^{* *} / / M M F F\right)=-3245.93338106$

01110

MM̄FF Geometry

C -2.299836 -4.055922 -0.143381

C $-1.522119-2.963381-0.151199$

C $-0.542977-2.6379890 .946637$

$\begin{array}{lllll}\text { O } & 0.745991 & -2.494347 & 0.308812\end{array}$

C $-0.947804-1.3162731 .624312$

C $-0.458309-1.1769543 .052650$

C $0.484236-0.3018143 .453508$

C -1.162232 -2.0416794 .067194$

C $1.278210 \quad 0.6327112 .587905$

C $0.562907 \quad 1.9745802 .394224$

C 1.4311892 .9939951 .643004

C 0.6263664 .2552911 .321264

C 1.4143675 .2474240 .451152

$\begin{array}{lllll}\text { O } & 1.888061 & 2.385310 & 0.434128\end{array}$

O $0.485798 \quad 6.201091-0.087446$

C $2.0258484 .490578-0.748715$

C 2.4234026 .0497751 .281083

O $2.8907695 .337493-1.501696$

C $2.7729343 .202594-0.340071$

C $3.1863582 .389065-1.593340$

O 3.9441623 .5708080 .393103

C $1.846090-2.9151390 .986748$

O $1.895048-3.3142222 .138800$

C $2.992323-2.8536170 .049364$

C $4.190028-3.3186030 .434909$

C $5.399051-3.401127-0.390437$

C $5.316967-2.896214-1.806685$

C $3.5185290 .911203-1.329726$

C $4.6758950 .685559-0.380262$

C $7.1373801 .021347-0.174372$

$\begin{array}{lllll}\text { O } & 4.508411 & 0.209086 & 0.739839\end{array}$

C $7.943232-0.137039-0.669917$

C $8.495422-1.0553280 .137708$

C $9.382322-2.163223-0.352064$

C $8.980374-3.5220120 .171717$

C $9.680926-4.1211631 .149904$

C $7.831306-4.230808-0.507680$

C $6.506933-3.9512220 .149641$

N $5.8897581 .087375-0.909398$

H $-2.215842-4.7650980 .676652$

H -1.612391 -2.249211 -0.965947

H $-0.514075-3.4673031 .663223$

H $-2.042826-1.2261261 .656594$

H - $-0.601517-0.4762301 .013575$

H $0.727991-0.2513484 .513574$

H -0.841912 -1.823963 5.091838

H -2.243929 -1.877899 4.021019

H $-0.962326-3.1008063 .880366$

H 2.2529630 .7960493 .064146

H 1.4928520 .1645821 .620887

H $-0.359616 \quad 1.7958841 .827193$

H $\quad 0.2675232 .3940643 .364198$

H 2.2953723 .2369412 .272670

H 0.2752494 .7395662 .240831

H -0.2786663 .9687090 .771342$

H 0.0189256 .6185530 .656668

H $1.1991484 .215013-1.418695$

H 1.9054296 .6372972 .048724

H $2.958537 \quad 6.7760270 .659545$

H 3.1570025 .4211561 .789920

H $2.3815536 .130562-1.741888$

H $2.3529962 .388630-2.307799$

H $4.0354462 .875992-2.088236$

H 4.6865633 .0030290 .137694

H $2.809226-2.464348-0.945624$

H $4.292982-3.7043611 .448508$

H $4.974097-1.857365-1.829196$

H $4.619138-3.506330-2.390751$

H $6.274279-2.908616-2.331288$

H $2.6428010 .385124-0.932514$
H $3.7612230 .420826-2.280536$

H $\quad \begin{array}{llll}6.934484 & 0.956927 & 0.899532\end{array}$

H $7.6749671 .954194-0.371167$

H $8.118530-0.197630-1.741586$

H $8.349255-0.9752131 .212561$

H $10.406699-1.929124-0.033202$

H $9.415076-2.194389-1.448332$

H $9.417709-5.1116841 .506313$

H $10.528315-3.6328551 .619472$

H $7.992696-5.316512-0.457076$

H $7.841417-4.002219-1.576666$

H $6.457822-4.2958501 .183930$

H $5.9248091 .425517-1.866730$

C -4.735521-4.246335 -0.592752

O $-5.019729-2.860797-0.314488$

C $-6.412602-2.609360-0.582839$

C -7.036757 -3.961253 -0.913528

C $-5.858295-4.701859-1.519127$

H -6.452781 -1.980196-1.478996

C $-7.043058-1.8785690 .608194$

H $-7.390819-4.476550-0.013104$

H -7.884634 -3.868219-1.599051

C $-6.050909-6.207111-1.570677$

H $-5.685872-4.322103-2.535158$

C $-6.338243-0.5535840 .973064$

O $-6.986477-2.729821 \quad 1.761184$

H -8.107646 -1.701560 0.419940

H $-5.325883-0.7645311 .335865$

O

C $-6.2807190 .477727-0.169301$

$\begin{array}{llll}\text { C } & -5.666916 & 1.829694 & 0.236747\end{array}$

H -5.718563 $0.076667-1.018325$

O $-7.6045230 .754668-0.636548$

H $-5.8346522 .530206-0.590765$

H -6.2316082 .2566491 .075007$

H $-5.174679-6.698899-2.004103$

H $-6.212239-6.625759-0.571593$

H $-6.919742-6.462583-2.186215$

C $-3.334333-4.361847-1.190920$

H $-6.051652-2.9459031 .921046$

H -7.078377 -0.6292712 .783452$

H -7.929694 -0.037231-1.096287

H $-3.230248-3.674058-2.039037$

H -3.155264 -5.376188 -1.563383

H $-4.790600-4.7841930 .363708$

C $-3.2848571 .798048-1.655966$

O $-3.3973551 .092923-0.415151$

C -4.1647891 .7704720 .586289$

C $-3.542127 \quad 3.1436990 .880315$

C $-3.3691003 .975848-0.390561$

C $-2.6299763 .173198-1.463960$

H $-4.2914601 .948091-2.066750$

C $-2.5812770 .883292-2.667951$

H -4.0729061 .1720931 .500636$

O -4.3346303 .8731691 .811300$

H -2.5554943 .0035251 .339157$

H $-4.3329394 .337563-0.767455$

O $-2.6007925 .142402-0.088126$

H - $1.5798993 .085373-1.166953$

O $-2.6688093 .912530-2.682127$

C $-1.1508900 .512113-2.335029$

H -3.139398 -0.060047 -2.725724

H -2.588862 1.352632 -3.657902

H -4.418969 3.3286422 .612632

H -3.0636135 .6168290 .623543$

H $-2.0477823 .493651-3.301366$

O $-0.666977-0.273521-3.344453$

O $-0.5366980 .847685-1.333430$

C $0.681463-0.726406-3.193011$

H $1.3684190 .103007-3.379598$

H $0.844071-1.148874-2.197611$

H $\quad 0.863348-1.507695-3.935703$

SCF Energy (B3LYP/6-31G**//MMFF) $=-3245.89265103$

01 111

MM̄FF Geometry

C -0.794576 -2.884662 0.258772

C $-1.869600-2.758665-0.534075$ 
C $-2.011763-1.796953-1.688975$

O $-0.769851-1.078211-1.893483$

C $-2.383132-2.484527-3.014697$

C $-3.826326-2.937441-3.110122$

C $-4.851910-2.125297-3.437721$

C $-4.064946-4.408727-2.894684$

C $-4.782658-0.649665-3.719553$

C $-5.9218910 .161152-3.084653$

C $-6.0162300 .075176-1.551858$

C -7.209852 $0.902758-1.059925$

C -7.3169420 .9155290 .470781$

O

O $\quad-8.247444 \quad 1.9532490 .816738$

C -5.9410991 .2956441 .057591$

C -7.916905 -0.3904591 .003904$

O

C $-4.770326 \quad 0.4810340 .462814$

C -3.3965390 .9766150 .972116$

O $-4.897304-0.8811120 .895680$

C $-0.8510190 .244088-2.198288$

$\begin{array}{lllll}\text { O } & -1.866541 & 0.906108 & -2.339077\end{array}$

C $0.5241430 .771677-2.331421$

C $0.6886042 .091528-2.505491$

C $1.9570212 .801530-2.660938$

C $3.2221851 .988374-2.688422$

C -3.0095862 .4090380 .595169$

C -1.6763542 .8032541 .215728$

C -0.1182354 .7227091 .401168$

O -1.0978532 .1114002 .050221$

C 0.6311385 .5426660 .400302

C 1.9680315 .5078480 .282781

C $2.7561106 .359926-0.672785$

C $3.6530795 .542311-1.576682$

C $4.9271565 .286498-1.234898$

C $3.0979815 .082402-2.905777$

C $1.9315434 .146952-2.755775$

N $-1.2240294 .034767 \quad 0.757121$

H $0.089166-2.2818910 .073268$

H -2.750624 -3.349650 -0.296301

H -2.796898 -1.090073 -1.405247

H -1.709149 -3.333152 -3.195592

H -2.171324 -1.803341 -3.850320

H $-5.844798-2.562725-3.532430$

H -3.758700 -4.710594-1.889609

H -5.119830 -4.679836 -3.010452

H -3.493136 -4.994767 -3.621744

H -3.828272 -0.223115 -3.408382

H -4.840264 - $0.515801-4.807032$

H -5.774876 $1.209415-3.376056$

H -6.874825 -0.160835 -3.524183

H -6.147236 $-0.975212-1.268872$

H -8.145134 $0.540930-1.505340$

H -7.096975 $1.940053-1.406274$

H -8.2658592 .0205491 .787032$

H -5.7918002 .3574160 .828588$

H $-8.923306-0.5409700 .594668$

H -8.041149 -0.3559572 .091788$

H -7.322993 -1.2712330 .751848$

H $-5.889887 \quad 0.2570612 .713079$

H $-3.351750 \quad 0.8499062 .062062$

H $-2.615131 \quad 0.3123250 .579479$

H -4.031806 -1.3133560 .811341$

H $1.3501220 .072234-2.281308$

H $-0.1971312 .725767-2.531177$

H $3.2016451 .270377-3.515573$

H $3.3410451 .440715-1.749325$

H $4.1243962 .591232-2.814584$

H -2.942188 $2.513356-0.492161$

H -3.754068 3.1209690 .965074

H 0.5267753 .9981451 .908453

H -0.5530935 .3784502 .162842$

H $0.056907 \quad 6.216724-0.230326$

H 2.5387724 .8511740 .936229

H $3.3651137 .053365-0.077737$

H $2.0997106 .994211-1.281432$

H $5.5857874 .721666-1.886188$

H $5.3383835 .632830-0.292354$

H $3.8773214 .628876-3.529153$
H $2.7668655 .961971-3.473319$

H $0.9677504 .655884-2.717059$

H $-1.8486264 .588506 \quad 0.177539$

C $-0.611801-3.0541612 .782089$

O $0.602020-2.2737612 .809092$

C $0.279475-0.9507873 .277085$

C $-1.056740-1.0859603 .996540$

C -1.758648 -2.092033 3.105981

H $0.135957-0.3309292 .384061$

C $1.414545-0.3732394 .125539$

H -0.936311 -1.494027 5.006696

H -1.588988 -0.1330504 .072231$

C -2.960264 -2.756588 3.752901

H -2.094210 -1.567850 2.204576

C $2.791638-0.3747263 .430172$

O $1.532256-1.1521005 .322623$

H 1.1484790 .6415964 .444333

H $3.154486-1.4060773 .354988$

$\begin{array}{lllll}\text { O } & 3.716290 & 0.298220 & 4.294181\end{array}$

C 2.8199650 .2858352 .039435

C 4.2287030 .4800221 .449680

H $2.223954-0.3131011 .343122$

O $2.192828 \quad 1.5697442 .098275$

H $4.1119920 .977914 \quad 0.480074$

H $4.787723 \quad 1.1991802 .062080$

H -3.416095 -3.481687 3.071287

H -2.682063 -3.283600 4.671654

H $-3.719487-2.0105004 .009727$

C $-0.729719-3.8016301 .448889$

H $2.256762-0.7699445 .847025$

H $3.398931 \quad 1.208190 \quad 4.421521$

H 2.7359242 .1447772 .663225

H $-1.603320-4.4635131 .477580$

H $\quad 0.152783-4.4400651 .317665$

H $-0.508158-3.7971913 .583270$

C $4.041971-1.656877-0.731137$

O $4.300833-1.8607540 .660176$

C $5.047368-0.8164751 .291669$

C $6.395280-0.6239530 .581211$

C $6.221901-0.397990-0.920519$

C $5.338624-1.486785-1.531596$

H $3.431780-0.752990-0.842088$

C $3.160858-2.808392-1.236217$

H $5.271767-1.1716262 .304824$

O 7.1022610 .4673901 .160681

H $7.007031-1.5220540 .736522$

H $5.8132550 .597932-1.124202$

O $7.503869-0.423711-1.553150$

H $5.902501-2.425719-1.566580$

O $5.017259-1.136589-2.875522$

C $3.810853-4.175329-1.173663$

H $2.257835-2.870506-0.620362$

H $2.863350-2.607601-2.271990$

H 7.9339470 .5703780 .667276

H $7.903378-1.294932-1.388456$

H $5.855671-0.992503-3.346962$

O $3.160029-5.015330-2.032502$

O $4.742774-4.482304-0.443247$

C $3.649291-6.359637-2.067439$

H $3.055932-6.918580-2.795858$

H $3.534182-6.829609-1.086037$

H $4.696857-6.373885-2.383154$

SCF Energy (B3LYP/6-31G**//MMFF)= -3245.91581602

01_112

MM̄FF Geometry

C -3.839268 -3.308251 1.151571

C $-3.004311-2.3151550 .811434$

C $-1.767305-1.9427421 .581785$

O $-0.718812-1.8154890 .594023$

C $-1.980025-0.5876852 .282565$

C $-1.124974-0.3972133 .518743$

C $-0.0695990 .434381 \quad 3.616642$

C $-1.573216-1.1788924 .729546$

C $0.541971 \quad 1.3031082 .551929$

C 2.0342310 .9966832 .411013

C 2.7987401 .9411901 .472949

C 2.2274871 .9661970 .053922 
C $3.1124922 .762364-0.917054$

O $4.147714 \quad 1.4573191 .458772$

O $2.6711942 .480775-2.254023$

C $4.5734552 .285866-0.778939$

C $2.9223694 .272333-0.738754$

O $5.4478073 .094005-1.563185$

C 5.0655832 .2383030 .685334

C 6.4414101 .5367650 .754265

O $\quad 5.1860253 .568273 \quad 1.188327$

C $0.541187-2.2041630 .914624$

O $0.939760-2.6194251 .989628$

C $1.362117-2.038328-0.307551$

C $2.696946-2.162437-0.258065$

C $3.612022-2.032404-1.397283$

C $2.996888-1.829401-2.758627$

C $6.980877 \quad 1.3438532 .178985$

C $8.137816 \quad 0.3659182 .171497$

C $8.618373-2.0664061 .908045$

O $\quad 9.306583 \quad 0.7437342 .157137$

C $8.711332-2.2788830 .430334$

C $8.146831-3.309382-0.216371$

C $8.179154-3.457385-1.710310$

C $6.804094-3.346206-2.331857$

C $6.280736-4.358230-3.044201$

C $6.061568-2.033928-2.183162$

C $4.941349-2.121059-1.178038$

N $7.714702-0.9545292 .137395$

H -3.639625 -3.903767 2.038252

H $-3.235697-1.712429-0.062942$

H -1.521250 -2.741513 2.291219

H -3.026502 -0.4872912 .603918$

H -1.817695 $0.225876 \quad 1.568787$

H $\quad 0.4276760 .5059354 .584069$

H $-0.971107-0.9552495 .616626$

H -2.613926 -0.9397734 .971970$

H -1.497405 -2.254917 4.546579

H 0.0476521 .1656051 .587862

H 0.4062282 .3518092 .840813

H $2.5184141 .036543 \quad 3.397136$

H $2.172166-0.0380062 .073995$

H 2.7862652 .9465511 .909948

H 1.2108822 .3746120 .046873

H $2.1395560 .937624-0.318062$

H $2.7547981 .524242-2.406427$

H $4.6324221 .271091-1.196211$

H $1.8845754 .553366-0.951403$

H $3.5351194 .836211-1.450890$

H 3.1569044 .6194830 .269574

H $5.1039673 .095085-2.472978$

H $\quad 6.358167 \quad 0.5536120 .273425$

H 7.1854892 .1085890 .185169

H 5.7719303 .5623941 .961234

H $0.835743-1.811549-1.228226$

H $3.165211-2.3749180 .701997$

H $2.457204-0.876961-2.797372$

H $2.296428-2.640175-2.988840$

H $3.723318-1.815630-3.572904$

H 7.3381262 .2903332 .598784

H $6.207391 \quad 0.9646512 .856262$

H $8.208373-2.9375892 .428078$

H $9.606273-1.8402272 .320827$

H $9.245069-1.521377-0.139419$

H $7.606619-4.0665110 .346788$

H $8.636270-4.427235-1.943299$

H $8.829573-2.700435-2.166913$

H $5.305403-4.272053-3.511899$

H $6.814171-5.293729-3.175715$

H $5.719227-1.703435-3.168036$

H $6.752987-1.246200-1.858031$

H $5.284893-2.266459-0.153223$

H $6.719184-1.1451092 .064691$

C $-6.306495-2.9292240 .946265$

O $-6.153765-1.5067600 .764029$

C -7.364619 -0.9694310 .206151$

C -8.426521 -2.0381190 .427251$

C $-7.610209-3.303468 \quad 0.240094$

H -7.195234 -0.860013 -0.871953

C -7.6661320 .3992330 .824423$
H $-8.838293-2.000921 \quad 1.442277$

H -9.256824 -1.952644 -0.279945

C $-8.277348-4.5479970 .799132$

H -7.428603 -3.447614 -0.833454

C $-6.485326 \quad 1.391586 \quad 0.740561$

$\begin{array}{llll}\text { O } & -7.977503 & 0.207008 & 2.210017\end{array}$

H -8.5637230 .8236260 .359328$

H -5.6682861 .0471081 .384422$

O -6.9243892 .6457651 .277250$

C $-5.9609881 .640419-0.688045$

C $-4.8835612 .738384-0.784115$

H $-5.5610470 .712526-1.106403$

O $-7.0534212 .029277-1.526068$

H $-4.7462142 .983678-1.843860$

H $-5.2655473 .661295-0.331748$

H -7.642605 -5.4280390 .655752$

H -8.478524 -4.448457 1.870983

H $-9.231019-4.7327710 .294199$

C $-5.080537-3.6365880 .368718$

H -8.3717561 .0324112 .538739$

H -7.0498832 .5272372 .233697$

H -7.437029 2.840148 -1.149933

H $-4.941791-3.339174-0.678041$

H $-5.220226-4.7234110 .377771$

H $-6.383006-3.1065082 .026748$

C $-2.4685381 .390315-2.098299$

O $-2.9597161 .189197-0.766677$

C $-3.5366372 .343578-0.144887$

C $-2.5068173 .479948-0.094699$

C $-1.9481733 .792404-1.477983$

C $-1.4272472 .518121-2.136633$

H -3.303635 $1.626185-2.768426$

C $-1.8325640 .070180-2.562805$

H -3.7402942 .0620930 .894271$

O -3.0754324 .6614340 .459227$

H -1.6734793 .1874550 .557850$

H -2.692459 $4.287935-2.112302$

O $-0.8579334 .706904-1.349390$

H $-0.5086532 .195928-1.631313$

O $-1.0881482 .799044-3.493466$

C $-2.852147-1.010091-2.852886$

H -1.264003 $0.225409-3.486494$

H $-1.161971-0.307696-1.783296$

H $-3.4148334 .436013 \quad 1.342261$

H $-1.1953335 .496220-0.892123$

H $-0.4361873 .521001-3.485362$

O $-2.188272-2.150376-3.209868$

O $-4.065854-0.879525-2.783152$

C $-3.014341-3.270674-3.540692$

H $-3.728652-3.004297-4.325737$

H $-2.368643-4.070277-3.913245$

H -3.535126 -3.627413 -2.648286

SCF Energy $(B 3 L Y P / 6-31 G * * / / M M F F)=-3245.91512770$

01113

MM̄FF Geometry

C $\quad 0.1734512 .109808 \quad 3.108827$

C $-0.754376 \quad 3.078078 \quad 3.070335$

C -1.250298 3.6642761 .776754

O -2.6381863 .2759481 .615361$

C -1.1962945 .2006601 .728191$

C 0.1393795 .7262151 .247821

C $0.4019026 .057976-0.032281$

C 1.2011855 .8839392 .303530

C $-0.5280825 .988837-1.213922$

C $-0.8408324 .582075-1.738164$

C $0.3703793 .662393-1.955810$

C $1.2947734 .175010-3.062260$

C $2.4430063 .195510-3.349238$

O $-0.1442862 .368527-2.308744$

O $3.0530333 .581000-4.590637$

C $1.8580941 .782979-3.554030$

C $3.5418673 .278981-2.282573$

O $2.8989770 .815256-3.674056$

C $0.8646551 .357157-2.447507$

C $0.1735470 .031707-2.852504$

O $1.5882881 .163185-1.231107$

C -2.8586492 .0807901 .002509$ 
O -2.0131691 .2848790 .624330$ C $-4.316073 \quad 1.8959490 .846121$ C $-4.7411751 .040248-0.094665$ C $-6.1250150 .675079-0.392641$ C $-7.196525 \quad 1.1579990 .546761$ C $-0.952981-0.412729-1.911904$ C $-1.525325-1.769762-2.288588$ C $-3.199764-3.447072-1.493361$ O $-1.171392-2.398162-3.282237$ C $-4.507152-3.198638-2.178073$ C $-5.701952-3.388847-1.596890$ C $-7.017487-3.151384-2.285651$ C $-7.865470-2.109502-1.586499$ C $-8.819000-2.470247-0.711186$ C $-7.665485-0.656703-1.961557$ C $-6.356945-0.109696-1.465211$ N $-2.480961-2.191841-1.373203$ H $0.611750 \quad 1.7586632 .177683$ H -1.220058 3.4181223 .991436 H $-0.673154 \quad 3.2669620 .932943$ H -1.437989 5.6329832 .708133 H $-1.9950215 .566717 \quad 1.070677$ H $1.3997796 .423510-0.271904$ H $2.109164 \quad 6.3544301 .911236$ H 0.8343846 .5138803 .120633 H 1.4881024 .9117342 .713431 H - $-1.4670816 .503055-0.975528$ H $-0.0824036 .578517-2.025189$ H $-1.5432274 .087223-1.058060$ H -1.399094 $4.673376-2.679816$ H $0.9147553 .566462-1.010571$ H $1.6985945 .164400-2.817495$ H $0.7150394 .308600-3.986308$ H $3.361558 \quad 4.498971-4.500677$ H $1.3241751 .784635-4.514732$ H $3.9664434 .289599-2.248875$ H $4.3771032 .611847-2.522223$ H $3.1850533 .040017-1.278450$ H $3.4906661 .112579-4.386277$ H $-0.2576940 .140543-3.856392$ H $0.920603-0.767402-2.907407$ H $1.1170870 .531303-0.667325$ H $-4.9817312 .455438 \quad 1.491859$ H $-3.9953800 .548930-0.718514$ H $-7.290605 \quad 2.2481110 .492675$ H -6.9601920 .8772281 .579385$ H -8.1814180 .7365730 .332416$ H $-0.590940-0.487656-0.881950$ H $-1.7696600 .316484-1.942958$ H -3.332169 $-3.843507-0.481593$ H $-2.605645-4.161516-2.071929$ H $-4.465506-2.852556-3.208194$ H $-5.742414-3.747582-0.570847$ H $-7.554049-4.108664-2.318853$ H $-6.873859-2.860747-3.333863$ H $-9.449443-1.732772-0.225493$ H -8.986391 -3.511670 -0.456936 H $-8.505425-0.044809-1.615482$ H $-7.689825-0.563660-3.055136$ H -5.507391 $-0.399440-2.084130$ H $-2.742332-1.571917-0.611864$ C $0.020517 \quad 0.005000 \quad 4.419037$ O $0.578663-0.8204593 .376014$ C $0.659184-2.1762963 .859463$ C $0.060089-2.1733125 .263811$ C $0.316205-0.7474795 .711660$ H $1.723275-2.4236133 .936976$ C $-0.039751-3.1304702 .883889$ H -1.016096 -2.383082 5.245007 H $\quad 0.530282-2.9181585 .913468$ C $-0.538185-0.3241306 .893582$ H $1.377910-0.6438815 .973027$ C $0.567651-3.1626651 .465618$ O $-1.416010-2.7466872 .758049$ H $-0.049039-4.1433423 .303007$ H $0.391275-2.2074690 .957751$ O $-0.143617-4.156698 \quad 0.716289$ C $2.068211-3.508544 \quad 1.422869$
C $2.618675-3.8405740 .023571$

H $2.644295-2.6805251 .848168$

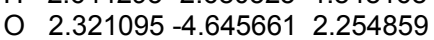

H $3.673650-4.1173110 .142200$

H $2.136039-4.751801-0.352243$

H $-0.3224510 .708871 \quad 7.183168$

H $-1.606361-0.392836 \quad 6.661759$

H $-0.340260-0.964258 \quad 7.759532$

C $\quad 0.617143 \quad 1.411350 \quad 4.361503$

H $-1.435887-1.8311002 .430253$

H $-1.086375-3.9191940 .743647$

H $1.785862-5.3833991 .914560$

H 1.7123501 .3649324 .356732

H $\quad 0.316428 \quad 1.988927 \quad 5.242590$

H -1.0640480 .0489664 .246391$

C $4.417227-1.416921-0.387269$

O $2.992221-1.465005-0.530571$

C $2.473914-2.707551-1.008715$

C $3.076872-3.030140-2.382533$

C $4.602399-2.999825-2.353735$

C $5.106068-1.698146-1.730139$

H $4.736162-2.1543380 .359076$

C $4.770890-0.0221210 .139706$

H $1.399473-2.554640-1.160999$

O $2.641143-4.304260-2.844173$

H $2.734518-2.289539-3.115972$

H $5.011743-3.870834-1.828706$

O $5.095725-3.074225-3.694202$

H $4.931224-0.875511-2.435112$

O $6.515449-1.817523-1.545714$

$\begin{array}{llll}\text { C } & 4.206826 & 0.200803 & 1.522215\end{array}$

H 5.8520850 .1452080 .187279

H $4.3552550 .754981-0.512057$

H $1.669160-4.286560-2.873942$

H $4.737830-3.888105-4.088489$

H $6.869439-0.929145-1.373450$

O $4.967549-0.4498842 .447980$

$\begin{array}{llll}\text { O } & 3.207576 & 0.869918 & 1.749087\end{array}$

C $4.536428-0.3096923 .804956$

H $4.569908 \quad 0.741724 \quad 4.106077$

H $3.527782-0.7134803 .926093$

H $5.220386-0.8790004 .439918$

SCF Energy (B3LYP/6-31G**//MMFF)= -3245.92026438

$01 \_114$

MM̄FF Geometry

C -0.7316653 .1650691 .597589$

$\begin{array}{llll}C & -0.488741 & 4.210212 & 0.792093\end{array}$

C $0.5819794 .282265-0.268068$

$\begin{array}{lllll}\text { O } & 1.713218 & 3.477428 & 0.140494\end{array}$

C $0.0924843 .782983-1.641646$

C -1.092061 $4.534915-2.204663$

C $-2.2961393 .991001-2.465865$

C $-0.8428965 .988054-2.521949$

C $-2.7542872 .571118-2.264464$

C $-3.0539181 .920036-3.621074$

C $-3.611548 \quad 0.489004-3.561393$

C $-4.9281950 .397732-2.785461$

C $-5.462036-1.040886-2.741584$

O $-2.629085-0.380469-2.985460$

O $-6.521075-1.095181-1.773294$

C $-4.328840-1.974566-2.270549$

C $-6.109405-1.447756-4.069251$

O $-4.733732-3.339848-2.345009$

C $-2.992243-1.769654-3.024554$

C $-1.883472-2.595966-2.324314$

O $-3.151075-2.239224-4.366527$

C $2.9298013 .806567-0.372172$

O $3.1943164 .740160-1.112316$

C 3.8894112 .7820510 .086899

C $4.9814732 .536919-0.650835$

C $5.954776 \quad 1.470549-0.411931$

$\begin{array}{llll}\text { C } & 5.863718 & 0.712220 & 0.885675\end{array}$

C $-0.463041-2.410766-2.872882$

C $0.345979-1.314240-2.196740$

C $2.682295-0.426197-2.146684$

O $-0.131867-0.492084-1.421811$

C $3.970707-1.154018-1.924235$ 
C $5.021849-1.094991-2.756933$

C $6.327157-1.800358-2.507033$

C $7.527054-0.879512-2.614054$

C $8.340749-0.928012-3.682267$

C $7.8223410 .049140-1.451688$

C $6.8431171 .189420-1.387854$

N $1.680794-1.380920-2.578374$

H -0.1509232 .2529861 .490149$

H -1.0930235 .1080280 .907781$

H $0.9100135 .327761-0.326371$

H $-0.0945592 .705464-1.579437$

H $0.9149083 .859615-2.365683$

H -3.065658 4.642769 -2.880081

H -1.686534 $6.450461-3.045528$

H $-0.6727706 .560642-1.605175$

H $\quad 0.0363126 .090439-3.166739$

H -2.031897 $1.968472-1.708815$

H -3.660216 2.611983-1.650214

H $-3.7628122 .546080-4.178875$

H -2.122025 $1.899212-4.202506$

H $-3.7733880 .182156-4.601990$

H -5.680079 $1.075648-3.207705$

H $-4.7727790 .725345-1.749805$

H $-6.159357-0.831221-0.910021$

H -4.149661 -1.764226 -1.209952

H $-6.952586-0.785493-4.300388$

H -6.530618 -2.457667 -4.010632

H $-5.419949-1.411652-4.915049$

H -5.565446 -3.423247 -1.847769

H -1.882749 -2.369053 -1.252776

H -2.131431 -3.660285 -2.429795

H -2.308869 -2.592329 -4.691113

H 3.6335822 .2115900 .970480

H $5.1421153 .126067-1.553115$

H 4.9732180 .0766470 .896154

H 5.8134221 .4023501 .735620

H 6.7269260 .0706011 .074518

H $0.080292-3.350312-2.704639$

H $-0.461386-2.219929-3.950310$

H $2.3616420 .070638-1.225432$

H $2.7681890 .334275-2.929837$

H 4.046931-1.742013-1.013180

H $4.939567-0.515593-3.673785$

H $6.410596-2.612108-3.241230$

H $\quad 6.335979-2.285093-1.522756$

H $9.214189-0.288735-3.757452$

H $8.149366-1.604841-4.508402$

H $8.8264840 .481027-1.551169$

H $7.850202-0.546395-0.535604$

H $6.837444 \quad 1.807131-2.287364$

H $1.934862-2.015014-3.330863$

C -2.977822 2.3313582 .401604

O $-2.602141 \quad 0.9424142 .330701$

C $-3.531248 \quad 0.164806 \quad 3.108218$

C $-4.715571 \quad 1.0809313 .390632$

C -4.0333032 .4271043 .506020$

H -3.027578 -0.0699604 .053709$

C -3.895534-1.132197 2.378136

H -5.4321141 .0932962 .561531$

H $\quad-5.255956 \quad 0.792547 \quad 4.297446$

C -4.9820103 .6038643 .352840$

H -3.545734 2.4870734 .488559

C $-2.689800-1.9703591 .908204$

O $-4.668315-0.8130811 .212845$

H -4.551436 -1.739605 3.012395

H $-2.190076-1.4534191 .081398$

O $-3.195728-3.1995261 .370648$

C -1.666325 -2.310089 3.006204

C $-0.577106-3.3084602 .566096$

H -1.194021 -1.390232 3.362536

O $-2.361634-2.8810734 .118085$

H $0.048236-3.5305173 .439245$

H -1.048019 -4.2657532 .310246$

H -4.4440804 .5533203 .435776$

H -5.4880613 .5872442 .381707$

H -5.7503703 .5823514 .132561$

C -1.751846 3.1940062 .700676

H $-4.140889-0.2006810 .671073$
H $-3.868241-2.9734570 .706120$

H -1.711785 -3.028799 4.826206

H -1.2588392 .8344603 .612192$

H -2.064476 4.2267572 .895615

H -3.4066602 .5962691 .426481$

C $1.964539-1.6014472 .654790$

O $0.917879-1.5567381 .677041$

C $0.294452-2.8131821 .395939$

C $1.338530-3.8253890 .908207$

C $2.491307-3.9643031 .894853$

C $3.062639-2.5912952 .238394$

H $1.549662-1.8887153 .628384$

C $2.543998-0.1827462 .765905$

H $-0.377744-2.6384540 .550812$

O $0.749372-5.1028010 .691004$

H $1.746086-3.492019-0.055083$

H $2.186313-4.5022752 .800081$

O $3.530811-4.7419611 .296160$

H $3.612798-2.1996591 .373896$

O $3.996717-2.7306713 .308027$

C 1.6858810 .7514523 .591491

H $3.528261-0.2164503 .246849$

H 2.6405310 .2587381 .768729

H $\quad 0.024845-4.9854740 .052999$

H $3.142541-5.597617 \quad 1.044673$

H $4.668960-3.373725 \quad 3.023922$

O 2.4062581 .8823563 .855517

O $0.5369650 .531073 \quad 3.945669$

C 1.7680802 .8777844 .662922

H 2.5487803 .4659285 .152717

H 1.1814403 .5405364 .022476

H 1.1359032 .4313115 .436554

SCF Energy (B3LYP/6-31G**//MMFF) $=-3245.91309988$

$01+115$

MM̄FF Geometry

C $-3.374708 \quad 3.1988720 .779433$

C $-2.9777013 .044584-0.492865$

C $-1.5764903 .331568-0.956286$

O $-1.0903412 .143961-1.627719$

C $-1.5047434 .486345-1.971411$

C -1.118226 $5.799893-1.324180$

C $0.1543546 .180296-1.090034$

C $-2.2664606 .689730-0.931150$

C $1.4122885 .414525-1.407432$

C $1.9773594 .739324-0.157376$

C $3.3450634 .064536-0.346590$

C $3.3647273 .037031-1.480597$

C $4.6905122 .260804-1.543687$

O $3.6338513 .421844 \quad 0.903174$

O $4.4975741 .125803-2.401967$

C $5.0174521 .712408-0.139632$

C $5.8099553 .095017-2.177192$

O $6.3072611 .106991-0.111832$

C 4.9132402 .7789620 .970277

C 5.0485392 .1839192 .393358

O $\quad 5.969410 \quad 3.733390 \quad 0.811990$

C $-0.2744651 .313799-0.925670$

$\begin{array}{lllll}\text { O } & 0.067346 & 1.423555 & 0.240524\end{array}$

C $0.1739290 .251541-1.851470$

C $1.253079-0.477234-1.532409$

C $1.860139-1.525157-2.352669$

C $1.135064-1.944128-3.604614$

C 4.0604911 .0577962 .725471

C $4.684688-0.3279312 .658446$

C $4.066634-2.7404862 .570947$

O $5.890455-0.5250812 .789627$

C $4.626385-3.2026821 .262869$

C $4.088469-4.1882750 .528788$

C $4.680031-4.691534-0.756236$

C $3.783067-4.455259-1.950294$

C $3.012678-5.438549-2.444899$

C $3.856624-3.108604-2.632968$

C $3.028956-2.061451-1.945168$

N $3.735024-1.3284442 .502847$

H -2.6538173 .5888841 .495851$

H $-3.6657402 .666276-1.242830$

H $-0.9327783 .554348-0.098182$ 
H $-2.4588184 .603125-2.502794$ H $-0.7882724 .245515-2.766306$ H $0.3241287 .141632-0.606955$ H -1.929467 $7.625856-0.473603$ H -2.863436 $6.949172-1.811556$ H $-2.9136676 .183957-0.208246$ H $1.2318364 .671660-2.188470$ H $2.1480236 .117581-1.816011$ H 2.0700765 .4754700 .653496 H 1.2653353 .9959220 .220074 H $4.0906794 .847990-0.526452$ H $3.1685993 .510598-2.449464$ H $2.5505682 .318313-1.327107$ H $4.2034031 .451804-3.269801$ H 4.2950330 .9113900 .060641 H $5.5447543 .374305-3.204041$ H $6.7375862 .517426-2.254724$ H $6.0202384 .016584-1.630819$ H $6.3262170 .433585-0.813342$ H 6.0877211 .8834842 .572053 H 4.8572842 .9990993 .105962 H 6.1172554 .1731181 .665795 H $-0.3679600 .127725-2.781872$ H $1.761509-0.268926-0.591371$ H $1.159859-1.140048-4.348080$ H $\quad 0.088814-2.186118-3.386484$ H $1.557801-2.836199-4.072183$ H 3.7268611 .1802193 .764051 H 3.1660791 .1082272 .095260 H $3.153712-3.2780112 .845049$ H $4.808966-2.8920533 .361653$ H $5.538110-2.7220710 .915845$ H $3.189398-4.6852480 .883504$ H $4.871289-5.765991-0.635541$ H $5.663334-4.242910-0.945177$ H $2.391867-5.283347-3.320978$ H $2.980001-6.418220-1.979708$ H $3.586965-3.202371-3.690302$ H $4.899610-2.766867-2.651409$ H $3.476048-1.682294-1.026348$ H $2.758410-1.0769072 .382851$ C $-5.7043232 .117908 \quad 0.469678$ $\begin{array}{lllll}\text { O } & -5.182619 & 0.804162 & 0.183605\end{array}$ C $-6.258462-0.1491950 .247666$ C -7.5434680 .6640570 .360361$ C -7.0645851 .8811751 .125694$ H $-6.121427-0.7151711 .176282$ C $-6.211940-1.093657-0.957042$ H $-7.9260830 .959252-0.623681$ H $-8.336694 \quad 0.1150550 .876966$ C -8.0125523 .0646231 .037007$ H -6.9317191 .6034832 .179970$ C $-4.891317-1.878433-1.107986$ O $-6.395687-0.318175-2.148951$ H -7.060895 -1.786213 -0.908139 H $-4.086435-1.190779-1.391731$ O $-5.048437-2.769624-2.220779$ C $-4.468028-2.6843310 .135411$ C $-3.357257-3.720810-0.109140$ H $-4.149449-1.9937730 .921424$ O $-5.598601-3.3903250 .658590$ H -3.202129 -4.2617350 .832298$ H $-3.727463-4.483253-0.806373$ H -7.622323 3.9210551 .595538 H -8.164897 $3.380228-0.000609$ H -8.9903652 .8059041 .456496$ C $-4.7235572 .876367 \quad 1.366999$ H $-6.363575-0.932433-2.902219$ H -5.779137 -3.377382 -2.016609 H -5.880396 -4.040871 -0.006511 H -4.5371882 .2750342 .266471$ H -5.1752573 .8191611 .696123$ H $-5.8355932 .635271-0.489286$ C -1.004154 -2.623560 1.507804 O $-1.480742-2.1438720 .244376$ C $-2.023284-3.144678-0.623182$ C $-0.964034-4.219862-0.908250$ C $-0.421746-4.8229020 .382156$
C $0.058721-3.715703 \quad 1.316258$

H -1.846592 -3.009942 2.094299

C $-0.397317-1.4330652 .268519$

H -2.231792 -2.644375 -1.576007

O $-1.493592-5.257882-1.725655$

H $-0.126719-3.774946-1.458348$

H $-1.163993-5.4608050 .875699$

O $0.689365-5.6678150 .078567$

H $0.977955-3.2737260 .910805$

O $0.381606-4.2835382 .584298$

C $-1.431448-0.4233432 .718657$

H $0.116554-1.7939783 .167036$

H $\quad 0.322626-0.904907 \quad 1.634503$

H - $1.817416-4.846850-2.545379$

H $0.380923-6.333590-0.559988$

H $1.044624-4.9784262 .430407$

O $-0.857066 \quad 0.4112243 .635964$

O $-2.582046-0.3555672 .310882$

C -1.7106041 .4273034 .170713$

H -2.6382970 .9930214 .555782$

H -1.1840401 .9129654 .996647$

H -1.922736 2.1744293 .402223

SCF Energy (B3LYP/6-31G**//MMFF) $=-3245.90751838$

01116

MM̄FF Geometry

C 4.9094811 .9452381 .907929

C 3.6092571 .6705941 .733595

C 2.5949931 .6256382 .841591

O 1.4731142 .4412842 .431138

C 2.0983530 .1853603 .047862

C 0.9790300 .0860484 .067547

C $-0.318952-0.0866003 .745650$

C 1.4056810 .1568105 .509069

C $-0.890343-0.1559192 .356794$

C $-1.152459-1.6021241 .921400$

C -2.549935 -2.102769 2.312878

C $-2.670389-3.6063352 .054816$

C $-4.101203-4.1180472 .275453$

O $-3.480329-1.3830091 .499377$

O $-4.183791-5.4439991 .730887$

C $-5.067627-3.2345511 .460879$

C $-4.447518-4.2366723 .763590$

O $-6.425359-3.5806361 .722706$

C $-4.858361-1.7227331 .691247$

C $-5.668752-0.8594700 .695165$

O $-5.288345-1.3794393 .011915$

C 1.4809353 .7506242 .786770

O $2.355277 \quad 4.3310293 .415937$

C 0.2491044 .4442152 .344978

C -0.6386103 .9224081 .483903$

C -1.8733164 .5635881 .024020$

C -2.2311545 .8996971 .622698$

C $-5.322598-1.114251-0.776036$

C $-5.7231770 .038246-1.679149$

C $-5.1750461 .004658-3.911891$

O $-6.5034530 .922944-1.339327$

C $-4.1420902 .035374-3.576433$

C $-4.4181423 .336962-3.410065$

C $-3.3909984 .357319-3.009502$

C $-3.8067435 .131494-1.776739$

C $-4.0621356 .448894-1.847667$

C $-3.9478634 .383843-0.468618$

C $-2.627928 \quad 3.935787 \quad 0.097237$

N $-5.103825-0.052002-2.919701$

H 5.2924392 .1343272 .907181

H 3.2441111 .4682950 .729729

H 3.0144422 .0041373 .782265

H $2.931777-0.4510043 .374860$

H $1.778813-0.2405652 .089210$

H - $-1.049635-0.1727544 .547842$

H $\quad 0.563546 \quad 0.029997 \quad 6.197684$

H $2.132723-0.6315865 .729136$

H 1.8658541 .1256885 .726388

H $-0.2121620 .312077 \quad 1.637455$

H -1.8063550 .4453772 .318620$

H -1.072485 -1.627662 0.828720

H $-0.376652-2.2679362 .318625$ 
H $-2.738630-1.877273 \quad 3.368597$ H -1.961796 -4.164971 2.679052 H -2.385882 -3.821705 1.015358 H -3.526190 -5.996804 2.186868 H -4.888192 -3.4648270 .402917$ H $-3.766652-4.9385374 .260136$ H -5.454012 -4.646704 3.902884 H $-4.384838-3.2858494 .296537$ H -6.516017 -4.536566 1.568012 H $-6.745681-0.9962720 .855778$ H $-5.4701020 .196740 \quad 0.925817$ H -5.502761 -0.4319763 .030498$ H $\quad 0.1198015 .4366102 .765388$ H -0.4530742 .9331551 .070218$ H -3.158943 6.3202721 .229266 H -1.447066 6.6364431 .415406 H -2.356621 5.8137632 .707693 H $-4.243386-1.269836-0.888945$ H -5.835637 -2.013653 -1.134400 H $-6.1880031 .420135-3.922059$ H $-4.9613080 .558166-4.887380$ H $-3.1200151 .689474-3.442502$ H $-5.4368293 .688778-3.556271$ H -3.247396 $5.037019-3.859145$ H -2.414882 $3.890543-2.829208$ H $-4.3682917 .013835-0.973529$ H -3.971797 $6.993689-2.781501$ H $-4.5793383 .498579-0.615632$ H -4.4999434 .9823820 .264986$ H -2.285385 $2.977945-0.295969$ H $-4.425230-0.791330-3.075084$ C 6.6354090 .7028760 .580240 O $5.726824-0.2820360 .041864$ C $6.373659-0.969091-1.044146$ C $7.852698-0.621108-0.935542$ C $7.7833520 .803481-0.424399$ H $5.978271-0.539756-1.972279$ C $6.069574-2.468005-0.984770$ H $8.368473-1.260716-0.209766$ H $8.372097-0.710351-1.894465$ C 9.0880221 .2978070 .175793 H $7.4991411 .461919-1.256365$ C $4.569174-2.826127-1.038982$ O $\quad 6.597102-3.0049450 .236142$ H $6.602764-2.984039-1.791796$ H $4.075171-2.508592-0.113354$ O $4.474906-4.256786-1.074440$ C $3.810484-2.256119-2.254148$ C $2.417859-2.873948-2.484924$ H $3.721677-1.170219-2.149915$ O $4.586108-2.502315-3.432095$ H $2.025224-2.476210-3.428414$ H $2.534207-3.949203-2.669092$ H 8.9848412 .3233660 .543914 H $9.4077040 .669597 \quad 1.014017$ H $9.884914 \quad 1.286991-0.574974$ C 5.8902922 .0226180 .771957 H $6.187557-2.5171700 .971457$ H $4.952214-4.598053-0.298769$ H 4.136560 -2.066582 -4.176142 H $5.3633462 .290747-0.152792$ H 6.5930202 .8357890 .986763 H 6.9947480 .3144291 .542087 C $0.436824-0.566464-2.145964$ O $1.168673-1.239406-1.112986$ C $1.410123-2.632684-1.344055$ C $0.079534-3.377069-1.523669$ C $-0.779607-2.745702-2.613885$ C $-0.920251-1.241307-2.392415$ H $1.026249-0.561201-3.070552$ C $0.2274780 .891171-1.700828$ H $1.861879-3.022317-0.424523$ O $0.316537-4.749203-1.820800$ H $-0.470447-3.352856-0.576540$ H $-0.370245-2.958442-3.608059$ O $-2.079491-3.342575-2.594512$ H -1.591966 -1.056169 -1.544718 O $-1.524975-0.659937-3.546695$
C $1.4936071 .718541-1.761358$

H $-0.5034981 .383756-2.352174$

H $-0.1347350 .916484-0.667463$

H $-0.548921-5.172837-1.952230$

H -2.471414 -3.182094-1.718959

H -2.348595 -1.146376 -3.721035

O $1.1962052 .998045-1.383781$

O $2.5955411 .308824-2.096953$

C $2.2872493 .923197-1.408677$

H $1.9093324 .901611-1.100151$

H $3.0659223 .611952-0.707334$

H $2.6907774 .005880-2.422168$

SCF Energy $\left(B 3 L Y P / 6-31 G^{* *} / / M M F F\right)=-3245.91420782$

01

MM̄FF Geometry

C $3.7882040 .669383-2.458650$

C $3.192744-0.389998-3.026535$

C $3.018498-1.718799-2.341294$

O $1.643352-2.108961-2.581701$

C $3.962849-2.795895-2.903407$

C $5.417865-2.597706-2.538134$

C $5.962259-2.984487-1.367523$

C $6.282986-1.992152-3.612430$

C $5.264542-3.633555-0.201231$

C $5.739166-3.0821281 .151528$

C $5.234739-1.6621681 .463745$

C $6.044630-1.0765882 .625654$

C 5.5213710 .2963273 .063139

O $3.846699-1.747121 \quad 1.819819$

O $6.129447 \quad 0.621686 \quad 4.322239$

C 4.0061180 .1821313 .310182

C 5.9272491 .4046292 .084239

O 3.4421981 .4612323 .595436

C $3.238056-0.4881092 .146662$

C $1.774712-0.7447912 .582520$

O 3.2368450 .4002321 .028869

C $1.094893-3.039804-1.757978$

O $1.652456-3.653202-0.862571$

C $-0.334442-3.164933-2.113118$

C $-1.161892-3.781107-1.256729$

C $-2.600104-3.980487-1.431104$

C $-3.229782-3.485453-2.705850$

C $0.965629-1.5982811 .600548$

C $-0.519103-1.3794201 .800218$

C $-2.373095-1.7875483 .409185$

O $-1.172806-0.6924041 .019104$

C $-3.246976-2.9121802 .951091$

C $-4.376917-2.7278132 .250178$

C $-5.327561-3.8292961 .873320$

C $-5.563836-3.9185280 .382360$

C $-6.521075-3.185531-0.210657$

C $-4.752699-4.921536-0.404118$

C $-3.287960-4.586399-0.441667$

N -1.014419-1.985213 2.941524

H $4.2098770 .563649-1.464112$

H $2.800793-0.301056-4.037255$

H $3.159153-1.600241-1.259625$

H $3.853331-2.854149-3.995283$

H $3.644362-3.789525-2.563478$

H $7.033815-2.840700-1.233787$

H $7.328666-1.897793-3.300450$

H $6.263000-2.618002-4.510727$

H $5.930639-0.992184-3.879712$

H $4.178171-3.548558-0.269064$

H $5.503477-4.703663-0.232366$

H $5.382742-3.7654061 .933499$

H $6.836039-3.1065561 .179471$

H $5.339898-1.0322600 .573769$

H $7.108230-1.0120722 .363621$

H $5.990222-1.7581293 .486597$

H 7.0941530 .6197974 .198587

H $3.865289-0.4268424 .214250$

H 7.0196271 .4825252 .025978

H 5.5749912 .3838652 .426802

H 5.5566371 .2396571 .070603

H 3.9461901 .8413894 .335317

H $1.766101-1.2612193 .551113$ 
H 1.2658360 .2175252 .726036 H $2.4130760 .296657 \quad 0.527875$ H - $0.665677-2.732261-3.049743$ H $-0.749184-4.172356-0.327307$ H -2.852873 -4.052809-3.563724 H $-3.004090-2.425564-2.862393$ H $-4.318982-3.568726-2.712748$ H $1.192005-1.3388700 .562770$ H $1.198555-2.6615621 .726001$ H $-2.738549-0.8166283 .060661$ H $-2.339659-1.7696904 .503028$ H $-2.964875-3.9170043 .255285$ H -4.667016 -1.717688 1.971020 H $-6.281031-3.6366402 .383047$ H $-4.986103-4.7999542 .253922$ H -6.716672 -3.263253 -1.274843 H $-7.126415-2.4851140 .355592$ H $-5.152889-5.045435-1.416568$ H $-4.868083-5.910618 \quad 0.058254$ H $-2.746297-4.9030630 .450372$ H $-0.400277-2.5702743 .500436$ C $3.296613 \quad 3.171305-2.401521$ O $1.8728132 .974773-2.298318$ C $1.4240403 .542550-1.050389$ C $2.6265374 .264847-0.443265$ C $3.7843853 .449609-0.982064$ H $1.1556232 .701408-0.400708$ C $0.2081614 .447958-1.274281$ H $2.6973815 .302372-0.790247$ H $2.586233 \quad 4.2829790 .650443$

C $5.1225714 .162683-0.910607$ H $3.8442892 .525498-0.399600$ C $-1.0048383 .754815-1.927165$ O $0.6026525 .528513-2.131183$ H $-0.0849434 .904246-0.320939$ H $-0.7701543 .490596-2.964838$ O $-2.0556104 .730049-2.001052$ C $-1.5263142 .510496-1.180921$ C $-2.8567661 .997774-1.773591$ H -0.774222 1.715271-1.237403 O -1.6950602 .8500910 .191763$ H -3.620762 2.774946 -1.670230 H -2.707912 $1.831446-2.847029$ H $5.9212303 .532486-1.314248$ H $5.1103115 .098585-1.479097$ H 5.3749224 .4028940 .127383 C $3.9527451 .999767-3.138144$ H $-0.1771826 .095295-2.259899$ H -2.325767 $4.938570-1.090078$ H -1.8661962 .0249940 .676834$ H $5.0246792 .196072-3.255856$ H $3.5269061 .947278-4.148407$ H $3.4332564 .070241-3.018095$ C -4.7629251 .6460250 .613196$ O -3.6275300 .8377620 .282066$ C $-3.3692900 .693555-1.118953$ C $-4.593724 \quad 0.074476-1.809013$ C $-5.8725320 .854291-1.513460$ C $-6.035408 \quad 1.062488-0.008498$ H -4.6057932 .6689930 .251646$ C -4.8716651 .6936732 .142193$ H -2.552663 -0.033720 -1.209837 O $-4.375846-0.001510-3.213382$ H -4.716341 -0.953411-1.445336 H -5.891264 $1.812970-2.045339$ O $-7.0009920 .110196-1.978002$ H -6.2847810 .1065240 .469239$ $\begin{array}{lllll}\text { O } & -7.123491 & 1.954195 & 0.225367\end{array}$ C $-3.7440472 .494978 \quad 2.747577$ H -5.8161072 .1384972 .475598$ H -4.8244290 .6856732 .569629$ H -5.096371 -0.529677 -3.595846 H $-6.9491730 .073847-2.948040$ H -7.909789 $1.569649-0.198902$ O -3.9825573 .8284472 .589709$ O -2.7646521 .9951963 .285560$ C -2.973115 4.6993893 .109288 H -2.015132 4.5090902 .616003
H $-3.274508 \quad 5.7298652 .902924$

H $-2.8829254 .569675 \quad 4.191921$

SCF Energy (B3LYP/6-31G**//MMFF)= -3245.92178519

$01 \_118$

MM̄FF Geometry

C $1.514482-1.945297-2.318027$

C $0.604672-2.907063-2.532665$

C $-0.393439-3.370383-1.505661$

O $-1.686923-2.950793-2.007840$

C $-0.372217-4.900286-1.326528$

C $0.839709-5.424570-0.586086$

C $0.902099-5.5696240 .752332$

C $1.990200-5.874598-1.447585$

C $-0.151421-5.1998081 .762661$

C $0.446569-4.6105253 .049392$

C $1.029942-3.1994392 .870196$

C $1.898102-2.8367074 .077195$

C $2.447854-1.4083303 .975414$

O $-0.059715-2.2732742 .760269$

O $2.989199-1.0532915 .256869$

C $1.274274-0.4495643 .695296$

C $3.604571-1.3137322 .974913$

O $1.745925 \quad 0.876114 \quad 3.457098$

C $0.341873-0.9123732 .548038$

C $-0.921396-0.0154502 .530155$

O $1.036790-0.7624151 .309116$

C $-2.714696-2.882194-1.121661$

O $-2.711253-3.2593290 .038895$

C -3.851601-2.218606 -1.795417

C $-4.912485-1.853713-1.060437$

C $-6.109010-1.163291-1.540596$

C $-6.160684-0.773702-2.994103$

C $-2.050452-0.5180641 .623296$

C $-3.1092270 .548847 \quad 1.413271$

C $-4.793643 \quad 1.9158532 .636028$

$\begin{array}{lllll}\text { O } & -3.271787 & 1.091152 & 0.321986\end{array}$

C $-6.149301 \quad 1.4281582 .231236$

C -6.8823102 .0154091 .272416$

C -8.2807851 .6068280 .903990$

C $-8.4178821 .235835-0.555778$

C $-8.6140172 .178439-1.493092$

C $-8.414691-0.230883-0.917637$

C $-7.092669-0.895595-0.656648$

$\begin{array}{llll}\mathrm{N} & -3.829407 & 0.835527 & 2.560440\end{array}$

H $1.551343-1.454184-1.350905$

H $0.556299-3.379535-3.511418$

H $-0.213293-2.867593-0.546654$

H $-0.452275-5.393579-2.305330$

H $-1.280561-5.229864-0.807414$

H $1.802489-6.0152271 .173672$

H $2.811047-6.297608-0.858555$

H $1.659203-6.646254-2.150290$

H $2.397845-5.037139-2.018588$

H $-0.883114-4.5006491 .352191$

H $-0.691924-6.1165342 .028067$

H $-0.350473-4.5752213 .803475$

H $1.218594-5.2924893 .427795$

H $1.626842-3.1669261 .952169$

H $2.720697-3.5534274 .195252$

H $1.298662-2.9150604 .995342$

H $3.685180-1.6958595 .477033$

H $0.683030-0.3956894 .619780$

H $4.407738-2.0078513 .249964$

H $4.055487-0.3168152 .992889$

H $3.306412-1.5432541 .950106$

H 2.1076490 .9040972 .554914

H $-1.325236 \quad 0.054983 \quad 3.548437$

H $-0.643721 \quad 1.001423 \quad 2.224944$

H $0.386557-0.6981990 .592873$

H $-3.775137-2.028293-2.859430$

H $-4.905582-2.0756430 .006402$

H $-6.156860-1.664650-3.631362$

H $-5.298752-0.149781-3.256386$

H $-7.048513-0.193384-3.255915$

H $-1.668257-0.7957940 .637236$

H -2.526277 -1.4068812 .052682$

H -4.4490972 .7449472 .008324$ 
H -4.8188692 .2567193 .675790$

H -6.5613170 .5847432 .779579$

H -6.4714752 .8754880 .747593$

H -8.9479032 .4495831 .129291$

H $-8.6342980 .780927 \quad 1.533881$

H $-8.7420651 .919553-2.538736$

H -8.646862 $3.231652-1.234044$

H -8.730149 -0.382035 -1.956001

H $-9.181432-0.743907-0.322221$

H $-6.957926-1.2021930 .381259$

H $-3.672661 \quad 0.2819983 .397386$

C $3.910591-1.242048-2.850420$

O $3.945284-0.079441-2.002304$

C $4.951264-0.280686-0.988958$

C $5.651941-1.596424-1.325986$

C $4.554778-2.363796-2.041104$

H $4.414545-0.405070-0.041963$

C $5.8795920 .940042-0.907033$

H $6.507346-1.444772-1.994192$

H $6.018909-2.107656-0.430493$

C $5.066496-3.517185-2.885361$

H $3.861318-2.750095-1.283796$

C $5.1384362 .283375-0.735382$

O $6.6303691 .014085-2.126426$

H $6.6101810 .793642-0.102733$

H $4.5994462 .514184-1.661359$

O $6.1137393 .318425-0.564434$

C 4.1704452 .3419980 .460284

C 3.4888213 .7095650 .656745

H 3.3932361 .5784990 .353585

O 4.8718652 .0597231 .673698

H 2.8251323 .6217201 .526531

H 4.2361734 .4562830 .955143

H $4.243073-4.013398-3.408295$

H $5.785138-3.176092-3.638283$

H $5.566333-4.261418-2.256802$

C $2.487613-1.478702-3.364409$

H $7.354270 \quad 1.647757-1.987124$

H $6.5832963 .421716-1.409086$

H 5.2038741 .1483351 .623032

H $2.524405-2.198289-4.191205$

H $2.102202-0.533876-3.767931$

H $4.541260-0.997383-3.716051$

C $0.7685402 .802905-0.384819$

O $1.899143 \quad 3.201019-1.162635$

C $2.7003384 .222754-0.564867$

C $1.8484605 .471654-0.290303$

C 0.5886255 .1465110 .512397

C $-0.1726873 .982256-0.123437$

H 1.1207232 .4148830 .579614

C $0.1043281 .611251-1.081598$

H $3.4443474 .506827-1.319296$

O $2.602756 \quad 6.4586670 .405639$

H $1.5409395 .915785-1.246107$

H $\quad 0.825090 \quad 4.932020 \quad 1.561287$

O $-0.270281 \quad 6.2892850 .522671$

H -0.641955 4.325769-1.052298

O $-1.216303 \quad 3.5655740 .753169$

C $-0.4891461 .898762-2.443101$

H $0.8534340 .827378-1.233155$

H $-0.6877891 .219500-0.438719$

H $3.3881436 .658644-0.131886$

H $0.239206 \quad 7.0297130 .894606$

H -1.780313 4.3396860 .921692

O $-1.359601 \quad 0.893339-2.760817$

O $-0.2059512 .852657-3.153650$

C -1.991944 $1.004810-4.039003$

H $-2.6469800 .139581-4.170884$

H $-2.5983121 .914462-4.082672$

H $-1.2413881 .000724-4.835058$

SCF Energy (B3LYP/6-31G**//MMFF) $=-3245.90114558$

01

MM̄FF Geometry

C $0.580414-2.2284380 .648872$

C $1.585094-2.799310-0.033149$

C $1.981283-2.497384-1.460211$

O $1.201635-1.383579-1.959662$
C $3.476445-2.128520-1.546400$

C $4.416061-3.314883-1.506951$

C $5.211115-3.634289-0.467198$

C $4.474135-4.128168-2.775280$

C $5.302346-2.9604350 .873850$

C $6.617646-2.1903901 .058684$

C $6.692085-0.9034110 .222269$

C $8.049926-0.2208830 .398385$

C $8.1133031 .123382-0.343077$

O $5.648942-0.0320810 .669928$

O $9.270804 \quad 1.8319750 .125549$

C 6.8799641 .9657080 .044494

C $8.3013790 .935561-1.853163$

O $6.8165393 .162688-0.727965$

C $5.5434671 .194371-0.063094$

C 4.4047192 .0281440 .569645

O $5.2446180 .965791-1.441362$

C $1.078124-1.256547-3.305348$

O $1.577454-1.983364-4.154075$

C $0.269560-0.071867-3.673486$

C $-0.5495950 .572445-2.827068$

C $-1.3998391 .719260-3.164691$

C $-1.1870762 .372886-4.505680$

C $3.0807381 .269624 \quad 0.737734$

C 2.0654332 .1501131 .437537

C 0.6024504 .1255831 .046614

O 1.8459762 .0508302 .642396

C $-0.614794 \quad 4.167170 \quad 0.179831$

C $-0.8325565 .122281-0.737836$

C $-2.0297615 .300510-1.633081$

C $-3.1798324 .342078-1.426263$

C $-4.0775784 .545534-0.447117$

C $-3.3491293 .219894-2.424296$

C $-2.3228492 .129568-2.269335$

N $1.473408 \quad 3.064310 \quad 0.582144$

H $-0.059520-1.4977100 .162489$

H $2.182770-3.5537300 .474174$

H $1.750454-3.384522-2.062252$

H $3.713714-1.402289-0.762258$

H $3.675733-1.576875-2.474611$

H $5.870344-4.495267-0.573796$

H $5.226673-4.922362-2.726241$

H $3.509131-4.603285-2.974946$

H $4.729375-3.487342-3.625703$

H $4.446160-2.3088701 .070846$

H $5.251949-3.7475791 .637126$

H $6.698183-1.9289432 .121955$

H $7.466865-2.8427010 .820052$

H $6.530803-1.152094-0.832266$

H $8.862855-0.8798920 .069018$

H $8.229291-0.0350501 .466709$

H $10.051283 \quad 1.277587-0.046081$

H 7.0146362 .2788201 .089408

H $9.2301750 .389584-2.058097$

H $8.4037621 .900693-2.361472$

H $7.4846950 .383046-2.322203$

H $7.6680963 .620654-0.622760$

H 4.7169862 .3657521 .567028

H $4.2287662 .928369-0.032603$

H $4.2843060 .889098-1.551984$

H $\quad 0.3510460 .217573-4.716612$

H $-0.6371600 .215491-1.802496$

H - $-1.512194 \quad 1.708077-5.313150$

H $-0.1282192 .614600-4.653433$

H -1.725636 $3.315619-4.621668$

H $2.6698650 .951893-0.226358$

H $3.2137360 .366098 \quad 1.343316$

H $1.1762815 .057050 \quad 0.996123$

H 0.3171163 .9648022 .090648

H -1.3491243 .3858550 .350912$

H $-0.0652165 .884375-0.868131$

H -1.693659 $5.256149-2.676487$

H -2.398439 $6.325864-1.492131$

H -4.928133 $3.887054-0.311285$

H -3.9764715 .3749430 .245197$

H $-3.3713923 .643904-3.431997$

H -4.335172 $2.754601-2.299764$

H $-2.396098 \quad 1.593410-1.322458$ 
H $1.7976553 .113109-0.379701$

C $0.087205-1.2476962 .891837$

O $-1.159933-0.6281352 .509454$

C -1.887207 -0.2769213 .699350$

C $-0.868077-0.3141834 .829564$

C $-0.002303-1.4817534 .399606$

H -2.625243 -1.070464 3.866398

C -2.596569 1.0672073 .517229

H $-0.272744 \quad 0.605501 \quad 4.869998$

H $-1.335297-0.4613805 .807764$

C $1.347674-1.5259635 .094784$

H $-0.542220-2.4161224 .604368$

C -3.561537 1.1274172 .314322

O $-1.6160552 .096623 \quad 3.333334$

H -3.133122 1.3269854 .437045

H -2.990766 1.1203081 .378584

$\begin{array}{llll}\text { O } & -4.235879 & 2.391175 & 2.367367\end{array}$

C -4.6245530 .0126942 .285338$

C $-5.748161 \quad 0.209972 \quad 1.250989$

H -4.137839 -0.9487932 .101063$

O $-5.245615-0.0878153 .570950$

H $-6.443849-0.6318791 .354500$

H $-6.3378161 .093767 \quad 1.525763$

H $1.943015-2.3721204 .737689$

H $1.921804-0.6107064 .914888$

H $1.219656-1.6350316 .176690$

C $0.277083-2.5358822 .089735$

H -1.065972 1.8444292 .571532

H -3.5524473 .0834522 .371019$

H -5.6449720 .7759293 .772458$

H $-0.640653-3.1328092 .126335$

H $1.083841-3.1346972 .527410$

H $0.883986-0.5303552 .661418$

C $-5.158860-2.013207-0.789273$

O $-4.458376-0.774998-0.615426$

C $-5.2677760 .332061-0.206703$

C $-6.3962010 .568651-1.220674$

C $-7.218142-0.692918-1.454165$

C $-6.301882-1.861799-1.803807$

H $-5.557360-2.3474750 .176467$

C $-4.141957-3.054872-1.288020$

H $-4.6220291 .214955-0.250019$

O $-7.2609091 .615241-0.792893$

H $-5.9642890 .880050-2.180470$

H -7.849445 $-0.931108-0.590108$

O $-8.106728-0.478402-2.553456$

H $-5.892879-1.715600-2.812027$

O $-7.071280-3.063298-1.825480$

C $-3.262870-3.591267-0.178891$

H $-4.671498-3.912580-1.718553$

H -3.484179 -2.620445 -2.049477

H $-6.7176092 .412033-0.671518$

H -8.655383 $0.294322-2.334317$

H -7.796101 -2.931980 -2.460724

O $-2.706331-4.769436-0.589601$

O $-3.072878-3.0431910 .897111$

C $-1.865676-5.440672 \quad 0.354822$

H $-0.863615-5.006706 \quad 0.321612$

H -2.276396 -5.3878551 .367744$

H -1.801797 -6.492269 0.062643

SCF Energy (B3LYP/6-31G**/MMFF) $=-3245.92394917$

01

MM̄FF Geometry

C -1.768934 4.4527871 .627632

C -0.9072913 .4256841 .653000$

C $-0.591408 \quad 2.613412 \quad 2.878620$

$\begin{array}{lllll}\text { O } & -1.108360 & 1.277900 & 2.679131\end{array}$

C $0.930775 \quad 2.4853503 .049608$

C 1.3160971 .7780084 .331910

C 1.7492830 .5039964 .400681

C 1.2382932 .6186925 .580087

C $1.896110-0.4954863 .284164$

C $3.329400-0.5087662 .744723$

C $3.733572-1.8533172 .119512$

C $5.175482-1.7666821 .610408$

C $5.629713-3.0611630 .928486$

O $2.845909-2.1823691 .044147$
O $6.845543-2.753064 \quad 0.229869$

C $4.567478-3.471291-0.109994$

C $5.976976-4.1473061 .953528$

O $4.866210-4.753675-0.660066$

C $3.118234-3.4609990 .442762$

C $2.135878-3.680419-0.732384$

O $2.974294-4.5271151 .382293$

C -2.4260201 .0599252 .929323$

O $-3.256474 \quad 1.870896 \quad 3.303944$

C -2.686103 -0.3641112 .635045$

C $-3.935441-0.7751982 .374455$

C $-4.326653-2.1427362 .023714$

C $-3.260664-3.2039942 .108812$

C $0.648703-3.687882-0.360849$

C $-0.231454-3.830814-1.595771$

C $-2.617510-4.086074-2.261484$

O $0.213616-3.853502-2.740375$

C $-3.823784-3.307267-1.845804$

C $-5.047081-3.836651-1.692642$

C $-6.252581-3.024186-1.310570$

C $-6.973764-3.560904-0.091495$

C $-8.266914-3.919479-0.165815$

C $-6.215677-3.681748 \quad 1.216472$

C $-5.602939-2.3785841 .653729$

N $-1.576929-3.906318-1.265811$

H -2.2707974 .7540532 .543569$

H $-0.433738 \quad 3.115760 \quad 0.726948$

H - -1.0412923 .0541653 .776690$

H 1.3980733 .4791563 .044405

H 1.3556201 .9529392 .190204

H 2.0313990 .1139495 .378290

H 1.5864302 .0793406 .467421

H 1.8609813 .5130925 .474661

H $\quad 0.2074732 .9323175 .770675$

H $1.631112-1.4804803 .688134$

H $1.188655-0.3040692 .470328$

H $3.4160490 .288424 \quad 1.995838$

H $4.039908-0.2723553 .547774$

H $3.650688-2.6224222 .896776$

H $5.862075-1.5039962 .425193$

H $5.252719-0.9476310 .881868$

H $7.184018-3.574173-0.165096$

H $4.624430-2.755362-0.941598$

H $6.799100-3.8134382 .597870$

H $6.334234-5.0584391 .460819$

H $5.136898-4.4130112 .598443$

H $5.727953-4.690743-1.104850$

H $2.296708-2.888252-1.474013$

H $2.359274-4.635381-1.225423$

H $2.030894-4.6576431 .564183$

H $-1.829582-1.0283282 .605157$

H $-4.740347-0.0415472 .389914$

H $-2.878568-3.277073 \quad 3.133447$

H $-2.425224-2.9626571 .445175$

H -3.604683 -4.2022661 .834248$

H $0.423482-4.526767 \quad 0.305914$

H $0.378558-2.7563630 .148048$

H $-2.818420-5.159844-2.333878$

H $-2.263849-3.734480-3.235883$

H -3.686836 -2.241129-1.684055

H $-5.198705-4.897429-1.876983$

H $-6.926897-3.012044-2.176777$

H $-5.980616-1.976410-1.131549$

H $-8.798717-4.3043270 .698072$

H $-8.825434-3.837478-1.092398$

H $-5.477044-4.4787371 .102433$

H $-6.890563-4.0104002 .017385$

H $-6.304420-1.5428781 .658209$

H $-1.824489-4.066072-0.294618$

C $-3.1281564 .457088-0.539952$

O $-2.471376 \quad 3.332218-1.162278$

C $-3.2799312 .161979-0.956551$

C $-4.6875192 .687633-0.709570$

$\begin{array}{llll}\text { C }-4.394910 & 3.915865 & 0.132594\end{array}$

H $-2.9143361 .683053-0.040787$

C -3.139746 $1.182060-2.124354$

H $-5.1821862 .981094-1.642641$

H $-5.3223301 .957513-0.198481$ 
C -5.5360114 .9172120 .176718$ H -4.1886863 .5817781 .155967$ C $-1.688100 \quad 0.842416-2.524440$ O $\quad-3.780137 \quad 1.726237-3.285809$ H $-3.6880230 .262632-1.889277$ H -1.252194 $1.683706-3.075884$ O $-1.741146-0.256760-3.443560$ C $-0.7577460 .456281-1.362340$ C $0.614645-0.096084-1.787280$ H $-0.6000831 .325539-0.719450$ O $-1.389914-0.536397-0.551259$ H $1.170412-0.331101-0.871147$ H $0.475640-1.061788-2.286368$ H $-5.263050 \quad 5.7888580 .780119$ H $-5.8006865 .269669-0.825871$ H $-6.4288724 .463980 \quad 0.619647$ C -2.1391945 .1967830 .373359$ H $-3.3650002 .585688-3.474330$ H -2.321105 $0.007458-4.178262$ H -1.566760 -1.307312 -1.116582 H $-2.568582 \quad 6.1622330 .666727$ H -1.222349 $5.418010-0.188244$ H $-3.3969075 .130429-1.363900$ C $2.4050942 .280385-1.020349$ O $1.5581612 .157219-2.166214$ C $1.4438150 .833628-2.694759$ C $2.8318820 .278833-3.051444$ C $3.7903860 .346113-1.865296$ C $3.8231021 .762642-1.295727$ H $1.9644861 .699631-0.200326$ C $2.3820473 .745888-0.558265$ H $0.8925050 .928986-3.638006$ O $2.743893-1.067003-3.506161$ H $3.2578840 .868281-3.873781$ H $3.527378-0.382306-1.091038$ O $5.108301 \quad 0.008327-2.302382$ H $4.3484392 .414444-2.003266$ O $4.5690591 .771539-0.081487$ C $2.9934844 .720690-1.544032$ H $1.3484784 .074850-0.409585$ H $2.912643 \quad 3.8288690 .397484$ H $2.131343-1.081578-4.261360$ H $5.061862-0.878043-2.700541$ H $5.4467551 .400615-0.276964$ O $3.4715085 .804756-0.864543$ O $3.0159904 .570986-2.758130$ C $4.0634256 .825915-1.673635$ H $3.3199437 .243798-2.358954$ H $4.4172137 .621356-1.012264$ H $4.9174776 .424034-2.227134$

SCF Energy (B3LYP/6-31G**//MMFF) $=-3245.92227460$

$01+120$

MM̄̄FF Geometry

C $-0.996358-2.9254620 .890259$

C $0.030548-2.983278 \quad 1.751193$

C $0.402430-1.9627512 .802891$

O $1.676081-1.3895752 .415914$

C $-0.584800-0.7923142 .974705$

C -0.2854210 .0536304 .196839$

C 0.3574591 .2370094 .169888

C $-0.809242-0.4927885 .500062$

C $0.968859 \quad 1.9274472 .983341$

C 0.1095783 .1082352 .520310

C 0.8391493 .9838811 .491066

C -0.0557095 .1398901 .039652$

C $0.6115605 .992612-0.047970$

O $1.195155 \quad 3.1742890 .366848$

O $-0.4015826 .811662-0.652666$

C $1.1567155 .064960-1.156147$

C 1.6433816 .9559490 .551153

O $1.9342055 .801669-2.096947$

C $1.9736763 .864027-0.621928$

C $2.2828732 .873738-1.764377$

O $3.2395414 .281307-0.102786$

C $2.814497-2.0194582 .806783$

O $2.907827-3.0276513 .487219$

C $3.945630-1.2764822 .209411$
C $5.130342-1.8876152 .064548$

C $6.305961-1.3187541 .406350$

C $6.3160700 .164198 \quad 1.140656$

C $3.0216981 .614052-1.292213$

C $3.5209450 .772828-2.455219$

C $4.738770-1.328878-2.951872$

O $3.3722691 .091166-3.631988$

C $5.598361-2.300815-2.211279$

C $6.925515-2.415407-2.378921$

C $7.761114-3.435340-1.656409$

C $8.866470-2.832881-0.810831$

C $10.139405-3.231671-0.976594$

C $8.528722-1.7867330 .237726$

C $7.319204-2.1419411 .064121$

N $4.177760-0.370227-2.019384$

H -1.659587 -2.065382 0.891521

H $\quad 0.682787-3.8542691 .707835$

H $\quad 0.505939-2.4892823 .759917$

H -1.610925 -1.169761 3.073524

H $-0.579657-0.1644672 .076252$

H $\quad 0.486757 \quad 1.7718665 .110103$

H $-0.599360 \quad 0.172138 \quad 6.344626$

H -1.894885 -0.624762 5.447440

H $-0.351330-1.4612785 .723300$

H 1.9630452 .2839533 .280572

H 1.1340321 .2355872 .151883

H -0.815205 2.7119172 .081137

H -0.1770493 .7270403 .380085$

H 1.7528634 .3688301 .959545

H -0.3463425 .7641511 .893864$

H -0.9920214 .7390690 .632671$

H -0.8163427 .3429380 .048665$

H $0.2920424 .670090-1.708105$

H 1.1579227 .6552701 .242710

H $2.1053157 .576012-0.224760$

H 2.4336426 .4451601 .105467

H $1.3770346 .527022-2.427959$

H $1.3511992 .560327-2.252051$

H $2.8950213 .377453-2.523358$

H $3.3824035 .210798-0.343399$

H $3.755333-0.2713261 .849728$

H $5.232352-2.9182182 .401902$

H 6.0072530 .7197382 .033652

H 5.6394760 .4181610 .321205

H $7.3083600 .545766 \quad 0.885994$

H $3.8941261 .889820-0.689586$

H $2.3589780 .999039-0.673069$

H $5.291450-0.785142-3.725834$

H $3.909111-1.852890-3.437774$

H $5.091982-2.964009-1.513380$

H $7.435033-1.766522-3.086978$

H $8.184838-4.105372-2.415948$

H $7.135146-4.072322-1.019018$

H $10.944918-2.816301-0.379888$

H $10.407513-3.981529-1.713517$

H $8.389730-0.838091-0.290806$

H $9.372555-1.6499010 .925208$

H $7.257449-3.1920041 .351776$

H $4.080575-0.640827-1.044499$

C $-2.681129-4.5884170 .041527$

O $-3.678596-3.560860-0.113193$

C $-4.710668-4.031566-0.996832$

C $-4.519464-5.539617-1.090375$

C $-3.009280-5.652056-1.008044$

H $-4.506940-3.586483-1.978568$

C $-6.086314-3.571333-0.502833$

H -4.981385 -6.064415 -0.246448

H -4.932562 -5.954842 -2.014492

C $-2.520206-7.044218-0.648259$

H $-2.584926-5.364923-1.979620$

C $-6.160460-2.067419-0.164882$

O $-6.407932-4.2913420 .694755$

H $-6.851150-3.844225-1.239331$

H $-5.571461-1.8818830 .739618$

O $-7.517771-1.7672470 .185153$

C $-5.709084-1.121421-1.292449$

C $-5.9069620 .372670-0.972134$

H $-4.657709-1.304154-1.529738$ 
O $-6.464478-1.428466-2.467063$ H $-5.6707300 .946882-1.876140$ H $-6.971640 \quad 0.563705-0.790045$ H -1.427669 -7.070246 -0.588128 H -2.918838 -7.372359 0.317597 H -2.834320 -7.769803 -1.405666 C -1.288185 -3.982553-0.139457 H -7.283096 -3.9861920 .989909$ H -8.057953 -1.881120 -0.615831 H -6.122760 $-0.873408-3.188659$ H -1.221600 -3.507221 -1.124436 H $-0.530347-4.773707-0.108268$ H -2.793768 -4.991572 1.056172 C $-3.0379911 .470660-0.972979$ O $-3.6602040 .644557 \quad 0.020236$ C $\begin{array}{lllll}-5.061019 & 0.875932 & 0.215061\end{array}$ C $-5.3135772 .342720 \quad 0.592156$ C $-4.6921663 .302969-0.415143$ C $-3.2229102 .955112-0.633632$ H -3.471929 $1.256188-1.957078$ C $-1.5422141 .112931-1.009781$ H -5.3486020 .2824151 .090168$ O -6.7073892 .6126130 .697430$ H -4.8729272 .5438151 .577545$ H $-5.2420913 .307994-1.363411$ O $\quad-4.7631334 .6348700 .099405$ H -2.656052 3.2018950 .271710 O $-2.7071113 .751730-1.697842$ C -1.264905 -0.190808 -1.728439 H $-0.9842571 .890158-1.543581$ H $-1.150261 \quad 1.021170 \quad 0.008970$ H $-7.0752161 .996827 \quad 1.354140$ H $-5.700548 \quad 4.8271310 .273857$ H -2.840144 $4.683744-1.453308$ O $0.086974-0.354333-1.824266$ O $-2.117169-0.959465-2.148950$ C $0.577509-1.514544-2.508733$ H $1.245976-1.182290-3.307111$ H $1.149299-2.117780-1.798475$ H $-0.215937-2.125153-2.948192$

SCF Energy $\left(B 3 L Y P / 6-31 G^{* *} / / M M F F\right)=-3245.92413251$

01

MM̄FF Geometry

C $-2.870963-3.640996-2.541785$

C $-1.850341-3.448630-1.694278$

C $-0.485654-2.984200-2.128751$

O $-0.201775-1.727552-1.469921$

C $0.581272-4.000374-1.684946$

C $1.963934-3.638758-2.188848$

C $2.866964-2.914097-1.498357$

C $2.293740-4.148152-3.567720$

C $2.698355-2.298144-0.135898$

C $3.818976-2.650315 \quad 0.855031$

C $5.204356-2.1213100 .452137$

C $6.227111-2.3463881 .567716$

C $7.598454-1.7428371 .217369$

O $5.085545-0.7220070 .184036$

O $8.378432-1.6969912 .422323$

C $7.402450-0.2833750 .747538$

C $8.372680-2.6303400 .235417$

$\begin{array}{lllll}\text { O } & 8.628617 & 0.271279 & 0.276299\end{array}$

C $6.284876-0.114906-0.307838$

C $5.9970651 .384765-0.554926$

O $6.715378-0.707718-1.536367$

C $-0.703346-0.589446-2.019398$

O $-1.395512-0.494922-3.019705$

C $-0.2694440 .537722-1.163011$

C $-0.8371591 .743796-1.309183$

C $-0.5527142 .933890-0.502674$

C 0.5835812 .8571950 .482561

C $4.6970341 .643552-1.332551$

C $4.5430153 .100971-1.730871$

C $2.9717404 .627407-2.920677$

O $5.3978393 .954091-1.507955$

C $2.2660315 .424900-1.869964$

C $1.089516 \quad 6.037253-2.071767$

C $0.4158626 .899768-1.044216$
C $-1.0246406 .513396-0.801744$

C $-2.0323647 .255368-1.291679$

C -1.2967455 .3225300 .088101$

C $-1.3263054 .026235-0.673610$

N $3.3394293 .330043-2.385899$

H $-2.715504-3.477594-3.605130$

H -2.004209-3.614360 -0.630858

H $-0.436574-2.845302-3.216420$

H $\quad 0.323212-5.002379-2.053257$

H $0.589080-4.088741-0.592140$

H $3.815911-2.698035-1.985848$

H $3.299832-3.859033-3.889671$

H $2.241959-5.241604-3.589801$

H $1.585706-3.751500-4.302229$

H $2.642359-1.210045-0.258358$

H $1.751495-2.5942570 .321628$

H $3.539161-2.2110891 .821782$

H $3.864464-3.7369090 .998742$

H $5.525437-2.640846-0.457198$

H $6.323219-3.4146421 .798423$

H $5.868806-1.8713012 .491800$

H $8.449841-2.6031082 .768166$

H 7.1295050 .3022861 .636033

H $8.513886-3.6333820 .655796$

H $9.380308-2.2383510 .058302$

H $7.870204-2.744310-0.727274$

H $8.793848-0.087905-0.611988$

H $5.909903 \quad 1.9071290 .406804$

H $6.8414321 .833745-1.093208$

H $\quad 6.272618-0.266203-2.277808$

H $0.4826590 .334519-0.409140$

H -1.608603 $1.865815-2.068498$

H $1.4984042 .506286-0.007644$

H $\quad 0.3380592 .169499 \quad 1.297732$

H 0.8354373 .8199990 .932051

H $4.6672661 .047131-2.250504$

H $3.8338381 .364975-0.716789$

H $2.3440034 .451998-3.799943$

H $3.8775885 .155438-3.235998$

H $2.7650355 .537332-0.910158$

H $0.6057115 .953357-3.042327$

H $0.4707407 .937680-1.397925$

H $0.9583706 .883643-0.090658$

H $-3.0684637 .000388-1.094378$

H -1.844436 $8.130083-1.905327$

H $-2.279695 \quad 5.437770 \quad 0.565251$

H -0.5870865 .3184410 .919769$

H -2.124359 $3.981185-1.416442$

H $2.6619452 .576664-2.459975$

C $-5.306243-2.984316-2.189125$

O $-5.064716-1.974130-1.189569$

C $-5.514193-0.705391-1.708572$

C $-6.181077-0.995212-3.053831$

C $-5.435583-2.232547-3.511738$

H $-4.614661-0.104796-1.886238$

C $-6.4330910 .000354-0.703539$

H -7.249420 -1.213872 -2.942092

H $-6.086799-0.154988-3.748861$

C $-6.150376-3.012327-4.600331$

H $-4.454095-1.913073-3.882371$

C $\begin{array}{rlll}-5.803511 & 0.254207 & 0.681669\end{array}$

O $-7.595929-0.816644-0.512023$

H $-6.7861920 .944031-1.137361$

H $-5.673186-0.696071 \quad 1.212781$

O

C -4.4674211 .0196490 .652178$

C -3.9954291 .5383492 .025585$

H -3.6924990 .3798670 .215699$

O $-4.6090202 .153281-0.209227$

H -3.0461232 .0664291 .872751$

H -4.6873022 .3146332 .376897$

H -5.574273 -3.897885 -4.886759

H -7.140694 -3.345344 -4.271953

H $-6.283407-2.392514-5.493169$

C $-4.241572-4.082021-2.114082$

H $-8.265255-0.272990-0.063656$

H -7.4943810 .4469831 .653847$

H $-3.7457882 .597014-0.253257$ 
H $-4.538867-4.924819-2.749220$

H -4.200428 -4.461529-1.085108

H $-6.271032-3.439608-1.926384$

C $-1.646298-0.3562572 .505620$

O $-3.039282-0.6468702 .652144$

C $-3.8341490 .451356 \quad 3.105843$

C -3.3043820 .9724644 .450466$

C -1.8134951 .3000894 .394116$

C -1.027037 0.1184863 .826470

H -1.5323750 .4362651 .756459$

C $-0.963933-1.5889321 .901740$

H -4.8341180 .0449663 .299341$

O -4.0208592 .1304074 .866441$

H -3.458552 0.2054935 .220773

H -1.623205 2.2126603 .816781

O -1.3413681 .5587335 .718810$

H -1.013990 -0.6815234 .575637$

O $0.320587 \quad 0.534423 \quad 3.619900$

C $-0.963862-2.8161462 .787874$

H -1.488041 -1.8731700 .983651$

H $0.071792-1.3393121 .648346$

H -4.9622081 .8907914 .911257$

H -1.8750952 .2882346 .078128$

H $\quad 0.850375-0.2588803 .434452$

O $0.033438-3.6554582 .378999$

O $-1.754922-3.0333543 .694942$

C $0.146647-4.8815273 .107393$

H $\quad 0.983999-5.4476652 .690698$

H $\quad 0.350967-4.678107 \quad 4.162925$

H $-0.767182-5.4728972 .996702$

SCF Energy $\left(B 3 L Y P / 6-31 G^{* *} / / M M F F\right)=-3245.90394316$

01 122

MMFF Geometry

C $-1.2025952 .249527-2.099883$

C $-0.2885843 .228506-2.007735$

C $0.5429293 .465728-0.775322$

O $1.9311323 .327565-1.161030$

C $0.3333354 .875194-0.186574$

C $-1.074026 \quad 5.1746870 .281194$

C -1.6440074 .6280871 .373030$

C -1.824332 $6.183944-0.546618$

C -1.0193653 .6450162 .324560$

C - -1.7738912 .3094272 .317620$

C -1.0454191 .1593263 .033406$

C -0.6366821 .5029514 .467944$

C $0.140868 \quad 0.3569545 .133482$

O $\quad 0.107035 \quad 0.809141 \quad 2.256694$

$\begin{array}{lllll}\text { O } & 0.760376 & 0.874370 & 6.321405\end{array}$

C $1.278936-0.0852284 .188711$

C $-0.787162-0.7754155 .588678$

O $1.944348-1.2376864 .701379$

C $0.810252-0.3458352 .739434$

C $2.033766-0.5862641 .818725$

O $-0.016630-1.5138192 .728168$

C $2.8085902 .990402-0.176523$

O 2.5588592 .8210021 .006599

C $4.1430672 .831300-0.791620$

C $5.0613902 .093103-0.150656$

C $6.4178151 .801556-0.612325$

C $6.9093172 .514512-1.844093$

C $1.674283-0.6335520 .328088$

C $2.828401-1.044429-0.566777$

C $4.341431-2.957347-1.085455$

O $3.237672-0.312655-1.465630$

C $5.684693-2.646870-0.501622$

C $6.731172-2.252408-1.243587$

C $8.110369-2.011256-0.696804$

C $8.687205-0.681559-1.129031$

C $9.351021-0.566337-2.292078$

C $8.5756780 .489942-0.181739$

C 7.1575120 .9115990 .081726

N $3.304095-2.316722-0.300326$

H -1.338982 $1.568003-1.263345$

H $-0.1474323 .910376-2.842680$

H $0.3092022 .696815-0.030538$

H $0.6481095 .624348-0.926943$

H 1.0262075 .0367270 .649751
H -2.6608774 .9232261 .627997$

H -2.857965 $6.314092-0.209115$

H $-1.3290117 .158923-0.490973$

H -1.856462 $5.873989-1.595717$

H 0.0391243 .4857962 .103925

H -1.059969 4.0847973 .328182

H -2.758659 2.4540442 .779795

H $-1.9486192 .004249 \quad 1.277562$

H -1.7317940 .3043523 .033641$

H -1.514060 1.7758635 .067040

H 0.0167892 .3850714 .470131

H 0.0595641 .2312836 .893618

H 2.0250950 .7217114 .170346

H -1.513738 -0.4031726 .320985$

H -0.225629 -1.565142 6.099688

H $-1.350412-1.2264514 .769056$

H $2.231439-1.0271805 .606593$

H 2.7656580 .2192241 .956316

H $2.527585-1.5184332 .115343$

H $0.089242-1.9827221 .886581$

H $4.3187113 .291503-1.756564$

H 4.7879671 .6355890 .799800

H $6.8654403 .600199-1.703744$

H $6.2979402 .246994-2.712996$

H $7.9428732 .271450-2.101460$

H $0.856927-1.3314860 .139003$

H $1.3265090 .350096-0.005339$

H $4.257882-2.630666-2.127350$

H $4.159471-4.035576-1.040482$

H $5.812805-2.7913680 .568392$

H $6.602718-2.144670-2.318593$

H $8.758913-2.821390-1.056437$

H $8.127158-2.0858820 .397526$

H $9.7962390 .373788-2.599872$

H $9.460700-1.411200-2.964104$

H $9.165223 \quad 1.344922-0.533844$

H 9.0377980 .2134550 .775133

H $\quad \begin{array}{llll}\text { H } & .713521 & 0.427743 & 0.952327\end{array}$

H $2.880061-2.8610570 .444611$

C -3.587422 $2.159704-2.979612$

O $-4.0003531 .115072-2.073648$

C $-4.7718571 .710763-1.014223$

C $-5.2416173 .054684-1.556956$

C $-4.0233953 .485017-2.348715$

H $-4.0762701 .887889-0.184649$

C $-5.8909770 .776177-0.544941$

H $-6.1046952 .947908-2.223809$

H $-5.5073903 .756485-0.760643$

C $-4.2987924 .585672-3.357349$

H $-3.2734013 .842282-1.635806$

C $-5.446818-0.663770-0.218718$

O $-6.897011 \quad 0.684759-1.562448$

H $\quad-6.390015 \quad 1.219306 \quad 0.324938$

H -5.269093 -1.209795 -1.152136

O $-6.544521-1.3203550 .428481$

C $-4.211684-0.7779270 .690090$

C -3.885552 -2.205731 1.168388

H $-3.337190-0.3790110 .165332$

O -4.3931830 .0358331 .851756$

H -2.931720 -2.157901 1.709150

H $-4.617880-2.5152191 .925552$

H -3.388156 $4.844533-3.906986$

H $-5.0588114 .283002-4.085214$

H $-4.6575415 .488698-2.852722$

C $-2.0906082 .033111-3.294544$

H $-6.4653310 .367129-2.374300$

H -7.312785 -1.252407 -0.164224

H $-5.199763-0.2695952 .301520$

H -1.831308 $2.734508-4.096491$

H $-1.8901471 .022976-3.671922$

H $-4.1465891 .984103-3.907751$

C -1.658803 -2.751461 -0.880955

O $-3.071606-2.825620-1.080133$

C $-3.817589-3.2692600 .054840$

C $-3.320223-4.6451100 .523427$

C $-1.810293-4.6633300 .751725$

C -1.078895 -4.110884 -0.471206

H $-1.456398-2.022997-0.086108$ 
C $-1.024706-2.169692-2.153261$

H -4.845766 -3.414105 -0.298800

O $-3.977954-5.0443121 .721320$

H -3.560784 -5.395410 -0.241142

H -1.532404 -4.111825 1.657536

O $-1.382048-6.0117010 .956597$

H -1.157719-4.834071 -1.290963

O $0.305284-3.964976-0.166551$

C -1.217751 -3.011719-3.397950

H -1.473896 -1.192701-2.366270

H $\quad 0.050147-2.031740-1.994759$

H -4.934027 -5.043802 1.543328

H $-1.884948-6.3626791 .711614$

H $0.632390-4.834466 \quad 0.121779$

O $-0.296144-2.634290-4.333638$

O $-2.080795-3.865045-3.549482$

C $-0.364281-3.331870-5.581121$

H $\quad 0.430648-2.950497-6.227610$

H - $0.206527-4.403462-5.425946$

H $-1.328457-3.148390-6.064601$

SCF Energy (B3LYP/6-31G**//MMFF) $=-3245.91782429$

01 123

MM̄FF Geometry

C $0.947582-0.180036-2.814487$

C $2.0093750 .473410-3.313202$

C $2.5297931 .833181-2.917392$

O $1.5327682 .565583-2.168957$

C $3.7978981 .722303-2.049422$

C $5.0316621 .268626-2.797121$

C $5.5399560 .021610-2.754634$

C $5.7246182 .342046-3.595888$

C $4.982410-1.181012-2.044735$

C $5.887043-1.672153-0.908054$

C $5.892566-0.7463040 .319289$

C $6.868236-1.2752621 .373478$

C $6.821973-0.4491322 .665904$

O $4.564513-0.7126830 .852523$

O $7.509976-1.1872953 .687178$

C $5.353448-0.315733 \quad 3.116907$

C 7.5641090 .8843142 .519096

O $5.2463830 .559624 \quad 4.238024$

C $4.3979240 .144971 \quad 1.990649$

C 2.9313620 .0098182 .467988

O 4.6719231 .5091131 .671145

C $1.5469843 .919972-2.298030$

O $2.2963624 .577847-3.008170$

C $0.4947844 .565942-1.484485$

C $-0.0858053 .982179-0.425861$

C -1.1237124 .5759960 .422074$

C $-1.7050045 .898563-0.004370$

C $1.885926 \quad 0.4482181 .433794$

C $0.511100-0.1308511 .705830$

C -1.274199-0.302199 3.436121

O $-0.097807-0.7533010 .838377$

C $-2.286650 \quad 0.749015 \quad 3.108538$

C -2.9788891 .4303054 .034177$

C -3.9842882 .5219883 .781922$

C -3.7518673 .3573682 .541681$

C $-4.6187713 .333878 \quad 1.515301$

C -2.5574864 .2895582 .540346$

C -1.517795 3.9007431 .521530

N 0.0286520 .1419512 .974119

H $\quad 0.3439290 .264042-2.028626$

H $2.590222-0.021227-4.090855$

H $2.7404032 .371854-3.849884$

H $3.5968781 .076748-1.188101$

H $4.0147652 .696342-1.589925$

H $6.450175-0.179039-3.318771$

H $6.629346 \quad 1.974017-4.091133$

H $5.0600522 .732408-4.372857$

H $6.0203713 .169666-2.942741$

H $3.968100-1.014841-1.672673$

H $4.896114-1.983627-2.787991$

H $5.521133-2.661789-0.604189$

H $6.910227-1.806428-1.280470$

H 6.1823990 .2631130 .007179

H $7.890189-1.3087150 .975557$
H $\quad 6.609715-2.3149121 .619963$

H $8.420157-1.3439883 .381952$

H $5.024952-1.3054353 .464502$

H 8.6178720 .7103372 .269965

H $7.568073 \quad 1.4403293 .463114$

H 7.1442561 .5259441 .741741

H $5.8513250 .227617 \quad 4.923419$

H $2.740565-1.0385222 .733324$

H 2.7843540 .6085943 .375086

H 3.9146971 .8790621 .191090

H $0.2512535 .580632-1.782770$

H $0.2278812 .978819-0.143160$

H -2.053097 5.853623 -1.042229

H -0.9531686 .6910310 .078452$

H -2.567786 6.2078720 .588894

H 1.7966841 .5396451 .403094

H 2.1773130 .1124810 .432876

H -1.194445 -0.481432 4.512795

H - $1.537838-1.2435012 .945364$

H -2.4558030 .9453092 .052978$

H -2.8199691 .1846265 .082509$

H -3.976247 3.2005124 .645140

H -4.983485 2.0685633 .773070

H -4.4741873 .9589840 .640709$

H -5.4932352 .6923791 .527514$

H -2.0763864 .2789003 .526944$

H -2.909386 5.3179222 .416854

H $-1.0225752 .955638 \quad 1.742347$

H 0.5461550 .7761133 .575006

C $0.830129-2.628768-2.242017$

O $-0.319596-2.771884-1.380502$

C $-0.598782-4.175792-1.208469$

C $0.636625-4.912244-1.707578$

C $1.070115-4.013577-2.846696$

H -1.437174 -4.414294-1.873532

C $-0.979310-4.4876050 .243236$

H $1.419213-4.959490-0.941433$

H $\quad 0.411623-5.934510-2.026357$

C $2.503796-4.250494-3.290891$

H $0.396576-4.173823-3.699555$

C $-2.176993-3.6802390 .779685$

O $0.141253-4.2072331 .093161$

H - $1.176627-5.5604940 .350056$

H -1.885998 -2.6328180 .896028$

O $-2.484075-4.1623832 .093299$

C $-3.450198-3.772185-0.076750$

C $-4.677036-3.0569490 .520539$

H $-3.253437-3.353700-1.068540$

O $-3.796491-5.144166-0.279075$

H $-5.504747-3.172349-0.190393$

H $-5.011651-3.5837081 .423469$

H $2.784040-3.562805-4.094793$

H $3.208341-4.106815-2.464794$

H $2.626792-5.272963-3.662689$

C $0.556796-1.552753-3.297580$

H $\quad 0.377266-3.2717120 .967350$

H -1.672906 -4.088470 2.624981

H $-3.928056-5.5454680 .597273$

H $-0.507284-1.533765-3.561147$

H $1.109102-1.781512-4.217025$

H $1.673956-2.346759-1.598737$

C -4.552035 -0.682183-1.392107

O $-3.767983-0.882667-0.213502$

C $-4.448946-1.5667860 .841703$

C $-5.714932-0.7980451 .245248$

C $-6.607914-0.4835410 .045766$

C $-5.7950800 .156141-1.081081$

H -4.866188 -1.658122 -1.783155

C $-3.649972-0.076760-2.472136$

H $-3.777044-1.5390231 .706893$

O $-6.466918-1.5257412 .210449$

H $-5.4246870 .149893 \quad 1.714674$

H -7.132039 -1.378280 -0.310058

\begin{tabular}{lllll}
\hline & -7.621525 & 0.441814 & 0.445849
\end{tabular}

H $-5.5149791 .174589-0.787140$

O $-6.6013830 .254019-2.251822$

C $-3.1764421 .317695-2.134374$

H $-2.750434-0.689054-2.607190$ 
H $-4.170590-0.063719-3.436843$

H $-5.882975-1.6920762 .970121$

H -8.1039260 .0382341 .187714$

H $-7.3972590 .760048-2.014192$

O $-3.6964162 .210003-3.024931$

O $-2.4428161 .582216-1.191107$

C $-3.3332633 .576731-2.813298$

H $-3.6489923 .904526-1.818901$

H -2.254615 $3.702893-2.941141$

$\mathrm{H}-3.8482044 .185456-3.561212$

SCF Energy (B3LYP/6-31G**//MMFF) $=-3245.91406646$

01

MMFF Geometry

C -1.574448 -3.102940 1.695423

C $-1.556498-3.9579580 .661994$

C $-1.894456-3.539565-0.745442$

O $-0.674252-3.583469-1.529761$

C -2.911593 -4.482371-1.416032

C $-4.351936-4.139591-1.097245$

C $-5.186434-3.506105-1.945109$

C $-4.856451-4.5879710 .249589$

C $-4.904089-3.011193-3.336676$

C -3.855354 -1.904040 -3.476501

C $-3.988912-0.716023-2.515285$

C $-5.2987530 .052463-2.687770$

C $-5.3469501 .293165-1.780826$

O $-2.877610 \quad 0.147659-2.791992$

O $-6.4393122 .116181-2.217104$

C $-4.0549972 .111815-1.982308$

C $-5.6370120 .924311-0.320358$

O $-3.9872653 .193322-1.054900$

C $-2.7621951 .264821-1.902466$

C -1.557204 2.116134-2.365400

O $-2.5584850 .843451-0.554295$

C $0.193948-2.544853-1.368230$

O $0.043439-1.560569-0.661394$

C $1.399249-2.827624-2.173906$

C $2.557431-2.249055-1.822103$

C $3.851932-2.435871-2.478761$

C $3.854701-3.109109-3.825674$

C $-0.2330361 .346855-2.461173$

C $0.8848382 .204329-3.029026$

C $3.3255012 .194435-3.432450$

O $0.7149823 .352019-3.432359$

C $4.3562751 .176680-3.799347$

C $5.5593391 .101679-3.210819$

C $6.6296640 .124035-3.604419$

C $7.064109-0.754065-2.453275$

C $8.064723-0.380098-1.637807$

C $6.393564-2.100561-2.301092$

C $4.961329-1.990015-1.852549$

N $2.0984821 .527667-3.042664$

H -1.871903 -2.071542 1.526141

H -1.224606 -4.9811270 .818653$

H -2.279159 -2.513771 -0.772762

H -2.714107 -5.526397 -1.137266

H $-2.753124-4.468778-2.501137$

H -6.207636 -3.323481-1.611908

H $-5.942968-4.4810770 .341210$

H $-4.621515-5.6445670 .413897$

H -4.402645 -3.997769 1.050093

H -4.620944 -3.861188 -3.970068

H -5.850206 -2.646516 -3.757435

H -2.851102 -2.330152 -3.362066

H $-3.873778-1.534296-4.510847$

H -3.896709 -1.081040 -1.488106

H $-6.166891-0.587787-2.493214$

H $-5.3945530 .380343-3.732231$

H $-7.2538441 .587723-2.160357$

H $-4.1090062 .564793-2.982266$

H $-6.5976750 .401389-0.241546$

H -5.7324811 .8210480 .301805$

H -4.8740010 .2792500 .120294$

H $-4.8083343 .706184-1.147498$

H - $1.7773712 .530724-3.358106$

H -1.418945 $2.963391-1.682912$

H $-1.6288730 .596070-0.432851$
H $1.309410-3.523744-2.999325$

H $2.569179-1.594875-0.950942$

H $3.132350-2.630302-4.497110$

H $3.592799-4.167990-3.726376$

H $4.818170-3.057408-4.336636$

H $0.0808811 .007515-1.469749$

H $\quad-0.3444140 .470949-3.109805$

H $3.6380232 .823543-2.591811$

H $3.1229562 .848433-4.287274$

H $4.1120430 .489983-4.606204$

H $5.8062451 .806186-2.419470$

H $7.4888240 .698305-3.975448$

H $6.311470-0.501925-4.447494$

H $8.401899-1.015917-0.826118$

H $8.5659290 .574203-1.762477$

H $6.920749-2.701365-1.547850$

H $6.506920-2.655812-3.235936$

H $4.848874-1.517557-0.875829$

H $2.1706080 .658554-2.520641$

C $0.378443-3.2544993 .235332$

O $0.729792-1.8754803 .011300$

C $1.880735-1.5601603 .819539$

C $2.241098-2.8334274 .583950$

C $0.915072-3.5683874 .627186$

H $1.563344-0.7975924 .538869$

C $3.011872-1.0189602 .936264$

H $2.992959-3.4277744 .051663$

H $2.641576-2.6146925 .578898$

C $1.054614-5.0504464 .925956$

H $0.278218-3.1007265 .389914$

C 2.6506230 .2453822 .128549

O $3.401969-2.0316161 .998258$

H $3.898262-0.8252673 .551269$

H 1.9112520 .0013041 .357205

O $3.833844 \quad 0.664690 \quad 1.435555$

C 2.1470991 .4252332 .981157

C 2.0607992 .7690372 .234346

H 1.1654221 .1814423 .399515

O $3.0254501 .617434 \quad 4.095047$

H 1.7799473 .5367012 .965786

H 3.0633523 .0642811 .899675

H $0.075752-5.5393714 .948173$

H $1.666808-5.5560794 .171625$

H $1.528682-5.2029905 .901156$

C $-1.129312-3.4548233 .084607$

H $2.617103-2.2606221 .471299$

H $4.136391-0.0874980 .898675$

H 3.9133821 .7973213 .740980

H -1.673862 -2.8121753 .786747$

H -1.403654 -4.490453 3.315263

H $0.911862-3.8480032 .479965$

C -0.9439663 .2242032 .267112$

O -0.2260252 .3317291 .406818$

C 1.0843452 .7784741 .042755

C 0.9966064 .1305320 .319835

C 0.2314925 .1670671 .140477

C -1.1105844 .5959511 .599946$

H -0.4050993 .3310563 .215837$

C -2.3109072 .5982562 .566697$

H $1.4663982 .054050 \quad 0.314126$

O $2.3043394 .602244 \quad 0.014701$

H $0.4765613 .989176-0.634287$

H $0.822346 \quad 5.5203401 .993684$

O $\quad-0.031404 \quad 6.3139910 .329090$

H -1.7771024 .5133670 .732190$

O -1.7239105 .5088432 .508441$

C $-2.184607 \quad 1.2965163 .320830$

H -2.947546 $3.267253 \quad 3.156352$

H -2.8453522 .3845061 .634250$

H $2.2107985 .380288-0.560349$

H $0.822175 \quad 6.7320620 .126092$

H -1.145928 5.5899083 .286212

O -1.7062821 .5234864 .577125$

$\begin{array}{llll}\text { O } & -2.494375 & 0.208757 & 2.852855\end{array}$

C -1.5580520 .3607195 .397780$

H $-2.539701-0.0679745 .620090$

H $-0.918270-0.3774244 .906510$

H $-1.085078 \quad 0.664021 \quad 6.335538$ 
H $\quad 6.859713 \quad 4.355434-0.066718$

H 8.1762303 .6937872 .045213

01

MM̄FF Geometry

C $-2.8661322 .721634-2.503730$

C $-2.2447891 .717795-1.867500$

C $-1.1657600 .865384-2.480870$

O $-0.0763480 .751721-1.536082$

C -1.716054 -0.548969-2.740003

C $-0.730807-1.471547-3.430875$

C $-0.245560-2.609460-2.896859$

C $-0.315086-1.064836-4.821565$

C $-0.560185-3.210192-1.556323$

C $0.382243-2.781134-0.422388$

C $1.849599-3.188952-0.628814$

C $2.659911-2.9438220 .646470$

C $4.149619-3.2654740 .451960$

O $2.395422-2.407709-1.694543$

O $4.846300-2.7118741 .578891$

C $4.658666-2.551308-0.820357$

C $4.405493-4.7764870 .490104$

O $5.995507-2.944103-1.119487$

C $3.743466-2.746883-2.047222$

C $4.141742-1.862425-3.253884$

O $3.833960-4.109886-2.480922$

C $0.764671 \quad 1.813393-1.414154$

O $\quad 0.7287002 .859785-2.040974$

C $1.7331611 .487982-0.344911$

C 2.4291332 .4739440 .239020

C 3.4027322 .3160781 .320907

C 3.8105660 .9148271 .692258

C $4.127527-0.351551-2.995941$

C $5.4837800 .225080-2.623002$

C $6.5455622 .345789-1.855042$

O $6.533272-0.405948-2.713050$

C $6.7566312 .282042-0.375278$

C $6.898227 \quad 3.371366 \quad 0.395274$

C 7.1564773 .3246431 .874269

C 6.1714254 .1562252 .665343

C 6.4836995 .3992653 .069242

C 4.8610423 .5143223 .060430

C 3.8922763 .4241931 .915306

N $5.387291 \quad 1.549121-2.215117$

H -2.602477 $2.955213-3.531654$

H -2.536945 $1.481611-0.847804$

H $-0.7959891 .310053-3.412691$

H -2.617249 -0.488635 -3.365056

H $-2.038548-0.977673-1.784843$

H $0.456328-3.193760-3.491146$

H $\quad 0.262447-1.846178-5.327392$

H $-1.196831-0.861653-5.438046$

H $0.307601-0.165707-4.791858$

H -1.592382 -2.986554-1.266085

H $-0.525674-4.302507-1.658226$

H $\quad 0.313172-1.694572-0.293992$

H $0.015106-3.2415670 .502083$

H $1.877021-4.250684-0.900562$

H $2.250622-3.5190371 .486516$

H $2.566938-1.8884250 .936255$

H $5.798756-2.8404561 .428441$

H $4.704328-1.484343-0.576183$

H $4.072924-5.1946481 .447857$

H $5.475926-5.0000670 .424487$

H $3.890262-5.318490-0.305656$

H $5.960796-3.823591-1.532837$

H $5.103104-2.194701-3.663648$

H $3.406729-2.052707-4.049426$

H $3.542467-4.158046-3.406618$

H $1.8268590 .449221-0.050260$

H $2.2558113 .498083-0.089116$

H 4.1384910 .3648660 .803983

H 2.9731080 .3771092 .148930

H 4.6453190 .8736482 .394766

H $3.8280360 .154811-3.922682$

H $3.392892-0.090131-2.229032$

H $6.357748 \quad 3.366471-2.203011$

H $7.4301191 .958191-2.370498$

H $6.823827 \quad 1.2958590 .078135$

H 7.1490802 .2943142 .250801

H 5.7941285 .9917173 .661273

H 7.4345945 .8540512 .811958

H 4.3740784 .1056573 .847762

H 5.0620612 .5462773 .527574

H 3.5399874 .3968241 .568978

H $4.4703641 .976651-2.122843$

C $-5.3251992 .947406-2.075878$

O $-5.4243121 .729214-1.308596$

C $-6.6659341 .729939-0.583981$

C $-7.5192732 .812382-1.230849$

C $-6.4637213 .842356-1.585317$

H -6.4344262 .0329650 .444071$

C $-7.2784240 .326697-0.590155$

H -8.016136 $2.450842-2.138512$

H $-8.2870753 .197708-0.553310$

C -6.929093 4.863606-2.608364

H $-6.1620404 .366098-0.668167$

C $-6.348243-0.778172-0.041190$

O $-7.622273-0.026579-1.937177$

H $-8.2224780 .333742-0.033246$

H $-5.519889-0.935174-0.741942$

O $-7.116507-1.988533-0.038910$

C $-5.805684-0.5096481 .377661$

C $-5.066024-1.6967872 .023697$

H $-5.1414150 .361072 \quad 1.355426$

$\begin{array}{llll}\text { O } & -6.908027 & -0.173715 & 2.226548\end{array}$

H -4.839910-1.425713 3.062033

H $-5.751715-2.5485682 .108938$

H $-6.1288085 .572116-2.843922$

H $-7.2394694 .383337-3.542426$

H $-7.7818575 .432346-2.223482$

C $-3.9397533 .557556-1.863899$

H $-6.8080180 .014153-2.467903$

H $-6.495640-2.7332160 .027167$

H $-6.5463150 .074136 \quad 3.094474$

H -3.733030 $3.655505-0.790196$

H $-3.8911144 .565024-2.292430$

H $-5.4723862 .674644-3.129016$

C $-2.243594-0.5985182 .385225$

O $-2.848673-1.032801 \quad 1.159930$

C $-3.775544-2.1151381 .291143$

C $-3.071788-3.3302031 .907558$

C $-2.392956-2.9859593 .230076$

C -1.501094 -1.753490 3.075972

H $-3.018641-0.1997843 .050361$

C -1.297299 0.5520792 .020266

H -4.060729 -2.400767 0.271957

O $-4.002841-4.3909812 .096412$

H -2.312977 -3.695551 1.204610

H $-3.133440-2.8482584 .026828$

O $-1.589420-4.1040643 .615433$

H -0.605989-2.021314 2.501875

O $-1.067476-1.3311114 .366893$

C -0.8724021 .3702943 .214233$

H $-0.4209160 .165780 \quad 1.486993$

H $-1.783171 \quad 1.2430241 .322510$

H -3.521826 -5.130480 2.505945

H $-1.243091-3.9241924 .505650$

H -0.491374 -2.025965 4.727274

O $0.485738 \quad 1.353486 \quad 3.330367$

O -1.6529991 .9542043 .954762$

C 1.0199192 .0875624 .436377

H $\quad 0.839478 \quad 3.157438 \quad 4.297007$

H 0.5806341 .7395315 .376268

H 2.0986581 .9138064 .471263

SCF Energy (B3LYP/6-31G**//MMFF) $=-3245.90505103$

01 126

MM̄FF Geometry

C $1.962466-4.939547-1.405790$

C $1.325789-3.890293-1.946149$

C $-0.165872-3.687123-1.856516$

O $-0.428051-2.449786-1.155677$

C $-0.752586-3.564960-3.274905$

C $-2.262076-3.424910-3.306395$ 
C $-2.920713-2.349148-3.780978$

C $-3.040611-4.607420-2.789184$

C $-2.342828-1.092017-4.368328$

C $-2.1199310 .042863-3.357556$

C $-3.4083020 .571023-2.706776$

C $-3.1249211 .841766-1.902393$

C $-4.3762592 .347136-1.166155$

O $-3.921522-0.437370-1.833150$

O $-3.9546123 .322005-0.199451$

C $-5.0090181 .177415-0.380758$

C $-5.3424083 .067034-2.114477$

O $-6.263849 \quad 1.557752 \quad 0.177755$

C $-5.173663-0.107824-1.216792$

C $-5.604570-1.333036-0.373566$

O $-6.1918390 .100853-2.203185$

C $-0.325436-2.463988 \quad 0.201174$

O $-0.034743-3.4147750 .909420$

C $-0.613310-1.0962450 .685059$

C $-0.253167-0.7481981 .929021$

C $-0.448696 \quad 0.5644112 .547419$

C -1.3260641 .5520991 .825458$

C $-4.641783-1.7291470 .751837$

C $-5.015859-1.1660092 .113324$

C -4.167820 -1.027793 4.453255

O $-6.081094-0.5990522 .340422$

C $-3.5617430 .323364 \quad 4.665531$

C -2.6499770 .5802565 .615715$

C -2.0692251 .9410595 .873294$

C -0.5571871 .9431255 .859743$

C 0.1446751 .8928947 .004585

C 0.1370692 .0929544 .525391

C $0.149093 \quad 0.8157553 .730671$

N $-4.029333-1.4094303 .060190$

H $1.389238-5.695902-0.875374$

H $\quad 1.901687-3.128870-2.466950$

H $-0.644582-4.516264-1.321416$

H - $0.482556-4.450644-3.865591$

H $-0.279144-2.712677-3.774272$

H $-4.009915-2.366910-3.757490$

H -4.114896 -4.513665 -2.981595

H $-2.702076-5.527995-3.275745$

H -2.910294 -4.713303 -1.707944

H - $1.397545-1.302850-4.879867$

H -3.020583 $-0.743537-5.158224$

H -1.430629 -0.306042 -2.580301

H -1.624095 $0.866091-3.886360$

H -4.137046 $0.775494-3.499869$

H -2.716352 2.629220 -2.548020

H $-2.3450811 .632776-1.157641$

H -3.486259 4.030704-0.673099

H -4.3437640 .9673710 .464025$

H -4.848348 $3.925977-2.584447$

H $-6.1994943 .477236-1.569263$

H -5.716871 $2.424632-2.914202$

H -6.1123572 .3536890 .715794$

H -6.627652 -1.195218 -0.003350

H -5.658308 -2.192301-1.057756

H $-6.528097-0.765172-2.487340$

H $-1.091866-0.407968-0.001719$

H $0.257604-1.4847192 .548072$

H -1.572057 2.4339662 .420657

H -2.284014 1.0908621 .564769

H $-0.843560 \quad 1.900670 \quad 0.906971$

H -4.667039 -2.8213060 .859060$

H $-3.612370-1.448906 \quad 0.508842$

H $-3.676405-1.8013395 .051825$

H -5.229216 -1.001436 4.720333

H -3.9099461 .1327954 .028339$

H -2.325331 -0.2253296 .270654$

H -2.4331302 .2772936 .853153$

H -2.4411662 .6794565 .152143$

H 1.2292431 .9235257 .004935

H -0.3502811 .8146517 .967050$

H 1.1848232 .3854814 .678170

H -0.3056752 .9315923 .980758$

H $\quad 0.7586690 .0307374 .180367$

H -3.154813 -1.837352 2.770102

C $4.211351-4.690008-0.238805$
O $4.189003-3.252114-0.150106$

C $4.095815-2.8791601 .238671$

C $4.306238-4.1607602 .041951$

C $3.713714-5.2022631 .111479$

H $3.068015-2.5329821 .398862$

C $5.082323-1.7504901 .559599$

H $5.369019-4.3670262 .213370$

H $3.810923-4.1231893 .017012$

C $4.141816-6.6238581 .427418$

H $2.621344-5.1335021 .181975$

C $4.973100-0.5218560 .633405$

O $6.415595-2.2643221 .431384$

H $4.969679-1.4522232 .608723$

H $5.334503-0.791483-0.366973$

O $5.887402 \quad 0.456879 \quad 1.144619$

C 3.5637170 .0922560 .530594

C $3.5359621 .357445-0.346096$

H $2.871741-0.6496110 .125183$

O 3.0961250 .4555151 .829940

H 4.0591952 .1641920 .183782

H $4.088878 \quad 1.177240-1.274852$

H $3.697940-7.3297690 .718326$

H $5.230167-6.7361941 .379090$

H $3.819454-6.9078732 .434505$

C $3.447706-5.149570-1.482978$

H $7.026714-1.5399561 .650414$

H 6.0245501 .1247640 .452008

H $2.945719-0.3617672 .333362$

H $3.631036-6.216733-1.655155$

H $3.843321-4.618322-2.358312$

H $5.266687-4.963070-0.373660$

C $2.7653823 .798087-1.943684$

O $2.0847083 .284108-0.793285$

C $2.1081521 .854537-0.670798$

C $1.4441511 .225063-1.905926$

C $2.0448831 .738488-3.213593$

C $2.1064633 .265327-3.221311$

H $3.821613 \quad 3.502075-1.915035$

C $2.6899325 .330246-1.878491$

H $1.477306 \quad 1.6283660 .195046$

O $1.540843-0.191764-1.865406$

H $\quad 0.375327 \quad 1.475100-1.889771$

H $3.0330791 .297848-3.389547$

O $1.2155871 .297981-4.291412$

H $1.0885373 .663405-3.317266$

O $2.8333373 .697376-4.369306$

C $3.5298335 .899050-0.753347$

H $3.0561105 .772929-2.811146$

H $1.6522305 .641258-1.708263$

H $1.101563-0.538409-2.660907$

H $1.5865831 .666465-5.111493$

H $3.7433143 .363548-4.290212$

O $3.3164007 .246885-0.681873$

O $4.2823955 .254617-0.036007$

C 4.0492567 .9321200 .338769

H 5.1247827 .8285200 .166822

H $3.787010 \quad 8.992470 \quad 0.291978$

H 3.7758897 .5461841 .325479

SCF Energy $\left(B 3 L Y P / 6-31 G^{* *} / / M M F F\right)=-3245.90753836$

01 127

MM̄MF Geometry

C $2.783616 \quad 3.7923940 .847968$

C 1.5763943 .2191990 .730980

C $0.6380583 .506603-0.412423$

O $0.2492922 .225208-0.956812$

C -0.6217534 .2231550 .105809$

C $-1.4116444 .894470-0.997779$

C $-2.5338444 .401931-1.556058$

C $-0.8731276 .229958-1.445928$

C $-3.2262133 .097272-1.274986$

C $-4.6372703 .333783-0.721969$

C $-5.4925842 .059489-0.760099$

C $-6.9312502 .365005-0.335180$

C $-7.8028771 .100405-0.316544$

O $-4.917378 \begin{array}{lll}1.081515 & 0.116045\end{array}$

O -9.0151551 .4105190 .388151$

C -7.0735420 .0111410 .496590$ 
C $-8.2215880 .668895-1.726711$

O $-7.766694-1.232151 \quad 0.426154$

C $-5.596753-0.1841250 .084455$

C $-4.847763-1.1636661 .014340$

O $-5.546889-0.755337-1.230576$

C $0.1660232 .075162-2.302212$

O $0.4363742 .913578-3.150706$

C $-0.3244860 .721901-2.664473$

C $-0.680803-0.193741-1.750800$

C -1.192706 -1.541667 -2.004465

C -1.344047 -1.989622 -3.431997

C $-4.835285-0.8045522 .500723$

C $-4.082935-1.8760963 .264933$

C -1.769051-2.637077 3.793990

O $-4.660398-2.855038 \quad 3.733059$

C $-0.920633-3.0521462 .635786$

C -1.038656-4.219056 1.983387

C $-0.216002-4.5611090 .771919$

C -1.042285 -4.752361 -0.486689

C $-0.873165-5.849354-1.244541$

C $-2.057756-3.699506-0.887555$

C -1.491274 -2.309083 -0.936705

N -2.717820 -1.651471 3.306590

H 3.0879854 .4993460 .077861

H 1.2276042 .5147741 .480621

H $1.1530164 .105893-1.172674$

H -0.3447054 .9973570 .834655$

H -1.2547143 .5168350 .655869$

H -3.010973 $4.992456-2.338048$

H -1.494888 $6.691714-2.220162$

H $-0.8313656 .924759-0.600628$

H $0.1353886 .121440-1.856821$

H $-2.6593532 .461773-0.588858$

H -3.287990 $2.548580-2.223011$

H -4.552956 3.6970580 .310440

H $-5.1385294 .116678-1.305527$

H -5.478805 $1.675576-1.787207$

H $-7.3777403 .126170-0.987195$

H -6.9275322 .7997130 .674293$

H $-9.4481572 .150277-0.071435$

H -7.1115830 .3302411 .545359$

H $-8.7983281 .463976-2.214531$

H -8.884458 $-0.202865-1.692133$

H -7.374685 $0.433258-2.374213$

H -8.685168 -1.0681230 .700839$

H $-5.253617-2.1762300 .884275$

H -3.805881 -1.229291 0.674076

H -4.677084 -1.171504 -1.352374

H $-0.3914630 .526115-3.729456$

H $-0.5974880 .062584-0.695681$

H -1.632522 -3.039454 -3.524884

H $-0.399086-1.882568-3.975989$

H -2.109438 -1.393545 -3.940692

H -4.3701300 .1730812 .668008$

H -5.845581 -0.7610562 .919198$

H $-2.292955-3.4849444 .246290$

H -1.151260 -2.159734 4.560640

H $-0.186922-2.3252222 .294062$

H -1.773787 -4.948785 2.313003

H $\quad 0.352830-5.470721 \quad 1.003310$

H $0.530948-3.7822560 .579853$

H -1.450803 -6.007769-2.149231

H $-0.150484-6.614283-0.980266$

H -2.876847 -3.705137 -0.156499

H -2.535261 -3.959008 -1.838287

H -1.342185 -1.8753760 .051567$

H -2.344057 -0.804679 2.887177

C 3.4876872 .5610203 .026362

O 3.4576651 .2367152 .456304

C 4.2450140 .3555223 .276587

C 4.4519671 .0879574 .595422

C 4.5579362 .5225804 .121140

H $\quad \begin{array}{llll}5.215070 & 0.245419 & 2.776969\end{array}$

C $3.571260-1.0141723 .405863$

H 3.5916840 .9747035 .265140

H 5.3435980 .7428605 .127564

C 4.3489813 .5443485 .225810

H 5.5530232 .6720433 .680748
C $3.205956-1.6866212 .067274$

O $2.354404-0.8759374 .153201$

H $4.209296-1.6808203 .997593$

H $2.356368-1.1621201 .615261$

O $2.751048-3.0140882 .362159$

C $4.357998-1.7856371 .051790$

C $4.035608-2.641496-0.187851$

H $4.651806-0.7815960 .733824$

O $5.491573-2.3670331 .702637$

H $4.942027-2.698236-0.802615$

H $3.850293-3.6750870 .130260$

H 4.4231864 .5637674 .834390

H 3.3648703 .4339265 .693566

H 5.1072323 .4264866 .006815

C 3.8047353 .5891921 .936114

H $1.794790-0.2320153 .685722$

H $2.052891-2.9405213 .034865$

H $6.228736-2.3621781 .068346$

H 4.7400623 .2986681 .441315

H 3.9786094 .5666792 .401752

H 2.5000172 .7491313 .465397

C $4.040302-0.585522-2.472114$

O $3.042611-0.769441-1.459766$

C $2.852912-2.122031-1.028824$

C $2.489585-3.019991-2.218801$

C $3.494803-2.892143-3.357521$

C $3.712258-1.423434-3.715646$

H $5.022641-0.873575-2.077852$

C $4.0777210 .911611-2.824063$

H $1.974764-2.109845-0.374476$

O $2.390594-4.377411-1.802738$

H $1.500903-2.729106-2.589712$

H $4.443201-3.378483-3.102545$

O $2.998007-3.586634-4.504469$

H $2.815585-1.027469-4.209523$

O $4.790919-1.329341-4.644375$

C $4.8590971 .724249-1.813486$

H $4.5674361 .051430-3.794663$

H $3.0661431 .328016-2.872447$

H $2.165607-4.907058-2.586709$

H $2.150348-3.182506-4.757274$

H $4.566687-1.888484-5.407939$

O $5.2755782 .884019-2.403152$

O $5.0653161 .396488-0.653726$

C $6.0775393 .747290-1.590775$

H $5.4481714 .253488-0.855101$

H $6.8781223 .188827-1.096126$

H $6.5307054 .499825-2.241650$

SCF Energy (B3LYP/6-31G**//MMFF) $=-3245.91071171$

01128

MM̄FF Geometry

C $-2.369013-3.054732-1.641695$

C $-1.509936-2.983077-2.668631$

C $-0.501533-1.874316-2.802395$

O $0.805005-2.491108-2.754582$

C $-0.640758-1.094610-4.122639$

C $-1.954924-0.365709-4.280093$

C $-2.2573460 .793157-3.663888$

C $-2.936849-1.000805-5.229522$

C $-1.4079411 .583802-2.705076$

C -1.072474 $2.962037-3.286471$

C $-0.5441483 .937451-2.223906$

C $-0.3674775 .329484-2.837772$

C $0.1922576 .335187-1.824420$

O $0.7088483 .462609-1.711179$

O $0.6227537 .498567-2.547230$

C $1.4426375 .724313-1.164187$

C $-0.8787476 .810904-0.835812$

O $1.9143386 .557657-0.106861$

C $1.2365764 .277633-0.651022$

C $2.6008183 .706702-0.189607$

O $0.354412 \quad 4.305048 \quad 0.476403$

C $1.835408-1.706172-2.339505$

O $1.781986-0.525813-2.033696$

C $3.053792-2.542359-2.301987$

C $4.218583-1.975115-1.957063$

C $5.503619-2.656385-1.808748$ 
C $5.543892-4.145039-2.030377$

C $2.607725 \quad 2.1962170 .083544$

C 3.6831671 .7894961 .081852

C $4.568953-0.2066212 .280800$

$\begin{array}{lllll}\text { O } & 4.390333 & 2.598137 & 1.678068\end{array}$

C $5.963022-0.3973991 .771498$

C $6.616612-1.5689161 .814717$

C $8.035964-1.7532241 .351370$

C $8.170273-2.8171090 .283292$

C $8.520338-4.0741090 .604129$

C $7.970409-2.409896-1.160527$

C $6.575562-1.926435-1.438475$

N $3.7412820 .413347 \quad 1.260401$

H -2.326643 -2.292834 - 0.867701

H -1.531344 -3.743582 -3.444856

H $-0.598949-1.191039-1.950237$

H $-0.484257-1.776123-4.970489$

H $0.182039-0.374019-4.217073$

H $-3.2357911 .232103-3.857887$

H -3.883915 $-0.452904-5.277841$

H $-2.517870-1.029317-6.240793$

H $-3.165990-2.025448-4.922572$

H - $1.9765601 .696926-1.773914$

H $-0.481316 \quad 1.065571-2.444941$

H $-0.3238382 .833698-4.079224$

$\mathrm{H}-1.9682603 .395275-3.750191$

H -1.274902 $3.975163-1.407139$

H -1.314175 5.696095-3.254215

H $0.3285445 .266723-3.686215$

H $-0.1438317 .851980-3.030343$

H $2.2376965 .708244-1.923044$

H -1.706591 7.291885-1.370679

H -0.479387 $7.572321-0.156772$

H $-1.3006386 .001347-0.236754$

H $2.0487747 .447003-0.476915$

H $3.3610813 .895454-0.958864$

H $2.913287 \quad 4.2518330 .709495$

H 0.5648803 .5636501 .065742

H $2.955750-3.597047-2.531431$

H $4.233033-0.907499-1.740274$

H $5.259064-4.387675-3.060105$

H $4.857446-4.655450-1.345665$

H $6.531873-4.580791-1.864340$

H 1.6459631 .8689940 .489736

H $2.7782601 .659134-0.855564$

H $4.094771-1.1531952 .556848$

H $4.587167 \quad 0.4420863 .162895$

H $6.4730790 .478347 \quad 1.377077$

H $6.117389-2.4405652 .231965$

H 8.639029-2.025934 2.227467

H $8.463476-0.8124120 .982949$

H $8.643839-4.838851-0.155427$

H $8.687614-4.3674161 .635252$

H $8.237568-3.222449-1.846121$

H $8.673579-1.601632-1.400165$

H $6.451333-0.853021-1.291708$

H $3.150547-0.1892550 .694754$

C $-4.817490-3.568826-1.253521$

O $-4.839176-2.695012-0.104878$

C $-5.578553-1.507412-0.449596$

C $-6.388831-1.875595-1.685731$

C $-5.400278-2.765085-2.416950$

H $-4.843323-0.745054-0.734202$

C $-6.395186-1.0196120 .748506$

H -7.291396 -2.441095 -1.426427

H $-6.688371-0.997414-2.265308$

C -6.027856 -3.618494 -3.504083

H $-4.633273-2.124754-2.868602$

C $-5.542685-0.7302202 .003486$

O $-7.349687-2.0330911 .086764$

H $-6.973590-0.1324020 .464955$

H -5.121996 -1.669401 2.378217

O $-6.427624-0.2592673 .028325$

C $-4.422350 \quad 0.307411 \quad 1.792059$

C -3.6808940 .7064263 .082564$

H -3.700509 -0.0685911 .059834$

O $-5.004587 \quad 1.490227 \quad 1.236059$

H -3.0272911 .5551572 .847134$
H -4.3986181 .1043423 .810386$

H $-5.274664-4.250820-3.984724$

H $-6.810175-4.270498-3.101723$

H $-6.480688-2.986598-4.275094$

C $-3.405315-4.126046-1.461234$

H -7.850576 -1.7136521 .856841$

H -6.7830740 .5979642 .736853$

H $-4.280372 \quad 2.1074151 .035189$

H $-3.402432-4.822515-2.307354$

H $-3.121490-4.697524-0.568528$

H $-5.483101-4.406589-1.008001$

C $-0.857359-0.2209322 .385291$

O $-1.966541-1.0400332 .769231$

C $-2.860221-0.4372083 .712017$

C -2.096286 -0.0382384 .983805$

C $-0.8859860 .840003 \quad 4.674126$

C $-0.0153790 .191192 \quad 3.598585$

H -1.2407040 .6811321 .891717$

C $-0.053044-0.9617541 .308317$

H -3.571987 -1.218810 4.002846

O $-2.948303 \quad 0.6468125 .895934$

H - $-1.742932-0.9446285 .492708$

H -1.1871201 .8516194 .377771$

$\begin{array}{lllll}\text { O } & -0.096960 & 0.987686 & 5.857081\end{array}$

H $0.498147-0.6755324 .030967$

$\begin{array}{lllll} & 0 & 0.986619 & 1.115363 & 3.182869\end{array}$

C $0.617534-2.2309761 .788932$

H $-0.715296-1.2507790 .487035$

H $0.711058-0.2892910 .905710$

H $-3.7005090 .061346 \quad 6.088736$

H -0.6743581 .3627596 .544160$

H $1.488373 \quad 1.376068 \quad 3.974214$

O $1.767784-2.4285141 .080256$

O $\quad 0.172097-2.9747242 .652284$

C $2.511089-3.602647 \quad 1.421823$

H $1.960135-4.4950051 .111459$

H $3.463353-3.5710090 .886248$

H $2.718367-3.6290632 .495988$

SCF Energy (B3LYP/6-31G**//MMFF)= -3245.92091856

01129

MMFF Geometry

C $-1.187345 \quad 3.269891-0.839201$

C $-0.361027 \quad 3.963859-0.041851$

C $0.379136 \quad 3.348993 \quad 1.117174$

$\begin{array}{llllll}\text { O } & 1.788341 & 3.552274 & 0.856265\end{array}$

C $0.012180 \quad 3.996708 \quad 2.465858$

C - $\begin{array}{llll}\text { - } & .457483 & 3.941330 & 2.821947\end{array}$

C -2.1125842 .8249553 .195441$

C $-2.182995 \quad 5.2600182 .754587$

C $-1.552647 \quad 1.437273 \quad 3.354290$

C $-2.100910 \quad 0.5013352 .270010$

C -1.694712 -0.9737582 .418637$

C $-2.412613-1.6522633 .584281$

C -2.000873 -3.1247163 .713651$

O $-0.281698-1.0565092 .598366$

O $-2.464889-3.5990214 .988399$

C $-0.459057-3.216973 \quad 3.733366$

C $-2.685468-4.0001582 .656835$

O $-0.063523-4.5865803 .682059$

C $0.244630-2.3945502 .620050$

C $1.756169-2.2658102 .928237$

O $\quad 0.126287-3.023342 \quad 1.344533$

C $2.6619122 .696582 \quad 1.451694$

$\begin{array}{llll}\text { O } & 2.402465 & 1.832082 & 2.272652\end{array}$

C 4.0020952 .9637030 .884966

C 4.9572432 .0289300 .997699

C 6.3071662 .0979740 .436393

C $6.7154963 .371290-0.256404$

C $2.544187-1.4739131 .876716$

C $3.086038-2.3147600 .732395$

C $4.354660-2.049577-1.405426$

O $3.035217-3.5407420 .706854$

C $5.827769-1.959350-1.159618$

C $6.658236-1.197595-1.887591$

C $8.135968-1.095617-1.635176$

C $8.5845720 .326971-1.377716$

C $9.1011001 .073044-2.368863$ 
$\begin{array}{llll}\text { C } & 8.515275 & 0.857309 & 0.038131\end{array}$

C $7.108402 \quad 1.0174610 .544388$

N $3.665380-1.517696-0.245434$ H - $-1.3283892 .207250-0.660822$ H $-0.2116365 .025884-0.220142$ H $\quad 0.186297 \quad 2.270279 \quad 1.146230$ H $0.356932 \quad 5.040545 \quad 2.469220$ H 0.5941173 .5322873 .272325 H -3.1734002 .9072843 .429286$ H -3.250883 5.1603642 .975782 H -1.757135 5.9614863 .479578 H -2.093535 $5.698521 \quad 1.755986$ H $-0.458676 \quad 1.4403223 .332106$ H $-1.839432 \quad 1.0785964 .348777$ H -3.194693 0.5732632 .226719 H -1.728352 0.8525161 .300153 H $-1.956819-1.4713461 .478633$ H -3.500970 -1.566053 3.476232 H $-2.162403-1.1469124 .526067$ H -3.429137 -3.4762795 .021722$ H $-0.132932-2.8317984 .709964$ H $-3.776021-3.9386662 .756248$ H -2.432455 -5.057218 2.793694 H $-2.434057-3.7134841 .634529$ H $0.873026-4.638723 \quad 3.934598$ H $1.865054-1.7348353 .883241$ H $2.211072-3.2543663 .051871$ H $-0.369336-3.8508811 .445737$ H $4.147757 \quad 3.893770 \quad 0.347753$ H 4.7218231 .1077291 .529039 H 6.6472494 .2220240 .430570 H $6.0695063 .563501-1.120423$ H $7.7416073 .351339-0.630620$ H $1.941121-0.6581161 .466764$ H $3.417915-1.0280682 .368760$ H $4.042169-1.455701-2.270059$ H $4.065047-3.091329-1.573857$ H $6.227774-2.550036-0.338667$ H $6.258033-0.617073-2.715928$ H $8.653411-1.497824-2.516009$ H $8.445718-1.731923-0.796845$ H $9.4541102 .083495-2.192331$ H $9.1792860 .690478-3.381273$ H $9.064325 \quad 1.8013480 .135223$ H 9.0460620 .1625490 .702178 H 6.7250330 .1325051 .053378 H $3.694751-0.512411-0.104072$ C $-3.4697303 .695879-1.872870$ O $-3.825762 \quad 2.297426-1.862358$ C -4.802266 $2.085331-0.824676$ C $-5.3612983 .464291-0.495704$ C $-4.114398 \quad 4.316610-0.631957$ H $-4.252818 \quad 1.7175560 .050435$ C $-5.8434741 .044506-1.248460$ H -6.118521 3.781509-1.221791 H $-5.804696 \quad 3.5068860 .503615$ C $-4.3943245 .802965-0.758874$ H -3.4924414 .1489100 .254386$ C $-5.245548-0.294531-1.730615$ O $-6.6202261 .583400-2.324972$ H $-6.5422490 .875558-0.420388$ H $-4.793668-0.147461-2.717789$ O $-6.325727-1.213578-1.932874$ C $-4.215308-0.920902-0.775780$ C $-3.741929-2.335491-1.150336$ H $-3.338941-0.267545-0.718737$ O $-4.757192-0.9813440 .546732$ H -2.950407 -2.608889 -0.443101 H $-4.547668-3.055670-0.957797$ H -3.461877 $6.365499-0.869544$ H -5.025874 6.020471-1.626550 H -4.9091956 .1742770 .133225$ C $-1.950163 \quad 3.859774-1.991829$ H -7.274120 $0.909035-2.577174$ H $-6.788543-1.323856-1.085275$ H $-5.504634-1.6025100 .538165$ H $-1.7072194 .921971-2.112617$ H $-1.6102563 .345957-2.899620$
H -3.927843 $4.112912-2.779291$

C $-1.099158-1.384466-2.407675$

O $-2.395902-1.433761-3.016494$

C $-3.235904-2.507368-2.593467$

C $-2.547765-3.851474-2.868716$

C $-1.144331-3.910890-2.266600$

C $-0.327730-2.685721-2.679610$

H $-1.210610-1.232054-1.327656$

C $-0.410159-0.153471-3.015823$

H $-4.123331-2.468524-3.237356$

O $-3.336119-4.937963-2.393814$

H $-2.460411-3.984559-3.954710$

H $-1.176480-4.008636-1.175240$

O $-0.478437-5.079078-2.752949$

H $-0.079461-2.773870-3.745226$

O $0.894347-2.707378-1.948971$

C $\quad 0.731630 \quad 0.403118-2.197687$

H $-0.050322-0.398300-4.022791$

H $-1.1387190 .659336-3.130062$

H $-3.281690-4.952236-1.423444$

H $-1.026716-5.845733-2.512988$

H $1.498583-2.069494-2.363477$

O $1.5354111 .143635-3.016987$

$\begin{array}{lllll}\text { O } & 0.878889 & 0.246043 & -0.994093\end{array}$

C $2.6494381 .787295-2.392392$

H $2.2967022 .636789-1.802721$

H $3.2097341 .085956-1.768820$

H $3.3126152 .157523-3.178657$

SCF Energy (B3LYP/6-31G**//MMFF) = -3245.89684328

0113

MMFF Geometry

C $-3.380800-0.138281-3.304275$

C $-2.492303-0.373436-2.326674$

C $-1.247045-1.202233-2.503778$

O $-0.206858-0.666211-1.653283$

C $-1.526090-2.647354-2.049933$

C $-0.355766-3.592541-2.243172$

C $0.246719-4.271291-1.247010$

C $0.112141-3.780646-3.663288$

C $-0.095710-4.254523 \quad 0.216058$

C $0.624118-3.166787 \quad 1.025698$

C $2.148390-3.3454151 .105941$

C $2.757374-2.3498032 .096054$

C $4.290756-2.4502062 .140229$

O $2.703823-3.121432-0.191947$

O $4.788282-1.2903902 .825558$

C $4.844103-2.3948430 .699319$

C $4.758738-3.6654662 .949639$

O $6.244901-2.656752 \quad 0.680232$

C $4.118425-3.343202-0.276935$

C $4.527539-3.127437-1.754851$

$\begin{array}{lllll}\text { O } & 4.445403 & -4.698493 & 0.054368\end{array}$

C $0.4390180 .453678-2.072946$

O $0.260816 \quad 1.061665-3.115771$

C $1.3919090 .836000-1.007839$

C $1.8995722 .077081-0.991162$

C 2.8213252 .6174040 .009990

C 3.4212781 .6530730 .998705

C $4.275069-1.720532-2.309450$

C $5.493183-0.811667-2.267280$

C $6.1736791 .542041-2.726668$

O $6.621766-1.206983-1.987978$

C $6.2783312 .269904-1.423756$

C $6.1957073 .604814-1.317237$

C $6.3497764 .353157-0.024081$

C $5.194127 \quad 5.2892830 .251236$

C $5.2853296 .600595-0.027942$

C 3.9695994 .7196250 .930396

C $3.0963523 .938291-0.012310$

N $5.1760680 .493377-2.621318$

H $-3.212274-0.553843-4.294136$

H $-2.6825580 .023994-1.333093$

H $-0.912440-1.182853-3.548007$

H $-2.380175-3.053703-2.608218$

H $-1.833027-2.625857-0.998113$

H $1.078330-4.926969-1.502975$

H $\quad 0.839637-4.594296-3.756150$ 
H $-0.734224-4.025138-4.313438$ H $\quad 0.590748-2.870169-4.036438$ H -1.177315 -4.145995 0.353574 H $\quad 0.145949-5.2394540 .635604$ H $0.389336-2.1876710 .592013$ H $\quad 0.210249-3.1813092 .041312$ H $2.357156-4.3743141 .421626$ H $2.335097-2.488753 \quad 3.099160$ H $2.485535-1.328173 \quad 1.797227$ H $4.387961-1.2711613 .711743$ H $4.716049-1.3640040 .351333$ H $4.385388-3.6063293 .979136$ H $5.851151-3.6904863 .029308$ H $4.420845-4.6156132 .530957$ H $6.666535-2.0230071 .285499$ H $5.566135-3.444152-1.908279$ H $3.922423-3.817670-2.360633$ H $4.283665-5.254248-0.726006$ H $1.6229880 .096832-0.249361$ H $1.5910812 .775683-1.767926$ H 3.8746840 .8051080 .475707 H 2.6563801 .2739221 .683617 H 4.2163612 .0889091 .607094 H $4.002045-1.811109$-3.368789 H $3.437934-1.237553-1.796836$ H $5.8673882 .200271-3.545856$ H $7.1423061 .098363-2.979089$ H $6.4602941 .674548-0.532074$ H $6.0433434 .199810-2.215174$ H $7.2856004 .924489-0.080620$ H 6.4738603 .6680690 .823790 H 4.4721657 .2840300 .193202 H $6.1737907 .019078-0.489174$ H 3.3538235 .5327531 .338519 H $4.2787484 .143146 \quad 1.806786$ H $2.6184674 .554679-0.775182$ H $4.2052590 .743650-2.784854$ C $-5.729402-0.187702-2.417288$ O $-5.361197-0.390227-1.038401$ C $-6.512246-0.176970-0.202749$ C $-7.708127-0.171878-1.144522$ C $-7.1034320 .479791-2.376571$ H $-6.4093120 .825757 \quad 0.228571$ C $-6.538669-1.2231110 .917100$ H -8.046401-1.187771 -1.378973 H $-8.5574210 .383919-0.736324$ C $-7.925690 \quad 0.277245-3.636891$ H $-6.9924131 .556331-2.188318$ C $-5.217628-1.2937961 .717661$ O $-6.759541-2.5104730 .327692$ H -7.388786 -1.0358811 .583029$ H -4.420094 -1.648221 1.056173 O $-5.363237-2.2992092 .728036$ C $-4.811310 \quad 0.0321932 .386031$ C -3.492919 -0.0397213 .178279$ H $-4.729656 \quad 0.8180291 .632781$ O H $-3.4040590 .877694 \quad 3.772775$ H $-3.550267-0.8488743 .916339$ H $-7.4415560 .747391-4.498452$ H $-8.057917-0.786104-3.863318$ H $-8.9194960 .722449-3.523100$ C $-4.6465400 .649663-3.099903$ H $-6.758554-3.162787 \quad 1.048970$ H $-6.041126-1.9889613 .352978$ H $-5.6271351 .296612 \quad 3.637174$ H -4.418778 $1.536713-2.498019$ H $-4.9916301 .002338-4.078214$ H -5.798403 -1.181265 -2.879776 C -1.8940622 .1061501 .692622$ $\begin{array}{llll}\text { O } & -2.178223 & 0.772806 & 1.250784\end{array}$ C -2.245037 -0.2129362 .287628$ C $-0.919033-0.2525323 .059481$ C $-0.517248 \quad 1.1245413 .576743$ C -0.5589872 .1502102 .446261$ H -2.6989012 .4591772 .348304$ C -1.8453043 .0040480 .444023$ H $-2.343943-1.1808831 .782267$
O $-0.989186-1.162187 \quad 4.151514$

H $-0.123204-0.6080162 .394035$

H - $1.145376 \quad 1.4398574 .417984$

$\begin{array}{lllll}\mathrm{O} & 0.821649 & 1.064983 & 4.075448\end{array}$

H $\quad 0.2673051 .9600531 .752731$

$\begin{array}{lllll} & 0.267305 & -0.367557 & 3.453905 & 2.991694\end{array}$

C $-3.2202603 .369571-0.076955$

H -1.3389203 .9485880 .671529$

H $-1.3110272 .490631-0.363002$

H $-1.236475-2.0314273 .792584$

H $0.8432040 .377344 \quad 4.762935$

H $0.4808143 .446873 \quad 3.467470$

O $-3.075022 \quad 4.107371-1.218433$

O $-4.282554 \quad 3.075893 \quad 0.451999$

C $-4.2883344 .595135-1.801293$

H $-5.0147993 .787907-1.931787$

H $-4.0496435 .011470-2.783635$

H $-4.7055415 .388042-1.173715$

SCF Energy (B3LYP/6-31G**//MMFF)= -3245.91495126

$01 \_130$

MM̄FF Geometry

C $2.6107230 .863366-3.106636$

C $2.0630320 .376193-1.983465$

C $0.7074020 .760248-1.461376$

O $-0.010443-0.487727-1.299272$

C $0.772051 \quad 1.455297-0.090463$

C $1.2691762 .883672-0.155282$

C $0.4906813 .939938-0.462326$

C $2.7213523 .087704 \quad 0.184105$

C $-0.9759013 .918025-0.791820$

C $-1.757723 \quad 4.8715350 .122064$

C $-3.2734644 .709733-0.054987$

C $-4.0262095 .811507 \quad 0.692365$

C -5.5470465 .6076090 .630280$

$\begin{array}{llll}\text { O } & -3.631123 & 3.429524 & 0.475558\end{array}$

O $-6.149642 \quad 6.480537 \quad 1.597808$

C $-5.8730464 .162642 \quad 1.060153$

C $-6.1232896 .006558-0.733237$

O $\begin{array}{llll}-7.259433 & 3.879183 & 0.882308\end{array}$

C $-5.016905 \quad 3.092501 \quad 0.342159$

C $-5.2353231 .716364 \quad 1.014743$

O $-5.4105553 .015780-1.028053$

C $-1.323026-0.531145-1.646000$

O $-2.0172440 .393130-2.035158$

C $-1.760377-1.939042-1.502809$

C $-2.975361-2.316714-1.928016$

C $-3.502313-3.684783-1.915488$

C $-2.626523-4.760729-1.325412$

C -4.3207530 .6030720 .486987$

C $-4.411804-0.6398451 .350544$

C -5.988151-2.453359 2.020114

O $-3.467730-1.0166262 .041342$

C $-5.702608-3.6605971 .185986$

C -6.656011-4.367020 0.561055

C $-6.396896-5.604869-0.247396$

C $-6.636648-5.392821-1.724721$

C $-7.878490-5.426752-2.237044$

C $-5.426678-5.235323-2.616288$

C $-4.725543-3.915703-2.438389$

N $-5.645175-1.2630901 .267343$

H $2.0780401 .612017-3.686697$

H $2.616461-0.361575-1.408910$

H $0.1888541 .394169-2.191512$

H 1.3965090 .8721880 .599682

H $-0.219001 \quad 1.4283140 .380974$

H $0.9483624 .927887-0.487301$

H 3.0119094 .1432330 .154129

H 2.9289402 .7192541 .193817

H $3.3620112 .552595-0.520559$

H $-1.1022744 .233001-1.834792$

H $-1.3901822 .908975-0.720705$

H -1.4914394 .6682791 .167819$

H $-1.4624295 .904713-0.099730$

H -3.509833 $4.740450-1.125164$

H -3.7555606 .8010170 .303458$

H -3.719870 5.8110541 .748015

H -5.8903317 .3924271 .381081$ 
H -5.6790654 .0919322 .139788$ H $-5.9122717 .061855-0.943837$ H -7.214759 $5.912094-0.743673$ H -5.717805 $5.418453-1.559178$ H -7.7584314 .5656401 .357415$ H -5.0557441 .8121032 .093923$ H -6.2799131 .4069300 .887182$ H -5.120356 2.164833-1.392162 H -1.047600 -2.640305 -1.083423 H -3.636640 -1.562874 -2.352700 H $-2.343676-4.505919-0.297654$ H -1.715145 -4.884279-1.920781 H $-3.110210-5.737791-1.270762$ H $-4.5789300 .328403-0.541188$ H -3.2738890 .9258670 .486578$ H -7.048212 -2.3775502 .283418$ H -5.406424 -2.492241 2.946640 H -4.665212 -3.9764431 .113987$ H -7.692468 -4.0474420 .645623$ H $-7.058847-6.3990250 .122389$ H $-5.379871-5.980295-0.081012$ H -8.056085 $-5.306268-3.300404$ H $-8.745608-5.573593-1.601275$ H $-5.724403-5.300410-3.671772$ H $-4.760156-6.089813-2.467252$ H -5.289901 -3.069639 -2.832748 H $-6.326292-0.9217360 .595627$ C $5.0978941 .253148-3.143650$ O $5.2890041 .007071-1.735201$ C $6.6793140 .739520-1.488220$ C $7.4187881 .243147-2.719983$ C $6.4273180 .901652-3.814938$ H $6.786357-0.350613-1.432344$

C $7.1168341 .369745-0.163785$ H $7.5825842 .326221-2.683408$ H $8.3898560 .756381-2.850905$ C $6.6754481 .646145-5.115523$ H $6.477268-0.178663-4.007622$ C 6.2399750 .9755841 .043369 O $7.0508132 .796087-0.295160$ H $8.168946 \quad 1.1273770 .027605$ H 5.2548411 .4395900 .935198 O 6.8258391 .5728512 .208258 C $6.084255-0.5403021 .275216$ C $5.383095-0.9012852 .599945$ H $5.541661-0.9892560 .437123$

O $7.389043-1.1262951 .296932$ H $5.475843-1.9833082 .749932$ H $5.942035-0.4579003 .432728$ H $5.9253851 .379135-5.866695$ H $6.6359682 .731002-4.970643$ H $7.6622121 .396556-5.519018$ C $3.9353910 .396981-3.644087$ H 7.3300993 .1783530 .554442 H 7.6873601 .1454422 .354031 H $7.276641-2.0899011 .363196$ H $4.096434-0.649554-3.355409$ H $3.884150 \quad 0.420242-4.738742$ H $4.8729752 .321520-3.255458$ C $2.891590-2.5234811 .845579$ O $3.115705-1.1210811 .648532$ C $3.901787-0.4782122 .658762$ C $3.248211-0.6637764 .035106$ C $2.999508-2.1342654 .347311$ C $2.230281-2.7917023 .204923$ H $3.844524-3.0618091 .780675$ C $1.978871-3.0125750 .708941$ H $3.860613 \quad 0.5946182 .441414$ O $4.051213-0.0956245 .064336$ H $2.284531-0.1380534 .053045$ H $3.934792-2.6682154 .552283$ O $2.209469-2.2364905 .534144$ H $1.197070-2.4207273 .200740$ O $2.176849-4.1996733 .429690$ C $2.692668-3.112391-0.621928$ H $1.591512-4.0126370 .933412$ H $1.140082-2.3177510 .586111$ H $4.186714 \quad 0.842146 \quad 4.844841$
H $2.690895-1.774616 \quad 6.241991$

H $1.770301-4.3385354 .302266$

O $1.789030-3.498579-1.572184$

O $3.880174-2.889665-0.810241$

C $2.309071-3.652609-2.895877$

H $2.661808-2.690474-3.276612$

H $3.115412-4.392054-2.905383$

H $1.499575-4.008124-3.539017$

SCF Energy (B3LYP/6-31G**//MMFF) $=-3245.92250943$

01131

MM̄FF Geometry

C $-0.8320284 .328457-0.894559$

C $0.2323054 .335549-0.077838$

C 0.9847943 .0814940 .280269

O $2.3648153 .292356-0.104157$

C 0.9445022 .7623511 .787320

C -0.4213922 .3655252 .308070$

C $-1.044178 \quad 1212317 \quad 1.992443$

C -1.0661673 .3506593 .244389$

C $-0.4956690 .120768 \quad 1.116846$

C $-0.803585-1.2758741 .670754$

C $-0.098438-2.3615570 .844845$

C $-0.642464-3.7485531 .184503$

C $0.119968-4.8556020 .442484$

O $1.298496-2.2959131 .155203$

O $-0.235197-6.1116921 .040017$

C $1.631954-4.6561980 .670299$

C $-0.288305-4.946101-1.032272$

O $2.391107-5.572010-0.116479$

C $2.118008-3.2085540 .414661$

C $3.576059-3.0618150 .915068$

O $2.072205-2.935043-0.986152$

C $3.0562782 .208428-0.551503$

O $2.6397851 .067120-0.670062$

C $4.4302232 .652555-0.876197$

C $5.3145681 .762655-1.351208$

C $6.7124122 .027527-1.698954$

C $7.2128563 .439220-1.536100$

C $4.111240-1.6227360 .924373$

C $5.498822-1.5468361 .541688$

C 7.1340480 .0921012 .464445

O $6.200665-2.5374641 .730183$

C 8.0186770 .7540221 .457460

C 9.0396930 .1360040 .844485

C $9.9583930 .799424-0.142568$

C $9.8019800 .246712-1.542287$

C $10.472391-0.850940-1.931183$

C $8.9290761 .015342-2.510754$

C $7.4776500 .997250-2.118455$

N $5.867311-0.242243 \quad 1.840605$

H -1.171195 $3.380777-1.307874$

H $\quad 0.587890 \quad 5.274888 \quad 0.337297$

H $\quad 0.5791472 .244555-0.299937$

H 1.3274073 .6241402 .351877

H 1.6621731 .9632522 .016780

H -2.0387511 .0325652 .397143$

H -2.097062 3.0771673 .492347

H $-0.498414 \quad 3.405244 \quad 4.178992$

H -1.0864694 .3490912 .799141$

H $-0.945720 \quad 0.2178880 .122131$

H $\quad 0.5863540 .2219860 .995511$

H $-0.473904-1.3345552 .716131$

H $-1.887070-1.4342461 .655807$

H $-0.245733-2.146538-0.220230$

H $-1.713782-3.8107090 .961724$

H $-0.548898-3.9241462 .265342$

H -1.199362 -6.216754 0.965910

H $1.837063-4.9075641 .720635$

H - $1.361946-5.150150-1.120324$

H $0.215788-5.781092-1.531291$

H $-0.078157-4.032423-1.591983$

H $2.066889-6.466250 \quad 0.086419$

H $3.638913-3.4417071 .943656$

H $4.242071-3.6798210 .299436$

H $2.683501-2.208798-1.185617$

H $4.6771403 .694497-0.707542$

H $4.9904420 .732336-1.491711$ 
H $7.0929903 .773369-0.499503$ H $6.6579094 .120023-2.190989$ H $8.2704703 .561217-1.776812$ H $4.183219-1.232767-0.095332$ H $3.440817-0.973807 \quad 1.499007$ H $7.596739-0.8068822 .884887$ H 6.9272720 .7822823 .288486 H $7.8193901 .800570 \quad 1.242623$ H $9.238429-0.9091341 .071898$ H 10.9902110 .6498800 .201805 H $9.8136701 .886883-0.153393$ H $10.387191-1.239004-2.940608$ H $11.119503-1.387509-1.245048$ H $8.9982880 .578947-3.515927$ H $9.3292732 .027390-2.615191$ H $7.0208620 .009297-2.194558$ H 5.2320850 .5183071 .616312 C $-3.0494935 .572987-0.851389$ O $-3.7153004 .341111-1.199448$ C $-4.4190383 .862286-0.037453$ C $-4.572561 \quad 5.073106 \quad 0.872517$ C $-3.2399695 .762693 \quad 0.653811$ H $-3.763123 \quad 3.1364360 .458417$ C $-5.7311083 .187128-0.442604$ H -5.3948595 .7219920 .549364$ H -4.7456884 .7923041 .915596$ C -3.220776 7.2137551 .100904 H -2.4715815 .2141561 .207937$ C $-5.5605852 .005336-1.420864$ O $-6.5835004 .154533-1.067939$ H -6.2639082 .8542900 .455605$ H -5.239226 $2.376400-2.401014$ O $-6.856164 \quad 1.426357-1.627295$ C $-4.5883380 .908593-0.942284$ C $-4.567305-0.305289-1.893461$ H $-3.5770601 .325948-0.878037$ $\begin{array}{lllll}\text { O } & -4.975601 & 0.505982 & 0.367892\end{array}$ H -5.559626 $-0.766908-1.912321$ H $-4.359747 \quad 0.059921-2.905981$ H -2.244356 7.6681060 .905151 H -3.9795957 .8054460 .578086$ H -3.4188157 .2873732 .175226$ C -1.594294 5.548216-1.330275 H -6.110157 $4.506292-1.841475$ H $-7.1437901 .042400-0.780901$ H $-4.276918-0.0750230 .713504$ H -1.082994 6.465661-1.016393 H -1.589727 $5.544679-2.427759$ H $-3.5766186 .367228-1.395692$ C -4.895642 -2.812994-0.145660 O $-3.755854-1.948055-0.219098$ C -3.511596-1.366577-1.503949 C $-3.314502-2.477183-2.544922$ C $-4.479364-3.462165-2.552483$ C $-4.758483-3.971523-1.139502$ H -5.806903 -2.241312 -0.358022 C $-4.992925-3.3359251 .292638$ H -2.555874 -0.834340 -1.421916 O $-3.150147-1.935383-3.850764$ H -2.396546 -3.030852 -2.311608 H $-5.378870-3.017885-2.994747$ O $-4.139847-4.584040-3.370770$ H -3.948943 -4.643457 -0.826651 O $-5.965662-4.730577-1.145064$ C $-5.398254-2.2321332 .240454$ H $-5.742912-4.1290581 .388752$ H $-4.038030-3.7593901 .624735$ H -2.390737 -1.328577 -3.821804 H -3.926104 -4.241634 -4.255656 H $-5.859721-5.439484-1.802615$ O $-4.309623-1.7801732 .925039$ O $-6.541846-1.8063962 .339730$ C $-4.555745-0.7088553 .841104$ H $-5.254141-1.0297994 .619753$ H -3.605937 $-0.438704 \quad 4.310346$ H -4.944109 0.1647653 .309111

SCF Energy (B3LYP/6-31G**//MMFF)= -3245.92096574
01132

MMFF Geometry

C $4.2760650 .594743-2.207851$

C $3.646223-0.207632-3.079739$

C $3.114444-1.563337-2.700267$

O $1.792186-1.735078-3.268764$

C $3.967944-2.720445-3.241713$

C $5.256021-2.946799-2.483319$

C $5.341717-3.662172-1.344256$

C $6.496471-2.375979-3.118049$

C $4.216564-4.320511-0.587968$

C $4.344424-4.1314290 .932191$

C $4.027414-2.7047121 .411818$

C $4.513479-2.5186162 .853123$

C $4.133117-1.1444293 .418889$

O $2.606530-2.517701 \quad 1.340727$

O $4.376522-1.1904324 .832956$

C $2.621091-0.9332863 .202904$

C $5.034549-0.0359572 .864295$

O $2.228778 \quad 0.376313 \quad 3.608161$

C $2.157572-1.2200901 .757703$

C $0.619047-1.1704411 .618582$

O $2.684219-0.1973700 .903536$

C $0.757123-1.155052-2.608379$

O $0.820196-0.361782-1.683771$

C $-0.491635-1.713486-3.159283$

C -1.445114 -2.077032 -2.289538

C $-2.730985-2.691459-2.616264$

C $-3.153170-2.687700-4.061560$

C $-0.166254-2.1932592 .443317$

C $-1.665351-2.0061342 .271238$

C -3.824416 -2.638141 3.329163

O $-2.156319-1.2324421 .454020$

C $-4.576609-3.3784972 .268789$

C $-5.398092-2.7776031 .393500$

C $-6.243769-3.5114070 .393819$

C $-5.908812-3.160535-1.037199$

C $-6.641028-2.268616-1.725190$

C $-4.788163-3.923772-1.703859$

C $-3.468680-3.209169-1.611405$

N $-2.394772-2.7988773 .145528$

H $4.4556800 .227843-1.201719$

H $3.4855930 .125628-4.101741$

H $3.029063-1.659141-1.610497$

H $4.178576-2.569001-4.309346$

H $3.374389-3.645081-3.223740$

H $6.329607-3.808174-0.908885$

H $7.399013-2.594666-2.537294$

H $6.638104-2.798514-4.118154$

H $\quad 6.421713-1.288930-3.208940$

H $3.233040-3.975705-0.920902$

H $4.265707-5.395642-0.800303$

H $3.650338-4.8317781 .415037$

H $5.357356-4.4169221 .243238$

H $4.519288-1.981670 \quad 0.751977$

H $5.598280-2.6707492 .920719$

H $4.063622-3.2935603 .490296$

H $4.068124-0.3493565 .212192$

H $2.117128-1.6244643 .888111$

H $6.079528-0.2225453 .139892$

H 4.7814410 .9379373 .297662

H 4.9904980 .0479101 .777014

H 2.4971020 .9957742 .908142

H $\quad 0.263337-0.154717 \quad 1.837759$

H $\quad 0.363565-1.3440800 .567118$

H $2.125557-0.1363560 .110957$

H -0.560049 -1.864498 -4.229417

H -1.254162 -1.937402 -1.226234

H $-2.525519-3.368431-4.646660$

H -3.068163 -1.680409-4.485579

H $-4.192274-2.988436-4.211573$

H $0.092006-3.2122682 .135739$

H $\quad 0.057097-2.0813663 .508619$

H $-4.059804-1.5686383 .329697$

H $-4.078153-3.0438874 .313610$

H -4.477125 -4.460938 2.254310

H -5.508219 -1.695752 1.425068

H $-7.292820-3.2634420 .603174$ 
H $-6.172807-4.5975750 .531845$

H -6.422563 -2.041833 -2.763491 H -7.468879 -1.740899-1.263470 H -5.063572 -4.152169-2.738331 H -4.678771 -4.907736 -1.229967 H -3.069245 -3.156579 -0.598378 H -1.900972 -3.4098003 .789407$ C $4.3096013 .063645-1.576377$ O $2.8708503 .140076-1.551196$ C $2.4560463 .472736-0.209733$ C 3.7270363 .8164260 .563102 C $4.7476392 .927888-0.119254$ H 2.0216362 .5635550 .220913

C $1.4138494 .596069-0.232172$ H 4.0016894 .8713120 .447188 H 3.6267163 .6122601 .633804 C 6.1861523 .3374360 .140120 H $4.598978 \quad 1.9051550 .242000$ C $0.1465924 .282615-1.052697$ O $2.0085955 .774931-0.791820$ H 1.1349934 .8600180 .794583 H $\quad 0.3900804 .227433-2.120703$ O $-0.7518005 .390130-0.902174$ C $-0.5895012 .998319-0.627296$ C -1.921353 2.817147-1.384035 H $0.0545052 .135443-0.826061$ O $\quad-0.8213193 .049278 \quad 0.777291$ H -2.602432 $3.631017-1.115492$ H -1.717093 $2.902326-2.457867$ H $6.8809442 .669123-0.378100$ H $6.3811944 .359286-0.201970$ H 6.4110533 .2927991 .210948 C $4.7806541 .969669-2.540978$ H $2.3198185 .549880-1.685485$ H $-0.2721626 .191298-1.174256$ H -1.1633182 .1807301 .048296$ H $5.8762481 .943583-2.555510$ H $4.4525952 .239990-3.552813$ H $4.6562914 .027874-1.972597$ C -4.0683922 .1168440 .710731$ O -2.9770601 .3006910 .271156$ C -2.602076 $1.458795-1.099612$ C $-3.8025771 .147776-2.002847$ C $-5.0186111 .991600-1.630268$ C $-5.3197721 .859543-0.137356$ H $-3.783376 \quad 3.1741430 .652292$ C $-4.332034 \quad 1.7772872 .182429$ H $-1.8448190 .692123-1.296832$ O $\quad-3.4602391 .349637-3.368911$ H $-4.0628710 .087726-1.891539$ H -4.870696 3.040854 -1.909880 O $\quad-6.151447 \quad 1.545774-2.379529$ H -5.7137830 .8559990 .069041$ $\begin{array}{lllll}\text { O } & -6.331407 & 2.797528 & 0.222367\end{array}$ C $-3.1908712 .241916 \quad 3.056201$ H -5.2410702 .2608982 .557334$ H -4.4672810 .6973942 .315212$ H $-4.2536581 .160655-3.898743$ H $-6.3112410 .613166-2.156186$ H $-7.0988912 .631451-0.351513$ O $-2.411379 \quad 1.181494 \quad 3.411079$ O -3.0013923 .4118933 .363060$ C -1.2707331 .4918474 .217001$ H -0.7675730 .5543834 .468153$ H -0.5753532 .1245333 .657546$ $\mathrm{H}-1.5805161 .9826265 .144367$

SCF Energy $\left(B 3 L Y P / 6-31 G^{* *} / / M M F F\right)=-3245.93079974$

01

MM̄FF Geometry

C $-2.814537-3.562888-2.444850$

C -1.672937 -3.176300 -1.857903

C $-0.538774-2.530175-2.611461$

O $-0.332159-1.210937-2.048602$

C $0.752997-3.349500-2.431024$

C $1.600404-3.399521-3.688158$

C $2.776539-2.763447-3.845983$

C $1.081467-4.301284-4.780587$
C $3.453667-1.846373-2.870601$

C $4.453301-2.608558-1.992815$

C $5.319246-1.664309-1.148653$

C $6.218700-2.453711-0.195381$

C $7.018690-1.5316640 .738707$

O $4.452715-0.808964-0.401770$

O $7.549632-2.3349741 .804019$

C $6.058324-0.5072091 .382599$

C $8.224503-0.9088910 .024711$

O $\quad 6.780643 \quad 0.463760 \quad 2.136509$

C 5.1333050 .1952640 .361372

C 4.0604201 .0323181 .099736

O $5.9210331 .050451-0.465091$

C -1.139671 -0.212795 -2.505019

O $-1.927014-0.276055-3.436504$

C $-0.9440210 .971398-1.636372$

C $-1.6019852 .105693-1.920776$

C $-1.5776543 .351035-1.149192$

C -0.9022613 .3272670 .195204$

C 2.9631381 .6169650 .196287

C $3.3358452 .923058-0.484061$

C $2.5593764 .457243-2.292623$

O $4.2754613 .624407-0.119074$

C $1.6524685 .476573-1.676835$

C $0.6825386 .114697-2.349487$

C $-0.2035257 .160733-1.735479$

C $-1.6763206 .881117-1.936337$

C $-2.4075537 .610597-2.796119$

C -2.321155 $5.816007-1.075865$

C $-2.1835934 .439064-1.669500$

N $2.4733073 .226785-1.528778$

H -2.935991 -3.421853 -3.516101

H -1.550006 -3.309732 -0.785846

H $-0.785543-2.432012-3.675416$

H $\quad 0.519511-4.390700-2.168404$

H $1.329825-2.957216-1.585814$

H $3.321316-2.908276-4.777970$

H $1.764657-4.354631-5.635039$

H $0.951541-5.320199-4.401169$

H $\quad 0.117187-3.945139-5.154967$

H $3.975074-1.068149-3.441679$

H $2.716612-1.321749-2.252654$

H $3.890013-3.280044-1.332104$

H $5.104549-3.237158-2.613142$

H $5.923740-1.057029-1.832839$

H $6.892116-3.115651-0.753929$

H $5.598355-3.1146010 .425890$

H $8.098739-3.0331951 .407889$

H $5.429338-1.0525872 .100359$

H $8.904652-1.691922-0.331368$

H $8.814237-0.290350 \quad 0.710087$

H $7.944974-0.300173-0.837721$

H $7.338638-0.0184292 .770457$

H $3.554637 \quad 0.3719831 .815730$

H 4.5360401 .8360081 .673777

H $5.3301751 .641431-0.955789$

H $-0.2888540 .871857-0.779283$

H -2.229831 2.129588 -2.811105

H 0.1559653 .0760940 .094267

H -1.3745802 .5838280 .844425$

H $-0.945158 \quad 4.2793280 .726877$

H $2.6401680 .886707-0.553152$

H $2.092397 \quad 1.8431840 .823666$

H $2.2787054 .221540-3.323825$

H $3.5906034 .824179-2.283791$

H $1.8160545 .714796-0.628226$

H $0.5342835 .896638-3.404639$

H $0.0616668 .125053-2.188409$

H $-0.0043167 .272372-0.662204$

H $-3.4706317 .434402-2.923640$

H $-1.9592738 .396046-3.395569$

H -3.395760 $6.020610-0.976641$

H -1.927579 $5.891256-0.059073$

H $-2.6616314 .343164-2.645829$

H $1.6824322 .616101-1.711067$

C $-5.116537-3.221240-1.421195$

O $-4.730391-2.282951-0.399099$

C $-5.432327-1.043974-0.628750$ 
C $-6.357309-1.278472-1.824326$

C $-5.627178-2.370598-2.581313$

H $-4.674728-0.302938-0.908653$

C $-6.161821-0.6000800 .645474$

H -7.348703 -1.627869-1.513687

H $-6.500588-0.368907-2.416013$

C $-6.501514-3.126684-3.564974$

H $-4.796403-1.902137-3.123112$

C $-5.254010-0.4621971 .884775$

O $-7.165560-1.5786470 .949003$

H $-6.693257 \quad 0.3398660 .452994$

H $-4.892413-1.4504572 .191767$

O -6.0624130 .0278622 .962919$

C -4.0607300 .4964661 .706654$

C -3.3206430 .8258073 .018975$

H -3.3560350 .0765400 .980728$

O $-4.542814 \quad 1.7271931 .158846$

H -2.5674661 .5910522 .798116$

H $-4.013377 \quad 1.3184403 .712562$

H -5.927972 -3.906829-4.075437

H -7.349821 $-3.604896-3.063606$

H -6.900404 -2.447780 -4.325895

C $-3.966669-4.187960-1.713702$

H -7.767604 -1.186676 1.603744

H $-6.671250-0.685028 \quad 3.219607$

H $-3.772713 \quad 2.2949920 .986449$

H -4.335201 -5.025271 -2.318373

H -3.611325 -4.615199-0.767401

H -5.943524 -3.805808 -0.995149

C $-0.574205-0.3952672 .497538$

O $-1.781752-1.0936652 .816202$

C -2.662852 -0.3914973 .699760$

C $-1.939935-0.0603255 .014269$

C $-0.635060 \quad 0.6939194 .773419$

C $0.229971-0.0486723 .756038$

H $-0.836110 \quad 0.532172 \quad 1.975544$

C $0.213665-1.2227551 .473376$

H -3.467103 -1.092439 3.952215

O -2.7725850 .7109515 .873940$

H -1.706573 -0.9930185 .544373$

H $-0.818777 \quad 1.726373 \quad 4.453794$

O $\quad 0.093345 \quad 0.7785596 .000735$

H $0.633361-0.9534804 .225116$

$\begin{array}{lllll}\text { O } & 1.336475 & 0.772321 & 3.393955\end{array}$

C $0.751611-2.5352801 .999456$

H $-0.436028-1.4724770 .628835$

H $1.052074-0.6249841 .099398$

H -3.5892780 .2040146 .021529$

H -0.4848541 .2147636 .650010$

H $1.8119620 .997244 \quad 4.212047$

O $1.884577-2.8623521 .309788$

O $\quad 0.226753-3.2083132 .875819$

C $2.494146-4.1020861 .681021$

H $3.374341-4.2512401 .050766$

H $2.812978-4.0680042 .727080$

H $1.799765-4.9310981 .514142$

SCF Energy (B3LYP/6-31G**//MMFF) $=-3245.90739106$

01134

MM̄FF Geometry

C $3.9016980 .855221-2.406038$

C $3.281433-0.152770-3.037371$

C $3.079930-1.518396-2.437547$

O $1.693918-1.859176-2.695418$

C $4.001032-2.578532-3.064790$

C $5.455964-2.441179-2.671105$

C $5.974790-2.908195-1.517927$

C $6.349757-1.795220-3.697317$

C $5.246224-3.605529-0.399332$

C $5.704174-3.1364350 .989854$

C $5.226696-1.7230501 .367850$

C $6.021406-1.2225172 .578698$

C 5.5301380 .1447213 .069552

O $3.828777-1.7886161 .688410$

O $6.1139390 .385736 \quad 4.358974$

C 4.0048470 .0685173 .269295

C $6.005171 \quad 1.2828282 .158474$

O $3.475478 \quad 1.348745 \quad 3.612765$
C $3.245498-0.5310012 .061036$

C $1.766492-0.7596502 .455933$

O 3.2962860 .4089340 .989326

C $1.125717-2.827047-1.929802$

O $1.677183-3.520433-1.090470$

C $-0.314610-2.878050-2.261022$

C $-1.135757-3.573227-1.460400$

C $-2.581251-3.729267-1.618843$

C $-3.240815-3.039647-2.783287$

C $0.939266-1.5265901 .419635$

C $-0.543359-1.3843491 .701132$

C $-2.294732-2.0047503 .359561$

O $-1.266518-0.6816630 .998309$

C $-3.137545-3.1145142 .815680$

C $-4.306199-2.9109732 .187945$

C $-5.215945-4.0121521 .720210$

C $-5.512912-3.9402100 .239406$

C $-6.519079-3.181294-0.225493$

C $-4.709833-4.817332-0.691899$

C $-3.248106-4.474056-0.712937$

N $-0.952207-2.0902062 .819814$

H $4.3364070 .676541-1.427786$

H $2.8686460 .015602-4.029624$

H $3.222807-1.469584-1.350886$

H $3.902456-2.558109-4.159069$

H $3.655097-3.584964-2.797000$

H $7.047551-2.799193-1.362706$

H $7.392734-1.743338-3.366880$

H $6.328102-2.367484-4.630629$

H $6.023374-0.773531-3.910314$

H $4.163356-3.498431-0.480835$

H $5.467477-4.676646-0.483977$

H $5.316130-3.8511521 .727408$

H $6.799389-3.1885731 .037710$

H $5.369808-1.0491200 .516112$

H $7.093711-1.1809272 .349093$

H $5.920828-1.9432853 .402749$

H 7.0812600 .3594294 .260269

H $3.828478-0.5736954 .143136$

H 7.1007461 .3049932 .114034

H 5.7038352 .2590522 .553827

H 5.6373091 .1947951 .134395

H $3.432417 \quad 1.8789892 .798675$

H $1.726919-1.3251383 .395934$

H 1.2867080 .2094752 .643308

H 2.5287540 .2825090 .411006

H -0.657656 -2.335175 -3.133830

H $-0.709252-4.080879-0.595503$

H -2.895107 -3.469659 -3.729468

H -3.009040 -1.968950 -2.780192

H $-4.330925-3.115030-2.769554$

H $1.112560-1.1487970 .408317$

H $1.204898-2.5892921 .421743$

H $-2.712170-1.0194063 .131270$

H -2.214383 -2.101541 4.446807

H $-2.793238-4.1313082 .987694$

H -4.656913 -1.891634 2.042896

H -6.154607 -3.933192 2.284765

H $-4.809932-5.0001951 .970317$

H $-6.760772-3.145934-1.282434$

H $-7.120840-2.5737860 .442534$

H -5.126775 -4.804322 -1.705303

H -4.812232 $-5.861221-0.366885$

H -2.685919 -4.9242020 .106040$

H $-0.287885-2.6919793 .297507$

C $3.2861253 .309276-2.184081$

O $1.8880772 .967199-2.100739$

C $1.4255413 .235075-0.764153$

C $2.4984324 .105066-0.118133$

C $3.7591843 .550280-0.750880$

H $1.3823622 .269814-0.244293$

C $0.0264973 .865143-0.772679$

H $2.3732645 .161857-0.381984$

H 2.4947814 .0257040 .973380

C $4.9576414 .476320-0.642768$

H $4.0037122 .606135-0.255267$

C -1.060261 $2.926248-1.348500$

O $0.0868445 .085220-1.509536$ 
H -0.2368604 .1190040 .260902$

H $-0.9901431 .961658-0.832453$

O $-0.8346512 .685246-2.736173$

C $-2.4918603 .472047-1.172409$

C $-3.5999602 .572747-1.750379$

H $-2.6773173 .641929-0.106683$

O $-2.6169084 .746750-1.808778$

H $-4.5507633 .104657-1.620412$

H $-3.4765602 .491152-2.838064$

H $5.8346964 .035101-1.126762$

H $4.7619865 .444562-1.115495$

H 5.2078554 .6595530 .407396

C $4.0431052 .238546-2.977551$

H $-0.7690825 .534011-1.406743$

H $0.0469162 .285931-2.827627$

H $-2.4060644 .625236-2.750752$

H $5.1077722 .493262-3.028536$

H $3.6661132 .244301-4.008255$

H $3.3345824 .248822-2.750411$

C $-4.821161 \quad 1.7616610 .913464$

O -3.6629621 .1896150 .296823$

C $-3.6909821 .163831-1.131269$

C $-4.9003630 .347067-1.608820$

C $-6.2056630 .864914-1.004709$

C -6.0801380 .9838220 .514785$

H -4.9177552 .8127770 .615113$

C -4.6034661 .7202862 .429353$

H $-2.7923650 .620241-1.447793$

O

H -4.761844 -0.698275 -1.305274

H $-6.5034731 .822382-1.447426$

O $-7.262558-0.052929-1.290578$

H $-6.060214-0.0194790 .958452$

O

C -3.489252 2.6548082 .837401

H -5.5023572 .0290332 .974943$

H -4.3565080 .7092152 .772172$

H -5.649004 -0.268156 -3.302649

H -7.402772 $-0.048888-2.252403$

H $-8.010316 \quad 1.1518750 .743382$

O -2.2907152 .0069892 .778081$

O $-3.664127 \quad 3.826248 \quad 3.146938$

C -1.140505 2.7940043 .101830

H -0.2508302 .2361432 .798651$

H -1.152738 3.7464562 .563555

$\mathrm{H}-1.1023192 .9629024 .181634$

SCF Energy (B3LYP/6-31G**//MMFF) $=-3245.91306112$

01135

MM̄FF Geometry

C $-3.197296-3.481503-2.319420$

C $-2.062940-3.003563-1.785847$

C $-1.099690-2.135899-2.547109$

O $-1.102171-0.833021-1.912536$

C $0.361066-2.620431-2.498048$

C $0.581348-4.106710-2.635623$

C $0.956897-4.903752-1.615432$

C $0.392438-4.663741-4.020162$

C $1.162477-4.511392-0.175629$

C $2.622218-4.6283210 .278450$

C $3.588537-3.644646-0.405470$

C $5.020469-3.9304230 .057138$

C $6.026907-2.933407-0.530205$

O $3.217791-2.304550-0.054691$

O $7.260035-3.0770670 .191497$

C $5.511530-1.504635-0.268876$

C $6.345130-3.242958-1.997725$

O $6.352483-0.543113-0.904958$

C $4.030031-1.287356-0.666953$

C $3.5650740 .096902-0.141559$

O $3.927646-1.318226-2.089740$

C $-1.9431780 .119798-2.388522$

O $-2.7457430 .014117-3.300608$

C $-1.6822451 .346726-1.604184$

C $-2.0402232 .538953-2.101565$

C $-1.7817503 .840853-1.482215$

C $-1.1668603 .846863-0.108351$

C $2.1132200 .464895-0.464096$
C $1.9901231 .242309-1.761042$

C $2.3692883 .507134-2.729474$

O $1.6783780 .694361-2.815452$

C $1.8737654 .851768-2.300364$

C $1.0312855 .600088-3.029203$

C $0.5353026 .951669-2.597649$

C $-0.9611587 .115578-2.762416$

C $-1.466197 \quad 7.932278-3.702631$

C -1.871502 $6.394299-1.790282$

C $-2.0878814 .955743-2.177856$

N $2.2830942 .588340-1.608193$

H -3.444297 -3.244983 -3.351434

H -1.828338 -3.220494 -0.746557

H -1.397495 -2.041350 -3.599401

H $0.832351-2.246185-1.585525$

H $0.921439-2.106373-3.291614$

H $1.112454-5.962139-1.819775$

H $0.588783-5.740171-4.064790$

H $-0.634349-4.500838-4.362683$

H $1.074784-4.173705-4.722354$

H $\quad 0.780633-3.5117760 .049059$

H $0.557594-5.1937090 .435523$

H $2.643128-4.4525061 .361879$

H $2.967490-5.6571110 .113818$

H $3.512920-3.766264-1.491468$

H $5.312133-4.958658-0.191761$

H $5.068396-3.8640741 .153487$

H $7.559294-3.9973370 .094039$

H $5.608921-1.3213130 .810137$

H $6.746262-4.259028-2.096030$

H $7.125695-2.577166-2.381861$

H $5.473286-3.168816-2.650900$

H $6.135316-0.541682-1.852796$

H $3.6629120 .093658 \quad 0.951567$

H $4.2287370 .880267-0.526813$

H $3.053307-0.983901-2.344017$

H -1.156266 $1.236733-0.662517$

H -2.536964 2.569410 -3.070531

H $-0.1822833 .369065-0.124037$

H -1.807119 3.3067560 .597520

H -1.0240214 .8464340 .305644$

H $1.461970-0.410663-0.503016$

H 1.7164641 .0987450 .337176

H $1.8099403 .108965-3.582221$

H $3.4260883 .571695-3.007635$

H $2.2342605 .236489-1.349556$

H $0.6967675 .232713-3.996790$

H $1.0636077 .704164-3.197491$

H $0.8016517 .157185-1.553193$

H -2.536308 $8.074379-3.811700$

H $-0.8220278 .473075-4.388057$

H -2.857033 $6.878381-1.769367$

H -1.474176 $6.515923-0.779564$

H $-2.5422834 .838903-3.163039$

H $2.6598872 .899901-0.716998$

C $-5.213234-3.520937-0.766367$

O $-4.552230-2.851750 \quad 0.324643$

C $-5.073652-1.5137980 .431008$

C $-6.360866-1.504985-0.385382$

C $-5.992669-2.443390-1.521554$

H $-4.346443-0.853179-0.056784$

C $-5.216328-1.1197311 .904360$

H -7.206721 -1.9111760 .180969$

H $-6.630201-0.500870-0.726359$

C $-7.188027-2.976734-2.290706$

H $-5.340646-1.897557-2.214462$

C -3.940326-1.364841 2.737135

O $-6.266084-1.9030132 .485958$

H $-5.534410-0.0732581 .977660$

H $-3.766593-2.4438112 .826318$

O $-4.190422-0.8988204 .069716$

C $-2.672463-0.6793802 .194674$

C -1.440506 -0.997537 3.065602

H -2.473899 -1.024609 1.174345

O -2.9086250 .7245002 .141423$

H -1.606968 -0.6198694 .078979$

H -1.350425 -2.087822 3.138104

H $-6.865855-3.661116-3.082031$ 
H -7.878412 -3.518544-1.635685 H -7.742494 -2.156032 -2.757419 C $-4.186721-4.325671-1.567940$ H -6.337416 -1.642995 3.420542 H -4.2907070 .0677554 .027040$ H -2.1328541 .1386411 .727826$ H -4.702924 -4.972381 -2.287089 H $-3.639828-4.989725-0.885954$ H -5.911139-4.229854 -0.301643 C $-0.030034 \quad 1.642436 \quad 3.758757$ O $-0.130506 \quad 1.0199932 .473810$ C $-0.128445-0.4122612 .494152$ C $1.145135-0.9252273 .182296$ C $1.319669-0.3262574 .575046$ C 1.2297831 .1982504 .510917 H $-0.9101611 .366814 \quad 4.352558$ C -0.1338893 .1644213 .579669$ H $-0.078922-0.7343141 .446450$ O $1.140596-2.3452813 .278260$ H $2.018879-0.6500962 .579282$ H $0.589917-0.7348425 .283844$ O $2.610695-0.6758825 .079227$ H 2.1338321 .5877234 .029478 O 1.1952531 .7258105 .835075 C 0.9644113 .7909822 .744993 H -1.079124 3.3971653 .074800 H -0.1362233 .6475994 .563412$ H $1.050389-2.6978332 .376630$ H $2.669756-1.6468685 .072977$ H $1.985706 \quad 1.3989836 .298231$ O $0.869625 \quad 5.1507822 .849508$

O 1.7779783 .1834152 .064201 C 1.8186515 .8957302 .080544 H 2.8398485 .6111532 .351553 H 1.6417835 .7360241 .013428 H 1.6794616 .9567452 .304060 SCF Energy (B3LYP/6-31G**//MMFF) $=-3245.93248900$

01136

MM̄̄FF Geometry

C $0.0592013 .861428-1.188426$

C $0.259074 \quad 3.386847-2.426395$

C $1.1418892 .202032-2.722366$

O $\quad 0.295636 \quad 1.118807-3.186998$

C $2.1565562 .514412-3.841785$

C $3.5709792 .641737-3.314416$

C $4.5327441 .716157-3.498254$

C $3.8675003 .906149-2.551740$

C $4.4175520 .419506-4.250744$

C $3.617129-0.673476-3.537316$

C $4.068620-1.009881-2.108984$

C $5.479607-1.601830-2.070915$

C $5.870720-2.070190-0.660991$

O $3.123461-1.963135-1.602393$

O $7.040282-2.894722-0.777204$

C $4.744498-2.960928-0.097232$

C $6.258254-0.8919950 .240328$

O $4.996881-3.2993561 .264842$

C $3.337255-2.335826-0.235847$

C $2.259134-3.3712980 .157005$

O $3.242103-1.2143920 .643895$

C $-0.3841820 .412472-2.241924$

O $-0.3460540 .562063-1.030924$

C - $-1.234355-0.569205-2.943417$

C $-2.436035-0.861411-2.424899$

C $-3.420510-1.779270-2.999510$

C $-2.940313-2.727266-4.066351$

C $0.823520-2.953092-0.193855$

C $-0.195373-3.9103330 .400973$

C $-2.630759-4.0939870 .897689$

O $0.109172-5.006107 \quad 0.865455$

C -3.576242 -4.442715 -0.206068

C $-4.874894-4.106976-0.194303$

C $-5.846198-4.478159-1.277628$

C $-6.551311-3.273555-1.858391$

C $-7.770860-2.911396-1.425564$

C $-5.875864-2.539159-2.995358$

C $-4.688922-1.732744-2.540832$
N -1.484683 -3.4019430 .339161$

H $0.5722723 .398384-0.350336$

H $-0.2847713 .826682-3.258991$

H $1.6688031 .857644-1.823086$

H $1.9002183 .444337-4.366946$

H $2.1046901 .738693-4.614162$

H $5.5163501 .902487-3.069626$

H $4.9169443 .969358-2.244923$

H $3.6533014 .782275-3.172311$

H $3.2562543 .962840-1.646201$

H $3.9778330 .604287-5.238515$

H $5.4304780 .053238-4.458151$

H $2.557248-0.395060-3.509179$

H $3.644124-1.585619-4.148667$

H $4.008189-0.104706-1.494877$

H $6.220784-0.886698-2.446571$

H $5.528145-2.466520-2.747492$

H $7.738834-2.366445-1.200169$

H $4.760573-3.905123-0.659601$

H $7.115997-0.353439-0.180109$

H $6.577638-1.2379821 .229472$

H $5.449628-0.1705800 .370092$

H $5.883665-3.6967971 .304917$

H $2.458650-4.319574-0.359300$

H $2.321197-3.5690281 .234964$

H $2.308469-1.0271590 .826110$

H $-0.870449-0.981215-3.877115$

H $-2.744726-0.356246-1.510519$

H -2.037011 -3.253092 -3.735886

H $-2.711320-2.183493-4.989148$

H $-3.665128-3.504311-4.317092$

H $\quad 0.609318-1.9470890 .178267$

H $\quad 0.685772-2.948938-1.281157$

H $-3.083960-3.4228601 .634131$

H -2.310406 -5.004599 1.413102

H $-3.179906-5.015354-1.041067$

H $-5.272283-3.5546530 .654548$

H $-6.581846-5.168624-0.844658$

H $-5.355594-5.038118-2.083471$

H -8.295553 -2.064355 -1.854780

H $-8.269736-3.451855-0.627774$

H $-6.581903-1.838463-3.460738$

H -5.630465 -3.260439 -3.779057

H -4.930964 -1.000701-1.769510

H -1.635722 -2.478541 -0.056529

C $-2.3217234 .409218-0.670609$

O -2.3360093 .4205390 .373771$

C -3.6156343 .4631661 .034346$

C -4.4306324 .5532150 .339167$

C $-3.3473725 .449163-0.233410$

H $-3.421623 \quad 3.7751492 .066937$

C -4.2590792 .0692561 .022545$

H $-5.0558584 .148600-0.464808$

H -5.0933625 .0762021 .035889$

C $-3.8358646 .353280-1.350943$

H $-2.936142 \quad 6.063690 \quad 0.578557$

C -3.3419810 .9439891 .547337$

O $-4.6050601 .738650-0.329463$

H -5.2006272 .0942371 .583755$

H -2.5266840 .7864530 .832779$

O $-4.121135-0.258558 \quad 1.548595$

C -2.7826371 .1935862 .958982$

C $-1.9489950 .040798 \quad 3.546306$

H -2.165426 2.0970722 .952825

O

H -1.6473960 .3376054 .558843$

H -2.586214 -0.839713 3.697034

H -3.022054 6.978047 -1.732139

H $-4.2370865 .774115-2.189425$

H $-4.6288457 .016468-0.990155$

C $-0.9080984 .961256-0.857498$

H $-5.0344030 .866366-0.310806$

H -3.508354 -1.007324 1.638317

H -4.4498890 .6747203 .843316$

H -0.5621505 .4438250 .064802$

H $-0.8962165 .724731-1.643743$

H -2.653188 $3.908148-1.590620$

C $0.762471 \quad 1.4421253 .381780$ 
$\begin{array}{llll}\text { O } & 0.056421 & 0.799390 & 2.316953\end{array}$

C $-0.709956-0.3424202 .712442$

C $0.196859-1.386562 \quad 3.381984$

C $1.016518-0.7902054 .524555$

C 1.7389420 .4749004 .061044

H $\quad 0.0356681 .798167 \quad 4.122913$

C 1.4363162 .7043892 .827144

H $-1.083550-0.791727 \quad 1.785442$

O $-0.565313-2.4829093 .875122$

H $0.895757-1.7902522 .640209$

H $0.394967-0.5876815 .404381$

O $2.001797-1.7391204 .939936$

H $2.5477450 .191803 \quad 3.376773$

O $2.334274 \quad 1.1199925 .183696$

C $2.561022 \quad 2.409611 \quad 1.858371$

H 0.7087183 .3230712 .288899

H 1.8240743 .3038783 .659351

H $-1.047047-2.8653723 .122243$

H $1.531759-2.5538545 .188155$

H 2.9217070 .4735285 .611660

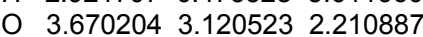

O $2.4674541 .649950 \quad 0.903720$

C 4.8282562 .9179781 .394836

H $5.285817 \quad 1.9572681 .644921$

H 4.5768102 .9596910 .331250

H 5.5422553 .7159101 .614783

SCF Energy (B3LYP/6-31G*//MMFF) $=-3245.90646687$

01_137

MM̄FF Geometry

C $-0.1121832 .951032-0.693218$

C $0.240754 \quad 1.795231-1.278599$

C $-0.6839540 .833725-1.989482$

O $-2.0156851 .401965-2.059747$

C $-0.2268480 .508251-3.424791$

C $0.974309-0.411205-3.517321$

C $0.907235-1.756217-3.451328$

C $2.291508 \quad 0.263599-3.792194$

C $-0.324988-2.594967-3.259408$

C $-0.137843-3.756821-2.274487$

C $0.125443-3.341446-0.817233$

C $0.310392-4.5957540 .045573$

C $0.469911-4.2677841 .535485$

O $-0.991594-2.577895-0.342890$

O $\quad 0.300462-5.499357 \quad 2.254840$

C $-0.665175-3.3112791 .956824$

C $1.885525-3.7806671 .856458$

O $-0.481681-2.8704193 .300387$

C $-0.845258-2.1071041 .005294$

C $-2.080168-1.2502001 .372092$

O $0.298100-1.2525961 .130800$

C $-3.0734540 .549376-2.022751$

O $-3.035909-0.668274-1.957034$

C $-4.3113401 .357138-2.073733$

C $-5.4954580 .732521-1.992894$

C $-6.8156801 .360101-2.041438$

C $-6.8800322 .859131-2.166329$

C -3.436767-1.949622 1.253334

C $-4.575767-1.0493331 .710442$

C -6.975697-1.179979 2.379616

O $-4.4193200 .131942 \quad 2.008492$

C -8.089078 -1.1137791 .383212$

C $-8.866830-0.030468 \quad 1.231422$

$\begin{array}{llll}\text { C }-10.042016 & 0.044340 & 0.297425\end{array}$

C $-9.953871 \quad 1.215664-0.655407$

C $-10.4624992 .414287-0.323146$

C $-9.3497650 .979934-2.020071$

C $-7.905690 \quad 0.569138-1.961894$

N -5.784557 -1.7310591 .758258$

H -1.147787 $3.279262-0.709735$

H $1.2800751 .486076-1.198249$

H $-0.716336-0.081145-1.389024$

H $-0.0275361 .440999-3.970335$

H - $-1.0598580 .059615-3.982342$

H $1.827550-2.323345-3.583714$

H $3.115386-0.452550-3.874191$

H $2.2428220 .817401-4.735570$

H $2.5443840 .965126-2.994462$
H $-1.181123-1.995027-2.949444$

H $-0.584739-3.022875-4.235989$

H $-1.055365-4.359059-2.310342$

H $0.679479-4.400187-2.624902$

H $1.028219-2.720934-0.782908$

H $1.162759-5.189016-0.309415$

H $-0.570102-5.244139-0.069438$

H $0.324988-5.2912793 .204809$

H $-1.584006-3.9091101 .957984$

H $2.622991-4.5452351 .584228$

H $2.014353-3.6150732 .931476$

H $2.149138-2.8626351 .330437$

H $0.212088-2.1890783 .295831$

H $-1.954205-0.8472312 .386109$

H -2.100376 -0.3665600 .720313$

H $0.051637-0.3561850 .849852$

H $-4.2160282 .431471-2.180254$

H $-5.504505-0.351862-1.885756$

H $-6.4349113 .185663-3.112682$

H $-6.341803 \quad 3.337912-1.340584$

H $-7.8992483 .252120-2.138908$

H $-3.626510-2.2477190 .216917$

H -3.459275 -2.846334 1.880496

H $-6.754823-0.2024092 .820001$

H $-7.252343-1.8639083 .188772$

H $-8.292128-2.0042140 .793381$

H $-8.6768080 .845556 \quad 1.848215$

H -10.949728 0.1361580 .908712

H $-10.164518-0.886572-0.270047$

H -10.428785 $3.253955-1.009316$

H -10.921170 2.5827290 .645714

H $-9.474973 \quad 1.855659-2.667437$

H $-9.9146350 .183375-2.522085$

H $-7.763643-0.507329-1.858604$

H $-5.801459-2.7153711 .507063$

C 0.9460013 .5030261 .507819

O $1.7862362 .339245 \quad 1.652740$

C 2.6982072 .5522272 .747661

C 2.1599153 .7574603 .505581

C $1.595603 \quad 4.5871192 .367017$

H 3.6645872 .8241012 .308124

C $2.833977 \quad 1.2644283 .567605$

H 1.3629413 .4759894 .203621

H $2.939764 \quad 4.275934 \quad 4.071139$

C 0.6343355 .6704402 .824327

H 2.4274975 .0521341 .821949

C 3.2701880 .0460512 .727292

$\begin{array}{lllll}\text { O } & 1.563460 & 0.953433 & 4.155091\end{array}$

H $3.526763 \quad 1.4180154 .402218$

H $2.510154-0.1629341 .970111$

O $3.315365-1.1000463 .585306$

C $4.649213 \quad 0.199568 \quad 2.062450$

C $5.038276-0.9877121 .161092$

H 4.6844841 .1149401 .468967

$\begin{array}{lllll}\text { O } & 5.645947 & 0.339844 & 3.077916\end{array}$

H $6.118711-0.9384860 .980673$

H $4.883952-1.9292611 .701648$

H $0.239463 \quad 6.2283261 .969477$

H $-0.214693 \quad 5.2486203 .372472$

H 1.1418776 .3801673 .485762

$\begin{array}{llll}\text { C } & 0.851382 & 3.857693 & 0.024091\end{array}$

H $0.9201450 .860393 \quad 3.431331$

H $2.442135-1.1813544 .005792$

H $5.614444-0.4599803 .630754$

H $1.8396503 .789323-0.446911$

H $0.5011864 .887748-0.107615$

H $-0.0404323 .237571 \quad 1.910694$

C $5.7116250 .490887-1.413903$

$\begin{array}{lllll}\text { O } & 4.402028 & 0.227507 & -0.894899\end{array}$

C $4.272803-1.007429-0.180153$

C $4.634374-2.179531-1.101014$

C $6.012225-2.000078-1.735265$

C $6.139280-0.619781-2.381326$

H $6.4307710 .562305-0.589390$

C $5.6618981 .850950-2.125762$

H $3.208095-1.1080330 .057328$

O $4.583062-3.405204-0.379889$

H $3.883003-2.245553-1.897978$ 
H $6.806775-2.159934-0.997611$

O $6.204667-3.004621-2.734358$

H $5.529123-0.582822-3.291993$

O $7.495118-0.408048-2.769073$

C $5.4874502 .995495-1.149704$

H $6.5842572 .045122-2.683253$

H $4.8195651 .862676-2.826871$

H $4.838909-4.115295-0.993134$

H $5.512167-2.895179-3.408318$

H $7.748173-1.142497-3.354306$

O $4.802202 \quad 4.008443-1.756451$

O $5.9325443 .014946-0.010094$

C $4.5921525 .176341-0.956309$

H $4.149076 \quad 4.9116440 .008292$

H $3.9001495 .833699-1.489318$

H $5.5403405 .702420-0.812861$

SCF Energy (B3LYP/6-31G**//MMFF) $=-3245.93164622$

01138

MM̄FF Geometry

C $2.888515-3.050454-1.599236$

C $2.095614-2.204839-0.924852$

C $0.954139-1.445101-1.544251$

O $-0.219403-1.645497-0.722137$

C $1.2698530 .060350-1.543376$

C $0.2047220 .877114-2.243796$

C $-0.8271701 .481469-1.622089$

C $0.3632940 .987267-3.737570$

C $-1.1604321 .453175-0.154621$

C -1.3183372 .8462630 .475008$

C $-2.4774173 .672711-0.103223$

C $-2.703093 \quad 4.9506760 .707382$

C -3.918789 5.7447290 .197699

O $-3.6577462 .867529-0.069798$

O -4.2584956 .7175321 .198018$

C -5.1272734 .7895940 .064624$

C $-3.5854096 .532874-1.074821$

O $-6.2371275 .449458-0.540350$

C $-4.8002903 .475262-0.681015$

C $-5.9823792 .483062-0.577126$

O -4.570698 3.771755 -2.061490

C -1.006666 -2.722434 -0.979991

O $-0.826550-3.583563-1.825077$

C $-2.139580-2.671408-0.027486$

C $-2.946364-3.7342800 .107493$

C $-4.074917-3.8466871 .035464$

C $-4.508694-2.6006541 .762430$

C $-5.6202021 .050347-0.999649$

C $-6.837803 \quad 0.147573-1.090695$

C -7.469993 -2.195061-1.663800

O $-7.9836620 .538988-0.886277$

C $-7.735609-2.911620-0.377950$

C $-7.697808-4.247182-0.255458$

C $-8.042327-4.9748091 .011494$

C $-6.983400-5.9687731 .428059$

C $-7.163925-7.2882951 .248223$

C $-5.760606-5.4406932 .142155$

C $-4.657778-5.0531891 .196015$

N $-6.488791-1.148881-1.447449$

H $2.715230-3.221415-2.658526$

H $2.282052-2.0336240 .132264$

H $\quad 0.755641-1.785394-2.568500$

H $2.2359540 .246071-2.031657$

H $1.3903510 .413960-0.514817$

H - $1.5415062 .019828-2.242702$

H - $0.4276221 .589308-4.197420$

H $1.3208761 .458139-3.982769$

H $0.334378-0.004130-4.200417$

H -2.089391 $0.883781-0.028945$

H -0.4050830 .9130420 .422828$

H $-1.491547 \quad 2.693621 \quad 1.548359$

H $-0.382125 \quad 3.4090410 .379607$

H -2.238962 $3.927511-1.141758$

H -1.8028195 .5778490 .704365$

H -2.8814914 .6878021 .759566$

H -3.480084 7.2820361 .344175

H $-5.447070 \quad 4.5374701 .084960$

H -2.752796 $7.222509-0.890671$
H $-4.4283857 .161087-1.383171$

H $-3.3035885 .893189-1.913645$

H -6.062046 $5.508598-1.494927$

H -6.3358122 .4365010 .461181$

H -6.817896 $2.845930-1.188930$

H $-4.7915942 .994250-2.597765$

H $-2.261300-1.7681620 .559911$

H -2.744039 -4.620044 -0.493391

H $-4.666162-1.7766661 .057585$

H $-3.749650-2.2973352 .491342$

H $-5.451940-2.7180582 .300393$

H $-5.1331531 .045720-1.980691$

H $-4.9257650 .612722-0.272783$

H $-7.071579-2.861173-2.435622$

H $-8.398168-1.746806-2.033076$

H $-8.013030-2.3066370 .482101$

H $-7.454080-4.854234-1.124466$

H -8.996161 -5.4926020 .845074$

H $-8.226544-4.2759971 .837068$

H $-6.421487-8.0089631 .574792$

H $-8.054635-7.6767190 .765535$

H $-5.358298-6.2201072 .803989$

H $-6.052203-4.6319572 .817760$

H $-4.267302-5.8945190 .621202$

H $-5.506451-1.396361-1.525031$

C $5.380893-3.254042-1.472291$

O $5.552745-1.890936-1.039670$

C $6.935331-1.678121-0.693351$

C $7.675174-2.956242-1.076497$

C $6.588561-4.002725-0.914186$

H $6.970408-1.5679780 .396636$

C $7.453503-0.399240-1.364884$

H $8.025918-2.931282-2.114558$

H $8.544699-3.137053-0.436838$

C $6.893613-5.309413-1.625065$

H $6.447565-4.1980740 .157468$

C $6.5684300 .839926-1.111049$

O $7.487083-0.618013-2.782206$

H $8.488048-0.208760-1.056519$

H $5.5961740 .676293-1.588378$

O $7.1794351 .938824-1.798497$

C $6.398126 \quad 1.200227 \quad 0.377113$

C 5.5146852 .4364530 .637512

H 5.9765820 .3476670 .914902

$\begin{array}{llllll}\text { O } & 7.691657 & 1.444709 & 0.934574\end{array}$

H 5.5864752 .6873601 .702708

H 5.9334243 .3064290 .118634

H $6.081456-6.029617-1.484929$

H $7.028402-5.158825-2.701355$

H $7.811971-5.756323-1.230479$

C $4.039278-3.784825-0.968238$

H $7.8190730 .199094-3.192408$

H $6.5179662 .647240-1.869213$

H 7.5769961 .5946621 .888476

H $3.988911-3.6860730 .121940$

H $3.936855-4.850287-1.201989$

H $5.402975-3.248792-2.570256$

C 3.2403331 .2117482 .288320

$\begin{array}{lllll}\text { O } & 3.479432 & 1.072798 & 0.882563\end{array}$

C $4.0404962 .223387 \quad 0.241379$

C 3.1345513 .4442140 .448560

C 2.8106873 .6743731 .920582

C 2.2942942 .3877092 .560793

H 4.1903021 .3719492 .811968

C $2.625456-0.1076002 .783138$

H $4.0251202 .011315-0.833696$

O $3.730616 \quad 4.619784-0.089114$

H $2.1912073 .287839-0.090710$

H $3.677400 \quad 4.064648 \quad 2.467014$

$\begin{array}{lllll} & & 1.787513 & 4.667122 & 2.026977\end{array}$

H 1.2914042 .1629322 .176407

O $2.1793792 .581773 \quad 3.969048$

C $3.641784-1.2278652 .870378$

H $2.205407 \quad 0.0118643 .787722$

H $1.831503-0.4219562 .095134$

H $3.9058734 .453767-1.031175$

H 2.1133655 .4667901 .579209

H 1.5936493 .3455204 .109603 
O $2.982294-2.4099663 .055349$

O $4.855575-1.0871712 .821368$

C $3.810971-3.5663893 .214908$

H $4.598398-3.5984102 .455969$

H $4.246844-3.5680494 .217999$

H $3.181811-4.4531413 .101263$

SCF Energy $\left(B 3 L Y P / 6-31 G^{* *} / / M M F F\right)=-3245.91325280$

01139

MM̄FF Geometry

C $-2.506077-1.556218-2.969030$

C $-1.884349-2.615633-3.509453$

C $-0.496295-3.097859-3.140276$

O $0.178820-2.145947-2.285106$

C $-0.623788-4.447281-2.402319$

C $0.617602-4.896485-1.658478$

C $0.622625-5.213749-0.347738$

C $1.880470-5.000812-2.471566$

C $-0.566900-5.2586590 .572008$

C $-0.999356-3.906813 \quad 1.156313$

C $-0.038256-3.3522722 .217115$

C $-0.666877-2.1430582 .915341$

C $0.287546-1.5044543 .933158$

O $1.179199-2.9609921 .577113$

O $-0.280634-0.2359494 .289537$

C $1.645925-1.2531833 .245983$

C $0.366993-2.3239715 .226282$

O $2.621000-0.802704 \quad 4.183641$

C $2.187153-2.4856522 .478780$

C $3.422747-2.0747451 .647596$

O $2.573378-3.4906813 .418348$

C $0.871271-1.151868-2.909458$

O $0.976751-0.980504-4.114269$

C $1.478897-0.287598-1.871438$

C $2.151490 \quad 0.807040-2.258844$

C $2.8350721 .776904-1.402159$

C 2.7434661 .6108230 .088926

C $3.896083-3.1240500 .636990$

C $5.200359-2.689749-0.002625$

C $6.143738-1.066449-1.646843$

O $6.275631-3.1788920 .337170$

C $6.3608320 .298530-1.070467$

C $6.506308 \quad 1.403639-1.818177$

C $6.7330832 .775122-1.246147$

C $5.7959313 .817801-1.819365$

C $6.2391974 .742878-2.687436$

C $4.3636543 .836795-1.326576$

C $3.5261912 .776650-1.989146$

N $5.033265-1.697798-0.956389$

H -1.990698 -0.950946-2.227973

H $-2.418032-3.221358-4.238703$

H $0.087754-3.250515-4.056721$

H $-0.898401-5.226742-3.126011$

H -1.471870 -4.382271 -1.711602

H $1.563134-5.5156330 .110296$

H $2.704838-5.441064-1.900360$

H $1.713864-5.635630-3.347954$

H $2.208429-4.014354-2.812418$

H -1.417659 -5.7061530 .043457$

H $-0.345673-5.957138 \quad 1.389132$

H -1.118274 -3.1759620 .346915$

H -1.987610 -4.046317 1.612579

H $0.170605-4.1458212 .943965$

H -1.612252 -2.418362 3.399409

H $-0.926967-1.3864242 .161618$

H $\quad 0.283927 \quad 0.1696624 .968757$

H $1.501561-0.4339412 .527483$

H $-0.620442-2.3845425 .699626$

H $1.022438-1.8427615 .960713$

H $\quad 0.721628-3.3444755 .067319$

H 2.3005890 .0318784 .564954

H $3.177574-1.1710711 .082597$

H $4.255159-1.8227252 .316985$

H $3.264402-4.0465023 .025596$

H $1.363593-0.578642-0.834148$

H $2.2222921 .015704-3.326238$

H 3.4747850 .8747340 .429876

H $1.743662 \quad 1.2912700 .398747$
H $2.9306412 .540400 \quad 0.632527$

H $4.062167-4.0978731 .108407$

H $\quad 3.149597-3.271009-0.150947$

H $5.884613-1.028291-2.709542$

H $7.048832-1.669299-1.524901$

H 6.4124220 .3801440 .012839

H $6.4840091 .317022-2.902194$

H $7.7746663 .052138-1.454789$

H $6.6376182 .771463-0.153214$

H $5.5798005 .508353-3.082997$

H $7.2697034 .752475-3.026512$

H $3.9004384 .808044-1.543998$

H $4.3746303 .755940-0.236024$

H $3.5201872 .842171-3.078161$

H $4.100023-1.345142-1.145414$

C $-4.775071-1.283341-2.002566$

O $-4.311965-0.357140-1.000629$

C $-5.4367940 .065557-0.206737$

C $-6.656949-0.672040-0.754850$

C $-6.247744-0.939403-2.192103$

H $-5.567841 \quad 1.137668-0.389832$

C $-5.148372-0.183101 \quad 1.279243$

H $-6.835035-1.614837-0.224686$

H -7.569275 -0.072606 -0.674292

C -7.064431 -2.031117 -2.860247

H $-6.345225-0.007849-2.765660$

C -3.8600290 .4986671 .790880$

O $-5.004877-1.595691 \quad 1.477248$

H -6.0121630 .1291851 .878122$

H -2.9824480 .0286541 .332215$

$\begin{array}{lllll}\text { O } & -3.763971 & 0.253221 & 3.199432\end{array}$

C -3.812281 2.0217631 .562922

C -2.6556232 .7370902 .286517$

H -3.7559952 .2298150 .489344$

O

H -2.7852243 .8162432 .138794$

H -2.761170 2.5949173 .369436

H $-6.734509-2.191342-3.891252$

H $-6.975013-2.982973-2.325984$

H $-8.124353-1.757681-2.884835$

C $-3.923194-1.151678-3.265234$

H -5.023591-1.760285 2.434926

H $-3.555730-0.6885553 .318944$

H $-5.022973 \quad 3.5397121 .817398$

H -3.914342 -0.112011-3.611975

H $-4.339523-1.766792-4.070482$

H -4.669877 -2.292835 -1.581335

C $-1.0654083 .732231-0.104714$

O

C -1.2488552 .3033611 .828803$

C -0.1055913 .0424512 .539917$

C 0.0261454 .4852362 .054641

C 0.0737034 .5405870 .527984

H -2.0274154 .2175110 .097573$

C $-0.8865183 .643627-1.624357$

H $-1.144627 \quad 1.2418942 .078892$

O -0.3111043 .0122373 .948382$

H 0.8351042 .5139852 .342973

H -0.7826975 .1154432 .442570$

O 1.2452055 .0430432 .551127

H $1.042547 \quad 4.162900 \quad 0.179808$

O $-0.0264455 .899447 \quad 0.105644$

C $-2.0576082 .964652-2.291610$

H $-0.7629894 .630949-2.083153$

H $0.0073573 .059076-1.868995$

H $0.485576 \quad 3.3736864 .371526$

H 1.1643615 .1121903 .517288

H $0.697046 \quad 6.3897190 .532429$

O $-3.1650693 .756420-2.221279$

O $-1.9882011 .866005-2.826711$

C $-4.3410153 .224576-2.838690$

H $-5.1666303 .915726-2.650176$

H $-4.1916703 .139659-3.919117$

H $-4.5937392 .252241-2.407413$

SCF Energy (B3LYP/6-31G**//MMFF) $=-3245.89670051$

0114

MM̄FF Geometry 
C $-0.411392-3.553782-0.667933$

C $0.227134-2.389556-0.476222$

C $0.694797-1.502067-1.598130$

O $0.314935-0.152293-1.230890$

C $2.224929-1.545842-1.756218$

C $2.780262-2.884805-2.185195$

C $3.496071-3.703954-1.389979$

C $2.529274-3.253963-3.623829$

C $3.832209-3.5174300 .064063$

C $5.317578-3.2085120 .296800$

C $5.742615-1.809631-0.179963$

C $7.243451-1.6041800 .039168$

C $7.687061-0.179845-0.326166$

O $5.004034-0.8413090 .572206$

O $9.006627 \quad 0.021007 \quad 0.202912$

C 6.7584450 .8284040 .381670

C $7.7936480 .014213-1.843296$

O $7.0396732 .161543-0.039534$

C 5.2534630 .5206440 .197159

C 4.4161891 .4309631 .128734

O $4.8898340 .773212-1.161000$

C $0.2306150 .773296-2.222794$

O $0.3419440 .580674-3.422438$

C $0.0290072 .089445-1.579170$

C $0.1657203 .210325-2.301765$

C $0.0516504 .566243-1.773096$

C $1.0844395 .513136-2.320509$

C 2.9183731 .0949721 .166406

C 2.1704871 .9368642 .186138

C -0.0710732 .1590093 .247346$

O 2.6938562 .8527212 .815237

C $-0.872928 \quad 3.2098742 .547254$

C -0.7185344 .5239702 .768472$

C -1.557359 5.5955622 .132338

C $-\begin{array}{llll}0.798170 & 6.407840 & 1.106767\end{array}$

C 0.1616567 .2741041 .472623

C -1.212172 $6.272815-0.340228$

C $-0.9209764 .911541-0.906613$

N 0.8449751 .5410652 .309134

H -0.579602 -3.882973-1.691735

H $\quad 0.433761-2.0392160 .531167$

H $0.190183-1.771872-2.534185$

H $2.687769-1.198001-0.827864$

H $2.538173-0.794878-2.493765$

H $3.872268-4.632841-1.817104$

H $3.013326-4.196183-3.901999$

H $1.457040-3.367833-3.810487$

H $2.918361-2.476052-4.288864$

H $3.204978-2.7599670 .543071$

H $3.591048-4.4580980 .575854$

H $5.502348-3.2871071 .376081$

H $5.934436-3.972463-0.192805$

H $5.499918-1.705159-1.243120$

H $7.824103-2.343986-0.526161$

H $7.484636-1.7797711 .097108$

H $9.589409-0.657408-0.179532$

H 6.9896990 .7876631 .455496

H $8.523115-0.685698-2.268445$

H $8.1629161 .016138-2.088507$

H $6.846440-0.141720-2.363821$

H 7.9887422 .3169390 .105624

H 4.8031511 .3431552 .152635

H 4.5304012 .4794570 .825579

H $3.9438180 .979837-1.206790$

H $-0.1916102 .111424-0.517574$

H $0.4212393 .149524-3.357792$

H $0.8855285 .727546-3.376144$

H $2.0862705 .074778-2.241321$

H $1.1222176 .464933-1.784911$

H $2.460673 \quad 1.2750510 .189319$

H 2.7729230 .0411931 .429410

H 0.4917302 .5689914 .092834

H $-0.733561 \quad 1.377612 \quad 3.628939$

H -1.636432 2.8694151 .853101

H 0.0421414 .8547143 .472549

H -1.924997 6.2627022 .923003

H -2.459616 5.1642551 .680947

H $0.6892457 .876646 \quad 0.740775$
H $\quad 0.441056 \quad 7.3987662 .513698$

H $-0.7580787 .060059-0.951918$

H -2.292535 $6.453438-0.416365$

H -1.622669 4.145920 -0.581270

H $0.484756 \quad 0.809612 \quad 1.702603$

C -1.014030 -3.949906 1.820992

O $-1.860462-2.7853891 .853447$

C $-2.809244-2.9173432 .924332$

C -2.261471-4.016305 3.824631

C $-1.635108-4.9471042 .803122$

H $-3.745980-3.2640142 .470489$

C -3.040752 -1.560607 3.597159

H -1.493325 -3.639823 4.509619

H -3.043972 -4.494940 4.420999

C $-0.637741-5.925495 \quad 3.398997$

H $-2.436928-5.5127952 .309825$

C $-3.321206-0.4101792 .606302$

O $-1.864471-1.2081274 .336395$

H $-3.849340-1.6492354 .332144$

H $-2.399384-0.1826272 .060074$

O $-3.6193650 .762333 \quad 3.374851$

C -4.470962 -0.670201 1.615940

C -4.8123160 .5358890 .720931$

H -4.229964 -1.530834 0.986056

O $-5.640296-1.0120702 .365111$

H $-5.718471 \quad 0.2900200 .154200$

H -5.1001941 .3843891 .353674$

H $-0.203342-6.5611082 .620895$

H $0.181898-5.4046953 .905378$

H -1.127212 -6.574880 4.132156

C $-0.949026-4.4855410 .387445$

H -2.043131 -0.3587244 .775195$

H $-4.4606140 .603173 \quad 3.836493$

H -6.338022 -1.2451361 .729171$

H -1.966514 -4.752046 0.075832

H $-0.354724-5.4063080 .362021$

H -0.019889 -3.640072 2.166412

C $-4.201867-0.510299-2.102492$

O $-3.264415-0.135794-1.084583$

C $-3.6786940 .944660-0.240840$

C $-3.9902442 .188615-1.083576$

C $-4.9965521 .891503-2.187754$

C $-4.5391360 .685821-3.004079$

H -5.119045 -0.889963-1.636005

C $-3.563623-1.643121-2.925079$

H -2.8093321 .1991890 .375054$

O $-4.4822723 .248972-0.271582$

H $-3.0677812 .545324-1.556075$

H $-6.0050501 .738132-1.786699$

O $-5.078756 \quad 3.018233-3.063975$

H $-3.6642650 .961763-3.607272$

O $-5.581361 \quad 0.314877-3.905149$

C $-3.703894-2.996283-2.260191$

H -4.057602 -1.719841-3.900545

H -2.494539-1.455981 -3.076850

H -3.8192023 .4210980 .418542$

H -5.324861 $3.787745-2.522285$

H $-5.785816 \quad 1.095127-4.448618$

O $-3.426022-3.968081-3.179969$

O $-4.005413-3.182198-1.089927$

C $-3.558431-5.319734-2.727855$

H -4.489194 -5.460412 -2.169838

H $-3.579951-5.969653-3.606868$

$\mathrm{H}-2.695368-5.589516-2.114217$

SCF Energy (B3LYP/6-31G**//MMFF) $=-3245.91093067$

01140

MM̄FF Geometry

C -0.382880 -4.469565 -2.226939

C $0.082654-3.808032-1.156513$

C $1.178982-2.783246-1.225685$

O $0.712456-1.529689-0.678901$

C $2.467637-3.173640-0.468067$

C $2.312574-3.7062240 .944526$

C $2.287030-2.9502162 .059916$

C $2.279802-5.2103081 .065530$

C $2.342259-1.4533002 .176961$

C $3.626969-0.9609822 .858799$ 
C $4.862852-1.0455211 .949125$

C $6.115505-0.5773722 .692494$

C $7.345394-0.5310991 .772175$

O $4.623999-0.2000030 .819988$

$\begin{array}{lllll}\text { O } & 8.373408 & 0.210984 & 2.445987\end{array}$

C $6.984497 \quad 0.2556310 .494087$

C $7.917985-1.9297711 .514188$

O $8.0472850 .203475-0.454948$

C $5.664831-0.207200-0.163933$

C $5.2543760 .779561-1.283123$

O $5.855091-1.505877-0.728222$

C $0.057243-0.678120-1.512155$

O $-0.350038-0.913780-2.637652$

C $-0.0191480 .630272-0.823996$

C $-0.5864271 .678967-1.437111$

C $-0.6695023 .042078-0.907055$

C 0.0922153 .3450910 .357110

C $3.8407690 .545111-1.834111$

C $3.5124711 .557265-2.912531$

C $2.9742043 .951144-3.256204$

O $3.597181 \quad 1.277594-4.106183$

C $2.1731834 .990820-2.540964$

C $1.0966185 .589067-3.073193$

C $0.3036956 .656297-2.374410$

C $-1.1747096 .342448-2.315437$

C $-2.0431096 .944619-3.145539$

C -1.655249 $5.393839-1.239713$

C $-1.3971903 .952843-1.586657$

N $3.1433792 .792225-2.399728$

H $0.034769-4.263748-3.209170$

H $-0.354304-4.008465-0.181261$

H $1.474623-2.609076-2.269430$

H $3.131316-2.301805-0.442567$

H $3.005925-3.925880-1.060775$

H $2.206512-3.4611273 .019048$

H $2.195391-5.5429122 .105689$

H $1.431215-5.6313420 .519897$

H $3.200400-5.6409960 .657790$

H $2.220549-0.9565381 .212480$

H $1.484842-1.1409482 .785435$

H $3.4658520 .087923 \quad 3.140266$

H $3.800831-1.5220273 .785303$

H $4.987317-2.0796051 .609247$

H $6.310988-1.2139673 .564366$

H 5.9463500 .4326673 .091803

H $8.559715-0.2342373 .290507$

H $6.876499 \quad 1.311347 \quad 0.780130$

H $8.221044-2.3992092 .457869$

H $8.824749-1.8784730 .901238$

H $7.208239-2.600878 \quad 1.026211$

H $8.8530330 .510106-0.004729$

H $5.2985761 .803676-0.890746$

H $5.969786 \quad 0.722636-2.113457$

H $5.259645-1.618526-1.485237$

H $\quad 0.4293630 .7049880 .160473$

H -1.026422 $1.531035-2.422331$

H 1.1471463 .0695830 .245610

H -0.3254142 .7899321 .203261$

H $0.082773 \quad 4.401783 \quad 0.630797$

H $3.737419-0.453420-2.270964$

H $3.0911980 .624455-1.039146$

H $2.5119033 .639128-4.198747$

H $3.9739824 .338411-3.479167$

H $2.5053415 .281522-1.547073$

H $0.7807225 .317538-4.077968$

H $0.4658287 .597741-2.915502$

H $\quad 0.6763756 .831229-1.357485$

H $-3.1080886 .744892-3.091347$

H -1.707684 $7.646581-3.901822$

H -2.739514 $5.503297-1.102341$

H -1.220818 $5.692155-0.282265$

H -1.907863 $3.629622-2.495204$

H $3.2680522 .952642-1.403943$

C $-2.871623-4.992680-2.376148$

O $-3.230903-4.103531-1.301668$

C $-3.972756-2.997399-1.849666$

C -4.347064 -3.405105 -3.270470

C $-3.131313-4.222961-3.669855$
H $-3.278907-2.149794-1.905436$

C $-5.146736-2.645485-0.931288$

H $-5.248245-4.028681-3.291921$

H -4.516929-2.540932 -3.919628

C $-3.359223-5.111248-4.879536$

H -2.309066 -3.529699 -3.885975

C $-4.735246-2.4042670 .537294$

O $-6.079516-3.733223-0.946631$

H $-5.679922-1.775455-1.332081$

H $-4.410274-3.3492720 .988349$

O $-5.909139-2.0094381 .259103$

C -3.643321 $-1.336120 \quad 0.727572$

C $-3.302106-1.1127642 .214563$

H $-2.732203-1.6487060 .205983$

O $-4.095347-0.1272580 .126770$

H $-4.193598-0.7636182 .744084$

H -3.025955 -2.081453 2.646787

H -2.459734 -5.690835 -5.110548

H $-4.180999-5.815642-4.712275$

H -3.608207 -4.507760 -5.758521

C $-1.454188-5.520916-2.153672$

H $-6.813138-3.493706-0.354701$

H $-6.182690-1.1399520 .919245$

H -3.3623830 .5107710 .156016$

H -1.225827 -6.297925 -2.892579

H $-1.409028-6.005386-1.169480$

H -3.563285 -5.842872 -2.305152

C -3.4336001 .8999152 .834094$

O -2.4944501 .2172941 .993122$

C $-2.145313-0.1064882 .414400$

C $-1.564911-0.0696763 .834641$

C $-2.4984990 .636563 \quad 4.811238$

C -2.8990362 .0063284 .266935$

H -4.3878861 .3598672 .834480$

C -3.6734003 .2895132 .229963$

H $-1.333852-0.4352531 .753285$

O $-1.291149-1.3819504 .313537$

H -0.6090980 .4705093 .819643$

H -3.3829450 .0278565 .033822$

O -1.8190220 .8315376 .053606$

H -2.0347062 .6820624 .299227$

O -3.9106032 .5623065 .104761$

C -4.7062523 .2259981 .130426$

H -4.060569 3.9958592 .973342

H -2.7515673 .7263861 .828965$

H $-0.697068-1.8096673 .673469$

H -1.537129 -0.0442916 .368905$

H -3.5535302 .5935446 .008904$

O $-4.1116442 .936515-0.059782$

O -5.9046723 .3957481 .315830$

C $-4.9930512 .841544-1.183490$

H -4.410465 $2.504073-2.044763$

H $-5.7853762 .111657-0.991190$

H $-5.4180883 .824149-1.408096$

SCF Energy $\left(B 3 L Y P / 6-31 G^{* *} / / M M F F\right)=-3245.91877106$

01_141

MM̄FF Geometry

C 3.6946112 .2006732 .519849

C 2.7089941 .4110032 .069599

C 1.6932550 .7318082 .946688

O $\quad 0.381901 \quad 1.0429162 .422505$

C $1.880699-0.7928182 .886090$

C $0.898732-1.5527363 .756744$

C $-0.036004-2.3958003 .275001$

C $1.046776-1.3571315 .242934$

C $-0.268767-2.7186871 .823072$

C $-1.462127-3.6603561 .598135$

C $-2.821423-2.9755031 .812532$

C $-3.936838-4.0202921 .898013$

C $-5.321610-3.3697132 .025688$

O $-3.053503-2.0949610 .703733$

O $-6.309086-4.386191 \quad 1.793827$

C $-5.475050-2.3255440 .901853$

C $-5.579376-2.8298143 .436799$

O $-6.687415-1.590078 \quad 1.045870$

C $-4.279368-1.3527660 .791120$

C $-4.375320-0.442179-0.455810$ 
O $-4.270614-0.490722 \quad 1.936425$

C -0.1859762 .2133832 .819236$

O 0.2697613 .0228153 .610753

C -1.463049 2.355609 2.088126

C -2.1751743 .4843952 .215394$

C $-3.4338773 .758783 \quad 1.529875$

C -4.4596924 .4329522 .399599$

C $-4.480939-1.170711-1.797301$

C $-4.538924-0.162743-2.926976$

C $-3.0763081 .268906-4.345858$

O $-5.6059540 .303204-3.320712$

C $-2.8690542 .526986-3.563937$

C $-3.7462243 .541368-3.537732$

C $-3.5762614 .756422-2.671095$

C $-4.6479284 .856190-1.603182$

C $-5.4398985 .938661-1.525505$

C $-4.8148443 .705970-0.629999$

C -3.5933043 .4814280 .220592$

N $-3.2860910 .188517 \quad-3.400119$

H 3.8090452 .3686103 .587175

H 2.6205181 .2414371 .000269

H 1.7749311 .0790043 .983874

H $2.898210-1.0560203 .204585$

H $1.798178-1.1235681 .844346$

H $-0.674651-2.9116463 .989902$

H $0.408732-2.0381695 .816510$

H $2.081298-1.5434855 .549626$

H $0.775562-0.3361555 .527726$

H $0.627480-3.2193061 .439797$

H $-0.414976-1.797713 \quad 1.249091$

H -1.407500 -4.031045 0.566257

H -1.365091 -4.529091 2.261257

H $-2.788049-2.3884332 .736727$

H -3.761446 -4.709485 2.733536

H -3.925670 -4.6403260 .990448$

H -6.173732 -5.0907712 .450631$

H -5.563729 -2.891587 -0.033167

H -5.523814 -3.641025 4.172735

H $-6.590592-2.4169363 .522316$

H -4.866057 -2.061255 3.740972

H -7.412838 $-2.235278 \quad 1.104439$

H $-5.2233470 .246788-0.344289$

H $-3.4847590 .201866-0.483874$

H $-3.803678 \quad 0.328721 \quad 1.704245$

H -1.7787531 .5411351 .444888$

H -1.8303524 .2748812 .878863$

H -4.8002563 .7474293 .183304$

H -5.3412854 .7699951 .849569$

H -4.035797 5.3216912 .881150

H -3.633282 -1.849070 -1.945168

H -5.392581 -1.772928 -1.861912

H $-3.9355291 .337045-5.021062$

H -2.183431 $1.035354-4.933090$

H -1.967472 $2.585455-2.958817$

H -4.652409 $3.482404-4.136070$

H -3.594291 $5.638286-3.323826$

H -2.590562 $4.761929-2.189435$

H $-6.211616 \quad 6.021858-0.767379$

H $-5.3343476 .768048-2.216869$

H $-5.0203882 .783946-1.187703$

H $-5.7066143 .849567-0.010829$

H -2.762101 $3.024802-0.316711$

H $-2.465590-0.208242-2.953368$

C 5.9402542 .0820651 .404221

O 5.6252550 .8936950 .648515

C $6.5685380 .758349-0.427865$

C $7.7308501 .676318-0.074340$

C 7.0024272 .8262670 .592720

H $6.0775671 .141089-1.330511$

C $6.943218-0.713499-0.620837$

H 8.4219561 .2046660 .633721

H $8.3029651 .981694-0.955524$

C 7.9052503 .7158971 .429513

H $6.5230953 .435681-0.185456$

C $5.741065-1.656925-0.844326$

O $7.642156-1.1743890 .544128$

H $7.653167-0.807188-1.450674$

H $5.168914-1.7372210 .087259$
O $6.291098-2.956202-1.098958$

C $4.813345-1.252102-2.009328$

C $3.758833-2.309157-2.391343$

H $4.311779-0.310341-1.764165$

O $5.620321-1.009419-3.166023$

H $3.253187-1.968119-3.302566$

H $4.270053-3.231400-2.692433$

H 7.3313784 .5187531 .902823

H $8.406416 \quad 3.1482872 .220788$

H 8.6781394 .1755440 .804874

C 4.6642302 .8976131 .606600

H $7.054037-1.0472341 .308514$

H $5.586369-3.609478-0.953434$

H $5.036106-0.672284-3.866447$

H 4.1840713 .0822250 .636904

H 4.8971953 .8775722 .038602

H $6.347117 \quad 1.7498282 .367967$

C $1.028294-0.994124-1.926058$

O $1.968579-1.431398-0.936901$

C $2.725710-2.596559-1.284226$

C $1.785030-3.768601-1.592785$

C $0.753753-3.407515-2.654738$

C $0.036288-2.112741-2.279044$

H $1.563624-0.675070-2.828122$

C $0.2809450 .220317-1.349823$

H $3.283192-2.878482-0.383942$

O $2.536467-4.906052-2.004621$

H $1.258506-4.054510-0.674210$

H $1.216772-3.339307-3.646229$

O $-0.198733-4.471365-2.723651$

H $-0.639708-2.298122-1.434681$

O $-0.760587-1.687734-3.382858$

C $1.0989881 .492688-1.382184$

H $-0.6224300 .421567-1.932755$

H $0.0034510 .030279-0.307333$

H $1.902725-5.615314-2.207766$

H $-0.789865-4.288717-3.473310$

H -1.458559-2.350985 -3.515969

O $\quad 0.3351862 .511306-0.885027$

O $2.248987 \quad 1.589417-1.786089$

C $0.9603323 .797291-0.850472$

H $1.8166863 .781925-0.170485$

H $1.2691524 .097960-1.856098$

H $0.2291614 .519690-0.477869$

SCF Energy $\left(B 3 L Y P / 6-31 G^{* *} / / M M F F\right)=-3245.90617261$

01_142

MM̄FF Geometry

C -1.530198 -2.842077 1.833409

C $-0.814088-3.2897080 .791730$

C $-1.278976-3.116038-0.630154$

O $-0.293217-2.329109-1.338442$

C $-1.457203-4.459392-1.353930$

C $-1.846696-4.327894-2.818322$

C $-3.004349-3.806176-3.274087$

C $-0.812398-4.827797-3.793242$

C $-4.139950-3.256738-2.455954$

C $-4.121619-1.723461-2.462236$

C $-4.969849-1.116591-1.335305$

C $-6.462842-1.143536-1.666853$

C $-7.306347-0.423862-0.602602$

O $-4.5318830 .237573-1.156619$

O $-8.607167-0.222245-1.176460$

C $-6.6865110 .963174-0.316158$

C $-7.509228-1.3031440 .637534$

O $-7.338301 \quad 1.5995440 .780966$

C $-5.1633480 .910961-0.057972$

C -4.5816032 .3439890 .026250$

$\begin{array}{lllll}\text { O } & -4.930603 & 0.242601 & 1.185537\end{array}$

C $-0.395941-0.977431-1.205281$

O $-1.193881-0.369046-0.508877$

C $0.628658-0.348216-2.064798$

C $0.6024140 .983651-2.216181$

C $1.5475411 .800369-2.976848$

C $2.7477421 .116279-3.573474$

C -3.0458342 .3935220 .052946$

C $-2.515974 \quad 3.793293 \quad 0.318072$

C $-0.379774 \quad 4.9932370 .810805$ 
$\begin{array}{llll}\text { O } & -3.231388 & 4.791118 & 0.334363\end{array}$

C $0.2141175 .509928-0.461570$

C $1.5328925 .551921-0.707138$

C $2.1331556 .086291-1.977233$

C $2.8981965 .034549-2.752117$

C $4.2291464 .908148-2.618490$

C $2.1326334 .185087-3.743738$

C $1.3090793 .125203-3.066422$

N -1.142898 3.7911890 .526456

H -2.512591 -2.415359 1.645645

H $\quad 0.165449-3.7280890 .956928$

H -2.232502 -2.582007 -0.635567

H - $-2.207247-5.072382-0.837575$

H - $0.516720-5.024271-1.292570$

H -3.160656 -3.754887 -4.350691

H -1.124314 -4.702145 -4.835434

H $\quad 0.127539-4.281639-3.661056$

H $-0.623038-5.893817-3.630297$

H -5.085233 -3.620629-2.875354

H -4.098097 -3.642263 -1.431814

H -3.092976 -1.356667 -2.362795

H -4.458462 -1.346418 -3.436905

H $-4.765188-1.668111-0.410321$

H $-6.817614-2.172186-1.803840$

H -6.626972 -0.640973 -2.630789

H $-9.1771680 .179640-0.499572$

H $-6.8673071 .594506-1.197370$

H $-8.048822-2.2196080 .369798$

H -8.130187 -0.7979491 .385403$

H -6.571141 -1.5986431 .112253$

H -8.2727841 .7169820 .541497$

H -4.915915 2.926061 -0.842423

H -4.9695372 .8439680 .922930$

H -4.1521950 .6233371 .619668$

H $\quad 1.369373-0.984347-2.534071$

H $-0.189473 \quad 1.543870-1.720117$

H $2.4382850 .406237-4.348056$

H $3.3051880 .572997-2.804040$

H $3.4576021 .806819-4.033839$

H -2.649283 1.7334040 .830853

H -2.646207 $2.063294-0.912582$

H $\quad 0.385421 \quad 4.726674 \quad 1.546570$

H -1.032638 5.7531251 .251813

H -0.479482 $5.877310-1.214303$

H 2.2267485 .1950430 .050683

H $2.8029456 .913404-1.707697$

H $1.3673536 .531979-2.624347$

H $4.7874394 .174199-3.190011$

H $4.7885165 .531815-1.929020$

H $2.8118613 .751534-4.485012$

H $1.4639594 .828787-4.329614$

H $0.4016343 .514840-2.605092$

H -0.6294172 .9193910 .432833$

C $-0.825526-1.4359673 .825963$

O $0.327290-0.8238963 .210396$

C 0.0831330 .5931303 .091667

C -1.214009 0.8758483 .848965

C $-1.963615-0.427548 \quad 3.668251$

H $-0.082130 \quad 0.7918372 .026370$

C 1.2803751 .4071753 .597204

H -1.035295 1.0657514 .913594

H -1.748350 1.7399393 .441834

C $-3.107429-0.6221234 .646175$

H -2.360657 -0.435713 2.647373

C 2.6252641 .0837492 .916162

O 1.4332501 .1529925 .000294

H 1.0545172 .4764143 .499408

H $2.976670 \quad 0.0958103 .236447$

O 3.5885142 .0333273 .393606

C 2.6095241 .1527201 .378389

C 4.0057031 .1324850 .724612

H 2.0074590 .3259200 .985910

O 1.9656122 .3650920 .978566

H $3.8636651 .174335-0.362045$

H 4.5333382 .0641870 .966890

H -3.608663 -1.5796004 .472535$

H -2.756324 -0.607411 5.683360

H -3.8507750 .1736834 .531483$
C $-1.061010-2.843533 \quad 3.258877$

H 2.0301511 .8313795 .358192

H 3.7609431 .8312844 .328609

H 1.9415822 .3815870 .006903

H -1.814476 -3.360406 3.864505

H $-0.130499-3.418783 \quad 3.341740$

H $-0.571617-1.5442954 .889315$

C $3.943136-1.701713-0.407176$

O $4.187051-1.3184260 .949158$

C $4.881052-0.0783271 .103111$

C $6.231997-0.1319440 .374159$

C $6.081081-0.554735-1.085885$

C $5.253623-1.836176-1.191059$

H $3.322288-0.933671-0.880128$

C $3.087210-2.971044-0.394819$

H 5.1053390 .0143402 .172522

O $\quad 6.891279 \quad 1.128845 \quad 0.428587$

H $6.884737-0.8579020 .876453$

H $5.6443190 .246505-1.693264$

O $7.376619-0.810876-1.634144$

H $5.858759-2.670569-0.816132$

O $4.980150-2.081937-2.568016$

C $3.810283-4.1826900 .142091$

H $2.196369-2.8144710 .223071$

H $2.726233-3.213320-1.400907$

H $6.989071 \quad 1.367291 \quad 1.366317$

H $7.9006170 .001616-1.528155$

H $4.607817-2.976876-2.640380$

O $3.790278-4.1751891 .505123$

O $4.338218-5.028596-0.568288$

C $4.461130-5.2699242 .136326$

H $5.522156-5.2688951 .868528$

H $4.372585-5.1442303 .218660$

H $3.991139-6.216607 \quad 1.853177$

SCF Energy $\left(B 3 L Y P / 6-31 G^{* *} / / M M F F\right)=-3245.90150605$

01143

MM̄FF Geometry

C $3.790355-3.275380-1.463553$

C $2.985838-4.155580-0.847297$

C $1.568016-3.890385-0.393203$

O $1.036850-2.753763-1.110528$

C $0.666326-5.109112-0.677745$

C $-0.617239-5.1301290 .133155$

C $-1.864080-5.045858-0.371405$

C $-0.438100-5.3230601 .620128$

C $-2.296209-4.911943-1.803444$

C $-2.795706-3.512367-2.193590$

C $-4.070340-3.054592-1.463706$

C $-4.630636-1.786422-2.114795$

C $-5.854782-1.240000-1.363979$

O $-3.752334-2.784976-0.093742$

O $-6.094500 \quad 0.098629-1.826110$

C $-5.522988-1.1430560 .140932$

C $-7.122273-2.040930-1.684892$

O $-6.682298-0.7981930 .894198$

C $-4.885552-2.4263870 .712779$

C $-4.396989-2.2728742 .175841$

O $\quad-5.863243-3.475278 \quad 0.716840$

C $0.357598-1.805605-0.415182$

O $0.127193-1.7880190 .782225$

C $-0.091632-0.788155-1.392962$

C $-1.0091980 .117193-1.025053$

C $-1.6187891 .134098-1.883548$

C $-1.076211 \quad 1.296644-3.277682$

C $-3.376982-1.1580142 .425782$

C -4.0003890 .1668822 .838543$

C -3.4656332 .6008552 .989612$

O $-5.1484550 .268848 \quad 3.261962$

C -4.0536723 .2010581 .751395$

C -3.4368274 .1250720 .998802$

C $-4.0207654 .693900-0.262489$

C $-3.2438364 .296962-1.500199$

C $-2.4477125 .176417-2.130437$

C $-3.4689982 .912149-2.067893$

C $-2.6424531 .857638-1.384876$

N $-3.106140 \quad 1.2207882 .714799$

H $3.428182-2.276799-1.693807$ 
H $3.395527-5.131078-0.591582$ H $1.621964-3.6847340 .681876$ H $1.203421-6.038763-0.445407$ H $0.454840-5.146683-1.751062$ H - $2.694385-5.1099810 .331240$ H $-1.389491-5.4949872 .135315$ H $0.198066-6.1921961 .816852$ H $0.022869-4.4419712 .075507$ H $-1.489303-5.191554-2.487225$ H -3.094870 -5.642041-1.985554 H $-1.991635-2.791806-2.017860$ H -2.988847 -3.522777 -3.273709 H $-4.806043-3.865802-1.516362$ H -4.878265 -1.966596 -3.168391 H -3.854566 - $1.010136-2.121148$ H -6.223686 $0.064868-2.789400$ H $-4.814546-0.3131520 .254939$ H -7.340281 -1.997800 -2.758840 H -7.998191-1.612599-1.185472 H -7.046356 -3.094302 -1.407603 H -7.0362160 .0267610 .520099$ H -5.257352 -2.185750 2.850493 H -3.905941 -3.217250 2.452186 H $-5.583078-4.1547621 .352304$ H $0.302685-0.846699-2.401001$ H $-1.3752140 .094746-0.000500$ H $-1.3550890 .437152-3.897053$ H $0.0159651 .375928-3.267172$ H -1.437286 2.197889-3.778619 H -2.730617 -1.4574013.260698 H $-2.734890-1.0173251 .551902$ H -2.557052 3.1194873 .310536 H -4.194649 2.6375503 .805295 H -5.0355392 .8412381 .451771$ H -2.455174 4.4871281 .294226 H $-4.0402195 .786395-0.157504$ H -5.069186 $4.394330-0.385532$ H - $1.9133224 .906225-3.035349$ H -2.304171 $6.182403-1.750442$ H -3.302258 2.916818-3.150012 H $-4.5280512 .646203-1.957509$ H -2.970397 $1.634699-0.371071$ H -2.196082 1.0477002 .298044 C $6.249640-2.924449-0.915657$ O $6.042420-1.498268-0.837021$ C $6.007509-1.1248010 .552439$ C $6.738140-2.2402031 .288779$ C $6.249227-3.4534540 .523056$ H $4.953070-1.1373140 .855508$ C 6.5842210 .2761810 .770127 H $7.826185-2.1473621 .194048$ H $6.487012-2.2733382 .353152$ C $7.107783-4.6906960 .720974$ H $5.230615-3.6791450 .859431$ C $5.9499781 .377971-0.102723$ $\begin{array}{lllll}\text { O } & 7.986109 & 0.239582 & 0.475405\end{array}$ H 6.4986750 .5369691 .832341 H $6.314803 \quad 1.297927-1.134393$ O 6.4132232 .6422740 .390530 C $4.4084841 .398491-0.117310$ C $3.8586102 .655985-0.818922$ H $4.0461680 .501204-0.631916$ O 3.9345101 .3513151 .224772 H $4.0087353 .523413-0.166717$ H $4.4393122 .824779-1.733344$ H $6.720677-5.5297180 .134168$ H $8.144349-4.5140250 .414736$ H $7.115407-4.9889081 .774348$ C $5.215538-3.565881-1.848629$ H $8.378722 \quad 1.063877 \quad 0.808547$ H 7.3656052 .6995460 .205907 H 2.9758451 .1966001 .187074 H $5.389743-4.647131-1.904136$ H $5.358977-3.172621-2.862838$ H $7.243192-3.059476-1.362140$ C 1.3470593 .4345410 .812797 O $1.5134572 .319760-0.071141$ C $2.3639682 .546990-1.196643$
C $1.8432143 .735654-2.019275$

C $1.6745354 .985925-1.162717$

C $0.823944 \quad 4.666667 \quad 0.064721$

H 2.3042293 .6663291 .294888

C $0.342823 \quad 3.0073191 .891538$

H $2.2737721 .654755-1.829499$

$\begin{array}{llll}\text { O } & 2.712302 & 4.023808 & -3.109493\end{array}$

H $0.8660213 .483134-2.448318$

H $2.6463985 .408817-0.882504$

O $1.0119965 .978415-1.949602$

H $-0.2142434 .499912-0.245495$

O $0.831996 \quad 5.782900 \quad 0.951936$

C 0.9381491 .9879292 .833096

H 0.0147903 .8520542 .506939

H $-0.5482972 .579504 \quad 1.417458$

H $2.7780753 .218603-3.650676$

H $0.9997936 .802132-1.433917$

H $0.378586 \quad 6.5172330 .504986$

$\begin{array}{llllll}\text { O } & 0.205380 & 0.838721 & 2.802133\end{array}$

O 1.9330992 .1897893 .517374

C $0.664850-0.2073283 .663278$

H $1.648424-0.5573543 .335655$

H 0.7023010 .1391414 .700501

H -0.043746 -1.037146 3.598774

SCF Energy (B3LYP/6-31G*//MMFF)= -3245.89777809

01144

MM̄FF Geometry

C $-2.344008-4.142622-2.249030$

C $-1.389479-3.281560-2.633923$

C $0.108069-3.432140-2.491751$

O $0.558932-2.276332-1.736956$

C $0.568291-4.685036-1.720403$

C $2.064495-4.742489-1.475521$

C $2.633948-4.733319-0.253301$

C $2.927675-4.830428-2.706832$

C $1.943653-4.6919431 .082738$

C $1.686599-3.279268 \quad 1.629364$

C $2.962619-2.5275152 .041217$

C $2.610922-1.2150092 .744232$

C $3.865317-0.4075723 .107963$

O $3.732914-2.245830 \quad 0.870907$

O $3.4222700 .910185 \quad 3.467974$

C $4.771546-0.2816071 .862513$

C $4.561301-0.9802994 .348736$

$\begin{array}{lllll}\text { O } & 6.010098 & 0.320209 & 2.231489\end{array}$

C $5.000138-1.6223221 .130669$

C $5.716026-1.470157-0.234638$

O $5.827500-2.4621361 .945331$

C $0.842374-1.137353-2.422583$

O $0.864952-0.986669-3.633132$

C $1.055622-0.069863-1.423438$

C $0.810026 \quad 1.202583-1.766617$

C $0.9085262 .364068-0.882856$

C 1.5550122 .1623390 .461386

C $5.003436-0.577917-1.257332$

C $5.502778 \quad 0.857773-1.285007$

C $5.0397413 .049859-2.381540$

O $6.4880551 .241751-0.660886$

C $4.2724933 .916506-1.433611$

C $3.4784304 .922758-1.830959$

C $2.7437455 .833319-0.888769$

C $1.2617425 .908722-1.181925$

C $0.7480496 .926505-1.893287$

C $0.3667814 .852511-0.575714$

C $0.4285593 .547575-1.317304$

N $4.7344831 .654774-2.123789$

H $-2.084466-5.110294-1.831226$

H $-1.712937-2.325052-3.041565$

H $0.557899-3.428524-3.491727$

H $0.274883-5.588637-2.270280$

H $0.033774-4.718757-0.765666$

H $3.720810-4.775454-0.193885$

H $3.982664-4.996441-2.463238$

H $2.603261-5.663437-3.339017$

H $2.867503-3.905074-3.287858$

H $0.992808-5.234106 \quad 1.037225$

H $2.553561-5.2550541 .800669$ 
H $\quad 1.135625-2.6972660 .881464$ H $1.033813-3.3747002 .506194$ H $3.539353-3.1763892 .710541$ H $2.001996-1.4004313 .638042$ H $1.984655-0.6044662 .079605$ H 4.2133971 .4517923 .633244 H 4.2715990 .4200621 .186203 H $3.879991-0.9655115 .208008$ H $5.421627-0.3677204 .640157$ H $4.902071-2.0087984 .212606$ H $6.5034650 .512801 \quad 1.416932$ H $6.758284-1.164049-0.086015$ H $5.773223-2.473978-0.680319$ H $6.238375-3.134747 \quad 1.377021$ H $1.352486-0.365609-0.424249$ H $\quad 0.470051 \quad 1.412102-2.779966$ H 2.5430811 .7071620 .342033 H $0.943974 \quad 1.5090571 .091220$ H 1.7151033 .0901091 .014235 H $5.191879-0.984044-2.259577$ H $3.921448-0.585722-1.099962$ H $4.7824523 .250251-3.426430$ H $6.1131673 .219033-2.248407$ H $4.4000303 .726449-0.370470$ H $3.3757985 .128591-2.894165$ H $3.1910626 .832219-0.976583$ H $2.8936645 .534803 \quad 0.156344$ H $-0.3175097 .002644-2.083061$ H $1.3829927 .703218-2.306553$ H $-0.6798035 .186050-0.599262$ H $0.596657 \quad 4.7500910 .488639$ H -0.014109 $3.593857-2.313279$ H $3.9039371 .263563-2.558760$ C $-4.334132-3.715357-0.818819$ O $-3.525025-2.806478-0.048830$ C $-4.367896-2.056050 \quad 0.841258$ C $-5.759542-2.6738440 .729608$ C $-5.758812-3.181707-0.701552$ H $-4.409416-1.0347390 .447149$ C -3.762332 -2.045666 2.249980 H $-5.895229-3.5086501 .426518$ H $-6.551332-1.9444490 .925936$ C $-6.833025-4.216669-0.983596$ H -5.896768 -2.325255 -1.375139 C $-2.274326-1.6347452 .326555$ O $-3.856316-3.3746192 .786379$ H -4.370416 -1.412064 2.906224 H -1.672001 -2.421994 1.854974 O $-1.912172-1.6300903 .714476$ C $-1.891388-0.2685441 .723743$ C -2.7339740 .8999832 .271929$ H -0.842666 -0.067102 1.976076 O $-1.971463-0.3123110 .306678$ H $-3.7777680 .764215 \quad 1.974508$ H -2.7013680 .8661643 .366621$ H $-6.782006-4.557978-2.022266$ H -6.726386 -5.092825 -0.335181 H -7.828609 -3.793136 -0.815557 C $-3.806635-3.785093-2.254373$ H -3.485909 -3.350172 3.685459 H $-0.954827-1.4666503 .766721$ H -1.434916 -1.063233 0.000737 H -3.953899 -2.816816 -2.748943 H -4.361503 -4.532982 -2.830971 H $-4.247780-4.699749-0.339237$ C $-3.7032022 .598970-0.113784$ $\begin{array}{lllll}\text { O } & -2.363296 & 2.452757 & 0.371620\end{array}$ C -2.2361182 .2820951 .786703$ C -2.8690813 .4759642 .515525$ C -4.3177263 .6901402 .088440$ C $-4.406995 \quad 3.780611 \quad 0.566538$ H -4.2657531 .6765230 .069584$ C -3.618152 2.830128 -1.630819 H -1.162006 2.3173322 .005317 O -2.7879043 .2865113 .923721$ H -2.2924314 .3800562 .278596$ H -4.974682 2.9020192 .474920 O $\quad-4.7969874 .9223022 .630774$
H $-3.963615 \quad 4.727377 \quad 0.231227$

O -5.7800563 .7917640 .179542$

C $-3.2824291 .566653-2.393845$

H $-4.5701383 .191752-2.034369$

H $-2.8427343 .577835-1.838356$

H -3.0735944 .1120354 .349728$

H -4.8487394 .8171973 .595688$

H -6.2047614 .5364240 .639165$

O $-2.6151721 .898131-3.538289$

O $-3.6097210 .436736-2.057875$

C $-2.2808090 .803219-4.396185$

H $-1.7400930 .031343-3.842971$

H $-3.1896730 .390488-4.843661$

H -1.633947 $1.179850-5.192931$

SCF Energy $(B 3 L Y P / 6-31 G * * / / M M F F)=-3245.91337391$

01 145

MM̄FF Geometry

C -3.763972 -3.414197-2.225577

C $-2.622165-3.391393-1.523844$

C $-1.267199-3.152583-2.138730$

O $-0.721410-1.934360-1.581844$

C $-0.316405-4.309052-1.785266$

C $1.090428-4.116530-2.318879$

C $2.181397-3.880303-1.561929$

C $1.232136-4.249226-3.814431$

C $2.268207-3.741781-0.066739$

C $2.857286-2.4065100 .403743$

C $4.290106-2.085315-0.054091$

C $5.293425-3.1712950 .338314$

C $6.731858-2.783871-0.030210$

O $4.648481-0.8506140 .581978$

O $7.593562-3.7135620 .645139$

C $7.029973-1.3719540 .516959$

C $6.988704-2.967321-1.530978$

O $8.288804-0.8952260 .046265$

C $5.929674-0.3270160 .201502$

C $6.1713650 .949074 \quad 1.045463$

O $5.9474480 .059324-1.171778$

C $-1.145086-0.759626-2.123978$

O $-1.956465-0.613374-3.024190$

C $-0.4478900 .335593-1.415561$

C $-0.8442331 .602865-1.605350$

C $-0.2726902 .793849-0.971315$

C $0.9046742 .591671-0.054597$

C 5.1384302 .0626620 .827156

C $5.4230652 .950566-0.374404$

C $4.3923204 .804371-1.684946$

O $6.4722552 .915639-1.010593$

C $3.0301104 .892212-2.293138$

C $2.2567825 .987351-2.245640$

C $0.8869856 .058982-2.857872$

C $-0.1949236 .349081-1.838842$

C $-0.9374377 .465796-1.924969$

C $-0.4248835 .349859-0.721503$

C $-0.8300273 .994596-1.236554$

N $4.3609833 .805152-0.633977$

H -3.726382 -3.284956 -3.304111

H $-2.662122-3.523801-0.445880$

H $-1.338463-3.055867-3.229333$

H $-0.714529-5.249636-2.189619$

H -0.302682 -4.447381 -0.699629

H $3.138751-3.798842-2.074953$

H $2.277490-4.208514-4.139207$

H $0.822596-5.207080-4.151477$

H $0.699108-3.441902-4.325686$

H $1.288058-3.844708 \quad 0.403557$

H $2.866988-4.5725420 .323229$

H $2.199445-1.5864500 .085576$

H $2.824354-2.3828801 .501695$

H $4.284996-1.928671-1.138221$

H $5.044417-4.133968-0.122634$

H $5.242808-3.3357191 .423866$

H $8.509470-3.5235940 .380925$

H $7.116838-1.4583331 .609311$

H $6.874953-4.021816-1.810391$

H $8.017114-2.697181-1.794642$

H $6.307574-2.386012-2.156313$ 
H $8.973911-1.5078470 .362356$ H $\quad 6.125227 \quad 0.6716472 .106938$ H $7.176341 \quad 1.3441780 .855125$ H $6.689620-0.382658-1.613503$ H $\quad 0.3608720 .067369-0.745638$ H -1.675986 $1.790296-2.283472$ H $1.7412612 .139374-0.598554$ H $0.634742 \quad 1.9355740 .779225$ H 1.2780063 .5146940 .391178 H 4.1277451 .6482030 .744338 H 5.1530412 .7212271 .705111 H $5.1291084 .528812-2.445959$ H $4.7069865 .748240-1.228128$ H $2.6630464 .003419-2.801472$ H $2.6243046 .881174-1.747196$ H $0.6421965 .130236-3.387682$ H $0.9094646 .840915-3.627808$ H -1.716800 $7.684723-1.202550$ H $-0.7838518 .188017-2.719974$ H -1.229136 $5.699378-0.060898$ H $0.4717505 .317734-0.098146$ H -1.698414 $4.024366-1.896657$ H 3.6136823 .8672850 .051542 C $-5.984868-2.339084-1.596366$ O $-5.432211-1.349780-0.703488$ C $-5.690701-0.043242-1.256735$ C $-6.610319-0.255635-2.458899$ C $-6.184340-1.629717-2.934259$ H $-4.7283730 .341131-1.613971$ C $-6.2753210 .896454-0.195510$ H -7.667108 $-0.264862-2.167376$ H $-6.4815850 .521528-3.218608$ C -7.192473 -2.303304 -3.847812 H $-5.233540-1.518152-3.469109$ C -5.4019481 .1078341 .057606$ $\begin{array}{lllll}\text { O } & -7.537247 & 0.377267 & 0.247282\end{array}$ H -6.498491 $1.867045-0.654271$ H $\quad-5.3915470 .1965281 .666760$ O -6.0376992 .1181461 .853144$ C $-3.958570 \quad 1.5587190 .773069$ C -3.1803702 .0671432 .002506$ H -3.4070530 .7305450 .315924$ O $-3.9689852 .618975-0.188075$ H $-2.1732112 .338857 \quad 1.663402$ H -3.618206 3.0136722 .345358 H $-6.840048-3.293531-4.153061$ H -8.162114 -2.427312 -3.354190 H -7.350263 - $1.706928-4.752380$ C -5.119760 -3.603392 -1.606506 H -7.378787 -0.5153960 .599962$ H -6.9429861 .8141712 .037549$ H -4.4906273 .3498510 .185897$ H - $-5.636825-4.397984-2.157288$ H -5.005274 -3.957560 -0.574051 H -6.961864 -2.609050 -1.172412 C $-1.319633-0.3290902 .374025$ O $-2.691296-0.2277832 .765768$ C -3.1001591 .0769473 .180474$ C -2.228979 1.5586954 .350069 C -0.7378871 .4802104 .026563$ C -0.3735620 .0887903 .506971$ H -1.154049 0.3317061 .515963 C $-1.083123-1.7495381 .849381$ H -4.1194990 .9680633 .570424$ O -2.5826432 .8911554 .705989$ H $-2.4326420 .927806 \quad 5.225343$ H -0.4468292 .2627523 .316079$ O $-0.004458 \quad 1.737956 \quad 5.226553$ H -0.411609 -0.6154114 .345808$ O $\quad 0.973110 \quad 0.1265393 .040113$ C -1.250233 -2.846654 2.879386 H -1.811975 -1.957268 1.059728 H $-0.077420-1.8189291 .422631$ H -1.997149 3.1626365 .433618 H 0.9399561 .6475785 .012395 H $1.253220-0.7882982 .869229$ O $-0.665034-3.9868352 .406356$ O $-1.844542-2.7368603 .942622$
C $-0.758247-5.129753 \quad 3.261899$

H $-1.806288-5.4072573 .409063$

H $-0.239996-5.9606622 .776007$

H $-0.273069-4.926337 \quad 4.221325$

SCF Energy (B3LYP/6-31G**//MMFF) $=-3245.91628006$

01146

MM̄FF Geometry

C $1.2227554 .011472-0.013272$

C 1.5939252 .7294730 .116054

C 1.9225902 .0983051 .442755

O 1.1812690 .8584021 .559191

C 3.4134371 .7391011 .547753

C 4.3224922 .9342281 .736616

C 5.1300513 .4450370 .787089

C 4.3221463 .5289883 .121830

C $5.2751793 .004376-0.642724$

C $6.6444862 .369629-0.923678$

C $6.8263320 .992748-0.265739$

C $8.2326820 .451176-0.529317$

C $8.413284-0.9675050 .032704$

O $5.8519830 .108376-0.826864$

O $9.620509-1.509904-0.524270$

C $7.247495-1.849596-0.462204$

C $8.604835-0.9566751 .553752$

O $7.297821-3.1432350 .136614$

C $5.851240-1.209708-0.265619$

C $4.786520-2.043178-1.016811$

O $5.542088-1.1935781 .128832$

C $-0.1096690 .935367 \quad 1.974889$

O $-0.731694 \quad 1.9577122 .231934$

C -0.732868 -0.403095 2.096089

C $-0.085093-1.5583411 .877384$

C $-0.658863-2.9042891 .979471$

C -2.094885 -3.013620 2.422225

C $3.389099-1.406794-1.055840$

C $2.424579-2.220980-1.900922$

C $0.053780-2.262946-2.652062$

O $2.743737-3.262827-2.468288$

C $-0.653908-3.187865-1.714658$

C $-0.502778-4.520572-1.726455$

C $-1.188927-5.460377-0.775628$

C $-0.237279-6.0249400 .257438$

C $0.558217-7.068220-0.032879$

C $-0.259884-5.4223161 .646807$

C $0.114080-3.9629891 .654150$

N $1.161935-1.643881-1.948737$

H 1.1834374 .6389420 .873343

H $1.6519152 .094896-0.765075$

H 1.6427002 .7497192 .279851

H 3.7093751 .1395650 .680446

H 3.5588591 .0556462 .396011

H 5.7575194 .2952521 .052882

H 5.0527124 .3381843 .227460

H 3.3385053 .9418723 .364796

H 4.5724232 .7635493 .863780

H $4.4720332 .331078-0.955462$

H $5.1713653 .897436-1.271971$

H $6.7389152 .260599-2.011961$

H $7.4414453 .048888-0.596092$

H $6.655478 \quad 1.0846600 .812242$

H $8.9933951 .126660-0.118628$

H $8.4143640 .419452-1.612934$

H $10.355050-0.921633-0.278631$

H $7.406092-2.010618-1.537407$

H $9.473174-0.3443201 .825743$

H $8.819977-1.9621231 .932074$

H $7.741088-0.5611422 .092027$

H $6.966723-3.0607791 .047183$

H $5.116335-2.185610-2.054693$

H $4.703847-3.038621-0.561854$

H $4.579256-1.1717651 .241948$

H -1.778756 -0.382592 2.387052

H $0.963206-1.5281921 .586595$

H -2.748769 -2.458480 1.743026

H $-2.215849-2.6088533 .433101$

H $-2.474750-4.0363252 .444449$

H $2.971286-1.333162-0.046692$ 
H $3.440275-0.399651-1.484585$ H $0.430781-2.788027-3.536281$ H $-0.623410-1.469881-2.983658$ H -1.316543 -2.729801-0.986909 H $\quad 0.167285-4.969954-2.456248$ H $-1.630726-6.277702-1.360400$ H $-2.036295-4.974809-0.277127$ H $1.226523-7.4949820 .707560$ H $\quad 0.558648-7.517459-1.020726$ H $0.451566-5.9430702 .301054$ H - $1.243068-5.6105912 .085613$ H $1.143695-3.7776591 .344237$ H $0.966189-0.829102-1.374039$ C $-0.617130 \quad 5.021785-1.448515$ O $-1.4263053 .828394-1.421933$ C $-2.5793014 .076450-0.598662$ C $-2.652907 \quad 5.589749-0.424190$ C $-1.1827325 .954482-0.376274$ H $-2.372265 \quad 3.6115270 .373700$ C $-3.8480783 .450127-1.191182$ H -3.135784 6.074265 -1.280897 H $-3.1978645 .877622 \quad 0.479926$ C $-0.9056367 .425399-0.632135$ H $-0.804806 \quad 5.6918260 .618412$ C $-3.7427251 .918140-1.366671$ O $-4.1305644 .076741-2.441508$ H -4.678986 $3.681142-0.513706$ H $-3.2932801 .491659-0.462487$ O $-2.8866221 .596592-2.461084$ C $-5.1123711 .246093-1.587926$ C $-5.068486-0.266428-1.867677$ H $-5.7338961 .430048-0.704757$ O $-5.794876 \quad 1.855037-2.687011$ H $-6.107923-0.615148-1.917396$ H $-4.671105-0.440696-2.876410$ H $0.1693667 .629225-0.600901$ H -1.281193 7.740928 -1.611349 H $-1.389898 \quad 8.0459620 .128916$ C $0.865324 \quad 4.656160-1.322748$ H $-5.002905 \quad 3.765277-2.735461$ H $-2.0245692 .013343-2.292694$ H $-5.2489551 .721328-3.481131$ H $1.4794095 .556172-1.443298$ H $1.1288183 .979700-2.145840$ H $-0.7719965 .463912-2.441673$ C $-5.843448-1.101575 \quad 0.974986$ O $-4.540501-0.7526290 .498950$ C $-4.273941-1.112217-0.856241$ C $-4.441758-2.627480-1.042796$ C $-5.797066-3.120946-0.542350$ C $-6.064769-2.6146520 .874153$ H $-6.604887-0.5695980 .391360$ C $-5.935052-0.6286842 .428977$ H $-3.214218-0.884673-1.026063$ O $-4.285661-2.996962-2.408514$ H $-3.662590-3.150149-0.476289$ H -6.607548 -2.832375 -1.221687 O $-5.791966-4.550488-0.510763$ H $-5.409753-3.1413501 .580035$ O $-7.411275-2.9162421 .233536$ $\begin{array}{llll}\text { C } & -5.925827 & 0.879829 & 2.501838\end{array}$ H $-6.857302-0.9708982 .911804$ H $-5.100107-1.0159833 .026050$ H -3.415082 -2.677564 -2.700819 H $-5.578844-4.858245-1.408543$ H $-7.529584-3.8765291 .134522$ O $-4.656596 \quad 1.3297702 .718234$ $\begin{array}{lllll}\text { O } & -6.924136 & 1.573636 & 2.358040\end{array}$ C -4.5108472 .7508842 .796302$ H -4.9174413 .2298461 .901184$ H $-5.008773 \quad 3.127828 \quad 3.694411$ H -3.444328 2.9808552 .861868 SCF Energy (B3LYP/6-31G*//MMFF)= -3245.92612665

01_147

MM̄FF Geometry

C -3.537451 1.6853352 .058927

C $-2.677477 \quad 1.0772242 .888943$
C $-2.349746-0.3889272 .804581$

O $-0.903786-0.4776892 .811869$

C $-2.894403-1.1938673 .996968$

C $-4.395809-1.3716883 .998428$

C $-5.043208-2.338043 \quad 3.317945$

C $-5.162829-0.4283594 .887838$

C $-4.450603-3.3781252 .402303$

C $-5.252410-3.5251861 .099642$

C $-5.054843-2.3536520 .121222$

C $-6.256996-2.254966-0.820860$

C $-6.078375-1.139524-1.856595$

O $-3.859428-2.600352-0.634849$

O $-7.094249-1.301830-2.858182$

C $-4.718994-1.335437-2.550796$

C $-6.2909420 .248786-1.245936$

O $-4.439603-0.247917-3.432270$

C $-3.529792-1.574759-1.582557$

C $-2.313616-2.039942-2.421374$

O $-3.210925-0.345541-0.931215$

C $-0.341632-1.5827092 .255522$

O $-0.920183-2.5094121 .712251$

C $1.121157-1.4832222 .445306$

C $1.896316-2.5136242 .077685$

C $3.351216-2.5909772 .202608$

C $4.069692-1.4108202 .797789$

C $-1.079804-2.496173-1.631667$

C $0.031320-2.936694-2.576014$

C $2.388623-3.654932-2.653134$

O $-0.103427-2.966206-3.797852$

C $3.384300-4.324787-1.763215$

C $4.633499-3.869656-1.579206$

C $5.674234-4.561912-0.744363$

C $6.110221-3.735556 \quad 0.445307$

C $7.101350-2.8349630 .336425$

C $5.447107-4.0079491 .776557$

C $3.974327-3.7034131 .763974$

N $1.191719-3.308251-1.907253$

H $-4.0599381 .084974 \quad 1.321059$

H $-2.163257 \quad 1.6609483 .648591$

H -2.722736 -0.8000641 .858750$

H $-2.570394-0.7301644 .939199$

H -2.417762 -2.1825294 .024272$

H -6.124258 -2.403868 3.436304

H $-6.239869-0.6270584 .874287$

H $-4.823820-0.5259965 .924450$

H -5.0181570 .6085814 .572293$

H -3.402981 -3.178192 2.167361

H $-4.481673-4.3376922 .932621$

H $-4.941566-4.4584540 .612699$

H $-6.314356-3.6346461 .354191$

H $-4.939525-1.4200580 .681498$

H $-7.184129-2.100541-0.254411$

H $-6.384923-3.208933-1.351937$

H -7.960241-1.253255 -2.417905

H -4.822131 -2.220666 -3.193519

H $-7.2910230 .324882-0.802566$

H $-6.244731 \quad 1.029849-2.012556$

H $-5.5655450 .483680-0.466310$

H $-4.1469720 .504096-2.889499$

H -2.625072 -2.884354 -3.051363

H $-2.008234-1.228984-3.095845$

H -2.287088 $-0.375108-0.636691$

H $1.515490-0.5772852 .890729$

H $1.421338-3.3918401 .641632$

H $3.803011-1.2923833 .853609$

H $3.803145-0.4926602 .266026$

H $5.157867-1.4910442 .743062$

H $-0.697413-1.683239-1.008068$

H $-1.333765-3.344484-0.987033$

H $2.779356-2.734479-3.099608$

H $2.110330-4.328232-3.471266$

H $3.074358-5.246540-1.277097$

H $4.945185-2.959046-2.085680$

H $6.539709-4.768469-1.387877$

H $5.325210-5.545405-0.405584$

H $7.435589-2.2544121 .189802$

H $7.595556-2.653433-0.612504$

H $5.951492-3.4712942 .587554$ 
H $5.577105-5.0694032 .025153$ H $3.368271-4.5076041 .345709$ H $1.282819-3.066361-0.924475$ C -3.3649253 .9103720 .836665$ $\begin{array}{lllll}\text { O } & -1.926660 & 3.840228 & 0.744834\end{array}$ C - $1.5746243 .455562-0.594437$ C $-2.7921733 .787685-1.448210$ C $-3.9187143 .420438-0.504144$ H -1.428938 2.367125 -0.575896 C $-0.2719114 .117674-1.060366$ H -2.838887 $4.855027-1.692782$ H -2.812919 $3.220208-2.383525$ C $-5.2580514 .032309-0.875130$ H $-4.0195002 .331267-0.517018$ C $0.9178713 .849623-0.110920$ O $-0.4847745 .521309-1.200667$ H $-0.0427703 .719414-2.056725$ H $\quad 0.885474 \quad 2.801408 \quad 0.206152$ O $\quad 0.813768 \quad 4.6533601 .062507$ C $2.2803814 .112225-0.780058$ C 3.5139683 .9561690 .127161 H $2.3774733 .433855-1.634500$ O $2.3223785 .435182-1.322073$ H $4.3977454 .040778-0.518551$ H 3.5851214 .8204670 .801097 H $-6.0299693 .740108-0.156165$ H -5.209273 $5.126066-0.893580$ H $-5.5738333 .693762-1.867337$ C -3.8470003 .1564332 .082638$ H $0.3096365 .898801-1.614191$ H -0.0480374 .4655181 .471384$ H $2.2205016 .057921-0.581565$ H -4.9305523 .2804582 .192034$ H -3.3824383 .6115582 .966567$ H -3.6023934 .9736410 .970883$ C $4.0574601 .098122-0.809303$ $\begin{array}{llllll}\text { O } & 3.177032 & 1.505077 & 0.240113\end{array}$ C 3.5920832 .6629460 .961010 C 4.9650292 .4157611 .605499 C 5.9959141 .9237990 .586684 C $5.4393600 .763823-0.241142$ H $4.1421371 .895765-1.557345$ C $3.420186-0.119945-1.481849$ H 2.8710802 .7823951 .779517 O $5.423303 \quad 3.6066792 .237089$ H 4.8563591 .6584822 .391571 H $6.3353742 .737403-0.064997$ O 7.1555241 .4508591 .276375 H $5.384329-0.1363690 .381979$ O $\quad 6.332496 \quad 0.481536-1.316097$ C $2.1194300 .259995-2.149828$ H $4.087246-0.563186-2.229158$ H $3.190203-0.904012-0.750645$ H 6.2291803 .3871362 .733836 H $7.5703792 .214761 \quad 1.711269$ H $7.2023540 .287773-0.926539$ O $2.3233090 .490805-3.477273$ O $1.0543630 .365960-1.555013$ C $1.159730 \quad 0.876554-4.215544$ H $\quad 0.395110 \quad 0.095804-4.156414$ H $\quad 0.7713121 .827126-3.837921$ H $1.4484231 .005919-5.261970$ SCF Energy (B3LYP/6-31G**//MMFF) $=-3245.92506079$

01148

MM̄FF Geometry

C $0.9260182 .908151-1.262582$

C $0.1471933 .995220-1.384341$

C -1.361115 $4.038490-1.461497$

O $-1.8743052 .695537-1.631647$

C $-1.9937264 .634187-0.181713$

C -1.432172 5.9601440 .288837

C $-0.724850 \quad 6.134411 \quad 1.424353$

C -1.730889 $7.135076-0.605673$

C -0.3494615 .0888292 .437889$

C 1.1493554 .7615192 .386467

C 1.4513003 .3941943 .018015

C 2.9595033 .1540223 .103562
C $3.290092 \quad 1.736338 \quad 3.594897$

O 0.8453872 .4008022 .183007

O $4.679507 \quad 1.4899143 .329716$

C 2.4848530 .7140872 .764846

C 3.1074911 .6030615 .111440

O $2.648994-0.6038723 .283722$

C 0.9802941 .0550572 .653228

C $0.3088670 .118494 \quad 1.615882$

O $\quad 0.363714 \quad 0.878743 \quad 3.927003$

C $-3.1439942 .552263-2.094803$

O $-3.8955703 .432609-2.480192$

C $-3.4807791 .116209-1.996335$

C $-4.7650630 .731747-1.999675$

C $-5.251549-0.642116-1.854146$

C $-4.224244-1.738741-1.752185$

C $-1.1463730 .465902 \quad 1.263810$

C $-2.183719-0.1733242 .171320$

C $-4.646911-0.2199622 .566961$

O $-1.897975-0.9506853 .077716$

C $-5.254598-1.3905201 .858992$

C $-6.555313-1.4640961 .536148$

C -7.188647 -2.656210 0.875953

C $-8.020474-2.288317-0.333383$

C $-9.355072-2.160760-0.240401$

C $-7.316700-2.157339-1.665296$

C $-6.583323-0.852512-1.801988$

N $-3.4718870 .229448 \quad 1.843114$

H $0.4840681 .918096-1.209844$

H $0.6361944 .967413-1.422032$

H -1.633177 $4.634230-2.341919$

H -1.9528043 .8738550 .604235$

H -3.071772 $4.767290-0.344752$

H -0.3693337 .1362341 .661660$

H -1.352593 $8.077266-0.194756$

H -1.271634 $6.997301-1.589448$

H $-2.8121597 .244845-0.738384$

H -0.5992305 .4687903 .436545$

H -0.9467314 .1823822 .320122$

H 1.4888834 .7543801 .343357

H 1.7076525 .5506162 .905158

H 0.9979013 .3579014 .015615

H 3.4355953 .9048543 .746722

H 3.4087453 .2882322 .110148

H 5.1979622 .1732003 .788273

H 2.9133970 .7034911 .752572

H 3.7655232 .3038495 .639305

H 3.3950790 .6044505 .458124

H 2.0850381 .8021745 .439173

H $3.603982-0.7849333 .317868$

H $0.8795110 .187216 \quad 0.680336$

H $\quad 0.367981-0.9209151 .958447$

H -0.5962220 .8209503 .803278$

H -2.661552 $0.417982-1.877936$

H $-5.5359551 .495314-2.095939$

H -3.544389 -1.554170 -0.913357

H $-3.638143-1.796888-2.675531$

H -4.652138 -2.728594 -1.582808

H $-1.303191 \quad 1.5487841 .247518$

H -1.3497440 .0915550 .253563$

H -5.3349200 .6292582 .627116$

H -4.361012 -0.5113523 .582590$

H -4.601276 -2.229172 1.629883

H -7.212694 -0.637555 1.796895

H -7.822616 -3.1503991 .623780$

H $-6.436604-3.4012030 .587775$

H $-9.962677-1.926246-1.108182$

H -9.869114 -2.282614 0.707300

H -8.047354 -2.205404 -2.484024

H $-6.673734-3.028118-1.820476$

H -7.247646 $0.009811-1.875175$

H -3.6140620 .8302401 .036338$

C $3.2331652 .200255-2.135719$

O $3.1323840 .788362-1.886827$

C $3.5028180 .101045-3.103434$

C $3.6341011 .171858-4.192427$

C $2.8727112 .349440-3.606443$

H $2.672708-0.563429-3.357791$

C $4.781292-0.718941-2.869171$ 
H $4.681111 \quad 1.437720-4.378820$

H $3.216006 \quad 0.832967-5.145924$

C $3.2571243 .687532-4.210218$

H $1.8012012 .172875-3.763092$

C $4.667902-1.723552-1.704047$

O $5.8471290 .189102-2.557339$

H $5.073082-1.228394-3.794424$

H $4.491641-1.171721-0.774833$

O $5.945801-2.355966-1.545602$

C $3.596451-2.816423-1.890521$

C $3.505392-3.779348-0.689455$

H $2.612833-2.373516-2.070820$

O $3.917958-3.603910-3.039917$

H $3.010476-4.699902-1.020047$

H $4.511515-4.094263-0.389123$

H $2.6870764 .501015-3.750392$

H $4.3220793 .900544-4.068938$

H $3.0517843 .697549-5.285542$

C $2.4196122 .995229-1.116958$

H $6.644884-0.344124-2.398570$

H $6.106035-2.894299-2.339748$

H $3.776209-3.050160-3.825957$

H $2.7400994 .043638-1.156145$

H $2.6661602 .626016-0.114686$

H $4.2890652 .464092-1.981040$

C $0.519598-3.833410-0.116727$

O $1.428230-2.758478 \quad 0.150205$

C $2.753409-3.1657680 .509666$

C $2.706322-4.053647 \quad 1.760569$

C $1.737022-5.2222351 .602258$

C $0.372545-4.726559 \quad 1.119452$

H $0.884847-4.427335-0.963118$

C $-0.821004-3.216166-0.523862$

H $3.287857-2.2513070 .791734$

O $4.001057-4.5546602 .076565$

H $2.378147-3.4509152 .617412$

H $2.147942-5.9834970 .928347$

O $1.585803-5.8491482 .878026$

H $-0.127236-4.1756281 .926695$

O $-0.445047-5.8465540 .787700$

C $-0.758449-2.712162-1.945679$

H -1.636725 -3.947219 -0.486784

H -1.105462 -2.391573 0.137905

H $4.589614-3.7893252 .194097$

H $1.058267-6.6550852 .748562$

H $-0.623415-6.3343971 .609152$

O $-0.370797-1.405985-1.969767$

O $-1.007974-3.408535-2.921613$

C $-0.285080-0.819595-3.272178$

H $\quad 0.0602980 .211082-3.159797$

H $0.432614-1.367012-3.890133$

H -1.270468 -0.809482 -3.747304

SCF Energy (B3LYP/6-31G**//MMFF) $=-3245.90844024$

$01 \_149$

MM̄FF Geometry

C -4.129669 1.0363302 .189198

C -3.2377520 .5405403 .060156$

C -2.492597 -0.7513482 .857436$

O $-1.093598-0.4230083 .041876$

C $-2.898993-1.8355613 .870504$

C $-4.287120-2.3959633 .654375$

C $-4.575652-3.4113902 .816871$

C -5.373471-1.792689 4.507695

C $-3.638744-4.1780211 .919693$

C $-4.188784-4.3079500 .489840$

C -3.902500 -3.075072 -0.384472

C $-4.888679-3.017841-1.553361$

C $-4.577208-1.856468-2.505752$

O $-2.561095-3.199086-0.878918$

O $-5.381345-2.019101-3.683218$

C -3.093504 -1.938088 -2.909417

C $-4.995240-0.509347-1.913209$

O $-2.720128-0.808022-3.696736$

C $-2.119710-2.121550-1.715848$

C $-0.725393-2.483127-2.284446$

O $-2.037328-0.896872-0.987842$

C $-0.167230-1.2585352 .504807$
O $-0.372577-2.310591 \quad 1.922847$

C $1.162351-0.6574182 .746906$

C $2.266498-1.3836842 .521950$

C $3.646141-0.9300462 .701776$

C 3.8737990 .5333272 .970734

C $0.348137-2.832473-1.247492$

C $1.637367-3.282374-1.919771$

C $3.913591-4.116220-1.427128$

O $1.769048-3.352628-3.139574$

C $4.911077-3.969838-0.325384$

C $5.949869-3.122261-0.374514$

C $6.971098-2.9715620 .716535$

C $6.858618-1.6449811 .435775$

C $7.456070-0.5418470 .955007$

C $6.119685-1.6196882 .755013$

C $4.640400-1.8351362 .595698$

N $2.632244-3.585205-0.999595$

H $-4.3672170 .462888 \quad 1.298789$

H $-3.007792 \quad 1.1026213 .962527$

H -2.630988 -1.108646 1.830595

H $-2.808477-1.4432314 .893198$

H -2.171613 -2.656629 3.850677

H -5.608010 -3.758959 2.783754

H -6.344962 -2.271511 4.344548

H -5.127183 -1.905573 5.568650

H $-5.495383-0.7280164 .292110$

H -2.633347 -3.7505611 .898511$

H -3.545395 -5.186414 2.341662

H $-3.721615-5.1874140 .027584$

H $-5.267317-4.5051300 .534034$

H $-3.985281-2.1670670 .221187$

H -5.921364 -2.943902 -1.189820

H $-4.837137-3.959630-2.117629$

H $-5.161547-2.876003-4.086672$

H -2.992977 -2.808880 -3.571472

H $-6.063138-0.513597-1.663994$

H $-4.8611710 .302317-2.637091$

H -4.442133 -0.257238 -1.007593

H -2.590446 -0.056921 -3.092762

H $-0.835000-3.350588-2.949427$

H $-0.352092-1.652585-2.896749$

H -1.217090 -0.894908 -0.469865

H $\quad 1.1958600 .364143 \quad 3.108541$

H $2.152717-2.4131122 .184773$

H 3.5392680 .7941153 .980458

H 3.3231591 .1485642 .251496

H $4.921436 \quad 0.8306152 .882131$

H $0.580903-1.959711-0.629401$

H $0.002324-3.644557-0.598944$

H $4.226775-3.601169-2.342112$

H $3.774092-5.174376-1.671810$

H $4.776100-4.6007430 .549683$

H $6.086330-2.501739-1.256993$

H $7.967747-3.0660430 .265571$

H $6.903380-3.7945671 .439130$

H 7.3996270 .4065491 .479014

H $8.009519-0.5628920 .022014$

H $6.329424-0.693513 \quad 3.300808$

H $6.518282-2.4133463 .400297$

H $4.372209-2.8740222 .404202$

H $2.375555-3.668414-0.019772$

C -4.5782783 .3619601 .245182$

O $-3.1604593 .548921 \quad 1.057162$

C $-2.8585973 .357066-0.336885$

C $-4.1683443 .621749-1.067870$

C $-5.1638872 .983422-0.118233$

H -2.599161 $2.299799-0.463754$

C $-1.6821744 .235751-0.771422$

H $-4.3725234 .694401-1.162449$

H $-4.1851313 .179075-2.068277$

C $-6.5935203 .454604-0.323431$

H $-5.1358781 .899668-0.264957$

C -0.4046864 .0979750 .083456$

O $-2.0986405 .607421-0.697583$

H -1.457439 $4.045342-1.826995$

H $-0.6154714 .508601 \quad 1.079178$

O $0.5870524 .957198-0.490716$

C 0.1846532 .6827980 .237432 
C $0.3574471 .858213-1.048331$

H $1.1627972 .758144 \quad 0.722703$

O

H $0.726800 \quad 0.869922-0.745698$

H $-0.6263571 .656249-1.487891$

H -7.2658802 .9802510 .398724$

H -6.680524 4.539768 -0.203796

H $-6.9418823 .198720-1.329400$

C -4.8313732 .3554322 .375517$

H $-1.3484346 .153393-0.989228$

H 1.3755634 .9178020 .077136

H -0.7072782 .4035121 .957362$

H -5.9075502 .1731482 .474671$

H -4.4981002 .8105113 .317089$

H $-4.9725384 .336392 \quad 1.560694$

C $3.4111411 .793652-1.174273$

O $2.5572102 .860439-1.604184$

C $1.2867952 .458362-2.121450$

C $1.4917141 .545855-3.337510$

C $2.3718810 .350158-2.991511$

C $3.6679200 .803390-2.322777$

H $2.9530181 .276774-0.322386$

C $4.7320382 .425714-0.701048$

H $0.8030363 .369141-2.491608$

O $\quad 0.2445591 .078587-3.840686$

H $1.9746082 .112105-4.144430$

H $1.839680-0.367004-2.358935$

O $2.706396-0.348800-4.192772$

H $4.3176521 .255731-3.083448$

O $4.327258-0.360436-1.827399$

$\begin{array}{llll}\text { C } & 4.582218 & 3.244933 & 0.563801\end{array}$

H $5.4642901 .638543-0.493219$

H $5.1355083 .093524-1.470903$

H $-0.292101 \quad 1.858526-4.062618$

H $1.871207-0.627156-4.606442$

H $5.247732-0.122166-1.628960$

O $5.800092 \quad 3.3454291 .175360$

O 3.5435653 .7578330 .955427

C $5.818776 \quad 4.1031012 .389293$

H 5.5413595 .1424732 .189796

H 5.1440393 .6571623 .126423

H 6.8365104 .0813172 .787829

SCF Energy (B3LYP/6-31G**//MMFF) $=-3245.91013366$

01

MM̄FF Geometry

C -3.218932 3.3512840 .011532

C $-2.843973 \quad 4.3258960 .853965$

C -1.620705 $4.326109 \quad 1.745709$

O $-0.841968 \quad 3.117317 \quad 1.595909$

C $-0.707249 \quad 5.5114901 .373914$

C $0.428681 \quad 5.733613 \quad 2.355771$

C $1.738140 \quad 5.5792192 .075444$

C $0.013668 \quad 6.192843 \quad 3.731651$

C $2.378095 \quad 5.1765770 .775193$

C $2.767138 \quad 3.6997010 .796006$

C $3.6364213 .251401-0.387819$

C $2.9417483 .435483-1.735819$

C $3.7676822 .859628-2.894990$

O $3.8986361 .860294-0.174415$

O $2.8938642 .805238-4.033856$

C $4.1919131 .414889-2.550237$

C $4.9163513 .800979-3.275938$

O $5.115197 \quad 0.905076-3.509092$

C $4.7806791 .254982-1.128327$

C $4.878567-0.241876-0.757131$

O $6.103537 \quad 1.785796-1.035533$

C -1.1798472 .0373412 .352450$

O $-2.094154 \quad 1.954183 \quad 3.155898$

C -0.2391550 .9492732 .002879$

C $-0.475889-0.3103232 .398090$

C $0.361263-1.4728542 .085938$

C $1.678962-1.2122511 .404252$

C $5.430233-0.4698530 .656184$

C $5.305111-1.9148991 .105659$

C $5.465798-3.2941583 .182395$

O $4.958858-2.8275140 .361513$

C $4.100460-3.3060693 .797144$
C $3.260315-4.3497003 .729221$

C $1.889327-4.3467294 .344926$

C $0.818506-4.8339013 .392724$

C $0.124049-5.9542393 .654716$

C $0.553797-4.0366872 .134213$

C $-0.086232-2.7029822 .414911$

N $5.630529-2.0533132 .448475$

H -2.613918 $2.456589-0.084308$

H $-3.477399 \quad 5.207493 \quad 0.935265$

H -1.9591694 .4219042 .784255$

H -1.2939776 .4389741 .326741$

H $-0.319715 \quad 5.349324 \quad 0.362596$

H 2.4550935 .7672922 .874362

H $\quad 0.8705796 .484299 \quad 4.348627$

H $-0.6448667 .064668 \quad 3.658985$

H $-0.516277 \quad 5.396863 \quad 4.263075$

H $1.7252405 .391958-0.074280$

H 3.2730465 .7947360 .632530

H $3.307033 \quad 3.466640 \quad 1.724444$

H 1.8611483 .0836730 .829572

H $4.5827113 .803490-0.353643$

H $2.7133414 .489829-1.929780$

H $1.9750182 .917680-1.708966$

H $3.4097632 .492724-4.796069$

H $3.2925340 .788538-2.619840$

H $4.5203424 .759162-3.633752$

H $5.5070123 .393643-4.103645$

H $5.5889434 .013886-2.442157$

H $4.6733020 .906700-4.374722$

H $3.881742-0.696431-0.823807$

H $5.526609-0.768490-1.469021$

H $6.3580192 .149082-1.898069$

H $0.619162 \quad 1.211467 \quad 1.395821$

H $-1.373782-0.5157802 .979034$

H $2.286497-0.5179501 .995913$

H $1.522760-0.7810600 .409918$

H $2.284713-2.1088291 .270205$

H $6.489634-0.1919870 .697132$

H $4.8862490 .151071 \quad 1.377425$

H $6.228849-3.3117003 .966295$

H $5.624574-4.1419582 .508308$

H $3.788560-2.4104474 .330160$

H $3.578149-5.2572463 .221287$

H $1.619599-3.3463814 .705121$

H $1.925867-4.9885905 .234658$

H $-0.644422-6.3151422 .979123$

H $\quad 0.306822-6.537327 \quad 4.551280$

H $-0.135240-4.5893011 .481611$

H $1.478168-3.9575491 .557298$

H $-1.054627-2.7837552 .910202$

H $5.842324-1.2189882 .988385$

C $-5.4854662 .325161-0.487270$

$\begin{array}{lllll}\text { O } & -4.952312 & 1.003660 & -0.717648\end{array}$

C -5.4156450 .1393840 .337798$

C $-6.4949390 .917751 \quad 1.088020$

C -5.9890952 .3382940 .955873$

H $-4.565082-0.0139641 .012099$

C $-5.905706-1.202957-0.212789$

H -7.4762110 .8171720 .609394$

H -6.5933950 .5904002 .127558$

C $-7.044410 \quad 3.394127 \quad 1.233488$

H -5.1596902 .4692131 .661661$

C $-4.852705-2.038331-0.970337$

O $-6.993604-0.953872-1.113547$

H $-6.323755-1.7936530 .611929$

H $-4.644569-1.579627-1.943726$

O $-5.464084-3.305040-1.258757$

C $-3.530435-2.275287-0.216693$

C $-2.671615-3.434406-0.755362$

H $-2.947752-1.348200-0.212637$

O $-3.834012-2.584655 \quad 1.148555$

H - $-1.762720-3.477768-0.144603$

H $-3.187336-4.380500-0.545353$

H $-6.629357 \quad 4.399988 \quad 1.114645$

H -7.8975403 .2983950 .553599$

H $-7.419643 \quad 3.3026702 .258008$

C $-4.4546013 .399841-0.842384$

H $-7.300830-1.816157-1.442222$ 
H $-5.617743-3.756925-0.411096$ H -2.991819 -2.642734 1.629942 H $-4.9246664 .388614-0.783579$ H $-4.1375783 .259028-1.882778$ H $-6.3328712 .429244-1.178164$ C $-0.304151-2.015807-2.075046$ O $-1.628703-2.153634-2.601805$ C $-2.302489-3.363827-2.249588$ C $-1.489848-4.574183-2.736121$ C $-0.065768-4.548057-2.190642$ C $0.586743-3.199124-2.484473$ H $-0.355526-1.934152-0.983701$ C $0.283864-0.711067-2.634264$ H -3.240791 -3.366505 -2.815981 O $-2.115931-5.795098-2.355117$ H -1.443023 -4.564899-3.832883 H $-0.046466-4.782224-1.120328$ O $0.685905-5.578074-2.837243$ H $0.808231-3.145492-3.558052$ O $1.838800-3.124920-1.804321$ C $-0.4684080 .517093-2.177284$ H $1.325723-0.591391-2.320645$ H $\quad 0.237443-0.737906-3.730153$ H -3.014396 -5.791019-2.727274 H $\quad 1.599090-5.520577-2.506898$ H $1.663604-3.188031-0.850122$ O $-0.1249301 .567318-2.979896$ O $-1.2428380 .556510-1.232444$ C $-0.7627332 .812483-2.681398$ H $-0.3545263 .572732-3.352734$ H $-1.8390092 .731531-2.855702$ H -0.554679 $3.110839-1.649636$

SCF Energy (B3LYP/6-31G**//MMFF) $=-3245.91119857$

01

MM̄FF Geometry

C $-3.0725413 .104542-0.672174$

C -3.3779963 .6035660 .535233$

C -3.6559002 .7839121 .772096$

O

C -2.3664952 .3746402 .503788$

C -1.5746313 .5275233 .079782$

C -0.3852593 .9528562 .611056$

C -2.1803444 .1797174 .296091$

C 0.3826343 .4444221 .422507

C 1.6732692 .7202351 .826583

C 1.4306471 .3525662 .484832

C 2.7607470 .7252512 .901146

C $2.576799-0.6842203 .478755$

O $0.7773990 .510699 \quad 1.530444$

O $3.871720-1.2992493 .551490$

C $1.731226-1.5127222 .490336$

C $2.033293-0.6476884 .911280$

O $1.425796-2.7956093 .033176$

C $0.443266-0.7964932 .014723$

C $-0.181529-1.5893850 .843401$

O $-0.484238-0.7390573 .098457$

C -5.7025851 .6543941 .249925$

O -6.3730922 .6780611 .296516$

C -6.3205860 .3426950 .951421$

C $-5.685535-0.8367031 .034863$

C $-6.274126-2.1511260 .753067$

C $-7.714594-2.184210 \quad 0.308409$

C -1.407355 -0.9255570 .200822$

C $-1.807077-1.616214-1.092156$

C $-3.522826-1.530445-2.892706$

O $-1.221943-2.601134-1.535737$

C $-4.619384-2.463881-2.487768$

C $-4.454042-3.790347-2.374750$

C $-5.520104-4.741091-1.911717$

C $-5.212289-5.312335-0.543604$

C $-4.407653-6.380220-0.407519$

C $-5.894101-4.6916090 .656737$

C $-5.503796-3.2530570 .882414$

N -2.899259 -1.002721 -1.692691

H $-2.9901992 .029591-0.803750$

H -3.429583 $4.683136 \quad 0.659311$

H -4.2892263 .3668082 .452198$
H -1.7542151 .7556651 .839703$

H -2.6273441 .6901453 .323206$

H 0.1008034 .7794463 .128474

H -1.526164 4.9452094 .727080

H -3.128249 4.6636564 .042448

H -2.3666413 .4326915 .074584$

H $-0.2241762 .808804 \quad 0.772231$

H 0.6528994 .3157750 .812419

H 2.2706752 .5770680 .917366

H 2.2610523 .3585742 .498223

H $\quad 0.782361 \quad 1.4826253 .358166$

H 3.2918381 .3679493 .614091

H 3.4106280 .6494352 .019505

H $4.440059-0.7387414 .107162$

H $2.363218-1.7020531 .612644$

H $2.705677-0.0746855 .561141$

H $1.988907-1.6537295 .342595$

H $1.041869-0.1957754 .982676$

H $\quad 0.712730-2.678973 \quad 3.683790$

H $\quad 0.581587-1.7210620 .066122$

H $-0.474355-2.5912041 .182690$

H -1.376579 -0.6046862 .743508$

H $-7.3672240 .403620 \quad 0.668933$

H $-4.642013-0.8524221 .340949$

H -7.854563 -1.570070 -0.588506

H $-8.369397-1.8054141 .100928$

H -8.073764 -3.1818040 .049649$

H -2.261092 -0.9685900 .884351$

H -1.204701 $0.125141-0.028691$

H -2.769735 -2.032033 -3.509915

H $-3.930627-0.689411-3.461594$

H -5.584679 -2.020380 -2.259746

H -3.482377 -4.222736 -2.604205

H $-5.597078-5.553424-2.646125$

H -6.507338 -4.263067 -1.904622

H $-4.199457-6.8131690 .565106$

H -3.936841 -6.843334 -1.268635

H $-5.633168-5.2426731 .570130$

H $-6.974101-4.8237200 .549726$

H -4.466999 -3.128444 1.197986

H $-3.378127-0.254840-1.199735$

C - $-1.3450114 .218169-2.123807$

O $-0.6453922 .982246-2.356881$

C $0.325277 \quad 3.180737-3.400768$

C $0.3494354 .679604-3.682464$

C $-1.0756235 .078221-3.357131$

H -0.059098 $2.654075-4.283474$

C $1.6938912 .592204-3.020729$

H $1.0500695 .205361-3.023414$

H $0.6328554 .901585-4.715852$

C $-1.2502016 .569220-3.125826$

H -1.724931 $4.762896-4.185052$

C $1.6356671 .077358-2.729950$

O $2.1919493 .297484-1.884071$

H $2.3862662 .775211-3.851449$

H $1.0994620 .585663-3.550602$

O $\quad 0.8797790 .839515-1.545719$

C $3.0189850 .404097-2.599226$

C $2.887422-1.089472-2.243817$

H $3.5508240 .522086-3.550824$

O $3.7789471 .064361-1.593100$

H $2.573763-1.179028-1.198636$

H $2.096824-1.526298-2.864940$

H $-2.2916846 .809370-2.890092$

H $-0.6272256 .922332-2.297166$

H $-0.969077 \quad 7.132626-4.021565$

C $-2.8319913 .951542-1.889273$

H $3.0821912 .957819-1.692529$

H $1.3475931 .268604-0.809372$

H $4.6893260 .729282-1.652451$

H $-3.2619543 .422731-2.748993$

H -3.372971 $4.900591-1.796969$

H $-0.8974614 .675615-1.231962$

C $5.195703-1.664821-0.269945$

O $5.286187-1.409877-1.677381$

C $4.191398-1.890500-2.461999$

C $4.032004-3.406202-2.269251$

C $3.892438-3.772164-0.793958$ 
C $5.043271-3.165828 \quad 0.006598$

H $4.344723-1.1137140 .147438$

C $6.478425-1.1274050 .378040$

H $4.476286-1.730579-3.509954$

O $2.909129-3.876622-3.006377$

H $4.918407-3.909676-2.676910$

H $2.923018-3.460215-0.387747$

O $3.955986-5.192384-0.648137$

H $5.974585-3.690677-0.243109$

O $4.803555-3.3657501 .397503$

C 6.5333840 .3796930 .310749

H $6.563133-1.4315431 .427688$

H $7.370162-1.507176-0.135543$

H $2.910367-4.847393-2.959562$

H $3.153534-5.564337-1.051500$

H $4.676318-4.3198201 .537186$

$\begin{array}{lllll}\text { O } & 6.009105 & 0.918600 & 1.447142\end{array}$

O $6.9635751 .002402-0.651709$

C 5.9573952 .3482241 .482663

H 6.9634212 .7681251 .389654

H 5.3061472 .7215600 .686566

H 5.5392962 .6493142 .446946

SCF Energy (B3LYP/6-31G**/MMFF)= -3245.91965981

01151

MM̄FF Geometry

C $3.326352-2.4386871 .478641$

C $2.796224-1.7361142 .490787$

C $2.948656-0.2443362 .619977$

$\begin{array}{lllll}\text { O } & 1.635976 & 0.353695 & 2.778753\end{array}$

C 3.7616140 .1616253 .860468

C $5.202222-0.2974763 .856034$

C $6.212547 \quad 0.376333 \quad 3.273715$

C $5.487372-1.5594444 .629976$

C 6.1421121 .6678682 .504691

C 6.9398851 .6008061 .193127

C 6.1826220 .9100640 .046449

C $7.1456860 .613760-1.106365$

C $6.4220670 .057428-2.339383$

O $5.1553021 .809501-0.384125$

$\begin{array}{lllll}\text { O } & 7.339738 & 0.097341 & -3.442927\end{array}$

C $5.2545371 .001868-2.685196$

C $6.028107-1.412585-2.155973$

O $4.4783990 .474419-3.759983$

C $4.3514541 .346252-1.476634$

C $3.3905982 .496423-1.867727$

O $3.5991550 .186399-1.126974$

$\begin{array}{llll}\text { C } & 0.829643 & 0.412733 & 1.684789\end{array}$

O $1.061121-0.037522 \quad 0.574795$

C $-0.393616 \quad 1.1499452 .069745$

C -1.2757111 .5089401 .124799$

C $-2.515543 \quad 2.258542 \quad 1.341238$

C - 2.8514242 .6502092 .756839

C $2.4893732 .991608-0.728882$

C $1.751004 \quad 4.270000-1.089456$

C $0.1594965 .919924-0.121219$

O $1.9161714 .865405-2.150680$

C $-1.215130 \quad 5.7162050 .430078$

C $-2.3297965 .886913-0.296743$

C -3.7239445 .7400320 .242498$

C $-4.4990744 .641006-0.448049$

C $-5.147298 \quad 4.870460-1.602585$

C $-4.591382 \quad 3.3016990 .246043$

$\begin{array}{lllll}C & -3.280341 & 2.566114 & 0.272386\end{array}$

N $0.885407 \quad 4.664505-0.076767$

H $3.918796-1.9219550 .729524$

H $2.207542-2.2451683 .249802$

H 3.4159940 .1919161 .728771

H $3.259714-0.2010514 .768775$

H 3.7215751 .2542383 .967782

H $7.218335-0.0292293 .380283$

H $6.552484-1.8152524 .630800$

H $5.178829-1.4402345 .673927$

H $4.948791-2.4105064 .204877$

H $5.113403 \quad 1.9824652 .305633$

H 6.5932122 .4426853 .136985

H $7.1679402 .632000 \quad 0.892008$

H 7.8996961 .0995091 .370694
H $5.725573-0.0164220 .410633$

H $7.935972-0.075596-0.783405$

H $7.6605011 .540500-1.397568$

H $8.117917-0.435687-3.205717$

H $5.6982631 .932693-3.064424$

H $6.916582-2.026081-1.963655$

H $5.577897-1.818818-3.068216$

H $5.335089-1.569815-1.327758$

H $3.917216-0.233359-3.399865$

H $3.9908173 .344839-2.222033$

H $2.7470422 .182448-2.699317$

H $2.8028790 .456223-0.644201$

H -0.5245831 .3925473 .118099$

H -1.0699031 .2373600 .090063$

H -2.0486413 .2585593 .188633$

H -2.9909941 .7585923 .378030$

H -3.7633183 .2435852 .844743$

H $1.7371932 .235180-0.492166$

H 3.0803333 .1909140 .172008

H $0.1417626 .308046-1.144973$

H $0.706894 \quad 6.636096 \quad 0.500279$

H $-1.2959645 .442792 \quad 1.479124$

H $-2.2394306 .177281-1.341379$

H -4.2425406 .6979090 .104170$

H -3.7172575 .5684651 .325978$

H $-5.7287854 .094824-2.089912$

H $-5.1066705 .840244-2.087673$

H $-5.3119302 .660173-0.274447$

H -5.0178803 .4476531 .241976$

H -2.948222 $2.240439-0.714484$

H $0.911376 \quad 4.1638120 .807371$

C $2.220853-4.295264 \quad 0.152274$

O $0.896597-3.7875440 .407885$

C $0.326655-3.341939-0.837417$

C $1.252187-3.859396-1.934270$

C $2.599912-3.794348-1.240512$

H $0.364901-2.245940-0.828697$

C $-1.133438-3.793220-0.970183$

H $1.013223-4.893295-2.210026$

H $1.204192-3.247516-2.840246$

C $3.682082-4.613050-1.920255$

H $2.913752-2.744266-1.210090$

C $-2.055101-3.1707990 .105436$

O $-1.172860-5.217810-0.912533$

H $-1.492983-3.494626-1.961759$

H $-1.902230-2.0854270 .099500$

O $-1.705922-3.6430281 .405646$

C $-3.551744-3.455051-0.130115$

C $-4.491498-2.853680 \quad 0.931835$

H -3.832202 -3.082001-1.120672

O $-3.790619-4.864616-0.149949$

H $-5.510794-3.1780440 .687557$

H $-4.283208-3.3111001 .907708$

H $4.626224-4.541270-1.371082$

H $3.406628-5.671109-1.982543$

H $3.855698-4.250451-2.938786$

C $3.155789-3.9197261 .304186$

H $-2.083382-5.495472-1.109094$

H $-0.779272-3.3994751 .569903$

H $-3.496828-5.2231960 .705412$

H $4.143008-4.3685021 .146085$

H $2.757401-4.3507582 .231669$

H $2.127296-5.3894810 .138174$

C $-5.783909-0.781101-0.869504$

O $-4.517266-0.664493-0.212402$

C $-4.436132-1.3181501 .055179$

C $-5.481736-0.7247362 .009537$

C $-6.887941-0.7787291 .412379$

C $-6.899282-0.1904490 .000691$

H $-5.993941-1.836443-1.082409$

C $-5.675279-0.043380-2.208241$

H -3.451194 -1.069868 1.468708

O $-5.444180-1.414820 \quad 3.253672$

H $-5.223783 \quad 0.3228842 .211769$

H $-7.287250-1.7997211 .412775$

O $-7.775850-0.000246 \quad 2.218371$

H -6.7956870 .8990230 .061967$

O $-8.160073-0.461194-0.609232$ 
C $-4.761125-0.784722-3.154836$

H $-6.6497890 .044751-2.701760$

H $-5.290468 \quad 0.974917-2.079216$

H -6.024459-0.940664 3.872576

H -7.852915 -0.4420833 .080708$

H -8.848200 -0.094385 -0.027954

O $-3.470615-0.386355-2.965525$

O $-5.147710-1.632480-3.948891$

C $-2.493789-1.039373-3.781960$

H -2.605904 -2.126472 -3.729981

H -2.584235 -0.692234 -4.815261

H -1.502756 -0.773363 -3.404313

SCF Energy (B3LYP/6-31G**//MMFF) $=-3245.92413628$

01

MM̄FF Geometry

C $-2.552333 \quad 3.1773441 .129682$

C -1.9840722 .4949722 .135469$

C -2.2264221 .0306842 .388364$

$\begin{array}{llll}\text { O } & -0.927328 & 0.384374 & 2.382105\end{array}$

C $-2.885106 \quad 0.779640 \quad 3.759252$

C -4.3867790 .9848953 .781596$

C $-5.284936-0.0095543 .630475$

C -4.8577782 .3841174 .079380$

C $-4.990180-1.4571343 .351474$

C $-5.987644-2.1198002 .390541$

C $-6.071252-1.4791250 .995278$

C $-7.078115-2.2480350 .131913$

C -7.144902 -1.709459-1.303308

O $-4.773729-1.507957 \quad 0.387514$

O $-7.857669-2.682166-2.082988$

C $-5.708573-1.614010-1.859469$

C $-7.964292-0.416439-1.381889$

O $-5.704741-1.005048-3.148455$

C $-4.726321-0.893608-0.909459$

C $-3.269648-0.937484-1.425794$

O

C $-0.893691-0.9233752 .006990$

O $-1.837698-1.5999051 .631137$

C $0.491838-1.4208802 .151315$

C $0.756260-2.6865251 .791887$

C $2.044690-3.3729951 .879141$

C $3.196950-2.6337702 .501200$

C $-2.652270-2.332166-1.564348$

C -1.215658 -2.260131-2.060863

C $0.625580-3.724768-2.859252$

O $-0.656783-1.202057-2.338540$

C $1.393311-4.822580-2.195609$

C $2.689286-4.710792-1.864364$

C $3.495347-5.806382-1.224868$

C $4.143818-5.3697300 .071205$

C $5.412057-4.9259530 .091894$

C $3.350140-5.5148441 .350531$

C $2.138444-4.6266101 .389795$

N -0.639090 -3.519782 -2.174656

H -3.2283792 .6592300 .456432$

H -1.320405 3.0135782 .823401

H $-2.8437130 .609711 \quad 1.584883$

H -2.417216 1.4121394 .526601

H -2.649843 -0.2375364 .097322$

H $-6.342167 \quad 0.233747 \quad 3.726426$

H -5.943681 2.4360964 .213589

H -4.398432 2.7527365 .002498

H -4.598444 3.0642443 .264416

H -3.981986 -1.591708 2.955211

H $-5.029896-1.9983334 .304740$

H -5.684667 -3.169871 2.284900

H $-6.984450-2.1211562 .849761$

H $-6.392146-0.437368 \quad 1.107289$

H $-8.074329-2.2368200 .592043$

H $-6.782792-3.3061490 .088798$

H -7.840179 -2.386652 -3.009757

H -5.368891 -2.645406 -2.011624

H -8.991346 $-0.596379-1.041667$

H -8.051425 $-0.063354-2.415261$

H $-7.5521940 .391810-0.774128$

H -5.814790 -0.046931 -3.023322

H $-3.206003-0.405229-2.384396$
H -2.639652 -0.356222 -0.738494

H $-4.3204430 .997364-0.539756$

H $1.240718-0.7495322 .554088$

H $-0.055340-3.2904041 .386550$

H $2.965803-2.3598163 .536401$

H $3.411657-1.7230741 .937400$

H $4.123864-3.2111982 .524140$

H $-2.663917-2.856186-0.603174$

H $-3.215163-2.927677-2.290368$

H $1.188762-2.787814-2.889309$

H $\quad 0.386745-4.010864-3.888773$

H $\quad 0.870957-5.755550-1.998845$

H $3.213915-3.784424-2.087784$

H $4.267875-6.114219-1.941834$

H $2.886485-6.701176-1.045869$

H $5.894337-4.6322241 .018369$

H $5.994249-4.839412-0.819582$

H $3.980405-5.3458202 .231231$

H $3.018812-6.5571581 .445229$

H $1.249807-5.0841820 .953490$

H $-1.232641-4.332636-2.034558$

C $-1.6115815 .010537-0.380239$

O $-0.2382684 .591860-0.277315$

C $0.2033404 .150653-1.576859$

C $-0.8933834 .549251-2.560682$

C $-2.1319064 .425813-1.692214$

H $0.2508713 .055908-1.534349$

C $1.5932754 .717976-1.888685$

H $-0.7752845 .581986-2.908455$

H $-0.9172003 .898733-3.440189$

C $-3.3480535 .142752-2.249621$

H -2.362838 $3.359071-1.585056$

C $2.6487474 .456124-0.794315$

O $1.5002116 .138637-2.056821$

H $1.9430984 .325112-2.850193$

H 2.4342065 .0686260 .090119

O $3.9183454 .897786-1.290558$

C $2.7777782 .982188-0.375160$

C $3.8982072 .758547 \quad 0.658464$

H 1.8306552 .6480420 .061359

O $3.0164942 .183571-1.529964$

H 4.8729672 .8859410 .175683

H 3.8114273 .5258071 .436421

H $-4.2055735 .032370-1.578498$

H $-3.1581016 .213176-2.382189$

H $-3.6258154 .727981-3.224105$

C -2.371152 4.6511910 .898694

H $1.1240526 .507094-1.238964$

H $3.8188985 .829573-1.551380$

H $3.8409442 .496489-1.940225$

H $-3.365697 \quad 5.1121410 .871869$

H -1.842570 5.0916151 .753885

H $-1.5837616 .106409-0.452437$

C $5.2622840 .100929-0.162048$

O 3.9458020 .2990830 .366243

C 3.8149371 .3614921 .314280

C 4.7791631 .1521252 .490798

C 6.2138120 .9440772 .019967

C $6.270233-0.1567420 .963640$

H $5.5644490 .986194-0.734738$

C $5.191898-1.097617-1.116873$

H 2.7997301 .2764891 .723297

O $4.7371972 .251956 \quad 3.393708$

H 4.4713170 .2644723 .058081

H 6.6529981 .8750671 .642519

O 7.0186440 .5389113 .129593

H $6.073175-1.1286431 .434068$

O $7.584888-0.2062230 .412156$

C $4.684998-0.674559-2.473876$

H $6.177499-1.547941-1.282563$

H $4.542915-1.889553-0.725041$

H 3.8171262 .3483363 .693602

H 6.9417441 .2303453 .809410

H $8.203564-0.3451491 .149645$

O $3.325681-0.756126-2.518709$

O $5.416276-0.295331-3.380136$

C $2.727299-0.349745-3.753901$

H $3.1037820 .628473-4.068990$ 
H $2.920030-1.103411-4.522681$

H $1.648578-0.269746-3.599836$

SCF Energy (B3LYP/6-31G**//MMFF) $=-3245.92230064$

01 153

MM̄FF Geometry

C -1.791697 $3.156450-0.721708$

C $-0.7336743 .841871-0.261544$

C $\quad 0.2851893 .2607350 .683675$

O 1.5819843 .4383890 .061164

C 0.2986713 .9808242 .045450

C -0.9255863 .7177892 .892175$

C -1.1254662 .5888413 .599996$

C -1.924074 4.8425772 .951354

C $-0.1976961 .409093 \quad 3.716891$

C $-0.8624990 .110848 \quad 3.242403$

C $0.039279-1.1376553 .302742$

C $0.641037-1.3729974 .691706$

C $1.562981-2.6000224 .722722$

O $1.080336-1.0121582 .323991$

O $2.326229-2.5490415 .938493$

C $2.567738-2.4912353 .556756$

C $0.770224-3.9115204 .766386$

O $3.358317-3.6750373 .463021$

C $1.901636-2.1857852 .193373$

C $2.994117-1.9170781 .126737$

O $1.137626-3.3268501 .797663$

C 2.5693382 .5788490 .432417

O 2.4751131 .6468241 .214767

C $3.8102882 .977086-0.267636$

C $4.9175562 .239718-0.094814$

C $6.2306242 .484795-0.690869$

C $6.3980223 .717043-1.539972$

C $2.446797-1.536035-0.253047$

C $3.532172-1.267936-1.279594$

C $5.329779-2.396138-2.587959$

O $3.638233-0.176749-1.835245$

C $6.635476-1.905323-2.045403$

C $7.407057-1.019419-2.694301$

C $8.758206-0.567188-2.217131$

C $8.9011680 .938507-2.207099$

C $9.3173721 .597177-3.302208$

C $8.651274 \quad 1.670744-0.910030$

C $7.2212131 .599675-0.453283$

N $4.318749-2.375195-1.548133$

H $-1.9384902 .131105-0.397344$

H $-0.5798414 .868096-0.586226$

H 0.0986042 .1868050 .812467

H $\quad 0.4255765 .0617291 .891957$

H 1.1961313 .6984612 .610749

H -2.0349662 .5089684 .193955$

H -2.839995 4.5595453 .480304

H -1.488525 5.7025903 .470681

H -2.209160 5.1623401 .945698

H 0.7348931 .5658603 .171245

H $\quad 0.070198 \quad 1.3259844 .776082$

H -1.759966 -0.074759 3.846596

H -1.195571 0.2476152 .205118

H $-0.593421-1.9880713 .020869$

H $-0.148665-1.4618685 .447789$

H $1.248179-0.5078434 .987700$

H $1.701782-2.5333246 .683992$

H $3.262079-1.6723333 .791943$

H $0.145203-3.9504035 .666712$

H $1.439984-4.7760964 .830037$

H $0.112348-4.0461163 .905508$

H $3.765588-3.8207674 .334137$

H $3.643119-1.0998031 .467358$

H $3.624691-2.8080221 .026581$

H $0.882171-3.2297360 .867688$

H $3.7804973 .859316-0.896105$

H $4.861177 \quad 1.3620690 .548445$

H $6.2077784 .619826-0.949284$

H $5.7032213 .697733-2.387118$

H $7.3996813 .818335-1.963950$

H $1.807081-2.322999-0.665671$

H $1.836839-0.631318-0.171120$

H $4.982378-1.795112-3.435039$
H $\quad 5.433515-3.435563-2.914705$

H $6.979383-2.325099-1.103205$

H $7.070308-0.631948-3.653690$

H $9.511722-0.999002-2.889450$

H $8.987828-0.968718-1.222527$

H $9.4535712 .673487-3.298574$

H $9.5277851 .073096-4.228648$

H $8.9807992 .714371-0.974289$

H $9.2862181 .232358-0.128834$

H $7.0041790 .724046 \quad 0.159562$

H $4.139219-3.244505-1.055280$

C $-4.2536163 .576586-1.248191$

O $-4.6440872 .191834-1.164213$

C $-5.5973072 .051672-0.088370$

C -5.9188543 .4659850 .389818$

C -4.6153704 .1859960 .102433$

H -5.0812531 .5206420 .719795$

C $-6.8160601 .245460-0.555410$

H $-6.7374343 .915021-0.184593$

H -6.2001593 .4904761 .447164$

C -4.7380975 .6983790 .090643$

H -3.8904013 .8861380 .867612$

C $-6.473262-0.145287-1.127790$

O $-7.4941961 .978465-1.584859$

H $-7.534270 \quad 1.1466540 .266422$

H $-5.902725-0.027230-2.055513$

O $-7.699633-0.794426-1.485330$

C $-5.716189-1.062583-0.151412$

C $-5.447651-2.482285-0.688013$

H -4.760745 -0.6042690 .120053$

O $-6.461392-1.1863931 .062772$

H -4.959439 -3.052935 0.112034

H -6.398212 -3.003003 -0.859981

H $-3.7741946 .165515-0.134310$

H $-5.4606676 .037120-0.659284$

H -5.0707646 .0646101 .067380$

C -2.794735 $3.704331-1.696719$

H $-6.8573192 .131488-2.304103$

H -8.173262 -0.202466 -2.094759

H -7.332180 -1.555886 0.835744

H -2.577236 $4.756246-1.916902$

H -2.668506 $3.145988-2.632992$

H $-4.8822814 .017286-2.034134$

C $-2.431930-2.126454-0.977254$

O $-3.449823-1.657706-1.865389$

C $-4.589941-2.514550-1.968172$

C -4.163762 -3.919235 -2.421437

C $-3.039406-4.493007-1.559508$

C $-1.893694-3.488178-1.424386$

H -2.855114 -2.233546 0.030026

C $-1.361856-1.036252-0.850032$

H -5.211314 -2.100492 -2.771553

O $-5.269173-4.816274-2.403845$

H -3.807059 -3.869067 -3.458563

H -3.407664 -4.800179 -0.573545

O $-2.526865-5.673926-2.180960$

H -1.366493 -3.411153 -2.381708

O $-0.959239-3.965185-0.460230$

C $-0.756317-0.571686-2.159091$

H -1.837758 -0.157789 -0.399193

H $-0.557544-1.369947-0.188450$

H -5.960503 -4.439161 -2.974464

H -3.273113 -6.287412 -2.294516

H $-0.668578-4.847249-0.749409$

O $0.072650 \quad 0.485160-1.905507$

O $-0.978637-1.054477-3.260107$

C $0.7046181 .064681-3.050791$

H $1.3789991 .852444-2.704778$

H $\quad 1.2904610 .311683-3.586567$

$\mathrm{H}-0.0477371 .509745-3.708601$

SCF Energy $\left(B 3 L Y P / 6-31 G^{* *} / / M M F F\right)=-3245.92474667$

01 154

MM̄MF Geometry

C $-0.8106215 .150895-0.794803$

C $-1.7702574 .623186-0.019875$

C $-2.9374593 .831461-0.551347$

O -2.8791572 .5117600 .038511$ 
C $-4.2617334 .487317-0.121971$

C $-5.4908953 .783966-0.665518$

C -6.4219423 .1718040 .092575$

C $-5.6559923 .819203-2.163636$

C -6.4696623 .0556561 .590120$

C $-5.814071 \quad 1.7886932 .160675$

C $-6.4746180 .476310 \quad 1.707307$

C $-5.936009-0.7061652 .516440$

C -6.510279-2.046484 2.030195

O $\quad-6.183373 \quad 0.274770 \quad 0.321553$

O $\begin{array}{llll}-5.721280 & -3.098894 & 2.606897\end{array}$

C $-6.339977-2.1432420 .498468$

C $-7.946336-2.2628162 .522392$

O $-7.008053-3.292115-0.015999$

C $-6.810121-0.880647-0.253393$

C $-6.450917-0.898100-1.759759$

O $-8.237505-0.785368-0.166446$

C $-2.054508 \quad 1.602623-0.547867$

O $-1.3953181 .751910-1.564256$

C -2.0632490 .3867110 .293933$

C $-1.069037-0.5042010 .168110$

C $-0.915793-1.7420700 .934399$

C $-2.074943-2.1797651 .789222$

C $-4.956626-1.015795-2.080254$

C $-4.492445-2.436479-2.358653$

C $-2.418587-3.746264-2.804886$

O $-5.261461-3.384213-2.492475$

C $-1.954812-4.407197-1.545245$

C $-0.688913-4.806343-1.348755$

C $-0.212299-5.512474-0.111491$

C $0.956202-4.8115390 .544287$

C $2.219482-5.2123670 .322343$

C $0.651192-3.6948381 .517270$

C $0.241294-2.4267870 .823122$

N -3.111103 -2.512987 -2.480335

H $-0.8756975 .039693-1.873737$

H -1.697805 4.7267311 .060559

H $-2.8936243 .752690-1.644437$

H $-4.2876155 .530857-0.463143$

H $-4.294791 \quad 4.5257010 .972257$

H $-7.2639052 .701522-0.414534$

H $-6.6308653 .435241-2.483165$

H $-5.5750674 .847456-2.530994$

H -4.889322 $3.210285-2.651992$

H -6.0147873 .9338912 .060496$

H $-7.523003 \quad 3.0841561 .897701$

H $-4.753147 \quad 1.783096 \quad 1.885675$

H -5.8632361 .8556203 .254728$

H -7.5583420 .5699041 .844694$

H -6.137337 -0.5700723 .586493$

H $-4.842350-0.7428202 .421307$

H -5.758154 -3.005472 3.574298

H $-5.270526-2.291903 \quad 0.308027$

H -7.980688 -2.253104 3.618510

H -8.323660 $-3.246186 \quad 2.220817$

H -8.641830 -1.500992 2.164707

H -6.665758 -4.0655560 .464182$

H -7.037848 -1.665679 -2.278492

H $-6.7889120 .059253-2.182602$

H -8.553090 $-0.218333-0.889553$

H $-2.870006 \quad 0.2709391 .007874$

H - $0.275985-0.298548-0.549617$

H $-2.993710-2.2209131 .194548$

H $-2.224817-1.4828642 .620816$

H -1.950044 -3.175339 2.219388

H $-4.753438-0.445558-2.995945$

H -4.347827 -0.580887 -1.282645

H -1.586166 -3.485679-3.466186

H -3.096011 -4.415752 -3.344562

H -2.698100 -4.589947 -0.772870

H $0.045400-4.645164-2.134958$

H $0.078343-6.531031-0.400374$

H $-1.022157-5.6331540 .618711$

H $3.060228-4.7280770 .808196$

H $2.441312-6.029220-0.356295$

H $1.543418-3.4624762 .114098$

H $-0.087720-4.0469672 .241758$

H $1.017811-2.0243730 .172596$
H $-2.548912-1.684656-2.307531$

C $1.6824565 .064784-0.303423$

O 1.5326153 .8517810 .463098

C $2.0602862 .751997-0.310233$

C $2.9496903 .390970-1.364596$

C $2.1621794 .644794-1.693425$

H $1.1922132 .275879-0.781712$

C 2.7548241 .7637620 .631959

H $3.9291983 .649974-0.944275$

H $3.1062502 .747083-2.234950$

C $2.9769775 .708299-2.408414$

H $1.3174104 .359762-2.331731$

C $3.5035480 .624655-0.090805$

O 1.7493521 .2108811 .486004

H 3.4538912 .3026441 .281674

H $4.2502331 .070418-0.756717$

O $2.599392-0.117797-0.907520$

C $4.235665-0.3532020 .854494$

C $4.930920-1.5240520 .133952$

H 4.9744000 .2026461 .442595

O $3.331563-0.9511251 .785384$

H $5.416635-2.1400600 .901495$

H $4.170887-2.182337-0.307200$

H $2.3689936 .597602-2.602999$

H $3.8453016 .015553-1.815901$

H $3.3430195 .331369-3.369009$

C $0.3861285 .881021-0.249805$

H 1.2928421 .9531661 .918159

H $2.2847950 .467594-1.616151$

H $2.938318-0.2385352 .316390$

H $0.5092596 .818242-0.804487$

H 0.1944326 .1570050 .795198

H 2.4681345 .6426750 .200697

C $7.794417-0.5429950 .502498$

O $6.854863-0.106295-0.486013$

C $5.952092-1.114946-0.944219$

C $6.736146-2.288768-1.548504$

C $7.799221-2.820452-0.589905$

C $8.661629-1.678601-0.052276$

H $7.258884-0.8836721 .397479$

C 8.6465420 .6699070 .889903

H $5.380635-0.664271-1.764767$

O $5.862972-3.352586-1.913286$

H $7.236972-1.954426-2.466531$

H $7.350343-3.3912570 .231280$

O $8.650091-3.730605-1.290902$

H $9.311584-1.301969-0.852547$

O $9.503331-2.1772090 .985223$

C 7.8464751 .6361561 .731071

H 9.5211370 .3794501 .482959

H $9.021847 \quad 1.1987770 .005362$

H $5.203742-2.991326-2.530265$

H $8.083676-4.431624-1.656815$

H $10.020291-2.9115740 .611583$

O 7.1931452 .5262260 .931030

O 7.7916971 .5876252 .953282

C 6.3805743 .4859071 .613620

H 7.0129474 .1687952 .188222

H 5.8306114 .0609830 .864156

H 5.6605342 .9851382 .267872

SCF Energy (B3LYP/6-31G**//MMFF) $=-3245.91009647$

01 155

MM̄FF Geometry

C $2.323794-0.537342-2.713107$

C $1.903281-1.745627-3.114305$

C $1.285438-2.753987-2.182635$

O $-0.139414-2.796850-2.439755$

C $1.815843-4.176964-2.426682$

C $3.243047-4.405632-1.981875$

C $3.585726-4.912317-0.781996$

C $4.311158-4.110884-3.003743$

C $2.672714-5.2863950 .355108$

C $3.236847-4.8326451 .709449$

C $3.065246-3.3261751 .966816$

C $3.989545-2.8898343 .106120$

C $3.768991-1.4246683 .495961$

O $1.694827-3.1056922 .323345$ 
O $4.449863-1.1907504 .737877$

C $2.266333-1.2156493 .756885$

C $4.380705-0.4595862 .474847$

O 1.9822320 .1571864 .016176

C $1.353690-1.7443862 .622360$

C $-0.115400-1.7237413 .113968$

O $1.492281-0.9048291 .477316$

C $-0.909170-1.864081-1.815900$

O $-0.528359-0.976760-1.069562$

C $-2.314365-2.103772-2.208626$

C $-3.277102-1.302444-1.728941$

C $-4.707359-1.393890-2.028257$

C $-5.162991-2.530989-2.905154$

C $-1.143633-2.2784452 .119887$

C $-2.507553-2.4783252 .761992$

C $-4.784789-3.3238132 .259768$

O $-2.728155-2.2625593 .951003$

C $-5.749076-3.0980031 .140132$

C $-6.775076-2.2365951 .217570$

C $-7.792694-2.0291230 .131930$

C $-7.790206-0.614225-0.402308$

C $-8.504697 \quad 0.3533270 .196978$

C $-7.024208-0.344353-1.677675$

C $-5.537416-0.471413-1.497218$

N $-3.452172-2.9260191 .845671$

H $2.184168-0.252659-1.673172$

H $2.001575-2.022922-4.161046$

H $1.454622-2.489704-1.130325$

H $1.714589-4.441225-3.488724$

H $1.148890-4.891796-1.926890$

H $4.643602-5.091167-0.591891$

H $5.302574-4.440345-2.674092$

H $4.093781-4.629229-3.943472$

H $4.375975-3.037819-3.202829$

H $\quad \begin{array}{llll}1.660822 & -4.890698 & 0.226447\end{array}$

H $2.594068-6.3803140 .366339$

H $2.711300-5.3907512 .495530$

H $4.296288-5.1119111 .771762$

H $3.295345-2.7642071 .054902$

H $5.040629-3.0596612 .840287$

H $3.800687-3.5162883 .989618$

H $5.392425-1.3905804 .605227$

H $2.018366-1.7597194 .679480$

H $5.458942-0.6358142 .379456$

H 4.2770890 .5798942 .803798

H $3.943412-0.554767 \quad 1.479094$

H 2.5704940 .4446254 .735396

H $-0.177928-2.3163684 .036426$

H $-0.411308-0.6973353 .366266$

H $0.722359-1.0279350 .900453$

H - $2.516166-2.924842-2.886989$

H -2.993952 -0.494859-1.054831

H -4.847198 -3.492372 -2.483901

H $-4.740972-2.432253-3.911270$

H -6.247314 -2.591604-3.016408

H $-1.272258-1.579521 \quad 1.289328$

H $-0.814169-3.2439361 .720910$

H $-5.067987-2.778043 \quad 3.166078$

H $-4.746193-4.3892232 .509640$

H -5.614096 -3.6915260 .239412$

H $-6.912653-1.6602792 .130081$

H -8.781726 -2.2638390 .547318$

H -7.649326 -2.739155 - -0.691775

H -8.529939 $1.364592-0.195086$

H $-9.0774990 .154352 \quad 1.096838$

H -7.220953 $0.676572-2.031294$

H -7.413523 -0.993069-2.467196

H $-5.110406 \quad 0.308686-0.866109$

H $-3.132458-3.217036 \quad 0.925911$

C $4.0094261 .353178-2.935359$

O $3.3548782 .207852-1.972198$

C $4.0697412 .116177-0.729696$

C $5.4609201 .614668-1.098052$

C $5.1275820 .617059-2.190636$

H $3.5658641 .346652-0.133747$

C 4.0430443 .4485240 .022198

H $6.0894422 .418269-1.498826$

H 5.980637 1.163185-0.247692
C $6.3082950 .247936-3.071092$

H $4.751604-0.296274-1.713506$

C $2.637457 \quad 4.0331730 .288807$

O $4.780965 \quad 4.427514-0.720752$

H 4.5805163 .3312760 .970718

H $2.2377514 .470327-0.633336$

$\begin{array}{llllll}\text { O } & 2.809464 & 5.130922 & 1.197033\end{array}$

C $1.607473 \quad 3.047656 \quad 0.871728$

C 0.3599273 .6958811 .497789

H 1.3065272 .3374710 .093929

$\begin{array}{llll}\text { O } & 2.242454 & 2.293382 & 1.908579\end{array}$

H -0.2943912 .8817541 .833486$

H $0.659298 \quad 4.2074182 .421959$

H $6.003874-0.456976-3.851485$

H $6.7367841 .129285-3.559606$

H $7.097929-0.224094-2.477399$

C $2.9669230 .468294-3.627215$

H $4.3675604 .501845-1.598087$

H 3.1159264 .7650942 .044616

H 1.6203811 .6020592 .193610

H $3.433895-0.040352-4.478866$

H $2.1730141 .115155-4.017721$

H $4.4371602 .029385-3.686700$

C $-1.886878 \quad 3.087629-0.502805$

O $-0.860208 \quad 4.078304-0.628139$

C -0.4222724 .6707750 .596408$

C $-1.6104795 .335207 \quad 1.307306$

C -2.7356894 .3367691 .556383$

C $-3.110427 \quad 3.633196 \quad 0.255141$

H -1.4791482 .2046440 .004209$

C $-2.3150802 .681922-1.923405$

H $\quad 0.265576 \quad 5.476617 \quad 0.315874$

O $\begin{array}{lllll} & -1.210630 & 5.907210 & 2.548330\end{array}$

H -1.9993516 .1525110 .686228$

H -2.471645 $3.614642 \quad 2.337287$

$\begin{array}{llll} & -3.887322 & 5.036766 & 2.034254\end{array}$

H $-3.6534264 .344479-0.380433$

$\begin{array}{llll}\text { O } & -4.016818 & 2.568510 & 0.541645\end{array}$

C $-1.2385611 .955374-2.697016$

H $-3.1862472 .019389-1.873836$

H -2.575503 $3.576716-2.501759$

H -0.4972856 .5411632 .361248$

H -3.621306 5.5149712 .838431

H $-3.552597 \quad 1.9237351 .102466$

O $-1.7762241 .512322-3.873159$

$\begin{array}{llll}0 & -0.079930 & 1.811322 & -2.335636\end{array}$

C $-0.8783870 .830809-4.753119$

H $-0.477863-0.063404-4.269653$

H -1.439153 $0.525569-5.640506$

H $-0.0716411 .501370-5.062453$

SCF Energy (B3LYP/6-31G**//MMFF) $=-3245.92516811$

01_156

MM̄FF Geometry

C -1.769323 2.7143360 .124609

C -1.2117212 .3320021 .284440$

C $-1.509597 \quad 1.0339051 .984034$

$\begin{array}{llll}\text { O } & -0.233111 & 0.364447 & 2.142784\end{array}$

C $-2.115671 \quad 1.264426 \quad 3.382738$

C $-3.552378 \quad 1.737214 \quad 3.383670$

C $-4.6137420 .907898 \quad 3.418714$

C $-3.756247 \quad 3.228643 \quad 3.441271$

C $-4.593944-0.597686 \quad 3.389332$

C $-5.749388-1.1822842 .564319$

C $-5.615008-0.9362171 .052394$

C $-6.963274-1.1821510 .369332$

C $-6.869945-1.027754-1.152210$

O $-4.624289-1.8367350 .533680$

O $-8.074882-1.557426-1.725127$

C $-5.706940-1.899714-1.657195$

C $-6.7945210 .442283-1.580452$

O $-5.487369-1.689551-3.051553$

C $-4.384075-1.698627-0.874722$

C $-3.382138-2.792021-1.321604$

O $-3.854824-0.409655-1.189001$

C $-0.257517-0.977302 \quad 2.356611$

O $-1.244111-1.6940582 .404235$

C $1.135101-1.4372812 .543687$ 
C $1.361844-2.7513232 .684734$

C $2.658967-3.3964862 .874605$

C $3.865333-2.5192283 .070360$

C $-2.058622-2.845589-0.547522$

C $-1.067008-3.770662-1.239803$

C $1.375531-4.148986-1.545523$

O $-1.419736-4.664450-2.006712$

C $1.940571-5.203603-0.647620$

C $3.251229-5.304465-0.375024$

C $3.861802-6.3805320 .480135$

C $4.645593-5.8165821 .645991$

C $5.957077-5.5487911 .528393$

C $3.928555-5.6189032 .963431$

C $2.712484-4.7438292 .846424$

N $0.250242-3.493723-0.901948$

H $-2.4793802 .041202-0.351719$

H -0.5009152 .9812521 .787708$

H -2.1685250 .4084121 .367985$

H -1.493254 1.9726593 .947949

H -2.0404240 .3432323 .974553$

H -5.6058481 .3527973 .484781$

H -4.8136633 .4997043 .531160$

H -3.236642 3.6498644 .308098

H -3.3730723 .7111952 .538394$

H -3.647607 -0.9861153 .006903$

H $-4.698488-0.9527334 .421689$

H -5.783613 -2.263331 2.751114

H $-6.693665-0.7613302 .932917$

H -5.2889610 .0946640 .877299$

H -7.732258 -0.5111740 .772469$

H -7.306691 $-2.201530 \quad 0.596030$

H -8.825695 -1.062419-1.354724

H -6.015565 -2.949055 -1.548223

H $-7.6826860 .986604-1.237482$

H $-6.7896350 .535521-2.672067$

H $-5.9182060 .961115-1.186310$

H -6.334141 -1.843379 -3.504400

H -3.857587 -3.776982 -1.219125

H $-3.156746-2.655184-2.386547$

H -2.895608 -0.413763 -1.048915

H $1.924509-0.6949422 .556851$

H $\quad 0.512266-3.4322642 .640215$

H $3.732876-1.8734473 .945439$

H $4.028337-1.8889092 .191550$

H $4.788008-3.0822803 .230468$

H - $-1.612327-1.850859-0.467403$

H -2.229598 -3.2312200 .462747$

H $2.109622-3.371633-1.775767$

H $1.048629-4.603740-2.486109$

H $1.250043-5.932938-0.231394$

H $3.938623-4.586510-0.817489$

H $4.526521-6.978343-0.157341$

H $3.102396-7.0809150 .849454$

H $6.533423-5.1637832 .363020$

H $6.482558-5.7058390 .592215$

H $4.602573-5.2289273 .735254$

H $3.609674-6.6008673 .336853$

H $1.788375-5.3017072 .690850$

H $0.449996-2.756142-0.232982$

C -0.5613724 .9984730 .030264$

O $0.7708324 .460720-0.046616$

C $1.7034165 .543962-0.230041$

C $0.8850906 .829810-0.168557$

C $-0.4703096 .354346-0.664554$

H $2.1054825 .441582-1.244259$

C 2.8375885 .4252010 .797468

H $\quad 0.8045557 .2142020 .854767$

H $1.3149727 .622684-0.788271$

C -1.604284 $7.306366-0.327508$

H - $0.4206416 .217110-1.753114$

C 3.5029664 .0306660 .811206

O 2.2914995 .6558942 .102427

H $3.581156 \quad 6.2119470 .626785$

H 2.7674373 .2919851 .146753

O $4.5353904 .033151 \quad 1.803969$

C $4.1090623 .611693-0.538358$

C $4.7237382 .200338-0.540033$

H $3.3494923 .666154-1.322549$
O $5.1284334 .551717-0.887427$

H $5.2277212 .056730-1.503996$

H 5.5275472 .1413610 .204602

H -2.560432 $6.922547-0.696528$

H $-1.695746 \quad 7.455166 \quad 0.753674$

H $-1.4339418 .284861-0.788307$

C -1.540904 $4.013282-0.612741$

H 3.0190545 .5629922 .741162

H 5.2212854 .6573711 .510639

H $5.4610434 .310960-1.768885$

H -1.184402 $3.754172-1.617756$

H -2.518521 $4.495572-0.727181$

H $-0.8015955 .143978 \quad 1.091738$

C $2.6674231 .074272-2.478415$

O $2.5019061 .250269-1.068262$

C $3.7005901 .067151-0.306333$

C $4.259589-0.345348-0.538313$

C $4.430420-0.663825-2.023782$

C $3.150132-0.344064-2.796681$

H $3.4111751 .794249-2.842164$

C $1.3569291 .458789-3.174720$

H $3.403414 \quad 1.1241150 .748006$

O $5.512307-0.5103790 .117724$

H $3.568158-1.081858-0.108868$

H $5.288007-0.132332-2.452402$

O $4.708892-2.057493-2.176514$

H $2.382798-1.082702-2.538593$

O $3.398466-0.454550-4.195213$

C $0.2318340 .478564-2.930245$

H $1.0067862 .437191-2.824804$

H $1.5335081 .542115-4.253899$

H $5.383876-0.2886741 .055761$

H $5.495943-2.255657-1.640393$

H $3.733186-1.352860-4.359974$

O $-0.3190180 .112038-4.123480$

O $-0.1209190 .092273-1.823885$

C -1.392251 -0.829512 -4.048006

H -1.051570 -1.753650 -3.572983

H -2.234764 -0.396697 -3.502178

H -1.716144 -1.059329 -5.066517

SCF Energy $\left(B 3 L Y P / 6-31 G^{* *} / / M M F F\right)=-3245.92224261$

01 157

MM̄FF Geometry

C $3.448783-0.076374-1.789360$

C $3.9493171 .026544-2.368450$

C $3.2295122 .343872-2.556581$

O $1.8106952 .127946-2.351021$

C $3.7173073 .431009-1.572482$

C $5.2188143 .590524-1.445344$

C $5.9300213 .300346-0.336267$

C $5.9111314 .132333-2.669104$

C 5.4078932 .7843450 .975960

C 5.7954081 .3175931 .212439

C 4.8863210 .6650922 .264481

C $5.382366-0.7314212 .638653$

C $4.412035-1.4439603 .595349$

O 3.5781830 .5723651 .689828

O $4.773014-2.8330523 .635811$

C $2.983374-1.3656463 .015064$

C $4.538922-0.9210875 .031039$

O $2.025858-1.8826563 .936351$

C 2.5719490 .0567412 .567301

C $1.232132-0.0066531 .788738$

O $2.4055490 .880757 \quad 3.720648$

C $0.9277503 .065369-2.778599$

O $1.1736714 .101607-3.372254$

C $-0.4152862 .607650-2.357008$

C $-1.4185413 .489485-2.240423$

C $-2.7850133 .186495-1.804074$

C $-3.1030121 .756231-1.454559$

C $0.896937 \quad 1.238882 \quad 0.960814$

C $0.2753782 .363177 \quad 1.760438$

C -1.8938883 .1456372 .690880$

$\begin{array}{llll}\text { O } & 0.953620 & 3.282734 & 2.212490\end{array}$

C -3.2865173 .1634332 .145275$

C -3.9027024 .2740541 .712846$

C -5.3185644 .2975551 .212962$ 
C $-5.457806 \quad 4.956325-0.140839$

C $-5.9365956 .206992-0.254537$

C $-5.1368064 .132313-1.367876$

C $-3.6796554 .194746-1.739310$

N $-1.0934232 .222593 \quad 1.910876$

H $2.439175-0.069213-1.389044$

H $4.9682260 .989870-2.749077$

H $3.3816312 .665233-3.594682$

H $3.2446123 .251528-0.602562$

H $3.3027204 .400795-1.878546$

H $7.0081523 .452570-0.360748$

H $6.9847404 .278593-2.508940$

H $5.7953163 .446820-3.514179$

H $5.4850945 .101970-2.946778$

H 5.8301753 .3992851 .780829

H 4.3265952 .9145291 .056756

H $\begin{array}{llll}5.714368 & 0.758128 & 0.272427\end{array}$

H 6.8432251 .2696601 .532990

H 4.8529381 .3116603 .149313

H $6.387473-0.6833023 .075772$

H $5.481140-1.3403581 .729488$

H $5.697944-2.8937683 .930376$

H $2.954584-2.0256022 .136252$

H $5.558589-1.0731015 .405101$

H $3.884744-1.4758545 .712671$

H 4.3101790 .1428165 .121878

H $2.312398-2.7798124 .178759$

H $1.291030-0.8375191 .073338$

H $0.410595-0.2245392 .480705$

H $1.8715321 .652973 \quad 3.478907$

$\mathrm{H}-0.531423 \quad 1.554903-2.125220$

H -1.220379 4.534276 -2.476931

H -2.435692 $1.394826-0.665333$

H $-2.9835511 .113306-2.333103$

H -4.119560 $1.609153-1.085275$

H $1.770489 \begin{array}{lll}1.632620 & 0.434957\end{array}$

H $\quad 0.1842940 .9516320 .177848$

H -1.427339 4.136116 2.681465

H -1.901062 2.7811143 .723187

H -3.8238362 .2182302 .127412$

H -3.3760145 .2248321 .754229$

H $-5.9211254 .838074 \quad 1.954827$

H -5.7440003 .2870751 .168210$

H -6.069908 $6.674381-1.224589$

H $-6.200051 \quad 6.7932570 .619596$

H $\quad-5.705440 \quad 4.514100-2.226839$

H -5.504870 $3.112839-1.226208$

H $-3.3470115 .196321-2.016642$

H -1.519630 1.3421941 .640200

C $3.525143-2.577633-2.231017$

O $2.289982-2.846100-1.538658$

C $1.303658-3.256025-2.505926$

C $2.063781-3.521864-3.802276$

C $3.171040-2.488857-3.715360$

H $0.641612-2.395589-2.661158$

C $0.493687-4.444810-1.979476$

H $2.488906-4.531974-3.826775$

H $1.430483-3.400988-4.686356$

C $4.339779-2.760257-4.645424$

H $2.742116-1.508990-3.958864$

C $-0.151947-4.211141-0.598334$

O $1.367598-5.574874-1.860488$

H $-0.268543-4.722012-2.717362$

H $0.627742-4.1812690 .172058$

O $-0.953064-5.362216-0.298056$

C -1.025784 -2.947629-0.495270

C - $1.728382-2.845728 \quad 0.873011$

H $-0.397758-2.061947-0.642610$

O $-1.989410-2.977248-1.543687$

H -2.449424 -3.663639 0.971535

H $-0.971893-2.9786231 .655275$

H $5.113716-1.994629-4.530518$

H $4.796325-3.734968-4.443564$

H $4.009936-2.753745-5.689439$

C $4.218470-1.356794-1.621973$

H $0.836387-6.318030-1.526304$

H -1.691403 -5.378136 -0.931103

H $-2.444254-2.118126-1.545920$
H $5.224288-1.261086-2.047464$

H $4.337958-1.522519-0.544370$

H $4.163821-3.452880-2.051918$

C $-4.664540-2.0834190 .327407$

O $-3.501415-1.2675090 .145983$

C $-2.444632-1.4927101 .082982$

C $-2.956862-1.2621932 .510333$

C $-4.192086-2.1050772 .812866$

C $-5.252341-1.8959891 .731916$

H $-4.402994-3.1362790 .167377$

C $-5.681751-1.676146-0.745312$

H $-1.694187-0.7201710 .882098$

O $-1.929178-1.5391963 .455276$

H -3.218386 -0.202830 2.626192

H -3.929209 -3.163982 2.921078

O $-4.714924-1.6845254 .074878$

H $-5.681133-0.8901551 .829902$

O $-6.309073-2.8350671 .918038$

C $-5.244650-2.155815-2.108749$

H $-6.670358-2.110803-0.558958$

H $-5.812013-0.587351-0.772622$

H -2.302750 -1.397790 4.342068

H $-5.434630-2.2932544 .312061$

H -6.744954 -2.625432 2.761039

O $-4.683856-1.129712-2.808640$

O $-5.365030-3.311914-2.493585$

C $-4.207405-1.461858-4.116476$

H $-3.766359-0.562974-4.555306$

H -3.437034 -2.236266 -4.056011

H $-5.037738-1.790990-4.748359$

SCF Energy $\left(B 3 L Y P / 6-31 G^{* *} / / M M F F\right)=-3245.91338844$

01 158

MMFF Geometry

C $-0.989056-2.4684251 .342539$

C $-2.121646-2.6487640 .645052$

C $-2.302470-2.359116-0.826451$

O $-1.058727-1.861575-1.374651$

C $-2.728151-3.580797-1.654453$

C $-4.149372-4.044531-1.418949$

C $-5.232597-3.475452-1.984764$

C $-4.299734-5.264984-0.549685$

C $-5.259549-2.271137-2.886442$

C $-6.399198-1.293326-2.560995$

C $-6.344979-0.682405-1.150577$

C $-7.5793490 .194867-0.914809$

C $-7.545151 \quad 0.8850840 .455271$

O $-5.1534090 .104702-1.028212$

O

C -6.1826501 .5912490 .616764$

C $-7.915600-0.0835351 .584268$

O -6.0484362 .1351301 .928664$

C -4.9740600 .6887340 .271009$

C $-3.643291 \quad 1.478100 \quad 0.280661$

O $-4.868130-0.3223331 .282383$

C $-1.110277-0.806122-2.226965$

O $-2.105338-0.274840-2.690665$

C $0.281855-0.366856-2.459532$

C $0.5070300 .868712-2.928442$

C $1.8171571 .477733-3.157451$

C $3.0395590 .643118-2.881256$

C $-3.4802202 .516164-0.832523$

C $-2.5727993 .649884-0.395792$

C -0.2477934 .1669380 .326789$

O $-3.0002934 .795367-0.263404$

C $0.5164744 .735070-0.827998$

C $1.8567854 .782268-0.866673$

C $2.6556915 .362250-1.999580$

C $3.5808684 .343773-2.630004$

C $4.8244414 .155643-2.156505$

C $3.0900013 .591892-3.847000$

C $1.8677792 .760066-3.572567$

N $-1.2652673 .253823-0.162354$

H $-0.087005-2.1397020 .835811$

H $-3.016376-2.9518611 .183998$

H $-3.064049-1.575839-0.894443$

H $-2.029227-4.409914-1.479658$

H $-2.600969-3.356040-2.722474$ 
H $-6.206611-3.923173-1.791894$ H $-3.894897-5.0818380 .449687$ H $-5.346572-5.563759-0.429994$ H $-3.763447-6.111700-0.990700$ H -4.310340 -1.731154-2.876303 H $-5.403458-2.627403-3.913951$ H $-6.359280-0.485439-3.303511$ H $-7.359060-1.807557-2.698681$ H $-6.317555-1.493974-0.415089$ H -8.500846 -0.391004-1.023835 H -7.625876 $0.970906-1.692163$ H -8.503124 2.389149 1.276176 H $-6.1999762 .452061-0.060924$ H $-8.923875-0.4845921 .425251$ H -7.9492230 .4287252 .552151$ H $-7.231572-0.930668 \quad 1.666225$ H -5.8357841 .4028082 .532187$ H -3.5034031 .9370001 .268419$ H -2.8119280 .7670420 .180998$ H -3.952614 -0.643903 1.304423 H $1.078854-1.053343-2.200141$ H $-0.3488671 .507465-3.143449$ H $3.064190-0.233151-3.538103$ H $3.0410600 .301700-1.840950$ H $3.9774291 .182418-3.032563$ H -3.064414 $2.057089-1.735750$ H $-4.4357312 .962629-1.121710$ H 0.3936583 .6007531 .009683 H $-0.726070 \quad 4.9772630 .886443$ H $-0.0546415 .139760-1.660029$ H $2.4219514 .403640-0.018853$ H $3.2434936 .200671-1.603443$ H $2.0055765 .798553-2.767954$ H $5.5031593 .443775-2.614082$ H $5.1890174 .707349-1.296188$ H $3.8835992 .972249-4.280979$ H $2.843648 \quad 4.317860-4.632917$ H $0.9311793 .295285-3.732287$ H $-0.9877712 .299055-0.370354$ C $-0.515737-1.3144563 .539357$ O $0.804316-0.8780263 .149326$ C $0.766410 \quad 0.5426552 .917317$ C -0.5398551 .0370883 .533775$ C -1.457433 -0.1378863 .271526$ H $\quad 0.718511 \quad 0.681097 \quad 1.830344$ C 2.0138441 .2303653 .477952 H $-0.444251 \quad 1.2079834 .612139$ H -0.8890531 .9668013 .073984$ C $-2.712852-0.1434634 .125060$ H -1.749566 -0.101177 2.216541 C 3.3595780 .6983492 .945005 O 2.0208971 .0644324 .902805 H 1.9380112 .3098043 .297759 H $3.549173-0.2982403 .359567$ O $4.3849231 .542346 \quad 3.490243$ C 3.5013120 .6488481 .410130 C 4.9509270 .5282350 .899641 H $2.904052-0.1815171 .020639$ O $2.953504 \quad 1.839154 \quad 0.836438$ H $4.9229160 .569015-0.195442$ H 5.5037231 .4301571 .191600 H -3.327964 -1.021826 3.905865 H -2.472268 -0.1573715 .193316$ H $-3.315787 \quad 0.7491693 .927776$ C $-0.875065-2.6276812 .832018$ H 2.8116571 .5165755 .243709 H 4.2430522 .4423513 .151073 H 3.4836772 .5920091 .147740 H -1.807413 -3.023779 3.250932 H $-0.090062-3.3686793 .025520$ H $-0.458079-1.5117764 .617636$ C $5.150569-2.211194-0.456466$ O $5.074183-1.9404000 .948845$ C $5.705581-0.7286651 .374047$ C $7.190241-0.7452310 .983729$ C $7.374372-0.980494-0.511007$ C $6.608257-2.228518-0.943006$ H $4.578871-1.454689-1.005484$
C $4.499809-3.582014-0.702127$

H $5.672817-0.7338182 .469545$

O 7.8139240 .4754341 .368701

H $7.694420-1.5499531 .534738$

H $7.074526-0.099609-1.090480$

O $8.766892-1.174753-0.768008$

H $7.124510-3.113378-0.549567$

O $6.646414-2.327726-2.366042$

C $2.997572-3.559236-0.530327$

H $4.699402-3.916318-1.726530$

H $4.901171-4.3167570 .005950$

H $8.7428250 .429441 \quad 1.084134$

H $8.861476-1.370885-1.716125$

H $6.182845-1.554921-2.731599$

O $2.507385-4.803951-0.809113$

O $2.337817-2.585585-0.197542$

C $1.088198-4.948211-0.703560$

H $\quad 0.823451-5.958301-1.026814$

H $\quad 0.583032-4.229623-1.355019$

H $\quad 0.775713-4.8197170 .336433$

SCF Energy $\left(B 3 L Y P / 6-31 G^{* *} / / M M F F\right)=-3245.91843639$

01_159

MMFF Geometry

C $1.1053555 .084449-0.347247$

C $1.7663403 .956886-0.047725$

C 2.2991283 .6307511 .320189

O 1.7742932 .3341191 .690071

C 3.8323753 .5274481 .281896

C 4.4391353 .2030912 .634801

C 4.8628781 .9753462 .997482

C 4.5919284 .3709153 .572229

C 4.7486830 .7161412 .185405

C 6.0695230 .3633561 .495842

C $5.882581-0.7387360 .441816$

C $7.232519-1.128562-0.162396$

C $7.084177-2.118699-1.325022$

O $5.019976-0.216525-0.574115$

O $8.355379-2.164127-1.990766$

C $6.039294-1.566266-2.318021$

C $6.802196-3.539527-0.821980$

O $5.758793-2.517834-3.342938$

C $4.719251-1.125081-1.641227$

C $3.842249-0.351448-2.660183$

O $4.029266-2.281280-1.171547$

C $0.5529422 .277748 \quad 2.277391$

O $-0.177963 \quad 3.2243322 .534722$

C 0.1826690 .8843342 .621638

C $0.865091-0.1913662 .197993$

C $0.571859-1.5908262 .516522$

C $-0.627757-1.8690863 .383430$

C $2.4604460 .075257-2.137333$

C $1.377152-0.962098-2.389341$

C $-1.003130-1.466233-1.884040$

O $1.568180-1.986730-3.039800$

C $-0.984632-2.399566-0.715807$

C $-0.502817-3.650107-0.771392$

C $-0.465007-4.5956620 .394498$

C $0.933261-4.7796970 .940970$

C $1.801508-5.6220930 .356124$

C $1.293276-4.0401762 .210759$

C $1.378114-2.5494262 .011658$

N $0.163761-0.605091-1.815520$

H $0.966688 \quad 5.8424760 .418398$

H $1.8993293 .198536-0.816608$

H 1.9939684 .3808252 .060536

H 4.2619024 .4725620 .923387

H 4.1369292 .7752690 .543586

H 5.3166111 .8476263 .978738

H 5.0688854 .0860904 .516077

H 5.2096755 .1489533 .112195

H 3.6143184 .7995523 .813602

H $4.449715-0.1039172 .849882$

H 3.9454450 .8031971 .449577

H 6.4687831 .2611601 .005350

H 6.8021240 .0466292 .248431

H $5.402827-1.6040310 .914249$

H $7.899975-1.5362030 .607278$ 
H $7.733436-0.226254-0.541635$ H $8.304502-2.832997-2.694192$ H $6.483314-0.692098-2.814707$ H $7.634352-3.895967-0.203112$ H $6.723659-4.247047-1.654930$ H $5.890922-3.610230-0.224266$ H $6.586385-2.686859-3.823478$ H $4.3789080 .566699-2.935329$ H $3.722152-0.937969-3.578216$ H $3.124410-2.029057-0.931247$ H $-0.698552 \quad 0.7933293 .248398$ H $1.728090-0.0453941 .552225$ H -1.526963 -1.418857 2.949626 H $-0.479136-1.4566464 .387275$ H $-0.847625-2.9319763 .500614$ H $2.497793 \quad 0.316651-1.069618$ H $2.1514130 .981284-2.673691$ H -1.002911 -2.008681 -2.835582 H -1.895868 -0.834939-1.850804 H -1.387466 -2.021885 0.218863 H $-0.100090-4.020356-1.711507$ H $-0.865769-5.5633360 .065285$ H -1.137116 -4.265016 1.194735 H $2.797873-5.7770200 .756451$ H $1.534125-6.172373-0.539936$ H $2.272399-4.3730272 .580314$ H $0.587093-4.3229662 .996441$ H $2.219892-2.2442151 .388506$ H $\quad 0.1154340 .218963-1.223189$ C -1.030159 5.361133-1.683359 O $-1.5360524 .168159-1.047296$ C $-2.5921204 .546053-0.144237$ C $-3.0142085 .945144-0.573445$ C -1.676366 $6.544273-0.962026$ H -2.1524694 .5995680 .860148$ C $-3.7256043 .514519-0.148000$ H -3.686572 $5.913091-1.439269$ H $-3.515104 \quad 6.4948630 .229030$ C -1.792753 $7.796374-1.813582$ H -1.127066 $6.790096-0.045068$ C -3.3128882 .1642490 .482950$ O $-4.1748783 .341575-1.490175$ H -4.5640303 .9248660 .428443$ H -2.926682 2.3577411 .491431 O $-2.2624391 .550936-0.261665$ C $-4.483559 \quad 1.1665300 .598687$ C -4.093123 -0.0996221 .387082$ H -5.3168321 .6589321 .113111$ O $-4.9431950 .806157-0.699472$ H -3.313112 $-0.637990 \quad 0.841059$ H $-3.661973 \quad 0.2120642 .345412$ H - $0.8026718 .176596-2.083952$ H -2.344607 $7.603035-2.739499$ H -2.320348 8.585045 -1.267279 C $0.5013455 .341012-1.700303$ H -4.946382 2.750658 -1.470596 H -1.507133 2.163012 -0.268587 H -4.191644 $0.415467-1.177675$ H $0.8898046 .285929-2.095992$ H $0.8240404 .554096-2.394419$ H $-1.3835975 .325664-2.722314$ C $-5.078849-2.510111-0.256675$ O $-5.876769-1.546057 \quad 0.441268$ C $-5.296961-1.0368941 .647229$ C $-5.007329-2.1945022 .614841$ C $-4.168035-3.2870671 .960532$ C -4.795551-3.721002 0.637557 H -4.134524 -2.049760 -0.567234 C $-5.847452-2.922131-1.517461$ H $-6.071327-0.4173322 .117810$ O $-4.344812-1.7329563 .787067$ H -5.957150 -2.639578 2.939083 H -3.133658 -2.9606391 .813359$ O $-4.112605-4.4230022 .825430$ H $-5.723473-4.2730120 .834971$ O $-3.905801-4.606453-0.037575$ C -5.856111 -1.809250 -2.538455 H -5.415854 -3.808354 -1.996113
H $-6.890856-3.159587-1.277309$

H $-4.910000-1.0539964 .193677$

H $-3.739015-4.1228843 .671896$

H $-3.712740-5.3423560 .568334$

O $-4.629650-1.708147-3.127191$

O $-6.833255-1.112589-2.779347$

C $-4.499611-0.675981-4.109490$

H $-5.170264-0.870601-4.951751$

H $-4.7098560 .301908-3.665864$

H -3.468338 -0.680768 -4.472172

SCF Energy $(B 3 L Y P / 6-31 G * * / / M M F F)=-3245.91062565$

0116

MM̄FF Geometry

C $-2.600196-2.7003561 .534769$

C $-1.995599-2.8910632 .717246$

C $-0.797944-2.1133313 .215960$

O $0.014642-1.6392332 .117716$

C -1.286120 -0.895092 4.021294

C $-0.170520-0.0442514 .593428$

C $0.064436 \quad 1.237327 \quad 4.248582$

C $0.692932-0.7117505 .632342$

C -0.6909142 .0859693 .262900$

C -0.1222412 .0686531 .835184$

C 1.2800752 .6871511 .711959

C 1.6879702 .8155960 .243562

C 3.1201963 .3490600 .083582

O $2.213278 \quad 1.8424932 .388091$

O $3.4897443 .122582-1.285446$

C 4.0733372 .5285790 .982549

C 3.1816454 .8647790 .306804

O 5.3767913 .1042241 .011415

C 3.5536442 .3503312 .424189

C 4.3973381 .3548243 .257670

O 3.6064903 .6129923 .100388

C $0.866242-2.5347601 .546560$

O $1.022062-3.7047961 .858149$

C $1.568578-1.8465870 .441760$

C $2.170096-2.577459-0.508408$

C $2.874309-2.059383-1.682831$

C $3.152329-0.581191-1.737407$

C $4.465760-0.0737702 .705554$

C $5.711800-0.3630831 .884344$

C $6.757536-2.1750370 .527215$

O $6.6545570 .417357 \quad 1.787550$

C $6.571444-1.842397-0.919208$

C $6.569477-2.763906-1.894526$

C $6.441051-2.436189-3.354393$

C $5.325232-3.200141-4.030982$

C $5.581284-4.307528-4.747870$

C $3.928750-2.627652-3.952726$

C $3.251260-2.927820-2.644396$

N $5.661020-1.6223751 .300216$

H $-2.228436-1.9328460 .860757$

H -2.394318 -3.639218 3.398539

H $-0.192234-2.7686553 .853812$

H -1.922088 -1.230075 4.851844

H -1.932480 -0.2904853 .376211$

H $\quad 0.893002 \quad 1.751867 \quad 4.734109$

H $1.394357-0.0129566 .100948$

H $0.071468-1.1338106 .428868$

H $1.283414-1.5167965 .184321$

H $-1.7455581 .793133 \quad 3.228022$

H -0.6969823 .1177553 .637208$

H $-0.106593 \quad 1.0353581 .468919$

H $-0.8151152 .627376 \quad 1.194478$

H $1.263463 \quad 3.6729322 .191368$

H $0.9818673 .453482-0.301663$

H $1.6293511 .828270-0.234634$

H $4.3790693 .490046-1.422757$

H 4.1773621 .5419460 .518891

H $2.5472055 .382916-0.422271$

H 4.1951995 .2486640 .147237

H 2.8539435 .1643871 .304566

H $5.7249493 .092878 \quad 0.104192$

H $5.396121 \quad 1.7683343 .441985$

H 3.9264151 .2853774 .249117

H 3.6115283 .4498274 .058074 
H $\quad 1.545242-0.7632730 .424655$

H $2.116994-3.663481-0.439518$

H $3.614180-0.245966-0.803816$

H $2.228059-0.017442-1.893327$

H $3.846685-0.297695-2.531247$

H $4.493905-0.7697003 .554114$

H $3.575918-0.3143562 .116187$

H $6.769271-3.2548420 .706195$

H $7.702611-1.7525800 .883515$

H $6.465824-0.791801-1.179072$

H $6.704692-3.811560-1.634822$

H $7.399649-2.675732-3.833260$

H $6.294452-1.360617-3.512704$

H $4.790469-4.843952-5.261552$

H $6.587195-4.705343-4.832202$

H $3.302686-3.058765-4.745889$

H $3.960473-1.558386-4.181378$

H $3.015886-3.984564-2.510403$

H $4.814350-2.1772321 .385878$

C -5.088657 -2.674505 1.217005

O $-5.011737-1.4527730 .460202$

C $-6.300169-1.180804-0.122281$

C $-7.255521-2.2368580 .426384$

C -6.320264 -3.4018990.682555

H -6.190695 -1.329138 -1.202867

C $-6.717057 \quad 0.2666140 .167080$

H -7.726809 -1.9161981 .362483$

H $-8.055435-2.475864-0.281236$

C $-6.890826-4.4409681 .631735$

H -6.086931-3.879775 -0.278409

C $-5.6715701 .328721-0.230652$

O

H $-7.6748290 .478995-0.321573$

H -4.8077321 .2610530 .440482$

O $-6.2554262 .618603-0.006512$

C $-5.2006291 .256283-1.693299$

C $-4.3187112 .439788-2.133822$

H $-4.6592670 .320401-1.856316$

O $\quad-6.3503921 .226256-2.543872$

H $-4.0865302 .304713-3.197324$

H -4.910212 $3.363156-2.093999$

H $-6.181319-5.259278 \quad 1.788132$

H -7.124383 -4.006042 2.609361

H -7.813224 -4.867975 1.224729

C $-3.796424-3.4765731 .063036$

H -6.1361830 .1714742 .038201$

H -6.544203 2.6485740 .921849

H $-6.0369311 .115626-3.457585$

H -3.633519 -3.7273610 .008209$

H -3.875290 -4.424070 1.608438

H $-5.233047-2.3904452 .267959$

C -1.617623 $1.062828-2.544546$

O $-2.2315121 .412021-1.298132$

C -3.019007 $2.606622-1.321404$

C $-2.1570893 .800017-1.755944$

C $-1.4680393 .539783-3.091619$

C $-0.7275462 .205035-3.056459$

H $-2.3918160 .825627-3.284364$

C $-0.768902-0.195561-2.303321$

H -3.312642 $2.800326-0.283073$

O $-2.9589274 .973742-1.831956$

H -1.392092 $3.982233-0.990526$

H $-2.1774513 .573108-3.926782$

O $-0.505750 \quad 4.568088-3.337851$

H $\quad 0.1623012 .299610-2.421749$

O $-0.2754301 .890746-4.373164$

C -1.588762 -1.462872 -2.194561

H $-0.071737-0.338772-3.136788$

H $-0.203672-0.092631-1.371031$

H $-2.3651125 .728218-1.982106$

H $-0.9920975 .398812-3.471845$

H $\quad 0.2777472 .631968-4.674378$

O $-0.747899-2.534041-2.303075$

O $-2.796614-1.512690-2.015282$

C $-1.346039-3.831357-2.216272$

H $-0.673772-4.545726-2.699014$

H -1.454520 -4.108625 -1.164917

H $-2.313075-3.866392-2.727042$
SCF Energy (B3LYP/6-31G**//MMFF) $=-3245.90210437$

01 160

MMFF Geometry

C -1.411834 -4.265519-0.295108

C $-1.491271-3.496725-1.392566$

C $-2.431993-2.330782-1.536247$

O $-1.649632-1.196776-1.980316$

C $-3.492099-2.619367-2.610364$

C $-4.614712-1.599076-2.604504$

C $-5.766097-1.747031-1.919874$

C $-4.403534-0.391650-3.479641$

C $-6.146076-2.896985-1.030982$

C $-6.841805-2.4842060 .274725$

C $-5.915265-1.8040571 .297269$

C $-6.593880-1.7872912 .671560$

C $-5.758843-1.0446903 .721594$

O $-5.651074-0.4634630 .867739$

O $-6.594004-0.8094434 .865573$

C $-5.371430 \quad 0.333674 \quad 3.154203$

C $-4.575550-1.8863224 .213394$

O $-4.477274 \quad 1.0110894 .035392$

C -4.7684070 .2710101 .729695$

C -4.6230411 .7098901 .176212$

O $-3.479061-0.3367861 .802881$

C $-1.009419-0.461894-1.034386$

O $-1.003852-0.6487790 .171405$

C $-0.3134640 .641467-1.727892$

C $-0.0513961 .768310-1.050578$

C $0.6516572 .944494-1.563955$

C $1.2293332 .856774-2.952203$

C $-4.2254501 .795014-0.302297$

C $-4.115696 \quad 3.235597-0.776532$

C $-3.6706254 .569977-2.833813$

O $-4.3250824 .201416-0.047100$

C $-2.2342844 .971047-2.949933$

C $-1.7168906 .052295-2.347136$

C $-0.3014676 .523745-2.524672$

C $0.4981826 .481506-1.242245$

C $0.4192467 .476347-0.342473$

C $1.4568485 .328824-1.051687$

C $0.7511994 .032020-0.770987$

$\mathrm{N}-3.7697443 .312494-2.118423$

H $-2.101896-4.0699070 .523660$

H -0.826393 -3.673617 -2.233087

H $-2.921705-2.093999-0.582915$

H -3.906562 -3.625822 -2.474504

H -3.030739 -2.625878 -3.607372

H $-6.526957-0.974532-2.021153$

H -4.251374 -0.698954 -4.519351

H $-5.2596510 .291105-3.460354$

H -3.527064 $0.174268-3.153779$

H $-6.843466-3.527417-1.596906$

H -5.284988 $-3.525250-0.782064$

H -7.688935 -1.825767 0.042833

H $-7.254917-3.3980400 .720540$

H $-4.972979-2.3625351 .345145$

H $-6.810521-2.8078883 .011436$

H -7.569474 -1.287378 2.587987

H $-6.908737-1.6709425 .189166$

H -6.2868660 .9410693 .115370$

H -4.932155 -2.817211 4.670591

H -4.017275 -1.363004 4.997351

H $-3.880149-2.1576573 .416363$

H -4.9000231 .0400514 .910833$

H -5.5812212 .2343461 .289703$

H -3.879965 2.2585481 .769119

H $-2.940887-0.0346401 .055212$

H $-0.0460520 .496058-2.767758$

H $-0.3857301 .838740-0.016145$

H $0.4406882 .657259-3.686228$

H $1.9723332 .053114-3.007675$

H $1.7266843 .771981-3.278743$

H $-3.2606571 .311423-0.473495$

H $-4.9790451 .296239-0.920813$

H $-4.2702835 .332402-2.325520$

H $-4.0928344 .415328-3.831688$

H $-1.6045274 .366221-3.597359$ 
H $-2.3589896 .663023-1.716067$ H $-0.3338577 .554935-2.900732$ H $0.2128015 .950326-3.305639$ H 1.0130567 .4675050 .565448 H $-0.2453478 .320726-0.494124$ H $2.1243685 .526508-0.202345$ H $2.1230305 .270350-1.917113$ H $0.291333 \quad 3.9973580 .217641$ H $-3.5635082 .458945-2.628283$ C $0.835749-5.362611-0.920284$ O $1.549081-4.130281-0.704859$ C $2.963756-4.392703-0.733796$ C $3.117522-5.851399-1.152613$ C $1.842969-6.463759-0.597460$ H $3.323628-4.2877140 .296313$ C $3.660544-3.367117-1.635663$ H $3.149493-5.962043-2.242746$ H $4.025817-6.305067-0.744347$ C $1.503021-7.813856-1.203189$ H $1.948971-6.5707490 .490364$ C $3.333433-1.900550-1.277074$ O $3.231339-3.586007-2.985878$ H $4.743290-3.537864-1.623029$ H $2.275737-1.699056-1.483892$ O $4.073447-1.057623-2.170339$ C $3.664710-1.5059420 .174392$ C $3.322311-0.0299170 .461073$ H $3.092923-2.1345990 .865271$ O $5.048229-1.7588680 .399190$ H $3.8915930 .612369-0.217646$ H 2.2616640 .1188930 .231323 H $\quad 0.572631-8.207304-0.781879$ H $1.379551-7.747246-2.289367$ H $2.298686-8.537784-0.999103$ C $-0.418794-5.374445-0.042216$ H $3.676144-2.923383-3.541782$ H $5.017273-1.171131-1.964296$ H $5.225476-1.6124321 .343813$ H $-0.105691-5.2776821 .005738$ H $-0.940513-6.333240-0.136351$ H $\quad 0.565966-5.406865-1.983085$ C 5.8053691 .3168981 .643150 O $4.979754 \quad 0.3279932 .271917$ C 3.5917640 .3859341 .926488 C 3.0284151 .7613562 .309195 C 3.8252412 .8927351 .667168 C 5.3136802 .7310971 .973962 H $5.800027 \quad 1.1643000 .557737$ C 7.2387651 .1040652 .143929 H $3.086917-0.3556392 .558709$ O 1.6604821 .8775981 .938998 H 3.0754371 .8819773 .399435 H 3.6497642 .9471620 .586372 O $3.390246 \quad 4.1417152 .209135$ H 5.4938082 .9549103 .033399 O 6.0561593 .6757571 .205781 C $7.814846-0.1854531 .609726$ H 7.9065391 .9210991 .848071 H 7.2641091 .0467583 .239038 H $1.178144 \quad 1.1472312 .362644$ H 2.4339324 .2099742 .044973 H 5.7062934 .5588271 .415366 O $8.176444-0.0294660 .304021$ O $7.918176-1.2105742 .271060$ C $8.717010-1.194048-0.327653$ H $9.634717-1.5079240 .178873$ H $7.979466-2.002319-0.328177$ H $8.957341-0.938427-1.363020$ SCF Energy $\left(B 3 L Y P / 6-31 G^{\star *} / / M M F F\right)=-3245.92315396$

\section{1}

MM̄̄FF Geometry

C $1.647462-2.604148-2.058408$

C $0.921724-1.609890-2.593688$

C $-0.549208-1.431403-2.316641$

O $-0.801822-0.043280-1.991056$

C $-1.373499-1.778855-3.566517$

C $-2.829374-2.039457-3.233241$
C $-3.326331-3.255049-2.929147$

C $-3.735908-0.840258-3.304947$

C $-2.563222-4.547314-2.845326$

C $-2.889331-5.401535-1.611248$

C $-2.357514-4.828729-0.285857$

C $-2.385590-5.9183210 .792146$

C $-1.961894-5.3869922 .167179$

O $-3.186882-3.7301510 .107167$

O $\quad-2.312951-6.3730883 .149978$

C $-2.788152-4.1250212 .479029$

C $-0.443434-5.1959632 .265749$

O $-2.342041-3.5127983 .687659$

C $-2.789886-3.0901721 .329248$

C $-3.813598-1.9726101 .645466$

O $-1.485681-2.5199561 .228094$

C $-0.4576310 .388326-0.751529$

O $0.077220-0.2744210 .126072$

C $-0.784471 \quad 1.817337-0.552211$

C $-1.5461682 .537178-1.389308$

C $-1.8797973 .955524-1.243841$

C $-1.2351824 .720905-0.117561$

C $-4.063573-0.9902170 .493732$

C $\begin{array}{llll}-5.090598 & 0.067737 & 0.863404\end{array}$

C $-6.3243911 .989761-0.095557$

O $-5.6793490 .085946 \quad 1.941255$

C $-6.0295583 .095520-1.057532$

C $-5.9070264 .378566-0.685510$

C $-5.6723035 .517839-1.636862$

C $-4.3860566 .257959-1.347145$

C $-4.3395957 .215708-0.405394$

C $-3.1771215 .948702-2.199176$

C $-2.7372074 .511864-2.124719$

N $-5.2754090 .989917-0.160084$

H $1.136661-3.322928-1.419208$

H $1.384595-0.878030-3.248574$

H $-0.867463-2.057993-1.474188$

H $-0.954474-2.655968-4.074730$

H $-1.317560-0.962523-4.299440$

H $-4.395358-3.341237-2.740272$

H $-3.728845-0.419341-4.315574$

H $-4.773560-1.086748-3.056419$

H -3.404978 -0.067697 -2.606173

H $-2.822878-5.133324-3.736088$

H -1.480806 -4.390568 -2.881343

H $-3.975610-5.545571-1.546463$

H $-2.441457-6.389641-1.777920$

H -1.332091 -4.473244 -0.439387

H -1.754786 -6.7681610 .502449$

H $-3.405867-6.3192120 .875822$

H -1.856179 -7.200646 2.920515

H -3.823893 -4.446094 2.658397

H $0.072895-6.1512802 .113095$

H $-0.152176-4.8547813 .265301$

H - $0.049556-4.4899021 .531569$

H -2.364488 -4.193103 4.382235

H $-4.777086-2.4332461 .901062$

H -3.481227-1.404655 2.523795

H -1.555318 -1.614813 0.888375

H -0.3705262 .2500920 .352073$

H $-1.9672742 .052338-2.267941$

H $-0.1433554 .685753-0.202638$

H -1.5267294 .2988180 .850633$

H -1.516391 $5.776452-0.094881$

H -3.139231 -0.4789850 .210932$

H $-4.444517-1.529144-0.380033$

H -6.4195342 .3464830 .935752$

H $-7.2639211 .498021-0.368904$

H $-5.9448892 .833097-2.109287$

H $-6.0143014 .635527 \quad 0.366253$

H $-6.5202206 .210269-1.550830$

H $-5.6809645 .177256-2.679721$

H -3.429834 $7.772619-0.207973$

H $-5.212218 \quad 7.4617580 .190787$

H -2.337953 $6.612387-1.958655$

H $-3.4200296 .178449-3.245138$

H $-3.1706413 .882054-2.902181$

H $-4.8717930 .782876-1.068749$

C $3.954247-1.729758-2.840669$ 
O $3.934785-0.578797-1.972512$

C $5.2472870 .014039-1.953841$

C $6.054575-0.702142-3.030912$

C $5.431680-2.084454-3.004770$

H $5.686946-0.228859-0.979717$

C $5.1477421 .535765-2.110467$

H $5.919752-0.248607-4.018521$

H $7.126095-0.708475-2.808882$

C $5.739895-2.911616-4.240715$

H $5.793351-2.616282-2.114479$

C $4.2809182 .221677-1.032471$

O $4.5468051 .847018-3.374159$

H $6.1501321 .980598-2.119842$

H $3.2344311 .925216-1.166535$

O $4.3169973 .634440-1.277454$

C $4.715721 \quad 1.9505750 .422118$

C 4.0172152 .8349201 .473313

H 4.5381070 .8979330 .664539

O $\quad 6.125351 \quad 2.159232 \quad 0.548694$

H 4.4798532 .6263082 .445639

H $4.2507903 .888697 \quad 1.276780$

H $5.264532-3.895596-4.181366$

H $5.383702-2.419813-5.152153$

H $6.819492-3.064545-4.341259$

C $3.123621-2.856559-2.222075$

H $5.2234411 .730349-4.061588$

H $5.2440203 .922884-1.230414$

H 6.3033343 .1030360 .398584

H $3.518038-3.069306-1.220455$

H $3.239933-3.772262-2.813172$

H $3.534149-1.420518-3.806048$

C $2.451061 \quad 0.7549223 .061218$

$\begin{array}{llll}\text { O } & 2.135592 & 1.266598 & 1.761050\end{array}$

C 2.4918602 .6358641 .548043

C 1.7857193 .5284722 .578163

C 2.0499813 .0652554 .007623

C $1.747361 \quad 1.574403 \quad 4.149964$

H 3.5358340 .7873473 .215010

C $1.999889-0.7110983 .108416$

H 2.0876662 .9108270 .567105

$\begin{array}{lllll} & \text { O } & 2.191769 & 4.885898 & 2.441830\end{array}$

H $0.702623 \quad 3.4986452 .402211$

H 3.0765183 .2894884 .320727

$\begin{array}{lllll}\text { O } & 1.192956 & 3.781607 & 4.899709\end{array}$

H 0.6622901 .4158934 .100542

O $2.187314 \quad 1.1277065 .430848$

C $2.786734-1.5848692 .159936$

H $2.100496-1.1387744 .111955$

H $0.943729-0.7887762 .822232$

H 1.9953295 .1589651 .529466

H 1.3677964 .7294944 .768989

H 1.7415231 .6773396 .098025

O $4.107240-1.5843302 .498429$

O $2.278862-2.219787 \quad 1.244859$

C $4.955090-2.3881661 .672656$

H $4.644402-3.436367 \quad 1.715127$

H $4.936720-2.0210250 .642902$

H $5.976358-2.3086632 .054462$

SCF Energy (B3LYP/6-31G**//MMFF) $=-3245.89407896$

01162

MMFF Geometry

C $-2.4831452 .639488-3.251717$

C $-2.1923821 .485201-2.635696$

C $-1.133137 \quad 0.512212-3.076389$

O $-0.1639510 .408112-2.008055$

C $-1.774071-0.872337-3.284260$

C $-0.786138-1.961027-3.651927$

C $-0.552604-3.057230-2.903351$

C $-0.057858-1.773594-4.957192$

C $-1.200545-3.436414-1.600468$

C $-0.495305-2.890040-0.349859$

C $0.908386-3.471201-0.117552$

C $1.459672-3.0260851 .239056$

C $2.892440-3.5319191 .470076$

O $1.775422-3.009717-1.156044$

O $3.412902-2.8099122 .596856$

C $3.758031-3.1812420 .238801$
C $2.905717-5.012721 \quad 1.866867$

O $5.053415-3.7653770 .348172$

C $3.100706-3.555664-1.106096$

C $3.870428-3.012835-2.334453$

O $3.073718-4.983422-1.227916$

C $0.7690231 .394503-1.918080$

O $0.9313742 .330085-2.685018$

C $1.5381651 .164872-0.677043$

C $2.2133382 .184464-0.126720$

C $2.9825672 .139073 \quad 1.118195$

C 3.1470230 .8011351 .789022

C $4.034457-1.489790-2.388335$

C $5.365298-0.991678-1.847911$

C $6.5813491 .123625-1.336772$

O $6.301819-1.735467-1.569623$

C $6.531366 \quad 1.3624210 .139200$

C 6.6946622 .5726340 .695209

C 6.7008242 .8207692 .176558

C 5.7277103 .9010732 .592910

C 6.1420435 .1628892 .797719

C 4.2949063 .4979392 .857093

C $3.5094743 .287157 \quad 1.592697$

N $5.3984530 .393558-1.754169$

H $-1.9411202 .919327-4.151059$

H $-2.7261051 .227746-1.726447$

H $-0.6399850 .843763-3.998259$

H -2.532194 - $0.812737-4.076562$

H -2.315468 -1.145337-2.371737

H $0.180428-3.777678-3.264174$

H $0.534525-2.653047-5.232051$

H $-0.771393-1.594102-5.767893$

H $0.627689-0.922567-4.898110$

H -2.248039 -3.116183-1.590007

H $-1.240641-4.531648-1.544412$

H $-0.439395-1.797131-0.417065$

H $-1.123360-3.1274930 .517144$

H $0.836538-4.564421-0.159993$

H $0.801842-3.3520822 .054563$

H $1.467049-1.9283431 .284336$

H $4.345590-3.0645032 .704639$

H $3.912149-2.0973830 .269801$

H $2.320751-5.1667652 .781655$

H $3.920412-5.3518492 .102929$

H $2.494108-5.666723 \quad 1.095294$

H $4.973749-4.707626 \quad 0.121930$

H $4.833099-3.528520-2.433771$

H $3.295778-3.299312-3.227228$

H $2.977849-5.210240-2.167793$

H $1.4943160 .177846-0.232021$

H $2.1810243 .155084-0.620324$

H 3.5449980 .0662381 .082132

$\begin{array}{llll}\text { H } & 2.185842 & 0.439728 & 2.168919\end{array}$

H 3.8439510 .8154242 .629328

H $3.998345-1.175478-3.439453$

H $3.214315-0.985770-1.869192$

H $6.6039552 .057720-1.907144$

H $7.4746510 .541556-1.585356$

H 6.3858600 .4965880 .780930

H $6.8703803 .431877 \quad 0.051677$

H 7.7211613 .1107142 .460192

H 6.4890601 .9024532 .738234

H $5.4563595 .937463 \quad 3.124643$

H 7.1757605 .4504392 .637241

H 3.7820364 .2846653 .426750

H 4.2818492 .6270993 .518476

H 3.3417094 .2064361 .029808

H $4.5578900 .927308-1.955708$

C $-3.1454994 .361886-1.467882$

O $-3.158512 \quad 3.467974-0.333275$

$\begin{array}{llll}\text { C }-1.954610 & 3.678675 & 0.422879\end{array}$

C -1.4412485 .0516360 .002818$

C $-1.7787005 .050969-1.474634$

H -1.2374772 .9199600 .090268$

C -2.2042203 .5321201 .926064$

H -1.9782205 .8599340 .512690$

H -0.3718665 .1740770 .199556$

C $-1.7929006 .433233-2.103355$

H -1.029707 $4.439898-1.992027$ 
C -2.814276 2.190662 2.388117 O $-3.089728 \quad 4.5726802 .362001$ H -1.261733 3.7049372 .459958 H -3.8850192 .1588012 .153541$ O $-2.7185012 .167963 \quad 3.820239$ C -2.137905 0.9249291 .832827 C $-2.433447-0.3740342 .604457$ H -2.4159840 .8022580 .780541$ O $-0.717994 \quad 1.1072791 .871694$ H -1.942030 -1.1887342.058799 H -1.907594 -0.3338003 .567735$ H $-2.0599676 .374448-3.163350$ H -2.514342 $7.092366-1.609080$ H $-0.8053516 .900263-2.028147$ C -3.521345 $3.606649-2.749356$ H -3.911256 4.4916991 .847342 H -3.1760192 .9588874 .153799$ H -0.3100890 .3176081 .477485$ H $-3.7187124 .331813-3.548201$ H -4.458006 $3.059693-2.585967$ H -3.934932 $5.097738-1.266681$ C $-4.384517-1.8380840 .751768$ O $-4.693770-0.7650401 .647865$ C -3.917392 -0.7157802 .845003$ C $-4.117599-2.0151633 .635756$ C $-3.741471-3.2311362 .797051$ C $-4.476555-3.204607 \quad 1.458775$ H $-3.383223-1.6812720 .334067$ C $-5.401095-1.787617-0.403257$ H -4.3421190 .0922963 .451678$ O $-3.345524-1.9870974 .831597$ H -5.170910 -2.096937 3.934108 H -2.656362 -3.300375 2.660956 O $-4.124763-4.4071023 .515545$ H -5.526903 -3.4698831 .637663$ O $-3.914421-4.2268530 .636806$ C -5.356531 -0.506902 -1.207779 H $-5.212532-2.619092-1.091322$ H $-6.422741-1.876336-0.014799$ H -3.479544 -2.837496 5.284156 H $-3.909107-5.1715212 .954028$ H $-4.509955-4.366573-0.117933$ O $-5.859723-0.752370-2.454101$ $\begin{array}{llll}O & -4.978959 & 0.576856 & -0.786864\end{array}$ C $-5.9521850 .374365-3.331252$ H $-6.3920371 .237198-2.821800$ H $-4.9609290 .617662-3.721113$ H $-6.5996990 .099435-4.168177$

SCF Energy (B3LYP/6-31G**//MMFF) $=-3245.91221342$

01163

MMFF Geometry

C $-3.5430112 .766345-2.157241$

C $-2.6822531 .948318-1.533962$

C $-1.3891951 .468995-2.132176$

O $-0.3323021 .753565-1.185094$

C $-1.424765-0.051874-2.356265$

C $-0.155690-0.560771-3.011964$

C $0.854267-1.174815-2.363859$

C $-0.076132-0.349535-4.502571$

C $0.963462-1.477630-0.893483$

C $1.040374-2.986465-0.612502$

C $2.392961-3.613690-0.983059$

C $2.359518-5.130400-0.787743$

C $3.731542-5.767726-1.055291$

O $3.384974-3.038111-0.127267$

O $3.698384-7.112668-0.554026$

C $4.801402-5.008513-0.242983$

C $4.033234-5.870823-2.555079$

O $6.111294-5.467259-0.570850$

C $4.725311-3.469371-0.394801$

C $5.658724-2.8008230 .647678$

O $5.139191-3.108304-1.711109$

C $0.2655272 .972436-1.259035$

O $-0.000723 \quad 3.876845-2.034132$

C $1.317502 \quad 3.015547-0.217615$

C $2.0861774 .108750-0.103020$

C $3.1640594 .319496 \quad 0.867413$
C 3.4489513 .2098661 .843725

C $5.514810-1.2775800 .783014$

C $6.367225-0.467507-0.178986$

C $6.8837241 .901384-0.764323$

O $7.103404-0.972040-1.021162$

C 7.2114663 .0686680 .110865

C $6.9132804 .341558-0.191234$

C 7.2831245 .5007520 .689895

C 6.1397746 .4603830 .930735

C 6.2120727 .7364350 .514395

C 4.9480905 .9708901 .726784

C 3.8279645 .4942940 .839681

$\begin{array}{llll}\mathrm{N} & 6.211717 & 0.895293 & 0.036071\end{array}$

H $-3.3319403 .102160-3.168969$

H $-2.9131891 .608347-0.528409$

H $-1.1848651 .977398-3.082767$

H $-2.280059-0.318600-2.991381$

H $-1.599143-0.567245-1.405882$

H $1.718540-1.478901-2.952304$

H $0.834751-0.776565-4.935720$

H $-0.928707-0.824865-4.998537$

H $-0.0843890 .717951-4.742738$

H $1.850081-0.969110-0.497014$

H $0.115204-1.070492-0.337294$

H $0.875598-3.1289840 .463055$

H $0.227197-3.501750-1.138462$

H $2.628752-3.375722-2.025240$

H $1.592685-5.588590-1.424655$

H $2.066531-5.359500 \quad 0.246623$

H $2.979125-7.584059-1.008345$

H $4.642512-5.258746 \quad 0.815497$

H $3.268305-6.474179-3.058662$

H $4.984220-6.385276-2.732008$

H $4.068134-4.900024-3.053873$

H $6.119282-6.432163-0.449004$

H $5.412490-3.2176101 .633538$

H $6.703894-3.0570970 .437305$

H $5.385355-2.170375-1.714351$

H 1.4280752 .1493300 .424813

H $1.9112224 .937606-0.788305$

H 3.6550252 .2735091 .315389

H 2.5885083 .0531522 .503919

H 4.3126903 .4002832 .483479

H $4.470201-0.9643270 .683471$

H $5.843445-0.9990451 .793192$

H $6.2103372 .171047-1.584201$

H $7.8000921 .480731-1.190060$

H 7.7431152 .8555761 .035780

H $6.4123714 .564581-1.130271$

H 8.1213876 .0233350 .211410

H 7.6576115 .1535411 .661230

H 5.4076798 .4385380 .708463

H $7.0730278 .107069-0.031860$

H 4.5564236 .7965592 .335988

H 5.2801955 .2180282 .446196

H 3.5176736 .2372930 .102766

H 5.4839521 .2047140 .672980

C -6.010862 $2.410284-1.848070$

O $-5.866057 \quad 1.138453-1.181725$

C $-7.0652610 .859825-0.440032$

C $-8.134348 \quad 1.755866-1.048339$

C $-7.3280593 .005403-1.344003$

H $-6.881334 \quad 1.1797520 .593034$

C $-7.373860-0.638341-0.469344$

H $-8.536874 \quad 1.337092-1.977875$

H $-8.9690401 .933746-0.363720$

C $-7.996736 \quad 3.942675-2.334531$

H $-7.1608073 .545097-0.401952$

C $-6.203696-1.529669-0.001421$

O $-7.690077-1.011849-1.816826$

H $-8.273525-0.8394790 .124152$

H $-5.400426-1.485694-0.744728$

O $-6.670796-2.885635-0.011674$

C $-5.647436-1.1961311 .397870$

C $-4.632182-2.2266261 .929797$

H $-5.191375-0.2011151 .387876$

O $-6.744037-1.1500732 .315611$

H -4.442394 -2.004466 2.986339 
H -5.099863 -3.218476 1.935943 H -7.367683 $4.817139-2.528837$ H -8.187761 $3.445488-3.291494$ H -8.955872 4.296127 -1.942184 C $-4.8009313 .281869-1.515539$ H -7.880481 -1.965629 -1.815649 H $-7.357818-2.9633790 .672647$ H $-6.394702-0.8583293 .175033$ H $-4.6642113 .325749-0.427573$ H -4.961514 4.310474-1.858496 H -6.060477 2.204288 -2.924718 C -2.033621 -0.6973722 .487456$ O $-2.606148-1.0115251 .211126$ C $-3.306885-2.2594491 .143532$ C $-2.373343-3.4120921 .536340$ C $-1.729721-3.1810272 .897853$ C $-1.074140-1.8038512 .948666$ H $-2.831783-0.5617453 .226655$ C $-1.2753320 .632663 \quad 2.344189$ H -3.556953 -2.411949 0.087810 O $-3.069156-4.6540731 .552151$ H $-1.578062-3.5032930 .787138$ H $-2.453349-3.3032233 .712288$ O $-0.714619-4.1651243 .110366$ H $-0.173221-1.8049542 .322415$ O $-0.659176-1.5354994 .287154$ C -2.1939391 .8295812 .226175$ H -0.6418260 .8078923 .220843$ H -0.6496090 .6040521 .445061$ H $-3.461590-4.7802800 .671342$ H $-1.137535-5.037475 \quad 3.032275$ H $-0.068419-2.2591874 .558129$ O -1.4302982 .9449072 .023580$ O $-3.413817 \quad 1.7998702 .305268$ C -2.1490634 .1748431 .894437$ H $-2.7837394 .149111 \quad 1.004712$ H $-2.746798 \quad 4.363228 \quad 2.791254$ H -1.421162 4.9827761 .781705

SCF Energy (B3LYP/6-31G**//MMFF)= -3245.92531686

\section{4}

MM̄FF Geometry

C $1.2031751 .980377-1.172553$

C $1.0393760 .790104-0.577435$

C 0.0264950 .5068260 .495286

$\begin{array}{llll}\text { O } & -0.713342 & -0.648576 & 0.028212\end{array}$

C $0.673804 \quad 0.1634551 .848542$

C 1.3209531 .3346552 .551049

C 0.6565352 .2130523 .326881

C 2.8152921 .4399172 .402387

C -0.8210932 .2572143 .616026$

C - $-1.386073 \quad 3.6806923 .498537$

C -1.5455984 .1592892 .044571$

C -1.573276 5.6894002 .003963

C $-1.827632 \quad 6.2207510 .589644$

O -2.7791893 .6272301 .538776$

O $-2.1251407 .621614 \quad 0.691166$

C -3.0840965 .5302440 .031814$

C $-0.589218 \quad 6.103312-0.305224$

O $-3.2940685 .888788-1.333251$

C -3.0766303 .9866390 .181834$

C $-4.4882083 .457254-0.172670$

O $-2.1234053 .435235-0.725715$

C $-1.944211-0.870598 \quad 0.556433$

O $-2.524031-0.189941 \quad 1.386294$

C $-2.465527-2.116967-0.044936$

C $-3.592942-2.6532340 .443217$

C $-4.243132-3.876540-0.024234$

C $-3.642511-4.589697-1.206518$

C -4.7243801 .9585530 .059122$

C $-6.1486281 .570546-0.314080$

C $-7.641377-0.366047-0.634948$

O $-6.9898992 .389825-0.678717$

C $-7.841138-1.723239-0.042445$

C $-8.077746-2.818722-0.780980$

C $-8.362214-4.181233-0.212248$

C $-7.337625-5.211265-0.633208$

C $-7.474703-5.891601-1.783809$
C $-6.193888-5.503594 \quad 0.310903$

C $-5.358311-4.2914290 .610773$

N $-6.3844830 .206250-0.186482$

H $0.6029302 .827449-0.855904$

H $1.662125-0.042162-0.893318$

H -0.6657321 .3511340 .595581$

H $1.405884-0.6452911 .716830$

H $-0.074994-0.2819752 .516089$

H 1.2373912 .9870483 .827426

H 3.2979090 .5292502 .771649

H 3.0888791 .5786221 .354249

H 3.2324562 .2832142 .963000

H $-1.392273 \quad 1.5798462 .977241$

H $-0.962519 \quad 1.917223 \quad 4.649271$

H -2.366401 3.6977683 .992105

H -0.7345414 .3655844 .056152$

H $-0.715613 \quad 3.7831201 .437228$

H -0.6398726 .1058442 .403211$

H -2.3718826 .0576122 .663592$

H -1.365283 8.0595491 .111622

H -3.9449215 .9256680 .589757$

H $\quad 0.250150 \quad 6.661351 \quad 0.126987$

H $-0.7696656 .548855-1.289739$

H $-0.2609535 .072807-0.448684$

H -3.311282 $6.860200-1.378122$

H -5.2282024 .0013730 .429863$

H $-4.7042183 .677727-1.226395$

H $-2.3538462 .509900-0.902248$

H $-1.905385-2.562988-0.858659$

H $-4.090541-2.1505861 .271922$

H $-2.637805-4.954059-0.965839$

H -3.575430 -3.917214 -2.069098

H $-4.227261-5.452997-1.533281$

H $-4.0403111 .360442-0.550817$

H $-4.569351 \quad 1.7083441 .114027$

H -7.617748 $-0.388782-1.730058$

H $-8.4607620 .294218-0.330715$

H -7.833639-1.800105 1.041908

H -8.107583 -2.728655 -1.864935

H $-9.357903-4.488868-0.558353$

H $-8.432397-4.1522780 .882222$

H $-6.758046-6.649169-2.082614$

H $-8.306933-5.702413-2.453917$

H $-5.567292-6.322396-0.061086$

H $-6.607218-5.8773551 .256865$

H $-5.729099-3.7042601 .451699$

H $-5.587493-0.412468-0.065415$

C $3.5164472 .734196-1.814656$

O $4.2050161 .671820-1.123044$

C $5.5417761 .562865-1.639795$

C $5.8057682 .872228-2.370259$

C $4.4386663 .157156-2.960263$

H $5.5322010 .745217-2.370924$

C $6.5255121 .236742-0.513589$

H $6.0934423 .673792-1.680364$

H $6.5901752 .776491-3.126959$

C $4.2519484 .599622-3.397854$

H $4.2877602 .499478-3.827090$

C $6.1390280 .000807 \quad 0.325791$

O $6.5855482 .358852 \quad 0.377265$

H $7.5332971 .120892-0.929572$

H 5.2529420 .2381810 .922262

O $7.195642-0.2203151 .269886$

C $5.900148-1.292127-0.478679$

C $5.720856-2.5485400 .396604$

H $5.027677-1.168258-1.128154$

O $7.032001-1.507159-1.326619$

H $5.732554-3.425728-0.260873$

H $6.605026-2.6691421 .034138$

H $3.2458894 .758197-3.798640$

H $4.3952585 .294047-2.563169$

H $4.9727164 .860651-4.179624$

C $2.1659632 .213489-2.303043$

H 7.2132282 .1334711 .085200

H $7.987633-0.4780810 .767440$

H $6.836632-2.277952-1.886324$

H $2.309816 \quad 1.278121-2.858829$

H $1.7063892 .927859-2.995794$ 
H $3.3837153 .552611-1.095722$

C $2.954444-3.687792-0.278758$

O $3.253089-2.4872700 .446442$

C $4.437587-2.5367381 .250718$

C $4.343275-3.6883792 .261038$

C $4.055512-5.0182381 .575011$

C $2.834147-4.8895670 .669089$

H $3.739078-3.875148-1.021134$

C $1.626547-3.464056-1.021155$

H $4.446405-1.6127031 .838920$

O $5.541590-3.8028313 .021025$

H $3.531725-3.4825012 .971239$

H $4.925538-5.3815911 .015635$

O $3.772781-6.0079802 .566951$

H $1.931066-4.7940151 .285918$

O $2.699599-6.082339-0.102242$

C $1.766675-2.550781-2.220020$

H $1.233253-4.415603-1.395903$

H $0.895918-3.011979-0.340696$

H $5.696086-2.9456903 .453688$

H $4.541299-6.0480003 .162029$

H $2.645843-6.8265780 .521749$

O $0.531362-2.371286-2.776975$

O $2.812626-2.061750-2.622607$

C $0.491095-1.537504-3.938785$

H $-0.537345-1.521046-4.309089$

H $0.786769-0.517031-3.680874$

H $1.139422-1.942168-4.721711$

SCF Energy (B3LYP/6-31G**/MMFF) $=-3245.92592936$

\section{5}

MM̄MF Geometry

C -0.2933973 .6398311 .093189$

C $0.724023 \quad 4.145596 \quad 0.378842$

C $1.0578853 .859464-1.069066$

O $2.3283313 .165086-1.109931$

C $0.0293572 .996372-1.822234$

C $0.2624872 .924559-3.319594$

C $0.6537581 .814302-3.975127$

C $-0.0545424 .180853-4.089183$

C $1.0059080 .483541-3.374485$

C $-0.223952-0.418847-3.225115$

C $0.143105-1.833971-2.755592$

C -1.121359 -2.654176 -2.485464

C $-0.804150-4.038700-1.900067$

O $0.923681-1.723624-1.562399$

O $-2.022816-4.581713-1.367868$

C $0.168793-3.868376-0.713086$

C $-0.328367-5.017973-2.978875$

O $0.609753-5.133065-0.226233$

C $1.384135-2.977584-1.042588$

C $2.256552-2.674074 \quad 0.193505$

O $2.222557-3.653567-1.987733$

C $3.4570973 .917184-1.159939$

O $3.5189415 .139781-1.150102$

C $4.6793753 .083190-1.214371$

C $4.6857541 .747699-1.350236$

C $5.8800010 .898511-1.416943$

C $7.2174251 .563106-1.210860$

C $1.547149-1.9230291 .322169$

C $2.552309-1.4808732 .364884$

C $4.430225 \quad 0.1287342 .695975$

O $2.713109-2.103038 \quad 3.412147$

C $5.689643-0.3098752 .006272$

C $5.919441-1.5485791 .541603$

C $7.141127-1.9611650 .772735$

C $6.786667-2.466030-0.610880$

C $6.526221-3.767046-0.824404$

C $6.799293-1.475463-1.754597$

C $5.726842-0.425314-1.635134$

N $3.267936-0.359314 \quad 1.974544$

H -0.9702322 .9186310 .646620$

H 1.3772694 .8689320 .864391

H $1.1445094 .826820-1.579310$

H -0.977153 $3.406641-1.673090$

H $0.0179241 .989660-1.389593$

H $0.7456041 .857851-5.059272$

H $0.0168874 .033370-5.172256$
H $-1.0750604 .513201-3.871628$

H $0.6389704 .983746-3.821865$

H $1.745620-0.000192-4.024452$

H $1.5029460 .622083-2.408225$

H $-0.9013940 .037819-2.493187$

H $-0.769481-0.480360-4.175095$

H $0.749078-2.306535-3.537763$

H -1.726394 -2.748532 -3.395771

H -1.751614 -2.118519-1.763043

H -2.675522 -4.616284 -2.088232

H $-0.401829-3.4037740 .100110$

H $-1.106120-5.156413-3.739589$

H $-0.142842-6.011828-2.556771$

H $\quad 0.576086-4.684990-3.491821$

H $-0.183487-5.658157-0.023751$

H $2.694787-3.6020090 .584006$

H $3.115164-2.075046-0.138810$

H $3.115712-3.274208-1.937118$

H $5.6031533 .650494-1.153029$

H $3.7377261 .219532-1.427866$

H $7.2299782 .119947-0.266823$

H $7.4315222 .256181-2.031659$

H $8.0525770 .862511-1.155552$

H $1.015749-1.0414550 .946670$

H $\quad 0.810637-2.5628691 .817576$

H $4.418125-0.2087573 .736757$

H 4.3669381 .2215042 .681273

H 6.4445320 .4579941 .855475

H $5.178784-2.3267761 .708455$

H $7.649045-2.7518671 .339969$

H $7.865878-1.1416030 .695106$

H $6.291729-4.143267-1.814486$

H $6.536773-4.484328-0.010123$

H $6.635609-1.994302-2.708681$

H $7.802711-1.049197-1.838110$

H $4.717186-0.817338-1.763737$

H $3.081076 \quad 0.0489541 .063141$

C -0.5304672 .9117503 .524568$

O -1.4432821 .8478623 .186093$

C -0.8254230 .5935193 .543851$

C 0.4140090 .9503474 .359797

C 0.8341412 .2499863 .700400

H $-0.500070 \quad 0.1267252 .607822$

C -1.823007 -0.3167694 .266135$

H $\quad 0.169404 \quad 1.1200605 .415210$

H 1.1814360 .1739624 .316918

C 1.8209513 .0603734 .520516

H 1.2820452 .0167982 .726084

C $-3.086533-0.6469283 .443029$

O -2.2402550 .3371085 .470939$

H $-1.318534-1.2403584 .574748$

H -3.677879 0.2650213 .305190

O $-3.903437-1.5257064 .227282$

C $-2.807142-1.3015912 .076718$

C $-4.053015-1.8337911 .345538$

H $-2.295499-0.5833161 .427958$

O $-1.906558-2.3992122 .247235$

H $-3.709781-2.3141850 .420617$

H -4.502636 -2.647706 1.928539

H 2.0821093 .9909484 .006943

H 1.4107733 .3196075 .502247

H 2.7437332 .4932104 .681213

C -0.6119124 .0474822 .504350$

H $-2.878577-0.2487745 .912796$

H $-3.383957-2.3239784 .421412$

H $-2.362804-3.0864842 .761317$

H 0.0335944 .8736322 .825118

H -1.638709 4.4352272 .496123

H -0.8745653 .3070624 .490325$

C $-4.1001250 .205465-0.923714$

$\begin{array}{llll}\text { O } & -4.555276 & 0.397536 & 0.418136\end{array}$

C $-5.118182-0.7654671 .031817$

C $-6.300154-1.2832080 .199010$

C $-5.911836-1.516970-1.261161$

C $-5.226577-0.279696-1.843350$

H $-3.300276-0.544101-0.916970$

C $-3.4371701 .503609-1.402020$

H $-5.527657-0.4362791 .994593$ 
O $-6.809295-2.485056 \quad 0.767066$ H $-7.109000-0.5418990 .236373$ H $-5.272061-2.400946-1.361432$ O $-7.087427-1.804138-2.022638$ H $-5.9739790 .507119-1.995301$ O $-4.681312-0.599625-3.121444$ C $-4.3569962 .705499-1.460297$ H $-2.6295391 .765028-0.709869$ H $-3.0084161 .346685-2.398174$ H $-7.536494-2.7908250 .198347$ H $-7.685421-1.040590-1.952207$ H $-5.408331-0.940606-3.670194$ O $-3.7793833 .664213-2.244231$ O $-5.4243772 .810858-0.872444$ C $-4.518326 \quad 4.882309-2.375026$ H $-3.9518885 .554907-3.024692$ H $-5.4921434 .687476-2.834368$ H $-4.6394735 .358163-1.397304$

SCF Energy (B3LYP/6-31G**/MMFF) $=-3245.90736748$

\section{6}

MM̄FF Geometry

C $3.9969762 .688677-0.632833$

C $3.2833173 .767633-0.990294$

C $1.7781153 .845725-1.120031$

O $1.2093492 .533169-0.914353$

C $1.2090274 .812832-0.065307$

C $-0.0972275 .462059-0.476338$

C -1.3119195 .1529140 .017525$

C $0.0377046 .582255-1.478655$

C $-1.6745654 .081911 \quad 1.009674$

C -2.8064853 .2108840 .459953$

C $-3.3767512 .193140 \quad 1.458021$

C -2.3279801 .2069921 .973818$

C -2.9453520 .0889142 .826355$

O $-4.405698 \quad 1.4931090 .747589$

O $-1.966686-0.9517942 .967066$

C $-4.140442-0.5221432 .069557$

C -3.271346 $0.572457 \quad 4.243993$

O $-4.831792-1.4646982 .886494$

C -5.1299190 .5315091 .522939$

C $-6.147698-0.1494210 .579563$

O $\begin{array}{llll}-5.823570 & 1.141013 & 2.610910\end{array}$

C $0.1850632 .127914-1.707537$

O $-0.3876392 .774723-2.568536$

C $-0.1170100 .725320-1.337915$

C -1.227960 $0.128591-1.796023$

C - $1.649837-1.245741-1.503923$

C $-0.718807-2.091691-0.674467$

C $-7.1193330 .819981-0.108873$

C $-7.7966580 .134133-1.277793$

C -7.310845 -0.700310 -3.577082

O $-8.918680-0.356516-1.181374$

C $-6.723265-2.061407-3.375146$

C $-5.648693-2.520833-4.033611$

C $-5.025406-3.858757-3.755263$

C -3.625602 -3.739754 -3.192982

C $-2.573100-4.280811-3.829610$

C $-3.453612-3.046790-1.856096$

C $-2.827932-1.682316-1.998155$

N $-6.9908090 .095427-2.406380$

H $3.4900371 .761319-0.380837$

H $3.8185944 .682915-1.237285$

H $1.5680784 .188926-2.140036$

H 1.9208335 .6269420 .129984

H 1.0997164 .2909880 .891006

H -2.161784 $5.738319-0.333374$

H $-0.9223077 .059447-1.703041$

H $0.7069087 .358280-1.092965$

H $\quad 0.4452696 .209445-2.422974$

H -0.8149953 .4533831 .251654$

H -1.999964 4.5654921 .937834

H -3.635105 3.8485820 .120556

H -2.470228 $2.686478-0.443130$

H -3.8305952 .7430112 .290943$

H -1.551645 1.7240002 .548003

H -1.813791 $0.744731 \quad 1.123055$

H - $-1.163536-0.558307 \quad 3.349509$
H -3.734469 -1.088898 1.219854

H $-2.3607050 .915494 \quad 4.749985$

H -3.665796 -0.2433974 .859551$

H -3.9877831 .3965524 .260323$

H -4.177383 -2.113902 3.196395

H -5.594034 -0.701134 -0.191160

H $-6.742142-0.8866941 .134153$

H -6.6430541 .5398252 .278803$

H $0.5854430 .222887-0.681807$

H -1.897903 $0.699239-2.437812$

H $-0.583941-1.6544130 .321102$

H $\quad 0.259123-2.167772-1.160729$

H -1.066978 -3.114527 -0.522537

H $-7.894987 \quad 1.160900 \quad 0.585257$

H $-6.6059881 .714155-0.479962$

H $-6.888064-0.190217-4.448157$

H -8.395973 $-0.770826-3.699626$

H -7.191866 -2.688136 -2.619545

H $-5.172668-1.897166-4.786236$

H -5.022691 -4.430548 -4.691806

H $-5.635136-4.437818-3.049980$

H -1.572025 -4.217807 -3.415437

H -2.688041 -4.797780 -4.776353

H $-2.890813-3.705178-1.188784$

H $-4.428430-2.926085-1.367211$

H -3.432242 $-0.983052-2.577351$

H $-6.0453800 .462103-2.341787$

C $6.1452931 .658168-1.510803$

O $5.7174110 .320343-1.184009$

C $5.321923-0.340258-2.400178$

C $5.9785900 .448940-3.525386$

C $5.8454431 .865119-2.998132$

H $4.233465-0.227423-2.480024$

C $5.679927-1.828095-2.350752$

H $7.0365870 .187679-3.642941$

H $5.4793740 .298962-4.487221$

C $6.7681962 .862751-3.675923$

H $4.8083212 .188451-3.149142$

C $5.165601-2.568103-1.098642$

O $7.105420-1.976166-2.376868$

H $5.312411-2.323560-3.256897$

H $5.753016-2.262936-0.225124$

O $5.416538-3.966870-1.284501$

C $3.666387-2.380021-0.807815$

C $3.114353-3.2625150 .327249$

H $3.469998-1.331077-0.562959$

O $2.911519-2.668858-1.987738$

H $2.051339-3.0206530 .445724$

H $3.126132-4.3142220 .013401$

H $6.6439083 .861184-3.244838$

H $7.8195402 .576230-3.567174$

H $6.5466582 .925742-4.746325$

C $5.4991072 .665317-0.553610$

H $7.466042-1.480420-1.621536$

H $\quad 6.367445-4.069442-1.461703$

H $3.108643-3.585941-2.245500$

H $5.9138213 .662580-0.741047$

H 5.7631362 .3953740 .476569

H $7.229747 \quad 1.673824-1.341511$

C $2.831686-1.0574902 .436381$

O $4.027982-1.7433122 .046861$

C $3.847172-3.1117101 .673870$

C $3.213448-3.8996852 .829134$

C $1.935877-3.2384763 .339538$

C $2.180240-1.7593323 .636386$

H $2.133864-1.0345651 .590385$

C 3.2444970 .3844302 .761113

H $4.853836-3.5229331 .530713$

O $2.922752-5.2371012 .437044$

H $3.926970-3.9568883 .661600$

H $1.106212-3.3642762 .633901$

O $1.530456-3.8764364 .553080$

H $2.823080-1.6688834 .521429$

O $0.937863-1.1364173 .947089$

C $2.073177 \quad 1.3352822 .819311$

H $3.799340 \quad 0.406078 \quad 3.707204$

H 3.9357630 .7662192 .000246

H $3.754114-5.6396282 .133120$ 
H $\quad 1.413850-4.8219804 .356875$

H $\quad 0.547018-1.6238954 .692577$

O $2.1740332 .123762 \quad 3.927375$

O $1.182366 \quad 1.382187 \quad 1.981637$

C 1.1069913 .0572834 .120852

H $\quad 0.1553892 .528178 \quad 4.227327$

H 1.3036973 .6114305 .042434

H 1.0662163 .7651093 .288611

SCF Energy (B3LYP/6-31G**//MMFF) $=-3245.92856676$

\section{1_167}

MM̄FF Geometry

C $-2.762618 \quad 1.3611512 .134403$

C -2.0267350 .5350062 .893959$

C $-1.928768-0.9509442 .676290$

O $-0.515073-1.2651332 .670560$

C $-2.587739-1.7512573 .816169$

C $-4.097121-1.6858593 .855113$

C $-4.903443-2.5066123 .154479$

C $-4.685385-0.6831704 .812975$

C $-4.497722-3.5729712 .170400$

C $-5.372203-3.5494610 .908096$

C $-5.089298-2.345493-0.006668$

C -6.303064 -2.074829 -0.898309

C $-6.045181-0.912736-1.862070$

O $-3.948916-2.660508-0.817538$

O $-7.094748-0.908469-2.842261$

C $-4.729501-1.184993-2.614912$

C $-6.1137030 .444055-1.151565$

O $-4.356171-0.057134-3.404868$

C $-3.544036-1.610713-1.708170$

C $-2.407824-2.151342-2.604183$

O $-3.005514-0.499482-0.992585$

C $-0.127529-2.4186982 .065149$

O $-0.833163-3.2098331 .461745$

C $1.327886-2.5599592 .280316$

C $1.991350-3.5615581 .686389$

C $3.430792-3.8055881 .768715$

C $4.263594-2.8551332 .588114$

C $-1.146857-2.606916-1.856967$

C $-0.004443-2.851386-2.833094$

C $2.463146-3.029670-2.952490$

O $-0.171930-2.900811-4.050231$

C $3.391083-4.061356-2.398243$

C $4.652206-3.793912-2.024688$

C $5.631352-4.833436-1.554074$

C $6.119494-4.579692-0.145501$

C $7.206493-3.8237340 .083023$

C $5.388665-5.2587800 .991064$

C $3.946607-4.8459461 .083316$

N $1.220975-3.014353-2.198882$

H $-3.3418680 .947619 \quad 1.314920$

H $-1.4775980 .941648 \quad 3.740492$

H -2.360335 -1.2223981 .704965$

H -2.176826 -1.426931 4.782825

H $-2.278298-2.8028583 .754799$

H -5.978278 -2.4126103 .305097$

H $-5.779878-0.7201014 .833047$

H $-4.331940-0.8789535 .830703$

H -4.3989920 .3351604 .536769$

H $-3.445414-3.4974481 .886288$

H -4.630221 -4.5445912 .661471$

H $-5.191964-4.4781360 .351498$

H $-6.426549-3.5572341 .213087$

H -4.863365 -1.4631370 .601319$

H $-7.195507-1.872881-0.292464$

H -6.534912 -2.974226 -1.485976

H -7.942826 -0.822771 -2.373891

H -4.926921 -2.004421 -3.320775

H $-7.1111110 .602548-0.724004$

H $-5.9564531 .267939-1.856114$

H $-5.3915030 .540529-0.338456$

H $-5.1103870 .155209-3.981079$

H - $2.777828-3.002615-3.191103$

H $-2.118460-1.369223-3.318132$

H $-3.5157310 .294528-1.215169$

H $1.818001-1.8233762 .906819$

H $1.432553-4.2608671 .065499$
H $3.952919-2.8798633 .638452$

H $4.158656-1.8311852 .214268$

H $5.331293-3.0852402 .567934$

H $-0.830529-1.842796-1.140190$

H -1.342795 -3.536529-1.312289

H $2.884415-2.020233-2.895469$

H $2.260953-3.250930-4.005462$

H $3.024853-5.083944-2.349136$

H $5.021965-2.773603-2.100668$

H $6.483137-4.829354-2.247089$

H $5.207787-5.843192-1.621987$

H $7.578064-3.6553281 .088192$

H $7.746528-3.352834-0.731874$

H $5.897125-5.0965611 .948565$

H $5.427832-6.3448850 .836119$

H $3.272168-5.4775080 .504134$

H $1.276960-2.847203-1.198291$

C $-2.630876 \quad 3.774176 \quad 1.245838$

O -1.2419383 .7626560 .870519$

C $-1.1509164 .238591-0.493998$

C $-2.5875664 .426813-0.991624$

C $-3.3819163 .532958-0.057203$

H $-0.6803993 .440920-1.076381$

C $-0.3086275 .523379-0.555467$

H -2.914056 $5.469749-0.902174$

H $-2.6944124 .143396-2.043795$

C $-4.8602883 .870639-0.000703$

H $-3.2557832 .497657-0.394365$

C $1.1541385 .334986-0.100935$

O $-0.920655 \quad 6.4969870 .301025$

H $-0.3402295 .939312-1.569200$

H 1.1663824 .9647060 .929168

O $1.7791006 .624980-0.067471$

C $1.9812794 .415651-1.019185$

C $3.4587504 .275610-0.600579$

H $1.5190493 .426682-1.066900$

O $1.9462684 .950198-2.345811$

H $3.9895103 .742976-1.399331$

H $3.9325045 .264553-0.566263$

H -5.3880793 .1971930 .681820$

H $-5.0250294 .897006 \quad 0.343790$

H $-5.3156873 .771438-0.991449$

C -2.9118352 .8294682 .416515$

H -0.3727867 .2996490 .264532$

H $1.8070316 .959907-0.980370$

H $2.4070534 .321761-2.927618$

H -3.9405102 .9940322 .760251$

H -2.252222 3.1102843 .247779

H -2.8404014 .7924021 .604217$

C $3.3684951 .295181-0.062617$

O 2.8940192 .3345660 .802608

C 3.6532273 .5454960 .742763

C 5.1162313 .2655321 .119278

C 5.7141152 .1076560 .319084

C $4.7866180 .891157 \quad 0.344789$

H $3.3720611 .645169-1.101650$

C 2.3816160 .1310520 .043026

H 3.2460814 .2019471 .521118

O 5.9209464 .4252730 .934693

H 5.1678153 .0052872 .184640

H $5.9350092 .407276-0.712297$

O $\quad 6.962981 \quad 1.7293930 .902553$

H 4.7844970 .4499641 .349265

O $5.276384-0.094720-0.560245$

C $1.0731210 .485541-0.624735$

H $2.761935-0.786421-0.418088$

H $2.166376-0.0885781 .093647$

H 5.5319465 .1387821 .468865

H 7.5285282 .5207230 .912503

H $6.182347-0.316638-0.284682$

O $1.230514 \quad 0.485274-1.978818$

O $\quad 0.0487370 .753169-0.011376$

C $0.1040960 .883067-2.769206$

H $\quad 0.206177 \quad 1.942176-3.020113$

H $\quad 0.1159180 .300148-3.693950$

$\mathrm{H}-0.8478650 .710418-2.260882$

SCF Energy (B3LYP/6-31G**//MMFF) $=-3245.91523592$ 
01 168

MM̄FF Geometry

C $-2.475629-4.290890 \quad 0.909432$

C $-1.898809-3.518117 \quad 1.843316$

C $-0.496561-2.9871971 .711208$

O $-0.531998-1.5856562 .084753$

C $0.499340-3.6971502 .649160$

C $0.794266-5.1420692 .307373$

C $1.721373-5.5273051 .407985$

C $0.040837-6.1715393 .107543$

C $2.563438-4.6369720 .537787$

C $3.972089-4.4538721 .112611$

C $4.869021-3.6070370 .195685$

C $6.247756-3.4163920 .826670$

C $7.140836-2.492606-0.011842$

O $4.246884-2.3351160 .008065$

O $8.265008-2.150740 \quad 0.813316$

C $6.368874-1.193145-0.339632$

C $7.704107-3.223374-1.237721$

O $7.117204-0.401313-1.259056$

C $4.943648-1.445239-0.883288$

C $4.071591-0.168818-0.923504$

O $5.018526-1.991187-2.201546$

C $0.416246-0.777478 \quad 1.536778$

O $1.229758-1.0826960 .679513$

C 0.3312460 .5463442 .194160

C 1.1553351 .5255511 .791432

C 1.2501262 .8777532 .345008

C 0.4473533 .1805713 .581488

C $4.5642810 .971691-1.814587$

C $3.4968892 .034792-2.020527$

C $3.1914594 .332576-2.932112$

O $2.3246861 .886213-1.687193$

C $3.2629095 .277065-1.773127$

C $2.1862815 .723299-1.107762$

C 2.2581816 .7049930 .026217

C 1.5954786 .2036811 .289888

C 0.4211976 .7114831 .700658

C 2.3404185 .1934432 .133412

C 2.0540913 .7719701 .730959

N $4.0035833 .164667-2.645580$

H -1.896112 -4.559395 0.030239

H -2.446607 -3.2683112 .748469$

H -0.171982 -3.0721900 .666568$

H $\quad 0.144741-3.625718 \quad 3.687137$

H $1.442937-3.1353462 .665446$

H $1.894465-6.5928601 .265390$

H $0.325644-7.1949172 .840591$

H $0.243332-6.0424104 .175984$

H -1.036550 -6.079651 2.945991

H $2.624996-5.091372-0.458667$

H $2.084565-3.6644750 .394768$

H $3.887138-3.9638622 .091639$

H $4.439399-5.4328451 .277841$

H $4.950704-4.115380-0.772080$

H $6.739734-4.3836320 .989372$

H $6.131673-2.9698641 .824577$

H $8.879850-1.6177070 .281687$

H $6.284771-0.6092660 .587392$

H $8.300907-4.088616-0.924419$

H $8.385907-2.580621-1.805485$

H $6.927160-3.582908-1.915784$

H $7.951519-0.154400-0.826221$

H $3.068541-0.467164-1.259512$

H 3.9293050 .2078770 .098210

H $4.173026-1.824995-2.650451$

H -0.3861640 .6714852 .996699$

H 1.8378401 .3194840 .969035

H -0.6184793 .0214713 .397110$

H $\quad 0.547968 \quad 4.211115 \quad 3.927727$

H 0.7590482 .5329354 .408382

H $5.4348761 .452350-1.355788$

H $4.8487540 .595216-2.803253$

H $2.1644074 .017691-3.145356$

H $3.6047154 .808309-3.826757$

H $4.2506165 .627869-1.482875$

H $1.1965635 .390939-1.411434$

H $1.7755887 .632049-0.310022$
H $3.296556 \quad 6.979209 \quad 0.251275$

H $-0.044356 \quad 6.3829372 .623874$

H -0.1058147 .4609891 .119704$

H 2.1300685 .3606203 .195141

H 3.4205655 .3677732 .044895

H 2.5955873 .4532530 .840162

H $5.0013543 .225460-2.826714$

C $-4.803223-4.510763-0.126043$

O $-5.046223-3.090814-0.143740$

C $-4.982207-2.635146-1.507610$

C $-5.106133-3.884802-2.372314$

C $-4.347355-4.895922-1.535384$

H $-3.980721-2.208607-1.645231$

C -6.037968 -1.558931-1.778794

H $-6.149303-4.200123-2.488865$

H $-4.679649-3.744339-3.370029$

C $-4.639718-6.340392-1.902518$

H $-3.276860-4.708225-1.673765$

C $-6.057168-0.392775-0.771470$

O $-7.343282-2.152073-1.758591$

H $-5.904782-1.171617-2.795712$

H $-6.516586-0.7157250 .171010$

O $-6.9146940 .625403-1.304955$

C $-4.6850240 .234775-0.470319$

C $-4.804124 \quad 1.5088810 .388255$

H $-4.062750-0.4991280 .052980$

O $\quad-4.0285260 .548241-1.695974$

H $-5.0713152 .356719-0.252663$

H $-5.6156761 .375787 \quad 1.113652$

H -4.078131 -7.025590 -1.259656

H $-5.704367-6.574355-1.797498$

H $-4.353425-6.538824-2.940634$

C $-3.856949-4.874758 \quad 1.023728$

H -7.460397 -2.573880 -0.889854

H -7.780701 $0.215515-1.472792$

H $-4.6030051 .155634-2.192961$

H -3.761149 -5.9655301 .079704$

H $-4.313663-4.5451301 .965559$

H $-5.776544-4.9742370 .083432$

C $-2.2663603 .022758-0.526259$

O -2.3504341 .8850590 .339138$

C -3.5106961 .8309601 .169313$

C -3.6023363 .0894972 .042807$

C $-3.491954 \quad 4.3692521 .219148$

C -2.2736774 .3148900 .298145$

H $-3.1100773 .020231-1.226421$

C $-0.9743502 .884086-1.338497$

H $-3.3521470 .983203 \quad 1.848852$

O -4.8158833 .0896952 .787653$

H -2.786143 3.0706192 .773431

H -4.4131104 .5540940 .653995$

O -3.3457645 .4691832 .121190$

H -1.3654304 .3886010 .908214$

O $-2.3190145 .454152-0.557135$

C $-1.0337711 .694134-2.266825$

H $-0.7902863 .764502-1.963358$

H $-0.1027292 .773998-0.684739$

H -4.8377453 .9114953 .307567$

H -3.230383 6.2708351 .582501

H -1.441657 $5.555989-0.962199$

O $-0.5956380 .574623-1.623077$

O $-1.4414221 .752186-3.419698$

C $-0.623725-0.631550-2.392012$

H $0.081542-0.560848-3.225077$

H $-0.319893-1.457059-1.743099$

$\mathrm{H}-1.635846-0.830331-2.757213$

SCF Energy (B3LYP/6-31G**//MMFF)= -3245.91563622

01_169

MM̄FF Geometry

C - $-1.0532872 .516977-1.829405$

C $-0.0617443 .348434-1.474135$

C $0.8064973 .167677-0.256463$

O $2.159108 \quad 3.040772-0.772339$

C 0.7629304 .3785750 .695968

C -0.3579824 .3477771 .715006$

C -0.2047373 .9952953 .006946$

C -1.7045124 .8120461 .233215$ 
C 1.0354313 .4633333 .667405 C 0.7495272 .3291634 .665006 C -0.0102891 .1282624 .076821$ C -0.2458880 .0705715 .160349$ C $-0.941701-1.1793954 .603884$ O $0.753887 \quad 0.5736932 .999003$ O $-0.829740-2.2021795 .605333$ C $-0.171713-1.651403 \quad 3.353061$ C $-2.442776-0.9472874 .395808$ O $-0.842207-2.7428432 .728933$ C $0.105693-0.5215512 .334967$ C $0.999984-0.9957651 .167885$ O $-1.138913-0.106108 \quad 1.756170$ C $3.0637432 .358658-0.022390$ O $2.8697791 .828901 \quad 1.058774$ C $4.3563042 .373743-0.741480$

C $5.4095761 .740952-0.203136$ C $6.7595561 .668925-0.763149$ C $7.0179962 .366744-2.072114$ C $2.405407-1.4645721 .550264$ C $3.199214-1.9091880 .330776$ C $5.361246-2.975356-0.276350$ O $2.730668-1.929788-0.804569$ C $6.780082-2.577098-0.024123$ C $7.615417-2.188286-0.999830$ C $9.049979-1.801057-0.775795$ C $9.374437-0.434917-1.340123$ C $9.916359-0.308228-2.563372$ C $9.1435030 .776486-0.464780$ C $7.7007270 .975282-0.089602$ N $4.489590-2.2988380 .668138$ H -1.287978 $1.665320-1.196369$ H $0.174114 \quad 4.191629-2.120165$ H $0.5371982 .245407 \quad 0.272769$ H 0.6998695 .3120840 .119438 H 1.7254154 .4708931 .214357 H -1.070243 4.0752593 .663844 H -2.1708414 .0288130 .631746$ H -2.3862985 .0403762 .059747$ H -1.612692 5.7212610 .630863 H 1.7592723 .1060642 .932556 H $\quad 1.5145374 .2856214 .212027$ H 1.7162281 .9888375 .058492 H 0.1840802 .7336715 .514340 H -0.9734231 .4790923 .689999$ H -0.8215780 .4890595 .995524$ H $\quad 0.721026-0.2323305 .586717$ H -1.199030 -3.021051 5.232213 H $0.783129-2.0509983 .714577$ H -2.923441 -0.6935085 .348335$ H $-2.941123-1.8575594 .044530$ H -2.660041 -0.1418593 .691613$ H -1.594777 -2.379864 2.232471 H $0.484437-1.7917720 .618721$ H $1.099861-0.1747760 .445511$ H -0.9536750 .3434040 .915191$ H $4.4081572 .907986-1.683031$ H $5.275404 \quad 1.2242030 .746819$ H $6.8380103 .443209-1.976369$ H $6.3641641 .968646-2.856066$ H $8.0431022 .247197-2.430779$ H $2.955411-0.6623812 .053047$ H $2.350655-2.3230212 .227504$ H $5.043834-2.755773-1.301073$ H $5.242259-4.050238-0.105247$ H $7.142933-2.6264500 .999657$ H $7.255604-2.165533-2.026359$ H $9.680006-2.561149-1.256118$ H $9.313376-1.8289990 .288850$ H $10.1778150 .663363-2.969154$ H $10.107186-1.174380-3.188345$ H $9.5436411 .685970-0.928741$ H $\quad 9.7256920 .6583360 .458637$ H $7.422490 \quad 0.4913280 .847298$ H $4.727627-2.3633051 .653959$ C -3.333492 $2.957597-2.909107$ O $-3.9612311 .899463-2.160076$ C $-4.6969552 .483569-1.071326$
C $-4.9398103 .933025-1.475410$

C $-3.6443894 .270067-2.186916$

H $-4.0317542 .460668-0.199196$

C $-5.9618201 .675737-0.761360$

H $-5.7815524 .027024-2.170687$

H $-5.1367314 .577984-0.613711$

C $-3.7491345 .468427-3.115435$

H $-2.8821244 .489593-1.434834$

C $-5.7425030 .154573-0.639700$

O $-6.9110451 .880304-1.816712$

H -6.4283412 .0733430 .147925$

H -5.572689 -0.256816-1.641268

O $-6.972737-0.427023-0.188297$

C $-4.599507-0.2824930 .294097$

C $-4.529900-1.8019330 .546904$

H $-3.6437720 .048633-0.125368$

O $-4.728181 \quad 0.3710651 .558693$

H -3.668772 -1.989074 1.199400

H -5.399692 -2.115132 1.138405

H -2.793334 $5.656284-3.614958$

H $-4.5095975 .313348-3.888163$

H $-4.0201076 .367998-2.553160$

C -1.843943 $2.655332-3.104973$

H -7.707548 $1.368246-1.594170$

H $-7.179218-0.0509580 .683898$

H $-5.5369340 .042692 \quad 1.986105$

H -1.402432 $3.425520-3.748890$

H -1.747455 $1.702723-3.641379$

H -3.815070 $2.949951-3.895276$

C $-2.083592-2.411734-1.198739$

O $-3.427665-2.180940-1.628307$

C $-4.434106-2.659435-0.731715$

C $-4.259251-4.164171-0.480189$

C $-2.839327-4.514713-0.038822$

C $-1.811542-3.906387-0.994316$

H $-1.925826-1.888877-0.248274$

C -1.131956-1.742024 -2.200250

H $-5.389393-2.543958-1.258081$

$\begin{array}{lllll}\text { O } & -5.197997 & -4.610397 & 0.492577\end{array}$

H -4.482115 -4.702386 -1.410684

H $-2.658351-4.1891480 .991089$

O $-2.686408-5.936259-0.026544$

H $-1.846820-4.444249-1.948380$

O $-0.503990-4.082129-0.456816$

C $-1.188554-2.311618-3.602632$

H $-1.386037-0.679649-2.287310$

H $-0.103253-1.824325-1.832368$

H $-5.039232-5.5589190 .636994$

H -2.841876 -6.263367 -0.929088

H $-0.380175-5.033395-0.296459$

O $-0.040796-1.975540-4.263505$

O $-2.126754-2.938870-4.074388$

C $0.045232-2.429611-5.617824$

H $1.007882-2.107798-6.023815$

H $-0.755074-1.985230-6.217247$

H $-0.003942-3.521959-5.655405$

SCF Energy (B3LYP/6-31G**//MMFF) $=-3245.91250763$

0117

MM̄̄F Geometry

C $-3.4729160 .454353 \quad 1.787342$

C $-3.961277 \quad 1.6625402 .109978$

C -3.2174402 .9733821 .982071$

O -1.8048782 .6793721 .852963$

C $-3.657610 \quad 3.796616 \quad 0.753752$

C -5.1499553 .9913670 .586174$

C $-5.8867163 .453615-0.407439$

C -5.8007754 .8729821 .620259$

C $-5.4079082 .570274-1.526174$

C $-5.9081361 .129112-1.361225$

C $-5.0729220 .143718-2.192131$

C $-5.690178-1.255031-2.156687$

C $-4.804892-2.291603-2.864342$

O $-3.759954 \quad 0.113575-1.620062$

O $-5.283353-3.597536-2.507781$

C $-3.365593-2.174765-2.321205$

C $-4.915152-2.190323-4.390115$

O $-2.476700-3.020500-3.048286$ 
C $-2.823426-0.725429-2.305553$

C -1.485249-0.683899-1.524094

O $-2.610603-0.288262-3.645994$

C -0.8930673 .6479752 .113331$

O

C 0.4311263 .0537651 .827403

C 1.5010043 .8386001 .639616

C 2.8469053 .3737241 .295567

C 3.0543131 .8881731 .155335

C $-0.9070350 .717541-1.273549$

C $0.0099141 .225818-2.373154$

C $1.4682873 .170605-2.935719$

O $0.246194 \quad 0.593810-3.398817$

C $2.8659482 .920691-2.460644$

C $3.7642683 .897118-2.259211$

C $5.1810243 .652997-1.820787$

C $5.5957574 .526496-0.656451$

C $6.3088055 .648179-0.855087$

C 5.2607174 .0545840 .740725

C 3.8181704 .2893721 .099168

N $0.5350682 .474952-2.068321$

H $-2.4734680 .372724 \quad 1.370145$

H -4.9706591 .7257692 .511093$

H -3.3706013 .5414772 .908169$

H -3.201141 $3.353952-0.136976$

H $-3.191905 \quad 4.7900180 .806229$

H $-6.9550573 .664167-0.428759$

H $-6.8674855 .025608 \quad 1.424558$

H $-5.709198 \quad 4.4304512 .617000$

H $-5.3242465 .858677 \quad 1.631715$

H $-5.7858572 .975108-2.473334$

H -4.320242 2.589938-1.619030

H $-5.8539810 .839410-0.304349$

H $-6.9625471 .078565-1.659676$

H $-5.0127260 .512800-3.222785$

H $-6.696438-1.246480-2.593966$

H -5.822865 -1.569848 -1.112347

H $-6.215399-3.659576-2.778692$

H $-3.374581-2.551104-1.288369$

H -5.950490 -2.357181-4.711028

H $-4.320820-2.968299-4.881909$

H -4.602871 -1.218963 -4.779221

H -2.844814 -3.920291-3.019660

H - $1.661953-1.126767-0.535807$

H -0.738305 -1.307139-2.027846

H $-2.0234310 .483329-3.632014$

H $0.4792491 .973561 \quad 1.741277$

H 1.3782634 .9174601 .724088

H 2.3759641 .4741720 .401593

H 2.8662051 .3834372 .109033

H $4.062496 \quad 1.6136460 .840473$

H -1.699989 $1.452810-1.103334$

H $-0.299450 \quad 0.671108-0.360997$

H $1.2064854 .232979-2.908332$

H $1.3565382 .807051-3.962115$

H $3.1603151 .886907-2.297419$

H $3.4779054 .928316-2.453981$

H $5.8326223 .850703-2.682017$

H $5.3442842 .599501-1.561286$

H $6.6352646 .265563-0.024733$

H $6.5772285 .977038-1.853599$

H 5.8681674 .5971131 .477861

H 5.5653293 .0101350 .850633

H 3.5663585 .3446891 .213007

H $0.3222482 .896060-1.168865$

C $-3.451218-1.8971382 .736985$

O $-2.260601-2.2846672 .020905$

C - $1.194424-2.4722442 .972141$

C $-1.850828-2.4720974 .350416$

C $-2.993230-1.4975594 .139087$

H $-0.544992-1.5921672 .894582$

C $-0.398078-3.7399672 .649332$

H -2.240568 -3.4616994 .616245$

H -1.158891 -2.160048 5.138668

C -4.081974 -1.589012 5.193080

H $-2.578813-0.481854 \quad 4.142441$

C $0.196859-3.7779321 .226688$

O $-1.272056-4.8685912 .784053$
H $\quad 0.393453-3.873468 \quad 3.396452$

H $-0.610447-3.8642850 .489875$

O $0.957815-4.989288 \quad 1.116175$

C $1.100719-2.5831920 .864417$

C $1.781313-2.774104-0.507036$

H $0.498305-1.6686250 .845818$

O $2.083386-2.4334931 .884788$

H $2.468166-3.625045-0.452815$

H $1.006524-3.027036-1.239799$

H $-4.885937-0.8761994 .984059$

H $-4.521051-2.5916115 .231439$

H -3.677302 -1.361624 6.184605

C $-4.224589-0.8364261 .947915$

H $-0.752164-5.6638462576129$

H $1.717977-4.9133881 .718448$

H $2.559142-1.6036031 .711819$

H $-5.195610-0.6614612 .425346$

H $-4.421130-1.2248260 .941848$

H -4.074464 -2.799767 2.790551

C $4.753490-2.045036-0.136337$

O $3.627006-1.160596-0.119586$

C $2.550990-1.523119-0.989699$

C $3.057284-1.611077-2.436870$

C $4.258991-2.542207-2.564747$

C $5.336031-2.161592-1.549455$

H $4.449725-3.0344450 .226057$

C $5.796016-1.4784310 .835748$

H $1.838724-0.691029-0.950716$

O $2.027883-2.053778-3.314604$

H $3.359368-0.611661-2.775778$

H $3.957362-3.590928-2.457431$

O $4.789335-2.404498-3.885174$

H $5.799645-1.211826-1.846023$

O $6.356533-3.157605-1.546700$

C $5.357868-1.6771552 .267370$

H $6.767567-1.9746050 .732419$

H $5.960076-0.4094200 .652748$

H $1.302200-1.408816-3.261915$

H $5.477492-3.081743-3.997263$

H $6.790922-3.136838-2.415875$

O $4.956877-0.4906362 .804332$

O $5.352737-2.7633412 .832463$

C $4.490741-0.5515164 .155914$

H $5.289264-0.9020054 .816650$

H 4.2002430 .4574834 .460604

H $3.615513-1.2042964 .225977$

SCF Energy $\left(B 3 L Y P / 6-31 G^{* *} / / M M F F\right)=-3245.92087649$

01170

MM̄FF Geometry

C $-3.084968-0.054109-2.753494$

C $-2.259786-0.191177-3.802771$

C $-0.749344-0.268453-3.745534$

O $\quad-0.244710 \quad 0.015235-2.420803$

C $-0.270245-1.676562-4.145805$

C $1.236519-1.857079-4.079182$

C $1.857269-2.803157-3.346211$

C $2.053644-0.906872-4.916298$

C $1.221669-3.881938-2.516603$

C $1.062331-3.536073-1.030517$

C $2.387451-3.464618-0.259040$

C $2.125875-3.5222781 .248651$

C $3.423589-3.3873572 .057138$

O $3.037866-2.231729-0.583949$

O $3.066663-3.1416903 .425891$

C $4.190224-2.1468641 .559011$

C $4.244349-4.6819772 .047360$

O $5.475658-2.0604052 .170288$

C $4.328990-2.0737580 .018519$

C $4.886548-0.688209-0.378914$

O $5.240188-3.082819-0.421755$

C $-0.0205311 .322955-2.116699$

O $-0.2709762 .291514-2.816881$

C $0.6038191 .376862-0.775812$

C $0.9175562 .573483-0.257140$

C 1.5683722 .8268391 .029540

C 1.9045821 .6411061 .892396

C $4.806261-0.369563-1.875974$ 
C $5.426566 \quad 0.987612-2.140048$

C $5.0228053 .422099-1.774291$

O $6.5701721 .098687-2.575683$

C $5.2030693 .836189-0.346097$

C $4.694914 \quad 4.965974 \quad 0.168933$

C $4.843675 \quad 5.3647251 .610313$

C $3.5236725 .727627 \quad 2.259017$

C 3.2434627 .0008962 .585519

C 25550654.6087822 .593052

C $1.827957 \quad 4.1055361 .374462$

N $4.5887682 .036787-1.794059$

H -2.674527 $-0.019522-1.747988$

H -2.691328 $-0.238322-4.800742$

H $-0.3465850 .464009-4.456570$

H $-0.599815-1.902031-5.168513$

H $-0.770440-2.402587-3.497420$

H $2.945868-2.835174-3.363050$

H $3.111957-1.187937-4.949414$

H $1.686735-0.898789-5.947859$

H $1.9977370 .109566-4.515048$

H $0.241251-4.151829-2.923838$

H $1.825942-4.792615-2.617829$

H $0.531241-2.581299-0.935111$

H $0.429797-4.313641-0.584763$

H $3.019106-4.305752-0.567522$

H $1.603547-4.4484021 .519826$

H $1.450491-2.7028351 .533343$

H $2.516797-3.8840693 .729982$

H $3.628764-1.2646441 .896372$

H $3.658814-5.5095852 .465384$

H $5.131364-4.5919112 .684266$

H $4.566821-4.9780981 .046966$

H $5.341477-2.0937063 .133012$

H 4.3281210 .0892570 .154456

H $5.932484-0.603665-0.056029$

H $5.633289-2.807924-1.264847$

H $0.8022780 .440882-0.266504$

H $0.688643 \quad 3.465449-0.839383$

H 2.6622121 .0192081 .409001

H 1.0139431 .0293702 .073725

H $2.287568 \quad 1.9153232 .877914$

H $5.340263-1.111058-2.478615$

H $3.767146-0.357349-2.222690$

H $4.2523704 .011731-2.280725$

H $5.9673193 .527439-2.316370$

H $5.773915 \quad 3.1703930 .297455$

H $4.1452105 .644709-0.479491$

H $5.5312396 .219428 \quad 1.648568$

H 5.3198804 .5688512 .196538

H 2.3101667 .2679273 .070162

H 3.9385357 .8060072 .371286

H 1.8019874 .9646353 .307718

H 3.1147393 .8199793 .104316

H $1.4938724 .896700 \quad 0.701287$

H $3.6670271 .829141-1.420791$

C $-5.1556001 .321752-2.227008$

O $-4.8194901 .372572-0.823898$

C -4.221229 $2.652676-0.551090$

C $-4.735603 \quad 3.572332-1.648779$

C -4.676966 $2.639925-2.845234$

H $-3.1382422 .530480-0.678664$

C -4.5147693 .1026510 .883189$

H $-5.7691543 .886894-1.463693$

H $-4.1176054 .466718-1.770303$

C -5.515396 $3.101721-4.024442$

H $-3.6319292 .563304-3.169942$

C -4.1530672 .0645841 .968544$

O $-5.910721 \quad 3.394651 \quad 1.017766$

H -3.992982 4.0461341 .081260

H -4.9029651 .2656051 .976451$

O -4.2404092 .7115953 .243390$

C -2.747293 1.4594681 .827115

C $-2.286417 \quad 0.553766 \quad 2.981159$

H $-2.6959380 .881936 \quad 0.898811$

O $-1.788432 \quad 2.5129621 .700930$

H -1.336462 0.0999972 .671021

H -2.024635 1.1700503 .851623

H $-5.4542432 .383700-4.848369$
H $-6.5696493 .211834-3.749361$

H $-5.161665 \quad 4.070512-4.392035$

C $-4.5822450 .047205-2.858927$

H -6.4034152 .5932360 .770123$

H -5.134908 3.0865663 .316966

H -1.8549243 .0691902 .495880$

H $-4.904910-0.018575-3.904496$

H $-5.000773-0.822208-2.337252$

H $-6.250643 \quad 1.262084-2.269751$

C -3.074658 -2.049060 1.537538

O $-3.915864-1.1916012 .316210$

C $-3.278282-0.541878 \quad 3.415284$

C $-2.677462-1.5849864 .369025$

C $-1.762456-2.5649913 .636127$

C -2.471082 -3.1541282 .416043$

H $-2.274069-1.4525121 .084460$

C -3.966834 -2.614055 0.420494

H -4.078076 -0.030417 3.965561

O $-1.946102-0.957702 \quad 5.417006$

H $-3.488375-2.1565624 .839011$

H $-0.814329-2.0954283 .348623$

O $-1.423923-3.639055 \quad 4.517099$

H -3.257504 -3.841218 2.753614

O $-1.537194-3.9156151 .656312$

C $-3.206134-3.101165-0.788906$

H $-4.581714-3.431173 \quad 0.816438$

H $-4.655263-1.8349930 .069503$

H -2.553840 -0.3525725 .875514$

H $-1.008483-3.2465245 .304166$

H $-1.150550-4.5804722 .251483$

O $-4.018982-3.900879-1.539577$

O $-2.057296-2.790139-1.066766$

C $-3.436034-4.407757-2.744392$

H $-2.530865-4.980213-2.519768$

H $-4.162085-5.072657-3.219624$

H -3.212949 -3.584341 -3.429267

SCF Energy (B3LYP/6-31G**//MMFF)= -3245.92102044

01171

MM̄FF Geometry

C $4.465804-2.5390631 .533599$

C $3.374926-2.7623380 .784891$

C $1.978669-2.8053531 .348411$

O $1.172931-1.9908830 .464210$

C $1.437592-4.246277 \quad 1.337960$

C $0.398507-4.5026522 .411802$

C $-0.926302-4.631608 \quad 2.204605$

C $0.955164-4.6766403 .804301$

C $-1.692215-4.5039560 .916545$

C $-2.805795-3.4645421 .059579$

C $-3.777483-3.395682-0.127238$

C $-3.083826-3.106322-1.459564$

C $-4.087660-2.869932-2.598137$

O $-4.696812-2.343013 \quad 0.190680$

O $-3.375334-2.287903-3.700829$

C $-5.132699-1.833449-2.140078$

C $-4.683864-4.185570-3.112576$

O $-6.163791-1.685942-3.114168$

C $-5.750304-2.149726-0.759503$

C $-6.593050-0.946623-0.276745$

O $-6.584985-3.302177-0.866707$

C $0.242892-1.1600740 .999724$

O $-0.043223-1.0262912 .177613$

C $-0.377936-0.428869-0.129063$

$\begin{array}{llll}C & -1.450207 & 0.350785 & 0.075214\end{array}$

C $-2.156167 \quad 1.127651-0.949573$

C $-1.6300571 .054410-2.360175$

C $-7.169442-1.1045991 .137563$

$\begin{array}{llll}\text { C }-7.679264 & 0.228735 & 1.645633\end{array}$

C -6.8558762 .4306672 .478438$

\begin{tabular}{lllll}
\hline & -8.866462 & 0.537423 & 1.578667
\end{tabular}

C $-6.608309 \quad 3.243874 \quad 1.247535$

C $-5.523255 \quad 4.009806 \quad 1.059492$

C $-5.251174 \quad 4.748273-0.219704$

C $-4.0382294 .213374-0.949584$

C $-2.982605 \quad 5.001117-1.214876$

C $-4.0753332 .776224-1.430078$

C $-3.2198411 .871480-0.578338$ 
N $-6.660901 \quad 1.034782 \quad 2.132454$ H $4.333262-2.4155872 .606920$ H $3.468155-2.910642-0.287090$ H $1.979636-2.3775242 .357893$ H $2.255176-4.9602821 .509874$ H $1.043669-4.4904350 .345321$ H -1.551378 -4.8591913 .068204$ H $0.177102-4.9127764 .538155$ H $1.681847-5.4954183 .822751$ H $\quad 1.453580-3.7621504 .139807$ H -1.038195 -4.2276590 .086594$ H -2.128398 -5.4805920 .677044$ H -3.395496 -3.670836 1.963978 H $-2.370738-2.4726231 .231544$ H -4.326115 -4.343574 -0.178192 H -2.396782 $-3.915567-1.732963$ H -2.462344 -2.208462 -1.357793 H -2.661375 -2.898544 -3.952487 H -4.624530 -0.860950 -2.078352 H -3.892396 -4.839806 -3.497678 H $-5.362166-4.009780-3.954672$ H -5.223727 -4.741093 -2.343039 H -5.734069 -1.477091 -3.961322 H $-5.964847-0.046786-0.301235$ H -7.429207 -0.771071 -0.965447 H -7.207192 $-3.312128-0.122754$ H $0.066482-0.560515-1.109523$ H $-1.849214 \quad 0.431748 \quad 1.085351$ H -1.671492 $0.024231-2.730789$ H $-0.5918161 .400859-2.404098$ H $-2.1895851 .662869-3.072206$ H -8.003239 -1.8147691 .148624$ H $-6.418296-1.4817261 .840650$ H -6.1547532 .6718673 .283397$ H -7.876783 2.5903852 .838619 H -7.3493113 .1677080 .454803$ H -4.7755204 .0846111 .845316$ H -5.123682 5.8103970 .024196 H $-6.1144564 .695552-0.895330$ H -2.122961 $4.624244-1.759122$ H $-2.9531206 .036968-0.894041$ H -3.795256 $2.753874-2.486658$ H -5.105272 2.398966 -1.407034 H -3.5264631 .8386490 .467757$ H $\quad-5.702232 \quad 0.7060372 .059378$ C $6.109061-2.235487-0.445070$

O $5.474603-1.015538-0.877300$ C $6.375138-0.293822-1.735376$

C $7.484886-1.273596-2.096578$

C $7.584694-2.091494-0.823689$

H $6.8038190 .514248-1.130572$

C $5.618040 \quad 0.298507-2.929288$

H $7.204291-1.917619-2.937967$

H $8.418550-0.765861-2.356866$

C $8.308387-3.414425-1.005003$

H $8.112131-1.495933-0.066325$

C $4.3877181 .145459-2.539430$

O $5.157778-0.781855-3.751244$

H $6.3101800 .884788-3.545703$

H $3.6004160 .491159-2.147102$

O $3.8661841 .740654-3.734219$

C $4.6769742 .274165-1.532171$

C $3.5045203 .245678-1.300371$

H $4.9725181 .842245-0.572729$

O $5.7910693 .046275-1.989929$

H $3.8513454 .025323-0.610970$

H $3.2873103 .777556-2.235247$

H $8.342779-3.972611-0.064165$

H $7.813425-4.043570-1.752434$

H $9.338539-3.246779-1.336221$

C $5.891208-2.4182031 .059611$

H $4.874613-0.404131-4.600792$

H $3.4885361 .027580-4.276152$

H $5.5599403 .405456-2.863836$

H $6.317608-1.5482971 .575104$

H $6.439988-3.2992271 .411850$

H $5.651873-3.058604-1.008173$

C 2.7182552 .6340191 .607778
O $2.4560291 .841801 \quad 0.442454$

C $2.2222842 .583766-0.759348$

C $1.0498053 .556777-0.569622$

C 1.2427614 .4520020 .647664

C 1.5616133 .6067871 .877922

H 3.6497823 .1961061 .468117

C 2.9007121 .6696902 .792972

H $1.8991441 .853590-1.510254$

O $\quad 0.863012 \quad 4.367265-1.724611$

H $0.1248662 .987415-0.426673$

H 2.0182125 .2075270 .475875

$\begin{array}{llll}\text { O } & 0.031751 & 5.168557 & 0.900735\end{array}$

H 0.6652493 .0515412 .183894

O $1.918148 \quad 4.4731142 .954081$

C 4.2938701 .0793662 .848892

H 2.7441202 .2068533 .735505

H 2.1905860 .8376822 .734778

H $\quad 0.713433 \quad 3.771026-2.478191$

H -0.1809805 .6689720 .094335$

H 1.1765115 .0876663 .089172

O 4.5469640 .6607214 .124503

O 5.0559830 .9807361 .897994

C 5.8504380 .1195334 .363198

H $6.627000 \quad 0.7423093 .908671$

H 6.0140790 .0962725 .443932

H $5.901011-0.902143 \quad 3.979277$

SCF Energy $(B 3 L Y P / 6-31 G * * / / M M F F)=-3245.90975790$

01172

MM̄FF Geometry

C $-4.442872-0.2727352 .822516$

C $-3.6588990 .742587 \quad 2.431634$

C -2.6920751 .4520763 .343951$

O -1.3671741 .1989142 .830188$

C -2.9350522 .9739793 .303798$

C -1.9379653 .7761754 .123979$

C -1.049019 4.6497653 .605736

C -1.9941943 .5559025 .613329$

C -0.8798395 .0210602 .156685$

C $0.1292864 .106331 \quad 1.462744$

C $0.1688374 .226499-0.068338$

C -1.161939 $3.820965-0.709899$

C $-1.0774763 .777335-2.241351$

O $1.2061163 .335241-0.504652$

O $-2.256247 \quad 3.125454-2.736679$

C $0.1445872 .933151-2.655697$

C -1.119526 $5.187624-2.839319$

O $0.3466872 .989336-4.065476$

C $1.4472773 .325213-1.918295$

C $2.5469632 .276362-2.196914$

O $1.9568784 .579632-2.374279$

C $-0.643727 \quad 0.190366 \quad 3.383418$

O $-0.980038-0.5564564 .287064$

C 0.6532060 .1565072 .673306

C $1.591111-0.7415563 .006513$

C $2.886417-0.8914312 .343782$

C 3.2786520 .1567761 .335788

C $3.8624522 .566357-1.464102$

C $4.9394511 .540622-1.779629$

C $7.2752870 .937092-1.119991$

O $4.8138930 .671105-2.637197$

C $7.134433-0.324116-0.328390$

C $7.531960-0.4529720 .946782$

C $7.438834-1.7310411 .731622$

C $6.223816-1.7655872 .634583$

C $6.312598-1.3513613 .909966$

C $4.968346-2.3271341 .999697$

C $3.668640-1.9453662 .656767$

N $6.0698221 .734650-0.997863$

H $-4.381766-0.5966243 .860124$

H $-3.680241 \quad 1.0813381 .399723$

H -2.783295 1.0908504 .375322

H $-3.945793 \quad 3.196320 \quad 3.670637$

H -2.918065 3.3020772 .259036

H -0.3686735 .1613774 .284608$

H -1.3417814 .2453906 .159613$

H -3.013712 3.7104965 .981418

H -1.6812782 .5382715 .865888$ 
H -1.851289 $5.017073 \quad 1.657516$ H -0.5302026 .0593472 .098881$ H 1.1339004 .3049331 .861078 H -0.0700843 .0612021 .716856$ H $0.4426075 .253156-0.336302$ H -1.976624 $4.489427-0.410986$ H $-1.4452982 .823736-0.344245$ H $-2.3715512 .295506-2.244058$ H $-0.0824511 .885180-2.415757$ H -2.074997 5.672581-2.604156 H -1.061428 $5.157701-3.932768$ H $-0.3235215 .835855-2.466394$ H $-0.4867912 .721511-4.489192$ H $2.1941811 .284110-1.888971$ H $2.7517252 .231458-3.274208$ H $1.4422024 .862829-3.146920$ H $\quad 0.796237 \quad 0.867430 \quad 1.867585$ H $1.382786-1.4480953 .808180$ H 3.2561401 .1532461 .791591 H 2.5958130 .1485440 .480967 H 4.2890480 .0236830 .945226 H $4.2496783 .549521-1.755820$ H $3.6955532 .573676-0.381516$ H $8.1113561 .547419-0.763204$ H $7.4509650 .698492-2.174037$ H $6.702929-1.179851-0.841951$ H $7.9585200 .405826 \quad 1.459993$ H $8.358336-1.8374592 .321840$ H $7.426041-2.6021111 .063776$ H $5.468983-1.3851094 .589841$ H $7.246490-0.9688754 .310064$ H $5.043342-3.4225661 .996835$ H $4.928941-2.0482550 .940194$ H $3.327011-2.6500593 .414633$ H $6.0483982 .441377-0.268390$ C $-5.601393-0.6232690 .522117$ O $-4.416715-0.916940-0.248588$ C $-4.815148-1.365400-1.559335$ C $-6.329735-1.195067-1.632705$ C $-6.731330-1.372990-0.183822$ H -4.584869 -2.435097-1.610528 C $-4.057135-0.609006-2.657436$ H $-6.610448-0.196056-1.986639$ H -6.795024 -1.926563 -2.300799 C $-8.122269-0.8449130 .123411$ H $-6.686349-2.4418460 .065189$ C -2.522010 -0.760906-2.611689 O $-4.3677840 .784455-2.531636$ H -4.435404 -0.924254 -3.637936 H -2.115402 -0.230262 -1.742930 O $-1.986015-0.116009-3.775565$ C $-2.025346-2.219131-2.618199$ C $-0.511361-2.382645-2.853755$ H -2.299423 -2.704363 -1.676200 O $-2.694023-2.943669-3.656452$ H $-0.306532-3.456359-2.947624$ H $-0.245144-1.968611-3.834455$ H -8.375062 -0.9934311 .177598$ H -8.202258 $0.225406-0.094490$ H $-8.872816-1.367423-0.478643$ C $-5.416608-1.0563841 .980624$ H $-4.121487 \quad 1.217020-3.366507$ H -2.180106 $0.832874-3.707774$ H -2.488954 -2.504670 -4.499765 H -5.091155 -2.103608 2.004917 H $-6.384532-1.0086812 .493401$ H $\quad-5.7724630 .4594390 .459960$ C $0.270686-3.578782-0.184014$ O $0.014277-2.194110-0.448315$ C $0.379863-1.760169-1.761468$ C $1.883174-1.984307-1.982395$ C $2.292286-3.428308-1.701482$ C $1.763099-3.888466-0.343573$ H $-0.317103-4.200918-0.869565$ C $-0.204687-3.8752501 .241550$ H $0.209830-0.677326-1.786595$ O $2.261208-1.641225-3.311312$ H $2.452155-1.331242-1.310680$
H $1.960498-4.102472-2.499788$

O $3.719773-3.513688-1.674114$

H $2.334979-3.3993350 .452558$

O $1.968110-5.293327-0.211182$

C -1.701101-3.716383 1.355081

H $0.059952-4.8890221 .562499$

H $0.251168-3.1849511 .959439$

H $2.015644-0.711274-3.454325$

H $4.045914-3.171412-2.524302$

H $2.918413-5.460340-0.334369$

O $-2.326228-4.8297210 .877292$

O $-2.246070-2.7148621 .800211$

C $-3.755831-4.7975410 .919917$

H -4.134488 -3.9809690 .299151$

H -4.129649 -5.743806 0.520005

H -4.102537 -4.691154 1.952269

SCF Energy $(B 3 L Y P / 6-31 G * * / / M M F F)=-3245.91588398$

01173

MM̄FF Geometry

C $-3.372600-2.858920-0.197677$

C $-2.899299-3.846244-0.974175$

C $-1.561929-3.894720-1.680908$

O $-0.729379-2.765608-1.332430$

C $-0.807997-5.176495-1.277927$

C $0.379177-5.478179-2.172344$

C $1.671921-5.302436-1.835176$

C $0.031609-6.060348-3.520214$

C $2.238242-4.750230-0.556089$

C $3.024871-3.467766-0.828749$

C $3.766800-2.9043670 .392488$

C $2.833959-2.5667551 .555697$

C $3.567368-1.8622432 .705612$

O $4.420663-1.715212-0.065300$

O $2.560454-1.3319683 .580264$

C $4.374520-0.6797102 .131923$

C $4.381129-2.8559123 .542391$

O $5.169787-0.0699783 .146304$

C $5.240356-1.0587730 .909288$

C 5.8050440 .2183110 .245504

O $6.320640-1.8875751 .338709$

C $-0.831900-1.640702-2.093250$

O $-1.553878-1.468129-3.061679$

C $0.075667-0.627436-1.508622$

C $-0.0065810 .654119-1.894801$

C $0.7820851 .766816-1.356064$

C $1.9055151 .430787-0.412179$

C $6.611028-0.057949-1.030605$

C $6.6198321 .142073-1.953844$

C $5.1923782 .365394-3.591496$

O $7.6013121 .874339-2.053548$

C $4.5808263 .533510-2.885360$

C $3.4622024 .148393-3.298914$

C $2.8999475 .370372-2.632852$

C $1.4261935 .254399-2.324627$

C $0.5237146 .017876-2.964554$

C $1.0064624 .328066-1.205753$

C $0.4419593 .024541-1.707621$

N $5.4239951 .295914-2.640476$

H -2.772708 -1.974109 -0.008560

H -3.544695 -4.705077 -1.153690

H -1.751525 -3.900937 -2.761077

H -1.483109 -6.042274 -1.320283

H $-0.497825-5.104431-0.230669$

H $2.429444-5.586148-2.565620$

H $0.921094-6.328282-4.100482$

H $-0.565133-6.970188-3.398171$

H $-0.542898-5.344042-4.114926$

H $1.455292-4.5544110 .178621$

H $2.902118-5.505623-0.119349$

H $3.763904-3.645535-1.622769$

H $2.355240-2.699795-1.235497$

H $4.525440-3.6324940 .702247$

H $2.326717-3.4613571 .933496$

H $2.042136-1.8996351 .192935$

H $3.011117-0.8098034 .266257$

H 3.6471020 .0827361 .822761

H $3.723314-3.6243863 .966299$ 
H $4.851548-2.3620724 .399682$ H $5.157993-3.3643992 .967599$ H $5.945024-0.6374933 .295642$ H $4.971914 \quad 0.891477 \quad 0.009937$ H $\quad \begin{array}{llll}6.457129 & 0.753133 & 0.947514\end{array}$ H $7.028343-1.8521960 .675847$ H $0.765313-0.958078-0.741699$ H $-0.7461030 .923614-2.647686$ H $2.5786040 .693962-0.864747$ H 1.5113961 .0171390 .522181 H $2.5280032 .288568-0.150400$ H $7.648370-0.309634-0.782024$ H $6.209481-0.908367-1.593618$ H $4.5419601 .968594-4.377268$ H $6.1450192 .663605-4.041087$ H $5.1074353 .918332-2.014909$ H $2.9531903 .787854-4.189742$ H $3.0795816 .221718-3.302422$ H $3.4389355 .601322-1.705252$ H $-0.5326605 .963804-2.721600$ H $0.8186996 .710155-3.745914$ H $0.219744 \quad 4.818979-0.616125$ H $1.8364194 .196053-0.507059$ H $-0.4019683 .152540-2.387417$ H $4.650000 \quad 0.670375-2.435811$ C $-5.737897-1.913884-0.165889$ O $-5.292686-0.553150 \quad 0.003493$ C $-5.4736900 .142040-1.244640$ C $-6.453507-0.701876-2.051076$ C $-6.019513-2.100513-1.659393$ H $-4.5004270 .129492-1.751049$ C -5.910472 $1.592403-1.013099$ H -7.491308 $-0.529068-1.743599$ H $-6.379831-0.512941-3.126218$ C -7.052213 -3.172179-1.960896 H $-5.099668-2.333981-2.208658$ C $-5.0266052 .390173-0.032934$ O $-7.2451471 .613070-0.490472$ H $-5.9616422 .112512-1.976811$ H -5.2400942 .0745190 .994685$ O $-5.4134503 .767257-0.119422$ C $-3.5168472 .293440-0.300888$ C -2.6416523 .2360320 .542867$ H $-3.1852631 .264182-0.130449$ O $-3.2532022 .578185-1.677747$ H $-1.598332 \quad 2.9867150 .323482$ H -2.760381 $4.267790 \quad 0.186830$ H -6.693636 -4.156902 -1.644835 H -7.998110 -2.975435 -1.445324 H -7.258543 -3.215922 -3.035289 C $-4.730504-2.8907930 .450246$ H -7.2475751 .0883820 .328577$ H -6.3677543 .8118370 .063500$ H -3.586195 $3.473319-1.861595$ H $-5.145825-3.9052450 .419825$ H -4.589059 -2.639262 1.508817 H -6.674549 -1.9918170 .401557$ C -1.8003541 .0791152 .548152$ O -3.0053821 .8541272 .561518$ C -2.8786623 .1856752 .063700$ C -1.8362853 .9584702 .883739$ C -0.5031113 .2137372 .954066$ C $-0.7160871 .763722 \quad 3.394423$ H -1.4534010 .9714071 .513900$ C -2.188947 -0.3075353 .086477$ H $-3.846813 \quad 3.6697972 .243022$ O -1.6501765 .2558252 .327723$ H -2.218862 4.0949323 .903738 H $\quad 0.0342793 .2562801 .999229$ O 0.3403823 .8489013 .918572 H -0.9923001 .7564564 .456675$ O $\quad 0.5284641 .0822773 .268228$ C -1.282582 -1.426201 2.630632 H -2.212423 -0.2882424 .182456$ H -3.199934 -0.562095 2.743214 H -1.0795325 .7578972 .933267$ H $\quad 0.576641 \quad 4.7247573 .569512$ H $\quad 0.4456880 .2248613 .717881$
O $-1.589487-2.5587893 .329144$

O $-0.428638-1.3309761 .761587$

C $-0.813747-3.7152523 .000061$

H $0.211254-3.5873543 .359193$

H $-1.258265-4.5776003 .503675$

$\mathrm{H}-0.825639-3.8969091 .921517$

SCF Energy $\left(B 3 L Y P / 6-31 G^{* *} / / M M F F\right)=-3245.92447552$

01174

MM̄FF Geometry

C $4.1603550 .317988 \quad 3.171009$

C $2.948654-0.1212722 .797834$

C 1.6515540 .4211383 .339839

$\begin{array}{llll}\text { O } & 0.916162 & 0.968468 & 2.221173\end{array}$

C $0.836780-0.7362823 .956150$

C $-0.514415-0.3292434 .512273$

C -1.697253 -0.752561 4.021674

C -0.4804680 .5767865 .715770$

C -1.928274 -1.727408 2.899792

C $-2.060532-1.0267841 .545629$

C $-2.205054-2.0244390 .388964$

C $-2.287718-1.283398-0.946933$

C $-2.510125-2.244458-2.122735$

O $-3.389449-2.7980970 .599727$

O $-2.872287-1.440293-3.255730$

C $-3.705845-3.163500-1.787576$

C -1.217444 -2.972458 -2.508846

O $-3.881125-4.157250-2.794164$

C $-3.614844-3.812428-0.387531$

C $-4.895672-4.594920-0.003052$

O $-2.541276-4.763865-0.390912$

C 0.3833492 .2125092 .360567

O 0.5476392 .9893093 .287899

C -0.5007492 .4600531 .199751$

C -1.5168193 .3249371 .340232$

C -2.5197623 .6687930 .330405$

C $-2.3791743 .052792-1.035099$

C $-6.212696-3.816435-0.005221$

C $-6.264775-2.7494991 .070366$

C $-6.320333-0.2984971 .409675$

O $-6.211180-3.0304442 .265828$

$\begin{array}{llll}\text { C } & -6.728857 & 0.902478 & 0.620874\end{array}$

C $-5.872810 \quad 1.8587230 .229460$

C $-6.2769653 .054584-0.583977$

C $-5.9690334 .365990 \quad 0.102929$

C -6.8863854 .9710760 .876319$

C $-4.6395335 .023045-0.193317$

C -3.5246054 .5017450 .674557$

N -6.375853 -1.4681830 .554292$

H $4.210771 \quad 1.1355193 .888204$

H $2.859094-0.9279752 .076153$

H 1.8530251 .1873134 .097638

H $1.414198-1.2022294 .766067$

H $\quad 0.712500-1.5137143 .194392$

H -2.610150 -0.3873814 .490311$

H $-1.4798020 .761766 \quad 6.124176$

H $\quad 0.118740 \quad 0.1232006 .512159$

H $-0.045426 \quad 1.5476065 .465854$

H -1.134872 -2.481380 2.863980

H -2.847371 -2.283972 3.122913

H $-2.929694-0.3585641 .573749$

H -1.177286 -0.407436 1.363638

H -1.333676 -2.689899 0.402188

H -1.389735 -0.675801-1.111868

H $-3.127026-0.573804-0.916306$

H -3.085008 -2.043405 -3.988768

H -4.598054 -2.528728 -1.832558

H $-0.445665-2.250323-2.799213$

H - $-1.368786-3.612702-3.384805$

H $-0.809558-3.583504-1.701074$

H $-3.201301-4.839104-2.658236$

H -5.001417 -5.459239 -0.671749

H $-4.751533-5.0174611 .001181$

H -2.677191 -5.3822880 .346164$

H $-0.318717 \quad 1.897624 \quad 0.291844$

H -1.6414603 .8228152 .301609$

H $-2.3584011 .960075-0.965948$

H $-1.4567693 .395557-1.511987$ 
H -3.199148 $3.298712-1.712898$ H $-6.424823-3.398836-0.993881$ H $-7.037448-4.5089450 .206248$ H $-5.300322-0.2159201 .798145$ H $-6.990426-0.4446132 .263412$ H $-7.781706 \quad 0.9869410 .363232$ H -4.8212121 .7541250 .480953$ H $-7.3481643 .009248-0.822178$ H $-5.7654533 .014241-1.553244$ H -6.6871065 .9290581 .345131$ H -7.8545664 .5176881 .061493$ H $-4.7098316 .104625-0.012752$ H $-4.4157654 .944698-1.261727$ H -3.5565924 .8829451 .696187$ H -6.215814 -1.335062 -0.440257

C 5.514341-1.494737 1.901292

O $4.906121-1.2702950 .618063$

C 5.583141-2.068205-0.370006

C $6.636207-2.8828820 .375872$

C $6.918308-2.0048931 .580255$

H $6.092464-1.366087-1.040122$

C $4.564815-2.899419-1.161704$

H $6.250422-3.8571100 .697220$

H $7.523749-3.067234-0.237492$

C $7.591278-2.7466292 .722183$

H $7.557755-1.1701361 .263159$

C $3.398582-2.073997-1.744838$

O $3.998872-3.879333-0.281216$

H $5.081864-3.454179-1.953810$

H $2.747685-1.732344-0.931658$

O $2.606491-2.943225-2.563518$

C $3.833892-0.873809-2.604242$

C $2.683226-0.157817-3.332251$

H $4.362779-0.147054-1.982698$

O $4.766303-1.312931-3.596700$

H $3.1262690 .631051-3.952583$

H $2.222968-0.849648-4.048909$

H $7.779683-2.0777203 .567498$

H $6.973417-3.5767733 .080751$

H $8.552967-3.1585362 .398902$

C $5.503422-0.2002852 .718632$

H $3.519793-4.523598-0.828934$

H $2.152046-3.567804-1.973628$

H $4.323480-1.990721-4.135644$

H 5.9815100 .5971692 .138550

H $6.106995-0.3480173 .622063$

H $4.932614-2.2741462 .410918$

C $2.6621672 .550528-1.906265$

O $2.149418 \quad 1.308747-1.409148$

C $1.6050050 .432843-2.401776$

C $0.4587491 .129280-3.146447$

C $0.9070822 .457790-3.746691$

C $1.5847783 .320468-2.684358$

H $3.5295022 .358831-2.549656$

C $3.1253873 .381802-0.696875$

H $1.157733-0.405910-1.855995$

O $-0.0508450 .274200-4.163485$

H $-0.359701 \quad 1.313481-2.440697$

H $1.5658642 .309991-4.610297$

O $-0.2342113 .170596-4.229213$

H $0.8281143 .703953-1.989110$

O $2.1846714 .449962-3.318269$

C $4.4233342 .889688-0.093684$

H $3.2911864 .420720-1.005150$

H $2.368328 \quad 3.3586210 .094745$

H $-0.8657640 .677267-4.507328$

H -0.601758 $2.665695-4.974041$

H $1.484076 \quad 4.912913-3.809175$

O 4.9049623 .8538990 .746186

O $4.9382691 .803066-0.311912$

C 6.1489453 .5781761 .399282

H 6.6272824 .5334961 .631364

H 5.9533713 .0486402 .334876

H 6.8256532 .9991750 .763423

SCF Energy (B3LYP/6-31G**//MMFF) $=-3245.91464058$

01175

MM̄FF Geometry
C $-3.810593-2.382741-2.800659$

C $-2.515754-2.302560-2.462367$

C $-1.491326-1.519567-3.239928$

O $-1.124921-0.404186-2.396635$

C $-0.247892-2.382972-3.515051$

C $0.791689-1.696882-4.380635$

C $2.006157-1.301397-3.949322$

C $0.419886-1.531034-5.832225$

C $2.572529-1.419922-2.559897$

C $3.461229-2.664675-2.454076$

C $4.178900-2.851404-1.108374$

C $3.214704-2.9866960 .069772$

C $3.955561-3.1496751 .404926$

O $5.071723-1.752102-0.899584$

O $2.988104-2.9426572 .446140$

C $5.019254-2.0352971 .516768$

C $4.481727-4.5768511 .589188$

O $5.819538-2.2115762 .682571$

C $5.906811-1.8940780 .259104$

C $6.873843-0.6846010 .330770$

O $6.734757-3.0610780 .146401$

C $-1.0764470 .833393-2.956766$

O $-1.2253861 .121384-4.133102$

C $-0.8332221 .801660-1.864742$

C $-0.6479793 .098429-2.151254$

C $-0.4244294 .174013-1.181741$

C $-0.5337843 .825608 \quad 0.279935$

C 6.2577750 .6808200 .638736

C $5.3889591 .240659-0.472228$

C $3.4531232 .752853-0.845116$

O $5.5550380 .960555-1.656265$

C $3.0284104 .051389-0.240090$

C $3.1018975 .230559-0.875105$

C $2.6492856 .529111-0.271278$

C $1.5379277 .184102-1.062254$

C $1.7841108 .238781-1.857601$

C $0.128316 \quad 6.667523-0.864141$

C $-0.1379255 .408921-1.645874$

N $4.4521822 .143210 \quad 0.010481$

H -4.153690 -1.888570 -3.705643

H $-2.176638-2.801909-1.558846$

H $-1.933950-1.170628-4.180480$

H $-0.547995-3.312045-4.018503$

H $0.194685-2.696901-2.562681$

H $2.686444-0.848935-4.669781$

H $1.239199-1.110852-6.425584$

H $\quad 0.163002-2.501230-6.270375$

H $-0.438631-0.862730-5.942045$

H $3.156073-0.516499-2.348898$

H $1.772562-1.442077-1.815444$

H $2.860061-3.559619-2.660516$

H $4.227641-2.608697-3.239327$

H $4.773737-3.768014-1.204219$

H $2.525226-3.822222-0.093433$

H $2.592408-2.0870250 .144944$

H $3.460349-2.9650433 .296216$

H 4.461918-1.103143 1.668412

H $3.651623-5.2933991 .567827$

H $4.956814-4.7013472 .568429$

H $5.195861-4.8747700 .818938$

H $6.454383-2.9252692 .500426$

H $7.639915-0.8905281 .090513$

H $7.417108-0.616622-0.621796$

H $7.485481-2.848405-0.432891$

H $-0.8242381 .420668-0.851330$

H $-0.6563153 .406403-3.196078$

H -0.3226584 .6624550 .948130$

H 0.1723353 .0320680 .547501

H -1.547435 3.4819130 .510568

H 5.7080620 .6528521 .584067

H $7.072837 \begin{array}{lll}1.402728 & 0.778268\end{array}$

H $2.6021442 .065656-0.896308$

H $3.8560332 .871872-1.856315$

H 2.6151204 .0155530 .765394

H $3.5122545 .269927-1.881550$

H $3.5211247 .194211-0.219274$

H 2.3203226 .3943950 .766741

H $\quad 0.987825 \quad 8.730758-2.406537$ 
H $2.7883038 .630379-1.981983$ H $-0.5973677 .418816-1.203886$ H $-0.064575 \quad 6.5584610 .206366$ H $-0.0885965 .554991-2.726128$ H 4.3040672 .1935931 .014667 C $-5.877372-2.230255-1.310891$ O $-5.270385-1.431817-0.273714$ C $-5.958415-0.164843-0.215823$ C $-7.151413-0.273773-1.166267$ C $-6.633288-1.248636-2.203770$ H $-5.2641230 .584661-0.612861$ C $\quad-6.352978 \quad 0.1905201 .223981$ H -8.036005 $-0.685130-0.666112$ H -7.430680 $0.696334-1.589151$ C -7.724331-1.887652 -3.043774 H $-5.946501-0.702852-2.861446$ C $-5.181962 \quad 0.2754082 .224854$ O $-7.260927-0.8131301 .697976$ H $-6.911479 \quad 1.134927 \quad 1.219727$ H -4.791384 -0.7285082 .428510$ O $\quad \begin{array}{llll}-5.709114 & 0.767173 & 3.464696\end{array}$ C $-4.030378 \quad 1.2031581 .796841$ C -3.0127121 .5273062 .910038$ H $-3.508466 \quad 0.7559840 .943494$ O $-4.5804672 .444160 \quad 1.343671$ H -2.2268222 .1502642 .466629$ H -3.485345 2.1762893 .659067 H -7.297430 -2.586328 -3.770091 H $-8.437908-2.439967-2.423113$ H -8.281041 -1.124059-3.596772 C $-4.837552-3.126203-1.993052$ H -7.676049 -0.4739182 .508655$ H $-6.267240 \quad 0.0678383 .843933$ H -3.8453362 .9903831 .017188$ H -5.345842 -3.832355 -2.660197 H $-4.335320-3.725249-1.222577$ H $-6.593353-2.887396-0.798370$ C $-0.807854-0.3316791 .904368$ O $-1.960411-0.6874672 .670945$ C $-2.3866980 .303038 \quad 3.604951$ C -1.2477460 .6384514 .580205$ C 0.0444131 .0150033 .852163 C $0.400342-0.0390612 .801119$ H $-1.0353190 .570921 \quad 1.325190$ C $-0.582264-1.4392110 .870051$ H $-3.184083-0.1609864 .198288$ O -1.6526201 .6929215 .446860$ H -1.053084 -0.2404395 .208905$ H -0.0259752 .0105583 .397799$ O $1.117617 \quad 1.078058 \quad 4.794811$ H $0.752134-0.940223 \quad 3.315404$ O $1.484113 \quad 0.4602412 .019246$ C $-0.377917-2.8253801 .446247$ H -1.484521 -1.4981550 .251345$ H $0.268026-1.1951420 .226750$ H $-0.970086 \quad 1.791103 \quad 6.131625$ H $0.942248 \quad 1.826681 \quad 5.389447$ H $1.832399-0.2780201 .492114$ O $-0.224309-3.7080940 .413951$ O $-0.371264-3.1111622 .634959$ C $-0.051349-5.076611 \quad 0.794751$ H $0.819100-5.1848951 .449034$ H $-0.954873-5.445651 \quad 1.289008$ H $0.117943-5.662442-0.112568$ SCF Energy (B3LYP/6-31G**//MMFF $)=-3245.90318931$

$01 \quad 176$

MM̄FF Geometry

C $1.444557-3.405379-0.115944$

C $0.596270-3.7290830 .871603$

C $-0.187108-2.7151681 .664724$

O $-1.581976-3.0210411 .405210$

C $0.053995-2.8100053 .183079$

C $1.240208-2.0098603 .678526$

C $1.139687-0.8279284 .319077$

C $2.591489-2.6422403 .487608$

C $-0.121935-0.0518754 .590157$

C 0.1402541 .4370824 .869746
C 0.6550452 .2191653 .649212

C 1.2374833 .5625244 .098603

C 1.7112694 .4065162 .909654

O -0.4422282 .4376502 .749176$

O 1.9613835 .7291793 .407741

C 0.5652904 .4902541 .884315

C 3.0441443 .8989222 .348439

O 0.9982105 .1583650 .700389

C -0.0770143 .1222021 .541660$

C -1.3468163 .3719220 .691857$

$\begin{array}{lllll}\text { O } & 0.854320 & 2.357486 & 0.774004\end{array}$

C $-2.473007-1.9990611 .492880$

O $-2.230784-0.8379041 .777404$

C -3.813585 -2.535751 1.174959

C $-4.869151-1.7100111 .219071$

C $-6.254800-2.0531640 .902908$

C $-6.555803-3.4685670 .487863$

C -2.2234582 .1380810 .449758$

C $-3.2747872 .405995-0.616249$

C $-4.8220041 .223218-2.171150$

O $-3.5645913 .537034-0.999283$

C $-6.2096621 .229953-1.611709$

C $-7.1560290 .351146-1.977206$

C $-8.5630450 .358411-1.446198$

C $-8.953032-0.963900-0.820662$

C $-9.603825-1.897163-1.535518$

C $-8.651560-1.1810210 .646325$

C $-7.186461-1.0797700 .966495$

N -3.856218 $1.237015-1.087089$

H $1.611909-2.355646-0.344105$

H $0.413432-4.7782441 .091802$

H $\quad 0.030295-1.7013681 .303711$

H $\quad 0.172348-3.8593703 .486341$

H $-0.850692-2.4899243 .715411$

H $2.057543-0.3698574 .685231$

H $3.376559-2.1257744 .050484$

H $2.582892-3.683863 \quad 3.824232$

H $2.873262-2.6128092 .433516$

H $-0.821525-0.1399863 .755540$

H $-0.602558-0.4822675 .476891$

H $-0.800879 \begin{array}{lll}1.885528 & 5.213415\end{array}$

H $0.857478 \quad 1.5168015 .696632$

H 1.4253421 .6301223 .139945

H 2.0597973 .4137724 .809911

H $0.468598 \quad 4.1278074 .644251$

H 2.1837726 .2933052 .647055

H -0.207848 5.1339522 .325963

H $3.816103 \quad 3.9140703 .127144$

H $3.4140384 .551447 \quad 1.549860$

H 2.9833772 .8811881 .959821

H 1.5357994 .5313780 .187306

H -1.975330 4.1235461 .187694

H -1.048636 $3.789411-0.279027$

H $\quad 0.369449 \quad 1.723220 \quad 0.223794$

H -3.893028 -3.5812730 .900744$

H $-4.703985-0.6707241 .502001$

H -6.294153 -4.168105 1.289440

H $-5.988860-3.735188-0.411173$

H -7.610257 -3.6353030 .254798$

H $-1.614356 \quad 1.2917190 .124177$

H -2.7403941 .8561741 .373569$

H $-4.6221420 .330125-2.771824$

H -4.674742 2.106314 -2.801437

H $-6.4537572 .005373-0.889503$

H $-6.915291-0.408324-2.718001$

H $-9.2348570 .586549-2.284056$

H $-8.7147771 .165702-0.719102$

H -9.909339 -2.839083 -1.092284

H $-9.842703-1.740618-2.582357$

H -9.051436 -2.138355 1.001183

H $-9.186844-0.4204581 .229762$

H -6.876647 -0.0779721 .266203$

H $-3.5919770 .347390-0.673814$

C $3.678087-4.343579-0.931279$

O $4.135337-3.004593-1.211557$

C $4.997964-2.592250-0.137322$

C $5.533569-3.8852720 .458724$

C $4.291593-4.7562050 .409890$ 
H $\quad 4.363235-2.100208 \quad 0.608927$

C $6.055154-1.601276-0.630330$

H $6.325568-4.319599-0.162189$

H $5.923169-3.7503451 .472056$

C $4.583957-6.2432860 .516592$

H $3.633080-4.4753241 .238967$

C $5.484067-0.382097-1.387871$

O $6.964701-2.271257-1.511185$

H $6.658649-1.2648870 .220723$

H $\quad 5.178017-0.693529-2.392810$

O $6.5537420 .552288-1.577407$

C $4.3112410 .325361-0.686744$

C $3.8734731 .651040-1.332257$

H $3.452440-0.353025-0.648045$

O $4.6848340 .602676 \quad 0.665871$

H $3.0042652 .014976-0.772770$

H $4.6462762 .412589-1.164326$

H $3.659329-6.8257600 .452115$

H $5.252071-6.580134-0.283171$

H $5.063504-6.4731521 .473558$

C $2.148115-4.403714-0.995864$

H $6.444346-2.641713-2.244764$

H $6.8120740 .884016-0.700272$

H $3.895196 \quad 0.9378201 .123389$

H $1.812087-5.423857-0.776164$

H $1.837264-4.184056-2.025353$

H $4.079625-4.972973-1.735591$

C $1.381470 \quad 0.492334-2.705148$

O $2.7361650 .441004-3.168608$

C $3.5383881 .575029-2.834101$

C $2.9064502 .855311-3.399433$

C $1.4463663 .005247-2.977155$

C $0.665411 \quad 1.725280-3.273470$

H $1.3788390 .526585-1.609686$

C $0.722911-0.820864-3.157012$

H $4.4921151 .434593-3.357638$

O $3.6319824 .008403-2.985684$

H $2.9466162 .825648-4.496097$

H $1.3584213 .281260-1.920650$

O $0.8508324 .073011-3.718277$

H $0.5411751 .622918-4.359201$

O $-0.6360261 .830333-2.704637$

C $-0.511532-1.196606-2.370058$

H $0.470308-0.757564-4.222346$

H $1.434642-1.648600-3.046829$

H $4.5517643 .896034-3.281193$

H $1.3765754 .872859-3.545124$

H -1.045250 $2.638797-3.058286$

O $-1.244666-2.095412-3.090651$

O $-0.780939-0.789933-1.248884$

C $-2.428916-2.580626-2.450209$

H -3.063195 -1.749943 -2.127297

H $-2.984701-3.182415-3.174115$

H $-2.157590-3.214413-1.601294$

SCF Energy (B3LYP/6-31G**//MMFF) $=-3245.93217620$

$01 \quad 177$

MM̄FF Geometry

C -2.382890 -5.129047 -1.785553

C $-1.607426-4.540806-0.860491$

C $-0.102074-4.488554-0.904440$

O $0.295114-3.105595-0.730846$

C $0.481987-5.2945290 .269225$

C $1.995403-5.244398 \quad 0.350024$

C $2.688364-4.6987251 .369543$

C $2.732448-5.877010-0.802198$

C $2.149802-4.0580182 .618645$

C $1.954884-2.5360632 .532529$

C $3.258916-1.7457892 .333816$

C $3.012682-0.2462472 .513413$

C 4.2757280 .5838942 .236982

O $3.744200-1.9920411 .011409$

O 3.8782101 .9578982 .106401

C $4.874906 \quad 0.155950 \quad 0.879744$

C $5.2620290 .527547 \quad 3.409474$

O $6.139213 \quad 0.7767370 .662151$

C $5.004380-1.3724110 .717033$

C $5.403209-1.799089-0.717638$
O $6.028865-1.8521731 .596663$

C $0.139720-2.266538-1.789569$

O $-0.240972-2.557298-2.912025$

C $0.476885-0.903497-1.325258$

C $0.0650940 .154205-2.038819$

C $0.2947181 .560417-1.705959$

C $1.2552761 .864462-0.587831$

C $4.427573-1.382733-1.824516$

C $4.804028-0.092134-2.534837$

C $3.9424651 .493092-4.257566$

O $5.8790010 .478978-2.373587$

C $3.3563652 .686917-3.572304$

C $2.4230093 .473998-4.129364$

C $1.8671934 .706468-3.474619$

C $0.3562254 .706515-3.408812$

C $-0.3755945 .386828-4.307223$

C $-0.2999403 .997244-2.247260$

C $-0.3437292 .505733-2.426447$

N $3.806206 \quad 0.325700-3.406412$

H -1.902294 -5.658876 -2.605341

H $-2.060574-4.015045-0.024860$

H $0.285765-4.863269-1.859386$

H $\quad 0.174616-6.3455530 .186067$

H $0.039155-4.9253211 .200497$

H $3.776337-4.7212621 .316455$

H $3.811948-5.931617-0.625050$

H $2.376594-6.899552-0.965238$

H $2.580426-5.301918-1.720584$

H $1.201460-4.5207822 .911835$

H $2.840356-4.2864483 .440777$

H $1.256297-2.3112121 .718101$

H $1.477571-2.2099103 .465312$

H $3.993237-2.1014603 .066036$

H $2.627833-0.0321213 .517909$

H 2.2280720 .0772291 .817213

H 3.4270382 .2187442 .927591

H 4.2035780 .5381070 .103100

H 4.7917740 .9092644 .323751

H $6.128876 \quad 1.1723853 .228140$

H $5.620130-0.4821743 .620358$

H 6.0091691 .7368540 .747095

H $6.427536-1.474683-0.936833$

H $5.439384-2.898143-0.728257$

H $6.342526-2.7101951 .265873$

H $1.029202-0.807278-0.397728$

H $-0.517450-0.022922-2.942180$

H $1.5009152 .924532-0.497506$

H $2.206444 \quad 1.348733-0.752857$

H $\quad 0.845464 \quad 1.5411420 .373261$

H $4.428502-2.163348-2.596399$

H $3.406321-1.305302-1.440813$

H $3.4356951 .267154-5.201116$

H $5.0024261 .671349-4.465635$

H $3.7405382 .932879-2.585101$

H $2.0617553 .242185-5.128897$

H $2.2142005 .573499-4.052068$

H $2.2753784 .842999-2.465365$

H -1.458602 $5.417590-4.247299$

H $0.0919545 .924166-5.125692$

H -1.338238 $4.337042-2.137358$

H $0.1901354 .300908-1.318560$

H -1.012003 2.184935 -3.226797

H $2.926794-0.182041-3.436197$

C $-4.536565-3.942181-1.057398$

O $-4.115636-2.690628-1.630378$

C $-5.108178-1.687524-1.309396$

C $-6.256636-2.431569-0.632226$

C $-6.055521-3.862129-1.111958$

H $-5.432211-1.264936-2.267788$

C $-4.399857-0.626547-0.458377$

H $-6.168716-2.3816700 .460106$

H -7.233496 -2.021260 -0.905874

C $-6.769515-4.893184-0.257058$

H $-6.402501-3.940423-2.150639$

C $-5.3153720 .414670 \quad 0.194973$

O $-3.4747690 .057524-1.313469$

H -3.804948 -1.132662 0.310199

H $-6.024739-0.0798590 .868527$ 
O $-6.098576 \quad 1.029535-0.834598$ C $-4.573274 \quad 1.5255800 .971980$ C -3.639675 $1.001172 \quad 2.083777$ H $-4.049983 \quad 2.1888230 .274559$ O $-5.589092 \quad 2.326156 \quad 1.595217$ H -3.750319 1.6303992 .974063 H -3.9742330 .0047732 .396142$ H $-6.589245-5.904869-0.633500$ H $-6.431290-4.8559260 .783941$ H -7.850259-4.717913 -0.265930 C $-3.894390-5.090191-1.837745$ H $-2.896239-0.615435-1.711681$ H $-6.6656421 .694963-0.408232$ H $-5.147914 \quad 3.0979631 .989959$ H -4.172507 -4.989965 -2.894888 H $-4.277312-6.055631-1.489612$ H $-4.213855-3.962614-0.008196$ C $-1.500905 \quad 3.1681542 .392855$ O $\begin{array}{llll}\text { O } & -1.660707 & 2.252124 & 1.303829\end{array}$ C -2.1534910 .9571341 .664681$ C -1.2177590 .3028302 .689705$ C -0.9762551 .2036283 .895918$ C -0.5387012 .5954413 .441842$ H -2.475186 3.3747382 .851563 C -0.9596414 .4842301 .814162$ H -2.0843130 .3499830 .758704$ O $-1.734515-0.947998 \quad 3.130358$ H $-0.250244 \quad 0.097241 \quad 2.214841$ H $-1.861638 \quad 1.2603934 .539757$ $\begin{array}{lllll}\text { O } & 0.068508 & 0.646293 & 4.696562\end{array}$ H 0.4790192 .5431693 .033309 O $-0.502730 \quad 3.4655094 .570947$ C $-1.979500 \quad 5.1988790 .952083$ H -0.6798555 .1723102 .619113$ H -0.0817124 .2771351 .191788$ H $-1.853556-1.5077412 .343931$ H $-0.201775-0.2567834 .936451$ H $0.0926003 .064517 \quad 5.227254$ $\begin{array}{llll}0 & -1.375515 & 6.265258 & 0.347408\end{array}$ O $-3.153695 \quad 4.8784620 .831366$ C $-2.2173227 .048978-0.504342$ H $-3.0058707 .527350 \quad 0.084035$ H $-1.6030837 .825795-0.967324$ H -2.648396 $6.426727-1.294438$ SCF Energy (B3LYP/6-31G**/MMFF) $=-3245.90998789$

01

MM̄FF Geometry

C $-0.114124-0.8586253 .265236$

C -1.186733 -0.1905163 .714630$

C $-1.630981 \quad 1.129247 \quad 3.142967$

O $-2.843890 \quad 0.9583912 .357305$

C -1.7972752 .2564994 .179985$

C $-0.495112 \quad 2.7948354 .734391$

C $0.294703 \quad 3.672674 \quad 4.085070$

C -0.1305162 .3180436 .114741$

C $0.089372 \quad 4.2100582 .694898$

C 1.3141073 .9106591 .819862

C 1.0410323 .9242050 .309103

C $0.3969585 .219070-0.186603$

C $0.0876525 .157393-1.691508$

$\begin{array}{lllll}\text { O } & 0.199132 & 2.803871 & 0.012265\end{array}$

O $-0.793798 \quad 6.246557-2.005933$

C $-0.6827953 .852684-1.991399$

C $1.3464445 .359959-2.543404$

O $-0.867397 \quad 3.682070-3.395170$

C $-0.0237222 .590407-1.387215$

C $-0.9666691 .372640-1.545730$

O $1.1971052 .325281-2.082232$

C $-4.021137 \quad 0.617188 \quad 2.936843$

$\begin{array}{llll}\text { O } & -4.224428 & 0.412495 & 4.125536\end{array}$

C $-5.113548 \quad 0.5271351 .938678$

$\begin{array}{llll}\text { C } & -4.922140 & 0.562899 & 0.610577\end{array}$

C $-5.9621790 .463887-0.416857$

C $-7.397017 \quad 0.4450540 .042525$

C $-0.5099050 .127061-0.775828$

C $-1.432718-1.051730-1.009060$

C $-2.141526-2.664111-2.776075$
O $-2.175130-1.477105-0.127392$

C $-3.262622-2.096141-3.587414$

C $-4.557831-2.359037-3.357325$

C $-5.676030-1.807481-4.194295$

C $-6.738979-1.109872-3.374882$

C $-7.918705-1.706508-3.133347$

C $-6.4788620 .310305-2.923553$

C $-5.5903080 .391607-1.712823$

$\mathrm{N}-1.323186-1.571386-2.288303$

H $0.473250-0.4408672 .451578$

H $-1.772229-0.6111674 .527750$

H $-0.889041 \quad 1.4568362 .406238$

H -2.429414 1.9225865 .012566

H $-2.368075 \quad 3.080178 \quad 3.730537$

H 1.1942304 .0284424 .585489

H 0.8170042 .7414806 .464244

H -0.9061932 .6046986 .832502$

H $-0.028536 \quad 1.2284996 .129004$

H -0.8131673 .79827722 .235610$

H $-0.058517 \quad 5.2945642 .758679$

H 2.1059154 .6334872 .053589

H 1.7012482 .9164122 .078238

H $2.0070303 .773860-0.187219$

H $1.033377 \quad 6.082510 \quad 0.043290$

H -0.5499895 .3939180 .340752$

H $-0.3506047 .073864-1.750798$

H $-1.6871443 .958384-1.557405$

H $1.7986286 .336300-2.331181$

H $1.1031525 .369170-3.611610$

H $2.1103694 .599295-2.370044$

H $-1.2934054 .489240-3.731243$

H $-1.9668321 .635767-1.177486$

H -1.070693 $1.128546-2.609848$

H $1.4337601 .392304-1.966132$

H -6.1028700 .4025472 .367665$

H -3.9090950 .6649770 .227656$

H $-7.625968 \quad 1.343608 \quad 0.626443$

H $-7.590598-0.4372680 .662505$

H $-8.1170950 .415196-0.777611$

H $0.500261-0.177496-1.060090$

H -0.4889440 .3285170 .299055$

H $-2.501261-3.263561-1.933686$

H - $1.506116-3.291749-3.408040$

H $-2.991880-1.447860-4.417920$

H - $-4.829464-3.025895-2.542223$

H $-6.118846-2.646377-4.747262$

H $-5.300469-1.116253-4.959152$

H $-8.704839-1.204528-2.579647$

H -8.124905 $-2.713088-3.482231$

H -7.422191 $0.840406-2.748745$

H $-6.0064260 .868935-3.741894$

H $-4.5261850 .404277-1.950426$

H $-0.687531-1.131958-2.947617$

C $0.107323-3.3189702 .845475$

O $\quad 0.897235-3.138898 \quad 1.652831$

C $1.433217-4.4140841 .252552$

C $0.755626-5.4599572 .131727$

C $0.516757-4.6793003 .407236$

H $2.503104-4.3928921 .490351$

C $1.231995-4.635752-0.250217$

H $-0.198388-5.7937851 .707120$

H $1.382471-6.3447102 .279276$

C $-0.519027-5.3128534 .319824$

H $1.468675-4.5916683 .948009$

C $1.829091-3.540076-1.155310$

O $-0.173297-4.700063-0.531969$

H $1.637577-5.613738-0.534295$

H $1.251043-2.616647-1.047020$

O $1.659221-3.965311-2.514023$

C $3.320465-3.242165-0.920880$

C $3.975420-2.366991-2.006350$

H $3.445367-2.7671260 .056654$

O $4.035526-4.480800-0.876742$

H $5.029361-2.233518-1.733252$

H $4.002630-2.928786-2.948651$

H $-0.662345-4.7141645 .224669$

H -1.489374 -5.4067983 .820980$

H $-0.200000-6.3146374 .625889$ 
C $0.355644-2.170443 \quad 3.823315$ H $-0.573888-3.871851-0.215850$ H $\quad 0.713585-4.152876-2.642648$ H $4.961766-4.277717-0.660888$ H $1.428498-2.0784384 .031334$ H $-0.142439-2.3755534 .777924$ H $-0.944974-3.3318222 .531547$ C $4.4515100 .270095-0.517598$ O $3.209220-0.230420-1.024376$ C $3.304132-0.997738-2.228169$ C $3.950305-0.159162-3.339551$ C $5.2999670 .401222-2.907164$ C $5.1682181 .128033-1.571622$ H $5.088861-0.568286-0.210856$ C $4.137672 \quad 1.1224690 .722791$ H $2.274513-1.193560-2.549064$ O $4.121358-0.926382-4.526460$ H $3.2907740 .680931-3.592940$ H $6.065939-0.381167-2.856259$ O $5.7529681 .342239-3.883544$ H $4.6220602 .068481-1.723582$ O $6.4701771 .464224-1.094808$ C 3.7246370 .3051401 .928056 H 5.0257201 .6959631 .012466 H 3.3180421 .8139870 .503342 H $3.246722-1.264075-4.784783$ H $5.7917090 .876627-4.736685$ H $6.9051421 .991674-1.786864$ O 3.7597071 .1110453 .031180 O $3.394318-0.8714931 .907070$ C 3.4031120 .5064414 .278887 H 2.3165990 .5219364 .390101 H $3.785124-0.5159244 .357066$ H 3.8451261 .1028285 .081676

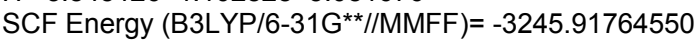

$01 \_179$

MM̄FF Geometry

C $3.930245-1.287600-2.115271$

C $3.006831-2.152232-2.565040$

C $2.139135-3.004882-1.675917$

O $0.800210-2.931597-2.230011$

C $2.625583-4.467624-1.645878$

C $3.919387-4.672709-0.886396$

C $4.003100-4.7940510 .453619$

C $5.156492-4.815941-1.735164$

C $2.884508-4.7426761 .461117$

C $3.228216-3.8735992 .680959$

C $3.062439-2.3634592 .436211$

C $3.783775-1.5808513 .537851$

C $3.560142-0.0695033 .414192$

O $1.659062-2.0647802 .459629$

O 4.0128780 .5464684 .628975

C 2.0481390 .1949683 .306960

C 4.3874590 .5425592 .280798

O $1.792513 \quad 1.5742343 .046668$

C $1.332687-0.6824972 .251217$

C $-0.198726-0.5107802 .419759$

O $1.718587-0.245166 \quad 0.946772$

C $-0.229036-3.361062-1.452720$

O $-0.148043-3.889442-0.355396$

C -1.497117 -3.046031-2.148005

C $-2.652191-3.238905-1.493000$

C $-4.000512-2.954940-1.985882$

C $-4.133613-2.383665-3.371975$

C $-1.045899-1.4718451 .584281$

C $-2.520058-1.1438271 .702080$

C $-4.435703-1.1937673 .282293$

O $-3.117788-0.5450760 .810920$

C $-5.445096-1.9259932 .452428$

C $-6.285397-1.3073791 .608047$

C -7.332553 -2.003826 0.786810

C -7.104309-1.852968 -0.701655

C $-7.486762-0.741915-1.353643$

C $-6.512193-3.027196-1.447191$

C $-5.045591-3.206775-1.169580$

N -3.089543 -1.570440 2.890203

H $4.076666-1.188239-1.044088$
H $2.884978-2.284526-3.638070$

H $2.109454-2.581059-0.664965$

H $2.728106-4.845489-2.672770$

H $1.857647-5.120180-1.213977$

H $4.985553-4.9869320 .883262$

H $\quad 6.056738-4.983048-1.134070$

H $5.051143-5.669074-2.413626$

H $5.327122-3.917742-2.334688$

H $1.942576-4.4097221 .021731$

H $2.723731-5.7694301 .812936$

H $2.561074-4.1719403 .500421$

H $4.252779-4.0959543 .004837$

H $3.472778-2.1042401 .454209$

H $4.857397-1.8080493 .535880$

H $3.411551-1.9069624 .519462$

H $4.953146 \quad 0.3261864 .744399$

H $1.609233-0.0208654 .291348$

H 5.4571580 .3663272 .445108

H 4.2647201 .6304352 .243874

H 4.1308420 .1321541 .303270

H 2.2359492 .0880143 .743196

H $-0.465013-0.6615313 .473842$

H -0.4816750 .5160872 .160228$

H $1.007518-0.4420180 .317836$

H -1.444973 -2.646513 -3.153808

H $-2.611116-3.637496-0.479540$

H -3.809597 -3.115608 -4.119755

H -3.522675 -1.481708 -3.479655

H -5.154272 -2.088758 -3.625326

H $-0.784536-1.4001020 .525727$

H $-0.886645-2.5099881 .895404$

H $-4.530078-0.1069313 .181194$

H $-4.561490-1.4544944 .337713$

H -5.494038 -3.0059642 .564760$

H $-6.233546-0.2246361 .512341$

H $-8.309749-1.5783931 .050812$

H -7.399257 -3.066769 1.049701

H -7.349947 -0.639261 -2.424975

H $-7.9394190 .092017-0.827240$

H -6.709485 -2.941506 -2.520550

H -7.037657 -3.943209-1.147233

H -4.841515 -3.617153 -0.179958

H -2.498863 -2.007770 3.591118

C $5.0407840 .966260-2.626569$

O $3.7781071 .641891-2.486555$

C $3.8246202 .456798-1.299624$

C $5.2976552 .564432-0.921887$

C $5.8098651 .195336-1.324855$

H $3.3013581 .896484-0.515547$

C $3.1163223 .795029-1.531706$

H $5.8107113 .342599-1.498656$

H 5.4405302 .7828520 .140523

C $7.3196281 .131000-1.480232$

H $5.5080000 .471779-0.561131$

C $1.6934393 .665975-2.110206$

O $3.8869274 .566824-2.461987$

H $3.1023184 .366572-0.596049$

H $1.7525113 .336281-3.154402$

O $1.1217934 .980556-2.148307$

C $0.7554652 .729383-1.328405$

C $-0.6617762 .710971-1.933958$

H $1.1620291 .712178-1.346803$

O $0.714412 \quad 3.157610 \quad 0.028746$

H -1.115136 $3.701592-1.826700$

H $-0.5683792 .508229-3.007190$

H $7.6372780 .129167-1.785779$

H $7.6774961 .842439-2.231915$

H $7.813677 \quad 1.367664-0.532113$

C $4.826383-0.495950-3.031002$

H $3.4223605 .411414-2.592336$

H $0.9950225 .270696-1.228371$

H 0.2185252 .4902920 .532686

H $\quad 5.798407-0.999687-3.086435$

H $4.400430-0.507161-4.042626$

H $5.5627321 .470084-3.451054$

C -2.7044413 .0178920 .357617$

O -1.8306101 .9150140 .093875$

C $-1.5760411 .646462-1.288351$ 
C $-2.894173 \quad 1.403321-2.038693$

C $-3.8889232 .539939-1.824865$

C $-4.0590142 .819176-0.333178$

H -2.240392 3.9456930 .004913

C -2.8888643 .1119081 .879504$

H -1.018165 $0.701185-1.317162$

O $-2.6376461 .223792-3.427536$

H -3.342812 $0.470643-1.674740$

H $-3.5875533 .446827-2.362491$

O $-5.1639662 .160258-2.347639$

H $-4.6050421 .989340 \quad 0.133911$

O $-4.8467883 .996516-0.165298$

C -1.6229103 .5091152 .602490$

H -3.661482 3.8390232 .153226

H -3.1961912 .1369232 .277726$

H $-3.4713250 .958473-3.850597$

H -5.077862 $2.095278-3.313534$

H $-5.6889713 .850184-0.629266$

O -1.1855894 .7245602 .167505$

O -1.1073392 .8332673 .484001$

C 0.0034895 .2126742 .796473

H 0.8241834 .5000832 .670180

H $\quad 0.2808706 .1539322 .314396$

H -0.1837395 .4021353 .857550$

SCF Energy (B3LYP/6-31G**//MMFF) $=-3245.91731020$

0118

MM̄FF Geometry

C 2.562997 -3.048602 1.666518

C $1.914628-2.2138732 .492634$

C $2.156057-0.7289152 .481021$

O $0.890218-0.0354412 .390243$

C $2.846928-0.2742423 .776765$

C 3.5956411 .0303293 .577463

C 4.9041501 .1096773 .262153

C 2.7851382 .2817493 .786184

C $5.856117-0.0331623 .045386$

C 6.7284010 .0904781 .787161

C $5.964879-0.1130000 .466499$

C $6.959939-0.403643-0.662190$

C $6.274950-0.518922-2.029012$

O $5.2271651 .077756 \quad 0.172882$

O $7.317610-0.495748-3.015411$

C $5.3873340 .724386-2.236024$

C $5.550823-1.861624-2.188181$

O $4.6139660 .607735-3.428763$

C $4.4556891 .019396-1.036639$

C $3.7743062 .395401-1.240911$

O $3.4555230 .002585-0.977292$

C 0.3457720 .1175251 .154061

$\begin{array}{lllll}\text { O } & 0.727515 & -0.385547 & 0.109992\end{array}$

C -0.7776441 .0698571 .280852$

C -1.1702371 .7580850 .198834$

C -2.242692 2.7532710 .146465

C -3.0333102 .9930571 .405856$

C $2.9869682 .903381-0.026202$

C $2.3869554 .278648-0.270402$

C 1.1721296 .0974530 .927382

O $2.4738144 .869746-1.343383$

C $-0.313628 \quad 6.0063770 .783331$

C $-0.9901006 .551004-0.239374$

C $-2.4875936 .554016-0.356894$

C $-2.9833695 .791178-1.563797$

C $-3.0082156 .359233-2.781374$

C $-3.5360544 .403541-1.336817$

C $-2.4653393 .398246-1.018063$

N 1.7508924 .7699350 .861359

H $3.300156-2.6402090 .979313$

H $1.184468-2.6039413 .196489$

H $2.777688-0.4491051 .621551$

H $3.538479-1.0434314 .139754$

H $2.109794-0.1420804 .580152$

H 5.3509232 .0985773 .169374

H 2.3868142 .3096114 .805571

H 3.3773983 .1908463 .637807

H 1.9466232 .3186423 .085276

H $6.525148-0.0632363 .914742$

H $5.344149-0.9995183 .015713$
H $7.2274451 .068063 \quad 1.784127$

H $7.512540-0.6733461 .867323$

H $5.269051-0.9514770 .583878$

H $7.535835-1.313242-0.448928$

H $7.6975290 .410387-0.711556$

H $6.910691-0.625644-3.888526$

H $6.0543831 .586180-2.378775$

H $\quad 6.266846-2.689913-2.124939$

H $5.082495-1.945048-3.175120$

H $4.783541-2.029762-1.429582$

H $5.2317770 .537967-4.175649$

H $4.5443263 .143784-1.471207$

H $3.0972292 .349362-2.103438$

H $2.6368540 .377014-0.617681$

H -1.222922 1.1997402 .260272

H $-0.6499221 .584317-0.742603$

H -2.3733443 .3186142 .217789$

H -3.5475602 .0784681 .718013$

H -3.7960413 .7672421 .303545$

H 2.1697722 .2205960 .220061

H $3.6494662 .978757 \quad 0.842761$

H 1.6225486 .7361740 .160165

H $1.4223996 .515321 \quad 1.907525$

H -0.8585775 .5086581 .581377$

H -0.438205 $7.064952-1.023601$

H $-2.8189227 .598758-0.424416$

H -2.958056 6.1623610 .553305

H $-3.3914365 .828484-3.646382$

H -2.641485 $7.368347-2.939037$

H $-4.0539644 .049224-2.238322$

H -4.316466 $4.449439-0.571801$

H -1.828013 $3.162719-1.871529$

H 1.6903794 .1861181 .689809

C $1.651417-5.035051 \quad 0.369301$

O $0.276977-4.6039450 .379483$

C $-0.116413-4.299079-0.972948$

C $1.010618-4.802843-1.871526$

C $2.219337-4.593531-0.979240$

H $-0.158078-3.205584-1.045646$

C -1.496209-4.890702 -1.277685

H $\quad 0.897622-5.865737-2.113817$

H $1.068694-4.247728-2.812784$

C $3.450570-5.365963-1.415730$

H $2.451854-3.521866-0.980057$

C $-2.591766-4.503471-0.263385$

O $-1.392080-6.320599-1.270719$

H -1.796316 -4.609376 -2.294102

H -2.406904 -5.012003 0.690310

O $-3.833250-5.030243-0.751398$

C $-2.745636-2.991298-0.017759$

C $-3.937384-2.6783370 .908865$

H -1.827003 -2.603618 0.436769

O $-2.914973-2.346614-1.275786$

H $-4.868220-2.9668530 .409978$

H -3.842945 -3.298776 1.807759

H $4.285068-5.185496-0.730753$

H $3.260723-6.444183-1.441094$

H $3.763441-5.055926-2.418125$

C $2.366037-4.5359061 .627360$

H $-2.275303-6.676237-1.469679$

H $-4.053740-4.549976-1.568021$

H -2.867943 -1.388138 -1.119953

H $3.351572-5.0108301 .701397$

H $1.793483-4.8529212 .508251$

H $1.620054-6.1321170 .415827$

C $-5.486460-0.372308-0.411073$

$\begin{array}{llll}\text { O } & -4.195056 & -0.313222 & 0.206747\end{array}$

C $-4.011024-1.190597 \quad 1.322143$

C $-5.055744-0.8846522 .405064$

C $-6.476055-0.9363991 .848620$

C $-6.589137-0.0553290 .605353$

H -5.644791 -1.369249 -0.839268

C $-5.4991680 .643938-1.558887$

H $-3.031037-0.9434401 .748698$

O $-4.913091-1.7939993 .490399$

H -4.8684700 .1213422 .802643$

H -6.787358 -1.964756 1.630295

O $-7.388859-0.4344772 .827127$ 
H -6.5373511 .0008840 .900510$

O $-7.861739-0.267321-0.003056$

C $-4.667765 \quad 0.159428-2.722423$

H $-6.5121010 .810047-1.943347$

H -5.121546 $1.619712-1.232569$

H $-5.499354-1.4952114 .205668$

H $-7.401799-1.0651373 .566643$

H $-8.539365-0.0792200 .668801$

O $-3.394266 \quad 0.632303-2.617559$

O $-5.096458-0.571043-3.606612$

C $-2.4991180 .243333-3.663791$

H $-2.443820-0.846907-3.737106$

H $-2.8223600 .678360-4.613993$

H $-1.5052450 .627980-3.420174$

SCF Energy (B3LYP/6-31G**//MMFF) $=-3245.91208080$

01180

MM̄FF Geometry

C $2.374560-4.313229-2.866666$

C $2.095431-3.027922-3.129649$

C $0.732562-2.523528-3.526786$

O $0.156602-1.863880-2.375359$

C $0.865069-1.486198-4.657131$

C $-0.450966-0.854651-5.068564$

C $-0.7396110 .456382-4.942906$

C $-1.465847-1.791533-5.670822$

C $0.1436251 .549589-4.406238$

C $0.0045441 .801897-2.897296$

C $-1.3502322 .395996-2.480903$

C -1.331929 2.789567-1.002296

C $-2.6978473 .305387-0.523084$

O $-2.364601 \quad 1.413510-2.697605$

O $-2.645273 \quad 3.336053 \quad 0.911625$

C $-3.7949472 .300675-0.942744$

C $-2.9442374 .747556-0.980827$

O $-5.0960562 .821142-0.683085$

C -3.696626 $1.865743-2.419639$

C $-4.6566440 .704288-2.776346$

O $-4.047606 \quad 2.973710-3.258259$

C $-0.471170-2.651197-1.460039$

O $-0.643544-3.858319-1.517074$

C $-0.894149-1.786421-0.339737$

C $-1.144196-2.343427 \quad 0.854543$

C $-1.557476-1.6333362 .065417$

C -1.959005 -0.1916021 .915436$

C $-4.444258-0.591856-1.985006$

C $-5.384876-0.759217-0.802279$

C $-5.864888-2.3217051 .081799$

O $-6.324434-0.002960-0.572496$

C $-5.330916-1.7117122 .338979$

C $-4.979214-2.4292533 .416878$

C $-4.500800-1.8234664 .705112$

C $-3.176394-2.3899325 .164687$

C $-3.122897-3.350406 \quad 6.102855$

C -1.914094-1.776270 4.604020

C $-1.574736-2.3034153 .236099$

N $-5.070444-1.884765-0.051179$

H $1.595366-5.064418-2.964071$

H $2.877637-2.284056-2.996643$

H $0.084628-3.346903-3.851817$

H $1.314880-1.958316-5.540771$

H $1.571759-0.712593-4.338058$

H $-1.7196810 .797966-5.273927$

H -2.337694 -1.260001-6.067656

H -1.020618 -2.352282 -6.499156

H $-1.828617-2.501498-4.921408$

H $1.1938251 .339242-4.635402$

H $-0.084620 \quad 2.473592-4.952507$

H $\quad 0.1721900 .860748-2.361859$

H $0.8033502 .492003-2.597747$

$\mathrm{H}-1.5492803 .272959-3.107951$

H $-0.5510363 .534977-0.807413$

H -1.061947 $1.911908-0.399727$

H -3.484058 $3.704188 \quad 1.236742$

H -3.677831 $1.418023-0.305035$

H $-2.1742085 .413175-0.572467$

H -3.899486 5.126589-0.601126

H $-2.9381734 .859048-2.067204$
H $-5.1681212 .972966 \quad 0.274073$

H $-5.6974761 .045953-2.726691$

H $-4.4841490 .457900-3.834220$

H $-4.3154992 .630271-4.126757$

H - $0.963952-0.720589-0.522271$

H $-1.012428-3.4190750 .965951$

H $-2.759344-0.0969491 .175320$

H -1.1075980 .4083191 .584657$

H -2.336461 0.2604542 .834546

H $-4.644444-1.439435-2.653363$

H $-3.408819-0.683873-1.643433$

H $-5.826561-3.4154331 .102760$

H $-6.905126-2.0126880 .936133$

H $-5.262962-0.6270652 .375870$

H $-5.076033-3.5123483 .387886$

H $-5.269975-2.0120665 .465640$

H $-4.423704-0.7314354 .630335$

H -2.175180 -3.740025 6.459581

H $-4.026198-3.7728086 .530716$

H -1.062672 -2.006445 5.259017

H $-1.996274-0.6863524 .636686$

H $-1.275613-3.3526083 .236562$

H $-4.238532-2.420569-0.281597$

C $3.949316-4.540066-0.874138$

O $4.050859-3.126022-0.612096$

C $3.357518-2.8300810 .616218$

C $3.114721-4.176064 \quad 1.290984$

C $2.887791-5.0742570 .087824$

H $2.387144-2.4033900 .340550$

C $4.160678-1.8201861 .443390$

H $3.991813-4.5125381 .855916$

H $2.260757-4.1489441 .974726$

C $3.023115-6.5551820 .392576$

H $1.877977-4.881418-0.294353$

C $4.509394-0.5061620 .708123$

O $5.399876-2.4312991 .833470$

H $3.633753-1.5992982 .377747$

H $5.159639-0.742850-0.143995$

$\begin{array}{lllll}\text { O } & 5.295245 & 0.311260 & 1.581880\end{array}$

C $3.3271810 .340723 \quad 0.198377$

$\begin{array}{llll}\text { C } 2.193403 & 0.616337 & 1.207282\end{array}$

H $3.7249751 .300490-0.150027$

O $2.769793-0.299605-0.949004$

H $1.373648 \quad 1.0999810 .661444$

H $1.767847-0.333873 \quad 1.550615$

H $2.868237-7.153172-0.511066$

H $4.014977-6.7946750 .790190$

H $2.280128-6.8644391 .134814$

C $3.713870-4.780743-2.370594$

H $5.865838-2.692607 \quad 1.020489$

H $6.045039-0.2273531 .887595$

H $2.1033370 .298831-1.325717$

H $3.807916-5.850533-2.590998$

H $4.498884-4.272126-2.945056$

H $4.930566-4.960599-0.616567$

C 2.4076063 .6559881 .376387

$\begin{array}{llll}\text { O } & 3.253937 & 2.692965 & 2.016767\end{array}$

C $2.598151 \quad 1.4853682 .415677$

C $1.4535601 .808715 \quad 3.386504$

C 0.4941562 .8489762 .811477

C 1.2651134 .0695712 .310310

H $1.996545 \quad 3.2326260 .451971$

C 3.2836984 .8620341 .010553

H 3.3317690 .9088722 .990146

$\begin{array}{lllll} & 0 & 0.758365 & 0.614935 & 3.725345\end{array}$

H 1.8830022 .2049374 .316124

H -0.1222122 .4307632 .010077$

$\begin{array}{lllll} & \text { O } & -0.407697 & 3.281447 & 3.831680\end{array}$

H 1.6588274 .6313983 .167086

$\begin{array}{lllll} & 0 & 0.372637 & 4.930815 & 1.606185\end{array}$

C $4.2426954 .528687-0.111893$

H 2.6843025 .7147810 .675037

H 3.8619065 .1711551 .890058

H 0.1169820 .8330104 .422098

H -0.9826132 .5292214 .052147$

H $-0.364593 \quad 5.1385432 .205595$

O $5.462845 \quad 5.082592 \quad 0.145562$

O $3.9442423 .883050-1.108136$ 
C $6.466928 \quad 4.846867-0.846556$

H $6.6465453 .773076-0.957019$

H $6.1666375 .289344-1.801069$

H $7.3923825 .324560-0.513916$

SCF Energy (B3LYP/6-31G**//MMFF) $=-3245.90766916$

01181

MM̄FF Geometry

C -2.035865 0.2599912 .690671

C $-2.552066-0.9226083 .058307$

C $-2.494225-2.1458352 .182235$

O $-1.161796-2.6896742 .340334$

C $-3.530844-3.2176932 .564720$

C $-4.965768-2.8576332 .243408$

C $-5.562815-3.1214761 .064448$

C $-5.760801-2.245506 \quad 3.367137$

C $-4.933890-3.725193-0.162951$

C $-5.467406-3.099898-1.461695$

C $-5.048905-1.634115-1.661539$

C $-5.873481-1.000775-2.785879$

C $-5.4352620 .440915-3.074961$

O $-3.654863-1.605158-1.998584$

O $-6.0328660 .844237-4.316392$

C $-3.909103 \quad 0.456176-3.281897$

C $-5.9409061 .422190-2.010227$

O $-3.427700 \quad 1.792846-3.412422$

C $-3.124349-0.283148-2.171987$

C $-1.637551-0.393432-2.587118$

O $-3.2123370 .474103-0.964505$

C $-0.663036-3.4298541 .315647$

O $-1.267971-3.8321530 .335167$

C $0.782614-3.6201241 .560908$

C $1.563031-4.0045980 .540282$

C $3.010694-4.2083850 .580916$

C $3.722139-3.9496011 .882456$

C $-0.795105-1.313596-1.696752$

C $0.682654-0.990325-1.799648$

C $2.564716-0.863324-3.428535$

O $1.312858-0.562639-0.835092$

C $3.441872-2.073405-3.358444$

C $4.610968-2.095719-2.699696$

C $5.552395-3.267314-2.690305$

C $5.880936-3.746697-1.294238$

C $6.899065-3.208424-0.601993$

C $5.094250-4.909784-0.736679$

C $3.635244-4.601840-0.548332$

N 1.206261-1.214949-3.060712

H $-1.5254830 .336027 \quad 1.733732$

H -3.017684 -1.014288 4.035800

H -2.638124 -1.842955 1.136691

H $-3.437975-3.4623083 .632119$

H -3.279405 -4.164111 2.068720

H $-6.620160-2.8790180 .964311$

H -6.819969 -2.131348 3.112124

H -5.707962 -2.876687 4.260235

H $-5.378056-1.2522043 .616898$

H -3.844575 -3.642580 -0.144411

H -5.179051 -4.793982 -0.173827

H -5.091752 -3.699114 -2.301200

H -6.561808 -3.181788 -1.470977

H $-5.205377-1.082457-0.728217$

H -6.944381 -1.032236 -2.548706

H -5.752458 -1.592305 -3.704403

H $-6.9979770 .768236-4.222933$

H -3.703597 -0.041878 -4.239994

H $-7.0364871 .406638-1.965583$

H -5.666542 $2.452883-2.261133$

H -5.567983 $1.201175-1.008066$

H -3.937538 $2.216154-4.124203$

H -1.575590 -0.782517 -3.611676

H -1.191696 $0.608175-2.596522$

H $-2.4154150 .324540-0.433386$

H $1.165406-3.4088042 .552264$

H $1.098755-4.180325-0.429776$

H $3.384946-4.6534152 .651123$

H $3.528485-2.9293652 .231362$

H $4.808026-4.0466591 .808806$

H -1.072704 -1.208134 -0.644449
H $-0.949344-2.363002-1.971152$

H $2.922137-0.056236-2.780719$

H $2.532669-0.492218-4.457808$

H $3.123447-2.954207-3.910528$

H $4.937663-1.200478-2.175590$

H $\quad 6.476398-2.957507-3.196765$

H $5.160405-4.101115-3.286097$

H $7.162725-3.5652780 .387921$

H $7.488943-2.394293-1.010255$

H $5.536555-5.2763290 .196716$

H $5.178981-5.756747-1.430435$

H $3.039464-4.747827-1.450076$

H $\quad 0.619573-1.638753-3.773194$

C -2.4083132 .7758132 .733352$

O -1.2758543 .1097991 .903576$

C -1.7527883 .4427700 .589561$

C $-3.245051 \quad 3.720518 \quad 0.744723$

C $-3.6304202 .718217 \quad 1.814551$

H -1.618761 $2.546914-0.026555$

C $-0.9497364 .600724-0.009711$

H -3.4349564 .7400091 .099699$

H -3.792478 $3.580802-0.192334$

C -4.9398163 .0364452 .514808$

H $-3.7229811 .734816 \quad 1.341458$

C $0.5824904 .410549-0.028759$

O

H -1.312643 $4.797852-1.025463$

H 0.9793684 .5405840 .984550

O $1.1262105 .491809-0.800894$

C $1.0875063 .074395-0.605106$

C $2.5884083 .049490-0.954790$

H 0.8555832 .2661450 .096611

O $0.3718012 .801937-1.814419$

H $2.8221512 .047416-1.333345$

H $2.7558323 .714003-1.812180$

H $-5.1579862 .290523 \quad 3.285762$

H -4.910572 4.0204702 .994706

H -5.7683303 .0362401 .798854$

C -2.1044331 .5038503 .533006$

H -0.9715795 .6284001 .659937$

H $0.8258375 .376351-1.718822$

H $0.6824061 .944296-2.151087$

H -2.8593391 .3862694 .319520$

H -1.130929 1.6260694 .021888

H -2.508402 3.6083683 .441871

C 3.9130671 .2249811 .141616

O 3.4220102 .5584351 .325137

C 3.5480703 .4222480 .192008

C $5.0184343 .513621-0.243413$

C $5.6053832 .134402-0.518771$

C 5.3793591 .2241460 .683461

H 3.2877950 .7035640 .408922

C 3.7995990 .4969102 .490812

H 3.2669974 .4228550 .539228

O $5.1610604 .323111-1.405870$

H 5.6058443 .9891150 .552841

H $5.1946911 .691663-1.433631$

O $7.0143112 .254797-0.728283$

H $6.029743 \quad 1.5471891 .506886$

O $5.759997-0.106876 \quad 0.341449$

C 2.3740440 .2208382 .913457

H $4.310965-0.4709442 .442623$

H $4.258881 \quad 1.1097393 .276108$

H $4.7846525 .196197-1.201434$

H $7.1441952 .874077-1.467043$

H $6.682579-0.0754220 .035174$

O $2.401540-0.3878824 .137584$

O 1.3735530 .4975392 .268681

C $1.130331-0.7130104 .705606$

H $0.534006-1.3014924 .004745$

H $1.302634-1.3119705 .603739$

H 0.6061420 .2029344 .990075

SCF Energy (B3LYP/6-31G*//MMFF)= -3245.91354545

01 182

MM̄FF Geometry

C -2.024890 $5.594060-0.280905$

C -2.6301394 .6861010 .499075$ 
C $-3.6139743 .663481-0.008235$

$\begin{array}{lllll}\text { O } & -3.065493 & 2.349724 & 0.248402\end{array}$

C -4.9387793 .7902520 .765440$

C -6.0168202 .8322090 .297310$

C -6.5592711 .8608391 .057713$

C $-6.5060033 .035407-1.113847$

C -6.2423671 .5154162 .486162$

C -5.1523140 .4466652 .662232$

C $-5.520850-0.9292602 .083776$

C $-4.490944-1.9831672 .493584$

C $-4.786864-3.3522661 .860109$

O $-5.549372-0.829610 \quad 0.658640$

O $-3.619881-4.173122 \quad 2.027933$

C $-5.002484-3.1766310 .339531$

C $-5.931369-4.0753912 .581251$

O $-5.443893-4.407376-0.228841$

C $-5.970159-2.025130-0.013480$

C $-6.020800-1.692443-1.524972$

O $-7.298515-2.393613 \quad 0.377535$

C $-2.1588501 .867361-0.644707$

O $-1.7807992 .403749-1.674015$

C $-1.6726970 .567862-0.130729$

C $-0.5584690 .030094-0.648791$

C $0.066845-1.228737-0.236879$

C $-0.692589-2.099176 \quad 0.728277$

C $-4.681109-1.298924-2.157306$

C $-3.958632-2.451498-2.834417$

C $-1.812620-2.993114-3.977381$

O $-4.465029-3.556826-3.007031$

C $-0.904033-3.683117-3.009713$

C $0.428185-3.729594-3.160241$

C $1.354591-4.452312-2.225227$

C $2.444096-3.555838-1.682780$

C $3.667622-3.537386-2.238156$

C $2.135783-2.740430-0.447999$

C $1.276392-1.543138-0.745828$

N -2.687828 -2.086805 -3.258339

H $-2.2545315 .626859-1.342558$

H -2.3841504 .6491151 .558257$

H -3.792882 $3.787544-1.083210$

H $-5.328051 \quad 4.8126880 .668715$

H -4.734952 3.6442791 .831765

H $-7.333093 \quad 1.2365110 .612021$

H -7.383759 2.420798 -1.341707

H $-6.7912174 .080796-1.270761$

H $-5.7265372 .771848-1.835126$

H -5.9553162 .4123023 .045519$

H $-7.167561 \quad 1.1682002 .964078$

H -4.2247800 .8058862 .201239$

H -4.959085 $0.342782 \quad 3.737276$

H $-6.516025-1.2029242 .453382$

H -4.436206 -2.070898 3.585901

H -3.492308 -1.660136 2.170103

H -3.432845 -4.237395 2.980213

H -4.015811-2.967306 -0.090552

H $-5.684627-4.2224353 .639669$

H -6.086954 -5.078888 2.169735

H $-6.877589-3.5323762 .535995$

H -5.439973 -4.308904 -1.195329

H -6.508226 -2.504734 -2.077160

H $-6.689023-0.826572-1.641266$

H -7.929528 -1.850959 -0.123820

H -2.2305340 .1027740 .673582$

H $-0.0372250 .573585-1.435872$

H -1.697757 -2.3051280 .345571$

H $-0.780663-1.6077811 .703190$

H - $0.229225-3.0729960 .897915$

H -4.874300 -0.557361 -2.943249

H -4.018626 -0.831904 -1.422267

H -1.260923 -2.397140 -4.711421

H $-2.414435-3.735462-4.511540$

H -1.363465 -4.188721 -2.163579

H $\quad 0.881057-3.245384-4.022780$

H $1.800908-5.289064-2.778428$

H $0.807734-4.909035-1.390973$

H $4.459478-2.912131-1.839644$

H $3.901626-4.140909-3.108794$

H $3.068000-2.362355-0.010209$
H $1.711818-3.3979420 .315270$

H $1.738064-0.846704-1.446096$

H $-2.329268-1.165555-3.024273$

C $0.4397116 .160554-0.025829$

O $0.7227194 .923314 \quad 0.659489$

C $1.4798934 .068637-0.225758$

C $2.0014734 .982124-1.324642$

C $0.8404565 .945405-1.485027$

H $\quad 0.7575873 .353659-0.638661$

C $2.550543 \quad 3.3315920 .586047$

H $2.9013925 .515746-0.994458$

H $2.2382034 .443328-2.246745$

C $1.2025397 .224709-2.218180$

H $0.0456565 .432037-2.039748$

C $3.5541632 .526180-0.264825$

O 1.8660162 .4664621 .497808

H 3.0968654 .0538941 .204033

H $4.0650563 .204487-0.958352$

O $2.8610131 .559040-1.051464$

C 4.6343261 .8017100 .567792

C $5.6351201 .040859-0.325286$

H 5.1726762 .5471341 .164413

O 4.0308020 .8880631 .477386

H $5.1371410 .168764-0.763495$

H $5.9346631 .696226-1.151375$

H $0.3366017 .890596-2.288138$

H $2.0056427 .767048-1.707929$

H $1.5416557 .002241-3.235193$

C -1.0107796 .5785540 .231796$

H $1.446987 \quad 1.7611690 .975570$

H $2.3151632 .035028-1.699294$

H 3.4801160 .2761850 .959407

H -1.207654 $7.554543-0.226593$

H - $1.1499586 .706812 \quad 1.313158$

H 1.0950856 .9132110 .431988

C $6.167158-1.573371 \quad 1.228327$

O $6.618688-0.2542201 .556989$

C 6.9035730 .5900580 .438153

C $7.975565-0.060744-0.448224$

C $7.588400-1.480391-0.856190$

C $7.206571-2.3051230 .372643$

H $5.216612-1.5113310 .687280$

C $5.923620-2.3179442 .546049$

H $7.347407 \quad 1.503744 \quad 0.854511$

O $8.2117060 .734386-1.604729$

H $8.919072-0.0984960 .111716$

H $6.774359-1.469993-1.589829$

O $8.695501-2.101682-1.513301$

H $8.104548-2.5153660 .967572$

O $6.670933-3.556954-0.049667$

C $4.725557-1.7580053 .274479$

H $5.756267-3.3898372 .389613$

H $6.787307-2.2241353 .215536$

H $8.8732560 .271260-2.146778$

H $9.440788-2.126208-0.889196$

H $7.335779-3.980702-0.619201$

O $3.581123-2.3130982 .783559$

O $4.798768-0.9149444 .159100$

C $2.364625-1.8519433 .379570$

H $2.401467-1.9609134 .467707$

H $2.183902-0.8099013 .100489$

H $1.545603-2.4661442 .996673$

SCF Energy (B3LYP/6-31G*//MMFF)= -3245.90186118

01_183

MM̄MF Geometry

C -3.239962 -4.162066 -1.040880

C $-2.070246-3.576306-0.741526$

C $-0.961866-3.371097-1.740083$

O $-0.692275-1.952093-1.825327$

C $0.321078-4.079206-1.270464$

C $1.506712-3.816245-2.179537$

C $2.542814-3.012251-1.866489$

C $1.488190-4.549996-3.494910$

C $2.724523-2.205680-0.609171$

C $3.788078-2.8160180 .315002$

C $5.227392-2.504551-0.127237$

C $6.231749-3.2688670 .738687$ 
C $7.682635-2.8730180 .425898$

O $5.414326-1.094750 \quad 0.024382$

O $8.517450-3.3969641 .470107$

C $7.796908-1.3357940 .485056$

C $8.180366-3.498010-0.882085$

O $9.090810-0.9003420 .075222$

C $6.712541-0.607889-0.338476$

C $6.7043750 .918079-0.082564$

O $6.961349-0.809430-1.733194$

C -1.336696 -1.256340 -2.796784

O $-2.193572-1.688532-3.556649$

C $-0.8464820 .136809-2.900261$

C $0.0042870 .714528-2.037746$

C $0.5451742 .072816-2.152278$

C $0.1543342 .879419-3.364752$

C 6.4545761 .3067001 .378644

C 5.9585962 .7341861 .524309

C 4.6601664 .1639703 .100649

O $6.0759803 .581644 \quad 0.643529$

C 3.2642664 .0442752 .571271

C 2.7020744 .9441641 .751258

C 1.3295184 .7860541 .161344

C $1.3412034 .911062-0.347240$

C $0.7295965 .941768-0.955008$

C $2.0782773 .862325-1.151007$

C $1.3651372 .537026-1.185215$

N 5.3766262 .9462782 .768444

H -3.391378 -4.510943 -2.061097

H -1.883301 -3.2066030 .262351$

H -1.242841 -3.762018 -2.726137

H $0.148392-5.162825-1.221150$

H $0.553723-3.782382-0.241819$

H $3.347208-2.904938-2.592531$

H $2.400272-4.381910-4.077418$

H $1.400546-5.628346-3.326729$

H $\quad 0.642491-4.222033-4.107059$

H $1.785312-2.117568-0.054937$

H $2.991442-1.178861-0.886532$

H $3.632344-2.3959361 .317550$

H $3.638665-3.9001870 .391695$

H $5.352983-2.774723-1.181341$

H $6.096599-4.3518730 .625110$

H $6.036794-3.0536321 .798936$

H $8.397431-4.3619391 .493869$

H $7.695251-1.0525831 .540397$

H $8.141066-4.592273-0.822295$

H $9.230611-3.245599-1.065425$

H $7.595173-3.195589-1.752911$

H $9.742383-1.3789350 .615738$

H $7.6392211 .371541-0.436035$

H $5.9122841 .359325-0.703962$

H $6.533393-0.092533-2.229908$

H $-1.2302650 .673828-3.762400$

H $0.3528060 .146416-1.177282$

H $0.5746963 .887006-3.374390$

H $-0.9331963 .002071-3.410985$

H $0.4932912 .384666-4.281631$

H 5.7052950 .6411891 .822614

H 7.3794751 .2135401 .958490

H 5.1929155 .0216062 .677296

H 4.6455784 .2538084 .190655

H 2.6992753 .1599152 .857631

H 3.2611885 .8352391 .475123

H 0.6854975 .5560271 .605062

H $0.8842843 .822906 \quad 1.438982$

H $0.7377856 .048198-2.034443$

H $\quad 0.2050476 .703273-0.387397$

H $3.0773243 .704261-0.725582$

H $2.2801114 .222988-2.166094$

H $1.5839021 .895448-0.331241$

H 5.2629702 .1559503 .396551

C -4.257797 -3.950700 1.334072

O $-4.174499-2.5142821 .411258$

C -4.816786 -2.076724 2.624364

C $-5.268113-3.3373993 .357100$

C $-5.449654-4.3177212 .215187$

H -5.704106 -1.508466 2.324953

C $-3.867687-1.1868113 .435516$
H $-4.503126-3.6990804 .054133$

H $-6.185304-3.1732153 .931326$

C $-5.474658-5.7681102 .665185$

H -6.388109-4.082033 1.695556

C -3.3607610 .0653332 .690062$

O $-2.716989-1.9540213 .816778$

H $-4.352266-0.8899094 .372782$

H $-2.686490-0.2304711 .877957$

O -2.5694690 .8243183 .614422$

C -4.4710880 .9782262 .138268$

C $-3.9792912 .353171 \quad 1.646614$

H $-4.9945810 .462871 \quad 1.325970$

O $\quad-5.432114 \quad 1.209075 \quad 3.173111$

H -4.8586432 .9313821 .337063$

H -3.5668202 .9157182 .493913$

H $-5.610646-6.4410091 .813007$

H -4.543856 -6.047642 3.170094

H $-6.300402-5.9395083 .363451$

C $-4.413660-4.393583-0.123113$

H -2.295101 -2.274485 3.001021

H $-1.8711740 .235817 \quad 3.949089$

H -6.1589901 .7281192 .788656$

H -5.273690 -3.875137 -0.565163

H $-4.635142-5.466801-0.151446$

H -3.332854 -4.359082 1.762218

C $-4.4930082 .023454-1.332456$

O $\quad-3.3964971 .479924-0.587562$

C -2.9506622 .2941710 .500946$

C $-2.5106773 .669885-0.017830$

C $-3.5882894 .335738-0.870902$

C $-4.1068493 .375554-1.941733$

H $-5.3628832 .142590-0.675284$

C $-4.8600291 .012789-2.422746$

H -2.0535821 .8071150 .901914$

O -2.1577634 .5155981 .071077$

H $-1.6100913 .542014-0.628513$

H $-4.4102574 .703787-0.246477$

O $-3.0367145 .492474-1.505431$

H $-3.3401723 .235341-2.713714$

O $-5.2486993 .950898-2.572430$

C $-5.405908-0.256277-1.816146$

H $-5.6073531 .408612-3.119604$

H $-3.9804880 .740988-3.017512$

H -1.9076695 .3792160 .700438$

H $-2.2943085 .204020-2.063622$

H $-4.9813404 .818240-2.921985$

O $-6.711086-0.083946-1.462030$

O $-4.744089-1.273615-1.661318$

C $-7.336919-1.220537-0.858733$

H $-8.369221-0.952944-0.618705$

H $-7.345149-2.063373-1.556468$

H -6.820666 -1.491056 0.066612

SCF Energy $\left(B 3 L Y P / 6-31 G^{* *} / / M M F F\right)=-3245.91046386$

01184

MM̄FF Geometry

C $2.313124-3.623452-0.525171$

C $1.724100-2.437045-0.315321$

C $0.629327-1.886111-1.191557$

O $-0.467730-1.454288-0.355563$

C $1.144040-0.663278-1.973642$

C $0.095663-0.008478-2.856365$

C $-0.2126951 .302998-2.817503$

C $-0.604536-0.908973-3.841343$

C $0.4337872 .365035-1.976052$

C $-0.2707632 .643606-0.642314$

C -1.664287 $3.270898-0.796878$

C -2.1097763 .9091570 .521502$

C -3.5281524 .4883050 .423338$

O $-2.5882472 .240745-1.165264$

O -3.9786434 .7810381 .754728$

C $-4.4660743 .405351-0.144011$

C $-3.5560085 .813999-0.346178$

O $-5.7651753 .935935-0.397009$

C $-3.9199592 .709530-1.414247$

C $-4.8120951 .493409-1.751461$

O $-3.9566793 .627745-2.508585$

C $-1.386040-2.396376-0.010529$ 
O $-1.348409-3.588547-0.271594$ C $-2.471003-1.7177850 .733751$ C $-3.468498-2.4550981 .244104$ C -4.641482 -1.958001 1.965015 C $-4.731421-0.4785562 .226124$ C $-4.2308380 .542741-2.803713$ C $-5.238231-0.544601-3.119768$ C $-6.313194-2.562353-2.117946$ O $-5.929307-0.505708-4.135197$ C $-7.266528-2.277807-0.998307$ C $-7.650212-3.196415-0.098804$ C $-8.579232-2.904671 \quad 1.046104$ C $-8.033002-3.3708192 .380107$ C $-8.573646-4.4240503 .016090$ C -6.905204-2.576885 3.012035 C $-5.579183-2.8481292 .351721$ N $-5.305994-1.516720-2.134036$ H $1.992175-4.239934-1.361331$ H $2.060884-1.8167730 .510706$ H $0.279502-2.649334-1.898240$ H $1.987764-0.962506-2.609406$ H $1.5410790 .059349-1.252330$ H $-0.989906 \quad 1.670750-3.486128$ H $-1.214607-0.346343-4.556452$ H $0.128418-1.481649-4.418948$ H $-1.268947-1.607774-3.323707$ H $1.4804472 .109119-1.779907$ H $0.4777173 .289702-2.565600$ H $-0.3473791 .710104-0.072789$ H $0.3707593 .325914-0.071459$ H $-1.6230914 .027325-1.589201$ H $-1.402694 \quad 4.6861570 .837971$ H -2.097239 3.1499861 .316463 H $-3.352242 \quad 5.4106792 .151117$ H -4.5907872 .6439520 .638766$ H -2.912875 6.5547970 .144078 H $-4.5623356 .247421-0.349277$ H -3.218975 $5.717464-1.380265$ H $-6.075065 \quad 4.3488130 .427280$ H $-4.9824280 .910200-0.839403$ H $-5.7960141 .840760-2.093302$ H $-4.0181553 .129148-3.338591$ H -2.428364 -0.6383920 .824015$ H -3.438718 -3.5348861 .101280$ H -3.827047 -0.1191852 .730283$ H -5.566196 -0.2023962 .874032$ H -4.8501870 .0698681 .287811$ H -3.991132 $1.064564-3.735587$ H $-3.3060650 .076936-2.445718$ H -5.790778 -3.514596 -1.982870 H $-6.850533-2.582312-3.070681$ H $-7.655425-1.264896-0.920335$ H -7.281639-4.215714 - -0.190955 H $-9.533747-3.403498 \quad 0.833929$ H -8.807279 -1.8334861 .110800$ H $-8.203973-4.7520723 .981881$ H $-9.395158-4.9854882 .583504$ H -6.808196 -2.839616 4.073095 H -7.181018 -1.518819 2.983366 H -5.394684 -3.905903 2.157320 H $-4.688122-1.448154-1.330932$ C $4.769568-4.021402-0.415156$ O $5.144134-2.629182-0.443214$ C $6.562676-2.521796-0.217473$

C $7.109680-3.943084-0.280023$

C $5.935575-4.7385320 .260770$ H $6.692279-2.1485510 .804832$ C $7.177703-1.537540-1.219160$ H $7.335370-4.247754-1.308669$ H $8.021044-4.0630090 .313715$

C $6.015003-6.223011-0.048838$

H $5.884052-4.5977901 .348896$

C $6.515580-0.142103-1.191645$

O $7.013141-2.080388-2.534892$

H $8.257332-1.457827-1.045549$

H $5.470449-0.221784-1.511222$

O $7.175347 \quad 0.688105-2.154574$

C 6.5995930 .5611230 .176290
C $6.016178 \quad 1.9860990 .195362$

H $6.090716-0.036806 \quad 0.937530$

$\begin{array}{lllll}\text { O } & 7.969878 & 0.645139 & 0.579340\end{array}$

H 6.2846982 .4434091 .155661

H $6.5218522 .604795-0.556393$

H $5.142740-6.7521880 .347192$

H $6.059259-6.406241-1.127796$

H $6.910160-6.6623820 .403328$

C $3.436114-4.162834 \quad 0.317251$

H $7.579682-1.567562-3.135418$

H $6.9421610 .353469-3.036969$

H $8.4451791 .150523-0.102438$

H $3.475857-3.6309501 .275974$

H $3.222541-5.2148910 .535613$

H $4.674453-4.349316-1.459248$

C 3.8038281 .5126602 .234427

$\begin{array}{lllll}\text { O } & 3.791440 & 1.138149 & 0.853255\end{array}$

C $4.4887252 .041701-0.012945$

C 3.8920243 .4530430 .103289

C 3.8273663 .9368631 .550909

C 3.1593232 .8879032 .440770

H 4.8446981 .5536172 .579362

C 3.1552310 .3913603 .058722

H $4.2943531 .696338-1.035430$

O $4.638762 \quad 4.387604-0.668656$

H $2.8728173 .449210-0.299994$

H $4.821257 \quad 4.198619 \quad 1.932403$

O $3.047783 \quad 5.1339301 .610988$

H 2.0874442 .8588052 .213472

$\begin{array}{llll}\text { O } & 3.294838 & 3.270758 & 3.806982\end{array}$

$\begin{array}{llll}\text { C } & 1.683003 & 0.171913 & 2.784735\end{array}$

H $3.657490-0.5589052 .840817$

H 3.2802930 .6138184 .124905

H $4.6463164 .067532-1.587050$

H 3.4616175 .7780751 .011017

H 2.9108434 .1596403 .898559

O $1.127093-0.484273 \quad 3.845898$

O 1.1009100 .4937091 .758886

C $-0.261479-0.806174 \quad 3.718249$

H -0.8412810 .0694363 .410278$

H $-0.382145-1.6231633 .001970$

H $-0.624472-1.1382074 .694505$

SCF Energy (B3LYP/6-31G**//MMFF) $=-3245.90951144$

01185

MM̄FF Geometry

C $-1.656371-3.386022-0.637192$

C $-0.797190-3.402128-1.667714$

C $-0.011680-2.181166-2.069542$

O $1.401985-2.498294-2.093646$

C $-0.367664-1.662282-3.475006$

C $-1.804452-1.222643-3.663320$

C $-2.329157-0.091359-3.151211$

C $-2.639186-2.120472-4.536847$

C $-1.6174860 .925469-2.305465$

C $-1.526576 \quad 2.287410-3.003496$

C $-0.534777 \quad 3.216596-2.286283$

C $-0.5513384 .611529-2.912898$

C $0.5235955 .525407-2.307513$

O $0.7665452 .635050-2.424079$

$\begin{array}{lllll} & 0 & 0.621614 & 6.677099 & -3.158780\end{array}$

C $1.880528 \quad 4.789205-2.343495$

C $0.104826 \quad 6.045138-0.927329$

O $2.886455 \quad 5.555074-1.683494$

C $1.8226343 .352128-1.774495$

C $3.1476652 .609292-2.083196$

O $1.6285293 .423688-0.363784$

C $2.062675-2.495283-0.904001$

O $1.579254-2.349933 \quad 0.207214$

C $3.502598-2.674379-1.192395$

C $4.357111-2.887754-0.181045$

C $5.809064-3.041787-0.295761$

C $6.437676-2.717141-1.625570$

C $3.185397 \quad 1.143715-1.622644$

C $3.7785010 .995846-0.234682$

C $5.936512 \quad 1.1741520 .998308$

$\begin{array}{lllll}\text { O } & 3.068558 & 0.823337 & 0.752834\end{array}$

$\begin{array}{llll}\text { C } 7.167670 & 0.337582 & 0.852642\end{array}$ 
C $7.603304-0.4992121 .806805$

C $8.845487-1.3348601 .683205$

C $8.602156-2.7935762 .003219$

C $8.988355-3.3162003 .179448$

C $7.982700-3.6628380 .930355$

C $6.501986-3.4358840 .793136$

N $5.1603641 .101207-0.226148$

H -1.788624 -2.462019-0.079369

H $-0.646094-4.315267-2.236391$

H $-0.160130-1.375769-1.343437$

H $-0.111552-2.428545-4.220464$

H $0.299615-0.825578-3.725818$

H $-3.3730310 .137650-3.359460$

H -3.695902 $-1.833107-4.540937$

H -2.275544 -2.082256 -5.568995

H -2.580075 -3.157405 -4.193811

H $-2.1655991 .035963-1.363285$

H $-0.6139910 .587463-2.040661$

H -1.194901 $2.139429-4.039796$

H -2.521801 $2.747436-3.036565$

H $-0.8003823 .265123-1.223648$

H -1.543965 $5.069418-2.818679$

H $-0.3640664 .526781-3.992809$

H $1.3511687 .227935-2.826107$

H $2.1868674 .734356-3.396989$

H $-0.8312066 .611691-1.002240$

H $0.8448366 .745857-0.525188$

H $-0.0480615 .247830-0.196994$

H $2.7580295 .448452-0.725527$

H $3.2869152 .604179-3.172217$

H $3.9897443 .157695-1.644982$

H 1.8239862 .5560530 .022641

H $3.821266-2.612191-2.226733$

H $3.959206-2.9599220 .830623$

H $\quad 6.136970-1.717423-1.959924$

H $6.131890-3.447143-2.382863$

H $7.528960-2.712943-1.604255$

H $2.1954570 .675958-1.638343$

H $3.8106020 .562744-2.312802$

H $\begin{array}{llll}5.323812 & 0.867937 & 1.852239\end{array}$

H $\quad 6.2168722 .2238621 .132826$

H $7.7359410 .433492-0.069480$

H $7.049846-0.5722982 .740661$

H $9.594058-0.9202992 .371076$

H $9.284430-1.2540690 .680992$

H $8.840749-4.3666723 .407364$

H 9.456135 -2.704031 3.943563

H $8.126823-4.7231891 .177189$

H $8.529748-3.513150-0.003863$

H $5.946445-3.6478681 .708217$

H $5.6316281 .346101-1.092520$

C -3.969453 -4.369333 -0.281910

O $-4.380875-3.1340010 .341522$

C $-5.296298-2.460055-0.544562$

C $-5.771627-3.520464-1.529776$

C $-4.504144-4.330791-1.713067$

H -4.714191 -1.711164 -1.094877

C $-6.413850-1.7759420 .245353$

H -6.564979-4.145134 -1.102865

H -6.143316 -3.085780 -2.462488

C $-4.735312-5.700534-2.325683$

H -3.825511-3.765185 -2.359022

C $-5.915631-0.7341851 .268916$

O $-7.142656-2.782430 \quad 0.959415$

H -7.125867 -1.315481 -0.450017

H $-5.366250-1.2454452 .066847$

O $-7.072018-0.1598671 .893071$

C $\begin{array}{llll}-5.053921 & 0.396900 & 0.672888\end{array}$

C $-4.747493 \quad 1.5421251 .658327$

H $-4.115816-0.0180910 .289499$

O $\quad \begin{array}{llll}-5.750224 & 0.957608 & -0.443976\end{array}$

H -4.2420492 .3405321 .101511$

H $-5.687410 \quad 1.995536 \quad 1.996857$

H -3.792295 -6.247788 -2.422540

H $-5.415023-6.304307-1.715196$

H $-5.174991-5.605661-3.323925$

C -2.454800 -4.560991-0.151228

H -7.843488 -2.331563 1.460722
H $-7.556957 \quad 0.336032 \quad 1.211115$

H $-5.1603601 .609559-0.859872$

H -2.149608 -5.482392 -0.660239

H $-2.210328-4.6896340 .910935$

H $-4.466020-5.1723090 .278283$

C $-1.7134121 .287344 \quad 1.859354$

O -2.6973550 .4429392 .464592$

C -3.8951941 .1142892 .868436$

C -3.5649512 .2518463 .847076$

C -2.4996803 .1970693 .295632$

C -1.2842142 .4063902 .812121$

H $-2.137145 \quad 1.7371130 .953435$

C $-0.553703 \quad 0.412402 \quad 1.377711$

H $-4.4844960 .379433 \quad 3.429510$

O -4.7315573 .0010494 .170568$

H $-3.189413 \quad 1.8219694 .784925$

H -2.8990553 .8312772 .495400$

O -2.0730894 .0819654 .333978$

H -0.7547061 .9981293 .681176$

O $-0.388948 \quad 3.2829612 .133967$

C $0.133798-0.3415042 .490846$

H $-0.922955-0.3237340 .655148$

H $\quad 0.200408 \quad 1.0041340 .848377$

H -5.3852252 .3805254 .536108$

H -2.8629894 .5469664 .659518$

H -0.1574073 .9969332 .752500$

O $-0.470868-1.5498522 .676582$

O $1.0852360 .094673 \quad 3.125425$

C $0.094125-2.3695213 .703700$

H $-0.466252-3.3077313 .736004$

H $1.140952-2.5937963 .477104$

H $0.005620-1.8733364 .674680$

SCF Energy $\left(B 3 L Y P / 6-31 G^{* *} / / M M F F\right)=-3245.91943413$

01 186

MM̄FF Geometry

C 4.8162610 .4587773 .449894

C $3.871579-0.4490893 .159518$

C $2.501993-0.4389263 .788631$

O $1.523472-0.1752972 .753947$

C $2.183951-1.8151984 .400323$

C $1.109906-1.7407595 .467061$

C $-0.188761-2.0519225 .292391$

C $1.596896-1.3019406 .826571$

C $-0.888348-2.5144124 .044636$

C $-1.856439-1.4423123 .543701$

C $-2.890595-1.9414922 .525543$

C $-2.275911-2.6052541 .292894$

C $-3.352420-2.9837580 .261578$

O $-3.640890-0.7866892 .129339$

O $-2.691503-3.310160-0.968233$

C $-4.235446-1.751776-0.018419$

C $-4.132324-4.2361190 .680966$

O $-5.332259-2.091322-0.864105$

C $-4.749645-1.0561021 .262078$

C -5.3941720 .3013950 .897191$

O $-5.724712-1.8847601 .895244$

C $1.375781 \quad 1.1181082 .353867$

O 1.9639612 .0958202 .788286

C $0.3753381 .143131 \quad 1.265542$

C 0.2666082 .2351390 .494496

C $-0.6696952 .410761-0.617560$

C $-1.7950081 .418780-0.732289$

C -5.8140201 .1367292 .113500$

C -5.9307152 .6027801 .751855$

C -4.5425164 .6209491 .283418$

O -7.0203473 .1375931 .561765$

C $-4.3187404 .710946-0.193213$

C $-3.3098785 .397388-0.751265$

C $-3.1311965 .539683-2.235188$

C $-1.7226885 .245135-2.695802$

C $-0.9323156 .222985-3.171191$

C $-1.2616293 .804624-2.698540$

C $-0.4784543 .444014-1.463981$

N -4.6955613 .2283441 .656706$

H $4.603780 \quad 1.2357784 .180198$

H $4.073683-1.2132902 .412800$

H 2.4314010 .3424444 .555942 
H $3.082402-2.2433774 .865480$ H $1.897981-2.5189933 .610455$ H $-0.849538-1.9645416 .155105$ H $\quad 0.794110-1.2825467 .571447$ H $2.367319-1.9888097 .192500$ H $2.023367-0.2954196 .778507$ H $-0.182022-2.7742823 .252032$ H -1.438831 -3.431078 4.288444 H -2.406998 -1.0046604 .388571$ H -1.291649 -0.6053763 .116589$ H -3.559168 -2.639702 3.043062 H -1.693052 $-3.492901 \quad 1.565347$ H -1.566908 -1.9125290 .819218$ H -2.128563 -4.086205 -0.807508 H -3.623475 -1.034007 -0.581644 H $-3.452316-5.088250 \quad 0.801100$ H $-4.848873-4.532365-0.093072$ H -4.672369 -4.111559 1.621638 H -4.964330 -2.509961 -1.661175 H -4.6836120 .8792530 .294467$ H -6.2818720 .1422940 .272097$ H -6.283929-1.334055 2.465585 H $-0.229003 \quad 0.257037 \quad 1.112687$ H $\quad 0.9396043 .0722090 .676056$ H $-2.306600 \quad 1.3092070 .230201$ H $-1.4143900 .438108-1.036686$ H -2.563603 $1.712385-1.449831$ H -6.7817540 .7982782 .500436$ H -5.0973761 .0499332 .938051$ H -3.699983 5.0218741 .855544 H -5.4478195 .1732191 .554661$ H -5.043155 $4.215938-0.836082$ H -2.604555 $5.922874-0.111533$ H $-3.4070636 .568133-2.502310$ H -3.826744 $4.891849-2.783449$ H $0.068166 \quad 6.011982-3.534452$ H -1.265039 $7.255182-3.204182$ H $-0.6029463 .641299-3.561967$ H -2.119905 $3.150219-2.870434$ H $0.3806014 .093999-1.289397$ H -3.8505552 .6757531 .770130$ C 6.2099171 .3323521 .510714 O $5.4387530 .669910 \quad 0.490873$ C $4.6755771 .656349-0.229519$ C 5.2861473 .0035310 .138160 C 5.6776882 .7640741 .586270 H 3.6543881 .6149150 .166576 C $4.6618841 .314582-1.723463$ H $6.1759713 .226240-0.461698$ H 4.5777753 .8282640 .014472 C 6.6844043 .7647972 .124791 H 4.7677472 .8149432 .195969 C $4.267841-0.146929-2.022222$ O $5.9733461 .519820-2.265163$ H $4.0021012 .005443-2.259876$ H $5.039393-0.825782-1.638743$ O $4.249603-0.321863-3.444991$ C $2.892797-0.563658-1.470201$ C $2.616925-2.061885-1.703671$ H $2.852735-0.385853-0.391084$ O $1.8809790 .240013-2.066960$ H $\quad 2.743617-2.292950-2.765150$ H $3.387568-2.626246-1.165434$ H 6.9439273 .5335193 .162725 H 7.6082703 .7617791 .536822 H 6.2717804 .7785702 .096309 C 6.1689620 .5038022 .798376 H $6.5900010 .960105-1.762374$ H $5.120983-0.050278-3.780982$ H $1.9078440 .078258-3.025436$ H $\quad \begin{array}{llll}6.886620 & 0.907018 & 3.522281\end{array}$ H $6.490610-0.5216102 .574725$ H $7.245073 \quad 1.3379551 .144621$ C $0.091541-2.488465-3.371388$ O $0.161822-1.966207-2.038475$ C $1.214136-2.504811-1.228872$ C $1.045665-4.026436-1.114361$ C $0.977361-4.689712-2.486421$
C $-0.079670-4.012700-3.357349$

H $1.004513-2.228723-3.917521$

C $-1.093341-1.813134-4.075590$

H $1.074128-2.088928-0.222699$

O $2.108345-4.606229-0.364855$

H $0.118651-4.250565-0.573119$

H $1.955033-4.687262-2.982650$

O $0.610398-6.062126-2.327395$

H -1.078983 -4.279420 -2.993278

O $0.028214-4.506198-4.691732$

C $-0.851082-0.344332-4.336279$

H - $1.318075-2.281889-5.040008$

H $-1.992477-1.888872-3.451885$

H $2.121804-4.1727830 .505716$

H $1.270634-6.471260-1.741868$

H $-0.061842-5.473775-4.651296$

O $0.249245-0.173012-5.122389$

O $-1.5818780 .545346-3.920549$

C $0.5698441 .182986-5.448784$

H $\quad 0.8190251 .739876-4.540939$

H $1.4430751 .176478-6.106440$

H $-0.2637371 .655906-5.976556$

SCF Energy (B3LYP/6-31G**//MMFF $)=-3245.92162700$

01187

MM̄FF Geometry

C $2.5801431 .966603-0.064784$

C 2.2326541 .2944851 .043581

C 0.9138861 .4807591 .746995

O $\quad 0.230323 \quad 0.2053271 .692164$

C 1.0959841 .8811423 .223471

C 1.6787783 .2597303 .437217

C 0.9477524 .3904183 .479924

C 3.1639933 .3108343 .687367

C -0.5379964 .5391873 .280020$

C -0.8726625 .7092792 .342579$

C -0.6193425 .3920100 .858303$

C $-0.424904 \quad 6.6923090 .074429$

C $-0.2678236 .436646-1.428204$

O -1.7631724 .6807650 .362740$

O $-0.3818427 .697627-2.104867$

C -1.444698 $5.563233-1.896281$

C $1.1154025 .878376-1.779307$

O $-1.2815485 .183125-3.261910$

C -1.686556 $4.307452-1.020005$

C $-3.0374103 .676122-1.435470$

O $-0.6367013 .369070-1.251467$

C -1.1225190 .2251301 .834944$

O $-1.827341 \quad 1.2058332 .013272$

C $-1.611606-1.1683901 .749941$

C $-2.891156-1.4221202 .062123$

C $-3.550477-2.7271212 .044801$

C $-2.770097-3.9055421 .530072$

C $-3.5331562 .518279-0.559278$

C $-4.8471441 .964354-1.091335$

C $-6.4009750 .061442-0.900744$

O $-5.4770992 .499472-2.001540$

C $-6.870161-0.8912390 .149686$

C -7.003299-2.209547 -0.062522

C -7.542430 -3.180357 0.950482

C $-6.538636-4.2496251 .319011$

C $-6.447264-5.3856330 .607206$

C $-5.693554-4.0352562 .553601$

C $-4.828063-2.8085802 .469218$

N $-5.2511730 .811193-0.428703$

H $1.8576972 .661564-0.485881$

H 2.9203330 .5836411 .492127

H $\quad 0.3096782 .2296291 .220386$

H 1.7144921 .1317683 .737405

H $\quad 0.133767 \quad 1.8118953 .746987$

H 1.4694225 .3223783 .695654

H 3.5152634 .3266543 .898090

H 3.4246272 .6890184 .550175

H 3.7192132 .9481992 .818028

H -1.0043953 .6231392 .908863$

H -0.9805004 .7492764 .261470$

H -1.931902 5.9620442 .480655

H -0.2911526 .5875352 .650471$ 
H $\quad 0.268597 \quad 4.758404 \quad 0.760482$ H $\quad 0.438713 \quad 7.2511290 .456469$ H $-1.295977 \quad 7.3450540 .228109$ H $0.3135138 .285236-1.762598$ H -2.347315 6.189305-1.856817 H $1.9010696 .576319-1.466042$ H $1.2313415 .763334-2.862765$ H $1.3214124 .916664-1.306899$ H -1.151221 $5.998591-3.775889$ H -3.811913 $4.454850-1.414761$ H $-2.9665393 .317912-2.471079$ H $-0.9499382 .479295-1.026312$ H $-0.911886-1.9386771 .446809$ H $-3.523533-0.5912122 .373788$ H -1.921215 -4.118907 2.188356 H -2.395346 -3.7051330 .520252$ H -3.361961 -4.821246 1.461216 H $-2.801146 \quad 1.706247-0.542324$ H -3.7000332 .8605310 .467814$ H -6.102376 -0.448680 -1.823334 H $-7.2039800 .763400-1.150424$ H -7.150027 -0.4729251 .113373$ H $-6.742856-2.617245-1.037045$ H $-8.440047-3.6476030 .524374$ H -7.885957 -2.665530 1.856335 H $-5.747647-6.1692120 .878405$ H -7.069054 -5.554855 -0.265701 H $-5.087576-4.9199812 .780993$ H $-6.359259-3.9255583 .419861$ H -5.323089 -1.899907 2.813391 H $-4.580541 \quad 0.3399240 .171981$ C $5.0303031 .132798-0.162684$ O $4.746182-0.277282-0.185480$ C $5.970185-0.999243-0.420112$ C $7.0903160 .035609-0.416589$ C $6.3634191 .277663-0.895444$ H $5.891778-1.425745-1.427000$ C $6.122958-2.1238840 .610686$ H 7.4967080 .1964980 .588377 H $7.921243-0.253776-1.067379$

C $7.1072312 .566204-0.589231$ H $6.2072001 .197792-1.979639$ C $4.889047-3.0436240 .714844$ O $\quad 6.326532-1.5341411 .901744$ H $7.025612-2.7061070 .391355$ H $4.058817-2.475748 \quad 1.148590$ O $5.192819-4.0769231 .660705$ C $4.454486-3.689194-0.612441$ C $3.286254-4.681549-0.469166$ H $4.181292-2.911817-1.332996$ O $5.572520-4.393085-1.159648$ H $3.133779-5.165951-1.441316$ H $3.578197-5.498074 \quad 0.203235$ H $6.5496333 .436180-0.949396$ H 7.2674512 .6909350 .486982 H $8.0872862 .569431-1.077603$ C $3.8826401 .902141-0.820627$ H $6.416058-2.2615112 .541324$ H $5.900581-4.6240251 .278741$ H $5.303793-4.742869-2.026362$ H $3.665674 \quad 1.466282-1.804110$ H $4.2003572 .938168-0.988010$ H $5.142467 \quad 1.4300560 .888345$

C $1.199174-3.160723-2.088047$

O $1.618416-2.878221-0.749320$

C $1.972015-4.0360960 .016977$ C $0.786077-5.008374 \quad 0.088150$ C $0.229578-5.348621-1.293080$ C $-0.033496-4.071679-2.093965$ H $2.018563-3.662550-2.617576$ C $0.995456-1.833215-2.827381$ H $2.149755-3.6826871 .039525$ O $1.153010-6.211366 \quad 0.755944$ H -0.014366 -4.547639 0.678074 H $0.900482-6.028440-1.831436$ O $-1.001372-6.053211-1.113201$ H $-0.899488-3.556287-1.661827$ O $-0.354327-4.408056-3.440592$
C $-0.157139-1.015487-2.295169$

H $1.892717-1.208163-2.735081$

H $\quad 0.848010-2.001500-3.900709$

H $1.478750-5.9676401 .639260$

H -1.296366 -6.357225 -1.988037

H -1.198125 -4.890039 -3.431046

O $-1.277634-1.251796-3.035843$

O $-0.074971-0.267860-1.330026$

C $-2.453640-0.555536-2.613955$

H -2.677935 -0.782490 -1.567314

H $-2.3217090 .520352-2.759689$

H -3.290267 -0.893659 -3.231056

SCF Energy (B3LYP/6-31G**//MMFF) $=-3245.90797904$

01188

MM̄FF Geometry

C $0.3260073 .628180-1.062414$

C $1.2707634 .044076-0.204842$

C 1.7641753 .2047110 .942649

O 3.1888173 .0286950 .761172

C 1.5202483 .8759052 .308408

C 0.0644814 .0936802 .659252

C -0.7906583 .1066262 .991242$

C -0.3903165 .5288332 .654223$

C -0.4789991 .6389063 .091650$

C -1.2951120 .8192592 .084489$

C $-0.834524-0.6408011 .931603$

C $-0.697553-1.3805303 .264446$

C $-0.176013-2.8145463 .078495$

O $0.415434-0.6407781 .230413$

O $0.235989-3.3020524 .365400$

C $1.084769-2.7724512 .186138$

C $-1.279445-3.7619362 .593434$

O $1.535825-4.0917911 .885298$

C $0.898791-1.9485840 .890530$

C $2.252134-1.7941450 .153587$

O $-0.009655-2.6512680 .039226$

C 3.7435131 .9176961 .317986

O 3.1753891 .0640491 .980646

C 5.1786161 .9008420 .964468

C 5.8478050 .7411961 .043370

C 7.2584790 .5291450 .722335

C $8.097918 \quad 1.7390470 .407829$

C $2.200814-0.830707-1.040948$

C $3.527136-0.724616-1.771659$

C $5.099936-2.034311-3.199951$

O $4.1407830 .338021-1.841848$

C $6.252101-2.520344-2.377154$

C $7.484205-1.995851-2.465430$

C $8.678610-2.515965-1.715639$

C $9.441486-1.423026-1.000683$

C $10.409893-0.736251-1.630866$

C $9.146932-1.1823630 .461348$

C $7.737000-0.7323450 .722296$

N $3.917551-1.911498-2.368552$

H $-0.0950272 .631921-0.948075$

H $1.6975555 .037770-0.316312$

H 1.2817362 .2213090 .902555

H 2.0607474 .8325342 .342776

H 1.9944823 .2859983 .103704

H -1.8173553 .3721953 .238365$

H -1.465091 5.6261012 .840346

H 0.1361586 .0927343 .431170

H -0.1790055 .9964541 .687811$

H $\quad 0.588210 \quad 1.4428332 .961907$

H $-0.7275891 .319646 \quad 4.110977$

H -2.348549 0.8329222 .387372

H -1.240111 1.3012791 .099373

H -1.579283 -1.139523 1.302782

H $-1.650269-1.3811393 .808063$

H $0.018381-0.8569743 .911356$

H -0.530947 -3.2590694 .961984$

H $1.884613-2.3086012 .779632$

H -2.111841 -3.775709 3.307352

H $-0.918303-4.7947482 .538691$

H $-1.686817-3.4851301 .619483$

H $0.945787-4.4579291 .204736$

H $3.010207-1.4185840 .851620$ 
H $2.592143-2.779939-0.184757$

H $0.147384-2.396182-0.882416$

H 5.6320142 .8258000 .628530

H $5.306588-0.1499251 .359936$

H $7.7216962 .245665-0.487668$

H 9.1464181 .4999250 .215982

H 8.0831252 .4461891 .244663

H $\quad 1.448047-1.135294-1.775667$

H $1.9259590 .175211-0.703606$

H $5.310263-1.071696-3.677612$

H $4.868043-2.768650-3.977847$

H $6.077592-3.364832-1.714879$

H $7.658085-1.174995-3.158194$

H $9.341488-3.005588-2.441522$

H $8.395124-3.299261-1.001699$

H $10.9850440 .029405-1.121195$

H $10.646453-0.923578-2.673166$

H $9.856002-0.4695860 .899434$

H $9.319645-2.1163741 .012363$

H $7.052757-1.5520520 .944335$

H $3.337075-2.737288-2.259222$

C $-1.7232514 .716481-2.098433$

O $-2.4518143 .477836-2.216879$

C -3.533769 $3.490532-1.264745$

C $-3.6649274 .935633-0.795590$

C $-2.2163695 .381064-0.813351$

H -3.212060 $2.873840-0.416580$

C $-4.8055152 .900092-1.881306$

H -4.255426 $5.540554-1.493312$

H -4.1268865 .0107660 .193461$

C $-2.0341716 .887551-0.786658$

H -1.722995 4.9464560 .062652

C $-4.6317801 .497911-2.496686$

O $-5.2720143 .766442-2.924323$

H -5.602921 $2.882182-1.129324$

H $-4.0361751 .560137-3.415692$

O $\quad-5.929777 \quad 1.033042-2.890965$

C $-4.0078190 .450902-1.555877$

C $-3.975823-0.952139-2.194796$

H $-2.9821180 .749266-1.313355$

O $-4.7361230 .415489-0.332145$

H -4.999389 -1.324801-2.305434

H -3.550026 -0.861564 -3.200960

H $-0.9721387 .151026-0.815231$

H -2.524509 7.366825 -1.640408

H $-2.462707 \quad 7.3103720 .127883$

C $-0.2147534 .460823-2.190817$

H $-4.5528883 .858062-3.573166$

H $-6.3063291 .700110-3.490443$

H $-5.6576390 .187467-0.543721$

H $0.3118235 .420870-2.246663$

H $-0.0024043 .921833-3.122378$

H -2.018240 5.319706-2.967296

C $-4.882994-2.912978-0.000537$

O $-3.653113-2.182611-0.063842$

C $-3.138282-1.963288-1.379909$

C -2.912925 -3.309782 -2.081398

C $-4.177620-4.162994-2.086923$

C $-4.737592-4.285098-0.670482$

H -5.677497 -2.336805 -0.489294

C $-5.248974-3.0712181 .479185$

H -2.151528 -1.502172 -1.249998

O $-2.453166-3.102325-3.412432$

H -2.122040 -3.857469 -1.552528

H -4.922346 -3.759011-2.782664

O $-3.839580-5.465039-2.569646$

H -4.070405 -4.924702 -0.079013

O $-6.002742-4.941403-0.722517$

C $-5.641368-1.7438502 .082442$

H $-6.098088-3.7492721 .620696$

H $-4.407503-3.4884702 .044800$

H -2.348771 -3.977607 -3.823684

H $-4.644529-6.008914-2.522658$

H -6.609198 -4.381096 -1.236159

O $-4.669938-1.3098592 .934829$

O $-6.682176-1.1568891 .814870$

C $-4.930116-0.0565113 .574060$

H $-5.850370-0.1139004 .163408$
H $-4.096531 \quad 0.161204 \quad 4.247143$

H -4.9976450 .7410762 .828025$

SCF Energy (B3LYP/6-31G**//MMFF) $=-3245.91988040$

01_189

MM̄̄F Geometry

C $4.8887321 .701202-0.784597$

C $5.6513060 .652035-0.438911$

C $5.154402-0.772797-0.318357$

O $3.728944-0.800013-0.075225$

C $5.440840-1.533162-1.625502$

C $4.946594-2.966437-1.624158$

C $4.003035-3.445787-2.459039$

C $5.594357-3.881727-0.617932$

C $3.272633-2.705289-3.545405$

C $1.974581-2.018718-3.093514$

C $0.855722-2.994065-2.694102$

C $-0.447833-2.238049-2.429943$

C $-1.570198-3.182956-1.974574$

O $1.246927-3.681102-1.505054$

O $-2.630664-2.379712-1.434450$

C $-1.054370-4.072500-0.819859$

C $-2.172580-3.948589-3.158355$

O $-1.995077-5.093434-0.499655$

C $0.331978-4.701522-1.080477$

C $0.947762-5.3662010 .172171$

O $0.254584-5.756689-2.047421$

C $3.311223-0.5202661 .189947$

O $4.010285-0.3112192 .168454$

C $1.834844-0.4579171 .165331$

C 1.1890730 .1726602 .156979

C -0.2598160 .3466332 .266488$

C $-1.123287-0.3976871 .284384$

C $1.174493-4.4380541 .371014$

C $0.070248-4.4917982 .415180$

C $-0.596991-3.4507934 .581429$

O $-0.854376-5.2988842 .380932$

C -1.698924 -2.474434 4.316505

C $-1.963529-1.4261185 .111478$

C $-3.105936-0.4767074 .890956$

C -2.6680500 .9693514 .838862$

C -2.7920551 .7694745 .911222$

C -2.1760151 .5092553 .516051$

C $-0.743176 \quad 1.1457663 .240049$

N $0.263734-3.5490883 .417047$

H $3.8447811 .539811-1.042163$

H $6.7022090 .812326-0.210113$

H $5.669570-1.2579050 .519904$

H $6.521253-1.539634-1.821684$

H $4.989891-0.977026-2.454513$

H $3.723421-4.494740-2.367898$

H $5.291716-4.926020-0.751199$

H $6.684223-3.842682-0.715268$

H $5.322521-3.5897070 .401048$

H $3.929497-1.958471-4.004659$

H $3.046786-3.415304-4.351380$

H $2.196401-1.345329-2.257323$

H $1.620621-1.394548-3.923690$

H $\quad 0.723250-3.716886-3.507647$

H $-0.759413-1.672823-3.317234$

H $-0.281612-1.490486-1.642820$

H -2.909684 -1.747215 -2.118447

H $-0.984073-3.4276520 .062825$

H -2.614982 $-3.250925-3.879823$

H $-2.990875-4.600656-2.834156$

H - $1.438256-4.552110-3.696140$

H -2.841869-4.656411-0.304959

H $\quad 0.367133-6.253586 \quad 0.452154$

H $1.934803-5.751499-0.123285$

H $-0.676708-5.900055-2.278989$

H $1.325169-0.9040370 .319391$

H 1.7759090 .6270232 .954456

H $-0.892398-1.4670531 .309619$

H $-0.953356-0.0288390 .268877$

H $-2.192330-0.3143601 .487212$

H $2.092051-4.7555501 .883097$

H $1.322211-3.4033551 .048898$

H $\quad 0.031600-3.1472485 .424568$ 
H $-1.022938-4.4350444 .801947$ H -2.329834 -2.658675 3.450097 H -1.349151-1.260742 5.993681 H -3.820114 -0.620399 5.712483 H -3.657885 -0.7217663 .975865$ H -2.509036 2.8160485 .871334 H $-3.174321 \quad 1.393788 \quad 6.854713$ H -2.2386082 .6058573 .510580$ H $-2.857146 \quad 1.1921182 .722792$ H -0.0339141 .6352393 .908948$ H $1.026729-2.8834043 .333616$ C 4.5356304 .0782810 .007701 O $3.1718264 .101527-0.460125$ C 2.2948704 .1671930 .682678 C 3.1841734 .4914621 .878981 C 4.4637013 .7676601 .502513 H 1.8820633 .1616090 .822704 C 1.1634785 .1690450 .427516 H 3.3683925 .5682731 .969501 H 2.7533714 .1417562 .822171 C 5.6804614 .2235592 .287354 H 4.3118742 .6947351 .672719 C $0.3337804 .859330-0.837242$ $\begin{array}{lllll}\text { O } & 1.748153 & 6.467435 & 0.267697\end{array}$ H 0.5142365 .2245241 .309400 H $\quad 0.961774 \quad 4.949007-1.731978$ O $-0.6880395 .857586-0.955595$ C $-0.3498313 .477340-0.821973$ C -1.202081 $3.243038-2.087710$ H $0.4117012 .692119-0.763661$ $\begin{array}{llll}\text { O } & -1.156187 & 3.389246 & 0.350239\end{array}$ H $-2.0025403 .988466-2.126848$ H $-0.5631953 .408070-2.963188$ H 6.5754243 .6811891 .966378 H 5.8677285 .2941082 .152824 H 5.5388874 .0392463 .357201 C $5.3715653 .121317-0.848224$ H 1.0298887 .1198730 .324307 H $-0.2519786 .696381-1.181552$ H -1.5123472 .4862680 .396877$ H $6.4246373 .188988-0.551754$ H $5.3075393 .438479-1.896421$ H $4.9232555 .094621-0.143446$ C $-3.9569692 .260686-1.140468$ O $-2.7118801 .559731-1.078128$ C $-1.8112011 .822357-2.158403$ C $-2.4865681 .482240-3.495262$ C $-3.8207342 .208757-3.659450$ C $-4.7116151 .963271-2.440540$ H $-3.7604873 .338809-1.088758$ C -4.7600051 .9385520 .129134$ H $-0.9740061 .122969-2.037221$ O $-1.6125041 .796762-4.573363$ H $-2.667510 \quad 0.399814-3.533158$ H $-3.6771973 .282157-3.830629$ O $-4.500503 \quad 1.701355-4.809574$ H $\quad-5.0673590 .927479-2.462532$ O $-5.8598292 .805357-2.517824$ C $-5.0233890 .465097 \quad 0.363840$ H -4.1845932 .2933810 .992830$ H -5.7217012 .4627160 .112005$ H -2.012389 $1.450696-5.388789$ H $-3.9885851 .965131-5.592735$ H $-6.2998462 .619097-3.365077$ O $-5.6603550 .319321 \quad 1.564553$ O $-4.712538-0.444359-0.391328$ C $-5.963294-1.0285091 .938605$ H $-6.395623-1.0135472 .942575$ H $-5.052236-1.6345281 .959234$ H $-6.694902-1.4544541 .245672$

SCF Energy (B3LYP/6-31G**//MMFF) $=-3245.90656940$

\section{19}

MM̄FF Geometry

C $0.549626-2.5450070 .601144$

C $1.486592-3.073654-0.200427$

C $1.751416-2.690949-1.637969$

O $0.841692-1.652990-2.076190$
C $3.174624-2.129377-1.819279$

C $4.259355-3.185287-1.819496$

C $5.106132-3.424161-0.799404$

C $4.388252-3.970044-3.100748$

C $5.148912-2.7621760 .549840$

C $6.448132-1.9739230 .767133$

C $6.523971-0.686859-0.068658$

C $7.890968-0.0196920 .095049$

C $7.9615551 .323782-0.645125$

O $5.4936490 .192420 \quad 0.392933$

O $9.1344282 .016744-0.191907$

C $6.7449082 .179527-0.237877$

C $8.125027 \quad 1.136943-2.158093$

O $6.6878143 .379920-1.005890$

C $5.3959971 .426845-0.328876$

C 4.2853082 .2752810 .334717

O $5.0690011 .210621-1.701805$

C $-0.273754-2.020603-2.758554$

O $-0.605400-3.158496-3.060926$

C $-1.078406-0.840674-3.154315$

C $-0.9319860 .371205-2.596997$

C $-1.6687461 .586172-2.952916$

C $-2.7133651 .493126-4.032611$

C 2.9200891 .5808680 .415404

C 1.9771052 .3256481 .340202

C 0.6552714 .4319531 .530647

O 1.5873801 .8306692 .395684

C -0.7101654 .0591181 .052510$

C -1.5329634 .8727080 .373566$

C $-2.8687354 .415685-0.141750$

C $-3.0241054 .533376-1.644764$

C $-4.1663685 .017982-2.162261$

C $-1.8952734 .116301-2.564438$

C -1.364635 $2.731323-2.307976$

N $1.619323 \quad 3.5764280 .860408$

H $-0.116451-1.7747320 .222614$

H $2.121021-3.8611020 .201304$

H $1.621990-3.579831-2.266119$

H $3.365535-1.352391-1.069799$

H $3.228016-1.585827-2.773305$

H $5.856904-4.202605-0.932050$

H $5.224363-4.677069-3.074007$

H $3.478563-4.546026-3.295079$

H $4.558991-3.293207-3.944410$

H $4.281162-2.1220130 .731341$

H $5.095637-3.5565911 .304912$

H $6.501839-1.7123811 .831969$

H $7.310451-2.6157020 .547192$

H $6.348118-0.930299-1.122088$

H $8.693071-0.687008-0.243994$

H $8.0833020 .162051 \quad 1.161818$

H $9.905598 \quad 1.453811-0.377778$

H 6.8976272 .4875560 .806171

H $9.0418880 .576641-2.377590$

H $8.2356972 .101637-2.665496$

H $7.2927760 .599172-2.616793$

H $7.5467543 .825855-0.910394$

H 4.5941602 .5301451 .357310

H $4.1669533 .218834-0.212191$

H $4.1176551 .037483-1.775807$

H -1.818013 -1.032063 -3.924755

H $-0.1915640 .498643-1.808533$

H $-3.4434140 .710261-3.799650$

H $-2.2488871 .262555-4.997380$

H $-3.2857592 .416105-4.156475$

H $2.4496121 .507506-0.571201$

H 3.0205270 .5605870 .802336

H 0.9115925 .4686251 .292142

H 0.7256334 .2880392 .613360

H -1.030206 3.0402491 .256716

H -1.232285 5.8956580 .163606

H -3.640065 5.0041500 .371558

H -3.046832 3.3720050 .137995

H -4.306778 $5.116587-3.233610$

H $-4.9909435 .324364-1.526929$

H - $1.0641284 .823611-2.443054$

H -2.186572 4.220054 -3.616537

H $-0.5939742 .696078-1.538375$ 
H $1.9082213 .843551-0.076200$ C $0.309306-1.7574122 .966829$ O $-0.863090-0.9653122 .683945$ C -1.576394 -0.7322813 .909685$ C $-0.567191-0.986798 \quad 5.019775$ C $0.209794-2.1533244 .441786$ H $-2.366289-1.4913123 .965621$ C $-2.2020380 .664822 \quad 3.905344$ H $0.097779-0.1285805 .170483$ H $-1.046961-1.2195375 .975018$ C $1.556428-2.3786475 .107722$ H $-0.395699-3.0638374 .546889$ C -3.076046 0.9666652 .670091 O -1.1629601 .6514253 .944939$ H -2.7843610 .8105264 .822343$ H -2.441081 1.0563411 .781891 O -3.6764052 .2529742 .869846$ C -4.196754 -0.056573 2.405256 C $-5.204481 \quad 0.366822 \quad 1.320442$ H -3.758937 -1.020303 2.130877 O $-4.934049-0.2725833 .612643$ H $-6.012534-0.3744061 .307704$ H -5.6901461 .3002801 .630242$ H $2.085028-3.2171774 .643395$ H $2.195773-1.4928545 .029802$ H $1.426563-2.6069776 .170542$ C $0.338829-2.9660302 .028910$ H $-0.572015 \quad 1.4867513 .189823$ H -2.956548 2.8870243 .030876 H -5.2982010 .5851093 .891755$ H $-0.619467-3.4963072 .081705$ H $1.123206-3.6640982 .343028$ H $1.179952-1.1121192 .793399$ C $-4.930135-1.728037-0.901331$ O $-4.004446-0.685495-0.571866$ C $-4.596870 \quad 0.524499-0.087688$ C $-5.5760961 .088305-1.126365$ C $-6.6210740 .055589-1.531074$ C $-5.945297-1.247649-1.949157$ H $-5.449846-2.0594560 .005760$ C $-4.124595-2.915709-1.453376$ H $-3.7790231 .245972-0.008036$ O $-6.2344632 .251288-0.635213$ H $-5.0224291 .391524-2.023049$ H -7.349177 -0.117888 -0.730046 O $-7.3632900 .551508-2.647865$ H -5.446382 -1.103427 -2.916456 O $-6.942832-2.251509-2.129877$ C $-3.312137-3.627640-0.393021$ H -4.806168 $-3.657002-1.886248$ H $-3.428723-2.572685-2.225971$ H $-5.5474682 .890462-0.380151$ H -7.752558 $1.401965-2.380772$ H -7.579791 -1.913305 -2.782600 O $-2.811356-4.780602-0.928951$ O $-3.129730-3.227927 \quad 0.747689$ C -2.035368 $-5.599458-0.048242$ H $-2.524494-5.7093130 .924588$ H -1.940942 -6.589500 -0.502339 H - $1.037331-5.1705880 .067223$

SCF Energy (B3LYP/6-31G**//MMFF) $=-3245.93172298$

01_190

MM̄FF Geometry

C $1.728192-2.358541-2.254288$

C $0.778732-2.386238-3.201731$

C $-0.304044-1.357431-3.422982$

O $-1.478868-1.847628-2.731234$

C $0.010997 \quad 0.086389-2.967815$

C $1.165160 \quad 0.739070-3.702366$

C $2.2527331 .276150-3.116585$

C $1.019340 \quad 0.823571-5.201623$

C $2.5794751 .367813-1.653424$

C $2.7202132 .820889-1.177801$

C $1.4177713 .629046-1.285822$

C $1.6514095 .084742-0.878342$

C $0.3496765 .898461-0.915991$

O $0.4431823 .031484-0.423930$
O $0.5854337 .139823-0.234532$

C $-0.731277 \quad 5.141870-0.118333$

C $-0.061067 \quad 6.260396-2.348121$

O $-1.9994745 .781696-0.246181$

C $-0.8523193 .643445-0.492374$

C -1.7778372 .9375820 .525119$

O $-1.420716 \quad 3.527846-1.797814$

C $-2.691177-1.394310-3.149511$

O $-2.911006-0.575627-4.027885$

C $-3.726649-2.083015-2.350043$

C $-4.970292-1.582897-2.311123$

C $-6.111957-2.163489-1.603478$

C $-5.929503-3.514953-0.963796$

C -1.8167051 .4098670 .407880$

C $-2.6627640 .846981 \quad 1.530981$

C $-5.0087450 .513116 \quad 2.241328$

$\begin{array}{lllll}\text { O } & -2.166790 & 0.524201 & 2.608023\end{array}$

C $-6.205469-0.1241601 .612391$

$\begin{array}{llll}C & -7.382819 & 0.502161 & 1.457017\end{array}$

C $-8.669647-0.050218 \quad 0.898886$

$\begin{array}{llll}C & -8.678033 & -1.518150 & 0.534938\end{array}$

C $-8.872153-2.4583981 .475749$

C $-8.566049-1.892481-0.925395$

C $-7.268088-1.469173-1.556917$

$\begin{array}{llll}\mathrm{N} & -4.014084 & 0.794484 & 1.223747\end{array}$

H $1.736210-1.556341-1.523487$

H $0.769403-3.222108-3.898916$

H $-0.524233-1.357998-4.498576$

H $0.1366880 .100545-1.881252$

H $-0.8729890 .716174-3.131107$

H $3.0097721 .720740-3.762429$

H $1.7785731 .468099-5.657987$

H $1.114422-0.166987-5.656627$

H $0.0412401 .239626-5.465183$

H $1.8585200 .845501-1.020719$

H $3.5376180 .859614-1.504154$

H $3.0470722 .797228-0.130267$

H $3.5127443 .317190-1.751950$

H $1.0592573 .587611-2.319956$

H $2.4128875 .551386-1.515541$

H 2.0531705 .1182260 .144149

H $1.3157267 .595783-0.686997$

H $-0.457049 \quad 5.2095170 .943959$

H $0.7282486 .844519-2.836520$

H $-0.9519226 .898285-2.353886$

H $-0.2589685 .386517-2.972152$

H $-1.881873 \quad 6.7118840 .012413$

H $-1.4401893 .179761 \quad 1.541999$

H -2.7999543 .3249610 .426533$

H $-1.8348602 .655285-1.886288$

H $-3.438159-2.985371-1.824211$

H $-5.176227-0.658267-2.849324$

H $-5.164070-3.469179-0.181824$

H $-5.627016-4.257404-1.710527$

H $-6.837000-3.896403-0.489979$

H $-2.2260121 .085663-0.554495$

$\begin{array}{llll}H & -0.813877 & 0.977322 & 0.494947\end{array}$

H $-5.253152 \quad 1.4638292 .727446$

H $-4.587815-0.1510693 .003510$

H $-6.089271-1.1620091 .316651$

H $-7.450023 \quad 1.5409051 .778059$

H -8.957056 0.5483020 .025503

H $-9.4565410 .130604 \quad 1.643884$

H $-8.915746-3.5125741 .223119$

H -8.988583 -2.1924862 .521300$

H $-9.387313-1.411982-1.473545$

H -8.728142 -2.966612 -1.075397

H -7.309656 -0.488341 -2.031253

H $-4.329983 \quad 1.243766 \quad 0.368633$

C $4.194865-2.748428-2.138036$

O $4.379246-1.960989-0.944657$

C $5.701175-2.195565-0.423729$

C $6.457643-2.932341-1.521062$

C $5.345008-3.754825-2.144985$

H $5.585559-2.8702500 .432860$

C $6.325924-0.8731280 .038878$

H $6.878635-2.241855-2.260800$

H $7.274600-3.542859-1.124675$ 
C $5.687917-4.292422-3.523447$

H $5.112143-4.595843-1.478108$

C $5.426362-0.0616000 .995065$

O $6.587294-0.049111-1.104752$

H $7.300528-1.0620930 .502557$

H $4.537703 \quad 0.278306 \quad 0.456904$

O $\quad 6.134261 \quad 1.1196051 .388187$

C $5.008454-0.8187642 .265181$

C $4.098378-0.0112343 .208593$

H $4.495002-1.7460341 .997146$

O $6.177345-1.2030242 .993246$

H $3.980730-0.5928724 .131352$

H 4.6079170 .9084003 .522775

H $4.852887-4.865698-3.938188$

H $5.921051-3.482780-4.223312$

H $6.559090-4.953669-3.472313$

C $2.808347-3.395903-2.109489$

H $5.7488500 .062806-1.584790$

H 6.3953481 .5844110 .574593

H $6.664323-0.3907623 .215123$

H $2.654676-3.907128-1.151478$

H $2.726787-4.145493-2.904486$

H $4.280383-2.061028-2.990217$

C $1.670373-1.8166182 .926285$

O $2.087457-0.8052842 .003939$

C 2.7152120 .3293232 .611098

C $1.746520 \quad 0.9888363 .603132$

C $1.223595-0.0083174 .636542$

C $0.675438-1.2602313 .951144$

H $2.552534-2.2006503 .453485$

C $1.122782-3.0029672 .124340$

H 2.8867111 .0520951 .804833

O 2.3727022 .0750834 .277373

H $0.8889491 .400173 \quad 3.054517$

H $\quad 1.995070-0.2697805 .370107$

O $\quad 0.159797 \quad 0.5964195 .376107$

H $-0.275437-1.0125543 .469268$

O $0.404970-2.2594654 .931158$

C -0.176865 -2.694559 1.415218

H $1.838760-3.3085031 .351829$

H $\quad 0.981062-3.8683442 .782634$

H 2.6799772 .7010103 .599613

H 0.5101831 .4137485 .770004

H -0.208155 -1.871333 5.578905

O $-1.182618-3.4474381 .945863$

O $-0.287941-1.8807300 .508008$

C $-2.472648-3.2611801 .356890$

H $-2.748558-2.2031011 .347634$

H -2.476302 -3.671007 0.343424

H $-3.203998-3.8063631 .959376$

SCF Energy (B3LYP/6-31G**//MMFF) $=-3245.91369381$

01

MM̄FF Geometry

C $0.782020 \quad 5.6222800 .246734$

C $1.3545474 .756776-0.602934$

C $2.5496943 .907667-0.253666$

O $2.1746632 .515239-0.373275$

C $3.6851494 .188365-1.255132$

C $4.9810643 .468468-0.938900$

C $5.5589412 .547519-1.734932$

C $5.6547293 .867537 \quad 0.349307$

C $5.0772692 .031295-3.062002$

C $4.193996 \quad 0.775829-2.980638$

C $4.893979-0.448360-2.367776$

C $4.030345-1.701434-2.520467$

C $4.660980-2.922793-1.831413$

O $5.111005-0.193586-0.979035$

O $3.653413-3.942822-1.741043$

C $5.056786-2.547769-0.384651$

C $5.803268-3.516322-2.665530$

O $5.792223-3.6166950 .205756$

C $5.838446-1.218846-0.289504$

C $6.046789-0.7164781 .160135$

O $7.140081-1.399381-0.860527$

C 1.4506001 .9748770 .645398

O $1.0883452 .533231 \quad 1.668925$

C 1.1550400 .5690040 .290886
C $0.229903-0.1081670 .987629$

C $-0.167400-1.5019160 .772157$

C $0.626948-2.302581-0.225379$

C $4.763489-0.4736431 .962029$

C $4.359749-1.6430912 .843534$

C $2.525665-2.3934264 .351423$

O $5.075848-2.6220993 .035183$

C $1.672908-3.3654863 .599543$

C $0.407222-3.6486753 .941882$

C $-0.447811-4.6562713 .229653$

C $-1.765384-4.0762102 .769041$

C $-2.885092-4.2423603 .493043$

C $-1.797274-3.3774451 .428798$

C -1.204408 -1.995289 1.481112

N $3.114082-1.4461213 .423960$

H 1.1920295 .7448981 .245729

H $0.9312974 .628286-1.596853$

H 2.8891964 .1073690 .769861

H $3.8980435 .265710-1.281696$

H $3.3365773 .924460-2.259608$

H $6.4985982 .105978-1.403972$

H 6.6569963 .4359920 .444860

H 5.7629444 .9558520 .400933

H 5.0709293 .5312331 .211358

H $4.5375722 .812008-3.608515$

H $5.9579631 .810674-3.678792$

H $3.2935391 .014196-2.402321$

H $3.8680540 .530731-3.999125$

H $5.857874-0.583834-2.872401$

H $3.838274-1.915834-3.579234$

H $3.045163-1.519941-2.070201$

H $3.346641-4.141971-2.642146$

H $4.120982-2.4602520 .179888$

H $5.437311-3.821223-3.653387$

H $\quad 6.200899-4.425678-2.201525$

H $6.628082-2.817998-2.821323$

H $5.906253-3.4156551 .149568$

H $6.743453-1.3731931 .694436$

H 6.5623050 .2529491 .093394

H $7.727335-0.706353-0.515776$

H $1.7036460 .132582-0.535405$

H $-0.2961820 .407079 \quad 1.790467$

H $1.691158-2.2831170 .032155$

H $\quad 0.499980-1.894381-1.234027$

H $\quad 0.345264-3.355945-0.270257$

H 4.9340720 .3727342 .639888

H $3.930689-0.2039271 .305545$

H $1.951330-1.8174155 .083884$

H $3.322132-2.9312064 .875847$

H $2.124587-3.8802052 .754731$

H $-0.032105-3.1528314 .804817$

H $-0.628724-5.4887743 .922180$

H $\quad 0.077685-5.0953502 .372383$

H $-3.839801-3.8519173 .157395$

H -2.870160 -4.767558 4.442456

H $-2.834053-3.2718301 .091403$

H -1.325696 -4.022555 0.683373

H -1.720095 -1.328295 2.173863

H $2.564490-0.6325953 .162464$

C -1.7385245 .8594640 .429955$

O $-1.9750934 .564799-0.156129$

C $-2.604198 \quad 3.7205750 .828031$

C $-2.963475 \quad 4.6314301 .998214$

C -1.8473125 .6575081 .938681$

H -1.8490172 .9949221 .155105$

C -3.8005192 .9773820 .218039$

H -3.9366615 .1139031 .847682$

H -3.0001114 .0896402 .948274$

C -2.1422276 .9290542 .713416$

H -0.9365935 .1956782 .339572$

C $-3.3949751 .939011-0.853722$

O $-4.7122713 .931409-0.322610$

H -4.3292282 .4509421 .020874$

H -2.703239 $1.229441-0.386441$

O $-2.6977962 .563338-1.930980$

C $-4.5921831 .163423-1.440632$

C $-4.1930910 .058936-2.439004$

H $\quad-5.1763040 .727711-0.623464$ 
O $-5.4685432 .057477-2.131482$ H -5.110423 $-0.306602-2.916806$ H -3.608492 $0.496505-3.257920$ H -1.3142117 .6393062 .624743$ H -3.0496977 .4211962 .347988$ H $-2.285326 \quad 6.707443 \quad 3.775959$ C $-0.4275306 .441876-0.101482$ H -4.257812 4.385027-1.053256 H -1.893507 $2.970782-1.567542$ H $-4.9452012 .510529-2.814716$ H $-0.284127 \quad 7.4583180 .282573$ H $-0.5008496 .528053-1.193435$ H -2.5577336 .5036530 .082655$ C $-5.311537-2.315919-0.916022$ O $-4.080018-1.634562-0.649783$ C $-3.418564-1.113606-1.806125$ C $-3.092072-2.257558-2.774753$ C $-4.329738-3.087341-3.113926$ C $-5.066542-3.512263-1.842372$ H $-6.026577-1.621801-1.374398$ C $-5.899666-2.768250 \quad 0.424438$ H -2.459447 -0.711929-1.459764 O $-2.506974-1.739829-3.964339$ H -2.344268 -2.911399 -2.307137 H -4.998206 -2.537264 -3.785909 O $-3.930382-4.254124-3.837432$ H -4.481483 -4.279149-1.318618 O $-6.317086-4.096579-2.198012$ C $-6.271342-1.5782151 .276007$ H $-6.807430-3.3664630 .285438$ H $-5.194615-3.3934560 .983134$ H -2.343445 -2.491436 -4.559566 H -3.334049-4.771085 -3.269364 H -6.132005 -4.830276 -2.809063 O $-5.222915-1.2330112 .076998$ O $-7.353132-1.0085021 .214170$ C -5.432835 -0.0900422 .911195$ H $-5.738128 \quad 0.773211 \quad 2.312325$ H $-6.184999-0.3170273 .672342$ $\mathrm{H}-4.487814 \quad 0.1460403 .407687$

SCF Energy $\left(\mathrm{B} 3 \mathrm{LYP} / 6-31 \mathrm{G}^{* *} / / \mathrm{MMFF}\right)=-3245.91635484$

01 192

MM̄MF Geometry

C $-1.983394 \quad 4.5749250 .751771$

C $-1.1911864 .327231-0.302879$

C $0.2377133 .865750-0.147401$

O $0.4802592 .707013-0.989327$

C $1.2051504 .981348-0.586666$

C 2.5299814 .9357830 .150510

C $3.6836064 .449435-0.346235$

C 2.5030095 .5224251 .540547

C $3.9267593 .829377-1.692603$

C $3.9545362 .301328-1.634630$

C $5.0462211 .683011-0.749554$

C $6.4492711 .885508-1.325448$

C $7.5181351 .126619-0.521430$

O $4.7471880 .283921-0.675606$

O $8.7188381 .089584-1.308036$

C $7.062115-0.335797-0.326652$

C $7.874591 \quad 1.8582640 .778402$

O $7.951040-1.0365620 .540324$

C $5.611853-0.4618110 .189435$

C $5.148448-1.9366170 .146229$

O 5.5553270 .0080051 .537811

C $-0.2313131 .575618-0.729921$

O $-1.094790 \quad 1.4336340 .121315$

C $0.1986050 .523734-1.677198$

C $-0.600190-0.537919-1.861335$

C $-0.367794-1.663805-2.766987$

C $1.010375-1.811190-3.352949$

C $3.646089-2.1177140 .413181$

C $3.220650-3.5715570 .328114$

C $1.182229-4.9983630 .423856$

O $4.010776-4.4954540 .154620$

C $0.771739-5.275237-0.987935$

C $-0.493713-5.519936-1.358478$

C $-0.897744-5.873381-2.759240$
C $-1.933648-4.932152-3.325888$

C $-3.237228-5.259654-3.330976$

C $-1.447703-3.658564-3.980780$

C -1.398719-2.494476-3.029934

N $1.847748-3.7121020 .482630$

H -1.5557614 .4859261 .749006$

H -1.571488 4.424455 - 1.315028

H $\quad 0.4362413 .5658620 .888969$

H $0.7657905 .970135-0.394717$

H $1.3573594 .935502-1.670268$

H 4.5682954 .4919270 .288389

H 3.4822595 .4823232 .029347

H 2.1974906 .5732321 .501994

H 1.7975404 .9792682 .176776

H $3.1640544 .136642-2.414506$

H $4.8731034 .213891-2.089567$

H $2.9826531 .939431-1.280103$

H $4.0489931 .906155-2.654797$

H 4.9738112 .1026570 .259139

H $6.7046142 .950214-1.380890$

H $6.4729141 .517693-2.360906$

H $8.9783752 .006523-1.502497$

H $7.126223-0.832886-1.304709$

H 8.2716282 .8567410 .558955

H 8.6681191 .3343861 .322446

H $7.0209821 .984778 \quad 1.447588$

H $8.847267-0.9410300 .174809$

H $5.362122-2.353728-0.846839$

H $5.714498-2.5271810 .877590$

H $4.869541-0.4769422 .021614$

H $1.1314560 .659885-2.210246$

H -1.542898 $-0.571809-1.319615$

H $1.769762-1.782830-2.563314$

H $1.214978-1.003303-4.063680$

H $1.158462-2.756151-3.879810$

H $3.383518-1.7549241 .412193$

H $3.060777-1.552709-0.321254$

H $0.329213-4.958516 \quad 1.107357$

H $1.866194-5.7805350 .768800$

H $1.561108-5.311254-1.735246$

H $-1.279080-5.513247-0.606852$

H -1.294082 -6.897298 -2.740969

H $-0.030455-5.910281-3.430118$

H $-3.982125-4.602621-3.767625$

H $-3.588219-6.188087-2.892750$

H $-2.137925-3.383398-4.790112$

H -0.493941 -3.846647 -4.480610

H $-2.359297-2.284740-2.556399$

H $1.267692-2.8805110 .552328$

C $-4.2660724 .348976-0.421769$

O $-4.2880732 .908902-0.300958$

C $-5.6490542 .455838-0.402492$

C $-6.4265693 .615989-1.012490$

C $-5.7289094 .801363-0.379992$

H -6.0049242 .2969490 .622302$

C $-5.7462621 .150542-1.198401$

H $-6.3179643 .650520-2.102851$

H -7.494548 $3.569879-0.778892$

C $-5.9885416 .115269-1.096865$

H -6.0672494 .8910080 .661148$

C $-4.917273-0.025009-0.640371$

O $-5.2963291 .406986-2.535405$

H $-6.8010600 .859397-1.278930$

H $-3.8518280 .143625-0.833157$

O $-5.285968-1.195473-1.383443$

C $-5.124786-0.3250090 .857244$

C $-4.606106-1.6986151 .329332$

H -4.6482470 .4628581 .450790$

O $-6.521743-0.2801631 .167974$

H $-4.786914-1.7636202 .409374$

H $-5.234945-2.4869660 .895731$

H $-5.4506086 .936438-0.612881$

H -5.667284 $6.072069-2.142892$

H $-7.0565406 .356090-1.083115$

C -3.4391994 .9488620 .719911$

H $-5.5528340 .646302-3.083091$

H -4.928435 -1.096937 -2.281911

H $-6.968510-0.9404690 .610713$ 
H -3.8689394 .6006671 .669009$ H -3.514055 6.0422000 .718105 H $-3.8263844 .583423-1.398173$ C -2.084649 -0.9344102 .922360$ O $-2.256895-0.9631991 .503466$ C $-3.126975-1.9841431 .014337$ C $-2.639063-3.3637161 .474637$ C $-2.472994-3.4327542 .989196$ C -1.597267 $-2.280110 \quad 3.477437$ H -3.049384 -0.689409 3.384945 C -1.147441 $0.235367 \quad 3.267790$ H -3.030311 -1.973279-0.076334 O $-3.530878-4.3891921 .051734$ H -1.666743 -3.5708451 .011794$ H -3.441170 -3.438511 3.503040 O $-1.827402-4.6603643 .335934$ H $-0.560495-2.4772443 .184307$ O $-1.627435-2.2345414 .902690$ C $\quad 0.2240520 .166901 \quad 2.620529$ H -1.612351 1.1643912 .914773 H -1.013293 0.3012894 .353046 H $-3.598889-4.335350 \quad 0.082910$ H $-2.370261-5.3833272 .977083$ H $-1.346583-3.1075835 .226828$ $\begin{array}{lllll}\text { O } & 0.936256 & 1.276674 & 2.982401\end{array}$ O $0.622064-0.7230691 .882270$

C $2.253364 \quad 1.3699362 .429482$ H 2.8864430 .5819022 .846562 H 2.2207841 .3028681 .337825 H 2.6714452 .3410052 .706759

SCF Energy (B3LYP/6-31G**//MMFF) $=-3245.90522199$

01_193

MMFF Geometry

C $-0.328082 \quad 3.3813670 .059353$

C $0.493987 \quad 3.736037 \quad 1.058235$

C $0.921986 \quad 2.8544732 .209541$

O $0.021091 \quad 1.7313102 .375833$

C 2.3184092 .2439081 .986520

C 3.4198923 .2356061 .687496

C $3.940016 \quad 3.4538850 .462858$

C 3.9339353 .9989132 .879240

C $3.5433282 .801198-0.833235$

C $4.7358742 .287204-1.650378$

C $5.6314291 .269445-0.925083$

C $6.6962870 .732426-1.886542$

C $7.576420-0.343402-1.234068$

O $4.8245680 .192531-0.437090$

O $8.298667-1.013490-2.278924$

C $6.664329-1.394187-0.567373$

C $8.6309670 .272721-0.307068$

O $7.435878-2.342420 \quad 0.167404$

C $5.555054-0.7825620 .322206$

C $4.577502-1.8943820 .774138$

O $6.165612-0.2044841 .479273$

C -1.160737 1.9451453 .014349

O -1.5549042 .9862143 .512822$

C -1.9117670 .6721772 .965826$

C -3.177572 0.6097693 .401999

C $-4.005949-0.5937463 .389443$

C $-4.880831-0.7395634 .603953$

C $3.315563-1.3718951 .468732$

C $2.447438-2.4911922 .017546$

C $0.403150-2.8291693 .415994$

O $2.684146-3.6819561 .837100$

C $-0.760267-3.0846702 .511562$

C -1.648207 -4.0641192 .736935$

C $-2.850319-4.3285981 .874911$

C $-4.143359-3.9646212 .571793$

C $-4.692374-4.7886523 .480430$

C $-4.839203-2.6842682 .167753$

C -3.993956-1.454336 2.351843

N $1.374631-1.9885592 .740533$

H -0.7330182 .3746190 .027546$

H 0.9007294 .7453531 .062859

H $0.922575 \quad 3.4440163 .134780$

H 2.2483931 .4792821 .206396

H 2.5952681 .6660712 .879534
H $4.7216554 .206598 \quad 0.366649$

H 4.7463224 .6843522 .615714

H 3.1327384 .5942173 .328268

H 4.3193443 .3066193 .635026

H $2.8346921 .981319-0.691583$

H $3.0178233 .552003-1.436910$

H $4.3278771 .828528-2.560136$

H $5.3485763 .137554-1.976303$

H $6.1100331 .772373-0.076886$

H $7.3150581 .549734-2.277665$

H $6.202797 \quad 0.286852-2.761980$

H $8.822726-0.347517-2.756200$

H $6.184701-1.960673-1.377294$

H $9.2713740 .967111-0.864448$

H $9.302997-0.4946220 .092532$

H 8.1973830 .8248850 .529213

H $7.708909-1.9202350 .999735$

H $4.256871-2.476998-0.099063$

H $5.096178-2.5844821 .451975$

H $5.517200-0.1640302 .198895$

H $-1.404830-0.1922052 .553723$

H -3.6537941 .4948473 .818904$

H -5.6495370 .0406854 .618247$

H $-4.283068-0.6510525 .518557$

H -5.386824 -1.707055 4.654736

H $3.577434-0.7173742 .306095$

H $2.712912-0.7963380 .759171$

H $0.075522-2.2778154 .302774$

H $\quad 0.884447-3.7632653 .723249$

H $-0.905101-2.4293001 .657673$

H $-1.511090-4.7122623 .599858$

H -2.858164 -5.3945831 .612652$

H -2.777759 -3.7973850 .918084$

H $-5.631133-4.5490843 .968637$

H $-4.212427-5.7217863 .756752$

H -5.797567 -2.5734152 .688028$

H $-5.107041-2.7557741 .105635$

H -3.333158 -1.247754 1.510475

H $1.224957-0.9838722 .766220$

C -2.246947 $4.464862-1.193732$

O $-2.861713 \quad 3.200403-1.509925$

C $-4.1001223 .098140-0.779699$

C $-4.3856804 .493175-0.233285$

C $-2.980728 \quad 4.998549 \quad 0.036162$

H -3.9132392 .4245920 .065250$

C $-5.1974242 .519663-1.678593$

H $-4.8819595 .128045-0.976308$

H -5.0133834 .4673410 .662592$

C -2.891135 $6.504695 \quad 0.202956$

H $-2.614615 \quad 4.5166620 .951244$

C $-4.840448 \quad 1.160777-2.317163$

O

H $-6.1332552 .435734-1.114524$

H $-4.0352181 .295824-3.048014$

$\begin{array}{llll}\text { O } & -5.983666 & 0.708304 & -3.053240\end{array}$

C $-4.455395 \quad 0.066182-1.306165$

C $-4.258249-1.332939-1.918696$

H $-3.5390800 .353515-0.781058$

O $-5.478901-0.036741-0.311953$

H $-4.055015-2.029369-1.096241$

H $-5.205268-1.681957-2.349540$

$\begin{array}{llll}H & -1.856397 & 6.815211 & 0.379155\end{array}$

H -3.254686 $7.029928-0.686665$

H -3.4929456 .8335151 .056482$

C $-0.7314024 .296128-1.061874$

H $-4.629583 \quad 3.582622-3.224778$

H $-6.2159481 .409007-3.686648$

H $-6.309089-0.260087-0.766979$

H $-0.2633715 .280900-0.947662$

H $-0.3423683 .859155-1.990074$

H $-2.4414315 .116972-2.055690$

C $-1.240926-1.596523-1.497364$

O $-1.912868-0.837060-2.506628$

C $-3.137010-1.413351-2.973821$

C $-2.888666-2.827079-3.522589$

C $-2.157238-3.713457-2.514487$

C $-0.904585-3.004669-1.999314$

H $-1.893216-1.672800-0.618917$ 
C $-0.013133-0.805845-1.033367$

H -3.459136 -0.799617 -3.823713

O $-4.125373-3.422503-3.899920$

H -2.277409 -2.749004 -4.431365

H $-2.810885-4.008228-1.685324$

O $-1.746103-4.922973-3.155308$

H $-0.164811-2.962918-2.807857$

O $-0.334233-3.760284-0.934647$

C $1.009368-0.600335-2.126286$

H $-0.3128150 .191416-0.693745$

H $0.463415-1.291569-0.175718$

H -3.924665 -4.255053 -4.359236

H -2.548506 -5.423578 -3.379646

H - $0.161524-4.655569-1.273153$

O $2.030631-1.492440-1.983801$

O $0.8996590 .235696-3.013801$

C $3.042515-1.437961-2.993481$

H $3.828188-2.149769-2.727182$

H $2.620962-1.725077-3.961311$

H $3.479779-0.437262-3.045502$

SCF Energy (B3LYP/6-31G**//MMFF) $=-3245.90384542$

01

MM̄̄FF Geometry

C $4.094437-3.760135-1.599391$

C $3.522372-2.603192-1.965606$

C $2.043122-2.440272-2.196501$

O $1.553409-1.701858-1.053766$

C $1.793178-1.639334-3.488940$

C $0.379325-1.738919-4.028831$

C $-0.489232-0.713739-4.134572$

C $-0.024979-3.103084-4.533595$

C $-0.2955050 .728883-3.763200$

C $-0.9750471 .151427-2.452571$

C $-2.5115121 .070851-2.461088$

C $-3.0834721 .722751-1.198068$

C $-4.6090351 .572877-1.099089$

O $-2.904100-0.303835-2.510512$

$\begin{array}{lllll}O & -4.996254 & 1.890304 & 0.247433\end{array}$

C $-4.9881650 .095933-1.339727$

C $-5.3371052 .572617-2.005253$

O $-6.402266-0.059494-1.416748$

C -4.322294 -0.510869-2.591282

C -4.568089-2.033604 -2.742085

O $-4.8670130 .110633-3.762580$

C $0.334421-2.032630-0.553855$

O $-0.473272-2.816015-1.025124$

C $0.154492-1.2778280 .705365$

C -1.080906 -1.059745 1.177774

C $-1.420138-0.3388412 .405752$

C $-0.2976430 .007578 \quad 3.348906$

C $-4.073590-2.901147-1.581567$

C $-5.116759-3.136100-0.502277$

C $-5.318545-3.7493691 .905365$

O $-6.324324-3.038828-0.703657$

C $-5.502082-2.4532442 .629926$

C $-4.995102-2.1999273 .845831$

C $-5.184759-0.8995634 .573215$

C $-3.874868-0.203773 \quad 4.871425$

C $-3.287280-0.3278016 .073459$

C $-3.2930940 .705702 \quad 3.812441$

C -2.714992 -0.044145 2.644181

N $-4.541016-3.5103150 .704084$

H $3.478431-4.646650-1.471273$

H $4.141124-1.713827-2.062996$

H $\quad 1.574099-3.430098-2.240273$

H $2.463604-1.998345-4.282037$

H $2.070017-0.595127-3.313096$

H -1.464054 -0.925357 -4.573189

H $-0.994861-3.087877-5.042728$

H $\quad 0.712556-3.475901-5.251825$

H -0.101900 -3.817930 -3.709556

H $\quad 0.767507 \quad 0.979097-3.696482$

H $-0.6842301 .344823-4.584185$

H $-0.5780210 .538155-1.636782$

H $-0.6791502 .188355-2.252630$

H -2.878565 $1.587990-3.355355$

H $-2.8038482 .782195-1.149500$
H $-2.632581 \quad 1.254382-0.313233$

H -4.6848522 .7903230 .444780$

H $-4.657260-0.461740-0.455276$

H -5.087169 $3.601265-1.718606$

H $-6.4237752 .487961-1.895239$

H $-5.0854462 .456160-3.061355$

H $-6.7794790 .311215-0.600634$

H $-5.625525-2.223187-2.963126$

H $-4.020212-2.363055-3.636621$

H $-4.706089-0.471533-4.523628$

H $1.045615-0.917647 \quad 1.204640$

H -1.928845 -1.424644 0.601367

H $-0.6431020 .420744 \quad 4.299322$

H $\quad 0.372678 \quad 0.747226 \quad 2.899751$

H $0.285163-0.8864203 .598106$

H $-3.816200-3.894472-1.970595$

H -3.165160 -2.478729-1.144319

H -4.776495 -4.487398 2.504886

H $-6.294123-4.1664101 .635748$

H $-6.087440-1.6864142 .127513$

H $-4.419162-2.9701174 .353743$

H -5.717370 -1.114829 5.508827

H $-5.839614-0.2191184 .014834$

$\begin{array}{llll}H & -2.364173 & 0.191264 & 6.308481\end{array}$

H -3.714779 -0.9555526 .848313$

H $-2.5492301 .381578 \quad 4.248305$

H -4.0811331 .3733613 .440136$

H $-3.458099-0.3411481 .904273$

H -3.529206 -3.5006790 .794640$

C $5.952538-3.5380120 .129600$

O $5.759170-2.1233530 .333510$

C $5.052566-1.9300311 .572081$

C $5.263492-3.2158362 .360976$

C $5.190063-4.2493411 .250605$

H $3.988045-1.8454781 .319234$

C $5.513970-0.6419732 .259976$

H $6.249819-3.2443642 .838010$

H $4.503575-3.360573 \quad 3.134547$

C $5.775775-5.5988651 .626861$

H $4.135096-4.3872200 .984140$

C 5.5003490 .6016881 .345406

O $6.860306-0.8246302 .716759$

H $4.906475-0.4682093 .156117$

H $\quad 6.3084050 .5162610 .608117$

O 5.8240721 .7258952 .173310

C 4.1604820 .8557370 .628057

C $4.1883652 .131675-0.235768$

H $3.9306660 .000463-0.015417$

O $3.1322960 .950783 \quad 1.609964$

H $4.171513 \quad 3.0105200 .417372$

H $5.1310882 .152512-0.794778$

H $5.715425-6.2957560 .784929$

H $6.827423-5.513117 \quad 1.919997$

H $5.227625-6.0350152 .468224$

C $5.561821-3.907142-1.306157$

H $7.130554-0.0002603 .156453$

H 6.0421642 .4711021 .588766

H 2.2855191 .0267911 .139853

H $5.853193-4.943210-1.514817$

H $6.126885-3.275678-2.004098$

H $7.030927-3.7089470 .242754$

C 1.3893803 .3730620 .117471

O $1.7332162 .182706-0.605057$

C $3.0137022 .203417-1.241092$

C $3.0925613 .382941-2.220239$

C $2.7599024 .708066-1.540040$

C $1.4259804 .601806-0.801966$

H $2.099436 \quad 3.5006380 .941624$

C -0.0122253 .1466110 .705408$

H $3.0688951 .286616-1.842054$

O $4.3852803 .446189-2.812644$

H $2.3755923 .214757-3.034790$

H $3.5686455 .021787-0.869840$

O $2.6574635 .716609-2.547492$

H $0.6204854 .542830-1.544629$

O $1.2082905 .792236-0.049940$

C -0.2648383 .8781692 .004429$

H $-0.7750163 .451862-0.018770$ 
H -0.1583322 .0795200 .903986$ H $4.3951614 .215707-3.407367$ H $2.399486 \quad 6.543016-2.104264$ H $\quad 1.903957 \quad 5.849168 \quad 0.627375$ O O $0.575294 \quad 4.4863492 .652333$ C -1.9790714 .3599843 .562175$ H -1.430198 3.9311414 .406038 H -1.814985 5.4401353 .501995 H -3.046665 4.174761 3.707919

SCF Energy (B3LYP/6-31G**//MMFF) $=-3245.91208563$

01 195

MM̄FF Geometry

C $0.277210-2.7950362 .028678$

C $1.574696-2.4851472 .179684$

C $2.216472-1.1498141 .868394$

O $1.309257-0.2825001 .150110$

C $2.617375-0.4597273 .183587$

C 3.5202220 .7402692 .982574

C 4.8665720 .6817832 .997288

C 2.8180452 .0640102 .837018

C $5.713396-0.5477023 .168641$

C $6.938720-0.6073472 .244015$

C $6.599507-0.8213680 .759131$

C $7.863610-1.228668-0.005531$

C $7.610040-1.367223-1.511969$

O $6.080218 \quad 0.4006860 .223698$

O $8.884103-1.436125-2.170405$

C $6.911581-0.088673-2.012571$

C $6.874428-2.668856-1.852021$

O $6.527287-0.222470-3.379592$

C $5.6914220 .326957-1.155660$

C $5.2073021 .728393-1.600878$

O $4.642636-0.619837-1.356978$

C $1.158465-0.493537-0.184622$

O $1.743787-1.329505-0.859898$

C $0.1172440 .383471-0.766766$

C $-0.4099911 .439658-0.129283$

C $-1.4774352 .307036-0.630211$

C $-2.0716781 .988795-1.976812$

C $4.1226312 .345745-0.709302$

C $3.7280393 .737120-1.177034$

C $2.2700255 .645558-0.564644$

O $4.2671284 .307271-2.122039$

C $0.8891785 .805520-0.014734$

C $-0.1512816 .219542-0.753235$

C -1.537124 $6.439611-0.216378$

C $-2.5609365 .545845-0.878595$

C $-3.1131275 .885024-2.055927$

C $-3.0051934 .303226-0.143652$

C $-1.887163 \quad 3.3375620 .137865$

N $2.7015794 .270054-0.406562$

H -0.424111 -2.042859 1.680960

H $2.255891-3.2571862 .532593$

H $3.109804-1.3226761 .254551$

H $3.108648-1.1766273 .853656$

H $1.720344-0.1324293 .724823$

H 5.4227291 .6126242 .896569

H $2.1929802 .261358 \quad 3.714157$

H 3.5197282 .8990632 .739174

H 2.1798512 .0649431 .950304

H $6.075205-0.5522554 .204461$

H $5.132803-1.4658023 .033908$

H 7.5263490 .3121452 .363062

H $7.564948-1.4390432 .591646$

H $5.837612-1.6057720 .681029$

H $8.279196-2.1609710 .397441$

H $8.640798-0.4659480 .145858$

H $9.367716-2.198562-1.808530$

H $7.6510950 .723807-1.977417$

H $7.464554-3.536328-1.532655$

H $6.744330-2.777801-2.934400$

H $5.894338-2.745500-1.376612$

H $7.322661-0.470634-3.881142$

H $6.0637432 .415809-1.603996$

H $4.8261081 .679011-2.628866$

H $3.791090-0.198754-1.164363$
H $-0.2003690 .102979-1.766035$

H -0.0442961 .6906890 .864192$

H -2.520674 $0.990204-1.971880$

H -1.301650 $2.025263-2.755314$

H -2.854209 $2.687487-2.281820$

H $3.2228251 .723532-0.703288$

H 4.4914762 .4358370 .317442

H $2.3344355 .926473-1.621450$

H $2.969876 \quad 6.276034-0.006116$

H $\quad 0.7458845 .5978191 .042777$

H $0.0045726 .442848-1.806709$

H -1.803262 $7.491205-0.386451$

H -1.571106 6.3048860 .871989

H -3.868974 $5.265262-2.526900$

H $-2.8186796 .790808-2.575828$

H $-3.8183043 .797245-0.677025$

H -3.4471444 .6029490 .815749$

H -1.3948473 .5040951 .096439$

H $2.445733 \quad 3.7879880 .450061$

C $-0.818870-4.8437591 .022512$

O $-1.948642-4.1289680 .479430$

C $-1.839229-4.129462-0.957310$

C $-0.742913-5.135251-1.302894$

C $0.189358-4.984851-0.119489$

H -1.493726 -3.128415 -1.241642

C $-3.190660-4.420457-1.618062$

H -1.121726 -6.161590 -1.349306

H $-0.263067-4.910859-2.260552$

C $1.158779-6.1407380 .051924$

H $\quad 0.761745-4.060345-0.260042$

C $-4.326804-3.443047-1.256986$

O $-3.645362-5.722719-1.223443$

H $-3.071222-4.449732-2.708449$

H $-4.647068-3.604886-0.220862$

O $-5.448814-3.791573-2.082996$

C $-4.016359-1.948485-1.471766$

C $-5.255891-1.031462-1.519142$

H $-3.334806-1.603504-0.687788$

O $-3.325512-1.776050-2.714192$

H $-4.904946-0.008823-1.701182$

H $-5.842409-1.282490-2.412041$

H $1.799518-5.9843840 .925512$

H $\quad 0.631554-7.0912080 .185661$

H $1.803681-6.235408-0.827817$

C $-0.299742-4.1571692 .290070$

H $-3.164042-6.378668-1.754727$

H -5.656662 -4.725720 -1.908886

H $-3.900520-2.117274-3.420646$

H $0.443204-4.8022912 .773745$

H -1.127898 -4.032991 2.998537

H $-1.202087-5.8329231 .306661$

C -5.1600090 .7289920 .993829$

O $-5.470960-0.6684000 .917570$

C $-6.156267-1.079878-0.269239$

C $-7.469926-0.298609-0.417515$

C $-7.2332681 .207357-0.384754$

C -6.4273121 .5858680 .855029$

H $-4.4379530 .987598 \quad 0.210483$

C -4.5040590 .9867202 .359613$

H $-6.438886-2.126708-0.111660$

O $-8.130271-0.665878-1.623801$

H $-8.138805-0.5721510 .408887$

H $-6.7351371 .550539-1.298466$

O $-8.4924751 .883400-0.351306$

H -7.0563191 .4692001 .747164$

O -6.0614432 .9611230 .772178$

C -3.1272770 .3736402 .481061$

H -4.3932382 .0647012 .522929$

H -5.1218030 .5595623 .158512$

H $-8.936605-0.126322-1.690597$

H $-8.967314 \quad 1.596640 \quad 0.447476$

H -6.8796273 .4739180 .655705$

O $-2.567710 \quad 0.792342 \quad 3.655441$

O $-2.612054-0.379998 \quad 1.668293$

C $-1.253737 \quad 0.297313 \quad 3.923570$

H -0.5628540 .6245543 .142529$

H $-0.921596 \quad 0.711147 \quad 4.879347$

H $-1.267890-0.7936323 .999840$ 
H $2.6158767 .661723-2.690934$

H $1.6476686 .993740-1.395160$

H $-0.1265507 .346003-5.115648$

H $1.5363048 .078654-4.755777$

H -1.251616 $6.093318-3.404544$

H $-0.5044015 .898883-1.848027$

H $0.0435674 .201118-4.389109$

H $2.7972172 .090057-1.216780$

C $-4.541956-3.405222-1.162677$

O $-4.697729-2.203278-0.387114$

C $-5.600679-1.322234-1.089253$

C $-6.080806-2.083266-2.325561$

C $-4.915519-3.018466-2.590730$

H $-5.003947-0.464039-1.419175$

C $-6.722934-0.853013-0.152164$

H -6.992354 -2.657734 -2.124857

H -6.292172 -1.412610 -3.164295

C $-5.265178-4.196655-3.481418$

H -4.113695 -2.435823 -3.061084

C $-6.215800-0.2183371 .159765$

O $-7.519925-1.9926210 .196129$

H -7.384103 -0.160980 -0.687195

H $-5.714711-0.9836081 .763129$

O $\quad-7.3505330 .218994 \quad 1.917039$

C $-5.2834890 .989490 \quad 0.959352$

C -4.854242 1.6759852 .271222

H -4.3901790 .6784420 .408736$

$\begin{array}{lllll}\text { O } & -5.954089 & 1.957184 & 0.147885\end{array}$

H -4.259438 2.5594062 .007776

H -5.7356012 .0795862 .785401$

H -4.394265 -4.842256 -3.632610

H $-6.065030-4.805380-3.046753$

H -5.602913 -3.849635 -4.463415

C $-3.152552-4.010349-0.948318$

H $-8.341030-1.6630680 .598577$

H -7.823919 -0.5742802 .219373$

H $-5.3314022 .685736-0.015942$

H -3.133286 -5.019437-1.377411

H $-2.974725-4.1184310 .129069$

H $-5.279005-4.116627-0.765156$

C -1.9229290 .8689552 .117754$

O -3.0188800 .0535722 .539753$

C -4.0563540 .7625513 .223786$

C -3.4870121 .4717914 .461451$

C -2.2797262 .3454104 .123758$

C $-1.251564 \quad 1.5591103 .308904$

H -2.2965181 .6410081 .433012$

C -0.9740710 .0106921 .273094$

H $-4.7561890 .002123 \quad 3.590845$

O -4.4816112 .2738645 .089670$

H $-3.1704770 .718450 \quad 5.194709$

H -2.5811253 .2578803 .595677$

O $-1.6551602 .773628 \quad 5.336200$

H -0.7560420 .8297443 .959489$

O -0.2505782 .4537982 .830025$

C $-0.397533-1.2028191 .970217$

H -1.531062 -0.3659230 .407313$

H -0.1447930 .6269020 .914260$

H -5.2295081 .6925445 .309836$

H -2.3301853 .2381365 .860336$

H $\quad 0.1377332 .896213 \quad 3.604382$

O $0.508760-1.7947551 .134082$

O $-0.708313-1.6006503 .083614$

C $1.118969-2.9876201 .635415$

H $1.674349-2.7733912 .553052$

H $\quad 0.359485-3.7550031 .812510$

H $1.817662-3.3556360 .880466$

SCF Energy (B3LYP/6-31G**//MMFF) $=-3245.90804018$

01197

MM̄FF Geometry

C 1.9279835 .6802930 .189118

C $2.2855864 .694896-0.648142$

C $3.2771743 .611579-0.312405$

O $2.5380292 .375762-0.178552$

C $4.2824483 .447040-1.466706$

C $5.2953662 .341300-1.242658$ 
C $5.3931991 .230716-2.000376$

C $6.2503382 .552336-0.096255$

C $4.5664020 .849480-3.197489$

C $3.3231150 .003367-2.879674$

C $3.638949-1.386517-2.303684$

C $2.370111-2.236294-2.209724$

C $2.643243-3.604195-1.563314$

O $4.194229-1.223787-0.997185$

O $1.377815-4.190499-1.219955$

C $3.417691-3.399914-0.241547$

C $3.313115-4.571464-2.546976$

O $3.823321-4.6642640 .277593$

C $4.624323-2.444702-0.377822$

C $5.270519-2.0568160 .975233$

O $5.641783-3.085173-1.157604$

C 1.9945682 .1053881 .039143

O 2.1193422 .7554252 .064690

C 1.1596290 .8939360 .893464

C $\quad 0.2046090 .6482651 .802529$

C $-0.751923-0.4589711 .772432$

C $-0.551563-1.5229640 .726629$

C $4.335002-1.3785031 .981990$

C $3.701771-2.3372532 .976322$

C $1.989506-2.4109254 .783891$

O $4.050278-3.5085463 .098162$

C $0.704995-2.9359684 .224451$

C $-0.486089-2.7335624 .807754$

C $-1.781760-3.2830904 .283389$

C $-2.838974-2.2150154 .116172$

C $-3.761884-2.0014795 .069291$

C $-2.877464-1.4558262 .810080$

C - $-1.754360-0.4666652 .675727$

N 2.722605-1.717937 3.741214

H 2.3793935 .7413731 .175516

H $1.8008514 .631039-1.620322$

H 3.8112013 .8380510 .618452

H $4.8266514 .388341-1.620390$

H $3.7252463 .268459-2.392738$

H $6.1644790 .504909-1.744327$

H $7.0382001 .791735-0.066593$

H $6.7410913 .527046-0.185179$

H 5.7206272 .5121160 .860334

H $4.2564121 .742384-3.750745$

H $5.2108960 .296476-3.892860$

H $2.6833170 .556963-2.182963$

H $2.755637-0.117580-3.810904$

H $4.374328-1.869579-2.957703$

H $1.912251-2.364527-3.198656$

H $1.623044-1.708480-1.601804$

H $\quad 0.839960-4.238985-2.028971$

H $2.700606-2.9800570 .474023$

H $2.679300-4.719088-3.429720$

H $3.439226-5.564445-2.101527$

H $4.287431-4.222361-2.895257$

H $4.182576-4.5205361 .168819$

H $5.780778-2.9220911 .415082$

H $6.070265-1.3347270 .754385$

H $6.491552-2.652702-0.970877$

H 1.3188770 .2704910 .021849

H $0.084828 \quad 1.3452592 .631301$

H $-0.709995-1.109059-0.274783$

H -1.223440 -2.376599 0.834784

H $0.464255-1.9286720 .783180$

H $4.923199-0.6684242 .577606$

H $3.553518-0.8082731 .471150$

H $1.825901-1.6952495 .595932$

H $2.592583-3.2412625 .164952$

H $\quad 0.759157-3.5286943 .314167$

H $-0.531765-2.1648625 .733930$

H -2.132091 -4.044533 4.992513

H -1.639749 $-3.809316 \quad 3.331377$

H -4.541576 -1.257109 4.945919

H -3.754849 -2.563961 5.997078

H -3.812689 -0.885673 2.736285

H -2.917717 -2.170237 1.983856

H -1.8106250 .3520023 .394684$

H $2.462423-0.7576463 .535791$

C $-0.539783 \quad 6.298600 \quad 0.251032$
O $-0.9114245 .081363-0.429016$

C -1.487668 4.1770870 .529895

C -1.9250965 .0568991 .691727$

C -0.7793386 .0524761 .741886$

H -0.6826383 .5143900 .868146$

C $-2.6008173 .328916-0.097838$

H -2.869691 5.5688121 .472646

H -2.0452404 .4930782 .621516$

C -1.095766 7.3120802 .528531

H $0.086417 \quad 5.5587812 .200537$

C $-2.0954462 .352475-1.180433$

O $-3.6002794 .184084-0.649544$

H -3.0758072 .7536340 .704381$

H -1.364588 $1.665743-0.739316$

O $-1.4032593 .069551-2.209938$

C $-3.1918731 .534266-1.890787$

C $-4.1501390 .791809-0.940520$

H $-3.7703462 .191727-2.550655$

O $\quad-2.535458 \quad 0.595088-2.747506$

H $-3.5613230 .170127-0.258644$

H $-4.6777801 .531853-0.330537$

H $-0.247098 \quad 8.0031982 .512163$

H -1.965491 7.8351632 .117184

H -1.314194 7.0682233 .573342

C $0.8814636 .704973-0.154460$

H $-3.1791134 .705186-1.354818$

H -0.622296 3.485091-1.807189

H -2.025114 $-0.012901-2.185849$

H $1.150997 \quad 7.6567650 .317408$

H $0.9000606 .878373-1.238421$

H $-1.2309607 .069056-0.114806$

C -4.079702 -2.220572 -1.573438

O $-4.600355-1.170549-2.397627$

C $-5.192076-0.074281-1.691449$

C $-6.346178-0.587594-0.818868$

C $-5.897903-1.7147470 .106515$

C $-5.180830-2.808609-0.683844$

H $-3.265276-1.832624-0.951005$

C $-3.498136-3.290421-2.504723$

H $-5.6386340 .575586-2.455271$

O $-6.9057020 .477973-0.059265$

H -7.142177 -0.963927 -1.474722

H $-5.260502-1.3280340 .907282$

O $-7.040049-2.2770640 .756505$

H $-5.907993-3.356050-1.297074$

O $-4.601200-3.7405400 .226499$

C $-2.183163-2.832986-3.088757$

H $-3.296414-4.228541-1.975142$

H -4.191250 -3.527254 -3.320966

H $-7.609920 \quad 0.101786 \quad 0.496173$

H -7.634301 -2.627087 0.071005

H -5.314045 -4.067499 0.801738

O $-2.368229-2.346071-4.347969$

O $-1.119765-2.882553-2.484481$

C -1.186711 -1.868019-4.999199

H $-0.726165-1.065175-4.415391$

H $-0.480106-2.690124-5.147949$

H -1.474193 -1.470270 -5.976049

SCF Energy (B3LYP/6-31G**//MMFF $)=-3245.91682764$

01 198

MM̄FF Geometry

C $1.369424-0.1650452 .348062$

C $0.154313-0.0605191 .790994$

C $-0.134148-0.5265390 .387728$

O $-1.273593-1.4229950 .410809$

C $-0.4948520 .641480-0.544924$

C $0.6423301 .601703-0.828324$

C $0.6573832 .903289-0.480283$

C $1.7968321 .029108-1.610510$

C -0.3742403 .6745390 .294080$

C $-1.1514244 .664523-0.583761$

C $-2.1424533 .986749-1.542571$

C $-2.8261505 .028443-2.431140$

C $-3.9061734 .399523-3.323544$

O $-3.1183723 .305171-0.749066$

O $-4.6984405 .462784-3.874281$

C $-4.8432793 .546850-2.443547$ 
C $-3.2988823 .649019-4.514484$ O $-5.7787482 .827534-3.244146$ C $-4.0949802 .574607-1.501398$ C $-5.0926251 .952247-0.495968$ O $-3.498078 \quad 1.537460-2.281189$ C -1.045835 -2.701271 0.811064 O $0.018673-3.1612141 .204038$ C $-2.263832-3.5387190 .734511$ C -3.433823 $-3.139003 \quad 0.212419$ C $-4.654267-3.948395 \quad 0.122553$ C $-4.592089-5.3572240 .656499$ C $-4.440458 \quad 1.1156990 .614961$ C $-5.4703330 .597606 \quad 1.604358$ C $-5.710731-0.9541523 .534357$ O C $-6.237762-2.2283032 .952985$ C -7.471633 -2.348525 2.440152 C $-8.010437-3.598096 \quad 1.804567$ C $-8.182679-3.4432870 .308080$ C $-9.279678-2.859101-0.203181$ C $-7.118850-4.029225-0.595209$ C $-5.767067-3.394287-0.405425$ N $-4.911920-0.2768642 .528730$ H $2.180180-0.5961181 .765576$ H -0.6661820 .3701462 .359033$ H $0.716396-1.072744-0.036923$ H - $1.3755021 .157150-0.148531$ H $-0.8403690 .230000-1.503623$ H $1.5243293 .497945-0.767430$ H $2.5206651 .795772-1.907113$ H $2.3356060 .286666-1.014590$ H $1.4351340 .551272-2.526478$ H -1.0588333 .0204280 .841883$ H 0.1584974 .2476961 .063476 H -1.706797 5.3320340 .088083 H $-0.4488345 .288251-1.150488$ H -1.604790 $3.259630-2.160728$ H -2.088251 $5.564412-3.040952$ H $-3.3021205 .791267-1.798848$ H -4.105364 $6.051494-4.371907$ H -5.432406 4.240495 -1.827086 H $-2.7041904 .331336-5.133787$ H $-4.080667 \quad 3.253088-5.172047$ H -2.648250 $2.824395-4.215925$ H -6.234422 $3.474930-3.809114$ H $-5.6605652 .759831-0.015036$ H -5.813713 $1.320338-1.030242$ H $-3.396210 \quad 0.743106-1.734788$ H -2.134103 $-4.540387 \quad 1.132999$ H -3.516062 -2.131035 -0.187993 H $-4.300070-5.355747 \quad 1.712775$ H -3.865411 -5.9500950 .090122$ H $-5.542810-5.8908680 .606425$ H -3.9148280 .2561920 .188842$ H -3.717644 1.7194621 .175129 H $-6.519259-0.2922683 .863130$ H -5.067721 -1.166174 4.393798 H -5.565073 -3.0814662 .943815$ H -8.135457 -1.486645 2.455791 H -8.978577 -3.828144 2.268190 H -7.373284 -4.463809 2.022118 H -9.424905 -2.764291-1.274128 H $-10.056498-2.4598330 .440863$ H -7.394667 -3.890821 -1.649160 H -7.099436 -5.112806 -0.451059 H $-5.719030-2.361645-0.754171$ H -3.944225 -0.565380 2.416567 C 2.6430971 .4733413 .805189 O 3.8043581 .2581632 .976429 C 4.0125442 .4343672 .171116 C 3.2400633 .5474682 .866023 C 2.0175552 .7912753 .346956 H 3.5476402 .2426241 .196739 C 5.5081832 .7042091 .993758 H 3.7955223 .9550973 .718771 H 2.9971614 .3720452 .189173 C 1.2360773 .5150944 .429044 H 1.3554762 .6215832 .491583
C $6.284174 \quad 1.5466821 .329229$

O $\quad 6.0946312 .946833 \quad 3.278777$

H 5.6503103 .6273581 .420337

H 6.3036570 .6854342 .005657

O $7.647812 \quad 1.966927 \quad 1.188991$

C $5.7453881 .119011-0.051056$

C $6.6531420 .126389-0.802760$

H $4.744760 \quad 0.6886150 .060871$

O $5.6108512 .287896-0.865355$

H $6.2590750 .011800-1.819781$

H $7.6451350 .573433-0.941651$

H 0.3761082 .9199024 .751738

H 1.8576903 .7150185 .308175

H $0.862444 \quad 4.4745124 .056298$

C $1.7142220 .257158 \quad 3.747441$

H 5.9358062 .1592593 .827090

H 7.6615472 .7143200 .566452

H $5.1945152 .016790-1.701255$

H $0.8092940 .449307 \quad 4.335335$

H $2.220774-0.5946904 .218659$

H 3.0158331 .5749914 .832546

C $4.817892-2.293319-1.075899$

O $5.501868-1.8585430 .108076$

C $6.777775-1.250173-0.121211$

C $7.703317-2.232735-0.852921$

C $7.066935-2.769187-2.131711$

C $5.668345-3.314679-1.845739$

H $4.610773-1.422474-1.708313$

C $3.474803-2.886725-0.622997$

H $7.218885-1.0800810 .867801$

O $8.948572-1.620830-1.172032$

H $7.922644-3.082823-0.193823$

H $7.037044-2.005944-2.918121$

O $7.867405-3.838426-2.641350$

H $5.755274-4.244052-1.268301$

O $5.034690-3.638515-3.079992$

C $2.430756-2.932039-1.716970$

H $3.633596-3.895948-0.224517$

H $3.057426-2.2854400 .192900$

H $9.337574-1.298981-0.340859$

H $8.763219-3.486534-2.782191$

H $5.611695-4.267043-3.547072$

O $1.505579-3.889566-1.415828$

O $2.404343-2.195463-2.693426$

C $0.422522-4.011631-2.343416$

H $\quad 0.799293-4.300679-3.328978$

H -0.249282 -4.793915 -1.980377

H $-0.134218-3.071255-2.402110$

SCF Energy (B3LYP/6-31G**//MMFF $)=-3245.91191392$

01199

MM̄FF Geometry

C $0.134458 \quad 4.931197 \quad 0.309734$

C 0.5406014 .4996581 .514361

C 1.6308753 .4825171 .763892

O 1.7251812 .5469610 .665235

C 2.9888744 .1882681 .923111

C 4.1032883 .2652342 .376499

C 5.1836822 .9445281 .637999

C 3.9666722 .7136343 .773222

C 5.5316253 .4019250 .249752

C $5.0482922 .473945-0.875857$

C $5.6053241 .042708-0.799711$

C $5.2920850 .270845-2.082592$

C $5.759243-1.191237-2.002289$

$\begin{array}{llll}\text { O } & 4.995816 & 0.377584 & 0.309540\end{array}$

O $5.146809-1.906420-3.087304$

C $5.238311-1.823855-0.691594$

C $7.274691-1.314126-2.205184$

O $5.805690-3.121460-0.526652$

C $5.490794-0.9468440 .554717$

C $4.785048-1.4608181 .834593$

O $6.896164-0.9252750 .834441$

C $0.799625 \quad 1.5487130 .632470$

O $-\begin{array}{llll}0.083426 & 1.341241 & 1.449494\end{array}$

C $1.0224010 .759493-0.598241$

C $0.071392-0.097082-0.998083$

C $0.104006-0.940613-2.192728$ 
C $1.415623-1.060689-2.920716$ C $3.260806-1.5923351 .742077$ C $2.782465-2.9885561 .380217$ C $0.705578-4.2505640 .833448$ O $3.528705-3.9618651 .318743$ C $0.594556-4.374395-0.653480$ C $-0.563163-4.623467-1.283350$ C $-0.695086-4.787411-2.770031$ C $-1.717070-3.847117-3.368483$ C $-2.973129-4.255721-3.615238$ C $-1.258476-2.459083-3.755917$ C $-1.030462-1.570525-2.563328$ N $1.411239-3.0265871 .164571$ H $0.6353224 .572804-0.585834$ H $0.053115 \quad 4.8916182 .404568$ H 1.3822492 .9329222 .679917 H 2.9050265 .0010592 .657007 H 3.2483344 .6660010 .972251 H 5.9206252 .2737512 .078929 H 4.8667172 .1813814 .099608 H 3.7919253 .5249384 .487404 H 3.1317632 .0087933 .831529 H 5.1508214 .4121570 .066276 H $6.623732 \quad 3.495552 \quad 0.190746$ H $3.9528502 .444729-0.863578$ H $5.3474602 .926966-1.829219$ H $6.688907 \quad 1.101520-0.643947$ H $5.7365610 .766958-2.954338$ H $4.2075870 .279709-2.255941$ H $5.413199-1.476808-3.918102$ H $4.159051-1.965984-0.823990$ H $7.564649-0.904653-3.180492$ H $7.588401-2.363780-2.217514$ H $7.853346-0.789034-1.442503$ H $5.361027-3.5491440 .223926$ H $5.251980-2.3914532 .178403$ H $4.988644-0.7280492 .629324$ H $7.021907-0.6695911 .763427$ H $1.9420670 .918490-1.148019$ H $-0.835930-0.176415-0.403104$ H $2.209271-1.359945-2.227714$ H $1.691282-0.105113-3.379355$ H $1.409241-1.810841-3.713698$ H $2.833535-1.3701492 .728493$ H $2.846012-0.8688781 .034760$ H $-0.273944-4.2034241 .318597$ H $1.254122-5.1085701 .235059$ H $1.508363-4.278231-1.235171$ H $-1.468242-4.746133-0.694351$ H - $0.985747-5.827827-2.966079$ H $0.268381-4.649860-3.276431$ H $-3.704860-3.594956-4.067971$ H $-3.299585-5.259291-3.364112$ H $-2.020424-1.971045-4.378426$ H - $0.380122-2.546054-4.400740$ H $-1.927404-1.416167-1.961343$ H $0.878038-2.1620611 .187947$ C $-1.9205505 .481488-1.025466$ O $-2.503503 \quad 4.193469-0.753606$ C $-3.8487734 .173969-1.269976$ C $-4.0684235 .514437-1.968221$ C $-3.1074546 .420693-1.225063$ H $-4.5153154 .114360-0.401998$ C $-4.0556772 .952099-2.174575$ H -3.805038 5.470674-3.031202 H $-5.1091785 .846577-1.901570$ C -2.773926 $7.697352-1.976759$ H $-3.5510276 .677694-0.253859$ C -3.695161 $1.599391-1.530695$ O $-3.238798 \quad 3.089895-3.346426$ H -5.091483 2.931889-2.532837 H -2.610563 $1.528436-1.387563$ O $-4.046168 \quad 0.570454-2.465655$ C $-4.404318 \quad 1.307509-0.194525$ C $-4.083190-0.101717 \quad 0.331215$ H -4.0879052 .0541650 .535766$ O $-5.813867 \quad 1.443740-0.359552$ H $-4.400466-0.841312-0.416179$
H -2.997452 -0.2059810 .415702$ H $-2.0797518 .319235-1.403093$ H -2.312078 $7.483202-2.946449$ H -3.680311 $8.284506-2.158309$ $\begin{array}{llll}\text { C } & -0.995958 & 5.903853 & 0.118387\end{array}$ H $-2.3145023 .164501-3.052734$ H $-3.5659860 .753718-3.291479$ H $-6.0932330 .804735-1.037618$ H $-1.575167 \quad 5.9869601 .046534$ H $-0.5701316 .889860-0.098455$ H $-1.3462315 .382625-1.956640$ C -3.480393 -2.527861 1.979058 O $-4.771268-1.9303531 .798236$ C $-4.758494-0.4983191 .663666$ C $-4.172800 \quad 0.1082642 .947055$ C $-2.790991-0.4604403 .263004$ C $-2.819362-1.988643 \quad 3.252915$ H -2.844451 -2.318176 1.110013 C $-3.683065-4.0481632 .060265$ H $-5.811482-0.1995291 .601900$ $\begin{array}{lllll}\text { O } & -4.092299 & 1.524190 & 2.879954\end{array}$ H -4.836386 -0.1306093 .788861$ H -2.033776 -0.0805782 .569522$ O $-2.390657-0.0311854 .566439$ H -3.357388 -2.349516 4.138932 O $-1.483299-2.4807183 .333905$ C $-4.074581-4.645066 \quad 0.724211$ H -2.753662 -4.5358362 .374468$ H $-4.480402-4.2848302 .774182$ H $-4.992533 \quad 1.8602132 .730208$ H -2.407723 0.9414654 .567205 H -1.082269 -2.101116 4.134547 O $-4.121654-6.0051210 .845900$ O $-4.310207-4.009141-0.293896$ C $-4.467393-6.718254-0.345718$ H $-3.691007-6.579961-1.103675$ H $-4.531379-7.781337-0.099049$ H $-5.439896-6.386682-0.721979$ SCF Energy (B3LYP/6-31G**//MMFF)= -3245.92730019

012

MMFF Geometry

C $-2.686567-3.811584-1.926736$

C $-1.740664-3.627719-0.994712$

C $-0.267902-3.545629-1.309199$

O $0.227044-2.263533-0.860205$

C $0.485335-4.648006-0.541937$

C $1.975864-4.685693-0.819392$

C $2.930103-4.4999870 .114153$

C $2.372266-4.983537-2.242594$

C $2.745315-4.232466 \quad 1.581769$

C $2.633333-2.7453471 .948672$

C $3.914551-1.9361301 .693532$

C $3.786819-0.5252032 .272140$

C 5.0167770 .3414531 .957832

$\begin{array}{lllll}\text { O } & 4.124501 & -1.850957 & 0.282507\end{array}$

$\begin{array}{lllll}\text { O } & 4.652000 & 1.696233 & 2.264248\end{array}$

$\begin{array}{llll}\text { C } & 5.325173 & 0.255616 & 0.445440\end{array}$

C $6.198641-0.0089122 .869671$

$\begin{array}{llllll}\text { O } & 6.561270 & 0.893610 & 0.135702\end{array}$

C $5.338464-1.189254-0.096505$

C $5.434996-1.262445-1.640159$

O $6.481968-1.8764540 .427154$

C $-0.015557-1.185548-1.655163$

O $-0.586917-1.176808-2.733630$

C $0.5123830 .007169-0.957922$

C $0.105356 \quad 1.228133-1.334916$

C $0.4989042 .496448-0.716981$

C 1.5639232 .4524420 .345905

C $4.305268-0.561602-2.403658$

C $4.6476790 .850712-2.849559$

C $3.6204192 .847992-3.928069$

O $5.778526 \quad 1.324649-2.782105$

C $3.3045203 .851206-2.864214$

C $2.4038714 .832741-3.024147$

C $2.1082245 .876456-1.985708$

C $0.6351465 .970616-1.657649$

C $-0.1404786 .911339-2.222429$ 
C $0.0830535 .029291-0.611688$ C $-0.1044823 .630408-1.129778$ N $3.5430321 .509393-3.373639$ H -2.389901 -3.927504 -2.966129 H $-2.033675-3.5173520 .046426$ H $-0.088674-3.649287-2.386381$ H $\quad 0.067937-5.630115-0.801327$ H $0.300113-4.5137620 .529209$ H $3.971730-4.553115-0.200809$ H $3.449005-5.156190-2.346156$ H $1.862358-5.884654-2.598666$ H $2.110411-4.148580-2.899686$ H $1.862564-4.7611811 .957127$ H $3.591959-4.6768712 .120476$ H $1.803412-2.3046711 .385505$ H $2.375037-2.6812263 .012899$ H $4.754321-2.4658082 .158399$ H $3.611046-0.5614483 .354562$ H $2.900186-0.0380181 .843568$ H 5.4294152 .2598052 .113264 H $4.5412620 .821410-0.070446$ H $5.9317770 .158704 \quad 3.920014$ H 7.0583610 .6410752 .672293 H $6.523217-1.0468692 .770878$ H 6.4788351 .8311340 .378277 H $6.422557-0.920959-1.972789$ H $5.388956-2.326078-1.916066$ H $\quad 6.678867-2.631947-0.151010$ H $1.194425-0.155230-0.131443$ H $-0.6063001 .308573-2.155810$ H $2.4580091 .945854-0.031963$ H 1.2035521 .9169971 .229604 H 1.8951833 .4386520 .676760 H $4.105147-1.128464-3.322235$ H $3.380028-0.549804-1.819462$ H $2.9155902 .894356-4.764129$ H $4.6304763 .024496-4.312046$ H $3.8611463 .785516-1.932276$ H $1.869944 \quad 4.913057-3.968604$ H $2.4661216 .840140-2.371489$ H $2.6765705 .698486-1.064331$ H -1.191964 $7.003572-1.971239$ H $0.2583137 .607323-2.953191$ H $-0.9014315 .379569-0.272868$ H 0.7147835 .0751520 .279568 H $-0.8553673 .559480-1.917666$ H $2.6321831 .060719-3.340472$ C $-4.967112-2.683203-2.111081$ O $-4.672418-1.518422-1.315880$ C $-4.851175-0.349252-2.142870$ C $-5.345959-0.844231-3.503416$ C $-4.771220-2.245611-3.559593$ H $-3.8584070 .097096-2.270933$ C $-5.7993780 .651877-1.470932$ H -6.440500 -0.878250 -3.553186 H $-4.999979-0.205580-4.322300$ C -5.447794 -3.146043 -4.576884 H -3.705732 -2.159064 -3.804982 C $-5.3598381 .121550-0.069293$ O $-7.0866830 .033961-1.333761$ H $-5.9432421 .514851-2.132242$ H -5.4475420 .2883010 .636642$ $\begin{array}{lllll}\text { O } & -6.308551 & 2.106262 & 0.365046\end{array}$ C -3.9459861 .7253190 .001459$ C -3.6174842 .4297851 .332985$ H $-3.2051160 .941833-0.191319$ O $-3.8147402 .693309-1.043713$ H -2.591102 2.8111041 .262385 H $-4.2387713 .329367 \quad 1.431471$ H -5.000216 -4.144938 -4.571110 H $-6.517578-3.254414-4.368845$ H $-5.341900-2.734153-5.585814$ C $-4.156370-3.889088-1.627777$ H -7.675474 $0.682297-0.910751$ H $-6.2174432 .878753-0.218993$ H -2.911749 3.049194-1.001690 H -4.545397 -4.800008 -2.098091 H $-4.306448-4.003352-0.546608$
H $-6.028119-2.906324-1.932019$

C $-1.6861160 .354110 \quad 2.470176$

$\begin{array}{lllll}\text { O } & -3.113313 & 0.285777 & 2.437746\end{array}$

C -3.7728421 .5452892 .585514$

C -3.3484042 .2235983 .897809$

C -1.8283312 .3231554 .030527$

C -1.1827790 .9589713 .784118$

H -1.3448950 .9829101 .638690$

C -1.128370 -1.043374 2.179994

H $-4.842005 \quad 1.3203672 .681797$

O $-3.9381803 .516374 \quad 3.984594$

H $-3.736807 \quad 1.6341674 .738962$

H -1.412535 3.0802243 .355048

O -1.4922332 .7359625 .356783$

H -1.404699 0.3004004 .631356

O 0.2334521 .1082123 .722620

C -1.505786 -2.110170 3.185676

H -1.516687 -1.381709 1.213339

H $-0.036363-0.9903932 .124496$

H -3.7638373 .8615994 .876120$

H $-1.810273 \quad 3.647473 \quad 5.469837$

H $\quad 0.517614 \quad 1.5271064 .553121$

O $-0.720578-3.2082742 .973043$

O $-2.389638-2.0154934 .025513$

C $-0.973992-4.3239513 .831917$

H $-0.791111-4.0480774 .874647$

H $-0.286823-5.1281653 .556011$

H -2.000407 -4.678717 3.698995

SCF Energy $\left(B 3 L Y P / 6-31 G^{* *} / / M M F F\right)=-3245.90651622$

0120

MMFF Geometry

C $-3.4199072 .313348-2.550998$

C $-2.6047131 .543366-1.815379$

C $-1.3656610 .874089-2.342001$

O $-0.2808631 .160472-1.427738$

C -1.562874 -0.651573 -2.369454

C $-0.377856-1.370537-2.981049$

C $0.659991-1.872894-2.282561$

C $-0.413217-1.494077-4.482259$

C $0.880479-1.815414-0.794708$

C $1.231358-3.173314-0.165442$

C $2.572838-3.759566-0.632547$

C $2.929360-5.0165420 .164532$

C $4.312455-5.565889-0.222888$

O $3.584291-2.765938-0.445864$

O $4.707799-6.5141140 .780298$

C $5.341678-4.414601-0.186333$

C $4.269228-6.332912-1.549961$

O $6.605197-4.842352-0.689492$

C $4.875631-3.145079-0.935651$

C $5.852438-1.974968-0.670106$

O $4.851910-3.413286-2.340277$

C $0.3619432 .351364-1.548747$

O $0.1418993 .230641-2.365357$

C $1.3741612 .408899-0.470277$

C $1.9183883 .587110-0.133556$

C 2.8748563 .8158600 .952292

C 3.4074432 .6093951 .680120

C $5.309312-0.603431-1.100982$

C $6.3644980 .485756-1.013723$

C $6.6663282 .928385-1.408081$

O $7.5308220 .269486-0.695303$

C $6.6963203 .579767-0.061561$

C 6.4461694 .8832750 .135210

C 6.5446725 .5547941 .474337

C 5.3066226 .3445311 .831092

C $5.2994467 .686867 \quad 1.766665$

C 4.1087855 .5839062 .352215

C 3.2158405 .0872521 .250139

N $5.849874 \quad 1.730197-1.354908$

H $-3.2083812 .472121-3.604815$

H $-2.8409431 .378789-0.767407$

H $-1.107707 \quad 1.238827-3.344244$

H -2.464194 -0.905378 -2.943446

H - $1.750762-1.028017-1.358074$

H $1.465351-2.337151-2.848666$

H $0.463834-2.016576-4.878794$ 
H -1.300076 -2.055030 -4.795051 H $-0.445531-0.504073-4.947852$ H $1.685274-1.098039-0.595833$ H $0.000485-1.429333-0.273309$ H $1.273305-3.0211320 .920949$ H $0.427281-3.894119-0.357460$ H $2.495460-4.004479-1.697204$ H $2.159142-5.7879320 .040636$ H $2.943033-4.7762531 .236957$ H $4.028633-7.2091730 .820003$ H $5.500961-4.1524670 .869098$ H $3.574385-7.178446-1.480713$ H $5.246313-6.768198-1.786964$ H $3.952490-5.715054-2.392809$ H $6.866537-5.630739-0.183431$ H $6.071718-1.9140710 .403948$ H $6.801773-2.170363-1.184762$ H $4.999894-2.587778-2.827456$ H 1.6079921 .4863790 .049116 H $1.6093654 .476751-0.681130$ H 3.8245031 .8838920 .973290 H 2.6086702 .1224032 .250331 H 4.2119642 .8431662 .380364 H $4.952314-0.633360-2.135931$ H $4.468383-0.317449-0.458388$ H $6.238368 \quad 3.581122-2.175359$ H $7.6838632 .655665-1.706862$ H 6.9741552 .9588470 .787050 H $6.2007775 .512334-0.717526$ H 7.4177556 .2198691 .446323 H 6.7514524 .8295212 .271248 H 4.4236498 .2608102 .050908 H $6.166358 \quad 8.2412291 .422930$ H 3.5015696 .2437422 .987074 H 4.4463754 .7901773 .024191 H 2.7686125 .8915020 .663535 H $4.8542571 .824981-1.533845$ C -5.891066 2.134972 -2.147067 O $-5.7720390 .967956-1.307253$ C $-6.9701140 .826843-0.526069$ C $-8.0155021 .684998-1.224514$ C -7.166405 $2.850027-1.695813$ H $-6.758907 \quad 1.2598790 .459355$ C -7.332360 $-0.652154-0.373879$ H $-8.4590991 .168663-2.083743$ H $-8.8247111 .985900-0.552596$ C -7.822336 $3.681941-2.784127$ H -6.950684 $3.496627-0.834503$ C $-6.186221-1.5250190 .181744$ O $-7.682650-1.169357-1.664342$ H $-8.229050-0.7489250 .249560$ H -5.390948 -1.592274 - 0.568389 O $-6.697343-2.8568230 .329035$ C $-5.603403-1.0552691 .529862$ C $-4.603960-2.0462212 .158875$ H $-5.125067-0.0785251 .406997$ O $-6.686297-0.8831332 .448890$ H $-4.403762-1.7222103 .186782$ H -5.088451-3.024059 2.266729 H -7.163617 $4.496265-3.102112$ H -8.058728 $3.075813-3.665144$ H -8.755551 4.124127 -2.420277 C $-4.6361072 .992151-1.984786$ H -7.905881 -2.108902 -1.548698 H -7.377781 -2.833658 1.023879 H $-6.319284-0.5081303 .267569$ H -4.470585 $3.208570-0.921747$ H -4.759146 $3.956082-2.491437$ H -5.987442 $1.777397-3.180197$ C $-1.986319-0.5006322 .539611$ $\begin{array}{llll}\text { O } & -2.565893 & -0.943648 & 1.305728\end{array}$ C -3.283661 -2.181440 1.374538 C -2.361018 -3.296328 1.884575 C -1.714321 -2.930348 3.214801 C $-1.042577-1.563567 \quad 3.119722$ H $-2.780884-0.2698303 .258978$ C -1.2058980 .7917332 .249738$ H -3.542522 -2.442279 0.342301
O $-3.069849-4.5222962 .030170$

H $-1.566002-3.4724441 .149495$

H $-2.438825-2.957368 \quad 4.037137$

O $-0.710565-3.898146 \quad 3.530842$

H $-0.141183-1.6434482 .499096$

O $-0.625276-1.158120 \quad 4.422500$

C $-2.103034 \quad 1.9772851 .967444$

H -0.5834351 .0634403 .109548$

H $-0.566705 \quad 0.642517 \quad 1.372524$

H $-3.464769-4.735803 \quad 1.167494$

H $-1.143862-4.7688723 .546222$

H $-0.043619-1.8556374 .770768$

O $-1.318112 \quad 3.0498791 .649157$

O -3.3245391 .9731952 .021331$

C -2.014346 4.2633101 .351014

H -2.6080124 .1415590 .441286$

H $-2.649597 \quad 4.5569892 .192134$

H -1.2717585 .0476051 .182108$

SCF Energy (B3LYP/6-31G*//MMFF) $=-3245.92104977$

01_200

MM̄FF Geometry

C $-0.155249-3.0703971 .957771$

C $-0.681556-3.057040 \quad 0.723897$

C $0.019684-3.308595-0.588033$

O $0.010774-2.050249-1.303193$

C $1.470426-3.817713-0.481065$

C $2.044422-4.298798-1.801103$

C $3.019185-3.670973-2.487514$

C $1.499331-5.611399-2.305405$

C $3.702732-2.379890-2.137551$

C $5.100948-2.644095-1.563736$

C $5.827027-1.343208-1.198141$

C $7.244710-1.629990-0.700541$

C $7.959761-0.344670-0.254763$

O $5.082264-0.701983-0.161292$

$\begin{array}{llll}\text { O } & 9.126673 & -0.723106 & 0.492463\end{array}$

C 7.0431190 .4376960 .713312

C $8.463050 \quad 0.462711-1.457451$

O 7.5865061 .7214011 .010007

C $5.585768 \quad 0.5852750 .217161$

C $4.679103 \quad 1.1255051 .343576$

O $5.4843591 .526772-0.855181$

C $-0.426955-2.039514-2.587927$

O $-0.813693-3.001737-3.237544$

C $-0.385616-0.679172-3.173756$

C $-0.0745290 .426505-2.480120$

C $-0.0293351 .794661-3.004493$

C $-0.361632 \quad 2.008425-4.457738$

C $3.193788 \quad 1.067652 \quad 0.972744$

C $2.341716 \quad 1.9189531 .888732$

C $1.534715 \quad 4.2609092 .141896$

O $1.613771 \quad 1.4227172 .744920$

C 0.3315864 .2458091 .254611

C $-0.009555 \quad 5.221026 \quad 0.399867$

C $-1.1515335 .076188-0.567771$

C $-0.7105485 .106851-2.018832$

C -1.354657 $5.886697-2.903722$

C $0.4659554 .258669-2.461779$

C $0.2991092 .793510-2.159533$

N $2.4637363 .275201 \quad 1.616357$

H $\quad 0.907617-3.2393712 .101732$

H $-1.748665-2.8706870 .632593$

H $-0.591038-4.053484-1.112770$

H $1.518941-4.660913 \quad 0.221019$

H $2.102485-3.033625-0.049987$

H $3.375662-4.128048-3.409783$

H $2.017829-5.958122-3.205752$

H $1.615320-6.388314-1.542532$

H $\quad 0.437812-5.523476-2.553650$

H $3.786669-1.778578-3.051338$

H $3.103642-1.782557-1.443764$

H $5.001566-3.269993-0.666843$

H $5.697975-3.206417-2.292721$

H $7.830841-2.148897-1.469186$

H $7.199261-2.3162550 .156777$

H $9.676480-1.289417-0.075829$ 
H $7.024601-0.1181881 .661411$

H $9.192644-0.120828-2.032034$

H $8.9911431 .366802-1.135522$

H $7.6641210 .751689-2.143663$

H 8.4903871 .5835831 .341898

H $4.815900 \quad 0.5305652 .255629$

H 4.9663312 .1561931 .585179

H $6.355381 \quad 1.928359-1.001913$

H - $-0.645036-0.635835-4.226807$

H $\quad 0.1681190 .332538-1.422869$

H -1.349460 $1.598679-4.695275$

H $\quad 0.3830911 .521498-5.096635$

H $-0.3954013 .062435-4.743565$

H $3.0242821 .407570-0.056291$

H 2.8255120 .0359231 .024305

H 2.0417755 .2303032 .142672

H 1.2545023 .9995523 .166916

H $-0.262796 \quad 3.3357901 .273683$

H 0.5800116 .1328740 .354265

H -1.868695 $5.880728-0.361709$

H -1.690930 4.136643 -0.394121

H -1.056554 $5.926688-3.946200$

H -2.198180 $6.501532-2.607628$

H $1.3719514 .610107-1.950851$

H $0.6812034 .414476-3.524564$

H $0.5153502 .534892-1.123394$

H $3.0275293 .556857 \quad 0.819321$

C $-0.795903-1.5615503 .880701$

O $-1.491402-0.5530443 .114964$

C $-2.4143780 .130045 \quad 3.981775$

C $-1.887486-0.1008415 .390227$

C -1.407894 -1.533074 5.285840

H $-3.383046-0.3738383 .874949$

C -2.5533881 .6022773 .586875$

H -1.046186 $0.561406 \quad 5.624914$

H -2.6583750 .0406836 .153468$

C $-0.436398-1.9318396 .383909$

H -2.281869 -2.197447 5.329631

C $-2.915707 \quad 1.8428252 .108100$

O $-1.3140652 .278408 \quad 3.837290$

H -3.2916962 .0872204 .235848$

H -2.061933 1.5816541 .473638

O -3.1313713 .2512491 .941908$

C -4.1790351 .1088661 .621370$

C $-4.606090 \quad 1.4824460 .187770$

H -4.0288180 .0273181 .681789$

O

H -5.6182951 .0948060 .023970$

H -4.7101882 .5714600 .111990$

H $-0.106314-2.9674456 .254625$

H $0.452680-1.2923056 .384723$

H $-0.911337-1.8479617 .366866$

C $-0.978508-2.9222423 .207454$

H -0.6201051 .8149093 .336973$

H -2.360651 3.7117532 .315130

H -5.3930662 .3850272 .474905$

H -2.039718 -3.076652 2.975420

H $-0.681210-3.7257183 .891818$

H $\quad 0.262061-1.2722243 .905844$

C $-4.717787-1.164874-1.338745$

O $-3.546982-0.471628-0.885047$

C $-3.6449890 .958561-0.897790$

C $-3.9645801 .453244-2.315097$

C $-5.2062990 .779968-2.887560$

C $-5.083722-0.737893-2.767855$

H $-5.555676-0.955095-0.663771$

C -4.421359 -2.672324 -1.283829

H $-2.6456281 .336335-0.655089$

O $-4.1402622 .865261-2.338682$

H -3.121240 $1.225526-2.977017$

H $-6.1203981 .143916-2.403990$

O $\quad-5.323210 \quad 1.112506-4.272919$

H -4.320572 -1.091798 -3.472738

O $-6.318534-1.337652-3.156810$

C $-4.471998-3.2274720 .124238$

H $-5.165572-3.226995-1.866105$

H -3.421611 -2.872788 -1.685469

H $-3.3201343 .266256-2.004078$
H $-5.3541742 .082704-4.335394$

H -7.005750 -1.034454 -2.539103

O $-4.233750-4.572460 \quad 0.072653$

O $-4.687122-2.5775021 .137401$

C $-4.256199-5.2524221 .331585$

H -3.448079 -4.887757 1.971495

H $-5.226526-5.1196281 .819543$

H -4.101305 -6.318332 1.144689

SCF Energy (B3LYP/6-31G**//MMFF) $=-3245.92800151$

01201

MM̄FF Geometry

C $4.639519-2.8534752 .303039$

C $3.326110-2.7457052 .050509$

C $2.368434-2.0450642 .976243$

O $1.898933-0.8708672 .274378$

C $1.166669-2.9452363 .315662$

C $0.232818-2.3285194 .340103$

C $-1.025443-1.9135774 .092786$

C $0.780365-2.2434815 .744007$

C $-1.800681-1.9715842 .803183$

C $-2.950340-2.9755492 .950333$

C $-3.847246-3.1488861 .717367$

C $-3.121326-3.7953810 .536367$

C $-4.056513-3.968065-0.671863$

O $-4.387371-1.8778311 .338051$

O $-3.246948-4.282407-1.815116$

C $-4.744046-2.615652-0.958052$

C $-5.020723-5.146824-0.494305$

O $-5.725274-2.746042-1.983145$

C $-5.370306-1.9594270 .293404$

C $-5.920114-0.5376680 .017680$

O $-6.492478-2.746548 \quad 0.719146$

C 1.9351630 .3138182 .942338

O 2.3069870 .5038814 .089710

C 1.4396151 .3661612 .028371

C 1.2416612 .6024382 .510649

C 0.7688303 .7660191 .759227

C $0.5314203 .588798 \quad 0.284695$

C $-4.9650830 .466917-0.625604$

C -3.7849280 .8502260 .240993$

C -3.1573682 .2526052 .196034$

O $-2.6408110 .496192-0.033524$

C -2.8164663 .6019091 .645056$

C -2.8173324 .7311622 .368475$

C -2.4710946 .0778881 .800362$

C -1.2309816 .6757862 .426455$

C -1.3232147 .6135153 .384414$

C 0.1137336 .2513131 .875583

C 0.5682084 .9247452 .422253

N -4.1413631 .6360881 .325984$

H $5.041941-2.4338523 .221958$

H $2.924870-3.1477841 .123267$

H $2.899097-1.7664923 .894451$

H $1.523131-3.9047813 .713747$

H $\quad 0.626497-3.1908262 .395496$

H $-1.592413-1.4933634 .923619$

H $0.031169-1.8935406 .462441$

H $1.115540-3.2307316 .079029$

H $1.627192-1.5540005 .794868$

H $-2.199030-0.9716652 .597612$

H $-1.164386-2.2305261 .954851$

H -2.551387 -3.956634 3.240007

H -3.588426 -2.638720 3.779304

H -4.672696 -3.797169 2.035693

H $-2.686029-4.7599070 .825530$

H $-2.278707-3.1651660 .225096$

H -2.756569 -5.099174 -1.618735

H $-3.968066-1.951358-1.353339$

H $-4.462049-6.081788-0.366802$

H $-5.639421-5.287425-1.387615$

H $-5.680863-5.0394060 .368404$

H $-5.285819-3.140623-2.755890$

H $-6.807922-0.620110-0.623618$

H $-6.282968-0.1194290 .966308$

H -7.063533 -2.191070 1.275511

H $1.254737 \quad 1.0964980 .995274$

H 1.4423922 .7863303 .565590 
H $0.0778864 .461945-0.186457$ H -0.1529532 .7544850 .096979$ H $1.4759123 .390667-0.233130$ H $-4.5947540 .107986-1.590643$ H -5.518844 $1.387719-0.851602$ H $-2.258526 \quad 1.6291702 .232659$ H -3.5928502 .3098273 .198209$ H -2.538252 3.6508730 .594576 H $-3.089251 \quad 4.6918803 .420595$ H -3.332710 6.7390881 .961038 H $-2.340030 \quad 6.031207 \quad 0.711817$ H -0.4384508 .0684523 .817119$ H -2.285941 7.9427963 .761184 H 0.8786156 .9917212 .146606 H 0.0802026 .2773410 .782908 H 0.7692134 .9475323 .494538 H $\quad-5.104160 \quad 1.9486101 .412029$ C $6.337395-2.5351310 .436425$ O $5.394401-2.018274-0.522343$ C $5.636769-0.609475-0.697613$ C $7.011423-0.345605-0.092115$ C $7.011724-1.3192361 .073037$ H $4.882958-0.085911-0.096550$ C $5.475791-0.219547-2.171844$ H $7.820423-0.592585-0.788905$ H $7.1360990 .696393 \quad 0.217324$ C $8.392751-1.6077141 .633818$ H $6.388585-0.892826 \quad 1.868275$

C $4.169507-0.734383-2.811459$

O $6.568398-0.782683-2.908636$

H $5.5580290 .868995-2.274932$

H $4.208233-1.827231-2.902299$

O $4.090158-0.220909-4.146950$

C $2.884573-0.329897-2.064353$

C $1.630287-0.911913-2.747290$

H $2.923717-0.707446-1.036926$

O $2.8167701 .090066-2.000829$

H $1.550507-0.510828-3.761876$

H $1.776626-1.994289-2.836977$

H $8.334676-2.3273712 .456539$

H $9.058797-2.0215960 .869261$

H $8.851322-0.6906642 .017640$

C $5.626090-3.5099231 .379644$

H $6.580828-0.359109-3.783401$

H $4.789635-0.649522-4.668114$

H $2.0141791 .328350-1.507408$

H $6.365861-4.0408521 .990337$

H $5.107821-4.2703830 .781277$

H $7.072047-3.105426-0.147419$

C $-0.5780821 .165852-3.319585$

O $-0.0679130 .748466-2.046058$

C $0.330708-0.625127-1.960855$

C $-0.843251-1.542755-2.328809$

C $-1.422506-1.197154-3.696303$

C $-1.7619650 .290434-3.756109$

H $\quad 0.217715 \quad 1.111320-4.070925$

C -1.020137 $2.633377-3.186787$

H $0.554369-0.813703-0.902650$

O $-0.428443-2.903529-2.290843$

H $-1.628107-1.429008-1.572464$

H $-0.739415-1.480723-4.505531$

O $-2.629631-1.931973-3.905562$

H $-2.6290060 .484605-3.113062$

O $-2.151961 \quad 0.627310-5.087018$

C $0.144366 \quad 3.600902-3.191632$ H $-1.6633372 .917547-4.027084$ H $-1.5683032 .771683-2.247151$ H $-1.222448-3.455985-2.384351$ H $-2.396639-2.874563-3.950542$ H $-1.3929040 .461491-5.671739$ O $-0.3331824 .864321-2.980895$ O $1.316397 \quad 3.300889-3.367941$ C $0.6537375 .900648-2.962140$ H $1.3940775 .708965-2.179422$ H $1.1368705 .977969-3.940629$ H $0.1495636 .845650-2.742858$

SCF Energy (B3LYP/6-31G**/MMFF) $=-3245.91237232$

\section{2}

MMFF Geometry

C $3.609841-3.087941-0.778892$

C $2.963956-3.2988600 .378741$

C $1.471088-3.2020360 .583113$

O $0.931419-2.304916-0.412713$

C $0.801133-4.580844 \quad 0.457415$

C $-0.595023-4.6367161 .049900$

C -1.735613 -4.797047 0.349963

C $-0.658914-4.5472152 .555590$

C $-1.916230-4.957591-1.132774$

C $-2.379986-3.689517-1.865461$

C $-3.780112-3.186549-1.472689$

C $-4.216373-2.052145-2.404185$

C $-5.578019-1.464639-2.005271$

O $-3.744028-2.701032-0.128331$

O $-5.716164-0.226012-2.719991$

C $-5.572497-1.143527-0.492077$

C $-6.726839-2.364485-2.475581$

O $-6.874127-0.783355-0.039046$

C $-5.021123-2.2908250 .384922$

C $-4.821455-1.8949491 .867232$

O $-5.933896-3.3958630 .433706$

C $0.062820-1.343582-0.008864$

O $-0.432782-1.208308 \quad 1.097587$

C $-0.163088-0.447358-1.164399$

C -1.198255 $0.402686-1.138889$

C $-1.5360521 .390788-2.161829$

C $-0.5273021 .646114-3.249561$

C $-3.879087-0.7150202 .120773$

C -4.5812700 .6307842 .210070$

C -4.100780 3.0785022 .181199

O $\begin{array}{lllll}-5.791979 & 0.749337 & 2.378472\end{array}$

C -4.4686943 .5431720 .807940$

C -3.7518814 .4294510 .100044$

C $-4.1369354 .904031-1.272550$

C $-3.1379614 .499783-2.334673$

C $-2.2125155 .366204-2.779484$

C $-3.2671493 .119735-2.942506$

C -2.708855 $2.047028-2.049562$

N -3.6865401 .6885852 .121234$

H $3.040919-2.895231-1.684970$

H $3.544856-3.5175711 .271579$

H $1.326296-2.7798131 .583664$

H $1.405621-5.3398230 .972437$

H $\quad 0.789383-4.873139-0.597579$

H -2.668152 -4.843956 0.911761

H -1.670344 -4.718178 2.940168

H $-0.007112-5.3013723 .008588$

H $-0.342585-3.5591742 .901843$

H $-0.993016-5.310130-1.602989$

H -2.647972 -5.757982 -1.300750

H $-1.643798-2.898133-1.696420$

H -2.372280 -3.909054 -2.940650

H -4.478761 -4.028636 -1.539275

H $-4.237329-2.390453-3.447821$

H -3.469474 -1.248101 -2.368711

H $-6.5914200 .144269-2.515548$

H $-4.934309-0.260939-0.361909$

H $-6.734699-2.430290-3.570377$

H -7.699999 -1.947467 -2.194198

H -6.660214 -3.382328 -2.085404

H $-7.1414290 .019872-0.516504$

H $-5.794658-1.7390552 .348649$

H $-4.385030-2.7671742 .375481$

H $-6.769576-3.1262870 .019912$

H $0.532600-0.503381-1.993818$

H $-1.8656480 .380120-0.280177$

H $-0.4332720 .768633-3.898472$

H $\quad 0.4556801 .872102-2.820989$

H $-0.7832812 .494973-3.887552$

H -3.388410 -0.863039 3.091367

H -3.094111 -0.6770511 .361874$

H -3.2702113 .6520732 .602071$

H -4.962058 3.1770492 .849673

H $-5.379930 \quad 3.1325940 .379048$

H -2.843600 $4.845640 \quad 0.529541$

H $-4.2256885 .997663-1.234114$ 
H $-5.1334354 .542156-1.555183$ H -1.511331 $5.092401-3.560891$ H -2.131769 $6.366633-2.367622$ H -2.806523 $3.091871-3.935739$ H $-4.3269942 .902807-3.128560$ H $-3.3699541 .777668-1.226385$ H -2.7088991 .4966451 .923179$

C $5.557670-1.856831-1.746792$

O $5.049321-0.629015-1.197081$

C $5.9694090 .434058-1.514803$

C $7.148698-0.210600-2.244706$

C $7.068009-1.656549-1.789684$

H $6.3262320 .830842-0.557601$

C $5.2414221 .539727-2.295764$

H $7.048217-0.149718-3.332983$

H $8.0991990 .264933-1.982023$

C $7.801551-2.622820-2.702465$

H $7.484961-1.726108-0.775991$

C $3.9660372 .063739-1.602747$

O $4.8163401 .028113-3.566726$

H $5.9243622 .372438-2.502977$

H $3.2045941 .275479-1.594560$

O $3.4363673 .127477-2.405231$

C $4.1819832 .602739-0.178242$

C 2.9456983 .2780080 .444763

H 4.5081781 .7916590 .476136

O $5.2416083 .563682-0.188850$

H 3.2429633 .6748701 .423080

H $2.6788804 .163415-0.145941$

H $7.716611-3.649690-2.333636$

H $7.399663-2.596226-3.720916$

H $8.865793-2.370734-2.753913$

C $5.107805-3.050342-0.900959$

H $5.5774321 .063192-4.169970$

H $3.2882722 .769301-3.297377$

H $4.9840714 .277578-0.797268$

H $5.560828-2.9868920 .095343$

H $5.450107-3.982077-1.364446$

H $5.150599-1.947762-2.763348$

C 2.2725051 .3269042 .708310

O 2.0261281 .1511971 .307627

C 1.7222162 .3515240 .588666

C 0.4853993 .0303501 .192892

C 0.6609413 .2840422 .685442

C $1.0714491 .995123 \quad 3.393891$

H 3.1726341 .9375912 .849804

C $2.529536-0.062553 \quad 3.316855$

H $1.4424842 .041512-0.423268$

O $\quad 0.2031104 .2598520 .534002$

H $-0.3913762 .385026 \quad 1.056253$

H 1.3818544 .0869302 .878031

O $-0.5815923 .721243 \quad 3.239008$

H 0.2184391 .3042673 .415730

O 1.4117212 .2967724 .746184

C $3.895688-0.6167312 .970998$

H $2.478063-0.0029754 .410124$

H $\quad 1.782198-0.7793282 .960535$

H $0.0610074 .060052-0.407291$

H -0.8516464 .5166612 .748375$

H $\quad 0.6415672 .7342035 .148255$

O $4.152442-1.6906993 .776488$

O $4.641296-0.1794012 .106664$

C $5.434323-2.3021373 .599688$

H $6.226490-1.5974763 .869832$

H $5.492219-3.1679834 .264553$

H $5.558329-2.6435442 .568491$

SCF Energy (B3LYP/6-31G**//MMFF) $=-3245.91292640$

\section{3}

MMFF Geometry

C -1.403855 -3.879618 -1.980197

C $-0.741534-3.446941-0.897917$

C $0.588828-2.742514-0.961681$

O $0.422867-1.402521-0.432298$

C $1.646484-3.441246-0.088070$

C $2.416964-4.516401-0.825916$

C $3.636832-4.326064-1.366525$

C $1.765786-5.872383-0.885151$
C $4.428986-3.047744-1.386621$

C $5.558588-3.100167-0.352751$

C $6.348716-1.785826-0.277512$

C $7.521359-1.9373230 .695386$

C $8.269329-0.615750 \quad 0.911801$

O $5.457798-0.7621310 .176755$

O $9.122285-0.7725712 .056089$

C 7.2439530 .4815061 .260331

C $9.185917-0.277898-0.269773$

O $7.877794 \quad 1.757245 \quad 1.337292$

C 6.0456130 .5428380 .283183

C 4.9609491 .4921530 .853100

O $6.4997551 .020620-0.980977$

C $-0.141489-0.472541-1.250863$

O $-0.535320-0.639894-2.394210$

C $-0.2041100 .803256-0.504311$

C $-0.8781801 .838424-1.026685$

C $-1.0180983 .172001-0.436582$

C $-0.319310 \quad 3.433160 \quad 0.871210$

C $3.7258701 .680079-0.040866$

C $3.8441222 .849870-1.006877$

C $2.5419924 .058408-2.756857$

O $4.8205333 .593344-1.049916$

C $1.7644895 .153465-2.094855$

C $0.6639545 .708341-2.625038$

C $-0.1170606 .807588-1.962105$

C $-1.5900316 .484027-1.830741$

C $-2.5012097 .081144-2.617508$

C $-2.0138355 .526015-0.736564$

C $-1.7481614 .092016-1.101413$

N $2.7196452 .970532-1.811917$

H - $0.956252-3.748460-2.961931$

H -1.186648 -3.5812380 .084634$

H $\quad 0.958707-2.660590-1.992361$

H $1.187615-3.8735910 .811741$

H $2.339360-2.6914350 .314141$

H $4.125769-5.168825-1.852958$

H $2.427904-6.631216-1.316049$

H $1.495181-6.2099200 .120765$

H $\quad 0.861689-5.841421-1.499117$

H $4.852713-2.918735-2.390116$

H $3.787411-2.176848-1.219731$

H $\quad 5.124447-3.314990 \quad 0.633187$

H $6.238576-3.926107-0.597590$

H $6.709190-1.532134-1.281291$

H $8.212079-2.7180020 .352584$

H $7.143529-2.2813401 .668822$

H $9.727015-1.5135121 .879842$

H $\quad 6.858481 \quad 0.2657382 .266858$

H $9.937887-1.064190-0.407746$

H $9.7482310 .643693-0.083194$

H $8.647426-0.169675-1.213585$

H 8.6136461 .6775281 .968103

H 4.6071391 .0651251 .801345

H 5.3974372 .4704761 .085042

H $5.728674 \quad 1.252473-1.521343$

H $\quad 0.307840 \quad 0.8566290 .449575$

H -1.373488 $1.701615-1.987395$

H 0.7615423 .2930010 .764634

H -0.6872042 .7516061 .645408$

H -0.4669754 .4444121 .253965$

H $3.4959640 .767249-0.601031$

H $2.867430 \quad 1.8982230 .606531$

H $2.0223683 .650409-3.629473$

H $3.5199624 .435601-3.071863$

H $2.1301625 .513325-1.135640$

H $\quad 0.3112735 .366618-3.595683$

H $0.0169927 .717525-2.561444$

H $0.2873087 .042301-0.969510$

H -3.560974 $6.873161-2.513262$

H $-2.2078027 .787355-3.387045$

H -3.091826 $5.620821-0.550690$

H $-1.5326395 .829678 \quad 0.196533$

H -2.226505 $3.791490-2.035119$

H $1.9362062 .342383-1.663322$

C $-3.896960-3.699570-2.468255$

O $-4.155222-2.579479-1.597921$

C $-4.615977-1.475209-2.404126$ 
C $-4.766682-2.008009-3.829668$

C $-3.722505-3.105707-3.864182$

H $-3.815698-0.726140-2.393972$

C $-5.907309-0.878787-1.829493$

H $-5.764505-2.425887-4.006817$

H $-4.596624-1.229325-4.579630$

C $-3.917977-4.103566-4.991299$

H -2.740783 -2.629214 -3.971356

C $-5.800361-0.381711-0.373202$

O $-6.923486-1.889671-1.872576$

H $-6.251173-0.066506-2.481694$

H $-5.697629-1.2360290 .306106$

O $-7.0408800 .256579-0.041459$

C $-4.663420 \quad 0.624728-0.117412$

C $-4.735615 \quad 1.342388 \quad 1.245608$

H -3.699673 $0.112192-0.210253$

O $-4.703258 \quad 1.629531-1.135240$

H -3.8732032 .0177861 .310026$

H -5.6075852 .0090421 .259297$

H -3.143723 $-4.876723-4.965018$

H $-4.892267-4.599134-4.924859$

H -3.861864 -3.602001-5.962942

C -2.745621 -4.552079-1.926709

H $-7.778760-1.452153-1.725355$

H $-7.720875-0.435742 \quad 0.014069$

H -3.957694 $2.233478-0.981042$

H -2.679615 -5.484759 -2.499286

H -2.974414 -4.832948 -0.890720

H $-4.806621-4.315251-2.439694$

C -2.423471-0.083069 2.643850

O $-3.743790-0.5841942 .422265$

C $-4.7729690 .406647 \quad 2.469267$

C $-4.750496 \quad 1.135383 \quad 3.822227$

C -3.3681741 .6961134 .151825$

C -2.3019450 .6109514 .003864$

H $-2.189017 \quad 0.6427821 .856420$

C $-1.430984-1.2344102 .445946$

H -5.723217 -0.1386232 .421923$

$\begin{array}{llll}\text { O } & -5.700998 & 2.195188 & 3.846003\end{array}$

H $-5.0336870 .431652 \quad 4.615921$

H -3.1288282 .5682813 .531866$

O -3.3559042 .1523205 .506518$

H -2.406733 -0.1076774 .824456$

O $-1.008314 \quad 1.1990124 .116797$

C $-1.589154-2.395747 \quad 3.404474$

H $-1.577989-1.6467401 .442798$

H $-0.406573-0.8540202 .526210$

H $-6.5724441 .810276 \quad 3.650107$

H -4.0665392 .8104945 .595682$

H $-0.974811 \quad 1.664124 \quad 4.970432$

O $-0.495318-3.2076113 .290068$

O $-2.551417-2.5915394 .133141$

C $-0.506684-4.3772514 .113909$

H $0.416046-4.9329443 .927197$

H $-0.539892-4.0959895 .170717$

H - $1.358263-5.0138583 .855416$

SCF Energy (B3LYP/6-31G**/MMFF) $=-3245.91055384$

01204

MM̄FF Geometry

C $-3.398495-3.008924-1.089608$

C $-2.401306-3.887526-1.272810$

C $-0.922379-3.610836-1.098943$

O $-0.716911-2.198474-0.866711$

C $-0.397157-4.407908 \quad 0.113228$

C $1.110633-4.560540 \quad 0.171094$

C $1.907276-4.0655731 .139325$

C $1.717596-5.396484-0.929535$

C $1.527225-3.2716882 .357650$

C $1.816832-1.766940 \quad 2.254974$

C $3.310000-1.4008962 .215030$

C 3.4887490 .1168552 .306914

C 4.9595190 .5367142 .172728

O $3.871427-1.872495 \quad 0.987777$

$\begin{array}{lllll}\text { O } & 4.969714 & 1.955270 & 1.948528\end{array}$

C $5.574950-0.1453140 .929514$

C $5.728505 \quad 0.305007 \quad 3.479213$

$\begin{array}{llll}\text { O } & 6.975893 & 0.113901 & 0.888955\end{array}$
C $5.286163-1.6606570 .861164$

C $5.731504-2.326371-0.465511$

O $6.001760-2.3204801 .913395$

C $0.360291-1.595340-1.432424$

O $1.238507-2.119179-2.097986$

C $0.287529-0.155160-1.093549$

C $1.380520 \quad 0.608574-1.235304$

C $1.4899872 .037065-0.935069$

C $0.2265132 .786685-0.611150$

C $5.105748-1.749251-1.738758$

C $5.933115-0.652558-2.389006$

C $5.742714 \quad 1.204012-4.044279$

O $7.124349-0.476413-2.147326$

C $5.6459902 .437028-3.202833$

C $4.8171353 .459022-3.464401$

C $4.7162614 .686780-2.605600$

C $3.3565994 .835624-1.957644$

C $2.4538485 .706665-2.438601$

C $3.0777994 .043095-0.698855$

C $2.7096742 .611997-0.981522$

N $5.192726 \quad 0.082713-3.304139$

H $-3.169454-1.990755-0.791701$

H -2.654925 -4.906069-1.561046

H $-0.432387-3.915538-2.030641$

H $-0.818411-5.4228130 .103574$

H $-0.776548-3.9372301 .025737$

H $2.972742-4.2824651 .072008$

H $2.784295-5.586770-0.768091$

H $1.218868-6.369726-0.985168$

H $1.619589-4.899208-1.898480$

H $0.468141-3.4040972 .595456$

H $2.067639-3.6868553 .217810$

H $1.316247-1.3721781 .365060$

H $1.358064-1.2805973 .125198$

H $3.806846-1.8955183 .057863$

H 3.0632200 .5040343 .241421

H 2.9177430 .5966461 .501496

H 5.8920102 .2216871 .790648

H 5.1352950 .3502740 .055405

H 5.2858730 .8956194 .290523

H 6.7666790 .6448703 .395380

H $5.732600-0.7401873 .795123$

H $7.316098-0.2089440 .038164$

H $6.825916-2.350567-0.528683$

H $5.423477-3.380972-0.415541$

H $6.085651-3.2614751 .686464$

H $-0.6532380 .229332-0.717346$

H $2.2958670 .139818-1.591094$

H -0.1524762 .4860360 .370415$

H $-0.5443182 .590400-1.363981$

H $0.3588123 .870869-0.591648$

H $5.031995-2.550777-2.484976$

H $4.090326-1.391908-1.545357$

H $5.175261 \quad 1.292052-4.976039$

H $6.7899661 .002587-4.291381$

H $6.2914882 .486789-2.328792$

H $4.1726363 .413679-4.339171$

H $4.9321495 .556302-3.240020$

H $5.4896274 .694899-1.827201$

H $1.4889565 .841956-1.961435$

H $2.6576636 .300831-3.323277$

H $2.3091174 .538537-0.095709$

H $3.9704004 .053701-0.060032$

H $3.5649801 .979725-1.216124$

H $4.195013-0.089562-3.386232$

C $-5.638016-2.342320-2.087787$

O $-5.560492-1.025136-1.503855$

C $-5.322612-0.073350-2.558769$

C $-5.677500-0.796017-3.852513$

C $-5.184603-2.197984-3.540941$

H $-4.2439420 .124499-2.567645$

C $-6.0811901 .229100-2.286208$

H $-6.757907-0.809780-4.032604$

H $-5.189974-0.352055-4.725469$

C $-5.740617-3.263984-4.468320$

H $-4.089642-2.203055-3.610818$

C $-5.7244361 .884393-0.933192$

O $-7.4894350 .966710-2.256010$ 
H $-5.9126221 .944625-3.100062$ H $-6.0955741 .262148-0.110827$ O $-6.4333003 .127705-0.850160$ C $-4.2237242 .170231-0.738873$ C -3.8867823 .0113240 .506495$ H $-3.6730101 .224737-0.694163$ O $-3.7448902 .878765-1.886243$ H $-2.805308 \quad 3.1944910 .499224$ H -4.3345844 .0083050 .407550$ H -5.370694 -4.254976 -4.186822 H $-6.835097-3.291240-4.439447$ H -5.436886 -3.070351 -5.502274 C $-4.857421-3.342636-1.231323$ H -7.7861480 .838548 -3.172488 H -7.378182 2.929919-0.969422 H $-2.7886563 .011221-1.773714$ H $-4.989817-4.353074-1.634958$ H $-5.283648-3.337701-0.219981$ H -6.699144 -2.622552 -2.054896 C $-2.473801 \quad 0.8382132 .118867$ O C -4.3069552 .3753241 .846823$ C -3.8612353 .1717593 .082888$ C -2.3590283 .0480303 .336012$ C -1.9360721 .5777213 .350104$ H -1.9745021 .2288181 .224929$ C -2.167311 -0.6651192 .130428$ H -5.4035372 .3565491 .858067$ O -4.2009274 .5482552 .946884$ H -4.3923492 .7948393 .966763$ H -1.7862323 .6228512 .598734$ O -2.0737453 .6309244 .610051$ H -2.2953521 .1123674 .274843$ O $-0.512628 \quad 1.4891343 .352799$ C $-2.790950-1.4368213 .274260$ H -2.555631 -1.113439 1.211637 H -1.083014 -0.814272 2.159732 H -5.1619674 .5993652 .807075$ H -1.108002 3.6420094 .718713 H $-0.194308 \quad 1.8747294 .186212$ O $-2.181438-2.6564743 .363656$ O $-3.711506-1.0446923 .977146$ C $-2.677381-3.5211134 .389842$ H $-2.543117-3.0590205 .372551$ H $-3.731484-3.7538934 .211698$ $\mathrm{H}-2.102530-4.4505274 .357574$ SCF Energy (B3LYP/6-31G**//MMFF) $=-3245.90668667$

01205

MM̄̄FF Geometry

C $-4.639929-2.834913-1.353939$

C -3.334795 -3.000565 -1.095751

C $-2.287779-3.104154-2.175372$

O $-1.316907-2.050535-1.975511$

C -1.553887 -4.452923 -2.077822

C $-0.561731-4.675216-3.203925$

C $0.776151-4.749782-3.058753$

C $-1.169690-4.858640-4.572912$

C $1.597160-4.651058-1.803082$

C $2.459525-3.387129-1.799007$

C $3.539585-3.384481-0.705606$

C $2.952824-3.4180350 .707227$

C $4.033522-3.2761491 .791175$

O $4.307315-2.186710-0.892348$

O $3.378061-2.9774523 .033566$

C $4.927412-2.0652061 .454625$

C $4.795759-4.5881582 .009620$

O $6.034119-1.9793722 .348509$

C $5.427472-2.058583-0.004622$

C $6.173287-0.755217-0.373458$

O $6.354816-3.135011-0.190464$

C -1.638426 -0.811256-2.438939

O $-2.663837-0.481570-3.013138$

C $-0.5171560 .093252-2.102863$

C $-0.6586651 .417339-2.264486$

C $0.3425912 .434056-1.937722$

C $1.7019811 .960610-1.494386$

C $5.3655960 .530991-0.184422$
C $6.0599861 .741069-0.787441$

C $5.7819174 .194400-1.065070$

O $7.1340331 .679014-1.379311$

C $4.6096215 .113350-1.182919$

C $4.0373805 .436404-2.352562$

C $2.8471356 .343428-2.482676$

C $1.5934905 .578668-2.854339$

C $1.1829085 .532039-4.133449$

C $0.8560384 .932333-1.697669$

C $0.0113613 .737485-2.053139$

N $5.3204352 .900694-0.598467$

H -4.971404 -2.808923 -2.388944

H $-2.995386-3.042804-0.064384$

H -2.741514 -2.997317 -3.168297

H -2.281614 -5.275249 -2.099204

H $-1.059270-4.518379-1.103656$

H $1.372889-4.921933-3.954624$

H $-0.429185-5.166809-5.319105$

H $-1.943000-5.633378-4.544660$

H -1.620295 -3.926315 -4.925533

H $0.976687-4.680617-0.905092$

H $2.242394-5.537261-1.758958$

H $2.957555-3.268405-2.771237$

H $1.822314-2.499399-1.691762$

H $4.192045-4.250130-0.870711$

H $2.375082-4.3339220 .877581$

H $2.240712-2.5912160 .816850$

H $2.738087-3.687473 \quad 3.213705$

H $4.319679-1.1687961 .626792$

H $4.108947-5.3802162 .331961$

H $5.534009-4.4878002 .812761$

H $5.306481-4.9422901 .111845$

H $5.676819-1.9798823 .253101$

H $7.112720-0.6869230 .190817$

H $\quad 6.472977-0.824088-1.428874$

H $6.910067-2.933850-0.961980$

H $\quad 0.386092-0.353741-1.703345$

H -1.604334 $1.798377-2.647733$

H $2.4281162 .769506-1.397687$

H $2.1258431 .260373-2.223238$

H $1.6379411 .460998-0.523241$

H $4.3835040 .436220-0.660942$

H 5.2174050 .7299210 .882522

H $6.3085514 .068733-2.017427$

H $6.4987944 .583155-0.334629$

H $4.2092495 .524484-0.259397$

H $4.4362055 .017190-3.273706$

H $3.0777277 .100779-3.242921$

H $2.6692826 .904816-1.556663$

H $0.2784725 .014430-4.432363$

H $1.7463856 .022642-4.920922$

H $0.2071175 .688924-1.237392$

H $1.5642924 .656783-0.908639$

H $-0.9881983 .995466-2.403657$

H 4.5359802 .8736220 .047577

C $-6.360204-1.307247-0.256535$

O $-5.437720-0.3139310 .233343$

C $-5.7353640 .940868-0.413396$

C $-7.0024940 .717424-1.240148$

C $-6.900636-0.756534-1.574807$

H $-4.9018461 .143434-1.096102$

C -5.8627222 .0739330 .612811$

H $-7.9112890 .912815-0.659331$

H $-7.0327791 .358463-2.126761$

C $-8.213492-1.379049-2.014362$

H $-6.164707-0.862518-2.380442$

C -4.6355132 .2674161 .525866$

O -6.9882191 .7896701 .455227$

H -6.0967043 .0091650 .089358$

H $-4.564731 \quad 1.4327162 .233227$

O -4.8621353 .4475092 .308595$

C $-3.2949802 .435304 \quad 0.787313$

C -2.1302142 .9121101 .679619$

H $-3.024975 \quad 1.4873180 .308795$

O $-3.4622323 .398453-0.256780$

H -1.2318972 .9706331 .052714$

H -2.314127 3.9477491 .993584

H $-8.084351-2.443161-2.236032$ 
H -8.981670 -1.286385 -1.239262 H $-8.588121-0.888502-2.918698$ C $-5.698779-2.689153-0.298669$ H -7.201188 2.6022071 .944123 H -5.5737593 .2486172 .939961$ H -2.622352 $3.452242-0.742685$ H -6.464133 -3.452399 -0.483064 H $-5.267925-2.9025590 .687906$ H -7.170004 -1.351186 0.484703 C $-0.585493 \quad 0.277444 \quad 1.865952$ O C -1.863149 2.029506 2.914254 C -0.6492922 .4563723 .753266$ C 0.6749602 .0646903 .098092 C 0.6612550 .5870902 .702112 H -0.5388120 .8486020 .931589$ C $-0.718234-1.1875241 .445425$ H -2.7394992 .1266793 .566251$ O -0.6833253 .8601493 .986939$ H $-0.714031 \quad 1.9674434 .734155$ H 0.8869762 .7003432 .230539

O $1.7423092 .298620 \quad 4.020247$ H $0.690092-0.0214183 .614053$ O $1.842894 \quad 0.3121791 .957651$ C $-0.746913-2.1507442 .607179$ H $-1.643610-1.3261460 .875511$ H $0.098290-1.4842680 .778900$ H $\quad 0.1111584 .0897954 .498809$ H 1.5938941 .7383804 .801058 H $1.919453-0.6528371 .877024$ O $-2.013425-2.2498653 .101861$ O $0.241127-2.7368213 .029922$ C $-2.170259-3.1287744 .219689$ H $-3.222143-3.1159134 .517113$ H -1.896932 -4.150527 3.939358 H -1.563097 -2.782806 5.061542 SCF Energy (B3LYP/6-31G**//MMFF) $=-3245.91049740$

01_206

MM̄̄FF Geometry

C $2.431177-4.1985670 .071233$

C $1.151070-4.290827-0.318855$

C $-0.011830-3.7878070 .498884$

O $-0.722958-2.803214-0.288047$

C $-0.981638-4.9433100 .803526$

C $-2.162002-4.5357351 .664870$

C -3.449355 -4.574756 1.268017

C $-1.826972-4.0857053 .064079$

C -3.998577 -5.020767 -0.058045

C $-4.166337-3.900714-1.096690$

C $-5.167245-2.806238-0.690325$

C $-5.450616-1.870289-1.868056$

C $-6.375917-0.708373-1.472134$

O $-4.605148-2.054986 \quad 0.388429$

O $-6.3206920 .274941-2.517602$

C $-5.826737-0.038064-0.194115$

C -7.839676 -1.154199-1.373872

$\begin{array}{lllll}\text { O } & -6.740184 & 0.936118 & 0.303371\end{array}$

C $-5.469330-1.0416550 .920668$

C $-4.729367-0.3883792 .113399$

O $-6.674713-1.6163301 .440011$

C $-0.183996-1.555881-0.356178$

O $0.820986-1.1563250 .209711$

C - $1.017266-0.758633-1.282190$

C $-0.5076300 .361094-1.814817$

C $-1.1760081 .260055-2.755988$

C $-2.6385061 .025718-3.023404$

C $-3.3994650 .290997 \quad 1.768911$

C -3.5094381 .7889751 .536398$

C $-2.138223 \quad 3.7467370 .829297$

O $-4.5224272 .437759 \quad 1.782712$

C $-2.4386044 .067315-0.600430$

C -1.605795 $4.764185-1.388270$

C $-1.9087305 .134428-2.811630$

C $-0.8258474 .691723-3.769818$

C $0.1295025 .544641-4.176806$

C $-0.9003963 .283762-4.315648$

C $-0.4545162 .253586-3.315895$
N -2.321355 2.3259131 .057648

H $2.666137-3.7657161 .040479$

H $0.920310-4.712527-1.294566$

H $\quad 0.333396-3.3290301 .433513$

H $-0.446168-5.7514301 .319528$

H -1.328553 -5.367332 -0.145028

H $-4.211978-4.2583351 .979165$

H -2.721018 -3.956087 3.683757

H -1.191942 -4.826890 3.560252

H -1.299962 -3.126944 3.046421

H $-3.375156-5.812808-0.486646$

H $-4.972833-5.4935630 .120882$

H -3.186568 -3.450756 -1.294795

H $-4.503315-4.364356-2.032281$

H $-6.092424-3.290696-0.356830$

H $-5.874875-2.425742-2.713824$

H $-4.503776-1.449457-2.232878$

H $-6.593210-0.153906-3.346924$

H $-4.9203390 .504782-0.485999$

H -8.183938 -1.552109-2.336224

H $-8.496756-0.306830-1.149034$

H -8.002983 -1.927794 -0.620793

H $-6.9195581 .561194-0.419720$

H $-5.403913 \quad 0.2882602 .651617$

H -4.496029 -1.191958 2.827104

H $-6.491787-1.9670942 .327428$

H -2.009978 -1.124287 -1.515804

H $0.5103520 .640945-1.552285$

H $-3.1973000 .984277-2.082513$

H -2.784772 $0.082786-3.560943$

H $-3.1063601 .813967-3.616351$

H -2.720084 0.1651192 .620842

H -2.926734 -0.1812290 .903181$

H -1.105634 3.9883651 .099646

H -2.811096 4.3107161 .483328

H $-3.3951113 .735453-0.997443$

H -0.662982 $5.119423-0.979986$

H -2.020983 $6.225702-2.855254$

H -2.873591 $4.725889-3.136510$

H $0.8944285 .241507-4.883916$

H $\quad 0.1709506 .563703-3.806644$

H $-0.2466183 .185182-5.192643$

H $-1.9083083 .100753-4.697936$

H $0.6007252 .338891-3.051908$

H $-1.541671 \quad 1.7076100 .852591$

C $4.431571-3.422081-1.202547$

O $5.191736-2.940063-0.071752$

C $6.544004-2.709131-0.493805$

C $6.515770-2.688666-2.014905$

C $5.456542-3.738582-2.292941$

H $7.114513-3.596370-0.184308$

C $7.174726-1.4921820 .193833$

H $6.193300-1.714614-2.399059$

H $7.485451-2.930938-2.459473$

C $4.896855-3.675567-3.703664$

H $5.890713-4.733193-2.122803$

C $6.607840-0.094590-0.142351$

O $8.573970-1.492853-0.124961$

H $7.115997-1.6370891 .279532$

H $6.8384790 .158423-1.183823$

O $7.3413520 .838430 \quad 0.666594$

C 5.1014370 .0871090 .123874

C $4.6269711 .542435-0.074371$

H $4.540619-0.554922-0.560818$

O $4.822896-0.339814 \quad 1.455115$

H 5.0769682 .1774940 .695273

H $4.9902391 .895418-1.046530$

H $4.125534-4.437525-3.852643$

H $4.449903-2.698285-3.914888$

H $5.688949-3.848658-4.439485$

C $3.591351-4.629773-0.782859$

H $8.665256-1.289762-1.071319$

H 7.2216121 .7223320 .280630

H $3.856584-0.3844541 .552179$

H $4.198982-5.333906-0.202090$

H $3.227742-5.161364-1.669661$

H $3.780873-2.598637-1.524909$

C 2.8011792 .1156532 .334380 
O $2.531751 \quad 1.2560351 .222394$

C $3.0868841 .686561-0.024525$

C $2.5623163 .087744-0.370776$

C 2.8161714 .0839160 .759432

C 2.2818803 .5349902 .083283

H 3.8849562 .1531232 .498682

C 2.2190561 .4694113 .598091

H $2.6973981 .000645-0.786332$

O $3.1542823 .551950-1.578556$

H $1.4807263 .024633-0.547821$

H 3.8817174 .3276260 .836863

O 2.1563865 .3162050 .461024

H 1.1860953 .5477932 .060306

O $2.6961494 .382542 \quad 3.151167$

C 0.7080811 .4017923 .591695

H 2.5783920 .4387463 .703629

H 2.5561422 .0205924 .483904

H $2.8013564 .441597-1.752098$

H 1.2016805 .1406790 .409012

H 2.3872395 .2815682 .945553

O $0.213217 \quad 2.0214524 .701236$

$\begin{array}{lllll}\text { O } & 0.048346 & 0.862999 & 2.712842\end{array}$

C -1.2125262 .0333054 .820837$

H -1.468782 24860145.782446

H -1.6498422 .6377984 .021546$

$\mathrm{H}-1.6078911 .0134864 .798633$

SCF Energy (B3LYP/6-31G**//MMFF) $=-3245.89770226$

01207

MM̄FF Geometry

C $-0.446318-3.1401851 .707825$

C $-0.910492-1.9171651 .412332$

C $-1.413984-0.9450482 .447526$

$\begin{array}{lllll}\text { O } & -0.771892 & 0.327450 & 2.202067\end{array}$

C $-2.927195-0.6994962 .304095$

C $-3.777939-1.9423152 .438560$

C $-4.346484-2.5946151 .404942$

C $-3.977610-2.4326343 .848331$

C $-4.251252-2.266052-0.061075$

C $-5.625887-2.071744-0.715236$

C $-6.424340-0.883633-0.156112$

C $-7.807732-0.823608-0.809012$

C $-8.6121030 .393067-0.331526$

$\begin{array}{llll}\text { O } & -5.701412 & 0.322771 & -0.428180\end{array}$

O $-9.7383260 .550088-1.208160$

C $-7.7392051 .653902-0.486283$

C $-9.183793 \quad 0.1868551 .076079$

$\begin{array}{lllll}\text { O } & -8.386129 & 2.792394 & 0.079352\end{array}$

C -6.3153491 .5048270 .102759$

C $-5.4669122 .741136-0.293847$

O

C 0.4581650 .5502862 .735411

O $1.079223-0.1629553 .504207$

C 0.9348681 .8205822 .153548

C 2.1756242 .2773002 .372794

C 2.6768523 .4849621 .725488

C 3.2259994 .5144272 .667528

C $-3.9652472 .620289-0.004788$

C $-3.1679841 .955526-1.117184$

C $-0.8164261 .296548-1.656557$

O $-3.651281 \quad 1.630245-2.197406$

C $0.1379072 .366851-2.105786$

C $0.1026783 .679295-1.818876$

C $1.1353124 .666634-2.294863$

C $1.8222145 .396060-1.156364$

C $1.4263526 .626066-0.787707$

C $3.0176624 .737572-0.497779$

C $2.616153 \quad 3.5918050 .383502$

N $-1.8362641 .803223-0.758354$

H $-0.419995-3.4388932 .754413$

H $-0.961057-1.5841180 .380004$

H $-1.189252-1.2785703 .468147$

H -3.124580 $-0.182493 \quad 1.359113$

H -3.241000 0.0322383 .061652

H -4.935744 -3.484164 1.625137

H $-4.626329-3.3138153 .894650$

H -3.018656 -2.705568 4.299581

H $-4.440611-1.6507624 .459339$
H $-3.626426-1.391541-0.259203$

H -3.751685 -3.108472 -0.556098

H -5.460459-1.921004-1.789981

H -6.211784 -2.993297 -0.605821

H -6.529136-1.005052 0.927583

H $-8.367318-1.748833-0.622814$

H $-7.693287-0.756990-1.900206$

H -10.258582 -0.271099-1.177541

H -7.645787 $1.854873-1.562909$

H $-9.845288-0.6875381 .095865$

H $-9.803957 \quad 1.037707 \quad 1.379039$

H -8.4134790 .0338591 .834666$

H $-9.2665932 .857582-0.328673$

H -5.605536 $2.959052-1.359805$

H $-5.8403703 .610573 \quad 0.262923$

H -5.579068 1.779556 1.915267

H $\quad 0.2536472 .3567351 .500092$

H 2.8600971 .7630823 .040980

H 4.1097464 .1250513 .184942

H 2.4767534 .7818773 .421251

H 3.5198365 .4409772 .169029

H -3.557166 3.6342700 .098636

H -3.7790152 .0963590 .937248$

H -1.283269 $0.829306-2.529045$

H $-0.2629840 .528193-1.107283$

H $\quad 0.958527 \quad 2.002777-2.721307$

H $-0.7039414 .083652-1.213074$

H $0.6281725 .388085-2.948852$

H $1.8929304 .182726-2.923733$

H 1.9275057 .1664130 .008594

H $0.5878197 .117291-1.270186$

H 3.6069205 .4779990 .052594

H $3.6927724 .360664-1.276390$

H $2.2361422 .733379-0.169012$

H -1.5403592 .1081830 .163749$

C $0.058515-3.827579-0.742388$

O $1.119612-2.882619-1.000353$

C $1.766742-3.240200-2.236987$

C $0.903847-4.325018-2.872281$

C $0.350348-5.023833-1.648561$

H $2.734812-3.679878-1.971845$

C $1.969303-2.011438-3.128736$

H $0.089661-3.897340-3.469362$

H $1.483752-4.987821-3.521816$

C $-0.861591-5.890526-1.945748$

H $1.141436-5.645957-1.208783$

C $2.874235-0.915446-2.528371$

O $0.686259-1.429046-3.392442$

H $2.365591-2.331078-4.100321$

H $2.363743-0.438732-1.684025$

O $3.0195790 .104273-3.527220$

C $4.273589-1.394235-2.094578$

C $5.282024-0.264402-1.809052$

H $4.177519-2.035165-1.211594$

O $4.825485-2.202135-3.139382$

H $6.234239-0.731491-1.528440$

H $5.5033710 .268773-2.742570$

H -1.232885 -6.371220 -1.035492

H $-1.680425-5.302417-2.373654$

H $-0.604107-6.678396-2.661281$

C $0.030924-4.1953390 .744430$

H $0.831796-0.647313-3.952325$

H $3.508742-0.283329-4.273295$

H $5.671810-2.558238-2.818943$

H $1.040161-4.4857251 .058708$

H $-0.614895-5.069550 \quad 0.889296$

H -0.883567 -3.354180 -1.046791

C $5.463225-0.5418571 .218200$

O 4.4133320 .0852200 .472174

C $4.8399970 .736381-0.726392$

C 5.884376 1.814499-0.399049

C 7.0392551 .2623460 .432198

C 6.5095070 .4932841 .641870

H $5.936138-1.3197900 .606348$

C $4.822433-1.2171372 .433660$

H $3.9612201 .256848-1.123159$

O $6.4007782 .403625-1.587463$

H 5.4102672 .6186430 .175986 
H $7.7050870 .634163-0.171066$

O 7.8366042 .3492990 .908445

H 6.0761151 .1970602 .364238

O $7.594386-0.1708362 .285545$

C $3.986896-2.3957191 .998920$

H $5.567956-1.5733833 .153425$

H $4.163137-0.5238922 .969024$

H $5.6472782 .764945-2.085093$

H 8.1328262 .8524020 .130569

H 8.2516740 .5069532 .519050

O $4.787490-3.4755361 .769906$

O $2.772586-2.3519761 .856633$

C $4.115935-4.6537491 .314759$

H $3.608866-4.4583250 .365271$

H $4.866112-5.4330641 .156682$

H $3.404555-4.9993502 .070625$

SCF Energy (B3LYP/6-31G**//MMFF) $=-3245.89966672$

01_208

MM̄FF Geometry

C $-0.9985463 .423144-0.861096$

C $0.0110544 .029080-0.218938$

C $0.842781 \quad 3.3314370 .825315$

O $2.224834 \quad 3.342228 \quad 0.396804$

C 0.7870954 .0340772 .194547

C -0.5936904 .1334462 .809262$

C -1.282896 3.0900783 .312465

C -1.1808075 .5199292 .845295$

C $-0.833172 \quad 1.6567013 .382422$

C -1.6120350 .7851282 .387765$

C $-1.068809-0.6447152 .234675$

C -1.278156 -1.477600 3.499108

C $-0.686286-2.8863873 .356592$

O $0.319908-0.5652741 .900779$

O $-0.667547-3.4875194 .661021$

C $0.780411-2.7587692 .894011$

C - $1.556622-3.7905612 .475513$

O $1.313190-4.0564432 .632252$

C $0.962052-1.8258301 .669319$

C $2.454779-1.5393891 .375498$

O $0.416964-2.4734720 .515790$

C $2.6423842 .293004-0.363406$

O $1.9722491 .345696-0.741754$

C $4.0774002 .495535-0.660116$

C $4.7254601 .600645-1.420737$

C $6.1340571 .659945-1.816724$

C $6.9655082 .788672-1.264627$

C $3.146854-0.6451682 .409292$

C $4.554368-1.1203202 .699849$

C $6.787657-1.4249651 .692093$

O $4.842382-1.6523683 .770426$

C $7.653581-0.6867340 .723680$

C $8.278494-1.278349-0.305705$

C $9.215253-0.572374-1.244154$

C $8.746295-0.618726-2.680632$

C $9.054285-1.657098-3.476072$

C $7.9983250 .580968-3.215052$

C $6.6127650 .704932-2.641962$

N $5.437526-0.8965441 .653506$ H -1.210294 2.381370 -0.640115 H $0.2389115 .070135-0.431545$ H $\quad 0.521307 \quad 2.2923440 .951665$ H 1.2281785 .0370892 .105815 H 1.4605323 .5171682 .891677 H $-2.272483 \quad 3.2749183 .727762$ H -2.1972505 .5318503 .252658$ H $-0.563798 \quad 6.174098 \quad 3.469929$ H -1.2249295 .9451201 .837834$ H 0.2436761 .5746453 .209033 H -1.003524 1.3000634 .404784 H -2.6698470 .7503392 .675505$ H -1.573583 1.2604121 .400930 H -1.603569 -1.1027181 .394224$ H -2.343338 -1.532023 3.756120 H $-0.787878-0.9921294 .353116$ H - $1.581033-3.5098524 .993886$ H $1.343330-2.3606003 .747364$ H $-2.565316-3.8797932 .896612$
H -1.156600 -4.809726 2.438026

H -1.659340 -3.423037 1.453215

H $2.280995-3.9792342 .594867$

H $2.992394-2.4845901 .231432$

H $2.532289-1.0304720 .405313$

H $\quad 0.805797-2.070048-0.277486$

H $4.5577373 .377245-0.251558$

H $4.1714730 .743152-1.801281$

H $6.9173182 .802917-0.169780$

H $6.6065943 .751177-1.644977$

H $8.0249292 .716637-1.518531$

H 3.1894420 .3902562 .051273

H $2.608872-0.6157373 .362222$

H $6.726090-2.4949551 .465264$

H $7.187598-1.3203042 .706378$

H 7.8040820 .3748750 .902910

H $8.141151-2.345689-0.464550$

H $10.198543-1.054469-1.163227$

H $9.3782550 .468484-0.938810$

H $8.747604-1.684552-4.516257$

H $9.618064-2.505171-3.101415$

H $7.8897930 .505384-4.305460$

H $8.6052121 .477383-3.059142$

H $5.932357-0.077916-2.979980$

H $5.058140-0.616440 \quad 0.753356$

C $-3.3736514 .056648-1.474011$

O $-3.8597582 .697870-1.392747$

C $-4.6693012 .577680-0.208172$

C -4.9905704 .0046730 .227096$

C $-3.7063824 .718281-0.135812$

H -4.0380822 .1118380 .557185$

C $-5.9123331 .719930-0.459363$

H $-5.8305294 .423720-0.339244$

H -5.2328654 .0692851 .292174$

C $-3.8389346 .228742-0.208357$

H $-2.9573374 .465390 \quad 0.622540$

C $-5.6573380 .271003-0.922595$

O $-6.7248792 .350753-1.459031$

H $-6.5240021 .704677 \quad 0.450911$

H $-5.3355990 .257006-1.970631$

O $-6.924336-0.403032-0.882246$

C $-4.656240-0.530060-0.069900$

C $-4.711763-2.059301-0.246450$

H $-3.641991-0.176303-0.281425$

O $-4.893441-0.272878 \quad 1.319218$

H $-3.913009-2.4847460 .373371$

H $-5.639552-2.4318840 .207414$

H $-2.8853986 .689594-0.485008$

H -4.588163 $6.531541-0.947271$

H $-4.141083 \quad 6.6349700 .762575$

C $-1.8933464 .081002-1.871422$

H $-6.1799792 .449178-2.258867$

H $-7.5375900 .099808-1.445639$

H $-5.811470-0.5283881 .513867$

H -1.579612 $5.116561-2.047668$

H -1.772565 $3.541099-2.817536$

H -3.950027 $4.530569-2.279352$

C $-2.197927-2.681561-1.891016$

O $-3.429997-2.128700-2.367732$

C $-4.604946-2.580686-1.692590$

C $-4.714371-4.109246-1.800979$

C $-3.462957-4.796758-1.265618$

C $-2.218591-4.217719-1.932200$

H -2.014612 -2.327850 -0.870309

C $-1.075173-2.156822-2.800611$

H $-5.453281-2.166405-2.249291$

O $-5.851706-4.593722-1.094810$

H -4.844305 -4.392681 -2.853465

H -3.395937-4.726643 -0.174019

O $-3.524514-6.191754-1.572601$

H $-2.176369-4.564701-2.972972$

O $-1.056831-4.718642-1.273409$

C $-0.908933-0.656069-2.727513$

H $-0.113350-2.606693-2.532330$

H -1.312547 -2.409965 -3.841591

H $-6.634127-4.146333-1.459806$

H -4.342674 -6.538292 -1.177029

H -1.108670 -5.689707 -1.290621 
O $-0.150622-0.247876-3.788101$

O $-1.3731150 .062906-1.854801$

C $0.062126 \quad 1.162279-3.897460$

H $-0.8810581 .660526-4.137620$

H $0.4886101 .563065-2.974705$

H $0.7690961 .337246-4.712731$

SCF Energy (B3LYP/6-31G*//MMFF) $=-3245.91625068$

01209

MM̄FF Geometry

C $2.086803-4.359358-0.385686$

C $0.858978-4.272100-0.917768$

C $-0.351191-3.849008-0.121988$

O $-0.965298-2.701655-0.753506$

C $-1.379577-4.994986-0.110175$

C $-2.569900-4.744964 \quad 0.794575$

C $-3.844445-4.631602 \quad 0.372789$

C $-2.264685-4.6584222 .268417$

C $-4.372179-4.712837-1.031741$

C $-4.513534-3.357525-1.743170$

C $-5.461069-2.372859-1.038313$

C $-5.758860-1.167613-1.932690$

C $-6.630514-0.122127-1.217359$

O $-4.829409-1.923352 \quad 0.163528$

O $-6.5839891 .090128-1.986089$

$\begin{array}{llll}\text { C } & -6.012794 & 0.195656 & 0.162439\end{array}$

C -8.103097 $-0.547133-1.167453$

O -6.8783861 .0290860 .928449$

C $-5.640440-1.0622510 .973610$

C $-4.833361-0.7489202 .257712$

O $-6.840522-1.7274161 .387292$

C $-0.346965-1.498270-0.602055$

O $0.701371-1.277686-0.017495$

C $-1.171052-0.480014-1.289558$

C $-0.6604570 .737899-1.525363$

C $-1.345274 \quad 1.842822-2.200067$

C $-2.8191881 .690371-2.466272$

C -3.507377 -0.0113532 .039669$

C -3.6118411 .4971712 .192100$

C -2.279117 3.5770861 .869027

O -4.6013162 .0623642 .649790$

C -2.580879 $4.182744 \quad 0.535147$

C -1.750402 $5.027772-0.094026$

C $-2.0547905 .676849-1.412983$

C -0.983906 $5.420203-2.448782$

C $-0.014796 \quad 6.321921-2.680143$

C -1.091184 $4.160730-3.277547$

C -0.630503 $2.936356-2.537151$

N $-2.447453 \quad 2.1384501 .792372$

H $2.227368-4.1172310 .665364$

H $0.709746-4.510371-1.967987$

H $-0.070924-3.5822590 .904299$

H $-0.897411-5.9242250 .222591$

H $-1.709803-5.177685-1.138403$

H $-4.615444-4.4630561 .124332$

H -3.172709 -4.6547742 .881279$

H -1.662896 -5.5166182 .584544$

H -1.714422 -3.740569 2.494593

H $-3.751051-5.373649-1.645378$

H -5.354586 -5.201109-0.994146

H $-3.519478-2.906256-1.843587$

H $-4.884347-3.552412-2.757145$

H $-6.388472-2.902687-0.791170$

H -6.233640 - $1.485180-2.869367$

H -4.813643 -0.690514 -2.224211

H $-6.9027830 .888961-2.882559$

H $-5.1036740 .778598-0.025532$

H -8.495615 $-0.686871-2.181926$

H $-8.7244110 .232007-0.711965$

H -8.262203 -1.479388 -0.621714

H $-7.067951 \quad 1.8191970 .394085$

H -5.469898 -0.2315682 .985667$

H $-4.583860-1.7126502 .725190$

H -6.632011 -2.294733 2.147875

H -2.171412 -0.764205 -1.594743

H $0.3639210 .942354-1.216106$

H $-3.2977222 .609240-2.811450$

H -3.345616 $1.405489-1.549233$
H -2.995402 $0.922427-3.226834$

H -2.794709 -0.3446552 .805149$

H -3.076007 -0.2547371 .063873$

H -1.2500303 .7662172 .187788$

H -2.958175 3.9877092 .622962

H -3.5357013 .9342800 .077724$

H -0.8093575 .2975550 .379585$

H -2.152756 $6.756287-1.238048$

H $-3.0273685 .353764-1.804564$

H $0.7406526 .157800-3.441308$

H $0.0492017 .240865-2.107034$

H $-0.4626494 .248712-4.174277$

H -2.110404 $4.070171-3.663204$

H $0.4321652 .951902-2.295987$

H -1.700596 1.5949911 .370116

C $4.281159-3.622559-1.350824$

O $4.714060-3.107438-0.076568$

C $6.131279-2.869359-0.128073$

C $6.530948-2.970344-1.592872$

C $5.558788-4.024984-2.089828$

H $6.602081-3.7059980 .406271$

C $6.473752-1.5732300 .619991$

H $6.358877-2.037420-2.135729$

H $7.579645-3.252935-1.723232$

C $5.415215-4.056232-3.601605$

H $5.901050-5.008757-1.741216$

C $5.874088-0.285127 \quad 0.008358$

O $7.889862-1.4506680 .726641$

H $6.070877-1.6738411 .636442$

H $4.862234-0.504396-0.349681$

$\begin{array}{llll}\text { O } & 6.660781 & 0.111018 & -1.114368\end{array}$

C $5.796658 \quad 0.874594 \quad 1.023958$

C $5.280698 \quad 2.208567 \quad 0.456054$

H 5.1562650 .5649451 .855659

O $7.0946951 .112802 \quad 1.574126$

H 5.2228762 .9184361 .290806

H $6.0412292 .631101-0.213276$

H $4.698968-4.824226-3.909998$

H $5.066417-3.094044-3.991473$

H $6.376639-4.281934-4.074393$

C $3.311505-4.786854-1.143089$

H $8.221620-2.2450241 .178425$

H $6.1753050 .801067-1.595382$

H $7.002626 \quad 1.7934672 .262443$

H $3.796526-5.580312-0.561206$

H $3.033355-5.219392-2.111066$

H $3.776477-2.797604-1.870988$

C $2.4457972 .193106 \quad 1.638320$

$\begin{array}{llll}\text { O } & 2.912948 & 1.476232 & 0.490690\end{array}$

C $3.9234342 .139506-0.272686$

C $3.4119963 .502178-0.757790$

C 2.8950034 .3584230 .394305

C 1.8944413 .5685901 .235726

H 3.2644182 .3079742 .359178

C $1.334891 \quad 1.3518482 .288705$

H $4.0742991 .527912-1.169940$

O $4.4414574 .193148-1.457773$

H $2.5962003 .339523-1.472166$

H 3.7212504 .7404551 .004881

$\begin{array}{lllll} & & 2.244789 & 5.501858 & -0.164616\end{array}$

H $0.963567 \quad 3.444317 \quad 0.668190$

$\begin{array}{lllll}\text { O } & 1.585628 & 4.305325 & 2.416847\end{array}$

C 1.8377860 .0464752 .865916

H $0.8564961 .902596 \quad 3.105711$

H $0.582126 \quad 1.108797 \quad 1.530096$

H $4.0804715 .054314-1.729862$

H 1.9972996 .0885590 .569632

H 1.1197685 .1143962 .146718

O $0.768279-0.7678103 .113379$

O $3.008817-0.2228643 .091512$

C $1.087398-2.0536613 .653428$

H $1.613391-1.9466344 .606922$

H $0.151597-2.5904803 .828788$

H $1.690713-2.6227762 .939864$

SCF Energy (B3LYP/6-31G**//MMFF) $=-3245.89000731$

0121

MM̄FF Geometry 
C $-0.5456463 .508729-1.242717$

C $0.3801114 .180051-0.541919$

C 1.0914983 .5925530 .648253

O $2.5007493 .592377 \quad 0.318573$

C 0.8638844 .4055571 .936921

C $-0.586673 \quad 4.5891102 .329844$

C -1.3770183 .6166152 .825185$

C -1.1257935 .9845662 .150405$

C -1.0095042 .1858043 .109675$

C -1.7489561 .2279772 .164892$

C $-1.505818-0.2658542 .442288$

C $-2.206914-0.7323933 .719910$

C $-1.915195-2.2081434 .025338$

O $-0.097544-0.4957822 .524730$

O $-2.341186-2.4724385 .370989$

C $-0.389308-2.425733 \quad 3.983145$

C $-2.717223-3.1532423 .122814$

O $-0.072351-3.8078874 .138357$

C $0.281457-1.8686972 .704754$

C $1.821864-1.9100082 .866252$

O $-0.103892-2.6726951 .593253$

C 3.2905962 .6927690 .963689

O 2.9670091 .9519841 .877567

C 4.6228532 .7285050 .321246

C 5.4699431 .7067830 .515866

C $6.7978081 .555386-0.080774$

C $7.3277812 .693190-0.912981$

C $2.608521-1.3156101 .689780$

C $3.012288-2.3220760 .625270$

C $4.301496-2.436255-1.513516$

O $2.751689-3.5194780 .691121$

C $5.777454-2.520613-1.281900$

C $6.689573-1.952798-2.085507$

C $8.171697-2.018712-1.846607$

C $8.802847-0.646883-1.741628$

C $9.383955-0.075822-2.810398$

C $8.8360670 .027362-0.387240$

C 7.4724950 .4049040 .126296

N $3.709856-1.709233-0.406411$

H $-0.7880642 .489311-0.957378$

H $0.6322535 .199409-0.823201$

H 0.7693872 .5542340 .793542

H 1.3478255 .3870051 .832993

H 1.4054723 .9394242 .770064

H -2.406859 3.8702333 .073981

H -2.1874886 .0581612 .408537$

H -0.5787526 .6848732 .790203$

H $-1.016756 \quad 6.308741 \quad 1.110928$

H 0.0707612 .0285933 .036410

H -1.280833 1.9780494 .150405

H -2.8271521 .4302612 .185438$

H - $1.4121681 .438303 \quad 1.142811$

H - $-1.901489-0.8200601 .582863$

H -3.288385 -0.5599153 .654325$

H -1.861218 -0.1386784 .575773$

H $-3.289903-2.2666835 .430589$

H $0.041024-1.9095764 .852868$

H -3.793080 -2.982139 3.249332

H -2.545873 -4.199960 3.396935

H -2.488287 -3.033256 2.062257

H $-0.504036-4.1142644 .954281$

H $2.080966-1.3134193 .751368$

H $2.156469-2.9361423 .056791$

H $\quad 0.521053-2.5242640 .867337$

H $4.8517293 .569277-0.323560$

H $5.1543340 .885863 \quad 1.158221$

H $7.3794193 .612255-0.318850$

H $6.6809452 .869989-1.779528$

H $8.3323372 .513796-1.302603$

H $2.053732-0.5016111 .211847$

H $3.542715-0.8972312 .085694$

H $4.057429-1.889072-2.429460$

H $3.872584-3.440608-1.581199$

H $6.108911-3.072252-0.405267$

H $6.356090-1.411729-2.968310$

H $8.620352-2.574565-2.680345$

H $8.408609-2.598798-0.945929$

H $9.8654800 .893993-2.742571$
H $\quad 9.387507-0.564440-3.779142$

H $9.4934830 .904871-0.396963$

H $9.301536-0.6554630 .335584$

H $7.006211-0.3711470 .734173$

H $3.891092-0.711174-0.352720$

C $-2.8173294 .083777-2.201779$

O $-3.3234682 .738821-2.063270$

C $-4.2980052 .726826-1.003207$

C $-4.6411774 .188111-0.727227$

C $-3.3066304 .857647-0.976824$

H $-3.7960062 .311657-0.121817$

C $-5.5077941 .865343-1.370844$

H $-5.3934684 .570093-1.427085$

H $-5.016120 \quad 4.3408340 .289535$

C $-3.3991196 .357750-1.188610$

H $-2.6671514 .661434-0.109375$

C $-5.1894010 .396361-1.714607$

O $-6.1485742 .447815-2.514110$

H $-6.2402671 .910083-0.555436$

H $-4.6780590 .349397-2.682759$

O $-6.447026-0.267106-1.911716$

C $-4.364547-0.372937-0.661978$

C $-4.411779-1.907169-0.800534$

H $-3.324519-0.032961-0.701534$

O $-4.841665-0.057700 \quad 0.650776$

H $-3.834861-2.3322580 .028995$

H -5.441059 -2.240364 -0.619554

H -2.409274 $6.785243-1.377308$

H -4.042053 $6.604718-2.040013$

H $-3.8144156 .846313-0.301119$

C $-1.3002104 .066524-2.415023$

H -6.917306 $1.892491-2.730021$

H $-6.937943-0.232871-1.073265$

H $-5.761668-0.3644790 .717553$

H $-0.9530915 .079216-2.650855$

H $-1.0694423 .434289-3.281299$

H -3.284624 $4.486235-3.110221$

C $-1.577455-2.789618-1.571055$

O $-2.540140-2.124041-2.400707$

C $-3.907992-2.459416-2.147766$

C $-4.109287-3.970997-2.323338$

C $-3.167016-4.770166-1.430108$

C $-1.724529-4.319904-1.645495$

H -1.700384 -2.451190 -0.536946

C $-0.185366-2.364620-2.068571$

H $-4.493684-1.975474-2.937553$

O $-5.462124-4.341895-2.078879$

H $-3.902255-4.235094-3.368381$

H $-3.448343-4.694527-0.373119$

O $-3.260489-6.155272-1.771848$

H $-1.380315-4.692640-2.619132$

O $-0.925310-4.941290-0.639073$

C $0.141604-0.922049-1.754199$

H $0.599261-2.968534-1.602586$

H -0.135854 -2.486793 -3.157420

H $-5.604987-4.362606-1.117760$

H -4.191979 -6.416300 -1.671134

H $\quad 0.006925-4.852984-0.897071$

O $1.299303-0.588814-2.398763$

O $-0.513110-0.190257-1.026461$

C $1.7814340 .738928-2.174880$

H $1.1690141 .450445-2.734642$

H $1.7816480 .977924-1.108023$

H $2.8097250 .793925-2.542166$

SCF Energy (B3LYP/6-31G**//MMFF) $=-3245.90655680$

01210

MM̄FF Geometry

C $6.3108240 .077740 \quad 0.131990$

C $5.407530-0.565233-0.622283$

C $4.942690-1.973445-0.360375$

O $3.548760-1.9213590 .020248$

C $5.059474-2.801890-1.654367$

C $4.623107-4.246934-1.503639$

C $3.538453-4.788294-2.094780$

C $5.512660-5.104489-0.640362$

C $2.545857-4.113934-3.003748$

C $1.299873-3.700560-2.223764$ 
C $0.223304-2.991010-3.056688$

C $0.696875-1.645997-3.610449$

C $-0.434661-0.879655-4.311842$

O $-0.874219-2.775309-2.162679$

O $0.033366 \quad 0.466282-4.494120$

C -1.670385 -0.831392 -3.386398

C $-0.695077-1.442246-5.714117$

O $-2.803427-0.295300-4.064432$

C -2.040797 -2.195984 -2.759666

C $-3.071630-1.999288-1.625634$

O $-2.643201-3.077207-3.708212$

C $3.262753-1.6609611 .326068$

$\begin{array}{llll}\text { O } & 4.057462 & -1.457549 & 2.229637\end{array}$

C $1.789624-1.6578971 .465327$

C $1.218399-1.3005992 .625238$

C $-0.222909-1.2378292 .884018$

C $-1.140727-1.7267041 .795237$

C $-3.443518-3.310301-0.919549$

C $-4.219865-3.0785490 .365575$

C $-4.840270-4.2711382 .472191$

O $-4.709922-1.9958540 .672375$

C $-3.726176-4.018618 \quad 3.441074$

C $-3.809424-3.1632184 .471375$

C $-2.672759-2.9023765 .421096$

C $-2.454953-1.4275475 .686129$

C $-2.615937-0.9204936 .920247$

C $-2.068998-0.5346564 .527793$

C $-0.652560-0.7527944 .068122$

N $-4.297179-4.2378851 .126134$

H $6.753240-0.4247270 .988102$

H $4.953534-0.043380-1.462105$

H $5.529300-2.4434360 .438353$

H $6.100200-2.793953-2.005833$

H $4.478868-2.307311-2.439920$

H $3.323068-5.839517-1.906815$

H $5.220023-6.159763-0.661418$

H $6.549331-5.046652-0.987890$

H $5.474125-4.7744750 .402173$

H $2.995202-3.258843-3.512818$

H $2.268113-4.819403-3.796303$

H $0.850010-4.587151-1.755383$

H $1.582569-3.051363-1.388115$

H $-0.096610-3.661224-3.862718$

H $1.540503-1.770838-4.298870$

H $1.074762-1.027996-2.784363$

H $-0.6441490 .957988-4.988066$

H $-1.433418-0.134729-2.570929$

H $0.191849-1.313843-6.346624$

H $-1.502457-0.899367-6.217427$

H $-0.943577-2.505784-5.708263$

H $-2.5772300 .604734-4.352823$

H -2.657439 -1.306266 -0.883692

H $-3.990025-1.545730-2.019211$

H -2.689123 -2.630396 -4.567838

H $1.206389-1.9364770 .596227$

H $1.859329-1.0108613 .456612$

H $-0.934559-2.7771811 .561208$

H - $-1.011342-1.1340130 .884365$

H $-2.196973-1.6686072 .058459$

H -4.055992 -3.934749 -1.579804

H $-2.536507-3.870609-0.664949$

H $-5.254318-5.2713092 .630486$

H $-5.642235-3.5313272 .561078$

H -2.799645 -4.5671553 .284897$

H $-4.742179-2.6330094 .648864$

H $-1.738390-3.3388965 .047472$

H -2.899466 -3.4257606 .358859$

H -2.467365 0.1352817 .122014

H -2.898932 -1.548672 7.758417

H -2.1489170 .5195244 .825785$

H $-2.800665-0.6449653 .723884$

H $0.092962-0.4293834 .795902$

H $-3.802127-5.0654870 .806361$

C 5.8714842 .5496290 .547014

O $4.5524812 .546139-0.033742$

C 3.5928212 .8353021 .002428

C 4.4023153 .2443212 .231151

C 5.6629942 .4183322 .053554
H $3.075103 \quad 1.8969151 .227468$

C 2.5970123 .8929420 .513622

H 4.6430354 .3137312 .222780

H $3.873723 \quad 3.0277403 .164519$

C 6.8368932 .9033252 .884492

H 5.4324991 .3817192 .329118

C $1.8213213 .519153-0.769432$

O $3.316515 \quad 5.1051120 .239659$

H $1.896776 \quad 4.142162 \quad 1.317590$

H $2.5363143 .405277-1.594249$

O $0.9812414 .621493-1.129621$

C $0.9401142 .256562-0.703646$

C 0.0370642 .1162060 .538976

H $0.3055312 .233265-1.598291$

$\begin{array}{llll}\text { O } & 1.786612 & 1.108330 & -0.782302\end{array}$

H -0.4634891 .1436700 .471033$

H $0.6602152 .040181 \quad 1.437741$

H 7.7213502 .2833902 .706826

H 7.0975403 .9398482 .645440

H 6.5985602 .8546123 .951924

C $6.7454531 .491393-0.129519$

H $3.9878924 .901061-0.434104$

H $1.5450555 .412412-1.179731$

H $1.2154530 .322195-0.809635$

H 7.7848081 .5992030 .201800

H $6.7439471 .669071-1.212945$

H 6.2984313 .5348590 .315672

C $-2.7192462 .396766-0.774460$

O $-1.782803 \quad 3.434906-0.474062$

C $-1.016594 \quad 3.227266 \quad 0.716435$

C $-1.943613 \quad 3.0373271 .928365$

C -2.9989781 .9605961 .688859$

C $-3.725177 \quad 2.2112040 .367095$

H $-2.169447 \quad 1.459308-0.920839$

C $-3.3754382 .704751-2.127411$

H -0.4698224 .1598160 .894568$

$\begin{array}{llll}\text { O } & -1.193244 & 2.710888 & 3.093521\end{array}$

H -2.461187 3.9833992 .135464

H $-2.552373 \quad 0.9612601 .714858$

$\begin{array}{lllll}\text { O } & -3.945928 & 2.007046 & 2.758050\end{array}$

H -4.3579753 .0984630 .480651$

$\begin{array}{lllll}\text { O } & -4.594937 & 1.118590 & 0.086179\end{array}$

C $-4.2013713 .974737-2.149707$

H $-2.5992122 .823516-2.892708$

H $-4.0185421 .864368-2.413087$

H $-0.548903 \quad 3.4250973 .235974$

H -4.6219811 .3321912 .574557$

H -4.0575830 .3095540 .045070$

O $-5.1046413 .899721-3.171949$

O $-4.0543314 .926244-1.395232$

C $-5.9498385 .043534-3.331259$

H $-6.6267754 .851966-4.168136$

H $-6.5454405 .203603-2.427429$

H -5.349216 $5.928704-3.561001$

SCF Energy (B3LYP/6-31G**//MMFF) $=-3245.90987908$

01211

MMFF Geometry

C 3.0682963 .0036841 .872810

C 2.3353731 .9647571 .445250

C $1.272506 \quad 1.2904172 .270238$

O 0.0625721 .2255331 .476328

C $1.695590-0.1510982 .604695$

C $0.996591-0.671213 \quad 3.843848$

C $-0.125151-1.415843 \quad 3.847520$

C $1.660002-0.3026845 .147320$

C $-0.940318-1.873962 \quad 2.670302$

C -1.161585-3.394158 2.716204

C $-2.150025-3.9247941 .664425$

C $-1.610546-3.8027590 .240368$

C $-2.630551-4.302911-0.792239$

O $-3.368932-3.1957661 .800596$

O $-2.179454-3.893362-2.093460$

C $-3.977379-3.589140-0.541258$

C $-2.700646-5.834011-0.827117$

O $-4.982271-4.137623-1.391119$

C $-4.436658-3.6099140 .939075$

C $-5.614174-2.6464451 .213483$ 
O $-4.924204-4.903508 \quad 1.313005$

C $-0.703542 \quad 2.3481281 .410345$

O -0.5372803 .3884912 .026876$

C $-1.7603892 .110694 \quad 0.399925$

C $-2.4665523 .153466-0.062806$

C $-3.4942513 .119764-1.107584$

C $-3.8027711 .787642-1.737025$

C $-5.275582-1.1639191 .034472$

C $-6.439924-0.2537901 .380430$

C -6.9751662 .1719111 .631436$

O $-7.584717-0.6617311 .553736$

C -7.3038552 .7551950 .293394$

C $-7.1181944 .045554-0.022611$

C $-7.455626 \quad 4.625209-1.365757$

C -6.291166 5.348882 -2.004022

C $-6.3163716 .681462-2.176250$

C $-5.1330124 .519899-2.519858$

C -4.081424 $4.281154-1.466988$

N $-6.044622 \quad 1.076437 \quad 1.437674$

H 2.9147433 .3952592 .874921

H 2.5109891 .5677780 .449888

H $1.074762 \quad 1.855526 \quad 3.189932$

H $2.777974-0.2035392 .785900$

H $1.509609-0.8122551 .750716$

H -0.531555 -1.713615 4.813870

H $1.120006-0.6940946 .015909$

H $2.676884-0.7073825 .181915$

H 1.7153890 .7848935 .257038

H -1.903519 -1.352713 2.717188

H -0.477259-1.595296 1.721185

H $-0.201148-3.9156202 .617690$

H -1.563104 -3.652773 3.705670

H -2.342982 -4.977047 1.904476

H $-0.661131-4.3412910 .136980$

H $-1.390143-2.7521260 .012600$

H $-1.303718-4.288483-2.244845$

H $-3.831413-2.550633-0.862113$

H - $1.728743-6.254824-1.112234$

H $-3.410606-6.180179-1.586223$

H -2.976356 -6.274150 0.133356

H $-5.753948-3.548124-1.359316$

H $-6.480689-2.9192320 .598367$

H -5.946597 -2.793923 2.251416

H $-4.866733-5.4951220 .546460$

H $-1.894107 \quad 1.0991740 .033860$

H -2.257728 4.1411820 .347383

H $-4.1511011 .076988-0.981874$

H -2.908705 $1.374742-2.218039$

H -4.577964 $1.833456-2.504064$

H -4.424865 -0.8905801 .668951$

H $-5.010071-0.957487-0.006654$

H $-6.491862 \quad 2.8975472 .292935$

H -7.886924 1.8067432 .114201

H -7.723596 $2.081093-0.449906$

H $-6.719307 \quad 4.7263290 .725927$

H -8.300547 $5.312130-1.227217$

H -7.810914 $3.849394-2.055647$

H $-5.4961537 .203366-2.658225$

H -7.153904 7.281005 - 1.835524

H -4.647484 $5.047582-3.351636$

H $-5.5271593 .596792-2.952492$

H -3.754673 $5.194829-0.967403$

H $-5.079615 \quad 1.3141701 .226720$

C 5.5055123 .1060061 .302253

$\begin{array}{lllll}\text { O } & 5.551409 & 1.752418 & 0.806057\end{array}$

C $6.706056 \quad 1.600627-0.035797$

C $7.628614 \quad 2.753106 \quad 0.332497$

C 6.6200453 .8605640 .574420

H $6.3663601 .738518-1.069616$

C $7.297274 \quad 0.199324 \quad 0.130669$

H $8.1917062 .547219 \quad 1.250088$

H $8.3429232 .986886-0.462453$

C $7.1794525 .034768 \quad 1.358679$

H $6.257045 \quad 4.222505-0.397141$

C $6.270834-0.935617-0.078638$

$\begin{array}{llll}\text { O } & 7.813178 & 0.077367 & 1.462114\end{array}$

H $8.152706 \quad 0.077565-0.544078$

H $5.544740-0.9179410 .741348$
O $6.966733-2.1821250 .050122$

C $5.532090-0.898613-1.431996$

C $4.643390-2.127381-1.700787$

H $4.9185160 .005171-1.493280$

O $6.490020-0.809046-2.490752$

H $4.347525-2.103517-2.756243$

H $5.246386-3.037835-1.600773$

H 6.4090065 .7944721 .524243

H 7.5583754 .7200912 .336871

H 8.0053675 .5029740 .813488

C 4.1152893 .6786001 .031082

H $8.176189-0.8206441 .551240$

H $7.667816-2.204146-0.622769$

H $6.984668-1.645403-2.519205$

H $3.8656903 .557743-0.030390$

H $4.0906564 .752774 \quad 1.246813$

H 5.7048013 .0587262 .380472

C $1.840680-1.017971-2.220135$

O $2.529198-1.079011-0.964065$

C $3.397796-2.205780-0.794230$

C $2.598385-3.506942-0.944595$

C $1.865079-3.559365-2.279431$

C $1.029430-2.297334-2.471076$

H $2.563570-0.863771-3.029916$

C $0.8949770 .194020-2.173573$

H $3.746118-2.1702110 .244127$

O $3.446168-4.644684-0.831124$

H $1.857933-3.575442-0.136868$

H $2.557724-3.704705-3.116553$

O $0.983698-4.684644-2.289320$

H $0.169773-2.326355-1.791838$

O $0.517289-2.273727-3.802262$

C $1.6177001 .520871-2.254874$

H $\quad 0.1951640 .164317-3.016151$

H $0.3311530 .180669-1.233700$

H $3.896303-4.5889260 .029124$

H $1.523645-5.475819-2.120646$

H $0.014848-3.095843-3.935038$

O $0.6964312 .523400-2.133980$

O $2.8221151 .666576-2.406219$

C $1.2128003 .856028-2.193226$

H $\quad 0.3657054 .546759-2.218233$

H $1.8084314 .064389-1.300903$

H $1.8084534 .000264-3.099684$

SCF Energy (B3LYP/6-31G**//MMFF) $=-3245.92038548$

$01 \_212$

MMFF Geometry

C -1.079951 $3.852305-0.860000$

C $-0.1845324 .406082-0.028791$

C $0.642992 \quad 3.587457 \quad 0.926611$

$\begin{array}{llllll}\text { O } & 2.031204 & 3.862157 & 0.621733\end{array}$

C 0.3411613 .9217562 .401577

C -1.0972993 .7185892 .833197$

C -1.7485852 .5379562 .822514$

$\begin{array}{llll}C & -1.801695 & 4.967209 & 3.296467\end{array}$

C $-1.1995391 .197537 \quad 2.414777$

C $-1.7227260 .066208 \quad 3.310310$

C $-1.260987-1.3142072 .815178$

C -1.936879-2.423679 3.622972

C $-1.436059-3.8164773 .215756$

O $0.160563-1.3983572 .965980$

O $-1.865196-4.7506154 .217841$

C $0.105995-3.8157093 .227567$

C $-2.055253-4.2831601 .894458$

$\begin{array}{lllll} & 0.618036 & -5.042568 & 2.710608\end{array}$

C $0.736771-2.6181572 .474750$

C $2.263089-2.5880862 .750959$

O $0.514773-2.7769671 .074811$

C $2.9348682 .934248 \quad 1.039377$

$\begin{array}{llll}\text { O } & 2.719004 & 1.985848 & 1.776643\end{array}$

C $4.2307773 .221558 \quad 0.388651$

C 5.1940842 .2899850 .452693

C $6.5079662 .343241-0.187451$

C $6.8416613 .561966-1.005734$

C $3.000311-1.3553242 .215624$

C $3.399328-1.4641650 .756925$

C $4.959796-2.673881-0.772954$ 
O $2.831428-0.808619-0.113250$

C $6.410108-2.307529-0.819806$

C $6.952987-1.532138-1.771411$

C $8.416633-1.194290-1.837827$

C $8.6769590 .294885-1.916448$

C $8.953725 \quad 0.883473-3.092357$

C $8.7026501 .086888-0.627557$

C $7.3327781 .285176-0.041395$

N $4.434418-2.3582850 .543144$ H $-1.2052612 .772764-0.855275$ H $-0.0502305 .484405-0.018738$ H $0.4676392 .523940 \quad 0.729783$ H 0.6454554 .9604522 .593297 H 0.9868113 .3345683 .066224 H -2.795511 $2.532343 \quad 3.123329$ H -2.848862 4.7836133 .559102 H $-1.302697 \quad 5.3744294 .181893$ H $-1.787216 \quad 5.7279852 .509183$ H -1.5090401 .0058161 .380801$ H $-0.105773 \quad 1.1926772 .445219$ H -1.3650110 .2318604 .334890$ H -2.8192530 .0958253 .334812$ H $-1.518159-1.4056121 .752983$ H -3.028370 -2.366133 3.526001 H -1.723297 -2.277680 4.691189 H $-2.835718-4.7103744 .268961$ H $0.423522-3.7668134 .278769$ H -3.148682 -4.3126181 .970995$ H $-1.744328-5.304671 \quad 1.649140$ H -1.801032 -3.634770 1.055321 H $0.207511-5.7643253 .217052$ H $2.410709-2.5885183 .838990$ H $2.728350-3.4980532 .354542$ H $1.047070-2.1180270 .602820$ H $4.3373734 .150939-0.157833$ H $4.9941131 .375577 \quad 1.010844$ H $6.8398614 .458754-0.376362$ H $6.1116543 .696711-1.811645$ H $7.823298 \quad 3.509822-1.481729$ H $2.405574-0.4490582 .354903$ H $3.922382-1.2078262 .792853$ H $4.376870-2.164159-1.546600$ H $4.848859-3.753994-0.911518$ H $7.050278-2.719697-0.043024$ H $6.318559-1.139973-2.563255$ H $8.830872-1.692112-2.724497$ H $8.963290-1.612206-0.983345$ H $9.1709521 .944444-3.156857$ H $8.9645740 .318007-4.018292$ H $9.2084772 .048793-0.768531$ H 9.3239850 .5578950 .106796 H $6.996563 \quad 0.4451450 .567290$ H $4.819075-2.8639991 .335954$ C $-3.4506124 .446352-1.510318$ O $-3.8265003 .053509-1.582952$ C $-4.6372572 .741676-0.434962$ C $-5.115515 \quad 4.082578 \quad 0.111178$ C $-3.9001704 .950292-0.137620$ H $-3.975196 \quad 2.2743190 .303513$ C $-5.770101 \quad 1.782807-0.805803$ H $-5.9761584 .466265-0.448940$ H $-5.394476 \quad 4.0253251 .167670$ C $-4.1873006 .440450-0.090475$ H -3.1539714 .7132240 .627608$ C $-5.3233100 .430938-1.400250$ O $-6.6243422 .417437-1.767433$ H $-6.396760 \quad 1.607498 \quad 0.076613$ H $-4.9279380 .586046-2.410290$ O $-6.508935-0.361678-1.564562$ C $-4.297013-0.360098-0.561579$ C $-4.180574-1.850741-0.937841$ H $-3.3171310 .124363-0.634039$ O $-4.692540-0.305095 \quad 0.813614$ H -3.485268 -2.318754-0.232098 H $-5.142603-2.337886-0.735806$ H $-3.2787757 .017444-0.289448$ H $-4.9401276 .728326-0.831840$ H $-4.561225 \quad 6.728590 \quad 0.897543$
C $-1.9571604 .612446-1.812150$

H -6.078469 $2.642230-2.540502$

H $-6.867891-0.540077-0.678185$

H $-4.002701-0.7495571 .335096$

H -1.697473 $5.677331-1.816387$

H $-1.7529444 .225966-2.818300$

H $-4.019497 \quad 4.952630-2.300886$

C $-1.357385-2.363880-2.012901$

O $-2.418454-1.647624-2.660569$

C $-3.739319-2.124258-2.388733$

C $-3.855640-3.596351-2.805617$

C $-2.804506-4.454747-2.114164$

C $-1.412171-3.868138-2.332104$

H $-1.419792-2.203796-0.931060$

C $-0.034967-1.762250-2.516353$

H $-4.409826-1.562226-3.048187$

O $-5.149560-4.111635-2.509877$

H $-3.716355-3.681514-3.891210$

H $-3.021830-4.575369-1.046925$

O $-2.829240-5.769273-2.677085$

H $-1.113629-4.050234-3.372824$

O $-0.504585-4.583610-1.494335$

C $0.179451-0.339400-2.051954$

H $\quad 0.823182-2.340473-2.159437$

H $-0.036807-1.758321-3.613344$

H $-5.799146-3.548628-2.964649$

H $-3.731076-6.115062-2.562749$

H $0.399158-4.374699-1.782484$

O $1.188193 \quad 0.212116-2.790596$

O $-0.4330550 .207196-1.146098$

C $1.5437471 .556509-2.456869$

H $1.7218311 .653152-1.383220$

H $2.4673481 .805647-2.985999$

H $\quad 0.7550252 .238897-2.784179$

SCF Energy (B3LYP/6-31G**//MMFF)= -3245.91479287

01213

MM̄FF Geometry

C $1.974107-4.2772951 .079974$

C $0.959652-3.818098 \quad 0.331703$

C $-0.300041-3.2347340 .916340$

O $-0.670578-2.0729940 .135651$

C $-1.455097-4.245770 \quad 0.811732$

C $-2.686865-3.7795551 .562093$

C $-3.702387-3.0805901 .017366$

C $-2.718055-4.1463813 .023536$

C $-3.830880-2.601078-0.403296$

C $-5.176883-2.950757-1.059205$

C $-6.391072-2.268375-0.408993$

C $-7.666655-2.528007-1.213731$

C $-8.873946-1.775704-0.628982$

O $-6.134730-0.862673-0.352506$

O $-9.923863-1.796665-1.608290$

C $-8.489981-0.295422-0.410485$

C $-9.430982-2.474160 \quad 0.617410$

$\begin{array}{lllll}\text { O } & -9.524748 & 0.405674 & 0.275332\end{array}$

C $-7.144591-0.108890 \quad 0.327862$

C $-6.723569 \quad 1.3799430 .311893$

O $-7.293725-0.5288541 .686749$

C $-0.055449-0.8906310 .398628$

$\begin{array}{lllll}\text { O } & 0.813481 & -0.676379 & 1.227465\end{array}$

C $-0.6300820 .108259-0.531788$

C $-0.0331601 .298286-0.688182$

C $-0.4609862 .361766-1.601074$

C - $-1.8058262 .217073-2.263045$

C $-5.261871 \quad 1.6142220 .724204$

C $-4.9353593 .089894 \quad 0.874317$

C -3.0299834 .6098561 .397105$

$\begin{array}{lllll}\text { O } & -5.776729 & 3.978996 & 0.776449\end{array}$

C -2.618721 5.2301840 .099271

C $-1.4073925 .765693-0.114746$

C $-1.0167746 .448918-1.393465$

C $0.2620045 .904225-1.986306$

C $1.4061276 .606979-1.929867$

C $0.1933684 .585028-2.722348$

C $0.3689443 .406018-1.802457$

N $-3.5899843 .293546 \quad 1.154758$

H $1.864535-4.2515212 .162840$ 
H $1.018251-3.850283-0.752036$ H $-0.150332-2.9362681 .961812$ H -1.146574 -5.218109 1.218982 H $-1.699510-4.435761-0.240057$ H -4.512381-2.778596 1.678835 H -3.620771 -3.782999 3.526049 H $-2.691015-5.2346233 .141024$ H $-1.855755-3.718723 \quad 3.544780$ H -3.689431-1.513862 -0.410140 H -3.039744 -3.010180 -1.037882 H -5.112986 -2.632611-2.108264 H $-5.320716-4.038181-1.062512$ H $-6.508380-2.6582120 .607732$ H -7.875725 -3.603181-1.276346 H -7.519685 -2.190230 -2.249263 H - $10.127027-2.726158-1.810077$ H -8.405595 $0.169491-1.402833$ H $-9.750413-3.4945870 .373600$ H -10.325057 -1.962733 0.990789 H -8.706362 -2.541996 1.431393 H - $-10.346616 \quad 0.273511-0.227675$ H -6.844154 $1.786533-0.700899$ H $-7.386374 \quad 1.9529160 .972708$ H $-6.664745-0.0436812 .243254$ H $-1.514827-0.177080-1.090117$ H $0.8706121 .504297-0.117006$ H -2.583187 2.012493 -1.518748 H $-1.7905691 .395747-2.987525$ H $-2.1276003 .115064-2.794587$ H -5.0452481 .1310851 .682796$ H $-4.590386 \quad 1.189721-0.031151$ H -2.186423 4.4848642 .083351 H -3.785924 5.2371391 .880712 H $-3.3653415 .274647-0.690321$ H -0.6694415 .7505370 .684184$ H $-0.9039257 .518879-1.174757$ H -1.816041 $6.382924-2.142094$ H $2.3202436 .234070-2.379928$ H $1.4560307 .567709-1.428136$ H $1.0005724 .536746-3.465997$ H $-0.7285844 .544492-3.308205$ H $1.3241133 .407977-1.279003$ H -2.953991 2.5012941 .144473

C $3.701389-4.475438-0.833674$

O $3.956676-3.057219-0.918377$

C $5.115798-2.843511-1.746859$

C $5.453395-4.195726-2.365864$

C $5.001326-5.146672-1.276152$

H $5.934767-2.555862-1.078199$

C $4.853654-1.732656-2.768022$

H $4.886449-4.372788-3.287446$

H $6.517520-4.287800-2.603880$

C $4.832047-6.578294-1.754226$

H $5.735652-5.122158-0.459668$

C $4.520499-0.358438-2.148466$

O $3.745527-2.126542-3.587850$

H $5.716028-1.643780-3.439546$

H $3.544320-0.406753-1.652967$

O $4.3658620 .569231-3.231659$

C $5.5816110 .184157-1.170946$

C $5.4032081 .665904-0.787980$

H $5.590177-0.432152-0.265655$

O $6.870724 \quad 0.052357-1.778760$

H $6.2435301 .946713-0.141131$

H $5.5255892 .289957-1.682456$

H $4.496465-7.226295-0.938633$

H $4.097310-6.647203-2.563470$

H $5.782173-6.973092-2.128954$

C $3.284476-4.8402300 .593369$

H $3.587354-1.409088-4.225029$

H $5.2320390 .660868-3.664879$

H $7.5341190 .324028-1.121600$

H $4.058097-4.4763701 .281412$

H $3.241563-5.9292610 .708116$

H $2.903774-4.708679-1.549634$

C $4.6652401 .346776 \quad 2.147781$

O 3.8086721 .1357811 .018887

C $4.0667721 .992136-0.096754$
C $3.917413 \quad 3.4613100 .325395$

C $4.7751713 .796501 \quad 1.542434$

C 4.5352112 .7844142 .661621

H 5.7047941 .1439841 .863645

C $4.2596490 .338459 \quad 3.226944$

H $3.2768021 .790180-0.828683$

$\begin{array}{lllll}\text { O } & 4.259063 & 4.337787 & -0.742975\end{array}$

H $2.869113 \quad 3.6597470 .583106$

H $5.838976 \quad 3.8499251 .282938$

$\begin{array}{lllll}\text { O } & 4.415693 & 5.092428 & 2.027606\end{array}$

H 3.5378422 .9438743 .091472

O 5.4888722 .9994193 .699782

C $4.580379-1.0709862 .794775$

H $4.7651020 .524770 \quad 4.181074$

H $3.1805750 .385947 \quad 3.417512$

H $3.6939674 .109789-1.500765$

H 4.5308285 .7183421 .291998

H 5.3990763 .9237613 .989045

O $5.919846-1.3014662 .901047$

O $3.739014-1.8672112 .400967$

C $6.352158-2.6050782 .500293$

H $7.434475-2.6632802 .642729$

H $5.874785-3.3706403 .119231$

H $6.128713-2.7685671 .442321$

SCF Energy (B3LYP/6-31G*//MMFF) $=-3245.90546047$

01214

MM̄FF Geometry

C $1.361105-4.025048-0.545825$

C $0.860004-4.0590440 .699292$

C $-0.486353-3.527318 \quad 1.136022$

O $-1.005980-2.5706860 .185829$

C $-1.498640-4.6791591 .264260$

C $-2.797368-4.2689271 .932320$

C $-4.001249-4.2448321 .327691$

C $-2.691794-3.897247 \quad 3.390187$

C $-4.339814-4.604640-0.090835$

C $-4.328732-3.427595-1.078706$

C $-5.294968-2.285488-0.721312$

C $-5.408777-1.294655-1.881701$

C $-6.301605-0.095131-1.529689$

O $-4.785101-1.603950 \quad 0.426921$

$\begin{array}{lllll}0 & -6.075076 & 0.896974 & -2.543706\end{array}$

C $-5.8505320 .497177-0.173282$

C $-7.786722-0.467162-1.615337$

\begin{tabular}{lllll}
\hline & -6.770258 & 1.481924 & 0.289479
\end{tabular}

C -5.635657 -0.5641290 .929172$

C $-4.961782-0.0009322 .202229$

O $-6.883124-1.0988841 .390769$

C $-0.501018-1.3085890 .250431$

O $0.318463-0.8839951 .049660$

C -1.099532 $-0.524027-0.849899$

C $-0.4851580 .600688-1.241602$

C $-0.929767 \quad 1.521098-2.287162$

C $-2.3114631 .319774-2.848239$

C -3.5713820 .6109552 .002182$

C $-3.5766292 .119403 \quad 1.814403$

C $-2.037743 \quad 4.0181091 .323697$

O -4.5783242 .8147091 .958956$

C -2.162389 $4.379291-0.122894$

C $-1.2137965 .043650-0.800754$

C $-1.3476355 .461692-2.237812$

C $-0.203644 \quad 4.971948-3.097341$

C $0.841305 \quad 5.768430-3.378814$

C $-0.3112053 .584756-3.691729$

C -0.092468 2.509486 -2.664109

N -2.313542 $2.604178 \quad 1.498557$

H $0.756660-3.634414-1.360054$

H $1.469649-4.4840401 .494481$

H $-0.354198-3.0320952 .105805$

H $-1.063543-5.4992431 .851129$

H $-1.690548-5.0894610 .267011$

H $-4.861730-3.9342711 .919964$

H -3.673789 -3.7655723 .857593$

H -2.170193 -4.6828463 .946382$

H -2.144121 -2.957963 3.512071

H $-3.670934-5.387667-0.463276$

H $-5.338418-5.060418-0.091832$ 
H $-3.307154-3.037426-1.148875$ H $-4.589667-3.824488-2.067765$ H $-6.274453-2.718627-0.487527$ H -5.778595 -1.793510 -2.786381 H $-4.408470-0.920115-2.136944$ H $-6.6628201 .650312-2.365316$ H $-4.9001781 .012741-0.356452$ H -8.048963 -0.751574 -2.641539 H -8.425967 $0.387873-1.369566$ H -8.057545 -1.300662 -0.963712 H $-6.7887562 .199953-0.365111$ H -5.6389780 .6960532 .710962$ H -4.841122 -0.8435602 .898949$ H -7.606971 -0.6016790 .977841$ H -2.012241 -0.889700-1.303203 H $\quad 0.4457110 .874206-0.749687$ H $-3.0530981 .315716-2.042098$ H $-2.3728870 .368491-3.387859$ H -2.620485 $2.102547-3.543482$ H -2.9759030 .4184842 .904017$ H -3.053962 $0.141573 \quad 1.161843$ H -1.031851 4.2041221 .712379 H -2.7521364 .6035291 .911500$ H -3.087359 4.109898 -0.627388 H $-0.3014215 .336522-0.285869$ $\mathrm{H}-1.3886016 .558643-2.262800$ H -2.298168 5.121991-2.667600 H $1.6536225 .432653-4.014740$ H $0.9098986 .772877-2.974332$ H $0.4498543 .448036-4.471422$ H -1.269569 $3.497323-4.209941$ H $0.8883172 .560923-2.190872$ H -1.5507661 .9495621 .351236$ C $3.422855-3.610808-1.928713$ O $3.615238-2.277406-1.416768$ C $4.823960-1.736439-1.990250$ C $5.398191-2.817184-2.905979$ C $4.822131-4.085446-2.311523$ H $5.520852-1.572862-1.161366$ C $4.539536-0.414083-2.714723$ H $5.063574-2.693211-3.942578$ H $6.492917-2.807454-2.914064$ C $4.846428-5.264796-3.268074$ H $5.392978-4.338471-1.407988$ C $3.9495750 .702004-1.829948$ O $3.600219-0.673546-3.768502$ H $5.456672-0.061167-3.201838$ H $2.9370240 .420390-1.519769$ O $3.8180651 .852580-2.676680$ C $4.8116671 .071258-0.606815$ C 4.3519092 .3254630 .163481 H $4.843366 \quad 0.2230140 .084132$ O $6.160357 \quad 1.299553-1.026805$ H 5.0776982 .5072970 .965712 H $4.4342583 .207556-0.483635$ H $4.430713-6.160730-2.796931$ H $4.264903-5.059333-4.173224$ H $5.873410-5.491727-3.572406$ C $2.742894-4.507194-0.890364$ H $3.4345710 .170544-4.222400$ H $3.2417732 .491052-2.225171$ H $6.1467042 .022425-1.677659$ H $3.358905-4.5497730 .016469$ H $2.658076-5.525183-1.286675$ H $2.789308-3.523414-2.821966$ C 3.4544591 .0073872 .753216 O 2.7450891 .0304441 .508902 C $2.9281392 .221196 \quad 0.739810$ C 2.4680803 .4422881 .546305 C 3.1359773 .5060742 .917453 C 3.0013582 .1678503 .645112 H 4.5326871 .0794892 .565611 C $3.179583-0.3467373 .413271$ H $2.2497272 .143275-0.115023$ O $2.727918 \quad 4.649257 \quad 0.838145$ H 1.3822623 .3857111 .696390 H 4.1880293 .8043152 .837881 O 2.4928734 .5098313 .706350
H 1.9586662 .0224623 .955564

O $3.8014442 .193646 \quad 4.824987$

C $3.845798-1.4557782 .635598$

H $3.541920-0.3857774 .446589$

H $2.104723-0.5627553 .440909$

H $2.2695924 .590121-0.017654$

H $2.5530765 .346583 \quad 3.214041$

H 3.5086462 .9532145 .357224

O $5.154630-1.5650623 .001491$

O $3.277203-2.1264421 .784941$

C $5.901379-2.5687902 .306757$

H $5.461981-3.5561592 .477943$

H $5.935627-2.341773 \quad 1.237118$

H $6.921840-2.5659652 .698851$

SCF Energy (B3LYP/6-31G**//MMFF)= -3245.89702402

01215

MM̄FF Geometry

C $2.3090620 .227869-2.437599$

C $1.103215-0.235921-2.795428$

C $0.223555-1.037060-1.868909$

O $-1.071892-0.400580-1.790298$

C $0.029836-2.452692-2.443148$

C $-0.721120-3.390996-1.518749$

C $-1.920082-3.940763-1.793945$

C $-0.022548-3.734965-0.227453$

C $-2.759210-3.770239-3.028932$

C $-3.798212-2.640994-2.952723$

C $-4.845731-2.821604-1.841872$

C $-5.980460-1.806510-1.998092$

C $-6.997423-1.897204-0.849077$

O $-4.203936-2.624660-0.579251$

O $-7.820703-0.721193-0.895632$

C $-6.243027-1.8657310 .498167$

C $-7.941864-3.093292-1.017643$

O $-7.130579-2.116041 \quad 1.584547$

C $-5.050657-2.8421720 .557585$

C $-4.175242-2.6634561 .822445$

O $-5.545010-4.1870310 .579016$

C $-1.1868400 .679429-0.970122$

O $-0.3135261 .174683-0.275748$

C $-2.5754601 .177506-1.079588$

C $-2.8672762 .415765-0.654882$

C $-4.1836633 .055624-0.701492$

C $-5.3652632 .189568-1.049633$

C $-3.550766-1.2750812 .001105$

C $-4.357539-0.3451772 .892619$

C -4.4281622 .0216693 .668583$

O $-5.309226-0.7194363 .572476$

C -5.4320052 .7745672 .853686$

C -5.4246994 .1105832 .730525$

C -6.4477124 .8869431 .951800$

C -5.8230955 .8137210 .932836$

C -5.6357697 .1159361 .206597$

C $-5.4951675 .248037-0.430361$

C $-4.2724254 .371377-0.415021$

$\begin{array}{llll}\mathrm{N} & -3.860917 & 0.951488 & 2.869967\end{array}$

H $2.6760620 .022040-1.434802$

H $\quad 0.731257-0.048224-3.799629$

H $0.662550-1.094740-0.865451$

H $1.007648-2.905169-2.656760$

H -0.482754 -2.370927 -3.407756

H -2.357608 -4.607285-1.051136

H $-0.539684-4.5260240 .326351$

H $0.993478-4.090023-0.428526$

H $\quad 0.033957-2.8611710 .428388$

H -2.126300 -3.612345 -3.908681

H -3.275856 -4.719896 -3.219122

H $-3.275387-1.687328-2.816304$

H $-4.307651-2.593882-3.923228$

H $-5.238054-3.843703-1.899054$

H -6.484001-1.930781-2.964856

H -5.559934 -0.791972 -2.009627

H $-8.246246-0.687610-1.769553$

H $-5.870641-0.8427090 .627968$

H -8.497104 -3.012584 -1.959952

H -8.698939 -3.113986 -0.226108

H -7.421595 -4.053369-1.023665 
H -7.851443 -1.465936 1.525592 H -4.732860 -2.974597 2.713953 H -3.340715 -3.3750471 .740214$ H -4.854659 -4.7635800 .946194$ H -3.313952 0.518951-1.521069 H $-2.0633383 .030512-0.251693$ H $-5.3891631 .300950-0.409906$ H $-5.3130441 .869493-2.095610$ H -6.327727 $2.685176-0.907994$ H -2.576112 -1.3944782 .491756$ H -3.373128 - 0.7955281 .033991 H -3.5991472 .6572673 .995200$ H -4.9144301 .5989484 .553580$ H -6.214126 2.1983202 .364931 H -4.6574404 .6856053 .244520$ H -7.0410585 .4688762 .669364$ H -7.161485 4.2212411 .450584 H -5.2141607 .7939250 .471884$ H -5.8954977 .5320202 .174398$ H -5.297496 $6.063965-1.138649$ H $-6.3793904 .742861-0.828443$ H -3.356615 $4.910044-0.167211$ H $-3.099517 \quad 1.1828312 .238297$ C $3.6174192 .349084-2.776538$ O $4.3112572 .164315-1.527051$ C $5.5463572 .897700-1.559746$ C $5.3850823 .915973-2.680065$ C $4.5784633 .118841-3.686601$ H $6.3284942 .180387-1.838264$ C $5.8596283 .482639-0.179123$ H $4.8159054 .794719-2.355937$ H $6.3466294 .259028-3.073227$ C $3.8818643 .977266-4.728338$ H $5.2540192 .419716-4.198021$ C 5.7458382 .4675370 .977499 O $4.931487 \quad 4.541120 \quad 0.093352$ H $6.8537873 .944432-0.195532$ H 4.6882032 .2263921 .130401 O $\quad 6.1682753 .1252552 .178994$ C 6.5524251 .1672350 .795827 C $\quad 6.567598 \quad 0.252578 \quad 2.036302$ H $6.1537280 .605505-0.053948$ O 7.9068831 .4873560 .467397 H $7.250040-0.5806111 .829901$ H 7.0281750 .7909142 .873840 H $3.3022313 .357216-5.419506$ H $3.1969644 .694805-4.264105$ H $4.6145564 .542275-5.313586$ C $3.2290390 .985917-3.351880$ H 5.1482974 .8982680 .971640 H 7.0893373 .4105642 .055615 H 8.3102351 .9160641 .240896 H $4.1303490 .375595-3.485664$ H $2.7726721 .115064-4.340052$ H $2.7209322 .942871-2.557916$ C $5.120382-2.2979321 .109758$ O $4.548066-1.0130491 .387807$ C $5.177989-0.2775622 .443010$ C 5.169149-1.094278 3.742791 C $5.781678-2.4755293 .547843$ C $5.123874-3.1816262 .365469$ H $6.146545-2.1722710 .744066$ C $4.276848-2.948101-0.001211$ H 4.5415680 .5952592 .627805 O $5.868224-0.4145954 .780215$ H $4.133356-1.2209914 .084054$ H $6.869120-2.4213333 .419752$ O $5.553440-3.2612354 .720039$ H $4.096518-3.4621132 .631984$ O $5.838200-4.3855642 .089217$ C $4.641774-2.450272-1.383779$ H $4.436878-4.032338-0.007221$ H $3.212048-2.7409430 .155320$ H 5.4491540 .4556914 .893084 H $5.941155-2.7789805 .470541$ H $5.842030-4.9142322 .905698$ O $4.042778-3.256958-2.310239$ O $5.348597-1.482314-1.625045$
C $4.323947-2.958726-3.681597$

H $3.746800-2.085883-3.995608$

H $5.394097-2.791764-3.837783$

H $4.015583-3.817297-4.284152$

SCF Energy (B3LYP/6-31G**//MMFF) $=-3245.91120953$

01216

MM̄FF Geometry

C -0.801924 -2.510717 0.899427

C $-1.259382-2.8790872 .105964$

C $-0.680573-2.4839053 .442676$

O $0.539606-1.7226533 .318775$

C $-1.663249-1.6627274 .300000$

C -2.265419-0.439605 3.631037

C $-3.548751-0.3332903 .233140$

C -1.3412730 .7403953 .463949$

C $-4.638912-1.3651543 .305334$

C $-5.227667-1.7679001 .941857$

C $-5.946620-0.6196851 .215220$

C $-6.940424-1.1641470 .187055$

C $-7.618301-0.038581-0.608097$

$\begin{array}{llll}\text { O } & -4.962029 & 0.179124 & 0.548052\end{array}$

O $-8.271403-0.661131-1.724715$

C $-6.5351070 .916259-1.152841$

C $-8.7161550 .648958 \quad 0.212653$

O $-7.1210452 .072513-1.747892$

C $-5.4944631 .345882-0.089882$

C $-4.3365822 .105192-0.779339$

$\begin{array}{llll}\text { O } & -6.121740 & 2.220625 & 0.851526\end{array}$

C $1.715693-2.3948193 .243607$

O $1.864171-3.6090353 .225177$

C $2.864772-1.4634993 .159335$

C $2.758667-0.1344373 .312855$

C 3.8486680 .8400093 .225118

C 5.2102190 .3239802 .842073

C -3.1022502 .3480250 .103390$

C $-2.0959623 .246888-0.596922$

C $0.2144074 .097278-0.469483$

O $-2.3726893 .906371-1.597009$

C 1.5292593 .6855710 .109476

C 2.4117594 .5692880 .600599

C 3.7770524 .2132101 .120787

C 3.9449194 .4551382 .606793

C 3.6657715 .6391663 .179126

C 4.5067933 .3162423 .431790

C 3.5727412 .1360043 .482979

N $-0.851874 \quad 3.244880 \quad 0.022133$

H $0.065024-1.8605710 .822119$

H -2.122822 -3.540154 2.153861

H $-0.460663-3.4033364 .001146$

H - $-1.142955-1.3219415 .205826$

H -2.448377 -2.333887 4.662004

H -3.8643220 .6225142 .818528$

H -1.878702 1.6532773 .185171

H -0.8128530 .9510754 .399350$

H -0.6056370 .5431462 .678743$

H $-4.297270-2.2803783 .795533$

H -5.440996 -0.967437 3.939630

H $-4.429616-2.1736481 .307390$

H -5.941005 -2.580099 2.130404

H $-6.473239-0.0099991 .958744$

H -7.696566 -1.794472 0.671830

H $-6.412948-1.822139-0.516407$

H $-8.7498980 .026791-2.217249$

H $-6.0077370 .386664-1.958033$

H $-9.489158-0.0752190 .496879$

H $-9.2288031 .417936-0.375923$

H $-8.341028 \quad 1.111778 \quad 1.127945$

H -7.680366 $1.774252-2.484503$

H -3.997405 $1.535439-1.654772$

H $-4.7123763 .068561-1.147041$

H -5.4490642 .7964391 .247565$

H $3.820633-1.9420292 .974003$

H 1.7816410 .2947113 .524463

H $5.164656-0.1930051 .878558$

H $5.581163-0.3770043 .598103$

H $5.966431 \quad 1.1042792 .741016$

H $\quad-3.3777262 .835297 \quad 1.043927$ 
H -2.6188621 .3928290 .337768$ H $-0.0475445 .130578-0.216476$ H $0.2481644 .014608-1.561136$ H 1.7815132 .6289280 .090644 H 2.1554145 .6265760 .587365 H 4.5276334 .8032060 .579658 H 4.0025923 .1667610 .881025 H 3.8105685 .8013484 .242222 H 3.2951006 .4783472 .600374 H 4.6811813 .6421504 .465172 H 5.4899943 .0643883 .026432 H 2.5552462 .3982183 .777738 H -0.7637842 .8281420 .945085$ C -1.830324 -1.815363 -1.290361 O $-0.664876-1.085401-1.725899$ C $-0.736114-0.894392-3.150183$ C $-2.182012-1.179102-3.534697$ C $-2.533029-2.288020-2.564906$ H -0.091356 -1.657630 -3.602408 C $-0.2376410 .500869-3.536235$ H $-2.829541-0.311224-3.363964$ H -2.282918 -1.475388 -4.583070 C $-4.027643-2.497647-2.400237$ H -2.078188 -3.222082 -2.921769 C $1.1642020 .850658-2.996631$ O $-1.156076 \quad 1.467009-3.014738$ H $-0.267217 \quad 0.611162-4.626874$ H $1.1203080 .938209-1.905016$ O $1.4775502 .155509-3.500998$ C $2.268769-0.136893-3.418313$ C $3.7033680 .308894-3.085449$ H $2.082991-1.111855-2.960031$ O $2.205077-0.337163-4.834606$ H $4.388026-0.407875-3.554510$ H $3.9146611 .258196-3.592549$ H -4.235407 -3.284910 -1.668962 H -4.520426 -1.582101-2.059655 H $-4.483346-2.789716-3.351877$ C $-1.404286-2.980150-0.395217$ H $-0.8344412 .346041-3.278942$ H $2.1954822 .517909-2.955083$ H $2.3284460 .529092-5.259491$ H $-0.646538-3.581429-0.911756$ H -2.263141 -3.633079 -0.201037 H -2.460644 -1.110046 -0.733826 C $4.710559-1.848736-1.191125$ O $3.791426-0.796950-0.870632$ C $4.0024960 .430235-1.577600$

C $5.4065340 .967244-1.268901$

C $6.480029-0.062438-1.607751$

C $6.164133-1.407219-0.953469$

H $4.570333-2.154012-2.234895$

C $4.380061-3.054240-0.295380$

H $3.2901071 .150435-1.161861$

O $5.6616952 .169707-1.986566$

H $5.4773991 .210753-0.201619$

H $6.593472-0.172309-2.692010$

O $7.7478620 .399792-1.133725$

H $6.361835-1.340750 \quad 0.123519$

O $7.044542-2.399536-1.479317$

C $3.047398-3.697192-0.614139$

H $5.148265-3.826815-0.415491$

H $4.346805-2.7399890 .752926$

H $4.9722932 .809446-1.739150$

H $7.6810560 .528823-0.172242$

H $7.954259-2.086655-1.335558$

O $2.922556-4.8310740 .138768$

O $2.220894-3.265318-1.404324$

C $1.717095-5.580958-0.042533$

H $0.904570-5.1039520 .510678$

H $1.461332-5.674608-1.102395$

H $1.877425-6.5827180 .364992$

SCF Energy (B3LYP/6-31G**//MMFF) $=-3245.90309842$

01217

MM̄MF Geometry

C $2.477988-3.323614-0.123205$

C $2.253539-2.508319-1.166677$
C $1.006926-1.688337-1.425505$

O $0.046835-1.920087-0.371693$

C $1.391268-0.192713-1.441349$

C $0.4024890 .719855-2.140420$

C $-0.2612021 .737439-1.556807$

C $0.2309620 .478366-3.620164$

C $-0.1909362 .207942-0.131817$

C $-1.369814 \quad 1.7648350 .746451$

C $-2.7234282 .386597 \quad 0.361582$

C -3.7832812 .0482031 .412742$

C -5.1747462 .5706181 .023599$

O $-3.1297571 .866399-0.906619$

O $-6.137818 \quad 1.9283691 .874718$

C $-5.4947042 .140212-0.425759$

C -5.3067864 .0765461 .282410$

O $-6.7084142 .754987-0.851546$

C $-4.3469532 .431473-1.417997$

C $-4.5719581 .833142-2.830042$

O $-4.2241933 .849650-1.586339$

C $-1.273754-1.938998-0.685530$

O $-1.780179-1.742229-1.777650$

C $-2.012732-2.2441140 .561018$

C $-3.329339-2.0002450 .624391$

C $-4.198886-2.2292081 .779455$

C $-3.625659-2.9835912 .949561$

C $-4.7549030 .313543-2.883376$

C $-6.203987-0.136047-2.796693$

C $-7.589200-2.143778-2.274456$

O $-7.1524630 .604764-3.041778$

C $-8.063927-1.920659-0.873325$

C $-8.152250-2.8898910 .050081$

C $-8.623533-2.6572351 .457230$

C $-7.554005-2.9503332 .487076$

C -7.556615 -4.103743 3.176012

C $-6.536261-1.8705762 .783373$

C $-5.467624-1.7733631 .728655$

N $-6.312388-1.476319-2.452505$

H $1.692274-3.4591450 .616791$

H $3.031136-2.384080-1.916429$

H $0.602142-2.019357-2.388611$

H $2.355009-0.056802-1.951342$

H $1.5513840 .126009-0.407021$

H $-0.9192382 .336169-2.185711$

H $-0.368327 \quad 1.258436-4.102084$

H $1.2064630 .465066-4.117623$

H $-0.268530-0.476454-3.806091$

H $\quad 0.736527 \quad 1.875486 \quad 0.341860$

H $-0.1324303 .303672-0.136131$

H $-1.441411 \quad 0.6725230 .715270$

H -1.134062 2.0427031 .781583

H $-2.5933843 .472596 \quad 0.286967$

H -3.491675 2.4338702 .397777

H $-3.844040 \quad 0.958287 \quad 1.527266$

H -5.9037032 .1282322 .797215$

H $-5.6810581 .059873-0.392424$

H -5.1403574 .2984822 .343445$

H -6.3199044 .4292781 .059899$

H -4.5975604 .6745340 .706515$

H $-6.9613782 .361042-1.702798$

H $-5.3917672 .355901-3.337159$

H $-3.6746522 .061552-3.423348$

H $-3.7531034 .021861-2.418459$

H -1.448051 -2.638312 1.398414

H $-3.819559-1.572692-0.247873$

H -2.844785 -2.393429 3.441340

H $-3.192417-3.9351122 .620736$

H $-4.369241-3.2350083 .709306$

H -4.386597 -0.048725 -3.851796

H $-4.160094-0.175545-2.107156$

H -7.438214 -3.204586 -2.497661

H $-8.317813-1.737992-2.983560$

H $-8.349122-0.905345-0.607070$

H -7.873194 -3.906867 -0.215112

H $-9.498004-3.2989991 .626359$

H $-8.985325-1.6303701 .593254$

H $-6.811850-4.3090523 .937707$

H -8.301147 -4.870956 2.990274

H $-6.099417-2.014774 \quad 3.777604$ 
H -7.047782 -0.9017292 .851396$ H $-5.782826-1.2315870 .837389$ H -5.474400 -1.992373 -2.200886

C $4.985052-3.717465-0.642516$

O $5.295322-2.320368-0.492270$

C $6.722125-2.145964-0.561736$

C $7.319309-3.532262-0.793872$

C $6.256131-4.441998-0.206125$

H $7.041417-1.7816190 .421271$

C $7.065945-1.108594-1.637930$

H $7.457212-3.745652-1.860017$

H $8.294201-3.645194-0.309125$

C $6.353082-5.878393-0.688731$

H $6.344787-4.4201860 .888373$

C $6.3119410 .231062-1.499386$

O $6.739831-1.642025-2.929567$

H $8.147777-0.934083-1.651367$

H $5.2492550 .080883-1.721065$

O $6.8127881 .116116-2.509993$

C $6.4671870 .922135-0.132726$

C $5.8732212 .343354-0.070493$

H $6.0080330 .307145 \quad 0.647310$

O $7.856887 \begin{array}{lll}1.019932 & 0.192521\end{array}$

H 6.1525722 .7790440 .896403

H $6.3700072 .979155-0.813709$

H $5.567822-6.495299-0.240953$

H $6.253018-5.943490-1.777405$

H $7.319993-6.312096-0.413427$

C $3.752159-4.0676250 .194951$

H $5.790840-1.855470-2.928215$

H $6.7010480 .669889-3.366933$

H $8.2872731 .535297-0.511264$

H $3.977686-3.8587851 .248739$

H $3.544762-5.1410100 .118613$

H $4.789070-3.901607-1.706973$

C 3.6891071 .9838322 .022344

O 3.6538041 .5482310 .658766

C $4.3442442 .396547-0.263335$

C $3.7490373 .811017-0.220390$

C 3.7350134 .3775461 .197559

C 3.0845123 .3862992 .161595

H 4.7252311 .9889292 .379609

C 2.8948320 .9689502 .854757

H $4.1332182 .000334-1.263513$

O $4.4792784 .668751-1.090345$

H $2.7181193 .771838-0.595965$

H 4.7427334 .6474621 .534487

O 2.9649295 .5815521 .220452

H 2.0037433 .3530911 .974776

O 3.2700553 .8445143 .499696

C $3.530545-0.4011272 .862976$

H 2.7874631 .2896623 .896744

H 1.8865190 .8532472 .437907

H $4.0030865 .514553-1.139843$

H 3.4429856 .2454520 .695586

H $2.8973204 .741363 \quad 3.553323$

O $4.793562-0.3403943 .371233$

O $2.950343-1.4150612 .496960$

C $5.487714-1.5892603 .448447$

H $5.000877-2.2434464 .177589$

H $5.526535-2.0686052 .466376$

H $\quad 6.510065-1.3900373 .780403$

SCF Energy (B3LYP/6-31G**//MMFF) $=-3245.91721818$

01218

MM̄MFF Geometry

C $3.910749-4.2422870 .344194$

C $3.251938-3.418645-0.485108$

C $1.920676-3.736581-1.116463$

O $0.967383-2.764345-0.624412$

C $2.019707-3.581374-2.644411$

C $0.708703-3.811222-3.370148$

C $0.059661-2.871011-4.085398$

C $0.142066-5.204095-3.273851$

C $0.473255-1.445781-4.328448$

C $-0.041040-0.439120-3.287938$

C $-1.571280-0.295562-3.255568$

C $-1.9824140 .873558-2.359699$
C $-3.5096220 .997203-2.240944$

O $-2.135500-1.504095-2.742593$

O $-3.7947161 .882058-1.145825$

C $-4.109019-0.379028-1.874820$

C $-4.1238411 .642151-3.489425$

O $-5.532075-0.314878-1.933265$

C $-3.570425-1.536891-2.743223$

C $-3.988302-2.940132-2.236644$

O $-4.086138-1.401748-4.073360$

C $0.473498-2.9548890 .629311$

O $0.674335-3.9071891 .365795$

C $-0.324834-1.7606020 .977765$

C $-0.578454-1.4932752 .267246$

C $-1.324158-0.3413332 .776823$

C -2.0111490 .5383091 .768264$

C $-3.554026-3.280446-0.806479$

C -4.611962 -2.995745 0.247286

C $-4.932279-2.9714222 .719805$

O $-5.776273-2.716578-0.025531$

C $-4.844405-1.5594063 .207046$

C $-4.544710-1.2343294 .474012$

C $-4.5031320 .175776 \quad 4.990812$

C -3.2056970 .5032755 .696268$

C -3.1130330 .4443107 .035572$

C -2.0449610 .9829244 .854741$

C $-1.371229-0.1370334 .109533$

N $-4.109708-3.1308831 .534906$

H $3.487033-5.2258580 .538834$

H $3.643952-2.427427-0.694538$

H $1.583641-4.748083-0.859091$

H $2.762151-4.287092-3.040092$

H $2.406714-2.581604-2.869323$

H $-0.877762-3.150009-4.565407$

H $-0.727452-5.344522-3.925264$

H $\quad 0.894545-5.941821-3.570707$

H $-0.177631-5.421123-2.250042$

H $1.564134-1.368184-4.390611$

H $\quad 0.112781-1.152284-5.322753$

H $0.325586-0.730055-2.296678$

H $0.4033280 .536352-3.521178$

H $-1.922828-0.132814-4.281248$

H -1.548687 $1.811575-2.726491$

H -1.566235 $0.726342-1.354350$

H $-3.3626232 .733131-1.329548$

H -3.846016 $-0.560854-0.826809$

H $-3.6979522 .639274-3.654093$

H $-5.201607 \quad 1.795645-3.366138$

H -3.958933 $1.058839-4.397730$

H $-5.884308-1.141472-1.563824$

H -5.064591 -3.091742 -2.380553

H $-3.504556-3.676523-2.895050$

H -4.034569 -2.266389 -4.513302

H $-0.651993-1.1202590 .167271$

H -0.185912 -2.168960 3.026150

H -2.689911 -0.0540961 .146759$

H $-1.2733491 .019506 \quad 1.119973$

H -2.617057 1.3306692 .211450

H $-3.356236-4.358832-0.752547$

H -2.625614 -2.765162 -0.545183

H -4.572138 -3.689294 3.463516

H $-5.971993-3.2127942 .476694$

H -5.067444 -0.769920 2.493167

H -4.352298 -2.027789 5.192987

H -5.3443730 .2995135 .685511$

H -4.6751580 .9028414 .187573$

H $-2.1949130 .705493 \quad 7.551175$

H -3.952506 0.1281747 .645955

H -1.282754 1.4495325 .493505

H -2.3862881 .7896434 .200352$

H $-0.846393-0.8378844 .760239$

H $-3.119106-3.3141881 .666681$

C $5.683604-2.4902671 .035506$

O $4.686981-1.5902651 .553783$

C $5.342075-0.4583482 .150218$

C $6.844230-0.7079612 .017794$

C $6.916524-2.2186091 .890790$

H $5.063687-0.4592423 .210996$

C 4.8646210 .8510491 .499391 
H $7.257701-0.2273931 .123383$

H $7.400184-0.3251932 .879636$

C $8.218320-2.7142601 .286727$

H $6.787296-2.6594752 .888277$

C 3.3456271 .1045051 .646452

O $5.224594 \quad 0.8485190 .117510$

H 5.4068151 .6799541 .969767

H 3.0654950 .9841632 .700232

$\begin{array}{lllll}\text { O } & 2.599347 & 0.149822 & 0.893379\end{array}$

C 2.9271772 .5197341 .193643

C 1.4195712 .7830961 .390149

H 3.4992743 .2514161 .775881

O $3.2637102 .717941-0.175645$

H 0.8505282 .2034440 .655834

H $\quad 1.1308072 .4303002 .386827$

H $8.225640-3.8063141 .215472$

H $8.373599-2.3100130 .280776$

H $9.069113-2.4116171 .905891$

C $5.172300-3.9319791 .112035$

H $4.7203090 .136506-0.312466$

H $2.816593-0.732477 \quad 1.238820$

H $2.8149742 .026236-0.691335$

H $4.964029-4.1735602 .162476$

H $5.953669-4.6203750 .770609$

H $5.875748-2.209377-0.008527$

C $0.5524004 .427544-1.096931$

O $1.4092714 .824462-0.019367$

C 1.0657864 .2844061 .260971

C -0.3833954 .6445861 .628775$

C -1.3700464 .3083490 .512893$

C $-0.8825454 .880359-0.816001$

H $0.5831013 .337529-1.212924$

C $1.0929935 .068535-2.380278$

H 1.7059774 .8048651 .985459

O -0.7586553 .9905712 .836167$

H -0.4332465 .7245091 .821970$

H -1.533993 3.2292890 .431154

O

H $-0.9372335 .976273-0.789407$

O $-1.739515 \quad 4.432631-1.862110$

C $2.3707884 .401614-2.831014$

H $0.3784125 .006794-3.208866$

H $1.3124176 .131280-2.221606$

H -1.629894 4.3312043 .099800

H $-3.0034414 .428106 \quad 1.580941$

H $-2.6298774 .774302-1.670637$

O $2.0929693 .181173-3.374085$

O $3.4804984 .904764-2.713516$

C $3.2255632 .440883-3.839878$

H $3.9251062 .256457-3.019097$

H $2.8702521 .479020-4.219193$

H $3.7189392 .979627-4.654231$

SCF Energy (B3LYP/6-31G**//MMFF) $=-3245.91495725$

01219

MM̄FF Geometry

C -3.726820 -1.687538 -3.013086

C $-2.484502-1.178400-3.012064$

C -1.192091 -1.943046-2.808526

O $-0.328146-1.151877-1.949803$

C $-1.360986-3.320665-2.133452$

C $-0.062823-4.073419-1.916664$

C $0.446866-4.396537-0.711742$

C $0.656480-4.501360-3.170589$

C $-0.107813-4.090857 \quad 0.652519$

C $0.509836-2.8548081 .326986$

C $2.006246-2.9971241 .653893$

C $2.496877-1.8100202 .483479$

C $4.005342-1.8897952 .765643$

O $2.737330-3.0374720 .428710$

O $4.399829-0.5968903 .252348$

C $4.763219-2.1526131 .441572$

C $4.304062-2.8845213 .893603$

O $6.137221-2.4435231 .681454$

C $4.142838-3.2743960 .579584$

C $4.737814-3.356054-0.844679$

O $4.389740-4.567397 \quad 1.147130$

C $0.453767-0.208603-2.543314$
O $\quad 0.4822420 .085023-3.728038$

C $1.2771630 .427081-1.491669$

C $1.8355481 .623289-1.730237$

C $2.6575272 .390611-0.793228$

C 3.2152781 .6624790 .400113

C $4.544546-2.110259-1.716351$

C $5.739998-1.172097-1.731708$

C $6.471873 \quad 1.010299-2.690638$

O $6.807186-1.425286-1.180117$

C $6.3616782 .048046-1.619303$

C $6.1746753 .352462-1.870597$

C $6.1161194 .408283-0.804898$

C $4.8541765 .237689-0.872126$

C $4.8557216 .454893-1.441521$

C $3.6161754 .694708-0.196440$

C $2.8802643 .696736-1.047717$

N $5.478870-0.023491-2.467804$

H $-3.879740-2.754090-2.878766$

H $-2.375710-0.108674-3.182066$

H $-0.714172-2.070959-3.787628$

H $-2.008030-3.962031-2.745229$

H -1.884186 -3.177094 -1.182538

H $1.378000-4.962087-0.692507$

H $1.513875-5.149131-2.958063$

H $-0.020160-5.059843-3.825450$

H $1.032507-3.630212-3.715747$

H -1.194270 -3.9637930 .615151$

H $0.054277-4.9693321 .289933$

H $\quad 0.351573-1.9834770 .680589$

H -0.042119 -2.670483 2.257275

H $2.152366-3.9362272 .200144$

H $1.934609-1.7280013 .422081$

H $2.297749-0.8808611 .933774$

H $5.344925-0.6344913 .476753$

H $4.735810-1.2165900 .872846$

H $3.832982-2.5536334 .827181$

H $\quad 5.377967-2.9378204 .103382$

H $3.942079-3.8929073 .681923$

H $6.532113-1.6683402 .114746$

H $5.791035-3.658538-0.797790$

H $4.225500-4.183686-1.357084$

H $4.952781-4.4625971 .930259$

H $1.383120-0.092277-0.546561$

H $1.6456452 .106470-2.687863$

H 3.6951390 .7299280 .086696

H $2.422236 \quad 1.422767 \quad 1.113811$

H 3.9812352 .2278150 .935470

H $4.400312-2.436945-2.754550$

H $3.650009-1.552724-1.422326$

H $6.2926421 .423304-3.688252$

H $7.4720530 .565700-2.672224$

H $6.470377 \quad 1.713980-0.590109$

H $6.0926603 .688448-2.901848$

H $6.9950285 .054520-0.929402$

H 6.2120653 .9724610 .197341

H $3.9613937 .068555-1.466115$

H $5.7503396 .862217-1.901073$

H 2.9167355 .5154580 .014025

H 3.8816234 .3029150 .789791

H $2.4402264 .131672-1.946315$

H $4.5442400 .129582-2.835624$

C $-5.939619-1.103838-2.024972$

O $-5.353809-0.627001-0.800390$

C $-6.411114-0.175406 \quad 0.070305$

C $-7.720270-0.392689-0.685699$

C $-7.261454-0.352100-2.132970$

H -6.2683430 .9023870 .200704$

C $-6.298944-0.885011 \quad 1.427262$

H $-8.173802-1.363028-0.458635$

H $-8.461606 \quad 0.377700-0.451028$

C $-8.255690-0.968403-3.100750$

H $-7.0779110 .693435-2.414803$

C $-4.918269-0.7091652 .095886$

O $-6.481075-2.2952351 .243203$

H -7.091409-0.548221 2.105985

H $-4.147316-1.1839871 .478850$

O $-4.932770-1.4192593 .341069$

C -4.5422510 .7539482 .387778$ 
C -3.190488 $0.925976 \quad 3.105234$ H -4.5244821 .3304191 .459213$ O $-5.553864 \quad 1.345470 \quad 3.208362$ H -3.1186641 .9699393 .433797$ H -3.1833300 .3417604 .033700$ H -7.883882 -0.917460 -4.128687 H -8.447642 -2.020262 -2.863665 H $-9.211140-0.435113-3.061717$ C $-4.977465-0.868459-3.188945$ H -7.434180 -2.462146 1.155232 H $-5.173104-2.3411703 .144446$ H -5.6031910 .8279944 .030513$ H $-4.7284200 .196182-3.261706$ H $-5.450365-1.162580-4.132331$ H -6.122434 -2.179996 -1.898311 C -1.817391 2.620464 1.001474 O $-2.000214 \quad 1.1995490 .957588$ C $-1.972780 \quad 0.5534592 .234585$ C $-0.616887 \quad 0.805402 \quad 2.907011$ C $-0.297753 \quad 2.2957373 .000726$ C -0.4691752 .9692411 .639584$ H -2.631540 3.0808731 .573940 C $-1.897264 \quad 3.136127-0.438921$ H -2.036466 -0.5229732 .036268$ $\begin{array}{lllll}\text { O } & -0.579032 & 0.233687 & 4.209452\end{array}$ H $\quad 0.1725790 .322147 \quad 2.318456$ H -0.9102042 .7913283 .762926$ O 1.0612722 .4607833 .412510 H $\quad 0.3445362 .6599340 .976819$ O $-0.373503 \quad 4.3827301 .798422$ C $-3.3102663 .041372-0.960870$ H -1.570913 4.178614 -0.525494 H $-1.2605142 .543644-1.106294$ H $-0.764483-0.7164364 .116555$ H $1.1675661 .986903 \quad 4.255295$ H 0.4875294 .5702332 .210262 O $-4.0422184 .105418-0.523622$ O $-3.7181832 .121788-1.657584$ C $-5.410876 \quad 4.121822-0.940729$ H -5.871092 $5.037730-0.560442$ H $-5.4767494 .123538-2.032943$ H $-5.942573 \quad 3.262386-0.522840$

SCF Energy (B3LYP/6-31G**/MMFF) $=-3245.90599086$

$01 \_22$

MMFF Geometry

C $-0.308228 \quad 2.2469812 .197940$

C $0.5880191 .878862 \quad 3.124749$

C 1.0259320 .4516883 .303414

$\begin{array}{lllll}\text { O } & 2.470527 & 0.458291 & 3.202950\end{array}$

C $0.627239-0.1452454 .661923$

C $-0.862687-0.3216104 .863427$

C $-1.602884-1.2739334 .261743$

C $-1.499163 \quad 0.6357505 .835174$

C -1.130459 -2.294333 3.261282

C $-1.902899-2.1523451 .943466$

C $-1.248519-2.8219620 .726815$

C $-0.871335-4.2855070 .957025$

C $-0.176436-4.892705-0.272869$

O $-0.088469-2.059757 \quad 0.374339$

O $0.432205-6.1295490 .129819$

C $0.965522-3.952672-0.718602$

C $-1.177731-5.243981-1.379953$

O $1.546466-4.402656-1.940805$

C $0.533407-2.473628-0.850780$

C $1.770417-1.575651-1.094576$

O $-0.352553-2.347325-1.965882$

C $3.065169-0.6423432 .671764$

O $2.529094-1.6877572 .341817$

C $4.501977-0.3361272 .513281$

C $5.188233-0.9556991 .542236$

C $6.598436-0.7525051 .214392$

C 7.4246750 .0847352 .154494

C $1.472782-0.073212-1.007661$

C $2.7249390 .766908-1.168193$

C $4.3990821 .487026-2.875291$

O $3.1852681 .422849-0.236774$

C $5.5982140 .593087-2.935828$
C $6.8012200 .945412-2.456376$

C $8.0370580 .098843-2.582621$

C $8.782747-0.049664-1.275123$

C $9.7291040 .836405-0.920686$

C $8.499650-1.264526-0.422074$

C $7.088103-1.3182220 .091538$

N $3.242707 \quad 0.720862-2.451514$

H $-0.779068 \quad 1.4804091 .585863$

H $1.0637382 .633667 \quad 3.745483$

H $0.620172-0.1516642 .483595$

H 1.0461400 .4704055 .470056

H $1.121745-1.1169084 .795402$

H -2.664776 -1.327797 4.498052

H $-2.5789580 .478374 \quad 5.928653$

$\begin{array}{llll}H & -1.058266 & 0.513884 & 6.829942\end{array}$

H $-1.344213 \quad 1.6695125 .511080$

H $-0.055738-2.2181973 .084017$

H $-1.304740-3.2921413 .681183$

H $-2.908670-2.5692242 .080225$

H -2.022708 -1.0852381 .712832$

H - $-1.970472-2.746497-0.093812$

H $-1.753445-4.8752931 .234526$

H $-0.176127-4.3649601 .803178$

H $-0.266232-6.7051350 .485963$

H $1.756803-4.0137370 .041915$

H $-1.913823-5.969765-1.013679$

H $-0.677109-5.727254-2.226218$

H - $1.728239-4.378090-1.753050$

H $1.814454-5.328419-1.810059$

H $2.541056-1.806142-0.350274$

H $2.199695-1.803763-2.077762$

H $-0.321021-1.436967-2.298114$

H $4.9369080 .414635 \quad 3.162419$

H $4.661870-1.6670450 .907188$

H $7.389668-0.3273273 .169156$

H 7.0527191 .1148422 .179948

H $8.4792670 .137061 \quad 1.874039$

H $\quad 0.7579490 .237803-1.776057$

H $1.0294030 .178716-0.038429$

H $4.5485662 .335752-2.199920$

H $4.1765461 .870723-3.875883$

H $5.484356-0.366670-3.434168$

H $6.9180601 .922613-1.992511$

H $8.6932320 .575842-3.322858$

H $7.805257-0.892599-2.990934$

H $\quad \begin{array}{lllll}\text { H } & 10.293543 & 0.725638 & -0.000942\end{array}$

H $9.9580781 .695604-1.542786$

H $9.207637-1.3404260 .412170$

H $8.683820-2.165602-1.021864$

H $6.413395-1.895333-0.541632$

H $2.7865290 .139694-3.148501$

C $-0.343945 \quad 4.021736 \quad 0.458062$

O $-1.1445393 .221677-0.433165$

C $-1.5723344 .035850-1.538977$

C $-0.804670 \quad 5.347584-1.422637$

C $-0.643392 \quad 5.470494 \quad 0.081355$

H -2.638809 $4.238785-1.386265$

C $-1.366936 \quad 3.272240-2.851010$

H $\quad 0.177150 \quad 5.289547-1.906473$

H $-1.3485436 .186609-1.867308$

C $0.434724 \quad 6.4553550 .497956$

H $-1.603766 \quad 5.780908 \quad 0.514470$

C $-2.030248 \quad 1.877698-2.878423$

$\begin{array}{lllll}\text { O } & 0.037468 & 3.081127 & -3.071528\end{array}$

H $-1.7227423 .876581-3.693133$

H $-1.5112411 .208728-2.183118$

O $-1.834512 \quad 1.326996-4.186820$

C $-3.5383401 .880132-2.568051$

C $-4.2358690 .527186-2.797039$

H $-3.7003602 .201181-1.535270$

O $-4.1733132 .846768-3.410775$

H $-5.3129620 .679051-2.657184$

H $-4.1315680 .243688-3.851733$

H $0.5225326 .500707 \quad 1.587985$

H 1.4127616 .1750800 .092551

$\begin{array}{lllll}H & 0.196938 & 7.461143 & 0.136494\end{array}$

C $-0.692705 \quad 3.6691501 .904315$

H $0.3974992 .625434-2.291354$ 
H $-0.878487 \quad 1.337407-4.365307$

H -5.109575 $2.892072-3.152081$ H -1.772133 3.7685632 .062659 H -0.1901964 .3560552 .594074$ H $0.706802 \quad 3.7794380 .248484$ C $-5.197609-0.2322050 .002262$ O $-3.855050-0.303406-0.493389$ C $-3.736438-0.613197-1.886951$ C $-4.396567-1.968049-2.177151$ C $-5.849661-1.989853-1.716892$ C $-5.950589-1.546596-0.259655$ H $-5.7187460 .608733-0.470784$ C $-5.137345 \quad 0.035231 \quad 1.515937$ H $-2.666100-0.736236-2.083124$ O $-4.339440-2.278568-3.565195$ H $-3.854277-2.762020-1.648586$ H $-6.488553-1.375093-2.361346$ O $-6.352522-3.324929-1.811923$ H $-5.551706-2.3401620 .385907$ O $-7.325261-1.3742770 .081012$ C -4.5780341 .3945691 .875308$ H -6.145823 -0.019805 1.942079 H $-4.503446-0.7137262 .001552$ H -3.403092 -2.269615 -3.827737 H $-6.243290-3.609228-2.735713$ H $-7.778557-2.213728-0.108185$ O $\quad-4.660374 \quad 1.5488503 .231176$ O $-4.1245142 .209170 \quad 1.085198$ C $-4.1906252 .793147 \quad 3.759210$ H -3.1019302 .7630753 .843925$ H -4.5074593 .6372753 .138908$ H -4.6161262 .9186994 .758455$

SCF Energy (B3LYP/6-31G**//MMFF)= -3245.91272168

01_220

MM̄FF Geometry

C $-0.704346 \quad 1.3107531 .334342$

C -0.4736660 .0967040 .814649$

C $0.511229-0.160034-0.293452$

O $1.401317-1.1938880 .194310$

C $-0.164350-0.657038-1.583839$

C $-1.0015060 .383271-2.290980$

C $-0.5079401 .308690-3.136362$

C $-2.4851380 .287791-2.058293$

C $0.9304221 .534565-3.522024$

C $1.3029623 .024676-3.502366$

C $1.5089443 .587267-2.084986$

C $1.3446545 .109102-2.104385$

C $1.6408635 .736308-0.738295$

O $2.8354093 .236553-1.662908$

O $1.7502457 .156570-0.919124$

C $3.0148375 .233554-0.262461$

C 0.5016135 .5130840 .261969

O 3.2850205 .6814121 .065119

C $3.1909953 .696058-0.351124$

C $4.6818223 .361400-0.098318$

O 2.3904643 .0771640 .654513

C $2.621599-1.297265-0.395078$

O $3.065386-0.602842-1.295057$

C $3.331639-2.4289200 .238995$

C $4.486554-2.850930-0.295378$

C $5.318598-3.9501240 .192266$

C $4.901750-4.6436231 .461844$

C $5.0840721 .891941-0.284878$

C $6.5730871 .701546-0.029537$

C $8.318970-0.0163020 .251687$

O $7.3349432 .637430 \quad 0.206285$

C $8.628000-1.384712-0.262987$

C $9.061362-2.3830980 .522458$

C $9.457297-3.7453310 .024091$

C $8.605448-4.8484660 .611801$

C $8.920692-5.4111741 .790576$

C $7.431125-5.347709-0.198544$

C $6.427730-4.271074-0.504540$

N $6.9652340 .369579-0.104202$

H -0.1711582 .1672100 .933174$

H -1.004394 -0.7631631 .212856$

H $1.0990410 .744945-0.489005$
H $-0.774792-1.544708-1.365567$

H $\quad 0.595157-1.036839-2.279271$

H $-1.217317 \quad 1.972795-3.629289$

H $-3.0403901 .085459-2.563153$

H -2.860271 -0.668033 -2.437535

H $-2.7168650 .352839-0.992122$

H $1.6251890 .970495-2.895383$

H $1.0543881 .165628-4.547552$

H $2.2311603 .148570-4.075309$

H $0.5241223 .591274-4.028353$

H $0.7820613 .139545-1.399106$

H $0.3372345 .386954-2.439003$

H $2.0349325 .540987-2.843081$

H $0.9102817 .476876-1.290097$

H $3.7720095 .704007-0.905924$

H $-0.4329855 .941652-0.119329$

H 0.7015796 .0256021 .209428

H $\quad 0.3183524 .4587640 .473324$

H 3.1827026 .6485961 .071051

H $5.2960823 .958698-0.785793$

H $4.953476 \quad 3.6621750 .922144$

H 2.7441582 .1934410 .840108

H $2.883596-2.8868841 .113152$

H $4.862292-2.345382-1.184462$

H $3.940877-5.1513391 .324559$

H $4.804949-3.9224542 .281188$

H $5.618426-5.3970091 .797991$

H 4.5364291 .2500340 .412083

H $4.8708761 .566092-1.308515$

H 8.4010360 .0472221 .342360

H $9.0188500 .707777-0.179586$

H $8.532449-1.548526-1.333417$

H $9.177224-2.2033481 .589233$

H $10.509357-3.9094630 .292931$

H $9.427533-3.795625-1.071552$

H $8.329501-6.2190972 .208622$

H $9.776691-5.0724672 .365020$

H $6.945278-6.2022860 .286312$

H $7.806126-5.744581-1.151041$

H $6.658157-3.705089-1.407973$

H $6.244324-0.346572-0.113125$

C -2.7918862 .4993992 .061248$

O -3.7606431 .7413401 .306092$

C -5.0799242 .0640781 .786669$

C -4.9202423 .3136052 .643012$

C -3.5545283 .0780023 .254020$

H -5.3910291 .2359122 .434485$

C -6.0629912 .2142430 .621970$

H -4.9124614 .2258192 .035000$

H -5.7150823 .4109303 .388686$

C $-2.9273494 .332386 \quad 3.838089$

H -3.647576 2.3208314 .044423

C $-6.1628820 .972135-0.286808$

O $-5.6364653 .315232-0.191115$

H -7.0524412 .4834951 .010305$

H $-5.2224070 .853077-0.833256$

O $-7.1612451 .243135-1.279589$

C $-6.521722-0.3364130 .441571$

C $-6.807440-1.525641-0.494219$

H $-5.717548-0.6031951 .132223$

O $-7.693463-0.1090141 .230013$

H -7.135335 -2.369354 0.125139

H -7.674452 -1.289150 -1.123497

H -1.9381454 .1181064 .254349$

H -2.8108335 .1135443 .079353$

H -3.5516544 .7356434 .642098$

C -1.6393481 .5848722 .477423$

H $-6.2681543 .393922-0.926456$

H -8.017255 $1.311640-0.822540$

H $-7.864343-0.918057 \quad 1.741688$

H -2.0377490 .6450882 .876546$

H -1.051043 2.0558083 .273244

H -2.436117 3.3056721 .406467

C $-4.511651-3.4445570 .175665$

O $-4.444263-2.237084-0.593072$

C $-5.610255-1.941380-1.369834$

C $-5.908627-3.094016-2.338028$

C $-6.017952-4.428517-1.609991$ 
C $-4.792162-4.653441-0.728486$

H $-5.298395-3.3544670 .934682$

C $-3.160607-3.6151710 .891592$

H -5.354385 -1.075967 -1.991420

O $-7.111340-2.856269-3.061626$

H -5.098806 -3.167357 -3.075724

H $-6.942006-4.495029-1.024026$

O $-6.076083-5.485107-2.571238$

H -3.919793 -4.859307 -1.362330

O $-5.010741-5.8055810 .084259$

C -3.019287 -2.718747 2.103595

H -3.056359 -4.647123 1.246052

H -2.332781 -3.3823610 .211769$

H -7.010346 -2.007188 -3.524896

H $-6.836631-5.306545-3.150744$

H -5.202945 -6.549182 -0.512616

O $-1.978333-3.1709282 .865232$

O $-3.710115-1.7394602 .344964$

C -1.717254 -2.438086 4.066648

H -1.024215 -3.023699 4.676615

H -1.246973 -1.482434 3.821709

H -2.637128 -2.282195 4.638697

SCF Energy (B3LYP/6-31G**//MMFF) $=-3245.92090860$

012221

MM̄FF Geometry

C $-0.756439-0.7741433 .917691$

C -0.3768840 .4324013 .470088$

C -1.2989961 .5150842 .963134$

O -2.6008120 .9455022 .675437$

C -1.4871012 .6556823 .979094$

C $-0.325703 \quad 3.6246564 .063748$

C -0.2033594 .7174883 .283753$

C 0.6804113 .3538665 .150735

C -1.1257935 .1367122 .173088$

C -0.3996455 .6630450 .927702$

C $0.595968 \quad 4.6816570 .287990$

C $1.2445655 .332171-0.939675$

C $2.1953204 .375395-1.669661$

O $-0.0929653 .481646-0.091478$

O $2.480824 \quad 4.962940-2.948479$

C $1.4572863 .043326-1.912499$

C $3.5411754 .254109-0.946383$

O $2.3349182 .082407-2.488848$

C $0.7696382 .476983-0.649634$

C $-0.0548641 .204742-0.953502$

O 1.7776572 .0866590 .292954

C -3.2859691 .4352831 .608874$

$\begin{array}{llll}\text { O } & -2.942219 & 2.338780 & 0.864610\end{array}$

C $-4.5458300 .669951 \quad 1.486203$

C -5.3410380 .8969090 .430036$

C -6.6166250 .2409880 .143441$

C $-7.121541-0.7812521 .126607$

C -1.204262 $1.367663-1.949349$

C $-1.9350760 .051611-2.174530$

C $-3.729332-0.942788-3.573318$

O $-1.570423-1.011328-1.677648$

C $-5.171989-0.606739-3.777608$

C $-6.171152-1.418037-3.396859$

C -7.628905 -1.123597-3.615653

C $-8.434413-1.211272-2.337432$

C $-9.057698-2.351450-1.994930$

C $-8.579606 \quad 0.035113-1.493517$

C -7.268851 $0.571502-0.990447$

N -3.029736 $0.202354-3.017465$

H $-1.813310-1.0258313 .958692$

H 0.6842350 .6680323 .447534

H -0.8648031 .8995332 .034789$

H -1.698594 2.2409814 .974631

H -2.402892 3.2115583 .737492

H $0.637227 \quad 5.3867163 .461151$

H 1.4539094 .1276555 .202044

H $\quad 0.185084 \quad 3.3206476 .126621$

H $\quad 1.1859172 .3988504 .985580$

H $-1.7883004 .323487 \quad 1.872672$

H -1.769456 5.9389132 .554352

H -1.169571 5.9259920 .190506

H 0.1243556 .5924201 .185835
H 1.3654814 .4346831 .028011

H $1.7696006 .254385-0.660518$

H $0.4574525 .639314-1.643249$

H $3.0213984 .328315-3.449848$

H $0.6941953 .249838-2.672628$

H $4.0330455 .232959-0.892662$

H $4.2307633 .604753-1.496971$

H 3.4493983 .8749360 .073445

H $2.8710901 .705618-1.771115$

H $0.6208680 .409955-1.295678$

H $-0.4775630 .826777-0.013368$

H $1.388821 \quad 1.4516450 .917111$

H -4.780140 -0.054489 2.257364

H $-5.0278391 .636699-0.306066$

H -7.278821 -0.3242892 .109860$

H $-6.403784-1.6025511 .230784$

H -8.071878 -1.232373 0.831070

H -1.920637 2.113464 -1.590855

H $-0.8241311 .692656-2.923241$

H -3.594086 -1.813030 -2.922616

H $-3.260789-1.162964-4.538209$

H $-5.4066750 .329051-4.278831$

H $-5.929050-2.366214-2.921141$

H $-8.010736-1.850475-4.344814$

H -7.776608 -0.138840 -4.075922

H $-9.658587-2.419929-1.094235$

H -8.977414 -3.243810 -2.607048

H $-9.266452-0.127663-0.654304$

H $-9.0608360 .813981-2.099460$

H $-6.8227221 .314537-1.652471$

H $-3.1562151 .098397-3.479821$

C $0.036619-3.1221653 .608082$

O $0.681192-2.9811922 .323866$

C $1.258699-4.253121 \quad 1.962021$

C $0.846819-5.2413293 .049719$

C $0.702826-4.3339814 .253752$

H $2.346657-4.1311891 .999189$

C $0.805224-4.6688680 .558538$

H $-0.109594-5.7238132 .814234$

H $1.590796-6.0311893 .192306$

C $-0.093982-4.9597005 .385402$

H $1.703688-4.0650334 .617351$

C $1.326385-3.770377-0.582359$

O $-0.627783-4.6607930 .511551$

H $1.102977-5.7064970 .367381$

H $0.900274-2.763779-0.500975$

O $0.839545-4.319489-1.814124$

C $2.863824-3.686943-0.666521$

C $3.414889-3.095785-1.975659$

H $3.243202-3.1075540 .179203$

O $3.420993-5.000251-0.537853$

H $4.507034-3.193748-1.947160$

H $3.105645-3.730366-2.815875$

H $-0.177267-4.273316 \quad 6.233596$

H -1.107568 -5.223340 5.064748

H $0.394579-5.8734125 .739611$

C $0.188229-1.8327554 .413929$

H $-0.921701-3.7556240 .713580$

H $-0.130569-4.353431-1.751891$

H $3.067779-5.541451-1.264959$

H $1.224075-1.4787844 .363887$

H -0.049044 -2.013426 5.468158

H - $1.023668-3.3344983 .413538$

C $4.793765-0.555104-1.010509$

O $3.384179-0.756908-1.173001$

C $3.015431-1.632809-2.244191$

C $3.549074-1.085322-3.575477$

C $5.054662-0.838582-3.519452$

C $5.4203160 .002331-2.297181$

H $5.272762-1.502657-0.736612$

C $4.9953280 .435317 \quad 0.147291$

H $1.921050-1.603984-2.304121$

O $3.239833-1.982973-4.636647$

H $3.040387-0.140967-3.801985$

H $5.608853-1.783903-3.523714$

O $5.463542-0.147495-4.702837$

H $5.093177 \quad 1.037433-2.456527$

O $6.8394080 .026098-2.153948$ 
C $4.552650-0.120121 \quad 1.483328$

H 6.0512520 .7091560 .246985

H $4.4111311 .341005-0.048525$

H $3.620239-1.612732-5.451609$

H $4.9842690 .697846-4.738287$

H $7.2104460 .356942-2.989904$

$\begin{array}{lllll}\text { O } & 4.373416 & 0.916763 & 2.354991\end{array}$

O $4.413908-1.3068501 .743453$

C 3.9762680 .5488623 .679311

H $3.092381-0.0938553 .651997$

H 3.7274101 .4621924 .226105

H 4.8029380 .0454454 .188807

SCF Energy (B3LYP/6-31G**//MMFF) $=-3245.93285882$

01222

MM̄FF Geometry

C -1.408267 4.8238110 .382003

C $-2.4814664 .904489-0.419682$

C $-3.4730693 .797316-0.703168$

O $-2.9913852 .513981-0.244676$

C $-4.7946884 .101413 \quad 0.026311$

C $-5.9163223 .135028-0.300180$

C -6.5078472 .3178280 .593150$

C -6.392953 $3.145782-1.730306$

C -6.2101582 .1723452 .059379$

C -5.1956591 .0695212 .400894$

C $-5.656444-0.3484492 .025061$

C $-4.700187-1.3986012 .595310$

C $-5.080614-2.8228192 .157819$

O $-5.679320-0.4540310 .599749$

O $-3.970094-3.6849742 .450761$

C $-5.277835-2.8469570 .625951$

C $-6.271580-3.3643922 .957579$

O $-5.795196-4.1040660 .198586$

C $-6.173454-1.7053940 .104353$

C $-6.202266-1.605535-1.440354$

O $-7.521790-1.9315700 .533681$

C $-2.1050691 .857286-1.043064$

O $-1.6721622 .225865-2.123046$

C $-1.7212120 .608442-0.349255$

C $-0.614892-0.046892-0.730935$

C $-0.096786-1.281920-0.137468$

C $-0.965668-1.9857360 .870607$

C $-4.844040-1.367611-2.109750$

C $-4.168275-2.636958-2.601265$

C $-2.032058-3.431858-3.606893$

O $-4.726273-3.730150-2.633872$

C $-1.190378-4.037702-2.528819$

C $0.140071-4.177910-2.628169$

C $0.995438-4.824391-1.577164$

C $2.137803-3.939496-1.133436$

C $3.371217-4.093545-1.643553$

C $1.867870-2.934628-0.037488$

C $1.114839-1.728650-0.529320$

N -2.873141 -2.394536 -3.039837

H -1.2003603 .9031250 .920141$

H -2.675835 $5.843371-0.935000$

H -3.640469 3.754177-1.786158

H -5.138016 $5.113066-0.228965$

H $-4.599217 \quad 4.112781 \quad 1.104070$

H $-7.312378 \quad 1.6738930 .239165$

H -7.298092 2.545118 -1.871117

H $-6.6283414 .167679-2.045150$

H -5.625304 2.740930 -2.396702

H $-5.858998 \quad 3.1206912 .479581$

H -7.1550681 .9653082 .578038$

H $-4.2461661 .294613 \quad 1.901223$

H -5.0099331 .1111363 .481358$

H $-6.667907-0.4984212 .420442$

H -4.657334 -1.333141 3.689617

H -3.680872 -1.1891612 .244036$

H -3.785662 -3.622909 3.403695

H $-4.282398-2.7503310 .177149$

H $-6.034949-3.3871244 .028205$

H $-6.496329-4.3999872 .679818$

H $-7.178112-2.7684542 .833877$

H $-5.190949-4.792410 \quad 0.525425$

H $-6.717738-2.475371-1.864860$
H $-6.834003-0.742270-1.695778$

H -8.118686 -1.433088 -0.048760

H -2.3488340 .2746410 .468714$

H $-0.0177620 .368268-1.542028$

H -1.961234 -2.169613 0.453135

H $-1.070581-1.3809081 .777723$

H $-0.582717-2.9616281 .174867$

H $-5.001745-0.744489-2.999578$

H $-4.165462-0.823062-1.446220$

H -1.428371 -2.966601 -4.392544

H $-2.661307-4.204221-4.060863$

H - $-1.700235-4.401652-1.639985$

H $0.642914-3.838008-3.530901$

H $1.389336-5.759152-1.996963$

H $\quad 0.402618-5.120899-0.702935$

H $4.200382-3.479596-1.309675$

H $3.577256-4.832657-2.410540$

H $2.816708-2.5722660 .376216$

H $1.375869-3.4402980 .797438$

H $1.663354-1.149784-1.273118$

H $-2.472523-1.468137-2.924323$

C 0.9864945 .6374000 .164800

O 1.5284424 .5631020 .956407

C 2.3305073 .7107590 .099072

C 2.496054 4.484928-1.201764

C $1.1735205 .221314-1.291804$

H $1.7195152 .814403-0.058691$

C 3.6230543 .3569920 .843352

H $3.3218005 .203264-1.127290$

H $2.6781823 .838781-2.064303$

C $1.1828036 .382452-2.269710$

H $0.4039674 .502341-1.598489$

C 4.6994232 .5475550 .087842

O 3.2492172 .6309612 .020533

H 4.0730754 .2965581 .189879

H 5.5462232 .4431110 .776720

O $5.1791493 .267682-1.042355$

C $4.2853501 .127161-0.345236$

C $5.4098450 .315990-1.021347$

H 3.9189390 .5744940 .525530

O $3.2005251 .186465-1.271500$

H $4.993609-0.665183-1.283174$

H $5.6639730 .775084-1.985503$

H $\quad 0.2112506 .886469-2.283895$

H $1.9443167 .124063-2.006356$

H $1.3951746 .028979-3.284096$

C $-0.4449055 .955966 \quad 0.603743$

H 4.0574952 .4655342 .535200

H $5.5046634 .127497-0.725621$

H $3.5088781 .672098-2.055490$

H $-0.788194 \quad 6.8617410 .090112$

H -0.446262 6.1765951 .678429

H $1.609006 \quad 6.5149820 .384734$

C $5.904371-1.5716471 .316937$

O $6.409363-0.2401721 .168869$

C $6.6895450 .144870-0.178561$

C $7.730813-0.804546-0.789088$

C $7.322596-2.269725-0.650331$

C $6.921623-2.5880660 .790406$

H $4.961954-1.6701800 .766756$

C $5.612749-1.7878492 .804790$

H $7.162517 \quad 1.132867-0.122620$

O $7.946552-0.508519-2.164756$

H $8.691161-0.665603-0.275463$

H $6.516977-2.527523-1.347120$

O $8.431670-3.101540-0.998913$

H $7.815660-2.5938561 .426935$

O $6.350092-3.8932320 .840546$

C $4.449594-0.9310123 .245824$

H $5.377314-2.8334913 .032299$

H $6.476511-1.5116393 .421535$

H $8.2215590 .422419-2.223759$

H $8.697475-2.863872-1.903902$

H $7.004941-4.5109960 .472191$

O $3.274710-1.5695522 .980816$

O 4.5727640 .1831643 .737866

C $2.086795-0.8443823 .313601$

H $2.119653-0.5001874 .351841$ 
H $\quad 1.234668-1.5191933 .197936$ H $1.963655-0.0001422 .629134$

SCF Energy (B3LYP/6-31G**//MMFF) $=-3245.91011085$

01223

MM̄FF Geometry

C -2.612292 $3.396182-1.303779$

C $-1.8868902 .333069-0.925003$

C $-0.8916601 .646806-1.823111$

O $0.3532261 .511344-1.096054$

C $-1.3916760 .236187-2.183942$

C $-0.795638-0.263575-3.484602$

C $0.276419-1.072074-3.585025$

C -1.512872 $0.194797-4.729527$

C $1.125607-1.620134-2.473397$

C $1.137394-3.155908-2.496974$

C $2.052504-3.795519-1.438896$

C $1.510898-3.610892-0.020573$

C $2.467442-4.1701681 .041138$

O $3.351651-3.218410-1.572214$

O $2.036360-3.6839052 .322026$

C $3.871686-3.5856200 .785402$

C $2.414044-5.6994931 .118282$

O $4.832637-4.1607301 .667534$

C $4.344439-3.735580-0.678366$

C $5.653402-2.956885-0.952805$

O $4.602942-5.118129-0.945761$

C $1.2035932 .574029-1.109114$

O $1.0696933 .613475-1.735389$

C $2.3178762 .272202-0.179654$

C 3.1799203 .2476920 .147148

C 4.2942313 .1516201 .095520

C 4.5418361 .8169331 .745851

C $5.520350-1.437222-0.821698$

C $6.780738-0.703915-1.242525$

C $7.5977231 .610787-1.699361$

O $7.865381-1.260469-1.388429$

C $8.0231012 .262057-0.421621$

C $7.9739073 .585498-0.208464$

C $8.418570 \quad 4.234228 \quad 1.069865$

C 7.3543015 .1146521 .684212

C 7.5052556 .4492401 .733442

C 6.1563404 .4435052 .322139

C $5.0130064 .264687 \quad 1.355817$

N $6.548224 \quad 0.655911-1.400710$

H $-2.4851703 .803254-2.303847$

H -2.027288 1.9174310 .069315

H $-0.7224382 .233920-2.734673$

H -2.484531 $0.231797-2.298004$

H -1.180190 -0.463772 -1.367519

H $0.606261-1.360485-4.582647$

H -1.050171 -0.191809 -5.643807

H -2.552439 $-0.149221-4.715561$

H $-1.509687 \quad 1.287268$-4.796053

H $2.142569-1.235923-2.615745$

H $0.793498-1.260349-1.497769$

H $\quad 0.117169-3.544050-2.384927$

H $1.493045-3.477906-3.485281$

H $2.119476-4.865033-1.672148$

H $0.521798-4.0730260 .071367$

H $1.365202-2.5443960 .192562$

H $1.122912-3.9856032 .464672$

H $3.818691-2.5195051 .037949$

H $1.403013-6.0339841 .380773$

H $3.069875-6.0775951 .910282$

H $2.687805-6.1874350 .180735$

H $4.499794-4.0453092 .574091$

H $\quad 6.457332-3.328446-0.304619$

H $5.982821-3.178320-1.977945$

H $5.215801-5.178943-1.697105$

H $2.372822 \quad 1.2733890 .238229$

H $3.0437474 .229471-0.305396$

H 4.6904261 .0415240 .988700

H 3.6890161 .5323672 .372174

H 5.4273611 .7970222 .383676

H $4.689237-1.075412-1.437605$

H $5.323865-1.1622520 .219108$

H $7.1897272 .330217-2.416223$
H $\quad 8.448998 \quad 1.099768-2.159689$

H $8.401683 \quad 1.610506 \quad 0.362757$

H $7.6163474 .240610-0.999573$

H 9.3173394 .8236200 .846753

H 8.7292943 .4852371 .809290

H 6.7587207 .0842042 .199220

H $8.374576 \quad 6.9372301 .305477$

H 5.7891625 .0665283 .148882

H 6.4786393 .5095052 .789255

H 4.7271055 .1874270 .847915

H $5.6221931 .025012-1.205052$

C $-5.0512323 .690954-0.869943$

O $-5.2697782 .289483-0.617486$

C $-6.6026342 .104190-0.106855$

C $-7.3086823 .445723-0.267745$

C $-6.1544574 .417868-0.104282$

H -6.5028921 .8889550 .962960$

C $-7.2715320 .917210-0.809306$

H -7.757989 $3.554800-1.261335$

H -8.0996643 .5887490 .474807$

C $-6.4556855 .811793-0.626183$

H -5.8956424 .4813850 .960784$

C $-6.421314-0.371667-0.787400$

O $-7.4880951 .271952-2.180918$

H $-8.2611650 .738989-0.372319$

H $-5.525662-0.228851-1.402192$

O $-7.179848-1.415457-1.410793$

C $-6.023050-0.8493930 .623056$

C $-5.256820-2.1868670 .639735$

H $-5.416250-0.0882251 .121212$

O $\quad-7.203828-1.0100181 .414082$

H -5.213261 -2.537816 1.677641

H -5.840804 -2.949502 0.110696

H $-5.5919296 .471713-0.498276$

H $-6.7124025 .795220-1.690742$

H -7.299492 $6.251040-0.083895$

C $-3.6361524 .063978-0.428581$

H $-8.0971970 .616289-2.560239$

$\mathrm{H}-7.245364-1.204481-2.357253$

H -7.766923 -1.6670930 .969874$

H -3.4787713 .7716030 .616226$

H $-3.4865235 .147588-0.487915$

H $-5.1696253 .843670-1.951111$

C $-2.631557-1.4873312 .056707$

O $-3.034984-1.1200160 .730771$

C $-3.836786-2.0892940 .045445$

C -3.090505-3.428055 -0.040089

C $-2.636988-3.9101831 .333219$

C -1.855716 -2.809567 2.046497

H -3.519292 -1.588539 2.692831

C $-1.764239-0.3446512 .605652$

H -3.941186 -1.727006 -0.983450

O $-3.903518-4.428672-0.643047$

H -2.202702 $-3.308948-0.675260$

H -3.483914 -4.248498 1.941785

O $-1.778436-5.0419561 .178232$

H -0.885490 -2.669109 1.556000

O $-1.602167-3.2144793 .390512$

C -2.5913160 .6303743 .408248$

H $-0.990592-0.7190173 .286558$

H -1.2487500 .2053321 .810035$

H -4.167787 -4.101154 -1.519773

H $-2.275610-5.7162180 .684128$

H -1.141056 -4.070057 3.354266

O

O $-2.527700 \quad 0.7255434 .627687$

C -4.2420692 .3036653 .256074$

H -4.9259861 .7852043 .934844$

H -3.6377713 .0365803 .799151$

$\mathrm{H}-4.8317472 .8290752 .502539$

SCF Energy $($ B3LYP/6-31G**//MMFF $)=-3245.91212841$

01224

MM̄MF Geometry

C -1.440170 $1.042563-1.021846$

C $-0.1969200 .840871-0.561489$

C $0.168581-0.3062010 .342817$

O $1.222595-1.029646-0.338451$ 
C $0.704036 \quad 0.1585821 .712133$

C -0.1854251 .1165582 .477483$

C 0.1743932 .3669992 .831953

C -1.538933 0.5801082 .866052

C 1.4903413 .0435782 .565999

C 1.3806094 .0509201 .416313

C 2.7553374 .3813030 .819940

C $2.6566085 .566007-0.140969$

C $3.9952805 .846406-0.839770$

O 3.1975003 .2169650 .111211

O $3.7419106 .749434-1.927039$

C $4.5179814 .530815-1.453654$

C 4.9866656 .5550860 .090292

O $5.8207704 .710756-2.006163$

C $4.4991943 .328820-0.476271$

C $4.7804672 .022970-1.264855$

O 5.5059673 .5229470 .514788

C $1.463343-2.3119640 .041429$

O $0.840734-2.9707540 .857830$

C $2.666456-2.761887-0.692541$

C $3.328391-3.850963-0.276651$

C $4.564189-4.382968-0.856306$

C $5.163430-3.641029-2.022745$

C $4.6446350 .720615-0.462104$

C 5.9079610 .2909210 .264076

C $6.765506-1.5218491 .749506$

$\begin{array}{lllll}\text { O } & 6.964639 & 0.913074 & 0.204036\end{array}$

C $7.407481-2.5814390 .909181$

C $7.568039-3.8512971 .312477$

C $8.249054-4.9092290 .491011$

C $7.427014-6.1726480 .358751$

C $7.688930-7.2495691 .118646$

C $6.360591-6.216566-0.713339$

C $5.108392-5.488036-0.305110$

N $5.711115-0.8787870 .987010$

H $-2.2271680 .349113-0.738187$

H $0.5984291 .524992-0.847179$

H $-0.689673-0.9746820 .479293$

H $1.7083930 .564454 \quad 1.559256$

H $0.873465-0.7217192 .346489$

H -0.5443032 .9777243 .376391$

H -2.099792 1.2830933 .491298

H $-2.146666 \quad 0.374519 \quad 1.980044$

H -1.428336 -0.3488933 .435042$

H 1.7993573 .5702643 .477809

H 2.2851902 .3181132 .376503

H 0.7453083 .6356420 .624953

H 0.8952414 .9622521 .786560

H 3.4542254 .6018911 .635275

H $2.300526 \quad 6.4628630 .381029$

H $1.9031475 .349641-0.911361$

H $3.3459547 .557965-1.559272$

H $3.8622574 .295818-2.303326$

H 4.5621727 .4981850 .455149

H $5.9032726 .828820-0.443992$

H 5.2592695 .9569060 .962262

H $6.4558484 .717393-1.269825$

H $4.0453801 .959815-2.078709$

H $5.7738732 .068694-1.726772$

H 5.6805492 .6743270 .950314

H $2.995675-2.160475-1.532039$

H $2.942345-4.3959870 .583994$

H $5.360219-2.596515-1.756619$

H 4.482035 -3.661729 -2.880424

H $6.115558-4.052816-2.362236$

H 3.8206590 .7804080 .255823

H $4.409072-0.084791-1.168984$

H $6.307742-1.9323732 .654963$

H $7.513327-0.7772672 .040591$

H $7.780438-2.283337-0.068015$

H $7.221538-4.1411502 .302038$

H $9.207396-5.1382540 .975503$

H $8.504874-4.537218-0.508886$

H $7.123236-8.1691131 .010691$

H $8.471592-7.2338841 .869924$

H $6.076131-7.257595-0.917843$

H $6.784012-5.855104-1.654045$

H $4.596909-5.9484810 .541575$
H $4.816515-1.3555890 .923785$

C $-2.8903383 .105377-1.329960$

O $-4.0504562 .363347-0.904003$

C -4.4895262 .8855550 .366156$

C -3.7640594 .2136370 .547201$

C -2.434432 $3.920995-0.121253$

H -4.1375032 .1883891 .135372$

C -6.0185512 .9800130 .399365$

H -4.2789545 .0308950 .028910$

H -3.6635594 .4918441 .600763$

C $-1.6508325 .170324-0.484856$

H -1.8340543 .3046330 .557776$

C $-6.737174 \quad 1.6401080 .134191$

O $-6.4489663 .908614-0.605025$

H $-6.3465313 .399348 \quad 1.357157$

H $-6.5312581 .311195-0.890277$

$\begin{array}{lllll}\text { O } & -8.149255 & 1.874741 & 0.207144\end{array}$

C -6.3828050 .5208961 .131343$

C -7.195565 -0.774758 0.934429

H -5.3144470 .2912621 .066170$

O $\quad-6.624796 \quad 0.9991752 .457408$

H -6.970114 -1.446397 1.771712

H -8.266259 -0.5580791 .033699$

H $-0.7216864 .911897-1.001747$

H -2.227928 $5.829431-1.142124$

H -1.3923065 .7374310 .415343$

C $-1.8444152 .163728-1.935782$

H $-6.1178303 .588170-1.461766$

H -8.356839 $2.589783-0.418616$

H $-6.3372380 .306932 \quad 3.076662$

H $-0.9703942 .740235-2.260070$

H -2.271172 $1.700355-2.834242$

H $-3.2378773 .781306-2.122525$

C $-4.906833-2.6630530 .215367$

O $-5.512796-1.673546-0.621816$

C $-6.915084-1.483558-0.405753$

C $-7.670176-2.807809-0.599767$

C $-7.075176-3.9403210 .235802$

C $-5.562746-4.0342150 .023805$

H $-5.022756-2.3611181 .264234$

C $-3.393458-2.661128-0.038977$

H -7.258246 -0.809440 -1.199276

O $-9.048215-2.659360-0.274357$

H -7.619494 -3.096017 -1.657847

H -7.311997-3.809832 1.297925

O $-7.678326-5.179604-0.144986$

H $-5.361150-4.428814-0.978297$

O $-5.012415-4.9551840 .962422$

C $-2.982650-2.911813-1.475507$

H -3.005916 -1.671431 0.226415

H $-2.905580-3.4073370 .597629$

H -9.404956 -1.945931-0.830519

H $-7.497399-5.324575-1.089446$

H $-5.477494-5.8013820 .845797$

O $-1.618050-2.965700-1.535939$

O $-3.743559-3.024073-2.425986$

C -1.066399 -3.162884 -2.841198

H $-1.331580-2.325200-3.493133$

H $0.022022-3.203821-2.748833$

H $-1.418166-4.109065-3.263422$

SCF Energy (B3LYP/6-31G**//MMFF) $=-3245.91414551$

01225

MM̄MF Geometry

C $-0.330572 \quad 3.2297780 .804969$

C $-0.522117 \quad 1.9427241 .135693$

C 0.5220330 .8480461 .170420

O $1.7884231 .310634 \quad 0.651915$

C 0.7257150 .4072882 .630820

C $1.583257-0.8321302 .780750$

C $1.097474-2.0890122 .785011$

C $3.048426-0.5815443 .019535$

C $-0.334766-2.5001032 .589818$

C $-0.513129-3.8001631 .789567$

C $-0.151623-3.6835110 .299112$

C $-0.670850-4.912377-0.456325$

C $-0.268891-4.892857-1.936597$

O $1.271828-3.6043040 .169861$ 
O $-0.517990-6.198297-2.480009$

C $1.248776-4.647572-2.030077$

C $-1.122313-3.913816-2.750427$

O $1.653676-4.480852-3.387603$

C $1.737116-3.451112-1.179366$

C $3.284770-3.437788-1.162811$

O $1.260624-2.240189-1.765958$

C $1.9770591 .251150-0.692210$

O $1.186128 \quad 0.818390-1.519609$

C $3.2767851 .838493-1.093678$

C $4.2336502 .210571-0.229271$

C $5.507222 \quad 2.848282-0.573427$

C $5.7730903 .156918-2.023598$

C $3.906616-2.414168-0.205942$

C $5.423353-2.507733-0.182574$

C $7.403427-1.5390120 .953992$

O $6.056375-3.341920-0.824361$

C $7.817456-0.1613001 .358619$

C $8.7286510 .557098 \quad 0.685979$

C $9.195977 \quad 1.925462 \quad 1.092048$

C 8.8690932 .9756300 .055261

C $9.6555853 .150002-1.020546$

C $7.683343 \quad 3.8780140 .303480$

C $6.373543 \quad 3.1460100 .417667$

N $5.986101-1.5468460 .648021$

H $0.655744 \quad 3.5805990 .515631$

H -1.5256541 .6278281 .412902$

H $0.165958-0.0021570 .574709$

H $-0.2456610 .232607 \quad 3.111984$

H $1.1896201 .222633 \quad 3.203363$

H $1.793204-2.9073962 .964252$

H 3.1883190 .0261903 .919477

H $3.612582-1.5095653 .159285$

H $3.489921-0.0514482 .171885$

H $-0.768901-2.6586833 .584809$

H $-0.921581-1.7102192 .109448$

H $0.084801-4.5943872 .255241$

H -1.566095 -4.095130 1.879592

H $-0.602999-2.768144-0.100636$

H $-1.759790-5.002588-0.358955$

H $-0.255872-5.8211390 .002614$

H -1.461186 -6.399932 -2.355585

H $1.749755-5.556504-1.667693$

H $-2.177277-4.209254-2.715735$

H $-0.843406-3.931566-3.809679$

H $-1.055411-2.884197-2.392527$

H $1.340407-5.258409-3.880774$

H $3.644450-4.431752-0.864969$

H $3.665209-3.243851-2.173939$

H $1.828618-1.506198-1.485653$

H $3.3999371 .959974-2.165407$

H 4.0705972 .0565320 .835765

H $5.017898 \quad 3.847014-2.415764$

H $5.7563592 .238615-2.621274$

H $6.7475423 .620051-2.196007$

H $3.634801-1.394991-0.496547$

H $3.550756-2.5936830 .813298$

H $7.965691-1.9072960 .089175$

H $7.565561-2.2369991 .781972$

H 7.3643610 .2531582 .255799

H $9.1886170 .128351-0.201912$

H $\quad 10.283033 \quad 1.882917 \quad 1.241457$

H 8.7816072 .2174192 .065026

H $9.4469463 .919486-1.756326$

H $10.5256492 .523914-1.189200$

H $7.6122814 .661739-0.460519$

H 7.8515174 .4233191 .241778

H 6.1115922 .8742361 .440649

H $5.368254-1.0134501 .252168$

C -1.983898 $4.523359-0.564228$

O $-2.7587583 .376465-0.969758$

C $-4.021563 \quad 3.825468-1.493905$

C $-3.8431935 .310201-1.776088$

C -2.941370 $5.714106-0.624270$

H $-4.754973 \quad 3.715917-0.686315$

C -4.439569 $2.953044-2.681574$

H -3.333894 5.490481-2.728871

H $-4.7947135 .849905-1.788973$
C $-2.2499857 .050402-0.831761$

H $-3.542295 \quad 5.7608950 .294099$

C $-4.4406521 .442786-2.361031$

O $-3.515585 \quad 3.137977-3.761045$

H -5.424368 $3.259111-3.054140$

H $-3.4132481 .104674-2.186502$

O $-4.900006 \quad 0.747302-3.527438$

C $-5.3405041 .041361-1.176078$

C $-5.413116-0.477406-0.929286$

H $-4.9978161 .532453-0.261246$

O $-6.669766 \quad 1.511002-1.416853$

H $-6.240918-0.670019-0.236656$

H $-5.703092-0.984683-1.857250$

H $-1.598087 \quad 7.287924 \quad 0.014727$

H $-1.6367257 .048627-1.739160$

H $-2.9876257 .853980-0.926392$

C $-1.417623 \quad 4.269266 \quad 0.831463$

H $-3.7213793 .988754-4.183209$

H $-4.3196441 .004890-4.264306$

H $-6.9795881 .101756-2.243211$

H $-2.219674 \quad 3.949411 \quad 1.506949$

H -0.9948445 .1900361 .249119$

H $-1.1753934 .639365-1.297732$

C $-4.520532-0.7507891 .978353$

O $-3.695355-0.4483890 .846232$

C $-4.101347-1.072891-0.378550$

C $-4.123481-2.597901-0.210506$

C $-4.955209-3.0346260 .990414$

C $-4.540005-2.2619812 .240478$

H -5.542606 -0.4010731 .796791$

C -3.9548700 .0040953 .191331$

H $-3.314355-0.851566-1.108683$

O $-4.622815-3.236990-1.380249$

H $-3.097957-2.954658-0.064337$

H $-6.028668-2.921790 \quad 0.797939$

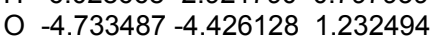

H -3.550567 -2.601671 2.570679

O $-5.461391-2.5456753 .291347$

C $-4.074806 \quad 1.5104003 .100507$

H $-4.458185-0.2973664 .116370$

H -2.886481 -0.2241713 .290571$

H $-4.093322-2.924296-2.133522$

H $-4.961683-4.9012430 .414975$

H $-5.469283-3.5101303 .417482$

O $-5.278132 \quad 1.882867 \quad 2.584355$

O -3.2090562 .2728753 .513980$

C $-5.502840 \quad 3.2925842 .485976$

H -5.5516893 .7351953 .485139$

H -4.7157213 .7655751 .891736$

H -6.4609173 .4493441 .983381$

SCF Energy (B3LYP/6-31G**//MMFF)= -3245.91406111

$01 \_226$

MM̄FF Geometry

C $-0.707738-4.3668220 .424013$

C $-0.084199-3.520724-0.409134$

C $-0.698116-2.247481-0.925256$

O $-0.081446-1.151334-0.211342$

C $-0.393112-2.080669-2.423246$

C $-1.123377-0.911327-3.053809$

C $-2.315372-1.013077-3.674363$

C $-0.3929640 .405766-3.022118$

C $-3.147503-2.255037-3.824702$

C $-4.659563-2.028505-3.674449$

C $-5.111107-1.698389-2.241553$

C $-6.632293-1.849406-2.133187$

C $-7.161843-1.444885-0.751820$

O $-4.735099-0.351524-1.935341$

O $-8.588438-1.339452-0.872888$

C $-6.603349-0.051856-0.399123$

C $-6.891102-2.529595 \quad 0.297372$

$\begin{array}{lllll}\text { O } & -6.938957 & 0.310385 & 0.939072\end{array}$

C $-5.0738620 .069389-0.606052$

C $-4.650747 \quad 1.549229-0.437876$

$\begin{array}{lllll}\text { O } & -4.412517 & -0.722703 & 0.380629\end{array}$

C -0.684385 -0.7392800 .936660$

O $-1.685131-1.2063801 .455753$

$\begin{array}{llll}\text { C } & 0.084722 & 0.406943 & 1.461622\end{array}$ 
C -0.4835451 .2408762 .344291$

C 0.1591192 .3993912 .967988

C 1.6449192 .5579012 .771458

C $-3.1984051 .847477-0.828721$

C $-2.8487523 .314494-0.640580$

C $-0.9589474 .909282-0.941197$

O $\quad-3.654524 \quad 4.152687-0.244804$

C $-0.233490 \quad 5.0982280 .352262$

C -0.6310995 .9491991 .309780$

C 0.1331936 .2095692 .576181

C -0.6070315 .7565863 .813966$

C $-1.564506 \quad 6.519816 \quad 4.367365$

C -0.1653524 .4639304 .461187$

C -0.6012903 .2476123 .691859$

N -1.529975 $3.577858-0.984512$

H - $-1.725186-4.1544720 .742251$

H $0.943762-3.726952-0.697963$

H -1.785243 -2.239681 -0.772029

H $-0.637795-3.004888-2.961371$

H $0.684456-1.933991-2.574692$

H $-2.728299-0.121365-4.143452$

H $\quad 0.5751630 .315080-3.525637$

H $-0.9509591 .203211-3.524299$

H $-0.2178910 .727164-1.992320$

H -2.967710 -2.646786 -4.833798

H -2.842290 -3.037294 -3.122203

H $-4.975526-1.229612-4.357898$

H -5.157505 -2.950031 -4.002090

H $-4.609287-2.383190-1.547941$

H -6.940105 -2.875355 -2.372325

H -7.112661 $-1.211108-2.888649$

H -8.952456 -1.137404 0.005545

H $-7.1006300 .677312-1.054139$

H -7.398351 -3.4617150 .020599$

H -7.297377 -2.2462691 .274616$

H $-5.829275-2.7539170 .419380$

H -7.9081600 .3360681 .003781$

H $-5.2958702 .179316-1.064478$

H $-4.8045491 .860670 \quad 0.603313$

H -3.523574 -0.3669850 .532864$

H 1.0939770 .5416921 .098269

H -1.518665 1.0653792 .635015

H 1.8824892 .6820561 .710187

H 2.1779141 .6776993 .148206

H 2.0652703 .4251193 .284127

H $-2.5051251 .256154-0.225470$

H -3.038130 $1.600965-1.883712$

H -1.750535 5.653174-1.080755

H -0.257149 4.996366 -1.776688

H 0.6844374 .5335720 .487920

H $-1.5436606 .522607 \quad 1.162316$

H 0.3248637 .2890442 .639739

H 1.1264215 .7453622 .541564

H -2.0774076 .2162155 .273879$

H -1.858124 7.4647913 .922110

H -0.5941464 .3790695 .468865$

H 0.9153514 .4952784 .626344

H -1.671390 $3.048972 \quad 3.767413$

H $-0.9182102 .807693-1.236352$

C $0.828730-5.3077252 .230418$

O $1.989843-4.5500841 .829283$

C $2.140640-3.4346862 .725550$

C $1.376201-3.8257973 .983040$

C $0.175491-4.5334513 .379236$

H $1.631762-2.5825792 .258253$

C $3.619804-3.0889912 .923755$

H $1.948939-4.5206594 .607998$

H $\quad 1.102697-2.9595364 .592468$

C $-0.575799-5.4155684 .360701$

H $-0.512941-3.7692472 .998703$

C $4.413159-2.9202831 .609488$

O $4.242784-4.1415863 .669790$

H $3.701098-2.1863103 .540903$

H $4.570349-3.9047561 .152481$

O $5.718518-2.4331471 .944915$

C $3.771588-1.9763540 .577508$

C $4.655434-1.783166-0.670206$

H $2.801660-2.3831620 .276539$
O $3.541685-0.7163711 .201951$

H $5.428361-1.036578-0.456829$

H $5.160347-2.729262-0.897890$

H -1.415567 -5.9156363 .867882$

H $0.074135-6.1870614 .786881$

H $-0.974251-4.8183075 .187467$

C $-0.053843-5.5892271 .007249$

H $5.177662-3.8997713 .786725$

H $5.613020-1.5320322 .295916$

H $3.077716-0.1559940 .559174$

H $-0.840535-6.3038471 .276965$

H $0.553556-6.0693690 .229267$

H $1.215469-6.2673152 .597248$

C $3.8494851 .046225-1.661356$

O $3.081794-0.163285-1.707015$

C $3.835773-1.360107-1.912733$

C $4.651326-1.259861-3.209640$

C $5.514788-0.003049-3.242669$

C $4.6646841 .231203-2.947887$

H $4.5255101 .008047-0.799273$

C $2.8466992 .190520-1.448689$

H $3.097146-2.156903-2.068525$

O $5.481750-2.402639-3.385161$

H $3.964950-1.228366-4.066086$

H $6.359107-0.074865-2.547082$

O $6.0788110 .145376-4.547781$

H $3.9957491 .421109-3.797115$

O $5.5173532 .365140-2.814590$

C $3.4767033 .437887-0.874506$

H $2.3470092 .424286-2.396768$

H $2.055648 \quad 1.883897-0.754832$

H $4.905252-3.185715-3.383084$

H $6.584513-0.663619-4.737353$

H $6.0381872 .435002-3.633004$

O $2.8853804 .539843-1.420048$

O $4.3565443 .442581-0.023892$

C $3.3648485 .798570-0.936855$

H 3.2049115 .8770420 .142846

H $4.4247965 .917835-1.180903$

H $2.7982226 .590967-1.432969$

SCF Energy $($ B3LYP/6-31G**//MMFF $)=-3245.91340882$

01227

MMFF Geometry

C 4.3149772 .2911751 .454752

C 3.3154211 .9827812 .296231

C 1.8559302 .1151601 .950550

O 1.2100940 .9047672 .421501

C 1.1960243 .3146542 .659694

C 1.5997954 .6724922 .125994

C 0.9799415 .2887931 .100334

C 2.7020475 .3752012 .874244

C $-0.1578424 .755686 \quad 0.274646$

C $-1.492713 \quad 5.343813 \quad 0.743213$

C $-2.6732514 .910540-0.138774$

C -3.9686045 .5379030 .376691$

C $-5.1917375 .065541-0.417027$

O $-2.7768823 .485830-0.096478$

$\begin{array}{lllll}\text { O } & -6.347628 & 5.470490 & 0.332466\end{array}$

C $-5.1827993 .522044-0.495176$

C $-5.2822565 .771012-1.776510$

O $-6.2154213 .077114-1.371732$

C $-3.8176672 .933868-0.925245$

C $-3.7261331 .402212-0.719661$

O $-3.5819123 .223556-2.304883$

C 0.0383320 .5422251 .833092

O $-0.5035691 .078603 \quad 0.880576$

C $-0.498862-0.6157332 .583050$

C - $-1.670278-1.1584792 .217852$

C $-2.347740-2.2812802 .872110$

C $-1.705662-2.8554714 .108673$

C $-4.7399950 .555868-1.492909$

C $-4.371804-0.917471-1.514366$

C $-5.238495-3.129387-2.253566$

O $-3.305695-1.353366-1.091068$

C $-5.613902-3.820238-0.980143$

C $-4.788532-4.640562-0.311513$

C $-5.178934-5.3797980 .935130$ 
C $-4.239049-5.1290922 .092673$

C $-3.358684-6.0669152 .481921$

C $-4.389876-3.8389872 .865662$

C $-3.503417-2.7391702 .344542$

N $-5.369095-1.694698-2.084539$

H 4.0670732 .6691020 .467275

H 3.5536461 .6387083 .300308

H 1.7323842 .1861560 .863046

H 1.3959263 .2655873 .739348

H 0.1040813 .2161912 .601812

H $1.314267 \quad 6.2855750 .816481$

H 2.9022896 .3752622 .474916

H 2.4280575 .4926773 .927987

H 3.6360464 .8107742 .820918

H $0.0225105 .025016-0.773268$

H -0.1906713 .6633820 .304306$

H -1.672703 5.0180071 .776304

H -1.4314426 .4396500 .753878$

H -2.470313 $5.227837-1.168237$

H $-3.903458 \quad 6.6333120 .365635$

H $-4.1126715 .259273 \quad 1.430394$

H -7.138868 5.240119 -0.182949

H -5.4245293 .1331530 .503780$

H $-5.3587426 .856224-1.637886$

H $-6.1870345 .475015-2.318821$

H $-4.4197145 .577082-2.417507$

H -7.065985 $3.352706-0.990964$

H $-2.7118291 .090816-1.005667$

H $-3.8021991 .169520 \quad 0.351110$

H -2.930131 $2.587905-2.644199$

H $0.078844-0.9808643 .424601$

H -2.190504 -0.738388 1.358945

H $-0.695957-3.2184673 .888147$

H -2.249595 -3.702545 4.531319

H -1.641166 -2.095153 4.895003

H $-5.728527 \quad 0.646252-1.029728$

H $-4.8061410 .890593-2.534131$

H $-4.212261-3.358989-2.559491$

H -5.917326 -3.430805 -3.057064

H -6.622242 -3.656994 -0.607261

H $-3.787986-4.819758-0.698665$

H -5.196902 -6.450069 0.691036

H $-6.202740-5.1322621 .242723$

H -2.705675 -5.9099913 .333700$

H -3.266471 -7.010046 1.953708

H $-4.223673-4.0197913 .933074$

H -5.432069 -3.498018 2.813328

H $-3.888240-2.2600801 .444879$

H $-6.248140-1.261055-2.351153$

C $6.671368 \quad 1.5498850 .773344$

O 6.1831650 .2367210 .436093

C $6.1287330 .133438-0.997863$

C $7.0881841 .194296-1.518855$

C $6.8212162 .322622-0.541109$

H $5.1094690 .410857-1.293378$

C $6.422300-1.295875-1.460715$

H $8.1324930 .869155-1.445672$

H $6.884768 \quad 1.468313-2.558265$

C $7.9146633 .376884-0.511563$

H $5.8792672 .806814-0.823466$

C $5.561730-2.384895-0.787611$

O $7.794502-1.613982-1.198447$

H $6.307652-1.352191-2.549649$

H $5.922571-2.5777430 .230236$

O $5.752560-3.603335-1.517911$

C $4.055616-2.076746-0.735424$

C $3.216179-3.271441-0.243685$

H $3.892528-1.220630-0.071987$

O $3.608267-1.694511-2.034806$

H $2.976594-3.924989-1.090260$

H $3.802702-3.8601780 .471948$

H 7.6835694 .1558750 .221866

H $8.8850662 .942481-0.248742$

H $8.0153053 .853810-1.491980$

C 5.7708962 .2003611 .829838

H $7.946102-1.495107-0.244914$

H $6.707457-3.788342-1.527425$

H $3.806398-2.426717-2.643550$
H $\quad 6.138816 \quad 3.2109342 .042558$

H 5.8650181 .6220792 .757985

H $7.661276 \quad 1.3953601 .222330$

C $0.497761-2.386440-1.447663$

O $1.169205-1.871882-0.293101$

C $1.914730-2.827240 \quad 0.460810$

C $1.010441-3.9811580 .912692$

C $0.225127-4.584831-0.247616$

C $-0.486749-3.490149-1.042108$

H $1.231289-2.789834-2.155691$

C $-0.211015-1.200747-2.110077$

H $2.236024-2.3015971 .370058$

O $1.779636-4.9878901 .561898$

H $\quad 0.298533-3.6006201 .653743$

H $0.875563-5.188405-0.891607$

O $-0.749448-5.4806760 .291689$

H -1.302701 -3.084064 -0.432133

O $-1.064272-4.093597-2.197133$

C $0.783540-0.255879-2.742292$

H $-0.893622-1.517078-2.905558$

H $-0.805641-0.640022-1.380606$

H $1.169690-5.7026971 .812829$

H -1.277704 -5.816004 -0.452926

H -1.704357 -3.465381-2.571079

O $1.0803180 .759073-1.881362$

O $1.255572-0.416114-3.860623$

C $2.0243101 .720144-2.363156$

H $1.6584732 .184646-3.283848$

H $2.1391312 .496865-1.602637$

H $2.9951831 .244595-2.529467$

SCF Energy $($ B3LYP/6-31G**//MMFF $)=-3245.91875218$

01_228

MMFF Geometry

C -1.792144 -4.206269 2.123044

C $-1.555547-2.8979231 .949078$

C $-0.570902-2.0973062 .755863$

O $0.503925-1.7751441 .844109$

C $-1.226464-0.7975073 .249880$

C $-0.475642-0.1298494 .383511$

C 0.2229521 .0156454 .266541

C $-0.611543-0.7942935 .729839$

C 0.4749031 .8117553 .018530

C -0.5983372 .8843212 .797004$

C -0.1744563 .9223551 .747630$

C -1.3233304 .8825311 .431937$

C $-0.9621335 .869605 \quad 0.309828$

O $0.227175 \quad 3.236286 \quad 0.559937$

O $-2.1798506 .466222-0.162729$

C $-0.3505655 .094638-0.879021$

C -0.0909527 .0196790 .830326$

O $\quad 0.1507085 .992631-1.866851$

C $0.7531424 .096673-0.460159$

C $1.1658293 .213826-1.664439$

O $1.8866204 .832951-0.002502$

C $1.782191-1.9134002 .283102$

O $2.153179-2.1491963 .420943$

C $2.662488-1.7874861 .098795$

C $3.965274-2.0891141 .197690$

C $4.935722-2.1010810 .098677$

C $4.439269-1.737896-1.276851$

C $2.1563582 .087869-1.326260$

C $3.5913822 .572572-1.218506$

C 5.6754172 .2611720 .109430

O $4.0549923 .426126-1.971632$

C $6.5802831 .334101-0.637949$

C $7.5497330 .614971-0.052136$

C $8.484791-0.287898-0.803198$

C $8.516783-1.693252-0.246875$

C $9.573134-2.1353530 .456501$

C $7.364099-2.613329-0.582188$

C $6.208176-2.4609700 .369915$

N $4.2950361 .942240-0.203376$

H -1.259972 -4.749506 2.899739

H -2.072544 -2.372749 1.152074

H $-0.203724-2.7041973 .591768$

H -2.244900-1.001811 3.608222

H - $1.342842-0.1050202 .407853$ 
H $0.685563 \quad 1.430415 \quad 5.161143$ H $-0.123784-0.2225346 .526624$ H -1.668093 -0.895074 5.999225 H $-0.158664-1.7901915 .717888$ H 1.4608472 .2835343 .116643 H $\quad 0.5443541 .1546762 .144343$ H -1.5195362 .3853752 .470641$ H -0.8276573 .4001323 .737970$ H 0.6834014 .4735302 .151409 H -1.637085 5.4223932 .334131 H -2.201212 $4.306147 \quad 1.112484$ H -2.616495 6.8951470 .593124 H -1.161212 $4.531731-1.359574$ H -0.6212147 .5783121 .611173$ H $\quad 0.1225497 .7450500 .037625$ H 0.8563846 .6812461 .254900 H $-0.5770746 .589545-2.111756$ H $\quad 0.2639282 .733059-2.062995$ H $1.5816993 .840561-2.462154$ H 2.5958624 .2052680 .203730 H $2.202565-1.4999760 .161637$ H $4.356579-2.3856292 .170076$ H $3.956320-0.755507-1.270561$ H $3.713486-2.479317-1.628808$ H $5.230885-1.680248-2.026258$ H $1.8493941 .561401-0.416639$ H $2.1464651 .351468-2.139429$ H 5.7922642 .1702461 .193971 H $5.8851323 .296013-0.179898$ H $6.4474961 .269231-1.715361$ H $7.7007640 .700468 \quad 1.021528$ H $9.4861920 .159409-0.755477$ H $8.229558-0.330003-1.869468$ H $9.614603-3.1519040 .833501$ H $10.416927-1.4895180 .675896$ H $7.696355-3.658678-0.520761$ H $7.083623-2.473331-1.629375$ H $6.462160-2.7171481 .399761$ H 3.8549331 .1920630 .321210 C -2.197591-5.408030 -0.099652 O $-2.070188-4.245122-0.944950$ C $-0.763169-4.258619-1.544900$ C $-0.298401-5.707529-1.465456$ C $-0.835184-6.107671-0.103301$ H -0.116291-3.648522 -0.902110 C $-0.793168-3.652994-2.952553$ H $-0.755411-6.325775-2.246704$ H $0.788656-5.802290-1.543973$ C $-0.919151-7.609100 \quad 0.108373$ H $-0.171163-5.6840400 .659827$ C $-1.471708-2.267788-3.044372$ O $-1.510740-4.544486-3.814837$ H $0.230692-3.603833-3.343046$ H -2.559942 -2.388822 -2.989873 O $-1.187713-1.723452-4.339183$ C $-1.013808-1.250435-1.986437$ C -1.516743 $0.188172-2.197994$ H -1.331056 -1.592116 -0.996990 O $0.415955-1.209343-1.974861$ H -1.199825 $0.770876-1.323748$ H $-0.9785890 .638700-3.042268$ H -1.331256 -7.8387031 .096209$ H -1.557136 -8.086199 -0.643023 H $0.074916-8.0631850 .041716$ C $-2.747585-4.998771 \quad 1.272812$ H -1.360888 -4.253817 -4.729960 H -1.646114 -2.272838 -4.996784 H $\quad 0.683740-0.579590-1.284440$ H -3.034437 -5.8969751 .832703$ H -3.664298 -4.4113981 .133690$ H -2.947147 $-6.043942-0.588247$ C $-3.8074070 .100264-0.140167$ O $-3.808920-0.407502-1.475911$ C $-3.0346710 .343948-2.412876$ C $-3.5067411 .806137-2.447713$ C $-3.5144232 .432031-1.055120$ C $-4.2941971 .553043-0.080367$ H $-2.7828920 .049340 \quad 0.249501$
C $-4.641692-0.848787 \quad 0.734139$

H $-3.255730-0.083396-3.398513$

O $-2.6812712 .588584-3.304375$

H $-4.5260471 .850267-2.852698$

H $-2.4979172 .610506-0.688913$

O $-4.1522593 .709396-1.114664$

H $-5.3615181 .621910-0.319741$

O -4.1255242 .0468081 .246206$

C -6.116717 -0.8791990 .389753$

H -4.283404 -1.877131 0.616579

H $-4.528617-0.559806 \quad 1.785499$

H -2.703675 2.178418 -4.185861

H $-3.6633634 .246595-1.761612$

H -4.4078782 .9775201 .247353$

O $-6.832979-1.1921401 .509530$

O $-6.581047-0.690036-0.726406$

C $-8.250239-1.2763241 .329714$

H $-8.701747-1.5184122 .295550$

H $-8.646744-0.3154360 .988023$

H $-8.494860-2.0711250 .618570$

SCF Energy (B3LYP/6-31G**//MMFF) $=-3245.90877421$

01229

MMFF Geometry

C $-1.362072-2.152018-2.650136$

C $-0.397557-2.073950-3.579531$

C $0.519799-0.895411-3.805615$

O $-0.1181140 .302028-3.302608$

C $1.882193-1.070760-3.105413$

C $2.630983-2.326471-3.491747$

C $2.875150-3.359801-2.662555$

C $3.144953-2.359586-4.908217$

C $2.477676-3.508477-1.219667$

C $3.697755-3.653107-0.300665$

C $4.586075-2.399199-0.237053$

C $5.842915-2.6956190 .584854$

C $6.726550-1.4530480 .744861$

O $3.836904-1.3388960 .369569$

O $7.700399-1.7315921 .762201$

C $5.853097-0.2936571 .259451$

C $7.511410-1.136157-0.533407$

O $6.599250 \quad 0.9219881 .294383$

C $4.543108-0.0905850 .457181$

C 3.6462210 .9173831 .215654

O $4.8589640 .425522-0.835070$

C $0.301416 \quad 1.496256-3.795348$

O $1.1750761 .690245-4.624791$

C $-0.4853042 .547860-3.118221$

C $0.0307053 .780915-3.018304$

C $-0.5872954 .920201-2.338861$

C $-2.0354124 .794608-1.945264$

C 2.3124521 .2492470 .536448

C 1.4001292 .0471191 .455584

C -0.8591873 .0932391 .507731$

O 1.6739392 .2995062 .626076

C -0.7785634 .5898541 .472477$

C 0.2716685 .3609031 .147911

C $0.2163356 .864467 \quad 1.155287$

C $0.6702487 .487198-0.147578$

C $1.7687458 .259735-0.198148$

C $-0.1888077 .276640-1.377707$

C $0.1744006 .008161-2.101153$

N 0.2302332 .4313520 .817898

H - $1.509919-1.327899-1.957241$

H $-0.251311-2.918761-4.249831$

H $0.649023-0.783799-4.889751$

H $1.735405-0.994689-2.022865$

H $2.523169-0.209061-3.334427$

H $3.428129-4.210883-3.059189$

H $3.756491-3.245872-5.108175$

H $2.314436-2.366034-5.620588$

H $3.767814-1.480942-5.105835$

H $1.842451-2.693147-0.866948$

H $1.870025-4.418093-1.134782$

H $3.330486-3.8830030 .706813$

H $4.295256-4.513593-0.628206$

H $4.866714-2.104389-1.253916$

H $6.416694-3.5169090 .137272$ 
H $5.550723-3.0452091 .585289$ H $8.213011-2.5090551 .481612$ H $5.586286-0.5224772 .301042$ H $8.154996-1.980976-0.806556$ H $8.183999-0.284527-0.382780$ H $6.869046-0.921050-1.389761$ H $7.400014 \quad 0.7552101 .820608$ H 3.4350870 .5069692 .212051 H $4.1860441 .861847 \quad 1.361616$ H $4.0453820 .757603-1.245336$ H -1.439188 $2.268018-2.686212$ H $1.0184513 .965778-3.439508$ H -2.175358 $3.986175-1.220846$ H $-2.6514774 .580057-2.826097$ H -2.445341 $5.699736-1.493279$ H $2.4837171 .841003-0.369429$ H 1.7864460 .3306410 .256651 H -0.8962842 .7513882 .547130$ H -1.778452 2.7720261 .009124 H -1.7058385 .0882111 .751653$ H 1.2233894 .9081690 .884652 H 0.8470037 .2131661 .983171 H -0.7967817 .2222811 .378070$ H $2.0862668 .728593-1.123780$ H 2.3775698 .4374680 .682053 H $-0.0468318 .109690-2.078917$ H -1.242201 $7.323554-1.089940$ H $1.2092415 .995750-2.448028$ H $\quad 0.1105392 .223404-0.169055$ C -3.762984 -2.964454 -2.482261 O $-4.071528-2.217595-1.289043$ C $-4.927275-1.115629-1.644562$ C $-5.462260-1.435568-3.036124$ C $-4.264543-2.126466-3.660648$ H -4.283495 -0.229467 -1.708695 C $-5.996305-0.894050-0.568666$ H $-6.315384-2.122566-2.998321$ H $-5.772846-0.537569-3.578718$ C $-4.599254-2.943853-4.895713$ H -3.535206 -1.354774 -3.935474 C $-5.445069-0.8051890 .870090$ O $-6.932011-1.980034-0.598258$ H $-6.5758010 .004482-0.809579$ H -5.120296 -1.802137 1.187481 O $-6.533496-0.455106 \quad 1.734449$ C $-4.300436 \quad 0.2039521 .070018$ C -3.861559 0.3797732 .536910 H $-3.434857-0.0986320 .471121$ $\begin{array}{lllll}\text { O } & -4.725807 & 1.477012 & 0.577367\end{array}$ H -3.0385501 .1039552 .554486$ H $-4.667591 \quad 0.8622113 .104618$ H -3.703391 -3.432184 -5.292085 H $-5.338411-3.721207-4.675234$ H - $-5.011232-2.302356-5.681564$ C -2.276474 -3.335857 -2.494872 H $-6.433508-2.801923-0.449466$ H $-6.815840 \quad 0.4456181 .499663$ H -3.9803512 .0937020 .671037$ H -2.096229 -4.066517 -3.292205 H -2.024122 -3.820927 -1.543854 H -4.344225 $-3.893051-2.408295$ C $-1.215667-1.1640802 .322815$ O $-2.538083-1.6995622 .434721$ C $-3.433490-0.9247453 .237364$ C $-2.864353-0.7305524 .651622$ C $-1.431684-0.2019104 .633277$ C $-0.562423-1.0507413 .704663$ H -1.272154 -0.1681811 .866303$ C $-0.436762-2.0325901 .331218$ H $-4.341135-1.5304143 .349328$ O H -2.865761 -1.696408 5.173851 H -1.4043800 .8570224 .350804$ O $-0.906839-0.2772325 .961022$ H -0.426886 -2.037176 4.164603 O $0.719160-0.4399613 .604701$ C -0.228943 -3.446596 1.817625 H $-0.985355-2.1072110 .385568$
H $\quad 0.532925-1.5853981 .088379$

H $-4.578199-0.2227615 .432356$

H $\quad 0.0147830 .0313975 .924785$

H $1.308675-1.0594613 .142877$

O $1.026672-3.5820592 .331110$

O $-1.086263-4.318771 \quad 1.765327$

C $1.343147-4.8783922 .846845$

H $1.287514-5.6260942 .050038$

H $2.366383-4.8505093 .230637$

H $\quad 0.666876-5.1354503 .667730$

SCF Energy $(B 3 L Y P / 6-31 G * * / / M M F F)=-3245.90389064$

0123

MMFF Geometry

C $-2.407089-3.476976-2.225789$

C $-1.559131-3.454153-1.186009$

C $-0.057704-3.450742-1.334942$

O $0.474426-2.317861-0.612232$

C $0.515942-4.733114-0.703052$

C $2.011324-4.899268-0.888747$

C $2.907948-4.9397150 .116333$

C $2.480559-5.063354-2.311930$

C $2.654659-4.8289071 .593789$

C $2.755320-3.4019462 .155946$

C $4.144660-2.7611822 .002081$

C $4.224779-1.4444262 .776101$

C $5.570874-0.7321812 .563369$

O $4.371870-2.5053050 .615069$

O 5.4336280 .6149953 .043056

C $5.872157-0.6455511 .049282$

C $6.688677-1.3746353 .394588$

O $7.190815-0.1421960 .849004$

C $5.673591-1.9869880 .309273$

C $5.754793-1.871011-1.232340$

O $6.699534-2.9019050 .713359$

C $0.471335-1.113887-1.246222$

O $0.072367-0.876039-2.374881$

C $1.033174-0.115964-0.308574$

C $0.9413611 .190226-0.598383$

C 1.4302722 .2968860 .227749

C 2.2344281 .9482071 .451425

C $4.750310-0.907191-1.873377$

C $5.3207420 .471025-2.162691$

C $4.6497312 .700051-3.048847$

O $6.5061820 .756006-2.016061$

C $4.4618293 .624608-1.888370$

C $3.6970354 .725415-1.942540$

C $3.5316825 .687291-0.801902$

C $2.0856225 .866547-0.400140$

C $1.3786366 .933843-0.808283$

C 1.4855694 .8479040 .543716

C $1.1311383 .558572-0.145451$

N $4.349607 \quad 1.340965-2.639929$

H -1.985960 -3.473950 -3.229844

H -1.938394 -3.453313 -0.168280

H $\quad 0.233305-3.387069-2.390409$

H $0.024893-5.613290-1.139931$

H $0.259072-4.7412610 .361876$

H $3.957623-5.075206-0.143032$

H $3.539019-5.339158-2.371055$

H $1.910287-5.852966-2.812039$

H $2.354542-4.131125-2.870610$

H $1.674980-5.2449881 .851797$

H $3.380013-5.4705442 .110400$

H $2.002332-2.7745421 .664332$

H $2.495331-3.4440863 .220859$

H $4.894385-3.4696462 .373678$

H $4.043535-1.6092913 .845595$

H $3.421397-0.7763042 .438116$

H 5.1778780 .5749313 .980572

H $\begin{array}{llll}5.183893 & 0.102731 & 0.637775\end{array}$

H $6.439238-1.3416134 .462033$

H $7.627654-0.8197283 .290951$

H $6.872399-2.4184443 .131995$

H $7.3039250 .029913-0.100517$

H $6.783503-1.654669-1.543860$

H $5.536905-2.868727-1.641101$

H $\quad 6.779769-3.5942240 .036407$ 
H $1.494421-0.4834930 .600590$ H $0.4457021 .482801-1.523087$ H $1.636398 \quad 1.3534092 .150649$ H 2.5913782 .8167332 .007274 H 3.1225451 .3737531 .168484 H $4.445441-1.320842-2.843374$ H $3.848137-0.808155-1.262012$ H $3.9853702 .942094-3.884425$ H $5.6855142 .754221-3.399285$ H $4.9993163 .393171-0.971745$ H $3.1796484 .967536-2.868282$ H $3.9570846 .650217-1.113655$ H 4.1166705 .3768010 .072749 H $0.3482817 .080687-0.501287$ H $1.8131187 .681532-1.463742$ H $0.562293 \quad 5.2465830 .982423$ H 2.1712694 .7118681 .383670 H $0.5281783 .696806-1.043880$ H $3.3805921 .037320-2.672587$ C -4.564992 -3.621639-0.800607 O $-4.441076-2.361111-0.110720$ C $-5.639996-2.1360840 .657126$ C $-6.484026-3.3989750 .520554$ C $-6.066534-3.899586-0.847373$ H $-6.175037-1.3095910 .177207$ C $-5.287724-1.7744132 .103754$ H $-6.235351-4.1374081 .292181$ H $-7.555678-3.1893870 .593674$ C $-6.427793-5.354811-1.090634$ H $-6.541988-3.273293-1.614161$ C $-4.464672-0.4786612 .262118$ O $-4.531035-2.8461752 .682389$ H -6.205444 -1.701362 2.698823 H $-3.458605-0.6156261 .849197$ O $-4.298512-0.2447273 .666708$ C $-5.120767 \quad 0.7681651 .640621$ C -4.4756702 .1086142 .035823$ H -5.1174120 .6757630 .550034$ O $-6.495598 \quad 0.8314462 .035806$ H -5.0791862 .9063951 .585088$ H -4.583206 2.2628723 .117093 H -6.107743 -5.677957 -2.085832 H -5.955621 -6.014066 -0.354495 H -7.511211 -5.497914 -1.023313 C -3.913321 -3.530347 -2.182471 H $-3.735060-2.9698852 .137170$ H -3.878350 -1.036489 4.044237 H -6.5198520 .8748273 .007236$ H -4.285503 -2.638621 -2.700754 H -4.213618 -4.399498 -2.779539 H $-4.067125-4.384016-0.187180$ C -3.347505 $2.872141-0.674451$ $\begin{array}{llll}\text { O } & -2.768629 & 1.949510 & 0.255370\end{array}$ C -2.9969922 .2624801 .631056$ C -2.4067513 .6425571 .948985$ C -2.9471094 .7221141 .011987$ C $-2.8078684 .290144-0.448519$ H $-4.4389432 .865635-0.567351$ C $-3.0058012 .373764-2.082354$ H $-2.428307 \quad 1.5273362 .213027$ O -2.6776293 .9902403 .302849$ H -1.315961 3.5942401 .843951 H $-3.985974 \quad 4.9710811 .257810$ O -2.1885415 .9144381 .227414$ H -1.754809 4.344268 -0.745969 O $-3.5310475 .194661-1.281101$ C $-3.7058831 .068629-2.373550$ H $-3.2876723 .096121-2.856645$ H -1.928656 2.197581-2.186330 H -2.304102 4.8751523 .455691 H $-2.593686 \quad 6.6210920 .697237$ H -3.101669 $6.064074-1.215179$ O $-4.991680 \quad 1.306275-2.760050$ O $-3.177063-0.028523-2.252974$ C $-5.7704910 .142993-3.054743$ H $-5.319356-0.416694-3.879668$ H -5.862094 -0.486209 -2.165227 H $-6.7689750 .469704-3.357029$
SCF Energy (B3LYP/6-31G**//MMFF) = -3245.91403315

01230

MMFF Geometry

C $3.0966760 .194203-3.173446$

C $3.7170421 .384203-3.169984$

C $3.0736672 .711450-2.825750$

O $1.6756092 .479770-2.522059$

C $3.7193363 .350907-1.578855$

C $5.2037443 .634597-1.677657$

C $6.1495612 .904858-1.051636$

C $5.5788804 .841065-2.494313$

C $5.9148741 .655155-0.248218$

C 5.7561161 .9498301 .247486

C 5.0300550 .8379292 .022551

C $5.750415-0.5093211 .958168$

C $4.960064-1.6099942 .684011$

O 3.7118370 .7206531 .475585

O $5.524069-2.8770862 .311504$

C $3.503487-1.5976822 .173802$

C $5.097894-1.5061444 .207166$

O $2.695415-2.5063632 .919030$

C $2.854197-0.1924942 .169712$

C $1.500959-0.2474131 .413026$

O $2.6366800 .217187 \quad 3.518482$

C $0.790823 \quad 3.504174-2.599148$

O $1.0123904 .652703-2.941943$

C $-0.5125752 .961539-2.150214$

C $-1.5471643 .779000-1.908713$

C $-2.8663833 .367069-1.419403$

C $-3.0635721 .912024-1.077615$

C 0.8586001 .1089601 .086979

C 0.0555791 .7290162 .216278

C -1.327890 3.7418512 .727190

O $-0.101607 \quad 1.1892823 .307599$

C -2.7589893 .4726962 .381326$

C -3.6442974 .4360972 .084263$

C -5.0880764 .1722521 .763648$

C $-5.534516 \quad 4.8368590 .479843$

C -6.2331515 .9845280 .503625$

C $-5.2481754 .126478-0.824274$

C $-3.8257114 .305996-1.279668$

N -0.4735882 .9602951 .852379$

H $2.0308590 .142616-2.966220$

H $4.7793021 .409620-3.402468$

H $3.1502443 .373562-3.696540$

H $3.4948782 .728450-0.703633$

H $3.2064744 .295113-1.350490$

H $7.1914053 .205480-1.144295$

H $6.6594125 .019026-2.499243$

H $5.2610124 .712914-3.533769$

H $5.0980085 .738017-2.090798$

H $5.0462301 .113444-0.634021$

H $6.7674760 .982526-0.403296$

H 6.7421512 .1309361 .692599

H $5.1746382 .873277 \quad 1.371550$

H 4.9496551 .1737993 .063243

H $6.765080-0.4275982 .366782$

H $5.871538-0.8202160 .911986$

H $6.465041-2.8700782 .557418$

H $3.518785-1.9734721 .142012$

H $6.150667-1.5907334 .502573$

H $4.579549-2.3314844 .707638$

H $4.717717-0.5650714 .609773$

H $3.122917-3.3787102 .872107$

H $1.679216-0.7316450 .443826$

H $0.785922-0.8694851 .964642$

H 1.9778460 .9281173 .526110

H $-0.5768921 .888443-2.004889$

H -1.416288 $4.847333-2.074412$

H $-2.2892831 .567659-0.383503$

H $-3.0229371 .295523-1.982025$

H -4.015202 $1.705470-0.585000$

H $1.607015 \quad 1.829496 \quad 0.741912$

H $\quad 0.153576 \quad 0.949803 \quad 0.261341$

H -1.059447 4.7937922 .588306

H -1.140444 3.4661323 .769674

H -3.0876772 .4364622 .395029$ 
H $-3.322517 \quad 5.4750112 .098645$ H -5.6877744 .5453142 .604226$ H -5.295996 3.0964751 .706018 H $-6.5822486 .453054-0.410618$ H $-6.466828 \quad 6.4842521 .437913$ H $-5.8942714 .525771-1.617891$ H $-5.5409643 .076880-0.734043$ H -3.583964 5.336210-1.545183 H $-0.338717 \quad 3.2951740 .902898$ C $3.491710-2.063335-2.218322$ O $2.088529-2.390421-2.216362$ C $1.925246-3.797632-1.978391$ C $3.281423-4.288146-1.488168$ C $4.224220-3.400529-2.281909$ H $1.706451-4.257689-2.950606$ C $0.749920-4.058595-1.023590$ H $3.408642-4.114260-0.413146$ H $3.431886-5.354901-1.679083$

C $5.634261-3.355723-1.719840$ H $4.259864-3.761112-3.318611$ C $-0.600059-3.558791-1.592307$ O $1.034224-3.436206 \quad 0.226495$ H $0.683431-5.138302-0.843136$ H $-0.746942-4.027057-2.572962$ O $-0.556653-2.147619-1.811152$ C -1.822242 -3.884259-0.708983 C $-3.139359-3.500326-1.418404$ H -1.828051 -4.958806 -0.490745 O $-1.698721-3.1901330 .527314$ H -3.117715 -2.433834 -1.663022 H -3.179357 -4.044369 -2.369461 H $\quad 6.269274-2.688994-2.311584$ H $5.642457-2.997425-0.685257$ H $6.085677-4.353143-1.733410$ C $3.782753-1.126571-3.394072$ H $0.313331-3.6565660 .839964$ H $-0.387907-1.726283-0.950608$ H -2.419918 -3.486937 1.107290 H $3.394658-1.557901-4.324357$ H $4.863282-0.987756-3.509436$ H $3.702509-1.555734-1.268022$ C $-4.737557-1.6529050 .434962$ O $-4.478818-3.0515910 .617646$ C $-4.405219-3.817775-0.589782$ C $-5.724075-3.684681-1.366391$ C $-6.100163-2.225076-1.607242$ C $-6.067370-1.442611-0.295199$ H -3.922633 -1.197031 -0.139452 C $-4.755850-1.0035191 .823063$ H -4.318412 -4.867761 -0.282017 O $-5.656321-4.363022-2.616037$ H -6.530866 -4.156353-0.790007 H $-5.449359-1.760777-2.357704$ O $-7.428655-2.161953-2.130714$ H $-6.903872-1.7579360 .341464$ O $-6.239053-0.054730-0.572054$ C $-3.358426-0.9566912 .394308$ H $-5.121990 \quad 0.0286121 .791655$ H -5.409061 -1.5637682 .502744$ H -5.435680 -5.292198 -2.432445 H -7.444078 -2.695382 -2.943836 H -7.073615 $0.042736-1.061908$ O $-3.269132-1.7595683 .491620$ O $-2.448917-0.2981151 .906976$ C $-1.978257-1.8194454 .106753$ H $-1.729660-0.8496714 .547362$ H -2.015939 -2.567794 4.902768 $\mathrm{H}-1.218027-2.1210763 .379580$ SCF Energy (B3LYP/6-31G**//MMFF) = -3245.93056295

$01 \_231$

MM̄FF Geometry

C $-5.829488 \quad 1.8116160 .771969$

C $-4.7125882 .445303 \quad 0.385285$

C -3.7937523 .1695871 .333858$

O $-2.5127452 .496167 \quad 1.311679$

C -3.5896284 .6199990 .858538$

C -2.7782865 .4689121 .819724$
C -1.5496605 .9620571 .564909$

C -3.4505205 .7834603 .132440$

C -0.7246355 .7973540 .318104$

C 0.3150424 .6947860 .503112

C $1.2513094 .492672-0.696252$

C $0.5103414 .043611-1.957044$

C $1.4766533 .706284-3.102360$

O $2.1734723 .473849-0.296316$

O $0.7083483 .009950-4.096522$

C $2.5632922 .742532-2.579774$

C $2.0055734 .978303-3.774894$

O $3.5789522 .528093-3.555300$

C $3.2066243 .179406-1.244526$

C $4.0377512 .020202-0.650028$

O $4.1010664 .279355-1.413090$

C $-2.372467 \quad 1.4001872 .108555$

O

C -1.0123030 .8538351 .894771$

C $-0.624615-0.2411732 .566798$

C $0.680278-0.9033652 .473043$

C $1.698533-0.2980311 .543602$

C 4.6650162 .3714800 .705186

C 5.2169481 .1537321 .425118

C 5.9642030 .4565723 .704610

O $5.339504 \quad 0.0527410 .896621$

C $4.742935-0.0474144 .409810$

C $4.511316-1.3437274 .665303$

C $3.278334-1.8369865 .369192$

C $2.653985-3.0355944 .688306$

C $2.578056-4.2206405 .317992$

C $2.127103-2.8739473 .278178$

C $0.895482-2.0112723 .214170$

N $5.557523 \quad 1.4577712 .736416$

H -6.1178331 .8240801 .819953$

H $-4.4179132 .412504-0.661329$

H -4.1982363 .1635822 .353117$

H -4.5647235 .1065840 .720235$

H -3.122159 $4.602495-0.131286$

H $-1.065736 \quad 6.5570842 .338852$

H -2.882834 6.5076753 .726700

H -4.4426946 .2126802 .958121$

H -3.561702 4.8790313 .737639

H -1.356709 $5.599348-0.550204$

H $-0.221424 \quad 6.7495360 .110503$

H 0.9288674 .9119081 .388604

H -0.1834003 .7452070 .730072$

H $1.8013565 .424147-0.872373$

H $-0.2076284 .801346-2.291074$

H $-0.0841583 .147651-1.727899$

H $1.2867582 .836283-4.858270$

H $2.0751121 .774272-2.414419$

H $1.1811985 .536109-4.235577$

H $2.6974604 .739115-4.589745$

H $2.5099525 .651871-3.078611$

H $3.1603392 .123191-4.333379$

H $3.3905501 .143432-0.522542$

H $4.842463 \quad 1.734677-1.339057$

H $4.1184744 .529826-2.349929$

H -0.3680751 .3649351 .189125$

H - $-1.330102-0.703016 \quad 3.256725$

H 1.9570500 .7159581 .868492

H $1.307944-0.2488380 .521281$

H $2.631789-0.8604701 .489031$

H 5.4833563 .0873300 .567803

H 3.9156812 .8319061 .359526

H 6.6316880 .9489494 .418111

H $6.508781-0.3448683 .195201$

H 4.0136550 .6925354 .732876

H $5.251643-2.0845874 .372561$

H $2.526892-1.0423125 .452971$

H $3.565563-2.0932316 .397192$

H $2.135529-5.0883534 .840006$

H $2.955707-4.3514856 .326588$

H $1.854155-3.8566262 .873049$

H $2.935351-2.5153292 .637062$

H $0.081398-2.375976 \quad 3.842641$

H 5.3552172 .3886163 .089765

C $-6.403181-0.457835-0.208543$ 
O - $-5.080931-0.671383-0.741707$

C $-4.455056-1.742852-0.011660$

C $-5.565619-2.3989850 .802579$

C $-6.448717-1.2077531 .121623$

H $-3.731399-1.2813780 .672189$

C -3.721917 -2.701430 -0.960270

H $-6.118690-3.1362980 .208616$

H -5.182757-2.902812 1.695366

C $-7.849688-1.5842541 .569008$

H $-5.963747-0.6290361 .917148$

C $-2.524739-2.043565-1.684640$

O $-4.652996-3.233906-1.900425$

H -3.346093 -3.546952 -0.373780

H $-1.873726-1.611905-0.916401$

O $-2.967922-0.961986-2.502018$

C $-1.698117-3.025853-2.539872$

C $-0.503012-2.377191-3.264671$

H -1.349317 -3.844991 -1.901367

O $-2.535722-3.607514-3.541650$

H $-0.025534-3.152100-3.877352$

H $-0.872456-1.640670-3.989774$

H -8.447302 -0.6891761 .768613$

H -8.369822 -2.174869 0.807511

H -7.813205 -2.178694 2.487766

C $-6.711993 \quad 1.041121-0.168152$

H $-5.063936-2.484279-2.363835$

H -3.484016 -1.340015-3.234345

H -2.013157 -4.282744 -4.007002

H -7.7561191 .2014490 .124303$

H -6.603216 $1.451254-1.180636$

H $-7.093607-0.925827-0.922923$

C $1.699162-3.719909-1.649856$

O $0.924725-2.579527-1.261673$

C $0.537836-1.721823-2.337440$

C $1.785780-1.196635-3.060656$

C $2.714792-2.325435-3.500611$

C $2.997176-3.273159-2.334849$

H $1.112198-4.352024-2.327420$

C $1.994834-4.529023-0.383962$

H $0.051099-0.852994-1.878122$

O $1.428436-0.416719-4.196608$

H $2.346288-0.543610-2.380363$

H $2.305876-2.868815-4.360367$

O $3.956783-1.765870-3.934648$

H $3.649125-2.766014-1.612165$

O $3.713055-4.409076-2.815740$

C $0.753955-5.233900 \quad 0.110991$

H $2.748962-5.302627-0.567875$

H $2.380883-3.8919290 .419416$

H $\quad 0.8338010 .289965-3.892037$

H $3.756455-1.135006-4.647271$

H $3.145402-4.871637-3.455408$

O $-0.013798-4.3760610 .840861$

O $0.494102-6.404663-0.134885$

C $-1.220933-4.9264491 .375597$

H - $1.786823-4.115147 \quad 1.841282$

H $-1.829502-5.3660860 .579584$

H -0.983914 -5.6741552 .137933$

SCF Energy (B3LYP/6-31G**/MMFF) $=-3245.90496904$

01_232

MM̄FF Geometry

C $2.4744552 .052246-2.704053$

C $3.5382801 .239984-2.604013$

C $3.510053-0.269016-2.593528$

O $2.157655-0.740734-2.809528$

C $4.411625-0.906347-3.665531$

C $5.896641-0.826952-3.374223$

C $6.550799-1.668054-2.547575$

C $6.6561210 .224492-4.139724$

C $5.957840-2.789654-1.739843$

C $6.485395-2.863879-0.299193$

C $6.216826-1.6180820 .561440$

C $6.828009-1.8073401 .953329$

C $6.539821-0.6144822 .872592$

O $4.803323-1.4195880 .680155$

O $6.874531-1.0244514 .208042$

C $5.025849-0.3083062 .838378$
C 7.4563030 .5719082 .550325

O 4.7623920 .8909503 .562353

C $4.440827-0.2320471 .406137$

C $2.901888-0.1033031 .389882$

$\begin{array}{lllll}\text { O } & 4.901666 & 0.944652 & 0.727528\end{array}$

C $1.747012-1.822924-2.096835$

O $2.403009-2.471701-1.298003$

C $0.335323-2.091878-2.446463$

C $-0.328660-3.028271-1.753035$

C $-1.717229-3.441904-1.949307$

C $-2.503074-2.783367-3.050896$

C $2.119447-1.2777141 .981281$

C $0.620529-1.0110191 .970172$

C $-1.508006-1.9554632 .844543$

O 0.1289440 .0342121 .552079

C $-2.244745-3.1764432 .394383$

C $-3.405260-3.1143231 .723531$

C $-4.212303-4.3086711 .299953$

C $-4.548181-4.285756-0.175732$

C $-5.688063-3.724933-0.613233$

C $-3.605058-4.974263-1.135319$

C $-2.223723-4.384156-1.127464$

N $-0.100566-2.0708892 .505240$

H $1.4775481 .636570-2.820905$

H $4.5139541 .695858-2.453422$

H $3.845107-0.576020-1.599148$

H $4.195625-0.459822-4.645889$

H $4.133928-1.961332-3.795190$

H $7.629676-1.556354-2.449159$

H $7.7267210 .215021-3.908619$

H $6.5505510 .057681-5.216782$

H $6.2804861 .224466-3.906330$

H $4.868302-2.741909-1.718117$

H $\quad 6.214697-3.731380-2.240866$

H $6.013941-3.7349210 .174749$

H $7.564994-3.060664-0.325382$

H $6.663379-0.7480630 .067703$

H $7.908675-1.9849451 .884889$

H $6.405329-2.7108492 .415362$

H $6.638018-0.2957914 .807588$

H $4.536706-1.1219753 .388208$

H 8.5047790 .2994642 .721915

H 7.2562791 .4210023 .213008

H 7.3707900 .9124431 .516204

H 3.8036620 .9446983 .711177

H 2.6079900 .8301821 .887710

H 2.5745510 .0261330 .349622

H 5.3264251 .5301441 .375009

H $-0.112501-1.520011-3.250450$

H $\quad 0.196417-3.549388-0.952788$

H $-2.075836-3.034057-4.027894$

H $-2.494717-1.694730-2.936147$

H $-3.553988-3.082411-3.070001$

H $2.316464-2.1940831 .415561$

H $2.409504-1.4446513 .023466$

H -1.926906 -1.038319 2.417465

H -1.562043 -1.877417 3.935320

H -1.829841 -4.145933 2.658037

H -3.822699 -2.138202 1.491945

H $-5.139470-4.3106991 .888136$

H $-3.702927-5.247717 \quad 1.548789$

H -5.951225 -3.722083 -1.665625

H $-6.381945-3.2518580 .073596$

H -4.013064 -4.989540 -2.152429

H $-3.526645-6.032069-0.851976$

H $-1.579564-4.799303-0.351359$

H $\quad 0.412351-2.8544682 .899420$

C $1.9672764 .152495-1.368831$

O $0.5655523 .834405-1.265106$

C 0.2565573 .5781990 .120733

C 1.4790324 .0201310 .919489

C $2.5985933 .702788-0.052019$

H $\quad 0.1480752 .4929970 .223060$

C - -1.0479694 .2782440 .513873$

H 1.4569825 .0941891 .137590

H 1.5685543 .4845751 .869572

C $3.9111784 .389686 \quad 0.277225$

H $2.7533082 .618277-0.047412$ 
C $-2.264583 \quad 3.881035-0.346267$ $\begin{array}{lllll}\text { O } & -0.872450 & 5.695788 & 0.385784\end{array}$ H $-1.2633474 .096048 \quad 1.572841$ H -2.123303 $4.217174-1.380473$ O -3.4050724 .5896880 .157173$ C -2.591439 $2.375589-0.330564$ C -3.840196 $2.044557-1.173374$ H - $-1.7471411 .812280-0.741503$ O -2.7672761 .9430031 .014802$ H $-4.6910042 .627456-0.808422$ H -3.644908 2.373714 -2.200697 H $4.6801614 .132028-0.457763$ H 3.8033325 .4794270 .286101 H 4.2702784 .0793191 .264010 C $2.5629103 .551796-2.645413$ H $-0.6221865 .878067-0.536432$ H -3.1848715 .5369770 .146887$ H -3.513955 2.4416451 .388669 H $3.602643 \quad 3.882493-2.753517$ H $2.0153573 .941258-3.512796$ H $2.0231655 .246087-1.452357$ C $-5.937161 \quad 0.572133 \quad 0.521314$ $\begin{array}{llll}\text { O } & -4.651309 & 0.086099 & 0.116537\end{array}$ C $-4.1974570 .540056-1.162968$ C $-5.2074850 .124985-2.241758$ C $-6.6117830 .624653-1.918473$ C $-7.0136740 .202920-0.506641$ H -5.8991841 .6603250 .645377$ C $-6.256537-0.053247 \quad 1.887289$ H -3.266388 -0.002758 -1.367057 O $-4.8202230 .609157-3.523036$ H -5.237336 -0.969988 -2.309937 H -6.691429 $1.711067-2.042788$ O $-7.540537 \quad 0.044730-2.837481$ H -7.198713 $-0.878117-0.489065$ O $-8.236361 \quad 0.847696-0.154625$ C $-5.355400 \quad 0.490552 \quad 2.974352$ H -7.287507 0.1506362 .195503 H $-6.124410-1.140298 \quad 1.829045$ H $-3.9279240 .268718-3.706502$ H $-7.2493380 .287076-3.733369$ H $-8.8922550 .616384-0.834514$ $\begin{array}{llll}\text { O } & -4.991902 & -0.518528 & 3.817688\end{array}$ O -5.0362831 .6670723 .084540$ C $-4.141050-0.1362754 .902773$ H $-3.868918-1.0394735 .455476$ H -3.2267590 .3319834 .525548$ H -4.673080 0.5420615 .576302

SCF Energy (B3LYP/6-31G**/MMFF)= -3245.92596166

01233

MM̄FF Geometry

C -3.925354 $0.043116-2.460052$

C $-3.2261601 .126223-2.828496$

C $-2.8119872 .219045-1.883558$

O $-1.3949842 .407597-2.121135$

C $-3.5405623 .546774-2.146806$

C $-5.0050803 .540589-1.772528$

C $-5.4665493 .753365-0.524626$

C $-5.9683903 .348796-2.914539$

C $-4.662302 \quad 3.9794220 .729380$

C $-5.203536 \quad 3.167411 \quad 1.916327$

C $-4.906512 \quad 1.6606991 .818854$

C $-5.878272 \quad 0.881226 \quad 2.708490$

C $-5.578178-0.6223012 .701241$

O \begin{tabular}{lllll}
\hline & -3.557370 & 1.442921 & 2.257247
\end{tabular}

O $-6.331777-1.2271173 .763224$

C $-4.087601-0.8203563 .031416$

C $-6.042711-1.2922921 .403952$

O $-3.721412-2.1947662 .912721$

C -3.1271250 .0755092 .206476$

C -1.712127 -0.0269772 .823061$

O $-3.086476-0.4115130 .866518$

C $-0.6519822 .982931-1.142024$

O $-1.039866 \quad 3.377275-0.054848$

C $0.738818 \quad 3.059448-1.638680$

C $1.653867 \quad 3.730572-0.926025$

C $3.0641903 .897692-1.272740$
C $3.5732113 .219981-2.517224$

C -0.6627280 .9361562 .252248$

C 0.6904520 .7041482 .906627

C 3.0596641 .3459142 .807362

O $0.874576-0.1490213 .773005$

C 3.8963332 .5421272 .494140

C 5.0528582 .4628741 .816834

C 5.9711863 .6260311 .562759

C 6.1843303 .8847490 .087873

C $7.1609003 .257038-0.588751$

C $5.3111194 .917410-0.587888$

C $3.8441434 .626660-0.449216$

N 1.6782801 .5530852 .418669

H $-4.279499-0.037430-1.438280$

H $-2.8853451 .212392-3.858034$

H $-2.9527631 .894029-0.845734$

H -3.423593 $3.833302-3.201330$

H -3.037993 4.359425 -1.606263

H $-6.5461933 .780428-0.380936$

H $-7.0131593 .382461-2.587754$

H $-5.8315894 .137798-3.661390$

H $-5.8086392 .381523-3.399350$

H -3.6011613 .7612280 .588993$

H -4.7391635 .0442840 .981076$

H -4.7501493 .5651322 .833618$

H -6.2852103 .3370581 .993314$

H $-5.003891 \quad 1.3329210 .778611$

H -6.9166121 .0614952 .402486$

H -5.8023611 .2479703 .741920$

H -7.273620 -1.042566 3.605671

H -3.965433 -0.569314 4.094155

H -7.120474 -1.147281 1.262001

H $-5.890471-2.3765411 .440667$

H $-5.538739-0.9008800 .519152$

H -3.623374 -2.393414 1.965787

H -1.7797110 .1695563 .901620$

H -1.341028 -1.053660 2.703685

H -2.249253 -0.1441760 .456044$

H $0.9694952 .568434-2.577163$

H 1.3424514 .2062750 .003349

H $3.0884893 .636910-3.406671$

H $3.3718432 .143686-2.480073$

H $4.6514413 .328384-2.658219$

H -0.5449910 .7884791 .174877$

H -0.9602041 .9740942 .435708$

H 3.4037150 .4537072 .278601

H $3.098651 \quad 1.1385663 .882251$

H 3.5664383 .5021492 .882852

H $5.3879681 .492748 \quad 1.455559$

H 6.9344523 .4038922 .040876

H 5.6085854 .5386872 .051847

H $7.3355333 .451349-1.641725$

H $7.8039762 .529588-0.104280$

H $5.5786355 .040826-1.643860$

H $5.5107665 .895751-0.131406$

H 3.3942815 .0716540 .439048

H 1.4867932 .0797751 .570970

C -3.534505 -2.404161 -2.981232

O $-2.115160-2.190407-2.829957$

C $-1.659593-2.937129-1.683433$

C $-2.796318-3.888722-1.323604$

C $-4.007301-3.048237-1.678950$

H -1.540309 -2.223322 -0.861948

C $-0.326961-3.624866-1.987771$

H $-2.771548-4.799889-1.932730$

H $-2.771569-4.184230-0.270250$

C $-5.290627-3.848263-1.810694$

H -4.140034 -2.292829 -0.897575

C $0.828137-2.667997-2.355212$

O $-0.535427-4.509084-3.099109$

H $-0.042421-4.262162-1.144020$

H $0.552112-2.130072-3.270796$

O $1.958187-3.477226-2.702727$

C $1.256617-1.651547-1.272713$

C $1.449443-2.2036450 .154955$

H $2.200062-1.185597-1.580766$

O $0.298030-0.594897-1.194797$

H $1.729869-1.3598720 .797977$ 
H $0.481174-2.5370710 .547984$ H $-6.129233-3.197813-2.078247$ H $-5.204466-4.622654-2.580199$ H -5.534699 -4.340985 -0.863762 C $-4.229092-1.099935-3.386379$ H $\quad 0.314210-4.944826-3.284559$ H $2.670186-2.881567-2.991889$ H $0.211484-0.208166-2.082778$ H -5.313850 -1.246413 -3.433111 H $-3.901917-0.839378-4.401166$ H -3.649082 -3.119776-3.806478 C $4.517715-2.0212990 .381115$ O $3.736509-2.992269-0.324670$ C $2.491406-3.3308100 .292243$ C $2.714460-3.8346261 .727625$ C $3.603853-2.9030952 .548152$ C $4.874870-2.5607741 .770420$ H $3.949063-1.0880930 .473377$ C $5.767892-1.737709-0.455900$ H $2.102777-4.188956-0.266536$ O $1.475736-4.0156342 .405863$ H $3.201565-4.8179301 .686821$ H $3.065691-1.9955532 .844372$ O $3.979912-3.5619203 .759172$ H $5.493175-3.4624651 .678226$ O $5.641739-1.6074682 .499827$ C $5.421234-0.850800-1.627788$ H $6.535587-1.2101910 .121479$ H $6.225167-2.661181-0.830616$ H $0.942461-4.6359191 .879916$ H $3.159781-3.8013584 .224145$ H $5.102752-0.8079452 .613421$ O $5.044522-1.608224-2.696637$ O $5.452311 \quad 0.372332-1.584283$ C $4.652616-0.874439-3.860995$ H $5.474017-0.239314-4.205916$ H $4.409125-1.592202-4.648786$ H $3.763050-0.274775-3.646211$ SCF Energy $\left(B 3 L Y P / 6-31 G^{* *} / / M M F F\right)=-3245.91265328$

\section{4}

MMFF Geometry

C $0.445894-3.641965-0.421391$

C $0.365833-2.904479-1.540085$

C $-0.828526-2.054132-1.881179$

O $-0.370373-0.697422-2.081331$

C -1.485011 -2.523228 -3.189363

C $-2.888382-1.967502-3.351680$

C $-4.010679-2.632197-3.011879$

C $-2.980879-0.600783-3.978868$

C $-4.106461-4.004164-2.407613$

C $-5.088953-4.111979-1.232127$

C $-4.607798-3.4199070 .054927$

C $-5.416394-3.9378831 .249388$

C $-5.041269-3.2259252 .554417$

O $-4.791316-2.005764-0.081364$

O $-6.040598-3.5443453 .534964$

C $-5.107751-1.7054732 .319028$

C -3.705723 -3.725347 3.117701

O $-4.631599-0.9947973 .460696$

C $-4.351945-1.2399431 .050887$

C $-4.672588 \quad 0.2522090 .788529$

O $-2.950683-1.3895901 .273468$

C $-0.2619290 .088706-0.978960$

O $-0.444875-0.2415040 .180851$

C $0.0774801 .452152-1.434637$

C $-0.1430862 .484229-0.607810$

C $0.1133463 .895094-0.897763$

C $0.7289844 .231025-2.231173$

C $-4.1472910 .802112-0.543467$

C $-4.5360932 .258406-0.745639$

C $-4.4251234 .074951-2.448551$

O -5.1731692 .9044930 .082266$

C $-3.2243394 .961165-2.347752$

C $-3.1636776 .036836-1.547617$

C $-2.0112657 .000854-1.511229$

C $-1.3242417 .051252-0.165383$

C -1.8126887 .8042890 .834559$
C $-0.020576 \quad 6.301170-0.014485$

C -0.2149534 .8122010 .036055$

N $-4.0970532 .746484-1.968573$

H $-0.403837-3.6336650 .259804$

H $\quad 1.188919-2.883453-2.247933$

H $-1.570790-2.080048-1.073442$

H -1.510585 -3.618260 -3.233016

H $-0.882179-2.210706-4.052732$

H $-4.969181-2.152638-3.205363$

H -2.499500 -0.600935 -4.962159

H $-4.017728-0.277603-4.120933$

H -2.489583 $0.147352-3.351341$

H -4.453561 -4.681683 -3.197902

H -3.132836 -4.380826 -2.079812

H $-6.064063-3.707635-1.532778$

H $-5.229693-5.181869-1.031490$

H -3.542196 -3.636194 0.195655

H $-5.294979-5.0228321 .360878$

H $-6.487227-3.7754101 .060707$

H -6.062096-4.511136 3.637964

H $-6.167866-1.4373022 .207314$

H -3.755786 -4.801337 3.323831

H -3.479162 -3.247831 4.077382

H -2.864311 -3.559279 2.441749

H $-5.140616-1.3062814 .228700$

H -5.7624810 .3877430 .793629$

H -4.2651830 .8650581 .602877$

H $-2.470162-0.7328510 .746658$

H $0.4698991 .568835-2.437922$

H -0.5613442 .2802960 .377320$

H $0.091953 \quad 3.877454-3.049548$

H $1.7150163 .763244-2.328546$

H $0.8684635 .301694-2.391808$

H $-3.0574740 .737652-0.587268$

H $-4.5634770 .225568-1.376575$

H $-5.2780064 .473308-1.889151$

H $-4.723893 \quad 3.978851-3.497297$

H -2.388123 $4.734391-3.003839$

H $-4.0143346 .269554-0.910461$

H -2.398845 $7.998048-1.759076$

H $-1.2800686 .775043-2.296933$

H -1.3082417 .8699761 .792800$

H $-2.731397 \quad 8.369447 \quad 0.716187$

H $0.481696 \quad 6.593716 \quad 0.917396$

H $0.6666626 .612466-0.806443$

H -0.6653934 .4669820 .967715$

H -3.546706 2.145688 -2.574878

C $2.882765-4.430993-0.798241$

O $3.476186-3.123657-0.664816$

C $4.905021-3.268735-0.560011$

C $5.208339-4.731368-0.865149$

C $3.960427-5.412561-0.338889$

H $5.165887-3.0718980 .486239$

C $5.612497-2.265746-1.476640$

H $5.314682-4.908909-1.941738$

H $6.125153-5.074861-0.376541$

C $3.777847-6.827247-0.860503$

H $4.006816-5.4348560 .758204$

C $5.252514-0.790399-1.197895$

O $5.247191-2.555498-2.832297$

H $6.697223-2.412543-1.412356$

H $4.207130-0.613247-1.473807$

O $6.036294 \quad 0.018284-2.085471$

C $5.501289-0.3316590 .250352$

C 5.3679321 .1843480 .475326

H $4.821432-0.8599290 .922870$

O $6.831170-0.707908 \quad 0.622858$

H $5.627217 \quad 1.387916 \quad 1.521520$

H $6.1407661 .702624-0.106210$

H $2.862110-7.275863-0.463160$

H $3.716022-6.847289-1.953773$

H $4.620227-7.459025-0.560040$

C $1.599072-4.4997670 .033154$

H $5.700933-1.908336-3.399086$

H $6.969379-0.089062-1.832154$

H $6.946461-0.4861571 .562694$

H $1.829840-4.1992241 .063380$

H $1.240210-5.5348860 .070586$ 
H $2.666856-4.587455-1.862858$

C 2.8834841 .3072612 .254121

$\begin{array}{lllll}\text { O } & 2.918046 & 1.100567 & 0.836729\end{array}$

C 3.9800641 .7707770 .149405

C 3.8838993 .2847130 .384528

C 3.8526653 .6248391 .870478

C 2.7807162 .8048462 .583951

H 3.7827010 .8767302 .711070

C 1.6551770 .5635332 .816167

H $3.8045571 .605708-0.920297$

O $4.9705973 .969704-0.229308$

H $2.9663843 .667526-0.075386$

H 4.8334773 .4831152 .338835

O 3.5309545 .0093882 .027683

H 1.7897983 .1831812 .300915

O 2.9167012 .9849713 .992586

C $1.741436-0.9442932 .669465$

H 1.5550670 .7881853 .882676

H $\quad 0.7672000 .9069692 .276684$

H $4.9583373 .747025-1.175891$

H $4.1954175 .518173 \quad 1.531929$

H 2.8621853 .9399224 .169340

O $0.654398-1.6511853 .081965$

O $2.721985-1.5200032 .208366$

C $-0.472514-1.0246123 .718613$

H -1.187351 -1.810955 3.975047

H $-0.161522-0.5290654 .642086$

H $-0.961493-0.3254393 .036094$

SCF Energy (B3LYP/6-31G**//MMFF) $=-3245.91051895$

\section{5}

MM̄FF Geometry

C $-0.339475-0.5145102 .683647$

C $-0.903687-1.7273582 .573345$

C $-0.561050-2.7470361 .516073$

O $-0.295201-2.042646 \quad 0.281187$

C $0.678694-3.557717 \quad 1.921673$

C $0.929914-4.7489881 .020855$

C $1.864271-4.7829650 .051060$

C $0.082094-5.9618561 .302087$

C $2.772650-3.665386-0.378558$

C $4.178492-3.8371050 .204469$

C $5.160797-2.794094-0.346376$

C $6.564819-3.0325610 .215326$

C $7.561258-1.960753-0.250854$

O $4.697227-1.4886930 .020394$

O $8.738449-2.0679250 .564694$

C $6.952115-0.5681540 .016907$

C $8.015734-2.190647-1.696988$

O $7.7717270 .461575-0.530765$

C $5.502148-0.420704-0.498816$

C $4.8601510 .920537-0.078631$

O $5.515885-0.437819-1.932848$

C $-0.904577-2.461732-0.854894$

O $-1.621897-3.444529-0.982450$

C $-0.629981-1.545810-1.987805$

C $0.145165-0.453282-1.899274$

C $0.4073290 .512547-2.971083$

C $-0.359517 \quad 0.354163-4.257628$

C 4.6589111 .1232341 .424369

C 4.2459662 .5550971 .708156

C $2.336944 \quad 4.1395501 .450904$

O $5.047548 \quad 3.3797192 .142204$

C 2.0788684 .7078990 .085078

C $2.5442224 .244136-1.085451$

C $2.2065004 .833983-2.424080$

C $1.2631443 .963706-3.227321$

C $0.0540934 .416901-3.598537$

C $1.7442872 .598311-3.669801$

C $1.2963981 .501567-2.740677$

N 2.9222252 .8119131 .392836

H $0.463943-0.2352852 .005998$

H -1.685442 -2.012327 3.272788

H -1.433914 -3.399902 1.400714

H $\quad 0.568551-3.9245052 .951255$

H $1.559740-2.9046771 .938002$

H $1.984612-5.703774-0.517946$

H $\quad 0.333225-6.8048280 .649558$
H $\quad 0.223516-6.2924532 .336259$

H $-0.977694-5.7342241 .151277$

H $2.364265-2.687733-0.104465$

H $2.820487-3.672140-1.474694$

H $4.118682-3.7445721 .296826$

H $4.553315-4.843932-0.019347$

H $5.169003-2.878643-1.439681$

H $6.926329-4.033460-0.051807$

H $6.524586-3.0124741 .313506$

H $9.090489-2.9693250 .466250$

H $6.962008-0.4261111 .104022$

H $8.510396-3.164815-1.792151$

H $8.760545-1.446586-1.999889$

H $7.193522-2.169113-2.414994$

H $8.6687490 .337624-0.175887$

H $5.4392891 .753485-0.499154$

H $3.8740290 .992678-0.556776$

H $4.693294-0.033602-2.254975$

H $-1.131103-1.813893-2.912522$

H $\quad 0.635920-0.230570-0.953322$

H $-0.201616 \quad 1.172386-4.963194$

H - $-1.4377570 .325372-4.063243$

H $-0.069181-0.571669-4.765998$

H $3.8956880 .440007 \quad 1.814121$

H 5.5768840 .9367451 .988928

H 2.9788784 .8172552 .021789

H 1.3853484 .0391681 .979557

H 1.4212845 .5751790 .069558

H $3.2155283 .390868-1.101001$

H $3.1351194 .974507-2.991133$

H $1.7853945 .839510-2.294654$

H $-0.6161793 .808157-4.196541$

H $-0.2953675 .402421-3.309338$

H $2.8410562 .589960-3.711690$

H $1.4322482 .415462-4.702925$

H $1.7887041 .524480-1.769019$

H 2.3456552 .0590621 .028092

C -0.9620691 .8786423 .007278$

O -1.9487331 .7838641 .964785$

C -2.6545003 .0370201 .878783$

C -2.0282453 .9619142 .921159$

C -1.479258 2.9735773 .933838

H -3.6915152 .8306732 .167289$

C -2.607096 3.5643330 .438191

H -1.2170784 .5655082 .498195$

H -2.7611554 .6521623 .350497$

C -0.4277703 .5650304 .855186$

H -2.3142872 .5910384 .536045$

C $-3.0753402 .547651-0.623716$

O -1.2576993 .9234380 .110493$

H $-3.189916 \quad 4.4891110 .361479$

H -2.354165 $1.724591-0.678839$

O $-3.0432573 .196344-1.900855$

C $-4.4919321 .991667-0.398963$

C $-4.9991031 .082183-1.533926$

H -4.5243561 .4411310 .543894$

O $-5.3991323 .089703-0.270543$

H $-6.0364460 .807810-1.307232$

H -5.060206 $1.662982-2.462671$

H -0.0625542 .8148515 .563549$

H 0.4321873 .9445584 .294310

H -0.8460174 .3966945 .431563$

C $-0.7735540 .520647 \quad 3.683314$

H -0.7090913 .1257360 .204545$

H -2.144392 $3.546300-2.023083$

H $-6.2767442 .723230-0.067917$

H -1.711490 $0.211500 \quad 4.159086$

H -0.0103330 .5964374 .465396$

H -0.0231592 .1884952 .531120$

C $-5.177192-1.619642-0.102084$

O $-3.992564-0.950541-0.551711$

C $-4.147138-0.184147-1.751329$

C $-4.629397-1.082961-2.897201$

C $-5.902294-1.835800-2.523694$

C $-5.717038-2.558355-1.190709$

H $-5.939743-0.8782830 .166417$

C $-4.813917-2.4196231 .160744$

H $-3.1443050 .155709-2.030734$ 
O $-4.838089-0.302757-4.068927$

H $-3.842800-1.812239-3.130831$

H $-6.772469-1.169748-2.491374$

O $-6.182980-2.819785-3.521778$

H $-5.033143-3.406212-1.327862$

O $-6.974392-3.087252-0.771933$

C $-4.663576-1.5467752 .388764$

H -5.606494 -3.144482 1.379457

H $-3.868870-2.9546761 .018314$

H $-5.014902-0.914040-4.803542$

H $-6.410046-2.349339-4.341477$

H $-7.297116-3.665985-1.484016$

O $-4.725748-2.3364043 .502660$

O $-4.494465-0.3361502 .381724$

C $-4.622917-1.6489834 .753629$

H $-3.638695-1.1825344 .848690$

H $-5.415931-0.9004204 .843390$

H $-4.741837-2.3829305 .555045$

SCF Energy (B3LYP/6-31G**//MMFF) $=-3245.90480304$

\section{6}

MM̄FF Geometry

C $0.0898544 .080590-0.244190$

C $0.3199723 .734850-1.519781$

C $1.3534992 .721569-1.941613$

O $0.6779231 .671348-2.681340$

C $2.4023183 .344128-2.886404$

C $3.7133153 .648411-2.189888$

C $4.8475652 .945655-2.377956$

C $3.7017234 .825993-1.251485$

C $5.0477131 .768603-3.290916$

C $4.4543280 .454816-2.776528$

C $4.9055010 .018928-1.374917$

C $6.409455-0.254665-1.303596$

C $6.826840-0.8288310 .059219$

O $4.176324-1.179054-1.075757$

O $8.156263-1.355499-0.071411$

C $5.904388-2.0150750 .408261$

C $6.896004 \quad 0.259879 \quad 1.136829$

O $6.160367-2.4873221 .729221$

C $4.400822-1.6971840 .240644$

C $3.567700-2.9901810 .396156$

O $4.008232-0.769226 \quad 1.251278$

C $0.0177070 .726639-1.957676$

O $-0.0551140 .646266-0.742205$

C $-0.647718-0.187980-2.909076$

C -1.714444 -0.883861-2.489083

C $-2.530188-1.795539-3.291437$

C $-2.004202-2.188262-4.646072$

C $2.090128-2.847683-0.001626$

C $1.349644-4.1665820 .141414$

C $-0.820032-5.250741-0.347230$

O $1.819180-5.1367190 .731601$

C $-1.741310-5.268982-1.523346$

C $-3.076767-5.327644-1.410495$

C $-4.013148-5.439076-2.579331$

C $-5.046673-4.337536-2.604804$

C $-6.230266-4.484190-1.985394$

C $-4.739452-3.103755-3.421766$

C $-3.701073-2.226655-2.777056$

N $0.096399-4.132128-0.457436$

H $0.696923 \quad 3.6337640 .538303$

H $-0.2736924 .184490-2.311724$

H $1.8513632 .263994-1.076662$

H $2.0265654 .269704-3.343131$

H $2.5693502 .671484-3.735443$

H $5.7363893 .241313-1.822529$

H $4.7022565 .066972-0.876593$

H $3.3217065 .716523-1.762710$

H $3.0679744 .622525-0.384152$

H $4.6325941 .991111-4.281218$

H $6.1243231 .644359-3.460223$

H $3.3588650 .517915-2.783246$

H $4.688968-0.342498-3.494816$

H $4.6148390 .790319-0.653244$

H $6.9893020 .649905-1.520816$

H $6.683131-0.980139-2.082575$

H $8.737577-0.632755-0.364099$
H $\quad 6.161071-2.840272-0.270844$

H 7.6309601 .0257470 .860686

H $7.237395-0.1510092 .093251$

H 5.9416220 .7645421 .297587

H $7.110561-2.6862841 .786679$

H $4.002591-3.775686-0.236217$

H $3.623513-3.3412691 .434591$

H $3.051470-0.8405291 .394185$

H $-0.273583-0.229634-3.925079$

H -2.049027 -0.746109-1.462905

H $-0.977927-2.564758-4.565786$

H $-2.009947-1.328520-5.324830$

H -2.580164 -2.983591 -5.123300

H $1.582911-2.1135660 .632544$

H $2.009229-2.518485-1.043778$

H $-1.354415-5.1436400 .602270$

H $-0.249453-6.184879-0.311317$

H -1.287772 -5.281001-2.511575

H $-3.523951-5.345763-0.419028$

H $-4.515414-6.413271-2.513942$

H $-3.467318-5.456101-3.530975$

H $-6.988849-3.709631-2.022818$

H $-6.463803-5.382011-1.422527$

H $-5.646740-2.496026-3.539057$

H -4.470935 -3.411772 -4.435616

H $-3.986628-1.878697-1.783572$

H $-0.274436-3.234173-0.756485$

C $-2.3661324 .760238-0.322709$

$\begin{array}{llll}\text { O } & -2.818897 & 3.490425 & 0.186835\end{array}$

C -4.2069973 .6034380 .555648$

C -4.6811984 .9498790 .019980$

C $-3.4145105 .777104 \quad 0.126167$

H -4.2379313 .6368781 .651002$

C $-4.9947462 .388790 \quad 0.049371$

H $-5.0043904 .882976-1.025250$

H -5.5123215 .3580750 .602975$

C $-3.4454617 .045458-0.708559$

H $-3.2552376 .042576 \quad 1.180036$

C $-4.398424 \quad 1.0338440 .485602$

O $-4.9977982 .422755-1.383523$

H -6.0411082 .4711790 .366627$

H -3.438833 $0.878356-0.019719$

O $-5.272513-0.0074230 .034809$

C $-4.224530 \quad 0.8790382 .007193$

C $-3.722961-0.5069522 .455421$

H $-3.530370 \quad 1.6381652 .379624$

O -5.4754121 .1229172 .656262$

H -3.707266 -0.5144393 .552383$

H $-4.458129-1.2746622 .182992$

H -2.507930 $7.601264-0.609427$

H $-3.5949616 .823693-1.770601$

H -4.261429 $7.699407-0.383638$

C -0.9618665 .0555280 .211005$

H -5.672400 $1.794111-1.690621$

H -5.241796 $-0.015570-0.936571$

H -6.1158360 .4778622 .309986$

H -0.9854795 .0133321 .307840$

H -0.644595 $6.068257-0.061936$

H -2.364028 $4.683077-1.416990$

C $-0.966006 \quad 0.402514 \quad 3.403247$

O -1.3859230 .1732122 .055554$

C $-2.336404-0.8837581 .897848$

C $-1.764793-2.1980072 .446489$

C -1.267263 -2.054476 3.883435

C $-0.329398-0.8530144 .010980$

H -1.8417440 .6793534 .004038$

C -0.0529451 .6377413 .424328$

H -2.462455 -1.024633 0.819086

O $-2.735090-3.2379062 .387432$

H $-0.918585-2.5104461 .822387$

H $-2.100261-1.9787364 .592132$

O $-0.538377-3.2296244 .246470$

H $0.621108-1.0910423 .520409$

O $-0.048129-0.616275 \quad 5.388361$

C 1.2267331 .4954232 .627096

H -0.5945182 .4885192 .992468$

H $\quad 0.210027 \quad 1.8783814 .460636$

H $-3.018532-3.3202701 .460702$ 
H $-1.131644-3.9893654 .116185$ H $\quad 0.321462-1.4383495 .754069$

O 2.0552622 .5382432 .932242

O 1.4646020 .6068361 .822205

C 3.3101122 .5586252 .243465

H 3.9724041 .7995622 .668315

H 3.1747422 .3957191 .169961

H 3.7621573 .5431032 .390244

SCF Energy (B3LYP/6-31G**//MMFF) $=-3245.90508491$

\section{7}

MM̄FF Geometry

C -3.2952403 .3044651 .289955$

C -2.6191213 .3629700 .132661$

C -1.1296913 .5571410 .048292$

O $-0.6123272 .485537-0.783058$

C $-0.7462774 .888720-0.628666$

C -0.3095315 .9439320 .370019$

C $0.976337 \quad 6.270707 \quad 0.611854$

C -1.4190446 .6447371 .108045$

C $2.1983415 .696575-0.051243$

C 2.5990124 .3458890 .542338

C $3.8988323 .759545-0.029134$

C $3.8537753 .585132-1.549802$

C $5.1053312 .874645-2.085702$

O 4.0739942 .4822280 .603449

O $4.8574022 .504233-3.450459$

C $5.3396011 .587985-1.271978$

C $6.3115263 .818551-2.134546$

O $6.5706800 .969283-1.639188$

C 5.2888991 .8051820 .258748

C 5.2941450 .4285220 .962236

O 6.4384522 .5452380 .670748

C $0.0138221 .446717-0.168587$

O 0.1849181 .2910561 .029895

C $0.4902990 .525216-1.224006$

C $1.394628-0.413195-0.908007$

C $1.982353-1.398467-1.818270$

C $1.434107-1.477399-3.217527$

C 4.9932090 .4647452 .465380

C $5.061476-0.9399293 .035499$

C $3.879263-3.1365842 .951249$

O $6.035507-1.3307563 .674613$

C $4.374300-3.8347261 .721802$

C $3.594545-4.566740 \quad 0.911349$

C $4.080239-5.202069-0.360525$

C $3.386906-4.658330-1.593930$

C $2.541711-5.422278-2.305984$

C $3.744376-3.263426-2.062507$

C $2.965843-2.193247-1.348011$

N $3.950123-1.7051552 .714418$

H -2.739115 3.4383462 .216275

H $-3.1399863 .240848-0.812422$

H -0.6737283 .5029701 .044791$

H -1.578109 5.286054-1.225328

H $0.0507554 .707863-1.359204$

H 1.1846987 .0334391 .360539

H -1.048708 7.4668941 .729809

H -2.138045 7.0698950 .400172

H -1.945234 5.9477291 .765775

H $2.0271595 .621971-1.129595$

H 3.0230206 .4096740 .071324

H 2.7088124 .4361981 .631802

H 1.7933333 .6186300 .398945

H 4.7265094 .4173040 .260621

H $3.7344384 .547186-2.060887$

H $2.9689382 .990673-1.817165$

H $4.0793861 .921119-3.471069$

H $4.5455190 .879419-1.544941$

H $6.1027934 .670113-2.793517$

H $7.1881813 .318886-2.561594$

H $6.5852174 .217503-1.155682$

H $6.5565420 .844793-2.603584$

H $4.539386-0.2088970 .488785$

H $6.264827-0.061526 \quad 0.811259$

H 6.6275952 .3451231 .600844

H $\quad 0.1010860 .655422-2.227093$

H $1.752781-0.4598230 .119991$
H $\quad 1.671487-0.564158-3.774090$

H $\quad 0.345780-1.604810-3.199962$

H $1.828779-2.319370-3.790430$

H 5.7148731 .0848263 .006739

H 3.9979520 .8792172 .661473

H $2.835968-3.380413 \quad 3.174885$

H $4.498426-3.4059333 .812273$

H $5.424773-3.7030801 .472269$

H $2.545341-4.6993311 .160930$

H $3.917273-6.284039-0.275151$

H $5.163520-5.074158-0.478801$

H $2.065083-5.048587-3.206330$

H $2.302365-6.435778-2.002139$

H $3.626729-3.187433-3.148412$

H $4.814420-3.087982-1.891710$

H $3.283954-2.044520-0.318134$

H $3.225470-1.2952562 .132254$

C -5.5391752 .5367810 .257272$

$\begin{array}{llll}\text { O } & -5.043619 & 1.238293 & -0.128939\end{array}$

C $-6.1545460 .421384-0.543789$

C $-7.3766671 .334859-0.562646$

C -7.0295742 .3332450 .523259$

H $-6.298966-0.3345770 .236099$

C -5.855276 -0.253686 -1.886877

H $-7.4895401 .843426-1.527266$

H $-8.3037350 .788209-0.364076$

C -7.8541133 .6070710 .460407$

H -7.1763971 .8546641 .500840$

C $-4.609321-1.165565-1.883835$

O $-5.6494880 .773045-2.865855$

H $-6.736403-0.820082-2.212456$

H -3.699028 $-0.562822-1.781954$

O $-4.536739-1.801510-3.166835$

C $-4.626338-2.267739-0.806843$

C $-3.567847-3.372050-0.989987$

H $-4.504910-1.8125840 .181536$

O $-5.905499-2.910992-0.801780$

H $-3.728701-4.114164-0.198279$

H -3.768383 -3.917977 -1.920716

H -7.5661654 .2994801 .257369$

H -7.724300 $4.122659-0.497195$

H -8.9190933 .3816050 .578762$

C -4.7674523 .0451501 .477547$

H $-5.6776780 .352893-3.741793$

H -4.299273 -1.120056 -3.817999

H $-6.047817-3.288957-1.686781$

H -4.8548232 .3003512 .278750$

H -5.2228703 .9704561 .848223$

H -5.401516 $3.209718-0.598714$

C $-1.784401-2.7943691 .412511$

O $-1.813062-2.0797840 .170608$

C $-2.107156-2.881891-0.976391$

C $-1.071960-4.010248-1.108856$

C $-0.955815-4.8271280 .175678$

C $-0.728052-3.9030361 .370559$

H $-2.772193-3.2282291 .609448$

C -1.478928 -1.782550 2.520290

H -1.980834 -2.228904 -1.848275

O $-1.395020-4.877962-2.189691$

H $-0.089013-3.577858-1.328602$

H $-1.837290-5.4597430 .332058$

O $\quad 0.157629-5.7152580 .075276$

H $\quad 0.276975-3.466043 \quad 1.311698$

O $-0.796001-4.6656902 .572969$

C -2.628391 -0.8211612 .694472$

H $-1.290541-2.2662553 .485338$

H -0.587707 -1.192678 2.275608

H -1.439903 -4.335337 -2.995482

H $\quad 0.013025-6.271520-0.709334$

H $-0.132230-5.3734452 .504879$

O $-3.644114-1.4228623 .376472$

$\begin{array}{lllll}\text { O } & -2.627798 & 0.322746 & 2.260671\end{array}$

C $-4.809661-0.6177773 .577784$

H $-4.5586980 .276550 \quad 4.156118$

H $-5.250805-0.3446612 .614759$

$\mathrm{H}-5.537404-1.2074304 .141507$

SCF Energy (B3LYP/6-31G**//MMFF) $=-3245.89993177$ 
01238

MM̄FF Geometry

C - $-1.707343-3.9885471 .964631$

C -2.681893 -4.091032 1.050364

C $-2.576213-3.780440-0.421925$

O $-3.247341-2.505743-0.606071$

C -1.160712 -3.661685-1.032907

C $-0.308953-4.912618-1.001899$

C $0.951732-4.970482-0.527594$

C $-0.926776-6.138643-1.624567$

C $1.770667-3.8732320 .095309$

C $2.940356-3.431309-0.794025$

C $2.507191-2.673028-2.059686$

C $3.730816-2.295914-2.898294$

C $3.354219-1.440134-4.117575$

O $1.810277-1.490978-1.653695$

O $4.562119-0.866204-4.640717$

C $2.461434-0.270558-3.651292$

C $2.755947-2.291594-5.243536$

O $1.977538 \quad 0.476413-4.765709$

C $1.293182-0.705419-2.735958$

C $0.603306 \quad 0.543842-2.137224$

O $0.342430-1.434067-3.515178$

C -3.788496 -2.246010-1.826324

O $-3.842738-3.001125-2.783654$

C $-4.275858-0.849635-1.807547$

C $-4.705748-0.285824-2.945199$

C $-5.172156 \quad 1.090332-3.077982$

C $-4.7107961 .761309-4.343430$

C $-0.4162940 .226734-1.035437$

C $-1.0019971 .481445-0.413119$

C -2.4622112 .1953601 .475312$

O $-0.7552502 .613506-0.818775$

C -3.8545872 .4428740 .989568$

C -4.2520413 .6115420 .464338$

C -5.6598673 .9150010 .036384$

C $-5.7965964 .077617-1.460615$

C $-5.2929315 .151157-2.093205$

C $-6.5894663 .035076-2.213596$

C $-5.9732051 .663538-2.158172$

N $-1.833302 \quad 1.1791600 .656637$

H $-0.723890-3.6425721 .667319$

H -3.665897 -4.416582 1.383564

H -3.142585 -4.555279 -0.954440

H $-0.655479-2.803782-0.579983$

H -1.262545 -3.375154 -2.088062

H $1.474184-5.925009-0.587482$

H $-0.209550-6.960136-1.726641$

H -1.758637 -6.503625 -1.014413

H -1.301415 -5.907525 -2.627300

H $1.171293-3.0053790 .380925$

H $2.179611-4.2656081 .035272$

H $3.577843-2.776094-0.187994$

H $3.545638-4.303518-1.071034$

H $1.832383-3.308801-2.643439$

H $4.276574-3.194068-3.213785$

H $4.435244-1.723665-2.279413$

H $5.164311-1.593867-4.872711$

H $3.1060050 .417273-3.087077$

H $3.465693-3.067685-5.554847$

H $2.565406-1.687842-6.137571$

H $1.827904-2.790543-4.956745$

H $1.254262-0.031697-5.170996$

H $1.3673111 .202943-1.707279$

H $0.0994531 .108374-2.932034$

H $-0.538507-1.333719-3.122498$

H -4.244944 -0.307697 -0.868555

H -4.691918 $-0.854461-3.873023$

H $-5.1747301 .291461-5.217449$

H -3.622379 $1.677803-4.444669$

H -4.945302 $2.828070-4.375388$

H -1.244755 -0.363118 -1.437540

H $\quad 0.061317-0.349332-0.234791$

H -1.852564 3.1035861 .458250

H -2.4881941 .8241082 .503534$

H $-4.573273 \quad 1.6359971 .108843$

H -3.527728 4.4167370 .361582

H -5.9787594 .8417960 .531100$
H $-6.351636 \quad 3.1443170 .398504$

H $-5.4120405 .285860-3.163101$

H $-4.7502735 .920241-1.553323$

H -6.755834 $3.339305-3.253721$

H -7.596287 2.977255-1.779346

H $-6.2601061 .086608-1.279251$

H -2.0441670 .2067810 .861248$

C $-1.738086-3.0792034 .332755$

O $-0.537937-2.3430214 .011656$

C $-0.907180-0.9768903 .752421$

C $-2.197881-0.7730114 .528903$

C $-2.901805-2.0863474 .243360$

H $-1.115891-0.9012862 .677809$

C $0.225400-0.0085324 .107323$

H -2.006400 -0.665634 5.603304

H -2.7644090 .0980224 .189148$

C $-4.040911-2.3903445 .200845$

H $-3.303991-2.0501143 .223173$

C $1.465095-0.1917623 .203317$

O $0.571983-0.1669395 .480218$

H $\quad-0.1462551 .0156683 .984037$

H $1.128261-0.1679692 .160947$

O $2.069250-1.4649043 .426616$

C 2.5350900 .9001703 .396905

C 3.7795760 .7359662 .505468

H 2.0839971 .8778523 .208436

O $2.9855610 .921702 \quad 4.753085$

H $4.467321 \quad 1.5562002 .745987$

H $4.316265-0.1749392 .800330$

H -4.496958 -3.357470 4.966672

H $-3.695216-2.422296 \quad 6.239459$

H $-4.818418-1.6226275 .129308$

C -1.861805-4.308803 3.425690

H $0.918744-1.0689545 .590110$

H $1.408852-2.1465633 .216393$

H 3.3103780 .0305494 .968238

H -2.808934 -4.824422 3.621952

H -1.059775 -5.013623 3.679626

H -1.616640 -3.4234765 .367547$

C $3.372973 \quad 3.0851900 .566985$

O $2.704074 \quad 1.8185920 .558467$

C 3.4935430 .7064370 .991279

C 4.7510010 .5755180 .120889

C $5.545951 \quad 1.8748200 .074626$

C $4.6372393 .040481-0.303843$

H 3.6327263 .3566081 .597650

C 2.3929794 .1364530 .017389

H $2.891242-0.1891120 .798669$

O $5.597254-0.4676150 .591843$

H $4.4563260 .314921-0.903047$

H 6.0603682 .0686081 .023032

O $6.5674051 .764604-0.919909$

H $4.3607392 .956689-1.363046$

O $5.3587814 .261150-0.146309$

C 1.2913584 .4928360 .992898

H $2.9335855 .063416-0.206527$

H $1.9148643 .769957-0.898080$

H $5.069044-1.2828330 .623519$

H $7.1123240 .992480-0.689621$

H $6.1592384 .197817-0.695133$

O $0.6842965 .641880 \quad 0.569442$

O $\quad 0.983693 \quad 3.845476 \quad 1.983115$

C $-0.3779906 .124868 \quad 1.397712$

H -0.6872897 .1022021 .017634$

H $-1.2308685 .443350 \quad 1.347081$

$\mathrm{H}-0.037856 \quad 6.2438892 .430976$

SCF Energy (B3LYP/6-31G**//MMFF) $=-3245.91492274$

01_239

MM̄FF Geometry

C $-0.955491-3.608188-0.340440$

C $0.275866-4.0189940 .002058$

C $1.288916-3.1882730 .759692$

O $0.587282-2.1455651 .484062$

C $2.068258-4.0123891 .808732$

C $3.401285-4.5323381 .304466$

C $4.601119-4.0815121 .721908$

C $3.337989-5.6386570 .284176$ 
C $4.882423-3.0125702 .740358$

C $4.686110-1.5808102 .234600$

C $5.403313-1.2269180 .922884$

C $6.926319-1.2801671 .061189$

C $7.636495-0.806730-0.216562$

O $4.992666 \quad 0.1032950 .572386$

O $9.016143-0.5729980 .105539$

C $7.0395860 .547732-0.652076$

C $7.623197-1.885604-1.306558$

O $7.5643660 .952495-1.914711$

C $5.4939980 .557952-0.692537$

C $4.9822162 .000285-0.929006$

O $5.052485-0.273815-1.768435$

C $0.913108-0.8500641 .243125$

O $1.776684-0.4330520 .489788$

C $0.014181-0.0100952 .066511$

C $0.230497 \quad 1.3109262 .144772$

C -0.5624582 .2742672 .910410$

C -1.817841 1.7787893 .578204

C $3.4681132 .166676-0.742455$

C $2.9929903 .568847-1.081912$

C $0.8904384 .899343-1.288220$

O $3.7563434 .491496-1.353029$

C 0.6372785 .5875830 .015718

C -0.5887545 .8092690 .512493$

C -0.8563256 .4934981 .821859$

C -1.5923105 .6018082 .798071$

C -2.9299955 .6582442 .910655$

C -0.7722364 .7064043 .699782$

C -0.1419013 .5551402 .966148$

N $1.6082233 .663917-1.035585$

H -1.270121 -2.595234 -0.102282

H $0.576661-5.024884-0.278729$

H $1.986181-2.7530370 .033646$

H $1.466496-4.8611522 .160821$

H $2.220398-3.3979832 .703726$

H $5.495443-4.5249131 .285593$

H $4.321677-6.0780450 .086197$

H $2.688096-6.4462260 .636707$

H $2.950851-5.262347-0.667323$

H $4.262746-3.1717043 .630981$

H $5.914894-3.1371893 .089476$

H $3.615024-1.3905672 .109674$

H $5.000256-0.8804693 .020016$

H $5.059476-1.9094530 .138140$

H $7.266995-2.2874801 .327486$

H $7.237636-0.6296501 .890605$

H $9.387454-1.4020790 .452963$

H 7.3702831 .3009850 .076806

H $8.133031-2.790799-0.955217$

H $8.174919-1.557628-2.194339$

H 6.615562 -2.174669-1.612463

H $8.5341130 .946088-1.840548$

H $5.4773982 .681523-0.224510$

H $5.2533182 .325343-1.941492$

H $4.1784070 .021932-2.065190$

H -0.797184 -0.502858 2.590512

H $\quad 1.0771341 .7310911 .604462$

H -1.572195 1.0813794 .386264

H -2.463708 1.2658822 .857617

H -2.4227422 .5787214 .011263$

H $2.9191021 .464927-1.377601$

H $3.199815 \quad 1.9705580 .301315$

H $-0.0371154 .636104-1.805756$

H $1.4828585 .546496-1.942560$

H 1.5075995 .9208390 .576199

H -1.457239 $5.485698-0.053958$

H $-1.450277 \quad 7.393367 \quad 1.614881$

H 0.0711296 .8550262 .283114

H -3.4643005 .0423413 .626329$

H -3.5212956 .3173472 .283620$

H -1.370695 4.351925 4.546522

H 0.0265765 .3006844 .162272

H 0.7813653 .8193292 .449874

H $1.0669322 .857730-0.736040$

C $-3.240288-4.582552-0.190596$

O $-3.881399-3.294585-0.109373$

C $-5.307401-3.473602-0.190814$
C $-5.551640-4.975719-0.102578$

C $-4.298236-5.522405-0.763077$

H $-5.609379-3.134176-1.188548$

C $-6.002610-2.6237440 .880438$

H $-5.614194-5.3188650 .936543$

H $-6.472447-5.276530-0.611402$

C $-4.048912-6.991089-0.468720$

H $-4.380005-5.379156-1.848921$

C $-5.581787-1.1368400 .856052$

O $-5.660128-3.153762 \quad 2.166679$

H $-7.090077-2.7217250 .780907$

H -4.533594 -1.046092 1.163447

O $-6.355669-0.4317241 .833059$

C $-5.793211-0.449610-0.504207$

C $-5.4559821 .052043-0.525464$

H $-5.193786-0.950334-1.269807$

O $-7.159195-0.596802-0.902645$

H $-5.7313571 .434266-1.516273$

H $-6.110807 \quad 1.5916380 .170473$

H $-3.130647-7.336436-0.953729$

H $-3.951185-7.1741630 .606639$

H -4.877359 -7.603286 -0.839795

C -1.974361 -4.464781-1.041204

H -6.256613 -2.748977 2.818556

H -6.067962 -0.736463 2.709956

H $-7.710796-0.186784-0.214338$

H -2.208667 -3.990747 -2.002008

H -1.563726 -5.458860 -1.249830

H $-2.983592-4.8773650 .835891$

C $-3.025610 \quad 0.796489-2.350599$

O $-3.0710730 .545661-0.942893$

C $-3.9798041 .380574-0.218005$

C $-3.6110882 .858193-0.422531$

C $-3.5402803 .223680-1.905517$

C -2.630632 $2.247300-2.652292$

H $-4.0208920 .609347-2.773562$

C -2.107671 -0.248957-3.000598

H $-3.823226 \quad 1.153567 \quad 0.842734$

$\begin{array}{llll}\text { O } & -4.549253 & 3.709959 & 0.225420\end{array}$

H -2.6300343 .0542080 .029436$

H $-4.5369213 .256071-2.360591$

O $-2.9984984 .537572-2.047647$

H -1.591289 2.443162 -2.366769

O $-2.7354892 .479630-4.054817$

C $-0.658063-0.184454-2.566096$

H $-2.466959-1.251750-2.738439$

H -2.145223 -0.136075 -4.090123

H $-4.5551393 .472768 \quad 1.168533$

H -3.561587 $5.139385-1.531025$

H $-2.515398 \quad 3.414019-4.211113$

O $0.082204-0.980373-3.394745$

O $-0.2279180 .456083-1.617838$

C $1.485486-1.023848-3.119402$

H $1.663622-1.449984-2.128673$

H $1.959008-1.666210-3.866553$

H $1.917559-0.021742-3.194979$

SCF Energy $(B 3 L Y P / 6-31 G * * / / M M F F)=-3245.90945134$

0124

MM̄FF Geometry

C $0.740410-3.729319-0.614386$

C $0.563010-2.931543-1.678833$

C $-0.724401-2.196541-1.940699$

O $-0.420055-0.799439-2.154069$

C $-1.408055-2.724011-3.212218$

C $-2.870659-2.324122-3.275349$

C $-3.885301-3.100451-2.845013$

C $-3.154281-0.986049-3.905552$

C $-3.781464-4.462942-2.220241$

C $-4.662115-4.661007-0.977701$

C $-4.182756-3.8907010 .265055$

C $-4.851362-4.4709601 .516474$

C $-4.487954-3.6884832 .784020$

O $-4.534150-2.5101250 .118627$

O $-5.383130-4.0982953 .828846$

C $-4.749139-2.1929172 .526418$

C $-3.071423-4.0132333 .272881$

O $-4.301093-1.4033833 .626648$ 
C $-4.123174-1.6727641 .210293$

C $-4.629156-0.2344160 .940701$

O $-2.703247-1.6534941 .353890$

C $-0.302819-0.010274-1.054402$

O $-0.333964-0.3620700 .113152$

C $-0.1791581 .385064-1.524550$

C $-0.4979782 .383291-0.688104$

C $-0.4519973 .813888-0.992766$

C $0.0342834 .219513-2.360043$

C $-4.2508570 .334936-0.432350$

C $-4.8172381 .730559-0.639867$

C $-5.0291703 .495895-2.385217$

O -5.4598552 .3295340 .218616$

C $-3.9449174 .523371-2.315153$

C $-3.9832045 .585187-1.495696$

C $-2.9547776 .680206-1.482432$

C $-2.2162476 .780686-0.167246$

C -2.7421557 .4477620 .874017$

C $-0.8274496 .189192-0.095833$

C $-0.8405934 .687286-0.040233$

N $-4.5285872 .222541-1.905134$

H $-0.094214-3.8579330 .073365$

H $1.363557-2.780788-2.396718$

H -1.410307 -2.295805-1.089708

H -1.319782 -3.815139-3.271570

H $-0.899014-2.340836-4.107068$

H -4.902582 -2.732145 -2.970029

H $-2.762231-0.957098-4.927338$

H $-4.226674-0.770040-3.958246$

H -2.687013 -0.181459-3.331444

H -4.101341 -5.190471 -2.976843

H $-2.750865-4.719222-1.957101$

H -5.696271 -4.380509-1.215543

H $-4.661358-5.735632-0.754307$

H -3.092830 $-3.981806 \quad 0.340119$

H -4.595291 -5.531074 1.638423

H $-5.943213-4.4402471 .391575$

H -5.282572 -5.0580843 .950656$

H $-5.838233-2.0553302 .469980$

H -2.983475 -5.0807103 .508486$

H -2.843230 -3.481841 4.203413

H -2.297897 -3.7742392 .540077$

H -4.725411 -1.755604 4.427800

H -5.725080 -0.2237081 .010556$

H $-4.247331 \quad 0.442173 \quad 1.716049$

H $-2.332905-0.9620670 .784060$

H $\quad 0.138954 \quad 1.547531-2.547689$

H -0.8308582 .1307740 .318095$

H $-0.5912343 .770845-3.139916$

H $1.0693543 .894923-2.512984$

H $0.0097475 .297330-2.531211$

H $-3.1650630 .393908-0.542912$

H $-4.646630-0.308254-1.225529$

H -5.911243 $3.789376-1.806184$

H $-5.3384013 .356152-3.425889$

H -3.114835 4.411508 -3.007672

H $-4.8281885 .700990-0.820170$

H -3.470338 $7.628732-1.684319$

H -2.239782 $6.563013-2.305717$

H -2.204094 7.5512911 .810383

H $-3.725721 \quad 7.9019240 .812562$

H $-0.311213 \quad 6.5408320 .807800$

H $-0.2262776 .578553-0.922639$

H -1.1874454 .2926980 .915943$

H -3.948773 $1.673082-2.532152$

C $3.258861-4.189007-1.020036$

O $3.667857-2.824999-0.794221$

C $5.104005-2.778609-0.695275$

C $5.605427-4.176869-1.043423$

C $4.449667-5.041914-0.583595$

H $5.341938-2.5726690 .354370$

C $5.669510-1.669696-1.587296$

H $5.764929-4.296249-2.121367$

H $6.547457-4.414702-0.539711$

C $4.466991-6.440002-1.176982$

H $4.479928-5.1138870 .512026$

C $5.122776-0.259030-1.284569$

O $5.343914-1.977180-2.949994$
H $\quad 6.764437-1.674508-1.525149$

H $4.065233-0.212053-1.566944$

O $5.8025510 .651835-2.160614$

C 5.2994290 .2166990 .171747

C 5.0395551 .7221010 .382228

H $4.648621-0.3685090 .828947$

O $6.647867-0.045638 \quad 0.573279$

H 5.2936291 .9677431 .420216

H $5.7576092 .293322-0.218851$

H $3.614885-7.027210-0.820874$

H $4.423329-6.412114-2.270956$

H $5.382600-6.967615-0.890466$

C $1.981960-4.490336-0.231583$

H $5.711703-1.266520-3.502843$

H $6.7412440 .658154-1.905359$

H $\quad \begin{array}{llll}6.723513 & 0.184826 & 1.515043\end{array}$

H $2.165122-4.2774320 .828443$

H $1.747548-5.558569-0.306301$

H $3.086360-4.306287-2.097632$

C 2.6152421 .9029932 .260331

O $2.636371 \quad 1.498070 \quad 0.884850$

C 3.5997482 .1674450 .064288

C 3.3857523 .6868990 .115366

C 3.3509474 .2137921 .544217

C $2.360773 \quad 3.4102662 .382416$

H 3.5734001 .6510472 .730891

C 1.5056911 .1034222 .961942

H $3.3863871 .863065-0.966647$

O $4.3973804 .374132-0.613693$

H $2.4314573 .933394-0.362154$

H $4.3481424 .209177 \quad 1.999931$

O 2.9196895 .5768371 .531341

H 1.3360193 .6470372 .069899

O $2.4913183 .797693 \quad 3.749494$

C $2.016924-0.2051253 .516610$

H 1.1044381 .6585303 .818063

H 0.6664340 .8885232 .292574

H $4.3839314 .032590-1.524146$

H $3.532705 \quad 6.0682650 .957882$

H $2.3414134 .757633 \quad 3.794154$

O $2.487580-1.0014162 .516614$

O $1.982306-0.4908274 .707221$

C $3.027028-2.2569092 .940448$

H $3.748531-2.1185593 .751893$

H $2.216723-2.9219393 .252411$

H $3.548197-2.7070852 .093374$

SCF Energy (B3LYP/6-31G**//MMFF) $=-3245.90838482$

$01 \_240$

MM̄FF Geometry

C -3.4120172 .3113651 .499762$

C -2.5323941 .8939462 .423482$

C -2.3032000 .4405892 .743886$

$\begin{array}{llll}\text { O } & -0.870980 & 0.221741 & 2.731184\end{array}$

C $-2.850356 \quad 0.053568 \quad 4.129850$

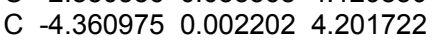

C $-5.100038-1.0599393 .824977$

C $-5.028067 \quad 1.2144604 .797762$

C $-4.612070-2.365911 \quad 3.257834$

C $-5.393033-2.7977302 .008770$

C $-5.029558-2.017926 \quad 0.732114$

C $-6.058566-2.329301-0.359593$

C $-5.693010-1.691836-1.702712$

O $-3.719447-2.4282090 .318171$

O $-6.559113-2.270995-2.690765$

C $-4.244300-2.085764-2.051074$

C $-5.977320-0.185460-1.712105$

O $-3.843975-1.409254-3.242042$

C $-3.245440-1.824633-0.895901$

C $-1.843646-2.406963-1.204050$

O $-3.091345-0.409281-0.747780$

C $-0.443726-1.0417362 .460256$

O $-1.138375-2.0104392 .196762$

C $1.032068-1.0622712 .555402$

C $1.681829-2.1943882 .245111$

C $3.126503-2.4166102 .305201$

C $3.986856-1.3006562 .831278$

C $-1.755073-3.933669-1.167942$ 
C $-0.659437-4.433649-2.087253$

C $1.797358-4.547568-2.368818$

O $-0.923298-4.920699-3.185349$

C $2.914160-5.001376-1.486125$

C $4.096348-4.372054-1.398846$

C $5.255004-4.875287-0.584610$

C $5.679289-3.9100440 .499102$

C $6.646968-3.0041450 .278936$

C $5.045230-4.0566971 .863653$

C $3.612880-3.6028371 .885554$

N $0.617319-4.265915-1.571691$

H $-4.001100 \quad 1.5722270 .964112$

H -1.9604172 .6258222 .988270$

H -2.762075 -0.1827981 .968822$

H $-2.460177 \quad 0.742198 \quad 4.892024$

H $-2.441241-0.9190814 .432379$

H -6.179548 -1.002451 3.960491

H $-6.116417 \quad 1.1052204 .852754$

H $-4.663171 \quad 1.3835105 .816240$

H $-4.819650 \quad 2.107718 \quad 4.202819$

H $-3.542750-2.3530213 .039791$

H -4.763125 -3.129095 4.031514

H -5.183274 -3.862579 1.841019

H -6.468576 -2.710799 2.209161

H -5.018482 -0.9445150 .949203$

H -7.062528 -2.013254 -0.048655

H -6.120633 -3.418220 -0.499315

H $-6.288036-1.924822-3.558533$

H -4.260720 -3.155581 -2.292760

H -7.047158 $0.000786-1.558772$

H $-5.7324420 .259535-2.682854$

H $-5.4338090 .359083-0.937588$

H -3.060068 -1.863033 -3.593306

H -1.487095 -2.014297 -2.163394

H -1.123859 -2.021861 -0.469743

H $-2.232637-0.229000-0.331429$

H $1.535841-0.1613642 .885200$

H $1.099045-3.0528221 .912094$

H $3.735272-1.0801603 .874345$

H $3.839543-0.3956342 .237501$

H $5.056375-1.5184182 .797040$

H -1.561234 -4.283720 -0.147166

H -2.686649 -4.410278-1.488103

H $2.047100-3.633571-2.915694$

H $1.568902-5.326041-3.104367$

H $2.756478-5.919600-0.925374$

H $4.260317-3.465992-1.977860$

H $\quad 6.092840-5.052510-1.272117$

H $5.035311-5.853864-0.139425$

H $6.977004-2.3270731 .059743$

H $7.124865-2.914269-0.690993$

H $5.636903-3.5400622 .627284$

H $5.079516-5.1122852 .163555$

H $2.910330-4.3531061 .521858$

H $\quad 0.732372-3.707974-0.729947$

C $-3.2730594 .198399-0.213287$

O $-1.8367694 .195495-0.317934$

C $-1.4746803 .733315-1.633789$

C $-2.7619183 .729746-2.453538$

C $-3.7871213 .374810-1.393510$

H $-1.1334972 .698097-1.514499$

C $-0.3423994 .589944-2.212432$

H $-2.9815884 .716803-2.876243$

H -2.726921 $3.009992-3.277081$

C $-5.2182833 .688084-1.789801$

H $-3.7007662 .301309-1.193415$

C $0.8810474 .748298-1.286968$

O $-0.8373875 .907970-2.485793$

H $-0.0317804 .183092-3.181557$

H $0.6237575 .400992-0.443380$

O $1.9036265 .432031-2.022819$

C $1.4694393 .433421-0.743873$

C 2.7253113 .6828180 .113964

H $0.7209722 .917341-0.132482$

O $1.8212322 .576590-1.825867$

H $3.5655783 .941866-0.539629$

H 2.5383564 .5413860 .769543

H $-5.9111663 .419606-0.986208$
H $-5.3508434 .752748-2.009263$

H $-5.5022253 .123076-2.683763$

C $-3.6997473 .752818 \quad 1.187943$

H -1.187113 $6.269083-1.652950$

H $1.5193946 .264095-2.348673$

H $1.0030522 .309184-2.275868$

H -4.7776543 .9144591 .307533$

H $-3.199948 \quad 4.392547 \quad 1.926491$

H $-3.5767475 .246884-0.336394$

C $4.5407631 .261557-0.538451$

O $3.327972 \quad 1.2787390 .223528$

C 3.0989502 .4644050 .988895

C 4.2683112 .7139881 .952574

C 5.6118852 .7138701 .228630

C 5.7600301 .4592350 .369492

H $4.5096772 .053762-1.296352$

C $4.608227-0.093306-1.253372$

H 2.2180972 .2558921 .610189

O 4.0855203 .9438372 .645897

H $4.275391 \quad 1.9224522 .712066$

H 5.7312273 .6195570 .623069

O 6.6667852 .7488982 .193143

H 5.8971960 .5830491 .015334

O $6.9300631 .581521-0.436806$

C $3.699705-0.110708-2.458099$

H $5.617034-0.313409-1.620902$

H $4.326830-0.914252-0.583330$

H $4.8612934 .074323 \quad 3.217822$

H 6.5916491 .9553272 .750065

H $7.680638 \quad 1.7372310 .161811$

O $2.485147-0.632682-2.126605$

O $4.0272690 .312899-3.559418$

C $1.531954-0.681570-3.192408$

H $\quad 0.595277-1.072697-2.790583$

H $1.3495190 .320040-3.592275$

H $1.883462-1.351487-3.982654$

SCF Energy $\left(B 3 L Y P / 6-31 G^{* *} / / M M F F\right)=-3245.90983951$

01241

MM̄MF Geometry

C $-3.230733-2.896109-2.521105$

C $-2.057709-2.949613-1.872020$

C $-0.730113-2.696741-2.538797$

O $-0.026788-1.696322-1.765294$

C $0.108591-3.986841-2.527667$

C $1.294219-3.920455-3.468789$

C $2.556619-3.638824-3.093780$

C $0.986944-4.238791-4.909691$

C $3.046398-3.271520-1.721552$

C $3.971517-4.353827-1.146637$

C $4.640045-3.9638590 .183595$

C $3.635440-3.8458421 .332857$

C $4.299500-3.3605722 .629387$

O $5.326773-2.729653-0.027613$

O $3.241566-3.0144403 .536616$

C $5.095422-2.0765932 .314644$

C $5.100311-4.4755343 .309749$

O $5.812166-1.6290083 .462932$

C $6.041857-2.2185511 .102136$

C $6.648923-0.8617070 .673224$

O $7.124663-3.0842651 .457670$

C $-0.199199-0.391716-2.109812$

O $-0.8692070 .043390-3.032654$

C $0.5648470 .429907-1.140957$

C $0.576011 \quad 1.765677-1.267531$

C $1.2272482 .722678-0.367489$

C 1.9877532 .1658890 .806330

C 5.6155810 .1551910 .179963

C $6.2565471 .359134-0.485235$

C $5.6653693 .278425-1.971450$

O $7.4263951 .682769-0.301611$

C $5.1081004 .418085-1.180657$

C $4.1655505 .253941-1.641416$

C $3.6184796 .400904-0.843300$

C $2.1205806 .325717-0.658719$

C $1.3107647 .265931-1.175246$

C 1.5660295 .2094950 .201824

C $1.0715864 .041737-0.610163$ 
N $5.3476482 .045069-1.279098$ H -3.217079 -2.646150 -3.580737 H -2.024424 -3.200215 -0.815751 H -0.868239 -2.347973 -3.569666 H $-0.510186-4.843993-2.826911$ H $0.442468-4.210999-1.507251$ H $3.331766-3.640105-3.859104$ H $1.878622-4.198321-5.544479$ H $\quad 0.568041-5.246829-4.994457$ H $0.262496-3.526022-5.315434$ H $3.585622-2.321060-1.811971$ H $2.216581-3.083746-1.037002$ H $3.418348-5.292987-1.020818$ H $4.767725-4.548051-1.878096$ H $5.380078-4.7388990 .415923$ H $3.119225-4.7999851 .496444$ H $2.850688-3.1237601 .073499$ H $3.649726-2.6310074 .332178$ H $4.351439-1.2991032 .103106$ H $4.443268-5.3180023 .557765$ H $5.522067-4.1364854 .262266$ H $5.912879-4.8606992 .690372$ H $6.593846-2.1984243 .564437$ H $7.244367-0.4348341 .490373$ H $7.363301-1.044446-0.142087$ H $7.873855-2.8999280 .867131$ H $1.075259-0.086657-0.335756$ H $0.0355092 .210186-2.102302$ H 2.7769741 .4882550 .466253 H 1.3146201 .6118631 .470201 H 2.4738402 .9294841 .416259 H $4.934862-0.314772-0.538589$ H 5.0214160 .5298921 .019958 H $5.2250423 .213192-2.971344$ H $6.7496193 .386341-2.072684$ H $5.5079004 .565094-0.179947$ H $3.7753445 .118506-2.647424$ H $3.8956877 .328238-1.360922$ H $4.0919216 .457200 \quad 0.145118$ H $\quad 0.2374057 .231809-1.018365$ H $1.7006598 .086877-1.767547$ H 0.7159295 .5947290 .779449 H $2.317961 \quad 4.9261360 .943034$ H $0.4643814 .331983-1.469229$ H $4.3858091 .721025-1.322852$ C -4.698137 -3.530928 -0.480706 O $-4.385130-2.376993 \quad 0.325411$ C $-5.302103-2.3217351 .434074$ C $-6.056486-3.6461501 .430045$ C $-6.102449-3.958637-0.051870$ H $-6.014225-1.5180451 .214074$ C -4.554402 -2.024100 2.737677 H $-5.508310-4.4286131 .967954$ H -7.048734 -3.559365 1.883019 C $-6.431087-5.410758-0.353036$ H $-6.853913-3.312611-0.525383$ C -3.759332 -0.7013012 .725467$ O $-3.631330-3.0935602 .978442$ H -5.264017 -2.032194 3.574081 H -2.903952 -0.7823382 .044562$ O $-3.220248-0.5058624 .039066$ C -4.5970680 .5398842 .364658$ C -3.9047161 .8890942 .622221$ H $-4.8947890 .487514 \quad 1.314083$ O $\quad \begin{array}{llll}5.808607 & 0.536115 & 3.128060\end{array}$ H -4.625105 2.677891 2.372726 H -3.732381 2.0049963 .699993 H -6.444977 -5.593724 -1.431842 H $-5.697130-6.0895770 .094397$ H -7.416770 -5.672037 0.045670 C $-4.605161-3.165284-1.964201$ H -3.316912 -3.008952 3.894178 H -2.524188 -1.1712184 .171678$ H $-5.5625310 .541140 \quad 4.069105$ H -5.208940 -2.268210 -2.148315 H $-5.038377-3.974613-2.563521$ H -3.975025 -4.316140 -0.225644 C $-3.5095902 .921343-0.224374$
O

C -2.5855972 .1057601 .854773$

C -1.9255143 .4617762 .143873$

C $-2.7015064 .607557 \quad 1.500441$

C -2.9336054 .3247550 .017709$

H -4.5471042 .8705780 .127702$

C $-3.4962772 .602057-1.729013$

H $-1.885071 \quad 1.3338532 .194972$

O -1.8212393 .6610823 .549387$

H -0.9046043 .4533581 .744990$

H -3.6503214 .7944632 .016648$

O -1.9446135 .8154321 .604192$

H -1.987285 $4.438597-0.524958$

O $-3.8378195 .297726-0.504425$

C $-4.3182561 .382425-2.086552$

H $-3.9122393 .449074-2.286662$

H -2.473635 $2.416304-2.073895$

H -1.2874854 .4599153 .695567$

H -1.891836 6.0487862 .546184

H $-3.4534756 .173995-0.329562$

O $-4.601012 \quad 1.420497-3.422731$

O $-4.6471150 .498315-1.308923$

C $-5.382160 \quad 0.335195-3.932326$

H $-4.748145-0.546996-4.049624$

H $-6.2327980 .117486-3.279082$

H $-5.7648750 .625196-4.914646$

SCF Energy $(B 3 L Y P / 6-31 G * * / / M M F F)=-3245.91703750$

01242

MM̄FF Geometry

C $-0.6287844 .708020-0.734918$

C -1.114223 3.8108350 .135997

C $-2.0200142 .679554-0.261312$

O -1.5320331 .4491830 .319165$

C $-3.4922502 .852914 \quad 0.171147$

C -3.7550963 .3094991 .593833$

C -3.9136142 .4911122 .652672$

C -3.9385444 .7971841 .771119$

C -3.8037720 .9935352 .701690$

C $-5.1474180 .311148 \quad 2.996182$

C -6.0916310 .2892961 .783854$

C $-7.426567-0.3632902 .149354$

C $-8.344976-0.5160510 .926927$

$\begin{array}{lllll}0 & -5.448357 & -0.465424 & 0.752594\end{array}$

O $-9.410984-1.409007 \quad 1.284387$

C $-7.552861-1.191756-0.212532$

C $-9.003788 \quad 0.812797 \quad 0.538554$

O $-8.317130-1.225928-1.415707$

C $-6.176704-0.539993-0.478436$

C $-5.353973-1.414887-1.454203$

O $-6.3761880 .750640-1.057795$

C $-0.601881 \quad 0.740877-0.374833$

O $-0.023767 \quad 1.079578-1.394375$

C $-0.442999-0.5583750 .316320$

C $0.380110-1.489791-0.186638$

C $0.608741-2.8253580 .370856$

C $-0.256992-3.2530001 .526826$

C $-3.887070-0.984370-1.592309$

C $-3.155835-1.889300-2.562680$

C $-2.226711-4.175437-2.790244$

O $-2.973018-1.564027-3.733670$

C -1.454709 -5.110903 -1.916207

C $-0.211853-5.528630-2.200764$

C $0.563366-6.490905-1.346557$

C $1.922113-5.956599-0.951233$

C $3.039233-6.383569-1.564046$

C $1.990877-4.9869330 .208420$

C $1.566262-3.597987-0.182559$

N $-2.761659-3.089692-1.989718$

H $-0.9053284 .631039-1.783225$

H $-0.8498113 .892249 \quad 1.187510$

H -2.028368 $2.561385-1.353607$

H $-4.015587 \quad 1.9047280 .002989$

H -3.972380 $3.568462-0.510151$

H -4.137855 2.9450753 .617710

H -4.1720205 .0675882 .806518$

H -3.0334975 .3416121 .489417$

H -4.7648405 .1526521 .146523$ 
$\begin{array}{llll}H & -3.365434 & 0.579478 & 1.791261\end{array}$ H $-3.1040140 .745573 \quad 3.509860$ H $-4.930284-0.7234183 .293048$ H -5.6382970 .7965223 .848742$ H $-6.253304 \quad 1.315828 \quad 1.436819$ H $-7.931914 \quad 0.202502 \quad 2.942040$ H -7.241153 -1.362777 2.567549 H -9.875135 -1.029949 2.050561 H -7.388880 -2.2385660 .079941$ H $-9.6095861 .196273 \quad 1.368436$ H $-9.6956560 .680428-0.300574$ H $-8.282243 \quad 1.5882080 .272895$ H $-9.167189-1.649956-1.207017$ H -5.368281-2.454635 -1.102232 H -5.819380 -1.405732 -2.448094 H $-5.6203580 .969922-1.624151$ H -1.027584 -0.7213051 .214886$ H $0.944547-1.254841-1.088184$ H -1.317833 -3.174749 1.262383 H $-0.065197-2.6232482 .402717$ H $-0.095422-4.2864761 .838236$ H $-3.7986790 .042368-1.961881$ H $-3.375393-1.019884-0.624184$ H -1.619181 -3.762399 -3.602371 H -3.079261 -4.699264 -3.234977 H -1.946860 -5.478782 -1.018929 H $0.269551-5.180618-3.112052$ H $\quad 0.677273-7.422649-1.915804$ H $0.005233-6.762777-0.442012$ H $4.019274-6.023985-1.268338$ H $2.998246-7.098305-2.379202$ H $3.021291-4.9204610 .580649$ H $1.414334-5.3973021 .040999$ H $2.143477-3.184977-1.011128$ H -3.110674 -3.313348 -1.061910 C $1.6811405 .783511-0.871838$ O $2.3811654 .684262-0.259290$ C $3.3166904 .150494-1.218367$ C $3.2864855 .088650-2.424076$ C $1.8603955 .602602-2.377218$ H $2.9245953 .174291-1.526849$ C $4.7011853 .982664-0.583342$ H $3.9940225 .918585-2.314373$ H $3.5258574 .568731-3.356866$ C $1.6378686 .868441-3.184780$ H $1.2049434 .811226-2.760149$ C 4.7175283 .1220760 .696187 O $5.2025825 .280262-0.235622$ H $5.3956163 .574351-1.327517$ H $4.218848 \quad 3.6599201 .510997$ O 6.0870922 .9848481 .100232 C 4.0936831 .7224310 .544180 C 4.2867760 .8638191 .810503 H 3.0220951 .8259840 .339307 O $4.6916791 .084168-0.579689$ H $\quad 5.3548240 .675576 \quad 1.959519$ H 3.9337741 .4415122 .672787 H $0.5974507 .200001-3.108978$ H $2.2787877 .685319-2.836674$ H $1.8607476 .694136-4.242489$ C $0.2473155 .864766-0.344408$ H $\quad 6.081417 \quad 5.154057 \quad 0.161457$ H 6.5411092 .4564880 .421086 H $4.1883180 .270930-0.753614$ H $-0.2242526 .784887-0.709529$ H $\quad 0.282802 \quad 5.9423180 .749873$ H $2.2061616 .691884-0.545384$ C $5.279230-1.8811300 .844227$ O $3.967616-1.3262290 .685342$ C $3.528294-0.4817581 .753812$ C $3.535272-1.2700583 .070564$ C $4.900063-1.8929273 .346652$ C $5.371115-2.6993032 .137881$ H $6.020829-1.0733810 .854349$ C $5.564413-2.765344-0.375420$ H $2.483228-0.2307131 .538247$ O $3.167235-0.4412104 .167534$ H $2.792512-2.0766433 .014768$
H $5.640338-1.1340983 .626017$

O $4.794376-2.7817814 .461210$

H $4.766102-3.6110102 .051429$

O $6.723600-3.1030742 .341633$

C $5.700007-1.935966-1.629348$

H $6.498181-3.327476-0.260091$

H $4.768953-3.503553-0.525854$

H $2.289760-0.0707143 .971751$

H $4.456559-2.2637905 .212016$

H $6.755688-3.5977153 .178443$

O $4.517303-1.919751-2.307640$

O $6.726624-1.352354-1.951645$

C $4.507367-1.150118-3.513772$

H $3.501658-1.199475-3.939447$

H $4.746817-0.103955-3.301023$

H $5.215574-1.569483-4.234474$

SCF Energy (B3LYP/6-31G**//MMFF) $=-3245.91712207$

01243

MM̄FF Geometry

C $-4.269898-3.272220-2.257168$

C $-3.471357-3.076629-1.196362$

C $-2.094813-3.676674-1.056937$

O $-1.149364-2.593161-0.893226$

C $-2.035501-4.5566990 .204603$

C $-0.682950-5.2013900 .439239$

C $0.093040-4.9758311 .518033$

C $-0.226389-6.172340-0.619137$

C $-0.194605-4.0860492 .694961$

C $0.329404-2.6480742 .559047$

C $1.861002-2.5409292 .485532$

C $2.308398-1.0801092 .587586$

C $3.831140-0.9354782 .428952$

O $2.296074-3.0855311 .237497$

O 4.1320120 .4565632 .249068

C $4.287191-1.6960901 .162862$

C $4.568678-1.3417773 .709627$

O $5.708821-1.729231 \quad 1.072247$

C $3.720313-3.1276541 .072356$

C $4.004570-3.811705-0.287128$

O $4.330786-3.9393102 .083450$

C $-0.784084-1.911386-2.012716$

O $-1.136105-2.134204-3.160123$

C $0.110020-0.811196-1.593401$

C $0.3302950 .211696-2.432601$

C $1.1800991 .373921-2.169001$

C $2.0553631 .325002-0.946529$

C $3.427987-3.100212-1.516401$

C $4.427503-2.222007-2.251539$

C $4.577998-0.684765-4.209287$

O $5.620112-2.167446-1.965264$

C $4.5717740 .725405-3.710786$

C $4.1706321 .768254-4.453738$

C $4.2147033 .194783-3.986967$

C $2.8755543 .888379-4.095361$

C $2.5978674 .701644-5.128328$

C $1.8970413 .710573-2.957280$

C $1.1513592 .407780-3.035558$

N $3.830039-1.529133-3.296796$

H $-3.917916-3.928408-3.051393$

H $-3.786587-2.425595-0.385911$

H -1.826503 -4.266731-1.941705

H -2.787228 -5.354249 0.134858

H -2.321564 -3.946163 1.067793

H $1.046682-5.4988911 .579403$

H $0.676403-6.716643-0.321477$

H -1.005678 -6.916370 -0.813902

H $0.000381-5.648320-1.552546$

H - $-1.270079-4.0544872 .899999$

H $0.248770-4.5485293 .586172$

H $-0.118638-2.1862891 .671046$

H - $0.024713-2.0856093 .431266$

H $2.285555-3.1275363 .308649$

H $1.980755-0.6370183 .535895$

H $1.815598-0.4955601 .798680$

H 3.6446320 .7763201 .471001

H $3.934241-1.1216880 .299375$

H $4.254952-0.7069724 .547354$ 
H $5.649220-1.1903153 .609391$ H $4.391802-2.3797943 .998179$ H $6.023944-0.8103011 .117200$ H $5.078429-4.005284-0.395140$ H $3.534754-4.805570-0.251290$ H $4.242887-4.8718651 .825336$ H $0.545765-0.864826-0.602287$ H $-0.1724440 .210464-3.398948$ H $2.7636582 .153057-0.881457$ H $2.6586350 .411530-0.945682$ H $1.4455021 .341601-0.038076$ H $3.106757-3.863882-2.236669$ H $2.546806-2.507372-1.254755$ H $4.110553-0.778167-5.194751$ H $5.609149-1.046497-4.277722$ H $4.9451190 .899959-2.704514$ H $3.8238261 .593741-5.469897$ H $4.9581053 .721587-4.599693$ H $4.5801873 .267801-2.955009$ H $1.6506185 .226192-5.195980$ H $3.3098734 .856257-5.932298$ H $1.1488034 .514756-2.975972$ H $2.4246603 .846967-2.009713$ H $\quad 0.4865362 .340970-3.898139$ H $2.824232-1.591803-3.425160$ C $-6.090914-1.599485-1.497644$ O $-5.196747-0.472016-1.514633$ C $-5.9427680 .722104-1.220481$ C $-7.3917550 .285850-1.010023$ C -7.460126 -1.002856-1.808758 H $-5.8902231 .353543-2.116110$ C $-5.3173411 .473400-0.032985$ H $-7.613867 \quad 0.0926630 .044770$ H $-8.1016461 .042251-1.359393$ C $-8.631422-1.890318-1.426241$ H $-7.527422-0.751028-2.875574$ C $-3.9096412 .032526-0.346595$ $\begin{array}{llll}\text { O } & -5.233980 & 0.606822 & 1.097814\end{array}$ H -5.9667042 .3146360 .239153$ H -3.987821 2.663775 -1.239871 O $-3.0073040 .974877-0.666969$ C -3.311763 2.8803390 .797623 C -1.9897363 .5556100 .378996$ H -4.031409 3.6582071 .079691 O -3.0728072 .0749101 .945902$ H -1.2441672 .7854630 .156808$ H -2.171746 4.110609 -0.548283 H -8.640542 $-2.806267-2.025127$ H $-8.588699-2.178457-0.370488$ H $-9.579415-1.368963-1.594481$ C -5.619657 -2.646934 -2.509452 H $-6.1316730 .475671 \quad 1.444478$ H -2.9526320 .3938610 .111219$ H -3.9250181 .7081862 .235116$ H -5.568377 -2.179749 -3.501558 H $-6.355208-3.457034-2.570272$ H -6.082804 -2.016358 -0.481782 C $0.186758 \quad 3.0825542 .529312$ O -1.0269523 .8366162 .650369$ C -1.447762 4.5191951 .462848 C -0.3394045 .4663190 .978689$ C $0.991134 \quad 4.742602 \quad 0.812670$ C 1.3436423 .9887332 .092009 H $\quad 0.0508712 .2785601 .797705$ C 0.4781882 .4506823 .899553 H -2.2922285 .1537231 .761506$ O $-0.6915646 .087161-0.253048$ H $-0.204204 \quad 6.270246 \quad 1.714084$ H $0.9704394 .065788-0.047482$ O 2.0213885 .6978710 .555056 H 1.5848954 .7065932 .886490 O 2.5060413 .1956991 .865277 C -0.4361921 .2816974 .197838$ H 1.5024132 .0662003 .952644 H 0.3407213 .2061424 .682722 H $-1.533217 \quad 6.553997-0.114317$ H $1.7667136 .187634-0.245951$ H $3.2088803 .792141 \quad 1.555077$
O $-\begin{array}{llll}0.541159 & 1.125933 & 5.550357\end{array}$

O $-0.958198 \quad 0.566376 \quad 3.353720$

C $-1.357338 \quad 0.0323295 .981221$

H $-0.921523-0.9157445 .652164$

H -1.3905400 .0427067 .073868$

$\mathrm{H}-2.3764220 .1436345 .598375$

SCF Energy $\left(B 3 L Y P / 6-31 G^{* *} / / M M F F\right)=-3245.90495533$

01244

MM̄FF Geometry

C $-3.930493-1.8065601 .688274$

C $-3.387619-2.9931591 .373993$

C $-2.864179-3.3211420 .000614$

O $-1.564187-3.9543250 .112279$

C $-3.751517-4.337431-0.737786$

C $-5.083671-3.799969-1.204808$

C $-5.267779-3.169617-2.380775$

C $-6.258982-4.083727-0.306271$

C $-4.225365-2.830860-3.413710$

C $-4.404378-1.406634-3.958677$

C $-3.927018-0.313992-2.986757$

C $-4.5305671 .032882-3.392589$

C $-4.0055402 .182167-2.525913$

O $-2.496493-0.261725-3.062842$

O $-4.3941563 .415972-3.147606$

C $-2.4680582 .129743-2.534712$

C $-4.6342272 .180620-1.129950$

O $-1.9246723 .109219-1.649613$

C $-1.8791160 .727860-2.228449$

C $-0.3696920 .756083-2.564786$

O $-2.067413 \quad 0.441019-0.844242$

C $-0.505183-3.1995410 .503051$

O $-0.519787-2.0336020 .860540$

C $0.694755-4.0601530 .406600$

C $1.861029-3.6462240 .921518$

C $3.126705-4.3818780 .881052$

C $3.172882-5.6460370 .062754$

C $0.401214-0.545098-2.313730$

C $1.829467-0.435330-2.826571$

C $4.103382-1.336600-2.545577$

O $2.1683840 .378013-3.684055$

C $4.794504-2.512485-1.936410$

C $5.833761-2.393886-1.095670$

C $6.605560-3.553660-0.533392$

C $6.603525-3.5778820 .978413$

C $7.530696-2.9078441 .683347$

C $5.573517-4.4451411 .665130$

C $4.185405-3.8819801 .551169$

N $2.683621-1.367187-2.249463$

H -4.016218 -1.054349 0.909948

H $-3.316212-3.7680012 .133428$

H -2.746789-2.419409-0.612987

H $-3.908092-5.225058-0.108451$

H $-3.197275-4.733953-1.599858$

H $-6.282770-2.875280-2.645260$

H $-7.200986-3.713229-0.724650$

H $-6.368802-5.162963-0.156494$

H $-6.128434-3.6104720 .670384$

H -3.205791-2.958151-3.037785

H -4.350236 -3.533469 -4.246871

H $-3.831430-1.329358-4.892145$

H -5.459825 -1.252102 -4.216859

H $-4.224345-0.569959-1.963919$

H $-5.6265600 .995620-3.351410$

H $-4.2783471 .244879-4.441352$

H $-5.364693 \quad 3.427997-3.209776$

H -2.147372 $2.427338-3.542660$

H $-5.7267362 .243844-1.198790$

H $-4.3200793 .058107-0.554424$

H $-4.3895691 .284200-0.558238$

H $-2.0179332 .772317-0.742113$

H $-0.2605411 .020394-3.625101$

H $0.118948 \quad 1.546794-1.984832$

H - $-1.459935-0.268232-0.582991$

H $0.583620-5.018222-0.089056$

H $1.893292-2.6811221 .423665$

H $2.831407-5.455345-0.961086$

H $2.533677-6.415600 \quad 0.509196$ 
H $4.174165-6.071686-0.028260$ H $\quad 0.432853-0.760488-1.243038$ H $-0.075982-1.386946-2.826177$ H $4.499711-0.384205-2.177408$ H $4.229263-1.350978-3.633827$ H $4.454875-3.501187-2.233852$ H $\quad 6.182877-1.399439-0.825199$ H $7.638733-3.476932-0.897384$ H $\quad 6.231607-4.509171-0.921138$ H $7.551530-2.9415032 .767524$ H $8.286768-2.3058261 .189800$ H $5.802435-4.5321092 .735680$ H $5.651561-5.465448 \quad 1.279612$ H $4.053736-2.9580902 .112234$ H $2.365515-1.882891-1.433918$ C -4.3993180 .0104953 .430306$ O -3.0569320 .5206223 .319698$ C -3.1119901 .8285152 .717554$ C -4.5753262 .2582512 .769053$ C -5.2904010 .9337162 .598187$ H -2.8146241 .7031001 .669729$ C -2.148502 2.7947523 .416024 H -4.8361282 .7028603 .736390$ H -4.822336 2.9858281 .990302 C -6.7414450 .9620603 .047566$ H -5.2606590 .6586991 .539116$ C -0.6925722 .3021093 .525316$ O $-2.612696 \quad 3.0398304 .751249$ H -2.1785393 .7657502 .908171$ H -0.6357721 .4821144 .251203$ O 0.0807293 .3717534 .087916 C -0.0419151 .8534972 .202995$ C 1.4516391 .5197742 .399905 H -0.5684090 .9701991 .824647$ O $-0.1903982 .895102 \quad 1.242529$ H 2.0049222 .4428952 .602856 H 1.5444660 .8807303 .285715 H -7.209693 -0.018272 2.913994 H $-6.830147 \quad 1.2376324 .103708$ H -7.3109891 .6908402 .461456$ C -4.450537 -1.477312 3.060888 H -2.655602 2.1826435 .208999 H 0.0935254 .0964043 .439027 H 0.1316852 .5557530 .390424 H -5.486267 -1.827948 3.136732 H -3.859792 -2.031883 3.801440 H $-4.668810 \quad 0.102246 \quad 4.490965$ C $2.9009462 .694744-0.060662$ O $2.022271 \quad 1.563667-0.011977$ C 2.0821870 .7948571 .191095 C 3.5140940 .2931661 .431634 C 4.5416131 .4183121 .366971 C 4.3597372 .2471210 .097433 H 2.6350863 .3930530 .741149 C $2.6487993 .391182-1.405711$ H $1.462790-0.0922321 .012959$ O $3.628787-0.3542222 .694769$ H $3.766807-0.4509170 .665916$ H 4.4947772 .0546392 .258715 O $5.8543120 .851277 \quad 1.345121$ H $4.6707551 .654417-0.771692$ O $\quad 5.2114413 .387496 \quad 0.162243$ C $3.1477224 .817059-1.446656$ H $3.1091112 .808406-2.212703$ H $\quad 1.5742923 .425947-1.620929$ H $2.931716-1.0298182 .744231$ H 5.9385740 .2867992 .132906 H 6.1202843 .0649340 .288379 O $3.6085445 .102178-2.699156$ O $3.1074585 .594767-0.502604$ C $4.1026106 .432316-2.884606$ H $4.4517316 .524553-3.916446$ H $3.3024307 .159809-2.718050$ H $4.9441786 .621659-2.211253$ SCF Energy (B3LYP/6-31G**//MMFF) $=-3245.93339935$

01245

MM̄FF Geometry
C $-1.5812152 .852440-1.537730$

C $-0.6861483 .694396-1.000593$

C 0.1202643 .3530520 .223052

O $1.5143963 .381567-0.159324$

C -0.0940594 .3684511 .362358$

C -1.5170394 .4848491 .858554$

C -2.1009183 .6028622 .691931$

C -2.2619225 .7152321 .410729$

C -1.4968712 .3482873 .262538$

C -2.4515851 .1594173 .098104$

C $-1.909719-0.1938753 .590314$

C $-1.270470-0.1304814 .980909$

C $-0.735431-1.4991785 .432573$

O $-0.965913-0.6982952 .632173$

O $0.098080-1.2924896 .584249$

C $0.138044-2.0887904 .301143$

C -1.866186-2.411138 5.920082

O $0.551333-3.4153144 .620298$

C $-0.540444-2.0429402 .911768$

C $0.396783-2.526521 \quad 1.783527$

O $-1.654683-2.9482702 .920845$

C 2.3744162 .6513150 .602050

O 2.0958541 .9614131 .569930

C 3.7340772 .8377860 .050696

C 4.7550212 .1535920 .586697

C 6.1493252 .1929140 .148399

C $6.5181373 .151396-0.952428$

C $1.637495-1.6726561 .514365$

C $2.521316-2.3212580 .458589$

C $4.376328-1.819622-1.126456$

O $2.422727-3.5064060 .146933$

C $5.777398-1.750767-0.606573$

C $6.783595-1.178073-1.285213$

C $8.200957-1.104216-0.788176$

C $8.7429420 .309531-0.796642$

C $9.4811580 .754522-1.827703$

C $8.4894231 .188040 \quad 0.409815$

C $7.030124 \quad 1.3529310 .730373$

N $3.451569-1.440042-0.072051$

H $-1.6983201 .861646-1.104482$

H $-0.5530084 .684197-1.429703$

H -0.1357202 .3400460 .558252$

H $\quad 0.2703755 .3556121 .044298$

H $\quad 0.5605004 .1169512 .207417$

H -3.118955 3.8081243 .020960

H -3.2933375 .7345641 .778522$

H $-1.760327 \quad 6.6152231 .781463$

H $-2.302647 \quad 5.768330 \quad 0.318877$

H -0.5307552 .1105012 .810555$

H -1.3185892 .5346854 .327653$

H $-3.378292 \quad 1.377758 \quad 3.645247$

H -2.7195691 .0641772 .037681$

H $-2.771054-0.8725113 .607840$

H $-1.976500 \quad 0.2712855 .717995$

H $-0.4203650 .563773 \quad 4.964285$

H $\quad 0.828053-0.7023256 .330849$

H $1.054456-1.4880824 .266312$

H -2.388593 -1.949259 6.766939

H -1.473639 -3.3618696 .297384$

H $-2.611734-2.6250205 .151822$

H $-0.206725-4.0057054 .470801$

H $0.684510-3.5677951 .980464$

H $-0.175650-2.5646880 .846742$

H $-1.890718-3.1567002 .002110$

H $3.8548743 .515950-0.786168$

H 4.5501201 .4802851 .418372

H $7.5853363 .147916-1.187093$

H $6.2636934 .178963-0.669720$

H $5.9869212 .898628-1.876688$

H $1.334428-0.678417 \quad 1.170525$

H $2.245491-1.5604782 .416913$

H $4.212675-1.130780-1.960977$

H $4.160524-2.836487-1.468052$

H $5.977350-2.2041060 .361385$

H $6.583688-0.749700-2.265151$

H $8.815617-1.742727-1.436159$

H $8.297060-1.5256860 .220167$

H $9.894683 \quad 1.757477-1.836988$ 
H $9.6858920 .125991-2.688212$ H $8.962413 \quad 2.1714830 .298859$ H $8.9826600 .733717 \quad 1.279121$ H $6.6754930 .682046 \quad 1.513533$ H $3.449357-0.4715780 .233751$ C -3.962904 $2.943728-2.402297$ O $-4.1849721 .540019-2.147086$ C $-4.9149691 .414015-0.913063$ C $-5.6197922 .751029-0.729695$ C $-4.5400373 .705580-1.204528$ H $-4.1698941 .299172-0.115866$ C $-5.824750 \quad 0.182312-0.932848$ H $-6.5063362 .836047-1.368636$ H $\quad-5.9225882 .925976 \quad 0.306907$ C $-5.0511315 .093032-1.549484$ H $-3.7970073 .797688-0.404677$ C $-5.110479-1.123640-1.341890$ O $-6.8812790 .414128-1.872031$ H -6.3014590 .0688920 .048291$ H -4.912787 -1.107812 -2.420001 O $-6.009865-2.214193-1.108591$ C $-3.805153-1.411798-0.578451$ C $-3.174338-2.785088-0.873106$ H $-3.068184-0.635486-0.807287$ O $-4.045214-1.3383880 .829342$ H $-2.239460-2.843757-0.304670$ H $-3.804569-3.578731-0.451340$ H $-4.2340805 .729915-1.902974$ H $-5.8153515 .056508-2.332988$ H $-5.4949735 .569026-0.669007$ C $-2.4770273 .191176-2.696474$ H $-7.551860-0.276351-1.737313$ H -6.744529 -2.129429-1.739050 H $-4.733532-1.9909611 .044556$ H -2.336705 $4.234412-3.002452$ H -2.174063 2.560009-3.540365 H $-4.535366 \quad 3.175950-3.309417$ C $-0.930545-1.721080-2.670258$ O $-2.294764-1.965945-3.032201$ C -2.905562 -3.071549-2.363059 C $-2.110606-4.359963-2.624896$ C $-0.623153-4.196408-2.313865$ C $-0.069450-2.946653-3.000973$ H $-0.875534-1.502389-1.597197$ C $-0.487194-0.473821-3.447044$ H $-3.886607-3.206192-2.835109$ O $-2.633361-5.443194-1.862587$ H - $2.211168-4.634501-3.683253$ H $-0.447031-4.170556-1.232113$ O $0.062734-5.349036-2.808023$ H $-0.047022-3.111027-4.086065$ O $1.267962-2.716640-2.575443$ C $0.6938140 .239927-2.828062$ H $-0.251155-0.749950-4.481939$ H $-1.3092250 .249483-3.495127$ H -3.570811-5.542421 -2.101134 H $0.981307-5.300528-2.493621$ H $1.808809-3.465863-2.876348$ O $1.382540 \quad 0.896884-3.806613$ $\begin{array}{lllll}\text { O } & 0.955739 & 0.254548 & -1.633119\end{array}$

C $2.5174741 .651563-3.370884$ H $2.1809862 .556836-2.858691$ H $3.160801 \quad 1.053373-2.719027$ H $3.0922571 .941293-4.254494$ SCF Energy (B3LYP/6-31G**/MMFF) $=-3245.91338003$

01246

MM̄FF Geometry

C $-1.768118-2.866014-0.631635$

C $-0.850613-2.361749-1.470239$

C $-1.025247-1.071163-2.226297$

O $0.201427-0.317897-2.042333$

C $-1.270459-1.315553-3.727084$

C $-2.630342-1.901216-4.042692$

C $-3.762824-1.176707-4.141058$

C $-2.652084-3.381437-4.322544$

C $-3.927473 \quad 0.306439-3.937330$

C $-5.1803280 .657125-3.120831$
C $-5.0583770 .349174-1.617793$

C $-6.4507190 .369106-0.978558$

C -6.3926750 .1610910 .539255$

O $-4.226643 \quad 1.353919-1.018081$

$\begin{array}{llll}0 & -7.682418 & 0.487700 & 1.078814\end{array}$

C $-5.386248 \quad 1.167066 \quad 1.128084$

C $-6.128218-1.3041440 .903010$

O $\begin{array}{lllll}-5.202411 & 0.939565 & 2.524888\end{array}$

C $-4.0226331 .196903 \quad 0.394451$

C -3.1983912 .4004330 .916734$

O $-3.323892-0.011307 \quad 0.693073$

C $0.1576681 .015335-2.309288$

O $-0.7899041 .641261-2.757801$

C $1.4579421 .612070-1.933880$

C $1.5641272 .949604-1.921414$

C $2.7464223 .735904-1.570545$

C $3.9780192 .996104-1.124358$

C $-1.899922 \quad 2.6713320 .151174$

C -1.0697313 .7243300 .859517$

C - -1.0096616 .1590191 .378916$

$\begin{array}{lllll}\text { O } & -0.084513 & 3.424547 & 1.530458\end{array}$

C $0.0850046 .769727 \quad 0.561445$

C 1.3373686 .9320261 .015841

C $2.4340247 .619562 \quad 0.252265$

C $3.6216136 .719643-0.006832$

C 4.5756616 .5470630 .923511

C $3.7463616 .089878-1.374551$

C $2.6673785 .080633-1.650143$

N -1.5621915 .0078180 .691092$

H -2.707638 -2.335929 -0.509827

H $0.075245-2.904159-1.634214$

H $-1.847244-0.493237-1.786397$

H $-0.478505-1.960566-4.132754$

H $-1.149250-0.381868-4.290067$

H $-4.676375-1.698900-4.423070$

H $-3.656160-3.743952-4.567243$

H -2.001996 -3.614436 -5.172321

H $-2.302995-3.947855-3.454759$

H $-3.0509150 .761140-3.473462$

H $-4.0329420 .760937-4.930273$

H $-5.3716331 .730698-3.248218$

H $-6.0408350 .125191-3.546368$

H $-4.592374-0.632732-1.482206$

H $-7.101048-0.384531-1.440502$

H $-6.9291051 .338523-1.178153$

H $-8.340186-0.0905620 .655841$

H $-5.8455312 .161654 \quad 1.044435$

H $-6.919991-1.9475590 .500688$

H $-6.145764-1.4534231 .988049$

H $-5.177209-1.6741860 .516248$

H -4.6205830 .1668332 .624509$

$\begin{array}{llll}H & -3.813341 & 3.308156 & 0.859638\end{array}$

H -2.953226 $2.244642 \quad 1.975407$

H -2.3693440 .1419960 .620502$

H $2.2729220 .948556-1.672672$

H $0.6898603 .539971-2.195683$

H $4.3585042 .361495-1.931802$

H $3.7535792 .368395-0.255788$

H $4.7953703 .654327-0.821010$

H -1.2853531 .7701410 .074338$

H $-2.113925 \quad 3.011327-0.867791$

H -0.6601875 .8493782 .369710$

H -1.8209056 .8819361 .510082$

H $-0.1758437 .130834-0.430303$

H 1.5835656 .5878402 .018172

H 2.7577798 .4904670 .837528

H $2.0627698 .029979-0.695023$

H 5.4448295 .9257710 .734964

H 4.5050607 .0216841 .896749

H $4.7410695 .654209-1.518253$

H $3.6825946 .879365-2.134977$

H $1.7266445 .528455-1.972454$

H -2.3517715 .1601540 .070605$

C -1.576607 -3.9975631 .652458$

O $-0.433190-3.2078092 .039357$

C $-0.891495-2.1296632 .872228$

C -2.203282 -2.618904 3.470953

C $-2.809701-3.3417562 .284106$ 
H $-1.097938-1.2848112 .203636$

C $0.175735-1.713945 \quad 3.889387$

H $-2.038485-3.3233934 .294211$

H $-2.827102-1.7986143 .838415$

C $-3.904124-4.3265982 .657036$

H -3.241012 -2.589448 1.618128

C $1.585564-1.4810313 .306372$

O $0.298298-2.7245604 .898765$

H $-0.166606-0.8147624 .415093$

H $2.067099-2.4506883 .137706$

O $2.365732-0.8303154 .318006$

C $1.641185-0.6514042 .013227$

C $3.056808-0.2414871 .568903$

H $1.161376-1.2113201 .203449$

$\begin{array}{lllll}\text { O } & 0.879817 & 0.544351 & 2.199816\end{array}$

H 2.9590310 .2319850 .585744

H 3.4280720 .5565172 .225209

H -4.293918 -4.8280731 .765436$

H $-3.535899-5.0956323 .344234$

H $-4.736241-3.8096873 .146109$

C - $-1.609317-4.1569890 .126255$

H $0.531074-3.5575714 .453734$

H $1.9947940 .059598 \quad 4.446868$

H 0.8913531 .0358411 .360616

H -2.434978 $-4.822144-0.152537$

H $-0.681895-4.651106-0.190009$

H $-1.414620-4.9893982 .093264$

C $3.401130-2.451795-0.541803$

O $3.586552-2.5546990 .870667$

C $4.094362-1.3791761 .502919$

C $5.432960-0.9678990 .870591$

C $5.327724-0.824757-0.646139$

C $4.701230-2.074264-1.261922$

H $2.645474-1.681061-0.740445$

C $2.795782-3.773192-1.038243$

H $4.310449-1.6640282 .539701$

O 5.9012440 .2584411 .421958

H $6.189388-1.7318471 .093270$

H $4.7653740 .071011-0.928503$

O $6.636922-0.663217-1.196416$

H $5.428269-2.893257-1.218545$

O $4.417335-1.832079-2.638231$

C $3.696411-4.980349-0.872506$

H $1.883994-3.993402-0.471158$

H $2.534322-3.673004-2.097977$

H $5.9753200 .136412 \quad 2.384025$

H $7.0364460 .113040-0.767296$

H $5.249784-1.562051-3.062799$

O $3.319647-5.942700-1.766055$

O $4.590155-5.085529-0.043879$

C $4.071408-7.159305-1.713372$

H $3.953538-7.634627-0.734879$

H $3.681266-7.833532-2.480375$

H $5.126935-6.962253-1.923347$

SCF Energy (B3LYP/6-31G**/MMFF) $=-3245.91396025$

01247

MM̄FF Geometry

C $0.342304 \quad 3.251573-1.542918$

C $-0.669770 \quad 2.631674-0.918435$

C - $-2.0557553 .195489-0.785155$

O $-2.9366862 .149402-1.260935$

C -2.414392 3.5206770 .680564

C $-1.374950 \quad 4.3233151 .437520$

C $-0.597193 \quad 3.8336702 .424877$

C $-1.252494 \quad 5.766278 \quad 1.021579$

C $-0.589235 \quad 2.4347492 .976303$

C $0.720164 \quad 1.6998362 .657071$

C $0.589318 \quad 0.193556 \quad 2.918575$

C $1.939311-0.5109822 .786444$

C $1.799271-2.0320112 .961844$

O $-0.321404-0.3299831 .944347$

O $3.025553-2.6406572 .531386$

C $0.684363-2.5406892 .022444$

C $1.619325-2.4223064 .433679$

O $0.426368-3.9261602 .241430$

C $-0.627629-1.7202712 .100026$

C $-1.571503-2.1351640 .940933$
O $-1.259640-1.9812983 .351842$

C $-4.2190462 .468477-1.568588$

O $-4.730492 \quad 3.575511-1.559702$

C $-4.9091181 .196173-1.876089$

C $-6.2484791 .144088-1.857688$

C $-7.065739-0.052837-2.072104$

C $-6.348006-1.343503-2.371754$

C $-2.830485-1.2694450 .767676$

C $-4.009486-1.6932531 .627280$

C $-6.346722-1.0193462 .183338$

O -3.993368 -2.686474 2.349077

C $-7.296676-1.7688011 .301571$

C $-8.555129-1.3696481 .060314$

C $-9.526346-2.1376990 .208702$

C -10.198191-1.279455 -0.841405

C $-11.453402-0.831918-0.667557$

C $-9.447725-1.012993-2.127861$

C $-8.4065020 .058349-1.967828$

N $-5.088356-0.8298861 .485031$

H $0.178740 \quad 4.219679-2.005874$

H $-0.4918001 .663560-0.454471$

H -2.174622 $4.082858-1.419596$

H -2.665462 $2.588521 \quad 1.196933$

H $-3.359642 \quad 4.0797790 .700312$

H $\quad 0.117177 \quad 4.5059042 .898534$

H -0.5080256 .3082191 .614663$

H $-0.9535875 .842152-0.028369$

H -2.211629 6.2790321 .147869

H -0.7112572 .4935114 .065112$

H -1.4415441 .8541212 .617880$

H $0.978479 \begin{array}{lll}1.854471 & 1.603544\end{array}$

H 1.5305822 .1235073 .262626

H $\quad 0.174746 \quad 0.0480973 .923098$

H $2.663790-0.1060463 .503930$

H $2.360791-0.3127811 .790578$

H $3.748280-2.2704203 .066500$

H $1.078059-2.4680821 .000457$

H $2.464333-2.0599375 .031510$

H $1.614277-3.5110904 .555597$

H $0.705606-2.0201554 .875987$

H $-0.105747-4.0031733 .051668$

H $-1.008728-2.0470490 .003187$

H - $1.858890-3.1878141 .048863$

H -2.185523 -1.6986363 .297366$

H $-4.2915250 .324808-2.063772$

H $-6.8005592 .057601-1.640259$

H $-5.648230-1.593427-1.566651$

H $-5.787620-1.260465-3.309640$

H -7.013711 -2.202250 -2.475491

H $-2.612853-0.2115820 .948197$

H $-3.162585-1.362028-0.274477$

H $-6.723926-0.0265902 .448073$

H $-6.171697-1.5860503 .103436$

H $-6.940470-2.6969930 .860607$

H -8.920749 $-0.457847 \quad 1.527487$

H $-10.283539-2.5682140 .877222$

H $-9.041545-2.992349-0.279548$

H -11.952917 -0.239808 -1.427239

H -12.007768 -1.0431050 .240905$

H $-10.144153-0.674072-2.906567$

H -9.044078 $-1.955198-2.507580$

H $-8.827857 \quad 1.039692-1.743900$

H - $-5.029929-0.0697520 .814041$

C $2.6953613 .268069-0.623383$

$\begin{array}{llll}\text { O } & 3.743833 & 2.304949 & -0.401348\end{array}$

C $5.0115182 .982912-0.376620$

C $4.6881484 .466391-0.265161$

C $3.3942294 .555855-1.058690$

H $5.4843232 .798702-1.348759$

C 5.8874832 .4014450 .740402

H 4.5138814 .7689580 .773732

H $5.4855925 .096579-0.670403$

C $2.6143835 .828300-0.774089$

H $3.6370584 .511994-2.128501$

$\begin{array}{llll}\text { C } 5.960748 & 0.858698 & 0.733777\end{array}$

O 5.3540582 .7920962 .012995

H 6.8933052 .8331470 .690266

H $4.9843090 .454677 \quad 1.023421$ 
$\begin{array}{llll}\text { O } & 6.881384 & 0.446211 & 1.750239\end{array}$

C $6.407498 \quad 0.247547-0.605241$

C $6.489801-1.291039-0.598571$

H $5.7233080 .558033-1.400839$

O $7.6952280 .769295-0.940119$

H $6.862258-1.610469-1.579718$

H $7.259152-1.6181320 .112186$

H $1.7178215 .889984-1.396374$

H 2.3026435 .8826530 .274503

H $3.2274416 .710067-0.988310$

C $1.7158482 .661508-1.628990$

H 4.4346662 .4768262 .056091

H $\quad \begin{array}{lll}6.590688 & 0.850422 & 2.585739\end{array}$

H $7.9418280 .408869-1.808936$

H $1.6587941 .578646-1.457123$

H $2.0954222 .765904-2.652492$

H 2.2072023 .4104390 .348534

C $4.080786-1.737620-2.430647$

O $4.063126-1.430799-1.033755$

C $5.149474-1.988104-0.286842$

C $5.172055-3.516532-0.437491$

C $5.158806-3.951198-1.902825$

C $4.033680-3.250722-2.668435$

H $5.007007-1.343269-2.868560$

C $2.946409-0.962217-3.117111$

H $4.932384-1.781087 \quad 0.767100$

O $6.313144-4.072628 \quad 0.206954$

H $4.286413-3.9381690 .055707$

H $6.128527-3.761198-2.376910$

O $4.964227-5.365949-1.977115$

H $3.070711-3.668729-2.355252$

O $4.177542-3.512535-4.062179$

C $1.554790-1.266359-2.600365$

H $3.1113180 .109485-2.952092$

H $2.964128-1.156335-4.195333$

H $6.283676-3.7997541 .139817$

H $4.115616-5.575656-1.551152$

H $4.188262-4.478420-4.176619$

O $0.646193-0.579651-3.356931$

O $1.289760-1.989352-1.651124$

C $-0.723707-0.743905-2.975747$

H -1.336812 - $0.120145-3.631576$

H $-1.026770-1.788001-3.097621$

H $-0.873288-0.416891-1.942961$

SCF Energy (B3LYP/6-31G**/MMFF) $=-3245.91685690$

01248

MM̄FF Geometry

C -3.172070 -0.406059-3.627390

C $-2.470242-1.122614-2.735791$

C $-1.049548-1.583019-2.945604$

O $-0.209868-0.962235-1.942908$

C $-0.983904-3.114932-2.784113$

C $0.419022-3.686178-2.730973$

C $0.868016-4.465643-1.726607$

C $1.316186-3.358088-3.894840$

C $0.079891-4.954390-0.543054$

C $-0.024929-3.9642150 .625058$

C $1.293116-3.7588541 .385345$

C $1.041547-2.9670132 .670936$

C $2.343449-2.6799903 .428756$

O $2.202695-3.0382060 .549399$

O $2.036864-1.6979564 .429893$

C $3.363597-2.0621272 .451358$

C $2.845705-3.9184934 .180121$

O $4.623764-1.9162023 .102415$

C $3.503534-2.8483441 .122972$

C $4.354199-2.0375090 .120314$

O $4.154743-4.093556 \quad 1.380963$

C $0.2946390 .263859-2.259034$

O $0.0498740 .914012-3.264479$

C $1.2239940 .687007-1.184163$

C $1.8491861 .867451-1.317296$

C $2.8158752 .482761-0.408230$

C 3.1604161 .7896650 .880381

C $4.326161-2.559087-1.319680$

C $5.302636-1.775170-2.173857$

C $5.6555130 .479162-3.181577$
O $6.392168-2.242421-2.497572$

C $6.1685011 .459828-2.172348$

C $6.0897852 .790732-2.324643$

C $6.5983973 .777569-1.311493$

C $5.5735664 .832000-0.948715$

C $5.7083546 .100046-1.372612$

C $4.4326894 .435502-0.033164$

C $3.3638563 .665002-0.761873$

N $4.841309-0.507991-2.495636$

H $-2.680363-0.117412-4.554728$

H $-2.922291-1.412518-1.792091$

H $-0.690700-1.319391-3.949045$

H -1.520131 -3.588724 -3.617327

H $-1.536615-3.391641-1.879766$

H $1.895380-4.823945-1.766962$

H $2.260473-3.912094-3.858787$

H $\quad 0.822508-3.615245-4.837686$

H $1.561610-2.291839-3.907301$

H $-0.930380-5.234249-0.865548$

H $0.531548-5.890514-0.190343$

H $-0.394591-2.9990370 .257078$

H $-0.778527-4.3546701 .320545$

H $1.714598-4.7434471 .619447$

H $0.328266-3.4893753 .320885$

H $0.567724-2.0081972 .417588$

H $2.869667-1.4568894 .870880$

H $3.008613-1.0471442 .226150$

H $2.096598-4.2510474 .908667$

H $3.746956-3.6921734 .760712$

H $3.064861-4.7608943 .520732$

H $5.179754-1.3404562 .551598$

H $3.983978-1.0082010 .097592$

H $5.396940-1.9999120 .459938$

H $4.601103-4.3924300 .573170$

H $1.374370 \quad 0.021722-0.342931$

H $1.6369322 .458535-2.208421$

H 3.9910621 .0964210 .728021

H 2.3106591 .2385351 .291256

H 3.4483452 .4967211 .664664

H $4.601417-3.617058-1.375192$

H $3.324642-2.460807-1.751082$

H $5.0218250 .956110-3.935680$

H $6.494789-0.013275-3.682190$

H $6.6325151 .055341-1.275470$

H $5.6508423 .195853-3.233892$

H $7.4936284 .252652-1.733161$

H $6.9301763 .272935-0.395580$

H $4.9918876 .867033-1.097599$

H $6.5349506 .399155-2.008443$

H 3.9636085 .3303370 .395742

H 4.8512303 .8802880 .811982

H $3.0349834 .134469-1.690046$

H $3.934117-0.209611-2.150071$

C $-5.430163-0.557530-2.372693$

O $-4.945817-0.112949-1.093562$

C $-6.038873-0.131190-0.155360$

C $-7.290646-0.503890-0.948997$

C $-6.898809-0.144437-2.371141$

H -6.1500520 .8913830 .220442$

C $-5.711514-1.0823991 .004593$

H $-7.521822-1.572433-0.870200$

H $-8.1724480 .043451-0.600529$

C $-7.749735-0.837236-3.420950$

H $-6.9821370 .943750-2.493408$

C $-4.372324-0.7867331 .713662$

O $-5.644543-2.4267900 .507823$

H $-6.532840-1.0734941 .730388$

H -3.543597 -1.015865 1.033689

O $-4.274697-1.7115432 .804456$

C -4.2450810 .6508452 .251903$

C -2.9978020 .9120853 .115501$

H $-4.2502211 .357667 \quad 1.416387$

$\begin{array}{lllll}\text { O } & -5.391879 & 0.959775 & 3.049957\end{array}$

H $-3.083874 \quad 1.927640 \quad 3.521271$

H -3.0197010 .2635313 .999858$

H $-7.444929-0.543614-4.430094$

H -7.668321 -1.926962 -3.347918

H $-8.804549-0.569701-3.298327$ 
C $-4.6016390 .063282-3.500290$ H -4.929401 -2.462146 -0.150411 H -3.345106 -1.738635 3.086799 H -5.4275580 .3128843 .775629$ H $-4.5816861 .153913-3.387787$ H -5.091243 $-0.153500-4.457049$ H -5.344604 -1.652204-2.401863 C -1.5655352 .9433591 .373064$ O $-1.604267 \quad 1.5249961 .167540$ C -1.6610220 .7451312 .365779$ C -0.4238091 .0306243 .226032$ C $-0.2596192 .521837 \quad 3.505865$ C $-0.341646 \quad 3.334412 \quad 2.213849$ H -2.485104 3.2681621 .873833 C $-1.5233073 .606391-0.007937$ H $-1.595349-0.3037302 .053256$ O $-0.490234 \quad 0.3291714 .463155$ H 0.4720100 .6739122 .703313 H $-0.995640 \quad 2.875342 \quad 4.237576$ $\begin{array}{lllll}\text { O } & 1.025402 & 2.745786 & 4.093387\end{array}$ H $\quad 0.581326 \quad 3.183631 \quad 1.642357$ O -0.4058534 .7149622 .567207$ C $-2.7650353 .303523-0.809534$ H -1.4208304 .6948190 .055848$ H $-0.669373 \quad 3.239572-0.587335$ H $-0.555489-0.6192544 .258245$ H 1.0748522 .1986954 .896127 H -0.2263795 .2359301 .766843$ O $-3.8096224 .053143-0.357424$ O $-2.7970042 .498643-1.730988$ C $-5.0428633 .875499-1.060809$ H $-4.9443974 .243373-2.086386$ H $-5.3402902 .823647-1.057106$ H -5.813462 4.458624 -0.549704 SCF Energy (B3LYP/6-31G**//MMFF) $=-3245.90146871$

01_249

MM̄FF Geometry

C -1.532746 $4.347831 \quad 1.067449$

C -1.277587 3.4094871 .991440

C $-2.053222 \quad 2.1214912 .090941$

O -1.1365161 .0042942 .047801$

C -2.8062872 .0794963 .430960$

C -3.7911370 .9318863 .529966$

C -5.0644920 .9919193 .091711$

C $-3.281003-0.299355 \quad 4.231067$

C -5.7237092 .1478832 .392360$

C -6.6075051 .7512381 .197780$

C $-5.841213 \quad 1.1381830 .012510$

C $-6.728497 \quad 1.130027-1.236609$

C $-6.0553800 .431253-2.426214$

O $-5.478618-0.203697 \quad 0.351310$

O $-7.077153 \quad 0.223179-3.412942$

C $-5.542378-0.948819-1.964943$

C $-4.9933511 .325060-3.077766$

O $-4.772352-1.579469-2.986266$

C $-4.722975-0.890356-0.655880$

C $-4.435685-2.326005-0.150134$

O $-3.480241-0.236591-0.917660$

C $-0.672714 \quad 0.633630 \quad 0.823131$

O $-0.8492721 .214533-0.236103$

C $0.050001-0.6482900 .983299$

C $0.351250-1.363952-0.110516$

C $1.023625-2.665122-0.147774$

C $1.471970-3.2581681 .160892$

C $-3.889537-2.3715541 .279736$

C $-3.583373-3.7876641 .718123$

C -1.857369-5.553519 1.589996

O $-4.322904-4.3981492 .486785$

C $-0.890323-6.1250840 .600629$

C $-0.854575-5.896027-0.721702$

C $0.163110-6.519379-1.634532$

C $0.947375-5.500082-2.429392$

C $0.883761-5.471345-3.771478$

C $1.867536-4.564083-1.674601$

C $1.202253-3.254664-1.348587$

N -2.416031 -4.2862131 .160569$

H $-2.335398 \quad 4.1838940 .352539$
H -0.4804653 .5690312 .713496$

H $-2.7694072 .025598 \quad 1.264606$

H -3.339591 3.0249953 .595952

H $-2.0901422 .000007 \quad 4.260526$

H $-5.7082200 .130207 \quad 3.261329$

H -2.963259 -0.0514895 .249215$

H $-4.043821-1.0812164 .307402$

H -2.426491 -0.7220833 .694461$

H -6.3617142 .6550253 .126788$

H -4.9920342 .8868512 .050707$

H -7.3789251 .0498901 .541880$

H -7.1228632 .6600060 .861873$

H $-4.9353911 .728859-0.166445$

H $-7.0229862 .150708-1.511879$

H $-7.6654940 .601267-1.010004$

H $-6.662025-0.167709-4.200250$

H $-6.420916-1.587046-1.796363$

H -5.453256 $2.242464-3.464276$

H $-4.5363790 .831621-3.942656$

H $-4.1972281 .618107-2.389863$

H $-5.348901-1.698735-3.759417$

H $-5.364009-2.912031-0.163306$

H $-3.731149-2.824340-0.827731$

H $-2.802007-0.591644-0.323119$

H $0.282878-0.9794801 .988528$

H $0.064050-0.964925-1.083007$

H $\quad 0.617279-3.4078091 .829318$

H $2.189272-2.5967721 .657720$

H $1.960793-4.2285391 .055898$

H -2.980844 -1.770749 1.385759

H $-4.625216-1.9654811 .982122$

H -2.660327 -6.268366 1.797500

H $-1.335158-5.3552282 .532733$

H $-0.148685-6.7984011 .026598$

H $-1.597812-5.250604-1.182784$

H $-0.370093-7.202811-2.308000$

H $0.870211-7.142219-1.072262$

H $1.464943-4.758674-4.347662$

H $0.248071-6.155354-4.323779$

H $2.745515-4.340527-2.295438$

H $2.266654-5.077360-0.796022$

H $0.846363-2.725313-2.234308$

H -1.795392 -3.630239 0.694555

C $0.1897885 .724224-0.206153$

O 1.2806504 .8082590 .004418

C $1.6906184 .283774-1.274387$

C $0.9296775 .080919-2.331679$

C $-0.3566045 .408674-1.596864$

H $1.3475543 .242912-1.309392$

C $3.2163794 .333994-1.406585$

H $1.4555366 .002502-2.606621$

H $0.767403 \quad 4.503128-3.246729$

C $-1.1493006 .542493-2.221450$

H $-0.975794 \quad 4.503504-1.581341$

C $3.9741243 .648227-0.251093$

O $3.6261545 .707616-1.433807$

H $3.5142063 .904730-2.370610$

H $3.851244 \quad 4.2325230 .668467$

O $5.373018 \quad 3.701108-0.562911$

C 3.5704152 .1854100 .008224

C 4.4352501 .5363301 .107410

H 2.5193192 .1480300 .315046

O $3.693348 \quad 1.463932-1.213188$

H 5.4776931 .5025390 .775266

H 4.3970552 .1799251 .993966

H $-2.0577846 .745018-1.645437$

H $-0.5633197 .466761-2.264095$

H - $1.4470226 .286136-3.243433$

$\begin{array}{llll}\text { C }-0.791839 & 5.650208 & 0.964627\end{array}$

H $4.5950335 .718959-1.518043$

H $5.5204023 .137544-1.342147$

H $3.3173640 .578887-1.069997$

H -1.5303616 .4558250 .877112$

H $-0.240375 \quad 5.829248 \quad 1.896592$

H $0.6367106 .727806-0.203852$

C $5.400939-1.1757240 .026944$

$\begin{array}{lllll}\text { O } & 4.065663 & -0.808629 & 0.395022\end{array}$

$\begin{array}{lll}\text { C } 3.964445 & 0.113387 & 1.485096\end{array}$ 
C $4.644342-0.4730342 .730363$

C $6.085225-0.8868622 .444736$

C $6.140345-1.7954201 .217435$

H $5.941511-0.290961-0.330139$

C $5.302597-2.173051-1.133123$

H $2.895817 \quad 0.196511 \quad 1.718624$

$\begin{array}{lllll}\text { O } & 4.598470 & 0.466610 & 3.798288\end{array}$

H $4.081006-1.3573163 .056402$

H $6.738131-0.0153302 .317301$

O $6.598918-1.6207993 .558191$

H $5.704468-2.7720241 .465266$

O $7.503802-2.0135330 .859623$

C $4.975958-1.465727-2.426724$

H 6.248432 -2.701969-1.297674

H $4.541554-2.938081-0.940824$

H 4.9195110 .0197834 .599455

H $6.674793-1.0041794 .305855$

H $7.952141-2.3856291 .638520$

O $3.628180-1.442785-2.622386$

O $5.821131-0.974417-3.163917$

C $3.186824-0.799290-3.821800$

H $3.5577290 .229063-3.866236$

H $3.519654-1.367959-4.695112$

H $2.093910-0.776161-3.812188$

SCF Energy (B3LYP/6-31G**//MMFF) $=-3245.91049542$

0125

MM̄FF Geometry

C -2.932346 -1.669651 -2.654098

C $-2.358238-2.792702-3.112486$

C $-0.979554-3.293231-2.736719$

O $-0.207391-2.268313-2.072826$

C $-1.142840-4.475524-1.761146$

C $0.160690-5.028980-1.222889$

C $0.508132-5.0202450 .079640$

C $1.085310-5.635258-2.245701$

C $-0.286638-4.4911801 .243410$

C $-0.009001-3.0190921 .587275$

C $1.384524-2.7723922 .187707$

C $1.536610-1.3153022 .628215$

C $2.948231-1.0166743 .159302$

O $2.369684-3.0713111 .198116$

O 3.0638440 .4126843 .234560

C $3.994892-1.5322402 .144543$

C $3.132852-1.5442874 .586972$

O $5.314259-1.4479592 .676863$

C $3.722790-2.9712691 .660737$

C $4.636666-3.4037490 .488519$

O $3.965528-3.8817702 .740611$

C $0.477272-1.391352-2.855857$

O $0.535661-1.379850-4.074705$

C $1.121016-0.408413-1.956941$

C $1.5590660 .756606-2.457139$

C 2.162276 1.848519-1.690112

C $2.5174311 .572932-0.254342$

C $4.524194-2.552013-0.781724$

C $5.607549-1.494195-0.915012$

C $6.3280110 .358236-2.419666$

O $6.565717-1.406662-0.152291$

C $5.9660291 .648181-1.754962$

C $5.7616872 .792014-2.425619$

C $5.4572414 .105051-1.764220$

C $4.1966254 .749390-2.293944$

C $4.2533955 .735799-3.204753$

C $2.8786254 .313944-1.697217$

C $2.3599483 .036395-2.299011$

N $5.394824-0.677715-2.018384$

H -2.383286 -1.020127 -1.977178

H -2.933300 -3.443857 -3.767667

H -0.457199 -3.623160 -3.642955

H - $1.685444-5.291458-2.257448$

H -1.785909-4.152109-0.935322

H $1.469864-5.4517990 .354639$

H $1.954347-6.118074-1.785739$

H $0.558446-6.397070-2.829536$

H $1.460988-4.867562-2.929235$

H -1.359570 -4.617822 1.063241

H $-0.072431-5.1177562 .118638$
H $-0.139902-2.4084980 .685969$

H $-0.767718-2.6926022 .309645$

H $1.510468-3.4453543 .043940$

H $\quad 0.781117-1.0529733 .379388$

H $1.343082-0.6581161 .769520$

H 3.9359940 .6260823 .606851

H $3.962405-0.8570241 .283245$

H $2.411414-1.0707435 .263916$

H $4.123358-1.2889934 .979478$

H $3.001260-2.6255684 .664300$

H $5.499999-0.5117722 .859754$

H $5.674702-3.4906760 .831239$

H $4.339454-4.4249910 .208714$

H $4.129848-4.7642092 .368976$

H $1.180108-0.654648-0.902934$

H $1.4309790 .947817-3.521746$

H $3.1512850 .683867-0.186320$

H 1.6136631 .4055070 .339146

H 3.0769852 .3798800 .222902

H $4.637967-3.213532-1.650409$

H $3.540373-2.078731-0.859388$

H $6.2866640 .427798-3.511271$

H $7.3417660 .065470-2.127444$

H $5.9018171 .648469-0.669359$

H $5.8545442 .796166-3.509340$

H $6.3152514 .768792-1.934134$

H $5.3806533 .997436-0.675100$

H $3.3542066 .223685-3.566210$

H $5.2000916 .073244-3.613294$

H $2.1153925 .083872-1.875621$

H $2.9705584 .269244-0.608406$

H $2.0726933 .135204-3.346763$

H $4.540852-0.782571-2.558560$

C $-5.221147-1.445168-1.715611$

O $-4.753978-0.598731-0.649094$

C $-5.877745-0.2294770 .173176$

C $-7.108912-0.882636-0.452752$

C $-6.682296-1.052666-1.900349$

H -5.9808880 .8582610 .098012$

C $-5.603791-0.6292691 .628710$

H $-7.328317-1.8564120 .000081$

H -8.003287 -0.261635 -0.341485

C -7.513301 -2.071162 -2.659973

H -6.749068 -0.078852 -2.403609

C $-4.283460-0.0680922 .200260$

O $-5.534311-2.0589681 .720091$

H -6.446806 -0.3292682 .261420$

H -3.430995 -0.531720 1.690782

O $-4.197602-0.4675153 .574101$

C $-4.163631 \quad 1.4649682 .135472$

C -2.9266732 .0370322 .852603$

H -4.1546751 .7931061 .091881$

O -5.3215612 .0541902 .735048$

H -3.032613 3.1284332 .876889

H -2.940279 1.7358023 .907451

H -7.170661 -2.161487 -3.695420

H -7.454163 -3.062357 -2.197915

H -8.566104 -1.771062 -2.678754

C $-4.346768-1.256991-2.954992$

H $-4.816214-2.3563111 .135391$

H $-4.276349-1.4365933 .599360$

H $-5.363316 \quad 1.7461383 .656734$

H $-4.336515-0.204515-3.260614$

H -4.747502 -1.837614 -3.793352

H $-5.146908-2.482730-1.360993$

C -1.5288243 .3466640 .497390$

O -1.5373821 .9472160 .809706$

C $-1.582661 \quad 1.6429582 .207355$

C -0.3550142 .2437362 .904069$

C -0.2285963 .7414252 .638480$

C $-0.3179074 .038517 \quad 1.141589$

H $-2.456713 \quad 3.8090180 .853627$

C $-1.4908893 .472169-1.029993$

H -1.4939240 .5531682 .288454$

O $-0.404040 \quad 2.018707 \quad 4.308714$

H $\quad 0.551477 \quad 1.7510942 .532438$

H $-0.9788894 .312808 \quad 3.197457$

O 1.0456754 .1913603 .107348 
H $\quad 0.612978 \quad 3.7149120 .661601$ O $-0.413570 \quad 5.4524840 .978959$ C -2.724083 2.895342 -1.682218 H -1.396362 $4.511755-1.360674$ H $-0.6324552 .923741-1.431394$ H -0.4606641 .0580994 .448663$ H 1.1030203 .9629884 .051119 H -0.2346995 .6574600 .046119$ O $-3.8162263 .652425-1.380104$ O $-2.7080971 .902655-2.398274$ C $-5.0476603 .227567-1.972650$ H $-5.2004512 .153434-1.836139$ H $-5.8642103 .760897-1.478796$ H -5.050267 3.482166 -3.036301 SCF Energy (B3LYP/6-31G**//MMFF) $=-3245.90946963$

01250

MM̄FF Geometry

C -3.317549 -2.835031 -2.752822

C $-2.552717-2.803561-1.651280$

C $-1.088224-3.173505-1.654930$

O $-0.317858-2.070645-1.128797$

C $-0.872672-4.392257-0.736855$

C $0.548899-4.920826-0.725478$

C $1.325659-4.9945850 .373384$

C $1.076590-5.420360-2.045841$

C $0.976877-4.588749 \quad 1.778219$

C $1.295003-3.1237322 .109843$

C $2.795283-2.7921712 .129027$

C $3.026856-1.3652242 .631950$

C $4.511752-0.9702412 .577381$

O $3.304128-2.9145880 .799639$

O 4.5946120 .4539132 .743488

C $5.072199-1.2941721 .174530$

C $5.307138-1.5882263 .733304$

O $6.483785-1.1030801 .131882$

C $4.718543-2.7122830 .683773$

C $5.092827-2.957978-0.798067$

O $5.440572-3.6735641 .463398$

C $0.015203-1.074212-1.994121$

O $-0.288245-0.987766-3.173132$

C $0.809484-0.078590-1.242260$

C $0.917726 \quad 1.172687-1.712800$

C $1.6291882 .278369-1.067100$

C 2.4352951 .9604430 .163893

C $4.426172-2.017263-1.808674$

C $5.295120-0.839970-2.219408$

C $5.2178361 .245665-3.579641$

O $6.482472-0.740490-1.922285$

C $5.0838252 .403575-2.642250$

C $4.6085553 .600974-3.016744$

C $4.5183844 .788677-2.102560$

C $3.1328305 .391839-2.065551$

C $2.8273596 .470259-2.807012$

C $2.1302924 .798564-1.102759$

C $1.5321713 .512261-1.605169$

N $4.5942630 .071759-2.998072$

H -2.842612 -3.103750 -3.695237

H $-2.987241-2.535637-0.693305$

H $-0.747446-3.409252-2.670549$

H $-1.531847-5.212622-1.051452$

H $-1.187582-4.1215380 .276883$

H $2.330653-5.4007340 .263650$

H $2.033703-5.942432-1.939645$

H $0.369882-6.125288-2.495782$

H $1.233183-4.588597-2.739168$

H $\quad-0.083742-4.777975 \quad 1.977264$

H $1.517786-5.2456832 .470983$

H $0.787848-2.4810951 .381879$

H $\quad 0.868060-2.9019863 .095388$

H $3.298943-3.5144932 .782060$

H $2.634412-1.2417163 .648986$

H $2.458591-0.6632632 .006452$

H 4.1749880 .6816293 .590958

H $4.638784-0.5625720 .483695$

H $4.898955-1.2603814 .696972$

H $6.349540-1.2512163 .720531$

H $5.296482-2.6800463 .725602$
H $6.662043-0.1927911 .424368$

H $6.183176-2.973793-0.913914$

H $4.761100-3.975959-1.049965$

H $5.492253-4.5029170 .960095$

H $1.253724-0.395053-0.305942$

H $0.416236 \quad 1.421433-2.647255$

H $3.146611 \quad 1.155377-0.045464$

H 1.7797591 .6465680 .982771

H 3.0292692 .8002600 .529258

H $4.228813-2.582739-2.728582$

H $3.463413-1.652548-1.437474$

H $4.7282081 .431188-4.540774$

H $6.2778731 .040298-3.761167$

H $5.4217722 .260845-1.618494$

H $4.2992473 .749398-4.049026$

H $5.2403995 .536260-2.456703$

H $4.8332694 .535384-1.082600$

H $1.8437396 .926145-2.763603$

H $3.5530496 .917595-3.478198$

H $1.3017215 .502364-0.950112$

H $2.5929984 .700903-0.117025$

H $0.9234903 .639399-2.501620$

H $3.598098-0.060610-3.147019$

C $-5.588862-2.412752-1.555868$

O $-5.386024-1.084323-1.024683$

C $-6.668627-0.478719-0.790441$

C $-7.649046-1.640227-0.704544$

C $-7.099219-2.556788-1.775279$

H $-6.905503 \quad 0.115722-1.681596$

C -6.6795180 .4249230 .449969$

H $-7.608211-2.130597 \quad 0.275345$

H -8.681761 -1.331148 -0.891696

C -7.603992 -3.985966-1.667886

H $-7.376987-2.154555-2.759137$

C $-5.817212 \quad 1.699242 \quad 0.358025$

O $-6.345141-0.3338361 .606800$

H -7.7199360 .7511050 .583531$

H -5.9628872 .2736841 .282006$

O $-6.3518562 .519372-0.691013$

C -4.2971561 .5592110 .136379$

C $-3.5704220 .765127 \quad 1.235872$

H $-4.0866991 .120916-0.844982$

$\begin{array}{lllll}\text { O } & -3.760053 & 2.885837 & 0.103774\end{array}$

H $-3.807751 \quad 1.2136512 .206646$

H $-3.953593-0.258897 \quad 1.244704$

H $-7.164640-4.615280-2.448183$

H -7.353462 $-4.428121-0.697732$

H $-8.692602-4.018378-1.780506$

C $-4.798339-2.582297-2.859705$

H -6.4220490 .2540302 .377553$

H $-6.0754452 .138527-1.541620$

H $-4.2204593 .369922-0.602940$

H $-4.934178-1.692601-3.487619$

H $-5.212259-3.434817-3.412632$

H $-5.251429-3.123151-0.791379$

C -1.3899622 .6470002 .333728$

O -1.4009151 .9831021 .064294$

C $-2.035096 \quad 0.700701 \quad 1.051455$

C $-1.320568-0.2259762 .045678$

C -1.2644540 .3783093 .444828$

$\begin{array}{llll}C & -0.682473 & 1.788978 & 3.389687\end{array}$

H -2.4194452 .8569912 .648084$

C $-0.666046 \quad 3.985608 \quad 2.149731$

H -1.8728650 .2888780 .048481$

O $-1.949320-1.4999772 .110831$

H $-0.290661-0.3907421 .707688$

H $-2.249796 \quad 0.376477 \quad 3.925195$

O $-0.412870-0.421630 \quad 4.268634$

H $0.391991 \quad 1.7318893 .172399$

$\begin{array}{llll}0 & -0.826728 & 2.404892 & 4.667923\end{array}$

C $-1.5201124 .963057 \quad 1.378588$

H -0.4352794 .4584513 .111179$

H $0.287558 \quad 3.856412 \quad 1.624335$

H $-1.908290-1.8883411 .220952$

H $-0.772782-1.3252784 .263208$

H $-0.390191 \quad 1.8285745 .318495$

O $-1.221074 \quad 4.900066 \quad 0.050803$

O -2.3648385 .6827401 .895701$ 
C $-1.9545375 .793161-0.792762$

H $-3.0305315 .625829-0.687535$

H -1.668261 $5.592812-1.828616$

H -1.699819 $6.829382-0.551511$

SCF Energy (B3LYP/6-31G**//MMFF) $=-3245.90286551$

01_251

MM̄FF Geometry

C -0.975682 -4.152276 -2.163470

C $-0.086811-4.027714-1.165794$

C $1.355938-3.638123-1.364672$

O $1.536496-2.354389-0.720715$

C $2.283720-4.653931-0.675951$

C $3.756793-4.321694-0.819689$

C $4.573832-4.0105880 .206092$

C $4.306130-4.375582-2.222178$

C $4.236966-3.9402191 .669164$

C $3.777064-2.5596492 .162155$

C $4.842593-1.4580622 .040686$

C $4.392075-0.1902992 .771283$

C 5.3956530 .9609452 .599440

O $5.040860-1.162940 \quad 0.655279$

O 4.7521212 .1731343 .022685

C 5.7238621 .1251571 .099484

C 6.6236620 .7855503 .500852

O 6.7751632 .0682140 .909659

C $6.075653-0.2026630 .397800$

C $6.207780-0.066208-1.139410$

O $7.340410-0.6702870 .883370$

C $1.192914-1.248827-1.434928$

O $0.855368-1.203340-2.607045$

C $1.246103-0.091111-0.517032$

C $0.5684761 .021681-0.833671$

C $0.4840552 .241193-0.029727$

C 1.3989902 .3529611 .160749

C $4.9625910 .453840-1.865779$

C $4.9724391 .953619-2.114534$

C $3.5159283 .790831-2.960573$

O $5.9612342 .660263-1.938683$

C $2.8886544 .510262-1.808115$

C $1.7779705 .254149-1.924055$

C $1.1557496 .018439-0.790077$

C $-0.3108855 .696720-0.607207$

C -1.262779 $6.479711-1.142578$

C -0.6724494 .5148280 .263564$

C $-0.3908973 .197985-0.404106$

N $3.7516472 .405575-2.597867$

H $-0.625476-4.014342-3.184823$

H $-0.404396-4.148028-0.133840$

H $1.604304-3.563796-2.430186$

H $2.113729-5.654971-1.094097$

H $2.009085-4.7185010 .382495$

H $5.616187-3.788050-0.020183$

H $5.396189-4.270073-2.245389$

H $4.061689-5.335111-2.689467$

H $3.886807-3.570535-2.833194$

H $3.471578-4.6817801 .922910$

H $5.125409-4.2466452 .236365$

H $2.876352-2.2658391 .611147$

H $3.489507-2.6639283 .215960$

H $5.777276-1.8346312 .472468$

H $4.222614-0.3936253 .836079$

H 3.4216070 .1337452 .371696

H 4.4727002 .0571833 .947039

H 4.8311651 .5511010 .625990

H 6.3226320 .7493024 .554806

H 7.3033071 .6404713 .414068

H $7.185662-0.1261123 .288074$

H 6.5038892 .8959041 .342204

H $7.097540 \quad 0.523973-1.389939$

H $6.406613-1.072316-1.536336$

H $7.710838-1.2889610 .232193$

H $\quad 1.808617-0.1982970 .402548$

H $-0.0030421 .036021-1.760537$

H $2.4371242 .166890 \quad 0.864847$

H 1.1175521 .6272381 .931476

H 1.3918623 .3404951 .626316

H $4.917622-0.016933-2.856382$
H $4.0510530 .175401-1.329969$

H $2.8732593 .788762-3.846520$

H $4.4664644 .268100-3.219541$

H $3.3839164 .437859-0.842647$

H $1.3026345 .346951-2.898206$

H $1.2773537 .088642-1.003340$

H 1.6886365 .8431840 .152646

H $-2.3171176 .271124-0.993957$

H -1.007905 $7.344444-1.746317$

H -1.7448674 .5349460 .497870$

H -0.1734964 .6194661 .230839$

H -1.009020 $3.018534-1.285140$

H $2.9732221 .757003-2.670396$

C $-3.041658-4.400568-0.616969$

O $-2.788817-3.1335750 .015098$

C $-3.911542-2.7793590 .840326$

C $-4.874566-3.9629870 .785242$

C $-4.559599-4.561474-0.573777$

H -4.391766 -1.918903 0.361890

C $-3.431590-2.3919502 .245188$

H $-4.673798-4.6938541 .576634$

H -5.918165 -3.648486 0.883734

C $-5.038684-5.993531-0.732618$

H $-5.026436-3.936143-1.346620$

C $-2.289648-1.3519372 .296201$

O $-2.942580-3.5770962 .893131$

H -4.285984 -2.053024 2.842695

H -1.376708 -1.821095 1.906566

O $-2.041312-1.0730073 .680924$

C $-2.521010-0.0155621 .567121$

C -3.8117580 .7080082 .001626$

H -1.674689 0.6426571 .801163

O $-2.492226-0.2420540 .165190$

H -4.6756870 .0794661 .769065$

H -3.789116 0.8319523 .090159

H $-4.786309-6.382357-1.724108$

H $-4.584673-6.6538180 .013945$

H $-6.125769-6.050402-0.615067$

C $-2.459075-4.399070-2.034543$

H -2.661341 -3.321895 3.788523

H $-1.261596-0.4936943 .727785$

H $-2.5005910 .623480-0.276816$

H $-2.954422-3.608769-2.613714$

H -2.687592 -5.350790 -2.527499

H -2.557702 -5.174482 -0.007045

C $-5.3453871 .431582-0.552354$

O $-4.1117381 .998428-0.092538$

C -3.9824392 .0912271 .330490$

C -5.1236932 .9447611 .901866$

C -6.4927142 .4300661 .463351$

C $-6.5348232 .259520-0.055323$

H $-5.4367880 .400564-0.191682$

C $-5.293013 \quad 1.394239-2.083604$

H -3.0512372 .6394241 .519571$

O $-5.0382232 .983598 \quad 3.321967$

H -5.0039143 .9760431 .544188$

H -6.7516401 .4927701 .970051$

O

H $-6.5373573 .246106-0.536329$

O $-7.7475621 .602105-0.417422$

C $-4.4238920 .251425-2.553836$

H $-6.2824591 .235320-2.526910$

H $-4.9062832 .336975-2.488845$

H -5.6896653 .6310693 .639764$

H $-7.546753 \quad 3.3969902 .797277$

H $-8.4821802 .136320-0.069434$

O $-3.2284190 .723444-3.006901$

O $-4.763039-0.924409-2.503556$

C $-2.317421-0.274477-3.477153$

H $-2.035107-0.942427-2.658153$

H $-2.763438-0.840816-4.300320$

$\mathrm{H}-1.4212160 .230691-3.846007$

SCF Energy (B3LYP/6-31G**//MMFF) $=-3245.90661691$

01252

MM̄MF Geometry

C -2.637777 $3.704072-0.105448$

C $-1.8258172 .637980-0.154932$ 
C $-0.8924572 .338408-1.297308$

O $0.4160332 .058795-0.748524$

C $-1.3816791 .086849-2.044004$

C $-0.4721030 .669787-3.181792$

C $0.360482-0.389944-3.144166$

C $-0.5757951 .508200-4.429009$

C $0.592451-1.330598-1.993785$

C $2.084130-1.502104-1.694722$

C $2.343104-2.437872-0.503494$

C $1.987376-1.7850750 .836492$

C $2.367369-2.6714482 .032901$

O $3.738485-2.767888-0.528876$

O $2.315370-1.8592623 .216738$

C $3.830820-3.1323011 .867400$

C $1.360145-3.8070012 .245480$

O $4.188765-4.0723702 .877024$

C $4.136522-3.7143030 .469941$

C $5.636009-4.0498480 .270192$

O $3.423331-4.950061 \quad 0.316699$

C $1.2441883 .120421-0.553410$

O $0.9932574 .295866-0.767787$

C $2.5293552 .600971-0.031705$

C 3.5260173 .4614410 .223390

C 4.8623663 .1328590 .728142

C 5.1722321 .6843350 .995327

C $6.633660-2.9002520 .421182$

C $6.714323-1.958385-0.768848$

C $7.8730240 .114339-1.499579$

O $6.170389-2.172609-1.847745$

C $8.5995091 .247306-0.851471$

C $8.2835062 .540990-1.013996$

C $9.0315173 .649546-0.328874$

C 8.1333904 .6334030 .387190

C 8.2476965 .9533680 .157900

C 7.1557534 .1050851 .417649

C 5.7337294 .1458960 .918333

N $7.511945-0.857362-0.483499$

H -2.634929 $4.420321-0.922963$

H $-1.841176 \quad 1.9204380 .661576$

H $-0.8345333 .182230-1.996206$

H $-2.3878601 .264295-2.448050$

H $-1.5031040 .263836-1.334014$

H $0.940854-0.616088-4.037811$

H $\quad 0.0540861 .127165-5.239888$

H -1.608221 $1.520703-4.793352$

H $-0.2644052 .537712-4.227431$

H $0.081958-0.993493-1.090678$

H $\quad 0.165481-2.305235-2.258118$

H $2.589229-1.908344-2.581720$

H $2.556426-0.528207-1.510748$

H $1.762205-3.355320-0.654948$

H $0.923957-1.5269470 .887792$

H $2.530272-0.8344090 .929450$

H $1.417484-1.4926173 .289695$

H $4.455457-2.2461532 .031360$

H $0.368163-3.3982142 .469266$

H $1.631439-4.4201373 .111715$

H $1.258208-4.4603071 .376546$

H $3.983720-3.6665303 .736792$

H $5.912306-4.8419720 .978883$

H $5.766539-4.495497-0.725659$

H $3.839756-5.458944-0.398588$

H $2.621772 \quad 1.5325840 .121041$

H 3.3504144 .5212990 .040282

H 4.4901191 .2778181 .750472

H 6.1853341 .5176911 .366357

H 5.0689651 .0936150 .078297

H $6.449000-2.3245731 .332236$

H $7.634743-3.3400120 .524651$

H $6.9636870 .433626-2.019586$

H $8.524329-0.387560-2.222714$

H $9.4403170 .988127-0.211292$

H $7.4637562 .815665-1.673048$

H $9.6317394 .163639-1.090274$

H $9.742513 \quad 3.2441630 .402477$

H 7.6289626 .6723340 .685078

H $8.9614046 .345281-0.559061$

H 7.2112524 .7335552 .316898
H 7.4647673 .1113851 .752875

H 5.3880925 .1587010 .702531

H $8.047254-0.8693130 .380663$

C -5.0424963 .7592210 .575939$

$\begin{array}{lllll}\text { O } & -5.248170 & 2.382312 & 0.206771\end{array}$

C $-6.5898941 .996750 \quad 0.565992$

C -7.261206 3.2453851 .132618

C -6.0754004 .0281181 .665532$

H $-6.499407 \quad 1.2526751 .364919$

C $-7.2955731 .382283-0.651342$

H -7.7792973 .8192930 .355670$

H -7.995988 3.0014441 .906258

C -6.3731035 .4995941 .892845$

H -5.7516203 .5726702 .611047$

C $-6.5233390 .214049-1.301351$

O $-7.4607752 .397746-1.651726$

H $-8.3070651 .063188-0.375950$

H $-5.5761980 .593767-1.700196$

O $-7.295213-0.225011-2.426168$

C $-6.282232-0.982123-0.360223$

C $-5.527135-2.161465-1.005303$

H $-5.728286-0.654790 \quad 0.523937$

O $-7.540335-1.4702970 .112813$

H $-5.562173-3.006198-0.306456$

H -6.069711 -2.514325 -1.890286

H -5.4904696 .0229982 .273262$

H -6.6847915 .9937040 .966353$

H -7.1778985 .6202072 .625350$

C -3.5956053 .9626031 .025325$

H $-6.5755762 .724654-1.887732$

H $-6.711168-0.751415-2.997295$

H -8.064386 -1.730616 -0.664326

H $-3.3702343 .297344 \quad 1.867187$

H $-3.4450804 .989277 \quad 1.377235$

H $-5.2529034 .367249-0.314294$

C $-3.076320-2.2253340 .795373$

O $-3.353589-1.285994-0.249870$

C $-4.066621-1.825555-1.367364$

C $-3.272338-2.982905-1.987567$

C $-2.872534-4.030733-0.952139$

C $-2.216173-3.3745770 .262215$

H -4.017154 -2.623354 1.193775

C $-2.368390-1.4636981 .920165$

H -4.099550 -1.031496 -2.122374

O $-4.014517-3.611757-3.026830$

H $-2.357063-2.585436-2.444269$

H -3.731674 -4.641021-0.649254

O $-1.927310-4.930437-1.536201$

H -1.217989-3.009378 -0.008798

O $-2.052322-4.3526201 .286660$

C $-3.345268-0.5709132 .648560$

H -1.913930 -2.135233 2.656946

H -1.567972 -0.8278551 .523219$

H $-4.233435-2.928931-3.683778$

H $-2.344107-5.313842-2.326917$

H $-1.517057-5.0747260 .914909$

O $-4.106637-1.3115803 .504102$

O -3.4343230 .6356872 .466525$

C $-5.101168-0.5806844 .228099$

H $-5.635812-1.2822514 .873889$

H $-4.6300810 .183648 \quad 4.853518$

H -5.816283 -0.127023 3.535224

SCF Energy (B3LYP/6-31G**//MMFF) $=-3245.90604949$

01_253

MM̄FF Geometry

C 2.0974894 .0545040 .337311

C 2.3318412 .7393570 .454560

C 2.7839942 .0735161 .724846

O $1.8932180 .960248 \quad 1.988215$

C 4.1930831 .4797141 .576234

C 5.3042282 .5057021 .568402

C 5.9808042 .8962610 .470834

C 5.6753623 .0591662 .919951

C $5.7493212 .483449-0.956125$

C $6.8619991 .577420-1.499672$

C $6.8125020 .146334-0.940905$

C $7.983158-0.678840-1.479291$ 
C $7.907127-2.145289-1.027666$

O $5.573116-0.440082-1.351585$

O $8.838499-2.897767-1.820215$

C $6.497922-2.689129-1.344134$

C $8.345315-2.3139740 .431868$

O $6.334716-4.009369-0.829124$

C $5.350183-1.768251-0.863106$

C $4.005148-2.265683-1.443856$

O $5.295398-1.8102450 .563725$

C $0.714371 \quad 1.2240932 .607059$

O 0.3185332 .3192332 .983447

C $-0.100946-0.0006882 .783690$

C $0.292542-1.2284452 .408693$

C -0.483585 -2.465242 2.542838

C $-1.838505-2.365763 \quad 3.193868$

C $2.809034-1.336039-1.184747$

C $1.541829-1.862286-1.838935$

C $-0.916297-1.484450-1.925455$

O $1.535493-2.822721-2.605403$

C - $1.516875-2.437581-0.940234$

C $-1.453767-3.771697-1.070023$

C $-2.011592-4.751691-0.078133$

C $-0.923975-5.484060 \quad 0.677581$

C $-0.339110-6.577400 \quad 0.160087$

C $-0.571339-4.9898062 .064614$

C $0.037337-3.6129072 .057704$

N $0.419204-1.126075-1.479806$

H 2.2571884 .7052951 .192305

H $2.1705352 .092242-0.405072$

H 2.7557502 .7604092 .579452

H 4.2294890 .8448740 .684665

H 4.3754210 .7764452 .400968

H 6.7849153 .6205820 .596434

H 6.5225593 .7514392 .868002

H 4.8332913 .6038523 .357568

H 5.9561772 .2471973 .598666

H $4.7712942 .015745-1.099925$

H $5.7252953 .400951-1.558070$

H $6.7459291 .535516-2.590715$

H $7.8417332 .026952-1.295544$

H 6.8505620 .1865990 .153093

H $8.941435-0.234649-1.182225$

H $7.970929-0.655237-2.578169$

H $9.723506-2.517913-1.684481$

H $6.431580-2.783071-2.436798$

H $9.370071-1.9476150 .568351$

H $8.367023-3.3712140 .718417$

H $7.707197-1.7764311 .136366$

H $6.188570-3.9369790 .129298$

H $4.110266-2.377807-2.531248$

H $3.769131-3.256774-1.035493$

H $4.394626-1.6035450 .856670$

H -1.0683930 .1710593 .245058$

H $1.271513-1.3507371 .950230$

H -2.468712 -1.649660 2.656428

H -1.740882 -2.0361914 .234047$

H -2.391767 -3.306543 3.208256

H $2.623847-1.239972-0.110925$

H $3.004153-0.338062-1.592826$

H $-0.862854-1.921144-2.928512$

H -1.514037 -0.569720 -1.980513

H -2.015974 -2.003800 -0.078638

H $-0.952199-4.193708-1.938402$

H -2.637286 -5.471039-0.622184

H -2.686526 -4.2613450 .631590$

H $0.423309-7.1239550 .704868$

H $-0.609617-6.947838-0.823408$

H $\quad 0.156897-5.663274 \quad 2.535715$

H -1.462629 -5.0543632 .694488$

H $1.023978-3.5795161 .593027$

H $0.506815-0.397685-0.777079$

C $0.1514495 .163405-0.850764$

O $-0.6996534 .082084-0.418124$

C -1.622074 4.5912860 .563285

C $-1.587986 \quad 6.1064180 .409472$

C $-0.121158 \quad 6.3325630 .095525$

H -1.220709 4.324613 1.549448

C -3.0108503 .9651820 .394571$
H $-2.2154306 .439064-0.426107$

H -1.9193016 .6251791 .313880$

C $0.1714347 .695147-0.507716$

H 0.4522026 .2254611 .024373

C -3.0345802 .4581960 .740666$

O $-3.4508394 .194953-0.942037$

H -3.706631 4.4868971 .063235

H -2.6456762 .3318181 .758816$

O $-2.1822281 .717555-0.131681$

C $-4.447301 \quad 1.8420530 .683429$

C -4.4638650 .3932921 .209482$

H $-5.1236102 .447248 \quad 1.298246$

O $-4.941181 \quad 1.882199-0.650832$

H $-3.814588-0.2252360 .582127$

H $-4.0332570 .392842 \quad 2.217565$

H $1.2370757 .799454-0.734564$

H $-0.3883187 .852608-1.435804$

H -0.1054638 .4919690 .190288$

C $1.6030974 .685262-0.934788$

H $-4.3620183 .864481-1.015004$

H $-1.2801702 .066217-0.032183$

H $-4.3132301 .394696-1.211335$

H $2.2630285 .518015-1.202317$

H $1.6760423 .954368-1.750788$

H $-0.1803055 .425048-1.864325$

C $-5.895651-1.331039-0.887679$

O $-6.483254-0.339058-0.036520$

C $-5.885516-0.2154031 .258099$

C $-5.972100-1.5601191 .994307$

C $-5.356520-2.6881071 .173503$

C $-5.956209-2.714756-0.230995$

H -4.853811 -1.064635 -1.101947

C $-6.668674-1.317245-2.210974$

H -6.5083870 .4955121 .815895$

O $-5.327737-1.4954343 .261744$

H $-7.025521-1.8049062 .182264$

H $-4.266525-2.5975131 .130469$

O $-5.633780-3.9394751 .804232$

H $-6.995355-3.063849-0.177912$

O $-5.232682-3.645165-1.033514$

C $-6.341262-0.074576-3.004434$

H -6.413098 -2.174348 -2.844261

H -7.750696 -1.358591 -2.036993

H $-5.748667-0.7790153 .767034$

H -5.269770 -3.895099 2.705269

H $-5.256985-4.503414-0.576601$

O $\quad-7.3450370 .839480-2.880933$

O $\quad-5.3032620 .073368-3.636945$

C -7.140984 $2.074380-3.574580$

H $-6.2363292 .570959-3.210873$

H $-7.0771781 .895935-4.652143$

H -7.999496 $2.721564-3.376050$

SCF Energy $\left(B 3 L Y P / 6-31 G^{* *} / / M M F F\right)=-3245.91910975$

01254

MM̄FF Geometry

C $2.626586-1.199597-2.910117$

C $3.931406-1.461823-2.739499$

C $4.897575-0.623993-1.936781$

O $4.4972150 .769010-1.919319$

C $4.971752-1.081943-0.470522$

C $5.579235-2.451968-0.265695$

C $4.887553-3.5536750 .085068$

C $7.075844-2.522076-0.427858$

C $3.403904-3.6934960 .284437$

C $3.025807-3.9262931 .752562$

C $3.192958-2.6768802 .630943$

C $2.868406-3.0039634 .089909$

C $2.939938-1.7575914 .984403$

O $2.297405-1.6698832 .146033$

O $2.325325-2.082486 \quad 6.240674$

C $2.095788-0.6380404 .340784$

C $4.388207-1.3652685 .299527$

O 22334790.5823295 .066485

C $2.388560-0.4170592 .836094$

C 1.3285920 .5396762 .241873

O $3.683330 \quad 0.171202 \quad 2.700379$

C $4.8564871 .529330-2.986131$ 
O $5.4553301 .146316-3.983048$

C $4.4294052 .939080-2.837517$

C $3.8611663 .453086-1.735274$

C $3.4333064 .845688-1.560447$

C $3.6301385 .782649-2.725533$

C $1.3919440 .712906 \quad 0.717190$

C 0.2882581 .6332520 .221972

C $-0.6634282 .597622-1.865948$

O $-\begin{array}{llll}0.499775 & 2.198047 & 0.976440\end{array}$

C $-0.1314233 .994689-1.916849$

C $-0.5338894 .965427-1.082995$

C $0.0159946 .362809-1.075620$

C 0.8588966 .6320610 .153004

C $0.287177 \quad 6.972126 \quad 1.320720$

C $2.364617 \quad 6.5753920 .009556$

C $2.8703635 .213454-0.389022$

N $0.2855341 .755524-1.160730$

H $2.187298-0.327804-2.437736$

H $4.348754-2.356914-3.196221$

H $5.893303-0.690875-2.392021$

H $3.976205-1.011227-0.019094$

H $5.563835-0.3523280 .099673$

H $5.441945-4.4803140 .230728$

H $7.474117-3.514760-0.191980$

H $7.363343-2.289632-1.457707$

H $7.564357-1.8059530 .240968$

H $2.847460-2.844093-0.120497$

H $3.078049-4.564315-0.298468$

H $1.974845-4.2407991 .777709$

H $3.619239-4.7537842 .161151$

H $4.223923-2.3161852 .546494$

H $3.534326-3.7881594 .470982$

H $1.851858-3.4175264 .153601$

H $2.804865-2.8373456 .623008$

H $1.042823-0.9332574 .447899$

H $4.911063-2.1947035 .790862$

H $4.426186-0.5295116 .006922$

H $4.962332-1.0940174 .411120$

H $3.0924730 .971616 \quad 4.829942$

H $0.3283560 .159192 \quad 2.489345$

H 1.4248991 .5288082 .708014

H 3.7286280 .6518911 .859540

H $4.6281693 .553022-3.710871$

H $3.6915862 .805629-0.877622$

H $3.1171335 .401936-3.616094$

H $4.6960995 .893616-2.952546$

H $3.2359276 .786156-2.556698$

H 2.3495421 .1472280 .415186

H $1.278581-0.2563290 .219122$

H -1.632209 2.550416 -1.359265

H $-0.7844802 .199525-2.877729$

H $0.626593 \quad 4.207374-2.665476$

H - $1.2940674 .739980-0.338127$

H $-0.8282007 .063851-1.107905$

H $0.5967176 .568385-1.983072$

H $0.882194 \quad 7.1902232 .201286$

H -0.7912887 .0374811 .421147$

H 2.8484596 .8323350 .961239

H $2.6767957 .360561-0.684245$

H 2.7573824 .4611590 .392867

H $1.0534311 .359891-1.693106$

C $0.917749-3.032416-2.841611$

O $-0.014215-2.319173-2.003186$

C $-1.209258-3.114860-1.867507$

C $-0.934687-4.437477-2.578491$

C $0.067495-4.021429-3.634730$

H $-2.003299-2.587778-2.408100$

C $-1.585935-3.281799-0.390704$

H $-0.492030-5.177301-1.900976$

H -1.845105 -4.876295 -2.998620

C $0.845607-5.188127-4.218442$

H $-0.464917-3.498947-4.440704$

C $-1.910123-1.9715150 .354830$

O $-0.500841-3.9177090 .297543$

H -2.435740 -3.969888 -0.308991

H $-0.999625-1.3752640 .478841$

O $-2.324980-2.3271001 .681195$

C $-3.004308-1.107124-0.302293$

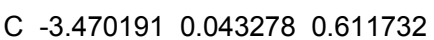

H $-2.614972-0.696276-1.240823$

O $-4.112585-1.944349-0.619833$

H $-4.012254-0.3742931 .466872$

H -2.584224 0.5517601 .004719

H $1.557099-4.845617-4.976043$

H $1.408102-5.722188-3.445241$

H $0.166451-5.903233-4.694253$

C $1.702399-2.061213-3.722211$

H $0.278206-3.3420780 .208770$

H $-3.160017-2.8199461 .605248$

H $-4.721293-1.422590-1.169402$

H $1.016439-1.399865-4.265395$

H $2.278492-2.613280-4.473873$

H $1.599826-3.574017-2.174149$

C -6.5442380 .1233590 .359505$

O $-5.5746440 .499789-0.626813$

C $-4.3678941 .073093-0.113022$

C $-4.696592 \quad 2.3207730 .720741$

C -5.7160762 .0182991 .814223$

C $-6.941568 \quad 1.3269621 .222000$

H $-6.134335-0.6730550 .992146$

C -7.763859-0.435822 -0.383515

H -3.800196 $1.413560-0.987911$

O $-3.5269952 .868877 \quad 1.317862$

H -5.1136293 .0951210 .063755$

H -5.2765651 .4182982 .619780$

O \begin{tabular}{llll}
\hline & -6.141956 & 3.244738 & 2.412508
\end{tabular}

H -7.5142192 .0478300 .624200$

$\begin{array}{llll}\text { O } & -7.786707 & 0.884215 & 2.282212\end{array}$

C $-7.471770-1.804759-0.949636$

H $-8.629884-0.5472370 .279071$

H -8.071881 $0.229129-1.199697$

H $-2.892118 \quad 3.0538890 .605345$

H $-5.347016 \quad 3.6918962 .750539$

H -8.0012611 .6616142 .826052$

O $-7.096437-1.701597-2.256289$

O $-7.544496-2.838959-0.298256$

C $-6.780960-2.937273-2.905618$

H $-5.955418-3.438948-2.391949$

H $-7.664217-3.582136-2.938234$

H -6.471099-2.713323 -3.929786

SCF Energy (B3LYP/6-31G**//MMFF) $=-3245.92031775$

01_255

MMMFF Geometry

C -1.029379 -3.162593 1.363164

C $-0.594441-3.1175500 .095668$

C $-1.348932-2.427755-1.007210$

O $-0.487678-1.368989-1.489104$

C $-1.679617-3.362131-2.182450$

C $-2.652804-4.472601-1.853317$

C $-3.976879-4.291428-1.680030$

C $-2.052974-5.850177-1.758349$

C $-4.727948-2.988951-1.734504$

C $-5.423886-2.708366-0.396100$

C $-5.918091-1.264263-0.214429$

C $-6.780791-0.761976-1.373761$

C $-7.2124490 .699644-1.172961$

O $-4.772810-0.418366-0.046251$

O $-7.7166091 .187424-2.426182$

C $-5.9674021 .545839-0.828468$

C $-8.3632810 .823598-0.167514$

O $-6.3382532 .880924-0.491156$

C $-5.0965630 .937694 \quad 0.295722$

$\begin{array}{llll}C & -3.779001 & 1.738819 & 0.437433\end{array}$

O -5.8142331 .0157851 .530473$

C $-1.083478-0.259039-1.998655$

O $-2.277385-0.066891-2.160857$

C $-0.015900 \quad 0.719115-2.291756$

C $-0.3087752 .026660-2.256822$

C $0.630815 \quad 3.127790-2.464204$

C $2.0286872 .784895-2.908067$

C $-2.738474 \quad 1.0727391 .346885$

C $-1.494076 \quad 1.924668 \quad 1.509282$

C -0.6665584 .0015302 .616028$

O -0.4113581 .5845901 .038615$

C -0.5059215 .0424871 .552536$ 
C $\quad 0.686542 \quad 5.396408 \quad 1.048283$

C 0.8847296 .5031210 .050526

C $1.7096416 .078329-1.143805$

C $3.0515776 .145702-1.112111$

C $0.9826445 .662572-2.401238$

C $0.2057264 .387916-2.236189$

N -1.7188303 .0737542 .248526$

H -1.990123 -2.715411 1.606069

H $0.358123-3.574496-0.157914$

H -2.262744 -1.981563 -0.598194

H $-0.749110-3.785048-2.587120$

H $-2.078086-2.774100-3.019997$

H $-4.594420-5.163646-1.469710$

H -2.800367 -6.614277 -1.520026

H -1.588469 -6.127162 -2.710473

H $-1.288127-5.882113-0.976097$

H $-4.074506-2.156065-2.003640$

H -5.478526 -3.064079-2.530306

H $-6.275055-3.393284-0.290622$

H $-4.727919-2.932710 \quad 0.423658$

H -6.496362 -1.2495960 .717310$

H -7.655346 -1.408357 -1.516288

H -6.212579 -0.811099-2.312019

H $-8.4542880 .613648-2.695918$

H $-5.355017 \quad 1.614583-1.738687$

H $-9.2376350 .260471-0.515886$

H $-8.6955941 .863244-0.073579$

H -8.107960 0.4511070 .826513

H $-6.8701993 .228180-1.227633$

H -3.319601 $1.867143-0.549885$

H -4.0052042 .7439410 .813640$

H $-5.184688 \quad 1.0251612 .267708$

H $0.9810710 .341128-2.479367$

H $-1.3306952 .323482-2.024588$

H $2.0081092 .217601-3.845133$

H $2.5367342 .185713-2.145247$

H $2.6553713 .661839-3.085145$

H -3.1397070 .8743902 .346451$

H -2.4295650 .1065990 .932773$

H 0.2581193 .4432792 .791584

H -0.9700334 .4769133 .554028$

H -1.4019315 .5592131 .217304$

H 1.5819924 .8988711 .414849

H 1.3924397 .3296590 .565414

H $-0.0744846 .914142-0.287676$

H $3.6532135 .873034-1.972496$

H $3.5783206 .471918-0.221166$

H $1.6710305 .584764-3.250911$

H $0.2852256 .460665-2.687661$

H $-0.8189684 .541472-1.896491$

H -2.6580203 .2762932 .577682$

C $0.252456-2.8112213 .535752$

O $1.310296-2.0016102 .981257$

C $1.199188-0.6729783 .529003$

C $0.163476-0.7520154 .648827$

C $-0.761192-1.8338554 .130536$

H $0.801088-0.0439222 .727763$

C $2.562262-0.1442463 .987713$

H $0.613340-1.0601005 .599921$

H -0.3419710 .2050584 .811510$

C $-1.655340-2.4419345 .195787$

H $-1.388444-1.3872073 .350193$

C $3.653504-0.0901102 .900293$

O $3.054341-0.9815745 .044241$

H 2.4373680 .8502604 .432068

H $3.954719-1.1088172 .629811$

$\begin{array}{lllll}\text { O } & 4.807878 & 0.528282 & 3.487254\end{array}$

C 3.2863050 .6935601 .625865

C $4.4753220 .968246 \quad 0.684708$

H 2.5053130 .1602611 .072312

O $2.759402 \quad 1.978053 \quad 1.971757$

H $4.0851501 .511946-0.184651$

H $5.161371 \quad 1.6783361 .165032$

H -2.293462 -3.2219114 .768742$

H -1.069109 -2.890450 6.004743

H $-2.304323-1.6773735 .635436$

C $-0.273546-3.8031212 .492210$

H $3.112316-1.8881704 .696301$
H $5.039503 \quad 0.0153384 .280468$

H 1.8808611 .8450312 .363306

H $-0.940721-4.5244522 .978679$

H $0.573709-4.3736872 .091087$

H $0.719155-3.3940034 .341655$

C $3.791179-1.021736-1.531211$

O $4.397305-1.307307-0.268237$

C $5.256270-0.2793160 .229210$

C $6.3738570 .017079-0.783014$

C $5.8199240 .330184-2.171565$

C $4.843534-0.759332-2.612822$

H $3.158865-0.133005-1.422718$

C $2.838002-2.169259-1.875603$

H $5.741787-0.6929111 .121254$

O $7.1821341 .105211-0.347607$

H $7.030397-0.859180-0.863685$

H $5.3468371 .318594-2.204869$

O $6.8976590 .370339-3.109833$

H $5.408350-1.672189-2.836887$

O $4.189620-0.352862-3.812263$

C $3.531430-3.495311-2.079190$

H $2.109083-2.297875-1.068794$

H $2.263141-1.947771-2.781956$

H 7.5305280 .8769720 .531190

H $7.5302361 .038563-2.794138$

H $4.881185-0.162150-4.469159$

O $3.739065-4.104665-0.877230$

O $3.856746-3.931884-3.175768$

C $4.402205-5.370828-0.935586$

H $4.504185-5.7483840 .085265$

H $3.808160-6.083499-1.515659$

H $5.400359-5.255760-1.368856$

SCF Energy (B3LYP/6-31G**//MMFF) $=-3245.90729220$

$01 \_256$

MMMFF Geometry

C $1.360801-2.9580061 .328184$

C $2.475793-2.2763521 .630223$

C $2.555784-0.8762212 .192409$

O $1.262186-0.226948 \quad 2.280956$

C $3.129806-0.8421213 .620591$

C $4.548057-1.3412113 .770903$

C $5.644144-0.5921873 .544720$

C $4.690628-2.7509254 .285622$

C 5.7090170 .8376893 .078057

C $6.720237 \quad 1.018647 \quad 1.935597$

C $6.173888 \quad 0.5893190 .563622$

C $7.3288640 .406948-0.422970$

C $6.8308680 .105387-1.841120$

$\begin{array}{lllll}\text { O } & 5.295757 & 1.626739 & 0.110866\end{array}$

\begin{tabular}{lllll}
\hline & 7.947329 & 0.235374 & -2.734829
\end{tabular}

C $5.8029551 .182533-2.238015$

C $6.327634-1.336873-1.979626$

O $5.2370040 .857918-3.507018$

C $4.6976801 .405653-1.174354$

C $3.8801632 .673554-1.531891$

$\begin{array}{llll}\text { O } & 3.837416 & 0.267152 & -1.164853\end{array}$

C $0.6849820 .226404 \quad 1.136720$

$\begin{array}{lllll}\text { O } & 1.080081 & 0.057455 & -0.004887\end{array}$

$\begin{array}{llll}\text { C } & -0.529394 & 0.976458 & 1.523445\end{array}$

C $-1.296493 \quad 1.533810 \quad 0.575466$

C -2.522110 $2.301462 \quad 0.803217$

C $-2.926813 \quad 2.5579342 .231812$

C $2.8725083 .118538-0.462420$

C $2.1706144 .411989-0.845242$

C $0.3776705 .948212-0.079286$

O $2.533895 \quad 5.119122-1.781702$

C -1.034604 5.7514210 .372569

C $-2.097104 \quad 6.019639-0.402450$

C -3.5269795 .8980250 .044402$

C $-4.3222404 .929920-0.803475$

C $-4.9121695 .330783-1.942374$

C $-4.500353 \quad 3.520500-0.286415$

C -3.213324 $2.745413-0.267302$

N $1.0987304 .690893-0.004713$

H $0.387774-2.5189721 .516085$

H $3.433384-2.7419921 .404806$

H $3.190540-0.2701001 .535632$ 
H $2.467287-1.4043104 .294159$ H 3.0652870 .1862574 .002790 H $6.615582-1.0464063 .738215$ H $5.738603-3.0489234 .397998$ H $4.215507-2.8437275 .267911$ H $4.219332-3.4657913 .605483$ H 4.7310351 .2286152 .781774 H 6.0461621 .4404453 .930493 H 6.9901512 .0820931 .892782 H 7.6376620 .4644312 .171102 H $5.610506-0.3446970 .665293$ H $8.009966-0.382894-0.081918$ H $7.9306801 .326455-0.454688$ H $8.633029-0.393667-2.451856$ H $6.3599762 .120351-2.373900$ H $7.130293-2.046248-1.744518$ H $6.029499-1.553978-3.011285$ H $5.485720-1.564149-1.322200$ H $4.7768311 .646251-3.839560$ H $4.5774673 .504863-1.700107$ H $3.3315132 .512823-2.468255$ H $2.9548550 .535245-0.865962$ H -0.7602201 .0471112 .580430$ H $-1.0053811 .414427-0.467194$ H -2.120670 3.0655912 .773693 H $-3.153899 \quad 1.6162332 .742785$ H -3.8074273 .1952872 .331260$ H $2.1081712 .352715-0.320157$ H 3.3769383 .2869360 .495345 H $0.435406 \quad 6.346216-1.097972$ H $\quad 0.885514 \quad 6.6543310 .585932$ H -1.188906 5.4080751 .392417 H -1.932906 $6.385131-1.413983$ H $-3.9832306 .895071-0.013374$ H -3.5932555 .6099511 .100721$ H -5.507296 $4.650359-2.542254$ H $-4.8089816 .349615-2.301227$ H $-5.2020912 .966095-0.923787$ H -4.9857743 .5612570 .692477$ H -2.832488 2.516370 -1.263359 H 0.9825394 .1254320 .831841 C $1.004106-4.297579-0.791817$ O $-0.316703-3.754786-1.005560$ C $-0.279824-2.929568-2.186296$ C $1.052438-3.223334-2.872455$ C $1.948693-3.488266-1.682238$ H $-0.273786-1.889062-1.842337$ C - $-1.493350-3.186677-3.084314$ H $0.993748-4.112928-3.510461$ H $1.393044-2.387024-3.490570$ C $3.240194-4.205115-2.032636$ H $2.192947-2.522056-1.225453$ C $-2.869526-2.875098-2.459890$ O $-1.498022-4.564832-3.481299$ H -1.378227 -2.612539-4.011602 H -3.117551 -3.631336 - 1.706508 O $-3.841602-3.032433-3.504800$ C $-3.012788-1.469514-1.843922$ C $-4.463298-0.998545-1.629891$ H -2.463018 -1.433695 -0.897617 O $-2.395520-0.520541-2.721860$ H $-4.4199270 .002979-1.186604$ H -4.928470 -0.841935 -2.611744 H $3.846496-4.374761-1.137221$ H $3.046880-5.176693-2.499445$ H $3.833251-3.607885-2.733082$ C $1.353085-4.3214640 .699356$ H -1.535837 -5.102025 -2.671395 H -3.667813 -2.348024 -4.173628 H -2.422158 $0.346303-2.282713$ H $2.320614-4.8168020 .842961$ H $0.607554-4.9224401 .234743$ H $0.961469-5.336669-1.144999$ C $-4.830298-1.0172421 .406460$ O $-4.823771-2.1528890 .533680$ C $-5.352372-1.921558-0.774195$ C $-6.806448-1.438377-0.666971$ C $-6.912849-0.1887800 .200149$
C $-6.240290-0.4234921 .549573$

H $-4.136259-0.2625031 .021566$

C $-4.325052-1.4839132 .781878$

H -5.380802 -2.898625 -1.269704

O $-7.350751-1.161826-1.953008$

H $-7.423290-2.228141-0.218950$

H $-6.4944480 .690934-0.301888$

O

H -6.868015 -1.093151 2.151987

O $-\begin{array}{llll}6.153095 & 0.817386 & 2.249228\end{array}$

C $-2.864546-1.8743572 .778232$

H $-4.444727-0.6793513 .516285$

H $-4.894198-2.3622203 .109264$

H -7.274291 -1.973558 -2.483104

H $-8.7171570 .209168-0.433159$

H $-7.053403 \quad 1.1803532 .309653$

O $-2.481203-2.1401754 .062749$

O $-2.155549-1.9514451 .785806$

C -1.119693 -2.543827 4.235041

H $-0.909811-3.4402113 .644041$

H $-0.448686-1.7268093 .956982$

H -0.965899-2.778345 5.291656

SCF Energy (B3LYP/6-31G**//MMFF) $=-3245.91737285$

01_257

MM̄̄F Geometry

C $0.105033-2.0216191 .151254$

C $1.081798-2.6226610 .453988$

C $1.454955-2.324331-0.981392$

O $0.769295-1.124837-1.414739$

C $2.970779-2.106015-1.146133$

C $3.798357-3.368505-1.031559$

C $4.598026-3.6718820 .009454$

C $3.725391-4.293968-2.219056$

C $4.809226-2.8868021 .274427$

C $6.227290-2.3087271 .375567$

C $6.515524-1.2048470 .345944$

C $7.966294-0.7319500 .459272$

C $8.2610010 .435554-0.493374$

O $5.624821-0.1143860 .603721$

O $9.5161221 .015387-0.106463$

C $7.1823881 .520387-0.298157$

C $8.428658-0.035413-1.942782$

O $7.3296102 .561806-1.261540$

C $5.7347950 .972003-0.325498$

C 4.7550222 .0825440 .120968

O $5.4121020 .565959-1.655980$

C $0.518455-0.973009-2.738556$

O $0.874855-1.722874-3.637377$

C $-0.2835810 .244595-3.007955$

C $-0.8084931 .026216-2.050537$

C $-1.6678162 .195228-2.263385$

C $-1.8903602 .650969-3.681309$

C $3.293197 \quad 1.6340860 .263544$

C 2.4656182 .7106520 .939190

C 1.6703925 .0538770 .631046

O 2.0009792 .5554032 .066332

C 0.2324625 .0337020 .220228

C $-0.2820235 .870579-0.694637$

C $-1.7133025 .996321-1.144930$

C $-2.7278405 .131409-0.433124$

C -3.2353175 .4949340 .757334$

C $-3.2340733 .905539-1.155602$

C $-2.2319512 .784441-1.188085$

N $2.325301 \quad 3.849158 \quad 0.162727$

H $-0.505168-1.2602810 .673462$

H $1.638882-3.4201910 .940501$

H $\quad 1.110608-3.168259-1.591639$

H $3.303228-1.338938-0.440198$

H $3.175649-1.656836-2.127107$

H $5.164412-4.601558-0.034357$

H $4.404815-5.147612-2.123473$

H $2.713257-4.692190-2.337630$

H $3.998864-3.759018-3.134515$

H $4.067212-2.0939191 .403710$

H $4.653898-3.5733512 .116336$

H $6.343009-1.8954632 .385897$

H $6.962101-3.1167481 .270672$ 
H $\quad 6.327527-1.595859-0.659764$

H $8.661741-1.5606390 .276909$

H $8.161952-0.3973451 .487863$

H $10.1947490 .320336-0.154633$

H 7.3574121 .9812590 .684291

H $9.252988-0.754793-2.018435$

H $8.6984580 .798393-2.600288$

H $7.534364-0.516176-2.344466$

H $8.2449682 .884932-1.201040$

H $5.0801602 .474147 \quad 1.094320$

H $4.7989472 .915071-0.592193$

H $4.4480470 .559601-1.760264$

H -0.457862 $0.441167-4.061289$

H $-0.6293140 .774411-1.006832$

H -2.461953 $1.902141-4.240271$

H $-0.9324112 .813862-4.188277$

H -2.432092 $3.596230-3.755285$

H $2.8427951 .408633-0.709076$

H 3.2198400 .7294080 .876796

H 2.2112355 .9039850 .202803

H 1.7437095 .1161821 .721811

H $-0.403166 \quad 4.3212350 .737267$

H $0.3967956 .570938-1.179692$

H $-1.756151 \quad 5.804997-2.224369$

H -2.006008 $7.048463-1.026515$

H -3.990895 $4.898301 \quad 1.257256$

H -2.900578 6.3968161 .259330

H -3.581664 4.196127 -2.150832

H $-4.1302423 .519112-0.651362$

H -2.007061 2.379487 -0.200190

H $2.6750753 .845010-0.790839$

C $-0.606703-1.1312573 .391763$

O $-1.806070-0.5422002 .846459$

C $-2.798644-0.4593603 .881021$

C $-2.010013-0.4606655 .181248$

C $-0.920335-1.4649174 .854315$

H $-3.397079-1.3771673 .823118$

C -3.701542 0.7558603 .653036

H $-1.5670620 .519858 \quad 5.390019$

H -2.620337 -0.7525356 .040988$

C $0.287003-1.3769275 .771895$

H $-1.344690-2.4755184 .927186$

C -4.289256 0.8478182 .228988

O $-2.949081 \quad 1.9558693 .878222$

H -4.5062780 .7640554 .396897$

H -3.487589 1.0960991 .525165

O $-5.203196 \quad 1.9513572 .202660$

C -5.034675 -0.4125811 .747677$

$\begin{array}{llll}C & -5.757412 & -0.248139 & 0.395047\end{array}$

H -4.334159-1.249532 1.674133

O $-6.014008-0.784038 \quad 2.721240$

H $-6.320328-1.1685780 .198967$

H -6.5208290 .5340310 .488933$

H $1.049615-2.1065755 .481368$

H $\quad 0.743283-0.3817935 .740584$

H $-0.001309-1.5820096 .808053$

C $-0.246816-2.372742 \quad 2.570737$

H -2.185648 $1.941693 \quad 3.275309$

H -4.7158532 .7377732 .502979$

H -6.623097 -0.0329112 .825946$

H -1.112395 -3.044983 2.539803

H $0.580130-2.9098593 .049153$

H $0.183909-0.3742903 .317181$

C $-4.291376-2.179375-1.509792$

O $-3.814344-0.945052-0.958603$

C $-4.811140 \quad 0.066358-0.778741$

C $-5.5122350 .375292-2.107834$

C $-6.064335-0.883526-2.763416$

C $-4.972999-1.945738-2.864827$

H -5.005036 -2.640237 -0.816217

C $-3.079084-3.114283-1.665362$

H $-4.2732670 .977296-0.506705$

O $-6.567897 \quad 1.312348-1.925384$

H $-4.7927260 .835275-2.797838$

H $-6.939988-1.267480-2.227054$

O $-6.506474-0.569795-4.086016$

H $-4.230691-1.640183-3.613533$

O $-5.557167-3.167905-3.312948$
C $-2.731163-3.834452-0.379630$

H $-3.297119-3.890090-2.408195$

H -2.194288 -2.550873 -1.982006

H $-6.1841052 .109584-1.521806$

H $-7.1711660 .136081-4.008111$

H $-5.999491-2.984313-4.159582$

O $-1.855334-4.844071-0.665528$

O $-3.163967-3.553627 \quad 0.728999$

C $-1.479586-5.674306 \quad 0.437778$

H $-0.884396-6.5049450 .049352$

H $-0.869883-5.108077 \quad 1.145735$

H $-2.366908-6.0819070 .931976$

SCF Energy (B3LYP/6-31G**//MMFF) $=-3245.92208854$

01_258

MM̄FF Geometry

C $-5.627558-1.759966-1.455783$

C $-4.405593-2.294443-1.310776$

C $-3.422218-2.396504-2.449733$

O $-2.299135-1.535766-2.145607$

C $-2.894258-3.835548-2.594940$

C $-2.138909-4.059062-3.892909$

C $-0.815723-4.294236-3.992399$

C $-2.982545-4.040479-5.144397$

C $0.202523-4.420497-2.894519$

C $0.947027-3.105921-2.665634$

C $2.266076-3.253423-1.892556$

C $2.085438-3.898730-0.517556$

C $3.408914-3.9547610 .263336$

O $2.787170-1.927630-1.738774$

O $3.104825-4.258591 \quad 1.634744$

C $4.055094-2.5525110 .254123$

C $4.301410-5.101106-0.226924$

O $5.352634-2.5756330 .842506$

C $4.082606-1.858227-1.129130$

C $4.407433-0.356721-0.952162$

O $5.097404-2.382630-1.984583$

C $-2.453156-0.206102-2.394515$

O $-3.418543 \quad 0.343370-2.900694$

C $-1.2428090 .484819-1.903309$

C $-1.2859751 .808229-1.689281$

C $-0.1885192 .633672-1.182095$

C $1.1591961 .976844-1.045087$

C $4.4612590 .414002-2.277396$

C $4.6193941 .910112-2.066167$

C $4.4813734 .056013-3.339375$

O $4.8486272 .416887-0.971691$

C $3.0654144 .541961-3.324482$

C $2.6286765 .549570-2.553502$

C $1.2074136 .039591-2.563656$

C $0.6190916 .180651-1.175789$

C $0.2098137 .377760-0.722480$

C $0.5063674 .946379-0.306968$

C $-0.4417913 .921813-0.868668$

N $4.4781632 .606985-3.257860$

H $-5.943352-1.417016-2.438510$

H $-4.072871-2.617405-0.327417$

H -3.885842 -2.069688 -3.387996

H $-3.729330-4.548212-2.566488$

H $-2.261764-4.072618-1.732715$

H $-0.397532-4.428456-4.989996$

H $-2.421470-4.362129-6.028500$

H $-3.834937-4.719522-5.037846$

H $-3.359984-3.033765-5.346102$

H $-0.256477-4.766795-1.963685$

H $0.911543-5.204254-3.189615$

H $1.169663-2.620785-3.626185$

H $0.292998-2.403974-2.134966$

H $2.959253-3.838159-2.508383$

H $1.661834-4.906271-0.600358$

H $1.359483-3.3128730 .061742$

H $2.600521-5.0899811 .655221$

H $3.441070-1.9333670 .922372$

H $3.816381-6.067488-0.041233$

H $5.246831-5.1336680 .324966$

H $4.519636-5.054282-1.295054$

H $5.972483-2.948558 \quad 0.193876$

H $3.6430630 .105310-0.315235$ 
H $5.373445-0.237209-0.445383$ H $5.274846-3.303637-1.749321$ H $-0.363036-0.114784-1.703271$ H -2.218741 2.337255 -1.880731 H $1.4789161 .562699-2.007461$ H $1.1224631 .165385-0.310628$ H $1.9484352 .657097-0.723298$ H $5.3065570 .068309-2.883172$ H $3.5401210 .246953-2.847322$ H $4.9566094 .324842-4.287613$ H $5.0703684 .470746-2.515087$ H $2.3654144 .048760-3.995674$ H $3.3304206 .065557-1.902373$ H $0.5661125 .377103-3.157821$ H $1.1980577 .008976-3.078796$ H -0.2123647 .4931780 .270329$ H $0.2864828 .271208-1.333120$ H 0.1344465 .2172140 .690101 H $1.5070814 .547342-0.138057$ H -1.459307 4.294663 -0.995720 H $4.2365232 .092955-4.100381$ C $-6.539191-0.1394770 .277478$ O $\quad-5.3323990 .0013461 .051154$ C $-4.805774 \quad 1.3284490 .857104$ C -5.9038822 .1211250 .153678$ C $-6.5624461 .044874-0.689761$ H -3.951546 1.2257990 .177670 C -4.3289641 .9143152 .193037$ H -6.6280612 .5378540 .862522$ H $-5.5013462 .947307-0.440359$ C $-7.9523681 .409882-1.179161$ H $-5.9181590 .861415-1.557454$ C -3.4341520 .9667673 .017003$ O -5.4698112 .2267983 .004587$ H -3.817067 2.8669082 .016102 H $-4.0356190 .119093 \quad 3.366913$ O $-3.020829 \quad 1.663824 \quad 4.199700$ C -2.1860360 .4413642 .284524$ C -1.405918 -0.5513893 .167482$ H -2.486988 -0.0679731 .363096$ O $-1.366216 \quad 1.5471991 .926808$ H -1.021900 -0.027140 4.047679 H -2.118008 -1.3050083 .522423$ H $-8.3831200 .593836-1.767948$ H -8.630268 $1.618895-0.344758$ H $-7.9151332 .301973-1.812874$ C $-6.600710-1.545051-0.332846$ H -5.9733451 .4046153 .133480$ H -2.4437002 .3954473 .920432$ H -0.5776601 .2004291 .477001$ H -7.609693 -1.735595 -0.717497 H -6.416729 -2.2872260 .454310$ H -7.363716 -0.0493350 .997254$ C $1.634495-0.0529793 .369582$ O $0.876310-0.3895922 .199512$ C $-0.248984-1.2503682 .416601$ C $0.208986-2.5577823 .076618$ C $0.963105-2.2939764 .372657$ C 2.103171-1.312395 4.118671 H 1.0263740 .5749114 .031093 C 2.8584440 .7650932 .921683 H -0.627404 -1.514923 1.420732 O $-0.893429-3.419700 \quad 3.338724$ H $\quad 0.873120-3.0957492 .392596$ H $0.294369-1.9324885 .162449$ O $1.524684-3.519544 \quad 4.847537$ H $2.885017-1.8211953 .540636$ O $2.689359-0.9452605 .367297$ C 2.5235182 .1859672 .522533 H 3.5806020 .8318063 .743688 H 3.3371520 .2862412 .061378 H -1.348799 -3.576688 2.493887 H $\quad 0.791370-4.1482584 .961206$ H $2.008879-0.4939805 .895365$ O 3.6931162 .8664592 .333462 O 1.3959212 .6368622 .386503 C $3.5962314 .251061 \quad 1.979947$ H 4.4576634 .7698242 .409553
H $3.6401434 .344006 \quad 0.892193$

H 2.6849974 .7164812 .367080

SCF Energy $($ B3LYP/6-31G**//MMFF $)=-3245.91108263$

01259

MMFF Geometry

C -0.932198 -4.041485 0.445224

C $0.110342-3.2171260 .256595$

C $0.785903-3.044035-1.081589$

O $1.011103-1.635640-1.326143$

C $2.160260-3.734258-1.068227$

C $2.757246-3.880606-2.454357$

C $3.733779-3.097512-2.952176$

C $2.222329-5.031533-3.267304$

C $4.369516-1.902847-2.299627$

C $5.726949-2.256968-1.683003$

C $6.459125-1.015231-1.154072$

C $7.800658-1.403913-0.529233$

C 8.520393-0.193654 0.087150

O $5.630274-0.385562-0.172676$

O $9.576321-0.6898940 .924494$

C 7.5331250 .5629751 .002397

C $9.1909570 .673000-0.985497$

O $\quad 8.113547 \quad 1.7711311 .489754$

C 6.1613090 .8417890 .342759

C 5.1693481 .3933751 .394378

O $6.3361911 .818830-0.686662$

C $-0.055699-0.879919-1.695757$

O $-1.211953-1.249093-1.814964$

C $0.4323480 .498298-1.919511$

C $-0.4542641 .495687-2.050453$

C $-0.1362122 .911621-2.234186$

C $1.2979703 .286203-2.497511$

C $3.714798 \quad 1.4483860 .907371$

C 2.7895322 .0931641 .925715

C 0.3859422 .7130792 .215470

O 3.1765112 .5491402 .997671

C 0.1387714 .0771911 .654289

C -0.9648294 .4063270 .965498$

C -1.1991195 .7582240 .352890$

C $-0.4666015 .949265-0.959415$

C $0.6322176 .721093-1.018452$

C $-1.0302495 .312360-2.213523$

C -1.133318 $3.815476-2.137048$

N $1.4757352 .100618 \quad 1.480514$

H -1.292733 -4.610801 -0.410238

H $\quad 0.520495-2.645217 \quad 1.083168$

H $\quad 0.164774-3.449033-1.889764$

H $2.077750-4.738072-0.629761$

H $2.847671-3.183013-0.414436$

H $4.114105-3.318141-3.948686$

H $2.718339-5.119633-4.239762$

H $2.374102-5.975797-2.734259$

H $1.151549-4.906692-3.455291$

H $4.499878-1.128804-3.066106$

H $3.710707-1.465236-1.543346$

H $5.561507-2.964267-0.859619$

H $6.357212-2.761828-2.425938$

H $6.612415-0.326260-1.993198$

H $8.444618-1.899889-1.266235$

H $7.630338-2.1445160 .264958$

H $\quad 10.167507-1.2306750 .373127$

H $7.369456-0.069443 \quad 1.885675$

H $9.9126900 .080160-1.560295$

H $9.7687211 .486152-0.532625$

H $8.480803 \quad 1.103975-1.694148$

H 8.0861452 .4240540 .769818

H 5.1919960 .7557172 .287835

H 5.4834592 .3983681 .703587

H $5.5049962 .301101-0.816462$

H $1.5044500 .658412-1.940742$

H - $1.5158011 .260274-1.986605$

H $1.6965582 .717438-3.345103$

H $1.9169123 .085144-1.617004$

H $1.4255604 .341633-2.750494$

H $3.6377562 .025422-0.019819$

H 3.3469150 .4344350 .710199

H 0.6352312 .7805643 .278755 
H -0.4809742 .0584102 .098673$ H 0.9135304 .8242831 .809341 H -1.738046 3.6578710 .809335 H -2.2753615 .9019440 .195803$ H -0.9084596 .5338581 .074119$ H $1.1564626 .889561-1.953221$ H $1.0347867 .199933-0.131956$ H $-0.4545715 .595892-3.103373$ H -2.035565 $5.718979-2.384944$ H -2.143501 $3.458589-1.940233$ H 1.2699431 .7428690 .552773 C -1.451857 -3.339473 2.887226 O $-2.087212-2.0807912 .577813$ C -2.854575 -1.655634 3.719991 C $-2.353565-2.4917824 .889152$ C $-2.082655-3.8141134 .199225$ H -3.897429 -1.930524 3.521990 C $-2.742357-0.1413293 .913993$ H -1.427324 -2.085668 5.312003 H -3.091242 -2.568392 5.693527 C $-1.199841-4.7500385 .006718$ H -3.041753 -4.310424 3.998502 C -3.1888690 .6814372 .686645$ $\begin{array}{llll}\mathrm{O} & -1.372737 & 0.179805 & 4.185365\end{array}$ H -3.3112030 .1579154 .802228$ H -2.4836890 .5172001 .864667$ O -3.0801172 .0684473 .032868$ C $-4.626640 \quad 0.3983282 .216340$ C -5.1146911 .3243221 .087446$ H -4.712630 -0.643902 1.893394 O $-5.506301 \quad 0.564067 \quad 3.332546$ H $-6.182131 \quad 1.125810 \quad 0.932090$ H $-5.0756772 .368578 \quad 1.422222$ H - $-1.016432 \quad-5.6812804 .461667$ H $-0.229539-4.2938585 .230050$ H -1.678273 -5.0041795 .958202$ C -1.674885 -4.314544 1.727761 H $-1.322761 \quad 1.1418774 .319041$ H -3.7302752 .2474553 .733865$ H $-6.399474 \quad 0.3115873 .042620$ H -2.744131 $-4.317276 \quad 1.477702$ H -1.414405 -5.332090 2.041806 H $-0.385496-3.1386723 .042810$ C $-5.471356-0.801620-1.058743$ O $-4.238428-0.203755-0.646528$ C $-4.3461881 .164817-0.240476$ C -4.913282 $2.007740-1.392376$ C $-6.2390071 .449711-1.909159$ C $-6.107975-0.040603-2.228660$ H -6.170898 $-0.783704-0.213139$ C $-5.220813-2.292172-1.333880$ H -3.322526 $1.513661-0.059545$ O $-5.1014813 .359855-0.987582$ H -4.197108 2.015419 -2.223966 H -7.055762 $1.628550-1.200383$ O $-6.5995692 .133584-3.111923$ H -5.518522 -0.151027 -3.144237 O $-7.399779-0.583683-2.491740$ C $-4.193599-2.593745-2.406693$ H -4.844761 -2.743165 -0.406090 H -6.156276 -2.790950 -1.609616 H $-4.2458603 .690812-0.664882$ H $-6.6377353 .082981-2.903756$ H $-7.791959-0.062408-3.213274$ O $-3.868809-3.920468-2.340559$ O $-3.740921-1.791091-3.209716$ C $-2.905329-4.367959-3.299616$ H $-1.983243-3.785031-3.218557$ H -2.675668 -5.415555 -3.087242 H $-3.320447-4.295418-4.309087$

SCF Energy $\left(\mathrm{B} 3 \mathrm{LYP} / 6-31 \mathrm{G}^{* *} / / \mathrm{MMFF}\right)=-3245.90598461$

0126

MM̄FF Geometry

C $2.719944-1.072750-2.361302$

C $2.113750-2.211575-2.727940$

C $1.316524-3.070258-1.783897$

O $-0.055193-2.988755-2.239828$
C $1.764399-4.540411-1.804606$

C $3.090936-4.786075-1.119464$

C $3.218841-5.0740210 .190477$

C $4.306349-4.762866-2.009450$

C $2.124016-5.1647631 .218701$

C $2.497598-4.4709622 .535777$

C $2.486604-2.9337912 .463696$

C $3.123092-2.3637843 .736633$

C $3.030079-0.8359393 .802747$

O $1.122924-2.5066942 .345228$

O $3.390765-0.4506655 .137604$

C $1.560502-0.4349123 .571933$

C $4.048438-0.1673562 .872470$

O 1.4248950 .9831603 .532018

C $0.935573-1.0847072 .314722$

C $-0.581911-0.7928432 .230317$

O $1.544864-0.5065051 .156185$

C $-1.038567-3.131123-1.311369$

O $-0.906610-3.426483-0.135040$

C $-2.321229-2.807924-1.974120$

C $-3.415126-2.590968-1.228864$

C $-4.737303-2.211588-1.730952$

C $-4.870550-1.933416-3.205948$

C -1.434425 -1.528083 3.267764

C $-2.573198-0.6559163 .753489$

C -4.6890330 .4099223 .025345$

O $-2.557156-0.1447704 .871477$

C $-5.903446-0.1190812 .334408$

C -6.5164830 .5090261 .320002$

C -7.7637340 .0041310 .654159$

C $-7.549439-0.342159-0.801760$

C $-7.7533230 .570798-1.766230$

C $-7.196138-1.771584-1.144047$

C $-5.758548-2.108347-0.854544$

N -3.582451 -0.504497 2.815685

H $2.636373-0.740648-1.331026$

H $2.166855-2.529969-3.766535$

H $1.380087-2.668536-0.765080$

H $1.804523-4.909343-2.838841$

H $0.989378-5.166206-1.343924$

H $4.217970-5.2834390 .570842$

H $4.463210-3.766160-2.430750$

H $5.220138-5.036336-1.471038$

H $4.187647-5.474707-2.832857$

H $1.175023-4.7665420 .852108$

H $1.961367-6.2289981 .429833$

H $1.773212-4.7938483 .295272$

H $3.483853-4.8247372 .862353$

H $3.043243-2.6031401 .579862$

H $4.168170-2.6864583 .825967$

H $2.609044-2.7792104 .615437$

H 3.2567130 .5099105 .212880

H $1.005530-0.7579864 .460596$

H $5.068325-0.4515673 .158870$

H 4.0094590 .9237662 .958480

H $3.914444-0.436807 \quad 1.822753$

H 1.7521591 .2873052 .668781

H -0.7464040 .2915332 .275205$

H -0.949095 -1.087543 1.238384

H $0.942864-0.6191530 .402565$

H -2.320756 -2.721275 -3.054651

H -3.337061 -2.691044 -0.146882

H $-4.695619-2.845625-3.786615$

H $-4.148372-1.171365-3.520515$

H -5.854104 -1.553365 -3.490315

H - $1.845821-2.4498422 .838968$

H -0.857070 -1.829819 4.147154

H $-4.381143 \quad 1.3871462 .638435$

H -4.8857450 .5184834 .096842$

H $-6.310607-1.0570512 .704662$

H -6.1179941 .4552750 .961264$

H -8.5302300 .7855610 .741766$

H $-8.175821-0.8638641 .183683$

H $-7.6345180 .321541-2.815403$

H $-8.0392861 .589594-1.526430$

H -7.451249 -1.993306 -2.186102

H -7.834638 -2.449459-0.562698

H $-5.562715-2.319050 \quad 0.197021$ 
H $-3.417669-0.8393501 .870281$

C $4.389714 \quad 0.833718-2.659517$

O $3.615566 \quad 1.772537-1.885402$

C $4.3412662 .076686-0.677844$

C $5.7474091 .518571-0.876945$

C $5.4616180 .287365-1.717827$

H $3.859827 \quad 1.510166 \quad 0.127172$

C $4.2730393 .578000-0.369842$

H $6.3877552 .216314-1.429002$

H $6.239369 \quad 1.2864530 .072575$

C $6.682373-0.268557-2.428697$

H $5.048890-0.488531-1.061116$

C $2.8348174 .136721-0.280847$

O $4.9552404 .281681-1.414450$

H 4.8222313 .7857070 .556321

H $2.3776944 .140051-1.276851$

$\begin{array}{llll}\text { O } & 2.908600 & 5.503242 & 0.140820\end{array}$

C 1.9327963 .3837290 .711526

C $\quad 0.535912 \quad 3.992670 \quad 0.931937$

H 1.8083962 .3502320 .375658

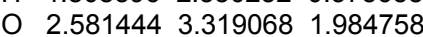

H 0.0028333 .3355871 .631233

H 0.6259554 .9475301 .466275

H $6.416393-1.143065-3.030892$

H $7.1337760 .475182-3.093778$

H $7.442752-0.575917-1.703329$

C $3.464952-0.191221-3.323266$

H $5.0799565 .198786-1.117554$

H $3.3048776 .013088-0.585549$

H 2.7368414 .2321612 .281839

H $4.048658-0.808412-4.016257$

H $2.7164310 .357877-3.908686$

H $4.862657 \quad 1.423753-3.456176$

C $-0.8675731 .903626-0.804010$

O $-0.1992933 .086385-1.245056$

C $-0.2993614 .194437-0.348097$

C $-1.7755614 .540824-0.093627$

C $-2.593317 \quad 3.3203730 .340179$

C $-2.3660832 .148291-0.616390$

H -0.4402251 .5936800 .157269$

C $-0.5359990 .772222-1.780712$

H $\quad 0.144238 \quad 5.049824-0.872864$

O $-1.874021 \quad 5.5685740 .886512$

H -2.205740 $4.940077-1.021370$

H -2.353942 3.0337151 .370588

O $-3.982043 \quad 3.6592940 .346654$

H -2.853979 2.364539-1.573226

O $-2.9703230 .972626-0.086516$

C $-1.0302300 .976709-3.196765$

H $0.5521250 .676476-1.846387$

H $-0.950007-0.164826-1.397390$

H -2.819705 5.7391381 .036001

H $-4.2329703 .917140-0.556736$

H $-3.917302 \quad 1.1603190 .027414$

O $-0.948052-0.212365-3.864692$

O $-1.4073072 .038312-3.671957$

C -1.368469 $-0.183766-5.231614$

H -2.414082 $0.131107-5.301053$

H $-1.280136-1.196055-5.634795$

H $-0.7234590 .483432-5.810962$

SCF Energy (B3LYP/6-31G**//MMFF)= -3245.92215212

01_260

MM̄FF Geometry

C $0.644002-1.8590292 .311473$

C $-0.200259-1.2963373 .187721$

C -0.5852890 .1582313 .131920$

$\begin{array}{lllll}\text { O } & -2.009829 & 0.274361 & 2.853661\end{array}$

C $-0.180054 \quad 0.981417 \quad 4.368253$

C 1.2769621 .3963714 .380250

C 1.7613962 .4552173 .700882

C $2.1822710 .568867 \quad 5.252073$

C 0.9945593 .3424242 .757403

C 1.7135323 .4539611 .404540

C $0.800344 \quad 3.815520 \quad 0.224884$

C $-0.016207 \quad 5.0890230 .447552$

C $-0.9716595 .362794-0.725899$

O $-\begin{array}{lll}0.066533 & 2.698058 & 0.002245\end{array}$
O $\begin{array}{llll}-1.915848 & 6.358883 & -0.303171\end{array}$

C $-1.7757354 .078811-1.027476$

C $-0.236778 \quad 5.949737-1.936746$

O $-2.575045 \quad 4.241529-2.197135$

C $-0.8959162 .813642-1.160127$

C $-1.7865831 .550303-1.233911$

O $-0.1207902 .909189-2.356946$

C -2.942155 $-0.073678 \quad 3.775173$

O $-2.739767-0.4608254 .917596$

$\begin{array}{llll}\text { C }-4.316436 & 0.076820 & 3.240185\end{array}$

C -4.5909860 .2299021 .935416$

$\begin{array}{llll}\text { C } & -5.921812 & 0.342790 & 1.333676\end{array}$

C -7.1078820 .3709842 .261620$

C $-1.0056960 .233294-1.139779$

C $-1.934732-0.961682-1.193972$

C $-3.327859-2.283532-2.788313$

O $-2.237157-1.593246-0.184698$

C $-4.671250-1.666154-3.017358$

C $-5.779229-2.013199-2.345373$

C $-7.126516-1.399311-2.598281$

C $-7.759606-0.825637-1.349697$

C $-8.790964-1.448817-0.754707$

C $-7.265528 \quad 0.512935-0.846125$

C $-6.0196230 .399663-0.011775$

N $-2.382713-1.228405-2.477092$

H $1.102604-1.2370551 .545733$

H $-0.656477-1.9126393 .957333$

H -0.1159770 .6122312 .250919$

H $-0.403300 \quad 0.4292515 .290207$

H -0.8100901 .8786564 .433679$

H 2.8169742 .7001543 .807957

H 3.2213950 .9130765 .217360

H $1.8517040 .615658 \quad 6.294724$

H $2.172168-0.4770104 .930649$

H -0.0250372 .9761872 .610435$

H 0.9103734 .3392263 .206170

H 2.5175764 .1960041 .486605

H 2.1866652 .4927301 .168300

H $1.4469943 .934036-0.652050$

H 0.6437945 .9481920 .619062

H $-0.6252624 .992071 \quad 1.355868$

H $-1.417297 \quad 7.150497-0.037192$

H $-2.4747653 .926438-0.192982$

H $0.245676 \quad 6.897471-1.668812$

H $-0.9361716 .188568-2.745540$

H $0.5363955 .288132-2.332636$

H $-3.1188495 .037425-2.067604$

H $-2.5062011 .567062-0.405831$

H -2.366967 $1.560874-2.164745$

H $0.1238102 .019079-2.652938$

H -5.1021130 .0102923 .985947$

H -3.7664430 .2572051 .227176$

H $-7.034021 \quad 1.2169512 .954039$

H -7.163800 -0.5561312 .842903$

H -8.064225 0.4725881 .744579

H $-0.2833900 .140295-1.955747$

H $-0.4389620 .175392-0.204758$

H -3.341754 -3.023084 -1.981185

H $-2.981980-2.771740-3.704563$

H $-4.736343-0.901056-3.787734$

H $-5.722287-2.792324-1.588672$

H $-7.770424-2.180582-3.022996$

H $-7.070978-0.614804-3.363481$

H $-9.276212-1.0305120 .120705$

H $-9.172072-2.393801-1.127519$

H $-8.058216 \quad 1.034253-0.297808$

H $-7.055693 \quad 1.168273-1.701243$

H $-5.1022880 .368031-0.600204$

H $-2.091701-0.620785-3.237462$

C $0.540199-3.9749450 .986662$

O $1.191848-3.333887-0.125215$

C $1.659023-4.335973-1.044367$

C $0.985102-5.635179-0.622427$

C $0.907796-5.454640 \quad 0.882915$

H $2.739812-4.429090-0.882524$

C $1.391051-3.882349-2.483774$

H $-0.022768-5.728583-1.042770$

H $1.559066-6.517254-0.921702$ 
C $-0.083748-6.385757 \quad 1.557973$

H $\quad 1.906497-5.6247091 .307364$

C $1.864131-2.443035-2.781795$

O $-0.019392-3.923988-2.739816$

H $1.844496-4.588201-3.188556$

H $\quad 1.232773-1.738407-2.231705$

O $1.640090-2.180074-4.171912$

C $3.345691-2.173412-2.463811$

C $3.823637-0.759930-2.846925$

H $3.530944-2.335825-1.398939$

O $4.142041-3.123628-3.176023$

H $4.909939-0.719914-2.701678$

H $3.679832-0.609264-3.923870$

H $-0.112700-6.2080942 .637670$

H -1.096716 -6.2456161 .165902$

H $\quad 0.198082-7.4311331 .394975$

C $0.998064-3.3191682 .290472$

H $-0.455389-3.363939-2.074687$

H $\quad 0.702315-2.363015-4.353738$

H $5.068297-2.985511-2.914232$

H $2.086473-3.3988552 .382549$

H $0.557288-3.8395643 .148190$

H $-0.539464-3.8327770 .847808$

C $4.6033760 .423083-0.126249$

O $3.280376 \quad 0.207975-0.635066$

C $3.1393190 .370733-2.051485$

C $3.5713261 .782800-2.467298$

C $4.9800472 .106958-1.985861$

C $5.1076541 .827811-0.490536$

H $5.282355-0.335304-0.534668$

C $4.5508940 .256897 \quad 1.402650$

H $2.0669580 .305036-2.260949$

O $3.5105061 .941724-3.880590$

H $2.8808462 .516258-2.032919$

H $5.7384661 .557164-2.555423$

O $5.2427423 .495346-2.203608$

H 4.5470102 .5862650 .070456

O $6.4784521 .946032-0.112410$

C $4.489254-1.1891761 .845437$

H 5.4516370 .6868751 .855416

H $3.667451 \quad 0.758288 \quad 1.810697$

H $2.5993681 .744821-4.157744$

H $5.1171483 .667300-3.152817$

H $6.7745832 .836003-0.369721$

O $4.687706-1.2370403 .196501$

O $4.283069-2.1460811 .113595$

C $4.694953-2.5333153 .804979$

H $5.322569-2.4824644 .698867$

H $3.677592-2.7936164 .106995$

H $5.105175-3.2978413 .138442$

SCF Energy (B3LYP/6-31G**//MMFF) $=-3245.91092210$

01261

MM̄̄FF Geometry

C $-2.1882303 .342193-1.387067$

C $-1.6065702 .241361-0.889756$

C $-0.6470131 .372748-1.661056$

O $0.5033171 .105315-0.826535$

C -1.325126 $0.027718-1.979529$

C $-0.479529-0.906113-2.822665$

C $-0.050248-2.118568-2.420550$

C $-0.146733-0.418373-4.209282$

C $-0.302169-2.798938-1.104480$

C $0.761603-2.531136-0.028749$

C $2.164379-3.045886-0.390484$

C $3.103313-2.9529500 .814714$

C $4.536615-3.3806170 .461866$

O $2.680902-2.244045-1.455517$

O $5.376463-2.9616011 .548554$

C $4.990790-2.627289-0.808757$

C $4.662633-4.9067940 .387008$

O $6.256970-3.105978-1.254672$

C $3.956832-2.667818-1.952852$

C $4.319056-1.741034-3.138542$

O $3.889657-4.002308-2.471201$

C $1.4473632 .079283-0.726350$

O $1.4538093 .161441-1.290502$

C $2.472891 \quad 1.604528 \quad 0.228543$
C $3.3308262 .482808 \quad 0.768348$

C 4.3821612 .1751481 .740356

C 4.6666280 .7235022 .021845

C $4.458957-0.254393-2.792417$

C $5.8893990 .184447-2.525297$

C $7.2017582 .156807-1.752591$

O $6.866307-0.524233-2.751486$

C $7.5484691 .972491-0.308938$

C 7.8725542 .9890200 .504571

C 8.2655612 .8182481 .943940

C 7.4452363 .6799272 .877550

C 7.9121944 .8606643 .317558

C 6.1239093 .1320913 .366949

C 5.0455723 .1978072 .319559

N $5.948471 \quad 1.484291-2.040112$

H -1.967078 $3.654524-2.404364$

H $-1.841702 \quad 1.932180 \quad 0.124937$

H $-0.3240821 .866469-2.585441$

H -2.269039 $0.203033-2.513172$

H -1.595895 -0.453469-1.034619

H $\quad 0.548137-2.705489-3.116588$

H $0.323644-1.195920-4.820893$

H -1.056437 -0.101127 -4.729476

H $0.5461480 .427255-4.165879$

H -1.285050 -2.520338 -0.711725

H $-0.366748-3.879355-1.286837$

H $\quad 0.802466-1.4536950 .170102$

H $\quad 0.429228-3.0176040 .896607$

H $2.074145-4.086072-0.724764$

H $2.721941-3.5477741 .654111$

H $3.128971-1.9149121 .173520$

H $6.297033-3.156521 \quad 1.301387$

H $5.149742-1.585720-0.508411$

H $4.383007-5.3591631 .346134$

H $5.699216-5.2115650 .206093$

H $4.032378-5.351320-0.386063$

H $6.110029-3.950900-1.712802$

H $5.206789-2.122377-3.657442$

H $3.501255-1.818399-3.869826$

H $3.512650-3.966026-3.365939$

H 2.4779690 .5498800 .478606

H 3.2433723 .5327750 .491378

H $4.8377580 .179427 \quad 1.086997$

H 3.8249960 .2618442 .549094

H 5.5598170 .5622522 .628672

H $4.1172430 .334860-3.653206$

H $3.8263890 .014123-1.941058$

H $7.0722423 .211112-2.016404$

H $7.9944301 .734666-2.378735$

H 7.5597570 .9560590 .077578

H 7.8883714 .0013910 .106871

H 9.3279873 .0811112 .032016

H 8.1943851 .7695042 .258104

H 7.3426875 .4720554 .009720

H 8.8729115 .2447302 .990846

H 5.7706643 .7158414 .227726

H 6.2791082 .1239933 .761148

H 4.7695094 .2166112 .043684

H $5.0857601 .978867-1.832812$

C $-4.5857664 .009652-1.158326$

O $-5.0409842 .665672-0.900573$

C -6.422089 $2.708665-0.494879$

C $-6.8904934 .140551-0.729425$

C $-5.6128114 .921146-0.489281$

H -6.4407282 .5050110 .582076$

C $-7.2250441 .640698-1.245592$

H -7.240448 4.291574-1.757228

H $-7.7027324 .425957-0.053923$

C $-5.6492976 .332019-1.049863$

H -5.4271584 .9668640 .592350$

C $-6.6768740 .205900-1.092076$

O $-7.2232951 .951806-2.645846$

H $-8.2746591 .677054-0.932217$

H $-5.7065360 .126135-1.595146$

O $-7.566212-0.676348-1.789159$

C $-6.553483-0.2814230 .363565$

C $-6.194241-1.7744630 .501137$

$\begin{array}{llll}\mathrm{H} & -5.808127 & 0.322858 & 0.891328\end{array}$ 
O $-7.805440-0.069576 \quad 1.022609$ H $-6.240908-2.0344041 .565666$ H -6.977442 -2.384159 0.033703 H $-4.7044516 .851600-0.862746$ H -5.824168 $6.329966-2.131010$ H $-6.4513896 .911284-0.580921$ C $-3.1679504 .180067-0.613162$ H $-6.296028 \quad 1.985007-2.937769$ H $-7.633562-0.355882-2.704973$ H -7.692964 -0.3206731 .955169$ H -3.142645 3.9019820 .448118 H -2.852858 $5.227099-0.682305$ H $-4.5990184 .148794-2.247494$ C $-3.461644-1.524498 \quad 1.825791$ O $-3.772719-1.3152040 .442294$ C $-4.814394-2.145057-0.077060$ C $-4.433080-3.6213520 .084678$ C $-4.071993-3.964667 \quad 1.527470$ C -3.038853 -2.979590 2.077502 H $-4.339361-1.2813352 .436002$ C $-2.353005-0.5223412 .169180$ H $-4.856180-1.948745-1.154932$ O $-5.498346-4.452446-0.364431$ H $-3.567865-3.835893-0.556057$ H $-4.968156-3.9986952 .158205$ O $-3.520059-5.2834711 .540419$ H -2.064846 -3.175535 1.613102 O $-2.896873-3.1949533 .479367$ C $-2.079846-0.3863913 .645173$ H $-1.419578-0.7927081 .660665$ H -2.610799 0.4731391 .788558 H $-5.223941-5.375646-0.228954$ H $-3.389668-5.5382232 .469334$ H $-2.501324-4.0738233 .604739$ O -3.1593210 .1585784 .274503$ O $-1.024461-0.7134154 .172784$ C $-3.021717 \quad 0.336228 \quad 5.687548$ H $-2.860083-0.6291336 .176655$ H -2.198182 1.0230485 .904629 H -3.9503130 .7705826 .067482$

SCF Energy (B3LYP/6-31G**//MMFF) $=-3245.91188173$

01_262

MM̄FF Geometry

C $2.4468730 .085607 \quad 3.231059$

C $1.580868 \quad 1.0612143 .541283$

C $\quad 0.555042 \quad 1.562217 \quad 2.560313$

$\begin{array}{lllll}\text { O } & -0.745514 & 1.327339 & 3.146592\end{array}$

C $0.721134 \quad 3.058176 \quad 2.239790$

C 2.0535393 .4407831 .632095

$\begin{array}{llll}\text { C } 2.491383 & 3.058809 & 0.415752\end{array}$

C $2.913772 \quad 4.3229252 .499778$

C $1.7954262 .189129-0.597973$

C $1.8401122 .784263-2.016326$

C $0.5190043 .392653-2.518149$

C $-0.0183864 .505922-1.617135$

C -1.374278 5.041619-2.104232

O $-0.4389942 .332982-2.642278$

O $-1.9518905 .813317-1.038889$

C $-2.3196503 .846013-2.356248$

C $-1.2187875 .997323-3.292478$

O $-3.5464904 .280111-2.937787$

C $-1.6870752 .738089-3.225367$

C -2.587796 $1.487402-3.349982$

O $-1.496158 \quad 3.239640-4.554838$

C $-1.794397 \quad 1.2431042 .282945$

O -1.7699251 .4400831 .078289$

C -2.984546 0.8207293 .052357

C $-4.136537 \quad 0.6099952 .398861$

C $-5.384403 \quad 0.1098712 .975228$

C $-5.403184-0.2078024 .446710$

C $-2.8966890 .769246-2.035369$

C $-3.636900-0.538628-2.263297$

C $-4.403706-2.575387-1.044423$

O $-4.100997-0.865985-3.352526$

C $-5.816835-2.366620-0.599343$

C $-6.341626-2.9226300 .503568$

C $-7.764587-2.720003 \quad 0.945084$
C $-7.867156-2.141740 \quad 2.340645$

C $-8.088997-2.9381873 .400011$

C $-7.790773-0.6383482 .501496$

C $-6.437344-0.0828542 .154105$

N $-3.730117-1.290957-1.100604$

H $2.392387-0.3701582 .245507$

H $1.615683 \quad 1.5257524 .522851$

H $\quad 0.620747 \quad 0.971417 \quad 1.642024$

H $0.5478083 .638593 \quad 3.157142$

H $-0.081817 \quad 3.389557 \quad 1.571126$

H 3.4859893 .3863940 .113546

H 3.8812264 .5480452 .038696

H 2.4068715 .2747052 .689447

H 3.1118983 .8373563 .460682

H $2.3355891 .234324-0.612074$

H $0.765827 \quad 1.957731-0.314853$

H $2.6385353 .531300-2.105008$

H $2.1009631 .965623-2.701108$

H $0.7203683 .791460-3.519944$

H $0.7112425 .320083-1.527833$

H $-0.1675214 .123419-0.601797$

H $-1.3268606 .522430-0.809874$

H -2.571628 $3.426687-1.373925$

H $-0.612578 \quad 6.865716-3.007601$

H -2.188623 $6.399350-3.605739$

H $-0.7402435 .534338-4.157734$

H -3.913039 $4.970615-2.359171$

H $-3.5226281 .749474-3.862614$

H $-2.085806 \quad 0.769992-4.015315$

H $-1.4203512 .485393-5.162278$

H -2.870783 0.6537004 .117047

H -4.1662630 .7960291 .325976$

H -6.361216 -0.6026244 .792583$

H -5.1997980 .6928335 .036482$

H $-4.647720-0.9642104 .687332$

H - $1.9649920 .549822-1.505830$

H $-3.5254971 .395051-1.393673$

H -3.840479 -3.210163-0.354169

H $-4.391336-3.042562-2.034354$

H $-6.442306-1.732809-1.223797$

H $-5.721396-3.5700691 .119384$

H $-8.267314-3.6952610 .906077$

H $-8.314547-2.078017 \quad 0.245624$

H -8.191091-2.533280 4.401219

H -8.169538 -4.0146623 .289744$

H $-8.085183-0.3314313 .511723$

H -8.536514 -0.1729331 .843906$

H -6.3281840 .1689591 .098811$

H -3.360668 $-0.912406-0.233275$

C $4.923051-0.311607 \quad 3.554453$

O $4.983604-0.9379112 .254534$

C $5.655621-0.0410751 .349006$

$\begin{array}{llll}\text { C } & 6.419110 & 0.934353 & 2.235267\end{array}$

C $5.428298 \quad 1.1208373 .369027$

H 4.8779230 .5174270 .814615

$\begin{array}{llll}\text { C } & 6.514898 & -0.823902 & 0.353387\end{array}$

H $7.3515890 .496862 \quad 2.610709$

H 6.6607741 .8673341 .717597

C $6.035187 \quad 1.7284024 .620897$

H 4.6210001 .7736393 .016589

C $5.712439-1.824470-0.507798$

O $7.499762-1.554677 \quad 1.092928$

H $7.063047-0.123709-0.288495$

H $5.313342-2.6264310 .123706$

O $6.621450-2.440812-1.427825$

C $4.572727-1.181244-1.323701$

C $3.895471-2.122546-2.337962$

H $3.811662-0.784981-0.645193$

O $5.086018-0.062616-2.054333$

H $3.224454-1.515312-2.957795$

H $4.644869-2.508368-3.040068$

H 5.2831721 .8231985 .410603

H 6.8562341 .1155765 .007642

H 6.4318292 .7268774 .409881

C $3.514029-0.4483574 .141422$

H $8.158368-1.8831460 .458156$

H $7.204879-3.025171-0.915068$

H $5.786860-0.393457-2.642134$ 
H 3.4715590 .0320625 .125490

H $3.299671-1.5137154 .292780$

H $5.617219-0.8723824 .193850$

C $1.054973-2.133970-1.232789$

O $2.185559-2.837332-0.708558$

C $3.119556-3.289432-1.695411$

C $2.414497-4.207214-2.705294$

C $1.185791-3.542913-3.324787$

C $0.269318-2.991191-2.232023$

H $1.411223-1.228537-1.740080$

C $0.205305-1.630556-0.059909$

H $3.854568-3.905957-1.164199$

O $3.325260-4.599703-3.726895$

H $2.097132-5.122815-2.189215$

H $1.477488-2.762881-4.037611$

O $0.474320-4.527225-4.078526$

H $-0.215745-3.834395-1.728196$

O $-0.767757-2.218697-2.831837$

C $-0.426941-2.7141740 .786861$

H $\quad 0.837182-1.044440 \quad 0.612775$

H $-0.587194-0.981382-0.444349$

H $2.836196-5.162409-4.351654$

H $-0.328016-4.101137-4.426163$

H $-0.356463-1.459200-3.278563$

O $-1.508284-2.1923901 .439157$

O $-0.007930-3.8580630 .897161$

C -2.202548 -3.093889 2.306946

H $-2.498996-3.9980991 .766763$

H $-1.571596-3.345138 \quad 3.164367$

H -3.104586 -2.592900 2.667481

SCF Energy (B3LYP/6-31G**//MMFF) $=-3245.91244550$

01_263

MMFF Geometry

C -5.432116 2.7649940 .306449

C $-4.3920142 .862651-0.534344$

C $-3.2816163 .868640-0.372938$

O $-2.0625713 .145759-0.081576$

C $-3.0904424 .648945-1.685841$

C -2.032013 5.732666-1.601155

C $-0.8136635 .671320-2.175883$

C $-2.4358096 .963851-0.830251$

C $-0.2265094 .545537-2.982594$

C $0.9171503 .875719-2.220411$

C $1.4706962 .610452-2.894760$

C $0.4384911 .480764-2.945999$

C $1.0363910 .159960-3.446533$

$\begin{array}{llll}\text { O } & 2.596069 & 2.206037 & -2.104431\end{array}$

O $0.078294-0.863862-3.146206$

C $2.323321-0.142235-2.652066$

C $1.198698 \quad 0.162136-4.970915$

O $2.972894-1.303325-3.164458$

C $3.2985161 .054459-2.590749$

C $4.443757 \quad 0.761187-1.593094$

O $3.846291 \quad 1.274233-3.890847$

C -1.8549812 .7907651 .218011$

O $-2.583217 \quad 3.0259742 .169554$

C -0.5769692 .0490331 .289728$

C $-0.183877 \quad 1.5135252 .454973$

C 1.0464360 .7518032 .685018

C 2.0247780 .6541601 .546365

C $5.4424631 .916258-1.452693$

C $6.188593 \quad 1.861276-0.136003$

C 5.9745132 .5119282 .261780

O $7.3299811 .413778-0.057329$

C 5.6341751 .3029213 .073196

C 4.9574981 .3564494 .230870

C 4.7033650 .1611845 .102510

C $3.239596-0.0358125 .419507$

C $2.7522290 .265437 \quad 6.635703$

C $2.377979-0.6815694 .357875$

C $1.238177 \quad 0.187853 \quad 3.895835$

N $5.445642 \quad 2.3672450 .919781$

H $-5.503376 \quad 3.4450241 .151592$

H $-4.3131672 .164586-1.364753$

H -3.499292 4.5700980 .442182

H $-4.0390215 .120986-1.976560$

H -2.861212 $3.945729-2.492607$
H $-0.1454996 .522869-2.051659$

H $-1.6714417 .747473-0.867143$

H -3.357287 $7.386181-1.244426$

H $-2.605687 \quad 6.721712 \quad 0.223212$

H -0.987143 $3.814504-3.255487$

H $0.1531094 .954205-3.926857$

H $1.7387704 .594343-2.093515$

H $0.6001953 .627948-1.199843$

H $1.8174152 .870720-3.901478$

H $-0.4238061 .750807-3.565740$

H $0.0376711 .316202-1.937540$

H $0.461378-1.712625-3.425527$

H $2.017008-0.397992-1.629361$

H $0.2252040 .300475-5.456859$

H $1.576121-0.800645-5.332474$

H $1.8625350 .951356-5.329962$

H $3.431856-1.049424-3.983268$

H $4.0108350 .535715-0.611567$

H $5.001867-0.129296-1.908375$

H $4.6534641 .805866-3.808764$

H $0.003806 \quad 1.954340 \quad 0.381255$

H -0.8282101 .6319463 .325252$

H $2.3121961 .653377 \quad 1.200654$

H $1.582594 \quad 0.1087140 .706787$

H 2.9514330 .1408761 .809050

H $6.1803481 .887629-2.262989$

H $4.9462512 .891627-1.518293$

H 5.5459463 .4271922 .682481

H 7.0620852 .6291612 .214370

H 6.0045290 .3451322 .715542

H 4.6135712 .3191454 .602382

H $5.2739090 .302277 \quad 6.029967$

H $5.101133-0.7562934 .650753$

H $1.7126510 .085878 \quad 6.888773$

H 3.3825280 .6962717 .406631

H $1.949804-1.6023784 .775464$

H $2.998344-1.0213243 .524067$

H 0.4630450 .3230154 .651602

H $4.4890222 .666257 \quad 0.755114$

C -6.2606830 .4439750 .941161$

$\begin{array}{lllll}0 & -5.182406 & -0.288051 & 0.326863\end{array}$

C $-4.429650-0.9542231 .360199$

C $-5.220925-0.7684932 .653861$

C -5.8877750 .5736222 .416082$

H $-3.479531-0.4173241 .454611$

C $-4.178698-2.4163320 .971763$

H $-5.977519-1.5504732 .786180$

H $-4.574631-0.7771013 .537128$

C $-7.0692200 .843465 \quad 3.329914$

H -5.1334931 .3558772 .564763$

C $-3.483065-2.627678-0.390695$

O $-5.441838-3.0972350 .910072$

H -3.613253 -2.917214 1.764489

H $-4.143494-2.256569-1.184947$

O $-3.350922-4.035108-0.619392$

C $-2.094987-1.986862-0.570548$

C $-1.080409-2.2016050 .570087$

H -1.659776 -2.376632 -1.497967

O $-2.266362-0.581844-0.765542$

H $-0.179890-1.6286790 .317519$

H $-1.455339-1.7298831 .486056$

H $-7.519193 \quad 1.8158613 .106037$

H -7.8455010 .0789263 .219861$

H -6.7513490 .8508834 .377630$

C $-6.520943 \quad 1.739927 \quad 0.169361$

H $-5.991115-2.6361210 .252799$

H $-4.234255-4.430000-0.520730$

H $-1.393332-0.204687-0.966428$

H -7.4593562 .1919220 .511670$

H $-6.6579591 .501107-0.893248$

H $-7.150301-0.1919950 .837116$

C $0.932563-3.957967-0.904610$

O $-0.312174-4.371765-0.331381$

C $-0.699370-3.6690780 .851138$

C $0.370673-3.8517101 .935732$

C $1.758338-3.4513521 .439613$

C $2.072860-4.128610 \quad 0.105701$

H $0.863458-2.910509-1.221072$ 
C $1.173398-4.822098-2.149587$

H -1.601867 -4.166730 1.223454

O $0.017164-3.0989273 .090162$

H $0.393962-4.9085042 .233169$

H $1.857965-2.3639721 .357735$

O $2.743265-3.8761332 .384875$

H $2.266720-5.1955370 .276085$

O $3.264121-3.559103-0.435226$

C $0.290316-4.394137-3.297112$

H $2.208989-4.736619-2.498726$

H $0.987354-5.884008-1.948924$

H $0.617216-3.3610983 .808214$

H $2.578787-3.4013603 .216350$

H $3.964564-3.6640320 .231348$

O $-0.984565-4.835210-3.102426$

O $0.687770-3.734299-4.248976$

C -1.929895 -4.446673 -4.103571

H -1.989134 -3.355795 -4.166564

H -1.653788 -4.876228 -5.071086

H -2.910331-4.833807 -3.813439

SCF Energy $\left(B 3 L Y P / 6-31 G^{* *} / / M M F F\right)=-3245.91628554$

\section{1_264}

MM̄FF Geometry

C $0.296793 \quad 3.0257682 .620772$

C 0.7312681 .9159322 .004828

C $1.295792 \quad 0.7317372 .745665$

O $0.517979-0.4356442 .395572$

C 2.7440410 .4226202 .319400

C 3.6947141 .5948722 .411546

C 4.1736252 .2742701 .350336

C 4.1058891 .9793513 .808381

C $3.8829032 .038455-0.107883$

C $5.1560791 .847131-0.943916$

C $6.0139920 .643214-0.522908$

C $7.2925200 .588013-1.363312$

C $8.151236-0.635281-1.015240$

O $5.253247-0.556856-0.708112$

O $9.143642-0.785209-2.041830$

C $7.260688-1.892240-1.059610$

C $8.914297-0.4459260 .301037$

O $7.975511-3.037615-0.599746$

C $5.930933-1.746225-0.278567$

C $5.035390-2.979598-0.564531$

O $6.216010-1.7057151 .123266$

C $-0.603854-0.7085963 .112419$

O $-1.061804-0.0788854 .050346$

C -1.181495-1.935845 2.529115

C -2.300707 -2.489413 3.016006

C $-2.877711-3.7061312 .454191$

C $-3.171809-4.7709963 .471231$

C $3.586064-2.862102-0.075856$

C $2.636153-2.251522-1.095275$

C $0.203183-1.753315-1.390990$

O $2.989518-1.862750-2.204444$

C $-0.789215-2.845478-1.673536$

C $-0.773747-4.117649-1.241561$

C $-1.848235-5.119376-1.573759$

C $-2.477656-5.742491-0.343388$

C $-2.102975-6.9625270 .077234$

C $-3.594176-4.9935730 .355206$

C -3.086545 -3.811601 1.126953

N $1.335498-2.216328-0.611664$

H $\quad 0.3228173 .0495023 .709002$

H $\quad 0.714929 \quad 1.844796 \quad 0.921284$

H $\quad \begin{array}{llll}1.265557 & 0.879320 & 3.832675\end{array}$

H $2.737412-0.0105511 .313852$

H $3.127245-0.3971502 .943221$

H 4.8452703 .1105971 .541281

H 4.8243512 .8057673 .818012

H 3.2357072 .2927884 .393404

H 4.5765161 .1285644 .312064

H $3.2119921 .192396-0.275381$

H $3.3527882 .920813-0.487999$

H $4.8477751 .723355-1.990240$

H $5.7596862 .762314-0.892879$

H 6.2716860 .7469080 .536791

H $7.8761831 .509870-1.246758$
H $7.0267940 .533860-2.428430$

H $9.6658810 .034591-2.075676$

H $7.018789-2.082123-2.114915$

H 9.5739020 .4280030 .238423

H $9.569175-1.3004530 .504153$

H $8.257914-0.3021151 .161691$

H $8.790399-3.101255-1.126869$

H $5.031487-3.194069-1.640151$

H $5.480341-3.851169-0.066549$

H $5.447058-2.0350631 .614526$

H $-0.661663-2.3797691 .686669$

H -2.807154 -2.066782 3.879358

H -3.962009-4.438898 4.153444

H $-2.276787-4.9921174 .063901$

H -3.496354 -5.714503 3.026235

H $3.214108-3.8753520 .125036$

H $3.519494-2.2966260 .858180$

H $\quad 0.551397-1.324055-2.335666$

H $-0.283186-0.960704-0.813235$

H -1.622074 -2.537274 -2.302281

H $0.044200-4.474032-0.621356$

H -1.391484 -5.900162 -2.195962

H -2.635873 -4.672590 -2.193321

H -2.564409 -7.4291530 .941148$

H -1.322200 -7.519692 -0.429898

H $-4.168422-5.6677440 .999905$

H $-4.317419-4.644757-0.392227$

H -2.857211 -2.947228 0.504464

H $1.158367-2.5314920 .337302$

C -0.0233844 .4345230 .471603$

O $-0.9458263 .576351-0.228800$

C -1.393523 $4.253055-1.419649$

C $-0.6007005 .553446-1.504854$

C $-0.3222515 .841435-0.044396$

H -2.449461 $4.500072-1.263713$

C $-1.2364163 .350274-2.647257$

H $0.3386785 .421901-2.054724$

H -1.165734 $6.349349-2.000036$

C 0.8046306 .8385510 .165765

H $-1.239973 \quad 6.2258680 .420879$

C $-2.0237252 .026198-2.576277$

O $\quad 0.1524383 .024703-2.794672$

H -1.518257 $3.908895-3.548078$

H -1.575137 $1.375391-1.817332$

O $-1.835891 \quad 1.354388-3.829744$

C $-3.5333492 .181139-2.309065$

C $-4.3584300 .901624-2.543408$

H $-3.6852572 .534710-1.283486$

O $-4.0517423 .190245-3.181266$

H $-5.4103091 .140381-2.344072$

H $-4.3327340 .645247-3.610337$

H $0.973737 \quad 7.0222801 .231091$

H $1.7442896 .479160-0.267067$

H $0.5622207 .796779-0.305701$

C -0.2108174 .2933161 .984695$

H $0.2342512 .449316-3.574410$

H -2.262616 $1.893255-4.517984$

H $-4.9864403 .326254-2.949757$

H -1.2740514 .3853872 .235553$

H 0.3105715 .1189822 .483436

H 0.9915864 .1357120 .178086

C $-5.091514 \quad 0.223175 \quad 0.332853$

O $-3.830286-0.001390-0.307293$

C $-3.914998-0.307937-1.700966$

C $-4.763921-1.568834-1.913290$

C $-6.128238-1.461859-1.237429$

C $-5.978575-1.0210250 .218343$

H $-5.5911641 .083261-0.129047$

C -4.8099750 .5690841 .797659$

H -2.897457 -0.555254-2.020390

O $-4.946982-1.836259-3.299511$

H -4.237783 -2.432314 -1.491223

H $-6.794655-0.784542-1.784441$

O $-6.760391-2.744128-1.251941$

H $-5.555748-1.8433690 .808763$

O $-7.267605-0.7294690 .753948$

C $-4.0880201 .889525 \quad 1.907187$

H $\quad-5.7274080 .6290122 .393715$ 
H $-4.173091-0.1914102 .263282$

H -4.064384 -1.921547 -3.699132

H -6.812780 -3.029766 -2.180279

H -7.813182 -1.5275980 .646794$

O -4.9684162 .9182621 .747619$

O $-2.883126 \quad 1.9908002 .095378$

C -4.3993164 .2295051 .808195$

H -3.6756254 .3648330 .999408$

H -5.2057664 .9564921 .681718$

H -3.927477 4.3943432 .781575

SCF Energy (B3LYP/6-31G**//MMFF) $=-3245.89985773$

01265

MMFF Geometry

C -1.845715 3.254212 -1.104309

C $-0.8000343 .948936-0.629816$

C 0.2529593 .3250380 .245616

O $1.5228053 .454279-0.434298$

C $0.3743194 .003487 \quad 1.623338$

C -0.8725573 .9396272 .477259$

C -1.3257982 .8233723 .080834$

C -1.601554 5.2464782 .646784

C -0.7033861 .4533543 .054793$

C - 1.5447570 .4867232 .214026

C - $1.000420-0.9488062 .143201$

C $-1.190629-1.7012423 .461862$

C $-0.634248-3.1314493 .383838$

O $0.384052-0.9154951 .775328$

O $-0.602133-3.6679644 .715783$

C $0.813627-3.0675792 .849063$

C $-1.568398-4.0606192 .601565$

O $1.338411-4.3772802 .645050$

C $0.967557-2.210094 \quad 1.569235$

C $2.451029-2.0274371 .162224$

O $0.324414-2.8919910 .485055$

C $2.4151512 .446810-0.233050$

O $2.248447 \quad 1.4433200 .441548$

C $3.6500432 .764351-0.977537$

C $4.5422821 .783903-1.181787$

C $5.8268081 .905978-1.869837$

C $6.285503 \quad 3.293014-2.235988$

C $3.321427-1.2653172 .165144$

C $4.786828-1.2298191 .753627$

C $6.979006-0.3955512 .605314$

O $5.247841-1.9090620 .840348$

C $7.5550480 .367031 \quad 1.453075$

C $8.483075-0.1534080 .634407$

C $9.1574260 .589087-0.485487$

C $8.888704-0.034252-1.838008$

C $9.560642-1.124934-2.244184$

C $7.8874880 .646477-2.745891$

C $6.5327720 .780628-2.108360$

N $5.527374-0.3840292 .570801$

H -1.924524 $2.194307-0.874937$

H $-0.7072635 .008097-0.854994$

H $\quad 0.024194 \quad 2.2623360 .373137$

H $0.6797305 .050347 \quad 1.485320$

H 1.2136183 .5621282 .177255

H -2.237112 2.8918893 .673429

H $-2.5306105 .135103 \quad 3.215533$

H $-0.9683545 .963873 \quad 3.179162$

H -1.860555 5.6728601 .672818

H $\quad 0.323210 \quad 1.4896012 .678755$

H -0.6378561 .0981474 .089140$

H -2.577262 0.4677162 .584432

H $-1.593347 \quad 0.8735881 .189298$

H -1.561005 -1.455087 1.348935

H $-2.248057-1.7175083 .753770$

H $-0.665538-1.1732264 .268215$

H $-0.034851-3.0971235 .261224$

H $1.416067-2.6242293 .649592$

H -2.559899 -4.086406 3.069786

H -1.204465 -5.0939562 .619621$

H $-1.703383-3.761618 \quad 1.560637$

H $\quad 0.951657-4.726314 \quad 1.824097$

H $2.890736-3.0102600 .947513$

H $2.490606-1.4832710 .208108$

H $0.666029-2.531493-0.350251$
H $3.7873173 .778193-1.333654$

H $4.3100980 .785349-0.812133$

H $7.2851023 .325554-2.672655$

H $6.3151083 .934731-1.348114$

H $5.605563 \quad 3.740292-2.969447$

H $2.957244-0.2399862 .282521$

H $3.291299-1.7490343 .146490$

H $7.314249-1.4383992 .619458$

H 7.2837540 .0773843 .544488

H 7.2306641 .3950821 .315166

H $8.814395-1.1765710 .801672$

H $10.2384600 .585905-0.292670$

H $8.8688121 .647415-0.492789$

H $9.390493-1.564498-3.221233$

H $10.296781-1.602229-1.605645$

H $7.7593320 .073605-3.673318$

H $8.3084691 .608750-3.047944$

H $6.098205-0.175609-1.811852$

H 5.0521850 .0867693 .335391

C $-4.3367413 .694227-1.281666$

O $-4.6736482 .301058-1.108078$

C -5.2184212 .1254920 .214156$

C $-5.611036 \quad 3.5202970 .688265$

C $-4.493788 \quad 4.351936 \quad 0.090878$

H $-4.398219 \quad 1.7612370 .845025$

C $-6.359464 \quad 1.1029190 .212839$

H -6.5773983 .8348150 .277851$

H -5.6635783 .5897531 .778994$

C -4.7941635 .8387030 .033260$

H $-3.597608 \quad 4.196257 \quad 0.700869$

C $-5.992855-0.262175-0.405505$

O $\quad-7.4494051 .645555-0.542847$

H $-6.727803 \quad 0.9710131 .237591$

H $-5.899189-0.159661-1.492874$

O $-7.085912-1.159292-0.172114$

C $-4.718811-0.9079740 .168256$

C $-4.459665-2.353553-0.295812$

H $-3.850325-0.294659-0.093245$

O $-4.790692-0.9237581 .596861$

H -3.510260 -2.675366 0.148954

H $-5.211257-3.0213040 .144828$

H $-3.9605976 .386095-0.418049$

H $-5.6933606 .044468-0.556951$

H -4.9546236 .2375511 .040334$

C $-2.9554183 .834543-1.933995$

H $-8.2311821 .095238-0.368018$

H -7.835857 -0.854538 -0.710158

H $-5.585375-1.4264711 .845665$

H -2.761269 $4.890485-2.155729$

H $-2.9592103 .297738-2.890788$

H $-5.0842284 .097471-1.977239$

C $-2.208519-1.707376-2.264655$

O $-3.617341-1.568043-2.479465$

C $-4.425959-2.546321-1.823499$

C $-4.023837-3.958078-2.274054$

C $-2.524999-4.207350-2.120149$

C -1.723854 -3.075136 -2.766264

H -1.990142 -1.602874 -1.195215

C $-1.542604-0.542963-3.005773$

H $-5.449834-2.383375-2.181714$

O $-4.741498-4.947979-1.544099$

H -4.286526 -4.086297 -3.332430

H $-2.253769-4.338306-1.065930$

O $-2.211562-5.439274-2.774490$

H -1.825987 -3.134121 -3.857509

O $-0.343974-3.238300-2.454679$

C $-0.131128-0.290454-2.539474$

H -1.566122 -0.715370 -4.088784

H $-2.1043290 .386353-2.848660$

H -5.689839-4.781228 -1.680479

H - $-1.285815-5.652972-2.569756$

H $-0.037756-4.053711-2.885868$

O $0.733676-0.427529-3.584159$

O $0.166687-0.008771-1.386371$

C $2.115370-0.219770-3.277888$

H $2.2985200 .847755-3.132022$

H $2.412666-0.790540-2.392705$

H $2.708182-0.565047-4.129159$ 
O $3.8452162 .539928-2.267318$

C $4.5976712 .309334-0.003919$

C $3.8935754 .574485-1.035915$

O $5.9490082 .698342-0.234537$

C 4.2501202 .4755041 .489783

C 5.0794771 .5522622 .416040

O $4.5250453 .824201 \quad 1.888434$

C $1.107415-1.9994692 .113923$

O $1.402447-3.0144992 .723880$

C $1.538830-1.6758990 .736743$

C $1.966861-2.660764-0.066445$

C $2.412638-2.508305-1.452251$

C $2.677498-1.112357-1.949045$

C 4.9240050 .0491312 .158726

C $5.997014-0.5397741 .257650$

C $6.608437-2.6637610 .104658$

O $\quad 6.9996590 .0770390 .908739$

C $6.181522-2.586044-1.327026$

C $5.956708-3.668217-2.087630$

C $5.571500-3.607892-3.537830$

C $4.316827-4.395824-3.841161$

C $4.386783-5.641552-4.339956$

C $2.985294-3.706598-3.648218$

C $2.583516-3.617181-2.201968$

N $5.718505-1.8575970 .919214$

H -1.804251 -2.208814 1.837468

H -1.906583 -2.109323 4.895127

H $\quad 0.490647-1.5652004 .540844$

H $-1.0756690 .296028 \quad 5.333696$

H -1.2463180 .8053603 .666770$

H 1.8571412 .8721484 .093256

H 2.4195941 .5028885 .828454

H 1.1012690 .6000956 .575671

H $2.134438-0.1709775 .349667$

H -0.9459622 .7490082 .916038$

H 0.2041384 .0527442 .878388

H $0.395696 \quad 1.478426 \quad 1.234709$

H -0.1875203 .0404460 .659512$

H 2.0994514 .0177931 .222888

H $1.4888013 .359004-1.133902$

H $1.9743501 .710411-0.798101$

H $3.1940422 .952442-2.860408$

H $4.5294481 .247679-0.267871$

H $3.2241455 .033747-1.773269$

H $4.9107194 .782435-1.385937$

H $3.7418945 .092230-0.086610$

H $6.1350602 .554373-1.178154$

H 6.1324551 .8584052 .409331

H 4.7327861 .7317723 .444214

H $4.634753 \quad 3.8444512 .853507$

H $1.470298-0.6435220 .414733$

H $1.968024-3.6810490 .315406$

H $3.193165-1.082325-2.911265$

H $3.322459-0.572489-1.247907$

H $\quad 1.740725-0.559014-2.060619$

H $5.012400-0.4765183 .118321$

H $3.935655-0.1812441 .750156$

H $6.568187-3.6857240 .494404$

H $7.633331-2.2920310 .204631$

H $6.078799-1.595994-1.764849$

H $6.411427-4.006651-4.121882$

H $5.442856-2.572265-3.876615$

H $3.491923-6.203060-4.587260$

H $5.341226-6.129767-4.507022$

H $2.194726-4.264943-4.167578$

H $3.008411-2.733451-4.146846$

H $2.376195-4.586991-1.747491$

H $4.837713-2.2713961 .211160$

C $-4.600554-2.4994152 .241613$

O $-4.388257-2.5394530 .815989$

C $-5.650776-2.7744910 .169849$

C $-6.722214-2.6883841 .247953$

C $-5.955705-3.1690492 .466617$

H -5.626085 -3.813810 -0.185608

C $-5.808382-1.865243-1.056794$

H -7.069648 -1.663870 1.404565

H -7.595202 -3.307388 1.018962

C -6.620021-2.801070 3.782153

H -5.849171 -4.260703 2.408095

C $-5.563773-0.361379-0.795327$

O $-7.094439-2.067287-1.634852$

H $-5.061463-2.186920-1.793627$

H $-4.671428-0.255565-0.170813$

O $-6.651474 \quad 0.203588-0.069042$

C $-5.3402050 .449538-2.088045$

C $-5.135517 \quad 1.959815-1.865820$

H $-4.4734040 .034110-2.614214$

O $\quad-6.464614 \quad 0.288737-2.955324$

H -4.938202 2.411061 -2.846387

H $-6.0813612 .411439-1.538682$

H $-6.031683-3.1605774 .632050$

H $-6.732653-1.7165603 .885186$

H -7.616414 -3.2501233 .848178$

C -3.440782 -3.199339 2.950336

H $-7.181844-3.014770-1.834858$

H -7.449482 $0.100325-0.615722$

H $-6.2649590 .754172-3.785543$

H -3.255576 -4.178108 2.491336

H -3.691674 -3.371052 4.003116

H $-4.644883-1.4406682 .530320$

C $-2.1170731 .916805-2.301871$

O $-2.8145441 .590637-1.095911$

C $-4.0144372 .333486-0.875744$

C $-3.6953273 .832990-0.819879$

C $-2.9245314 .301358-2.053532$

C $-1.7181783 .397116-2.311273$

H $-2.7518891 .695053-3.168614$

C $-0.8839801 .011050-2.364880$

H $-4.3711642 .049330 \quad 0.121958$

O $-4.8974714 .583010-0.680147$

H -3.086374 4.0316570 .072046

H $-3.5801994 .353952-2.930577$

O $-2.4714615 .635083-1.809279$

H $-0.952973 \quad 3.587478-1.549776$

O $-1.1488783 .713871-3.579528$

C -1.285062 -0.420899 -2.630526

H $-0.195850 \quad 1.306341-3.164642$

H $-0.3205151 .051432-1.425566$

H $-4.6528245 .524224-0.665487$

H $-2.0724955 .965226-2.631846$

H -0.799466 $4.619335-3.528188$

O $-1.263403-1.137186-1.470527$

O $-1.598979-0.844644-3.735538$

C $-1.618596-2.517423-1.594625$

H $-0.915950-3.030747-2.257830$

H -1.562513 -2.974945 -0.603687

H -2.641493 -2.613736 -1.968695

SCF Energy $($ B3LYP/6-31G**//MMFF $)=-3245.89820378$

01267

MM̄MF Geometry

C $-1.7720893 .453552-0.435861$

C -1.189906 2.295246 -0.093221

C $-0.2574331 .524946-0.991874$

O $0.9543751 .233823-0.258011$

C $-0.9254150 .196417-1.393924$

C $-0.065758-0.704406-2.260409$ 
C $0.197412-1.994310-1.971059$

C $0.482379-0.093042-3.522873$

C $-0.316650-2.780976-0.798324$

C $0.531941-2.6541580 .473276$

C $1.887758-3.3733740 .394270$

C $2.598492-3.3055791 .748718$

C $3.992697-3.9476991 .701261$

O $2.688535-2.740483-0.605853$

O $4.693705-3.5632502 .894348$

C $4.770235-3.3546020 .507341$

C $3.915301-5.4787361 .719432$

O $6.021371-4.0182050 .336487$

C $3.967108-3.349682-0.816722$

C $4.699558-2.558316-1.926961$

O $3.831653-4.700553-1.273102$

C $1.8912302 .221275-0.213166$

O $1.8098123 .329893-0.719285$

C $3.049247 \quad 1.7246180 .565474$

C 4.0529402 .5717580 .841006

C 5.2938832 .2664101 .556667

C 5.4841590 .8671782 .077214

C $4.674968-1.038617-1.740443$

C $5.799215-0.334788-2.479725$

C $6.9217641 .891470-2.578112$

O $6.578590-0.910399-3.233384$

C $7.8936821 .967623-1.441019$

C $8.2515873 .111514-0.838491$

C 9.2050393 .1755280 .320965

C 8.6225503 .8899881 .522111

C 9.0754975 .1001701 .891386

C 7.5678953 .1709292 .341650

C 6.2020493 .2533591 .712254

N $5.8307341 .021119-2.180449$

H -1.559786 $3.892578-1.407554$

H $-1.403561 \quad 1.8576250 .878474$

H $-0.0155682 .101338-1.893727$

H -1.854828 $0.405926-1.940734$

H -1.221207 -0.326860 -0.478059

H $0.828233-2.560111-2.655058$

H $\quad 0.950487-0.838283-4.175094$

H $-0.3216230 .381560-4.094833$

H $1.2410690 .660475-3.289818$

H -1.343417 -2.474623 -0.572662

H $-0.392337-3.835921-1.090231$

H $0.688857-1.5915750 .698176$

H $\quad-0.044447-3.0772301 .305655$

H $1.708395-4.4164370 .108015$

H $\quad 1.989039-3.7722142 .532724$

H $2.713215-2.2533342 .046237$

H $4.175238-3.8687383 .658430$

H $5.018418-2.3231910 .781885$

H $3.389778-5.8244822 .617784$

H $4.914751-5.9244601 .769941$

H $3.394294-5.892173 \quad 0.853484$

H $5.846336-4.874160-0.090437$

H $5.724756-2.937056-2.021477$

H $4.229280-2.772116-2.896976$

H $3.623805-4.686319-2.221899$

H 3.0505490 .6851340 .872222

H 3.9694713 .6002380 .490397

H 5.6286750 .1671681 .249529

H 4.6152980 .5461962 .662729

H $\quad 6.3444320 .7679072 .743270$

H $3.719930-0.636212-2.098610$

H $4.769714-0.778205-0.683672$

H $6.4902312 .867162-2.821623$

H $7.4162311 .489343-3.467533$

H $8.3189721 .029184-1.092064$

H $7.8414384 .053192-1.196115$

H $10.112006 \quad 3.689231-0.023541$

H 9.5281632 .1726710 .627098

H 8.6809525 .6087092 .764855

H 9.8472555 .6121511 .326343

H 7.5005743 .6205403 .340672

H 7.9008532 .1402002 .494471

H 5.9500154 .2469371 .338090

H $5.1634241 .389260-1.509777$

C $-4.1279064 .246822-0.200576$
O $-4.6707842 .917514-0.316992$

C $-6.1006802 .983001-0.145757$

C -6.4483744 .4588080 .035440$

C $-5.1618925 .018750 \quad 0.612518$

H $-6.3315362 .450507 \quad 0.783251$

C $-6.7990382 .306378-1.333228$

H $-6.6819304 .942507-0.920145$

H -7.3107014 .5996790 .694589$

C $-5.051138 \quad 6.5283470 .492559$

H -5.0982244 .7323151 .670896$

C $-6.3736020 .839896-1.562748$

O $-6.4960743 .037261-2.529573$

H $-7.8858972 .369546-1.208630$

H $-5.3111730 .809878-1.827373$

O $-7.0741850 .346635-2.711634$

C $-6.643657-0.096702-0.369984$

C $-6.331661-1.580267-0.648013$

H -6.0629570 .2337150 .495922$

$\begin{array}{lllll}\text { O } & -8.018540 & 0.000957 & 0.012009\end{array}$

H $-6.710263-2.1665930 .198068$

H $-6.917083-1.926865-1.508698$

H -4.1089106 .8866630 .918620$

H $-5.0929906 .852076-0.552865$

H -5.8708017 .0175501 .029082$

C $-2.7436304 .189246 \quad 0.445206$

H $-5.5302853 .024256-2.644585$

H $-8.0264980 .460380-2.553079$

H $-8.556165-0.373406-0.706176$

H -2.8100403 .7009301 .424721$

H -2.358841 5.2014370 .611116

H $-4.0552994 .656062-1.217518$

C $-4.165712-1.9871981 .438555$

O $-4.018780-1.3333630 .171906$

C $-4.834089-1.870406-0.874334$

C $-4.499241-3.353629-1.087358$

C $-4.575422-4.1530570 .211896$

C -3.782677 -3.465408 1.323892

H $-5.204168-1.9012121 .780558$

C -3.275000-1.247049 2.440552

H $-4.541924-1.345109-1.791283$

O $-5.370939-3.940370-2.047982$

H -3.480278 -3.440578-1.485405

H $-5.614992-4.3182640 .519003$

O $-4.010783-5.449147-0.001119$

H $-2.708660-3.5674821 .128543$

O $-4.053799-4.1176262 .562789$

C -3.8900310 .0860112 .795510$

H $-3.131750-1.8123883 .368146$

H -2.279620 -1.054498 2.022350

H $-5.290776-3.424268-2.868448$

H $-4.500742-5.860536-0.733816$

H -3.828752 -5.0573142 .451050$

O $-4.840371-0.0796273 .759688$

$\begin{array}{llll}\text { O } & -3.585268 & 1.142796 & 2.259755\end{array}$

C $-5.530716 \quad 1.1117474 .149320$

H -4.8265881 .8410344 .561167$

H $-6.068870 \quad 1.5333563 .294973$

H -6.2551450 .8469864 .924040$

SCF Energy (B3LYP/6-31G**//MMFF $)=-3245.91423350$

01268

MM̄FF Geometry

C $2.012793-3.240094-0.324291$

C $1.219379-3.3332440 .753659$

C $1.494155-2.5646852 .019182$

O $0.281984-1.8969362 .432407$

C $1.962616-3.4870273 .155932$

C $2.274169-2.7559234 .451860$

C $3.289308-1.8845444 .624263$

C $1.342935-3.0646265 .594595$

C $4.297056-1.4530693 .594998$

C $3.990746-0.0353953 .096697$

C $4.5718860 .246877 \quad 1.704831$

C 6.0977630 .3392251 .714608

C 6.6568520 .7140930 .332625

O $4.010377 \quad 1.4871541 .252934$

O 8.0298061 .0834040 .531505

C $5.897570 \quad 1.949527-0.201944$ 
C $6.662006-0.491658-0.616172$

O $6.2658352 .234887-1.549693$

C $4.3595411 .818409-0.098203$

C $3.6830553 .160829-0.467771$

O $3.9177780 .818277-1.020806$

C $0.078682-0.647825 \quad 1.930883$

O $0.763628-0.0673861 .102778$

C -1.124996 -0.0880892 .583031$

C $-1.439715 \quad 1.1940212 .350469$

C -2.6018151 .9195642 .864627$

C $-3.555198 \quad 1.1780833 .761558$

C $2.1885793 .225940-0.117306$

C $1.4893684 .405464-0.776460$

C $-0.7791995 .376163-1.076546$

O $2.054378 \quad 5.182195-1.541598$

C $-1.8736765 .740822-0.126610$

C -3.131767 $5.282469-0.220888$

C -4.2318385 .6632740 .729027$

C -4.6954954 .5056181 .586485$

C $-5.836665 \quad 3.8508401 .314480$

C $-3.873928 \quad 4.1585852 .810539$

C -2.758646 3.2043602 .485554

N $0.1493964 .476803-0.415387$

H $2.909792-2.632106-0.246770$

H $\quad 0.333813-3.9610250 .724621$

H $2.265632-1.8158711 .825694$

H $2.850795-4.0529122 .845970$

H $1.184327-4.2370943 .353483$

H $3.410383-1.4151845 .599437$

H $1.595923-2.5071556 .502476$

H $0.313567-2.8068965 .324408$

H $1.383462-4.1312505 .838472$

H $5.295776-1.4801914 .046812$

H $4.327364-2.1610782 .760367$

H $2.9061210 .130003 \quad 3.053856$

H 4.3604420 .7053243 .818142

H $4.238465-0.5503991 .030741$

H $6.550159-0.5964882 .064339$

H $6.408487 \quad 1.1093332 .434611$

H $8.4214691 .271344-0.338013$

H 6.2121452 .8145210 .398579

H $7.299055-1.289010-0.214466$

H $7.090074-0.228705-1.589913$

H $5.668373-0.913485-0.781154$

H $7.2194472 .421616-1.562030$

H $4.177623 \quad 3.9838330 .064217$

H $3.8149123 .343374-1.541818$

H $3.0121761 .018270-1.303327$

H $-1.715326-0.7381043 .217825$

H -0.7867011 .7743921 .699171$

H -3.047486 0.8619154 .679494

H -3.949566 0.2902223 .259208

H -4.420493 1.7702384 .065212

H $1.6733402 .317389-0.444743$

H 2.0654003 .3119700 .968649

H $-1.1581404 .856073-1.962882$

H $-0.2547926 .275898-1.414274$

H -1.614749 6.4249300 .677996

H -3.391284 $4.598023-1.025422$

H $-5.067226 \quad 6.052790 \quad 0.132546$

H -3.927294 6.4943361 .377608

H $-6.182445 \quad 3.0360441 .942164$

H -6.444904 4.1086110 .453895

H $-4.527733 \quad 3.7738453 .598796$

H -3.440246 5.0736493 .233205

H - 1.9868833 .6416251 .854153

H $-0.243707 \quad 3.7039590 .113793$

C $1.749952-3.002950-2.854344$

O $0.625887-2.100949-2.791372$

C $1.068693-0.791738-3.197130$

C $2.418547-0.995130-3.880305$

C $2.994368-2.137463-3.069617$

H $1.222915-0.221615-2.273286$

C $0.025590-0.079878-4.067960$

H $2.307494-1.298146-4.927609$

H $3.037130-0.092719-3.853456$

C $4.139633-2.862401-3.753995$

H $3.360321-1.720503-2.126138$
C $-1.402497-0.034539-3.488332$

O $-0.048679-0.757076-5.330029$

H $0.3793460 .935699-4.284961$

H $-1.877670-1.014827-3.611295$

O $-2.1632240 .887564-4.280583$

C -1.502577 $0.404109-2.018449$

C $-2.9347490 .687842-1.525123$

H $-1.048004-0.364260-1.383726$

O $-0.739923 \quad 1.599083-1.836766$

H $-2.8770450 .884040-0.447558$

H $-3.2890411 .633631-1.956404$

H $4.509753-3.681193-3.128903$

H $3.830915-3.284143-4.716321$

H $4.972433-2.176120-3.939702$

C $1.769290-3.935699-1.634310$

H $-0.541743-0.185745-5.942449$

H -2.268562 $0.498989-5.165066$

H $-0.7668701 .819306-0.890220$

H $2.555510-4.687270-1.770882$

H $0.812755-4.471605-1.590567$

H $1.577688-3.623887-3.743617$

C $-3.307989-2.007689-0.117817$

O $-3.455991-1.726967-1.511031$

C $-3.962900-0.424808-1.808224$

C $-5.324528-0.211357-1.129529$

C $-5.274182-0.5150210 .367146$

C $-4.649091-1.888702 \quad 0.616631$

H -2.603942 $-1.285698 \quad 0.310674$

C $-2.629072-3.376166 \quad 0.020718$

H $-4.145846-0.412357-2.889688$

O $-5.784624 \quad 1.122930-1.318652$

H $-6.066436-0.876484-1.590335$

H -4.7364810 .2671120 .914199$

O $-6.605386-0.523436 \quad 0.888281$

H $-5.358446-2.656395 \quad 0.286302$

O $-4.460477-2.0538982 .018892$

C $-3.466486-4.542290-0.460628$

H $-1.709620-3.389822-0.576188$

H $-2.361016-3.541677 \quad 1.070256$

H $-5.8234481 .285328-2.276710$

H -6.9960260 .3462200 .695295$

H $-4.230725-2.9844182 .179333$

O $-3.066560-5.6763550 .187168$

O $-4.334156-4.478515-1.320738$

C $-3.758784-6.873023-0.182776$

H $-4.827968-6.7732880 .027395$

H $-3.591339-7.094762-1.241109$

H $-3.359013-7.6958880 .415692$

SCF Energy (B3LYP/6-31G**//MMFF) = -3245.89507971

$01 \_269$

MM̄FF Geometry

C $-3.883072-0.815739-3.743777$

C $-3.005452-1.167178-2.790864$

C $-1.600902-1.643942-3.063447$

O $-0.704038-0.953398-2.162168$

C $-1.500274-3.149423-2.756727$

C $-0.124424-3.734392-3.010926$

C $0.662358-4.279755-2.062181$

C $0.344591-3.705251-4.442998$

C $0.371121-4.442987-0.596627$

C $0.868027-3.291024 \quad 0.289752$

C $2.394654-3.1102210 .293161$

C $2.815462-2.1262301 .385469$

C $4.325597-1.8459961 .361709$

O $2.796161-2.602928-0.981000$

O $4.567665-0.6894492 .178966$

C $4.756782-1.483934-0.077153$

C $5.123972-2.9944731 .991106$

O $6.177956-1.391969-0.143706$

C $4.214736-2.460083-1.144562$

C $4.453258-1.986695-2.600251$

O $4.877098-3.722838-1.002387$

C $-0.3509310 .322575-2.469793$

O $-0.6700430 .959874-3.459954$

C $0.4706120 .828404-1.348396$

C $0.6328772 .149850-1.188819$

C $1.3650432 .798845-0.099902$ 
C $2.164891 \quad 1.9180170 .822386$

C $3.837887-0.630247-2.961125$

C $4.7936110 .541785-2.812406$

C $4.8406453 .031885-2.905964$

O $5.9979940 .408109-2.613468$

C $4.7660853 .575363-1.513955$

C $4.3474634 .818424-1.231146$

C 4.3060875 .3941940 .155389

C 2.9498595 .9616830 .510719

C 2.7013987 .2777790 .401379

C 1.9130505 .0162631 .073521

C 1.2938494 .1413250 .017690

N $4.145098 \quad 1.759856-2.966247$

H $-3.571733-0.895864-4.783615$

H -3.286893 -1.118765 -1.744017

H -1.307520 -1.442164 -4.100628

H -2.224478 -3.702591 -3.369570

H -1.798709 -3.312732 -1.715283

H $1.632641-4.670722-2.367014$

H $1.269625-4.273569-4.588696$

H $-0.413653-4.143075-5.100403$

H $0.537959-2.677735-4.765634$

H $-0.702087-4.582937-0.428991$

H $\quad 0.833982-5.380107-0.261529$

H $\quad 0.386554-2.361116-0.034455$

H $0.528904-3.4915131 .313787$

H $2.859014-4.0888850 .461860$

H $2.515171-2.4957992 .372727$

H $2.281874-1.1764891 .245692$

H $4.232809-0.8784913 .072322$

H $4.366894-0.478504-0.272895$

H 4.811352 -3.153589 3.030299

H $6.192485-2.7563492 .034869$

H $5.001756-3.9416391 .461781$

H $6.413655-1.026436-1.012409$

H $5.521940-2.024644-2.842717$

H $3.983046-2.729743-3.260978$

H $4.797048-4.209313-1.839609$

H $\quad 0.8818680 .100125-0.658964$

H $0.1576622 .822499-1.901610$

H $2.827457 \quad 1.262890 \quad 0.247389$

H 1.5004051 .2983451 .433704

H 2.8135272 .4736831 .502163

H $3.547719-0.652399-4.019547$

H $2.932010-0.442368-2.377800$

H $4.3666983 .695658-3.635833$

H $5.8880842 .890103-3.190808$

H $5.0953002 .926897-0.705397$

H $4.0461405 .473794-2.045397$

H $5.065966 \quad 6.1848980 .209939$

H $4.599278 \quad 4.651456 \quad 0.907724$

H 1.7406797 .6973340 .681054

H $3.452197 \quad 7.9644110 .024467$

H 1.0975155 .5863341 .538949

H 2.3555654 .4471181 .895844

H $0.6914614 .693576-0.704952$

H $3.1352901 .776308-3.076108$

C $-5.655360 \quad 0.143970-2.126677$

O $-4.9240301 .351607-1.820204$

C $-5.8027122 .262209-1.144973$

C $-7.0351581 .458001-0.756235$

C -7.129494 $0.498456-1.930651$

H $-6.1164122 .997827-1.899240$

C $-5.1039203 .031713-0.018461$

H $-6.874650 \quad 0.9058210 .176681$

H $-7.9266012 .081193-0.638779$

C -8.025100 -0.698504-1.663586

H -7.505831 $1.043615-2.806559$

C -4.5306442 .2230261 .167137$

O $\quad-6.0581413 .9684230 .499880$

H $-4.2937903 .627500-0.458292$

H $\quad-5.3499691 .789847 \quad 1.752557$

O -3.8875513 .1793612 .024182$

C $-3.514870 \quad 1.1307210 .787387$

C $-2.783816 \quad 0.506113 \quad 1.987878$

H $-4.037867 \quad 0.344890 \quad 0.244974$

O $-2.5373341 .677617-0.097694$

H -2.0936861 .2496152 .407771$
H $-3.506340 \quad 0.2624142 .774445$

H -8.046393 -1.370748 -2.526920

H -7.679867 -1.273332 -0.797521

H $-9.051169-0.373206-1.463163$

C $-5.294593-0.316697-3.541086$

H $-5.608472 \quad 4.488224 \quad 1.188175$

H -3.7510602 .7563942 .888733$

H -2.0972932 .4114040 .364768$

H $-5.4341930 .527615-4.228540$

H $-5.976220-1.112723-3.861933$

H $-5.371958-0.614648-1.387869$

C -1.029609-1.126416 3.805502

O $-0.780650-0.8457872 .424135$

C -1.959792 -0.7506631 .612914$

C $-2.720381-2.0862211 .660958$

C $-3.005936-2.5327303 .095478$

C -1.736691-2.478404 3.945585

H $-1.645895-0.3313484 .243573$

C $0.324555-1.1370804 .528028$

H -1.596529 -0.6174330 .588125$

O $-3.935926-1.9965740 .929858$

H -2.107971 -2.853975 1.171045

H -3.804777 -1.935972 3.551119

O $-3.469903-3.8850293 .087468$

H -1.061968 -3.290586 3.645128

O $-2.079490-2.6903595 .313236$

C $0.998868 \quad 0.2186594 .508545$

H $0.204115-1.4273165 .577180$

H $0.992107-1.8505364 .032795$

H -4.304571 -2.892647 0.855369

H $-4.335214-3.8959072 .644859$

H -2.544352 -3.543086 5.367135

O $2.312045 \quad 0.065218 \quad 4.852421$

O 0.4453231 .2780564 .248296

C 3.0820721 .2704704 .896009

H 2.6773811 .9510915 .651016

H 4.1069791 .0088975 .172108

H 3.0955661 .7480793 .912307

SCF Energy $(B 3 L Y P / 6-31 G * * / / M M F F)=-3245.91053984$

0127

MM̄FF Geometry

C $-1.868103-2.8574500 .207589$

C $-1.167553-2.533900-0.888533$

C $-1.548474-1.393234-1.794742$

O $-0.442883-0.457721-1.874960$

C -1.826352 -1.852582 -3.234401

C $-3.057250-2.714647-3.392048$

C $-4.302853-2.229460-3.557805$

C $-2.813228-4.201183-3.429647$

C $-4.735084-0.787454-3.588289$

C $-5.981775-0.542817-2.724856$

C $-5.676512-0.419172-1.222022$

C $-6.975642-0.545626-0.420730$

C $-6.760904-0.2919971 .074893$

O $-5.082840 \quad 0.867982-1.010244$

O $-8.061126-0.1574251 .667925$

C $-6.021675 \quad 1.0494571 .236939$

C $-6.095338-1.4892631 .762681$

O

C $-4.757996 \quad 1.176156 \quad 0.352161$

C -4.2535792 .6399690 .399219$

$\begin{array}{lllll}\text { O } & -3.755777 & 0.302545 & 0.867477\end{array}$

C $-0.1789220 .308262-0.782377$

$\begin{array}{lllll}\text { O } & -0.745388 & 0.271971 & 0.297643\end{array}$

C $0.9366221 .213439-1.132341$

C $1.3690682 .109342-0.233252$

C $2.4699613 .056583-0.419399$

C $3.1963213 .029123-1.739054$

C $-3.0328212 .934523-0.482120$

C $-2.7050264 .418616-0.526173$

C -1.043589 $6.012738-1.455522$

O $-3.4327195 .281505-0.041811$

C $0.4513426 .042326-1.470597$

C $1.1814146 .760605-0.603552$

C $2.6806626 .857939-0.633323$

C $3.322797 \quad 6.349697 \quad 0.638532$

C 3.4782867 .1536861 .704000 
C $3.860124 \quad 4.936397 \quad 0.641471$ C $2.769427 \quad 3.9026730 .588506$ N -1.502198 $4.664305-1.178047$ H -2.774994 -2.304419 0.435082 H $-0.266039-3.089249-1.130345$ H -2.422418 $-0.850020-1.415124$ H $-0.948288-2.378133-3.635701$ H $-1.909790-0.968309-3.880777$ H $-5.108896-2.946008-3.712283$ H $-3.733853-4.769284-3.601087$ H $-2.118974-4.450608-4.238994$ H $-2.385226-4.551127-2.486198$ H $-3.934781-0.101994-3.294188$ H $-4.990071-0.549607-4.628500$ H $-6.4459720 .391284-3.068078$ H $-6.709718-1.345034-2.900453$ H $-4.967258-1.198694-0.922982$ H -7.435592 -1.529355 -0.578876 H $-7.7053890 .186851-0.794985$ H -7.9352410 .0835582 .602063$ H -6.7286621 .8404830 .951303$ H $-6.717278-2.3856341 .652038$ H $-5.999578-1.3250212 .841659$ H $-5.107056-1.720351 \quad 1.360556$ H -4.9351610 .7358412 .832532$ H $-5.071287 \quad 3.299572 \quad 0.079687$ H -3.999116 2.9140721 .431131 H -2.885875 0.5990890 .558830 H $1.3775721 .111232-2.117424$ H $0.8747492 .153510 \quad 0.736425$ H $2.4972213 .188257-2.567736$ H $3.6962712 .065116-1.880429$ H $3.9612983 .801857-1.835398$ H -2.154662 2.416165 -0.090080 H -3.208549 2.599248 -1.510036 H -1.463864 $6.705155-0.718371$ H -1.432005 $6.295067-2.439508$ H $0.9519615 .472814-2.249586$ H $\quad 0.670741 \quad 7.3433430 .160379$ H $2.9465627 .912801-0.782215$ H $3.0986166 .329459-1.499020$ H 3.9633176 .8064852 .610084 H 3.1195338 .1777101 .693406 H 4.4381064 .7503951 .556678 H $4.5849724 .831123-0.170609$ H 2.1782973 .8501211 .503976 H -1.056204 3.898104 -1.674928 C $-0.972622-3.4172142 .517759$ O $0.317828-2.7912532 .364699$ C $0.412280-1.7057403 .309822$ C $-0.835450-1.7854394 .189448$ C $-1.846613-2.391752 \quad 3.237413$ H $\quad 0.373500-0.7787402 .726773$ C $1.728396-1.7780374 .093553$ H $-0.685136-2.4428375 .053675$ H $-1.135894-0.8032594 .567585$ C -3.062113 -2.986009 3.925041 H -2.173761 -1.599697 2.553676 C $3.007725-1.7718613 .231462$ O $1.743403-2.9820964 .873540$ H $1.764566-0.9551244 .817003$ H $3.088455-2.7226172 .692848$ O $4.125922-1.7344124 .129730$ C $3.124962-0.6003972 .238725$ C $4.517803-0.4479321 .593941$ H $2.366912-0.7120471 .455772$ $\begin{array}{lllll}\text { O } & 2.835430 & 0.616559 & 2.933053\end{array}$ H 4.4768850 .4121490 .914077 H $5.243533-0.1550562 .363529$ H -3.748891 -3.4219443 .192617$ H -2.779152 -3.772579 4.632491 H -3.605622 -2.214301 4.479902 C -1.479988 -3.9411591 .170107$ H $1.655106-3.7307224 .258798$ H $4.099648-0.8806534 .595081$ H 2.8744221 .3409602 .285981 H -2.356111 -4.5802931 .331385$ H $-0.703313-4.5729910 .720830$
H $-0.806495-4.2853003 .170256$

C $3.850148-1.393703-1.233951$

O $4.050763-2.203047-0.072714$

C $5.022581-1.6970070 .845572$

C $6.377425-1.5114910 .143019$

C $6.257235-0.673698-1.129641$

C $5.146006-1.216971-2.029984$

H $3.498096-0.405574-0.914960$

C $2.697925-1.992304-2.048151$

H $5.166717-2.4804831 .599032$

O $7.324337-0.904306 \quad 1.015557$

H $6.777085-2.497288-0.128753$

H $6.0838660 .381825-0.890372$

O $7.496208-0.710045-1.842350$

H $5.470473-2.166225-2.470163$

O $4.918769-0.306363-3.102662$

C $2.919053-3.404875-2.546393$

H $1.813995-2.033549-1.404195$

H $2.479578-1.350517-2.908960$

H $7.394765-1.4656091 .806591$

H $7.690749-1.637346-2.060601$

H $5.768141-0.176837-3.558449$

O $1.891078-3.734633-3.385462$

O $3.844662-4.137290-2.227695$

C $1.931775-5.061146-3.919886$

H $1.884780-5.796616-3.111227$

H $1.059554-5.193916-4.565570$

H $2.837082-5.200924-4.518346$

SCF Energy (B3LYP/6-31G**//MMFF)= -3245.92767179

$01 \_270$

MM̄FF Geometry

C $-2.372167 \quad 1.259904 \quad 3.174963$

C $-1.733088 \quad 0.1017892 .943751$

C $-0.236173-0.1079832 .957065$

$\begin{array}{lllll}\text { O } & 0.419767 & 1.180007 & 2.837930\end{array}$

C $0.240122-0.7762124 .263410$

C $0.226595-2.2927274 .254892$

C $1.335374-3.0574284 .185374$

C -1.119467-2.945922 4.426620

C $2.750454-2.5819974 .003089$

C $3.645927-3.5629303 .228418$

C $3.108362-3.9831351 .851613$

C $4.120715-4.8789101 .130674$

C $3.641794-5.273272-0.274791$

O $2.847914-2.8114441 .070632$

O $4.774956-5.823763-0.963785$

C $3.202398-3.997046-1.025563$

C $2.592440-6.389330-0.219529$

O $2.622335-4.323007-2.286474$

C $2.254636-3.094039-0.205171$

C $1.949031-1.756631-0.914914$

O $0.999639-3.767445-0.046168$

C 1.6447621 .2125652 .251079

$\begin{array}{lllll}\text { O } & 2.271797 & 0.264409 & 1.809208\end{array}$

C 2.1054772 .6181552 .241568

C 3.2951442 .9134291 .696718

C 3.8996424 .2427401 .601566

C 3.1372845 .4065842 .177510

C $3.161861-0.867746-1.199072$

C $2.7580050 .464409-1.812763$

C $3.7518242 .473806-2.883461$

O $1.5905910 .782895-2.022381$

C $4.8626943 .404179-2.515497$

C $4.6702354 .697252-2.213256$

C $5.7753995 .643516-1.836556$

C $5.5178536 .340044-0.517309$

C $5.0311807 .592346-0.486378$

C 5.8911255 .6209710 .760964

C $5.0956124 .365168 \quad 0.988967$

N $3.864183 \quad 1.253615-2.103473$

H $-1.8029742 .153214 \quad 3.418174$

H $-2.330443-0.7781562 .723085$

H $0.023206-0.7175082 .081768$

H $-0.365871-0.4235935 .109975$

H $1.246867-0.4170404 .509238$

H $1.217592-4.1372164 .264897$

H -1.035731 -4.015799 4.646286 
H -1.667622 -2.487946 5.256302 H $-1.712514-2.8468273 .513744$ H $2.776869-1.6170763 .490586$ H $3.193167-2.4306874 .994987$ H $4.623495-3.0792103 .103758$ H $3.811719-4.4598883 .838734$ H $2.171499-4.5313422 .001727$ H $4.343216-5.7738271 .725203$ H $5.074266-4.3408681 .032131$ H $4.502104-6.008299-1.879120$ H $4.121261-3.442180-1.249455$ H $3.010458-7.2802930 .264634$ H $2.298646-6.708553-1.225572$ H $1.691833-6.1064220 .329213$ H $1.719658-4.645485-2.122344$ H $1.412451-1.954735-1.850877$ H $\quad 1.241430-1.188890-0.295146$ H $0.341983-3.1114930 .236773$ H $1.456783 \quad 3.3675002 .680337$ H 3.8877512 .1037671 .271873 H $2.9729505 .265073 \quad 3.251432$ H 2.1651695 .5125131 .683212 H 3.6538256 .3622192 .061310 H $3.718193-0.667969-0.277329$ H $3.833951-1.362580-1.908224$ H $2.7640192 .920630-2.728374$ H $3.8369172 .189988-3.937507$ H $5.8723983 .000417-2.504046$ H $3.6623755 .105618-2.247901$ H $5.8672826 .385536-2.640513$ H $6.7451785 .132393-1.792862$ H 4.8668078 .1128840 .451072 H $4.7829848 .124377-1.398829$ H $5.809104 \quad 6.2843181 .630317$ H 6.9550775 .3537750 .716288 H 5.5741863 .4684530 .593925 H $4.7846770 .830182-2.024349$ C -4.3118012 .5162222 .198201$ O $-4.0655742 .110783 \quad 0.837513$ C -5.1707302 .5388680 .017318$ C -6.0408143 .4271220 .899567$ C -5.8079762 .8167522 .267259$ H $-5.7393351 .637645-0.239431$ C $-4.6546283 .216520-1.257246$ H $-5.704053 \quad 4.4702270 .886197$ H $-7.091596 \quad 3.4113000 .594087$ C -6.2079343 .7297353 .413098$ H -6.3767821 .8796162 .335941$ C -3.688899 2.348767 -2.089749 O $-3.9534644 .415624-0.900248$ H -5.500422 $3.536508-1.876309$ H -2.754185 2.205409-1.536290 O $-3.3506633 .080319-3.274499$ C $-4.2649830 .986664-2.514485$ C $-3.3655990 .179030-3.466553$ H $-4.4700230 .381827-1.628835$ O $-5.5220721 .184272-3.167943$ H $-3.914532-0.726391-3.753423$ H $-3.236122 \quad 0.735145-4.403590$ H -6.0168393 .2524374 .379214$ H -5.6519094 .6729423 .387422$ H -7.2755083 .9674703 .360247$ C $-3.8686691 .410868 \quad 3.157337$ H -3.230818 4.164626 -0.299475 H $-2.9859023 .936386-2.991570$ H $-5.3644951 .754585-3.939991$ H $-4.341017 \quad 0.4641402 .871311$ H -4.1931251 .6476864 .176858$ H -3.729575 3.4313642 .370161 C $-2.628214-2.183293-1.658500$ O $-2.099276-0.851934-1.610093$ C -1.990953 -0.192565 -2.876972 C $-1.102695-1.006170-3.827460$ C -1.575934 -2.451178 -3.949295 C -1.770296 -3.074202 -2.568237 H $-3.660242-2.157216-2.029214$ C $-2.639217-2.730928-0.220381$ H $-1.4612000 .748550-2.687299$
O $-1.064407-0.393470-5.112005$

H $-0.076057-1.000448-3.443226$

H $-2.496363-2.515217-4.540653$

O $-0.599301-3.207212-4.670258$

H $-0.792167-3.250832-2.106177$

O $-2.405494-4.342913-2.717796$

C $-3.828723-2.2513920 .584367$

H -2.686419 -3.825732 -0.240176

H - $1.739331-2.4205180 .320799$

H $-0.512265-0.952592-5.685130$

H $0.236879-3.169086-4.174981$

H -1.854284 -4.875152 -3.316828

O $-4.028383-3.107908 \quad 1.630365$

O $-4.480122-1.2435390 .351615$

C $-5.122895-2.7906772 .495745$

H $-4.883380-1.9029273 .087031$

H -5.273323 -3.634004 3.175064

H $-6.041541-2.6375811 .921165$

SCF Energy (B3LYP/6-31G**//MMFF) $=-3245.92523887$

01_271

MM̄FF Geometry

C $0.8744172 .875371-1.383128$

C $2.1113673 .387565-1.279579$

C $3.0715223 .222961-0.126889$

O 2.3944082 .8618311 .099859

C $4.1098522 .119852-0.391763$

C $5.1137972 .441839-1.475815$

C $5.0966651 .913117-2.714700$

C $6.2154983 .386841-1.071994$

C $4.0763800 .978181-3.300637$

C $4.638272-0.422024-3.572281$

C $4.937436-1.231667-2.299806$

C $5.533243-2.591463-2.674697$

C $5.759542-3.479466-1.444371$

O $3.712520-1.413503-1.578295$

O $6.005994-4.817178-1.904058$

C $4.459604-3.518661-0.616630$

C $7.003362-3.056335-0.654071$

O $4.662513-4.2185220 .609716$

C $3.857057-2.119859-0.337224$

C $2.452733-2.2862240 .292951$

O $4.708114-1.4233240 .573669$

C 1.8701423 .8723371 .840805

O $1.7869235 .048543 \quad 1.512490$

C 1.3867783 .3978293 .157496

C 1.6515902 .1810433 .658840

C 1.2112531 .6684854 .960156

C 0.4485352 .6109575 .855679

C $1.693142-0.9783590 .553948$

C $0.304028-1.2413841 .113232$

C -1.676966 -0.053486 2.043638

O $-0.168577-2.3691621 .232471$

C -1.5594680 .0073593 .532069$

C -1.446267-1.075417 4.316078

C $-1.300839-1.0239135 .810536$

C $0.079147-1.4502806 .265125$

C $0.361511-2.7439246 .493783$

C $1.106852-0.3680916 .523393$

C 1.4835120 .3837065 .274905

N $-0.351602-0.0660791 .453682$

H $0.4769942 .243052-0.594973$

H $2.4853963 .999260-2.098979$

H 3.5902514 .1759960 .036549

H $3.5928261 .175483-0.590902$

H 4.6598231 .9172740 .538266

H $5.8893042 .197859-3.405669$

H $6.9460363 .540962-1.873258$

H $5.8052374 .366546-0.809056$

H $6.7565012 .991431-0.206099$

H $3.1764680 .910714-2.685052$

H $3.7472631 .410140-4.254251$

H $3.895051-0.963585-4.171132$

H $5.545786-0.342185-4.183926$

H $5.642428-0.669623-1.677365$

H $6.468812-2.465500-3.233885$

H $4.846512-3.114187-3.355431$

H $6.782088-4.794626-2.489823$ 
H $3.724254-4.102596-1.188242$

H $7.898309-3.123284-1.284420$

H $7.182444-3.7296360 .191467$

H $6.944412-2.033972-0.275199$

H $5.025150-5.093170 \quad 0.387373$

H $1.837908-2.903776-0.375542$

H $2.538363-2.8240561 .245910$

H $4.209639-0.7025380 .987365$

H $\quad 0.8158204 .1328943 .715867$

H 2.2419571 .4843183 .066678

H -0.4659122 .9579185 .361531$

H 1.0641503 .4818916 .107215

H 0.1358652 .1644696 .801100

H $2.231836-0.3586361 .277456$

H $1.581253-0.410091-0.374751$

H $-2.238660-0.936328 \quad 1.723697$

H -2.1921710 .8380651 .676803$

H -1.5718270 .9959593 .982141$

H -1.442678 -2.0622013.858308

H -2.060088 -1.6846096 .249178$

H -1.528134 -0.0239456 .200447$

H $1.339439-3.0576306 .843120$

H $-0.380873-3.5188736 .332607$

H $2.028460-0.8053496 .929654$

H $\quad 0.7320390 .2852607 .315689$

H $2.035456-0.2215804 .554152$

H $\quad 0.142886 \quad 0.817887 \quad 1.377971$

C -1.391235 $3.713605-2.233367$

O $-2.2910672 .677660-1.793365$

C $-3.2312353 .259723-0.869215$

C $-2.9196404 .757339-0.805833$

C -1.445932 $4.791934-1.154556$

H -3.0172242 .8154340 .108899$

C $-4.6783312 .959375-1.284229$

H $-3.4981905 .327398-1.542017$

H -3.1369645 .1796410 .180362$

C $-0.9530896 .152660-1.612324$

H $-0.8862434 .486882-0.261874$

C $-5.0564381 .470500-1.431718$

O $-4.9477923 .587010-2.546475$

H $-5.3585073 .436225-0.567873$

H $-4.6344291 .060895-2.357161$

O $-6.4825301 .417310-1.584135$

C $-4.6592470 .572058-0.250378$

C $-5.370276-0.791336-0.186207$

H $-3.5757010 .420838-0.273869$

O $-4.962851 \quad 1.255503 \quad 0.971652$

H -4.932035 -1.3413200 .655998$

H $-6.416263-0.6289030 .106317$

H $\quad 0.112058 \quad 6.117743-1.861669$

H -1.495531 $6.499192-2.498278$

H -1.090586 $6.897404-0.821592$

C $-0.0139043 .134615-2.570270$

H $-4.3113913 .232174-3.191010$

H $-6.7131541 .974348-2.347461$

H -4.6602990 .6950891 .706471$

H $0.4880663 .823578-3.261228$

H $-0.1460382 .187301-3.103956$

H $-1.8243424 .097722-3.167538$

C -3.155703 -2.677375-1.146004

O $-4.001845-1.859794-1.961882$

C $-5.323179-1.652406-1.463633$

C $-6.031537-3.005364-1.309847$

C $-5.242859-3.942081-0.400403$

C $-3.793861-4.051310-0.869660$

H -2.945244 -2.155880 -0.205977

C -1.831937 -2.861077-1.906543

H -5.859483 -1.100663 -2.244270

O $-7.343766-2.839132-0.783215$

H $-6.136177-3.479798-2.294179$

H $-5.294979-3.6276850 .648346$

O $-5.828764-5.246072-0.451917$

H -3.768166 -4.673732 -1.773836

O $-3.057148-4.738390 \quad 0.141458$

C $-1.127414-1.560314-2.221690$

H -1.134224 -3.478280 - 1.331543

H -2.040104 -3.353481 -2.865037

H $-7.825146-2.242287-1.381385$
H $-6.758963-5.155147-0.182818$

H -2.195076 -4.985828 -0.231931

O $-0.138859-1.816298-3.130040$

O $-1.396889-0.469971-1.739452$

C $0.599632-0.678729-3.579527$

H $1.020367-0.134404-2.730852$

H $1.417628-1.033039-4.211288$

H -0.046669 -0.028595-4.175834

SCF Energy (B3LYP/6-31G**//MMFF) $=-3245.90753961$

01272

MM̄FF Geometry

C -2.484472 2.4043541 .294630

C -1.525595 1.9657482 .124293

C -1.4507360 .5462012 .620087$

$\begin{array}{lllll}\text { O } & -0.057233 & 0.148214 & 2.542447\end{array}$

C -1.9143370 .3997654 .080381$

C $-3.4175930 .439575 \quad 4.259389$

C $-4.219219-0.638214 \quad 4.142426$

C -3.9940391 .7695494 .668679$

C $-3.809449-2.0429073 .792936$

C $-4.764823-2.7297622 .807335$

C $-4.760471-2.1406401 .385996$

C $-5.862027-2.8109500 .555822$

C $-5.854545-2.351335-0.906742$

O $-3.476330-2.3789020 .792213$

O $-6.707107-3.249800-1.632979$

C $-4.422705-2.504397-1.458257$

C $-6.471495-0.956318-1.062655$

O $-4.336237-1.977870-2.781422$

C $-3.343523-1.877566-0.546003$

C $-1.914553-2.178283-1.055802$

O $-3.503681-0.455155-0.583857$

C $0.200645-1.1712972 .335549$

O $-0.617482-2.0590522 .156161$

C $1.664704-1.3736192 .381923$

C $2.152870-2.5920012 .104684$

C $3.558470-2.9936982 .113571$

C $4.575794-2.0008062 .605646$

C $-1.451243-3.630363-0.920444$

C $-0.302340-3.925001-1.867506$

C $2.041392-3.274659-2.406996$

O $-0.431557-4.713087-2.802723$

C $3.010547-4.261814-1.838328$

C $4.294015-3.967389-1.578475$

C $5.292096-4.955758-1.041747$

C $5.932214-4.4921920 .249004$

C $7.110098-3.8456390 .239658$

C $5.244980-4.8371951 .552136$

C $3.876295-4.2279151 .671418$

N $0.852844-3.217414-1.574475$

H -3.275199 1.7153221 .016373

H -0.7554142 .6528862 .462661$

H $-2.042922-0.1016811 .962746$

H $-1.441427 \quad 1.1701964 .705005$

H $-1.526829-0.5393064 .497283$

H -5.282180 -0.509749 4.342885

H -3.8079782 .5292593 .905363$

H -5.0770951 .7224874 .825060$

H -3.5412882 .1043685 .607795$

H $-2.792884-2.0871413 .399892$

H -3.811204 -2.624868 4.723079

H -4.472069 -3.786694 2.752439

H $-5.782862-2.700283 \quad 3.216545$

H $-4.934252-1.0602811 .444325$

H $-6.848028-2.6368401 .005261$

H -5.715907 -3.900628 0.575084

H $-6.647657-3.014978-2.575138$

H $-4.237347-3.580606-1.552341$

H -7.512871 -0.961418 -0.718763

H $-6.507718-0.653093-2.114835$

H $-5.941797-0.185133-0.499488$

H -4.298739 -1.008547 -2.712406

H -1.824752 -1.841712 -2.096869

H -1.203598 -1.552663 -0.499363

H $-2.655527-0.042436-0.353758$

H $2.291220-0.5307072 .646245$

H $1.453158-3.3817561 .832006$ 
H $4.326721-1.6599103 .616523$

H $4.613627-1.1338311 .941137$

H $5.589020-2.4064752 .654234$

H $-1.132713-3.8473900 .104904$

H $-2.254079-4.333965-1.159682$

H $2.458891-2.263969-2.445396$

H $1.763113-3.573750-3.422611$

H $2.647449-5.270328-1.655900$

H $4.659757-2.963694-1.784804$

H $6.064055-5.100744-1.808990$

H $4.838955-5.943454-0.890921$

H $7.589783-3.5294901 .159962$

H $7.618911-3.614124-0.690370$

H $5.861447-4.5620292 .416049$

H $5.144687-5.9285071 .618196$

H $3.075291-4.8838711 .328921$

H $0.887965-2.645353-0.735929$

C -2.683424 $3.892210-0.772787$

O $-1.5064203 .336991-1.394113$

C $-1.9227652 .463288-2.459152$

C $-3.3591702 .859776-2.782497$

C $-3.892243 \quad 3.193961-1.403767$

H -1.921904 $1.448597-2.044091$

C $-0.9579262 .519893-3.649484$

H $-3.4042503 .745733-3.425960$

H $-3.9126122 .053397-3.273360$

C $-5.1507014 .044613-1.422153$

H $-4.1212192 .252103-0.895432$

C $0.5337072 .354356-3.292776$

O $-1.104496 \quad 3.792664-4.293187$

H $-1.2600111 .766400-4.387469$

H $0.9116093 .293102-2.871349$

O $1.2529332 .139041-4.514189$

C $0.8577731 .194058-2.337156$

C $2.3602040 .899204-2.160045$

H $0.418396 \quad 1.401162-1.355231$

O $\quad 0.2371550 .003839-2.829640$

H $2.4533950 .154919-1.358745$

H $2.7387220 .390379-3.056507$

H -5.483333 $4.267402-0.403394$

H $-4.9859154 .995607-1.939763$

H $-5.9622813 .519203-1.936049$

C -2.575637 $3.805796 \quad 0.756705$

H $-0.645833 \quad 3.745142-5.148620$

H $1.2084222 .960533-5.031457$

H $0.432822-0.710728-2.200285$

H -3.449643 4.2902851 .207304

H -1.691965 4.3740151 .073782

H -2.688665 $4.955055-1.047954$

C 2.7009332 .4568330 .471419

O $2.6712632 .975511-0.859259$

C $3.2445452 .123053-1.852301$

C $4.6987371 .777632-1.496582$

C $4.8266341 .231280-0.074911$

$\begin{array}{llll}\text { C } & 4.136127 & 2.164225 & 0.921021\end{array}$

H 2.1191521 .5264340 .500320

C $1.958906 \quad 3.441518 \quad 1.384919$

H $3.2828332 .717359-2.773574$

O $5.221674 \quad 0.837967-2.429581$

H $5.3071172 .687334-1.581796$

H $4.4190080 .216853-0.009785$

$\begin{array}{lllll}\text { O } & 6.209779 & 1.117201 & 0.266422\end{array}$

H 4.7176463 .0893391 .004669

O 4.1136201 .5606612 .210366

C 2.6143244 .8014101 .511576

H $0.954633 \quad 3.6218720 .984764$

H 1.8645813 .0023682 .384761

H $6.1295480 .626465-2.152472$

H 6.6065612 .0035550 .214479

H 5.0325511 .3485422 .448108

O $2.201297 \quad 5.3873962 .674560$

O $3.361446 \quad 5.3047120 .683953$

C $2.723608 \quad 6.6962592 .923218$

H 2.3313487 .0419353 .883397

H 2.3974357 .3883142 .140948

H $3.815707 \quad 6.6644382 .981194$

SCF Energy (B3LYP/6-31G**//MMFF) $=-3245.91541801$

\section{3}

MMFF Geometry

C -1.080792 -2.779086 0.781640

C $-1.544532-2.0248611 .790264$

C $-0.722878-1.3169722 .846517$

O $0.674623-1.6697922 .721058$

C -0.8863230 .2072762 .703954$

C $-0.4551790 .986868 \quad 3.931073$

C $0.638132 \quad 1.771128 \quad 3.998578$

C -1.3881330 .8990545 .112448$

C 1.6665442 .0002992 .929396

C 1.4689733 .3685362 .263375

C 2.4984513 .6160911 .153536

C 2.3107884 .9970600 .523396

C $3.2599355 .219147-0.665985$

O $2.3152792 .606494 \quad 0.158949$

O $2.7970546 .368670-1.391346$

C $3.1613144 .011711-1.623870$

C $4.6853055 .543459-0.203219$

O $4.1370534 .097204-2.659432$

C $3.2694712 .647845-0.907487$

C $2.9405521 .495015-1.885084$

O $4.6050332 .471128-0.427364$

C $1.345989-1.9811453 .859455$

O $0.919911-1.9007035 .004399$

C $2.691117-2.5337273 .581672$

C $3.349562-2.4249282 .416688$

C $4.665477-3.0112802 .124856$

C $5.284417-3.8851923 .187999$

C $2.6451560 .169802-1.170715$

C $2.483811-0.993692-2.130682$

C $1.946478-3.427710-2.087334$

O $2.840983-0.953066-3.304366$

C $3.122055-4.158634-1.518756$

C $4.331209-4.161108-2.098602$

C $5.560296-4.760393-1.479325$

C $6.536654-3.690015-1.036676$

C $7.396770-3.139874-1.910833$

C $6.566410-3.3058830 .429962$

C $5.256810-2.7557800 .937205$

N $1.939795-2.102713-1.494201$

H $-0.006141-2.9065950 .680549$

H -2.617474 -1.893074 1.903313

H $-1.116410-1.6614103 .810377$

H -1.9406700 .4525932 .521973$

H $-0.3486850 .550748 \quad 1.812782$

H 0.8283422 .3118334 .924761

H -1.0787921 .5515995 .935963$

H -2.399722 1.1996774 .819947

H -1.427494 -0.122217 5.502133

H 2.6596121 .9567653 .393667

H 1.6524891 .1984772 .184978

H $0.4574773 .410727 \quad 1.839321$

H 1.5410424 .1625103 .017204

H 3.5014053 .5239861 .586770

H 2.4394015 .7881631 .272411

H 1.2786595 .0935810 .160249

H $2.7845557 .124227-0.779033$

H $2.1805974 .065636-2.116909$

H 4.6909756 .4527650 .409872

H $5.3395585 .754281-1.056358$

H 5.1360264 .7444850 .389013

H $4.0261314 .961659-3.091131$

H $2.0537781 .752034-2.477521$

H $3.7711331 .360800-2.589179$

H $4.8059931 .522984-0.383483$

H $3.127736-3.0531574 .429583$

H $2.890702-1.8689261 .602061$

H $4.653476-4.7597813 .381250$

H $5.409137-3.3256024 .121852$

H $6.277424-4.2579482 .929968$

H $3.461380-0.088223-0.487909$

H $1.7303210 .262610-0.575246$

H $1.998108-3.353995-3.178329$

H $1.014962-3.931083-1.813339$

H $2.984042-4.646475-0.557229$

H $4.461628-3.652225-3.051373$

H $6.034711-5.417900-2.219151$ 
H $5.307064-5.409080-0.631559$ H $8.116870-2.389577-1.602123$ H $7.397002-3.428001-2.956980$ H $7.333732-2.5412950 .605146$ H $6.880172-4.1961970 .982298$ H $4.759827-2.0712690 .250256$ H $1.740648-2.040619-0.500358$ C -3.405223 -3.542142 -0.103576 O $-3.956592-2.222553-0.245780$ C $-5.255133-2.316194-0.866468$ C $-5.527110-3.803299-1.073632$ C $-4.121732-4.370522-1.168567$ H -5.158116 -1.842525 -1.850277 C $-6.294201-1.547795-0.032226$ H $-6.053779-4.252605-0.225627$ H $-6.126409-3.990764-1.969963$ C $-4.060840-5.870969-0.941851$ H $-3.716220-4.136777-2.162029$ C $-5.826625-0.1366610 .381201$ O $-6.543590-2.2527621 .191134$ H -7.250248 -1.491091 -0.565941 H $-4.985939-0.2354571 .075990$ O $-\begin{array}{llll}6.880677 & 0.496984 & 1.115920\end{array}$ C $-5.4480340 .778401-0.794648$ C $-4.8994322 .154202-0.371880$ H -4.698931 $0.290965-1.419152$ O $-6.5914650 .983148-1.626754$ H -4.762067 2.755140 -1.279301 H -5.6570852 .6904760 .212741$ H -3.033579 -6.238793 -1.025112 H $-4.436476-6.1425580 .050419$ H $-4.667340-6.396966-1.686505$ C -1.883162 -3.500948 -0.273397 H $-7.179599-2.9618830 .997685$ H -7.112682 -0.0923981 .854125$ H -7.282954 $1.391438-1.078035$ H $-1.638843-3.031150-1.232644$ H -1.503871 -4.528903 -0.312646 H $-3.666570-3.903200 \quad 0.900107$ C -2.037923 $1.799851-1.425769$ O $-2.612848 \quad 1.238149-0.239534$ C $-3.577048 \quad 2.0683150 .417556$ $\begin{array}{llll}C & -2.956705 & 3.420579 & 0.792565\end{array}$ C $-2.2837344 .093576-0.400401$ C -1.331778 $3.120177-1.094643$ H $-2.8220191 .980725-2.170611$ C $-1.0462090 .767874-1.987403$ H -3.8121741 .5712551 .365756$ O H -2.203239 3.2559681 .574240 H -3.021321 $4.490025-1.108289$ O H $-0.4628952 .932033-0.452463$ O $-0.854516 \quad 3.716765-2.298711$ C -1.706868 $-0.300163-2.834423$ H $-0.3134841 .259432-2.635701$ H $-0.5295750 .262573-1.164334$ H -3.508038 5.0639251 .691230 H $-2.141693 \quad 5.875827 \quad 0.392771$ H $-0.4248274 .554939-2.055861$ O $-0.740885-1.141046-3.312135$ O $-2.903358-0.386683-3.067779$ C $-1.202018-2.182846-4.178192$ H $-1.606040-1.751960-5.099147$ H $-0.348652-2.816962-4.431790$ H $-1.956843-2.795416-3.676578$

SCF Energy (B3LYP/6-31G**//MMFF) $=-3245.90990740$

01274

MMFF Geometry

C $1.646127-3.130133 \quad 1.335657$

C $1.386138-2.2215472 .288323$

C $2.205374-0.9709402 .461998$

$\begin{array}{llll}\text { O } & 1.276050 & 0.126554 & 2.618574\end{array}$

C $3.108070-1.0208833 .708197$

C $4.219562-2.0467853 .652537$

C $5.336093-1.9150622 .909184$

C $4.029536-3.2532444 .532979$
C $5.677205-0.782913 \quad 1.979077$

C $5.893903-1.297940 \quad 0.549817$

C $5.855515-0.213881-0.539511$

C $6.7844180 .969198-0.261221$

C $6.6707992 .051570-1.347395$

$\begin{array}{lllll}\text { O } & 4.501765 & 0.239118 & -0.664579\end{array}$

O $7.300373 \quad 3.243181-0.851037$

C $5.1791912 .387999-1.565822$

C $7.4274371 .662939-2.623000$

O $5.015697 \quad 3.282263-2.664604$

C $4.2848851 .141153-1.759742$

C $2.7919001 .552769-1.766060$

$\begin{array}{lllll}\text { O } & 4.599281 & 0.534529 & -3.015724\end{array}$

C $1.698927 \quad 1.3562002 .220829$

O $2.7832021 .652917 \quad 1.746025$

C 0.5863472 .2993362 .439215

C $0.4285383 .311614 \quad 1.575197$

C $-0.646466 \quad 4.3013951 .594923$

C $-1.557032 \quad 4.3266742 .794327$

C $1.8207850 .366446-1.736271$

C $0.3752530 .809308-1.622833$

C $-1.4790451 .830713-2.940729$

O $-0.2876620 .580226-0.614231$

C $-1.6404543 .300421-2.714842$

C $-2.6442823 .821976-1.991920$

C $-2.8900525 .296759-1.834360$

C $-3.0338925 .719300-0.389431$

C $-4.237240 \quad 5.7294530 .208607$

C - $-1.801034 \quad 6.208572 \quad 0.335348$

C $-0.775457 \quad 5.1290280 .537608$

N $-0.0924701 .444546-2.760250$

H $2.495974-2.9624490 .679322$

H $\quad 0.568457-2.3884542 .984820$

H $2.799691-0.8028031 .556364$

H $2.488801-1.186904 \quad 4.600925$

H $3.549223-0.0306683 .884611$

H $6.087930-2.7011832 .962982$

H $4.860270-3.9623684 .452444$

H $3.955160-2.9478985 .581910$

H $3.113764-3.7868414 .260714$

H $4.908408-0.0074791 .989942$

H $6.598298-0.3110792 .341393$

H $6.859501-1.8178400 .504550$

H $5.118764-2.0397460 .314048$

H $6.142688-0.704617-1.477306$

H $7.8229670 .631958-0.156947$

H $6.520807 \quad 1.4353090 .697234$

H $8.2231913 .026008-0.633796$

H $4.8299342 .926379-0.673405$

H $8.4924081 .515432-2.406095$

H $7.3827482 .464439-3.368613$

H $7.0555130 .744096-3.080633$

H $5.5817754 .054848-2.495437$

H $2.5827612 .178289-0.890480$

H $2.5847352 .165276-2.651999$

H $3.849069-0.007751-3.304236$

H $-0.0929222 .101806 \quad 3.259783$

H 1.1351943 .4053430 .751530

H -0.9820834 .5208863 .706498$

H -2.078026 3.3702262 .907453

H -2.329506 5.0967522 .736160

H $1.913202-0.251635-2.635902$

H $2.031520-0.284534-0.880355$

H $-2.1138581 .241680-2.272232$

H $-1.7430101 .589366-3.975139$

H $-0.9359963 .965670-3.207982$

H -3.366913 $3.151436-1.531332$

H $-3.8121695 .540015-2.379145$

H $-2.1014265 .888564-2.315467$

H $-4.3565606 .057054 \quad 1.235851$

H $-5.1297795 .403589-0.315445$

H -2.061679 $6.676618 \quad 1.291608$

H $-1.3426207 .016839-0.249224$

H $-0.0765045 .028745-0.293414$

H $0.5395281 .590120-3.541806$

C $0.383355-4.704860-0.248317$

O $-0.717996-3.844831-0.592388$

C $-0.749117-3.713854-2.031405$ 
C $0.419378-4.538395-2.577390$

C $1.373795-4.561956-1.400156$

H $-0.568124-2.656126-2.253286$

C $-2.113369-4.149656-2.584612$

H $0.112702-5.556682-2.843278$

H $\quad 0.855792-4.085135-3.473195$

C $2.410179-5.668186-1.472776$

H $1.880838-3.590272-1.367731$

C $-3.321469-3.376275-2.020439$

O $-2.319214-5.538407-2.288554$

H - $2.105267-4.076971-3.678469$

H -3.460835 -3.626188 -0.962452

O $-4.492745-3.842364-2.703772$

C $-3.239125-1.850684-2.192771$

C $-4.536728-1.097163-1.842775$

H - $2.417319-1.460837-1.582937$

O $-2.920038-1.560901-3.557101$

H $-4.346148-0.026215-1.985524$

H $-5.311608-1.338488-2.582024$

H $3.070663-5.640902-0.600328$

H $1.940540-6.656890-1.509737$

H $3.029763-5.556664-2.368591$

C $0.895191-4.4193701 .166940$

H -2.293727 -5.639028 -1.321508

H $-4.531983-4.806963-2.586448$

H -2.861092 $-0.595444-3.648889$

H $1.570771-5.2309691 .463739$

H $0.043075-4.4428491 .858050$

H $-0.025317-5.725459-0.241662$

C -3.5560650 .0498150 .807689$

O $-4.044296-1.2715690 .568846$

C $-5.072012-1.356559-0.420838$

C $-6.267375-0.475360-0.026205$

C $-5.844451 \quad 0.963168 \quad 0.274172$

C $-4.6822260 .980628 \quad 1.267997$

H $-3.1274730 .444141-0.121475$

C $-2.388829-0.0401151 .794006$

H -5.426418 -2.394491-0.404047

O $-7.244658-0.498047-1.060979$

H $-6.736373-0.899400 \quad 0.871478$

H $-5.5841391 .507755-0.641117$

O $-6.938662 \quad 1.6676520 .864949$

H -5.0537130 .6839702 .256188$

O $-4.176527 \quad 2.308121 \quad 1.377935$

C $-2.783573-0.527097 \quad 3.166854$

H $-1.616191-0.7188001 .411847$

H -1.9133650 .9377801 .911623$

H $-8.036301-0.041816-0.729996$

H $-7.633123 \quad 1.750352 \quad 0.189917$

H -4.9166432 .8808021 .642183$

O $-2.831426-1.8895513 .183899$

O -3.0251210 .2165714 .108938$

C -3.197814 -2.476617 4.435874

H $-3.196732-3.5631124 .314988$

H $-2.469659-2.2085785 .207379$

H $-4.203472-2.1562784 .724506$

SCF Energy (B3LYP/6-31G**//MMFF) $=-3245.90245252$

01275

MM̄FF Geometry

C -1.178929 -2.922300 -0.590939

C $-1.217193-2.3322970 .614023$

C $-0.000310-1.8329941 .347458$

O $-0.141407-0.3932941 .432590$

C $0.081874-2.4138322 .773947$

C $0.598309-3.8366752 .844286$

C $1.873924-4.163702 \quad 3.132785$

C $-0.429107-4.9230812 .657508$

C $3.023516-3.2248733 .373931$

C $4.338473-3.7011162 .740264$

C $4.319378-3.7681581 .204345$

C $5.657636-4.3126440 .693907$

C $5.744038-4.308894-0.837753$

O $4.091828-2.4499790 .690705$

O $7.121716-4.537435-1.172220$

C $5.351114-2.906564-1.348496$

C $4.948949-5.466262-1.452914$

O $5.269141-2.883269-2.771747$
C $4.028651-2.382171-0.741399$

C $3.740537-0.917937-1.150614$

O $2.945997-3.174923-1.246275$

C $0.996217 \quad 0.330975 \quad 1.610524$

O $2.129809-0.103257 \quad 1.736175$

C $0.631597 \quad 1.7637391 .632839$

C 1.5974132 .6738821 .829640

C 1.4241994 .1247071 .895232

C 0.0515384 .6798451 .626253

C $4.7262670 .125968-0.619053$

C $4.4165041 .517227-1.152556$

C $5.4931403 .751918-1.430991$

O $3.3870101 .788718-1.764455$

C $5.3368414 .770499-0.347348$

C $4.5000505 .815455-0.442637$

C 4.3869626 .9030710 .587950

C 2.9693607 .1012281 .075067

C 2.1364647 .9492710 .448245

C 2.5453376 .3852022 .335670

C 2.4984104 .8908672 .176551

N $5.4356892 .415644-0.867588$

H $-0.213809-3.021373-1.083353$

H -2.165699 -2.204306 1.127412

H $0.910037-2.0689850 .782282$

H $-0.901744-2.358751 \quad 3.261721$

H $0.702964-1.7631163 .401296$

H $2.124054-5.2205523 .216911$

H $-0.028480-5.9161072 .888846$

H $-1.285384-4.7577243 .319516$

H $-0.785356-4.9496641 .624537$

H $2.806030-2.2151503 .020114$

H $3.175897-3.1551484 .458057$

H $5.124017-3.0025363 .057298$

H $4.601255-4.6852543 .148559$

H $3.500762-4.4237510 .887133$

H $5.841127-5.3218411 .084239$

H $6.474053-3.6894481 .086324$

H $7.192300-4.596267-2.139867$

H $6.167530-2.229002-1.071177$

H $5.358121-6.427782-1.120035$

H $5.031458-5.467524-2.545402$

H $3.889523-5.449138-1.188740$

H $6.145474-3.114757-3.121967$

H $3.674978-0.850371-2.244671$

H $2.739691-0.644240-0.792060$

H $2.124601-2.664158-1.162163$

H $-0.4106572 .027172 \quad 1.494464$

H 2.6204452 .3254221 .968238

H $-0.6550214 .351627 \quad 2.396285$

H $-0.309103 \quad 4.3426040 .648446$

H 0.0206085 .7715831 .604127

H 4.6916670 .1643560 .475028

H $5.747208-0.123856-0.923977$

H $4.7373583 .861402-2.215601$

H $6.4814013 .858313-1.890151$

H 5.9706744 .6718910 .530454

H $3.8923485 .925069-1.338541$

H 4.7511187 .8337850 .132994

H 5.0500086 .7172331 .442036

H $1.127196 \quad 8.1210520 .806937$

H $2.4459688 .487911-0.441427$

H 1.5858146 .7666272 .703455

H 3.2584016 .6241833 .135690

H $3.459642 \quad 4.4026952 .340598$

H $6.2439902 .093023-0.343354$

C $-3.648755-3.692713-0.595880$

O $-4.265220-2.412769-0.369419$

C $-5.697169-2.564373-0.434022$

C $-5.965593-4.052258-0.634217$

C $-4.703518-4.501260-1.348280$

H $-6.027701-2.028224-1.330844$

C $-6.342681-1.9455930 .814619$

H $-6.072615-4.5805510 .320073$

H $-6.875049-4.232532-1.215683$

C $-4.490264-6.004429-1.302861$

H $-4.753627-4.171947-2.394743$

C $-5.901473-0.4906421 .084820$

O $-5.982573-2.7227741 .964443$ 
H -7.433866 -2.006805 0.737755

H $-4.835760-0.4864761 .337208$

O $-6.578218-0.0168872 .254396$

C $-6.1702220 .477710-0.079841$

C $-5.723194 \quad 1.9273140 .183185$ H $-5.6638130 .119485-0.980135$ O $\quad-7.5660540 .484100-0.387267$ H $-6.0340452 .530825-0.678913$ H $-6.2825292 .343728 \quad 1.030551$ H -3.572952 -6.286935 -1.828220 H -4.413842 -6.367869-0.272553 H $-5.325813-6.525473-1.781814$ C $-2.336262-3.507678-1.362884$ H -5.012013 -2.720620 2.028889 H -7.533833 -0.1213882 .109975$ H -8.0339790 .9000980 .356222$ H -2.504592 -2.868258 -2.238222 H $-2.002689-4.484506-1.732517$ H -3.458855 -4.146906 0.386021 C $-3.4467111 .846036-1.844961$ O $-3.4287901 .330859-0.510783$ C -4.2071052 .0836410 .425865$ C -3.7185713 .5385790 .473309$ C $-3.6538004 .172727-0.917356$ C $-2.8891423 .272103-1.891394$ H $-4.4834481 .865731-2.205146$ C $-2.7239970 .851882-2.763673$ H -4.0019071 .6486621 .411814$ O $\quad-4.559944 \quad 4.309350 \quad 1.324233$ H $-2.715083 \quad 3.5540860 .915486$ H $-4.6596194 .387775-1.295614$ O $-2.9976875 .439645-0.830078$ H -1.823695 $3.287639-1.636598$ O $-3.0202533 .787148-3.213468$ C $-1.2625870 .623219-2.442296$ H -3.214754 -0.125979-2.681256 H -2.799186 $1.194955-3.801769$ H -4.2296655 .2241381 .309297$ H -2.091268 $5.288564-0.513279$ H -2.698198 $4.704795-3.201035$ O $-0.701375-0.097116-3.459571$ O $-0.6882110 .997178-1.430237$ C $0.682768-0.416664-3.295453$ H $0.997965-1.019480-4.151100$ H $\quad 1.2805240 .499022-3.270853$ H $\quad 0.830162-1.000307-2.383037$

SCF Energy $\left(B 3 L Y P / 6-31 G^{* *} / / M M F F\right)=-3245.90318254$

\section{6}

MM̄FF Geometry

C $0.1240434 .829721-1.800601$

C $-0.7349044 .360878-0.881963$

C $-2.0009623 .617332-1.230272$

O $-2.0884712 .442624-0.388531$

C $-3.2263974 .501079-0.935747$

C $-4.5381183 .895110-1.397455$

C $-5.5504493 .541937-0.581568$

C $-4.6911943 .723188-2.887782$

C -5.6246003 .6610380 .914167$

C -5.1572222 .4170301 .685422$

C -5.9522501 .1390971 .368780$

C -5.6049610 .0283972 .361613$

C $-6.309540-1.2934322 .017524$

$\begin{array}{lllll}\text { O } & -5.613559 & 0.712835 & 0.046908\end{array}$

O $-5.684930-2.3349092 .785179$

C $-6.072758-1.6255150 .526811$

C -7.785290 -1.274898 2.435089

O $-6.856128-2.7575090 .156328$

C $-6.342170-0.432939-0.416750$

C $-5.900297-0.684064-1.880100$

O $-7.750155-0.168439-0.445414$

C $-1.3197281 .370748-0.720583$

O $-0.5767541 .267130-1.683452$

C -1.5201460 .3394040 .322226$

C $-0.678303-0.7021330 .385311$

C $-0.722241-1.7974331 .355110$

C -1.960448 -1.925217 2.201006

C $-4.413751-1.003251-2.073679$
C $-4.099998-2.489242-2.127316$

C $-2.168738-4.062048-2.232511$

O $-4.964544-3.359326-2.186465$

C $-1.920251-4.599354-0.858404$

C $-0.749509-5.132981-0.477220$

C $-0.498741-5.7153580 .884711$

C $0.712203-5.1136331 .562352$

C $1.898817-5.7438061 .543431$

C $0.524884-3.8233842 .330036$

C $0.329879-2.6396981 .422305$

$\mathrm{N}-2.731901-2.727911-2.138000$

H $-0.1238074 .691823-2.851706$

H -0.5332824 .4945330 .176754$

H - $-1.9948473 .308841-2.282569$

H -3.113366 $5.473580-1.433471$

H -3.2563644 .7115410 .138827$

H $-6.4468733 .120016-1.035277$

H $-5.7118293 .443666-3.170753$

H $-4.4557044 .659040-3.405018$

H -4.023596 $2.939532-3.257962$

H -5.0567974 .5304961 .262483$

H -6.6667103 .8770021 .183075$

H -4.0940772 .2514161 .476305$

H -5.2443522 .6397412 .756140$

H $-7.021734 \quad 1.3747301 .417104$

H $-5.845454 \quad 0.333757 \quad 3.387657$

H -4.520389 -0.142964 2.348888

H -5.767166 -2.104165 3.726426

H $-5.022254-1.9274750 .439782$

H -7.875675 -1.100683 3.514084

H $-8.261364-2.2440742 .249050$

H -8.365074 -0.5012691 .927337$

H $-6.581872-3.032291-0.734337$

H $-6.542294-1.439911-2.347677$

H $-6.096420 \quad 0.243071-2.438237$

H $-7.9540450 .329565-1.254381$

H -2.3388250 .4743691 .019020$

H $0.133714-0.753662-0.336838$

H $-2.853357-1.946067 \quad 1.567175$

H -2.041311 -1.082525 2.895840

H $-1.992007-2.8393832 .796737$

H -4.094134 -0.590304 -3.039217

H $-3.806337-0.528853-1.298103$

H -1.248377 -3.985175 -2.820027

H -2.869254 -4.715346 -2.762461

H $-2.747930-4.572991-0.153487$

H $0.067931-5.183598-1.192987$

H $-0.358544-6.7974220 .762785$

H $-1.373688-5.6026731 .536938$

H $2.766644-5.3322742 .048151$

H $2.031274-6.6821651 .015074$

H $1.412317-3.6172542 .943239$

H $-0.290340-3.9559553 .046068$

H $1.179328-2.4564930 .764465$

H $-2.089379-1.947842-2.034508$

C $2.0259105 .410410-0.145360$

O $2.3596034 .030404 \quad 0.105102$

C $3.6570253 .965840 \quad 0.725102$

C 3.9894985 .3909591 .148475

C 3.3297886 .1883580 .039045

H $4.3649443 .661928-0.055155$

C 3.6534302 .9311111 .854586

H 3.5412205 .6441442 .116141

H 5.0678265 .5624231 .219768

C 3.1327737 .6552620 .379380

H $3.9473486 .109296-0.865859$

C 3.1723451 .5306251 .420588

O 2.7707673 .3915632 .886416

H 4.6513712 .8725302 .305135

H $2.104951 \quad 1.5717151 .170368$

O 3.2893220 .6804102 .568917

C 3.9696790 .9201860 .250116

C $3.493960-0.499119-0.110784$

H $3.8545831 .553980-0.635122$

O $5.355108 \quad 0.903486 \quad 0.580170$

H $3.708557-1.1780530 .720173$

H $2.408100-0.465178-0.235278$

H $2.6402428 .184004-0.442542$ 
H 2.5176497 .7801721 .276867

H 4.0971398 .1400390 .563217

C $1.4325155 .537174-1.551442$

H 2.7825582 .7253583 .595061

H $2.746750-0.1097842 .407096$

H $5.4595590 .372201 \quad 1.388117$

H $2.1526475 .120109-2.267885$

H $1.2972886 .594002-1.808433$

H 1.2992455 .7159090 .617411

C $6.009706-2.224041-0.441305$

O $5.552249-1.185488-1.315817$

C $4.131482-1.048833-1.408027$

C $3.515191-2.367388-1.900399$

C $3.925901-3.540871-1.014703$

C $5.446391-3.584850-0.866793$

H $5.706809-1.9996370 .588359$

C $7.541607-2.226049-0.493755$

H $3.945586-0.298275-2.187020$

O $2.097424-2.254793-1.952889$

H $3.860697-2.559092-2.924733$

H $3.440507-3.494179-0.033494$

O $3.505437-4.765226-1.619144$

H $5.896578-3.899735-1.817121$

O $5.792105-4.5609160 .114224$

C $8.101120-1.0116350 .208276$

H $7.967002-3.1052520 .003273$

H $7.902096-2.238796-1.529542$

H $1.750798-3.061953-2.368741$

H $2.534133-4.788858-1.587699$

H $5.400416-5.405814-0.166620$

O $8.439886-0.056960-0.703945$

O $8.199412-0.9156601 .424945$

C $8.965541 \quad 1.156782-0.158060$

H $9.896073 \quad 0.9565220 .381442$

H $\quad 9.1794601 .837312-0.986434$

H 8.2302771 .6276480 .501278

SCF Energy (B3LYP/6-31G**//MMFF) $=-3245.91143251$

01277

MM̄̄FF Geometry

C -1.416917 $3.465752-0.378048$

C $-0.9054102 .254836-0.111850$

C $0.0645051 .540842-1.019176$

O $1.257847 \quad 1.239822-0.260645$

C $-0.5541580 .217735-1.513241$

C $0.290547-0.613103-2.466822$

C $0.165311-1.950918-2.594331$

C $1.252200 \quad 0.120012-3.366833$

C $-0.805334-2.855411-1.888322$

C $-0.198856-4.163545-1.358920$

C $0.906695-4.056035-0.295423$

C $0.522599-3.178510 \quad 0.896453$

C $1.654861-3.0688701 .928269$

O $2.104137-3.570524-0.912472$

O $1.305467-1.9853282 .804607$

C $2.958009-2.6900011 .190880$

C $1.748824-4.3228852 .803979$

O $4.076762-2.7139422 .073013$

C $3.245822-3.576650-0.042643$

C $4.470858-3.095838-0.852808$

O $3.547772-4.9060020 .403302$

C $2.1935082 .227229-0.181619$

O $2.1146323 .352358-0.649537$

C $3.3584601 .692698 \quad 0.557156$

C 4.4732562 .4332680 .645876

C 5.7329532 .0424641 .283116

C $5.825228 \quad 0.652917 \quad 1.855897$

C $4.355789-1.694635-1.454851$

C $5.644305-1.286284-2.148133$

C $6.969630 \quad 0.728142-2.770785$

O $6.454041-2.101578-2.583533$

C $7.9765770 .863805-1.670304$

C $8.5443772 .025294-1.312297$

C $9.5697072 .143871-0.220031$

C 9.1938123 .1609950 .835114

C 9.8305924 .3417900 .914495

C 8.1351802 .7774691 .849036

C 6.7403542 .9405431 .306745
N $5.7757390 .092678-2.243644$

H -1.127003 $3.977865-1.292330$

H $-1.187379 \quad 1.7461430 .805987$

H $0.3189702 .168425-1.882700$

H -1.501946 $0.439999-2.021728$

H $-0.792024-0.381961-0.628476$

H $0.788092-2.454877-3.332694$

H $1.714415-0.540432-4.108649$

H $0.7301360 .908159-3.919174$

H $2.0637450 .569664-2.787328$

H $-1.338700-2.350167-1.080010$

H -1.574889 -3.127873 -2.622293

H -1.019998 -4.760326 -0.940897

H $\quad 0.195713-4.729736-2.213195$

H $1.094289-5.0807800 .048256$

H $-0.393872-3.5469851 .371807$

H $0.299052-2.1629680 .549214$

H $1.983271-1.9276753 .498899$

H $2.838054-1.6516190 .863017$

H $\quad 0.820277-4.460773 \quad 3.371022$

H $2.544716-4.2259843 .550643$

H $1.924918-5.2355972 .231098$

H $3.913397-2.0622302 .775138$

H $5.372780-3.162241-0.229774$

H $4.639753-3.802915-1.677682$

H $4.033103-5.365565-0.301729$

H $3.277304 \quad 0.7005680 .983241$

H 4.4742163 .4226930 .189516

H 5.0499480 .4960362 .614254

H 6.7785300 .4432952 .344411

H $5.701195-0.0968931 .067730$

H $3.550184-1.659473-2.195210$

H $4.137971-0.962806-0.674741$

H $6.6712031 .696719-3.183449$

H $7.3821560 .112223-3.576151$

H $8.266156-0.047145-1.151051$

H $8.2771452 .935814-1.843723$

H $10.5223612 .421346-0.689735$

H $9.749199 \quad 1.176385 \quad 0.265359$

H 9.5845705 .0655291 .684495

H $10.608226 \quad 4.613825 \quad 0.208445$

H 8.2174153 .4194062 .735906

H 8.3439641 .7640212 .201146

H 6.5556183 .9295050 .883684

H $5.0865410 .688876-1.795543$

C $-3.7138854 .439574-0.165625$

O $-4.4023853 .186571-0.347303$

C $-5.8024823 .379244-0.068137$

C -6.0055684 .8829650 .081155$

C -4.6669005 .3128750 .648343$

H -5.9975772 .9048820 .900663$

C $-6.6570582 .719954-1.156912$

H $-6.1829205 .369119-0.885093$

H -6.8481215 .1236950 .736629$

C $-4.4054476 .803508 \quad 0.521437$

H -4.6260845 .0279451 .708254$

C $-6.3465701 .223604-1.370667$

O $-6.4091813 .400574-2.393591$

H -7.719678 $2.864906-0.928568$

H $\quad-5.339198 \quad 1.112144-1.787531$

O $-7.2552250 .717803-2.356131$

C $-6.4909100 .360728-0.103060$

C $-6.339247-1.152482-0.339749$

H -5.7589260 .6753720 .644001$

$\begin{array}{llll}\mathrm{O} & -7.781132 & 0.581283 & 0.474754\end{array}$

H $-6.589817-1.6627380 .598240$

H -7.104625 -1.486519-1.051104

H -3.4266267 .0656370 .934584$

H $-4.4272607 .127229-0.524599$

H -5.1647717 .3745921 .065585$

C -2.3744684 .1906360 .526136$

H -7.098956 $3.127307-3.021540$

H -7.013194 $1.116072-3.209119$

H $-8.4464340 .336744-0.191515$

H -2.536312 3.6138351 .444037

H -1.9128625 .1421450 .812685$

H -3.559039 $4.868544-1.164541$

C $-3.867035-1.8371951 .331572$ 
$\begin{array}{lll}\text { O } & -3.899873-1.160678 & 0.067042\end{array}$

C $-4.939507-1.578852-0.824379$

C $-4.812705-3.080097-1.114123$

C $-4.787995-3.8998480 .169560$

C $-3.734833-3.3562291 .133350$

H $-4.780416-1.6105911 .894720$

C $-2.665282-1.2819982 .115492$

H $-4.755143-1.061556-1.772983$

O $-5.880686-3.531827-1.940642$

H -3.882296 -3.267939 -1.663691

H $-5.779600-3.935050 \quad 0.635773$

O $-4.455387-5.249955-0.166040$

H -2.741321 $-3.609240 \quad 0.744465$

O $-3.898196-4.0358752 .377081$

C $-2.895997 \quad 0.1130892 .657066$

H $-2.448845-1.9204392 .978125$

H $-1.782199-1.2354181 .470229$

H $-5.861348-3.004205-2.757223$

H $-4.414547-5.752390 \quad 0.665731$

H -3.083188 -3.9168852 .892455$

O -1.8913380 .4112463 .534820$

O $-3.817412 \quad 0.853542 \quad 2.346034$

C $-1.966103 \quad 1.6987214 .155195$

H -2.9584791 .8686314 .583928$

H -1.2288261 .7293624 .961910$

H - 1.7208412 .4756113 .426541

SCF Energy (B3LYP/6-31G**//MMFF)= -3245.90184793

01278

MM̄FF Geometry

C $1.5735354 .245652-1.854256$

C $1.560507 \quad 3.169114-2.654748$

C $2.3801391 .936284-2.372462$

O $1.581598 \quad 0.759077-2.628212$

C $3.6027431 .902362-3.303795$

C $4.6052570 .827599-2.933394$

C $5.5738300 .986396-2.009114$

C $4.505012-0.449100-3.725496$

C $5.813168 \quad 2.198399-1.152389$

C $6.1433821 .888857 \quad 0.317923$

C 4.9985631 .2306341 .108661

C $5.287463 \quad 1.3097702 .611602$

C 4.2358930 .5688403 .449859

O $4.903606-0.140173 \quad 0.711276$

$\begin{array}{llllll}\text { O } & 4.772084 & 0.457296 & 4.776888\end{array}$

C $4.060032-0.8544422 .880212$

C $2.9378901 .376827 \quad 3.569342$

O $2.984060-1.5362583 .521439$

C $3.851765-0.8771301 .349373$

C $3.900743-2.3351720 .828386$

O $2.568240-0.3261501 .052442$

C $0.7084390 .380135-1.656683$

O $0.4395870 .985564-0.631243$

C $0.163322-0.942500-2.036003$

C $-0.427304-1.692861-1.094230$

C $-1.023438-3.018304-1.277962$

C $-0.995384-3.610763-2.660940$

C $4.005776-2.430908-0.696607$

C $3.979905-3.866671-1.174725$

C $2.440400-5.721264-1.733361$

O $5.002894-4.443420-1.537411$

C $1.198214-6.350995-1.185434$

C $0.662581-6.1506020 .029163$

C $-0.594495-6.828763 \quad 0.494094$

C -1.663467 -5.8532190 .931305$

C $-2.107007-5.8353702 .199883$

C $-2.275450-4.951413-0.119896$

C $-1.573533-3.622740-0.204466$

N $2.710234-4.422330-1.147732$

H $2.215596 \quad 4.233810-0.977347$

H $0.9525653 .172844-3.555716$

H $2.7123011 .918372-1.326378$

H $4.1091102 .876629-3.303042$

H $3.2788821 .741446-4.341274$

H $6.2786920 .170308-1.857157$

H $4.629190-0.243084-4.793748$

H $5.271680-1.177136-3.440909$

H $3.528851-0.919573-3.574431$
H $6.6675312 .738206-1.579868$

H $4.9641192 .888749-1.178495$

H $7.033698 \quad 1.2476680 .355044$

H 6.4101422 .8402710 .795581

H $4.061448 \quad 1.7474170 .872088$

H 5.3671722 .3543482 .938053

H 6.2700260 .8594652 .813881

H 4.0996620 .0394065 .340630

H $4.973289-1.4189393 .114957$

H 3.1306412 .3345964 .067395

H $2.2036110 .856798 \quad 4.194436$

H 2.4752121 .5928922 .603978

H $3.196765-1.5994324 .467569$

H $4.777088-2.8462501 .248151$

H $3.013953-2.882537 \quad 1.171347$

H $2.212920-0.7555120 .259385$

H $\quad 0.279635-1.265485-3.063787$

H $-0.484813-1.296332-0.080652$

H $0.036018-3.710327-3.016798$

H $-1.547404-2.974202-3.361384$

H $-1.442036-4.605197-2.719361$

H $3.196803-1.885366-1.192909$

H $4.947021-1.989747-1.042055$

H $3.299764-6.385734-1.597124$

H $2.316029-5.551714-2.808695$

H $0.699525-7.042768-1.861729$

H $1.152856-5.486800 \quad 0.736824$

H $-0.324042-7.4978041 .321116$

H $-1.009891-7.473633-0.290543$

H -2.892677 -5.1555682 .512640$

H $-1.693107-6.4961072 .954284$

H $-3.324167-4.7527130 .138048$

H -2.313727 $-5.483461-1.073892$

H $-1.544413-3.0910430 .747529$

H $1.921559-3.798814-1.000502$

C $-0.2029735 .880454-1.022817$

O $-1.2681814 .913209-0.960002$

C $-1.661988 \quad 4.7604150 .418010$

C $-0.938196 \quad 5.855128 \quad 1.200055$

$\begin{array}{llll}\text { C } & 0.340060 & 6.004622 & 0.399961\end{array}$

H -1.2787313 .7859580 .743708$

C -3.1868014 .7943230 .559088$

H $-1.497766 \quad 6.797517 \quad 1.199773$

H $-0.763152 \quad 5.570902 \quad 2.242306$

C 1.0843827 .3003610 .667681

H $\quad 0.991043 \quad 5.1594940 .652222$

C $-3.943407 \quad 3.778127-0.318517$

O -3.6510546 .1016910 .195909$

H -3.4531004 .6580841 .614414$

H $-3.9078394 .094924-1.367653$

$\begin{array}{lllllllll} & \text { O } & -5.326240 & 3.843717 & 0.059234\end{array}$

C $-3.4550722 .321386-0.209160$

C $-4.4053531 .341772-0.926209$

H $-2.4522352 .247353-0.645110$

O $-3.356906 \quad 1.980081 \quad 1.169741$

H $-5.3546431 .301640-0.381505$

H $-4.617801 \quad 1.737598-1.926459$

H 1.9925347 .3599520 .059522

H $\quad 0.465817 \quad 8.174274 \quad 0.437686$

H 1.3779537 .3646911 .720526

C $0.7998415 .506334-2.118047$

H $-4.617642 \quad 6.1062150 .303786$

H $-5.397422 \quad 3.5067640 .969034$

H -2.8773591 .1363351 .221861$

H $1.5197376 .324045-2.242183$

H $0.2586125 .411351-3.067961$

H $-0.6776036 .826484-1.317597$

C $-4.650881-1.041458 \quad 1.002608$

O $-3.522016-0.6959150 .191947$

C $-3.832741-0.087484-1.063961$

C $-4.708813-1.037505-1.892878$

C $-5.965530-1.448537-1.128843$

C $-5.594349-1.989500 \quad 0.251367$

H $-5.183658-0.1291441 .296112$

C $-4.128417-1.7099322 .281106$

H -2.881132 $0.019803-1.598452$

O $-5.057085-0.424150-3.128771$

H $-4.128467-1.938182-2.131929$ 
H -6.678382 -0.619853-1.046509

O $-6.636163-2.490026-1.841900$

H $-5.124857-2.974753 \quad 0.137700$

O $-6.781972-2.1721751 .019815$

C $-3.260009-0.7706553 .083245$

H -4.950708 -2.031518 2.930441

H -3.545617 -2.608354 2.049762

H $-5.500447-1.093447-3.676371$

H $-6.973732-2.108219-2.669580$

H -7.367888 -2.7602750 .512876$

O $-1.952578-0.9309882 .728337$

O

C -1.018000 -0.0695953 .385636$

H -1.261649 0.9787223 .188858

H -1.012186 -0.2704534 .460922$

$\mathrm{H}-0.023387-0.2789562 .982873$

SCF Energy (B3LYP/6-31G**//MMFF) $=-3245.90849345$

\section{9}

MM̄FF Geometry

C $-0.6129232 .902155-1.690469$

C $0.4792103 .652122-1.475306$

C $1.3976223 .488635-0.292754$

O $2.7047113 .102293-0.791574$

C 1.6168984 .8042440 .474506

C 0.4458505 .2501741 .318133

C 0.2865204 .9307712 .616980

C $-0.529586 \quad 6.1806160 .646465$

C 1.1755764 .0646383 .469875

C 0.3651993 .0933194 .340446

C $-0.131911 \quad 1.8592983 .570342$

C -1.2308531 .1576024 .370681$

C $-1.677128-0.1482193 .705328$

O $\quad 0.9882150 .982903 \quad 3.395838$

O $-2.482961-0.8716874 .647605$

C $-0.429757-1.0048653 .423619$

C -2.5605590 .1045152 .480732$

O $-0.774599-2.1755362 .685222$

C $0.703100-0.2379922 .698763$

C $1.986767-1.1046312 .721917$

O $\quad 0.309151 \quad 0.0081001 .350429$

C $2.9060371 .791464-1.089721$

O $2.0967290 .883611-0.996258$

C $4.3015301 .651648-1.561172$

C $4.7662390 .444572-1.916653$

C $6.1113380 .151446-2.417955$

C $7.0879081 .296153-2.499836$

C $3.229529-0.4408902 .115420$

C $4.493353-1.2410172 .387745$

C $6.945571-1.1855781 .994296$

O $4.508026-2.2615773 .071092$

C $7.747531-1.0329190 .742224$

C $8.225939-2.0744540 .044928$

C $9.077581-1.950002-1.185893$

C $8.428403-2.564777-2.405470$

C $8.569566-3.872873-2.678886$

C $7.691918-1.643605-3.351127$

C $6.407618-1.117071-2.772329$

N $5.604830-0.6763301 .774410$

H $-0.8759962 .125155-0.977742$

H $0.7528984 .411392-2.204630$

H 1.0384012 .7180920 .400997

H $1.8953575 .606298-0.223800$

H 2.5095904 .7014081 .105831

H $-0.566825 \quad 5.3585213 .142186$

H -1.3341806 .4975301 .318520$

H -0.0150207 .0833810 .301123$

H $-0.9963255 .699035-0.214828$

H 1.9104633 .5089622 .879773

H $1.736506 \quad 4.7321424 .135447$

H 1.0122742 .7613125 .163178

H -0.4799633 .6274764 .792601$

H -0.5103702 .1636732 .588271$

H -2.090172 1.8238744 .518054

H $-0.857607 \quad 0.9242945 .378012$

H $-3.238699-0.3092844 .889499$

H $-0.047398-1.3524894 .393774$

H $\quad-3.4486490 .6822712 .762847$
H $-2.933423-0.8392482 .070595$

H -2.052270 0.6516621 .684166

H -1.479822 -2.631858 3.175310

H $2.213288-1.3585173 .766079$

H $1.811602-2.0476472 .188489$

H 1.1008140 .1526940 .809608

H $4.9062532 .550020-1.611275$

H $4.097909-0.412673-1.844136$

H $7.1932861 .783465-1.523804$

H $6.7474692 .039471-3.229014$

H $8.0943250 .993389-2.795246$

H $3.118992-0.3604651 .031203$

H 3.3725650 .5630062 .529708

H $6.899265-2.2266232 .330932$

H $7.393853-0.5932732 .798675$

H $7.966648-0.0205940 .412446$

H $8.016241-3.0839210 .392416$

H $10.034085-2.451013-0.986115$

H $9.336748-0.903676-1.388934$

H $8.132160-4.316573-3.566916$

H $9.123870-4.529713-2.016339$

H $7.432950-2.177269-4.275585$

H $8.370080-0.848074-3.672695$

H $5.635179-1.880045-2.666159$

H $5.508557 \quad 0.239026 \quad 1.343455$

C $-2.982483 \quad 3.127548-2.634426$

O $-3.4921251 .869730-2.147656$

C $-4.5818472 .146091-1.244768$

C $-4.8161613 .656615-1.291373$

C $-3.4330204 .176875-1.624345$

H $-4.2315841 .879963-0.241249$

C $-5.8256941 .327648-1.615001$

H -5.528396 $3.932655-2.077895$

H $-5.2038604 .042442-0.343345$

C $-3.4258465 .597166-2.159828$

H $-2.8247504 .128294-0.712962$

C $-5.664150-0.206686-1.547277$

O $-6.2283721 .663312-2.949851$

H $-6.6580541 .633418-0.969686$

H $-5.043989-0.555753-2.380542$

O $-6.964006-0.775477-1.761258$

C $-5.094638-0.737773-0.220871$

C $-5.288488-2.2426210 .031329$

H $-4.030129-0.488549-0.167417$

O $-5.742032-0.0512510 .857980$

H $-4.787613-2.4744120 .979615$

H $-6.351285-2.4342660 .230226$

H $-2.4083055 .915920-2.407124$

H -4.036281 $5.688716-3.064390$

H $-3.8253126 .291247-1.413180$

C $-1.4802343 .041810-2.911487$

H -5.485298 $1.452244-3.540983$

H -7.525658 -0.516317 - 1.010572

H $-5.356222-0.3751161 .689271$

H -1.177183 $3.923856-3.488658$

H $-1.2954582 .166786-3.544708$

H -3.486129 $3.298361-3.596062$

C $-2.449193-3.247604-0.494939$

O $-3.447033-2.951269-1.477344$

C $-4.794318-3.196556-1.072923$

C $-4.959178-4.679517-0.707470$

C $-3.969645-5.097138 \quad 0.377392$

C $-2.548357-4.706941-0.020344$

H -2.556569 -2.557859 0.349750

C -1.072927 -3.009401-1.137154

H $-5.416158-3.012975-1.957064$

O $-6.283144-4.952740-0.260943$

H $-4.784047-5.298502-1.596926$

H $-4.234752-4.677198 \quad 1.354308$

O $-4.014090-6.5180550 .532052$

H $-2.206356-5.382800-0.815237$

O $-1.677459-4.8941301 .093452$

C $-0.937034-1.632817-1.747888$

H $-0.266255-3.139304-0.408011$

H $-0.932528-3.741580-1.942987$

H $-6.891583-4.684829-0.970719$

H $-4.932940-6.7565160 .744171$

H $-1.768118-5.8188371 .380941$ 
O $-0.116189-1.713392-2.836776$

O $-1.475489-0.620806-1.322569$

C $0.048874-0.508394-3.587531$

H $\quad 0.4366080 .290473-2.952142$

H $0.770604-0.700674-4.385791$

$\mathrm{H}-0.904272-0.218128-4.038573$

SCF Energy (B3LYP/6-31G**//MMFF) $=-3245.92273636$

0128

MM̄FF Geometry

C $4.129650-3.9755920 .313347$

C $3.158237-3.632495-0.545564$

C $1.709519-4.002030-0.354219$

O $0.961215-2.785727-0.122726$

C $1.164825-4.670094-1.629419$

C $-0.283918-5.107087-1.522330$

C $-1.279589-4.641883-2.302407$

C $-0.580260-6.153529-0.478878$

C -1.183790 -3.639018 -3.417662

C $-1.351395-2.176180-2.981679$

C $-2.753271-1.829671-2.455621$

C $-2.882774-0.324395-2.209204$

C $-4.2487730 .043742-1.607440$

O $-2.969066-2.529953-1.228920$

O $-4.1618821 .388256-1.109756$

C $-4.533103-0.869417-0.394428$

C $-5.3553650 .045349-2.668788$

O $-5.861416-0.6817170 .086468$

C $-4.282401-2.364511-0.676657$

C $-4.358326-3.2459820 .593842$

O $-5.284024-2.846297-1.581159$

C $0.984491-2.2806581 .141197$

O $1.552094-2.7535362 .113336$

C $\quad 0.210427-1.0214491 .141043$

C $0.380388-0.1567732 .151982$

C $-\begin{array}{llll}0.296979 & 1.130023 & 2.313204\end{array}$

C -1.4580801 .4222001 .401831$

C $-3.366466-2.8848831 .705970$

C $-3.960300-2.0226382 .808610$

C -3.316122 -0.870207 4.923670

O $-5.155450-1.7523222 .886325$

C $-3.213166 \quad 0.5958214 .645136$

C -2.4712641 .4361595 .382774$

C -2.4062062 .9185675 .151829$

C -0.9909853 .4238394 .983613$

C $-0.334393 \quad 3.9984746 .005479$

C -0.3745743 .3458383 .606050$

C $0.136171 \quad 1.9693043 .276702$

N -2.995922 -1.6220353 .724477$

H $3.884184-4.5663811 .192143$

H $3.404953-3.018994-1.409310$

H $1.587486-4.6787350 .500525$

H $1.769922-5.554026-1.871420$

H $1.292951-3.975758-2.467036$

H -2.284046 -5.029673 -2.136648

H -1.600941 -6.542839 -0.559810

H $0.101741-7.003102-0.587994$

H $-0.467320-5.7389150 .527409$

H $-0.229577-3.746972-3.945281$

H -1.949106 -3.884517 -4.165156

H $-0.600379-1.941457-2.218296$

H -1.135776 -1.547283 -3.853133

H -3.488282 -2.159311 -3.199388

H $-2.7086490 .239240-3.134085$

H -2.098483 -0.001613 -1.511063

H $-3.9105221 .966104-1.850607$

H -3.863973 -0.5453990 .409638$

H $-5.121590 \quad 0.763245-3.464228$

H $-6.3106110 .371903-2.243360$

H -5.500956 -0.930060 -3.137665

H $\quad \begin{array}{llll}-5.969510 & 0.265881 & 0.277060\end{array}$

H $-5.389650-3.2847920 .964513$

H $-4.123933-4.2754830 .285676$

H - $-5.339802-3.812305-1.493273$

H $-0.458619-0.8355370 .309307$

H $1.101075-0.4026302 .931196$

H -1.999636 2.3350911 .657085

H -2.1910570 .6113861 .452804$
H -1.121503 1.5237040 .366698

H -3.039513 -3.814092 2.190571

H -2.474642 -2.397677 1.300587

H -2.620830 -1.193504 5.704821

H -4.335063 -1.113061 5.242398

H $-3.8022460 .989528 \quad 3.820130$

H $-1.905591 \quad 1.0441646 .225318$

H -2.8769793 .4096376 .013703$

H -3.004824 3.2155394 .281593

H $\quad 0.669748 \quad 4.3905495 .883062$

H $-0.7847144 .084126 \quad 6.989002$

H 0.4790984 .0334763 .536193

H -1.092279 3.7201592 .872333

H 0.9818121 .6598023 .892465

H -2.020105 -1.845128 3.550501

C $5.893208-2.2182630 .829556$

O $5.224353-1.1471950 .135138$

C $4.759034-0.1871721 .103804$

C $5.432984-0.5568882 .421936$

C $5.515696-2.0679252 .302559$

H $3.679328-0.3455331 .208832$

C $5.0282971 .239260 \quad 0.610044$

H $6.438450-0.1280382 .501571$

H $4.855245-0.2246163 .289741$

C $6.505587-2.7069513 .259682$

H $4.517253-2.4776972 .497367$

C $4.4817651 .522263-0.805553$

O $\quad 6.446867 \quad 1.440928 \quad 0.577637$

H $4.626307 \quad 1.9601791 .331842$

H $5.0301400 .924859-1.544486$

O $4.7440892 .895853-1.119856$

C $2.9695771 .275168-0.966752$

C $2.4881531 .595226-2.396714$

H $2.7467030 .224733-0.751398$

O $2.2763632 .071601-0.013070$

H $2.6532562 .656614-2.605210$

H $3.114121 \quad 1.024782-3.092395$

H $6.536253-3.7920563 .119122$

H $7.518277-2.3181363 .109596$

H $6.220065-2.5078224 .297772$

C $5.563615-3.5554850 .159862$

H 6.6043042 .3953070 .481940

H $5.7053272 .993280-1.225299$

H $1.3258361 .895347-0.111229$

H $6.199988-4.3459040 .574760$

H $5.803782-3.487997-0.909115$

H $6.967737-2.0322560 .699824$

C $0.019248 \quad 3.456219-2.468380$

O $0.1087562 .126525-1.938987$

C $1.0001841 .240418-2.626800$

C $0.6006071 .139900-4.104430$

C $0.5507612 .510564-4.765568$

C $-0.3534223 .438973-3.959278$

H $0.9776143 .970001-2.329752$

C $-1.0564304 .207871-1.663206$

H $0.8425730 .246616-2.188596$

O $1.497900 \quad 0.284116-4.802590$

H $-0.3942320 .683619-4.174100$

H $1.5566882 .927969-4.890222$

O $0.0097542 .357464-6.079418$

H -1.394651 $3.114303-4.081495$

O $-0.2646524 .756384-4.500721$

C $-0.5277964 .759385-0.355966$

H -1.422907 $5.061989-2.244816$

H -1.901646 $3.551452-1.428085$

H $1.2209560 .270380-5.734826$

H $-0.0607633 .246300-6.468441$

H $0.6517885 .062941-4.391551$

O

O $0.414216 \quad 4.298115 \quad 0.271813$

C -0.9032326 .5012131 .219407$

H -1.324651 5.9750902 .079150

H $\quad 0.1849806 .5762591 .308138$

$\mathrm{H}-1.317414 \quad 7.5127201 .190568$

SCF Energy (B3LYP/6-31G**//MMFF)= -3245.90772876

01280

MM̄MF Geometry 
C $-3.015682-3.888957-1.416899$

C $-2.076050-3.558494-0.518135$

C $-0.600621-3.554245-0.830795$

O $-0.054241-2.259192-0.491773$

C $0.117847-4.6102340 .029250$

C $1.585033-4.783522-0.316048$

C $2.606646-4.5338000 .526389$

C $1.875432-5.306260-1.700309$

C $2.540755-4.0529201 .948014$

C $2.610307-2.5278642 .111785$

C $3.915241-1.8930721 .605575$

C $4.006698-0.4301192 .046187$

C $5.2566830 .263721 \quad 1.482108$

O $3.927636-1.9596230 .177178$

O $5.0948631 .679017 \quad 1.664924$

C $5.3317010 .011363-0.039483$

C $6.522929-0.1305812 .251625$

O $6.5614230 .492365-0.575515$

C $5.130652-1.467159-0.429234$

C $4.996064-1.682056-1.957136$

O $6.268360-2.2251280 .000743$

C $-0.270159-1.238530-1.364725$

O $-0.880768-1.285365-2.420347$

C $0.357390-0.028027-0.792520$

C $0.0004401 .180547-1.250135$

C $0.5081632 .465705-0.768036$

C 1.7103442 .4407340 .137372

C $3.832721-0.941622-2.626822$

C $4.2193220 .396039-3.237160$

C $3.2065462 .399145-4.321781$

O $5.3822740 .768704-3.365230$

C $3.0749273 .492287-3.308701$

C $2.2102304 .510893-3.432946$

C $2.0958875 .638857-2.447589$

C $0.6795385 .845789-1.958617$

C $-0.1075286 .790664-2.500409$

C $0.2077095 .015801-0.786294$

C $-0.1071663 .596410-1.171367$

N $3.1066381 .110903-3.661035$

H -2.709111-4.186462 -2.416327

H $-2.372971 \quad-3.266010 \quad 0.486329$

H -0.427614 -3.751764 -1.895495

H $-0.372271-5.585724-0.091938$

H $0.000049-4.3393521 .084279$

H $3.620159-4.7014110 .162872$

H $2.929593-5.574170-1.830193$

H $1.284851-6.206610-1.898775$

H $1.634094-4.553672-2.457039$

H $1.634963-4.4232562 .439802$

H $3.370863-4.5121702 .500044$

H $1.757133-2.0792751 .590594$

H $2.495037-2.3040713 .179408$

H $4.758374-2.4668382 .007809$

H $3.986108-0.3503393 .140289$

H 3.1192030 .1112391 .691550

H 4.9692511 .8459622 .614820

H $4.5362550 .609795-0.498136$

H $\quad \begin{array}{lll}6.427428 & 0.140363 & 3.310029\end{array}$

H 7.3970390 .4162131 .881394

H $6.738387-1.2000462 .203811$

H $6.6370691 .430508-0.330726$

H $5.950789-1.469797-2.453318$

H $4.819118-2.755656-2.117241$

H $6.306992-3.044826-0.519459$

H $1.075170-0.1635980 .008127$

H $-0.7541901 .238744-2.033260$

H $2.5197981 .863203-0.321177$

H $1.458171 \quad 1.9854911 .099433$

H 2.1252473 .4292520 .343522

H $3.463146-1.557287-3.456942$

H $3.001873-0.801160-1.929270$

H $2.4162392 .437857-5.078131$

H $4.1756402 .477637-4.824836$

H $3.7389193 .458957-2.448086$

H $1.5677924 .556466-4.309715$

H $2.4551206 .550572-2.942898$

H $2.7603835 .489388-1.587512$

H -1.114888 $6.964846-2.136939$
H $\quad 0.2368897 .407898-3.323589$

H $-0.7112125 .445916-0.365501$

H 0.9409935 .0871790 .021755

H -0.965069 $3.509610-1.839271$

H $2.1777960 .747031-3.468938$

C $-5.274765-2.784099-1.827566$

O $-4.868614-1.499523-1.320550$

C $-4.834333-0.556091-2.407119$

C $-5.541128-1.247378-3.571384$

C $-5.152332-2.695526-3.346514$

H -3.781095 -0.397934 -2.669123

C $-5.4841260 .774733-2.008483$

H $-6.629561-1.128241-3.505729$

H $-5.216731-0.860097-4.542092$

C $-6.022624-3.688812-4.095045$

H $-4.111400-2.819341-3.667798$

C $-4.6899271 .683175-1.045039$

O $-6.7852820 .540776-1.469952$

H -5.629646 $1.348359-2.932995$

H $-5.2970092 .584250-0.885857$

O $-3.4784032 .099663-1.674044$

C -4.3554661 .1096880 .346284$

C -3.8023292 .1991851 .291343$

H -5.2588650 .6799620 .791759$

$\begin{array}{lllll}\text { O } & -3.389722 & 0.069742 & 0.232021\end{array}$

H -2.8116182 .5114360 .943330$

H -4.4581263 .0760101 .238797$

H -5.709605 -4.716170 -3.883609

H -7.076575 -3.592094 -3.813449

H $-5.947913-3.527729-5.175450$

C $-4.490635-3.898322-1.129090$

H -6.696614 -0.115723 -0.758117

H $-3.7140672 .534729-2.510915$

H $-2.6241100 .430626-0.245876$

H -4.891587 -4.873547-1.428805

H $-4.649797-3.810427-0.046553$

H -6.331433 -2.894461 -1.548327

C -1.5032540 .7763602 .784198$

O -2.9095070 .5577632 .920981$

C $-3.719086 \quad 1.7258002 .762323$

C -3.2899742 .8134263 .760741$

C -1.7911643 .1033323 .688477$

C $-0.996726 \quad 1.8017453 .803340$

H -1.299444 1.1571821 .776355

C $-0.789646-0.5801402 .857428$

H -4.7379641 .4260953 .040621$

O -4.0364604 .0039753 .533982$

H -3.5320252 .4710964 .775653$

H -1.527897 3.6435912 .771230

O -1.4158873 .9461214 .779767$

H $-1.083706 \quad 1.4178774 .825905$

O $\quad 0.3828792 .0660913 .566072$

C $-0.999623-1.3481594 .145982$

H -1.170264 -1.220548 2.052594

H $\quad 0.286326-0.4348902 .712965$

H -3.8412644 .6182064 .261449$

H -1.834639 4.8119124 .638925

H 0.6608422 .7495664 .199598

O $-0.197141-2.4544414 .128507$

O $-1.774563-1.0430295 .041032$

C $-0.286769-3.3016525 .277900$

H $0.406450-4.1355275 .139390$

H $\quad 0.001833-2.7509246 .178157$

$\mathrm{H}-1.300903-3.7000765 .376022$

SCF Energy (B3LYP/6-31G**//MMFF) $=-3245.90240215$

01281

MM̄FF Geometry

C $4.325544-1.1907613 .054134$

C $3.324871-1.8116332 .411016$

C $2.046693-2.2552973 .076058$

O $0.932692-1.6232792 .404886$

C $1.905659-3.7812752 .923060$

C $0.644072-4.3444273 .546200$

C $-0.442496-4.7456162 .855643$

C $0.675521-4.4757975 .047134$

C $-0.670358-4.6807841 .368596$

C $-1.887529-3.8119731 .050003$ 
C $-2.156654-3.614972-0.450169$ C $-1.025090-2.872502-1.168457$ C $-1.384116-2.529254-2.622245$ O $-3.366860-2.851008-0.528334$ O $-0.426300-1.572383-3.099367$ C $-2.763490-1.840299-2.649484$ C $-1.266255-3.751607-3.540380$ O $-3.198322-1.626901-3.990592$ C $-3.846107-2.605324-1.856716$ C $-5.122643-1.740084-1.727984$ O $-4.159948-3.818386-2.540632$ C $0.592926-0.3666352 .806126$ $\begin{array}{llllllll}\text { O } & 1.164501 & 0.322147 & 3.636034\end{array}$ C - 0.6267380 .0234702 .064675 C $-1.186501 \quad 1.2264742 .258223$ C $-2.398472 \quad 1.7216301 .598823$ C -3.2161690 .7326450 .810586$ C $-6.240521-2.409124-0.919440$ C $-7.237025-1.392802-0.401674$ C $-7.616668 \quad 0.188177 \quad 1.490126$ O $-8.295214-1.169834-0.984752$ C $-7.183008 \quad 1.5733591 .130363$ C -6.7231632 .4596162 .026756$ C $-6.360245 \quad 3.8770991 .691148$ C -4.9333704 .2155852 .057739$ C -4.6632914 .9441023 .154887$ C -3.8532243 .7812901 .091072$ C -2.7219543 .0243571 .736196$ N $-6.811679-0.7746730 .764132$ H $4.198426-0.9756294 .113769$ H $3.411014-2.0362221 .351888$ H $2.034633-1.9900124 .140636$ H $2.769750-4.2789303 .384869$ H $1.960424-4.0506981 .862724$ H -1.279053 -5.160842 3.416880 H $-0.235045-4.9375345 .443807$ H $1.521228-5.0986995 .356289$ H $0.776597-3.4926525 .516924$ H $\quad 0.206626-4.2960140 .847647$ H $-0.837745-5.6979160 .995664$ H $-2.782466-4.2580431 .506458$ H $-1.788499-2.8318731 .531118$ H -2.326071 -4.597954-0.904811 H $-0.092629-3.447466-1.146249$ H $-0.810197-1.935093-0.638864$ H $0.459811-1.962571-3.008169$ H -2.643546 -0.844317 -2.201896 H $-0.238528-4.134673-3.537528$ H -1.483442 $-3.486923-4.581107$ H $-1.923187-4.572974-3.247424$ H $-2.495783-1.134096-4.448338$ H $-4.858691-0.786656-1.254177$ H $-5.521991-1.503738-2.722222$ H $-5.020053-4.143129-2.231967$ H $-1.040509-0.6967381 .371420$ H $-0.708846 \quad 1.9199852 .948737$ H -3.485067 -0.1273541 .434534$ H -2.657021 $0.370753-0.057299$ H -4.1556591 .1449470 .438404$ H -6.785313 -3.133305 - 1.535865 H $-5.842896-2.965025-0.062396$ H -7.487782 -0.0214352 .556915$ H -8.673243 $0.053913 \quad 1.237162$ H $-7.288273 \quad 1.8711890 .089961$ H $-6.6414772 .166027 \quad 3.070998$ H -7.0554254 .5339292 .230299$ H $-6.520874 \quad 4.0918240 .627222$ H -3.649951 $5.234463 \quad 3.410211$ H $-5.453240 \quad 5.273376 \quad 3.822134$ H -3.434423 4.6786670 .615655 H -4.2907713 .2145040 .263678$ H -2.063661 3.6545152 .335875 H $-5.891159-0.9932531 .133880$ C $5.958145-1.1853441 .044264$ O $5.108177-0.4776830 .123359$ C $5.818839-0.295843-1.117185$ C $7.203910-0.910228-0.922310$ C $7.375490-0.8544210 .584123$
H $5.931100 \quad 0.784244-1.261095$

C $5.014144-0.900772-2.275427$

H $7.244475-1.947007-1.275694$

H $7.977744-0.353406-1.460362$

C $8.441490-1.8042921 .102534$

H 7.6351950 .1728770 .870755

C $3.577438-0.350235-2.394805$

O $4.922406-2.316075-2.064312$

H $5.560541-0.760080-3.215624$

H $2.988781-0.659483-1.523688$

O $2.964363-0.959895-3.537834$

C $3.4961731 .178678-2.570332$

C $2.0806751 .708085-2.870595$

H $3.8810801 .676882-1.675517$

O $4.343877 \quad 1.576194-3.652260$

H $2.1666232 .773357-3.117128$

H $1.7017691 .240950-3.787995$

H $8.538824-1.7292982 .189813$

H $8.205611-2.8449500 .856292$

H $9.415065-1.5667340 .661484$

C $5.650003-0.7521392 .480295$

H $4.628194-2.717813-2.899075$

H $2.838780-1.902084-3.334628$

H $4.0329051 .118120-4.452137$

H 5.6853900 .3414502 .550331

H $6.428196-1.1446543 .145293$

H $5.764376-2.2591830 .918547$

C $1.5796233 .495768-0.444167$

O $1.5547412 .063353-0.487964$

C $1.0806441 .503096-1.717120$

C $-0.3385572 .004901-2.016573$

C $-0.428512 \quad 3.526472-1.982877$

C $0.1831574 .073468-0.695924$

H $2.2731693 .871460-1.206570$

$\begin{array}{llll}\text { C } & 2.105128 & 3.901176 & 0.940037\end{array}$

H $1.0011080 .423058-1.548578$

O $-0.792261 \quad 1.541435-3.283737$

H -1.027154 $1.603102-1.264942$

H $\quad 0.0444163 .976217-2.863878$

O $-1.803810 \quad 3.915812-2.022672$

H $-0.473592 \quad 3.8449250 .149803$

$\begin{array}{lllll}\text { O } & 0.274500 & 5.494035 & -0.791413\end{array}$

C 3.5891714 .1684450 .888446

H $1.6416874 .827997 \quad 1.298251$

H 1.9086503 .1360181 .700315

H $-0.7342710 .570686-3.277418$

H $-2.1914843 .524885-2.824442$

H $-0.621233 \quad 5.830742-0.965317$

$\begin{array}{lllll}\text { O } & 4.286212 & 2.998973 & 0.944553\end{array}$

$\begin{array}{llllll}\text { O } & 4.069427 & 5.290688 & 0.786930\end{array}$

$\begin{array}{llll}\text { C } & 5.707917 & 3.143276 & 0.875034\end{array}$

H 6.0686023 .7799341 .688842

H 6.1582372 .1553150 .982953

H $5.9970303 .554934-0.096678$

SCF Energy (B3LYP/6-31G**//MMFF) $=-3245.91472708$

$01 \_282$

MM̄FF Geometry

C $-2.147842-4.810500-0.764740$

C $-1.207177-4.141738-0.083927$

C $0.100472-3.704461-0.687641$

O $0.285288-2.298343-0.399937$

C $1.268918-4.474193-0.047691$

C $2.592539-4.163423-0.716936$

C $3.454373-3.210614-0.309223$

C $2.911637-5.009483-1.922259$

C $3.291214-2.2543220 .841259$

C $4.473744-2.2526631 .823559$

C $5.802253-1.7825721 .210508$

C $6.882944-1.6292902 .283232$

C $8.190863-1.0665641 .701214$

O $5.575970-0.523947 \quad 0.571211$

O $9.014849-0.6413682 .797601$

C $7.871897 \quad 0.191006 \quad 0.862100$

C $8.988446-2.1404480 .951581$

$\begin{array}{llll}\text { O } & 9.031312 & 0.655202 & 0.174630\end{array}$

C $6.710476-0.012767-0.137057$

C $6.3044291 .335963-0.777516$ 
O $7.134456-0.903174-1.172860$

C $-0.314339-1.394081-1.218916$

O $-1.029724-1.627957-2.179213$

C $0.049596-0.047437-0.722056$

C $-0.5806081 .033973-1.203383$

C $-0.3654792 .417988-0.773227$

C 0.7922932 .6889310 .151160

C $4.9597761 .284765-1.519837$

C $4.6810042 .558119-2.298550$

C $2.9521093 .588116-3.769463$

O $5.4770303 .490320-2.373202$

C $2.2238954 .599138-2.941662$

C $0.9981455 .054723-3.241447$

C $0.2953246 .123940-2.456516$

C -1.105065 5.730886-2.047939

C $-2.178256 \quad 6.238834-2.677476$

C $-1.2633324 .825479-0.848517$

C $-1.2177973 .367334-1.213053$

N $3.4384412 .518557-2.918733$

H -1.950687 -5.087314 -1.797142

H $-1.390268-3.8794440 .953582$

H $\quad 0.107147-3.857474-1.774603$

H $1.088172-5.555570-0.112627$

H $1.328791-4.2564451 .024280$

H $4.359428-3.068600-0.897050$

H $3.873899-4.746110-2.373847$

H $2.957577-6.066766-1.641073$

H $2.142237-4.887010-2.691137$

H $3.152074-1.2475740 .429396$

H $2.385747-2.466527 \quad 1.415875$

H $4.204335-1.5764612 .645747$

H $4.600140-3.2513042 .259369$

H $6.121556-2.5179510 .464074$

H $7.065921-2.5844812 .790932$

H $6.528453-0.9389003 .061511$

H $9.164375-1.4093903 .375467$

H 7.5874830 .9879921 .563486

H $9.243665-2.9675371 .625197$

H $9.943674-1.7435670 .590824$

H $8.446970-2.5630540 .102786$

H 9.7306200 .7816960 .838647

H $6.2162392 .103490 \quad 0.002413$

H $7.0918451 .663704-1.468059$

H $6.643848-0.706923-1.986064$

H 0.8046580 .0139340 .053716

H -1.351109 $0.892416-1.959972$

H $1.7218532 .288004-0.268046$

H $\quad 0.621403 \quad 2.224628 \quad 1.128239$

H $0.9731493 .751994 \quad 0.322723$

H $4.939610 \quad 0.452892-2.231753$

H $4.1440871 .138994-0.801856$

H $2.3092593 .133418-4.529819$

H $3.8020984 .065023-4.268346$

H $2.7449685 .005450-2.077794$

H $0.4904394 .673941-4.124687$

H $0.2632017 .025424-3.082535$

H $0.8662936 .405535-1.562902$

H -3.186711 $5.984743-2.367804$

H -2.072818 $6.914960-3.519475$

H -2.239684 $5.009549-0.380653$

H $-0.5345615 .102644-0.082254$

H -2.027485 $3.063647-1.878394$

H $2.8171631 .735029-2.738862$

C $-4.683829-4.544035-0.807111$

O $-4.743364-3.155237-0.419136$

C $-5.260514-2.397984-1.529676$

C $-5.726028-3.415382-2.573127$

C $-4.771707-4.563995-2.332467$

H -4.414480 -1.835620 -1.940468

C $-6.374658-1.440150-1.096138$

H $-6.758848-3.738484-2.398364$

H -5.670970 -3.015787 -3.590479

C $-5.243569-5.887481-2.907498$

H -3.813063 -4.296040 -2.791419

C $-6.008548-0.394943-0.023597$

O $-7.464361-2.217634-0.577751$

H -6.764161 -0.932486 -1.987682

H $\quad-5.938377-0.8781360 .957859$
O $\quad \begin{array}{llll}-7.134249 & 0.495060 & 0.064008\end{array}$

C $-4.7303260 .427056-0.278836$

C -4.6610421 .7687620 .476769$

H -3.854471 -0.187782 -0.042976

O $\quad-4.657890 \quad 0.742183-1.674674$

H -3.7025192 .2363980 .225120$

H -5.4134632 .4426490 .046766$

H -4.520727 -6.682212 -2.698134

H $-6.208157-6.188240-2.484914$

H $-5.360972-5.814993-3.993670$

C $-3.466758-5.234051-0.182096$

H $-8.173883-1.597747-0.336362$

H $-7.1962040 .974623-0.779986$

H $-3.8083881 .188328-1.831117$

H $-3.558044-6.318632-0.315822$

H $-3.471738-5.0452950 .898836$

H $-5.583489-5.007245-0.379362$

C -2.5082691 .5380742 .644517$

O

C -4.8369861 .7013162 .007317$

C -4.9554723 .0844262 .664783$

C -3.6279603 .8301962 .616969$

C $-2.5207542 .967486 \quad 3.212911$

H -2.1211521 .5337741 .620024$

C -1.5908700 .6621003 .512690$

H $\quad-5.777109 \quad 1.177629 \quad 2.211282$

O

H -5.2553522 .9687413 .714371$

H -3.378052 4.1483071 .599327

O -3.7289695 .0254593 .394537$

H $-2.6455962 .933386 \quad 4.303157$

O -1.2587193 .5815182 .954364$

C $-1.400940-0.7385062 .977379$

H -0.5973951 .1175073 .592618$

H $-2.026647 \quad 0.566328 \quad 4.515075$

H -6.7912833 .3734962 .073384$

H -4.4670665 .5444323 .031185$

H -1.2987154 .4872323 .306547$

O $-0.668209-1.4475683 .888186$

O $-1.830551-1.1582491 .912943$

C $-0.401964-2.8102303 .549248$

H $\quad 0.209875-2.8556592 .645649$

H $\quad 0.155833-3.2638204 .372780$

H $-1.338567-3.3594363 .415965$

SCF Energy (B3LYP/6-31G**//MMFF) $=-3245.91153124$

01283

MM̄FF Geometry

C $1.659645-3.3417690 .936721$

C $0.691716-3.8972371 .682421$

C $-0.752943-3.4552121 .776545$

O $-1.037342-2.3640920 .873525$

C $-1.671145-4.6301861 .386345$

C $-3.147419-4.3559021 .600267$

C $-4.073596-4.3347680 .621745$

C $-3.570424-4.1293143 .029562$

C $-3.877056-4.567057-0.849928$

C $-3.658527-3.289228-1.674876$

C $-4.853253-2.321051-1.665399$

C $-4.649529-1.200151-2.685914$

C $-5.786149-0.166262-2.639531$

O $-4.966978-1.752844-0.359164$

O $-5.3559940 .989453-3.376491$

C $-6.0086130 .285457-1.178145$

C $-7.049275-0.680900-3.341321$

O $-7.1680061 .111618-1.101433$

C $-6.099587-0.890495-0.180518$

C $-6.096700-0.4504281 .304445$

O $-7.324896-1.599146-0.403941$

C $-0.874691-1.0955551 .335494$

O $-0.519882-0.7541182 .451816$

C $-1.173697-0.1792180 .213068$

C $-0.708622 \quad 1.0778930 .242300$

C $-0.8967472 .089445-0.799174$

C $-1.9127211 .801399-1.871766$

C -4.8665430 .3462991 .752895$

C -5.0591271 .8531611 .718846$

C -3.8073003 .9757232 .093299$ 
O -6.1391592 .3908491 .490456$ C -3.377378 4.5305860 .772311 C $-2.341937 \quad 5.3716880 .629826$ C -1.924804 $5.973717-0.681037$ C $-0.4772485 .692925-1.015523$ C $0.4783226 .609232-0.785165$ C $-0.1537734 .380503-1.693507$ C $-0.1655103 .221311-0.736162$ N -3.8817182 .5293162 .009933$ H $1.444674-2.4691920 .327151$ H $\quad 0.950337-4.757363 \quad 2.298230$ H $-0.949945-3.1530742 .811917$ H -1.409558 -5.520961 1.973350 H $-1.474347-4.8892490 .340477$ H $-5.107170-4.1359960 .903600$ H -4.659868 -4.0983963 .140359$ H -3.201359 -4.9382493 .668483$ H -3.177191 -3.178987 3.403085 H -3.040571 -5.251201-1.026750 H -4.760567 $-5.095255-1.230900$ H $-2.763257-2.776889-1.303266$ H $-3.451599-3.592699-2.708768$ H $-5.760528-2.890427-1.898930$ H $-4.545653-1.609213-3.698695$ H -3.702986 -0.685034 -2.474359 H $-5.149040 \quad 0.706676-4.283771$ H $-5.1532270 .919075-0.916007$ H -6.833788 $-0.921644-4.389347$ H $-7.8290100 .088323-3.366787$ H -7.462982 -1.577746 -2.875748 H $-7.203762 \quad 1.494197-0.209042$ H -7.029976 0.0704321 .549420 H $-6.113731-1.3678171 .910982$ H $-7.540976-2.1025790 .398476$ H -1.743485 -0.573342 -0.620247 H -0.1124181 .3930291 .096864$ H -2.872896 $1.527059-1.422270$ H - $1.5752650 .978948-2.511595$ H -2.118485 $2.654196-2.521373$ H $-4.651993 \quad 0.0902362 .798527$ H $-3.9854870 .077713 \quad 1.162794$ H $-3.101940 \quad 4.2186252 .894444$ H -4.7899114 .3778262 .360050$ H $-3.9602674 .249401-0.101663$ H -1.7765645 .6710421 .509750$ H -2.090523 $7.057189-0.617704$ H -2.562750 $5.626157-1.503276$ H $1.5147346 .424665-1.047865$ H $0.245737 \quad 7.562968-0.323322$ H $0.8494194 .425211-2.137345$ H $-0.8298014 .245991-2.541636$ H $0.544448 \quad 3.3291310 .084523$ H -3.0224192 .0019392 .134559$ C $3.572635-3.965655-0.555346$ O $3.912540-2.643054-1.029633$ C $5.234693-2.682408-1.596783$

C $5.505804-4.149775-1.891872$

C $4.845751-4.803686-0.693710$

H $5.927192-2.353951-0.812364$

C $5.336092-1.745684-2.804286$

H $5.019330-4.474077-2.819140$

H $\quad 6.574547-4.370455-1.968845$

C $4.586738-6.289109-0.877533$

H $5.488083-4.6594780 .185674$

C $4.966183-0.276591-2.505141$

O $4.460475-2.210584-3.838871$

H $6.347614-1.795857-3.223501$

H $3.885520-0.191483-2.342782$

O $5.262016 \quad 0.497171-3.673829$

C $5.724375 \quad 0.336362-1.315526$

C $5.5144901 .846583-1.109440$

H $5.435920-0.183997-0.397097$

O $7.1289770 .116831-1.477930$

H $6.0917282 .136374-0.222004$

H $5.9823392 .401150-1.933562$

H $4.089966-6.7102550 .002117$

H $3.950257-6.480879-1.747923$

H $5.528338-6.828095-1.024805$
C $3.069505-3.8663570 .887455$

H $3.561202-2.242949-3.469278$

H $4.7758690 .098373-4.415836$

H $7.3953780 .531884-2.316325$

H $3.706688-3.1781541 .456091$

H $3.119399-4.8489841 .370332$

H $2.786710-4.349450-1.218813$

C $3.655463 \quad 1.4977751 .286936$

O $3.2942951 .430877-0.098189$

C $4.0469672 .290972-0.956787$

C $3.8788173 .752024-0.516108$

C $4.1853413 .945341 \quad 0.969737$

C 3.4380832 .9178021 .822545

H $4.710173 \quad 1.2201551 .392161$

C 2.7928770 .4604042 .023053

H $3.5908472 .201816-1.950695$

O $4.7073414 .601719-1.302186$

H $2.8436074 .057793-0.703100$

H 5.2638313 .8954591 .157215

$\begin{array}{lllll}\text { O } & 3.785677 & 5.260912 & 1.363457\end{array}$

H $2.3680903 .160554 \quad 1.828484$

O 3.8998792 .9942303 .167734

C $3.502650-0.2352213 .163752$

H 1.8845380 .9259252 .420411

H $2.487351-0.3201301 .315825$

H $4.5866205 .509706-0.975473$

H 2.8284265 .3423891 .211851

H 3.7823483 .9130103 .464347

O $2.676142-1.2019593 .665016$

$\begin{array}{lllll}\text { O } & 4.627155 & 0.024073 & 3.567793\end{array}$

C $3.212257-1.9749624 .742688$

H $4.113529-2.5035044 .417687$

H $3.428918-1.3304705 .599860$

H $2.461167-2.7123515 .038000$

SCF Energy (B3LYP/6-31G**//MMFF)= -3245.91621705

01284

MM̄FF Geometry

C $2.443962-3.455428 \quad 0.043603$

C $1.364012-3.8010690 .760070$

C $0.287037-2.814651 \quad 1.120242$

$\begin{array}{lllll}\text { O } & -0.938398 & -3.286184 & 0.512867\end{array}$

C $0.065524-2.6852812 .637814$

C $1.244783-2.1290613 .405761$

C $1.596940-0.8282033 .412755$

C $2.007802-3.1304404 .231336$

C $0.938138 \quad 0.290040 \quad 2.655377$

C 0.3163421 .3280563 .593381

C -0.4080632 .4339112 .808966$

C $-0.886682 \quad 3.5305703 .762626$

C $-1.6994224 .609818 \quad 3.033575$

O $-1.523933 \quad 1.842142 \quad 2.131053$

O $-2.326034 \quad 5.438707 \quad 4.024734$

C $-2.805140 \quad 3.9208292 .204950$

C $-0.7927895 .553442 \quad 2.235987$

$\begin{array}{llll}\text { O } & -3.509731 & 4.874181 & 1.411674\end{array}$

C -2.288609 $2.752301 \quad 1.330106$

C $-3.457142 \quad 1.9705560 .678920$

$\begin{array}{lllll}\text { O } & -1.498108 & 3.297436 & 0.267634\end{array}$

C -1.845184 -2.341596 0.141266

O $-1.755321-1.1342550 .294185$

C $-2.973536-3.036663-0.513998$

C $-3.907911-2.319168-1.155478$

C $-5.056870-2.858572-1.885220$

C $-5.307164-4.341288-1.786964$

C $-4.254991 \quad 1.0882271 .643982$

C $-5.590586 \quad 0.643251 \quad 1.069702$

C $-7.611853-0.656323 \quad 1.737254$

$\begin{array}{lllll}0 & -6.056832 & 1.083039 & 0.022471\end{array}$

C $-7.772125-1.6188530 .603162$

C $-8.566302-1.371520-0.449835$

C $-8.807129-2.326326-1.583480$

C $-8.312766-1.786557-2.906735$

C $-9.035953-0.894663-3.605014$

C $-7.018657-2.341804-3.454553$

C $-5.829048-2.009346-2.595704$

N $-6.220162-0.2778831 .899209$

H $2.544474-2.427202-0.296283$ 
H $1.242354-4.8279441 .094202$ H $0.540152-1.8402790 .689226$ H $-0.224336-3.6619353 .050304$ H - $0.813986-2.0551982 .827394$ H $2.455173-0.5276244 .012110$ H $2.880693-2.6869664 .721882$ H $1.361714-3.5454645 .011945$ H $2.364775-3.954303 \quad 3.606312$ H 1.6998940 .7729662 .031644 H $\quad 0.170622-0.0839891 .975221$ H $-0.397673 \quad 0.824737 \quad 4.258390$ H 1.1003171 .7690424 .221682 H 0.2851592 .8467212 .066441 H -0.0402173 .9831414 .294156$ H -1.520480 3.0813784 .540153 H -2.898973 4.8773074 .574046 H -3.5376573 .5327262 .921742$ H -0.0741606 .0449722 .903191$ H -1.370600 6.3624321 .775307 H -0.2235365 .0467191 .454048$ H -2.9513585 .0923710 .646054$ H -4.1112682 .6746850 .149817$ H $-3.0607591 .314094-0.107897$ H $-1.4563072 .647310-0.453196$ H -2.988286 -4.119973 -0.475323 H -3.813086 -1.234120 -1.171401 H $-6.246226-4.658349-2.244615$ H -5.364031-4.653162 -0.737813 H -4.502501 -4.900022 -2.277103 H -3.6683040 .1992451 .902720$ H -4.4771241 .6253162 .571410$ H $-8.200796 \quad 0.2559261 .592110$ H -7.934266 -1.129639 2.670084 H -7.240517 -2.5646960 .667387$ H -9.105690 -0.427181 -0.490838 H $-9.887038-2.514483-1.646715$ H -8.355598 -3.305368 -1.382068 H -8.701299 -0.522526 -4.567464 H $-9.979683-0.515807-3.226485$ H -6.819416 -1.930159-4.452999 H -7.138222 $-3.417053-3.614261$ H $-5.572025-0.949157-2.596822$ H $-5.772489-0.5253102 .776946$ C $4.926485-3.9710770 .121274$

O $5.238724-2.641944-0.343947$

C $5.934403-1.9421840 .708687$

C $6.318893-3.0022821 .735027$ C $5.134896-3.9457761 .634175$ H $5.208580-1.2648781 .173073$

C $7.108282-1.1462560 .131556$ H $7.245310-3.5188321 .457498$ H $6.450931-2.5821432 .736578$ C $5.382993-5.3099702 .251293$ H $4.279228-3.4767212 .134087$

C $6.686843-0.090071-0.913300$ O $8.002870-2.069040-0.502897$ H $7.674287-0.6747720 .943477$ H $6.239671-0.594768-1.777069$

O $7.8897220 .535256-1.377868$

C $5.7394160 .991696-0.356037$

C $5.3860722 .120212-1.342390$

H $4.8108760 .529180-0.008891$

O $6.340831 \quad 1.596336 \quad 0.793330$

H $4.8463842 .893936-0.782285$

H $6.3016572 .618979-1.682517$

H $4.504078-5.9528762 .140260$

H $6.233089-5.8153991 .781055$

H $5.598107-5.214513 \quad 3.320602$

C $3.538994-4.397082-0.364923$

H $8.737923-1.550387-0.872817$

H $7.6810000 .993305-2.209378$

H $7.188071 \quad 1.9824860 .511525$

H $3.325281-5.417850-0.027913$

H $3.546093-4.417210-1.462116$

H $5.666591-4.636728-0.342854$

C $2.3778711 .686855-1.503078$

O $3.3924190 .909976-2.148767$

C $4.5486371 .654864-2.550288$
C $4.1405402 .784952-3.507185$

C $3.0401983 .667729-2.919781$

C $1.8773322 .809440-2.419330$

H $2.7965252 .124301-0.588322$

C $1.2694300 .742764-1.029455$

H $5.1763010 .962795-3.124406$

O $5.2763563 .575681-3.841237$

H $3.7733862 .340406-4.441509$

H $3.4348644 .312999-2.126497$

O $2.5681904 .541051-3.948228$

H $1.3526532 .396358-3.288784$

O $\quad 0.9459543 .633197-1.723619$

C $0.5057350 .097463-2.161161$

H $1.697688-0.072059-0.435503$

H $0.569770 \quad 1.274148-0.374729$

H $4.9722804 .285991-4.431956$

H $1.8381495 .062418-3.572247$

H $1.3943753 .999568-0.942307$

O $-0.7642320 .592624-2.205330$

O $0.982969-0.735295-2.920725$

C $-1.5963210 .064803-3.242466$

H $-2.6041090 .466630-3.106736$

H $-1.2194430 .379395-4.220020$

H -1.643351 -1.026591-3.179846

SCF Energy (B3LYP/6-31G**//MMFF) $=-3245.91946503$

01285

MM̄FF Geometry

C -0.614361 -3.170377 0.039209

C $0.099592-3.193301-1.097239$

C $-0.077828-2.309515-2.310157$

O $-1.258855-1.485845-2.210871$

C $1.133897-1.369474-2.428921$

C $1.223907-0.668636-3.769164$

C $0.8302550 .600456-3.990795$

C $1.858226-1.472612-4.873520$

C $0.156028 \quad 1.520524-3.014068$

C $1.1511252 .472534-2.344716$

C $0.4458203 .450740-1.394556$

C $1.4570704 .385918-0.728896$

C 0.7886375 .3169480 .294589

O $-0.2465472 .691449-0.397194$

O $1.8234255 .907026 \quad 1.096175$

C -0.0923014 .4754821 .242689$

C $0.0537656 .477504-0.386871$

O -0.8558845 .3136272 .107684$

C -1.025034 3.4859310 .506428

C -1.7046662 .5391321 .525543$

O $-2.0383994 .226192-0.180391$

C -2.432133 -2.003707 -2.660449

O $-2.622156-3.123587-3.106148$

C $-3.465372-0.956692-2.497422$

C $-4.760336-1.300494-2.545869$

C $-5.901464-0.401116-2.373802$

C $-5.6350431 .078229-2.295155$

C $-2.419477 \quad 1.3378310 .887771$

C -3.2995600 .6038371 .886932$

C $-4.997696-1.2142592 .011200$

O -3.3150420 .8650643 .086828$

C $-6.219210-1.4367811 .178306$

C $-7.320304-0.6754581 .268525$

C $-8.562921-0.8735820 .445626$

C $-8.7082520 .176562-0.635101$

C $-9.1144201 .422083-0.334583$

C $-8.441607-0.220864-2.071256$

C $-7.135067-0.938206-2.276032$

N -4.070874 -0.3745911 .274906$

H -1.436678 -2.469741 0.141979

H $\quad 0.908774-3.915933-1.180837$

H $-0.145437-2.948218-3.199604$

H $2.062944-1.935604-2.286737$

H $1.111520-0.631487-1.616898$

H $0.9793721 .024105-4.982757$

H $1.930099-0.909036-5.809714$

H $2.872517-1.772712-4.590748$

H $1.272432-2.374510-5.076338$

H $-0.6020412 .098023-3.557827$

H $-0.3918400 .952935-2.256242$ 
H $1.8812891 .877178-1.782543$ H $1.7046993 .034131-3.107327$ H -0.282030 $4.026756-1.978295$ H $2.0011954 .969882-1.481733$ H $2.2184863 .789361-0.207411$ H 2.4287196 .3827200 .501797 H 0.5826023 .8989261 .890000 H $0.7527917 .074521-0.984939$ H $-0.369896 \quad 7.1659890 .352615$ H - $0.7478236 .147104-1.050766$ H -0.2320015 .9054222 .562106$ H $-0.953343 \quad 2.1397532 .219054$ H -2.424091 3.1125772 .124137 H -2.843360 $3.689025-0.243650$ H $-3.133070 \quad 0.058852-2.314347$ H -5.013226 -2.348718 -2.701696 H -5.086908 1.419155 -3.180399 H $-5.0464601 .316193-1.403229$ H $-6.5482041 .676821-2.243288$ H $-3.053577 \quad 1.6614210 .056557$ H $-1.681851 \quad 0.6270180 .496943$ H $-5.240365-0.7547332 .975146$ H - $4.501372-2.1687872 .209629$ H $-6.193554-2.2646430 .474659$ H -7.3321150 .1512501 .975897$ H $-9.430493-0.832643 \quad 1.117047$ H $-8.590281-1.8784170 .006345$ H -9.241196 $2.177492-1.102888$ H $-9.329618 \quad 1.7128980 .688432$ H -8.499576 $0.641975-2.746044$ H $-9.248935-0.891285-2.394646$ H -7.237888 -2.022522 -2.329668 H $-3.880906-0.6100550 .305416$ C $-0.075821-3.3738712 .534868$ O $1.025813-2.4472492 .418739$ C $0.629676-1.2023263 .018926$ C $-0.545521-1.5410493 .930197$ C $-1.259985-2.5895353 .104663$ H $0.261166-0.5685152 .204114$ C $1.790519-0.5074403 .739380$ H $-0.212101-1.9724634 .881179$ H $-1.166522-0.6683904 .151566$ C $-2.233061-3.4373673 .905513$ H -1.811879 -2.0804902 .307736$ C $3.045630-0.2020532 .891759$

$\begin{array}{lllll} & \text { O } & 2.199872 & -1.335386 & 4.836327\end{array}$ H $1.412736 \quad 0.422535 \quad 4.183645$ H $3.666201-1.1008002 .793394$ O $3.825050 \quad 0.7484463 .632995$ C $2.7778220 .382356 \quad 1.495762$ C $3.9678101 .086676 \quad 0.819746$ H $2.402171-0.4147700 .845086$ $\begin{array}{lllll}\text { O } & 1.737172 & 1.359611 & 1.597511\end{array}$ H $3.6610671 .307013-0.209871$ H 4.0980062 .0740701 .283215 H $-2.716400-4.1827773 .266235$ H -1.728646 -3.9671074 .720488$ H -3.015478 -2.811161 4.346779 C $-0.337322-4.0873091 .202365$ H $2.791046-0.8089645 .399881$ H 4.1630100 .2987854 .425449 H 1.4812111 .6090870 .693399 H -1.187342 -4.7702411 .315169$ H $\quad 0.537707-4.707706 \quad 0.974407$ H $0.247595-4.1296683 .262726$ C $4.918342-1.213863-0.974729$ O $5.235917-1.0094840 .404398$ C $5.322910 \quad 0.353782 \quad 0.816685$ C $6.4068801 .070057-0.004677$ C $6.1419870 .954435-1.504685$ C $5.925287-0.505666-1.893719$ H $3.902851-0.850745-1.168893$ C $4.959614-2.728861-1.238248$ H $5.675436 \quad 0.333502 \quad 1.854456$ $\begin{array}{llllll}\text { O } & 6.520423 & 2.438068 & 0.373668\end{array}$ $\begin{array}{lllll}\text { H } & 7.378198 & 0.607456 & 0.212228\end{array}$ H $5.2938921 .573484-1.818548$ O $7.277815 \quad 1.443432-2.221994$
H $\quad 6.890079-1.028899-1.865630$

O $5.450146-0.568385-3.237725$

C $3.976184-3.512324-0.398129$

H $4.738786-2.938304-2.290725$

H $5.960160-3.108694-0.995961$

H 5.7552432 .9187750 .015614

H $7.4355222 .356552-1.926543$

H $6.096458-0.103055-3.796026$

O $4.203263-4.845964-0.588048$

$\begin{array}{llll}\text { O } & 3.120785 & -3.025348 & 0.326046\end{array}$

$\begin{array}{lllll}\text { C } 3.372347 & -5.739272 & 0.160417\end{array}$

H $2.392641-5.813762-0.318410$

H $3.842754-6.726164 \quad 0.152228$

H $3.271453-5.4122461 .200035$

SCF Energy (B3LYP/6-31G**//MMFF) $=-3245.89807720$

01286

MMFFF Geometry

C $\quad 0.230820-3.2718551 .249303$

C $0.244620-3.019226-0.068530$

C $-0.956271-2.531665-0.835656$

O $-0.804660-1.124284-1.175723$

C $-1.277311-3.363016-2.093313$

C $-1.989293-4.668143-1.806034$

C $-3.326151-4.777204-1.672805$

C $-1.111151-5.888101-1.729257$

C $-4.322751-3.650174-1.699495$

C $-5.281831-3.729193-0.502837$

C $-6.015091-2.414031-0.194539$

C $-6.785162-1.850336-1.390423$

C $-7.432336-0.493800-1.066089$

O $-5.034061-1.4726320 .253596$

$\begin{array}{lllll}0 & -7.838319 & 0.109212 & -2.304611\end{array}$

C $-6.365561 \quad 0.433203-0.442589$

C -8.704769 $-0.653560-0.225978$

\begin{tabular}{lllll}
\hline & -6.950497 & 1.654704 & 0.003480
\end{tabular}

C $-5.572878-0.2255360 .709575$

C $-4.3870890 .676688 \quad 1.127487$

O $-6.442572-0.4013431 .829994$

C $0.146021-0.702057-2.046213$

O $0.990183-1.388529-2.605108$

C $0.038403 \quad 0.754458-2.301331$

C $-0.7457851 .580612-1.591576$

C $-0.8865373 .025136-1.789510$

C $-0.2338203 .631246-3.003728$

C -3.4150590 .0051712 .106728$

C $-2.255400 \quad 0.9189102 .442087$

$\begin{array}{llll}\text { C } & -1.674520 & 2.960797 & 3.752547\end{array}$

O $-1.137642 \quad 0.737513 \quad 1.967013$

C $-1.923815 \quad 4.209092 \quad 2.966845$

$\begin{array}{llll}\text { C }-0.978866 & 4.847865 & 2.260684\end{array}$

C $-1.231293 \quad 6.109512 \quad 1.487102$

C $-0.892245 \quad 5.977000 \quad 0.019165$

C $0.1892056 .585835-0.496184$

C $-1.853508 \quad 5.216170-0.867731$

C $-1.5849273 .736626-0.879421$

N $-2.6046541 .938356 \quad 3.311017$

H $-0.696705-3.1034061 .794304$

H $1.154921-3.168519-0.640126$

H - $-1.833977-2.549873-0.177682$

H $-0.362292-3.571736-2.663015$

H - $-1.882777-2.760513-2.783403$

H $-3.752108-5.766950-1.515746$

H $-1.686237-6.802854-1.551078$

H $-0.562605-6.020942-2.667477$

H $-0.387827-5.791068-0.914124$

H $-3.821579-2.678189-1.705102$

H $-4.896913-3.720603-2.630758$

H $-6.017353-4.523183-0.683541$

H $-4.713514-4.0144620 .393020$

H $-6.703432-2.6198890 .633918$

H -7.539233 -2.566729 -1.739088

H $-6.103448-1.696038-2.237088$

H $-8.452788-0.500833-2.747583$

H $-5.6576270 .701266-1.239453$

H $-9.445720-1.256055-0.765184$

H $-9.1825200 .315397-0.044327$

H $-8.526383-1.1371340 .736498$ 
H $-7.4281912 .039573-0.751262$ H -3.8132220 .9589540 .234958$ H -4.7672331 .6043251 .572649$ H -5.915869 -0.436240 2.643076 H $0.669026 \quad 1.118616-3.106324$ H $-1.3344181 .172141-0.772925$ H $-0.5941513 .145042-3.917142$ H $0.8530223 .519511-2.951886$ H -0.432194 $4.698391-3.120591$ H -3.910681 -0.2778673 .041336$ H $-2.999174-0.9130231 .676152$ H -0.6464552 .6001613 .643777$ H -1.870762 3.1445804 .813304 H -2.933023 4.6140742 .990964 H 0.0352994 .4568282 .249420 H -0.6292776 .9049091 .945454$ H -2.274388 6.4358361 .584735 H $0.4243096 .521968-1.553171$ H 0.8684797 .1564920 .128272 H -1.844294 5.628959-1.882334 H -2.881649 5.382209-0.521203 H -2.021584 $3.206767-0.032723$ H -3.5700272 .0173763 .617720$ C $2.671108-4.1403911 .381206$ O $3.297536-2.9681190 .825605$ C $4.727317-3.1345410 .880991$ C $4.982606-4.5142561 .482934$ C $3.729043-4.7398582 .304720$ H $5.107580-2.3736051 .571119$ C $5.338959-2.939603-0.511674$ H $5.077212-5.2849360 .708999$ H $5.898111-4.5376362 .082478$ C $3.498253-6.1984612 .660493$ H $3.802209-4.1476033 .226752$ C $5.050002-1.570321-1.160424$ O $4.825837-3.947813-1.394047$ H $6.421307-3.106887-0.464843$ H $3.985462-1.491601-1.409848$ O $5.761753-1.525976-2.404646$ C $5.480338-0.355960-0.317509$

C $5.4424560 .991063-1.065227$ H $4.850430-0.2898530 .575237$ O $6.820474-0.5465610 .146677$ H $5.8183591 .761242-0.380442$ H $\quad 6.1711250 .974398-1.885757$ H $2.589302-6.3178603 .257841$ H $3.396037-6.8186841 .763643$ H $4.337779-6.5887833 .245190$ C $1.373991-3.7615502 .101840$ H $3.858144-3.851167-1.417900$ H $5.473146-2.291993-2.930069$ H $7.389740-0.651164-0.635078$ H $1.585417-2.9775702 .839461$ H $1.005892-4.6335962 .654934$ H $2.459947-4.8276790 .551224$ C 3.1395692 .3034030 .425303 O $3.0410051 .308069-0.599779$ C $4.0546461 .383141-1.605090$ C $4.0100982 .754304-2.294793$ C $4.0562283 .906326-1.293266$ C $3.0254883 .703858-0.183597$ H 4.0943152 .1973650 .954630 C 2.0159892 .0458941 .430754 H $3.7873320 .639670-2.365423$ O $5.0826852 .897671-3.220262$ H $3.0809742 .835048-2.871439$ H $5.0614494 .032141-0.873988$ O $3.7480505 .127939-1.969084$ H $2.0176863 .859182-0.587280$ O $3.232754 \quad 4.679676 \quad 0.834812$ C $2.219041 \quad 0.7214812 .123117$ H 1.9567242 .8314522 .191864 H 1.0414092 .0102980 .931927 H $5.0215372 .162705-3.854173$ H $4.3886535 .226031-2.694446$ H 3.1711985 .5544720 .414225 O 3.0087950 .8819653 .222098 O $1.753497-0.3344591 .716054$
C $3.291357-0.3159043 .951991$

H $3.925951-0.0541834 .802673$

H $2.363976-0.7581614 .328349$

H $3.829370-1.0273813 .318950$

SCF Energy (B3LYP/6-31G**//MMFF) $=-3245.90988807$

01287

MM̄̄F Geometry

C $1.995772-4.221487-0.872048$

C $0.995287-4.042275-1.746802$

C $-0.372480-3.566372-1.325259$

O $-0.605588-2.262745-1.917499$

C $-1.457869-4.531630-1.841129$

C $-2.704958-4.539804-0.976543$

C $-3.869654-3.932889-1.278028$

C $-2.586237-5.3377390 .299308$

C $-4.206057-3.118312-2.495405$

C $-4.043493-1.616326-2.261225$

C $-4.958767-1.003152-1.192196$

C $-6.409574-0.888392-1.664954$

C $-7.285719-0.118920-0.661328$

O $\quad-4.430397 \quad 0.301524-0.925224$

O $-8.5083410 .237410-1.324895$

C $-6.5805731 .201462-0.277918$

C $-7.681341-0.9966730 .532519$

O -7.2850721 .8737880 .763096$

C $-5.101906 \quad 1.0100130 .123054$

C -4.3943462 .3764190 .284670$

$\begin{array}{lllll}\text { O } & -5.045398 & 0.308507 & 1.367308\end{array}$

C $0.089415-1.208528-1.405105$

O $0.865434-1.224899-0.462702$

C $-0.214389-0.014896-2.221888$

C $0.7069030 .956706-2.301243$

C $0.6068422 .187318-3.087372$

C $-0.7518642 .564745-3.613770$

C -2.8668312 .2591850 .408455$

C $-2.1954573 .604600 \quad 0.608710$

C 0.0720624 .6279910 .755643

O -2.8169424 .6489520 .782870$

C $0.3320555 .253931-0.577557$

C $1.5610365 .438532-1.082148$

C $1.8256316 .109326-2.398706$

C $2.6315265 .246750-3.341900$

C $3.9369455 .485606-3.553790$

C $1.9028304 .162201-4.105135$

C $1.7306692 .904919-3.296539$

N $-0.811073 \quad 3.4908940 .588048$

H $1.814329-4.0491870 .185761$

H $1.176801-4.183454-2.809201$

H $-0.433104-3.467868-0.234977$

H - $-1.075186-5.561421-1.862237$

H -1.704480 -4.287558 -2.879914

H -4.691283 -4.027085 -0.568605

H $-3.509310-5.3215270 .888492$

H $-2.360287-6.3842890 .070211$

H $-1.787124-4.9423570 .933631$

H -3.583301 -3.411513 -3.346368

H $-5.234621-3.347793-2.796785$

H -3.005249 -1.409989-1.982222

H -4.191010 -1.087171 -3.211928

H $-4.892991-1.595209-0.273530$

H -6.839246 -1.877182 -1.863827

H $-6.437236-0.352095-2.623933$

H $-8.928843-0.581575-1.638570$

H $-6.6189211 .861645-1.155692$

H $-8.255192-1.8676670 .193351$

H -8.339236 -0.4550621 .220960$

H $-6.822477-1.3689741 .094650$

H -8.2051851 .9819540 .467052$

H $-4.6074003 .004349-0.590245$

H $-4.7895982 .897993 \quad 1.165467$

H $-4.277850 \quad 0.6110611 .876484$

H -1.152708 $0.012553-2.762173$

H $1.6469110 .824527-1.767953$

H -1.490496 $2.568994-2.804201$

H - $-1.0781451 .854573-4.381297$

H $-0.7848503 .560601-4.059854$

H -2.5925051 .6274561 .259742$ 
H $-2.4522221 .806832-0.499671$ H $\quad 0.9892654 .261846 \quad 1.225597$ H $-0.397695 \quad 5.357873 \quad 1.422704$ H - $0.5317025 .596044-1.142835$ H $2.4270385 .118070-0.507312$ H $2.3615917 .044988-2.192146$ H $0.8918016 .407809-2.891372$ H $4.5146664 .887657-4.250871$ H $4.4582186 .280261-3.030526$ H $2.4768653 .894388-5.002366$ H $0.9589084 .567043-4.478985$ H $2.6652522 .525745-2.880269$ H -0.3898382 .5940420 .362480$

C $4.182635-3.356666-1.748581$

O $4.145854-2.298164-0.772230$

C $5.420370-1.629960-0.761242$

C $6.242907-2.243764-1.892448$

C $5.664160-3.641519-1.980606$

H $5.892688-1.8832230 .194901$

C $5.241225-0.110029-0.866973$

H $6.096696-1.713024-2.840157$

H $7.315008-2.236181-1.672284$

C $5.964914-4.343378-3.293038$

H $6.067190-4.238139-1.151225$

C 4.2917600 .5135420 .176570

O $4.706837 \quad 0.198718-2.161923$

H $6.2260350 .370583-0.820795$

H $3.2592550 .244761-0.068497$

O 4.3644021 .9380380 .024786

C $4.5769130 .138673 \quad 1.641200$

C 3.8342670 .9930362 .686036

H $4.322461-0.9154981 .795731$

O $5.977583 \quad 0.255710 \quad 1.908271$

H 4.1024140 .6031623 .675956

H 4.2393182 .0132932 .672772

H $5.524253-5.345103-3.310251$

H $5.566692-3.785318-4.147117$

H $7.045610-4.448037-3.434279$

C $3.401705-4.576641-1.253779$

H $4.6199231 .165905-2.216210$

H 5.2781242 .2124940 .211241

H 6.2259421 .1898031 .805446

H $3.886757-4.998684-0.364829$

H $3.398084-5.363684-2.016803$

H $3.719168-2.959612-2.661833$

C $1.728367-1.0496673 .577940$

O $1.719948-0.2541482 .389553$

C 2.3015371 .0442902 .527389

C 1.5880161 .8334293 .636676

C 1.5490881 .0601494 .953058

C $0.998069-0.3466514 .728913$

H $2.771550-1.2305523 .866238$

C $1.145311-2.4301143 .240279$

H 2.0973401 .5694671 .586399

O $2.2196053 .090273 \quad 3.859163$

H $\quad 0.5558412 .0418013 .326259$

H 2.5352121 .0245355 .430496

O $0.6851351 .736735 \quad 5.869653$

H $-0.076590-0.2730834 .522884$

O $1.153661-1.1147465 .919178$

C $-0.356475-2.449303 \quad 3.059073$

H $1.593721-2.8266132 .322284$

H $\quad 1.383159-3.1348674 .046197$

H 2.2519203 .5579603 .007689

H 1.0245192 .6426825 .970766

H $\quad 0.706688-0.6328196 .636055$

O $-0.724526-1.6481112 .021619$

O $-1.112273-3.1139313 .757052$

C -2.129463 -1.565052 1.766644

H $-2.619812-1.0223782 .579542$

H $-2.272910-1.0132960 .834335$

H -2.565075 -2.561307 1.652520

SCF Energy (B3LYP/6-31G**//MMFF) $=-3245.89723774$

01288

MM̄MF Geometry

C -2.368082 -3.915968 -0.558916

C $-1.577091-2.833007-0.550012$
C $-0.671411-2.4627280 .595755$

O $0.670083-2.4359710 .058835$

C $-1.057374-1.0669771 .118223$

C $-0.664049-0.8040212 .556924$

C 0.3048060 .0498592 .939478

C $-1.501509-1.5076743 .594126$

C 1.2330200 .8314102 .055113

C 0.6607622 .2081831 .697736

C 1.7022163 .1128471 .022045

C 1.0595344 .4161730 .541382

C $2.0433655 .296776-0.244592$

O $2.2616582 .405370-0.085965$

O $1.2829626 .296964-0.940145$

C $2.7410144 .434492-1.319552$

C 2.9990736 .0510580 .687115

O $3.7714595 .169431-1.975807$

C $3.3081833 .107852-0.766462$

C $3.8053242 .205582-1.925927$

O 4.4029023 .4113600 .102250

C $1.685622-2.8562210 .858349$

O $1.617538-3.1628922 .037523$

C $2.905841-2.9310020 .020440$

C $4.027067-3.4611250 .532019$

C $5.290214-3.683751-0.178362$

C $5.369162-3.254648-1.619357$

C $3.9807610 .719912-1.572389$

C $4.9983710 .447774-0.485330$

C $7.4406230 .585166-0.006178$

O $4.6634330 .040765 \quad 0.624674$

C $8.182932-0.664553-0.358738$

C $8.550016-1.5883070 .542569$

C $9.375484-2.7949990 .201837$

C $8.790025-4.0839000 .729273$

C $9.327763-4.7030421 .794091$

C $7.647339-4.708926-0.037894$

C $6.299108-4.2890670 .483216$

N $6.2945460 .724566-0.882752$

H -2.350683 -4.599966 0.285851

H -1.604529 -2.152515 -1.397339

H $-0.748872-3.2237111 .381386$

H $-2.146038-0.9382031 .061899$

H $-0.636607-0.2999590 .460023$

H 0.4692760 .2000724 .005556

H -1.229786 -1.216894 4.614565

H -2.560355 -1.265093 3.455404

H -1.380113 -2.592260 3.519597

H 2.1854640 .9497972 .586587

H 1.4667050 .2656041 .146491

H -0.1892182 .0605311 .019489$

H $\quad 0.2748732 .7088782 .594532$

H $2.494453 \quad 3.321178 \quad 1.750929$

H 0.6282924 .9722901 .383043

H $0.2186494 .179936-0.122826$

H $0.7684586 .794221-0.281232$

H $1.9910004 .197644-2.087278$

H 2.4368076 .7122721 .357654

H 3.6721086 .7031920 .119783

H 3.6023845 .3886941 .311067

H $3.3719395 .989410-2.313109$

H $3.0695012 .236608-2.740032$

H $4.7461892 .602484-2.326193$

H $5.1179842 .771429-0.030863$

H $2.835050-2.587914-1.005605$

H $4.013985-3.7947641 .569199$

H $5.114905-2.195712-1.723486$

H $4.675835-3.841289-2.231951$

H $6.363135-3.369935-2.056111$

H $3.0266550 .279238-1.263182$

H $4.2894040 .170247-2.470418$

H 7.1137620 .5931971 .038554

H $8.0828121 .454762-0.176786$

H $8.471025-0.793593-1.399518$

H $8.292231-1.4423151 .589147$

H $10.376099-2.6387750 .626063$

H $9.525316-2.884007-0.881434$

H $8.933098-5.6466262 .156486$

H $10.170008-4.278316 \quad 2.330240$

H $7.702971-5.8023310 .055555$ 
H $7.777210-4.517550-1.106144$ H $6.133278-4.5722741 .523999$ H $6.467498 \quad 1.011097-1.841833$ C $-4.769717-4.202810-1.153857$ O $-5.097272-2.872528-0.711697$ C $-6.499438-2.637040-0.945511$ C -7.057919 -3.917277 -1.564348 C $-5.824754-4.528737-2.204935$ H -6.563772 -1.830054 -1.683734 C -7.172143 -2.2094920 .365732$ H -7.469526 -4.592030 -0.804925 H -7.855630 -3.710202 -2.284562 C $-5.969665-6.009775-2.506207$ H -5.608101 -3.987468 -3.135480 C $-6.498741-1.0014371 .051177$ O $-7.126742-3.304467 \quad 1.292275$ H $-8.235252-2.0078080 .194725$ H $-5.487882-1.2801061 .368741$ O $-7.229925-0.7144542 .250913$ C -6.4470940 .2826800 .200306$ C $\quad-5.800419 \quad 1.473716 \quad 0.934789$ H $-5.908820 \quad 0.106938-0.736224$ O H $-6.0763752 .393080 \quad 0.405175$ H -6.2504091 .5822591 .928901$ H -5.057982 -6.406782 -2.963297 H -6.169473 -6.586619-1.597048 H $-6.797601-6.181270-3.202006$ C $-3.331906-4.231248-1.670429$ H $-6.191551-3.531073 \quad 1.434448$ H $-7.256377-1.5319342 .777201$ H $-8.1274940 .023292-0.772703$ H -3.215975 -3.507384 -2.486645 H $-3.089289-5.221418-2.071180$ H -4.873665 -4.868235 -0.285786 C $-3.6852042 .404948-1.043308$ O C $-4.2650451 .360507 \quad 1.051889$ C -3.6074872 .5002311 .842379$ C -3.5989463 .8089701 .056092$ C $-3.0132593 .589970-0.338437$ H $-4.7360812 .652464-1.235469$ C -3.101214 2.073306 -2.421927 H $-4.053596 \quad 0.4347941 .598209$ O $-4.267442 \quad 2.7059003 .086771$ H -2.5693002 .2284582 .072188$ H -4.6002144 .2516500 .998035$ O $\quad-2.778254 \quad 4.758843 \quad 1.741422$ H - $1.9379413 .420265-0.228137$ O $-3.1904544 .783170-1.096115$ C $-1.6002241 .901209-2.437111$ H -3.557349 $1.155540-2.814271$ H $-3.3415672 .863025-3.143651$ H -4.2441911 .8636773 .572240$ H -3.1404874 .8588402 .638495$ H -2.667202 $4.696271-1.910633$ O $-1.273130 \quad 0.583078-2.329063$ O $-0.8111042 .832578-2.536984$ C $0.128187 \quad 0.298111-2.379854$ H $0.5871020 .754276-3.262600$ H $0.6089280 .656658-1.466798$ H $\quad 0.256667-0.785193-2.444336$ SCF Energy (B3LYP/6-31G**//MMFF) $=-3245.89962359$

$01 \_289$

MM̄MF Geometry

C -1.860560 $3.694995-0.747521$

C $-1.3146422 .498855-0.483379$

C $-0.3133511 .823158-1.385939$

O $0.8159161 .401369-0.589330$

C $-0.9553530 .575012-2.025681$

C $-0.113675-0.153455-3.061164$

C $-0.185126-1.482361-3.285494$

C $0.772046 \quad 0.690140-3.942814$

C -1.074685 -2.485328 -2.606635

C $-0.415327-3.847839-2.339260$

C $0.774459-3.879667-1.365176$

C $0.451979-3.269990-0.000013$
C $1.660996-3.3232390 .946705$

O $1.889216-3.212713-1.963474$

O $1.383650-2.4722552 .069137$

C $2.880586-2.7237830 .215883$

C $1.878045-4.7284621 .519168$

O $4.060893-2.8615191 .004703$

C $3.102462-3.296173-1.203778$

C $4.204912-2.526344-1.966925$

O $3.543631-4.654310-1.080552$

C $1.7989652 .319836-0.379595$

O $1.8328173 .467746-0.794024$

C $2.840961 \quad 1.6832260 .456867$

C 3.9187342 .3915890 .824895

C 5.0369421 .9138131 .642839

C 4.9957730 .4841212 .114032

C $3.814185-1.115620-2.409929$

C $5.023604-0.360776-2.921033$

C $7.0267150 .886178-2.149052$

O $5.248285-0.230538-4.121770$

C $7.8530930 .924351-0.904035$

C $8.2992792 .057655-0.341751$

C 9.1201202 .0937820 .915714

C 8.5323043 .0008531 .975136

C 9.1292084 .1602032 .300224

C 7.2954392 .5288372 .712772

C 6.0354502 .7760421 .928799

N $5.8272010 .111440-1.895516$

H -1.568281 $4.229642-1.648047$

H -1.625269 1.9608210 .407610

H $\quad 0.0176702 .514325-2.170734$

H -1.889786 $0.871852-2.521085$

H -1.222492 -0.111451-1.215072

H $\quad 0.423093-1.895775-4.089475$

H $1.2240860 .111976-4.756144$

H $0.1916591 .495856-4.404331$

H $1.5906921 .128561-3.363937$

H -1.498889 -2.101559-1.677393

H -1.927361 -2.662132 -3.275102

H -1.191687 -4.522193 -1.954972

H $\quad-0.086774-4.264674-3.300689$

H $1.038051-4.937410-1.243158$

H $-0.412037-3.7689490 .454048$

H $\quad 0.168390-2.216685-0.119146$

H $\quad 0.567397-2.7893222 .491708$

H $2.695830-1.6470310 .140288$

H $0.983239-5.0635202 .057712$

H $2.688256-4.7334932 .256645$

H $2.100902-5.4753270 .754749$

H $\quad 4.356339-3.7853890 .935874$

H $5.126868-2.515554-1.371804$

H $4.468025-3.086967-2.875305$

H $3.980778-4.913105-1.908646$

H $2.6895440 .652050 \quad 0.751946$

H 3.9965413 .4261710 .492337

H 5.8372430 .2089352 .752617

H $5.006033-0.2019861 .261469$

H 4.0898890 .2982622 .702103

H $3.075137-1.159678-3.216843$

H $3.364466-0.537678-1.598251$

H $6.715375 \quad 1.883225-2.478004$

H $7.5854470 .414497-2.964135$

H $8.097813-0.029790-0.442713$

H $8.0735493 .011124-0.814018$

H 10.1283112 .4321690 .643700

H 9.2459541 .0890851 .338322

H 8.7277784 .8041453 .075735

H $10.0295094 .494316 \quad 1.795199$

H 7.2014913 .0672473 .664966

H 7.4342591 .4784152 .981084

H 5.9482063 .8005901 .562953

H $5.4583530 .094180-0.949313$

C $-4.2813964 .235947-0.534147$

O $-4.7265602 .866346-0.458815$

C $-6.1386252 .848887-0.174041$

C $-6.6114274 .294219-0.284428$

C -5.3713675 .0500910 .157082$

H -6.2459532 .5309680 .869226$

C $-6.8467801 .855586-1.103283$ 
H $-6.8732994 .559456-1.315314$ H $-7.483920 \quad 4.4938150 .345182$ C $-5.387907 \quad 6.518922-0.227762$ H -5.2728214 .9619711 .247497$ C $-6.2633850 .426834-1.032343$ O $-6.704138 \quad 2.326062-2.449100$ H $-7.921507 \quad 1.846232-0.886562$ H $-5.2277680 .431919-1.390461$ O $-7.003582-0.406899-1.931657$ C $-6.337017-0.2075320 .369109$ C $-5.828665-1.6596850 .437936$ H $-5.771327 \quad 0.396526 \quad 1.083847$ $\begin{array}{llll}\text { O } & -7.694668 & -0.198258 & 0.820329\end{array}$ H $-6.078954-2.0536131 .430813$ H -6.397794 -2.286731 -0.259741 H $-4.471003 \quad 7.019106 \quad 0.098730$ H -5.475013 $6.648889-1.311627$ H $-6.235613 \quad 7.0293290 .240943$ C $-2.903168 \quad 4.349492 \quad 0.116549$ H -7.318253 $1.816028-3.003462$ H $-6.780508-0.130579-2.836461$ H -8.219616 -0.7077710 .179258$ H -2.9169273 .8822871 .109212$ H $-2.622849 \quad 5.3996340 .252651$ H $-4.2282704 .498151-1.599571$ C $-3.508642-1.1728332 .349665$ O $-3.538518-0.8891010 .946680$ C $-4.316290-1.8082720 .171001$ C $-3.783111-3.2362990 .354322$ C $-3.671269-3.6303841 .825867$ C $-2.914946-2.5615092 .617310$ H $-4.534682-1.1469812 .737789$ C $-2.784268-0.0229063 .062801$ H $-4.151813-1.538735-0.879365$ O $-4.612861-4.161533-0.340212$ H $-2.787309-3.301241-0.098138$ H $-4.660358-3.823267 \quad 2.257575$ O $-2.952858-4.8644581 .904184$ H $-1.856658-2.6071952 .337918$ O $-3.008274-2.8847084 .002032$ $\begin{array}{llll}\text { C } & -1.313966 & 0.106781 & 2.728477\end{array}$ H -3.2509870 .9297962 .784015$ H -2.885900 -0.1508714 .146550$ H $-4.249185-5.050382-0.186462$ H $-2.858183-5.0839582 .847053$ H $-2.390536-2.3084614 .482582$ O -0.6970110 .8149453 .720495$ O $-0.781464-0.314767 \quad 1.711870$ C $0.695603 \quad 1.077793 \quad 3.523700$ H $0.818413 \quad 1.8219622 .732501$ H 1.0995451 .4851174 .454294 H 1.2377650 .1590013 .281383 SCF Energy (B3LYP/6-31G*//MMFF) $=-3245.91072673$

0129

MMFF Geometry

C 3.4125022 .1156871 .954390

C $2.7728153 .255387 \quad 1.648361$

C $2.2751573 .571935 \quad 0.262333$

$\begin{array}{llllllll}\text { O } & 0.901331 & 4.031664 & 0.330329\end{array}$

C $3.0602414 .719224-0.395553$

C $4.4682964 .368190-0.815519$

C $4.7812973 .818274-2.004536$

C $5.557573 \quad 4.750178 \quad 0.152211$

C $3.8350583 .399533-3.099171$

C $4.2202992 .038185-3.696154$

C $3.866948 \quad 0.848945-2.786807$

C $4.660102-0.385228-3.221816$

C $4.274959-1.627176-2.411531$

O $2.460800 \quad 0.604760-2.917892$

O $4.844633-2.772356-3.062777$

C $2.745589-1.785159-2.463889$

C $4.861252-1.593176-0.997865$

O $2.322048-2.856643-1.621440$

C $1.959166-0.489008-2.137163$

C $0.478500-0.708365-2.528748$

O $2.061633-0.226636-0.739360$

C $-0.066023 \quad 3.130180 \quad 0.640152$ $\begin{array}{llll}\text { O } & 0.086896 & 1.962462 & 0.957897\end{array}$

C -1.365079 $3.827203 \quad 0.512010$

C $-2.482905 \quad 3.238603 \quad 0.959690$

C $-3.837458 \quad 3.788977 \quad 0.883112$

C -4.0310525 .0579710 .095195$

C $-0.4603180 .477186-2.274364$

C $-1.8252870 .233825-2.899482$

C $-4.2248540 .731066-2.657125$

O $-1.986192-0.491285-3.879526$

C $-5.1059751 .768191-2.041626$

C $-6.1476951 .470090-1.249673$

C $-7.106163 \quad 2.485876-0.695304$

C -7.160160 2.4766470 .815503

C -7.9984021 .6587771 .474266$

C -6.2983013 .4750531 .554166$

C $-4.838412 \quad 3.1216321 .493476$

N -2.842587 $0.944400-2.273378$

H 3.5964691 .3975711 .161162

H 2.5965343 .9962872 .424245

H $2.3013692 .688768-0.387849$

H 3.0771545 .5928960 .271105

H $2.4993485 .079195-1.269281$

H $5.8356683 .661335-2.229180$

H $6.5561904 .519679-0.234203$

H $5.524647 \quad 5.825975 \quad 0.353712$

H 5.4439604 .2167841 .099549

H $2.793333 \quad 3.375078-2.765278$

H $3.9001454 .152089-3.894402$

H $3.691027 \quad 1.927829-4.651705$

H $5.2941132 .038487-3.922447$

H $4.0941511 .097818-1.744424$

H $5.739214-0.200968-3.145316$

H $4.466259-0.588843-4.284561$

H $5.809018-2.650930-3.093964$

H $2.495581-2.092590-3.488645$

H $5.952940-1.498582-1.034787$

H $4.660486-2.529214-0.465692$

H $4.476297-0.764508-0.401894$

H $2.332989-2.534837-0.703792$

H $0.441978-0.957383-3.597411$

H $0.076160-1.570315-1.984340$

H $1.3456260 .372275-0.476392$

H -1.3653434 .8083650 .050460$

H $-2.3998012 .261144 \quad 1.430465$

H $-3.6254754 .948980-0.917151$

H -3.5258795 .8959270 .587669$

H $-5.0784485 .340445-0.028084$

H $-0.5855530 .626071-1.199123$

H $-0.0569741 .398233-2.708218$

H $-4.499602-0.284161-2.351689$

H $-4.2968180 .780644-3.749134$

H $-4.9071112 .805948-2.297590$

H $-6.3551830 .427040-1.019552$

H -8.102392 2.261763-1.099191

H -6.869597 $3.495887-1.052433$

H -8.0613601 .6651502 .557319$

H $-8.6364320 .959917 \quad 0.943068$

H -6.5793603 .5081682 .615171$

H $-6.511796 \quad 4.479023 \quad 1.177063$

H -4.5961302 .2099192 .037734$

H -2.653276 $1.367803-1.369416$

C $4.0823330 .297687 \quad 3.629791$

O $2.846645-0.4153743 .428483$

C $3.134368-1.6445902 .734323$

C $4.643375-1.8454012 .841814$

C $5.146853-0.4173572 .795727$

H $2.868741-1.4786921 .684020$

C $2.303954-2.8042963 .295819$

H $4.926645-2.3102113 .793089$

H $5.039001-2.4692242 .035140$

C $6.560637-0.2518243 .327129$

H $5.126627-0.0766901 .755686$

C $0.788518-2.544426 \quad 3.387726$

O $2.758047-3.1104174 .622210$

H $2.495046-3.7055192 .701736$

H $0.589423-1.8235704 .189897$

O $\quad 0.169740-3.769796 \quad 3.806856$

C $0.110149-2.0575292 .092809$ 
C $-1.419478-1.9683242 .269674$ H $0.509469-1.0727261 .826261$ O $0.429528-2.9575321 .038938$ H -1.834475 -2.979366 2.339072 H -1.616057 -1.465032 3.223230 H $\quad 6.872578 \quad 0.796376 \quad 3.283513$ H $6.641222-0.5850934 .367378$ H $7.266258-0.8393942 .730751$ C $3.903117 \quad 1.7936313 .339487$ H $2.659608-2.3053765 .159206$ H $0.281829-4.4147823 .087294$ H $0.016571-2.6198590 .226395$ H 4.8601032 .3035403 .500187 H 3.1856562 .1977074 .065227 H 4.3197650 .1888344 .696478 C $-2.889088-3.034308-0.249069$ O $-2.069969-1.864655-0.126515$ C $-2.127243-1.1941191 .136023$ C $-3.575611-0.8038311 .465081$ C $-4.511479-2.0032771 .406016$ C $-4.358296-2.7266520 .073638$ H -2.511784 -3.8164380 .420101$ C - $2.776329-3.531085-1.700892$ H -1.571095 -0.258092 1.007004 O $-3.671589-0.2146022 .758007$ H $-3.922200-0.0543890 .742391$ H -4.346913 -2.686349 2.247694 O $-5.863200-1.5489971 .512703$ H $-4.798850-2.114047-0.722902$ O $-5.091617-3.949925 \quad 0.120251$ C $-1.425681-4.128050-2.028058$ H -3.519693 -4.309261-1.905763 H -2.945996 -2.688642 -2.381771 H -3.0311260 .5153082 .798375$ H -5.932944 -1.040557 2.339002 H $-6.012141-3.727990 \quad 0.342795$ O $-1.283310-4.177319-3.386127$ O $-0.613747-4.530562-1.207538$ C $-0.063880-4.755306-3.861337$ H $\quad 0.066257-5.764207-3.458278$ H $-0.120522-4.818978-4.951311$ H $0.782213-4.118424-3.591265$ SCF Energy (B3LYP/6-31G**//MMFF) $=-3245.93492148$

01_290

MM̄MF Geometry

C $5.168889-2.334754-1.927255$

C $4.035306-1.769515-2.368672$

C $2.753127-2.531238-2.568466$

O $1.712178-1.938764-1.757084$

C $2.267711-2.587105-4.031319$

C $2.061025-1.260381-4.736630$

C $0.889168-0.598063-4.798617$

C $3.248375-0.736969-5.508469$

C $-0.435812-0.977554-4.196095$

C $-1.0591330 .199459-3.431578$

C -2.552244 -0.025510 -3.154364

C $-3.1838621 .224894-2.541424$

C $-4.6760841 .014193-2.244998$

O $-2.703193-1.130558-2.256514$

O $-5.1158092 .093333-1.406690$

C $-4.839501-0.288140-1.435876$

C $-5.5274211 .082195-3.518182$

O $-6.218004-0.605417-1.253131$

C $-4.070948-1.500140-2.023140$

C $-4.112416-2.663802-1.007054$

O $-4.710622-1.926096-3.228127$

C $1.752116-2.197823-0.420851$

O $2.588752-2.856100 \quad 0.177310$

C $0.583555-1.5437390 .203128$

C $0.521302-1.4624681 .540415$

C $-0.586368-0.9122702 .322649$

C $-1.805845-0.4644921 .567548$

C $-3.166173-3.829176-1.317392$

C -3.132481-4.770048 -0.128145

C $-2.180179-4.9510042 .175303$

O $-3.873355-5.747589-0.057593$

C $-2.782728-3.984316 \quad 3.149239$
C $-2.184162-3.5995684 .287066$

C $-2.785693-2.6149655 .251187$

C $-1.817092-1.5292655 .673795$

C $-1.311899-1.5034416 .918965$

C $-1.490828-0.4317014 .681151$

C $-0.471311-0.8760243 .666043$

N $-2.241674-4.3575950 .851567$

H $5.190831-3.401482-1.719462$

H $4.012534-0.696329-2.539275$

H $2.876070-3.574195-2.244541$

H $1.329349-3.155376-4.060188$

H $2.977904-3.185614-4.617933$

H $\quad 0.8595700 .317594-5.389207$

H $3.0116940 .174658-6.067771$

H $4.083548-0.500166-4.845499$

H $3.585251-1.484344-6.234698$

H -1.097011 -1.267598 -5.021721

H $-0.360061-1.842592-3.531316$

H $-0.5174760 .330986-2.487437$

H $-0.940738 \quad 1.124742-4.009609$

H $-3.039535-0.264095-4.107221$

H -3.043660 $2.095948-3.193526$

H -2.671197 $1.467265-1.600142$

H $-4.9399502 .927733-1.874452$

H $-4.444060-0.084110-0.431098$

H $-5.3797322 .042751-4.026385$

H $-6.5960831 .032421-3.281449$

H $-5.2943040 .290255-4.232838$

H -6.548533 -0.978545 -2.088048

H $-3.868290-2.278463-0.011703$

H $-5.134339-3.060155-0.939084$

H -4.478243 -2.853939 -3.392969

H $-0.196803-1.170459-0.448978$

H $1.354255-1.8517872 .124906$

H -2.217647 -1.295806 0.993045

H -1.5554520 .3408060 .870987$

H -2.610665 -0.098019 2.205291

H -3.498124 -4.391580 -2.196207

H -2.147961 -3.480408 -1.522728

H -1.127013 -5.153287 2.393053

H $-2.735671-5.8933512 .191009$

H -3.762039 -3.579178 2.903595

H -1.217435 -4.024250 4.548172

H -3.124453 -3.1785776 .130248$

H $-3.683473-2.1448984 .830621$

H $-0.636594-0.7166117 .238475$

H -1.557890 -2.270524 7.645657

H $-1.0722460 .437815 \quad 5.204921$

H -2.422800 -0.075663 4.234951

H $0.464901-1.2166674 .111472$

H $-1.679930-3.5278870 .687055$

C $6.547291-1.018138-0.243689$

O $5.5783930 .029817-0.049316$

C $5.077567-0.0467581 .299787$

C $5.966192-1.0522132 .028158$

C $6.334795-2.0033880 .904139$

H $4.063864-0.4589021 .234997$

C 5.0298221 .3522331 .926019

H $6.867776-0.5814322 .436481$

H $5.442461-1.5443232 .853401$

C $7.544725-2.8696511 .204109$

H $5.470982-2.6512410 .712711$

C 4.2755762 .3918111 .070892

O 6.3768831 .8159732 .085054

H 4.5991091 .2909822 .932412

H 4.8206322 .5732240 .136209

O 4.2702653 .6355191 .783895

C 2.8150542 .0190660 .749114

C $2.1315073 .108454-0.103076$

H $2.789788 \quad 1.0756390 .193442$

O 2.1169471 .8219821 .975201

H 2.1311364 .0516620 .451766

H $2.7407583 .264628-1.001260$

H $7.773953-3.5232020 .356480$

H $8.432243-2.2626181 .412159$

H $7.357934-3.5019802 .078223$

C $6.435271-1.570500-1.667266$

H 6.3524592 .5872962 .676192 
H 5.1816653 .9733111 .789477 H $1.216587 \quad 1.5265761 .759155$ H $7.284008-2.232137-1.876450$ H $6.503565-0.738464-2.380159$ H $7.530198-0.538050-0.145343$ C $-0.515802 \quad 3.8735591 .247873$

$\begin{array}{llll}\text { O } & -0.201741 & 2.639419 & 0.595387\end{array}$

C $0.6863272 .750122-0.521233$

C $0.0842693 .700083-1.566633$

C $-0.2394615 .065624-0.965544$

C $-1.111867 \quad 4.903780 \quad 0.279423$

H $0.405428 \quad 4.2859401 .677367$

C $-1.415848 \quad 3.5687142 .456166$

H $\quad 0.7335191 .753207-0.976099$

O $\quad 0.964532 \quad 3.868580-2.672441$

H $-0.8441663 .267804-1.960322$

H $0.670675 \quad 5.633107-0.738628$

O $-0.9676535 .839765-1.921681$

H $-2.1202774 .614586-0.034774$

$\begin{array}{llll}\text { O } & -1.224987 & 6.159366 & 0.945894\end{array}$

C -2.762810 $2.962340 \quad 2.118769$

H -0.9031452 .8455353 .102691$

H -1.5887504 .4893513 .024770$

H $1.1377372 .986747-3.044355$

H $-0.4192005 .898724-2.722969$

H $-1.572318 \quad 6.799670 \quad 0.301288$

O $-3.499022 \quad 2.862527 \quad 3.266133$

O -3.1240032 .6003781 .008351$

C -4.8008842 .2881853 .112445$

H -5.2791092 .2624364 .095157$

H -5.408771 2.9027572 .441588

H $-4.722724 \quad 1.2646832 .733777$

SCF Energy (B3LYP/6-31G**/MMFF) $=-3245.91515885$

$01 \_291$

MMFF Geometry

C $-3.5902782 .607727 \quad 0.841754$

$\begin{array}{llll}C & -3.612410 & 2.989511 & 2.128007\end{array}$

C -2.399434 $3.086793 \quad 3.024757$

O $\begin{array}{llll}\text { O } & -1.354514 & 2.195488 & 2.572072\end{array}$

C -1.837592 4.5205073 .015325

C $-0.643074 \quad 4.7020483 .934289$

C $0.6171084 .948963 \quad 3.522348$

C -0.9411024 .6036605 .409064$

C $1.1191195 .126752 \quad 2.115153$

C 1.7472053 .8403341 .578052

C 2.4262923 .9950910 .208257

C $1.4402794 .394954-0.892306$

C $2.0966984 .414983-2.279948$

O $3.0108912 .720415-0.099907$

O $1.0379694 .495637-3.245401$

C $2.8383943 .081253-2.497377$

C $2.9505285 .672880-2.477594$

$\begin{array}{llll}\text { O } & 3.561270 & 3.100229 & -3.726427\end{array}$

C $3.7611472 .686738-1.321050$

C $4.2832161 .244627-1.532431$

O $4.8701543 .587104-1.286401$

C $-1.397723 \quad 0.904553 \quad 3.000637$

$\begin{array}{llll}\text { O } & -2.239028 & 0.394408 & 3.722334\end{array}$

C $-0.2214390 .212233 \quad 2.429442$

C $-0.010091-1.0856802 .691673$

C $1.106266-1.8877262 .188213$

C $2.197970-1.1689671 .439341$

C $5.049530 \quad 0.668425-0.335227$

C $5.553386-0.741739-0.598831$

C $6.744037-2.6093670 .558889$

O $5.462315-1.299904-1.688625$

C $5.694902-3.6348450 .849631$

C $5.470862-4.1423912 .071144$

C $4.449000-5.2026112 .367343$

C $3.181549-4.6247352 .959725$

C $3.038993-4.5280924 .292745$

C $2.124238-4.224262 \quad 1.950690$

C $1.114945-3.2158382 .427930$

N $6.141361-1.2903010 .532254$

H -2.6367842 .3879590 .371102$

H -4.565065 3.2406262 .588728

H -2.6985892 .8083614 .042574$
H -2.618780 5.2308503 .316958

H $-1.573572 \quad 4.7863751 .986426$

H 1.3928885 .0460104 .281278

H $-0.074840 \quad 4.864615 \quad 6.026456$

H -1.751301 5.2888565 .679048

H -1.2397803 .5857725 .676926$

H $0.3207415 .474556 \quad 1.454974$

H 1.8689555 .9275762 .122511

H 2.4900273 .4604692 .292936

H 0.9846163 .0563281 .510450

H 3.2264914 .7383270 .302793

H $0.9824475 .369155-0.686590$

H $0.6131843 .672525-0.908860$

H $1.438773 \quad 4.426776-4.129117$

H $2.0705672 .305038-2.612776$

H $2.3310996 .572437-2.377217$

H $3.3707705 .713393-3.488581$

H $3.7692295 .753375-1.759614$

H $4.3683303 .624383-3.586861$

H $3.4331330 .578991-1.731058$

H $4.9342591 .214507-2.415461$

H $5.6107683 .164589-0.825198$

H $0.4420440 .790357 \quad 1.797353$

H $-0.724955-1.6161303 .318807$

H $2.602689-0.3505452 .045644$

H $1.817008-0.7532950 .500899$

H $3.047011-1.8084831 .189991$

H $5.9226981 .285590-0.099661$

H 4.4022590 .6436130 .547638

H $7.525835-2.5970741 .325196$

H $7.214018-2.819779-0.407367$

H $5.105453-3.988582 \quad 0.007229$

H $6.058650-3.7825402 .912504$

H $4.898763-5.9282783 .057478$

H $4.205372-5.7790691 .465519$

H $2.136783-4.1415034 .752769$

H $3.831473-4.8436514 .964093$

H $1.584546-5.1288541 .640771$

H $2.606938-3.8603441 .037679$

H $\quad 0.290660-3.6510402 .993725$

H $6.101670-0.7754001 .407099$

C $-4.866714 \quad 1.065769-0.652428$

$\begin{array}{llllllllll}\text { O } & -5.083402 & 0.072324 & 0.373863\end{array}$

C $-6.097290-0.833826-0.078574$

C $-6.241244-0.603465-1.575730$

C $-6.0100620 .893246-1.654355$

H $-7.031297-0.503742 \quad 0.397783$

C $-5.839542-2.2792050 .357246$

H -5.468821 -1.134896 -2.142356

H $-7.220177-0.907896-1.957917$

C $-5.6918451 .381943-3.056302$

H $-6.9089531 .409121-1.290556$

C $-4.598025-2.992411-0.221403$

O $-6.983352-3.057453-0.026127$

H -5.786184 -2.322987 1.452173

H $-4.710046-3.120773-1.302764$

O $-4.587331-4.319973 \quad 0.331145$

C $-3.235626-2.3437790 .096259$

C $-2.017498-3.226058-0.251245$

H -3.143828 -1.388032 -0.425805

O $-3.168255-2.0587711 .496562$

H -1.134892 $-2.778500 \quad 0.216098$

H $-2.124383-4.1996790 .241210$

H $-5.5162802 .462047-3.062943$

H $-4.7996100 .890994-3.459498$

H $-6.5251121 .171791-3.734762$

C $-4.8094272 .460045-0.023908$

H $-7.759897-2.6754620 .417293$

H $-5.438434-4.7335420 .105911$

H $-3.260786-2.9015321 .973157$

H -5.7160992 .6395340 .566600$

H $-4.7652893 .223191-0.809043$

H $-3.9098950 .822336-1.129679$

C $-0.169377-1.637793-2.105829$

O $-1.468403-2.166625-2.408348$

C $-1.788250-3.406140-1.764751$

C $-0.742060-4.469393-2.125700$

C $0.675195-4.001902-1.813857$ 
C $0.935854-2.642581-2.458627$

H $-0.113045-1.389491-1.040328$

C $0.000219-0.342333-2.915200$

H -2.735937 -3.743272 -2.199502

O $-1.020511-5.684998-1.438488$

H $-0.813580-4.686451-3.199653$

H $0.846821-3.973697-0.733164$

O $1.588967-4.962652-2.345987$

H $1.006213-2.760063-3.547651$

O $2.186305-2.134564-1.997828$

C $-0.827490 \quad 0.790013-2.348940$

H $1.041309-0.003638-2.913146$

H $-0.316157-0.518308-3.950424$

H $-0.322840-6.319701-1.675886$

H $2.480271-4.717363-2.045663$

H $2.885660-2.722077-2.330267$

O $-1.0900541 .694235-3.337066$

O $-1.1715120 .884274-1.179114$

C -1.870183 $2.830386-2.951942$

H $-1.4617773 .303621-2.054727$

H -1.844306 $3.554007-3.770960$

H -2.905648 $2.526566-2.784703$

SCF Energy (B3LYP/6-31G**//MMFF) $=-3245.91434273$

01292

MM̄FF Geometry

C -1.874857 2.0537174 .038514

C $-1.018184 \quad 1.237708 \quad 3.407228$

C $-0.742257-0.1724663 .861789$

O $-1.161123-1.0880022 .823041$

C $0.767113-0.3709004 .077748$

C $1.093649-1.6387084 .840351$

C $1.504997-2.7865294 .267657$

C $0.984901-1.5342106 .339182$

C $1.642067-3.0743282 .799566$

C $3.101670-2.9971162 .341970$

C $3.261133-3.4300430 .878154$

C $4.723175-3.3408250 .443466$

C $4.895404-3.734110-1.030788$

O $2.470098-2.5666290 .058943$

O $6.207698-3.321220-1.445625$

C $3.883130-2.944533-1.891815$

C $4.847789-5.256352-1.214277$

O $3.903465-3.432725-3.231384$

C $2.438877-2.934352-1.326652$

C $1.576585-1.886236-2.063588$

O $1.786101-4.191565-1.522101$

C -2.478999-1.418352 2.772219

O $-3.372682-0.9943943 .487551$

C -2.652965 -2.399965 1.678204

C $-3.888904-2.6605631 .227546$

C $-4.246988-3.5986370 .163240$

C $-3.169670-4.494185-0.386673$

C $0.181353-1.697972-1.450189$

C $-0.717629-0.850219-2.334855$

C $-3.091973-0.137687-2.573347$

O $-0.314433-0.266626-3.337515$

C $-4.369784-0.889181-2.382075$

C $-4.838544-1.776581-3.272247$

C $-6.109552-2.561576-3.105122$

C $-5.842726-4.011742-2.762911$

C $-5.417758-4.873764-3.702695$

C $-6.117835-4.486967-1.352642$

C $-5.521697-3.615245-0.278250$

$\mathrm{N}-2.027187-0.835058-1.876010$

H -2.4006391 .6896054 .918202$

H $-0.494411 \quad 1.5937322 .524766$

H - $-1.284238-0.4009424 .788375$

H $1.1889150 .478322 \quad 4.632175$

H $1.281329-0.3783363 .110347$

H $1.749870-3.6276154 .914766$

H $1.260320-2.4672326 .842250$

H $1.649682-0.7490306 .714125$

H $-0.040623-1.2919436 .634310$

H $1.247588-4.0814002 .615068$

H $1.024526-2.3986392 .200646$

H $3.451722-1.9638452 .460719$

H $3.729246-3.6311162 .980751$
H $2.890399-4.4575510 .783939$

H $5.360900-3.9628181 .083825$

H $5.081055-2.3095350 .572315$

H $6.856330-3.749259-0.860808$

H $4.247471-1.908978-1.931640$

H $5.660568-5.734717-0.654341$

H $5.004232-5.535058-2.262117$

H $3.910816-5.700429-0.871726$

H $3.438665-2.788810-3.791153$

H $2.084905-0.914708-2.056139$

H $1.458191-2.184166-3.113011$

H $2.397577-4.796561-1.970189$

H -1.765369-2.869575 1.269920

H $-4.726370-2.1237331 .672171$

H $-2.704419-5.0765630 .416270$

H $-2.395848-3.902642-0.886097$

H $-3.542660-5.213951-1.119792$

H $-0.301655-2.671203-1.310815$

H $0.258487-1.208871-0.472524$

H $-2.835048-0.030284-3.632656$

H $-3.1797690 .865074-2.147168$

H $-4.927632-0.693889-1.470044$

H -4.267285 -1.964059-4.179153

H $-6.676907-2.503517-4.043271$

H $-6.760270-2.104033-2.349867$

H $-5.241682-5.919592-3.474116$

H $-5.237416-4.550970-4.722854$

H -5.794737 -5.525175-1.207245

H $-7.205953-4.503076-1.205798$

H $-6.237027-2.9236790 .168346$

H $-2.226684-1.219863-0.957218$

C $-2.9312603 .651806 \quad 2.313793$

O $-2.0423013 .529007 \quad 1.189919$

C $-2.823063 \quad 3.1387410 .039724$

C $-4.2604472 .935107 \quad 0.527841$

C -4.0751382 .6897562 .014070$

H $-2.4213212 .176810-0.294102$

C $-2.689154 \quad 4.190882-1.071987$

H -4.8799853 .8232090 .357892$

H -4.7485962 .0988420 .016785$

C -5.3260492 .9359512 .837307$

H $-3.756493 \quad 1.6483172 .142134$

C $-1.235424 \quad 4.465626-1.508791$

O $-3.235603 \quad 5.425961-0.588197$

H -3.301398 $3.898578-1.933143$

H $-0.6800174 .880621-0.661378$

O $-1.264377 \quad 5.497397-2.504408$

C $-0.500588 \quad 3.243572-2.087156$

C $0.9115163 .555777-2.619873$

H $-0.4404062 .452845-1.332651$

O $-1.2741452 .729455-3.174707$

H $1.3027812 .644405-3.088617$

H $0.8441234 .281303-3.440322$

H -5.1357512 .7517493 .899486$

H -5.6795793 .9671592 .732003$

H -6.1337942 .2692452 .518157$

C -2.1712843 .4675383 .630050$

H $-3.1386106 .083380-1.298368$

H $-1.722628 \quad 5.138688-3.283942$

H $-0.8301191 .926835-3.497814$

H $-2.7644383 .916434 \quad 4.436999$

H $-1.227538 \quad 4.026575 \quad 3.593621$

H $-3.298626 \quad 4.6874552 .291583$

C $2.491406 \quad 1.943692-0.574795$

$\begin{array}{lllll}\text { O } & 1.886950 & 3.225717 & -0.382210\end{array}$

C $1.8903234 .063098-1.542863$

C $3.3277504 .280012-2.040441$

C $4.0773322 .963328-2.237795$

C $3.9578722 .087276-0.990969$

H $1.9440481 .408201-1.359766$

$\begin{array}{llll}\text { C } & 2.296308 & 1.116086 & 0.697054\end{array}$

H $1.5212415 .042239-1.215185$

O $3.3394425 .011177-3.261990$

H $3.8769994 .879230-1.302438$

H $3.7243292 .428601-3.127485$

O $5.4622403 .239432-2.459113$

H $4.5505032 .530939-0.181561$

O $4.4989700 .797979-1.262704$ 
C $2.9939731 .700697 \quad 1.903128$

H $1.231501 \quad 1.0530700 .947601$

H 2.6410820 .0888800 .538931

H $2.8740275 .850407-3.104384$

H $5.5193103 .828607-3.230969$

H $5.4172110 .924473-1.557294$

O $3.981511 \quad 0.8583912 .320766$

O 2.6973802 .7728832 .413882

C 4.7071151 .2858973 .476958

H 4.0319571 .3848524 .332447

H $5.456600 \quad 0.5244353 .708329$

H 5.2179552 .2322213 .274999

SCF Energy (B3LYP/6-31G**//MMFF) $=-3245.91974913$

01_293

MM̄FF Geometry

C 1.8111094 .7411751 .109534

C 2.0833263 .4793220 .746346

C $2.4861442 .399007 \quad 1.712251$

O $1.3776161 .468048 \quad 1.717507$

C 3.7727681 .6775351 .274794

C 4.9706002 .5831071 .092041

C $5.5575252 .841141-0.093043$

C 5.5298933 .1763952 .358826

C $5.1542762 .338223-1.452117$

C $6.1806721 .364592-2.045945$

C $6.3127810 .044404-1.268067$

C $7.420510-0.817820-1.877681$

C $7.537462-2.177424-1.175030$

O $5.060898-0.648474-1.332810$

O $8.362428-3.023815-1.990245$

C $6.138717-2.823725-1.117914$

C $8.243338-2.0601220 .180950$

O $6.170913-4.033105-0.361526$

C $5.027815-1.878419-0.594321$

C $3.648988-2.543423-0.819581$

O $5.224950-1.6621570 .803375$

C 1.2770800 .5887742 .746601

O $2.081046 \quad 0.4022823 .644669$

C -0.027681 -0.0932392 .604497$

C $-0.286987-1.2006333 .314367$

C - $-1.559721-1.9244973 .332871$

C $-2.732306-1.2755582 .645223$

C $2.439467-1.686416-0.422599$

C $1.154596-2.317996-0.924702$

C $-0.242915-4.369621-0.679081$

O $0.511373-1.819135-1.845486$

C -1.415830 -4.199229 0.232435

C -1.885957 -5.1831601 .013769$

C $-3.116416-5.0747581 .867597$

C $-2.822133-5.2255703 .342994$

C -2.640763 -6.438071 3.893705

C $-2.837359-3.9767754 .192813$

C - $-1.627683-3.1065073 .980471$

N $0.835094-3.493172-0.264028$

H 1.8995175 .0306562 .153334

H $1.9719203 .191873-0.297065$

H 2.6002042 .8109492 .723092

H $3.563171 \quad 1.0923540 .374776$

H 4.0343480 .9153572 .019741

H $6.4284643 .495498-0.101672$

H 6.4430243 .7541032 .179585

H 4.8023363 .8483282 .824293

H 5.7783282 .3831283 .071590

H $4.1599481 .883688-1.455662$

H $5.0781903 .209062-2.115876$

H $5.8691011 .144975-3.075439$

H $7.1586251 .859133-2.103596$

H $6.5481190 .267512-0.221769$

H $8.382514-0.290732-1.853702$

H $7.202456-0.994262-2.940489$

H $\quad 9.227037-2.590499-2.093388$

H $5.883483-3.119773-2.144838$

H $9.242807-1.6263630 .055784$

H $8.401714-3.0466510 .630412$

H $7.700534-1.4370690 .894835$

H $6.197018-3.7920490 .580159$

H $3.546736-2.795431-1.883883$
H $3.603541-3.486232-0.260211$

H $4.389736-1.3665051 .197488$

H $-0.7520300 .354704 \quad 1.932914$

H $\quad 0.496409-1.6086373 .951520$

H -3.659636 -1.843579 2.732932

H -2.536019 -1.162710 1.573784

H -2.928632 -0.2857043 .072332$

H $2.369643-1.5679980 .663536$

H $2.508495-0.684878-0.860094$

H $\quad 0.149372-5.391694-0.656670$

H $-0.535194-4.142503-1.709091$

H -1.927012 -3.2406190 .211023$

H -1.380638 -6.146685 1.009807

H -3.815826 -5.8616691 .555122$

H -3.645904 -4.132494 1.681767

H -2.461839 -6.556239 4.957102

H -2.665071 -7.339553 3.290050

H -2.857056 -4.243294 5.258412

H -3.777855 -3.441908 4.030213

H $-0.720934-3.4988154 .442798$

H $1.406802-3.7919130 .520542$

C -0.1716515 .9443130 .074057$

O $-0.7556014 .734507-0.450369$

C - $-1.947958 \quad 4.434250 \quad 0.297774$

C -2.2819375 .7040871 .072809$

C $-0.895719 \quad 6.233473 \quad 1.388631$

H -1.6841453 .6374271 .005333$

C $-3.0713253 .940091-0.624389$

H $-2.831654 \quad 6.4221840 .452761$

H -2.8792205 .5004041 .966577$

C -0.8743327 .6965281 .793532$

H -0.4812525 .6326432 .207283$

C $-2.7288482 .618958-1.350848$

O $-3.3823194 .960883-1.571203$

H $-3.9693443 .782820-0.016257$

H $-2.3885381 .893063-0.603239$

O $-1.6568502 .818208-2.271419$

C $-3.9234472 .011561-2.115805$

C $-3.5934540 .725416-2.897272$

H $-4.7331821 .809841-1.406664$

O $-4.4427342 .951036-3.059686$

H $-4.5030080 .420784-3.430168$

H -2.871865 $0.955806-3.691599$

H $\quad 0.1494008 .0299001 .990978$

H $-1.292780 \quad 8.3368281 .009708$

H -1.4627847 .8522692 .703629$

C 1.3513635 .7978510 .144921

H $-2.5927385 .094887-2.123420$

H $-0.8850753 .122505-1.764720$

H -3.711552 $3.212799-3.645347$

H $1.8072096 .752706 \quad 0.431471$

H $1.7297935 .557767-0.857234$

H $-0.4022596 .729354-0.658156$

C $-5.175568-1.232843-1.178972$

O $-3.885490-0.681795-0.890455$

C $-3.065922-0.437642-2.035954$

C $-2.843918-1.744037-2.810727$

C $-4.157008-2.447246-3.143259$

C $-5.028613-2.579785-1.894885$

H $-5.744792-0.534710-1.805074$

C $-5.920109-1.3892990 .150767$

H -2.086224 -0.128827 -1.651512

O $-2.118596-1.509942-4.012762$

H $-2.240339-2.425000-2.200546$

H -4.697403 -1.931530 -3.945683

O $-3.874183-3.761350-3.630567$

H $-4.588523-3.322588-1.217013$

O $-6.318281-3.054981-2.273786$

C $-6.334226-0.0420440 .693647$

H $-6.835031-1.9813830 .034272$

H $-5.305537-1.8984470 .901519$

H - $1.273186-1.096773-3.766745$

H -3.286604 -3.663570 -4.399550

H $-6.190079-3.894377-2.748258$

O $\quad-5.308748 \quad 0.5150101 .398010$

$\begin{array}{llll}\text { O } & -7.431691 & 0.462535 & 0.494557\end{array}$

C $-5.564173 \quad 1.8138011 .940703$

H $\quad-5.8885632 .5028381 .154897$ 
H $-6.318027 \quad 1.7462802 .730351$

H -4.6329822 .1905322 .372284$

SCF Energy (B3LYP/6-31G**//MMFF)= -3245.92103835

$01 \_294$

MM̄FF Geometry

C $4.3588972 .968452 \quad 0.151027$

C 3.0271782 .9388870 .310840

C $2.0443943 .173525-0.805499$

O $1.0848052 .096356-0.717245$

C $1.3065404 .507429-0.599752$

C $0.4415504 .907984-1.779338$

C $-0.9063144 .879899-1.799000$

C $1.1967745 .424945-2.978487$

C $-1.8465504 .418953-0.717188$

C $-2.9506273 .518479-1.283906$

C $-4.0764343 .205318-0.284093$

C -3.5840462 .4339420 .941679$

C -4.7348121 .9946041 .859963$

O

O $-4.221696 \quad 1.0105032 .771515$

C -5.8187381 .2980441 .010987$

C -5.2470323 .1545762 .721997$

O -6.9712821 .0098741 .798256$

C $-6.2246502 .102643-0.240886$

C $-7.1872471 .327906-1.176503$

O $\quad-6.9134493 .291780 \quad 0.166372$

C $0.6826401 .473688-1.851397$

O $1.0557841 .707266-2.991884$

C $-0.3592050 .463260-1.554513$

C $-0.6030350 .004482-0.316838$

C -1.657184 $-0.934216 \quad 0.067470$

C $-2.614163-1.388286-1.002504$

C $-6.664520-0.012074-1.705722$

C -7.119614 -1.218933-0.898663

C $-6.657532-3.660401-0.638446$

O $-8.014899-1.173959-0.059802$

C $-5.862377-3.7407470 .625592$

C $-4.723472-4.438657 \quad 0.751986$

C $-3.910830-4.4788722 .015713$

C $-2.642953-3.6564621 .900951$

C - $-1.449692-4.2611031 .773133$

C $-2.832529-2.1546381 .981805$

C - $1.748829-1.3153621 .359406$

N -6.428498 -2.367948 -1.259293

H $4.752970 \quad 3.212375-0.833879$

H 2.6009302 .7158431 .285030

H $2.5651053 .151152-1.769985$

H $2.0346935 .312266-0.429600$

H $\quad 0.707301 \quad 4.4589680 .316524$

H -1.403834 5.244098 -2.697609

H $0.5286895 .773775-3.773259$

H $1.8326496 .269039-2.691791$

H $1.8299874 .641220-3.404694$

H -1.313572 3.8800930 .069793

H $-2.3006415 .306497-0.261182$

H -3.404091 $3.993815-2.164819$

H $-2.5180742 .581637-1.657417$

H $-4.536994 \quad 4.1532700 .018173$

H -2.854082 3.0179551 .513969

H -3.0527591 .5365420 .606553$

H -3.475164 1.4071163 .252492

H $\quad-5.403794 \quad 0.3321100 .695869$

H -4.4444103 .5376573 .363711$

H -6.0395522 .8227303 .401637$

H $-5.623883 \quad 3.9932792 .133278$

H -6.676456 0.4854492 .562333

H $-8.1675831 .217122-0.697526$

H -7.365391 $1.965780-2.054286$

H $-7.4483273 .609528-0.579917$

H $-0.9217480 .121470-2.416912$

H $\quad 0.011723 \quad 0.360487 \quad 0.508411$

H -2.078129 -1.914650 -1.799968

H -3.140903 -0.531707 -1.436669

H $-3.375660-2.078070-0.634433$

H -7.061568 $-0.162425-2.717994$

H -5.572424 -0.005943 -1.782370

H $-6.363694-4.428785-1.360523$
H $-7.723279-3.779037-0.418270$

H $-6.240612-3.1810761 .478117$

H $-4.336793-4.991207-0.101013$

H $-3.678831-5.5283732 .237100$

H -4.493489-4.119262 2.873440

H $-0.523088-3.7017371 .715224$

H -1.369485 -5.3425961 .730531$

H -2.922395 -1.876276 3.040022

H -3.791067 -1.866043 1.535807

H -1.008732 -0.9499402 .071474$

H $-5.656935-2.294033-1.915857$

C 4.9373801 .9503072 .461299

O $4.384556 \quad 0.6683342 .103542$

C $4.889485-0.3324103 .004799$

C 5.5333210 .4289874 .156932

C 6.0746231 .6510773 .439875

H $5.671765-0.8761832 .461569$

C $3.772170-1.3012513 .406685$

H $4.796467 \quad 0.7292804 .910647$

H $6.310349-0.1558964 .658342$

C 6.4098162 .8042394 .369560

H 6.9793311 .3603742 .888988

C $3.007264-1.9111682 .212950$

O $2.818245-0.5905984 .207174$

H $4.184013-2.0905184 .046634$

H $2.416764-1.1233631 .732313$

O $2.054066-2.8461612 .732954$

C $3.889693-2.6146901 .164225$

C $3.108676-3.3609910 .065399$

H $4.545062-1.8795350 .690905$

O $4.747920-3.5567921 .812689$

H $3.838726-3.864852-0.579676$

H $2.528593-4.1729090 .521623$

H 6.7822783 .6646073 .804980

H 5.5324183 .1287904 .939034

H 7.1841212 .5085585 .084984

C 5.4063342 .6741051 .194913

H $2.116714-1.2193864 .448898$

H $2.541463-3.5284553 .224586$

H $4.193389-4.2679262 .175411$

H 6.1641502 .0488240 .706908

H 5.8946433 .6182781 .461947

H 4.1453462 .5186392 .964596

C $3.773569-1.679419-2.421516$

O $2.882082-1.347618-1.349098$

C $2.184494-2.457119-0.772282$

C $1.375369-3.195629-1.847096$

C $2.244191-3.602469-3.030472$

C $3.023612-2.398324-3.551548$

H $4.578864-2.321691-2.044454$

C $4.392090-0.367541-2.935929$

H $1.454210-2.032224-0.076523$

O $0.745154-4.352778-1.309992$

H $\quad 0.577553-2.539379-2.217678$

H $2.918629-4.427336-2.772402$

O $1.406891-4.082483-4.085115$

H $2.334804-1.702940-4.048689$

O $3.962807-2.843507-4.529036$

C $5.5502750 .104832-2.082894$

H $4.783947-0.514419-3.949107$

H $3.6466230 .434854-2.954447$

H $0.159434-4.056295-0.593184$

H $0.885916-4.821434-3.726391$

H $3.462131-3.308793-5.221117$

O $6.310230 \quad 0.973874-2.813655$

O $5.754778-0.227040-0.924211$

C $7.4812421 .488824-2.170754$

H $8.0038200 .710071-1.606885$

H $8.1545501 .864497-2.946028$

H $7.2036312 .318645-1.516291$

SCF Energy (B3LYP/6-31G**//MMFF) $=-3245.91250492$

01_295

MM̄FF Geometry

C $-4.5913642 .575190-0.863319$

C $-3.6820442 .554274-1.850063$

C -2.262765 $3.026306-1.682253$

O $-1.3923312 .007989-2.239986$ 
C $-1.982386 \quad 4.323447-2.470027$ C $-2.1557235 .584905-1.646715$ C $-1.1451126 .238335-1.037873$ C $-3.5609356 .117463-1.549274$ C $0.3154345 .878000-1.051397$ C $0.683174 \quad 4.9974250 .141719$ C $2.170315 \quad 4.615100 \quad 0.220126$ C $2.6474883 .858300-1.023614$ C $4.072873 \quad 3.310945-0.872760$ O $2.296257 \quad 3.7932981 .389252$ O $4.2789852 .379312-1.945653$

C $4.1646012 .535567 \quad 0.455330$ C $5.1202794 .414318-1.058159$ O $5.499738 \quad 2.0958300 .694562$ C 3.6206383 .3256521 .666378 C 3.5555872 .4499012 .940577 O $4.507238 \quad 4.415740 \quad 1.940997$ C $-0.5870641 .309902-1.395590$ O $-0.591351 \quad 1.337318-0.175906$ C $0.360046 \quad 0.535966-2.231916$ C $1.438295-0.026473-1.664933$ C $2.501810-0.757172-2.359914$ C $2.348128-0.969825-3.843187$ C $2.500504 \quad 1.3389182 .899499$ C 3.0333340 .0125553 .402779 C $4.615576-1.8139092 .826338$ O $2.626915-0.497634 \quad 4.443927$ C $5.347072-2.2547521 .600773$ C $4.929046-3.2427080 .795446$ C $5.652237-3.656492-0.452704$ C $4.877395-3.334411-1.711573$ C $4.350697-4.309538-2.470810$ C $4.799867-1.886864-2.145240$ C $3.564184-1.187075-1.646061$ N $3.974173-0.5428232 .552032$ H -4.2959692 .9636160 .107204$ H $-3.9722672 .199598-2.836283$ H -2.029594 $3.177704-0.621582$ H -2.619755 4.383337 -3.362952 H $-0.9668414 .280906-2.879729$ H $-1.3806827 .131721-0.461051$ H -3.600548 $7.093739-1.053860$ H -3.990992 $6.243166-2.548302$ H $-4.1954205 .435849-0.977780$ H $0.5795625 .393324-1.994203$ H $0.9000786 .805569-1.019798$ H $\quad 0.405977 \quad 5.5058501 .075543$ H $0.082015 \quad 4.0798770 .130719$ H 2.7588535 .5286050 .365058 H $2.5907694 .487588-1.919156$ H $1.9707613 .014844-1.209865$ H $5.1456211 .959016-1.809146$ H 3.5711541 .6244490 .321901 H $5.0353924 .854769-2.059043$ H $6.1371084 .011667-0.996266$ H $5.0235375 .223952-0.331950$ H 6.0124112 .8615841 .004963 H 4.5550862 .0640313 .177123 H 3.3010963 .0973953 .791850 H 4.3703884 .7009242 .859558 H $0.1680050 .491313-3.297834$ H $1.5737820 .081377-0.589385$ H $2.412706-0.014076-4.374690$ H $1.382171-1.432597-4.072688$ H $3.105174-1.631583-4.269122$ H $1.647483 \quad 1.6214173 .528402$ H 2.0959741 .1749191 .894939 H $3.853360-2.5335813 .143795$ H $5.312587-1.6739263 .658905$ H $6.260927-1.7182651 .356422$ H $4.013726-3.7776991 .035781$ H $5.846397-4.734611-0.384238$ H $6.640846-3.183924-0.512845$ H $3.822824-4.083734-3.391337$ H $4.428060-5.353422-2.185627$ H $4.893872-1.821694-3.234048$ H $5.676345-1.344049-1.768010$ H $3.579917-0.978215-0.576884$
H $4.3344490 .023451 \quad 1.789679$ C $-6.5270631 .119550-0.033068$ O $-5.786340-0.107815-0.169097$ C $-5.540927-0.6382991 .147599$ $\begin{array}{llll}\text { C } & -6.455683 & 0.132856 & 2.093848\end{array}$ C -6.4619901 .5034021 .445913$ H $-4.499995-0.3939941 .392031$ C $-5.734780-2.1577341 .168931$ H $-7.469952-0.2823752 .110503$ H $-6.0772860 .142922 \quad 3.120487$ $\begin{array}{llll}\text { C }-7.604490 & 2.392426 & 1.904356\end{array}$ H $-5.512312 \quad 1.994443 \quad 1.687174$ C $-4.929960-2.9304820 .105138$ O $-7.119093-2.4653550 .958559$ H $-5.493898-2.5397572 .167884$ H $-5.378011-2.789870-0.886159$ O $-5.048293-4.3279620 .403181$ C $-3.435385-2.5714850 .039206$ C $-2.644967-3.510310-0.892742$ H $-3.330696-1.539892-0.314315$ O $-2.880283-2.6276311 .350875$ H $-2.451041-4.456921-0.375928$ H -3.255825 -3.734861 -1.775206 H $-7.569577 \quad 3.3625891 .398675$ H -8.5779171 .9366501 .694186$ H -7.5437442 .5711082 .982869$ C $-6.0277572 .163281-1.036597$ H $-7.382005-2.0689520 .109983$ H $-5.997485-4.5388340 .429880$ H -3.019794 -3.5272131 .693279$ H -6.650922 $3.062096-0.959854$ H $-6.1697461 .761854-2.048131$ H $-7.5636250 .872008-0.299461$ C $0.120427-3.466104 \quad 0.490009$ O $-0.488532-2.437208-0.295767$ C $-1.311310-2.893165-1.369762$ C $-0.501207-3.790845-2.314209$ C $0.196643-4.925216-1.568688$ C $0.992054-4.371628-0.386925$ H $-0.654767-4.0623970 .985814$ C $0.961175-2.7764301 .569705$ H $-1.585260-1.996516-1.940848$ O $-1.340900-4.318107-3.335611$ H $0.261542-3.180904-2.812924$ H $-0.523536-5.686852-1.247212$ O $1.087802-5.569795-2.481044$ H $1.857251-3.810651-0.758641$ $\begin{array}{llllll}\text { O } & 1.483089 & -5.452113 & 0.402145\end{array}$ C $0.080444-2.1077682 .597223$ H $1.594122-3.4903972 .108396$ H $1.628570-2.0231191 .135240$ H $-0.791506-4.893008-3.895616$ H $1.462878-6.343664-2.028024$ H $2.142607-5.928612-0.129615$ O $-0.177386-0.821307 \quad 2.227281$ O $-0.346564-2.6720023 .596330$ C -1.021242 $-0.081443 \quad 3.114966$ H -1.1126990 .9366852 .727492$ H $-2.016838-0.5326823 .154591$ H $-0.576701-0.0369074 .113802$ SCF Energy (B3LYP/6-31G**//MMFF)= -3245.91215701

01_296

MM̄FF Geometry

C 0.2817052 .8999562 .277694

C -0.0956501 .6269492 .476096$

C 0.8275580 .4516202 .707048

$\begin{array}{llllll}\text { O } & 2.201964 & 0.880449 & 2.525123\end{array}$

C $0.699820-0.1255874 .131224$

C $-0.460396-1.0799084 .336245$

C $-0.367258-2.4181774 .199076$

C $-1.746305-0.463026 \quad 4.817724$

C $0.845274-3.1953713 .770659$

C $0.531187-4.4013482 .875134$

C $-0.200768-4.0718371 .563810$

C $-0.405847-5.356898 \quad 0.752524$

C $-1.050338-5.089678-0.613856$

O $0.575464-3.1288320 .813011$ 
O $-0.888620-6.285270-1.393008$

C $-0.268229-3.957754-1.311914$

C $-2.560620-4.863473-0.490527$

O $-0.890601-3.594479-2.540804$

C $-0.045033-2.718675-0.415927$

C $0.845511-1.653634-1.096400$

O $-1.309513-2.091603-0.165913$

C $3.086827-0.0083332 .004047$

O $2.862231-1.1588351 .667769$

C $4.402645 \quad 0.661119 \quad 1.910327$

C $5.435607-0.0081071 .376709$

C 6.8011410 .4891941 .209996

C 7.1084321 .8827411 .690027

C $2.250914-2.113767-1.490798$

C $3.062471-0.981658-2.102649$

C $5.237033-0.556313-3.224537$

O $2.6044750 .140570-2.302178$

C $6.659664-0.815106-2.844050$

C $7.5463110 .166604-2.617479$

C $8.985210-0.065422-2.249998$

C $9.3841110 .666473-0.986592$

C $9.9822671 .868188-1.048953$

C $9.158460-0.0192010 .342652$

C $7.711723-0.3148970 .622955$

N $4.355367-1.382579-2.418091$

H 1.3364913 .1617402 .283268

H $-1.157732 \quad 1.3991882 .451819$

H $0.585162 \quad-0.310647 \quad 1.957044$

H $\quad 0.636327 \quad 0.6915334 .863252$

H $1.634512-0.6335714 .403085$

H - $1.242354-3.0222224 .434145$

H $-2.510193-1.2151355 .042913$

H - 1.5741460 .1119915 .733432

H -2.162064 0.2044204 .061312

H $1.566750-2.5581153 .257396$

H $1.342296-3.5680964 .674791$

H $1.488603-4.8845712 .639650$

H -0.059479 -5.129583 3.445672

H -1.171124 -3.6246011 .807951$

H $-0.998731-6.0851521 .320347$

H $0.569382-5.8358620 .583935$

H $-1.220689-6.100347-2.288482$

H $0.705997-4.382627-1.582314$

H $-3.042412-5.744003-0.048359$

H $-3.022830-4.737008-1.475438$

H -2.818391 -4.0014810 .127980$

H -1.634124 -3.005109-2.332394

H $0.326589-1.253287-1.977958$

H $0.946197-0.798156-0.415017$

H $-1.150370-1.1719650 .104890$

H 4.4855031 .6761872 .280702

H $5.268360-1.0247501 .022175$

H 6.9292251 .9665682 .767514

H 6.4812372 .6158691 .170692

H 8.1454892 .1803591 .517655

H $2.788968-2.500263-0.619313$

H $2.193440-2.908887-2.241614$

H $4.9619430 .497701-3.113165$

H $5.074832-0.842895-4.268849$

H $6.981128-1.850893-2.767824$

H $7.227207 \quad 1.201509-2.720782$

H $9.6037330 .277486-3.089907$

H $9.205149-1.134287-2.137915$

H $10.2957062 .391940-0.151921$

H $10.1686812 .357125-1.999434$

H $9.5928020 .556813 \quad 1.168671$

H $9.710592-0.9682770 .345339$

H $7.399090-1.3070520 .295724$

H $4.580000-2.370740-2.340998$

C $-0.582114 \quad 4.5840730 .639685$

O $-1.3068293 .704759-0.247130$

C $-2.078788 \quad 4.505849-1.163312$

C $-1.5696225 .933138-1.007280$

C $-1.223357 \quad 5.9603050 .467779$

H $-3.1177694 .472500-0.815688$

C - $-1.9783143 .945986-2.585804$

H $-0.6742746 .113618-1.613624$

H -2.322850 $6.676007-1.287076$
C -0.3203257 .1183710 .855690$

H -2.153658 6.0173291 .048958

C $-2.4848232 .494453-2.722128$

O $-0.601728 \quad 3.979573-2.981419$

H -2.517651 $4.603541-3.278097$

H $-1.8281301 .816456-2.164894$

O $-2.3875712 .119803-4.101655$

C $-3.9470202 .295067-2.278732$

C $-4.5385050 .911634-2.599565$

H $-4.0331262 .472799-1.204637$

O $-4.775760 \quad 3.272107-2.916857$

H $-5.5936390 .926489-2.299399$

H $-4.5612790 .770155-3.687569$

H $-0.084653 \quad 7.0902031 .924043$

H $0.624186 \quad 7.093847 \quad 0.301831$

H -0.8093498 .0746950 .643310$

C $-0.668346 \quad 4.0463042 .067693$

H $-0.568393 \quad 3.818298-3.939265$

H $-1.4431702 .032003-4.314670$

H $-4.6822443 .153078-3.877706$

H -1.6953713 .7352502 .289105$

H -0.4021174 .8278502 .788322$

H $0.458727 \quad 4.6035240 .290266$

C $-4.917943-0.227576 \quad 0.222114$

O $-3.692736-0.105769-0.510848$

C $-3.808822-0.266973-1.928699$

C $-4.412832-1.638134-2.257704$

C $-5.735370-1.864343-1.533105$

C $-5.578544-1.589149-0.039010$

H $-5.5995890 .582866-0.064159$

C $-4.584274-0.0764951 .716068$

H $-2.784774-0.273762-2.320886$

O $-4.591388-1.775175-3.662790$

H $-3.707341-2.416195-1.947980$

H $-6.532800-1.249078-1.964513$

O $-6.149330-3.220387-1.719333$

H $-4.982362-2.3880150 .420452$

O $-6.866463-1.6118370 .573895$

C -4.3191591 .3604442 .112062$

H $-5.428220-0.4295992 .319995$

H $-3.692800-0.6585941 .976587$

H $-4.999452-2.643628-3.821607$

H $-5.463181-3.798962-1.345151$

H $-7.269309-2.473890 \quad 0.372091$

O -4.5684091 .5075593 .447151$

$\begin{array}{llll}0 & -3.912915 & 2.234443 & 1.359999\end{array}$

C $-4.3378632 .813551 \quad 3.985265$

H -3.262548 2.9987364 .053897

H -4.7641712 .8456284 .991514$

H -4.8255953 .5803803 .375689$

SCF Energy (B3LYP/6-31G**//MMFF) $=-3245.91561156$

01_297

MM̄FF Geometry

C $1.835484-4.323944 \quad 0.046624$

C $0.570879-4.185881-0.378266$

C $-0.527167-3.598421 \quad 0.473591$

O $-1.115584-2.473563-0.221074$

C $-1.620924-4.656958 \quad 0.706354$

C $-2.779004-4.1815041 .563671$

C $-4.068703-4.2073001 .173403$

C $-2.425846-3.6854552 .941297$

C $-4.617137-4.714592-0.130545$

C $-4.663055-3.664460-1.248766$

C $-5.704057-2.556647-1.021972$

C $-5.811210-1.668655-2.264776$

C $-6.792281-0.506137-2.056932$

O $-5.302948-1.7680950 .101250$

$\begin{array}{llll}0 & -6.598041 & 0.431395 & -3.127165\end{array}$

C $-6.429236 \quad 0.222844-0.747053$

C $-8.252331-0.965805-2.142697$

O $-7.406548 \quad 1.211181-0.427080$

C $-6.225328-0.7307320 .455418$

C $-5.656693 \quad 0.017243 \quad 1.685910$

O $-7.495840-1.2732990 .833824$

C $-0.408407-1.309853-0.207710$

O $0.691475-1.1279140 .291509$

C $-1.205370-0.276317-0.907087$ 
C $-0.6355560 .913311-1.149811$ C -1.252243 $2.083207-1.776841$ C $-2.6866431 .975379-2.216715$ C $-4.186861 \quad 0.432617 \quad 1.553790$ C $-3.861949 \quad 1.716907 \quad 2.296817$ C -2.0774453 .4528432 .440387$ O -4.6517912 .2829283 .047325$ C $-2.2177504 .410374 \quad 1.297603$ C -1.190024 5.0734080 .746190 C $-1.3398866 .034254-0.398765$ C $-0.4689645 .678957-1.584107$ C $0.6239056 .402865-1.880181$ C $-0.9087884 .533923-2.475010$ C $-0.502173 \quad 3.196237-1.921021$ N -2.576345 2.159088 2.013155 H $2.094447-4.0093461 .054901$ H $\quad 0.306569-4.497205-1.385956$ H $-0.132533-3.2578951 .439186$ H -1.181063 -5.5381211 .192499$ H -1.983325 -4.995299 -0.270534 H $-4.824528-3.8417191 .867259$ H -3.312125 -3.5231093 .564061$ H -1.793583 -4.414907 3.457968 H -1.889333 -2.734285 2.881209 H -4.036246 -5.578259-0.473533 H $-5.626801 \quad-5.1059290 .047563$ H -3.667370 -3.219258 -1.367234 H -4.897912 -4.184895 -2.185696 H $-6.669123-3.030206-0.806884$ H -6.100455 -2.259639 -3.142798 H -4.822112 -1.249385 -2.498395 H -6.753050 -0.035432 -3.966209 H $-5.497336 \quad 0.767945-0.934679$ H -8.455226 -1.414612 -3.122461 H -8.939582 -0.117079 -2.055013 H $-8.515765-1.703315-1.381824$ H -7.494771 $1.792978-1.201470$ H -6.3066850 .8708821 .912981$ H -5.728199 -0.6333182 .568654$ H -7.435306 -1.591859 1.749505 H $-2.226803-0.512777-1.180459$ H $0.3988551 .056165-0.842888$ H -3.019939 2.824656 -2.817166 H -3.346792 $1.912053-1.347144$ H -2.839209 $1.088253-2.841447$ H $-3.542259-0.3640991 .944562$ H -3.917874 0.5854290 .505903 H -1.0361133 .3205602 .746057$ H -2.651528 3.8119413 .300021 H -3.221019 4.5677950 .907758 H -0.1891224 .9330641 .147089$ H -1.082639 $7.034639-0.026988$ H -2.383792 $6.100736-0.729956$ H $1.2394666 .168878-2.742606$ H $0.9328437 .239608-1.262583$ H $-0.4473134 .635315-3.465931$ H -1.986832 4.618622 -2.637460 H $0.5400223 .155546-1.602302$ H $-2.008110 \quad 1.6329501 .355937$

C $4.012948-3.848991-1.101032$

O $4.658518-3.4282760 .119713$

C $6.078618-3.514433-0.056649$ C $6.316646-3.560833-1.557989$ C $5.123868-4.382892-2.009991$ H $6.375093-4.4875790 .359681$ C $6.819419-2.4216980 .721816$ H $6.278060-2.563933-2.009350$ H $7.276168-4.017414-1.818156$ C $4.820415-4.250095-3.492538$ H $5.320618-5.438988-1.781009$ C $6.725378-0.9754420 .192337$ O $8.213216-2.7646180 .732230$ H $6.485922-2.4329391 .767254$ H $7.303124-0.890842-0.736233$ O $7.401408-0.1516731 .155771$ C $5.300990-0.430014-0.025340$ C $5.3006971 .055083-0.439628$ H $4.813101-1.012774-0.811531$
O $4.554372-0.600751 \quad 1.173731$

H 5.5975801 .6685750 .417281

H $6.0553381 .189636-1.223113$

H $3.944942-4.847413-3.766228$

H $4.618358-3.209733-3.768733$

H $5.668332-4.598158-4.091449$

C $2.945427-4.899425-0.786475$

H $8.300133-3.6253261 .176508$

H 7.6079430 .6959380 .727642

H $3.633726-0.3513960 .985565$

H $3.388922-5.727456-0.219830$

H $2.547204-5.317478-1.718304$

H $3.548651-2.956591-1.539844$

C 3.0097362 .5012941 .054042

O $2.9042661 .510896 \quad 0.024318$

C $3.9305291 .536777-0.971322$

C $3.9723242 .915573-1.642846$

C $4.1540064 .031387-0.619930$

C $3.0897683 .919308 \quad 0.468805$

H 3.8935312 .2965391 .669375

C 1.7560282 .3777661 .935616

H $3.6256550 .817454-1.742265$

O $5.0153042 .961864-2.610819$

H $3.0303573 .077989-2.182332$

H $5.1662884 .022665-0.199377$

O $4.0063185 .280773-1.298253$

H 2.1169094 .2077260 .052739

O 3.3895264 .8340701 .521273

C 1.7265841 .1022602 .747198

H 1.6856143 .2071152 .647433

H 0.8684712 .3929391 .292106

H $5.0191303 .856991-2.991192$

H $4.2143245 .986467-0.663026$

H 3.3056275 .7335871 .162916

O 0.4288450 .7286922 .948786

O $2.7137820 .528602 \quad 3.185473$

C $0.239101-0.4509593 .735393$

H $0.664872-0.3143294 .733945$

H $-0.836038-0.6219453 .833915$

H $\quad 0.689509-1.314953 \quad 3.237915$

SCF Energy $\left(B 3 L Y P / 6-31 G^{* *} / / M M F F\right)=-3245.89908040$

01_298

MM̄FF Geometry

C 1.4340623 .5793723 .485840

C 1.4427672 .3815422 .882116

C $0.4663451 .274848 \quad 3.189335$

$\begin{array}{lllll}\text { O } & -0.278116 & 0.973661 & 1.985524\end{array}$

C 1.2407840 .0094393 .602353

C $0.353644-1.1546213 .997579$

C $0.315592-2.3387393 .355362$

C $-0.505735-0.9396695 .216970$

C $1.108812-2.7671992 .152893$

C $0.425189-2.496606 \quad 0.804174$

C $-0.892513-3.2611390 .599661$

C $-1.389632-3.108908-0.839722$

C $-2.749539-3.793267-1.053974$

O $-1.871351-2.7307941 .495888$

O $-3.283633-3.328643-2.303589$

C $-3.727303-3.3376970 .051708$

C $-2.606367-5.314434-1.182670$

O $-4.943840-4.077681-0.006350$

C $-3.130584-3.4168551 .471735$

C $-4.029230-2.7619382 .549117$

O $-2.972469-4.793044 \quad 1.838604$

C $-1.3034371 .809516 \quad 1.665124$

O -1.6769982 .7896432 .290139$

C $-1.894318 \quad 1.335256 \quad 0.394703$

C $-2.6834572 .162142-0.307537$

C $-3.3506451 .855275-1.573908$

C $-3.3650340 .419529-2.025297$

C $-4.329492-1.2731912 .340399$

C $-5.660586-0.9997491 .658910$

C -7.0321320 .8830370 .771199$

O $-6.498303-1.869948 \quad 1.438180$

C $-6.8981950 .908651-0.718473$

C $-7.1184982 .005492-1.459125$

C $-7.0388982 .033323-2.958655$ 
C $-6.1175023 .115468-3.475125$

C $-6.6047004 .293612-3.899875$

C $-4.6436612 .796971-3.581366$

C $-3.9390672 .861174-2.254404$

N $-5.819187 \quad 0.347449 \quad 1.360672$

H 0.6939473 .7652424 .261356

H 2.1845192 .1659462 .120749

H -0.2274361 .5700033 .985904$

H $1.8973520 .237990 \quad 4.452615$

H $1.902538-0.2777012 .777882$

H $-0.359126-3.104473 \quad 3.736985$

H -1.000080 -1.860852 5.544331

H $\quad 0.103570-0.5824396 .053594$

H -1.289105 -0.2034195 .013156$

H $2.096568-2.2943552 .153754$

H $1.310544-3.8421752 .244853$

H $0.249823-1.4190090 .702471$

H $1.128184-2.7860570 .015140$

H $-0.715890-4.3178340 .832670$

H - $0.649996-3.497024-1.551011$

H -1.498957 -2.041377 -1.074512

H -2.642183 -3.543732 -3.002185

H -3.986912 -2.295563 -0.164857

H $-1.954888-5.567298-2.027884$

H -3.571659 -5.785466 -1.398723

H - $-2.185088-5.782586-0.290705$

H -5.298993 -3.981380 -0.906544

H $-4.947247-3.3469862 .681389$

H -3.494706 -2.843095 3.506967

H -2.927623 -4.850632 2.807459

H $-1.656446 \quad 0.3294980 .068707$

H -2.846345 3.1709650 .070291

H $-4.0441750 .231587-2.859639$

H $-3.702168-0.232837-1.213565$

H $-2.365906 \quad 0.103199-2.337423$

H $-4.388753 \quad-0.791928 \quad 3.325304$

H $-3.525661-0.7809931 .784834$

H $-7.1829691 .882581 \quad 1.191090$

H $-7.882516 \quad 0.254021 \quad 1.053637$

H -6.638012 -0.024568 -1.212501

H $-7.4071992 .930207-0.964287$

H -8.054865 2.195765 -3.342098

H $-6.7240441 .063011-3.362103$

H -5.953662 $5.064175-4.299113$

H -7.665773 $4.515677-3.852396$

H $-4.1498123 .522169-4.242353$

H -4.517559 $1.835013-4.086257$

H $-3.8880933 .868334-1.838097$

H -5.0498470 .9891491 .529164$

C 2.6205405 .1010601 .733385

O 3.3852624 .0887571 .053872

C $2.8368553 .912522-0.267425$

C $1.9464635 .124684-0.514511$

C $1.3809185 .357244 \quad 0.875423$

H $2.1986113 .021365-0.229284$

C $3.9552133 .692778-1.289653$

H $2.5264865 .996110-0.839509$

H $1.1753664 .928560-1.265511$

C $\quad 0.770848 \quad 6.7342601 .066657$

H $0.610536 \quad 4.599650 \quad 1.063343$

C $4.9308022 .554488-0.919417$

O $4.7190984 .899880-1.399251$

H $3.5155133 .515846-2.278426$

H $5.5544282 .866890-0.073492$

O $5.8289902 .377567-2.022410$

C $4.2587451 .208245-0.592851$

C $5.2863300 .091118-0.327975$

H $3.628466 \quad 1.326546 \quad 0.294594$

O $3.414517 \quad 0.851201-1.682219$

H $5.816039-0.140945-1.257685$

H $\quad \begin{array}{llll}6.026536 & 0.468330 & 0.387189\end{array}$

H 0.3951776 .8545532 .087604

H $1.502386 \quad 7.5276280 .879887$

H $-0.067612 \quad 6.8819470 .378195$

C $2.375596 \quad 4.7191193 .199091$

H $5.4232634 .738800-2.050523$

H $5.3086772 .040925-2.772267$

H $2.8962430 .076966-1.405207$
H 1.9770735 .6034263 .712595

H 3.3339084 .4864323 .680301

H 3.2575185 .9960201 .730581

C $4.270693-2.406555-1.820424$

O $3.701443-1.791999-0.657647$

C $4.639780-1.1939680 .242156$

C $5.644805-2.2492030 .722879$

C $6.328619-2.951138-0.445848$

C $5.284540-3.487579-1.424068$

H $4.756220-1.641355-2.437889$

C $3.121762-3.016029-2.634035$

H $4.061882-0.8822431 .121694$

O $6.616635-1.6531591 .574972$

H $5.111303-2.9989231 .322144$

H $7.042806-2.283862-0.942389$

O $7.090466-4.0447300 .070153$

H $4.767147-4.337169-0.960853$

O $5.946285-3.987795-2.584330$

C $2.327365-1.944830-3.341823$

H $3.493569-3.696378-3.408978$

H $2.441564-3.598101-2.000919$

H $7.241365-2.3511861 .836546$

H $7.483509-4.507510-0.689780$

H $6.402739-3.243362-3.012379$

O $1.367141-1.449916-2.511500$

O $2.550152-1.581244-4.489251$

C $0.558455-0.394940-3.041958$

H $1.1609240 .333466-3.593472$

H $-0.217166-0.819099-3.685405$

H $0.0842160 .118282-2.201642$

SCF Energy $\left(B 3 L Y P / 6-31 G^{* *} / / M M F F\right)=-3245.91482278$

01_299

MMFF Geometry

C $-0.0352085 .291627-0.178353$

C $1.0351474 .742660-0.772379$

C $1.9998653 .832597-0.054837$

O $2.0914242 .596130-0.802428$

C $3.4012594 .464723-0.004147$

C 4.3479163 .7364350 .931283

C 5.4386393 .0478840 .543173

C 4.0238393 .8437552 .400749

C $5.9680712 .845521-0.846918$

C $5.4931051 .561390-1.544232$

C $5.7641100 .270700-0.753732$

C $5.593969-0.958573-1.649311$

C $5.773119-2.269309-0.866851$

O $4.830607 \quad 0.1993990 .331021$

O $5.282846-3.341797-1.686826$

C $4.886720-2.2267790 .396890$

C $7.250802-2.574046-0.594284$

O $5.143036-3.3488991 .236654$

C $5.029357-0.9206161 .206650$

C $4.003976-0.7975192 .363417$

O $6.331486-0.8750991 .804165$

C $1.1039731 .681738-0.607793$

O $\quad 0.170237 \quad 1.763604 \quad 0.174063$

C $1.3338830 .552846-1.534297$

C $0.320777-0.290093-1.779268$

C $0.358018-1.472606-2.638857$

C $1.703503-1.950346-3.113150$

C $2.530986-0.9212361 .958625$

C $1.989263-2.3380012 .064603$

C $0.111773-3.7727381 .297592$

O $2.501177-3.1972562 .777759$

C $-0.429701-4.028064-0.072968$

C $0.057596-4.975764-0.889001$

C $-0.404928-5.357714-2.270275$

C $-1.481939-4.502174-2.898162$

C $-2.779442-4.836028-2.794112$

C $-1.043372-3.324861-3.742127$

C $-0.806600-2.092665-2.918470$

N $0.849431-2.5232051 .294161$

H -0.1993825 .1121800 .880780$

H $1.2109304 .918295-1.830904$

H 1.6447143 .6187040 .960156

H 3.3324255 .5067540 .335995

H $3.8112894 .503151-1.019185$ 
H $\quad 6.0395572 .569401 \quad 1.316229$ H 4.8245933 .4443723 .032736 H 3.8802494 .8917402 .683440 H 3.1124753 .2878572 .639518 H $5.7342813 .704535-1.484635$ H $7.0641062 .828851-0.785165$ H $4.4208421 .649530-1.749570$ H $6.0025991 .508332-2.514515$ H $6.785920 \quad 0.314173-0.358903$ H $6.289481-0.922450-2.497135$ H $4.587448-0.950358-2.088756$ H $5.783696-3.334769-2.520524$ H $\quad 3.849337-2.3213100 .052927$ H $7.803703-2.661654-1.537351$ H $7.368717-3.538713-0.088465$ H $7.743968-1.8065930 .005756$ H $5.018321-4.1488380 .697860$ H $4.259452-1.5011273 .164927$ H 4.1301230 .2025842 .802736 H $6.305643-0.2528302 .550030$ H $2.3121060 .448553-1.986451$ H - $0.639271-0.095234-1.303603$ H $2.376030-2.102430-2.261472$ H $2.155363-1.219664-3.792568$ H $1.663522-2.903771-3.644247$ H $1.924089-0.3145302 .642636$ H $2.374153-0.5325030 .949632$ H $-0.710076-3.6722302 .013102$ H $\quad 0.757016-4.587387 \quad 1.642923$ H -1.273416 -3.417497 -0.377232 H $0.894340-5.571746-0.525829$ H $-0.749406-6.399545-2.223512$ H $0.468626-5.374194-2.934292$ H $-3.554367-4.246784-3.272954$ H -3.095026 -5.700644 -2.219593 H -1.823990 -3.081065 -4.475694 H $-0.177380-3.605164-4.348482$ H -1.725939 -1.667946 -2.515050 H $\quad 0.409541-1.7113010 .869931$ C $-2.4130175 .458958-1.066864$ O $-2.2652984 .263269-1.857243$ C $-2.9538583 .173631-1.199463$ C $-3.9450423 .858941-0.268756$ C -3.1344275 .0466610 .214790$ H -2.191819 2.636181 -0.623945 C $-3.5580812 .243669-2.259294$ H -4.828482 4.197994 -0.823721 H -4.2789923 .2364050 .562371$ C -3.975132 6.1530360 .828148 H $-2.426724 \quad 4.6869940 .970442$ C $-3.9064520 .820074-1.781553$ O $-2.5715472 .058598-3.293069$ H $-4.4183802 .713158-2.750111$ H $-2.974030 \quad 0.283683-1.577933$ O $\quad-4.5309840 .135239-2.876952$ C $-4.8283880 .699052-0.563255$ C $-5.261718-0.739606-0.220954$ H -4.3269381 .1154390 .311646$ O $-6.0111221 .470819-0.777362$ H $-5.889521-0.6876310 .677505$ H $-5.929101-1.116544-1.006464$ H -3.344657 6.9914051 .140859 H -4.7174296 .5342530 .118830$ H -4.511276 5.7849361 .709101 C -1.050628 6.141595 -0.891229 H $-2.3198812 .942456-3.611823$ H -3.919862 $0.180912-3.632454$ H $-6.4375591 .137179-1.585435$ H -1.173589 $7.078768-0.336233$ H $-0.6658226 .408749-1.883841$ H $-3.0534976 .128662-1.655274$ C -3.509721 -1.1597802 .269380$ O $-3.107144-1.2283880 .896429$ C $-4.099982-1.729842-0.003891$ C $-4.543547-3.1370720 .419404$ C $-4.996785-3.1763071 .875176$ C -3.940010 -2.543549 2.778558 H $-4.339749-0.4507582 .373659$
C $-2.308103-0.6409013 .077236$

H $-3.601436-1.843500-0.973071$

O $-5.597491-3.612459-0.411166$

H $-3.704270-3.8341980 .302366$

H -5.970753 -2.690621 2.006138

O $-5.171081-4.5379712 .274594$

H -3.071755 -3.211732 2.831972

O $-4.460587-2.4351614 .102679$

C -2.0656390 .8428462 .894434$

H -2.462799 -0.7997504 .149960$

H -1.401892 -1.170978 2.762566

H -5.278554 -3.589680 -1.329597

H $-5.815767-4.9372601 .665580$

H -5.232720 -1.844835 4.072866

O $-0.825406 \quad 1.1505803 .378066$

O -2.8707041 .6383702 .432356$

C -0.4643262 .5359513 .346573$

H -0.9313993 .0517494 .190099$

H 0.6220692 .6053833 .446634

H -0.7571283 .0064502 .403664$

SCF Energy $(B 3 L Y P / 6-31 G * * / / M M F F)=-3245.91298635$

013

MMFF Geometry

C $-2.7537112 .684217-2.282674$

C $-2.1303621 .652196-1.695377$

C $-1.0280730 .854750-2.340491$

O $0.0547480 .720200-1.391187$

C -1.543411 -0.556596-2.675115

C $-0.530988-1.420624-3.401700$

C $-0.029977-2.576189-2.923010$

C $-0.105621-0.931340-4.762441$

C $-0.351812-3.253721-1.621597$

C $0.552740-2.853884-0.446869$

C $2.035680-3.207594-0.639803$

C $2.813047-2.9957770 .661569$

C $4.316721-3.2596640 .482665$

O $2.578508-2.364376-1.658945$

O $4.996754-2.7288591 .630912$

C $4.825825-2.474096-0.745820$

C $4.631240-4.7600800 .448833$

O $6.174190-2.821973-1.049282$

C $3.942893-2.649950-1.998029$

C $4.335601-1.706019-3.161320$

O $4.080190-3.990634-2.485362$

C $0.8679571 .794805-1.208110$

O $0.8180362 .866552-1.789696$

C $1.8199881 .447724-0.131067$

C 2.4794882 .4255510 .506841

C 3.4216792 .2493421 .613555

C 3.8134890 .8411891 .976358

C $4.277866-0.207760-2.842946$

C $5.6118180 .385380-2.418960$

C $6.6057442 .491299-1.531389$

O $6.679113-0.213539-2.519768$

C $6.7940262 .358435-0.053042$

C 6.9167673 .4113850 .769551

C 7.1502243 .2979012 .249052

C 6.1469164 .0880323 .059623

C 6.4425375 .3161373 .518060

C 4.8363653 .4195373 .408497

C 3.8948363 .3477962 .238592

N $5.4742271 .685656-1.951157$

H $-2.4763902 .978188-3.291539$

H $-2.4306201 .353326-0.694767$

H $-0.6603611 .353105-3.245457$

H -2.440131 -0.485332 -3.305257

H -1.863688 -1.039696 -1.745279

H $\quad 0.691876-3.113853-3.537073$

H $\quad 0.490815-1.674409-5.302823$

H $-0.983236-0.708790-5.378129$

H $0.501742-0.025472-4.675444$

H -1.396757 -3.076405-1.344921

H $-0.280807-4.337940-1.777015$

H $\quad 0.447777-1.777729-0.266520$

H $\quad 0.180452-3.3705800 .445365$

H $2.100931-4.255073-0.957015$

H $2.407310-3.6235281 .464784$ 
H $2.680335-1.9586560 .998421$

H $4.632424-3.1622402 .421731$

H $4.832451-1.415171-0.462855$

H $4.306811-5.2401331 .379986$

H $5.710543-4.9363380 .382672$

H $4.144295-5.282050-0.377410$

H $6.700131-2.672439-0.245054$

H $5.311577-1.996015-3.568633$

H $3.617932-1.882437-3.975806$

H $3.814604-4.007711-3.419803$

H $1.925457 \quad 0.4008700 .128924$

H 2.2953843 .4569850 .208445

H $4.1805180 .307118 \quad 1.093701$

H 2.9554100 .2970752 .385943

H $4.615406 \quad 0.7857122 .715047$

H $3.9845590 .328759-3.754573$

H $3.5213120 .003637-2.081948$

H $6.3982613 .523305-1.831494$

H $7.5081032 .151129-2.049675$

H $6.859604 \quad 1.3527110 .355194$

H $6.880170 \quad 4.4156960 .353222$

H 8.1648403 .6638082 .454648

H 7.1420662 .2512862 .577928

H 5.7396795 .8784424 .123710

H 7.3930925 .7886663 .293109

H 4.3242443 .9841154 .199361

H 5.0420602 .4425513 .854268

H 3.5525294 .3258081 .897125

H $4.5452912 .085751-1.856010$

C $-5.2230932 .901945-1.901474$

O $-5.3563411 .643010-1.207917$

C $-6.5871361 .645865-0.465839$

C $-7.4285292 .756855-1.077840$

C $-6.3609813 .787741-1.389910$

H $-6.334517 \quad 1.9235260 .564511$

C $-7.2266120 .255894-0.489550$

H -7.922762 2.431887 -2.000762

H $-8.1959643 .125682-0.390930$

C $-6.8081684 .847608-2.381606$

H $-6.0634214 .278950-0.453612$

C $-6.300687-0.870600 \quad 0.017699$

O $-7.586725-0.053556-1.842345$

H $-8.1630530 .270750 \quad 0.080303$

H -5.483845 -1.010054 -0.698697

O $-7.057009-2.088841-0.010522$

C $-5.727003-0.6682311 .435066$

C $-4.968238-1.8906921 .984124$

H $-5.066303 \quad 0.203791 \quad 1.458427$

O $-6.797212-0.4178652 .351902$

H -4.753495 -1.701910 3.042896

H -5.641152 -2.7570431 .989925$

H -5.998261 $5.554199-2.588270$

H -7.117627 $4.402033-3.333005$

H -7.657098 $5.412956-1.983478$

C -3.838392 $3.480641-1.613207$

H -7.984399 $-0.941139-1.840491$

H -7.762234 -2.010967 0.654864

H $\quad-7.1558190 .4642602 .158867$

H -3.661787 $3.500164-0.530388$

H -3.770705 $4.515507-1.967421$

H $-5.3436602 .691947-2.971928$

C $-2.131366-0.8817082 .524466$

O $-2.725799-1.1510561 .247797$

C -3.668148 -2.229375 1.227690

C $-2.993769-3.5232821 .704712$

C $-2.353013-3.3508893 .076899$

C -1.436937 -2.130372 3.088063

H -2.901109 -0.5323923 .222884$

C - $-1.104106 \quad 0.2459382 .330368$

H -3.933897 -2.3848290 .175925$

O $-3.925763-4.598574 \quad 1.755940$

H -2.213217 -3.8128160 .990960$

H -3.107473 -3.284135 3.869494

O $-1.561158-4.503673 \quad 3.373708$

H -0.531296 -2.349249 2.509187

O $-1.025356-1.8699864 .429592$

C -1.740555 1.6030132 .120698

H -0.4631420 .3315013 .215027$
H $-0.4843030 .031891 \quad 1.452663$

H -4.313443 -4.691419 0.868900

H $-2.148377-5.277643 \quad 3.323865$

H $-0.604894-2.6798804 .766383$

O -0.7467852 .5315531 .987164$

O -2.9416621 .8262792 .075957$

C -1.1749563 .8803021 .777702$

H -1.8901944 .1848142 .547897$

H -0.2964844 .5281911 .841334$

$\mathrm{H}-1.6122903 .9802940 .781122$

SCF Energy $(B 3 L Y P / 6-31 G * * / / M M F F)=-3245.91417853$

01_30

MM̄FF Geometry

C $-3.891611-3.0339431 .986125$

C $-2.796634-2.5419501 .389770$

C -1.585472 -2.005059 2.098107

O $-0.458285-2.6764091 .488688$

C -1.490027 -0.4774201 .887055$

C -1.7422240 .3245323 .148612$

C -0.8785331 .2052373 .690225$

C -3.1049460 .1464313 .772515$

C $0.486151 \quad 1.6041023 .202214$

C 0.4634543 .0587092 .715321

C 1.8444003 .5466072 .258804

C 1.7882525 .0319421 .893614

C 3.1307435 .5399281 .349374

O $2.2410852 .779904 \quad 1.117377$

O 2.9047236 .8207050 .741294

C $3.6065074 .587613 \quad 0.233937$

C 4.1536925 .7653672 .468815

O $4.9197874 .931564-0.202817$

C 3.5533583 .0927130 .628766

C $3.8277962 .220003-0.617871$

O 4.5577082 .8311051 .610760

C $0.731094-2.6525132 .144474$

O $1.000598-2.0403193 .164403$

C $1.651427-3.5409911 .399172$

C $2.954616-3.5796981 .712990$

C $3.966473-4.4284271 .076395$

C $3.483045-5.4755430 .106088$

C $3.5766130 .721285-0.409059$

C $3.787579-0.068505-1.689801$

C $3.668472-2.407372-2.550927$

O $4.1908510 .434231-2.734729$

C $4.904521-3.228992-2.327044$

C $5.961740-2.912332-1.561756$

C $7.136531-3.823670-1.346732$

C $7.420744-4.0701040 .120100$

C $8.516605-3.5593460 .706628$

C $6.476348-4.9721450 .887008$

C $5.263994-4.2362881 .395383$

N $3.482098-1.409811-1.515113$

H $-3.949138-3.0842323 .069434$

H -2.784008 -2.478052 0.304322

H $-1.608967-2.2787923 .160249$

H -2.235786 -0.144361 1.153067

H $-0.525130-0.2263801 .438194$

H $-1.189525 \quad 1.7300124 .593550$

H $-3.2973180 .876419 \quad 4.566247$

H -3.8884830 .2758153 .018186$

H -3.203141 -0.8492844 .214204$

H 1.1903991 .5051734 .037210

H 0.8560230 .9460132 .412486

H -0.2472353 .1391161 .882746$

H 0.0972343 .7082403 .520752

H 2.5573443 .3784863 .074610

H 1.4719125 .6337032 .754684

H 1.0210965 .1877821 .121690

H 2.5340077 .4126381 .418150

H $2.9434524 .739862-0.629439$

H 3.7781916 .5040443 .187221

H 5.0871236 .1808412 .073307

H 4.3887414 .8556063 .025187

H $4.9041585 .870560-0.455786$

H $3.1819542 .555642-1.439031$

H $4.8657042 .359193-0.946653$

H $4.7853471 .888737 \quad 1.589727$ 
H $1.223953-4.1491490 .609657$ H $3.319507-2.9245792 .502945$ H $2.945917-5.014888-0.729696$ H $2.807807-6.1771890 .608607$ H $4.285505-6.070727-0.333442$ H 4.2582590 .3168680 .346765 H $2.5484830 .549783-0.070630$ H $3.703579-1.935240-3.537513$ H $2.789855-3.059451-2.510550$ H $4.907847-4.191542-2.835293$ H $6.000265-1.950635-1.056991$ H $8.007511-3.363398-1.830746$ H $6.990156-4.788883-1.847298$ H $8.739914-3.7522901 .750721$ H $9.212175-2.9329920 .158111$ H $6.990902-5.3919651 .762044$ H $6.228494-5.8383370 .268195$ H $5.505903-3.4555472 .118472$ H $3.197428-1.734323-0.595710$ C $-6.138085-2.3631571 .120158$ O $-5.575011-1.2238130 .437351$ C $-6.553864-0.683424-0.465945$ C $-7.866210-1.389431-0.140142$ C -7.376880 -2.750202 0.315621 H -6.239605 $-0.968487-1.476922$ C $-6.6031600 .842479-0.344770$ H -8.407654 $-0.890450 \quad 0.671827$ H -8.534342 -1.440226 -1.005434 C -8.408375 -3.5351961 .106598$ H -7.084795 -3.330644 -0.569838 C $-5.2465661 .550301-0.553345$ O $\quad-7.062694 \quad 1.176803 \quad 0.972474$ H -7.352622 $1.239246-1.040049$ H -4.5856211 .3218290 .290165$ O $-5.4954682 .961732-0.481677$ C $-4.5303411 .230736-1.881463$ C -3.354161 2.174072 -2.209907 H $-4.1841470 .191967-1.869945$ O $\quad-5.4815341 .347106-2.944624$ H $-3.0086771 .942535-3.224364$ H $-3.7316713 .201737-2.275946$ H -8.005057 -4.502678 1.421626 H -8.723359 -2.992992 2.004520 H -9.298709 -3.7227730 .497560$ C $-5.094371-3.478010 \quad 1.201025$ H -7.0910782 .1471881 .031631$ H -6.050255 $3.200074-1.244526$ H $-5.041551 \quad 1.062756-3.763963$ H -4.784509 -3.7704880 .189398$ H -5.516006 -4.370537 1.676377 H $-6.416538-2.0256222 .127202$ C $-0.815000 \quad 0.451138-2.357781$ O $-1.5786180 .797529-1.196458$ C $-2.1806172 .095612-1.214893$ C -1.107215 $3.174068-1.418799$ C $-0.2694752 .907889-2.663021$ C $\quad 0.2842731 .487206-2.623457$ H -1.478286 $0.376746-3.227489$ C $-0.187973-0.929550-2.100320$ H $-2.5915112 .257164-0.211866$ O $-1.6909724 .468629-1.512805$ H - $0.4362393 .188191-0.550663$ H $-0.8392193 .085790-3.582475$ O $\quad 0.8338593 .816269-2.689166$ H $1.0490751 .422437-1.841931$ O $0.9170801 .194825-3.867116$ C -1.192591-2.058165 -2.178686 H $\quad 0.582627-1.143085-2.849265$ H $0.260448-0.952167-1.099741$ H -2.207084 $4.615577-0.701762$ H $0.4675794 .716904-2.660175$ H $1.6115211 .862434-4.002722$ O $-0.554968-3.230276-1.881565$ O $-2.373387-1.938535-2.472630$ C $-1.362258-4.409699-1.948137$ H $-0.743907-5.260906-1.650828$ H -2.209051 -4.335708 -1.260076 H $-1.710933-4.568862-2.972798$
SCF Energy (B3LYP/6-31G**//MMFF) $=-3245.91956960$

01300

MMFF Geometry

C $-0.436978-2.6770241 .196405$

C -1.301009-2.730982 2.222619

C $-1.582524-1.6385513 .227686$

O $-0.532289-0.6419923 .228915$

C -2.898905 -0.903481 2.915450

C $-4.144352-1.7375753 .119682$

C $-4.833330-2.3441142 .133185$

C $-4.617078-1.8411184 .546551$

C $-4.511987-2.3748020 .664236$

C $-5.644567-1.808860-0.201333$

C $-5.857525-0.295270-0.037056$

C $-7.0375270 .162262-0.898578$

C $-7.2222471 .684735-0.860936$

O $-4.6591350 .370576-0.448310$

O $-8.1198932 .047433-1.921221$

C $-5.8679172 .354648-1.166775$

C -7.8804492 .1493210 .443412$

O $-5.9582023 .769619-1.009000$

C $-4.6965071 .799868-0.322553$

C $-3.3581892 .352567-0.871443$

O -4.8612822 .2074921 .035552$

C $0.504412-0.8344324 .085344$

O $0.648819-1.7757794 .854549$

C 1.5157410 .2418433 .989183

C 1.3563621 .3780433 .293235

C 2.3373492 .4639823 .198247

C 3.7145402 .2120143 .756376

C $-2.1207201 .923555-0.072259$

C $-0.8246681 .973786-0.859806$

C $0.5177333 .382124-2.419160$

O $-0.0073441 .057981-0.779633$

C $1.5159234 .287254-1.761924$

C $1.4461034 .832489-0.537447$

C 2.5001735 .7389520 .029529

C 3.3563655 .0655321 .078428

C 4.6293844 .7290770 .810566

C 2.7815734 .8823292 .464642

C 1.9701633 .6234082 .612931

N $-0.6498383 .132579-1.595133$

H $\quad 0.153050-1.7811201 .024684$

H -1.884379 -3.640345 2.355990

H -1.650852 -2.088704 4.225735

H -2.860863 -0.4924371 .902104$

H $-2.968327-0.0091303 .550712$

H -5.721002 -2.913553 2.406261

H -5.535839 -2.429950 4.639385

H $-3.855414-2.3195185 .170020$

H -4.822964 -0.844707 4.951002

H -3.575974 -1.862821 0.427200

H $-4.357660-3.4247620 .384463$

H -5.392036 -2.022049-1.248416

H $-6.578009-2.3418190 .019369$

H -6.050258 -0.0714731 .018087$

H -7.961640 -0.344209-0.592688

H $-6.864504-0.137802-1.941883$

H $-8.9565021 .571451-1.781663$

H $-5.6435502 .175219-2.227871$

H -8.8691841 .6889120 .558208$

H $-8.0536793 .231108 \quad 0.435104$

H -7.2966491 .8996191 .331709$

H -6.696216 $4.076219-1.563155$

H $-3.2399872 .021220-1.911559$

H -3.390409 $3.448980-0.876916$

H -4.0231142 .0772251 .506295$

H 2.4206420 .0488034 .557765

H $\quad 0.4215201 .5465612 .761724$

H 4.1432471 .2995853 .326123

H 3.6749192 .1041414 .845732

H 4.4253193 .0115583 .537819

H -1.997403 $2.545950 \quad 0.820914$

H $-2.2369890 .886557 \quad 0.257632$

H $0.1623563 .843083-3.346184$

H $0.9994932 .432802-2.669032$

H $2.3913264 .507714-2.370655$ 
$\begin{array}{llll}H & 0.582617 & 4.643613 & 0.092999\end{array}$ H 2.0150506 .6223260 .463044 H $3.1317716 .128960-0.780076$ H $5.267313 \quad 4.2797661 .563907$ H $5.0623624 .891251-0.170881$ H 2.1390265 .7386182 .707681 H $3.5786114 .927863 \quad 3.215412$ H $0.951849 \quad 3.7086302 .234197$ H $-1.3304083 .881467-1.516291$ C $-0.737133-3.435515-1.175761$ O $0.210813-2.545774-1.798403$ C $0.410075-2.956003-3.166700$ C $-0.694448-3.960254-3.468426$ C $-0.859287-4.632285-2.118991$ H $1.372939-3.478704-3.200042$ C $0.449351-1.731121-4.090510$ H - $1.627413-3.465245-3.761649$ H $-0.416702-4.656439-4.265582$ C $-2.165169-5.396141-1.979461$ H $-0.018748-5.321850-1.962538$ C $1.452429-0.649736-3.643590$ O $-0.849430-1.122750-4.114107$ H $0.657987-2.042324-5.120055$ H $1.126183-0.244097-2.683195$ O $1.4018820 .433958-4.578604$ C $2.909368-1.130615-3.548362$ C $3.875913-0.073982-2.977261$ H $2.972849-2.026917-2.926761$ O $3.369595-1.512809-4.846114$ H $4.897918-0.457171-3.088173$ H $3.8467500 .833030-3.593966$ H - $-2.247760-5.852976-0.988295$ H -3.030828 -4.740001-2.120164 H -2.224448 -6.195485 -2.725343 C $-0.247632-3.8115360 .224724$ H - $-1.080211-0.887472-3.198728$ H $0.475240 \quad 0.723927-4.635203$ H $3.294802-0.733910-5.423928$ H $\quad 0.819878-4.060758 \quad 0.199802$ H $-0.781492-4.6999120 .581707$ H -1.691835 -2.895949 -1.120839 C $4.581706-1.657357-0.470618$ O $3.395136-0.892169-0.700553$ C $3.5947770 .278633-1.500187$ C $4.6336581 .199372-0.845294$ $\begin{array}{llll}\text { C } & 5.926248 & 0.465245 & -0.492097\end{array}$ C $5.628364-0.8244090 .275538$ H $5.001787-1.965032-1.436731$

C $4.196985-2.9666540 .230890$ H $2.645270 \quad 0.824377-1.483370$ O $4.9410332 .300068-1.694022$ H $4.212144 \quad 1.6127100 .080493$ H $6.5250600 .257083-1.386873$ $\begin{array}{lllll}\text { O } & 6.729074 & 1.305704 & 0.340626\end{array}$ H $5.284914-0.5709951 .285013$ O $6.827687-1.5825220 .408949$ C $3.548916-2.7965411 .587109$ H $3.471735-3.504389-0.392393$ H $5.088922-3.5926230 .347547$ H $4.1082322 .766467-1.878652$ H $6.8695892 .139365-0.140425$ H $7.482386-1.0138940 .849383$ O $3.526594-4.008362 \quad 2.217796$ O $3.089109-1.7526172 .025941$ C $2.908195-4.0250933 .507948$ H $3.324915-3.2433434 .150369$ H $1.827071-3.903663 \quad 3.399641$ H $3.107796-4.9966353 .967679$ SCF Energy (B3LYP/6-31G**//MMFF)= -3245.92153090

01301

MMFF Geometry

C $-2.629229-1.643841-2.595865$

C $-2.105179-2.796872-3.041662$

C $-0.788948-3.399859-2.615130$

O $0.042519-2.433433-1.932781$

C $-0.943431-4.643354-1.710120$

C $-1.957626-4.539487-0.586097$
C -1.696957 -4.0798710 .652506$

C $-3.330793-5.085109-0.898205$

C $-0.394088-3.587827 \quad 1.213423$

C $-0.518436-2.1594481 .761878$

C $0.614842-1.8351032 .745558$

C $0.463108-0.4242793 .311613$

C $1.603963-0.0784284 .280028$

O $1.869910-1.9476402 .067821$

$\begin{array}{lllll}\text { O } & 1.570520 & 1.337067 & 4.515885\end{array}$

C $2.950647-0.3905123 .596117$

C $1.420677-0.7500725 .645820$

O $4.035055-0.2410244 .510159$

C $3.007133-1.7872392 .929349$

C $4.288688-1.8973102 .073111$

O $3.043876-2.7930013 .944093$

C $0.913237-1.719659-2.699570$

O $1.091936-1.828232-3.902939$

C $1.602532-0.745581-1.824609$

C $2.4346100 .148364-2.380669$

C $3.1620251 .212532-1.687037$

C $2.8740991 .414682-0.224453$

C $4.342573-3.125719 \quad 1.157245$

C $5.573380-3.0446520 .277088$

C $6.482367-1.803993-1.687609$

O $6.609698-3.6408110 .560965$

C $6.854974-0.392732-1.352481$

C $6.9587930 .585321-2.264762$

C $7.2953002 .009049-1.921881$

C $6.3102323 .002324-2.502189$

C $6.6769573 .842831-3.484598$

C $4.9204673 .072623-1.898370$

C $4.0260271 .968566-2.396306$

N $5.396349-2.203563-0.811361$

H $-2.083506-1.039358-1.876349$

H -2.684268 $-3.394920-3.742656$

H $-0.266552-3.737570-3.519684$

H $0.036790-4.907651-1.293992$

H $-1.225067-5.498212-2.340406$

H $-2.510355-4.0925991 .377986$

H $-4.025761-4.970343-0.059307$

H $-3.775949-4.578476-1.757818$

H $-3.265551-6.154010-1.127198$

H $-0.115294-4.2711432 .025470$

H $0.417499-3.6295820 .481466$

H $-0.501198-1.4572450 .918728$

H $-1.480595-2.0289342 .273681$

H $\quad 0.574570-2.5733353 .555449$

H $-0.509498-0.2988443 .803033$

H $0.4815820 .297922 \quad 2.484758$

H $0.6891191 .563114 \quad 4.859658$

H 3.0993860 .3695292 .817069

H $0.468994-0.4449566 .097432$

H $2.199661-0.4343956 .348735$

H $1.426527-1.8407175 .591741$

H 3.9717070 .6514194 .891326

H $4.379429-1.0031921 .446311$

H $5.171771-1.9121632 .725283$

H $3.443325-3.5969823 .576468$

H $1.386199-0.769910-0.763102$

H $2.6000670 .103951-3.456616$

H 3.1931880 .5423470 .349914

H $1.8011091 .565635-0.061106$

H 3.3724002 .2876610 .202497

H $4.393644-4.0552151 .733453$

H $3.454735-3.1902030 .517869$

H $6.124090-1.903416-2.716911$

H $7.343758-2.463209-1.544449$

H $7.040255-0.162984-0.305487$

H $6.7967190 .352505-3.314819$

H $8.3053722 .211432-2.300723$

H $7.3460342 .154956-0.835610$

H $5.9849424 .572953-3.891122$

H $7.6754263 .817173-3.908168$

H $4.4470824 .027554-2.160552$

H $5.023823 \quad 3.071607-0.809264$

H $4.1172421 .775698-3.466446$

H $4.502434-1.736149-0.931754$

C $-4.969875-1.323567-1.815835$ 
O $-4.654182-0.381414-0.773723$

C $-5.879710 \quad 0.023033-0.129054$

C $-6.995712-0.794650-0.772007$

C $-6.430926-1.051761-2.157810$

H $-6.0349651 .077103-0.383912$

C $-5.743750-0.139324 \quad 1.391874$

H $-7.162586-1.741080-0.244675$

H -7.946432 $-0.252859-0.789485$

C -7.122688 -2.189005 -2.888759

H -6.513716 - $0.131438-2.751268$

C $-4.5243520 .600478 \quad 1.983489$

O $-5.596179-1.5320231 .700971$

H $-6.665490 \quad 0.1828251 .888158$

H $-3.613623 \quad 0.1348151 .592649$

$\begin{array}{llll}\text { O } & -4.499904 & 0.378391 & 3.398053\end{array}$

C -4.5040752 .1173061 .718118$

C -3.245232 2.8273392 .248717

H -4.5852102 .3202520 .646887$

$\begin{array}{lllll}\text { O } & -5.632018 & 2.734433 & 2.342683\end{array}$

H -3.371549 3.9037272 .075685

H -3.190116 2.723078 3.339661

H -6.683387 -2.343156 -3.878974

H $-7.042040-3.130221-2.334619$

H -8.186716 -1.968247 -3.023430

C $-3.997460-1.150626-2.983171$

H $-4.802863-1.854041 \quad 1.239133$

H -5.2883650 .8052683 .774956$

H -6.4309152 .4250021 .883765$

H -3.913683 $-0.093356-3.261514$

H $-4.358865-1.694872-3.862672$

H $-4.867700-2.327023-1.381211$

C -2.143197 $3.410501-0.541036$

O -2.0612292 .1822460 .187464$

C -1.9330692 .3376391 .604318$

C $-0.690964 \quad 3.178083 \quad 1.937094$

C $-0.6474294 .496367 \quad 1.162953$

C $-0.883358 \quad 4.256022-0.329519$

H -3.011522 $3.980567-0.186095$

C $-2.4445653 .085574-2.011401$

H $-1.749257 \quad 1.3337252 .004074$

O -0.6012353 .4234863 .336611$

H 0.2019942 .6005641 .667822

H -1.369923 5.2192661 .559278

O $\quad 0.642970 \quad 5.090395 \quad 1.321650$

H $-0.0019523 .765936-0.758907$

\begin{tabular}{lllll}
\hline & -1.040120 & 5.508978 & -0.990368
\end{tabular}

C $-1.398367 \quad 2.232148-2.693378$

H -3.384814 $2.522853-2.065305$

H $-2.5602364 .016750-2.577166$

H $-1.287847 \quad 4.068288 \quad 3.576567$

H $0.799741 \quad 5.1916232 .276281$

H $-0.2420316 .034402-0.808357$

O $-1.6316992 .232198-4.039201$

O $-0.509754 \quad 1.616360-2.123339$

C $-0.731605 \quad 1.437546-4.818105$

H $-0.985847 \quad 1.571414-5.872869$

H $0.3009121 .764914-4.662439$

H $-0.8457060 .380638-4.560992$

SCF Energy (B3LYP/6-31G**//MMFF) $=-3245.91227664$

01_302

MMFF Geometry

C $2.475140-3.4910552 .175866$

C $1.618657-3.465921 \quad 1.143281$

C $0.118766-3.4586031 .306839$

O $-0.420759-2.3270150 .587594$

C $-0.464216-4.741573 \quad 0.684535$

C -1.959264 -4.8998240 .879578$

C $-2.861110-4.944513-0.120638$

C $-2.421805-5.0499382 .306490$

C $-2.614328-4.848496-1.600432$

C $-2.709054-3.426035-2.174620$

C $-4.097713-2.781189-2.033143$

C $-4.166082-1.465358-2.808871$

C $-5.521346-0.764015-2.623503$

O $-4.333104-2.516635-0.650469$

O $-5.3842940 .586858-3.094873$

C $-5.858684-0.680379-1.115125$
C $-6.607550-1.411765-3.491506$

O $-7.192653-0.209959-0.941517$

C $-5.640751-2.008606-0.351836$

C $-5.713980-1.8711951 .185801$

O $-6.653741-2.969373-0.675185$

C $-0.391339-1.117466 \quad 1.210194$

O $0.045434-0.8715232 .323121$

C $-0.978640-0.125948 \quad 0.281236$

C $-0.883398 \quad 1.181788 \quad 0.562371$

C $-1.3873742 .283306-0.261222$

C $-2.2540911 .929489-1.439627$

C $-4.712074-0.8945541 .810783$

C $-5.2861790 .487214 \quad 2.075359$

C -4.6249142 .7268952 .942332$

O $\begin{array}{lllll}-6.468883 & 0.771157 & 1.907059\end{array}$

C -4.4146513 .6368311 .774161$

C $-3.644653 \quad 4.7340701 .826994$

C -3.4576945 .6818770 .677947$

C $-2.004523 \quad 5.854956 \quad 0.300233$

C -1.3019206 .9230220 .713891$

C $-1.391503 \quad 4.829482-0.626945$

C -1.0483453 .5448750 .076188$

N $-4.320791 \quad 1.3622612 .554915$

H $2.061871-3.4878183 .183182$

H $1.989067-3.4653440 .122296$

H $-0.161782-3.3910502 .364894$

H $0.025712-5.621763 \quad 1.122643$

H $-0.213692-4.755558-0.381741$

H $-3.910103-5.0727380 .145103$

H -3.480789 -5.3219382 .373358$

H $-1.851424-5.8368812 .810665$

H -2.289882 -4.1131102 .856238$

H - $1.638251-5.272501-1.858768$

H $-3.345607-5.490581-2.108057$

H $-1.956971-2.796210-1.684719$

H $-2.444382-3.477295-3.238081$

H $-4.847953-3.488288-2.405710$

H -3.962445 -1.629192 -3.874385

H $-3.374125-0.792476-2.453798$

H $-5.0933080 .553496-4.022219$

H $-5.1974350 .087619-0.696245$

H $-6.346034-1.332983-4.553859$

H -7.565693 -0.892520 -3.381830

H $-6.757000-2.470822-3.270721$

H $-7.324715-0.0306460 .004423$

H $-6.741767-1.6535611 .500160$

H $-5.493759-2.8638731 .605697$

H $-7.310506-2.548419-1.252194$

H $-1.457107-0.498365-0.616974$

H $-0.3684591 .479798 \quad 1.474244$

H -2.664758 $2.796223-1.960262$

H $-3.1129001 .334201-1.112359$

H $-1.685697 \quad 1.353425-2.177855$

H $-4.407218-1.2911862 .787866$

H -3.809774 -0.8030581 .198251$

H $-3.9745562 .977598 \quad 3.786200$

H $-5.6664642 .787809 \quad 3.274072$

H -4.939675 3.3976340 .852438

H -3.140016 4.9843672 .757587

H -3.887268 6.6489950 .970410

H $-4.0273675 .361647-0.203276$

H $-0.266073 \quad 7.0652630 .423665$

H -1.7454797 .6758581 .357100$

H $-0.4610085 .224884-1.053961$

H -2.059681 4.687513 -1.479936

H $-0.409014 \quad 3.6868750 .948379$

H $-3.352858 \quad 1.0579582 .605495$

C $4.621871-3.6570490 .736570$

O $4.501069-2.3998150 .040359$

C $5.715273-2.161678-0.697622$

C $6.547619-3.433960-0.578777$

C $6.121531-3.947708 \quad 0.781273$

H $6.245835-1.354203-0.180645$

C $5.397703-1.754697-2.140242$

H $6.292828-4.160201-1.359781$

H $7.621395-3.233674-0.646616$

C $6.468872-5.4088991 .008509$

H $6.601026-3.3356291 .556971$ 
C $4.573084-0.456108-2.266981$

O $4.655123-2.817536-2.750754$

H $6.332520-1.664089-2.707089$

H $3.558096-0.611944-1.883070$

O $4.442740-0.159191-3.663016$

C $5.2106410 .765211-1.577525$

C $4.5832582 .121365-1.940508$

H $5.1736060 .633504-0.493021$

$\begin{array}{llll}\text { O } & 6.597781 & 0.839928 & -1.927266\end{array}$

H $5.1742372 .896540-1.437044$

H $4.7341392 .314274-3.010334$

H $6.140249-5.7411631 .997894$

H $5.995113-6.0549230 .261737$

H $7.551471-5.560590 \quad 0.945303$

C $3.980628-3.5473302 .122078$

H $4.638201-2.650747-3.708086$

H $3.844804-0.821815-4.048217$

H $6.6525480 .918772-2.895218$

H $4.358759-2.6484112 .624006$

H $4.284269-4.4090622 .728141$

H $4.114317-4.4193650 .131236$

C 3.3533032 .9342500 .720746

O $2.8082821 .995224-0.212806$

C $3.0903762 .272181-1.587388$

C $2.5130263 .643240-1.959939$

C $3.0599194 .744011-1.055062$

C $2.883182 \quad 4.367217 \quad 0.415375$

H 4.4484792 .8790690 .698065

C 2.8828012 .5240692 .126715

H $2.5429981 .523339-2.171945$

O $2.8004803 .942290-3.322081$

H $1.4209743 .609099-1.863880$

H $4.1073624 .965186-1.290394$

O $2.3285345 .942317-1.326637$

H $1.8279974 .479948 \quad 0.689403$

O $3.6263625 .275392 \quad 1.227657$

C 3.4527821 .2028602 .596948

H 3.1842083 .2853532 .855287

H 1.7915472 .4262442 .139080

H $2.4379184 .825378-3.508241$

H $2.7370736 .659430-0.813379$

H 3.2370616 .1588021 .115322

O $3.067542 \quad 1.0025743 .893160$

$\begin{array}{lllll}\text { O } & 4.130732 & 0.439781 & 1.924557\end{array}$

C $3.514945-0.2143964 .498142$

H $3.339769-0.1424415 .574814$

H $2.935584-1.052804 \quad 4.103649$

H $4.585180-0.3681574 .329480$

SCF Energy (B3LYP/6-31G**//MMFF) $=-3245.91092712$

01_303

MMFF Geometry

C $2.993310-2.9562811 .418604$

C $2.313897-2.2100932 .302472$

C $2.445088-0.7120792 .350055$

$\begin{array}{llll}\text { O } & 1.129891 & -0.111730 & 2.322617\end{array}$

C $3.139125-0.2559693 .643362$

C $3.782278 \quad 1.1082253 .472929$

C 5.0732381 .2979643 .133716

C 2.8842302 .2874673 .737561

C 6.1054560 .2401642 .861699

C 6.9321870 .4663611 .587444

C $\begin{array}{llll}6.156123 & 0.228046 & 0.279749\end{array}$

C $7.1458360 .036390-0.874502$

C $6.436460-0.110045-2.226041$

$\begin{array}{lllll}\text { O } & 5.321659 & 1.362671 & 0.024405\end{array}$

O $7.428590-0.019862-3.260275$

C $5.4485641 .061850-2.395735$

C $5.810930-1.500535-2.387140$

O $4.688206 \quad 0.876602-3.588276$

C $4.5257161 .268192-1.168530$

C $3.7378652 .593264-1.320806$

O $3.6045310 .179804-1.105176$

C $0.539367 \quad 0.047952 \quad 1.108635$

$\begin{array}{lllll}\text { O } & 0.922427 & -0.391031 & 0.036626\end{array}$

C $-0.638517 \quad 0.919803 \quad 1.302389$

C $-1.126027 \quad 1.594344 \quad 0.250909$

C -2.2605692 .5191810 .265028$
C $-2.985126 \quad 2.715163 \quad 1.571341$

C $2.9656723 .027529-0.067759$

C $2.2520554 .354395-0.273677$

C $0.948873 \quad 6.0663840 .985039$

O $2.2599934 .963273-1.340413$

C $-0.531640 \quad 5.877155 \quad 0.889669$

C $-1.2777506 .382451-0.104525$

C $-2.7762426 .295203-0.166296$

C $-3.2729495 .515588-1.362296$

C $-3.3804936 .093092-2.570787$

C $-3.7333044 .095304-1.130591$

C $-2.5924153 .146081-0.883377$

N 1.6156004 .7827230 .882805

H $3.677256-2.4631740 .731943$

H $1.635216-2.6835723 .006526$

H $3.018037-0.3516971 .486769$

H $3.895605-0.9845253 .956667$

H $2.418968-0.2084374 .471237$

H $5.4406512 .320844 \quad 3.063979$

H 2.5002912 .2492194 .762117

H 3.4054423 .2429863 .615971

H 2.0351442 .2875583 .048666

H 6.7972090 .2407343 .713747

H $5.671081-0.7627072 .817342$

H 7.3471981 .4824331 .596814

H $7.779537-0.2299171 .631272$

H $5.530256-0.6639810 .395314$

H $7.796309-0.828447-0.692214$

H $7.8152250 .907041-0.922929$

H $7.8681820 .844127-3.186419$

H $6.0471651 .971399-2.543988$

H $6.586073-2.275035-2.337203$

H $5.340833-1.612215-3.370549$

H $5.066708-1.730794-1.622052$

H $4.2372461 .713540-3.788716$

H $4.4404433 .399069-1.571577$

H $3.0265552 .509529-2.151995$

H $2.7680790 .488026-0.723768$

H -1.0455091 .0070732 .302963$

H $-0.6418141 .466191-0.716739$

H -2.296716 3.0804502 .341545

$\begin{array}{llll}H & -3.425548 & 1.773366 & 1.914957\end{array}$

H -3.7964913 .4433591 .516491$

H 2.2154162 .2805560 .203896

H 3.6558943 .1478990 .774266

H $1.328194 \quad 6.743151 \quad 0.212157$

H $1.203523 \quad 6.490247 \quad 1.961697$

H -1.0149895 .3429181 .703630$

H $-0.7880946 .934777-0.903847$

H $-3.1721347 .318774-0.209143$

H $-3.187246 \quad 5.8682810 .756625$

H -3.766031 $5.548708-3.426366$

H $-3.0812337 .123824-2.730402$

H $-4.2756123 .723620-2.010622$

H $-4.4751954 .084602-0.326923$

H $-1.9968312 .946120-1.775328$

H $\quad 1.629445 \quad 4.189308 \quad 1.706764$

C $2.180184-4.933094 \quad 0.043921$

O $0.778553-4.609405 \quad 0.121275$

C $0.319561-4.243916-1.195084$

C $1.443728-4.616102-2.158691$

C $2.667355-4.377003-1.294481$

H $0.207878-3.152919-1.193039$

C $-1.032309-4.899265-1.495575$

H $1.393625-5.668512-2.460584$

H $1.430766-4.004496-3.065909$

C $3.930745-5.036854-1.815878$

H $2.828060-3.293742-1.239418$

C $-2.106770-4.664526-0.414459$

O $-0.839698-6.315687-1.605776$

H $-1.390844-4.561195-2.475215$

H $-1.851261-5.2358230 .485900$

O $-3.333665-5.228425-0.897565$

C $-2.342100-3.190559-0.036681$

C $-3.500025-3.038718 \quad 0.970714$

H -1.427131 - 2.7795920 .404336

O $-2.619773-2.457714-1.226953$

H $-4.439816-3.3238620 .486451$ 
H $-3.332214-3.7399931 .796635$ H $4.775274-4.840356-1.147651$ H $3.811946-6.122047-1.901139$ H $4.186872-4.647874-2.806896$ C $2.899255-4.4499141 .306293$ H -1.706510 -6.711686-1.800127 H $-3.618307-4.698258-1.661795$ H -2.635661 -1.514271 -0.992783 H $3.917367-4.8563041 .325987$ H $2.379212-4.8512992 .185220$ H $2.231681-6.0301840 .028951$ C $-5.171350-0.673821-0.063382$ O $-3.866415-0.6294430 .528065$ C $-3.621692-1.6078611 .540696$ C $-4.649996-1.4578282 .670569$ C $-6.083221-1.4916802 .147115$ C $-6.257461-0.4886621 .005506$ H $-5.304701-1.636332-0.569908$ C $-5.2064430 .436242-1.124142$ H $-2.641245-1.3658301 .969639$ O $-4.483222-2.4757413 .652021$ H $-4.491013-0.4965153 .176736$ H $-6.365221-2.5052191 .838039$ O $-6.956431-1.1365893 .222037$ H $-6.2180190 .530816 \quad 1.410543$ O $-7.540892-0.6665880 .413605$ C $-6.2803890 .248235-2.170861$ H $-5.3408411 .406165-0.631131$ H $-4.2483090 .478529-1.656270$ H -3.571093 -2.415174 3.983849 H $-7.868713-1.2768922 .917341$ H $-8.208646-0.4279841 .078256$ O $-6.6425371 .468236-2.665875$ O $-6.720957-0.833162-2.536144$ C $-7.644811 \quad 1.445197-3.686710$ H $-7.2820660 .895509-4.560514$ H $-8.566710 \quad 0.997857-3.303248$ H $-7.8523902 .477047-3.982517$

SCF Energy $\left(B 3 L Y P / 6-31 G^{* *} / / M M F F\right)=-3245.90425124$

\section{4}

MM̄FF Geometry

C -1.903451-2.980045 -2.176015

C $-0.904187-3.501339-2.904129$

C $0.548787-3.078678-2.851950$

O $0.762944-2.046112-1.862576$

C $1.428360-4.286457-2.475945$

C $2.903121-3.959525-2.325884$

C $3.627919-4.245878-1.226110$

C $3.562593-3.295428-3.506399$

C $3.164076-4.981626-0.001558$

C $2.599785-4.0890701 .111544$

C $3.655027-3.1913581 .775468$

C $3.129759-2.6462973 .105093$

C $4.152922-1.7256483 .782593$

O $3.943840-2.0959890 .902413$

O $3.457104-1.0452654 .839213$

C $4.627464-0.6665742 .764359$

C $5.271931-2.5318274 .452824$

O 5.7243150 .0868843 .275783

C $4.993857-1.2383371 .369897$

C $5.134928-0.0776330 .363829$

O $6.254485-1.9113731 .382760$

C $0.658509-0.754701-2.278660$

O $0.396100-0.365539-3.405732$

C $0.9088040 .117470-1.108936$

C $0.7819561 .444584-1.253701$

C $1.0207352 .462233-0.230679$

C 1.3450942 .0020211 .164605

C $5.384167-0.524920-1.080174$

C $5.7151260 .679423-1.938700$

C $4.6990722 .768083-2.847911$

O $6.8670560 .918978-2.293821$

C $4.4945123 .812627-1.793403$

C $3.6481564 .845254-1.925770$

C $3.4089425 .871254-0.853384$

C $1.9390096 .057542-0.536008$

C $1.2929177 .180796-0.891905$
C 1.2301294 .9720490 .251699

C $0.9315913 .760227-0.587522$

N $4.6061801 .462695-2.218475$

H -1.697719 -2.170676 -1.481289

H -1.135872 -4.299589-3.606636

H $\quad 0.837623-2.717865-3.847056$

H $1.326309-5.068293-3.240093$

H $1.035644-4.712816-1.546251$

H $4.677834-3.957267-1.208704$

H $4.649862-3.229372-3.390711$

H $3.366355-3.865716-4.420219$

H $3.185603-2.276966-3.640145$

H $2.412663-5.732359-0.271733$

H $4.009674-5.5622740 .389285$

H $1.783610-3.4753850 .709628$

H $2.165785-4.7536061 .869094$

H $4.564752-3.7811931 .936493$

H $2.849061-3.4635973 .781331$

H $2.208077-2.0745062 .925546$

H $4.097947-0.4902285 .314854$

H 3.7983160 .0423732 .632943

H $4.859557-3.1680865 .245347$

H $5.996558-1.8738544 .944868$

H $5.810299-3.1795903 .757822$

H 5.4266110 .5279724 .088931

H 4.2213870 .5273890 .376799

H 5.9589520 .5790730 .672580

H $6.586083-1.9424742 .293364$

H $1.200278-0.349908-0.175426$

H $\quad 0.495683 \quad 1.832609-2.230795$

H 2.3266351 .5223001 .194201

H 0.5938961 .2877681 .520638

H 1.3543542 .8153201 .894598

H $6.223147-1.226490-1.141931$

H $4.504496-1.029889-1.493800$

H $3.9299662 .810177-3.625212$

H $5.6824222 .892073-3.311274$

H $5.0641073 .709119-0.872449$

H $3.0956324 .962768-2.855403$

H $3.8442166 .818380-1.197207$

H 3.9415805 .6132300 .070504

H $0.2457757 .331324-0.650495$

H $1.7945767 .973486-1.436872$

H 0.2779925 .3520820 .641263

H 1.8477134 .7301441 .122332

H $0.6271273 .992783-1.609147$

H $3.6993301 .178721-1.860110$

C $-3.879153-3.743262-0.853475$

O $-3.948329-2.528509-0.078624$

C $-5.132177-2.5635580 .739566$

C $-5.740389-3.9509510 .556287$

C $-5.301722-4.296084-0.853973$

H $-5.824807-1.8229990 .324363$

C $-4.785859-2.2044562 .189117$

H $-5.329736-4.6735251 .271063$

H $-6.827928-3.9439500 .678440$

C $-5.394366-5.778484-1.170766$

H $-5.923449-3.733887-1.563657$

C $-4.100929-0.8299312 .355403$

O $-3.893110-3.2049802 .695941$

H $-5.692106-2.2565272 .804548$

H -3.095770 -0.856987 1.918600

O $-3.928267-0.597361 \quad 3.759333$

C $-4.891790 \quad 0.353928 \quad 1.765742$

C $-4.358637 \quad 1.741823 \quad 2.170927$

H -4.9121550 .2720800 .673794$

$\begin{array}{llll}\text { O } & -6.247466 & 0.265926 & 2.216632\end{array}$

H -5.0579902 .4928951 .783932$

H -4.4187391 .8500943 .261217$

H $-5.063051-5.981628-2.193837$

H $-4.774473-6.373557-0.491735$

H $-6.427793-6.127806-1.075744$

C $-3.335002-3.434088-2.248940$

H $-3.852879-3.0995153 .661264$

H $-3.252513-1.2190494 .078150$

H -6.7504590 .9711821 .774847$

H -3.919480 -2.626937 -2.705129

H $-3.424455-4.315601-2.893356$ 
H $-3.201334-4.425103-0.322309$

C $-3.4815302 .813069-0.532851$

$\begin{array}{lllll}\text { O } & -2.776366 & 1.863687 & 0.276057\end{array}$

C -2.927433 $2.048128 \quad 1.686854$

C -2.4215723 .4428592 .083732$

C -3.104586 $4.543992 \quad 1.276573$

C -3.025822 $4.243071-0.219022$

H $-4.5591532 .716829-0.355815$

C -3.207227 $2.465832-2.000282$

H -2.262460 1.3197192 .165961

O $-2.6275223 .685243 \quad 3.471345$

H -1.340962 3.5027401 .905168

H -4.1442084 .6884071 .592779$

O -2.4391645 .7862051 .517148$

H $-1.9981304 .396540-0.568238$

O $-3.8543685 .163690-0.926544$

C $-3.7876281 .122180-2.367453$

H -3.622015 $3.212940-2.686095$

H -2.128196 $2.417527-2.188571$

H -2.175415 2.9774313 .961608

H -2.459236 5.9434262 .476848

H $-3.5551316 .059809-0.695083$

O $-5.148012 \quad 1.195667-2.417880$

O $-3.1087780 .127887-2.586510$

C $-5.818111-0.023870-2.750016$

H $-6.8919770 .177041-2.786244$

H -5.494336 $-0.381742-3.732040$

H -5.628569-0.778621 -1.981935

SCF Energy (B3LYP/6-31G**//MMFF) $=-3245.89986672$

01305

MMFF Geometry

C $3.188337-3.161437-0.118796$

C $2.469062-3.657654-1.136917$

C $1.171528-3.061946-1.637623$

O $0.491572-2.449003-0.518303$

C $1.485921-2.001594-2.710555$

C $0.285970-1.510960-3.494339$

C $-0.208881-0.257845-3.450439$

C $-0.332640-2.523375-4.426281$

C $0.2761830 .916351-2.645644$

C $-0.567609 \quad 1.221497-1.399833$

C $-1.9781931 .756834-1.695241$

C $-2.6634352 .196505-0.399144$

C $-4.1118202 .652964-0.634897$

O $-2.7485320 .723725-2.312739$

O $-4.765123 \quad 2.714575 \quad 0.643077$

C $-4.8557801 .587171-1.471991$

C $-4.169048 \quad 4.070837-1.217317$

O $-6.1446172 .076241-1.834977$

C $-4.065744 \quad 1.127754-2.717118$

C $-4.704228-0.073488-3.459153$

O $-4.0064522 .207785-3.657678$

C $-0.855762-2.583263-0.416873$

O $-1.616878-3.087556-1.225612$

C $-1.251286-2.0053630 .887078$

C -2.527477-1.657294 1.101675

C -3.065968-1.066035 2.327825

C $-2.175844-1.0359423 .541746$

C $-4.897354-1.342120-2.622864$

C $-6.268166-1.448927-1.974892$

C $-7.479408-2.690498-0.181733$

O $-7.236329-0.785093-2.336084$

C $-7.562461-1.7117680 .946498$

C $-7.372226-2.0354692 .234384$

C $-7.443481-1.0424903 .358804$

C $-6.115976-0.8617044 .062669$

C $-5.881369-1.4368155 .253970$

C $-5.0952210 .059326 \quad 3.430102$

C $-4.331584-0.5993252 .313719$

N -6.293693 -2.405473 -0.969589

H $2.837127-2.2715660 .397295$

H $2.842996-4.524750-1.676337$

H $0.567406-3.883697-2.039117$

H $2.202872-2.411231-3.435800$

H $2.000872-1.163453-2.229774$

H -1.057716 $-0.030282-4.094245$

H - $-1.138071-2.094273-5.032130$
H $\quad 0.422626-2.913668-5.116246$

H $-0.757883-3.361289-3.866987$

H $1.3147990 .776214-2.331490$

H $\quad 0.291593 \quad 1.797257-3.299734$

H $-0.6341270 .317234-0.786974$

H $-0.0273161 .969432-0.809057$

H $-1.8847822 .604796-2.383797$

H -2.088038 2.9886990 .096028

H $-2.677364 \quad 1.355854 \quad 0.306432$

H $-4.2627303 .326572 \quad 1.207889$

H $-5.0180290 .729608-0.807574$

H $-3.6883284 .783871-0.536538$

H $-5.2040804 .413280-1.324838$

H $-3.673014 \quad 4.156678-2.186248$

H -6.640626 $1.344156-2.237270$

H $-5.6365820 .234783-3.947004$

H $-4.025163-0.341013-4.281869$

H -3.810109 $1.841545-4.535928$

H $-0.474251-1.8638711 .628665$

H -3.246022 -1.8000620 .297143$

H $-1.352347-0.3299483 .394115$

H $-1.756623-2.0289313 .740345$

H -2.698221 -0.7405234 .454417$

H $-4.810911-2.212590-3.285839$

H $-4.113514-1.432618-1.865510$

H $-7.398574-3.723598 \quad 0.170546$

H $-8.368787-2.606113-0.814388$

H $-7.787430-0.6800570 .685626$

H -7.149617 -3.0665402 .498949$

H -8.204864 -1.394618 4.067103

H $-7.801573-0.0664773 .007755$

H $-4.943898-1.2866285 .778948$

H -6.619788 -2.074448 5.728719

H -4.4183710 .4622084 .191470$

H $-5.6062970 .943978 \quad 3.028410$

H $-4.888130-0.6593891 .379487$

H $-5.426309-2.855621-0.692352$

C $5.685259-2.977724-0.235737$

O $5.565459-1.563154-0.006644$

C $6.880777-1.005709 \quad 0.170424$

C $7.870546-2.1606450 .020465$

C $7.023549-3.3640130 .386898$

H $6.922244-0.6303311 .199301$

C $7.1006350 .149640-0.815857$

H $8.240299-2.254360-1.006909$

H $8.742907-2.0386430 .670273$

C $7.588123-4.681021-0.115381$

H $6.931424-3.4032411 .480624$

C $5.9943741 .225004-0.812898$

O $7.168551-0.373067-2.150974$

H $8.0765550 .611980-0.628087$

H $5.0792910 .810539-1.251392$

O $6.4161132 .280216-1.687388$

C 5.6785951 .8322050 .564766

C $4.746568 \quad 3.058578 \quad 0.516554$

H 5.2403141 .0650511 .209240

$\begin{array}{lllll}\text { O } & 6.905499 & 2.240341 & 1.176933\end{array}$

H 4.6365943 .4349951 .540852

H $5.2514513 .869527-0.023374$

H $6.944326-5.5176140 .173698$

H $7.680154-4.687931-1.206585$

H $8.582436-4.859302 \quad 0.307210$

C $4.493209-3.7229580 .364738$

H $6.333079-0.838950-2.326730$

H $6.6035211 .880868-2.554192$

H 6.6952392 .5609882 .070543

H $4.503682-3.633827 \quad 1.457860$

H $4.562412-4.7920970 .133246$

H $5.705673-3.124523-1.323991$

C 2.2068232 .0269611 .877750

$\begin{array}{lllll}\text { O } & 2.663862 & 1.726327 & 0.553495\end{array}$

C $3.3609132 .788893-0.105059$

C $2.4645354 .031758-0.199598$

C 1.9372994 .4508041 .169076

C $1.2930193 .261106 \quad 1.877282$

H 3.0679832 .1967322 .535271

C $1.4365110 .799798 \quad 2.392139$

H $3.5296052 .452561-1.134660$ 
O $3.1832655 .102218-0.803072$

H $1.6135453 .807060-0.855215$

H 2.7254274 .8957141 .787593

O 0.9356455 .4573231 .004516

H $0.3382863 .022140 \quad 1.392054$

O $1.001307 \quad 3.6262793 .225676$

C $2.341997-0.3476322 .785955$

H $\quad 0.860313 \quad 1.0699193 .284342$

H $\quad 0.754128 \quad 0.430879 \quad 1.618310$

H $2.5548505 .825512-0.965950$

H $1.373603 \quad 6.2530780 .658183$

H 0.4347754 .4162913 .196128

O $1.599533-1.2811453 .453166$

O $3.535597-0.4290192 .535047$

C $2.311283-2.4372563 .904521$

H $3.191600-2.1485864 .486933$

H $1.643191-3.0172714 .546645$

H $2.598055-3.0540193 .048943$

SCF Energy (B3LYP/6-31G**//MMFF) $=-3245.91096681$

01306

MM̄FF Geometry

C -1.090765 4.2260830 .418111

C $-0.4946063 .879300-0.733572$

C $0.7743213 .066450-0.781111$

O $0.6095031 .929891-1.670721$

C $1.9615533 .879663-1.326435$

C $2.5372984 .867266-0.334243$

C 3.4676824 .5441350 .585538

C $2.0560816 .287861-0.462962$

C $4.066963 \quad 3.1855150 .823087$

C $5.5287063 .151240 \quad 0.367763$

C 6.1560881 .7573020 .518170

C $7.6385621 .813246 \quad 0.140690$

C 8.2924590 .4257360 .149743

O $5.4553650 .862034-0.351371$

O $9.5614050 .533343-0.513803$

C $7.422388-0.532934-0.687928$

C $8.588300-0.0538131 .575436$

O $7.927922-1.865252-0.612484$

C $5.919282-0.495539-0.319753$

C $5.110138-1.282940-1.381872$

O $5.746368-1.0816330 .968463$

C $-0.2754580 .965423-1.300870$

O $-1.0490501 .005357-0.357694$

C $-0.126147-0.177073-2.229426$

C $-0.964371-1.217186-2.109583$

C $-0.939532-2.461424-2.880275$

C $0.198100-2.659843-3.845391$

C $3.612273-1.439201-1.079748$

C $3.282632-2.711856-0.312430$

C $1.336325-4.0150030 .542112$

O $4.133551-3.520970 \quad 0.047774$

C $0.817572-4.923940-0.528141$

C $-0.426806-5.425054-0.533629$

C $-0.955922-6.336523-1.603518$

C -2.226474 -5.814961 -2.238452

C $-3.421611-6.348182-1.933292$

C $-2.102521-4.724865-3.283225$

C -1.919000 -3.364452-2.663776

N $1.920411-2.841955-0.083722$

$\mathrm{H}-0.6128613 .939616 \quad 1.353309$

H $-0.9285064 .167370-1.686309$

H 1.0281382 .6664230 .209202

H $1.6735994 .399714-2.250551$

H $2.7509543 .190587-1.656499$

H 3.8429445 .3276911 .241985

H 2.5463676 .9580940 .251090

H $2.2661396 .668829-1.467832$

H $0.979214 \quad 6.353194-0.285782$

H 4.0095202 .9650361 .895552

H 3.4944402 .4013820 .319350

H $5.5763473 .455365-0.686619$

H 6.1085823 .8817980 .945963

H 6.0326431 .4232911 .555132

H 8.1824462 .4984050 .803122

H $7.7403992 .234175-0.869819$

H $10.0946011 .192134-0.036679$
H $7.529533-0.229723-1.738616$

H 9.2526200 .6533312 .086686

H $9.121899-1.0105481 .568304$

H $7.689858-0.1613812 .186743$

H $7.655362-2.2370140 .243708$

H $5.192761-0.741764-2.334240$

H $5.554153-2.272588-1.539614$

H $4.800145-1.2340371 .116609$

H $0.676943-0.144886-2.956200$

H -1.746422 -1.161685-1.355222

H $1.158711-2.618732-3.320571$

H $\quad 0.186631-1.882696-4.617564$

H $\quad 0.167663-3.618395-4.366777$

H $3.221255-0.572750-0.535846$

H $3.075959-1.502727-2.034829$

H $0.541844-3.6697261 .210586$

H $2.094645-4.5348341 .135593$

H $1.499961-5.187057-1.333118$

H - $1.099896-5.1830640 .285281$

H -1.138742 -7.316824 -1.144202

H $-0.207744-6.511113-2.386702$

H -4.334294 -5.995049-2.401602

H -3.515022 -7.145120 -1.202808

H $-3.014974-4.685608-3.892727$

H -1.302607 -4.995158 -3.977134

H $-2.705699-3.107725-1.952960$

H $1.287058-2.138084-0.450973$

C $-3.4569374 .689763-0.480262$

O $-3.8801043 .314252-0.360579$

C $-5.3183503 .268392-0.368776$

C $-5.7754414 .628330-0.882200$

C $-4.7180195 .535802-0.287832$

H $-5.636271 \quad 3.166230 \quad 0.675533$

C $-5.8255182 .077689-1.188550$

H $-5.7481104 .680605-1.976812$

H $-6.7897194 .876826-0.555791$

C $-4.6501436 .903658-0.944659$

H -4.9272175 .6634380 .782882$

C $-5.3045910 .703343-0.716841$

O $-5.4117282 .261700-2.548428$

H $-6.9223802 .084249-1.194978$

H $-4.2391880 .606453-0.957795$

O $-5.987501-0.302317-1.477146$

C $-\begin{array}{llll}-5.525438 & 0.406218 & 0.779337\end{array}$

C $-5.291258-1.0623011 .186472$

H -4.8765311 .0575671 .375526$

O -6.8760440 .7345021 .120552$

H -5.470526-1.139667 2.265855

H $-6.066686-1.6918590 .731395$

H $-3.8621007 .514617-0.493385$

H $-4.4424796 .824305-2.017161$

H -5.600057 $7.435052-0.825036$

C $-2.380674 \quad 4.9851330 .568428$

H $-5.9062371 .627453-3.094121$

H $-5.664048-0.247890-2.392026$

H -6.9769720 .6036912 .078813$

H -2.784717 4.7219801 .555222

H $-2.147901 \quad 6.0557180 .594134$

H -3.070415 4.820454-1.497697

C $-2.659056-0.7805012 .735098$

O $-2.846563-0.770894 \quad 1.317074$

C $-3.896738-1.6107720 .835935$

C $-3.655764-3.0607161 .273567$

C $-3.473088-3.1761672 .784189$

C $-2.403087-2.1984373 .270368$

H $-3.563546-0.3789703 .209395$

C $-1.5373780 .218503 \quad 3.067053$

H -3.823455 -1.599447 -0.256852

O $-4.727803-3.9025300 .863855$

H -2.747331 -3.4371360 .787290$

H $-4.418346-3.023748 \quad 3.317754$

O $-3.043779-4.5031053 .101500$

H -1.424533 -2.578293 2.956202

O $-2.418162-2.1938204 .696481$

C $-0.181504-0.1522692 .499793$

H -1.7925431 .1990992 .646659$

H -1.445315 0.3195544 .153991

H $-4.802212-3.830227-0.103195$ 
H $-3.713164-5.1148962 .749674$

H -1.624004 -1.722650 4.999312

O $0.780736 \quad 0.605431 \quad 3.104271$

O $\quad 0.013625-0.975971 \quad 1.617365$

C 2.1166310 .3718982 .645659

H 2.7870491 .0359043 .197476

H $2.409257-0.6641602 .841180$

H 2.1953770 .5991791 .578532

SCF Energy (B3LYP/6-31G**//MMFF)= -3245.89675512

\section{7}

MM̄MF Geometry

C $2.009012-2.6715321 .870257$

C $3.019269-1.8197982 .107962$

C $2.897420-0.3585662 .471098$

O $1.508916 \quad 0.045007 \quad 2.419091$

C $3.445878-0.0427303 .875145$

C $4.928727-0.2725904 .058342$

C 5.8885580 .5648303 .619401

C $5.298912-1.5036604 .844010$

C 5.7183561 .8416042 .837914

C 6.6422821 .8903771 .610829

C 6.2131420 .9417160 .477379

C $7.4085870 .635322-0.428371$

C $7.011535-0.250887-1.613408$

O $5.1757231 .586862-0.277178$

O $8.119333-0.251844-2.525711$

C $5.8092430 .399498-2.321176$

C $6.801501-1.708248-1.187785$

O $5.325608-0.443041-3.366156$

C $4.6586730 .808848-1.366612$

C $3.6435021 .666819-2.160486$

O $4.007629-0.373507-0.903153$

C 1.2472191 .3599052 .195521

O 2.0598082 .2589932 .051594

C -0.2215391 .5215352 .150841$

C -0.7347472 .7518152 .008585$

C -2.1533213 .0947661 .922125$

C -3.1521731 .9700191 .934645$

C $2.5366382 .333976-1.331507$

C $1.6073773 .142335-2.226853$

C $-0.0818574 .933919-2.215500$

O $1.6026813 .032742-3.451851$

C -1.052359 $5.578061-1.279957$

C $-2.3840695 .496447-1.427120$

C $-3.3793256 .213663-0.557534$

C -4.2489925 .2671530 .239620$

C $-5.366517 \quad 4.743390-0.291232$

C -3.8648484 .9886561 .675665$

C $-2.490754 \quad 4.394598 \quad 1.802882$

N $0.7622773 .984175-1.512484$

H $0.979266-2.3369831 .959508$

H $4.036801-2.1888811 .993848$

H 3.4473860 .2046941 .710124

H $2.882714-0.6198234 .622026$

H 3.2192750 .9996364 .135813

H $6.923400 \quad 0.3162063 .852423$

H $6.382711-1.6199994 .950352$

H $4.871758-1.4517815 .850922$

H $4.919118-2.4044714 .352736$

H 4.6840292 .0110092 .530361

H 5.9883872 .6713893 .502706

H 6.6527922 .9213851 .234481

H 7.6645631 .6562111 .934609

H 5.8221530 .0121400 .903529

H $8.220094 \quad 0.1650340 .141341$

H $7.8223381 .576632-0.817224$

H $7.847050-0.744335-3.319341$

H $6.1869001 .304354-2.817132$

H $7.716985-2.108518-0.735894$

H $6.592554-2.347883-2.052393$

H $5.992458-1.831832-0.464848$

H $4.801178-1.151189-2.955221$

H $4.1853312 .469274-2.679321$

H $3.1732291 .043694-2.932460$

H $3.089752-0.159541-0.673761$

H $-0.835071 \quad 0.6315302 .230280$

H -0.0493943 .5961411 .942138$
H -3.1580221 .4718502 .910081$

H -2.9032641 .2306011 .166885$

H -4.1745252 .2955201 .728199$

H $1.9288911 .584376-0.815738$

H $2.9785513 .003628-0.585880$

H $-0.5821484 .414612-3.040611$

H $0.5717015 .696716-2.652925$

H $-0.6393756 .179828-0.474150$

H $-2.7906744 .910241-2.248437$

H -4.010106 $6.833353-1.208796$

H $-2.882294 \quad 6.9212340 .117857$

H -6.0113704 .0863190 .282538$

H $-5.6593574 .959170-1.313546$

H -4.6109094 .3585662 .172594$

H -3.882422 5.9347172 .232677

H -1.6944035 .1395631 .796318$

H $0.9536304 .139491-0.526416$

C $1.709043-4.4340650 .060582$

O $0.279994-4.264305-0.020860$

C $-0.035739-3.653466-1.287221$

C $1.231058-3.749389-2.132620$

C $2.304441-3.599909-1.073402$

H $-0.238579-2.595570-1.080622$

C -1.272183-4.302804-1.915512

H $1.320427-4.723439-2.627045$

H $1.275355-2.973631-2.903158$

C $3.680070-4.058311-1.521651$

H $2.354642-2.541745-0.793922$

C $-2.513846-4.338490-1.002364$

O $-0.952891-5.657890-2.257989$

H $-1.506339-3.795634-2.859282$

H $-2.361740-5.072807-0.202256$

O $-3.604886-4.840115-1.787272$

C $-2.914322-2.985466-0.384730$

C $-4.255890-3.0756910 .369725$

H $-2.129060-2.6603950 .306985$

O $-3.003378-2.019943-1.427752$

H $-5.061923-3.248027-0.351437$

H -4.217878 -3.9470041 .033985$

H $4.409667-3.944848-0.713860$

H $3.673888-5.110419-1.825417$

H $4.024032-3.464755-2.374861$

C $2.209182-4.1083111 .471363$

H -1.746291 -6.049176 -2.662410

H -3.804726 -4.175465 -2.468920

H $-3.107004-1.149570-1.007635$

H $3.267142-4.3853981 .551837$

H $1.662145-4.7250242 .195272$

H $1.893599-5.500446-0.125413$

C $-5.831831-0.551700-0.430212$

O $-4.680547-0.6302130 .417223$

C $-4.575182-1.8161911 .207952$

C $-5.815277-1.9548572 .102597$

C $-7.103858-1.9178951 .284295$

C $-7.123421-0.6820180 .385958$

H $-5.782259-1.339604-1.191317$

C $-5.7851050 .805234-1.143932$

H $-3.715407-1.6633621 .873230$

O $-5.739533-3.1594012 .856620$

H -5.827845 -1.124624 2.821191

H $-7.236223-2.8332590 .695684$

O $-8.225387-1.8368992 .166465$

H -7.2695370 .2130391 .004576$

O $-8.232681-0.768059-0.506494$

C $-4.6315030 .873219-2.115879$

H $-6.7001000 .996859-1.715632$

H -5.685568 $1.622693-0.420545$

H $-6.468397-3.1521553 .499349$

H $-8.277230-2.6772642 .652342$

H $-9.033789-0.8699880 .035527$

O $-3.571126 \quad 1.504262-1.536483$

O $-4.661766 \quad 0.396230-3.242816$

C $-2.386618 \quad 1.593147-2.333944$

H $-1.5860051 .990705-1.705312$

H $-2.0868670 .604353-2.694097$

$\mathrm{H}-2.5518102 .276151-3.172034$

SCF Energy (B3LYP/6-31G**//MMFF) $=-3245.92479330$ 
01_308

MM̄FF Geometry

C $1.950267-2.9761790 .248952$

C $1.036721-2.4494081 .075072$

C $1.201197-1.1203521 .759376$

O $0.084506-0.3132491 .315705$

C $1.176477-1.2487513 .291908$

C $2.385454-1.9513353 .867668$

C $3.567858-1.3510664 .107152$

C $2.182085-3.3986574 .234201$

C 3.9501930 .0822843 .843000

C $5.3164070 .199623 \quad 3.148917$

C $5.269071-0.0869031 .637463$

C $6.675134-0.4150951 .128609$

C $6.707998-0.611378-0.391620$

O $4.771174 \quad 1.0901200 .984384$

O $8.083768-0.614700-0.803483$

C $6.0380380 .606189-1.053301$

C $6.128833-1.965810-0.811731$

O $5.9272660 .418265-2.463630$

C $4.6651720 .989403-0.442243$

C $4.2473232 .368973-1.008786$

O $3.6992550 .012325-0.827426$

C 0.1721181 .0328931 .469245

O 1.0809951 .6602241 .987468

C $-1.052721 \quad 1.6236280 .890463$

C -1.3648652 .8941831 .180506$

C $-2.530863 \quad 3.6307960 .694231$

C -3.443278 $2.944282-0.286957$

C $2.9952572 .996891-0.382098$

C $2.7150384 .368178-0.980294$

C $1.3757516 .414419-0.668196$

O $3.3304664 .812993-1.947278$

C $0.0917346 .837833-0.032541$

C $-0.9916827 .208006-0.733221$

C $-2.2658797 .718745-0.119722$

C $-3.4369306 .789291-0.349317$

C $-4.152786 \quad 6.845303-1.484837$

C $-3.821338 \quad 5.845126 \quad 0.767477$

C $-2.715796 \quad 4.893598 \quad 1.129333$

N $1.686417 \quad 5.037977-0.326783$

H $2.880068-2.4435700 .080511$

H $\quad 0.108181-2.9877801 .245672$

H $2.126555-0.635778 \quad 1.427538$

H $0.256518-1.7602523 .608236$

H $1.085728-0.2573173 .752595$

H $4.354153-1.9485134 .567614$

H $3.078018-3.8450214 .678641$

H $1.370990-3.4935854 .963829$

H $1.925011-3.9909873 .351456$

H 3.1925400 .6195543 .267607

H 4.0204440 .5813454 .817603

H 5.6850641 .2219853 .304646

H $6.027059-0.4753743 .642515$

H $4.592468-0.9253431 .441324$

H $7.073736-1.3035501 .634841$

H $7.3571910 .407197 \quad 1.387636$

H $8.535170-1.338025-0.335521$

H $6.7239831 .453832-0.917869$

H $\quad 6.667823-2.783862-0.318971$

H $6.253115-2.132588-1.887508$

H $5.071851-2.070776-0.566155$

H $5.183751-0.187746-2.623312$

H $5.0744673 .076445-0.860402$

H $4.0857172 .283051-2.091323$

H $2.8147140 .407602-0.780061$

H -1.6621410 .9990180 .248921$

H -0.7049743 .4516681 .844571$

H -3.933806 2.0853810 .183291

H $-2.8801172 .594485-1.159339$

H $-4.2323573 .594303-0.672444$

H $2.1163582 .370619-0.562637$

H 3.1327123 .1124680 .698480

H $1.3502626 .509143-1.759597$

H $2.1961157 .040921-0.301293$

H 0.0677536 .8834941 .053335

H $-0.9543237 .176909-1.820034$

H $-2.4831048 .700960-0.560054$
H -2.1435097 .9067380 .954304$

H $-5.0086406 .198823-1.647226$

H $-3.8951697 .538624-2.278939$

H -4.7428785 .3015690 .530652$

H -4.0692146 .4374451 .658039$

H -1.9974345 .3108191 .836093$

H 1.3612694 .6726460 .564217

C $1.402301-4.083199-1.964061$

O $0.085330-3.504044-2.078048$

C $0.098440-2.546615-3.153843$

C $1.402996-2.778286-3.913118$

C $2.332931-3.196515-2.792208$

H $0.130902-1.553739-2.689672$

C -1.159698 -2.680724-4.017452

H $1.309554-3.585789-4.648551$

H $1.740102-1.880472-4.440167$

C $3.593911-3.897354-3.264522$

H $2.616287-2.293101-2.239951$

C -2.497268 -2.538897-3.260771

O $-1.159505-3.968987-4.648686$

H $-1.116279-1.950684-4.834305$

H -2.656670 -3.420965 -2.630435

O $-3.541506-2.569169-4.244723$

C $-2.645111-1.262932-2.408914$

C $-4.086442-0.974113-1.942510$

H $-1.978153-1.325379-1.541709$

O $-2.216470-0.141687-3.188095$

H $-4.075066-0.024248-1.395245$

H -4.714736 -0.775867 -2.820138

H $4.215364-4.194120-2.413714$

H $3.360940-4.797511-3.843044$

H $4.187439-3.232968-3.901013$

C $1.767732-4.271035-0.488565$

H -1.135996 -4.640068 -3.944952

H -3.445424 -1.775727 -4.799128

H $-2.2410470 .642000-2.612780$

H $2.695858-4.848361-0.405320$

H $0.981866-4.861941-0.001194$

H $1.330321-5.078167-2.423299$

C $-3.899429-1.3969691 .081890$

O $-3.920303-2.4007330 .063174$

C $-4.720939-2.081459-1.078355$

C $-6.167568-1.790859-0.648381$

C $-6.238236-0.7130060 .430248$

C $-5.306196-1.0599801 .589893$

H $-3.450804-0.4888790 .663959$

C -2.943083 -1.849759 2.192814

H $-4.754147-2.988839-1.692532$

O $-6.959575-1.389166-1.761422$

H $-6.617888-2.708920-0.248405$

H -6.0093550 .2792870 .024509$

O $-7.572892-0.6402310 .937068$

H $-5.732959-1.8978622 .152376$

$\begin{array}{lllll}\text { O } & -5.227069 & 0.051361 & 2.480073\end{array}$

C -3.335632 -3.132724 2.895393

H $-1.959630-2.0357081 .752130$

H -2.848085-1.055774 2.942041

H -6.910125 -2.097715 -2.425849

H $-8.159193-0.4623270 .181590$

H -6.1341990 .2650272 .758654$

O $-2.564905-3.2709634 .015911$

O $-4.177636-3.9288212 .504881$

C $-2.795851-4.4586824 .779758$

H $-2.573953-5.3449274 .177591$

H $-2.124300-4.4437055 .642370$

$\mathrm{H}-3.828937-4.4833075 .139177$

SCF Energy $\left(B 3 L Y P / 6-31 G^{* *} / / M M F F\right)=-3245.91237733$

01_309

MM̄FF Geometry

C 1.0089302 .8839770 .654924

C $0.0802053 .434373-0.142425$

C $-0.6195602 .688020-1.247560$

O $-2.0353912 .955272-1.072743$

C $-0.2101503 .153807-2.657835$

C $1.1912852 .771041-3.083347$

C $1.5812091 .516995-3.386041$

C $2.1569173 .914919-3.231726$ 
C $0.7322310 .276616-3.383305$

C $1.266014-0.768523-2.397284$

C $0.321452-1.959234-2.153453$

C $-0.164500-2.624514-3.443647$

C $-1.151757-3.768130-3.165478$

O $-0.792946-1.495368-1.380604$

O $-1.798693-4.104595-4.402904$

C $-2.249805-3.254012-2.209492$

C $-0.436422-5.039232-2.692935$

O $-3.116028-4.316537-1.815753$

C $-1.691614-2.534019-0.960758$

C $-2.847216-1.885961-0.158837$

O $-1.042086-3.499716-0.131207$

C -2.915468 $2.024840-1.527620$

O $-2.6539030 .978657-2.098132$

C $-4.2774352 .494751-1.194778$

C $-5.2856921 .610512-1.185421$

C $-6.6849141 .894695-0.869142$

C $-7.0853553 .334799-0.686166$

C $-2.378199-0.9835750 .990389$

C $-3.534425-0.3512661 .741999$

C $-5.425570-0.9093893 .272713$

O -3.7136610 .8644871 .745818$

C $-6.702483-1.0451502 .503641$

C -7.672660 -0.118199 2.525629

C $-8.995366-0.2673491 .827625$

C -9.3565210 .9416260 .993778$

C -10.0294341 .9719281 .534398$

C $-9.0201710 .922625-0.478983$

C $-7.5447950 .860852-0.757855$

N $-4.305194-1.2750772 .427276$

H $1.317624 \quad 1.8569860 .477866$

H -0.2246384 .4639100 .030774$

H $-0.4502551 .612384-1.121078$

H $-0.340764 \quad 4.242710-2.731796$

H $-0.9187212 .760341-3.398885$

H $2.6109091 .359379-3.704209$

H $3.1763983 .577571-3.446431$

H $1.8403114 .569179-4.050707$

H $2.192674 \quad 4.508102-2.317013$

H $-0.3129720 .503554-3.164541$

H $0.751665-0.134542-4.399721$

H $2.228870-1.146038-2.764864$

H $1.458204-0.279120-1.433359$

H $0.880333-2.681965-1.548761$

H $0.684214-2.980546-4.040401$

H $-0.687505-1.889991-4.069831$

H $-1.109988-4.353122-5.043116$

H -2.869384 -2.540522 -2.771003

H $0.263395-5.391646-3.460418$

H $-1.147308-5.858205-2.537869$

H $\quad 0.132676-4.896310-1.772332$

H $-3.450298-4.733420-2.628417$

H $-3.463233-1.277306-0.831261$

H -3.496713 -2.674102 0.240286

H $-1.041850-3.1903120 .787279$

H $-4.406616 \quad 3.541380-0.945207$

H -5.065664 $0.569424-1.417971$

H $-6.8484253 .917380-1.583274$

H -6.5593093 .7744590 .168418$

H $-8.1536443 .466404-0.499094$

H -1.779688 -1.5398611 .718580$

H $-1.746022-0.173604 \quad 0.608926$

H -5.2789130 .1060053 .655170$

H -5.428828 -1.605279 4.117495

H $-6.853827-1.9619191 .938972$

H -7.5291510 .7803013 .122158$

H -9.763289 -0.4205592 .597459$

H -9.021195-1.169156 1.203536

H -10.322095 2.8315910 .941000

H -10.299920 1.9788822 .585222

H $-9.4670641 .777054-1.001467$

H $-9.4931260 .043311-0.935847$

H -7.164990 $-0.152886-0.889283$

H $-4.075972-2.2619142 .357629$

C 3.0729434 .0463981 .627189

O 3.9110302 .9424941 .234700

$\begin{array}{llll}\text { C } 4.653508 & 3.326496 & 0.063995\end{array}$
C 4.6332044 .8503520 .045768

C 3.2440575 .1416840 .573785

H $4.0931172 .948668-0.799948$

C 6.0561662 .7104800 .073850

H 5.3852105 .2766800 .719199

H $4.8060315 .256299-0.955537$

C $3.080607 \quad 6.5498381 .121670$

H $2.5273125 .008054-0.240103$

C 6.1042921 .1914670 .326909

$\begin{array}{lllll}\text { O } & 6.841001 & 3.328767 & 1.103610\end{array}$

H $6.5639152 .949522-0.867917$

H 5.8798020 .9889491 .380487

$\begin{array}{lllll}\text { O } & 7.456964 & 0.764208 & 0.114904\end{array}$

C $5.1844970 .342150-0.567650$

C $5.421824-1.177997-0.458898$

H $4.1384890 .558253-0.325517$

O $5.3678650 .711900-1.936796$

H $4.706536-1.673677-1.126950$

H $6.406478-1.424436-0.876569$

H 2.0660576 .7022891 .503526

H $3.782303 \quad 6.7472561 .938917$

H 3.2620267 .2911610 .336600

C 1.6295873 .5774801 .838550

H 6.3731313 .1992321 .946731

H $8.023857 \quad 1.2972140 .698584$

H $6.3052910 .572479-2.155749$

H 1.0115194 .4276612 .151498

H 1.6117612 .8591672 .668402

H $3.464708 \quad 4.3923922 .592349$

C $2.912930-1.9540571 .110516$

O $4.095976-1.3287931 .614562$

C $5.305748-1.7411460 .971663$

C $5.478050-3.2648431 .072208$

C $4.245156-4.0198980 .581620$

C $2.986265-3.478144 \quad 1.257704$

H $2.812452-1.7060330 .047584$

C $1.694866-1.3195861 .793610$

H $6.124431-1.3000281 .552597$

O $\quad 6.615467-3.699693 \quad 0.334571$

H $5.652265-3.5384292 .121105$

H $4.153679-3.976930-0.510213$

$\begin{array}{lllll}\text { O } & 4.374981 & -5.402621 & 0.919861\end{array}$

H $2.993350-3.7714502 .313704$

$\begin{array}{llll}\text { O } & 1.837923 & -4.069692 & 0.659594\end{array}$

C $1.605735-1.568503 \quad 3.284346$

H $1.726462-0.2322181 .662501$

H $\quad 0.783180-1.6980511 .319903$

H $7.388097-3.2203000 .679789$

H $5.200894-5.7219050 .517281$

H $1.935651-5.034544 \quad 0.732159$

O $0.306050-1.4265233 .680441$

O $2.553283-1.8176664 .016495$

C $0.062147-1.6135475 .077839$

H $-1.009214-1.4869285 .255199$

H $\quad 0.351143-2.6242705 .381114$

H $0.606002-0.8638595 .660288$

SCF Energy (B3LYP/6-31G*//MMFF) $=-3245.91247960$

0131

MM̄FF Geometry

C $3.578110-3.260982-1.091488$

C $2.498302-2.476623-0.967116$

C $1.578532-2.100072-2.095809$

O $0.235040-2.406815-1.661110$

C $1.652146-0.581940-2.321633$

C $1.135204-0.144029-3.673354$

C $-0.0976520 .347649-3.901216$

C $2.139010-0.224992-4.794589$

C $-1.241393 \quad 0.466451-2.928086$

C $-2.545166 \quad 0.028077-3.607852$

C $-3.752432-0.038198-2.660371$

C $-5.012043-0.364689-3.471792$

C $-6.288230-0.322178-2.623845$

O $-3.896192 \quad 1.219908-1.994698$

O $-7.412613-0.291480-3.516707$

C $-6.309793 \quad 0.998257-1.827954$

C $-6.446327-1.587437-1.772117$

O $-7.4122141 .016255-0.922359$ 
C $-4.9932951 .276548-1.067107$ C $-5.0233832 .703939-0.461864$ O $-4.853213 \quad 0.311221-0.029522$ C $-0.260766-3.646515-1.892662$ O $0.251029-4.524190-2.572918$ C -1.548534 -3.844328 -1.183228 C $-1.968385-3.029804-0.201567$ C $-3.192855-3.1661910 .589790$ C $-4.086086-4.3437560 .304998$ C $-3.875283 \quad 3.0183140 .508873$ C -4.1561312 .5642491 .935865$ C -3.0148142 .1801184 .116786$ O -5.2637572 .1980172 .320900$ C -2.7302040 .7110624 .142917$ C $-3.569997-0.2095494 .640562$ C $-3.280369-1.6848104 .625266$ C $-4.329200-2.4852123 .878361$ C $-4.976633-3.4956844 .482767$ C $-4.642153-2.1229102 .441416$ C -3.448199 -2.2372491 .534785$ N -3.0252282 .6303662 .736150$ H $3.836283-3.678552-2.060747$ H $2.264260-2.0565790 .007635$ H $1.821010-2.647147-3.014746$ H $2.689849-0.231623-2.233246$ H $1.097265-0.063576-1.532829$ H $-0.3244020 .691661-4.910129$ H $\quad \begin{array}{llll}1.724653 & 0.112817 & -5.750443\end{array}$ H $3.0090200 .401399-4.571560$ H $2.478704-1.256594-4.929991$ H -1.079604 -0.138215 -2.031822 H -1.322550 $1.512393-2.610857$ H $-2.7614850 .741609-4.414970$ H -2.408954 -0.955051-4.077435 H -3.562336 -0.816521 -1.912659 H -4.911477 -1.338705 -3.967460 H $-5.1196430 .369983-4.282705$ H -7.368255 -1.081611 -4.082106 H $-6.4781901 .817976-2.540501$ H -6.517025 -2.474376 -2.413281 H $-7.376062-1.562147-1.193261$ H -5.614427 -1.747700 -1.084385 H $-8.2167320 .848244-1.442243$ H -4.965354 $3.425154-1.287748$ H -5.9776042 .8800060 .047938$ H $-4.054417 \quad 0.5225350 .478585$ H -2.106359 -4.726740 -1.478138 H -1.352189 -2.173362 0.067311 H $-3.529957 \quad-5.282558 \quad 0.405743$ H -4.489912 -4.281999-0.711482 H $-4.936208-4.4191700 .986704$ H -2.9358632 .5793810 .154772$ H -3.7405454 .1062650 .551740$ H -3.975763 2.4224624 .582716 H -2.223193 2.7262974 .638398 H $-1.7815490 .387247 \quad 3.723517$ H -4.516224 0.1067265 .073071 H -3.212290 -2.019378 5.668413 H -2.297839 -1.8876404 .181280$ H -5.730814 -4.076598 3.962004 H $-4.767256-3.7676165 .512163$ H -5.003701-1.087721 2.405444 H -5.483036 -2.714405 2.065070 H -2.731359 -1.425229 1.664505 H -2.137303 2.8897522 .316440 C $5.808536-2.826143-0.045249$ O $5.559549-1.4079110 .020000$ C $6.581605-0.7869820 .820356$ C $7.624522-1.8658861 .093906$ C $6.774288-3.1212461 .100060$ H $6.108896-0.5017601 .767493$ C 7.1207410 .4579230 .107544 H $8.374842-1.9203600 .296869$ H $8.152878-1.6996602 .037676$ C $7.575010-4.3993130 .922475$ H $6.227189-3.1680462 .051246$ C $6.0398121 .480861-0.300313$ O $7.7971640 .055704-1.092174$
H 7.8811230 .9418210 .731144

H $5.4107591 .058098-1.091591$

O $6.7106772 .608420-0.879024$

C 5.1576721 .9861370 .857658

C 4.2329313 .1616050 .483534

H $4.553443 \quad 1.1627341 .249488$

O 5.9960022 .4244191 .931244

H 3.7886473 .5468321 .409138

H 4.8410673 .9907070 .102075

H $6.918408-5.2749830 .925138$

H $8.129995-4.397354-0.021692$

H $8.297389-4.5165621 .736863$

C $4.484924-3.5819540 .064401$

H $7.159400-0.436325-1.637614$

H $7.2602662 .275195-1.609133$

H 6.5640403 .1357281 .588435

H $3.989261-3.3227411 .008664$

H $4.659906-4.6633830 .079576$

H $6.283783-3.023609-1.015285$

C 1.3817942 .1860791 .028963

O $2.2549831 .778381-0.030662$

C $3.1318222 .793747-0.530325$

C $2.3153293 .990887-1.035792$

C 1.3762214 .5261590 .038658

C 0.5275673 .3926170 .608540

H 1.9744162 .4332111 .917680

C $0.472427 \quad 0.9955421 .373431$

H $3.6275422 .365067-1.408423$

O $3.1677965 .041714-1.477849$

H $1.7128873 .680867-1.899953$

H 1.9265215 .0471410 .830745

O $0.4983655 .492676-0.543553$

H $-0.2073203 .085785-0.146788$

O -0.2101473 .8801441 .728296$

C $1.205994-0.1393572 .053910$

H $\quad-0.3175291 .3166532 .058454$

H $\quad 0.0161180 .597123 \quad 0.460293$

H $3.7481444 .676696-2.167569$

H $1.0540226 .185250-0.940706$

H $0.427236 \quad 4.1576182 .408233$

O $0.321380-1.1571412 .276883$

O $2.389413-0.1379902 .360943$

C $0.851027-2.3056862 .945628$

H $0.055096-3.051498 \quad 3.020217$

H $1.679815-2.7338582 .374508$

H $1.175872-2.0357213 .954801$

SCF Energy (B3LYP/6-31G**//MMFF)= -3245.92346400

01310

MM̄MF Geometry

C $0.6665415 .145643 \quad 0.224132$

C 1.8476745 .1426050 .861219

C 2.8698184 .0286590 .816456

$\begin{array}{llll}\text { O } & 2.243077 & 2.743917 & 0.595110\end{array}$

C $3.8613834 .295171-0.331580$

C $5.0108203 .309879-0.404655$

C $5.2508362 .493093-1.449427$

C 5.9436243 .3016120 .779051

C $4.4669292 .368213-2.726772$

C $3.3457381 .318371-2.683134$

C $3.843553-0.130187-2.552177$

C $2.680095-1.118342-2.664660$

C $3.134829-2.571109-2.447390$

O $4.469480-0.281522-1.277395$

O $1.961431-3.373262-2.240880$

C $3.971346-2.652720-1.150532$

C $3.834394-3.139012-3.687872$

O $4.555852-3.943609-1.000249$

C $5.062806-1.567127-1.055476$

C $5.756683-1.5121370 .326760$

O $6.082301-1.838034-2.025240$

C 1.6965322 .1275261 .680480

O 1.6627702 .5512592 .825206

C $1.1221900 .838417 \quad 1.232371$

C 0.3033150 .1610822 .051734

C $-0.370384-1.1066201 .755709$

C $0.008680-1.8152040 .483450$

C $4.829107-1.2389481 .516451$ 
C $4.394312-2.4943502 .253673$

C $2.898493-3.2399764 .098714$

O $4.873316-3.6044972 .038933$

C $1.685464-3.8564903 .477996$

C $0.521750-4.0009054 .128942$

C $-0.690163-4.6610173 .538586$

C $-1.922490-3.7904363 .621546$

C $-2.820057-3.9549654 .608142$

C -2.157638 -2.792411 2.511829

C -1.291334 -1.568541 2.628360

N $3.430742-2.2201053 .215199$

H $0.3952304 .299510-0.401983$

H $2.125726 \quad 6.0103671 .455710$

H 3.4013514 .0080211 .775631

H $4.2879815 .302027-0.226906$

H $3.3035294 .301083-1.274154$

H $6.1169991 .834562-1.393675$

H 6.8325692 .6862400 .602997

H $6.288464 \quad 4.3173070 .998721$

H 5.4399722 .9023691 .664733

H $4.0383963 .335661-3.009769$

H $5.1662712 .120074-3.535248$

H $2.6731521 .554267-1.850418$

H $2.7623611 .411124-3.607395$

H $4.578413-0.312563-3.345154$

H $2.172300-1.015221-3.631685$

H $1.925640-0.877467-1.903857$

H $1.385204-3.267203-3.017195$

H $3.273534-2.527969-0.314643$

H $3.159268-3.116492-4.551845$

H $4.100474-4.192097-3.544512$

H $4.736985-2.588343-3.960923$

H $3.834636-4.594818-1.041789$

H $6.363111-2.4126890 .480267$

H $\quad 6.477110-0.6818280 .293123$

H 6.897128 -1.385376 -1.751079

H 1.3733830 .4934320 .236558

H $\quad 0.0787540 .5873643 .028907$

H $1.093292-1.9561070 .434984$

H $-0.310263-1.235311-0.388039$

H $-0.428541-2.8110190 .388584$

H $5.376352-0.6316232 .248997$

H $3.946902-0.6664041 .211963$

H $2.675780-2.7576015 .055721$

H $3.659323-4.0097914 .263366$

H $1.775848-4.2265412 .459287$

H $0.443162-3.6561785 .157677$

H $-0.858388-5.5982624 .085081$

H $-0.520089-4.9549882 .495378$

H -3.722280 -3.354387 4.658392

H $-2.669670-4.6911065 .390897$

H -3.200463 -2.449212 2.539640

H $-2.057222-3.298303 \quad 1.547717$

H -1.495082 -0.9797703 .523970$

H $3.045006-1.2826173 .282881$

C -1.7434125 .8463270 .654621$

O $-2.2746675 .004821-0.388931$

C -2.8174653 .8091250 .216302$

C -3.1019864 .2031881 .657236$

C -1.8981385 .0735161 .965741$

H -2.0079013 .0713400 .163275$

C $-4.0221143 .336414-0.602194$

H $-4.0262394 .790454 \quad 1.724342$

H -3.1848723 .3513262 .335897$

C -2.079402 5.9637023 .182521

H -1.0376254 .4157262 .139631$

C $-4.7457382 .063046-0.113168$

O $-3.5602063 .100582-1.938158$

H $-4.7410744 .163259-0.663124$

H $-5.5112771 .824676-0.860540$

O $-5.4272632 .310644 \quad 1.112623$

C -3.8370530 .8278460 .045266$

C $-4.597066-0.4919240 .263371$

H $-3.188906 \quad 0.732418-0.830507$

O

H $-3.859005-1.2499630 .547563$

H -5.256040 $-0.400277 \quad 1.135965$

H -1.1922706 .5843763 .344165$
H $-2.941746 \quad 6.629080 \quad 3.069038$

H $-2.238378 \quad 5.358828 \quad 4.081221$

C $-0.318348 \quad 6.281357 \quad 0.296048$

H -4.334453 $2.882896-2.484531$

H -6.0170483 .0709420 .973094$

H -3.5335301 .0682141 .962782$

H $\quad 0.0180877 .0380941 .014555$

H $-0.3358946 .755771-0.693019$

H $-2.379757 \quad 6.7405560 .666616$

C $-3.553388-1.863766-2.229746$

O $-4.643629-0.936179-2.167528$

C $-5.401448-0.984869-0.955229$

C $-6.001755-2.387514-0.778570$

C $-4.934471-3.478406-0.857661$

C $-4.068029-3.301940-2.105050$

H $-2.839805-1.652037-1.424586$

C $-2.838102-1.646300-3.568090$

H $-6.239589-0.290731-1.087769$

O $-6.686748-2.4951770 .464974$

H $-6.739928-2.568856-1.570644$

H $-4.316179-3.5023500 .047091$

O $-5.573323-4.755161-0.935719$

H -4.650498 -3.573749-2.994755

O $-2.955618-4.191225-2.033007$

C $-2.229275-0.266070-3.652432$

H $-2.042288-2.380836-3.734506$

H -3.546233 -1.740226 -4.400639

H -7.367785 -1.801227 0.484368

H $-6.146441-4.839366-0.154316$

H $-3.309664-5.090745-1.925021$

O $-1.146766-0.178825-2.828989$

$\begin{array}{lllll}\text { O } & -2.666726 & 0.626272 & -4.367637\end{array}$

C $-0.4941301 .094254-2.795840$

H $0.0740651 .240892-3.718432$

H $\quad 0.1955541 .099410-1.949087$

H $-1.2156401 .903465-2.654752$

SCF Energy $(B 3 L Y P / 6-31 G * * / / M M F F)=-3245.90562418$

01311

MM̄FF Geometry

C $2.394369-3.9566972 .027664$

C $1.547859-2.9513782 .301143$

C $0.036715-2.9890122 .335387$

O $-0.435886-2.0258321 .358191$

C $-0.593141-4.3506161 .981070$

C $-2.107874-4.3693922 .067272$

C $-2.930634-4.5953081 .024045$

C $-2.688767-4.1448143 .440000$

C $-2.560556-4.872371-0.406405$

C $-2.491646-3.626159-1.302701$

C $-3.837789-2.904943-1.481473$

C $-3.730490-1.815631-2.550342$

C $-5.036391-1.019374-2.691393$

O $-4.204831-2.308437-0.235765$

O $-4.731160 \quad 0.146951-3.471899$

C $-5.502554-0.548495-1.294804$

C $-6.088060-1.803494-3.485991$

O $-6.793748 \quad 0.046821-1.398491$

C $-5.491193-1.672436-0.236119$

C $-5.744670-1.1737601 .208022$

O $-6.529072-2.612127-0.541062$

C -0.529397 -0.7231461 .739989$

O $-0.313867-0.2608402 .848680$

$\begin{array}{llll}\text { C } & -0.897611 & 0.063134 & 0.542525\end{array}$

C $-0.674347 \quad 1.3851720 .531965$

C $-0.9300142 .294541-0.586246$

C $-1.7228141 .760433-1.748796$

C $-4.752200-0.1280161 .727912$

C -5.2249001 .3065621 .562327$

C -4.4510573 .6537461 .877707$

O -6.3582121 .6053151 .195809$

C $-4.0076384 .196376 \quad 0.555985$

C $-3.1702205 .237317 \quad 0.433251$

C $-2.7430955 .819392-0.883622$

C $-1.2393705 .877014-1.031729$

C $-0.5621897 .010196-0.781024$

C $-0.5328694 .643734-1.547501$

C $-0.4471363 .552617-0.515869$ 
N -4.2371502 .2190051 .907945$ H $2.022454-4.9541611 .815913$ H $1.980042-1.9732032 .508948$ H $-0.285651-2.6883103 .339583$ H $-0.212206-5.1256322 .658863$ H $-0.267616-4.6319010 .974234$ H -4.004035 -4.591890 1.211201 H $-3.769416-4.3218213 .467710$ H -2.228825 -4.825505 4.163672 H -2.517857 -3.1150763 .768183$ H - $1.604626-5.403902-0.462418$ H $-3.298150-5.572947-0.818562$ H -1.750504 -2.931642 -0.889847 H -2.123312 -3.943709 -2.286236 H -4.590466 -3.648833 -1.767792 H -3.436984 -2.243980 -3.516891 H -2.926393 -1.118845 -2.277579 H $-5.5345960 .693934-3.509928$ H $-4.8125240 .247016-0.989659$ H -5.714665 -2.028339-4.492356 H $-6.998887-1.212238-3.631422$ H -6.363786 -2.749548 -3.015439 H $-7.0075740 .448822-0.540190$ H $-6.782814-0.8384431 .318169$ H -5.654720 $-2.047648 \quad 1.869844$ H $-6.739210-3.1120070 .265144$ H -1.300911 -0.471596 -0.309170 H $-0.2308431 .844272 \quad 1.414141$ H -2.661862 $1.319029-1.398657$ H -1.151863 $0.994908-2.285144$ H $-2.0036592 .525267-2.475407$ H -4.620715 -0.2823922 .806618$ H -3.771466 $-0.247107 \quad 1.258235$ H -3.8882404 .0834552 .712414$ H -5.5145243 .8674082 .025942$ H $-4.4112473 .728500-0.338916$ H -2.7891115 .7196581 .330699$ H -3.164071 $6.831105-0.953057$ H -3.171397 $5.263282-1.726657$ H $0.5136347 .066891-0.909925$ H -1.068658 $7.905414-0.435625$ H $0.4974424 .893049-1.835221$ H -1.014034 4.322795 -2.475049 H 0.1170643 .8400210 .371975 H -3.309354 1.8821852 .148360 C $4.426426-4.0761050 .555886$

O $3.763529-3.240370-0.414709$ C $4.749765-2.475882-1.123112$

C $6.033965-3.283947-0.997142$

C $5.925157-3.7799060 .432465$

H $4.870616-1.533156-0.576728$

C $4.292967-2.184382-2.555970$

H $\quad 6.050737-4.135038-1.687330$

H $6.927961-2.679109-1.175405$

C $6.810475-4.9766750 .734650$

H $\quad 6.204960-2.9585001 .105878$

C $2.865891-1.612542-2.701969$

O $4.328959-3.412208-3.299192$

H $5.020389-1.520557-3.038159$

H $2.148357-2.410359-2.470496$

O $2.685212-1.300150-4.090333$

C $2.517024-0.359234-1.876207$

C $3.5263070 .793543-2.045544$

H $1.5356580 .007058-2.203709$

O $2.391264-0.704344-0.502154$

H $4.4808000 .510036-1.592572$

H $3.7098940 .953216-3.114196$

H $6.679180-5.304411 \quad 1.770748$

H $6.577209-5.8236160 .080631$

H $7.865250-4.7212830 .590381$

C $3.885179-3.7680551 .956110$

H $4.062616-3.206091-4.211622$

H $1.759158-1.029195-4.211842$

H $1.732093-1.415955-0.433853$

H $4.139828-2.7356672 .227836$

H $4.361738-4.4234322 .693923$

H $4.203416-5.1145140 .280928$

C 3.8179561 .8144220 .843272
O $2.702926 \quad 1.988947-0.034227$

C $3.0369072 .120534-1.417509$

C $3.9890683 .309651-1.615754$

C $5.2211603 .211339-0.716025$

C 4.8042542 .9820130 .738212

H 4.3451310 .8907630 .572667

C 3.2811131 .5724512 .261698

H $2.1021102 .370952-1.935150$

O $4.3785073 .395322-2.982160$

H $3.4499864 .235555-1.375095$

H $5.9057292 .424746-1.055066$

O $5.9529254 .437691-0.775009$

H 4.3690693 .9058811 .134148

O 5.9601702 .6901161 .520418

C 2.3626872 .6545902 .792647

H 2.6900870 .6476442 .242586

H 4.1081881 .4431972 .967732

H $4.8657784 .228019-3.098689$

H $6.3135364 .520854-1.673693$

H 6.5805193 .4307401 .408192

O 1.8013092 .2170263 .959964

O 2.1537013 .7373322 .264917

C 0.8726213 .1183444 .570640

H 0.4852002 .6448415 .476613

H 1.3757394 .0494654 .848023

H 0.0359833 .3167093 .893588

SCF Energy $\left(B 3 L Y P / 6-31 G^{* *} / / M M F F\right)=-3245.91432717$

01312

MM̄FF Geometry

C $-0.300517-3.5176941 .262278$

C $-0.733427-2.2754610 .996423$

C $-0.992369-1.2363612 .053752$

O $-0.314982-0.0277181 .627183$

C $-2.493003-0.9222942 .177086$

C $-3.321196-2.0189732 .807112$

C $-4.198838-2.7947762 .141259$

C $-3.157399-2.1703274 .297417$

C $-4.483687-2.8158160 .664935$

C $-5.847155-2.206750 \quad 0.306649$

C $-5.881271-0.6725840 .401920$

C $-7.269977-0.139548 \quad 0.042494$

C $-7.314037 \quad 1.3974890 .052378$

O $-4.923346-0.159171-0.526042$

O $-8.5146391 .806111-0.626069$

C $-6.1295631 .946488-0.777393$

C -7.4326841 .9445791 .479724$

O $-6.0247993 .362851-0.660920$

C $-4.7690591 .264707-0.497347$

C $-3.7542551 .622311-1.607764$

O $-4.1782091 .713540 \quad 0.722630$

C 0.0604430 .8488842 .597395

O $-0.0581490 .702908 \quad 3.803924$

C 0.6310432 .0434291 .935225

C 1.2395712 .9749292 .684462

C 1.7953034 .2397482 .206398

C 1.6872805 .3476193 .221531

C $-2.4182030 .885340-1.456604$

C $-1.4785171 .238465-2.588488$

C $0.3185832 .802461-3.239842$

O $-1.4288860 .569482-3.618144$

C $0.7091814 .208084-2.923443$

C $1.8935764 .537589-2.387798$

C $2.3085905 .942842-2.068227$

C $2.1366016 .290511-0.606724$

C $1.2719327 .246615-0.226883$

C 3.0178595 .5943960 .406721

C 2.3693394 .3683820 .993089

N $-0.7391112 .384337-2.340997$

H $-0.129027-3.7892322 .302217$

H $-0.928353-1.970716-0.027660$

H $-0.576369-1.5626613 .014026$

H -2.880629 -0.6467421 .191869$

H -2.628938 -0.0087322 .771568$

H $-4.767681-3.5284622 .711273$

H $-3.835091-2.9217994 .716327$

H $-2.135890-2.4763174 .543592$

H $-3.368089-1.2213304 .801224$ 
H $-3.685663-2.3454970 .082875$ H $-4.484949-3.868498 \quad 0.353450$ H $-6.072836-2.500817-0.726887$ H $-6.628727-2.6395180 .943255$ H $-5.606018-0.3694371 .417911$ H -8.031309 -0.5495790 .717842$ H -7.543734 -0.486352 -0.963997 H $-9.2704821 .389578-0.177568$ H $-6.3833901 .757016-1.829863$ H -8.3763851 .6195021 .935116$ H -7.4634513 .0393311 .485659$ H -6.6289841 .6119962 .138806$ H -5.616581 3.5656490 .197236 H -4.174886 $1.365835-2.588812$ H $-3.5650282 .703273-1.608343$ H -4.8741591 .9603371 .346842$ H 0.5160282 .1390020 .861286 H 1.3160472 .8283183 .760936 H 2.3050155 .1241594 .098473 H 0.6483845 .4613603 .552310 H 2.0008226 .3216882 .840576 H -1.941720 $1.128793-0.500668$ H $-2.558247-0.201563-1.472307$ H $-0.0308332 .724112-4.274634$ H $1.1514892 .103420-3.112576$ H $-0.0137784 .988092-3.149101$ H $2.6128033 .752823-2.167743$ H $3.3602756 .074804-2.350990$ H $1.7484636 .645339-2.699559$ H 1.1604177 .5263630 .815173 H $\quad 0.6537667 .770418-0.948496$ H 3.3202786 .3006971 .187619 H $3.9621945 .297104-0.067404$ H 2.4086903 .4968950 .342096 H $-0.7379272 .759730-1.396569$ C $-0.164511-4.276111-1.209407$ O $0.847908-3.319254-1.572162$ C $1.391901-3.671400-2.857215$ C $0.486077-4.762130-3.415293$ C $0.050982-5.467000-2.143458$ H $2.381662-4.104550-2.669367$ C $1.534148-2.417456-3.727549$ H $-0.384759-4.345519-3.934020$ H $1.011151-5.420005-4.114415$ C -1.176669 -6.342634 -2.324823 H $\quad 0.883979-6.084068-1.780682$ C $2.275631-1.257295-3.027535$ O $0.223348-1.949696-4.066332$ H $2.022914-2.677770-4.673593$ H $1.647628-0.869548-2.217978$ O $2.406526-0.186815-3.970400$ C $3.669434-1.625375-2.492129$ C $4.421890-0.455128-1.833278$ H $3.586853-2.445847-1.773211$ O $4.458313-2.107692-3.583366$ H $5.435060-0.803317-1.597354$ H $4.5692890 .350602-2.563443$ H -1.459480 -6.819657 -1.381331 H -2.034968 -5.762065 -2.679401 H $-0.980179-7.132833-3.056795$ C $-0.021875-4.6232520 .274823$ H $\quad 0.329822-1.137319-4.590595$ H $2.995283-0.493251-4.681460$ H $5.312896-2.398771-3.222085$ H $1.004937-4.9629310 .462500$ H $-0.689511-5.457360 \quad 0.520141$ H -1.140421 -3.815133 -1.408968 C $4.402722-1.6292860 .980145$ O $3.330640-0.936792 \quad 0.333177$ C $3.7452490 .100520-0.562122$ C $4.590227 \quad 1.1371530 .193732$ C 5.7629240 .4955050 .933489 C $5.280766-0.6715391 .795503$ H $5.024433-2.1101190 .213985$ C $3.810664-2.7832671 .803373$ H $2.8291350 .608665-0.887258$ O $5.0881112 .133007-0.692930$ H 3.9603901 .6462110 .933566
H $\quad 6.5494390 .1734810 .241138$

O $6.3629871 .467007 \quad 1.794141$

H $4.735351-0.2721582 .657683$

O $6.407373-1.3841402 .300767$

C $2.916041-2.3533112 .947137$

H $3.198664-3.4151641 .148459$

H $4.626673-3.3895422 .212979$

H $4.3237042 .535127-1.138980$

H $\quad 6.6234312 .2224791 .239546$

H $6.961418-0.7463562 .782857$

O $2.716348-3.4198513 .777596$

O $2.431468-1.2398393 .088122$

C $1.886081-3.1668324 .915564$

H $\quad 0.876252-2.8951644 .595559$

H $1.831187-4.0847475 .506753$

H $2.320956-2.3763035 .534788$

SCF Energy (B3LYP/6-31G**//MMFF) $=-3245.89993464$

01313

MM̄FF Geometry

C $-6.319416 \quad 0.401697 \quad 0.189965$

C $-5.4217331 .370652-0.040332$

C $-4.8716352 .272661 \quad 1.032891$

O $-3.457082 \quad 1.9917751 .147272$

C $-5.043497 \quad 3.7476110 .628150$

C $-4.5286224 .725072 \quad 1.669498$

C $-3.419754 \quad 5.4819051 .539584$

C -5.3704714 .8385582 .915118$

C $-2.461993 \quad 5.529580 \quad 0.380429$

C $-1.145606 \quad 4.8346860 .731151$

C $-0.1713944 .695929-0.449668$

C $-0.7118883 .769381-1.540945$

C $0.3168093 .532959-2.651863$

$\begin{array}{llllll}\text { O } & 1.035701 & 4.144429 & 0.091472\end{array}$

O $-0.1756592 .446177-3.450731$

C $1.6519903 .092735-2.019389$

C $0.4172624 .744816-3.586664$

O $2.6518513 .001775-3.031345$

C $2.1153053 .983042-0.845993$

C $3.2562553 .333133-0.028600$

O $2.5574055 .245367-1.344260$

C $-3.095697 \quad 0.9313301 .921981$

O -3.8329750 .2057232 .569885$

C -1.6259380 .7948831 .832310$

C $-0.999092-0.173872 \quad 2.515777$

C $0.443459-0.4181562 .521361$

C 1.3183910 .5461821 .765264

C $4.5634263 .057919-0.769629$

C 5.6474782 .6365220 .203369

C $6.383436 \quad 0.7639641 .649531$

O $6.578915 \quad 3.383795 \quad 0.494394$

C $5.733070-0.3902972 .341293$

C $5.279189-0.3364383 .602847$

C $4.664439-1.4989734 .329554$

C $3.162407-1.3600124 .465952$

C $2.630116-0.8122735 .571960$

C $2.361690-1.9117833 .304219$

C $0.927930-1.4692063 .215040$

N $5.446276 \quad 1.3647780 .720688$

H $-6.7140970 .264103 \quad 1.193175$

H $-5.014013 \quad 1.489712-1.041779$

H $-5.3655632 .092283 \quad 1.995538$

H $-6.1062953 .961763 \quad 0.451847$

H $-4.5506573 .912939-0.334911$

H $-3.155134 \quad 6.1487092 .359692$

H -5.0110905 .6226653 .590014$

H $-6.405247 \quad 5.082292 \quad 2.653127$

H -5.3638333 .8974503 .473280$

H $-2.9069475 .096104-0.515236$

H -2.2658756 .5806770 .135638$

H -0.6455045 .3898331 .536800$

H $-1.3404563 .841557 \quad 1.152319$

H $0.0406485 .694776-0.847864$

H $-1.6393194 .155220-1.977869$

H $-0.9733022 .798482-1.096808$

H $0.5098722 .226897-4.105179$

H $1.5051812 .074896-1.636325$

H $-0.5623524 .970605-4.024986$ 
H $1.0804364 .539853-4.434454$ H $0.772395 \quad 5.645387-3.080944$ H $2.9586623 .903651-3.226408$ H 3.4768093 .9835430 .830273 H $2.8885352 .401508 \quad 0.415467$ H $3.1554475 .640276-0.688064$ H -1.095082 1.4950991 .198555 H $-1.592677 \quad-0.8542083 .124645$ H 2.3861280 .3410061 .870989 H $1.165547 \quad 1.5681202 .130935$ H 1.0896530 .5204910 .695241 H $4.4449712 .257747-1.507531$ H $4.9119913 .949948-1.301106$ H $6.727223 \quad 1.5286212 .354988$ H $7.2543720 .422743 \quad 1.080265$ H $5.651427-1.3192481 .782376$ H $5.362400 \quad 0.5955534 .157623$ H $5.134188-1.5687645 .319316$ H $4.901032-2.447778 \quad 3.831038$ H $1.559893-0.7163645 .715073$ H $3.262878-0.4455426 .374101$ H $2.373438-3.0070693 .374492$ H $2.870952-1.6794412 .362408$ H $\quad 0.232172-2.1078773 .759209$ H $4.7494470 .767757 \quad 0.283114$ C $-6.098178-1.919252-0.810850$ O $-4.686394-1.772425-1.066290$ C -3.967818-2.708540 -0.239009 C $-5.021317-3.6298540 .370018$ C $-6.206581-2.6929900 .501198$ H $-3.492733-2.1262020 .560382$ C $-2.889078-3.444876-1.046595$ H $-5.264370-4.462377-0.300908$ H $-4.698329-4.0522041 .326465$ C -7.533534-3.405801 0.690206 H $-6.026832-2.0368251 .361493$ C $-1.749096-2.524380-1.540732$ O $-3.507218-4.111139-2.146094$ H $-2.451631-4.220805-0.408353$ H $-1.337370-1.997383-0.672417$ O $-2.252385-1.537006-2.439053$ C $-0.607794-3.289285-2.245426$ C $0.549449-2.397074-2.734426$ H $-0.217775-4.054543-1.566241$ O $-1.108614-3.985190-3.389639$ H $1.239810-3.029607-3.306589$ H $0.167628-1.672754-3.465083$ H $-8.354426-2.6856120 .765121$ H -7.750485 $-4.079532-0.145546$ H $-7.522105-4.0026131 .608079$ C $-6.791011-0.554380-0.868250$ H -3.879976 -3.426305 -2.727643 H $-2.910644-1.007380-1.958049$ H $-1.537032-3.329686-3.966461$ H -7.875313 $-0.678889-0.768240$ H -6.615192 - $0.113715-1.858233$ H $-6.490830-2.530291-1.634828$ C $2.723443-3.475842-0.900548$ O $1.697661-2.538047-0.552372$ C $1.308772-1.660870-1.614015$ C $2.520146-0.849771-2.096149$ C $3.717190-1.734656-2.434633$ C $3.997463-2.726315-1.305752$ H $2.379106-4.111951-1.725160$ C $2.974528-4.3809300 .308220$ H $0.603450-0.943204-1.179122$ O $2.163141-0.069673-3.232083$ H $2.813696-0.150474-1.302161$ H $3.564621-2.249565-3.390501$ O $4.858728-0.891895-2.610627$ H $4.405836-2.181876-0.445260$ O $4.999609-3.646692-1.731126$ C $1.777382-5.260013 \quad 0.580534$ H $3.830862-5.044557 \quad 0.142365$ H $3.198150-3.8001891 .208345$ H $2.9484130 .442027-3.492190$ H $5.620658-1.470125-2.787430$ H $4.643756-4.149251-2.483956$
O $\quad 0.897473-4.612374 \quad 1.395954$

O $1.633347-6.3786520 .104545$

C $-0.300420-5.3287021 .709869$

H $-0.979140-4.6451042 .226917$

H $-0.790528-5.6837600 .798396$

H $-0.068246-6.1662792 .373752$

SCF Energy (B3LYP/6-31G**//MMFF) $=-3245.92361001$

01314

MM̄FF Geometry

C 1.0062271 .8911052 .372911

C 0.4290110 .6943292 .562780

C $1.164393-0.6210882 .680352$

O $2.591293-0.3773952 .762706$

C $0.760588-1.4465743 .914123$

C $-0.535384-2.2173953 .755522$

C $-0.632377-3.4061293 .125201$

C $-1.739670-1.6136054 .425787$

C $0.477333-4.1485602 .433715$

C $0.076525-4.7955361 .099637$

C $-0.553721-3.8528590 .061046$

C $-0.862881-4.628862-1.224622$

C $-1.453729-3.726950-2.316615$

O $0.345059-2.773715-0.227790$

O $-1.401896-4.467056-3.545874$

C $-0.555451-2.481800-2.472059$

C $-2.934016-3.433122-2.057793$

O $-1.135534-1.547705-3.379579$

C $-0.204186-1.798512-1.130808$

C $0.809879-0.645321-1.313802$

O $-1.392616-1.210717-0.584204$

C $3.415700-1.1321821 .990233$

O $3.101868-2.0561881 .258195$

C $4.792922-0.6217552 .163908$

C $5.756660-1.0777871 .350236$

C $7.162503-0.6754111 .354755$

C 7.6125110 .3096702 .400239

C $2.167193-1.032193-1.904503$

C $3.1127890 .159042-1.959179$

C $5.3517820 .809976-2.802159$

O $2.7791631 .296041-1.634173$

C $6.7295460 .234297-2.719210$

C $7.7552300 .874406-2.136113$

C $9.1564700 .333852-2.062348$

C $9.6973400 .318015-0.648559$

C $10.438646 \quad 1.338710-0.185478$

C $9.441469-0.9039250 .205815$

C $7.979660-1.1879280 .411542$

N $4.370075-0.195968-2.435007$

H $2.089037 \quad 1.9696342 .318826$

H -0.6565920 .6441082 .571642$

H $0.940528-1.1853851 .771089$

H $0.708563-0.7985524 .799596$

H $1.563262-2.156514 \quad 4.157150$

H -1.598759 -3.907597 3.114185

H $-2.640730-2.2210004 .289311$

H -1.565713 -1.516983 5.502452

H -1.948791 -0.6216014 .019196$

H $1.342410-3.5065202 .263909$

H $0.816135-4.9471453 .105521$

H $0.983213-5.2455630 .674201$

H $-0.619888-5.6196791 .301485$

H - $1.479663-3.4471310 .484144$

H $-1.534761-5.471925-1.019949$

H $0.065455-5.074250-1.609980$

H - $1.704838-3.877252-4.257701$

H $\quad 0.369316-2.830243-2.947606$

H -3.510240 -4.365965 -2.034944

H $-3.367899-2.836490-2.867667$

H $-3.106913-2.914615-1.112771$

H -1.844506 -1.078847 -2.907870

H $\quad 0.3553030 .146528-1.924031$

H $\quad 0.988070-0.178818-0.337169$

H - $-1.132665-0.5262780 .054830$

H 4.9702710 .1201222 .933554

H $5.490088-1.8118880 .590169$

H $7.437504-0.0895093 .405410$

H 7.0688631 .2554422 .297663 
H 8.6765830 .5500472 .337288

H $2.634447-1.824033-1.311260$

H $2.045299-1.395190-2.930368$

H $5.2299681 .691206-2.163365$

H $5.1349881 .104748-3.834319$

H $6.899176-0.737674-3.176069$

H $7.5866391 .859375-1.705814$

H $9.7920560 .967036-2.695193$

H $9.222792-0.673650-2.491244$

H 10.8520531 .3290720 .817522

H $10.6454692 .209560-0.799077$

H $9.952208-0.8311921 .173486$

H $9.895639-1.774653-0.285049$

H $7.565516-1.887308-0.315618$

H $4.484901-1.128659-2.822267$

C $0.292612 \quad 3.622592 \quad 0.727991$

$\begin{array}{llllll}\text { O } & -0.755924 & 2.941784 & 0.001761\end{array}$

C $-1.4039453 .892586-0.866386$

C $-0.5054755 .122531-0.887127$

C $0.029638 \quad 5.1144970 .530093$

H $-2.3491234 .169569-0.385848$

C -1.665763 $3.281814-2.246094$

H $0.3138445 .012678-1.607517$

H $-1.0555816 .035305-1.135124$

C 1.2566425 .9900230 .717912

H -0.7630605 .4511961 .211815$

C -2.638433 2.083209-2.229739

O $-0.4125812 .844003-2.783996$

H -2.040289 $4.058270-2.924354$

H -2.186084 $1.238112-1.699764$

O $-2.8363721 .660408-3.584140$

C -4.019401 $2.402900-1.623214$

C $-5.1017361 .333630-1.850327$

H $-3.9149222 .576048-0.549390$

O $-4.515237 \quad 3.625312-2.180678$

H -6.030253 $1.709651-1.403010$

H -5.321092 $1.263529-2.923497$

H 1.6200615 .9390471 .748950

H 2.0744535 .6820140 .057611

H 1.0204107 .0354400 .494562

$\begin{array}{llll}\text { C } & 0.261495 & 3.183127 & 2.192187\end{array}$

H $-0.5513372 .642704-3.724642$

H -2.008168 $1.250868-3.886355$

H $-4.5783743 .504133-3.143733$

H -0.7740783 .0799252 .534325$

H 0.7443083 .9296842 .832751

H 1.2414923 .3414520 .251473

C -5.4540940 .2784791 .000905$

$\begin{array}{llll}\text { O } & -4.394666 & -0.034144 & 0.088487\end{array}$

C $-4.767586-0.062260-1.292470$

C $-5.878410-1.098163-1.514276$

C $-7.074401-0.842006-0.602440$

C -6.619382 -0.7120460 .850016$

H -5.8016021 .3035590 .823029$

$\begin{array}{llll}\text { C } & -4.880438 & 0.202235 & 2.425879\end{array}$

H -3.888592 $-0.418128-1.841167$

O $-6.289072-1.095579-2.876834$

H $-5.481898-2.098219-1.300651$

H $-7.6319810 .045906-0.920334$

O $-7.992825-1.932873-0.710583$

H $-6.321195-1.6987121 .227826$

O $-7.718706-0.267441 \quad 1.643111$

C $-3.966131 \quad 1.3621902 .757747$

H -5.7007110 .2147993 .153112$

H $-4.300282-0.7180592 .558901$

H $-7.010795-1.741441-2.964784$

H -7.530372 -2.744106 -0.439289

H $-8.446818-0.8986731 .510658$

O -3.9017191 .4993084 .115522$

O

C -3.052875 2.5436824 .602430

H -2.008194 2.2303294 .526567

H -3.292873 2.7119135 .655712

H -3.219014 3.4765054 .054951

SCF Energy (B3LYP/6-31G**//MMFF)= -3245.91750332

01315

MM̄FF Geometry
C $3.938383-4.450421-0.036379$

C $3.018832-4.001216-0.904483$

C $1.540853-4.272447-0.783778$

O $0.886186-3.028883-0.437894$

C $0.979805-4.744396-2.137609$

C $-0.508521-5.035334-2.112689$

C $-1.433804-4.355570-2.818810$

C $-0.933809-6.188921-1.240727$

C $-1.221268-3.207514-3.766391$

C $-1.344971-1.813699-3.130751$

C $-2.747316-1.487564-2.590479$

C $-2.838840-0.017223-2.177786$

C $-4.1992280 .322714-1.549354$

O $-3.000393-2.313364-1.452665$

O $-4.075903 \quad 1.600483-0.904437$

C $-4.529013-0.710589-0.448542$

C $-5.2907640 .480319-2.615161$

O $-5.860668-0.5083200 .019098$

C $-4.315205-2.173497-0.895053$

C $-4.421396-3.198007 \quad 0.261483$

O $-5.320152-2.520098-1.855711$

C $0.854193-2.6892370 .879243$

O $1.293450-3.333462 \quad 1.818724$

C $0.217421-1.359048 \quad 0.985903$

C $0.444966-0.6141722 .077906$

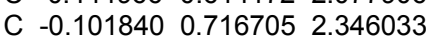

C -1.1900591 .2155431 .433911$

C $-3.427047-2.9979751 .410714$

C $-3.986821-2.2162662 .587606$

C $-3.296618-1.2758814 .791316$

$\begin{array}{llll}\text { O } & -5.169245 & -1.900970 & 2.687374\end{array}$

C $-3.068734 \quad 0.195066 \quad 4.641647$

C -2.2748330 .9040335 .458664$

C -2.0835292 .3903125 .359766$

C $-0.629136 \quad 2.787161 \quad 5.242744$

C 0.0641803 .2039196 .315538

C $-0.009393 \quad 2.788277 \quad 3.864140$

C 0.3738521 .4085503 .402417

N $-3.009529-1.9445423 .536201$

H $3.628080-5.0670060 .803197$

H $3.336524-3.360509-1.724296$

H $1.340993-5.027638-0.013614$

H $1.503323-5.654659-2.458816$

H $1.206181-3.984619-2.893371$

H $-2.475088-4.661084-2.720740$

H $-1.991706-6.440885-1.372142$

H $-0.352853-7.084270-1.484954$

H $-0.783390-5.950575-0.183449$

H $-0.245210-3.287679-4.256645$

H $-1.953677-3.298756-4.578916$

H $-0.607073-1.723479-2.325132$

H - $-1.077532-1.075312-3.896695$

H -3.479481 -1.710086 -3.375661

H $-2.6424820 .638985-3.034026$

H -2.051806 $0.204615-1.444555$

H $-3.7867792 .247033-1.571164$

H $-3.864552-0.488490 \quad 0.393778$

H $-5.021303 \quad 1.271979-3.324679$

H $-6.2399930 .793189-2.166367$

H $-5.463236-0.432441-3.189257$

H $-5.989947-1.0668520 .803531$

H $-5.454600-3.2592170 .623196$

H $-4.209443-4.188616-0.166942$

H $-5.400209-3.487909-1.883025$

H $-0.400257-1.0248830 .160940$

H $1.110080-1.0078632 .845787$

H -1.9897450 .4726861 .354055$

H -0.793122 1.4126910 .432940

H $-1.6680782 .132752 \quad 1.783247$

H -3.152923 -3.9852221 .804877$

H $-2.508036-2.5204161 .059238$

H -2.646775 -1.720555 5.551800

H $-4.338367-1.4600685 .072742$

H -3.6072410 .7051953 .846389$

H -1.7596970 .3953296 .270553$

H -2.521555 2.8420356 .259591

H -2.645484 $2.811617 \quad 4.516820$

H 1.0990213 .5191866 .232269 
H $-0.3863653 .233703 \quad 7.302122$ H $0.9045463 .397353 \quad 3.861860$ H -0.686209 3.2988913 .174803 H 1.1601840 .9533624 .006556 H -2.044505 -2.197172 3.343430 C $5.776501-2.7747350 .536903$ O $5.124538-1.693340-0.161508$ C $4.374250-0.9255810 .791827$ C $5.097253-1.1425792 .111022$ C $5.410422-2.6270712 .018244$ H $3.378028-1.3773360 .849782$ C $4.214418 \quad 0.534656 \quad 0.353712$ H $6.023697-0.5585452 .160830$ H $4.478172-0.8887122 .976416$ C $6.508348-3.0813952 .964238$ H $4.496861-3.1858632 .254693$ C $3.4808430 .686486-0.996037$ O $5.4911001 .164493 \quad 0.276191$ H 3.6502181 .0489921 .138114 H $2.5198270 .158498-0.956959$ O $4.2462330 .068369-2.038096$ C $3.2376512 .142581-1.441812$ C $2.4256632 .968889-0.427325$ H $4.1955412 .637378-1.639444$ O $2.5486892 .098521-2.694240$ H $1.5042692 .426156-0.193613$ H 2.9998053 .0429100 .501552 H $6.710245-4.1499892 .838017$ H $7.442342-2.5376192 .786973$ H $6.213986-2.9120204 .005136$ C $5.401217-4.107692-0.125705$ H $6.002140 \quad 0.701259-0.409948$ H $4.302389-0.881467-1.839005$ H $1.7062401 .633935-2.554191$ H $5.977859-4.9225920 .327148$ H $5.688977-4.070852-1.184582$ H $6.852751-2.6142290 .395513$ C $-0.0914703 .967158-1.878067$ O $1.254398 \quad 4.415372-2.081118$ C $2.0865734 .398477-0.916107$ C 1.4690325 .3013210 .161946 C 0.0258314 .9067110 .463683 C $-0.7956964 .825727-0.822013$ H $-0.0884252 .916441-1.565593$ C $-0.8162734 .056375-3.226292$ H $3.0392314 .856120-1.212676$ O $2.247807 \quad 5.255576 \quad 1.352527$ H $1.4867916 .339847-0.193150$ H -0.0079163 .9568051 .004515$ O $-0.5590985 .874475 \quad 1.337208$ H $-0.9684875 .837204-1.211327$ O $-2.069927 \quad 4.256129-0.530321$ C $-0.2652203 .039477-4.198288$ H $-1.8895273 .858435-3.128810$ H $-0.7074595 .060305-3.654375$ H 1.7995715 .8077762 .015789 H $-0.538358 \quad 6.7365520 .887815$ H -2.4755924 .7936800 .171409$ O $0.3786363 .668983-5.221756$ O $-0.3777671 .828777-4.054438$ C $0.9701362 .806426-6.198279$ H $1.7294352 .171081-5.732364$ H $0.2001482 .197919-6.682127$ H $1.4517373 .430322-6.955841$ SCF Energy (B3LYP/6-31G**//MMFF) $=-3245.92054013$

$01 \_316$

MM̄FF Geometry

C $1.224542-2.196696-2.198444$

C $0.327034-3.192602-2.128052$

C $-0.488660-3.481072-0.895998$

O $-1.882204-3.354442-1.265698$

C $-0.250184-4.903706-0.352148$

C $1.166189-5.191130 \quad 0.095561$

C $1.734153-4.6687971 .200252$

C $1.928781-6.159220-0.769532$

C $1.097939-3.7298242 .187965$

C $1.828750-2.3815852 .224349$
C $1.085918-1.2714432 .987540$

C $0.689232-1.6770894 .408344$

C $-0.102789-0.5706815 .121417$

O $-0.076330-0.9110922 .231263$

O $-0.704906-1.1468256 .291725$

C $-1.254510-0.1077164 .201847$

C 0.8115400 .5563365 .617472

O $\begin{array}{llll}-1.918916 & 1.008445 & 4.790982\end{array}$

C -0.7962940 .2145922 .758540$

C -2.0188590 .4692641 .840065$

O $0.012367 \quad 1.3949292 .788828$

C $-2.756691-3.058036-0.265557$

O $-2.501005-2.9209410 .920474$

C $-4.096468-2.894492-0.867585$

C $-5.013261-2.172309-0.206706$

C $-6.373274-1.875737-0.654735$

C $-6.871667-2.570776-1.894073$

C -1.6534660 .5550040 .352274$

C $-2.8027450 .989916-0.537275$

C $-4.3121822 .916002-1.015121$

O $-3.2097740 .280662-1.455000$

C $-5.6567552 .588447-0.443772$

C $-6.6976582 .201246-1.197316$

C $-8.0777061 .942466-0.660556$

C $-8.6455930 .617580-1.118637$

C $-9.3073830 .520484-2.284489$

C $-8.528422-0.570831-0.193182$

C $-7.109573-0.9970610 .057075$

N $-3.2768742 .256511-0.242877$

H $1.359273-1.539738-1.342342$

H $\quad 0.187448-3.849793-2.982815$

H $-0.260589-2.731215-0.130181$

H $-0.557339-5.635698-1.112611$

H $-0.933583-5.1026680 .483905$

H $2.758306-4.9526401 .438336$

H $2.967431-6.279955-0.444486$

H $1.452654-7.144863-0.740593$

H $1.945913-5.816118-1.808512$

H $0.035606-3.5814481 .978010$

H $1.151591-4.2034753 .175431$

H $2.819370-2.5254182 .674095$

H $1.990141-2.0358331 .194887$

H $1.758350-0.4059763 .017467$

H $1.573464-1.9598694 .992793$

H $\quad 0.049275-2.5686944 .378673$

H $0.006351-1.5100666 .846811$

H -1.986817 -0.9266644 .165916$

H 1.5513170 .1645446 .325978

H $\quad 0.242528 \quad 1.313570 \quad 6.168073$

H 1.3590751 .0519574 .813227

H $-2.7610801 .134813 \quad 4.323223$

H -2.746316 -0.3434081 .956521$

H -2.5184201 .3927122 .152448$

H -0.1020011 .8923311 .964780$

H -4.276119-3.336087-1.840566

H $-4.735312-1.7319650 .750523$

H -6.823653 -3.658397 -1.771132

H $-6.267263-2.287958-2.763009$

H -7.907756 -2.326794 -2.140139

H $-0.834538 \quad 1.256917 \quad 0.186113$

H - $-1.304624-0.419507-0.005639$

H -4.224336 $2.615924-2.064740$

H -4.131883 $3.992988-0.942288$

H -5.7905932 .7133030 .627910$

H $-6.5641232 .112905-2.273470$

H $-8.7291752 .755424-1.008017$

H -8.0990311 .9970340 .434979$

H -9.746673 -0.416189-2.610771

H $-9.4213571 .377275-2.940509$

H -9.118639-1.419908 -0.558206

H $-8.986787-0.3125870 .770569$

H $-6.660550-0.5254690 .931939$

H -2.8577362 .7812710 .518692$

C $3.597221-2.037425-3.102025$

O $4.001910-1.016653-2.165501$

C $4.796234-1.634131-1.135833$

C $5.284193-2.949397-1.729992$

C $4.064270-3.374967-2.521899$ 
H $4.113644-1.853023-0.305432$

C $5.903463-0.695561-0.646403$

H $6.137013-2.803428-2.402753$

H $5.572631-3.672858-0.961577$

C $4.347020-4.435698-3.570338$

H $3.329198-3.769603-1.813013$

C $5.4363430 .722620-0.261274$

O $6.894187-0.547491-1.672232$

H $6.422133-1.1604810 .200181$

H $5.238296 \quad 1.300529-1.171227$

O $6.528702 \quad 1.374544 \quad 0.399318$

C $4.209378 \quad 0.7783220 .663583$

C 3.8628602 .1788121 .203383

H 3.3368220 .3849850 .131645

O $4.416932-0.0784221 .789554$

H 2.9145982 .0916071 .749204

H 4.5958102 .4689061 .967592

H $3.434769-4.692190-4.118405$

H $5.093236-4.095067-4.295856$

H $4.727397-5.348634-3.100586$

C $2.094860-1.927885-3.395610$

H $6.446164-0.208649-2.466462$

H $7.2915121 .343737-0.203601$

H 5.2230890 .2222732 .243036

H $1.838213-2.608213-4.216341$

H $1.872539-0.910257-3.738766$

H $4.141961-1.820053-4.029901$

C $1.6175302 .755819-0.811369$

O $3.0272982 .882459-1.014881$

C 3.7675633 .2887390 .137563

C 3.2401814 .6297970 .668417

C 1.7298984 .6037940 .896300

C $1.0096094 .086615-0.349068$

H $1.4406961 .991502-0.045157$

C $1.0091572 .205479-2.106279$

H $4.7912413 .471769-0.211711$

O 3.8894294 .9889231 .883500

H $3.4633895 .419384-0.061044$

H 1.4653704 .0089231 .778482

O $1.2747705 .933690 \quad 1.158690$

H $1.0826904 .851090-1.132283$

O $-0.3694923 .922239-0.033329$

C $\quad 1.1072343 .175003-3.260539$

H $1.5352441 .294370-2.416250$

H $-0.0384551 .921595-1.961561$

H 4.8452175 .0178401 .706329

H 1.7669986 .2597591 .931569

H $-0.8439353 .744200-0.862898$

O $-0.1132863 .733290-3.503168$

O $2.146013 \quad 3.422189-3.859085$

C $-0.1498884 .695289-4.561526$

H -1.175618 $5.062869-4.651344$

H $0.5061115 .539324-4.328452$

H $0.140408 \quad 4.229027-5.507773$

SCF Energy (B3LYP/6-31G**/MMFF) $=-3245.90755142$

01317

MM̄FF Geometry

C $3.962383-0.339448-2.375266$

C $3.101386-1.243705-2.868259$

C $2.527294-2.373154-2.055243$

O $1.128888-2.476647-2.421208$

C $3.209533-3.721106-2.352315$

C $4.607829-3.846930-1.786968$

C $4.879708-4.210803-0.518015$

C $5.731258-3.610966-2.762711$

C $3.896494-4.5398820 .572591$

C $4.241594-3.8696161 .909820$

C $3.955978-2.3582601 .956012$

C $4.551245-1.7684713 .239238$

C $4.203379-0.2869873 .421059$

O $2.534905-2.171048 \quad 1.935385$

O $4.563534 \quad 0.0586574 .767077$

C $2.678316-0.1215653 .271318$

C 5.0400140 .6077462 .501328

O $2.311581 \quad 1.2571603 .281176$

C $2.108648-0.8024842 .002936$

C $0.560973-0.7763371 .999271$
O $2.549273-0.0655370 .857156$

C $0.278667-3.007324-1.502503$

O $0.552935-3.379036-0.373125$

C -1.065578-3.072669-2.115595

C $-2.108862-3.431124-1.353484$

C $-3.500542-3.554856-1.786729$

C $-3.806104-3.355206-3.247827$

C $-0.104910-1.7013583 .020575$

C - $1.448208-1.1465233 .446508$

C $-3.758889-0.7370352 .643723$

O $-1.587094-0.5691284 .523189$

C $-4.804395-1.6995912 .181195$

C $-5.670064-1.4339271 .191417$

C $-6.775140-2.3590980 .766360$

C $-6.685493-2.744181-0.693341$

C $-7.327363-2.034266-1.636359$

C $-5.916210-3.996612-1.047972$

C $-4.435871-3.833974-0.855572$

N -2.446028 -1.339093 2.503883

H $4.280992-0.439246-1.342492$

H $2.806963-1.183867-3.913433$

H $2.598053-2.133303-0.988564$

H $3.224814-3.901544-3.436192$

H $2.590685-4.541865-1.966612$

H $5.925415-4.314437-0.230699$

H $6.716293-3.741255-2.301828$

H $5.662046-4.318740-3.595483$

H $5.692669-2.596626-3.168017$

H $2.869457-4.2977630 .293405$

H $3.931509-5.626470 \quad 0.721479$

H $3.644648-4.3637992 .687947$

H $5.296354-4.0588662 .146362$

H $4.395837-1.8773281 .075349$

H $5.639296-1.9094853 .262607$

H $4.162100-2.3226214 .105739$

H 4.4005631 .0090034 .889030

H $2.218491-0.5687574 .160640$

H 6.1064460 .5056952 .735509

H 4.7994691 .6661992 .649434

H $4.9086300 .369611 \quad 1.444978$

H 2.5828751 .6294174 .136760

H $\quad 0.2154390 .2581742 .119524$

H $0.199236-1.0758521 .007221$

H $1.905562-0.1834570 .139684$

H -1.159162 -2.825025 -3.166461

H $-1.931222-3.649059-0.300681$

H -3.302801 -4.119759 -3.849893

H $-3.472189-2.369778-3.587478$

H -4.871504 -3.413863 -3.481029

H $-0.241107-2.7045382 .599395$

H $0.495598-1.8253093 .926711$

H -3.753234 0.1847712 .054033

H -3.940663 -0.468357 3.689151

H -4.876751 -2.644789 2.713928

H $-5.607649-0.4784690 .677068$

H $-7.729204-1.8500940 .956836$

H -6.800183 -3.264403 1.385573

H $-7.289217-2.318596-2.682643$

H $-7.900807-1.148650-1.382378$

H -6.148532 -4.324702 -2.066935

H $-6.261480-4.821331-0.411127$

H -4.115920 -3.965264 0.178530

H -2.191913 -1.715804 1.594940

C $4.7400992 .100262-2.457820$

O $3.4792392 .582906-1.958664$

C $3.6967313 .174454-0.662471$

C $5.2089313 .291982-0.491201$

C $5.7009742 .088705-1.269369$

H 3.3123782 .4615110 .075461

C $2.9442874 .503178-0.537367$

H $5.6003294 .214087-0.936133$

H 5.5086403 .2763840 .561037

C $7.1672272 .170869-1.657585$

H $5.5522191 .197820-0.651383$

C $1.4405394 .436355-0.864169$

O $3.5261285 .461839-1.432399$

H 3.0892014 .9104490 .469999

H $1.2938634 .291194-1.941686$ 
O $0.877985 \quad 5.719162-0.554305$

C $0.649827 \quad 3.359342-0.097787$

C $-0.8653703 .479969-0.343357$

H $1.0048632 .372774-0.403620$

O $0.903612 \quad 3.4784051 .301611$

H -1.221252 4.4132560 .113355

H - $1.0524213 .570029-1.419413$

H $7.4710501 .283755-2.222076$

H $7.3707633 .051118-2.276438$

H $7.7978542 .235148-0.764723$

C $4.5617900 .769005-3.196827$

H $3.4757935 .092328-2.330880$

H $1.3789346 .384178-1.057246$

H $0.637945 \quad 4.373316 \quad 1.574568$

H $5.5374530 .434547-3.568139$

H $3.9244040 .947819-4.072196$

H $5.0775902 .845496-3.190766$

C -3.846699 $3.150651-0.509399$

O $-3.017824 \quad 2.798606 \quad 0.602584$

$\begin{array}{llll}\text { C } & -1.708275 & 2.324024 & 0.243229\end{array}$

C $-1.8406151 .078089-0.648720$

C $-2.7616341 .318444-1.846957$

C $-4.0898431 .934751-1.406657$

H $-3.3459003 .934844-1.091796$

C $-5.134015 \quad 3.794373 \quad 0.018649$

H -1.2412542 .0151851 .185189$

O $-0.5658910 .642730-1.103442$

H -2.258121 $0.261489-0.047385$

H -2.270937 $1.946846-2.598692$

O $-3.019726 \quad 0.072741-2.496967$

H $-4.6810231 .176721-0.880658$

O $-4.8315732 .334840-2.555244$

C -6.0248972 .8339490 .773670$

H -4.8981654 .6121300 .710154$

H $-5.6970464 .234690-0.812874$

H $-0.708360-0.120204-1.688939$

H -3.466024 -0.510944 -1.859875

H -4.936985 $1.550364-3.120603$

O

$\begin{array}{llll}\text { O } & -5.690257 & 2.243870 & 1.792493\end{array}$

$\begin{array}{llll}\text { C } & -8.187229 & 1.872883 & 0.777272\end{array}$

H -8.349215 2.1679721 .818404

H -9.133545 1.9623590 .237303

H -7.8467080 .8359940 .717646$

SCF Energy (B3LYP/6-31G**//MMFF) $=-3245.91810464$

01_318

MMFF Geometry

C $1.258917-2.126074-2.404463$

C $0.244234-2.978440-2.618144$

C $-0.781084-3.345700-1.578590$

O $-2.068008-2.828453-2.002786$

C $-0.972490-4.868810-1.447226$

C $0.195761-5.625383-0.858316$

C $0.325783-5.9274620 .447404$

C $1.210374-6.138450-1.846833$

C $-0.595865-5.5654451 .579981$

C $0.176154-5.0047162 .782648$

C $0.565684-3.5276372 .613634$

C $1.603421-3.1411413 .670405$

C $1.925119-1.6423423 .641859$

O $-0.628934-2.7545572 .777964$

O $2.663420-1.3272934 .831949$

C $0.598727-0.8621503 .709005$

C $2.826668-1.2726022 .458912$

$\begin{array}{lllll}\text { O } & 0.833657 & 0.539880 & 3.589389\end{array}$

C $-0.455967-1.3354492 .678008$

C $-1.817481-0.6764383 .007550$

O $-0.024069-0.9482351 .374988$

C -2.323685-1.507893 -1.806705

O $-1.572600-0.672436-1.330757$

C $-3.703858-1.256307-2.277487$

C $-4.222695-0.023018-2.190587$

C $-5.567193 \quad 0.379019-2.608757$

C $-6.466032-0.685768-3.182136$

C $-2.981027-1.0921802 .097599$

C $-4.298856-0.4949632 .565449$

C $-6.687396-0.3098591 .929003$ $\begin{array}{llll}\text { O } & -4.430804 & 0.070999 & 3.647916\end{array}$

C $-7.4023090 .040402 \quad 0.663838$

C $-7.907770 \quad 1.259256 \quad 0.419158$

C $-8.699023 \quad 1.618967-0.806294$

C -8.053368 $2.720120-1.617246$

C $-8.2645874 .013607-1.320473$

C $-7.236902 \quad 2.318168-2.824207$

C $-5.933617 \quad 1.668133-2.450424$

N $-5.314947-0.6767341 .634825$

H $1.356407-1.642734-1.435504$

H $0.135425-3.438465-3.597751$

H $-0.535500-2.926979-0.595283$

H -1.226401 -5.297274-2.427455

H $-1.876822-5.057526-0.853599$

H $1.184777-6.5288600 .743876$

H $1.991592-6.739929-1.369755$

H $\quad 0.723849-6.770205-2.597302$

H $1.705651-5.311625-2.358475$

H $-1.378440-4.8633761 .277835$

H -1.099353 -6.487468 1.895866

H $-0.467410-5.1024063 .667015$

H $1.068454-5.6165692 .965688$

H $0.967582-3.3634881 .607606$

H $2.520415-3.7310743 .547240$

H $1.218800-3.3935874 .668803$

H $3.469044-1.8719824 .838260$

H $\quad 0.190438-1.0121774 .717932$

H $3.778071-1.8148642 .516493$

H $3.086468-0.2094302 .482109$

H $2.378736-1.4971391 .488652$

H 0.9698530 .7383692 .647050

H -2.086853 -0.9358214 .040227$

H $-1.726922 \quad 0.4153852 .964967$

H $-0.792418-0.8819700 .787964$

H $-4.257154-2.096513-2.681722$

H $-3.6052480 .771873-1.775910$

H $-6.585608-1.512479-2.472736$

H -6.046623 -1.080363 -4.114193

H $-7.473811-0.333125-3.409326$

H $-2.804361-0.7508221 .074519$

H -3.091448 -2.1820532 .087350$

H -6.7022340 .5122042 .652658$

H $-7.164818-1.1763002 .398674$

H $-7.544762-0.754712-0.063385$

H $-7.7794772 .042748 \quad 1.163083$

H $-9.6964201 .942293-0.479641$

H $-8.8738390 .741404-1.440560$

H -7.829134 4.809719-1.915111

H $-8.8758354 .303813-0.472181$

H $-6.9959873 .201182-3.431437$

H $-7.8507901 .692663-3.478473$

H $-5.2137642 .363144-2.017130$

H $-5.144166-1.2947650 .846193$

C $3.731929-1.819876-2.966990$

O $3.991965-0.703447-2.098104$

C $4.974800-1.097901-1.121639$

C $5.390234-2.528632-1.465089$

C $4.171142-3.059975-2.194589$

H $4.465590-1.096602-0.151791$

C $6.137396-0.095501-1.101534$

H $6.266996-2.554282-2.121906$

H $5.638007-3.108239-0.570086$

C $4.470950-4.256915-3.080827$

H $3.423442-3.342066-1.443509$

C $5.7199281 .380664-0.944274$

O $6.867951-0.195143-2.333091$

H $6.845700-0.374713-0.313143$

H $5.221457 \quad 1.711377-1.862030$

O $6.9210832 .159124-0.849318$

$\begin{array}{llll}\text { C } & 4.827880 & 1.687446 & 0.271726\end{array}$

C $4.610162 \quad 3.1927750 .525394$

H 3.8586811 .1910950 .152607

$\begin{array}{lllll}\text { O } & 5.446376 & 1.133307 & 1.436388\end{array}$

H 3.9930973 .2942191 .426577

H 5.5685803 .6556690 .792696

H $3.580678-4.566983-3.635902$

H $5.255412-4.029386-3.810575$

H $4.809377-5.105929-2.478142$ 
C $2.279768-1.781468-3.454096$

H $6.249177-0.002168-3.058367$

H $7.3666811 .909916-0.021262$

H 4.8680131 .3208702 .194998

H $2.178173-2.457376-4.311882$

H $2.054830-0.767811-3.808398$

H $4.381675-1.674312-3.840811$

C $1.6451803 .487071-0.170769$

O $2.7431123 .348150-1.082219$

C $3.9669443 .951288-0.652013$

C $3.7597925 .451009-0.400562$

C 2.6047855 .7121180 .559585

C 1.3498574 .9702680 .102100

H 1.8875422 .9741970 .767832

C $0.4501112 .765833-0.812041$

H $4.6595063 .874755-1.498402$

O $4.942526 \quad 6.051123 \quad 0.117052$

H $3.5364695 .950410-1.352575$

H 2.8672815 .4411611 .589037

$\begin{array}{lllll}\text { O } & 2.314805 & 7.112117 & 0.575127\end{array}$

H $0.9553995 .448282-0.803787$

$\begin{array}{llll}\text { O } & 0.353958 & 5.089125 & 1.114929\end{array}$

C -0.6830902 .5427840 .158826$

H $0.0999723 .330843-1.684598$

H $0.7534711 .782375-1.192055$

H $5.6584825 .881606-0.518894$

H $3.1320017 .573147 \quad 0.831993$

H 0.2108006 .0381651 .272343

O $-1.864677 \quad 2.926437-0.402402$

$\begin{array}{llll}\text { O } & -0.538286 & 2.083862 & 1.283824\end{array}$

C $-3.011863 \quad 2.811950 \quad 0.445986$

H -3.860668 $3.273172-0.065124$

H -3.238500 1.7579930 .625545

H -2.847705 3.3382781 .391366

SCF Energy (B3LYP/6-31G*//MMFF) $=-3245.92663474$

01_319

MMFF Geometry

C -2.865538 $4.405634-0.599779$

C -1.611482 4.278035-1.060532

C $-0.439997 \quad 3.952490-0.166818$

O $0.432306 \quad 3.016668-0.843147$

C $0.372493 \quad 5.2285940 .118630$

C 1.4466435 .0405831 .173067

C 2.7705715 .1525490 .950905

C 0.9508354 .7423092 .565861

C $3.4747875 .479399-0.334884$

C $3.9171934 .258938-1.156824$

C $4.8632473 .303602-0.410609$

C $5.4944962 .301277-1.379468$

C $6.3743591 .272557-0.651705$

O 4.1045822 .5962340 .575635

O $6.6497480 .201848-1.568446$

C $5.576430 \quad 0.665770 \quad 0.522889$

C $7.7327201 .863503-0.255529$

O $6.408391-0.1674811 .325661$

C 4.8813311 .7222001 .406608

C 3.9213511 .1081132 .456653

O $5.876082 \quad 2.456214 \quad 2.131896$

C $0.0238551 .722409-0.927513$

O $-1.0159611 .249048-0.499311$

C $1.071356 \quad 0.972338-1.654874$

C $0.772157-0.207785-2.216122$

C $1.694981-1.073525-2.952653$

C $3.157447-0.717122-2.926169$

C 2.7819300 .2517331 .892363

C $3.099120-1.2332991 .830218$

C $2.219002-3.3724480 .906897$

O $4.065580-1.7395262 .393843$

C $2.815371-3.664845-0.433575$

C $2.242718-4.483727-1.328630$

C $2.841681-4.826222-2.661876$

C $1.899406-4.546986-3.811309$

C $1.118192-5.516562-4.316437$

C $1.927214-3.166226-4.426345$

C $1.195230-2.150407-3.593469$

N $2.150470-1.9364941 .099989$

H $-3.035415 \quad 4.2937890 .466801$
H -1.410325 $4.417707-2.119792$

H $-0.786156 \quad 3.4999790 .770284$

H $-0.295424 \quad 6.0291070 .464070$

H $0.8100425 .583000-0.820970$

H 3.4429594 .9997181 .794825

H 1.7531754 .7866953 .310589

H 0.1910215 .4721152 .863979

H 0.5145243 .7403372 .616062

H $2.8569346 .127910-0.964738$

H $4.3587126 .082099-0.088729$

H $3.0253523 .713332-1.486016$

H $4.4176664 .633211-2.058531$

H $5.641198 \quad 3.8994740 .080920$

H $6.0737692 .819685-2.153770$

H $4.6995481 .762929-1.912239$

H $7.0877880 .581193-2.349455$

H 4.8129930 .0143410 .081667

H $8.2737312 .208948-1.144756$

H $8.373813 \quad 1.105447 \quad 0.207819$

H 7.6491232 .7106470 .428287

H $6.804762-0.8334420 .738173$

H 4.4942880 .5653403 .218159

H 3.4499471 .9455522 .991270

H 5.4538262 .8658452 .905016

H $2.0584811 .415448-1.713423$

H $-0.251200-0.571748-2.144692$

H $3.500258-0.581805-1.894843$

H $3.3366800 .209605-3.481611$

H $3.802290-1.485283-3.357853$

H 1.9131130 .3478342 .556176

H 2.4790230 .6067860 .903461

H $1.201112-3.7605161 .000843$

H $2.836698-3.8224941 .690377$

H $3.771886-3.203275-0.667431$

H $1.301425-4.966187-1.076797$

H $3.101637-5.892895-2.646545$

H $3.787933-4.295661-2.825405$

H $0.459594-5.331281-5.158415$

H $1.115778-6.516108-3.894129$

H $1.443812-3.184422-5.412486$

H $2.963456-2.883932-4.632439$

H $\quad 0.119812-2.323414-3.550305$

H $1.397112-1.4310930 .642521$

C $-5.3579594 .047774-1.063367$

O $-5.2143272 .613780-1.043915$

$\begin{array}{llll}\text { C } & -5.904310 & 2.103988 & 0.113644\end{array}$

C -6.7786993 .2453150 .625007$

C $-5.920656 \quad 4.4507300 .301355$

H -5.1351851 .8865050 .864430$

C $-6.6811930 .826488-0.221145$

H -7.7306253 .3036630 .084556$

H $-7.002663 \quad 3.1499691 .691891$

C -6.6890555 .7612240 .289706$

H -5.1279254 .5155971 .054965$

C $-5.850397-0.308734-0.849480$

O $\begin{array}{llll}\text { O } & -7.733130 & 1.140654 & -1.144465\end{array}$

H -7.1806160 .4602150 .683572$

H -5.597584 -0.062858 -1.888248

O $-6.690905-1.470156-0.909334$

C $-4.560945-0.676363-0.092211$

C $-3.924059-1.970448-0.639043$

H $-3.8514620 .154317-0.180633$

$\begin{array}{lllll}\text { O } & -4.868386 & -0.840636 & 1.290014\end{array}$

H $-4.512349-2.830998-0.301191$

H -3.970011 -1.947111 -1.734332

H -6.0282936 .5978450 .041938$

H $-7.5021925 .744233-0.443618$

H -7.128367 5.9577161 .273170

C $-4.0455414 .726618-1.475667$

H $-7.3228631 .539275-1.931184$

H $-7.491800-1.222891-1.402865$

H $-4.024982-0.9040421 .769005$

H $-4.1917975 .813016-1.477219$

H -3.814911 $4.426184-2.505743$

H $-6.102923 \quad 4.262417-1.841467$

C -2.726339 -3.3018991 .891780$

O $-2.271238-2.1339221 .203169$

C $-2.450357-2.153437-0.213721$ 
C $-1.762330-3.383100-0.826154$

C $-2.207730-4.680530-0.154861$

C $-2.051267-4.5703761 .360808$

H $-3.811685-3.3913131 .758898$

C $-2.501735-3.0838793 .393198$

H $-1.920407-1.273308-0.593468$

O $-2.019188-3.440750-2.224694$

H $-0.677873-3.273306-0.702432$

H $-3.237750-4.941879-0.424765$

O $-1.384661-5.758310-0.605088$

H $-0.984394-4.5779441 .610814$

O $-2.641571-5.7092641 .982749$

C $-1.040704-2.9261243 .751192$

H $-3.003310-2.1663703 .724872$

H -2.937673 -3.904570 3.974454

H $-1.451441-4.134310-2.600758$

H $-1.563781-5.889216-1.551430$

H $-2.216593-6.4986891 .605717$

O $-0.600819-4.0316214 .417157$

O $-0.367992-1.9453223 .460260$

C $0.777333-4.0090414 .801424$

H $1.416147-3.9539603 .915386$

H $0.971362-3.1656115 .470777$

H $0.996900-4.9378175 .334576$

SCF Energy (B3LYP/6-31G*//MMFF)= -3245.91209447

0132

MMFF Geometry

C $2.090536-4.345848-0.294505$

C $0.863149-4.256845-0.828064$

C $-0.353448-3.856491-0.029948$

O $-0.965360-2.693328-0.634800$

C $-1.382573-5.001638-0.052849$

C $-2.601228-4.750520 \quad 0.813919$

C $-3.860847-4.6310510 .350114$

C $-2.343132-4.669246 \quad 2.297037$

C $-4.340392-4.715768-1.071916$

C $-4.439618-3.364937-1.797976$

C $-5.447583-2.386101-1.173642$

C $-5.659322-1.173067-2.082567$

C $-6.592652-0.131004-1.444676$

O $-4.933408-1.948617 \quad 0.086512$

O $-6.472328 \quad 1.086820-2.196405$

C $-6.1060860 .174765-0.011065$

C -8.063961 -0.553036 - 1.536633

$\begin{array}{llll}\text { O } & -7.037674 & 1.005492 & 0.676645\end{array}$

C -5.815536 -1.089780 0.821576

C $-5.133109-0.7853312 .177271$

O $-7.051117-1.7534301 .115508$

C $-0.332607-1.500034-0.469946$

O $0.730193-1.3032200 .097785$

C -1.159313 $-0.456664-1.114903$

C $-0.618100 \quad 0.748778-1.340595$

C $-1.2739921 .901126-1.958637$

C -2.746295 $1.794417-2.248734$

C $-3.781066-0.0685342 .089755$

C $-3.868702 \quad 1.4401672 .249847$

C -2.4404473 .4804342 .179342$

O -4.8957912 .0262722 .580092$

C -2.615659 4.1653600 .861504

C $-1.692804 \quad 4.9776020 .324138$

C $-1.8896695 .733330-0.958407$

C $-0.7947065 .474077-1.967153$

C $0.2388636 .322192-2.101187$

C $-0.947714 \quad 4.279547-2.880564$

C $-0.5286762 .994313-2.222300$

N $-2.645040 \quad 2.053843 \quad 2.018094$

H $2.226808-4.1278010 .762366$

H $0.719370-4.470872-1.884248$

H $-0.081070-3.6154121 .004885$

H $-0.911119-5.9322550 .290904$

H -1.681108 -5.180426 -1.091508

H -4.656051 -4.460474 1.075223

H -3.270582 -4.6405832 .879202$

H $-1.775105-5.5430032 .632745$

H $-1.775950-3.7666472 .542466$

H -3.703234 -5.387097-1.657042

H $-5.327872-5.194926-1.067724$
H $-3.445275-2.904115-1.826231$

H $-4.731711-3.566551-2.836081$

H $-6.394293-2.917272-1.019721$

H $-6.044150-1.482323-3.062199$

H -4.690336 -0.695186 -2.279946

H $-6.7052940 .892873-3.120560$

H $-5.1820420 .756020-0.107408$

H -8.359934 -0.683123 -2.584596

H $-8.7239940 .223417-1.134369$

H -8.274998 -1.489675 -1.016616

H $-7.173638 \quad 1.8002990 .132998$

H -5.829516 -0.2578822 .840102$

H -4.944403 -1.751774 2.667106

H -6.917310 -2.326001 1.888858

H -2.176923 -0.707793-1.389055

H $0.4207070 .906945-1.058448$

H -3.195122 $2.732635-2.580891$

H $-3.2927091 .500437-1.346618$

H -2.930584 $1.049679-3.030174$

H -3.152304 -0.4225702 .916558$

H $-3.261680-0.3100021 .157092$

H -1.436519 3.6219472 .590630

H -3.166291 3.8750182 .897703

H -3.561542 4.0178830 .345547

H $-0.759695 \quad 5.1464130 .857166$

H $-1.9213956 .802785-0.711257$

H -2.863855 $5.508811-1.410515$

H $1.0135966 .156512-2.842513$

H $0.3376437 .196486-1.466303$

H $-0.3152144 .406634-3.769662$

H $-1.9692274 .250197-3.269375$

H $0.5309682 .968053-1.966920$

H -1.8582501 .4932241 .703574$

C $4.309367-3.579883-1.183713$

O $4.781778-3.197960 \quad 0.125735$

C $6.214822-3.1561140 .105374$

C $6.614487-3.068447-1.359557$

C $5.554586-3.946564-1.998087$

H $6.551529-4.1303480 .487707$

C $6.767385-2.0713561 .034603$

H $6.536363-2.046863-1.745368$

H $7.632898-3.425016-1.539927$

C $5.398240-3.722516-3.492417$

H $5.820997-4.997680-1.822979$

C $6.630166-0.6019520 .583848$

O $8.166897-2.3451251 .197402$

H $6.309614-2.1867312 .025270$

H $7.301073-0.419587-0.264314$

O 7.1341430 .1963801 .666134

C $5.203255-0.1392390 .235394$

C $5.1443331 .362155-0.112946$

H $4.843688-0.711260-0.624655$

O $4.345749-0.4205881 .335860$

H $\quad 5.3106991 .9516810 .794451$

H $5.9630431 .582377-0.807520$

H $4.614104-4.366396-3.903146$

H $5.133306-2.683768-3.716640$

H $6.332599-3.951232-4.015357$

C $3.319255-4.741090-1.062840$

H $8.519147-1.6863051 .820471$

H 7.3132921 .0864571 .319349

H $3.434920-0.2293541 .055150$

H $3.786168-5.577838-0.528543$

H $3.046575-5.103777-2.060827$

H $3.810783-2.700341-1.610711$

C 2.6146572 .5794921 .190765

O 2.6907641 .6277560 .122706

C $3.8041391 .777619-0.762560$

C $3.8094303 .186091-1.372556$

C $3.7660694 .269714-0.300689$

C $2.6005104 .017150 \quad 0.652942$

H 3.4661572 .4409631 .867249

C 1.3199082 .2928111 .967025

H $3.6312611 .077012-1.589861$

O $4.9531973 .361579-2.202138$

H $2.9305193 .295217-2.020210$

H $4.720403 \quad 4.3330870 .235336$

O $3.5801175 .528043-0.952115$ 
H $\quad 1.654954 \quad 4.214520 \quad 0.132753$

O 2.6825394 .9222601 .751686

C 1.3958221 .0006692 .748916

H 1.0972173 .0852332 .689478

H 0.4849172 .2273231 .259809

H $4.9245954 .270043-2.548297$

H $3.6494546 .222381-0.275452$

H 2.5380525 .8190251 .405961

O $\quad 0.199187 \quad 0.3488162 .676209$

O $2.378144 \quad 0.628237 \quad 3.376630$

C $0.132691-0.8978563 .375804$

H $0.946703-1.5601323 .064480$

H $\quad 0.168226-0.7218764 .454668$

H -0.817595 -1.376701 3.127054

SCF Energy (B3LYP/6-31G**//MMFF)= -3245.90523905

01320

MM̄FF Geometry

C -2.721874 -2.269361 -3.502877

C $-1.782317-2.574724-2.596827$

C $-0.308804-2.333765-2.811229$

O $0.149214-1.384728-1.819877$

C $0.463985-3.649003-2.619259$

C $1.948841-3.538630-2.906465$

C $2.912379-3.792413-1.999154$

C $2.324143-3.191464-4.323776$

C $2.701223-4.180144-0.560159$

C $3.987388-4.1786320 .283362$

C $4.556150-2.8022160 .674875$

C $3.679632-2.0784851 .700792$

C $4.265648-0.7106992 .082039$

O $4.721102-2.001534-0.496216$

O $3.249889-0.0057802 .810820$

C $4.549906 \quad 0.0730570 .784974$

C $5.458088-0.8461213 .034607$

O $5.196317 \quad 1.311576 \quad 1.069307$

C $5.343450-0.731564-0.269628$

C $5.4126210 .008315-1.626008$

O $6.688258-0.8944720 .196521$

C $-0.116002-0.070869-2.061132$

O $\quad-0.6566390 .398950-3.050228$

C $\quad 0.340216 \quad 0.710319-0.889930$

C $-0.0336661 .994123-0.784352$

C 0.3110302 .9222170 .294536

C 1.2218782 .4228601 .382339

C $4.0675340 .167291-2.347015$

C $3.8884241 .566067-2.903706$

C $3.7319153 .943979-2.229477$

O $3.8869411 .784942-4.113058$

C 3.371666 4.731811-1.010803

C $2.4450505 .702088-1.000530$

C 2.1022926 .5137390 .217028

C $0.619528 \quad 6.5256970 .518756$

C $-0.1317957 .607168 \quad 0.251428$

C 0.0242825 .3132591 .202990

C $-0.194447 \quad 4.1726690 .246778$

N $3.7317892 .527248-1.914977$

H -2.421399 -1.847788 -4.458681

H -2.085532 -2.995178 -1.641624

H $-0.120421-1.934137-3.815112$

H $0.051074-4.418758-3.285355$

H $\quad 0.298890-4.015074-1.598726$

H $3.947578-3.738405-2.331563$

H $3.399455-3.293587-4.505317$

H $1.810275-3.854519-5.027411$

H $2.051190-2.156960-4.553061$

H $1.951909-3.533915-0.094223$

H $2.304790-5.203470-0.547898$

H $3.803694-4.7533371 .200138$

H $4.762010-4.725089-0.271263$

H $5.545387-2.9902551 .109640$

H $3.531697-2.6954862 .595562$

H $2.679486-1.9103831 .282368$

H 3.5817850 .8916002 .986589

H $3.571346 \quad 0.3367120 .371386$

H $5.154440-1.3650793 .951793$

H 5.8227570 .1360653 .355365

H $6.294775-1.3984382 .602002$
H $6.129480 \quad 1.119751 \quad 1.264175$

H $5.9253200 .969490-1.493458$

H $6.068292-0.564237-2.297707$

H $7.258497-1.081702-0.567534$

H $\quad 0.9529790 .209553-0.149697$

H $-0.6644532 .413876-1.567525$

H 2.1953832 .1497370 .964836

H 0.7904861 .5462741 .876832

H 1.4137473 .1559712 .167623

H $4.013421-0.539711-3.183789$

H $3.209717-0.056224-1.706755$

H $3.0454484 .118744-3.064882$

H $4.7430924 .206438-2.557929$

H $3.9146714 .508595-0.095156$

H $1.9212075 .946266-1.921972$

H 2.4581337 .5375930 .043059

H 2.6453376 .1566431 .101015

H -1.1902857 .6327590 .488428$

H $0.2933908 .489544-0.215321$

H -0.9521305 .5669971 .636650$

H 0.6550255 .0480522 .055069

H -0.858533 $4.427979-0.580846$

H $3.9038862 .253861-0.951913$

C $-4.960667-1.166366-3.008750$

O $-4.604223-0.627013-1.720017$

C $-4.6791020 .811714-1.792565$

C $-5.2409901 .150584-3.173833$

C $-4.762688-0.024569-4.002676$

H -3.648906 $1.181590-1.733045$

C $-5.5111871 .373514-0.632881$

H $-6.3363531 .193310-3.170818$

H $-4.8738512 .113785-3.541776$

C $-5.516339-0.202330-5.308215$

H $-3.6989720 .131094-4.219538$

C -5.0023490 .9921910 .772351$

O $-6.849751 \quad 0.875552-0.761866$

H $-5.5760762 .464343-0.729267$

H $-5.159130-0.0782650 .948978$

O -5.8120001 .6879241 .729686$

C -3.5306231 .3547571 .044687$

C -3.1001861 .2405082 .520769$

H $-2.880490 \quad 0.7218390 .431688$

O $-3.289710 \quad 2.7043450 .635800$

H -2.047344 1.5426192 .583063

H $-3.6365111 .990833 \quad 3.115641$

H $-5.137168-1.067379-5.861450$

H $-6.587224-0.354218-5.136809$

H $-5.4000050 .682112-5.943066$

C -4.193995 -2.464322 -3.278706

H $-7.4165701 .401909-0.173432$

H $-6.698318 \quad 1.2894741 .708181$

H -3.886758 3.2779041 .146397

H -4.609095 -2.958763 -4.164884

H -4.349366 -3.147805 -2.434054

H $-6.027905-1.418663-2.941745$

C $-1.287150-1.2133312 .342216$

O $-2.717225-1.1884612 .337227$

C $-3.297027-0.1565813 .140764$

C $-2.818394-0.2752624 .597354$

C $-1.296028-0.3417304 .703367$

C $-0.747508-1.4139693 .761417$

H -0.918846 -0.259927 1.946226

C $-0.815595-2.2767651 .349491$

H $-4.376587-0.3488823 .150814$

O $-3.296191 \quad 0.8139245 .380400$

H -3.232833 -1.191982 5.037205

H $-0.843174 \quad 0.6371264 .506249$

O $-0.950639-0.6816076 .048019$

H -1.035003 -2.397556 4.151284

O $0.675478-1.3658193 .761106$

C $-1.120485-3.6929991 .771511$

H -1.287085 -2.112797 0.375906

H $0.265156-2.2021711 .189998$

H -4.2664710 .8160565 .316261$

H $0.017797-0.7644556 .085618$

H $0.947643-0.5012193 .408759$

O $-2.426764-3.9876981 .516898$

O $-0.293619-4.4454812 .270730$ 
C $-2.841708-5.3073821 .881022$

H $-3.900338-5.4123291 .629278$

H $-2.271321-6.0524581 .318372$

H -2.720293 -5.458976 2.957949

SCF Energy (B3LYP/6-31G**//MMFF) $=-3245.90703966$

01_321

MM̄FF Geometry

C $2.7686863 .866726 \quad 0.250443$

C 2.0702352 .7226050 .200356

C 1.0895082 .2974551 .260806

O $\quad-0.1537292 .020700 \quad 0.575145$

C 1.5905141 .0194261 .956177

C $\quad 0.897286 \quad 0.759540 \quad 3.276932$

C $-0.132168-0.0907753 .451636$

C 1.4601721 .5154864 .453350

C $-0.841740-0.9042792 .406397$

C $-0.758750-2.4061992 .716285$

C -1.563166 -3.290709 1.747701

C $-0.926656-3.3608200 .358815$

C $-1.779863-4.172503-0.627653$

O $-2.887402-2.7635631 .676072$

O $-1.271909-3.932312-1.949488$

C $-3.220369-3.621447-0.588753$

C $-1.659522-5.680672-0.384352$

O $-4.090067-4.400765-1.406796$

C -3.793895 -3.4895880 .840059$

C $-5.131648-2.716606 \quad 0.863684$

O $-4.039967-4.7963821 .368736$

C -1.3037382 .5406491 .079952$

O $-1.444528 \quad 3.1478152 .128842$

C $-2.3706492 .291638 \quad 0.081944$

C $-3.493103 \quad 3.0263270 .117879$

C $-4.5871672 .991592-0.859253$

C $-4.4989771 .974917-1.966786$

C $-5.037524-1.2918360 .310744$

C $-6.146639-0.411290 \quad 0.843635$

C $-8.599220-0.036630 \quad 0.706852$

O $\quad-5.9203650 .502422 \quad 1.633963$

C $-8.8140671 .069769-0.276125$

C -8.8356632 .3682130 .060325$

C $-9.1176843 .471509-0.916149$

C $-8.0185194 .505850-0.970749$

C $-8.2126175 .745073-0.487226$

C $-6.7352024 .138608-1.686885$

C $-5.576543 \quad 3.904700-0.750810$

N $-7.393607-0.7568920 .351503$

H 2.6275964 .5456151 .087824

H $2.2285622 .040195-0.631322$

H $\quad 0.958631 \quad 3.110179 \quad 1.985747$

H 2.6687271 .0949242 .155072

H 1.4815010 .1565121 .290463

H $-0.543340-0.1960934 .455167$

H $\quad 0.936463 \quad 1.283875 \quad 5.386800$

H 2.5159181 .2626514 .596528

H 1.3802392 .5948164 .290968

H -1.889328 -0.580163 2.400193

H $-0.454511-0.7063871 .405265$

H $0.288204-2.7335082 .735915$

H -1.160438 -2.566807 3.726124

H -1.607994 -4.295557 2.184510

H $\quad 0.085320-3.7755750 .423786$

H $-0.811862-2.350786-0.053039$

H $-0.352587-4.247474-1.979254$

H -3.181511 -2.628556-1.053817

H $-0.614451-6.000546-0.475225$

H -2.211583 -6.247861-1.141890

H -2.011841 -5.9862110 .602949$

H $-4.286917-5.224152-0.928622$

H $-5.903692-3.2797630 .323953$

H -5.482704 -2.666685 1.904226

H -4.704611 -4.723119 2.073639

H -2.172554 $1.560027-0.692595$

H -3.6035313 .7624970 .913411$

H $-5.3697571 .966750-2.625015$

H $-4.4107200 .964130-1.556893$

H -3.625696 $2.171738-2.598435$

H $-4.088398-0.8173500 .585043$
H $-5.088428-1.298543-0.783953$

H -8.5120230 .3341121 .733696$

H -9.433400 -0.7435030 .662511$

H $-8.9892070 .783252-1.310375$

H -8.6804982 .6534651 .098423$

H $-10.0655193 .939865-0.620116$

H -9.287563 $3.077250-1.925984$

H -7.441693 $6.505151-0.560721$

H $-9.1434096 .026502-0.006154$

H $-6.4610474 .965773-2.355392$

H $-6.925293 \quad 3.282302-2.339335$

H -5.5151554 .6237230 .067431$

H -7.462719 -1.472187 -0.366429

C $5.2009084 .067291-0.232481$

O $5.4481722 .655103-0.086350$

C $6.8003352 .370987-0.489484$

C $7.4764653 .722779-0.686188$

C $6.3097994 .576659-1.149557$

H $6.7412041 .866784-1.461048$

C 7.4586891 .4484980 .541790

H 7.8857654 .1138120 .252430

H $8.2918913 .676350-1.414486$

C $6.5685366 .068874-1.040530$

H $6.0803724 .324342-2.193675$

C 6.6616600 .1540760 .816788

O $7.5824842 .149672 \quad 1.786418$

H $8.480557 \quad 1.2060820 .229414$

H $5.7099410 .400174 \quad 1.300755$

O $7.404229-0.6280461 .760532$

C $6.411798-0.709228-0.433024$

C $5.700401-2.044460-0.147656$

H $5.827842-0.147394-1.167840$

O $7.665687-1.006758-1.054887$

H $5.771850-2.659599-1.052780$

H $\quad 6.257572-2.6051280 .612871$

H $5.6966406 .641668-1.371311$

H $6.7935436 .362989-0.009836$

H $7.4195356 .358084-1.665802$

C $3.7887244 .268065-0.779492$

H 6.6903092 .4267342 .057353

H $7.558798-0.0692122 .541531$

H $8.209110-1.484002-0.404296$

H $3.6526073 .675387-1.692757$

H $3.6196905 .318008-1.042491$

H 5.2949274 .5145550 .766317

C $3.244986-1.721790-1.920133$

O $3.469605-1.101469-0.648710$

C $4.224696-1.8902660 .279104$

C $3.506462-3.2228780 .532489$

C $3.232007-3.974151-0.768512$

C $2.517831-3.065182-1.768838$

H $4.215004-1.888659-2.404292$

C $2.505858-0.720240-2.820751$

H $4.220367-1.3389461 .226911$

O $4.277403-4.0333741 .412885$

H $2.551015-3.0214701 .034425$

H $4.155910-4.387041-1.189654$

O $2.394510-5.094478-0.472694$

H $1.489870-2.919108-1.422904$

O $2.455081-3.740934-3.021853$

C $1.067688-0.482505-2.412786$

H $3.0065320 .254953-2.800356$

H $2.528206-1.083183-3.854988$

H $3.799288-4.8723561 .528932$

H $2.193966-5.535874-1.315845$

H $1.864160-3.233347-3.603188$

O $0.272647-0.452367-3.520957$

O $0.692524-0.323156-1.259495$

C $-1.119340-0.227638-3.275016$

H $-1.659453-0.379802-4.213212$

H $-1.503239-0.934506-2.532944$

H $-1.2735950 .802912-2.943482$

SCF Energy (B3LYP/6-31G**//MMFF)= -3245.91735447

01322

MM̄FF Geometry

C $1.755315-0.0047562 .161856$

C 0.4514650 .1386131 .884552 
C $-0.148996-0.168145 \quad 0.537933$

O $-1.168524-1.1671330 .779036$

C $-0.768238 \quad 1.087245-0.108351$

C $0.2016752 .229663-0.330556$

C $0.059750 \quad 3.4675130 .182086$

C $1.3652271 .929643-1.241874$

C $-1.0387183 .990381 \quad 1.065198$

C -1.902291 5.0371970 .346956

C $-2.708703 \quad 4.469015-0.833268$

C -3.430841 $5.594637-1.576498$

C $-4.3064205 .052518-2.714514$

O $-3.6604653 .532469-0.316744$

O $-5.1688376 .113608-3.151860$

C $-5.2095153 .936857-2.153249$

C $-3.4744794 .638133-3.933758$

O $-5.942756 \quad 3.302433-3.199424$

C $-4.4502642 .875623-1.319026$

C $-5.4766431 .967737-0.600613$

O $-3.6440012 .082133-2.189862$

C $-1.674342-1.835727-0.289023$

O $-1.425743-1.654084-1.469636$

C $-2.595116-2.8646680 .231978$

C $-3.688819-3.181962-0.474804$

C $-4.669714-4.206468-0.115379$

C $-4.249596-5.2350470 .903337$

$\begin{array}{lllll}\text { C } & -4.873784 & 0.956546 & 0.384881\end{array}$

C -5.9588870 .2629051 .193172$

C $-6.273739-1.2235273 .160553$

$\begin{array}{lllll}\text { O } & -7.158175 & 0.405977 & 0.966926\end{array}$

C -6.536285 -2.617144 2.687977

C $-7.694545-3.008292 \quad 2.135169$

C $-8.000749-4.4192351 .721427$

C -8.167303 -4.5701960 .225826$

C $-9.331601-4.272106-0.375299$

C $-7.012793-5.158477-0.553390$

C $-5.873295-4.192134-0.725352$

N $-5.433098-0.5316932 .202343$

H $2.426361-0.3704391 .389692$

H -0.2217450 .5039662 .656678$

H $0.604620-0.608215-0.126425$

H -1.6404061 .3898740 .479399$

H $-1.1905090 .822712-1.085428$

H $0.818743 \quad 4.210403-0.062266$

H $1.9411272 .827764-1.490103$

H $2.0534891 .220667-0.773013$

H $1.0087751 .500745-2.184102$

H $-1.6706413 .196747 \quad 1.471436$

H -0.5665524 .4647581 .934987$

H -2.594911 5.4596961 .086358

H -1.262043 $5.857170-0.002348$

H -2.028489 $3.951124-1.517623$

H -2.713719 $6.329764-1.962727$

H $-4.0738846 .143040-0.873810$

H -4.609209 $6.854122-3.442054$

H -5.953533 $4.418087-1.502791$

H -2.902116 $5.492444-4.314657$

H $-4.118262 \quad 4.317243-4.760143$

H -2.765402 $3.836221-3.717861$

H $-6.4158173 .999560-3.685405$

H -6.173133 $2.605965-0.040016$

H -6.067639 $1.414508-1.341820$

H $-3.484430 \quad 1.219721-1.776218$

H $-2.346045-3.3237441 .181401$

H -3.893575 -2.633964 -1.393925

H $-4.047685-4.7661941 .871952$

H $-3.341631-5.7514060 .571721$

H -5.001438 -6.0058251 .082851$

H $-4.305636 \quad 0.187475-0.148135$

H $-4.201373 \quad 1.4632601 .085912$

H -7.203220 -0.6617003 .303858$

H $-5.742378-1.2467664 .117133$

H -5.746256 -3.344655 2.848482

H -8.486338 -2.275522 1.994257

H $-8.926757-4.726307 \quad 2.225452$

H -7.234096 -5.113993 2.085467

H -9.468834 -4.407790 -1.442896

H -10.172196 -3.8814940 .188850$

H -7.343276 -5.449447 -1.559671
H $-6.704247-6.097754-0.085462$

H $-6.079350-3.399960-1.446506$

H $-4.426355-0.6478892 .274601$

C $3.3846111 .457608 \quad 3.443553$

O $4.441257 \quad 1.1602242 .509833$

C 4.7772222 .3688611 .797500

C 4.0968123 .5059392 .552501

C 2.8314142 .8214673 .034017

H $4.3161872 .289142 \quad 0.806054$

C 6.2972902 .4991911 .660325

H 4.6955143 .8440893 .406108

H 3.9013364 .3708701 .911612

C $2.117243 \quad 3.5636224 .148591$

H 2.1515292 .7219472 .180932

C 6.9618521 .2855540 .976115

$\begin{array}{llll}\text { O } & 6.857503 & 2.623263 & 2.973762\end{array}$

H 6.5448203 .4253051 .128633

H 6.8280630 .4019331 .608839

$\begin{array}{lllll}\text { O } & 8.374270 & 1.526191 & 0.930361\end{array}$

C $6.4581041 .003998-0.452315$

C $7.179724-0.166918-1.147891$

H $5.3815450 .808251-0.438317$

O $6.6568232 .181611-1.239394$

H $6.880543-0.172066-2.202981$

H $8.2599340 .022250-1.166136$

H 1.2201393 .0216184 .464163

H $2.7618003 .690103 \quad 5.024829$

H $1.8087504 .558706 \quad 3.811447$

C $2.3768120 .306267 \quad 3.493175$

H 7.8223162 .6914542 .871603

H 8.5223072 .2859290 .341105

H $6.2733172 .016138-2.117519$

H 1.6067170 .5234654 .242463

H $2.895091-0.6027193 .823627$

H 3.8629381 .5322674 .429288

C $4.783779-2.006331-1.588088$

O $5.480480-1.767937-0.361201$

C $6.886564-1.538225-0.506359$

C $7.550365-2.728420-1.215165$

C $6.857979-3.076934-2.531138$

C $5.350882-3.225953-2.322134$

H $4.888349-1.123938-2.232087$

C $3.281632-2.111777-1.290332$

H $7.298163-1.5090150 .509322$

O $8.927137-2.467426-1.467330$

H $7.505001-3.608511-0.560362$

H $7.074355-2.334180-3.308049$

O $7.366895-4.322171-3.014728$

H $5.154766-4.147284-1.761277$

O $4.709380-3.353034-3.588773$

C $2.896710-3.242180-0.358646$

H $2.948163-1.185647-0.810409$

H $2.731653-2.229979-2.230952$

H $9.347565-2.267363-0.613418$

H $8.330629-4.225039-3.103766$

H $5.112759-4.112640-4.042906$

O $1.558269-3.485210-0.487333$

O $3.656010-3.8152750 .410118$

C $1.034609-4.518625 \quad 0.352664$

H $1.617065-5.4388850 .245062$

H $1.029545-4.1827521 .393607$

H $\quad 0.006506-4.7215790 .042079$

SCF Energy (B3LYP/6-31G**//MMFF)= -3245.90630570

01_323

MMMFF Geometry

C $2.2409961 .581774-2.855499$

C $3.3799130 .874842-2.773255$

C $3.497810-0.601066-2.476277$

O $2.196355-1.228525-2.583659$

C $4.452480-1.343606-3.430578$

C $5.927455-1.136720-3.145239$

C $6.620148-1.820186-2.211118$

C $6.638767-0.161455-4.046257$

C $6.084812-2.857111-1.262637$

C $6.618910-2.7258750 .171843$

C $6.301830-1.3925830 .868765$

C $6.909931-1.3800802 .276159$ 
C $6.561369-0.0995823 .048153$

O $4.880009-1.2285090 .950698$

O $6.897973-0.3358064 .424118$

C $5.036678 \quad 0.1290222 .964532$

C 7.4249551 .0829982 .594824

O $4.677631 \quad 1.3747623 .557210$

C $4.475990 \quad 0.0229871 .528924$

C 2.9324690 .1224691 .486686

$\begin{array}{lllll}\text { O } & 4.976429 & 1.126274 & 0.762047\end{array}$

C $1.876210-2.181148-1.668838$

O $2.567133-2.580680-0.745993$

C $0.514250-2.667648-1.973578$

C $-0.109428-3.434499-1.067353$

C -1.450001-4.006566-1.182859

C $-2.186777-3.820302-2.481546$

C $2.170670-0.9588372 .258257$

C $0.663109-0.7804462 .135927$

C - $-1.450054-1.4486883 .283975$

O $0.143317-0.0001301 .342580$

C -2.150134 -2.751457 3.059479

C $-3.309543-2.8512222 .390460$

C $-4.068906-4.1322782 .188789$

C $-4.306856-4.4409620 .726540$

C $-5.409868-4.0005650 .098094$

C $-3.301885-5.317898 \quad 0.014177$

C $-1.958417-4.660803-0.118426$

N -0.028732 -1.5966993 .021595$

H $1.2806351 .092546-2.718224$

H $4.3167301 .416524-2.873708$

H $3.864730-0.686724-1.449528$

H $4.228410-1.068627-4.470338$

H $4.236998-2.420523-3.396241$

H $7.689988-1.634658-2.126498$

H $7.710782-0.101632-3.829723$

H $\quad 6.532856-0.466581-5.092539$

H $\quad 6.227913 \quad 0.845126-3.935788$

H $4.994595-2.856066-1.237828$

H $6.383943-3.841538-1.643495$

H $6.184046-3.5475620 .756075$

H $7.705466-2.8809730 .163603$

H $6.725771-0.577990 \quad 0.271043$

H $7.997898-1.5158882 .231967$

H $6.525400-2.2400062 .843030$

H $6.611546 \quad 0.4409934 .934885$

H $4.576023-0.6467993 .587598$

H 8.4865230 .8646602 .763259

H 7.2096451 .9813283 .183691

H 7.3017101 .3256641 .537389

H 4.9046072 .0790942 .926215

H 2.6202991 .1149961 .839024

H 2.6091070 .0832910 .438427

H $4.4435791 .198974-0.046185$

H $0.066499-2.378234-2.916719$

H $0.407693-3.659045-0.134707$

H -1.676076 -4.356411-3.288945

H -2.240708 -2.761297 -2.749466

H -3.217551-4.180792 -2.451741

H $2.429162-1.9554011 .886518$

H $2.423984-0.9096543 .322212$

H -1.865590 -0.6431682 .671354$

H -1.545260 -1.161002 4.336105

H -1.704312 -3.644877 3.489649

H -3.766903 -1.9501621 .990130$

H -5.032378 -4.0360692 .706754$

H -3.562295 -4.978887 2.668379

H $-5.599330-4.234416-0.944120$

H -6.144070 -3.387792 0.611041

H -3.679901 $-5.638380-0.962928$

H -3.179068 $-6.250140 \quad 0.580945$

H -1.346260 -4.745043 0.780105

H $0.501965-2.1874413 .655507$

C $1.8306283 .919930-1.919385$

O $0.4917223 .631133-1.472036$

C $0.4652103 .722588-0.032181$

C 1.8041004 .3238010 .386392

C $2.7215523 .775253-0.687342$

H $\quad 0.4151292 .694748 \quad 0.345274$

C -0.7588684 .5145800 .439006$
H 1.7891575 .4193610 .352060

H 2.0952804 .0221451 .397298

C $4.0522784 .497142-0.788557$

H $2.9021722 .718602-0.463983$

C $-2.1121783 .963174-0.051948$

O $-0.6532185 .866832-0.025481$

H -0.7574974 .5733841 .533727$

H -2.223395 4.133421 -1.129731

O -3.1478854 .7228030 .584378$

C -2.3424122 .4727590 .254933$

C $-3.7642262 .007546-0.102953$

H $-1.6087131 .882939-0.297688$

O -2.1148702 .2292971 .644020$

H -4.4747962 .4847800 .584915$

H -4.022297 2.355811-1.108941

H $4.6703694 .064962-1.581724$

H $3.9162955 .561359-1.007593$

H 4.6061014 .4153110 .152510

C $2.2017723 .060227-3.130920$

H $-0.5860525 .839033-0.995461$

H -2.9895395 .6594860 .375803$

H -2.7321772 .7887172 .145949$

H $3.1656603 .397976-3.530689$

H $1.4603283 .221188-3.923512$

H $1.8176024 .968850-2.245421$

C $-6.2939210 .471442-0.630964$

$\begin{array}{lllll}\text { O } & -5.314107 & 0.165695 & 0.368304\end{array}$

C $-3.9625710 .476934-0.001245$

C $-3.587375-0.334833-1.250437$

C $-4.578030-0.110314-2.392588$

C $-6.012547-0.323284-1.911351$

H $-6.2814021 .546863-0.848635$

C $-7.6694270 .112228-0.049640$

H -3.3419530 .1181930 .826368$

O $-2.275843-0.029279-1.703420$

H $-3.598322-1.402715-0.997430$

H $-4.458950 \quad 0.883278-2.839834$

O $-4.317798-1.048974-3.438441$

H $-6.184664-1.393093-1.738280$

O $-6.9158650 .094923-2.932286$

C -8.0784181 .0404141 .075580$

H $-8.4422670 .180288-0.822796$

H -7.644365 -0.9070240 .354127$

H -1.658018 $-0.289422-0.998468$

H -3.400715 -0.907287 -3.730037

H $-6.696527-0.406261-3.736556$

O

O -7.4559512 .0280951 .440599$

C -9.7787431 .3791182 .684822$

H -9.9907252 .3977282 .346365$

H $-10.712744 \quad 0.918778 \quad 3.018039$

H -9.073796 1.3878723 .521629

SCF Energy (B3LYP/6-31G**//MMFF) $=-3245.93622819$

01_324

MM̄FF Geometry

C -0.6470401 .5141682 .563245$

C 0.4139791 .0958263 .269538

C $1.247467-0.1032522 .904660$

O 2.6113120 .3536942 .707555

C $1.304292-1.1469664 .033559$

C $0.055777-1.9873074 .187195$

C $-0.090132-3.2299453 .687685$

C - $-1.040985-1.3931855 .033948$

C $0.879366-4.0237272 .854374$

C $0.195182-4.6680511 .638315$

C $-0.010451-3.6895490 .471397$

C $-0.948812-4.301520-0.569919$

C $-1.082423-3.413299-1.811352$

O $1.274315-3.447965-0.112857$

O $-1.746694-4.195466-2.815153$

C $0.328452-3.068709-2.328285$

C $-1.979756-2.200331-1.545784$

O $0.227037-2.142491-3.408309$

C $1.277876-2.540972-1.223709$

C $2.727430-2.471025-1.765283$

O $0.855684-1.234583-0.835302$

C 2.9168870 .9308591 .514729 
O $2.167441 \quad 1.1063650 .567550$

C 4.3440121 .3193021 .558757

C 4.9552071 .7093580 .430196

C 6.3460842 .1504770 .305155

C 7.1700992 .2217731 .564305

C $3.794556-2.192845-0.697393$

C $5.201658-2.278232-1.265609$

C $7.581983-2.368060-0.532095$

O $5.434983-2.395412-2.465735$

C $8.268056-1.046375-0.401030$

C $8.846142-0.408232-1.429854$

C $9.6207450 .871818-1.299994$

C $8.9984072 .015875-2.066713$

C $9.2483102 .190011-3.375435$

C $8.1673113 .010293-1.289645$

C $6.8115282 .475426-0.919480$

N $6.164933-2.208688-0.268431$

H $-0.9579990 .957578 \quad 1.682611$

H $\quad 0.724744 \quad 1.659764 \quad 4.146174$

H $0.896720-0.5840811 .982908$

H $1.534277-0.6567174 .990002$

H $2.174857-1.7948813 .869763$

H -1.013891 -3.759485 3.917058

H -1.823744 -2.122186 5.270055

H $-0.637667-1.0351145 .986914$

H -1.521503 -0.5570424 .520177$

H $1.737287-3.4297432 .526753$

H $1.271636-4.8265873 .490729$

H $\quad 0.832450-5.4949251 .298061$

H $-0.764030-5.1053641 .942483$

H $-0.420662-2.747174 \quad 0.850865$

H -1.935933 -4.503846 -0.136509

H $-0.556231-5.279277-0.883850$

H -1.775749 $-3.665048-3.630109$

H $\quad 0.748833-3.993881-2.747305$

H -2.975071 -2.529261 -1.224805

H -2.134208 -1.615071-2.458755

H -1.586906 -1.534176 -0.775681

H $1.093800-2.096542-3.844908$

H $2.974920-3.430140-2.239508$

H $2.802181-1.695634-2.537675$

H $1.616980-0.744045-0.488440$

H 4.8486101 .2767382 .517094

H $4.3815521 .712685-0.496043$

H 7.1999581 .2459452 .061947

H 6.7471682 .9564882 .258324

H 8.2098262 .5060041 .391128

H $3.665661-1.191919-0.279740$

H $3.715300-2.9209270 .117711$

H $7.731166-2.812789-1.521533$

H $7.977915-3.0641340 .214288$

H $8.330822-0.6200260 .596678$

H $8.797030-0.851325-2.422217$

H $10.6367350 .689942-1.674841$

H $9.750114 \quad 1.153640-0.247839$

H $8.8312673 .027404-3.925047$

H $9.8731851 .494872-3.926574$

H $8.0033953 .915648-1.889782$

H $8.7371233 .357127-0.422801$

H $6.1383482 .380182-1.772732$

H $5.873095-2.0775530 .695877$

C - -1.3445373 .7878241 .800035$

O $-2.171305 \quad 3.3820820 .689924$

C $-2.8804924 .534046 \quad 0.193713$

C -2.2356725 .7443980 .856810$

C $-1.854615 \quad 5.1695872 .207312$

H -3.9105304 .4517930 .559651$

C $-2.8688384 .550262-1.338857$

H -1.340881 6.0758710 .317247

H -2.9215016 .5940150 .927965$

C $-0.835605 \quad 6.0106032 .956761$

H -2.761396 5.0704772 .819020

C $-3.4431053 .266510-1.975659$

O $-1.5116704 .692276-1.774729$

H -3.407836 $5.433650-1.701677$

H -2.778595 2.420453 -1.766302

O $-3.4529743 .437752-3.397652$

C $-4.8779862 .933624-1.528041$
C $-5.5143411 .733039-2.250653$

H $-4.8940982 .741420-0.451178$

O $-5.7211324 .068442-1.747578$

H $-6.5485421 .643072-1.895122$

H $-5.607801 \quad 1.954242-3.321354$

H -0.5794605 .5520603 .916885$

H 0.0893506 .1270532 .381992

H -1.235061 7.0104403 .156224

C -1.438664 2.7441242 .913753

H -1.528944 $4.899853-2.724046$

H $-2.5287523 .434042-3.698948$

H $-5.6862744 .280174-2.696314$

H -2.482299 2.4438603 .066060

H -1.075704 3.1662023 .857738

H $-0.3155153 .859700 \quad 1.422637$

C $-5.528439-0.1249820 .169888$

O $-4.4179420 .172974-0.681871$

C $-4.7654480 .400084-2.053421$

C $-5.492132-0.826888-2.628206$

C $-6.673683-1.263493-1.761162$

C $-6.245743-1.394311-0.298902$

H -6.2342840 .7146480 .140383$

C $-5.029824-0.1838491 .617915$

H $-3.8174570 .491245-2.597455$

O $-5.921642-0.589264-3.964931$

H $-4.783081-1.661782-2.680454$

H -7.522318 -0.576501-1.858716

O $-7.137435-2.539522-2.207908$

H $-5.594134-2.270510-0.197844$

O $-7.395058-1.6128900 .513984$

C $-4.082778-1.3291481 .888106$

H $-4.486500 \quad 0.7368631 .866536$

H $-5.870092-0.2421522 .319801$

H $-6.6813070 .016641-3.937260$

H $-7.358773-2.453036-3.151031$

H $-7.844272-2.4057200 .173731$

O $-4.774093-2.3941992 .384978$

O $-2.878609-1.2843071 .675013$

C -4.002209-3.565042 2.667709

H $-4.691855-4.4042712 .790867$

H $-3.317717-3.7962621 .846486$

H $-3.452577-3.421653 \quad 3.601281$

SCF Energy $\left(B 3 L Y P / 6-31 G^{* *} / / M M F F\right)=-3245.91023560$

01325

MM̄FF Geometry

C $-2.9643613 .430905-0.101706$

C -1.9431174 .2246300 .255726$

C -0.5822103 .7514350 .712842$

O $-0.6730872 .361848 \quad 1.112604$

C $0.4677133 .888013-0.407941$

C $1.3402835 .119077-0.272556$

C 2.6386215 .1009480 .088766

C $0.678306 \quad 6.427606-0.618946$

C 3.4778503 .9136460 .470368

C $4.5710743 .651487-0.571263$

C $5.4432762 .445091-0.193295$

C $6.6064132 .299557-1.177195$

C $7.4464481 .046200-0.893327$

O $4.6194201 .274066-0.222830$

O $8.3007740 .821938-2.025677$

C $6.500657-0.168484-0.793612$

C $8.370766 \quad 1.235302 \quad 0.314964$

O $7.211842-1.337372-0.392584$

C 5.2948310 .0635890 .145658

C $4.270075-1.0938980 .075990$

O $5.766341 \quad 0.1285461 .497198$

C 0.2103911 .9122082 .042213

O 1.1170422 .5416312 .562500

C -0.1328950 .5026652 .335541$

C $0.704576-0.2397223 .075040$

C $0.496193-1.6295083 .489626$

C $-0.834013-2.2572753 .167299$

C $3.529791-1.216251-1.257393$

C $2.675102-2.466368-1.310776$

C $0.634292-3.504484-0.332253$

O $2.965371-3.412753-2.039832$

C $0.921467-4.3703340 .858601$ 
C $2.036948-4.3936121 .605599$

C $2.214389-5.2856002 .801080$

C $2.583052-4.5192034 .052436$

C $3.797731-4.6510114 .611881$

C $1.516400-3.6623424 .699993$

C $1.491274-2.2606894 .147946$

N $1.576170-2.411713-0.470582$

H -2.831186 2.352397-0.101794

H -2.088750 5.3019410 .230600

H $-0.3099364 .344116 \quad 1.596007$

H $-0.0163153 .913298-1.394065$

H $1.0855732 .985762-0.443236$

H 3.1697426 .0512490 .134218

H $1.3904817 .259470-0.645610$

H $\quad 0.2100116 .369070-1.607024$

H $-0.088936 \quad 6.677177 \quad 0.119750$

H 3.9410584 .1240131 .442383

H $2.875148 \quad 3.0141270 .612844$

H $4.0965213 .468402-1.544455$

H $5.2008544 .544071-0.673820$

H 5.8209052 .5942790 .825151

H $7.2396673 .195380-1.166233$

H $6.2104852 .225994-2.200078$

H $8.8506241 .614813-2.148008$

H $\quad 6.133934-0.364044-1.808188$

H 9.0645152 .0663570 .139435

H 8.9975940 .3510450 .474700

H 7.8313101 .4480901 .240024

H $7.954156-1.451427-1.010731$

H $4.759102-2.0431370 .330435$

H $3.520873-0.9418640 .865398$

H $5.022274-0.0568242 .093700$

H -1.0693020 .1208561 .944579$

H $1.635727 \quad 0.2123943 .414981$

H -1.015379 -2.246027 2.087684

H -1.644066 -1.709809 3.662145 H $-0.916148-3.2987523 .483448$

H $2.887016-0.345585-1.423485$

H $4.224767-1.267699-2.100588$

H $\quad 0.631627-4.114952-1.240807$

H $-0.354845-3.050078-0.218958$

H $\quad 0.107936-5.0399301 .132997$

H $2.877827-3.753746 \quad 1.353525$

H $2.996533-6.0159592 .557046$

H $1.308429-5.8720442 .998989$

H $4.058798-4.1239055 .523717$

H $4.559630-5.2837594 .168995$

H $1.711806-3.5825255 .778124$

H $\quad 0.551527-4.1715024 .630257$

H $2.412562-1.7076184 .338977$

H $1.437076-1.5931510 .114618$

C $-5.467819 \quad 3.326213 \quad 0.284271$

$\begin{array}{llll}\text { O } & -5.494288 & 1.895383 & 0.103074\end{array}$

C $-5.639547 \quad 1.274806 \quad 1.393900$

C -6.1812722 .3636752 .311313$

C -5.4280763 .5754941 .794300$

H -4.6306531 .0044931 .729926$

C -6.5003030 .0133571 .292536$

H -7.260482 2.5053142 .182166

H -5.9869452 .1543413 .367368$

C -6.0373034 .9020842 .212439$

H -4.3998203 .5262862 .173349$

C $-6.014443-0.9938190 .228470$

$\begin{array}{lllll}\text { O } & -7.836235 & 0.398556 & 0.945969\end{array}$

H $-6.564144-0.4661882 .276433$

H $-6.198803-0.576555-0.767534$

O $-6.844597-2.1585690 .325487$

C $-4.538721-1.4151920 .357293$

C $-4.118629-2.552369-0.593877$

H -3.891306 -0.5475190 .190919$

O $-4.311523-1.8578411 .698156$

H -3.070196 -2.795542 -0.381594

H -4.672321 -3.464471 -0.337824

H -5.4673515 .7389491 .796291$

H -7.073063 4.9948521 .869160

H -6.0350284 .9995303 .302992$

C $-4.3168913 .931493-0.526308$

H $\quad-8.365575-0.4151950 .884697$
H $-6.665736-2.5745241 .186266$

H $-3.368734-2.0797501 .777537$

H $-4.3644105 .025094-0.467721$

H -4.445780 $3.655828-1.580359$

H $-6.4126523 .698170-0.132722$

C $-2.257940-0.981700-2.447275$

O $-3.691738-0.981799-2.449391$

C $-4.297687-2.226698-2.089293$

C $-3.832219-3.338317-3.040174$

C $-2.311079-3.415557-3.127490$

C $-1.719582-2.037851-3.423731$

H -1.896817 -1.188291-1.432661

C $-1.8212350 .439043-2.830686$

H $-5.373730-2.103026-2.260024$

O $-4.341700-4.604293-2.634396$

H $-4.224889-3.141404-4.046365$

H -1.878104 -3.841565 -2.215445

O $-1.945894-4.296459-4.192576$

H -1.961651 -1.754717-4.456079

O $-0.300509-2.110852-3.324643$

C $-0.3686930 .714554-2.521125$

H -2.023543 $0.613227-3.894707$

H $-2.4163291 .182916-2.288717$

H $-5.311116-4.532906-2.604516$

H $-2.358783-5.157893-4.009275$

H $-0.001114-2.811575-3.929043$

O $\quad 0.2249791 .305251-3.597245$

O $0.1705240 .443056-1.456597$

C $1.6236131 .586461-3.476799$

H $2.1932690 .668544-3.644804$

H $1.8908432 .312793-4.249026$

H $1.8657992 .014577-2.500393$

SCF Energy $\left(B 3 L Y P / 6-31 G^{* *} / / M M F F\right)=-3245.91412235$

01_326

MM̄FF Geometry

C -1.594382 -3.590975 0.381673

C $-0.630079-3.891563-0.501765$

C $0.226730-2.835237-1.149295$

O $1.621995-3.192593-0.987152$

C $-0.018308-2.699644-2.664227$

C $-1.425390-2.311313-3.068371$

C $-2.005460-1.128568-2.782947$

C $-2.170664-3.350112-3.863351$

C $-1.3789530 .024872-2.051597$

C $-1.6386291 .362179-2.757034$

C $-0.8909092 .513181-2.067092$

C $-1.2196293 .850320-2.731574$

C $-0.3952775 .004135-2.138420$

O $0.5099802 .246243-2.180539$

O $-0.5211686 .131637-3.018590$

C $1.0957954 .600021-2.127797$

C $-0.9460885 .452340-0.779589$

O $1.8855305 .586168-1.465858$

C $1.3560713 .198087-1.527722$

C $2.8227162 .776353-1.776370$

O $1.1188533 .247894-0.121864$

C $2.224136-2.8345700 .179011$

O $1.698678-2.3089281 .146857$

C $3.657802-3.1830470 .070436$

C $4.515696-2.7238090 .993730$

C $5.955395-2.9855211 .048023$

C $6.537954-3.891165-0.005388$

C $3.1464641 .331286-1.367109$

C $4.5274730 .945293-1.858508$

C $6.9441891 .393882-1.448850$

O $4.6854220 .257011-2.864372$

C $7.5506890 .155812-0.868283$

C 8.5177700 .1771850 .061623

C $9.230780-1.0433450 .569491$

C $8.945815-1.3397192 .023686$

C $9.461278-0.5801853 .005253$

C $8.140616-2.5796172 .335820$

C $6.677521-2.4123752 .034059$

N $5.5437041 .484950-1.086720$

H $-1.770568-2.5473910 .633070$

H -0.442759-4.928540 -0.767294

H $0.065680-1.864436-0.668824$ 
H $\quad 0.264076-3.641048-3.156647$ H $0.687976-1.967085-3.079342$ H -3.031981-0.966691-3.107809 H -3.218058 -3.076908 -4.029394 H -1.698126 -3.482265 -4.842151 H $-2.158546-4.313631-3.345568$ H $-1.7954230 .063307-1.038969$ H $-0.299408-0.117372-1.954461$ H $-1.3050771 .283634-3.800192$ H -2.715326 $1.571416-2.768541$ H $-1.1734102 .529443-1.007733$ H -2.292818 4.066949-2.659576 H $-0.9993003 .786703-3.806586$ H -1.465772 $6.351065-3.091604$ H $1.4326764 .597317-3.173572$ H $-1.992750 \quad 5.765775-0.874494$ H -0.406276 $6.327862-0.402806$ H $-0.9055784 .667162-0.022904$ H $1.7541345 .476254-0.508666$ H $3.0464242 .878459-2.846786$ H $3.4989943 .453819-1.239941$ H $1.522117 \quad 2.467616 \quad 0.289537$ H $3.965890-3.801202-0.764782$ H $4.128067-2.0976721 .796395$ H $6.359629-3.480885-1.005821$ H $6.086370-4.8878440 .051735$ H $7.617625-4.0269840 .082205$ H $3.1102311 .205773-0.280534$ H $2.4301910 .622751-1.797539$ H $7.4328432 .303092-1.083664$ H $7.0372021 .371525-2.539763$ H $7.219640-0.796250-1.275430$ H $8.858412 \quad 1.1356820 .447335$ H $\quad 10.310120-0.8883510 .438937$ H $8.993057-1.917201-0.048890$ H $9.284824-0.8099994 .050747$ H $10.0713090 .289307 \quad 2.783248$ H $8.224539-2.8273403 .402728$ H $8.583948-3.4369451 .820766$ H $6.163456-1.7614412 .742793$ H $5.3068742 .001146-0.244519$ C $-3.932733-4.5528010 .660820$ O $-4.461088-3.2164790 .795718$ C $-5.132656-2.870010-0.429835$ C $-5.448223-4.195736-1.109161$ C $-4.201273-4.990895-0.779974$ H -4.407939-2.323983-1.045070 C -6.351189-1.984408-0.158502 H $-6.330683-4.676613-0.671604$ H -5.615838 -4.084056 -2.184664 C $-4.368774-6.490937-0.947202$ H -3.395024 -4.654678 -1.438908 C $-6.046195-0.6911880 .625585$ O $-7.318002-2.7287850 .593321$ H $-6.838326-1.737880-1.109068$ H $-5.810382-0.9459491 .664838$ $\begin{array}{llllll}\text { O } & -7.253795 & 0.079460 & 0.680474\end{array}$ $\begin{array}{llll}\text { C } & -4.914222 & 0.176436 & 0.041228\end{array}$ C -4.7929641 .5824090 .658776$ H -3.961380 -0.3490490 .163633$ O $-5.107177 \quad 0.339431-1.366593$ H $-3.967647 \quad 2.0969280 .150652$ H -5.6850532 .1685060 .403482$ H -3.446358 -7.017049 -0.681675 H -5.173287 -6.878504 -0.313157 H $-4.611487-6.737108-1.986093$ C $-2.461166-4.5954421 .087676$ H $-6.889403-3.0179731 .417344$ H $-7.5223410 .281234-0.231748$ H $-5.9281190 .842189-1.501027$ H $-2.067259-5.6104090 .959015$ H -2.400058 -4.3673322 .159263$ H $-4.510040-5.1775621 .354446$ C -2.182451 $1.274796 \quad 2.232877$ O -3.4778190 .7697732 .581603$ C -4.5773461 .5930402 .183894$ C -4.441013 2.9958392 .792252 $\begin{array}{lll}\text { C }-3.084902 & 3.621262 & 2.482837\end{array}$
C -1.957342 2.6604202 .856422

H -2.098349 1.3377391 .141622

$\begin{array}{lll}\text { C }-1.165810 & 0.239576 & 2.730174\end{array}$

H $-5.471283 \quad 1.1458762 .634465$

$\begin{array}{llll}\text { O } & -5.471518 & 3.859405 & 2.324025\end{array}$

H -4.5486632 .9294453 .882843$

H -3.0110653 .9234701 .431604$

O $-2.9301314 .814155 \quad 3.255186$

H -1.8978752 .5771373 .949141$

O $-0.721125 \quad 3.2028542 .402999$

$\begin{array}{llll}\text { C } & 0.214314 & 0.453492 & 2.159495\end{array}$

H -1.1322250 .2425843 .826755$

H $-1.479846-0.7735742 .451109$

H -6.322816 3.4469552 .549705

H $-3.6746015 .399806 \quad 3.034002$

H -0.6309404 .0892312 .792816$

$\begin{array}{lllll}\text { O } & 1.119181 & 0.581446 & 3.171278\end{array}$

$\begin{array}{llll}\text { O } & 0.457956 & 0.508340 & 0.961346\end{array}$

C 2.4703590 .8337522 .773259

H $2.556726 \quad 1.8517412 .383130$

H 3.1076650 .7404983 .656566

H 2.8014520 .1061892 .027380

SCF Energy (B3LYP/6-31G**//MMFF)= -3245.91668928

01_327

MM̄FF Geometry

C $-0.870856 \quad 4.990662 \quad 0.351114$

$\begin{array}{llll}\text { C }-2.103449 & 4.813457 & 0.846001\end{array}$

C $-2.5533393 .701031 \quad 1.760817$

O -3.3312932 .8149730 .911760$

C -1.4563642 .8700692 .468345$

C $-0.603107 \quad 3.617186 \quad 3.472680$

C 0.7451373 .6374113 .476164

C $-1.3578874 .313307 \quad 4.577119$

C 1.6989992 .9925572 .508057

C 2.4693041 .8208983 .130891

C 1.5952540 .5832133 .390628

C $2.421174-0.5260504 .045535$

C $1.615440-1.8231174 .210139$

$\begin{array}{lllll}\text { O } & 1.082168 & 0.135819 & 2.132148\end{array}$

O $2.538131-2.8806954 .512471$

C $0.951660-2.1748072 .863028$

C $0.642665-1.7438125 .392431$

O $0.076185-3.2917863 .002236$

C $0.198861-0.989602 \quad 2.215659$

C $-0.228123-1.3676310 .779115$

O $-0.966761-0.6943862 .986140$

C -4.2464421 .9980651 .494513$

$\begin{array}{llll}\text { O } & -4.518267 & 1.916548 & 2.681048\end{array}$

C $-4.8528391 .185630 \quad 0.417494$

$\begin{array}{llll}C & -5.398913 & -0.001555 & 0.718174\end{array}$

C $-6.015884-0.933221-0.229382$

C $-6.241227-0.445231-1.636302$

C $-0.778366-0.201584-0.047545$

C -1.036864 -0.646566 -1.472252

C $-2.692623-1.800622-2.902914$

O $-0.245737-0.400278-2.379942$

C $-3.766989-2.841460-2.815757$

C $-4.042555-3.647346-1.777752$

C $-5.155143-4.657340-1.790633$

C $-6.094769-4.518002-0.612855$

C $-6.186257-5.4849490 .315966$

C $-6.987751-3.296325-0.553721$

C $-6.351585-2.1648370 .208620$

N -2.213272 -1.369141 -1.604316

H $-0.078763 \quad 4.3031200 .620860$

H $-2.886524 \quad 5.502438 \quad 0.534315$

H $-3.227623 \quad 4.1411682 .506262$

H $-0.8558762 .357867 \quad 1.710784$

H $-1.936727 \quad 2.047844 \quad 3.014371$

H 1.2423334 .1890364 .273904

H $-0.6943174 .684350 \quad 5.365727$

H -1.9117095 .1713214 .184223$

H -2.066228 3.6229405 .046876

H 1.2115262 .6683341 .585547

H 2.4217713 .7597742 .202306

H 3.2714031 .5503592 .432198

H 2.9482902 .1435714 .063555 
H $\quad 0.760603 \quad 0.8643424 .042122$ H $2.817657-0.1948565 .013502$ H $3.300162-0.7418943 .423399$ H $3.018502-2.6370065 .322412$ H $1.750572-2.4919812 .177913$ H $\quad 1.189287-1.5660836 .326437$ H $0.112346-2.6922505 .532308$ H $-0.096241-0.9466815 .287237$ H $0.592657-4.0170463 .393402$ H $\quad 0.642647-1.760540 \quad 0.243332$ H $-0.976898-2.1691170 .811121$ H -1.635373 -0.2900052 .411730$ H $-4.8032931 .573790-0.593305$ H $-5.372527-0.336077 \quad 1.754711$ H -5.292113 -0.176773 -2.111611 H $-6.8902860 .437659-1.633854$ H -6.717547 -1.182762 -2.284961 H -1.7076090 .1907910 .377027$ H $-0.0672350 .630573-0.083185$ H -1.852458 -2.168865 -3.501230 H -3.090435 -0.908864 -3.399978 H $-4.381110-2.930985-3.710367$ H -3.433543 -3.605291 -0.878602 H $-4.696074-5.654313-1.795734$ H -5.740466 -4.591406 -2.716439 H $-6.877412-5.4029431 .148446$ H $-5.570312-6.3767640 .268162$ H -7.924827 -3.550073 -0.039929 H -7.291074 -3.020514 -1.566920 H -6.154046 -2.408591 1.254056 H $-2.881312-1.328053-0.839529$ C $-0.0559285 .501039-1.984928$ O $0.9660294 .495018-1.810591$ C $0.5594743 .309020-2.517082$ C $-0.4140093 .798508-3.578439$ C $-1.1796634 .848985-2.796803$ H $0.0219062 .674038-1.800547$ C $1.7678002 .541315-3.062893$ H $\quad 0.1120244 .257395-4.423900$ H -1.053216 2.997679-3.961745 C -1.947313 $5.824343-3.672100$ $\mathrm{H}-1.8915174 .337667-2.137086$ C $2.6494851 .933155-1.947489$ O $2.5328783 .403330-3.901213$ H $1.3992851 .724397-3.694505$ H $2.002124 \quad 1.344541-1.288762$ O $3.2413752 .958367-1.151916$ C $3.7647101 .016589-2.487089$ C $4.6245950 .360386-1.389933$ H $3.3127760 .241772-3.116875$ O $4.6414641 .773455-3.324729$ H $5.421601-0.205143-1.888518$ H $5.1510621 .138503-0.822401$ H -2.461501 $6.572703-3.060776$ H -1.282655 $6.352168-4.364266$ H -2.700312 $5.296853-4.266703$ C $-0.4771726 .065437-0.622064$ H $2.8921624 .110951-3.338606$ H $2.5217523 .480049-0.758009$ H $5.2847701 .155915-3.712495$ H -1.280986 $6.798793-0.752715$ H $0.3748226 .598304-0.181601$ H $0.4079896 .308585-2.565392$

C $3.662412-2.502645-1.837985$

O $2.989431-1.470151-1.111772$

C $3.850347-0.559357-0.424206$

C $4.742009-1.3253960 .563040$

C $5.511836-2.455061-0.118935$

C $4.563322-3.345505-0.923744$

H $4.290036-2.037912-2.609385$

C $2.601728-3.303401-2.609268$

H $3.197867 \quad 0.0878240 .173174$

O $5.665429-0.4491041 .200034$

H 4.116989-1.764618 1.350619

H $6.318974-2.068245-0.751658$

O $6.141552-3.2604770 .881326$

H $3.981037-3.947207-0.218572$

O $5.348735-4.245087-1.702891$
C $1.548536-3.977668-1.752470$

H $2.066908-2.604007-3.265331$

H $3.076687-4.068028-3.232443$

H 5.1507390 .2487451 .639885

H $6.713039-2.6740451 .406159$

H $4.752177-4.923321-2.061649$

O $0.558162-4.450698-2.568057$

O $1.580089-4.091175-0.535961$

C $-0.517636-5.118217-1.901310$

H $-0.159199-6.049525-1.453196$

H $-1.282612-5.357033-2.644711$

H $-0.958362-4.468471-1.139165$

SCF Energy (B3LYP/6-31G**//MMFF) $=-3245.90710298$

01328

MM̄FF Geometry

C -0.670416 $2.070225-2.166046$

C $0.2915542 .999245-2.282558$

C $1.2054813 .434808-1.168714$

O $2.5261592 .993369-1.574425$

C $1.2328994 .962656-0.980027$

C $-0.0381385 .559370-0.421075$

C -0.2912645 .7055950 .893822$

C -1.012262 $6.091616-1.438686$

C 0.5696735 .2729932 .051787

C -0.2674044 .6818943 .197064$

C -0.7686633 .2574632 .907324$

C -1.9789822 .9370003 .786544$

C -2.4515791 .4914753 .596881$

$\begin{array}{lllll}\text { O } & 0.302931 & 2.350917 & 3.201442\end{array}$

O -3.3858351 .1899934 .644090$

C -1.2451750 .5530513 .781301$

C -3.2014071 .3001062 .274932$

O $-1.602234-0.7950813 .478072$

C 0.0019240 .9675902 .962022

C 1.2125260 .1219483 .438254

$\begin{array}{lllll}\text { O } & -0.243947 & 0.727051 & 1.579364\end{array}$

C $3.4703352 .832317-0.612024$

O 3.3749233 .1093930 .571847

C $4.6420052 .195801-1.250540$

C $5.4704151 .460751-0.494420$

C $6.6195890 .693136-0.972118$

C $7.0543750 .895012-2.399788$

C 2.5410790 .4392442 .744279

C $2.804960-0.4733431 .562579$

C $3.512535-2.8039431 .001652$

O $2.643381-0.0929980 .406305$

C $4.992233-3.0272880 .981066$

C $5.717498-3.113195-0.144799$

C $7.196892-3.380806-0.161823$

C $7.965777-2.399331-1.019671$

C $8.338035-2.726905-2.268745$

C $8.379232-1.078817-0.407489$

C $7.216034-0.170430-0.123792$

N $3.209097-1.7420701 .942943$

H $\quad-0.8394291 .587735-1.207565$

H $\quad 0.4660343 .455389-3.255124$

H $0.9337202 .931545-0.231911$

H $1.4825665 .450018-1.933120$

H $2.0746875 .238344-0.332203$

H -1.2144816 .2096091 .177430$

H -1.898934 $6.536986-0.975091$

H $-0.536617 \quad 6.865222-2.050503$

H -1.356356 $5.292474-2.098064$

H 1.3401554 .5576931 .751044

H 1.0837276 .1648282 .430403

H $\quad 0.3564034 .6674954 .100292$

H -1.113126 5.3505783 .401766

H -1.0364113 .1689631 .848741$

H -2.8013983 .6352983 .586348$

H -1.7126623 .0816634 .843032$

H -4.1202541 .8246204 .582223$

H $-0.9784820 .569496 \quad 4.847530$

H -4.0811651 .9532482 .233187$

H -3.5823990 .2780382 .189623$

H -2.5890961 .5138461 .396380$

H -2.375955 -1.019526 4.022807

H 1.3590280 .3167304 .508964 
H $0.984291-0.9446813 .326347$ H 0.6015180 .7584531 .104505 H $4.7508582 .294292-2.324252$ H $5.2640101 .376060 \quad 0.571890$ H $7.2563471 .954302-2.594439$ H $6.2766630 .552123-3.090955$ H $7.9682980 .353646-2.654324$ H 2.5946911 .4805032 .416324 H 3.3652940 .3009123 .455951 H $3.117138-2.5538950 .012505$ H $3.009437-3.7061271 .362829$ H $5.488609-3.1508251 .940983$ H $5.217934-3.020516-1.106649$ H $7.343666-4.400384-0.542274$ H $7.616485-3.3809530 .851977$ H $8.916736-2.046407-2.884228$ H $8.069371-3.683064-2.705804$ H $9.118094-0.564169-1.033390$ H $\quad 8.906707-1.2745650 .535177$ H $6.825574-0.2615350 .890109$ H $3.317860-1.9482462 .931840$ C $-3.0202841 .636884-3.107860$ O $-3.4194270 .586218-2.211971$ C $-4.6286840 .996667-1.540694$ C $-4.9667312 .398460-2.052463$ C -3.619201 2.912118 -2.526430 H $-4.3837111 .061588-0.475425$ C $-5.740538-0.042409-1.754830$ H $-5.6798862 .370399-2.883968$ H $-5.4081413 .023120-1.269174$ C $-3.7225994 .052920-3.523590$ H $-3.0534793 .243919-1.647069$ C $-5.332380-1.491692-1.418810$ O $-6.125134-0.022092-3.136559$ H -6.629509 $0.251476-1.184151$ H $-4.613576-1.838349-2.169030$ O $-6.490594-2.321697-1.573667$ C $-4.762366-1.689527-0.005953$ C $-4.450840-3.1536120 .357694$ H -3.851151 -1.093658 0.105133 O $-5.709201-1.1878040 .940977$ H $-4.025435-3.157881 \quad 1.369277$ H $-5.386904-3.7196960 .448675$ H $-2.7321804 .356279-3.876177$ H -4.315912 $3.768045-4.399095$ H $-4.2005854 .923545-3.063016$ C -1.505935 $1.610496-3.330599$ H -6.831193 $-0.681393-3.250346$ H $-7.129492-2.063416-0.887052$ H $-5.329325-1.3052801 .828254$ $\mathrm{H}-1.2741982 .213860-4.216972$ H -1.203802 $0.579966-3.556942$ H -3.498598 $1.405851-4.069848$ C -1.429220 -2.8384010 .037295$ O $-2.390612-3.052417-0.997096$ C $-3.499489-3.871430-0.620354$ C $-3.007510-5.244927-0.137747$ C $-1.941503-5.1257290 .952570$ C $-0.831792-4.1600190 .529140$ H -1.920362 -2.341773 0.884104 C $-0.388653-1.836022-0.473896$ H $-4.073264-4.046398-1.538939$ O $-4.091162-6.0288150 .350250$ H - $-2.573627-5.788454-0.987151$ H -2.387075 -4.811511 1.903333 O $-1.370670-6.412842 \quad 1.201779$ H $-0.220939-4.630402-0.249139$ O $0.021052-3.9037891 .642524$ C $0.356818-2.248620-1.725993$ H $-0.904873-0.900012-0.718261$ H $0.345950-1.6423210 .312743$ H $-4.743224-6.104759-0.367113$ H $-0.972845-6.7309110 .373567$ H $0.352421-4.762046 \quad 1.958024$ O $1.276489-1.280706-2.019314$ O $0.161078-3.267390-2.372564$ C $2.081987-1.518943-3.177097$ H $2.660154-2.439605-3.053083$
H $1.453118-1.572942-4.070653$

H $2.776194-0.681153-3.285295$

SCF Energy (B3LYP/6-31G**//MMFF) $=-3245.91461781$

01_329

MM̄FF Geometry

C $-2.285548-3.060984-1.695218$

C $-1.420175-2.932359-2.711255$

C $-0.438904-1.794499-2.819076$

O $0.873955-2.394974-2.698402$

C $-0.524001-1.061780-4.171831$

C $-1.4441130 .141827-4.155939$

C $-1.0148291 .416854-4.237082$

C $-2.919066-0.149641-4.092827$

C $0.418961 \quad 1.872159-4.305492$

C $0.571532 \quad 3.398562-4.211265$

C $0.2180603 .973536-2.829160$

C $0.1169475 .499225-2.908631$

C $-0.1643856 .125067-1.537098$

O $1.246278 \quad 3.596046-1.902535$

O $0.0577737 .539434-1.642735$

C $0.8648085 .579302-0.530026$

C $-1.6257905 .945475-1.109969$

$\begin{array}{lllll}\text { O } & 0.562299 & 6.019256 & 0.792894\end{array}$

C $1.0110984 .037034-0.556845$

$\begin{array}{llll}\text { C } 2.222717 & 3.634023 & 0.318999\end{array}$

O $-0.1732103 .447858-0.012219$

C $1.865694-1.619716-2.184650$

O $1.777309-0.458600-1.820118$

C $3.095438-2.439175-2.134883$

C $4.250206-1.861609-1.773397$

C $5.544088-2.528040-1.629712$

C $5.605760-4.011507-1.880020$

C 2.6501392 .1656940 .197413

C 3.6114991 .7765301 .310378

C $4.568612-0.2161202 .457926$

$\begin{array}{lllll}\text { O } & 4.176020 & 2.602041 & 2.024552\end{array}$

C $5.980572-0.3725521 .989295$

C $6.641893-1.5402272 .001628$

C $8.068103-1.6989321 .552014$

C $8.219392-2.7189980 .443923$

C $8.593844-3.980338 \quad 0.717063$

C $8.008828-2.262950-0.984002$

C $6.606505-1.791264-1.245052$

N 3.7717630 .4017341 .412935

H $-2.297353-2.303647-0.916255$

H $-1.386847-3.696687-3.483715$

H $-0.585599-1.090413-1.989674$

H $-0.851780-1.748047-4.964624$

H $0.482851-0.759058-4.483855$

H $-1.7663692 .204571-4.256912$

H $-3.5267710 .737377-4.301574$

H $-3.193385-0.910799-4.830373$

H -3.192621 $-0.505944-3.096071$

H $1.0058261 .401533-3.510764$

H $\quad 0.837725 \quad 1.553312-5.267751$

H $1.6155163 .644824-4.444499$

H $-0.0523963 .866185-4.983630$

H $-0.7365213 .553061-2.495334$

H $-0.6527105 .801872-3.629811$

H $1.0630445 .908067-3.290715$

H $-0.5364817 .885482-2.330531$

H $1.8376586 .021379-0.787651$

H $-2.2978976 .409126-1.841973$

H $-1.8240586 .452117-0.158999$

H $-1.9192874 .898478-1.010456$

H 0.4987296 .9893550 .767950

H $3.092025 \quad 4.2470580 .046792$

H 1.9893023 .8521661 .369387

H 0.0514652 .6030370 .407674

H $3.014825-3.491741-2.380741$

H $4.249599-0.796123-1.546393$

H $5.325277-4.238605-2.914474$

H $4.926339-4.544642-1.205696$

H $6.599844-4.436183-1.721891$

H $1.779808 \quad 1.5077750 .248077$

H $3.1508161 .995233-0.762183$

H $4.102641-1.1760262 .699356$ 
H 4.5450330 .4158393 .351725

H 6.4905140 .5223151 .640257

H $\quad 6.139360-2.4316262 .370651$

H $8.660633-2.0032452 .424886$

H $8.495286-0.7425101 .225823$

H $8.730063-4.713720-0.070720$

H $8.768954-4.3088821 .736190$

H $8.288350-3.045645-1.699089$

H $8.698199-1.434983-1.194720$

H $6.467986-0.722512-1.078404$

H $3.309562-0.206500 \quad 0.743254$

C $-4.691917-3.791073-1.329080$

O $-4.798285-2.874707-0.220132$

C -5.608672 -1.757287 -0.628902

C $-6.390511-2.244863-1.841429$

C $-5.346809-3.105924-2.528942$

H $-4.922045-0.966346-0.954027$

C $-6.457900-1.2568320 .541119$

H -7.253678 -2.854275 -1.549140

H $-6.747871-1.421116-2.466561$

C $-5.924793-4.071917-3.548374$

H -4.632469 -2.448200 -3.036559

C $-5.633675-0.8641531 .787134$

O $-7.362393-2.3003910 .923323$

H -7.079287 -0.4157210 .211719$

H -5.185324 -1.763992 2.222074

O $-6.551074-0.3676982 .770848$

C $-4.546557 \quad 0.1980201 .531672$

C $-3.859423 \quad 0.7193472 .808437$

H $-3.790319-0.2025960 .848565$

O

H -3.217109 1.5616302 .523898

H -4.611371 1.1598093 .474753

H -5.134319-4.681603 -3.997355

H -6.655264 -4.748361 -3.092217

H -6.429196 -3.525900 -4.352407

C $-3.232324-4.214930-1.518207$

H $-7.886386-1.9692981 .672829$

H -6.9312870 .4584842 .425874$

H $-4.447527 \quad 1.9442650 .651793$

H $-3.156715-4.919052-2.354825$

H -2.909424 -4.750636 -0.616519

H -5.272045 -4.678348 -1.044231

C $-0.988196-0.1839372 .300352$

O $-2.089797-0.9995482 .711119$

C $-3.036685-0.344738 \quad 3.562567$

C $-2.335327 \quad 0.182598 \quad 4.823611$

C -1.1426571 .0736124 .483963$

C -0.2099200 .3668063 .501355$

H -1.375642 0.6562041 .709966

C $-0.115914-0.9922921 .330147$

H -3.740074 -1.118656 3.891789

O $-3.242706 \quad 0.911003 \quad 5.644157$

H -1.973346 -0.6660005 .418786$

H -1.464014 2.0453454 .091106

O $-0.405870 \quad 1.349067 \quad 5.677827$

H $0.318943-0.4363934 .027293$

O $0.772326 \quad 1.2898443 .038336$

C $0.566742-2.1977681 .940392$

H $-0.732625-1.3676150 .508575$

H $0.650928-0.3360070 .908931$

H -3.983434 0.3182055 .857862

H -1.021481 1.7616496 .308016

H $1.232721 \quad 1.6416063 .819521$

O $1.697122-2.477236 \quad 1.225992$

O $0.146402-2.8366532 .895043$

C $2.447119-3.6114721 .671190$

H $1.872621-4.5273891 .505518$

H $3.368228-3.6618811 .084400$

H $2.712191-3.5060872 .727657$

SCF Energy (B3LYP/6-31G**//MMFF) $=-3245.91692725$

01333

MM̄FF Geometry

C $1.674793-1.714690-2.563769$

C $0.808622-2.725773-2.721680$

C $-0.115922-3.202750-1.635389$

O $-1.448507-2.800434-2.032394$
C $-0.089424-4.735242-1.476832$

C $1.149939-5.275820-0.795916$

C $1.236217-5.5241000 .525802$

C $2.301268-5.623950-1.702828$

C $0.182071-5.2769721 .569987$

C $0.759679-4.7320722 .884105$

C $1.315293-3.3021512 .783022$

C $1.985061-2.9141844 .104531$

C 2.465831-1.457899 4.109172

O $0.224847-2.4174032 .502361$

O $2.794865-1.1391155 .470026$

C $1.295471-0.5526163 .671132$

C $3.754846-1.2852163 .297373$

O $1.727098 \quad 0.796932 \quad 3.503271$

C $0.597097-1.0383722 .379068$

C $-0.680519-0.2199962 .078153$

O $1.495127-0.8429991 .279586$

C $-2.357142-2.607615-1.039014$

O $-2.178161-2.7545920 .159209$

C $-3.629144-2.184492-1.663376$

C $-4.697032-1.974960-0.879799$

C $-6.027444-1.554318-1.316941$

C $-6.242162-1.300031-2.785091$

C -1.865666 -0.477992 3.012076

C -2.8504090 .6753732 .975791$

C -4.3909331 .9214101 .466399$

O -3.0269521 .3944243 .957731$

C $-5.807618 \quad 1.447648 \quad 1.545576$

C -6.7239321 .7332740 .607339$

C $-8.162197 \quad 1.2997310 .667731$

C $-8.5876630 .542623-0.571927$

C $-9.1611881 .182542-1.605063$

C $-8.411874-0.959391-0.592080$

C $-6.987983-1.393395-0.383522$

N $-3.4858280 .822700 \quad 1.752984$

H $1.701467-1.182496-1.617828$

H $\quad 0.764513-3.243857-3.676824$

H $\quad 0.139921-2.718799-0.684157$

H $-0.210443-5.217281-2.457144$

H $\quad-0.982559-5.061030-0.928691$

H $2.155910-5.9699550 .902569$

H $3.130816-6.091373-1.161288$

H $1.976896-6.328862-2.475284$

H $2.695772-4.730560-2.191829$

H $-0.598612-4.6015191 .212115$

H $-0.301513-6.2376601 .785935$

H $-0.047817-4.7478963 .627948$

H $1.542451-5.4109803 .245547$

H $2.039548-3.2533891 .962269$

H $2.817914-3.5916424 .331892$

H $1.265419-3.0424104 .925989$

H $3.151883-0.2353025 .491125$

H $0.569711-0.5454874 .493041$

H $4.555747-1.9041433 .719554$

H $4.117935-0.2534043 .347929$

H $3.643580-1.5602442 .246552$

H 2.0710821 .1062274 .357795

H $-0.434004 \quad 0.8487732 .053820$

H -1.013720 -0.4564881 .059441$

H $0.973582-0.7841250 .463375$

H -3.653166 -2.059657 -2.739594

H $-4.590329-2.1251340 .194114$

H $-6.071376-2.214886-3.363011$

H $-5.558216-0.523379-3.145380$

H -7.253371 $-0.959522-3.020684$

H $-2.387404-1.4009382 .734664$

H -1.543467 -0.595200 4.051069

H -4.1388972 .2915110 .467464$

H -4.2311222 .7281982 .188841$

H -6.0984430 .8679332 .418132$

H $-6.4315012 .337646-0.248920$

H -8.7788972 .2008500 .781967$

H -8.3627810 .6905011 .557805$

H $-9.4913470 .648029-2.489540$

H -9.310342 2.257238 -1.590057

H -8.810524 -1.397597 -1.514775

H -9.022942 -1.3928530 .210609$

H $-6.740113-1.5874090 .660770$ 
H $-3.3108390 .146857 \quad 1.015106$

C $4.051656-0.999336-3.094098$

O $4.039197 \quad 0.151735-2.228118$

C $4.916391-0.098476-1.112977$

C $5.720491-1.342595-1.477736$

C $4.709928-2.124841-2.296434$

H $4.273600-0.332681-0.255820$

C $5.7507441 .151049-0.808623$

H $6.595438-1.098766-2.091114$

H $6.069626-1.884078-0.593329$

C $5.327163-3.209980-3.160392$

H $3.996776-2.584814-1.601590$

C $4.9203182 .442026-0.652708$

O $6.6782411 .371936-1.880018$

H $\quad 6.3609570 .9816070 .085641$

H $4.4941012 .720233-1.623017$

O $5.8143973 .500493-0.286583$

C 3.8080292 .3639440 .407338

C 3.0508493 .6872630 .627893

H 3.0875191 .5864970 .135430

O 4.3752951 .9711011 .659393

H 2.3558753 .5367521 .463349

H 3.7481194 .4569590 .982080

H $4.559751-3.721575-3.749696$

H $\quad 6.066937-2.798338-3.855194$

H $5.830363-3.957065-2.537928$

C $2.638095-1.262485-3.624034$

H $6.1635051 .460149-2.700752$

H $6.5108693 .538550-0.964539$

H 5.0444732 .6348641 .899738

H $2.688454-2.000087-4.433529$

H $2.243372-0.330972-4.048682$

H $4.686942-0.723348-3.946193$

C $0.3214772 .822729-0.429264$

O $1.4737843 .173820-1.200236$

C $2.2934854 .192887-0.617199$

C $1.4704125 .468055-0.384925$

C 0.2019865 .1928440 .421007

C $-0.5869354 .033694-0.189762$

H 0.6546122 .4351990 .541432

C $-0.3908311 .646669-1.111479$

H $3.0515984 .443639-1.368665$

O $2.264497 \quad 6.4495860 .272848$

H $1.1865635 .885550-1.359754$

H $0.440674 \quad 4.991979 \quad 1.471568$

O $-0.616468 \quad 6.3653150 .428714$

H -1.051886 4.365279-1.124871

O -1.6362513 .6632520 .700050$

C $-0.8798841 .909827-2.519406$

H $0.3073960 .805511-1.181008$

H $-1.2510501 .341474-0.506398$

H 1.7001467 .2269520 .425035

H $-0.8459836 .578047-0.492060$

H $-2.1717304 .457940 \quad 0.866435$

O $-1.6869010 .872813-2.898425$

O $-0.5807572 .873519-3.209747$

C -2.219728 $0.957151-4.223307$

H $-2.8354361 .855486-4.327957$

H $-1.4087160 .955399-4.957684$

H -2.848784 $0.079227-4.393141$

SCF Energy (B3LYP/6-31G**//MMFF) $=-3245.91696602$

01_330

MM̄FF Geometry

C $-1.210359-0.379676-1.220982$

C $0.088341-0.191251-0.945018$

C 0.6118750 .1190590 .431224

O $1.627351-0.8754790 .710246$

C 1.2643111 .5115430 .493165

C 0.3254842 .6702910 .246503

C $0.3651933 .465195-0.840340$

C $-0.6635232 .943921 \quad 1.348923$

C $1.2573173 .340844-2.044278$

C $2.3256844 .440491-2.112638$

C $3.4295604 .303739-1.050325$

C $4.4234115 .462394-1.163553$

C $5.6000135 .308880-0.187558$

O $4.1052643 .061241-1.269316$
O $6.6202406 .240327-0.579893$

C $6.1936603 .892764-0.343388$

C 5.2014565 .6819101 .245168

O 7.2078393 .6566190 .631295

C $5.1339732 .765519-0.314730$

C $5.7880721 .421763-0.719721$

O $4.6147902 .657159 \quad 1.011771$

C $1.962550-1.0886362 .010627$

O $1.464132-0.5696662 .996090$

C $3.081782-2.0544752 .022466$

C $3.746097-2.2918973 .162199$

C $4.891881-3.1868873 .281643$

C $6.010900-2.6242654 .114451$

C $4.7965170 .262108-0.888689$

C $5.466799-0.996945-1.411637$

C $4.910140-3.277924-2.250275$

O $6.681259-1.100227-1.561311$

C $4.977536-4.299104-1.159098$

C $6.120132-4.877347-0.758765$

C $6.202505-5.9645700 .275435$

C $6.841987-5.5065811 .567085$

C $8.154215-5.2251961 .635501$

C $5.971157-5.4542272 .801037$

C $4.897469-4.4076312 .710637$

N 4.539619-1.992420-1.691162

H $-1.942294-0.295167-0.421116$

H $0.819879-0.263784-1.746694$

H $-0.1892040 .026413 \quad 1.175225$

H $2.1176101 .532474-0.191305$

H 1.7257861 .6545841 .479270

H $-0.3395004 .294224-0.894283$

H -1.274995 3.8303291 .149308

H -1.3447792 .0967601 .472469$

H -0.1400893 .1112452 .295932$

H $1.7200242 .352606-2.119678$

H $0.6166993 .427636-2.931544$

H $2.7841254 .388085-3.108976$

H $1.8480395 .424697-2.028722$

H $2.9702994 .296748-0.055764$

H $3.9187516 .424217-1.008437$

H $4.8261375 .497977-2.185701$

H $6.2360267 .133508-0.556256$

H $6.701513 \quad 3.865707-1.317408$

H $4.8349856 .714953 \quad 1.283764$

H 6.0658055 .6481581 .917389

H 4.4185435 .0396621 .653592

H 6.7670603 .4756901 .478963

H $6.312398 \quad 1.555327-1.675151$

H 6.5386361 .1324610 .026915

H $4.272306 \quad 1.7606991 .149592$

H $3.355738-2.5311321 .087647$

H $3.468712-1.7720524 .076718$

H $5.710177-2.5597415 .165890$

H $6.275509-1.6175703 .770010$

H $6.926187-3.2190034 .064150$

H 4.3317640 .0139970 .069760

H $4.0070400 .540035-1.595979$

H $5.860690-3.186258-2.786275$

H $4.137885-3.558886-2.973195$

H $4.036727-4.602146-0.706276$

H $7.055534-4.576638-1.225569$

H $6.786335-6.795407-0.141982$

H $5.208870-6.3857380 .473866$

H $8.620526-4.9220392 .567099$

H $8.791387-5.2922780 .759743$

H $6.576779-5.317758 \quad 3.704019$

H $5.491225-6.4322172 .937366$

H $4.024484-4.7202132 .137671$

H $3.558618-1.830387-1.481783$

C $-2.463935-2.030089-2.637062$

O $-3.647452-1.967326-1.816708$

C $-4.747058-2.557968-2.532359$

C $-4.130907-3.296606-3.713679$

C $-2.948826-2.404908-4.038037$

H $-5.355799-1.728886-2.912609$

C $-5.585328-3.430424-1.592436$

H -3.783333 -4.297603 -3.433265

H $-4.831356-3.403941-4.547289$ 
C $-1.894665-3.081558-4.896899$ H -3.317076 -1.511359-4.559999 C $-6.062789-2.713108-0.312822$ O $-4.807159-4.562098-1.179280$ H -6.443585 -3.841019 -2.136717 H $-5.205490-2.5212980 .342010$ O $\quad-6.919690-3.615730 \quad 0.398254$ C $-6.836153-1.405137-0.558299$ C $-7.474691-0.7935150 .702325$ H -6.173063 -0.665475 -1.013976 O $-7.885673-1.644431-1.500714$ H $-8.061290 \quad 0.0780220 .387211$ H -8.214512 $-1.494821 \quad 1.108404$ H -1.057929 -2.404269 -5.094591 H -1.494923 -3.977536-4.410184 H -2.319302 $-3.383485-5.859879$ C $-1.737267-0.685312-2.595342$ H -4.000216 $-4.223774-0.754170$ H -6.417312 -4.4352410 .547133$ H -8.464427 -2.331275 -1.127305 H $-2.4311610 .116462-2.871832$ H -0.921596 -0.678036 -3.327356 H -1.828774 -2.823040 -2.221172 C $-5.931507 \quad 1.8362291 .027047$ O C $-6.465879-0.394558 \quad 1.797646$ C $-7.115605 \quad 0.160516 \quad 3.072784$ C -7.6684381 .5643142 .856902$ C -6.5946352 .4696932 .259550$ H -6.6435801 .8004700 .193464$ C -4.7145582 .6717300 .592800$ H -5.934764 -1.306704 2.093183 O $-8.163647-0.6898873 .525932$ H $-6.366641 \quad 0.2022413 .874331$ H $\quad-8.5680141 .5533942 .230565$ O $\quad-8.0640152 .112724 \quad 4.116588$ H -5.8367932 .6876803 .023305$ O $\quad-7.1904803 .710893 \quad 1.884423$ C $-4.2614722 .366876-0.819281$ H $-4.967703 \quad 3.7377620 .623290$ H -3.8651792 .4850701 .259008$ H -7.783009 -1.573933 3.664983 H -8.7168721 .5044374 .503769$ H -7.616760 4.0791482 .677281 O $-3.4432693 .375011-1.245346$ O $-4.567511 \quad 1.372604-1.461364$ C -2.952226 $3.261086-2.584728$ H $-3.7595683 .013007-3.280535$ H -2.529354 $4.227020-2.873614$ H $-2.1622062 .507378-2.624322$

SCF Energy (B3LYP/6-31G**//MMFF) $=-3245.91834369$

01_331

MM̄FF Geometry

C 3.0026163 .6652421 .088852

C 2.2250082 .5880440 .908721

C 1.2978662 .0255171 .953280

O $0.035807 \quad 1.7127751 .315336$

C 1.8787180 .7168122 .517298

C $1.155697 \quad 0.2381763 .760965$

C $0.266371-0.773273 \quad 3.803680$

C $1.524424 \quad 0.9640245 .031028$

C $-0.231954-1.6314542 .673674$

C -1.722209-1.390046 2.427625

C $-2.365642-2.3663221 .431854$

C $-1.714922-2.3346320 .049591$

C $-2.454303-3.228464-0.956919$

O $-3.739891-1.9777131 .324060$

O $-1.978658-2.901379-2.271020$

C $-3.956844-2.885590-0.920363$

C $-2.138302-4.713117-0.740220$

O $-4.706435-3.790094-1.728381$

C $-4.546069-2.8382920 .508246$

C $-5.968911-2.2335790 .469429$

O $-4.609451-4.1620921 .039422$

C -0.8263242 .7411931 .088001$

O -0.6825223 .9082061 .415141$

C $-1.975708 \quad 2.198700 \quad 0.328728$
C $-2.8693673 .036167-0.218181$

C $-4.0290652 .650930-1.027663$

C $-4.3813761 .188875-1.085080$

C $-6.597075-2.0403041 .855949$

C $-7.660148-0.9618551 .832812$

C $-7.935278 \quad 1.5162101 .765506$

O $-8.858193-1.2336981 .833076$

C $-8.154512 \quad 1.9172580 .340931$

C -7.915844 $3.151104-0.129554$

C $-8.2169873 .566852-1.540252$

C $-7.0697494 .289104-2.206895$

C $-7.1539475 .599015-2.497044$

C $-5.867373 \quad 3.475153-2.629414$

C $-4.7073773 .617724-1.680414$

N $-7.121806 \quad 0.316777 \quad 1.805948$

H 2.9930694 .1842942 .043643

H $2.2536822 .077687-0.048779$

H 1.1296872 .7501322 .759269

H 2.9375630 .8540372 .776447

H $1.867296-0.0559871 .742184$

H $-0.167800-1.0265394 .770755$

H $1.021156 \quad 0.547567 \quad 5.910338$

H 2.6028350 .8948565 .207864

H 1.2495072 .0210684 .966744

H $0.329851-1.4533561 .754964$

H $-0.066039-2.6811242 .943820$

H -2.273749 -1.461576 3.375626

H $-1.881667-0.3599002 .086858$

H -2.312263 -3.3736821 .861096$

H $-0.665047-2.6349180 .103416$

H $-1.716913-1.304788-0.331801$

H -1.015500 -3.036638 -2.284371

H $-4.076002-1.893639-1.377021$

H -1.061480 -4.894482 -0.842844

H -2.620099 -5.334817 -1.502768

H $-2.441050-5.0765220 .243917$

H -4.311689-3.780152 -2.617216

H $-5.926879-1.266343-0.045352$

H -6.637076 -2.876177 -0.117860

H $-5.272789-4.1867331 .747135$

H $-2.037475 \quad 1.1238510 .208631$

H $-2.7252234 .108052-0.088335$

H $-4.4562310 .772271-0.074547$

H $-3.6171630 .631239-1.636681$

H $-5.3417280 .990210-1.565414$

H -7.057769 -2.9721522 .203279$

H -5.852124 -1.759269 2.608985

H $-7.4093202 .287428 \quad 2.336927$

H $-8.898806 \quad 1.3180132 .245817$

H $-8.569478 \quad 1.165900-0.327330$

H -7.5355253 .9146450 .544997$

H -9.102282 4.215459-1.509495

H $-8.5007602 .704899-2.157338$

H $-6.3478216 .115373-3.007904$

H $-8.0269476 .184351-2.228229$

H $-5.5287913 .825337-3.614348$

H -6.164196 $2.435754-2.792608$

H $-4.3583564 .646244-1.575304$

H -6.1138860 .4290151 .746345$

C 5.3760703 .8934530 .311873

O 5.5954782 .4712550 .202149

C $6.8172402 .240349-0.523404$

C $7.5179543 .591127-0.610492$

C $6.3373234 .537792-0.686437$

H $6.5296011 .925680-1.533415$

C $7.642111 \quad 1.1410200 .153417$

H 8.1138163 .7995630 .285698

H $8.1809853 .658942-1.478285$

C $6.6923345 .978653-0.362484$

H $5.9152654 .490908-1.699428$

C $6.902887-0.2003250 .336847$

O 8.0414351 .5898501 .456172

H $8.5717010 .981125-0.404913$

H $6.104049-0.0845901 .078250$

O $7.837923-1.1346570 .892805$

C $6.329617-0.806902-0.958213$

C $5.811936-2.252305-0.812615$

H $5.520257-0.172163-1.333802$ 
O $7.342247-0.815013-1.968865$

H $5.504167-2.598493-1.807020$

H $6.644762-2.911028-0.535937$

H $5.8081716 .621131-0.418950$

H $7.112876 \quad 6.0717740 .644460$

H $7.4331236 .360953-1.072485$

C $3.909191 \quad 4.209108 \quad 0.020094$

H 7.2329891 .8061481 .952125

H $8.188062-0.7419211 .710797$

H $8.086717-1.344177-1.634359$

H $3.6282873 .790548-0.954906$

H $3.7545075 .292455-0.038472$

H 5.6360014 .1785101 .339873

C $2.810476-1.852355-1.266515$

O $3.571052-1.523765-0.094492$

C $4.656322-2.409808 \quad 0.192892$

C $4.143199-3.8483970 .354619$

C $3.291290-4.290400-0.832559$

C $2.205149-3.255281-1.127471$

H $3.461535-1.807104-2.147394$

C $1.744477-0.758392-1.405923$

H $5.044629-2.1088361 .173059$

O $5.222586-4.7606850 .524091$

H $3.528231-3.9076701 .262190$

H $3.911396-4.469269-1.718263$

O $2.672443-5.543882-0.531373$

H $1.470697-3.267704-0.314193$

O $1.525933-3.618877-2.324236$

C $1.081112-0.692994-2.761163$

H $0.984643-0.883897-0.626527$

H $2.2079230 .219367-1.241196$

H $5.737424-4.4649691 .294192$

H $2.114679-5.4230610 .255941$

H $1.193286-4.525419-2.207309$

O $-0.157865-0.134585-2.625055$

O $1.598375-1.044989-3.812237$

C $-0.8995430 .024190-3.838153$

H $-1.033227-0.942038-4.333721$

H $-1.8837230 .426702-3.584695$

H $-0.3904030 .730733-4.500112$

SCF Energy (B3LYP/6-31G**//MMFF) $=-3245.91752471$

01_332

MM̄FF Geometry

C $-0.021098 \quad 4.201473 \quad 3.356020$

C $0.625538 \quad 3.0390893 .180279$

C -0.0002641 .6973543 .461110$

O -0.3007941 .0928222 .181140$

C $0.982804 \quad 0.776648 \quad 4.204241$

C $0.359816-0.5400104 .629447$

C $0.713125-1.7501104 .152587$

C $-0.716466-0.4420395 .680865$

C $1.766339-2.0789573 .133910$

C $1.269615-2.142275 \quad 1.681507$

C $0.147279-3.1624381 .426409$

C $-0.093979-3.329638-0.076290$

C $-1.278782-4.259163-0.379323$

O $-1.050858-2.6890192 .049618$

O $-1.597262-4.077744-1.767759$

C $-2.498177-3.812438 \quad 0.457104$

C $-0.894972-5.733773-0.211083$

O $-3.566482-4.748300 \quad 0.342105$

C $-2.165205-3.5872741 .946700$

C -3.338146 -2.970735 2.749071

O $-1.864883-4.8486952 .557885$

C -1.4744771 .4491841 .592784$

O $-2.356917 \quad 2.1464462 .068400$

C -1.488799 0.8760920 .231033

C $-2.2963331 .426003-0.687828$

C $-2.4536790 .992804-2.076229$

C $-1.765391-0.281771-2.487143$

C $-3.851033-1.6205082 .238028$

C $-5.037557-1.7248941 .293749$

C $-6.436191-0.332449-0.229068$

O $-5.676868-2.7600471 .125781$

C $-5.953578-0.491213-1.637006$

C $-6.2409680 .382253-2.614329$

C $-5.8041270 .221526-4.043331$
C $-5.0610801 .432442-4.564522$

C $-5.6991572 .385635-5.264194$

C $-3.5658971 .499159-4.336772$

C $-3.2200491 .742821-2.894899$

N -5.325421 -0.5083020 .688472$

H -1.0293664 .1970553 .762956$

H 1.6185013 .0432652 .736812

H $-0.9197251 .814524 \quad 4.047296$

H $1.3608581 .280597 \quad 5.103771$

H 1.8547670 .5999003 .564883

H $0.188608-2.6228604 .540144$

H - $1.012904-1.4240056 .065556$

H -0.3645020 .1488126 .532946$

H $-1.6141810 .031883 \quad 5.272210$

H $2.598815-1.3688883 .193597$

H $2.201755-3.0504113 .403598$

H $\quad 0.935626-1.1438001 .379184$

H $2.131886-2.3965851 .054236$

H $0.441636-4.1189201 .874281$

H $0.809414-3.695191-0.577101$

H $-0.300759-2.346605-0.520307$

H $-2.321248-4.685858-1.992773$

H $-2.852781-2.8695630 .022511$

H $-0.098040-6.002495-0.914178$

H $-1.736986-6.392944-0.449502$

H $-0.545024-5.9735910 .794925$

H $-3.836815-4.775401-0.590944$

H -4.151599 -3.699867 2.847213

H -2.974696 -2.8036373 .773436$

H $-1.968107-4.7557013 .519398$

H -0.8309480 .0425700 .020607$

H -2.901095 $2.284859-0.397870$

H -2.100289 -1.114534-1.859425

H $-0.678769-0.181039-2.388827$

H $-1.959921-0.574160-3.520391$

H $-4.197283-1.0327253 .097754$

H -3.047880 -1.056077 1.757617

H -6.854237 $0.662376-0.044866$

H $-7.206638-1.080785-0.017473$

H $-5.365526-1.375952-1.869105$

H $-6.853647 \quad 1.251185-2.383408$

H $-6.7036550 .049698-4.648895$

H -5.183717 - $0.673516-4.176073$

H $-5.1711223 .246468-5.660620$

H $-6.7666392 .329190-5.451162$

H $-3.1299172 .320053-4.920914$

H $-3.1158190 .587346-4.737343$

H $-3.6600082 .657941-2.494171$

H $-4.7153750 .287016 \quad 0.853284$

C 0.3614395 .8213741 .431954

$\begin{array}{llllll}\text { O } & 1.251037 & 4.970524 & 0.680933\end{array}$

C $0.5369404 .433923-0.448419$

C $-0.6933875 .316741-0.613859$

C -1.0337715 .6182640 .834852$

H $\quad 0.2122603 .427269-0.163582$

C $1.4562354 .343238-1.669209$

H $-0.4609976 .245265-1.147352$

H -1.501447 $4.809065-1.148576$

C $-1.960624 \quad 6.8065801 .014471$

H $-1.519047 \quad 4.730876 \quad 1.257067$

C $2.7929383 .626723-1.390099$

O $1.7653385 .674284-2.102445$

H $0.9241923 .863300-2.498682$

H $3.3949224 .239522-0.708787$

O $3.5266483 .579809-2.621637$

C $2.6687552 .197729-0.824676$

C $4.0593861 .589661-0.553486$

H 2.1071862 .2181960 .115136

O $1.9371011 .402521-1.752094$

H $4.6264951 .565243-1.488935$

H 4.5919592 .2670740 .124594

H -2.159110 6.9859462 .075876

H $-1.531327 \quad 7.7201660 .590102$

H -2.9189696 .6242760 .517114$

C 0.5230105 .5358752 .931139

H $2.3606345 .599085-2.867899$

H $3.0458682 .986425-3.224526$

H $1.7985910 .529331-1.347710$ 
H 0.0115356 .3139863 .509766

H 1.5858455 .5888533 .199620

H $0.703824 \quad 6.847112 \quad 1.243324$

C $4.304937-1.126322-1.950376$

O $3.477194-0.808423-0.826555$

C $4.0150650 .173160 \quad 0.066970$

C $5.371216-0.3091310 .602055$

C $6.346495-0.594509-0.538270$

C $5.716163-1.568214-1.534013$

H $4.382928-0.235022-2.584956$

C $3.573937-2.168399-2.813601$

H $3.328466 \quad 0.2258130 .921427$

O $5.9142750 .661478 \quad 1.490192$

H $5.219194-1.2289351 .182102$

H $6.6684850 .326523-1.037945$

O $7.528318-1.203920-0.014591$

H $5.699374-2.564891-1.080102$

O $6.536647-1.654602-2.697856$

C $3.415171-3.528787-2.164149$

H $2.563466-1.810290-3.044336$

H $4.122938-2.299288-3.753579$

H $6.693920 \quad 0.2673881 .916145$

H $7.984666-0.5388860 .528046$

H $7.430720-1.902445-2.405704$

O $3.161734-4.456828-3.134771$

O $3.479146-3.751809-0.963359$

C $2.998388-5.802722-2.676274$

H $2.212912-5.859742-1.917068$

H $3.945970-6.179218-2.279819$

H $2.704442-6.419407-3.529854$

SCF Energy $\left(B 3 L Y P / 6-31 G^{* *} / / M M F F\right)=-3245.91281200$

01_333

MMFF Geometry

C $-3.598447-2.7324472 .221102$

C $-2.407198-2.2045791 .903139$

C $-1.250303-2.1152962 .863796$

O $-0.046356-2.5178712 .171230$

C -1.073896 -0.6616313 .340599$

C $0.103612-0.4537954 .274988$

C $1.0660690 .475074 \quad 4.106897$

C $0.132775-1.3329835 .500447$

C 1.2059631 .4965523 .011071

C 0.9655502 .9053673 .568109

C 1.0637934 .0394222 .535923

C $-0.086974 \quad 4.0180281 .527411$

C 0.0398515 .1553460 .503453

O 2.3230023 .9489091 .863858

O $-0.8999944 .879101-0.546535$

C $1.4598105 .110145-0.100947$

C $-0.3708386 .506451 \quad 1.099289$

O $1.671554 \quad 6.210057-0.982009$

C 2.5883845 .0234060 .952684

C 3.9815204 .7943930 .308869

O $2.656904 \quad 6.275016 \quad 1.649817$

C $0.178767-3.8538912 .040985$

O $-0.523020-4.7645392 .450661$

C $1.429077-4.0200641 .271685$

C $1.690715-5.1973210 .686176$

C $2.848869-5.443053-0.164226$

C $2.490460-6.018263-1.506421$

C $4.1563453 .506224-0.502681$

C 4.2254722 .2533230 .352483

C $3.688824-0.1820030 .295687$

$\begin{array}{lllll}\text { O } & 4.717688 & 2.247446 & 1.478377\end{array}$

C $4.823811-0.983946-0.255418$

C $4.665106-2.147199-0.904475$

C $5.806334-2.966604-1.434831$

C $6.216764-4.079374-0.494955$

C $7.325393-3.969456 \quad 0.256841$

C $5.391441-5.349049-0.481794$

C $4.097890-5.1844650 .270637$

N $3.716712 \quad 1.140784-0.299841$

H -3.765452 -3.108269 3.227408

H -2.248992 -1.8128820 .901583$

H $-1.412216-2.7722043 .727823$

H -1.982686 -0.3409533 .867618$

H $-0.991243-0.0154802 .461434$
H $1.854145 \quad 0.530826 \quad 4.857993$

H $0.890325-1.0133856 .224240$

H $-0.835488-1.3046356 .011024$

H $\quad 0.356867-2.3688335 .227886$

H $2.223676 \quad 1.4237982 .613865$

H 0.5377651 .2942212 .172968

H -0.0132242 .9527444 .062561$

H 1.7201993 .0935704 .344283

H 1.0373194 .9775193 .103394

H -1.056516 4.0667012 .038321

H -0.0851123 .0683140 .977171$

H $-0.7988575 .572556-1.221389$

H $1.4923304 .213612-0.726701$

H -1.409703 6.4674521 .448560

H $-0.340676 \quad 7.298788 \quad 0.343371$

H $\quad 0.251207 \quad 6.8124691 .942758$

H $1.8337366 .998663-0.436524$

H $4.2185415 .649938-0.337078$

H 4.7407584 .8120291 .102701

H 3.5326246 .3476262 .064364

H $2.082941-3.1615911 .159906$

H $0.990330-6.0249250 .772236$

H $1.905099-5.294896-2.084722$

H $1.890508-6.927917-1.388003$

H $3.358089-6.291958-2.109844$

H $3.3813693 .414069-1.268192$

H $5.1137663 .559500-1.036546$

H $2.714776-0.6240010 .064213$

H $3.784660-0.1066461 .383275$

H $5.828159-0.603160-0.084655$

H $3.661732-2.531883-1.068931$

H $6.663908-2.312870-1.643831$

H $5.525261-3.387688-2.408019$

H $7.643979-4.7704870 .915522$

H $7.936307-3.0730650 .234626$

H $5.952961-6.1584120 .002985$

H $5.244071-5.672993-1.515548$

H $4.220928-4.8096581 .287714$

H $3.4089661 .227184-1.263933$

C $-5.556910-1.5624361 .160799$

$\begin{array}{lllll}\text { O } & -4.814448 & -0.596831 & 0.384494\end{array}$

C $-5.674564-0.058936-0.634961$

C $-7.091747-0.377516-0.180781$

C $-6.892110-1.7477360 .436388$

H $-5.468885-0.620615-1.554061$

C $-5.3840241 .427763-0.856249$

H $-7.445640 \quad 0.3319990 .576044$

H $-7.805704-0.378176-1.009697$

C -8.030536 -2.177476 1.345435

H $-6.782694-2.484729-0.370948$

C $-3.9171441 .731590-1.230266$

O -5.6868892 .1291190 .355775$

H $-6.0628271 .822704-1.621754$

H $-3.266506 \quad 1.540247-0.369039$

O $-3.8194343 .133094-1.513136$

C $-3.3979050 .958111-2.459416$

C $-2.0360121 .437410-2.994964$

H -3.332072 $-0.107175-2.221103$

O $-4.3408551 .078877-3.529407$

H $-1.823657 \quad 0.879572-3.915123$

H $-2.1265422 .481782-3.318568$

H -7.830987 -3.161194 1.781794

H $-8.177989-1.4681582 .166769$

H $-8.968170-2.2405130 .783586$

C $-4.744445-2.8540271 .256262$

H -5.6929593 .0792270 .150731$

H -3.918890 $3.609148-0.671436$

H $-4.4398002 .025862-3.727851$

H $-4.359119-3.1291780 .266815$

H $-5.378027-3.682481 \quad 1.593740$

H $-5.713060-1.1227032 .154157$

C $-0.171586-0.959687-2.551533$

O $-0.693664-0.063253-1.561919$

C $-0.872748 \quad 1.288977-1.996853$

C $0.4573281 .866090-2.499375$

C $1.0979660 .985364-3.564576$

C $1.177280-0.460409-3.086914$

H $-0.889153-1.060005-3.374678$ 
C $0.004298-2.333741-1.885181$ H $-1.1410261 .863274-1.102992$ O $0.2831253 .175234-3.031353$ H $1.1514101 .951433-1.654557$ H $0.5643651 .055328-4.519808$ O $2.4292491 .445297-3.812809$ H $1.944543-0.545153-2.308454$ O $1.585906-1.287543-4.175452$ C -1.301739-3.074742 -1.695797 H $0.643182-2.972564-2.505271$ H $0.462249-2.212134-0.897894$ H $-0.1629643 .709811-2.352964$ H $2.3672902 .379918-4.075458$ H $2.434392-0.940487-4.500672$ O $-1.023760-4.346383-1.280876$ O $-2.416644-2.605839-1.873622$ C $-2.151738-5.196228-1.052119$ H -1.788864 -6.224038 -0.967077 H $-2.637017-4.917769-0.113525$ H -2.859516 -5.144738 -1.884989

SCF Energy $\left(B 3 L Y P / 6-31 G^{* *} / / M M F F\right)=-3245.92564132$

\section{1_334}

MM̄FF Geometry

C 0.6326431 .4844912 .511422

C 1.7558301 .2201303 .197034

C $2.514875-0.0808713 .275881$

O $1.723016-1.1744952 .759444$

C $3.829985-0.0204562 .475704$

C 4.8216011 .0113902 .967709

C 5.1812702 .1154582 .284220

C $5.458904 \quad 0.709487 \quad 4.299757$

C 4.6821512 .5851580 .944449

C $5.7540072 .437879-0.145418$

C $5.9877610 .977387-0.563745$

C $7.2362850 .856192-1.438011$

C $7.445136-0.585181-1.924094$

O $4.8361170 .541611-1.295777$

O $8.440510-0.562211-2.958194$

C $6.135644-1.083811-2.565646$

C $7.989861-1.494743-0.816541$

O $6.228374-2.466016-2.905120$

C $4.875383-0.833431-1.699775$

C $3.614803-1.126251-2.548091$

O $4.895009-1.710154-0.572383$

C $1.960591-2.4041783 .291365$

O $2.748560-2.6713654 .189490$

C $1.109806-3.4465312 .678480$

C $\quad 0.519357-3.309974 \quad 1.482122$

C $-0.335685-4.3028600 .825568$

C $-0.686139-5.5473101 .598722$

C $2.286134-0.697132-1.910104$

C $1.123245-0.931978-2.862252$

C -1.286521 $-0.391231-3.172471$

O $1.220168-1.606779-3.884481$

C $-2.097832-1.528737-2.638922$

C $-2.529223-2.548679-3.396936$

C $-3.327619-3.736450-2.932302$

C $-3.026841-4.220617-1.531204$

C $-3.948590-4.146970-0.556139$

C $-1.681617-4.875030-1.291111$

C $-0.782897-4.039331-0.419673$

N $-0.040330-0.310938-2.432132$

H $\quad 0.1563810 .7169751 .908881$

H 2.1940972 .0297713 .779814

H $2.715499-0.2621414 .339243$

H 3.5926230 .1147791 .414502

H $4.318811-1.0037282 .506183$

H 5.9240422 .7741322 .733536

H 6.2426131 .4295224 .558662

H 4.7127190 .7353275 .099640

H $5.919819-0.2835434 .283777$

H 3.7550972 .0891970 .642799

H 4.4290183 .6482871 .044288

H $5.4253323 .013827-1.020051$

H $\quad \begin{array}{llll}6.692727 & 2.885147 & 0.205042\end{array}$

H $6.103004 \quad 0.358257 \quad 0.331546$

H $8.125046 \quad 1.210335-0.900856$
H $7.1344911 .509874-2.315587$

H $9.253858-0.183445-2.582759$

H $6.014228-0.541129-3.513799$

H $8.944901-1.109336-0.439856$

H $8.203059-2.498725-1.200110$

H $7.312387-1.5884080 .034764$

H $7.019005-2.575429-3.460892$

H $3.701471-0.596527-3.506404$

H $3.565195-2.199156-2.774717$

H $3.985950-1.844231-0.261056$

H $1.024592-4.3601023 .258137$

H $0.672205-2.3874620 .924364$

H -1.112687 -5.291648 2.574844

H $0.206719-6.1620041 .757768$

H -1.426391 -6.1771701 .101617$

H $2.091036-1.265686-0.995199$

H $2.3145890 .368490-1.656295$

H -1.069538 $-0.503203-4.240003$

H $-1.8188230 .550744-3.028560$

H -2.343337 -1.499075 -1.580528

H $-2.302997-2.533467-4.461457$

H -3.134613 -4.569882 -3.620592

H -4.392396 -3.498755 -3.050788

H $-3.746379-4.5261700 .439908$

H $-4.924915-3.708399-0.731642$

H $-1.168309-5.034158-2.248033$

H $-1.840358-5.881675-0.893514$

H $-0.457692-3.112870-0.891819$

H $-0.0512110 .156348-1.529921$

C 0.1206703 .5676661 .194968

$\begin{array}{lllll}\text { O } & -1.003612 & 3.228307 & 0.354582\end{array}$

C -1.568793 $4.445871-0.171627$

C -0.4939565 .5080350 .004985$

C 0.0995495 .0924051 .334573

H -2.4217114 .7022250 .468174$

C $-2.0410154 .250301-1.614896$

H $0.2652365 .454064-0.783919$

H $-0.905763 \quad 6.5216320 .014486$

C 1.4653165 .7003971 .606988

H -0.5910655 .3875742 .136216$

C $-3.0575013 .107364-1.796488$

O $-0.9090613 .955884-2.444049$

H $-2.4595455 .187663-1.998825$

H $-2.5595002 .150817-1.611322$

O $-3.4720903 .097097-3.167434$

C $-4.3130163 .213292-0.914847$

C $-5.3904542 .150305-1.203912$

H $-4.025453 \quad 3.1410160 .138725$

O $-4.9120854 .500218-1.082538$

H $-6.2143932 .314663-0.498094$

H $-5.8325262 .329550-2.192431$

H 1.8644525 .3515612 .564669

H 2.1858295 .4346670 .826008

H 1.3990836 .7925191 .645321

C -0.0090232 .8472132 .539639$

H $-0.4802343 .160310-2.084175$

H -2.669538 $3.026576-3.712486$

H -5.132111 $4.601013-2.024752$

H -1.0644252 .7238652 .810237$

H 0.4547093 .4483723 .331315

H 1.0241533 .2488670 .658805

C $-4.822467 \quad 0.4682321 .279157$

$\begin{array}{lllll}\text { O } & -4.090187 & 0.470040 & 0.050881\end{array}$

C $-4.8881860 .696186-1.114248$

C $-5.988446-0.368879-1.223349$

C $-6.806429-0.4889750 .061428$

C $-5.887015-0.6333311 .274934$

H $-5.3129291 .441823 \quad 1.404204$

C -3.8226610 .3594492 .434564$

H $-4.2242820 .547388-1.973369$

O $-6.863708-0.088043-2.310402$

H -5.526262 -1.342532 -1.428325

H $-7.486994 \quad 0.3617850 .185328$

O $-7.631091-1.654127-0.014887$

H $-5.421433-1.6260791 .254058$

O $-6.659135-0.5455422 .469161$

C -3.097696 -0.965485 2.464362

H -3.0531621 .1360652 .349295$ 
H $-4.3277150 .522318 \quad 3.393756$

H $-6.321725-0.040094-3.116569$

H $-8.181943-1.566145-0.811735$

H -7.346266 -1.232361 2.421460

O $-3.502671-1.6895873 .546400$

O $-2.283118-1.3184841 .621729$

C $-2.903346-2.9804203 .684867$

H $-3.104884-3.5902662 .799627$

H -1.827377 -2.878876 3.851365

$\mathrm{H}-3.347796-3.4704204 .555058$

SCF Energy $\left(\mathrm{B} 3 \mathrm{LYP} / 6-31 \mathrm{G}^{* *} / / \mathrm{MMFF}\right)=-3245.91476377$

01_335

MMFF Geometry

C $2.645657-2.7482022 .039638$

C $2.237375-1.4764702 .171948$

C $0.822443-0.9671242 .044598$

O $-0.056007-2.036261 \quad 1.621420$

C $0.277553-0.3959193 .367459$

C 0.8832640 .9308063 .776398

C 0.5916862 .1024833 .176790

C 1.8564370 .8789224 .922856

C -0.3497122 .2805282 .019390$

C -0.9331273 .6975151 .938995$

C -1.8429873 .8411470 .710840$

C $-2.298416 \quad 5.2872230 .523724$

C $-3.2659515 .425222-0.663049$

O $-2.9807222 .995961 \quad 0.906090$

O $-3.8815936 .719512-0.579190$

C $-4.3892914 .375825-0.515617$

C $-2.5280515 .382781-2.006673$

O $-5.238004 \quad 4.372876-1.661794$

C $-3.8771142 .946587-0.210070$

C -5.0676452 .0340180 .169799$

O $-3.2395922 .421933-1.376286$

C $-1.063921-1.7208690 .763116$

O $-1.330444-0.6192380 .310353$

C $-1.796143-2.9713120 .459111$

C $-2.823196-2.947185-0.403716$

C $-3.645610-4.091196-0.805816$

C $-3.304628-5.433770-0.212795$

C $-4.659724 \quad 0.6585310 .716689$

C $-5.865543-0.1644121 .138171$

C $-6.460617-2.2497852 .366449$

O $\quad-7.019510 \quad 0.1431020 .850915$

C $-6.424634-3.476041 \quad 1.512034$

C -7.336782 -3.749845 0.567379

C $-7.326862-4.990253-0.279731$

C $-7.041460-4.694228-1.735470$

C $-8.028297-4.338127-2.575038$

C $-5.626078-4.894036-2.231089$

C $-4.665088-3.883378-1.666505$

N -5.496208 -1.292467 1.858407

H $1.924564-3.5404441 .859573$

H $2.992438-0.7103632 .334697$

H $0.842826-0.1964441 .265308$

H $0.413741-1.1342054 .169934$

H $-0.813102-0.2864473 .301799$

H 1.0988033 .0012003 .522846

H 2.2648941 .8654895 .165942

H 1.3621040 .4946135 .821047

H 2.6994920 .2238064 .682910

H $\quad 0.2029232 .0771981 .095964$

H -1.171966 1.5607862 .079228

H -1.5037003 .9025752 .853699$

H $-0.1184034 .429797 \quad 1.882623$

H -1.287804 $3.502488-0.171371$

H -1.436988 5.9544760 .395883

H -2.811585 5.6297281 .433322

H -3.176329 $7.389061-0.600463$

H -5.0193004 .7031160 .323031$

H -1.771848 6.175718 -2.053133

H -3.212900 $5.576322-2.839657$

H -2.021338 $4.432716-2.188560$

H $-4.7718563 .902621-2.373787$

H -5.6681362 .5307740 .943407$

H $-5.7166311 .889959-0.703328$

H $-3.2295661 .453570-1.322643$
H -1.467603 $-3.878490 \quad 0.953661$

H -3.092809 -1.998038 -0.864058

H $-3.342703-5.3925590 .881668$

H $-2.298823-5.743063-0.517809$

H -3.987286 -6.231586 -0.510855

H $-4.1230450 .085962-0.045242$

H -4.0038630 .7771141 .586620$

H -7.458880 -1.800319 2.392829

H $-6.174163-2.5010323 .392378$

H $-5.615830-4.1783381 .695748$

H $-8.147399-3.0451200 .394781$

H $-8.308247-5.473576-0.185475$

H $-6.606754-5.7259060 .099307$

H -7.837843 -4.151161-3.626495

H $-9.049456-4.222450-2.226452$

H $-5.589169-4.798484-3.324602$

H -5.325231 -5.926104-2.031467

H -4.836636 -2.869895 -2.032161

H -4.509969-1.4882572.004194

C $4.553656-3.6174770 .693542$

O $4.433799-2.528384-0.239628$

C $5.494722-2.622125-1.209225$

C $6.281786-3.885217-0.867824$

C $6.020092-4.0294020 .621195$

H $6.140289-1.750888-1.050528$

C $4.900477-2.583166-2.624648$

H $5.904460-4.761052-1.407763$

H $7.344749-3.784188-1.107855$

C $6.302884-5.4219661 .155728$

H $6.642149-3.3009781 .158101$

C $3.936554-1.400874-2.866553$

O $4.155792-3.788022-2.854848$

H $5.703554-2.582367-3.369609$

H $3.044805-1.534049-2.244501$

O $3.488065-1.460982-4.226130$

C $4.546146-0.008734-2.620783$

C $3.5531511 .148415-2.842212$

H $4.9416410 .060789-1.604973$

O $5.6436230 .201061-3.512572$

H $4.1249652 .083962-2.869589$

H $3.1056451 .064321-3.840029$

H $6.094867-5.4781792 .228706$

H $5.688906-6.1770650 .653415$

H $7.354100-5.6866121 .001739$

C $4.093470-3.1571242 .076295$

H $3.462601-3.840064-2.174464$

H $3.108386-2.345187-4.367471$

H $6.352919-0.413084-3.259354$

H $4.710180-2.3165902 .413533$

H $4.213020-3.9626462 .808922$

H $3.906975-4.4275340 .329489$

C $3.6823842 .485059-0.106714$

O $2.9900101 .274229-0.440109$

C $2.4498861 .230797-1.765305$

C $1.4544302 .381879-1.959849$

C $2.0747783 .734471-1.618119$

C $2.7478673 .695649-0.245345$

H $4.5480302 .612199-0.767128$

C 4.1910262 .3280341 .335265

H $1.8697610 .302845-1.828655$

O $0.9710612 .382095-3.298662$

H $\quad 0.5890432 .212808-1.307217$

H $2.7830514 .056345-2.390957$

O $1.0469934 .727937-1.574869$

H 1.9742443 .6779920 .530806

O $3.4846724 .907852-0.089468$

C $5.355803 \quad 1.3645891 .436717$

H 4.5424263 .2804901 .743457

H 3.3796141 .9469091 .964753

H $0.2498163 .031352-3.352144$

H $0.7000754 .831103-2.476863$

H 3.7000175 .0075410 .852604

O $5.450858 \quad 0.9124382 .721993$

O $6.1151001 .079422 \quad 0.521227$

C $6.544816 \quad 0.0285732 .988344$

H 7.4778540 .5983943 .018748

H $6.379085-0.4316713 .965991$

H $6.603990-0.7635642 .235676$ 
C $1.904304-2.9406353 .200352$

C $3.096853-3.7295862 .700047$

C $4.343623-3.2240262 .611244$

C $2.839352-5.1740012 .363913$

C $4.747730-1.8064392 .912350$

C $6.091492-1.3924642 .294604$

C $6.148496-1.4786470 .761382$

C $7.517944-1.0096340 .260335$

C $7.599792-1.003410-1.271267$

O $5.112485-0.6580240 .208829$

O $8.781036-0.266457-1.623734$

C $6.379197-0.241621-1.830860$

C $7.794131-2.416468-1.833586$

O $6.365681-0.338418-3.253106$

C $5.034260-0.710038-1.224296$

C $3.8385750 .158318-1.676575$

O $4.763182-2.043864-1.676668$

C $0.815851-0.2203272 .016550$

O $1.822299-0.1303641 .332371$

C 0.0234790 .9193572 .527433

C 0.4450242 .1712122 .296263

C $-0.228443 \quad 3.4010412 .716592$

C -1.5153383 .2665163 .486225$

C $3.8735041 .625233-1.241057$

C $2.7537912 .427857-1.885867$

C $1.8186474 .720415-1.984218$

O $2.0334831 .979843-2.774353$

C $1.4628185 .758621-0.970831$

C $0.1997266 .056234-0.629578$

C -0.1690337 .1189310 .365608$

C -0.9587656 .5757661 .535760$

C $-2.295576 \quad 6.7019311 .583516$

C -0.1920835 .9652952 .688186$

C 0.3207594 .5856942 .377809

N $2.6737573 .717339-1.375271$

H $-0.288154-3.914809-0.012866$

H $-0.697700-3.9161913 .022797$

H $1.330868-2.5262101 .128443$

H $1.443048-3.4950734 .029462$

H $2.239376-2.0086353 .670900$

H $5.146380-3.8910492 .301871$

H $3.764461-5.7227172 .156585$

H $2.341428-5.677963 \quad 3.198718$

H $2.205496-5.2547031 .476156$

H $3.978372-1.1065792 .571450$

H $4.831398-1.6968744 .000452$

H $6.283514-0.3563302 .603080$

H $6.892643-2.0072072 .724437$

H $5.979105-2.5190260 .462989$

H $8.320653-1.6259560 .684450$

H $7.704846 \quad 0.0121530 .620167$

H $8.806806-0.198804-2.593937$

H $6.5420750 .815958-1.590098$

H $8.731194-2.848044-1.461263$

H $7.885960-2.400280-2.925311$

H $6.986612-3.101130-1.566793$

H $5.7318890 .316673-3.590091$

H $3.7235440 .080080-2.765223$

H $2.918433-0.278333-1.266089$

H $3.814546-2.220667-1.563365$

H $-0.884073 \quad 0.6966843 .077014$

H 1.3677252 .3142581 .734862

H -1.3349822 .7824984 .452196$

H -2.237598 2.6671862 .923715

H -2.003505 4.221606 3.691063

H $3.7796571 .696193-0.151591$

H $4.8130852 .099684-1.539618$

H $0.9354314 .238323-2.415361$

H $2.3844285 .177299-2.802993$

H $2.282436 \quad 6.304167-0.509264$

H $-0.7569027 .880384-0.163734$

H $\quad 0.719154 \quad 7.6434410 .739294$

H -2.8683006 .3408102 .431211$

H -2.8488817 .1621820 .771442$

H -0.7983185 .9640423 .600895$

H 0.6632576 .6080622 .933522

H $1.2563924 .581617 \quad 1.818637$

H $3.4225664 .038262-0.767686$

C $-3.168031-4.5843140 .597574$

O $-3.184083-4.137382-0.775426$

C $-4.409923-4.571556-1.383502$

C $-5.335136-4.972806-0.244229$

C $-4.339858-5.5587040 .738582$

H $-4.159335-5.482396-1.945281$

C $-4.968848-3.546072-2.377441$

H $-5.833932-4.1042780 .199105$

H $-6.102687-5.687087-0.556485$

C $-4.881432-5.6735032 .153321$

H $-4.042944-6.5559780 .386774$

C $-5.525219-2.224512-1.805385$

O $-6.046210-4.170013-3.090631$

H $-4.198421-3.306136-3.121040$

H $-6.446887-2.412725-1.242573$

O $-5.908447-1.404258-2.920795$

C $-4.548338-1.411495-0.939805$

C $-5.119309-0.039269-0.526766$

H $-4.319779-1.971917-0.030155$

O $-3.328441-1.234212-1.651487$

H -5.224195 $0.594835-1.413296$

H $-6.123649-0.195120-0.115809$

H $-4.126129-6.0871182 .828711$

H $-5.186639-4.6978612 .546302$

H $-5.754567-6.3336712 .178224$

C $-1.816830-5.2181990 .930760$

H $-5.684307-4.949478-3.545627$

H $-6.538313-1.918670-3.454639$

H -3.534521 -0.767599-2.479589

H - $-1.559260-5.9812550 .186050$

H -1.868839 -5.7232761 .902616$

H $-3.333957-3.6986961 .224371$

C $-2.7992031 .941667-0.939363$

$\begin{array}{llll}\text { O } & -2.911528 & 0.932479 & 0.070788\end{array}$

C -4.2446140 .6817340 .527862$

C -4.8618951 .9728411 .088280$

C -4.7604903 .1351270 .102347$

C $-3.3289913 .278895-0.413250$

H $-3.3652651 .641270-1.829109$

C $-1.3233002 .045929-1.335142$

H -4.148451 -0.014862 1.371005

O -6.2198531 .7477561 .451495$

H -4.3299752 .2455312 .007645$

H -5.465095 $3.017822-0.729701$

O $\quad-5.1120834 .3519090 .764708$

H -2.682861 3.6514990 .391443

O $-3.3057044 .243016-1.463274$

C $-0.896674 \quad 0.830710-2.122624$

H -1.134115 2.917101-1.971499

H $-0.6790322 .148233-0.455791$

H -6.5369612 .5398491 .916711$

H $\quad-6.057070 \quad 4.302770 \quad 0.986462$

H -3.653872 $5.075242-1.099779$

O $-0.232108-0.044763-1.316436$

O $-1.1490420 .669753-3.310078$

C $0.212774-1.247407-1.951418$

H $0.840358-1.015294-2.817428$

H $0.810123-1.811546-1.231061$

H $-0.645441-1.856329-2.249147$

SCF Energy (B3LYP/6-31G**//MMFF) $=-3245.90230788$

01_337

MM̄FF Geometry

C $-2.334963 \quad 3.9631170 .456585$

C -1.9342803 .4347081 .622715$

C -2.3598622 .0763922 .107141$

O -1.1590181 .2980492 .337805$

C -3.1136922 .1369753 .450381$

C -4.6123472 .2789463 .289851$ 
C $-5.482906 \quad 1.2589493 .423115$ C $-5.119143 \quad 3.6649092 .991361$ C $-5.176344-0.1747283 .756887$ C $-4.531213-0.9964412 .636343$ C $-5.206303-0.9182801 .258979$ C $-6.664283-1.3801281 .290204$ C $-7.286388-1.398581-0.113835$ O $-4.445815-1.7658870 .384155$ O $-8.522449-2.125529-0.039615$ C $-6.356828-2.183473-1.060454$ C $-7.6409780 .011799-0.600370$ O $-6.820354-2.106642-2.407143$ C $-4.875074-1.744189-0.983917$ C $-4.001813-2.743299-1.782069$ O $-4.742664-0.442075-1.556262$ C $-0.824530 \quad 0.358677 \quad 1.413275$ $\begin{array}{lllll}\text { O } & -1.364511 & 0.150982 & 0.338725\end{array}$ C $0.298722-0.4261771 .971418$ C $0.506139-1.6734101 .524274$ C $1.527343-2.6082582 .000806$ C $2.542679-2.0939942 .985667$ C $-2.489418-2.537990-1.626252$ C $-1.691378-3.570057-2.407790$ C $0.669592-4.189922-2.939855$ O $-2.210373-4.487681-3.037548$ C $1.210067-5.129630-1.910069$ C $2.477674-5.101529-1.472191$ C $3.021305-6.027955-0.423190$ C $3.467103-5.2957930 .824161$ C $4.760180-4.9855331 .016392$ C $2.432006-5.0082101 .889827$ C $1.514990-3.8726231 .529235$ $\mathrm{N}-0.326353-3.339861-2.313598$ H -3.015906 $3.397454-0.174044$ H $-1.257398 \quad 3.9991172 .259841$ H -2.988420 1.5733171 .361556 H -2.733787 2.9552284 .076972 H -2.884731 1.2330714 .028259 H $-6.542946 \quad 1.468971 \quad 3.286877$ H -6.2108453 .7289503 .056210$ H -4.7099914 .3840663 .708296$ H -4.8338853 .9731081 .982474$ H $-4.541147-0.2187514 .649947$ H $-6.114768-0.6577814 .057763$ H -3.481381 -0.6999052 .523785$ H -4.484836 -2.046458 2.955745 H -5.1329440 .1115390 .892632$ H -7.269581 -0.7546971 .956287$ H $-6.714456-2.3962011 .706253$ H $-9.093599-1.6801650 .609530$ H $-6.417672-3.242556-0.772562$ H -8.356016 0.4842270 .084103 H -8.137691 $-0.020155-1.576267$ H $-6.7735730 .670897-0.675610$ H -7.745889 -2.405488-2.412295 H $-4.232768-3.763890-1.448757$ H -4.254085 -2.684376 -2.848556 H $-3.820146-0.304330-1.821412$ H 0.8864200 .0228652 .763765 H $-0.153584-2.0616490 .749845$ H $2.069220-1.8863323 .951273$ H $3.003637-1.1722122 .617795$ H $3.364003-2.7912293 .165691$ H -2.194982 -1.546143 -1.984211 H $-2.205190-2.625358-0.571879$ H $1.445352-3.535624-3.349490$ H $\quad 0.220366-4.753845-3.763201$ H $0.519788-5.863427-1.500540$ H $3.168311-4.373125-1.889865$ H $3.866919-6.572441-0.863482$ H $2.289139-6.798593-0.151348$ H $5.094156-4.4888781 .921269$ H $5.512860-5.214268 \quad 0.269146$ H $2.913931-4.8351992 .858297$ H $1.822271-5.9071132 .048393$ H $0.732466-4.1500970 .823311$ H $0.006471-2.589956-1.714497$ C $-0.9432565 .282668-1.214294$
O $0.281238 \quad 4.636062-0.813388$

C $0.6774993 .725400-1.852933$

C $-0.1037604 .145491-3.091812$

C $-1.4249904 .562338-2.475076$

H $0.3424752 .728263-1.538163$

C $2.1999243 .706323-2.033378$

H $0.3660084 .997676-3.596759$

H $-0.2043743 .331895-3.816375$

C -2.282832 $5.423762-3.384799$

H $-1.9824563 .652787-2.222449$

C $2.9423203 .270707-0.750128$

O $2.6349164 .998679-2.450942$

H $2.4320263 .005466-2.843675$

H $2.4205442 .406281-0.323825$

$\begin{array}{lllll}\text { O } & 2.912831 & 4.310931 & 0.225121\end{array}$

C $4.4070222 .870379-1.007601$

C $5.209125 \quad 2.487917 \quad 0.249544$

H $4.4187672 .030584-1.710658$

O $5.1090593 .937047-1.649901$

H $6.1969212 .148832-0.087779$

H 5.4144863 .3898600 .841092

H -3.212516 $5.711181-2.883792$

H $-1.7609286 .340120-3.680376$

H $-2.5443514 .877691-4.297060$

C -1.918726 $5.321533-0.035009$

H $3.5819844 .936357-2.659685$

H 1.9809564 .5306460 .393383

H $5.0882004 .703585-1.051138$

H $-2.8193155 .879671-0.316212$

H -1.4485205 .8756910 .787645$

H $-0.6684326 .316761-1.460722$

C $4.990377-0.525523-0.198111$

O 4.0197870 .3255140 .417420

C 4.5614271 .4218911 .154438

C 5.4802640 .9055492 .271905

C $6.546015-0.051922 \quad 1.737517$

C $5.911226-1.1344820 .863874$

H $5.5797070 .047840-0.924303$

C $4.227072-1.614010-0.957402$

H 3.7089241 .9079921 .645144

$\begin{array}{lllll} & & 6.091614 & 2.003762 & 2.940561\end{array}$

H 4.8708650 .3759253 .015153

H 7.3289540 .4829891 .187289

O $7.194912-0.6989272 .834820$

H $5.347801-1.8290001 .499001$

O $6.940125-1.8827930 .220431$

C $3.443788-1.022837-2.105175$

H $4.902859-2.367533-1.377013$

H $3.532476-2.142751-0.295851$

H 6.5663271 .6530853 .712616

H $7.691166-0.0209723 .323587$

H $7.515471-2.2429480 .917006$

O $2.153643-0.797725-1.724289$

O $3.929355-0.767280-3.199516$

C $1.311226-0.206036-2.717839$

H $1.7397240 .734321-3.073883$

H $1.168485-0.900163-3.550902$

H $0.3401020 .001942-2.260950$

SCF Energy (B3LYP/6-31G**//MMFF) = -3245.91383086

01_338

MMFF Geometry

C $2.705157-0.146434-3.017866$

C $2.4332430 .776274-3.953190$

C $1.2564351 .723895-3.937059$

$\begin{array}{lllll}\text { O } & 0.256170 & 1.308551 & -2.971921\end{array}$

C $1.632748 \quad 3.195439-3.622149$

C $2.967573 \quad 3.439140-2.939797$

C $3.1817503 .367581-1.612063$

C $4.0992173 .822638-3.860440$

C $2.1875663 .031253-0.538153$

C 2.0132504 .2209420 .415393

C $0.9691094 .005407 \quad 1.520694$

C 1.3908822 .9405692 .534248

C 0.3058092 .7083243 .596021

O $-0.264547 \quad 3.643018 \quad 0.899675$

$\begin{array}{lllll}\text { O } & 0.671418 & 1.517874 & 4.309233\end{array}$

$\begin{array}{llll}\text { C }-1.034628 & 2.446434 & 2.876326\end{array}$ 
C $\quad 0.268268 \quad 3.842328 \quad 4.627645$ O -2.1068952 .3491953 .811194$ C -1.3728293 .5044751 .796815$ C -2.578735 3.0250010 .957491 $\begin{array}{llll}\text { O } & -1.695693 & 4.740141 & 2.433605\end{array}$ C $-0.686582 \quad 0.420042-3.393707$ O $-0.792042-0.062101-4.510732$ C -1.568974 $0.109120-2.245117$ C $-2.555668-0.786678-2.403060$ C $-3.501457-1.243228-1.381080$ C $-3.381477-0.660247-0.000628$ C $-2.951353 \quad 3.950108-0.207657$ C $-3.8700153 .267023-1.204847$ C -6.200339 2.408999-1.469222 O $-3.5075543 .036713-2.356590$ C $-6.6895351 .151243-0.822268$ C $-7.0047420 .041871-1.509273$ C $-7.548587-1.204085-0.869482$ C $-6.885137-2.469742-1.366590$ C $-7.543020-3.327430-2.165274$ C $-5.492707-2.786625-0.867303$ C $-4.425851-2.164049-1.725266$ N $-5.1156112 .965934-0.679959$ H $2.026150-0.284213-2.180289$ H $3.1322080 .906387-4.776361$ H $\quad 0.794515 \quad 1.715681-4.932914$ H $0.8331713 .671198-3.039110$ H $1.6437653 .747697-4.572481$ H $4.1871223 .567964-1.243462$ H $5.0456183 .955153-3.325436$ H $4.2589283 .054203-4.622422$ H $3.8699974 .767117-4.365079$ H $1.2218432 .745118-0.961826$ H 2.5636232 .1585700 .006337 H 2.9761894 .4849240 .870749 H $1.6958825 .087674-0.180774$ H 0.8379684 .9696902 .025941 H 2.3437893 .2068813 .008013 H 1.5668621 .9862602 .021790 H $\quad 0.073090 \quad 1.427603 \quad 5.070044$ H -0.9586541 .4654932 .386873$ H $1.226803 \quad 3.9067875 .156405$ H $-0.487327 \quad 3.6522865 .398046$ H $0.066503 \quad 4.8203934 .186141$ H $-1.908515 \quad 1.6103104 .410598$ H -2.332322 2.0434610 .539038 H -3.457038 2.8984001 .601765 H -2.199787 5.2915061 .815065 H - $1.3728980 .612136-1.305702$ H -2.681461 -1.248178 -3.382162 H $-3.7092370 .381586-0.008249$ H $-2.347822-0.7051750 .357317$ H -3.981215 -1.1782700 .749931$ H $-3.449375 \quad 4.8572040 .152190$ H -2.057353 $4.269035-0.755420$ H $-5.8742802 .243898-2.500899$ H -7.001444 3.155048 -1.472191 H $-6.821415 \quad 1.160858 \quad 0.257163$ H $-6.9097490 .044181-2.592815$ H -8.625135 -1.239465 -1.082029 H $-7.460186-1.160446 \quad 0.223280$ H -7.083085 -4.250888 -2.501619 H $-8.552874-3.123471-2.505280$ H $-5.330683-3.872913-0.881803$ H $-5.419923-2.5084370 .187090$ H $-4.425337-2.536569-2.750804$ H -5.3340653 .2682090 .265007$ C $4.820957-0.583737-1.795587$ O $4.129182-0.916259-0.576723$ C $5.084609-1.3935660 .387523$ C $6.461329-1.180802-0.232957$ C $6.153997-1.320317-1.712857$ H $4.912514-2.4707650 .494575$ C $4.858812-0.6886931 .729663$ H $6.854614-0.180445-0.017857$ H $7.193355-1.9109670 .125193$ C $7.235799-0.750602-2.613229$ H $6.011225-2.384167-1.944984$
C $3.417933-0.8122072 .271396$

$\begin{array}{lllll}\text { O } & 5.141225 & 0.709667 & 1.581171\end{array}$

H $5.574132-1.0621222 .471044$

H $2.733249-0.2450871 .630973$

O $3.380014-0.1845463 .558843$

C $2.914982-2.2593012 .419002$

C $1.558622-2.392963 \quad 3.133590$

H $2.850874-2.7275081 .433446$

O $\quad 3.880250-3.001548 \quad 3.170726$

H $1.356575-3.4630083 .265380$

H $1.648479-2.0004764 .154241$

H $\quad 6.966901-0.864917-3.668007$

H $7.3980360 .315279-2.420772$

H $8.185460-1.270250-2.449168$

C $3.958310-0.976496-2.995174$

H 4.5644881 .0523450 .876971

H $3.6871870 .731046 \quad 3.443579$

H $3.586846-3.9283893 .191725$

H $3.668204-2.029968-2.922516$

H $4.522180-0.851061-3.926118$

H $4.9846410 .502443-1.784517$

C $-0.193018-3.516028 \quad 0.895294$

O $0.234445-2.157508 \quad 1.052187$

C $0.378924-1.7166792 .406752$

C $-0.959919-1.854306 \quad 3.145055$

C $-1.500842-3.2777603 .064434$

C $-1.527272-3.761771 \quad 1.617362$

H $0.579777-4.1912921 .282303$

C $-0.360294-3.785526-0.609642$

H $0.604087-0.6455522 .356299$

O $-0.831458-1.4839594 .513519$

H -1.696033 -1.1746592 .698259$

H -0.927068 -3.964165 3.697905

O $-2.840426-3.299607 \quad 3.564659$

H -2.341142 -3.257106 1.082872

O $-1.817523-5.1590311 .599490$

C $0.949026-3.844018-1.366373$

H $-0.861737-4.748536-0.759982$

H $-0.959424-2.992128-1.067107$

H $-0.466780-0.5832844 .537557$

H $-2.814855-2.9535434 .473356$

H -2.659463 -5.2864322 .069651$

O $0.696746-4.296220-2.631072$

O $2.041151-3.522155-0.922307$

C $1.824006-4.425111-3.504248$

H $2.011965-3.465400-3.991934$

H $2.715515-4.767942-2.970439$

H $1.574931-5.164385-4.270380$

SCF Energy (B3LYP/6-31G**//MMFF) $=-3245.90595487$

01_339

MMFF Geometry

C $0.9262115 .336151 \quad 0.775189$

C $1.880792 \quad 4.771577 \quad 0.021147$

C $2.868375 \quad 3.764866 \quad 0.552431$

O $2.6316962 .511249-0.130385$

C $4.307658 \quad 4.213827 \quad 0.246035$

C 5.3636513 .3011090 .840270

C 6.2294002 .5594710 .121606

C $5.431808 \quad 3.2728992 .346164$

C $6.3585202 .470655-1.372497$

C $5.5386611 .348345-2.027307$

C $5.908191-0.065897-1.550573$

C $5.253708-1.123602-2.442565$

C $5.529395-2.550380-1.942202$

O $5.447708-0.228757-0.205405$

O $4.627327-3.438034-2.621182$

C $5.195043-2.633016-0.436489$

C $6.945656-3.015307-2.302723$

O $5.599911-3.8881950 .103697$

C $5.804221-1.4833910 .392699$

C $5.308974-1.4555201 .860131$

O $7.228195-1.6416350 .439375$

C $1.582326 \quad 1.7612930 .304333$

$\begin{array}{llllll}\text { O } & 0.846967 & 2.003103 & 1.248328\end{array}$

C $1.4586620 .594510-0.595600$

C $0.342072-0.146076-0.551247$

C $0.054108-1.332812-1.357461$ 
C $1.143696-1.852818-2.255187$

C $3.793881-1.3197662 .051071$

C $3.076382-2.6425642 .270694$

C $0.795526-3.5720682 .651852$

O $3.656909-3.721706 \quad 2.346115$

C $-0.041740-3.808576 \quad 1.435107$

C $0.232210-4.7547560 .523408$

C $-0.542189-5.116691-0.716854$

C $-1.799985-4.325060-0.998868$

C $-2.989669-4.731371-0.524714$

C $-1.692760-3.132718-1.924394$

C $-1.156206-1.913598-1.227773$

N $1.704370-2.4698642 .398153$

H 0.8719145 .0986211 .834528

H $1.9220954 .993677-1.042733$

H 2.7410863 .6232501 .632576

H 4.4739585 .2261050 .638057

H $4.4293494 .285895-0.840196$

H $6.944905 \quad 1.942268 \quad 0.664340$

H 6.3068762 .7247682 .712128

H 5.4969014 .2912172 .743148

H 4.5447462 .7884802 .765368

H $6.0941413 .424496-1.841510$

H $7.4199832 .323431-1.610334$

H $4.4742591 .533808-1.844467$

H $5.6955331 .415193-3.111074$

H $6.999545-0.165670-1.579984$

H $5.583233-1.013630-3.483318$

H $4.167238-0.964119-2.456402$

H $4.774685-3.341136-3.577658$

H $4.102349-2.592152-0.350936$

H $7.088399-2.999545-3.389980$

H $7.111462-4.053738-1.995299$

H $7.726998-2.394371-1.859545$

H $5.173076-4.583025-0.426190$

H $5.708205-2.3174972 .408584$

H $5.765153-0.5749722 .335625$

H $7.568581-1.1448071 .201682$

H $2.2800620 .382284-1.269248$

H -0.4463240 .1463630 .141516$

H $2.043392-2.073861-1.671294$

H $1.394665-1.115321-3.025120$

H $0.879412-2.777421-2.772400$

H $3.613009-0.7193342 .952021$

H $3.334861-0.7945101 .208961$

H $0.156658-3.2900823 .494622$

H $1.354856-4.4681512 .939933$

H $-0.924226-3.1852051 .336950$

H $1.125534-5.359126 \quad 0.678121$

H -0.799719 -6.181988 -0.641063

H $0.131274-5.045607-1.580001$

H -3.902712 -4.193249 -0.753332

H -3.074561 $-5.608198 \quad 0.108513$

H -2.684362 -2.872081 -2.319002

H $-1.110483-3.406937-2.809037$

H - $-1.868305-1.465424-0.535156$

H $1.310439-1.5380652 .307509$

C -1.478575 5.5772620 .004327

O $-1.3606624 .562049-1.009946$

C -2.234454 $3.450324-0.676070$

C -3.0718313 .9396210 .498203$

C -2.114916 $4.884009 \quad 1.202376$

H $-1.5719902 .635083-0.361685$

C -2.988224 $3.047767-1.950375$

H -3.952393 4.4905590 .148498

H -3.403271 3.1341721 .156388

C -2.793663 $5.829817 \quad 2.176580$

H -1.3793994 .2774821 .744896$

C $-3.8317241 .757086-1.906153$

O $-1.9898892 .812912-2.965756$

H -3.589321 $3.886342-2.322160$

H -3.169500 $0.894480-1.765856$

O $-4.4233721 .601328-3.207401$

C $-4.9703351 .700446-0.878250$

C $-5.9729080 .545730-1.067595$

H $-4.551421 \quad 1.6273970 .126358$

O

H $-6.6874320 .600267-0.236603$
H $-6.5775320 .737272-1.963768$

H -2.0624846 .4963092 .644890$

H -3.545167 6.4504261 .677250

H $-3.296248 \quad 5.2679892 .970798$

C $-0.125746 \quad 6.2592250 .230740$

H $-1.4502923 .619712-3.030928$

H $-3.6980151 .598371-3.855621$

H $-6.0552963 .019311-1.836422$

H $-0.2419627 .101318 \quad 0.922699$

H $0.2193906 .679885-0.722608$

H $-2.1694326 .329414-0.400253$

C $-5.080934-1.307722 \quad 1.208831$

O $-4.468253-1.155525-0.074831$

C $-5.360144-0.865820-1.152163$

C $-6.405144-1.983042-1.282304$

C $-7.149203-2.2108810 .030265$

C $-6.159367-2.402587 \quad 1.177621$

H $-5.514473-0.3503221 .522516$

C $-3.976694-1.6934462 .207951$

H $-4.753261-0.895061-2.064814$

O $-7.347532-1.685898-2.307398$

H $-5.906761-2.918501-1.566585$

H -7.848909-1.394802 0.243905

O $-7.941357-3.396059-0.080067$

H $-5.688313-3.3881201 .073220$

O $-6.870847-2.4181192 .414505$

C $-2.960174-0.5958942 .436963$

H -4.419811 -1.933644 3.180839

H $-3.429635-2.5666191 .833560$

H $-6.849863-1.555909-3.132737$

H $-8.538258-3.274870-0.838465$

H $-7.298262-1.5515762 .523969$

O $-2.055459-1.0252223 .367182$

O $-2.9485110 .490452 \quad 1.877060$

C $-1.019887-0.093906 \quad 3.694514$

H $-0.387042-0.5451974 .463234$

H $-0.407503 \quad 0.1111952 .812840$

$\mathrm{H}-1.4498810 .8307574 .090947$

SCF Energy (B3LYP/6-31G**//MMFF)= -3245.91245324

0134

MMFF Geometry

C $-4.154986 \quad 1.096715-2.153614$

C $-3.3310452 .106868-2.473321$

C $-2.5958132 .941619-1.460630$

O $-1.231338 \quad 3.044446-1.937322$

C $-3.179176 \quad 4.358460-1.321948$

C $-4.5013504 .407903-0.587656$

C $-4.6204594 .440077 \quad 0.754485$

C $-5.7285824 .489530-1.457588$

$\begin{array}{llll}C & -3.517317 & 4.416926 & 1.778727\end{array}$

C $-3.772607 \quad 3.4007952 .901390$

C -3.5315311 .9366222 .493224$

C $-4.0910841 .006797 \quad 3.574164$

C $-3.796505-0.4680413 .276718$

O $-2.118213 \quad 1.7407112 .355111$

O $-4.098844-1.2256084 .458086$

C $-2.286373-0.618916 \quad 3.012957$

C $-4.698688-1.0156282 .166895$

O $-1.979716-1.9490822 .597771$

C -1.7207950 .4079902 .001768$

C $-0.1721690 .355543 \quad 1.967191$

$\begin{array}{llllll}\text { O } & -2.193623 & 0.055763 & 0.697658\end{array}$

C $-0.256116 \quad 3.257925-1.015085$

O $-0.394996 \quad 3.361504 \quad 0.192652$

C $1.0309823 .361632-1.736651$

C $2.1552803 .565241-1.034669$

C $3.5048713 .717154-1.578632$

C $3.6784033 .631875-3.071967$

C 0.5095590 .9689153 .191405

C $1.897636 \quad 0.3977193 .387017$

C $4.2289410 .512788 \quad 2.584654$

O $2.103610-0.5196884 .180009$

C $5.158811 \quad 1.5842852 .116389$

C $6.0180851 .419813 \quad 1.098764$

C 7.0133672 .4539370 .655021

C $6.8037802 .887226-0.778640$

C $7.3934932 .233115-1.793350$ 
C $5.976847 \quad 4.127692-1.027149$

C $4.5215513 .923160-0.716394$ N 2.8623680 .9992872 .592724 H $-4.3372820 .879447-1.105509$ H -3.168435 2.348746 -3.521245 H -2.594756 $2.433459-0.490796$ H -3.281475 $4.821303-2.313357$ H -2.457508 $5.008512-0.810505$ H $-5.622817 \quad 4.5179851 .174271$ H $-6.6509074 .554702-0.870619$ H -5.681334 5.379129-2.094302 H $-5.8123823 .608855-2.099830$ H $-2.537500 \quad 4.2372041 .332353$ H -3.476285 5.4161902 .229986 H -3.098714 3.6497563 .731878 H -4.7980313 .5243783 .272227$ H $-4.019027 \quad 1.7403241 .532236$ H -5.1703921 .1615013 .698448$ H $-3.639717 \quad 1.2613924 .543766$ H $-5.034705-1.0784214 .677762$ H - $1.786870-0.4879473 .979796$ H $-5.754767-0.9151232 .444610$ H -4.531917 -2.086911 2.010562 H $-4.556862-0.4990001 .216838$ H -2.230818 -2.0337981 .662179$ H $0.156780-0.6795811 .811058$ H $\quad 0.1909330 .897464 \quad 1.084714$ $\begin{array}{llll}\mathrm{H} & -1.573442 & 0.404106 & 0.036253\end{array}$ H $1.0166843 .281642-2.817300$ H $2.083706 \quad 3.635090 \quad 0.050432$ H $4.7242283 .631706-3.387541$ H $3.1892334 .481131-3.561453$ H $3.2436432 .707345-3.464875$ H 0.5737302 .0587733 .088333 H -0.0469240 .7786954 .114365$ H $4.254325-0.3688451 .939617$ H $4.505074 \quad 0.1982933 .596843$ H 5.1521442 .5236592 .663489 H 6.0385030 .4692570 .571477 H 8.0174162 .0236980 .767292 H $6.9987943 .331131 \quad 1.313768$ H $7.2687672 .553929-2.822094$ H $8.0094291 .357450-1.616502$ H $6.1160024 .492360-2.050436$ H $6.3553094 .941163-0.394364$ H 4.2954153 .9731010 .349130 H 2.5606741 .6067691 .835746 C $-4.733090-1.254840-2.957443$ O $-3.346681-1.628570-2.843652$ C $-3.223250-2.622582-1.806871$ C $-4.641551-3.088813-1.493987$ C $-5.434628-1.817206-1.722012$ H $-2.823667-2.111244-0.924145$ C $-2.266256-3.737001-2.241554$ H $-4.974576-3.872142-2.184584$ H $-4.734439-3.476970-0.475066$ C $-6.923558-2.052750-1.907378$ H $-5.288566-1.158974-0.860228$ C $-0.867957-3.237133-2.657815$ O $-2.846056-4.407731-3.367744$ H $-2.185174-4.482970-1.441875$ H $-0.938248-2.638396-3.574201$ O $-0.071129-4.384240-2.982727$ C $-0.125478-2.430009-1.573254$ C $1.295817-2.041876-2.030971$ H $-0.685177-1.515011-1.352441$ O $-0.077832-3.208002-0.381160$ H $1.866269-2.951411-2.242686$ H $1.205675-1.493081-2.975848$ H -7.449270 -1.108330 -2.079506 H $-7.123526-2.710643-2.759818$ H -7.352457-2.520122 -1.014835 C $-4.867427 \quad 0.255637-3.177595$ H -2.359132 $-5.239387-3.495146$ H $-0.423311-4.761122-3.806567$ H $\quad 0.300735-2.6512780 .319932$ H $-5.927748 \quad 0.532487-3.185905$ H $-4.4688390 .489368-4.173308$
H $-5.112522-1.762066-3.854547$

C $3.252963-2.9489470 .137997$

$\begin{array}{llll}\text { O } 2.321252 & -1.862924 & 0.223974\end{array}$

C $2.068688-1.177556-1.007269$

C $3.383644-0.599175-1.546481$

C $4.448565-1.679420-1.709169$

C $4.604424-2.477215-0.415686$

H $2.838405-3.736154-0.502396$

C $3.421077-3.526688 \quad 1.547977$

H $1.418625-0.328305-0.762238$

$\begin{array}{llll}\text { O } & 3.159052 & 0.053738 & -2.790745\end{array}$

H $3.7507370 .162453-0.846872$

H $4.218759-2.331915-2.559390$

O $5.690478-1.042496-2.018120$

H $5.118798-1.8546590 .324856$

O $5.450671-3.600145-0.659043$

C $2.183190-4.2782601 .974203$

H $4.255474-4.2348551 .603278$

H $3.626370-2.7315962 .274309$

H $4.0191750 .381596-3.105137$

H $6.367383-1.738733-2.072942$

H $5.006229-4.174302-1.306015$

O $1.515286-3.5766092 .932893$

O $1.845607-5.355982 \quad 1.500967$

C $0.328098-4.198892 \quad 3.432573$

H $-0.115759-3.5343034 .178495$

H $-0.391019-4.3514652 .622235$

H $0.574404-5.1502553 .913506$

SCF Energy (B3LYP/6-31G**//MMFF) $=-3245.91871675$

01340

MMFF Geometry

C $0.8206730 .313654-2.377201$

C $1.2250601 .432286-3.000510$

C $0.7860032 .851126-2.723672$

O $-0.1268592 .879594-1.602090$

C $1.9445343 .805002-2.364820$

C $3.1564293 .761415-3.269240$

C $4.3710823 .312734-2.892735$

C $2.9433754 .308865-4.656268$

C $4.7582472 .749295-1.552751$

C $5.5750701 .455520-1.682004$

C $5.8726320 .854443-0.299617$

C $6.855236-0.311674-0.404353$

C $7.077140-0.9986790 .953217$

$\begin{array}{lllll}\text { O } & 4.630853 & 0.400613 & 0.250256\end{array}$

O $7.723617-2.257246 \quad 0.707329$

C $5.706295-1.3101291 .592954$

C $8.027335-0.1917611 .846149$

O $5.864824-1.8213772 .914899$

C $4.725878-0.1134371 .583320$

C $3.318479-0.5820772 .025235$

$\begin{array}{lllll}\text { O } & 5.192306 & 0.877999 & 2.500675\end{array}$

C -1.123517 $3.802713-1.608830$

O $-1.4404554 .551626-2.517599$

C $-1.7546593 .758398-0.275533$

C $-2.9788394 .265361-0.078984$

C $-3.645813 \quad 4.237872 \quad 1.217111$

C $-5.002498 \quad 3.594166 \quad 1.184671$

C $2.2087120 .456464 \quad 1.804242$

$\begin{array}{llll}\text { C } & 0.844483 & -0.078675 & 2.208237\end{array}$

C $-1.586698 \quad 0.369222 \quad 1.879173$

O $0.695394-1.0674162 .921081$

C $-2.248419 \quad 1.142373 \quad 2.979249$

C $-1.7107942 .078958 \quad 3.776455$

C -2.479145 2.7629704 .873488

C $-2.549970 \quad 4.263780 \quad 4.700551$

C $-1.797000 \quad 5.082918 \quad 5.453930$

C $-3.5580314 .818252 \quad 3.714547$

C -3.0509654 .7740782 .301428$

N $-0.1864770 .697207 \quad 1.698327$

H $0.1095770 .365654-1.558167$

H $1.9105121 .318881-3.838960$

H $0.2769473 .210441-3.627265$

H $2.2177593 .648893-1.315488$

H $1.5565664 .833671-2.366233$

H $5.1832723 .364661-3.616618$

H $3.8633114 .305515-5.250364$ 
H $2.2013583 .712035-5.195634$

H $2.5877485 .343149-4.605358$

H $5.3592123 .500948-1.026813$

H $3.8825782 .548967-0.931131$

H $\quad 5.010250 \quad 0.732097-2.284518$

H $6.5139551 .668177-2.207800$

H 6.2874561 .6418640 .341025

H $7.8115350 .023975-0.824949$

H $6.467414-1.055357-1.112347$

H $8.558490-2.0797040 .241258$

H $5.260674-2.1262931 .008147$

H $8.993992-0.0506911 .347727$

H $8.248297-0.7283982 .775416$

H 7.6397780 .7971592 .099728

H $6.063824-1.0711213 .500462$

H $3.045006-1.4805651 .457784$

H $3.338507-0.8625223 .085903$

H $4.443587 \quad 1.4268762 .781839$

H -1.195282 3.3003950 .532440

H $-3.5484384 .686684-0.903108$

H -5.5188203 .6204202 .146423$

H -4.9164682 .5420790 .889465$

H $-5.650944 \quad 4.1009490 .461010$

H 2.3945011 .3593362 .394912

H 2.1689950 .7414970 .746700

H -1.679335 -0.6991772 .091884$

H -2.092158 0.5769680 .932022

H -3.2963640 .8872543 .130459$

H -0.6670642 .3595173 .665123$

H -1.988933 2.5145975 .824070

H -3.4978342 .3639114 .957697$

H -1.863230 6.1612845 .355202

H -1.0933594 .6933586 .182186$

H -3.7754565 .8676083 .953215$

H -4.5045174 .2863543 .844898$

H -2.0780605 .2500352 .167794$

H 0.0402631 .5213851 .150465

C $1.859464-1.864502-1.635817$

O $0.780858-2.378134-0.823266$

C $0.944148-3.803095-0.688540$

C $2.412575-4.067365-0.981462$

C $2.653708-3.085939-2.109991$

H $0.341679-4.267552-1.478794$

C $0.460325-4.2900920 .680825$

H $3.049866-3.822543-0.123676$

H $2.602641-5.106411-1.266232$

C $4.123874-2.800743-2.358797$

H $2.208999-3.489946-3.029618$

C - $0.994296-3.8890621 .002037$

O $1.313010-3.7307811 .684319$

H $\quad 0.580442-5.3780920 .745162$

H -1.059305 -2.801765 1.112248

O $-1.345769-4.4523522 .270701$

C $-2.023860-4.373176-0.035861$

C $-3.493329-4.1116860 .343864$

H -1.822334 -3.900394 -1.002078

O $-1.873075-5.781507-0.236354$

H -4.120581-4.548719-0.443019

H -3.749217 -4.6785531 .248005$

H $4.251190-2.052328-3.147072$

H $4.610984-2.425807-1.454184$

H $4.646344-3.711971-2.667465$

C $1.280374-1.057149-2.799708$

H $1.121430-4.1863072 .521091$

H $-0.840778-3.9761732 .951443$

H -2.008331 -6.2159720 .623350$

H $\quad 0.415121-1.583710-3.221252$

H $2.030012-0.967614-3.594906$

H $2.476269-1.229421-0.989382$

C $-4.042763-1.982493-1.771392$

O $-3.355008-1.807381-0.528663$

C -3.841488 -2.623911 0.541093

C $-5.332627-2.3484150 .783955$

C $-6.157531-2.483846-0.494619$

C $-5.534500-1.661921-1.622979$

H $-3.928440-3.023893-2.097373$

C -3.336867 -1.137020 -2.837538

H $-3.311920-2.2975881 .443107$
O $-5.858301-3.2262401 .773767$

H $-5.453199-1.3254801 .164313$

H $-6.272764-3.532887-0.791077$

O $-7.477036-1.987468-0.256798$

H $-5.684856-0.596503-1.410264$

O $-6.201643-1.953832-2.847744$

C $-3.4479250 .346315-2.577187$

H $-2.265767-1.371991-2.864436$

H $-3.731752-1.364013-3.834521$

H $-5.327470-3.1086672 .580086$

H $-7.845699-2.4930400 .487805$

H -7.147817 -1.773616 -2.712737

O $-4.308494 \quad 0.914811-3.468561$

O $-2.857306 \quad 0.925460-1.675409$

C $-4.5266362 .319153-3.302952$

H $-4.8983092 .532587-2.296077$

H -3.601301 $2.867737-3.499855$

H -5.282011 $2.633532-4.027975$

SCF Energy $\left(B 3 L Y P / 6-31 G^{* *} / / M M F F\right)=-3245.90772334$

01341

MM̄FF Geometry

C $0.786361-0.8331601 .862190$

C 0.7338890 .0200860 .828861

C $-0.171658-0.162693-0.357980$

O $-0.9438441 .057966-0.470861$

C $0.607179-0.375061-1.667908$

C $1.346935-1.692182-1.748629$

C $0.759010-2.877558-2.003021$

C $2.836907-1.606707-1.563988$

C $-0.707074-3.133458-2.221497$

C $-1.248027-4.139868-1.197792$

C $-2.781368-4.246448-1.149485$

C $-3.417111-4.509361-2.516314$

C $-4.951018-4.558579-2.434039$

O $-3.296395-3.032055-0.588564$

O $-5.468005-4.473295-3.771246$

C $-5.450181-3.308267-1.677865$

C $-5.451323-5.890289-1.862514$

O $-6.856509-3.379860-1.452302$

C $-4.710481-3.055900-0.342695$

C $-5.130540-1.6852430 .243769$

O $-5.069808-4.0832660 .584831$

C $-2.1623410 .972035-1.067619$

O $-2.673028-0.020128-1.561772$

C $-2.7928452 .307526-0.997697$

C $-4.1208972 .404597-1.157088$

C $-4.9142273 .631567-1.100509$

C $-4.1778564 .940918-0.995184$

C $-4.301282-1.2445131 .457476$

C -4.7278520 .1104991 .992268$

C $-6.610567 \quad 1.2627623 .158538$

O -3.9705781 .0781691 .973706$

C -7.4812581 .9855112 .178772$

C -7.4802423 .3209352 .045081$

C -8.4015414 .0761261 .128454$

C -7.6717685 .0755680 .258854$

C $-7.462803 \quad 6.3329220 .685151$

C $-7.2621044 .650371-1.131902$

C $-6.2592383 .532314-1.141782$

N $-6.008120 \quad 0.1080742 .519812$

H $0.157149-1.7201091 .853770$

H 1.3604220 .9069610 .838160

H $-0.857353-0.998469-0.171088$

H $1.3067150 .458925-1.820745$

H $-0.075376-0.294487-2.524068$

H $1.389383-3.763468-2.065729$

H $3.318841-2.589656-1.587984$

H $3.278766-1.000173-2.361011$

H $3.077626-1.145729-0.601837$

H - $1.288550-2.210116-2.181864$

H $-0.828603-3.536626-3.233811$

H $-0.827551-5.129891-1.416379$

H $-0.895742-3.854250-0.197392$

H $-3.014385-5.069024-0.462592$

H $-3.022672-5.432868-2.957611$

H -3.152166 -3.703679 -3.213496

H $-5.097828-5.212818-4.283320$ 
H $-5.284893-2.440419-2.332068$ H -5.123497 -6.724662 -2.494259 H $-6.546129-5.931667-1.857391$ H -5.093342 -6.085464 -0.849704 H -7.280954 -3.525481 -2.315199 H $-5.029711-0.911018-0.526542$ H $-6.190845-1.7210520 .520868$ H $-4.926972-3.7630351 .488594$ H -2.157065 $3.160492-0.791207$ H $-4.6911031 .492799-1.330473$ H -3.491806 5.065838 -1.840339 H -3.601243 $4.983884-0.064587$ H $-4.8390885 .810590-0.995089$ H -4.377063 -1.962916 2.280517 H -3.240478 -1.175976 1.190205 H -5.8246991 .9065693 .566865$ H -7.2211630 .8872403 .985765$ H -8.1716601 .3909441 .585428$ H -6.8128473 .9122802 .668491$ H -9.134282 4.6042531 .753126 H $-8.988207 \quad 3.3959350 .498715$ H -6.9677007 .0683850 .059870$ H -7.780076 6.6534211 .671986 H -6.887962 $5.500451-1.714923$ H -8.156044 4.316893-1.675086 H $-6.7096342 .540899-1.200358$ H $-6.556618-0.7459462 .487587$ C $2.732837-1.6959453 .197859$ O $3.785198-1.4189372 .248985$ C $5.056884-1.5554892 .912582$ C $4.769104-2.2700774 .226268$ C $3.404968-1.7102064 .571796$ H $5.408136-0.5412543 .135791$ C $6.064607-2.2740442 .009869$ H $4.711799-3.3567724 .094114$ H $5.526546-2.0611504 .987782$ C $2.661964-2.5262195 .615698$ H $3.528978-0.6828884 .940499$ C $6.307703-1.5899470 .649472$ O 5.591872 -3.602086 1.745217 H $7.015890-2.3950142 .540776$ H $5.406980-1.6715890 .033415$ O $7.329744-2.327566-0.033369$ C $6.749444-0.117740 \quad 0.739731$ C $7.1817480 .499210-0.604005$ H $\begin{array}{llll}5.940853 & 0.479821 & 1.168647\end{array}$ O $7.858048-0.027848 \quad 1.639312$ H $7.5544851 .511319-0.404827$ H $8.052197-0.047123-0.988021$ H $1.678312-2.0936605 .823141$ H $2.512441-3.5597345 .285286$ H $3.224272-2.5518906 .554940$ C $1.643668-0.6293523 .079644$ H $4.714455-3.5236211 .332515$ H $7.036771-3.253843-0.079492$ H $8.0789900 .913626 \quad 1.740204$ H $2.103166 \quad 0.3658583 .057041$ H $0.982968-0.6630383 .953587$ H $2.336058-2.6900362 .953901$ C $5.0407342 .646965-1.057325$ O $4.8985601 .228124-1.202319$ C $6.0666670 .541853-1.666507$ C $6.5101691 .107118-3.022636$ C $6.7056802 .617646-2.968208$ C $5.4669033 .291798-2.384126$ H $5.7832112 .865423-0.280166$ C $3.6811973 .206507-0.603607$ H $5.761789-0.494367-1.850507$ O $7.717741 \quad 0.494892-3.462620$ H $5.7438460 .884243-3.776599$ H $7.6049752 .888765-2.403038$ O $6.9005183 .114623-4.294517$ H $4.645093 \quad 3.236588-3.109898$ O $5.7507694 .672587-2.164317$ C 3.4104762 .9749100 .868187 H $3.6533344 .290430-0.764138$ H $2.8657382 .737799-1.166240$ H $7.563498-0.464705-3.496371$
H $7.6676812 .647546-4.667942$

H $6.0348285 .049154-3.014994$

O $2.3709243 .773918 \quad 1.254269$

O 4.0106032 .1859481 .583824

C 1.9936443 .6870852 .632217

H 1.3059604 .5092772 .847613

H 1.4775652 .7412652 .815889

H 2.8674793 .7846373 .283668

SCF Energy $\left(B 3 L Y P / 6-31 G^{* *} / / M M F F\right)=-3245.91181211$

01342

MM̄FF Geometry

C $1.0201103 .995473-0.140803$

C $0.8037563 .812648-1.453183$

C $-0.3634523 .071474-2.060878$

O $-1.0265022 .226478-1.092606$

C $-1.4023403 .982526-2.740575$

C $-2.1281904 .964347-1.842175$

C $-3.3215014 .726945-1.262336$

C $-1.4905866 .324223-1.702641$

C $-4.1539633 .475870-1.331169$

C -4.6430843 .0587420 .062633$

C $-5.6588751 .909817-0.010072$

C -6.2833721 .6594281 .363826$

C $-7.2704490 .484711 \quad 1.331800$

$\begin{array}{lllll}\text { O } & -4.988158 & 0.728032 & -0.462848\end{array}$

$\begin{array}{llllll}0 & -7.568255 & 0.121197 & 2.688309\end{array}$

C $-6.575537-0.7264970 .680543$

C $-8.6045290 .871103 \quad 0.682467$

O $-7.495583-1.7995080 .489511$

C $-5.858703-0.397366-0.653347$

C $-5.006688-1.614314-1.080671$

O $-6.836614-0.138642-1.662417$

C $-0.4137271 .043205-0.808868$

O $0.5945620 .596926-1.334695$

C -1.1555710 .3858920 .288205$

C $-0.550804-0.6198970 .936374$

C -1.106891 -1.4475942.004999

C $-2.509654-1.1586392 .463123$

C $-4.049621-1.359422-2.251168$

C $-3.250513-2.617852-2.535045$

C $-1.454151-4.046355-1.540328$

O $-3.526474-3.359562-3.474685$

C $-1.680537-4.675203-0.199804$

C $-0.693134-5.0260230 .638121$

C $-0.928922-5.6110792 .003012$

C $-0.195439-4.8640553 .098775$

C $0.851946-5.4209883 .730130$

C $-0.710423-3.4956433 .506900$

C $-0.336499-2.4361092 .504943$

N $-2.248037-2.832516-1.603520$

H 0.2809743 .6523230 .578416

H $1.5388874 .188639-2.161827$

H $\quad 0.0394432 .424927-2.852858$

H -2.140650 $3.344684-3.242693$

H $-0.916914 \quad 4.540929-3.552517$

H $-3.7742655 .534515-0.687634$

H -2.117179 $7.020418-1.134474$

H $-0.5297246 .260895-1.187245$

H $-1.3261256 .768597-2.689814$

H $-5.0196833 .687450-1.970490$

H -3.613820 2.643019-1.789565

H $-3.7786372 .757016 \quad 0.667777$

H -5.1087793 .9196450 .559100$

H $-6.4355182 .184403-0.733620$

H -6.7789722 .5635851 .738963$

H -5.4898331 .4287902 .088631$

H -7.943962 0.9009043 .131990

H $-5.823236-1.0894201 .394932$

H $-9.0689851 .701743 \quad 1.227508$

H -9.3212650 .0439000 .731088$

H -8.501726 $1.177215-0.360593$

H -7.908764 -1.983826 1.350475

H $-4.409707-1.947700-0.223990$

H $-5.665435-2.450849-1.348746$

H $-6.444955-0.310609-2.533009$

H -2.1556930 .7304250 .519056$

H $\quad 0.459436-0.888710 \quad 0.632724$ 
H $-3.223755-1.3504131 .657774$ H $-2.606423-0.1120702 .773485$ H -2.816048 -1.7601123 .322139$ H -4.591854 -1.087526 -3.162564 H $-3.355898-0.540575-2.030272$ H $-0.409141-3.759227-1.691278$ H - $-1.751735-4.736622-2.335193$ H $-2.713258-4.8370860 .101541$ H $0.340494-4.883770 \quad 0.331118$ H $-0.602898-6.6587741 .975248$ H -1.998571 -5.635245 2.246146 H $1.372191-4.9017964 .528259$ H $1.217157-6.4062423 .459721$ H -0.283227 -3.205733 4.475158 H -1.791543 -3.570347 3.656682 H $0.686123-2.5257972 .135511$ H -2.086391-2.130311 -0.888391 C 2.7413273 .9361481 .679066 O 3.1327682 .5810081 .383385 C 4.2055952 .2097342 .273817 C 4.4767503 .4235003 .161873 C 3.9809544 .5692212 .302482 H 5.0866392 .0266651 .649725 C 3.8362650 .9444653 .059552 H 3.9114273 .3742674 .099998 H 5.5360693 .5118413 .422998 C 3.7097955 .8398563 .088486 H 4.7293454 .7742551 .525362 C $3.562553-0.3011252 .193280$ O 2.6533361 .2024973 .829455 H 4.6239940 .7206693 .788287 H $2.657988-0.1475371 .595070$ O $3.283060-1.3913993 .081841$ C $4.730269-0.7168001 .279359$ C $4.562784-2.0957230 .609588$ H 4.8764920 .0433870 .505766 O $5.942149-0.7570552 .038908$ H $5.473056-2.2976810 .031448$ H $4.546281-2.8761261 .380799$ H 3.3596276 .6407612 .429968 H 2.9482825 .6800203 .859350 H 4.6222126 .1873513 .584014 C 2.2522424 .6512110 .417020 H 1.9434901 .4355653 .206448 H $2.532455-1.1243203 .640053$ H $5.814848-1.3963482 .761015$ H $3.0481704 .655007-0.337602$ H 2.0079545 .6921830 .655390 H 1.9287773 .8820262 .416762 C $4.197356-1.142586-2.257340$ O $3.215454-1.134819-1.214194$ C $3.318203-2.219522-0.288194$ C $3.184535-3.555597-1.032628$ C $4.159930-3.665619-2.203385$ C $4.077890-2.423096-3.090483$ H $5.201341-1.073426-1.820969$ C $3.9654510 .105275-3.113866$ H $2.448261-2.1448790 .372605$ O $3.348270-4.656505-0.145031$ H $2.164786-3.630654-1.431081$ H $5.187388-3.829753-1.857724$ O $3.816039-4.804581-2.995292$ H $3.129776-2.428447-3.643255$ O $5.133834-2.461598-4.047775$ C $4.335177 \quad 1.348797-2.342737$ H $4.5515100 .088108-4.039619$ H $2.9114640 .197698-3.402515$ H $4.290187-4.7242340 .085162$ H $3.826917-5.579623-2.407639$ H $\quad 5.045614-3.293897-4.543397$ O $5.6746181 .582454-2.442179$ O $3.5326992 .016982-1.705155$ C $6.1502872 .726155-1.725626$ H $5.9832292 .595449-0.652488$ H $7.2247532 .817140-1.904823$ H $5.6560583 .633666-2.085701$ SCF Energy (B3LYP/6-31G**//MMFF) $=-3245.91162532$
01343

MM̄FF Geometry

C $-2.2626803 .650058-0.516861$

C $-1.5900982 .501231-0.356940$

C $-0.7287541 .874448-1.420640$

O $0.5887541 .680932-0.854227$

C $-1.3007050 .497742-1.799192$

C $-0.684545-0.072146-3.059380$

C $0.322637-0.966137-3.081806$

C $-1.3076380 .401564-4.346702$

C $1.080932-1.521111-1.908646$

C $0.851108-3.030336-1.749464$

C $1.704698-3.679893-0.645285$

C $1.315449-3.2023860 .753895$

C $2.218175-3.8115011 .834275$

O $3.070121-3.371228-0.918036$

O $1.980785-3.0951363 .057743$

C $3.692090-3.5610991 .445325$

C $1.856921-5.2739772 .117354$

O $4.555412-4.2383692 .355443$

C $4.030463-3.939278-0.019001$

C $5.408934-3.404771-0.471952$

O $4.110898-5.358846-0.184974$

C $1.4836442 .697873-0.981639$

O $1.3280913 .740545-1.597065$

C $2.6868602 .339230-0.193315$

C $3.6746583 .237313-0.055715$

C 4.8957663 .0819950 .741954

C 5.0894621 .7806311 .473090

C $5.508224-1.877480-0.514291$

C $6.809335-1.394108-1.128439$

C $7.8579880 .694004-2.012249$

O $7.804109-2.104830-1.239355$

C $8.4406911 .496107-0.892069$

C $8.5177872 .835284-0.895885$

C $9.130103 \quad 3.6309080 .219530$

C 8.2032534 .6931630 .764132

C 8.4634055 .9986710 .578185

C 7.0265024 .2471371 .605685

C 5.7563424 .1203760 .806862

N $6.730751-0.058173-1.498244$

H -2.191240 $4.187775-1.458841$

$\mathrm{H}-1.668416 \quad 1.967270 \quad 0.586513$

H $-0.6698972 .513455-2.310491$

H $-2.3860370 .568552-1.954696$

H $-1.170576-0.203867-0.968365$

H $0.672518-1.321749-4.050266$

H $-0.837528-0.048739-5.227308$

H $-2.3710610 .141643-4.371182$

H $-1.2126251 .487740-4.442219$

H $2.144435-1.317798-2.082303$

H $\quad 0.821077-1.003489-0.983135$

H $-0.210772-3.231536-1.562645$

H $1.104754-3.517321-2.700890$

H $1.568629-4.764992-0.723620$

H $0.264701-3.4304240 .963250$

H $1.405828-2.1109330 .821922$

H $1.034566-3.1691253 .270217$

H $3.864603-2.4878401 .592098$

H $0.827455-5.3485572 .488606$

H $2.490577-5.6924912 .906802$

H $1.931582-5.9134631 .235341$

H $5.458913-3.9126332 .207854$

H $6.207446-3.8233690 .153174$

H $5.610259-3.787238-1.483153$

H $3.934145-5.7860480 .667761$

H 2.7076921 .3649720 .281950

H $3.5708174 .199263-0.556810$

H 5.0196290 .9355950 .782281

H 4.3233631 .6596832 .247056

H 6.0607351 .6939511 .963708

H $4.676665-1.459993-1.092885$

H $5.462604-1.4657250 .498885$

H $7.4852961 .326036-2.824520$

H $8.6122770 .011748-2.416066$

H $8.8325670 .942567-0.041706$

H $8.1462103 .388971-1.754964$

H $10.0486044 .090642-0.167982$ 
H 9.4481552 .9808911 .044387 H 7.8190026 .7662280 .994169 H $\quad 9.322024 \quad 6.3291280 .003224$ H 6.8445694 .9924582 .392017 H 7.2922193 .3316632 .140225 H 5.5026125 .0282270 .256788 H $5.8709750 .453614-1.323582$ C -4.6104824 .2274450 .117923$ O $-5.0566602 .863053-0.003281$ C -6.4045152 .7647110 .493636$ C -6.8627634 .1920360 .776845$ C $-5.5536024 .870337 \quad 1.131925$ H $-6.3487312 .219202 \quad 1.442942$ C -7.277470 $1.992952-0.502109$ H -7.306279 4.662269-0.108372 H -7.6021894 .2360001 .582332$ C $-5.613387 \quad 6.3855201 .051378$ H -5.2699694 .5733232 .150443$ C $-6.7112630 .611357-0.893260$ O $-7.3881942 .767711-1.703710$ H $-8.2937191 .895815-0.102514$ H -5.791184 $0.752463-1.470516$ O $-7.641443-0.004231-1.793669$ C $-6.446272-0.3373290 .291707$ C $-6.041773-1.765566-0.118694$ H $-5.666016 \quad 0.0841410 .930452$ O $-7.622628-0.4280641 .100231$ H $-6.054414-2.389800 \quad 0.782685$ H $-6.820376-2.191143-0.763389$ H -4.6462356 .8289351 .307724$ H $-5.883114 \quad 6.7236600 .045211$ H -6.3599336 .7783461 .749348$ C $-3.1434894 .248196 \quad 0.544845$ H $-7.9402302 .262193-2.324724$ H -8.495215 -0.081159-1.335265 H -8.306653 -0.8876030 .584565$ H -3.025851 3.6955091 .484765 H $-2.812903 \quad 5.2765510 .727975$ H $-4.7292684 .693214-0.869605$ C -3.285193 -2.148498 1.151693 O $-3.610337-1.344258 \quad 0.010717$ C $-4.667550-1.850800-0.812726$ C $-4.309738-3.253563-1.322468$ C $-3.970779-4.198843-0.175315$ C $-2.908356-3.5743550 .724656$ H $-4.138559-2.1746051 .839892$ C -2.104231-1.480683 1.876333 H $-4.707672-1.200133-1.693770$ O $-5.375151-3.807832-2.086502$ H -3.437889-3.187484-1.986635 H -4.862286 -4.475765 0.399301 O $-3.442651-5.415402-0.709267$ H -1.950795 -3.5638190 .191158$ O $-2.741195-4.3850401 .885829$ C -2.486244 -0.191284 2.571175 H -1.705643 -2.151049 2.646225 H $-1.311425-1.2449721 .157837$ H -5.563393 -3.191631 -2.814937 H $-4.111862-5.779409-1.314242$ H -2.518633 -5.2823821 .583523$ O O $-3.602525 \quad 0.3067492 .583077$ C -1.5769371 .5356753 .910805$ H -1.8935672 .3331093 .232875$ H -2.3152871 .3873644 .704604$ H -0.6247371 .8204784 .366094$ SCF Energy (B3LYP/6-31G**//MMFF)= -3245.91113578

01344

MM̄MF Geometry

C -1.219660 $3.521280-0.866536$ C $-0.8627132 .329875-0.363245$ C $0.0002301 .330869-1.090131$ O $1.1611841 .046843-0.276232$ C $-0.7877490 .019763-1.272423$ C $-0.043330-1.038536-2.061847$ C $0.349183-2.229134-1.567443$ C $0.226242-0.705705-3.507119$
C $0.150834-2.772151-0.179746$

C $1.321115-2.5202440 .783486$

C $2.647830-3.1643530 .348908$

C $3.683400-3.0796911 .472677$

C $5.052051-3.6288391 .038486$

O $3.135367-2.464384-0.798434$

O $6.022096-3.2140882 .012993$

C $5.455604-2.979078-0.303317$

C $5.073884-5.1619661 .031144$

O $6.635936-3.582283-0.826372$

C $4.335095-3.010986-1.362556$

C $4.665387-2.181957-2.628048$

O $4.130988-4.363242-1.790611$

C $2.1907041 .935349-0.325462$

O $2.2561912 .951944-0.997855$

C 3.2326091 .4641230 .613313

C 4.1869012 .3141801 .020518

C 5.2728562 .0088791 .954463

C 5.4799060 .5660452 .332346

C $4.926339-0.690540-2.389186$

C $6.397177-0.336027-2.244087$

C $7.8835341 .584901-1.686556$

O $7.308473-1.123747-2.482360$

C $8.3031691 .478034-0.254599$

C 8.7357512 .5250420 .464294

C 9.2018042 .4289541 .888711

C 8.4866903 .3997162 .801390

C 9.0434044 .5779563 .129445

C 7.1678592 .9661773 .400196

C 6.0345913 .0240742 .413329

N $6.5729120 .984725-1.852653$

H $-0.8661153 .818593-1.850387$

H -1.2186982 .0418390 .623587$

H $0.3183021 .719455-2.065109$

H -1.735674 $0.223605-1.789170$

H $-1.061779-0.363350-0.283467$

H $\quad 0.870470-2.911624-2.238197$

H $0.628264-1.560170-4.062248$

H $-0.699450-0.400021-4.005795$

H $0.9536840 .107554-3.587699$

H $-0.765394-2.3735950 .267765$

H $-0.021324-3.853051-0.261861$

H $1.456143-1.4386590 .900090$

H $1.033723-2.9173021 .765117$

H $2.454427-4.2109560 .086055$

H $3.328773-3.6028952 .369530$

H $3.809838-2.0300561 .770241$

H $\quad 5.741087-3.5549602 .879587$

H $5.708464-1.934879-0.085687$

H $4.850360-5.5526552 .031329$

H $6.069673-5.5420640 .778062$

H $4.350885-5.5979140 .338708$

H $7.322313-3.518788-0.140361$

H $5.484530-2.653173-3.184566$

H $3.790873-2.245214-3.292130$

H $3.695612-4.349482-2.659095$

H 3.1739240 .4385890 .958628

H 4.1607523 .3398070 .654486

H $5.552858-0.0572811 .434913$

H 4.6468360 .2053542 .944833

H 6.3990390 .3887622 .894300

H $4.564874-0.132095-3.262357$

H $4.373379-0.327658-1.517632$

H $7.8088292 .624708-2.020279$

H $8.6071281 .064850-2.322595$

H $\quad 8.2753740 .491960 \quad 0.203158$

H $8.789173 \quad 3.504640-0.005645$

H $\quad \begin{array}{lll}10.280628 & 2.632932 & 1.900301\end{array}$

H $9.090355 \quad 1.4100822 .280002$

H 8.5501415 .2681513 .805868

H $\quad \begin{array}{llll}10.003168 & 4.880789 & 2.724020\end{array}$

H 6.8995923 .6267954 .235719

H 7.2896651 .9793833 .855087

H 5.8076144 .0358122 .073996

H $5.7597791 .550213-1.627069$

C $-3.4077934 .751067-0.914599$

O $-4.1088333 .509818-1.126498$

C $-5.4624933 .650268-0.667331$ 
C $-5.7208815 .149977-0.624792$

C $-4.3738235 .665174-0.155015$

H $-5.486917 \quad 3.2547450 .356022$

C $-6.4204872 .838749-1.545407$

H $-5.9500045 .553959-1.617414$

H $-6.541506 \quad 5.413247 \quad 0.049239$

C -4.156752 $7.144405-0.422997$

H -4.2918395 .4862140 .925603$

C $-5.9701591 .387512-1.813669$

O $-6.5411703 .484806-2.819364$

H -7.421827 2.857362 -1.098750

H $-5.1405541 .390981-2.531105$

O $-7.051444 \quad 0.717379-2.475560$

C $-5.5583960 .580874-0.568988$

C $-5.242199-0.886284-0.919362$

H $-4.6744151 .043311-0.116143$

$\begin{array}{llll}\text { O } & -6.616957 & 0.640650 & 0.380869\end{array}$

H -6.172425 -1.403808 -1.176872

H $-4.601391-0.897563-1.808807$

H -3.166096 7.460219-0.080607

H $-4.2335417 .374341-1.491031$

H -4.9049557 .7447570 .104598$

C -2.115047 $4.484690-0.138708$

H $-7.1620662 .959653-3.353076$

H -7.781389 $0.639124-1.837285$

H $-6.283751 \quad 0.254644 \quad 1.208135$

H -2.359095 4.0834770 .853247

H - 1.5709085 .4232470 .016271

H -3.179956 $5.157899-1.907899$

C -6.439495 -2.562118 1.379579

O $-5.293989-1.7018571 .425565$

C $-4.523844-1.642487 \quad 0.221419$

C $-4.041134-3.048794-0.165245$

C $-5.195969-4.041271-0.250544$

C $-6.031472-3.997304 \quad 1.026370$

H $-7.154526-2.182003 \quad 0.639988$

C $-7.106981-2.5149882 .759607$

H -3.629029 -1.054179 0.463008

O $-3.351719-3.034392-1.410692$

H $-3.330615-3.4081070 .590547$

H $-5.818968-3.859526-1.134250$

O $-4.670137-5.362893-0.393966$

H -5.460797 -4.4421001 .851859$

O $-7.205414-4.786620 \quad 0.844235$

C $-7.811354-1.1972672 .976325$

H -7.863866 -3.299422 2.873639

H $-6.373178-2.6627793 .561405$

H -2.636744 -2.379828 -1.341746

H $-4.108536-5.365276-1.188160$

H $-6.917333-5.6824310 .598304$

O $-7.002093-0.340603 \quad 3.661952$

O $-8.937000-0.9520942 .561932$

C -7.554742 $0.953651 \quad 3.922373$

H $-8.4406910 .864206 \quad 4.558100$

H -7.8019141 .4590432 .984002$

H -6.8015381 .5443964 .450380$

SCF Energy (B3LYP/6-31G*//MMFF) $=-3245.91782553$

01345

MM̄FF Geometry

C $1.2923993 .543631 \quad 1.967544$

C 1.6934982 .3227621 .581158

C $2.227211 \quad 1.2737632 .519925$

$\begin{array}{lllll}\text { O } & 1.451016 & 0.079913 & 2.254598\end{array}$

C 3.7134600 .9804132 .255076

$\begin{array}{llll}\text { C } 4.632829 & 2.142506 & 2.559811\end{array}$

C 5.2184342 .9194231 .627635

C $4.9055282 .378498 \quad 4.022406$

C $5.0604002 .845583 \quad 0.133885$

C $6.3505672 .411985-0.574309$

C $6.7623310 .961375-0.275706$

C $8.0868280 .631830-0.967531$

C $8.490297-0.835006-0.759292$

O $5.7301210 .093831-0.757329$

O $9.548365-1.134790-1.682564$

C $7.293462-1.733981-1.131765$

C $9.058640-1.0762620 .644091$

O $7.574052-3.100529-0.833909$
C $5.954151-1.296579-0.487811$

C $4.795509-2.101202-1.121193$

O $6.006566-1.5640530 .914038$

C $1.376828-0.8652013 .223989$

O $1.955513-0.8488714 .301976$

C $0.456936-1.9654392 .846807$

C $-0.228278-2.0105281 .692990$

C $-1.206443-3.0323591 .307628$

C $-1.410309-4.1986372 .238365$

C $3.390575-1.694417-0.658431$

C $2.360906-2.491713-1.433766$

C $1.256198-2.582506-3.663447$

O $1.944018-3.574260-1.027888$

C $-0.194478-2.625437-3.302177$

C $-0.874554-3.774288-3.167776$

C $-2.341946-3.864440-2.866059$

C $-2.623140-4.561570-1.554273$

C $-2.604523-5.901901-1.461016$

C $-3.019068-3.706480-0.375782$

C -1.889852 -2.869893 0.155249

N $2.016229-1.894850-2.637395$

H 1.3697323 .7999593 .022706

H 1.6417302 .0369260 .534210

H 2.0650141 .5768733 .561877

H 3.8360840 .6301971 .225501

H 4.0325210 .1186492 .856048

H 5.8780643 .7174761 .966443

H 5.6183223 .1936314 .186557

H 3.9821502 .6384354 .548996

H $5.326207 \quad 1.4770874 .480134$

H $4.2317332 .199359-0.168518$

H $4.7919813 .849837-0.218156$

H $6.1858582 .523028-1.653988$

H $7.1658373 .093354-0.300198$

H 6.8647210 .8347360 .807520

H $8.884177 \quad 1.301582-0.621820$

H $7.9899030 .814238-2.047148$

H $10.284580-0.525045-1.503265$

H $7.184965-1.681630-2.223942$

H $9.927621-0.4310610 .821119$

H $9.424585-2.1033920 .750993$

H $8.335914-0.8812401 .439136$

H $7.477690-3.2196270 .126347$

H $4.840003-1.985093-2.212332$

H $4.927639-3.169926-0.907804$

H $5.102680-1.6249681 .259586$

H $\quad 0.345677-2.7347093 .604538$

H $-0.095223-1.2089180 .968431$

H $-1.858040-3.865253 \quad 3.180741$

H $-0.455173-4.6900282 .455729$

H $-2.058343-4.9748561 .824469$

H $3.237508-1.8966750 .406477$

H $3.214251-0.624543-0.813932$

H $1.680618-3.584131-3.792807$

H $1.387917-2.028568-4.598184$

H $-0.708516-1.676325-3.176210$

H $-0.350491-4.716582-3.314072$

H -2.824624 -4.417203 -3.682809

H $-2.809659-2.872166-2.872778$

H $-2.836016-6.407786-0.529620$

H $-2.353504-6.522088-2.315427$

H $-3.470332-4.3158930 .414295$

H $-3.825952-3.030600-0.688397$

H $-1.664338-2.000068-0.461894$

H $2.474045-1.027523-2.901963$

C $0.4340504 .273419-0.365456$

O $-0.583505 \quad 3.255094-0.407541$

C $-1.5525833 .594249-1.414228$

C $-0.9201344 .704371-2.243183$

C $-0.1178765 .440711-1.185731$

H $-2.4219904 .004171-0.885753$

C $-1.9704302 .340132-2.189171$

H $-0.2516064 .307487-3.015691$

H $-1.6677925 .335023-2.733431$

C $0.9523896 .352803-1.759376$

H $-0.8080006 .037631-0.574438$

C -2.380844 $1.156060-1.288786$

O $-0.862413 \quad 1.903409-2.987336$ 
H -2.774550 $2.592957-2.890130$ H -1.491531 $0.755924-0.786806$ O $-2.852003 \quad 0.105210-2.142897$ C -3.462626 $1.478311-0.240875$ C -3.8202250 .2382060 .599465$ H -3.1003112 .2638630 .430763$ O $-4.6106971 .977900-0.917936$ H $-4.246343-0.528938-0.054130$ H -2.889337 -0.1627301 .014122$ H $1.5123876 .848121-0.959869$ H $1.6662735 .795914-2.375580$ H $0.5007197 .128398-2.386301$ C $0.7253954 .635573 \quad 1.094421$ H $-1.146214 \quad 1.104671-3.464430$ H -3.681938 $0.408891-2.549500$ H $-5.240607 \quad 2.280990-0.242415$ H -0.2101134 .9601071 .568413$ H $1.4180505 .484438 \quad 1.130644$ H $1.3291023 .862259-0.849538$ C $-6.878725 \quad 0.007423 \quad 0.650734$ $\begin{array}{llll}\text { O } & -6.073527 & 0.994974 & 1.307048\end{array}$ C $-4.796026 \quad 0.535917 \quad 1.759522$ C -4.981141 -0.6342862 .737253$ C $-5.820373-1.7522402 .126501$ C -7.128903-1.192824 1.571195 H $-6.378750-0.323687-0.267016$ C $-8.200093 \quad 0.6790220 .257591$ H -4.3572531 .3649192 .329574$ O $-3.726379-1.1647193 .150595$ H -5.490133 -0.2744833 .641052$ H $-5.266151-2.2948911 .352456$ O $-6.137015-2.710277 \quad 3.138825$ H -7.783619 -0.9016712 .402774$ O $-7.800192-2.214020 \quad 0.835956$ C $-7.989698 \quad 1.692073-0.842072$ H $-8.946012-0.045133-0.088792$ H $-8.637782 \quad 1.2083341 .112838$ H -3.213442 -0.4354393 .538678$ H $-5.295078-3.0307583 .505677$ H -7.922292 -2.970243 1.435425 O $-7.844674 \quad 1.056724-2.040091$ O $-7.9458672 .900997-0.654979$ C -7.615883 $1.910643-3.165236$ H $-8.4692282 .579566-3.312268$ H $-6.6945332 .484019-3.025915$ H $-7.5060921 .281544-4.052517$

SCF Energy (B3LYP/6-31G**//MMFF)= -3245.92272001

01346

MMFF Geometry

C $-0.391112-3.4552390 .258493$

C $-0.185313-2.640848-0.787854$

C $-1.149257-1.559000-1.197550$

O $-0.491134-0.285876-1.007611$

C $-1.497201-1.692115-2.689956$

C $-2.736002-0.903377-3.068959$

C $-3.972509-1.433502-3.153352$

C $-2.5060650 .544000-3.416618$

C $-4.369578-2.855044-2.871106$

C $-5.658708-3.002768-2.049333$

C $-5.522210-2.566482-0.580423$

C $-6.701367-3.1152570 .230314$

C $-6.675501-2.6369151 .686966$

O $-5.514696-1.135822-0.523789$

O $-7.952184-2.9336462 .272855$

C $-6.519490-1.1047661 .696295$

C -5.630167 -3.3883282 .519464$

O $-\begin{array}{llll}-6.353030 & -0.620634 & 3.027764\end{array}$

C $-5.368599-0.5928800 .797017$

C -5.4493390 .9500620 .698356$

O $-4.123445-0.9668401 .385783$

C -0.6304440 .3187090 .201950$

$\begin{array}{llll}\text { O } & -1.228102 & -0.105513 & 1.177516\end{array}$

C $0.064224 \quad 1.620093 \quad 0.128705$

C $-0.1986122 .561893 \quad 1.046279$

C $0.441402 \quad 3.8721541 .102442$

C $0.709908 \quad 4.3499532 .504420$

C $-4.4677361 .585388-0.293988$
C $-4.6548123 .091083-0.388292$

C $-3.7459105 .088153-1.546723$

O $-5.570913 \quad 3.6899620 .169223$

C $-2.3653475 .605774-1.793383$

C $-1.8277346 .622051-1.101783$

C $-0.4625157 .193050-1.361293$

C $0.4494177 .079866-0.160328$

C 0.4605228 .0303870 .789506

C $1.4049825 .911409-0.101018$

C $0.7123064 .580945-0.011707$

N $-3.6808143 .685649-1.180740$

H -1.302955 -3.3213690 .838803$

H $0.716403-2.729726-1.386161$

H $-2.069613-1.603591-0.601344$

H -1.633217 -2.746673 -2.956940

H $-0.658822-1.342708-3.308160$

H $-4.787124-0.787426-3.477250$

H $-1.7899680 .627603-4.240646$

H $-3.4267291 .048255-3.728977$

H -2.110151 $1.090061-2.556295$

H $-4.530877-3.345566-3.839207$

H $-3.574780-3.412088-2.365861$

H $-6.464972-2.437072-2.533743$

H $-5.940936-4.062817-2.083245$

H $-4.575469-2.950790-0.183103$

H $-6.723934-4.2117200 .190968$

H -7.643243 - $2.780980-0.227496$

H -8.097602 -3.8927902 .204645$

H $-7.462722-0.677322 \quad 1.327427$

H $-5.847927-4.4631652 .527070$

H -5.657182 -3.070481 3.567597

H -4.611716 -3.2623952 .146444$

H -7.101271 -0.9532093 .552626$

H $-6.464674 \quad 1.2309370 .388117$

H $-5.2767401 .394474 \quad 1.687008$

H $-3.432894-0.3535931 .091159$

H $0.7731091 .774218-0.677574$

H $-0.9130452 .360876 \quad 1.842117$

H $1.3859453 .659693 \quad 3.020999$

H -0.2239694 .4065913 .075088$

H 1.1661035 .3418542 .544115

H $-3.434073 \quad 1.3904940 .005205$

H $-4.6251451 .171341-1.295065$

H $-4.2670105 .649254-0.763866$

H $-4.3401705 .159331-2.463562$

H $-1.7934505 .140753-2.592702$

H -2.412741 $7.094568-0.315501$

H $-0.5849868 .249780-1.633188$

H $0.0100296 .722120-2.232370$

H $1.1305267 .972774 \quad 1.640847$

H $-0.204523 \quad 8.8862190 .737464$

H 2.1225116 .0272890 .719845

H $2.0237225 .915273-1.008194$

H $0.4371524 .163118-0.979776$

H -3.065239 $3.083352-1.719589$

C $1.739190-4.893069-0.085934$

O $2.661985-3.790163-0.099234$

C $3.989443-4.306216-0.320999$

C $3.884277-5.830986-0.257757$

C $2.566977-6.0514380 .462819$

H $4.608741-3.9549570 .511197$

C $4.541752-3.772539-1.650624$

H $3.861670-6.282072-1.256479$

H $4.733571-6.2738860 .272663$

C $1.969690-7.425612 \quad 0.215141$

H $2.732650-5.9157011 .540033$

C $4.529523-2.234744-1.770385$

O $3.734436-4.290886-2.717627$

H $5.553169-4.163933-1.812365$

H $3.492393-1.883352-1.789072$

O $5.078348-1.901387-3.053361$

C $5.323400-1.492102-0.678880$

C $5.4484190 .024555-0.927651$

H $4.867429-1.6710150 .300124$

O $6.644044-2.039970-0.633392$

H $6.1798920 .426914-0.217068$

H $5.898547 \quad 0.197161-1.912710$

H $1.031289-7.5506590 .764012$ 
H $\quad 1.762253-7.586835-0.848080$

H $2.658659-8.2095790 .546415$

C $0.510339-4.5537850 .761623$

H $4.097346-3.943172-3.550299$

H $6.014018-2.167551-3.049201$

H $7.111690-1.6173140 .107165$

H $0.830039-4.2665951 .770398$

H $-0.109004-5.4525840 .864909$

H $1.439129-5.093655-1.123234$

C 4.1777491 .2260361 .566173

O 3.5020830 .5913800 .472865

C $4.1120520 .783510-0.807957$

C $4.2033632 .282203-1.127026$

C $4.9091953 .057543-0.019654$

C $4.2866762 .737411 \quad 1.337332$

H $5.1815650 .797741 \quad 1.675203$

C 3.3817490 .9122512 .838787

H $3.4248830 .349433-1.543356$

O $4.8839042 .501917-2.357972$

H $3.1914212 .690830-1.243233$

H $5.9874582 .859584-0.013777$

O $4.7596724 .459189-0.258551$

H 3.2965773 .1998341 .400716

O $5.090671 \quad 3.3110042 .366369$

C $3.812416-0.4141003 .415590$

H 3.5529661 .6591063 .622718

H 2.3014570 .8869322 .653847

H $4.4083862 .006975-3.047076$

H $5.1200364 .641514-1.143336$

H 5.1538844 .2649442 .187070

O $3.130446-1.4345882 .823352$

O $4.676710-0.5274354 .276131$

C $3.482273-2.7427543 .283593$

H $4.523431-2.9635493 .029601$

H $3.321184-2.8249124 .362747$

H $2.838688-3.4671462 .780956$

SCF Energy (B3LYP/6-31G**//MMFF) $=-3245.90784299$

01347

MM̄FF Geometry

C $3.998021-3.530323-0.248791$

C $2.913023-3.611192-1.035098$

C $1.502901-3.450462-0.530159$

O $0.916484-2.396278-1.328391$

C $0.695850-4.749275-0.723443$

C $-0.548347-4.8386980 .143530$

C -1.818073 -4.877915 -0.307426

C $-0.295766-4.9405841 .628561$

C -2.318207-4.852688 -1.722958

C $-2.865092-3.495645-2.189898$

C $-4.105442-2.992586-1.429899$

C $-4.688194-1.760821-2.130029$

C $-5.885172-1.173957-1.367659$

O $-3.729687-2.641314-0.094757$

O $-6.1403240 .140232-1.889386$

C $-5.503774-0.9964910 .119282$

C -7.160013 -1.989229-1.614266

O $-6.639491-0.6266590 .895536$

C $-4.826794-2.2380310 .741462$

C $-4.267298-1.9874522 .162462$

O $-5.762376-3.3112010 .912774$

C $0.075241-1.525219-0.714899$

O $-0.292780-1.551496 \quad 0.447763$

C $-0.326689-0.514367-1.719735$

C $-1.2884590 .365613-1.409091$

C $-1.7956421 .444602-2.256291$

C -1.113057 $1.683040-3.576234$

C $-3.230581-0.8674732 .282955$

C -3.8140370 .4872232 .653787$

C -3.2098342 .9094662 .710226$

O $-4.940040 \quad 0.6311893 .121638$

C -3.8302613 .5196811 .493622$

C $-3.222243 \quad 4.4341290 .722567$

C $-3.8493675 .045696-0.498051$

C $-3.1332544 .666791-1.776565$

C $-2.2477985 .500410-2.347563$

C $-3.5061793 .356753-2.437567$

C $-2.8256512 .181858-1.792541$
N $-2.905829 \quad 1.5158322 .440222$

H $3.868775-3.3776350 .818727$

H $3.038821-3.772077-2.103351$

H $1.533559-3.1522870 .523015$

H $1.321685-5.617226-0.474618$

H $\quad 0.441956-4.853551-1.783279$

H -2.612156 -4.9653010 .433830$

H -1.200314 -5.205124 2.187068

H $\quad 0.449989-5.7146711 .836991$

H $0.065514-3.9887082 .026952$

H $-1.535660-5.169232-2.419629$

H $-3.108340-5.608709-1.815847$

H -2.064715 -2.753954 -2.118941

H -3.117181 -3.592652 -3.253537

H $-4.843477-3.802593-1.400780$

H $-4.972500-1.998019-3.162867$

H -3.914222 -0.986130 -2.205970

H $-6.2923130 .059126-2.846606$

H $-4.803204-0.1535090 .163914$

H -7.427204 -1.969700 -2.677844

H $-8.015914-1.557915-1.083885$

H -7.060896 -3.036947 -1.322468

H -7.0151080 .1762960 .495133$

H $-5.092311-1.8449122 .871140$

H -3.771860 -2.916759 2.479463

H $-6.658005-2.9664660 .766284$

H $0.177106-0.521027-2.679302$

H $-1.7518520 .297108-0.427537$

H -1.278059 $0.834520-4.249218$

H $-0.034144 \quad 1.814792-3.440255$

H $-1.4662472 .578514-4.092051$

H -2.530903 -1.124276 3.088703

H $-2.649557-0.7809651 .362654$

H -2.272520 3.4015622 .987310

H -3.901389 2.9796113 .555921

H -4.8344373 .1898201 .236887$

H -2.2202074 .7680070 .981192$

H $-3.8393416 .135806-0.367879$

H $-4.9091614 .773940-0.580719$

H -1.750536 $5.245246-3.277534$

H -1.994455 $6.452457-1.893214$

H -3.301496 $3.400099-3.512291$

H $-4.5921963 .212298-2.372715$

H $-3.2557081 .908122-0.829665$

H -2.0251691 .3096221 .978095$

C $6.236882-2.359757-0.573606$

O $5.584130-1.242601-1.208603$

C $5.705541-0.092138-0.348257$

C $6.787685-0.4443070 .665259$

C $6.521434-1.9264100 .864865$

H 4.7605670 .0051760 .193916

C 5.949608 1.173368-1.172215

H $7.793625-0.2895670 .258348$

H $\quad \begin{array}{llll}6.699144 & 0.137108 & 1.587967\end{array}$

C $7.671786-2.6712631 .518408$

H $5.627302-2.0342421 .490685$

C $4.918917 \quad 1.425037-2.294631$

O $7.2393571 .057626-1.790813$

H $6.0099402 .041757-0.506440$

H $5.0343910 .637656-3.050410$

O $5.2848652 .654797-2.934373$

C $3.4349661 .513968-1.879823$

C $3.1387072 .628256-0.853372$

H $2.8496341 .744398-2.779764$

O $2.9628320 .263319-1.400012$

H 3.7455842 .4640020 .040214

H $3.4555593 .581422-1.289633$

H $7.436623-3.7350441 .623282$

H $8.592183-2.5850990 .931119$

H $7.871963-2.2690832 .516900$

C $5.408711-3.635876-0.758917$

H $7.3869551 .869943-2.304921$

H $4.6896512 .778993-3.693225$

H $3.154829-0.407167-2.077473$

H $5.902773-4.475981-0.257796$

H $5.386774-3.875839-1.829843$

H $7.186785-2.496892-1.107592$

C 1.7514821 .9096771 .823193 
$\begin{array}{llll}\text { O } & 1.282831 & 1.682431 & 0.487661\end{array}$

C $1.6392622 .685307-0.472311$

C $1.1342424 .056566-0.010286$

C 1.6258504 .3999761 .389359

C 1.2769413 .2693892 .354797

H 2.8468141 .8708911 .843167

C 1.2160520 .7679582 .705763

H $1.0784042 .450839-1.383794$

O $1.5255375 .065091-0.935398$

H $0.0367594 .041680-0.004422$

H 2.7009374 .6150981 .387981

$\begin{array}{lllll}\text { O } & 0.967463 & 5.597462 & 1.807209\end{array}$

H $0.192542 \quad 3.2498442 .520763$

O $1.9016553 .517873 \quad 3.612814$

C $2.033907-0.4972812 .562015$

H 1.2610271 .0612543 .760936

H 0.1801610 .5249162 .446718

H $1.1926805 .914067-0.597171$

H $1.361218 \quad 5.8688132 .653441$

H 1.4937334 .3144863 .992011

O $1.847616-1.2795313 .665879$

O $2.733322-0.7757241 .598794$

C $2.596608-2.4985713 .692554$

H $3.666705-2.2927963 .591258$

H $2.423250-2.9826604 .657278$

H $2.256124-3.1676772 .898463$

SCF Energy (B3LYP/6-31G*//MMFF) $=-3245.90030588$

01348

MM̄FF Geometry

C $-1.095410-3.132172 \quad 0.201856$

C $-0.456766-2.698716-0.894131$

C $-1.020811-1.648862-1.811232$

O $-0.007790-0.616446-1.925320$

C $-1.321849-2.183900-3.220739$

C $-2.662869-2.880535-3.337836$

C $-3.807188-2.248429-3.669451$

C $-2.649514-4.371712-3.134769$

C $-3.971569-0.773576-3.909655$

C $-5.316699-0.200610-3.442480$

C $-5.652360-0.415646-1.956935$

C $-6.9874820 .265456-1.629808$

C -7.350915 $0.156955-0.143333$

O $-4.605358 \quad 0.128712-1.142011$

O $-8.418802 \quad 1.0869110 .095793$

$\begin{array}{llll}\text { C } & -6.134286 & 0.605494 & 0.693568\end{array}$

C $-7.908157-1.229125 \quad 0.199795$

O -6.3773940 .4091602 .084395$

C $-4.811518-0.068924 \quad 0.266834$

C -3.5903650 .4865551 .037384$

O $-4.892408-1.465410 \quad 0.584308$

C $-0.439086 \quad 0.671431-1.991754$

O $-1.5948191 .061734-1.955417$

C $0.742211 \quad 1.552244-2.112865$

C $0.5649322 .876057-1.989670$

C $1.5967173 .909924-2.052775$

C $3.0121093 .485275-2.336962$

C -3.3185931 .9846440 .876661$

C -2.1162902 .4254921 .698851$

C $-0.768621 \quad 4.4281742 .247627$

$\begin{array}{llll}\text { O } & -1.576479 & 1.704325 & 2.534450\end{array}$

C $-0.2148765 .607006 \quad 1.513432$

C $1.099728 \quad 5.849824 \quad 1.392494$

C $1.672047 \quad 7.053047 \quad 0.694839$

C $2.6523286 .684659-0.398428$

C $3.9746976 .666926-0.160613$

C $2.1083976 .407462-1.782794$

C $1.2311355 .188864-1.827530$

N $-1.738717 \quad 3.7340261 .418375$

H -2.087566 -2.745077 0.405548

H $0.526420-3.090981-1.132250$

H $-1.928345-1.221906-1.367070$

H -0.521076 -2.858034 -3.554576

H -1.277914 -1.357382 -3.942529

H $-4.710677-2.843807-3.789870$

H -2.347846 -4.621834 -2.114219

H -3.633379 -4.822064 -3.304693

H - $-1.948226-4.843637-3.830835$
H -3.170639 -0.203846 -3.435236

H -3.887538 $-0.596131-4.988943$

H $-5.2962430 .876972-3.652771$

H $-6.120062-0.626858-4.057366$

H -5.726527 -1.493065 -1.771340

H $-7.794694-0.141533-2.251945$

H $-6.9210681 .331700-1.889832$

H -8.6056501 .0813581 .050464$

H $-6.047015 \quad 1.6893250 .551351$

H -8.817553 -1.426997 -0.380563

H -8.208809 -1.2875461 .251611$

H -7.204445 $-2.038564-0.004226$

H $-6.269814-0.538642 \quad 2.272717$

H $-3.692713 \quad 0.2385832 .102521$

H -2.692562 -0.0533670 .705698$

H $-3.991190-1.8170830 .657925$

H $1.7094131 .095733-2.283808$

H $-0.4429553 .247219-1.804510$

H $3.0685532 .933384-3.281605$

H $3.3895352 .846932-1.532795$

H $3.7067374 .324053-2.423553$

H $-3.1436052 .231330-0.174909$

H -4.172799 $2.570812 \quad 1.230365$

H $0.0116723 .725272 \quad 2.557974$

H $-1.2956954 .765342 \quad 3.146501$

H -0.9266896 .3087301 .085245$

H $1.8096435 .160017 \quad 1.844081$

H 2.1743047 .6690641 .452378

H 0.8814697 .6883990 .276316

H $4.6874226 .427798-0.942959$

H $4.373823 \quad 6.8867330 .824074$

H $2.9151666 .328264-2.520403$

H $1.5181047 .272907-2.111008$

H $0.1811625 .403889-1.625716$

H $-2.363675 \quad 4.296503 \quad 0.847487$

C $-0.300247-3.556607 \quad 2.583287$

O $0.730601-2.5476192 .526049$

C $0.228782-1.3549813 .152306$

C $-0.861419-1.8325114 .103187$

C -1.514228 $-2.920242 \quad 3.272799$

H $-0.230686-0.7572212 .355448$

C $1.350477-0.542973 \quad 3.808811$

H $-0.443979-2.2626855 .020624$

H $-1.552801-1.0317074 .382041$

C -2.337761 -3.9042024 .086179$

H $-2.178180-2.4337042 .550516$

C $2.583458-0.2871522 .917438$

O $1.803483-1.2479474 .971876$

H 0.9358450 .4069374 .168504

H $3.204094-1.1904272 .882552$

$\begin{array}{lllll} & \text { O } & 3.375951 & 0.725931 & 3.550772\end{array}$

C $2.2755200 .176678 \quad 1.484709$

$\begin{array}{llll}\text { C } 3.496834 & 0.650858 & 0.678157\end{array}$

H $1.788631-0.6366010 .936892$

$\begin{array}{lllll}\text { O } & 1.341976 & 1.259246 & 1.514202\end{array}$

H $3.1466730 .847091-0.340190$

H 3.8262241 .6291891 .051995

H -2.769859 -4.6744663 .439547$

H $-1.730231-4.4038394 .848237$

H $-3.158673-3.3893984 .595970$

C $-0.546097-4.132146 \quad 1.180896$

H $2.366263-0.6430335 .483495$

H $3.7295940 .351334 \quad 4.374910$

H 1.7352651 .9729852 .045098

H -1.249836 -4.9699161 .248330$

H $0.399113-4.5365810 .797676$

H $\quad 0.107418-4.3590253 .211333$

C $3.864435-1.916811-0.960135$

O $4.308793-1.6645830 .374501$

C $4.695541-0.3153210 .640614$

C $5.8125480 .119686-0.320263$

C $5.427562-0.107540-1.782556$

C $4.937698-1.541363-1.989780$

H $2.960345-1.323611-1.146685$

C $3.430871-3.385191-1.046761$

H $5.127950-0.3167921 .648686$

O $6.1351591 .489941-0.108224$

H $6.716376-0.461973-0.096809$ 
H $4.6725420 .616145-2.107990$

O $\quad 6.568127 \quad 0.127633-2.611749$

H $5.798953-2.214871-1.913340$

O $4.416525-1.681102-3.308996$

C $4.572847-4.365809-0.913705$

H $2.706265-3.617991-0.257140$

H $2.930982-3.583660-2.001944$

H $6.8204151 .731339-0.754832$

H $7.261877-0.503955-2.356075$

H $3.656920-1.080068-3.395684$

O $4.932193-4.494814 \quad 0.395041$

O $5.086848-4.942693-1.863527$

C $6.020766-5.3877190 .649271$

H $6.209467-5.3955691 .726033$

H $\quad 6.923177-5.039797 \quad 0.137531$

H $5.761596-6.4017720 .330552$

SCF Energy (B3LYP/6-31G**//MMFF) $=-3245.90250704$

01349

MM̄FF Geometry

C -1.2465452 .4444650 .705437$

C $-1.3749391 .777341-0.452478$

C $-0.4812040 .653578-0.932043$

O $\quad 0.797448 \quad 0.661367-0.258550$

C $-1.168282-0.692991-0.638837$

C $-0.446060-1.892294-1.218667$

C $0.117205-2.875670-0.489469$

C $-0.407864-1.964824-2.723836$

C $0.163433-3.0175831 .006102$

C $1.427814-2.4501081 .669535$

C $2.734566-3.1237351 .219500$

C $3.902796-2.6816512 .104111$

C $5.241665-3.2640811 .625535$

O $2.995157-2.744916-0.134458$

O $\quad 6.278302-2.5470712 .313560$

C $5.398723-2.9923510 .112545$

C $5.391553-4.7333172 .036963$

O $6.557200-3.645251-0.400143$

C $4.150475-3.365463-0.713569$

C $4.231485-2.901865-2.188296$

O $4.014246-4.791966-0.731035$

C $1.7343631 .538377-0.712323$

O $1.6224512 .320769-1.642617$

C 2.9280191 .4037610 .151038

C 3.8443362 .3830290 .165670

C 5.0659462 .4091130 .972979

C 5.4539221 .1418891 .687646

C $4.389118-1.391600-2.395931$

C $5.828733-0.939869-2.578245$

C $7.2013361 .130628-2.777159$

O $6.764904-1.717698-2.740436$

C $7.8123291 .444819-1.448255$

C $8.2395432 .669980-1.107139$

C $8.893566 \quad 2.994687 \quad 0.205082$

C $8.221297 \quad 4.1442250 .921577$

C 8.7094215 .3931940 .834345

C 7.0364443 .8295311 .806412

C 5.7831053 .5513121 .022833

N $5.938566 \quad 0.444480-2.578117$

H $-0.4355482 .166188 \quad 1.375531$

H $-2.1906082 .021410-1.127296$

H $-0.3216050 .773174-2.010354$

H -2.190724 -0.688189-1.040448

H -1.270960 $-0.796851 \quad 0.446279$

H $0.601668-3.691734-1.024989$

H $-0.006479-2.918286-3.083977$

H -1.417309 -1.860455 -3.135118

H $0.222116-1.169489-3.133520$

H - $0.713255-2.5545871 .469056$

H $0.080291-4.0853271 .246386$

H $1.478651-1.3735431 .469886$

H $1.315663-2.5719632 .754165$

H $2.601507-4.2104861 .277485$

H $3.721887-2.9495663 .152606$

H $3.974572-1.5857482 .086032$

H $7.131903-2.8533521 .961570$

H $5.586605-1.9175660 .010975$

H $5.332768-4.8308993 .127830$
H $\quad 6.375023-5.1264041 .756590$

H $4.625129-5.3797241 .604277$

H $6.341913-4.586794-0.512021$

H $5.006548-3.467429-2.719282$

H $3.284383-3.187813-2.668703$

H $3.457735-5.038651-1.488427$

H 3.0087430 .5147440 .765304

H $3.6762473 .262438-0.454958$

H 5.4666720 .2981350 .989364

H 4.7453020 .9220092 .493099

H $6.451192 \quad 1.1774642 .130609$

H $3.864879-1.112753-3.319166$

H $3.929085-0.830789-1.576970$

H $6.9918492 .032696-3.360716$

H $7.8784800 .491447-3.352738$

H $7.9323940 .623462-0.745679$

H $8.1436023 .483147-1.823435$

H 9.9427293 .2460670 .000953

H 8.9235802 .1197890 .866355

H $8.2502726 .218978 \quad 1.367720$

H 9.5767845 .6185320 .222507

H 6.8203634 .6832352 .462904

H 7.3052463 .0173222 .487283

H 5.4209974 .4147900 .463060

H $5.1166181 .009642-2.385642$

C -3.2226624 .0843180 .316472$

O $-4.2112623 .065900 \quad 0.077556$

C $-5.511523 \quad 3.6792780 .013545$

C $-5.2894665 .184567 \quad 0.130927$

C -3.9996425 .2519110 .923553$

H -6.0632853 .3324220 .894842$

C -6.239717 $3.247611-1.265373$

H $-5.1585215 .657034-0.849300$

H -6.1256435 .6859060 .628536$

C $-3.296007 \quad 6.5939230 .817426$

H $-4.2263075 .044473 \quad 1.978103$

C $-6.3452591 .721614-1.467197$

O $-5.5476623 .778684-2.404528$

H -7.237888 $3.699995-1.286336$

H -5.354542 $1.318152-1.704847$

O $-7.1489171 .499108-2.633930$

C $-6.9503620 .945829-0.283627$

C $-7.289530-0.523548-0.594884$

H $-6.268413 \quad 0.9923260 .570294$

$\begin{array}{lllll}\text { O } & -8.162627 & 1.594479 & 0.113147\end{array}$

H $-7.747414-0.9553430 .303495$

H -8.084161 -0.553762 -1.351148

H $-2.373421 \quad 6.600817 \quad 1.405881$

H $-3.0356936 .829322-0.220016$

H $-3.939927 \quad 7.3960331 .192650$

C -2.1243283 .5479691 .238099$

H $-4.6373503 .436776-2.380527$

H $-8.0476011 .813575-2.435361$

H -8.4948391 .1369230 .904247$

H -2.583267 3.1718102 .161228

H $-1.4618084 .374393 \quad 1.521264$

H -2.801771 $4.369503-0.656619$

C $-5.271890-2.046126 \quad 1.124068$

O $-5.013741-1.367980-0.111057$

C $-6.092935-1.383176-1.050517$

C $-6.465358-2.831448-1.397861$

C $-6.796242-3.637780-0.145742$

C $-5.669409-3.5095090 .876766$

H $-6.064128-1.5225631 .672695$

C $-3.985485-1.9928731 .964445$

H $-5.704524-0.933028-1.971472$

O $-7.565011-2.845516-2.301538$

H $-5.617559-3.303164-1.911986$

H $-7.757080-3.3381840 .288639$

O $-6.922539-5.019681-0.489059$

H $-4.798409-4.0791500 .526804$

O $-6.090892-4.0936432 .108737$

C $-3.671039-0.6109122 .495756$

H -4.082884 -2.657026 2.830961

H -3.130103 -2.313633 1.360377

H -7.690343 -3.761861 -2.600192

H -7.709884 -5.111319-1.051619

H $-6.346186-5.0132411 .921348$ 
O $-2.699359-0.722533 \quad 3.450206$

O $-4.187624 \quad 0.4298792 .116078$

C $-2.288022 \quad 0.495812 \quad 4.078286$

H -1.7657410 .2395525 .003953$

H $-1.596157 \quad 1.0283753 .421753$

H -3.147145 1.1258864 .328099

SCF Energy (B3LYP/6-31G*//MMFF)= -3245.91037795

0135

MMFF Geometry

C $-0.759677 \quad 3.895048-0.291217$

C -0.5981913 .5214510 .987156$

C -1.472690 2.5074921 .671665

O $-0.594497 \quad 1.4776252 .188554$

C -2.2379223 .0866832 .876184$

C -3.6176103 .5914072 .511905$

C -4.7540072 .9004092 .729044$

C -3.677697 4.9601611 .887859

C $-4.900579 \quad 1.542548 \quad 3.358445$

C $-4.478253 \quad 0.3567242 .484540$

C $-5.031458 \quad 0.3343191 .051473$

C $-6.560812 \quad 0.3071501 .012168$

C $-7.0922580 .177923-0.422944$

O $-4.506883-0.848010 \quad 0.426153$

O $-8.487264-0.154247-0.349246$

C $-6.382514-1.003623-1.113186$

C $-7.0004891 .504565-1.186634$

O $-6.736956-1.073690-2.493070$

C $-4.842317-0.981264-0.962095$

C $-4.257740-2.318268-1.480305$

O $-4.311910 \quad 0.094973-1.737701$

C -0.5404580 .2988091 .514901$

$\begin{array}{llllll}\text { O } & -1.124582 & 0.009571 & 0.483147\end{array}$

C $0.340265-0.6070392 .283224$

C $0.289683-1.9240752 .039791$

C $1.061163-2.9618202 .723667$

C $2.133121-2.5226813 .686321$

C $-2.758779-2.511744-1.216183$

C $-2.259854-3.849914-1.740329$

C $-0.157245-5.181561-1.956553$

O $-2.994880-4.686168-2.257692$

C $0.130622-5.998543-0.737581$

C $1.367984-6.214988-0.266151$

C $1.670293-7.025600 \quad 0.961538$

C $2.312025-6.2023902 .057802$

C $3.646725-6.1714442 .209944$

C $1.404089-5.4882073 .034613$

C $0.778930-4.2525652 .449926$

N $-0.892012-3.994437-1.559441$

H $-1.5881293 .462380-0.849354$

H 0.2110883 .9353541 .581419

H -2.187052 2.0674010 .964661

H -1.666475 3.8926973 .355821

H -2.3170372 .3172243 .653913$

H -5.6959013 .3557562 .425613$

H $-4.705811 \quad 5.3244261 .786894$

H -3.135574 5.6853602 .503128

H -3.236413 4.9490070 .888072

H $-4.3475291 .509947 \quad 4.304925$

H $-5.952598 \quad 1.416590 \quad 3.644621$

H -3.383842 0.3143532 .433910

H $-4.762830-0.5733742 .995280$

H -4.6447691 .2082280 .515661$

H -6.9904821 .1961731 .488504$

H -6.923135 -0.5503671 .596468$

H -8.9376140 .5486750 .149593$

H -6.760624 -1.926124-0.650060

H -7.586752 2.280245 -0.679104

H -7.430417 1.412951-2.190072

H -5.977387 $1.874925-1.281482$

H $-7.707757-1.107685-2.541459$

H $-4.787746-3.150371-0.997645$

H $-4.434622-2.402404-2.560388$

H $-3.375387-0.077829-1.921992$

H $0.976681-0.1715323 .044899$

H $-0.400073-2.2836961 .277383$

H $1.683498-2.0872634 .585171$

H $2.788936-1.7739123 .228510$
H $2.786686-3.3378894 .005795$

H $-2.174856-1.723372-1.702300$

H $-2.559215-2.478529-0.139525$

H $0.757633-4.844927-2.452833$

H $-0.741844-5.768649-2.671287$

H $-0.722117-6.428438-0.217727$

H $2.218252-5.791552-0.794655$

H $2.339708-7.8443820 .666070$

H $\quad 0.768450-7.5144221 .350307$

H 4.111139-5.606435 3.011115

H $4.304442-6.7053231 .532161$

H $1.939521-5.2643823 .963535$

H $0.600520-6.169783 \quad 3.342307$

H $-0.027365-4.4624851 .747285$

H $-0.379626-3.266477-1.071002$

C $1.3899235 .329261-0.427013$

O $2.288095 \quad 4.209327-0.293520$

C $3.6183194 .633052-0.641937$

C $3.5632296 .152060-0.764008$

C $2.1468466 .363366-1.260247$

H $3.8337844 .211953-1.630828$

C $4.6325834 .116177 \quad 0.384207$

H 3.7042506 .6441030 .205352

H $4.3220306 .540183-1.450196$

C $1.6483277 .787265-1.083180$

H $2.1035016 .095341-2.324459$

C $4.6404082 .582308 \quad 0.556182$

O $4.3189604 .699071 \quad 1.655853$

H 5.6347364 .4739320 .117542

H 3.7169682 .2548901 .048045

O 5.7159112 .2504381 .443919

C $4.8418111 .793300-0.751151$

C $5.1490450 .296923-0.564113$

H $3.9562321 .900253-1.383648$

O $5.9338632 .357636-1.485511$

H $5.318434-0.128238-1.561086$

H $6.1142040 .189638-0.052347$

H $0.6209307 .889675-1.445754$

H $1.6662048 .091283-0.031174$

H $2.2756068 .486737-1.645183$

C $0.0846564 .864084-1.077484$

H $5.070734 \quad 4.5322292 .248639$

H 5.4694432 .5606422 .331506

H $6.7257222 .302644-0.923128$

H $\quad 0.3247704 .371997-2.028758$

H $-0.5416275 .732227-1.312673$

H 1.2055795 .7240370 .579863

C $2.637793-1.032187-1.692497$

O $2.774667-0.391287-0.418358$

C $4.067082-0.5053950 .185062$

C $4.427274-1.9859790 .376138$

C $4.346221-2.754692-0.937055$

C $2.989893-2.524910-1.598241$

H $3.285205-0.534373-2.424623$

C $1.178862-0.864271-2.147720$

H $3.976999-0.0696391 .186998$

O $5.733121-2.1300620 .924186$

H $3.729470-2.4459671 .085305$

H $5.165798-2.489067-1.614353$

O $4.484762-4.152304-0.672834$

H $2.216779-3.055308-1.027733$

O $3.012241-3.084637-2.910151$

C $0.8449490 .552726-2.562342$

H $0.986838-1.511109-3.011654$

H $0.492814-1.136206-1.339574$

H $5.753883-1.6370801 .762216$

H $5.328423-4.275958-0.204761$

H $3.258489-4.021448-2.821315$

$\begin{array}{llll} & -0.268368 & 0.529326 & -3.354021\end{array}$

$\begin{array}{lllll}0 & 1.459093 & 1.552685 & -2.218972\end{array}$

C $-0.7077751 .795856-3.854606$

H $\quad 0.1286182 .365849-4.270858$

H $-1.4332361 .612101-4.651628$

H $-1.1988242 .356187-3.055572$

SCF Energy (B3LYP/6-31G**//MMFF) = -3245.90843801

01350

MM̄FF Geometry 
C $-0.893408-2.8849740 .554471$ C $-0.792449-1.750144 \quad 1.263459$ C $-0.153221-1.6760482 .624167$ O $0.771092-0.5668632 .583609$ C -1.165271 -1.5206223 .779018$ C $-2.150942-0.3698823 .686425$ C $-3.488981-0.5145513 .606650$ C -1.5624681 .0171013 .742445$ C $-4.288109-1.7878603 .568024$ C $-4.588680-2.2953482 .150107$ C $-5.579684-1.4268181 .357976$ C $-5.854299-2.048220-0.014367$ C $-6.765218-1.163957-0.881492$ O $-5.023855-0.1210511 .187902$ O $-6.692496-1.646543-2.232229$ C $-6.212800 \quad 0.280297-0.884745$ C $-8.236146-1.284020-0.463762$ O $-7.1207921 .144741-1.562805$ $\begin{array}{llll}\text { C } & -5.894428 & 0.808061 & 0.531247\end{array}$ C -5.1985592 .1857870 .536492$ $\begin{array}{lllll}\text { O } & -7.122414 & 0.969747 & 1.253727\end{array}$ C $1.813942-0.5546133 .448988$ O $2.057448-1.3874974 .310951$ C 2.6808110 .6283023 .232061 C 2.5119441 .4962642 .221631 C 3.3806182 .6315771 .902086 C 4.4417693 .0049862 .903365 C $-3.8279172 .256430-0.144900$ C $-3.2606083 .666597-0.068956$ C $-1.4774435 .105349-0.968839$ O $-3.8353364 .586451 \quad 0.510244$ C $-0.0254945 .011490-1.311129$ C $0.9040735 .791952-0.737697$ C $2.3474625 .831773-1.151274$ C 3.3054385 .6665450 .006796 C 3.5681106 .6841200 .844419 C 4.0436864 .3563580 .124997 C 3.2023903 .2645720 .722967 N $-2.0408153 .789919-0.726267$ H $-0.537485-3.8074541 .009644$ H -1.154399 -0.8151880 .845253$ H $0.427462-2.5922752 .797483$ H - $0.617001-1.3964524 .721848$ H -1.694710 -2.4741403 .874331$ H $-4.0955650 .389664 \quad 3.571377$ H $-2.327526 \quad 1.7862893 .895333$ H -0.853036 1.0990054 .572387 H -1.0447951 .2552582 .808799$ H -3.778015 -2.578872 4.127407 H -5.230396 -1.620971 4.104970 H $-3.648102-2.3752641 .595173$ H -4.998478 -3.3098262 .232259$ H $-6.506770-1.3546831 .938651$ H -6.281714 -3.0532300 .091081$ H -4.901265 -2.183768 -0.543569 H $-6.958833-2.581862-2.231763$ H $-5.292277 \quad 0.261587-1.480965$ H -8.581515 -2.319207 -0.573661 H -8.882201 -0.683473 -1.113435 H -8.414994 -0.9884660 .571945$ H $-6.665454 \quad 1.988117-1.722759$ H -5.8717162 .9402280 .108633$ H -5.0601782 .4927261 .583698$ H $-6.9665191 .573171 \quad 1.998970$ H 3.5047190 .7202573 .932487 H 1.6909881 .3414071 .523549 H 4.0083853 .1091023 .904642 H 4.9224573 .9620152 .687516 H 5.2250562 .2402772 .937195 H $-3.122073 \quad 1.5727850 .339613$ H $-3.9101091 .985835-1.202337$ H -1.655938 $5.740575-0.094215$ H -2.026174 5.536987 -1.813187 H $0.2633624 .331564-2.107974$ H $0.594886 \quad 6.5010040 .027554$ H $2.5344586 .802485-1.629828$ H $2.5562845 .081406-1.923771$ H 4.2827136 .5798921 .654176
H $3.070628 \quad 7.642608 \quad 0.739359$

H 4.9796984 .4947190 .675548

H $4.3662634 .033881-0.873890$

H 2.4013192 .9228170 .066706

H -1.746223 $3.027008-1.329294$

C $-1.507911-1.773867-1.702371$

O $-0.190211-1.200764-1.825499$

C $0.041704-0.842782-3.199411$

C $-1.319926-0.893933-3.878194$

C $-1.971679-2.044453-3.135907$

H $0.677289-1.627214-3.627207$

C $0.7574860 .508633-3.280153$

H $-1.8895180 .029373-3.720949$

H -1.241366 -1.065089-4.955863

C $-3.477889-2.098429-3.306494$

H $-1.537871-2.985434-3.500692$

C $2.0593340 .586122-2.455884$

O $\quad-0.118117 \quad 1.532941-2.789487$

H $0.9584020 .760915-4.327523$

H $1.8140850 .578571-1.387908$

O $2.6715971 .854295-2.721681$

C $3.084918-0.520157-2.766593$

C $4.445556-0.349501-2.065120$

H $2.669569-1.493272-2.492796$

O $3.325485-0.563078-4.175761$

H $5.102736-1.157461-2.408725$

H $4.9225590 .571816-2.422179$

H $-3.904998-2.932859-2.742068$

H $-3.950323-1.174259-2.960098$

H $-3.741593-2.234317-4.360439$

C $-1.432605-3.040899-0.844747$

H $-0.3618531 .295947-1.878022$

H $2.0162822 .541783-2.512223$

H $3.6576030 .311122-4.443374$

H $-0.770289-3.754027-1.351772$

H $-2.419999-3.512434-0.782104$

H -2.149931 -1.020212-1.229209

C $4.480190-2.717917-0.104838$

O $3.718423-1.508493-0.005244$

C $4.360444-0.341206-0.527475$

C $5.699674-0.1057280 .182357$

C $6.597428-1.3352160 .114039$

C $5.838295-2.5688500 .598299$

H $4.632700-2.968451-1.161693$

C $3.656358-3.8395360 .550865$

H $3.7190690 .501391-0.259136$

O $6.3857941 .009945-0.374941$

H $5.515451 \quad 0.126401 \quad 1.239194$

H $6.996925-1.486256-0.895537$

O $7.726540-1.1394170 .968412$

H $5.692189-2.4939901 .683350$

O $\quad 6.637402-3.728284 \quad 0.366443$

C $2.565391-4.366602-0.357316$

H $4.309583-4.6866640 .789835$

H $3.177960-3.4846281 .470860$

H $5.798471 \quad 1.781224-0.299162$

H $8.174324-0.3276570 .673997$

H $\quad 6.774723-3.809492-0.592781$

O $2.187998-5.6051980 .078525$

O $2.091377-3.767671-1.311959$

C $1.186622-6.270421-0.698595$

H $1.385549-6.173286-1.770371$

H $\quad 1.206415-7.332030-0.438021$

H $0.201794-5.867155-0.450635$

SCF Energy $\left(B 3 L Y P / 6-31 G^{* *} / / M M F F\right)=-3245.90341890$

01351

MM̄FF Geometry

C -3.8661104 .6439890 .539159$

C $-2.7159444 .431080-0.117660$

C -1.3590714 .6407070 .503386$

O -0.6095873 .4054620 .428067$

C $-0.5925195 .714000-0.290074$

C $0.658212 \quad 6.196547 \quad 0.416293$

C 1.9168345 .8349920 .099489

C 0.4212947 .1909511 .525619

C $2.3758164 .870275-0.959698$

C $3.1407913 .707964-0.326150$ 
C $3.8298422 .777114-1.333751$

C $2.8564322 .094888-2.297207$

C $3.5634411 .061052-3.189651$

O $4.5066251 .789711-0.547678$

O $2.5560290 .256936-3.819865$

C $4.4009270 .117855-2.301960$

C $4.3521761 .730463-4.321644$

O $5.184513-0.768857-3.097525$

C $5.3044520 .863162-1.295370$

C $5.908311-0.134083-0.279392$

O $6.359072 \quad 1.518299-2.000484$

C -0.8663852 .4604501 .374812$

O -1.6959092 .5204462 .268343$

C $\quad 0.056792 \quad 1.3272601 .139219$

C $-0.0238490 .225922 \quad 1.900734$

C $0.826637-0.963711 \quad 1.792218$

C $2.019242-0.8847340 .876966$

C 6.7689840 .5393480 .798834

C $6.744067-0.2294052 .102902$

C $5.298361-0.6822614 .083029$

O $7.699564-0.9072932 .472971$

C $4.690283-2.0330613 .877845$

C $3.555311-2.4357344 .469328$

C $2.999934-3.8225144 .322025$

C $1.539033-3.8413483 .940562$

C $0.610586-4.3198964 .786961$

C $1.166071-3.4029912 .541633$

C $0.496930-2.0562352 .512333$

N $5.552829-0.0667712 .795276$

H -3.835052 4.9789021 .572916

H $-2.7435964 .096360-1.151893$

H -1.450281 4.9447501 .553665

H -1.237574 $6.586680-0.461757$

H $-0.3496605 .332645-1.288146$

H 2.7306016 .2817000 .670746

H 1.3556507 .5507091 .969484

H -0.1175148 .0641891 .143588$

H $-0.170225 \quad 6.7408132 .328449$

H $1.5403804 .483165-1.546586$

H $3.0306335 .410487-1.653584$

H 3.9111834 .0968380 .354830

H 2.4692833 .1235630 .314912

H $4.5737213 .362623-1.886847$

H $2.3337022 .829089-2.921016$

H $2.0757441 .578406-1.722911$

H $1.9737050 .847648-4.327713$

H $3.699226-0.512171-1.738590$

H $3.6820372 .321809-4.957234$

H $4.8076830 .983655-4.981455$

H $5.1373452 .398188-3.961229$

H $4.577978-1.224149-3.706439$

H $5.092159-0.6908950 .196873$

H $6.534916-0.869904-0.799009$

H $7.0985091 .674969-1.392388$

H $\quad 0.779851 \quad 1.4273640 .339320$

H $-0.792771 \quad 0.1817902 .671050$

H $2.641555-0.0191471 .130468$

H $1.697334-0.790058-0.165357$

H $2.673261-1.7568960 .933386$

H 7.8094150 .6148750 .461679

H 6.4366071 .5619861 .011885

H $4.639413-0.0107084 .642255$

H $6.242794-0.7821414 .627893$

H $5.234083-2.7299763 .244060$

H $3.026737-1.7543045 .131837$

H $3.147978-4.3394105 .279139$

H $3.565652-4.3999983 .579892$

H $-0.435708-4.3719954 .503741$

H $0.874021-4.6686615 .779813$

H $0.461093-4.1324602 .126260$

H $2.042074-3.4610931 .890730$

H -0.401073 -2.005669 3.130370

H $4.800426 \quad 0.4671752 .370019$

C $-5.950188 \quad 3.1949520 .336522$

O $-5.2637282 .047051-0.195155$

C -5.4259570 .9496540 .726912$

C -6.3873611 .4333811 .811012$

C -6.1044372 .9245561 .830285$
H $-4.4445410 .774593 \quad 1.182555$

C -5.887909-0.301305 -0.029865

H -7.4334881 .2472801 .541904$

H -6.2005570 .9477352 .773801$

C -7.1907913 .7464482 .499419$

H -5.1558953 .0824312 .358048$

C $-4.973387-0.699198-1.206439$

O $-7.197145-0.066889-0.566678$

H $-5.994095-1.1406820 .666675$

H $-5.0061370 .069078-1.988378$

O $-5.506916-1.894602-1.791855$

C $-3.511715-0.972498-0.808610$

C $-2.646286-1.374060-2.020669$

H $-3.073742-0.071596-0.366613$

O $-3.480817-1.9870410 .190294$

H $-3.092071-2.241204-2.516608$

H -2.676066 -0.547464 -2.740202

H -6.9432344 .8122842 .474134$

H -8.1584373 .6128372 .004169$

H -7.306183 3.4508323 .547323

C $-5.2257794 .476609-0.075973$

H -7.140769 $0.710263-1.148916$

H -6.430517 -1.710520 -2.034405

H -3.879655 -2.786409-0.193763

H $-5.8326885 .348000 \quad 0.196854$

H $-5.1325954 .490357-1.169624$

H $-6.9394143 .203247-0.141730$

C $-1.340242-4.091324-1.480026$

O $-1.058630-2.851964-0.817216$

C -1.180422 -1.685441-1.639505

C $-0.223375-1.795300-2.834457$

C $-0.429115-3.091821-3.611538$

C $-0.392740-4.292572-2.666938$

H -2.378809 -4.097374 -1.829483

C $-1.186269-5.217179-0.451776$

H $-0.833558-0.841387-1.031763$

O $-0.379556-0.689874-3.717628$

H $0.812565-1.772775-2.471544$

H $-1.363167-3.071996-4.185530$

O $0.626337-3.241070-4.563866$

H $0.633650-4.447333-2.310148$

O $-0.779007-5.463277-3.383484$

C $-2.291750-5.1698590 .576908$

H -1.205629 -6.208703 -0.918382

H $-0.231432-5.1321430 .079103$

H $-0.2126020 .118914-3.203878$

H $0.619836-2.449290-5.128833$

H $-0.177778-5.549744-4.143216$

O $-3.449674-5.6491530 .038152$

O $-2.147352-4.7507231 .717700$

C $-4.582478-5.6460330 .912512$

H -4.806277 -4.627085 1.242562

H $-5.441833-6.0302590 .356665$

H $-4.398620-6.2988411 .771053$

SCF Energy (B3LYP/6-31G**//MMFF) $=-3245.92011091$

01352

MM̄FF Geometry

C $4.334252-2.6639481 .805643$

C $3.053400-3.0001121 .594235$

C $1.982919-2.8835072 .644230$

O $1.097647-1.8052292 .265590$

C $1.143800-4.1725512 .716551$

C $0.097320-4.1331763 .814240$

C $-1.234820-4.0938233 .613860$

C $0.636554-4.1601495 .223024$

C -1.997150-4.115405 2.317673

C $-2.504572-2.7236751 .934506$

C $-3.652773-2.7424740 .913516$

C $-3.265447-3.396543-0.412562$

C $-4.403220-3.323575-1.442601$

O $-4.026157-1.3768150 .684407$

O $-3.826512-3.664937-2.713648$

C $-4.920038-1.868830-1.528331$

C $-5.477189-4.380283-1.158590$

O $-6.096998-1.780452-2.326460$

C $-5.182752-1.215013-0.151352$

C $-5.4743170 .299903-0.245678$ 
O $-6.347670-1.764833 \quad 0.477605$

C $1.378223-0.5651012 .749373$

O $2.347101-0.231877 \quad 3.412407$

C $0.257895 \quad 0.3204412 .364509$

C 0.1282451 .5201812 .950546

C $-0.9684792 .467753 \quad 2.736490$

C -2.050678 2.0612491 .772527

C $-4.4205201 .118930-0.992928$

C $-4.5661322 .616436-0.768602$

C $-3.4436314 .763348-1.402857$

O $-5.3962823 .111428-0.012230$

C $-2.2764885 .018833-0.501405$

C $-2.289903 \quad 5.8947300 .514326$

C $-1.110928 \quad 6.125431 \quad 1.417878$

C -1.4700416 .0328442 .885755$

C -1.3265907 .0955623 .695767$

C -2.0035534 .7199243 .417918$

C -0.9656453 .6302563 .422645$

$\mathrm{N}-3.634004 \quad 3.328620-1.509419$

H $4.638774-2.2930242 .781076$

H $2.754333-3.3461620 .608584$

H $2.428466-2.6796753 .625510$

H $1.797862-5.0368512 .892029$

H $0.671472-4.344376 \quad 1.744392$

H $-1.877947-4.0562744 .493232$

H $1.169446-3.2327635 .452847$

H $-0.156675-4.2757395 .969568$

H $1.325918-5.0014605 .349505$

H $-1.401285-4.5368801 .503531$

H -2.846787 -4.7969282 .450756$

H $-2.851904-2.1846252 .826653$

H -1.674556 -2.124802 1.540862

H -4.499006 -3.2684841 .370766$

H $-2.959294-4.439579-0.270369$

H $-2.389819-2.880453-0.825812$

H $-4.539103-3.677604-3.374585$

H $-4.143968-1.295930-2.050109$

H -5.052873 -5.387736 -1.249053

H $-6.288418-4.330345-1.893120$

H -5.909202 -4.294054-0.159285

H $-5.874693-2.099024-3.217347$

H $-6.4556670 .466458-0.709633$

H $-5.568147 \quad 0.6871160 .778667$

H $-6.779408-2.372926-0.143127$

H -0.453412 -0.0567411 .639276$

H $0.878129 \begin{array}{llll}1.828597 & 3.677808\end{array}$

H -2.528050 1.1291962 .095285

H $-1.631703 \quad 1.9139750 .771082$

H -2.847494 $2.798921 \quad 1.674144$

H -3.414789 $0.829635-0.668655$

$\mathrm{H}-4.5022020 .932790-2.069811$

H $-4.3620085 .234051-1.037933$

H -3.225450 5.136091-2.408101

H -1.369192 $4.450596-0.693788$

H -3.185183 6.4831950 .700395

H -0.707541 7.1197091 .186761

H $-0.302976 \quad 5.4153201 .202554$

H - 1.5824747 .0433094 .748866

H -0.9489818 .0430013 .325638$

H -2.907296 4.4487462 .867023

$\mathrm{H}-2.339847 \quad 4.8438584 .456306$

H $-0.135578 \quad 3.8267884 .102825$

H -2.964703 $2.815552-2.074239$

C $5.293784-1.544716-0.223564$

O $5.489627-0.3007710 .483515$

C $6.3502190 .537914-0.299378$

C $6.428720-0.095655-1.682554$

C $6.348330-1.568816-1.329966$

H 7.3468480 .4592710 .157554

C $5.9330802 .011830-0.253315$

H $5.5745030 .186291-2.307631$

H $7.3463440 .169495-2.215938$

C $5.993872-2.457287-2.509425$

H $7.316254-1.886517-0.919268$

C $4.5979472 .392300-0.929720$

O $6.9800612 .752725-0.896623$

H 5.9144452 .3438200 .791762

H $4.6865342 .225725-2.007328$
O $4.4355893 .811868-0.777569$

C $3.3338971 .701057-0.385696$

C $2.0242922 .202912-1.026183$

H $3.3931540 .620648-0.529140$

$\begin{array}{llll}\text { O } & 3.227470 & 1.936922 & 1.019579\end{array}$

H $1.1867681 .769205-0.466342$

H $1.927613 \quad 3.284921-0.873474$

H $5.918609-3.504791-2.201558$

H $5.038074-2.167774-2.957757$

H $6.762320-2.387977-3.286271$

C $5.394305-2.7246600 .743426$

H $6.7369483 .693600-0.854235$

H 4.3016513 .9905100 .169261

H 3.9231621 .4180741 .457959

H $6.370366-2.7202131 .244118$

H $5.317764-3.6737320 .200032$

H $4.289578-1.505665-0.663150$

C $1.075449-0.399090-2.300983$

O $2.1140640 .467566-2.780251$

C $1.8994701 .860374-2.524842$

C $0.5882332 .317733-3.177660$

C $-0.5908821 .438492-2.774105$

C $-0.259522-0.040666-2.969025$

H $0.992400-0.291840-1.213410$

C $1.521061-1.836364-2.611142$

H $2.7063562 .393345-3.041320$

$\begin{array}{lllll}\text { O } & 0.300337 & 3.672262 & -2.848367\end{array}$

H $0.6946592 .269358-4.269419$

H $-0.900804 \quad 1.643822-1.742978$

O $-1.7047421 .774998-3.606045$

H $-0.223421-0.253248-4.045338$

O $-1.328611-0.800441-2.414951$

C $0.882339-2.878579-1.720878$

H $1.305337-2.073426-3.659750$

H $2.605162-1.924152-2.475299$

H $1.0596834 .210155-3.130895$

H $-2.412517 \quad 1.135734-3.415396$

H -1.238287 -1.714204 -2.733184

O $1.076240-4.113381-2.270658$

O $0.308402-2.647997-0.665932$

C $0.531253-5.209406-1.529195$

H $1.061352-5.318464-0.578784$

H $-0.540195-5.064732-1.360844$

H $0.672550-6.120499-2.116588$

SCF Energy (B3LYP/6-31G**//MMFF)= -3245.91858301

01353

MM̄FF Geometry

C $0.976208-4.083897-0.280435$

C $0.294485-3.770861-1.393070$

C $-0.933587-2.900894-1.388419$

O $-0.660818-1.710733-2.173253$

C $-2.143132-3.577751-2.052905$

C $-2.794153-4.672135-1.236723$

C $-3.758530-4.443764-0.323570$

C $-2.361851-6.077796-1.555560$

C $-4.270020-3.101543 \quad 0.116915$

C $-5.536212-2.684179-0.636984$

C $-6.058698-1.326203-0.146403$

C $-7.318492-0.917383-0.911757$

C $-7.8109910 .479123-0.499948$

O $-5.030689-0.348697-0.346461$

$\begin{array}{lllll}0 & -8.767723 & 0.916213 & -1.477407\end{array}$

C $-6.6258981 .466980-0.554810$

$\begin{array}{llll}\text { C } & -8.547824 & 0.449557 & 0.844492\end{array}$

\begin{tabular}{lllll}
\hline & -6.998548 & 2.740506 & -0.032839
\end{tabular}

C $-5.3575810 .950948 \quad 0.162164$

C $-4.164278 \quad 1.894879-0.117536$

$\begin{array}{llll}\text { O } & -5.594260 & 0.925054 & 1.573015\end{array}$

C $0.105997-0.745262-1.600216$

O $0.625187-0.776024-0.496412$

C $0.2132010 .373279-2.561120$

C $1.1571911 .304318-2.364340$

C $1.4053182 .484405-3.191296$

C $0.4097832 .793988-4.277389$

C $-2.802921 \quad 1.321604 \quad 0.308323$

C $-1.876682 \quad 2.414305 \quad 0.804345$

$\begin{array}{llll}\text { C } & -0.833202 & 4.552432 & 0.143653\end{array}$ 
O

C $-0.2122145 .153796-1.075349$

C $1.0409955 .633230-1.099339$

C $1.6624746 .297801-2.295130$

C $2.9869175 .680041-2.684069$

C $4.1481536 .219060-2.276000$

C $2.9632784 .484262-3.609925$

C $2.4912873 .237610-2.918836$

N -1.486799 $3.299982-0.191118$

H $\quad 0.616664-3.7247070 .680553$

H $\quad 0.653007-4.119930-2.358160$

H -1.200369 -2.587706 -0.371487

H -1.862440 -3.967348 -3.041564

H -2.890336 -2.807274 -2.287837

H $-4.209857-5.294597 \quad 0.183813$

H $-2.911370-6.823097-0.970549$

H $-2.535214-6.297874-2.614010$

H -1.297726 -6.213290 -1.341836

H -4.481999 -3.1540481 .192162$

H $-3.493529-2.3380380 .005159$

H $-5.307189-2.621643-1.708499$

H $-6.314688-3.446946-0.512198$

H -6.272224 -1.411111 0.925851

H $-8.114294-1.660692-0.778267$

H -7.100717 -0.902324 -1.988960

H $-9.4867150 .261882-1.506584$

H $-6.3816301 .626201-1.614632$

H $-9.418899-0.2144370 .788907$

H $-8.942426 \quad 1.4389561 .100849$

H $-7.9220420 .102493 \quad 1.669228$

H $-7.7900313 .031728-0.517091$

H -4.106286 2.101968 -1.194065

H -4.3393292 .8538100 .387194$

H -4.7566821 .0680162 .041738$

H $-0.4763440 .396586-3.396818$

H $1.8258561 .188602-1.514288$

H $-0.6047842 .846851-3.865933$

H $\quad 0.4342772 .019864-5.052007$

H $\quad 0.5863353 .750915-4.772293$

H -2.903835 0.5875621 .115378

H $-2.3274250 .801553-0.530598$

H -0.1047804 .3774640 .942010$

H -1.606134 5.2233630 .533666

H $-0.8294295 .233090-1.966965$

H $1.6449815 .581415-0.196109$

H $1.8056377 .358243-2.049019$

H $0.9858136 .284671-3.158731$

H $5.1029035 .796997-2.571507$

H $4.1692667 .092048-1.632051$

H $3.9727454 .281591-3.991952$

H $2.3714734 .733529-4.494639$

H $3.1467682 .924207-2.104820$

H $-1.9486723 .233819-1.093756$

C $3.388836-4.205123-1.038088$

O $3.690816-2.919542-0.461149$

C $5.114847-2.707090-0.517658$

C $5.695604-3.879498-1.303254$

C $4.702040-4.982416-0.994601$

H $5.479253-2.7616470 .514442$

C $5.424236-1.327810-1.114008$

H $5.717706-3.677794-2.380408$

H $6.716363-4.120311-0.990218$

C $4.768073-6.148105-1.965659$

H $4.889389-5.3482270 .023793$

C $4.742450-0.149104-0.389990$

O $4.983210-1.298450-2.479346$

H $6.508364-1.171916-1.147787$

H $3.661746-0.200740-0.560560$

O $5.1911281 .064249-1.006626$

C $5.023789-0.0544001 .120517$

C $4.4425501 .205551 \quad 1.792013$

H $4.628315-0.9385581 .631504$

O $6.433877-0.027316 \quad 1.356778$

H 4.7159151 .1693162 .854098

H 4.9521232 .0976421 .405703

H $4.031079-6.914671-1.706955$

H $4.572128-5.825671-2.993708$

H $5.759469-6.612205-1.942172$
C $2.253485-4.875853-0.260950$

H $4.029072-1.488544-2.483758$

H $4.9913790 .997546-1.956153$

H $6.794398-0.8967001 .115929$

H $2.551349-4.987616 \quad 0.789427$

H $2.058565-5.883333-0.645640$

H $3.090810-4.025337-2.079252$

C $2.197719-0.1973173 .322691$

O 2.2162010 .1580151 .937768

C 2.9186391 .3657971 .638438

C 2.3162932 .5392502 .421599

C 2.2338222 .2511683 .919113

C 1.5523890 .9050574 .173147

H $3.230651-0.3452513 .663123$

C $1.526097-1.5717463 .450754$

H 2.7336891 .5740800 .579355

O $3.066645 \quad 3.7305572 .211567$

H 1.3007952 .7305792 .054599

H 3.2234372 .2847564 .389653

O $1.456506 \quad 3.2732404 .548438$

H 0.4837181 .0102053 .953825

O $1.677814 \quad 0.5954045 .559047$

C $0.070429-1.6098613 .033023$

H $2.050545-2.2885782 .806755$

H $1.600898-1.9192004 .487103$

H 3.0826943 .9001281 .253998

H 1.8794674 .1248384 .343901

H $\quad 1.089407-0.1545745 .748518$

O $-0.490565-2.7633213 .504918$

O $-0.497036-0.7664612 .353569$

C $-1.860276-2.9698663 .148564$

H -2.477392 -2.133286 3.490315

H -1.945354 -3.097378 2.066468

H -2.206152 -3.8840673 .638149$

SCF Energy $\left(B 3 L Y P / 6-31 G^{* *} / / M M F F\right)=-3245.88971639$

01354

MM̄FF Geometry

C $4.336981-1.3530013 .631044$

C $3.278314-1.8621822 .982672$

C $1.854163-1.4660123 .270036$

O $1.374713-0.6870022 .147937$

C $0.968393-2.7185923 .394910$

C $-0.438794-2.3861743 .850851$

C $-1.482902-2.1632233 .027581$

C $-0.620612-2.3007445 .344401$

C -1.472393 -2.173778 1.523454

C $-2.714134-2.8089340 .876490$

C $-4.041134-2.1055561 .204545$

C $-5.156407-2.5598260 .259801$

C $-6.472049-1.8033490 .516639$

O $-3.852182-0.6948041 .078723$

O $-7.334432-2.027552-0.609600$

C $-6.182705-0.2853210 .570629$

C $-7.210252-2.3561141 .741720$

O $-7.346550 \quad 0.4450860 .950881$

C -4.9858530 .0840701 .475744$

C -4.6176251 .5763591 .314339$

$\begin{array}{llll}\text { O } & -5.346860 & -0.152084 & 2.839649\end{array}$

C 1.6367530 .6485882 .159147

O 2.2302161 .2756553 .022106

C $1.093997 \quad 1.2242600 .908853$

C 1.4947462 .4453580 .523176

C $1.0974633 .137449-0.704925$

C $-0.0306542 .542953-1.502385$

C -3.2529181 .9342901 .919819$

C -3.0620503 .4341661 .994182$

C -2.7139115 .4465210 .596022$

$\begin{array}{llll}\text { O } & -3.143381 & 4.041327 & 3.059747\end{array}$

C $-2.0006595 .759825-0.678557$

C $-0.8925716 .513273-0.740289$

C $-0.2093486 .882383-2.024215$

C $1.2553226 .512589-2.038698$

C $2.2075497 .450853-1.899923$

C $1.6173025 .071603-2.316481$

C $1.7673454 .252840-1.063507$

N -2.800602 4.0091810 .760826

H $4.182123-0.6205464 .419141$ 
H $3.441978-2.5737662 .176340$ H $1.786135-0.8736274 .191544$ H $1.410550-3.4223964 .112837$ H $\quad 0.942326-3.2570772 .440557$ H -2.432494 -1.895655 3.486541 H -1.649640 -2.053752 5.626072 H $-0.372285-3.2595495 .811126$ H $\quad 0.030832-1.5292375 .766690$ H -1.372194 -1.139840 1.173015 H $-0.601809-2.7122831 .142385$ H -2.554862 -2.779874 -0.208902 H $-2.783819-3.8684141 .152670$ H -4.307959 -2.344713 2.240000 H $-5.312172 \quad-3.6431890 .333773$ H -4.851542 -2.371259-0.779410 H -7.466221 -2.986920 -0.700119 H $-5.9507610 .031342-0.455456$ H $-7.428057-3.4227441 .608185$ H -8.182886 -1.868809 1.870704 H -6.642368 -2.2499102 .668259$ H $-7.4618830 .339374 \quad 1.910613$ H -4.5878621 .8316720 .248393$ H -5.3938282 .2024751 .772590$ H $-4.838712 \quad 0.4416843 .414214$ H $\quad 0.4132350 .615808 \quad 0.324554$ H 2.2128022 .9780461 .145615 H $-0.8950902 .336838-0.862114$ H $0.2905591 .605460-1.962738$ H $-0.3943863 .195515-2.298831$ H -3.1439831 .5379382 .934955$ H -2.4383191 .5045621 .324408$ H -2.219533 5.8811891 .471553 H -3.7367715 .8355410 .557991$ H $-2.4343975 .373339-1.597880$ H -0.4737016 .9203990 .177255$ H $-0.3251177 .965629-2.160450$ H $-0.7058316 .423035-2.888131$ H $3.2611577 .195454-1.943581$ H $1.9553168 .492879-1.733885$ H $2.5818925 .034934-2.841651$ H $0.9036504 .643560-3.025863$ H $2.5731594 .600126-0.414836$ H $-2.9223473 .439587-0.071760$ C $6.371332-0.9000392 .163690$ O $5.697531-1.2219100 .931148$ C $5.452345-0.0017520 .206391$ C $6.3733451 .038336 \quad 0.831713$ C 6.3260380 .6234502 .292814 H $4.4155290 .287540 \quad 0.415807$ C $5.624605-0.235827-1.298910$ H 7.3972450 .9596270 .448480 H 6.0221772 .0607650 .663262 C 7.4508811 .2018043 .132375 H 5.3669370 .9538282 .710408

C $4.817166-1.441686-1.829560$ O $7.010304-0.487363-1.559227$ H $5.363620 \quad 0.677791-1.845977$ H $5.234603-2.373194-1.427482$ O $4.976648-1.506645-3.251269$ C $3.311112-1.378005-1.517311$

C $2.557001-2.620441-2.034007$ H $3.167210-1.316720-0.433741$ O $2.776143-0.190183-2.094570$ H $2.582959-2.634329-3.127911$ H $3.087315-3.511691-1.680146$ H $7.380330 \quad 0.854198 \quad 4.167914$ H $8.432814 \quad 0.9105972 .744473$ H 7.4031912 .2956503 .138029 C $5.760880-1.7120053 .310675$ H $7.142403-0.431691-2.520598$ H $5.901665-1.743954-3.431868$ H $1.841282-0.133007-1.835791$ H $6.362357-1.5826054 .218063$ H $5.806021-2.7787753 .055473$ H $7.409513-1.2314092 .030421$ C $-0.049136-1.747760-3.457640$ O $0.306327-1.596907-2.079413$ C $1.094013-2.660574-1.534110$
C $0.383022-4.008442-1.724967$

C $-0.014555-4.248061-3.177179$

C $-0.807171-3.055561-3.709405$

H $0.866771-1.750822-4.061571$

C $-0.827852-0.502767-3.909437$

H $1.137954-2.481112-0.452781$

O $1.196274-5.086831-1.275467$

H $-0.529150-4.024667-1.115617$

H $0.860124-4.453115-3.805831$

O $-0.847910-5.406737-3.252031$

H $-1.794140-3.034608-3.232618$

O $-1.014516-3.217537-5.110820$

C $-2.050468-0.175385-3.075726$

H $-0.1615230 .364709-3.840830$

H $-1.138795-0.613281-4.954262$

H $1.412182-4.919885-0.341945$

H $-0.346626-6.147733-2.870215$

H $-1.463612-4.070516-5.240757$

O $-2.6823380 .901720-3.631849$

O $-2.399126-0.758747-2.059420$

C $-3.8358601 .368736-2.925824$

H $-4.2882982 .175351-3.508524$

H $-4.5691450 .564412-2.811510$

H -3.538783 $1.764088-1.950650$

SCF Energy (B3LYP/6-31G**//MMFF) $=-3245.90615367$

01355

MM̄FF Geometry

C $3.036340 \quad 1.0728803 .095325$

C 3.4176212 .3157112 .761814

C 4.2432322 .7115681 .560828

O $4.882053 \quad 1.566443 \quad 0.947759$

C 3.3623873 .3456060 .467554

C 2.8070784 .7104110 .811220

C 1.5352394 .9498711 .186867

C 3.7784335 .8524180 .661441

C 0.4349003 .9506851 .416206

C -0.7030584 .0726360 .395069$

C $-0.3174063 .575924-1.006962$

C $-1.4668003 .808157-1.989589$

C $-1.1586863 .230465-3.378729$

O $-0.0296372 .176014-0.914032$

O $-2.3759473 .233387-4.140169$

C $-0.6867631 .768831-3.221083$

C $-0.1938974 .131080-4.157398$

O $-0.2603951 .235000-4.472994$

C $0.4061041 .577345-2.140904$

C $0.6190100 .066265-1.886902$

O $1.6241922 .155820-2.611721$

C 6.0953651 .1911581 .426042

O 6.6924051 .6906242 .370503

C 6.6589460 .0524720 .664451

C $6.111911-0.455332-0.451673$

C $6.640548-1.575267-1.237669$

C $7.898480-2.239610-0.739159$

C $1.570188-0.268512-0.728380$

C $1.682493-1.770045-0.519111$

C $2.928977-3.4646770 .813216$

O $1.009547-2.585881-1.144815$

C $4.123470-3.9038830 .028037$

C $4.038998-4.578226-1.128690$

C $5.222098-5.008982-1.947670$

C $5.333271-4.230759-3.241347$

C $4.627657-4.591092-4.326735$

C $6.316415-3.081201-3.295470$

C $5.969924-1.967572-2.342423$

N $2.612433-2.0926390 .460071$

H 3.3442430 .2257202 .491030

H 3.0733603 .1423193 .380713

H 5.0148983 .4253701 .873508

H 2.5633612 .6473990 .193179

H $3.9505603 .434786-0.456871$

H 1.2453645 .9813561 .383854

H 3.3180026 .8220340 .878954

H 4.6242295 .7299171 .344900

H $4.1616155 .893042-0.363521$

H 0.8072352 .9238791 .440549

H 0.0252034 .1407882 .416354 
H -1.543372 3.4716130 .766590 H -1.046162 5.1133650 .344372 H $0.578974 \quad 4.108939-1.342072$ H -1.709418 4.875682-2.062907 H -2.373070 $3.323359-1.604399$ H -3.030964 2.689315 -3.670802 H $-1.564503 \quad 1.177907-2.929351$ H - $0.6189535 .135642-4.272078$ H $-0.0375223 .757524-5.175407$ H $0.7798664 .236968-3.674658$ H $0.6241591 .592399-4.661122$ H -0.352608 $-0.394644-1.662799$ H $1.003113-0.410265-2.798006$ H $2.3713391 .749073-2.146684$ H $7.582453-0.3431091 .075763$ H $5.192792-0.017166-0.835614$ H $7.760751-2.6048500 .284973$ H $8.735934-1.533322-0.752024$ H $8.204144-3.104782-1.330091$ H $2.5738220 .119830-0.927982$ H 1.2054250 .1748680 .204851 H $2.063562-4.1072590 .620423$ H $3.147391-3.4959781 .884837$ H $5.099074-3.6510830 .433957$ H $3.056313-4.827540-1.523553$ H $5.115188-6.079554-2.165864$ H $6.155038-4.923042-1.377206$ H $4.718527-4.055346-5.265623$ H $3.940602-5.430478-4.297956$ H $6.342299-2.648272-4.304294$ H $7.322818-3.476582-3.133812$ H $5.060850-1.430351-2.616757$ H $3.182174-1.3555470 .863709$ C $0.7273350 .418792 \quad 3.738678$ O $0.721925-0.9252853 .219428$ C $-0.588240-1.5006003 .448910$ C -1.421794 -0.4041354 .101469$ C -0.3639210 .4258724 .802296$ H $-0.432478-2.3265054 .154068$ C -1.120074-2.032122 2.111941 H -1.934655 0.2027413 .344671 H -2.177714 -0.8041484 .783839$ C $-0.850353 \quad 1.8115765 .190721$ H $-0.023713-0.1099045 .698291$ C -2.620808 -2.382951 2.115408 O $-0.339337-3.1886241 .800497$ H $-0.918829-1.2934411 .326143$ H -3.175026 -1.479876 2.384048 O $-2.893063-3.3504603 .126558$ C $-3.141055-2.8856610 .753988$ C $-4.666982-3.090928 \quad 0.700518$ H -2.838002 -2.174112 -0.022449 O $-2.521787-4.1337410 .436430$ H $-4.916938-3.466766-0.299760$ H $-4.953179-3.9017741 .382680$ H -0.0590142 .3809385 .687595$ H $-1.1763072 .382914 \quad 4.314930$ H -1.697696 1.7415025 .880578 C $2.120795 \quad 0.777804 \quad 4.252664$ H $-0.574007-3.4699200 .900172$ H $-2.399439-4.1563812 .896404$ H -2.814315 $-4.389069-0.455084$ H $2.547464-0.051876 \quad 4.828388$ H $2.070552 \quad 1.6474804 .917268$ H 0.4424891 .0812092 .910494 C $-5.167093-0.640303-1.056555$ O $-4.974439-0.667138 \quad 0.359750$ C $-5.496879-1.8283231 .010599$ C $-7.001902-1.9672970 .733592$ C $-7.324816-1.899978-0.760121$ C $-6.658492-0.679746-1.399546$ H $-4.675398-1.514119-1.502615$ C $-4.4304530 .578889-1.619888$ H $-5.394407-1.6463252 .087634$ O $-7.482404-3.1860741 .290157$ H $-7.528539-1.1494431 .242879$ H $-7.030507-2.820645-1.277662$ O $-8.737561-1.771836-0.935132$
H $-7.1635480 .228438-1.049332$

O $-6.813952-0.741901-2.814475$

C $-4.9793621 .896445-1.126789$

H $-3.3688600 .538162-1.344843$

H $-4.4642260 .588191-2.715428$

H -8.451069 -3.1852381 .212161$

H -9.147668 - $2.597731-0.628051$

H $-7.767361-0.796194-2.999429$

$\begin{array}{lllll}\text { O } & -4.440315 & 2.201967 & 0.088133\end{array}$

O $-5.7920482 .570345-1.746519$

C -4.8750753 .4381940 .662018$

H $-4.599874 \quad 4.2743350 .012090$

H $-5.955808 \quad 3.4179620 .831314$

H -4.370828 3.5617411 .624077

SCF Energy (B3LYP/6-31G*//MMFF) $=-3245.91024578$

01_356

MM̄FF Geometry

C $0.5642303 .313434-1.690300$

C $1.6883293 .873180-1.216396$

C 2.4148693 .3923290 .011632

O $3.7097462 .881731-0.398936$

C 2.7113054 .5292981 .005743

C 1.5118195 .0453511 .766432

C 1.1401234 .6012992 .982602

C $0.768817 \quad 6.188536 \quad 1.125637$

C 1.7723113 .5060833 .799897

C 0.7189682 .5677614 .407104

C $0.144975 \quad 1.567416 \quad 3.391277$

C $-1.140836 \quad 0.946528 \quad 3.940624$

C $-1.688772-0.1406333 .010474$

$\begin{array}{lllll}\text { O } & 1.134044 & 0.552220 & 3.182118\end{array}$

O $-2.710604-0.8577293 .718187$

C $-0.559053-1.1433652 .712248$

C $-2.352507 \quad 0.4543751 .765118$

O $-0.978630-2.1091321 .749584$

C $0.762320-0.4778262 .255348$

C $1.887398-1.5423712 .261089$

$\begin{array}{lllll}\text { O } & 0.591321 & 0.038957 & 0.936573\end{array}$

C $3.7591041 .635818-0.941198$

O $2.8201500 .885236-1.150090$

C $5.1777211 .341835-1.243197$

C $5.5186630 .146029-1.746347$

C $6.871565-0.282635-2.109841$

C $7.9979890 .688364-1.866783$

C $3.285866-1.0125681 .917611$

C $4.365972-2.0501152 .181041$

C $6.828776-2.3529652 .093444$

O $4.132614-3.1521522 .671216$

C $7.823118-2.1491950 .996225$

$\begin{array}{llll}C & 8.254443 & -3.141850 & 0.203285\end{array}$

C $9.294542-2.979583-0.868101$

C $8.763852-3.304032-2.246451$

C $8.765558-4.565627-2.709239$

C $8.315119-2.152884-3.117331$

C $7.040723-1.515364-2.633543$

N $5.624377-1.5964691 .805099$

H $0.1340232 .458654-1.174491$

H $2.1314714 .709359-1.752405$

H 1.8667462 .5936080 .526642

H 3.2030895 .3641060 .486200

H 3.4803004 .1886101 .711718

H 0.2924235 .0897153 .462272

H $-0.0367446 .570784 \quad 1.761923$

H 1.4512327 .0214870 .926822

H $0.3165945 .877846 \quad 0.181713$

H $2.5003582 .923276 \quad 3.227762$

H 2.3183903 .9853334 .621668

H 1.1960332 .0117335 .224800

H -0.0869253 .1652874 .851588$

H -0.0543122 .0783292 .442809$

H -1.9016051 .7176634 .115695$

H -0.9397300 .4958694 .922929$

H -3.390806 -0.2188973 .991294$

H $-0.363844-1.6986383 .640584$

H -3.189112 1.1043092 .046779

H -2.781198 -0.3348561 .142582$

H -1.6696151 .0440921 .150011$ 
H -1.808371 -2.500277 2.072827 H $1.931408-1.9919143 .262098$ H $1.645886-2.3462231 .554137$ H 1.4599570 .1213010 .513249 H $5.9037312 .123116-1.048260$ H $4.733724-0.589658-1.917259$ H $8.0211970 .996350-0.815306$ H $7.8774711 .580004-2.491741$ H $8.9858910 .276684-2.082138$ H $3.336031-0.7437040 .859383$ H $3.520651-0.1257362 .516380$ H $6.580724-3.4102112 .234670$ H $7.234092-1.9755193 .037933$ H $8.227471-1.1481580 .870625$ H $7.857708-4.1443810 .349156$ H $10.131326-3.649173-0.629260$ H $9.721793-1.969445-0.861130$ H $8.414205-4.803619-3.707589$ H $9.115857-5.389388-2.095928$ H $8.131157-2.501854-4.142446$ H $9.137015-1.437661-3.212844$ H $6.163155-2.150581-2.760896$ H $5.728076-0.6219361 .535651$ C -1.635438 $3.955567-2.822670$ O $-2.2786782 .677790-2.663291$ C $-3.4634612 .860590-1.861354$ C $-3.5929914 .362728-1.610734$ C -2.146306 4.817324 -1.672859 H -3.273203 $2.362252-0.903807$ C $-4.6746152 .222311-2.555566$ H $-4.1821634 .857279-2.391586$ H $-4.0699544 .579714-0.649821$ C -1.983528 $6.309632-1.899014$ H $-1.661150 \quad 4.537694-0.729546$ C $-4.4903100 .733865-2.921461$ O $-4.9475252 .931877-3.771458$ H -5.564763 2.349035 - 1.928834 H -3.742305 $0.638623-3.717256$ O $-5.722413 \quad 0.264937-3.484444$ C $-4.102451-0.171811-1.739419$ C $-4.016633-1.656131-2.147178$ H -3.130886 $0.146727-1.346917$ O $-5.0716440 .001587-0.709954$ H $-5.019847-2.024525-2.385657$ H $-3.413953-1.727640-3.060040$ H $-0.9253536 .580988-1.966562$ H -2.472909 $6.631439-2.824320$ H -2.425394 $6.874054-1.071374$ C $-0.1203513 .778888-2.945256$ H $-4.1537082 .873168-4.330349$ H -6.394663 $0.292765-2.781983$ H $-4.775426-0.5086630 .061744$ H $0.3200104 .722783-3.288616$ H $0.080128 \quad 3.027253-3.719297$ H -2.000848 $4.356713-3.778253$ C $-5.379012-3.2598690 .107935$ O $-4.134579-2.551597 \quad 0.164735$ C $-3.379822-2.542445-1.051003$ C $-3.080210-3.982337-1.491209$ C $-4.351038-4.819492-1.593196$ C $-5.156600-4.720523-0.299396$ H -6.047842 -2.770668 -0.610314 C $-6.019419-3.1707041 .498456$ H -2.415031-2.079791-0.806799 O $-2.400983-4.007465-2.741844$ H - $2.414944-4.453988-0.756123$ H $-4.957916-4.526631-2.458207$ O $-3.997028-6.189696-1.794201$ H $-4.633448-5.2607850 .500213$ O $-6.421377-5.352393-0.486817$ C $-6.615897-1.8022811 .726569$ H -6.836042 -3.8912871 .621351$ H $-5.286749-3.3807212 .287162$ H $-1.587625-3.483651-2.642727$ H $-3.450583-6.231719-2.597925$ H $-6.249434-6.267190-0.768833$ O $-5.778264-1.0426802 .487765$ O $-7.690105-1.444762 \quad 1.260030$
C $-6.238226 \quad 0.283627 \quad 2.766470$

H -7.2010180 .2518703 .285409$

H -5.5069650 .7691393 .417764$

H -6.317922 0.8581521 .838890

SCF Energy (B3LYP/6-31G**//MMFF)= -3245.92960767

01357

MM̄FF Geometry

C $-1.550315-4.720358-1.626630$

C $-0.679253-4.032181-2.380349$

C $0.796234-3.825940-2.125926$

O $0.940204-2.511993-1.527413$

C $1.451125-4.842803-1.170243$

C $2.942365-4.629510-0.978272$

C $3.524607-4.346900 \quad 0.204538$

C $3.793650-4.776501-2.213082$

C $2.863469-4.2100841 .548037$

C $2.362951-2.7965251 .878329$

C $3.479255-1.7559552 .054366$

C $2.905194-0.4374242 .579618$

C 3.9804290 .6551572 .688665

O $4.103026-1.5339650 .787666$

O 3.3124731 .9147862 .862235

C $4.756598 \quad 0.7356251 .355669$

C $4.8655490 .460874 \quad 3.925526$

$\begin{array}{lllll}\text { O } & 5.871315 & 1.616160 & 1.468307\end{array}$

C $5.222876-0.6383180 .830461$

C $5.818059-0.578731-0.597846$

O $6.252152-1.1454731 .689208$

C $0.874730-1.424574-2.340555$

O $0.808870-1.415033-3.558939$

C $0.833905-0.229551-1.470269$

C $0.3451870 .920334-1.957025$

C $0.1930342 .174795-1.217984$

C $0.783166 \quad 2.2409490 .165344$

C $4.882664-0.025970-1.678892$

C $5.0689941 .457729-1.952621$

C $4.0472553 .343304-3.219798$

O $6.017132 \quad 2.108962-1.522604$

C $3.2121714 .158304-2.283462$

C $2.2447654 .990095-2.698359$

C $1.4227215 .846502-1.778296$

C $-0.0636215 .672157-1.996108$

C $-0.7531436 .526860-2.770602$

C $-0.7626424 .558228-1.250608$

C $-0.453966 \quad 3.199101-1.812634$

N $4.0694181 .962685-2.773474$

H $-1.218955-5.246795-0.737526$

H $-1.072904-3.491727-3.240177$

H $1.314445-3.852145-3.092229$

H $1.296369-5.861059-1.549914$

H $0.945342-4.791440-0.201994$

H $4.605305-4.210900 \quad 0.224214$

H $4.864213-4.777225-1.981144$

H $3.569929-5.721358-2.718830$

H $3.610690-3.952891-2.909680$

H $2.027630-4.9137131 .632043$

H $3.579509-4.5299812 .315800$

H $1.674037-2.4696941 .091476$

H $1.786710-2.8561492 .809534$

H $4.215795-2.1587802 .759585$

H $2.410590-0.5856073 .547851$

H $2.120647-0.0849011 .896467$

H 2.7543861 .8510163 .656105

H 4.0770041 .1838740 .621585

H 4.2582180 .4856034 .838372

H 5.5888591 .2775864 .026247

H $5.410733-0.4851553 .918501$

H 5.5329872 .4744411 .775951

H $6.778124-0.049009-0.583668$

H $6.061515-1.610897-0.889260$

H $6.763996-1.8092971 .197726$

H $1.168387-0.340974-0.445625$

H $-0.0030620 .941038-2.989055$

H 0.7300943 .2326610 .618423

H 1.8451921 .9759430 .139310

H $0.264715 \quad 1.5491350 .837540$

H $5.104670-0.537772-2.624142$ 
H $3.835525-0.229079-1.435827$ H $3.6506023 .348080-4.239991$ H $5.0688853 .736339-3.239352$ H $3.4293394 .081279-1.220654$ H $2.0510185 .086595-3.764509$ H $1.7040396 .892599-1.956974$ H $1.6631205 .651883-0.725699$ H -1.824185 $6.425901-2.910899$ H $-0.2599347 .344422-3.286140$ H $-1.8506174 .691821-1.314383$ H $-0.5338224 .642411-0.184841$ H $-0.8430293 .050131-2.821066$ H $3.2931931 .364282-3.040380$ C $-3.695072-3.573070-1.109501$ O $-3.328486-2.317793-1.719773$ C $-4.481668-1.449047-1.743992$ C $-5.532085-2.132127-0.882805$ C $-5.220169-3.597675-1.125493$ H $-4.810814-1.419352-2.790552$ C $-4.037254-0.054648-1.286991$ H $-5.395397-1.880948 \quad 0.175439$ H $-6.552251-1.858829-1.168121$

C $-5.833586-4.525756-0.091942$ H $-5.582007-3.876422-2.124201$ C $-5.1833650 .924670-0.994702$ O $-3.208457 \quad 0.495455-2.318064$ H -3.398262 -0.154129-0.408809 H $-5.7655900 .580359-0.132969$ O $-6.078247 \quad 0.898130-2.116817$ C $-4.7444132 .385362-0.786990$ C -3.7209442 .6573210 .334689$ H $-4.3392942 .771447-1.730857$ O $-5.9249403 .157780-0.530483$ H -2.792621 2.1260960 .112044 H $-3.487888 \quad 3.7269400 .275749$ H $-5.567928-5.567677-0.295747$ H $-5.490905-4.2823780 .919503$ H $-6.925676-4.447001-0.104661$ C $-3.034166-4.717142-1.882183$ H -2.489240 -0.138743 -2.481358 H $-6.8023911 .518154-1.923605$ H $-5.6574374 .090996-0.472973$ H $-3.227598-4.610913-2.956859$ H $-3.440213-5.685819-1.570877$ H -3.326898 $-3.544998-0.075556$ C $-2.877096 \quad 0.394517 \quad 2.278995$ $\begin{array}{llll}\text { O } & -4.182977 & 0.952346 & 2.070903\end{array}$ C -4.2196812 .3496561 .767222$ C -3.5103993 .1436102 .877731$ C -2.097631 2.631854 3.134013 C $-2.127493 \quad 1.1279793 .397651$ H -2.2992420 .4748791 .353424$ C $-3.086943-1.0939482 .598189$ H -5.2758212 .6403051 .813953$ O -3.4590994 .5325232 .569629$ H -4.0834933 .0472143 .809447$ H -1.424877 2.8762742 .304292 O $-1.564936 \quad 3.277148 \quad 4.292406$ H -2.607833 0.9387894 .366035 O $-0.791880 \quad 0.6385563 .480970$ C -1.920042 -1.9796202 .222318$ H -3.302834 -1.219692 3.665846 H -3.956489-1.469067 2.045479 H $-4.372574 \quad 4.8336202 .425893$ H -1.592315 4.235303 4.127075 H $-0.336102 \quad 1.1519284 .169931$ O $-2.133044-3.2224032 .748006$ O $-0.963654-1.6404291 .540391$ C -1.137570 -4.212555 2.471100 H $-0.345427-4.1450973 .221026$ H $-1.608717-5.1964022 .542873$ H $-0.723335-4.1006091 .465850$ SCF Energy (B3LYP/6-31G**//MMFF) $=-3245.90222356$

01358

MM̄FF Geometry

C -2.417590 -3.386945 -2.422972

C $-1.493919-3.557098-1.465109$
C $-0.011103-3.409837-1.693424$

O $0.464471-2.284336-0.921246$

C $0.709965-4.675704-1.193259$

C $2.204750-4.676452-1.445387$

C $3.144006-4.698144-0.479146$

C $2.624443-4.687650-2.893140$

C $2.944981-4.721403 \quad 1.010840$

C $2.943375-3.336548 \quad 1.677581$

C $4.276503-2.5798571 .557580$

C $4.264482-1.3165712 .420104$

C $5.550567-0.4934132 .250051$

O $4.467974-2.213420 \quad 0.190114$

O $5.3042910 .791552 \quad 2.842164$

C $5.826917-0.2782150 .744148$

C $6.717359-1.109428 \quad 3.031945$

$\begin{array}{llllll}\text { O } & 7.100755 & 0.338666 & 0.575149\end{array}$

C $5.722348-1.575161-0.086787$

C $5.775328-1.344380-1.616773$

O $6.820808-2.4332640 .245247$

C $0.372183-1.053940-1.496825$

O $-0.076500-0.789441-2.600832$

C $0.904217-0.064580-0.533695$

C $0.691828 \quad 1.242092-0.749533$

C $1.1128892 .341152 \quad 0.121307$

C 2.0962372 .0227711 .215239

C $4.688147-0.422869-2.181251$

C $5.132102 \quad 1.020675-2.346648$

C $4.2529063 .258725-2.995677$

O $6.2913251 .392887-2.187284$

C $3.9750854 .037288-1.749173$

C $3.1242875 .073531-1.704863$

C $2.8614955 .889352-0.472273$

C $1.3922445 .952522-0.121775$

C $0.6428277 .012516-0.469032$

C $0.814614 \quad 4.825694 \quad 0.703594$

C $0.5981923 .569900-0.096630$

N $4.0811431 .843154-2.729261$

H -2.107779 $-3.106956-3.426345$

H $-1.817296-3.819362-0.460036$

H $0.206686-3.253697-2.756984$

H $0.285043-5.562009-1.683552$

H $0.502904-4.794388-0.124278$

H $4.189479-4.708483-0.785918$

H $3.701977-4.844675-3.012154$

H $2.116633-5.495445-3.430172$

H $2.377819-3.736477-3.374513$

H $2.016885-5.241403 \quad 1.270193$

H $3.744034-5.332257 \quad 1.450269$

H $2.135800-2.7349651 .243974$

H $2.706607-3.4778682 .739465$

H $5.082724-3.2517261 .874209$

H $4.106336-1.5662363 .476856$

H $3.409749-0.6897012 .131716$

H 6.0876991 .3450702 .680212

H 5.0796160 .4421460 .391622

H $6.478025-1.1526464 .101441$

H $7.618838-0.4917862 .953252$

H $6.964699-2.1219402 .706206$

H $7.1939330 .580542-0.361342$

H $6.779071-1.022494-1.918769$

H $5.634005-2.325023-2.094651$

H $6.946478-3.068059-0.479359$

H $1.430244-0.4372790 .337394$

H $0.1227601 .538990-1.629762$

H $2.961461 \quad 1.4909730 .805844$

H 1.6297851 .3971501 .983526

H 2.4979292 .9078091 .712609

H $4.422829-0.776739-3.185905$

H $3.780265-0.459423-1.571051$

H $3.5701313 .521104-3.810002$

H $5.279743 \quad 3.445675-3.326197$

H $4.5149993 .751985-0.849311$

H $2.6055825 .373077-2.612897$

H $3.2461496 .901436-0.654237$

H 3.4257775 .5099340 .388690

H $-0.4056347 .077656-0.197136$

H $1.0603457 .836811-1.037941$

H -0.1635425 .1205411 .107301$ 
H $\quad 1.444913 \quad 4.6710451 .583430$ H $-0.1112973 .694721-0.916108$ H $3.1413421 .460538-2.780597$ C $-4.559277-2.311898-1.662609$ O $-4.524320-1.267189-2.657691$ C $-5.809353-0.627997-2.694537$ C $-6.559711-1.095468-1.453899$ C $-6.034067-2.510006-1.310959$ H -6.326523 -1.041948 -3.571578 C $-5.7029020 .890780-2.889222$ H $-6.291111-0.507428-0.570107$ H -7.645880 -1.051715 -1.577023 C $-6.270837-3.1061830 .066279$ H -6.517952 -3.144622 -2.065678 C $-5.1527841 .725646-1.710820$ O $-7.0186131 .374853-3.197829$ H $-5.0916591 .092187-3.777112$ H $-5.8496141 .663139-0.868865$ O $-5.1687973 .095764-2.140156$ C $-3.7235661 .366322-1.266919$ C $-3.1509262 .253053-0.145722$ H $-3.6883680 .330114-0.928947$ O $-2.8419871 .457546-2.388893$ H $-2.0952511 .990337-0.026382$ H -3.139518 $3.301686-0.465614$ H $-5.851722-4.114746 \quad 0.134360$ H -5.812632 $-2.496646 \quad 0.851975$ H $-7.343352-3.171276 \quad 0.276712$ C $-3.890336-3.578228-2.193758$ H $-7.569470 \quad 1.271622-2.403429$ H $-5.1090283 .654188-1.346947$ H -2.883168 2.370876 -2.720975 H -4.342639 -3.875201 -3.148031 H -4.045546 -4.409613 -1.495889 H -4.011222 -1.937459 -0.790584 C $-2.746656 \quad 0.1389082 .018540$ O C $-3.8828542 .105796 \quad 1.202658$ C -3.2942422 .9557982 .338043$ C -1.970703 2.391359 2.851089 C $-2.1035370 .904848 \quad 3.181167$ H -2.055618 $0.140349 \quad 1.166300$ C $-2.996512-1.3434122 .335100$ H $-4.9102292 .455347 \quad 1.047126$ O $\quad-3.115344 \quad 4.298717 \quad 1.900127$ H -4.0131332 .9815723 .167186$ H -1.168641 2.5573362 .126597 O -1.5775853 .1019174 .027661$ H -2.693076 0.7918504 .097810 O $-0.810291 \quad 0.3625993 .439929$ C $-3.993408-1.5961543 .447822$ H -3.398553 -1.837099 1.444100 H -2.048403 -1.8252582 .599923$ H -2.7088224 .7926782 .632720$ H -2.271601 2.9811624 .697976 H $-0.406305 \quad 0.8968974 .144966$ O $-3.911150-2.9083283 .821421$ O $-4.766696-0.7694853 .910826$ C -4.816583 -3.3120304 .853259$ H -5.850850 -3.186349 4.519265 H -4.642162 -4.370617 5.063675 H -4.632021 -2.737659 5.766117 SCF Energy (B3LYP/6-31G**//MMFF) $=-3245.91046384$

\section{1_359}

MM̄FF Geometry

C $0.867502-2.1866922 .044304$

C $2.130406-1.8344962 .332987$

C $2.654084-0.4370322 .556450$

O 1.5728950 .5240062 .481694

C $3.345776-0.2618693 .921967$

C $4.726297-0.8801064 .022968$

C $5.854376-0.283123 \quad 3.587031$

C $4.800169-2.2033074 .738524$

C 5.9627141 .0461412 .892890

C 6.9413291 .0571181 .709565

C 6.6299350 .0512990 .589041

C $7.6848780 .163935-0.517945$
C $7.389942-0.771687-1.698039$

$\begin{array}{lllll}\text { O } & 5.327346 & 0.320894 & 0.053108\end{array}$

O $8.235335-0.357254-2.782313$

C $5.923355-0.568960-2.134054$

C $7.782431-2.219099-1.380170$

O $5.562625-1.507912-3.144433$

C $4.918862-0.611754-0.960507$

C $3.478806-0.275999-1.412779$

O $4.878559-1.948230-0.441445$

C $1.842996 \quad 1.7204131 .894109$

O $2.9004172 .074787 \quad 1.397982$

C 0.6209252 .5503431 .947349

C 0.6005063 .6987791 .254700

C -0.5120024 .6419451 .158105$

C -1.7404814 .3741341 .984688$

C $3.2545891 .133638-1.965119$

C $1.8169151 .323766-2.427760$

C $0.3196823 .012222-3.453603$

$\begin{array}{llll}\text { O } & 1.018326 & 0.394537 & -2.523837\end{array}$

C $-0.0482854 .427693-3.141250$

C $-1.2816894 .797397-2.761329$

C -1.694704 6.219474-2.498968

C $-2.3110496 .404928-1.129038$

C $-3.6405206 .316711-0.954326$

C -1.3983996 .7620680 .022862$

C $-0.3888005 .690056 \quad 0.317977$

N $1.5397262 .646107-2.754803$

H $\quad 0.091918-1.4323041 .970085$

H $2.879349-2.6223912 .367335$

H $3.369017-0.2423611 .751719$

H $2.699343-0.6546944 .718944$

H 3.4217440 .8086364 .157319

H $6.803890-0.7877213 .759443$

H $5.826962-2.5768894 .816196$

H $4.408831-2.1048425 .756455$

H $4.215307-2.9642104 .215237$

H 4.9904321 .4006472 .549429

H $6.316317 \quad 1.7791863 .628579$

H 6.9334292 .0727101 .292294

H 7.9565840 .8755552 .085380

H $6.642305-0.9580401 .015492$

H $8.689540-0.027115-0.120172$

H $7.7048681 .196980-0.893574$

H $8.003262-0.895439-3.558740$

H $5.8769420 .418278-2.608941$

H $8.854282-2.281699-1.156145$

H $7.621195-2.873409-2.243884$

H $7.242146-2.634172-0.526911$

H $5.404478-2.363677-2.710369$

H $3.148833-1.024020-2.146158$

H $2.801971-0.404692-0.557958$

H $4.058526-2.0533140 .068385$

H -0.2194422 .1931482 .530030$

H 1.4840413 .9722160 .678277

H -1.488268 4.3459583 .050490

H -2.1905333 .4169071 .705392$

H -2.517906 5.1319511 .863607

H $3.4867701 .883818-1.202864$

H $3.8945891 .314691-2.834469$

H $-0.4790742 .308909-3.196415$

H $0.5243822 .912272-4.524748$

H $0.7207865 .186012-3.265693$

H -2.054912 $4.037794-2.665988$

H -2.421095 $6.503598-3.271919$

H $-0.8520956 .911653-2.619497$

H -4.0952486 .4712420 .018480$

H -4.303382 $6.082926-1.780875$

H -1.9704027 .0090840 .924534$

H $-0.8611497 .686545-0.226313$

H $0.5366085 .799653-0.248607$

H $2.3163423 .300726-2.789553$

C $-0.017689-3.9142560 .380523$

O $-1.310972-3.3401810 .117006$

C $-1.437923-3.164806-1.311885$

C $-0.090679-3.554009-1.928466$

C $0.866324-3.421998-0.759365$

H -1.614215 -2.098696 -1.486468

C $-2.612594-3.996623-1.849528$ 
H $-0.099264-4.582588-2.307710$

H $0.174657-2.903795-2.768480$

C $2.152386-4.209196-0.934538$

H $1.102512-2.357992-0.637470$

C $-3.987997-3.642340-1.248843$

O $-2.366546-5.383500-1.575196$

H -2.653324 -3.912725 -2.941653

H $-3.996785-3.866140-0.176278$

O $-4.959247-4.508159-1.852371$

C $-4.433347-2.188013-1.489443$

C $-5.894841-1.896815-1.093209$

H $-3.768768-1.506385-0.951019$

O $-4.296219-1.872270-2.878422$

H $-6.141230-0.886695-1.442379$

H -6.567542 -2.553174 -1.659155

H $2.795062-4.110200-0.054514$

H $1.952621-5.275374-1.084880$

H $2.710117-3.845722-1.803807$

C $0.445854-3.6101161 .807687$

H -2.322161 -5.488008 -0.609247

H $-4.667491-5.422973-1.697627$

H $-4.854659-2.493496-3.376982$

H $1.269876-4.2892332 .059952$

H $-0.370734-3.8319482 .505972$

H $-0.148527-5.002948 \quad 0.306823$

C $-5.450033 \quad 0.1726501 .078808$

O $-5.269379-1.2436181 .200262$

C $-6.174340-2.0278940 .416495$

C $-7.622642-1.7178350 .824918$

C -7.923012 -0.2201420 .782684$

C $-6.855305 \quad 0.5710341 .541135$

H -5.3065440 .4770890 .035762$

C -4.3680790 .8525901 .924747$

H $-5.987722-3.0742290 .686065$

O $-8.546916-2.405485-0.011439$

H $-7.789563-2.0751441 .849433$

H $-8.0069110 .132974-0.251475$

O -9.1988790 .0256501 .379647$

H $-6.962842 \quad 0.3969722 .619294$

O $\quad-7.0504501 .9639181 .309096$

C -2.9848820 .5714231 .384620$

H -4.4984521 .9393151 .966141$

H -4.4002150 .4747982 .954034$

H -8.342721 -3.3545160 .045198$

H $-9.164796-0.2838172 .300752$

H -7.9609222 .1781101 .575767$

O $-2.858457 \quad 1.0836110 .129160$

O $-2.121593-0.0166792 .021852$

C -1.616960 $0.877754-0.555212$

H $-1.0998521 .836567-0.632403$

H $-0.9696550 .146491-0.062842$

H -1.843037 $0.521626-1.563858$

SCF Energy (B3LYP/6-31G**//MMFF) $=-3245.92689947$

01_36

MM̄FF Geometry

C $4.8008201 .944466-0.788026$

C $5.6450230 .978355-0.392393$

C $5.264273-0.475448-0.208866$

O $3.843255-0.6024770 .029131$

C $5.622835-1.270094-1.477372$

C $5.207711-2.727786-1.429652$

C $4.329084-3.292978-2.281835$

C $5.856266-3.560736-0.355311$

C $3.618639-2.635992-3.433336$

C $2.296617-1.948095-3.062208$

C $1.168292-2.921604-2.686003$

C $-0.145560-2.165010-2.481929$

C -1.283198 -3.092927 -2.030556

O $1.520569-3.594884-1.475900$

O $-2.343819-2.241102-1.574031$

C $-0.799605-3.945391-0.835561$

C $-1.848007-3.901574-3.203955$

O $-1.753792-4.949152-0.499554$

C $0.580031-4.597419-1.064849$

C $1.153321-5.2709260 .206206$

O $0.454320-5.621118-2.060275$

C $3.399265-0.3104531 .282529$ $\begin{array}{llll}\text { O } & 4.076726 & -0.033368 & 2.259327\end{array}$

C $1.922052-0.3246821 .243376$

$\begin{array}{lrrr}\text { C } & 1.237712 & 0.308208 & 2.207574\end{array}$

$\begin{array}{llll}\text { C }-0.218235 & 0.425217 & 2.295741\end{array}$

C $-1.037187-0.364566 \quad 1.311322$

C $1.348129-4.3445871 .412548$

C $0.222534-4.4099402 .433284$

C $-0.490275-3.3908944 .595570$

O $-0.694160-5.2248932 .378372$

C -1.614292 -2.444056 4.316108

C $-1.915795-1.4015985 .105797$

C $-3.081503-0.4846354 .871044$

C -2.6844240 .9733654 .825198$

C $-2.843586 \quad 1.768619 \quad 5.896538$

C $-2.193778 \quad 1.528316 \quad 3.508225$

$\begin{array}{llll}\text { C }-0.746147 & 1.217896 & 3.251273\end{array}$

N $0.387590-3.4696023 .442579$

H $3.7756461 .686004-1.041869$

H $6.6775361 .234465-0.167085$

H $5.810176-0.8797330 .652501$

H $6.706389-1.222908-1.649149$

H $5.161900-0.772866-2.337851$

H $4.097546-4.349783-2.155700$

H $5.615248-4.624811-0.453057$

H $6.945826-3.466348-0.407821$

H $5.523784-3.2392440 .636451$

H $4.278533-1.905513-3.914935$

H $3.431821-3.396746-4.202010$

H $2.472808-1.247364-2.237571$

H $1.974968-1.352888-3.926116$

H $1.065886-3.654196-3.495136$

H $-0.431351-1.624099-3.392866$

H $-0.001056-1.394890-1.711749$

H $-3.094522-2.801901-1.316079$

H $-0.734522-3.2744220 .027209$

H -2.247315 $-3.228847-3.972695$

H $-2.690366-4.526031-2.886426$

H $-1.103744-4.544344-3.678702$

H $-2.577732-4.500531-0.246062$

H $0.559031-6.1555580 .464619$

H $2.148192-5.658873-0.056602$

H $\quad 1.197990-6.238733-1.962741$

H $1.443543-0.820008 \quad 0.406664$

H 1.7951610 .8128272 .996004

H $-0.784163-1.4268621 .375539$

H $-0.845331-0.0220580 .290402$

H -2.113365 -0.2974941 .479481$

H $2.256645-4.657693 \quad 1.943535$

H $1.496609-3.3077961 .097247$

H $\quad 0.119544-3.070355 \quad 5.446174$

H $-0.893310-4.3856024 .812191$

H -2.230906 -2.646848 3.443571

H $-1.316176-1.2183175 .994714$

H -3.802052 -0.6490835 .683060$

H -3.614355 -0.744252 3.948796

H -2.5902282 .8230015 .860316$

H -3.2254251 .3812906 .835252$

H -2.2974492 .6218743 .498934$

H $-2.850578 \quad 1.1845592 .705592$

H $-0.066094 \quad 1.7429843 .923488$

H $1.146214-2.7970783 .377352$

C $4.2471694 .316198-0.100791$

$\begin{array}{lllll}\text { O } & 2.888368 & 4.212860 & -0.572934\end{array}$

C 2.0013574 .2783330 .562475

C 2.8604384 .7094691 .747579

C 4.1890014 .0615711 .405018

H $1.646907 \quad 3.2576390 .745629$

C $0.815561 \quad 5.1997790 .259406$

H 2.9693055 .7991671 .796164

H 2.4490394 .3686602 .702614

C 5.3664694 .6323412 .174508

H $4.1101912 .988342 \quad 1.618041$

C $0.001475 \quad 4.785128-0.984793$

$\begin{array}{lllll}\text { O } & 1.321526 & 6.521497 & 0.031410\end{array}$

H $\quad 0.164205 \quad 5.265025 \quad 1.138935$

H $0.6254304 .877215-1.881249$

O $-1.0604865 .735830-1.145760$

C $-0.6046933 .369874-0.912526$ 
C $-1.4472293 .040715-2.163197$ H $0.1987332 .630729-0.823903$ $\begin{array}{llllll} & \text { O } & -1.404469 & 3.289427 & 0.264853\end{array}$ H $-2.2947153 .731195-2.219373$ H $-0.8257173 .216982-3.048808$ H 6.2988134 .1402541 .880003 H 5.4806745 .7067391 .995986 H 5.2309194 .4832523 .250708 C $5.1632603 .395820-0.913765$ H $0.5599157 .092450-0.168372$ H -1.660016 $5.635354-0.386281$ H -1.7131082 .3718880 .350060$ H $6.2057923 .563721-0.619874$ H $5.0775203 .660453-1.974909$ H $4.5541845 .353229-0.291456$ C $-4.1254231 .905220-1.157024$ O $-2.8339251 .295458-1.083481$ C $-1.9629561 .581684-2.181839$ C -2.623736 $1.152576-3.500257$ C $-4.0075461 .776577-3.673074$ C $-4.8679241 .508179-2.437322$ H $-4.0038282 .995511-1.146600$ C $-4.894246 \quad 1.5756340 .131700$ H -1.079967 $0.944472-2.047156$ O $-1.7834391 .493769-4.597065$ H -2.727686 $0.059611-3.502707$ H -3.942502 $2.851373-3.879657$ O $-4.659498 \quad 1.185275-4.799099$ H $-5.1462340 .448762-2.419974$ O $-6.075574 \quad 2.260762-2.530457$ C $-5.064894 \quad 0.0976550 .419089$ H -4.3358181 .9942520 .977672$ H $-5.8868172 .038550 \quad 0.106563$ H -2.162378 $1.090497-5.396043$ H $-4.1727741 .456496-5.595543$ H -6.507372 $2.013700-3.366197$ O $-5.720425-0.0439261 .610389$ O $-4.674618-0.817894-0.290468$ C $-5.947421-1.3934002 .029585$ H -6.635494 -1.889479 1.338718 H $-6.402395-1.3685773 .023300$ H $-5.001400-1.9401282 .091590$

SCF Energy (B3LYP/6-31G*//MMFF) $=-3245.91270014$

01_360

MMMFF Geometry

C -2.572309 -3.056067 2.835388

C $-1.795776-2.0087552 .518695$

C $-1.119725-1.1156473 .524600$

O $0.299139-1.315167 \quad 3.342889$

C $-1.4644010 .352815 \quad 3.220095$

C $-1.051874 \quad 1.3149824 .312997$

C $-0.027762 \quad 2.1844304 .216069$

C -1.9178601 .3024655 .546392$

C $0.9408702 .347671 \quad 3.077818$

C 0.4790323 .4402532 .107969

C 1.6117273 .9476461 .202896

C 1.0790565 .0054580 .230977

C $2.1517505 .487676-0.755285$

$\begin{array}{llll}\text { O } & 2.176380 & 2.844790 & 0.482522\end{array}$

O $1.4892536 .185752-1.821603$

C $2.8389814 .256615-1.382468$

C $3.1086486 .496047-0.109047$

O $3.9412644 .650005-2.196266$

C $3.2957203 .212341-0.339901$

C $3.8744151 .938620-0.995943$

$\begin{array}{lllll}\text { O } & 4.352915 & 3.775599 & 0.449319\end{array}$

C $1.094723-1.3719794 .439623$

O $0.749271-1.2227565 .603607$

C $2.500367-1.6703444 .073784$

C $2.916719-1.8421192 .809408$

C $4.276053-2.1746692 .377690$

C $5.318311-2.407243 \quad 3.438744$

C $2.9008601 .121144-1.847309$

C $3.597454-0.070328-2.486521$

C $3.119647-2.251075-3.587050$

O $4.819945-0.184811-2.535089$

C $3.368086-3.207696-2.464031$
C $4.567756-3.746445-2.197634$

C $4.835017-4.609335-0.997135$

C $5.912204-4.041000-0.091889$

C $6.946354-4.806795 \quad 0.295615$

C $5.814642-2.5985410 .365231$

C $4.517220-2.2814611 .054725$

N $2.696773-0.982440-3.019106$

H -2.715304 -3.290069 3.888881

H $-1.619610-1.7589601 .478267$

H $-1.427095-1.3971934 .538567$

H -2.547778 $0.460447 \quad 3.073021$

H -1.007199 0.6444112 .266498

H 0.1527612 .8593565 .051939

H -1.6201542 .0679386 .271033$

H -2.962325 1.4928395 .278706

H $-1.859015 \quad 0.3336006 .051045$

H 1.0940151 .4039322 .543211

H 1.9155942 .6111643 .506902

H -0.3316043 .0304641 .492332$

H 0.0630874 .2906842 .663579

H 2.3837674 .3839861 .848119

H $0.6499845 .854793 \quad 0.777414$

H $0.2523804 .578833-0.350407$

H $0.9816456 .917614-1.430882$

H $2.106213 \quad 3.791666-2.053989$

H $2.559305 \quad 7.3842520 .225713$

H $3.8490036 .857432-0.831125$

H $3.6372906 .093147 \quad 0.757241$

H $3.6087915 .297519-2.841353$

H $4.7613852 .206775-1.585180$

H $4.2502211 .277234-0.201791$

H $4.844340 \quad 3.050708 \quad 0.869851$

H $3.179608-1.7566574 .915840$

H 2.196270-1.739064 1.999582

H $6.276207-2.747203 \quad 3.037948$

H $4.989084-3.1819184 .140360$

H $5.509368-1.4848143 .997782$

H $2.0762050 .760348-1.223073$

H $2.4855841 .723908-2.660767$

H $4.014241-2.087409-4.196919$

H $2.315604-2.619132-4.230486$

H $2.526565-3.429195-1.811442$

H $5.410700-3.526193-2.848473$

H $5.120754-5.604949-1.359315$

H $3.921065-4.751289-0.406706$

H $7.727665-4.4187140 .940729$

H $7.035519-5.840592-0.021120$

H $5.907424-1.939497-0.507190$

H $6.667359-2.3288590 .997896$

H $3.691729-2.1027350 .365092$

H $1.704389-0.854580-2.845124$

C $-3.309973-3.5618300 .406850$

O $-2.008501-3.777774-0.180241$

C $-2.183652-4.284747-1.511551$

C $-3.668657-4.162059-1.830893$

C $-4.283695-4.360959-0.458391$

H $-1.946284-5.356522-1.461198$

C $-1.219745-3.645000-2.521797$

H $-3.917416-3.167301-2.216218$

H $-4.004257-4.903898-2.561716$

C $-5.725142-3.887953-0.372502$

H $-4.233701-5.426172-0.196388$

C $-1.351701-2.125335-2.777701$

O $-1.389302-4.325580-3.773366$

H $-0.190919-3.853936-2.205291$

H $-2.333021-1.903604-3.209227$

O $-0.391606-1.770436-3.783429$

C $-1.098319-1.228190-1.554564$

C $-1.0393330 .279094-1.862261$

H $-1.877888-1.398515-0.812320$

O $0.145023-1.603002-0.956040$

H $-0.7471910 .795601-0.939492$

H $-0.2216880 .483259-2.563749$

H $-6.128097-4.037810 \quad 0.633701$

H $-5.813911-2.823532-0.615460$

H $-6.354551-4.445635-1.073829$

C $-3.301732-3.9720451 .882276$

H $-2.265372-4.092403-4.124787$ 
H $-0.550889-2.343454-4.552867$ H $0.253345-1.077930-0.144790$ H -2.859476 -4.970965 1.983249 H $-4.336349-4.0358742 .239775$ H $-3.537568-2.4941850 .313722$ C $-3.5279211 .129224-0.310200$ O $-3.4839170 .504230-1.596637$ C $-2.357967 \quad 0.865269-2.404064$ C -2.323890 $2.385559-2.615495$ C $-2.3625813 .141736-1.288977$ C -3.523322 $2.658732-0.420019$ H -2.648485 0.8113430 .263345 C -4.7399850 .5835810 .460119$ H $-2.5267800 .410597-3.387582$ O $-1.1599812 .750545-3.350259$ H $-3.192158 \quad 2.679555-3.218971$ H -1.412785 $3.037301-0.756027$ O $-2.5141024 .539755-1.543755$ H $-4.4650923 .024377-0.844203$ O -3.3997593 .2199760 .885163$ C $-6.0806120 .871287-0.184688$ H $-4.663139-0.5062100 .534122$ H -4.7428211 .0004811 .473835$ H - $1.1558823 .720046-3.427732$ H $-3.3482794 .668867-2.026499$ $\mathrm{H}-3.3498854 .1859130 .783046$ O -7.0660170 .6860730 .743896$ O $-6.2520041 .183435-1.354800$ C -8.4010720 .8995980 .275070$ H -8.639720 $0.189951-0.522879$ H $-9.0856450 .731192 \quad 1.110585$ H -8.520187 $1.929696-0.074192$

SCF Energy (B3LYP/6-31G**//MMFF)= -3245.91509648

01_361

MMFFF Geometry

C -3.960056 -3.440453-1.845921

C $-2.769920-3.435203-1.229302$

C $-1.452993-3.313840-1.952023$

O $-0.830188-2.071242-1.553300$

C $-0.515231-4.466108-1.545322$

C $0.857844-4.398858-2.190055$

C $2.025950-4.337230-1.518696$

C $0.871317-4.440254-3.697727$

C $2.255227-4.354736-0.032883$

C $2.703063-3.0062280 .537679$

C $4.014402-2.426538-0.016975$

C $5.207288-3.3641630 .172842$

C $6.520573-2.720384-0.294695$

O $4.249447-1.2079320 .702429$

$\begin{array}{llll}\text { O } & 7.583877 & -3.545849 & 0.206825\end{array}$

C $6.657996-1.322708 \quad 0.347792$

C $6.636913-2.747689-1.823733$

O $7.764333-0.610534-0.201508$

C $5.380475-0.4519540 .246127$

$\begin{array}{llll}C & 5.512906 & 0.783594 & 1.170607\end{array}$

O $5.1806630 .040269-1.078629$

C $-1.237836-0.936726-2.185624$

O $-2.085525-0.841161-3.058220$

C $-0.4684330 .188186-1.611654$

C $-0.7984541 .451386-1.920364$

C $-0.1438922 .659061-1.410004$

C $1.0242982 .466134-0.482086$

C $4.259751 \quad 1.6702521 .219906$

C 4.4530803 .0283880 .568105

C $4.8091754 .116548-1.653179$

O $4.3862524 .068414 \quad 1.220258$

C $3.5566344 .265719-2.458784$

C $2.8258915 .389767-2.513186$

C $1.5793375 .523271-3.343121$

C $0.3925096 .040660-2.556950$

C $-0.199187 \quad 7.199322-2.894192$

C $-0.115643 \quad 5.227877-1.384141$

C $-0.6116343 .866298-1.791076$

N $4.6728682 .953550-0.795266$

H -3.994905 -3.388942 -2.931041

H -2.737620 -3.490429 -0.144487

H $-1.599463-3.321167-3.039277$
H $-0.974485-5.426126-1.816013$

H $-0.427279-4.469099-0.454466$

H $2.943983-4.299577-2.104566$

H $1.882256-4.561453-4.101725$

H $\quad 0.274153-5.283663-4.059779$

H $0.461141-3.514966-4.113766$

H $1.361013-4.6763970 .507894$

H $3.004569-5.1233150 .189438$

H $1.909768-2.2650500 .377601$

H $2.788275-3.0996141 .628880$

H $3.869286-2.185054-1.075732$

H $5.058070-4.315584-0.350368$

H $5.302188-3.6184661 .237898$

H $8.425344-3.188822-0.123705$

H $6.882632-1.4763661 .412559$

H $6.659806-3.782966-2.185183$

H $7.575131-2.292880-2.159587$

H $5.808643-2.242704-2.325588$

H $8.567835-1.125186-0.017354$

H $5.6945670 .426322 \quad 2.193055$

H 6.3829701 .3830420 .876293

H $5.909466-0.265081-1.641220$

H $0.341299-0.056667-0.933196$

H $-1.632503 \quad 1.622073-2.599603$

H $1.8096571 .886760-0.979298$

H $\quad 0.714997 \quad 1.9338280 .422489$

H $1.4846143 .397300-0.150410$

H 3.3887231 .1863850 .767496

H $3.999637 \quad 1.8575052 .269460$

H $5.6571183 .929854-2.319234$

H $5.0243775 .004688-1.050829$

H $3.2414693 .401745-3.040131$

H $3.1485616 .264434-1.953472$

H $1.3106034 .566670-3.807755$

H $1.8098806 .203998-4.172956$

$\mathrm{H}-1.0503207 .582825-2.341270$

H $0.1530647 .792730-3.731456$

H $-0.9567105 .743623-0.901570$

H $0.6637565 .188349-0.619594$

H $-1.4696753 .900207-2.463890$

H $4.6609342 .041787-1.242922$

C $-6.077289-2.199423-1.162602$

O $-5.419306-1.167715-0.398012$

C $-5.6644380 .100097-1.041174$

C $-6.673136-0.167776-2.157903$

C $-6.338940-1.595099-2.540378$

H $-4.7150120 .410364-1.492570$

C -6.137848 $1.150033-0.028378$

H $-7.706692-0.102438-1.798229$

H -6.565072 $0.536804-2.988325$

C $-7.435125-2.293008-3.324997$

H $-5.423459-1.573373-3.143483$

C $-5.170971 \quad 1.432727 \quad 1.139096$

$\begin{array}{llll}\text { O } & -7.382556 & 0.722829 & 0.542827\end{array}$

H -6.358992 $2.086253-0.554590$

H $-5.150513 \quad 0.578394 \quad 1.825646$

O

$\begin{array}{llll}\text { C } & -3.736536 & 1.795415 & 0.718118\end{array}$

C -2.8519512 .3703821 .841878$

H -3.2517140 .9081490 .297723$

O $-3.7749812 .769838-0.329429$

H -1.8600282 .5582191 .412674$

H -3.219389 3.3656712 .124320

H -7.146154 -3.320668 -3.566988

H $-8.372593-2.329991-2.759976$

H $-7.630201-1.766557-4.264997$

C $-5.274670-3.504054-1.121179$

H $-7.230678-0.1419240 .961725$

H -6.6116912 .2872112 .153056$

H $-4.240727 \quad 3.5519630 .013361$

H $-5.867114-4.313383-1.563752$

H $-5.102747-3.775527-0.071774$

H $-7.033114-2.382603-0.652499$

C -1.140415 $-0.114306 \quad 2.278566$

$\begin{array}{llll}\text { O } & -2.455920 & 0.123020 & 2.785398\end{array}$

C $-2.7326251 .488812 \quad 3.100858$

C $-1.723548 \quad 2.005570 \quad 4.137504$

C -0.2773751 .7769723 .697552$ 
C $-0.064604 \quad 0.320504 \quad 3.280759$ H $-1.0150390 .460773 \quad 1.354734$ C -1.057256 -1.583909 1.857116 H -3.7170571 .4968403 .584386$ O $-1.945606 \quad 3.3896934 .386705$ H -1.889874 1.4777855 .085745 H 0.0037362 .4688782 .895120 O 0.5805562 .0773014 .800962 H -0.077424 -0.302927 4.182205 O $1.231522 \quad 0.2065792 .698655$ C -1.114566 -2.5758872 .997844$ H -1.900597 -1.817604 1.200280 H $-0.131047-1.744194 \quad 1.297987$ H -1.275314 3.6805045 .028645 H $\quad 1.4925371 .8977884 .514239$ H $1.420569-0.7389792 .577126$ O $-0.466433-3.7189072 .625234$ O $-1.684709-2.3905864 .063946$ C $-0.451602-4.7660313 .599876$ H -1.472264 -5.096224 3.815502 H $\quad 0.109675-5.6076353 .185175$ H $0.047151-4.4276424 .512999$

SCF Energy (B3LYP/6-31G**//MMFF) $=-3245.91883668$

01_362

MM̄FF Geometry

C 1.7222683 .3622950 .907939

C $1.7629153 .905999-0.317658$

C $2.1183913 .112840-1.548382$

O $0.9183152 .979564-2.353028$

C $3.1859633 .808467-2.413960$

C $4.6039033 .523364-1.964658$

C $5.4365232 .661243-2.581146$

C $5.0894644 .304293-0.771124$

C $5.1747611 .819228-3.799179$

C $4.0878090 .747266-3.679555$

C $4.144330-0.144452-2.432884$

C $5.428427-0.965617-2.337368$

C $5.397626-1.916080-1.129365$

O $3.014937-1.022856-2.521191$

O $6.477431-2.850751-1.280342$

C $4.085295-2.728847-1.158072$

C $5.648317-1.1724840 .189196$

O $3.976023-3.5067710 .032999$

C $2.820710-1.859782-1.374192$

C $1.598840-2.763603-1.662897$

O $2.575701-1.089576-0.197783$

C $0.0142432 .042666-1.948723$

O $\quad 0.1227201 .270784-1.008639$

C $-1.1715802 .143159-2.824018$

C $-2.3499661 .699359-2.361051$

C $-3.6319981 .750693-3.064618$

C $-3.6032412 .076241-4.534525$

C $0.324483-2.003978-2.057643$

C $-0.802667-2.945204-2.445824$

C $-3.223838-2.973752-2.945701$

O $-0.660188-4.160963-2.547567$

C $-4.225282-2.040316-3.543900$

C -5.430789-1.809162 -3.002905

C $-6.472384-0.918437-3.617228$

C $-6.8867370 .207355-2.697697$

C -7.898478 $0.052228-1.826999$

C $-6.1824571 .536502-2.849016$

C $-4.7590891 .497387-2.366628$

N -1.989548 -2.261508 -2.680046

H 1.9832262 .3148651 .033265

H $1.4678794 .943447-0.453462$

H $2.4656852 .107349-1.285412$

H $3.0204344 .894221-2.434960$

H $3.0542723 .505221-3.459746$

H $6.4411252 .546657-2.175101$

H $6.1687004 .196420-0.617072$

H $4.8879745 .372144-0.904926$

H 4.5947423 .9643660 .142303

H $4.9440892 .476389-4.646857$

H $6.1175571 .326668-4.070630$

H $3.0982111 .218479-3.722500$

H $4.1225150 .114193-4.576859$
H $4.0281910 .483031-1.544406$

H $6.313243-0.320684-2.284292$

H $5.547545-1.565406-3.250410$

H $7.307317-2.345464-1.324207$

H $4.171148-3.441206-1.990787$

H $6.618790-0.6625890 .162365$

H $5.700756-1.8701841 .032288$

H $4.887293-0.4211540 .410621$

H $3.272624-4.163216-0.101617$

H $1.851584-3.447845-2.483456$

H $1.372043-3.377639-0.783343$

H $1.647102-0.810565-0.184613$

H -1.051887 2.609957 -3.794565

H $-2.3903231 .282938-1.355298$

H $-2.886775 \quad 1.432314-5.057678$

H $-3.3148823 .121130-4.691112$

H -4.562535 $1.923414-5.033166$

H $-0.024654-1.392609-1.220677$

H $0.516343-1.346256-2.912587$

H -3.565514 -3.403779-1.997833

H $-3.018695-3.798828-3.636084$

H -3.956845 -1.555567-4.479295

H $-5.701767-2.314228-2.078358$

H -7.345653 -1.539003 -3.857861

H $-6.133841-0.507309-4.576503$

H $-8.2207240 .863323-1.182569$

H -8.423830 -0.892924 -1.736805

H $-6.7008632 .306691-2.262223$

H $-6.2722111 .865526-3.887761$

H $-4.6691591 .272480-1.302959$

H -2.049605 -1.288745 -2.391965

C $-0.2653984 .044202 \quad 2.251338$

O -0.7222022 .6825202 .360443$

C - -1.9234202 .6662493 .157144$

C -2.1997684 .1135823 .562804$

C -0.8189934 .7370663 .491334$

H -1.6975102 .0873984 .059008$

C -3.0654812 .0057602 .374255$

H -2.8822284 .6089602 .862289$

H -2.6483534 .1813274 .558852$

C -0.8433736 .2526883 .404559$

H -0.2489164 .4314974 .378849$

C $-2.7847980 .557620 \quad 1.919155$

O -3.3387432 .7835621 .199945$

H -3.9843202 .0357832 .971505$

H $-2.0066770 .559478 \quad 1.147171$

O

C $-2.403747-0.4028333 .062940$

C $-2.372475-1.8943152 .683940$

H -1.427366 -0.118002 3.467540

O $-3.342739-0.2601294 .134245$

H -2.183200 -2.464242 3.601964

H -3.374483 -2.211851 2.369300

H 0.1719586 .6582563 .356306

H -1.385522 6.5952392 .516754

H $-1.334724 \quad 6.6801914 .284685$

C 1.2579724 .0911702 .134834

H -2.524122 2.8091560 .669202

H -3.758987 -0.6825990 .751001$

H -4.225246 -0.4650563 .780223$

H 1.7231273 .6150753 .006451

H 1.6094065 .1289642 .108485

H $-0.7232204 .464742 \quad 1.345178$

C $0.584756-2.5057853 .039655$

O $-0.017172-1.8158971 .937932$

C $-1.336316-2.2597691 .603224$

C -1.312621 -3.752699 1.244525

C $-0.675946-4.5942242 .348941$

C $0.679560-4.0090282 .747188$

H $-0.008405-2.3363333 .945801$

C $1.979097-1.9106873 .267164$

H -1.620634 -1.719371 0.693087

O $-2.633492-4.2059970 .970081$

H $-0.730233-3.8873190 .326352$

H -1.337427 -4.684368 3.218533

O $-0.459143-5.922258 \quad 1.867047$

H $1.396110-4.1891251 .935765$

O $1.170765-4.6994383 .894650$ 
C $1.916522-0.4561293 .666586$

H $2.532081-2.4486844 .045333$

H $2.574020-1.9716572 .348705$

H $-2.568395-5.1082270 .614646$

H $-1.330338-6.3263641 .717041$

H $\quad 0.548043-4.545277 \quad 4.625546$

O $1.355092-0.3294404 .902329$

$\begin{array}{lllll}\text { O } & 2.337628 & 0.457018 & 2.968727\end{array}$

C 1.2584161 .0087235 .399482

H $0.714568 \quad 1.642993 \quad 4.694178$

$\mathrm{H} \quad 0.706466 \quad 0.982326 \quad 6.342743$

H $2.258267 \quad 1.4110965 .586592$

SCF Energy (B3LYP/6-31G**/MMFF) $=-3245.91150709$

01_363

MMFF Geometry

C $-1.468048 \quad 4.7083780 .112015$

C $-2.3788174 .735556-0.873647$

C $-3.5109903 .741400-1.014767$

O $-3.1002252 .447904-0.509373$

C $-4.7436854 .207345-0.221603$

C $-5.9422753 .290444-0.373434$

C $-6.4883502 .572851 \quad 0.628143$

C $-6.5443823 .224500-1.753568$

C -6.0675232 .5256242 .070254$

C -5.0442461 .4313612 .411317$

C $-5.549632-0.0017822 .177671$

C $-4.575061-1.0209962 .773548$

C $-5.005253-2.4677052 .483143$

O $-5.664483-0.221843 \quad 0.768974$

O $-3.892840 \quad-3.3261942 .779657$

C $-5.297613-2.6143830 .973983$

C $-6.152447-2.9151943 .396766$

O $-5.860086-3.8918260 .686949$

C $-6.206985-1.5016100 .412849$

C $-6.333899-1.535934-1.130260$

O $-7.528986-1.6626120 .942428$

C -2.277129 $1.708635-1.303580$

O $-1.923760 \quad 1.970488-2.442510$

C -1.824668 $0.540828-0.516661$

C $-0.760700-0.155752-0.941506$

C $-0.155298-1.310432-0.276429$

C $-0.873512-1.8849170 .914982$

C $-5.016905-1.407368-1.903672$

C $-4.411391-2.738078-2.320658$

C $-2.375627-3.689775-3.399773$

O $-5.000812-3.810297-2.216191$

C $-1.440210-4.205597-2.352139$

C $-0.127324-4.384038-2.564069$

C $0.820770-4.945320-1.543096$

C $2.001576-4.036780-1.283665$

C $3.182961-4.242644-1.890088$

C $1.833662-2.939075-0.258100$

C $1.003018-1.795939-0.769306$

$\mathrm{N}-3.143278-2.580612-2.864589$

H -1.5728193 .9769990 .910535$

H -2.287096 $5.475338-1.665046$

H -3.760217 $3.644532-2.078474$

H $-5.0374395 .214262-0.546303$

H -4.465852 4.3003190 .833683

H $-7.349676 \quad 1.9467620 .397148$

H -7.484582 $2.662670-1.770084$

H $-6.7607884 .232456-2.122347$

H $-5.8587982 .734960-2.451852$

H $-5.669756 \quad 3.4958352 .387073$

H -6.9679222 .3772342 .680343$

H -4.1324591 .6020521 .827601$

H $-4.7756641 .548298 \quad 3.468690$

H $-6.536261-0.0994982 .645761$

H -4.461308 -0.8659213 .853659$

H -3.576744 -0.8633652 .343178$

H -3.647438 -3.1883413 .710637$

H $-4.330123-2.5754940 .459432$

H $-5.849853-2.853527 \quad 4.449090$

H -6.410811 -3.9652953 .221680$

H $-7.055387-2.312583 \quad 3.278341$

H $-5.246489-4.5629041 .032065$

H -6.901317 -2.421156 -1.441979
H $-6.955372-0.676577-1.421529$

H -8.153407 -1.2021370 .357562$

H -2.3555100 .3076280 .398415$

H $-0.2672260 .160710-1.859489$

H - $0.426101-2.8054681 .294170$

H $-1.907521-2.1343730 .653683$

H $-0.882748-1.1662421 .741747$

H $-5.214425-0.859524-2.834154$

H $-4.281823-0.831195-1.335163$

H $-1.844782-3.319674-4.282721$

H $-3.055992-4.488807-3.711604$

H $-1.865844-4.470891-1.387311$

H $0.289206-4.142891-3.539711$

H $1.172688-5.916901-1.913998$

H $0.309960-5.159040-0.595982$

H $4.040594-3.609418-1.687774$

H $3.316511-5.045542-2.607394$

H $2.814895-2.5288780 .013681$

H $1.443098-3.3753070 .664836$

H $1.438183-1.302031-1.638718$

H $-2.713783-1.659981-2.869692$

C 1.0020324 .7178750 .273947

$\begin{array}{lllllll}\text { O } & 1.172557 & 3.967080 & -0.943064\end{array}$

C $2.5859273 .729104-1.142691$

C 3.3001364 .4112510 .019687

C 2.3080855 .4852030 .428824

H $2.8412354 .218037-2.090590$

C $2.7976452 .212500-1.246119$

H 3.4612463 .7100180 .847904

H $4.2749074 .814790-0.270950$

C $2.5517206 .031348 \quad 1.824281$

H $2.3534256 .305566-0.299567$

C $4.2752401 .775393-1.250726$

$\begin{array}{llll}\text { O } & 2.160320 & 1.771543-2.449088\end{array}$

H $2.2766161 .714749-0.420559$

H $4.7485322 .162782-0.343303$

O $4.9318162 .377349-2.364421$

C $4.4526470 .242703-1.284873$

C $5.907553-0.251768-1.217813$

H $3.893166-0.190631-0.448880$

O $3.884595-0.302981-2.477176$

H $5.877658-1.348779-1.231213$

H $\quad 6.4308130 .021089-2.143153$

H 1.8130566 .7975422 .078870

H 2.4923905 .2413022 .580581

H 3.5452856 .4865521 .890800

C $-0.251307 \quad 5.588408 \quad 0.176030$

H $1.2337892 .065321-2.411920$

H $5.8863772 .224996-2.265412$

H $4.2864720 .159993-3.232226$

H $-0.196476 \quad 6.228478-0.712574$

H $-0.3357806 .235907 \quad 1.055025$

H $0.891785 \quad 3.9896281 .089742$

C $5.804381-1.2463591 .670556$

$\begin{array}{llll}\text { O } & 5.995743 & 0.101578 & 1.228568\end{array}$

$\begin{array}{llll}\text { C } & 6.712571 & 0.241018 & 0.000022\end{array}$

C $8.105943-0.3909120 .127795$

C $8.028691-1.8418460 .600343$

C $7.156438-1.9504981 .851549$

H $5.189227-1.7887350 .942281$

C $5.042660-1.1903873 .000319$

H $6.8683961 .317361-0.142012$

O $8.787736-0.311255-1.119267$

$\begin{array}{llll}\text { H } & 8.691404 & 0.187346 & 0.854595\end{array}$

H $7.663024-2.504617-0.192548$

O $9.340255-2.3009800 .935702$

H $7.696907-1.5117142 .699999$

O $6.950028-3.3281102 .160319$

C $3.588859-0.8357542 .796846$

H $5.061401-2.1591903 .512887$

H $5.484847-0.4589153 .687625$

H $9.702797-0.604935-0.975006$

H $9.857137-2.339608 \quad 0.113434$

H $6.464975-3.7309591 .419919$

O 3.4433050 .5152042 .685879

O $2.689763-1.6648832 .743576$

C $2.098626 \quad 0.975122 \quad 2.520041$

H 1.5060490 .7332363 .407272 
H 2.1228632 .0610872 .399532 H 1.6510630 .5321651 .626173

SCF Energy (B3LYP/6-31G**//MMFF) $=-3245.89278929$

013364

MM̄FF Geometry

C $2.071237-1.504500-2.480425$

C $1.239507-2.503156-2.808834$

C $0.215226-3.070406-1.864060$

O $-1.073181-2.623111-2.347531$

C $0.247198-4.610661-1.832987$

C $1.419162-5.190860-1.067478$

C $1.375742-5.5345030 .235054$

C $2.665263-5.455200-1.871965$

C $0.216936-5.382117 \quad 1.180971$

C $0.634064-4.8727342 .567606$

C $1.136197-3.4192732 .588888$

C $1.605406-3.0623064 .003770$

C $2.052634-1.5999394 .114097$

O $0.056265-2.5636132 .200209$

O $2.151777-1.2987845 .516811$

C $0.946323-0.6984093 .523506$

C $3.463874-1.4062323 .548640$

O 1.3663830 .6620453 .465716

C $0.389533-1.1697262 .157842$

C $-0.889610-0.3946151 .765363$

O $1.302622-0.8877721 .092295$

C $-2.082553-2.556193-1.438227$

O $-2.045166-2.913218-0.271713$

C $-3.256623-1.950433-2.101318$

C $-4.380664-1.774808-1.391033$

C $-5.632606-1.194925-1.876094$

C $-5.644080-0.611301-3.264253$

C -2.142739-0.709405 2.587038

C -3.2896240 .2294852 .242052$

C $-5.4449131 .059706 \quad 3.180673$

O

C -6.6116650 .4896552 .438077$

C $-7.274272 \quad 1.1686091 .488334$

C $-8.5181910 .674056 \quad 0.805333$

C $-8.3853330 .626654-0.700072$

C $-8.6017021 .720752-1.449514$

C $-8.093305-0.709240-1.340781$

C $-6.701933-1.200074-1.053383$

N $-4.327727 \quad 0.134626 \quad 3.157900$

H $1.987753-1.049655-1.498026$

H $1.302829-2.943982-3.800852$

H $0.373652-2.669382-0.855027$

H $0.241461-5.009281-2.857131$

H $-0.692279-4.991076-1.413634$

H $2.261391-5.9939560 .671959$

H $3.438403-5.963656-1.285767$

H $2.434701-6.093713-2.731004$

H $3.097910-4.521586-2.238734$

H $-0.559616-4.7296960 .777357$

H $-0.237344-6.3726891 .307565$

H $-0.244585-4.9535333 .221267$

H $1.403192-5.5369672 .981759$

H $1.958446-3.3097111 .873178$

H $2.411164-3.7330804 .327966$

H $\quad 0.779393-3.2256384 .710824$

H $2.369519-0.3542255 .600061$

H $0.129116-0.7187314 .254158$

H $4.191513-1.9652974 .150169$

H $3.776430-0.3587733 .605982$

H $3.570600-1.7483882 .517558$

H 1.9664090 .7619792 .707967

H $-0.6833920 .683100 \quad 1.792397$

H -1.118254 -0.6093620 .713331$

H $2.206496-0.8691601 .433752$

H $-3.165778-1.667550-3.143390$

H $-4.393649-2.095774-0.349860$

H -5.514442 -1.399675 -4.013480

H $-4.8371640 .121176-3.381719$

H -6.569584 -0.082638 -3.504073

H -2.475518 -1.736022 2.398216

H -1.936228 -0.6062193 .656608$

H -5.1247022 .0231822 .769746$
H $-5.716907 \quad 1.2075654 .230789$

H $-6.959887-0.4971602 .732566$

H $-6.9370762 .168427 \quad 1.222990$

H -9.3405391 .3499881 .075336$

H -8.816362 -0.3110431 .185206$

H $-8.5378931 .687257-2.532018$

H -8.843452 $2.675819-0.994712$

H -8.287632 -0.677251 -2.418332

H -8.807061 -1.450760 -0.958284

H -6.591171 -1.635327 -0.059555

H $-4.268154-0.5510353 .905008$

C $4.464847-0.686133-2.673494$

O $4.2996910 .425857-1.772643$

C $5.0178970 .147843-0.555535$

C $5.914355-1.048246-0.859304$

C $5.056388-1.824701-1.841973$

H $4.269171-0.1520790 .187424$

C $5.7457521 .408562-0.073417$

H $6.853220-0.744924-1.336299$

H $6.162147-1.6194050 .040501$

C $5.828374-2.844125-2.660352$

H $4.276479-2.344103-1.271750$

C 4.8483762 .6626060 .000123

O $6.8205511 .706452-0.974445$

H $6.213698 \quad 1.2152060 .898281$

H $4.5681972 .956043-1.017156$

O $5.633073 \quad 3.746434 \quad 0.512173$

C 3.5828022 .5027530 .862715

C 2.7382163 .7864360 .987901

H $2.953944 \quad 1.7073420 .451458$

O 3.9519512 .0813232 .177421

H 1.9275123 .5864111 .699350

H 3.3373244 .5750431 .459682

H $5.168825-3.352468-3.370643$

H $6.637296-2.373304-3.229066$

H $6.273542-3.602785-2.008381$

C $3.139315-0.967701-3.389688$

H $6.4368441 .829172-1.859910$

H 5.9869243 .4775991 .376639

H 4.4303792 .8123832 .603610

H $3.318301-1.662593-4.218521$

H $2.764978-0.029085-3.816890$

H $5.192644-0.353659-3.425408$

C $0.2540092 .828303-0.518416$

O $1.5066003 .239340-1.074007$

C $2.1644244 .287760-0.353000$

C $1.2498895 .518882-0.249848$

C -0.1339465 .1739820 .300856$

C $-0.7391863 .993523-0.461444$

H 0.4239872 .4619320 .501637

C $-0.2612181 .614536-1.303077$

H $3.0206654 .590508-0.967417$

O $1.856157 \quad 6.5408790 .534478$

H $1.1230715 .938111-1.256163$

H $-0.0878754 .958406 \quad 1.374457$

$\begin{array}{lllll}\text { O } & -0.993565 & 6.308348 & 0.169827\end{array}$

H -1.021293 $4.318183-1.469308$

O $-1.928475 \quad 3.569948 \quad 0.198816$

C $-0.4522981 .832833-2.788512$

H $0.4699620 .805101-1.202621$

H $-1.2149341 .278746-0.882464$

H 1.8051666 .2775361 .468734

$\mathrm{H}-1.0531246 .533385-0.774298$

H $-2.521746 \quad 4.3386440 .252600$

O

O $-0.1374792 .837662-3.409374$

C -1.239506 $0.736511-4.734040$

H -1.681976 $-0.218927-5.028057$

H $-1.9369901 .540032-4.988843$

H $-0.2916230 .862504-5.265940$

SCF Energy (B3LYP/6-31G**//MMFF) $=-3245.90740706$

01_365

MM̄MF Geometry

C $0.3126360 .406309 \quad 3.147715$

C -0.5718691 .2901553 .635533$

C -1.212969 2.428965 2.880490

O -0.3477912 .8337791 .793966$ 
C -2.5925492 .0490412 .313975$

C -3.596950 1.5987353 .351016

C $-4.054304 \quad 0.337413 \quad 3.472841$

C $-4.1186172 .683495 \quad 4.257108$

C $-3.666186-0.8691172 .663294$

C $-4.817844-1.3665781 .781170$

C $-5.196308-0.4007120 .646369$

C $-6.403694-0.944020-0.121199$

C $-6.770181-0.052723-1.315209$

O $-4.072200-0.271411-0.231617$

O $-7.688705-0.782695-2.142842$

C $-5.499410 \quad 0.192796-2.152516$

C $-7.5017971 .220355-0.873945$

O $-5.761191 \quad 1.117387-3.207201$

C $-4.272403 \quad 0.643252-1.319193$

C $-3.0129980 .604462-2.216404$

O $-4.4911161 .975897-0.857542$

C $-0.467170 \quad 4.1140901 .349236$

O -1.2783224 .9488941 .716658$

C $0.596773 \quad 4.3395010 .348239$

C $0.7237075 .545874-0.222750$

C $1.7568795 .912195-1.185127$

C $1.2610016 .779015-2.309601$

C $-1.701547 \quad 1.010670-1.529880$

C $-0.5023250 .733375-2.422739$

C $1.9663430 .829369-2.459043$

$\begin{array}{lllll}\text { O } & -0.598090 & 0.206549 & -3.528848\end{array}$

C $3.0059061 .817405-2.038777$

C $3.7142882 .539735-2.920023$

C $4.8229813 .486896-2.558234$

C $4.5217974 .913500-2.960412$

C $4.5854975 .299002-4.246571$

C $4.2215995 .912729-1.868705$

C $3.0402545 .535447-1.019071$

N $0.6919491 .143970-1.842748$

H 0.6087420 .4540102 .103328

H $-0.869243 \quad 1.2021274 .678918$

H -1.298828 3.2701653 .580302

H -2.458206 1.3014301 .526242

H -3.016699 2.9087181 .778573

H -4.7921700 .1333414 .248031$

H -4.9026602 .3212714 .930719$

H -3.313232 3.0879174 .877650

H -4.5465373 .4990723 .665058$

H -2.770650 -0.702316 2.058549

H -3.398336 -1.6667533 .367794$

H $-4.511298-2.3246431 .345261$

H -5.696905 -1.565804 2.407147

H -5.4348010 .5792891 .073455$

H -7.266966 -1.064808 0.545313

H -6.173736 -1.950697 -0.497976

H -8.465945 -1.006838 -1.602833

H -5.252678 -0.759144 -2.642939

H $-8.4120230 .965345-0.317880$

H -7.835644 $1.804148-1.738856$

H $-6.894363 \quad 1.864486-0.234740$

H -5.782692 $2.009070-2.819901$

H -2.895902 $-0.417802-2.600496$

H $-3.1533341 .263071-3.083160$

H -3.641781 2.359603 -0.590464

H $1.270644 \quad 3.5206610 .123135$

H 0.0217606 .3413120 .019592

H $0.9360647 .753360-1.928563$

H $0.4098746 .303565-2.810565$

H $2.0167396 .959003-3.078297$

H $-1.7072302 .080516-1.295188$

H $-1.5659540 .452404-0.598512$

H $1.8472790 .792102-3.547315$

H $2.248294-0.171040-2.123177$

H $3.2134381 .910159-0.975913$

H $3.5119442 .416182-3.982185$

H $5.7371573 .154077-3.067271$

H $5.0539743 .434640-1.487104$

H $4.3961826 .326006-4.540562$

H $4.8292374 .594027-5.034634$

H $4.0881446 .921505-2.277475$

H $5.1012425 .990790-1.216050$

H $3.2989784 .917924-0.158939$
H $\quad 0.6863711 .391029-0.857054$

C $2.455261-0.738804 \quad 3.923869$

O $2.880635-1.2810152 .660739$

C $4.104737-0.6289952 .268580$

C $4.541698 \quad 0.2232363 .458676$

C 3.2077120 .5808024 .087030

H 3.8456950 .0353541 .435208

C $5.136880-1.6639891 .800522$

H $5.152709-0.3453214 .168891$

H 5.1197081 .0991543 .148571

C $3.310867 \quad 1.0573755 .524478$

H 2.7528191 .3726223 .479162

C $4.605584-2.6808300 .769472$

O $5.601741-2.4183482 .928380$

H $6.018918-1.1482861 .403666$

H $3.922436-3.3791371 .266355$

O $5.714260-3.461957 \quad 0.307618$

C $3.914728-2.054972-0.451942$

C $3.492506-3.060935-1.539133$

H $3.027981-1.502679-0.125808$

O $4.788997-1.098264-1.056074$

H $2.996471-2.495952-2.337840$

H $4.385655-3.486218-2.014911$

H $2.320204 \quad 1.2869325 .929767$

H $3.771157 \quad 0.299477 \quad 6.167103$

H 3.9202681 .9648025 .586085

C $0.925919-0.6823053 .985583$

H $4.826201-2.8320473 .345189$

H $6.137682-3.8547521 .090163$

H $5.602558-1.565507-1.312875$

H $0.621827-0.5665995 .033014$

H $0.518990-1.6381363 .633469$

H $2.793915-1.4581264 .681887$

C $0.492310-2.996403-0.878799$

O $1.523344-3.719490-0.202560$

C $2.576061-4.197285-1.043714$

C $2.012368-5.089629-2.160318$

C $0.884599-4.402948-2.931324$

C $-0.164453-3.856366-1.962521$

H $0.928833-2.105742-1.345987$

C $-0.497091-2.477846 \quad 0.170070$

H $3.198659-4.843219-0.412233$

O $3.058586-5.470534-3.047274$

H $1.619839-6.010475-1.709377$

H $1.265244-3.609027-3.584525$

O $0.243271-5.355119-3.782773$

H $-0.708624-4.697335-1.516471$

O $-1.107883-3.065026-2.680097$

C $-1.123365-3.580660 \quad 0.990186$

H $0.020203-1.8200330 .878590$

H $-1.287164-1.875375-0.289950$

H $2.698764-6.131805-3.661961$

H $0.882339-5.612336-4.468496$

H $-1.481894-3.624450-3.382315$

O $-2.351589-3.8959770 .490102$

O $-0.576679-4.111008 \quad 1.948841$

C $-3.038323-4.951768 \quad 1.168991$

H $-4.005411-5.0970980 .680192$

H $-2.465910-5.8814441 .099109$

H -3.211330 -4.680673 2.214968

SCF Energy (B3LYP/6-31G**//MMFF) = -3245.90533454

01_366

MM̄FF Geometry

C -2.953580 -3.020499 2.149354

C - $-1.766275-3.6453502 .116134$

C $-0.945620-3.7500220 .858678$

O $0.443599-3.479812 \quad 1.162986$

C $-1.023784-5.160898 \quad 0.257014$

C $-0.602919-5.170564-1.201133$

C $-1.455297-5.005790-2.232478$

C $0.860751-5.417800-1.455857$

C $-2.938308-4.772938-2.154563$

C $-3.453661-3.641354-3.057205$

C $-3.075012-2.228320-2.579529$

C $-3.943411-1.190142-3.300212$

C $-3.5527040 .249105-2.940946$

O $-1.693043-1.995563-2.871875$ 
O $-4.2162491 .105822-3.882131$

C $-2.0323200 .405358-3.136091$

C $-4.0663030 .644009-1.551463$

O $-1.5858561 .678392-2.675543$

C $-1.207848-0.711274-2.454938$

C $0.273571-0.585452-2.883181$

O $-1.299888-0.545071-1.039733$

C $0.798417-2.1970801 .424238$

O $0.043926-1.2415411 .539016$

C $2.265158-2.0401791 .506475$

C $3.131663-3.0634471 .516648$

C $4.584863-2.9248781 .555199$

C $5.301963-3.9540330 .723917$

C $1.156919-1.772246-2.480409$

C $2.596562-1.514893-2.878586$

C $4.540214-0.054270-2.325107$

O $3.097736-2.048784-3.865366$

C $5.559676-0.678529-1.427320$

C $6.2611770 .013195-0.515922$

C $7.384311-0.5630320 .298410$

C $7.138431-0.4908901 .787524$

C 7.4181580 .6237952 .483773

C $6.662997-1.7470822 .479450$

C $5.189557-1.9769012 .298697$

N $3.228629-0.604738-2.046557$

H $-3.360196-2.6266091 .221878$

H -1.347820 -4.064182 3.027630

H -1.286094 -3.015364 0.118715

H -2.039003 -5.5654350 .347635$

H $-0.381183-5.8526020 .817953$

H -1.054799 -5.061255 -3.243746

H $1.165314-6.375137-1.020480$

H $1.096004-5.453099-2.524909$

H $1.470224-4.625105-1.012983$

H -3.426782 -5.702907 -2.472110

H -3.273538 -4.580404-1.130930

H -3.091023 -3.799116-4.081085

H -4.547623 -3.726432 -3.084285

H $-3.232274-2.168211-1.496537$

H -5.007175 -1.358025 -3.088722

H -3.832129 -1.318399-4.386419

H -4.023674 2.027000 -3.639366

H -1.833343 $0.375283-4.216611$

H -5.162102 $0.610536-1.526231$

H -3.791288 $1.675044-1.303569$

H -3.697574 $-0.010185-0.759214$

H -2.020465 2.356411 -3.219062

H $\quad 0.329402-0.498318-3.976595$

H $\quad 0.6951620 .338699-2.468122$

H $-0.477733-0.851099-0.627014$

H $2.610497-1.0123961 .546845$

H $2.773757-4.0891071 .463745$

H $4.889855-3.980125-0.291750$

H $5.187040-4.9496831 .166178$

H $\quad 6.371249-3.7581540 .619322$

H $1.118951-1.961565-1.405210$

H $0.826721-2.688296-2.981692$

H $4.4721491 .028867-2.179478$

H $4.808872-0.237243-3.370541$

H $\quad 5.773930-1.733030-1.581237$

H $6.0596091 .074642-0.387683$

H $8.296453-0.0019680 .054102$

H $7.598392-1.5975890 .003740$

H 7.2745080 .6707653 .558094

H 7.7923061 .5157111 .991898

H $6.852741-1.6781873 .559243$

H $7.266323-2.5987482 .149916$

H $4.570763-1.2920382 .878864$

H $2.733670-0.255317-1.231472$

C $-4.321578-1.3916033 .544962$

O $-3.286567-0.399827 \quad 3.380361$

C -3.8187860 .6846942 .593011$

C -5.3308660 .4905962 .574370$

C -5.434495 -1.0214102 .562348$

H -3.4406150 .5490591 .574162$

C -3.3663852 .0395333 .143270$

H -5.8028440 .8982843 .475980$

H $\quad-5.799010 \quad 0.960911 \quad 1.704233$
C $-6.806548-1.5402852 .956423$

H -5.200033 -1.374732 1.551159

C -1.8377412 .2430593 .157604$

O -3.8415802 .1602054 .490003$

H -3.847563 2.8437352 .573639

H -1.379013 1.5615643 .883406

O -1.5877723 .5671743 .649857$

C -1.1519402 .0805111 .786102$

C 0.3448982 .4495461 .840010

H -1.2553991 .0429061 .455512$

O

H 0.4452393 .5139732 .076089

H $0.803516 \quad 1.8875562 .661892$

H $-6.828122-2.6344032 .944104$

H -7.088617 -1.208332 3.961264

H $-7.568819-1.1798492 .257815$

C $-3.750230-2.8081023 .405436$

H -3.541315 3.0218414 .826715

H -1.932843 4.1942592 .990899

H -1.478053 $2.686373-0.039892$

H $-4.566129-3.538675 \quad 3.439074$

H -3.106891 -3.002175 4.273294

H -4.690819-1.276912 4.572955

C $0.9124674 .289161-0.577179$

O $0.6046002 .889257-0.588251$

C 1.1017872 .1423810 .527174

C 2.6262552 .2970220 .624828

C 3.0561943 .7596160 .650946

C $2.4286034 .514652-0.519488$

H $0.4373124 .763900 \quad 0.289011$

C $0.3209254 .897719-1.859465$

H $\quad 0.904552 \quad 1.0883370 .296827$

O 3.1236851 .6225151 .775090

H $3.0838321 .817181-0.247864$

H 2.8085914 .2258061 .612025

O 4.4802383 .8019240 .532346

H $2.8951554 .190435-1.458720$

O $2.6822695 .910023-0.369972$

C $-1.1792085 .077358-1.763425$

H $0.7374665 .888793-2.068581$

H $0.5447874 .241264-2.709254$

H $4.0889221 .741271 \quad 1.787974$

H 4.7577334 .7249810 .657056

H $3.6408426 .046183-0.454752$

O $-1.7520144 .812450-2.973547$

O $-1.7714385 .444923-0.757397$

C $-3.1748874 .959734-3.024989$

H -3.652656 $4.299265-2.294914$

H -3.452632 $6.002296-2.843997$

H -3.509702 $4.674605-4.025832$

SCF Energy (B3LYP/6-31G**//MMFF $)=-3245.90967911$

01_367

MM̄MF Geometry

C $-0.5668555 .094343-0.560961$

C $0.4885994 .500466-1.138675$

C $1.5655063 .798380-0.348850$

O $1.7963062 .487338-0.919859$

C $2.8831764 .588762-0.430328$

C 3.9488434 .0838910 .524003

C 5.1171663 .5276930 .149375

C 3.6525994 .2690321 .991496

C $5.6306923 .283174-1.240663$

C $5.3198391 .890694-1.811466$

C $5.8361200 .723171-0.954044$

C $5.762120-0.593288-1.730952$

C $6.178474-1.795924-0.868982$

O $5.018154 \quad 0.629283 \quad 0.216618$

O $5.771199-2.990873-1.553950$

C $5.402216-1.7550510 .465531$

C $7.701376-1.884145-0.713081$

O $5.879065-2.7548591 .362140$

C $5.433984-0.3747351 .152700$

C $4.492279-0.2805412 .379089$

O $6.761560-0.1150791 .625876$

C $0.8377241 .542698-0.721201$

O $-0.2141861 .681209-0.118145$

C $1.2835580 .295963-1.381133$ 
C $0.400175-0.695292-1.566944$

C $0.666207-1.997803-2.178689$

C $2.099593-2.355983-2.461582$

C $3.009482-0.5462892 .095407$

C $2.573586-1.9777352 .364236$

C $0.602582-3.483876 \quad 2.113779$

O $3.282430-2.8037952 .932265$

C $0.731958-4.3048620 .870612$

C $-0.318302-4.842648 \quad 0.231731$

C $-0.200178-5.719083-0.982193$

C $-1.017524-5.213033-2.148854$

C $-2.234408-5.718021-2.412879$

C $-0.387865-4.176873-3.053544$

C $-0.381875-2.807687-2.433924$

N $1.276255-2.2122611 .928186$

H $-0.626967 \quad 5.1119700 .523832$

H $0.573214 \quad 4.484376-2.222357$

H 1.2576543 .6748810 .696590

H $2.7016295 .646451-0.196667$

H $3.2455314 .563959-1.463761$

H 5.8032023 .2087740 .933930

H 4.5202854 .0451452 .621667

H 3.3636055 .3055502 .193783

H 2.8398393 .6094712 .310014

H $5.2575214 .044117-1.934328$

H $6.7193393 .424410-1.223787$

H $4.2358111 .799448-1.943955$

H $5.7721171 .835579-2.809591$

H $6.8715220 .936537-0.663715$

H $6.374863-0.544147-2.639665$

H $4.731583-0.753124-2.075174$

H $6.194852-2.993710-2.429427$

H $4.361960-2.0128370 .234217$

H $8.181295-1.984036-1.694126$

H $7.989971-2.777063-0.147617$

H $8.133874-1.009642-0.222962$

H $5.818906-3.6095240 .901835$

H $4.868194-0.9131473 .192126$

H 4.5600450 .7492792 .758851

H 6.7134670 .5597082 .323268

H $2.3183850 .227131-1.693384$

H $-0.627859-0.538331-1.246494$

H $2.699996-2.270847-1.549588$

H $2.519692-1.690730-3.223578$

H $2.233474-3.378272-2.820178$

H 2.4099810 .0840322 .765078

H $2.751060-0.2710581 .069468$

H $-0.441834-3.2680252 .358029$

H $1.052056-4.0184342 .956744$

H $1.736441-4.4927120 .498613$

H $-1.319914-4.674307 \quad 0.621552$

H - $0.536937-6.725169-0.699760$

H $0.845530-5.835589-1.292916$

H -2.816353 -5.376074-3.262244

H -2.679382 -6.478691-1.779947

H $-0.952673-4.105481-3.992660$

H $0.607610-4.521217-3.345649$

H $-1.379554-2.444718-2.188734$

H $0.782248-1.4830551 .422357$

C $-3.0585395 .089793-1.074215$

O $-3.0203693 .693982-1.434241$

C -3.671990 $2.935115-0.400138$

C -4.4969743 .9433310 .390348$

C $-3.589057 \quad 5.1567450 .359300$

H -2.877945 2.5348620 .241254

C -4.488456 $1.768394-0.972638$

H $-5.4470594 .165961-0.109520$

H $-4.7182593 .597738 \quad 1.404573$

C $-4.296200 \quad 6.458820 \quad 0.692924$

H -2.7853304 .9985041 .086845$

C $-3.6431250 .727115-1.736541$

O $-5.5072432 .275799-1.835902$

H $-5.002152 \quad 1.285643-0.137344$

H -2.817924 $0.373644-1.113392$

O $-3.045766 \quad 1.350513-2.881483$

C $-4.459979-0.470435-2.261093$

C $-5.098792-1.384931-1.194175$

H $-5.260844-0.092013-2.908427$
O $-3.631598-1.276408-3.104019$

H $-5.625099-0.784650-0.447349$

H $-5.874888-1.961330-1.715003$

H -3.6013297 .3028830 .639433$

H -5.122072 6.6565340 .001327

H $-4.7086916 .423397 \quad 1.706532$

C $-1.6916675 .739437-1.322187$

H $-5.0627722 .726429-2.574498$

H $-2.4382552 .037411-2.557965$

H $-3.234558-0.688606-3.769450$

H $-1.732916 \quad 6.801444-1.054911$

H $-1.4768645 .688773-2.397166$

H $-3.7819685 .555369-1.756324$

C $-3.419646-1.1365291 .400834$

O $-3.063928-1.7981240 .180487$

C $-4.148737-2.408168-0.520484$

C $-4.853790-3.4291420 .388676$

C $-5.276274-2.8127071 .720193$

C $-4.086449-2.1130082 .375339$

H $-4.113862-0.3245781 .167757$

C $-2.130085-0.5363041 .985818$

H -3.695342 -2.991695 -1.330571

O $-5.979688-3.985532-0.280652$

H $-4.159462-4.256148 \quad 0.589258$

H -6.119206 -2.1224261 .597429$

O $-5.719668-3.8452832 .602932$

H -3.363516 -2.867816 2.710865

O $-4.530950-1.4036673 .528049$

C -2.319484 0.8279002 .613077

H -1.695152 -1.197438 2.742916

H $-1.393158-0.4221981 .182752$

H $-6.314335-4.718436 \quad 0.262905$

H -6.544208 -4.2085792 .238065$

H $-4.973770-2.0443634 .110604$

$\begin{array}{llll}\text { O } & -1.089973 & 1.383472 & 2.832302\end{array}$

$\begin{array}{lllll}0 & -3.394964 & 1.341806 & 2.885992\end{array}$

C $-1.0975242 .702318 \quad 3.386564$

H $-1.648428 \quad 3.3859472 .733883$

H $-1.5349962 .687247 \quad 4.389174$

H -0.0622403 .0463183 .458364$

SCF Energy (B3LYP/6-31G**//MMFF) $=-3245.91527960$

01_368

MMFF Geometry

C $2.077341-4.290928-0.272769$

C $0.781056-4.312055-0.613560$

C $-0.324037-3.833153 \quad 0.293235$

O $-1.002190-2.740367-0.368144$

C $-1.342344-4.964804 \quad 0.518244$

C $-2.478279-4.5880191 .449618$

C $-3.775739-4.5332591 .089211$

C $-2.086152-4.2873282 .873618$

C $-4.376238-4.829137-0.256473$

C $-4.514597-3.610294-1.181736$

C $-5.457347-2.519946-0.647269$

C $-5.738888-1.470979-1.725616$

C $-6.613401-0.323337-1.195692$

O $-4.834530-1.8895030 .475151$

$\begin{array}{llll}O & -6.554110 & 0.750823 & -2.147184\end{array}$

C -6.0069240 .2077350 .121506$

C $-8.088112-0.730667-1.094106$

O $-6.875643 \quad 1.1560490 .735459$

C $-5.649397-0.905483 \quad 1.127665$

C $-4.855581-0.3915652 .354382$

O $-6.857059-1.4918651 .629433$

C $-0.413529-1.514466-0.311250$

O $0.607346-1.2107240 .284921$

C $-1.218411-0.600028-1.149416$

C $-0.6680060 .539319-1.592910$

C $-1.3153261 .530775-2.453720$

C $-2.7888971 .367677-2.718906$

C $-3.528604 \quad 0.3070752 .036864$

$\begin{array}{llll}\text { C } & -3.639127 & 1.819696 & 1.936060\end{array}$

C -2.3163543 .8266531 .285680$

$\begin{array}{llll}\text { O } & -4.635392 & 2.447339 & 2.284987\end{array}$

C $-2.6024004 .204152-0.133471$

C $-1.771347 \quad 4.950189-0.876709$

C $-2.0544095 .377498-2.287696$ 
C $-0.9413085 .003598-3.240403$

C $0.0221685 .884425-3.558816$

C $-0.9953043 .638176-3.885954$

C $-0.5684722 .537950-2.952406$

N $-2.4738002 .393183 \quad 1.445650$

H $2.362314-3.9352660 .713746$

H $\quad 0.495610-4.652940-1.606129$

H $\quad 0.074989-3.489646 \quad 1.255509$

H $-0.834138-5.842946 \quad 0.938527$

H - $-1.734393-5.279657-0.454929$

H -4.504946 -4.2518661 .848214$

H -2.956626 -4.173804 3.528985

H -1.476642 -5.101135 3.280117

H -1.512206 -3.357362 2.928597

H -3.801623 -5.605148 -0.773490

H $-5.367247-5.272405-0.094796$

H -3.520427 -3.184909-1.362146

H -4.892411 -3.968935 -2.147387

H $-6.390915-2.998330-0.328378$

H $-6.204757-1.931512-2.605675$

H $-4.788069-1.048350-2.077797$

H $-6.8649140 .410038-3.003465$

H $-5.0929520 .749454-0.148807$

H -8.470937 -1.030205 -2.077200

H $-8.7103810 .112972-0.776029$

H -8.257108 -1.562871 -0.407722

H -7.055132 1.8517510 .080067

H -5.4999750 .2327972 .985001$

H -4.610200 -1.268817 2.970464

H -6.658787 -1.9309802 .473043$

H $-2.227261-0.904044-1.402800$

H $\quad 0.3643850 .756883-1.323178$

H $-3.2374182 .222524-3.229327$

H -3.338419 $1.258502-1.777743$

H $-2.9725260 .482971-3.337807$

H -2.8269320 .1086772 .857376$

H -3.082944 -0.0926491 .121438$

H -1.2935654 .0739301 .582799$

H -3.0083414 .3503621 .952787$

H -3.546004 $3.871591-0.559698$

H $-0.8433075 .306158-0.437206$

H -2.190342 $6.466948-2.285437$

H $-3.0033454 .963561-2.650443$

H $0.8090615 .632545-4.261926$

H $\quad 0.0487716 .875343-3.117437$

H $-0.3199983 .604540-4.751710$

H -1.991435 $3.478082-4.308177$

H $0.4914982 .573382-2.696921$

H -1.712065 1.7941371 .140763

C $3.983245-3.482708-1.665729$

O $4.676479-2.889787-0.549708$

C $5.987477-2.491993-0.982316$

C $6.015311-2.643481-2.496704$

C $5.066982-3.812609-2.691984$

H $6.685227-3.232233-0.566660$

C $6.359417-1.122656-0.397609$

H $5.625264-1.759746-3.008311$

H $7.021748-2.838488-2.879236$

C $4.554140-3.945490-4.115285$

H $5.585194-4.738027-2.406814$

C $5.3447800 .015245-0.665932$

O $7.656289-0.752701-0.858227$

H $6.420330-1.2465280 .691075$

H $4.332606-0.371291-0.504023$

O $5.4388530 .425846-2.027144$

C 5.5613531 .2291780 .263759

C $4.6156202 .419947 \quad 0.022237$

H 5.4611660 .8906341 .301442

O $6.899690 \quad 1.710895 \quad 0.117414$

H 4.8642723 .1860190 .767471

H $4.8553662 .888301-0.941263$

H $3.869227-4.794154-4.206800$

H $4.019047-3.044927-4.434854$

H $5.385322-4.106729-4.809665$

C $3.190862-4.702287-1.193998$

H $8.270908-1.465078-0.613013$

H $4.6795770 .999829-2.221208$

H 7.0324512 .4171800 .772469
H $3.843765-5.392097-0.645699$

H $2.789307-5.247383-2.055879$

H $3.303602-2.715980-2.061855$

C 2.8605551 .9436452 .478204

O 2.7649151 .2877351 .209414

C 3.1095472 .0966040 .083295

C 2.2026003 .3348630 .032940

C $2.2358014 .113317 \quad 1.347357$

C 1.9498783 .1767022 .521989

H 3.9003662 .2422542 .660559

C 2.4587200 .9216473 .546414

H $2.8796781 .496520-0.805295$

O $2.5837394 .177743-1.048493$

H $1.1706763 .013107-0.157094$

H 3.1920094 .6341331 .473257

O $1.2339095 .128811 \quad 1.292339$

H 0.8964852 .8694872 .499934

O 2.1726223 .8721723 .746343

C $3.549014-0.1060113 .734245$

H 2.2951191 .3925304 .522376

H 1.5267490 .4084113 .279568

H $1.9869234 .945800-1.041913$

H 1.3269355 .6781812 .088538

H 1.5031344 .5735663 .812846

O $3.301588-1.2028412 .963139$

O $4.518306 \quad 0.0602824 .463778$

C $4.275719-2.2464553 .055632$

H $4.281790-2.6651134 .066337$

H $4.000189-3.0350422 .351522$

H $5.267389-1.8696052 .788816$

SCF Energy (B3LYP/6-31G**//MMFF $)=-3245.88791179$

01_369

MM̄FF Geometry

C $3.192092-3.1984600 .439812$

C $2.539969-4.3307190 .748084$

C $1.038844-4.4975210 .784359$

O $0.361283-3.2312860 .613906$

C $0.486197-5.501454-0.246173$

C $0.847352-5.254815-1.696886$

C $0.097490-4.549164-2.565628$

C $2.095262-5.948029-2.186946$

C $-1.196353-3.826884-2.314051$

C $-1.114515-2.372336-2.798549$

C $-2.493547-1.699907-2.820792$

C $-2.409551-0.320360-3.473561$

C $-3.7840970 .360540-3.510711$

O $-2.963184-1.559798-1.477823$

O $-3.584873 \quad 1.743854-3.842804$

C $-4.4004070 .325008-2.096587$

C $-4.674290-0.219799-4.616050$

O $\quad-5.756956 \quad 0.764420-2.117851$

C $-4.300612-1.047328-1.378341$

C $-4.615320-0.8575160 .120251$

O $-5.258989-1.983604-1.873834$

C $0.145930-2.4926821 .738162$

O $0.391402-2.8190252 .889169$

C $-0.408513-1.1847391 .325963$

C $-0.774443-0.3113822 .275503$

C -1.3237301 .0260092 .062532$

C -1.3304881 .5702040 .659811$

C $-4.515385-2.1396610 .956660$

C $-4.498893-1.8040732 .436701$

C $-5.823070-0.6061564 .176270$

O $-3.512648-2.0206763 .136954$

C -5.5980610 .8666464 .016813$

C -4.7201821 .5779264 .740395$

C -4.5189473 .0578434 .571991$

C -3.0658273 .4495024 .411595$

C -2.4314804 .1518535 .366012$

C -2.3654203 .1005853 .113507$

C -1.7622401 .7220123 .132065$

N -5.680764 -1.227918 2.872915

H $2.630236-2.3140780 .155707$

H $3.118444-5.2083821 .028444$

H $0.779516-4.9021931 .771907$

H $-0.606336-5.533827-0.148141$

H $\quad 0.823940-6.5106870 .026407$ 
H $\quad 0.442075-4.491964-3.598144$ H $2.252414-5.809992-3.262292$ H $2.984508-5.566314-1.679472$ H $2.026815-7.025597-2.004771$ H -1.982024 -4.353561 -2.869103 H -1.486552 -3.845714 -1.260033 H $-0.437363-1.817472-2.137489$ H $-0.685327-2.343059-3.808225$ H -3.180739 -2.346404 -3.378880 H -1.990027 $-0.387708-4.485042$ H $-1.718847 \quad 0.314328-2.900745$ H $-3.1052051 .783815-4.687951$ H $-3.8513421 .061702-1.494315$ H -4.211596 -0.067025 -5.598783 H $-5.640340 \quad 0.295054-4.658388$ H $-4.856329-1.290488-4.504172$ H $-5.7708041 .641606-2.537735$ H -3.929189 -0.1167390 .546245$ H -5.628696 -0.4508280 .228604$ H -5.692279-1.607601-2.655131 H $-0.492432-0.9740790 .266662$ H $-0.674020-0.606776 \quad 3.319375$ H -0.3206201 .5442170 .239635$ H -1.667732 2.6087150 .602500 H -1.9825550 .9783190 .012915$ H $-5.354831-2.8126960 .750561$ H -3.592864 -2.686087 0.730980 H $-5.121803-1.0592174 .884007$ H $-6.845292-0.7926444 .518498$ H $-6.200702 \quad 1.3765313 .268236$ H $-4.134248 \quad 1.0785395 .508477$ H -4.9471803 .5505195 .454754$ H -5.0838153 .4398243 .712293$ H -1.397414 4.4585005 .247193 H -2.9286814 .4313336 .288882$ H $-1.562193 \quad 3.8251342 .926372$ H -3.081947 3.2029762 .289979 H -1.671731 1.2629814 .116766 H -6.433473 -1.084428 2.206521 C $5.151923-1.9680401 .464299$ O $4.685291-0.6601441 .066141$ C $4.267953 \quad 0.0515902 .246948$ C $4.795178-0.7493993 .435555$ C $4.699472-2.1661092 .911667$ H 3.1722410 .0217722 .260375 C 4.7598141 .5021622 .228901 H $5.837976-0.4995243 .664093$ H $4.203395-0.5839304 .341041$ C $5.536437-3.1669613 .688209$ H $3.647174-2.4717142 .959062$ C 4.2707392 .3731631 .053936 O $\quad 6.1943411 .5099142 .197134$ H 4.4835531 .9848313 .174132 H 4.7778242 .0828660 .126324 O $4.689216 \quad 3.717310 \quad 1.336191$ C 2.7479462 .3798610 .824466 C $2.2178623 .548624-0.028398$ H $2.449481 \quad 1.4304230 .369036$ O 2.0704102 .4520472 .084770 H $1.1340773 .416789-0.125687$ H 2.3280364 .4799260 .542692 H $5.438828-4.1699063 .260747$ H $6.597693-2.8970063 .678086$ H $5.211344-3.2113954 .732788$ C $4.684859-3.0379010 .470954$ H 6.4713591 .0206641 .403261 H 5.6547193 .7014381 .452394 H 2.3585243 .2683612 .528135 H $5.170207-3.9919630 .707759$ H $5.006552-2.753229-0.537683$ H $6.247952-1.9185231 .418560$ C $1.4772282 .183329-2.675169$ O $2.7878152 .538415-2.219679$ C $2.8609213 .719317-1.418504$ C $2.3094604 .917251-2.205605$ C $0.8875044 .655392-2.695166$ C $0.8219823 .332784-3.455523$ H $0.8568401 .902097-1.816924$
C $1.6251700 .957552-3.590568$

H $3.9277593 .913240-1.257548$

O $2.3424276 .092155-1.402747$

H $2.9577925 .101869-3.071934$

H $0.1732514 .667166-1.864477$

O $0.4838875 .716387-3.564811$

H $1.3147533 .452108-4.429003$

O $-0.5422973 .004022-3.710455$

C $2.170844-0.252429-2.867925$

H $0.6603300 .674178-4.024188$

H $2.3252501 .200130-4.399957$

H $1.9553656 .814203-1.926756$

H $1.1047035 .745652-4.312671$

H $-0.9411173 .758088-4.177656$

O $2.618994-1.150296-3.794722$

O $2.190527-0.398499-1.654342$

C $3.200181-2.347721-3.272494$

H $2.559791-2.788650-2.505717$

H $3.304219-3.062723-4.092881$

H $4.191458-2.127568-2.867269$

SCF Energy $(B 3 L Y P / 6-31 G * * / / M M F F)=-3245.91600061$

01_37

MMFF Geometry

C 1.8268560 .6009652 .527597

C $1.950413-0.4303033 .377728$

C $1.023936-1.6207063 .473712$

O $-0.142566-1.3755502 .652653$

C $1.699369-2.9108532 .968209$

C $2.955755-3.3239803 .705927$

C $4.197733-3.2045893 .194635$

C $2.738716-3.9438665 .059498$

C $4.552788-2.5681471 .879572$

C $4.559078-3.5808140 .729078$

C $4.430080-2.937261-0.660841$

C $5.577213-1.979897-0.984050$

C $5.386457-1.313130-2.354572$

O $3.178120-2.242780-0.706285$

O $6.310369-0.217305-2.443661$

C $3.966924-0.712267-2.423395$

C $5.727421-2.263365-3.508212$

O $3.692576-0.215000-3.731749$

C $2.847257-1.688037-1.985232$

C $1.510128-0.911128-1.839492$

O $2.711318-2.705242-2.975904$

C $-1.281907-2.0632772 .917952$

O $-1.468746-2.8708803 .812406$

C $-2.266472-1.6667041 .887281$

C $-3.408124-2.3553911 .748425$

C $-4.452664-2.0919280 .755871$

C $-4.233959-0.945373-0.195925$

C $0.349380-1.694165-1.205306$

C $-0.405706-2.594004-2.168215$

C $-2.091056-4.433034-2.192580$

O $-0.256710-2.547589-3.386258$

C $-3.461825-3.877971-2.420204$

C $-4.586317-4.520063-2.067783$

C $-5.968574-3.995057-2.334131$

C $-6.843458-3.989715-1.099864$

C $-7.739884-4.967313-0.884175$

C $-6.740849-2.806380-0.164110$

C $-5.541463-2.8888280 .737192$

N -1.276561 $-3.448081-1.504818$

H 0.9886580 .6388561 .837015

H $2.794652-0.4332904 .064336$

H $0.708096-1.7226324 .519305$

H $1.885200-2.8140531 .891304$

H $0.983282-3.7418603 .024140$

H $5.042950-3.5661133 .777134$

H $3.677369-4.2623805 .525034$

H $2.261287-3.2280825 .735993$

H $2.095591-4.8259634 .975221$

H $3.872150-1.7382521 .667813$

H $5.545505-2.1113701 .975893$

H $5.473686-4.184500 \quad 0.776449$

H $3.714604-4.2717530 .852734$

H $4.404129-3.755567-1.390234$

H $6.542420-2.498901-0.937131$ 
H $5.625967-1.183721-0.229220$

H $7.208541-0.572578-2.328709$

H $3.9502570 .155265-1.750065$

H $6.766886-2.603923-3.428945$

H $5.652928-1.753281-4.474962$

H $5.091145-3.150405-3.536107$

H $4.3938180 .422082-3.951602$

H $1.690989-0.052849-1.179997$

H $1.198453-0.514403-2.813032$

H $\quad 1.858290-3.148592-2.852885$

H -2.011324 -0.8254971 .252307$

H $-3.593428-3.1965272 .415141$

H -3.298806 -1.074302 -0.751987

H $-4.184364-0.0006930 .354576$

H -5.021090 -0.838519 -0.944511

H $0.695804-2.281970-0.349115$

H $-0.383499-0.968934-0.828647$

H -2.107495 -5.332179 -1.568548

H -1.630230 -4.683294 -3.153391

H $-3.533423-2.916741-2.923541$

H $-4.512591-5.495768-1.592523$

H -6.420816 -4.630396 -3.107064

H - $-5.938081-2.985521-2.762287$

H $-8.391398-4.955941-0.016760$

H -7.837924 -5.804348 -1.567538

H $-7.627596-2.7605060 .482464$

H $-6.778109-1.882048-0.746986$

H $-5.602989-3.6985281 .465809$

H -1.380142 $-3.368669-0.497438$

C 3.3450061 .9164601 .032703

O 2.2827352 .3619150 .163400

C $2.7171523 .534684-0.544318$

C $4.2368103 .523847-0.451966$

C 4.4420052 .9801830 .947700

H $2.331327 \quad 4.397544 \quad 0.012673$

C $2.1560343 .553315-1.969309$

H $4.6857012 .845767-1.186907$

H $4.6706994 .517889-0.595757$

C 5.8394122 .4343611 .188974

H 4.2437623 .7844731 .668866

C $0.6316163 .336755-2.060137$

O $2.7811302 .508514-2.722765$

H $2.4414684 .492179-2.458792$

H $0.3993752 .299516-1.794960$

O $0.2635203 .478628-3.439120$

C $-0.2182244 .298058-1.209052$

C -1.728883 4.234818-1.504093

H $-0.0404404 .102092-0.147634$

O $0.2177835 .636201-1.467958$

H -2.218362 $5.020876-0.916680$

H $-1.8998004 .524460-2.548270$

H 5.9321672 .0360702 .204311

H 6.0818361 .6283240 .487793

H 6.5875403 .2239581 .064763

C 2.8013661 .7428012 .453089

H $2.4048612 .529636-3.619384$

H $0.4191264 .405482-3.689866$

H $-0.2734846 .225439-0.870450$

H 2.2749792 .6532202 .763163

H 3.6300371 .5848593 .152941

H 3.7004250 .9568190 .636406

C $-2.969115 \quad 3.225410 \quad 1.105337$

O $-2.2226442 .462436 \quad 0.147283$

C $-2.3807752 .867431-1.215966$

C $-3.8584742 .783773-1.619915$

C $-4.7423513 .596182-0.679988$

C $-4.471455 \quad 3.215357 \quad 0.774787$

H $-2.599140 \quad 4.257700 \quad 1.121049$

C -2.7081252 .5959332 .485252$

H -1.852579 2.121850-1.821222

O $-4.019618 \quad 3.229672-2.962137$

H -4.177177 $1.734395-1.590557$

H -4.611531 $4.673830-0.834504$

O $-6.1156553 .312528-0.960635$

H -4.9036492 .2257270 .968925$

O $\quad \begin{array}{llll}-5.161679 & 4.154543 & 1.599662\end{array}$

C -1.344691 2.9482423 .042330

H -3.4447722 .9574443 .210142$
H -2.7685851 .5035232 .425722$

H $-4.9358093 .043186-3.227477$

H $-6.3147713 .670256-1.842206$

H -5.2334113 .7751032 .491008$

O -1.3156602 .6208594 .368716$

O -0.4239383 .4365532 .403560$

C -0.1106502 .9390275 .072926$

H -0.3330252 .9320026 .143451$

H $\quad 0.6454482 .1780374 .867717$

H 0.2582663 .9331944 .802692

SCF Energy (B3LYP/6-31G**//MMFF) $=-3245.91359721$

01_370

MMFF Geometry

C $2.086961-3.3818190 .517795$

C $1.100675-3.5960751 .401816$

C $0.111458-2.5316141 .793092$

O $-1.204379-3.0395911 .462541$

C $0.144222-2.1989183 .295959$

C $1.419602-1.5587373 .791729$

C $1.727721-0.2574363 .626096$

C $2.323576-2.4597384 .590202$

C 0.9395730 .7850252 .882112

C -0.0122841 .5545563 .802592$

C -0.5835662 .8117953 .128476$

C -1.5589543 .5247414 .068027$

C -2.1944484 .7589693 .410665$

O -1.2540612 .4430431 .915468$

O -3.3163715 .1554874 .215755$

C -2.7494244 .3597532 .025595$

C -1.2338405 .9543083 .392644$

O -3.2123345 .5194231 .337481$

C $-1.737688 \quad 3.5693371 .163531$

C $-2.3431873 .048387-0.160021$

O $-0.657898 \quad 4.4392190 .795516$

C $-2.194190-2.1187741 .304550$

O $-2.090887-0.9061881 .401082$

C $-3.444288-2.8439560 .997830$

C $-4.581133-2.148960 \quad 0.849371$

C $-5.884870-2.7301520 .550831$

C $-7.008074-2.1133331 .339545$

C $-3.5850062 .163734-0.034045$

C $-4.0575601 .674953-1.395090$

C $-5.9510720 .545700-2.509664$

O $-3.4573031 .915555-2.440077$

C $-6.999105-0.466586-2.177796$

C -7.128312 -1.633580 -2.827716

C $-8.211336-2.636731-2.546375$

C $-7.668303-4.020123-2.263698$

C $-7.589148-4.943476-3.236536$

C $-7.296144-4.355552-0.836801$

C $-6.029672-3.676649-0.398001$

N $-5.2332320 .937724-1.310098$

H $2.185420-2.3993780 .063886$

H $\quad 0.985984-4.5786351 .852359$

H $0.292201-1.634377 \quad 1.191627$

H $-0.066891-3.1089713 .875351$

H $-0.696953-1.5402463 .547120$

H 2.6517720 .1120504 .068778

H $3.273093-1.9774514 .845270$

H $1.832944-2.7505545 .525055$

H $2.556120-3.3696084 .030021$

H $1.654770 \quad 1.4760892 .418439$

H 0.3749000 .3439302 .057807

H -0.8341680 .8865334 .090271$

H $0.507393 \quad 1.8459054 .724188$

H $\quad 0.2595913 .4695692 .885674$

H -1.063450 3.8029205 .006612

H $-2.3637542 .830823 \quad 4.349123$

H -2.990785 5.3410685 .113254

H $-3.630483 \quad 3.7343462 .217658$

H -0.9457126 .2288544 .414582$

H -1.715989 6.8425692 .969722

H -0.3156585 .7600642 .834756$

H -3.7200785 .2242190 .563493$

H $-2.5611913 .895906-0.823519$

H - $1.5712912 .470215-0.685109$

$\mathrm{H}-0.2034364 .0564430 .026401$ 
H $-3.406934-3.9236950 .903449$ H $-4.579659-1.0685040 .976409$ H -7.005578 -1.0228501 .228757$ H -7.997930 -2.454296 1.029778 H $-6.896800-2.3496742 .403547$ H $-3.372190 \quad 1.2926730 .592340$ H -4.4090342 .7262270 .416695$ H $-5.2315190 .176908-3.249119$ H $-6.4213971 .447164-2.916897$ H -7.705999 -0.214513-1.390905 H $-6.438023-1.869310-3.634846$ H -8.870395 -2.669510 -3.423968 H -8.848325 -2.316699-1.712436 H -7.226724 -5.946521 -3.037100 H -7.880642 $-4.714737-4.256308$ H $-7.137068-5.436929-0.730212$ H -8.145488 $-4.132107-0.184983$ H $-5.142067-4.019026-0.931239$ H $-5.739567 \quad 0.946025-0.428835$ C $4.529961-4.0668520 .330912$ O $4.868541-2.803205-0.275894$ C $5.752508-2.0922860 .616308$ C $6.186855-3.1020781 .673470$ C $4.934419-3.9484281 .797869$ H $5.150518-1.3194831 .108281$ C $6.899661-1.447989-0.167342$ H $7.029567-3.7133681 .329906$ H $6.477012-2.6210522 .612456$ C $5.159432-5.2790132 .492148$ H $4.187926-3.3732072 .356428$ C $6.429072-0.453239-1.249945$ O $7.641349-2.489477-0.814406$ H $7.593484-0.9584980 .526115$ H $5.862200-0.994716-2.014603$ O $7.5944420 .057333-1.910146$ C $5.6040990 .730334-0.708983$ C $5.2404011 .783610-1.772835$ H $4.6879480 .360580-0.238176$ $\begin{array}{lllll}\text { O } & 6.364857 & 1.380142 & 0.313709\end{array}$ H $4.7851572 .638512-1.258185$ H $6.1561612 .187085-2.222523$ H $4.228634-5.8522602 .546318$ H $5.901144-5.8874961 .964080$ H $5.519184-5.1229133 .514516$ C $3.066306-4.4234800 .058526$ H $8.355240-2.064889-1.320405$ H $8.0949480 .578668-1.258959$ H 5.8074242 .0799790 .694575 H $2.835357-5.399248 \quad 0.501215$ H $2.930781-4.526467-1.025694$ H $5.157821-4.819039-0.165564$ C $2.2098681 .462788-1.673629$ O $3.1497300 .597900-2.318910$ C $4.2929591 .260079-2.870556$ C $3.8502292 .322496-3.886782$ C $2.8233753 .289656-3.297634$ C $1.6733952 .524975-2.639765$ H $2.7079251 .962942-0.833478$ C $1.1104520 .600797-1.038606$ H $4.848070 \quad 0.498480-3.431225$ O $4.981413 \quad 3.042453-4.365031$ H $3.4027891 .815713-4.751855$ H $3.2945643 .975393-2.584526$ O $2.3013194 .116588-4.340692$ H $1.0531882 .066767-3.418754$ O $0.8492623 .439784-1.922441$ C $0.279691-0.185000-2.031616$ H $1.566167-0.130540-0.362961$ H $\quad 0.4473531 .241902-0.448244$ H $4.656343 \quad 3.716811-4.985934$ H $1.8753353 .539688-4.997415$ H $0.5484114 .116277-2.553306$ O $-0.953657-0.424635-1.497066$ O $0.672263-0.573785-3.123148$ C -1.842913 -1.187097 -2.319211 H $-2.812465-1.238433-1.817667$ H -1.978792 -0.701203 -3.290260 H $-1.457827-2.203270-2.444582$
SCF Energy (B3LYP/6-31G**//MMFF)= -3245.90623717

01_371

MMFF Geometry

C $3.564185-0.1758611 .909155$

C $3.589304-0.8617963 .063615$

C $2.408544-1.5279023 .736062$

O $1.196638-1.2807922 .992003$

C $2.622705-3.0535203 .794914$

C $1.497353-3.8062654 .486484$

C $0.668296-4.6787523 .875868$

C $1.348086-3.5375395 .961800$

C $0.690162-5.0903322 .429029$

C $-0.250992-4.2229851 .591039$

C $0.081305-4.1835690 .091420$

C $1.431909-3.511226-0.175906$

C $1.703029-3.320367-1.672314$

O $-0.966712-3.425788-0.533157$

O $2.813445-2.422272-1.798402$

C $0.483846-2.631786-2.313446$

C $2.125803-4.628692-2.350277$

O $0.633712-2.546131-3.728657$

C $-0.859505-3.310613-1.959001$

C $-2.031898-2.434345-2.465086$

O $-0.922207-4.587516-2.596114$

C $0.340640-0.3270513 .444849$

$\begin{array}{lllll}\text { O } & 0.466534 & 0.375426 & 4.433963\end{array}$

C $-0.798981-0.2905922 .501431$

C -1.8678260 .4785612 .753856$

C -3.0483820 .5970131 .896724$

C $-3.139015-0.3229370 .707558$

C $-3.414609-2.893433-1.984338$

C $-4.521251-1.956032-2.441000$

C $-6.951963-1.513915-2.060640$

O $-4.357143-1.077632-3.283006$

C $-6.985463-0.273523-1.224983$

C -7.525099 -0.2167100 .002096$

C $-7.604874 \quad 1.0349760 .829033$

C -6.5069161 .1080611 .868909$

C -6.7014140 .5967543 .096647$

C -5.2448161 .8191511 .423721$

C -4.0003421 .5003972 .210650$

N -5.722969 -2.241685 -1.808114

H $2.630094-0.0621151 .366000$

H $4.536315-0.9516833 .592693$

H $2.326998-1.1264114 .753042$

H $3.559458-3.2779644 .322325$

H $2.761621-3.4218742 .773451$

H $-0.113340-5.1527704 .467523$

H $\quad 0.606537-4.1935346 .430263$

H $2.300535-3.7024736 .476042$

H $1.029777-2.5053696 .136665$

H $1.713834-5.0834022 .050899$

H $0.372936-6.1383912 .358268$

H -1.280737 -4.583818 1.719752

H $-0.270390-3.1957901 .968432$

H $\quad 0.064570-5.207236-0.299785$

H $2.259859-4.069010 \quad 0.273740$

H $1.444318-2.5226090 .304431$

H $3.571695-2.813405-1.331473$

H $0.457309-1.597755-1.941537$

H $3.047131-5.014542-1.897238$

H $2.358023-4.467885-3.408839$

H $1.370807-5.413929-2.276711$

H $1.486240-2.112064-3.903966$

H -1.885069 -1.402649 -2.119816

H -2.030845 -2.411837 -3.562354

H -1.850631 -4.848054 -2.697473

H $-0.723956-0.9016681 .608992$

H -1.8767891 .0845383 .658519$

H $-3.048026-1.3679131 .024957$

H -2.343161 -0.104157 -0.011553

H $-4.088414-0.2495840 .173844$

H $-3.649817-3.888915-2.375129$

H -3.435212 -2.943720 -0.890387

H -7.786014 -2.183054 -1.825232

H -7.012107 -1.251258 -3.121865

H $-6.5614730 .626177-1.664293$ 
H -7.943656 -1.119648 0.440716 H $-8.5904981 .064463 \quad 1.311570$ H $-7.572418 \quad 1.9292220 .193252$ H -5.9477580 .6520423 .873962$ H $-7.6355390 .109242 \quad 3.357517$ H -5.4206882 .9011841 .485994$ H $-5.065856 \quad 1.6258980 .360252$ H -3.8688262 .1139443 .102165$ H -5.738246 -2.944204 -1.074572 C $5.0182310 .019637-0.134932$ O $3.9728070 .516349-0.998021$

C $4.5728581 .152747-2.141430$ C $6.002740 \quad 0.634817-2.190951$

C $6.3315480 .552933-0.713203$ H $4.5943842 .228074-1.925520$

C $3.7426310 .901949-3.406607$

H $6.059829-0.361045-2.645301$

H $6.669723 \quad 1.302291-2.744531$

C $7.537153-0.318845-0.406308$

H $6.5216751 .568204-0.339626$

C $2.2450461 .243501-3.257093$

O $3.836294-0.478044-3.776216$

H $4.1739191 .460622-4.244982$

H $1.7740650 .545881-2.555778$

O $1.6128871 .031177-4.524906$

C $1.9741272 .692877-2.820356$

C $0.4875003 .089973-2.798675$

H $2.4008762 .864043-1.827561$

O $2.6486173 .589944-3.707942$

H $0.4375594 .160225-2.562212$

H $0.0675153 .012190-3.809649$

H $7.724152-0.3606370 .671289$

H $7.392192-1.343987-0.763117$

H $8.4341840 .081375-0.890006$

C $4.7688390 .489672 \quad 1.301376$

H $3.521773-1.004333-3.021587$

H $1.7992480 .114735-4.791663$

H $2.3086023 .425894-4.604500$

H 4.5869521 .5716611 .309400

H $5.6565480 .298972 \quad 1.915236$

H $4.986076-1.075788-0.192347$

C 0.2868053 .4154620 .215012

O $0.2217262 .181891-0.513684$

C $-0.3776402 .283507-1.808915$

C $-1.8188712 .798703-1.677240$

C $-1.8872124 .100666-0.879218$

C -1.1237443 .9749600 .441523$

H $0.8867364 .136200-0.352101$

C $1.0157253 .112371 \quad 1.532276$

H $-0.4368071 .260426-2.200059$

O $-2.4061363 .000753-2.958006$

H -2.426902 $2.045237-1.162556$

$\mathrm{H}-1.5065114 .942464-1.468472$

O $-3.2541054 .414696-0.600641$

H $-1.681930 \quad 3.322557 \quad 1.122227$

O $-1.0403175 .256543 \quad 1.057328$

C 1.6741424 .3181832 .163425

H $\quad 0.3147662 .6705852 .248783$

H 1.8079262 .3755831 .355596

H $-2.3691262 .153053-3.433384$

H $-3.6301753 .682479-0.083902$

H -1.947781 5.5927571 .154382

O 1.9307184 .0452603 .477268

O 1.9605135 .3533791 .578332

C 2.5776425 .0917214 .207941

H 2.7147754 .7525225 .238205

H 3.5594845 .3045883 .774663

H 1.9542565 .9909524 .214722

SCF Energy (B3LYP/6-31G**//MMFF) $=-3245.91712949$

01_372

MM̄MF Geometry

C $-1.881900-4.141888-1.374653$

C $-0.684546-4.063092-0.776646$

C $0.540416-3.488291-1.438924$

O $0.905188-2.278715-0.734488$

C $1.713599-4.480834-1.349799$

C $3.025196-3.935803-1.885504$
C $4.172647-3.909682-1.178745$

C $3.005736-3.421540-3.301483$

C $4.400063-4.4560680 .201744$

C 4.155451-3.456601 1.339994

C $5.163758-2.2978631 .369335$

C $5.130784-1.5970712 .730130$

C $6.086808-0.3968762 .775764$

O $4.819811-1.3624670 .340777$

$\begin{array}{lllll}\text { O } & 5.772820 & 0.373454 & 3.945908\end{array}$

C $5.8102120 .501347 \quad 1.554925$

C $7.549836-0.8313282 .921348$

O 6.7702201 .5518571 .465450

C $5.740030-0.2699880 .214116$

C $5.2276640 .679666-0.892185$

O $7.048874-0.724098-0.137060$

C $0.297217-1.132375-1.147739$

O $-0.539209-1.024482-2.031375$

C $0.826537-0.012153-0.338767$

C $0.3149151 .212546-0.532263$

C 0.7199862 .4485740 .136142

C 1.7518242 .3572251 .226822

C $4.831962-0.010248-2.202145$

C $4.5045251 .034636-3.250890$

C $2.8109852 .784267-3.804263$

O $5.2947351 .313026-4.149787$

C $2.7991433 .975916-2.896608$

C $1.7508884 .804188-2.771896$

C $1.7225075 .980455-1.836053$

C $0.5028515 .987164-0.937040$

C $-0.4829376 .882605-1.117245$

C 0.4531564 .9993890 .212835

C $0.1418413 .603544-0.253901$

N $3.273861 \quad 1.637229-3.043365$

H -1.993951 -3.790170 -2.397269

H $-0.582294-4.3955460 .253414$

H $0.343085-3.262828-2.494837$

H $1.464524-5.393132-1.907527$

H $1.827305-4.782137-0.302914$

H $5.058839-3.483546-1.646945$

H $4.010190-3.190104-3.672173$

H $2.576173-4.173190-3.971785$

H $2.411511-2.505491-3.373894$

H $3.777966-5.3432530 .366405$

H $5.432157-4.8255100 .256710$

H $3.133658-3.0629651 .267362$

H $4.223195-4.0165932 .281210$

H $6.164671-2.7015471 .176756$

H $5.365900-2.3009543 .538286$

H $4.111044-1.2387992 .930713$

H $5.874618-0.2047324 .721462$

H 4.8382890 .9852641 .724542

H $7.685844-1.4168203 .838655$

H 8.2113660 .0364103 .020081

H $7.902170-1.4401232 .086198$

H 6.7697302 .0182372 .318937

H $4.3441621 .213535-0.524808$

H $5.9906121 .439311-1.107714$

H $7.102538-0.823361-1.100575$

H $1.618598-0.2195830 .371175$

H $-0.474674 \quad 1.331550-1.273917$

H 2.7280272 .0967160 .810701

H 1.4700601 .5977231 .965051

H 1.8702033 .2879541 .786932

H $5.639063-0.638870-2.591466$

H $3.958760-0.655819-2.057660$

H $1.8121022 .542684-4.180535$

H $3.4765542 .967283-4.653131$

H $3.6952034 .162564-2.308774$

H $0.8636504 .631796-3.377026$

H $1.7457946 .891539-2.447878$

H $2.6241736 .014502-1.211804$

H -1.350153 $6.907426-0.465674$

H $-0.4479787 .607706-1.923614$

H $-0.3235855 .294470 \quad 0.929388$

H 1.4049255 .0586140 .749399

H $-0.6403153 .556350-1.013324$

H $2.6967401 .324406-2.268566$

C $-4.042191-3.523223-0.280397$ 
O $-4.532557-2.826271-1.444767$

C $-5.949198-2.652866-1.310673$

C $-6.269190-2.8927000 .157646$

C $-5.278184-3.9923830 .489146$

H $-6.410472-3.465137-1.890243$

C $-6.424457-1.325375-1.909006$

H $-6.063824-2.0070270 .768457$

H $-7.309430-3.1894170 .320363$

C $-5.046277-4.1695781 .979845$

H -5.646442 -4.9377840 .068591$

C $-6.063373-0.025875-1.156207$

O $-7.854587-1.389387-2.013905$

H $-6.051112-1.250183-2.937456$

H $-6.594143-0.007692-0.199418$

O $-6.6136921 .067099-1.909169$

C $-4.560470 \quad 0.237760-0.924120$

C $-4.2470001 .649548-0.379847$

H $-4.157045-0.507488-0.233828$

O $\quad-3.852320 \quad 0.079163-2.155952$

H $-3.1640911 .806103-0.444917$

H $-4.6759162 .397750-1.056677$

H $-4.313700-4.9603172 .169566$

H -4.674700 -3.248011 2.440633

H $-5.978481-4.4446022 .483991$

C $-3.117784-4.666426-0.700372$

H -8.220089 -1.401074 -1.112995

H $-6.2188731 .046890-2.797140$

H $-4.1366010 .786391-2.759268$

H -3.629475 $-5.330496-1.407433$

H -2.846091 -5.2698830 .173640$

H $-3.486144-2.7897300 .312752$

C -2.7076801 .3588662 .264755$

O $-4.074593 \quad 1.0178221 .995050$

C -4.7430881 .8766751 .062709$

C -4.6863163 .3339531 .541927$

C -3.2637583 .7829501 .855330$

C -2.599877 2.7896852 .807621

H $-2.118731 \quad 1.2746831 .345593$

C -2.1674340 .3404023 .281690$

H -5.8004131 .5913411 .090442$

O $\quad-5.2705354 .1946880 .569947$

H -5.2917243 .4271722 .453307$

H $-2.683785 \quad 3.9123470 .934881$

$\begin{array}{lllll}\text { O } & -3.330451 & 5.066611 & 2.478989\end{array}$

H -3.070474 2.8554623 .797033

O -1.2241413 .1288272 .961010$

C -1.840325 -0.9916432 .641421$

H $-1.2393240 .701423 \quad 3.737954$

H -2.914946 0.1659954 .064430

H $-5.197320 \quad 5.1045690 .905667$

H -2.420812 5.3980672 .565470

H -1.180261 3.9935533 .402279

O $-1.533871-1.8924863 .621345$

O $-1.825221-1.2119831 .438935$

C -1.153807 -3.193333 3.161880

H $-0.986631-3.8276944 .036240$

H -1.951353 -3.634016 2.556804

H $-0.223326-3.1315712 .589461$

SCF Energy (B3LYP/6-31G**//MMFF) $=-3245.89255092$

01_373

MMFF Geometry

C $-0.1386334 .702907-0.195123$

C $-1.0453634 .824563-1.177234$

C $-2.3008663 .990027-1.295597$

O $-2.0637392 .645997-0.813601$

C $-3.4321424 .619186-0.463194$

C $-4.7540043 .884341-0.567790$

C -5.3726443 .2754920 .463302$

C $-5.3956873 .882931-1.931459$

C -4.9207853 .1906281 .894887$

C -4.0764681 .9506792 .227746$

C -4.8317310 .6198522 .082301$

C $-4.005722-0.5388732 .647126$

C $-4.702556-1.8936822 .435784$

$\begin{array}{llll}\text { O } & -5.082427 & 0.390229 & 0.693711\end{array}$

O $-3.756195-2.9330452 .728439$

C $-5.122539-2.0245510 .953690$
C $-5.845309-2.099436 \quad 3.436259$

O $-5.917884-3.189107 \quad 0.750490$

C $-5.861308-0.7815910 .416024$

C $-6.094968-0.822787-1.113787$

O $-7.151885-0.6982791 .033243$

C $-1.3818681 .805251-1.640680$

O $-0.9794262 .047941-2.767507$

C $-1.1719770 .532814-0.916804$

C $-0.255304-0.336133-1.368462$

C $0.096611-1.618645-0.755450$

C $-0.747821-2.0929340 .396901$

C $-4.827908-0.915404-1.971375$

C $-4.479397-2.328862-2.407802$

C $-2.729514-3.596828-3.648664$

O $-5.213873-3.294890-2.221178$

C $-1.836712-4.275579-2.659057$

C $-0.585225-4.665261-2.945100$

C $0.306596-5.393143-1.981230$

C $1.640419-4.707977-1.791243$

C $2.732523-5.119662-2.457495$

C $1.726096-3.604464-0.762926$

C $1.145725-2.305839-1.253722$

N -3.259045 -2.376887 -3.069224

H -0.3183293 .9929760 .607897$

H $-0.8783675 .551821-1.968075$

H $-2.5919213 .942462-2.352108$

H -3.592786 $5.658228-0.780413$

H -3.1072434 .6709220 .581424$

H -6.3241902 .7820710 .268090$

H -6.407898 $3.464582-1.913709$

H $-5.4721994 .904950-2.316722$

H -4.807366 $3.285812-2.634738$

H -4.3603304 .0884892 .176309$

H -5.8129043 .2026362 .534188$

H -3.1882041 .9432361 .585758$

H -3.7277152 .0531373 .262774$

H -5.7846680 .7056332 .617488$

H $-3.787814-0.3827843 .710978$

H $-3.029487-0.5644242 .143903$

H -2.993597 -2.827036 2.135315

H $-4.204798-2.1685900 .372312$

H -5.461115 -2.075249 4.463284

H $-6.306186-3.0859103 .313710$

H $-6.627877-1.3420463 .358952$

H $-5.411871-3.9486111 .086727$

H $-6.814426-1.611527-1.364385$

H $-6.5919550 .118924-1.389003$

H -7.726439 -0.1488970 .474607$

H $-1.7563420 .359516-0.020762$

H $\quad 0.312275-0.075819-2.261023$

H $-0.514754-3.1087640 .722167$

H -1.805707 -2.102072 0.113993

H $-0.621857-1.4367541 .264418$

H $-4.993984-0.344879-2.894459$

H -3.969656 -0.468186-1.461027

H $-2.197680-3.317183-4.563596$

H $-3.556881-4.262273-3.915560$

H -2.247713 -4.482445 -1.673805

H $-0.187342-4.482004-3.940907$

H $\quad 0.461027-6.407600-2.371911$

H $-0.178379-5.524080-1.005893$

H $3.698926-4.650730-2.303841$

H $2.682563-5.931226-3.175891$

H $2.777313-3.411995-0.513557$

H $1.279114-3.9498040 .173157$

H $1.693311-1.876355-2.094074$

H -2.691629 -1.536477 -3.130886

C 2.3341154 .5260630 .035840

O $2.4390493 .665799-1.112922$

C $3.8339203 .365364-1.342436$

C $4.6126984 .077412-0.242167$

C 3.6871155 .2225090 .121741

H $4.0727463 .788888-2.325128$

C $4.0004471 .841567-1.352080$

H 4.7601223 .4182000 .622825

H $5.598474 \quad 4.411770-0.579721$

C 3.9881225 .8365911 .477297

H $3.7629655 .996023-0.654124$ 
C $5.4560931 .363843-1.469150$

O $3.253527 \quad 1.317381-2.455045$

H $3.5468731 .431230-0.445035$

H $6.0381991 .720135-0.612732$

O $6.038951 \quad 1.977925-2.625914$

C $5.610408-0.163453-1.613026$

C $4.902333-0.997890-0.527975$

H $5.238785-0.471082-2.598140$

O $7.009389-0.462018-1.606387$

H $3.830134-0.788042-0.573736$

H $5.015074-2.052344-0.807560$

H 3.2956816 .6546941 .698532

H 3.9033025 .0971232 .280700

H 5.0053376 .2410941 .498655

C $1.1482895 .476226-0.132555$

H $2.3369481 .628393-2.357608$

H $6.9569781 .662891-2.688606$

H $7.104195-1.424329-1.709950$

H $1.2747436 .072024-1.044697$

H $1.097127 \quad 6.1681360 .714986$

H 2.1800813 .8789130 .910387

C $3.426567-1.3163152 .121283$

O $4.427577-0.3422821 .804369$

C $5.452332-0.7883030 .905552$

C $6.170630-2.0073441 .504568$

C $5.189692-3.1192871 .869358$

C $4.059739-2.5694512 .737596$

H $2.872622-1.5861841 .216471$

C $2.442324-0.6693853 .109073$

H 6.1864430 .0239990 .878888

O $7.149958-2.4903800 .591674$

H $6.702561-1.6931842 .412288$

H $4.791365-3.6169500 .978042$

O $5.870686-4.1258342 .621884$

H $4.447819-2.3415273 .738789$

O $3.056720-3.5732482 .887228$

C $1.597294 \quad 0.4159162 .476186$

H $1.753624-1.4254373 .502312$

H $2.994057-0.2118833 .938890$

H $7.672384-3.1693361 .050672$

H $6.511122-4.5527352 .028104$

H $3.488198-4.3610853 .260176$

$\begin{array}{lllll}\text { O } & 0.700365 & 0.868548 & 3.402745\end{array}$

$\begin{array}{lllll}O & 1.706281 & 0.822594 & 1.328582\end{array}$

C $-0.171596 \quad 1.9122552 .958924$

H -0.9040452 .1028043 .747781$

H 0.4023812 .8264332 .782830

H -0.7047231 .6092202 .053558$

SCF Energy (B3LYP/6-31G**//MMFF) $=-3245.90333516$

\section{4}

MMFF Geometry

C $4.462103 \quad 3.337826-0.659447$

C $3.1867623 .355884-0.245692$

C $2.0554853 .870323-1.100227$

O $1.0290842 .855948-1.180464$

C $1.4400585 .118958-0.446548$

C $0.4067185 .798935-1.322959$

C $-0.926798 \quad 5.727195-1.142365$

C $0.9695506 .643535-2.438143$

C $-1.6821734 .951006-0.098115$

C $-2.7459454 .056628-0.741044$

C $-3.662556 \quad 3.3581290 .275504$

C -2.9082292 .3750861 .173181$

C -3.8522601 .5797312 .087244$

O $-4.6583142 .663515-0.487604$

O $-3.111452 \quad 0.4825832 .640784$

C -4.9861630 .9745001 .232999$

C -4.3279862 .4186863 .279623$

O $\begin{array}{llll}-5.967420 & 0.353897 & 2.059891\end{array}$

C $-5.6593192 .002632 \quad 0.300445$

C $-6.6693721 .356972-0.678004$

O $\begin{array}{lllll}-6.391876 & 2.945136 & 1.093610\end{array}$

C $1.2422451 .813199-2.028571$

O $2.209698 \quad 1.626138-2.748076$

C $0.0897700 .900056-1.889988$

C $0.253871-0.409753-2.124397$

C $-0.763418-1.440843-1.917904$
C $-2.180534-0.989429-1.675511$

C $-6.0832030 .335016-1.659028$

C $-6.387243-1.113205-1.305222$

C $-6.285295-3.396877-2.321563$

O $-6.897801-1.461999-0.245294$

C $-5.028289-4.133137-1.986474$

C $-4.252860-4.725936-2.907195$

C $-3.013322-5.511903-2.589539$

C -1.751932-4.710293 -2.828285

C $-1.140898-4.745221-4.024845$

C $-1.243607-3.937158-1.629548$

C $-0.388051-2.736926-1.938379$

N $-6.020932-1.970843-2.333913$

H $4.6982113 .724662-1.647905$

H 2.9378752 .9883120 .746754

H $2.402374 \quad 4.110182-2.113135$

H $2.2282035 .850247-0.220793$

H 1.0069324 .8532840 .524326

H -1.561402 $6.296402-1.821134$

H $0.187767 \quad 7.162613-3.002992$

H $1.6429867 .406473-2.033872$

H $1.5291826 .025058-3.146360$

H -1.012722 4.3382980 .508708

H -2.165660 5.6673690 .576377

H -3.375356 $4.653893-1.415356$

H $-2.2695393 .306148-1.385065$

H $-4.155416 \quad 4.1302170 .877867$

H -2.1571302 .8913541 .780273$

H -2.3531751 .6652840 .546075$

H $-2.339270 \quad 0.845122 \quad 3.107958$

H -4.5412300 .1771150 .623953$

H -3.472782 2.7375003 .888042

H $-4.966774 \quad 1.8302273 .947460$

H -4.872148 3.3175582 .983437

H -5.506879 -0.2958792 .618142$

H $-7.5237170 .948495-0.124839$

H -7.088577 2.170380 -1.288261

H -7.0687923 .3605510 .534122$

H $-0.8400541 .318484-1.524384$

H $1.234862-0.767244-2.434580$

H $-2.904957-1.805277-1.723312$

H $-2.493040-0.267077-2.438245$

H $-2.273760-0.522006-0.690131$

H $-6.5311500 .518255-2.644721$

H $-4.9998370 .450441-1.767660$

H $-6.659793-3.664203-3.315199$

H -7.065078 -3.633474 -1.590735

H $-4.759036-4.198790-0.935191$

H -4.526057 -4.653394 -3.957628

H -3.013693 -6.417630 -3.209880

H $-3.030971-5.875515-1.554068$

H -0.222632 -4.203767 -4.222041

H $-1.548110-5.329913-4.843629$

H $-0.658033-4.621562-1.001617$

H -2.087229 -3.635028 -0.999531

H $0.658140-2.976674-2.131238$

H -5.560896 -1.587716 -3.154788

C $6.3041671 .591621-0.356577$

O $5.4930230 .429301-0.104573$

C $5.797321-0.559685-1.111052$

C $6.8671980 .049008-2.021448$

C $6.6326031 .536065-1.845454$

H $4.879516-0.704271-1.692383$

C $6.225509-1.882247-0.459888$

H $7.880530-0.218489-1.700794$

H $6.756491-0.281542-3.059266$

C $7.8148082 .396328-2.252950$

H $5.7602881 .806804-2.452512$

C $5.208931-2.4691160 .538991$

O $7.449312-1.6550580 .252989$

H $6.455227-2.614173-1.244058$

H $5.163780-1.8415651 .436652$

O $5.702222-3.7482200 .959260$

C $3.791768-2.670504-0.026897$

C $2.853565-3.4881940 .882030$

H $3.336717-1.695451-0.229397$

O $3.867870-3.350103-1.283582$

H $\quad 1.898079-3.5981280 .354837$ 
H $3.233273-4.5137110 .974998$ H $7.5948713 .457744-2.100720$ H $8.7098002 .150648-1.671491$ H $8.0510862 .248786-3.311822$ C 5.6187902 .8566640 .168419 H $7.828653-2.5235180 .468552$ H $6.479465-3.5919051 .521615$ H $4.309733-4.202807-1.129947$ H $6.353592 \quad 3.6699380 .208689$ H 5.2873632 .6777041 .199048 H 7.2215841 .4479240 .231213 C $0.962546-1.2538051 .698590$ O $2.268228-1.5013122 .225011$ C $2.630890-2.8839562 .283230$ C $1.623868-3.6563903 .147530$ C $0.188060-3.4566122 .667663$ C -0.124004-1.9672912.512053 H $0.929995-1.6249070 .668481$ C $0.758863 \quad 0.261907 \quad 1.587040$ H $3.594258-2.9273602 .805311$ O $1.948705-5.0426293 .153079$ H $1.702021-3.3034474 .184211$ H $0.005518-4.0053881 .736439$ O $-0.694611-4.0197773 .640762$ H $-0.222161-1.5252663 .509271$ O $-1.388544-1.8232041 .870670$ C $\quad 0.820907 \quad 1.0240522 .893051$ H $\begin{array}{llll}1.551537 & 0.676284 & 0.952964\end{array}$ H -0.2111770 .4637301 .121104$ H $1.279078-5.495773 \quad 3.693644$ H -1.603702 -3.8436513 .342973$ H -1.326122 -2.2306900 .990419$ O 0.4146242 .3088682 .663113 $\begin{array}{lllll}\text { O } & 1.195190 & 0.573161 & 3.965724\end{array}$ C $0.4158603 .172160 \quad 3.803625$ H -0.2688942 .7908614 .567025$ H 1.4292023 .2689504 .204383 H 0.0692374 .1581013 .482568 SCF Energy (B3LYP/6-31G*//MMFF) $=-3245.89959759$

\section{5}

MM̄FF Geometry

C $-0.872842-2.228325 \quad 0.321075$

C $-2.012386-2.152008-0.382656$

C $-2.245236-1.355644-1.645114$

O $-1.083823-0.544384-1.941666$

C $-2.542399-2.263730-2.855163$

C $-3.796783-3.100462-2.750124$

C $-5.045277-2.620540-2.910174$

C $-3.572613-4.570573-2.509161$

C $-5.459774-1.196017-3.169527$

C $-6.602677-0.749389-2.245626$

C $-6.196194-0.597922-0.768399$

C $-7.450884-0.592370 \quad 0.108917$

C $-7.116904-0.3590911 .585655$

O $-5.483402 \quad 0.638415-0.617984$

O $-8.346060-0.0840992 .275330$

C -6.2426120 .9034291 .693902$

C $-6.517237-1.606998 \quad 2.245476$

O $-5.8154761 .070398 \quad 3.045410$

C -5.0346340 .9111050 .719954$

C $-4.378604 \quad 2.313667 \quad 0.737202$

O $-4.085918-0.0572991 .162873$

C - $1.2533610 .508903-2.782555$

O $-2.2853390 .849202-3.342886$

C $0.0247461 .206620-3.013193$

C $0.8965831 .419079-2.016679$

C $2.1483772 .168230-2.128244$

C $2.6343582 .503152-3.514750$

C $-3.2497302 .541283-0.279229$

C $-2.6990373 .957159-0.166168$

C -0.975433 $5.476398-1.082304$

O -3.2092704 .8154720 .551038$

C $0.4584945 .357745-1.488257$

C $1.4816575 .719220-0.697826$

C $2.9278685 .688718-1.108871$

C $3.7543344 .729413-0.279430$

C 4.2823305 .1077420 .896843
C $4.0326143 .359353-0.858455$

C $2.7827522 .533893-0.995019$

N - $1.5743654 .159128-0.956933$

H $0.009775-1.693403-0.013044$

H -2.878963 -2.695124 -0.009668

H $-3.084687-0.681206-1.446413$

H $-1.674702-2.912505-3.038342$

H -2.605351 -1.663204 -3.771630

H $-5.871992-3.328300-2.858904$

H -4.511846 -5.130144 -2.444470

H -2.985792 $-5.002408-3.326721$

H -3.030185 -4.729544 -1.572265

H $-4.626604-0.494427-3.091667$

H $-5.817717-1.141479-4.205052$

H -6.978381 $0.213308-2.615770$

H -7.424641 -1.471272 -2.336209

H $-5.541494-1.426951-0.479744$

H -8.012937 - $1.527435-0.009555$

H $-8.126906 \quad 0.207160-0.226057$

H -8.934806 -0.8489242 .155450$

H -6.8905551 .7606501 .461471$

H -7.219165 -2.447329 2.183102

H $-6.341668-1.4430533 .314418$

H $-5.579322-1.9277211 .787548$

H $-5.5039661 .984636 \quad 3.150014$

H $-5.155383 \quad 3.0657320 .544136$

H -3.971432 2.5145701 .736269

H -3.199958 0.2091670 .873712

H $\quad 0.1633841 .612625-4.008789$

H $0.6536731 .051499-1.020569$

H $1.9493873 .203300-4.005167$

H $2.705687 \quad 1.595462-4.125034$

H $3.6242542 .962097-3.534983$

H -2.425933 $1.840886-0.109076$

H -3.623408 $2.403066-1.299387$

H $-1.0907106 .019666-0.137894$

H $-1.5360086 .022275-1.848475$

H $0.6590785 .000067-2.494719$

H 1.2689806 .0960910 .300522

H $3.3293996 .705205-1.002326$

H $3.0346615 .448086-2.173916$

H 4.8999534 .4361381 .483413

H 4.1056916 .0999241 .299377

H $4.7272272 .802988-0.217237$

H $4.5640653 .484803-1.805277$

H $2.3477752 .241339-0.038436$

H $-1.3439863 .458194-1.655121$

C $-0.307729-2.2341622 .803765$

O $1.024435-1.7158442 .634778$

C $1.108323-0.4423623 .308169$

C $-0.215166-0.2547014 .047988$

C $-1.174352-1.030745 \quad 3.164858$

H 1.1819030 .3210662 .525098

C $2.354338-0.4002974 .202973$

H $-0.186520-0.6899625 .053553$

H -0.4839340 .8012344 .150347$

C -2.481883 $-1.390776 \quad 3.846815$

H -1.384500 -0.421393 2.277571

C $3.670524-0.7280683 .466141$

O $2.204235-1.3567635 .260936$

H 2.4262920 .5782354 .690901

H $3.653769-1.7780623 .155184$

O $4.744817-0.6143794 .407812$

C 3.9685410 .1775312 .258385

C $5.330471-0.0926111 .589916$

H 3.1745940 .0720601 .512427

$\begin{array}{lllll}\text { O } & 3.949580 & 1.537325 & 2.699571\end{array}$

H $\begin{array}{llll}5.463756 & 0.645479 & 0.790059\end{array}$

H 6.1396940 .1194792 .299862

H $-3.123416-1.9726743 .177829$

H $-2.312794-1.9845954 .751387$

H -3.027000 -0.486096 4.135654

C $-0.726679-3.044417 \quad 1.574966$

H $2.090584-2.2319864 .852017$

H 4.7993330 .3183354 .678400

H 4.0779752 .1014311 .918990

H -1.659318 -3.5765621 .797044$

H $0.037451-3.8065021 .376475$ 
H $-0.255792-2.9241463 .657080$

C $4.217350-1.252992-1.007393$

O $4.342570-1.913687 \quad 0.254517$

C $5.481825-1.5193111 .024514$

C $6.773822-1.756782 \quad 0.226951$

C $6.723634-1.115888-1.160120$

C $5.438979-1.506233-1.894986$

H $4.132537-0.173620-0.834604$

C $2.886131-1.667841-1.646856$

H $5.516819-2.1963531 .886569$

O $7.904930-1.2576520 .932914$

H $6.923416-2.8372150 .102647$

H $6.815800-0.025949-1.091179$

O $7.850411-1.550485-1.925701$

H $5.496800-2.558563-2.194315$

O $5.323449-0.729611-3.085119$

C $2.689269-3.159080-1.823386$

H $2.079541-1.328437-0.987481$

H $2.767988-1.185647-2.623070$

H $7.924061-1.6950731 .801277$

H $7.808552-2.519196-1.999235$

H $6.131373-0.875318-3.606449$

O $1.450972-3.372662-2.363392$

O $3.495178-4.026914-1.520268$

C $1.094011-4.744081-2.560815$

H $1.062204-5.267316-1.600353$

H $0.097926-4.775986-3.009886$

H $1.801236-5.226683-3.241978$

SCF Energy (B3LYP/6-31G**/MMFF) $=-3245.89956719$

01_376

MMFF Geometry

C -2.419111 4.131185-0.200192

C $-1.704780 \quad 4.273551 \quad 0.925511$

C $-0.772562 \quad 3.198377 \quad 1.416325$

\begin{tabular}{lllll}
\hline & 0.570923 & 3.734930 & 1.347536
\end{tabular}

C - -1.0508742 .7658492 .867791$

C -2.3915102 .1009553 .095604$

C $-2.637964 \quad 0.7972652 .858499$

C $-3.4535742 .974975 \quad 3.707435$

C $-1.681665-0.2136132 .290498$

C $-0.996556-1.0183593 .400395$

C $-0.035397-2.0803302 .845977$

C $0.669568-2.8099403 .989088$

C $1.719659-3.8049583 .477590$

O $0.931708-1.4254502 .025074$

O $2.512173-4.1840364 .613512$

C $2.644031-3.0972722 .458499$

C $1.061876-5.0892012 .955647$

O $3.512207-4.047938 \quad 1.846724$

C $1.871669-2.3033321 .380808$

C $2.772641-1.3662620 .544357$

O $1.209756-3.2098560 .498693$

C 1.5738252 .8410381 .125333

O $1.458917 \quad 1.634244 \quad 0.982831$

C 2.8532443 .5834511 .083905

C 3.9869532 .9165620 .817582

C 5.3363303 .4811890 .745906

C $5.4879054 .955861 \quad 1.013499$

C $3.865676-2.031463-0.290298$

C $4.484929-1.069909-1.293137$

C $6.365578-0.919024-2.921924$

O $4.0359120 .049300-1.524425$

C $7.456981-0.144344-2.251982$

C $7.6462891 .172484-2.428307$

C $8.7764371 .942495-1.808346$

C $8.3151313 .172928-1.059824$

C $8.4241194 .394326-1.609710$

C $7.828567 \quad 3.002862 \quad 0.360972$

C 6.3658112 .6609380 .443898

N $5.591617-1.625542-1.919414$

H -2.291709 $3.220902-0.782528$

H $-1.795506 \quad 5.1812851 .515471$

H -0.8447292 .3309020 .748562$

H $-0.9462023 .631373 \quad 3.536905$

H -0.2527572 .0888383 .200518$

H -3.6242070 .4029293 .098347$

H -4.4219342 .4680423 .774827$
H -3.159090 3.2699904 .719991

H -3.598889 3.8832033 .116781

H -2.242445 -0.8868971 .631977$

H -0.9339230 .2699011 .656664$

H -0.435844 -0.3226714 .038535$

H $-1.750846-1.5018664 .033560$

H $-0.610694-2.7819792 .233174$

H -0.058037 -3.316076 4.635991

H $1.177020-2.0750744 .630294$

H $3.145036-4.8641954 .327763$

H $3.281657-2.3950323 .013165$

H $0.489243-5.5756183 .754548$

H $1.813402-5.8209892 .639021$

H $0.382066-4.9122752 .119965$

H $4.071749-4.4290772 .543891$

H $2.117245-0.796811-0.129751$

H $3.228360-0.6141381 .202302$

H $1.003357-2.736279-0.323360$

H $2.827336 \quad 4.650204 \quad 1.273614$

H 3.9272991 .8445070 .636961

H 4.8842155 .5386130 .308549

H 6.5135985 .3155210 .909363

H 5.1674895 .1960522 .033294

H $4.664820-2.3946840 .364441$

H $3.459190-2.878223-0.854307$

H $5.699359-0.274426-3.504924$

H $6.798622-1.669942-3.589986$

H $8.141601-0.700673-1.616189$

H $6.976887 \quad 1.721697-3.086606$

H $9.4591652 .233753-2.617589$

H $9.3711901 .310680-1.136999$

H $8.1310855 .287011-1.067460$

H $8.8050534 .528801-2.616809$

H 8.0617353 .8955360 .951824

H 8.4004482 .2037580 .850185

H $6.153182 \quad 1.6079520 .256863$

H $5.928247-2.534780-1.616065$

C $-4.8092574 .580769-0.924409$

O $-4.764002 \quad 3.313099-1.615248$

C $-5.4194142 .331817-0.790297$

C $-6.419563 \quad 3.1300030 .028957$

C $-5.574046 \quad 4.341668 \quad 0.381993$

H $-4.6654801 .919262-0.108540$

C $-5.996968 \quad 1.208436-1.650792$

H $-7.2863383 .430216-0.571050$

H -6.7746642 .5841340 .907904$

C $-6.3846305 .539604 \quad 0.844645$

H -4.8818424 .0603371 .182997$

C $-4.9488670 .509923-2.544513$

O $-7.0156111 .742914-2.503960$

H $-6.4950550 .470346-1.011942$

H $-4.6107691 .192614-3.333559$

O $-5.595899-0.583890-3.206640$

C $-3.726746-0.039860-1.781876$

C $-2.735756-0.773101-2.707359$

H $-3.1915150 .787872-1.305458$

O $-4.169995-0.906540-0.742289$

H $-3.231488-1.638281-3.158141$

H $-2.469470-0.091613-3.523644$

H -5.7302816 .3895991 .062556$

H -7.1061195 .8548320 .083509$

H $-6.941616 \quad 5.298243 \quad 1.755863$

C $-3.387548 \quad 5.136005-0.757754$

H $-6.6104902 .446718-3.039387$

H $-6.363653-0.221518-3.681388$

H $-4.663627-1.634680-1.157127$

H -3.410166 $6.048509-0.151270$

H -3.009392 $5.419193-1.748253$

H $-5.374160 \quad 5.262672-1.572282$

C $-2.091619-3.503496-1.456471$

O $-1.702336-2.212989-0.966592$

C $-1.450052-1.227315-1.973421$

C $-0.332292-1.716705-2.903906$

C $-0.647235-3.088335-3.491591$

C $-1.014546-4.079018-2.386390$

H $-3.041800-3.422968-1.996533$

C $-2.317277-4.401304-0.232536$

H $-1.063345-0.344652-1.447353$ 
O $-0.110673-0.774118-3.947618$

H $0.602971-1.782047-2.333940$

H $-1.439083-3.018832-4.246878$

O $\quad 0.517687-3.561021-4.172869$

H $-0.108911-4.335723-1.822868$

O $-1.484961-5.271475-3.011989$

C $-3.545872-3.9688730 .535778$

H -2.473699 $-5.449000-0.507844$

H $-1.444860-4.3606620 .429125$

H $0.589338-1.133127-4.519500$

H $\quad 0.312699-4.449111-4.512607$

H $-1.509680-5.971743-2.339067$

O $-3.213802-3.6736941 .824330$

O $-4.667677-3.9039110 .048974$

C $-4.292399-3.2254242 .650691$

H $-5.066209-3.9961732 .717316$

H $-3.897686-3.0376403 .652701$

H -4.707238-2.292774 2.256553

SCF Energy (B3LYP/6-31G**//MMFF) $=-3245.92175215$

\section{7}

MM̄FF Geometry

C $3.330986-1.0358072 .873205$

C $2.680675-1.7003283 .841057$

C $1.184712-1.7169314 .066195$

O $0.548616-0.746767 \quad 3.203518$

C $0.638794-3.121582 \quad 3.734865$

C $-0.827048-3.3101964 .069327$

C $-1.798662-3.5034373 .155441$

C $-1.165938-3.3184165 .538424$

C $-1.651814-3.6469051 .666124$

C $-1.695616-2.3035840 .930874$

C $-1.784118-2.487350-0.590413$

C $-1.682914-1.140447-1.307394$

C $-1.885095-1.282694-2.823877$

O $-3.043650-3.098925-0.874055$

O $-2.0893730 .031413-3.364828$

C $-3.181146-2.079454-3.083107$

C $-0.641662-1.849533-3.517066$

O $-3.338701-2.372921-4.469043$

C $-3.284088-3.379081-2.256922$

C $-4.677398-4.051439-2.360227$

O $-2.325548-4.323744-2.750037$

C $-0.4278610 .044007 \quad 3.722040$

$\begin{array}{llll}\text { O } & -0.858599 & 0.036870 & 4.863556\end{array}$

C -0.8853190 .9499292 .643293$

C -1.904141 1.7915992 .872811

C -2.438403 2.7818751 .934652

C -1.7976302 .8678210 .574705$

C $-5.897086-3.185014-2.027764$

C $-5.843203-2.586320-0.634180$

C $-5.711682-0.411310 \quad 0.565277$

O $-5.811208-3.2871200 .374926$

C -5.3587580 .9940540 .195660$

C $-6.116592 \quad 2.057168 \quad 0.502734$

C -5.8058963 .4610500 .068815$

C -5.5573214 .3944511 .230750$

C -6.576274 5.0047961 .859249

C $-4.121502 \quad 4.6946201 .594133$

C $-3.458673 \quad 3.5678882 .338624$

N $-5.800768-1.201641-0.648196$

H $2.776405-0.4290012 .162317$

H $3.261765-2.3093984 .531288$

H $1.015369-1.4642975 .119255$

H $1.209855-3.8840124 .281741$

H $0.815869-3.3198192 .671585$

H -2.822386 -3.6142263 .511427$

H -2.226004 -3.526669 5.718900

H $-0.588837-4.0941216 .052702$

H -0.939462 -2.355689 6.003264

H $-0.731468-4.1846651 .412478$

H -2.468605 -4.293207 1.320852

H -2.561019-1.727370 1.282075

H $-0.793615-1.7321071 .170254$

H $-0.975362-3.154712-0.910782$

H $-0.723533-0.654652-1.090603$

H -2.454971 -0.462433 -0.918337

H $-1.3058800 .566697-3.152291$
H $-4.010156-1.415153-2.815179$

H $0.219099-1.191089-3.352152$

H $-0.779596-1.893643-4.602952$

H $-0.368372-2.845454-3.162216$

H -3.270373 -1.532129 -4.953141

H $-4.808498-4.462273-3.369887$

H $-4.687482-4.919106-1.685637$

H -2.609365 -5.214652 -2.485589

H $-0.355244 \quad 0.914173 \quad 1.698069$

H -2.3855951 .7699333 .849697$

H $-0.750571 \quad 3.1781290 .663779$

H $-2.2872003 .575996-0.096739$

H -1.834076 1.8961410 .069662

H $-6.026282-2.407388-2.787785$

H $-6.803238-3.801518-2.074874$

H $-4.943014-0.8434011 .213810$

H $-6.674651-0.4827251 .081838$

H $-4.4409501 .140594-0.368781$

H $-7.037926 \quad 1.9082841 .061366$

H -6.657256 $3.827338-0.520118$

H $-4.9507463 .485264-0.618194$

H -6.4038355 .7019712 .672404$

H -7.606541 4.8162351 .575634

H -4.0752015 .5794492 .243637$

H -3.5759644 .9880340 .692758$

H -3.8576673 .4231013 .343672$

H $-5.996604-0.716715-1.519376$

C $5.162456-1.9057831 .422547$

O $4.679332-1.2226150 .251455$

C $5.591990-1.463568-0.837606$

C $6.661108-2.413327-0.304474$

C $6.656922-2.0886321 .178491$

H $6.063780-0.502209-1.069944$

C $4.815017-1.978012-2.056766$

H $6.391655-3.463471-0.466486$

H $7.633744-2.245719-0.777566$

C $7.302872-3.163768 \quad 2.034277$

H $7.181940-1.1362861 .332300$

C $3.637508-1.071764-2.474224$

O $4.271828-3.270946-1.755481$

H $5.497907-2.126927-2.900592$

H $2.876649-1.071323-1.685914$

O $3.020806-1.650227-3.631056$

C $4.045607 \quad 0.370455-2.820382$

C $2.8731781 .265954-3.261377$

H $4.5424050 .837259-1.965380$

$\begin{array}{llll}\text { O } & 5.006328 & 0.346309 & -3.879582\end{array}$

H $3.2930032 .195144-3.666052$

H $2.3524730 .804555-4.109674$

H $7.274754-2.8903513 .093599$

H $6.793395-4.1266491 .921331$

H $8.351145-3.3025691 .750261$

C $4.820981-1.0942712 .671663$

H $3.697248-3.174178-0.976486$

H $2.766947-2.560190-3.399394$

H $4.590769-0.092811-4.641700$

H $5.186982-0.0653172 .570341$

H $5.307986-1.5301793 .551077$

H $4.659631-2.8820661 .456894$

C $3.1016913 .345782-1.054725$

O $2.5211922 .042324-0.939778$

C $1.8700811 .580932-2.130616$

C $0.7527782 .554608-2.534208$

C $1.2457193 .999514-2.625422$

C $2.0218204 .388180-1.365095$

H $3.8336553 .337796-1.871910$

C 3.9225983 .6269810 .207230

H $1.3851850 .634397-1.865323$

$\begin{array}{lllll}\text { O } & 0.184516 & 2.148672 & -3.774745\end{array}$

H $-0.0449112 .501581-1.783601$

H $1.8542204 .160229-3.523314$

$\begin{array}{llll}\text { O } & 0.120119 & 4.874179 & -2.737682\end{array}$

H $1.3094884 .483236-0.536796$

O $2.6149945 .665845-1.579701$

C 3.0922503 .8667801 .444446

H 4.6049372 .7914850 .409925

H 4.5584054 .5093730 .066992

H $-0.5857622 .715575-3.947610$ 
H $-0.2941974 .710557-3.601487$

H $2.9654585 .975647-0.727655$

O 2.8960312 .6982112 .117625

O 2.6719754 .9684581 .775081

C 2.1813432 .8228953 .350625

H 2.1056061 .8318483 .803867

H 2.7249353 .4780954 .038325

H $\quad 1.1744183 .2088113 .167998$

SCF Energy (B3LYP/6-31G**//MMFF)= -3245.91762827

01378

MM̄FF Geometry

C $3.210158-3.284253-1.452940$

C $2.332408-2.592402-0.711874$

C $1.068312-1.974984-1.247395$

O $-0.003906-2.467089-0.410584$

C $1.164439-0.441648-1.121455$

C $0.1168060 .307297-1.916772$

C $-0.9970920 .860416-1.398594$

C $0.4057910 .434744-3.390815$

C $-1.473171 \quad 0.8238440 .027177$

C -1.5996712 .2123810 .671346$

C $-2.5972063 .148926-0.029219$

C $-2.793522 \quad 4.437206 \quad 0.773036$

C $-3.860375 \quad 5.346703 \quad 0.141975$

O $-3.8440742 .461344-0.155797$

O $-4.2194296 .343800 \quad 1.110833$

C $-5.1305364 .514306-0.141245$

C $-3.3156706 .102897-1.075846$

O $-6.0908525 .279960-0.865451$

C $-4.8469923 .185301-0.879550$

C $-6.1246712 .312731-0.918693$

O $-4.4349033 .472038-2.217033$

C $-1.235633-2.619104-0.962353$

O $-1.565221-2.413761-2.119114$

C $-2.147136-3.0537890 .114939$

C $-3.438475-2.699014 \quad 0.060324$

C $-4.412080-3.041294 \quad 1.087537$

C $-5.058927-1.8495771 .734765$

C $-5.8952950 .889831-1.450346$

C $-7.1523150 .039225-1.371851$

C -7.915732 -2.319056-1.664631

O $-8.2560620 .494748-1.085100$

C $-8.010389-2.905556-0.290562$

C $-7.839695-4.209023-0.021738$

C $-8.000400-4.8042391 .348224$

C $-6.799484-5.6107881 .789053$

C $-6.804541-6.952824 \quad 1.718478$

C $-5.633782-4.8680442 .401928$

C $-4.686097-4.3303341 .367250$

N -6.893152 -1.289989-1.681078

H $3.014658-3.439444-2.510893$

H $2.538965-2.4360540 .343291$

H $\quad 0.910772-2.286189-2.287252$

H $2.148008-0.101442-1.472959$

H $1.116852-0.155814-0.064972$

H -1.674913 $1.363692-2.086643$

H $-0.3635531 .008432-3.918503$

H $1.3615840 .946026-3.546679$

H $0.458675-0.552405-3.860104$

H -2.4483340 .3228110 .045467$

H $-0.820170 \quad 0.2138310 .657949$

H -1.924874 2.0559671 .708246

H -0.6157962 .6933410 .714733$

H -2.210796 3.386927-1.026516

H -1.8436574 .9752590 .884045$

H -3.116407 4.1856001 .793066

H -3.412722 6.8292521 .354383

H -5.5901234 .2784950 .829020$

H -2.462839 $6.729156-0.787348$

H $-4.0660706 .789624-1.482841$

H -2.981254 $5.441364-1.877585$

H $-6.2494926 .096577-0.361739$

H -6.5305762 .2267160 .098000$

H -6.889947 $2.800102-1.535855$

H $-4.5888232 .692477-2.772482$

H -1.734440 -3.618464 0.944164

H -3.809361 -2.079832 -0.752599
H $-5.889551-2.1120302 .393616$

H $-5.463538-1.1688700 .979792$

H $-4.324175-1.2974542 .331119$

H $-5.5818720 .914010-2.499253$

H $-5.1097830 .393566-0.870369$

H -7.639880 -3.065555 -2.416012

H -8.878256 -1.879298 -1.945252

H $-8.265173-2.2258810 .519377$

H -7.612367 -4.894304 -0.835251

H $-8.891469-5.4454421 .326777$

H $-8.213100-4.0319012 .097811$

H -5.959485 -7.539655 2.062742

H -7.651333 -7.493871 1.309288

H $-5.059177-5.5453513 .048292$

H $-6.008529-4.0945573 .078611$

H $-4.157437-5.1030560 .807550$

H -5.933768 -1.582397-1.842103

C $5.707263-3.165820-1.523239$

O $5.736477-1.784725-1.116641$

C $7.105048-1.386862-0.904044$

C $7.968550-2.567351-1.337587$

C $7.045577-3.741196-1.066248$

H $7.225248-1.2450520 .175467$

C $7.386753-0.066738-1.631527$

H $8.220857-2.520869-2.403034$

H $8.906550-2.620135-0.776267$

C $7.454615-5.013495-1.786998$

H $7.024527-3.9284190 .015873$

C $6.3647531 .045408-1.314145$

O $7.332538-0.307296-3.044064$

H $8.4076050 .267289-1.413072$

H $5.391307 \quad 0.755447-1.722963$

O $6.7562472 .220329-2.036097$

C 6.2359711 .3954390 .180111

C 5.2312312 .5300690 .462867

H 5.9471680 .5072720 .748492

O $7.520891 \quad 1.799131 \quad 0.659153$

H 5.3630652 .8560311 .501357

H $5.4889813 .408026-0.141525$

H $6.758405-5.828661-1.566753$

H $7.474166-4.871179-2.872736$

H $8.454198-5.329664-1.471282$

C $4.490445-3.854877-0.905236$

H $7.5064100 .539931-3.488952$

H $7.6014162 .524259-1.662654$

H 7.4428941 .9494121 .616699

H $4.516168-3.7419800 .185636$

H $4.508799-4.928374-1.122922$

H $5.637598-3.177311-2.619368$

C 3.2887181 .1569122 .380675

O 3.4075260 .9509580 .968451

C 3.7664842 .1159910 .215614

C 2.7360473 .2306960 .439942

C 2.5120973 .5157911 .921429

C 2.2237232 .2203972 .678174

H $4.251851 \quad 1.4803712 .790928$

C $2.910177-0.187383 \quad 3.021547$

H $3.6880141 .833871-0.840127$

O $3.1317704 .432085-0.213067$

H $1.7778942 .927728-0.001508$

H 3.3648734 .0455742 .361857

O 1.3826714 .3797722 .068698

H 1.2290291 .8476042 .402684

O 2.2075422 .4912354 .078023

C $3.986615-1.2478972 .927805$

H $2.674370-0.0683924 .084626$

H $2.023323-0.5895962 .515797$

H $3.2500634 .226404-1.156281$

H 1.5620475 .1807671 .546801

H 1.5420533 .1836814 .231628

O $5.218942-0.7288153 .183410$

O $3.741010-2.4303982 .719913$

C $6.304543-1.6612953 .154140$

H $7.239022-1.0940013 .157931$

H $6.270719-2.2942624 .045302$

H $6.271825-2.2723482 .246926$

SCF Energy (B3LYP/6-31G**//MMFF) $=-3245.90885629$ 
01379

MM̄FF Geometry

C $1.069747-3.563223-0.195137$

C $-0.110062-3.0412800 .168960$

C $-0.556757-2.8851321 .596685$

O $-0.823641-1.4784161 .800201$

C - $-1.863803-3.6564771 .840266$

C $-2.362216-3.5315773 .268554$

C -3.344362 -2.692955 3.656567

C $-1.708297-4.4525414 .263764$

C $-4.067712-1.7006792 .790044$

C $-5.469483-2.1932832 .416674$

C $-6.068642-1.3728491 .264636$

C -7.510637-1.805533 0.994245

C $-8.095987-1.116013-0.245977$

O $-5.257721-1.6131850 .109965$

O $-9.304718-1.803118-0.604278$

C $-7.112677-1.289523-1.421822$

C $-8.491244 \quad 0.337278 \quad 0.041641$

O $-7.550947-0.550831-2.560624$

C $-5.655567-0.906724-1.071732$

C $-4.706145-1.360960-2.210155$

O $-5.5730290 .505838-0.896345$

C $0.190661-0.6894232 .232806$

O $1.326993-1.0462782 .512725$

C $-0.233784 \quad 0.7257942 .340282$

C $-1.3993931 .193187 \quad 1.865723$

C -1.8522322 .5864681 .898929$

C -0.9177773 .6059382 .496942$

C $-3.223488-1.016843-1.990741$

C $-2.8188840 .323224-2.583379$

C $-0.9004561 .898179-2.718860$

O $-3.5716261 .008688-3.270753$

C -1.206694 2.977618-1.729847

C $-2.1669413 .897212-1.908441$

C $-2.4965464 .983116-0.923871$

C $-3.7939444 .718434-0.189568$

C $-4.9809374 .961037-0.770705$

C $-3.709576 \quad 4.2358821 .242778$

C -3.0538522 .8866181 .361036$

N -1.507379 $0.658261-2.270994$

H $1.749413-3.9378510 .566108$

H $-0.779641-2.658440-0.598116$

H $\quad 0.213121-3.2302752 .297965$

H -1.718623 -4.720605 1.609969

H -2.636771 -3.3141661 .141000$

H -3.652131 -2.697244 4.700746

H -2.128343 -4.3429605 .269258$

H -1.843690 -5.496162 3.961556

H $-0.635725-4.2457034 .330979$

H $-4.145896-0.751411 \quad 3.334474$

H -3.488901-1.473332 1.891311

H -5.412019 -3.246651 2.110739

H $-6.120466-2.1415843 .298126$

H $-6.024173-0.3085081 .524048$

H $-8.142715-1.6210661 .871911$

H -7.540082 -2.891997 0.829356

H $-9.908010-1.7653790 .157543$

H -7.131405 -2.349137-1.713557

H -9.2488820 .3780150 .833522$

H $-8.9515080 .803009-0.836762$

H -7.649750 $0.956038 \quad 0.360214$

H -8.463180 -0.828089 -2.752236

H -4.771416 -2.455003 -2.284396

H -5.044109 -0.953860 -3.169752

H $-4.6402770 .769770-0.911951$

H $\quad 0.4949101 .3788192 .809099$

H -2.0879540 .5020261 .383910$

H 0.0340093 .6171331 .954229

H -0.7212743 .3766893 .550114$

H -1.3008224 .6273892 .464261$

H -2.963055 -1.039824 -0.926999

H $-2.613863-1.774440-2.499358$

H $-1.2760852 .144672-3.717882$

H $\quad 0.179814 \quad 1.740757-2.788518$

H $-0.6061402 .999650-0.825518$

H -2.764637 $3.867268-2.816951$

H $-2.5686635 .932532-1.470491$
H -1.678934 $5.127719-0.207079$

H $-5.9146904 .794755-0.243956$

H $-5.0435685 .329684-1.789341$

H -4.7141414 .1481241 .677487$

H -3.209558 5.0031421 .839755

H -3.6526042 .0766240 .942667$

H $-0.9815280 .072307-1.628815$

C $2.783078-2.748101-1.806870$

O $3.928153-3.421742-1.238253$

C $5.013899-3.343244-2.171713$

C $4.634716-2.262916-3.174659$

C $3.135834-2.479151-3.270039$

H $5.025842-4.303340-2.707206$

C $6.371472-3.182889-1.480781$

H $4.846531-1.260007-2.788100$

H $5.147315-2.378270-4.134228$

C $2.390546-1.302342-3.877369$

H $2.944472-3.376596-3.873558$

C $6.623888-1.871710-0.705120$

O $7.370571-3.292845-2.503695$

H $6.523228-4.032603-0.803593$

H $6.653425-1.035196-1.410626$

O $7.948163-1.947442-0.154456$

C $5.633592-1.5638850 .432558$

C $6.027865-0.3685261 .320705$

H $4.644112-1.3697750 .012593$

O $5.497444-2.7088071 .278271$

H $5.295187-0.3046832 .134790$

H $6.980946-0.5795041 .821897$

H $1.312421-1.489858-3.897756$

H $2.560936-0.382004-3.309066$

H $2.722976-1.127185-4.905773$

C $1.538795-3.617864-1.622871$

H $8.238161-3.212291-2.071774$

H $7.982899-2.7188790 .436015$

H $6.346725-2.8525731 .728430$

H $1.761635-4.662321-1.870952$

H $\quad 0.740699-3.280461-2.294166$

H $2.670272-1.798664-1.267621$

C 3.7671631 .5323520 .550089

O $4.9391421 .227371-0.212098$

C 6.1117240 .9740430 .568605

C 6.4282862 .1807401 .465031

C 5.2260742 .5947472 .312961

C 3.9896142 .7668521 .430477

H $3.5226310 .673508 \quad 1.187614$

C $2.5877321 .677300-0.417160$

H $6.9406630 .886019-0.144041$

O $7.543577 \quad 1.883116 \quad 2.298208$

H 6.7178813 .0263970 .827376

H 5.0343491 .8789113 .120991

O 5.4968293 .8500612 .940477

H 4.1169543 .6655360 .815105

O 2.8447882 .9688622 .254088

C $2.7624002 .814187-1.396236$

H $2.4741180 .765210-1.015069$

H $1.645324 \quad 1.8070640 .125913$

H 7.7982502 .7021882 .754999

H 6.2110653 .7078753 .584394

H 3.0251083 .7402412 .818367

O $2.0472003 .898793-0.983074$

O $3.4620922 .749612-2.398697$

C $2.1229665 .047730-1.832085$

H $1.7585694 .804049-2.834635$

H $1.4838075 .826440-1.407101$

H $3.1512065 .419341-1.873115$

SCF Energy $\left(B 3 L Y P / 6-31 G^{* *} / / M M F F\right)=-3245.90075580$

01_38

MM̄FF Geometry

C -3.5022971 .8379841 .939345$

C -2.6479001 .2424162 .784244$

C $-2.364241-0.2350742 .764673$

O $-0.921878-0.3679222 .738760$

C $-2.900665-0.9602314 .011143$

C $-4.406700-1.0789014 .066010$

C -5.111037-2.048566 3.450044

C $-5.109371-0.0674054 .933578$ 
C $-4.586713-3.1514582 .567002$

C $-5.429944-3.3216081 .294120$

C $-5.204121-2.2104340 .253090$

C $-6.421910-2.110654-0.669072$

C $-6.216049-1.073119-1.777161$

O $-4.033989-2.545815-0.508565$

O $-7.260235-1.248247-2.746820$

C $-4.885110-1.377564-2.485444$

C $-6.3436320 .361792-1.256720$

O $-4.572984-0.359077-3.435265$

C $-3.686731-1.592883-1.524011$

C $-2.503369-2.154778-2.350273$

O $-3.313379-0.338128-0.956599$

C $-0.408549-1.5234332 .239777$

O $-1.029935-2.4644131 .773597$

C $1.061389-1.4585752 .382768$

C $1.795350-2.5365052 .070707$

C $3.250262-2.6496042 .165854$

C $4.016677-1.4517282 .655769$

C $-1.259336-2.577320-1.557473$

C $-0.180564-3.105926-2.493775$

C $2.187468-3.788660-2.588282$

O $-0.361615-3.259122-3.700387$

C $3.171071-4.473851-1.696948$

C $4.440917-4.065431-1.549815$

C $5.467930-4.772992-0.711358$

C $5.947926-3.9313860 .450090$

C $6.964084-3.0648550 .303333$

C $5.295781-4.1482281 .796875$

C $3.832442-3.8078851 .794864$

N $1.007257-3.401481-1.835541$

H -4.0500101 .2233991 .232270$

H -2.108563 1.8441513 .511890

H $-2.774739-0.6817431 .851037$

H -2.529950 -0.464339 4.919142

H -2.460685 -1.964167 4.073712

H -6.189681 -2.066622 3.603112

H $-6.192851-0.2247094 .961688$

H $-4.741249-0.1311105 .962875$

H $-4.9361830 .947903 \quad 4.565997$

H -3.538605 -3.006672 2.296470

H -4.642478 -4.0845143 .141012$

H $-5.176767-4.291348 \quad 0.846403$

H $-6.488759-3.3670871 .579337$

H $-5.039697-1.2548540 .761869$

H $-7.326998-1.876264-0.094474$

H -6.607757 -3.088409-1.135886

H -8.112919 -1.131805 -2.293482

H -5.031946 -2.301616 -3.062530

H $-7.3361400 .525056-0.819909$

H $-6.2519471 .087908-2.071870$

H $-5.6036310 .604230-0.493441$

H -5.338376 -0.272435 -4.029246

H -2.851333 -3.037751 -2.903628

H -2.195947 -1.408244 -3.094587

H $-2.389849-0.385472-0.664430$

H $1.495428-0.5363492 .751775$

H $1.283416-3.4285351 .711242$

H $3.761291-1.2317913 .697924$

H $3.780767-0.5753132 .045325$

H $5.100786-1.5780192 .607656$

H - $0.845121-1.729218-1.004117$

H -1.512571 -3.372184 -0.848032

H $2.597979-2.881126-3.043852$

H $\quad 1.889267-4.463880-3.397501$

H $2.833099-5.367174-1.177570$

H $4.779400-3.184664-2.090495$

H $6.316840-5.026548-1.359944$

H $5.089142-5.734366-0.342403$

H $7.329133-2.4734711 .136407$

H $7.448960-2.922818-0.656960$

H $5.822433-3.6001762 .586213$

H $5.403516-5.2047482 .075340$

H $3.196353-4.6226071 .447288$

H $\quad 1.129847-3.064208-0.884815$

C -3.2562594 .0137770 .644427$

O $-1.8196153 .901666 \quad 0.576744$

C $-1.4575293 .479411-0.748557$
C $-2.6560593 .816463-1.626608$

C $-3.8024193 .494798-0.688504$

H $-1.3365252 .389037-0.703305$

C $-0.1336114 .104306-1.207042$

H -2.675837 $4.878511-1.896976$

H $-2.6764773 .227303-2.548307$

C $-5.1252444 .119496-1.095230$

H $-3.9224322 .407148-0.670467$

C $1.0376073 .800263-0.245757$

O $-0.3075625 .513337-1.346909$

H $0.094345 \quad 3.702541-2.201383$

H $0.9813132 .747696 \quad 0.055221$

O $0.9270674 .589508 \quad 0.937725$

C $2.4210084 .043590-0.880505$

C 3.6190863 .8783300 .072372

H $2.5379493 .358592-1.727654$

O $2.4947765 .363917-1.423691$

H $4.5297913 .987048-0.530556$

H 3.6484084 .7246350 .771309

H -5.911495 $3.863226-0.378094$

H $-5.0558235 .211117-1.145904$

H -5.434829 $3.756844-2.080962$

C -3.7743603 .3161271 .907955$

H $0.4979095 .868968-1.758173$

H $\quad 0.0581114 .4062831 .333349$

H $2.3840375 .990330-0.687435$

H -4.8555753 .4716561 .998620$

H -3.3100793 .7894352 .782556$

H -3.4667185 .0869260 .739324$

C $4.4090821 .079176-0.883514$

O 3.3866841 .4171790 .058331

C 3.6626422 .5602130 .867875

C 4.9626452 .3503551 .659185

C 6.1255651 .9488260 .754010

C $5.7291930 .790960-0.161045$

H $4.5434131 .903378-1.595044$

C $3.921207-0.151007-1.655100$

H 2.8504232 .6128241 .603362

O 5.3178673 .5255042 .380725

H 4.8129661 .5563002 .400685

H 6.4913562 .8007550 .168957

O 7.2231511 .5199041 .563738

H $5.645270-0.129496 \quad 0.428337$

O $6.757604 \quad 0.584341-1.127044$

C $2.8379740 .228097-2.636111$

H $4.728352-0.611330-2.235959$

H $3.531243-0.922308-0.979989$

H 4.5699783 .7491652 .960889

H 7.4434512 .2534702 .163316

H $7.5834250 .419762-0.640337$

O $1.6160150 .175486-2.033468$

O $3.057824 \quad 0.554411-3.795568$

C $0.5091560 .542377-2.862418$

H $\quad 0.6587341 .540646-3.283965$

H $\quad 0.377697-0.195224-3.659325$

H $-0.3909150 .556442-2.242833$

SCF Energy (B3LYP/6-31G**//MMFF)= -3245.92487722

01_380

MM̄FF Geometry

C -3.0024681 .0320802 .552464$

C -1.7144811 .0317392 .176277$

C -0.6210240 .3911342 .990103$

O $0.059029-0.5281392 .098332$

C 0.4192201 .4084673 .503394

C -0.1209682 .5715934 .309592$

C 0.1029193 .8664584 .005965

C -0.9080822 .2115035 .542486$

C 0.9597554 .3943102 .890568

C 0.1349894 .8388111 .677198

C 1.0288084 .9886300 .437928

C $0.2941765 .703971-0.693994$

C $1.1493505 .769159-1.969091$

O 1.3914453 .6680360 .017370

O $0.296610 \quad 6.167927-3.052957$

C $1.6679444 .353789-2.297753$

C $2.2491556 .832761-1.868136$

O $2.5892164 .387436-3.385688$ 
C $2.3004433 .621019-1.089558$

C $2.5278392 .131164-1.454226$

O $3.5486064 .231615-0.768140$

C $0.789357-1.5278632 .660189$

O $0.854607-1.8173993 .843743$

C $1.551724-2.1790601 .571820$

C $2.541583-3.0311521 .875794$

C $3.430467-3.7030810 .924371$

C $3.260422-3.379728-0.536644$

C $2.9480831 .223469-0.288032$

C $4.4491381 .099414-0.094936$

C $6.122055-0.1481591 .273979$

O $5.2697901 .718918-0.765385$

C $6.498708-1.4065580 .555528$

C $6.998912-2.4900431 .168947$

C $7.411460-3.7414670 .446764$

C $6.802805-4.9926301 .041152$

C $7.524939-5.8021641 .834232$

C $5.386334-5.3504130 .646592$

C $4.361490-4.5530441 .406501$

N $4.759270 \quad 0.2072620 .924314$

H -3.2559910 .5751813 .504268$

H $-1.425861 \quad 1.5124451 .245514$

H -1.050124 -0.174166 3.827381

H $\quad 0.997530 \quad 1.7488852 .638500$

H $\quad \begin{array}{llll}1.154240 & 0.881902 & 4.126707\end{array}$

H -0.3357694 .6333464 .642299$

H -1.163352 3.0916046 .142438

H -1.8443401 .7120495 .280123$

H $-0.323105 \quad 1.5404306 .180221$

H 1.5257015 .2541283 .272122

H 1.7232603 .6657102 .602025

H -0.6492454 .0993451 .470090$

H -0.3586295 .7900601 .910512$

H 1.9313235 .5441220 .719367

H $-0.0118436 .711341-0.385827$

H $-0.6351995 .165232-0.923615$

H $-0.0946237 .028812-2.825436$

H $0.8071853 .765569-2.642915$

H $1.8101007 .821614-1.688828$

H $2.8032476 .918257-2.809417$

H $2.9624536 .637316-1.064724$

H $2.1465984 .836267-4.126357$

H $1.5762871 .732700-1.826713$

H $3.2532952 .048823-2.272167$

H $4.0820423 .601216-0.259276$

H $1.303972-1.9074200 .553028$

H $2.735770-3.2464332 .926011$

H $3.376356-2.304013-0.708447$

H $2.268058-3.687281-0.883497$

H $3.988000-3.872761-1.183715$

H 2.4949381 .5430690 .655487

H $2.5809590 .211905-0.499474$

H $6.158938-0.2653762 .361458$

H $\quad 6.799260 \quad 0.6612160 .983744$

H $6.373929-1.418384-0.524826$

H $7.150701-2.4674812 .245868$

H $8.506789-3.8024830 .490532$

H $7.159977-3.689280-0.619848$

H $7.105397-6.7160492 .241425$

H $8.550570-5.5663832 .098676$

H $5.194238-6.4104710 .860742$

H $5.287205-5.261367-0.438255$

H $4.398902-4.7189512 .484366$

H $4.008183-0.3018431 .380965$

C $-5.343913 \quad 0.7553881 .552820$

O $-4.991342-0.4007910 .766862$

C $-5.560161-1.5664751 .391190$

C $-6.654356-1.0480072 .318640$

$\begin{array}{llll}\text { C } & -6.032510 & 0.239236 & 2.820477\end{array}$

H $-4.760234-2.0115421 .996071$

C $-6.038548-2.5898780 .354168$

H -7.581086 $-0.827787 \quad 1.776724$

H -6.889214 -1.754269 3.120568

C $-7.034873 \quad 1.214703 \quad 3.413522$

H $-5.304448-0.0234393 .594902$

C -5.032623 -2.929252 -0.762802

O $-7.222944-2.101048-0.291510$
H $-6.344517-3.5067210 .871736$

H -5.010812 -2.108835 -1.489201

O $-5.538424-4.072193-1.466005$

C $-3.601292-3.240054-0.296903$

C $-2.668435-3.775760-1.402530$

H $-3.159737-2.3352650 .135215$

O $-3.652017-4.2162190 .746306$

H -1.657763 -3.836595 -0.978775

H -2.935300 -4.815376-1.633538

H $-6.5350312 .129328 \quad 3.747839$

H -7.8020851 .4960202 .684488$

H -7.5399130 .7696114 .277023$

C -4.1214971 .6605491 .767594$

H -7.004355 -1.243491 -0.695436

H -6.436490 -3.852395 -1.768184

H $-2.742318-4.359927 \quad 1.058329$

H -4.4386902 .5677192 .295140$

H $-3.744666 \quad 1.9725450 .785279$

H -6.0678861 .3135840 .944458$

C $-1.304242-1.086978-1.979673$

O $-2.574862-1.542989-2.446476$

C $-2.651466-2.946284-2.701861$

C $-1.580254-3.371388-3.718193$

C $-0.183165-2.894619-3.322934$

C $-0.200933-1.399851-2.997440$

H $-1.069433-1.595865-1.035711$

C -1.435879 $0.398793-1.622753$

H -3.621144 -3.111054 -3.187417

O $-1.562309-4.785985-3.877927$

H -1.823551 -2.940534 -4.698272

H $0.214232-3.475878-2.482905$

O $\quad 0.712333-3.106319-4.416497$

H $-0.351618-0.842774-3.928140$

O $1.068640-1.009356-2.486089$

C $-1.8342771 .305382-2.769049$

H $-2.2265950 .499892-0.869852$

H $-0.4956790 .763555-1.197425$

H -2.454485 -5.060306 -4.150923

H $0.679664-4.052844-4.637799$

H $1.233371-1.520657-1.676735$

O $-1.8289272 .598816-2.325756$

O $-2.1379350 .939713-3.895580$

C $-2.2290963 .578470-3.289330$

H -2.267846 $4.550570-2.791164$

H $-1.4968013 .626747-4.100656$

H $-3.2250853 .348044-3.679719$

SCF Energy $\left(B 3 L Y P / 6-31 G^{* *} / / M M F F\right)=-3245.91097868$

01381

MM̄FF Geometry

C $-5.0640401 .015910-0.059158$

C $-5.8788980 .068719-0.550221$

C $-5.491882-1.373001-0.793155$

O $-4.053442-1.508276-0.838543$

C $-6.027789-2.2604050 .344856$

C $-5.707359-3.7347940 .171790$

C $-4.849370-4.4306080 .946411$

C $-6.437525-4.427667-0.949742$

C $-4.053864-3.9176932 .115603$

C $-2.628186-3.5594671 .695306$

C $-1.853717-2.7324432 .731494$

C -2.440329-1.327709 2.910611

C $-1.584331-0.4585193 .843101$

O $-0.508933-2.6286422 .239544$

O -2.0167830 .9032003 .696894$

C $-0.118504-0.517778 \quad 3.375313$

C -1.793032 -0.826017 5.316799

O $0.736540 \quad 0.153456 \quad 4.297326$

C $0.396772-1.9526723 .121245$

C $1.785898-1.8882022 .440961$

O $0.524238-2.6367414 .369038$

C $-3.437849-1.312648-2.036725$

O $-3.968286-1.063963-3.107204$

C $-1.981247-1.447109-1.811712$

C $-1.141583-1.491336-2.856489$

C $0.314683-1.633317-2.783319$

C $0.932307-1.783122-1.417104$

C $2.275990-3.227174 \quad 1.876528$ 
C $3.621877-3.0854221 .184727$

C $5.102882-4.274125-0.435035$

O $4.380875-2.1401291 .380504$

C $4.944659-3.515425-1.714977$

C $4.609203-4.088068-2.880813$

C $4.491586-3.346031-4.181409$

C $3.050925-3.062738-4.550371$

C $2.348527-3.956623-5.267643$

C $2.517927-1.718970-4.093799$

C $1.025296-1.633591-3.931039$

N $3.888292-4.1600550 .349123$

H $-4.051177 \quad 0.754296 \quad 0.236197$

H $-6.8966280 .338274-0.823455$

H -5.922108 -1.693302 -1.749745

H -7.118019 -2.1513140 .420751$

H $-5.629112-1.8877191 .293818$

H $-4.689030-5.4843650 .722749$

H $-6.249291-5.506580-0.963737$

H $-7.518126-4.285887-0.843797$

H -6.125609-4.027555 -1.919223

H -4.562254 -3.076122 2.588213

H -4.020149 -4.699879 2.884159

H -2.071111 -4.483827 1.488407

H $-2.630992-3.0169310 .744509$

H -1.846751 -3.2810583 .680277$

H -3.468995 -1.362936 3.285160

H -2.496998 -0.8319901 .931064$

H -2.9629740 .9429773 .918459$

H -0.0581820 .0394612 .430333$

H -2.844638 -0.6940355 .599069$

H -1.221598 -0.1621015 .974672$

H -1.518218 -1.858196 5.543840

H $\quad 0.398743 \quad 1.0599524 .398834$

H $1.748671-1.1754331 .606719$

H $2.527842-1.5112043 .156124$

H $1.185355-3.3407174 .278253$

H -1.636939-1.508984 -0.785922

H - $-1.555884-1.420225-3.861304$

H $\quad 0.473439-2.619121-0.877767$

H $\quad 0.794587-0.867800-0.831152$

H $2.003192-1.993787-1.442282$

H $2.394301-3.9674092 .674564$

H $\quad 1.550377-3.6175801 .154745$

H $5.281151-5.339967-0.611122$

H $5.948086-3.8736120 .134198$

H $5.136385-2.445618-1.680469$

H $4.414722-5.157832-2.910382$

H $4.973783-3.947189-4.963381$

H $5.059040-2.406921-4.153251$

H $1.323593-3.778981-5.572262$

H $2.792316-4.898121-5.576124$

H $2.828097-0.960125-4.824095$

H $3.012847-1.430371-3.162281$

H $\quad 0.489270-1.525185-4.874613$

H $3.167974-4.8604370 .199948$

C $-4.5489653 .410609-0.692017$

O $-3.1876593 .325248-0.221971$

C $-2.3096403 .294545-1.361647$

C $-3.1480713 .764224-2.544960$

C $-4.5007083 .172378-2.202711$

H $-2.0265242 .244750-1.513105$

C -1.044709 4.127804-1.118553

H -3.211106 4.858011 -2.586792

H $-2.7523943 .409491-3.501452$

C $-5.6565593 .800345-2.960934$

H $-4.4690972 .100350-2.432334$

C -0.1867713 .6101730 .057468$

O $-1.4106735 .489641-0.899925$

H -0.441793 4.102393 -2.033105

H $-0.0665442 .525512-0.047082$

O -0.8482543 .8493501 .298605$

C 1.2153834 .2514180 .109362

C 2.0709673 .8480401 .323864

H $1.7510673 .994149-0.810712$

O $1.115807 \quad 5.6773640 .124635$

H 3.0446324 .3424411 .215094

H 1.6395924 .2845732 .234336

H $-6.6076553 .347584-2.663322$
H $-5.7219634 .877438-2.774289$

H -5.534296 $3.653367-4.039014$

C -5.4455272 .4593820 .108366$

H -1.939683 $5.520650-0.084246$

H -1.6987743 .3790301 .274391$

H $\quad 0.556970 \quad 5.924107 \quad 0.881578$

H $-6.4924952 .620050-0.173943$

H $-5.3559632 .698370 \quad 1.175161$

H $-4.8725414 .439266-0.485214$

C $3.936306 \quad 1.950377-0.172306$

O $2.621507 \quad 1.6599860 .313078$

C 2.2625502 .3324191 .520388

C 3.2454121 .9621672 .640090

C 4.6961922 .2144982 .231854

C $4.990373 \quad 1.5721680 .875412$

H $4.0127053 .017738-0.413784$

C $4.1309101 .149318-1.464437$

H 1.2849361 .9330291 .812853

O 2.9278882 .6897213 .821336

H 3.1293160 .8971552 .874658

H 4.9290303 .2855982 .218169

O 5.5692691 .6246223 .197904

H 5.0250400 .4831830 .996994

O $6.2843241 .980108 \quad 0.434109$

C $3.3904891 .788728-2.615325$

H $5.1870941 .104685-1.753747$

H $3.7833520 .115292-1.356514$

H 3.4747492 .3353544 .542417

H 5.4533852 .1086214 .032829

H 6.2696212 .9453190 .317001

O $2.0613241 .495701-2.543054$

O $3.9319782 .476877-3.471127$

C $1.2498972 .059259-3.577892$

H $0.2074861 .803086-3.370384$

H $1.3475693 .148934-3.589061$

H $1.5301591 .637285-4.547433$

SCF Energy $(B 3 L Y P / 6-31 G * * / / M M F F)=-3245.90974716$

01382

MM̄FF Geometry

C $-2.9928673 .786762-0.004659$

C -2.0084292 .8909940 .161379$

C $-0.8660342 .727202-0.805542$

O $\quad 0.3498652 .924483-0.050098$

C $-0.8715721 .303026-1.389875$

C $0.0679911 .121103-2.566653$

C $1.1813500 .361800-2.545323$

C $-0.3549541 .797121-3.845979$

C $1.740762-0.411215-1.382773$

C $1.129231-1.813935-1.317918$

C $1.805512-2.780620-0.335104$

C $1.937250-2.2289541 .084485$

C $2.623272-3.2353002 .022696$

O $3.098410-3.124133-0.848259$

O $2.965790-2.5138203 .216594$

C $3.934273-3.7169711 .358670$

C $1.668187-4.3548972 .451764$

O $4.539667-4.7665752 .108639$

C $3.754268-4.166021-0.109644$

C $5.092897-4.507649-0.814601$

O $2.972519-5.370199-0.134818$

C $1.3397683 .660944-0.621500$

O $1.3282264 .188353-1.722103$

C 2.4657303 .7120650 .338127

C $3.6117324 .309069-0.021903$

C 4.8243644 .4213510 .792251

C 4.7668513 .8948412 .202578

C $6.211131-3.466798-0.738073$

C $5.930098-2.182573-1.496092$

C $6.4414270 .241124-1.460724$

O $5.198620-2.132098-2.481294$

C $7.5696261 .103769-0.994741$

C $7.447601 \quad 1.9931560 .001712$

C 8.5521372 .8870900 .484703

C 8.3532524 .3362860 .094614

C $9.1404484 .914482-0.828322$

C 7.2986625 .1391780 .829424

C 5.9263144 .9623200 .230263 
N $6.645107-1.110395-0.979247$ H -2.972079 $4.448595-0.867330$ H -2.027135 2.2299421 .023814 H $-0.9545283 .476631-1.600639$ H -1.883210 $1.042185-1.730334$ H $-0.6326610 .587816-0.594643$ H $1.7617570 .270783-3.462492$ H $\quad 0.299187 \quad 1.541592-4.686637$ H -1.370663 $1.491839-4.118307$ H $-0.3360772 .885194-3.736805$ H $2.828555-0.474360-1.505371$ H $1.5706410 .131334-0.446737$ H $\quad 0.068919-1.726089-1.051472$ H $1.168504-2.265053-2.318744$ H $1.187186-3.686012-0.329444$ H $\quad 0.961051-1.9326271 .484765$ H $2.548048-1.3163531 .073169$ H $3.353338-3.1421933 .848842$ H $4.626460-2.8664651 .389120$ H $0.817329-3.9367773 .002898$ H $2.158440-5.0511423 .140978$ H $1.269788-4.9279431 .612037$ H $4.732650-4.4223032 .996687$ H $5.479489-5.448241-0.399493$ H $4.883705-4.717929-1.872610$ H $3.119700-5.812547-0.987497$ H 2.3254893 .2452941 .306380 H $3.6798764 .743199-1.018738$ H 4.5445402 .8218312 .204770 H 3.9901654 .4152292 .774376 H 5.6990404 .0213662 .756099 H $6.464931-3.2532620 .304646$ H $7.113511-3.896084-1.192352$ H $5.4720940 .583962-1.081733$ H $6.387504 \quad 0.236231-2.554485$ H $8.5180361 .007000-1.516969$ H 6.4964772 .0624850 .521282 H 9.5172152 .5222790 .108721 H 8.6193132 .8108001 .576983 H $9.0230775 .959384-1.095957$ H $9.9157914 .355051-1.341260$ H 7.5401606 .2093810 .776259 H $7.3414104 .895421 \quad 1.893935$ H $5.8577815 .317092-0.799483$ H $7.095122-1.225141-0.075377$ C -5.4722193 .6114080 .211193$ O $-5.5066842 .207579-0.111268$ C -6.8489741 .7192550 .075386$ C -7.713400 2.9413910 .368116 C -6.7198293 .8589341 .055521$ H $-6.831431 \quad 1.0859960 .969752$ C -7.282315 $0.895827-1.143549$ H $-8.0883613 .405944-0.551139$ H $-8.5768992 .696307 \quad 0.994227$ C $-7.164648 \quad 5.310178 \quad 1.098760$ H -6.5581083 .4985672 .080335$ C -6.316973 -0.259803 -1.484974 O $-7.338421 \quad 1.770828-2.277254$ H $-8.2999520 .517383-0.990226$ H $-5.3548790 .147552-1.815783$ O $-6.859732-0.981079-2.597282$ C $-6.099437-1.257212-0.332489$ C $-5.248879-2.485237-0.700574$ H $-5.634118-0.7498440 .515038$ O $\quad-7.368890-1.7312380 .127456$ H $-5.286036-3.1785220 .148640$ H -5.728480 -3.028695 -1.524009 H -6.4146555 .9343591 .594359$ H -7.324887 5.7112310 .092309 H -8.1039135 .4077361 .652899$ C $-4.162463 \quad 3.9360370 .928065$ H -7.811056 $1.302158-2.985458$ H $-6.798396-0.404738-3.377573$ H -7.812004 -2.159346 -0.625219 H -4.0413773 .2751131 .793936$ H -4.1753754 .9667591 .298669$ H -5.537378 4.160658 -0.737786 C $-2.872873-2.1291201 .178466$
O $-3.117572-1.412292-0.037992$

C -3.785181-2.159036-1.061086

C $-2.949300-3.391784-1.430725$

C $-2.654222-4.253772-0.207931$

C $-2.062255-3.4065480 .914773$

H -3.827651 -2.379954 1.656201

C -2.090238-1.1947192.117766

H -3.807074 -1.514001-1.947148

O $-3.612705-4.183551-2.410334$

H -1.995920 -3.069810-1.868796

H -3.546636 -4.791042 0.133204

O $-1.696073-5.255566-0.559424$

H -1.030277 -3.144451 0.659134

O $-2.014437-4.1847402 .109835$

C $-2.947453-0.1038592 .723403$

H $-1.671301-1.7722082 .950024$

H $-1.275752-0.7037151 .573025$

H $-3.780836-3.614400-3.180760$

H -2.067069-5.761621-1.302670

H -1.501273 -4.987174 1.913082

O -2.2516200 .4845393 .742101$

$\begin{array}{lllll}0 & -4.067075 & 0.210845 & 2.347012\end{array}$

C -2.9284881 .5399344 .432057$

H -2.970154 2.4288823 .797483

H $-3.934451 \quad 1.229610 \quad 4.731137$

$\mathrm{H}-2.3558441 .7781975 .332398$

SCF Energy $\left(B 3 L Y P / 6-31 G^{* *} / / M M F F\right)=-3245.90454411$

01383

MM̄FF Geometry

C $-1.333773-1.4422632 .282883$

C $-2.463403-1.2040112 .967283$

C -3.1064510 .1464663 .191690$

$\begin{array}{llll}\text { O } & -2.327380 & 1.173586 & 2.530997\end{array}$

C -4.5412310 .1746422 .627988$

C $-5.557688-0.5834323 .454134$

C $-6.115594-1.7572373 .098593$

C -5.9844990 .0988924 .728583$

C $-5.820911-2.5923601 .883337$

C $-6.977798-2.5988130 .874630$

C $-7.165109-1.2580870 .146799$

C $-8.362005-1.324959-0.804244$

C $-8.511754-0.036385-1.626522$

O $-5.970231-0.989851-0.593330$

O $-9.433503-0.299082-2.695448$

C $-7.1528990 .302678-2.274372$

C $-9.1197601 .102340-0.799093$

O $-7.2036391 .568017-2.930412$

C $-5.9667570 .267223-1.282296$

C $-4.6327600 .376504-2.058001$

O $-6.0782421 .369021-0.380427$

C -2.4626562 .4503012 .970482$

O -3.2166702 .8407573 .851784$

C - -1.5790623 .3830262 .233033$

C -0.5371772 .9946371 .480210$

C 0.4029263 .8855240 .791501

C 0.0695965 .3544550 .751748

C $-3.3766430 .190391-1.194519$

C $-2.1425090 .078754-2.066634$

C $-0.7062851 .412471-3.609195$

O $-1.563988-0.993076-2.227727$

C $0.3981492 .212010-2.994312$

C $0.6609043 .482335-3.339281$

C $1.7635104 .379501-2.844631$

C $2.7751633 .763717-1.906125$

C $3.8145893 .057407-2.382347$

C $2.6522284 .076030-0.432977$

C 1.5138863 .3548440 .237500

N $-1.7864601 .281416-2.651690$

H $-0.777285-0.616178 \quad 1.849347$

H $-2.982289-2.0525783 .408725$

H -3.097114 0.3426224 .270766

H -4.525212 $-0.176631 \quad 1.591935$

H $-4.889244 \quad 1.2121222 .539077$

H -6.861762 -2.191332 3.763113

H $-6.774913-0.4511135 .250523$

H -5.1401030 .1925555 .417987$

H -6.3704321 .1005144 .512170$ 
H $-4.888529-2.298751 \quad 1.392567$ H -5.656805 -3.621493 2.227838 H $-6.761335-3.3793090 .133447$ H -7.909121 -2.8828361 .380412$ H $-7.318099-0.4667630 .888770$ H $-9.286206-1.538338-0.252729$ H -8.228287 -2.165096 -1.500326 H $-10.275148-0.586140-2.301514$ H $-6.969161-0.445305-3.058593$ H $-10.112460 \quad 0.818717-0.429152$ H -9.273679 1.997347 -1.411785 H -8.5124451 .3762080 .066140$ H -7.952402 $1.542611-3.550621$ H -4.612849 -0.390190 -2.844160 H $-4.576728 \quad 1.351694-2.557494$ H $-5.1995081 .588272-0.034190$ H -1.8040784 .4327702 .394068$ H $-0.321271 \quad 1.9320861 .385414$ H 0.1434015 .7928661 .752763 H $-0.947571 \quad 5.5086330 .373549$ H 0.7226505 .9315350 .093581 H $-3.2383171 .024015-0.497812$ H -3.440511 -0.725472 -0.596645 H -1.121609 $1.900806-4.496922$ H $\quad-0.333577 \quad 0.427807-3.908285$ H $1.0142301 .697711-2.263684$ H $0.0127133 .949306-4.080090$ H $1.3106675 .260910-2.374270$ H $2.2937064 .765424-3.726162$ H $4.5739832 .652543-1.722716$ H $3.9274002 .868455-3.444897$ H $2.6024285 .160710-0.301116$ H 3.5706463 .7786820 .089764 H 1.6639502 .2770540 .310006 H -2.327879 $2.114222-2.438730$ C $-0.506173-3.0581110 .565293$ O $0.619963-2.2552160 .151232$ C $1.564528-3.096880-0.532951$ C $0.773947-4.324055-0.960108$ C $-0.135571-4.5068830 .240664$ H $2.312466-3.4032120 .208775$ C $2.249108-2.324264-1.664145$ H $0.179085-4.130923-1.860383$ H $1.415104-5.189642-1.150618$ C -1.334176 -5.398783 -0.033604 H $0.449744-4.9386461 .063749$ C $2.896724-0.996698-1.218384$ O $1.288239-2.014468-2.679996$ H $2.997482-2.963527-2.146323$ H $2.121649-0.269857-0.946886$ O $3.588011-0.446833-2.346645$ C $3.897814-1.136087-0.056095$ C $4.581236 \quad 0.2000490 .291490$ H $3.371989-1.4958420 .834472$ O $4.877258-2.114384-0.392414$ H $5.2030560 .517070-0.551450$ H 3.8019140 .9577330 .426600 H -1.969784 -5.4808960 .853633$ H -1.947266 -5.006785 -0.852091 H -1.008122 -6.406504 -0.311079 C $-0.771326-2.8188512 .053798$ H $\quad 0.572853-1.504460-2.262084$ H $2.937555-0.346357-3.062990$ H $5.323335-1.816839-1.203853$ H $\quad 0.166509-2.8966202 .616976$ H - $1.458862-3.5802532 .439228$ H -1.366310 -2.737228 -0.035761 C $7.580493-0.3960830 .571608$ O $6.531615-0.7981651 .461207$ C 5.4424280 .1226221 .574014 C 5.9645921 .4809782 .063572 C 7.0795792 .0111201 .167182 C 8.1695310 .9541500 .996018 H $7.190769-0.327725-0.450692$ C $8.652707-1.4917410 .601610$ H $4.789145-0.2723572 .362784$ O 4.9170482 .4420022 .129126 H 6.3623371 .3713573 .081003
H $6.6933352 .340026 \quad 0.195210$

O 7.6666463 .1626861 .777222

H 8.7229450 .8455241 .937760

$\begin{array}{lllll}\text { O } & 9.096024 & 1.392487 & 0.004294\end{array}$

C $8.154573-2.764727-0.039849$

H $9.568815-1.1884320 .082238$

H $8.932871-1.7328931 .634345$

H 4.2251512 .0816942 .709658

H 6.9538793 .8094041 .918161

H 9.4351182 .2591510 .287232

O $8.164190-2.637819-1.397797$

O $7.794711-3.7499950 .591351$

C $7.706151-3.781407-2.125728$

H $8.362471-4.636267-1.937280$

H $6.673740-4.019061-1.852410$

H $7.739651-3.541259-3.191699$

SCF Energy $\left(B 3 L Y P / 6-31 G^{* *} / / M M F F\right)=-3245.92404575$

01384

MM̄FF Geometry

C $2.860237 \quad 1.1115462 .668892$

C 1.8343691 .8136143 .172392

C 0.5969322 .1449962 .381148

O -0.5107821 .4916153 .044813$

C 0.3344643 .6621332 .324923

C 1.2624534 .3871241 .371320

C 1.1029244 .4009380 .032462

C 2.4057215 .1268352 .012484

C $-0.0149583 .756124-0.741670$

C $-0.5827504 .707115-1.803849$

C $-2.0208924 .379612-2.242479$

C $-3.0412004 .841914-1.195455$

C $-4.4792644 .488697-1.598585$

O $-2.1389882 .972735-2.483796$

O $-5.3159024 .659486-0.443547$

C $-4.5275762 .993948-1.973058$

C $-5.0274695 .435727-2.671793$

O $-5.8114522 .637478-2.480498$

C $-3.4266192 .569783-2.973993$

C $-3.4070801 .039510-3.204773$

O $-3.699973 \quad 3.174688-4.245105$

C -1.5467281 .0857882 .260256$

O $-1.680764 \quad 1.2774121 .062165$

C -2.4881130 .3199053 .106855$

C $-3.550018-0.2635402 .531032$

C $-4.536942-1.1211663 .188297$

C $-4.375425-1.3745694 .663604$

C $-3.0695530 .189424-1.977140$

C $-3.236820-1.296786-2.251222$

C $-3.258000-3.489485-1.057432$

O $-3.524457-1.749535-3.355879$

C $-4.605555-3.734400-0.453924$

C $-4.798675-4.4006070 .694567$

C $-6.149570-4.6250451 .313289$

C $-6.248534-4.0602912 .714847$

C $-6.090975-4.8540533 .787780$

C $-6.611186-2.5994922 .876523$

C $-5.514519-1.6691992 .436350$

N $-3.034291-2.055619-1.106923$

H 2.8011170 .7491181 .646649

H 1.8798672 .1659214 .199920

H $\quad 0.692121 \quad 1.7379581 .366375$

H 0.4122834 .0965113 .331105

H -0.7088293 .8537812 .044851$

H $1.8399994 .928424-0.571014$

H 3.0773355 .5763221 .273472

H 2.0222065 .9327202 .647077

H 3.0036164 .4557242 .634929

H $0.3725572 .856011-1.233989$

H $-0.8128373 .430638-0.074013$

H $-0.5397545 .750692-1.466469$

H $\quad 0.069340 \quad 4.640037-2.685190$

H -2.195478 $4.915327-3.183372$

H $-2.9498415 .920337-1.015162$

H $-2.8324774 .361431-0.230481$

H $-5.2310605 .581516-0.145870$

H $-4.3979312 .435738-1.038346$

H $-5.0367326 .468150-2.301972$ 
H $-6.0684345 .195950-2.915179$ H -4.445206 $5.423817-3.595177$ H -6.470864 2.914449-1.821545 H $-4.3691070 .725142-3.631060$ H $-2.662527 \quad 0.810244-3.980504$ H $-3.2381222 .668067-4.933466$ H -2.2607520 .2217804 .161883$ H $-3.702850-0.1224701 .461976$ H -5.133334 -2.045688 5.073840 H -4.444988 -0.4358345 .224208$ H $-3.403922-1.8371634 .870887$ H -2.034309 $0.369792-1.667883$ H -3.729986 $0.436071-1.140844$ H -2.451251 -3.927669 -0.462281 H -3.217136 -3.912536 -2.065822 H $-5.464673-3.335288-0.988247$ H $-3.940743-4.8113751 .221709$ H $-6.332887-5.7073931 .330683$ H -6.950299 -4.201567 0.694315 H -6.187806 -4.467659 4.796796 H $-5.857292-5.9081623 .680675$ H -6.912062 -2.379925 3.907396 H -7.507673 -2.387106 2.279557 H -5.545522 -1.434060 1.372056 H -2.821124 -1.583763 -0.232873 C 5.3992211 .0561132 .661266 O $5.452030 \quad 0.2516621 .466039$ C 5.9912191 .0539180 .395980 C 6.5905122 .2886491 .060973 C 5.6197342 .4986402 .209069 H $5.1407041 .372945-0.217973$ C $6.963266 \quad 0.225557-0.451074$ H 7.5988022 .0964871 .445497 H $\quad 6.643522 \quad 3.143087 \quad 0.379664$ C 6.1437073 .4154663 .299019 H 4.6957582 .9228411 .798685 C $6.344054-1.082300-0.989899$ O $8.085136-0.1210730 .369656$ H $7.3531030 .837472-1.273072$ H $6.105265-1.745530-0.151444$ O $7.331821-1.758145-1.776867$ C $5.098478-0.871819-1.872812$ C $4.508543-2.172184-2.453013$ H $4.318039-0.356086-1.305950$ O $5.435488-0.014710-2.967006$ H $3.744705-1.895176-3.189904$ H $5.275862-2.701804-3.030865$ H 5.4113743 .5171424 .106310 H 7.0741963 .0339343 .732456 H 6.3445314 .4140502 .897389 C 4.1073380 .7668763 .431746 H $8.781604-0.461981-0.216431$ H $8.019953-2.078676-1.169650$ H $6.149816-0.445927-3.467134$ H 4.1305751 .2914144 .394022 H $4.064892-0.3084173 .646248$ H $\quad 6.243658 \quad 0.7273693 .281844$ C $1.740388-2.064266-1.160267$ O $2.979445-2.416441-0.536304$ C $3.905849-3.105716-1.383409$ C $3.268830-4.387554-1.938329$ C $1.923969-4.120575-2.609808$ C $1.015945-3.308109-1.685771$ H $1.943517-1.384816-1.997354$ C $0.902382-1.253016-0.165888$ H $4.733075-3.423346-0.737731$ O $4.157555-5.017492-2.855145$ H $3.113349-5.090400-1.109210$ H $2.060580-3.624146-3.577677$ O $1.303006-5.377635-2.888000$ H $0.693095-3.948299-0.856302$ O $-0.156356-2.928093-2.401059$ C $0.632390-1.9903071 .125326$ H $1.434445-0.3351930 .106091$ H $-0.043782-0.934080-0.617122$ H $3.710889-5.812541-3.193283$ H $\quad 0.429693-5.189941-3.273021$ H $\quad 0.113477-2.351870-3.136394$
O $-0.675545-2.366998 \quad 1.187107$

O $1.486681-2.2163191 .972973$

C $-1.047307-3.1018002 .357292$

H $-2.123307-3.2869472 .315815$

H $-0.526378-4.0636112 .377025$

H $-0.826333-2.5237693 .259691$

SCF Energy (B3LYP/6-31G**//MMFF) $=-3245.91049455$

01385

MM̄FF Geometry

C 3.6605771 .5888302 .827623

C 2.6812490 .9883402 .134065

C 1.4611070 .3749512 .766785

O $\quad 0.3249660 .6041061 .896146$

C $1.641200-1.1478372 .904820$

C $0.781364-1.7184374 .014702$

C $-0.414611-2.3132583 .845517$

C $1.369903-1.5967135 .398749$

C -1.175124-2.532373 2.567425

C -1.542637 -4.015788 2.401909

C $-2.489379-4.3109321 .226580$

C $-1.824248-4.091024-0.132044$

C $-2.805951-4.324260-1.289862$

O $-3.639391-3.4763681 .370565$

O $-2.197160-3.827868-2.491254$

C $-4.068499-3.473527-1.036272$

C $-3.074571-5.814131-1.529872$

O $-5.045641-3.749405-2.037410$

C $-4.659779-3.6597850 .381149$

C $-5.778287-2.6389040 .701193$

O $-5.233939-4.9678780 .476001$

C $-0.285423 \quad 1.8178401 .960528$

O -0.0161082 .7354412 .719042$

C -1.326602 $1.853010 \quad 0.905910$

C -1.9263153 .0179660 .616692$

C $-2.9329383 .253241-0.423067$

C $-3.4107682 .068314-1.218838$

C $-5.309805-1.1816100 .738498$

C $-6.410281-0.2303751 .172583$

C -6.7399362 .1485401 .855300$

O $\quad-7.599357-0.5362851 .175709$

C -6.8912173 .0043850 .637410$

C $-6.5472004 .300050 \quad 0.589873$

C $-6.7172115 .155925-0.631673$

C $-5.4434545 .860665-1.039623$

C $-5.3286477 .195604-0.934292$

C $-4.3349995 .031212-1.652675$

C $-3.3607134 .517844-0.625126$

N $-5.9015591 .014726 \quad 1.517340$

H $3.5949471 .646903 \quad 3.910884$

H $2.7722740 .913513 \quad 1.055180$

H $1.265626 \quad 0.8329863 .744567$

H $2.686066-1.3950813 .137299$

H $1.428703-1.6446151 .951148$

H $-0.931821-2.6699854 .736092$

H $\quad 0.720263-2.0274976 .168071$

H $2.330234-2.1204485 .448657$

H $1.533767-0.5462225 .658226$

H $-2.084972-1.9231552 .619234$

H $-0.615381-2.1871861 .695395$

H $-0.631559-4.6192392 .303762$

H -2.045517 -4.3483903 .320521$

H $-2.803284-5.3573881 .321047$

H $-0.938675-4.728979-0.241419$

H -1.462023 -3.058364 -0.205372

H - $-1.353095-4.295099-2.615327$

H -3.765764 -2.428110 -1.174413

H -2.141134 -6.336346 -1.771781

H $-3.732585-5.961994-2.393292$

H $-3.517862-6.317084-0.668281$

H $-5.734293-3.066606-1.977830$

H -6.615009 -2.7574300 .001725$

H $-6.196830-2.8800881 .689005$

H $-5.891889-4.9664041 .190782$

H -1.5420590 .9324390 .375643$

H -1.6359573 .9013881 .184764$

H $-3.7200491 .253502-0.557911$

H $-2.6130611 .700597-1.871983$ 
H $-4.2737212 .287081-1.850883$ H -4.465306 -1.074132 1.428460 H -4.985616 $-0.859844-0.256247$ H -6.2531402 .6805782 .678511$ H $-7.721371 \quad 1.8004482 .191944$ H $-7.3166782 .534581-0.246535$ H $-6.1433004 .778027 \quad 1.479411$ H -7.504055 $5.889932-0.414243$ H -7.084379 $4.567548-1.482007$ H -4.428228 $7.710827-1.252462$ H $-6.1287907 .802403-0.523716$ H -3.764432 $5.651612-2.357132$ H $-4.7751834 .239977-2.264911$ H -2.932091 $5.307762-0.005879$ H $-4.902591 \quad 1.1804241 .436989$ C 5.9742691 .1229521 .974464 O 5.5491450 .2176660 .935948 C 6.6299230 .0202790 .007069 C 7.8765790 .5235920 .720304 C 7.3081781 .6980481 .497884 H $6.4350090 .675858-0.849854$ C $6.659323-1.441631-0.455408$ H $8.283212-0.2259591 .408967$ H 8.6684690 .8115040 .022555 C 8.2092192 .1771092 .622369 H 7.1313942 .5290740 .801733 C $5.292916-1.937436-0.981048$ O $7.022090-2.2572950 .664798$ H $7.442039-1.577807-1.210701$ H $4.567042-1.934274-0.160683$ O $5.429133-3.300880-1.397086$ C $4.752847-1.128830-2.175621$ C $3.370404-1.586980-2.677093$ H $4.690498-0.071252-1.915003$ O $5.676583-1.222374-3.262821$ H $3.182600-1.100546-3.642018$ H $3.395933-2.660478-2.900029$ H 7.7484283 .0094073 .163555 H 8.4117381 .3773993 .342877 H 9.1692842 .5223392 .225006 C 4.8866342 .1771582 .184527 H $7.240998-3.1409010 .324058$ H $5.577819-3.836345-0.599618$ H $5.762053-2.163359-3.493880$ H 4.6069052 .6230261 .223189 H 5.2528382 .9894012 .822269 H 6.1142640 .5240692 .883928 C $1.7819181 .015757-2.349998$ O $2.2011840 .112293-1.320701$

C $2.227667-1.270460-1.690140$

C $0.836955-1.715191-2.159901$ C $0.289077-0.812022-3.260198$ C $0.3746390 .652704-2.838215$ H $2.4874570 .977474-3.188355$ C $1.7999462 .434618-1.755446$ H $2.437345-1.826828-0.769192$ O $0.861459-3.060456-2.623142$ H $\quad 0.143300-1.677878-1.310480$ H $\quad 0.804655-0.978913-4.213078$ O - $-1.087089-1.124644-3.486770$ H $-0.3573810 .845414-2.044917$ O $\quad 0.032016 \quad 1.479362-3.948367$ C $3.1965493 .009017-1.636348$ H $1.2335013 .123520-2.391759$ H $1.3591492 .422146-0.752182$ H $1.187878-3.614404-1.893521$ H -1.137877 -2.072482 -3.699299 H $-0.8562011 .214738-4.243646$ O $3.1186824 .229114-1.025026$ O $4.2202572 .479344-2.043748$ C $4.3560664 .937132-0.895466$ H $5.1136534 .315656-0.409208$ H $4.7017455 .261837-1.881210$ H $4.1788345 .820177-0.275865$ SCF Energy (B3LYP/6-31G**//MMFF) $=-3245.91525627$

01386

MM̄FF Geometry
C $-1.0596401 .922876 \quad 1.194128$

C -0.9481540 .8113570 .451350$

C $0.0692980 .631247-0.642623$

O $0.792954-0.604767-0.402280$

C $-0.5696420 .477744-2.033065$

C $-1.2381351 .720984-2.572692$

C $-0.6037552 .682987-3.269816$

C $-2.7272591 .805502-2.363636$

C $0.8569932 .760461-3.623706$

C $1.4380004 .156043-3.348723$

C $1.7748244 .393188-1.867642$

C $2.0034015 .885474-1.614901$

C 2.474636 6.161149-0.181661

O $2.9680323 .654276-1.580231$

O $2.9441467 .516684-0.124048$

C $3.6808565 .251708 \quad 0.119129$

C 1.3293716 .0539650 .831583

O $4.081598 \quad 5.373547 \quad 1.482645$

C $3.4404493 .763806-0.231357$

C $4.7812162 .994426-0.133930$

O 2.5081003 .2143340 .698768

C $1.673331-0.6438340 .632794$

O 1.9330890 .2552331 .415796

C $2.268227-1.9967630 .654315$

C $3.166979-2.3139641 .596954$

C $3.787771-3.6267051 .735277$

C $3.864110-4.1104403 .157525$

C $4.7331411 .549853-0.649877$

C $6.1013100 .888681-0.609014$

C $7.270760-1.196864-1.250097$

O $7.1140931 .462181-0.215848$

C $6.937686-2.651324-1.330965$

C $7.417718-3.567385-0.476237$

C $7.132752-5.039360-0.572620$

C $6.440276-5.5796400 .658611$

C $7.149337-6.0598801 .694049$

C $4.930134-5.6435280 .654032$

C $4.282406-4.2874390 .669630$

N $6.056645-0.424389-1.063138$

H -0.4130062 .7719790 .990198$

H $-1.606643-0.0282340 .653525$

H $0.7944851 .453028-0.663429$

H -1.287352 -0.354882 -2.023804

H $\quad 0.1998080 .138934-2.740226$

H -1.203593 $3.506942-3.655200$

H $-3.1639392 .698159-2.824240$

H -3.221907 $0.934491-2.805260$

H $-2.9674391 .839258-1.299322$

H $1.452970 \quad 1.997867-3.113493$

H $0.9401522 .562415-4.699472$

H $2.3566914 .257498-3.941445$

H $\quad 0.7384434 .921469-3.707624$

H $0.9576174 .022144-1.239453$

H $1.0949496 .460025-1.835517$

H $2.7690526 .259784-2.309257$

H $2.2106448 .099176-0.386017$

H $4.5235555 .617767-0.484278$

H 0.5395396 .7766730 .594116

H 1.6713096 .3052451 .841562

H 0.8711635 .0634520 .858730

H 4.2333626 .3183361 .656693

H $5.5391603 .529460-0.721554$

H 5.1268532 .9816170 .907749

H 2.6633562 .2607740 .782055

H $1.942545-2.711588-0.094428$

H $3.452402-1.5831972 .350494$

H $2.856834-4.2646043 .560249$

H $4.377620-3.3755303 .787947$

H $4.408085-5.0515393 .265973$

H $4.0553510 .947321-0.040867$

H $4.380514 \quad 1.527408-1.687147$

H $7.968252-0.972616-0.435775$

H $7.731565-0.865970-2.186799$

H $6.292432-2.968656-2.146544$

H $8.077943-3.2478830 .327223$

H $8.090051-5.557696-0.716603$

H $6.538065-5.274996-1.463940$

H $6.662243-6.4762032 .569505$ 
H $8.234295-6.0427721 .690152$ H $4.560732-6.2593601 .481987$ H $4.599695-6.173206-0.249093$ H $4.205078-3.823556-0.313586$ H $5.209241-0.743523-1.525017$ C -3.3440812 .6815191 .927654$ O -4.0812231 .7180351 .147374$ C -5.4137361 .6064971 .674169$ C -5.6223422 .8542052 .520270$ C -4.2398803 .0318353 .117783$ H $-5.4215790 .728724 \quad 2.331925$ C $-6.420127 \quad 1.408527 \quad 0.538474$ H -5.8833303 .7242091 .906745$ H -6.4038292 .7211713 .274349$ C -3.9938814 .4200363 .682972$ H -4.1071432 .2924973 .919184$ C $-6.0739790 .241502-0.410377$ O $-6.4597422 .605890-0.249085$ H -7.4257581 .2820400 .956564$ H $\quad-5.1865700 .507130-0.992804$ O $-7.143141 \quad 0.129521-1.359434$ C $-5.863728-1.1208810 .278605$ C $-5.694787-2.297344-0.702248$ H $-4.995706-1.0709560 .943253$ O $-7.006823-1.3898451 .095503$ H -5.740191 -3.229335 -0.126743 H -6.566802 -2.339069-1.365860 H -2.9788244 .5031974 .083814$ H -4.1179995 .1930252 .917109$ H -4.6969264 .6349994 .494343$ C -2.0138372 .0589842 .347641$ H -7.100289 $2.459492-0.966310$ H -7.937176 -0.151907 -0.873172 H $-6.829253-2.2098721 .587214$ H -2.191396 1.0718152 .792409 H - 1.5277512 .6719553 .115523 H -3.182483 3.5580031 .287382 C $-2.966420-3.531478-0.055026$ O $-3.223783-2.275429-0.697257$ C $-4.392260-2.240455-1.525895$ C $-4.294582-3.313143-2.619243$ C $-4.051934-4.696907-2.028826$ C $-2.852859-4.664413-1.085352$ H $-3.769187-3.7523430 .658329$ C -1.648572 -3.3950910 .725681$ H $-4.373696-1.273389-2.040035$ O $-5.475499-3.343743-3.413863$ H -3.461173 -3.071798 -3.291974 H $-4.943592-5.082278-1.520801$ O $-3.763088-5.615345-3.085476$ H - $-1.931948-4.547891-1.671637$ O $-2.766334-5.912937-0.400179$ C $-1.783990-2.5612861 .981754$ H -1.289929 -4.3816581 .040067$ H $-0.891934-2.9192540 .091587$ H - $-5.602442-2.452974-3.782880$ H -4.516032 -5.593079 -3.700882 H -2.714305 -6.611175 -1.075385 O $-0.568675-2.5164472 .605347$ O $-2.809174-2.0185002 .368246$ C $-0.516313-1.7573333 .816682$ H -1.274897 -2.106267 4.523837 H $0.470986-1.9006914 .263890$ H -0.650339 -0.694927 3.597138 SCF Energy (B3LYP/6-31G**//MMFF)= -3245.92093155

$01 \_387$

MM̄FF Geometry

C $0.351904-3.8518050 .377303$

C $-0.709517-4.026927 \quad 1.180102$

C $-1.389010-2.9469351 .993168$

O $-0.621224-1.7195011 .885314$

C $-2.813459-2.6498941 .491323$

C $-3.775125-3.814101 \quad 1.418127$

C $-4.278228-4.314750 \quad 0.271527$

C $-4.210578-4.3713492 .747295$

C -3.936331-3.902856-1.133022

C $-5.070492-3.190971-1.883029$
C $-5.595636-1.903559-1.222545$

C $-6.554687-1.174413-2.171167$

C $-7.0303810 .172626-1.604365$

O $-4.489902-1.054490-0.903319$

O $-7.6278350 .896432-2.691350$

C $-5.8019510 .975209-1.118240$

C $-8.137817-0.021062-0.562175$

O $-6.2057132 .175752-0.464673$

C $-4.8565180 .152966-0.218154$

C -3.5594320 .9144540 .143241$

O $-5.527866-0.1287821 .016919$

C $-0.795109-0.7496232 .819364$

O $-1.512849-0.7938303 .804536$

C 0.0458670 .4002862 .411324

C $0.009613 \quad 1.5433943 .112092$

C 0.8096032 .7426772 .846389

C 1.6707412 .7481841 .611017

C $-2.6525471 .264040-1.037871$

C $-2.6583382 .749321-1.329749$

C $-1.9274704 .926319-0.401822$

O $-3.262741 \quad 3.219002-2.290639$

C -0.6930605 .4241750 .276491$

C -0.7067786 .1408521 .410308$

C 0.5227236 .7197492 .046944

C 0.7131836 .2728993 .477527

C 0.2410787 .0065034 .499914

C 1.5498375 .0400443 .724685

C 0.7460303 .7695323 .719858

N -1.926176 $3.477837-0.402374$

H $\quad 0.782895-2.8625930 .262632$

H $-1.119271-5.0296351 .283257$

H -1.399167 -3.265763 3.042661

H $-2.726629-2.1414770 .527785$

H -3.266219 -1.890569 2.141662

H $-4.993583-5.1332570 .336593$

H -4.957695 -5.165026 2.640729

H -3.355678 -4.794018 3.284350

H $-4.653998-3.5821303 .363183$

H -3.032295 -3.290932 -1.170268

H -3.680179-4.814425 -1.688533

H $-4.684520-2.948778-2.882234$

H $-5.907068-3.885869-2.030558$

H $-6.115123-2.179725-0.297721$

H -7.412002 -1.811393 -2.422393

H -6.040332 -0.979980 -3.123288

H $-7.8641641 .780680-2.361519$

H -5.257772 $1.288974-2.017346$

H -9.003197 $-0.519468-1.015695$

H $-8.5096200 .941053-0.193099$

H -7.822506 -0.6216560 .293460$

H $-6.517474 \quad 1.9380320 .425190$

H -3.808862 1.7997420 .741852

H -2.9728190 .2734310 .813511$

H -4.861936 -0.3044161 .701555$

H $\quad 0.6850040 .268858 \quad 1.545210$

H $-0.648774 \quad 1.6018213 .977812$

H 2.1442163 .7123371 .413196

H 1.0678612 .5216640 .724325

H 2.4692102 .0029191 .694257

H -1.620032 $0.981049-0.798627$

H $-2.9161180 .721944-1.951671$

H -2.842382 5.2519160 .104740

H -1.965188 $5.293489-1.432845$

H $0.2555535 .217095-0.212645$

H -1.659508 6.3667821 .883966

H $0.430693 \quad 7.8136272 .011967$

H 1.4227116 .4874401 .464121

H $0.407728 \quad 6.7118715 .530761$

H $-0.327428 \quad 7.914540 \quad 4.327544$

H 2.0274405 .1137364 .711734

H 2.3895055 .0145173 .023870

H 0.0825143 .6762984 .580866

H -1.609838 3.0042520 .439600

C $2.491263-5.148412-0.026566$

O $3.258634-3.955335-0.301234$

C $4.135091-3.7141300 .814922$

C $4.169907-5.0169831 .609439$

C $2.747277-5.5060481 .438749$ 
H $3.655145-2.9427891 .428567$

C $5.515309-3.2368810 .356033$

H $4.873091-5.7384241 .177085$

H $4.447919-4.8579482 .655713$

C $2.561856-6.979793 \quad 1.754247$

H $2.104536-4.9190932 .106086$

C $5.533140-1.937856-0.475918$

O $6.110292-4.269911-0.441321$

H $6.161004-3.1230211 .235784$

H $5.140468-2.136965-1.479710$

O $6.912822-1.588366-0.665278$

C $4.781750-0.7383970 .136276$

C $5.1670140 .639294-0.437167$

H $3.704861-0.8951610 .018445$

O $5.025143-0.6813721 .546694$

H 4.5612721 .3905970 .084188

H $6.1993360 .866207-0.140912$

H $1.521080-7.2821711 .600939$

H $3.193707-7.6096021 .118977$

H $2.823806-7.1856642 .797225$

C $1.021112-4.944916-0.406963$

H $6.989929-3.956253-0.712997$

H $7.296499-1.4016230 .208162$

H $5.977785-0.5414391 .681094$

H $0.478989-5.890926-0.293729$

H $0.964365-4.665819-1.466140$

H $2.907336-5.926660-0.679711$

C $2.720157 \quad 1.530610-2.049514$

O $3.6904330 .545809-2.423586$

C $5.0205380 .794659-1.963689$

C $5.5081172 .150315-2.493779$

C $4.5617303 .280422-2.100507$

C $3.1323962 .936103-2.513034$

H $2.589727 \quad 1.513501-0.962042$

C $1.3870931 .137628-2.706158$

H $5.6533170 .030905-2.429810$

O $6.8211712 .421914-2.014600$

H $5.5698762 .103394-3.589036$

H $4.6311343 .501314-1.028936$

O $4.9785304 .464454-2.784477$

H $3.0433093 .011773-3.604694$

O $2.233883 \quad 3.886941-1.944026$

C $0.814187-0.141376-2.141849$

H $\quad 0.635501 \quad 1.920565-2.557026$

H $1.5441420 .984833-3.780848$

H $7.0797923 .295480-2.355113$

H $4.4340795 .199994-2.457148$

H $2.4294394 .752522-2.340404$

O $-0.216801-0.550426-2.938985$

O $1.201571-0.698644-1.125285$

C $-0.887743-1.740618-2.517210$

H $-0.228993-2.603973-2.644444$

H $-1.212053-1.649438-1.476687$

H -1.771378 -1.874700 -3.146731

SCF Energy (B3LYP/6-31G**/MMFF)= -3245.91168865

01_388

MM̄FF Geometry

C $4.011490-2.698399-1.673654$

C $3.354286-3.565262-0.887098$

C $1.963234-3.362583-0.322997$

O $1.257095-2.407522-1.148230$

C $1.184056-4.695877-0.331493$

C $-0.023020-4.7266680 .588806$

C -1.301931 -4.8879030 .194480$

C $0.286057-4.6285312 .063676$

C $-1.843123-5.078493-1.194042$

C $-2.537859-3.843778-1.787178$

C $-3.835349-3.428928-1.072269$

C $-4.571654-2.359500-1.885035$

C $-5.830719-1.848582-1.167714$

O $-3.510831-2.9058630 .220126$

O $-6.250735-0.640321-1.821337$

C $-5.466324-1.4705380 .284262$

C $-6.996926-2.835933-1.293414$

O $-6.634598-1.147761 \quad 1.033456$

C $-4.654431-2.5589091 .017023$

C $-4.142242-2.1113432 .409918$
O $\begin{array}{ccc}-5.491091 & -3.702220 & 1.236874\end{array}$

C $0.413649-1.523153-0.557445$

O $0.146098-1.430800 \quad 0.628548$

C $-0.154300-0.681495-1.635595$

C $-1.2162360 .096048-1.380263$

C $-1.9132680 .951860-2.342047$

C $-1.3022431 .109996-3.709409$

C $-3.268094-0.8540212 .423803$

C $-4.047143 \quad 0.4327992 .650135$

C $-3.855303 \quad 2.900726 \quad 2.328347$

$\begin{array}{lllll}\text { O } & -5.164576 & 0.461991 & 3.158782\end{array}$

C $-4.518938 \quad 3.218776 \quad 1.026213$

C $-4.027528 \quad 4.091364 \quad 0.133768$

C $-4.6750224 .387621-1.187248$

C $-3.8454253 .910326-2.359311$

C $-3.0988334 .768038-3.074799$

C $-3.954532 \quad 2.455126-2.758885$

C $-3.052321 \quad 1.561127-1.952222$

N -3.325195 1.5513552259621

H $3.542758-1.756974-1.948966$

H $3.866150-4.480267-0.595280$

H $2.086271-2.9774270 .695264$

H $1.841191-5.516972-0.013503$

H $0.897700-4.924035-1.363042$

H -2.065762 $-4.922727 \quad 0.970739$

H $-0.596156-4.8118692 .686740$

H $1.040270-5.3713012 .343428$

H $\quad 0.663315-3.6346672 .319905$

H $-1.054379-5.393523-1.883588$

H $-2.553372-5.914690-1.169364$

H $-1.831739-3.008047-1.786146$

H $-2.768723-4.069112-2.836088$

H $-4.464062-4.319874-0.959098$

H $-4.827313-2.736755-2.883116$

H $-3.900347-1.507408-2.053061$

H $-6.406354-0.845252-2.759279$

H $-4.864665-0.5549220 .228916$

H -7.252057 -2.994196 -2.348219

H $-7.903564-2.439314-0.823263$

H $-6.780367-3.813491-0.857838$

H $-7.102246-0.4438650 .552216$

H $-4.984217-2.0210743 .106984$

H $-3.524091-2.9303502 .805188$

H $-5.107182-4.2306481 .956303$

H $0.297953-0.749879-2.618590$

H $-1.6300570 .093779-0.374253$

H $-1.3552700 .166779-4.263676$

H $-0.2517831 .412709-3.632621$

H - $1.7916691 .873576-4.317938$

H -2.562571 $-0.927158 \quad 3.261427$

H -2.675753 -0.7843341 .507398$

H $-3.016068 \quad 3.5706702 .539979$

H -4.5778662 .9778993 .146674$

H -5.4433142 .6909720 .802995$

H $-3.104197 \quad 4.6199920 .356924$

H $-4.8314455 .472527-1.247890$

H $-5.6776913 .946031-1.248245$

H $-2.525154 \quad 4.436437-3.933960$

H $-3.0382625 .819005-2.812489$

H $-3.7755142 .342847-3.833404$

H $-4.9898182 .117120-2.622286$

H $-3.4130101 .371838-0.941951$

H -2.4498281 .4212651 .762392$

C $6.506110-2.186939-1.494585$

O $6.167201-0.793223-1.332141$

C $6.329890-0.4554890 .057379$

C $7.319884-1.4740650 .601585$

C $6.836005-2.728962-0.099466$

H $5.360442-0.6259330 .541886$

C $6.7383521 .009308 \quad 0.229577$

H $8.348019-1.2416580 .300622$

H $7.286461-1.5508021 .692278$

C $7.853251-3.857036-0.104284$

H $5.928451-3.0792480 .406673$

C $5.7821102 .031807-0.422784$

O $8.0406841 .209896-0.332596$

H $6.8392881 .229792 \quad 1.299133$

H $5.9446842 .051725-1.507589$ 
$\begin{array}{lllll}\text { O } & 6.168888 & 3.321079 & 0.069107\end{array}$ C $4.2900241 .785222-0.128450$ C $3.3737782 .928743-0.600587$ H $3.9895340 .852455-0.619903$ $\begin{array}{lllll}\text { O } & 4.129932 & 1.608813 & 1.276396\end{array}$ H 3.4206883 .7524210 .120614 H $3.7409123 .303550-1.563077$ H $7.463978-4.728939-0.639448$ H $8.787857-3.553586-0.587681$ H $8.089839-4.1656370 .919345$ C $5.388675-2.932196-2.232958$ H $7.9988120 .951219-1.269334$ H $5.7339923 .993084-0.481956$ H $3.255242 \quad 1.2093041 .413270$ H $5.617489-4.003897-2.270542$ H $5.365818-2.585719-3.274293$

H $7.405697-2.208568-2.122903$

C 1.0442012 .7767241 .462099

$\begin{array}{lllll}\text { O } & 1.356555 & 1.875270 & 0.393918\end{array}$

C $1.9057162 .476860-0.780686$

C $0.9681623 .567754-1.319140$

C $0.582292 \quad 4.577262-0.243778$

C $0.056717 \quad 3.850597 \quad 0.993114$

H 1.9646103 .2509261 .824575

C $0.442157 \quad 1.941122 \quad 2.599776$

H $1.9315861 .682915-1.538556$

O $1.555340 \quad 4.251742-2.420837$

H $0.0478173 .102308-1.689710$

H $1.424997 \quad 5.2340830 .001801$

O $-0.4478315 .415895-0.771181$

H $-0.914097 \quad 3.3929770 .765663$

O -0.1414054 .7916932 .045184$

C $1.5136481 .206398 \quad 3.368921$

H -0.0928162 .5629013 .326694$

H $-0.276746 \quad 1.207403 \quad 2.217895$

H $1.7485393 .588937-3.105760$

H $-0.6153816 .119307-0.121776$

H $-0.867105 \quad 5.3813291 .779716$

O $1.703742-0.0343732 .837515$

O $2.124262 \quad 1.688654 \quad 4.313908$

C $2.701809-0.8366613 .476455$

H $2.404409-1.0563414 .505975$

H $3.672020-0.3314513 .453898$

H $2.785022-1.7774302 .925897$

SCF Energy (B3LYP/6-31G**/MMFF) $=-3245.89162820$

01_389

MM̄FF Geometry

C $4.606930 \quad 0.6814482 .414483$

C 3.4617550 .0166712 .196902

C $2.591252-0.5215873 .301409$

O $1.228515-0.1318083 .014156$

C $2.635784-2.0593333 .336881$

C $1.838651-2.6403644 .490046$

C $0.740262-3.4081834 .351440$

C $2.357668-2.3256705 .870391$

C $0.104298-3.900627 \quad 3.082127$

C $-0.909311-2.9111892 .504289$

C $-1.934120-3.563075 \quad 1.562713$

C $-1.287584-4.2745080 .372823$

C $-2.340939-4.879196-0.567928$

O $-2.789433-2.5143751 .087438$

O $-1.661478-5.228178-1.786283$

C $-3.388085-3.797071-0.912743$

C $-2.894182-6.196428-0.012683$

O $-4.476023-4.341892-1.653144$

C -3.894471 -2.9720530 .294205$

C $-4.684507-1.715969-0.144242$

O $-4.827789-3.7052001 .095298$

C $0.811703 \quad 1.0908413 .435743$

$\begin{array}{lllll}\text { O } & 1.450825 & 1.912116 & 4.072094\end{array}$

C -0.5752101 .2625362 .950340$

C -1.2060212 .4343863 .115043$

C -2.554334 2.754467 2.638910

C -3.2963671 .6742681 .897093$

C $-3.918066-0.749030-1.049458$

C $-4.640402 \quad 0.575419-1.235923$

C $-4.2989912 .809057-2.309842$
O $-5.7407740 .821875-0.751205$

C $-3.6178793 .734546-1.350694$

C $-4.2504844 .713381-0.685570$

C $-3.556254 \quad 5.657449 \quad 0.255274$

C -4.2551705 .7823621 .593382$

C $-4.746843 \quad 6.9645322 .001793$

C $-4.386957 \quad 4.5491192 .463596$

C $-3.049023 \quad 3.988306 \quad 2.872606$

N -3.894940 $1.447417-2.015814$

H $4.9451160 .800796 \quad 3.442208$

H $3.101602-0.1387251 .184321$

H $2.898949-0.1109314 .270540$

H $3.674198-2.4044003 .426370$

H $2.263957-2.4473622 .382376$

H $\quad 0.240051-3.751807 \quad 5.256394$

H $1.849125-2.9070996 .647067$

H $3.425068-2.5601705 .937271$

H $2.215229-1.2672886 .107823$

H $\quad 0.868403-4.1453182 .336211$

H $-0.394862-4.8496743 .316383$

H $-1.459225-2.4091813 .311930$

H $-0.382389-2.1096111 .971267$

H -2.531837 -4.265698 2.155105

H $-0.591690-5.0561740 .698652$

H $-0.685007-3.550366-0.191396$

H $-2.329669-5.560151-2.410662$

H $-2.877524-3.117294-1.603014$

H -2.097368 -6.948686 0.040442

H $-3.657466-6.619649-0.674471$

H $-3.315264-6.1021970 .989688$

H $-5.061465-4.806421-1.032119$

H $-5.619739-2.014074-0.636703$

H -4.993731 -1.176777 0.762583

H $-4.643658-4.6516081 .019859$

H -1.0312840 .4219232 .439386$

H -0.6844773 .2404063 .629302$

H -3.4286350 .7904212 .531204$

H -2.740085 1.3828790 .999815

H $-4.293211 \quad 1.9697951 .567562$

H -2.927524 $-0.539899-0.629831$

H -3.786596 -1.189044 -2.043946

H $-5.389623 \quad 2.888934-2.259742$

H $-3.973074 \quad 3.028119-3.331147$

H -2.547024 $3.604624-1.210342$

H $-5.3155014 .864513-0.846102$

H $-3.5086886 .636599-0.239080$

$\begin{array}{llll}H & -2.514797 & 5.355003 & 0.420512\end{array}$

H -5.2489597 .0693282 .958004$

H -4.6594687 .8563321 .390006$

H -5.0162893 .8228691 .944136$

H -4.9302334 .7918673 .386379$

H -2.432251 4.6951623 .429588

H $-2.979274 \quad 1.151267-2.340307$

C 4.8536721 .6281360 .025650

$\begin{array}{lllll}\text { O } & 3.808016 & 2.609756 & 0.209094\end{array}$

C $3.9395433 .614988-0.804664$

C $4.8670993 .031150-1.861441$

C $5.8236792 .239961-0.987772$

H $4.4657384 .457003-0.332634$

C $2.5857314 .138422-1.296672$

H $4.3322732 .358607-2.541137$

H $5.3662553 .800730-2.457434$

C $6.651647 \quad 1.222489-1.752071$

H $6.5007562 .941637-0.482105$

C $1.662043 \quad 3.142537-2.031014$

O $2.8636815 .227413-2.187289$

H $2.0430604 .563715-0.443289$

H $2.1345762 .838888-2.970514$

O $0.4760713 .855518-2.415834$

C $1.2454031 .900568-1.224418$

C $0.187271 \quad 1.015316-1.908957$

H $2.124567 \quad 1.286973-1.019079$

$\begin{array}{lllll}\text { O } & 0.726997 & 2.301799 & 0.045124\end{array}$

H $-0.0754610 .210200-1.211069$

H $-0.7412831 .583855-2.037964$

H $7.2952470 .653258-1.074071$

H $\quad 6.0186920 .512002-2.291897$

H $7.2921411 .722882-2.485679$ 
C 5.4993121 .3156411 .378430

H $2.0091825 .582525-2.486752$

H $\quad 0.0615334 .203927-1.608525$

H $-0.1141002 .765643-0.104487$

H 5.8589892 .2580241 .812194

H $6.3733990 .668906 \quad 1.241679$

H $4.3913190 .731710-0.403403$

C $2.020976-1.359485-2.429222$

O $1.942320-0.150485-3.196165$

C $0.636640 \quad 0.429922-3.262275$

C $-0.350556-0.569421-3.880882$

C $-0.322662-1.922124-3.170812$

C $1.115177-2.432831-3.046502$

H $1.702384-1.149691-1.401429$

C $3.498368-1.783729-2.404291$

H $\quad 0.712277 \quad 1.272931-3.959831$

O $-1.676140-0.052155-3.859019$

H $-0.086475-0.730226-4.934451$

H $-0.810855-1.860573-2.191478$

O $-1.086565-2.845279-3.949635$

H $1.485494-2.713639-4.040745$

O $1.143829-3.599734-2.232420$

C $3.931389-2.367136-1.075784$

H $3.719352-2.514451-3.189856$

H $4.121541-0.901290-2.592671$

$\mathrm{H}-1.6696830 .788285-4.348278$

H -1.162295 -3.668349 -3.438161

H $0.629653-4.290000-2.683585$

O $5.290025-2.269476-0.969254$

O $3.184242-2.865775-0.245661$

C $5.852736-2.7886850 .239569$

H $5.420661-2.2866981 .109913$

H $5.689241-3.8688090 .296314$

H $6.928599-2.5954290 .223569$

SCF Energy (B3LYP/6-31G**//MMFF) $=-3245.90887912$

0139

MM̄FF Geometry

C -0.269667 -3.145082 0.828427

C $0.596890-3.6419681 .723263$

C $1.435596-2.8667292 .708732$

O $2.699357-2.5508882 .065926$

C $0.859471-1.5385263 .239797$

C -0.267906-1.678756 4.241480

C -1.505691-1.167493 4.095036

C $0.090748-2.3785195 .529534$

C -2.083814 -0.419536 2.929131

C -2.2990601 .0710273 .220762$

C -0.9948641 .8827653 .273972$

C -1.2961243 .3574073 .552196$

C -0.0266814 .2209453 .513483$

O -0.3327181 .7526212 .011054$

O $-0.4306745 .597527 \quad 3.455748$

C $\quad 0.742287 \quad 3.9213772 .209839$

C 0.8074384 .0668584 .790669

O 2.0028104 .5882292 .197470

C 0.9393062 .4113541 .940669

C 1.5094832 .2023070 .517092

O 1.8629741 .8868982 .896761

C $3.683699-3.4849972 .112098$

O $3.618194-4.5873782 .640425$

C $4.903675-3.0407421 .400637$

C $5.098912-1.7993300 .928886$

C $6.285188-1.333418 \quad 0.202413$

C $7.365817-2.346189-0.080424$

C 1.5493530 .7378680 .057773

C $2.1108040 .602460-1.345849$

C $2.814019-1.114776-3.006026$

O $2.3640221 .568111-2.061817$

C $4.308586-1.103721-2.934894$

C $5.059656-0.104578-3.422233$

C $6.556900-0.036031-3.322907$

C $7.0106611 .048586-2.368066$

C $7.0896602 .329519-2.766861$

C $7.4347200 .640034-0.973162$

C $6.337236-0.045666-0.201452$

N $2.276471-0.727476-1.713775$

H $-0.413030-2.0734780 .745848$
H $\quad 0.745182-4.7204401 .747959$

H $1.638066-3.5170803 .568140$

H $0.589513-0.8875092 .404887$

H $1.671517-0.9911333 .740079$

H -2.212478 -1.308244 4.912936

H $-0.689492-2.274516 \quad 6.291315$

H $0.240418-3.4489055 .359454$

H $1.010189-1.9571695 .949196$

H -1.497780 -0.545698 2.016861

H -3.059747 -0.8710582 .715691$

H $-2.935924 \quad 1.4728042 .422885$

H $-2.852115 \quad 1.1919964 .160626$

H $-0.354919 \quad 1.471198 \quad 4.062415$

H -1.811412 $3.474743 \quad 4.513622$

H -1.9930413 .7361942 .791376$

H $-0.979014 \quad 5.7820674 .237677$

H $\quad 0.1598014 .3476351 .381361$

H $\quad 0.2225004 .3674805 .668294$

H 1.6817524 .7270504 .773899

H 1.1499913 .0438494 .959056

H 1.8321825 .5328822 .353895

H $0.8953362 .758849-0.202193$

H 2.5254812 .6142080 .462029

H 2.3239631 .1243222 .515304

H $5.657121-3.8146891 .290768$

H $4.324598-1.0500331 .078217$

H $6.958080-3.198257-0.636252$

H $7.802724-2.7117880 .855413$

H $8.188453-1.958022-0.683180$

H 2.1722900 .1404390 .729656

H 0.5387810 .3144610 .052363

H $2.437814-0.428017-3.772012$

H $2.451807-2.120980-3.237133$

H $4.786713-1.949515-2.448572$

H $4.5674940 .736164-3.907112$

H $6.9586090 .160170-4.325553$

H $6.982735-1.002085-3.025258$

H $7.4345983 .111312-2.098573$

H $6.8084092 .623980-3.772660$

H $7.7356571 .521523-0.391580$

H $8.3390630 .031275-1.053439$

H $5.500000 \quad 0.6100440 .042189$

H 2.114386-1.457706-1.026356

C $-2.548729-3.9238530 .123812$

O $-3.041880-2.608971-0.195663$

C $-4.363922-2.729690-0.760733$

C $-4.732166-4.208556-0.687119$

C $-3.370953-4.874224-0.743256$

H -4.282261 -2.439159-1.814052

C $-5.333769-1.793944-0.025259$

H $-5.239508-4.4567220 .252271$

H $-5.391230-4.509713-1.507271$

C $-3.382476-6.312473-0.255030$

H $-3.008575-4.846533-1.779470$

C $-4.877787-0.320797-0.000570$

O $-5.459518-2.2295521 .335389$

H -6.335990 -1.873289 -0.460685

H -3.924132 -0.2430460 .529013$

$\begin{array}{lllll}\text { O } & -5.827583 & 0.431856 & 0.763649\end{array}$

C $-4.7643890 .325986-1.392119$

C $-4.3154891 .799455-1.358439$

H -4.069802 -0.242345 -2.014748

O $-6.0351530 .264406-2.044791$

H -4.491806 $2.231670-2.350616$

H $-4.9673742 .369262-0.685136$

H $-2.382723-6.753824-0.311558$

H -3.722574 -6.3806370 .783791$

H -4.054051 -6.921267 -0.869167

C -1.044954 -3.995313 -0.141191

H -4.565872 -2.262839 1.717038

H -5.8941680 .0105201 .637827$

H $-6.6736910 .742528-1.488181$

H $-0.822879-3.630185-1.150330$

H $-0.703496-5.035230-0.088688$

H -2.757121 -4.0973601 .188213$

C $-1.8419331 .654472-3.122449$

O $-1.9566701 .199629-1.768296$

C $-2.8384591 .976943-0.950286$ 
C $-2.362743 \quad 3.434677-0.900521$

C $-2.1586654 .021711-2.294885$

C $-1.3009353 .089828-3.152182$

H -2.821632 $1.616141-3.612506$

C $-0.9036430 .690085-3.858313$

H $-2.736346 \quad 1.5801890 .064647$

O $-3.2807374 .248166-0.178310$

H -1.406911 $3.482584-0.365970$

H -3.117379 $4.233496-2.782623$

O $-1.4812395 .275216-2.179608$

H $-0.2682403 .112237-2.782200$

O $-1.2743023 .575544-4.492647$

C - $1.537723-0.670369-4.052710$

H $-0.6359861 .063632-4.852218$

H $\quad 0.0133290 .566036-3.275069$

H $-3.381608 \quad 3.8590180 .707210$

H -2.018715 $5.842062-1.599994$

H $-2.1843743 .559138-4.834789$

O $-0.585780-1.640349-3.929932$

O $-2.718343-0.855093-4.316719$

C $-1.046459-2.981699-4.118996$

H -1.322555 -3.136282 -5.166236

H $-0.228109-3.660907-3.865883$

H -1.894504 -3.195077 -3.461468

SCF Energy (B3LYP/6-31G**//MMFF) $=-3245.91879775$

01_390

MM̄FF Geometry

C $2.881783-2.7511251 .009470$

C $2.220381-2.1474562 .007944$

C $2.442342-0.7051992 .377069$

O $1.169932-0.0086032 .393146$

C $3.036042-0.5461093 .788220$

C $4.402173-1.1645613 .985350$

C $5.565809-0.5423983 .715657$

C $4.413422-2.5409494 .601380$

C $5.770230 \quad 0.839608 \quad 3.158082$

C 6.8053240 .8554172 .022534

C 6.2271750 .4428870 .658798

C $7.3645310 .229047-0.344138$

C $6.842152-0.070644-1.754214$

$\begin{array}{lllll}\text { O } & 5.383437 & 1.511275 & 0.220805\end{array}$

O $7.9592240 .057559-2.649665$

C $5.8101861 .009875-2.139808$

C $6.372807-1.524051-1.877773$

O $5.1899590 .705430-3.386677$

C $4.7534971 .314474-1.050950$

C $4.0351512 .642426-1.387727$

O $3.739827 \quad 0.314970-0.991606$

C $0.5851340 .284953 \quad 1.200678$

O $0.964052-0.049671 \quad 0.090341$

C -0.6029551 .1203281 .485268$

C -1.2450631 .7314210 .478216$

C -2.4210812 .5968970 .603201$

C $-2.9816472 .812103 \quad 1.985083$

C $2.9388433 .020415-0.382320$

C $2.3975234 .419555-0.619885$

C $0.801266 \quad 6.0634420 .346949$

O $2.8234975 .164196-1.498683$

C -0.6673375 .9483280 .599502$

C $-1.5959516 .381266-0.266444$

C $-3.0762846 .322748-0.020897$

C $-3.7961495 .462663-1.034746$

C $-4.1825805 .966321-2.219144$

C $-4.1426444 .045999-0.638092$

C $-2.9330043 .159187-0.512386$

N $1.382092 \quad 4.736107 \quad 0.273898$

H $3.615555-2.1849830 .442997$

H $1.490769-2.7073052 .587430$

H $3.097918-0.2005021 .657171$

H $2.336543-0.9535854 .532091$

H 3.0731250 .5233464 .036400

H $6.488626-1.0733233 .947981$

H $5.429720-2.9116074 .772937$

H $3.903525-2.5253115 .570403$

H $3.908458-3.2649183 .956426$

H 4.8354141 .3002282 .825241

H 6.1549091 .4613373 .976203
H 7.1920821 .8803181 .943428

H 7.6545550 .2123712 .285491

H $5.631748-0.4697100 .769776$

H $8.035318-0.571477-0.007250$

H $7.9836031 .136560-0.389640$

H $7.625570-0.062785-3.555605$

H $6.3844071 .930521-2.315026$

H $7.220241-2.208153-1.745527$

H $5.976229-1.732870-2.876955$

H $5.618936-1.799016-1.138469$

H $4.517140 \quad 0.021899-3.230788$

H $4.7769043 .451371-1.419593$

H $3.5759802 .580876-2.382765$

H $4.109774-0.537144-1.259814$

H $-0.907837 \quad 1.2208172 .520750$

H $-0.8737501 .591557-0.536356$

H -2.220064 3.2409282 .646050

H $-3.324650 \quad 1.8640802 .412943$

H -3.8301783 .4980822 .013348$

H $2.1042132 .317403-0.462574$

H 3.3275142 .9825850 .641536

H $1.0211346 .620939-0.569500$

H 1.2820526 .5851411 .180772

H -0.9778725 .5135661 .546252$

H -1.274993 $6.830395-1.204001$

H $-3.4671417 .347896-0.064567$

H -3.3001215 .9708610 .993737$

H $-4.7247985 .364884-2.941142$

H $-3.9608606 .992450-2.493627$

H $-4.7972683 .594313-1.394658$

H $-4.747421 \quad 4.0710150 .272601$

H -2.449361 $2.949310-1.467428$

H 1.1858884 .0908911 .034338

C $1.887833-4.394880-0.654363$

O $0.513510-4.035600-0.421928$

C $-0.030346-3.491377-1.641284$

C $1.040560-3.678747-2.715129$

C $2.314703-3.610944-1.893500$

H $-0.167588-2.417451-1.470158$

C $-1.379774-4.147265-1.955346$

H $\quad 0.958627-4.653435-3.209806$

H $0.987055-2.907348-3.489449$

C $3.532134-4.181309-2.597742$

H $2.501394-2.558030-1.649597$

C $-2.395674-4.088608-0.795850$

O $-1.151119-5.531102-2.255226$

H -1.804072 -3.701178 -2.862699

H $-2.047335-4.7215840 .029494$

O $-3.606519-4.681908-1.283325$

C $-2.695134-2.665774-0.286318$

C $-3.749517-2.6464760 .838702$

H -1.774833 -2.218848 0.104255

O $-3.122771-1.858453-1.379147$

H $-4.699275-3.0315860 .455967$

H -3.416378 -3.330124 1.628195

H $4.414072-4.131109-1.951695$

H $3.379669-5.228571-2.879236$

H $3.748331-3.616009-3.510037$

C $2.703547-4.1899750 .622894$

H -2.014611 -5.928657 -2.460853

H $-4.151169-4.916051-0.513180$

H -3.924186 -2.266527 -1.748974

H $3.698491-4.6338530 .499800$

H $2.216666-4.7305951 .444589$

H $1.883346-5.470209-0.879241$

C $-5.849426-0.5384430 .086505$

O $-4.491137-0.3063360 .479780$

C $-3.968242-1.2361291 .434228$

C $-4.828450-1.2105032 .706019$

C $-6.303938-1.4466002 .398365$

C $-6.778963-0.4926121 .303637$

H -5.930162 -1.514200 -0.406035

C $-6.2271140 .540416-0.934115$

H $-2.975805-0.8630701 .715058$

O $-4.384364-2.1821473 .646637$

H $-4.730696-0.2297043 .189385$

H -6.494968 -2.490021 2.120710

O $-7.075793-1.1950393 .574860$ 
H $-6.828967 \quad 0.5284251 .703273$

O $-8.096603-0.8609230 .901584$

C $-5.5110620 .324517-2.246236$

H -7.303491 $0.558154-1.139285$

H $-5.954611 \quad 1.535724-0.565493$

H -3.448788 -1.997451 3.837271

H $-6.735857-1.7800144 .273573$

H $-8.656829-0.8463781 .696619$

O $-6.104706-0.689551-2.939071$

O $-4.537903 \quad 0.972082-2.608599$

C $-5.510827-1.000289-4.203394$

H -5.579618 -0.139044 -4.874809

H $-4.468249-1.304105-4.070045$

H -6.064855 -1.833795 -4.643424

SCF Energy (B3LYP/6-31G**//MMFF) $=-3245.90822804$

01_391

MM̄FF Geometry

C -4.834660 $2.819241 \quad 0.939215$

C -3.5310043 .0134510 .698184$

C -2.5158073 .3042811 .771602$

O -1.4198082 .3722191 .627818$

C $-1.948714 \quad 4.7247501 .591102$

C -0.9731465 .1273822 .681775$

C 0.3458015 .3402112 .500134

C -1.5762075 .3152384 .051445$

C 1.1393565 .2536951 .225103

C 1.8968393 .9292881 .136417

C $2.8721723 .836170-0.046264$

C $2.1682473 .936896-1.400072$

C $3.1303783 .705294-2.574398$

$\begin{array}{lllll}\text { O } & 3.519237 & 2.560758 & 0.066472\end{array}$

O $2.3147383 .515138-3.740629$

C $3.9259032 .407436-2.326002$

C $3.9967404 .941783-2.842403$

O $4.9476932 .234928-3.305917$

C $4.5413342 .321099-0.909497$

C $5.1014710 .896550-0.675484$

O $5.6045743 .269006-0.803097$

C -1.5770881 .1188302 .133028$

O $-2.560142 \quad 0.6644792 .694786$

C $-0.319916 \quad 0.385976 \quad 1.867557$

C $-0.221959-0.9241752 .133730$

C $0.962242-1.7510801 .890328$

C $2.229188-1.0456571 .480484$

C 5.5618320 .6256880 .762733

C $5.983777-0.8211310 .962712$

C $6.570217-2.4286242 .780696$

O $6.125958-1.6162550 .037887$

C $5.329004-3.2314223 .023412$

C $5.156140-4.4813682 .564505$

C $3.953836-5.3673372 .767420$

C $2.646025-4.6489243 .034186$

C $2.171110-4.5561484 .288590$

C $1.939152-4.1189121 .800894$

C $0.870618-3.0875032 .052986$

N $6.198247-1.1094742 .303385$

H -5.1995032 .9082291 .958964$

H -3.166810 $2.942562-0.321299$

H -2.9573583 .1959892 .769629$

H -2.7686815 .4552131 .580104$

H -1.474685 4.7921620 .606440

H $\quad 0.9417895 .618521 \quad 3.368919$

H -0.8614335 .7377304 .765938$

H -2.428240 6.0011514 .000568

H -1.9198794 .3595924 .458220$

H 0.4999785 .3933490 .350318

H 1.8514046 .0882321 .212301

H 2.4608573 .7579822 .063725

H 1.1827893 .1006131 .076840

H 3.6225704 .6276900 .063522

H $1.6649224 .903072-1.520002$

H $1.3760563 .177982-1.445755$

H $2.9024643 .425009-4.509550$

H $3.2304871 .567194-2.452474$

H $3.3652545 .801068-3.098113$

H $4.6540614 .786482-3.705171$

H $4.6138695 .225654-1.987271$
H $4.5174592 .183787-4.175789$

H $4.3212570 .163034-0.917570$

H $5.9440300 .711555-1.353832$

H $6.2104182 .989074-0.099737$

H $\quad 0.4993570 .946623 \quad 1.433960$

H -1.086627 -1.441774 2.545157

H $2.500719-0.2854082 .221772$

H $2.105346-0.5569720 .508437$

H $3.089615-1.7116751 .398000$

H 6.4188641 .2566961 .020320

H $4.7506150 .844958 \quad 1.465717$

H $7.116243-2.2928173 .719145$

H $7.233364-2.9019602 .049113$

H $4.552882-2.7588783 .619444$

H $5.968881-4.9394812 .003194$

H $4.183968-6.0714793 .577463$

H $3.825485-5.9874931 .870583$

H $1.228538-4.0735114 .519332$

H $2.722100-4.9707925 .126916$

H $1.472089-4.9674811 .283587$

H $2.678590-3.7313561 .092489$

H -0.084602 -3.510940 2.365498

H $5.979860-0.4031473 .000137$

C $-6.4614541 .105583-0.021774$

O $-5.493160 \quad 0.089097-0.357713$

C $-5.730287-1.0556850 .483541$

C -7.047588 -0.7930821 .215066$

C $-7.036183 \quad 0.7143871 .339029$

H $-4.917468-1.0737141 .218088$

C $-5.750676-2.358309-0.323116$

H $-7.913781-1.1180930 .626890$

H -7.091151-1.304742 2.181625

C -8.3980291 .3182941 .632128$

H -6.3479910 .9726782 .152117$

C $-4.473619-2.714818-1.109600$

O $-6.827496-2.305557-1.271226$

H $-5.996013-3.1868450 .352877$

H -4.389579 -2.091667 -2.008017

O $-4.653885-4.062295-1.575501$

C $-3.153401-2.643768-0.319932$

C $-1.994753-3.472308-0.908451$

H $-2.853983-1.594597-0.218113$

O $-3.379101-3.1438191 .003996$

H -1.114650 -3.290321 -0.281493

H -2.222758 -4.535699 -0.757392

H -8.3327432 .4085161 .703429$

H -9.1231051 .0738310 .848708$

H -8.7900660 .9403802 .582020$

C $-5.8456922 .504529-0.127453$

H -6.676686 -1.531877-1.841157

H $-5.471924-4.080014-2.101470$

H -2.552646 -3.035979 1.504316

H $-6.6417323 .256095-0.065960$

H -5.384286 2.610931-1.117438

H -7.244602 $1.029402-0.788454$

C $-0.020416-1.454177-2.113589$

O $-1.276556-1.871387-2.662190$

C $-1.650656-3.222970-2.390370$

C $-0.576086-4.176280-2.931977$

C $0.791102-3.860003-2.338064$

C $1.126613-2.386195-2.547820$

H -0.097648 -1.420464 -1.021736

C $0.249407-0.029988-2.624415$

H -2.557518 -3.411471-2.975339

O $-0.930691-5.526885-2.654924$

H $-0.523816-4.075493-4.023890$

H $0.840419-4.140048-1.279905$

O $1.768379-4.660874-3.007502$

H $1.362315-2.233192-3.609253$

O $2.309861-2.099814-1.804690$

C $-0.8104410 .971014-2.224471$

H $1.2029310 .344335-2.240914$

H $0.284189-0.046934-3.721032$

H $-0.210527-6.090149-2.986365$

H $2.637194-4.421866-2.640929$

H $2.644508-1.235714-2.096500$

O $-0.6683002 .083190-3.005961$

O $-1.6341320 .813162-1.335433$ 
C $-1.5792443 .155056-2.749813$

$\mathrm{H}-1.3412033 .618860-1.788697$

H $-1.454673 \quad 3.901726-3.538517$

$\mathrm{H}-2.6133262 .798127-2.768033$

SCF Energy (B3LYP/6-31G**//MMFF) $=-3245.90528131$

01_392

MM̄̄F Geometry

C $\quad 1.3714975 .327208 \quad 0.671353$

C $2.1164904 .632356-0.201144$

C 3.1292933 .5966270 .217495

O $2.7478352 .322724-0.353764$

C $4.5165043 .974919-0.333156$

C $5.6423833 .107980 \quad 0.195975$

C $6.4034232 .291880-0.558841$

C 5.9210433 .2264881 .673258

C $6.3228342 .046854-2.038832$

C $5.4238180 .870245-2.450442$

C $5.831728-0.482656-1.843478$

C $5.053312-1.626311-2.498586$

C $5.354371-2.983098-1.841015$

O $5.542602-0.451798-0.443148$

O $4.355918-3.917412-2.280126$

C $5.203120-2.848929-0.309302$

C $6.704892-3.548146-2.297227$

O $5.632582-4.0366890 .350341$

C $5.942515-1.6272890 .273441$

C $5.635638-1.3791911 .770918$

O $7.356097-1.8374710 .168089$

C 1.7586951 .6287570 .274120

O 1.1621091 .9527861 .288976

C $1.5085390 .394455-0.503451$

C $0.391104-0.311713-0.275963$

C $-0.003985-1.554955-0.941777$

C $1.009844-2.210759-1.840498$

C $4.161304-1.1353272 .110662$

C $3.418842-2.3801992 .566981$

C $1.126114-3.1793683 .124479$

O $3.979256-3.4381672 .840155$

C $0.615779-3.9467981 .945953$

C $-0.685636-4.198788 \quad 1.739287$

C -1.206952 -4.9980310 .579225$

C $-2.291725-4.273452-0.186024$

C $-3.588584-4.5472090 .033532$

C -1.870749 -3.295742 -1.261495

C -1.235082 $-2.053121-0.700551$

N $2.051996-2.1573452 .672393$

H 1.5038825 .1587691 .737236

H $1.9766014 .789906-1.268039$

H 3.1670013 .5078291 .309844

H $4.7492985 .016605-0.073542$

H $4.4821263 .935514-1.427407$

H $7.1900511 .725061-0.061032$

H 6.8437112 .7126441 .964412

H 6.0321894 .2783321 .956622

H 5.1059622 .7893902 .257761

H $5.9930252 .949264-2.564725$

H $7.3412181 .861591-2.404233$

H $4.3902851 .103016-2.169261$

H $5.451698 \quad 0.798477-3.544937$

H $6.909069-0.619499-1.993426$

H $5.257891-1.669336-3.575686$

H $3.976902-1.429105-2.408833$

H $4.386202-3.954224-3.251557$

H $4.130540-2.746488-0.104244$

H $\quad 6.711948-3.691330-3.384618$

H $6.885940-4.538348-1.864722$

H $7.547865-2.901286-2.045802$

H $5.127798-4.776929-0.027806$

H $6.065219-2.1822532 .381723$

H $6.179760-0.4698602 .064836$

H $7.802710-1.2586100 .807982$

H $2.2461170 .104920-1.242304$

H $-0.313757 \quad 0.0602540 .464829$

H $1.938293-2.398118-1.290443$

H $\quad 1.232677-1.571928-2.701974$

H $0.689764-3.176802-2.234510$

H $4.111054-0.4283782 .948901$
H $3.633693-0.6803941 .267807$

H $\quad 0.323054-2.6733243 .669405$

H $1.643122-3.8582203 .810372$

H $1.350342-4.3340221 .243564$

H -1.417238 -3.835288 2.457724

H $-1.598860-5.9435500 .976448$

H $-0.401232-5.278322-0.110617$

H $-4.373434-4.055237-0.529715$

H $-3.894830-5.2665210 .785880$

H -2.745131 -2.975619-1.843750

H $-1.228934-3.820286-1.973784$

H -1.891719-1.501072 -0.027467

H $1.667080-1.2664862 .372293$

C -1.0938565 .7636100 .229902$

O $-1.2073104 .818156-0.852436$

C $-1.9544283 .672232-0.390354$

C -2.6310414 .1166030 .897463$

C -1.5838825 .0418541 .485363$

H -1.207770 $2.895131-0.186966$

C $-2.9110833 .218570-1.497891$

H -3.5553114 .6676090 .683579$

H -2.8747293 .2802301 .556579$

C -2.1192415 .9720972 .559127$

H -0.7924304 .4196021 .919780$

C -3.814265 2.030240-1.101663

O $-2.1193752 .850152-2.631822$

H -3.534309 $4.064307-1.812993$

H $-4.3911412 .295565-0.208336$

O $-2.9957860 .912247-0.780540$

C $-4.8119581 .637305-2.209385$

C $-5.9178460 .645090-1.787485$

H $-5.3255152 .551121-2.535520$

O $-4.1480051 .109802-3.355602$

H $-6.4408121 .053757-0.917559$

H $-6.6418450 .646971-2.612039$

H -1.3272446 .6281172 .934291$

H -2.928495 6.6035422 .176940

H -2.512589 5.3979283 .404357

C $\quad 0.328185 \quad 6.332048 \quad 0.272222$

H -1.556534 $3.611881-2.853021$

H $-2.538648 \quad 1.1078000 .054083$

H $-3.5025581 .771326-3.655901$

H $\quad 0.371813 \quad 7.1702790 .977444$

H $0.5779126 .738412-0.716327$

H -1.778743 $6.584392-0.020359$

C $-5.668883-0.9091210 .874788$

O $-4.829574-1.006289-0.281140$

C $-5.476228-0.818777-1.543624$

C $-6.609420-1.847899-1.692722$

C $-7.589495-1.776432-0.527592$

C $-6.839126-1.8977180 .795860$

H $-6.051540 \quad 0.1134790 .966561$

C $-4.792279-1.2169662 .099443$

H -4.730665 -1.068553 -2.307575

O $-7.316854-1.668139-2.914958$

H $-6.181315-2.857928-1.721948$

H -8.189413 -0.859287 -0.559787

O $-8.510241-2.865888-0.618206$

H $-6.474239-2.9260650 .914542$

O $-7.740321-1.639921 \quad 1.871169$

C $-3.856263-0.0750542 .424637$

H -5.394745 -1.391865 2.997290

H -4.197350 -2.117490 1.905980

H $-6.668543-1.722610-3.637922$

H -8.947283 -2.802861-1.484905

H $-8.482029-2.2629201 .783465$

O $-2.627042-0.5648672 .757333$

$\begin{array}{lllll}\text { O } & -4.182547 & 1.104551 & 2.423279\end{array}$

C $-1.6533350 .414180 \quad 3.133375$

$\mathrm{H}-1.9332240 .8700504 .087556$

H $-0.691319-0.0911693 .252684$

H -1.552583 1.1784342 .357101

SCF Energy (B3LYP/6-31G**//MMFF) $=-3245.91334846$

01393

MM̄FF Geometry

C -3.505826 $1.537924-2.926573$

C $-2.5868041 .065395-2.071627$ 
C $-1.2606150 .486752-2.482004$ O $-0.2437951 .115421-1.664431$ C $-1.230482-1.024452-2.196670$ C $0.002600-1.691120-2.771641$ C $1.133074-1.946623-2.083862$ C $-0.100715-2.075557-4.225529$ C $1.429959-1.619051-0.646304$ C $1.975191-2.8080230 .163285$ C $3.353812-3.304737-0.301442$ C $3.919500-4.3526990 .659116$ C $5.345220-4.777527 \quad 0.266517$ O $4.230731-2.178499-0.352849$ O $5.917286-5.4835191 .378328$ C $6.207861-3.5148010 .041657$ C $5.342184-5.757547-0.912963$ O $7.491945-3.857378-0.474197$ C $5.535284-2.463679-0.869440$ C $6.339394-1.142017-0.857849$ O $5.480943-2.965314-2.205968$ C $0.346828 \quad 2.244808-2.130367$ $\begin{array}{llll}\text { O } & 0.151739 & 2.775235 & -3.215512\end{array}$ C $1.2717352 .830332-1.131201$ C $1.547723 \quad 2.2665930 .054400$ C 2.4127712 .8483031 .075464 C 3.3851421 .8686901 .670832 C $5.6124600 .026692-1.541320$ C $6.3653821 .335926-1.388553$ C $6.0313993 .798883-1.590632$

O $7.5542001 .393928-1.085933$

C $5.8970494 .295986-0.185623$

C $5.196675 \quad 5.3882890 .154925$

C 5.1268365 .9246091 .555634

C 3.7105666 .0906342 .055066

C 3.1411067 .3054522 .130773

C $2.990096 \quad 4.8602952 .558687$

C 2.2605704 .1298651 .464742

N $5.5564292 .430655-1.666114$ H -3.316609 $1.502355-3.996081$ H $-2.7988041 .089651-1.006491$ H -1.061777 $0.672894-3.544654$ H -2.118328 -1.508989-2.624707 H $-1.292603-1.205454-1.118928$ H $1.956775-2.404476-2.628581$ H $0.808641-2.562373-4.593990$ H - $0.930739-2.773950-4.374232$ H $-0.274961-1.191085-4.846062$ H $2.156521-0.798805-0.628957$ H $0.544075-1.242866-0.128076$ H $2.053205-2.4734511 .206156$ H $1.257499-3.6372210 .145396$ H $3.252553-3.734078-1.303986$ H $3.257830-5.225806 \quad 0.716434$ H $3.953423-3.9351991 .675268$ H $5.342870-6.2424331 .578928$ H $6.381000-3.0578971 .026179$ H $4.775705-6.661459-0.657983$ H $6.357080-6.097335-1.147203$ H $4.900448-5.337386-1.818821$ H $7.883954-4.5078530 .133390$ H $6.534841-0.8488440 .182081$ H $7.311679-1.290447-1.344139$ H $5.417218-2.220928-2.824148$ H $1.7152543 .781273-1.413516$ H $1.122188 \quad 1.3039220 .324771$ H 4.1231572 .3348122 .326978 H 3.9492851 .3616360 .881386 H 2.8532221 .1076652 .252606 H $5.499308-0.160142-2.614313$ H $4.6125980 .151160-1.112080$ H $5.4416514 .389440-2.298708$ H $7.0824013 .833881-1.895191$ H 6.4347353 .7491700 .585725 H $4.6804365 .953052-0.617860$ H $5.643723 \quad 6.8933001 .564043$ H 5.6837055 .2893622 .255781 H 2.1362057 .4344382 .519053 H $3.664408 \quad 8.1969651 .801251$ H $2.241712 \quad 5.1536953 .307526$
H $3.6941504 .224856 \quad 3.102991$

H 1.4857704 .7265660 .981322

H $4.5656252 .285266-1.837059$

C $-5.939162 \quad 1.101029-2.488837$

$\begin{array}{llll}\text { O } & -5.691426 & 0.128691 & -1.453476\end{array}$

C $-6.908084-0.102241-0.720974$

C -8.021727 $0.501305-1.566045$

C $-7.3123531 .696068-2.175826$

H $-6.8283180 .468772 \quad 0.211354$

C -7.055166 $-1.592500-0.398629$

H $-8.353734-0.184040-2.354264$

H $-8.8946560 .778095-0.967348$

C -8.024570 $2.273971-3.386165$

H $-7.2177542 .476794-1.408871$

C $-5.810409-2.2048760 .278787$

O $-7.271359-2.302941-1.624785$

H $-7.950300-1.7528750 .213473$

H $-4.984562-2.211633-0.440209$

O $-6.097690-3.5819430 .555125$

C $-5.376838-1.5102271 .584222$

C $-4.168811-2.1805102 .268946$

H $-5.145429-0.4593941 .388876$

O $-6.480431-1.5353872 .492630$

H $-4.079367-1.7774583 .284535$

H $-4.375193-3.2482982 .408761$

H $-7.4682303 .121406-3.798539$

H $-8.138288 \quad 1.527383-4.179347$

H $-9.0239282 .627168-3.111681$

C $-4.8133902 .134743-2.482381$

H -7.354272 -3.246094 -1.402212

H $-6.800904-3.6044581 .226930$

H $-6.221897-1.0299323 .282207$

H -4.700227 $2.554850-1.475350$

H $-5.0528612 .969491-3.150846$

H $-5.9596320 .556356-3.441768$

$\begin{array}{llll}\text { C }-2.146627 & 0.106074 & 2.492361\end{array}$

O $-2.544625-0.5935831 .307399$

C $-2.848658-1.981314 \quad 1.497442$

C $-1.639525-2.7138352 .094954$

C -1.107421 -2.026804 3.348020

C $-0.889938-0.536728 \quad 3.091240$

H $-2.9559870 .079863 \quad 3.230323$

C -1.8821101 .5706952 .111396$

H -2.996583 -2.400139 0.495703

O $-1.958939-4.066578 \quad 2.402221$

H $-0.834847-2.739448 \quad 1.350690$

H $-1.772827-2.1839994 .205119$

O $0.150516-2.606323 \quad 3.702292$

H $-0.034958-0.4006862 .416373$

$\begin{array}{llll}\text { O } & -0.571796 & 0.107813 & 4.322605\end{array}$

C -3.1080902 .3400251 .667893$

H -1.4453992 .1257962 .948550$

H $-1.1775811 .597002 \quad 1.272513$

H -2.275497 -4.4852441 .583404$

H $0.005695-3.5609203 .820265$

H $0.213480-0.3356254 .687356$

O $-4.203600 \quad 2.0023992 .401320$

\begin{tabular}{lllllll}
\hline & -3.070988 & 3.202897 & 0.797889
\end{tabular}

C $-5.411077 \quad 2.6943782 .067837$

H -6.2296692 .2387492 .631217$

H -5.3281363 .7471492 .352779$

H -5.6279802 .5994750 .999558$

SCF Energy (B3LYP/6-31G**//MMFF)= -3245.91194385

01_394

MM̄FF Geometry

C $2.853986-2.3884182 .312969$

C $2.214867-1.4285751 .627121$

C $0.752876-1.4435341 .278741$

O $0.282952-0.096888 \quad 1.534880$

C $0.513925-1.770288-0.208658$

C $0.755639-3.221596-0.569493$

C $-0.116031-4.217241-0.312091$

C $2.049946-3.511421-1.281533$

C $-1.463821-4.0802820 .338148$

C $-2.542866-4.859564-0.428654$

C $-3.950515-4.417394-0.006021$

C $-5.013808-5.376425-0.539935$ 
C $-6.434077-4.887202-0.214094$

O $-4.159517-3.111234-0.553771$

O $-7.355225-5.660668-0.998342$

C $-6.570047-3.418636-0.668967$

C $-6.799941-5.1351431 .253920$

O $-7.827326-2.874976-0.271457$

C $-5.408391-2.506594-0.204202$

C $-5.496745-1.142309-0.930092$

O $-5.514125-2.2968441 .204650$

C -1.0284020 .0896541 .833957$

$\begin{array}{llll}\text { O } & -1.895863 & -0.762561 & 1.925717\end{array}$

C -1.2231241 .5455862 .018150$

C -2.4581222 .0527992 .139481$

C -2.7925203 .4717962 .283878$

C -1.650885 4.4397552 .463548

C $-4.264427-0.245891-0.743078$

C $-4.3718221 .038768-1.547061$

C $-3.0139812 .994419-2.279033$

O $-5.4142061 .408977-2.081242$

C $-3.0899704 .109457-1.285785$

C $-4.1860924 .858949-1.094292$

C $-4.2742775 .995046-0.114846$

C $-5.2414445 .712348 \quad 1.014602$

C -6.5551875 .9584030 .877500$

C $-4.670716 \quad 5.2219982 .327807$

C $-4.090848 \quad 3.8377512 .227375$

N $-3.1681411 .730333-1.584180$

H $2.310392-3.2661652 .650774$

H $2.786158-0.5694131 .283748$

H $\quad 0.215547-2.141851 \quad 1.932392$

H $1.139318-1.121680-0.835665$

H $-0.507171-1.486739-0.494472$

H $\quad 0.159765-5.232059-0.594554$

H $2.157543-4.571447-1.534973$

H $2.102522-2.942996-2.215959$

H $2.904298-3.234867-0.660192$

H -1.401724 -4.4704101 .361122$

H -1.757859 -3.0310810 .418730$

H -2.420050 -4.693467 -1.507047

H -2.408011 -5.931862 -0.240504

H $-3.993438-4.3673561 .088495$

H $-4.857576-6.389174-0.148069$

H -4.916702 -5.459229-1.631702

H -7.230546 -6.597656 -0.769724

H -6.582421 -3.429017 -1.767549

H $-6.713616-6.2014041 .495100$

H -7.844376 -4.869315 1.450977

H $-6.164970-4.5860611 .952335$

H -7.773498 -2.668543 0.677252

H -5.614417 -1.316922 -2.007917

H $-6.386830-0.598210-0.589517$

H -5.047576 -1.478913 1.437922

H -0.3370102 .1697772 .023373$

H -3.306808 1.3719322 .108111

H $-0.9876974 .421348 \quad 1.591790$

H -1.0665994 .1818993 .353997$

H -1.968522 5.4764172 .587542

H -4.1508830 .0316460 .308192$

H -3.358837 -0.774358 -1.061865

H -3.780442 $3.088401-3.055671$

H $-2.0350202 .991119-2.767357$

H -2.191450 4.315621-0.710395

H $-5.0775874 .649927-1.681720$

H -4.597119 $6.890323-0.662639$

H $-3.287825 \quad 6.2474690 .293326$

H -7.2521605 .7846221 .690542$

H -6.962536 $6.336510-0.054573$

H -5.4545085 .1885213 .096308$

H -3.951173 5.9587912 .694821

H -4.8433133 .0609612 .083588$

H $-2.3743891 .376722-1.057708$

C $5.194613-2.7727151 .463462$

O $5.052946-1.8400150 .374734$

C $6.337650-1.611243-0.230168$

C $7.280472-2.6381220 .386599$

C $6.689224-2.796040 \quad 1.776431$

H $6.657511-0.6103580 .082548$

C $6.209212-1.661521-1.758657$
H $7.250363-3.594335-0.148157$

H $8.318668-2.2927110 .397361$

C $7.152114-4.0512882 .494712$

H $6.961220-1.9164732 .375481$

C $5.089480-0.753631-2.314779$

O $5.901304-3.009595-2.136560$

H $7.173738-1.419231-2.219786$

H $4.117681-1.129552-1.977505$

O $5.091726-0.858076-3.743365$

C $5.2366760 .735280-1.947876$

C $4.1362011 .643545-2.534069$

H $5.2442520 .850974-0.862049$

O $6.5006851 .212578-2.415634$

H $4.4365562 .686017-2.374844$

H $4.1034031 .522982-3.623628$

H $6.691717-4.1268573 .484790$

H $6.892212-4.9543841 .932473$

H $8.238812-4.0392582 .627939$

C $4.322938-2.3166422 .634778$

H $6.029262-3.080249-3.097506$

H $4.781124-1.750469-3.971706$

H $6.5201301 .091622-3.380589$

H $4.574453-1.2856092 .908106$

H $4.512831-2.9353653 .519029$

H $4.860040-3.7513811 .095146$

C 2.9088162 .8281310 .013230

O $2.7571381 .496222-0.496050$

C $2.7432781 .388323-1.923511$

C $1.6109942 .243266-2.508320$

C $1.6655883 .684372-2.008802$

C $1.7609273 .717696-0.483141$

H $3.8652853 .247305-0.320437$

C 2.9306992 .7283991 .547236

H $2.4877520 .347613-2.150514$

O $1.6623902 .207054-3.930031$

H $\quad 0.6496341 .804320-2.209363$

H $2.4957634 .236734-2.465134$

O $0.4677194 .363872-2.391281$

H $0.802943 \quad 3.391525-0.058423$

O $1.9759275 .071557-0.089954$

C 4.2475502 .1982802 .078485

H 2.7885713 .7098562 .009637

H 2.1366112 .0527851 .882872

H $0.8630282 .645717-4.266172$

H $0.4719434 .444284-3.359840$

H 1.7542225 .1467930 .853119

O $4.094501 \quad 1.8756113 .397183$

O 5.2867082 .1166441 .439168

C 5.2760401 .4272194 .070208

H 5.8205820 .6904333 .471965

H 5.9161802 .2853914 .293680

H 4.9738210 .9573235 .009861

SCF Energy (B3LYP/6-31G**//MMFF)= -3245.90818393

01_395

MM̄FF Geometry

C $0.3077323 .210006-0.324950$

C 1.3196363 .5052150 .505308

C 1.7262112 .7101791 .726323

O 0.7379521 .7043912 .056939

C 3.0609561 .9690071 .519251

C 4.2607462 .8818551 .385482

C 4.9466173 .1001020 .246668

C 4.6959183 .5603002 .660358

C $4.7075112 .513701-1.117619$

C $5.9877921 .913163-1.718762$

C $6.4902250 .663773-0.977432$

C $7.8655350 .250663-1.505990$

C $8.365111-1.042181-0.846058$

O $5.547050-0.390768-1.196161$

O $9.489332-1.515521-1.603420$

C $7.258992-2.110507-0.957097$

C $8.874889-0.7965550 .578897$

O $7.623339-3.292422-0.246101$

C $5.857891-1.616210-0.516635$

C $4.797985-2.671383-0.924471$

O $5.850049-1.4450140 .899332$

C -0.3260032 .0925622 .814207$ 
O $-0.548737 \quad 3.206356 \quad 3.260559$

C -1.190487 0.9081242 .996888 C -2.3093780 .9891363 .730686$ C -3.209378 -0.1306873 .991244$ C $-3.685910-0.2034725 .415791$ C $3.341255-2.277630-0.649806$ C $2.819648-2.7100200 .710284$ C $0.745091-2.6602392 .078473$ O $3.525923-3.2131861 .578769$ C $-0.710251-2.8392081 .786453$ C $-1.481243-3.7587772 .385436$ C $-2.947174-3.9381642 .105301$ C -3.819042 -3.477588 3.252329 C $-4.015681-4.2582604 .328160$ C $-4.525610-2.1469243 .116841$ C $-3.577483-0.9842243 .015402$ N $1.459425-2.4571910 .832403$ H $-0.2775862 .310521-0.161733$ H $1.892788 \quad 4.4117050 .321644$ H 1.8186893 .3960612 .577066 H $2.9678301 .282307 \quad 0.670788$ H 3.2310641 .2991542 .373875 H 5.7845563 .7958540 .287524 H $5.623410 \quad 4.1292012 .533821$ H 3.9289764 .2580483 .009646 H 4.8748102 .8165893 .443886 H $3.9146681 .762801-1.121282$ H $4.3682763 .325530-1.772800$ H $5.7731461 .650388-2.762812$ H $6.7734562 .679337-1.733489$ H 6.5506650 .8791030 .094791 H $8.5954561 .057811-1.366080$ H $7.8052860 .086063-2.591125$ H $10.168314-0.819161-1.599317$ H $7.205291-2.402189-2.015010$ H $9.681143-0.0531300 .574015$ H $9.312310-1.7066391 .004042$ H $8.098041-0.4363961 .256421$ H $7.480489-3.1236440 .700928$ H $4.879540-2.823302-2.009253$ H $5.016512-3.632606-0.444483$ H $4.929284-1.399438 \quad 1.201017$ H $-0.882294-0.0164882 .525121$ H -2.5829201 .9260624 .210949$ H -4.3507530 .6377185 .640033$ H -2.835441 -0.1636526 .106277$ H $-4.226613-1.1259755 .641750$ H $3.198084-1.197578-0.750027$ H $2.708984-2.769256-1.400753$ H $0.900841-1.7633192 .686277$ H $1.173870-3.5158192 .610597$ H -1.161195 -2.162525 1.065574 H - $1.037042-4.4271733 .119618$ H -3.131036 -5.0015731 .903457$ H $-3.240090-3.4168421 .186325$ H $-4.660068-3.9471265 .143483$ H -3.533141 -5.226122 4.416710 H $-5.239702-1.9952513 .934059$ H $-5.144245-2.1682512 .210319$ H -3.184146 -0.827358 2.011131 H $1.020407-1.8728150 .126407$ C -1.557087 $4.549457-1.393536$ O $-2.4706603 .434336-1.475622$ C -3.519281 $3.634253-0.509768$ C $-3.4152365 .091518-0.066636$ C $-1.9185495 .314403-0.119058$ H -3.2852062 .9883910 .344600$ C $-4.8867723 .264150-1.091300$ H -3.923248 $5.767085-0.764624$ H -3.840055 5.253060 0.928816 C -1.514285 $6.777787-0.143905$ H -1.4768254 .8410390 .766006$ C $-5.0298161 .819305-1.611244$ O $-5.1852754 .140654-2.187201$ H $-5.6607723 .458678-0.339081$ H $-4.485373 \quad 1.700725-2.555497$ O $-6.4181091 .625823-1.920869$ C $-4.5955900 .716473-0.628136$
C $-5.106102-0.698426-0.963900$

H $-3.5029240 .702604-0.561222$

O $-5.078162 \quad 1.0288820 .683517$

H $-4.703413-1.379728-0.205111$

H $-6.192509-0.725322-0.809168$

H $-0.425565 \quad 6.879107-0.194621$

H $-1.9426227 .298649-1.006734$

H -1.8596997 .2875520 .761432$

C $-0.107328 \quad 4.063824-1.489908$

H $-4.4696674 .047307-2.839529$

H $-6.6710962 .314979-2.558996$

H -6.0475751 .0917720 .634240$

H $0.5591384 .928472-1.590307$

H $0.0056993 .460785-2.398326$

H $-1.7667295 .169890-2.274852$

C $-2.620398-2.222378-1.925873$

O $-3.366464-1.232590-2.644437$

C $-4.771003-1.213077-2.377571$

C $-5.385807-2.585025-2.694754$

C $-4.680364-3.704025-1.936332$

C $-3.175747-3.632397-2.178100$

H $-2.646007-1.988607-0.855810$

C $-1.163211-2.146434-2.408471$

H $-5.206656-0.505369-3.091960$

O $-6.774059-2.610816-2.380544$

H $-5.296411-2.784547-3.770563$

H $-4.911195-3.677256-0.865250$

O $-5.149366-4.966345-2.415976$

H $-2.962300-3.940143-3.210145$

O $-2.515542-4.557465-1.316459$

C $-0.486165-0.846234-2.040888$

H $-0.567297-2.955056-1.971280$

H $-1.141729-2.231845-3.502030$

H $-7.200141-1.894168-2.881364$

H $-6.114394-4.980137-2.295639$

H $-2.904993-5.433943-1.477905$

O $0.685711-0.753241-2.736798$

O $-0.914709-0.029713-1.238604$

C $1.455706 \quad 0.429710-2.507378$

H $1.5866890 .606778-1.436288$

H $2.4410930 .287162-2.959038$

H $0.9693391 .283064-2.986612$

SCF Energy (B3LYP/6-31G**//MMFF)= -3245.91989252

01_396

MM̄FF Geometry

C -2.0602501 .4679242 .396107$

C $-0.740823 \quad 1.394506 \quad 2.166305$

C $0.2425280 .649677 \quad 3.026168$

$\begin{array}{lllll}\text { O } & 0.919050 & -0.266813 & 2.129995\end{array}$

C $1.292946 \quad 1.5952633 .649287$

C 0.7327922 .7808724 .408290

C 0.7681004 .0579643 .974859

C $0.1187042 .450140 \quad 5.743487$

C $1.324598 \quad 4.568478 \quad 2.673627$

C 0.2095945 .0944131 .758584

C $0.647737 \quad 5.1450890 .286958$

C $-0.4549515 .750693-0.583099$

C $-0.1048065 .693783-2.077677$

O $0.9079913 .800153-0.129880$

O $-1.3012055 .970614-2.821856$

C $0.3275924 .256765-2.438361$

C $0.909146 \quad 6.775999-2.466794$

O $0.8012564 .189587-3.781823$

C $1.3835213 .665861-1.473927$

C $1.5661232 .152412-1.764655$

O $2.6211014 .347657-1.664107$

C $1.663236-1.2693872 .663524$

O $1.770860-1.5683733 .840896$

C $2.382690-1.9089291 .540533$

C $3.511828-2.5930201 .771187$

C $4.363265-3.2118890 .752082$

C $3.924989-3.109608-0.686566$

C $2.4512941 .389117-0.766853$

C $3.9405891 .454794-1.059002$

C $6.1398610 .804882-0.073948$

O $4.4056101 .974832-2.069475$

C $6.588606-0.494669-0.665477$ 
C $7.462855-1.318094-0.066176$

C $7.962269-2.596886-0.677473$

C $7.845377-3.782594 \quad 0.255213$

C $8.905303-4.2142330 .959516$

C $6.526723-4.5213580 .301365$

C $5.502179-3.8178711 .147734$

N $4.6893670 .855766-0.054105$

H $-2.4907340 .952821 \quad 3.250537$

H $-0.330622 \quad 1.9098901 .301870$

H -0.2771430 .0745853 .802736$

H 1.9955191 .9074092 .869274

H 1.9278521 .0215464 .337768

H $\quad 0.339168 \quad 4.830046 \quad 4.612247$

H -0.2413093 .3413336 .268282$

H $-0.733837 \quad 1.7754295 .617514$

H $\quad 0.856118 \quad 1.962606 \quad 6.389674$

H 2.0279455 .3821422 .889134

H 1.9105983 .8037492 .160416

H $-0.669515 \quad 4.444767 \quad 1.843550$

H -0.0939116 .0931792 .096194$

H 1.5691965 .7344020 .214314

H -0.670026 $6.781842-0.276693$

H -1.389176 $5.192565-0.427604$

H -1.627176 $6.845765-2.550544$

H $-0.5702363 .624749-2.395660$

H $0.5057917 .773058-2.252487$

H $1.1106766 .760303-3.543585$

H $1.8588206 .684579-1.935750$

H $\quad 0.104758 \quad 4.556129-4.353045$

H $\quad 0.5750281 .681447-1.717892$

H $1.9492312 .007453-2.781664$

H $3.3301363 .824474-1.260261$

H $1.990779-1.7535950 .543062$

H $3.860898-2.6873732 .798719$

H $3.759811-2.064677-0.971476$

H $2.995642-3.666093-0.843316$

H 4.656822 -3.501738 -1.395566

H 2.2694871 .7263620 .258506

H $2.1789600 .328043-0.816720$

H 6.4827670 .9298040 .958021

H $6.525732 \quad 1.635569-0.673473$

H $6.200159-0.754759-1.647372$

H $7.874869-1.0398770 .901259$

H $9.014583-2.443487-0.950802$

H $7.443217-2.821012-1.617559$

H $8.838179-5.0815781 .607756$

H $9.862521-3.7060200 .907775$

H $\quad 6.670616-5.5209900 .733491$

H $6.182162-4.711781-0.718918$

H $5.740191-3.8297962 .212389$

H 4.2105780 .3925610 .712873

C -3.851292 1.3667670 .646544

$\begin{array}{llll}\text { O } & -4.880740 & 0.729931 & 1.432724\end{array}$

C $-6.099136 \quad 0.735206 \quad 0.673871$

C $-5.7305491 .164219-0.741500$

C $-4.5814672 .114354-0.467988$

H -6.7218151 .5307291 .106809$

C $-6.879245-0.5796830 .791406$

H $-5.3831860 .318256-1.344118$

H $-6.5641531 .638535-1.267982$

C $-3.7237842 .395278-1.690420$

H $-4.9864503 .060410-0.084282$

C $-6.208622-1.8543650 .230758$

O $-8.119038-0.3842680 .097391$

H -7.134090 -0.745058 1.845500

H $-6.065881-1.746492-0.848614$

O $-7.142518-2.935314 \quad 0.381202$

C $-4.883184-2.257910 \quad 0.901439$

C $-4.316618-3.6115550 .434600$

H $-4.128307-1.491980 \quad 0.721811$

O $\quad-5.062181-2.3209302 .318690$

H -3.470731 -3.860824 1.086927

H $-5.053169-4.4026990 .620488$

H -2.905054 $3.078386-1.445266$

H $-3.2855431 .476096-2.093821$

H $-4.3224122 .858018-2.481825$

C -2.9938912 .2688311 .529513$

H $-8.630362-1.2074540 .179060$
H -7.360184 -3.013307 1.325403

H -5.653791 -3.067053 2.513838

H -3.627532 2.8780062 .185209

H -2.416006 2.9603830 .905800

H -3.2388600 .5666940 .216508$

C $-1.713182-2.597650-0.779049$

O $-3.015830-2.533122-1.369080$

C $-3.875270-3.633441-1.043264$

C $-3.223855-4.951296-1.489851$

C $-1.816642-5.119300-0.917278$

C $-0.974791-3.874820-1.197020$

H $-1.820208-2.5821170 .312809$

C $-0.953615-1.313523-1.133154$

H $-4.781326-3.504616-1.647234$

O $-4.036025-6.067730-1.141769$

H $-3.150958-4.952283-2.585027$

H $-1.839613-5.3422140 .155772$

O $-1.184065-6.237043-1.544490$

H $-0.726744-3.849093-2.264991$

O $0.250243-3.962142-0.475112$

C $-0.593246-1.211828-2.596610$

H - $1.554411-0.428588-0.900596$

H $-0.043022-1.237205-0.530538$

H $-3.962741-6.210988-0.183181$

H $-1.755082-7.011190-1.400331$

H $\quad 0.682387-4.793813-0.735342$

O $0.761773-1.260120-2.743383$

O $-1.414119-1.107874-3.498572$

C $1.242385-1.158717-4.086801$

H $2.334812-1.192788-4.059436$

H $0.878610-2.000831-4.682912$

H $0.932200-0.207403-4.529588$

SCF Energy (B3LYP/6-31G**//MMFF) $=-3245.90747783$

01_397

MM̄FF Geometry

C $2.816589-4.079389-2.775460$

C $2.394991-2.839877-3.066922$

C $0.982300-2.498209-3.463294$

O $0.344937-1.870901-2.326234$

C $0.994017-1.486567-4.624480$

C $-0.384438-1.010634-5.040559$

C $-0.8080940 .266336-4.953909$

C -1.299998 -2.067982 -5.600833

C $-0.0404651 .463163-4.462107$

C $-0.1887811 .743609-2.959022$

C -1.590857 $2.214398-2.541905$

C $-1.6017302 .628385-1.069329$

C $-3.0049213 .041723-0.600164$

O $-2.5079331 .137290-2.730875$

O -2.9616593 .0903410 .834961$

C $-4.0246671 .951261-1.008449$

C $-3.3468884 .461111-1.067916$

O $-5.3631662 .384929-0.783933$

C $-3.8778431 .470095-2.469944$

C $-4.7115440 .206769-2.784686$

O $-4.3531722 .454982-3.396602$

C $-0.187719-2.693717-1.382146$

O $-0.220359-3.913875-1.398156$

C $-0.709396-1.845331-0.291041$

C $-0.915366-2.3893410 .917448$

C -1.412164 -1.689195 2.102575

C $-1.961742-0.3031591 .904617$

C $-4.369619-1.035882-1.954259$

C $-5.293335-1.262100-0.768008$

C $-5.636115-2.8236001 .146086$

O $-6.293908-0.584708-0.550090$

C $-5.168454-2.1336522 .388134$

C $-4.748438-2.7863873 .482521$

C $-4.336495-2.1045714 .755522$

C $-2.959602-2.517528 \quad 5.224000$

C -2.804747 -3.441665 6.187219

C $-1.769255-1.7904814 .642449$

C -1.367955 -2.319744 3.294320

N $-4.882098-2.3414430 .003859$

H $2.124110-4.914104-2.843576$

H $3.092449-2.011594-2.964005$

H $0.425358-3.395994-3.758600$ 
H $\quad 1.485106-1.934144-5.498739$ H $1.616504-0.632792-4.335174$ H -1.821275 $0.492307-5.284683$ H -2.226049 -1.644167 -6.004209 H $-0.805067-2.605889-6.416006$ H - $-1.579962-2.787110-4.824967$ H $1.0235281 .357927-4.700322$ H $-0.3715632 .341003-5.031369$ H $0.0747080 .838616-2.400542$ H $0.5412812 .516328-2.687428$ H - $1.8774143 .056925-3.181974$ H $-0.8825473 .436092-0.885498$ H $-1.264077 \quad 1.783624-0.454176$ H -3.828491 3.3941241 .152928 H $-3.8555171 .099623-0.340616$ H $-2.6502915 .184689-0.627035$ H $-4.3444154 .762134-0.729942$ H -3.299228 $4.577324-2.152870$ H -5.4608032 .5628510 .166541$ H $-5.7822790 .440597-2.742007$ H $-4.516900-0.050318-3.836361$ H -4.698757 $3.212414-2.898840$ H $-0.886082-0.798169-0.505998$ H - $0.675598-3.4419561 .063411$ H $-2.751728-0.3129381 .147439$ H -1.1720450 .3756001 .573139$ H -2.405429 0.1289082 .803655 H -4.478351 -1.919233-2.596936 H -3.332160 -1.010353 -1.607418 H $-5.493743-3.907788 \quad 1.195227$ H $-6.700403-2.6190480 .990342$ H -5.210076 -1.047106 2.399240 H -4.735422 -3.874105 3.479593 H -5.081459 -2.355896 5.521987 H -4.374880 -1.012553 4.656169 H -1.821112 -3.7204246 .550212$ H -3.658321 -3.944162 6.630088 H $-0.900560-1.9050295 .305149$ H -1.968454 -0.7151864 .637911$ H $-0.960115-3.3310113 .330181$ H $-4.006527-2.806808-0.217192$ C $4.435753-4.086211-0.804848$ O $4.374255-2.664900-0.571202$ C $3.669246-2.4274460 .663154$ C $3.599816-3.7781761 .367880$ C $3.460188-4.7215290 .186044$ H $2.650253-2.1266320 .396573$ C $4.357796-1.3109261 .455709$ H $4.520388-3.9964721 .921548$ H $2.760692-3.8381602 .067711$ C $3.774026-6.1697080 .515600$ H $2.428821-4.656237-0.181367$ C 4.5333420 .0195230 .689119 O $5.667637-1.7598901 .834779$ H $3.822593-1.1354432 .395095$ H $5.195681-0.154226-0.168822$ O $\quad 5.224810 \quad 0.945623 \quad 1.533750$ C $3.2489930 .703540 \quad 0.182527$ C 2.1058350 .8633721 .206335 H $3.5203121 .695949-0.195554$ O $2.756261-0.026756-0.940318$ H 1.2284831 .2409040 .666362 H $1.795338-0.1229331 .570873$ H $3.675985-6.800385-0.373660$ H $4.793570-6.2829500 .899227$ H $3.084736-6.5486281 .277246$ C $4.206437-4.384087-2.291527$ H $6.148548-1.9817081 .018776$ H 6.0398110 .5101541 .836895 H $2.0090950 .471376-1.311280$ H $4.416794-5.441044-2.492685$ H $4.921433-3.803461-2.888687$ H $5.463521-4.383278-0.557325$ C 2.0531783 .9026421 .292730 O 2.9736543 .0421781 .974186 C 2.4238391 .7913802 .396552 C 1.2481592 .0397053 .352037 C 0.2092142 .9840702 .747819
C 0.8773834 .2505812 .210924

H 1.6851043 .4086760 .384784

C 2.8252045 .1552210 .867861

H 3.2012891 .2936542 .987362

O $0.6524850 .799872 \quad 3.713481$

H 1.6355252 .4912194 .274906

H -0.3693542 .4943391 .958708$

O -0.7275963 .3694293 .755184$

H 1.2200114 .8693623 .049973

O -0.0811115 .0112801 .478734$

C $3.7653004 .828134-0.268159$

H 2.1556585 .9467440 .512621

H 3.4046205 .5740601 .699224

H -0.0115170 .9790124 .399929$

H -1.2376082 .5785003 .998694$

H -0.8342895 .1773032 .071114$

O $4.981653 \quad 4.4565320 .223297$

O $3.4428894 .864129-1.448799$

C $5.9472264 .063093-0.756386$

H $5.5850713 .196690-1.318241$

H $6.1656894 .897604-1.429542$

H $6.8658623 .782353-0.234488$

SCF Energy (B3LYP/6-31G**//MMFF) $=-3245.90165760$

01_398

MM̄FF Geometry

C -4.680125 -0.8323962 .520484$

C $-3.526621-1.5190002 .526909$

C $-2.346633-1.3009673 .448133$

O $-1.147231-1.2361922 .634641$

C $-2.394690-0.013324 \quad 4.295691$

C $-1.172414 \quad 0.178820 \quad 5.174112$

C -0.2209441 .1142074 .980726$

C $-1.083068-0.7478446 .360313$

C -0.1233752 .1346663 .878559$

C 1.2154602 .0285233 .143773

C 1.4389543 .1366792 .103161

C 0.4580913 .0579650 .930008

C $0.7725014 .111098-0.144720$

O 2.7784992 .9723041 .620811

O $0.0015403 .800284-1.315664$

C $2.2696524 .023168-0.517637$

C 0.2950915 .5022360 .285471

O $2.6465545 .084729-1.389551$

C 3.2153743 .9862870 .703872

C 4.6737473 .6527840 .320339

O 3.2918665 .2633611 .348299

C $-0.445881-2.3900512 .461066$

O $-0.768144-3.5091792 .827530$

C $0.812628-2.0532571 .759047$

C $1.663273-3.0328691 .417502$

C $2.943936-2.8573510 .732114$

C $3.522089-1.4678650 .666478$

C $4.8620952 .309193-0.388198$

C $6.3182721 .879643-0.445709$

C $7.741331-0.023379-1.179588$

O $7.2445592 .564597-0.021642$

C $7.520951-1.491268-1.348329$

C $7.659211-2.380052-0.352921$

C $7.389608-3.850509-0.501163$

C $6.102191-4.2578680 .187947$

C $6.139620-4.8581931 .389951$

C $4.831987-3.963256-0.587342$

C $3.561760-3.9412220 .216869$

N $6.4523760 .623138-1.019711$

H $-4.839047-0.0258793 .230334$

H -3.415997 -2.337207 1.817131

H -2.292277 -2.163660 4.123895

H $-3.277547-0.0290604 .948316$

H -2.5322940 .8488883 .635867$

H $0.592103 \quad 1.1660155 .704560$

H $-0.235707-0.5095197 .012128$

H -1.991919 -0.677509 6.966820

H $-0.961773-1.784396 \quad 6.031495$

H -0.9388802 .0347223 .160754$

H $-0.2147723 .130373 \quad 4.328830$

H 2.0397412 .0666943 .869503

H 1.3047861 .0481312 .660220 
H 1.3636094 .1044232 .612092 H -0.5800583 .1683841 .262921$ H $\quad 0.5232232 .0604780 .474509$ H $0.2778142 .925886-1.637976$ H $2.3979283 .094698-1.086094$ H $-0.794698 \quad 5.508697 \quad 0.410878$ H $0.5113386 .251447-0.484112$ H $\quad 0.734525 \quad 5.836314 \quad 1.227678$ H $2.0350595 .070655-2.145614$ H $5.0970654 .456907-0.295934$ H 5.2707683 .6514161 .243509 H 2.8047825 .9114680 .814842 H $1.005603-1.0105981 .537319$ H $1.390305-4.0640931 .637054$ H $2.962623-0.847415-0.038129$ H $4.569899-1.4561750 .356720$ H $3.496263-0.9885861 .651902$ H $4.304514 \quad 1.5237800 .134094$ H $4.4915422 .370663-1.417397$ H $\quad 8.3703330 .205423-0.312337$ H $8.2266130 .395904-2.066635$ H $7.210605-1.833523-2.332479$ H $7.957231-2.0328030 .633838$ H $8.244852-4.397628-0.084623$ H $7.341781-4.142308-1.558080$ H $5.242378-5.1812671 .905559$ H $7.083040-5.0482101 .892108$ H $4.725476-4.721832-1.373855$ H $4.933587-3.013911-1.124912$ H $3.092623-4.9187680 .331182$ H $5.6453120 .212124-1.481436$ C $-6.192540 \quad 0.1278090 .775316$ O $-5.152900 \quad 0.394186-0.184784$ C $-5.7582550 .846588-1.412184$ C $-7.2425271 .030766-1.115998$ C $-7.469506-0.022658-0.047975$ H $-5.6507980 .029863-2.134964$ C $-5.0348842 .097901-1.922503$ $\mathrm{H}-7.4587952 .029221-0.718820$ H -7.863183 $0.883090-2.005082$ C $-8.749091 \quad 0.1840500 .743086$ H -7.494292 -1.011010 -0.526313 C $-3.5105591 .911021-2.075091$ O $-5.2497213 .158393-0.981767$ H -5.481692 $2.427053-2.867963$ H $-3.0698791 .764250-1.082711$ O $-2.9872063 .146527-2.577342$ C $-3.1156490 .767571-3.028392$ C $-1.603177 \quad 0.588291-3.236408$ H -3.524417 -0.176624-2.664005 O $-3.710100 \quad 0.996167-4.309291$ H -1.463063 -0.156378 -4.029520 H -1.176406 $1.512016-3.645223$ H -8.867480 -0.591809 1.505803 H $-8.756548 \quad 1.156053 \quad 1.247466$ H -9.6206490 .1419640 .081708$ C -5.828066 -1.113319 1.588798 H $-4.7810453 .941580-1.317791$ H -2.027101 $3.143007-2.425781$ H $-3.3858971 .853885-4.633878$ H $-5.573798-1.9375370 .913260$ H -6.682741 -1.431876 2.195751 H -6.2619021 .0093091 .426951$ C -1.181443 -2.226923 -2.123080 O $-1.381570-1.025097-1.369485$ C $-0.8443080 .161988-1.963458$ C $0.6679690 .007284-2.162923$ C $1.003019-1.238743-2.976935$ C $0.312183-2.465847-2.386176$ H -1.726979 -2.162121 -3.072260 C - $-1.766772-3.392887-1.306912$ H $-0.9847080 .958873-1.225254$ O $1.196278 \quad 1.167643-2.795118$ H $1.143569-0.067692-1.178120$ H $\quad 0.741031-1.113161-4.034037$ O $2.413839-1.467928-2.936012$ H $0.812713-2.747088-1.453526$ O $0.459381-3.561551-3.288447$
C $-3.280652-3.417847-1.300419$

H $-1.433240-4.346262-1.732669$

H $-1.438235-3.329794-0.264057$

H $2.1653321 .093979-2.785307$

H $2.843948-0.738004-3.412458$

H $1.412151-3.688660-3.436851$

O $-3.696784-4.617696-0.794837$

O $-4.007772-2.508416-1.671906$

C $-5.113547-4.813347-0.744328$

H $-5.302496-5.854773-0.470801$

H $-5.552366-4.1652920 .018635$

H -5.566311-4.622000-1.722133

SCF Energy (B3LYP/6-31G**//MMFF) $=-3245.91946220$

01_399

MM̄FF Geometry

C -3.0340731 .2888902 .441352$

C -1.8314920 .7907062 .118613$

C -1.032870 -0.116929 3.014449

O $-0.585678-1.2194152 .187843$

C 0.2083200 .5944603 .597356

C -0.1017681 .7564564 .518187$

C 0.3143003 .0216014 .313126

C -0.9025241 .4221285 .750773$

C 1.1951113 .5304903 .207228

C 0.4065503 .8764051 .941823

C 1.2502784 .6531920 .921404

C $0.4396444 .909170-0.351301$

C $1.2724375 .614868-1.430358$

O $2.422327 \quad 3.890557 \quad 0.614072$

O $0.5567075 .521225-2.671288$

C $2.6003864 .849553-1.614354$

C $1.4351267 .111351-1.139199$

O $3.4697835 .550398-2.501008$

C $3.3275894 .544680-0.284085$

C $4.5688473 .640075-0.492581$

$\begin{array}{lllll}\text { O } & 3.803332 & 5.778777 & 0.269756\end{array}$

C $-0.220971-2.3654442 .822185$

O $-0.314590-2.6137424 .013489$

C $0.390892-3.2519971 .810629$

C $1.335733-4.1174142 .206709$

C $2.130726-4.9805111 .332804$

C $1.678364-5.135957-0.093951$

C $4.2905332 .188394-0.896822$

C 4.1347641 .2390780 .282335

C $3.866266-1.1786490 .794394$

O 4.0753791 .6104131 .450813

C $4.387500-2.4457180 .194420$

C $5.320422-3.2191110 .771500$

C $5.822162-4.5073690 .179330$

C $5.632001-5.6932311 .103455$

C $6.653740-6.1690321 .835023$

C $4.277838-6.3746121 .118296$

C $3.242167-5.5542661 .839414$

N $4.081495-0.085348-0.134854$

H $-3.457904 \quad 1.042598 \quad 3.410874$

H -1.3885911 .0505701 .162028$

H -1.670811 -0.5144703 .813723$

H 0.8597840 .8944862 .767699

H $0.810354-0.1300844 .161442$

H 0.0209993 .7827905 .035026

H -0.9686982 .2674276 .444310$

H -1.924242 1.1343925 .487600

H $-0.4346480 .593384 \quad 6.292344$

H 1.7109274 .4258023 .577168

H 1.9860982 .8061352 .984625

H $\quad 0.0624872 .943378 \quad 1.482256$

H -0.4847934 .4628652 .197598$

H 1.5505075 .6016611 .382469

H $-0.4665595 .485624-0.126923$

H $0.0898773 .949887-0.758609$

H $-0.3173755 .928446-2.545163$

H $2.3576843 .902872-2.108455$

H $0.4552717 .602554-1.102383$

H $1.9900937 .611892-1.940329$

H $1.9386197 .313099-0.191666$

H $2.9772085 .708492-3.324732$

H $5.2129194 .102301-1.252122$ 
H $5.173756 \quad 3.6424130 .424121$ H 4.4952895 .5779290 .921422 H $\quad 0.112682-3.1107370 .773028$ H $1.598874-4.1526753 .263469$ H $1.842591-4.207379-0.648181$ H $0.612522-5.387505-0.137778$ H $2.196729-5.932863-0.631058$ H $3.4062692 .101697-1.534070$ H $5.1508441 .830914-1.477544$ H $2.787536-1.2572080 .962673$ H $4.342044-0.9422861 .752002$ H $3.955876-2.756997-0.753864$ H $5.755129-2.9098231 .719258$ H $6.888272-4.375667-0.047493$ H $5.340352-4.719720-0.783279$ H $6.533631-7.0331212 .479782$ H $7.632340-5.7011531 .809385$ H $4.343464-7.3441681 .629215$ H $3.998287-6.6102760 .088265$ H $3.478668-5.3853862 .891205$ H $3.955644-0.270846-1.126535$ C -5.1538851 .6236631 .058368$ $\begin{array}{llll}\text { O } & -4.894382 & 0.484561 & 0.212082\end{array}$ C $-5.690852-0.6159680 .681142$ C $-6.8473880 .021217 \quad 1.440857$ C -6.1387451 .1649892 .139983$ H $-5.064447-1.1749371 .387554$ C $-6.101813-1.539540-0.468811$ H $-7.613927 \quad 0.4142910 .763269$ H -7.326872 -0.6758852 .134385$ C -7.0715542 .2636892 .620291$ H -5.6144620 .7534563 .009275$ C $-4.960510-1.975002-1.410347$ O $-7.086931-0.887140-1.281560$ H -6.601622 -2.423534-0.055486 H $-4.717959-1.154423-2.096008$ O $-5.465204-3.041226-2.226055$ C $-3.672862-2.454365-0.719468$ C $-2.656576-3.133899-1.656605$ H -3.192272 -1.604151 -0.222752 O $-4.014527-3.3955730 .301879$ H -1.756699 -3.343227 -1.065157 H -3.036574 -4.123671 -1.941807 H -6.506455 3.0696393 .099097 H -7.6427652 .6969151 .792344$ H -7.7861001 .8699683 .350655$ C -3.830046 2.2131931 .559982 H $-6.698417-0.054850-1.602316$ H -6.265223 -2.712058 -2.670762 H -3.194381 -3.6351770 .766322$ H -4.029882 3.1335782 .121275 H -3.224026 2.4964810 .689675 H -5.6324702 .3694890 .410906$ C $-0.686564-0.795726-1.982062$ O $-1.942950-0.978493-2.640082$ C $-2.288219-2.336420-2.923445$ C $-1.200023-2.999338-3.781156$ C $0.178487-2.874557-3.138649$ C $0.464819-1.416855-2.784881$ H $-0.738606-1.266885-0.992654$ C $-0.4980510 .706062-1.728892$ H -3.191065 -2.295083 -3.544309 O $-1.489714-4.376213-3.999099$ H -1.168209 -2.516582 -4.766600 H $\quad 0.267987-3.520514-2.259763$ O $1.172976-3.316591-4.064737$ H $\quad 0.628175-0.868092-3.719435$ O $1.675297-1.334186-2.038485$ C $-0.3791321 .503681-3.007287$ H -1.364328 $1.112535-1.193694$ H $\quad 0.3780820 .893420-1.096926$ H -2.364784 -4.427334 -4.420282 H $\quad 0.951202-4.230715-4.312405$ H $1.544858-1.816545-1.204409$ O $\quad 0.9296341 .788954-3.260397$ O $-1.3331451 .806137-3.712649$ C $1.1841062 .475682-4.489791$ H $2.2481242 .724369-4.526210$
H $\quad 0.945258 \quad 1.824955-5.336161$

H $0.6057653 .402647-4.541326$

SCF Energy (B3LYP/6-31G**//MMFF) $=-3245.90346234$

014

MM̄FF Geometry

C $4.661405-3.504490-0.350026$

C $3.684147-3.047941-1.147618$

C $2.264737-3.551333-1.099469$

O $1.405140-2.471463-0.666645$

C $1.819695-3.977961-2.510138$

C $0.418977-4.555549-2.561466$

C $-0.618949-3.995730-3.213852$

C $0.227163-5.866019-1.841234$

C $-0.641851-2.723361-4.014142$

C $-1.117076-1.483369-3.239826$

C $-2.555935-1.586405-2.707583$

C $-3.044515-0.230969-2.192817$

C $-4.449142-0.326352-1.577386$

O $-2.573755-2.531500-1.635093$

O $-4.679710 \quad 0.898595-0.865344$

C $-4.480518-1.482357-0.552389$

C $-5.532014-0.402163-2.660559$

O $-5.816359-1.681724-0.096502$

C $-3.875317-2.795909-1.091990$

C $-3.695751-3.888449-0.008558$

O $-4.748769-3.335806-2.091388$

C $1.372140-2.1971360 .666013$

O $2.004929-2.7505281 .550998$

C $0.423825-1.0827500 .869858$

C $0.472132-0.3730072 .006731$

C -0.4029310 .7482882 .349266$

C -1.6393470 .9380031 .514041$

C $-2.784632-3.5099631 .164640$

C $-3.523242-2.9690522 .377761$

C $-3.090456-1.9839004 .628361$

O $-4.746727-2.9731702 .480525$

C $-3.213891-0.4956054 .538602$

C -2.5911610 .3494795 .374185$

C $-2.745874 \quad 1.8426285 .323464$

C -1.4201512 .5668115 .250169$

C -0.8574313 .0918946 .351729$

C $-0.7955292 .761793 \quad 3.887982$

C $-0.0780051 .532753 \quad 3.397264$

N -2.645345 -2.510713 3.351551

H $4.443590-4.2865750 .372794$

H $3.903623-2.248792-1.852245$

H $2.168863-4.394357-0.404265$

H $2.513317-4.731685-2.906437$

H $1.901339-3.113898-3.178277$

H -1.576783 -4.514690 -3.183885

H -0.758374 -6.304604 -2.031655

H $0.976325-6.593718-2.169894$

H $\quad 0.320671-5.730366-0.759610$

H $\quad 0.342340-2.514116-4.445617$

H $-1.301460-2.880822-4.877315$

H $-0.426510-1.299375-2.408610$

H -1.044918 -0.623535 -3.917239

H $-3.200093-1.941250-3.520614$

H $-3.0261770 .521316-2.991302$

H $-2.3509660 .134945-1.424247$

H -5.538946 $0.821391-0.415796$

H -3.901375 -1.142879 0.313456

H $-5.4965790 .490449-3.296923$

H $-6.535115-0.414221-2.219876$

H $-5.430873-1.275631-3.308083$

H -5.788474 -2.309804 0.644402

H $-4.672375-4.2490210 .335370$

H $-3.228250-4.753960-0.500385$

H -4.560064 -4.284173 -2.186233

H $-0.280856-0.8694670 .074522$

H $\quad 1.235047-0.6165772 .744967$

H -2.198189 -0.0002831 .440123$

H $-1.373019 \quad 1.2634140 .505601$

H -2.3365641 .6699311 .925795$

H -2.265936 -4.415844 1.504168

H -2.019593 -2.792673 0.853822

H $-2.358062-2.2942565 .380310$ 
H $-4.060899-2.4198514 .886345$ H -3.868415 -0.0963653 .767329$ H -1.959633 $-0.053816 \quad 6.162750$ H -3.2881252 .1507436 .227141$ H -3.3773992 .1525294 .481581$ H $\quad 0.0780803 .6391096 .302929$ H -1.317321 2.9808757 .328263 H -0.0545793 .5719523 .927458$ H -1.554725 3.1224513 .188020 H 0.8320521 .3049203 .953800 H -1.648842 -2.4945113 .154647$ C $6.286629-1.7693470 .550367$ O $5.529100-0.6423910 .068678$ C 4.9947140 .0729931 .199541 C $5.701179-0.4915092 .428240$ C $5.901060-1.9398622 .018902$ H $3.930987-0.1841121 .261321$ C 5.1537191 .5819950 .988949 H $6.670110-0.0083572 .599674$ H $5.102958-0.3799143 .337729$ C $6.939987-2.6744832 .846907$ H $4.938519-2.4565512 .115322$ C $4.5575712 .090286-0.340953$ O 6.5535531 .8912310 .982100 H $4.7211912 .122423 \quad 1.838955$ H $5.1216001 .667895-1.180793$ O $4.7712193 .507362-0.399844$ C $3.0548761 .803739-0.524977$ C $2.5354052 .342332-1.873704$ H $2.8774530 .723786-0.489655$ O $2.3530982 .398757 \quad 0.562918$ H $2.6975313 .423891-1.914394$ H $3.1413501 .896971-2.671562$ H $7.055757-3.7056452 .498720$ H $7.918770-2.1865422 .788939$ H $6.641617-2.704148 \quad 3.899951$ C $6.065778-2.971936-0.370741$ H 6.6375002 .8496570 .839541 H 4.2343093 .9129340 .302708 H 1.4152922 .1592980 .475888 H $6.747977-3.784226-0.094255$ H $6.321868-2.684783-1.398907$ H $7.342170-1.4755300 .474427$ C $0.0771414 .080237-1.291165$ O $0.1768502 .657208-1.177995$ C $1.0419192 .029984-2.129538$ C $0.5661002 .359687-3.551630$ C $0.4724533 .866694-3.779427$ C $-0.3800844 .516712-2.689385$ H $1.0627394 .518222-1.090727$ C $-0.8252244 .594250-0.158344$ H $0.9209310 .948382-1.988881$ O $1.4332571 .789533-4.525666$ H $-0.4286561 .924093-3.712509$ H $1.4648654 .329298-3.832071$ O $-0.1533564 .114253-5.040709$ H -1.429736 4.256071-2.861893 O $-0.2785745 .935537-2.791336$ C $-2.2465934 .069923-0.183287$ H $-0.394760 \quad 4.2773330 .799952$ H $-0.8628585 .688925-0.183023$ H $1.4580230 .830087-4.368185$ H $0.375283 \quad 3.659896-5.719122$ H $-0.5421286 .180854-3.694942$ O $-2.945294 \quad 4.6502630 .838201$ O $-2.6893753 .247576-0.972236$ C -4.3080354 .2321900 .966590$ H $-4.3551713 .163111 \quad 1.194414$ H -4.8633494 .4574390 .051197$ H -4.7568774 .7868951 .794798$

SCF Energy $\left(\mathrm{B} 3 \mathrm{LYP} / 6-31 \mathrm{G}^{* *} / / \mathrm{MMFF}\right)=-3245.92893140$

0140

MM̄FF Geometry

C 3.0665722 .9831451 .886118

C 2.3338301 .9450981 .456426

C 1.2769541 .2630382 .282762

O 0.0630351 .2017471 .494487
C $1.705121-0.1798512 .604588$

C $1.014646-0.7101483 .844204$

C $-0.106097-1.4562743 .849153$

C $1.685366-0.3502195 .146338$

C $-0.927543-1.9067682 .673359$

C $-1.157010-3.4258532 .715622$

C $-2.149392-3.9477941 .662912$

C $-1.600603-3.8455600 .239136$

C $-2.630791-4.304793-0.803659$

O $-3.362419-3.204376 \quad 1.796840$

O $-2.159847-3.905890-2.099821$

C -3.947857 -3.543732 -0.550899

C $-2.771349-5.830036-0.846319$

O $-4.982002-4.011690-1.413557$

C $-4.422329-3.6009110 .919031$

C $-5.615013-2.6497721 .182570$

O $-4.859735-4.9316401 .215003$

C $-0.7044562 .324226 \quad 1.439289$

O -0.5386643 .3592322 .064897$

C $-1.7606232 .095590 \quad 0.426133$

C $-2.4614263 .143672-0.033025$

C $-3.4848433 .119948-1.082271$

C $-3.795140 \quad 1.792832-1.721338$

C $-5.277733-1.1627891 .041127$

C $-6.453539-0.2617821 .371400$

C $-7.0060672 .160061 \quad 1.624183$

O $-7.598062-0.6776371 .526749$

C -7.3143292 .7506210 .284360$

C -7.124570 $4.042865-0.021337$

C $-7.4438244 .630565-1.365430$

C $-6.2712005 .358692-1.983114$

C $-6.2913596 .693313-2.140325$

C $-5.1102534 .532637-2.497365$

C $-4.0660884 .285642-1.437733$

N $-6.0667391 .070527 \quad 1.439660$

H 2.9181773 .3683372 .891365

H 2.5045251 .5548100 .457459

H 1.0829851 .8212503 .207458

H $2.788678-0.2311872 .778977$

H $1.515635-0.8353911 .747057$

H $-0.506369-1.7612434 .815818$

H $1.150536-0.747926 \quad 6.015389$

H $2.702613-0.7546565 .172120$

H $\quad \begin{array}{llll}1.740907 & 0.736487 & 5.263369\end{array}$

H -1.887769 -1.380509 2.725062

H $-0.466344-1.6279611 .723494$

H -0.199918 -3.953042 2.616152

H -1.560292 -3.684057 3.704478

H -2.354542 -4.997176 1.906309

H -0.670205 -4.417479 0.140146

H - $-1.339900-2.8036510 .014842$

H -1.297549-4.330870 -2.248077

H $-3.762928-2.499124-0.829012$

H -1.811655 -6.295192 -1.101993

H -3.472476 -6.139584 -1.629224

H $-3.101625-6.2590920 .101761$

H -4.645624 -3.959183 -2.324625

H $-6.459048-2.9162180 .533635$

H $-5.976133-2.8147492 .207672$

H $-5.468058-4.8965981 .971531$

H $-1.896670 \quad 1.0865820 .054044$

H -2.249775 4.1283770 .382916

H $-4.1495411 .079376-0.971964$

H $-2.9003931 .379749-2.200698$

H -4.566606 $1.845717-2.491531$

H -4.446255 -0.8990961 .704698$

H -4.983637 -0.9361540 .012060$

H -6.5381032 .8837652 .298700$

H -7.9243401 .7875112 .088376$

H -7.722792 $2.080597-0.468846$

H -6.7370844 .7193270 .736891$

H -8.290759 $5.316419-1.234088$

H -7.789960 $3.859284-2.064832$

H $-5.4650937 .218512-2.607954$

H -7.130890 7.290939-1.801152

H -4.618482 $5.066795-3.321307$

H $-5.5021423 .613860-2.941123$

H $-3.7386695 .195017-0.930793$ 
H $-5.1001591 .314301 \quad 1.243311$

C 5.5003283 .0984361 .302538

$\begin{array}{lllll}\text { O } & 5.549176 & 1.746241 & 0.802837\end{array}$

C $6.6974121 .602474-0.048965$

C 7.6179202 .7578900 .316073

C 6.6065513 .8601390 .569206

H $6.3491001 .743205-1.079529$

$\begin{array}{llll}\text { C } 7.297033 & 0.202808 & 0.107344\end{array}$

H $8.1890272 .551844 \quad 1.228660$

H $8.3250332 .997397-0.483592$

C $7.166642 \quad 5.033833 \quad 1.353547$

H $6.2350184 .223983-0.398412$

C $6.272484-0.934745-0.097746$

$\begin{array}{lllll}\text { O } & 7.823781 & 0.084943 & 1.434582\end{array}$

H $8.1463670 .087836-0.576559$

H $5.550798-0.9352270 .726844$

O $6.977572-2.179951-0.021574$

C $5.527728-0.886780-1.447574$

C $4.634019-2.111155-1.721475$

H $4.9175790 .019211-1.506863$

O $6.485701-0.805065-2.507465$

H $4.334457-2.078557-2.775550$

H $5.234112-3.024419-1.631199$

H $\quad 6.394427 \quad 5.7897231 .527457$

H 7.5541074 .7172692 .327775

H 7.9866315 .5075230 .803764

C $4.1060113 .666127 \quad 1.041539$

H $8.381545-0.7107311 .458790$

H $7.264695-2.297950 \quad 0.899523$

H $7.053244-1.593009-2.451031$

H $3.8512173 .548793-0.019197$

H $4.0779914 .739215 \quad 1.261708$

H 5.7066493 .0494432 .379357

C $1.829854-0.998081-2.227162$

O $2.522852-1.064597-0.973836$

C $3.391529-2.192410-0.811496$

C $2.590882-3.492389-0.964474$

C $1.855020-3.539420-2.298103$

C $1.018462-2.276820-2.481881$

H $2.549852-0.839130-3.038599$

C $0.8832830 .212973-2.171460$

H $3.742930-2.161506 \quad 0.225972$

O $3.438164-4.631200-0.856898$

H $1.852150-3.563221-0.155509$

H $2.545771-3.680629-3.137363$

O $0.974336-4.665342-2.310991$

H $0.160433-2.309105-1.800735$

O $0.502617-2.247503-3.811629$

C $1.604707 \quad 1.540975-2.245145$

H $0.1819610 .188183-3.013071$

H $0.3208890 .193226-1.230844$

H $3.891027-4.5784620 .002108$

H $1.515509-5.456935-2.147719$

H $0.000136-3.069281-3.946330$

O $0.6828052 .541791-2.116274$

O $2.8088511 .688528-2.396853$

C $1.197824 \quad 3.875413-2.166634$

H $1.7948214 .026152-3.071148$

H $0.3500054 .565364-2.188446$

H $1.7916794 .078926-1.271889$

SCF Energy (B3LYP/6-31G**//MMFF) $=-3245.92469217$

01_400

MM̄FF Geometry

C 1.3351491 .7069992 .615936

$\begin{array}{llll}\text { C } & 0.582122 & 2.807413 & 2.770237\end{array}$

C $-0.2310843 .493131 \quad 1.695841$

$\begin{array}{llll}\text { O } & -0.284143 & 2.658531 & 0.517062\end{array}$

C -1.672056 3.7347532 .182797

C -2.392974 4.7996861 .380398

C $-3.294054 \quad 4.556302 \quad 0.409476$

C -2.065301 $6.220676 \quad 1.763644$

C $-3.7402353 .225778-0.126744$

C $-5.211413 \quad 2.9631350 .213135$

C $-5.7394981 .694105-0.469727$

C $-7.2316011 .511190-0.181794$

C $-7.776624 \quad 0.206108-0.780708$

$\begin{array}{lllll}\text { O } & -4.999233 & 0.572187 & 0.024167\end{array}$
O $-9.067109-0.040517-0.200875$

C $-6.858231-0.956685-0.349505$

C $-7.9972370 .316735-2.293813$

O $-7.233997-2.171194-0.994344$

C $-5.357093-0.675786-0.585517$

C $-4.450116-1.7761970 .008532$

O $-5.104260-0.649185-1.996700$

C $-0.105600 \quad 3.224608-0.701568$

$\begin{array}{lllll}\text { O } & 0.103367 & 4.406793 & -0.936065\end{array}$

C $-0.1952782 .215676-1.785854$

C $-0.4030650 .906992-1.570269$

C $-0.501112-0.142092-2.590429$

C $-0.3915660 .275184-4.032792$

C $-4.503757-1.9284991 .530290$

C -3.676013 -3.119931 1.972336

C $-1.319800-3.9148442 .155131$

O $-4.201384-4.1790852 .308658$

C $-0.772513-4.4860040 .876551$

C $-1.241036-4.285866-0.365302$

C $-0.627121-4.852800-1.613019$

C $0.029273-3.798779-2.481186$

C $1.322350-3.901157-2.830750$

C $-0.825978-2.664446-3.012995$

C $-0.699507-1.413412-2.181251$

N -2.312351 -2.8820291 .918636$

H $1.398913 \quad 1.2271231 .643129$

H $0.5770473 .294078 \quad 3.743983$

H 0.2730374 .4419951 .476135

H $-1.673216 \quad 4.055926 \quad 3.233297$

H -2.233812 2.7932262 .166355

H -3.756603 $5.410666-0.083819$

H $-2.6357426 .951548 \quad 1.180646$

H -2.2960446 .3914102 .820249$

H -1.0034096 .4289761 .601560$

H -3.1177542 .4050370 .240193$

H $-3.610753 \quad 3.243093-1.216039$

H $-5.307228 \quad 2.863708 \quad 1.302419$

H $-5.8226123 .821308-0.093886$

H $-5.5700641 .790483-1.548863$

H $-7.8073332 .370245-0.548406$

H $-7.391592 \quad 1.4861520 .905412$

H $-9.6358990 .723089-0.399205$

H $-7.030115-1.1093830 .722620$

H $-8.720558 \quad 1.110057-2.517862$

H $-8.429715-0.604945-2.698274$

H $-7.0828920 .541072-2.846691$

H $-8.179218-2.314090-0.815210$

H $-4.668807-2.737834-0.474430$

H -3.412105-1.548314 -0.269902

H $-4.155822-0.800681-2.144061$

H $-0.0754452 .617467-2.787002$

H $-0.5110990 .555026-0.545607$

H $-0.490574-0.552770-4.737264$

H $0.5787760 .743887-4.227720$

H $-1.1814210 .992175-4.283889$

H $-4.132135-1.0268352 .030186$

H $-5.524848-2.0974291 .884483$

H $-1.748668-4.7161872 .764928$

H $-0.509307-3.4445262 .720474$

H $0.108556-5.1133570 .997141$

H $-2.129582-3.680727-0.515649$

H $-1.414878-5.348656-2.193892$

H $0.092145-5.639934-1.352629$

H $1.788622-3.164921-3.476264$

H $1.941738-4.720538-2.482426$

H $-1.878212-2.974001-3.025978$

H $-0.558792-2.490650-4.059533$

H $-0.796879-1.585450-1.109124$

H - $-1.981307-1.9818051 .583885$

C $2.031649-0.4067523 .804200$

O $2.559591-1.0019432 .600582$

C $3.601511-1.9254122 .954738$

C $3.304887-2.3195024 .393645$

C $2.840659-0.9945014 .966121$

H $4.545900-1.3677512 .919958$

C $3.653538-3.0842291 .955234$

H $2.497169-3.0576424 .456558$

H $4.181931-2.7260594 .905625$ 
C $2.046545-1.1307156 .254064$

H $3.724380-0.3721115 .162564$

C $3.687089-2.6483780 .475659$

O $2.493504-3.9078222 .125327$

H $4.510938-3.7284872 .181094$

H $2.715090-2.2245560 .199307$

O $3.850951-3.828851-0.320210$

C $4.810461-1.6576490 .118193$

C $4.932877-1.366770-1.390452$

H $4.658659-0.7186940 .658707$

O $6.054963-2.2024830 .565231$

H $5.837789-0.768022-1.549086$

H $5.124642-2.306769-1.922052$

H $1.721061-0.1500716 .615375$

H $1.154963-1.7508246 .111840$

H $2.657195-1.5945167 .035462$

C 2.1727301 .1152013 .716459

H $1.714868-3.3412761 .990002$

H $3.127249-4.436432-0.089461$

H $6.743929-1.5374020 .396823$

H $3.2183271 .366933 \quad 3.502753$

H 1.9133191 .5674024 .680873

H $0.975381-0.6960163 .867715$

C $4.3817661 .645024-1.624318$

O $3.4414890 .600420-1.342179$

C $3.707248-0.649267-1.990694$

C $3.768222-0.463101-3.513545$

C $4.7474940 .634282-3.915019$

C $4.456727 \quad 1.913047-3.133533$

H $5.3726451 .362026-1.249347$

C $3.9187002 .905936-0.875036$

H 2.835711-1.283307-1.795505

O $4.119528-1.691401-4.141185$

H $2.768502-0.193323-3.875140$

H $5.7882260 .317855-3.776950$

O 4.5920180 .924058 -5.305974

H $3.5145752 .352735-3.485937$

O $5.4944012 .857764-3.391410$

C 4.2893642 .8870100 .592664

H $4.3949073 .793910-1.305993$

H $2.8313693 .012020-0.941286$

H $4.010577-1.575437-5.099972$

H $4.886890 \quad 0.142480-5.802934$

H $5.5295852 .994228-4.353746$

O $4.062043 \quad 4.128726 \quad 1.116694$

O 4.7190381 .9188441 .203026

C 4.4256174 .3065362 .489372

H 5.4364843 .9321362 .678273

H 4.4058085 .3774272 .708696

H $3.7011573 .803923 \quad 3.134427$

SCF Energy (B3LYP/6-31G**//MMFF) $=-3245.91296133$

014401

MM̄̄FF Geometry

C $0.011457 \quad 0.2922150 .964580$

C $-0.443315-0.7831890 .303027$

C $-0.119736-1.151409-1.125193$

O $0.473083-0.021994-1.807994$

C $0.906504-2.293727-1.190841$

C $0.365220-3.649249-0.796231$

C $0.627263-4.2694740 .370761$

C $-0.473847-4.334079-1.844384$

C $1.426920-3.7557391 .536885$

C $2.778265-4.4701301 .681680$

C $3.823950-3.9951770 .660244$

C $5.090718-4.8483670 .744157$

C $6.187580-4.331805-0.199224$

O $4.137340-2.630326 \quad 0.961472$

O $7.418387-4.977808 \quad 0.159581$

C $6.381803-2.8211300 .043958$

C $5.910780-4.705572-1.660177$

O $7.286597-2.262883-0.906552$

C $5.060198-2.0165710 .052950$

C $5.334708-0.5733020 .542626$

O $4.534743-1.962651-1.274949$

C $-0.3215810 .759108-2.583186$

O $\quad-1.492570 \quad 0.555470-2.872209$

C $0.406118 \quad 1.959974-3.060324$
C $1.5784562 .352040-2.538208$

C $2.3314103 .557093-2.883504$

C $1.8172594 .431254-3.994274$

C 4.0723410 .2316680 .881718

C 4.4081101 .6445951 .333418

C 3.3388193 .6571772 .353290

O 5.5115122 .1584091 .169181

C 2.7916194 .5070861 .251677

C 3.4690935 .5014350 .656415

C $2.9008396 .307660-0.478178$

C $3.7737676 .323796-1.718507$

C $4.0403507 .489730-2.332902$

C $4.3406455 .035809-2.285100$

C $3.4380923 .840865-2.167138$

N $3.3167002 .272841 \quad 1.916140$

H $\quad 0.6897000 .9849080 .474687$

H -1.109755 -1.465661 0.822566

H -1.044959-1.445625 -1.633951

H $1.785984-2.025180-0.594876$

H $1.299872-2.364177-2.214716$

H $0.204280-5.2611920 .528271$

H $-0.821761-5.320564-1.519938$

H -1.359315 -3.737391-2.083058

H $0.107223-4.474521-2.761684$

H $1.566727-2.6716251 .500402$

H $\quad 0.835630-3.9453642 .441708$

H $3.153570-4.2667112 .693189$

H $2.635011-5.5547851 .600257$

H $3.396756-4.057246-0.346293$

H $4.866516-5.9010520 .531002$

H $5.478674-4.8263961 .772276$

H $7.286822-5.9382090 .080395$

H $6.859565-2.7099711 .027638$

H $5.846943-5.794692-1.770967$

H $\quad 6.731816-4.386494-2.311659$

H $4.980873-4.280498-2.043648$

H $8.105385-2.786263-0.868916$

H $5.948598-0.6096881 .452372$

H $5.913323-0.032180-0.216912$

H $4.000867-1.158691-1.373534$

H $-0.1054312 .538193-3.822693$

H $2.0273331 .751755-1.748629$

H $0.8355404 .843821-3.737103$

H $1.7259713 .858975-4.924178$

H $2.4750345 .277384-4.210883$

H 3.4154210 .3120720 .009480

H $3.516451-0.2655801 .684940$

H 4.3596193 .9408682 .628308

H 2.7004623 .7340753 .238525

H $1.7814184 .279440 \quad 0.917236$

H 4.4740725 .7449010 .991866

H $2.7468627 .329372-0.107582$

H $1.9074715 .935723-0.759939$

H $4.6592717 .531230-3.223214$

H $3.6471728 .430202-1.961219$

H $5.2664934 .806484-1.740399$

H $4.6431955 .159261-3.332721$

H $3.7413643 .146245-1.383376$

H 2.4253701 .7877341 .941236

C -1.1180881 .9515752 .441135$

O $-2.364791 \quad 1.8150281 .734969$

C -3.4124122 .4362012 .500233$

C -2.7148153 .2417593 .589314$

C -1.4910842 .3847403 .857679$

H -3.9803151 .6248452 .970870$

C -4.3284953 .2348591 .565874$

H -2.410964 4.2330133 .233917

H -3.3460373 .3794824 .472414$

C -0.3801603 .1186174 .588243$

H -1.7968161 .5119924 .450216$

C -4.8134752 .4363800 .336580$

O -3.6182544 .3790961 .073005$

H -5.1825583 .6299072 .126992$

H -3.963706 $2.235732-0.324884$

O $-5.7117093 .269808-0.406622$

C $-5.541721 \quad 1.1230020 .676247$

C $-6.0526680 .353704-0.556363$

H -4.8844820 .4670941 .251902$ 
O -6.6631891 .4128701 .514638$ H -6.746163 $-0.421039-0.208296$ H -6.664531 $1.021326-1.174778$ H $\quad 0.4842722 .464964 \quad 4.741297$ H -0.0426413 .9967754 .027350$ H -0.7249613 .4588455 .570049$ C -0.3651870 .6235922 .381703$ H -2.817937 4.0569610 .623493 H $-5.2402294 .096327-0.607927$ H $-7.2458642 .018707 \quad 1.024952$ H $-0.990270-0.1741612 .799453$ H 0.5484130 .6746812 .983525 H $-0.5476812 .740811 \quad 1.933625$ C $-4.763431-2.402998-0.257921$ O $-4.114962-1.181919-0.637704$ C $-4.923413-0.275732-1.399571$ C $-5.405339-0.958283-2.686646$ C $-6.127182-2.269470-2.395008$ C $-5.268923-3.158206-1.497131$ H $-5.599636-2.1823360 .415831$ C $-3.742473-3.260750 \quad 0.506861$ H $-4.2589450 .538416-1.709386$ O $-6.270018-0.102719-3.426226$ H $-4.541068-1.174326-3.328319$ H -7.114558 -2.094611-1.951852 O $-6.356184-2.962492-3.624250$ H $-4.418062-3.534807-2.079123$ O $-6.032607-4.295474-1.097818$ C $-3.449372-2.7406841 .898089$ H $-4.119259-4.2828120 .625097$ H -2.795362 -3.284699 -0.042368 H $-5.7816760 .718120-3.609260$ H $-6.868316-2.368553-4.199497$ H $-6.791597-3.978292-0.579082$ O $-2.535757-3.5687612 .488709$ O $-3.948448-1.7471972 .406433$ C $-2.161735-3.223783 \quad 3.825870$ H $-3.040782-3.2173454 .477413$ H $-1.461879-3.9811714 .188707$ H -1.663112 -2.250909 3.839902

SCF Energy (B3LYP/6-31G**//MMFF) $=-3245.91565153$

01 402

MM̄FF Geometry

C $-4.248181-1.5495861 .770483$

C $-3.746079-2.753517 \quad 1.456047$

C $-3.238306-3.1063780 .083500$

O $-1.963133-3.787966 \quad 0.208568$

C $-4.160287-4.089098-0.656435$

C $-5.446812-3.485602-1.168839$

C $-5.565221-2.869529-2.360943$

C $-6.657056-3.680093-0.293383$

C $-4.482372-2.611162-3.376093$

C $-4.542087-1.176381-3.921193$

C $-3.985777-0.129728-2.941309$

C $-4.493038 \quad 1.262425-3.324349$

C $-3.8893972 .355536-2.435243$

O $-2.554675-0.175265-3.023785$

O $-4.187805 \quad 3.625200-3.034642$

C $-2.3583942 .195218-2.444227$

C $-4.5218892 .373614-1.039689$

O $-1.750563 \quad 3.114773-1.537033$

C $-1.8715220 .749539-2.166983$

C $-0.3593300 .673081-2.485680$

O $-2.0936320 .448941-0.791664$

C $-0.880600-3.0760720 .618039$

O $-0.848299-1.9034840 .952565$

C $0.275892-3.9981990 .584440$

C $1.467063-3.6019901 .054620$

C $2.688648-4.4097681 .078507$

$\begin{array}{lllll}\text { C } 2.644038 & -5.765497 & 0.422187\end{array}$

C $0.298364-0.695345-2.273695$

C $1.749782-0.690946-2.727115$

C $3.755499-2.130561-2.871453$

O $2.272528 \quad 0.265935-3.293741$

C $4.377061-3.215298-2.052409$

C $5.464825-3.030846-1.288399$

C $6.157813-4.123253-0.524644$
C $6.197554-3.8643270 .964694$

C $7.183544-3.1351171 .513880$

C $5.144314-4.5280341 .822433$

C $3.789109-3.8963221 .665551$

N $2.398420-1.881944-2.422788$

H $-4.343982-0.8018240 .988355$

H $-3.662713-3.5166662 .226172$

H $-3.080550-2.212459-0.531411$

H $-4.382458-4.952835-0.013910$

H $-3.608394-4.533658-1.496162$

H $-6.554501-2.520639-2.655240$

H $-7.565623-3.264543-0.742278$

H $-6.833846-4.747322-0.123701$

H $-6.520619-3.1933940 .675832$

H -3.481809 -2.817000 -2.983949

H $-4.647294-3.301797-4.212164$

H $-3.957687-1.143688-4.849941$

H $-5.579685-0.937141-4.186603$

H $-4.296508-0.376720-1.920465$

H $-5.5888881 .301646-3.283831$

H $-4.224601 \quad 1.474811-4.368999$

H $-5.1547863 .706337-3.100331$

H -2.014200 $2.490519-3.445071$

H $-5.6057442 .524294-1.111000$

H $-4.143110 \quad 3.212114-0.445077$

H $-4.352872 \quad 1.449922-0.483469$

H $-1.8592702 .759367-0.638461$

H $-0.2099490 .962053-3.534805$

H $0.179641 \quad 1.402482-1.871007$

H $-1.525304-0.292091-0.531645$

H $0.112004-4.9887480 .174449$

H $1.558008-2.5973381 .465690$

H $2.298419-5.681868-0.614418$

H $1.965722-6.4321870 .965816$

H $3.615515-6.2617630 .377691$

H $0.289232-0.959395-1.213315$

H $-0.234265-1.469715-2.835942$

H $4.326955-1.196964-2.824818$

H $3.704659-2.438352-3.921283$

H $3.937365-4.206508-2.126727$

H $5.913664-2.041293-1.239202$

H $7.183135-4.202640-0.909658$

H $5.699920-5.100523-0.720774$

H $7.235895-2.9690242 .584712$

H 7.957305-2.683330 0.901991

H $5.415420-4.4510522 .883960$

H $5.137947-5.602451 \quad 1.616997$

H $3.718670-2.9071582 .116292$

H $1.838649-2.673015-2.116306$

C $-4.2244920 .233885 \quad 3.590371$

O $-2.8175590 .421604 \quad 3.329912$

C -2.649163 1.6725922 .639393

C -3.9059602 .4759942 .943873$

C -4.9607391 .3883632 .904553$

H -2.625890 1.4413671 .568519

C $-1.3408882 .372882 \quad 3.023034$

H $-3.8623852 .928498 \quad 3.941587$

H $-4.081276 \quad 3.2718752 .214179$

C $-6.262387 \quad 1.7781943 .584272$

H $-5.1746551 .146293 \quad 1.857244$

C $-0.083758 \quad 1.5592732 .637808$

$\begin{array}{lllll}\text { O } & -1.361187 & 2.643485 & 4.422977\end{array}$

H -1.3134213 .3374042 .500741$

H $-0.195327 \quad 1.2208381 .601146$

$\begin{array}{llll}\text { O } & 0.034055 & 0.397143 & 3.455589\end{array}$

C $1.2195582 .377346 \quad 2.743264$

C 2.5054951 .6019902 .403329

H 1.1396673 .2488692 .084004

$\begin{array}{lllll} & 1.377534 & 2.891469 & 4.068047\end{array}$

H 3.3442362 .3038582 .496182

H 2.6910850 .8448103 .176530

H $-6.9823440 .954626 \quad 3.547834$

H $-6.104048 \quad 2.0420614 .635247$

H -6.7121102 .6430153 .085415$

C $-4.672164-1.1700493 .163218$

H $-0.582306 \quad 3.1871264 .628860$

H $-0.771992-0.1323903 .332893$

H 1.4098492 .1313594 .674609 
H $-5.762524-1.2474083 .240097$

H -4.246608 -1.8908993 .873305$

H $-4.345754 \quad 0.3150864 .678428$

C $2.9210182 .890332-0.342846$

O $2.0498461 .802900-0.014604$

C 2.5252810 .9355301 .014938

C 3.8841890 .3376720 .622507

C 4.8891811 .4082830 .201383

C $4.2756082 .359774-0.825360$

H 3.0649353 .5208760 .542476

C $2.1972453 .716763-1.412793$

H 1.8146630 .1008331 .061311

O $4.409781-0.418518 \quad 1.706945$

H $3.734804-0.355258-0.213929$

H 5.2687401 .9641891 .067082

O $6.023356 \quad 0.781790-0.403167$

H $4.1603181 .837960-1.783622$

O $5.1706273 .448904-1.034960$

C $2.7651465 .101493-1.594918$

H $2.2140693 .198131-2.379192$

H $1.1337703 .824419-1.163481$

H $5.193995-0.8923971 .383750$

H $\quad \begin{array}{lll}6.470698 & 0.258001 & 0.282628\end{array}$

H $6.0200823 .074867-1.325603$

O $2.5331595 .853914-0.481857$

O $3.3355705 .473555-2.612316$

C $3.0299457 .194759-0.532136$

H $4.1164097 .191870-0.662826$

H $2.5441587 .748142-1.341489$

H 2.7922967 .6810710 .417774

SCF Energy $\left(B 3 L Y P / 6-31 G^{* *} / / M M F F\right)=-3245.93391053$

01403

MM̄FF Geometry

C $-3.442517-1.084638-2.589953$

C $-3.074176-2.060899-3.434511$

C $-1.731499-2.755817-3.482132$

O $-0.747216-2.056446-2.687621$

C $-1.871574-4.183663-2.924388$

C $-0.657217-5.050512-3.193023$

C $0.279094-5.379714-2.281593$

C $-0.557073-5.591031-4.597687$

C $0.370429-4.959036-0.840203$

C $1.623924-4.115406-0.607904$

C $1.896108-3.7697370 .863214$

C $0.762990-2.9785071 .520573$

C $1.130876-2.5237232 .942235$

O $3.097498-2.9889820 .862919$

O $0.167618-1.5450933 .357558$

C $2.502305-1.8196172 .903627$

C $1.037831-3.6745293 .951650$

O $2.946827-1.4978434 .219485$

C $3.587671-2.6302972 .161157$

C $4.848132-1.7592411 .949541$

O $3.931901-3.7802202 .933960$

C $-0.042920-1.063540-3.298479$

O $-0.123195-0.713944-4.465185$

C $0.827960-0.439221-2.277455$

C $1.468073 \quad 0.705287-2.559313$

C $2.2958891 .476039-1.628404$

C $2.7046210 .806532-0.343988$

C $5.950124-2.4572321 .141768$

C $6.874151-1.4539080 .484030$

C $7.0217090 .084610-1.472939$

O $7.980490-1.1912160 .949289$

C $6.6561181 .460959-1.015638$

C $6.2094572 .422428-1.838296$

C $5.9233403 .825636-1.388357$

C $4.5682144 .326751-1.828612$

C $4.4559655 .301426-2.747460$

C $3.3472713 .771664-1.131829$

C $2.6202662 .743770-1.957825$

N $6.325285-0.893547-0.660114$

H -2.745571 -0.719606 -1.840475

H -3.800498 -2.413442 -4.165028

H -1.403222 -2.793549 -4.527972

H -2.743911 -4.679824 -3.371595

H $-2.085131-4.137045-1.851019$
H $1.092012-6.031171-2.601981$

H $\quad 0.300919-6.259482-4.726937$

H $-1.457021-6.161366-4.849909$

H $-0.447954-4.775496-5.318833$

H $-0.514001-4.402072-0.525297$

H $0.412204-5.862720-0.220766$

H $2.504888-4.642480-1.000760$

H $1.565466-3.191442-1.195430$

H $2.079420-4.7046561 .405614$

H $-0.165739-3.5598621 .547134$

H $0.542656-2.0870240 .917371$

H $-0.713688-1.9542563 .314698$

H $2.365184-0.8627342 .381196$

H $0.016840-4.0739633 .983274$

H $1.254394-3.3271914 .967915$

H $1.707926-4.5047473 .719834$

H $2.241557-0.9808264 .645206$

H $4.557114-0.8339081 .438287$

H $5.273291-1.4670332 .918004$

H $4.808560-4.0940162 .661759$

H $0.879796-0.909265-1.302822$

H $1.3340211 .149392-3.544786$

H $3.148022-0.174293-0.548236$

H 1.8383040 .6667530 .311146

H $3.454319 \quad 1.366894 \quad 0.218517$

H $6.551920-3.1047951 .789796$

H $5.536414-3.0990350 .355404$

H $6.737929-0.095272-2.514593$

H $8.101931-0.062654-1.372826$

H 6.8056791 .6901110 .037079

H $6.0926282 .205947-2.897609$

H $6.7137374 .469229-1.796333$

H $5.9984203 .916974-0.297446$

H $3.4859725 .689940-3.040428$

H $5.3287315 .730093-3.228637$

H $2.6435274 .593869-0.941871$

H $3.6194223 .407337-0.137593$

H $2.258803 \quad 3.132762-2.911108$

H $5.375295-1.136989-0.925269$

C $-5.625880-0.848875-1.381697$

O $-5.048869-0.272673-0.194103$

C -6.1098320 .1921190 .663272$

C $-7.406643-0.3337450 .059932$

C -7.067625 -0.344768 -1.418250

H $-6.111758 \quad 1.2862390 .594191$

C $-5.845713-0.2392802 .111158$

H -7.635410 -1.348780 0.404613

H $-8.263506 \quad 0.3028610 .300332$

C -8.005885 -1.204043 -2.247932

H $-7.0953600 .687061-1.793806$

C -4.4549130 .1652782 .642131$

O $-5.936777-1.6675622 .201005$

H -6.6307410 .1541622 .766682$

H -3.683749 -0.4004292 .107490$

O $-4.368089-0.2324044 .015582$

C -4.1594581 .6726452 .558971$

C -2.800832 2.088409 3.153262

H -4.1983602 .0007041 .516147$

$\begin{array}{llll}\text { O } & -5.181688 & 2.397080 & 3.247652\end{array}$

H -2.737236 3.1823053 .100765

H -2.7775641 .8585994 .225873$

H -7.719653 -1.188809 -3.304025

H -7.998212 -2.246393 -1.911910

H $-9.033724-0.834171-2.171991$

C $-4.804333-0.444152-2.606026$

H $-5.293792-2.0429031 .575038$

H -4.567826 -1.183560 4.055001

H -5.1858072 .0915554 .171219$

H $-4.6618340 .643306-2.623886$

H $-5.338558-0.717081-3.523386$

H $-5.605437-1.939162-1.250857$

C -1.508162 2.8891740 .516529

O -1.6648631 .5589651 .026762$

C -1.5893951 .4459532 .449487$

C -0.2248141 .9455852 .940230$

C 0.0730793 .3580282 .443469

C -0.1491973 .4687090 .933753$

H $-2.316648 \quad 3.5279480 .892118$ 
C $-1.6666012 .774865-1.003366$ H $-1.631805 \quad 0.3742182 .671942$ O $-0.155452 \quad 1.920874 \quad 4.361524$ H 0.5601901 .2748672 .567895 H -0.5310754 .0979522 .980393$ $\begin{array}{lllll}\text { O } & 1.434509 & 3.685449 & 2.737564\end{array}$ H 0.6548082 .9413730 .411273 O -0.0710264 .8415640 .559879$ C -1.771272 4.110051-1.694067 H - $0.8435832 .190208-1.427702$ H $-2.5729072 .207752-1.251797$ H $-0.326713 \quad 1.0068704 .646170$ H 2.0041093 .0458502 .277098 H 0.7937865 .1749910 .854630 O $-0.7046194 .286020-2.525310$ O $-2.6824264 .906033-1.507966$ C -0.662546 $5.536016-3.219623$ H $-0.623696 \quad 6.364903-2.506384$ H $\quad 0.2451015 .555599-3.828801$ H -1.530689 $5.632053-3.878364$ SCF Energy (B3LYP/6-31G**//MMFF) $=-3245.91793801$

\section{1_404}

MMFF Geometry

C -2.660980 $1.707876-2.706509$

C $-2.640606 \quad 3.030569-2.483597$

C -1.983349 $3.653186-1.281355$

O $-0.6424494 .019954-1.684028$

C $-2.7190334 .912956-0.786802$

C $-3.9956404 .634641-0.020162$

C $-4.065096 \quad 4.5513641 .323569$

C $-5.2501484 .523699-0.846504$

C -2.9232614 .6267172 .299864$

C $-3.0198293 .584783 \quad 3.425114$

C -3.024056 2.126642 2.940401

C $-3.175798 \quad 1.1736584 .129253$

C $-3.165450-0.2953293 .685444$

O $-1.791147 \quad 1.8596472 .262070$

O $-2.977027-1.0864284 .871284$

C -1.943845 -0.5321932 .768343$

C $-4.526409-0.717543 \quad 3.119287$

O $-1.978146-1.8341342 .193529$

C -1.7272930 .5489921 .681516$

C $-0.3555850 .406150 \quad 0.981086$

$\begin{array}{lllll}\text { O } & -2.670546 & 0.420874 & 0.612247\end{array}$

C $0.3091304 .023250-0.710988$

O 0.1414013 .8205040 .481116

C $1.6161534 .296231-1.345918$

C $2.7244644 .213300-0.594837$

C $4.0992274 .437821-1.039742$

C $4.3242264 .770038-2.490893$

C $0.8752680 .632224 \quad 1.863568$

C 2.1654420 .4501281 .076945

C $4.535071-0.262661 \quad 1.399148$

O $2.2260430 .559132-0.144906$

$\begin{array}{llll}\text { C } & 5.512721 & 0.866589 & 1.471368\end{array}$

C 6.3404431 .1788360 .461706

C 7.4016102 .2407820 .535906

C $7.310963 \quad 3.240633-0.595215$

C $7.9401543 .021347-1.762190$

C $6.5649124 .530162-0.345345$

C $5.092534 \quad 4.321669-0.134098$ N 3.2413700 .1588251 .903021 H -2.137394 $1.047870-2.019935$ H -3.128487 $3.695346-3.192304$ H $-1.9298352 .913787-0.473297$ H -2.935639 $5.580605-1.632324$ H -2.040087 $5.511636-0.166575$ H -5.0443574 .4075181 .778227$ H -6.150484 $4.454408-0.226426$ H $-5.3639165 .403702-1.487954$ H -5.220911 3.631421 -1.477952 H $-1.957273 \quad 4.517199 \quad 1.802534$ H -2.933134 5.6239982 .756027 H -2.162119 3.7413014 .092198 H -3.924930 3.7764874 .015307 H -3.8574501 .9903332 .242550$ H -4.0901491 .3947564 .694182$
H -2.3439041 .3323454 .830132$

H $-2.931465-2.0188994 .598213$

H $-1.074851-0.5289453 .436544$

H -5.291122 -0.6779153 .904953$

H -4.510331 -1.756589 2.773156

H $-4.873237-0.0831232 .301744$

H -2.595253 -1.820360 1.443199

H $-0.290375-0.5800200 .502965$

H $-0.316977 \quad 1.1208590 .148305$

H $-3.474649-0.0070030 .935752$

H $1.6319574 .544485-2.400578$

H 2.6194353 .9517870 .457894

H $3.8492335 .724009-2.744749$

H $3.9071713 .987391-3.134559$

H $5.3803174 .855563-2.757187$

H $0.875316 \quad 1.6461132 .277682$

H $0.878349-0.0798542 .694619$

H $4.424495-0.6487680 .380893$

H $4.873702-1.0808172 .042992$

H 5.5796371 .4162812 .406977

H $6.2908060 .600231-0.458346$

H $8.378198 \quad 1.7396250 .501851$

H 7.3765312 .7661821 .498691

H $7.9051253 .745261-2.569487$

H $8.5012992 .109173-1.936824$

H $6.7437775 .252876-1.149514$

H 6.9784525 .0108530 .550928

H $4.833964 \quad 4.0625870 .893347$

H 3.1012780 .1365142 .908968

C $-4.137954-0.209933-3.492050$

O $-3.202627-1.258822-3.170791$

C $-3.665965-1.944576-1.993232$

C $-5.131799-1.557310-1.838535$

C $-5.104746-0.113818-2.310079$

H $-3.103112-1.530368-1.149580$

C $-3.379943-3.446450-2.097224$

H $-5.781714-2.154669-2.488141$

H $-5.484413-1.666727-0.808504$

C $-6.469071 \quad 0.441432-2.677425$

H $-4.6833120 .498411-1.503791$

C $-1.925411-3.791445-2.482356$

O $-4.231941-4.025918-3.093262$

H $-3.646306-3.936745-1.153775$

H - $1.751528-3.546498-3.537531$

O $-1.760888-5.209935-2.367638$

C $-0.851742-3.106223-1.619225$

C $0.577325-3.516360-2.025245$

H $-0.942954-2.020568-1.728031$

O $-1.075688-3.412649-0.247670$

H $0.754503-4.559013-1.741894$

H $0.654719-3.451957-3.116610$

H $-6.3852331 .475261-3.027477$

H $-6.942308-0.146873-3.470638$

H $-7.1351600 .430924-1.808505$

C $-3.3733721 .066468-3.864656$

H $-4.065431-3.558826-3.930185$

H $-2.443779-5.626367-2.921163$

H $-1.005367-4.377169-0.144189$

H $-4.0699901 .779185-4.321899$

H $-2.6156850 .813850-4.616656$

H $-4.672904-0.556700-4.386021$

C $2.257895-3.9318170 .547764$

O $1.694254-2.7163670 .040767$

C $1.648428-2.603643-1.384949$

C $3.055457-2.763090-1.976942$

C $3.716263-4.056316-1.517550$

C $3.676250-4.1533440 .004636$

H $1.619571-4.7776650 .267944$

C $2.289258-3.8148172 .080686$

H $1.336979-1.573475-1.598495$

O $3.023326-2.732508-3.399750$

H $3.681948-1.921423-1.655053$

H $3.252624-4.934558-1.981881$

O $5.085437-4.057049-1.928092$

H $4.365546-3.4159570 .435747$

O $4.131760-5.4458260 .401467$

C $\quad 0.932513-4.0299372 .716732$

H $2.956108-4.5633272 .522461$ 
H $2.645497-2.8161192 .359986$

H $2.615053-1.890197-3.663900$

H $5.096618-3.949156-2.894725$

H $5.020674-5.567878 \quad 0.025861$

O $0.912278-3.4150793 .935914$

O $\quad 0.030517-4.700762 \quad 2.234937$

C $-0.270459-3.5932354 .723326$

H $-1.172346-3.6330854 .106450$

H $-0.174336-4.5105805 .310490$

H $-0.353922-2.7434175 .405916$

SCF Energy (B3LYP/6-31G**//MMFF) $=-3245.92386431$

$01 \_405$

MMFF Geometry

C $5.5089661 .693784-1.138889$

C $4.2699442 .171177-0.951987$

C $3.3670702 .609942-2.076513$

O $2.1133471 .900838-1.958399$

C $3.0754354 .117173-1.940498$

C $2.2089644 .679066-3.052530$

C $0.9608435 .161809-2.886389$

C $2.8434974 .701916-4.419791$

C $0.1675675 .257274-1.610996$

C $-0.7942334 .077810-1.485145$

C $-1.6588964 .087435-0.216882$

C -0.8284273 .9510461 .061217$

C - -1.7142803 .8143692 .308443$

O $-2.5382332 .962777-0.337460$

O -0.8853593 .3703403 .393852$

C -2.7628582 .7089242 .065512$

C -2.2940845 .1679672 .735410$

O -3.7042522 .6521803 .133640$

C -3.5032942 .8298690 .714301$

C -4.3109911 .5439330 .433488$

O -4.4412953 .9065890 .710949$

C $2.0256480 .659462-2.509164$

O $2.9016790 .045325-3.095096$

C $0.6559670 .165509-2.244422$

C $0.332586-1.117525-2.464276$

C $-0.962277-1.731782-2.151524$

C $-2.065838-0.818113-1.687414$

C $-5.0038941 .555415-0.935615$

C $-5.5797990 .197081-1.295347$

C $-6.323745-1.111399-3.286813$

O $-5.737784-0.704485-0.477287$

C $-5.111121-1.804009-3.828329$

C $-4.833860-3.100834-3.623223$

C $-3.607192-3.778202-4.168725$

C $-2.885523-4.614009-3.132810$

C $-2.783711-5.946514-3.275638$

C $-2.291484-3.918136-1.928178$

C -1.097525 -3.069082 -2.273000

N $-5.902727 \quad 0.119921-2.643051$

H $5.8994321 .615042-2.150277$

H 3.8796592 .2601970 .059371

H $3.8207102 .400673-3.052817$

H $4.0197664 .678067-1.929528$

H $2.6118774 .295976-0.964819$

H $0.4337655 .534186-3.764190$

H $2.2302025 .236068-5.153584$

H $3.8148935 .205468-4.379382$

H $2.9915793 .684839-4.794864$

H $0.8267965 .324552-0.742910$

H -0.399555 6.196171-1.626912

H -1.458401 $4.049878-2.360304$

H $-0.2346333 .137125-1.522866$

H -2.254347 $5.007499-0.205187$

H -0.1431864 .7970641 .185618$

H $-0.1923893 .058048 \quad 0.986433$

H $-0.1644454 .013543 \quad 3.504891$

H -2.2266931 .7502932 .071468$

H -1.488454 5.8626613 .002490

H -2.912988 5.0687873 .633755

H -2.888955 5.6441531 .952930

H -3.2002602 .5423113 .958301$

H -3.6383960 .6786480 .476711$

H $-5.0754341 .399703 \quad 1.207653$

H -4.4687854 .2997301 .597719$
H $-0.0492070 .869858-1.819714$

H $1.091805-1.787403-2.864696$

H $-2.277231-0.056140-2.446023$

H $-1.786395-0.316107-0.755956$

H -3.007513 -1.332451-1.495003

H $-5.8219262 .284349-0.941757$

H -4.288669 $1.837299-1.716775$

H $-6.994793-0.837332-4.106347$

H -6.869817 - $1.732316-2.569585$

H -4.425653 -1.205685 -4.424708

H $-5.529154-3.710125-3.050522$

H -2.906082 $-3.046894-4.589229$

H -3.925627 -4.409272 -5.008543

H $-2.271797-6.557157-2.539238$

H -3.209077 -6.458659-4.132373

H $-1.950763-4.661622-1.194652$

H -3.075613 -3.362972 -1.408091

H $-0.239006-3.647021-2.617955$

H $-5.6774810 .905940-3.246122$

C $6.644918-0.2201320 .108785$

O $5.429354-0.8616070 .541010$

C $5.323583-2.133956-0.126490$

C $6.672532-2.380095-0.797505$

C $7.096343-0.966525-1.145885$

H $4.562486-2.013984-0.906792$

C $4.892081-3.2276590 .856764$

H $7.395272-2.833297-0.109254$

H $6.586802-3.032115-1.672154$

C $8.577165-0.825467-1.449030$

H $6.519878-0.649763-2.023314$

C $3.605314-2.9197581 .651247$

O $5.939570-3.4465241 .811494$

H $4.780791-4.1746490 .315951$

H $3.813932-2.1616902 .414091$

O $3.256352-4.1102342 .372706$

C $2.404684-2.4752290 .796655$

C $1.096585-2.3412141 .602637$

H $2.642929-1.5370770 .286592$

O $2.205982-3.458567-0.224218$

H $\quad 0.350211-3.0444681 .209137$

H $1.254202-2.6383282 .644809$

H $8.8293730 .214622-1.678714$

H $9.193179-1.143053-0.601145$

H $8.852976-1.439144-2.312695$

C $6.4293111 .289211-0.021618$

H $6.105268-2.6016842 .264189$

H $3.000687-4.7840071 .719399$

H $1.472477-3.153840-0.783961$

H $7.3943621 .784035-0.183289$

H 6.0364881 .6699030 .930069

H $7.370523-0.3848060 .916921$

C -1.321146-1.396570 3.099394

O $-0.931841-0.9811071 .786101$

C $0.485093-0.9218891 .572946$

C 1.0930770 .1017162 .541076

C $0.726465-0.2002293 .994545$

C $-0.782268-0.4118694 .144838$

H $-0.936924-2.4040643 .301231$

C $-2.854993-1.4612363 .123571$

H $0.610763-0.5332120 .558178$

O 2.5071270 .1650782 .416960

H $0.707306 \quad 1.0990772 .295069$

H $1.284348-1.0634934 .375592$

O $1.105556 \quad 0.909767 \quad 4.812530$

H $-1.2887200 .555398 \quad 4.047087$

O $-1.062878-0.8919515 .458164$

C -3.391112 -2.561386 2.229202

H -3.214728 -1.659109 4.138796

H $-3.270750-0.5120652 .767205$

H $2.7088780 .438078 \quad 1.505843$

H 2.0582491 .0542854 .679728

H $-0.620848-1.7517215 .562884$

O $-4.753322-2.4719882 .183646$

O $-2.710220-3.3993401 .653939$

C $-5.406346-3.4610041 .380955$

H $-6.478614-3.2481361 .385039$

H $-5.044564-3.4168740 .349238$

H $-5.243611-4.4563381 .804821$ 
H -9.076140 $2.688045-1.166189$

H -7.991002 $1.749685-2.167925$

H -6.978532 $5.687205-1.456584$

H -8.632232 $5.000094-0.982023$

H $-5.3964644 .182027-2.549559$

H -5.698765 $2.472132-2.597763$

H $-4.7425694 .095632-0.133673$

H -5.019996 -0.582506 1.079813

C $2.4204774 .937840 \quad 0.487086$

O $2.3591613 .846699-0.455811$

C $1.0358463 .801420-1.016988$

C $0.4495735 .188835-0.786052$

C 1.0046115 .5137490 .588308

H $0.4676603 .077007-0.421787$

C $1.0691173 .339307-2.477251$

H $0.8166635 .914461-1.520850$

H $-0.6440195 .189985-0.819362$

C 0.9704046 .9925340 .932070

H 0.4070624 .9701371 .329600

C $1.8557302 .033352-2.722760$

O $1.6831174 .367917-3.263851$

H $0.040593 \quad 3.241286-2.845653$

H $2.9318182 .234780-2.663155$

O $1.5940861 .611875-4.067472$

C $1.4979350 .869655-1.781646$

C $2.102400-0.492032-2.168385$

H $1.8135361 .115617-0.763874$

O $0.078077 \quad 0.706332-1.737575$

H $1.802261-1.209415-1.395062$

H $1.622102-0.855295-3.086154$

H 1.3997197 .1699541 .923321

H 1.5364247 .5870250 .207194

H -0.0600997 .3624520 .937650$

C 3.0554994 .4595031 .800358

H $1.5183024 .161428-4.199048$

H $2.0071812 .258412-4.664073$

H $-0.2268940 .534883-2.645178$

H 3.3090405 .3296592 .417672

H 3.9991723 .9430651 .581911

H 3.0951135 .6763000 .034881

C $4.291767-0.661422-0.027876$

O $4.3199670 .092384-1.242617$

C $3.633338-0.507903-2.343633$

C $4.211101-1.901298-2.637565$

C $4.211496-2.790500-1.397406$

C $4.870842-2.068568-0.223026$

H $3.250263-0.7399580 .307878$

C $5.0242240 .142538 \quad 1.054784$

H $3.8572110 .115789-3.217498$

O $3.478577-2.546647-3.674521$

H $5.245808-1.797784-2.989774$

H $3.197660-3.118040-1.148592$

O $4.952246-3.977267-1.690759$

H $5.949104-2.022029-0.414391$

O $4.692787-2.836624 \quad 0.965071$

C $6.5149020 .249270 \quad 0.831380$

H 4.6326091 .1641981 .111255

H $4.866552-0.3103072 .040656$

H $3.511068-1.970511-4.457192$

H $4.976866-4.511350-0.878174$

H $3.738883-2.888787 \quad 1.147548$

O $6.7799421 .157608-0.149801$

O $7.343273-0.4020701 .454849$

C $8.1638141 .335714-0.466156$

H $8.5958050 .394406-0.819141$

H $8.2367862 .076784-1.266516$

H 8.7072121 .7075350 .407642

SCF Energy (B3LYP/6-31G**//MMFF) $=-3245.90532318$

$01 \_407$

MM̄FF Geometry

C $-1.883470 \quad 4.2107110 .078537$

C $-0.9812315 .096107 \quad 0.530583$

C $0.490044 \quad 4.834668 \quad 0.774510$

O 0.7829473 .4220330 .661503

C $1.3310335 .597279-0.265455$

C 2.7554615 .8454910 .188566 
C $3.8355035 .152330-0.219978$

C $2.9263306 .999767 \quad 1.145242$

C $3.8935643 .978793-1.157715$

C $4.3273282 .725468-0.399901$

C $4.602508 \quad 1.501380-1.285137$

C $3.3794651 .071187-2.096975$

C $3.605175-0.257861-2.829825$

$\begin{array}{lllll}\text { O } & 4.993676 & 0.467836 & -0.376626\end{array}$

O $2.321853-0.697077-3.299757$

C $4.122080-1.299260-1.817791$

C $4.479725-0.072594-4.074448$

O $4.453376-2.521677-2.473525$

C $5.307128-0.797710-0.963054$

C $5.627576-1.7701770 .199947$

O $6.467274-0.707816-1.795279$

C 0.6140252 .6561311 .776725

O $\quad 0.4161253 .0488992 .915442$

C 0.6206301 .2364251 .350921

C $0.302008 \quad 0.2741532 .230853$

C $0.153274-1.1531301 .934959$

C $0.335009-1.5965110 .507754$

C $4.579373-1.757848 \quad 1.316190$

C $4.876959-2.7415782 .431445$

C $3.956213-3.4120594 .657132$

O $5.703480-3.6433502 .336828$

C $2.774983-4.2951704 .410087$

C $1.592907-4.1497885 .027486$

C $0.389188-4.9859714 .703662$

C $-0.731304-4.1751574 .089779$

C $-1.947012-4.1277474 .660399$

C $-0.463340-3.4765932 .770468$

C $-0.188215-2.0024852 .927850$

N $4.060064-2.5053243 .529837$

H $-1.5754943 .201658-0.177797$

H -1.3195106 .1010840 .776388$

H 0.7319605 .1817161 .786225

H $0.8810656 .577482-0.475437$

H $1.3148685 .062714-1.220509$

H 4.8081475 .4514260 .170391

H 3.9746887 .1666751 .414592

H 2.5527347 .9247390 .693713

H 2.3750556 .8183872 .072858

H $2.934328 \quad 3.805727-1.648924$

H $4.6173584 .205537-1.949424$

H $5.2375592 .936410 \quad 0.179274$

H 3.5691302 .4658460 .351533

H $5.446457 \quad 1.728153-1.946937$

H $3.0888291 .845957-2.815945$

H $2.5209180 .952007-1.422633$

H $2.438283-1.577095-3.697693$

H $3.278182-1.535642-1.162131$

H $4.0073150 .631615-4.770069$

H $4.586112-1.011504-4.628749$

H $5.4772130 .307573-3.843855$

H $5.312191-2.398413-2.912843$

H $5.778935-2.785574-0.187232$

H $6.590296-1.4879190 .648614$

H $7.256385-0.735537-1.229459$

H $0.824839 \begin{array}{lll}1.028196 & 0.307411\end{array}$

H $\quad 0.0942700 .5584243 .261682$

H $1.346468-1.3679090 .160402$

H $-0.381016-1.085638-0.142916$

H $\quad 0.182760-2.6696350 .364465$

H $4.513830-0.7547021 .753792$

H $3.593857-2.0223800 .921706$

H $3.836744-2.8038545 .559088$

H $4.866887-4.0109124 .752546$

H $2.891582-5.0682813 .654059$

H $1.470550-3.3719845 .777478$

H $\quad 0.057621-5.4728285 .629340$

H $0.649216-5.7975174 .012080$

H -2.758824 -3.567597 4.207793

H $-2.156988-4.6445225 .590799$ H - $1.338279-3.6076412 .120657$ H $0.376614-3.9688922 .266056$ H $-0.322379-1.5974243 .930858$

H $3.362009-1.7692533 .466688$

C $-4.248592 \quad 3.6726610 .815372$
O $-4.222338 \quad 2.287503 \quad 0.415355$

C $-4.295117 \quad 1.4704091 .600188$

C -4.5933622 .4165212 .762518$

C $-3.911613 \quad 3.6891332 .306253$

H $-3.297843 \quad 1.0384261 .743330$

C $-5.3360830 .358323 \quad 1.435916$

H -5.6694372 .5860912 .885730$

H -4.2050982 .0370803 .712774$

C $-4.384716 \quad 4.935180 \quad 3.033004$

H -2.8339393 .5677382 .470117$

C $-5.115662-0.592413 \quad 0.242582$

$\begin{array}{lllll}\text { O } & -6.633543 & 0.949321 & 1.272993\end{array}$

H -5.391116 -0.2244172 .363114$

H $-5.335830-0.074398-0.698195$

O $-6.086724-1.6425740 .360909$

C $-3.717838-1.233498 \quad 0.162638$

C $-3.613557-2.453520-0.773623$

H $-2.989843-0.475951-0.143721$

O $-3.322869-1.6739721 .465942$

H $-2.578360-2.813485-0.731643$

H $-4.207577-3.273432-0.350077$

H $-3.867001 \quad 5.8241202 .658671$

H -5.460848 5.0916202 .903736

H $-4.183664 \quad 4.8540354 .106223$

C -3.345065 $4.515459-0.089909$

H $-6.594437 \quad 1.530666 \quad 0.494113$

H $-6.964199-1.2236950 .387789$

H -3.980856 -2.322060 1.770879

H -3.5376835 .5783600 .098379$

H -3.603354 $4.321601-1.138087$

H -5.2837504 .0063000 .662415$

C -1.904336 -1.526678 -3.152560

O $-3.262865-1.176132-2.862976$

C $-4.038752-2.199154-2.233433$

C $-4.052853-3.461030-3.106801$

C $-2.641753-3.939980-3.428521$

C $-1.821355-2.794606-4.016895$

H -1.357869 -1.677902 -2.215210

C $-1.272934-0.340914-3.900153$

H $-5.070355-1.830011-2.210478$

O $-4.787349-4.496689-2.462494$

H $-4.573909-3.236670-4.046890$

H $-2.158491-4.371545-2.544166$

O $-2.740899-4.992764-4.390438$

H -2.174585 - $2.579063-5.033720$

O $-0.455205-3.195468-4.113197$

C $-0.998466 \quad 0.845350-3.002711$

H $-0.314684-0.641518-4.337778$

H $-1.942536 \quad 0.000734-4.698277$

H $-4.750879-5.280070-3.037764$

H $-1.850282-5.361622-4.515062$

H $-0.405403-3.920231-4.758819$

O $-0.2645251 .760172-3.704352$

O $-1.3852110 .967564-1.849455$

C $0.0794752 .952507-2.994795$

H $-0.8137523 .564423-2.842389$

H $0.551202 \quad 2.708842-2.039072$

H $0.7935183 .516183-3.601011$

SCF Energy (B3LYP/6-31G**//MMFF) $=-3245.93010200$

$01 \_408$

MMFF Geometry

C $2.582487-2.805525-1.487647$

C $2.078408-2.916108-2.726344$

C $0.870366-2.181218-3.257836$

O $0.044908-1.640260-2.200318$

C $1.214497-1.048816-4.246584$

C $2.0580460 .089794-3.704188$

C $1.5629311 .238069-3.201843$

C $3.550070-0.061919-3.863922$

C $0.122497 \quad 1.638526-3.039976$

C $-0.1319232 .255738-1.657752$

C $-1.5013592 .946014-1.584870$

C $-1.665193 \quad 3.682292-0.254528$

C $-3.0441264 .345674-0.137178$

O $-2.528336 \quad 1.958347-1.723833$

O $-3.233012 \quad 4.730416 \quad 1.233052$

C $-4.1269643 .300019-0.467882$ 
C $-3.1348875 .632256-0.965951$

O $-5.417733 \quad 3.903732-0.524528$

C $-3.8546562 .505825-1.769543$

C $-4.8652351 .341776-1.874684$

O $-4.0276573 .370408-2.894091$

C $-0.809774-2.504005-1.584645$

O $-0.951155-3.692850-1.825960$

C $-1.543192-1.756179-0.540048$

C $-2.271723-2.4407620 .354197$

C $-3.074707-1.8797131 .442059$

C $-3.068907-0.3856771 .620789$

C $-4.5686450 .326351-2.984618$

C $-5.592861-0.790011-2.924782$

C $-6.244617-2.749202-1.518798$

O $-6.575249-0.804743-3.662284$

C $-6.701397-2.428010-0.128873$

C $-6.644486-3.2951970 .892940$

C $-7.046192-2.9503522 .299505$

C $-5.966147-3.270046 \quad 3.313278$

C $-6.129558-4.2636684 .203266$

C $-4.724143-2.3982563 .341948$

C $-3.771141-2.7294632 .225848$

N -5.313997-1.714035 -1.930369

H $2.128689-2.114765-0.783460$

H $2.561364-3.594640-3.426565$

H $0.266280-2.907953-3.818263$

H $\quad 0.275015-0.639997-4.639683$

H $1.723453-1.476524-5.121073$

H $2.2757822 .006029-2.901907$

H $4.0978430 .826534-3.531183$

H $3.925520-0.906168-3.284912$

H $3.803211-0.224689-4.916856$

H -0.100863 $2.381632-3.815279$

H $-0.5616450 .799550-3.194281$

H $-0.0658661 .466370-0.898119$

H $0.6493892 .992914-1.435689$

H $-1.5681053 .655615-2.418131$

H -0.872238 $4.427931-0.119557$

H -1.5531602 .9682910 .572596$

H -2.508018 5.3305401 .478380

H $-4.156128 \quad 2.5888300 .369472$

H -2.373835 $6.351542-0.640436$

H -4.099433 6.130219-0.817548

H -2.994396 $5.464582-2.035714$

H $-5.554406 \quad 4.3750550 .315189$

H $-4.8895830 .802086-0.920851$

H $-5.8753961 .742001-2.033490$

H -4.233099 2.834259-3.676032

H $-1.462918-0.675774-0.534025$

H -2.294652 -3.5274810 .278398$

H -3.493718 $0.107547 \quad 0.742895$

H -2.046758 -0.0194981 .765031$

H -3.637763 -0.0486932 .489999$

H $-4.6213360 .785005-3.977204$

H -3.565826 - $0.102385-2.878237$

H $-5.712386-3.704053-1.568606$

H -7.101209 -2.782173 -2.198342

H -7.080883 -1.423954 0.048296

H $-6.283621-4.305490 \quad 0.713565$

H -7.961423 -3.510313 2.530375

H -7.311321 -1.889416 2.388771

H $-5.371320-4.4869944 .946519$

H $-7.022625-4.8796484 .206331$

H $-4.188400-2.5395014 .289332$

H $-5.045052-1.3525493 .323227$

H -3.660925 -3.8000192 .045024$

H $-4.466453-1.603175-1.381897$

C $5.074877-2.819035-1.141851$

O $5.016437-1.571924-0.427100$

C $6.318167-1.2886960 .122227$

C $7.248657-2.392566-0.373618$

C $6.287124-3.548888-0.569704$

H $6.221322-1.372878 \quad 1.210790$

C $6.7538370 .133948-0.255265$

H $7.725566-2.129182-1.324619$

H $8.044643-2.6132240 .344315$

C $6.834404-4.647063-1.464321$

H $6.042191-3.9709910 .414105$
C $5.725474 \quad 1.2286980 .098502$

$\begin{array}{llll}\text { O } & 6.974637 & 0.200140 & -1.670820\end{array}$

H 7.7207880 .3570890 .209719

H $4.847247 \quad 1.114088-0.546756$

O $6.2915242 .500337-0.241260$

C $5.286923 \quad 1.248346 \quad 1.573877$

C 4.3614782 .4220281 .948571

H 4.7777590 .3096701 .813358

O $\quad 6.4380411 .3038112 .419812$

H 4.1411962 .3435903 .020667

H 4.9066393 .3687141 .844298

H $6.107399-5.457219-1.578519$

H $7.076056-4.268067-2.463035$

H $7.747914-5.072244-1.035923$

C $3.763197-3.586836-0.983181$

H $6.143402-0.049796-2.109826$

H 7.1261072 .5968720 .247352

H 6.8717502 .1623972 .280129

H $3.581190-3.8093860 .075683$

H $3.826296-4.549905-1.503170$

H $5.241878-2.576569-2.199436$

C $1.840752 \quad 0.7374242 .282326$

O 2.4171891 .2004771 .053762

C 3.0530542 .4793131 .136659

C 2.0464623 .5403531 .604013

C 1.3019723 .1270872 .872885

C $\quad 0.7453041 .7090312 .739886$

H 2.6209920 .6640733 .048955

C $1.308584-0.6734162 .005332$

H 3.3336852 .7468200 .110454

$\begin{array}{lllll} & & 2.678323 & 4.802516 & 1.790075\end{array}$

H 1.3049873 .6854690 .810330

H 1.9416533 .2107843 .759393

$\begin{array}{lllll}O & 0.205037 & 4.019934 & 3.082374\end{array}$

H -0.0825841 .7078952 .020766$

$\begin{array}{lllll} & 0.219010 & 1.297286 & 3.997253\end{array}$

C $1.033104-1.4840673 .250231$

H $0.400316-0.6036201 .398072$

H $2.042168-1.2369821 .417385$

H 3.2182104 .7559292 .597125

H 0.5690404 .9210333 .123214

H $-0.454516 \quad 1.948682 \quad 4.258015$

O $0.068144-2.4103532 .978069$

O $1.615633-1.3480434 .317522$

C $-0.292896-3.2647164 .067519$

H $0.580997-3.8185154 .423738$

H $-0.734859-2.6755914 .876627$

H $-1.036839-3.9801543 .706961$

SCF Energy (B3LYP/6-31G**//MMFF)= -3245.90741159

01409

MMFF Geometry

C $1.888003-3.278432-0.227101$

C $1.061883-3.7392450 .724109$

C $0.151912-2.8606381 .544389$

O $-1.190908-3.304671 \quad 1.214034$

C $0.380565-3.002307 \quad 3.062240$

C $1.479201-2.1133353 .611411$

C $1.266088-0.9824494 .313972$

C $2.887898-2.5974853 .399294$

C $-0.058532-0.3433654 .633317$

C $0.0517001 .167461 \quad 4.895713$

C 0.3957112 .0007723 .648284

C 0.8078403 .4148324 .070812

C 1.0823794 .3203772 .864828

O $\begin{array}{lllll} & -0.764744 & 2.058751 & 2.805289\end{array}$

$\begin{array}{lrrrr}O & 1.167319 & 5.664783 & 3.360251\end{array}$

C $-0.123215 \quad 4.238306 \quad 1.909829$

C 2.4393194 .0118462 .223133

O $\quad 0.1294794 .972917 \quad 0.714016$

C $-0.5705102 .791579 \quad 1.585256$

C $-1.910108 \quad 2.842742 \quad 0.807805$

$\begin{array}{lllllll}\text { O } & 0.420218 & 2.175265 & 0.759198\end{array}$

C -2.209944 -2.4321741 .426215$

O $-2.134802-1.3370371 .956989$

C $-3.447596-3.0253530 .876918$

C $-4.575648-2.299630 \quad 0.890223$

C $-5.879659-2.7103050 .369105$ 
C $-5.964674-4.036740-0.338976$

C -2.6114681 .4888790 .665204$

C $-3.6807841 .517330-0.409891$

C $-5.8455102 .596549-1.009318$

O $-3.5718580 .858890-1.442253$

C $-7.0318451 .781049-0.602398$

C $-7.6466640 .911164-1.418647$

C $-8.892150 \quad 0.153419-1.054787$

C -8.718558 -1.345753-1.146935

C $-8.963009-2.000692-2.294466$

C $-8.356074-2.0950880 .114106$

C $-6.928306-1.8763970 .531454$

N $-4.7451302 .346550-0.098515$

H $1.943817-2.207262-0.403919$

H $0.992044-4.8117640 .890542$

H $\quad 0.256307-1.8115961 .237082$

H $0.599836-4.0482313 .317826$

H $-0.555331-2.8004773 .596932$

H $2.137137-0.4601324 .707990$

H $3.612509-2.0549944 .016175$

H $2.976555-3.6583173 .654527$

H $3.178300-2.4573582 .356717$

H $-0.782104-0.5135433 .834579$

H -0.449790 -0.815684 5.542482

H $-0.912003 \quad 1.5071565 .297024$

H $\quad 0.8034131 .3354595 .677652$

H 1.2134251 .5175243 .102449

H 1.6840333 .3847124 .730863

H 0.0007883 .8645424 .666905

H $\quad 1.2631296 .2558172 .593545$

H $\quad-0.956238 \quad 4.7540562 .407038$

H 3.2459574 .1456282 .953866

H 2.6607364 .7057551 .404852

H 2.5052932 .9933131 .838318

H $\quad 0.741811 \quad 4.448973 \quad 0.170944$

H -2.6025303 .5181551 .326549$

H -1.736905 $3.264565-0.190809$

H $-0.002223 \quad 1.5025830 .202299$

H -3.393476 -4.025783 0.463862

H -4.546675 -1.3012841 .325762$

H $-5.767188-4.8577940 .358713$

H -5.234774 -4.085463 -1.155118

H $-6.940268-4.225039-0.792955$

H -1.8985490 .7078050 .390533$

H -3.073792 $1.191617 \quad 1.613185$

H $-5.5303442 .384362-2.036290$

H $-6.0897013 .661169-0.937882$

H $-7.428121 \quad 1.9466650 .396589$

H -7.261176 $0.766573-2.425524$

H -9.689172 $0.473168-1.739141$

H $-9.2460920 .424192-0.052272$

H -8.872082 -3.079750 -2.360606

H -9.254924 -1.470078 -3.194842

H -8.579905 -3.162465 0.010534

H -9.009982 -1.7605490 .929982$

H $-6.764586-0.9304531 .048730$

H $-4.7737532 .797180 \quad 0.811298$

C $4.223670-3.938659-1.034960$

O $4.542943-2.548551-1.247159$

C $5.339905-2.097437-0.138470$

C $5.997062-3.3531790 .414076$

C 4.854791-4.346089 0.299786

H $4.644991-1.7062360 .613814$

C $6.295846-0.981475-0.567245$

H $6.841514-3.674773-0.206337$

H $6.351804-3.2237541 .440840$

C $5.300170-5.7978530 .347649$

H $4.156375-4.1744461 .125750$

C $5.6135350 .198945-1.293514$

O $7.290188-1.515594-1.449158$

H $6.841822-0.6168930 .310618$

H $5.363874-0.104711-2.316266$

O $6.5800831 .248754-1.422366$

C $4.3572470 .749805-0.596455$

C $3.7880522 .040601-1.210331$

H $3.577344-0.018811-0.598537$

O 4.6738051 .0197290 .771903

H $2.8709042 .285757-0.661651$
H $4.4673872 .876887-0.999347$

H $4.443226-6.4700790 .237532$

H $6.013522-6.026345-0.451341$

H $5.784778-6.0198681 .304033$

C $2.709579-4.154476-1.135227$

H $6.828063-1.909824-2.208874$

H $6.7834631 .571367-0.527655$

H $3.843441 \quad 1.2448501 .224444$

H $2.478968-5.213611-0.969812$

H $2.395218-3.920101-2.160503$

H $4.702087-4.485904-1.857107$

C $1.4603690 .679251-2.667449$

O $2.8201000 .778886-3.107389$

C $3.4974361 .976528-2.722241$

C $2.7515653 .201776-3.269028$

C $1.2781583 .194840-2.869232$

C $0.6305211 .852503-3.207010$

H $1.4383000 .674695-1.571881$

C $0.943582-0.676450-3.172949$

H $4.4717901 .948369-3.225701$

O $3.3524024 .409665-2.813829$

H $2.8117703 .204530-4.365172$

H $1.1493013 .432779-1.808295$

O $\quad 0.5898394 .217742-3.592865$

H $0.5216321 .769167-4.295911$

O $-0.6771671 .813518-2.643170$

C $-0.272172-1.184989-2.433520$

H $0.718382-0.606533-4.244101$

H $1.725930-1.437558-3.063154$

H $4.2836044 .396310-3.094248$

H $1.0339445 .060199-3.394921$

H -1.166391 $2.580917-2.986541$

O $-0.939037-2.073898-3.226555$

O $-0.577088-0.876292-1.290185$

C $-2.099833-2.672442-2.641941$

H $-2.788414-1.905410-2.275102$

H $-2.607591-3.255684-3.414661$

$\mathrm{H}-1.801103-3.345646-1.833536$

SCF Energy $\left(B 3 L Y P / 6-31 G^{* *} / / M M F F\right)=-3245.92445134$

0141

MMFF Geometry

C $1.084349-2.415568-2.383575$

C $0.198625-3.422092-2.418488$

C $-0.756583-3.732674-1.297442$

O $-2.066281-3.334859-1.771347$

C $-0.769695-5.230267-0.940062$

C $0.467254-5.707477-0.210055$

C $0.600652-5.691811 \quad 1.130834$

C $1.551239-6.300072-1.071008$

C $-0.386342-5.1568872 .133644$

C $0.293842-4.4990313 .342660$

C $1.009306-3.1775773 .016012$

C $1.895277-2.7705964 .196169$

C $2.575090-1.4177073 .960610$

O $0.016219-2.1701832 .777839$

O $3.131631-0.9805605 .209884$

C $1.497111-0.3925803 .561497$

C $3.746723-1.5302412 .979666$

O 2.0870740 .8535883 .199083

C $0.550713-0.8811042 .437070$

C -0.6249350 .1201312 .294372$

O $1.272741-0.924178 \quad 1.204417$

C $-3.014001-3.073035-0.832456$

O $-2.933694-3.2728530 .368793$

C $-4.165464-2.441273-1.511188$

C $-5.109759-1.856303-0.759566$

C $-6.293241-1.146507-1.242572$

C $-6.478622-1.019350-2.731295$

C $-1.797440-0.3919611 .448298$

C -2.7696950 .7197541 .096100$

C -4.2207832 .4696362 .113373$

O $-2.9766271 .048650-0.069853$

C -5.6523542 .0664381 .947398$

C -6.4367662 .5385440 .965775$

C -7.9019962 .2317040 .829539$

C $-8.2525551 .620139-0.508651$

C $-8.4797372 .393264-1.584042$ 
C $-8.4239720 .120863-0.579387$

C -7.142695 -0.625919 -0.332908 N $-3.374083 \quad 1.2949082 .200601$ H $1.116266-1.766719-1.513678$ H $\quad 0.146047-4.052378-3.303276$ H $-0.505004-3.130582-0.414132$ H $-0.922270-5.831823-1.847064$ H -1.656334 -5.460492 -0.335963 H $1.507509-6.1173141 .558266$ H $2.392368-6.677578-0.479676$ H $1.156982-7.138613-1.654289$ H $\quad 1.948990-5.553232-1.762238$ H -1.078351-4.443734 1.680874 H $-0.982397-6.0023182 .498246$ H $-0.480052-4.3091684 .097924$ H $1.003923-5.211613 \quad 3.781427$ H $1.617156-3.3052312 .113481$ H $2.646349-3.5431244 .404441$ H $1.281632-2.6979765 .105481$ H $3.764011-1.6564065 .509003$ H $0.891258-0.1968644 .457532$ H $4.476990-2.2652303 .338878$ H $4.291490-0.5837962 .909212$ H $3.441564-1.8335041 .976313$ H $2.647723 \quad 1.1344023 .942697$ H $-1.016951 \quad 0.353765 \quad 3.292383$ H -0.2572391 .0614501 .865299$ H $\quad 0.670890-0.7070370 .475094$ H $-4.186867-2.444383-2.594472$ H -5.002795 -1.8877560 .324516$ H -6.616202 $-2.006021-3.186766$ H $-5.605948-0.539410-3.188562$ H -7.343043 -0.412210 -3.010068 H $-1.438307-0.8233600 .508697$ H -2.346524 -1.1747681 .982938$ H $-3.871752 \quad 3.1021461 .289960$ H -4.1008333 .0242153 .049314$ H -6.0732371 .3969092 .693303$ H -6.0140703 .2308610 .240526$ H -8.4551903 .1720620 .955028$ H -8.2504961 .5800081 .640384$ H -8.759564 $1.964829-2.540676$ H -8.386613 $3.473051-1.530851$ H -8.873035 -0.181380 -1.532041 H $-9.156379-0.1878270 .178026$ H $-6.915365-0.7548760 .726015$ H -3.2163640 .8943023 .120208$ C $3.475752-1.838988-3.032175$ O $3.547197-0.577386-2.338296$ C $4.501112-0.699450-1.267038$

C $5.187876-2.052787-1.447409$

C $4.092337-2.875276-2.094819$ H $3.921944-0.721443-0.337060$ C $5.4683130 .488977-1.256618$ H $6.055371-1.984641-2.114088$ H $5.532614-2.469482-0.495857$ C $4.597812-4.120155-2.802145$ H $3.384426-3.170630-1.310717$

C $4.8063341 .879373-1.156369$ O $6.2322850 .465046-2.470523$ H $6.1896300 .355039-0.441038$ H $4.3214352 .125729-2.107777$ O $5.8677242 .833145-1.000073$ C $3.8011722 .049436-0.003838$ C 3.4358363 .5066450 .332056 H $2.8929691 .479644-0.227368$ O $4.374027 \quad 1.4843581 .179256$ H 2.7023843 .4794501 .147358 H 4.3151913 .9974090 .769149 H $3.774009-4.657537-3.282019$ H $5.332910-3.872626-3.575194$ H $5.076041-4.799659-2.089139$ C $2.042106-2.106272-3.499570$ H $6.8487551 .216689-2.439726$ H $6.2855672 .668248-0.137196$ H 3.6987281 .5250601 .877738 H $2.050875-2.926104-4.227657$ H $1.669705-1.212875-4.015447$
H $\quad 4.104282-1.721107-3.925224$

C $0.5518293 .786543-0.669983$

O $1.7473443 .769841-1.458438$

C $2.8987554 .348246-0.840356$

C $2.6137595 .809766-0.465115$

C 1.3865755 .9237630 .432002

C $0.1997755 .209390-0.207241$

H $\quad 0.674274 \quad 3.122870 \quad 0.194734$

C $-0.5832113 .243434-1.552120$

H $3.6797264 .369348-1.609169$

O $3.730625 \quad 6.3967210 .194442$

H $2.4349266 .393914-1.377230$

H $1.5823355 .540671 \quad 1.440145$

O 1.0444607 .3031320 .588936

H $-0.1582135 .801814-1.059452$

O $-0.868411 \quad 5.1393000 .736820$

C $-0.4479261 .771268-1.869250$

H $-1.5497623 .376376-1.053458$

H -0.592058 $3.783973-2.506775$

H $4.4960226 .316098-0.400053$

H 1.8249307 .7539120 .954734

H -1.0636016 .0484451 .021963$

O $-1.4337581 .432428-2.754028$

O $0.3883051 .015439-1.396796$

C $-1.4975850 .052520-3.121400$

H $-0.671345-0.187550-3.795126$

H -1.475935 -0.583954 -2.232975

H $-2.441395-0.116982-3.646564$

SCF Energy $\left(B 3 L Y P / 6-31 G^{* *} / / M M F F\right)=-3245.92958259$

01410

MM̄FF Geometry

C $-5.4183432 .542815 \quad 1.282338$

C -4.1442112 .8851661 .038873$

C -3.0981213 .0049322 .115341$

O $-2.0152352 .096374 \quad 1.815498$

C -2.5202254 .4323102 .138485$

C -1.4750204 .6367123 .219770$

C -0.1633174 .8591223 .000949$

C -1.9984294 .6081184 .634372$

C 0.5685624 .9714281 .691384

C 1.5416503 .8068601 .492346

C 2.4810623 .9883360 .289458

C $1.7340944 .018046-1.045937$

C 2.699323 4.100991-2.238919

O 3.3923222 .8811320 .308122

O $1.9568873 .806634-3.432333$

C $3.7717322 .999653-2.095325$

C $3.2517235 .520174-2.418916$

O $4.7835453 .134884-3.089452$

C $4.4194262 .939260-0.693477$

C $5.3221631 .701349-0.504659$

O $5.2893744 .056652-0.472178$

C $-2.092351 \quad 0.8407452 .334107$

O -3.0335420 .3453762 .932355$

C -0.7920640 .1815332 .081887$

C $-0.532503-1.0201182 .617808$

C $0.721737-1.7634612 .485070$

C $1.870845-1.0777501 .793702$

C $4.6172030 .352986-0.661581$

C $5.510166-0.790822-0.221365$

C $6.465727-1.8586851 .824503$

O $6.035559-1.549540-1.032545$

C $5.595585-3.0135862 .207615$

C $5.163261-3.2325173 .458744$

C $4.307052-4.3989543 .862397$

C $2.875146-3.9868544 .131320$

C $2.471770-3.7101565 .383251$

C $1.966853-3.9593692 .918852$

C $0.801651-3.0069753 .003440$

N $5.658526-0.8569161 .154381$

H -5.7276672 .3325082 .303061$

H -3.8332283 .0945200 .018032$

H -3.524199 2.7674363 .098032

H -3.328692 5.1580442 .299593

H -2.1073404 .6648391 .151881$

H 0.4789274 .9949903 .871100

H -1.2348384 .8924235 .366493$ 
H $-2.8319575 .309544 \quad 4.745088$ H -2.347287 3.6052024 .898026 H -0.1191015 .0319160 .846035$ H 1.1253065 .9167191 .700838 H 2.1570663 .6758682 .393038 H 0.9849992 .8677551 .384490 H 3.0489714 .9141250 .437953 H $1.0225914 .850471-1.088711$ H $1.1336583 .103571-1.144398$ H $\quad 1.2198104 .438399-3.491841$ H $3.2687082 .046100-2.293162$ H $2.4374836 .224016-2.630079$ H $3.9262405 .576641-3.280286$ H $3.7807775 .889676-1.537989$ H $4.3398853 .142923-3.954930$ H $6.1762541 .749116-1.193506$ H 5.7665841 .7595680 .498676 H $5.3990724 .535019-1.309553$ H $-0.0694920 .715837 \quad 1.476235$ H -1.305953 -1.506850 3.210265 H $2.094367-0.1186772 .274778$ H $1.637454-0.8940990 .740888$ H $2.797282-1.653641 \quad 1.821637$ H $3.6930200 .317444-0.076300$ H $4.3438510 .176266-1.707082$ H $6.919512-1.3796722 .698124$ H $7.268421-2.1981171 .162220$ H $5.318101-3.7060701 .416610$ H $5.440101-2.5352964 .246357$ H $4.750688-4.8532294 .757810$ H $4.323898-5.1881513 .099907$ H $1.448114-3.4372045 .613721$ H $3.163949-3.7574966 .217961$ H $1.573692-4.9719092 .758939$ H $2.553991-3.7451352 .019621$ H $-0.071303-3.4202253 .509551$ H $5.117904-0.2251491 .738035$ C $-6.8301461 .032860-0.209737$ O $\quad-5.709130 \quad 0.437005-0.889143$ C $-5.657597-0.962604-0.548026$ C $-6.960293-1.2719150 .184293$ C -7.2292820 .0470240 .885985$ H $-4.825834-1.0827680 .156277$ C -5.401937-1.802359-1.804824 H -7.774696 -1.504877 -0.511502 H -6.857158 -2.114258 0.875013 C $-8.659742 \quad 0.205623 \quad 1.368323$ H $-6.551273 \quad 0.1182521 .745298$ C $-4.168173-1.361543-2.619905$ O $-6.539777-1.713087-2.672491$ H $-5.314957-2.860193-1.531626$ H -4.353942 -0.386999-3.087585 O $-3.991275-2.295459-3.692418$ C $-2.861605-1.302335-1.810317$ C -1.652060 -0.899120 -2.676711 H -2.965289 -0.566383 -1.006035 O $-2.629890-2.566576-1.197077$ H -1.470903 -1.668098 -3.434342 H $-1.9060570 .026819-3.205236$ H $-8.801504 \quad 1.177827 \quad 1.850891$ H $-9.373557 \quad 0.1320670 .540965$ H -8.908579 -0.5732412 .096510$ C -6.4809942 .4578540 .223395$ H -6.679180 -0.772642 -2.878104 H -4.825367 -2.323782 -4.192052 H -2.556887 -3.228494 -1.905940 H -7.379769 2.9589750 .601776 H -6.156356 $3.022984-0.660009$ H -7.632303 $1.098199-0.957531$ C $0.654149-2.855914-2.071905$ O $0.076470-1.879362-1.197932$ C $-0.375087-0.679864-1.833927$ C $0.783065-0.012263-2.588393$ C $1.473996-0.975033-3.546813$ C $1.854275-2.264569-2.820681$ H $-0.100933-3.202018-2.787557$ C $1.079823-4.051702-1.213871$ H $-0.6559150 .006384-1.025194$
O $\quad 0.3322201 .126606-3.313307$

H $1.5244900 .342918-1.861164$

H $\quad 0.844576-1.176697-4.421727$

O $2.660758-0.348998-4.039040$

H $2.671280-2.053291-2.119303$

O $2.352913-3.204202-3.770529$

C $-0.120069-4.874342-0.809366$

H $1.752268-4.725680-1.756813$

H $1.614067-3.728159-0.313372$

H $-0.0936971 .727994-2.679194$

H $3.113721-0.994143-4.608573$

H $1.634560-3.410229-4.392845$

O $-0.566995-4.4830350 .416046$

O $-0.613206-5.746281-1.513422$

C $-1.709448-5.1868540 .912663$

H $-1.447627-6.2303871 .110969$

H $-2.017050-4.7166701 .850423$

H $-2.539544-5.1253900 .202425$

SCF Energy $\left(B 3 L Y P / 6-31 G^{* *} / / M M F F\right)=-3245.91233987$

01411

MMFF Geometry

C $-1.790315-1.8464022 .155735$

C $-0.866981-2.3348981 .315636$

C $-1.085388-2.449549-0.167694$

O $0.019561-1.748795-0.788198$

C -1.104437-3.900337-0.668462

C $-2.307798-4.691118-0.206579$

C $-3.514213-4.656408-0.805617$

C $-2.072896-5.6036350 .969124$

C $-3.928494-3.844689-2.004206$

C $-5.273846-3.132710-1.790934$

C $-5.175061-1.849793-0.946791$

C $-6.575006-1.393332-0.535292$

C $-6.541812-0.0604800 .216872$

O $-4.557633-0.844864-1.756431$

$\begin{array}{llll}\text { O } & -7.899283 & 0.396919 & 0.314752\end{array}$

C $-5.7510300 .967834-0.619195$

C $-6.043983-0.2396861 .656523$

O -5.5944942 .1669380 .137662$

C $-4.3893360 .445577-1.136229$

C $-3.7929901 .338735-2.256789$

O $-3.4780720 .364299-0.046311$

C $-0.223374-1.114059-1.964354$

O $-1.250304-1.138383-2.622551$

C $0.977287-0.324743-2.301513$

C $0.809206 \quad 0.887393-2.849435$

C $1.8740681 .829878-3.188897$

C $3.2681951 .279253-3.330505$

C $-3.5070892 .810349-1.957563$

C $-2.3157193 .075635-1.052667$

C -1.1568874 .9750220 .084407$

O $-1.4995952 .216907-0.733588$

C $-0.1734605 .587451-0.863807$

C $1.1463305 .351698-0.819908$

C $2.1372515 .979583-1.757586$

C $3.0204494 .964015-2.448781$

C $4.2778474 .747713-2.026289$

C $2.4842804 .268824-3.681261$

C $1.5568703 .131227-3.354119$

N $-2.2533794 .411871-0.681233$

H -2.763668 -1.565240 1.765508

H $\quad 0.106337-2.6204121 .704456$

H $-2.021678-1.949380-0.439668$

H $-0.182274-4.413577-0.365471$

H -1.058986 -3.910045 -1.765278

H $-4.295707-5.303541-0.408486$

H -2.969201 -6.1711421 .241221$

H $-1.284788-6.3267340 .734522$

H $-1.767527-5.0325831 .850379$

H $-3.165279-3.122477-2.305503$

H $-4.045482-4.545242-2.840500$

H $-5.669979-2.871655-2.781375$

H $-5.986742-3.831417-1.335002$

H -4.556536 -2.038236 -0.063206

H $-7.073819-2.1612840 .069459$

H -7.197764 -1.270341 -1.432914

H $\quad-7.884034 \quad 1.2795050 .723353$ 
H $-6.3732571 .230466-1.485163$ H $-6.674524-0.9600222 .191374$ H -6.1169140 .6954302 .222719$ H -5.013211 -0.5939971 .710457$ H -4.868072 2.0255740 .768756 H $-2.8640450 .876676-2.612503$ H $-4.4753921 .298454-3.117443$ H $-2.5739010 .386696-0.401247$ H $1.945956-0.728615-2.034607$ H $-0.2046101 .244515-3.025499$ H $3.2762220 .423801-4.015598$ H $3.6553650 .953009-2.361387$ H $3.9815762 .001939-3.732099$ H -3.269440 $3.299931-2.912013$ H $-4.3937303 .317445-1.569063$ H -0.7026604 .1966130 .705444$ H $-1.578310 \quad 5.7475830 .734490$ H $-0.5656876 .268369-1.615726$ H $1.5411814 .696146-0.048518$ H $2.7541186 .672794-1.170612$ H $1.6377606 .598514-2.513272$ H $4.9325204 .046658-2.532746$ H $4.6795975 .263733-1.160526$ H $3.3055043 .928785-4.322323$ H $1.9426784 .994681-4.301361$ H $0.5173313 .433738-3.225280$ H -2.937693 $5.059282-1.061530$ C $-1.248596-0.1668543 .972946$ $\begin{array}{lllll}\text { O } & 0.047917 & 0.184381 & 3.445201\end{array}$ C -0.0478251 .4704782 .807965$ C $-1.3323062 .100693 \quad 3.337487$ C -2.2317940 .8872743 .457356$ H $-0.157014 \quad 1.2801601 .733336$ C 1.2161142 .2965603 .060354 H -1.187770 2.5589214 .322440 H -1.7300442 .8647872 .663260$ C -3.4363541 .0968144 .357389$ H -2.5883610 .6407032 .452481$ C 2.5435491 .5915032 .715112 O 1.2796222 .6503904 .449597 H 1.1451893 .2459852 .516814 H 2.7471050 .8042663 .450135 O 3.5880402 .5634102 .869859 C 2.6346591 .0038351 .293592 C 4.0600050 .5972820 .866858 H $1.966728 \quad 0.1401541 .207644$ O $2.1713021 .973300 \quad 0.350015$ H $4.0193260 .298687-0.185650$ H 4.7022211 .4866010 .875754 H -4.073615 1.8969423 .966598 H $-4.038106 \quad 0.1843764 .418383$ H -3.135803 1.3724665 .373612 C $-1.567724-1.6255003 .624223$ H 1.2696181 .8242414 .963131 H 3.5301762 .9089463 .777273 H 2.7602642 .7450470 .409011 H -2.466810 -1.948498 4.161578 H $-0.743780-2.2633333 .968566$ H -1.154930 -0.090158 5.063950 C $3.996046-2.4255620 .385137$ O $3.936919-1.7400211 .644635$ C $4.688414-0.5245501 .715873$ C $6.165630-0.7999501 .401997$ C $6.333337-1.4966040 .056054$ C $5.447238-2.739555-0.010117$ H $3.525084-1.804064-0.383887$ C $3.156436-3.7004530 .549938$ H $4.651702-0.2002572 .761944$ O $6.901595 \quad 0.4181791 .426204$ H $6.581158-1.4432602 .188821$ H $6.122053-0.818978-0.779707$ O $7.691110-1.916630-0.096312$ H $5.857637-3.5134940 .651182$ O $5.482796-3.252957-1.340122$ C $2.908666-4.464955-0.727258$ H $3.640688-4.3734531 .268713$ H $2.181552-3.4605720 .988570$ H 7.8448970 .1949381 .355956
H $8.238381-1.117036-0.173301$

H $6.413803-3.431550-1.558011$

O $2.316658-3.664058-1.656456$

O $3.182220-5.650240-0.868362$

C $2.018879-4.286756-2.909643$

H $2.940061-4.631949-3.388518$

H $1.322750-5.118305-2.764022$

H $1.545141-3.542281-3.554945$

SCF Energy (B3LYP/6-31G**//MMFF)= -3245.92322117

\section{2}

MM̄FF Geometry

C $-1.585502-3.6544550 .284321$

C $-0.724238-4.096809-0.643921$

C $0.132355-3.160758-1.455708$

O $1.510206-3.543698-1.241701$

C $-0.202640-3.215145-2.959999$

C $-1.646329-2.914866-3.304965$

C $-2.260954-1.735865-3.083543$

C $-2.402345-4.059701-3.927326$

C $-1.655865-0.485100-2.504161$

C $-2.1751720 .764559-3.225262$

C $-1.6326932 .067753-2.616416$

C $-2.2732323 .266725-3.322308$

C $-1.7122704 .601639-2.816172$

O $-0.2138592 .092397-2.794672$

O $-2.1324775 .633385-3.725125$

C $-0.1689804 .540433-2.846407$

C $-2.3383194 .988722-1.472118$

O $0.4018155 .701999-2.248667$

C $0.4409233 .248640-2.247823$

C $1.9315043 .139533-2.661642$

O $0.4088393 .246953-0.823985$

C $2.446591-2.586802-1.487461$

O $2.251376-1.475247-1.953746$

C $3.761697-3.103739-1.050754$

C $4.837216-2.314072-1.186773$

C $6.196395-2.600833-0.731193$

C $6.455834-3.928878-0.072398$

C $2.6939202 .004986-1.959524$

C $3.3569082 .426991-0.655310$

C $4.6508251 .475073 \quad 1.258491$

O $3.4124953 .592779-0.273429$

C $6.110428 \quad 1.313376 \quad 0.966907$

C 6.9012370 .4367081 .605534

C 8.3671060 .2614751 .316159

C $8.732435-1.1754401 .003198$

C $9.250760-1.9739351 .951640$

C $8.564025-1.669400-0.418332$

C $7.134244-1.642381-0.880018$

N $3.904995 \quad 1.3440380 .019038$

H -1.646300 -2.586454 0.477877

H $-0.652553-5.160567-0.853638$

H $0.006139-2.141212-1.077604$

H $\quad 0.076581-4.205065-3.347446$

H $\quad 0.442689-2.524618-3.517682$

H -3.317528 -1.659049 -3.337599

H -3.450488 -3.810730 -4.123934

H $-1.942898-4.340428-4.880807$

H -2.388822 -4.931604 -3.265368

H $-1.926670-0.428067-1.443780$

H -0.563762 -0.507133 -2.569874

H -1.883199 $0.704672-4.282243$

H $-3.2720610 .778533-3.188302$

H - $1.8610452 .079528-1.544431$

H $-3.3650853 .245876-3.214098$

H -2.079896 $3.193887-4.401918$

H -1.795147 $5.416556-4.610880$

H $\quad 0.1233894 .581847-3.904786$

H -3.414782 $5.164467-1.592148$

H -1.925062 $5.930208-1.095201$

H $-2.2235514 .222977-0.703235$

H $0.3525225 .601410-1.283445$

H $1.9740282 .949813-3.742393$

H $2.4461814 .090632-2.483501$

H $-0.3268933 .790838-0.511902$

H $3.801403-4.085316-0.593695$

H $4.707707-1.338351-1.653666$ 
H $\quad 6.230012-4.749831-0.761877$

H $5.836503-4.0423200 .824328$

H $7.493703-4.0588440 .243375$

H $2.0342641 .151986-1.772557$

H $3.5014061 .666844-2.621109$

H 4.2805610 .7051471 .941832

H 4.4669022 .4573971 .704497

H 6.5372751 .9520200 .197249

H $6.476638-0.1858742 .390019$

H 8.9236820 .6046282 .198119

H 8.6935010 .9061620 .490645

H $9.538287-2.9973421 .734907$

H $9.395502-1.6227642 .967983$

H $8.987780-2.672331-0.546530$

H $9.155588-1.029298-1.085780$

H $6.851651-0.705482-1.361442$

H $3.8221100 .416578-0.385912$

C $-3.992244-4.1557720 .878992$

O $-4.214010-2.7712961 .222541$

C -4.981031-2.155416 0.169658

C $-5.636373-3.307329-0.581386$

C $-4.528854-4.342808-0.541845$

H $-4.265392-1.664146-0.499960$

C $-5.948855-1.1158890 .741736$

H $-6.524030-3.681886-0.058693$

H $-5.928892-3.029226-1.598316$

C $-4.996222-5.755492-0.843212$

H $-3.772221-4.060221-1.280925$

C $-5.261695-0.0387601 .607224$

O $-6.903209-1.7999181 .562851$

H $-6.514687-0.651186-0.074473$

H $-4.855079-0.5029112 .512523$

$\begin{array}{lllll}\text { O } & -6.264278 & 0.889510 & 2.039233\end{array}$

C $-4.1588690 .758753 \quad 0.881027$

C -3.5614811 .9089571 .717552$

H -3.3516930 .0867570 .575238$

O $-4.693991 \quad 1.322698-0.319424$

H -2.932328 2.5172601 .057244

H -4.3632272 .5847022 .038650$

H $-4.161522-6.461211-0.785029$

H -5.766277 -6.083301-0.136954

H -5.418471 -5.813512 -1.851857

C -2.516217 -4.5199131 .083854$

H $-7.631180-1.1814201 .742580$

H -6.837082 0.4269672 .673846

H $-5.4312351 .904617-0.066467$

H -2.360791 -5.5803850 .854817$

H -2.260667 -4.375306 2.140931

H $-4.592312-4.7422461 .586588$

C -0.6087001 .1093921 .867462$

O -1.7166120 .5075492 .547653$

C $-2.749651 \quad 1.4218142 .934409$

C -2.176884 2.5417573 .816385

C $-0.9544493 .210242 \quad 3.192210$

C 0.0657872 .1562032 .761689

H -0.9732051 .5968600 .955659$

C $0.325339-0.0015481 .383119$

H -3.4381060 .8524643 .569473$

O $-3.162420 \quad 3.5321194 .090447$

H -1.8752942 .1160114 .782619$

H -1.233893 3.8538132 .349311

O -0.3408814 .0606184 .163885$

H 0.4942381 .6960713 .660521

$\begin{array}{llll}\text { O } & 1.119929 & 2.8112012 .065084\end{array}$

C $0.934696-0.8083392 .503322$

H $-0.226364-0.6905580 .735280$

H 1.1391910 .3985090 .769422

H -3.917103 3.0849234 .510006

H -1.012751 4.7028604 .450695

H $1.8401972 .169571 \quad 1.947100$

O $0.103845-1.8340012 .844736$

O $2.013979-0.5506823 .019983$

C $0.568366-2.6789583 .901158$

H $0.683032-2.102533 \quad 4.824002$

H $-0.179209-3.4595584 .065113$

H $1.513990-3.1522773 .620021$

SCF Energy (B3LYP/6-31G**//MMFF)= -3245.90650301
01413

MM̄FF Geometry

C $-3.544062-2.7887180 .626813$

C $-2.436726-3.4361971 .019156$

C $-1.273712-2.8391021 .774986$

O $-0.311626-2.470740 \quad 0.758007$

C -1.617450 -1.596462 2.622652

C $-0.663768-1.3649503 .778532$

C $0.278560-0.4038833 .833734$

C $-0.871422-2.2729464 .966393$

C 0.6571900 .5991322 .779070

C 2.1621550 .5391682 .508895

C 2.6928241 .6387001 .578825

C $2.020827 \quad 1.6391730 .204968$

C $2.6826132 .628634-0.765122$

O $4.096417 \quad 1.382433 \quad 1.443482$

O $2.2126812 .329120-2.088150$

C $4.2060272 .389346-0.760229$

C $2.2703614 .076640-0.473666$

O $4.8768433 .371582-1.546800$

C 4.8158092 .3406310 .659108

C 6.2851391 .8650160 .584316

O $4.7678323 .643621 \quad 1.239220$

C $1.001746-2.7540460 .949798$

O $1.518820-3.2508961 .935936$

C $1.708370-2.349678-0.287946$

C $3.048511-2.349990-0.346049$

C $3.854391-1.978961-1.514877$

C $3.115401-1.637479-2.783791$

C 6.9639991 .6976611 .951506

C 8.2526180 .9148151 .804753

C $9.080623-1.4024581 .402017$

O $9.343508 \quad 1.472306 \quad 1.714581$

C $9.084713-1.557926-0.085575$

C $8.640318-2.648930-0.726909$

C $8.570280-2.749073-2.223729$

C $7.147080-2.833855-2.730469$

C $6.726242-3.890045-3.446817$

C $6.226500-1.660576-2.459178$

C $5.199850-1.976041-1.401873$

N $8.037078-0.453878 \quad 1.743828$

H -3.645760 -1.727998 0.839485

H -2.327129 -4.485474 0.751536

H -0.869855 -3.636973 2.409850

H -2.618332 -1.705226 3.062094

H $-1.670426-0.7097711 .981657$

H $0.861056-0.3222634 .751414$

H $-0.202793-2.0300835 .799372$

H -1.898620 -2.186424 5.335721

H $-0.686559-3.3162374 .693584$

H $\quad 0.1110140 .427704 \quad 1.848906$

H $0.387042 \quad 1.598566 \quad 3.138748$

H 2.7140740 .6066993 .457202

H $2.429867-0.4429822 .100627$

H 2.5543772 .6064462 .075205

H $\quad 0.951520 \quad 1.859998 \quad 0.289011$

H $2.0844990 .634955-0.231955$

H $1.2435852 .412785-2.088504$

H $4.3888181 .422533-1.250005$

H $1.1852524 .194375-0.569764$

H $2.7108764 .767104-1.201214$

H 2.5459764 .4079530 .529477

H $4.4709403 .361188-2.430591$

H 6.3162060 .9034470 .055558

H $6.8815182 .573407-0.004582$

H $5.4040973 .688517 \quad 1.969611$

H $1.094717-2.051560-1.130331$

H $3.608370-2.6450810 .540302$

H $2.486564-0.752447-2.634527$

H $2.478441-2.473197-3.095004$

H $3.768113-1.417923-3.629984$

H 7.2060412 .6694882 .394960

H $\quad 6.315727 \quad 1.1738742 .662961$

H $8.854687-2.341143 \quad 1.917317$

H $10.051795-1.0342641 .746936$

H $9.441350-0.708782-0.664431$

H $8.276129-3.498162-0.154043$

H $9.147900-3.630022-2.530537$ 
H $9.057751-1.888224-2.698930$

H $5.712739-3.941066-3.831352$

H $7.383613-4.725069-3.664510$

H $5.779230-1.341436-3.404494$

H $6.810398-0.794719-2.122722$

H $5.632992-2.225343-0.432407$

H $7.080648-0.796937 \quad 1.740710$

C -6.031865 -3.0867620 .397111$

O $-6.229403-1.6596790 .343135$

C $-7.430206-1.378620-0.391924$

C $-8.254971-2.653450-0.302422$

C $-7.169782-3.707748-0.420457$

H $-7.132981-1.214817-1.435319$

C $-8.098102-0.1135730 .154103$

H -8.762283 -2.7442160 .664849$

H $-9.008167-2.720898-1.092842$

C $-7.595513-5.0807360 .070169$

H $-6.875610-3.786922-1.475766$

C $-7.143724 \quad 1.090590 \quad 0.305040$

O $-8.642879-0.3853491 .452453$

H $-8.9525560 .155286-0.477086$

H -6.4499830 .9022951 .131713$

$\begin{array}{llll}\text { O } & -7.930148 & 2.224736 & 0.691449\end{array}$

C $-6.353992 \quad 1.457204-0.965931$

C $-5.5213762 .748426-0.844299$

H $-5.6903890 .632998-1.242110$

O $\quad-7.266673 \quad 1.628532-2.053782$

H -5.123805 $2.986248-1.838236$

H $-6.1886373 .584760-0.602890$

H -6.773108 -5.797478 -0.019717

H $-7.905586-5.0532231 .120198$

H -8.438323 $-5.456058-0.519417$

C -4.650470 -3.422382 -0.170712

H $-7.914364-0.6947632 .017850$

H -8.4195511 .9777631 .494963$

H $-7.8856472 .337631-1.808436$

H -4.577392 -3.035104 -1.194044

H $-4.521461-4.509822-0.218306$

H $-6.095983-3.3781631 .452859$

C $-2.6129211 .842773-1.257284$

O $-3.4507221 .611516-0.116811$

C -4.3768672 .6622910 .185870$

C -3.628608 3.9849100 .408970

C $-2.7214864 .325884-0.767747$

C $-1.813858 \quad 3.143124-1.097000$ H $-3.2301791 .897982-2.162102$

C -1.659915 $0.641189-1.385730$

H -4.8288412 .4012971 .149394$

O

H -3.0100613 .9002411 .312084$

H -3.306776 $4.627585-1.643765$

O $-1.9116465 .455929-0.433424$

H -1.066436 $3.026859-0.303039$

O $-1.113973 \quad 3.416137-2.309653$

C -2.312944 -0.553965 -2.046143

H $-0.7984860 .910309-2.007606$

H $-1.3062640 .323803-0.398544$

H -5.0960904 .8184371 .387399$

H $-1.383322 \quad 5.227123 \quad 0.350211$

H -0.659777 $4.268854-2.198589$

O $-1.343384-1.464814-2.357287$

O $-3.510484-0.680477-2.257430$

C $-1.785190-2.648560-3.028659$

H $-0.901607-3.184596-3.385384$

H -2.326814 -3.291885 -2.330970

H -2.411816 -2.397608 -3.889969

SCF Energy (B3LYP/6-31G**/MMFF) $=-3245.92104290$

01414

MM̄FF Geometry

C -1.980037 -4.106312 1.522086

C $-2.875947-4.448470 \quad 0.584774$

C $-2.759470-4.244720-0.906167$

O $-3.720049-3.231905-1.328569$

C $-1.371695-3.969688-1.535929$

C $-0.393292-5.124744-1.469551$

C $0.831353-5.072578-0.909115$

C $-0.835105-6.394199-2.154942$
C $1.509048-3.924802-0.211794$

C $2.774271-3.470636-0.952024$

C $2.479461-2.646135-2.214608$

C $3.776316-2.366335-2.977098$

C $3.541362-1.461283-4.194811$

O $1.880315-1.411296-1.804423$

O $4.822142-1.003669-4.655245$

C $2.751049-0.217810-3.739560$

C $2.909672-2.228902-5.361912$

O $2.396104 \quad 0.595844-4.856071$

C $1.504133-0.545650-2.882811$

C $0.9368540 .764765-2.284798$

O $0.512667-1.147916-3.716921$

C $-3.530353-1.939229-0.957476$

O $-2.629140-1.488671-0.267798$

C $-4.619295-1.127218-1.536178$

C $-4.3363380 .141874-1.860950$

C $-5.265017 \quad 1.164978-2.335644$

C $-6.7311580 .827901-2.341123$

C $-0.1631080 .559649-1.236603$

C $-0.6621341 .876986-0.664314$

C $-2.1626452 .775170 \quad 1.117187$

O $-0.2875872 .971302-1.076534$

C -3.4837723 .1461590 .519860$

C $-3.7151144 .307569-0.112316$

C $-5.0429194 .709193-0.696749$

C $-5.0041044 .813708-2.208042$

C $-4.5939595 .942009-2.811349$

C $-5.4962043 .629302-3.019668$

C $-4.7558972 .366521-2.679898$

N -1.576193 1.6823710 .362601

H -1.052124 -3.623266 1.238008

H $-3.809238-4.9048980 .910878$

H $-3.144826-5.164894-1.361484$

H $-0.939826-3.058112-1.116056$

H -1.522207 -3.723066 -2.596581

H $1.440852-5.975721-0.946276$

H $-0.020473-7.120189-2.252070$

H -1.638945 -6.877995 -1.592058

H -1.195091 -6.175566 -3.165702

H $0.846285-3.072735-0.044921$

H $1.799927-4.2783590 .785539$

H $3.359591-2.855870-0.259030$

H $3.388712-4.343462-1.205964$

H $1.777025-3.197517-2.849352$

H $4.257828-3.303395-3.283549$

H $4.491997-1.867551-2.308162$

H $5.357332-1.784451-4.878199$

H $3.437300 \quad 0.388817-3.132399$

H $3.546821-3.072113-5.654806$

H $2.826524-1.595907-6.252274$

H $1.921123-2.628739-5.127080$

H $1.6436740 .172480-5.303260$

H $1.7504591 .324022-1.806355$

H $\quad 0.541123 \quad 1.396190-3.090668$

H $-0.357660-1.040372-3.302822$

H -5.597688 -1.577038 -1.647675

H $-3.3113290 .484686-1.736125$

H $-7.0802260 .612780-1.325359$

H $-6.921531-0.048973-2.970112$

H $-7.3595061 .627928-2.738113$

H -1.016675 $0.046940-1.685681$

H $0.212073-0.050905-0.407602$

H -1.467236 3.6200691 .133414

H -2.3031172 .4286402 .145834$

H -4.2925142 .4281220 .631046$

H -2.903394 $5.025060-0.211531$

H $-5.3222555 .679853-0.266173$

H $-5.8390624 .018483-0.391917$

H $-4.5816086 .034397-3.892238$

H $-4.2594126 .798544-2.235081$

H $-5.3660683 .816997-4.092527$

H $-6.5722273 .539248-2.847021$

H $-3.6709602 .486037-2.678993$

H -1.8801190 .7380580 .582051$

C $-2.350381-3.0272273 .795257$

O $-1.154491-2.2282093 .713125$

C - $-1.535951-0.8390323 .647337$ 
C $-3.030353-0.7886923 .952639$ C -3.500576 -2.110942 3.378701 H - $1.381155-0.5214012 .609962$ C $-0.665686 \quad 0.0016624 .588917$ H -3.225478 -0.7516225 .030594$ H -3.5167550 .0774173 .493313$ C $-4.860286-2.5528333 .889143$ H $-3.546823-2.0100062 .288540$ C $0.852054-0.133808 \quad 4.348006$ O $-0.923983-0.3923805 .942989$ H -0.9644191 .0540704 .520866$ H $1.183160-1.1397604 .631773$ O 1.5182030 .7649375 .244825 C $1.303601 \quad 0.1689782 .907698$ C 2.8369380 .1162442 .759340 H $0.851379-0.5612532 .227713$ $\begin{array}{lllll}\text { O } & 0.821359 & 1.458378 & 2.548580\end{array}$ H 3.2847190 .9506263 .308782 H $3.189690-0.8128463 .221006$ H -5.146068 -3.514864 3.452138 H $-4.864638-2.6620604 .978773$ H -5.627874 -1.819128 3.622138 C $-2.189482-4.3234392 .995111$ H $-0.716075-1.3396586 .017711$ H 1.2813621 .6707964 .980883 H 1.0730021 .6230891 .624385 H -3.059284 -4.9678573 .169701$ H - $1.312303-4.8661923 .368745$ H -2.457564 -3.297789 4.854403 C 3.8089212 .5177361 .041456 O 2.9934331 .4067140 .646716 C 3.2913380 .1587551 .282699 C $4.763721-0.2171121 .062308$ C 5.7026420 .9016771 .493540 C 5.2979512 .2129230 .829263 H 3.6261822 .7507452 .096798 C 3.3910863 .7268380 .183904 H $2.693982-0.5966310 .758001$ O $5.109342-1.4043751 .768314$ H $4.929527-0.418730-0.004005$ H $5.733374 \quad 1.0049822 .584731$ $\begin{array}{llll}\text { O } & 7.033656 & 0.584372 & 1.079789\end{array}$ H $5.5247912 .164580-0.243757$ $\begin{array}{lllll}\text { O } & 6.075509 & 3.273255 & 1.383467\end{array}$ C 2.1320414 .4085450 .676428 H 4.1752554 .4923260 .194112 H $3.2146143 .404589-0.849316$ H $4.482994-2.0988041 .504016$ H $7.262553-0.2733541 .477353$ H 7.0119713 .0447161 .253262 O $1.8067995 .409176-0.196608$ O $1.522146 \quad 4.1260361 .697326$ C $0.6579446 .187298 \quad 0.154092$ H $-0.238545 \quad 5.5618730 .149697$ H $0.7987376 .654247 \quad 1.133586$ H $0.5394496 .974419-0.595231$

SCF Energy (B3LYP/6-31G**//MMFF)= -3245.92276159

01415

MM̄FF Geometry

C $4.950525 \quad 1.556783-0.150273$

C $5.7878201 .155324 \quad 0.819024$

C $5.557792-0.0388611 .720971$

O $4.169718-0.4413091 .684435$

C $6.419238-1.220568 \quad 1.238187$

C $6.218511-2.4957102 .036586$

C $5.605231-3.6040621 .571482$

C $6.772993-2.4721083 .437241$

C $5.004220-3.8112010 .207332$

C $3.486095-3.6229470 .239890$

C $2.857177-3.338004-1.132736$

C $3.257184-1.962552-1.678547$

C $2.522908-1.616916-2.981937$

O $1.434298-3.377843-0.943955$

O $2.708714-0.215127-3.233199$

C $1.012776-1.843023-2.778931$

C $3.117693-2.356866-4.185214$

O $0.301703-1.676872-4.003310$
C $0.671985-3.213101-2.146846$

C $-0.832104-3.251823-1.790111$

O $0.944660-4.253794-3.087099$

C 3.2977420 .2142342 .499053

O 3.5598641 .0800663 .317754

C $1.943513-0.2956372 .189848$

C $0.875776 \quad 0.1506632 .866974$

C $-0.509887-0.2682972 .643261$

C $-0.759379-1.3237651 .597083$

C $-1.238989-4.403312-0.864329$

C $-2.720562-4.326553-0.533497$

C $-4.322960-4.6295881 .353571$

O $-3.567558-4.021468-1.370134$

C $-4.572817-3.2708571 .930140$

C -4.505171 -3.002669 3.242920

C $-4.794421-1.6596943 .849678$

C $-3.533159-0.9443194 .284474$

C $-3.010735-1.1692095 .502522$

C $-2.968570 \quad 0.044113 \quad 3.285499$

C -1.488253 0.2945753 .382393

$\begin{array}{llll}\mathrm{N}-2.990340 & -4.667737 & 0.782275\end{array}$

H $4.0457750 .987688-0.345868$

H $6.702661 \quad 1.7166910 .993919$

H 5.8331690 .2305112 .747897

H $7.481901-0.946548 \quad 1.282059$

H $6.210218-1.3952770 .178029$

H $5.509988-4.4600952 .238021$

H $6.674464-3.4403103 .939576$

H $7.838039-2.2192383 .419334$

H $6.248062-1.7304614 .047077$

H $5.478821-3.164118-0.531212$

H $5.233836-4.832183-0.122729$

H $3.023741-4.5246990 .664665$

H $3.208734-2.8186880 .929124$

H $3.149760-4.137578-1.822874$

H $4.338544-1.884965-1.833801$

H $3.007834-1.195214-0.933670$

H $3.664675-0.042468-3.280933$

H $0.653340-1.053451-2.103444$

H $4.174327-2.092457-4.313133$

H $2.620873-2.061584-5.115858$

H $3.059443-3.443370-4.091417$

H $0.522417-0.794703-4.348575$

H $-1.107756-2.318977-1.284238$

H -1.424971 -3.305895 -2.711877

H $0.394154-5.023657-2.872783$

H $1.855586-1.0240521 .392978$

H 1.0252370 .8918363 .650573

H $-0.203579-2.2385151 .832278$

H $-0.443115-0.9651270 .612226$

H $-1.807667-1.6163511 .510404$

H - $-1.058252-5.374661-1.335924$

H $-0.651161-4.3648530 .058949$

H $-4.374100-5.4139482 .115641$

H -5.067501 -4.8504800 .581916$

H $-4.839757-2.479147 \quad 1.234325$

H $-4.236287-3.7996243 .932913$

H $-5.459446-1.8089494 .710404$

H $-5.362312-1.0258603 .156354$

H -2.125469 -0.6533745 .856463$

H -3.467977 -1.8791696 .184576$

H -3.484469 1.0010443 .430010

H $-3.227567-0.2675612 .268030$

H $-1.216201 \quad 1.0421104 .128149$

H -2.218320 -4.844069 1.418149

C $4.0398573 .795223-0.889398$

O $2.7618373 .199814-1.198055$

C $1.7945153 .668000-0.238822$

$\begin{array}{llll}\text { C } 2.437849 & 4.867943 & 0.446339\end{array}$

C $3.890827 \quad 4.4376090 .490190$

H 1.6612052 .8650240 .497956

C $0.448643 \quad 3.975813-0.906970$

H $2.3260925 .779936-0.152251$

H $2.014791 \quad 5.057074 \quad 1.437563$

C 4.8597345 .5777190 .748266

H 4.0034803 .6891921 .284426

C $-0.2713892 .710262-1.427823$

O $0.6698814 .913303-1.958194$ 
H $-0.1960504 .461586-0.165168$ H $-0.3476042 .001149-0.595749$ O $0.4882162 .078966-2.456525$ C -1.687971 $2.993338-1.968912$ C $-2.4580081 .737655-2.419959$ H $-2.2659293 .519522-1.202023$ O $-1.6175693 .873210-3.093849$ H -3.394045 2.070267 -2.886084 H $-1.9059571 .239651-3.227262$ H 5.8928075 .2162550 .754223 H $4.7782976 .355692-0.018277$ H 4.6580306 .0411001 .719554 C $5.1587412 .758059-1.025649$ H -0.198249 $5.173049-2.309857$ H $1.3531391 .839049-2.083260$ H -1.054662 $3.447251-3.763203$ H $6.1279243 .228420-0.824551$ H $5.1829622 .399286-2.062250$ H $4.2016734 .573058-1.647285$ C $-4.6558491 .849451-0.317594$ O $-3.3218491 .360300-0.136873$ C $-2.755506 \quad 0.732506-1.290514$ C $-3.620938-0.462244-1.713485$ C $-5.089099-0.079254-1.888901$ C $-5.5961840 .693079-0.671373$ H -4.669171 2.605200-1.112487 C -5.0893812 .5327120 .982674$ H $-1.789590 \quad 0.322732-0.974839$ O $-3.113932-1.022367-2.919243$ H -3.549501 -1.235093 -0.937529 H $\quad-5.247016 \quad 0.495834-2.808917$ O $-5.876445-1.265810-2.013965$ H -5.6918420 .0091270 .181988$ O $-6.8944391 .210826-0.953616$ C $-4.2795803 .782554 \quad 1.231499$ H -6.1430242 .8325120 .948418$ H -4.9820401 .8643151 .843468$ H -3.578877 -1.861820 -3.073299 H $-5.613044-1.707748-2.838643$ H $-7.4599190 .458281-1.198278$ O $-3.157838 \quad 3.478526 \quad 1.944326$ O C -2.2857704 .5762932 .229471$ H -2.7400705 .2211302 .987084$ H $-1.349375 \quad 4.1722782 .623478$ $\mathrm{H}-2.0653185 .1458701 .321706$ SCF Energy (B3LYP/6-31G**//MMFF) $=-3245.91987473$

01416

MM̄FF Geometry

C $-2.264057-0.535996-2.746520$

C $-2.475229-1.725435-3.332824$

C $-1.628433-2.966223-3.172836$

O $-0.324364-2.587433-2.671081$

C $-2.251407-3.979337-2.195149$

C $-3.643010-4.459244-2.551294$

C $-4.752338-4.107841-1.870052$

C $-3.723496-5.422597-3.704114$

C $-4.810005-3.134503-0.726244$

C $-4.592240-3.8190170 .629180$

C $-4.108660-2.862801 \quad 1.731492$

C -5.101844 -1.735098 2.015443

C $-4.558525-0.7510653 .061504$

O $-2.847027-2.327488 \quad 1.315313$

O

C $-3.141707-0.3124612 .638489$

C $-4.649662-1.3145884 .483934$

O $-2.543103 \quad 0.5028773 .643520$

C $-2.200860-1.4868832 .278741$

C $-0.904703-0.9314351 .630306$

O $-1.879745-2.2046963 .468083$

C $0.722169-3.418488-2.917509$

O $0.699355-4.476234-3.525006$

C $1.917215-2.807255-2.294795$

C $3.059177-3.504538-2.208407$

C $4.307923-3.037246-1.601366$

C $4.312819-1.659989-0.991383$

C $0.067738-1.9832501 .076753$
C $1.025436-2.5615862 .104375$

C $2.735630-4.3599672 .363375$

O $1.127241-2.1270123 .248173$

C $4.105080-3.8225092 .089586$

C $5.136231-4.5900081 .704052$

C $6.526853-4.070521 \quad 1.473375$

C $7.087546-4.470060 \quad 0.126321$

C $7.919579-5.5187630 .007947$

C $6.755735-3.600536-1.065305$

C $5.375742-3.862416-1.603767$

N $1.761484-3.6183211 .584729$

H -1.426320 -0.403802 -2.067240

H -3.326095 -1.827446 -4.003982

H - $1.503237-3.410701-4.168352$

H -2.220410 -3.556939-1.183857

H -1.597664 -4.858609 -2.119226

H $-5.709333-4.531561-2.167684$

H $-4.744811-5.778585-3.876301$

H -3.381015 -4.945046 -4.627231

H -3.096392 -6.299221 -3.511554

H $-4.084621-2.330403-0.884205$

H -5.791605 -2.644900 -0.738795

H $-5.520277-4.3120680 .943915$

H -3.834834 -4.606558 0.519912

H -3.952189 -3.464501 2.634701

H $-6.070509-2.1410862 .331752$

H -5.299703 -1.168277 1.095992

H $-6.300310 \quad 0.147715 \quad 3.225279$

H $-3.2523680 .319872 \quad 1.747695$

H -5.692049-1.538475 4.740520

H -4.311292 -0.579146 5.222154

H -4.072881 -2.231601 4.620521

H -3.1508241 .2423353 .815666$

H -1.199253 -0.3104940 .773862$

H $-0.377527-0.2826232 .339686$

H $-1.111850-2.7695663 .292584$

H $1.818675-1.798151-1.910470$

H $3.083775-4.515389-2.613290$

H $3.518401-1.570474-0.242378$

H $4.156169-0.897331-1.762053$

H $5.242743-1.410836-0.476416$

H $-0.472447-2.797350 \quad 0.582762$

H $\quad 0.694322-1.4952970 .319982$

H $2.640941-5.4134442 .081649$

H $2.506475-4.2616843 .429197$

H $4.261960-2.7573492 .242814$

H $4.983413-5.6599571 .580914$

H $7.165698-4.4674312 .273340$

H $6.569431-2.9790901 .576298$

H $8.349544-5.794356-0.949230$

H $8.184663-6.1286800 .865369$

H $7.462663-3.797389-1.882742$

H $6.923636-2.551933-0.804569$

H $5.269049-4.846408-2.062743$

H $1.651440-3.8660470 .605660$

C $-3.8623901 .158139-1.752649$

O $-2.9198101 .680536-0.791965$

C $-3.1917373 .078248-0.597031$

C $-4.6764813 .223449-0.892468$

C $-4.8239452 .307017-2.089938$

H $-2.6136693 .617976-1.358698$

C -2.7534583 .5585620 .791020$

H $-5.2946282 .855612-0.065541$

H $-4.9635834 .257567-1.104618$

C $-6.2519421 .848296-2.334661$

H $-4.4731732 .843558-2.982006$

C $-1.3503473 .094647 \quad 1.224574$

O -3.6809963 .0729631 .769466$

H -2.8254514 .6522930 .827368$

H -1.3889182 .0342691 .491753$

O -1.0235363 .7849872 .438120$

C -0.2271173 .3119900 .196982$

C 1.1425712 .8928400 .765809

H $-0.4404692 .724542-0.702500$

O $-0.2082244 .685883-0.173525$

H 1.4465623 .6082391 .537339

H 1.0284121 .9159421 .250073

H $-6.301081 \quad 1.176912-3.197909$ 
H $-6.655248 \quad 1.313370-1.468127$

H $-6.9031882 .705631-2.533809$

C -3.118906 $0.673175-3.002537$

H -3.388274 3.4030252 .636255

H -0.9122774 .7258872 .217950$

H $0.4155554 .776675-0.913286$

H $-2.456463 \quad 1.466831-3.371029$

H -3.842753 $0.460667-3.798826$

H $-4.3873200 .327295-1.265927$

C $3.1488745 .028487-0.189419$

O $2.4915534 .029976-0.979605$

C $2.2439542 .788573-0.313529$

C 3.5637502 .1889800 .194935

C 4.3358813 .1781861 .063771

C $4.493101 \quad 4.511746 \quad 0.335444$

H $2.503752 \quad 5.3168150 .649291$

C $3.3533026 .259833-1.080615$

H $1.8584042 .103667-1.079963$

$\begin{array}{lllll}\text { O } & 3.305920 & 0.991831 & 0.920487\end{array}$

H $4.184267 \quad 1.915614-0.668556$

H 3.8565093 .3187162 .039756

O 5.6434242 .6626751 .323538

H $5.2018474 .393271-0.494369$

$\begin{array}{lllll}\text { O } & 5.044536 & 5.472368 & 1.234347\end{array}$

C $2.0467316 .977944-1.319332$

H $4.0332716 .986882-0.622077$

H $3.7896645 .983001-2.048046$

H 4.1617260 .5807561 .127875

H $5.542961 \quad 1.8810541 .892538$

H $5.883368 \quad 5.1098921 .567397$

O $1.4978676 .572952-2.499721$

O $1.5613427 .783891-0.535906$

C $0.2449247 .177425-2.835569$

H $0.3691178 .257013-2.961669$

H $-0.0956236 .749675-3.782333$

H $-0.5016306 .961828-2.065212$

SCF Energy (B3LYP/6-31G*//MMFF) $=-3245.91889643$

01417

MM̄FF Geometry

C $-0.386258-2.350103 \quad 0.727705$

C $0.450408-2.3906331 .774778$

C $0.595287-1.3605362 .874963$

O $1.912752-0.7739702 .731096$

C $-0.427309-0.2077852 .833915$

C $-0.369964 \quad 0.695528 \quad 4.052420$

C $0.230505 \quad 1.902213 \quad 4.073710$

C $-1.098622 \quad 0.186648 \quad 5.267958$

C 1.0243972 .5259672 .962193

C $0.194638 \quad 3.519782 \quad 2.140520$

C $0.9703353 .992903 \quad 0.903315$

C $0.186901 \quad 5.045948 \quad 0.121330$

C $0.9175125 .450058-1.169958$

$\begin{array}{lllll}O & 1.185005 & 2.849146 & 0.073474\end{array}$

O $-0.012546 \quad 6.165224-1.998676$

C $1.325896 \quad 4.178817-1.951027$

C $2.0688586 .420652-0.879650$

O $2.1676624 .502917-3.054598$

C $1.9982393 .092267-1.079110$

C $2.1050111 .759740-1.851909$

O $3.3334793 .451933-0.716439$

C $2.977129-1.4571823 .227198$

O $2.961216-2.475698 \quad 3.898412$

C $4.190067-0.7880112 .714831$

C $5.224754-1.5580652 .348885$

C $6.447573-1.1009211 .691167$

C $6.686074 \quad 0.3823351 .594756$

C $2.6116450 .604121-0.978489$

C $2.785121-0.686645-1.759110$

C $3.670250-3.006560-1.447589$

O $2.498053-0.803253-2.946550$

C $5.159433-3.162780-1.563064$

C $6.042905-2.195476-1.860141$

C $7.530666-2.408500-1.933012$

C $8.297131-1.430632-1.065754$

C $8.798861-0.299298-1.589859$

C $8.543135-1.786013 \quad 0.385866$

C $7.279989-2.0252871 .168698$
N $3.308380-1.690456-0.956789$

H $-1.060736-1.5086940 .599223$

H $1.114638-3.2494221 .858340$

H $\quad 0.522638-1.8751703 .841256$

H -1.447161 -0.6088862 .764319$

H -0.2773580 .3872351 .924950$

H $\quad 0.1784922 .4948884 .985347$

H -1.0561530 .8931046 .103770$

H $-2.1546250 .015672 \quad 5.034188$

H $-0.661081-0.7557645 .611338$

H 1.8855933 .0445173 .402095

H 1.4512651 .7541692 .315270

H $-0.7349413 .031648 \quad 1.820624$

H -0.0796824 .3780582 .765709$

H $1.9370964 .393403 \quad 1.230524$

H -0.0105445 .9271640 .744308$

H $-0.7988544 .645038-0.148751$

H - $0.3451536 .924876-1.490489$

H $0.4076113 .753291-2.378346$

H $1.6866187 .343529-0.426703$

H $2.5716106 .728051-1.803039$

H $2.8150946 .007383-0.197723$

H $1.6974045 .162014-3.593485$

H $1.1219331 .479704-2.247879$

H $2.7800851 .879819-2.708858$

H $3.5582604 .292453-1.145727$

H 4.1560230 .2834802 .558294

H $5.143105-2.6360232 .486741$

H 6.6466350 .8441582 .587703

H $\begin{array}{llll}5.930010 & 0.853671 & 0.957317\end{array}$

H $7.6630550 .633793 \quad 1.174651$

H $3.5805550 .864237-0.536666$

H $1.9041240 .408071-0.164197$

H $3.188417-3.211414-2.408393$

H $3.290250-3.720807-0.709703$

H $5.534930-4.166997-1.376706$

H $5.688091-1.191286-2.078079$

H $7.833583-2.291932-2.981838$

H $7.805979-3.434055-1.657207$

H $9.3696790 .399468-0.987446$

H $8.646468-0.044782-2.633621$

H $9.153822-1.026398 \quad 0.888873$

H $9.147204-2.7021410 .420419$

H $7.022095-3.0799111 .270913$

H $3.526639-1.4783930 .011623$

C $-1.745660-4.201505-0.285182$

O $-2.873199-3.316168-0.387058$

C $-3.911693-3.956658-1.150718$

C $-3.418392-5.369626-1.452864$

C $-1.911449-5.190901-1.435844$

H $-3.991451-3.398876-2.091022$

C $-5.245790-3.874536-0.396974$

H $-3.721728-6.086128-0.681392$

H $-3.794867-5.739839-2.411467$

C $-1.146713-6.490032-1.253412$

H $-1.605740-4.723498-2.381074$

C $-5.567493-2.4718620 .159401$

O $-5.194397-4.7667690 .725108$

H $-6.055469-4.237183-1.040840$

H $-4.873428-2.2552630 .978170$

O $-6.869133-2.5288340 .757957$

C $-5.539044-1.331214-0.873677$

C $-5.9419620 .038563-0.290797$

H $-4.543210-1.255736-1.320769$

O $-6.447875-1.655949-1.928076$

H $-6.0234040 .750797-1.120498$

H $-6.955936-0.0254250 .122666$

H $-0.067234-6.310089-1.245912$

H $-1.412624-6.983617-0.312600$

H $-1.366984-7.184028-2.071260$

C $-0.442628-3.403506-0.344153$

H $-6.048498-4.7018871 .186027$

H $-7.512713-2.6763410 .043543$

H $-6.376394-0.958501-2.602029$

H $-0.355048-2.890607-1.308881$

H $0.414162-4.082792-0.267244$

H $-1.827978-4.713944 \quad 0.682637$

C $-3.3633301 .643699-0.683891$ 
$\begin{array}{llll}\text { O } & -3.615358 & 0.619044 & 0.285819\end{array}$

C $-4.9603950 .568840 \quad 0.774966$

C $-5.355274 \quad 1.9174101 .393743$

C $-5.1056643 .081466 \quad 0.440859$

C $-3.6801313 .026607-0.103728$

H -3.989188 $1.469634-1.567866$

C $-1.8899701 .533593-1.097980$

H $-4.958424-0.1546471 .597973$

O -6.7239281 .9191001 .785433$

H $-4.7614712 .084356 \quad 2.302231$

H $-5.8378653 .097908-0.375048$

$\begin{array}{llll}\text { O } & -5.265568 & 4.312273 & 1.149894\end{array}$

H -2.971597 3.2747400 .696158

O $-3.5352084 .007994-1.128664$

C $-1.7528810 .813704-2.416631$

H -1.437463 2.519713 - 1.244984

H -1.280492 $1.015081-0.349341$

H $-6.851803 \quad 1.1791832 .403593$

H $-6.163264 \quad 4.314871 \quad 1.524434$

H -3.758034 $4.870340-0.737699$

O $-1.907876-0.528739-2.245129$

O $-1.543416 \quad 1.385865-3.479239$

C -1.810995-1.304262 -3.443494

H $-0.807643-1.213745-3.870791$

H $-1.989267-2.351423-3.190240$

H -2.570249 -0.987572 -4.164961

SCF Energy (B3LYP/6-31G*//MMFF) $=-3245.91044493$

01418

MM̄FF Geometry

C $-1.896757-0.5702232 .211650$

C $-0.571316-0.7031372 .057309$

C $0.175975-0.1566600 .869980$

$\begin{array}{lllll}\text { O } & 1.192050 & 0.730297 & 1.395209\end{array}$

C $0.860571-1.2658060 .050910$

C $-0.097843-2.235317-0.607870$

C $-0.219454-3.538565-0.287276$

C $-0.927530-1.659806-1.727766$

C $0.500244-4.3045300 .787546$

C $1.408730-5.4052800 .221729$

C $2.600806-4.869564-0.586229$

C $3.439562-6.022757-1.135246$

C $4.681618-5.512407-1.877484$

O $3.402952-4.067596 \quad 0.282937$

O $5.559874-6.629371-2.085218$

C $5.434760-4.505044-0.979393$

C $4.330204-4.972334-3.270040$

O $6.500560-3.909805-1.713339$

C $4.521663-3.421185-0.354897$

C $5.224833-2.6327470 .781902$

O $4.077300-2.521098-1.366196$

C $1.611487 \quad 1.747566 \quad 0.594916$

O $1.2367341 .994833-0.540002$

C $2.619488 \quad 2.5215601 .354355$

C 3.2035933 .5904060 .792206

C 4.2291914 .4379031 .407641

C 4.6550294 .1005692 .813421

C $6.319574-1.6368390 .389503$

C $5.791741-0.317909-0.148250$

C $6.5515021 .758687-1.293939$

O $4.6230330 .037869-0.020713$

C $6.9239932 .777605-0.263709$

C $7.5480283 .928282-0.556756$

C $7.898095 \quad 4.9771180 .461136$

C 7.1039016 .2517920 .270469

C $7.5506247 .228938-0.536305$

C 5.8328456 .4227491 .076390

C $4.754724 \quad 5.4478090 .681359$

N $6.7894740 .431837-0.756578$

H -2.467942 -0.0449161 .451486$

H $0.007212-1.2245492 .815886$

H $\quad-0.5040950 .4230890 .234601$

H $1.597199-1.7769420 .680560$

H $1.472123-0.804568-0.736330$

H $-0.937529-4.136402-0.847995$

H $-1.515619-2.425180-2.245488$

H -1.628937 -0.912580 -1.344418

H $-0.282204-1.183992-2.473247$
H $1.068285-3.6539891 .457941$

H $-0.261339-4.7805601 .417637$

H $1.784321-5.9892561 .072064$

H $0.816014-6.087410-0.400728$

H $2.223885-4.251688-1.408458$

H $2.838310-6.666647-1.789104$

H $3.770626-6.662921-0.305423$

H $5.074993-7.300993-2.595080$

H $5.898149-5.074568-0.161779$

H $3.843642-5.750360-3.870609$

H $5.232128-4.689067-3.823982$

H $3.659589-4.111061-3.239241$

H $7.054936-4.630892-2.057513$

H $4.455936-2.0979851 .357287$

H $5.648440-3.3573511 .490569$

H $3.721992-1.730451-0.927730$

H 2.8588592 .1833852 .356268

H $2.9158623 .867417-0.221229$

H $5.043653 \quad 3.0772202 .863471$

H 3.8069304 .1882893 .501602

H 5.4436794 .7471873 .201817

H $6.888970-1.3856211 .293970$

H $7.028553-2.070666-0.318122$

H $5.4922451 .862172-1.550559$

H $7.1504541 .855128-2.204799$

H 6.6440492 .5752460 .767157

H $7.8185254 .137239-1.589460$

H 8.9718025 .1889130 .373489

H 7.7592954 .6046191 .483793

H $7.0024318 .156998-0.659580$

H $8.4746107 .119568-1.094633$

H 5.4242877 .4319010 .933920

H 6.0847946 .3678522 .138640

H $4.3811585 .606438-0.331236$

H $7.7460630 .090030-0.743960$

C $-3.731776-2.1294313 .016062$

O $-4.648330-1.5950762 .039605$

C -4.929586 -2.621119 1.066064

C $-4.419066-3.9244661 .670771$

C -3.201094 -3.437171 2.431600

H $-4.325669-2.3907360 .180456$

C -6.416987-2.619268 0.701196

H $-5.147399-4.3658102 .360831$

H $-4.179508-4.6706610 .907176$

C -2.693994 -4.420136 3.471435

H $-2.402157-3.2393961 .708919$

C $-6.926694-1.2628790 .169406$

O $-7.169093-2.939516 \quad 1.878535$

H $-6.621303-3.416890-0.022784$

H $-6.868609-0.5192470 .971341$

O $-8.320852-1.409416-0.131172$

C $-6.196738-0.751453-1.087444$

C $-6.7964370 .540484-1.677965$

H $-5.137464-0.591029-0.863051$

O $-6.261092-1.768218-2.091482$

H $-6.3017720 .736915-2.636971$

H $-7.8487130 .370659-1.936932$

H $-1.820292-4.017643 \quad 3.993523$

H $-3.461203-4.6451644 .219910$

H -2.399969 -5.3627612 .998346$

C $-2.675937-1.0845133 .388122$

H $-8.110327-2.9237951 .634034$

H $-8.396370-2.038038-0.869733$

H $-5.736950-1.463580-2.851766$

H -2.005477 -1.493018 4.153003

H -3.181472 -0.2197973 .836196$

H $-4.331903-2.3397583 .911291$

C $-4.3957292 .390076-1.282622$

O $-5.3197951 .934412-0.289386$

C $-6.6642811 .765716-0.752242$

C $-7.1909613 .083395-1.341707$

C $-6.2558213 .663638-2.402291$

C $-4.824348 \quad 3.737943-1.870984$

H $-4.3517941 .650645-2.092448$

C $-2.9874472 .405113-0.671594$

H $-7.271178 \quad 1.5571840 .136860$

$\begin{array}{llll}\text { O } & -8.500752 & 2.917984 & -1.874899\end{array}$

H -7.279766 $3.816082-0.529163$ 
H $-6.2964913 .087556-3.334143$

O $-6.6808324 .988032-2.730427$

H $-4.7599704 .533780-1.120320$

O $-3.9445084 .086813-2.937188$

C $-2.838558 \quad 3.2524230 .575573$

H $-2.7315061 .379867-0.383038$

H -2.259336 $2.748642-1.414536$

H -8.429693 2.422666 -2.708202

H -7.607912 $4.931420-3.019327$

H $-4.2596144 .928381-3.309502$

O $-1.529752 \quad 3.224717 \quad 0.969211$

O $-3.742323 \quad 3.8454461 .147213$

C -1.2306833 .9579922 .160871$

H -1.4738175 .0163602 .026647$

H -1.779398 $3.538748 \quad 3.009517$

H -0.1594833 .8677092 .357875$

SCF Energy (B3LYP/6-31G**//MMFF) $=-3245.92140251$

01419

MM̄FF Geometry

C $-1.037910-3.418272-0.267399$

C $-0.007328-3.607755-1.105988$

C $0.689612-2.547128-1.930625$

O $-0.015977-1.291896-1.781059$

C $2.159989-2.340712-1.512298$

C $2.991454-3.601219-1.429601$

C $3.473862-4.120125-0.283636$

C $3.291259-4.263746-2.748735$

C $3.300485-3.5821251 .109457$

C $4.631391-3.1985471 .768087$

C $5.478553-2.1878620 .975157$

C $6.804505-1.9469551 .702060$

C $7.673105-0.9153890 .974075$

O $4.755668-0.9563100 .840180$

O $8.730803-0.5230451 .862092$

C $6.820610 \quad 0.3376840 .703668$

C $8.346999-1.504387-0.270775$

O $7.542814 \quad 1.273318-0.096097$

C 5.4412410 .0372710 .059672

C 4.6087131 .3423390 .058723

O $5.641062-0.397834-1.285455$

C $0.189684-0.318656-2.704368$

O $0.921749-0.365505-3.679069$

C $-0.6457270 .836304-2.308786$

C $-0.4291302 .033544-2.871971$

C $-1.1848923 .259340-2.608258$

C $-2.3558793 .165969-1.666024$

C $3.1874701 .245996-0.508817$

C $2.5676892 .632748-0.496442$

C 1.7380294 .3334791 .115661

O $2.6511783 .383678-1.466139$

C $0.510070 \quad 4.8728540 .453837$

C $0.5138515 .993583-0.284529$

C $-0.7160376 .636029-0.858497$

C $-0.6730016 .752408-2.364695$

C $-0.0271397 .767353-2.963482$

C -1.464003 5.747319-3.167699

C $-0.8046324 .398159-3.223973$

N $1.9977732 .959547 \quad 0.725782$

H -1.438290 -2.420174 -0.113253

H $\quad 0.355840-4.624572-1.243126$

H $\quad 0.630018-2.855261-2.982399$

H $2.179365-1.774799-0.576124$

H $2.647544-1.663421-2.225553$

H $4.064214-5.033634-0.341456$

H $3.948364-5.132821-2.637449$

H $2.368810-4.607745-3.226485$

H $3.790388-3.558647-3.421586$

H $2.617279-2.7319871 .150072$

H $2.826881-4.3648571 .715160$

H $4.401769-2.7828322 .757844$

H $5.219275-4.1107861 .933554$

H $5.675581-2.597878-0.021321$

H $7.356024-2.8868851 .830227$

H $6.602229-1.5771702 .717129$

H $9.234680-1.3206012 .098659$

H 6.6467550 .8290031 .671574

H $8.981282-2.3555840 .004327$
H $\quad 9.014543-0.774019-0.741187$

H $7.634468-1.853351-1.020955$

H $8.388108 \quad 1.446596 \quad 0.352683$

H 4.5332281 .7117881 .090368

H $5.1434832 .110553-0.515465$

H $4.801168-0.331216-1.765111$

H $-1.4158580 .666903-1.564537$

H $\quad 0.3756512 .127500-3.600182$

H -2.036205 $2.782908-0.690620$

H -3.121213 2.497481-2.073962

H $-2.8403854 .124686-1.471284$

H $3.1789320 .890603-1.543658$

H 2.5744890 .5576600 .079190

H 2.6279514 .9285370 .882201

H 1.5973144 .3404842 .201459

H -0.4283564 .3597880 .640560$

H $1.4540866 .518369-0.441116$

H $-0.8061747 .639149-0.420446$

H - $1.6241166 .105850-0.547313$

H $-0.0198997 .875795-4.042925$

H $0.5123618 .510306-2.385206$

H -1.577020 $6.095225-4.203504$

H -2.487754 $5.700810-2.784235$

H $0.0671974 .369244-3.878895$

H 2.0714402 .2828621 .479245

C $-3.144867-4.8019060 .063628$

O $-3.948266-3.613473 \quad 0.215232$

C $-4.653201-3.385331-1.018472$

C $-4.696283-4.739369-1.712821$

C $-3.312608-5.268345-1.386302$

H $-4.033331-2.703886-1.614679$

C $-6.015866-2.737275-0.759972$

H $-5.462619-5.393753-1.281607$

H $-4.881486-4.653028-2.787568$

C $-3.170903-6.769780-1.566209$

H $-2.592447-4.768935-2.045459$

C $-5.964930-1.4690110 .118267$

O $-6.875604-3.678776-0.105600$

H $-6.497090-2.512187-1.718719$

H $-5.786507-1.7612421 .159096$

O $-7.268410-0.8735460 .097673$

C $-4.923181-0.420409-0.310353$

C -5.0103270 .9158850 .448300$

H $-3.917407-0.836666-0.188576$

O $-5.095928-0.144738-1.702958$

H $-4.194616 \quad 1.552207 \quad 0.086465$

H $-5.926126 \quad 1.4445720 .153772$

H -2.161707 -7.098841 -1.299016

H $-3.882231-7.318653-0.940114$

H $-3.354312-7.051396-2.608360$

C $-1.703589-4.5247160 .505309$

H -6.439485 -3.950094 0.720434

H $-7.434322-0.562920-0.809041$

H $-4.3812250 .456228-1.973896$

H -1.118383 -5.449784 0.443062

H $-1.707101-4.2216671 .559926$

H -3.575775 $-5.543826 \quad 0.748011$

C -2.5674820 .4636562 .241939$

O $-3.889302-0.0576582 .423031$

C -4.9454450 .7984591 .982632$

C -4.8774772 .1468332 .713878$

C -3.4979042 .7909412 .598437$

C -2.4067931 .7952702 .991801$

H -2.3779700 .6089631 .171555$

C -1.605985 -0.614413 2.759312

H -5.8785150 .3166152 .299485$

O -5.8573483 .0494622 .211849$

H $-5.097378 \quad 1.992571 \quad 3.778412$

H -3.3232713 .1893881 .592257$

O $-3.4274213 .908423 \quad 3.487590$

H -2.4558891 .6262114 .074684$

O -1.1333902 .3743322 .720745$

C $-0.170708-0.3668992 .362693$

H -1.691229 -0.6906043 .850438$

H -1.888647 -1.599341 2.369016

H -6.7258762 .6237052 .313541$

H $-4.1500434 .514016 \quad 3.248383$

H -1.0477212 .4647291 .756555$ 
O $0.637911-0.523982 \quad 3.449244$

O $0.186871-0.070598 \quad 1.230534$

C $2.033673-0.281308 \quad 3.245334$

H $2.370571-0.6572542 .276797$

H 2.2293540 .7911023 .327389

H $2.587527-0.8024204 .030823$

SCF Energy (B3LYP/6-31G**//MMFF) $=-3245.90653602$

0142

MM̄FF Geometry

C $2.3914592 .211107-2.616040$

C $3.4747651 .420522-2.561955$

C $3.481701-0.087901-2.612930$

O $2.136731-0.581378-2.826683$

C $4.377272-0.657942-3.727168$

C $5.865139-0.568924-3.453184$

C $6.545936-1.448042-2.690040$

C $6.5953560 .539342-4.164918$

C $5.981721-2.626049-1.944804$

C $6.551347-2.798086-0.528853$

C $6.318340-1.6113820 .421187$

C $6.953392-1.9068151 .785053$

C $6.689382-0.7899722 .804006$

O $4.909041-1.3954340 .572924$

O $7.041805-1.3095924 .095362$

C $5.177779-0.4784292 .813098$

C 7.6041040 .4168892 .565558

O 4.8971950 .6391843 .652643

C $4.582811-0.2686011 .402164$

C $3.046351-0.0938631 .434406$

O 5.1201150 .9447720 .857353

C $1.758292-1.694790-2.145124$

O $2.437680-2.353891-1.374962$

C $0.349015-1.983642-2.488555$

C $-0.286573-2.957543-1.820652$

C $-1.668848-3.392688-2.014821$

C $-2.479739-2.718090-3.088319$

C $2.242352-1.2912071 .946939$

C $0.750610-0.9885851 .975674$

C -1.401733 -1.972766 2.727071

O $0.285996 \quad 0.115019 \quad 1.701078$

C -2.144459-3.196462 2.294468

C $-3.311870-3.1388531 .635484$

C $-4.119469-4.3364361 .221497$

C $-4.468897-4.316386-0.251285$

C $-5.624101-3.778155-0.676812$

C $-3.519802-4.980624-1.222337$

C -2.148409-4.367281-1.214995

N $0.001371-2.0902862 .370848$

H $1.4029831 .777630-2.739645$

H $4.4416321 .892789-2.404513$

H $3.841917-0.428052-1.637656$

H $4.140476-0.168293-4.681795$

H $4.113379-1.709612-3.904013$

H $7.624381-1.325312-2.601635$

H $7.6700360 .531786-3.953527$

H $6.4726190 .437784-5.248268$

H $6.2102731 .517067-3.863501$

H $4.893184-2.584113-1.886573$

H $6.225513-3.529625-2.517294$

H $6.087386-3.696258-0.100332$

H $7.628061-2.999038-0.599789$

H $6.774378-0.717379-0.018524$

H $8.031408-2.0842251 .682709$

H $6.534595-2.8422702 .183090$

H $6.807218-0.6365734 .757445$

H $4.689169-1.3399623 .283790$

H 8.6560760 .1196342 .654805

H $7.448607 \quad 1.1898243 .326158$

H 7.4712910 .8695751 .580944

H 5.1464291 .4440923 .167089

H $2.797100 \quad 0.8022402 .018474$

H 2.6964720 .1269420 .417335

H $4.536574 \quad 1.2458420 .141901$

H $-0.120793-1.394381-3.267030$

H $0.258744-3.493956-1.044549$

H -2.054435 -2.926491-4.076148

H $-2.497099-1.633850-2.938348$
H -3.522807 -3.042946 -3.110010

H $2.412680-2.1669661 .312587$

H $2.537692-1.5425382 .970538$

H $-1.823420-1.0584902 .296345$

H -1.443127 -1.884456 3.817618

H -1.725724 -4.164335 2.557595

H $-3.733235-2.1643971 .403526$

H $-5.040597-4.3404671 .819084$

H $-3.604443-5.2736361 .466065$

H $-5.896637-3.777094-1.726819$

H $-6.321925-3.3224660 .017579$

H $-3.931799-4.992147-2.238103$

H $-3.423710-6.039761-0.949611$

H -1.488117 -4.792555 -0.458171

H $\quad 0.497269-2.9178082 .689835$

C $1.8361754 .241221-1.195178$

O $0.4378523 .901487-1.115605$

C 0.1227723 .5846650 .256262

C 1.3355514 .0048341 .081117

C 2.4637363 .7384600 .104132

H $\quad 0.0256572 .4951140 .312199$

C $-1.1917594 .253305 \quad 0.669454$

H 1.3011625 .0687271 .343458

H 1.4244703 .4307832 .008625

C 3.7697444 .4186060 .470943

H 2.6255492 .6556510 .063127

C $-2.3999673 .870576-0.209082$

O -1.0340095 .6763750 .593512$

H -1.408396 4.0296681 .720297

H -2.260840 $4.246511-1.229791$

O -3.5521964 .5430310 .316506$

C $-2.7039762 .360710-0.249570$

C $-3.9488362 .039974-1.102253$

H -1.851472 $1.826541-0.682172$

O -2.8698731 .8755191 .079119$

H $-4.8091172 .592594-0.713199$

H -3.762524 2.411555-2.116693

H $4.5441634 .199777-0.271039$

H 3.6541925 .5059620 .528634

H 4.1262434 .0658801 .444349

C $2.4468593 .708352-2.494392$

H $-0.7834875 .895180-0.320550$

H -3.3463215 .4932490 .342046$

H -3.6223342 .3494071 .473299$

H $3.4796004 .065536-2.584830$

H $1.8926974 .122465-3.345888$

H $1.8784215 .338231-1.227522$

C -6.0060910 .4619240 .545570$

$\begin{array}{llll}\text { O } & -4.713536 & 0.017930 & 0.115018\end{array}$

C $-4.277100 \quad 0.529478-1.148494$

C $-5.2854990 .135363-2.236484$

C $-6.6979540 .592193-1.886033$

C $-7.0814460 .108255-0.489005$

H -5.9897651 .5454450 .709013$

C $-6.301140-0.2194671 .889941$

H $-3.3370910 .013490-1.378472$

O $-4.916271 \quad 0.676334-3.500131$

H -5.293103 -0.956766 -2.346677

H $-6.8012911 .680749-1.967768$

O $-7.6203440 .028111-2.821168$

H -7.242611 $-0.976606-0.511650$

O $-8.3153450 .712329-0.105333$

C $\begin{array}{llll}-5.406173 & 0.309367 & 2.989276\end{array}$

H -7.334726 -0.0529242 .211540$

H $-6.142383-1.2999691 .792965$

H $-4.0194090 .359361-3.702426$

H $-7.3393770 .309387-3.708910$

H $-8.9706900 .492823-0.789652$

O $-4.984608-0.722108 \quad 3.776458$

O $-5.135181 \quad 1.492048 \quad 3.151299$

C $-4.128467-0.3562994 .862918$

H -3.808795 -1.272289 5.366979

H $-3.2418160 .166816 \quad 4.491846$

H -4.6741930 .2668465 .577556$

SCF Energy (B3LYP/6-31G**//MMFF) $=-3245.93308120$

01420

MM̄MF Geometry 
C 3.2221753 .3749101 .067262 C 2.3822722 .3368920 .943316 C 1.5543531 .7819042 .072954 O $\quad 0.1860951 .6552441 .622685$ C 2.0638430 .3756662 .429505 C $1.352163-0.2473253 .611958$ C $0.419182-1.2161133 .521951$ C 1.7954160 .2530724 .962468 C - $0.158922-1.8326812 .275245$ C -1.689107 -1.836303 2.330883 C $-2.358065-2.5116311 .122822$ C $-2.146382-1.732063-0.177491$ C $-2.962292-2.301293-1.348037$ O $-3.756272-2.5813141 .433955$ O $-2.967700-1.321563-2.398577$ C $-4.427022-2.488088-0.899895$ C $-2.309762-3.558643-1.934011$ O $-5.183941-3.157819-1.905173$ C $-4.560510-3.2339890 .442059$ C $-6.014405-3.2619930 .971361$ $\begin{array}{lllll}\text { O } & -4.152291 & -4.595878 & 0.264484\end{array}$ C -0.5759722 .7815931 .590924$ O -0.2414973 .9107631 .909516$ C -1.902951 2.394144 1.065420 C -2.7383823 .3306490 .593163$ C -4.0553083 .0830990 .002989$ C -4.6164041 .6868160 .089699$ C $-6.634929-1.892721 \quad 1.273620$ C $-7.633416-1.4153520 .229830$ C $-9.3723790 .354726-0.087713$ O $-7.830339-1.989403-0.836574$ C $-8.8603141 .561472-0.805822$ C $-9.0122762 .812810-0.345945$ C $-8.5570414 .043430-1.075902$ C $-7.2252754 .551720-0.567254$ C -7.1772235 .4127280 .463746$ C $-6.0144194 .050653-1.327906$ C $-4.706694 \quad 4.107096-0.587847$ N -8.288850 -0.2654790 .649839$ H 3.3287563 .8685972 .029689 H $2.2900901 .842406-0.020074$ H 1.5897292 .4330182 .955240 H 3.1388890 .4114502 .653419 H $1.973283-0.2759031 .555756$ H $0.014442-1.6175834 .450517$ H $1.291329-0.2656805 .784816$ H 2.8723950 .1011315 .089176 H 1.5794561 .3206675 .066747 H $0.168829-1.3045111 .378155$ H $\quad 0.206130-2.8634312 .198137$ H $-2.020585-2.3538823 .241946$ H -2.066808 -0.8099712 .432105$ H -1.965283 -3.5311271 .032139$ H -1.086197-1.693989-0.447946 H -2.451950 -0.690283 -0.025192 H -2.043987 -1.131928 -2.636786 H $-4.863559-1.486787-0.796552$ H -1.317615 -3.320838 -2.334124 H -2.886195 -3.947080 -2.780617 H -2.185974 -4.359057 -1.201714 H -5.088894 -2.647461 -2.727522 H -6.648204 -3.8605270 .305923$ H -5.998497 $-3.814558 \quad 1.922255$ H -4.547495 $-5.132200 \quad 0.971580$ H $-2.143407 \quad 1.338671 \quad 1.052011$ H -2.4176124 .3714280 .608740$ H -4.5359201 .2942411 .109701$ H -4.079231 $1.018323-0.590046$ H $-5.6780001 .632635-0.163426$ H -7.186649 -1.973806 2.219587 H -5.870591 -1.119958 1.408110 H -10.149981 0.6143870 .638190 H -9.801087 -0.354124 -0.803331 H -8.365559 $1.396675-1.759681$ H -9.5050322 .9703560 .610967$ H $-9.3283964 .816213-0.960995$ H -8.497809 $3.859669-2.156388$ H $\quad-6.243974 \quad 5.8160570 .839817$
H $-8.085301 \quad 5.742880 \quad 0.958641$

H $-5.9147774 .647209-2.244310$

H -6.197033 $3.028781-1.677353$

H -4.238258 $5.091715-0.595999$

H $-8.0216370 .159369 \quad 1.533217$

C 5.5344083 .5602810 .128530

O 5.7080912 .1322160 .041804

C $6.8977471 .848029-0.717977$

C $7.6138063 .181761-0.898307$

C $6.4448664 .147030-0.948477$

H $6.5644371 .493858-1.700718$

C $7.7189780 .756621-0.022579$

H $8.2622213 .415901-0.046279$

H $8.2286463 .202518-1.803311$

C $6.8399855 .593101-0.705771$

H $5.9643894 .065108-1.932811$

C $6.912458-0.5193030 .300292$

O 8.2103881 .2807351 .218228

H $8.6008850 .515851-0.627496$

H $\quad 6.157447-0.279431 \quad 1.056377$

O $7.798964-1.4571370 .923699$

C $6.244881-1.185462-0.918166$

C $5.532905-2.514397-0.600225$

H $5.523681-0.496223-1.364052$

O $7.232014-1.438604-1.921211$

H $5.261807-2.986988-1.551905$

H $6.244720-3.206750-0.134930$

H $5.9641956 .248449-0.740730$

H 7.3171655 .7201720 .271785

H $7.5460015 .932215-1.470852$

C $4.0578583 .902379-0.066239$

H 8.7228510 .5748421 .648401

H $8.532189-1.6294030 .309139$

H $7.825768-2.132999-1.589171$

H $3.7052003 .482540-1.015807$

H $3.9189254 .987805-0.119964$

H 5.8718923 .8644181 .128134

C $2.637501-1.971042-1.443033$

O $3.322436-1.464071-0.291805$

C $4.285699-2.3480420 .290723$

C $3.613891-3.6669250 .693686$

C $2.876696-4.304914-0.477546$

C $1.931143-3.296288-1.123990$

H $3.351424-2.114872-2.262886$

C $1.609858-0.909876-1.871910$

H $4.614073-1.871176 \quad 1.221306$

O $4.568275-4.5908901 .205342$

H $2.889425-3.4750301 .496138$

H $3.573359-4.720625-1.214920$

O $2.095768-5.4028890 .000619$

H $1.078387-3.118222-0.456781$

O $1.417490-3.852772-2.333105$

C $2.2415090 .292558-2.540535$

H $0.901706-1.337191-2.590882$

H $1.062655-0.545550-0.994829$

H $5.014252-4.164127 \quad 1.956830$

H $2.704236-6.0177420 .445444$

H $0.987425-4.694752-2.104932$

O $1.2479161 .151930-2.918972$

O $3.4397530 .464683-2.711099$

C $1.6804222 .340755-3.587419$

H $0.7933632 .916587-3.864240$

H $2.2971982 .947063-2.918790$

H $2.2316232 .086347-4.497644$

SCF Energy (B3LYP/6-31G**//MMFF) $=-3245.90889218$

01421

MM̄FF Geometry

C -1.357134-1.271994 2.277223

C $-1.634795-0.3741643 .236346$

C $-0.8291650 .868823 \quad 3.549136$

O $\quad 0.2438391 .0035242 .583959$

C -1.6635902 .1640803 .495425$

C $-2.8404202 .237644 \quad 4.446838$

C -4.1190952 .4241724 .061987$

C -2.5051432 .1207585 .911103$

C -4.6233752 .6165902 .660423$

C -5.2755321 .3415882 .114037$ 
C $-5.328916 \quad 1.359230 \quad 0.580568$

C -6.2051790 .2234680 .055430$

C $-6.1716220 .138323-1.476940$

O -3.9843651 .1949110 .114173$

O $-6.747299-1.119724-1.861286$

C $-4.7020840 .123945-1.943532$

C -7.030355 $1.227548-2.129822$

O $-4.6196670 .190851-3.365676$

C $-3.8321511 .235058-1.309852$

C $-2.3376680 .968569-1.629285$

O $-4.2256922 .497313-1.843200$

C 1.3811501 .6369402 .971277

O 1.6265982 .1066414 .070157

C 2.2839721 .6674201 .799946

C 3.4747342 .2764461 .892943

C 4.4370782 .3875390 .801162

C 5.8569132 .1222351 .219245

C $-1.3366691 .919991-0.957592$

C -1.033581 $3.176710-1.756328$

C $0.3846135 .228404-1.668007$

O $-1.5364573 .420159-2.849533$

C $1.6363384 .924497-2.429609$

C $2.8427545 .417205-2.110300$

C $4.0999235 .089832-2.863795$

C $5.1308384 .388703-2.005353$

C $6.2554985 .016293-1.622908$

C $4.9004372 .932875-1.662489$

C $4.0481092 .754600-0.436422$

$\mathrm{N}-0.1259153 .995136-1.096506$

H -0.494048 -1.124132 1.633350

H $-2.483700-0.5678993 .888704$

H $-0.396541 \quad 0.7354084 .548359$

H -1.9652112 .3203162 .455707$

H -1.008126 3.0186223 .711821

H -4.8921712 .4563184 .828162$

H $-3.3723882 .313170 \quad 6.552012$

H -2.1398601 .1162916 .145850$

H -1.7316102 .8467946 .181886$

H -5.3657023 .4249242 .667650$

H -3.8308912 .9700211 .995650$

H -4.7046990 .4624022 .440483$

H -6.2860961 .2515742 .530987$

H -5.7159572 .3296420 .248013$

H $-7.237326 \quad 0.3307210 .411824$

H $-5.846151-0.7300580 .464955$

H -7.652949 -1.155213 - 1.508455

H -4.282781 -0.848542 -1.654742

H $-8.0738781 .138705-1.804373$

H -7.046799 $1.117155-3.219722$

H -6.695205 2.238786-1.889954

H $-5.154761-0.539319-3.721039$

H -2.094012 -0.036278 -1.262784

H $-2.1767740 .968154-2.713714$

H $-3.5248173 .142847-1.664343$

H 1.9479151 .1977770 .881046

H 3.7908212 .7201342 .834736

H 5.9652761 .0834321 .551125

H 6.1449402 .7799292 .047312

H 6.5832142 .2858550 .420247

H -1.671594 2.2058690 .044202

H $-0.3834661 .386148-0.846967$

H $\quad 0.5604875 .923155-0.840639$

H $-0.3636065 .663488-2.338096$

H $1.5432544 .259964-3.285660$

H $2.9374966 .088325-1.259714$

H $4.5061786 .030253-3.257863$

H $3.8871194 .469212-3.743223$

H $7.0112904 .509516-1.032178$

H $6.4438346 .051339-1.887894$

H $5.8581282 .410959-1.560839$

H $4.4074672 .433414-2.506305$

H $2.9884532 .939152-0.606686$

H $0.2948663 .667640-0.231433$

C -2.661449 -2.644237 0.619065

O $-1.554584-2.660137-0.302536$

C - $-1.779464-3.672218-1.300887$

C $-3.235246-4.093726-1.147677$

C $-3.437017-3.9335430 .347897$
H $-1.137795-4.520229-1.033176$

C $-1.384720-3.147498-2.687140$

H $-3.914258-3.428549-1.693094$

H -3.410514 -5.115067-1.498673

C $-4.897329-3.8811450 .759814$

H $-2.951485-4.7762200 .858423$

C $0.016532-2.501226-2.726205$

O $-2.331334-2.150140-3.085481$

H -1.462890 -3.954999-3.424057

H $0.018211-1.607701-2.093075$

O $0.246359-2.032169-4.061583$

C $1.163583-3.446006-2.323672$

C $2.551386-2.779097-2.383740$

H $0.991568-3.845701-1.319934$

O $1.158379-4.559122-3.221839$

H $3.314335-3.566193-2.390267$

H $2.671177-2.268726-3.346572$

H $-4.995385-3.7200861 .837746$

H $-5.429532-3.0743420 .246666$

H $-5.399607-4.8219460 .511551$

C $-2.134959-2.5407962 .051930$

H -2.053157 -1.814213 -3.954692

H $\quad 0.288890-2.811705-4.641807$

H $1.835467-5.186314-2.915664$

H -1.454288 -3.377261 2.254612

H $-2.966921-2.6150842 .761532$

H $-3.270447-1.7648920 .376487$

C $3.580637-3.3969660 .407698$

O $2.601577-2.4113370 .060011$

C $2.809491-1.796426-1.220684$

C $4.188805-1.121969-1.251885$

C $5.315184-2.078831-0.863584$

C $4.984386-2.7827680 .452691$

H $3.561187-4.193665-0.346014$

C $3.154813-4.0791931 .712110$

H $2.062460-0.998078-1.302398$

O $4.448242-0.540695-2.524688$

H $4.184274-0.297285-0.530627$

H $5.519555-2.807318-1.656662$

O $6.521070-1.333113-0.681338$

H $5.064714-2.0551881 .269347$

O $5.940138-3.8111540 .694450$

C $3.320541-3.2190612 .941213$

H $2.105925-4.3965691 .655789$

H $3.742593-4.9906971 .875342$

H $4.658530-1.255146-3.149131$

H $6.685642-0.842528-1.504981$

H $\quad 6.819497-3.3956090 .694773$

O $2.211405-2.4529893 .138914$

O $4.324835-3.227943 \quad 3.642423$

C $2.244117-1.6317334 .309970$

H $2.962176-0.8181664 .173297$

H $1.248893-1.2078214 .457060$

H $2.497546-2.2249925 .194259$

SCF Energy $\left(B 3 L Y P / 6-31 G^{* *} / / M M F F\right)=-3245.91202364$

01422

MM̄FF Geometry

C -3.642834 -1.092033 -2.728180

C $-2.465401-1.091583-2.086570$

C $-1.150555-0.776606-2.744660$

O $-0.638048 \quad 0.396013-2.060135$

C $-0.121702-1.907741-2.573529$

C $-0.555503-3.289238-3.007369$

C $-0.721736-4.332008-2.168811$

C $-0.743308-3.467753-4.490506$

C $-0.596749-4.331133-0.670616$

C $0.599365-5.130509-0.135903$

C $1.977625-4.642591-0.616379$

C $3.094031-5.3662020 .145274$

C $4.490267-4.837897-0.220078$

O $2.072785-3.233047-0.395598$

O $5.399663-5.3373530 .772860$

C $4.484038-3.295062-0.124593$

C $4.968751-5.406763-1.560799$

O $5.720500-2.752130-0.580830$

C $3.295303-2.645161-0.863335$

C $3.207184-1.118363-0.632244$ 
O $3.466465-2.843492-2.272694$ C $0.334278 \quad 1.124139-2.670368$ $\begin{array}{llll}\text { O } & 0.797267 & 0.949343 & -3.785377\end{array}$ C $0.7543002 .175993-1.716920$ C $1.6875463 .073941-2.064414$ C $2.1600764 .186963-1.235527$ C $1.641733 \quad 4.2780710 .176589$ C $2.961779-0.6908120 .816266$ C $4.181443-0.0248811 .416216$ C 5.5613582 .0285691 .264132 $\begin{array}{lllll}\text { O } & 4.900897 & -0.602426 & 2.227563\end{array}$

C 5.2417553 .4869741 .212709 C $5.8084514 .341452 \quad 0.347795$ C $5.557266 \quad 5.8207750 .350790$ C $5.0377456 .333642-0.971585$ C $5.8725296 .850296-1.889337$

C $3.5418416 .352016-1.180982$ C $3.0149215 .078546-1.779842$ N $4.372842 \quad 1.2651350 .942235$ H -3.666107 $-0.874154-3.792764$ H $-2.441580-1.307545-1.022730$ H $-1.295472-0.539644-3.806223$ H $0.216650-1.901042-1.533819$ H $0.782552-1.649029-3.139318$ H $-1.005384-5.293973-2.593437$ H $-0.993354-4.500285-4.756619$ H $-1.553487-2.828499-4.855054$ H $0.175812-3.203482-5.023802$ H $-0.568929-3.318002-0.261420$ H $-1.514431-4.775874-0.264401$ H $0.558731-5.0654730 .959616$ H $0.481075-6.190695-0.392966$ H $2.059690-4.844383-1.690613$ H $3.036608-6.449632-0.017543$ H $2.946580-5.2201621 .225010$ H $6.273261-4.9485400 .594000$ H $4.421923-3.0489190 .942218$ H $5.024675-6.501096-1.510826$ H $5.983487-5.068472-1.796958$ H $4.314197-5.147799-2.395511$ H $5.717378-2.783835-1.552607$ H $4.093481-0.631018-1.057733$ H $2.362198-0.740699-1.222239$ H $2.974582-2.154013-2.747574$ H $\quad 0.2692502 .190528-0.748835$ H $2.127647 \quad 3.008405-3.058584$ H $1.803798 \quad 3.3344710 .710244$ H 0.5697014 .5026190 .179095 H $2.138345 \quad 5.0473190 .772201$ H 2.1368260 .0319070 .850862 H $2.666338-1.5250491 .460064$ H $6.335162 \quad 1.745346 \quad 0.542540$ H $5.916597 \quad 1.7564852 .263423$ H 4.5374983 .8630211 .951132 H $6.5302543 .965852-0.374171$ H 6.5061746 .3174810 .593485 H $4.867366 \quad 6.1084411 .153993$ H $5.5019837 .255255-2.825248$ H $6.9450326 .873940-1.726845$ H $3.2798447 .164800-1.872749$ H $3.0406916 .623206-0.247332$ H $3.3384824 .921991-2.809929$ H 3.8080291 .5789800 .157661 C $-5.806971-0.137295-1.769419$ O $-5.150860 \quad 0.678398-0.775074$ C $-5.0948812 .029537-1.264089$ C $-6.2144522 .128760-2.292863$ C $-6.1075910 .772795-2.964824$ H $-4.1325812 .133681-1.781003$ C $-5.1642933 .049345-0.121340$ H -7.196695 2.235783 -1.818332 H $-6.0735082 .963982-2.985232$ C $-7.3503710 .370791-3.740033$ H $-5.2612120 .807844-3.660259$ C $-4.1878252 .791775 \quad 1.047137$ $\begin{array}{lllll}\text { O } & -6.491951 & 3.039681 & 0.418374\end{array}$ H $-5.0086064 .053941-0.533599$ H -4.5908192 .0013581 .690608$
O -4.1368213 .9802451 .846511$

C $-2.7507742 .436853 \quad 0.632162$

C -1.7229512 .3775631 .777307$

H $-2.752348 \quad 1.468477 \quad 0.122303$

O $-2.2767393 .393275-0.320590$

H $-0.798265 \quad 1.964102 \quad 1.356881$

H -1.4531483 .3976032 .081886$

H $-7.224757-0.621060-4.185927$

H $-8.2349150 .341977-3.094887$

H $-7.5472381 .083349-4.547689$

C $-4.961410-1.383181-2.064091$

H $-6.5755423 .804958 \quad 1.011466$

H -5.0093534 .0964212 .258320$

H $-2.3192694 .270456 \quad 0.097607$

H $-5.525433-2.066651-2.709722$

H $-4.782059-1.916903-1.122146$

H $-6.745438-0.467600-1.305842$

C $-1.948761-0.6736492 .163697$

$\begin{array}{llll}\text { O } & -2.792758 & 0.344224 & 2.700821\end{array}$

C -2.138129 $1.570503 \quad 3.022915$

C -0.9913391 .3191114 .013666$

C $-0.0252570 .244993 \quad 3.512261$

C $-0.792538-1.015668 \quad 3.110915$

H $-1.533994-0.3195821 .211159$

C -2.831736 -1.8826371 .820631$

H -2.8836912 .1799223 .548836$

O $-0.2869302 .531814 \quad 4.261664$

H -1.4160920 .9918984 .971738$

H $\quad 0.5878600 .6230672 .686871$

O $0.879037-0.0688894 .573554$

$\mathrm{H}-1.160015-1.5108084 .017134$

O $0.083802-1.9265552 .452719$

C $-3.486788-2.5432023 .016367$

H $-3.646548-1.5675011 .158660$

H -2.228309 -2.631034 1.297669

H 0.4388282 .3255514 .875408

H $1.552406-0.6724364 .216512$

H $\quad 0.753439-2.2106303 .097294$

O $-3.764456-3.8450892 .709415$

O $-3.755043-1.9849004 .071262$

C $-4.407239-4.5974543 .743116$

H $-3.773182-4.6365634 .633901$

H $-5.381359-4.159106 \quad 3.980213$

H -4.560634 -5.615978 3.376831

SCF Energy (B3LYP/6-31G**//MMFF)= -3245.90771892

01423

MM̄FF Geometry

C 4.3585571 .5747342 .832510

C 3.2412361 .2192092 .181760

C 2.0518220 .5686642 .830214

$\begin{array}{lllll}\text { O } & 0.893333 & 1.316349 & 2.395232\end{array}$

C $1.908115-0.8837852 .336134$

C $0.779441-1.6342423 .015449$

C $-0.447083-1.8309602 .489900$

C $1.112656-2.1838044 .378865$

C $-0.977458-1.3477851 .166126$

C $-1.551022-2.4764480 .295861$

C $-2.894513-3.0310690 .797806$

C $-3.383683-4.159159-0.114549$

$\begin{array}{llll}\text { C }-4.773198 & -4.670240 & 0.297515\end{array}$

O $-3.845011-1.9586860 .803971$

O $-5.282924-5.475736-0.776741$

C $-5.722758-3.461960 \quad 0.444871$

C $-4.707993-5.582231 \quad 1.528007$

O $-6.988414-3.863342 \quad 0.962755$

C $-5.138622-2.3218621 .307571$

C $-6.022189-1.0517911 .300018$

O $-5.052541-2.7584892 .669820$

C $-0.068597 \quad 1.617078 \quad 3.303913$

$\begin{array}{lllll} & -0.027305 & 1.425257 & 4.510665\end{array}$

C - -1.2844192 .1259472 .627837$

C $-1.2690252 .642731 \quad 1.389534$

C -2.437470 $3.047931 \quad 0.609045$

C -3.799217 2.7349091 .172519

C $-6.290397-0.450534-0.081975$

C $-6.994156 \quad 0.895217-0.001295$

C $-7.9899172 .645873-1.479863$ 
O $\quad-7.179740 \quad 1.496016 \quad 1.053496$

C $-6.9627463 .558731-2.070142$

C $-6.4422434 .616079-1.429063$

C $-5.3739055 .498093-2.013200$

C $-4.0474175 .334285-1.296703$

C $-3.5856486 .311438-0.497612$

C $-3.3119074 .037130-1.578699$

C $-2.2488883 .642559-0.588183$

N $-7.373572 \quad 1.350271-1.257153$

H 4.4454801 .3871083 .899355

H 3.1785851 .3957351 .111427

H 2.1530780 .6012843 .921542

H $2.843776-1.4321892 .510130$

H $1.767939-0.8936291 .250491$

H -1.171090 -2.370843 3.097673

H $0.276230-2.7341904 .822633$

H $1.961097-2.8726894 .312592$

H $1.374955-1.3739755 .066678$

H $-0.202592-0.8384480 .587313$

H -1.752849 -0.5980051 .357178$

H -1.701337 -2.065342 -0.710461

H $-0.818732-3.2883190 .210037$

H -2.766197 -3.405954 1.818494

H $-2.661333-4.984807-0.133622$

H -3.444158 -3.790840 -1.148437

H $-4.654174-6.201476-0.931423$

H -5.906424 -3.084339 -0.568686

H $-4.080756-6.457991 \quad 1.322175$

H $-5.698633-5.9764301 .780158$

H -4.298768 -5.0859542 .410204$

H $-7.322180-4.5766160 .391974$

H $-6.976350-1.2566051 .803457$

H $-5.526315-0.2907231 .918811$

H $-5.013487-1.9747363 .242718$

H -2.200930 2.0228663 .199142

H -0.3143902 .7583090 .880556$

H -4.617045 3.0196530 .507281

H -3.959194 3.2710882 .114347

H -3.9011591 .6597181 .356672$

H -5.348370 -0.300643 -0.621525

H $-6.924601-1.124612-0.667709$

H $-8.3977373 .034425-0.541205$

H -8.814792 2.505847 -2.185021

H -6.617912 $3.323597-3.074579$

H $-6.7798604 .850699-0.422273$

H $-5.7242436 .536545-1.956903$

H -5.228340 $5.291864-3.081276$

H $-2.632668 \quad 6.2396040 .014191$

H -4.154099 $7.222414-0.339248$

H -2.848228 4.114757 -2.570849

H $-4.0225213 .206493-1.662033$

H -1.231605 $3.839798-0.925990$

H $-7.1392140 .793855-2.074426$

C 6.6320721 .2177591 .841181

O 6.1595850 .3069560 .827420

C $7.1766010 .154179-0.178374$

C $8.451057 \quad 0.7242190 .429937$

C 7.8953751 .8599171 .267302

H $6.8793330 .781380-1.027234$

C $7.265173-1.310400-0.617298$

H $8.958813-0.0056591 .070948$

H $9.1621071 .058357-0.331662$

C 8.8623642 .3684452 .322187

H 7.6260092 .6889380 .598984

C $5.924258-1.912120-1.090064$

O $7.724433-2.1039550 .485683$

H $8.024207-1.418573-1.400344$

H $\quad 5.240390-1.997950-0.238420$

O $6.181749-3.250233-1.535121$

C $5.241125-1.140548-2.235790$

C $4.004499-1.850030-2.822240$

H $4.958549-0.141936-1.889411$

O $6.189865-0.965776-3.292046$

H $3.701831-1.311775-3.728202$

H $4.294971-2.846675-3.175591$

H 8.4108383 .1743402 .909203

H 9.1568301 .5719043 .013919

H 9.7714092 .7597231 .853844
C 5.5308042 .2292282 .155319

H $7.100128-1.9740531 .220331$

H $6.612709-3.721709-0.801813$

H $5.771411-0.413165-3.974012$

H 5.1988712 .7121641 .227956

H 5.9088333 .0206612 .812210

H 6.8701630 .6150832 .727205

C $1.7051990 .129007-2.390320$

O $2.374721-0.661993-1.400021$

C $2.819755-1.949873-1.841052$

C $1.632033-2.767081-2.365117$

C $0.858899-2.015109-3.443135$

C $0.488987-0.615120-2.958130$

H $2.4031810 .378150-3.198442$

C $1.2573431 .434587-1.713432$

H $3.181853-2.471222-0.947823$

O $2.079932-4.021429-2.866895$

H $\quad 0.956937-2.981747-1.527649$

H $1.430356-1.968223-4.377071$

O $-0.336649-2.734752-3.755213$

H $-0.295627-0.685679-2.194996$

O $\quad-0.047930 \quad 0.128798-4.050471$

C $2.4005472 .394490-1.463123$

H $0.5348641 .962449-2.345843$

H $0.796966 \quad 1.208879-0.744986$

H $1.302043-4.491793-3.212725$

H $-0.866969-2.804854-2.943173$

H $-0.800615-0.375889-4.403610$

O $1.8984373 .528827-0.889031$

O $3.5755442 .186153-1.729072$

C $2.8559324 .549567-0.593019$

H $2.3167745 .419102-0.207917$

H 3.5518174 .2004920 .174430

H $3.3941994 .844228-1.499074$

SCF Energy $\left(B 3 L Y P / 6-31 G^{* *} / / M M F F\right)=-3245.91613447$

01424

MM̄FF Geometry

C $0.3104823 .146064-0.211720$

C 1.5022463 .4218210 .341661

C 2.2264912 .5943371 .379100

O 1.3875571 .4904211 .790964

C 3.5630972 .0281510 .865986

C 4.5623333 .0679900 .407688

C $5.0193693 .188636-0.854076$

C 5.0870523 .9807201 .486366

C $4.6552952 .359754-2.054208$

C $5.8415671 .535063-2.572142$

C $6.3552840 .484535-1.573024$

C $7.597191-0.213269-2.131542$

C $8.102961-1.314104-1.189057$

O $5.315163-0.472938-1.344321$

O $9.056619-2.107336-1.912134$

C $6.920389-2.236452-0.832229$

C $8.848096-0.7349840 .019161$

O $7.308668-3.2031660 .142541$

C $5.644561-1.482061-0.378420$

C $4.468055-2.483836-0.304333$

O $5.873479-0.9212560 .914525$

C 1.5556241 .0020293 .048095

O $2.3982991 .336242 \quad 3.864551$

C 0.4897640 .0027943 .272265

C $0.327252-0.5390244 .487458$

C $-0.722573-1.4881584 .842400$

C $-0.266774-2.5865405 .762519$

C $3.101399-1.8681760 .027408$

C $1.987902-2.896309-0.098693$

C $-0.469772-3.123106-0.049800$

O $2.188087-4.060672-0.437618$

C $-1.591896-2.6262290 .803651$

C $-2.368276-3.4489231 .525154$

C $-3.561123-3.0155782 .329600$

C $-3.409596-3.3246963 .802401$

C $-3.535270-4.5815014 .262316$

C $-3.185071-2.1740634 .754735$

C $-1.988738-1.3321374 .407295$

N $0.740148-2.3649640 .207895$

H -0.2142242 .2313990 .048859$ 
H 1.9895104 .3553500 .066109 H 2.3943043 .2458102 .246238 H 3.3534331 .2865200 .089545 H 4.0328331 .4393111 .665010 H $5.7490843 .970755-1.061000$ H 5.8930524 .6292431 .126554 H 4.2908774 .6273761 .867150 H 5.4882723 .3928282 .318490 H $3.800657 \quad 1.705130-1.870512$ H $4.3349393 .046038-2.848218$ H $5.5171381 .030366-3.491365$ H $6.6596782 .212805-2.847582$ H $6.6030420 .980041-0.628274$ H $8.3946440 .512598-2.334104$ H $7.354324-0.670348-3.101251$ H $9.775057-1.520420-2.203908$ H $6.679283-2.807005-1.739686$ H $9.692610-0.118455-0.311656$ H $9.283621-1.5311580 .633150$ H $8.215206-0.1135580 .656142$ H $7.334617-2.7576661 .006496$ H $4.381795-2.988947-1.275881$ H $4.684210-3.255848 \quad 0.445334$ H $5.019621-0.7628141 .345965$ H $-0.163363-0.2401922 .441157$ H $1.005250-0.2780395 .297500$ H $0.036313-2.1749556 .731578$ H $0.590082-3.1153475 .329397$ H $-1.038605-3.3375195 .950070$ H $3.089368-1.4773131 .050094$ H $2.880029-1.048384-0.662988$ H $-0.271815-4.1888450 .108042$ H $-0.715406-2.983460-1.106577$ H $-1.805898-1.5604730 .791282$ H -2.161296 $-4.517148 \quad 1.503873$ H $-4.444698-3.5379411 .939683$ H $-3.770044-1.9486882 .185207$ H $-3.452493-4.8090625 .319847$ H -3.724541 -5.411298 3.589222 H -3.115176 -2.521834 5.792588 H $-4.072864-1.5281334 .734210$ H $-2.220493-0.4903423 .754617$ H $0.659522-1.3558910 .299215$ C -1.738459 $4.581847-0.659047$ O $-2.721746 \quad 3.526878-0.670401$ C -3.5158923 .6296290 .525334$ C $-3.1957114 .992608 \quad 1.136595$ C -1.7399635 .1615310 .756914$ H $-3.1582352 .843603 \quad 1.201024$ C $-5.004162 \quad 3.4198840 .228029$ H -3.7951175 .7942290 .690131$ H $-3.363920 \quad 5.010698 \quad 2.217738$ C -1.243504 6.5943820 .834417 H -1.1460434 .5441991 .441204$ C $-5.3685392 .139508-0.547813$ O $-5.4939864 .525176-0.545962$ H $-5.563666 \quad 3.4512211 .170635$ H -5.100056 2.254591-1.604688 O $-6.7991242 .023829-0.508238$ C -4.762595 $0.830777-0.010908$ C $-5.415326-0.454232-0.558485$ H $-3.6903210 .813224-0.229154$ O $-4.885590 \quad 0.789882 \quad 1.414410$ H $-4.863470-1.305458-0.142071$ H -6.423894 $-0.545348-0.134888$ H -0.1915726 .6576310 .538466$ H -1.819371 7.2553420 .177932 H -1.331287 6.9772101 .856553 C $-0.3898044 .065950-1.174649$ H $-4.9586264 .575065-1.356666$ H $-7.1662302 .844451-0.879666$ H -5.8314770 .8590251 .630424$ H $0.2511234 .923744-1.411617$ H - $0.5475663 .513268-2.108558$ H -2.106981 $5.332020-1.371266$ C $-3.333678-1.565593-2.526027$ O $-4.206790-0.447340-2.720475$ C $-5.488007-0.559087-2.095487$
C $-6.214753-1.815356-2.597791$

C $-5.372546-3.069019-2.394594$

C $-3.985122-2.873691-2.998465$

H $-3.076276-1.639446-1.464895$

C $-2.048963-1.295045-3.326354$

H $-6.0740680 .296253-2.450344$

O $-7.466501-1.981710-1.940456$

H -6.426634 -1.709520 -3.669840

H $-5.306750-3.346598-1.336164$

O $-5.997595-4.169358-3.059668$

H $-4.060471-2.881721-4.093647$

O $-3.156201-3.972045-2.620148$

C $-1.137823-0.293943-2.650046$

H $-1.477591-2.223630-3.438064$

H $-2.290782-0.901100-4.320219$

H $-7.986079-1.173952-2.093172$

H $-6.894790-4.255525-2.693933$

H $-3.600861-4.785756-2.913581$

O $0.103279-0.380923-3.214735$

O $-1.469818 \quad 0.475487-1.759954$

C $1.0957850 .500248-2.682142$

H $1.1224060 .443239-1.590253$

H $2.0690260 .187753-3.069744$

H $0.8971321 .523310-3.012575$

SCF Energy (B3LYP/6-31G**//MMFF)= -3245.91679966

01425

MM̄FF Geometry

C $0.0453324 .771467 \quad 0.458169$

C $0.5117154 .462041 \quad 1.678655$

C $0.072476 \quad 3.3037462 .546630$

O $-0.999296 \quad 2.5612151 .924913$

C 1.2679902 .3462602 .729039

C $0.986517 \quad 1.176688 \quad 3.652263$

C $0.909307-0.111693 \quad 3.262358$

$\begin{array}{llll}\text { C } & 0.814200 & 1.530608 & 5.107358\end{array}$

C $1.070788-0.6697191 .874969$

C $-0.288319-1.0109341 .270247$

C $-0.236607-1.547337-0.167477$

C $0.329774-0.529285-1.160056$

C $0.208413-1.002782-2.616521$

O $-1.593387-1.854231-0.511233$

O $0.467797 \quad 0.141950-3.442245$

C $-1.238819-1.467750-2.879081$

C $1.283677-2.039096-2.959748$

O $-1.362300-2.061565-4.169556$

C $-1.774989-2.440798-1.805125$

C $-3.293971-2.661589-2.008045$

O $-1.098014-3.692186-1.918786$

C -2.278475 2.9204922 .220233

O $-2.640861 \quad 3.8006672 .983525$

C -3.174061 2.0574841 .416179

C -4.4994142 .2649621 .419716$

C -5.4884951 .5028290 .650438$

C $-4.9965860 .294460-0.102429$

C $-3.959414-3.472119-0.886855$

C $-5.474475-3.356334-0.912767$

C $-7.451230-3.6987930 .574569$

O $-6.101137-2.912789-1.870663$

C -7.671606 -2.407612 1.300785

C $-8.653472-1.542476 \quad 1.004746$

C $-8.866029-0.2511111 .744209$

$\begin{array}{llll}\text { C } & -9.075521 & 0.928847 & 0.819329\end{array}$

$\begin{array}{llll}\text { C }-10.243674 & 1.593198 & 0.808163\end{array}$

C $-7.9432441 .339157-0.097832$

C $-6.772291 \quad 1.9204040 .650205$

N $-6.038063-3.8215780 .267494$

H -0.7559184 .1866670 .017600$

H 1.3193145 .0655562 .090063

H -0.2479753 .6949303 .519338$

H 2.1285852 .8961053 .133969

H 1.5856631 .9943951 .742195

H $\quad 0.700525-0.8653404 .020790$

H $\quad 0.724567 \quad 0.643427 \quad 5.743234$

H 1.6776932 .1017135 .464072

H -0.0879192 .1319195 .254539$

H 1.6186050 .0198461 .232201

H $1.685673-1.5742541 .938780$ 
H $-0.794257-1.7547161 .901607$ H $-0.938932-0.1296801 .295797$ H $0.352683-2.470921-0.175861$ H $1.376532-0.295431-0.936562$ H $-0.2176820 .418361-1.056528$ H $0.456691-0.145111-4.370718$ H -1.875311 -0.571598 -2.884646 H $2.282353-1.601442-2.842966$ H $1.214999-2.352054-4.007345$ H $1.233028-2.930195-2.331284$ H -1.126099-1.387639-4.828520 H -3.786138-1.682301-2.061282 H -3.479786 -3.170575 -2.962002 H -1.640654 -4.383102 -1.507595 H $-2.713001 \quad 1.2816410 .816626$ H -4.8987503 .0778372 .024616$ H $-4.495990-0.4051240 .576689$ H $-4.2900960 .590837-0.885610$ H $-5.791619-0.270624-0.590740$ H -3.702906 -4.533186 -0.977634 H $-3.614259-3.1201240 .091789$ H -7.716879 -4.5407771 .220749$ H $-8.033606-3.758738-0.350425$ H -6.993055 -2.176416 2.119102 H $-9.352669-1.7830070 .207297$ H -8.024759 -0.0361652 .414471$ H -9.742239 -0.3834092 .392221$ H -10.407383 $2.439422 \quad 0.149127$ H -11.064256 1.3073951 .457921 H -8.289245 2.111459-0.798057 H $-7.6717080 .490875-0.730306$ H -7.024072 2.8140991 .223281 H -5.423278 -4.1135851 .021833$ C $1.3745555 .342327-1.620982$ O $2.3413354 .350347-1.212646$ C $2.1276463 .160967-1.993948$ C $1.4563903 .652517-3.267106$ C $0.5049374 .690746-2.700948$ H $1.4253792 .529918-1.433215$ C $3.4341582 .386519-2.203611$ H $2.1780084 .124491-3.944307$ H $0.9441602 .854276-3.810922$ C $-0.0173165 .670287-3.737971$ H $-0.3501324 .170035-2.253703$ C $4.0331161 .855631-0.880215$ O $4.3713473 .218361-2.882516$ H $3.226641 \quad 1.532822-2.859531$ H $3.2458731 .311341-0.347807$ O $4.4396172 .937413-0.042886$ C $5.2335390 .907523-1.077888$ C 5.8481310 .3967990 .240899 H $4.9183290 .059146-1.696397$ O $6.2654421 .586321-1.796731$ H $6.735848-0.196228-0.011030$ H $6.237786 \quad 1.2479220 .813652$ H $-0.6767676 .410850-3.274228$ H $0.7997086 .207361-4.231415$ H $-0.5878445 .144513-4.510542$ C $0.6244225 .870928-0.391401$ H $4.5820603 .961105-2.290844$ H 3.6524613 .4745150 .149675 H $6.9741920 .944107-1.972220$ H $-0.1767846 .552195-0.698812$ H $1.327047 \quad 6.460870 \quad 0.211105$ H $1.9510346 .169257-2.054738$ C $5.135111-2.5643960 .020773$ O $4.262636-1.488294 \quad 0.378519$ C $4.886645-0.4318151 .115268$ C $5.518129-0.9828542 .402228$ C $6.476437-2.1382762 .121186$ C $5.794809-3.1971391 .254165$ H $5.919917-2.173044-0.639097$ C $4.349668-3.569312-0.835100$ H $4.0827390 .243376 \quad 1.429323$ O $\quad 6.2145680 .0407653 .105151$ H $4.724201-1.3470633 .067371$ H $7.405068-1.7858321 .657652$ O $6.853366-2.7468313 .358660$
H $\quad 5.056116-3.7232631 .868159$

O $6.755923-4.1695310 .848259$

C $3.156238-4.201143-0.147385$

H $3.961281-3.049663-1.718467$

H $5.019609-4.370364-1.167502$

H $5.5791530 .754293 \quad 3.287021$

H $7.245516-2.0517983 .914802$

H $7.433499-3.717250 \quad 0.317322$

O $2.653889-5.182923-0.954953$

O $2.704030-3.8734670 .940109$

C $1.504788-5.868721-0.447711$

H $1.141729-6.545609-1.225591$

H $\quad 1.780200-6.4575960 .432058$

H $0.708904-5.159170-0.202880$

SCF Energy (B3LYP/6-31G**//MMFF) $=-3245.90701575$

01426

MM̄FF Geometry

C -0.939447 -1.895437 2.782898

C $-0.700918-2.7639841 .787255$

C $-1.605848-3.0213600 .603232$

O $-2.931682-2.517167 \quad 0.903988$

C $-1.126782-2.343984-0.692511$

C $0.279320-2.677854-1.141381$

C $1.266110-1.765025-1.239123$

C $0.514382-4.105322-1.555146$

C $1.168611-0.317654-0.850132$

C $0.6584000 .561968-1.997319$

C $0.1160921 .924438-1.537276$

C $1.1549642 .765178-0.794276$

C $0.5580274 .081265-0.273227$

O $-1.0162651 .674962-0.697287$

O $1.481879 \begin{array}{llll}4.638587 & 0.674697\end{array}$

C -0.7454793 .7719320 .494718$

C $0.4051665 .120317-1.389960$

O -1.4090194 .9760020 .874161$

C $-1.7197982 .850086-0.276523$

C -2.8720392 .4032260 .662119$

O $-2.2521403 .569428-1.387021$

C $-3.993996-3.0764550 .268428$

O $-3.974217-3.990548-0.539160$

C $-5.202612-2.3557410 .728212$

C $-6.394947-2.6289200 .178916$

C $-7.667841-1.9873840 .518007$

C $-7.642737-0.8872211 .547453$

C -3.8223901 .3429580 .082236$

C $-4.9245081 .903100-0.801199$

C $-6.6889031 .173483-2.405757$

O $-5.1760013 .102435-0.880771$

C $-7.9966471 .047536-1.689826$

C $-9.0079100 .281764-2.127282$

C $-10.3384300 .179549-1.437664$

C $-10.748885-1.250472-1.163565$

C $-11.635483-1.878651-1.954219$

C $-10.198134-1.9202240 .075693$

C $-8.786058-2.402520-0.113398$

N -5.603746 $0.901300-1.481206$

H -1.827200 -1.269044 2.749802

H $\quad 0.193135-3.3820801 .840227$

H -1.669540 -4.108904 0.471030

H $-1.274046-1.262423-0.591554$

H -1.809887 -2.616152 -1.508865

H $2.237169-2.078758-1.616717$

H $1.521484-4.259769-1.957109$

H $\quad 0.394899-4.778204-0.700485$

H $-0.198973-4.398291-2.332385$

H $0.546436-0.2114120 .045141$

H $2.1667130 .021718-0.555197$

H $1.4615940 .710051-2.729235$

H $-0.1545470 .039708-2.519034$

H $-0.2241472 .453660-2.435314$

H $2.0234192 .961642-1.434026$

H 1.5390922 .2104730 .070685

H 2.3341844 .7648030 .223480

H -0.4645143 .2682421 .428923$

H $1.3800355 .344611-1.839486$

H $0.0315986 .071856-0.996082$

H $-0.259414 \quad 4.793352-2.192252$ 
H $-0.768273 \quad 5.5170581 .366562$ H -2.4223311 .9552121 .558394$ H $-3.450363 \quad 3.2756730 .988417$ H -3.018021 $3.086209-1.732381$ H $-5.074773-1.6086021 .503548$ H $-6.447490-3.392636-0.596036$ H $-6.935615-0.1018531 .258086$ H -7.345142 -1.283998 2.524246 H -8.606000 -0.3913531 .681803$ H $-3.2662940 .575598-0.466544$ H -4.3276880 .8452250 .919495$ H $-6.5985180 .460201-3.230977$ H $-6.5832562 .186778-2.806308$ H $-8.1264531 .633028-0.782509$ H -8.886466 $-0.280494-3.050551$ H -11.083761 $0.664438-2.081844$ H $-10.3473930 .745714-0.497864$ H -11.956671 -2.894327 -1.748149 H -12.055829 -1.392969-2.828785 H -10.807974 -2.797679 0.330390 H $-10.313710-1.2467920 .929178$ H -8.697796 -3.204867 -0.847380 H $-5.368947-0.070189-1.299746$ C $0.774806-0.5150684 .111213$ O $1.969913-0.6729053 .324468$ C 2.3983400 .6344402 .892422 C 1.4349111 .6418643 .519490 C 0.1720910 .8126753 .662801 H $2.2649390 .658074 \quad 1.806843$ C $3.874306 \quad 0.8657793 .235179$ H 1.7805451 .9822174 .502522 H 1.3027012 .5283552 .892900 C -0.8402751 .3976254 .629361$ H -0.2843950 .7205302 .669112$ C $4.833398-0.2270532 .719936$ O 4.0120640 .9178964 .661193 H 4.1806121 .8503932 .861439 H $4.708030-1.1391233 .315522$ $\begin{array}{lllll}\text { O } & 6.171082 & 0.222997 & 2.974296\end{array}$ C $4.688009-0.5677541 .226489$ C $5.788512-1.5275620 .736090$ H $3.707529-1.0274151 .062012$ O $4.736103 \quad 0.6458280 .483063$ H $6.746211-0.9964190 .716520$ H $5.880705-2.3465801 .458989$ H $-1.721913 \quad 0.753872 \quad 4.706880$ H -0.4175561 .5131455 .632959$ H -1.171253 2.3840574 .288145 C $-0.095778-1.7687354 .019820$ H 4.9520851 .0740604 .855931 H 6.3322490 .9969882 .407460 H $4.4840020 .434269-0.431100$ H $-0.782997-1.7826204 .875287$ H $\quad 0.543826-2.6548654 .121829$ H $1.117959-0.4327175 .152330$ C $6.572380-0.458423-2.046731$

O $5.359706-1.128111-1.680085$ C $5.496172-2.123443-0.661461$ C $6.499116-3.196304-1.110632$

C $7.840649-2.582558-1.503591$

C $7.630951-1.461715-2.520556$

H $6.9511840 .109607-1.188732$

C $6.2318710 .533245-3.166875$

H $4.518291-2.615622-0.583903$

O $6.676963-4.159160-0.077921$

H $6.084563-3.726689-1.978066$

H $8.391733-2.219951-0.627980$

O $8.660488-3.581540-2.113927$

H $7.334094-1.896070-3.483965$

O $8.865367-0.776559-2.724017$

C $5.3389831 .645354-2.672655$

H $7.1290770 .987095-3.602809$

H $5.6998690 .027410-3.982187$

H $7.210704-4.886038-0.440357$

H $8.887057-4.233366-1.429403$

H $9.521587-1.437920-3.003170$

O $6.0688232 .556354-1.968383$

O $4.1330741 .690341-2.878626$
C $5.3261183 .656709-1.433113$

H $4.5846243 .298830-0.712734$

H $6.0252914 .319235-0.916311$

H $4.8419924 .216941-2.238876$

SCF Energy $\left(B 3 L Y P / 6-31 G^{* *} / / M M F F\right)=-3245.92008042$

01427

MM̄FF Geometry

C -1.558846 -0.331685-3.155988

C $-2.5456520 .471040-3.584804$

C $-3.4379121 .318487-2.713849$

O $-3.9153360 .571932-1.569180$

C $-2.7275462 .565394-2.160354$

C $-2.3497183 .592214-3.203630$

C $-1.0906273 .840255-3.612661$

C $-3.4940054 .405132-3.751826$

C $0.1713343 .137722-3.198283$

C $1.0938964 .026311-2.356584$

C $0.5755994 .289119-0.932703$

C $1.5422215 .216275-0.190681$

C 1.1264755 .4317491 .269890

O $0.4719313 .034583-0.247745$

O 2.2333696 .0316291 .960144

C 0.8795424 .0571981 .921521

C $-0.045544 \quad 6.412347 \quad 1.393155$

O $0.3573454 .209030 \quad 3.240185$

C -0.0383463 .1284671 .089960$

C $-0.028025 \quad 1.711829 \quad 1.715414$

O -1.3675533 .6498321 .113898$

C $-4.982718-0.245074-1.762473$

O $-5.494447-0.532571-2.836556$

C $-5.511162-0.774870-0.484882$

C $-5.141298-0.3227650 .723659$

C $-5.654982-0.7910462 .014621$

C $-6.743667-1.8334021 .991989$

C -0.8115320 .6465100 .936427$

C $-0.717855-0.7167091 .604713$

C $-1.763281-2.9778131 .580802$

O $0.069128-0.9613682 .516167$

C $-2.932645-3.0102742 .511373$

C $-2.842931-2.7530623 .825128$

C $-4.009333-2.7677424 .771804$

C $-4.354366-1.3824755 .275968$

C $-3.741680-0.8681996 .355629$

C $-5.461056-0.6271544 .570824$

C $-5.116709-0.2877713 .146238$

N -1.605426 -1.633146 1.054951

H -1.340239-0.394831 -2.094503

H -2.726946 $0.551305-4.654688$

H -4.310232 $1.633042-3.300518$

H $-1.8625642 .257330-1.563757$

H -3.384580 $3.048956-1.423513$

H $-0.9379134 .626655-4.351080$

H $-3.1565595 .187100-4.440548$

H $-4.1937713 .766155-4.298919$

H $-4.0349844 .897611-2.937068$

H $-0.0255342 .197145-2.678767$

H $0.7031262 .859936-4.116913$

H $2.0669093 .523770-2.294235$

H $1.2604204 .979810-2.873475$

H $-0.4168324 .749000-0.993612$

H $1.6329566 .178949-0.709212$

H $2.5494074 .776147-0.200858$

H 2.4478256 .8681591 .512732

H 1.8566353 .5668472 .031008

H 0.2269987 .3886650 .974495

H $-0.297424 \quad 6.5970012 .443192$

H $-0.945615 \quad 6.072926 \quad 0.876521$

H $0.9724384 .780623 \quad 3.731191$

H 1.0124321 .3704871 .796283

H $-0.435221 \quad 1.7561602 .733722$

H -1.992207 2.9412490 .896503

H -6.265029 -1.547395 -0.598823

H -4.3932360 .4652490 .785576$

H $-6.405004-2.7342901 .468283$

H -7.634014 -1.445571 1.484616

H -7.063025 -2.158499 2.983582

H -1.8691170 .9193070 .870323$ 
H $-0.4144600 .550164-0.078083$ H $-0.848295-3.2918402 .090206$ H -1.935984 -3.652568 0.737300 H -3.895475 -3.265901 2.078426 H -1.872339 -2.505617 4.249943 H -3.753009-3.419453 5.617355 H $-4.892582-3.2270814 .311058$ H -3.999095 0.1131416 .739687 H -2.965311 -1.418283 6.877261 H -5.6687590 .3185635 .089014$ H $-6.386337-1.2029674 .656738$ H -4.3247750 .4574763 .058329$ H $-2.248384-1.3282010 .329923$ C $-0.364669-2.550829-3.629759$ O $0.641675-2.536047-2.596107$ C $0.406345-3.658919-1.724508$ C $-0.740121-4.460716-2.343645$ C $-1.515506-3.384211-3.075177$ H $0.065351-3.243084-0.772266$ C $1.681227-4.486217-1.515428$ H $-0.372363-5.213990-3.050501$ H -1.335429 -4.979898-1.586025 C $-2.461706-3.918114-4.134782$ H -2.090114 -2.818418 -2.331166 C $2.869739-3.757122-0.851095$ O $2.134250-4.978910-2.783874$ H $1.433450-5.376612-0.924485$ H $3.337506-3.063459-1.559544$ O $3.856870-4.755615-0.554768$ C $2.528857-3.0117580 .449491$

C $3.721291-2.6416831 .349820$ H $1.961606-2.1084250 .199383$ O $1.666346-3.8382501 .240632$ H $3.322388-2.0152662 .158030$ H $4.077624-3.5473301 .859478$ H -2.984073 -3.098611-4.638465 H -1.927514 -4.496359-4.896041 H $-3.214993-4.572008-3.683354$ C $-0.697655-1.130484-4.094166$ H $2.300010-4.208214-3.353865$ H $4.071963-5.206590-1.389335$ H $1.436322-3.3380722 .042297$ H -1.183409 -1.192733 -5.075898 H $\quad 0.237210-0.577739-4.229929$ H $0.098227-3.073465-4.478382$ C $3.979150 \quad 0.2735200 .476906$ O $4.542496-0.866884-0.177436$ C $4.917565-1.9391890 .682363$

C $5.971440-1.4466851 .685093$

C $5.471813-0.2364302 .472191$

C 4.9460290 .8433191 .527481

H $3.027059-0.0073670 .942906$

C $3.6893051 .318182-0.607665$

H $5.406280-2.683513 \quad 0.042821$

O $\quad 6.331578-2.4793072 .596638$

H $6.883255-1.1587321 .146000$

H $4.710563-0.5184783 .208509$

O $6.559677 \quad 0.3148113 .220055$

H $5.8038001 .323874 \quad 1.039155$

O 4.2879581 .8382682 .309552

C $2.9009960 .723409-1.751051$

H $3.1295712 .170875-0.212407$

H $4.6329061 .712445-1.006287$

H $6.651459-3.2333202 .072330$

H $6.903493-0.3921423 .792733$

H 4.1166432 .6025861 .734512

O $3.6386550 .791209-2.896609$

O $1.7820040 .242605-1.637460$

C $3.0439140 .212682-4.060557$

H $2.184770 \quad 0.810670-4.374743$

H $3.7875330 .220737-4.861838$

H $2.751150-0.823915-3.868696$

SCF Energy (B3LYP/6-31G**//MMFF)= -3245.91303283

01428

MM̄MF Geometry

C -4.483414 -1.761626 -3.431880

C $-3.351706-1.999556-2.751991$
C $-2.037839-1.340229-3.072713$

O $-1.780594-0.367989-2.031642$

C $-0.893840-2.368485-3.047582$

C $0.376202-1.844184-3.689355$

C $1.405993-1.292075-3.018392$

C $0.451235-2.011927-5.185110$

C $1.491952-1.007816-1.546451$

C $2.441915-1.938967-0.785474$

C $3.906993-1.896899-1.249557$

C $4.780037-2.718026-0.294838$

C $6.272314-2.617231-0.637825$

O $4.337999-0.533358-1.271018$

O $6.996056-3.1465620 .483699$

C $6.645875-1.125911-0.783695$

C $6.633967-3.502632-1.835634$

O $7.996775-0.984090-1.216468$

C $5.688296-0.339231-1.705834$

C $5.9933371 .180628-1.715763$

O $5.865297-0.803467-3.050908$

C $-2.2223160 .903316-2.227255$

O $-2.8142941 .336915-3.202411$

C $-1.8554681 .689754-1.027747$

C $-2.0440233 .017917-1.020511$

C -1.7108553 .9310520 .075977$

C $-1.334653 \quad 3.3194471 .398867$

C $5.7905561 .929907-0.394858$

C $4.3331572 .072164-0.000108$

C $2.1977623 .138454-0.725811$

O 3.8717881 .4905450 .978953

C 1.9708394 .3661110 .096435

C $1.4940595 .516911-0.401792$

C $1.177506 \quad 6.720908 \quad 0.438917$

C $-0.237101 \quad 7.2240530 .232095$

C $-0.4682398 .409850-0.356344$

C $-1.380990 \quad 6.3870250 .770672$

C $-1.7464745 .259748-0.158518$

N $3.6203712 .896813-0.854739$

H $-4.466331-1.078784-4.277587$

H -3.379818 -2.659254-1.888019

H $-2.075277-0.847208-4.052302$

H - $-1.192728-3.281735-3.579675$

H $-0.689711-2.682188-2.016189$

H $2.275156-0.969131-3.589899$

H $1.388304-1.626946-5.601229$

H $\quad 0.382809-3.071769-5.451713$

H $-0.369676-1.476830-5.672663$

H $1.8096380 .033555-1.418871$

H $\quad 0.508024-1.066869-1.074404$

H $2.398912-1.6410560 .269424$

H $2.070515-2.970079-0.837598$

H $3.966371-2.307491-2.263897$

H $4.458017-3.766880-0.278212$

H $4.639823-2.348760 \quad 0.731370$

H $7.944499-3.0179780 .309181$

H $6.606062-0.6976850 .224833$

H $6.409625-4.552913-1.613039$

H $7.708348-3.466858-2.046696$

H $\quad 6.093516-3.235740-2.746089$

H $8.024619-1.178679-2.168793$

H $7.0260091 .335481-2.054223$

H $5.3673791 .652947-2.484616$

H $5.510503-0.132587-3.657417$

H -1.421516 $1.156015-0.190103$

H -2.455465 $3.490025-1.911805$

H -0.3928442 .7717661 .309939$

H -2.1127452 .6268151 .738560$

H -1.205681 4.0492662 .200181

H $6.336175 \quad 1.4577340 .427317$

H $6.2041112 .942045-0.490011$

H $1.7178052 .279177-0.246819$

H $1.7908313 .238387-1.737250$

H $2.1587144 .283903 \quad 1.163915$

H $1.3084215 .596686-1.470561$

H 1.9016687 .5049720 .183302

H 1.3248026 .5099121 .505516

H -1.476806 $8.787882-0.487975$

H $0.3445219 .027286-0.724040$

H -2.2759457 .0105440 .896699$ 
H -1.122823 6.0500841 .777773 H -2.050345 $5.599398-1.149991$ H $4.1162423 .399239-1.585171$ C -6.548679-1.559894-1.954305 O $-5.846866-1.722524-0.704579$ C $-5.679223-0.427941-0.099759$ C $-6.7397640 .456161-0.742760$ C $-6.709918-0.052509-2.173535$ H -4.689549-0.064024-0.401555 C $-5.733533-0.5321891 .427925$ H $-7.7304560 .289431-0.304642$ H $-6.5021691 .520832-0.659935$ C $-7.939430 \quad 0.318234-2.984089$ H $-5.8267580 .372859-2.664862$ C $-4.810455-1.6208182 .016518$ O H -5.5061630 .4464361 .867389$ H $-5.216715-2.6148651 .791415$ O $-4.821043-1.4919273 .443570$ C $-3.346601-1.5519191 .542273$ C $-2.469605-2.6311532 .207835$ H $-3.308523-1.6945240 .458377$ O $-2.819779-0.256818 \quad 1.817067$ H -2.316350 -2.375139 3.261605 H -3.008125 -3.585582 2.177249 H $-7.872911-0.090343-3.997503$ H -8.855638 $-0.066554-2.523931$ H $-8.0347221 .406145-3.062676$ C $-5.819848-2.338777-3.057112$ H -7.146732 -0.7142572 .772684$ H -5.708088 -1.739349 3.754251 H -2.892415 -0.1061112 .775171$ H $-6.443688-2.372904-3.957964$ H $-5.678911-3.377565-2.731872$ H -7.531465 -2.024904-1.803161 C $0.197195-1.1818202 .732986$ O $-0.330593-1.6086811 .470502$ C -1.103049-2.809815 1.505142 C $-0.251491-3.9595842 .059178$ C $0.357344-3.6105623 .415586$ C $1.090142-2.2700763 .348084$ H $-0.637374-0.9632813 .407508$ C $0.973110 \quad 0.1186092 .465847$ H -1.328265 -3.0560710 .459398$ O $-1.030596-5.1461832 .161539$ H $0.560051-4.1697501 .350071$ H -0.409386 -3.599297 4.198666 O $1.281226-4.6354463 .791789$ H $2.005971-2.3986632 .757128$ O $1.479135-1.9087504 .670273$ C 0.9155961 .1147503 .603596 H $2.024189-0.1046382 .252505$ H 0.5570520 .6163391 .581962 H $-0.462228-5.8356382 .545422$ H $1.983624-4.6690243 .120170$ H $2.065979-1.1367964 .606270$ O $1.6696022 .204563 \quad 3.270273$ O $0.289474 \quad 0.9704254 .643854$ C $1.6974103 .255544 \quad 4.240616$ H 0.6913023 .6593594 .388045 H 2.1105662 .8896125 .185312 H 2.3426924 .0520323 .860742 SCF Energy (B3LYP/6-31G**//MMFF) $=-3245.90852727$

$01 \_429$

MM̄FF Geometry

C $0.8282593 .541857-0.998416$

C $1.7447683 .905263-0.089003$

C 2.1722973 .0031581 .036316

O 3.5864062 .7522140 .862356

C 1.9567993 .6376712 .422989

C 0.5224643 .9800512 .760662

C -0.4251223 .0701993 .059324$

C 0.1996835 .4506522 .784678

C -0.2692701 .5744703 .092851$

C -1.2082170 .9128822 .077281$

C $-0.929704-0.5728631 .809269$

C $-0.880285-1.4236523 .079824$
C $-0.549609-2.8930152 .772239$

O $\quad 0.309524-0.6722481 .095776$

O $-0.191985-3.5334484 .006762$

C $0.695129-2.9457221 .859709$

C $-1.766183-3.6510532 .230129$

O $0.951853-4.2798321 .426443$

C $0.607519-1.9999850 .639898$

C $1.960053-1.967123-0.110701$

O $-0.394340-2.491363-0.256294$

C 4.0685441 .5756341 .346824

O $3.441091 \quad 0.7058401 .930017$

C 5.5117811 .5184441 .029580

C 6.1617270 .3524041 .155807

C 7.5787170 .1182310 .880277

C 8.4396991 .3078580 .547670

C $2.059789-0.845467-1.154588$

C $3.001206-1.206678-2.286094$

C $5.360747-1.711816-2.829790$

O $2.602297-1.292092-3.446259$

C $6.496018-2.397396-2.139960$

C $7.775528-2.015260-2.272330$

C $8.936216-2.714844-1.621486$

C $9.783322-1.777058-0.789537$

C $10.844130-1.151280-1.327268$

C $9.450668-1.6167630 .677195$

C $8.045306-1.1467020 .928349$

N $4.307752-1.420420-1.874563$

H $0.3900422 .547968-0.937764$

H $2.1984974 .891348-0.144845$

H 1.6318642 .0523670 .961596

H 2.5844314 .5359252 .510626

H 2.3564522 .9706293 .198473

H -1.423373 3.4277963 .308711

H -0.8579605 .6418382 .994637$

H 0.7891205 .9535923 .558256

H 0.4316095 .9110451 .819297

H $\quad 0.764171 \quad 1.2685502 .912228$

H $-0.521028 \quad 1.2336594 .104068$

H -2.241407 1.0249362 .426645

H -1.130576 1.4466091 .121334

H $-1.734157-0.9253311 .153314$

H -1.822990 -1.346509 3.635490

H $-0.100062-1.0452283 .753035$

H -0.940195 -3.439242 4.620817

H $1.560604-2.6447302 .466650$

H -2.582857 -3.638799 2.961706

H -1.532131 -4.707838 2.061172

H $-2.151330-3.2300841 .300456$

H $1.011372-4.8360242 .221971$

H $2.776081-1.8195600 .608360$

H $2.128015-2.937512-0.595325$

H -0.162675 -2.246702 -1.166107

H 5.9932912 .4280190 .689808

H $5.600468-0.5236741 .479388$

H 8.3955472 .0522161 .350503

H $8.1055311 .775458-0.385080$

H $9.494231 \quad 1.0537340 .415852$

H $1.084376-0.626934-1.603087$

H $2.4060830 .084984-0.692436$

H $5.660514-0.762795-3.286913$

H $4.958255-2.358261-3.617058$

H $\quad 6.258706-3.262737-1.525707$

H $8.009801-1.165569-2.910035$

H $9.548601-3.158315-2.417816$

H $8.603920-3.557685-1.002757$

H $11.474830-0.495927-0.735882$

H $11.103474-1.278681-2.373190$

H $10.161521-0.949988 \quad 1.180473$

H $9.580236-2.5892381 .170257$

H $7.350504-1.9527961 .165909$

H $4.573334-1.135192-0.936068$

C -1.163799 $4.666334-2.095052$

O $-1.873243 \quad 3.411774-2.163813$

C $-2.8864743 .406601-1.142867$

C $-3.1158974 .870298-0.794820$

C $-1.6960965 .400619-0.862047$

H -2.454616 $2.898131-0.271521$

C $-4.1360802 .640536-1.590632$ 
H -3.746285 $5.368469-1.540681$ H -3.5768334 .9990600 .188946$ C -1.607646 $6.912909-0.959401$ H -1.168004 5.0755260 .041761 C $-3.8369361 .153119-1.885557$ O $-4.6887543 .284466-2.735789$ H $-4.8775042 .706921-0.786207$ H $-3.2308370 .755906-1.062915$ O $-3.0713451 .022306-3.082197$ C $-5.1034050 .285018-2.012888$ C $-4.835692-1.194549-2.349912$ H $-5.667140 \quad 0.345706-1.075647$ O $-5.9614090 .804848-3.030695$ H $-5.806747-1.704758-2.378322$ H -4.451415 -1.271955 -3.375341 H $-0.5640887 .237188-1.024204$ H -2.134694 $7.290506-1.841946$ H -2.053372 $7.381679-0.075881$ C $0.3484074 .416770-2.122105$ H $-5.5274612 .840543-2.946235$ H -2.254495 $1.536830-2.968182$ H $-5.4656200 .788762-3.867635$ H $0.8789715 .376014-2.125028$ H $\quad 0.6031413 .903952-3.057924$ H -1.436378 $5.216324-3.004963$ C $-5.412647-2.3052870 .436164$ O $-4.204885-1.684010-0.013851$ C $-3.878784-1.920391-1.384188$ C $-3.738719-3.427328-1.642985$ C $-4.968302-4.203457-1.173377$ C $-5.327522-3.8253080 .264020$ H $-6.264900-1.911578-0.131539$ C $-5.598028-1.9223391 .908421$ H $-2.885300-1.482907-1.541627$ O $-3.504337-3.659246-3.027668$ H -2.860241 -3.799671 -1.100837 H -5.822505 -4.047973-1.842732 O $-4.684263-5.604192-1.200484$ H $-4.580834-4.2436910 .950504$ O $-6.586992-4.405356 \quad 0.598101$ C $-6.064967-0.4918292 .034957$ H $-6.354249-2.5469602 .397495$ H $-4.668887-2.0393412 .478790$ H -3.292256 -4.601297 -3.137905 H $-4.563304-5.861118-2.130077$ H $-6.510790-5.3647850 .457270$ $\begin{array}{lllll}\text { O } & -4.997227 & 0.355600 & 2.015385\end{array}$ O $-7.242597-0.1680572 .121635$ C -5.3137821 .7477782 .112303$ H $-4.3987592 .318981 \quad 1.934691$ H -6.0529852 .0306341 .356616$ H -5.6826371 .9753643 .116460$ SCF Energy (B3LYP/6-31G**//MMFF) $=-3245.91625461$

01443

MM̄FF Geometry

C $1.748308-3.454064-1.601900$

C $1.386470-3.708053-0.334701$

C $0.029189-3.427754 \quad 0.271252$

O $-0.687734-2.432115-0.494775$

C $-0.818365-4.7110210 .310657$

C $-2.073680-4.5745781 .151404$

C $-3.331696-4.6067220 .670457$

C $-1.850850-4.4234062 .635576$

C $-3.782903-4.780785-0.751450$

C $-4.001440-3.474047-1.529792$

C $-5.010567-2.507399-0.887138$

C $-5.370386-1.380896-1.859654$

C $-6.295944-0.335062-1.218066$

O $-4.416304-1.9495790 .289307$

O $-6.307614 \quad 0.823443-2.066999$

$\begin{array}{llll}\text { C } & -5.698424 & 0.105130 & 0.136324\end{array}$

C -7.745441 -0.829132 -1.141512

$\begin{array}{lllll}\text { O } & -6.604164 & 0.950827 & 0.839925\end{array}$

C $-5.277709-1.0767401 .033761$

C $-4.508142-0.6370482 .304496$

O $-6.450286-1.7705721 .478422$

C $-0.320592-1.136604-0.298218$
O $0.509572-0.723850 \quad 0.495405$

C $-1.080667-0.291152-1.241414$

C $-0.6238830 .940508-1.508944$

C $-1.2500471 .931678-2.383080$

C $-2.6484871 .648186-2.861957$

C -3.2096490 .1378232 .056639$

C -3.3680731 .6488552 .098816$

C $-2.090508 \quad 3.747423 \quad 1.678225$

O -4.3902522 .2095712 .484157$

C $-2.4594454 .271803 \quad 0.325922$

C $-1.6980535 .138354-0.359888$

C $-2.0741055 .709897-1.698146$

C $-0.9929775 .517453-2.739107$

C $-0.1103746 .494352-3.007825$

C $-0.9844704 .221261-3.519868$

C $-0.5571643 .051434-2.677478$

N -2.2120122 .3013301 .689334$

H $1.015100-3.062812-2.302540$

H $2.123801-4.1239840 .348101$

H $\quad 0.187422-3.0490451 .288362$

H $-0.227838-5.5406440 .721810$

H - $1.070200-4.998325-0.715957$

H $-4.150725-4.5005281 .381497$

H -2.784815 -4.487213 3.204380

H -1.191615 -5.216193 3.003957

H $-1.395971-3.4547582 .863302$

H -3.082076 -5.413083 -1.307078

H $-4.724017-5.345800-0.736381$

H -3.035488 -2.971064 -1.649307

H -4.348902 -3.743603 -2.535117

H $-5.907585-3.074424-0.611971$

H -5.825864 -1.785553 -2.772145

H $-4.451377-0.875829-2.185466$

H $-6.6095360 .545795-2.948875$

H $-4.8132270 .712169-0.089769$

H $-8.128897-1.047209-2.145581$

H $-8.405544-0.056877-0.731360$

H -7.859074 -1.735193 -0.542858

H $-6.824767 \quad 1.6928610 .251137$

H $-5.178774-0.0960202 .982945$

H $-4.228273-1.5534462 .844355$

H $-6.224421-2.2783442 .275391$

H -1.980453 -0.699163 -1.684057

H $\quad 0.306606 \quad 1.260423-1.042991$

H -3.310388 $1.453042-2.010990$

H $-2.6595750 .775219-3.523551$

H -3.099089 $2.474236-3.415103$

H -2.497496 -0.1150082 .852792$

H -2.756295 -0.155635 1.106835

H -1.0578863 .9890631 .948026$

H -2.7585474 .1740382 .433268$

H -3.407604 3.946669-0.095906

H -0.7638045 .4836780 .077918$

H -2.269412 $6.781331-1.559335$

H -3.015391 $5.283682-2.066857$

H $\quad 0.652573 \quad 6.374280-3.769732$

H $-0.1281247 .434966-2.467111$

H $-0.2816094 .289739-4.360772$

H $-1.9666834 .083908-3.979260$

H $\quad 0.4526493 .153682-2.276802$

H $-1.4277751 .756277 \quad 1.343008$

C $3.630052-2.422849-2.887256$

O $3.719324-1.292922-1.997762$

C $4.829003-0.468465-2.408292$

C $5.416598-1.119976-3.658457$

C $5.031333-2.574118-3.470584$

H $5.570912-0.518045-1.603785$

C $4.3667150 .980132-2.617522$

H $4.967218-0.720578-4.575197$

H $6.498331-0.968878-3.730362$

C $5.091301-3.386341-4.752200$

H $5.702637-3.021260-2.725146$

C $3.7169481 .629289-1.378785$

O $3.3994241 .011692-3.677174$

H $5.2080181 .590700-2.964211$

H $2.7738331 .116183-1.165189$

O $3.3643542 .974891-1.725957$

C $4.5933631 .657702-0.112324$ 
C 3.9822222 .4498531 .058667 H $4.806036 \quad 0.639675 \quad 0.222914$ O $5.8500862 .274404-0.406213$ H 4.7101822 .4400231 .879402 H 3.8956973 .5067060 .775681 H $4.807282-4.427248-4.569617$ H $4.417709-2.982076-5.515291$ H $6.106270-3.381641-5.163103$ C $3.139777-3.660074-2.131981$ H $2.6501580 .453095-3.407518$ H $4.1908113 .461855-1.887145$ H $6.3496551 .671990-0.982341$ H $3.826831-3.885133-1.307858$ H $3.127688-4.525736-2.803251$ H $2.914197-2.154289-3.676146$ C $3.3965410 .177807 \quad 3.036141$ $\begin{array}{lllll}\text { O } & 2.659389 & 0.526680 & 1.860398\end{array}$ C $2.6207291 .920937 \quad 1.546857$ C 2.0193502 .7091892 .717132 C 2.7553272 .4268494 .022016 C 2.8502260 .9219694 .263603 H 4.4571810 .4165582 .889684 C $3.261532-1.3428903 .231285$ H 1.9194302 .0286740 .711783 $\begin{array}{lllll}\text { O } & 2.043384 & 4.108427 & 2.457085\end{array}$ H 0.9671042 .4234832 .844868 H 3.7482192 .8912214 .035394 O 2.0270923 .0072775 .106829 H 1.8578320 .5334824 .527037 O $3.710477 \quad 0.6827795 .376489$ C $4.093495-2.1398562 .248232$ H $3.599599-1.6155114 .237567$ H $2.219025-1.6574443 .106107$ H $1.559770 \quad 4.2596311 .626900$ H 1.9379073 .9574774 .918919 H 3.3525311 .1802096 .131730 O $4.288044-3.3965652 .748387$ O $4.502550-1.7296751 .171582$ C $5.084410-4.2679091 .939308$ H $6.064537-3.8210961 .745863$ H $5.228446-5.2027362 .487430$ H $4.568184-4.4872831 .001090$ SCF Energy (B3LYP/6-31G**//MMFF) $=-3245.90542754$

01430

MM̄FF Geometry

C $3.334252-3.768346-1.658401$

C $2.248703-4.378036-1.156506$

C $0.990794-3.672403-0.708114$

O $0.849825-2.433554-1.441227$

C $-0.268971-4.526084-0.961386$

C $-1.347488-4.2653890 .073230$

C $-2.407712-3.450943-0.090303$

C $-1.172082-5.013126 \quad 1.372386$

C $-2.761286-2.608548-1.283897$

C $-4.141581-2.931306-1.877243$

C $-5.315064-2.716332-0.907383$

C $-6.654584-2.943403-1.610963$

C $-7.841956-2.663288-0.676055$

O $-5.250723-1.377209-0.406439$

O $-9.027314-2.577477-1.481712$

C $-7.644584-1.283132-0.013549$

C $-8.073538-3.8110980 .314005$

$\begin{array}{llll}0 & -8.641700 & -1.046240 & 0.977974\end{array}$

C $-6.234890-1.0786220 .592152$

$\begin{array}{llll}\text { C } & -6.059036 & 0.396962 & 1.023987\end{array}$

O $-6.092399-1.9164531 .741465$

C $0.431761-1.328520-0.772701$

O $0.184480-1.2311320 .417692$

C $0.293473-0.243649-1.769418$

C $-0.4185490 .848526-1.457350$

C $-0.6490772 .008407-2.321064$

C $0.036658 \quad 2.031761-3.661458$

C $-4.619612 \quad 0.786423 \quad 1.393578$

C -4.5287422 .2372601 .839715$

C $-2.8573594 .030548 \quad 2.297032$

O -5.5150752 .9304982 .075895$

C -2.8980884 .8604601 .052284$
C -1.816766 $5.402817 \quad 0.472675$

C $-1.872115 \quad 6.190451-0.804694$

C $-1.178558 \quad 5.492074-1.955105$

C $-0.0465175 .985683-2.483581$

C $-1.8461214 .273034-2.555772$

C $-1.4475142 .996397-1.866109$

N -3.2125412 .6632531 .958366$

H $3.330926-2.690506-1.799955$

H $2.299256-5.449325-0.973403$

H $1.116505-3.4728170 .363636$

H $-0.030900-5.597365-0.919878$

H $-0.649435-4.351558-1.974196$

H $-3.087281-3.3419840 .753766$

H $-1.975178-4.8032522 .086846$

H $-1.169095-6.0928911 .189790$

H $-0.227018-4.7393101 .851315$

H $-2.732916-1.555933-0.977746$

H -2.019092 -2.708362 -2.080994

H $-4.276884-2.279121-2.750016$

H $-4.152744-3.965079-2.244418$

H $-5.213227-3.420946-0.075019$

H $-6.715244-3.961484-2.015265$

H $-6.730722-2.271239-2.477230$

H $-9.124342-3.417528-1.962383$

H $-7.797024-0.523370-0.793141$

H $-8.271674-4.746058-0.223697$

H $-8.960188-3.6277170 .930903$

H -7.222659 -3.985682 0.975813

H $-9.507863-1.1682910 .552749$

H $-6.372815 \quad 1.0542330 .202049$

H -6.7161460 .6082541 .877256$

H $-5.422183-1.5369342 .330941$

H $\quad 0.767440-0.381692-2.734485$

H $-0.8827950 .899474-0.472917$

H $-0.372658 \quad 1.252099-4.312986$

H $1.1135221 .863936-3.551455$

H $-0.0686622 .983289-4.186990$

H $-4.242697 \quad 0.1656742 .212841$

H -3.9618180 .6498290 .527377$

H $-1.861943 \quad 4.0095532 .751124$

H -3.569439 4.4225733 .030113

H $-3.873480 \quad 5.0073350 .593648$

H -0.8391825 .2625980 .926328$

H $-1.4129257 .168893-0.613590$

H -2.909067 $6.403447-1.093919$

H $0.4388725 .505461-3.326666$

H $0.4246536 .876013-2.080693$

H $-1.6495014 .230105-3.632017$

H $-2.9360994 .381665-2.484404$

H $-1.8998082 .870230-0.883433$

H -2.464069 2.0418701 .664536

C $5.713001-4.292638-0.936376$

O $5.913584-2.890787-0.651998$

C $5.764279-2.696730 \quad 0.765714$

C $6.103887-4.0451541 .384505$

C $5.430507-4.9844390 .403404$

H $4.703764-2.4804390 .946859$

C $6.609816-1.5247031 .265893$

H $7.184860-4.2261201 .398101$

H $5.723796-4.1457322 .405412$

C $5.943645-6.4128980 .472476$

H $4.356919-4.9933270 .623239$

C $6.396545-0.2020310 .504287$

O $7.995359-1.8710111 .145875$

H $6.423567-1.3816212 .337353$

H $6.887792-0.260498-0.474492$

$\begin{array}{lllll}\text { O } & 7.097282 & 0.820126 & 1.227545\end{array}$

$\begin{array}{llll}\text { C } & 4.929532 & 0.229217 & 0.306686\end{array}$

C $4.8319691 .654698-0.269450$

H $4.436638-0.480297-0.367261$

$\begin{array}{llll}\text { O } & 4.262533 & 0.173821 & 1.562689\end{array}$

H $5.0598352 .375800 \quad 0.523285$

H $5.5954731 .764335-1.048357$

H $5.438886-7.042113-0.267427$

H $7.020718-6.4627870 .280414$

H $5.759320-6.8403461 .463385$

C $4.619572-4.471775-1.999075$

H $8.512183-1.1185371 .481472$ 
H 6.6394810 .9433322 .077002 H 3.3189510 .3408601 .399795 H $4.445651-5.539634-2.176619$ H $4.972984-4.049006-2.947987$ H $6.660759-4.655407-1.353512$ C 2.3291682 .9654521 .027657 $\begin{array}{lllll}\text { O } & 2.379705 & 1.912214 & 0.058022\end{array}$ C $3.4518761 .979358-0.885216$ C $3.4148473 .320649-1.632582$ C $3.4512814 .495119-0.662868$ C $2.332923 \quad 4.350242 \quad 0.363289$ H 3.1813822 .8710981 .711134 C 1.0270052 .7881831 .827420 H $3.2539231 .194650-1.626535$ O $4.5001253 .432319-2.546473$ H $2.4921073 .388649-2.221748$ H $4.4279754 .591909-0.175100$ O $3.2407955 .710609-1.384597$ H $1.3701824 .528355-0.130388$ O $2.483437 \quad 5.350073 \quad 1.369278$ C $1.018987 \quad 1.5410332 .681521$ H 0.8542313 .6348412 .500474 H $\quad 0.1863152 .725864 \quad 1.125760$ H $4.4429062 .680425-3.160747$ H $3.9306815 .763480-2.068268$ H 2.4919576 .2132360 .920923 O $-0.257582 \quad 1.064347 \quad 2.769445$ $\begin{array}{llll}\text { O } & 2.000898 & 1.069160 & 3.237322\end{array}$ C $-0.430545-0.1026573 .579351$ H $-1.460130-0.4500773 .457785$ H $\quad 0.247315-0.9001383 .260284$ H $-0.2637480 .146954 \quad 4.631249$

SCF Energy (B3LYP/6-31G**//MMFF) $=-3245.91994648$

01431

MM̄FF Geometry

C 4.0472201 .8899041 .240548

C 3.5948962 .8038142 .111594

C 2.3755822 .6745842 .992371

O $1.271295 \quad 3.2973992 .293038$

C 1.9613701 .2333233 .350620

C $\quad 0.708379 \quad 1.170588 \quad 4.206497$

$\begin{array}{lllll}\text { C } & -0.499008 & 0.778627 & 3.750598\end{array}$

C $0.8900391 .543843 \quad 5.653740$

C -0.8464110 .3818732 .341336$

C $-0.972792-1.1401772 .188667$

C $-2.350978-1.6728742 .609811$

C $-2.356674-3.2031362 .618916$

C $-3.756602-3.7739222 .889826$

O $-3.293973-1.1814811 .652929$

O $-3.727566-5.1761692 .582139$

C $-4.752329-3.1180771 .911204$

C $-4.148227-3.661067 \quad 4.367242$

O $-6.091662-3.5103562 .200164$

C -4.652942 -1.577972 1.876024

C $-5.490673-0.9596500 .731461$

O $-5.145016-1.041616 \quad 3.108074$

C 1.0312854 .6123572 .522295

O 1.6848905 .3598303 .237676

C -0.1801595 .0810101 .810244$

C $-0.876700 \quad 4.3413940 .932535$

C $-2.096355 \quad 4.758574 \quad 0.234281$

C -2.6415836 .1300410 .542439$

C $-5.102185-1.454752-0.665422$

C -5.527607 -0.499779-1.766456

C $-4.9680690 .061465-4.132032$

O $-6.3306760 .413010-1.597909$

C $-3.955326 \quad 1.152734-3.970824$

C $-4.245292 \quad 2.457713-4.075520$

C -3.242427 $3.550791-3.836254$

C $-3.7353474 .562188-2.823786$

C -3.991509 $5.830096-3.187914$

C $-3.9586694 .104225-1.399370$

C $-2.6743913 .905595-0.638268$

N $-4.896140-0.800819-2.966897$

H 3.5084980 .9560381 .111577

H 4.1242493 .7517472 .187898

H 2.5816053 .2091023 .928625
H $2.780836 \quad 0.736433 \quad 3.886717$

H 1.8117450 .6429422 .441951

H $-1.332306 \quad 0.748315 \quad 4.450572$

H $-0.027906 \quad 1.408002 \quad 6.235638$

H $1.664700 \quad 0.920676 \quad 6.112400$

H 1.1855712 .5932535 .746875

H $-0.093957 \quad 0.747088 \quad 1.636629$

H -1.7762720 .8857032 .051807$

H $-0.818053-1.3780881 .129118$

H $-0.177147-1.6432792 .751914$

H -2.604853 -1.284605 3.602351

H -1.635682 -3.5900303 .349896$

H $-2.019464-3.5744041 .641484$

H $-3.049498-5.591893 \quad 3.141913$

H $-4.521604-3.5151430 .914404$

H $-3.438398-4.2138814 .994371$

H -5.127813 -4.115835 4.551461

H $-4.172059-2.6308814 .728040$

H $-6.112539-4.4826332 .213995$

H $-6.561070-1.1302910 .903615$

H -5.3580670 .1307410 .769988$

H $-5.419075-0.1219532 .956666$

H -0.4757386 .0954592 .059352$

H $-0.538155 \quad 3.332362 \quad 0.705290$

H -3.529435 $6.388763-0.037913$

H -1.8951316 .9002390 .317489$

H -2.918654 6.2051191 .599867

H $-4.015324-1.578980-0.732628$

H $-5.568724-2.426123-0.864314$

H $-5.9866620 .451157-4.227930$

H $-4.732062-0.547638-5.009353$

H $-2.9375360 .855153-3.730466$

H $-5.2587722 .759552-4.330163$

H $-3.042917 \quad 4.037919-4.799161$

H -2.281721 $3.143348-3.497961$

H $-4.3547626 .561563-2.473764$

H $-3.8445606 .166746-4.208844$

H $-4.5102273 .155626-1.395876$

H $-4.6253114 .795592-0.871755$

H -2.203611 $2.937919-0.814791$

H $-4.202199-1.541974-2.980015$

C $6.196551 \quad 0.895526 \quad 0.420409$

O $5.514678-0.284881-0.047601$

C $6.351535-0.971621-0.991842$

C $7.747299-0.393225-0.802329$

C $7.4249951 .053049-0.477213$

H $5.987356-0.701519-1.990535$

C $6.234118-2.485023-0.790081$

H $8.272329-0.8604840 .038640$

H $8.369349-0.505845-1.695438$

$\begin{array}{llll}\text { C } 8.573278 & 1.802112 & 0.176320\end{array}$

H $7.1475091 .566696-1.407617$

C $4.783337-3.014657-0.775804$

O $6.827449-2.8427970 .465660$

H $6.819921-3.006541-1.555633$

H $4.281203-2.6865250 .141358$

O $4.847313-4.445191-0.707342$

C $3.936757-2.619437-2.000649$

C $2.573655-3.332760-2.081721$

H $3.783647-1.536034-2.008922$

O $4.672378-2.948653-3.183121$

H $2.127585-3.095219-3.054700$

H $2.739078-4.416686-2.112212$

H 8.2855202 .8328670 .405821

H 8.8846791 .3230621 .110666

H $9.4409811 .833550-0.490695$

C $5.2426412 .089133 \quad 0.350216$

H $6.368822-2.339853 \quad 1.160677$

H $5.380895-4.6744880 .072827$

H $4.162580-2.625723-3.945606$

H $4.8711322 .200248-0.675964$

H 5.7738463 .0109050 .612767

H $6.4931660 .701591 \quad 1.459224$

C $0.499962-1.092935-2.005817$

O $1.283114-1.574368-0.906030$

C $1.596054-2.972167-0.943823$

C $0.298765-3.790128-0.958489$

C $-0.598721-3.390065-2.123804$ 
C $-0.813500-1.878420-2.146765$

H $1.086243-1.163304-2.929859$

C $0.1864460 .391705-1.749973$

H $2.098260-3.2011700 .002816$

O $0.594051-5.181005-1.022124$

H $-0.237929-3.620129-0.017430$

H $-0.187755-3.742693-3.076452$

O $-1.866947-4.037824-1.987318$

H -1.504252 -1.598522 -1.341417

O $-1.435383-1.519267-3.380007$

C $1.3984751 .291275-1.855956$

H $-0.5432030 .749752-2.485399$

H $-0.2267360 .518158-0.743617$

H $-0.252882-5.657537-1.059240$

H -2.258656 -3.758373 -1.142083

H $-2.207727-2.097734-3.497645$

O $0.9846372 .588919-1.740753$

O $2.5512710 .915506-2.011262$

C $2.0061813 .586331-1.834588$

H $2.6534133 .406269-2.698395$

H $1.5200894 .557528-1.960270$

H $2.5896913 .603937-0.911019$

SCF Energy (B3LYP/6-31G**//MMFF) $=-3245.91384643$

01432

MM̄FF Geometry

C $0.643370-1.3794662 .622288$

C $-0.505690-0.9513442 .079963$

C -1.831817 -0.9887292 .789123$

O -2.4342150 .3248692 .679354$

C $-2.798157-1.9819452 .124928$

C $-2.469283-3.4352392 .390372$

C $-1.941325-4.2829541 .486316$

C $-2.825848-3.9335253 .767626$

C $-1.506300-3.9866590 .078491$

C $-2.425178-4.630271-0.969051$

C $-3.796913-3.946507-1.079775$

C $-4.684247-4.678945-2.088427$

C $-6.021465-3.954744-2.305187$

O $-3.577496-2.600223-1.511704$

O $-6.642362-4.514114-3.472711$

C $-5.739591-2.469082-2.611106$

C $-6.993513-4.188035-1.142558$

O $-6.953272-1.723515-2.678817$

C $-4.764721-1.803913-1.611094$

C $-4.350829-0.407931-2.134607$

O $-5.416665-1.661672-0.348249$

C -2.0749341 .2548443 .600990$

O -1.3033341 .0917174 .537176$

C -2.7170782 .5648973 .347645$

C -3.5785322 .8099442 .347971$

C -4.2054934 .1025752 .051627$

C $-3.825840 \quad 5.2727052 .923477$

C $-3.232990 \quad 0.269829-1.327388$

C $-2.8025451 .583856-1.956686$

C $-1.2576673 .501227-1.596583$

O $-3.2889922 .020757-2.996734$

C $-2.0757924 .577091-0.954631$

C $-3.0272905 .263988-1.604796$

C $-3.8900806 .323282-0.980702$

C $-5.3296135 .874419-0.842616$

C $-6.1789785 .950922-1.881136$

C -5.8009535 .4030330 .516288$

C -5.0576494 .1884541 .007322$

N -1.803831 $2.209738-1.221350$

H $0.644204-1.7812643 .631656$

H -0.499536 -0.5487071 .069334$

H -1.716765 -1.239987 3.850436

H -2.858516 -1.7725301 .051836$

H -3.817726 -1.785943 2.485296

H -1.775624 -5.3160161 .790546$

H -2.642627 -5.007292 3.882242

H $-2.234418-3.4174884 .529984$

H -3.887425 -3.7590713 .971698$

H -1.403219 -2.914030 -0.108065

H $-0.498501-4.403221-0.044408$

H -1.914850 -4.562636 -1.938780

H $-2.554509-5.696549-0.745058$
H $-4.274826-3.938495-0.094161$

H -4.855645 -5.716218 -1.774876

H $-4.164301-4.740304-3.054819$

H -6.759012 -5.467881-3.322139

H -5.291199-2.421895-3.613583

H $-7.202332-5.258512-1.027552$

H -7.961893 -3.713577 -1.336129

H $-6.614405-3.822279-0.186021$

H -7.529071-2.165509 -3.326155

H -3.999356 -0.508416 -3.170074

H $-5.2231630 .257884-2.149759$

H $-5.020204-0.9209640 .135590$

H -2.421928 3.3427194 .045385

H -3.850164 2.0000831 .674273

H -2.7412375 .4293452 .905679$

H -4.1402475 .0976373 .958290$

H -4.2726776 .2185892 .612361$

H $-3.5683150 .478609-0.306868$

H $-2.351430-0.379165-1.275853$

H -1.252731 $3.590500-2.688328$

H $-0.2231723 .548911-1.244526$

H -1.878052 4.7894350 .092512

H -3.213990 $5.041517-2.653420$

H -3.840551 $7.220763-1.611146$

H -3.497763 $6.634319-0.004836$

H $-7.2182005 .654401-1.786881$

H -5.850885 $6.311262-2.850759$

H -6.8654795 .1360320 .479974$

H $-5.745026 \quad 6.2440991 .212396$

H $-5.260864 \quad 3.2876600 .426514$

H $-1.526148 \quad 1.815947-0.326994$

C $2.419285-2.6341731 .306205$

O $3.566084-2.3462520 .477818$

C $4.636287-3.2404060 .825648$

C $3.968020-4.4087681 .531009$

C $2.868818-3.7020782 .308159$

H $5.272693-2.7112651 .545194$

C $5.451588-3.593116-0.424843$

H $3.528924-5.1156580 .817611$

H $4.661160-4.9601772 .173317$

C $1.771020-4.6549652 .754675$

H $3.313149-3.2263673 .192395$

C $5.901311-2.361218-1.240153$

O $4.654757-4.409968-1.292942$

H $6.317376-4.204642-0.147342$

H $5.018937-1.879616-1.675326$

O $6.695738-2.813572-2.342502$

C $6.723068-1.336542-0.439452$

C $7.156951-0.104713-1.257767$

H $6.151030-0.9983960 .429609$

O $7.894086-1.9833020 .064138$

H $7.8093710 .507925-0.623045$

H $7.795185-0.418570-2.093099$

H $1.000188-4.1361843 .330290$

H $1.286221-5.1360861 .898675$

H $2.184939-5.4442613 .391013$

C $1.957967-1.3070411 .911510$

H $3.846109-3.912310-1.504520$

H $6.169095-3.470845-2.828908$

H $8.376053-1.3374960 .608205$

H $1.910603-0.5548601 .112335$

H $2.717997-0.9259162 .605170$

H $1.647506-3.0285300 .633711$

C 5.4183191 .8941940 .260363

O $4.9997380 .985105-0.761312$

C $5.977510 \quad 0.743377-1.778947$

C $6.3855992 .063321-2.451059$

C $6.8104693 .125903-1.437788$

C $5.7636443 .265505-0.330458$

H $\quad 6.311471 \quad 1.487797 \quad 0.751695$

C 4.3430681 .9378991 .354123

H $5.475406 \quad 0.143687-2.547578$

O $7.4447011 .854375-3.379419$

H $5.5323412 .455653-3.019945$

H $7.7989362 .904179-1.018426$

O $6.9314284 .385557-2.102486$

H $4.8730563 .748586-0.747381$

O $6.263214 \quad 4.1273030 .689520$ 
C 2.9765032 .4061250 .897849

H $4.2058100 .925606 \quad 1.752265$

H 4.6785832 .5893442 .169147

H $7.1418371 .192326-4.024182$

H $7.5701694 .270037-2.826790$

H 7.0643813 .7197001 .060399

O 2.2057062 .6401972 .002529

O $2.6134622 .521090-0.264037$

C 0.8647283 .0665281 .743840

H 0.3055682 .2644851 .254135

H $\quad 0.3904663 .2926692 .702154$

H $0.8621123 .972801 \quad 1.130883$

SCF Energy (B3LYP/6-31G**//MMFF)= -3245.90292026

$01 \_433$

MM̄FF Geometry

C $1.7562023 .315286-0.724995$

C $1.6737812 .861968-1.984463$

C $2.2075631 .520534-2.404876$

O $1.0952160 .719056-2.873211$

C $3.2139021 .610706-3.567226$

C $4.6421711 .791215-3.100488$

C $5.5284290 .782065-2.986483$

C $5.0572043 .201340-2.775182$

C $5.308763-0.674558-3.291301$

C $4.447553-1.444967-2.284042$

C $4.813669-1.265073-0.803200$

C $6.234753-1.738201-0.491327$

C $6.548377-1.6510081 .009375$

O $3.865737-2.034595-0.046748$

O $7.752389-2.3945851 .252798$

C $5.420417-2.3475561 .795981$

C $6.832016-0.2099361 .450376$

O $5.594092-2.1666443 .200061$

C $3.999560-1.9012551 .375359$

C $2.952193-2.8181822 .053453$

O $3.789364-0.5535291 .801044$

C $0.492460-0.092404-1.962469$

O $0.716196-0.149906-0.763623$

C $-0.468377-0.948233-2.691710$

C $-0.858382-2.098289-2.123840$

C $-1.774547-3.088567-2.690360$

C $-2.466113-2.751391-3.983600$

C $1.509630-2.6118261 .573342$

C $0.533272-3.5184232 .306790$

C $-1.915715-3.9503482 .533532$

O $0.888473-4.4016603 .082805$

C $-2.335748-5.0049181 .559585$

C -3.499065 -4.982491 0.891291

C $-3.919756-6.033909-0.095544$

C $-4.073584-5.486896-1.498649$

C $-5.281370-5.152570-1.982872$

C $-2.838286-5.407646-2.369534$

C -1.958521 -4.242202 -2.015763

N $-0.784215-3.225673 \quad 1.983934$

H 2.2417452 .6994980 .028902

H $1.1823563 .465583-2.742365$

H $2.685071 \quad 1.009553-1.559328$

H $2.9447922 .421039-4.257866$

H $3.1354930 .703526-4.179553$

H $6.5330231 .019051-2.638655$

H $6.1295593 .279602-2.565303$

H $4.8418023 .865924-3.618233$

H $4.5247133 .569274-1.894304$

H $4.876709-0.782029-4.293673$

H $6.293102-1.155296-3.358196$

H $3.394406-1.171020-2.418753$

H $4.483540-2.513298-2.537170$

H $4.689605-0.209581-0.537782$

H $6.980068-1.171116-1.060848$

H $\quad 6.347070-2.784071-0.809672$

H $8.458308-2.0094770 .705696$

H $5.511561-3.4271491 .609879$

H $7.688230 \quad 0.197378 \quad 0.899246$

H $7.110895-0.1685352 .509079$

H $\begin{array}{llll}5.987423 & 0.462840 & 1.288934\end{array}$

H $6.489965-2.4724263 .422915$

H $3.216689-3.8665961 .861901$
H $\quad 2.982667-2.668488 \quad 3.140471$

H $2.836722-0.3819061 .857098$

H $-0.796201-0.629910-3.674143$

H $-0.453664-2.351974-1.145073$

H $-1.741473-2.708200-4.803924$

H -2.971646 -1.783152 -3.915312

H -3.234593 -3.474540 -4.265760

H $1.193280-1.577327 \quad 1.735675$

H $1.433647-2.8335690 .503213$

H -2.708474 -3.221013 2.721540

H -1.638568 -4.4116623 .486585$

H -1.643205 -5.8272831 .396009$

H $-4.193730-4.1638581 .059217$

H -4.872199 -6.4557450 .251287$

H -3.215968 -6.875433 -0.107752

H $-5.401427-4.784591-2.996587$

H $-6.175465-5.231798-1.373507$

H -3.116062 -5.392534 -3.428608

H $-2.256336-6.331178-2.253912$

H -1.389944 -4.392739-1.098665

H $-0.975052-2.5152811 .283236$

C $-0.010927 \quad 4.4246810 .639573$

O $\quad 0.3725193 .703698 \quad 1.831947$

C -0.3033664 .2846512 .955603$

C -1.360487 5.2207592 .384344

C -0.6519835 .7197561 .136915$

H 0.4441954 .9075463 .466871

C -0.7915243 .2322533 .959560$

H -2.2731374 .6793282 .111431$

H -1.630159 6.0239453 .076288

C $-1.573631 \quad 6.392840 \quad 0.136750$

H $\quad 0.1294586 .431513 \quad 1.436369$

C -1.8660122 .2302723 .483775$

O -1.3384573 .9257165 .089942$

H 0.0699412 .6624814 .329813

H $-2.8064672 .758363 \quad 3.293706$

O $-2.128921 \quad 1.333173 \quad 4.574211$

C -1.4843401 .3743972 .265256$

C -2.4735680 .2444041 .924008$

H -1.3856532 .0137421 .387118$

O $-\begin{array}{llll}0.204155 & 0.774821 & 2.479644\end{array}$

H -2.076462 -0.2812121 .046453$

H $-2.468060-0.5083042 .722699$

H -1.023961 $6.700906-0.758052$

H -2.382072 $5.727037-0.176991$

H -2.0295927 .2859300 .576653$

C $1.2064144 .633822-0.259483$

H -0.6359814 .4897795 .455897$

H -2.3835721 .8762445 .339960$

H $-0.267693 \quad 0.224425 \quad 3.279127$

H 2.0008095 .1517970 .291325

H $0.9388905 .262041-1.116670$

H $-0.756398 \quad 3.811548 \quad 0.117015$

C $-3.6038131 .596666-0.564601$

O -3.9768801 .8499320 .796134$

C -3.9180500 .7123861 .658687$

C $-4.851970-0.3858821 .133637$

C $-4.548760-0.738177-0.323106$

C $-4.5191630 .529321-1.180607$

H $-2.5634191 .252864-0.589041$

C $-3.6996202 .944271-1.298763$

H -4.3227981 .0400692 .624198$

O $-4.751600-1.5389971 .959156$

H $-5.888393-0.0297221 .201323$

H -3.611222 -1.297984 -0.417207

O $-5.578809-1.591551-0.827310$

H $-5.5444020 .908913-1.278180$

O $-4.065851 \quad 0.178652-2.483639$

C -2.650205 $3.137968-2.371885$

H $-4.6889153 .069522-1.753301$

H $-3.5665313 .755591-0.573106$

H -5.448184 -2.158981 1.685331

H $-5.507248-2.442318-0.362704$

H $-4.1763490 .955439-3.056869$

O $-2.748024 \quad 4.413292-2.853394$

O $-1.8515682 .291813-2.746341$

C -1.810067 $4.763262-3.875480$

H $-2.0747505 .753980-4.254304$ 
H $-0.8016244 .806650-3.455141$

H -1.855210 4.049104 -4.703388

SCF Energy (B3LYP/6-31G**//MMFF) $=-3245.90334722$

$01 \_434$

MM̄FF Geometry

C -1.872886 -3.050030 -0.435156

C $-1.214001-2.629892-1.525435$

C -1.634882 -1.415989 -2.311909

O $-0.499645-0.534124-2.503306$

C $-2.146330-1.775526-3.715799$

C $-3.510445-2.427850-3.726353$

C $-4.671071-1.743878-3.707759$

C -3.517213 -3.930493 -3.829592

C $-4.854868-0.251005-3.646285$

C $-5.919073 \quad 0.165349-2.620374$

C $-5.441990 \quad 0.098594-1.158923$

C $-6.6403330 .285588-0.221812$

C $-6.222567 \quad 0.370316 \quad 1.250314$

O $-4.4840831 .146565-0.966338$

O

C -5.1234391 .4436891 .379259$

C $-5.863392-1.0084881 .815442$

O $-4.621999 \quad 1.4918972 .714172$

C $-3.968679 \quad 1.266270 \quad 0.366580$

C -2.9920952 .4692510 .392839$

$\begin{array}{lllll}\text { O } & -3.230353 & 0.101144 & 0.745263\end{array}$

C $-0.0526330 .162033-1.424140$

$\begin{array}{lllll}\text { O } & -0.420929 & 0.043277 & -0.266821\end{array}$

C $0.9654241 .122982-1.899638$

C $1.6046971 .890801-1.005174$

C $2.6446612 .876433-1.297782$

C $2.8999093 .213292-2.743469$

C $-3.5590143 .758183-0.202293$

C $-2.787704 \quad 4.9767150 .259950$

C $-0.8022756 .356064-0.185814$

O -3.2512325 .7400401 .106186$

C $0.5839626 .223658-0.730001$

C $1.6724676 .597371-0.038963$

C $3.0696876 .609748-0.592163$

C 4.0358055 .7842290 .226785

C 4.5838016 .2717701 .352752

C $4.4320734 .429578-0.310987$

C $3.3041033 .438640-0.263533$

N -1.550671 $5.124038-0.354577$

H $-2.774760-2.520623-0.141587$

H - $0.334109-3.167424-1.865745$

H -2.408898 -0.847158-1.783511

H -1.417763 -2.416921 -4.230952

H -2.170544 -0.867877 -4.334095

H $-5.598180-2.313027-3.765468$

H -4.532391 -4.339281 -3.874190

H -2.994854 -4.249460 -4.737571

H -3.020656 -4.383455 -2.967292

H $-3.9188940 .278854-3.446740$

H $-5.1951970 .073396-4.637531$

H $-6.2080371 .199963-2.848185$

H -6.815095 $-0.454194-2.753033$

H -4.960336 -0.867685 -0.973356

H -7.373741 $-0.518267-0.364768$

H $-7.1649351 .215174-0.485561$

H -7.093600 0.9524192 .910061

H -5.6120472 .4099531 .210815$

H -6.729200 -1.679455 1.759313

H -5.602342 -0.9477062 .877647$

H $-5.039338-1.4890481 .284382$

H $-4.020306 \quad 0.7369602 .831615$

H -2.631844 $2.633177 \quad 1.416593$

H -2.094072 2.214477 -0.185114

H -2.3214540 .1846770 .414832$

H $\quad 1.1675001 .165565-2.963683$

H 1.3599991 .7739470 .049627

H $3.5857094 .051686-2.881378$

H $1.9668733 .501833-3.241087$

H $3.3271562 .354856-3.271800$

H -3.541271 $3.717051-1.297878$

H -4.6030373 .9164730 .084088$

H $-0.809423 \quad 6.6301600 .875128$
H $-1.3383997 .137457-0.736062$

H $0.6931305 .872041-1.752857$

H 1.5469946 .9825620 .971019

H $3.4142967 .652292-0.611932$

H $3.0861326 .280888-1.638266$

H 5.3004835 .6966661 .929494

H 4.3242837 .2579241 .723687

H 5.2564504 .0101660 .281262

H $4.8462954 .551823-1.315803$

H $3.0364173 .137097 \quad 0.750220$

H $-1.3485894 .545867-1.165719$

C $-1.216490-3.9152381 .877324$

O $-0.019304-3.1260232 .028855$

C -0.225696-2.187839 3.104298

C $-1.530632-2.5938873 .788380$

C $-2.317225-3.1642542 .626761$

H $-0.368148-1.2076922 .633607$

C $0.982617-2.1388814 .048900$

H $-1.373038-3.3645174 .551603$

H -2.026615 -1.744286 4.268079

C $-3.489514-4.0339963 .043064$

H -2.692669 -2.319318 2.040492

C $2.341978-1.8802823 .368665$

O $1.076133-3.399628 \quad 4.726175$

H $\quad 0.797758-1.3855974 .824799$

H $2.671204-2.7875052 .848425$

O $3.304338-1.6326964 .402551$

C $2.370084-0.6895152 .396297$

C $3.777729-0.2785121 .921402$

H $1.748782-0.9244041 .525382$

O 1.7796840 .4462893 .034816

H 3.6516070 .5128241 .172796

H 4.3138800 .2085252 .746594

H -4.018216 -4.419364 2.165627

H -3.162131 -4.889158 3.643699

H $-4.202900-3.4571593 .640680$

C -1.478363 -4.2351230.399596

H $1.697199-3.2910755 .465826$

H $3.440175-2.4659234 .883878$

H 1.7644241 .1724832 .388610

H $-2.283872-4.9757630 .331025$

H $-0.578048-4.698745-0.022962$

H -1.008499-4.865503 2.388085

C $3.592121-1.648638-0.804139$

O $3.913214-2.256746 \quad 0.447604$

C $4.642292-1.4182311 .346147$

C $5.951461-0.9475740 .693692$

C $5.716478-0.299891-0.670826$

C $4.854784-1.206383-1.551759$

H $2.968423-0.769245-0.611442$

C $2.704648-2.614431-1.596917$

H $4.923225-2.0548932 .194043$

O $6.626676-0.0375101 .555875$

H $6.611414-1.8150250 .561876$

H $5.2716890 .695907-0.562364$

O $6.984588-0.103677-1.300118$

H $5.456447-2.070335-1.854593$

O $4.501129-0.510998-2.744113$

C $3.371686-3.917079-1.985466$

H $1.838468-2.884997-0.983023$

H $2.350819-2.116560-2.506942$

H 7.4380960 .2461461 .100806

H $6.815000 \quad 0.267198-2.183092$

H $3.9621910 .257260-2.490913$

O $2.665930-4.486818-3.007536$

O $4.358528-4.396252-1.444624$

C $3.162929-5.745941-3.471606$

H $4.183440-5.634573-3.850326$

H $3.127254-6.486528-2.667048$

H $2.520674-6.084408-4.288940$

SCF Energy (B3LYP/6-31G**//MMFF) $=-3245.91588122$

014435

MM̄FF Geometry

C $1.600374-4.3869720 .331986$

C $0.700694-3.961634-0.567916$

C $-0.719759-3.604243-0.200512$

O $-1.072094-2.363270-0.860397$ 
C $-1.682248-4.697441-0.707076$ C -2.975896 -4.7725970 .081859$ C $-4.181168-4.355517-0.352711$ C -2.856681 -5.414309 1.442956 C $-4.529711-3.716219-1.667330$ C $-4.554652-2.188574-1.592084$ C $-5.548600-1.587949-0.587403$ C $-7.000178-1.728903-1.049954$ C $-7.976730-0.996053-0.114524$ O $-5.210297-0.200359-0.467022$ O $-9.237846-0.884674-0.792223$ C -7.4668090 .4420320 .126929$ C $-8.243020-1.797473 \quad 1.165731$ O $-8.258687 \quad 1.1057201 .109307$ C -5.9736220 .5064580 .519216$ $\begin{array}{llll}C & -5.480561 & 1.972274 & 0.534897\end{array}$ O $-5.811479-0.0532231 .824456$ C $-0.512042-1.214745-0.393285$ O $0.216809-1.0878320 .577431$ C $-0.893232-0.126311-1.319269$ C $-0.0137380 .860466-1.542809$ C $-0.1795221 .981379-2.470144$ C - $-1.5517132 .214060-3.043623$ C $-3.957110 \quad 2.109058 \quad 0.678451$ C $-3.506417 \quad 3.5583630 .688691$ C $-1.4315504 .935910 \quad 0.790413$ O $-4.2848304 .506774 \quad 0.640449$ C -1.087179 $5.329683-0.611039$ C $0.1603535 .611393-1.014590$ C $0.5081176 .053120-2.405682$ C $1.5192925 .147771-3.069523$ C $2.8158675 .493139-3.143092$ C $1.0096923 .885800-3.728909$ C $0.900614 \quad 2.734956-2.765302$ N -2.123612 3.6618820 .770753 H $1.274124-4.5001971 .364358$ H $0.972499-3.860485-1.614060$ H $-0.819527-3.4633490 .882413$ H - $-1.207957-5.686247-0.637132$ H $-1.882957-4.541289-1.772446$ H $-5.033495-4.4822760 .314309$ H -3.825144 -5.506654 1.946396 H -2.439113 -6.422399 1.351223 H -2.204409 -4.8261032 .095176$ H -3.828886 -4.020975 -2.450986 H $-5.506289-4.095071-1.990473$ H -3.551393 -1.829465 -1.340892 H $-4.757514-1.782687-2.591938$ H $-5.403822-2.0602690 .389603$ H $-7.284925-2.783445-1.143413$ H $-7.103245-1.300493-2.056753$ H -9.537261 -1.782153-1.017684 H $-7.602367 \quad 1.000290-0.810084$ H -8.682213 -2.7725970 .922395$ H -8.975353-1.291332 1.804189 H $-7.340578-1.9816611 .752177$ H $-9.185286 \quad 1.0517330 .819039$ H -5.770392 $2.462333-0.403955$ H -5.9662492 .5192691 .352804$ H -5.0199910 .3211552 .240304$ H $-1.845185-0.197905-1.830477$ H $0.943123 \quad 0.817687-1.027370$ H $-2.300058 \quad 2.265193-2.245608$ H -1.824873 $1.403701-3.728009$ H -1.638166 $3.151499-3.597171$ H -3.611314 1.6505621 .610777 H -3.455626 $1.606621-0.155668$ H $-0.543709 \quad 4.815402 \quad 1.419021$ H -2.077950 5.6964531 .239979 H -1.907256 $5.410557-1.320832$ H $0.975268 \quad 5.556177-0.297350$ H $0.9044727 .075003-2.340394$ H $-0.3852836 .126098-3.038423$ H $3.5411734 .860506-3.644024$ H $3.1817806 .412069-2.697111$ H $1.7067053 .578226-4.520423$ H $0.0739794 .102927-4.250812$ H $1.8526932 .471539-2.304116$
H -1.5596272 .8177300 .728476$

C $3.706400-4.155201-1.157309$

O $3.834670-2.725742-0.996350$

C $5.169511-2.334494-1.368808$

C $5.756948-3.521728-2.121231$

C $5.127435-4.680240-1.375658$

H $5.731991-2.201005-0.437202$

C $5.155608-1.026527-2.166337$

H $5.445461-3.531446-3.172304$

H $6.850680-3.533359-2.091400$

C $5.191594-5.995931-2.132299$

H $5.638987-4.796649-0.410647$

C $4.5309790 .169336-1.418017$

O $4.400467-1.243104-3.365052$

H $6.177136-0.782472-2.482735$

H $3.4530050 .010526-1.298910$

$\begin{array}{llll}\text { O } & 4.686210 & 1.330379-2.244336\end{array}$

C $5.1685140 .473396-0.047526$

C $4.746962 \quad 1.815106 \quad 0.582141$

H $4.934093-0.3347590 .650342$

O $6.5937300 .494836-0.176489$

H $5.275803 \quad 1.9144261 .537993$

H $5.1308362 .637585-0.034670$

H $4.711391-6.797878-1.563093$

H $4.690641-5.925395-3.103656$

H $6.232236-6.285693-2.311543$

C $3.040183-4.7521770 .085599$

H $4.567594-0.490608-3.956841$

H $4.0930531 .227625-3.007481$

H $6.8192651 .180334-0.828694$

H $3.603735-4.4201270 .967006$

H $3.104317-5.8461940 .059745$

H $3.103618-4.328971-2.056382$

C 3.0063100 .9060082 .940683

$\begin{array}{llll}\text { O } & 2.660208 & 0.909515 & 1.550523\end{array}$

$\begin{array}{llll}\text { C } 3.229983 & 1.975841 & 0.786657\end{array}$

C 2.8083963 .3306561 .368346

C 3.1420793 .4363132 .851198

C 2.5953002 .2273343 .606728

H 4.0864710 .7489053 .050135

C $2.277256-0.2766463 .600652$

H $2.7760981 .917515-0.205934$

$\begin{array}{lllllllllll}\text { O } & 3.431547 & 4.403551 & 0.670338\end{array}$

H 1.7243793 .4543501 .247716

H 4.2201823 .5482173 .015651

$\begin{array}{lllll} & & 2.529544 & 4.611829 & 3.386925\end{array}$

H 1.5009952 .2960513 .660942

$\begin{array}{lllll}\text { O } & 3.091613 & 2.252879 & 4.944074\end{array}$

C $2.924203-1.6059953 .274329$

H $2.297572-0.1595404 .690289$

H $1.233471-0.3291613 .271539$

H 3.198764 4.314344 -0.269771

H 2.8541635 .3680942 .868435

H 2.8326153 .1059125 .333174

O $2.714727-2.4726354 .308829$

O $3.514226-1.8617382 .234277$

C $3.288349-3.7753904 .160097$

H $4.333048-3.710753 \quad 3.840509$

H $3.252951-4.2741335 .132366$

H $2.700943-4.3573573 .445618$

SCF Energy (B3LYP/6-31G**//MMFF) = -3245.90322905

$01 \_436$

MM̄FF Geometry

C $4.2855723 .349413-1.053045$

C $3.0789053 .476168-0.483814$

C $1.8298783 .834089-1.245695$

O $0.859392 \quad 2.779622-1.041947$

C $1.2424685 .143631-0.692146$

C $0.1081765 .690533-1.535725$

C $-1.1997005 .614744-1.219907$

C $0.5341686 .396674-2.798604$

C $-1.8358544 .967724-0.020202$

C $-2.7083713 .787579-0.447508$

C -3.5340413 .1652540 .687448$

C $-2.6789642 .614046 \quad 1.829143$

C -3.5311121 .8803052 .877506$

$\begin{array}{lllll}\text { O } & -4.280452 & 2.100434 & 0.087977\end{array}$ 
O $-2.641100 \quad 1.144114 \quad 3.730397$

C $-4.434260 \quad 0.8511932 .165231$

C $-4.2844252 .860818 \quad 3.785400$

O $-5.325622 \quad 0.2596353 .108406$

C $-5.206828 \quad 1.440566 \quad 0.961173$

C $\begin{array}{llll}-5.873038 & 0.312563 & 0.138007\end{array}$

$\begin{array}{llll}\text { O } & -6.213388 & 2.333599 & 1.438008\end{array}$

C $1.0011661 .649450-1.789622$

O $1.8475801 .431474-2.641783$

C $-0.0546010 .700741-1.371153$

C $-0.054011-0.554066-1.845015$

C $-1.011346-1.606081-1.491389$

C $-2.228289-1.198968-0.706387$

C $-6.568368 \quad 0.807370-1.137887$

C $-6.697505-0.301729-2.160936$

C $-5.384693-1.540599-3.882492$

O $-7.752360-0.909140-2.327643$

C $-4.908737-2.820934-3.272635$

C $-3.836837-3.499527-3.709943$

C $-3.416627-4.827356-3.149410$

C $-1.952365-4.882484-2.782657$

C $-1.094385-5.648973-3.477810$

C $-1.502762-4.132375-1.549477$

C $-0.745788-2.872704-1.872461$

N $-5.514804-0.533600-2.847594$

H $4.3859673 .549127-2.116609$

H 2.9694063 .2991210 .581130

H $2.0356753 .936985-2.318294$

H $2.0254045 .912711-0.640474$

H $0.917172 \quad 4.992296 \quad 0.342727$

H $-1.9147036 .068813-1.905819$ H $-0.3141436 .825361-3.342919$

H $1.2192497 .217383-2.561692$

H $1.0417825 .704031-3.476563$

H $-1.0891514 .633168 \quad 0.702210$

H -2.451829 5.7191120 .488033

H -3.403507 4.105894 -1.237395

H -2.087424 $3.014236-0.915606$

H $-4.2316813 .925344 \quad 1.058172$

H -2.099575 3.4082972 .313592

H -1.941508 1.9071111 .425009

H -2.025496 1.7766694 .138877

H -3.7796880 .0454891 .806263$

H -3.578712 3.5163224 .309837

H -4.8366442 .3298024 .568608$

H -4.983675 3.4991123 .241457

H -5.721381 -0.5251102 .694445$

H -5.109268 -0.426872 -0.130700

H $-6.623841-0.2092850 .743581$

H $-6.8941152 .437130 \quad 0.754644$

H $-0.7913711 .051779-0.659081$

H $0.737900-0.852203-2.530993$

H -2.760963 -0.391356-1.220546

H $-1.942810-0.8452240 .288482$

H -2.951731-2.004974 -0.570088

H $-7.5724401 .182617-0.909313$

H -6.024826 $1.635528-1.606623$

H $-4.685726-1.152327-4.629901$

H $-6.356965-1.698804-4.360241$

H -5.500987 -3.231571-2.457952

H $-3.263114-3.108819-4.547108$

H -3.644500 -5.590009 -3.905452

H $-4.013650-5.094398-2.268195$

H $-0.048992-5.721814-3.195843$

H $-1.415953-6.217966-4.343749$

H $-0.828880-4.777908-0.968985$

H -2.355152 -3.959683 -0.887135

H $0.171638-3.054349-2.434839$

H $-4.682465-0.010672-2.591851$

C $6.1407791 .622322-0.729918$

O $5.3210020 .515562-0.296450$

C $5.401514-0.522520-1.293358$

C $6.480523-0.089246-2.287491$

C $6.371046 \quad 1.418055-2.226538$

H $4.434403-0.533556-1.808054$

C $5.692504-1.893763-0.671553$

H $7.479931-0.410194-1.970837$

H $6.303765-0.493815-3.289104$
C $7.5869612 .145570-2.771426$

H $5.4885921 .707908-2.808702$

C $4.658810-2.4441430 .330988$

O $6.954810-1.8412640 .009347$

H $5.825493-2.623665-1.479888$

H $4.747478-1.930323 \quad 1.295467$

O $5.018031-3.8138720 .571920$

C $3.193126-2.405630-0.133812$

C $2.229858-3.3442840 .617516$

H $2.826298-1.376545-0.064608$

O $3.112372-2.765927-1.518218$

H $1.221139-3.1211910 .254555$

H $2.414991-4.3769020 .292419$

H $7.457033 \quad 3.229817-2.697555$

H $8.4957041 .876207-2.222907$

H $7.7445291 .895380-3.825647$

C $5.5329292 .965154-0.308361$

H $6.892059-1.1454750 .686167$

H $5.934346-3.8196490 .898527$

H $3.488151-3.658086-1.613016$

H $6.2721173 .759613-0.466372$

H 5.3268702 .9348450 .767973

H $7.0923791 .509119-0.192328$

C $0.863380-1.3423462 .493929$

O $2.140413-1.9645462 .675485$

C $2.255728-3.2880052 .156359$

C $1.211892-4.1990222 .818569$

C $-0.199715-3.6412842 .660547$

C $-0.253181-2.1881503 .127980$

H $0.676789-1.1971181 .423430$

$\begin{array}{llll}\text { C } & 0.934176 & 0.039723 & 3.160851\end{array}$

H $3.239194-3.6536682 .473410$

O $1.284097-5.5106292 .269576$

H $1.441715-4.2854843 .888658$

H $-0.555916-3.7452931 .629383$

O $-1.083543-4.4244443 .467026$

H $-0.172940-2.1725474 .222732$

O $-1.533460-1.6642602 .787527$

C $2.111303 \quad 0.8526502 .671605$

H $\quad 0.027087 \quad 0.6224122 .976294$

H $1.046061-0.0956444 .244108$

H $0.591195-6.0429322 .696765$

H $-1.970481-4.0333333 .386280$

H $-1.632098-0.8042873 .229241$

O 2.6034391 .5953363 .705481

$\begin{array}{llll}\text { O } & 2.533913 & 0.840617 & 1.524112\end{array}$

C $3.7704802 .375483 \quad 3.428762$

H 4.4970911 .8071312 .840301

H 3.4822923 .2935252 .910941

H 4.2327992 .6425794 .382820

SCF Energy (B3LYP/6-31G**//MMFF) $=-3245.92222655$

01_437

MM̄FF Geometry

C $-0.524420 \quad 5.305484-0.282521$

C $0.3915534 .557938-0.916813$

C $1.5715103 .934808-0.212834$

O $1.7569212 .590236-0.711495$

C $2.8474914 .736057-0.531865$

$\begin{array}{llll}C & 4.052857 & 4.318959 & 0.288991\end{array}$

C $5.1867573 .798586-0.219634$

C $3.947948 \quad 4.5567291 .774530$

C $5.5189093 .515827-1.657002$

C $5.2020142 .085714-2.120886$

C $5.9351550 .987663-1.332069$

C $5.784743-0.367737-2.025952$

C $6.413230-1.510372-1.212468$

O $5.3632960 .916172-0.023123$

O $5.935672-2.735309-1.790266$

C $5.904611-1.4354280 .245229$

C $7.939694-1.527256-1.356100$

O $6.596360-2.3623041 .078043$

C $6.001688-0.0212100 .853956$

$\begin{array}{llll}\text { C } 5.312414 & 0.098724 & 2.235954\end{array}$

$\begin{array}{lllll}\text { O } & 7.381872 & 0.313760 & 1.046177\end{array}$

C $0.9918811 .602193-0.174759$

$\begin{array}{lllll}\text { O } & 0.152191 & 1.711819 & 0.703619\end{array}$

C $1.3549930 .343927-0.863976$ 
C $0.540523-0.719720-0.799857$

C $0.781277-2.019421-1.431785$

C $2.163664-2.281735-1.968658$

C $3.813323-0.2198882 .255509$

C $3.489066-1.6537472 .641516$

C $1.571337-3.2400962 .780674$

O $4.321200-2.4365193 .091307$

C $1.610468-4.1170761 .569068$

C $0.546536-4.7972731 .115703$

C $0.585660-5.707071-0.078885$

C $-0.488501-5.387626-1.094656$

C $-1.615358-6.116412-1.158353$

C $-0.216285-4.288693-2.097150$

C $-0.225743-2.916190-1.480020$

N $2.143458-1.9456912 .458678$

H -0.4017525 .4830510 .782533$

H $0.3079824 .399177-1.989005$

H 1.4006883 .9025360 .869798

H $2.6744835 .804644-0.345069$

H $3.0589104 .645217-1.602823$

H 5.9873923 .5467420 .475350

H 4.9040154 .4062832 .287776

H 3.6312065 .5856541 .974015

H 3.2238913 .8721182 .226309

H $5.0140174 .225691-2.320644$

H $6.5903653 .707848-1.798617$

H $4.1192151 .926412-2.055599$

H $5.4761182 .013327-3.180723$

H $6.9937901 .262341-1.256442$

H $6.212156-0.337907-3.036039$

H $4.717144-0.584870-2.162661$

H $6.354531-3.475071-1.318791$

H $4.853429-1.7464020 .231599$

H $8.221682-1.676453-2.405373$

H $8.380882-2.365663-0.805857$

H $8.412664-0.604550-1.013498$

H $6.414966-3.2553390 .740434$

H $5.860868-0.4871902 .983384$

H 5.4147801 .1455732 .557185

H 7.4379101 .0209741 .710041

H $2.2848020 .332014-1.420890$

H $-0.395163-0.630463-0.250303$

H $2.916025-2.065271-1.202929$

H $2.361570-1.656235-2.845610$

H $2.331430-3.319050-2.263960$

H 3.3345780 .4133873 .013462

H $3.3495870 .014903 \quad 1.293219$

H $0.549906-3.0658463 .133471$

H $2.148384-3.7013523 .588528$

H $2.563389-4.2157171 .054204$

H $-0.398982-4.7202781 .648036$

H $\quad 0.462298-6.735927 \quad 0.283912$

H $1.566492-5.682470-0.570018$

H -2.375836 -5.918010 -1.906084

H $-1.804654-6.926047-0.461456$

H $-0.984802-4.295112-2.881193$

H $0.716416-4.516139-2.619631$

H $-1.189775-2.633314-1.056747$

H $1.536727-1.2519642 .031512$

C $-3.0640915 .582766-0.424416$

O $-3.342570 \quad 4.200134-0.717367$

C $-4.028597 \quad 3.624642 \quad 0.411257$

C $-4.443653 \quad 4.794914 \quad 1.300204$

C $-3.298150 \quad 5.761218 \quad 1.076122$

H -3.286972 3.0259550 .950526

C $-5.1937352 .739662-0.044614$

H $-5.3873315 .245170 \quad 0.971301$

H -4.5591664 .4985672 .347365$

C -3.609114 $7.189377 \quad 1.487096$

H -2.441561 5.4026491 .658084

C $-4.8438831 .628521-1.055385$

O $-6.188753 \quad 3.568810-0.665660$ H -5.6832832 .3051610 .833336$ H -4.577181 2.096335 -2.011809 O $-6.0295850 .867125-1.313706$ C $-3.7282190 .644211-0.662923$ $\begin{array}{llll}C & -3.777356 & 0.061328 & 0.761767\end{array}$ H -3.736974 -0.193412 -1.370470
O $-2.459723 \quad 1.283170-0.814501$

H $-2.890710-0.5752390 .879270$

H -3.629822 $0.863177 \quad 1.494070$

H -2.7537027 .8444461 .293954$

H -4.469891 7.5872910 .939199

H -3.8394107 .2403422 .556289$

C -1.684776 $5.979559-0.960761$

H -5.763795 $4.028811-1.410073$

H $-6.7248351 .492561-1.580563$

H $-2.3883291 .585864-1.735616$

H $-1.5552777 .062881-0.850425$

H $-1.6549765 .762750-2.036118$

H $-3.8145676 .158963-0.982523$

C $-4.489950-2.8073150 .019549$

O $-5.389822-1.699726 \quad 0.124204$

C $-5.044843-0.737691 \quad 1.121961$

C $-4.992700-1.4112432 .502087$

C $-4.076666-2.6350862 .505581$

C $-4.425388-3.5695971 .346472$

H -3.491202 -2.446289 - 0.250928

C $-5.002809-3.701053-1.113997$

H $-5.873340-0.0214401 .155672$

O $-4.568230-0.4709793 .482799$

H $-6.006886-1.7296062 .777180$

H -3.019063 -2.347779 2.470308

O $-4.256719-3.3602773 .724014$

H $-5.386029-4.0604291 .548359$

O $-3.433968-4.589396 \quad 1.253859$

C $-4.889505-3.000354-2.447223$

H $-4.455900-4.647215-1.177528$

H $-6.060676-3.948826-0.963279$

H $-4.666704-0.8883434 .354869$

H $-3.927381-2.8025284 .448879$

H $-3.394516-5.0361392 .116886$

O $-3.586818-2.964799-2.847294$

O $-5.844280-2.531649-3.053346$

C $-3.340946-2.288289-4.083618$

H $-3.857022-2.797803-4.902835$

H $-3.660322-1.243926-4.014352$

H -2.265165 -2.312860 -4.276996

SCF Energy (B3LYP/6-31G**//MMFF) $=-3245.89437009$

01_438

MM̄FF Geometry

C $-6.1346430 .051038-0.235236$

C $-5.1981640 .591258-1.029290$

C $-4.491628 \quad 1.889545-0.738082$

O $-3.1269881 .567101-0.384130$

C $-4.4641702 .773701-1.997949$

C $-3.6640054 .051224-1.828662$

C $-2.5495434 .349357-2.526325$

C $-4.196398 \quad 5.027473-0.811982$

C $-1.8912193 .532463-3.603827$

C $-0.8214042 .549986-3.103683$

C $0.4434793 .227155-2.551869$

C $1.5304202 .186416-2.272305$

C $2.7847302 .816938-1.647573$

O $0.1123373 .907369-1.338970$

O $3.596517 \quad 1.754219-1.123835$

C $2.3660993 .698155-0.450337$

C $3.6401433 .539401-2.694959$

O $3.476370 \quad 4.437740 \quad 0.051168$

C $1.1963634 .653374-0.766116$

C 0.6461815 .3770610 .487698

O $1.6520225 .669473-1.668325$

C $-2.889302 \quad 1.2110470 .907051$

$\begin{array}{llll}\text { O } & -3.688928 & 1.190926 & 1.829036\end{array}$

$\begin{array}{llll}\text { C } & -1.475888 & 0.792158 & 0.996648\end{array}$

C -1.098344 -0.0119932 .001179$

C $0.244429-0.550762 \quad 2.206326$

$\begin{array}{llll}\text { C } & 1.359520 & 0.042685 & 1.391094\end{array}$

C $0.1113034 .463577 \quad 1.596682$

C $1.106247 \quad 4.1941602 .714730$

C 1.3409672 .8815084 .823255

$\begin{array}{llllll}\text { O } & 2.189449 & 4.764611 & 2.808278\end{array}$

C 2.1849521 .6784844 .543186

C $2.1147200 .543716 \quad 5.255972$

C $2.994130-0.6505245 .017783$ 
C $2.209501-1.9310834 .839234$

C $2.050168-2.7921925 .858367$

C $1.692539-2.2566473 .457360$

C $0.421997-1.5248103 .122570$

N $0.6194723 .262596 \quad 3.622935$

H -6.4466130 .5751870 .664010$

H -4.870400 $0.038581-1.907278$

H -4.9754452 .4307740 .084487$

H $-5.4900073 .039552-2.285327$

H $-4.0635832 .183750-2.829183$

H $-2.0528165 .296241-2.317843$

H -3.655756 $5.979903-0.828273$

H $-5.2502845 .246927-1.011905$

H -4.1088234 .6185450 .199255$

H -2.643713 $2.977563-4.174563$

H -1.441044 $4.222225-4.329138$

H -1.259251 $1.899493-2.337652$

H $-0.539377 \quad 1.908045-3.947745$

H $0.7945193 .953588-3.294122$

H $1.792608 \quad 1.643447-3.188858$

H $1.1393331 .429823-1.578216$

H $3.8138881 .153213-1.856974$

H 2.0595963 .0178960 .351254

H $3.9577952 .840405-3.478063$

H $4.5628553 .929393-2.251282$

H $3.1154694 .363756-3.182198$

H 4.1784953 .7988110 .262233

H $1.389912 \quad 6.0865780 .870061$

H -0.1996945 .9966770 .155836$

H $1.0537206 .431953-1.601451$

H $-0.799812 \quad 1.119586 \quad 0.215548$

H -1.848087 -0.3357022 .722214$

H $2.348284-0.2397711 .752269$

H 1.3293221 .1353641 .437942

H $1.282620-0.2635220 .344476$

H -0.7465204 .9606112 .068129$

H -0.2462043 .5138461 .188517$

H 0.5963272 .6928395 .603040

H 1.9782973 .7117505 .144341

H $2.9090871 .756158 \quad 3.735565$

H $1.4100590 .479526 \quad 6.082193$

H $3.666445-0.7442465 .880808$

H $3.648992-0.5001674 .150501$

H $1.515119-3.7273395 .730111$

H $2.446474-2.5783336 .845500$

H $1.475035-3.3307883 .380965$

H $2.484687-2.0880322 .723087$

H $-0.446107-1.8791023 .679990$

H -0.2645792 .7989483 .434419$

C $-5.982145-2.454188 \quad 0.163890$

O $-4.657643-2.547246-0.400652$

C -3.714689-2.767183 0.664673

C $-4.545881-3.2244731 .856883$

C $-5.797561-2.3838181 .680184$

H $-3.268328-1.7938540 .894575$

C $-2.605953-3.7401220 .239674$

H $-4.789455-4.2915041 .791999$

H -4.039420 -3.046370 2.810210

C $-6.992378-2.8893952 .468685$

H -5.569406 -1.360053 2.000840

C $-1.716898-3.183952-0.900893$

O $-3.217806-4.971648-0.138514$

H -1.971609 $-3.942146 \quad 1.111571$

H -1.323556 -2.211884 -0.583204

O $-2.495001-2.959186-2.076754$

C $-0.526745-4.097159-1.270087$

C $0.356835-3.531488-2.400570$

H $\quad 0.075749-4.286395-0.374560$

O $-1.027151-5.362059-1.711784$

H $0.979828-4.346453-2.787051$

H $-0.276397-3.245195-3.248982$

H -7.870112 -2.260588 2.288873

H -7.250590 -3.917282 2.192570

H $-6.778825-2.874043 \quad 3.542549$

C $-6.747284-1.297945-0.490439$

H $-2.508656-5.604933-0.339640$

H -3.186920 -2.313131-1.856196

H $-0.264231-5.947972-1.853297$
H -7.784225 -1.284612 -0.135461

H $-6.788893-1.470377-1.573712$

H $-6.488095-3.390376-0.106204$

C $3.139027-3.607257-1.173697$

O $2.099132-2.669261-0.870879$

C $1.244540-2.342543-1.974572$

C $2.083510-1.751104-3.115059$

C $3.212464-2.690173-3.533069$

C $4.037781-3.107272-2.315023$

H $2.671604-4.553618-1.471831$

C $3.894922-3.9278520 .125023$

H $\quad 0.574447-1.548463-1.626132$

O $1.251106-1.453667-4.230594$

H $2.521224-0.802831-2.778530$

H $2.824040-3.560768-4.074220$

O $4.057200-1.992796-4.452146$

H $4.637750-2.244440-2.002529$

O $4.941003-4.133993-2.717266$

C $4.930797-2.9064300 .536366$

H $3.198513-4.0495170 .963059$

H $4.422898-4.8819900 .003735$

H $1.823050-1.102947-4.934744$

H $4.790261-2.590155-4.680454$

H $5.547486-4.302973-1.976589$

O $4.367861-1.6763280 .687425$

O $6.108658-3.1847320 .726899$

C $5.266400-0.6359501 .086716$

H $5.700128-0.8657572 .064644$

H $\quad 6.050643-0.5040790 .335299$

H 4.6993610 .2950201 .166777

SCF Energy $($ B3LYP/6-31G**//MMFF $)=-3245.90459150$

01439

MM̄FF Geometry

C $1.359229-2.5612870 .939954$

C $2.192444-1.8033611 .669703$

C $1.872485-0.4579352 .279941$

O $0.464965-0.1616942 .110525$

C $2.195362-0.3890633 .785673$

C $3.664364-0.4537674 .138651$

C 4.5203270 .5807564 .022958

C $4.132321-1.7617054 .722141$

C 4.2348691 .9635443 .497360

C 5.2852632 .4256892 .476085

C 5.2027651 .6918631 .125592

C 6.5480871 .7865250 .402640

C $6.4996871 .126661-0.979009$

O $4.1754042 .309746 \quad 0.333849$

O $7.6897961 .505365-1.687753$

C $5.3035031 .706968-1.754985$

C $6.515564-0.404328-0.889242$

O $5.159801 \quad 1.012947-2.994000$

C $3.9739001 .703572-0.953141$

C $2.9151242 .521040-1.732867$

O $3.5186500 .358588-0.826245$

C $0.095779 \quad 1.1467182 .109957$

O 0.8276272 .1186252 .204441

C $-1.376307 \quad 1.2010791 .997179$

C -1.9933892 .3847592 .123104$

C -3.4334922 .6204382 .049810$

C -4.3337981 .4282311 .876297$

C $1.5874272 .773698-1.005124$

C $0.6065003 .507522-1.909502$

C $-1.7334604 .284281-2.051473$

O $0.8868123 .852080-3.055963$

C $-2.6990424 .969337-1.139070$

C $-4.0176834 .719047-1.145556$

C $-5.0245975 .452419-0.302759$

C -5.7963254 .5294030 .613994$

C -6.9189293 .9215950 .194318$

C -5.3073594 .3617712 .035106$

C -3.883752 3.8895792 .118589

N $-0.6193853 .739301-1.296198$

H $\quad 0.341698-2.2267640 .758323$

H $3.214888-2.1521331 .795561$

H $2.4557380 .288048 \quad 1.729679$

H $1.648367-1.1836144 .311626$

H 1.7776110 .5321884 .212995 
H $\quad 5.546513 \quad 0.4298364 .356390$ H $5.200089-1.7510704 .965496$ H $3.585434-1.9800395 .645445$ H $3.964751-2.5828644 .019278$ H 3.2377672 .0472713 .062299 H 4.2636702 .6512044 .351587 H 5.1485683 .5022132 .311352 H 6.2806692 .2924202 .919200 H $4.943718 \quad 0.6412681 .293434$ H $7.347780 \quad 1.3399571 .007087$ H 6.8219002 .8435920 .276638 H $8.4557981 .201350-1.171258$ H $5.5615242 .745795-2.005872$ H $7.418026-0.747751-0.369690$ H $6.554313-0.857305-1.886061$ H $5.653967-0.813719-0.357927$ H $4.5943001 .549548-3.573724$ H $3.3394593 .501128-1.988620$ H $2.6890542 .007833-2.676391$ H $2.556140 \quad 0.356207-0.710062$ H $-1.909493 \quad 0.274571 \quad 1.823449$ H -1.386679 3.2735162 .292825 H $-4.206117 \quad 0.722497 \quad 2.704534$ H $-4.101517 \quad 0.9164690 .937574$ H -5.3950241 .6858991 .841915$ H $1.122622 \quad 1.829267-0.705960$ H $1.7560723 .384349-0.111866$ H -2.193249 $3.452753-2.596501$ H -1.353245 $5.001438-2.786866$ H -2.303892 $5.737266-0.478657$ H -4.409176 $3.968672-1.829391$ H -5.720586 $5.964032-0.980751$ H $-4.552576 \quad 6.249936 \quad 0.284431$ H -7.4925193 .2756390 .850628$ H -7.287042 4.054974 -0.817683 H -5.9602713 .6943132 .610205$ H -5.3776115 .3320462 .544041$ H -3.1602334 .6975752 .230998$ H $-0.8187403 .266205-0.419562$ C $1.927628-3.816018-1.205514$ O $0.755368-3.254263-1.830849$ C $1.155498-2.084876-2.566860$ C $2.609500-2.338665-2.936229$ C $3.128517-2.980242-1.663681$ H $1.101623-1.237229-1.872123$ C $0.215056-1.824090-3.747265$ H $2.699024-3.041956-3.771911$ H $3.141053-1.420236-3.202267$ C $4.395486-3.795393-1.864711$ H $3.347927-2.191250-0.938746$ C $-1.286247-1.850557-3.393708$ O $0.429337-2.817695-4.758510$ H $0.477540-0.867067-4.212950$ H -1.591860 -2.890524 -3.234310 O $-2.011722-1.403004-4.546354$ C -1.698304 -0.999717 -2.179040 C -3.221395 -0.923336-1.950731 H -1.219929 -1.395261 -1.276972 O $-1.2092120 .331309-2.360469$ H -3.390946 -0.328764 -1.045166 H -3.682770 -0.338688 -2.756985 H $4.720305-4.244502-0.920697$ H $4.243201-4.602920-2.588557$ H $5.206829-3.161004-2.235712$ C $1.737663-3.8793970 .317048$ H $\quad 0.268973-3.687963-4.354460$ H -1.791261 -0.465850 -4.685224 H -1.436225 $0.839483-1.562863$ H $2.643168-4.2853520 .783731$ H $\quad 0.926349-4.5825170 .543409$ H $2.012201-4.841623-1.585213$ C -3.331852 -2.811975 0.452910 O $-3.243030-3.173598-0.931382$ C $-3.921239-2.292163-1.829318$ C $-5.412760-2.221613-1.474355$ C $-5.639046-1.883244-0.003649$ C $-4.799777-2.787140 \quad 0.899922$ H $-2.877779-1.8244710 .594642$
C $-2.502078-3.8484901 .227768$

H $-3.862112-2.762253-2.818394$

O $-6.085012-1.261724-2.282817$

H -5.877964 -3.195099-1.677568

H -5.433595 -0.8274920 .197476$

O $-7.017460-2.0866760 .318052$

H -5.217119 -3.801993 0.879736

O $-4.887150-2.3170952 .241476$

C $-1.929400-3.3401002 .532778$

H $-3.103738-4.7423421 .429480$

H $-1.651508-4.1606180 .608580$

H -5.941453 -1.510669-3.211888

H $-7.539844-1.531987-0.286580$

H $-5.829981-2.2865602478310$

O $-1.230433-4.3529753 .127711$

O $-2.056106-2.2067482 .973190$

C $-0.598518-4.0184064 .367039$

H $-1.347663-3.7183655 .105831$

H $-0.079200-4.9073314 .734744$

H $\quad 0.135323-3.2219644 .214270$

SCF Energy $(B 3 L Y P / 6-31 G * * / / M M F F)=-3245.91263023$

01444

MMFF Geometry

C $3.6212161 .309236-1.702995$

C $3.7354582 .619171-1.978697$

C $2.6493573 .664060-1.848877$

O $1.3988983 .043622-1.484077$

C $3.0115144 .685248-0.753254$

C $1.9944305 .805478-0.600098$

C 1.2369826 .0090040 .498082

C $1.8730046 .738911-1.777090$

C 1.2560175 .2089591 .771495

C 0.2337494 .0721221 .731042

C 0.4865372 .9512872 .750416

C 1.7918732 .2024132 .460859

C 1.9783600 .9827013 .370086

O -0.6248392 .0483252 .638795$

$\begin{array}{llll}\text { O } & 3.040230 & 0.190987 & 2.820040\end{array}$

C $0.6991190 .128043 \quad 3.319605$

C 2.4223231 .3820124 .781795

O $\quad 0.772287-0.952928 \quad 4.245995$

C $-0.5949760 .941603 \quad 3.550499$

C -1.824114 0.0440523 .264521

O -0.6379091 .3774654 .909888$

C $0.4365232 .915737-2.435342$

O $\quad 0.4878113 .278753-3.598638$

C $-0.7096282 .232039-1.796970$

C - $-1.8343891 .999682-2.489023$

C $-3.0114001 .294500-1.978761$

C $-3.0349360 .954523-0.511621$

C $-3.165727 \quad 0.787016 \quad 3.261712$

C $-4.327220-0.1336582 .920938$

C $-6.764788-0.0431542 .378192$

O $-4.229446-1.3568792 .880168$

C $-6.848848-0.2936430 .905710$

C $-7.4261210 .553156 \quad 0.039929$

C $-7.5623240 .290241-1.432799$

C $-6.4992570 .999680-2.243893$

C $-6.7105732 .248230-2.694827$

C $-5.2513200 .187082-2.522940$

C $-4.0104640 .982870-2.830605$

N -5.4951640 .5813102 .696351$

H $2.6807570 .909182-1.333954$

H $4.6871582 .987630-2.357584$

H $2.5625894 .173926-2.815624$

H $3.9879835 .137348-0.972699$

H 3.1369724 .1466650 .191846

H $0.528762 \quad 6.8361180 .494797$

H $1.2273407 .596947-1.561280$

H $2.8569177 .132549-2.052354$

H $1.4493146 .219219-2.641649$

H $2.268368 \quad 4.853738 \quad 1.972874$

H 1.0202235 .8771392 .609221

H -0.7709074 .4855501 .895006$

H $0.1901623 .626670 \quad 0.732351$

H 0.4948743 .3854093 .756847

H 2.6644402 .8578012 .550966 
H $\quad 1.786371 \quad 1.857237 \quad 1.416961$ H $3.834547 \quad 0.7497602 .765463$ H $0.647814-0.3231152 .319342$ H 3.3806991 .9139614 .745781 H 2.5943540 .4987005 .406523 H 1.7059842 .0310595 .289577 H $1.598444-1.4332104 .065577$ H -1.703919 -0.4229092 .278617$ H -1.871504 -0.7652774 .004135$ H -1.558493 1.5489725 .160966 H $-0.586396 \quad 1.911974-0.768235$ H -1.889180 $2.323265-3.527288$ H -2.876144 1.8557520 .091164 H $-2.2515610 .228704-0.271500$ H $-3.984510 \quad 0.531024-0.178804$ H $-3.372330 \quad 1.218824 \quad 4.246538$ H -3.1431211 .6019032 .530486$ H -7.557202 0.6299212 .721494 H -6.857642 -0.9880782 .923448$ H $-6.432764-1.2297280 .541236$ H $-7.836935 \quad 1.4906330 .407978$ H $-8.562507 \quad 0.615109-1.747853$ H -7.533246 $-0.786491-1.644338$ H $-5.9821962 .776916-3.298991$ H -7.633354 2.774444 -2.471480 H $-5.454558-0.474883-3.374926$ H $-5.056920-0.490630-1.684729$ H $-3.9208411 .279741-3.875771$ H -5.4574591 .5963562 .685857$ C $5.134267-0.414800-0.639900$ O $4.062807-1.273490-0.194574$ C $4.593801-2.5849960 .073003$ C $6.102668-2.4073980 .176450$ C $6.345131-1.326945-0.855890$ H $4.365791-3.200086-0.806000$ C $3.942458-3.2006321 .316720$ H $6.405793-2.0536351 .168620$ H $6.645837-3.333820-0.033275$ C $7.682490-0.623790-0.696776$ H $6.299096-1.782705-1.854323$

C $2.400839-3.2186071 .293433$

O $4.345739-2.4637672 .477580$ H $4.326570-4.2162961 .466120$ H $2.018110-2.1939131 .341681$ O $1.955242-3.8740102 .487926$ C $1.788717-3.9511280 .086016$ C $0.263798-4.1493210 .167463$ H $2.033274-3.414155-0.833801$ O $2.383735-5.247481-0.031275$ H $-0.031575-4.801634-0.663195$ H $0.021736-4.7251541 .069378$ H $7.8082010 .153457-1.456904$ H $7.772509-0.1506840 .286855$ H $8.506622-1.336745-0.803111$ C $4.726257 \quad 0.313077-1.923377$ H $4.070881-1.5400842 .347118$ H $2.372150-3.4209073 .240681$ H $2.204753-5.7282230 .795162$ H $4.367811-0.417257-2.658673$ H $5.599916 \quad 0.810859-2.360440$ H $\begin{array}{llll}5.323925 & 0.303209 & 0.168047\end{array}$ C $-0.764259-2.618164-2.258023$ O $-0.287745-2.054385-1.029197$ C $-0.550471-2.8401240 .139412$ C $-2.063374-3.0456290 .300773$ C $-2.681901-3.659562-0.950681$ C -2.279417 -2.867241 -2.193299 H $-0.232239-3.554101-2.466082$ C $-0.445418-1.627818-3.389632$ H $-0.223474-2.2384260 .994358$ O $-2.349888-3.8725751 .423205$ H -2.543819 -2.077450 0.486656 H $-2.403748-4.714603-1.052161$ O $-4.107433-3.645539-0.831193$ H $-2.813377-1.909780-2.198557$ O $-2.687819-3.584879-3.356408$ C $1.032577-1.497627-3.687893$ H $-0.933003-1.951621-4.316137$
H $-0.807146-0.631443-3.121466$

H -1.969422 -3.4417302 .207787$

H $-4.390343-2.723492-0.707283$

H -3.644342 -3.743558 -3.278765

O $1.182157-0.695793-4.784628$

O $1.935587-2.014256-3.046647$

C $2.523058-0.467186-5.230627$

H $3.133825-1.372036-5.155111$

H $2.482744-0.164245-6.280344$

H $2.9637940 .345660-4.648554$

SCF Energy $(B 3 L Y P / 6-31 G * * / / M M F F)=-3245.91958866$

01440

MM̄FF Geometry

C $0.545943-3.2444670 .984235$

C $-0.216185-3.207761-0.119504$

C $0.249604-3.117109-1.554058$

O $-0.223167-1.849449-2.068065$

C $1.776419-3.179415-1.763702$

C $2.221329-3.139977-3.215445$

C $3.159933-2.298810-3.694656$

C $1.619783-4.178497-4.126854$

C $3.939519-1.262531-2.931443$

C $5.156787-1.896226-2.248681$

C $6.094166-0.865932-1.602243$

C $7.251943-1.588479-0.906402$

C $8.174597-0.616489-0.161038$

O $5.351716-0.101441-0.650062$

O $9.011303-1.3843550 .718153$

C 7.3148510 .3041640 .730596

C $9.1174040 .113304-1.125158$

O 8.1143241 .3281321 .318650

C $6.1074450 .935082-0.002846$

C 5.1659701 .6194641 .011792

O $6.526281 \quad 1.941857-0.926593$

C $-1.253002-1.851430-2.952663$

O $-1.845020-2.830185-3.386836$

C $-1.599390-0.479354-3.390465$

C $-1.1433440 .626148-2.782592$

C $-1.4525382 .010130-3.148316$

C $-2.3130462 .249515-4.360609$

C 3.9984282 .3596300 .347536

C 3.0728952 .9925461 .372461

C 1.3705464 .8103431 .582517

O 2.9367112 .5509472 .510514

C 0.0382364 .4141721 .034219

C -0.8132745 .2638020 .440350$

C $-2.1220364 .830817-0.156787$

C $-2.1944215 .069332-1.651851$

C $-3.2012365 .784964-2.180739$

C $-1.1169094 .484404-2.542675$

C $-0.9579552 .998275-2.375045$

N 2.3882474 .0797290 .849115

H $1.627933-3.1945700 .905476$

H -1.295687 -3.258301 0.003832

H $-0.214476-3.954957-2.088277$

H $2.164879-4.109124-1.327166$

H $2.237942-2.355567-1.209162$

H $3.434145-2.377749-4.745814$

H $2.129448-4.225931-5.095303$

H $1.694985-5.171895-3.672398$

H $0.566370-3.961100-4.322722$

H $4.269846-0.492394-3.639386$

H $3.302038-0.747625-2.204989$

H $4.798712-2.591069-1.477450$

H $5.724864-2.489351-2.977143$

H $6.468080-0.197083-2.386273$

H $7.824998-2.187634-1.625060$

H $6.847370-2.304630-0.176931$

H $9.498069-2.0319490 .179798$

H $6.930662-0.3059301 .560310$

H $9.772035-0.603134-1.636180$

H $9.7842450 .795846-0.587237$

H $8.5882580 .678009-1.895739$

H $8.8453580 .890924 \quad 1.788314$

H 4.7661800 .8659551 .702530

H 5.7258572 .3480891 .611908

H $7.4723902 .112803-0.793479$ 
H -2.279692 -0.429352-4.234472 H $-0.4860560 .517176-1.921313$ H $-3.2736501 .732128-4.266832$ H -1.808998 1.888981 -5.263854 H -2.549883 $3.303860-4.521978$ H $4.3849183 .141482-0.316038$ H $3.3971261 .669348-0.255094$ H 1.5738305 .8772071 .447652 H 1.4249794 .5732172 .649326 H -0.2269073 .3621541 .110591$ H $-0.554636 \quad 6.3168420 .361499$ H -2.9217995 .3743260 .362120$ H -2.307945 3.7677870 .038546 H -3.269346 $5.962639-3.248923$ H -3.983035 $6.208062-1.558643$ H $-0.1570114 .959548-2.302758$ H -1.291708 $4.741385-3.593164$ H $-0.3490032 .722245-1.513675$ H $2.5378294 .337016-0.122319$ C $0.216121-2.111473 \quad 3.202274$ O $-0.744100-1.1116872 .795736$ C $-1.386667-0.5836743 .970915$ C $-0.484167-0.9609055 .136836$ C $-0.010687-2.3317784 .699984$ H -2.338819-1.117045 4.078946 C -1.6459310 .9178413 .822963$ H $0.367546-0.2771155 .229949$ H -1.018596 -0.9732256 .091451$ C $1.222013-2.8145965 .444969$ H $-0.826048-3.0515614 .854972$ C -2.4726901 .3067472 .580421$ O -0.3919651 .6081753 .737146$ H -2.1340891 .2968984 .728200$ H -1.8730011 .1551741 .676254$ O -2.7398592 .7130712 .662008$ C -3.8210090 .5747262 .452975$ C -4.7045521 .0684591 .292560$ H $-3.650735-0.4980862 .337134$ $\begin{array}{lllll}\text { O } & -4.562739 & 0.740970 & 3.664922\end{array}$ H $-5.6958930 .614373 \quad 1.409100$ H -4.8775232 .1463291 .399645$ H $1.536743-3.7983245 .082818$ H $2.063040-2.1244545 .319105$ H $1.014716-2.8994016 .516716$ C $0.002028-3.3827742 .379277$ H $\quad 0.094947 \quad 1.2408582 .979298$ H -1.892119 3.1626132 .818902 H -4.6936881 .6953443 .799707$ H -1.066475 -3.6254962 .346997$ H $0.514591-4.2305082 .848384$ H $1.212729-1.6884453 .021260$ C $-5.078789-1.451383-0.401356$ O $-3.897516-0.645564-0.294963$ C $-4.1323720 .755442-0.104248$ C $-4.9720841 .311862-1.262084$ C $-6.2646520 .527904-1.455888$ C $-5.965808-0.966072-1.558149$ H $-5.637732-1.4113880 .541255$ C $-4.637692-2.905085-0.640130$ H $-3.152126 \quad 1.241006-0.166722$ O $-5.2845682 .685013-1.057085$ H -4.393856 1.249691-2.190946 H $-6.984751 \quad 0.734557-0.655635$ O $\quad-6.887080 \quad 0.944765-2.673613$ H $-5.472350-1.165743-2.517794$ O $-7.196725-1.687749-1.566512$ C $-4.127621-3.5829130 .614013$ H $-5.486977-3.499325-0.995926$ H -3.834553 -2.940410 -1.383892 H $-4.4439083 .164062-0.964422$ H $-7.0302011 .904828-2.610840$ H -7.645503 -1.522723 -0.719798 O $-3.958085-4.913836 \quad 0.352638$ O $-3.894645-3.026868 \quad 1.677403$ C $-3.493038-5.7115531 .445994$ H -2.434764 -5.507986 1.627782 H $-4.084490-5.5235972 .347298$ H -3.605440 -6.763395 1.169773
SCF Energy (B3LYP/6-31G**//MMFF)= -3245.92645416

014441

MMFF Geometry

C $-1.355600-3.880375-1.942723$

C $-0.730135-3.438854-0.842241$

C $0.615390-2.761495-0.866554$

O $0.460849-1.416062-0.347614$

C $1.631462-3.4768790 .041889$

C $2.387132-4.585204-0.661384$

C $3.615066-4.431549-1.195615$

C $1.711021-5.929884-0.691992$

C $4.431918-3.169367-1.239960$

C $5.561003-3.224609-0.205672$

C $6.377880-1.925257-0.156007$

C $7.548001-2.0825020 .819012$

C $8.326214-0.7746971 .006201$

O $5.508719-0.8750880 .279597$

O $9.180786-0.9547752 .145472$

C 7.3251330 .3516251 .334722

C $9.250674-0.496003-0.184381$

O 7.9932371 .6102551 .403103

C 6.1232040 .4187970 .362796

C 5.0617401 .4003050 .920367

O $6.5857060 .866625-0.909499$

C $-0.063360-0.479163-1.184448$

O $-0.433177-0.644345-2.336100$

C $-0.1154120 .801872-0.446099$

C $-0.7600331 .846680-0.985903$

C $-0.8835303 .186060-0.405354$

C -0.2000343 .4414580 .911740$

C 3.8318501 .6060340 .023517

C $3.9791622 .759922-0.957665$

C $2.7058473 .979638-2.721042$

O $4.9740433 .477881-1.009915$

C $1.9502715 .097834-2.072467$

C $0.8630875 .670268-2.611706$

C $0.1027376 .791583-1.961840$

C $-1.3771776 .499952-1.833791$

C $-2.2726267 .109566-2.628989$

C $-1.8253835 .559294-0.734019$

C -1.585243 4.116044-1.086639

N $2.8579712 .898334-1.764087$

H $-0.863749-3.778540-2.906716$

H -1.217463 -3.544593 0.123513

H $1.019114-2.691094-1.885207$

H $1.139158-3.8793840 .937862$

H $2.337520-2.7412740 .447934$

H $4.091942-5.295386-1.656608$

H $2.360680-6.710720-1.101774$

H $1.429856-6.2384200 .320212$

H $\quad 0.810183-5.896865-1.310448$

H $4.857444-3.067499-2.245813$

H $3.807882-2.283019-1.088646$

H $5.123399-3.4116210 .784284$

H $6.224049-4.068562-0.435058$

H $6.742301-1.698056-1.164695$

H $8.220454-2.8865450 .494037$

H $7.162555-2.3967701 .799608$

H $9.623617-0.1060672 .318488$

H 6.9438850 .1579422 .346788

H $9.973018-1.312491-0.304042$

H $9.8484190 .407463-0.020707$

H $8.713152-0.388025-1.128772$

H $8.152712 \quad 1.910240 \quad 0.491877$

H 4.6981960 .9943191 .874041

H 5.5213722 .3709411 .139547

H $5.817778 \quad 1.096539-1.455514$

H 0.3808470 .8509980 .516270

H -1.240852 $1.714079-1.954418$

H 0.8799543 .2843840 .821201

H -0.5894342 .7685111 .683227$

H $-0.3383864 .456245 \quad 1.288862$

H $3.5804440 .691663-0.524774$

H $2.978595 \quad 1.8534160 .667245$

H $2.1796083 .573509-3.590561$

H $3.6923034 .332470-3.037468$

H $2.3206195 .459398-1.115804$ 
H $\quad 0.5061825 .326196-3.579862$ H $0.2580947 .693030-2.568818$ H $0.5079227 .026578-0.969645$ H -3.336928 $6.924627-2.527576$ H -1.961518 $7.803277-3.403070$ H -2.902468 $5.675895-0.555427$ H -1.343290 5.8617450 .198774 H -2.055370 $3.818901-2.025702$ H $2.0595692 .291131-1.608244$ C $-3.819073-3.660388-2.547736$ O $-4.103638-2.525283-1.705339$ C $-4.489709-1.420232-2.547979$ C $-4.578001-1.967342-3.973225$ C -3.559837 -3.088571-3.939945 H -3.671278 -0.692418 -2.502019 C $-5.792516-0.780953-2.053908$ H -5.574455 -2.365047 -4.198092 H -4.350599 -1.202202 -4.722078 C -3.719485 -4.097108 -5.063060 H - $2.563555-2.634921-4.002683$ C $-5.769282-0.276295-0.596483$ O $-6.840185-1.756715-2.152845$ H $-6.0722350 .036183-2.730425$ H $-5.757743-1.1350180 .084938$ O C $-4.6144790 .687910-0.266866$ C -4.7283151 .4026251 .094066$ H $-3.6655610 .142080-0.311199$ O $\quad-4.564401 \quad 1.697627-1.279788$ H -3.858942 2.0642171 .194646 H -5.5886132 .0836511 .074644$ H -2.966083 -4.887426 -4.986036 H -4.707484 -4.569202 -5.041892 H -3.600618 -3.610632 -6.036722 C $-2.712278-4.524403-1.935234$ H $-7.662080-1.328985-1.856734$ H -7.1545360 .5004210 .561703$ H -3.804793 2.271581-1.085436 H -2.639795 -5.470221 -2.485223 H -2.993461 -4.778179 -0.905166 H $-4.739588-4.260280-2.559639$ C -2.480540 -0.006992 2.620360 O $-3.784113-0.5164992 .323705$ C -4.824102 0.4637592 .312350 C -4.8860971 .1911123 .664138$ C -3.5300051 .7630484 .071868$ C -2.450370 $0.684403 \quad 3.988665$ H $-2.203516 \quad 0.718521 \quad 1.846846$ C -1.482554 -1.159846 2.483953 H $-5.762940-0.0932312 .214572$ O $\quad \begin{array}{llll}-5.846340 & 2.241819 & 3.634598\end{array}$ H -5.2076410 .4838514 .439880$ H -3.2614772 .6362553 .465654$ O -3.6039822 .2210805 .424296$ H -2.614412 -0.0325404 .802196$ O $-1.182839 \quad 1.3002454 .201499$ C -1.656926 -2.240004 3.524168 H -1.589520 -1.628116 1.499816 H $-0.449715-0.7990292 .544054$ H $-6.702841 \quad 1.8481203 .395920$ H -4.3187022 .8793035 .467537$ H $-0.5268340 .593025 \quad 4.321001$ O -2.635268 -3.107908 3.139228 O $-1.005957-2.2977304 .559572$ C $-2.903646-4.1764894 .051765$ H $-3.699544-4.7941863 .627146$ H -2.011085 -4.795610 4.183286 H $-3.243259-3.7776185 .012410$

SCF Energy (B3LYP/6-31G**//MMFF) $=-3245.91499625$

\section{2}

MM̄FF Geometry

C $-2.197094-2.112128 \quad 1.744605$

C $-0.970594-2.6555581 .742398$

C $-0.010540-2.5399260 .584599$

O $1.207260-1.9061861 .040656$

C $0.345222-3.9511830 .078781$

C $1.175244-3.962069-1.189523$
C $2.433256-4.435554-1.281373$

C $0.491437-3.429420-2.422424$

C $3.284882-5.035928-0.199019$

C $4.194638-4.0373240 .533917$

C $5.190532-3.300345-0.377232$

C $6.217709-2.5298090 .455602$

C $7.168403-1.700577-0.422072$

O $4.458065-2.383018-1.194224$

$\begin{array}{lllll}\text { O } & 7.859504 & -0.794678 & 0.451897\end{array}$

C $6.335247-0.860166-1.415964$

C $8.246440-2.581074-1.064928$

O $7.183127-0.188925-2.344258$

C $5.252579-1.674465-2.153789$

C $4.290410-0.797975-2.992005$

O $5.891044-2.579713-3.063158$

C $1.169848-0.5611201 .245574$

$\begin{array}{lllll}\text { O } & 0.211798 & 0.179447 & 1.093662\end{array}$

C $2.507612-0.1346981 .710006$

C 2.6540191 .0812922 .256833

C 3.9014411 .6599192 .759836

C 5.1792120 .9211742 .463044

C $3.4973750 .250395-2.204045$

C $4.1359331 .629161-2.192768$

C $3.8841863 .876602-1.146964$

O $5.0798821 .941094-2.913630$

C $4.8788933 .993782-0.035190$

C 4.7642504 .8832690 .962722

C 5.7741285 .0443822 .063083

C 5.1527044 .9672803 .439870

C 4.8381236 .0829944 .119590

C 4.9736843 .5995844 .059880

C 3.8441532 .8260243 .436843

N $3.4964922 .487673-1.308144$

H -2.529842 -1.5412980 .881219$

H $-0.633451-3.2180732 .609904$

H $-0.444570-1.944082-0.227397$

H - $0.574691-4.517812-0.119695$

H $\quad 0.863879-4.4889730 .880102$

H $2.921751-4.396070-2.254757$

H $1.067327-3.622622-3.334094$

H $-0.487910-3.901732-2.550444$

H $\quad 0.352981-2.347756-2.346926$

H $2.666401-5.5608050 .536814$

H $3.906960-5.816789-0.655331$

H $3.566202-3.3084441 .058547$

H $4.749650-4.5942581 .299040$

H $5.691427-4.039772-1.013191$

H $6.787556-3.2107691 .100130$

H $5.691254-1.8481631 .136959$

H $8.406213-0.210817-0.101777$

H $5.850724-0.074777-0.825051$

H $8.834330-3.088973-0.290863$

H $8.963160-1.979572-1.634689$

H $7.836068-3.347147-1.725967$

H $7.468370-0.838715-3.008920$

H $4.827881-0.349819-3.836417$

H $3.551414-1.474126-3.445927$

H $5.244518-2.844503-3.738359$

H $3.329053-0.8318541 .597096$

H $1.773375 \quad 1.7143232 .360507$

H 5.2620500 .7187251 .390013

H $5.208071-0.0303143 .004618$

H 6.0795321 .4761672 .733627

H $2.5194580 .376017-2.685900$

H $3.315948-0.081143-1.177506$

H $2.9715324 .447646-0.948622$

H $4.3291004 .239776-2.078910$

H $5.7453583 .337342-0.066489$

H 3.9114335 .5586100 .974811

H 6.2600386 .0197781 .928556

H 6.5772514 .3012491 .983248

H 4.4173076 .0375425 .118517

H 4.9901447 .0675043 .689389

H 4.7456333 .6974925 .129772

H 5.9278123 .0668954 .025421

H 2.8672073 .2854563 .594252

H $2.7538322 .130016-0.714180$

C $-4.504476-2.8649172 .498607$ 
O $-5.232762-1.9773551 .628954$

C $-5.930447-2.7686090 .646571$

C $-5.805751-4.2214791 .099051$

C $-4.449561-4.2113381 .778263$

H - $-5.384802-2.646082-0.296599$

C -7.370407 -2.269245 0.483391

H $-6.587052-4.494547 \quad 1.817687$

H $-5.862149-4.9220510 .260295$

C $-4.213658-5.3946322 .699397$

H $-3.680088-4.2088790 .996398$

C $-7.495709-0.760080 \quad 0.188514$

O $-8.099014-2.5266631 .691849$

H -7.875158 $-2.848348-0.298326$

H -7.216265 -0.1885521 .080850$

O $-8.879940-0.476742-0.053450$

C $-6.680719-0.268862-1.020784$

C $-6.9581921 .194673-1.417001$

H -5.611921 $-0.392064-0.815955$

O $-6.987683-1.095320-2.146807$

H $-6.3648551 .416750-2.312446$

H $-8.0006891 .293402-1.745238$

H -3.228206 -5.3291903 .171361$

H -4.966133 -5.4421293 .493745$

H $-4.258011-6.3336782 .138121$

C -3.162049 -2.241277 2.889284

H -7.632381 -2.076965 2.417358

H $-9.379533-0.7906010 .719758$

H $-6.423614-0.807771-2.884847$

H -2.716489 -2.8295793 .700264$

H -3.339391 -1.232129 3.281455

H $-5.111951-2.9537423 .409412$

C $-4.2647742 .384598-0.617735$

O $-5.3499662 .040143 \quad 0.253918$

C $-6.6533972 .220812-0.307623$

C $-6.8482383 .684057-0.725250$

C $-5.7269844 .172755-1.638076$

C $-4.3588413 .861519-1.028985$

H $-4.3036821 .750512-1.511209$

C $-2.9744912 .059246 \quad 0.144861$

H -7.366002 2.0304350 .503846

O $-8.1087803 .846297-1.366836$

H $-6.8648604 .309577 \quad 0.176999$

H -5.823706 $3.745805-2.643288$

O $-5.8601195 .588160-1.790500$

H -4.200673 $4.517303-0.163157$

O $-3.3684124 .180185-2.002410$

C $-1.7788181 .886367-0.757659$

H -2.7515312 .8384270 .883574$

H $-3.088002 \quad 1.1350820 .726223$

H -8.182373 4.779341-1.631342

H $-5.1197905 .889656-2.344720$

H -2.513138 4.239466 -1.544396

O $-1.908408 \quad 0.748341-1.496674$

O $-0.8390432 .670388-0.796826$

C $-0.8433400 .489866-2.415840$

H $-0.700341 \quad 1.340971-3.088644$

H $0.077940 \quad 0.270907-1.868592$

H -1.115643 -0.383997 -3.013221

SCF Energy $\left(B 3 L Y P / 6-31 G^{* *} / / M M F F\right)=-3245.92408888$

01443

MMFF Geometry

C $3.131115-3.1406090 .596215$

C $2.193711-4.0047581 .017041$

C $0.700516-3.7658480 .949390$

O $0.434132-2.6892120 .022682$

C $-0.057508-5.0157420 .461696$

C $-1.538040-4.9917030 .804096$

C $-2.548107-4.897394-0.083280$

C $-1.861530-5.1330232 .272355$

C $-2.482638-4.807105-1.580532$

C $-2.693114-3.397767-2.152837$

C $-4.073535-2.775784-1.875313$

C $-4.248640-1.495469-2.698953$

C $-5.570080-0.775338-2.392539$

O $-4.178871-2.463503-0.481650$

O $\quad-5.4613620 .539744-2.959974$

C $-5.722435-0.626836-0.862460$
C $-6.753525-1.454679-3.091797$

O $-7.012039-0.124287-0.523355$

C $-5.458736-1.938376-0.092862$

C $-5.450904-1.7512241 .446181$

O $-6.505360-2.873912-0.385616$

C $-0.358971-1.6662510 .428095$

O $-0.958483-1.5690451 .485946$

C $-0.358767-0.660848-0.660026$

C $-1.2222230 .361268-0.603644$

C $-1.3740061 .432930-1.586619$

C $-0.3583941 .526963-2.691792$

C $-4.432762-0.7437401 .988265$

C -4.9854740 .6595822 .190924$

C -4.2238253 .0020152 .619759$

O

C -4.3629873 .6991841 .303247$

C -3.4522954 .5484750 .802133$

C $-3.5946165 .229622-0.530193$

C $-2.5518864 .783480-1.534028$

C $-1.4936955 .558696-1.824310$

C $-2.7817363 .477757-2.266952$

C $-2.4072352 .285330-1.433346$

N -3.973195 1.5896742 .389770

H $2.825778-2.1950810 .156392$

H $2.513975-4.9237281 .503080$

H $\quad 0.390123-3.4948591 .964987$

H $0.367011-5.9192890 .919331$

H $0.094009-5.125517-0.616998$

H -3.565165 -4.9120450 .308205$

H -2.934700 -5.260499 2.451729

H -1.357100 -6.010823 2.689540

H -1.541935 -4.247867 2.829615

H -1.528986 -5.193275 -1.953830

H -3.245827 -5.479068 -1.993200

H -1.909839 -2.742437 -1.761986

H $-2.544637-3.460421-3.238340$

H $-4.841352-3.510513-2.144575$

H $-4.171581-1.709943-3.772429$

H -3.421919 -0.808454 -2.475510

H $-6.3049811 .000800-2.817414$

H $-4.9933080 .127638-0.542225$

H $-6.617644-1.430283-4.179773$

H -7.690176 -0.921312 -2.895470

H $-6.883660-2.498111-2.797586$

H $-7.0991560 .758193-0.920891$

H $-6.464011-1.5281181 .802283$

H -5.196197 -2.725749 1.887254

H $-6.525279-3.5432470 .318373$

H $0.333562-0.799955-1.482453$

H $-1.8969430 .418540 \quad 0.247313$

H $-0.4867690 .699342-3.397812$

H $\quad 0.6591391 .485069-2.291065$

H $-0.4225302 .457905-3.259746$

H -4.103055 -1.081518 2.979186

H -3.549779 -0.7072691 .346632$

H $-3.3859123 .391376 \quad 3.206656$

H -5.1423753 .1208983 .203285$

H -5.2624123 .4862120 .729673$

H -2.552221 4.7640121 .373155

H $-3.5206686 .311621-0.360615$

H $-4.5939805 .069346-0.954303$

H $-0.7578805 .256337-2.562279$

H -1.334631 $6.508350-1.324445$

H $-2.2532553 .481823-3.225742$

H $-3.8423563 .396521-2.537124$

H $-3.0991582 .094228-0.614949$

H -3.005642 1.2942082 .298165

C $5.368986-2.982913-0.533256$

O $5.200495-1.575730-0.808424$

C $6.487410-0.987038-1.059667$

C $7.419251-2.153688-1.358361$

C $6.877697-3.211177-0.416234$

H $6.803896-0.510190-0.124144$

C $6.3891080 .066074-2.168376$

H $7.328667-2.492617-2.396800$

H $8.468674-1.907041-1.171465$

C $7.315271-4.622444-0.767816$

H $7.215667-2.9772530 .602470$ 
C $5.3057911 .142930-1.943802$

O $6.088621-0.582869-3.411677$

H $7.3665890 .541371-2.310002$

H $4.3128390 .697015-2.073651$

O $5.4457282 .121169-2.982532$

C $5.3749691 .863664-0.585420$

C $4.4346423 .079790-0.459642$

H $5.153571 \quad 1.1499550 .215656$

O $6.7134242 .322879-0.377051$

H 4.5832173 .5148950 .536359

H $4.7597333 .864527-1.154789$

H $6.886376-5.348856-0.070304$

H $7.000271-4.899891-1.779380$

H 8.405578-4.710226-0.719546

C $4.611897-3.3469420 .747275$

H $5.256731-1.073174-3.292953$

H $5.3935331 .651027-3.832466$

H 6.7524102 .7227180 .508484

H $4.948731-2.6969811 .564686$

H $4.829513-4.3827621 .030997$

H $4.955301-3.526614-1.392186$

C 2.4204811 .8824761 .499690

O 2.5027801 .6423970 .088327

C $2.9436602 .762340-0.685328$

C $1.9975153 .953187-0.475952$

C 1.7977824 .2769231 .003529

C 1.4288063 .0173341 .788224

H 3.4155482 .1478241 .874429

C $1.989926 \quad 0.5608702 .151707$

H $2.8442382 .467240-1.736577$

O $2.4858705 .097477-1.167044$

H $1.0229983 .707064-0.915441$

H 2.6904434 .7524121 .425504

O $\quad 0.746738 \quad 5.2371791 .137056$

H $0.4111232 .707088 \quad 1.518011$

O $1.4290913 .314573 \quad 3.180856$

C 2.4528820 .3921793 .581168

H 0.8986170 .4718362 .123392

H $2.406514-0.2788661 .584578$

H $1.8682155 .828338-0.992990$

H $\quad-0.066054 \quad 4.844841 \quad 0.775115$

H 0.8178374 .0581533 .320169

O $1.829489-0.700274 \quad 4.116534$

O 3.2694961 .1013554 .151652

C $2.179811-1.0077505 .469174$

H $3.247057-1.2383885 .540347$

H $1.919072-0.1736576 .127640$

H $1.609480-1.8885515 .776121$

SCF Energy (B3LYP/6-31G**//MMFF) $=-3245.90794279$

\section{4}

MM̄FF Geometry

C - $-1.317147 \quad 4.4579890 .747469$

C $-0.319526 \quad 4.3299191 .636205$

C $-0.223516 \quad 3.3032032 .740904$

O -1.4396712 .5321042 .858477$

C 0.9237192 .3270842 .422455

C 1.2222961 .3639673 .554093

C 0.8082960 .0821223 .599269

C 2.0804531 .9219254 .659440

C $-0.058651-0.6364712 .603912$

C $\quad 0.693287-1.7885741 .929460$

C $-0.165308-2.4719830 .855580$

C $0.532833-3.7188640 .319125$

C $-0.269293-4.370798-0.813389$

O $-0.357123-1.538006-0.209082$

O $0.576714-5.340412-1.450290$

C $-0.612098-3.301703-1.874049$

C - $-1.485437-5.137476-0.279129$

O $-1.496171-3.854920-2.845566$

C -1.200194 -1.999657 -1.282267

C -1.201269 -0.829661 -2.297255

O $-2.526425-2.233453-0.813912$

C -2.429132 3.0214493 .649471

O -2.4143134 .0803884 .262553$

C $-3.5942022 .106073 \quad 3.702397$

C -3.6335710 .9101153 .094420$

C $-4.732473-0.0567223 .149062$
C -6.0278880 .3934493 .771383$

C $-2.017073-0.972774-3.582726$

C $-3.518375-1.103513-3.412218$

C $-5.5099130 .055266-2.446810$

O $-4.144961-2.007453-3.962392$

C $-6.048423-1.062702-1.612497$

C $-6.296926-0.976560-0.297135$

C $-6.886729-2.1052770 .498814$

C $-5.845932-2.9069841 .244617$

C $-5.335336-4.0316440 .715249$

C $-5.470116-2.4615902 .638281$

C $-4.528648-1.2906972 .641135$

N -4.079365 -0.100593 -2.637338

H -2.173905 3.7927990 .792345

H 0.5280925 .0092371 .563135

H -0.0202013 .8213153 .686109$

H 1.8420822 .8866122 .197335

H $0.692971 \quad 1.773881 \quad 1.504525$

H $1.100132-0.5220684 .457384$

H 2.2953421 .1790555 .434960

H 3.0397462 .2650204 .257834

H 1.5824262 .7677985 .143133

H $-0.926245-1.0394743 .140420$

H -0.4583880 .0435811 .847493$

H $1.607988-1.3947131 .469694$

H $\quad 0.990597-2.5213262 .689494$

H -1.136275 -2.729573 1.294358

H $0.720499-4.4405541 .123708$

H $1.519882-3.443418-0.074611$

H $\quad 0.853503-5.985318-0.776915$

H $0.317626-3.048239-2.402415$

H -1.169470 -5.9092060 .433155$

H -2.004664 -5.669059 -1.084078

H -2.204813 -4.4926820 .228918$

H -1.068941 -4.649462 -3.208793

H $-1.5253960 .075863-1.767772$

H $-0.161585-0.623384-2.584569$

H $-2.975793-1.375772-0.732352$

H $-4.4225922 .465607 \quad 4.304740$

H -2.7723860 .5858102 .515653$

H $-6.3675301 .331813 \quad 3.318006$

H $-5.9035290 .548525 \quad 4.848494$

H $-6.846077-0.3169523 .635876$

H -1.848680 -0.088616 -4.209876

H -1.657043 -1.819989-4.174232

H $-5.998814 \quad 0.061370-3.426587$

H $-5.6746671 .029196-1.974221$

H $-6.270659-1.992757-2.131071$

H $-6.082755-0.0502690 .228997$

H -7.460365 -2.767837 -0.163665

H $-7.626237-1.7019291 .201400$

H -4.612284 -4.633511 1.255381

H $-5.624711-4.371910-0.273760$

H $-4.963433-3.2796213 .168395$

H $-6.381185-2.2816973 .215442$

H $-3.553972-1.5179172 .206282$

H $-3.4947300 .676765-2.347617$

C $-1.0950884 .890078-1.743602$

O $0.1740174 .204648-1.789389$

C $-0.0017982 .967845-2.508043$

C $-1.3263433 .109138-3.249267$

C -2.141934 3.890291-2.236998

H $-0.1045402 .178368-1.754211$

C $1.2117032 .680011-3.395689$

H -1.215915 $3.686568-4.174488$

H $-1.7703442 .144505-3.502987$

C $-3.3875314 .533584-2.819222$

H $-2.4401123 .202971-1.436104$

C $2.5561752 .674747-2.638987$

O $1.2840073 .695653-4.404305$

H $1.0639381 .730201-3.923476$

H $2.7779923 .686425-2.279574$

O $3.5850592 .362320-3.588351$

C $2.6386181 .677809-1.466153$

C $4.0257501 .707379-0.793053$

H $1.8783501 .928860-0.719458$

O $2.3501680 .373460-1.961370$

H $4.7863101 .416578-1.524580$ 
H $4.2380902 .742570-0.501224$

H -3.927475 $5.098570-2.052801$

H $-3.1412635 .220886-3.635440$

H -4.064353 $3.769646-3.216154$

C -1.319542 $5.484886-0.350980$

H $2.0616933 .501738-4.955261$

H $3.4446181 .445009-3.880169$

H $2.291131-0.225704-1.197901$

H -2.271161 $6.027710-0.323419$

H $-0.5304546 .223223-0.158821$

H $-1.0143235 .721106-2.456983$

C $5.014143-1.197854-0.589957$

O $3.943951-0.5905350 .140531$

C $4.1282950 .794006 \quad 0.451234$

C 5.4209180 .9778251 .259568

C 6.6280960 .3885530 .534108

C $6.352767-1.0638240 .146173$

H $5.089275-0.706196-1.567504$

C $4.628841-2.652726-0.897856$

H $3.296170 \quad 1.0706291 .108369$

O 5.6624532 .3535791 .533480

H 5.3182320 .4713642 .228360

H $\quad 6.904414 \quad 0.985728-0.342638$

$\begin{array}{llll}\text { O } & 7.758921 & 0.409351 & 1.408172\end{array}$

H $6.361300-1.6769111 .055340$

O $7.398649-1.539144-0.697271$

C $4.703826-3.5970910 .281727$

H $3.608825-2.706165-1.296082$

H $5.300930-3.047737-1.669512$

H 4.8859562 .6972442 .007713

H 7.8964861 .3331321 .679926

H $8.236541-1.413578-0.219447$

O $3.939077-3.145304 \quad 1.313980$

$\begin{array}{llll}\text { O } & 5.346937 & -4.639617 & 0.268575\end{array}$

C $3.930681-3.9684262 .484152$

H $3.361260-3.4503213 .260165$

H $3.442265-4.9228102 .266352$

H $4.950116-4.1285482 .848002$

SCF Energy $\left(B 3 L Y P / 6-31 G^{* *} / / M M F F\right)=-3245.90920894$

\section{5}

MMFF Geometry

C $0.261702-2.5673650 .475969$

C $1.180592-3.187150-0.280117$

C $1.617667-2.790957-1.672722$

O $0.820395-1.688436-2.166749$

C $3.079426-2.303021-1.694542$

C $4.093636-3.311231-1.202665$

C $4.667474-3.2831790 .016882$

C $4.458182-4.387984-2.190159$

C $4.418828-2.3018151 .131437$

C $5.695302-1.5750991 .574113$

C $6.320276-0.6947170 .481157$

C $7.655686-0.1226740 .963009$

C $8.2848450 .808053-0.081971$

O $5.412432 \quad 0.3713160 .172188$

O $\quad 9.3469981 .5340540 .555199$

C $7.2292981 .841473-0.519716$

C $8.9243440 .028297-1.236974$

O $7.7161512 .639186-1.597327$

C $5.8596801 .220666-0.893951$

C $4.8296712 .360427-1.100170$

O $5.9966830 .495296-2.119150$

C $-0.318408-1.962082-2.851894$

O $-0.714526-3.065779-3.199625$

C $-1.094965-0.726987-3.122927$

C $-0.771130 \quad 0.473255-2.616381$

C $-1.5179041 .724040-2.778065$

C $-2.8203241 .671917-3.530001$

C $3.3707461 .910957-1.252763$

C 2.6004311 .8098980 .052985

C 1.6549653 .2323961 .879346

O 2.0872200 .7546550 .418050

C $0.4134894 .011577 \quad 1.540477$

C $0.138160 \quad 4.625803 \quad 0.378337$

C -1.1250595 .3841920 .089153$

C $-2.0680724 .653378-0.843933$

C $-3.3463394 .437838-0.492134$
C $-1.572074 \quad 4.240981-2.214971$

C $-1.0095732 .843184-2.220324$

N 2.5005053 .0221340 .717853

H -0.267047 -1.701258 0.088804

H $1.673253-4.0646370 .134249$

H $1.510088-3.652943-2.342003$

H $3.156489-1.361321-1.140912$

H $3.339264-2.011469-2.721919$

H $5.386042-4.0644690 .261911$

H $5.215704-5.074288-1.796955$

H $3.577770-4.983514-2.450793$

H $4.861097-3.942152-3.105418$

H $3.647854-1.5684590 .883758$

H $4.029085-2.8660591 .988056$

H $5.438321-0.9499062 .439133$

H $6.429285-2.3152761 .917179$

H $6.480754-1.302801-0.415595$

H $8.353208-0.927718 \quad 1.226098$

H $7.4953690 .449610 \quad 1.887687$

H $9.9859450 .887606 \quad 0.901324$

H 7.0760122 .5263200 .326421

H $9.705248-0.642611-0.859346$

H $9.4249770 .703643-1.939550$

H $8.208512-0.579761-1.793837$

H $8.565173 \quad 3.020483-1.314973$

H $4.9086863 .065988-0.265605$

H $5.0989272 .908446-2.012497$

H $5.1416360 .476115-2.576993$

H -1.979944 -0.870115 -3.734292

H $\quad 0.1230350 .557791-2.000906$

H $-3.4838200 .911490-3.104575$

H -2.643523 $1.429792-4.583575$

H -3.376188 2.611446 -3.502328

H $2.8361202 .646201-1.868909$

H $3.2900880 .956576-1.780807$

H 2.2435693 .7822412 .620095

H 1.3745232 .2645712 .306404

H -0.3262664 .0632292 .336890$

H $0.8651984 .607454-0.428991$

H $-0.8543116 .345050-0.366386$

H -1.6313615 .6343981 .030662$

H -4.036905 $3.946015-1.168583$

H -3.7313064 .7470990 .473759$

H $-0.7912874 .936813-2.547681$

H $-2.3734774 .358199-2.951585$

H $-0.0531842 .758978-1.704887$

H $2.934163 \quad 3.843778 \quad 0.307627$

C $-0.101286-1.8012482 .827204$

O $-1.240608-0.9681932 .523500$

C $-1.945114-0.6803733 .743259$

C $-0.967644-0.9986404 .866049$

C $-0.253308-2.2067894 .293821$

H $-2.785401-1.3826743 .798059$

C $-2.4669150 .758483 \quad 3.730915$

H $-0.256779-0.1803905 .030404$

H -1.474089-1.205953 5.813461

C $1.063714-2.5188254 .983347$

H $-0.915795-3.0790044 .376493$

C -3.3644531 .1044842 .524066$

O $-1.354617 \quad 1.6620693 .711271$

H -3.0019570 .9641734 .665184$

H $-2.761736 \quad 1.1410491 .609162$

O -3.8671442 .4313902 .728592$

C -4.5615640 .1560052 .320653$

C $-5.6106960 .644257 \quad 1.305730$

H $-4.198583-0.8285102 .012892$

O $-5.236980-0.0327443 .568604$

H $-6.425571-0.0899081 .292713$

H $-6.072304 \quad 1.5643801 .685677$

H $1.549796-3.3845904 .522560$

H $1.757189-1.6731354 .925346$

H $\quad 0.899218-2.7464096 .041625$

C $-0.086990-2.997426 \quad 1.873708$

H $-0.822851 \quad 1.4512702 .924424$

H -3.0984163 .0186812 .830992$

H -5.5281720 .8414663 .880229$

H -1.079569-3.461465 1.847795

H $0.625742-3.7506262 .228558$ 
H $\quad 0.793897-1.1812122 .687246$

C $-5.361985-1.355308-1.014187$

O $-4.460745-0.294840-0.673888$

C $-5.073020 \quad 0.873837-0.119228$

C $-6.1183151 .441459-1.090902$

C -7.140362 $0.386959-1.497617$

C $-6.435155-0.869210-1.999485$

H $-5.833018-1.743255-0.102817$

C $-4.536178-2.484974-1.652662$

H $-4.2780331 .622771-0.043865$

O $-6.8025432 .552878-0.521380$

H $-5.616016 \quad 1.805027-1.996088$

H -7.827252 $0.151455-0.676281$

O $-7.9472710 .900201-2.560542$

H -5.981378 $-0.664820-2.977992$

O $-7.402612-1.901296-2.185452$

C $-3.703816-3.250467-0.647193$

H -5.207376 -3.208052 -2.130246

H -3.851681 -2.080004 -2.405753

H $-6.1330513 .205661-0.255610$

H $-8.3582901 .721449-2.239980$

H -8.076885 -1.559901 -2.797626

O $-3.297875-4.423423-1.218061$

O $-3.429494-2.8670990 .480654$

C $-2.518062-5.295572-0.393521$

H $-2.570042-6.300539-0.820891$

H $-1.476535-4.966477-0.399705$

H $-2.905499-5.3341940 .629169$

SCF Energy (B3LYP/6-31G**//MMFF) $=-3245.91954824$

\section{6}

MM̄FF Geometry

C - $-1.034716-3.702121-0.378138$

C $-0.923510-3.1896020 .857688$

C $0.274865-2.4084721 .328930$

O $-0.239804-1.2045691 .949684$

C $1.119856-3.2158532 .335624$

C $1.758103-4.4596331 .754117$

C $2.784021-4.4533650 .879883$

C $1.170209-5.7637692 .225871$

C $3.499641-3.2587200 .306783$

C $5.020735-3.4119220 .430658$

C $5.787679-2.290482-0.290399$

C $7.292044-2.570422-0.229539$

C $8.117230-1.434184-0.845700$

O $5.489148-1.0455080 .349104$

O $9.488044-1.626680-0.464846$

C $7.659443-0.099983-0.225449$

C $8.083250-1.467713-2.378056$

O $8.3041261 .000334-0.864881$

C $6.1248020 .100061-0.244465$

C 5.7582861 .3361720 .616961

O $5.6970590 .303261-1.590386$

C $0.640742-0.1906582 .160849$

O $1.846939-0.2200891 .978216$

C $-0.116230 \quad 0.9952422 .614400$

C 0.5590472 .1241902 .874189

C -0.0262543 .4062023 .247476$

C 0.7711684 .1463514 .286330

C 4.2582681 .6571000 .670546

C $3.8084842 .720979-0.319355$

C $1.7470013 .946378-1.027300$

O $4.5767363 .308056-1.075586$

C $1.1913704 .982919-0.100120$

C $-0.0911175 .377569-0.113256$

C $-0.654974 \quad 6.4341960 .795655$

C -1.9142425 .9860971 .507808$

C $-3.126868 \quad 6.316706 \quad 1.032662$

C -1.769921 5.2298992 .812863

C -1.126563 3.8826732 .631742

N $2.4397702 .942284-0.241041$

H - $0.214824-3.540150-1.076011$

H -1.710477 $-3.343716 \quad 1.589883$

H $0.877751-2.1085350 .464313$

H $\quad 0.496527-3.4803453 .201376$

H $1.908563-2.5899262 .769755$

H $3.145521-5.4141110 .514487$

H $1.659557-6.6310091 .770154$
H $\quad 1.278925-5.855717 \quad 3.311574$

H $0.105604-5.8182021 .977711$

H $3.226648-3.176920-0.751932$

H $3.191767-2.3296270 .793698$

H $5.285891-3.4130711 .496160$

H $5.327308-4.3805300 .015546$

H $5.447529-2.249132-1.331985$

H $7.530223-3.524169-0.717089$

H $7.597203-2.6879900 .819992$

H $9.768626-2.500606-0.786525$

H $7.995752-0.0903790 .820995$

H $8.491611-2.415471-2.749197$

H $8.716409-0.681878-2.804824$

H $7.076596-1.360952-2.787586$

H $9.2625390 .841529-0.818227$

H $6.078057 \quad 1.130771 \quad 1.647621$

H 6.3164782 .2154690 .276643

H $4.8178410 .711980-1.583389$

H -1.1964440 .9302362 .682363$

H 1.6452862 .1346892 .804871

H 1.7098834 .5134503 .857177

H 1.0132853 .4846635 .126354

H 0.2393194 .9993644 .713420

H $3.662154 \quad 0.7547380 .511617$

H 4.0241882 .0449861 .670064

H $0.9619113 .432139-1.590435$

H $2.437074 \quad 4.413695-1.736151$

H 1.8817755 .4423330 .603552

H $-0.7707724 .935160-0.837224$

H $-0.872117 \quad 7.3182770 .181771$

H 0.0844326 .7623241 .536909

H -4.0358736 .0262861 .548778$

H -3.2380526 .8803040 .112216$

H -2.753764 5.0666533 .271021

H -1.217520 5.8679453 .508357

H -1.6157763 .2566601 .885646$

H 1.8820492 .3665210 .382405

C $-3.434958-4.620228-0.070409$

O $-4.067951-3.326134-0.011856$

C $-5.475521-3.484404-0.262809$

C $-5.762732-4.968063-0.082682$

C $-4.488018-5.574260-0.639032$

H $-5.639217-3.227448-1.316340$

C $-6.285447-2.5413710 .630703$

H $-5.881051-5.2344490 .974056$

H $-6.661672-5.287308-0.618446$

C $-4.283133-7.026692-0.244669$

H $-4.510883-5.500428-1.734709$

C $-5.865792-1.0593560 .515331$

O $-6.108128-2.9395811 .995480$

H $-7.353625-2.6571740 .412352$

H $-4.880359-0.9258950 .975406$

O $-6.776578-0.287187 \quad 1.307613$

C $-5.854643-0.509347-0.921667$

C $-5.6092731 .005359-1.026086$

H $-5.097582-1.037415-1.510385$

O $-7.122281-0.788875-1.523929$

H $\quad-5.676067 \quad 1.277479-2.086802$

H $-6.4395571 .542804-0.550389$

H $-3.343221-7.412545-0.651625$

H $-4.253878-7.1470530 .843503$

H -5.098512 -7.647919 -0.629428

C $-2.174033-4.519573-0.932658$

H $-6.622504-2.3244532 .545922$

H $-7.653467-0.3508020 .891482$

H $-7.075489-0.503996-2.452485$

H -2.442943 -4.071976-1.898323

H - $1.786365-5.524399-1.137383$

H -3.188269 -4.9118790 .957829$

C $-2.8110940 .828073-2.244665$

O $-3.1627310 .682710-0.865698$

C $-4.2672701 .488629-0.440797$

C $-3.9666712 .972099-0.705557$

C $-3.5782713 .230921-2.161578$

C $-2.4588462 .281611-2.588921$

H $-3.6667070 .517211-2.857867$

C -1.691808 -0.173414 -2.569257

H -4.3352231 .3650220 .646895$ 
O $-5.071247 \quad 3.790406-0.335527$ H -3.127218 $3.276982-0.069588$ H $-4.440578 \quad 3.144470-2.832524$ O $-3.105096 \quad 4.572625-2.293505$ H -1.529276 $2.589454-2.097969$ O $-2.252652 \quad 2.393717-3.994867$ C $-0.3728900 .102325-1.875596$ H $-2.002344-1.180137-2.265661$ H $-1.518777-0.174016-3.651815$ H $-5.7693133 .680473-1.002765$ H $-3.8046875 .160841-1.961343$ H $-2.0609053 .327945-4.186169$ O $0.626173-0.533141-2.557581$ O $-0.234566 \quad 0.767323-0.858916$ C $1.950701-0.347953-2.047445$ H $2.605330-1.081846-2.525113$ H $2.3024720 .655414-2.301606$ H $1.984408-0.505176-0.966236$ SCF Energy (B3LYP/6-31G*//MMFF) $=-3245.91204308$

\section{7}

MM̄FF Geometry

C -1.795298 -3.444965 -0.586647

C $-0.968413-3.441276-1.643121$

C $-0.203796-2.207926-2.050008$

O $1.211900-2.510815-2.117351$

C $-0.603182-1.677404-3.439371$

C $-2.038047-1.210909-3.564840$

C $-2.515236-0.068896-3.031302$

C $-2.929440-2.092421-4.397353$

C $-1.7428540 .934653-2.223638$

C -1.673048 $2.296055-2.924705$

C $-0.642898 \quad 3.217312-2.253438$

C $-0.6790754 .612556-2.877554$

C $0.4257835 .520818-2.316692$

O $0.6470402 .628050-2.451347$

O $0.5195126 .674441-3.166502$

C $1.7760534 .777380-2.409569$

C $0.0758406 .040405-0.916981$

O $2.8138555 .532864-1.787385$

C $1.7341523 .336848-1.845550$

C $3.0410282 .588960-2.213720$

O $1.600306 \quad 3.401492-0.427721$

C $1.897629-2.549300-0.942587$

O $1.434442-2.4559140 .182521$

C $3.332930-2.704011-1.265090$

C $4.211324-2.923976-0.275718$

C $5.660716-3.071160-0.425250$

C $6.258383-2.732422-1.765656$

C $3.1034921 .130132-1.736405$

C $3.7560531 .008566-0.372896$

C 5.9658791 .1864190 .761842

$\begin{array}{lllll}\text { O } & 3.088233 & 0.877717 & 0.649658\end{array}$

C $7.161316 \quad 0.302446 \quad 0.602334$

C $7.592959-0.5286101 .563161$

C $8.805454-1.4044161 .428166$

C $8.525107-2.8486371 .780149$

C $8.917779-3.3610732 .958714$

C $7.862992-3.7182600 .734096$

C $6.379354-3.478180 \quad 0.642136$

N $5.137973 \quad 1.092327-0.426681$

H -1.916795 -2.527332 -0.015438 H $-0.825980-4.346287-2.226831$ H $-0.338875-1.412152-1.309024$ H $-0.391557-2.444704-4.197464$ H $0.068386-0.851730-3.713743$ H -3.562997 $0.180907-3.190982$ H -3.980684 -1.790518 -4.344758 H -2.619093 -2.053930 -5.446747 H -2.866800 -3.131674-4.063030 H -2.236692 $1.050997-1.253045$ H $-0.7308740 .582420-2.014222$ H -1.390116 $2.145842-3.975147$ H -2.665026 $2.764051-2.912516$ H $-0.8595083 .266738-1.179581$ H -1.664508 $5.074953-2.739868$ H $-0.5380684 .529485-3.964604$ H $-0.3484147 .113312-3.173470$
H $2.0398134 .724575-3.474831$

H $-0.8682296 .597997-0.940977$

H $0.8304156 .749157-0.558336$

H $-0.0316915 .243894-0.177892$

H $2.7202855 .427277-0.825274$

H $3.1242792 .571217-3.308416$

H $3.9052553 .141111-1.825992$

H $1.7520372 .516110-0.061427$

H $3.627926-2.624855-2.305171$

H $3.836639-3.0134320 .743302$

H $5.938949-1.735031-2.089427$

H $5.946675-3.462704-2.520261$

H $7.349908-2.712868-1.764749$

H $2.1152150 .659348-1.702741$

H $3.7006010 .541460-2.444524$

H $5.3795030 .936437 \quad 1.651808$

H 6.2865332 .2300080 .842278

H $7.7085240 .360361-0.335562$

H $7.061712-0.5637252 .511881$

H $9.581438-1.0010492 .091888$

H $9.224805-1.3548170 .415588$

H $8.744087-4.4025483 .208489$

H $9.416765-2.7491563 .702888$

H $8.003267-4.7782540 .985530$

H $8.382071-3.585028-0.218623$

H $5.846634-3.6980141 .568743$

H $5.5761651 .302691-1.319091$

C $-4.087723-4.446798-0.154061$

$\begin{array}{llll}\text { O } & -4.474549 & -3.199927 & 0.463273\end{array}$

C $-5.359663-2.505282-0.435896$

C $-5.905794-3.574996-1.372044$

C $-4.675471-4.438603-1.565778$

H $-4.742729-1.813553-1.022116$

C $-6.425658-1.7260750 .337411$

H $-6.707905-4.155218-0.901284$

H $-6.288275-3.155489-2.307339$

C $-4.977945-5.820895-2.116796$

H $-3.999147-3.926541-2.257218$

C $-5.855345-0.6754691 .314287$

O $-7.204099-2.6626231 .092088$

H -7.114435 -1.249897 -0.370976

H $-5.349673-1.1768642 .147687$

O $-6.9626840 .038636 \quad 1.878891$

C -4.9012940 .3499590 .668076$

C -4.5556011 .5534921 .567434$

H $-3.978720-0.1544030 .360869$

O $-5.5134160 .861675-0.519990$

H -3.9547372 .2534520 .973963$

H -5.4730392 .1086351 .799752$

H -4.059744 -6.407661 -2.220534

H $-5.660186-6.373817-1.462360$

H $-5.445708-5.747215-3.104113$

C $-2.567408-4.631245-0.084662$

H -7.996083 -2.196862 1.409282

H -7.434295 -0.572169 2.469730

H $-4.8666821 .441598-0.957010$

H -2.276206 -5.545448 -0.614220

H -2.281800 -4.769255 0.965894

H $-4.558516-5.2398010 .440923$

C $-1.564613 \quad 1.1210472 .004243$

O -2.6422860 .4023372 .610973$

C -3.8140681 .1830162 .866934$

C -3.4693532 .3839663 .761548$

C -2.3236913 .2160043 .186865$

C -1.1311272 .3182162 .857644$

H -1.8940801 .4856751 .024593$

C -0.4242070 .1368721 .711728$

H -4.4887640 .5432443 .447942$

O -4.6248423 .1943633 .947165$

H -3.1731762 .0120834 .751400$

H -2.6399943 .7863502 .305530$

O -1.8976254 .1748914 .157234$

H $-0.6669721 .984993 \quad 3.793152$

O $-\begin{array}{llll}0.154898 & 3.072609 & 2.143957\end{array}$

C $0.215695-0.4574172 .949577$

H $-0.802913-0.7044291 .122151$

H 0.3452950 .6471191 .121751

H -4.4120263 .8627924 .619991$ 
H -2.6229834 .8099524 .280290$

H 0.0786923 .8400682 .693861

O $1.526272-0.7345442 .689344$

O $-0.368002-0.6838554 .000871$

C $2.261021-1.3314853 .761788$

H $3.288979-1.4872553 .422993$

H $2.273969-0.6652784 .629576$

H $1.826656-2.3009084 .023705$

SCF Energy (B3LYP/6-31G**//MMFF) $=-3245.90016225$

\section{8}

MM̄MF Geometry

C $0.862296 \quad 3.015864-2.569512$

C $0.8102502 .170008-3.609178$

C $1.278214 \quad 0.739498-3.531714$

O $0.103097-0.110842-3.605806$

C $2.2021190 .364248-4.708528$

C $3.6626820 .333403-4.306866$

C $4.372006-0.796608-4.115110$

C $4.3191031 .678447-4.139525$

C $3.898704-2.215296-4.275186$

C $2.898674-2.701873-3.223267$

C $3.334161-2.546029-1.759821$

C $4.525555-3.438347-1.406349$

C $4.856798-3.3912570 .094306$

O $2.198523-2.915931-0.967091$

O $5.736881-4.487403 \quad 0.387099$

C $3.564659-3.6206880 .906791$

C $5.617854-2.1144520 .466470$

O $3.796489-3.4294152 .300759$

C $2.378300-2.7445960 .444023$

C $1.077430-3.2030411 .145459$

O $2.640380-1.3820830 .783852$

C $-0.623532-0.257001-2.461588$

O $-0.377513 \quad 0.226710-1.367926$

C $-1.806469-1.087108-2.775796$

C $-2.844217-1.093479-1.924912$

C $-4.106621-1.812274-2.108879$

C $-4.169957-2.835803-3.211654$

C $-0.190770-2.5521310 .581478$

C -1.431244 -3.045925 1.294909

C $-2.931137-5.0273191 .470906$

O $-2.023622-2.3507312 .116945$

C $-4.096894-4.858741 \quad 0.548459$

C $-5.262847-4.314607 \quad 0.927348$

C $-6.469342-4.2111290 .040070$

C $-6.960890-2.791242-0.115537$

C $-7.807099-2.2503050 .777577$

C $-6.552011-2.037820-1.358992$

C $-5.142297-1.517978-1.294801$

N $-1.784026-4.3327190 .923148$

H $1.2978332 .683549-1.631007$

H $\quad 0.3324492 .490360-4.532074$

H $1.7868690 .533049-2.580791$

H $2.0824141 .062858-5.547502$

H $1.900867-0.606585-5.118875$

H $5.417267-0.703718-3.823666$

H $5.3832371 .593969-3.894686$

H $4.2398572 .256314-5.066129$

H $3.8420052 .244733-3.335024$

H $3.462161-2.340817-5.273727$

H $4.778482-2.870527-4.266608$

H $\quad 1.940973-2.186374-3.357676$

H $2.668183-3.757773-3.419865$

H $3.563767-1.493194-1.565850$

H $5.410215-3.170024-1.995631$

H $4.291508-4.478268-1.674317$

H $6.527648-4.394349-0.171452$

H $3.283810-4.6759770 .781380$

H $6.560313-2.052603-0.090931$

H $5.896004-2.1123821 .526068$

H $5.050794-1.2070960 .255384$

H $4.539742-4.0040602 .552369$

H $0.977568-4.2906961 .038033$

H $1.134436-2.9899552 .220360$

H $\quad 1.804841-0.8900900 .795487$

H -1.806785 -1.635416 -3.710665

H -2.782753 -0.484201-1.024036
H $-3.321628-3.526868-3.145161$

H $-4.149278-2.347679-4.191843$

H -5.065506 -3.459800 -3.172675

H $-0.159636-1.4654180 .689381$

H $-0.299958-2.765956-0.487594$

H -3.141017 -4.650434 2.477351

H -2.667797 -6.0869391 .545531$

H $-3.986960-5.235246-0.465632$

H $-5.374077-3.9614561 .950304$

H -7.264628 -4.8263620 .481468$

H $-6.278146-4.649835-0.947153$

H -8.191230 -1.243044 0.655611

H -8.130034 -2.803035 1.653730

H -7.206978 -1.166890-1.499134

H $-6.737435-2.664222-2.236157$

H $-4.994775-0.764220-0.520812$

H $-1.264100-4.7938220 .182125$

C $-1.2092644 .315823-2.120150$

O $-1.2827353 .818309-0.772318$

C $-2.4575284 .369150-0.143422$

C $-3.0987265 .310232-1.163901$

C -1.927764 $5.658192-2.066747$

H -2.1071544 .9638520 .707406$

C -3.3664473 .2312120 .347207$

H -3.891463 $4.814277-1.735768$

H -3.544042 $6.188805-0.686227$

C $-2.3483986 .198019-3.421954$

H -1.299404 $6.400608-1.556822$

C -2.6454842 .2078261 .250482$

O $-3.8602712 .525059-0.798897$

H -4.2415163 .6511260 .857126$

H -1.8955501 .6667130 .663186$

O -3.6035331 .2357341 .684835$

C - -1.9947012 .8204212 .503124$

C -1.3392661 .7904543 .442652$

H -1.2410293 .5570972 .210029$

O

H -1.017095 2.321599 4.346688

H -2.0932241 .0755143 .795154$

H -1.474683 $6.434990-4.036916$

H -2.959865 $5.473600-3.970395$

H $-2.9361457 .114394-3.305264$

C $0.2484844 .387352-2.577327$

H $-4.5768331 .941839-0.497262$

H -3.8407860 .6945590 .913698$

H -3.6796692 .8964193 .494748$

H $0.8331615 .024098-1.902776$

H $0.3151854 .826439-3.579091$

H $-1.7659773 .612034-2.754621$

C 1.5352182 .7301643 .093679

O $\quad 0.7808601 .9242032 .183112$

C -0.1512141 .0367292 .810550$

C 0.5842640 .0918733 .772160

C 1.4548550 .8520254 .771673

C 2.3551031 .8537894 .046833

H $0.840493 \quad 3.3454173 .679058$

C 2.3739783 .7279962 .286515

H -0.5662170 .4150372 .010228$

O $-0.335550-0.7304754 .481915$

H $1.233614-0.577803 \quad 3.195041$

H 0.8478421 .3517935 .535636

O $2.290042-0.0763795 .467578$

H 3.1302521 .3033403 .499915

O 3.0089812 .6836575 .002770

C 3.5450893 .1095881 .561556

H 1.7551214 .2414241 .540166

H 2.7743234 .5028722 .951378

H $-0.861547-1.2158253 .823198$

H $1.706224-0.7319445 .886547$

H 3.4944272 .0992925 .610067

O 3.1094742 .3354430 .528959

O 4.7129363 .3059081 .873387

C $4.1368041 .704818-0.240516$

H $4.8407561 .177240 \quad 0.409493$

H $3.6653070 .978720-0.906706$

H $4.6586162 .452801-0.844002$

SCF Energy (B3LYP/6-31G**//MMFF $)=-3245.91099340$ 


\section{9}

MM̄MF Geometry

C $-0.0363685 .084328-1.412040$

C $-0.4169294 .216648-0.462625$

C $-1.4690913 .167027-0.679316$

O $-0.8802021 .861349-0.486917$

C -2.6980873 .2690320 .248385$

C $-2.4433393 .474441 \quad 1.729668$

C -2.26090624886052 .630451$

C -2.4974824 .9083612 .197242$

C -2.1875941 .0060682 .396960$

C -3.3764310 .2464653 .002358$

C $-4.659193 \quad 0.3625122 .162921$

C -5.812191-0.385779 2.835356

C $-7.078583-0.3967781 .964261$

O $-4.389302-0.214248 \quad 0.881299$

O $-7.978876-1.3731482 .510403$

C $-6.702761-0.8708780 .543507$

C -7.814204 0.9473942 .016846

O $-7.819413-0.776402-0.338330$

C $-5.474862-0.142200-0.049979$

C $-5.021679-0.839758-1.355602$

O $-5.8384531 .208643-0.341638$

C $-0.1902471 .314935-1.522818$

O $0.1015261 .846922-2.581125$

C $0.108498-0.084451-1.151548$

C $0.834284-0.855472-1.972906$

C $1.134964-2.272700-1.765815$

C $0.395586-2.981280-0.661472$

C $-3.670351-0.343334-1.886219$

C $-3.301916-1.046186-3.177223$

C $-2.484370-3.209532-4.075230$

O $-3.489941-0.520908-4.272401$

C -1.489853 -4.238608 -3.641110

C $-0.361052-4.510457-4.314266$

C $0.624459-5.566877-3.900199$

C $2.046300-5.053135-3.830794$

C $2.925776-5.319616-4.811120$

C $2.471176-4.306093-2.586179$

C $2.013878-2.873548-2.593917$

N $-2.764668-2.308175-2.972371$

H $-0.5206435 .061369-2.385044$

H $\quad 0.0936314 .2253890 .497309$

H -1.852757 $3.213098-1.707927$

H -3.3026582 .3634350 .120863$

H $-3.3346614 .090858-0.106690$

H -2.126743 2.7725443 .674023

H -2.326709 5.0022803 .275063

H -1.739115 5.5142081 .693682

H -3.481682 5.3381191 .982485

H $-2.0876790 .757216 \quad 1.339223$

H -1.267302 0.6488552 .876280

H -3.090118 -0.8119823 .061839$

H -3.562289 0.5896074 .027492

H $-4.912317 \quad 1.4214402 .039805$

H $-6.028260 \quad 0.0402283 .823121$

H -5.512331 -1.427227 3.018964

H -8.167434 -1.125224 3.431778

H $-6.467913-1.9415370 .617385$

H -8.091996 $1.191423 \quad 3.049334$

H $-8.7556870 .904903 \quad 1.458149$

H -7.218525 1.7775451 .631515

H $-7.9278590 .159298-0.579311$

H -4.936551 -1.919286 -1.175832

H -5.780594 -0.702231 -2.136257

H $-5.2964801 .536562-1.075840$

H $-0.308484-0.454783-0.221662$

H $1.238931-0.415533-2.883201$

H $-0.686377-2.855946-0.778807$

H $\quad 0.682874-2.5775140 .313524$

H $0.574706-4.057538-0.634305$

H -3.683638 $0.733070-2.086183$

H -2.877443 -0.520138-1.151712

H -2.138449 -2.630030 -4.937899

H -3.428211 -3.693790 -4.346678

H -1.719159-4.803456 -2.740422

H -0.145732 -3.965419 -5.230716

H $0.558852-6.384371-4.630119$
H $\quad 0.353586-6.010033-2.933830$

H $3.954453-4.979106-4.755437$

H $2.635401-5.879326-5.694088$

H $3.566751-4.296151-2.508079$

H $2.136423-4.858644-1.705166$

H $2.485785-2.271627-3.372051$

H -2.817339 -2.704035 -2.037928

C $2.4400625 .571772-1.678760$

O $2.8660324 .501961-0.812094$

C $3.5205643 .494852-1.607667$

C $3.7572464 .126120-2.976444$

C $2.5439975 .028995-3.103944$

H $2.8071452 .670263-1.720546$

C $4.7808672 .993900-0.895778$

H $4.6766554 .722483-2.998159$

H $3.8222043 .377645-3.772094$

C $2.6884186 .106482-4.163563$

H $1.6796244 .400770-3.350812$

C 4.5354462 .5200660 .551374

O $5.7265134 .070417-0.841610$

H $5.2497392 .200185-1.488930$

H 4.2436573 .3756981 .171734

O 5.7920622 .0681661 .076114

C 3.4962231 .3912120 .701511

C 3.3256170 .9826412 .179100

H $2.527426 \quad 1.7273190 .317183$

O $3.9195840 .283938-0.088368$

H 4.2948270 .6666302 .576644

H $3.027947 \quad 1.8759902 .740912$

H $1.7944396 .737020-4.200609$

H $3.549746 \quad 6.753058-3.964776$

H $2.8271315 .657234-5.152370$

C $1.0685716 .085057-1.230229$

H $6.5174773 .738020-0.383528$

H 6.0452441 .2703590 .580390

H $3.221758-0.391528-0.048749$

H $0.8087006 .992661-1.787145$

H $1.1235496 .370539-0.171729$

H $3.1677326 .381415-1.534546$

C $3.774022-2.0381522 .382497$

O $2.653760-1.3751481 .788191$

C $2.283220-0.1381032 .406866$

C $1.949556-0.3853233 .885769$

C $3.116232-1.0464654 .619302$

C $3.559871-2.3087893 .878088$

H $4.659009-1.4011492 .263270$

C $4.083995-3.3015081 .563078$

H 1.3586090 .1897701 .915051

O $1.597974 \quad 0.8423124 .514100$

H $1.072076-1.0423383 .946973$

H $3.955510-0.3539134 .752929$

O $2.697706-1.4315705 .930572$

H $2.806485-3.0874994 .035653$

O $4.778786-2.7871664 .443286$

C $3.007720-4.3682101 .585852$

H $4.219345-3.0178860 .511952$

H $5.014349-3.7503471 .929413$

H 1.2666430 .6342555 .403775

H $2.528266-0.6179976 .434729$

H $4.624718-2.9214525 .394171$

O $3.491511-5.4912040 .974242$

O $1.886348-4.2413222 .056143$

C $2.585952-6.5979300 .913091$

H $3.073374-7.4054550 .360452$

H $2.355645-6.9510761 .922696$

H $1.670419-6.3151980 .384759$

SCF Energy (B3LYP/6-31G**//MMFF) $=-3245.91675017$

01445

MM̄̄FF Geometry

C $-3.517761-1.914949-3.261866$

C $-2.443034-2.317700-2.568881$

C $-1.027507-2.137274-3.046851$

O $-0.277577-1.402519-2.050794$

C $-0.288862-3.454705-3.355572$

C $-0.148234-4.449959-2.219758$

C $0.927420-4.541638-1.412832$

C $-1.256148-5.467116-2.094845$ 
C $2.171057-3.695307-1.415834$

C $2.542476-3.2469920 .004802$

C $3.946590-2.6306030 .064189$

C $4.348518-2.3520111 .513716$

C $5.731174-1.6912801 .600980$

O $3.946203-1.409585-0.683393$

O $5.895859-1.1864252 .934860$

C $5.760284-0.4782270 .650742$

C $6.865306-2.6976001 .374131$

O 7.0699690 .0818060 .579086

C $5.235126-0.784708-0.774456$

C $5.0851820 .540586-1.554684$

O $6.175365-1.618296-1.454567$

C $-0.483636-0.057374-1.996978$

O $-1.2365420 .598439-2.700440$

C $0.3651370 .492955-0.918014$

C $0.2043791 .776285-0.562618$

C 0.9567782 .5018200 .461606

C 2.0095491 .7462131 .225575

C $4.3455170 .423606-2.892561$

C $4.2059791 .802593-3.507452$

C $2.9737293 .952428-3.190010$

O $4.9741052 .201111-4.379590$

C $3.2684344 .694522-1.922670$

C $2.4265615 .576378-1.362785$

C $2.6998016 .276067-0.060946$

C 1.5664276 .1292500 .934063

C $0.807074 \quad 7.184746 \quad 1.273737$

C 1.3521984 .7763051 .587095

C 0.6659383 .8035030 .665674

N $3.1797942 .538204-2.936017$

H -3.377810 -1.469764 -4.243485

H $-2.587392-2.758679-1.588104$

H -1.014745 -1.553242 -3.977735

H $\quad 0.710496-3.203600-3.733087$

H $-0.787954-3.953875-4.197259$

H $\quad 0.936125-5.342876-0.674034$

H -1.020994 -6.249030-1.364480

H -2.191504 -5.000669 -1.778271

H -1.424196 -5.965735 -3.055253

H $2.984315-4.304934-1.828066$

H $2.072142-2.815903-2.058350$

H $1.803527-2.5135440 .347504$

H $2.497712-4.1047990 .687773$

H $4.649504-3.335953-0.394597$

H $4.326710-3.2725842 .110121$

H $3.611572-1.6784361 .973550$

H $5.808340-1.9338573 .551007$

H 5.1184280 .2955321 .094972

H $6.816726-3.5028512 .117220$

H $7.844769-2.2254781 .508639$

H $\quad 6.838131$-3.159229 0.384987

H $7.3531320 .273887 \quad 1.489511$

H $4.5440281 .262210-0.931735$

H $\quad 6.076718 \quad 0.972643-1.744437$

H $\quad 6.064257-1.503717-2.411447$

H $1.089149-0.163686-0.450929$

H $-0.5499342 .365787-1.082932$

H 2.8084631 .4180150 .555582

H 1.5752720 .8639081 .707659

H 2.4726142 .3309572 .023277

H $4.887353-0.212532-3.599746$

H $3.348173-0.011723-2.762980$

H $1.9346684 .077645-3.509986$

H $3.6372704 .295209-3.989416$

H $4.2162164 .481829-1.432776$

H $1.4874945 .805573-1.861498$

H $2.8738247 .336625-0.284283$

H 3.6251035 .9095640 .400941

H 0.0066867 .0945302 .000693

H 0.9648498 .1619930 .829529

H $\quad 0.7236884 .8854602 .480161$

H 2.3227534 .4128441 .937144

H -0.1541354 .2413090 .094061$

H $2.6021622 .102566-2.223273$

C $-5.594428-0.708714-2.404572$

O $-5.000077-0.145928-1.216670$

C $-5.0072131 .290791-1.342092$
C $-5.814271 \quad 1.613341-2.599686$

C $-5.5461240 .395067-3.459236$

H -3.966335 $1.592418-1.507162$

C $-5.5520121 .958535-0.073534$

H $-6.8862811 .699187-2.387366$

H -5.491999 $2.549102-3.067043$

C $-6.5392570 .211962-4.592585$

H $-4.5382130 .499348-3.878226$

C -4.8124661 .5990441 .230803$

O

H $-5.5567563 .046120-0.216107$

H -5.0464950 .5646881 .507488$

O -5.3662292 .4127572 .274496$

C -3.2858441 .7905651 .195216$

C -2.5888591 .7295022 .568341$

H -2.8489961 .0311870 .537970$

O -2.9693183 .0567350 .609104$

H -1.511282 1.8344342 .393502

H -2.858608 2.6170583 .155406

H $-6.304631-0.684951-5.174331$

H $-7.5633300 .112868-4.217486$

H -6.511882 $1.071197-5.270650$

C $-4.930698-2.041888-2.766281$

H -7.255198 2.0120440 .892009

H $-5.200593 \quad 3.3434992 .048081$

H -3.3114023 .7532321 .194306$

H -5.517234 -2.541106 -3.546709

H $-4.954108-2.696053-1.885358$

H -6.639433 -0.920877-2.139821

C -1.398761 -1.070572 2.270141

O $-2.742231-0.7336592 .621328$

C $-2.874397 \quad 0.460212 \quad 3.394513$

C -2.0488930 .3524804 .686886$

C $-0.593510-0.0252844 .411620$

C $-0.523233-1.259548 \quad 3.512410$

H $-0.982261-0.2582621 .663596$

C -1.439007 -2.294087 1.349213

H $-3.9277110 .511407 \quad 3.695612$

O $-2.083174 \quad 1.5747335 .416553$

H -2.487748 -0.4198475 .332100$

H -0.0355820 .8111003 .974524$

O $0.054040-0.3358725 .647384$

H $-0.839552-2.1374064 .087512$

O $0.825300-1.4794443 .107778$

C -1.930801 -3.563564 2.010461

H $-2.118530-2.0955340 .515150$

H $-0.437902-2.4723450 .944856$

H $-3.016477 \quad 1.7808575 .596581$

H -0.0349650 .4429696 .223426$

H $1.362971-1.5513373 .915052$

O $-1.370058-4.6398821 .384821$

O $-2.732555-3.6098832 .933365$

C $-1.760552-5.9199341 .890388$

H -1.213643 -6.686166 1.334435

H -1.503207 -6.005424 2.950453

H -2.833091 -6.073598 1.738389

SCF Energy (B3LYP/6-31G**//MMFF)= -3245.91194188

01450

MM̄MF Geometry

C $-0.4906594 .803975-0.613320$

C 0.0937764 .7938370 .595360

C 1.2472033 .9219681 .037278

O 1.4409442 .7978020 .151272

C 2.5412454 .7558451 .050087

C 3.7321864 .0338341 .649185

C 4.8530073 .7121600 .974076

C 3.6249763 .7009943 .115671

C $5.1764293 .973624-0.470135$

C $4.8350252 .815562-1.420971$

C $5.6114091 .519401-1.136188$

C $5.4011270 .501711-2.257899$

C $6.100901-0.834128-1.958814$

O 5.1357250 .9662720 .092905

O $5.590368-1.810843-2.880336$

C $5.717256-1.307706-0.538020$

C $7.611833-0.750998-2.209863$

O $6.494336-2.450178-0.186561$ 
C $5.843552-0.201393 \quad 0.532924$

C $5.250639-0.590677 \quad 1.909878$

$\begin{array}{lllll}\text { O } & 7.231157 & 0.088414 & 0.743497\end{array}$

C $\quad 0.6622401 .7006190 .361766$

O $\begin{array}{llll}-0.197749 & 1.561455 & 1.216418\end{array}$

C $1.0254080 .690073-0.654971$

C $0.202344-0.343658-0.886466$

C $0.412224-1.401094-1.877845$

C $1.772580-1.494716-2.516908$

C $3.766135-0.9732061 .906796$

C $3.518175-2.4678921 .795447$

C $1.672057-4.1174891 .531284$

O $4.412936-3.3063401 .861719$

C $1.538101-4.4747130 .084956$

C $0.411737-4.965760-0.453372$

C $0.283004-5.371391-1.893196$

C $-0.879797-4.700950-2.588422$

C $-2.052135-5.339129-2.742953$

C $-0.652258-3.333932-3.191345$

C $-0.609891-2.234525-2.163893$

N $2.169326-2.759396 \quad 1.644684$

H $-0.1244974 .152632-1.401953$

H $-0.283993 \quad 5.471757 \quad 1.358894$

H $1.027822 \quad 3.5538492 .046897$

H 2.3857485 .6789601 .624875

H 2.7586105 .0728460 .024550

H 5.6469053 .2026321 .519560

H 4.5681093 .3241013 .526246

H 3.3536434 .5930933 .689556

H 2.8655292 .9312583 .283064

H $4.6770974 .881795-0.823262$

H $6.2492794 .193912-0.542197$

H $3.7574672 .620898-1.369707$

H $5.055448 \quad 3.148838-2.442827$

H $6.6746791 .768899-1.040896$

H $5.7417970 .904940-3.219656$

H $4.3262040 .311255-2.378436$

H $5.767591-1.493498-3.782356$

H $4.674538-1.641519-0.594435$

H $7.812043-0.470303-3.250957$

H $8.090891-1.725892-2.066950$

H $8.113169-0.021884-1.569926$

H $6.142886-2.8062700 .646318$

H $5.867109-1.3632652 .384625$

H 5.3454880 .2906912 .561012

H 7.3289810 .5150851 .610901

H $1.9484800 .837987-1.203316$

H $-0.726965-0.407637-0.321976$

H $2.554857-1.499104-1.750289$

H $1.942216-0.646766-3.188808$

H $1.920353-2.408534-3.096186$

H $3.327432-0.6623542 .863570$

H $3.224572-0.4508701 .112348$

H $\quad 0.716357-4.1614862 .061887$

H $2.370701-4.8059352 .017398$

H $2.417062-4.348400-0.543015$

H $-0.457515-5.1189610 .182085$

H $0.155182-6.461580-1.920647$

H $1.205961-5.169931-2.451237$

H -2.881624 -4.879008 -3.269494

H $-2.211475-6.333451-2.339024$

H -1.469487 -3.090107 -3.883724

H $0.241847-3.366303-3.820225$

H -1.554775 -2.090799 -1.640458

H $1.501739-1.9993431 .549256$

C $-2.8698594 .885330-1.421705$

O $-3.254382 \quad 3.948694-0.399875$

C $-4.6804153 .983888-0.231243$

C $-5.2230294 .757702-1.426927$

C $-4.1041675 .750049-1.678861$

H -4.8648584 .5562510 .686966$

C $-5.2581172 .567230-0.056219$

H $-5.3574334 .112915-2.302941$

H -6.182762 $5.236672-1.210656$

C $-4.142046 \quad 6.375138-3.062326$

H $-4.1701486 .546176-0.925143$

C -4.5508891 .7651351 .059596$

O -5.166537 1.863287 -1.292279
H -6.3238392 .6685820 .173973$

H -4.4743642 .4293911 .930775$

O -3.2197721 .4516140 .657961$

C $-5.266004 \quad 0.483727 \quad 1.531314$

C $-5.606584-0.5978320 .492090$

H -4.6396170 .0185002 .299719$

$\begin{array}{llll}\text { O } & -6.478951 & 0.855247 & 2.195876\end{array}$

H -6.124636 -1.400756 1.032960

H $-6.362409-0.211095-0.202900$

H -3.309226 $7.072439-3.197716$

H $-4.0765435 .615085-3.848133$

H $-5.074664 \quad 6.929828-3.208437$

C $-1.6572625 .692473-0.952340$

H $-4.2255801 .829696-1.536845$

H -2.7780441 .0152501 .406439$

H -7.1192151 .1380651 .521587$

H $-1.9336896 .290693-0.074840$

H -1.348763 $6.391862-1.737491$

H $-2.6129724 .296126-2.311532$

C -3.488554 -2.643921 1.389990

O $-3.309122-1.5193660 .525901$

C $-4.426721-1.194177-0.299662$

C $-4.809574-2.396705-1.176647$

C $-5.030797-3.657519-0.342816$

C $-3.839415-3.8984940 .582771$

H $-4.281630-2.4298232 .116587$

C -2.163707-2.839662 2.139912

H $-4.073919-0.415369-0.982656$

O $-5.975862-2.091637-1.933654$

H $-4.000692-2.584900-1.893988$

H $-5.965086-3.6068540 .228124$

O $-5.150996-4.787238-1.209774$

H -2.974807-4.215893 -0.012412

O $-4.149984-4.9608851 .482012$

C $-1.890819-1.7118783 .110582$

H $-2.154293-3.7708802 .715716$

H $-1.345088-2.8745451 .410943$

H -6.114212 -2.815116 -2.567710

H $-5.970759-4.679258-1.720765$

H $-4.384771-5.7365290 .944055$

O $-0.566425-1.3853153 .072250$

O $-2.734235-1.2040833 .837381$

C $-0.159266-0.3442063 .965601$

H $-0.207165-0.7017634 .998199$

H $0.875318-0.0803353 .730882$

H -0.7841110 .5447963 .836234$

SCF Energy (B3LYP/6-31G**//MMFF)= -3245.91477135

01451

MM̄FF Geometry

C $-0.804055 \quad 2.516274 \quad 2.340557$

C $0.0772662 .214173 \quad 3.304795$

$\begin{array}{lllll}\text { C } & 0.703772 & 0.853739 & 3.444887\end{array}$

$\begin{array}{lllll}\text { O } & 2.140198 & 1.027040 & 3.353900\end{array}$

C $0.409937 \quad 0.1898804 .803144$

C $-0.785980-0.7381704 .750029$

C $-0.696221-2.0756184 .608574$

C $-2.134172-0.079620 \quad 4.869688$

C $0.553978-2.9032104 .487280$

C $1.239164-2.8507073 .117492$

C $0.339672-3.1295611 .903666$

C $-0.224446-4.5507481 .915693$

C $-1.024416-4.8608160 .642006$

O $1.151809-2.940720 \quad 0.735148$

O $-1.232118-6.281055 \quad 0.589674$

C $-0.180795-4.479700-0.592459$

C -2.416934 -4.221202 0.677567

O $-0.965792-4.625939-1.774532$

C $0.439183-3.062468-0.504898$

C $1.445731-2.849529-1.662745$

O $-0.602675-2.090513-0.611235$

C $2.7133440 .819616 \quad 2.137914$

$\begin{array}{lllll}\text { O } & 2.146337 & 0.540803 & 1.093348\end{array}$

C $4.176000 \quad 0.9686182 .295993$

C $4.980506 \quad 0.4067551 .382158$

C 6.4423410 .4563841 .356651

C 7.1311221 .3583632 .345713

C $2.304809-1.584919-1.530705$ 
C $3.247508-1.403358-2.709676$

C $4.9679230 .183359-3.583463$

O $3.337357-2.210111-3.630987$

C $6.314432-0.302018-3.147647$

C $7.3270190 .510994-2.809056$

C $8.6805550 .024457-2.373246$

C $9.0374500 .470095-0.971023$

C $9.8093901 .550633-0.766557$

C $8.571833-0.3827370 .189131$

C $7.090976-0.278707 \quad 0.429837$

N $3.977545-0.226956-2.604525$

H $-1.112171 \quad 1.7394701 .644018$

H 0.3907382 .9835014 .005977

H $\quad 0.367813 \quad 0.1869262 .640252$

H $0.2553020 .943176 \quad 5.586877$

H $1.296877-0.3643215 .133549$

H $-1.619940-2.6517304 .570964$

H -2.949815 -0.8088324 .921234$

H -2.1822250 .5295965 .778057$

H $-2.3248450 .562645 \quad 4.006603$

H $1.272228-2.6102135 .262393$

H $0.296166-3.9432844 .723598$

H $1.711303-1.8708892 .983999$

H $2.077882-3.5599543 .115852$

H $-0.467394-2.3889931 .885685$

H $-0.848231-4.7250532 .800093$

H $0.604018-5.2694491 .987369$

H $-1.709126-6.5444401 .395306$

H $0.631728-5.216779-0.663040$

H -2.984039 -4.5828301 .543679$

H -3.003381 $-4.506056-0.202807$

H $-2.386372-3.1313520 .737049$

H $-0.365508-4.594815-2.537751$

H $2.127536-3.708723-1.710813$

H $0.907123-2.805362-2.617627$

H $-0.229022-1.253774-0.928360$

H $4.544616 \quad 1.5170683 .154654$

H $4.524392-0.1556490 .568915$

H 6.9842270 .9908013 .367094

H 6.7351652 .3780662 .279228

H 8.2083551 .4375542 .181839

H $1.672644-0.693205-1.469060$

H $2.914908-1.642403-0.622753$

H $4.9238501 .274860-3.652967$

H $4.718912-0.238338-4.562399$

H $6.462576-1.378965-3.114291$

H $7.1819501 .588080-2.853016$

H $9.4195570 .408417-3.088672$

H $8.753113-1.068386-2.436947$

H $\quad 10.093811 \quad 1.8617370 .233107$

H $10.1700662 .151504-1.594995$

H $9.134650-0.1453301 .098644$

H $8.818136-1.432417-0.017439$

H $6.499161-0.903492-0.239444$

H $3.8769930 .343439-1.769904$

$\begin{array}{llll}C & -1.041821 & 4.440798 & 0.764748\end{array}$

O $-1.614912 \quad 3.610198-0.263637$

C $-2.1479354 .449576-1.303439$

C $-1.6552925 .860467-1.003642$

C -1.5934745 .8424370 .511790$

H $-3.2388604 .427793-1.196201$

C $-1.746973 \quad 3.900061-2.676802$

H $-0.6584256 .042101-1.421613$

H -2.329239 $6.626986-1.398000$

C -0.7461916 .9577821 .098529$

H -2.6158315 .9208960 .905635$

C -2.113004 2.417019-2.898857

O $-0.3259944 .015043-2.834330$

H $-2.1867774 .519613-3.466564$

H $-1.4894511 .783078-2.258312$

O $-1.7746272 .081889-4.250509$

C -3.599086 $2.084978-2.676319$

C -4.002407 $0.652507-3.069377$

H -3.860991 2.250537-1.628365

O $-4.405430 \quad 2.985412-3.442154$

H -5.090432 $0.573461-2.953783$

H -3.825901 $0.507822-4.142578$

H -0.7228726 .8974092 .191014$
H $\quad 0.2863626 .910346 \quad 0.736524$

H -1.1542247 .9358260 .823310$

C -1.404869 3.8788122 .139763

H $\quad 0.0906373 .527008-2.103180$

H $-0.8330602 .292933-4.374011$

H $-4.1565372 .879400-4.376685$

H $-2.492740 \quad 3.781312 \quad 2.225607$

H $-1.073132 \quad 4.5656262 .926538$

H $\quad 0.046698 \quad 4.4222820 .620439$

C $-4.787656-0.522970-0.361346$

O $-3.459265-0.300028-0.849509$

C $-3.295458-0.455832-2.262944$

C $-3.691974-1.878411-2.679761$

C $-5.115862-2.212195-2.244096$

C $-5.298506-1.917613-0.756235$

H $-5.4566990 .250352-0.757435$

C $-4.747247-0.3934351 .171560$

H -2.221529 $-0.360018-2.460809$

O $-3.557321-2.024316-4.089246$

H $-3.001193-2.591949-2.213357$

H $-5.858070-1.676103-2.846912$

O $-5.360721-3.605505-2.450223$

H $-4.770603-2.683856-0.177281$

O $-6.681669-2.039054-0.423480$

C $-4.660267 \quad 1.043453 \quad 1.639938$

H $-5.663017-0.8161091 .600939$

H $-3.880235-0.9247721 .580980$

H -3.683703 -2.964099-4.301923

H $-5.348600-3.767059-3.408525$

H $-7.169719-1.365023-0.926520$

\begin{tabular}{llll}
\hline & -5.032395 & 1.100784 & 2.953334
\end{tabular}

$\begin{array}{llll}\text { O } & -4.293630 & 1.988456 & 0.956526\end{array}$

C $-5.0173612 .398016 \quad 3.558442$

H $-5.6066142 .348902 \quad 4.478047$

H $-3.9902262 .669507 \quad 3.814796$

H -5.4638253 .1493892 .899958$

SCF Energy (B3LYP/6-31G**//MMFF) $=-3245.90844716$

01452

MM̄FF Geometry

C $-0.5629415 .229420-1.186809$

C $-1.612024 \quad 4.583477-0.656208$

C $-2.6143913 .804928-1.466488$

O $-2.556152 \quad 2.432439-1.006472$

C $-4.0337554 .339823-1.213260$

C $-5.027758 \quad 3.851603-2.247832$

C $-5.8432822 .791191-2.091030$

C $-5.0837244 .666940-3.514461$

C $-5.910716 \quad 1.848775-0.922265$

C $-7.2887301 .891822-0.246713$

$\begin{array}{llll}\text { C } & -7.464076 & 0.864137 & 0.885381\end{array}$

C $-6.599862 \quad 1.187424 \quad 2.107218$

C $-6.7145450 .108597 \quad 3.193555$

$\begin{array}{llll}0 & -7.144161 & -0.423546 & 0.356098\end{array}$

$\begin{array}{llll}0 & -5.665396 & 0.357336 & 4.141981\end{array}$

C $-6.450824-1.2650982 .541963$

C $-8.0303020 .221434 \quad 3.970529$

O $-6.637634-2.3181593 .484717$

C $-7.289501-1.5169691 .268667$

C -6.845901-2.796540 0.520298

O $-8.659638-1.6913901 .645202$

C $-1.6723151 .600182-1.621696$

O $-0.996416 \quad 1.845765-2.608207$

C -1.620457 $0.342027-0.839099$

C $-0.700988-0.585150-1.148750$

C $-0.435467-1.832966-0.424902$

C $-1.288993-2.134592 \quad 0.777280$

C $-5.422131-2.734332-0.039960$

C $-5.090035-3.926147-0.919339$

C $-3.345740-4.703761-2.529234$

O $-5.752847-4.959514-0.928260$

C $-2.191327-5.323665-1.808008$

C $-0.931312-5.307510-2.268143$

C $0.221765-5.941056-1.545625$

C $1.345593-4.969280-1.266522$

C $2.544473-5.106809-1.858223$

C $1.108334-3.886858-0.234449$

C $0.583596-2.613809-0.843358$ 
N -3.941563 -3.701894 -1.666773 H $-0.4246265 .228219-2.264569$ H -1.741742 4.5768250 .423911 H -2.379097 $3.856260-2.536931$ H -4.034588 5.438199-1.235448 H $-4.3677194 .067954-0.204644$ H $-6.5241992 .543868-2.904649$ H $-5.8204474 .280462-4.226737$ H -5.357096 $5.702538-3.286801$ H -4.110584 4.666457 -4.015169 H $-5.710040 \quad 0.840499-1.303895$ H -5.128369 $2.059259-0.190678$ H -7.490689 2.9001390 .135566 H -8.049807 $1.682347-1.010641$ H -8.5232070 .8715551 .169104$ H -6.8560672 .1736292 .513895$ H $-5.5440201 .251617 \quad 1.814299$ H $-5.677818-0.3669514 .791283$ H -5.385754 -1.280431 2.281764 H $-8.102211 \quad 1.2026104 .455166$ H -8.077064 -0.5147254 .780605$ H $-8.913606 \quad 0.097756 \quad 3.340843$ H -7.594664 -2.451307 3.593929 H $-6.963850-3.674367 \quad 1.168214$ H -7.533160 -2.963260 -0.321331 H $-9.113546-2.1814320 .939766$ H -2.299500 $0.239171-0.000423$ H $-0.046790-0.398148-1.999798$ H -1.065837 -3.0959541 .243493$ H -2.347003 -2.162205 0.500352 H -1.150182 -1.367455 1.547351 H -5.287561 -1.821598 -0.631403 H $-4.693621-2.7266560 .776829$ H $-3.036323-4.198555-3.449537$ H -4.084885 -5.470377 -2.781141 H $-2.407217-5.818721-0.863983$ H $-0.716721-4.829648-3.221282$ H $0.581945-6.774698-2.162275$ H $-0.101649-6.389063-0.597595$ H $3.362407-4.428388-1.638744$ H $2.733793-5.896591-2.577536$ H $2.058483-3.6455790 .260571$ H $0.463501-4.2842370 .553542$ H $1.158788-2.281113-1.709039$ H -3.438151 -2.827317 -1.549541 C $1.7856945 .175495-0.219781$ $\begin{array}{llllll}\text { O } & 1.536998 & 3.894912 & 0.396838\end{array}$ C $2.2264702 .879257-0.362993$ C $3.2904323 .623696-1.152667$ C $2.5487464 .896221-1.515392$ H $1.4781472 .451352-1.042431$ C $2.742008 \quad 1.8012180 .595373$ H $4.1585473 .849757-0.521082$ H $3.6348063 .067548-2.029560$ C $3.4593026 .032374-1.947239$ H $1.8587524 .667645-2.336484$ C $3.635190 \quad 0.746764-0.087916$ O $1.5968861 .162811 \quad 1.168364$ H 3.2891432 .2645061 .424098 H $4.5038651 .262166-0.510232$

O $2.9291210 .168534-1.184543$

C $4.145436-0.3742740 .842307$ C $4.978405-1.4543090 .126617$ H 4.7380030 .0700671 .649763 O $3.058163-1.0680711 .457781$ H $5.291390-2.1833480 .884793$ H $4.328950-2.023725-0.551466$ H $2.8772796 .930752-2.175966$ H $4.1819566 .287815-1.164944$ H $4.0212315 .754984-2.844946$

C $0.4695685 .947710-0.361640$ H 1.0517411 .8560071 .578782 H $2.147776-0.275630-0.815191$ H $2.566480-0.4271311 .998183$ H $\quad 0.6561916 .931477-0.807275$ H $0.064504 \quad 6.1292600 .642306$ H 2.4292925 .7253260 .479243 C $7.698302-0.6100691 .216868$
O $6.987718-0.0195550 .122930$

C $6.203363-0.932195-0.647359$

C $7.098562-2.035644-1.229406$

C $7.936570-2.719713-0.151525$

C $8.664486-1.6842560 .705555$

H $6.986677-1.0521381 .925242$

C 8.4486710 .5163161 .935089

H $5.817223-0.359822-1.499566$

O $6.323107-3.016579-1.910319$

H $7.781168-1.595421-1.968079$

H $7.325479-3.3830440 .471895$

O $8.917511-3.550845-0.776839$

H $9.467979-1.2219590 .118029$

O $9.269216-2.339757 \quad 1.818124$

C 7.4879251 .3890922 .707446

H 9.1749930 .1249002 .656523

H 9.0059341 .1443641 .229565

H $5.807994-2.557763-2.595814$

H $8.441889-4.180367-1.345725$

H $9.854868-3.0309971 .464084$

O $7.0109992 .384823 \quad 1.907155$

O 7.1814131 .1922723 .876406

C 6.0683743 .2698822 .519788

H 6.5669083 .8745343 .282846

H 5.6725593 .9337121 .746647

H 5.2376132 .7071962 .955994

SCF Energy $(B 3 L Y P / 6-31 G * * / / M M F F)=-3245.93262837$

01453

MM̄FF Geometry

C $0.2386204 .660385-0.200602$

C $-0.0594974 .220687-1.433633$

C $-1.2164433 .322887-1.808692$

O $-1.6756662 .552196-0.675155$

C $-2.3929594 .164740-2.333388$

C $-3.4804563 .339775-2.994342$

C $-4.7358973 .202061-2.525460$

C $-3.0945722 .668157-4.288557$

C $-5.3407483 .800870-1.287887$

C $-5.2680082 .911487-0.036703$

C $-5.9343021 .534122-0.194749$

C $-6.059657 \quad 0.8362531 .161830$

C $-6.626775-0.5864501 .030383$

O $-5.1243030 .738340-1.065278$

O $-6.399747-1.2683142 .273957$

C $-5.833613-1.349704-0.053188$

C $-8.144982-0.5755780 .814622$

O $-6.431368-2.614624-0.324437$

C $-5.666828-0.555141-1.364448$

C $-4.710506-1.235121-2.375930$

O $-6.940949-0.440028-2.010312$

C $-0.9361131 .459445-0.337514$

O $0.0674551 .050277-0.899896$

C $-1.548007 \quad 0.8496730 .863234$

C $-0.833655-0.0283871 .581511$

C $-1.273556-0.7358232 .783806$

C $-2.738317-0.6792443 .123947$

C $-3.278750-1.465236-1.880268$

C $-3.034693-2.853091-1.310713$

C $-1.287696-4.187539-0.135002$

O $-3.842626-3.772923-1.403693$

C -1.615391 -42184311324647

C $-0.709432-4.4891692 .276681$

C - $-1.029935-4.5628003 .742751$

C $-0.140120-3.6696284 .578654$

C $0.942634-4.1625435 .203321$

C $-0.550630-2.2236134 .751839$

C $-0.352972-1.4171293 .497597$

N $-1.783691-2.953895-0.716074$

H -0.4095534 .4113100 .635311$

H $0.5908114 .503128-2.259430$

H $-0.8683432 .633620-2.587606$

H -2.033734 $4.895138-3.070924$

H -2.802636 $4.750192-1.503272$

H $-5.4278402 .585548-3.098862$

H $-3.9558022 .222690-4.798423$

H -2.650136 $3.394778-4.976687$

H $-2.3718931 .866641-4.109269$ 
H -4.885892 4.771026-1.061730

H -6.393473 4.024205-1.504305 H $-4.215877 \quad 2.781557 \quad 0.240632$ H -5.7522213 .4554870 .783803$ H $-6.9246561 .677318-0.642841$ H $-6.673878 \quad 1.4305791 .849840$ H $-5.0678690 .772182 \quad 1.628814$ H $-6.830997-0.7564642 .979499$ H -4.841297 -1.5556840 .365897$ H -8.647783 -0.0870981 .657896$ H -8.546448 -1.5942910 .777363$ H -8.445367 -0.051659-0.095061 H $-6.495195-3.0922150 .520420$ H -5.160444 -2.159735 -2.756950 H -4.637414 -0.568588 -3.247681 H $-6.793705-0.251190-2.951838$ H $-2.556596 \quad 1.143387 \quad 1.126612$ H $\quad 0.183525-0.249318 \quad 1.266221$ H -3.339797 -1.004064 2.268145 H -3.0322640 .3406913 .394151$ H $-3.021078-1.3261433 .956377$ H -2.595896 -1.363523 -2.733679 H -2.993798 -0.711056-1.141959 H $-0.209195-4.222950-0.318661$ H - $-1.756615-5.040163-0.636515$ H $-2.648596-4.034741 \quad 1.609957$ H $0.317606-4.6996361 .986124$ H $-0.909692-5.6076554 .057718$ H -2.080942 -4.3149523 .937187$ H $1.572325-3.5348785 .825212$ H $1.226693-5.2047945 .102081$ H $0.052233-1.7512715 .538778$ H - $-1.577922-2.1940735 .123923$ H $0.685048-1.3887423 .161648$ H -1.195868 -2.128132 -0.647999 C 2.2499554 .9110471 .285798

O 2.6045783 .5391331 .035168 C 3.9433973 .3034831 .508357

C 4.3629794 .5645292 .258282

C 3.5719545 .6354451 .533038

H 4.5701443 .1957240 .615233

C 3.9994902 .0121922 .336580

H 4.0711324 .5316343 .313990

H 5.4444424 .7274402 .218176

C 3.4336906 .9280432 .318034

H 4.0698265 .8469200 .577179

C $3.339454 \quad 0.789774 \quad 1.668777$

O 3.3229162 .2204523 .584934

H 5.0401831 .7891402 .597315

H 2.2530260 .9319051 .663190

O $3.576994-0.3491312 .506612$

C 3.8112300 .4685190 .238562

C $3.104321-0.785101-0.312533$

H $3.5969251 .314158-0.423910$

$\begin{array}{llll}\text { O } & 5.217706 & 0.247236 & 0.230413\end{array}$

H $3.464122-1.6684460 .225305$

H $2.033054-0.696780-0.109008$

H 2.8518077 .6655751 .756441

H 2.9324506 .7631453 .277851

H 4.4184097 .3605422 .523169

C 1.4609305 .4819100 .103984

H 2.4063862 .4766663 .383755

H $4.531211-0.5357882 .482535$

H 5.6544251 .0885130 .443803

H $2.1079275 .522992-0.781326$

H 1.1484036 .5076050 .329936

H 1.6282204 .9169522 .190913

C $5.237925-2.426402-1.787532$

O $4.676478-1.172203-2.192986$

C $3.307084-0.973864-1.832777$

C $2.441074-2.084519-2.444774$

C $2.946342-3.469524-2.052837$

C $4.436067-3.598546-2.367740$

H $5.251758-2.487402-0.692718$

C $6.688599-2.460141-2.281686$

H $2.999720-0.032556-2.306268$

O $1.079724-1.952863-2.050127$

H $2.466416-2.003596-3.539193$
H $2.746527-3.687845-0.997033$

O $2.241922-4.458118-2.807595$

H $4.575416-3.648893-3.455294$

O $4.927016-4.819506-1.817688$

C $7.547476-1.504927-1.487581$

H $7.134317-3.455234-2.172590$

H $6.751718-2.197503-3.344807$

H $\quad 0.784924-1.062137-2.306348$

H $1.293639-4.337026-2.627495$

H $4.386410-5.540415-2.183730$

O $7.695311-0.331905-2.166567$

O $8.010779-1.764353-0.384311$

C $8.4818360 .665653-1.507983$

H $9.5072700 .309133-1.373025$

H $8.5002491 .556605-2.141191$

H $8.0348330 .929541-0.544860$

SCF Energy (B3LYP/6-31G**//MMFF) $=-3245.91097092$

01454

MM̄FF Geometry

C 0.0501834 .5128340 .780102

C $-0.2393434 .278975-0.509154$

C $0.4849663 .331751-1.436546$

O $1.7314842 .905525-0.843029$

C $-0.4193582 .110633-1.680655$

C $0.1579691 .089511-2.635464$

C $0.695765-0.084859-2.251904$

C $0.0510901 .438682-4.096213$

C $0.870778-0.593844-0.846118$

C $-0.115347-1.731113-0.561156$

C $-0.075941-2.2801890 .873132$

C $-0.685069-1.3187471 .893080$

C $-0.641539-1.9064623 .311960$

O $1.276472-2.573327 \quad 1.228826$

O $-0.945061-0.8498944 .234794$

C $0.797770-2.3717113 .610943$

C $-1.709274-2.9851153 .525547$

O $0.864993-3.0632344 .856121$

C $1.424597-3.2373352 .490260$

C $2.933450-3.4517442 .785789$

O $0.790827-4.5204892 .468571$

C $2.8319952 .871283-1.643553$

O $2.8872593 .146647-2.831711$

C $3.9842452 .441739-0.817503$

C $5.1921942 .325295-1.390914$

C $6.4426011 .940055-0.730348$

C $6.387143 \quad 1.652767 \quad 0.746854$

C $3.769582-3.8586181 .564368$

C $4.197493-2.6687040 .718405$

C $5.406453-2.098267-1.376527$

O $3.986500-1.5017691 .040422$

C $6.757173-1.659773-0.905790$

C $7.817217-1.510815-1.713170$

C $9.161416-1.046375-1.231894$

C $9.5277790 .322285-1.760092$

C $10.387658 \quad 0.461640-2.783185$

C $8.9676721 .531242-1.039243$

C $7.567351 \quad 1.869577-1.474722$

N $4.863373-3.060446-0.435197$

H $\quad 0.897127 \quad 4.0189881 .247217$

H $-1.1044974 .777932-0.943036$

H $0.6680783 .871398-2.373563$

H -1.384125 2.448967 -2.081925

H $-0.6624091 .636394-0.722357$

H $1.061255-0.761185-3.023497$

H $\quad 0.4700000 .659243-4.741545$

H $-0.9990811 .570619-4.377240$

H $0.5870042 .367616-4.312345$

H $1.899894-0.957957-0.749489$

H $\quad 0.7658770 .205949-0.109461$

H -1.139310 -1.416455 -0.798508

H $\quad 0.123715-2.558568-1.243291$

H $-0.649263-3.2140820 .861221$

H $-1.713023-1.0606841 .621072$

H $-0.128937-0.3721351 .898254$

H - $1.832947-0.5147494 .021527$

H $1.410106-1.4661513 .726986$

H $-2.708570-2.5806263 .324730$ 
H -1.726194 -3.321644 4.568150 H -1.570751 -3.857891 2.885739 H $0.478891-2.4804435 .532166$ H $3.364759-2.5386103 .214238$ H $3.036470-4.2350333 .548029$ H $1.404145-5.1626322 .075469$ H 3.8093342 .2456560 .234169 H $5.2820052 .540310-2.455307$ H 5.6627150 .8589190 .959293 H 6.0927142 .5512321 .300398 H 7.3390641 .3187901 .163862 H $4.689727-4.3406801 .917326$ H $3.231374-4.5781050 .938337$ H $4.735164-1.234715-1.428223$ H $5.444070-2.580539-2.358103$ H $\quad 6.865930-1.4220570 .150132$ H $7.718106-1.729593-2.773713$ H $9.904889-1.789543-1.548301$ H $9.211111-1.035777-0.135671$ H $10.678514 \quad 1.441376-3.147229$ H $10.815345-0.401282-3.282859$ H $9.5925772 .409780-1.249356$ H 9.0569781 .3760430 .039092 H $7.4960692 .107804-2.537314$ H $5.083146-4.042354-0.574499$ C -1.6982934 .6579102 .615396$ O $-2.6341573 .847856 \quad 1.872849$ C -2.7819552 .5830972 .549109$ C -2.1691492 .7773213 .932215$ C -1.0241463 .7207813 .616727$ H -2.1758561 .8554111 .996056$ C -4.2501522 .1431522 .553028$ H $-2.872493 \quad 3.2531474 .625590$ H -1.839580 1.8361134 .379608 C -0.4571124 .4253594 .836143$ H -0.2263193 .1404243 .137761$ C -4.8972682 .0566251 .154184$ O -5.0158303 .0766653 .326193$ H -4.3420331 .1803163 .068722$ H $-4.995123 \quad 3.0629550 .730136$ O $\quad-6.236302 \quad 1.5716291 .322730$ C $-4.158467 \quad 1.1473650 .153596$ C $-4.9178561 .018104-1.180596$ H -3.159870 $1.555063-0.033952$ O $-4.005824-0.1366860 .744960$ H $-5.8261750 .427161-1.021830$ H $-5.2248732 .022359-1.493979$ H 0.3527085 .1044184 .551153 H -1.223198 5.0116645 .354413 H -0.0533613 .6974155 .547555$ C $-0.7749505 .414977 \quad 1.655549$ H -4.9072193 .9534672 .919294$ H -6.1773120 .6592341 .654694$ H -3.509545 -0.6922920 .120626$ H -0.1007896 .0696322 .219598$ H -1.3906196 .0683541 .023888$ H -2.3042965 .3987803 .153833$ C $-4.814054-1.929701-2.161539$ O $-3.734019-0.993716-2.055559$ C $-4.0676850 .375996-2.303470$ C $-4.6797270 .521471-3.702759$ C $-5.901105-0.373664-3.861759$ C $-5.537419-1.813300-3.514187$ H -5.518936 -1.765812 -1.337638 C $-4.217702-3.344320-2.027756$ H $-3.1162310 .917749-2.319737$ O $-5.0474721 .870870-3.966708$ H $-3.9367730 .237686-4.459641$ H -6.746031 -0.020919-3.258995 O $-6.335889-0.340658-5.223134$ H $-4.905437-2.225539-4.311985$ O $-6.728807-2.598181-3.472457$ C $-3.788599-3.702476-0.621566$ H -4.963396 -4.089348 -2.328999 H -3.333948 -3.439304 -2.670009 H $-4.2482412 .415919-3.867169$ H -6.521353 $0.588240-5.444262$ H $-7.172764-2.497415-4.331933$
O $-3.478641-5.033446-0.587897$

O $-3.716588-2.9271750 .319725$

C $-3.057818-5.5518030 .678247$

H -3.697624 -5.1900711 .488981$

H $-3.131697-6.6418080 .639285$

H $-2.015109-5.2772970 .856057$

SCF Energy (B3LYP/6-31G**//MMFF)= -3245.92122237

01455

MM̄MF Geometry

C $-0.4007193 .855248-1.414124$

C $-0.6891562 .942986-2.355587$

C $-0.1194921 .544651-2.459620$

O $0.9482321 .329085-1.511354$

C $-1.2425190 .539092-2.145540$

C $-0.836770-0.914430-2.276911$

C $-0.826928-1.797718-1.259201$

C $-0.457956-1.364721-3.663843$

C $-1.196333-1.5582980 .178411$

C $-0.016943-1.1733431 .084368$

C $1.034402-2.2825411 .249708$

C $2.055506-1.9047902 .325385$

C $3.171357-2.9536892 .453606$

O $1.705444-2.460158 \quad 0.000515$

O $4.214537-2.3586103 .240850$

C $3.746356-3.2547121 .051100$

C $2.694943-4.1843583 .233914$

O $4.680032-4.3300071 .107531$

C $2.663421-3.526056-0.013779$

C $3.228322-3.615482-1.451925$

O $2.038274-4.7841420 .269394$

C $2.2016601 .709635-1.879598$

O $2.5413882 .200764-2.943793$

C $3.0957081 .459884-0.727546$

C $4.2768882 .091900-0.660799$

C $5.252591 \quad 1.9831300 .425908$

C 5.0219250 .9176461 .464644

C $3.949840-2.359731-1.954202$

C $5.460219-2.409726-1.793760$

C $7.489300-0.995691-2.098559$

O $6.070742-3.419852-1.455165$

C $7.904092-0.455242-0.766914$

C $8.6567720 .645919-0.622664$

C $9.121757 \quad 1.1746190 .703408$

C 8.7620022 .6285800 .909357

C 9.6583013 .6023790 .676626

C 7.3936762 .9448861 .468705

C 6.3035592 .8297390 .437671

N $6.052509-1.195377-2.114754$

H $0.3293883 .626060-0.644237$

H $-1.4280583 .197751-3.113326$

H $\quad 0.251343 \quad 1.390540-3.479935$

H -2.094635 $0.713602-2.816470$

H $-1.6104670 .742373-1.134226$

H $-0.526239-2.822255-1.475813$

H $-0.305601-2.447819-3.721872$

H -1.249033 -1.107918 -4.375921

H $\quad 0.472169-0.885923-3.984615$

H $-1.967419-0.7854150 .257326$

H -1.667916 -2.471041 0.563868

H $0.457596-0.2689130 .685731$

H -0.422750 -0.915322 2.069902

H $0.516856-3.209723 \quad 1.523191$

H $1.563189-1.7483953 .293492$

H $2.513916-0.9407532 .065742$

H $4.959406-2.9839043 .263618$

H $4.321796-2.3697240 .757839$

H $2.363761-3.8936094 .238172$

H $3.511321-4.8989863 .386140$

H $1.866511-4.7048842 .748922$

H $4.174621-5.1587591 .164908$

H $3.850986-4.512074-1.557582$

H $2.373286-3.776713-2.124591$

H $1.615228-5.107543-0.543407$

H 2.7428970 .7904320 .048328

H $4.5456942 .778039-1.463003$

H $4.861162-0.0541150 .986249$

H 4.1453391 .1588012 .075124 
H $\begin{array}{lll}5.865825 & 0.7795912 .143627\end{array}$ H $3.762599-2.261812-3.031373$ H $3.559450-1.458485-1.471663$ H $7.731143-0.311122-2.917721$ H $7.992528-1.950576-2.281711$ H $7.595659-1.0098560 .116222$ H $8.9873401 .181991-1.509726$ H $10.211899 \quad 1.050938 \quad 0.746981$ H $8.724715 \quad 0.579127 \quad 1.534898$ H 9.4175364 .6459940 .849565 H $10.651667 \quad 3.377246 \quad 0.302486$ H $7.371783 \quad 3.9770851 .843924$ H 7.2159262 .3262462 .352692 H $6.3856523 .564311-0.364791$ H $5.464699-0.395750-2.331842$ C -1.998966 5.364338 -0.139395 O $-3.0584694 .387345-0.207295$ C $-3.277422 \quad 3.8584591 .114917$ C -2.5767134 .8187182 .070344$ C -1.3684745 .2220541 .247023$ H -2.7632682 .8903831 .155704$ C $-4.772392 \quad 3.663187 \quad 1.383465$ H -3.194460 5.6967682 .291864 H -2.311819 4.3417233 .018806 C -0.6811716 .4818061 .743492$ H $-0.6499454 .394405 \quad 1.271269$ C -5.5013752 .7873090 .343588$ O -5.4046374 .9500621 .384474$ H $-4.911134 \quad 3.2525992 .390897$ H $-5.5709243 .329920-0.607508$ O -6.8442662 .6276560 .819966$ C $-4.866591 \quad 1.4011350 .113979$ C $-5.7070200 .514392-0.826670$ H $-3.8710131 .537054-0.321950$ $\begin{array}{llll}\text { O } & -4.703117 & 0.760329 & 1.375662\end{array}$ H $-6.6458220 .249533-0.329813$ H -5.958809 1.102695 -1.716579 H $\quad 0.172140 \quad 6.735568 \quad 1.106613$ H $-1.364767 \quad 7.3374501 .749135$ H -0.3113326 .3409772 .764370$ C -1.056949 $5.206474-1.337521$ H -6.3490714 .8076221 .568064$ H -7.3877652 .3206150 .074903$ H $-4.179840-0.0453081 .228286$ H -0.275356 $5.973972-1.301823$ H $-1.6322275 .383740-2.255321$ H -2.488571 $6.343767-0.222038$ C $-5.788267-2.2983310 .431385$ O $-4.664700-1.640312-0.165589$ C $-4.974275-0.776204-1.262120$ C $-5.688963-1.569720-2.364744$ C $-6.919563-2.297687-1.832630$ C $-6.551776-3.131753-0.605318$ H $-6.456877-1.5522350 .877048$ C $-5.249185-3.1925901 .555560$ H $-4.012217-0.455933-1.677365$ O $-6.072372-0.718959-3.440109$ H $-4.997762-2.316225-2.778016$ H -7.729691 -1.592422 -1.612161 O $-7.403314-3.163900-2.861770$ H -5.946077 $-3.992608-0.916772$ O $-7.741401-3.637751-0.003222$ C $-4.773394-2.3619652 .724406$ H $-6.014958-3.8737801 .942744$ H $-4.419745-3.8102491 .189588$ H $-5.266321-0.289277-3.773892$ H -8.247329 -3.538242 -2.557973 H $-8.150691-4.254947-0.632619$ O $-3.421912-2.4744672 .860817$ O $-5.514680-1.6835693 .423999$ C $-2.844902-1.7182163 .929751$ H -2.933756 -0.6482143 .719899$ H -3.324542 -1.967831 4.881134 H $-1.784917-1.9777643 .995877$ SCF Energy (B3LYP/6-31G**//MMFF) $=-3245.90518884$

01456

MM̄FF Geometry
C $4.8475950 .562583-1.824705$

C $4.228648-0.219598-2.721766$

C $3.459680-1.460438-2.359799$

O $2.177813-1.383648-3.032517$

C $4.150136-2.747352-2.857459$

C $4.955144-3.441527-1.778246$

C $4.545034-4.541765-1.116338$

C $6.305796-2.846562-1.479096$

C $3.259331-5.302845-1.287920$

C $1.976669-4.575065-0.874134$

C $1.994398-3.895360 \quad 0.502172$

C $2.189524-4.889813 \quad 1.648155$

C $2.084429-4.2084023 .021502$

O $0.730468-3.228743 \quad 0.639155$

O $1.976562-5.254043 \quad 3.999667$

C $0.782952-3.3786993 .066177$

C $3.362054-3.4329793 .363247$

O $0.697571-2.6306104 .276482$

C $0.595548-2.4582121 .840286$

C $-0.791706-1.7779181 .814237$

O $1.571510-1.4099591 .895165$

C $1.097241-0.985558-2.310121$

O $1.076786-0.608366-1.150496$

C $-0.085797-1.130642-3.185293$

C $-1.298962-1.304170-2.640130$

C $-2.549373-1.501131-3.377828$

C $-2.509161-1.312163-4.872594$

C $-1.984565-2.7331931 .722049$

C $-3.300458-1.9828611 .617336$

C $-5.758938-2.3420501 .727017$

O $-3.370247-0.7733421 .419361$

C $-6.278480-2.427230 \quad 0.327894$

C $-6.499262-1.354107-0.446414$

C $-7.069769-1.427343-1.832076$

C $-6.057407-1.085350-2.899678$

C $-6.1214430 .072987-3.577083$

C $-5.029809-2.137619-3.248545$

C $-3.665252-1.830163-2.692757$

N $-4.392959-2.826101 \quad 1.760671$

H $4.8201390 .279342-0.776651$

H $4.2695650 .043903-3.776273$

H $3.300357-1.519668-1.276149$

H $4.804408-2.537112-3.714319$

H $3.396920-3.436209-3.259121$

H $5.209987-4.960999-0.362101$

H $6.902580-3.485252-0.818926$

H $6.877728-2.711840-2.402855$

H $6.199815-1.876950-0.986315$

H $3.169370-5.631189-2.330861$

H $3.339584-6.229812-0.705669$

H $1.722160-3.823734-1.629968$

H $1.141862-5.288189-0.912543$

H $2.784981-3.1379240 .510873$

H $3.148191-5.4145991 .563230$

H $1.416197-5.6684081 .586704$

H $1.835603-4.8336514 .865543$

H $-0.038702-4.103641 \quad 3.101058$

H $4.224775-4.1102103 .379365$

H $3.306462-2.9985654 .367396$

H $3.582906-2.6334622 .653146$

H $1.288981-1.8633534 .192145$

H $-0.903281-1.1261222 .690273$

H $-0.828504-1.1024710 .948467$

H $1.271926-0.6817441 .325479$

H $0.083405-1.132265-4.256208$

H $-1.383933-1.332435-1.554818$

H $-1.916973-2.103927-5.344107$

H $-2.066263-0.342227-5.126561$

H $-3.495455-1.320759-5.340991$

H $-1.894387-3.3752070 .838858$

H $-2.031670-3.3681322 .612555$

H $-5.792178-1.3180282 .114544$

H $-6.356301-2.9772962 .388161$

H $-6.503754-3.419527-0.055276$

H $-6.276331-0.363505-0.057811$

H -7.924693 $-0.740202-1.877981$

H $-7.486426-2.421696-2.036881$

H $-5.4156050 .303250-4.368146$ 
H $-6.8777530 .817873-3.353147$

H $-5.016137-2.285988-4.332856$

H $-5.343897-3.110643-2.849146$

H -3.596812 -1.933340 -1.609611

H -4.243018 -3.827517 1.842740

C $5.3220993 .013356-1.297744$

O $3.9082503 .284119-1.235194$

C 3.5793623 .6661420 .115399

C 4.9070823 .9227550 .821200

C 5.8034912 .9021950 .148928

H 3.0995802 .7978290 .581999

C 2.6250974 .8632280 .119350

H 5.2772764 .9374450 .633490

H 4.8342703 .7854191 .904303

C 7.2856353 .1792970 .332009

H $5.573077 \quad 1.9149790 .566024$

C $1.2928884 .628349-0.620797$

O $3.2732925 .982023-0.500498$

H 24244915.1692871 .152770

H $1.4638674 .551597-1.701365$

O $0.4839895 .796519-0.425154$

C $0.4994003 .397414-0.139439$

C $-0.9122113 .338885-0.756753$

H $1.0493782 .488735-0.409703$

O $0.410993 \quad 3.443423 \quad 1.281471$

H -1.490285 $4.200971-0.408266$

H -0.813767 $3.430330-1.844990$

H $7.8895112 .422947-0.178852$

H $7.5636884 .160132-0.067857$

H 7.5513213 .1623601 .394153

C $5.6050521 .806757-2.197670$

H $3.5194975 .714780-1.402778$

H $0.9983376 .560819-0.736993$

H $\quad 0.0453002 .5926991 .576642$

H $6.6778971 .583736-2.181491$

H $5.3503692 .085362-3.228321$

H $5.7792033 .895641-1.766072$

C -2.8139242 .7637651 .591742$

$\begin{array}{lllll}\text { O } & -1.890369 & 1.854248 & 0.980747\end{array}$

C -1.682459 $2.039443-0.422497$

C $-3.0194671 .896669-1.162523$

C $-4.0693762 .855583-0.606932$

C -4.1865912 .6968610 .908779$

H -2.414002 3.7833351 .536331

C -2.9302692 .3831013 .073096$

H $-1.0421621 .210925-0.746891$

O $-2.8393472 .116839-2.556596$

H -3.382081 $0.868066-1.043713$

H $-3.8380673 .892208-0.876123$

O $-5.3351532 .574012-1.207404$

H $-4.676171 \quad 1.742167 \quad 1.138865$

O -5.0174863 .7341661 .426135$

C -1.6386302 .6493373 .808341$

H -3.713293 2.9598623 .578504

H $-3.1902801 .324913 \quad 3.194440$

H $-3.7106772 .029602-2.979598$

H $-5.5657071 .652108-1.003146$

H -5.8679953 .6862020 .956792$

O $-0.877193 \quad 1.518358 \quad 3.836210$

O

C 0.3905221 .6403104 .488297

H $\quad 0.912416 \quad 0.684123 \quad 4.396533$

H 0.9947422 .4160504 .008506

H $\quad 0.2474201 .8630805 .549773$

SCF Energy (B3LYP/6-31G**//MMFF) $=-3245.92649117$

01457

MM̄FF Geometry

C -0.9102122 .7112673 .277367$

C -2.087952 2.1604073 .604004

C $-2.4487210 .696303 \quad 3.606688$

O

C $-1.316797-0.330553 \quad 3.376723$

C $-0.260431-0.4342944 .454915$

C $1.068121-0.407954 \quad 4.227101$

C $-0.772104-0.6664955 .853351$

C $1.791678-0.2211412 .921541$

C $2.457304-1.5094642 .422140$
C $1.471520-2.5662751 .897462$

C $2.236938-3.7942031 .396561$

C $1.303287-4.8416210 .771901$

O $\quad 0.721791-1.9913750 .821749$

O $2.116712-5.781767 \quad 0.053179$

C $0.394593-4.144177-0.262175$

C $0.555853-5.645177 \quad 1.842575$

O $-0.581084-5.049709-0.774980$

C $-0.279313-2.8568670 .267862$

C $-0.970022-2.108505-0.898242$

O $-1.263370-3.2229291 .237671$

C $-4.307987-0.4779542 .614531$

O $-4.459744-1.2515473 .545749$

C $-5.055950-0.5104671 .339481$

C $-5.849601-1.5536401 .059160$

C $-6.588832-1.724944-0.186983$

C $-6.553774-3.132239-0.717842$

C $-1.490633-0.713569-0.526604$

C $-2.1246630 .001346-1.706749$

C $-3.3035812 .122034-2.276307$

O $-2.149031-0.464457-2.842499$

C $-4.7802381 .882208-2.246613$

C $-5.463124 \quad 1.355552-3.274324$

C $-6.9547301 .173888-3.297033$

C $-7.373859-0.278049-3.239921$

C $-7.171014-1.104170-4.280456$

C -8.103295 -0.751762 -2.004103

C $-7.254336-0.704777-0.763983$

N -2.648812 $1.233226-1.336531$

H -0.0788462 .0836222 .979995$

H -2.9021912 .8280903 .882519$

H -2.955930 0.4886824 .557204

H $-0.882397-0.1520032 .389000$

H -1.768481 -1.3273543 .287435$

H $1.733307-0.5300265 .081543$

H $0.032480-0.9069376 .556723$

H -1.281262 0.2259426 .230215

H -1.476447 -1.504806 5.866050

H $1.154816 \quad 0.2029692 .141037$

H 2.5754510 .5286593 .090849

H $3.134353-1.2300101 .607908$

H $3.077682-1.9411993 .217491$

H $0.788085-2.8469792 .706450$

H $2.828465-4.2406992 .205625$

H $2.963903-3.4826310 .632647$

H $2.744696-6.1771940 .681693$

H $1.031111-3.875988-1.115967$

H $1.266839-6.1494412 .508134$

H $-0.044825-6.4424141 .390980$

H $-0.096478-5.0285112 .464212$

H $-1.274496-5.150159-0.100701$

H $-0.256340-1.992463-1.723361$

H $-1.808838-2.705943-1.277954$

H -1.974531-2.564162 1.240923

H -4.9103010 .3063910 .640992$

H $-5.944660-2.3734641 .768255$

H -7.115646 -3.803709 -0.059564

H -5.520157 -3.492885 -0.776535

H $-6.971945-3.223603-1.723326$

H $-2.243041-0.7873210 .262343$

H $-0.669822-0.085772-0.161725$

H -2.881502 $1.970801-3.275417$

H -3.092154 3.149817 -1.966795

H $-5.3134052 .189141-1.350215$

H -4.922535 $1.061979-4.171601$

H $-7.3442871 .626577-4.218403$

H -7.426002 $1.736067-2.481126$

H -7.494260 -2.139434 -4.253089

H $-6.676819-0.762050-5.183876$

H -8.517830 -1.755668 -2.150902

H $-8.981548-0.112087-1.845700$

H $-7.2237410 .275727-0.289052$

H $-2.6325661 .505600-0.357857$

C -0.2543344 .7249611 .881412$

O $0.9483204 .072403 \quad 1.420911$

C $0.7310643 .625280 \quad 0.071675$

C $-0.4177654 .473779-0.452634$

C -1.3013764 .5443510 .777699$ 
H $\quad 0.4073832 .5774280 .134436$

C $2.012936 \quad 3.698434-0.764624$

H $-0.0808705 .477686-0.736826$

H $-0.9109114 .020232-1.316203$

C $-2.3387335 .651796 \quad 0.725815$

H $-1.820348 \quad 3.583804 \quad 0.882521$

C $3.1464832 .818022-0.196876$

O $2.4451355 .052426-0.872675$

H $1.7702763 .356129-1.777667$

H 2.7209911 .8463670 .076519

O 3.6881363 .3941140 .989802

C $4.2870432 .589363-1.206969$

C $5.4514341 .721230-0.693771$

H $3.8661642 .122599-2.103761$

O $4.8382783 .837022-1.632696$

H $6.1172741 .533361-1.545878$

H 6.0614442 .3013240 .011200

H -2.932205 5.6706471 .645372

H -1.8716176 .6349600 .604761$

H -3.022309 $5.498737-0.115674$

C $-0.645478 \quad 4.1920303 .264183$

H 2.6647505 .3598590 .023541

H 2.9632123 .4804431 .631834

H $5.1273684 .316075-0.837155$

H $-1.512894 \quad 4.7480763 .638426$

H 0.1811624 .3768673 .961413

H 0.0021935 .7872941 .984312

C $4.371148-0.824556-2.006495$

O $3.994544-0.274381-0.742114$

C $5.041814 \quad 0.390100-0.036207$

C $6.200215-0.5835040 .221524$

C $6.658831-1.283392-1.057473$

C $5.467316-1.875005-1.812837$

H $4.730245-0.027468-2.669479$

C $3.107287-1.426764-2.626141$

H 4.6268520 .6523960 .945045

$\begin{array}{lllll}\text { O } & 7.308708 & 0.086922 & 0.811545\end{array}$

H $5.872826-1.3523800 .933740$

H $7.230516-0.605085-1.701979$

O $7.544999-2.351526-0.715032$

H $5.073882-2.738366-1.260983$

O $5.903417-2.340328-3.087502$

C $2.129256-0.331065-2.983254$

H $3.332029-2.010246-3.525918$

H $2.599909-2.099306-1.924935$

H 6.9941040 .5066241 .630465

H $8.279793-1.965552-0.207835$

H $6.617479-2.982342-2.932961$

O $2.226057-0.028008-4.308502$

O $1.392766 \quad 0.215319-2.171956$

C $1.3644991 .022430-4.758330$

H $1.6093301 .957486-4.245280$

H $1.5251061 .157952-5.831142$

H $0.3172000 .752696-4.591292$

SCF Energy (B3LYP/6-31G**//MMFF) $=-3245.91286266$

$01 \_458$

MMFF Geometry

C $-1.275868-1.4757442 .383169$

C $-0.064281-1.7362941 .872440$

C $0.136624-2.4377280 .553509$

O $0.681670-1.473643-0.378233$

C $1.155417-3.577375 \quad 0.708747$

C $1.296475-4.423164-0.542059$

C $2.300029-4.297765-1.432817$

C $0.246806-5.487103-0.725531$

C $3.393243-3.267390-1.422111$

C $4.700261-3.823335-0.847956$

C $5.804527-2.756529-0.831147$

C $7.110767-3.332730-0.287179$

C $8.207032-2.260353-0.200537$

O $5.372563-1.6851420 .010879$

O $9.272861-2.7853740 .607574$

C $7.652899-1.0194730 .538209$

C $8.816455-1.970405-1.577992$

$\begin{array}{llll}\text { O } & 8.597708 & 0.046032 & 0.467935\end{array}$

C $6.258858-0.5583450 .040548$

C 5.6449420 .4767051 .008989
O $6.338356 \quad 0.085275-1.233385$

C $-0.183408-0.589956-0.944937$

O $-1.395232-0.545976-0.807376$

C $0.6198860 .365692-1.734979$

C $0.1514941 .609255-1.915090$

C $0.8367072 .691671-2.620408$

C $2.0656472 .344010-3.418075$

C 4.2085240 .8753810 .640027

C 3.6684321 .9756261 .538498

C 1.6341253 .3995801 .826506

$\begin{array}{lllll}\text { O } & 4.315619 & 2.479696 & 2.451769\end{array}$

C 1.4444444 .5706450 .908723

C $2.2798844 .962632-0.066450$

C $2.0138916 .128693-0.977362$

C $2.1217725 .751385-2.439620$

C $3.2625535 .965158-3.117304$

C $0.8979915 .198981-3.139195$

C $0.3463543 .944805-2.518632$

N 2.3749572 .3298851 .184673

H -2.161143 -1.804760 1.847040

H $0.821319-1.3689252 .385675$

H $-0.808219-2.8316540 .159449$

H $0.861381-4.234444 \quad 1.538376$

H $2.132128-3.1663200 .993978$

H $2.331281-4.981757-2.279505$

H $0.430400-6.103831-1.611761$

H $0.225867-6.155298 \quad 0.141455$

H $-0.742158-5.033186-0.840341$

H $3.556661-2.933049-2.454273$

H $3.085119-2.374906-0.869708$

H $4.516158-4.1741840 .176063$

H $5.028033-4.687592-1.438561$

H $5.940090-2.383456-1.853063$

H $7.448531-4.179839-0.897236$

H $6.940947-3.737210 \quad 0.720579$

H $9.582165-3.6111550 .197247$

H 7.569509-1.295609 1.598990

H $9.281450-2.875018-1.988656$

H $9.619323-1.228297-1.508718$

H $8.081634-1.622300-2.306913$

H 8.3333810 .7229891 .112813

H $5.6343790 .069523 \quad 2.028212$

H 6.2625871 .3831981 .022958

H $7.2680660 .124037-1.507670$

H $1.5876120 .037919-2.096495$

H $-0.8103101 .872343-1.476330$

H $1.8531721 .530366-4.120652$

H $2.8792862 .032303-2.754198$

H $2.4380513 .177880-4.018050$

H $4.170314 \quad 1.238441-0.393493$

H $3.5421650 .009050 \quad 0.725531$

H 2.1217003 .7154872 .753731

H 0.6555122 .9821272 .078211

H 0.5284455 .1370041 .064115

H $3.2146824 .429542-0.221277$

H $2.7450076 .912357-0.739197$

H $1.0294536 .573969-0.787574$

H $3.3485175 .725240-4.171845$

H $4.1384196 .382072-2.631008$

H $1.0895215 .024200-4.205420$

H $0.1128995 .966242-3.115826$

H $-0.5450014 .110209-1.912565$

H 1.9320881 .8558180 .403543

$\begin{array}{llll}\text { C }-1.609318 & 0.827022 & 3.271520\end{array}$

O -2.6729801 .0457362 .328542$

C -3.2758242 .3264612 .592427$

C -2.481461 $2.953192 \quad 3.736205$

C $-1.938661 \quad 1.730748 \quad 4.454277$

H $-4.295322 \quad 2.1227352 .939952$

C $-3.3249793 .158226 \quad 1.302417$

H -1.6575093 .5761093 .370043$

H -3.1088523 .5818304 .375834$

C -0.7538702 .0329105 .354728$

H $-2.746432 \quad 1.2880205 .052041$

C $-3.928112 \quad 2.4158460 .091269$

O $-1.993985 \quad 3.5458090 .936333$

H -3.867715 4.0919991 .487572

H -3.235761 $1.627635-0.222063$ 
O $-3.9906713 .337694-1.004203$

C -5.3262501 .8203550 .328009$

C $-5.953964 \quad 1.176397-0.923010$

H -5.2797521 .0770271 .130436$

$\begin{array}{llll}\text { O } & -6.194931 & 2.864184 & 0.775382\end{array}$

H -6.961547 $0.836755-0.653143$

H $-6.1161151 .943982-1.690348$

H $-0.394731 \quad 1.123488 \quad 5.846341$

H 0.0804462 .4624294 .790022

H -1.036011 2.748408 6.134000

C -1.509678 $-0.658443 \quad 3.620849$

H -1.4756302 .7328510 .808438$

H $-4.628054 \quad 4.032600-0.765468$

H -7.0579042 .4626090 .974319$

H -2.441668 -1.0072254 .081823$

H $-0.706604-0.8274414 .347147$

H -0.6814811 .1554162 .785720$

C $-5.814271-1.7271310 .020416$

O $-4.746254-0.934155-0.504671$

C $-5.1374530 .006652-1.509178$

C $-5.818706-0.716988-2.680304$

C $-6.970506-1.606166-2.215571$

C $-6.515154-2.527007-1.083480$

H $-6.547394-1.0621440 .494809$

C $-5.260975-2.5992591 .156509$

H $-4.2088510 .436217-1.904046$

O $-6.3067320 .215201-3.639501$

H $-5.081498-1.349337-3.192206$

H $-7.840382-1.012082-1.911841$

O $-7.404869-2.421513-3.306708$

H $-5.851812-3.296472-1.494186$

O $-7.648315-3.193861-0.532233$

C $-4.180864-3.5778680 .741266$

H $-4.813607-1.9490071 .918233$

H -6.081133 -3.162284 1.616464

H $-5.5525330 .755620-3.931191$

H -7.651161 -1.823715 -4.033562

H $-8.091257-3.662808-1.260269$

O $-3.976817-4.4626661 .762853$

O $-3.566435-3.553306-0.315670$

C $-2.946006-5.4310281 .546306$

H $-1.970141-4.9377291 .537070$

H -2.970499 -6.144582 2.374151

H -3.115256 -5.974241 0.611434

SCF Energy (B3LYP/6-31G**/MMFF)= -3245.91043735

01459

MM̄FF Geometry

C -2.443124 -3.565627 0.770598

C $-1.975359-4.1524861 .882902$

C $-0.650395-3.8649062 .550581$

O $0.259304-3.2581281 .604133$

C $-0.869656-2.9042723 .734746$

C $0.364455-2.6404124 .575832$

C $0.841407-1.4160274 .877114$

C $1.041860-3.8577655 .153883$

C $0.273087-0.0717184 .515648$

C $1.1772190 .676553 \quad 3.536076$

C 0.7614892 .1418283 .330648

C -0.5786632 .2801132 .603651$

C -0.9194803 .7512042 .317118$

O 1.7993532 .7518632 .557233

O $\begin{array}{lllll}1.2 .009219 & 3.784057 & 1.382820\end{array}$

C $0.287395 \quad 4.4153831 .619578$

C $-1.416827 \quad 4.4717443 .575514$

$\begin{array}{lllll}\text { O } & 0.083903 & 5.816911 & 1.463488\end{array}$

C 1.6377764 .1562612 .325067

C 2.8514304 .6409191 .496829

O $1.729986 \quad 4.8872523 .555196$

C $1.378470-3.9557501 .267812$

O $1.751149-5.022377 \quad 1.731220$

C $2.083683-3.208730 \quad 0.201320$

C $3.336045-3.565658-0.123784$

C $4.167173-2.985028-1.181706$

C $3.508516-2.019986-2.130490$

C 3.0201434 .0472930 .095174

C 3.1686692 .5392070 .069495

$\begin{array}{llll}\text { C } 4.678260 & 0.690817 & 0.776397\end{array}$
O $2.3214201 .817630-0.452924$

C $5.5294130 .327300-0.398046$

C $6.693860-0.330668-0.299605$

C $7.531581-0.711995-1.485402$

C $7.660097-2.209573-1.652116$

C $8.799567-2.846346-1.333277$

C $6.498621-2.953236-2.276724$

C $5.460466-3.363712-1.265562$

$\begin{array}{lllll}N & 4.327462 & 2.095343 & 0.686217\end{array}$

H $-1.854435-2.801445 \quad 0.270427$

H -2.595792 -4.896331 2.379261

H $-0.260014-4.8241112 .909390$

H -1.642063 -3.313345 4.400139

H $-1.271787-1.9674503 .336518$

H $1.734458-1.3580485 .499190$

H $1.791953-3.5970865 .908409$

H $\quad 0.305811-4.5057075 .641374$

H $1.549729-4.4295954 .373172$

H $-0.739325-0.1499364 .114484$

H 0.1831150 .5069815 .443592

H 2.2141150 .6592703 .899571

H 1.2015310 .1569542 .568540

H 0.7247982 .6291954 .311750

H $-1.391984 \quad 1.813838 \quad 3.171378$

H -0.5322231 .7413451 .646785$

H -2.747576 3.2771261 .762285

H 0.3314213 .9939220 .609353

H -2.340905 4.0079853 .941842

H $-1.6709745 .515177 \quad 3.359745$

H -0.6956564 .4518534 .395392$

H -0.7653645 .9370591 .004945$

H 2.8126045 .7345941 .406322

H 3.7620314 .4334902 .075554

H 0.9769475 .4971973 .611402

H $1.550409-2.404803-0.291267$

H $3.806864-4.3705600 .440049$

H $2.699452-2.518693-2.674906$

H $4.187677-1.607967-2.879204$

H $3.092348-1.164082-1.588756$

H $2.1825684 .328794-0.551209$

H $3.9153634 .476675-0.372031$

H $3.765305 \quad 0.0865840 .775277$

H 5.1976210 .5430841 .728201

H $5.1552040 .594300-1.383830$

H $7.069236-0.6098700 .682015$

H $8.521648-0.256189-1.354357$

H $7.131944-0.280467-2.411761$

H $8.908018-3.915966-1.479479$

H $9.646560-2.313761-0.913796$

H $6.863561-3.872404-2.755129$

H $6.088772-2.359338-3.097940$

H $5.835393-4.079089-0.531667$

H $5.0102102 .774528 \quad 1.009031$

C $-4.819393-2.8264700 .455060$

O $-4.447302-1.579586-0.163362$

C $-5.628319-0.957722-0.704075$

C $-6.813210-1.779840-0.208312$

C $-6.201999-3.161960-0.101584$

H $-5.563168-1.057134-1.793812$

C $-5.680514 \quad 0.523754-0.313443$

H $-7.161802-1.4418800 .774501$

H $-7.662989-1.736684-0.896429$

C $-7.007455-4.1148920 .764568$

H $-6.108455-3.583175-1.111579$

C $-4.4350241 .339285-0.717139$

$\begin{array}{llll}0 & -5.820010 & 0.631599 & 1.109776\end{array}$

H $-6.5820650 .980958-0.737501$

H -3.583555 $1.028384-0.101412$

O $-4.6803992 .709486-0.374286$

C $-4.0543691 .247156-2.206816$

C $-2.979423 \quad 2.250962-2.659334$

H $-3.7098730 .233412-2.429047$

O $-5.214651 \quad 1.461491-3.015957$

H $-2.8268572 .107815-3.736099$

H $-3.376278 \quad 3.269926-2.567117$

H $-6.527559-5.0969440 .817155$

H -7.112817 -3.7362311 .786702$

H -8.012367 -4.2528090 .352069$ 
C $-3.772407-3.898007 \quad 0.152666$ H -5.061432 0.1761261 .514057 H $-5.4665523 .004613-0.863825$ H $-5.5140602 .375581-2.875345$ H -3.624765 -3.987017 -0.930196 H $-4.120082-4.8742040 .510180$ H $-4.874745-2.6423621 .536302$ C $-0.5954710 .414986-3.241409$ O $-1.0866390 .821337-1.958901$ C $-1.6401802 .138496-1.906671$ C $-0.592825 \quad 3.169212-2.345534$ C $-0.0287292 .845367-3.723314$ C $0.4607091 .400125-3.767215$ H $-1.4301430 .338940-3.948876$ C $0.031069-0.977475-3.069250$ H -1.848771 2.336220 -0.850581 O $-1.1387214 .483519-2.355162$ H $0.2356223 .171976-1.628592$ H $-0.759553 \quad 3.039070-4.516782$ O $1.0858193 .701170-3.987344$ H $1.3820481 .311924-3.176859$ O $0.7869561 .060072-5.114158$ C $-0.986766-2.083906-2.895459$ H $0.628851-1.229205-3.952711$ H $0.671434-0.986681-2.180941$ H $-1.4725494 .666748-1.460103$ H $0.7703404 .617983-3.909418$ H $1.4450591 .705610-5.424523$ O $-0.338292-3.283928-2.982458$ O $-2.181566-1.924134-2.694326$ C $-1.143444-4.461601-2.862898$ H $-0.628803-5.276019-3.379999$ H $-1.241299-4.724674-1.807174$ H $-2.128915-4.330449-3.320216$ SCF Energy (B3LYP/6-31G**/MMFF) $=-3245.92023152$

0146

MM̄FF Geometry

C -3.904432 $0.080151-2.496776$

C $-3.1576871 .106965-2.928718$

C $-2.6835692 .228632-2.046908$

O $-1.262762 \quad 2.349729-2.306528$

C $-3.3609113 .570206-2.374227$

C $-4.8132803 .654106-1.962979$

C $-5.228737 \quad 3.977645-0.722627$

C $-5.8168563 .422913-3.062218$

C $-4.378633 \quad 4.260048 \quad 0.489115$

C -4.9158403 .5620241 .747718$

C -4.6844672 .0405311 .754684$

C -5.6747861 .3712312 .711538$

C $-5.435969-0.1385992 .815033$

O -3.3377051 .7949092 .187609$

O $-6.198802-0.6332493 .925919$

C $-3.951746-0.3729413 .144943$

C $-5.937235-0.8864731 .575500$

O $-3.641020-1.7656203 .116726$

C -2.968911 0.4085212 .235485

C -1.550184 0.2919092 .844713

O $-2.974334-0.1785510 .935620$

C $-0.492108 \quad 2.945413-1.360550$

O $-0.856483 \quad 3.391386-0.285259$

C $0.8933842 .977069-1.876265$

C $1.8304743 .647511-1.191398$

C $3.2394923 .780560-1.558017$

C $3.7173093 .073628-2.798004$

C -0.4492701 .1158072 .163633$

C $0.8852490 .917107 \quad 2.869028$

C 3.2713061 .5226712 .783118

$\begin{array}{lllll}\text { O } & 1.026135 & 0.162399 & 3.829616\end{array}$

C 4.1185202 .6742882 .350513

C 5.2799102 .5202321 .695577

C 6.1995043 .6452991 .311805

C $6.3732263 .763297-0.186146$

C $7.3012083 .039092-0.833939$

C $5.5152464 .767072-0.922042$

C $4.0446684 .507271-0.757083$

N 1.9070391 .6790312 .313484

H $-4.2396700 .069025-1.464819$
H -2.836509 $1.129642-3.967775$

H $-2.8263521 .965096-0.992065$

H $-3.2616713 .786372-3.447178$

H $-2.807903 \quad 4.391020-1.899241$

H $-6.301517 \quad 4.062162-0.552943$

H $-6.8491783 .529611-2.712377$

H $-5.6669654 .147191-3.869806$

H $-5.7150122 .416076-3.476522$

H -3.3325413 .9853290 .337053$

H -4.4031095 .3428160 .662276$

H -4.4190844 .0060172 .619973$

H $-5.986697 \quad 3.7840861 .840886$

H -4.8136681 .6437920 .742295$

H $-6.709046 \quad 1.5708962 .403760$

H -5.5696331 .8113113 .713293$

H $-7.134380-0.4214733 .765306$

H -3.795668 -0.0438364 .182251$

H -7.015403 -0.7343691 .445308$

H $-5.797551-1.9680191 .680785$

H $-5.446446-0.5619680 .656749$

H $-4.264637-2.2137443 .713582$

H $-1.5922350 .610804 \quad 3.895274$

H $-1.240850-0.7605662 .840281$

H $-2.144945 \quad 0.043880 \quad 0.485114$

H $1.1011122 .458716-2.805359$

H $1.5402324 .151354-0.270034$

H $3.2461123 .502654-3.688965$

H $3.4723882 .007792-2.751582$

H $4.7987193 .134725-2.942269$

H $-0.3251170 .812808 \quad 1.119368$

H -0.6996292 .1812742 .197171$

H 3.6395240 .5694422 .393873

H 3.2639071 .4590173 .876642

H 3.7859873 .6722252 .624973

H 5.6166251 .5163351 .447644

H $7.173913 \quad 3.4602161 .782608$

H 5.8559204 .6021441 .724412

H $7.4468223 .132610-1.904846$

H $7.9334972 .332852-0.305850$

H $5.7824284 .820199-1.983751$

H $5.7322795 .769142-0.529161$

H $3.614617 \quad 4.976893 \quad 0.128187$

H 1.7501312 .1152221 .409284

C -3.753874 -2.425562 -2.879892

O $-2.321274-2.353341-2.719131$

C $-1.972083-2.978709-1.470591$

C $-3.178509-3.822585-1.080408$

C $-4.314119-2.936598-1.551744$

H $-1.847415-2.173348-0.735798$

C $-0.661575-3.765347-1.578812$

H $-3.190852-4.779152-1.616056$

H $-3.212233-4.030137-0.006602$

C $-5.643844-3.658678-1.676900$

H $-4.425951-2.116565-0.835066$

C $0.561773-2.854959-1.830791$

O $-0.796292-4.740547-2.609914$

H $-0.512960-4.304006-0.635956$

H $0.528603-2.029007-1.110973$

O $0.508953-2.285051-3.136670$

C $1.904631-3.591435-1.663177$

C $3.153474-2.721970-1.895267$

H $1.945977-4.021118-0.656745$

O $1.981793-4.693087-2.571170$

H $4.029399-3.365498-1.743897$

H $3.205279-2.429395-2.952093$

H $-6.423576-2.978558-2.034118$

H $-5.581002-4.498486-2.376913$

H $-5.959235-4.053699-0.705555$

C $-4.302625-1.079726-3.366627$

H $0.001996-5.294766-2.597021$

H $-0.329152-1.798341-3.213263$

H $1.896537-4.336105-3.472186$

H $-5.395835-1.120851-3.427934$

H $-3.934244-0.909718-4.386489$

H -3.933616 -3.170875 -3.666038

C $3.922209-2.5386331 .047227$

$\begin{array}{llll}\text { O } & 2.958441 & -1.730088 & 0.364147\end{array}$

C $3.258375-1.466018-1.007631$ 
C $4.604824-0.734766-1.114098$

C $5.720747-1.485178-0.389421$

C $5.298474-1.8629511 .029864$

H $3.983319-3.5246190 .570251$

C $3.421127-2.7355072 .482156$

H $2.488381-0.768221-1.359507$

O $4.982831-0.553171-2.474947$

H $4.5105160 .264037-0.669122$

H $6.035529-2.372855-0.950505$

O $6.872439-0.642100-0.301528$

H $5.287999-0.9654241 .658794$

O $\quad 6.264688-2.754556 \quad 1.583374$

C $2.212094-3.6401802 .515535$

H $4.186125-3.1984333 .115949$

H $3.154404-1.7819712 .952098$

H $4.242166-0.119553-2.931209$

H $7.103134-0.372473-1.207192$

H $7.128654-2.3101391 .538580$

O $1.075855-2.9114712 .322980$

O $2.276628-4.8518592 .678563$

C $-0.151497-3.6480242 .325166$

H $-0.054634-4.5910151 .778870$

H $-0.461323-3.8337883 .357165$

H $-0.913642-3.0399401 .830521$

SCF Energy (B3LYP/6-31G**//MMFF)= -3245.92515109

01460

MM̄FF Geometry

C -1.572255 -4.408039-0.095600

C $-1.216843-3.3085390 .583705$

C $0.076977-2.5717150 .374104$

O $-0.291942-1.268163-0.135989$

C $0.857864-2.3932901 .690211$

C $1.488750-3.6728522 .198258$

C $2.655136-4.1700111 .740568$

C $0.735598-4.382413 \quad 3.292205$

C $3.531868-3.5664770 .679533$

C $5.003970-3.5316931 .114107$

C $5.834808-2.6395080 .181402$

C $7.331365-2.8366290 .420489$

C $8.173259-1.879514-0.437760$

O $5.474422-1.2824970 .463450$

O $9.519573-1.9126150 .060100$

C $7.648408-0.442256-0.237046$

C $8.240585-2.333830-1.900450$

O $8.3105890 .469650-1.111113$

C $6.111676-0.308983-0.370266$

C 5.6724291 .0953570 .109596

O $5.745798-0.472183-1.741229$

C $0.597418-0.615833-0.929786$

O $1.713865-0.988226-1.250762$

C $-0.0372800 .656140-1.342382$

C $0.6900171 .596293-1.962998$

C $0.2133232 .912229-2.395102$

C -1.253199 $3.212198-2.221666$

C 4.1545921 .2714970 .259932

C 3.8003082 .6451260 .805570

C $1.8822213 .942121 \quad 1.722543$

O $4.612793 \quad 3.563176 \quad 0.884731$

C 1.1321334 .6505730 .640790

C $1.6204425 .697091-0.041835$

C $0.8659416 .430418-1.114432$

C $1.4765386 .240216-2.485844$

C $24781157.028282-2.911983$

C $0.8679255 .190370-3.390329$

C $1.1085863 .788540-2.898713$

N 2.4658482 .7294601 .180156

H $-0.894594-4.822761-0.837163$

H -1.906573 $-2.898917 \quad 1.317965$

H $0.690189-3.089217-0.374820$

H $0.201226-1.9692732 .462194$

H $1.628069-1.6217111 .565647$

H $3.016660-5.1083462 .158597$

H $1.255058-5.2826403 .637482$

H $\quad 0.613716-3.7214304 .156780$

H $-0.256252-4.6877632 .946672$

H $3.443394-4.171148-0.231244$

H $3.203940-2.5575560 .418576$
H $\quad 5.074247-3.1492502 .140948$

H $5.399590-4.5548701 .112501$

H $5.578310-2.877748-0.857715$

H $7.622816-3.8774830 .232005$

H $7.560696-2.6480911 .478734$

H $9.838179-2.8296290 .002373$

H $7.934044-0.1422320 .780676$

H $8.652518-3.347998-1.967730$

H $8.918998-1.698278-2.480438$

H $7.265949-2.339704-2.392685$

H $7.9276980 .359803-1.998181$

H 6.1235351 .2956731 .090626

H $6.0507991 .857494-0.583577$

H $4.886943-0.047042-1.891306$

H -1.086357 $0.790252-1.107419$

H $1.7418811 .394875-2.158096$

H - $1.5424613 .133184-1.168004$

H -1.857702 2.510397 -2.805872

H -1.537855 4.215976-2.542248

H $3.6588191 .162570-0.708616$

H 3.7510460 .5156970 .943243

H 2.6665184 .5736132 .153310

H 1.1981713 .6543862 .526767

H $0.130346 \quad 4.2910100 .423565$

H 2.6215046 .0565940 .186073

H $0.8606397 .497580-0.855663$

H -0.190232 $6.134124-1.132811$

H $2.9037196 .917384-3.903625$

H $2.8968607 .800122-2.274521$

H $1.2993645 .258554-4.397927$

H $-0.1936475 .414401-3.525974$

H $2.1522963 .479767-2.972132$

H 1.8526731 .9352251 .020582

C -3.928631-4.792234-0.967162

O $-4.318263-3.405093-0.886132$

C $-4.512371-2.907083-2.224120$

C $-4.490961-4.127760-3.142132$

C $-3.515854-5.034227-2.418099$

H $-3.642300-2.281138-2.454433$

C $-5.797856-2.078672-2.320073$

H $-5.475256-4.605644-3.208792$

H $-4.171276-3.875258-4.157886$

C $-3.594076-6.488375-2.847362$

H $-2.502777-4.659942-2.608719$

C $-5.866854-0.870712-1.362191$

O $-6.905837-2.938532-2.021595$

H -5.935364 -1.746064 -3.356457

H $-5.986128-1.217197-0.328840$

O $-7.057115-0.138020-1.685041$

C $-4.6697870 .097189-1.446090$

C $-4.9031701 .463905-0.770584$

H -3.784502 -0.383342 -1.014399

O $\quad-4.379550 \quad 0.349287-2.824795$

H $-4.0050222 .072049-0.931224$

H $-5.6952901 .999252-1.309522$

H $-2.880235-7.098522-2.285151$

H -4.594694 -6.902571 -2.684057

H $-3.359390-6.588127-3.912152$

C $-2.883850-5.113294 \quad 0.104751$

H -7.716229 -2.480573 -2.301111

H $-7.817155-0.677836-1.410218$

H $-3.5726270 .890182-2.860666$

H -2.694152 -6.192696 0.128435

H -3.294235 -4.845205 1.086921

H $-4.834117-5.369978-0.737883$

C $-3.0009571 .339407 \quad 1.624564$

$\begin{array}{lllll}\text { O } & -4.256851 & 0.669841 & 1.473747\end{array}$

C $-5.240498 \quad 1.395180 \quad 0.731496$

C -5.4929532 .7644101 .381112$

C -4.2010693 .5558821 .569315$

C -3.1592512 .7088602 .298877$

H -2.5637801 .4848180 .630326$

C -2.0419480 .3925052 .357254$

H -6.1740600 .8279040 .825051$

O -6.4044423 .5365690 .606226$

H -5.9536402 .6175582 .366915$

H -3.810298 3.9236890 .613110

O $-4.473141 \quad 4.7137552 .362811$ 
H -3.4657082 .6094863 .346562$

O -1.9194953 .4120142 .280327$

C $-2.434600 \quad 0.066232 \quad 3.782797$

H -2.012191 -0.563296 1.826051

H -1.0335590 .8211192 .357824$

H -7.2219973 .0178390 .514171$

H -5.1639175 .2221051 .904161$

H -1.3163672 .9652182 .897740$

O $-1.336989-0.4057124 .445795$

O $-3.560826 \quad 0.1705294 .248056$

C $-1.557506-0.7944805 .805099$

H -0.607789 -1.147593 6.215507

H $-1.898190 \quad 0.062530 \quad 6.393761$

H -2.285049-1.610418 5.852001

SCF Energy (B3LYP/6-31G**//MMFF) $=-3245.89961558$

$01 \_461$

MM̄MF Geometry

C -0.5623754 .8565562 .237313$

C -0.0594164 .3621331 .095586$

C 1.3947844 .0078250 .906795

O 1.4809282 .6074250 .552047

C $1.9775474 .832705-0.255374$

C $3.4606644 .614328-0.481724$

C $3.9949884 .091311-1.602874$

C 4.3663395 .0570490 .638992

C $3.2825593 .620964-2.840019$

C $2.9184362 .127826-2.843829$

C $4.1301721 .183592-2.775719$

C $3.702233-0.265279-3.014492$

C $4.877806-1.244291-2.863314$

O $4.715727 \quad 1.293098-1.477026$

O $4.333774-2.571282-2.779989$

C $5.604997-0.968242-1.527582$

C $5.791269-1.228194-4.095332$

O $6.792857-1.753322-1.455064$

C $5.9150620 .527369-1.296032$

C 6.4352190 .8444800 .127818

O $\quad 6.932931 \quad 0.942064-2.215208$

C 1.3674221 .7004831 .560593

O 1.2212001 .9353432 .749529

C 1.4307430 .3500880 .959721

C $1.027563-0.7093521 .676929$

C $1.026373-2.1047531 .232059$

C $1.717944-2.418355-0.068139$

C $5.4922280 .459501 \quad 1.273125$

C $5.785796-0.9010541 .882207$

C $4.879289-2.5187413 .545910$

O $6.788925-1.5572871 .615149$

C $4.140104-3.5930212 .812736$

C $3.205665-4.3651473 .387658$

C $2.486943-5.4778652 .680480$

C $0.983286-5.3557332 .781684$

C $0.294606-6.0438093 .708005$

C $0.272721-4.5050241 .754054$

C $0.428831-3.0309392 .011041$

N 4.808514 -1.281008 2.792420

H 0.1209735 .0230153 .068465

H $-0.708166 \quad 4.1774710 .244330$

H $1.968221 \quad 4.188596 \quad 1.823987$

H $1.8189885 .902955-0.066463$

H $1.4135394 .599840-1.165203$

H $5.0789293 .990583-1.651581$

H 5.4242085 .0207600 .357036

H 4.1410546 .0897100 .924761

H 4.2396634 .4143761 .515461

H $2.3747024 .209091-3.011379$

H $3.9259673 .835709-3.702942$

H $2.2442441 .925472-2.003228$

H $2.3567231 .924764-3.764086$

H $4.8562011 .498212-3.534513$

H $3.235952-0.376596-4.001426$

H $2.927188-0.539400-2.286188$

H $3.815361-2.734074-3.586648$

H $4.940895-1.328815-0.733609$

H $5.224617-1.495167-4.995544$

H $6.584695-1.978819-4.009442$

H $6.253194-0.255022-4.273675$
H $7.151821-1.670044-0.556056$

H 7.4387430 .4259460 .268746

H 6.5692391 .9347530 .185648

H $7.3550441 .744874-1.866892$

H $1.7947810 .267048-0.057515$

H $0.646018-0.5411912 .683377$

H $2.744064-2.036041-0.055578$

H $1.183719-1.962699-0.908642$

H $1.797339-3.487066-0.275852$

H 5.6176751 .1884892 .084164

H 4.4462950 .5014440 .954818

H $4.452332-2.3220994 .534439$

H $5.927127-2.8109623 .669317$

H $4.404381-3.7618281 .771430$

H $2.964936-4.2125164 .437450$

H $2.814468-6.4243003 .130338$

H $2.778330-5.5372841 .624350$

H $-0.787026-5.9842993 .769299$

H $0.798464-6.6783124 .429790$

H $\quad-0.803477-4.7221301 .770349$

H $\quad 0.601115-4.8038750 .754979$

H - $0.039716-2.7012492 .939507$

H $3.982903-0.7006392 .909005$

C $-3.017145 \quad 4.9541291 .415563$

O -3.1072083 .5432601 .144459$

C $-4.467216 \quad 3.2138210 .813698$

C $-5.239797 \quad 4.529510 \quad 0.801597$

C -4.4407055 .3736251 .775450$

H -4.8424172 .5808831 .627481$

C $-4.5331282 .434879-0.508614$

H $-5.2395354 .990188-0.193214$

H -6.2833064 .3968681 .103963$

C $-4.705643 \quad 6.863144 \quad 1.642825$

H -4.681108 5.0518952 .797575

C $-3.7696351 .091918-0.466247$

O $-4.0281293 .251716-1.564656$

H $-5.5867342 .236687-0.733416$

H $-4.060470 \quad 0.5559510 .444451$

O $-2.3618661 .317044-0.399878$

C $-4.0674120 .189416-1.680952$

C $-3.276903-1.131478-1.712727$

H $-5.140177-0.030860-1.699264$

O $-3.7812720 .878229-2.900197$

H $-3.597663-1.683216-2.605468$

H -2.214052 -0.917609 -1.886540

H -4.1085097 .4326512 .361564$

H -4.4613947 .2264300 .639027$

H -5.7612257 .0840021 .832542$

C -2.0003125 .2023422 .532502$

H -3.088105 $3.418836-1.378200$

H $-2.182421 \quad 1.8276090 .407770$

H -2.853739 $1.168676-2.861888$

H -2.2911134 .6168153 .414159$

H -2.029459 6.2593692 .821463

H -2.699295 5.4494940 .488639

C $-5.609547-2.936628-0.915044$

O $-4.788332-2.150243-0.044675$

C $-3.427336-2.013557-0.459291$

C $-2.771999-3.396164-0.578600$

C $-3.585845-4.344602-1.457056$

C $-5.053277-4.360310-1.028184$

H -5.650850 -2.470007 -1.907107

C $-7.025080-2.940143-0.328623$

H -2.910713 -1.489909 0.354664

O $-1.451994-3.270524-1.094233$

H $-2.687873-3.8301940 .425585$

H -3.492470 -4.075657 -2.515331

O $-3.049083-5.665255-1.345807$

H $-5.151370-4.881331-0.067350$

O $-5.813078-5.087638-1.990637$

C $-7.690103-1.598335-0.525104$

H -7.663655 -3.686228 -0.815240

H -7.017975 -3.175855 0.742423

H -1.079689-4.166118 -1.165341

H $-3.113077-5.938737-0.414749$

H $-5.417585-5.973118-2.064535$

O $-7.385009-0.7669160 .511486$

O $-8.381665-1.319424-1.496114$ 
C -7.9449370 .5477880 .435350$

H $-7.7354111 .005037-0.536399$

H -9.0229640 .5012710 .613882$

H -7.483249 1.1596691 .214933

SCF Energy (B3LYP/6-31G**//MMFF) $=-3245.91587601$

014462

MM̄FF Geometry

C $2.4418844 .670651-2.312280$

C $1.7100674 .172413-1.302802$

C $0.2067444 .225774-1.213800$

O $-0.2671262 .880128-0.961401$

C $-0.2007585 .105300-0.017792$

C -1.696108 $5.172690 \quad 0.219760$

C -2.3091594 .7367341 .338337$

C $-2.5076925 .796763-0.885548$

C -1.680308 4.1192252 .557414

C -1.5628962 .5874062 .514905$

C -2.9145571 .8547942 .500846$

C -2.7140820 .3461802 .649301$

C $-4.040551-0.4238462 .552898$

O $-3.5646002 .123076 \quad 1.258006$

O $-3.725698-1.8141272 .373752$

C $-4.808591 \quad 0.0343411 .292074$

C $-4.847240-0.3273553 .853735$

O $-6.111557-0.544068 \quad 1.284398$

C $-4.8833651 .570347 \quad 1.143374$

C $-5.4439462 .038212-0.222168$

O $\quad-5.7509542 .0966082 .155075$

C $-0.2841772 .012482-2.009319$

O $-0.0072902 .257391-3.172493$

C $-0.664140 \quad 0.683699-1.480383$

C $-0.453679-0.408709-2.229674$

C $-0.758447-1.790330-1.850362$

C -1.510009-2.007135-0.564252

C $-4.6587681 .565956-1.451091$

C $-5.2234240 .313239-2.099504$

C $-4.737368-1.313410-3.923055$

O $-6.301885-0.182082-1.784359$

C $-4.188382-2.557708-3.300356$

C $-3.439814-3.446957-3.970261$

C $-2.928896-4.727340-3.375463$

C $-1.434816-4.890776-3.537484$

C $-0.924437-5.632591-4.535127$

C $-0.539028-4.270008-2.490470$

C $-0.369908-2.786913-2.673333$

N $-4.393536-0.166197-3.104311$

H $\quad 1.9278215 .189732-3.118664$

H $2.1964633 .655279-0.480586$

H $-0.2398044 .605887-2.140429$

H $0.1696426 .128131-0.169061$

H 0.3083554 .7318280 .877565

H $-3.3920404 .839417 \quad 1.400255$

H -3.555525 $5.941106-0.600749$

H -2.101932 6.779831-1.146246

H $-2.4954185 .163862-1.778172$

H -0.6880364 .5476082 .735700$

H -2.275249 4.4117853 .432159

H -0.9752772 .2986861 .635327$

H $-0.995301 \quad 2.2711363 .399019$

H -3.5243522 .2426973 .325330$

H -2.204747 0.1128383 .591989

H -2.048003 -0.0117601 .852896$

H -3.179470 -2.097558 3.126914

H $-4.273803-0.3871280 .433632$

H $-4.266849-0.7259764 .694567$

H -5.755300 -0.9382013 .802418$

H $-5.132350 \quad 0.6961564 .105929$

H $-6.515718-0.3586570 .420409$

H $-6.5098151 .793540-0.300597$

H $-5.4021043 .137305-0.227020$

H -6.0608252 .9725011 .870827$

H - $-1.0861140 .640120-0.483056$

H $\quad 0.000375-0.284960-3.212178$

H -1.812612 -3.043464 -0.402191

H -2.433216 $-1.419257-0.559998$

H $-0.899215-1.7085330 .293859$

H $-4.7088942 .351985-2.215821$
H $-3.6024911 .411084-1.211158$

H $-4.328029-1.133988-4.922253$

H $-5.826801-1.390479-4.000528$

H $-4.444293-2.752337-2.261469$

H -3.210587 -3.264452 -5.017856

H $-3.449627-5.555937-3.873328$

H $-3.192254-4.810251-2.313570$

H $0.145374-5.779452-4.640776$

H $-1.565709-6.105007-5.271890$

H $0.461624-4.718024-2.546543$

H $-0.907553-4.539574-1.497188$

H $\quad 0.164610-2.523925-3.587459$

H $-3.4903180 .272890-3.257456$

C $4.6242973 .559021-1.535641$

O $4.1547292 .232763-1.838694$

C $5.1585661 .285548-1.405730$

C $6.3547602 .116598-0.948249$

C $6.1357373 .438593-1.669943$

H $5.4163200 .693494-2.291928$

C $4.5026720 .403755-0.335353$

H $\quad 6.3362192 .269200 \quad 0.137826$

H $7.3083621 .643154-1.201201$

C $6.9143304 .593275-1.066436$

H $6.4143043 .317743-2.725225$

C $5.454637-0.513246 \quad 0.436205$

O $3.522862-0.407975-0.994995$

H $3.9656391 .047607 \quad 0.369595$

H $6.1659150 .092277 \quad 1.009859$

O $6.232665-1.249170-0.517142$

C $4.774632-1.5309141 .377582$

C $3.811027-0.9471132 .431415$

H $4.290555-2.3170460 .791759$

O $5.829447-2.1980232 .093858$

H $3.850606-1.5916623 .318701$

H 4.1904490 .0245182 .772062

H $6.7210015 .522150-1.611897$

H $6.6439114 .755792-0.017768$

H $7.9903084 .395227-1.109503$

C $3.9425094 .557450-2.471618$

H $2.9104250 .191605-1.455175$

H $6.830410-1.830927-0.016736$

H $6.271136-1.5372392 .654590$

H $4.1307404 .252758-3.509037$

H $4.3740955 .555711-2.339985$

H $4.3705573 .780960-0.490356$

C $1.690087-3.1200612 .198743$

O $1.849410-1.9870001 .338023$

C $2.338030-0.8091341 .983607$

C $1.368591-0.3911303 .099432$

C $1.070831-1.5375704 .063620$

C $0.672410-2.8005053 .298758$

H $2.656220-3.3862542 .645237$

C $1.233099-4.2983481 .333348$

H $2.294168-0.014791 \quad 1.234695$

O $1.879858 \quad 0.717743 \quad 3.830726$

H $0.421554-0.0688702 .648261$

H $1.921567-1.7349464 .726480$

O $-0.018335-1.1671404 .911963$

H $-0.326673-2.6664172 .863995$

O $0.602245-3.895804 \quad 4.208398$

C $2.359234-4.7774850 .447651$

H $\quad 0.920028-5.1512551 .945976$

H $\quad 0.380940-4.0302940 .699759$

H $2.0312601 .439983 \quad 3.197320$

H $\quad 0.231781-0.3402635 .358804$

H $-0.029190-3.6526134 .907039$

O $2.430942-4.007674-0.675219$

O $3.100580-5.708757 \quad 0.733666$

C $3.486039-4.334459-1.584235$

H $3.347666-5.347224-1.974102$

H $3.446175-3.628209-2.417727$

H $4.457489-4.238831-1.089853$

SCF Energy (B3LYP/6-31G**//MMFF) $=-3245.90857886$

01463

MM̄MF Geometry

C 3.1249870 .6094382 .961687

C 2.6310950 .2684204 .162666 
C $1.251815-0.2964784 .427370$ O $0.380616-0.087958 \quad 3.291253$ C $1.336037-1.8060404 .706717$ C $0.060155-2.3689995 .302261$ C $-0.872638-3.0683084 .625925$ C $-0.113329-2.1251106 .780771$ C $-0.897741-3.4264053 .165188$ C $-2.058748-2.7258862 .458664$ C $-2.279667-3.1647361 .003257$ C $-1.057745-2.9460630 .110377$ C $-1.354276-3.260845-1.363383$ O $-3.377181-2.3801430 .520474$ O $-0.268298-2.721299-2.130320$ C $-2.641843-2.527976-1.789173$ C $-1.356790-4.771813-1.621752$ O $-3.026639-2.903750-3.109963$ C $-3.817373-2.708148-0.802666$ C $-4.959181-1.732066-1.168676$ O $-4.299431-4.049494-0.883004$ C $-0.189628 \quad 1.1440553 .168748$ O -0.1163002 .0708543 .959961$ C -0.8790851 .1924501 .861062$ C -1.2618782 .3759381 .359155$ C -1.904999 2.5932300 .061262 C $-2.3655551 .377910-0.697031$ C $-6.120626-1.730129-0.166580$ C $-6.890837-0.426917-0.226899$ C $-6.743412 \quad 1.968426 \quad 0.454735$ O $-7.979394-0.343982-0.790261$ C $-6.1950672 .750972-0.696851$ C $-5.5989223 .946289-0.567265$ C $-5.1264524 .762583-1.735571$ C $-3.7241145 .297384-1.562500$ C $-3.5042886 .609606-1.370422$ C $-2.5709144 .331294-1.712969$ C $-2.0354243 .858535-0.388851$ $\begin{array}{llll}\mathrm{N} & -6.225411 & 0.614966 & 0.403497\end{array}$ H 2.5369610 .4322042 .065298 H 3.2401560 .4434735 .047179 H $\quad 0.833570 \quad 0.221817 \quad 5.299189$ H $2.156494-2.0166145 .406152$ H $1.597321-2.3358843 .786177$ H -1.736551 -3.432118 5.181963 H -1.024312 -2.587424 7.175813 H $\quad 0.733090-2.5426397 .335795$ H $-0.172751-1.0529806 .991197$ H $0.042333-3.1710192 .674166$ H -1.012516 -4.513454 3.080079 H -2.992505 -2.903572 3.010721 H -1.915192 -1.639331 2.495031 H $-2.570390-4.2218851 .006063$ H - $0.207984-3.5480700 .449429$ H $-0.735260-1.8987840 .184360$ H - $0.475727-2.855875-3.071069$ H -2.394033 -1.460002 -1.843650 H $-0.387500-5.206421-1.349091$ H -1.491856 -4.993141 -2.686229 H -2.129313 -5.299435 -1.058277 H $-3.443437-3.780892-3.058403$ H -4.546720 -0.719106 -1.242458 H -5.365258 -1.979694 -2.157760 H $-5.211923-4.075136-0.553799$ H $-1.0141130 .260436 \quad 1.326266$ H -1.055997 3.2771221 .935399 H $-2.9820940 .738340-0.055977$ H - $-1.5066290 .795928-1.047755$ H -2.979927 $1.613791-1.568324$ H $-6.813403-2.552692-0.377408$ H -5.774795 -1.8667940 .864303$ H -6.4523932 .3932231 .420544$ H $-7.836244 \quad 1.9422070 .396429$ H $-6.3324512 .331892-1.691129$ H -5.4940994 .3848380 .422463$ H -5.831822 $5.594544-1.860782$ H $-5.1757514 .187578-2.668848$ H -2.497659 $7.004689-1.280705$ H -4.323818 $7.316308-1.294701$ H -1.746686 $4.835636-2.236293$
H -2.859352 $3.512991-2.378168$

H $-1.6569194 .666940 \quad 0.238790$

H -5.2995570 .4506930 .788024$

C 4.3442552 .6233022 .023653

O 3.9320182 .4085590 .657988

C 2.9024413 .3611930 .333651

C 2.9954034 .4508641 .396190

C 3.3656943 .6384412 .621388

H 1.9474082 .8347830 .448186

C $3.0473783 .856228-1.109964$

H 3.7875135 .1744061 .172477

H 2.0558094 .9998761 .511256

C 3.9535294 .4625223 .753147

H 2.4555173 .1464342 .983247

C $3.1858602 .742982-2.168065$

O $4.2180944 .679127-1.213039$

H $2.2041824 .510572-1.359543$

H $4.1764602 .283499-2.074238$

O $3.1515463 .361874-3.460628$

C $2.1046601 .648453-2.128921$

C $2.1964540 .628084-3.280721$

H $2.1516541 .108833-1.176931$

O $0.8051972 .238088-2.217068$

H $1.412136-0.122219-3.117576$

H $1.9187741 .117112-4.223658$

H 4.2149093 .8237914 .602766

H 4.8584714 .9917593 .436899

H 3.2319935 .2093214 .100255

C 4.4550181 .2804632 .758570

H $4.9786924 .145024-0.925698$

H $3.8482854 .040306-3.475946$

H $0.6629932 .773422-1.419549$

H $4.956148 \quad 1.437508 \quad 3.720901$

H 5.0823540 .5999202 .170300

H 5.3489553 .0612321 .962492

C $3.389895-1.665488-1.648753$

O $4.083867-0.537801-2.194312$

C $3.568015-0.058038-3.437140$

C $3.607070-1.171712-4.492806$

C $2.919612-2.446450-4.006061$

C $3.443220-2.849196-2.626013$

H $2.345943-1.389833-1.456583$

C $4.061794-1.976495-0.306398$

H $4.2665750 .715869-3.778449$

O $3.006570-0.714100-5.699597$

H $4.655762-1.403153-4.721463$

H $1.829736-2.334200-3.994741$

O $3.205064-3.512580-4.915181$

H $4.472366-3.215507-2.733813$

O $2.647260-3.931206-2.150573$

C $3.136170-2.6932570 .642876$

H $4.984689-2.550123-0.453874$

H $4.373761-1.0517030 .195322$

H $3.157148-1.391237-6.380190$

H $2.764065-3.306539-5.756520$

H $3.115274-4.335111-1.400760$

O $3.606724-3.9467240 .897088$

O $2.110597-2.2004841 .093229$

C $2.800802-4.7447881 .769474$

H $1.794621-4.8635421 .357025$

H $3.263486-5.7319161 .850649$

H $2.761750-4.2931982 .764714$

SCF Energy (B3LYP/6-31G**//MMFF) $=-3245.91597759$

01464

MM̄FF Geometry

C -1.724417 4.7395240 .225105

C $-2.7269374 .820456-0.663360$

C $-3.7401923 .734031-0.959355$

O $-3.3898252 .491303-0.308366$

C $-5.1278874 .160397-0.447534$

C $-6.2335183 .186717-0.809635$

C -6.9528112 .4763410 .081589$

C $-6.5313153 .054758-2.281525$

C -6.8464012 .4876291 .580474$

C -5.9171221 .4149182 .168910$

C $-6.363061-0.0299311 .889494$

C $-5.544243-1.0173472 .724712$ 
C $-5.904204-2.4775342 .407457$

O $-6.167388-0.307680 \quad 0.500029$

O $-4.885770-3.3156392 .975573$

C $-5.859881-2.6880830 .878421$

C -7.224824 -2.8945883 .065512$

O $-6.350144-3.9797190 .528402$

C $-6.620056-1.6048530 .086060$

C $-6.401730-1.703625-1.444090$

O $-8.026709-1.753693 \quad 0.316794$

C $-2.4773401 .693336-0.926968$

O $-1.948528 \quad 1.879696-2.010944$

C $-2.1888500 .564596-0.015350$

C $-1.061033-0.140949-0.181817$

C $-0.608389-1.2563820 .651558$

C - $-1.575664-1.8074251 .664354$

C $-4.946819-1.585523-1.911808$

C $-4.253965-2.921139-2.130906$

C $-2.025654-3.875028-2.717222$

O $-4.840499-3.999364-2.100873$

C -1.310485-4.313893-1.478455

C $0.023433-4.440680-1.405767$

C $0.757024-4.926171-0.188208$

C $1.802548-3.9445570 .291754$

C $3.099047-4.098384-0.026831$

C $1.350886-2.8287631 .206712$

C $0.640373-1.7306150 .463870$

N -2.901946 -2.760933 -2.405033

H -1.615109 3.8463770 .834413

H -2.824933 $5.727105-1.256946$

H -3.770388 $3.581380-2.044959$

H $-5.3931045 .143741-0.858311$

H -5.0713994 .2921940 .638339$

H $-7.7240431 .809043-0.302004$

H $-7.4301542 .458197-2.471536$

H $-6.6961284 .041772-2.726120$

H $-5.7007442 .569166-2.802493$

H -6.5240443 .4712521 .938292$

H -7.8552892 .3541331 .991896$

H -4.9038291 .5716091 .781739$

H -5.8760021 .5736243 .253792$

H -7.428365 -0.1157962 .134133$

H -5.668109 $-0.817773 \quad 3.796475$

H -4.475946 -0.8703062 .515900$

H -4.850532 -3.1386003 .931275$

H -4.803401 -2.6655260 .586508$

H $-7.160498-2.7861124 .154836$

H -7.440249 -3.953345 2.883342

H -8.078569 -2.3046782 .725849$

H -5.828941 -4.6323621 .026558$

H -6.880643 -2.609102 -1.835607

H -6.948297 -0.866265 -1.901969

H -8.505564 -1.321977 -0.410065

H -2.8925330 .3680060 .784746$

H $-0.387700 \quad 0.141052-0.990183$

H -1.223818 -2.716391 2.156079

H $-2.522843-2.071671 \quad 1.182409$

H -1.773520 -1.069600 2.449166

H -4.936816 -1.075738-2.883901

H -4.358975 -0.978276 -1.218112

H -1.334842 -3.534705 -3.495109

H $-2.616425-4.706039-3.115694$

H -1.918528 -4.565763 -0.612843

H $0.626194-4.211730-2.282002$

H $1.232195-5.882033-0.444965$

H $0.065469-5.1525010 .632995$

H $3.856405-3.4098320 .333673$

H $3.430847-4.912689-0.662303$

H $2.220082-2.3735301 .700195$

H $\quad 0.756075-3.2545662 .018967$

H $1.258640-1.260988-0.301034$

H $-2.492286-1.832146-2.365207$

C 0.7184505 .3945220 .040264

O 1.0901374 .1632210 .696460

C $1.5696483 .242147-0.307236$

C $2.0130184 .119191-1.465107$

C $0.9197725 .170610-1.461477$

H $\quad 0.7012592 .637048-0.597893$

C 2.6517832 .3447240 .300559
H $2.9889954 .574993-1.257976$

H $2.0759613 .576285-2.412572$

C $1.2824366 .432080-2.225624$

H $0.0212724 .734056-1.914828$

C $3.3730371 .448518-0.722659$

O 2.0207251 .5114921 .279686

H 3.3814092 .9606710 .838177

H $3.9953422 .069941-1.377718$

O $2.3860880 .841552-1.566894$

C $4.2238400 .315045-0.113064$

C 5.3057370 .8018850 .866618

H $3.569455-0.4075070 .386097$

O $4.838006-0.392434-1.193290$

H 6.0319921 .4117920 .318467

H $4.844691 \quad 1.4449481 .622093$

H $0.4701307 .164101-2.173931$

H $2.1861096 .900842-1.822038$

H $1.4658946 .203279-3.280517$

C -0.6975015 .8130470 .457065$

H 1.5647292 .0964861 .908813

H $2.8558520 .274440-2.202232$

H $5.4191600 .228392-1.664880$

H $-0.9751816 .741687-0.054752$

H -0.6993596 .0291321 .532775$

H 1.4220096 .1545540 .403393

C $7.860846-0.7256960 .063899$

O $6.694851-1.2566690 .703769$

C $6.026878-0.3596291 .594372$

C 6.9938940 .0912332 .699732

C 8.2733630 .6892532 .119424

C $8.891317-0.2693701 .102286$

H $7.5785200 .117417-0.577619$

C $8.437795-1.831599-0.827278$

H $5.238757-0.9482212 .082254$

O $6.352503 \quad 1.0279653 .557849$

H $7.257963-0.7797883 .314072$

H $8.092011 \quad 1.6732631 .671174$

O 9.2235530 .8894003 .167950

H $9.309334-1.1383071 .626839$

O $9.968374 \quad 0.3858730 .435463$

C $7.540685-2.085270-2.015364$

H $9.424075-1.565071-1.223501$

H $8.560786-2.765819-0.265765$

H $6.9417531 .187876 \quad 4.314026$

H 8.8750861 .5849593 .750662

H $10.594912 \quad 0.681849 \quad 1.117957$

O $6.790552-3.204474-1.806697$

O $7.495036-1.358129-2.999530$

C $5.882544-3.542442-2.859817$

H $6.423139-3.678835-3.801435$

H $5.393946-4.484718-2.598139$

H $5.118253-2.766327-2.961508$

SCF Energy (B3LYP/6-31G**//MMFF) $=-3245.90955509$

01465

MM̄FF Geometry

C $1.879956-3.4394291 .049824$

C $0.809086-4.1797361 .376858$

C $-0.645821-3.8053261 .201591$

O $-0.781577-2.4791760 .644246$

C $-1.336792-4.7999410 .248225$

C $-2.853151-4.7554240 .305671$

C $-3.657471-4.469648-0.736610$

C $-3.471395-5.1118401 .635039$

C $-3.271761-4.136253-2.148799$

C $-3.149282-2.633061-2.437195$

C $-4.443781-1.831947-2.214775$

C $-4.295154-0.413221-2.768525$

C $-5.5208140 .461417-2.464601$

O $-4.699436-1.771260-0.809327$

O $\quad-5.158159 \quad 1.828024-2.718939$

C $-5.8587630 .356309-0.960478$

C $-6.6908350 .144104-3.403905$

O $-7.0810091 .039759-0.694439$

C $-5.914794-1.100047-0.448140$

C $-6.058169-1.2190771 .090820$

O -7.053474 -1.753939-1.022006

C $-0.922016-1.4409351 .508717$ 
O $-1.008119-1.5109682 .727473$

C $-0.959252-0.139767 \quad 0.805512$

C $-0.7404550 .017800-0.508102$

C $-0.7631801 .300362-1.204907$

C $0.331841 \quad 1.456640-2.222805$

C $-4.926577-0.5906921 .909804$

C -5.1741090 .8584292 .292880$

C -3.9512342 .8988183 .045153$

O -6.2861601 .3788232 .276409$

C -3.8328213 .7136591 .795903$

C $-2.7315504 .393941 \quad 1.443708$

C $-2.634677 \quad 5.2191130 .193024$

C $-1.5748594 .719951-0.764153$

C $-0.4029215 .364128-0.890851$

C $-1.9131613 .543523-1.653388$

C $-1.7241612 .216533-0.970260$

N -4.0055531 .4938682 .687659$

H $1.751091-2.4613130 .595387$

H $\quad 0.978026-5.1471751 .847436$

H -1.111906 -3.849582 2.192340

H -1.035242 -5.8272060 .493740$

H $-0.979715-4.611003-0.769852$

H -4.733979 -4.484282 -0.566912

H $-4.553033-5.2691121 .561241$

H -3.035616 -6.0387742 .021945$

H -3.307392 -4.3137392 .365240$

H -2.332331 -4.628814 -2.421411

H $-4.026187-4.570111-2.817465$

H -2.352444 -2.223389-1.806673

H -2.828944 -2.519690 -3.480344

H $-5.267698-2.353786-2.715495$

H $-4.098227-0.435213-3.847785$

H $-3.4146820 .059849-2.316196$

H $-4.8673601 .894155-3.644584$

H $-5.0681990 .900233-0.428703$

H $-6.4010470 .316460-4.447593$

H -7.540542 $0.809793-3.216888$

H -7.036103 $-0.888926-3.325785$

H $-7.191772 \quad 1.090597 \quad 0.269386$

H -7.040843 -0.8526431 .410805$

H $-6.058873-2.2921121 .332429$

H -7.275407 -2.525062 -0.474351

H $-1.1590010 .717722 \quad 1.441628$

H $-0.495625-0.835632-1.135222$

H $\quad 0.067170 \quad 0.933187-3.147689$

H $1.271457 \quad 1.033002-1.852217$

H $0.5389252 .502156-2.465004$

H -4.832549 -1.139933 2.855319

H -3.970904 -0.681593 1.384524

H -3.094495 3.0357153 .712378

H -4.8626643 .1746013 .584956$

H -4.707629 3.7496341 .150193

H -1.856621 4.367354 2.089035

H $-2.417735 \quad 6.252446 \quad 0.493927$

H -3.597988 $5.265125-0.330407$

H $0.3443315 .038384-1.605842$

H $-0.1634666 .229883-0.282461$

H -1.336522 $3.594176-2.583936$

H -2.958963 $3.622826-1.976337$

H -2.496546 $1.977592-0.240705$

H -3.1250670 .9920752 .626157$

C $4.143463-3.8921130 .050649$

O $4.394300-2.539258-0.386538$

C $5.796063-2.398378-0.680175$

C $6.345745-3.815151-0.779320$

C $5.518549-4.5248860 .271498$

H $6.251978-1.9031580 .185268$

C $6.005518-1.552592-1.939160$

H $6.162437-4.254997-1.766643$

H $7.421364-3.858798-0.583328$

C $5.531734-6.0377150 .134233$

H $5.902953-4.252324 \quad 1.263791$

C $5.382298-0.143023-1.886399$

O $5.431704-2.232738-3.064040$

H $7.077839-1.475415-2.153741$

H $4.289541-0.218176-1.932326$

O $5.7892590 .547753-3.075576$

C $5.7917330 .703940-0.666878$
C $5.4233582 .196108-0.771156$

H $5.344576 \quad 0.2778920 .236342$

O $7.2118210 .623635-0.507122$

H 5.7809342 .6922740 .139300

H $6.0082252 .649954-1.581379$

H $4.910763-6.505750 \quad 0.904330$

H $5.152706-6.355228-0.843001$

H $6.550541-6.4245090 .241227$

C $3.294205-3.8735211 .322005$

H $4.489940-2.378531-2.868682$

H $5.5148060 .004716-3.834349$

H 7.4436301 .1060830 .304710

H $3.724746-3.1691492 .044282$

H $3.300617-4.8652641 .788737$

H $3.610590-4.397976-0.764884$

C 3.2181822 .5293161 .333440

O 3.1087091 .9058920 .048812

C $3.9230432 .477884-0.979371$

C $3.5844553 .963877-1.157904$

C 3.6990924 .7283630 .156530

C 2.8919084 .0277461 .246529

H 4.2309032 .3824691 .728158

C $2.224577 \quad 1.8321532 .277818$

H $3.6315041 .983166-1.912781$

O $4.4334424 .540828-2.143872$

H $2.5563824 .047584-1.530476$

H 4.7443484 .8551920 .461863

O $3.163866 \quad 6.043346-0.009417$

H 1.8207494 .1659951 .051001

O 3.1765094 .6424672 .502381

C 2.6605220 .4376982 .673333

H 2.1255862 .4138763 .201643

H 1.2418651 .7473971 .802297

H $4.1068515 .437747-2.326802$

H $3.7471726 .522236-0.621955$

H 2.9717825 .5893262 .414692

O 1.9620050 .0588413 .784904

O $3.486407-0.2401562 .079250$

C $2.273964-1.2333344 .315012$

H $3.355769-1.3902914 .365554$

H $1.867643-1.2904685 .328395$

H $1.799740-2.0043353 .703809$

SCF Energy (B3LYP/6-31G**//MMFF) = -3245.90955177

01466

MM̄FF Geometry

C -3.629652 -1.132537 -3.500983

C $-2.608903-1.724415-2.864619$

C $-1.170996-1.582032-3.284110$

O $-0.402350-1.064364-2.172799$

C $-0.518838-2.889511-3.775760$

C $-0.468382-4.046569-2.796605$

C $0.580270-4.326420-1.997326$

C $-1.643000-4.993354-2.836028$

C $1.874891-3.572823-1.857434$

C $2.223203-3.337601-0.381199$

C $3.653454-2.810852-0.202553$

C $4.010749-2.7286631 .282047$

C $5.432143-2.1885481 .486967$

O $3.742999-1.508854-0.787334$

O $5.579654-1.8454602 .873970$

C $5.592296-0.8809330 .683442$

C $6.491377-3.2579491 .194191$

O $6.948176-0.4399550 .689163$

C $5.067038-0.953904-0.773722$

C $4.9845660 .470507-1.359860$

O $5.951995-1.687645-1.622723$

C $-0.4977110 .272248-1.929038$

O $\quad-1.165741 \quad 1.085706-2.548192$

C $0.3570140 .584574-0.763004$

C $0.387367 \quad 1.842282-0.298751$

C 1.1886022 .3393000 .820808

C 1.9932501 .3375021 .603406

C $4.3924620 .531982-2.771692$

C $4.4451391 .955668-3.290833$

C $3.3908844 .197178-2.967286$

O $5.2945472 .313467-4.103505$

C $3.5788705 .008623-1.716436$ 
C $3.8208054 .555155-0.475785$

C $3.9576395 .436848 \quad 0.733347$

C 2.6532135 .6401441 .476563

C 2.1354216 .8712041 .627289

C 1.9723434 .4332522 .095381

C 1.1684973 .6633561 .080854

N $3.4806732 .770087-2.720477$

H -3.428641 -0.551534 -4.397298

H -2.813776 -2.300913 -1.968692

H -1.089374 -0.867562 -4.115176

H $\quad 0.502710-2.657170-4.102477$

H - $1.033737-3.224001-4.686637$

H $0.520285-5.224616-1.383071$

H -1.478444 -5.884568 -2.220755

H -2.553813 -4.513221 -2.471620

H - -1.818228 -5.338638 -3.860391

H $2.662088-4.177238-2.324074$

H $1.854392-2.613513-2.382091$

H $1.510887-2.6178770 .038110$

H $2.113010-4.2746130 .179294$

H $4.337200-3.490169-0.724736$

H $3.900068-3.7063101 .767416$

H $3.305585-2.057261 \quad 1.791717$

H $5.396256-2.6417063 .401520$

H $5.016791-0.1088241 .212803$

H $6.371727-4.1106531 .873565$

H $7.501932-2.8743391 .372359$

H $6.441226-3.6441490 .173996$

H $7.227694-0.3742021 .618408$

H $4.3724911 .097682-0.702156$

H $5.9865970 .918624-1.386848$

H 6.692025 -2.021449-1.092394

H $0.937905-0.218539-0.326086$

H $-0.2204702 .594056-0.800909$

H 2.7502460 .8693540 .968677

H $1.341750 \quad 0.5526972 .001720$

H $2.511846 \quad 1.7684242 .462121$

H $4.954634-0.103433-3.464908$

H $3.3532050 .184073-2.782669$

H $2.3933884 .387346-3.375928$

H $4.1324594 .497703-3.713921$

H $3.4820136 .084058-1.856235$

H $3.9221613 .489415-0.293839$

H $4.3875496 .401898 \quad 0.434248$

H $4.692426 \quad 4.989083 \quad 1.414113$

H 1.2110077 .0321732 .172257

H 2.6207077 .7453861 .206204

H 1.2853094 .7556072 .888349

H 2.7349593 .8244592 .588647

H 0.5158614 .2949650 .475455

H $2.7922412 .356019-2.098819$

C $-5.6269920 .095238-2.499917$

O $-5.0170420 .426062-1.235273$

C $-4.9143401 .861684-1.142161$

C $-5.6632202 .430679-2.347422$

C $-5.4696251 .336076-3.376786$

H $-3.8500172 .103878-1.244305$

C -5.4409622 .3721480 .204582$

H $-6.7301702 .570019-2.138001$

H $-5.2582713 .396520-2.665382$

C $-6.4474941 .402987-4.535932$

H $-4.4473121 .421171-3.763734$

C $-4.763628 \quad 1.767183 \quad 1.450777$

$\begin{array}{lllll}\text { O } & -6.840799 & 2.069754 & 0.288795\end{array}$

H $\quad-5.363785 \quad 3.4660840 .227789$

H $-5.083107 \quad 0.7255151 .567560$

O -5.2817032 .4661002 .591977$

C -3.2273621 .8478641 .469707$

C -2.5806021 .5140942 .829028$

H -2.823422 1.1832870 .698155

O $-2.835731 \quad 3.1796901 .122806$

H -1.492518 1.5484452 .692937

H -2.7934512 .3229013 .540302$

H $-6.2684620 .587004-5.243383$

H -7.484553 $1.329953-4.191813$

H $-6.3390702 .348895-5.076588$

C -5.060689-1.218378 -3.050570

H -7.1599422 .4102201 .142245$
H -4.9654983 .3845902 .541620$

H -1.864222 3.2064771 .107462

H -5.663891 -1.545452 -3.905709

H $-5.157871-1.993080-2.279416$

H $-6.691014-0.069235-2.280947$

C -1.619384 -1.297193 2.117032

O $-2.940670-0.9042822 .494441$

C $-2.9969880 .162456 \quad 3.442113$

C -2.229395 -0.216904 4.718055

C $-0.802114-0.6769544 .416565$

C $-0.804514-1.7557273 .331602$

H $-1.117327-0.4385591 .656704$

C $-1.742887-2.3464581 .007887$

H -4.0514670 .2597063 .727746$

O $-2.213871 \quad 0.8870045 .616822$

H $-2.763761-1.0346005 .219483$

H $-0.160048 \quad 0.1645054 .130957$

O $-0.227103-1.2399365 .598161$

H -1.209864 -2.678972 3.762187

O $0.544475-2.0155822 .951511$

C $-2.323020-3.6685601 .460615$

H -2.405463 -1.969370 0.222986

H $-0.757796-2.5229220 .565721$

H -1.8410440 .5770336 .459145$

H $-0.119852-0.5210346 .243499$

H $\quad 0.546363-2.8096782 .391157$

$\begin{array}{llll}\text { O } & -1.765006 & -4.678288 & 0.731029\end{array}$

O $-3.182791-3.7972202 .321398$

C $-2.236868-5.993844 \quad 1.036438$

H $-2.051484-6.2302432 .088485$

H $-3.302584-6.0753370 .802633$

H -1.685796 -6.7050620 .415315$

SCF Energy $\left(B 3 L Y P / 6-31 G^{* *} / / M M F F\right)=-3245.90381066$

01467

MM̄FF Geometry

C $-1.8328344 .333348-0.331829$

C -3.0018794 .0718800 .272207$

C $-3.2016353 .594117 \quad 1.694468$

O -3.6801762 .2276861 .656548$

C -1.9301243 .5998402 .566640$

C -2.1965563 .3224184 .033860$

C -1.9708392 .1366574 .632841$

C -2.6834354 .4996984 .836790$

C -1.5307730 .8617103 .971892$

C -0.0118610 .6634194 .032530$

C $0.399551-0.6792113 .411614$

C $1.915002-0.8775243 .467701$

C $2.339801-2.1893372 .787102$

O $-0.035316-0.6947192 .047959$

O $3.761619-2.1486462 .595099$

C $1.694760-2.2629641 .385792$

C $2.059407-3.4081213 .674552$

O $1.920653-3.5360520 .785092$

C $0.182888-1.9433501 .380174$

C $-0.324762-1.805094-0.075138$

O $-0.520721-3.0137302 .016553$

C -5.0013032 .0049601 .436628$

O -5.8653312 .8335641 .202572$

C -5.2123590 .5438301 .522680$

C $-6.264071-0.0111010 .903680$

C $-6.582094-1.4389220 .874531$

C $-5.827101-2.3476031 .808674$

C $-1.735066-1.209206-0.185520$

C $-2.270811-1.251694-1.606378$

C $-4.295678-0.585514-2.916004$

O $-1.663627-1.762148-2.543007$

C $-5.425596-1.571891-2.922751$

C $-5.430848-2.783724-2.344586$

C $-6.611268-3.715034-2.364179$

C $-7.017452-4.156120-0.974197$

C $-6.574725-5.321043-0.470659$

C $-7.988815-3.295354-0.194237$

C $-7.525246-1.8778220 .015206$

N -3.521819 -0.658338 -1.691404

H $-0.899004 \quad 4.1848350 .202511$

H -3.916563 $4.233723-0.295236$

H $-3.960596 \quad 4.2331392 .163652$ 
H -1.429393 4.5747612 .498667 H -1.213978 2.8660222 .176698 H -2.149992 2.0532155 .703555 H -2.817843 4.2534425 .895447 H -1.964106 5.3231094 .778766 H -3.647218 4.8518284 .456502 H -2.0325790 .0276844 .478509$ H - 1.8766430 .8249352 .934341 H 0.4751011 .4831813 .489055 H 0.3312810 .7095425 .073460 H $-0.111557-1.4770093 .963719$ H $2.274856-0.8474324 .503668$ H $2.412217-0.0436792 .952220$ H $4.178690-2.0239063 .464724$ H $2.203983-1.5243540 .752222$ H $2.596101-3.3196614 .626742$ H $2.427557-4.3290003 .208948$ H $0.999788-3.5371323 .904353$ H $2.875658-3.7116490 .826574$ H $\quad 0.350754-1.148043-0.638669$ H $-0.306228-2.787752-0.563101$ H -1.415758 -3.0748431 .648459$ H -4.474828 -0.0384552 .063897$ H -6.9374080 .6332010 .339706$ H -5.881091 -1.975466 2.837935 H $-4.774532-2.4146311 .513228$ H -6.223834 -3.3654991 .830649$ H -2.440516 -1.7568130 .447769$ H -1.723368 -0.1628330 .142683$ H -3.655121 $-0.730680-3.791155$ H $-4.7082470 .427924-2.952298$ H -6.320007 -1.246695 -3.450440 H $-4.539496-3.141945-1.835707$ H $-6.336780-4.589824-2.968334$ H -7.473906 -3.264527 -2.870665 H $-6.877060-5.6641880 .513136$ H $-5.896638-5.956741-1.030595$ H $-8.235946-3.7452600 .775597$ H $-8.939111-3.267047-0.743545$ H -8.020921 -1.153695 -0.632033 H $-3.949519-0.293058-0.846616$ C $-1.0156273 .916077-2.705232$ O $0.240823 \quad 3.454332-2.170937$ C $0.3700772 .047502-2.456365$ C $-0.6806371 .747401-3.517963$ C -1.806798 2.664120 -3.079705 H $0.1114241 .510905-1.533995$ C $1.8059161 .693042-2.864400$ H $-0.3301232 .024928-4.519397$ H $-0.9646210 .692593-3.540523$ C $-2.8545612 .897649-4.153857$ H $-2.2885042 .229577-2.194844$ C $2.8197301 .883508-1.712065$ O $2.1615282 .481893-3.997357$ H $1.8234280 .642064-3.176924$ H $2.4605061 .306509-0.852526$ O $2.8812713 .251212-1.310018$ C $4.2485171 .417874-2.059115$ C $5.2506941 .547015-0.894991$ H $4.2154040 .378686-2.402014$ O $4.7646182 .185740-3.148967$ H $6.2494831 .320979-1.288358$ H $5.3085262 .595045-0.575058$ H -3.645980 $3.559152-3.787797$ H -2.418436 $3.355910-5.047867$ H $-3.3159541 .952459-4.455893$ C -1.699345 $4.874334-1.727831$ H $3.0328632 .180688-4.304992$ H $1.9919683 .516166-1.020667$ H $4.7443763 .121336-2.882914$ H -2.672606 5.181885-2.127346 H -1.090922 $5.784845-1.652092$ H $-0.7689714 .480638-3.614339$ C $5.885516-1.403393-0.537467$ O $4.718650-0.715162-0.074294$ C 4.9370810 .6434190 .313761 C $5.975726 \quad 0.706038 \quad 1.442537$ C $7.263101-0.0310811 .076335$
C $6.951521-1.4373910 .562259$

H $6.281767-0.903110-1.429718$

C $5.458331-2.817385-0.942358$

H $3.9910540 .997604 \quad 0.739998$

O 6.2557622 .0638591 .764982

H 5.5478310 .2426942 .341204

H 7.8533740 .5303170 .342508

O $8.083158-0.1606432 .239909$

H $6.614767-2.0661881 .396487$

O $8.144829-2.0191900 .041680$

C $4.649608-2.785656-2.217610$

H $6.323456-3.463882-1.128953$

H $4.864860-3.300204-0.158268$

H 6.8171652 .0699492 .558317

H 8.3731820 .7311482 .495222

H $8.808096-2.0060670 .752915$

O $3.329882-2.596549-1.933220$

O $5.139817-2.890722-3.334665$

C $2.460887-2.520501-3.067224$

H $2.779429-1.718803-3.740340$

H $2.443306-3.480742-3.591119$

H $1.452772-2.296140-2.710045$

SCF Energy (B3LYP/6-31G**//MMFF)= -3245.91286232

01468

MM̄FF Geometry

C -3.4352572 .2684951 .578727$

C -2.6525431 .9045202 .605094$

C -2.372926 0.4660672 .940622

O -0.9424000 .3075553 .104666$

C -3.0043460 .0149904 .266307$

C $-4.506069-0.1446414 .226805$

C $-5.134672-1.2497803 .780393$

C -5.2982261 .0016574 .797963$

C $-4.510865-2.4859853 .183128$

C $-5.302294-2.9953381 .966975$

C $-5.157872-2.0973090 .724034$

C $-6.454576-2.109057-0.088706$

C $-6.331312-1.277644-1.370874$

O $-4.079795-2.613882-0.071398$

O $-7.466262-1.575321-2.198399$

C $-5.077401-1.742703-2.132822$

C $-6.3856080 .226853-1.088120$

O $-4.836654-0.910772-3.267334$

C $-3.800576-1.858982-1.258571$

C $-2.718911-2.602592-2.080657$

O $-3.335858-0.546722-0.942715$

C $-0.229195-0.0907542 .020088$

$\begin{array}{llll}\text { O } & -0.623706 & -0.204234 & 0.871439\end{array}$

C $1.123805-0.4438092 .493279$

C $1.708047-1.5276991 .963641$

C $3.012632-2.0795102 .330006$

C $3.889916-1.2608193 .236556$

C $-1.439626-2.976774-1.322949$

C $-0.444815-3.672034-2.243000$

C $1.916549-4.402194-2.373179$

O $-0.716125-4.005769-3.394176$

C $2.918925-4.969883-1.422646$

C $4.157048-4.477649-1.265652$

C $5.167220-5.038589-0.306329$

C $5.518787-4.0696550 .802208$

C $6.622309-3.3066840 .730752$

C $4.638161-4.0507572 .032990$

C $3.365926-3.2775161 .820599$

N $0.780891-3.888908-1.627517$

H -3.9173021 .4917210 .993436$

H -2.173824 2.6658373 .215306

H -2.717902 -0.197342 2.137673

H -2.7199470 .7050905 .072955$

H $-2.549729-0.9381504 .571050$

H $-6.221338-1.2786893 .853409$

H -6.3773960 .8165534 .769469$

H $-5.020627 \quad 1.1700925 .843713$

H $-5.109167 \quad 1.9210614 .236597$

H $-3.464859-2.3320482 .901650$

H $-4.526028-3.2680783 .951939$

H $-4.952933-4.0085311 .731024$

H $-6.357424-3.0829052 .256765$ 
H $-4.916828-1.0736521 .028712$ H $-7.296078-1.7486160 .516293$ H $-6.703723-3.143441-0.364779$ H -8.269527 -1.350547 -1.698207 H $-5.309056-2.738138-2.536587$ H $-7.3150090 .486817-0.567385$ H $-6.3939870 .804147-2.019131$ H $-5.5543010 .573129-0.473308$ H -4.438871 -0.083154 -2.947365 H -3.149997 -3.535190 -2.469126 H $-2.438786-1.990061-2.947802$ H $-2.393940-0.591937-0.714603$ H $1.5592010 .144768 \quad 3.291472$ H $1.164052-2.0838701 .200913$ H $3.477789-1.2389564 .251020$ H $3.967762-0.2331872 .870370$ H $4.913915-1.6350203 .303780$ H $-0.957561-2.083932-0.917706$ H -1.669748 $-3.655242-0.494321$ H $2.322739-3.573250-2.962892$ H $1.575537-5.175686-3.069201$ H $2.608065-5.829238-0.833127$ H $4.469691-3.624876-1.862995$ H $6.066571-5.297120-0.880537$ H $4.820883-5.9834040 .130963$ H $6.894421-2.6304241 .534402$ H $7.272963-3.334832-0.136926$ H $5.197197-3.6735482 .895670$ H $4.378027-5.0813932 .306569$ H $2.644953-3.7940321 .188500$ H $0.963933-3.417147-0.746499$ C -3.121722 $4.062024-0.192877$ O $-1.6874993 .914836-0.166356$ C -1.281991 $3.180515-1.333599$ C $-2.4331703 .317501-2.321607$ C $-3.6261453 .248970-1.388671$ H -1.192226 2.130485-1.025406 C $0.0762353 .659122-1.861519$ H -2.411934 4.284213-2.838009 H -2.431591 2.525075 -3.076148 C $-4.9126953 .780979-1.994719$ H -3.778473 2.199795-1.117526 C $1.1971553 .558887-0.802537$ O $-0.0529205 .002281-2.323969$ H $\quad 0.333714 \quad 3.038009-2.727301$ H $1.1093082 .593994-0.289567$ O $1.0449394 .581261 \quad 0.181142$ C $2.6131233 .655373-1.403645$ C $3.7571843 .681832-0.373548$ H $2.7597592 .810307-2.085174$ O $2.7373804 .835837-2.200402$ H $4.7007253 .678313-0.934105$ H $3.751844 \quad 4.6463470 .151129$ H -5.734499 $3.720491-1.274061$ H -4.809572 $4.826545-2.303590$ H $-5.1924253 .196238-2.877123$ C $-3.7057263 .690676 \quad 1.175422$ H $\quad 0.7787445 .241515-2.766339$ H $0.160571 \quad 4.4835590 .572959$ H $2.5974435 .600375-1.615059$ H $-4.789713 \quad 3.8524551 .171603$ H -3.284146 4.367691 1.929218 H $-3.3096995 .129851-0.364211$ C $4.6024290 .769006-0.734536$ $\begin{array}{lllll}\text { O } & 3.521027 & 1.260483 & 0.062553\end{array}$ C 3.7474272 .5385060 .658982 C 4.9932602 .4968921 .557315 C 6.2108841 .9324450 .825390 C $5.861960 \quad 0.619690 \quad 0.123197$ H $4.7939761 .458117-1.566439$ C $4.166932-0.576543-1.321665$ H 2.8899232 .7214261 .318584 O 5.2757443 .8009972 .054596 H 4.7807091 .8651602 .427723 H 6.6224512 .6569660 .112808 O $7.2484831 .661606 \quad 1.770645$ H $5.718311-0.1690000 .872023$ O $6.953571 \quad 0.228870-0.706593$
C $3.121727-0.383895-2.393731$

H $5.007055-1.105154-1.785929$

H $3.758864-1.239961-0.552144$

H 5.9885713 .7199572 .710114

H 7.5455652 .5133042 .132506

H $7.7389800 .156760-0.137486$

O $1.875729-0.366243-1.839784$

O $3.386639-0.243075-3.580805$

C $0.799578-0.152945-2.758101$

H $\quad 0.9720440 .748769-3.352899$

H $0.686886-1.024762-3.408804$

H $-0.119616-0.019294-2.182020$

SCF Energy (B3LYP/6-31G**//MMFF) $=-3245.91639681$

01469

MM̄FF Geometry

C $3.033694-3.456478-1.592690$

C $2.344651-2.384876-1.170012$

C $1.214429-1.767996-1.954177$

O $0.153629-1.388616-1.048184$

C $1.718440-0.489403-2.650988$

C $0.7171330 .126019-3.609643$

C $0.205640 \quad 1.366717-3.490232$

C $0.322008-0.729483-4.786567$

C $0.4949172 .389402-2.428751$

C $-0.4490702 .336253-1.219500$

C -1.923059 2.618130-1.550644

C $-2.7407712 .786651-0.267802$

C $-4.2367482 .982690-0.561044$

O $-2.4386881 .515036-2.299497$

O -4.9537472 .8052310 .670658$

C $-4.7129711 .872840-1.523653$

C $-4.5412514 .407617-1.038080$

O $-6.0496872 .111996-1.955977$

C $-3.7913831 .681164-2.745883$

C $-4.1502280 .434701-3.591888$

O $-3.9106822 .820078-3.607353$

C $-0.661910-2.373578-0.586091$

O $-0.594214-3.566110-0.836412$

C $-1.660890-1.7405530 .302529$

C $-2.354234-2.4988491 .164237$

C $-3.369190-2.0244622 .106948$

C $-3.840289-0.6006951 .969145$

C $-4.090572-0.904761-2.848670$

C $-5.433621-1.377190-2.316004$

C $-6.441870-3.186597-0.929449$

O $-6.500175-0.839099-2.600006$

C $-6.694425-2.6563450 .446438$

C $-6.817999-3.4423531 .526753$

C $-7.119827-2.9299812 .905911$

C $-6.125796-3.4120893 .938638$

C $-6.402798-4.4657514 .725489$

C $-4.851287-2.6190264 .117282$

C $-3.844836-2.8855293 .030931$

N $-5.302691-2.502579-1.512808$

H $2.768783-3.926048-2.536677$

H $2.632420-1.896047-0.242052$

H $\quad 0.822877-2.478750-2.691773$

H $2.629974-0.711107-3.222526$

H $2.0102650 .233581-1.880982$

H $-0.502627 \quad 1.702222-4.247359$

H -0.252469 -0.169586 -5.532484

H $1.212712-1.120110-5.289422$

H $-0.296812-1.571591-4.462575$

H $1.5291402 .300587-2.078276$

H $0.4356963 .383857-2.889249$

H $-0.3586851 .351898-0.745886$

H $-0.0991653 .081300-0.496291$

H $-1.9725843 .529658-2.157717$

H $-2.355988 \quad 3.6200030 .333293$

H $-2.625591 \quad 1.8900270 .356121$

H -4.610338 3.4499831 .312777

H $-4.7340840 .941511-0.946438$

H -4.240826 $5.138275-0.277256$

H $-5.6171334 .554128-1.184198$

H -4.027712 4.668514 -1.965688

H -6.600341 2.201696-1.159306

H $-5.1173020 .581298-4.087901$ 
H -3.413729 $0.375099-4.406355$ H -3.617803 2.565046 -4.497854 H $-1.788258-0.6667230 .231306$ H -2.148128 -3.567935 1.198157 H $-4.116024-0.3863750 .931329$ H -3.0546890 .0968872 .275256$ H -4.727909 -0.376207 2.564291 H -3.756384 -1.676519-3.554271 H -3.362875 -0.872002 -2.032314 H $-6.210731-4.256405-0.925349$ H $-7.325067-3.022680-1.555294$ H $-6.809456-1.5803330 .554247$ H $-6.731553-4.5206431 .410295$ H -8.128786 -3.270644 3.173409 H -7.164237 -1.833966 2.926108 H $-5.707655-4.8004235 .488376$ H -7.329266 -5.020723 4.620107 H $-4.373025-2.8862845 .069407$ H -5.098592 -1.558892 4.221759 H -3.449508 -3.902300 3.044098 H $-4.374663-2.849190-1.287689$ C $5.517478-3.402753-1.374820$ O $5.546406-2.018966-0.968848$ C $6.783474-1.755916-0.287885$ C $7.729873-2.849893-0.754728$ C $6.782559-4.038323-0.794005$ H $6.595081-1.8965290 .783733$ C $7.220275-0.311097-0.543534$ H $8.124801-2.645771-1.756627$ H $8.573022-2.994192-0.073056$ C $7.306824-5.209942-1.605547$ H $6.595812-4.3742060 .234970$ C $6.1094610 .724859-0.252758$ O $7.585328-0.181113-1.922523$ H $8.123251-0.0883030 .036305$ H $5.3211410 .630061-1.009274$ O $6.6688312 .031231-0.432390$ C 5.4847960 .6281131 .154055 C 4.3314591 .6238041 .350603 H $5.088561-0.3793341 .298037$ O $6.482250 \quad 0.8627012 .143628$ H 4.6648242 .6294351 .061058 H 3.5330371 .3673310 .645406 H $6.578432-6.026940-1.623688$ H $7.514425-4.920252-2.641030$ H $8.235840-5.593615-1.171347$ C $4.218209-4.027961-0.862245$ H $7.8432430 .745337-2.067853$ H 7.3248992 .1680890 .272447 H 7.1201420 .1308032 .103383 H $4.109540-3.8440710 .214167$ H $4.226674-5.113932-1.007691$ H $5.547044-3.412911-2.472075$ C 1.5961532 .6308082 .199797 O 2.8480232 .8437652 .866000 C 3.7724231 .7440142 .789255 C 3.1210390 .5016473 .416196 C 1.7880030 .1724722 .751967 C 0.8811041 .4019092 .774246 H 1.7660232 .4914541 .125944 C 0.7512203 .9000542 .388550 H 4.6146422 .0287003 .431759 O $3.966675-0.637153 \quad 3.376253$ H $2.925696 \quad 0.7042994 .477815$ H $1.925382-0.2006031 .730401$ O $1.139338-0.8718093 .480690$ H $\quad 0.560421 \quad 1.6004773 .805109$ O -0.2896391 .1332842 .009407$ C 1.2527365 .0520521 .542202 H $-0.286473 \quad 3.7113192 .092789$ H 0.7799654 .2206483 .436323 H $4.796593-0.4001843 .824404$ H $1.744560-1.6330523 .493517$ H -0.6955240 .3293512 .376428$ O $0.393450 \quad 6.105615 \quad 1.678883$ O 2.2628555 .0368950 .852352 C 0.7303347 .2750590 .925918 H 1.7016617 .6643381 .245357
H $-0.0323998 .034468 \quad 1.117857$

H $0.7371627 .047456-0.144327$

SCF Energy (B3LYP/6-31G**//MMFF)= -3245.91055579

0147

MM̄FF Geometry

C $-3.755941 \quad 1.2948192 .064057$

C -2.8463830 .7715502 .899990$

C $-2.298474-0.6247412 .766192$

O $-0.856085-0.5030362 .847571$

C $-2.790770-1.5564613 .888923$

C $-4.255573-1.9229453 .799484$

C $-4.742377-2.9168993 .030476$

C $-5.177790-1.1439394 .701044$

C $-3.981322-3.8204522 .095151$

C $-4.690759-3.9860170 .741991$

C $-4.559546-2.757911-0.175521$

C $-5.661235-2.781824-1.237949$

C $-5.525697-1.616516-2.223973$

O $-3.271513-2.810091-0.805976$

O $-6.400165-1.870166-3.333759$

C $-4.088325-1.606248-2.773582$

C $-5.978839-0.290014-1.608081$

O $-3.862202-0.454733-3.585324$

C $-2.988388-1.711769-1.685856$

C $-1.632932-1.976210-2.389197$

O $-2.913024-0.477828-0.972487$

C $-0.111485-1.5275802 .353761$

O $-0.524434-2.5582371 .846966$

C $1.315254-1.1856652 .541502$

C $2.243177-2.1178472 .281231$

C $3.692441-1.9579642 .392556$

C $4.230443-0.5860692 .694341$

C $-0.460454-2.332302-1.465684$

C $0.772381-2.707428-2.276114$

C $2.913133-3.924064-2.147054$

O $0.883563-2.456918-3.474677$

C $3.932912-4.303374-1.123671$

C $5.171296-3.787740-1.088693$

C $6.236948-4.201628-0.112171$

C $6.598729-3.0986490 .858408$

C $7.501587-2.1585930 .532624$

C $5.969989-3.1297822 .233680$

C $4.471448-3.0393052 .187537$

N $1.748180-3.335741-1.510444$

H $-4.1472910 .673778 \quad 1.264663$

H $-2.466474 \quad 1.376106 \quad 3.720257$

H $-2.555840-1.0345821 .782157$

H -2.575790 -1.104682 4.867432

H $-2.194380-2.4777123 .899977$

H $-5.810533-3.1238693 .087196$

H $-6.218600-1.4743344 .617281$

H -4.877191 -1.267313 5.746723

H $-5.153034-0.0783044 .457459$

H -2.955135 -3.483707 1.933885

H $-3.922010-4.8068072 .571360$

H $-4.255919-4.8584880 .237141$

H $-5.748732-4.2142430 .923354$

H $-4.631600-1.8439760 .423091$

H $-6.653847-2.769254-0.770135$

H $-5.606451-3.724344-1.800884$

H -7.305416 -1.956251-2.988706

H -3.995202 -2.475061 -3.440465

H $-7.032581-0.344348-1.309406$

H $\quad-5.914490 \quad 0.527712-2.334088$

H $-5.400364-0.016902-0.725047$

H -4.558292 -0.437736 -4.264382

H -1.758418 -2.813667 -3.089084

H $-1.351812-1.097606-2.984520$

H $-2.022279-0.384623-0.598905$

H $1.561993-0.1892692 .888951$

H $1.911256-3.1030741 .955066$

H $3.969045-0.2869303 .715061$

$\begin{array}{llll}H & 3.816096 & 0.149558 & 1.998165\end{array}$

H $5.316785-0.5163692 .599493$

H $-0.196775-1.483363-0.826994$

H - $0.729241-3.183137-0.830799$

H $3.307167-3.218523-2.886251$ 
H $2.579441-4.816738-2.687132$ H $3.650290-5.064744-0.400868$ H $5.453680-3.042502-1.828502$ H $7.125982-4.494913-0.685936$ H $5.942278-5.1025950 .440346$ H $7.784013-1.3781591 .231158$ H $7.973800-2.146391-0.444263$ H $6.390975-2.3516222 .879882$ H $6.242471-4.0745992 .722226$ H $3.987952-3.9925831 .971475$ H $1.497012-3.652688-0.577959$ C -3.9601953 .6031770 .994717$ O $-2.529647 \quad 3.7478520 .886664$ C $-2.1501213 .495325-0.478115$ C $-3.4118373 .739760-1.296457$ C $-4.4631333 .147884-0.377965$ H -1.895905 $2.431197-0.541387$ C $-0.9399144 .339876-0.882265$ H -3.601575 $4.808653-1.447067$ H $-3.3746163 .254497-2.276349$ C $-5.8796513 .594224-0.693390$ H -4.412024 $2.059551-0.472194$ C 0.2773504 .2463200 .060507 O $-1.3458785 .716464-0.916133$ H $-0.6492934 .087046-1.908128$ H $0.011916 \quad 4.7331201 .008112$ O $1.3171895 .047242-0.513103$ C 0.8299342 .8407580 .362091 C $1.0570651 .904054-0.839632$ H 1.7811952 .9561850 .894064 O -0.0568632 .1951891 .276898$ H $1.4116540 .944865-0.440396$ H $0.0961651 .659504-1.307093$ H -6.5919733 .1467080 .007106$ H $-5.9815454 .682673-0.630772$ H $-6.1627223 .288346-1.705870$ C -4.305805 2.6908002 .178112 H $-0.5725826 .239448-1.189346$ H $2.063475 \quad 5.0529870 .110576$ H 0.3295351 .3344051 .507907 H -5.3948482 .6260562 .285107$ H -3.923379 3.1559223 .095793 H -4.3454954 .6067791 .216690$ C $4.0989291 .973626-0.745560$ O $3.2408062 .945091-1.351423$ C $2.0384852 .413764-1.912430$ C $2.3808421 .375498-2.991787$ C $3.3366900 .302425-2.471917$ C $4.5430440 .939905-1.782973$ H 3.5682881 .4748360 .072822 C $5.2954622 .723587-0.152546$ H $1.5445723 .248249-2.423215$ O $1.2023370 .751207-3.489520$ H $2.8605811 .880890-3.840206$ H $2.828626-0.395605-1.796758$ O $3.810143-0.476831-3.572210$ H $5.189917 \quad 1.409630-2.534771$ O $5.303061-0.075501-1.134275$ C 4.8584963 .5945611 .001348 H 6.0748242 .0424010 .207119 H $5.758576 \quad 3.377997-0.900894$ H $\quad 0.6207201 .452458-3.829704$ H $3.030351-0.857091-4.012505$ H $5.576555-0.711047-1.817393$

O 4.7138122 .8383352 .126537

O 4.6478274 .7966550 .906436

C 4.2529373 .5396283 .285156 H 4.9759924 .3078953 .574629 H 3.2712573 .9838193 .094133 H 4.1583662 .8195244 .102353 SCF Energy (B3LYP/6-31G**//MMFF) $=-3245.91264410$

01470

MM̄FF Geometry

C $-2.146668 \quad 4.4705240 .818277$

C $-1.1547564 .300409-0.069493$

C $\quad 0.155102 \quad 3.652798 \quad 0.299997$

O $0.4266242 .549679-0.602187$
C $1.3119814 .657767 \quad 0.166851$

C 2.5317214 .2122920 .948406

C 3.5111083 .4214800 .467600

C 2.5849954 .7032472 .372618

C $3.6040572 .819005-0.907866$

C $5.0257512 .821780-1.494571$

C $6.0134681 .911259-0.747138$

C $7.3672281 .865475-1.458141$

C $8.3427400 .901684-0.761333$

O $5.4547950 .595482-0.690279$

O $9.4418520 .667446-1.655463$

C $7.637695-0.455359-0.542106$

C 8.9422591 .5197590 .507476

O $8.453073-1.3361160 .228080$

C $6.225708-0.3336830 .079021$

C $5.511620-1.7050970 .043332$

O $6.351384 \quad 0.083043 \quad 1.441273$

C $-0.3899301 .464710-0.540722$

$\begin{array}{lllll}0 & -1.381980 & 1.333071 & 0.157241\end{array}$

C $0.1243380 .449991-1.485875$

C $-0.658817-0.592885-1.797037$

C $-0.335854-1.690293-2.709046$

C $1.075381-1.774089-3.226079$

C $4.006134-1.6402630 .344590$

C $3.390853-3.0263200 .440273$

C $1.189185-4.1725670 .643657$

O $4.059729-4.0564020 .471792$

C $0.924630-4.750433-0.711516$

C $-0.289067-5.142642-1.126803$

C $-0.549932-5.760991-2.469970$

C $-1.628217-5.040147-3.246449$

C $-2.859648-5.563502-3.370851$

C $-1.253723-3.752244-3.946398$

C -1.314705 -2.564039-3.025445

N $2.004822-2.9787710 .513912$

H $-1.9781074 .173903 \quad 1.851718$

H -1.290256 $4.596386-1.105769$

H 0.1186423 .2530351 .321988

H 1.0081685 .6444810 .541490

H $1.5680014 .809488-0.888180$

H 4.3066383 .1441331 .155568

H $3.461927 \quad 4.3286302 .911241$

H 2.6261335 .7971622 .394135

H 1.6962374 .3778042 .922677

H $3.2304191 .789717-0.858923$

H $2.9595023 .350894-1.614051$

H $4.9446172 .475455-2.533465$

H $5.4155343 .846702-1.526394$

H 6.1445782 .2947840 .269870

H $7.8037862 .869339-1.530967$

H $7.2248531 .524109-2.493139$

H $9.8524391 .525722-1.857282$

H $7.540003-0.924389-1.530848$

H 9.4593852 .4570690 .269187

H $\quad 9.703074 \quad 0.863944 \quad 0.944977$

H $\quad 8.194057 \quad 1.740727 \quad 1.271564$

H 8.395886-1.055459 1.157234

H $5.622546-2.150029-0.954333$

H $5.996365-2.3841150 .756398$

H $5.576582-0.2203681 .939989$

H $1.1134340 .593007-1.904195$

H -1.650251 -0.643481-1.351060

H $1.791478-1.767629-2.396920$

H $1.293998-0.925072-3.882795$

H $1.277776-2.683475-3.795168$

H $3.818474-1.1321841 .296024$

H $3.491200-1.084994-0.447847$

H $0.268415-3.8858261 .160818$

H $1.725604-4.9041651 .256702$

H $1.776435-4.878230-1.375572$

H $-1.138267-5.047699-0.455253$

H $-0.841351-6.806073-2.302505$

H $\quad 0.363621-5.801983-3.076260$

H $-3.629992-5.063429-3.948540$

H $-3.126968-6.499939-2.892696$

H - $1.954885-3.560281-4.769809$

H $-0.280651-3.875737-4.428837$

H $-2.305963-2.402579-2.598254$ 
H $1.529549-2.0859620 .416613$

C $-4.1844554 .411246-0.726638$

O $-4.3331662 .983215-0.567919$

C $-5.6720582 .611099-0.937706$

C $-6.2481393 .808677-1.685541$

C $-5.5945794 .960816-0.952102$

H $-6.2295302 .481434-0.002557$

C -5.688095 $1.305696-1.740913$

H $-5.9485903 .814039-2.739902$

H -7.341316 $3.836960-1.644846$

C $-5.6316456 .271387-1.718971$

H -6.1058045 .0959660 .010637$

C -5.029327 $0.093274-1.049658$

O $-4.993405 \quad 1.530659-2.975190$

H -6.724418 $1.066151-2.010172$

H -3.939161 $0.203243-1.064582$

O $-5.331971-1.063441-1.842796$

C $-5.493127-0.1782730 .393836$

C $-5.131946-1.5741590 .942165$

H -5.0796520 .5937041 .052855$

$\begin{array}{llll}H & -5.07962 & 0.593704 & 1.052855\end{array}$

H -5.466574 -1.612764 1.986168

H $-5.742555-2.3278060 .427814$

H $-5.1365837 .067776-1.154517$

H -5.130106 $6.183617-2.688507$

H $-6.6658806 .580353-1.902554$

C $-3.516571 \quad 5.007743 \quad 0.516502$

H -5.186481 $0.780649-3.562384$

H $-4.829437-0.990581-2.671611$

H -7.184557 -0.1656181 .381099$

H -4.1430064 .7780921 .388787$

H -3.461474 6.0998920 .444600

H -3.567784 $4.569511-1.619622$

C $-2.812220-0.9064492 .857785$

O $-2.789108-0.9756101 .429345$

C $-3.643937-1.9574440 .846104$

C $-3.314916-3.3473371 .405657$

C $-3.373955-3.3743442 .929374$

C $-2.504018-2.2622943 .513356$

H -3.810305 -0.5774393 .174149$

C $-1.843396 \quad 0.2103363 .284851$

H -3.392251 -1.987275 -0.219572

O $-4.199902-4.3313800 .881976$

H -2.300956 -3.629347 1.097618

H -4.404656 -3.304416 3.296005

O $-2.867980-4.6286903 .394214$

H -1.453144 -2.544021 3.382698

O $-2.752295-2.1950434 .916676$

C $-0.403723-0.0114622 .857687$

H -2.164148 1.1512542 .821189

H -1.8695840 .3344434 .372480$

H $-4.121455-4.308022-0.087174$

H -3.405222 -5.327017 2.982078

H $-2.064251-1.6347395 .312726$

$\begin{array}{lllll}\text { O } & 0.379675 & 0.959488 & 3.415241\end{array}$

O $-0.011667-0.897652 \quad 2.111244$

C $1.767872 \quad 0.8933153 .072135$

H 2.2802151 .7241783 .563665

H $2.201152-0.0461213 .428507$

H 1.8964910 .9907721 .989900

SCF Energy (B3LYP/6-31G**//MMFF) $=-3245.89473663$

01_471

MM̄FF Geometry

C -2.594464 -2.230659 2.371918

C $-1.958385-1.1159331 .979460$

C $-0.862583-0.4353612 .753384$

O $0.195360-0.2181191 .787230$

C -1.2857510 .9374593 .315443$

C -2.619462 $0.958772 \quad 4.033342$

C $-3.776623 \quad 1.354230 \quad 3.463077$

C $-2.583157 \quad 0.508305 \quad 5.468153$

C -3.9671591 .7728772 .030719$

C $-4.458412 \quad 3.2193341 .894091$

C -4.0108853 .8988060 .587031$

C $-4.3698513 .099854-0.670862$

C $-3.8035123 .741830-1.949186$

$\begin{array}{lllll} & -2.593307 & 4.088315 & 0.679874\end{array}$
O $-3.8859482 .769436-3.003451$

C $-2.3029474 .044628-1.732293$

C $-4.6464594 .938910-2.402963$

O $-1.757728 \quad 4.747294-2.846572$

C $-2.0147614 .801729-0.419260$

C $-0.5044044 .954992-0.134438$

O $-2.554614 \quad 6.125455-0.516780$

C $1.466458-0.0517672 .229238$

O $1.863673-0.1120033 .380343$

$\begin{array}{llll}\text { C } & 2.293380 & 0.237027 & 1.035143\end{array}$

C 3.6189310 .3966251 .152811

C $4.544808 \quad 0.713616 \quad 0.062362$

C $3.9547991 .026779-1.288515$

C 0.2719513 .6437290 .011204

C 1.5842743 .8880320 .732539

C 3.7820905 .0392250 .458913

O 1.7450153 .5534641 .904043

C $4.9040004 .527014-0.387301$

C $6.060665 \quad 4.0744810 .121199$

C $7.209013 \quad 3.592526-0.718814$

C $7.7648372 .264524-0.253366$

C $8.9374132 .197002 \quad 0.399760$

C $7.0050731 .008941-0.618912$

C $5.8678310 .731768 \quad 0.326730$

N $2.5198524 .537890-0.055772$

$\mathrm{H}-2.304756-2.684323 \quad 3.317549$

H $-2.229922-0.6374361 .042904$

H $-0.499514-1.0857363 .558606$

H -1.2599941 .6881812 .516653$

H $-0.507081 \quad 1.2913734 .005051$

H -4.6849141 .3418404 .062997$

H -3.5722080 .5362965 .937449$

H -2.214152 -0.5202475 .535244$

H $-1.920294 \quad 1.1547516 .052368$

H -3.0487101 .6334131 .456538$

H $-4.705917 \quad 1.100874 \quad 1.576057$

H $-5.553453 \quad 3.2329431 .958193$

H -4.078275 3.8165352 .733407

H $-4.490087 \quad 4.8846620 .555641$

H $-5.4567482 .972410-0.748584$

H -3.956888 $2.084805-0.606966$

H $-4.8165952 .501840-3.093148$

H -1.787955 $3.075841-1.705062$

H $-5.6785024 .625823-2.602550$

H $-4.2734735 .350409-3.347130$

H $-4.6854635 .741879-1.664365$

H $-2.0553185 .671224-2.788648$

H -0.040265 $5.580687-0.907900$

H $-0.391514 \quad 5.5252710 .799157$

H -2.109880 6.6891220 .137818

H 1.7809700 .3228540 .083078

H $4.071044 \quad 0.284196 \quad 2.137236$

H $4.688276 \quad 1.386988-2.012592$

H $3.1984581 .814906-1.207975$

H $3.4838440 .138210-1.719446$

H -0.2928012 .9064860 .591712$

H $0.477433 \quad 3.195840-0.967322$

H 3.8984514 .7650381 .512104

H $3.7381006 .130703 \quad 0.387435$

H $4.7721164 .550830-1.466512$

H 6.2050364 .0830331 .199342

H $7.9927674 .360296-0.678282$

H $6.9244613 .512111-1.775314$

H $9.355851 \quad 1.2460030 .712386$

H 9.5026083 .0909300 .641532

H $7.6771800 .140985-0.578583$

H $6.692907 \quad 1.066297-1.665128$

H 6.1952640 .4976591 .340870

H $2.2588824 .810315-0.999136$

C $-4.121016-2.3474750 .291313$

O $-3.035030-2.491705-0.641259$

C $-3.570983-2.759796-1.949651$

C $-5.085852-2.639619-1.824178$

C $-5.304633-3.043047-0.375640$

H $-3.323637-3.802320-2.181208$

C $-2.910208-1.828005-2.973666$

H $-5.428799-1.611759-1.989484$

H -5.611992 -3.284768 -2.534271 
C $-6.659014-2.6269440 .170299$ H -5.197621 -4.132825 -0.292119 C $-1.365919-1.836573-2.918453$ O $-3.336767-0.480662-2.731801$ H -3.256807 -2.077189 -3.982792 H -1.030696 -1.366073 -1.987071 O $-0.875474-1.015059-3.983913$ C $-0.739418-3.233954-3.057531$ C $0.799449-3.253230-3.063681$ H -1.088699 -3.877826 -2.245500 O $-1.191809-3.838657-4.272088$ H $1.112672-4.292305-3.225796$ H $1.175293-2.710612-3.940625$ H -6.768390 -2.928978 1.216494 H $-6.797441-1.5418490 .116723$ H -7.465230 -3.097215 -0.402315 C $-3.707601-2.9425621 .639981$ H -3.088693 $-0.252216-1.819325$ H -1.284134 -0.137959-3.884727 H $-0.922953-3.258902-5.005533$ H $-3.379434-3.9788531 .489339$ H -4.575682 -2.968775 2.308535 H $-4.331588-1.2746280 .391524$

C $1.066936-4.568792-0.317467$ O $0.828458-3.184656-0.591949$ C $1.452582-2.694604-1.782420$ C $2.971024-2.907617-1.706273$ C $3.334519-4.359032-1.398831$ C $2.566836-4.859621-0.173814$ H $\quad 0.671967-5.166469-1.148904$ C $0.223649-4.9638500 .904045$ H $1.281950-1.611106-1.792755$ O $3.582846-2.497672-2.924573$ H $3.378066-2.271250-0.910533$ H $3.162603-5.002025-2.269716$ O $4.737413-4.420506-1.128629$ H $2.989604-4.3809010 .716374$ O $2.790098-6.262193-0.052519$ C $\quad 0.618123-4.2783372 .195740$ H $-0.824282-4.6988750 .718038$ H $0.285812-6.0475351 .053448$ H $4.538486-2.655892-2.836998$ H $4.946569-5.341716-0.896744$ H $2.448872-6.541940 \quad 0.813559$ O $0.038194-4.9390553 .241642$ O $1.318659-3.2802132 .283759$ C $0.317452-4.4029504 .538814$ H $-0.059047-3.3789944 .615891$ H -0.195610 -5.021761 5.279710 H $1.392795-4.435248 \quad 4.738483$

SCF Energy $\left(B 3 L Y P / 6-31 G^{* *} / / M M F F\right)=-3245.90849625$

01472

MM̄FF Geometry

C $-2.361776-3.0018201 .361227$

C $-1.135412-3.5288041 .490066$

C $-0.057468-3.3574370 .448506$

O $0.922935-2.4274970 .961540$

C $0.642858-4.7031380 .184660$

C $1.616544-4.664162-0.977578$

C $2.948348-4.838184-0.871605$

C $1.002191-4.445965-2.337477$

C $3.758205-5.1002940 .366392$

C $4.322572-3.8432941 .046751$

C $5.258040-3.0056090 .158704$

C $5.965425-1.9285440 .985258$

C $6.835171-1.0090490 .113932$

O $4.474123-2.381890-0.862322$

$\begin{array}{lllll}\text { O } & 7.182696 & 0.125994 & 0.922017\end{array}$

C $5.996016-0.503191-1.081266$

C $8.158179-1.685120-0.264591$

O $6.807650 \quad 0.234548-1.991157$

C $5.232616-1.625371-1.815140$

C $4.242146-1.097658-2.881635$

O $6.176159-2.458762-2.500367$

C $0.695765-1.1047290 .733312$

O $-0.230266-0.6119850 .108402$

C $1.767530-0.337511 \quad 1.403573$
C 1.5734510 .9666351 .647049

C 2.5156421 .8801352 .293253

C 3.9380251 .4126522 .445930

C $3.145726-0.163443-2.358803$

C $3.4684231 .314310-2.507825$

C $2.5797823 .564428-1.909203$

O $4.4320001 .734525-3.142200$

C $3.3198184 .063315-0.708340$

C 2.8455215 .0212660 .102542

C 3.5929045 .5611181 .288791

C 2.7865885 .4877662 .566340

C 2.1092236 .5565013 .018567

C 2.8298164 .1984913 .357336

C 2.0669403 .0904442 .686570

N $2.5247232 .114574-1.876693$

H $-2.581659-2.4035840 .480326$

H $-0.888114-4.0966612 .383469$

H $-0.485725-2.975627-0.486143$

H $-0.106058-5.477197-0.030838$

H $1.151194-5.0204381 .101620$

H $3.541310-4.794939-1.784826$

H $1.724672-4.591771-3.147825$

H $\quad 0.182161-5.152564-2.502682$

H $0.611848-3.427851-2.427650$

H $3.175467-5.6695361 .098404$

H $4.590231-5.7610430 .090549$

H $3.486691-3.2230011 .389267$

H $4.869842-4.1680611 .940424$

H $5.992239-3.677661-0.300829$

H $6.567269-2.3814941 .783049$

H $5.212582-1.3127171 .495403$

H 7.6673680 .7522190 .356973

H $5.2733270 .211519-0.670431$

H $8.722093-1.9495880 .638198$

H $8.804979-1.004828-0.829368$

H $8.021713-2.597052-0.849400$

H $7.317691-0.403810-2.518337$

H $4.792182-0.649391-3.717727$

H $3.731606-1.973543-3.307938$

H $5.707753-2.942005-3.201136$

H $2.669882-0.8635991 .689722$

H $0.623843 \quad 1.4123451 .356498$

H 4.3374251 .0886881 .478867

H 3.9954800 .5747693 .148863

H 4.6171642 .1852542 .810936

H $2.234628-0.336106-2.946012$

H $2.908026-0.382232-1.314325$

H $1.5478193 .928000-1.941114$

H $3.0937853 .888801-2.819670$

H $4.3003003 .636199-0.511154$

H $1.8761875 .465939-0.112872$

H 3.8527666 .6054801 .071622

H 4.5493065 .0426141 .431258

H $1.5490656 .520513 \quad 3.946973$

H 2.0961297 .4908102 .466899

H 2.3807814 .3482114 .348181

H 3.8747393 .9439163 .552216

H $1.012583 \quad 3.3242032 .532454$

H $1.7856081 .679129-1.332496$

C $-4.845982-3.3776501 .754062$

O $-5.222041-2.2222440 .971063$

C $-5.598926-2.673851-0.339596$

C $-6.015121-4.127292-0.154042$

C $-4.969285-4.5993480 .838115$

H -4.693851 -2.644637 -0.957899

C $-6.667947-1.769183-0.954527$

H -7.016379 -4.2097260 .284275$

H $-6.001796-4.689818-1.092244$

C $-5.348694-5.8747231 .570593$

H $-4.034633-4.7776910 .292397$

C $-6.315252-0.266789-1.021733$

O $-7.882316-1.888161-0.201766$

H $-6.908048-2.134217-1.960436$

H $-6.4233230 .189651-0.030514$

O $\quad-7.3023690 .354734-1.857517$

C $-4.9191800 .065409-1.580779$

C $-4.7189531 .529718-2.015701$

H $-4.161706-0.205345-0.837108$ 
O $-4.679800-0.746906-2.736157$

H -3.676034 $1.631677-2.340926$

H $-5.3102581 .706843-2.923650$

H $-4.570690-6.1556602 .287485$

H -6.288751 -5.758236 2.120308

H $-5.473548-6.7016190 .863868$

C $-3.462179-3.153398 \quad 2.375354$

H $-7.681472-1.6434020 .718068$

H -8.173713 $0.156370-1.473387$

H -3.766989 $-0.581033-3.027401$

H $-3.236645-3.9730023 .067480$

H $-3.496576-2.2271702 .960090$

H $-5.581534-3.4373752 .566271$

C $-2.914173 \quad 2.7248640 .171952$

$\begin{array}{lllll}\text { O } & -4.318169 & 2.448042 & 0.252664\end{array}$

C $-5.0536402 .603396-0.961710$

C $-4.9005024 .039506-1.479603$

C $-3.4338174 .402218-1.676928$

C -2.641931 4.127774 -0.400837

H $-2.4274001 .958905-0.444123$

C -2.3590312 .6326151 .604050$

H $-6.1098772 .478494-0.697174$

O $-5.6140434 .200334-2.700926$

H -5.345522 4.732359 -0.753602

H -3.006587 $3.876286-2.538499$

O $-3.3539195 .796915-1.983372$

H -2.893936 4.8993210 .338482

O $-1.2562304 .271793-0.714325$

C -2.366416 1.2273992 .162405

H -1.3223552 .9816121 .627694$

H -2.9617473 .2510502 .279923$

H -5.466798 $5.112197-3.005515$

H $-2.4111656 .019356-2.072149$

H $-0.762515 \quad 4.3289440 .120300$

$\begin{array}{lllll}\text { O } & -1.659623 & 1.221608 & 3.332373\end{array}$

$\begin{array}{lllll}0 & -2.927473 & 0.267195 & 1.655941\end{array}$

C -1.568791-0.039758 4.000338

H $-2.560119-0.3589344 .334040$

H $-1.118560-0.7888833 .343521$

H -0.9271290 .0855394 .876498$

SCF Energy (B3LYP/6-31G**//MMFF) $=-3245.91661582$

01_473

MM̄FF Geometry

C $3.268335-2.713035-1.944614$

C $2.099864-3.043556-1.372364$

C $0.743429-2.530832-1.791275$

O $0.902069-1.183811-2.300578$

C $0.099738-3.397124-2.892061$

C $-0.804617-4.491417-2.361017$

C $-2.123960-4.574926-2.622012$

C $-0.147324-5.548838-1.512683$

C -2.952184 -3.662755-3.482299

C $-3.388448-2.361008-2.804962$

C $-4.032407-2.503864-1.418470$

C $-5.309663-3.343771-1.445127$

C $-5.974253-3.407697-0.062490$

O $-4.346475-1.177798-0.973363$

O $-7.291759-3.943514-0.264012$

C $-6.121674-1.9749380 .496245$

C $-5.243846-4.3932210 .858099$

O $-6.553486-1.993762 \quad 1.854744$

C $-4.842091-1.1108220 .370279$

C -5.1762620 .3662690 .671571$

O $-3.844504-1.4972101 .318885$

C $0.072848-0.213357-1.832763$

O $-0.852339-0.340253-1.047542$

C $0.507617 \quad 1.061484-2.446803$

C $-0.2252892 .172334-2.279634$

C $0.0881723 .495366-2.824852$

C $1.4269943 .675144-3.492897$

C -3.9908601 .3166510 .461476$

C -4.301070 $2.727400 \quad 0.934395$

C $-3.185186 \quad 4.9382101 .261695$

O -5.3952193 .0629541 .379622$

C -2.923299 5.8298930 .092313

C -1.773562 $6.496589-0.089275$

C $-1.5110737 .403007-1.255897$
C $-0.3916096 .902628-2.140866$

C $0.8497527 .405714-2.035097$

C $-0.7338025 .893527-3.213885$

C $-0.8202624 .484792-2.692658$

N -3.202862 3.5632030 .801156

H $3.275767-2.058533-2.812702$

H $2.109795-3.717003-0.519936$

H $0.106456-2.506018-0.899354$

H $0.872028-3.858697-3.522141$

H $-0.453274-2.740592-3.573018$

H $-2.682534-5.400165-2.181821$

H $-0.808797-6.402335-1.327973$

H $0.747560-5.936127-2.010673$

H $0.137642-5.140243-0.538681$

H $-2.415032-3.427167-4.408850$

H $-3.840508-4.217945-3.808632$

H -2.523167 -1.692850 -2.716505

H $-4.080060-1.830155-3.473057$

H $-3.293075-2.932816-0.733102$

H $-5.117725-4.358789-1.811344$

H $-6.021342-2.898935-2.154813$

H $-7.714244-4.0418460 .605827$

H $-6.920313-1.485037-0.078571$

H $-5.296028-5.4082680 .446144$

H $-5.720240-4.445376 \quad 1.843213$

H $-4.188411-4.1489480 .996980$

H -7.417359 -2.437900 1.884053

H -5.9967090 .7010140 .023514$

H -5.5211140 .4574501 .709592$

H $-4.179042-2.2424381 .841600$

H $1.4242801 .049343-3.026032$

H -1.146533 2.108175 -1.702097

H $1.4782413 .086858-4.415500$

H $2.2365083 .356513-2.825952$

H $1.6451154 .711898-3.757935$

H -3.1170280 .9590591 .016264$

H $-3.7271901 .362466-0.601451$

H -2.399312 5.0140072 .020144

H $-4.1377305 .202047 \quad 1.731083$

H $-3.723503 \quad 5.934511-0.636351$

H $-0.976377 \quad 6.3942340 .643751$

H $-1.263663 \quad 8.396014-0.858267$

H -2.415275 $7.550106-1.859741$

H $1.6506437 .077512-2.689211$

H $1.0938068 .154109-1.288392$

H $-0.0256315 .965861-4.046712$

H $-1.7004946 .156338-3.663130$

H $-1.7603854 .258573-2.190007$

H $-2.3380943 .182740 \quad 0.428778$

C $5.544745-1.992577-1.217691$

O $4.967094-1.082346-0.266945$

C $6.027775-0.4459870 .471131$

C $7.338885-1.026332-0.058492$

C $6.896667-2.366023-0.619385$

H $5.904201-0.7502781 .516766$

$\begin{array}{lllll}\text { C } & 5.898187 & 1.079947 & 0.352688\end{array}$

H $7.773612-0.405459-0.849888$

H $8.091762-1.1219800 .730290$

C $7.876665-2.961463-1.614365$

H $6.757351-3.0650230 .216231$

C 4.4861091 .6109810 .681286

O $6.1921241 .454100-1.000075$

H $6.654737 \quad 1.5639500 .981549$

H $3.7854461 .281640-0.094485$

$\begin{array}{lllll}\text { O } & 4.518789 & 3.041740 & 0.616453\end{array}$

C 3.9612261 .2046432 .069889

C $2.602103 \quad 1.8296102 .434832$

H 3.8831270 .1164872 .131605

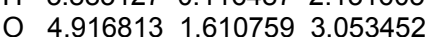

H 2.3885931 .5806333 .481233

H 2.6892412 .9229492 .420479

H $7.515148-3.923532-1.990738$

H $8.028684-2.299100-2.473218$

H $8.850265-3.128554-1.142147$

C $4.605770-3.178628-1.438149$

H $6.3227442 .417165-1.015163$

H $4.6414113 .290004-0.315168$

H 4.6060561 .2867223 .916001 
H $\quad 4.479637-3.728512-0.498270$

H $5.038937-3.868442-2.170559$

H $5.677850-1.438253-2.156919$

C $0.817834-0.6428822 .714435$

O $1.294683-0.0509731 .499042$

C 1.4386811 .3741161 .530888

C 0.0926792 .0285491 .867269

C $-0.481899 \quad 1.4862973 .171080$

C $-0.532855-0.0387043 .126602$

H $1.556041-0.4907113 .511219$

C $0.670197-2.1548322 .473686$

H 1.6867501 .6806640 .508719

O $0.218994 \quad 3.4429201 .958061$

H -0.6209531 .8187371 .061378$

H $\quad 0.086230 \quad 1.8367714 .040466$

O

H -1.315155 -0.356296 2.425425

O $-0.892224-0.529714 \quad 4.416931$

C $1.998150-2.8797772 .417187$

H $0.096603-2.6078263 .290388$

H $\quad 0.152527-2.337411 \quad 1.525634$

H 0.5593643 .7609081 .104257

H -1.772771 2.9439253 .319123

H -1.741948 -0.1209854 .655793$

O $1.761459-4.2259362 .394309$

O $3.098396-2.3476452 .388901$

C $2.925691-5.0572912 .364523$

H $3.541571-4.8793473 .251260$

H $2.598574-6.1003952 .368283$

H $3.499822-4.8735001 .452318$

SCF Energy $\left(B 3 L Y P / 6-31 G^{* *} / / M M F F\right)=-3245.89975526$

01474

MM̄FF Geometry

C $-1.375362-1.5360402 .810227$

C $-2.472675-1.1659203 .488963$

C -3.1165610 .2024093 .461700$

O $-2.247056 \quad 1.1166212 .751354$

C $-4.476051 \quad 0.1766822 .734878$

C $-5.520224-0.7441903 .331293$

C $-5.894389-1.9133332 .773120$

C $-6.178971-0.2482864 .589725$

C $-5.289679-2.5394251 .547213$

C $-6.010711-2.1127590 .263646$

C $-5.171276-2.305684-1.009583$

C $-4.779282-3.763395-1.251756$

C -3.893867 -3.909951-2.498540

O $-3.998751-1.492362-0.886392$

O $-3.316719-5.224903-2.476822$

C $-2.727273-2.904562-2.403135$

C $-4.706767-3.813829-3.794705$

O $-1.964744-2.899439-3.608480$

C $-3.165962-1.462129-2.051800$

C $-1.913326-0.605612-1.722454$

O $-3.853408-0.904671-3.170405$

C -2.3823412 .4495142 .967720$

O -3.1567433 .0012853 .731285$

C -1.416226 3.1251822 .072685

C -1.480058 4.4520951 .892591

C -0.6094075 .2430411 .018820$

C 0.4564924 .5089870 .246763

C $-2.1935420 .790817-1.144403$

C $-2.4932561 .857444-2.183264$

C $-3.3084764 .207733-2.373214$

O $-2.3470891 .679611-3.389496$

C $-2.1530805 .156570-2.436326$

C $-2.2469786 .456607-2.116874$

C $-1.1062437 .428673-2.221844$

C $-0.8887148 .219092-0.950315$

C $-1.3612839 .470963-0.825554$

C -0.0484927 .5914750 .139123$

C $-0.812615 \quad 6.5748250 .941761$

N $-2.9330643 .038297-1.599857$

H $-0.852303-0.8144282 .188453$

H -2.970008 -1.909438 4.108657

H -3.2272450 .5484384 .496689$

H -4.306301-0.050074 1.675078

H $-4.893626 \quad 1.1921702 .708802$
H $-6.683577-2.4932613 .247509$

H $-6.955096-0.9324814 .948850$

H -5.440691 -0.1377975 .390004$

H $-6.651018 \quad 0.723928 \quad 4.414288$

H $-4.222207-2.3061201 .493301$

H $-5.342742-3.6295981 .656293$

H $-6.955682-2.6623810 .173450$

H $-6.267143-1.0472060 .331938$

H $-5.771462-1.931503-1.847484$

H $-5.670385-4.397827-1.333134$

H $-4.213195-4.147688-0.392996$

H $-4.043748-5.870808-2.463162$

H -2.055781 -3.262705-1.611381

H -5.477292 -4.593629-3.821805

H $-4.073708-3.988797-4.671610$

H $-5.207249-2.851139-3.917137$

H - $1.675477-3.813653-3.771230$

H -1.334996 -1.138263 -0.956880

H $-1.275220-0.516158-2.609658$

H $-3.882510 \quad 0.058975-3.069997$

H -0.6864572 .5027641 .566977$

H -2.246173 5.0130822 .426181

H $0.0135853 .720111-0.371352$

H $1.177844 \quad 4.0527480 .933029$

H $1.0224005 .146395-0.435022$

H $-3.0064560 .756292-0.411597$

H -1.291639 $1.125678-0.616250$

H -4.181883 $4.651887-1.885323$

H -3.591362 $3.902678-3.385777$

H -1.202575 $4.762115-2.787897$

H $-3.2066676 .855249-1.795332$

H -1.329632 8.113016-3.050925

H $-0.1730796 .923108-2.499761$

H $-1.184928 \quad 10.0553380 .071457$

H -1.940365 9.937276-1.615770

H $\quad 0.295847 \quad 8.3639850 .840095$

H $0.8685837 .190386-0.300655$

H -1.6158747 .0110261 .537583$

H $-2.9611083 .111891-0.587114$

C $-0.728583-3.5047861 .407829$

O $0.267088-2.7793960 .656558$

C $1.220132-3.7119120 .121288$

C $0.494644-5.0489360 .094995$

C $-0.296108-4.9734451 .387279$

H $2.044637-3.767900 \quad 0.843330$

C $1.761224-3.226518-1.227166$

H $-0.188501-5.125461-0.758756$

H $\quad 1.183280-5.8983150 .060747$

C $-1.458873-5.9498531 .446315$

H $\quad 0.382500-5.1778702 .226305$

C $2.246775-1.762222-1.227003$

O $0.722562-3.331514-2.206631$

H $2.561193-3.898872-1.559283$

H $1.383186-1.090217-1.155208$

O $2.835452-1.502760-2.508490$

C $3.261743-1.412463-0.123707$

C $3.7685720 .037703-0.251635$

H $2.789529-1.5415710 .856326$

O $4.350501-2.326350-0.206832$

H $4.3563380 .134375-1.170325$

H $2.8977720 .696638-0.349635$

H -2.011293 -5.840702 2.384966

H $-2.161026-5.7877150 .621467$

H - $-1.098315-6.9817321 .382832$

C $-0.810409-2.9293862 .825314$

H $1.083857-3.010544-3.050666$

H $3.642502-2.041936-2.574059$

H $4.914832-2.1776750 .570602$

H $\quad 0.192443-2.8778383 .266450$

H -1.420192 -3.583620 3.459014

H $-1.683099-3.3718670 .883138$

C $6.819661-0.0661320 .133712$

O $5.808938-0.277256 \quad 1.127514$

C 4.6159920 .4947490 .959101

C 4.9592281 .9917030 .959036

C $6.0194562 .325808-0.086997$

C $7.231314 \quad 1.4096940 .078007$

H $6.445793-0.389033-0.845313$ 
C $8.017937-0.9497260 .501158$

H $4.003881 \quad 0.308351 \quad 1.851021$

O 3.7826902 .7607850 .737224

H 5.3415982 .2665451 .950933

H $5.6167432 .264228-1.104790$

O $6.459268 \quad 3.6734500 .094775$

H 7.7740551 .6843410 .991761

O $8.1189811 .606243-1.021099$

C $7.718978-2.4044770 .228905$

H $8.907980-0.695259-0.085600$

H $8.289137-0.832597 \quad 1.557426$

H 4.0106093 .6961190 .871341

H $5.7142974 .257198-0.127114$

H $8.3466822 .551522-1.044567$

O $7.308808-3.021181 \quad 1.373587$

O $7.815073-2.922687-0.876255$

C $6.983476-4.4085811 .243048$

H $7.870465-4.9774160 .948542$

H $6.641194-4.7704162 .216302$

H $\quad 6.177756-4.5454870 .515637$

SCF Energy (B3LYP/6-31G**//MMFF) $=-3245.92413638$

\section{5}

MMFF Geometry

C -1.5847740 .5676854 .178056$

C -0.3427850 .1484234 .465541$

C $0.204919-1.238914 \quad 4.222557$

O $-0.812087-2.1101273 .677821$

C $1.419325-1.2209103 .265672$

C $1.219338-0.4366611 .978710$

C $1.8834170 .687817 \quad 1.646586$

C $0.199641-0.9962411 .020516$

C 2.9133081 .4393632 .437599

C 4.2278551 .6818571 .679772

C 4.9956030 .3952671 .330598

C 6.4529200 .7279020 .996865

C $7.239617-0.5129030 .553918$

O $4.368955-0.213514 \quad 0.195238$

O $8.475977-0.068632-0.025108$

C $6.449624-1.226400-0.560177$

C $7.613034-1.4095331 .740093$

O $7.067154-2.464091-0.908185$

C $4.957807-1.455498-0.216319$

C $4.213935-1.950811-1.481759$

O $4.861304-2.446560 \quad 0.807516$

C $-0.725823-3.4396913 .935244$

O $0.083015-3.9905564 .669269$

C $-1.769829-4.2012643 .209111$

C -2.428393 -3.713765 2.145778

C $-3.449757-4.406758 \quad 1.357528$

C $-3.870543-5.780713 \quad 1.809000$

C $2.692723-2.054403-1.325709$

C $2.007397-2.481954-2.612134$

C $-0.189300-3.186637-3.506895$

O $2.595137-2.584286-3.685825$

C - $1.632137-3.025639-3.149228$

C $-2.523258-4.026201-3.221382$

C $-3.996229-3.865459-2.966370$

C $-4.536697-4.832400-1.935499$

C $-4.639983-6.146730-2.198199$

C $-5.057044-4.261216-0.638045$

C -3.960082 -3.784138 0.273826

N $0.648340-2.708495-2.424696$

H -2.308204 -0.127345 3.760065

H $\quad 0.363847 \quad 0.8633774 .883084$

H $0.524114-1.6336945 .195690$

H $1.683075-2.2494502 .986523$

H $2.282054-0.8435123 .822346$

H 1.6450351 .1496510 .691088

H $0.257035-0.532400 \quad 0.031046$

H $0.348138-2.0718700 .885445$

H $-0.811578-0.8218201 .396025$

H 2.4821782 .4170782 .688011

H $3.141190 \quad 0.9605153 .393102$

H 4.0150852 .2478070 .763358

H 4.8539942 .3192612 .316744

H $4.947455-0.2890712 .185722$

H 6.9485351 .2075231 .850198
H $\quad 6.480050 \quad 1.465275 \quad 0.181999$

H 8.9529910 .4481390 .646723

H $6.503408-0.591607-1.455937$

H $8.228994-0.8561142 .459017$

H $8.223426-2.2597141 .415790$

H $6.744122-1.7936762 .278305$

H $7.994308-2.272680-1.131112$

H $4.417419-1.256599-2.307887$

H $4.599922-2.933970-1.779582$

H $\quad 3.967097-2.821388 \quad 0.802755$

H $-1.933394-5.2105863 .572072$

H -2.200605 -2.706619 1.804886

H $-4.313116-5.7373572 .810184$

H -3.009598 -6.4581681 .833620$

H -4.607356 -6.248304 1.152216

H $2.433775-2.777484-0.545946$

H $2.283682-1.078011-1.050892$

H $0.081152-4.230718-3.698341$

H $0.039523-2.606956-4.407856$

H -1.967390 -2.032069 -2.865172

H $-2.183110-5.008717-3.541859$

H $-4.527258-4.027956-3.913580$

H -4.229620 -2.834072 -2.673767

H $-5.059518-6.837400-1.474339$

H -4.307602 -6.560572 -3.144499

H $-5.708314-4.979645-0.128311$

H -5.717054 -3.412507-0.860945

H $-3.577254-2.7981000 .010216$

H $0.292054-2.768123-1.474871$

C -2.3938032 .6021172 .993080$

O -3.5760361 .9657322 .458345$

C -4.4490502 .9799611 .941648$

C -3.6081154 .2427041 .825128$

C -2.7170634 .0956653 .046879$

H -5.2037223 .1565682 .721506$

C -5.1901382 .5240400 .681446$

H -3.0033454 .2419630 .911208$

H -4.2113265 .1551321 .840271$

C -1.496943 4.9987293 .026619

H -3.3099184 .3176543 .944624$

C $-4.3592842 .274834-0.596345$

O $-6.163993 \quad 3.5339030 .383596$

H $-5.752166 \quad 1.6124980 .921243$

H $-3.9980123 .232612-0.985994$

O $-5.2643511 .772495-1.592502$

C $-3.1883031 .289051-0.458157$

C $-2.5468250 .850907-1.787059$

H $-2.417264 \quad 1.7303910 .178699$

$\begin{array}{llll}\mathrm{O} & -3.658382 & 0.107993 & 0.197500\end{array}$

H -1.708659 $0.187915-1.539414$

H $-3.2492060 .212211-2.338325$

H -0.881989 4.8441923 .918618

H -0.8704454 .8093182 .150001$

H -1.798255 6.0510703 .002196

C -2.0520281 .9873124 .351705$

H $-6.6604523 .236089-0.397932$

H -5.565255 $0.895143-1.298828$

H $-2.890874-0.4689890 .348520$

H -2.938056 1.9761894 .997571

H -1.282434 2.5846354 .853192

H -1.5795192 .4175992 .279681$

C $-0.0206352 .569645-1.555324$

O $-1.3191262 .986028-1.983294$

C $-2.0639801 .996821-2.696528$

C $-1.2778431 .523059-3.929249$

C $0.1263481 .048075-3.560613$

C $0.8491942 .108524-2.731505$

H $-0.1393951 .735829-0.853681$

C $0.6095453 .710193-0.742864$

H $-2.9633032 .503112-3.067819$

O $-1.9649750 .470222-4.597524$

H - $1.1857222 .352533-4.642600$

H $\quad 0.1001010 .090790-3.029836$

O $0.8783810 .822393-4.754340$

H $1.1114282 .951335-3.381212$

O $2.0679891 .566314-2.227781$

C $0.8304924 .991721-1.520293$

H -0.0517343 .9638790 .092752$ 
H $\quad 1.5726523 .380412-0.338156$ H $-2.8479880 .802044-4.834446$ H $\quad 0.392653 \quad 0.164358-5.281026$ H $2.575414 \quad 1.234482-2.988407$ O $1.7391805 .763019-0.851931$ O $0.2607015 .297627-2.558433$ C $2.0398077 .023961-1.458073$ H $1.1399587 .644441-1.506347$ H $2.7830267 .531177-0.837055$ H $2.4607726 .874080-2.456871$ SCF Energy (B3LYP/6-31G**//MMFF) $=-3245.90118655$

\section{6}

MMFF Geometry

C $3.496589-1.1006562 .627068$

C $3.147466-1.9807823 .578096$

C $1.743400-2.4579573 .877710$

O $0.768672-1.7641283 .065838$

C $1.629195-3.9624643 .579619$

C $0.250507-4.5221623 .871899$

C $-0.660802-4.8433352 .931827$

C $-0.055793-4.747706 \quad 5.329897$

C $-0.533448-4.6909731 .440691$

C - $1.752542-3.987094 \quad 0.835712$

C $-1.682600-3.894217-0.696383$

C $-0.618621-2.904165-1.178698$

C $-0.622206-2.769958-2.709522$

O $-2.973091-3.467544-1.151909$

O $0.156345-1.613820-3.053670$

C $-2.064834-2.495235-3.187563$

C $0.066506-3.963733-3.381979$

O $-2.146914-2.526380-4.609956$

C $-3.117417-3.452484-2.577744$

C $-4.570182-3.050511-2.922169$

O $-2.982576-4.774156-3.120965$

C $0.218396-0.6335003 .586081$

O $0.458501-0.1331134 .673425$

C $-0.727998-0.0907432 .584969$

C -1.3463041 .0718172 .839329$

C $-2.298431 \quad 1.7679911 .970241$

C -2.6798061 .0989410 .678016$

C $-5.056509-1.694237-2.409266$

C $-5.507207-1.682581-0.957563$

C $-6.475173-0.1409320 .734353$

O $-5.565288-2.683409-0.250277$

C $-6.587413 \quad 1.3362270 .926291$

C $-6.152176 \quad 1.9939542 .011457$

C $-6.264553 \quad 3.4855262 .153197$

C -4.9659054 .1563852 .541160$

C -4.9222695 .0108803 .578278$

C -3.7352673 .8989381 .695356$

C -2.770193 2.9648002 .378627

N -5.866848 $-0.401502-0.557654$

H $2.733185-0.6620561 .990245$

H $3.928889-2.4048764 .205726$

H $1.539370-2.2835994 .941476$

H $2.364062-4.5181564 .178034$

H $\quad 1.910417-4.1535112 .538209$

H -1.605891 -5.2724313 .263030$

H -1.037370 -5.209094 5.482571

H $0.691810-5.4110885 .776908$

H -0.050956 -3.799069 5.875384

H $\quad 0.368250-4.1389371 .170258$

H $-0.440559-5.6928421 .004364$

H -2.666110 -4.530956 1.112354

H $-1.870124-2.9836881 .264753$

H -1.486896 -4.898862 -1.088586

H $\quad 0.382057-3.187272-0.833975$

H $-0.817404-1.914950-0.742854$

H $\quad 1.052508-1.742643-2.698629$

H $-2.300303-1.467876-2.885963$

H $1.118217-4.021343-3.075395$

H $\quad 0.079036-3.851596-4.471553$

H $-0.396967-4.921405-3.135969$

H -1.480813 -1.906083 -4.952727

H -4.685461 -3.068634 -4.014352

H -5.246908 -3.833096 -2.551489

H $-2.386009-4.734844-3.885827$
H $-0.880504-0.651547 \quad 1.670522$

H $-1.127323 \quad 1.576717 \quad 3.779845$

H -1.7936830 .9292230 .056998$

H -3.378149 1.6823520 .074894

H $-3.155790 \quad 0.1318750 .873828$

H -4.308636 -0.911155 -2.560174

H -5.937094 -1.412108 -3.001733

H $-5.874914-0.6268911 .510741$

H -7.469884 -0.5984760 .731288$

H $-7.057012 \quad 1.9005140 .123160$

H -5.7062501 .4396032 .833425$

H -7.0395203 .6895962 .902909$

H -6.6137423 .9388601 .216564$

H -4.0030535 .5189343 .850979$

H -5.8022445 .2228204 .176385$

H $-3.2137974 .850578 \quad 1.523972$

H -4.0302903 .5589050 .699041$

H -2.402762 3.3564593 .329104

H $-5.9358680 .317172-1.273207$

C $5.339174-1.0055690 .939809$

O $4.552262-0.259730-0.010146$

C $5.3894430 .104624-1.124075$

C $6.740747-0.563188-0.885997$

C $6.787772-0.6399170 .627530$

H 5.522714 1.191111-1.077980

C $4.711488-0.286389-2.441897$

H $6.780506-1.568842-1.320686$

H $7.5665750 .014516-1.312585$

C $7.809435-1.6339211 .151275$

H $7.0171900 .358608 \quad 1.023228$

C $3.3060410 .317673-2.645865$

O $4.577050-1.714202-2.491905$

H $5.360139-0.017846-3.283795$

H $2.610322-0.132564-1.928747$

O $2.877214-0.092121-3.951113$

C $3.2506731 .854964-2.551155$

C $1.9089912 .484893-2.970572$

H $3.4853362 .167275-1.528636$

O $4.2655022 .416651-3.389412$

H $2.0374053 .574212-2.961411$

H $1.7045342 .244856-4.021281$

H $7.807740-1.6575192 .245319$

H $7.603631-2.6478980 .792166$

H $8.816828-1.3587550 .822264$

C $4.908392-0.6548852 .363981$

H $4.048479-1.986342-1.722036$

H $1.9144500 .031652-3.999654$

H $4.0971322 .113250-4.298117$

H 4.9490390 .4308192 .511247

H $5.593912-1.1100503 .087477$

H $5.159522-2.0707220 .740117$

C $0.990823 \quad 3.727608-0.351084$

O $0.9440272 .339075-0.700554$

C $0.7116722 .078480-2.088618$

C $-0.6237772 .700331-2.521788$

C $-0.6959854 .186393-2.181204$

C $-0.3273804 .420328-0.717212$

H $1.8255764 .210603-0.872407$

C 1.2547313 .8220791 .155664

H $0.5980210 .992811-2.179760$

O $-0.8380932 .528534-3.918741$

H -1.447315 2.188985-2.008466

H $-0.0608574 .783344-2.845974$

O $-2.0337084 .649284-2.382593$

H -1.137130 $4.050577-0.077492$

O $-0.2042565 .821154-0.481770$

C 2.6001773 .2488221 .528274

H 1.2158664 .8557681 .516962

H 0.4985063 .2567521 .711028

H $-0.8069341 .574262-4.103540$

H $-2.2678904 .460988-3.307714$

H -1.047137 $6.233332-0.737918$

O $3.6002594 .039414 \quad 1.046019$

O 2.7413482 .2249032 .184080

C 4.9287303 .6002361 .345143

H $5.6304844 .288747 \quad 0.867271$

H 5.0972633 .6189602 .425922

H 5.0971142 .5962160 .946252 
C $1.134165-0.687750-1.217354$

C $1.611430-1.947598-1.790071$

C $0.964083-2.427660-3.061452$

C 4.0552690 .8783833 .007803

C $4.352759-0.5934663 .251754$

C $3.199202-2.7931803 .486469$

O $5.466382-1.0093023 .561815$

C $3.701533-3.5968302 .330124$

C $2.964338-4.5097801 .679980$

C $3.491439-5.356460 \quad 0.557973$

C $2.800924-5.085364-0.758851$

C $1.865913-5.923710-1.236264$

C $3.265694-3.894980-1.567197$

C $2.597316-2.613621-1.153420$

N $3.214748-1.3808203 .153748$

H -2.151156 4.7769510 .398023

H $-2.971323 \quad 3.583125-2.298964$

H $-0.2903793 .854010-0.752334$

H -1.010127 4.637007 -3.606642

H $0.4899353 .815323-3.378912$

H $2.0508876 .845331-1.773466$

H $0.0015397 .841330-2.079555$

H $-1.0088117 .081110-3.310533$

H -1.306208 $6.764449-1.587247$

H $2.5081494 .002206-2.808779$

H $3.6861715 .254950-2.491823$

H 3.1586085 .0517350 .003048

H $2.1061493 .711909-0.359890$

H $5.1153943 .853276-0.845930$

H $4.0704262 .371009-2.595083$

H $3.1345331 .565608-1.354877$

H $4.7019470 .029591-2.916482$

H 4.4389860 .1502070 .448573

H $6.4112661 .627197-3.047563$

H $7.3256390 .762305-1.833208$

H $6.8975472 .465295-1.572934$

H $6.378568-0.878403-0.098003$

H $6.220471 \quad 1.2180952 .918543$

H 5.2390852 .6477543 .114576

H 6.7930023 .2499331 .627221

H $-0.264938-0.069817-2.705534$

H $1.600091-0.398479-0.275832$

H $1.187552-1.744307-3.887885$

H $-0.121478-2.487502-2.939741$

H $1.288561-3.423906-3.368429$

H 3.6952651 .2679283 .968842

H 3.2349510 .9921572 .291145

H $2.171159-3.0539623 .757150$

H $3.840510-2.9617534 .357991$

H $4.735704-3.4405982 .032233$

H $3.359875-6.4078120 .845609$

H $4.573485-5.2245250 .433006$

H $1.390184-5.752102-2.195964$

H $1.549236-6.796310-0.674561$

H $3.134222-4.088071-2.637296$

H $4.350401-3.773848-1.449288$

H $3.003612-2.181840-0.239006$

H $2.346281-0.9618742 .836771$

C -4.9614573 .9984260 .920453$

O -5.0079432 .5933320 .607382$

C -4.7902171 .8484321 .821429$

C -4.8844532 .8555012 .964729$

C -4.3303794 .1050672 .308180$

H -3.7616251 .4710741 .772931$

C $-5.7681340 .673327 \quad 1.922116$

H -5.9210473 .0213433 .280015$

H -4.3123722 .5399543 .842593$

C -4.670453 5.3884443 .044331

H -3.240134 4.0012142 .256490

C $-5.783444-0.2550560 .691004$

O -7.0946851 .1921732 .083307$

H -5.5505390 .1001572 .831445$

H $-6.2711580 .255190-0.148231$

O $-6.625417-1.3739050 .999755$

C $-4.401704-0.7621770 .237649$

C $-4.504091-1.837477-0.861268$

H $-3.8208840 .084762-0.143748$

O $-3.694791-1.2819991 .359435$

H -4.844125 -2.779502 -0.417078

H $-5.262262-1.524468-1.588795$

H -4.2578896 .2571812 .521612$

H -5.7532465 .5283103 .129433$

H -4.2535615 .3733204 .056536$

C $-4.2832614 .772841-0.212472$

H -7.6972010 .4318562 .152912$

H -6.248425 -1.828962 1.771496

H -4.179266 -2.056637 1.690516

H $-4.3798675 .848859-0.025533$

H $-4.8204184 .564812-1.146652$

H -6.0086674 .3268450 .967286$

C $-2.152746-3.714237-0.160603$

O $-2.088966-2.404074-0.734337$

C $-3.169155-2.066260-1.607247$

C $-3.259264-3.078763-2.759708$

C $-3.314673-4.518282-2.254814$

C $-2.183050-4.780280-1.261902$

H $-3.047906-3.7995430 .466520$

C $-0.923699-3.8878310 .737870$

H -2.903215 -1.099387 -2.054008

O $-4.396551-2.827442-3.578797$

H -2.377499 -2.974992 -3.403673

H $-4.290248-4.749057-1.810868$

O $-3.155384-5.409067-3.361430$

H -1.228796 -4.792048 -1.802689

O $-2.351025-6.074008-0.686117$

C -1.024443 -3.016619 1.966704

H $-0.816212-4.9214531 .085821$

H $-0.002480-3.636017 \quad 0.203197$

H -4.324025 -1.914770 -3.906707

H -3.859012 $-5.204052-4.000978$

H -3.194560 -6.078988 -0.202396

O $-0.392642-1.827581 \quad 1.752513$

O $-1.621893-3.3444222 .983812$

C $-0.462132-0.8893222 .830620$

H $-0.012464-1.3127993 .733561$

H $\quad 0.1010350 .0019812 .542402$

$\mathrm{H}-1.500977-0.6016003 .016726$

SCF Energy $(\mathrm{B} 3 \mathrm{LYP} / 6-31 \mathrm{G} * * / / \mathrm{MMFF})=-3245.89876883$

01478

MM̄FF Geometry

C $-1.853889-3.9286440 .147186$

C -1.332757 -2.694995 0.086023

C $-0.327299-2.1598611 .071581$

O $0.793407-1.6207660 .334396$

C $-0.968637-1.0321191 .902073$

C $-0.006494-0.3056462 .823495$ 
C $0.119808 \quad 1.0359892 .861422$

C $0.815182-1.1642173 .748720$

C -0.6896662 .0363412 .087180$

C -0.1761132 .3350260 .673435$

C 1.1593363 .0914290 .642410

C $1.4324403 .621894-0.767766$

C $2.7904964 .331168-0.858460$

O 2.2070972 .1964491 .029486

O $3.1025544 .511871-2.248137$

C $3.8721863 .405268-0.268584$

C $2.7494155 .731871-0.237069$

O $5.1295024 .073581-0.191191$

C 3.4996262 .8120461 .111727

C $4.533477 \quad 1.7310601 .498968$

O 3.5270673 .8504942 .093300

C $1.773787-2.500177-0.012001$

O $1.793528-3.700176 \quad 0.214242$

C $2.835926-1.750415-0.720865$

C $3.889791-2.425089-1.204678$

C $5.044718-1.863199-1.906496$

C $5.021060-0.399389-2.253224$

C $4.137618 \quad 0.8630012 .697760$

C $5.293222-0.0377273 .085850$

C $6.592422-2.0125172 .286910$

O $6.000687 \quad 0.2085604 .060033$

C $7.532693-1.6906571 .166330$

C $8.058518-2.6176280 .350733$

C $8.988780-2.297962-0.785812$

C $8.562838-2.935440-2.092379$

C $9.244285-3.972842-2.607639$

C $7.391422-2.326992-2.838735$

C $6.070187-2.685728-2.212353$

N $5.462798-1.1029792 .215200$

H -1.541593 -4.604637 0.939203

H -1.662723 -2.018618 -0.698743

H $\quad 0.018002-2.951248 \quad 1.749385$

H -1.780320 -1.449120 2.513076

H -1.440621 -0.3239491 .211780$

H $\quad 0.846837 \quad 1.4709173 .545346$

H $1.369714-0.5691374 .482543$

H $0.166655-1.8477874 .306349$

H $1.545841-1.7534673 .186408$

H -1.7295781 .6949192 .024843$

H -0.7334962 .9679602 .665499$

H $-0.084613 \quad 1.396386 \quad 0.115045$

H -0.9387082 .9396910 .168755$

H 1.1047233 .9212831 .356716

H $0.6286764 .293021-1.095820$

H $1.4316802 .783072-1.478130$

H $2.3857215 .031383-2.650887$

H $4.0071142 .574225-0.975113$

H $2.0026936 .354068-0.745032$

H $3.7067016 .248998-0.365610$

H 2.5022885 .7227850 .826561

H $5.3303804 .416626-1.078839$

H $4.6823591 .059070 \quad 0.647330$

H 5.5022442 .2039661 .706368

H 3.7136003 .4620172 .962430

H $2.729885-0.675656-0.811812$

H $3.925387-3.503858-1.054896$

H $4.066922-0.120637-2.714650$

H $5.790801-0.116866-2.975494$

H $5.1695270 .207914-1.357290$

H 3.8735831 .4684963 .570578

H $3.268010 \quad 0.2413162 .459533$

H $6.195602-3.0297322 .212611$

H $7.103910-1.8990313 .247521$

H $7.794945-0.6452931 .019606$

H $7.818066-3.6657250 .514342$

H $9.989438-2.650285-0.503861$

H $9.082016-1.214630-0.931852$

H $8.960992-4.421355-3.554110$

H $10.098017-4.400631-2.092506$

H $7.375578-2.687859-3.875089$

H $7.552276-1.246482-2.895659$

H $5.974468-3.744020-1.964211$

H $4.828445-1.2032641 .428445$

C $-4.268367-4.455233-0.151343$
O $-4.721926-3.097948 \quad 0.026742$

C $-6.145446-3.050877-0.188696$

C $-6.598295-4.495175-0.382628$

C $-5.356589-5.129216-0.981257$

H $-6.305598-2.508393-1.127227$

C $-6.823105-2.3154290 .972928$

H $-6.850904-4.9737250 .570699$

H -7.474923 -4.568678 -1.033651

C $-5.352337-6.645561-0.900491$

H -5.274216 -4.820942 -2.032183

C $-6.304404-0.8754471 .181495$

O $-6.572834-3.0563222 .173971$

H -7.909820 -2.315533 0.826767

H -5.245639-0.894109 1.464130

O $-7.013561-0.3080612 .290073$

C $-6.5097850 .047273-0.036264$

C $-6.153903 \quad 1.5238490 .219250$

H $-5.928745-0.319224-0.887679$

O $-7.884448-0.000598-0.433116$

H $-6.5545582 .114360-0.613398$

H -6.7001821 .8867891 .098354$

H -4.436328 -7.057936 -1.334504

H $-5.420015-6.9925010 .136097$

H -6.202747 -7.061932-1.450257

C $-2.891563-4.441452-0.812697$

H -7.174496 -2.716320 2.857481

H -6.708269 -0.7610413 .094299$

H -8.4182360 .2989590 .322848$

H -2.913847 $-3.814423-1.712922$

H -2.599574 -5.450206 -1.124590

H $-4.212166-4.9103070 .846991$

C -4.071494 $2.018058-1.943882$

O $-3.8782351 .294183-0.723756$

C $-4.6425021 .788648 \quad 0.385450$

C $-4.2998163 .264500 \quad 0.646855$

C $-4.4508374 .121219-0.608847$

C $-3.6905693 .492760-1.777124$

H $-5.1309551 .960748-2.223356$

C $-3.3342941 .292825-3.075681$

H -4.3116821 .2214271 .263341$

O $-5.116147 \quad 3.803847 \quad 1.681222$

H -3.2614423 .3416330 .989614$

H $-5.5048144 .280192-0.865236$

O $-3.8983865 .416573-0.362072$

H -2.617249 $3.603777-1.592066$

O $-3.9962194 .197007-2.977284$

C -1.829197 $1.236150-2.928894$

H $-3.7107550 .262940-3.132402$

H $-3.5499301 .753772-4.046488$

H -4.9869883 .2528602 .472100$

H -4.3629855 .7865110 .408404$

H -3.774672 $5.132406-2.829313$

O $-1.421222-0.060956-2.818831$

O $-1.0958392 .215666-2.949479$

C $-0.004608-0.259278-2.786232$

H $\quad 0.4512860 .113271-3.708534$

H $0.4294610 .238576-1.915511$

H $0.186157-1.332922-2.711760$

SCF Energy (B3LYP/6-31G**//MMFF) $=-3245.91071572$

01479

MM̄FF Geometry

C $1.2642750 .514024 \quad 4.210617$

C 2.4008531 .2025674 .031417

C 2.6876712 .2167862 .952638

O 3.4234031 .5076381 .922348

C 1.4790872 .9136062 .287321

C 0.6597103 .8222793 .179363

C -0.6702543 .7167643 .372842$

C 1.4149994 .9630533 .812870

C -1.6092252 .6767762 .831212$

C -2.5922073 .2189331 .787817$

C -1.9367683 .6099820 .452440$

C $-2.9996724 .138607-0.515178$

C $-2.4154274 .455555-1.897586$

O $-1.3035582 .450356-0.102876$

O $-3.5255734 .637321-2.789486$

C $-1.6080573 .234828-2.384705$ 
C -1.647073 $5.781973-1.892399$

O $-0.9452443 .527563-3.613248$

C $-0.6084852 .689933-1.335465$

C $-0.0202541 .348104-1.834628$

O $0.449706 \quad 3.636088-1.174465$

C 4.2672102 .2314111 .138584

O $4.5370893 .416977 \quad 1.244510$

C 4.7825691 .3265280 .089316

C $5.4801031 .833506-0.937017$

C $6.0006111 .057951-2.057589$

C $5.8321091 .737620-3.389084$

C $0.8685430 .619796-0.818077$

C $1.339701-0.727894-1.337416$

C $2.492552-2.800865-0.580893$

O $1.150384-1.107603-2.489896$

C $3.951485-2.757956-0.906766$

C $4.449115-3.116559-2.099826$

C $5.914613-3.165200-2.428546$

C $6.331989-2.101901-3.419170$

C $5.967256-2.170969-4.710784$

C $7.240919-0.999632-2.927263$

C $6.601371-0.134697-1.877494$

N 2.000149-1.456210 - 0.357526

H $\quad 0.437818 \quad 0.647787 \quad 3.523619$

H 3.2229921 .0242774 .722479

H 3.3485942 .9732173 .394542

H 0.8666902 .1606021 .784941

H 1.8470843 .5338951 .458756

H -1.153916 4.4558914 .010800

H 0.7494325 .6815894 .303408

H 2.1108784 .5907024 .570893

H 1.9828425 .5113423 .053950

H -1.0773201 .8202892 .418275$

H -2.185347 2.2836693 .678594

H -3.3312832 .4292401 .600475$

H -3.1355064 .0774852 .201751$

H -1.179436 4.3788530 .641180

H $-3.5023435 .020613-0.098964$

H -3.784916 $3.379666-0.640239$

H -3.166515 4.762171 -3.684936

H $-2.3344202 .443404-2.616281$

H -2.310309 $6.604314-1.598077$

H -1.286106 $6.036068-2.895025$

H $-0.7956205 .782059-1.208506$

H $-0.1645094 .069011-3.406248$

H $-0.846550 \quad 0.676082-2.099032$

H $0.5651421 .517724-2.747462$

H $1.2402873 .179287-0.848811$

H $4.5529110 .269598 \quad 0.165879$

H $5.6626772 .904724-0.996648$

H $4.7888532 .041680-3.533371$

H $6.0888301 .095898-4.235546$

H $6.4621302 .631954-3.445122$

H $1.7582421 .212944-0.589269$

H $\quad 0.3166120 .4500460 .112054$

H $1.908669-3.278623-1.374387$

H $2.338432-3.3674830 .340856$

H $4.628880-2.457265-0.111600$

H $3.763531-3.429423-2.884716$

H $6.142446-4.155664-2.843826$

H $6.520211-3.091689-1.516573$

H $6.286676-1.424374-5.430210$

H $5.340439-2.977445-5.077036$

H $7.609730-0.389934-3.760138$

H $8.143654-1.452813-2.496798$

H $6.682179-0.533614-0.866446$

H $2.155799-1.0371410 .555062$

C $0.720420-1.8873024 .857491$

O $-0.462517-1.9015954 .030882$

C $-0.217742-2.7668162 .903950$

C $0.993273-3.6077063 .291430$

C $1.816644-2.5853404 .052169$

H $0.066426-2.1206892 .066162$

C - $-1.473345-3.5681112 .546029$

H $0.716726-4.4383633 .951449$

H $1.511862-4.0231082 .423962$

C $2.916375-3.1943634 .903056$

H $2.268075-1.8963663 .327040$
C $-2.735631-2.7269492 .255765$

O $-1.787619-4.4448963 .637943$

H $-1.261720-4.2239741 .694198$

H -3.052235 -2.236169 3.185196

O $-3.798373-3.6173921 .898756$

C $-2.624404-1.6581291 .153493$

C $-1.977060-2.105684-0.171392$

H -3.630311 -1.275019 0.945523

O $-1.879107-0.5560611 .668439$

H -1.779421 -1.204804 -0.763296

H $-0.983868-2.5254890 .020435$

H $3.464853-2.4169005 .444029$

H $2.512674-3.8975465 .639129$

H $3.630508-3.7380344 .275966$

C $1.032223-0.4639525 .328618$

H $-1.919532-3.8929714 .428081$

H -3.878074 -4.275125 2.610785

H -1.9206010 .1580761 .009473$

H $1.888949-0.4844846 .012177$

H $\quad 0.174873-0.0907665 .902857$

H $0.472801-2.4881015 .742797$

C -4.277129-1.483811-2.040157

O $-4.155551-2.636131-1.196517$

C $-2.814380-3.095453-1.002946$

C $-2.193030-3.462932-2.358628$

C $-2.296205-2.318797-3.365085$

C $-3.727540-1.786906-3.437862$

H $-3.729685-0.641667-1.599626$

C $-5.760962-1.107023-2.091942$

H -2.882623 -4.024269 -0.425519

O $-0.826864-3.833318-2.209682$

H -2.718655 -4.333261 -2.772645

H -1.593502 -1.512100 -3.129352

O $-1.930801-2.798672-4.661106$

H -4.366324 -2.520876 -3.945553

O $-3.743347-0.592152-4.216186$

C $-6.224749-0.583186-0.753472$

H $-5.968864-0.341376-2.847811$

H -6.378736 -1.979404 -2.337009

H $-0.790656-4.568515-1.574280$

H $-1.028898-3.156617-4.591935$

H $-3.362457-0.806898-5.084954$

O $-5.8172390 .708487-0.591345$

O $-6.856553-1.2488850 .056521$

C $-6.173698 \quad 1.3125490 .655746$

H -5.6577120 .8071541 .477567$

H -5.8559372 .3581450 .630776$

$\mathrm{H}-7.2577141 .2806180 .801216$

SCF Energy (B3LYP/6-31G**//MMFF $)=-3245.91298263$

0148

MM̄FF Geometry

C $-1.9804923 .089233-1.079300$

C $-1.4104982 .030918-0.484636$

C $-0.4892081 .069113-1.189764$

O $0.7239760 .935731-0.415255$

C -1.165456 -0.312938 -1.259319

C $-0.376157-1.349022-2.034290$

C $0.118984-2.485122-1.505264$

C $-0.184443-1.064159-3.501753$

C $0.006420-2.971757-0.088111$

C $1.166014-2.5568250 .830253$

C $2.536023-3.1082990 .402992$

C $3.585313-2.8505611 .486680$

C $4.986536-3.3140841 .054412$

O $2.931803-2.451571-0.803464$

O $5.937286-2.7456971 .968882$

C $5.301065-2.735545-0.343556$

C $5.137944-4.8359141 .164500$

O $6.524686-3.263649-0.849147$

C $4.160539-2.930892-1.364797$

C $4.388564-2.165859-2.691200$

O $4.066252-4.322014-1.695956$

C $1.6617091 .912234-0.547593$

O $1.6050962 .896862-1.266567$

C 2.7773561 .5835330 .366852

C 3.6692832 .5321180 .688517

C 4.8128952 .3709111 .589193 
C $5.1542130 .974208 \quad 2.036916$

C $4.530195-0.645477-2.557581$

C $5.970208-0.163517-2.492980$

C $7.3085241 .905184-2.120885$

O $6.935513-0.888625-2.717456$

C $7.7901891 .926128-0.704596$

C $8.161566 \quad 3.049140-0.071497$

C 8.6886443 .0839951 .334340

C $7.933648 \quad 4.0541952 .214930$

C $8.406694 \quad 5.290898 \quad 2.444847$

C 6.6774843 .5602122 .895792

C $5.5048863 .469546 \quad 1.957394$

N $6.0482431 .190118-2.193327$

H $-1.7631973 .297681-2.123970$

H $-1.632748 \quad 1.8206960 .558427$

H $-0.2453341 .423877-2.198436$

H $-2.153052-0.220427-1.731334$

H $-1.349473-0.658936-0.236847$

H $\quad 0.665437-3.159428-2.163997$

H $0.249546-1.915842-4.036722$

H $-1.146171-0.838987-3.974174$

H $0.486047-0.211547-3.645869$

H $-0.934752-2.6369680 .358170$

H $-0.063484-4.066823-0.109329$

H $1.206318-1.4621940 .877685$

H $0.933636-2.9152231 .840896$

H $2.433297-4.1842680 .219057$

H $3.297375-3.3312712 .430003$

H $3.627459-1.7741981 .701505$

H $5.707087-3.0445422 .865239$

H $5.471003-1.662431-0.200986$

H $4.945459-5.1664142 .192347$

H $6.163546-5.1486260 .939353$

H $4.456517-5.3827080 .509581$

H $6.348788-4.164435-1.170100$

H $5.230001-2.605559-3.239999$

H $3.506284-2.345233-3.322664$

H $3.605129-4.405248-2.547214$

H 2.8213790 .5769310 .766146

H 3.5402563 .5303960 .271880

H 5.2439630 .3072531 .172994

H 4.3785470 .5836782 .704217

H 6.1061270 .9055342 .567171

H $4.093998-0.177849-3.449770$

H $3.978213-0.271779-1.689987$

H $7.1336422 .911623-2.514137$

H $8.0487291 .406108-2.754437$

H $7.8620110 .972904-0.186078$

H $8.1154263 .997169-0.603136$

H $9.747108 \quad 3.371537 \quad 1.285783$

H 8.6747412 .0876561 .793409

H $7.8861975 .983543 \quad 3.097789$

H 9.3230815 .6398781 .980206

H 6.3907954 .2500003 .701078

H 6.8939922 .6164923 .403769

H 5.1815384 .4379251 .572727

H $5.1990151 .701149-1.970362$

C -4.341895 $3.891684-1.017058$

O $-4.8551512 .560578-0.820554$

C $-6.2857742 .631614-0.659604$

C $-6.6664284 .102443-0.812875$

C $-5.3895334 .806347-0.391242$

H $-6.5022792 .317247 \quad 0.367138$

C $-6.9662751 .683610-1.657279$

H -6.916814 4.353264 -1.850098

H $-7.5279044 .370341-0.193005$

C $-5.3131336 .250654-0.852838$

H $-5.314146 \quad 4.7697870 .703857$

C $-6.467368 \quad 0.224374-1.574248$

O $-6.714016 \quad 2.149639-2.989987$

H -8.053134 $1.722358-1.524706$

H $-5.4073550 .192817-1.847016$

O $-7.148425-0.541668-2.575662$

C $-6.677650-0.444135-0.202831$

C $-6.229955-1.918279-0.148068$

H -6.1440110 .1177840 .568674$

$\begin{array}{lllll}0 & -8.062566 & -0.384861 & 0.148446\end{array}$

H $-6.590445-2.3446520 .795978$
H $-6.745979-2.492178-0.927663$

H $-4.3766206 .715571-0.529562$

H $-5.3680506 .326752-1.944010$

H $-6.140408 \quad 6.832546-0.433265$

C $-2.9522534 .008141-0.391112$

H $-5.7504082 .155630-3.121804$

H $-8.104347-0.436264-2.433953$

H -8.550749 $-0.963962-0.460895$

H -3.0034183 .7706740 .677937$

H -2.579353 $5.034511-0.478466$

H $-4.2839664 .058634-2.101251$

C $-4.126826-1.6668052 .026195$

O $-3.986353-1.2829930 .653029$

C $-4.703982-2.104714-0.273007$

C $-4.225625-3.558715-0.157773$

C $-4.284479-4.0717601 .279362$

C $-3.606343-3.0917402 .237082$

H $-5.182182-1.6125142 .319124$

C $-3.347939-0.6508462 .867187$

H $-4.421113-1.758544-1.274019$

O $-4.996819-4.416648-0.991947$

H -3.186212 $-3.625084-0.504098$

H $-5.316863-4.2702061 .590994$

$\begin{array}{llll}\text { O } & -3.595377 & -5.321738 & 1.359678\end{array}$

H -2.519883 -3.1255082 .092177$

O $-3.869697-3.4934213 .579968$

C $-4.082938 \quad 0.6679152 .911415$

H -3.200230 -0.9895293 .898707$

H -2.354182 -0.4644832 .442373$

H $-4.927991-4.078811-1.901280$

H $-4.013696-5.9236610 .720421$

H -3.554340 -4.4085793 .675792$

O $-5.0951100 .608013 \quad 3.823783$

O $-3.806549 \quad 1.627826 \quad 2.204923$

C $-5.893438 \quad 1.7906293 .931328$

H -5.2747642 .6403764 .235738$

H $-6.393091 \quad 1.9969472 .980100$

H -6.654516 1.6186064 .697001

SCF Energy (B3LYP/6-31G**//MMFF) $=-3245.90944247$

01480

MMFF Geometry

C $-0.944354-4.820755-0.195018$

C $-0.539100-4.326426-1.375234$

C $0.668954-3.448581-1.612169$

O $1.100152-2.787817-0.399211$

C $1.838599-4.290062-2.151733$

C $2.960906-3.458278-2.741675$

C $4.205741-3.372042-2.234371$

C $2.625027-2.715915-4.011422$

C $4.764134-4.037863-1.009265$

C $4.669984-3.206590 \quad 0.280469$

C $5.319695-1.8153140 .191160$

C $5.424855-1.1759051 .577823$

C $5.968300 \quad 0.260987 \quad 1.512843$

O $4.503417-0.994046-0.649146$

$\begin{array}{lllll}\text { O } & 5.722220 & 0.883626 & 2.783448\end{array}$

C $5.1688851 .058260 \quad 0.458178$

C 7.4876790 .2848231 .307332

$\begin{array}{lllll}\text { O } & 5.745283 & 2.344211 & 0.247388\end{array}$

C $5.0259350 .319408-0.887940$

C $4.0640211 .023932-1.876292$

O $6.306193 \quad 0.254426-1.528191$

C $0.336654-1.7442490 .029622$

O $-0.676143-1.314682-0.499592$

C $0.930479-1.2145621 .275960$

C $0.217819-0.3510372 .014377$

$\begin{array}{lllll}\text { C } & 0.640639 & 0.293595 & 3.257658\end{array}$

C 2.0942170 .1884603 .632065

C $2.6251621 .203413-1.380590$

C $2.3458852 .567297-0.771235$

C 0.5661923 .8302090 .431186

O $3.1353663 .506190-0.827666$

C 0.9048473 .8329581 .888758

C $0.001993 \quad 4.0658232 .853532$

C 0.3320324 .1054254 .319005

C $-0.535793 \quad 3.1735335 .136090$

C -1.6182343 .6312005 .787749$ 
C $-0.104421 \quad 1.727747 \quad 5.258189$ C -0.2839750 .9660163 .974846$ N $1.0880432 .624435-0.184577$ H - $0.351231-4.6489980 .698955$ H -1.143707 -4.523420 -2.258679 H $0.385251-2.680858-2.342446$ H $1.483235-4.970347-2.937628$ H $2.212987-4.929367-1.345008$ H $4.925697-2.744404-2.759363$ H $3.509655-2.268187-4.477119$ H $2.181957-3.397401-4.745104$ H $1.916800-1.906053-3.812440$ H $4.289186-5.010468-0.842156$ H $5.819520-4.266999-1.206298$ H $3.613563-3.1013240 .551893$ H $5.151590-3.7818561 .080939$ H $6.315109-1.924416-0.255316$ H $6.044962-1.7896862 .243095$ H $4.429414-1.1492872 .040417$ H $6.1572250 .348072 \quad 3.468942$ H $4.170051 \quad 1.2279120 .878322$ H $7.993565-0.2306362 .132639$ H 7.8719061 .3108011 .316130 H $7.802781-0.1947400 .378367$ H $5.7969532 .784177 \quad 1.113247$ H $4.4985701 .972347-2.214241$ H $4.0094910 .394313-2.776506$ H $\quad 6.1687440 .102523-2.477948$ H $1.924251-1.5455461 .550409$ H $-0.782893-0.0846781 .679676$ H $2.723601 \quad 0.5580172 .815641$ H $2.364354-0.8521083 .842172$ H 2.3642740 .7711894 .514476 H $1.9494791 .107296-2.239440$ H $2.3562230 .422018-0.665227$ H -0.5142023 .8439920 .257831$ H $1.0098474 .706179-0.052771$ H 1.9428693 .6586612 .162229 H -1.030490 4.2669452 .575552 H 0.1959025 .1390934 .663072 H 1.3885313 .8706494 .499390 H -2.232107 2.9754826 .396191 H -1.917292 4.6722645 .722994 H $-0.702001 \quad 1.2184536 .025732$ H $\quad 0.923083 \quad 1.7033855 .629771$ H -1.313782 0.9696823 .613105 H $0.5157041 .786026-0.147862$ C $-3.121803-4.9109911 .035664$ O $-3.355737-3.532578 \quad 0.692173$ C $-4.718155-3.1923381 .005998$ C $-5.302780-4.394641 \quad 1.743012$ C $-4.508169-5.5415941 .148479$ H $-5.236583-3.0793600 .046425$ C $-4.774449-1.8687291 .779729$ H $-5.130903-4.3378362 .823756$ H $-6.381197-4.4910431 .583529$ C -4.547351 -6.808697 1.984215 H $-4.905967 \quad-5.7576590 .147921$ C -3.970994 -0.7162821 .143564$ O $-4.231960-2.0829363 .090852$ H $-5.820894-1.5754261 .925463$ H -2.901212 -0.9221371 .256047$ $\begin{array}{lllll}\text { O } & -4.243968 & 0.452770 & 1.926787\end{array}$ C $-4.293388-0.436013-0.336209$ C $-3.5581870 .801855-0.882520$ H $-4.024168-1.314675-0.925146$ O $-5.700079-0.246602-0.489133$ H $-3.9470251 .692838-0.372072$ H -2.494193 $0.740011-0.629982$ H -3.955862 -7.602436 1.517256 H $-4.148698-6.6386382 .990007$ H -5.575770 -7.170071 2.087031 C $-2.233693-5.574345-0.020313$ H $-4.287060-1.2374113 .568552$ H -3.544278 1.1017951 .741910 H -5.9585720 .5046910 .072013$ H -2.770964 -5.617959-0.976087 H $-2.006778-6.6048220 .274586$
H $-2.613738-4.9129182 .009271$

C $-2.3462403 .006173-2.537491$

O $-3.6500002 .435038-2.701598$

C $-3.7300611 .031771-2.402600$

C $-2.7687070 .261558-3.324479$

C $-1.3471220 .824588-3.290472$

C -1.362362 $2.338898-3.503529$

H $-2.0042232 .866282-1.504919$

C -2.463177 $4.513313-2.805819$

H $-4.7516280 .735595-2.668633$

O $-2.728524-1.122761-3.005334$

H $-3.1343630 .338329-4.357380$

H $-0.8411410 .562890-2.355196$

O $-0.597721 \quad 0.207434-4.339889$

H -1.638347 2.562718 -4.541808

O $-0.0575082 .864592-3.280108$

C $-3.2058275 .234282-1.699520$

H $-1.4698174 .969780-2.874924$

$\mathrm{H}-3.0103134 .682367-3.740783$

H $-3.621173-1.480135-3.150328$

H $\quad 0.3306390 .474937-4.234150$

H $\quad 0.5261842 .509279-3.971228$

O $-3.3460496 .548546-2.046466$

O $-3.6040414 .721720-0.662617$

C $-4.0256287 .367634-1.089596$

H $-4.0729278 .385483-1.485895$

H -5.045742 $7.003145-0.935360$

$\mathrm{H}-3.4734047 .383869-0.145108$

SCF Energy $\left(B 3 L Y P / 6-31 G^{* *} / / M M F F\right)=-3245.90495652$

01481

MMFF Geometry

C $1.744754-3.626490-1.433852$

C $1.195654-3.180965-2.573507$

C $-0.059227-2.353251-2.592814$

O $0.318631-1.005901-2.981460$

C -1.099812 -2.823009 -3.618844

C $-1.698374-4.194316-3.393648$

C -2.684007 -4.463295 -2.513977

C $-1.173552-5.280227-4.295866$

C $-3.283586-3.525904-1.506006$

C $-4.423483-2.664420-2.057066$

C $-4.977712-1.712334-0.983414$

C $-6.054384-0.798801-1.572336$

C $-6.5509690 .238985-0.553436$

O $-3.892507-0.926697-0.477715$

O $-7.2904901 .240765-1.269129$

C -5.3319030 .9460140 .080064$

C $-7.525591-0.3772340 .457357$

O

C $-4.250268-0.0307120 .583756$

C $-2.953606 \quad 0.6766341 .050371$

O $-4.761081-0.7487571 .714421$

C $0.710221-0.157158-1.992348$

O $0.737750-0.387627-0.794427$

C $1.1321401 .110196-2.629218$

C $1.6067102 .107610-1.868099$

C $2.1036053 .400404-2.344060$

C $2.0399503 .667271-3.825469$

C $-2.2067851 .479294-0.019971$

C $-2.5523862 .958351-0.044754$

C $-2.1376605 .021299-1.365170$

O $-3.281668 \quad 3.4969220 .782799$

C $-0.9005765 .606519-1.965231$

C $-0.1196266 .490912-1.326805$

C $1.1054417 .120691-1.924378$

C $2.3662926 .768690-1.167545$

C $2.7663737 .498625-0.112590$

C $3.2075935 .627722-1.692561$

C $2.5905134 .277983-1.441134$

N $-1.9143703 .614973-1.089713$

H $1.248530-3.424148-0.488015$

H $1.710163-3.342596-3.517174$

H $-0.520897-2.319119-1.601366$

H $-0.665806-2.772834-4.627814$

H - $1.906003-2.079015-3.657221$

H $-3.077894-5.477777-2.473992$

H -1.651969 -6.247015 -4.106387 
H $-1.353602-5.022435-5.344851$ H $-0.097027-5.412406-4.149803$ H $-3.662040-4.127908-0.670678$ H -2.502175 -2.894841-1.076988 H -4.051866 -2.074654 -2.903204 H -5.229006 -3.304543 -2.436996 H $-5.390122-2.319523-0.168693$ H $-6.893535-1.388136-1.962668$ H $-5.640573-0.260012-2.436331$ H $-8.0200730 .798377-1.735983$ H $-4.8972001 .587972-0.696321$ H -8.404294 -0.783935 -0.057644 H $-7.909478 \quad 0.380463 \quad 1.149357$ H -7.083843 -1.187337 1.041170 H -6.4040892 .4177010 .778850$ H -3.1534461 .2819701 .943020$ H -2.262281 -0.1092081 .386170$ H -4.012536 -1.059736 2.249764 H $1.0625141 .178424-3.708826$ H $1.652827 \quad 1.962177-0.790142$ H $1.0164683 .541095-4.196361$ H $2.6974912 .979225-4.367811$ H $2.3366694 .681516-4.100261$ H -1.133365 1.4266940 .201546 H -2.358085 $1.051816-1.016883$ H $-2.4348725 .536415-0.445505$ H -2.969543 $5.093643-2.073214$ H $-0.6498095 .302839-2.978376$ H $-0.3854746 .799495-0.318030$ H $0.9623888 .209310-1.917408$ H $1.2185056 .850986-2.981678$ H 3.6854657 .2718960 .417383 H $2.1793778 .337600 \quad 0.246574$ H $4.1908515 .627416-1.202759$ H $3.4274335 .802752-2.749648$ H $2.5847203 .995689-0.387304$ H -1.445292 $3.058374-1.799102$ C $4.209437-3.271386-1.310221$

O $4.012320-2.353601-0.219299$

C $5.299840-1.9079340 .248677$

C $6.346542-2.627086-0.602478$

C $5.594632-3.859988-1.071003$

H $5.389668-2.2516651 .285075$

C $5.374467-0.374514 \quad 0.194244$

H $6.661940-2.021978-1.460140$

H $7.245596-2.868908-0.026827$

C $6.211571-4.520919-2.290505$

H $5.558145-4.582251-0.244536$

C 4.2204030 .3484820 .919895

O $5.3405310 .050656-1.176217$

H $6.339701-0.0355510 .586656$

H $3.288354 \quad 0.176523 \quad 0.371297$

O 4.4717331 .7578680 .852615

C $4.028975-0.0381192 .397091$

C $2.947110 \quad 0.7832553 .125459$

H $3.778540-1.1009392 .479071$

O $\quad 5.247868 \quad 0.163250 \quad 3.117265$

H 2.9341930 .4578774 .173433

H 3.2515311 .8369463 .167754

H $5.635503-5.401535-2.591122$

H $6.248091-3.834944-3.143480$

H $7.235058-4.845155-2.075669$

C $3.081450-4.304322-1.349660$

H $4.505454-0.265863-1.561966$

H $4.5718061 .989653-0.086749$

H $5.895900-0.4851742 .795383$

H $3.090381-4.913581-0.437568$

H $3.216151-4.988474-2.195112$

H $4.200959-2.676797-2.234209$

C $0.903477-1.4624063 .440793$

O $1.163635-0.6898942 .265141$

C $1.536240 \quad 0.6683302 .514444$

C $0.4377381 .382686 \quad 3.314528$

C $0.054163 \quad 0.6176584 .580019$

C $-0.240513-0.8479764 .257298$

H $1.811364-1.4882244 .056182$

C $0.657628-2.9131603 .011794$

H $1.577747 \quad 1.1593131 .535674$
O $0.8405862 .701610 \quad 3.668188$

H $-0.458356 \quad 1.4798662 .689595$

H 0.8298250 .7013325 .350370

O -1.1275021 .2001865 .136359$

H -1.191915 -0.899261 3.713795

O $-0.405031-1.5538065 .484462$

C $-0.639030-3.0995452 .257958$

H $1.459696-3.2597782 .348670$

H $0.667719-3.5706023 .889030$

H 1.0553653 .1719062 .844391

H -0.9364692 .1396785 .300696$

H $-0.749072-2.4379255 .273369$

O $-1.455302-3.9494502 .943883$

O $-0.895979-2.5573661 .191555$

C $-2.717640-4.2276692 .330696$

H $-3.341355-4.7521373 .059392$

H -3.224570 -3.303231 2.039431

H -2.568576 -4.876463 1.463177

SCF Energy $\left(B 3 L Y P / 6-31 G^{* *} / / M M F F\right)=-3245.89680234$

01482

MM̄FF Geometry

C $3.776928-0.503608-2.821384$

C $3.434532-1.800675-2.823244$

C $3.245831-2.596832-1.558544$

O $1.967627-3.278178-1.635445$

C $4.329415-3.675743-1.362777$

C $5.614407-3.153148-0.752256$

C $5.958841-3.2926650 .543820$

C $6.582754-2.496873-1.703289$

C $5.210998-3.9768691 .653461$

C $3.827028-3.4373612 .016793$

C $3.710515-1.9236272 .233498$

C $4.577248-1.4022543 .377870$

C 4.3290030 .0946503 .632106

O $2.328004-1.6841532 .523728$

$\begin{array}{lllll}\text { O } & 4.933723 & 0.434219 & 4.889068\end{array}$

C 2.8115440 .3351423 .779999

C 5.0033920 .9754572 .572753

O $2.5213381 .727933 \quad 3.875413$

C $1.967031-0.3046712 .653265$

C $0.467193-0.2544293 .030634$

O $2.1753200 .411933 \quad 1.436363$

C $0.848186-2.534290-1.411475$

O $0.794343-1.346115-1.137240$

C $-0.319229-3.424646-1.593221$

C $-1.539857-2.894480-1.764743$

C $-2.774793-3.644860-2.007571$

C $-2.720612-5.142625-1.852942$

C $-0.450415-1.0141082 .062272$

C $-1.847553-1.1935362 .629416$

C $-3.879265-2.5877712 .384831$

O $-2.282250-0.5282213 .565830$

C $-4.526060-3.4039841 .313901$

C $-5.633718-3.0238980 .660518$

C $-6.312934-3.846114-0.395572$

C $-6.323688-3.160317-1.742607$

C $-7.315335-2.319085-2.081770$

C $-5.236380-3.524352-2.727427$

C $-3.889216-2.966522-2.354198$

N $-2.563872-2.1664591 .943717$

H $3.966153-0.021323-1.866356$

H $3.257222-2.309750-3.767273$

H $3.222060-1.940320-0.683126$

H $4.551294-4.172074-2.317537$

H $3.924422-4.483252-0.740252$

H $\quad 6.923118-2.8909440 .855094$

H $7.580821-2.386113-1.264937$

H $6.698280-3.098792-2.610567$

H $6.239931-1.498816-1.983647$

H $5.129348-5.0452061 .416231$

H $5.841379-3.9340972 .551334$

H $3.101111-3.7341521 .250026$

H $3.482639-3.9543072 .923294$

H $3.968236-1.4089601 .303863$

H $5.641661-1.5795233 .184851$

H $4.341329-1.9558384 .297363$

H 5.8814080 .2229644 .832368 
H $2.504654-0.1106524 .736612$ H 6.0854650 .7985882 .560604 H 4.8764302 .0386422 .804829 H 4.6286950 .7964441 .562685 H 3.0606042 .0873814 .600630 H $0.341234-0.6949074 .028711$ H $\quad 0.1282410 .7859453 .089879$ H 1.4316740 .2407230 .837388 H $-0.135087-4.492761-1.619883$ H -1.643174 -1.809997 -1.760863 H $-2.285930-5.414846-0.884241$ H -2.114671 -5.589597 -2.648388 H $-3.702815-5.618914-1.882761$ H $-0.546376-0.4687621 .117827$ H $-0.038013-2.0058871 .847330$ H $-4.465376-1.7011632 .650386$ H -3.752740 -3.1884633 .291697$ H $-4.069299-4.362167 \quad 1.078367$ H -6.096320 -2.0722120 .910115$ H -7.343863 -4.032723 -0.066658 H $-5.854612-4.838554-0.487293$ H -7.347722 -1.845837 -3.057473 H -8.113845 -2.080636 -1.386813 H -5.481792 -3.128328 -3.722234 H -5.224122 -4.610229 -2.859104 H $-3.829689-1.880873-2.435306$ H -2.063135 -2.763366 1.291004 C $3.4292031 .771402-3.905757$ O $2.0853191 .797985-3.383615$ C $2.0279582 .770955-2.325227$ C $3.2516243 .659430-2.513702$ C $4.2792842 .651387-2.984957$ H $2.1264292 .218739-1.381318$ C $0.7002843 .536756-2.326387$ H $3.0826814 .419773-3.285343$ H $3.541694 \quad 4.169857-1.590222$ C $5.4797563 .280168-3.671817$ H $4.6323882 .086814-2.114193$ C $-0.5179562 .629544-2.045947$ O $0.5581514 .201636-3.580776$ H $\quad 0.7557204 .309465-1.550822$ H $-0.2822651 .999611-1.180839$ O $-0.7454571 .753722-3.147414$ C -1.803267 $3.421407-1.733759$ C $-3.0566382 .556912-1.515196$ H -1.621951 4.040601 -0.848039 O $-2.0877364 .318555-2.809976$ H $-3.8803893 .235507-1.259367$ H -3.357730 $2.105436-2.469720$ H $6.1827432 .511350-4.007463$ H $5.1806473 .868776-4.545498$ H $6.0105463 .946758-2.984107$ C $3.9152440 .325912-4.066966$ H $-0.2533764 .735006-3.541666$ H $-0.9223092 .307607-3.927084$ H -2.858612 $4.852397-2.552440$ H $4.9654760 .327794-4.380023$ H $3.334341-0.142522-4.871719$ H $3.3762512 .226639-4.903424$ C -3.0630412 .8433331 .527067$ $\begin{array}{lllll}\text { O } & -2.293406 & 1.920456 & 0.750071\end{array}$ C $-2.9285301 .458249-0.443313$ C $-4.255110 \quad 0.766351-0.100257$ C -5.1524951 .6459010 .769155$ C $-4.3811792 .198400 \quad 1.967924$ H $-3.265973 \quad 3.7455860 .936824$ C -2.2135863 .2455402 .736640$ H $-2.2671380 .689585-0.862053$ O $-4.942206 \quad 0.402104-1.292078$ H $-4.037479-0.1613240 .440277$ H $\quad \begin{array}{llll}-5.591056 & 2.459728 & 0.180830\end{array}$ O $\quad-6.2593530 .8721911 .238190$ H -4.1859471 .3909112 .684189$ O $\quad-5.183745 \quad 3.1732042 .630371$ C -1.057849 4.1236432 .318526 H -2.7977313 .8136993 .469569$ H -1.816495 2.3680543 .259872 H -5.780354 -0.014374 -1.028230
H $-5.9100790 .157452 \quad 1.797228$

H -6.0219792 .7434722 .872538$

O 0.0195713 .3560991 .988939

O -1.1154045 .3458272 .275360$

C 1.1906404 .0691831 .579570

H $0.9472634 .826675 \quad 0.828843$

H 1.6628914 .5308802 .451222

H 1.8885693 .3530801 .137781

SCF Energy (B3LYP/6-31G**//MMFF) $=-3245.91752933$

01483

MM̄MF Geometry

C $-2.0209455 .372293-0.521600$

C -2.7421104 .6001950 .305085$

C $-3.8147223 .647846-0.157718$

O -3.4242712 .3172180 .254143$

C -5.1540623 .9827260 .521936$

C -6.2988083 .0945800 .071771$

C -6.9662912 .2399870 .872042$

C $-6.7004853 .228057-1.375190$

C -6.7564751 .9865942 .338287$

C -5.7887710 .8386492 .663847$

C $-6.251603-0.5395922 .163396$

C $-5.376330-1.6483142 .753296$

C $-5.756810-3.0340742 .208069$

O $-6.151882-0.5648180 .736599$

O $-4.701072-3.9463732 .548362$

C $-5.818075-2.9708340 .666482$

C -7.028663 -3.5772162 .870183$

O $-6.330846-4.1864710 .127776$

C $-6.631973-1.7742710 .131415$

C -6.520869-1.598442 -1.403355

O $-8.019080-1.9790760 .427668$

C $-2.5608791 .643260-0.552211$

O $-2.1247822 .005324-1.633113$

C -2.1992030 .3865600 .138255$

C -1.093533 -0.271201-0.238645

C $-0.576128-1.5036370 .357607$

C $-1.462829-2.232351 \quad 1.331448$

C $-5.101678-1.389008-1.942988$

C $-4.431995-2.661556-2.437017$

C $-2.249008-3.490101-3.311731$

O $-5.022779-3.730613-2.562831$

C $-1.463111-4.131128-2.211702$

C $-0.129429-4.274515-2.249484$

C $0.674347-4.954346-1.177859$

C $1.774510-4.073639-0.628907$

C $3.039071-4.184919-1.070007$

C $1.419840-3.1182150 .488017$

C $0.654681-1.921998-0.003161$

N $-3.098062-2.450192-2.760612$

H -2.229483 $5.350894-1.587684$

H -2.5252584 .6156401 .370940$

H $-3.9274193 .683191-1.248039$

H -5.4273645 .0254210 .311848$

H -5.0220823 .9172411 .607382$

H -7.772839 1.6536980 .432507

H -7.623190 2.682951-1.601854

H $-6.8741004 .279572-1.626148$

H -5.918765 2.834584 -2.031809

H -6.4109462 .8959292 .841460$

H -7.734507 1.7699522 .787136

H $-4.804114 \quad 1.0737492 .243852$

H -5.6740410 .8036043 .754469$

H -7.297314 -0.6811792 .460578$

H $-5.425920-1.6424983 .849259$

H -4.324988 -1.453288 2.501606

H -4.600241 -3.940739 3.515705

H $-4.784083-2.8834520 .312481$

H $-6.889098-3.6622503 .954537$

H -7.256042 -4.5895792 .518387$

H -7.903901 -2.947070 2.699540

H $-5.776956-4.9103300 .467164$

H -7.030214 -2.424018 -1.914964

H -7.093851 -0.697489-1.666634

H $-8.547354-1.432096-0.177101$

H -2.8318130 .0556790 .953165$

H $-0.4911750 .145928-1.044576$ 
H $-2.446584-2.4175960 .886934$ H -1.595154 -1.645020 2.246322 H -1.077130 -3.210205 1.626515 H -5.157297 -0.719135 -2.810814 H -4.465006 -0.905665 -1.196965 H -1.604538 -3.018480 -4.060318 H $-2.867825-4.243540-3.809709$ H -2.017655 -4.517992 -1.360082 H $0.418341-3.909065-3.115463$ H $1.107455-5.864546-1.612988$ H $\quad 0.036006-5.296785-0.353914$ H $3.836644-3.572371-0.662446$ H $3.303249-4.887063-1.853862$ H $2.334696-2.7417660 .965307$ H $\quad 0.898028-3.669746 \quad 1.274397$ H $1.211858-1.321854-0.722675$ H -2.679938 -1.541858-2.581859 C $0.4936645 .732932-0.398901$ O $\quad 0.692574 \quad 4.4403490 .211181$ C $1.2661833 .552564-0.773249$ C $1.8492484 .462237-1.842898$ C $0.8100365 .566388-1.886196$ H $0.4258652 .974789-1.176724$ C $2.2694362 .618445-0.089196$ H $2.8220054 .860369-1.528126$ H $1.9772193 .961682-2.806646$ C $1.2974156 .839070-2.556187$ H $-0.0625795 .192088-2.434606$ C $3.0471451 .701521-1.052294$ O 1.5453371 .7988930 .834960 H 2.9680303 .2177410 .505069 H $3.6973542 .297256-1.704107$ O $2.1228631 .018024-1.909385$ C $3.8882870 .606463-0.363465$ C 4.9291991 .1549830 .627647 H $3.233898-0.1095800 .146062$ O $4.570442-0.122444-1.388986$ H 5.6514801 .7668350 .076423 H 4.4323851 .8080341 .351205 H $0.5186497 .608003-2.542202$ H $2.1819757 .244664-2.053683$ H $1.5641486 .646020-3.600405$ C $-0.9029316 .265200-0.057187$ H 1.0501822 .3930601 .424818 H $1.7512301 .667592-2.528870$ H $3.892989-0.482272-1.987088$ H -1.042390 $7.260244-0.494722$ H $-0.968706 \quad 6.3882201 .031613$ H 1.2327096 .3994610 .064475 C $7.525168-0.361788-0.072417$ O $6.354996-0.8813860 .565136$ C $5.666040 \quad 0.041476 \quad 1.412312$ C 6.6170770 .5622502 .501808 C 7.8920181 .1561651 .907748 C 8.5432440 .1617650 .947296 H $7.2300830 .462928-0.733187$ C $8.093309-1.446909-0.999749$ H $4.885136-0.5394561 .920122$ O $5.980190 \quad 1.541995 \quad 3.314721$ H $6.899326-0.2680503 .162521$ H 7.6970132 .1137231 .410608 O 8.8188631 .4313182 .960705 H $8.977427-0.6610191 .526905$ $\begin{array}{lllll}\text { O } & 9.612494 & 0.802530 & 0.255381\end{array}$ C $8.562912-2.698799-0.286318$ H $7.318750-1.764812-1.707780$ H $8.933438-1.031283-1.567969$ H $5.184570 \quad 1.1325013 .695588$ H 8.3755502 .0304443 .585738 H $\quad 10.222874 \quad 1.153494 \quad 0.926359$ O $9.445292-3.363781-1.090048$ O $8.182701-3.069047 \quad 0.815870$ C $9.963634-4.585964-0.555597$ H $10.505094-4.3932390 .375623$ H $9.152023-5.301017-0.390487$ H $10.659804-5.007897-1.285259$

SCF Energy (B3LYP/6-31G**//MMFF) $=-3245.90811105$

\section{4}

MM̄FF Geometry

C $-1.358329-2.7293441 .414281$

C $-0.723114-2.7194960 .234562$

C $-1.213986-1.948410-0.959038$

O $-0.204307-0.938986-1.211092$

C $-1.365138-2.818372-2.216721$

C $-2.679770-3.570050-2.280501$

C $-3.785052-3.097200-2.891858$

C $-2.679542-4.945164-1.668628$

C $-3.938139-1.750288-3.541938$

C $-5.321636-1.109887-3.359528$

C $-5.790717-0.943992-1.904337$

C $-7.132390-0.201361-1.873465$

C $-7.6215080 .061481-0.442523$

O $-4.806222-0.213869-1.161344$

O $\quad-8.6822861 .025444-0.533054$

C -6.4693050 .6974820 .364286$

C $-8.240175-1.1956560 .179281$

O

C $-5.132227-0.0631730 .230361$

C -3.9658640 .6595010 .941850$

O $-5.267483-1.3409160 .867324$

C $-0.617750 \quad 0.272489-1.665673$

O $-1.7583520 .607527-1.938517$

C $0.5708171 .145856-1.766693$

C $0.4130912 .426254-2.132367$

C $1.4736023 .423691-2.271439$

C $2.8943512 .981080-2.042532$

C -3.6641942 .0810320 .463477$

C -2.4374432 .6549431 .158316$

C -1.1618924 .7681251 .391465$

O -1.8119182 .0493322 .025297$

C -0.6074275 .7855820 .446334$

C $0.702126 \quad 6.0665770 .360629$

C $1.2840507 .098055-0.565616$

C $2.3996836 .541175-1.424008$

C $3.6845566 .686420-1.058215$

C $2.0375565 .878694-2.735173$

C $1.1291084 .691968-2.576182$

N -2.134045 3.9340370 .706073

H -2.301754 -2.202298 1.512327

H $\quad 0.225351-3.241550 \quad 0.139645$

H $-2.165544-1.458013-0.723955$

H $-0.528045-3.525236-2.298949$

H $-1.257268-2.190733-3.110924$

H -4.664945 -3.736975 -2.936140

H -2.504685 -4.888935 -0.590665

H -3.631247 -5.466024 -1.819108

H -1.894063 -5.561744 -2.117936

H $-3.184808-1.048339-3.179514$

H -3.762334 -1.872033 -4.617791

H $-5.283080-0.122852-3.839214$

H $-6.065947-1.698914-3.910685$

H -5.905082 -1.941200-1.464208

H $-7.893370-0.748113-2.444271$

H -7.019500 $0.767430-2.380792$

H $-8.949505 \quad 1.2550860 .373805$

H $-6.3429671 .714100-0.027780$

H $-9.100639-1.528471-0.413852$

H $-8.631873-0.9918031 .181846$

H -7.541494 -2.032082 0.244567

H $-6.763652-0.0278642 .155537$

H $-4.144971 \quad 0.6662242 .025528$

H -3.0564250 .0602750 .808820$

H $-4.379302-1.6882451 .048311$

H $1.5366370 .718642-1.528119$

H $-0.5932362 .787080-2.342416$

H $3.1580162 .158377-2.716474$

H $3.0261372 .646206-1.008569$

H $3.6268693 .772916-2.215650$

H -3.493923 $2.096895-0.617581$

H -4.5062252 .7434220 .690821$

H $-0.3807944 .136861 \quad 1.828583$

H -1.692030 5.2700092 .207609

H -1.310376 $6.329146-0.180263$

H 1.3990925 .5404701 .009623

H 1.6656417 .9225710 .050782 
H $\quad 0.5145107 .541534-1.209638$

H $4.4947446 .313531-1.675918$

H $3.9548307 .178978-0.130011$

H $2.9321645 .590521-3.300507$

H $1.5306956 .617128-3.370319$

H $\quad 0.0736974 .929221-2.714058$

H -2.809873 $4.397010 \quad 0.104593$

C $-0.266414-2.4028513 .675399$

O $0.942722-1.7989853 .169847$

C $0.973714-0.4192613 .584781$

C $-0.155670-0.2504704 .599907$

C -1.177024 -1.2447714.086656

H $\quad 0.7408480 .1792842 .696058$

C 2.356443-0.040462 4.125629

H $0.160612-0.5220675 .613631$

H $-0.532390 \quad 0.7766804 .631106$

C $-2.235878-1.6233635 .106222$

H -1.667683 -0.7948523 .215748$

C $3.526633-0.2887663 .152294$

O $2.601804-0.8166935 .306389$

H $2.344172 \quad 1.0095814 .443257$

H $3.693512-1.3658643 .034453$

O $4.710464 \quad 0.242336 \quad 3.763833$

C 3.3741170 .3648331 .764573

C 4.6681730 .3904110 .925483

H $2.586920-0.1493251 .202556$

O 2.9431931 .7210831 .917896

H $4.4501530 .927177-0.004992$

H 5.4151081 .0147091 .432282

H -2.938462 -2.349440 4.685107

H $-1.790127-2.0653906 .003653$

H -2.806018 -0.7407445 .414361$

C $-0.830461-3.3943822 .651811$

H $3.359323-0.4185475 .767079$

H $4.935644-0.3326784 .514327$

H 3.6132632 .1842792 .449499

H -1.644094 -3.972669 3.104603

H $-0.043939-4.1093812 .378962$

H $0.034825-2.9727094 .564657$

C $3.984758-1.522614-1.358161$

O $4.313221-1.864528-0.008094$

C $5.263886-0.9968160 .617265$

C $6.565001-0.955955-0.198796$

C $6.312340-0.596123-1.662276$

C $5.230158-1.501277-2.252219$

H $3.523424-0.528787-1.364133$

C $2.899876-2.483464-1.853428$

H $5.514100-1.4583661 .579791$

O $7.479664-0.0322180 .381250$

H $7.038415-1.945440-0.154480$

H $6.041856 \quad 0.460854-1.765399$

O $7.523226-0.760377-2.404062$

H $5.642791-2.510452-2.364091$

O $4.886835-1.044063-3.557809$

C $3.361363-3.917352-1.960179$

H $2.043215-2.463731-1.172653$

H $2.522416-2.176526-2.835644$

H $8.274814-0.019166-0.178501$

H $7.791031-1.693043-2.341006$

H $4.527765-0.144076-3.475053$

O $3.304415-4.520077-0.738566$

O $3.735520-4.435528-3.004406$

C $3.731000-5.885296-0.705340$

H $3.639549-6.2451790 .322973$

H $4.778406-5.962189-1.012425$

H $3.092809-6.497427-1.349593$

SCF Energy (B3LYP/6-31G**//MMFF) $=-3245.90778193$

\section{5}

MM̄FF Geometry

C $-1.101164-4.832954-1.907922$

C $-0.210738-4.244940-1.095396$

C $0.985145-3.471744-1.592693$

O $0.934626-2.131969-1.052664$

C $2.276458-4.159701-1.103033$

C $3.595150-3.527924-1.518500$

C $4.736692-3.665345-0.810659$

C $3.629062-2.788026-2.831036$
C $4.948156-4.4531850 .453267$

C $5.738370-3.7196011 .548474$

C $5.145468-2.4104862 .096314$

C $3.682795-2.5366232 .525676$

C $3.108438-1.2028653 .028623$

O $5.298168-1.3884321 .106152$

O $1.678550-1.3327993 .067312$

C $3.431169-0.1044851 .992899$

C $3.553129-0.8908344 .461179$

O 3.0217641 .1775532 .461899

C $4.912002-0.0811771 .552124$

C 5.1775820 .9142860 .400531

O 5.7142780 .3470162 .660372

C $0.163729-1.233301-1.728461$

O $-0.564082-1.461238-2.682225$

C $0.3968700 .102649-1.136771$

C $-0.0557641 .191168-1.776355$

C $0.1421882 .586525-1.376332$

C $0.9661742 .857253-0.145403$

C $4.4527030 .606035-0.910421$

C $4.7343481 .664250-1.962563$

C $3.7507592 .691471-4.007301$

O $5.7420202 .367167-1.948091$

C $3.1435433 .967277-3.511805$

C $2.0849224 .564246-4.079521$

C $1.5038895 .859670-3.588417$

C $0.0350775 .754574-3.244477$

C $-0.9003116 .334879-4.015251$

C $-0.3445755 .045432-1.960888$

C $-0.4161093 .551665-2.136923$

N $3.744397 \quad 1.713493-2.934932$

H $-0.973316-4.753446-2.984546$

H $-0.336831-4.324480-0.018163$

H $\quad 0.991237-3.427911-2.689301$

H $2.281449-5.195190-1.469394$

H $2.226596-4.203575-0.009649$

H $5.642875-3.208471-1.207302$

H $4.643924-2.486496-3.112558$

H $3.247292-3.424530-3.635836$

H $3.024808-1.877513-2.781460$

H $4.012836-4.8290980 .875517$

H $5.523499-5.3475230 .179471$

H $5.862391-4.4173612 .386744$

H $6.745438-3.5103951 .163365$

H $5.759984-2.1359912 .962589$

H $3.566353-3.3199853 .284607$

H $3.070096-2.8504431 .673095$

H $1.459262-2.0695063 .663074$

H $2.805686-0.3207391 .120779$

H $3.234664-1.6889575 .142758$

H 3.0774360 .0237044 .832226

H $4.635183-0.7856744 .561815$

H 3.6743331 .4746713 .118737

H $4.939657 \quad 1.9343360 .730248$

H $\quad 6.2573340 .9240890 .193847$

H 6.5647750 .6698722 .319160

H $0.9617240 .156977-0.215280$

H $-0.613117 \quad 1.051126-2.702084$

H $\quad 0.972144 \quad 3.907775 \quad 0.151908$

H $2.0071292 .562248-0.310017$

H 0.5797022 .3028670 .715796

H $4.782655-0.356964-1.312808$

H $3.3745960 .557465-0.746360$

H $3.1931502 .263119-4.845930$

H $4.7827722 .872202-4.325248$

H $3.6138274 .434804-2.649505$

H $1.6234864 .113614-4.955115$

H $1.6566226 .610063-4.375023$

H $2.0485386 .234153-2.712525$

H -1.953397 $6.284714-3.758895$

H $-0.6352036 .868133-4.922095$

H - $1.3343255 .385479-1.629003$

H $0.3509745 .353313-1.175708$

H -1.017703 $3.244238-2.994085$

H $2.9113041 .142992-2.824793$

C $-3.616325-4.916844-1.505070$

O $-3.649668-3.827150-0.563990$

C $-4.410175-2.747323-1.142254$ 
C $-5.048648-3.304590-2.412263$

C $-4.006228-4.315655-2.853652$

H -3.684705 -1.978500 -1.434037

C $-5.385274-2.170411-0.108972$

H $-5.998443-3.810954-2.210446$

H $-5.235612-2.525219-3.157325$

C $-4.520965-5.331362-3.857348$

H $-3.167522-3.765275-3.297323$

C $-4.715042-1.730271 \quad 1.209679$

O $-6.353465-3.1658850 .245973$

H -5.942055 -1.330334 -0.541679

H -4.360463 -2.607555 1.764509

O $-5.723516-1.1202982 .027071$

C $-3.557893-0.7294121 .038368$

C $-3.015753-0.2319782 .393964$

H -2.743039 -1.211112 0.486124

$\begin{array}{lllll}\text { O } & -4.022516 & 0.366879 & 0.257677\end{array}$

H $-3.798300 \quad 0.3307082 .913186$

H -2.780366 -1.108381 3.008915

H $-3.734387-6.042638-4.128515$

H -5.366663 -5.900011 -3.456503

H -4.855630 -4.832061 -4.772500

C $-2.271718-5.640426-1.423777$

H -7.013259-3.203855 -0.466506

H $-6.440843-1.7687552 .132389$

H $-3.253707 \quad 0.9236280 .047591$

H -2.314377 -6.563523 -2.014018

H -2.097985 -5.942434 -0.382839

H -4.393439 -5.619862 -1.175250

C -2.7907972 .8361922 .200992$

O -2.0033621 .8529361 .517670$

C -1.7533590 .6490142 .251433$

C -1.0510880 .9760563 .576838$

C -1.815052 2.0185834 .385669

C -2.1233723 .2395423 .521115$

H -3.7926252 .4345682 .394256$

C -2.932153 4.0464861 .270614

H $-1.0408840 .067977 \quad 1.652397$

O $-0.878097-0.2067594 .349921$

H $-0.047872 \quad 1.3647393 .357549$

H -2.7269701 .5896474 .817538$

O -0.9973682 .4199325 .487127$

H -1.190533 3.7821023 .322901

O $-2.972562 \quad 4.1233384 .250770$

C $-3.9796043 .796003 \quad 0.212216$

H -3.2509304 .9431621 .814681$

H $-1.982151 \quad 4.2936770 .782939$

H -0.4307680 .0484485 .174977$

H -1.478449 3.1167035 .965689

H -3.810889 3.6604324 .420129

O $-3.407813 \quad 3.246005-0.894730$

$\begin{array}{lllll}\text { O } & -5.168916 & 4.044547 & 0.362787\end{array}$

C $-4.2990432 .966421-1.978844$

H -5.115565 2.316778 -1.649408

H -4.691031 3.901211 -2.390099

$\mathrm{H}-3.7334782 .448523-2.758089$

SCF Energy (B3LYP/6-31G**//MMFF) $=-3245.92111152$

01486

MM̄FF Geometry

C $-3.359889-1.225942-3.724487$

C $-2.539040-1.891343-2.899631$

C $-1.083200-2.137505-3.205240$

O $-0.305575-1.422090-2.213151$

C $-0.768281-3.640007-3.085662$

C $0.375897-4.073127-3.979753$

C $1.645280-4.274289-3.578180$

C $-0.000451-4.310113-5.421724$

C $2.226721-4.096745-2.204276$

C $3.105193-2.848256-2.164322$

C $3.938153-2.687797-0.885123$

C $3.085331-2.681766 \quad 0.384413$

C $3.911961-2.3464771 .633486$

O $4.621547-1.439867-1.039038$

O $2.997926-2.0714862 .705537$

C $4.699077-1.0490451 .370175$

C $4.771901-3.5309312 .088176$

O $5.582459-0.7597432 .451966$
C $5.482147-1.0610550 .038617$

C $6.0482830 .340991-0.302803$

O $6.584800-1.9646780 .157946$

C $-0.125795-0.085852-2.414980$

O $-0.3923830 .545374-3.425708$

C $0.3782730 .502017-1.151746$

C $0.3611801 .835582-0.999819$

C 0.7234872 .5808140 .207952

C 1.1831871 .7972201 .408446

C $4.9787461 .346048-0.740696$

C $5.5198082 .746803-0.951900$

C $4.7064485 .009500-1.631347$

O $6.6567523 .088622-0.642780$

C $4.0616875 .647199-0.441917$

C 2.910846 6.333814 -0.496149

C 2.2428116 .9208210 .712798

C $0.887323 \quad 6.306203 \quad 0.983274$

C -0.2318207 .0491450 .942990$

C 0.8357334 .8433731 .379809

C 0.5823883 .9240090 .214061

N $4.5476323 .574575-1.499206$

H -2.977670 -0.856669 -4.673048

H -2.916482 -2.248040 -1.944255

H $-0.824181-1.770182-4.205935$

H -1.643765 -4.240587 -3.368847

H $-0.564704-3.896675-2.041655$

H $2.370912-4.596051-4.324900$

H $0.852519-4.633983-6.027721$

H $-0.766603-5.089480-5.489726$

H $-0.395116-3.394999-5.873717$

H $1.447109-4.033886-1.441512$

H $2.824861-4.984569-1.966983$

H $3.796071-2.847567-3.019394$

H $2.482767-1.954467-2.305708$

H $4.679178-3.494739-0.847981$

H $2.575159-3.6420050 .524980$

H $2.289997-1.9313360 .283805$

H $2.446163-2.8616612 .837908$

H $3.966603-0.2349511 .349799$

H $4.138751-4.3937072 .327946$

H $5.315522-3.2937013 .009336$

H $5.493392-3.8519501 .333948$

H $5.051839-0.7574893 .267066$

H $\quad \begin{array}{llll}6.630657 & 0.727056 & 0.543023\end{array}$

H $6.7686770 .247858-1.127522$

H $7.256368-1.722651-0.500721$

H $0.688346-0.175223-0.365146$

H $0.0127622 .447637-1.831216$

H 1.4141322 .4255522 .272582

H 2.0886151 .2301281 .174380

H 0.4061981 .0970041 .730466

H $4.5182001 .013841-1.678697$

H 4.1921121 .4255710 .014846

H $4.2299725 .307412-2.570575$

H $5.7675575 .273423-1.674743$

H 4.5592355 .5140240 .516103

H $2.4110726 .467506-1.452596$

H 2.1535738 .0030340 .554246

H 2.8672096 .7999101 .607233

H $-1.202605 \quad 6.616437 \quad 1.162074$

H -0.2033228 .1028920 .686872$

H 0.0311524 .7003242 .113151

H 1.7725034 .5828251 .886001

H $0.2035424 .406495-0.687171$

H $3.6172123 .196561-1.655055$

C $-5.0407230 .487097-2.884173$

O $-4.542810 \quad 0.607249-1.537813$

C $-4.1407831 .975917-1.320827$

C $-4.4657252 .738482-2.606832$

C $-4.4055831 .642355-3.652947$

H $-3.0538201 .964728-1.182397$

C $-4.8282482 .542593-0.071566$

H $-5.4668683 .183536-2.577586$

H $-3.7529393 .548002-2.793279$

C $-5.1171841 .989203-4.947951$

H -3.3482981 .441541 -3.864593

C $-4.585711 \quad 1.726670 \quad 1.214565$

O $-6.2419452 .564551-0.312207$ 
H -4.5215673 .5857290 .072600$ H $\quad-5.0668600 .745563 \quad 1.128776$ O $\quad-5.2377372 .4091082 .293748$ C -3.1027851 .5519431 .593029$ C -2.8708360 .9421642 .989885$ H -2.599212 0.9340350 .842569 O $-2.4562412 .827924 \quad 1.571357$ H -1.7919740 .9595113 .186119$ H -3.2988421 .6046033 .752809$ H $-5.0504701 .162175-5.661900$ H $-6.1776492 .204089-4.778540$ H -4.665106 $2.872574-5.410647$ C $-4.798276-0.928175-3.416138$ H -6.6466543 .1226820 .373124$ H -6.1962512 .3101062 .165577$ H $-2.913871 \quad 3.4007812 .210541$ H $-5.377998-1.079837-4.334415$ H -5.173295 -1.653919-2.683373 H -6.127249 $0.635714-2.816967$ C -1.558699 -1.7172202 .262170$ O $-2.942442-1.3731562 .143581$ C -3.426514 -0.486092 3.157901 C $-3.198040-1.0930554 .550380$ C -1.742376 -1.498262 4.769396 C -1.257836 -2.374483 3.614374 H $-0.962739-0.8025272 .166431$ C -1.162664 -2.585139 1.062186 H -4.512353 -0.4242823 .019242$ O $-3.607061-0.1738425 .557971$ H -3.830670 -1.9844404 .654379$ H -1.104037 -0.6171604 .902948$ O $-1.660671-2.2419005 .987157$ H -1.747392 -3.3520653 .691088$ O $\quad 0.142267-2.6017203 .749021$ C -1.825804 -3.944997 1.033825 H -1.445768 -2.0817920 .132794$ H $-0.074825-2.7186121 .059590$ H -3.430222 -0.5891896 .419461$ H $-0.737622-2.5312606 .088160$ H $0.591191-1.7408103 .692003$ O $-0.952787-4.862411 \quad 0.524227$ O $-2.977340-4.167717 \quad 1.382125$ C $-1.459619-6.1945660 .401340$ H $-1.742374-6.5848921 .383669$ H $-2.313110-6.212646-0.283131$ $\mathrm{H}-0.666004-6.822964-0.011428$ SCF Energy (B3LYP/6-31G**//MMFF) $=-3245.91525733$

01487

MM̄MF Geometry

C $-2.370014-4.828408-1.712036$

C $-1.311465-4.148432-2.179015$

C $0.122408-4.196036-1.705137$

O $0.465196-2.858823-1.257545$

C $0.400347-5.155779-0.532100$

C $1.863726-5.215671-0.133030$

C $2.351840-4.8169731 .058222$

C $2.799453-5.793245-1.164672$

C $1.598829-4.2554042 .231006$

C $1.526910-2.7214132 .278164$

C $2.894039-2.0264942 .395629$

C $2.714697-0.5392222 .709626$

C 4.0541220 .2127442 .741451

O $3.588925-2.1710221 .153510$

O 3.7720861 .6204242 .705733

C $4.849795-0.1184131 .460970$

C $4.817392-0.0413184 .046544$

O $\quad 6.1661810 .4232531 .526939$

C $4.917347-1.6280621 .152272$

C $5.545825-1.940970-0.228715$

O $5.735322-2.2731752 .136699$

C $0.807389-1.939670-2.199992$

O $0.942115-2.121950-3.399056$

C $0.934152-0.636813-1.515222$

C $0.7444300 .491726-2.213899$

C $0.7960061 .851878-1.675891$

C $1.2829142 .015883-0.261414$

C $4.828426-1.326789-1.435517$
C $5.3976630 .009878-1.882726$

C $4.9246651 .896419-3.443103$

O $6.4645310 .459337-1.473438$

C $4.2194873 .001105-2.720674$

C $3.4814733 .932761-3.343610$

C $2.8009585 .073525-2.641854$

C $1.3260575 .161926-2.966998$

C $0.8759576 .000457-3.915579$

C $0.3683564 .341103-2.133390$

C $0.4201942 .876159-2.469588$

N $4.5885070 .625180-2.828854$

H $-2.261917-5.543486-0.902805$

H -1.489874 -3.447226 -2.993698

H $\quad 0.751589-4.470950-2.560173$

H $0.083016-6.172692-0.796931$

H $-0.214230-4.8517220 .321510$

H $3.424170-4.9126171 .226765$

H $3.807234-5.954976-0.767027$

H $2.428657-6.762246-1.514461$

H $2.890989-5.121555-2.023455$

H $0.581465-4.6591932 .268273$

H $2.081257-4.6198943 .147049$

H $1.004139-2.3628291 .383981$

H $0.910013-2.4473893 .143148$

H $3.463764-2.5174423 .193286$

H $2.183134-0.4060633 .659374$

H $2.076688-0.0808481 .942383$

H $3.185278 \quad 1.8282683 .453000$

H 4.3415460 .3909330 .633322

H 4.2231550 .2881564 .907532

H 5.7441660 .5416774 .082973

H $5.064496-1.0937414 .200847$

H $\quad 6.078848 \quad 1.3771121 .695688$

H $6.613197-1.689105-0.223031$

H $5.506250-3.031938-0.361244$

H $6.048424-3.117573 \quad 1.772054$

H $1.138727-0.645221-0.451447$

H $\quad 0.5039280 .414421-3.273399$

H $2.2741331 .564886-0.143144$

H $0.591036 \quad 1.531870 \quad 0.435453$

H 1.3820123 .0560200 .053940

H $4.944068-2.005874-2.290032$

H $3.757344-1.224662-1.242028$

H $4.6293871 .835586-4.495367$

H $6.0066262 .054282-3.392217$

H $4.3427763 .051959-1.641382$

H $3.3847923 .894400-4.426526$

H $3.3047836 .000012-2.947447$

H $2.9320185 .011548-1.554375$

H $-0.1827336 .090457-4.135352$

H $1.5575246 .612113-4.497445$

H $-0.6639974 .674947-2.303854$

H $0.5578084 .544985-1.076522$

H $0.0833972 .650017-3.482253$

H $3.7012460 .198172-3.078826$

C $-4.711306-4.079406-1.164139$

O $-4.138088-2.933068-0.507027$

C $-5.124924-1.893270-0.414789$

C $-6.461822-2.557598-0.730611$

C $-6.049828-3.611701-1.738213$

H $-4.890982-1.171409-1.205336$

C $-5.060140-1.2049680 .954254$

H $-6.902155-3.0369410 .150805$

H -7.193730 -1.846746 -1.126202

C $-7.067940-4.725168-1.911682$

H $-5.894165-3.118395-2.707068$

C -3.663606 -0.746782 1.424925

O $-5.549773-2.116926 \quad 1.952038$

H $-5.757030-0.3599240 .966347$

H $-3.062921-1.641377 \quad 1.634791$

O $-3.843589-0.0955582 .688197$

C -2.8595670 .1779950 .490750$

C $-3.588247 \quad 1.408400-0.074625$

H -1.9731820 .5330251 .030218$

O $-2.373429-0.570107-0.625645$

H $-2.873201 \quad 1.933916-0.721097$

H $-4.3808001 .079209-0.757286$

H $-6.717620-5.462826-2.640576$ 
H -7.256306 -5.247255 -0.967538 H $-8.021368-4.322648-2.269114$ C $-3.759865-4.604723-2.243450$ H -4.987844 -2.910436 1.925156 H -2.967232 0.1732543 .009990 H -1.868052 -1.325105 -0.278761 H $-3.723968-3.889473-3.075228$ H $-4.142710-5.546855-2.652148$ H $-4.859691-4.842720-0.389646$ C $-2.150516 \quad 3.5558321 .586524$ O $\quad-3.2470402 .7305601 .991184$ C -4.1731772 .3910750 .957644$ C $-4.764500 \quad 3.666840 \quad 0.339041$ C $-3.6728064 .626314-0.131575$ C -2.6627754 .8721810 .988460$ H $-1.537073 \quad 3.0193570 .852905$ C -1.295142 3.8261582 .831493 H $-5.0034491 .872326 \quad 1.450577$ O $-5.6255873 .326371-0.742013$ H $-5.3789514 .173781 \quad 1.094585$ H $-3.1728354 .260376-1.035883$ O $-4.2609175 .882628-0.476518$ H -3.1305185 .4836641 .771065$ O -1.5606495 .6139930 .471106$ C $-0.5089512 .607258 \quad 3.252435$ H -0.5655154 .6233422 .647062$ H -1.910393 4.1476413 .680790 H $-6.0781814 .138071-1.026426$ H -4.815328 $5.741019-1.262241$ H $-1.917833 \quad 6.4270730 .074532$ O O $\quad 0.6776972 .4447002 .999725$ C -0.6707650 .5448464 .393983$ H $0.2097750 .781448 \quad 4.998916$ H -1.383307 -0.0049595 .014534$ H $-0.398541-0.0777723 .536744$

SCF Energy (B3LYP/6-31G**//MMFF) $=-3245.89853486$

01488

MM̄FF Geometry

C $-0.228458 \quad 4.4804802 .376319$

C 0.6603173 .5666221 .956265

C 1.5899042 .8370072 .892098

O 1.4572961 .4114972 .681302

C 3.0473923 .2242002 .585465

C 4.0393272 .6434653 .576385

C 4.8226531 .5748063 .328451

C 4.1506523 .3791604 .885849

C 4.8150210 .7221832 .091811

C 5.9694581 .0752001 .150859

C $5.8926900 .287170-0.165935$

C $7.0780640 .643911-1.064945$

C $6.978570-0.022619-2.444432$

O $4.6625110 .632235-0.811300$

O $7.9327980 .612867-3.309130$

C $5.5784410 .257200-3.030191$

C $7.365867-1.505078-2.386773$

O $5.397734-0.445219-4.258894$

C $4.420157-0.048008-2.049975$

C $3.0904830 .505559-2.624607$

O $4.330481-1.459650-1.868361$

C $\quad 0.456093 \quad 0.7665203 .332556$

O $-0.447903 \quad 1.2853523 .973876$

C $0.611231-0.7060943 .256795$

C $1.518430-1.3314672 .490376$

C $1.784243-2.7730982 .462311$

C $0.912113-3.6584013 .314588$

C $1.8365670 .171202-1.800850$

C $1.196278-1.150963-2.195384$

C $-0.586753-2.740506-1.507378$

O $1.569800-1.816132-3.158319$

C $0.020707-3.768246-0.608836$

C $0.984384-4.612415-1.005390$

C $1.631606-5.633326-0.117221$

C $3.055866-5.2616560 .230194$

C $4.068831-5.539529-0.607950$

C $3.309474-4.6421291 .585370$

C $2.798575-3.2313341 .696928$
N $\quad 0.145451-1.496557-1.356679$

H -0.2761124 .6917743 .443663$

H $\quad 0.747110 \quad 3.3248580 .901184$

H 1.3599753 .0705193 .939529

H 3.1549434 .3173942 .593314

H 3.3062662 .9151071 .565242

H 5.5290421 .2582324 .093999

H 4.9135842 .9465835 .541804

H 4.4210704 .4257594 .711752

H 3.1990773 .3505425 .425458

H $4.893531-0.3291332 .394732$

H $3.8606430 .810057 \quad 1.566576$

H 5.9289092 .1503420 .930204

H $\quad 6.926848 \quad 0.8781611 .648844$

H $5.890085-0.7845020 .065595$

H $8.0266910 .383456-0.579025$

H $7.1084851 .732959-1.211607$

H $8.8145530 .506759-2.912646$

H $5.5438581 .325111-3.286143$

H $8.390044-1.618506-2.011050$

H $7.365021-1.953411-3.386441$

H $6.711970-2.094600-1.740919$

H $5.210140-1.374668-4.043269$

H $3.1697541 .600461-2.661857$

H $2.9515060 .160646-3.655596$

H $3.519160-1.657670-1.375154$

H $-0.052543-1.2572363 .915549$

H $2.154919-0.7374641 .839856$

H $-0.145465-3.5055373 .072720$

H $1.065085-3.4403884 .377118$

H $\quad 1.100557-4.724477 \quad 3.173175$

H $2.0575580 .169465-0.728077$

H $1.0830550 .947198-1.985553$

H - $0.568647-3.057773-2.555565$

H -1.627266 -2.562496 -1.224789

H $-0.348878-3.8111310 .411866$

H $1.345315-4.559206-2.030292$

H $1.615213-6.600175-0.636931$

H $1.049439-5.7895530 .799100$

H $5.096113-5.299464-0.354834$

H $3.890431-6.010018-1.569333$

H $4.388492-4.6072871 .788371$

H $2.906002-5.3014402 .359268$

H $3.370684-2.5133861 .107416$

H $-0.044833-0.927277-0.537191$

C -1.1926455 .0477250 .032829$

O $-1.6487193 .710710-0.246950$

C $-2.6536133 .760142-1.274228$

C $-2.5203745 .133783-1.916452$

C $-2.1486425 .981394-0.715269$

H $-3.6239273 .702354-0.765740$

C $-2.5045812 .569643-2.226869$

H -1.715838 $5.162242-2.660030$

H -3.445221 $5.457571-2.403149$

C $-1.5386147 .323057-1.083335$

H $-3.0527366 .153304-0.115649$

C $-2.3959971 .206817-1.514084$

O $-1.3078092 .749521-2.994621$

H -3.335772 $2.568544-2.942168$

H -1.419083 $1.120523-1.022555$

O $-2.4427720 .181507-2.514104$

C $-3.5059440 .922842-0.484719$

C $-3.362069-0.4786990 .138275$

H $-3.458714 \quad 1.6645450 .319303$

O $-4.7778131 .056996-1.110464$

H $-3.539518-1.234079-0.633350$

H -2.327676 -0.5955260 .481839$

H -1.274191 $7.890934-0.185824$

H $-0.6311047 .199937-1.683943$

H $-2.2476287 .921243-1.664952$

C -1.1973935 .2824861 .546441$

H -1.322913 2.101841 -3.719101

H -1.613860 $0.226534-3.019733$

H $-4.8117400 .423064-1.847302$

H -2.2002535 .0653691 .936695$

H $-0.995544 \quad 6.341725 \quad 1.745947$

H $-0.1767795 .137124-0.371980$

C $-6.173567-1.7136200 .140008$ 
O $-5.698021-0.641503 \quad 0.963188$

C $-4.317477-0.720317 \quad 1.328763$

C -4.053692 -2.033095 2.081310

C $-4.512159-3.2455371 .276794$

C $-5.961755-3.0673000 .828722$

H $-5.648981-1.699579-0.822419$

C $-7.663041-1.463183-0.124108$

H -4.1419670 .0978962 .039009$

O $-2.673005-2.1761362 .395994$

H $-4.600424-2.0224413 .033222$

H -3.854992 -3.432461 0.419307

O $-4.438820-4.4114562 .100295$

H -6.625872 -3.156800 1.698011

O $-6.303349-4.112531-0.079492$

C $-7.868090-0.258665-1.011345$

H -8.148567 -2.321311 -0.602747

H $-8.197519-1.2735520 .814808$

H $-2.412528-1.4002062 .921199$

H $-3.519941-4.4888212 .409847$

H -6.138506 -4.9566940 .374778$

O $-7.582013-0.578751-2.305921$

O $-8.2326350 .835457-0.600948$

C $-7.7343200 .485328-3.250537$

H $-8.778370 \quad 0.810013-3.287629$

H -7.078373 $1.322038-2.991839$

H -7.448379 $0.108152-4.236073$

SCF Energy (B3LYP/6-31G**/MMFF) $=-3245.91037968$

01489

MM̄FF Geometry

C $-0.9124624 .903079-0.647078$

C $-0.943877 \quad 4.0906510 .419595$

C -1.905456 2.9394780 .545266

$\begin{array}{lllll}\text { O } & -1.144871 & 1.706932 & 0.620837\end{array}$

C $-2.7496803 .022351 \quad 1.829017$

C -3.8594714 .0506951 .770953$

C $-5.077130 \quad 3.806776 \quad 1.246631$

C -3.551431 5.3859172 .393545

C -5.5338412 .5316460 .594657$

C -6.5860521 .8134721 .448476$

$\begin{array}{llll}\text { C } & -6.925409 & 0.429645 & 0.876900\end{array}$

C $-8.072292-0.215748 \quad 1.655340$

C -8.357027 -1.6469641 .172409$

O $-5.750655-0.3797530 .987820$

O $-9.203769-2.2781892 .145237$

C $-7.030764-2.4373621 .143264$

C $-9.134537-1.655775-0.149109$

O $-7.222309-3.7319270 .576040$

C $-5.874622-1.6933890 .431388$

C $-4.539875-2.4405130 .679412$

O $-6.144913-1.651587-0.968647$

C $-0.6534511 .209565-0.547765$

O $-0.7462241 .709929-1.657551$

C $0.014888-0.075214-0.247095$

C $0.735325-0.677303-1.204700$

C $1.410115-1.972209-1.097618$

C $1.123935-2.8042000 .124030$

C $-3.288451-1.7199960 .158227$

C $-2.976207-2.047366-1.288487$

C $-2.145478-3.890641-2.745978$

O $-3.215134-1.251475-2.193570$

C $-0.864176-4.658360-2.670525$

C $0.141785-4.508463-3.545586$

C $1.426671-5.283063-3.476543$

C $2.650515-4.394065-3.504032$

C $3.440423-4.338262-4.589725$

C $3.004671-3.633067-2.244613$

C $2.218818-2.358140-2.106813$

N -2.421206 $-3.306550-1.446380$

H -1.623166 $4.749356-1.455331$

H $-0.2248864 .232157 \quad 1.222734$

H -2.573785 $2.877812-0.323341$

H -2.104379 3.2143862 .697516

H -3.177508 2.0333632 .043511

H -5.822748 4.5998491 .270588

H -4.4090456 .0664472 .360997$

H -3.272999 5.2586783 .444882

H -2.724468 5.8758821 .871983
H $-5.9643182 .778880-0.383593$

H $-4.6908851 .865026 \quad 0.390549$

H $-6.200246 \quad 1.6982032 .470088$

H -7.4925542 .4288921 .504510$

H -7.192751 $0.542542-0.180396$

H -8.979327 0.3983511 .593712

H -7.811054 -0.2568162 .722256$

H $-10.015675-1.7486362 .224824$

H $-6.739759-2.6080972 .188983$

H -10.081992 -1.114428 -0.039453

H $-9.407415-2.676078-0.440414$

H $-8.585162-1.194959-0.972698$

H -7.284157 -3.625848 -0.388576

H $-4.408589-2.5488551 .764138$

H $-4.594515-3.4480500 .250743$

H $-5.343132-1.358038-1.428736$

H $-0.118797-0.4970740 .742241$

H $0.840763-0.177449-2.167010$

H $0.045299-2.9449100 .252200$

H $1.518514-2.3174921 .022054$

H $1.556676-3.8052650 .082102$

H $-3.359519-0.632897 \quad 0.268633$

H -2.423392 -2.030262 0.758521

H $-2.119448-3.108299-3.511244$

H $-2.972841-4.569787-2.974844$

H $-0.761962-5.380218-1.863464$

H $0.031012-3.805101-4.367841$

H $1.442982-5.974149-4.329232$

H $1.464494-5.914216-2.579847$

H $4.334135-3.723277-4.606971$

H $3.208632-4.901563-5.487606$

H $4.068144-3.360859-2.262302$

H $2.894983-4.300525-1.387331$

H $2.359601-1.671721-2.943440$

H $-2.376066-3.919766-0.637312$

C $1.2760205 .648274-1.694302$

O $2.0951034 .678524-1.013326$

C $2.6568853 .780358-1.991622$

C $2.2995304 .356658-3.360579$

C $0.9817545 .050024-3.068662$

H $2.1401092 .821384-1.869294$

C $4.1594643 .600192-1.743175$

H $3.0425835 .084677-3.705652$

H $2.2155503 .578785-4.125806$

C $0.5813906 .074710-4.114486$

H $0.2037804 .279751-3.005675$

C $4.5080143 .171742-0.303056$

O $4.8054024 .855881-1.991174$

H $4.5700392 .888520-2.469455$

H $4.241728 \quad 3.970340 \quad 0.400479$

O $5.9310823 .016198-0.219152$

C $3.8563101 .850016 \quad 0.149550$

C 4.2706551 .4863581 .590702

H 2.7657191 .9459200 .113302

O $4.2335170 .822795-0.762600$

H 5.3596951 .3913051 .637919

H 3.9949572 .3253512 .240789

H $-0.3673756 .551178-3.848078$

H $1.3370386 .860671-4.216987$

H $0.4577085 .597950-5.092345$

C $0.0743176 .020563-0.821711$

H $5.7601744 .685224-2.056491$

H $6.3262123 .901937-0.283366$

H $3.7581750 .013507-0.509839$

H $-0.4513206 .877535-1.258699$

H $\quad 0.4363486 .3418540 .163394$

H $1.9012166 .544733-1.804723$

C $5.375600-1.3744531 .580473$

O $4.015703-0.9724001 .387172$

C $3.615814 \quad 0.189895 \quad 2.121287$

C $3.833861-0.0483163 .623261$

C $5.277566-0.4406093 .928127$

C $5.699412-1.624898 \quad 3.059277$

H $6.031855-0.5804381 .203565$

C $5.657757-2.5864070 .679319$

H $2.5353940 .298001 \quad 1.962942$

$\begin{array}{lllll}\text { O } & 3.493899 & 1.108773 & 4.379894\end{array}$

H $3.175363-0.8587083 .962420$ 
H $5.9618590 .406128 \quad 3.799930$

O $5.379436-0.8351435 .298323$

H $5.197145-2.5264263 .426531$

O $7.102112-1.8399453 .199502$

C $4.857992-3.8291211 .013788$

H $5.413128-2.325661-0.357146$

H $6.723146-2.8379480 .735079$

H $2.568581 \quad 1.3287674 .177054$

H $5.067554-0.0881675 .837495$

H $7.283536-1.9619904 .147308$

O $5.394484-4.891691 \quad 0.342027$

O $3.877710-3.8690751 .743644$

C $4.738577-6.1465220 .549948$

H $5.239669-6.897859-0.066076$

H $4.817508-6.4429891 .600154$

H $3.689850-6.0852750 .243854$

SCF Energy (B3LYP/6-31G**//MMFF) $=-3245.91834353$

0149

MM̄FF Geometry

C $-1.7249704 .056982-0.198107$

C -0.7607294 .7535090 .422576$

C $0.557961 \quad 4.1968770 .918088$

O $\quad 0.662521 \quad 2.771376 \quad 0.692004$

C 1.7261234 .8871110 .188415

C 3.0967764 .3513110 .562674

C $4.0002793 .909360-0.334804$

C 3.4352344 .3494852 .030661

C $3.8686603 .925882-1.830834$

C $3.1752382 .696976-2.431061$

C $3.9959041 .403663-2.324761$

C $3.4096340 .334501-3.250580$

C $4.156672-0.999365-3.130755$

O $3.9513380 .942660-0.970151$

O $3.356715-1.987856-3.797015$

C $4.244496-1.381080-1.640365$

C $5.499985-0.963007-3.867675$

O $5.043697-2.548632-1.462671$

C $4.735135-0.231945-0.725144$

C $4.548748-0.6479890 .750227$

O $6.123328-0.000338-0.971036$

C 0.1219611 .9577201 .640822

O -0.4785472 .3051932 .646044$

C 0.3811380 .5543991 .244741

C $-0.216655-0.4267881 .936839$

C $-0.062440-1.8689551 .751358$

C $0.813380-2.3812940 .641330$

C $4.7327760 .477501 \quad 1.773029$

C $4.651750-0.0899133 .177473$

C $3.039583-1.1252104 .779686$

O $5.658802-0.2594163 .861253$

C $2.662963-2.5326054 .429114$

C $1.561649-3.1449764 .891004$

C $1.176469-4.5531234 .531045$

C $-0.262170-4.6747274 .070977$

C $-1.190700-5.2501004 .853650$

C $-0.611266-4.1955492 .675816$

C $-0.705711-2.6943792 .604064$

N $3.357875-0.4153033 .553718$

H -1.580552 $3.001019-0.395953$

H $-0.927087 \quad 5.811144 \quad 0.617290$

H $0.633736 \quad 4.405378 \quad 1.992933$

H 1.7077945 .9638920 .402965

H $1.5589304 .787163-0.889832$

H 4.9520633 .5280030 .031768

H 4.4846494 .0941432 .213500

H 3.2613935 .3412642 .460478

H 2.8226863 .6204872 .569955

H $3.3318144 .828144-2.147460$

H $4.8715294 .029599-2.264426$

H $2.2027272 .555266-1.945022$

H $2.9839542 .915473-3.489030$

H $5.0333621 .622047-2.603410$

H $3.4054710 .676891-4.292981$

H $2.3562680 .168297-2.986123$

H $3.779857-2.852449-3.657102$

H $\quad 3.229737-1.662827-1.328683$

H $5.345838-0.733916-4.929045$
H $5.992658-1.941281-3.843327$

H $6.190327-0.219617-3.463616$

H $5.976133-2.282225-1.535790$

H $3.534363-1.0386690 .878844$

H $5.239884-1.4652820 .994207$

H $6.5216290 .398942-0.181593$

H 1.0625630 .3750660 .422049

H $-0.883334-0.1442682 .751725$

H $1.844498-2.4809480 .989604$

H $0.801514-1.726612-0.234576$

H $0.481652-3.3573550 .273941$

H 5.7029010 .9720961 .662740

H 3.9607221 .2456571 .655684

H $2.218177-0.5870495 .262785$

H $3.906041-1.1262245 .447747$

H $3.331568-3.0754053 .764551$

H $0.907195-2.6122325 .577431$

H $1.340575-5.1777575 .418653$

H $1.834477-4.9591153 .752557$

H -2.217981 -5.3623184 .523008$

H $-0.946329-5.6175145 .844869$

H -1.580994 -4.604776 2.364548

H $0.125396-4.6074081 .979258$

H -1.353695 -2.255889 3.364266

H $2.597733-0.2409352 .903787$

C $-4.2423544 .002790 \quad 0.061353$

O $-4.3128622 .582266-0.193918$

C -4.6429121 .9169561 .039904$

C -5.1391623 .0065941 .986325$

C -4.2641284 .1734441 .581278$

H -3.7067861 .5075141 .436993$

C $-5.661200 \quad 0.7953600 .816316$

H $-6.195077 \quad 3.2453181 .812692$

H -5.0241272 .7264583 .037991$

C -4.7869195 .5221922 .042911$

H -3.2672634 .0147132 .010271$

C $-5.216789-0.347372-0.119616$

O

H $-5.947360 \quad 0.373775 \quad 1.787578$

H -5.211535 -0.007883 -1.162013

O $-6.224564-1.366146-0.033365$

C $-3.856002-0.983980 \quad 0.219801$

C $-3.602074-2.370983-0.400340$

H $-3.055664-0.299460-0.078228$

O $-3.742885-1.1442861 .639004$

H $-2.594434-2.681265-0.099176$

H $-4.267700-3.0988050 .081949$

H -4.1252456 .3293621 .713040$

H -5.7878935 .7226451 .646261$

H $-4.8456545 .558303 \quad 3.135637$

C $-3.0384004 .625269-0.653138$

H $-6.6258781 .801801-0.561238$

H -7.072854 -0.955265 -0.274033

H -4.473969 -1.715886 1.930439

H -3.056169 $5.713555-0.523701$

H $-3.1225444 .428026-1.728872$

H $-5.1549164 .429821-0.375086$

C -1.520179-1.822209-2.579504

O $-2.924211-1.547498-2.639223$

C $-3.756379-2.468186-1.930795$

C $-3.541279-3.886735-2.477234$

C $-2.073508-4.298175-2.406230$

C $-1.198313-3.240740-3.075483$

H -1.172660 -1.691221-1.549302

C $-0.808165-0.790261-3.469807$

H $-4.789970-2.192559-2.169865$

O $-4.345586-4.816802-1.759410$

H -3.869887 -3.919503 -3.524186

H -1.762618 -4.484811-1.371741

O $-1.928568-5.539313-3.100520$

H $-1.335052-3.296263-4.163419$

O $\quad 0.174270-3.520127-2.803450$

C $-0.9207110 .619862-2.938762$

H $\quad 0.258347-1.023817-3.552326$

H -1.260272 -0.801919-4.469243

H $-4.160472-5.700664-2.120619$

H -1.014746 -5.843710 -2.970668

H $\quad 0.393920-4.365266-3.229935$ 
O $-0.448294 \quad 1.485487-3.883997$

O $-1.360344 \quad 0.924919-1.840039$

C $-0.525276 \quad 2.873874-3.548162$

H $-1.5719823 .188129-3.505378$

H $-0.0242443 .069762-2.596719$

H $-0.0183533 .442349-4.332350$

SCF Energy (B3LYP/6-31G*//MMFF) $=-3245.92478814$

01_490

MM̄FF Geometry

C $-0.707353-3.627487-0.856166$

C $0.461953-2.971643-0.818649$

C $0.863344-2.0418540 .297068$

O $2.208937-2.4272010 .671498$

C $0.842260-0.566987-0.154186$

C $-0.533350-0.036571-0.500692$

C $-0.8709690 .520264-1.679826$

C $-1.550708-0.0898370 .611429$

C $0.000514 \quad 0.775923-2.877016$

C $0.161480 \quad 2.279487-3.148317$

C $0.8637453 .041136-2.011450$

C $0.872338 \quad 4.543312-2.299139$

C $1.6240145 .322168-1.211031$

O $2.2051772 .553876-1.901715$

O $1.8506046 .653466-1.697818$

C $3.0070344 .672875-1.005596$

C $0.795162 \quad 5.463547 \quad 0.071284$

O 3.6905825 .2709140 .094281

C $2.9582333 .135140-0.826680$

C $4.3976542 .570559-0.890487$

$\begin{array}{lllll}\text { O } & 2.385339 & 2.828497 & 0.444800\end{array}$

C $2.704346-1.9521211 .843610$

O $2.162029-1.1910842 .628370$

C $4.053402-2.5246652 .017474$

C $5.003915-1.7805422 .600351$

C $6.378435-2.2039632 .869902$

C $6.672396-3.6805782 .800275$

C $4.4969141 .038111-0.879366$

C $5.917754 \quad 0.577025-1.163684$

C $7.228418-1.459379-1.725746$

O $6.872367 \quad 1.347912-1.228102$

C $7.933197-1.961268-0.506803$

C $9.011887-1.3664330 .024946$

C $9.780126-1.9006931 .199968$

C $9.675849-1.0170572 .422819$

C 10.4464910 .0755572 .556366

C $8.744814-1.4582963 .529447$

C $7.294900-1.2634563 .180848$

N $5.996162-0.797037-1.343547$

H -1.422059 -3.488150 -0.048833

H $1.170761-3.103856-1.633155$

H $\quad 0.216921-2.1956541 .170184$

H $1.557134-0.443527-0.973456$

H 1.2444290 .0679860 .644313

H -1.896209 $0.867232-1.802587$

H -2.4531250 .4813810 .373462$

H $-1.856364-1.1198920 .813583$

H - 1.1329180 .3379581 .528863

H $0.9840880 .307528-2.794883$

H $-0.4793930 .313857-3.748436$

H $0.7428232 .390300-4.072582$

H $-0.8264442 .720631-3.332447$

H $0.3348472 .850608-1.071890$

H $-0.1504364 .926181-2.405958$

H $1.364998 \quad 4.728719-3.263987$

H $0.9857087 .049880-1.899714$

H $3.6062714 .896306-1.899718$

H $-0.1489965 .980809-0.138420$

H 1.3163296 .0802880 .811794

H 0.5497204 .5047580 .532508

H $3.713776 \quad 6.229957-0.065877$

H $4.8711072 .929256-1.814425$

H $4.9877502 .959553-0.050728$

H 2.7017701 .9589140 .734319

H $4.224657-3.5286311 .647750$

H $4.759385-0.7602922 .894129$

H $6.506947-4.0638031 .787848$

H $6.024287-4.2317223 .491066$
H $7.700638-3.9352673 .063797$

H 4.2000620 .6365020 .095003

H $3.8425200 .609966-1.646699$

H $7.853390-0.766385-2.299584$

H $6.968387-2.298433-2.378665$

H $7.560019-2.883604-0.071317$

H $9.388147-0.452169-0.429011$

H $10.833452-1.9910820 .903052$

H $9.465633-2.9220161 .446876$

H $\quad 10.3995820 .6965953 .444690$

H $11.1393620 .374071 \quad 1.776455$

H $8.938454-0.8791714 .442465$

H $8.978773-2.4905503 .805733$

H $6.980464-0.2189033 .198764$

H $5.166812-1.367786-1.206868$

C $-2.141575-4.009870-2.927548$

O $-3.294387-3.510207-2.219724$

C $-3.637332-2.221912-2.765630$

C $-2.946029-2.149774-4.120328$

C $-1.645577-2.867457-3.815996$

H $-3.187723-1.470321-2.107208$

C $-5.155732-2.030906-2.802088$

H $-3.507307-2.689975-4.891706$

H $-2.800406-1.120043-4.460183$

C $-0.896410-3.327071-5.054280$

H $-1.000271-2.188285-3.248171$

C $-5.850733-2.199341-1.434583$

O $-5.730675-2.984173-3.703948$

H $-5.388732-1.044826-3.220433$

H $-5.840670-3.255970-1.142131$

O $-7.231547-1.853256-1.601695$

C $-5.250067-1.349077-0.298933$

C $-6.064971-1.4577321 .003622$

H $-4.224471-1.682687-0.108935$

O $-5.1977760 .007700-0.730433$

H $-7.020742-0.9385950 .875320$

H $-6.285457-2.5160511 .185282$

H $0.024190-3.851610-4.779500$

H $-1.503361-4.005740-5.662593$

H $-0.623681-2.469648-5.678179$

C $-1.110672-4.579036-1.948137$

H $-5.487009-3.871229-3.387871$

H -7.272562 - $0.903928-1.809634$

H $-4.7225110 .512146-0.049647$

H $-0.231233-4.931536-2.499884$

H $-1.541759-5.459944-1.455521$

H $-2.506206-4.833774-3.554933$

C -6.1480371 .3901152 .194939$

$\begin{array}{llll}0 & -5.020069 & 0.510682 & 2.105136\end{array}$

C -5.317397 $-0.883322 \quad 2.230920$

C $-6.014924-1.1507603 .572446$

C $-7.253263-0.2779843 .745932$

C $-6.901163 \quad 1.1896613 .516028$

H -6.8208551 .2101111 .348047$

C $-5.618327 \quad 2.8264292 .094055$

H $-4.348159-1.3972552 .268290$

O $-6.385642-2.519447 \quad 3.696268$

H $-5.319524-0.9315454 .393268$

H $-8.067893-0.5963873 .084970$

O $-7.742294-0.4148485 .082191$

H -6.2943561 .5538714 .355124$

O $-8.100783 \quad 1.960783 \quad 3.491255$

C $-5.139425 \quad 3.1357800 .695390$

H -6.3907493 .5658392 .333670$

H -4.7930702 .9837552 .799624$

H $-5.577328-3.0510173 .598169$

H -7.924230 -1.3592765 .227449$

H -8.568311 1.7968694 .328330

O $-3.779791 \quad 3.233671 \quad 0.675433$

O $-5.886173 \quad 3.262926-0.266565$

C -3.202096 $3.532933-0.599115$

H -3.507837 $4.531674-0.924499$

H -2.114671 $3.516499-0.491107$

H $-3.4936872 .780423-1.337722$

SCF Energy (B3LYP/6-31G**//MMFF) $=-3245.90928003$

01491

MM̄FF Geometry 
C $2.609381-3.149388 \quad 1.566904$

C $1.830908-3.6574142 .535371$

C $0.550771-3.0453223 .058771$

O $0.149031-1.9492622 .207305$

C $-0.579335-4.0880563 .068068$

C $-1.816719-3.6201283 .808870$

C $-2.984007-3.2986713 .217381$

C $-1.700895-3.5923965 .311658$

C $-3.279790-3.2906751 .742264$

C $-4.341887-2.244608 \quad 1.385654$

C $-4.827288-2.353128-0.067374$

C $-3.769406-1.908213-1.079071$

C $-4.313391-1.918791-2.517937$

O $-5.986803-1.517849-0.177744$

O $-3.388643-1.192423-3.342848$

C $-5.651652-1.149281-2.556220$

C $-4.381458-3.338691-3.092353$

O $-6.273249-1.259561-3.834083$

C -6.641211-1.578290 -1.452059

C $-7.906767-0.685464-1.386093$

O $-7.097464-2.910954-1.722352$

C $-0.077746-0.7369672 .776421$

O $-0.053159-0.4590973 .964203$

C $-0.3249250 .221046 \quad 1.674657$

C -0.3653261 .5350531 .939800$

$\begin{array}{llll}\text { C } & -0.525184 & 2.612320 & 0.960068\end{array}$

C $-0.733606 \quad 2.216588-0.478088$

C -7.690015 $0.826153-1.289981$

C $-6.903826 \quad 1.240379-0.060628$

C -4.7987342 .3704200 .620427$

$\begin{array}{llll}\text { O } & -7.253559 & 0.925349 & 1.074446\end{array}$

C $-4.016845 \quad 3.5351240 .106181$

C -3.7788824 .6496900 .812673$

C -3.0090695 .8197090 .272261$

C -1.6959696 .0388810 .986790$

C $-1.547976 \quad 7.035873 \quad 1.875254$

C $-0.526533 \quad 5.162136 \quad 0.594985$

C $-0.461813 \quad 3.8887381 .394777$

N $-5.7881042 .000269-0.374398$

H $2.336284-2.2118071 .090167$

H $2.148571-4.5764603 .024306$

H $0.759924-2.6989654 .077783$

H $-0.233705-5.0148283 .545913$

H $-0.828817-4.3663572 .037233$

H -3.820893 -3.0305153 .860864$

H $-2.649219-3.3384735 .797339$

H $-1.393730-4.5740555 .687185$

H $-0.962195-2.8508355 .629850$

H $-2.373428-3.090347 \quad 1.163857$

H -3.646545 -4.2855631 .463371$

H $-5.211743-2.3608532 .047030$

H $-3.962123-1.2328321 .580363$

H -5.125771 -3.392038 -0.250259

H -2.869168 -2.530169-1.014435

H $-3.445119-0.885599-0.839155$

H $-2.519628-1.623029-3.269759$

H -5.403402 -0.089922 -2.423689

H -3.383842 -3.794011-3.106377

H $-4.719401-3.327369-4.134469$

H -5.036878 -4.001435 -2.523633

H -5.621603 $-0.977857-4.498878$

H -8.536158 $-0.886799-2.262982$

H -8.502391 - $1.000082-0.517607$

H -7.930843 $-3.052751-1.243214$

H -0.423762 $-0.178840 \quad 0.671755$

H -0.2377841 .8597552 .971939$

H $-0.9046713 .064798-1.143899$

H $-1.6094581 .565224-0.576098$

H $0.141057 \quad 1.679852-0.860062$

H -7.225601 $1.202134-2.207220$

H $-8.664533 \quad 1.325518-1.222324$

H $-4.139948 \quad 1.5071260 .760960$

H -5.2983692 .5818831 .571456$

H -3.614163 $3.455494-0.901307$

H -4.1696364 .7372871 .823543$

H $-3.646794 \quad 6.7086580 .364238$

H -2.819826 $5.709749-0.802989$

H $-0.597417 \quad 7.2175612 .366038$
H -2.374699 7.6884012 .135529

H $0.412648 \quad 5.703524 \quad 0.773203$

H $-0.5450314 .991015-0.484580$

H -0.3005624 .0611182 .460244$

H -5.508675 $2.057806-1.349416$

C $3.928985-3.951455-0.410826$

O $3.941175-2.654817-1.043482$

C $4.955039-2.639863-2.063139$

C $5.329868-4.097283-2.298872$

C $5.187791-4.668443-0.902330$

H $5.820554-2.116440-1.639530$

C $4.453139-1.897695-3.305224$

H $4.630859-4.592449-2.982869$

H $6.338670-4.206079-2.708454$

C $5.086938-6.183695-0.872413$

H $6.055770-4.355689-0.306091$

C $3.943382-0.465920-3.038735$

O $3.368799-2.633504-3.888326$

H $5.242559-1.878064-4.065399$

H $2.998679-0.505710-2.484310$

O $3.6411220 .126264-4.309066$

C $4.9391010 .449849-2.303279$

C $4.5401681 .936887-2.264840$

H $5.0787650 .091055-1.280016$

$\begin{array}{llll}\text { O } & 6.218652 & 0.368959 & -2.939538\end{array}$

H $5.3531702 .484254-1.772287$

H $4.5231862 .328964-3.289454$

H $4.972046-6.5471810 .153554$

H $4.230408-6.540531-1.454209$

H $5.990744-6.637438-1.292267$

C $3.895839-3.7787701 .108630$

H $2.679914-2.728553-3.208074$

H $3.004491-0.457381-4.756507$

H $6.1040420 .644739-3.865257$

H $4.716009-3.1214181 .420916$

H $4.043959-4.7477001 .598766$

H $3.025499-4.468166-0.759635$

C 3.9938912 .4489160 .709651

O $3.159593 \quad 1.743841-0.219882$

C $3.1967942 .226927-1.566680$

C $2.7807423 .702584-1.606333$

C $3.6332704 .550768-0.671823$

C 3.6503313 .9483420 .731435

H 5.0467932 .3086680 .436339

C 3.7654901 .8205512 .095503

H $2.4251401 .672715-2.113337$

$\begin{array}{llll}\text { O } & 2.864477 & 4.201825 & -2.936891\end{array}$

H $1.731023 \quad 3.786842-1.300517$

H $4.6476144 .677314-1.067735$

O $3.0621635 .860462-0.610801$

H $2.6714864 .112666 \quad 1.199096$

O $4.618674 \quad 4.667992 \quad 1.493874$

C 4.4078920 .4572562 .242298

H 4.2007132 .4582552 .871994

H 2.6944221 .6999402 .289746

H $2.618158 \quad 5.142347-2.910510$

H 3.5934156 .3779370 .018592

H 4.4516644 .4907332 .434352

$\begin{array}{lllll}\text { O } & 4.455085 & 0.137630 & 3.569950\end{array}$

O $4.801201-0.2426941 .320672$

C $5.069567-1.1112593 .904863$

H $4.337063-1.9149073 .801377$

H $5.948930-1.3078963 .283940$

H $5.389089-1.0610574 .949329$

SCF Energy (B3LYP/6-31G*//MMFF)= -3245.91743594

$01 \_492$

MMFF Geometry

C $1.531803-1.086949-3.443700$

C $0.438978-1.659274-3.970004$

C $-0.803085-1.944748-3.170062$

O $-1.840016-1.093021-3.710955$

C $-1.230365-3.423331-3.250814$

C $-0.194992-4.408962-2.752084$

C $0.115080-4.603919-1.455318$

C $0.490710-5.216080-3.823492$

C $-0.460159-3.905490-0.253688$

C $-1.023353-4.9095510 .759526$ 
C $-1.456018-4.2350592 .072274$ C $-1.933503-5.2917163 .071843$ C $-2.471963-4.6629764 .363791$ O $-2.512575-3.3140161 .785450$ O $-3.179443-5.6803505 .089011$ C -3.499333 -3.574660 3.994183 C $-1.340853-4.1832855 .280954$ O $-3.926639-2.8670705 .156656$ C -2.990864 -2.579122 2.924563 C $-4.166507-1.6897172 .445364$ O $-1.970322-1.7616353 .495499$ C $-2.900908-0.826686-2.902930$ O $-3.160589-1.358489-1.835859$ C $-3.6623690 .289165-3.506721$ C $-4.6013070 .899561-2.768549$ C $-5.3659052 .089559-3.140303$ C $-5.1754552 .646768-4.525429$ C $-3.802476-0.6878571 .340141$ C $-3.470850 \quad 0.7101961 .838747$ C $-2.961406 \quad 2.9921050 .955932$ O $-3.477463 \quad 1.0256873 .024785$ C $-4.2554503 .702138 \quad 0.702739$ C $-4.4273574 .620367-0.260866$ C $-5.7327375 .317925-0.528659$ C $-6.1987665 .155563-1.961133$ C $-5.9818116 .124561-2.866545$ C $-6.9786493 .910484-2.329749$ C $-6.164302 \quad 2.653091-2.209634$ N $-3.176264 \quad 1.566868 \quad 0.785557$ H $1.524355-0.790149-2.398655$ H $0.435507-1.948000-5.018159$ H $-0.634634-1.657274-2.125831$ H - $-1.508135-3.663436-4.286774$ H $-2.162694-3.576651-2.693048$ H $0.885688-5.337586-1.220998$ H $1.252036-5.889630-3.416115$ H $-0.241183-5.828483-4.360447$ H $0.985847-4.557814-4.543466$ H $\quad 0.346188-3.3300080 .215185$ H -1.243295 $-3.195134-0.531889$ H $-1.884049-5.4173850 .304650$ H $-0.269371-5.675830 \quad 0.980012$ H $-0.599506-3.683382 \quad 2.477677$ H -1.130356 -6.004319 3.298161 H -2.738787 -5.885173 2.616006 H $-2.559798-6.4091825 .264492$ H $-4.389130-4.0835503 .597282$ H $-0.694331-5.0232035 .562554$ H $-1.736864-3.7827696 .220688$ H -0.708057 -3.421978 4.820036 H $-4.248599-3.5262975 .795028$ H $-4.938026-2.3521712 .030160$ H $-4.615789-1.1632713 .294939$ H -1.896678 -0.9423992 .982126$ H $-3.3844030 .619404-4.500744$ H $-4.8042780 .517575-1.768925$ H -5.432952 $1.895424-5.280238$ H $-4.1353442 .955649-4.677415$ H $-5.7974623 .521637-4.728573$ H $-2.964907-1.0528120 .738921$ H $-4.670754-0.5820110 .677106$ H $-2.182807 \quad 3.290513 \quad 0.247405$ H -2.612512 3.1980621 .972576 H $-5.0943813 .442907 \quad 1.344679$ H -3.584559 $4.891275-0.892840$ H -5.594676 $6.382482-0.298349$ H $-6.521192 \quad 4.9691360 .149806$ H $-6.3324996 .033054-3.889117$ H -5.444682 $7.030672-2.605973$ H -7.408498 $3.991995-3.334965$ H $-7.8469073 .827591-1.662935$ H $-6.2321632 .184340-1.227252$ H -3.232016 $1.213860-0.164872$ C $4.047165-1.391953-3.507916$ O $4.328743-0.637900-2.312315$ C $4.891067-1.530215-1.329722$ C $5.088462-2.876958-2.026037$ C $3.993385-2.855043-3.073591$
H $4.137903-1.643973-0.541535$

C $6.180939-0.939630-0.748750$

H $6.071670-2.948325-2.505477$

H $4.999592-3.716638-1.329483$

C $4.207438-3.842982-4.206058$

H $3.044892-3.083535-2.572687$

C $6.0298980 .455326-0.107013$

O $7.154818-0.825492-1.796626$

H $6.607617-1.640502-0.021853$

H $5.8134541 .193907-0.886469$

$\begin{array}{lllll}\text { O } & 7.312577 & 0.815324 & 0.426242\end{array}$

C 4.9743350 .5550641 .012906

C 5.0582151 .8546641 .840464

H 3.9718620 .4484170 .583683

O $5.165507-0.5404951 .913282$

H 4.3602601 .7660772 .681359

H $6.046557 \quad 1.9156962 .312317$

H $3.405749-3.765814-4.947038$

H $5.158362-3.665705-4.719440$

H $4.217753-4.869066-3.824154$

C $2.801362-0.837412-4.205181$

H $6.781343-0.245004-2.481969$

H 7.5185750 .1896361 .142076

H $4.448039-0.5102352 .568938$

H $2.732154-1.265934-5.211994$

H $2.9102640 .248244-4.317792$

H $4.906102-1.229689-4.173032$

C 2.3821093 .2516991 .383435

$\begin{array}{llllll}\text { O } & 3.462618 & 3.121609 & 0.450583\end{array}$

C $4.7696193 .136532 \quad 1.035027$

C 4.9803794 .4387291 .821698

C 3.8748624 .6710822 .846284

C 2.5041354 .5538022 .183882

H 2.3837452 .3949032 .067052

C $1.074025 \quad 3.2271190 .584053$

H 5.4790363 .1613360 .200096

$\begin{array}{lllll}\text { O } & 6.240109 & 4.438356 & 2.485025\end{array}$

H 4.9864995 .2858941 .123372

H 3.9615633 .9833743 .695773

O 4.0000065 .9901303 .382602

H 2.3391015 .4181241 .527805

$\begin{array}{lllll}\text { O } & 1.494523 & 4.581463 & 3.190841\end{array}$

$\begin{array}{lllll}\text { C } & 0.800897 & 1.855203 & 0.018078\end{array}$

H $\quad 0.2110403 .511667 \quad 1.195943$

H $1.1230273 .928389-0.257844$

H 6.9260314 .3001981 .809615

H $4.8917716 .063146 \quad 3.764139$

H 1.6114645 .4043803 .696090

$\begin{array}{lllll}\text { O } & 0.485072 & 0.990498 & 1.024169\end{array}$

O $0.855902 \quad 1.588417-1.175021$

C $0.229961-0.355767 \quad 0.618193$

H $0.028818-0.9494551 .513217$

H $-0.647344-0.392327-0.033026$

H $1.106489-0.7732570 .113757$

SCF Energy (B3LYP/6-31G**//MMFF) = -3245.91692930

01493

MM̄FF Geometry

C -2.128407 -3.101696 0.187919

C $-1.676234-3.712345-0.916066$

C $-1.041928-2.952014-2.049723$

O $0.361241-3.299267-2.047750$

C $-1.663390-3.296375-3.416258$

C $-3.167993-3.137647-3.493051$

C $-3.815830-1.956738-3.514007$

C $-3.947171-4.425719-3.560123$

C $-3.219572-0.576744-3.479285$

C $-3.5491290 .125731-2.154842$

C $-3.0656811 .582027-2.059571$

C $-3.854740 \quad 2.514968-2.981054$

C -3.333863 $3.957397-2.913101$

O $-1.6712721 .625605-2.371332$

O $-3.903097 \quad 4.684975-4.012510$

C $-1.8068913 .938620-3.126315$

C $-3.7888144 .674305-1.636719$

O $-1.255203 \quad 5.241407-2.944646$

C -1.066041 $2.919995-2.227686$

C $0.4070322 .797390-2.690745$ 
O $\quad-1.1064903 .374900-0.875319$

C $1.224145-2.372859-2.546692$

O $0.937061-1.339559-3.129142$

C $2.595433-2.806962-2.199365$

C $3.600710-1.920798-2.262799$

C $4.996222-2.164678-1.890362$

C $5.340382-3.517820-1.324586$

C $1.2324321 .772657-1.903568$

C $2.1793982 .457948-0.938697$

C $4.5554773 .126866-0.654039$

O 1.7839252 .9449860 .117759

C $5.8483632 .461723-1.000246$

C $6.5314541 .677621-0.152784$

C $7.8345421 .014152-0.492890$

C $7.740713-0.495495-0.481930$

C $8.064567-1.1987930 .616137$

C $7.370013-1.193981-1.771253$

C $5.892474-1.167643-2.049811$

N $3.4881022 .479670-1.392741$

H -1.990090 -2.027446 0.280886

H -1.781268 -4.788953 -1.019995

H -1.134766 -1.875196 -1.861386

H -1.384499-4.323895 -3.689101

H -1.194355 -2.685692 -4.198714

H -4.903742 -1.970144 -3.571264

H $-5.028136-4.257397-3.611558$

H -3.659103 -4.995321 -4.449717

H $-3.752341-5.040259-2.675887$

H $-2.136752-0.602947-3.634487$

H -3.638397 -0.018116 -4.322946

H -4.628833 $0.089561-1.961357$

H -3.067940 -0.439285 -1.347262

H $-3.2013231 .892833-1.016828$

H $-4.9254382 .480897-2.744259$

H $-3.7673252 .177656-4.021663$

H $-4.8713174 .640234-3.931898$

H -1.625103 $3.669093-4.176234$

H $-4.8839484 .709778-1.587812$

H $-3.4551865 .717849-1.629793$

H -3.434572 4.192889-0.723057

H -1.739805 $5.845668-3.532848$

H $0.4072412 .474700-3.740467$

H $\quad 0.8963793 .778068-2.648559$

H $-0.3954732 .944308-0.375179$

H $2.729852-3.826305-1.855961$

H $3.381823-0.910408-2.605982$

H $5.180260-4.299872-2.074691$

H $4.719943-3.737988-0.448471$

H $6.377029-3.599875-0.990949$

H $\quad 0.614701 \quad 1.066881-1.337909$

H $1.8175551 .170844-2.608315$

H 4.3390573 .0873830 .418736

H $4.5798164 .179643-0.953100$

H $6.2407372 .637288-1.999075$

H $6.141141 \quad 1.5088510 .848130$

H $\quad 8.581121 \quad 1.3478220 .239837$

H $8.213647 \quad 1.352212-1.465322$

H $8.033059-2.2832130 .623100$

H $8.363323-0.7044091 .534589$

H $7.755444-2.219319-1.776408$

H $7.890947-0.709387-2.607292$

H $5.543972-0.219348-2.459202$

H $3.672267 \quad 2.216599-2.356867$

C $-4.022701-2.9675821 .854161$

O $-3.530219-1.7396562 .431277$

C $-4.323929-0.646657 \quad 1.933744$

C $-5.617474-1.2806041 .438781$

C $-5.093035-2.570760 \quad 0.832799$

H -3.786577 -0.2292461 .073900$

C -4.4786380 .4331663 .007565$

H $-6.303825-1.5020222 .264077$

H -6.141799 -0.6485090 .715874$

C $-6.159033-3.6306990 .620416$

H -4.642075 -2.332141 -0.137998

C -3.1370370 .9043243 .611066$

O $-5.272303-0.0988494 .075260$

H -5.0389321 .2822082 .598819$

H -2.7014010 .0810884 .187421$
O $-3.419011 \quad 1.9366704 .564233$

C $-2.110857 \quad 1.4274072 .586133$

C $-0.818067 \quad 1.988158 \quad 3.214213$

H -1.844615 0.6271991 .888485

O $-2.7091052 .460444 \quad 1.799746$

H -0.2212172 .4384622 .411738$

H -1.0684902 .8230173 .880162$

H $-5.720842-4.5422070 .201214$

H $-6.655793-3.8952321 .559829$

H $-6.924738-3.271984-0.075002$

C $-2.841180-3.785617 \quad 1.317656$

H -5.3497910 .5948634 .752541$

H -3.8803162 .6548874 .099109$

H -2.852144 3.2311822 .374452

H -3.191692 -4.777080 1.009207

H $-2.114339-3.9232752 .127694$

H $-4.478753-3.5249872 .682437$

C $1.198426-0.0445572 .117494$

O $0.249345-0.2301173 .172823$

C $0.0131530 .934086 \quad 3.973743$

C 1.3321501 .4499674 .573118

C 2.4283651 .6283683 .523122

C 2.5659520 .3624362 .676038

H $\quad 0.8350620 .7486461 .453336$

C $1.223762-1.3082661 .250918$

H $-0.599764 \quad 0.602273 \quad 4.820233$

O $1.1266192 .663801 \quad 5.288958$

H 1.6852530 .7164355 .309457

H 2.2368782 .5081722 .898052

O $3.663928 \quad 1.876433 \quad 4.197471$

H $2.999845-0.4280203 .299217$

O 3.4823580 .6273381 .619449

C $1.641200-2.5733671 .969214$

H $\quad 0.214513-1.4868370 .868627$

H $1.891583-1.1542380 .397431$

H 1.0104313 .3811304 .643266

H 4.3545091 .9493943 .516354

H $3.716006-0.2213711 .207514$

O $1.822632-3.5606891 .042216$

O $1.767109-2.6976523 .178946$

C $2.203425-4.8368261 .564245$

H $2.325772-5.5244320 .723224$

H $3.156529-4.7597842 .095952$

H $1.421355-5.2241222 .224110$

SCF Energy $\left(B 3 L Y P / 6-31 G^{* *} / / M M F F\right)=-3245.90734729$

01494

MM̄FF Geometry

C 4.7000540 .9651301 .491933

C 3.7794030 .6936562 .430697

C 2.4302751 .3614982 .466383

$\begin{array}{llll}\text { O } & 1.446881 & 0.334485 & 2.747862\end{array}$

C 2.3288272 .4326923 .570228

C 3.1032963 .7011093 .277598

C 2.6474924 .7093902 .507717

C 4.4497923 .8113433 .945089

C 1.3151064 .8101341 .816691

C 1.4509934 .6374360 .298815

C $0.1403224 .835031-0.483441$

C $-0.2476906 .308373-0.592834$

C $-1.5662286 .484462-1.359182$

O -0.9003364 .1033650 .164211$

O $-2.0069567 .837710-1.165695$

C $-2.6372755 .562594-0.734401$

C $-1.3756206 .303379-2.870382$

O $-3.8332865 .617991-1.506580$

C $-2.1672364 .100693-0.519458$

C -3.1241583 .3153980 .416162$

O $-2.0605313 .438842-1.776346$

C 0.2144400 .4914582 .191702

O $-0.1308501 .363967 \quad 1.411082$

C $-0.669673-0.5691982 .725776$

C $-1.928675-0.6587532 .270452$

C $-2.955670-1.6052222 .711509$

C $-2.611818-2.5281293 .850298$

C $-4.4670632 .854082-0.156069$

C $-4.3942991 .542838-0.920813$

C $-5.860701-0.094175-2.099325$ 
O $\begin{array}{lll}\text { O 3.357440 } & 0.901422 & -1.060561\end{array}$

C $-6.446132-1.065257-1.122708$

C $-5.881611-2.238355-0.797356$

C $-6.492208-3.2068450 .174859$

C $-5.595393-3.5136771 .353901$

C $-5.085570-4.7440041 .531767$

C $-5.369642-2.4273282 .382076$

C $-4.153105-1.5912582 .087815$

N $-5.633721 \quad 1.167319-1.418226$

H $4.462876 \quad 1.7115830 .739058$

H $4.008038-0.0188743 .219111$

H 2.2198191 .8014051 .484962

H $2.6543732 .009414 \quad 4.530778$

H 1.2742122 .6792273 .747133

H 3.2891365 .5782182 .365508

H 4.9534734 .7554713 .711401

H 4.3359753 .7607515 .032982

H 5.1122243 .0011883 .629683

H $0.606664 \quad 4.0771032212867$

H 0.8960225 .7951992 .050300

H $2.2213365 .309746-0.099755$

H 1.8032923 .6160700 .107072

H $0.3000414 .417001-1.484576$

H $0.5529086 .888950-1.067578$

H -0.3766956 .7388290 .408890$

H -1.310699 $8.430085-1.497764$

H -2.891042 5.9893520 .245376

H $-0.6143806 .997604-3.246304$

H -2.293838 $6.546118-3.416744$

H $-1.0667255 .292619-3.144994$

H -3.696717 $5.075744-2.302239$

H -2.590105 2.4377600 .802796

H -3.3144293 .9324791 .304942$

H -2.007693 2.483568 -1.605890

H $-0.269681-1.2279863 .487623$

H -2.248068 0.0368601 .495835

H $-1.719790-3.1180673 .618926$

H -3.399919 -3.247237 4.082730

H $-2.420747-1.9527034 .762951$

H $-5.1478772 .679140 \quad 0.687561$

H -4.928763 $3.613386-0.790009$

H $-4.923079-0.457279-2.532716$

H $-6.5714910 .092781-2.909941$

H $-7.394051-0.788617-0.666387$

H -4.941762 -2.527320 -1.261237

H $-6.724843-4.126516-0.377437$

H -7.453879 -2.8362060 .551823$

H $-4.468859-4.9798732 .392604$

H $-5.266041-5.540780 \quad 0.818065$

H -5.326584 -2.864918 3.384914

H $-6.245413-1.7662002 .415239$

H $-4.300951-0.8784531 .276550$

H $-6.4453861 .738952-1.201673$

C $6.455061-0.2797140 .109316$

O $5.562125-1.367620-0.197547$

C $5.166712-1.255516-1.576606$

C $6.192493-0.336317-2.229318$

C $6.4561570 .649236-1.107969$

H $4.190385-0.754896-1.580180$

C $5.034739-2.636382-2.225199$

H $7.115010-0.869674-2.485658$

H $5.8089320 .137208-3.138124$

C $7.7423701 .439603-1.275400$

H $5.6164971 .351920-1.074680$

C $4.172760-3.645780-1.442392$

O $6.334819-3.222441-2.374851$

H $4.643406-2.521046-3.242760$

H $4.727456-4.022029-0.573843$

O $3.952672-4.777647-2.295251$

C $2.806314-3.116437-0.972133$

C $1.929511-4.232748-0.372601$

H $2.963092-2.335897-0.219896$

O $2.127282-2.514237-2.070851$

H $1.486685-4.820889-1.184384$

H $2.565029-4.9074110 .213656$

H $7.8906472 .122704-0.433060$

H $8.6148900 .780386-1.333603$

H $7.7106372 .035813-2.193305$
C $6.0754440 .357203 \quad 1.451363$

H $6.740666-3.265716-1.491813$

H $4.825651-5.109238-2.567109$

H $2.038174-3.186253-2.768279$

H 6.8025061 .1407181 .695449

H $6.155023-0.4096942 .232274$

H $7.447924-0.7346210 .223795$

C $-0.891423-3.202151-1.087043$

O $-0.009324-2.710447-0.072912$

C $0.813970-3.6959860 .551655$

C $-0.056270-4.7925251 .181589$

C $-1.041884-5.385716 \quad 0.178757$

C $-1.826363-4.272818-0.514444$

H $-0.306783-3.616881-1.916542$

C -1.693809-2.009649-1.615532

H $1.325915-3.1815371 .375450$

O $\quad 0.761800-5.8194321 .731657$

H $-0.622598-4.3584072 .013341$

H $-0.528189-6.027866-0.545948$

O $-1.951149-6.2290150 .888775$

H $-2.513918-3.8198670 .208813$

O $-2.625131-4.836783-1.551868$

C $-0.804296-1.040666-2.356702$

H $-2.479075-2.325278-2.311602$

H -2.191214 $-1.477177-0.797496$

H $0.169294-6.4974492 .099325$

H -2.606198 -6.5534490 .247060$

H -2.026262 $-5.230005-2.209507$

O $-0.332708-0.088779-1.501763$

O $-0.539438-1.136962-3.548125$

$\begin{array}{llll}\text { C } & 0.553193 & 0.875279 & -2.078541\end{array}$

H $1.455346 \quad 0.383178-2.453765$

H $0.0488841 .422837-2.880357$

H $0.8404291 .583895-1.297791$

SCF Energy (B3LYP/6-31G**//MMFF)= -3245.92734247

01495

MM̄FF Geometry

C $0.209513-4.2037301 .831575$

C $0.249444-2.8642021 .795820$

C $0.466539-2.0073173 .017944$

O $1.488615-1.0304492 .715576$

C $-0.827576-1.244413 \quad 3.337201$

C $-0.787375-0.485144 \quad 4.647359$

C -0.6318620 .8500304 .741607$

C $-1.007938-1.315857 \quad 5.883644$

C $-0.365567 \quad 1.8064703 .614392$

C -1.6534332 .3966743 .028081$

C -1.3503993 .4270241 .930531$

C -2.6388423 .9811951 .322223$

C $-2.3589404 .948706 \quad 0.161344$

O $-\begin{array}{llll}0.579111 & 2.783331 & 0.911586\end{array}$

O $-3.6069205 .140886-0.522129$

C $-1.368047 \quad 4.285238-0.821454$

C $-1.922835 \quad 6.3272190 .671457$

O $-0.942565 \quad 5.203998-1.825180$

C $-0.1335143 .664709-0.126180$

C $0.6921742 .840225-1.140676$

$\begin{array}{llllll}\text { O } & 0.694394 & 4.711679 & 0.387805\end{array}$

C $2.787048-1.4294702 .766078$

O $3.208947-2.5376053 .053199$

C $3.613976-0.2638922 .381348$

C $4.920178-0.4257282 .123544$

C 5.8439280 .6246561 .690807

C 5.3498522 .0477791 .718325

C $1.7107821 .898881-0.487919$

C $2.6049241 .268684-1.535766$

C $4.6807981 .672008-2.856418$

O $2.3417490 .178610-2.038187$

C $5.6828720 .748865-2.237171$

C $7.0030290 .987508-2.192395$

C $7.9948560 .012039-1.622086$

C $8.833640 \quad 0.585837-0.498375$

C $10.1755090 .559509-0.569293$

C $8.143242 \quad 1.181180 \quad 0.715021$

C $7.086790 \quad 0.2767651 .295895$

N $3.6953232 .059369-1.863903$

H $0.317101-4.6906992 .799557$ 
H $\quad 0.114303-2.337903 \quad 0.856340$ H $0.778515-2.6078003 .881241$ H -1.671788 -1.945353 3.382289 H -1.054856 -0.5571702 .513180$ H $-0.6658301 .308955 \quad 5.728269$ H -1.019299 $-0.709038 \quad 6.795294$ H -1.968139-1.838818 5.824056 H $-0.212675-2.0594545 .993707$ H $\quad 0.2691942 .6149403 .998479$ H 0.2231451 .3189132 .830483 H -2.257499 1.5825182 .607651 H -2.2467672 .8675093 .821466$ H -0.7584564 .2342982 .377996$ H -3.257251 4.4655392 .087983 H -3.240209 3.1484370 .933745 H -3.466388 $5.790898-1.231014$ H $-1.9112763 .483371-1.341217$ H $-2.7125106 .771875 \quad 1.289069$ H -1.763027 $7.024936-0.158129$ H -1.011208 6.2933901 .271853 H -1.730975 $5.492137-2.314854$ H $0.0155712 .220145-1.739413$ H $1.2059643 .515503-1.836440$ H 1.6115324 .3983180 .434126 H 3.1154280 .6949242 .291644 H $5.348206-1.4241442 .203007$ H 4.8834622 .2785402 .683034 H 4.6165992 .2154220 .925578 H 6.1464162 .7851591 .594582 H 2.3310622 .4253570 .244054 H $1.205537 \quad 1.0897880 .052626$ H $5.1410022 .588584-3.238272$ H $4.1738121 .163544-3.683131$ H $5.302171-0.186889-1.833492$ H $7.3968331 .908535-2.614786$ H $8.638956-0.317863-2.447728$ H $7.490715-0.893340-1.261562$ H $\quad 10.7927120 .9632080 .226653$ H $10.689760 \quad 0.134548-1.424968$ H 7.7307552 .1473920 .409143 H $8.874402 \quad 1.3872701 .506715$ H $7.378548-0.7720521 .361221$ H $3.8564442 .921203-1.351138$ C $0.453827-4.738798-0.732009$ O $-0.369715-3.692695-1.276473$ C $0.448984-2.885580-2.149559$ C $1.811195-3.574882-2.235527$ C $1.897443-4.268177-0.889768$ H $0.581276-1.921081-1.647638$ C $-0.234556-2.685418-3.508298$ H $1.843705-4.311408-3.046912$ H $2.623878-2.861319-2.403774$ C $2.925627-5.384337-0.842241$ H $2.154197-3.516154-0.134947$ C -1.625962 -2.022234-3.433175 O $-0.391586-3.970442-4.123210$ H $0.426273-2.105664-4.164499$ H -2.344002 -2.710941-2.973006 O $-2.076987-1.794335-4.774331$ C $-1.644172-0.672714-2.693383$ C $-2.956547 \quad 0.124740-2.811514$ H -1.425326 $-0.835097-1.632835$ O $-0.597908 \quad 0.158243-3.203873$ H -2.842585 $1.031801-2.204440$ H $-3.0710970 .493084-3.839204$ H $2.944121-5.8546490 .145886$ H $2.709597-6.162078-1.582453$ H $3.927273-4.993234-1.048517$ C $0.005854-5.1466480 .675999$ H $-0.619492-3.823072-5.056342$ H $-2.268305-2.661371-5.169844$ H $-0.7500290 .271320-4.157825$ H $0.536191-6.0734800 .930735$ H -1.062321 -5.3969540 .650785$ H $0.286185-5.608144-1.383787$ C $-4.027019-0.4694570 .001383$ O $-4.059949-1.320958-1.145994$ C $-4.224029-0.643010-2.392849$
C $-5.5015320 .211475-2.378341$

C $-5.5554381 .147620-1.171769$

C -5.3066370 .3654420 .117415$

H $-3.1666540 .207264-0.084781$

C $-3.747771-1.3380891 .234034$

H $-4.377988-1.426037-3.145415$

O $-5.6141680 .981191-3.570961$

H $-6.377105-0.449742-2.338988$

H $-4.8456421 .977308-1.271271$

O $-6.8546921 .738277-1.092740$

H $-6.175355-0.2709810 .320618$

O

C $-4.811925-2.3707441 .542596$

H -2.816845 -1.894785 1.075539

H -3.628589 -0.6908732 .110085$

H $-5.5978130 .361876-4.320585$

H -7.014012 2.198445 -1.934651

H $-5.984898 \quad 1.819753 \quad 1.226147$

O $-4.658220-2.7883492 .834823$

O $-5.646698-2.787198 \quad 0.751741$

C $-5.581863-3.7898223 .272326$

H -5.467644 -4.697883 2.672798

H -5.356547 -4.027509 4.315419

H $-6.607022-3.4121073 .211889$

SCF Energy $\left(B 3 L Y P / 6-31 G^{* *} / / M M F F\right)=-3245.90494249$

01496

MM̄FF Geometry

C $2.237840-4.2490840 .071248$

C $0.957858-4.285538-0.329346$

C $-0.200431-3.7839810 .496534$

O $-0.866947-2.731571-0.239671$

C $-1.214015-4.9239400 .713088$

C $-2.412273-4.5312361 .556265$

C $-3.686489-4.5102191 .116909$

C $-2.113402-4.1756222 .989569$

C $-4.204136-4.871233-0.247394$

C $-4.318915-3.692868-1.226110$

C $-5.346096-2.624309-0.815219$

C $-5.547920-1.613553-1.947567$

C $-6.492141-0.470474-1.543127$

O $-4.860887-1.9486070 .347186$

O $-6.3604880 .575770-2.518171$

C $-6.0277180 .113021-0.191401$

C $-7.961322-0.908211-1.573200$

O $-\begin{array}{llll}-6.966456 & 1.064709 & 0.302536\end{array}$

C $-5.755603-0.9621320 .879426$

C $-5.100808-0.3889112 .159405$

O $-6.998307-1.5559571 .274725$

C $-0.275140-1.506727-0.254210$

O $0.762101-1.1812080 .301017$

C - $-1.105785-0.621172-1.098759$

C $-0.5678020 .506483-1.584739$

C $-1.2349671 .491285-2.436974$

C $-2.714518 \quad 1.333224-2.660733$

C $-3.7312960 .269473 \quad 1.960596$

C -3.7780601 .7792121 .793294$

C -2.2879783 .7196851 .309294$

O -4.7862892 .4497681 .996572$

C $-2.5318254 .156465-0.100698$

C $-1.6462494 .869628-0.813145$

C $-1.8989145 .372440-2.205749$

C $-0.8159634 .967231-3.180310$

C $0.1840665 .808280-3.493535$

C $-0.9409463 .617845-3.850891$

C $-0.4976162 .494993-2.955321$

N -2.539373 2.2962931 .434139

H $2.483449-3.8507911 .052373$

H $0.722094-4.674225-1.317711$

H $\quad 0.141141-3.3924741 .462692$

H $-0.717245-5.7733431 .200712$

H -1.541817 $-5.288624-0.266540$

H $-4.463593-4.2132201 .820563$

H $-3.023688-4.0474083 .585254$

H -1.522752 -4.966635 3.463106

H -1.552834 -3.238769 3.043780

H -3.581099 -5.649486 -0.701077

H $-5.190980-5.335471-0.124396$ 
H $-3.331647-3.231186-1.343612$ H -4.602067 -4.101045 -2.204519 H $-6.291693-3.126185-0.578449$ H $-5.916324-2.111806-2.853033$ H $-4.577118-1.179160-2.221226$ H $-6.5774610 .202376-3.389636$ H $-5.0995010 .662657-0.384562$ H $-8.241352-1.238796-2.580696$ H -8.628114 -0.071617-1.337363 H -8.178658 -1.728390 -0.885947 H $-7.091373 \quad 1.735282-0.390634$ H -5.8009560 .2780992 .676532$ H -4.944876 -1.233033 2.846939 H -6.882024 -1.962428 2.149409 H -2.122809 -0.928650-1.311105 H $\quad 0.4714720 .726850-1.348174$ H $-3.238347 \quad 1.239516-1.704077$ H $-2.9177130 .441015-3.262512$ H -3.172318 2.182001-3.172828 H -3.137561 0.0890272 .865210 H $-3.192210-0.1786941 .120343$ H -1.254365 3.8957931 .623935 H -2.9561174 .2649151 .983920$ H $-3.4932533 .906138-0.542775$ H $-0.6992845 .145063-0.355545$ H -1.963101 $6.467461-2.156621$ H -2.873452 $5.037848-2.582224$ H $\quad 0.9500375 .533613-4.211069$ H $0.262726 \quad 6.787717-3.033382$ H $-0.3097713 .584664-4.749224$ H $-1.9609293 .491968-4.224429$ H $0.5702482 .518022-2.736481$ H $-1.7726231 .661076 \quad 1.233034$ C $4.263207-3.551699-1.227817$ O $5.071750-3.093915-0.120104$ C $6.427771-2.953543-0.571323$ C $6.368906-2.942519-2.091744$ C $5.244871-3.929161-2.339409$ H $6.945965-3.873416-0.265105$ C $7.153860-1.7732540 .087303$ H $6.095253-1.955089-2.478755$ H $7.313215-3.244810-2.554062$ C $4.657815-3.839770-3.737745$ H $5.625260-4.946352-2.174756$ C $6.683088-0.345946-0.272030$ O $8.544503-1.880629-0.251747$ H 7.101215-1.893286 1.176244 H $6.894472-0.140615-1.327772$ O 7.5100830 .5532020 .482761 C $5.205633-0.0484480 .042105$ C $4.8141341 .418067-0.240187$ H $4.573274-0.693832-0.573628$ O $4.962352-0.370576 \quad 1.409255$ H 5.3609832 .0786390 .439427 H $5.1246281 .666676-1.261569$ H $3.840380-4.556179-3.865299$ H 4.263487 -2.838978 -3.944152 H $5.421905-4.061467-4.490102$ C $3.382303-4.720986-0.782525$ H $8.635655-1.697652-1.202110$ H 7.4389421 .4327500 .075423 H $4.002629-0.3226941 .556941$ H $3.969653-5.438128-0.196642$ H $2.996552-5.254255-1.659277$ H $3.642640-2.702545-1.542808$ C 3.2223702 .3806562 .216911 O $2.832241 \quad 1.4092401 .240372$ C $3.2948121 .666606-0.089659$ C $2.8153733 .048962-0.555812$ C $3.184712 \quad 4.1512870 .433774$ C 2.7162943 .7768511 .840920 H 4.3169392 .4089102 .282552 C 2.7455211 .9058953 .593336 H $2.8025820 .926312-0.732545$ O $3.3403103 .346682-1.844175$ H $1.7227103 .023623-0.652219$ H 4.2625624 .3492700 .417734 O 2.5572525 .3725590 .037037
H 1.6217213 .8186371 .880886

O 3.2184634 .7290972 .774100

C 1.2757131 .5577803 .624925

H 3.3090081 .0078423 .879354

H 2.9437372 .6465184 .375838

H $3.0164234 .229372-2.093373$

H 1.5954535 .2314750 .041151

H 2.9139745 .6068682 .486087

O 1.1252030 .2047253 .540751

O $0.3747292 .382342 \quad 3.697573$

C $-0.220783-0.2754363 .550421$

H -0.7937620 .1725874 .368017$

H $-0.690101-0.0640222 .586895$

H $-0.191034-1.3573133 .698728$

SCF Energy (B3LYP/6-31G**//MMFF)= -3245.89992495

01497

MM̄FF Geometry

C -1.689431 -4.7172260 .061320$

C $-0.874204-3.6525750 .039611$

C $0.331575-3.4753350 .920235$

O $1.408589-3.059198 \quad 0.051055$

C $0.037628-2.3644641 .945045$

C $1.147836-2.1228302 .946122$

C $1.885705-0.9961022 .997102$

C $1.345018-3.2044593 .976043$

C 1.7921850 .1961012 .083027

C 0.8045831 .2297902 .638333

C 0.7573452 .5638661 .877719

C 0.3297882 .4126190 .416794

C $0.334453 \quad 3.761404-0.322260$

O 2.0474653 .1798221 .961978

O $0.2470413 .492900-1.730530$

C $1.6943334 .451830-0.078295$

C $-0.8854024 .620246 \quad 0.027439$

O $1.7101275 .764983-0.631729$

C 2.1028224 .5026191 .410289

C 3.5280275 .0729141 .631301

O 1.2078925 .3791082 .110559

C $2.642385-3.5901870 .255662$

O $2.972212-4.3851761 .120587$

C $3.541213-3.030984-0.779508$

C $4.853613-3.303707-0.731399$

C $5.871764-2.843198-1.678931$

C $5.404884-2.043243-2.867054$

C 4.6670494 .4255560 .841658

C 4.9922323 .0036461 .262077

C 5.8656080 .8613410 .354079

O 4.8270582 .5921192 .407432

C $6.8781470 .494955-0.682144$

C $8.058384-0.079544-0.405839$

C $9.067991-0.447366-1.455318$

C $9.353330-1.932188-1.498273$

C $10.473221-2.437258-0.954207$

C $8.382446-2.817266-2.250747$

C $7.162407-3.157252-1.437305$

N 5.5514602 .2718220 .224094

H -1.487451 -5.5449580 .735037$

H $-1.113979-2.825721-0.624826$

H $0.572423-4.4213931 .419294$

H $-0.873119-2.6135592 .507444$

H $-0.196927-1.4371311 .410017$

H $2.633680-0.9022613 .783147$

H $2.111785-2.9410664 .712505$

H $0.412376-3.3806754 .522075$

H $1.652459-4.1421173 .505084$

H $2.7928050 .633747 \quad 1.993559$

H $1.510291-0.1143461 .072477$

H -0.2043400 .7982702 .662659$

H 1.0820021 .4468083 .679046

H $0.035373 \quad 3.1956722 .409270$

H -0.6548731 .9345800 .345292$

H $1.0287761 .748178-0.106447$

H $-0.5583522 .969983-1.885590$

H $2.4402013 .869317-0.633262$

H -1.808277 $4.115410-0.277005$

H $-0.8719595 .568122-0.521626$

H -0.9671214 .8401931 .093728$ 
H $1.4510705 .688902-1.565998$ H 3.5186426 .1458431 .396783 H 3.7655985 .0093232 .702122 H 1.6361805 .6577222 .937085 H $3.097490-2.413540-1.552259$ H $5.224835-3.9236880 .083971$ H $4.862320-1.148040-2.543337$ H $4.739782-2.645921-3.495525$ H $6.217747-1.694334-3.506841$ H $4.4700824 .478794-0.233147$ H 5.5812975 .0081041 .014729 H 4.9390460 .3017320 .188713 H $6.2109090 .656507 \quad 1.372821$ H $6.6186980 .697782-1.718792$ H $8.322105-0.2871550 .628571$ H $9.9894050 .109406-1.239946$ H $8.750414-0.112371-2.450693$ H $10.698696-3.497271-1.006729$ H $11.186781-1.802687-0.439061$ H $8.869477-3.765607-2.515197$ H $8.142904-2.350891-3.209904$ H $7.391644-3.746151-0.547595$ H $5.5060462 .658301-0.714646$ C -4.184069-4.533578 0.068914 O $-4.077202-3.2417010 .700122$ C $-5.299006-2.5135020 .501868$ C $-6.328617-3.5514980 .070995$ C $-5.472113-4.493440-0.754626$ H $-5.125116-1.826890-0.334519$ C $-5.654744-1.7184501 .762902$ H $-6.755404-4.0855010 .927552$ H -7.152150 -3.108874 -0.497277 C $-6.102402-5.856871-0.977413$ H $-5.281735-4.025245-1.729835$ C $-4.518297-0.8324442 .322073$ O $-6.008226-2.6554002 .790855$ H -6.552854 -1.118618 1.573974 H $-3.716420-1.4770812 .704670$ O $-5.028934-0.1087203 .450082$ C -3.9060290 .2090041 .364288$ C -4.9571911 .1054340 .682663$ H $-3.2395850 .856923 \quad 1.948239$ O $-3.104259-0.432010 \quad 0.381830$ H $-5.5285980 .509774-0.035638$ H -5.6610411 .4642051 .441898$ H $-5.438533-6.501526-1.561902$ H $-6.312982-6.361615-0.028544$ H -7.046635 -5.759650 - 1.523105 C $-2.935426-4.790919-0.777776$ H $-6.460773-2.1610683 .494721$ H $-5.189843-0.7510724 .161612$ H $-2.455615-0.9881340 .844844$ H -2.878507 -4.050055 - 1.585752 H -2.989354 -5.777073-1.252046 H -4.256876 -5.2741690 .875592$ C $-4.017256 \quad 1.373333-2.239804$ O $-3.4213461 .960805-1.077124$ C $-4.3352532 .324583-0.038485$ C $-5.3672363 .323089-0.582319$ C $-6.0829062 .775786-1.813222$ C $-5.0665802 .309283-2.852876$ H $-4.4781870 .414978-1.972573$ C $-2.890304 \quad 1.102917-3.245406$ H -3.7425802 .8566440 .716602$ O $-6.331974 \quad 3.6577890 .409453$ H $-4.8588434 .254878-0.862840$ H -6.777469 $1.969045-1.551257$ O $-6.8738563 .813168-2.397781$ H $-4.5762003 .184409-3.298798$ O $-5.7486081 .623376-3.901065$ C $-1.9671410 .012183-2.754602$ H $-3.2776100 .779677-4.217954$ H $-2.3084542 .017483-3.413365$ H $-5.851293 \quad 4.0162231 .175108$ H -7.487380 4.129134-1.712289 H $-6.4203052 .230728-4.255886$ O $-0.705452 \quad 0.505097-2.599572$ O $-2.325731-1.137507-2.534849$
C $0.272452-0.425011-2.124695$

H $0.082200-0.649358-1.072126$

H $\quad 0.266602-1.340231-2.724371$

H $1.2570950 .042227-2.211143$

SCF Energy $\left(B 3 L Y P / 6-31 G^{* *} / / M M F F\right)=-3245.91006259$

01498

MM̄FF Geometry

C $0.764340-3.737292-0.517561$

C $0.543049-2.789767-1.441495$

C $-0.774340-2.071613-1.598393$

O $-0.544062-0.649452-1.464413$

C $-1.337429-2.343652-3.003625$

C $-2.753743-1.839833-3.191092$

C $-3.856300-2.560322-2.905982$

C $-2.876869-0.470879-3.804142$

C $-3.902689-3.945717-2.326296$

C $-4.967717-4.140368-1.235758$

C $-4.680436-3.3852100 .073394$

C $-5.576390-3.9296011 .191461$

C $-5.402946-3.1530052 .503288$

O $-4.948385-1.993059-0.127750$

O $-6.482553-3.515173 \quad 3.377654$

C $-5.547498-1.6479262 .206793$

C $-4.108285-3.5371753 .229636$

O $-5.255899-0.8710383 .366733$

C $-4.685665-1.1672671 .016047$

C -5.0605070 .2934620 .664015$

O $-3.309874-1.2157141 .394059$

C $-0.378269-0.163741-0.206806$

O $-0.387033-0.8133630 .830424$

C $-0.1596451 .298081-0.199071$

C $-0.2478212 .086557-1.280187$

C $0.0238193 .521860-1.272974$

C $0.8263263 .977362-2.461765$

C $-4.4583820 .803804-0.651105$

C $-4.8364232 .249677-0.929574$

C $-4.6326273 .995574-2.699980$

O $-5.4628332 .946333-0.135683$

C $-3.3948244 .820098-2.544340$

C $-3.3066965 .869380-1.713480$

C $-2.0928916 .743871-1.582918$

C $-1.4395036 .630390-0.224669$

C -1.9163367 .3085810 .832916$

C $-0.1826775 .798277-0.117893$

C $-0.4371944 .327016-0.293830$

N -4.388082 $2.666578-2.175257$

H $-0.054122-4.0009290 .150509$

H $1.333009-2.493403-2.125414$

H -1.500346 -2.392363 -0.840264

H - $-1.304207-3.418897-3.222509$

H -0.696158 -1.872632 -3.761577

H $-4.828384-2.122482-3.127952$

H -2.399091-0.451585 -4.789109

H -3.920186 -0.167426 -3.939097

H $-2.393506 \quad 0.277208-3.170773$

H -4.134902 -4.635096 -3.147685

H -2.932042 -4.253179 -1.924201

H $-5.946937-3.839997-1.630536$

H -5.019939-5.216041-1.024835

H $-3.623760-3.5194100 .332817$

H $-5.389159-4.9985091 .354762$

H $-6.628526-3.8548870 .881802$

H $-6.448917-4.4777723 .512645$

H -6.603714 -1.461142 1.966463

H $-4.110268-4.6060123 .475268$

H -4.021065 -3.010629 4.186444

H -3.209812 -3.337353 2.642030

H $-5.825650-1.197114 \quad 4.084375$

H $-6.152107 \quad 0.3750180 .576934$

H -4.7490480 .9593811 .479042$

H -2.819222 $-0.541806 \quad 0.899632$

H $\quad 0.092987 \quad 1.7141850 .772165$

H $-0.4866461 .667216-2.253969$

H $0.3412223 .668493-3.394883$

H $1.8290553 .536581-2.433383$

H $0.9458805 .061067-2.519373$

H $-3.3663710 .742858-0.630737$ 
H $-4.8247100 .195239-1.484721$

H -5.492935 $4.443028-2.191373$

H $-4.8786913 .892412-3.761460$

H -2.542816 $4.555535-3.165528$

H $-4.1675586 .134551-1.103458$

H -2.401678 $7.783121-1.756105$

H -1.360693 $6.526763-2.370558$

H $-1.4358407 .255075 \quad 1.804136$

H -2.801372 $7.931000 \quad 0.749458$

H $\quad 0.276090 \quad 5.928756 \quad 0.871547$

H $0.558364 \quad 6.186338-0.822638$

H - 1.0373883 .8875430 .503280

H $-3.8307112 .032888-2.739172$

C $3.228525-4.186489-1.183475$

O $3.701328-2.850389-0.924251$

C $5.138795-2.838938-1.012195$

C $5.551752-4.224138-1.498943$

C $4.437984-5.088855-0.941586$

H $5.512621-2.7104010 .010246$

C $5.608397-1.671975-1.889464$

H $5.568014-4.290824-2.591790$

H $6.542739-4.511128-1.133850$

C $4.342983-6.452229-1.604341$

H $4.600375-5.2204580 .136742$

C $5.131463-0.286829-1.401777$

O $5.091211-1.834571-3.216816$

H $6.701861-1.680147-1.974555$

H $4.046320-0.209598-1.532383$

O $\quad 5.702778 \quad 0.696829-2.275737$

C 5.5113690 .0546950 .051131

C 5.2572591 .5200240 .448223

H $4.974722-0.607730 \quad 0.735768$

O $6.907951-0.2044430 .227275$

H $5.639297 \quad 1.6593841 .466847$

H $5.8826082 .173980-0.172363$

H $3.526315-7.040906-1.175129$

H $4.164428-6.363673-2.681151$

H $5.272495-7.013526-1.463001$

C $2.039568-4.503255-0.273330$

H $5.639863-2.495932-3.670236$

H $6.667277 \quad 0.674331-2.151718$

H $7.117153-0.0586621 .165648$

H $2.330749-4.3170240 .767794$

H $1.795068-5.568687-0.355361$

H $2.930366-4.231871-2.239035$

C 3.0736161 .2503552 .566715

O $2.916123 \quad 1.1332651 .148227$

C $3.782914 \quad 1.9622410 .367510$

C 3.5593733 .4356900 .728893

C 3.7353093 .6834592 .223208

C 2.8791122 .7055453 .026001

H 4.0669510 .8867732 .856653

C 2.0211430 .3508843 .237482

H $3.4663521 .843256-0.675325$

O $4.4464874 .263961-0.014789$

H 2.5405213 .7218940 .443090

H 4.7911253 .6281012 .512386

O 3.3104005 .0202512 .500281

H 1.8226272 .9886172 .929494

O 3.2236882 .8023994 .406970

C $2.241576-1.1274452 .997605$

H 2.0381030 .5073954 .322218

H 1.0236370 .5962282 .855951

H 4.2807465 .1834310 .255498

H 3.5162185 .2069583 .431607

H 2.9761483 .6916144 .711290

O $1.336832-1.826223 \quad 3.746980$

O $3.077722-1.6102502 .248250$

C $1.394113-3.2518813 .637530$

H $\quad 0.912345-3.5647312 .708004$

H $2.426086-3.6134063 .680231$

H $0.842148-3.6786624 .479124$

SCF Energy (B3LYP/6-31G**//MMFF) $=-3245.89155132$

01499

MM̄FF Geometry

C 1.4057174 .3937440 .446491

C $0.4013274 .429666-0.441764$
C $-0.421527 \quad 3.212147-0.768547$

O $-1.7898583 .514889-0.407831$

C $-0.3851112 .836395-2.261146$

C $0.9775472 .437458-2.783525$

C $1.5601071 .246499-2.543265$

C $1.6542643 .453109-3.664339$

C $1.0069990 .122080-1.714254$

C $0.696868-1.108310-2.571482$

C $0.079719-2.237946-1.734338$

C $-0.045467-3.511804-2.571403$

C $-0.749967-4.637906-1.801719$

O $-1.211744-1.802421-1.293319$

O $-1.094454-5.665146-2.744317$

C $-2.071161-4.087418-1.225567$

C $0.176249-5.291090-0.769756$

O $-2.704459-5.059586-0.395329$

C $-1.911096-2.744279-0.471381$

C $-3.286780-2.137872-0.095439$

O $-1.203311-2.9883190 .749906$

C $-2.5625342 .476338 \quad 0.014148$

O $-2.234510 \quad 1.3046900 .109109$

C $-3.8921643 .021250 \quad 0.364283$

C -4.7842722 .2387130 .990119$

C -6.1210172 .6412621 .432975$

C -6.6023584 .0112581 .029896$

C $-4.066077-1.543856-1.273221$

C $-5.534460-1.311216-0.954870$

C $-7.625885-0.544458-2.076947$

O $-6.072730-1.6971130 .078859$

C $-8.1312820 .524789-1.160813$

C $-9.0328440 .285097-0.196226$

C $-9.619681 \quad 1.3323400 .705998$

C -9.2702771 .1043892 .159305$

C -9.9320640 .1970752 .897468$

C -8.2081831 .9857492 .774105$

C $-6.848698 \quad 1.7732592 .167332$

N -6.181411 $-0.652702-1.994327$

H 1.6224183 .4548180 .952097

H $0.1581705 .359603-0.948296$

H $-0.0711982 .372656-0.156486$

H $-0.7865753 .667523-2.857865$

H -1.094556 $2.019080-2.449257$

H $2.5381661 .050681-2.980026$

H $2.6698883 .152378-3.942390$

H $1.0812483 .590870-4.587215$

H $1.7239304 .419835-3.157617$

H $1.751118-0.136561-0.952566$

H $\quad 0.1060370 .422691-1.175602$

H $-0.002921-0.820459-3.367121$

H $1.615507-1.460017-3.054372$

H $0.716128-2.417396-0.860644$

H $0.939674-3.845324-2.921161$

H $-0.627206-3.295917-3.478714$

H $-0.274319-5.970332-3.168577$

H -2.744257 -3.942626 -2.078580

H $1.068089-5.699580-1.259725$

H $-0.311446-6.142944-0.283322$

H $0.512636-4.5966920 .002414$

H $-2.237209-5.0698410 .457393$

H -3.881022 -2.891557 0.436034

H $-3.140411-1.3314090 .636228$

H -1.366443 -2.244786 1.353688

H $-4.090404 \quad 4.058804 \quad 0.120448$

H -4.5053281 .2116801 .222894$

H -7.6473834 .2004171 .282780$

H $-6.5278274 .141212-0.055918$

H -6.0044904 .7878371 .519160$

H -3.615431 -0.589708 -1.570159

H $-4.035329-2.213422-2.138476$

H $-8.058034-1.524593-1.846898$

H $-7.877510-0.291442-3.111750$

H -7.768041 $1.536642-1.320008$

H -9.401078 -0.730136 -0.061679

H $-10.710936 \quad 1.312495 \quad 0.586113$

H $-9.314410 \quad 2.3394840 .397419$

H -9.7055950 .0464873 .947726$

H -10.715195 -0.416953 2.464897

H $-8.118111 \quad 1.7804323 .849175$ 
H -8.538671 3.0265462 .715095 H $-6.417157 \quad 0.7986022 .399453$ H $-5.651153-0.425900-2.830652$ C 3.7548435 .3498310 .556015 $\begin{array}{llllll}\text { O } & 4.193881 & 4.096997 & 1.123904\end{array}$ C $4.903333 \quad 3.3649490 .106575$

C $5.366508 \quad 4.417325-0.889887$ C $4.1473325 .320614-0.922930$ H $4.1727752 .718215-0.394634$ C 6.0097932 .5098700 .727205 H $6.2402374 .967776-0.522116$ H $5.6117013 .990222-1.866770$ C $4.4167406 .691993-1.516204$ H $3.3685074 .828486-1.515872$ C $5.497528 \quad 1.507132 \quad 1.784508$ $\begin{array}{lllll}\text { O } & 6.953053 & 3.383780 & 1.356506\end{array}$ H $6.5532141 .982448-0.066199$ H 5.1478542 .0405402 .676917 O $6.604774 \quad 0.6979132 .199685$ C $4.3843450 .568251 \quad 1.276555$ C $3.974166-0.4893062 .321550$ H 3.4974591 .1593001 .024887 O $4.819566-0.0691360 .078782$ H $4.823608-1.1494582 .524075$ H 3.7343780 .0294863 .256971 H $3.5121227 .308151-1.496650$ H $5.1990707 .222565-0.963107$ H $4.7432156 .602902-2.557388$ C $2.262798 \quad 5.564173 \quad 0.837276$ H 7.7417802 .8581951 .571591 H 7.2109131 .2671272 .703050 H $5.627204-0.5688950 .288336$ H 1.9199936 .4862210 .354028 H 2.1305595 .7026681 .917941 H $4.3192456 .134148 \quad 1.076403$ C $3.915569-3.1874020 .845031$ O $3.009479-2.0895080 .686562$ C $2.754687-1.3314911 .873381$ C $2.180406-2.2500982 .960708$ C $3.075815-3.459158 \quad 3.215242$ C $3.395528-4.1699581 .900498$ H $4.902076-2.8109801 .140333$ C $4.054870-3.868470-0.520616$ H $1.964315-0.6150071 .614291$ O $1.983492-1.5218894 .167655$ H $1.193096-2.6052602 .637163$ H $3.988216-3.1664703 .747906$ O $2.378134-4.3615054 .076418$ H $2.490832-4.6721041 .537610$ O $4.364893-5.1875772 .143049$

C $4.899860-3.028290-1.448242$ H $4.545338-4.845245-0.445181$ H $3.073606-4.035456-0.979844$ H $1.638336-2.1447984 .829930$ H $2.942173-5.1455094 .191934$ H $5.178372-4.7568042 .456804$ O $4.109786-2.391717-2.357618$ O $6.117200-2.931436-1.356866$ C $4.796578-1.543525-3.283325$ H $5.524864-2.121184-3.860545$ H $4.058929-1.123129-3.971809$ H $5.287430-0.723137-2.751681$ SCF Energy (B3LYP/6-31G**//MMFF) $=-3245.91745576$

\section{1_5}

MM̄FF Geometry

C -1.559140 3.311643 -1.019149

C $-0.7373024 .057041-0.265991$

C $0.0764593 .489587 \quad 0.866403$

$\begin{array}{lllllll}\text { O } & 1.464689 & 3.729181 & 0.540324\end{array}$

C $-0.262584 \quad 4.1519862 .216860$

C -1.718527 $4.053762 \quad 2.621444$

C $-2.3178772 .928154 \quad 3.056497$

C -2.499666 5.3387582 .524722

C -1.694716 1.5754623 .264606

C $-2.204723 \quad 0.558174 \quad 2.234887$

C -1.757772 -0.8908112 .496185$

C $-2.542802-1.524797 \quad 3.646933$
C -2.065469 -2.952602 3.942389

O $-0.354800-0.9039682 .771549$

O $-2.624760-3.3537905 .202454$

C $-0.533553-2.9310474 .114456$

C $-2.575581-3.9559262 .900876$

O $-0.025222-4.2551324 .268967$

C $0.211499-2.2089842 .966600$

C $1.701853-2.0217363 .355906$

O $\quad 0.119301-2.9995191 .784095$

C $2.3844222 .917792 \quad 1.128673$

$\begin{array}{llll}\text { O } & 2.176678 & 2.069051 & 1.980646\end{array}$

C $3.699808 \quad 3.2129770 .519856$

C $4.7303922 .388708 \quad 0.760338$

C $6.0746872 .493148 \quad 0.191308$

C $6.3729073 .672983-0.695874$

C $2.556705-1.2911012 .310510$

C $3.213072-2.2048931 .287280$

C $4.528022-2.094579-0.835746$

O $3.221407-3.4286651 .383792$

C $5.986715-1.820284-0.651905$

C $6.726640-1.114828-1.520813$

C $8.191116-0.833509-1.334869$

C $8.5032860 .647647-1.331029$

C $8.944884 \quad 1.258807-2.443357$

C $8.3844811 .403074-0.024929$

$\begin{array}{llll}\text { C } & 6.968146 & 1.519402 & 0.464126\end{array}$

N $3.803863-1.480590 \quad 0.261443$

H $-1.6220502 .242786-0.835164$

H $-0.6661875 .126881-0.445961$

H $-0.0811202 .406307 \quad 0.925964$

H 0.0458185 .2065602 .188889

H 0.3624183 .7234093 .010435

H -3.3748712 .9771083 .315649$

H $-3.553678 \quad 5.2071212 .791541$

H -2.076876 $6.088192 \quad 3.201777$

H -2.4660395 .7358501 .505673$

H $-0.6023421 .629862 \quad 3.226272$

H -1.9489681 .2511094 .279629$

H -3.2991510 .5941152 .164156$

H $-1.8224950 .854597 \quad 1.250618$

H $-1.940967-1.4538321 .573634$

H -3.618991-1.516956 3.433382

H $-2.415675-0.9301714 .560828$

H $-3.593290-3.2987005 .131316$

H $-0.315129-2.4037315 .053855$

H -3.672116 -3.9658472 .884636$

H -2.270388 -4.9770513 .154411$

H $-2.234647-3.7354801 .887047$

H $-0.509651-4.6674025 .004575$

H $1.734655-1.4154684 .271124$

H $2.156063-2.9907703 .592302$

H $\quad 0.772202-2.6706721 .147908$

H $3.7728254 .070690-0.138806$

H $4.5711151 .536317 \quad 1.419606$

H $6.2303284 .611184-0.148343$

H $5.7144233 .672801-1.571510$

H $7.3973873 .682493-1.074648$

H $1.966773-0.5311521 .790072$

H $3.375597-0.7778362 .830378$

H $4.139278-1.663232-1.763511$

H $4.348795-3.173853-0.853640$

H $6.455764-2.2251930 .241916$

H $6.258983-0.722328-2.421118$

H $8.731969-1.328381-2.152294$

H $8.573648-1.287645-0.412341$

H $9.1998922 .313305-2.446968$

H $9.0579020 .718381-3.377377$

H $8.8459012 .395034-0.097040$

H 8.9742150 .8819930 .740481

H $6.6612720 .696121 \quad 1.109887$

H $3.745182-0.4665780 .275060$

C -3.924253 $3.506698-1.901308$

O $\begin{array}{llll}-4.104628 & 2.076705 & -1.945448\end{array}$

C $-4.9975751 .692507-0.881584$

C $-5.6840162 .977409-0.432904$

C $-4.5555613 .979062-0.590616$

H $-4.3693511 .327693-0.060536$

C $-5.9375780 .575430-1.345866$ 
H -6.520951 $3.244496-1.088205$ H -6.0607362 .9115510 .592182$ C $-5.0139535 .425916-0.613213$ H -3.8636293 .8409790 .247539$ C $-5.203609-0.634271-1.961302$ O $-6.8107831 .113340-2.346693$ H $-6.5753290 .259353-0.511922$ H $-4.726842-0.330368-2.899745$ O $-6.182657-1.623114-2.303144$ C $-4.167029-1.294772-1.030833$ C $-3.496171-2.545835-1.634492$ H -3.390702 -0.573202 -0.759339 O $-4.804543-1.6807730 .189610$ H -2.933774 -3.046537 -0.837285 H -4.264820 -3.270011 -1.930291 H -4.162603 $6.100748-0.746383$ H $-5.7223615 .610568-1.427649$ H -5.5084255 .6871680 .328090$ C $-2.4448783 .854281-2.104053$ H -7.529972 $0.473664-2.482211$ H $-6.697336-1.278573-3.052265$ H -5.514473 -2.306370 -0.036150 H $-2.3335704 .941496-2.189067$ H -2.104782 $3.416101-3.050490$ H $-4.4884653 .906451-2.753967$ C $-0.562527-1.704236-1.607224$ O $-1.589626-1.240746-2.489596$ C $-2.572726-2.226925-2.828751$ C -1.903114 -3.452115 -3.468206 C $-0.765620-4.004357-2.613023$ C $0.199933-2.883723-2.222143$ H -1.025963 -2.030380 -0.668985 C $0.331907-0.519566-1.221502$ H -3.200616 -1.777808 -3.606880 O $-2.871213-4.467741-3.710651$ H -1.495892 -3.159409-4.444985 H -1.156649 -4.525315 - 1.731222 O $-0.060966-4.983579-3.379126$ H $\quad 0.750707-2.568715-3.115561$ O $1.161729-3.387954-1.300552$ C $1.1128530 .082433-2.369192$ H $-0.280627 \quad 0.284592-0.801644$ H $\quad 1.037529-0.845597-0.451814$ H -2.404521 -5.227340 -4.099191 H $0.685934-5.292029-2.837518$ H $0.681739-3.806626-0.566181$

O $2.2455880 .668208-1.879566$ O $\quad 0.760880 \quad 0.072703-3.540618$ C $3.0971451 .277096-2.855056$ H $4.0276541 .567754-2.361209$ H $3.3367770 .569619-3.654908$ H $2.6169752 .172339-3.260490$ SCF Energy (B3LYP/6-31G**//MMFF) $=-3245.91773883$

01 _50

MM̄FF Geometry

C $-3.497997 \quad 1.7539742 .091227$

C -2.8971440 .9341812 .966594$

C $-2.865178-0.5605832 .792569$

O $-1.519319-1.0432653 .035575$

C $-3.758947-1.2942363 .806808$

C $-5.243771-1.0745373 .630577$

C $-6.021540-1.7979572 .802237$

C $-5.860126-0.0251334 .519344$

C $-5.600152-2.9070931 .875207$

C $-6.252546-2.7749100 .490604$

C $-5.592106-1.707392-0.397681$

C $-6.512583-1.367167-1.572681$

C $-5.853739-0.390219-2.552190$

O $-4.358074-2.252778-0.878814$

O $-6.673868-0.357376-3.729277$

C $-4.475245-0.952605-2.946375$

C $-5.8381191 .037042-1.997331$

O $-3.769363-0.024544-3.769031$

C $-3.604351-1.391266-1.742891$

C $-2.395831-2.201163-2.273362$

O $-3.146667-0.226518-1.058207$

C $-0.561853-0.8059462 .100647$
O $-0.669606-0.1459991 .080824$

C $0.653298-1.5260102 .543263$

C $1.738654-1.5833801 .757433$

C $2.987732-2.2809862 .079117$

C $3.043745-3.0431953 .378407$

C $-1.465844-2.765286-1.191960$

C $-0.472746-3.764428-1.763344$

C $1.277420-5.374377-1.066588$

O $-0.431324-4.063889-2.954066$

C $2.447298-5.311027-0.138901$

C $3.711383-5.165407-0.563763$

C $4.911991-5.1560340 .338210$

C $5.675274-3.8526770 .271133$

C $6.629575-3.655435-0.654035$

C $5.386903-2.8155771 .332074$

C $4.011626-2.2178211 .202612$

N $0.366715-4.280947-0.783377$

H $-4.016149 \quad 1.317912 \quad 1.241992$

H -2.397387 1.3481683 .838494

H -3.151333 -0.854521 1.775266

H $-3.462422-1.0219894 .829752$

H -3.540427 -2.369992 3.758224

H -7.090632 -1.588085 2.799764

H -6.9434880 .0544624 .379084$

H -5.683140 -0.269907 5.571963

H -5.4326170 .9604514 .316988$

H -4.513194 -2.975528 1.770833

H $-5.938147-3.8500032 .322275$

H -6.176941-3.749591-0.009281

H -7.321597 -2.559442 0.613044

H $-5.383982-0.8107860 .196006$

H -7.467712 -0.962177 -1.214911

H $-6.764671-2.288670-2.116911$

H $-6.2238910 .201240-4.386398$

H -4.662096 -1.831489 -3.578841

H $-6.8612371 .386457-1.813235$

H $-5.4079321 .740822-2.718118$

H $-5.286138 \quad 1.120410-1.059970$

H $-3.4075580 .667823-3.189824$

H -2.779393 -3.043492 -2.864654

H $-1.794272-1.579376-2.948162$

H $-2.352447-0.450395-0.548311$

H $0.614080-2.0085083 .513950$

H $1.713213-1.0779170 .792956$

H $2.233228-3.779453 \quad 3.427612$

H $2.950927-2.3589064 .228979$

H $3.968497-3.6051673 .520448$

H $-0.890723-1.957165-0.733043$

H -2.044369 -3.272323 -0.411996

H $1.580081-5.338193-2.118618$

H $0.727093-6.307792-0.908784$

H $2.248169-5.4202080 .924214$

H $3.903372-5.075382-1.631001$

H $5.567467-5.9833680 .036127$

H $4.633682-5.3712421 .377373$

H $7.202747-2.735021-0.690352$

H $6.856347-4.411650-1.398451$

H $6.107834-1.9908461 .262771$

H $5.568732-3.2609592 .313513$

H $3.869146-1.6496570 .283497$

H $0.150643-4.0818720 .189634$

C -2.8540763 .9937511 .067095$

O $-1.447933 \quad 3.6790821 .029947$

C $-1.0546193 .508636-0.343514$

C $-2.1937354 .077281-1.183695$

C $-3.3948983 .716433-0.335644$

H $-0.9834682 .427155-0.515625$

C $0.3054474 .156775-0.628373$

H $-2.1168585 .165725-1.289777$

H $-2.2276183 .640347-2.186408$

C $-4.6502074 .496951-0.680623$

H -3.590091 $2.649275-0.479050$

C 1.4589823 .5576650 .205466

O $0.2170985 .564603-0.411315$

H $0.5302214 .012786-1.691781$

H 1.3799202 .4656330 .163360

O $1.327273 \quad 3.9297501 .575335$

C $2.8493783 .967873-0.318406$ 
C 4.0367573 .4707500 .522920

H $2.9490373 .606101-1.347952$

O $2.9407205 .393803-0.375691$

H $4.9519893 .764019-0.007239$

H $4.0749904 .031781 \quad 1.466226$

H -5.482787 4.197904-0.036176

H -4.499114 $5.574942-0.559922$

H $-4.9440684 .313722-1.719314$

C -3.535286 3.2504132 .220789

H $-0.098921 \quad 5.7016700 .498135$

H $1.501698 \quad 4.884427 \quad 1.637971$

H $3.7924835 .618225-0.787869$

H $-4.583493 \quad 3.5637432 .289642$

H -3.0505973 .5472023 .159554$

H -2.9134545 .0696861 .278950$

C $4.6928201 .161731-1.361690$

O $3.7302311 .156802-0.304194$

C 4.0659121 .9607200 .825631

C $5.3902621 .476703 \quad 1.433314$

C 6.5057161 .4166820 .390015

C $6.0500250 .659596-0.857296$

H $4.7928092 .175869-1.767001$

C $4.152100 \quad 0.255806-2.473349$

H 3.2827831 .7770341 .571832

O 5.7989122 .3210612 .504554

H $5.2536660 .472747 \quad 1.852511$

H 6.8645852 .4187130 .127715

O 7.6285120 .7255160 .943655

H $5.993366-0.411057-0.631839$

O $7.021917 \quad 0.822154-1.888061$

C $2.8638580 .806233-3.039018$

H $4.8635280 .162655-3.301582$

H $3.963933-0.759280-2.102697$

H 5.0795222 .3311563 .158873

H 7.8853151 .1958551 .755550

H $7.8733890 .511898-1.534950$

O $1.797705 \quad 0.240381-2.403059$

O $2.8148241 .654930-3.919773$

C $0.5062790 .695408-2.818906$

H $\quad-0.2242420 .366729-2.075294$

H $\quad 0.472517 \quad 1.787176-2.878238$

H $\quad 0.2535320 .249879-3.785128$

SCF Energy (B3LYP/6-31G**//MMFF) $=-3245.92263399$

01 500

MM̄FF Geometry

C -1.039924 -3.305028 0.856102

C $-0.796041-2.789781-0.359028$

C -1.815296-1.996918 -1.136681

O $-1.556212-0.569104-1.083588$

C $-1.966645-2.459261-2.600667$

C $-3.375108-2.923943-2.913637$

C $-4.366545-2.106903-3.319822$

C $-3.611233-4.402598-2.764472$

C $-4.298066-0.611035-3.456944$

C $-5.5576590 .104050-2.945394$

C $-5.911628-0.168081-1.472792$

C $-7.1807850 .604376-1.095114$

C -7.5422750 .4392810 .386618$

O

$\begin{array}{lllll}\text { O } & -8.535566 & 1.431221 & 0.688195\end{array}$

C $-6.289753 \quad 0.747277 \quad 1.232751$

C $-8.197899-0.9189240 .659760$

O -6.5377540 .4965922 .614050$

C $-5.023987-0.001366 \quad 0.758325$

C $-3.758485 \quad 0.4513901 .523497$

O $-5.191385-1.4003741 .022830$

C $-0.411823-0.029461-1.575765$

O $0.577862-0.615480-1.980183$

C $-0.6073831 .438665-1.580391$

C $0.4251552 .265339-1.798141$

C $0.3527903 .728835-1.852698$

C $-1.0156894 .359678-1.859865$

C -3.3910991 .9273521 .358702$

C -2.059066 2.261307 2.011219

C -0.5526924 .1914672 .447095$

O -1.3925091 .4432092 .638254$

C -0.1100715 .3769261 .650230$
C $1.1557475 .578598 \quad 1.252738$

C $1.5971956 .790846 \quad 0.483010$

C $2.417356 \quad 6.456432-0.743764$

C $3.7364576 .710381-0.779535$

C 1.695345 5.921941-1.962210

C $1.5040984 .429921-1.907822$

N -1.7099753 .5900651 .810050$

H -2.001986 -3.090918 1.319259

H $0.158327-2.968958-0.843523$

H -2.782971 -2.105971 -0.632238

H $-1.274526-3.281035-2.827564$

H -1.693957 -1.659631 -3.300098

H $-5.330810-2.549942-3.564427$

H $-4.646776-4.683006-2.984167$

H -2.963406 -4.962167 -3.446960

H $-3.393575-4.718850-1.739086$

H -3.427254 -0.195229 -2.943466

H $-4.179057-0.372574-4.520872$

H $-5.3970141 .181404-3.083615$

H -6.409516 -0.174351 -3.579079

H $-6.077842-1.243445-1.343401$

H -8.023506 $0.303375-1.730336$

H $-7.0255201 .674283-1.294793$

H -8.7163531 .3861411 .642985$

H -6.1206541 .8274171 .147698$

H $-9.119024-1.0207760 .073326$

H -8.502056 -1.009523 1.708388

H -7.553616 -1.765193 0.412831

H $-6.502511-0.4654372 .751242$

H -3.8621150 .2107032 .589871$

H $-2.909960-0.1534261 .178954$

H -4.314043 -1.811766 1.088073

H -1.614986 $1.801959-1.413625$

H $1.4155431 .835005-1.938314$

H -1.569536 $4.103080-0.952346$

H -1.589992 $4.012639-2.726315$

H -0.996966 $5.449678-1.911856$

H $-3.3281112 .188246 \quad 0.297001$

H -4.1515222 .5616081 .826612$

H 0.2361523 .4411632 .556836

H -0.8632584 .5091003 .447718$

H -0.8658456 .1194891 .404710$

H 1.9200354 .8548101 .524765

H 2.1843147 .4177101 .166909

H $\quad 0.7388907 .403798 \quad 0.180242$

H $4.3267066 .506485-1.666810$

H 4.2574937 .1226350 .078123

H $2.2800756 .139575-2.866206$

H $\quad 0.7617616 .474287-2.096310$

H $2.4423723 .875134-1.920999$

H -2.4144924 .2135821 .425406$

C $\quad 0.978977-4.923070 \quad 0.935545$

O $2.022665-4.0049920 .561364$

C $3.295187-4.6667290 .688264$

C $2.995938-6.1165361 .056586$

C $1.675452-5.9842791 .787581$

H $3.811986-4.1930451 .530605$

C $4.115014-4.501600-0.596654$

H $2.878943-6.7478740 .168239$

H $3.786196-6.5565511 .672695$

C $0.917462-7.2961791 .898650$

H $1.868475-5.5902702 .794439$

C $4.355431-3.035801-1.012884$

O $3.411051-5.150061-1.663961$

H $5.070439-5.029225-0.489289$

H $3.414182-2.580752-1.343147$

O $5.227764-3.043965-2.150251$

C $5.002589-2.1618460 .076959$

C $5.492462-0.785558-0.405890$

H $4.297377-2.0202010 .900582$

O $6.134286-2.8446210 .627274$

H $5.966770-0.2893540 .449682$

H $6.306203-0.924611-1.129075$

H $-0.026422-7.1609152 .435362$

H $0.687130-7.7111200 .911506$

H $1.510539-8.0373262 .444531$

C $-0.136342-4.1846121 .681281$

H $4.022238-5.223768-2.415901$ 
H $4.722694-3.392835-2.903893$

H $6.755804-3.016590-0.101068$

H $0.291758-3.5682472 .480055$

H $-0.784914-4.9275442 .161030$

H $\quad 0.591002-5.3728190 .011833$

C 3.5368161 .0584251 .037999

O $3.2786520 .291568-0.143849$

C $4.4009720 .116946-1.013632$

C $4.9188761 .485360-1.474807$

C $5.2484892 .385953-0.287424$

C 4.0673302 .4544070 .679195

H 4.2592750 .5273501 .669824

C 2.2180151 .1774811 .817963

H $4.021636-0.394253-1.906309$

O $6.0667221 .324798-2.300915$

H $4.147676 \quad 1.968187-2.088155$

H 6.1545672 .0462750 .226366

O $5.5466193 .701018-0.760764$

H 3.2647303 .0516040 .232265

O $4.4779543 .123406 \quad 1.870180$

C $1.800049-0.1330822 .447723$

H 2.3316451 .9168112 .619295

H 1.3997781 .4971341 .163418

H $6.3785662 .213759-2.542282$

H $4.7740554 .024053-1.254300$

H 4.8143753 .9981021 .610030

O 1.1975330 .1122863 .647606

O $1.948878-1.2325501 .933423$

C $0.704974-1.0379374 .341551$

H $\quad 0.389389-0.7230635 .339723$

H $1.488649-1.7945884 .445593$

H -0.160737 -1.446058 3.812249

SCF Energy (B3LYP/6-31G**//MMFF)= -3245.90580769

01 501

MM̄FF Geometry

C $2.798165-3.009466-1.916963$

C $2.113154-1.992527-1.372949$

C $1.032605-1.232522-2.098317$

O $-0.060248-0.996618-1.180346$

C $1.5666810 .137335-2.554729$

C $0.5786370 .925793-3.395041$

C $0.0792612 .131617-3.059425$

C $0.177390 \quad 0.294393-4.703931$

C $0.3872342 .948979-1.837756$

C $-0.5190922 .670941-0.630109$

C -1.990486 $3.053885-0.852582$

C $-2.778606 \quad 2.9582210 .455673$

C -4.2729753 .2546090 .248491$

O $-2.5540072 .157435-1.812943$

O -4.9740852 .8282881 .427083$

C $-4.8012482 .399692-0.924600$

C $-4.540702 \quad 4.7576910 .105727$

O $-6.1364212 .766538-1.261293$

C $-3.9073472 .458482-2.180446$

C $-4.3212261 .443875-3.274579$

O $-4.0017173 .763803-2.764403$

C $-0.928721-2.018642-0.957335$

O $-0.910845-3.127289-1.466717$

C $-1.900585-1.5562300 .057692$

C $-2.636638-2.457500 \quad 0.724189$

C $-3.601759-2.1666411 .786181$

C $-3.951367-0.7229542 .031891$

C $-4.311495-0.027887-2.846088$

C $-5.668984-0.549052-2.403014$

C $-6.742672-2.559513-1.396381$

O $-6.7148830 .075608-2.556751$

C $-6.956743-2.3109420 .063209$

C -7.126979 -3.2954620 .958698$

C -7.386548 -3.0646202 .419772$

C $-6.417740-3.8124393 .308158$

C $-6.751861-4.9935413 .855114$

C $-5.097267-3.149734 \quad 3.629119$

C $-4.129764-3.1978382 .478275$

N -5.579568 -1.819526 -1.849491

H $2.586360-3.309869-2.940144$

H $2.337247-1.684561-0.355393$

H $0.668798-1.806470-2.958991$
H $2.4805560 .002267-3.148558$

H $1.8604490 .709176-1.668072$

H $-0.6242622 .603515-3.744537$

H $-0.3879240 .983381-5.340982$

H $\quad 1.065230-0.012953-5.265949$

H $-0.452535-0.583868-4.533264$

H $1.4305312 .805380-1.543387$

H $0.3142514 .010041-2.108547$

H $-0.4478831 .609284-0.366959$

H $-0.126303 \quad 3.242503 \quad 0.219470$

H $-2.0236964 .077777-1.243266$

H -2.3570013 .6287291 .214816$

H -2.6796591 .9440680 .865778$

H $-4.599742 \quad 3.3050832 .187664$

H $-4.8428861 .364476-0.567093$

H $-4.2043265 .293073 \quad 1.001812$

H -5.6137074 .9617940 .021334$

H $-4.0352595 .203703-0.753254$

H -6.670469 $2.691708-0.451884$

H $\quad-5.2850691 .732789-3.710797$

H $-3.5933651 .538099-4.093665$

H -3.732244 $3.704807-3.696019$

H -1.955098 -0.4918720 .254649$

H $-2.499323-3.5132920 .493923$

H -4.261076 -0.2412931 .098676$

H $-3.089910-0.1821722 .438519$

H $-4.780521-0.5855982 .728787$

H -4.020787 -0.638249 -3.710896

H $-3.573223-0.205188-2.058323$

H -6.560024 -3.616490 -1.614528

H $-7.623709-2.233658-1.958680$

H -7.000943 -1.275951 0.394108

H -7.111372 -4.328850 0.619002

H -8.413319 -3.3913232 .630985$

H -7.360180-1.996485 2.668810

H $-6.073652-5.5233094 .515874$

H -7.709834 -5.459631 3.649512

H -4.616551 -3.659087 4.475260

H -5.285916 -2.134259 3.987929

H -3.813668 -4.209806 2.220820

H $-4.665420-2.241025-1.712654$

C $5.265903-3.309317-1.596181$

O $5.512959-2.021218-0.994986$

C $6.750876-2.067902-0.264763$

C $7.509409-3.239588-0.872863$

C $6.380883-4.228273-1.094235$

H $6.512040-2.3036270 .779756$

C $7.494855-0.729809-0.344754$

H $7.971415-2.963596-1.828441$

H $8.291266-3.621479-0.209604$

C $6.732152-5.348917-2.057314$

H $6.101019-4.665805-0.126277$

C 6.9136340 .4378420 .486358

O $7.582637-0.335820-1.714934$

H $8.519211-0.9057620 .009278$

H $7.508312 \quad 1.3273190 .242482$

O $7.129624 \quad 0.177857 \quad 1.874191$

C $5.4327050 .790506 \quad 0.247284$

C 5.0493362 .1267330 .917122

H $5.2454190 .853072-0.830985$

O $4.618630-0.2429490 .787303$

H 5.1293132 .0214752 .004113

H 5.7735792 .8879910 .605743

H $5.879091-6.019402-2.201829$

H $7.024103-4.958562-3.038054$

H $7.567295-5.941423-1.669656$

C $3.867389-3.781965-1.195078$

H $8.1355080 .462754-1.757185$

H $6.490774-0.5013822 .149991$

H $3.698504-0.0528170 .539866$

H $3.733207-3.676797-0.110900$

H $3.736849-4.843320-1.435743$

H $5.335540-3.172606-2.682904$

C 2.3613611 .7025392 .405295

O $2.603108 \quad 1.697320 \quad 0.992133$

C 3.6241022 .5931980 .537128

C 3.2892334 .0310320 .957511

C 3.0385004 .1382092 .457020 
C 1.9972333 .1105622 .893153 H 3.2567941 .3536082 .932441 C 1.2081920 .7199962 .678919 H $3.5831612 .568181-0.559123$ O $4.329026 \quad 4.931067 \quad 0.590313$ H $2.382774 \quad 4.3602460 .432996$ H 3.9666024 .0269473 .030029 O 2.5317235 .4406622 .756980 H 1.0111023 .4015292 .509239 O 1.9161543 .1054714 .317438 C $1.654832-0.7269712 .664498$ H $\quad 0.7660430 .8936933 .665953$ H $0.432366 \quad 0.847210 \quad 1.914431$ H $4.4535974 .856311-0.371375$ H $3.186476 \quad 6.0855542 .438299$ H 1.7105664 .0132884 .599488 O $\quad 0.571970-1.5295942 .444011$ O $2.797442-1.1132792 .867436$ C $0.838005-2.9360992 .456713$ H $1.502219-3.2017531 .629411$ H $-0.110007-3.4646802 .329878$ H $1.276213-3.2327853 .414546$

SCF Energy (B3LYP/6-31G**//MMFF)= -3245.90472261

01_502

MM̄FF Geometry

C 3.3449561 .4490173 .030495

C 2.4492210 .8575652 .225009

C $1.130800 \quad 0.3038212 .696139$

O $0.153866 \quad 0.457070 \quad 1.640040$

C $1.271654-1.2003922 .995161$

C $-0.018588-1.8780723 .420193$

C $-0.485542-3.0171372 .871513$

C $-0.780325-1.2251374 .544551$

C $0.177625-3.8595091 .819694$

C $-0.262028-3.5543210 .382084$

C - $1.738722-3.8780840 .106963$

C $-1.993981-3.991038-1.396647$

C $-3.467810-4.291607-1.693613$

O $-2.542626-2.8201710 .635871$

O $-3.659548-4.088320-3.102976$

C $-4.351950-3.270145-0.945640$

C $-3.802669-5.764324-1.428493$

O $-5.722617-3.644596-1.061373$

C $-3.956024-3.0470840 .538564$

C $-4.662864-1.7889811 .085673$

O $-4.371994-4.1303111 .372094$

C $-0.442277 \quad 1.672787 \quad 1.518034$

O -0.2055012 .6752842 .173235$

C $-1.453858 \quad 1.570430 \quad 0.442017$

C -2.1088622 .6762160 .058580$

C -3.171499 $2.759277-0.945451$

C $-3.5648811 .495184-1.661023$

C $-4.197778-1.3694992 .484236$

C $-5.069011-0.2461723 .010872$

C -5.6047912 .1637732 .632574$

O $-5.911946-0.4417313 .883462$

C -6.2742692 .5678151 .355446$

C -6.2719343 .8231640 .881326$

C $-6.9301284 .228844-0.407904$

C $-6.0135355 .026212-1.312881$

C $-6.2063686 .342446-1.502991$

C $-4.9174554 .285631-2.053834$

C $-3.7546783 .957398-1.159531$

N -4.8243670 .9649272 .383313$

H 3.1275901 .5516924 .090375

H $2.6850630 .737726 \quad 1.171207$

H $\quad 0.7890590 .8340813 .593972$

H $2.010856-1.3475203 .793484$

H $1.678020-1.6853812 .100126$

H -1.428871 -3.4183533 .239339$

H -1.603175 -1.851208 4.906593

H $-0.115644-1.0384455 .394346$ H -1.212407 -0.2737454 .219849$ H $\quad 1.267797-3.7685961 .884934$ H $-0.029161-4.9132522 .047311$ H $-0.062061-2.4986560 .162728$

H $\quad 0.371165-4.154479-0.282961$
H -1.991939-4.818331 0.609986 H -1.350198 -4.755202 -1.849907 H -1.727994 -3.043513-1.884429 H $-4.604803-4.217361-3.293192$ H $-4.242799-2.315891-1.480790$ H $-3.210649-6.412614-2.086082$ H $-4.850904-5.982429-1.660340$ H -3.605033 -6.072105 -0.399679 H $-6.264476-2.891681-0.772383$ H $-4.486330-0.9507700 .403250$ H $-5.747167-1.9548351 .118338$ H $-4.725957-4.8399170 .814504$ H -1.6432590 .5930910 .012820$ H -1.8595503 .6188190 .545189$ H $-4.0432990 .798872-0.967386$ H $-2.6890941 .006246-2.100795$ H -4.259816 $1.660579-2.486830$ H -4.258510 -2.206074 3.189072 H -3.156004-1.0313572.467868 H -4.9134462 .9306302 .995757$ H -6.3568591 .9705443 .403360$ H -6.7882081 .7902150 .794630$ H -5.7819304 .6062911 .455710$ H -7.817807 $4.822331-0.153886$ H -7.300632 $3.354739-0.957917$ H $-5.5692606 .916944-2.167110$ H -7.002422 $6.876811-0.995077$ H $-4.5369794 .901156-2.879251$ H -5.359681 $3.401950-2.521179$ H -3.373974 $4.820649-0.611081$ H -4.0750241 .0241561 .700513$ C 5.6853140 .8270762 .352537 O $5.295050 \quad 0.0604521 .196233$ C $6.443715-0.156606 \quad 0.359312$ C 7.6478260 .1520161 .236192 C 7.1090991 .3050602 .066338 H $6.3988930 .588549-0.443564$ C $6.382184-1.566975-0.236777$ H $7.907370-0.6933741 .883794$ H 8.5328350 .4189630 .651100 C 7.9256651 .5897323 .314425 H 7.0830482 .2078851 .441092 C $5.045080-1.874902-0.949349$ O $6.541015-2.5279800 .814830$ H $7.223945-1.724134-0.920209$ H $4.233848-1.886839-0.213373$ O $5.118396-3.199857-1.488690$ C $4.708418-0.905701-2.097384$ C $3.377087-1.201906-2.810797$ H $4.6795510 .119221-1.724579$

O $5.754409-0.952089-3.071519$ H $3.349216-0.606412-3.731318$

H $3.365939-2.245373-3.148145$

H 7.4873122 .4125743 .887946

H 7.9775290 .7130383 .968780

H 8.9497381 .8708573 .047877

C 4.6778441 .9608462 .554294

H $5.838159-2.3671791 .468030$

H $5.347268-3.798008-0.756717$

H $5.811222-1.867274-3.396101$

H 4.5318802 .5071591 .615437

H 5.0518972 .6798713 .291765

H 5.6731980 .1417063 .210025

C $1.9012481 .457731-2.435854$

O $2.1199190 .438675-1.451373$

C $2.133543-0.904413-1.947047$

C $0.799960-1.215946-2.635732$

C $0.470873-0.198239-3.721342$

C $0.5732051 .224468-3.174259$

H $2.730197 \quad 1.461749-3.153892$

C $1.8906802 .806976-1.695205$

H $2.192017-1.553700-1.065763$

O $0.812122-2.520364-3.205056$

H $-0.006597-1.193583-1.892071$

H $1.109955-0.328538-4.602518$

O $-0.872190-0.410528-4.165691$

H $-0.2755931 .408782-2.505018$

O $0.4567472 .121702-4.277641$ 
C $3.2728043 .272909-1.285491$

H $1.4790543 .591569-2.338125$

H $1.2869342 .731304-0.784466$

H $1.012431-3.149294-2.491130$

H $-0.932756-1.331778-4.472236$

H $\quad 0.2373783 .000716-3.926893$

O $3.1691114 .521388-0.737966$

O $4.3090142 .640538-1.428300$

C $4.4044335 .132456-0.352078$

H $4.181378 \quad 6.1239880 .050929$

H $4.896924 \quad 4.5413960 .424573$

H $5.0579225 .246638-1.222244$

SCF Energy (B3LYP/6-31G**//MMFF)= -3245.91600262

01_503

MM̄FF Geometry

C -0.483712 5.202091-0.458627

C -1.2609224 .5855890 .444270$

C -2.617153 $4.010068 \quad 0.126834$

O -2.5662002 .5909190 .402119$

C -3.6874604 .6345141 .039032$

C $-5.093364 \quad 4.1466960 .743459$

C -5.8546573 .4375861 .599977$

C $-5.6473804 .526343-0.606279$

C -5.5143522 .9935572 .994552$

C -4.8815361 .5970693 .092436$

C -5.7852920 .4568502 .595104$

C $-5.192351-0.9033082 .971587$

C -6.024054 -2.069028 2.413806

O

O $-5.237413-3.2652652 .527308$

C $-6.274267-1.8354860 .908220$

C $-7.295127-2.307327 \quad 3.237574$

O $-7.180696-2.8038270 .387056$

C $-6.777961-0.4145670 .581415$

C $-6.832172-0.121079-0.938459$

O $-8.111839-0.2620021 .082969$

C $-2.0231941 .790646-0.554502$

O $-1.6325682 .123824-1.661762$

C $-1.948530 \quad 0.423090 \quad 0.001905$

C $-1.105156-0.462409-0.547720$

C $-0.900326-1.843853-0.109791$

C $-1.861135-2.4011530 .906770$

C $-5.497968-0.246113-1.682391$

C $-5.280874-1.597106-2.344964$

C $-3.546437-2.895652-3.579082$

O $-6.160002-2.448351-2.446222$

C $-2.852877-3.835432-2.643988$

C -1.632592-4.340834 -2.881556

C $-0.938917-5.321986-1.980022$

C $0.438250-4.856636-1.562271$

C $1.539013-5.287328-2.201297$

C $0.540481-3.955704-0.352431$

C $0.118108-2.545051-0.649405$

N -3.992923 -1.723646 -2.848884

H $-0.8505905 .338082-1.472336$

H -0.8889774 .4402361 .456011$

H $-2.8774614 .174374-0.925883$

H -3.675948 5.7274420 .932175

H -3.4221314 .4295852 .081763$

H -6.8517853 .1436831 .273170$

H $-6.7115174 .284144-0.701533$

H -5.543158 $5.603900-0.770695$

H -5.119121 $3.996648-1.404665$

H -4.8522263 .7187593 .479684$

H -6.4368663 .0117703 .589179$

H -3.9395471 .5938572 .532006$

H $-4.630324 \quad 1.4219904 .146043$

H -6.7762470 .5790163 .048068$

H -5.087923 -0.9950374 .059947$

H -4.173977 -0.9794922 .567119$

H -5.007631 -3.385085 3.464749

H $-5.316588-1.9981200 .399796$

H -7.038780 -2.537375 4.278783

H -7.848923 -3.1770912 .867518$

H -7.967876 -1.447346 3.246983

H -6.815267 -3.681842 0.590301

H -7.616828 - $0.724071-1.411182$
H $-7.1599030 .922306-1.053701$

H $-8.539750 \quad 0.4668540 .603732$

H -2.5693780 .1915520 .859173$

H $-0.488941-0.141683-1.386760$

H -2.895004 -2.257918 0.574685

H -1.729954 -1.903210 1.873479

H -1.746446 -3.473555 1.075911

H $-5.4829540 .494302-2.492641$

H $-4.656871-0.022530-1.020342$

H $-2.886520-2.546190-4.379417$

H $-4.408820-3.395714-4.031412$

H $-3.389275-4.135408-1.746920$

H -1.111437 -4.059343 -3.794072

H $-0.861713-6.274179-2.521290$

H -1.537685 -5.538676 -1.086629

H $2.531201-4.977690-1.890685$

H $1.471316-5.954994-3.053860$

H $1.579328-3.913945-0.000608$

H $-0.015494-4.4056070 .474349$

H $\quad 0.755122-2.049721-1.382925$

H $-3.316716-0.984700-2.679310$

C $2.0123704 .833129-0.761357$

O $1.9351973 .491343-0.235817$

C $2.1263012 .560382-1.323911$

C $2.7344813 .374168-2.455727$

C $2.0198004 .700708-2.284571$

H $1.1229502 .214152-1.599887$

C $2.9762481 .381353-0.837758$

H $3.8150403 .497646-2.310835$

H $2.5674662 .923281-3.438398$

C $2.6962735 .857324-2.999041$

H $1.0013274 .591002-2.675430$

C $3.3770290 .399163-1.953436$

$\begin{array}{lllll}\text { O } & 2.221224 & 0.670961 & 0.149541\end{array}$

H $3.8710641 .763179-0.333049$

H $4.0362690 .904992-2.669027$

O $2.1943840 .036361-2.675979$

C $4.052308-0.897914-1.457619$

C $5.281219-0.630869-0.576582$

H $3.320106-1.508609-0.921383$

O $4.469312-1.649065-2.600139$

H $5.9195100 .106463-1.083265$

H $4.956236-0.1526820 .352866$

H $2.1517896 .791399-2.828190$

H $3.7254355 .997604-2.651754$

H $2.7282065 .676684-4.078465$

C $0.9023505 .700639-0.157307$

H 1.9631891 .3115440 .834394

H $2.474091-0.417008-3.488762$

H $3.669224-1.985591-3.037625$

H $0.9916836 .730581-0.520949$

H 1.0445195 .7371160 .930536

H $2.9765165 .232887-0.420443$

C $7.370600-0.8250851 .584894$

O $7.436542-1.4133050 .279522$

C $6.174745-1.851751-0.252636$

C $5.589357-2.9174960 .683420$

C $5.454370-2.3969522 .112061$

C $6.784744-1.8188152 .596124$

H $\quad 6.7567380 .0829751 .554700$

C $8.799038-0.4176501 .977434$

H $6.416946-2.341586-1.202927$

O $4.330308-3.3917520 .232378$

H $6.263215-3.7844560 .701802$

H $4.648570-1.6586872 .197537$

O $5.099925-3.4792892 .976328$

H $7.494037-2.6377612 .772692$

O $6.581801-1.1549663 .841983$

C 9.3130430 .7435291 .151001

H $8.833641-0.1082713 .027667$

H $9.480553-1.2625741 .821961$

H $4.453369-3.735834-0.668855$

H $4.275386-3.8642532 .632698$

H $6.188902-1.8008684 .454017$

O $10.610873 \quad 0.991606 \quad 1.499058$

$\begin{array}{lllll}\text { O } & 8.665921 & 1.359327 & 0.315244\end{array}$

C $11.2389922 .071746 \quad 0.801340$

H 10.7116443 .0093871 .001361 
H 12.2654252 .1615381 .166960

H $11.2658841 .864351-0.272688$

SCF Energy (B3LYP/6-31G $\left.{ }^{* *} / / M M F F\right)=-3245.91353500$

01_504

MM̄FF Geometry

C $1.487884-4.0123131 .241373$

C $0.344749-4.5832131 .652900$

C -1.051880-4.152374 1.267235

O $-1.093310-2.7515360 .913109$

C $-1.552683-4.971900 \quad 0.065210$

C $-3.010292-4.722004-0.274537$

C $-3.448658-4.177289-1.426673$

C $-4.008372-5.1559730 .768999$

C $-2.636908-3.718032-2.604404$

C $-2.244886-2.233396-2.575133$

C $-3.435112-1.261668-2.617357$

C $-2.9477190 .171843-2.840106$

C $-4.1016861 .185703-2.800266$

O $-4.130225-1.338142-1.370013$

O $-3.5302182 .498639-2.689384$

C $-4.9386510 .949049-1.524562$

C $-4.9143101 .174881-4.100472$

O $-6.1153161 .753179-1.530057$

C $-5.313439-0.530197-1.299171$

C $-5.964836-0.7878930 .082377$

O $-6.266095-0.929358-2.292452$

C $-1.051519-1.855148 \quad 1.937434$

O $-0.976363-2.1006543 .130929$

C $-1.088258-0.500751 \quad 1.349396$

C -0.6282730 .5374252 .062570$

C -0.5991581 .9332621 .624350$

C -1.3555762 .2812650 .370775$

C $-5.101050-0.4262131 .296364$

C $-5.380180 \quad 0.9535331 .871088$

C -4.5430292 .5326783 .611635$

O -6.3281511 .6514971 .522246$

C -3.7052603 .5822212 .952153$

C -2.7531034 .2779983 .592665$

C -1.9432605 .3716732 .956319$

C $-0.453020 \quad 5.171206 \quad 3.120497$

C 0.2282055 .8105204 .086281

C 0.2592104 .3020472 .109296

C 0.0706392 .8338262 .372786

N $-4.461974 \quad 1.2965192 .855534$

H $1.457392-3.2007490 .520380$

H $0.396503-5.4106492 .357622$

H - $-1.707376-4.3139102 .131897$

H -1.431222 -6.0445280 .266559$

H $-0.913800-4.751165-0.796513$

H -4.523404 -4.051577 - 1.554521

H -5.040262 -5.1001510 .405397$

H $-3.823560-6.1942631 .063658$

H -3.940529 -4.5213291 .657638$

H -1.730217 -4.323611 -2.707602

H -3.216281 -3.919182 -3.514553

H -1.645547 -2.043040 -1.677485

H -1.599644 -2.045061 -3.442083

H $-4.104041-1.568406-3.429823$

H $-2.3975970 .254940-3.785669$

H $-2.2286670 .434961-2.053127$

H -2.938151 $2.632991-3.449224$

H -4.331269 $1.292495-0.680019$

H $-4.2718101 .418244-4.955293$

H $-5.6947911 .943622-4.085456$

H $-5.3830410 .210170-4.305093$

H -5.834457 $2.676769-1.647763$

H $-6.950996-0.3106150 .129570$

H $-6.162809-1.8677020 .148556$

H -6.744742 -1.708855 -1.964905

H $-1.476165-0.3990360 .342733$

H -0.2180640 .3484343 .053825$

H -2.388644 1.9261220 .438406

H $-0.8850091 .819571-0.501413$

H -1.417955 3.3541490 .178846

H $-5.322073-1.1391502 .101370$

H -4.036484 -0.517709 1.063366

H -4.2057932 .3129454 .629544$
H -5.5848242 .8664943 .654264$

H -3.913360 3.8014891 .907514

H -2.5678204 .0771484 .645584$

H -2.2435976 .3198043 .421558$

H -2.1812415 .4787131 .890601$

H $1.3018705 .695357 \quad 4.192143$

H $-0.273976 \quad 6.459196 \quad 4.796545$

H 1.3397504 .4975922 .139271

H -0.0437774 .5976811 .100842$

H $\quad 0.5751132 .4857993 .275374$

H -3.6777300 .6782353 .041918$

C $3.684983-3.2418722 .273539$

O $4.253292-2.5106151 .172303$

C $4.418629-1.1347291 .570647$

C $3.968051-1.0436693 .031111$

C $2.983057-2.1931603 .132853$

H $3.726356-0.5454780 .961162$

C $5.860733-0.6751701 .317393$

H $4.797711-1.1929043 .730920$

H $3.517414-0.0726733 .260156$

C $2.703666-2.6428164 .554913$

H $2.045375-1.8657452 .668241$

C $6.443056-0.980911-0.079185$

O $6.724756-1.3253432 .265330$

H $5.9459410 .395746 \quad 1.532887$

H $6.555334-2.066956-0.190564$

O $7.770612-0.434197-0.123294$

C $5.698500-0.434148-1.309123$

C $5.3800021 .073751-1.236053$

H $6.357065-0.584578-2.174471$

O $4.538802-1.213802-1.566408$

H $4.6414541 .253073-0.449283$

H $6.2929591 .609126-0.952816$

H $1.996245-3.4780164 .566977$

H $3.619078-2.9685705 .060252$

H $2.270081-1.8243385 .138596$

C $2.841511-4.4132391 .756211$

H $6.631355-2.2850592 .137396$

H $8.260454-0.8018640 .632366$

H $3.801084-0.854849-1.049453$

H $2.729423-5.1454232 .565242$

H $3.377917-4.9096930 .938426$

H $4.539180-3.6641652 .820692$

C $2.4747711 .463058-2.208769$

O $3.6132001 .044731-2.972342$

C $4.8588171 .630358-2.581981$

C $4.7682163 .162183-2.654206$

C $3.5736003 .703832-1.876302$

C $2.2951692 .981580-2.297278$

H $2.6050661 .172428-1.159441$

C $1.2580860 .715637-2.767657$

H $5.5883261 .324820-3.343169$

O $5.9589153 .772899-2.168806$

H $4.6592333 .466021-3.703686$

H $3.7305013 .626708-0.793830$

O $3.4157815 .094961-2.163967$

H $2.024153 \quad 3.274075-3.319777$

O $1.2314463 .382364-1.438421$

C $1.263836-0.715403-2.283920$

H $\quad 0.3116351 .156064-2.439408$

H $1.2598260 .731230-3.864144$

H $6.7000053 .426464-2.694662$

H $4.2510105 .534661-1.929016$

H $1.1642684 .351244-1.490478$

O $1.499286-1.566949-3.320883$

O $1.104908-1.034171-1.112474$

C $1.578715-2.952862-2.971412$

H $\quad 0.679273-3.269587-2.435809$

H $1.657847-3.532337-3.894891$

H $2.469992-3.134436-2.363734$

SCF Energy (B3LYP/6-31G**//MMFF) $=-3245.90788603$

01 505

MM̄MF Geometry

C $-3.7269481 .373594-2.268486$

C $-2.8367042 .353445-2.490733$

C -2.157325 $3.140730-1.400840$

O $-0.765143 \quad 3.253068-1.793679$ 
C $-2.781064 \quad 4.542479-1.242577$

C -4.171282 $4.538312-0.642511$

C -4.4301534 .4677610 .678516$

C $-5.3037754 .701028-1.623958$

C -3.4464184 .3708831 .815231$

C $-3.849726 \quad 3.3128422 .855030$

C -3.452907 1.8787782 .464299

C $-4.205957 \quad 0.874626 \quad 3.339924$

C -3.777937 -0.567221 3.043852

O -2.0440701 .7412152 .683440$

O $-4.324617-1.4159764 .064913$

C -2.240142 -0.6621613 .111335$

C $-4.390740-1.0708201 .735510$

O $-1.791845-1.9371802 .660008$

C $-1.493763 \quad 0.4588812 .344504$

$\begin{array}{llll}C & -0.005568 & 0.461830 & 2.772429\end{array}$

$\begin{array}{lllll}\text { O } & -1.508785 & 0.241359 & 0.932641\end{array}$

C $0.1249903 .657820-0.849756$

O -0.1264364 .0024390 .293553$

C $1.4804073 .609659-1.437886$

C $2.5328123 .901751-0.659462$

C $3.9206863 .940301-1.103872$

C $4.6645195 .151096-0.606965$

C $0.804036 \quad 1.593968 \quad 2.139947$

C 2.2140311 .6459812 .688950

C $4.467194 \quad 0.617322 \quad 2.436511$

O 2.5270492 .4286813 .583701

C 5.2718721 .5022311 .536268

C 6.1405601 .0407550 .623809

C $7.0019951 .914940-0.243450$

C $6.6855801 .774417-1.715997$

C $7.1990980 .765016-2.438770$

C $5.8601642 .857797-2.372365$

C $4.4584542 .943696-1.835280$

N 3.0630240 .7299182 .092715

H $-3.9860301 .126007-1.243634$

H -2.604032 $2.634674-3.515574$

H -2.198826 2.585489-0.456341

H -2.793092 $5.054032-2.215299$

H -2.136022 $5.183434-0.630049$

H -5.4723934 .5218030 .991981$

H -6.282431 4.712351-1.132241

H -5.198288 $5.645857-2.167278$

H $-5.3141673 .884726-2.350877$

H -2.426906 4.187099 1.470492

H -3.439539 5.3482082 .314086

H -3.353123 3.5692503 .800262

H -4.9305873 .3731213 .034399$

H -3.676133 1.7124791 .405271

H $-5.2907930 .982663 \quad 3.215324$

H -4.0030641 .0915774 .398124$

H -3.993376 -1.108661 4.925958

H -1.957671 -0.5917304 .171076$

H -5.485708 -1.0645191 .796805$

H $-4.108598-2.1109621 .538989$

H -4.105716 -0.4642210 .874777$

H -2.249712 -2.611186 3.191069

H $0.0618330 .563807 \quad 3.863503$

H $0.461908-0.4942942 .506942$

H -2.035876 -0.5499810 .742181$

H $1.5848543 .350894-2.485767$

H $2.381665 \quad 4.176857 \quad 0.382692$

H 4.5951755 .2245970 .484467

H $4.2370576 .062365-1.040143$

H $5.7286595 .142454-0.850116$

H 0.8468751 .4779311 .051855

H $\quad 0.3419742 .5638852 .350532$

H $4.745222-0.4358952 .338456$

H $4.6177490 .915740 \quad 3.479145$

H 5.1546832 .5752801 .666857

H $6.266446-0.0336970 .510690$

H $8.049758 \quad 1.637570-0.067147$

H 6.9304802 .9674260 .056400

H $7.0062630 .672743-3.502298$

H $7.8229380 .005063-1.979473$

H $5.7809812 .675550-3.452540$

H $6.3979273 .806600-2.287506$

H $3.8287792 .095856-2.103432$
H 2.7176080 .1614251 .325287

C $-4.602688-0.861005-3.165435$

O $-3.323842-1.479287-2.933967$

C $-3.463072-2.427364-1.858954$

C $-4.961300-2.655594-1.690906$

C $-5.511053-1.278903-2.008318$

H $-3.077047-1.935826-0.958748$

C $-2.647810-3.692186-2.139339$

H $-5.345150-3.388377-2.410019$

H $-5.220166-3.002783-0.686384$

C $-6.990540-1.277909-2.353404$

H $-5.357384-0.633991-1.137203$

C $-1.172074-3.428592-2.494543$

O $-3.238813-4.380417-3.249284$

H -2.722655 -4.371005 - 1.281357

H $-1.112764-2.960142-3.484385$

O $-0.525879-4.701527-2.633423$

C $-0.393740-2.567754-1.479658$

C $1.092707-2.444507-1.865316$

H $-0.828921-1.563187-1.449214$

O $-0.530019-3.120250-0.175285$

H $1.550332-3.438749-1.850745$

H $1.143592-2.081956-2.898892$

H $-7.334798-0.265782-2.587959$

H $-7.202488-1.915477-3.218175$

H $-7.581829-1.648876-1.509860$

C $-4.439657 \quad 0.647144-3.378194$

H $-2.709545-5.180579-3.409784$

H $-0.605037-5.171530-1.786187$

H $-0.076347-3.979580-0.163886$

H $-5.4292031 .099800-3.510685$

H $-3.8875270 .798752-4.314737$

H -4.981937 -1.293927 -4.100856

C $2.763868-3.1193140 .618483$

O $1.970307-1.9453410 .405764$

C $1.896242-1.492479-0.950194$

C $3.310995-1.193020-1.469157$

C $4.220803-2.409495-1.329203$

C $4.197050-2.9240660 .107332$

H $2.302595-3.970504 \quad 0.105607$

C $2.771669-3.4076212 .126348$

H $1.349396-0.541188-0.927612$

O $3.283784-0.782825-2.831900$

H $3.743848-0.365401-0.894321$

H $3.947178-3.201897-2.035520$

O $5.562647-2.038716-1.646809$

H $4.736408-2.2211350 .752976$

O $4.888297-4.170527 \quad 0.170473$

C $1.418401-3.8295282 .647564$

H $3.483777-4.1980772 .388341$

H $3.063790-2.5054552 .677865$

H $2.699557-0.007811-2.891864$

H $5.556203-1.685123-2.552941$

H $5.784456-4.023013-0.177785$

O $1.018167-4.9871502 .049431$

O $0.808462-3.2194923 .516464$

C $-0.243564-5.5028152 .485191$

H $-0.207659-5.7393693 .552911$

H $-1.040901-4.7841992 .274850$

H $-0.445143-6.4216841 .928296$

SCF Energy (B3LYP/6-31G**//MMFF)= -3245.90662779

01_506

MM̄FF Geometry

C $-1.499819 \quad 4.0712312 .106030$

C $-0.6579593 .323112 \quad 2.837997$

C $-0.743282 \quad 1.829295 \quad 3.056351$

$\begin{array}{lllll}\text { O } & -1.951276 & 1.322788 & 2.444592\end{array}$

C 0.4735891 .1255322 .414320

C 1.4452310 .5605773 .432114

C $1.623339-0.7522813 .679589$

C 2.2811751 .5828094 .159090

C $0.894485-1.9081343 .056790$

C $1.832860-2.7704122 .201310$

C $1.040035-3.7800941 .361044$

C $1.965360-4.780838 \quad 0.669457$

C $1.188858-5.720982-0.267068$

O $0.318956-3.0309170 .378208$ 
O $2.141352-6.396532-1.102307$

C $0.291127-4.876800-1.196990$

C $0.441817-6.8117840 .509323$

O $-0.563481-5.708153-1.978730$

C $-0.545383-3.812271-0.451865$

C $-1.222347-2.860224-1.467558$

$\begin{array}{llll}O & -1.561501 & -4.461515 & 0.316268\end{array}$

C -2.518595 0.2042252 .966857

O $-2.118639-0.4608903 .907255$

C $-3.732940-0.0704572 .169251$

C $-4.423319-1.2061262 .342559$

C $-5.604225-1.5838091 .569683$

C $-5.654535-3.0469691 .223489$

C $-1.747824-1.564022-0.834893$

C $-2.535177-0.716245-1.817117$

C $-3.6165201 .521127-2.004006$

O $-2.922371-1.133870-2.905075$

C $-4.997968 \quad 1.444542-1.432652$

C $-6.0068230 .785225-2.021606$

C $-7.3992900 .692982-1.464261$

C $-7.740753-0.702790-0.988647$

C $-8.014795-1.684011-1.865256$

C $-7.841026-0.9454630 .500582$

C $-6.555245-0.6878601 .236490$

N $-2.7792940 .556465-1.316900$

$\mathrm{H}-2.3623793 .6147621 .629788$

H 0.2011283 .8115703 .294298

H -0.7946751 .6550844 .138716$

H $1.033522 \quad 1.8229261 .777357$

H $\quad 0.1366640 .349203 \quad 1.719783$

H $2.366828-1.0399364 .421919$

H 3.0301591 .1202394 .810806

H 2.8168632 .2153653 .443592

H 1.6497112 .2210704 .784511

H $0.473691-2.5236373 .861736$

H $0.039647-1.5741122 .464136$

H $2.412799-2.1202991 .533994$

H $2.541574-3.2934642 .854848$

H $0.332241-4.3023422 .015548$

H $2.536104-5.3581151 .407448$

H $2.710939-4.2390090 .072502$

H $2.759278-6.876369-0.524488$

H $0.953190-4.361101-1.906587$

H $1.145738-7.4138721 .096278$

H - $0.054611-7.511742-0.171821$

H $-0.304861-6.4123931 .198674$

H $0.001268-6.351693-2.440104$

H $-0.500833-2.572184-2.242913$

H $-2.044032-3.385511-1.970523$

H $-2.308513-3.8534180 .432363$

H -4.0265890 .6686841 .432560$

H $-4.089255-1.9405833 .072285$

H -5.765960 -3.652009 2.129807

H $-4.731263-3.3509810 .717166$

H $-6.478004-3.3024170 .552031$

H -2.411059-1.790035 0.005010

H - $-0.909136-0.966976-0.457756$

H -3.607841 $1.320193-3.080479$

H -3.193571 $2.516070-1.836557$

H $-5.1710531 .957319-0.489959$

H $-5.8237120 .276085-2.965645$

H $-8.1055350 .994650-2.248863$

H -7.546909 $1.415065-0.651592$

H $-8.285417-2.680968-1.533821$

H -7.973409 -1.510776 -2.935739

H $-8.207482-1.9565830 .713745$

H -8.610905 $-0.279500 \quad 0.912146$

H $-6.429558 \quad 0.349621 \quad 1.545524$

H $-2.4524780 .793329-0.384732$

C $-0.735688 \quad 5.8241730 .441690$

$\begin{array}{lllll}\text { O } & 0.487638 & 5.081740 & 0.249788\end{array}$

C $0.355703 \quad 4.297013-0.947136$

C $-0.6562755 .051833-1.793267$

C $-1.6585795 .463390-0.728383$

H $-0.0747953 .330160-0.651791$

C $1.7166904 .046693-1.606094$

H $-0.2087165 .936935-2.260341$

H - $-1.0958674 .429027-2.577937$
C $-2.5737346 .597922-1.155803$

H $-2.2789394 .593230-0.483297$

C $2.6609413 .245299-0.682082$

O $2.2978315 .295430-1.969201$

H $1.5466223 .481537-2.529497$

H $2.0930142 .406210-0.266591$

O 3.0981924 .0501970 .411730

C $3.8973592 .669745-1.396057$

C $4.8686281 .908323-0.471257$

H $3.5619642 .000834-2.196038$

O $4.6368353 .714925-2.029608$

H $5.6662131 .496335-1.101934$

H 5.3778572 .6216500 .189980

H $-3.2563026 .871203-0.344762$

H -2.004092 $7.491078-1.433731$

H $-3.1757146 .301034-2.020837$

C $-1.287433 \quad 5.538693 \quad 1.844729$

H $3.1185485 .107192-2.454567$

H 2.3080694 .3509490 .891555

H $4.9160324 .338017-1.336542$

H -2.232772 6.0715911 .996823

H -0.5787475 .9363002 .582859$

H $-0.459006 \quad 6.8846080 .389016$

C $4.057725-0.910297-1.321696$

O $3.375104-0.059263-0.394895$

C $4.223416 \quad 0.793173 \quad 0.375664$

C $5.218285-0.048527 \quad 1.185700$

C $5.993581-1.0208930 .300302$

C $5.039063-1.825945-0.581229$

H $4.596068-0.299967-2.057286$

C $2.991002-1.722678-2.062545$

H $3.5739831 .291691 \quad 1.104164$

$\begin{array}{lllll}\text { O } & 6.136938 & 0.782261 & 1.886877\end{array}$

H $4.671346-0.6304751 .939079$

H $6.743446-0.501117-0.307669$

O $6.714514-1.936421 \quad 1.128445$

H $4.491566-2.5457290 .039182$

O $5.795867-2.570401-1.533038$

C $2.257094-0.864181-3.064471$

H $3.431290-2.558013-2.618747$

H $2.257811-2.150070-1.370597$

H $5.617701 \quad 1.3767042 .455191$

H $7.289208-1.4089971 .709393$

H $6.423695-3.122716-1.036318$

O $1.262307-0.165997-2.446352$

O $2.546567-0.810577-4.252952$

C $\quad 0.501108 \quad 0.702323-3.291039$

H $-0.1284631 .329216-2.654412$

H $1.1591361 .352278-3.875889$

H - $0.1396720 .109536-3.949801$

SCF Energy (B3LYP/6-31G**//MMFF) $=-3245.90559709$

01_507

MM̄FF Geometry

C $-0.3808124 .995434-2.055073$

C $-1.0403784 .374511-1.064926$

C $-2.353878 \quad 3.657778-1.257765$

O $-2.2940922 .391594-0.559279$

C $-3.4899864 .487683-0.632558$

C $-4.8706203 .898624-0.847796$

$\begin{array}{llll}\text { C } & -5.687823 & 3.482739 & 0.139748\end{array}$

C $-5.334158 \quad 3.820640-2.280140$

C -5.4395113 .5070821 .621692$

C $-4.821823 \quad 2.2205132 .191199$

C -5.7013490 .9709462 .020226$

C $-5.137163-0.2021322 .823885$

C $-5.943320-1.4905442 .601617$

$\begin{array}{lllll}\text { O } & -5.728610 & 0.623204 & 0.634244\end{array}$

O $-5.162066-2.5689643 .139237$

C $-6.109782-1.7342761 .084461$

C $-7.256011-1.4746313 .394602$

O $-6.991660-2.8336750 .871439$

C $-6.583655-0.4833050 .313015$

C $-6.543405-0.646551-1.226531$

O $\begin{array}{llll} & -7.942972 & -0.201928 & 0.668381\end{array}$

C $-1.6148321 .372662-1.151332$

O $-1.0605601 .389128-2.238254$

C $-1.6398680 .225307-0.216641$ 
C $-0.811446-0.810852-0.413211$ C $-0.698045-1.997548 \quad 0.438671$ C $-1.711320-2.1654751 .539126$ C $-5.168929-0.980068-1.817343$ C $-4.935593-2.465803-2.034199$ C $-3.158657-4.071946-2.720402$ O $-5.818144-3.311306-1.916024$ C $-2.566850-4.688216-1.492399$ C -1.351721 -5.256567 -1.467991 C $-0.759228-5.915503-0.255613$ C $0.603978-5.3648340 .098195$ C $1.725722-6.006864-0.269253$ C $0.662404-4.1184150 .952109$ C $0.298609-2.8731820 .193137$ N -3.626695 -2.733295 -2.412572 H $-0.8293104 .997858-3.047097$ H $-0.6368194 .367229-0.056718$ H -2.550662 $3.477744-2.321435$ H -3.486959 $5.500808-1.056624$ H -3.2858024 .6065310 .437164$ H $-6.6618883 .081795-0.139338$ H $-6.3904193 .541711-2.360218$ H $-5.2163144 .791962-2.771518$ H -4.756378 $3.074462-2.833713$ H $-4.805316 \quad 4.357430 \quad 1.894032$ H -6.3978223 .6975182 .122010$ H -3.847375 2.0549461 .716949 H -4.6372092 .3860303 .260016$ H $-6.715637 \quad 1.2134742 .358269$ H $-5.0923880 .041874 \quad 3.892671$ H -4.098578 -0.3845452 .516752$ H -5.631002 -3.3983272 .942777$ H -5.126224 -2.0423970 .710898$ H -7.051060 -1.380658 4.467876 H -7.802760 -2.416757 3.277120 H $-7.918927-0.6547273 .110412$ H -6.977785 -3.049055 -0.075887 H -7.310045 -1.358643 -1.554070 H $-6.8453140 .318262-1.659850$ H $-8.336780 \quad 0.347818-0.029398$ H $-2.323876 \quad 0.2761490 .622637$ H $-0.131179-0.778639-1.263373$ H $-2.726987-2.1050571 .133871$ H -1.587752 -1.3865272 .299161$ H -1.648986 -3.128888 2.048791 H -5.095969 -0.513265 -2.808159 H -4.364048 -0.568048 -1.201517 H -2.429616 -3.982202 -3.531941 H -3.998559 -4.680678 -3.070227 H -3.174254 -4.690141 -0.590338 H $-0.757653-5.277328-2.379132$ H $-0.684576-6.990776-0.464417$ H -1.423464 -5.8290910 .613393$ H $2.707000-5.6350020 .006494$ H $1.688083-6.917095-0.858381$ H $1.681963-3.9734891 .334469$ H $\quad 0.047521-4.2712001 .842864$ H $0.972265-2.663234-0.637652$ H -2.948644 -1.977018 -2.431184 C $1.7792745 .452244-0.706578$ O $2.2122524 .076893-0.710456$ C $3.5998274 .020462-0.335695$ C 3.9348015 .3964970 .223438 C $3.0596466 .285342-0.639018$ H $4.1716523 .859325-1.258732$ C 3.8594552 .8581430 .632088 H 3.6471975 .4826621 .278034 H 5.0003255 .6310810 .141689 C $2.8626917 .679474-0.069759$ H $3.5115596 .364361-1.636912$ C $3.6310891 .472336-0.013338$ O 3.0384603 .0144161 .787273 H 4.9015842 .9159370 .968155 H $4.2282931 .426794-0.930613$ O $2.2639871 .301181-0.384529$ C 4.0350830 .3009160 .903102 C $3.906849-1.0857130 .244583$ H 5.0652940 .4491441 .241986
O $3.221543 \quad 0.2895342 .078472$

H $4.117915-1.8394411 .013331$

H $2.862075-1.258912-0.036500$

H $2.2108208 .276464-0.715203$

H 2.4107537 .6461570 .927179

H $3.822728 \quad 8.1994090 .014035$

C 0.940075 5.717316-1.959605

H 2.1127532 .9449081 .496791

H $2.0371872 .003863-1.016809$

H $2.2938770 .229705 \quad 1.791774$

H $1.5256095 .416715-2.838340$

H $0.7401596 .790681-2.056326$

H 1.1891125 .6082550 .204652

C $6.875527-1.7302930 .195498$

O $6.166716-0.902840-0.734844$

C $4.816145-1.303064-0.981756$

C $4.794097-2.730382-1.545844$

C $5.553621-3.709083-0.650875$

C $6.947707-3.171940-0.319946$

H $6.371227-1.708247 \quad 1.169124$

C $8.273631-1.1282420 .370072$

H $4.440082-0.645786-1.774910$

O $3.452302-3.166771-1.724181$

H $5.263075-2.722882-2.538400$

H $4.990098-3.921610 \quad 0.264697$

O $5.680414-4.963511-1.324948$

H $7.582788-3.221490-1.213536$

O $7.544646-3.9982730 .676491$

C 8.1965290 .2283391 .029886

H $8.927324-1.7669940 .974423$

H $8.762510-0.992192-0.602274$

H $3.483956-4.079411-2.058899$

H $6.177211-4.814179-2.147417$

H $7.545679-4.9093820 .336154$

O $7.953127 \quad 0.086132 \quad 2.364131$

O $8.321826 \quad 1.2865850 .427121$

C 7.8195861 .3076653 .097276

H 8.7488961 .8829273 .047165

H $\quad \begin{array}{lll}6.980417 & 1.8929932 .708821\end{array}$

H 7.6177371 .0568444 .141992

SCF Energy $(B 3 L Y P / 6-31 G * * / / M M F F)=-3245.92720730$

01_508

MM̄FF Geometry

C 2.6062902 .3852673 .459176

C 3.7395182 .7411572 .835241

C 3.8648983 .5921951 .593862

O $3.9528952 .708521 \quad 0.450655$

C 2.6856074 .5491831 .340637

C 2.9646155 .5426400 .228526

C $2.4322725 .468808-1.007049$

C 3.8552136 .6999490 .599898

C $1.5286844 .397250-1.551205$

C $0.0578864 .801185-1.416441$

C $-0.8904063 .835934-2.142458$

C $-2.3416534 .285981-1.946596$

C $-3.342373 \quad 3.304767-2.570735$

O $-0.7038542 .517706-1.615108$

O $-4.6467293 .615318-2.056624$

C $-2.9990201 .879790-2.095420$

C $-3.4266143 .464944-4.092914$

O $-3.8156700 .915658-2.756054$

C $-1.5040811 .513887-2.256365$

C $-1.2313820 .158688-1.563908$

O $-1.2031351 .401837-3.648404$

C 5.1501432 .1320410 .169629

O 6.2041002 .2819150 .765134

C $4.9337891 .238679-0.988539$

C $5.7845700 .226057-1.208185$

C $5.654619-0.781460-2.260848$

C $4.656721-0.520854-3.359635$

C $0.244082-0.258436-1.503052$

C $0.394618-1.646642-0.900512$

C $1.998798-3.529406-0.733953$

O $-0.516178-2.221749-0.310653$

C $3.478879-3.659530-0.576588$

C $4.238602-4.498739-1.298105$

C $5.720650-4.648298-1.097670$ 
C $6.531026-4.399113-2.354433$

C $7.395876-5.326901-2.799536$

C $6.368127-3.087147-3.101665$

C $6.432931-1.882383-2.202981$

N $1.672330-2.158291-1.078434$

H 1.6439222 .7086533 .073604

H 4.6823252 .3752843 .238918

H 4.7893824 .1772131 .678420

H 2.4548265 .1147652 .253030

H $1.7827583 .973417 \quad 1.106091$

H $2.6701916 .257882-1.719321$

H $3.9577667 .423682-0.215807$

H 3.4432867 .2346461 .462127

H $4.859700 \quad 6.3491370 .854834$

H $\quad 1.7800104 .245650-2.608211$

H $1.7105363 .436073-1.059398$

H $-0.1972134 .829341-0.348754$

H $-0.0894785 .814622-1.811353$

H $-0.629302 \quad 3.835592-3.207417$

H $-2.4933605 .295982-2.347782$

H $-2.5567554 .357331-0.870910$

H $-4.8464474 .539543-2.284379$

H $-3.2520481 .822820-1.028183$

H $-3.7546284 .478597-4.353463$

H -4.175415 $2.789310-4.520622$

H -2.473772 $3.288987-4.596278$

H -4.742232 $1.173004-2.611101$

H $-1.5997210 .207015-0.531619$

H -1.794597 -0.632275 -2.075661

H $-0.4254370 .835535-3.765206$

H $4.0508921 .406857-1.595099$

H $6.6229970 .090697-0.526221$

H $4.817800 \quad 0.472790-3.794166$

H $3.632715-0.569693-2.980014$

H $4.735588-1.225679-4.191027$

H $\quad 0.685907-0.271992-2.504211$

H $0.8144660 .443005-0.883842$

H $1.606578-4.170120-1.530893$

H $1.491412-3.799813 \quad 0.197844$

H $3.945964-3.044276 \quad 0.189312$

H $3.772918-5.130846-2.050302$

H $5.898050-5.666056-0.726077$

H $6.077971-3.978039-0.305887$

H $7.986882-5.166698-3.695232$

H $7.533108-6.269537-2.280083$

H $5.415027-3.137818-3.637763$

H $7.154992-2.980004-3.858628$

H $7.172978-1.953349-1.404907$

H $2.296733-1.672051-1.713658$

C 2.0983490 .0977824 .454162

O $0.757774 \quad 0.084230 \quad 3.925811$

C $0.663090-0.9746012 .952243$

C $1.932860-1.8054323 .105000$

C $2.938944-0.7399933 .491101$

H $0.669518-0.4922021 .966577$

C $-0.634258-1.7784513 .116369$

H $1.835128-2.5486083 .905281$

H $2.197764-2.3329702 .185120$

C $4.215025-1.2938254 .098230$

H $3.191077-0.1679552 .589652$

C - $-1.897392-0.9348232 .833317$

O $-0.671510-2.3242554 .433145$

H $-0.603296-2.6196932 .413600$

H -1.749533 -0.4152861 .880824$

O $-2.075470 \quad 0.061383 \quad 3.838542$

C $-3.187761-1.7739902 .736180$

C $-4.460434-0.9564712 .444452$

H -3.059085 -2.532861 1.958612

O $-3.408745-2.4814283 .958308$

H -5.310035 -1.650477 2.457185

H -4.647985 -0.2683693 .278494$

H $4.900716-0.4845864 .368211$

H 4.009911-1.878359 5.001276

H $4.727938-1.9468843 .384466$

C 2.5733701 .5322164 .696808

H -1.447043 -2.9074914 .487681$

H -1.272151 0.6084183 .858795

H -3.480432 -1.821647 4.669568
H $3.5590841 .513627 \quad 5.176968$

H 1.8901512 .0191795 .404017

H $2.042175-0.4058585 .428787$

C $-5.096677-1.948075-0.390367$

O $-4.089728-1.0075940 .004471$

C $-4.429381-0.1759161 .116801$

C -5.7097340 .6141590 .816387$

C $-6.853011-0.2984640 .387632$

C $-6.407001-1.225782-0.741673$

H $-5.274582-2.6610040 .423509$

C $-4.542043-2.711833-1.604277$

H -3.6251980 .5638151 .203532$

O $-6.117412 \quad 1.375866 \quad 1.947989$

H $-5.515821 \quad 1.3264430 .004257$

H -7.248946 -0.871922 1.234079

O $\quad-7.9346660 .504268-0.093113$

H $-6.291744-0.637382-1.661035$

O $-7.449657-2.174711-0.961124$

C -3.448731-3.690499-1.228515

H $-5.321620-3.299386-2.098560$

H -4.126568 -1.994989 -2.322793

H $-5.377877 \quad 1.959725 \quad 2.188721$

H $-8.185775 \quad 1.1101660 .625067$

H -7.297002 -2.591842 -1.825133

O $-2.692559-3.959824-2.333786$

O $-3.305174-4.193595-0.122956$

C -1.652161 -4.928051-2.160122

H -2.087143 -5.931194 -2.138782

H $-0.974120-4.854485-3.014612$

H -1.080975 -4.740102 -1.246317

SCF Energy $\left(B 3 L Y P / 6-31 G^{* *} / / M M F F\right)=-3245.91818069$

01 509

MMFF Geometry

C $4.638797-0.175883-1.871964$

C $4.033435-1.071098-2.667633$

C $3.219629-2.223056-2.147128$

O $1.995655-2.320880-2.917621$

C $3.934383-3.574424-2.310948$

C $5.054539-3.809041-1.322997$

C $4.872400-4.312800-0.086656$

C $6.444140-3.516759-1.825210$

C $3.569578-4.6982430 .563054$

C $3.484368-4.2291632 .023296$

C $3.212299-2.7232952 .179504$

C $3.436998-2.3087943 .637883$

C $3.054594-0.8464313 .894120$

O $1.852346-2.4839201 .793486$

O $3.032659-0.6620595 .317763$

C $1.625318-0.6211843 .363336$

C 4.1145190 .1194933 .351953

O $1.254780 \quad 0.751277 \quad 3.481458$

C $1.422488-1.1217951 .914972$

C $-0.061931-1.0421951 .488200$

O $2.165045-0.265377 \quad 1.038247$

C $0.976522-1.488555-2.583027$

O $1.003779-0.557530-1.795552$

C $-0.223417-1.952371-3.303766$

C $-1.372206-2.037110-2.617972$

C $-2.647954-2.535021-3.130397$

C $-2.785766-2.679565-4.622895$

C -1.007829 -2.028880 2.180341

C $-2.463412-1.7317111 .856472$

C $-4.766108-2.3524302 .549344$

O $-2.815004-0.8206201 .112633$

C $-5.419431-2.9317761 .334994$

C $-6.017278-2.1852390 .392886$

C $-6.771840-2.750716-0.774835$

C $-6.102386-2.475938-2.100594$

C $-6.540912-1.504238-2.918040$

C $-4.985503-3.401647-2.523031$

C -3.624283 -2.828158 -2.245936

N $-3.334634-2.5858562 .517413$

H $4.568154-0.297028-0.794855$

H $4.121877-0.975572-3.747112$

H $2.953184-2.079167-1.092751$

H $4.309842-3.682426-3.338054$

H $3.196882-4.383923-2.221805$ 
H $5.756049-4.4915670 .524721$

H $7.212480-3.747488-1.079489$

H $6.658912-4.118418-2.714539$

H $6.551285-2.461126-2.087522$

H $2.701108-4.3344050 .005885$

H $3.515404-5.7938810 .555933$

H $2.668485-4.7864492 .502450$

H $4.409695-4.5018752 .546445$

H $3.878984-2.1594671 .517547$

H $4.477390-2.4890313 .936860$

H $2.825534-2.9456614 .293346$

H 2.7149430 .2407235 .492618

H $0.954891-1.1706354 .034206$

H $5.078580-0.0600163 .842985$

H $3.8557611 .160173 \quad 3.575898$

H 4.2706700 .0273332 .275213

H 1.6820741 .2372712 .755520

H $-0.418563-0.0116201 .609838$

H $-0.130245-1.2414300 .412832$

H $1.768660-0.3063410 .152457$

H $-0.110827-2.273623-4.331981$

H -1.372232 -1.750229-1.567543

H -2.177345 -3.514830 -4.985688

H $-2.462084-1.763638-5.130795$

H -3.813171 -2.854236 -4.949408

H $-0.784101-3.0527031 .860168$

H -0.898242 -1.9761683 .267562$

H $-4.946433-1.2750272 .634459$

H - -5.161285 -2.834778 3.448969

H -5.443084 -4.015661 1.254863

H $-6.001406-1.1011620 .483821$

H -7.778974 -2.313545 -0.762005

H $-6.927703-3.830960-0.661491$

H -6.079771-1.331509-3.884859

H -7.364627 -0.857065 -2.635782

H $-5.117445-3.672231-3.575109$

H -5.070901-4.353712 -1.983786

H -3.416304 -2.687721 -1.184910

H $-2.957460-3.3104283 .121221$

C $5.1113092 .325843-1.745430$

O $3.6981882 .597945-1.817843$

C $3.3062063 .277191-0.606791$

C 4.5978113 .6385630 .119854

C $5.4951912 .481759-0.275093$

H 2.7622712 .5484240 .004789

C $2.4098424 .474765-0.936111$

H $5.0135044 .586978-0.240730$

H 4.4548403 .7229241 .201489

C $6.9712632 .749981-0.041631$

H 5.1962421 .5997410 .303686

C $1.0953954 .104010-1.654393$

O $3.1315805 .380498-1.780559$

H $2.1858695 .032923-0.019623$

H $1.3034403 .709492-2.655974$

O $0.3450625 .311088-1.841167$

C $0.2165683 .107733-0.875091$

C -1.151936 $2.864037-1.545142$

H $0.7380242 .148135-0.798663$

O $\quad 0.032802 \quad 3.591634 \quad 0.454184$

H -1.734128 $3.791287-1.537090$

H $-0.9806792 .595000-2.593924$

H $7.5771841 .892034-0.349037$

H $7.3148303 .624802-0.603871$

H 7.1645482 .9360021 .020001

C $5.4416620 .980966-2.396861$

H $3.3914404 .891165-2.580041$

H $0.9127455 .931919-2.329281$

H $-0.409261 \quad 4.4558300 .393374$

H $6.506910 \quad 0.760433-2.264160$

H $5.2678621 .076679-3.476582$

H $5.6039513 .111940-2.334069$

C -3.1688153 .0303390 .786669$

O $-2.1939182 .016350 \quad 0.533655$

C $-1.9580381 .740845-0.851861$

C $-3.2695641 .352837-1.557460$

C $-4.3986262 .345286-1.284631$

C -4.5271972 .6226680 .214515$

H -2.8520923 .9642790 .305972$
C $-3.182703 \quad 3.3387532 .285913$

H $-1.3148250 .852504-0.875038$

O $-3.0838591 .235599-2.964220$

H $-3.5886810 .366674-1.197911$

H $-4.2423243 .276709-1.841115$

O $-5.6315291 .805305-1.767020$

H -4.9112941 .7276040 .717491$

O -5.4648713 .6745660 .421656$

C -3.4996232 .1487843 .161674$

H -2.1988933 .7236352 .584859$

H -3.8996054 .1324272 .525389$

H $-2.3673120 .595934-3.113129$

H $-5.8012280 .974163-1.291659$

H -6.3020553 .4026980 .007939$

O $-2.3504201 .654934 \quad 3.705679$

O -4.6309381 .7229903 .353396$

C -2.5072930 .5885374 .646927$

H -2.8320200 .9974605 .607810$

H -3.222305 -0.1577074 .291529$

H $-1.5352400 .106498 \quad 4.779656$

SCF Energy $(B 3 L Y P / 6-31 G * * / / M M F F)=-3245.92709630$

0151

MMFF Geometry

C $3.156663-3.445671-1.079722$

C $2.503476-2.375323-0.604911$

C $1.248899-1.797982-1.200504$

O $0.325560-1.670437-0.094891$

C $1.554213-0.406414-1.786033$

C $0.4728770 .157414-2.686121$

C $-0.2394671 .274668-2.438302$

C $0.254208-0.588577-3.979473$

C $-0.1508562 .205505-1.261441$

C -1.277225 $2.041197-0.230433$

C $-2.6758152 .429195-0.740411$

C -3.6813782 .4329700 .413656$

C $-5.1122752 .725328-0.063725$

O $-3.090601 \quad 1.481501-1.727405$

O

C $-5.4512591 .803015-1.256490$

C $-5.3236044 .219747-0.336281$

O $-6.7108272 .177612-1.808955$

C $-4.3534081 .779869-2.343006$

C $-4.5870610 .718368-3.448190$

O $-4.3175673 .054418-2.997673$

C $-0.993507-1.901523-0.312995$

O $-1.536379-2.164155-1.372945$

C $-1.675892-1.7742040 .995022$

C $-3.005900-1.6122471 .034016$

C $-3.823400-1.4545422 .237738$

C $-3.162032-1.6902753 .569599$

C $-4.685621-0.731272-2.964439$

C $-6.104124-1.184689-2.660538$

C -7.357801-2.951057-1.423093

O $-7.099794-0.611967-3.095402$

C $-7.800041-2.284702-0.158691$

C -7.795017 -2.881152 1.043021

C $-8.232051-2.2026342 .309875$

C -7.108955 -2.063770 3.314890

C $-7.008363-2.9035584 .358743$

C $-6.156582-0.8978223 .161179$

C $-5.124265-1.1278942 .091472$

N $-6.126506-2.331005-1.878408$

H $2.791566-3.955883-1.966534$

H $2.901920-1.8694810 .270465$

H $0.849705-2.483534-1.956586$

H $2.477442-0.448698-2.380543$

H $1.7620490 .277794-0.959538$

H $-0.964194 \quad 1.585132-3.190227$

H -0.437212 -0.066305 -4.649601

H $1.202014-0.703441-4.515659$

H -0.162875 -1.582051 -3.792357

H $0.8064852 .094252-0.745065$

H $-0.1537443 .235281-1.640123$

H $-1.285124 \quad 1.0041680 .120103$

H -1.0297502 .6705210 .633602$

H -2.611652 3.425491-1.193590

H -3.3824603 .1505581 .188113$ 
H $-3.675007 \quad 1.4500860 .902148$ H $-5.7597142 .898557 \quad 1.786449$ H $-5.5737630 .795137-0.841850$ H -5.1370254 .8046280 .572605$ H $-6.3619214 .428184-0.616588$ H $-4.6685974 .609818-1.117982$ H $-6.9669861 .500855-2.457180$ H $-5.4498150 .996962-4.064918$ H $-3.722348 \quad 0.763897-4.126245$ H -3.882965 $2.946366-3.859910$ H -1.061632 -1.7917321 .888013$ H -3.550631 -1.571193 0.093213 H -2.417290 -0.9125453 .771132$ H -2.663946 -2.666304 3.586201 H -3.863312 -1.6931314.407176 H -4.325363 -1.391363 -3.763814 H -4.042285 -0.894069-2.095451 H -7.154598 -4.017090 -1.280929 H -8.129967 -2.842299 -2.191403 H -8.140413 -1.254775 -0.239235 H $-7.459822-3.9123891 .125785$ H $-9.053021-2.7925172 .738163$ H -8.659955 -1.213038 2.106288 H -6.224211 -2.792085 5.100152 H -7.705058 -3.724528 4.492348 H $-5.686032-0.6564174 .120454$ H -6.7283200 .0038912 .906280$ H -5.502082 -0.9700171 .081956$ H -5.253321 -2.695133 -1.508532 C $5.667136-3.452837-1.089052$ O $5.758384-2.032092-0.854895$ C $7.091306-1.710777-0.422064$ C $7.938740-2.935138-0.743376$ C $6.945158-4.054709-0.504615$ H $7.048784-1.5838770 .666238$ C $7.562657-0.411774-1.081604$ H $8.269147-2.939524-1.788587$ H $8.826701-3.004272-0.107923$ C $7.350605-5.374419-1.137648$ H $6.833085-4.1973450 .578803$ C $6.6231200 .794255-0.868380$ O $7.675846-0.618292-2.496742$ H $8.572893-0.166427-0.734553$ H $5.6892560 .635298-1.419205$ O $7.2513511 .937049-1.464789$ C 6.3095381 .1234740 .604858 C 5.5899852 .4725410 .810248 H $5.7125160 .316347 \quad 1.041548$ O 7.5419621 .1744651 .329924 H 5.5475742 .6740271 .887141 H 6.2159683 .2760060 .403627 H $6.596231-6.144978-0.950322$ H $7.472625-5.278629-2.221806$ H $8.301247-5.724232-0.722056$ C $4.397915-3.991412-0.429664$ H $6.804216-0.902539-2.822019$ H $7.4354311 .714843-2.393705$ H 7.3250731 .3064602 .268488 H $4.396471-3.7315070 .636719$ H $4.366293-5.085177-0.490294$ H $5.629361-3.595917-2.176529$ C $2.896216 \quad 1.818123 \quad 2.130574$ O $3.302776 \quad 1.5542470 .782138$ C $4.1753202 .528626 \quad 0.200377$ C 3.5232703 .9180810 .237687 C 3.0842634 .2983031 .647660 C 2.2198013 .1905262 .244355 H 3.7686381 .7774072 .793381 C 1.9173930 .7061142 .543465 H $4.2673512 .263233-0.858808$ O $4.4235334 .890387-0.282135$ H $2.6427343 .913181-0.417902$ H 3.9421594 .5194392 .293575 O 2.2967445 .4898101 .595333 H 1.2469423 .1745781 .737265 O 1.9779153 .4794183 .620506 C $2.608635-0.6119912 .818725$ H 1.3903970 .9821873 .463583
H $\quad 1.1869410 .539845 \quad 1.742774$

H $3.9378345 .727969-0.366647$

H 2.8824726 .2168221 .324851

H 1.5665934 .3596803 .664129

O $1.658930-1.5501943 .111947$

O $3.816550-0.8001522 .790865$

C $2.153795-2.8580073 .415133$

H $1.296635-3.5099343 .603158$

H $2.718931-3.2582152 .568720$

H $2.775862-2.8264234 .314507$

SCF Energy (B3LYP/6-31G**//MMFF) = -3245.91757389

01_510

MMFF Geometry

C $0.0537803 .183348-1.361786$

C $1.2370133 .139545-0.732032$

C $1.7825441 .901475-0.068915$

O $3.1805551 .769997-0.416662$

C 1.6789462 .0599141 .457189

C 1.9464600 .7716442 .207394

C $0.995200-0.1330992 .512096$

C 3.3639560 .5770232 .673816

C $-0.460363-0.0673252 .142882$

C $-1.061880-1.4100201 .699963$

C $-0.551408-1.9161420 .340180$

C $-1.440185-3.062548-0.152311$

C $-0.938487-3.653334-1.475409$

O $0.793714-2.3834030 .490683$

O $-1.639238-4.885292-1.703200$

C $0.553189-4.002255-1.317094$

C $-1.254210-2.745999-2.670172$

O $1.109246-4.430221-2.558858$

C $1.399674-2.849976-0.724371$

C $2.815204-3.380930-0.392170$

O $1.502981-1.807083-1.693332$

C $3.4837191 .273191-1.643294$

O $2.6873510 .902895-2.495422$

C $4.9454201 .257928-1.885434$

C $5.8659291 .503553-0.940150$

C $7.3170631 .548691-1.138329$

C $7.8462981 .325422-2.530918$

C $3.705738-2.3981620 .376927$

C $5.058705-3.004540 \quad 0.711868$

C $7.168849-2.4881941 .907316$

O $5.361493-4.1640750 .442576$

C $8.055221-1.2897582 .015144$

C $9.226084-1.1834691 .369491$

C $10.158075-0.0124291 .495040$

C $10.367572 \quad 0.700727 \quad 0.178813$

C $11.2650150 .249560-0.713999$

C $9.6030971 .980031-0.067905$

C $8.1101751 .800435-0.075903$

N $5.887777-2.093640 \quad 1.353914$

H $-0.5583532 .286303-1.414952$

H $1.8399144 .042476-0.666325$

H $1.2414501 .003009-0.392493$

H $0.6859902 .437507 \quad 1.734402$

H 2.3880572 .8233881 .805628

H $1.282470-1.0023113 .101898$

H 3.6597421 .3931753 .341058

H $3.497146-0.3614983 .222162$

H 4.0481190 .5618801 .821559

H $-1.006070 \quad 0.2669203 .033520$

H $-0.6512930 .674906 \quad 1.361073$

H $-0.870145-2.1643592 .474015$

H $-2.148995-1.2736591 .643257$

H $-0.570373-1.083285-0.372466$

H $-2.480615-2.736927-0.253710$

H $-1.452834-3.8621510 .602112$

H -2.591187 -4.687744 -1.730016

H $0.617263-4.863261-0.636796$

H -2.336366 -2.603741 -2.768646

H $-0.927402-3.203663-3.610427$

H $-0.799104-1.756574-2.591670$

H $0.556577-5.159750-2.887646$

H $2.719603-4.2884240 .219041$

H $3.330619-3.665573-1.318481$

H $2.300746-1.285772-1.516275$ 
H $\quad 5.2269801 .032234-2.909200$ H 5.5355651 .7099220 .076161 H $7.4703502 .094868-3.214098$ H $7.5414950 .341732-2.905093$ H $8.937011 \quad 1.352677-2.589027$ H $3.877083-1.488567-0.206454$ H $3.225803-2.1224941 .321289$ H $7.607028-3.2774531 .287033$ H $6.983072-2.9069312 .901887$ H $7.730444-0.4859032 .671149$ H $9.551399-1.9997210 .727894$ H $11.121537-0.3840491 .868487$ H 9.8081310 .6980962 .254198 H $11.445003 \quad 0.769448-1.649054$ H $11.838635-0.652760-0.529009$ H $9.9328422 .468479-0.992804$ H 9.8560862 .6962200 .725122 H 7.6472571 .9341930 .902252 H $5.483257-1.2184671 .672781$ C -1.693366 $5.029045-1.262959$ O $-2.8000664 .106309-1.247074$ C $-3.411786 \quad 4.1511770 .057311$ C -2.8652825 .4034960 .734778$ C -1.4489445 .4323840 .192711$ H -3.0396763 .2759330 .604221$ C $-4.9380894 .070686-0.053005$ H -3.400235 6.3076620 .425306 H -2.9084545 .3386341 .826100$ C $-0.756000 \quad 6.773728 \quad 0.353515$ H $-0.868133 \quad 4.667160 \quad 0.721713$ C $-5.4501132 .868494-0.871553$ O $-5.4381335 .243496-0.707413$ H -5.3866794 .0552780 .947964$ H $-5.2322823 .016788-1.936432$ O $-6.8792762 .841334-0.754567$ C $-4.9027551 .500832-0.424040$ C -5.559272 $0.333109-1.187295$ H $-3.8218981 .470170-0.598651$ O $\quad-5.107922 \quad 1.344196 \quad 0.977081$ H $-6.6090530 .247757-0.887432$ H $-5.5417490 .567026-2.258103$ H $0.2545386 .741874-0.065684$ H -1.306647 $7.573983-0.152296$ H -0.6744977 .0397121 .412484$ C $-0.4986444 .424076-2.005548$ H $-5.4351595 .967377-0.059044$ H -7.210083 3.704515-1.057271 H -6.0639781 .4088041 .144196$ H $0.2854395 .183659-2.109376$ H $-0.8096504 .150186-3.021475$ H -2.039596 $5.900012-1.835309$ C $-6.093426-1.8214190 .951111$ O $-4.827808-1.4106200 .420805$ C $-4.839365-1.014712-0.953561$ C $-5.356516-2.168550-1.824398$ C $-6.713993-2.675327-1.344787$ C $-6.666341-2.9953230 .148877$ H $-6.793589-0.9778440 .929549$ C $-5.867102-2.2205572 .413938$ H -3.790390 -0.850746 -1.232329 O $-5.435104-1.763604-3.186298$ H -4.636392 -2.995586 -1.777939 H -7.505410 -1.950255 -1.565978 O $-7.058125-3.859554-2.067931$ H $-6.059919-3.895246 \quad 0.312877$ O $-7.985679-3.2785820 .609333$ C -5.593597 -1.003110 3.265189 H $-6.742901-2.7185682 .844658$ H -5.024495 -2.917714 2.499928 H -5.792104 -2.512755 -3.693329 H $-6.372751-4.527539-1.895827$ H -8.334707 $-4.003787 \quad 0.063248$ O $-4.284392-0.9792603 .643582$ O $-6.441140-0.1642363 .543329$ C $-3.8950490 .140475 \quad 4.444703$ H $-4.536293 \quad 0.2252805 .327374$ H -2.866278 -0.0208604 .777071$ H -3.9335351 .0569293 .848494$
SCF Energy (B3LYP/6-31G**//MMFF)= -3245.90885961

01511

MMFF Geometry

C $0.0263255 .361689-0.655993$

C -0.6753684 .6476910 .236712$

C $-1.9769283 .960006-0.091000$

O -1.8779892 .5613970 .261764$

C -3.1014834 .5887090 .753723$

C -4.4910954 .0907690 .410100$

C -5.3002853 .4304001 .261341$

C $-4.9780934 .413870-0.979611$

C -5.0295573 .0346072 .686003$

C -4.4403241 .6252062 .857404$

C -5.3518390 .4958262 .349005$

C $-4.797559-0.8731652 .748426$

C -5.627666 -2.028392 2.164566

$\begin{array}{lllll}\text { O } & -5.418063 & 0.580415 & 0.924149\end{array}$

O $-4.844296-3.2205482 .330335$

C $-5.832657-1.7938430 .649865$

C $-6.922216-2.2446452 .957465$

O $-6.745886-2.7406380 .102205$

C $-6.304599-0.3645090 .312755$

C $-6.315138-0.060662-1.205300$

O $-7.647107-0.1899200 .782826$

C $-1.2161291 .741130-0.599769$

O $-0.6657182 .056604-1.642548$

C $-1.2723870 .370996-0.042224$

C $-0.483424-0.589277-0.547765$

C $-0.424681-1.981720-0.094941$

C $-1.471119-2.4357140 .888213$

C $-4.961274-0.192534-1.911579$

C $-4.743964-1.543135-2.572121$

C $-2.985295-2.887444-3.710993$

O $-5.635869-2.374670-2.718014$

C $-2.445887-3.865806-2.716226$

C $-1.258036-4.473549-2.854122$

C $-0.720287-5.485074-1.883791$

C $0.678658-5.153775-1.415454$

C $1.748438-5.738008-1.980938$

C $0.828030-4.219101-0.236252$

C $0.542973-2.784551-0.585651$

N $-3.438008-1.696064-3.018241$

H $-0.3441595 .451728-1.674041$

H -0.3119254 .5621331 .257999$

H $-2.2093654 .048980-1.159086$

H -3.098298 $5.679410 \quad 0.622255$

H $-2.8813904 .411243 \quad 1.812111$

H -6.2856783 .1358540 .900912$

H $-6.0399884 .179792-1.112678$

H $-4.8520595 .481168-1.188886$

H -4.421552 $3.841007-1.727372$

H -4.3671503 .7591123 .171343$

H -5.9748743 .0984573 .240101$

H -3.4746421 .5813772 .339862$

H -4.2419871 .4736503 .925731$

H $-6.352131 \quad 0.6420142 .773174$

H -4.730595 -0.963462 3.839851

H -3.767194 -0.9656632 .379568$

H -5.367954 -3.973257 2.007853

H $-4.864950-1.9727980 .166759$

H -6.692590 -2.497431 3.999532

H -7.492504 -3.092722 2.562654

H -7.572103 -1.367115 2.964634

H $-6.362247-3.6252810 .223129$

H $-7.092999-0.652082-1.702982$

H $-6.6293140 .986747-1.322720$

H -8.0509450 .5478180 .296344$

H $-1.955571 \quad 0.1931900 .780263$

H $0.200146-0.330734-1.355516$

H $-2.472716-2.1967680 .515743$

H -1.328261 -1.944964 1.856830

H -1.468915 -3.513015 1.065541

H $-4.9179130 .549861-2.718991$

H $-4.1342530 .021197-1.227104$

H $-2.228786-2.574128-4.437407$

H -3.826105 -3.335491-4.250325

H -3.069172 -4.103765 -1.857420 
H $-0.649736-4.256528-3.729316$ H $-0.723609-6.461817-2.385354$ H -1.378967 -5.597904-1.013712 H $2.754634-5.536024-1.632209$ H $1.641076-6.431830-2.808167$ H $1.855803-4.2573020 .146514$ H $\quad 0.212328-4.5885280 .588212$ H $1.249778-2.364470-1.302452$ H -2.749215 -0.981610 -2.800580 C $2.5632165 .466005-0.891133$ O $2.8289954 .212729-0.235421$

C $3.5158053 .350094-1.166073$ C $3.7467714 .172482-2.434452$ C $2.5930135 .156619-2.384289$ H $2.8282782 .532077-1.404739$ C $4.7986562 .805454-0.526800$ H $4.7048594 .704661-2.407498$ H $3.7408793 .549556-3.334374$ C $2.7882486 .375519-3.267227$

H $1.683744 \quad 4.627054-2.694097$

C 4.5975702 .0416360 .799532

O $5.6783303 .907092-0.252503$

H $5.3317102 .177052-1.247296$

H 4.1961282 .7351931 .549272

O $5.880921 \quad 1.639393 \quad 1.291301$

C 3.6976790 .7917450 .748060

C $3.961985-0.199731-0.402172$

H 3.8013850 .2478901 .694284

$\begin{array}{lllll}\text { O } & 2.327273 & 1.186697 & 0.661634\end{array}$

H $3.212923-0.997015-0.321968$

H $3.740707 \quad 0.284131-1.361164$

H $1.9347077 .055908-3.185439$

H $3.6906756 .931333-2.991257$

H $2.8844376 .078666-4.316718$

C $1.2916136 .100616-0.328067$

H 5.2114724 .5122520 .349136

H 6.4400862 .4342391 .330085

H $2.1376021 .763573 \quad 1.420980$

H $1.1934137 .124036-0.709509$

H 1.3941436 .1815250 .761835

H $3.403611 \quad 6.125527-0.633552$

C $5.086559-2.4993141 .261150$

O $5.779529-1.3220730 .833929$

C $5.374779-0.814906-0.439255$

C $5.578932-1.884774-1.522590$

C $4.924501-3.214280-1.149874$

C $5.326601-3.6359670 .264292$

H $4.012442-2.2910171 .341850$

C $5.606899-2.8555502 .657018$

H $6.068423-0.001167-0.678514$

O $5.073023-1.414622-2.767325$

H $6.656702-2.049101-1.653864$

H $3.834377-3.163466-1.246759$

O $5.365050-4.229669-2.054038$

H $6.384042-3.9304070 .272518$

O $4.562092-4.774293 \quad 0.652428$

C $5.101932-1.8593633 .673989$

H $5.265175-3.8453742 .979895$

H $6.703091-2.8689742 .687581$

H $5.326011-2.057150-3.451102$

H $5.001991-4.019247-2.930790$

H $4.719876-5.468953-0.009768$

O $5.986854-0.8297333 .800116$

O $4.031895-1.9685154 .258812$

C 5.5938790 .2135254 .696824

H 4.6611030 .6736794 .356705

H $5.484053-0.1802745 .711564$

H $\quad 6.3791560 .9742114 .697891$

SCF Energy (B3LYP/6-31G**//MMFF) $=-3245.90177884$

01512

MM̄FF Geometry

C -3.826047 -2.184304 -2.999383

C $-2.564014-2.156367-2.548052$

C -1.446641 -1.427334 -3.245561

O $-1.032396-0.375663-2.344369$

C $-0.259044-2.370349-3.498417$

C $0.832244-1.748817-4.348631$
C $2.052179-1.402585-3.892366$

C $0.502975-1.587271-5.810835$

C $2.572709-1.514848-2.485575$

C $3.330100-2.833805-2.298595$

C $4.101679-2.965333-0.977446$

C $3.221996-2.8009530 .261193$

C $4.037466-2.9316091 .559119$

O $5.156471-1.996591-0.965233$

O $3.224596-2.4477562 .640165$

C $5.265126-1.9979781 .466821$

C $4.362305-4.3930451 .886596$

O $6.128526-2.1730592 .587126$

C $6.055445-2.1315340 .145240$

C $7.183347-1.077995-0.000784$

O $6.699408-3.4138680 .118252$

C $-0.8479630 .870579-2.853488$

O $-0.937420 \quad 1.215227-4.020371$

C $-0.5304541 .764967-1.717857$

C $-0.2831023 .063713-1.941662$

C $0.0260054 .072901-0.925536$

C -0.0091493 .6407840 .517442$

C 6.8087520 .3828550 .252295

C $5.8269850 .959470-0.750625$

C $3.9384132 .564033-0.922157$

O $5.8193780 .635679-1.935352$

C $3.5612133 .831300-0.225747$

C $3.6108115 .043805-0.797346$

C $3.2214896 .310940-0.090875$

C $2.0741177 .028932-0.766589$

C $2.2881788 .122502-1.517463$

C $0.6705206 .527333-0.499919$

C $0.3238095 .324376-1.334292$

N $5.0082921 .924042-0.180978$

H -4.063157 -1.688561 -3.937014

H -2.324855 -2.658379-1.614285

H $-1.816537-1.008737-4.188984$

H $-0.606944-3.279535-4.007169$

H $\quad 0.149079-2.707620-2.538211$

H $2.771462-0.995941-4.602188$

H $1.356937-1.221194-6.391041$

H $0.203994-2.548877-6.240636$

H -0.315295 -0.874826 -5.949596

H $3.236287-0.662624-2.298988$

H $1.757323-1.427406-1.761012$

H $2.623388-3.669506-2.381591$

H $4.050501-2.943577-3.120723$

H $4.549581-3.966485-0.984530$

H $2.396237-3.5220860 .247673$

H $2.759038-1.8059900 .259182$

H $2.408751-2.9769632 .662124$

H $4.870246-0.9778711 .544166$

H $3.439045-4.9773321 .982353$

H $4.869305-4.4775092 .854148$

H $4.980735-4.8767881 .127937$

H $6.625326-2.9978322 .451830$

H $8.002175-1.3386450 .683369$

H $7.606190-1.160445-1.011500$

H $7.422268-3.378545-0.530301$

H $-0.5168931 .330245-0.726030$

H $-0.3063483 .427214-2.968365$

H $0.2734394 .428397 \quad 1.218286$

H 0.6828242 .8097890 .692767

H -1.019048 3.3175540 .791065

H 6.4445340 .5106261 .276371

H $7.7173330 .993707 \quad 0.174406$

H $3.0877521 .874717-0.937788$

H $4.2608762 .729903-1.955476$

H 3.2105553 .7432950 .800111

H $3.9597545 .136360-1.823177$

H $4.1070556 .958888-0.057697$

H $2.958836 \quad 6.1198760 .957149$

H $1.4672198 .658562-1.982104$

H $3.2897448 .502824-1.689061$

H $-0.0595807 .311074-0.742751$

H $\quad 0.550226 \quad 6.356818 \quad 0.573078$

H $0.3146475 .532523-2.405518$

H 4.9907392 .0025470 .832033

C $-5.998980-1.906325-1.697687$ 
O - $-5.439368-1.151982-0.603010$

C $-6.0243640 .166318-0.612836$

C $-7.1338110 .141236-1.664518$

C $-6.600650-0.876449-2.651928$

H $-5.2381620 .852541-0.948780$

C -6.5109550 .5681510 .785017$

H -8.087522 $-0.200290-1.245914$

H $-7.3007991 .126824-2.110234$

C $-7.657708-1.440343-3.584442$

H $-5.822272-0.385289-3.247549$

C $-5.435512 \quad 0.530913 \quad 1.889323$

O $-7.558465-0.3331341 .168863$

H -6.9648861 .5656510 .734335$

H $-5.175674-0.5088962 .120828$

O -6.0220591 .0783843 .078211$

C -4.1532741 .3263721 .580072$

C $-3.227366 \quad 1.5404702 .795426$

H $-3.599950 \quad 0.8216170 .780314$

O $-4.5167722 .618397 \quad 1.084363$

H -2.351982 2.1037462 .451482

H -3.718875 2.209934 3.513188

H -7.220846 -2.174178 -4.268962

H -8.461999-1.933768 -3.028662

H $-8.105686-0.642321-4.185510$

C $-4.957772-2.866342-2.283541$

H -8.012073 0.0540371 .936280

H $-6.683283 \quad 0.4423263 .398840$

H $-3.697013 \quad 3.0776800 .833354$

H $-5.445390-3.546234-2.992110$

H $-4.558139-3.487355-1.471665$

H $-6.801116-2.515107-1.258500$

C $-1.004390-0.4064902 .017160$

O $-2.278769-0.7221542 .584674$

C $-2.781763 \quad 0.247402 \quad 3.504844$

C $-1.7844310 .462962 \quad 4.653222$

C $-0.379947 \quad 0.7870874 .143416$

C $0.063282-0.2443953 .104242$

H - 1.0859730 .5343501 .460350

C $-0.678956-1.479107 \quad 0.975921$

H -3.681095 -0.193502 3.951563

O -2.2520951 .4996005 .509558$

H $-1.737033-0.4549875 .253827$

H $-0.323881 \quad 1.803186 \quad 3.734844$

$\begin{array}{lllll}\text { O } & 0.541786 & 0.738620 & 5.234988\end{array}$

H $0.260792-1.1930633 .617185$

$\begin{array}{lllll}\text { O } & 1.287766 & 0.202884 & 2.527008\end{array}$

C $-0.655360-2.8782371 .545478$

H $-1.441740-1.4534340 .190311$

H $\quad 0.276168-1.291000 \quad 0.475636$

H $-1.672215 \quad 1.5229906 .289161$

H $0.328315 \quad 1.4751005 .832161$

H $1.674804-0.5443092 .040901$

O $-1.869531-3.4738081 .366245$

$\begin{array}{lllll}0 & 0.315917 & -3.377010 & 2.097786\end{array}$

C $-1.983443-4.8054021 .876634$

H $-1.262839-5.4639561 .382358$

H $-1.831598-4.8093562 .960240$

H -2.992882 -5.1661331 .662174$

SCF Energy (B3LYP/6-31G**/MMFF) $=-3245.90589671$

01513

MMFF Geometry

C $2.631310-4.134442-0.415575$

C $1.896879-5.1018220 .155877$

C $0.519497-4.9407160 .765369$

O $0.107635-3.556307 \quad 0.800443$

C $-0.501923-5.716440-0.088997$

C $-1.918892-5.6686940 .449796$

C $-2.947117-5.012265-0.125866$

C $-2.149646-6.4592601 .713054$

C $-2.942421-4.184099-1.383344$

C $-3.059788-2.683085-1.096907$

C $-4.436339-2.220661-0.592365$

C $-5.484836-2.235423-1.710360$

C $-6.830075-1.649750-1.256488$

O $-4.271945-0.883410-0.092087$

O $-7.605840-1.415542-2.442119$

C $-6.576486-0.286209-0.578200$
C $-7.624993-2.658672-0.419483$

$\begin{array}{llll}\text { O } & -7.783326 & 0.237593 & -0.029366\end{array}$

C $-5.461537-0.3305050 .488641$

C -5.1297831 .0676101 .055486$

O $-5.914978-1.1221251 .595284$

C $0.547328-2.7864051 .833559$

O $1.252929-3.1316152 .766894$

C $0.045177-1.4163601 .595265$

C $0.608940-0.3680802 .212321$

C $0.244386 \quad 1.0330801 .996541$

C -0.9891151 .3189011 .178963$

C $-4.555215 \quad 2.075712 \quad 0.056553$

C -4.2719343 .4123040 .724920$

C $-3.2384815 .634028 \quad 0.295302$

O $\begin{array}{llll}\text {-4.637618 } & 3.679140 & 1.867511\end{array}$

C $-1.886855 \quad 5.6292550 .936939$

C -1.6985525 .6076332 .265487$

$\begin{array}{llll}\text { C } & -0.351675 & 5.631926 & 2.929140\end{array}$

C $0.098414 \quad 4.2508373 .357772$

C $-0.1742913 .799672 \quad 4.593846$

C 0.8824393 .4861392 .312576

C 1.0161472 .0019502 .531346

N $-3.5901844 .285968-0.111657$

H $2.244033-3.122656-0.473140$

H $2.308456-6.108566 \quad 0.196933$

H $0.537192-5.342874 \quad 1.785627$

H $-0.200336-6.770439-0.161613$

H $-0.466327-5.335689-1.114411$

H $-3.921090-5.0761020 .357965$

H -3.204255 -6.4736692 .008847$

H $-1.835389-7.4987511 .573382$

H $-1.582169-6.0312882 .544969$

H -2.033975 -4.351270 -1.966728

H $-3.762412-4.521557-2.026320$

H $-2.295619-2.394600-0.366728$

H $-2.807902-2.123976-2.007826$

H $-4.746222-2.8618490 .239162$

H -5.631559 -3.246940 -2.106266

H $-5.117797-1.636094-2.555669$

H -8.431491 $-0.977688-2.172074$

H $-6.2822410 .406342-1.376037$

H -7.831446 -3.561101-1.007676

H $-8.604719-2.257514-0.137827$

H -7.103947 -2.969023 0.488502

H $-7.964227-0.2402390 .798104$

H -6.0218651 .4846121 .541637$

H -4.3953650 .9482081 .864839$

H $-5.374734-0.9036092 .372712$

H $-0.750625-1.2981220 .870426$

H $1.435527-0.5445392 .898847$

H -1.8515370 .7814981 .588823$

H $-0.848806 \quad 1.0109370 .138307$

H -1.2670572 .3753301 .172353$

H -3.624858 $1.692500-0.376444$

H $-5.264742 \quad 2.264451-0.755161$

H -4.0060156 .0206340 .974675$

H $-3.2276296 .264277-0.599338$

H $-1.025025 \quad 5.662393 \quad 0.275501$

H $-2.563652 \quad 5.5700702 .923894$

H -0.4083236 .3033803 .795804$

$\begin{array}{lllll}H & 0.403624 & 6.078621 & 2.269647\end{array}$

H 0.1506392 .8254164 .940692

H -0.7340004 .4059975 .299115$

H 1.8927093 .9135852 .265157

H $\quad 0.4536623 .6692951 .320809$

H 1.8768601 .7165193 .136162

H $-3.261473 \quad 3.957344-1.014876$

C $5.101075-3.558446-0.321162$

O $4.905157-2.137665-0.482753$

C $5.325159-1.4832130 .730769$

C $6.007358-2.5513951 .585115$

C $5.249222-3.7973741 .180829$

H $4.413378-1.1565201 .243529$

C $6.231021-0.2845600 .429280$

H $7.069986-2.6539061 .335302$

H $5.935789-2.3317042 .654846$

C $5.960183-5.0909671 .535482$

H $4.273403-3.7765501 .681581$ 
C $5.597566 \quad 0.858002-0.392716$

O $7.387884-0.742848-0.285268$

H $6.6108170 .121268 \quad 1.375043$

H $5.4844010 .550149-1.438906$

O $\quad 6.563724 \quad 1.919734-0.378241$

C 4.2559621 .3797480 .157621

C $3.7973552 .747187-0.378177$

H $3.4751680 .636695-0.031968$

$\begin{array}{lllll}\text { O } & 4.349536 & 1.512639 & 1.581777\end{array}$

H 2.8630032 .9962180 .136053

H $4.5056903 .515281-0.041993$

H $5.374766-5.9579191 .213596$

H $6.944394-5.1523541 .059012$

H $6.106103-5.1652292 .618172$

C $3.990566-4.347598-1.018312$

H $7.076597-1.165990-1.103924$

H $6.3386642 .541168-1.089993$

H 5.0706602 .1368901 .772658

H $4.247749-5.413407-1.017412$

H $3.934020-4.032428-2.067540$

H $6.047102-3.788249-0.830010$

C $1.3128072 .078850-2.029125$

O $2.6777391 .887366-2.416867$

C $3.6002242 .850195-1.902758$

C $3.1873214 .254987-2.365176$

C $1.7581814 .584697-1.941308$

C $0.8065873 .482141-2.400851$

H $1.2179211 .912078-0.950560$

C $0.4707401 .022390-2.762892$

H $4.5645332 .629291-2.373322$

O $4.0885685 .227050-1.845510$

H $3.2555014 .306209-3.459659$

H $1.6921744 .752222-0.860200$

O $1.3855495 .817623-2.561395$

H $\quad 0.673700 \quad 3.554949-3.488244$

O $-0.4736923 .678090-1.802553$

C $0.802145-0.394302-2.354382$

H $-0.596033 \quad 1.176690-2.566611$

H $0.655323 \quad 1.101465-3.841560$

H $3.7757376 .099792-2.139135$

H $0.5162566 .072769-2.209239$

H $-0.830023 \quad 4.518832-2.135084$

O $0.160055-1.251755-3.202964$

O $1.519371-0.711641-1.417015$

C $0.385644-2.642784-2.964119$

H $1.439752-2.885876-3.125183$

H $0.075561-2.906440-1.950162$

H $-0.218725-3.213325-3.674111$

SCF Energy (B3LYP/6-31G**//MMFF) $=-3245.91493037$

\section{4}

MMFF Geometry

C $1.071352-0.9478361 .541004$

C $-0.139015-0.6766241 .031096$

C $-0.415877-0.554012-0.442720$

O $-1.616657-1.328672-0.689923$

C $-0.666256 \quad 0.907996-0.854915$

C $0.523167 \quad 1.837886-0.745986$

C $0.5302422 .985336-0.039542$

C $1.7299161 .457194-1.565370$

C -0.5593873 .5489540 .830030$

C $-1.333440 \quad 4.691080 \quad 0.157036$

C $-2.3101474 .220001-0.933639$

C $-3.0022345 .418093-1.587872$

C $-4.0732264 .980984-2.598725$

O $-3.2839273 .375826-0.312102$

O $-4.8739876 .129225-2.917855$

C $-5.0057773 .953410-1.923973$

C $-3.4536514 .503434-3.917350$

O $-5.932640 \quad 3.412512-2.863182$

C $-4.2506812 .810149-1.207348$

C $-5.2411051 .975781-0.359111$

O $-3.6436351 .966914-2.186811$

C -1.904848 -1.675180 -1.970000

O $-1.249965-1.401287-2.966280$

C $-3.181261-2.420390-2.057222$

C $-3.762173-3.017576-1.005014$

C $-5.006620-3.789564-1.032082$
C $-5.832632-3.747582-2.290883$

C $-4.573120 \quad 0.924846 \quad 0.541151$

$\begin{array}{llll}\text { C } & -5.564899 & 0.290172 & 1.501274\end{array}$

C $-5.646603-1.1294613 .545793$

$\begin{array}{lllll}\text { O } & -6.779788 & 0.446952 & 1.412170\end{array}$

C $-5.882433-2.562023 \quad 3.190014$

C $-7.084940-3.0512362 .851578$

C $-7.354618-4.4981442 .552020$

C $-7.642201-4.7475521 .088724$

C $-8.851008-4.485606 \quad 0.563909$

C $-6.551858-5.3815430 .256116$

C $-5.359478-4.4839770 .069564$

N $-4.928712-0.4754372 .469427$

H $1.914323-1.0829240 .868644$

H $-0.977755-0.5242871 .706832$

H $0.402612-0.995754-1.022509$

H $-1.527273 \quad 1.280216-0.291942$

H $-1.0007760 .937123-1.899575$

H $1.4310143 .597880-0.068642$

H $2.4866352 .248994-1.583509$

H $2.2063970 .560298-1.158971$

H $1.4390791 .260315-2.602413$

H -1.2411762 .7736851 .192515$

H -0.0763093 .9478591 .731516$

H $-1.904008 \quad 5.204267 \quad 0.942336$

H $-0.6297705 .422000-0.260378$

H $-1.7590773 .647102-1.686722$

H -2.267653 $6.077328-2.067089$

H $-3.4885196 .026465-0.812210$

H $-4.2848926 .814948-3.276604$

H $-5.6028784 .495098-1.176788$

H $-2.8656245 .307098-4.376805$

H $-4.228813 \quad 4.245246-4.647283$

H -2.793636 $3.642157-3.794687$

H $-6.3920514 .161244-3.280667$

H -5.8116302 .6561000 .287193$

H $-5.9605191 .467759-1.013821$

H -3.549623 $1.072117-1.825389$

H $-3.600581-2.486925-3.055923$

H -3.275471-2.961478 -0.032666

H $-5.320736-4.272733-3.104297$

H $-6.013760-2.711719-2.599745$

H $-6.819150-4.203161-2.175892$

H $-4.1337250 .123838-0.061006$

H $-3.7767321 .386206 \quad 1.135870$

H $-6.583635-0.5966163 .740033$

H $-5.027538-1.0662494 .446178$

H $-5.027289-3.230906 \quad 3.243797$

H -7.937245 -2.3766302 .806766$

H $-8.214420-4.818047 \quad 3.155349$

H $-6.521652-5.1304152 .884388$

H $-9.071748-4.688823-0.478676$

H $-9.645744-4.0588521 .166820$

H $-6.944399-5.724814-0.707087$

H $-6.215286-6.2994680 .755853$

H $-4.700921-4.4475570 .937740$

H -3.921454 $-0.600322 \quad 2.423699$

C $2.087456 \quad 0.1193623 .616254$

$\begin{array}{lllll}\text { O } & 3.340321 & 0.339861 & 2.937867\end{array}$

C $3.4682491 .748032 \quad 2.664779$

C 2.4665132 .4419343 .579984

C 1.3255821 .4453663 .572861

H 3.1563951 .8952431 .623919

C 4.9177762 .2100402 .838850

H 2.8573132 .5604734 .597166

H 2.1787643 .4299413 .208665

C $0.340211 \quad 1.630571 \quad 4.713160$

H $\quad 0.7860521 .5513132 .626609$

C $5.953121 \quad 1.4062482 .026224$

$\begin{array}{lllll}\text { O } & 5.284782 & 2.106999 & 4.221152\end{array}$

H 4.9910263 .2752312 .590544

H $6.0704510 .413303 \quad 2.474634$

O 7.2209772 .0579132 .180953

C 5.6399961 .2632320 .525601

C $6.7897420 .663880-0.307006$

H $4.7401570 .651503 \quad 0.398331$

$\begin{array}{llll}\text { O } & 5.345069 & 2557712-0.006469\end{array}$

H $6.4544890 .617146-1.350581$ 
H $7.6341391 .364844-0.319229$ H -0.4535230 .8782504 .666805$ H $\quad 0.832081 \quad 1.543616 \quad 5.687709$ H -0.1277002 .6190024 .659582$ C $1.353587-1.0816373 .011702$ H 5.1647831 .1794304 .488290 H 7.1567522 .9291151 .753136 H $5.0848462 .440321-0.936125$ H $\quad 0.425237-1.2614043 .567022$ H $\quad 1.973347-1.9783003 .138041$ H $2.346892-0.1264304 .654148$ C $5.557823-2.068550-0.885922$ O $6.182437-1.6418130 .331493$ C $7.265536-0.7237870 .164514$ C $8.357082-1.350429-0.713464$ C $7.799463-1.857212-2.040111$ C $6.579299-2.748678-1.809000$ H $5.120064-1.199812-1.391209$ C $4.418678-3.011097-0.475257$ H $7.706317-0.5872501 .159411$ O $9.401913-0.418494-0.971819$ H $8.806874-2.199207-0.182025$ H $7.556096-1.029152-2.716263$ O $8.799977-2.633701-2.703709$ H $6.902755-3.703385-1.375183$ O $5.975870-3.033460-3.068692$ C $3.468075-3.355327-1.594151$ H $4.830324-3.942357-0.066683$ H $3.831813-2.5712550 .340144$ H $9.748559-0.122706-0.112690$ H $9.582573-2.065960-2.810294$ H $6.654096-3.448662-3.628663$ O $2.893108-2.225514-2.094038$ O $3.249067-4.501024-1.966726$ C $1.994992-2.428008-3.188895$ H $2.520211-2.891130-4.029560$ H $1.148680-3.045536-2.874201$ H $1.620944-1.451068-3.505983$ SCF Energy (B3LYP/6-31G**//MMFF) $=-3245.91053803$

\section{5}

MMFF Geometry

C $0.934812-4.033264-0.457537$

C $1.252740-3.319423-1.549661$

C $0.303913-2.446445-2.344425$

O $-0.873464-2.178162-1.549349$

C $0.968518-1.103443-2.698435$

C $0.349236-0.410399-3.896874$

C $-0.3530280 .737430-3.840460$

C $0.635732-1.047133-5.233495$

C $-0.7266041 .523157-2.617195$

C $0.3516432 .550647-2.255508$

C $-0.1291703 .565831-1.208178$

C $1.0339424 .428563-0.712288$

C 0.6144575 .3809670 .418651

O $-0.7128862 .857752-0.112238$

O 1.8079795 .8601011 .058098

C -0.1790524 .5906571 .483697$

C $-0.1097996 .618183-0.125749$

O -0.7221865 .4678482 .467650$

C -1.2974103 .7074630 .884526$

C -1.9250522 .8108281 .981790$

O $-2.306946 \quad 4.556218 \quad 0.338179$

C -2.092613 -2.256827 -2.141204

O $-2.331885-2.435433-3.323896$

C $-3.103114-2.136720-1.063806$

C $-4.392045-2.394305-1.330493$

C $-5.494691-2.397026-0.364407$

C -5.171382 -2.055498 1.066807

C -2.8797921 .7247591 .457986$

C -4.2608302 .2543681 .116117$

C $-6.1322901 .959688-0.500944$

O $-4.8036153 .144708 \quad 1.766782$

C $-7.161328 \quad 1.069246 \quad 0.120031$

C $-8.0526860 .361409-0.589948$

C $-9.106241-0.5093830 .030813$

C $-9.088457-1.923666-0.503630$

C $-10.051865-2.362766-1.331340$
C $-8.002432-2.853744-0.009594$

C $-6.729186-2.725537-0.800676$

N $-4.822909 \quad 1.6152430 .021473$

H $-0.089990-4.018347-0.100560$

H $2.268991-3.388501-1.931460$

H $0.042138-3.010263-3.247330$

H $2.029432-1.255458-2.937090$

H $0.950345-0.448863-1.819442$

H $-0.711711 \quad 1.168642-4.774257$

H $0.265572-0.443400-6.069192$

H $1.714936-1.169456-5.372709$

H $0.161185-2.029971-5.305833$

H -1.676862 2.032926 -2.819664

H $-0.9195240 .853290-1.771585$

H $1.2233102 .007880-1.870914$

H $0.6848953 .092033-3.150008$

H $-0.8965274 .191718-1.679305$

H $1.4856094 .986937-1.541651$

H $1.8278533 .776664-0.327111$

H $2.359554 \quad 6.2883620 .381257$

H 0.5324483 .9378852 .008748

H $\quad 0.5476747 .180512-0.799905$

H -0.3722927 .3109000 .681320$

H -1.016976 $6.373743-0.682098$

H 0.0137935 .9940622 .824288

H -1.1160292 .2905082 .508573$

H -2.444227 3.4335822 .720144

H -3.0638354 .0103820 .077508$

H $-2.750244-1.884725-0.069872$

H -4.667038 -2.656466 -2.351494

H $-4.669366-1.0850121 .132279$

H -4.514105 -2.816529 1.501729

H $-6.051240-1.9844191 .708981$

H $-2.439947 \quad 1.199748 \quad 0.603758$

H $-3.029503 \quad 0.9769862 .246828$

H $-6.088396 \quad 1.847063-1.588749$

H $-6.3565423 .004939-0.265297$

H -7.187795 1.0205061 .206116

H $-8.0441800 .430622-1.675411$

H -10.080122 -0.043096 -0.167426

H $-9.007724-0.5384851 .123193$

H -10.060179 -3.384691-1.695791

H -10.850298 -1.709488 -1.667109

H $-8.337173-3.895766-0.104777$

H $-7.861889-2.7035081 .063870$

H $-6.853199-2.969375-1.857133$

H -4.333209 $0.837411-0.410750$

C $1.922369-4.6507161 .819816$

O $2.225757-3.2681762 .098445$

C $1.162914-2.7318022 .903739$

C $0.586959-3.9325553 .641625$

C $0.614422-4.9850822 .550064$

H $0.407776-2.3443012 .207955$

C $1.648099-1.5879013 .798365$

H $1.226836-4.2428514 .475586$

H $-0.418098-3.7432814 .030048$

C $\quad 0.559549-6.410613 \quad 3.074997$

H $-0.255091-4.8287281 .902620$

C $2.496293-0.5133633 .088213$

O $2.452138-2.1297684 .854352$

H $0.779530-1.1294624 .286618$

H $3.515693-0.8945442 .958821$

O $2.608740 \quad 0.5992203 .986076$

C $1.959219-0.0200641 .734998$

C 2.6876291 .2123951 .168849

H $2.012570-0.8354651 .005794$

O $\quad 0.577998 \quad 0.3170451 .877623$

H 2.2705921 .4018090 .173589

H 2.4173992 .0991681 .756687

H $0.601845-7.1287182 .249827$

H $1.394673-6.6227893 .751024$

H $-0.370585-6.5818863 .626651$

C $1.909797-4.8945420 .302957$

H $2.728603-1.3875915 .418880$

H $1.726206 \quad 1.0003034 .067354$

H $\quad 0.2468310 .5778831 .000906$

H $1.669630-5.9464620 .109470$

H $2.922632-4.717959-0.080802$ 
H $2.748508-5.2308612 .249925$

C $4.396570-0.302716-0.867980$

O $4.646095-0.1529430 .530459$

C 4.2222391 .0935971 .084882

C 4.9034082 .2597540 .352554

C $4.6940662 .186752-1.158973$

C $5.0714460 .803254-1.687964$

H $3.314339-0.255753-1.034012$

C $4.821070-1.718535-1.282825$

H $4.595044 \quad 1.1087372 .116268$

O 4.4229053 .5117960 .830496

H 5.9825652 .2317360 .552052

H $3.6664362 .444016-1.437314$

O $5.5319613 .153271-1.797353$

H $6.1621990 .699437-1.666103$

O $4.6613300 .689778-3.048995$

C $6.301953-2.000846-1.131782$

H $4.302463-2.452905-0.655433$

H $4.537135-1.888752-2.327632$

H $4.5775633 .537306 \quad 1.790179$

H $5.3076354 .022112-1.421935$

H $5.0741331 .422083-3.538152$

O $6.638001-3.051707-1.937722$

O $7.061792-1.405568-0.380445$

C $8.011220-3.451337-1.886627$

H $8.149363-4.283261-2.582417$

H $8.658640-2.624867-2.194962$

H $8.269099-3.789984-0.878614$

SCF Energy (B3LYP/6-31G**//MMFF) $=-3245.91380847$

\section{6}

MM̄FF Geometry

C -0.5032084 .3468202 .577069$

C -0.0321943 .1339552 .253263$

C -0.3153691 .8868423 .046323$

O $-1.083496 \quad 1.0167642 .180835$

C 0.9967931 .1772513 .418222

C 0.8594240 .2770924 .631036

C $0.856530-1.0690034 .590789$

C 0.8012790 .9875695 .960137

C $0.916278-1.9468193 .374948$

C $2.357229-2.3606943 .054378$

C $2.421600-3.4533531 .977764$

C $3.873067-3.7503381 .592546$

C $3.978605-4.7315140 .414058$

O $1.689558-3.0018510 .836580$

O $5.313103-4.650193-0.110511$

C $3.024483-4.281131-0.715268$

C $3.782238-6.1823570 .870929$

O $2.978035-5.252299-1.758139$

C $1.595458-3.966832-0.218755$

C $0.748505-3.347291-1.358431$

O $0.984662 \quad-5.1796380 .219178$

C $-2.439710 \quad 1.1004392 .265045$

O -3.0890461 .6884663 .115448$

C -3.0106620 .4047921 .088718$

C -4.3420650 .2782830 .983924$

C $-5.081480-0.292258-0.145040$

C $-4.318344-0.570273-1.412541$

C $-0.637586-2.839401-0.928298$

C $-1.678252-3.937924-0.792162$

C $-3.788293-4.493468 \quad 0.413511$

O $-1.630350-4.980595-1.440236$

C $-4.938116-4.161608-0.484315$

C $-6.173039-3.885483-0.038056$

C $-7.336585-3.593627-0.941264$

C -8.042975 -2.302277 -0.595034

C $-9.248379-2.310706-0.000898$

C -7.393735 -1.003635 -1.019624

C $-6.408528-0.495494-0.005038$

N $-2.676087-3.6108280 .114445$

H -1.110056 4.4651013 .471112

H $0.5479873 .018241 \quad 1.341955$

H -0.886672 2.1292293 .949815

H 1.7773051 .9151913 .649754

H 1.3697410 .6112322 .556338

H $\quad 0.797516-1.6133035 .532390$

H $\quad 0.7881650 .2897906 .804432$
H 1.6767941 .6334756 .083846

H -0.0991321 .6046826 .032715$

H $0.306221-2.8375503 .570030$

H $0.456288-1.4518052 .512816$

H $2.901442-1.4718732 .708470$

H $2.863520-2.7176703 .960085$

H $1.941719-4.3540952 .379172$

H $4.433471-4.1252852 .458242$

H $4.370040-2.8152751 .305278$

H $5.931901-4.8571410 .610881$

H $3.445255-3.368683-1.159466$

H $4.555687-6.4632131 .596095$

H $3.891429-6.8799700 .033484$

H $2.813015-6.3553191 .343322$

H $3.893182-5.411136-2.046631$

H $1.290073-2.479324-1.754897$

H $\quad 0.637869-4.067240-2.177764$

H $0.059981-4.9955350 .443291$

$\begin{array}{llll}H & -2.324366 & 0.054897 & 0.327352\end{array}$

H $-4.9620150 .641070 \quad 1.803239$

H $-3.471950-1.236624-1.226315$

H -3.938263 $0.365693-1.835590$

H -4.917399 -1.052560 -2.187358

H $-0.567719-2.259970-0.001679$

H -1.015127 -2.162531-1.704506

H $-4.035577-4.3584751 .471234$

H -3.482499 -5.5325010 .254550$

H -4.750865 -4.172980 -1.555613

H $-6.368213-3.9009491 .031777$

H -8.034986 -4.437128 -0.862993

H -7.027095 -3.561800 -1.993534

H $-9.773534-1.3880930 .223205$

H $-9.734386-3.2393110 .279662$

H -8.162664 -0.228534 -1.140745

H -6.963593 -1.123425 -2.017370

H $-6.869606-0.2441620 .951609$

H -2.673481 -2.693321 0.549897

C -1.1621495 .6538120 .485450$

O $-0.7017274 .703184-0.496810$

C $-1.8451464 .047444-1.073399$

C $-3.0499704 .907040-0.703333$

C -2.6570875 .3891950 .679758$

H -1.936805 $3.078040-0.569032$

C -1.649240 $3.828895-2.577022$

H $-3.1669995 .760742-1.380617$

H $-3.9841134 .337310-0.711033$

C $-3.4372496 .602327 \quad 1.153987$

H $-2.8267214 .564716 \quad 1.382758$

C $-0.3378643 .109443-2.959120$

O $-1.6429005 .109792-3.221039$

H $-2.5140273 .286004-2.977775$

H $0.5114203 .788674-2.818855$

O $-0.398216 \quad 2.818716-4.362043$

C $-0.071511 \quad 1.792198-2.207109$

C $1.0677950 .937540-2.795516$

H $0.1399322 .009490-1.154296$

O $\quad-1.260060 \quad 0.998185-2.242072$

H $\quad 1.1524370 .032829-2.181622$

H $\quad 0.7670650 .571782-3.785723$

H $-3.100240 \quad 6.9162472 .147087$

H -3.3153347 .4505010 .472092$

H -4.5059506 .3723831 .215718$

C $-0.2847205 .576195 \quad 1.740463$

H -1.738495 $4.958713-4.176252$

H $-0.3456143 .663644-4.839452$

H - $-1.0931010 .197969-1.716365$

H -0.4765456 .4519892 .372079$

H 0.7727535 .6231521 .450548

H -1.0126586 .6413020 .029489$

C $3.2457501 .234698-0.653597$

O $2.8425732 .197154-1.632240$

C $2.4316071 .648157-2.887531$

C $3.5489040 .778994-3.485570$

C $4.017908-0.294953-2.508774$

C $4.3780240 .336575-1.166390$

H $2.3786940 .611392-0.399333$

C $3.614357 \quad 1.9796890 .638668$

H $2.3036172 .499877-3.565812$ 
O $3.1213210 .156456-4.692443$

H $4.4071831 .414957-3.738695$

H $3.268403-1.084168-2.385145$

O $5.186332-0.930910-3.031227$

H $5.3078430 .905329-1.282102$

O $4.627958-0.690029-0.211422$

C $4.8155972 .894778 \quad 0.515524$

H 2.7792572 .6148980 .949491

H 3.8080741 .2485031 .432075

H $2.8413130 .861156-5.301335$

H $4.952524-1.292010-3.903629$

H $5.317449-1.268636-0.579887$

O $5.3748253 .065011 \quad 1.750126$

O $5.1951303 .423925-0.520171$

C 6.5149083 .9286741 .795505

H $\quad 6.8702413 .9686692 .828571$

H 7.3158973 .5329071 .163795

H 6.2368004 .9389571 .480453

SCF Energy (B3LYP/6-31G**//MMFF) $=-3245.90586175$

\section{7}

MM̄FF Geometry

C $0.0215594 .642781-1.227552$

C $0.6333604 .790965-0.042010$

C 1.7783973 .9558920 .484951

O $1.9167102 .713252-0.242227$

C 3.0961074 .7406750 .363556

C 4.2596074 .0942451 .089889

C 5.3640613 .6075090 .491375

C 4.1458134 .0440982 .592753

C $5.6953763 .584445-0.973549$

C $5.2860752 .297094-1.706323$

C $5.9187741 .017309-1.135310$

C $5.696625-0.165400-2.080651$

C $6.235356-1.480311-1.494341$

O 5.3069320 .7357650 .127283

O $5.700283-2.561697-2.273609$

C $5.697642-1.652177-0.056487$

C $7.761171-1.573688-1.614696$

O $6.306903-2.7702290 .583346$

C $5.863135-0.3920490 .817130$

C $5.147063-0.4943732 .187316$

O $7.255383-0.1896271 .090505$

C 1.0121611 .7296570 .026796

O $0.091215 \quad 1.773406 \quad 0.826524$

C $1.3192230 .585384-0.857856$

C $0.397667-0.369095-1.056839$

C $0.557876-1.548362-1.910831$

C $1.938289-1.837012-2.440909$

C $3.634540-0.7365282 .128841$

C $3.242185-2.2023902 .213076$

C $1.277012-3.7091011 .951315$

O $4.028747-3.0934872 .522102$

C $1.276511-4.2701040 .564164$

C $0.185846-4.782149-0.026106$

C $0.187210-5.381916-1.403380$

C $-0.892239-4.809476-2.294871$

C $-2.051322-5.464244-2.477451$

C $-0.592595-3.536029-3.052357$

C $-0.519346-2.320610-2.166147$

N $1.895244-2.3963621 .936788$

H $\quad 0.3796973 .903199-1.938352$

H $0.253428 \quad 5.5400170 .650381$

H 1.5705663 .7234451 .536495

H $2.969478 \quad 5.7527270 .770962$

H $3.3275054 .869785-0.699208$

H 6.1364803 .1722831 .125103

H 5.0766933 .7177783 .069071

H 3.9083585 .0363762 .989725

H 3.3608243 .3461132 .898800

H $5.2518044 .443540-1.487790$

H $6.7796583 .722543-1.074574$

H $4.1936342 .210450-1.683966$

H $5.5796042 .407904-2.757603$

H $6.9911961 .195105-0.993279$

H $6.148740 \quad 0.029321-3.061224$

H $4.620824-0.282975-2.266800$

H $5.969861-2.426645-3.198292$
H $4.629792-1.884632-0.144552$

H $8.066208-1.516982-2.666594$

H $\quad 8.128357-2.539107-1.249347$

H $8.283213-0.779230-1.077523$

H $6.156767-3.5459400 .016348$

H $5.649958-1.2357472 .819940$

H 5.2911100 .4684642 .698938

H 7.3362270 .3712381 .879746

H $2.2916320 .569448-1.335722$

H $-0.571475-0.270140-0.569229$

H $2.028113-2.813749-2.920435$

H $2.668272-1.839690-1.624196$

H $2.234094-1.081626-3.176528$

H $3.170237-0.2480002 .995148$

H $3.201109-0.2894901 .229932$

H $0.266346-3.5899212 .351975$

H $1.840008-4.3717232 .616399$

H $2.223312-4.2777470 .029017$

H $-0.753419-4.8030030 .521818$

H $0.042185-6.464797-1.293110$

H $1.162231-5.263437-1.892180$

H -2.818783 -5.081379 -3.141851

H $-2.261733-6.395122-1.961294$

H -1.381173 $-3.342416-3.792196$

H $\quad 0.312968-3.683832-3.647396$

H - $1.477249-2.038918-1.727173$

H $1.325863-1.6062631 .647299$

C $-2.4098624 .445492-1.702931$

O $-2.5857763 .784575-0.435598$

C $-3.9925573 .696627-0.136074$

C $-4.7184274 .134300-1.399969$

C $-3.7407485 .134371-1.989131$

H -4.1667784 .4108090 .677904$

C $-4.2994202 .264733 \quad 0.320729$

H -4.857785 3.287011-2.083159

H $-5.7014044 .566562-1.190302$

C $-3.9809255 .428907-3.458994$

H $-3.8112246 .069617-1.417633$

C -5.7844052 .0188890 .639102$

O -3.5301682 .0082311 .501503$

H $-3.9348881 .572274-0.438749$

H $-6.3994122 .184034-0.252678$

O -6.1691753 .0244651 .591106$

C $-6.1072790 .634593 \quad 1.243219$

C $-6.227762-0.5289970 .243034$

H $\quad \begin{array}{lllll}-5.404639 & 0.391447 & 2.045588\end{array}$

O

H $-6.580095-1.4000250 .810588$

H -7.050764 -0.305406 -0.449653

H -3.242791 $6.142244-3.839070$

H $-3.9152834 .519976-4.066446$

H $-4.9760335 .860957-3.606977$

C $-1.2182005 .401530-1.614353$

H -2.5961872 .1668681 .280036$

H -7.1061042 .8753751 .805319$

H $-7.558332-0.0869962 .355631$

H -1.424210 $6.183972-0.873425$

H -1.051156 $5.897718-2.576421$

H $-2.2120173 .660761-2.445291$

C $-3.788260-2.1640461 .129451$

O $-3.807889-1.0443090 .236703$

C $-4.982696-0.925009-0.566635$

C $-5.173314-2.195136-1.411827$

C $-5.191863-3.450378-0.545125$

C $-3.965613-3.4818020 .362620$

H $-4.583338-2.052341 \quad 1.876528$

C -2.428179-2.153028 1.843787

H $-4.800976-0.114252-1.279850$

O $-6.379167-2.131328-2.165994$

H $-4.347576-2.282244-2.130279$

H $-6.115601-3.5272310 .039510$

O $-5.158693-4.604865-1.386442$

H -3.073599-3.682752 -0.241835

O $-4.097876-4.553276 \quad 1.294093$

C -2.282207 -0.994102 2.804010

H $-2.276237-3.0682872 .425748$

H -1.632028 -2.077515 1.093179

H $-6.334799-1.333837-2.720962$ 
H - $-5.920722-4.546188-1.987955$

H $-4.216196-5.370693 \quad 0.780308$

O $-0.965086-0.6437022 .887682$

O $-3.200849-0.4877693 .433249$

C $-0.665856 \quad 0.425133 \quad 3.790590$

H -0.9105650 .1314884 .815639$

H $\quad 0.4064250 .6302053 .729907$

H -1.212588 1.3291703 .506238

SCF Energy (B3LYP/6-31G**//MMFF $)=-3245.90763100$

\section{8}

MM̄FF Geometry

C -3.592153 -3.499659-2.465427

C $-2.422953-3.307609-1.835733$

C $-1.274049-2.507573-2.388808$

O $-1.224854-1.248646-1.669834$

C $0.068584-3.226551-2.141928$

C $0.643122-3.879923-3.383039$

C $1.807510-3.514150-3.954859$

C $-0.139736-5.042863-3.933803$

C $2.710802-2.394865-3.517967$

C $3.759221-2.890233-2.514754$

C $4.639013-1.749718-1.985134$

C $5.708867-2.296893-1.037595$

C $6.538875-1.175641-0.397440$

O $3.793408-0.831805-1.285641$

O $7.278822-1.7662250 .681610$

C $5.578803-0.1263650 .201259$

C $7.578111-0.617881-1.376559$

O $6.304560 \quad 0.9961980 .698139$

C $4.4654710 .326149-0.773675$

C $3.4226651 .177004-0.009694$

O $5.0497371 .103536-1.816778$

C $-2.006647-0.235078-2.133103$

O $-2.742363-0.261553-3.107198$

C $-1.8210480 .928085-1.236336$

C $-2.5119942 .053260-1.473963$

C $-2.4505943 .296838-0.702163$

C -1.5303403 .3363660 .487539$

C $2.1914721 .589037-0.828608$

C $2.3428642 .915326-1.554707$

C $1.0619194 .474446-3.020946$

O $3.3450063 .619699-1.470660$

C $0.3520845 .455395-2.140503$

C $-0.7620316 .109722-2.502486$

C $-1.4643957 .105173-1.623643$

C $-2.9372336 .803818-1.453046$

C $-3.8700177 .537972-2.083125$

C $-3.3314345 .706544-0.487548$

C $-3.2138594 .336754-1.100284$

N $1.2140543 .222407-2.302652$

H -3.751344 -3.070256 -3.450806

H -2.292007 -3.724638 -0.839398

H -1.401089 -2.301274 -3.459232

H - $0.034121-4.004033-1.372581$

H $0.783450-2.517091-1.710429$

H $2.158218-4.069161-4.823325$

H $0.385013-5.544638-4.753890$

H $-0.313567-5.788985-3.151569$

H -1.107496-4.708439-4.319646

H $3.212799-1.988089-4.404624$

H $2.126881-1.565230-3.105409$

H $3.246085-3.368593-1.669954$

H $4.388307-3.653800-2.989394$

H $5.101395-1.239866-2.838437$

H $6.361316-3.010755-1.556034$

H $5.222795-2.867619-0.233153$

H $7.749006-1.0504581 .142857$

H $5.105986-0.5904551 .077733$

H $8.258735-1.413539-1.702797$

H $8.2113540 .136603-0.896875$

H $7.131630-0.176396-2.269985$

H $6.5591331 .544440-0.063688$

H 3.0505290 .5738250 .826050

H 3.8987952 .0656490 .420475

H $4.3468251 .594666-2.269788$

H -1.124863 $0.827887-0.412034$

H $-3.1923962 .072247-2.324670$
H $-0.5025053 .108400 \quad 0.191651$

H -1.847899 $2.608249 \quad 1.241214$

H -1.494226 4.3097360 .978992

H $1.9089860 .813092-1.548034$

H $1.3535751 .711559-0.132339$

H $0.5028944 .259206-3.936966$

H $2.0477224 .864790-3.292524$

H $0.7844475 .645943-1.160897$

H -1.181495 $5.938926-3.491300$

H -1.333345 $8.096723-2.076457$

H $-0.9943377 .166245-0.634005$

H $-4.9288337 .345672-1.944727$

H $-3.5959808 .344054-2.755542$

H -4.378496 $5.836382-0.182481$

H -2.7545965 .8203110 .433726$

H $-3.8548564 .200369-1.972900$

H $0.4077362 .606815-2.254262$

C $-5.523076-3.519940-0.786869$

$\begin{array}{lllll}\text { O } & -4.660290 & -3.204417 & 0.324880\end{array}$

C $-4.857291-1.8230540 .679917$

C $-6.218614-1.4553960 .107905$

C -6.176051-2.203812 -1.215078

H $-4.097471-1.2411550 .145388$

C $-4.680015-1.6345322 .189497$

H -7.036546 -1.831597 0.733121

H $-6.344022-0.375472-0.013152$

C -7.537264 -2.377561-1.864804

H $-5.524137-1.652043-1.904169$

C $-3.320419-2.1532962 .712990$

O $-5.721053-2.3566602 .855803$

H $-4.815013-0.5775702 .448187$

H -3.269116 -3.244166 2.610220

O $-3.236326-1.8699954 .114063$

C -2.101494-1.511042 2.024327

C $-0.757502-2.0680962 .535042$

H $-2.146854-1.7074320 .950072$

O $-2.154209-0.0986102 .194308$

H $-0.677436-1.8985773 .612655$

H $-0.764634-3.1526072 .376923$

H -7.452244 -2.942336 -2.798470

H $-8.230898-2.912999-1.207977$

H -7.979487 -1.403227 -2.097007

C $-4.731479-4.274959-1.861550$

H -5.722539 -2.076179 3.786454

H -3.885719 -2.434462 4.566224

H $-2.0969460 .085056 \quad 3.147792$

H -5.406080 -4.587875 -2.667075

H $-4.325027-5.195550-1.422080$

H $-6.286159-4.201177-0.388113$

C $1.1686850 .142303 \quad 3.501913$

O $0.659266-0.0685312 .177698$

C $0.451247-1.4347881 .804520$

C $1.764234-2.2168101 .931519$

C $2.369836-2.0804173 .322373$

C $2.492683-0.6082723 .712080$

H $0.435744-0.2022334 .239917$

C 1.3532091 .6603883 .670290

H $0.201285-1.4227720 .736651$

O $1.572439-3.5956401 .634206$

H $2.487890-1.8337611 .200996$

H $1.791104-2.6388284 .067878$

O $3.681028-2.6515783 .317626$

H $3.299105-0.1493493 .126672$

O $2.874359-0.5557985 .086400$

C $0.0424422 .384368 \quad 3.902405$

H 1.9842901 .8886774 .534647

H 1.8144522 .0767942 .766746

H $1.211238-3.6521490 .733150$

H $3.592177-3.5766583 .030438$

H 3.2040950 .3391715 .271631

O 0.2516353 .7288053 .776804

O -1.0178931 .8508384 .196548$

C -0.8826104 .5613944 .040933$

H -0.6441175 .5752893 .708931$

H $-1.763694 \quad 4.2135483 .493441$

H -1.0825004 .5791835 .116166$

SCF Energy (B3LYP/6-31G**//MMFF) $=-3245.92465240$ 
01_519

MM̄FF Geometry

C $-3.126968-3.674756-2.606474$

C $-2.414393-3.372431-1.509840$

C $-0.942859-3.668058-1.358965$

O $-0.265661-2.443562-0.991998$

C $-0.736067-4.684452-0.221047$

C $0.708006-5.105114-0.028263$

C $1.425993-4.8853191 .090759$

C $1.334944-5.844569-1.182695$

C $0.985789-4.2073432 .357873$

C $1.266468-2.6977202 .410623$

C $2.758122-2.3305312 .349475$

C $2.960527-0.8419852 .635337$

C $4.432918-0.4243032 .498419$

O $3.245991-2.6275961 .040035$

O 4.4770351 .0112222 .460626

C $4.984668-0.9311671 .146439$

C $5.259167-0.8509303 .718065$

O $6.390099-0.7001341 .083125$

C $4.653891-2.4136130 .865698$

C $4.998588-2.867540-0.574197$

O $5.413013-3.238868 \quad 1.757664$

C $-0.009874-1.550230-1.985450$

O $-0.270897-1.671874-3.171593$

C $0.632564-0.369686-1.368414$

C $0.7009590 .777601-2.059309$

C $1.2963402 .030855-1.592851$

C $2.0878691 .987425-0.312722$

C $4.295929-2.092118-1.694226$

C $5.125621-0.959576-2.275466$

C $4.9761840 .907692-3.918800$

O $6.305600-0.773100-1.991536$

C $4.7385882 .183332-3.174282$

C $4.1869893 .270116-3.735110$

C $3.9865574 .573324-3.016586$

C $2.5567735 .060790-3.081679$

C $2.1803545 .982859-3.983692$

C $1.5873064 .543698-2.043854$

C $1.1184463 .144291-2.334494$

N $4.397668-0.199643-3.181290$

H -2.615601-4.170927 -3.429631

H -2.887164 -2.879171 -0.665439

H - $0.519653-4.054247-2.294115$

H -1.327726 $-5.588350-0.418961$

H -1.136842 -4.2568830 .704525$

H $2.455729-5.240771 \quad 1.113676$

H $2.316019-6.259228-0.926434$

H $0.698328-6.680729-1.489523$

H $1.475723-5.177588-2.038483$

H $-0.081249-4.3772322 .536845$

H $1.494972-4.6969383 .197965$

H $0.729934-2.2074321 .589610$

H $0.843338-2.3155673 .347581$

H $3.293386-2.9383723 .088322$

H $2.580196-0.5822303 .630749$

H $2.367169-0.2517971 .924094$

H 4.0754441 .3454383 .280858

H $4.528299-0.3046910 .371538$

H $4.848576-0.4068844 .632936$

H $6.289376-0.4849143 .645544$

H $5.285669-1.9331803 .860468$

H $6.684614-0.8948260 .177831$

H $6.085582-2.892003-0.716175$

H $4.674626-3.914529-0.667150$

H $5.470136-4.1317021 .378730$

H $1.016766-0.473096-0.360288$

H $\quad 0.2632530 .811855-3.056321$

H $2.8647191 .218283-0.371982$

H 1.4351061 .7608690 .536490

H $2.6039642 .921791-0.084258$

H $4.102083-2.784017-2.524072$

H $3.329234-1.703545-1.360210$

H $4.5136330 .914124-4.910735$

H $6.052397 \quad 0.744891-4.036480$

H $5.0609952 .224200-2.136477$

H $3.8916913 .234782-4.781466$

H $4.6537525 .313410-3.477811$
H $4.3015604 .506220-1.967825$

H $1.1631466 .358858-4.017425$

H $2.8803376 .378138-4.712391$

H $0.6944575 .182744-2.013440$

H $2.0364744 .648218-1.052467$

H $0.5306893 .067056-3.250385$

H $3.408899-0.392383-3.312590$

C $-5.331189-2.612820-1.769776$

O $-4.842223-1.255599-1.746642$

C $-5.951603-0.370328-1.524398$

C $-7.155872-1.246814-1.202249$

C $-6.836823-2.495323-2.004405$

H $-6.1560520 .115979-2.488394$

C $-5.5947640 .736159-0.519052$

H -7.217274 -1.480576-0.134461

H $-8.101136-0.780893-1.497756$

C $-7.632200-3.713209-1.567508$

H -7.037081 -2.294049 -3.065371

C -4.6958690 .3115260 .665835$

O $-6.7905361 .372924-0.081026$

H -5.028152 $1.496900-1.071076$

H -3.754651 -0.0728190 .258050$

O $-5.301726-0.7422331 .409578$

C -4.3787441 .4662691 .638579$

C $-3.349086 \quad 1.0944202 .723965$

H -4.0211052 .3324541 .072730$

O

H $-3.366608 \quad 1.881783 \quad 3.487327$

H -3.6791290 .1895663 .249264$

H -7.364766 -4.590359-2.164835

H $-7.450803-3.954343-0.514696$

H -8.705498 -3.535802 -1.691463

C $-4.591342-3.404767-2.850387$

H -7.2010140 .8016940 .590220$

H $-5.213474-1.5568140 .888139$

H -5.9322321 .1085612 .772938$

H -4.661376 -2.859793 -3.800557

H $-5.081236-4.374625-2.994661$

H -5.147398 -3.049909-0.780542

C -1.2791183 .2281552 .133059$

O -1.4751072 .0120601 .400416$

C -1.9136890 .9020382 .190608$

C -0.8707610 .6009743 .274639$

C -0.5406061 .8370054 .109926$

C $-0.208783 \quad 3.0305043 .212295$

H -2.2248263 .5417062 .591635$

C $-0.8655124 .314593 \quad 1.136641$

H $-1.9321850 .034707 \quad 1.520333$

O $-1.327319-0.4523054 .115356$

H 0.0466760 .2494182 .787332

H -1.3632962 .0795234 .791941$

O $\quad 0.583272 \quad 1.5527094 .946997$

H 0.7726942 .8777192 .746240

O -0.1229954 .2078354 .011587$

C -2.0061974 .6520520 .206881$

H -0.5770815 .2416951 .645076$

H -0.0043454 .0020490 .538142$

H $-0.648875-0.5955814 .797365$

H $1.342781 \quad 1.3545434 .373200$

H 0.5397474 .0417324 .703922

O $-1.9685863 .844159-0.890857$

O $-2.844620 \quad 5.512261 \quad 0.443000$

C $-3.0195444 .042977-1.840595$

H $-3.9954313 .907882-1.364498$

H $-2.9403755 .039631-2.284720$

$\mathrm{H}-2.9090733 .295438-2.630575$

SCF Energy (B3LYP/6-31G**//MMFF)= -3245.90392606

$01 \_52$

MM̄MF Geometry

C $-2.517045-3.493693-2.450560$

C $-1.567294-3.305330-1.521625$

C $-0.167922-2.853515-1.844350$

O $0.133149-1.695339-1.031733$

C $0.837530-3.958047-1.471754$

C $2.248775-3.619342-1.906718$

C $3.173466-3.030723-1.122645$

C $2.585570-3.992025-3.327324$ 
C $3.012680-2.5585280 .296968$

C $4.029250-3.1664161 .277098$

C $5.495996-2.8449870 .949844$

C $6.427080-3.2898962 .080004$

C $7.887392-2.8877581 .810273$

O $5.611033-1.4332490 .759123$

O $8.617967-3.027558 \quad 3.038493$

C $7.938913-1.3928021 .422056$

C $8.557713-3.826008 \quad 0.799343$

O $9.250197-1.0160191 .008653$

C $6.910096-1.0029680 .337660$

C 6.8666810 .5325290 .155277

O $7.290436-1.600660-0.905094$

C $-0.127321-0.464299-1.545130$

O $-0.660767-0.205724-2.611305$

C $0.3750200 .529307-0.567916$

C $0.156871 \quad 1.837914-0.764829$

C $0.582750 \quad 2.924145 \quad 0.122800$

C $1.507108 \quad 2.572146 \quad 1.258713$

C $5.6466031 .018606-0.642780$

C $5.7392772 .489216-1.007944$

C $4.4752424 .261404-2.222694$

O $6.7073193 .192783-0.732942$

C $3.8514035 .142789-1.188042$

C $2.7721585 .905016-1.420124$

C $2.1881546 .846536-0.407760$

C $0.7183386 .604039-0.158471$

C $-0.2134917 .460349-0.610980$

C $0.334176 \quad 5.4177990 .697101$

C $0.1135894 .167671-0.111038$

N $4.6122802 .916094-1.698980$

H -2.260806 -3.301215 -3.491127

H -1.781498 $-3.486766-0.472429$

H $-0.066036-2.602105-2.907693$

H $0.545575-4.907828-1.939902$

H $0.809967-4.147096-0.392025$

H $4.145834-2.819764-1.564779$

H $3.614919-3.729916-3.594573$

H $2.471979-5.071242-3.472898$

H $1.921873-3.474836-4.027290$

H $3.105904-1.4657090 .302117$

H $2.011354-2.7688750 .683054$

H $3.790811-2.7670192 .271590$

H $3.894081-4.2537841 .329722$

H $5.764747-3.3612100 .021488$

H $6.348668-4.3713692 .246372$

H $6.109583-2.8179893 .020494$

H $8.533791-3.9504263 .333757$

H $7.720691-0.8118412 .329186$

H $8.553228-4.857207 \quad 1.172946$

H $9.612230-3.5650230 .656372$

H $8.066635-3.827680-0.175893$

H $9.862152-1.267344 \quad 1.721603$

H 6.8288651 .0218201 .137279

H $7.7873870 .867223-0.339260$

H $6.969519-1.051821-1.637551$

H $\quad 0.907520 \quad 0.1520930 .298154$

H $-0.4062462 .145070-1.644996$

H 2.3994192 .0583890 .882853

H 1.0012781 .9180121 .977297

H 1.8678243 .4394571 .814813

H $5.542406 \quad 0.454675-1.575950$

H $4.7343450 .871165-0.052746$

H $3.8707254 .197011-3.132948$

H $5.4651374 .647938-2.486747$

H $4.3335625 .186378-0.214245$

H $2.3069455 .885255-2.403007$

H $2.3448667 .868230-0.777853$

H $2.7273916 .792244 \quad 0.546243$

H $-1.2680147 .305170-0.407122$

H $0.0559818 .331149-1.199099$

H $-0.606027 \quad 5.637379 \quad 1.219353$

H 1.0773245 .2958811 .488984

H $-0.563176 \quad 4.307769-0.955091$

H $3.8293182 .279142-1.816115$

C $-4.359780-4.249172-0.792882$

O $-4.426294-3.020771-0.041339$

C $-5.524963-3.105234 \quad 0.886937$
C $-6.091701-4.5162490 .760793$

C $-5.756477-4.856313-0.677512$

H $-6.284812-2.3950230 .542597$

C $-5.061630-2.7389372 .300721$

H -5.596984 -5.2156461 .445038$

H $-7.165182-4.5497790 .971611$

C $-5.819756-6.344507-0.974722$

H $-6.454094-4.323588-1.337661$

C $-4.468964-1.3197632 .431424$

$\begin{array}{llll}\text { O } & -4.051298 & -3.673664 & 2.701981\end{array}$

H -5.894779 -2.867392 3.002230

H $-3.513312-1.2706751 .897480$

O $-4.156319-1.115538 \quad 3.816257$

C $-5.397385-0.1879291 .953638$

C $-4.932684 \quad 1.227756 \quad 2.341664$

H $-5.519312-0.2511870 .867187$

O $-6.691540-0.3871312 .531900$

H -5.7054761 .9321242 .009900$

H $-4.928021 \quad 1.323232 \quad 3.434993$

H $-5.570945-6.546751-2.020993$

H $-5.121723-6.909127-0.347492$

H $-6.827093-6.730844-0.788117$

C $-3.934687-3.959658-2.234623$

H $-3.767187-3.4258203 .598533$

H $-4.995030-1.1134074 .308710$

H -7.2862090 .2863752 .159723$

H $-4.594201-3.190994-2.655275$

H $-4.068055-4.864151-2.839404$

H $-3.628022-4.900620-0.297593$

C $-4.2938932 .290449-0.430476$

$\begin{array}{llllllllll} & \text { O } & -3.469873 & 1.425802 & 0.360548\end{array}$

C -3.5606241 .6370701 .771840$

C $-3.153008 \quad 3.078903 \quad 2.108503$

C $-3.961258 \quad 4.0982041 .308633$

C $-3.9356853 .759378-0.181107$

H $-5.3490772 .115764-0.188396$

C $-4.074148 \quad 1.915585-1.899249$

H -2.8134320 .9780432 .230008$

$\begin{array}{llll}\text { O } & -3.312870 & 3.343763 & 3.498082\end{array}$

H -2.090551 $3.219571 \quad 1.876277$

H -4.9918244 .1698691 .675310$

$\begin{array}{lllll}\text { O } & -3.387808 & 5.396063 & 1.480724\end{array}$

H -2.942384 $3.979084-0.588905$

O $-4.8686604 .591887-0.867061$

C $-4.6109570 .535458-2.187271$

H $-4.5580102 .621085-2.584030$

H $-3.005678 \quad 1.909400-2.145960$

H -2.778838 $2.691153 \quad 3.982707$

H -3.3673525 .5787462 .435896$

H $-4.6283555 .514892-0.676116$

O $-5.9657810 .580926-2.333023$

O $-3.909013-0.464249-2.258389$

C $-6.597397-0.675488-2.596443$

H $-7.671833-0.501518-2.697391$

H $-6.220936-1.100933-3.531498$

H $-6.430871-1.364599-1.763763$

SCF Energy (B3LYP/6-31G**/MMFF)= -3245.91263544

01520

MM̄FF Geometry

C $4.676628 \quad 2.777078-0.674766$

C $5.7046552382997 \quad 0.093457$

C $5.809493 \quad 1.0382950 .782074$

$\begin{array}{lllll}\text { O } & 4.502929 & 0.426846 & 0.894055\end{array}$

C $6.7201510 .101814-0.029238$

C $7.004349-1.2101490 .676066$

C $6.430016-2.392004 \quad 0.374849$

C $8.035424-1.1348911 .773783$

C $5.390783-2.682275-0.673893$

C $4.062676-3.072620-0.024818$

C $2.962603-3.474672-1.018388$

C $2.597718-2.361542-2.002614$

C $1.409452-2.752554-2.895989$

O $1.815888-3.805515-0.224521$

O $0.938254-1.562170-3.546417$

C $0.257535-3.268425-2.010836$

C $1.828477-3.720303-4.009180$

O $-0.800910-3.798415-2.806535$ 
C $0.699563-4.315998-0.964311$

C $-0.449949-4.5628710 .040963$

O $1.021255-5.539733-1.625822$

C 3.7079330 .8509051 .916359

O 4.0196101 .5959722 .831563

C $2.351927 \quad 0.3093331 .678512$

C 1.3093580 .8284362 .343240

C -0.0978120 .4699282 .152456$

C $-0.402589-0.6910071 .245339$

C $-0.077289-5.5008851 .195333$

C $-1.017805-5.3188922 .369016$

C $-1.467117-3.808224 \quad 4.303189$

O $-1.945781-6.0957132 .580835$

C -2.491903 -2.788994 3.915637

C $-2.627144-1.6039244 .530683$

C $-3.713005-0.6196964 .204949$

C $-3.196640 \quad 0.783958 \quad 3.993445$

C -3.4040921 .7422494 .912818$

C -2.5341921 .1008882 .672900$

C $-1.037253 \quad 1.2068632 .779862$

N $-0.710802-4.2004293 .130011$

H $3.8635132 .084377-0.877284$

H 6.5261743 .0731050 .271589

H 6.2255011 .1903891 .785565

H $7.6796900 .596165-0.233207$

H $6.274418-0.077595-1.013190$

H $6.731417-3.2665290 .950879$

H $8.245193-2.1146162 .216297$

H $8.979648-0.7436561 .381123$

H $7.693958-0.4777522 .579291$

H $5.245424-1.829921-1.339208$

H $5.753359-3.507040-1.298884$

H $4.221683-3.9118830 .666830$

H $3.700058-2.2541750 .607536$

H $3.297855-4.370675-1.553792$

H $3.453168-2.085601-2.629557$

H $2.328952-1.454600-1.443721$

H $1.675898-1.188090-4.058364$

H $-0.158603-2.401294-1.479954$

H $2.605925-3.268638-4.636958$

H $0.990723-3.936551-4.681301$

H $2.219619-4.666232-3.628798$

H - $1.041419-3.121392-3.461639$

H $-0.770418-3.599177 \quad 0.454369$

H $-1.318811-4.990189-0.475743$

H $0.959302-6.268552-0.988427$

H $2.237337-0.4537620 .918900$

H 1.4960041 .6227123 .064948

H $\quad 0.151307-1.5800901 .565360$

H $-0.117631-0.4569630 .214404$

H $-1.456849-0.975047 \quad 1.235153$

H $-0.124846-6.5479990 .876196$

H $0.944818-5.3258861 .549834$

H $-0.752959-3.4194455 .035828$

H -1.962952 -4.686417 4.729233

H -3.183872 -3.0576083 .120716$

H -1.957839 -1.3506235 .349805$

H -4.429152 -0.632967 5.037152

H -4.282662 -0.9308203 .320336$

H -3.069581 2.761279 4.748795

H -3.910932 1.5297445 .848213

H -2.902284 2.0707202 .312072

H $-2.856700 \quad 0.383763 \quad 1.914573$

H $-0.704746 \quad 2.0326823 .410877$

H $\quad 0.050826-3.5957072 .836957$

C $3.2558014 .862211-0.880849$

O $2.103098 \quad 4.103610-1.301779$

C $1.1413534 .094928-0.229526$

C $1.564574 \quad 5.216510 \quad 0.710519$

C 3.0762555 .1199400 .616145

H $1.261415 \quad 3.136962 \quad 0.291123$

C $-0.2950384 .192208-0.764888$

H 1.2260956 .1960380 .355755

H 1.1872845 .0717661 .727365

C 3.8030896 .3541201 .119577

H 3.4011514 .2533261 .205256

C $-0.7142172 .940887-1.573120$

O $-0.427623 \quad 5.356107-1.576825$
H -0.9838334 .2958940 .082850$

H $-0.5403842 .060761-0.944580$

O $0.0972202 .795722-2.738316$

C $-2.1957752 .952380-2.010454$

C -2.626556 $1.698028-2.796304$

H $-2.8300223 .068539-1.126546$

O $-2.454937 \quad 4.081397-2.847914$

H $-3.6192971 .889627-3.220906$

H $-1.969787 \quad 1.574233-3.665959$

H $4.8861846 .237737 \quad 1.012293$

H 3.5032567 .2507280 .566912

H 3.5841816 .5239622 .178943

C $4.5443714 .140084-1.292284$

H $-0.414796 \quad 6.131980-0.992301$

H $1.0213472 .714847-2.448422$

H $-1.8317244 .041789-3.593690$

H $5.4102574 .765700-1.046762$

H $4.5401804 .003081-2.380687$

H $3.2073405 .811017-1.431125$

C $-4.9078900 .548524-1.109937$

O $-3.5052120 .518868-0.814310$

C -2.646090 $0.407489-1.954542$

C $-2.982942-0.861879-2.748220$

C $-4.460621-0.930316-3.117256$

C $-5.326872-0.712833-1.878200$

H $-5.1408521 .440014-1.704131$

C -5.6643690 .6440110 .225061$

H $-1.6335120 .264298-1.563125$

O $-2.201196-0.946609-3.934830$

H -2.740106 -1.741573 -2.138387

H $-4.710884-0.211431-3.906209$

O $-4.751549-2.225994-3.646452$

H $-5.247028-1.593091-1.227939$

O $-6.693628-0.611989-2.274788$

C -5.5743352 .0201540 .850301$

H -6.7299000 .4352810 .079468$

H $-5.247016-0.0772010 .937482$

H -1.266389 -0.875762 -3.675856

H $-4.162522-2.366306-4.407577$

H $-6.7806310 .165422-2.852559$

\begin{tabular}{llll}
\hline & -6.138838 & 1.977317 & 2.094014
\end{tabular}

$\begin{array}{lllll}\text { O } & -5.092767 & 3.010302 & 0.318381\end{array}$

C $-6.155466 \quad 3.2190972 .805177$

H $-6.534732 \quad 3.028645 \quad 3.812744$

H $-5.1454813 .632713 \quad 2.883444$

H -6.823537 3.9264022 .304940

SCF Energy (B3LYP/6-31G**//MMFF)= -3245.91416740

01521

MM̄FF Geometry

C $0.532821 \quad 0.191068 \quad 3.660881$

C $-0.201257 \quad 1.230694 \quad 4.086009$

C $-0.5725372 .380917 \quad 3.189593$

$\begin{array}{llll}\text { O } & -2.004836 & 2.314816 & 2.968954\end{array}$

C $-0.271543 \quad 3.765143 \quad 3.792405$

C 1.1019094 .2760453 .413865

C $1.3414545 .091246 \quad 2.366718$

C 2.2297753 .8273614 .303937

C $0.338721 \quad 5.680750 \quad 1.410924$

C $-0.224082 \quad 4.721168 \quad 0.357169$

C $0.8127273 .959565-0.482230$

C $1.6186014 .892407-1.389147$

C $2.557648 \quad 4.119355-2.328475$

O $0.0821593 .014815-1.280690$

O $3.0003675 .023741-3.352283$

C $1.7628972 .998222-3.029002$

C $3.8186103 .637858-1.603375$

O $2.6297982 .159266-3.789526$

C $0.9113492 .142921-2.061553$

C $0.0008511 .189934-2.875126$

$\begin{array}{lllll}\text { O } & 1.779768 & 1.363908 & -1.236309\end{array}$

C $-2.421126 \quad 1.5825051 .900472$

$\begin{array}{llll}\text { O } & -1.720810 & 0.945413 & 1.129425\end{array}$

C $-3.891734 \quad 1.6939861 .808372$

C $-4.471911 \quad 1.4383180 .626943$

$\begin{array}{llll}\text { C } & -5.903340 & 1.450848 & 0.329793\end{array}$

C $-6.858942 \quad 1.600026 \quad 1.482744$

C $-1.0645280 .465710-2.042654$ 
C $-1.874701-0.519933-2.869174$ C $-3.771243-2.131653-2.646721$ O $-1.682902-0.712378-4.066636$ C -5.029151-1.428545-3.050969 C $-6.227967-1.665561-2.495843$ C -7.494448 -0.965995-2.905044 C $-8.137238-0.204854-1.764476$ C $-9.133210-0.750484-1.046245$ C $-7.6830391 .215956-1.507595$ C $-6.2878121 .283602-0.952397$ $\mathrm{N}-2.838366-1.161676-2.101817$ H $\quad 0.9172900 .1918092 .643397$ H $-0.6157321 .221575 \quad 5.090737$ H -0.0585452 .2954292 .223555$ H -0.3752423 .7505284 .885399$ H - 1.0358124 .4784703 .459382 H 2.3706865 .3925032 .176242 H $3.1965804 .231346 \quad 3.985274$ H 2.0553724 .1620505 .331844 H 2.3140342 .7374134 .305121 H $-0.4926856 .119667 \quad 1.975995$ H 0.8135326 .5315090 .906333 H $-0.888514 \quad 3.9968650 .840261$ H -0.884768 5.286168 -0.314539 H $1.473867 \quad 3.4037590 .190659$ H $2.1907985 .619794-0.801277$ H $0.9267415 .483870-2.005199$ H $3.4441345 .772935-2.919004$ H $1.0864363 .478704-3.749805$ H $4.3693844 .488253-1.183841$ H $4.5103333 .144484-2.294911$ H $3.5992472 .951073-0.785608$ H $3.1331922 .732873-4.392291$ H $-0.524476 \quad 1.764491-3.649413$ H $0.6178440 .442949-3.389444$ H $1.2821710 .618633-0.866317$ H $-4.444107 \quad 1.9788262 .695665$ H -3.831485 $1.191557-0.218565$ H $-6.7632782 .593579 \quad 1.933966$ H -6.6579050 .8450782 .251271$ H -7.9055831 .4745611 .195763$ H $-0.604480-0.092231-1.221344$ H - $-1.7598361 .198607-1.619205$ H -3.950072 -2.883001-1.871204 H -3.325081 -2.626272 -3.515181 H $-4.947946-0.692162-3.847222$ H $-6.310826-2.411667-1.708727$ H $-8.188350-1.727561-3.284679$ H -7.321236 $-0.283268-3.746179$ H -9.617854 - $0.203525-0.244394$ H -9.482219-1.759241 - 1.241296 H -8.391434 $1.742577-0.858776$ H -7.708227 $1.774892-2.452000$ H $-5.5137601 .188108-1.714289$ H -2.953202 -0.893869 -1.128499 C $-0.060961-2.1986283 .970892$ O $0.353146-2.5814422 .645588$ C $0.126071-3.9966732 .482331$ C $-0.489517-4.4900613 .789869$ C $0.044866-3.4797874 .790288$ H $1.112906-4.4606042 .377231$ C $-0.709335-4.246171 \quad 1.217273$ H - $1.583912-4.4605313 .768568$ H $-0.193489-5.5182624 .020776$ C $-0.730888-3.4544506 .095434$ H $1.098964-3.7089294 .996237$ C $-0.068765-3.668436-0.063646$ O $-1.984460-3.6050841 .355593$ H $-0.904049-5.3174201 .088413$ H $-0.012908-2.5771490 .015792$ O $-0.939901-3.949848-1.166107$ C $1.315274-4.253881-0.394231$ C $1.936623-3.717046-1.697640$ H $2.008768-4.0609080 .428649$ O $1.214168-5.675953-0.508078$ H $2.854385-4.286586-1.889333$ H $1.282930-3.950064-2.547485$ H $-0.314339-2.7130766 .784319$
H -1.784975 -3.206740 5.931104

H $-0.687771-4.4317626 .586951$

C $0.803428-1.0421924 .475897$

H $-2.535072-4.1603741 .932041$

H -1.811023 -3.578587 -0.945030

H $0.576819-5.868047-1.217462$

H $1.868125-1.2867594 .381455$

H $0.601132-0.8492235 .535330$

H -1.110781-1.880634 3.900206

C $4.263964-2.281651-0.362389$

O $2.912792-1.818822-0.460381$

C $2.241809-2.205991-1.664394$

C $3.007029-1.682816-2.888270$

C $4.485533-2.068555-2.857775$

C $5.110593-1.713316-1.506651$

H $4.271477-3.377061-0.424583$

C $4.785318-1.957013 \quad 1.040352$

H $1.273489-1.693562-1.653878$

O $2.422346-2.158707-4.095758$

H $2.944342-0.588161-2.911744$

H $4.627271-3.131125-3.087402$

O $5.177664-1.342689-3.877075$

H $5.194296-0.621744-1.436843$

O $6.427318-2.255752-1.464573$

C $4.988565-0.4830291 .291030$

H $4.092213-2.3332201 .803621$

H $5.742740-2.4593491 .222269$

H $1.491386-1.877548-4.100153$

H $4.744166-1.548787-4.723094$

H $6.873544-1.887793-0.683249$

$\begin{array}{lllll}\text { O } & 3.797481 & 0.112469 & 1.578839\end{array}$

$\begin{array}{lllll}\text { O } & 6.081139 & 0.067322 & 1.243000\end{array}$

C 3.8557171 .5104791 .874896

H 4.5261752 .0385541 .190936

H 2.8498951 .9223801 .762403

H 4.1837041 .6479182 .908743

SCF Energy (B3LYP/6-31G**//MMFF)= -3245.91142173

$01 \_522$

MM̄FF Geometry

C $-3.5256602 .942470-0.887720$

C $-3.100892 \quad 3.293317 \quad 0.336529$

C -3.550142 2.6957581 .650890

O $-4.402482 \quad 1.5494991 .415429$

C -2.361224 2.2151002 .503197

C $-1.576503 \quad 3.3254893 .167065$

C -0.3695993 .7653892 .760425$

C -2.2174993 .9125664 .398495$

C $0.4222283 .324873 \quad 1.560520$

C 1.7182242 .5990241 .943523

C 1.4883721 .2003942 .538489

C 2.8254500 .5520712 .896556

C $2.651477-0.8882803 .398515$

$\begin{array}{llll}\text { O } & 0.816302 & 0.407118 & 1.557270\end{array}$

O $3.946304-1.5069923 .410007$

C $1.781337-1.6637932 .386214$

C $2.141473-0.9290644 .843511$

O $1.485132-2.9732892 .867094$

C $0.485074-0.9210511 .982583$

C $-0.184548-1.6438820 .788930$

O $-0.410106-0.9146143 .095145$

C $-5.489815 \quad 1.396694 \quad 2.214844$

$\begin{array}{lllll}\text { O } & -5.847453 & 2.137755 & 3.119774\end{array}$

C $-6.2794650 .211208 \quad 1.817447$

C $-5.719009-0.8958951 .306130$

C $-6.430394-2.1137020 .906456$

C $-7.934173-2.039493 \quad 0.835077$

C $-1.416180-0.9192940 .228324$

C $-1.879344-1.502083-1.094842$

C $-3.553814-1.103243-2.899175$

O $-1.408248-2.526007-1.582066$

C $-4.756133-1.931198-2.573541$

C $-4.799168-3.261976-2.734764$

C $-5.977295-4.121383-2.374985$

C $-5.688326-5.001292-1.177930$

C $-5.027775-6.163167-1.319545$

C $-6.221068-4.5682340 .171568$

C $-5.713046-3.2174040 .603730$ 
N -2.893558 -0.739403 -1.659896 H -4.280022 2.164936 -0.981625 H -2.391722 4.1111930 .425899 H -4.1121143 .4747772 .179690$ H $-1.711677 \quad 1.5762991 .897328$ H -2.7266191 .5393533 .288966$ H 0.1076134 .5556183 .339058 H -1.589371 4.6748754 .871574 H -3.1731084 .3834064 .148708$ H -2.399024 3.1292485 .141790 H -0.1672352 .7119590 .873053$ H 0.6860594 .2276390 .994911 H $2.3226722 .504021 \quad 1.032533$ H 2.2945593 .2129812 .646902 H $\quad 0.856867 \quad 1.2887343 .429286$ H 3.3678731 .1551673 .635109 H 3.4617750 .5281552 .001815 H $4.530458-0.9722483 .974600$ H $2.391059-1.8073841 .484546$ H $2.832883-0.3971275 .508316$ H $2.100246-1.9575185 .218907$ H $1.155226-0.4761854 .963771$ H $0.788784-2.8903873 .540588$ H $\quad 0.550620-1.744609-0.019704$ H $-0.485285-2.6573441 .083074$ H -1.311511 -0.7546732 .775753$ H -7.342359 0.2776552 .024445 H $-4.638325-0.9263011 .184785$ H -8.249242 -1.1856040 .224308$ H -8.360551 -1.930246 1.838156 H $-8.397212-2.918255 \quad 0.382590$ H -2.249999 -0.9914130 .933642$ H $-1.197511 \quad 0.1408210 .065568$ H -2.849251 -1.637902 -3.545081 H -3.855230 -0.180938 -3.404916 H $-5.622112-1.409588-2.174346$ H -3.925663 -3.774103 -3.132834 H $-6.225685-4.744802-3.243786$ H -6.872472 -3.515494 -2.187989 H $-4.834880-6.812848-0.472365$ H -4.664002 -6.489035 -2.288525 H $-5.923980-5.2879890 .945568$ H -7.312178 -4.6162110 .134295$ H -4.626582 -3.1685930 .691557$ H $-3.2797980 .034822-1.128644$ C -1.645624 $4.065789-2.268870$ O $-0.7335182 .946466-2.238992$ C $0.2092213 .086507-3.317517$ C $0.1313774 .546903-3.737940$ C $-1.3505264 .814313-3.571568$ H $-0.1566272 .457968-4.140267$ C $1.6151122 .619384-2.915657$ H $0.7139615 .190031-3.067646$ H $0.4855814 .706888-4.760681$ C -1.700287 $6.291898-3.526035$ H -1.888877 4.345455-4.406334 C $1.6691371 .107109-2.613560$ O $2.045373 \quad 3.380379-1.789432$ H $2.2992802 .840208-3.743981$ H $1.2104930 .574742-3.455831$ O $0.884210 \quad 0.808344-1.464715$ C $3.0948180 .550168-2.423172$ C $3.075471-0.968888-2.164760$ H $3.6752780 .771696-3.326667$ O $3.7228731 .208695-1.329440$ H $2.738143-1.153666-1.139532$ H $2.344833-1.426785-2.841468$ H $-2.7761236 .433893-3.383396$ H -1.181650 $6.802295-2.707516$ H -1.415498 $6.783253-4.462012$ C $-3.0870453 .548048-2.196823$ H $2.9517263 .103173-1.573873$ H $1.2842921 .271093-0.709090$ H $4.6460230 .906163-1.300151$ H -3.206508 2.766234 -2.959042 H -3.793674 4.348201 -2.445992 H -1.399703 $4.703866-1.413007$ C $5.350755-1.563127-0.145351$
O $5.466786-1.150517-1.512855$

C $4.450974-1.636754-2.394591$

C $4.413674-3.171515-2.364929$

C $4.238930-3.697223-0.944220$

C $5.304156-3.093088-0.032726$

H $4.447000-1.1229060 .291030$

C $6.570227-1.0126530 .608670$

H $4.765580-1.348708-3.406133$

O $3.370333-3.651695-3.205215$

H $5.357753-3.556535-2.772456$

H $3.231694-3.500767-0.557963$

O $4.408127-5.116258-0.937984$

H $\quad 6.283686-3.517985-0.288397$

O $5.023370-3.4579291 .316597$

C $6.4447400 .464706 \quad 0.894083$

H $6.692711-1.5068981 .579768$

H $7.498668-1.1769200 .048327$

H $3.454282-4.618557-3.257375$

H $3.654783-5.503027-1.415128$

H $4.970612-4.4283811 .351182$

O $6.719940 \quad 1.187914-0.227971$

O $6.142800 \quad 0.9210391 .989413$

C $6.6380082 .609246-0.078658$

H 5.6425052 .9008730 .270423

H 7.4085992 .9585580 .614889

H $6.8120813 .063806-1.057514$

SCF Energy $(B 3 L Y P / 6-31 G * * / / M M F F)=-3245.91608244$

01523

MM̄FF Geometry

C $0.0445313 .329938-1.092129$

C $1.0723333 .819674-0.382619$

C 1.6465163 .1171650 .823398

O 3.0305032 .7254470 .589800

C 1.5059313 .8889782 .153007

C 0.0977304 .3382832 .483777

C -0.8778433 .5315032 .944550$

C -0.1682725 .8083652 .284394$

C -0.7854742 .0601853 .237291$

C $-1.643411 \quad 1.2459012 .258970$

C $-1.602817-0.2739312 .491808$

C $-2.346114-0.6814583 .764074$

C $-2.237414-2.1891604 .029841$

O $-0.235652-0.6808392 .554243$

O $-2.689013-2.4306835 .371736$

C $-0.749744-2.5963013 .970974$

C -3.156921 -3.002068 3.109767

O $-0.639797-4.0131784 .097977$

C $-0.021299-2.0916322 .699914$

C $1.508073-2.3012742 .837576$

O $-0.507154-2.8174501 .574473$

C 4.0004663 .6510780 .385033

O $3.8626094 .866822 \quad 0.359547$

C 5.3294843 .0274230 .180148

C 5.5669761 .7058930 .179844

C 6.874951 1.069517 -0.019009

C $8.0598011 .968612-0.263989$

C $2.341738-1.7513141 .669148$

C $2.573725-2.7519250 .548312$

C $3.720409-2.929276-1.662157$

O $2.213594-3.9241770 .602049$

C $5.168553-3.234796-1.450349$

C $6.155780-2.681971-2.170477$

C $7.611595-2.938940-1.914607$

C $8.363930-1.680618-1.537504$

C $9.228040-1.114050-2.396483$

C $8.187924-1.134521-0.136218$

C $6.958305-0.2775660 .015471$

N $3.252295-2.174595-0.514573$

H $-0.3844142 .373447-0.809505$

H $1.4947994 .780665-0.661636$

H 1.1260602 .1596130 .946645

H 2.1663414 .7645132 .159922

H 1.9003063 .2670422 .967420

H $-1.8536503 .969718 \quad 3.150327$

H -1.210111 6.0755632 .490891

H 0.4651776 .3997582 .953738

H 0.0484656 .1041141 .253457 
H $\quad 0.251870 \quad 1.714256 \quad 3.207821$ H -1.132613 1.9059554 .264719 H -2.6832591 .5939792 .286308$ H $-1.274588 \quad 1.438917 \quad 1.244236$ H -2.071140 -0.747723 1.621136 H $-3.397983-0.3736823 .714333$ H -1.922537 -0.1582094 .630849$ H -3.606459-2.115494 5.440334 H $-0.262592-2.1624154 .855534$ H -4.201645 -2.697954 3.246942 H -3.120576 -4.068639 3.357647 H -2.917724 -2.886083 2.051030 H $0.286999-4.2250114 .297463$ H $1.839351-1.7690253 .739368$ H $1.738092-3.3622632 .982755$ H $\quad 0.109258-2.7002450 .835794$ H 6.1300523 .7443470 .024298 H 4.7370561 .0200360 .340052 H 8.2296442 .6239760 .597400 H $7.8953152 .588504-1.152626$ H $8.9916821 .426503-0.435815$ H $1.898622-0.8393551 .254562$ H $3.335780-1.4880742 .053816$ H $3.554952-2.312716-2.550713$ H $3.148818-3.856194-1.767800$ H $5.414629-3.924737-0.646555$ H $5.909125-1.992414-2.974558$ H $8.037279-3.382212-2.824018$ H $7.752869-3.689290-1.126657$ H $9.799104-0.232391-2.126043$ H $9.382840-1.517787-3.391600$ H $9.086516-0.5924050 .179091$ H $8.118729-1.9704390 .572187$ H $6.047257-0.8455230 .201017$ H $3.552031-1.206820-0.438245$ C $-2.0807474 .286445-2.086838$ O $-2.8093823 .044862-1.974146$ C $-3.7865093 .185668-0.925953$ C $-3.8894594 .681535-0.643376$ C $-2.4577825 .121998-0.862887$ H $-3.3720672 .688329-0.041709$ C $-5.1155552 .536270-1.318437$ H $-4.5544185 .185980-1.354024$ H -4.2536124 .8886910 .367549$ C $-2.2956636 .618101-1.061418$ H -1.8759044 .8162540 .013253$ C $-5.0465761 .036341-1.669021$ O $-5.6521313 .217269-2.461292$ H -5.844132 2.690269-0.513594 H $-4.5550690 .897841-2.639130$ O $\quad-6.3988900 .585178-1.835714$ C $-4.3637540 .142485-0.615567$ C $-4.643065-1.365307-0.764916$ H $-3.2830740 .317632-0.637453$ O H -4.119993 -1.878389 0.050703 H -5.706922 -1.547478 -0.566102 H -1.245119 $6.876305-1.228266$ H -2.872061 $6.974324-1.921717$ H $-2.6409807 .162695-0.176556$ C $-0.5839424 .015428-2.271462$ H $-4.9902973 .161890-3.172239$ H $-6.8092221 .145848-2.516608$ $\begin{array}{llll}\text { H } & -5.777737 & 0.410318 & 0.714934\end{array}$ H $-0.0671144 .956993-2.491050$ H $-0.4470533 .359849-3.140178$ H $-2.4544884 .769096-2.999510$ C $-1.979388-2.627329-1.616967$ O $-2.860455-1.829070-2.416962$ C $-4.253091-1.976039-2.124907$ C $-4.672685-3.444989-2.284351$ C $-3.817394-4.367078-1.423472$ C $-2.338178-4.118182-1.700631$ H -2.028729 -2.286292 -0.577389 C $-0.552142-2.407985-2.144072$ H $-4.788383-1.414994-2.899302$ O $-6.043438-3.627111-1.945545$ H $-4.558952-3.744805-3.334275$
H $-4.044333-4.254170-0.356981$

O $-4.110567-5.726428-1.754661$

H -2.085848 -4.509804 -2.694787

O $-1.556433-4.840067-0.750097$

C $-0.010072-1.032697-1.827400$

H $0.142988-3.130330-1.702581$

H $-0.549055-2.526786-3.234716$

H $-6.565740-3.034225-2.512505$

H $-5.063629-5.858760-1.612157$

H -1.818949 -5.774793 -0.807742

O $1.089191-0.808177-2.606434$

O $-0.467811-0.270044-0.989036$

C $1.7697420 .433586-2.401341$

H $2.7626490 .354617-2.852123$

H $1.2204831 .234931-2.901277$

H $1.8911390 .646787-1.335309$

SCF Energy (B3LYP/6-31G**//MMFF) $=-3245.91722565$

01524

MM̄FF Geometry

C $-0.6479062 .377816-1.843453$

C $0.5802012 .916500-1.783366$

C $1.5374512 .705269-0.638900$

O $2.6932161 .979896-1.137859$

C $2.0852534 .028352-0.071547$

C 1.0789524 .8858250 .662926

C 0.8841164 .8613971 .995537

C $0.3338925 .889135-0.179813$

C 1.5668433 .9969863 .020036

C 0.5648323 .3511983 .988974

C -0.1245472 .1084413 .405686$

C -1.2789141 .6729364 .311336$

C $-1.9090370 .354156 \quad 3.846248$

O $0.861478 \quad 1.0729193 .333482$

O $-2.756457-0.1274854 .900216$

C $-0.789946-0.687626 \quad 3.653876$

C -2.8078180 .5485502 .621296$

O $-1.307986-1.8926113 .092522$

C $0.393562-0.1729272 .801787$

C $1.564028-1.1828582 .883410$

O $-\begin{array}{llll}0.033818 & -0.037231 & 1.448649\end{array}$

C $2.5488430 .658426-1.416396$

O $1.533855-0.009996-1.282207$

C $3.7885100 .090952-1.990442$

C $5.0040190 .605282-1.749833$

C $6.2708320 .116791-2.297158$

C $6.216367-0.962972-3.345720$

C $2.850497-0.7237172 .183553$

C $4.027730-1.6320622 .494025$

C $6.423076-1.9604341 .970879$

O $3.950384-2.6084323 .235102$

C $7.546523-1.1867521 .360294$

C $8.358889-1.6935210 .420827$

C $9.516992-0.952523-0.185165$

C $9.424382-0.877603-1.692465$

C $9.928824-1.859106-2.459795$

C $8.8227220 .360390-2.316973$

C $7.4228190 .662732-1.855021$

N $5.186318-1.2130321 .851530$

H $-0.9962011 .748585-1.028256$

H $0.9359663 .521951-2.613869$

H 1.0844002 .1253190 .173404

H $2.5452144 .616619-0.878428$

H 2.9351053 .8017210 .585716

H $\quad 0.1677905 .5679602 .414497$

H $-0.319654 \quad 6.5333380 .418335$

H $1.0394206 .539809-0.707163$

H $-0.2939045 .388637-0.918793$

H 2.1984313 .2279082 .566149

H 2.2305524 .6491333 .601072

H 1.1187493 .0568014 .890267

H $-0.1832424 .091670 \quad 4.298708$

H -0.4923172 .3323502 .398021$

H -2.0406112 .4600914 .375001$

H $-0.906146 \quad 1.534887 \quad 5.336249$

H -3.4235110 .5559205 .084464$

H -0.411048 -0.9491824 .651897$

H -3.6228651 .2456222 .849553$ 
H -3.288603 -0.3939302 .339933$ H -2.2759380 .9389361 .751143$ H -2.038021 -2.1871723 .663519$ H $1.798550-1.3599283 .941460$ H $1.261469-2.1449812 .450649$ H $0.728578-0.1406090 .858737$ H $3.647788-0.796305-2.598881$ H $5.087308 \quad 1.465754-1.087750$ H $5.593479-0.649529-4.190895$ H $5.801613-1.885769-2.925286$ H $7.196743-1.214153-3.757480$ H $2.709259-0.7244341 .099717$ H 3.1209210 .2903062 .498492 H $6.272766-2.9328631 .489309$ H $6.619615-2.1367513 .033878$ H $7.712141-0.1747591 .721767$ H $8.200826-2.7154280 .082176$ H $10.438599-1.4760790 .101427$ H $9.612798 \quad 0.057576 \quad 0.232148$ H $9.894198-1.806230-3.542852$ H $10.385954-2.739690-2.020179$ H $8.8451230 .308195-3.412702$ H $9.4601031 .218137-2.064309$ H $7.3750001 .433742-1.085457$ H $5.116232-0.4914611 .139145$ C $-2.9999592 .963858-2.656227$ O $-3.6794131 .879394-1.998419$ C $-4.6322972 .434514-1.069168$ C $-4.5855843 .952125-1.246929$ C $-3.1670914 .170676-1.738872$ H $-4.2665632 .188573-0.065472$ C -6.017651 $1.812018-1.288916$ H $-5.3063104 .298474-1.996471$ H $-4.8018254 .481623-0.313761$ C -2.962151 5.505679-2.432634 H -2.494098 4.101825 -0.875432 C $-6.0342590 .269470-1.260495$ O $-6.502228 \quad 2.219913-2.575559$ H $-6.7213462 .218574-0.552819$ H -5.522642 -0.110677 -2.151604 O $-7.399037-0.152085-1.381308$ C $-5.426471-0.3570230 .004966$ C $-5.596518-1.8838580 .107598$ H $-4.361646-0.1092830 .056961$ O $-6.054044 \quad 0.2334021 .146968$ H $-5.136420-2.2048231 .050656$ H $-6.660790-2.1263690 .221703$ H - $1.9368525 .600345-2.803164$ H -3.639179 $5.623166-3.285448$ H -3.149419 6.331718-1.738806 C $-1.5695802 .556612-3.018043$ H -7.378863 $1.816315-2.696265$ H -7.865716 $0.132302-0.576525$ H -5.653875 -0.1591491 .941042$ H -1.155897 $3.296726-3.713635$ H $-1.6045081 .596907-3.549111$ H -3.545431 $3.131911-3.595035$ C $-2.678956-2.650880-0.418355$ O $-3.664419-2.268007-1.380807$ C $-4.999534-2.678167-1.070743$ C $-5.063913-4.202886-0.898209$ C $-4.041013-4.7096060 .116913$ C $-2.649748-4.171098-0.216263$ H $-2.915853-2.1652970 .536254$ C $-1.323344-2.076819-0.851675$ H $-5.605750-2.435940-1.952105$ O $-6.375772-4.595248-0.507608$ H -4.862480 -4.676988 -1.867877 H $-4.338500-4.4512731 .139646$ O $-4.017183-6.1375050 .055896$ H $-2.289108-4.677654-1.118912$ O $-1.747847-4.5090890 .835042$ C $-0.796401-2.652798-2.149360$ H $-1.402798-0.993503-0.999216$ H $-0.589075-2.260710-0.059191$ H $-6.368753-5.561405-0.396063$ H $-3.328053-6.441700 \quad 0.671290$ H $-2.041219-4.0496341 .640514$
O $0.560489-2.780117-2.071022$

O $-1.486107-2.926942-3.122416$

C $1.203340-3.280709-3.247055$

H $1.037841-2.599693-4.087222$

H $2.277012-3.338072-3.048956$

H $0.835226-4.283830-3.482672$

SCF Energy (B3LYP/6-31G**//MMFF)= -3245.91921736

01525

MM̄FF Geometry

C $-1.9960990 .635518 \quad 3.226174$

C $-2.242256-0.6508682 .933631$

C $-1.203471-1.7392852 .775071$

O $0.121661-1.1569812 .838056$

C $-1.302720-2.8165653 .872897$

C $-2.548961-3.6709553 .833739$

C $-2.710678-4.7384593 .027991$

C $-3.617011-3.3094984 .833422$

C $-1.752945-5.2729021 .995053$

C $-2.449185-5.532156 \quad 0.650453$

C $-2.762711-4.243678-0.129815$

C $-3.932659-4.482744-1.086354$

C $-4.229008-3.245500-1.940013$

O $-1.586588-3.875557-0.867306$

O $-5.131541-3.636915-2.985426$

C $-2.919776-2.800660-2.613876$

C $-4.937566-2.145666-1.141589$

O $-3.105739-1.578698-3.325806$

C $-1.717946-2.674048-1.641576$

C $-0.433839-2.483282-2.485691$

O $-1.908754-1.536001-0.803260$

C $1.139892-1.8234302 .234032$

O $1.070986-2.8743291 .618585$

C $2.375866-1.0450312 .463641$

C $3.548326-1.5229622 .023345$

C $4.850484-0.8698342 .155989$

C 4.8878640 .5200882 .734570

C $0.888066-2.451896-1.708239$

C $2.069923-2.316587-2.658135$

C $4.497973-1.908441-2.731237$

O $1.950354-2.332150-3.881608$

C $5.694463-2.079002-1.854159$

C $6.563880-1.089746-1.596379$

C $7.816225-1.254554-0.782021$

C $7.810547-0.407447 \quad 0.470627$

C 8.2439650 .8641140 .447356

C $7.373579-1.0578641 .763163$

C $5.946219-1.5291951 .728135$

N $3.281520-2.185004-1.990314$

H $-0.9725710 .969780 \quad 3.376717$

H $-3.275218-0.9545162 .778400$

H -1.341321 -2.183300 1.783892

H $-1.208024-2.3412374 .859475$

H $-0.424531-3.474123 \quad 3.825719$

H -3.632709 -5.3111523 .124577$

H $-4.485182-3.9746224 .773662$

H -3.219752 -3.3775905 .851652$

H $-3.975174-2.2893774 .669667$

H $-0.890858-4.6191921 .845487$

H $-1.365850-6.2279022 .370982$

H $-1.793909-6.1715510 .045049$

H $-3.369468-6.100617 \quad 0.836023$

H $-3.014707-3.4400980 .569403$

H $-4.831274-4.786305-0.534422$

H $-3.693468-5.321511-1.755307$

H $-5.938458-3.985948-2.569478$

H $-2.671501-3.560337-3.368814$

H $-5.879196-2.522085-0.724249$

H $-5.212661-1.305220-1.788360$

H $-4.339529-1.761899-0.312278$

H $-3.858207-1.705433-3.928865$

H $-0.369501-3.305129-3.211577$

H $-0.510199-1.550424-3.059753$

H $-1.047737-1.241576-0.467885$

H $2.288941-0.1019762 .990625$

H $3.559756-2.4868981 .516126$

H 4.5539420 .5125893 .777806

H 4.2393381 .1920252 .161827 
H 5.8838160 .9691052 .719200 H $0.909278-1.602959-1.017743$ H $\quad 1.016375-3.377335-1.136974$ H $4.417749-0.892510-3.132102$ H $4.556659-2.596718-3.581374$ H $5.874139-3.070434-1.446034$ H $6.386995-0.105197-2.024302$ H $8.668785-0.973158-1.414303$ H $7.986681-2.305976-0.518761$ H 8.2670101 .4711711 .346222 H $8.5823311 .326904-0.473935$ H $7.545020-0.3959892 .619752$ H $8.015103-1.9281191 .954179$ H $5.823181-2.5285991 .309515$ H $3.263669-1.983772-0.994296$ C -2.994239 2.715141 2.266025 O $-3.8659142 .293593 \quad 1.192600$ C -4.5378793 .4509450 .673589$ C $-3.857130 \quad 4.6643551 .294289$ C -3.4685824 .1143552 .652853$ H $-5.5617953 .412511 \quad 1.071535$ C $-4.6402033 .456458-0.856583$ H $-2.963800 \quad 4.9550870 .730185$ H $-4.519272 \quad 5.533167 \quad 1.357607$ C -2.415974 $4.949646 \quad 3.361780$ H -4.3663784 .0480463 .281828$ C $-3.3215963 .387178-1.662614$ O $-5.3314934 .659681-1.220125$ H $-5.2868472 .626003-1.166138$ H -2.713971 4.273228 - 1.451113 O $-3.6988533 .476157-3.046143$ C $-2.5034272 .098850-1.458175$ C $-1.3169511 .905066-2.419671$ H -2.123206 $2.060848-0.435640$ O $-3.3820020 .983517-1.622605$ H $-0.8683810 .929050-2.196989$ H -1.686066 1.807149 -3.448056 H -2.1501384 .5080104 .327181$ H -1.5001975 .0315172 .766338$ H -2.788891 5.9625983 .545265 C -3.0506201 .6941673 .402431$ H $-5.4251494 .657044-2.188302$ H -2.902231 $3.695297-3.557584$ H -2.875665 $0.176763-1.428811$ H -4.0470191 .2396523 .460369$ H -2.8563202 .1779664 .366599$ H -1.9839092 .7758151 .844544$ C $0.9416002 .233551-0.384851$ O $0.163956 \quad 3.269331-0.991164$ C $-0.242858 \quad 3.007170-2.337627$ C $0.9851932 .749953-3.224164$ C $1.8766811 .645618-2.656441$ C $2.2097111 .931407-1.192293$ H $\quad 0.3299811 .324085-0.323698$ C $1.232462 \quad 2.634617 \quad 1.068632$ H $-0.7088263 .931124-2.700250$ O $0.565253 \quad 2.417822-4.543030$ H $1.5708183 .676096-3.294214$ H $1.4157250 .657870-2.767940$ O $3.104247 \quad 1.603940-3.386578$ H $2.9120402 .771851-1.147106$ O $2.866417 \quad 0.801060-0.624625$ C $2.1098403 .859998 \quad 1.217152$ H 0.2964912 .8602991 .589375 H 1.7142661 .7954081 .579383 H $1.3574932 .379405-5.104697$ H $2.9005951 .293420-4.284846$ H $3.6554730 .621700-1.164048$ $\begin{array}{lllll}O & 2.790634 & 3.781426 & 2.398689\end{array}$

$\begin{array}{lllll}\text { O } & 2.166860 & 4.785307 & 0.418867\end{array}$ C $3.6611184 .880902 \quad 2.682587$ H 4.1547384 .6853313 .638304 H $4.425593 \quad 4.970021 \quad 1.904827$ H 3.0850485 .8071832 .767452 SCF Energy (B3LYP/6-31G**//MMFF) $=-3245.91120911$

01526

MMMFF Geometry
C -1.261903 $2.760042-1.677783$ C $-2.0784042 .459387-0.657516$ C $-3.4557493 .028978-0.466220$ O $-4.3450111 .886845-0.465903$ C $-3.6263213 .733898 \quad 0.890946$ C -2.657882 4.8627001 .159200 C -1.6071894 .7806911 .999851$ C -2.961548 $6.151424 \quad 0.441976$ C $-1.132166 \quad 3.5681962 .757116$ C $0.373143 \quad 3.359316 \quad 2.547644$ C 0.8643712 .0064893 .082153 C 2.3930941 .9482453 .017453 C $2.9359650 .573158 \quad 3.424191$ $\begin{array}{lllll}\text { O } & 0.296002 & 0.975374 & 2.264425\end{array}$ $\begin{array}{lllll}\text { O } & 4.317677 & 0.511413 & 3.040595\end{array}$ C $2.190988-0.5059802 .615158$ C $2.8997710 .367504 \quad 4.942600$ O $2.572715-1.8123373 .040489$ C $0.650571-0.3596302 .653361$ C $0.019562-1.343707 \quad 1.637998$ O $0.180808-0.6689933 .965155$ C $-5.6166302 .040655-0.913986$ $\begin{array}{lllll}0 & -6.124024 & 3.038544 & -1.395649\end{array}$ C $-6.2876130 .743244-0.696170$ C $-7.4782890 .465332-1.243756$ C $-8.135257-0.828622-1.082610$ C $-8.616565-1.425155-2.375127$ C $-1.504980-1.2385201 .495457$ C $-2.011191-2.096661 \quad 0.346335$ C $-3.895090-2.259826-1.282107$ O $-1.399879-3.078123-0.068982$ C $-5.076249-3.107326-0.912096$ C $-5.564496-3.3655840 .311844$ C $-6.748792-4.2620040 .555156$ C $-7.875328-3.5722101 .293927$ C $-8.054416-3.7622982 .612003$ C $-8.845681-2.7310300 .488644$ C $-8.270813-1.3864870 .137469$ $\mathrm{N}-3.234636-1.658814-0.137824$ H $-1.5885553 .464408-2.436241$ H $-1.743693 \quad 1.7404670 .085039$ H $-3.713183 \quad 3.704754-1.291255$ H -3.6011492 .9881021 .695037$ H $-4.649564 \quad 4.1298980 .955586$ H -1.0028395 .6744772 .150515$ H -2.2516246 .9459110 .695044$ H -2.917959 $6.004531-0.641846$ H $-3.962948 \quad 6.5066890 .705741$ H -1.331171 3.7188793 .824517 H -1.6697132 .6658272 .456567$ H 0.5942053 .4169751 .476435 H 0.9185834 .1748303 .039069 H $\quad 0.5154721 .8847994 .114197$ H 2.8392482 .7353603 .638133 H 2.7211642 .1579711 .989316 H $4.783345 \quad 1.2465053 .475042$ H $2.516314-0.4114831 .570200$ H 3.5137111 .1242315 .445784 H $3.331162-0.6006685 .220275$ H 1.8932360 .4302375 .360905 H $3.542292-1.8631952 .983183$ H $\quad 0.460451-1.1582660 .649650$ H $0.268023-2.3753121 .917903$ H $-0.767280-0.867817 \quad 3.921379$ H $-5.7688750 .012574-0.084404$ H $-7.9798261 .186840-1.883135$ H $-7.768064-1.633014-3.036487$ H $-9.291523-0.731918-2.889977$ H $-9.165272-2.360051-2.245061$ H -2.006842 -1.5857992 .404608$ H $-1.796225-0.1984341 .322841$ H -3.177113 - $2.866202-1.843716$ H $-4.223993-1.439478-1.927863$ H $-5.580689-3.559212-1.765020$ H $-5.086197-2.9425451 .191029$ H $-6.395328-5.1257081 .133674$ H -7.135948 $-4.678310-0.383272$ H -8.875862 -3.2943993 .144268$ 
H $-7.379812-4.3857943 .189587$ H $-9.762689-2.5563001 .066338$ H $-9.156448-3.304255-0.389059$ H -7.916746 -0.8232791 .002300$ H -3.678127 -0.8543110 .294993$ C $1.2593243 .054739-1.581938$ O $2.4137112 .235030-1.296509$ C $3.5486642 .762058-2.002567$ C $3.1436374 .156830-2.458594$ C $1.6635313 .967497-2.740702$ H $3.6910622 .131221-2.887927$ C $4.7975182 .710581-1.117751$ H $3.2850924 .900465-1.665441$ H $3.7093574 .487364-3.335190$ C $0.9093435 .284901-2.810193$ H $1.5548503 .438322-3.696499$ C $5.1256581 .306655-0.567214$ O 4.5957133 .5792520 .005083 H $5.6539983 .115437-1.669879$ H 4.3621501 .0192210 .163622 O $\quad 6.350822 \quad 1.4052440 .170274$ C $5.2592760 .204039-1.634843$ C $5.835632-1.127172-1.119631$ H $4.281040 \quad 0.017022-2.085822$ O $6.106557 \quad 0.660744-2.693891$ H $5.932205-1.799598-1.980728$ H $6.865396-0.964295-0.777398$ H $-0.1392475 .128533-3.075594$ H $0.9405605 .816657-1.853258$ H $\quad 1.3483855 .938287-3.571634$ C $0.0822582 .118523-1.852388$ H 5.3925663 .5254170 .560130 H $7.0457281 .700249-0.441986$ H $7.0009490 .779860-2.332424$ H $0.1649491 .246923-1.188432$ H $\quad 0.1528501 .707388-2.866762$ H $1.0764943 .646615-0.678402$ C $3.442315-3.019388-1.383103$ O $3.647385-2.017320-0.380176$ C $5.010690-1.797943-0.003867$ C $5.622717-3.1005040 .526800$ C $5.485373-4.234218-0.484473$ C $4.034795-4.367307-0.943555$ H $3.895630-2.692700-2.326898$ C $1.926106-3.162923-1.598897$ H $4.988606-1.0994160 .839491$ O $6.992732-2.9016620 .857412$ H $5.106489-3.383453 \quad 1.453414$ H $6.155343-4.085358-1.338732$ O $5.900483-5.4608840 .121818$ H $3.433418-4.790445-0.128407$ O $3.974636-5.283546-2.035377$ C $1.303445-1.973717-2.298663$ H $1.723744-4.044482-2.218041$ H $1.417768-3.276379-0.634792$ H $7.346883-3.7559111 .158398$ H $5.329504-5.6245730 .891847$ H $4.371779-6.119178-1.735788$ O $0.048394-2.319678-2.713640$ O $1.829853-0.879722-2.441259$ C $-0.703543-1.297752-3.375470$ H $-0.091958-0.767105-4.111588$ H $-1.537567-1.774975-3.896764$ $\mathrm{H}-1.106022-0.604076-2.632858$ SCF Energy (B3LYP/6-31G**//MMFF) $=-3245.90833913$

\section{7}

MM̄FF Geometry

C $-0.704607 \quad 5.0715220 .107388$

C $0.342314 \quad 4.588598-0.579809$

C 1.5061053 .8828380 .071555

O $1.7250052 .612495-0.586685$

C $2.7836124 .726310-0.092454$

C 3.9727504 .1876120 .679447

C 5.1070153 .7237900 .119510

C 3.8471804 .2233912 .181884

C $5.4560483 .628673-1.338789$

C $5.1379252 .274004-1.991269$
C $5.8542031 .076515-1.344898$

C $5.703637-0.177204-2.209562$

C $6.327790-1.414629-1.543891$

O $5.2669400 .839172-0.062611$

O $5.861022-2.575959-2.248243$

C $5.806933-1.527337-0.094134$

C $7.856099-1.417824-1.668695$

O $6.488290-2.5599300 .613265$

C $5.893724-0.2051350 .694115$

C $5.182909-0.2653672 .068286$

O $7.270270 \quad 0.101258 \quad 0.948545$

C $0.8332311 .619347-0.324884$

O $-0.154815 \quad 1.6940800 .387375$

C $1.2577690 .414983-1.071414$

C $0.432055-0.639652-1.129891$

C $0.677019-1.898188-1.834689$

C $2.075546-2.165788-2.319283$

C $3.685353-0.5899342 .022752$

C $3.362542-2.0579802 .248659$

C $1.434631-3.6378402 .264537$

O $4.196839-2.8861462 .603036$

C $1.381828-4.3837900 .969319$

C $0.260943-4.9400930 .485270$

C $0.203487-5.739307-0.784911$

C $-0.812649-5.201141-1.766124$

C $-2.037966-5.743999-1.863247$

C $-0.377464-4.083672-2.687662$

C $-0.356466-2.749867-1.995354$

N $2.015260-2.3266672 .045867$

H -0.7162744 .9729091 .189442$

H $0.3722954 .698283-1.661147$

H $1.3049123 .701617 \quad 1.134508$

H 2.5999205 .7533070 .250760

H $3.0188534 .801574-1.159646$

H 5.8946093 .3703970 .784695

H 4.7897473 .9784642 .683362

H 3.5520075 .2236122 .515406

H 3.0983593 .5033782 .525573

H $4.9640334 .422742-1.910295$

H $6.5303213 .829999-1.440533$

H $4.0539022 .115062-1.962151$

H $5.4262872 .339822-3.047746$

H $6.9136671 .331072-1.223121$

H $6.137677-0.018611-3.204703$

H $4.636626-0.375057-2.378039$

H $6.117995-2.483760-3.181739$

H $4.756488-1.833918-0.157440$

H $8.153838-1.409080-2.724170$

H $8.285578-2.332406-1.245106$

H $\quad 8.327597-0.558935-1.186777$

H $\quad 6.387687-3.3790030 .098532$

H $5.724028-0.9390442 .743476$

H 5.2741440 .7324502 .521958

H 7.3157190 .7138421 .701328

H $2.2226250 .433093-1.563618$

H $-0.530756-0.574091-0.626158$

H $2.792058-2.041503-1.500767$

H $2.341589-1.476410-3.127640$

H $2.220617-3.179948-2.696725$

H $3.190410-0.0427422 .835229$

H $3.236019-0.2560971 .082695$

H $0.440734-3.4853962 .696051$

H $2.044000-4.1930832 .984775$

H $2.313814-4.4977460 .420618$

H $-0.664504-4.8465101 .049272$

H $-0.048362-6.773672-0.516089$

H $1.186093-5.793027-1.269914$

H $-2.763550-5.377231-2.581590$

H $-2.345346-6.562074-1.220211$

H $-1.077180-3.999123-3.529988$

H $0.580949-4.348931-3.141718$

H -1.330254 -2.450577 -1.609126

H $1.406150-1.5904071 .701256$

C $-3.2295835 .143474-0.294046$

O $-3.2877543 .827293-0.872097$

C $-4.2724723 .059060-0.143158$

C -4.8270153 .9795540 .946894$

C $-3.6844224 .958703 \quad 1.149337$ 
H -3.7394622 .2391790 .346389$ C $-5.3310332 .511850-1.110117$ H $-5.730716 \quad 4.5054270 .617929$ H -5.0833003 .4276811 .856906$ C $-4.0980696 .243471 \quad 1.843369$ H -2.9098804 .4579841 .742916$ C $-4.7744581 .655479-2.268041$ O $-6.0065753 .636965-1.695301$ H $-6.0934391 .956103-0.553339$ H $-4.0803512 .271468-2.853192$ O $-5.8723631 .348023-3.138431$ C $-4.0830930 .330739-1.882226$ C $-5.003053-0.644997-1.118427$ H -3.779765 -0.168689-2.811477 O $-2.8988330 .568818-1.138976$ H $-5.284568-0.197459-0.161472$ H -5.921027 -0.775799-1.700870 H $-3.241763 \quad 6.914474 \quad 1.963474$ H -4.8679886 .7769561 .275848$ H -4.501407 6.0303662 .838713 C -1.861153 $5.787565-0.529312$ H $-6.6799133 .285162-2.302591$ H $-5.5117240 .879856-3.910649$ H -2.340193 $1.174358-1.655506$ H -1.875101 $6.816140-0.150217$ H -1.695616 $5.852763-1.612498$ H $-3.9706005 .748303-0.836039$ C -3.862531-1.863605 1.494346 O $-3.351568-1.9785820 .160055$ C $-4.334888-2.021080-0.880640$ C $-5.315384-3.176534-0.634265$ C $-5.948696-3.0888320 .748697$ C $-4.856318-2.9891951 .810004$ H $-4.357411-0.8937511 .618737$ C -2.660659-1.929265 2.452560 H $-3.795288-2.270994-1.802953$ O $-6.317902-3.191308-1.644192$ H $-4.768623-4.125575-0.712977$ H -6.648976 -2.248299 0.822344 O $-6.703325-4.2749741 .003789$ H -4.328616 -3.949039 1.883453 O $-5.461237-2.7360183 .077292$ C -1.906783 -0.6200632 .542230$ H -2.992963 -2.164139 3.470001 H -1.958009 -2.699158 2.113468 H $-6.835683-4.006444-1.534436$ H -7.466553 -4.2715820 .401751$ H $-6.098558-3.451898 \quad 3.242479$ O $-0.819329-0.796737 \quad 3.350656$ O C -0.0209170 .3658663 .595193$ H $-0.645116 \quad 1.226753 \quad 3.853539$ H $\quad 0.6404640 .150445 \quad 4.438811$ H $\quad 0.5923570 .5823252 .717356$ SCF Energy (B3LYP/6-31G**//MMFF)= -3245.90106542

01528

MM̄FF Geometry

C $-0.268578-2.608082-0.266077$

C $-1.260734-2.8407060 .608197$

C $-1.789468-1.8776241 .647847$

O $-1.204269-0.5702981 .431998$

C $-3.321129-1.7207101 .537863$

C $-4.132436-2.9523471 .870567$

C $-4.824839-3.6750770 .968199$

C $-4.181534-3.3145823 .331735$

C $-4.877522-3.482893-0.522742$

C $-6.232698-2.956411-1.013354$

C $-6.508821-1.492191-0.632629$

C $-7.890497-1.065318-1.135027$

C $-8.1663210 .422445-0.869711$

O $-5.498866-0.677149-1.236453$

O $-9.3137130 .801589-1.645493$

C $-6.9722861 .249836-1.391046$

C -8.5320740 .6789460 .596964$

O $-7.1228862 .627368-1.053324$

C $-5.5952030 .719406-0.925013$

C $-4.4656461 .456991-1.685429$ $\begin{array}{llll}\text { O } & -5.460492 & 0.963937 & 0.476246\end{array}$

C - -1.2299550 .3233572 .455523$

$\begin{array}{lllll}\text { O } & -1.598149 & 0.124594 & 3.601208\end{array}$

C -0.7705481 .6183241 .909118$

C -0.8847892 .7373382 .637559$

C -0.5265924 .0706382 .163195$

C -1.5322205 .1275452 .530045$

C $-3.0582590 .903113-1.422143$

C $-1.9991871 .596658-2.261801$

C $0.4479091 .533719-2.728517$

O $-2.2512272 .509683-3.043368$

C $1.1396822 .568357-1.898282$

C $1.1058173 .880908-2.175336$

C $1.8606494 .929934-1.409975$

C $0.9759745 .768800-0.516168$

C $0.1300326 .678659-1.028094$

C 1.1390005 .6118900 .978805

C 0.6154914 .2985041 .484551

N $-0.7359391 .065036-2.035566$

H $\quad 0.268544-1.664147-0.248270$

H $-1.752730-3.8104000 .565614$

H -1.499886 -2.245589 2.638786

H -3.562503 -1.341158 0.540500

H -3.656732 -0.9136722 .202771$

H -5.404303 -4.524600 1.327513

H $-4.842530-4.1656883 .527099$

H -3.184788 -3.582366 3.695139

H $-4.551986-2.4690503 .920688$

H $-4.065748-2.848149-0.889812$

H $-4.704046-4.465280-0.980986$

H $-6.238944-3.044980-2.107758$

H -7.037756 -3.597946 -0.633667

H $-6.454624-1.3896640 .456716$

H -8.676046 -1.687437 -0.687872

H -7.953301 -1.239019-2.218597

H $-10.0576230 .235290-1.377437$

H -7.007584 $1.204209-2.488230$

H -9.4165210 .0948130 .878554$

H $-8.8049331 .727486 \quad 0.758927$

H -7.7306610 .4172091 .291006$

H $-6.8966932 .727826-0.112949$

H -4.657122 $1.384799-2.764191$

H -4.474606 2.522436 -1.422687

H $-4.519790 \quad 1.0082580 .705718$

H $-0.378550 \quad 1.632500 \quad 0.897934$

H $-1.3138152 .698973 \quad 3.636554$

H -1.514425 5.3135473 .609391

H -2.543301 4.8068522 .253016

H -1.361177 6.0800722 .022670

H $-2.7866491 .037227-0.371437$

H $-3.021825-0.166634-1.656861$

H $\quad 0.165276 \quad 1.923244-3.712534$

H $1.1089730 .674509-2.879852$

H $1.7242902 .213789-1.053505$

H $0.5227164 .225951-3.026619$

H $2.3719275 .581886-2.130500$

H $2.6652824 .473773-0.822945$

H $-0.4853407 .302260-0.388213$

H $\quad 0.0342486 .818357-2.099791$

H 0.6778906 .4516441 .510136

H 2.2054385 .6812021 .230381

H $1.278273 \quad 3.4575301 .286597$

H $-0.6179880 .352399-1.321506$

C $1.460118-4.257715-1.191395$

O $2.533525-3.302003-1.133026$

C $3.619565-3.894669-0.382836$

C $3.154305-5.2919090 .028375$

C $1.644322-5.1328300 .041995$

H $3.741761-3.2913130 .522127$

C $4.903357-3.879111-1.228188$

H $3.456460-6.050117-0.703838$

H $3.562174-5.5911360 .998961$

C $0.892845-6.450529-0.006632$

H $1.361718-4.5862460 .950174$

C $5.367276-2.456251-1.607496$

O $4.664017-4.607947-2.439035$

H $5.706970-4.409823-0.705799$

H $4.559272-1.934799-2.130970$ 
O $6.455832-2.555516-2.532910$

C $5.851988-1.636489-0.398417$

C $6.224931-0.180901-0.731130$

H $5.090577-1.6331760 .381686$

O $7.000624-2.2707610 .171879$

H 6.7819420 .2287690 .120269

H $6.932268-0.158754-1.569076$

H $-0.188802-6.2834310 .000231$

H $1.140265-7.021259-0.907906$

H $1.143580-7.0682460 .861909$

C $0.108747-3.566800-1.360104$

H $3.937955-4.164026-2.909905$

H $6.153537-3.102692-3.277875$

H $7.684913-2.311283-0.518372$

H $-0.672218-4.326085-1.488747$

H $\quad 0.127890-2.985968-2.291583$

H $1.633336-4.858224-2.095743$

C 4.3342931 .1708351 .233070

O $3.9882440 .595527-0.033611$

C $5.0005070 .704796-1.041017$

C $5.3311732 .182544-1.290462$

C $5.6824732 .917738 \quad 0.000856$

C $4.624648 \quad 2.666818 \quad 1.074981$

H $5.217732 \quad 0.6678801 .643490$

C 3.1491010 .9307352 .180802

H $4.5537530 .319276-1.965072$

O $6.4088612 .315344-2.211108$

H $4.4613632 .676554-1.741762$

H 6.6794532 .6391450 .362119

O $5.7289104 .323164-0.257890$

H 3.7049123 .2026970 .816906

$\begin{array}{lllll}\text { O } & 5.087318 & 3.190219 & 2.318148\end{array}$

C $3.083082-0.5019582 .668299$

H 3.2160341 .5632143 .072158

H 2.2164751 .1529161 .650741

H $6.153766 \quad 1.853837-3.028234$

H $6.3784984 .464584-0.967811$

H 5.2811074 .1335592 .181400

O $1.809837-0.7807693 .074358$

O $4.033035-1.2704562 .725019$

C $1.605823-2.0986733 .593544$

H $2.307873-2.3061844 .406906$

H $0.589531-2.1546923 .991318$

H $1.713702-2.8350552 .792255$

SCF Energy (B3LYP/6-31G**/MMFF) $=-3245.91950853$

01_529

MMFF Geometry

C -2.510828 $3.123947 \quad 0.769252$

C -2.436126 $2.922366-0.556430$

C $-1.1797122 .717280-1.372217$

O $-0.0066942 .836769-0.536714$

C - -1.192278 1.308671 -2.003867

C $-0.035678 \quad 1.057870-2.951942$

C $0.9408910 .153511-2.740418$

C $-0.0422041 .868884-4.222797$

C $1.055956-0.832285-1.613045$

C $1.624234-0.234870-0.320428$

C $2.032072-1.338767 \quad 0.666210$

C $2.248570-0.7775062 .070265$

C $2.758674-1.8566333 .037793$

O $3.250935-1.9062650 .181216$

O $3.222756-1.1983434 .226165$

C $3.976905-2.5600912 .403830$

C $1.641614-2.8097063 .477109$

O $4.397305-3.6694293 .194558$

C $3.748131-3.0094130 .941227$

C $5.092745-3.4684510 .317970$

O $2.834867-4.109134 \quad 0.916675$

C $1.0070583 .637121-0.962238$

O $1.0242344 .347193-1.954649$

C $2.132828 \quad 3.474413-0.015053$

C $3.3533203 .905949-0.367665$

C $4.576635 \quad 3.8022120 .431687$

C $4.462096 \quad 3.1791831 .798198$

C $5.076367-3.634089-1.211198$

C $5.777618-2.476118-1.901953$

C $5.488356-0.051217-2.419348$
O $6.941446-2.563417-2.287400$

C $6.0344370 .656885-1.217146$

C $7.2663321 .184857-1.164556$

C 7.8486381 .8485270 .051285

C $8.0701643 .332052-0.139833$

C $9.1360123 .792618-0.816328$

C 7.1008084 .2789410 .531809

C $5.7334494 .255542-0.096816$

N $4.985675-1.345334-1.998554$

H -1.5905393 .1460841 .348810$

H $-3.3514132 .905596-1.142736$

H -1.182097 $3.487202-2.152657$

H -2.127611 $1.153074-2.558126$

H -1.192385 $0.565215-1.199124$

H $1.7309960 .068494-3.485602$

H $\quad 0.757775 \quad 1.571014-4.909243$

H $-0.9917261 .732659-4.751091$

H $0.0876102 .933536-4.013854$

H $0.080952-1.291785-1.414996$

H $1.695837-1.653133-1.960646$

H $2.4955890 .389080-0.555532$

H 0.8663520 .4077590 .139170

H $1.253142-2.1090850 .680949$

H $1.328533-0.3203242 .455162$

H 2.9912530 .0303182 .029443

H $2.479673-0.6928864 .598302$

H $4.807097-1.8396022 .415618$

H $\quad 0.834568-2.2529713 .968284$

H $2.004701-3.5275794 .220859$

H $1.202623-3.3664692 .646850$

H $4.546202-3.3414154 .097882$

H $5.882376-2.754800 \quad 0.585723$

H $5.368430-4.4351140 .759000$

H $3.009946-4.6447310 .125900$

H 1.9312582 .9815170 .929128

H $3.4757664 .374252-1.343734$

H 3.7879953 .7649592 .432859

H 5.4129873 .1053872 .328982

H 4.0742442 .1580701 .721234

H $5.624024-4.544542-1.484647$

H $4.064052-3.746213-1.614099$

H $4.6456600 .509665-2.834210$

H $6.244243-0.192139-3.198402$

H $\quad 5.3868660 .728337-0.346398$

H $7.9120601 .109930-2.036755$

H $8.806431 \quad 1.3621990 .278707$

H 7.2224631 .6728160 .934710

H $9.3175564 .855940-0.932072$

H $9.8499083 .113715-1.271412$

H 7.4694445 .3113040 .464003

H 7.0793224 .0615371 .603264

H $5.7129074 .682003-1.100803$

H $4.063633-1.364511-1.574889$

C -5.1040393 .2113290 .871569$

O $-5.266717 \quad 1.877508 \quad 0.358015$

C -6.6402261 .4755580 .509398$

C -7.3935002 .7031581 .010293$

C $-6.3109083 .434951 \quad 1.781641$

H -6.6559890 .7081141 .292121$

C $-7.1547730 .884468-0.808969$

H $-7.757586 \quad 3.3256300 .184952$

H -8.2553282 .4346331 .629047$

C -6.6333374 .8962702 .039969$

H -6.1583562 .9232562 .741411$

C $-6.244663-0.210624-1.404675$

O $-7.254061 \quad 1.927725-1.788387$

H -8.172799 $0.502216-0.673751$

H $-5.3064210 .241386-1.745185$

O $-6.887783-0.732488-2.573851$

C $-5.949157-1.382452-0.453069$

C $-5.124550-2.519718-1.082182$

H -5.424369 -1.018394 0.432911

O $-7.183219-1.9390780 .008467$

H $-5.082089-3.339531-0.354656$

H $-5.669547-2.933821-1.939449$

H -5.8241815 .3835502 .592721$

H -6.7809955 .4461461 .104513$

H -7.5494634 .9886192 .632414$ 
C -3.7594423 .3202741 .595399$ H $-6.3729142 .329345-1.880185$ H $-7.0716290 .019238-3.163133$ H -7.680071-2.238094-0.772601 H -3.730828 2.5673162 .391710 H -3.683786 4.3019682 .077277 H $-5.139813 \quad 3.9004740 .018032$ C $-2.577735-2.4234480 .615091$ O $-2.955173-1.522563-0.434404$ C $-3.700552-2.106397-1.507128$ C $-2.887647-3.235207-2.153284$ C $-2.462587-4.276004-1.125122$ C $-1.768116-3.6087650 .060783$ H $-3.475885-2.7878361 .128005$ C $-1.735713-1.6174611 .618346$ H $-3.805726-1.324134-2.267678$ O $-3.633332-3.871190-3.185952$ H $-1.985703-2.815547-2.617790$ H $-3.315794-4.883416-0.801143$ O $-1.545746-5.178318-1.750642$ H $-0.770361-3.280944-0.256466$ O $-1.592033-4.6029511 .069631$ C $-2.551719-0.6755552 .477200$ H -1.218930 -2.295736 2.304022 H $-0.998769-1.0073151 .086571$ H $-3.878147-3.187803-3.832952$ H $-1.263877-5.818110-1.074501$ H $-0.928250-4.2773271 .699539$ O $-1.760616-0.2274283 .497596$ O $-3.715001-0.3600012 .276602$ C $-2.366544 \quad 0.6622544 .442022$ H -2.245616 1.6910454 .094232 H -3.4243080 .4325154 .602114$ H -1.8415090 .5509135 .394747$ SCF Energy (B3LYP/6-31G*//MMFF) $=-3245.90832352$

0153

MMFF Geometry

C -4.035762 -1.586988 1.969060

C $-3.471005-2.7944911 .814297$

C $-2.933280-3.3010370 .502507$

O $-1.613083-3.862908 \quad 0.726058$

C $-3.781200-4.437695-0.089253$

C $-5.122519-4.003641-0.633203$

C -5.315139-3.541807-1.883809

C $-6.296330-4.1893330 .292826$

C $-4.281812-3.327839-2.958421$

C $-4.451758-1.968975-3.654274$

C $-3.875164-0.792329-2.848605$

C $-4.430660 \quad 0.527765-3.391352$

C $-3.7873441 .744078-2.715470$

O $-2.448867-0.831241-2.992847$

O $-4.1664182 .917621-3.449763$

C $-2.2566101 .595550-2.797338$

C $-4.3291281 .959548-1.298976$

O $-1.6087952 .637713-2.070903$

C $-1.739260 \quad 0.217456-2.318481$

C $-0.2416650 .092999-2.691659$

O $-1.897827 \quad 0.133025-0.904951$

C -0.594192 -3.0068091 .003411$

O $-0.659572-1.7928121 .103869$

C $0.631603-3.817257 \quad 1.170865$

C $1.777349-3.2222681 .533912$

C $3.067115-3.8870751 .732141$

C $3.141885-5.3647001 .447219$

C $0.424185-1.234570-2.308583$

C $1.837838-1.329657-2.859538$

C $3.905023-2.683134-2.716455$

O $2.270953-0.574866-3.726951$

C $4.625297-3.443131-1.650088$

C $5.708183-2.973437-1.011778$

C $6.492165-3.7488590 .008662$

C $6.503233-3.0867041 .368361$

C $7.402468-2.1338401 .667068$

C $5.528099-3.5951622 .406307$

C $4.119094-3.1458142 .137706$

N $2.561756-2.361063-2.273525$

H $-4.169134-0.9511811 .098423$
H -3.347402 -3.441629 2.679509

H $-2.836129-2.493706-0.233082$

H $-3.920015-5.2315390 .658265$

H $-3.207785-4.934618-0.883724$

H $-6.335645-3.307881-2.185593$

H -7.244366 $-3.903503-0.175375$

H $-6.381320-5.2400170 .589614$

H -6.183158 -3.5829021 .195212$

H $-3.258870-3.431052-2.584559$

H $-4.427941-4.115091-3.708281$

H $-3.935618-2.021966-4.621949$

H $-5.515027-1.800319-3.867117$

H $-4.134805-0.906814-1.790598$

H $-5.5219840 .565258-3.283300$

H $-4.2364850 .584249-4.471790$

H $-3.8629932 .814056-4.367787$

H $-1.967212 \quad 1.725882-3.849404$

H $-5.4099082 .143561-1.327337$

H $-3.8861832 .848420-0.836089$

H $-4.1575701 .106546-0.639940$

H $-1.9510543 .483470-2.407519$

H $-0.1450670 .222393-3.777923$

H $0.3283350 .902082-2.218252$

H $-1.295313-0.540890-0.555154$

H $\quad 0.556422-4.8841030 .992884$

H $1.774455-2.1460191 .703296$

H $2.802796-5.5798560 .427658$

H $2.513733-5.9224322 .150568$

H $4.150938-5.7745071 .524013$

H $0.483868-1.323057-1.220908$

H $-0.146665-2.083349-2.699952$

H $4.426198-1.758793-2.989456$

H $3.818367-3.296119-3.619652$

H $4.261131-4.441377-1.422477$

H $6.079444-1.981570-1.261434$

H $7.522080-3.844055-0.360171$

H $6.122507-4.7776380 .097753$

H $7.435419-1.6743342 .649298$

H $8.122365-1.7892380 .932069$

H $5.803378-3.2203093 .401390$

H $5.621110-4.6820352 .486651$

H $3.963777-2.0824412 .321740$

H $2.063756-3.041858-1.706442$

C $-4.025546 \quad 0.398305 \quad 3.561874$

$\begin{array}{llll}\text { O } & -2.633888 & 0.576382 & 3.221512\end{array}$

C -2.5160531 .7596612 .410263$

C $-3.7733482 .568988 \quad 2.691624$

C -4.8067931 .4644282 .789540$

H -2.5319871 .4339871 .363470$

C -1.2086872 .5112322 .682631$

H -3.6964863 .1092663 .642794$

H -3.9913063 .2929831 .900953$

C $-6.095676 \quad 1.8961893 .467794$

H $-5.043768 \quad 1.1139261 .777738$

C 0.0367521 .7428012 .190045

$\begin{array}{lllll}\text { O } & -1.110097 & 2.812669 & 4.071377\end{array}$

H -1.2482583 .4687312 .151624$

H -0.1372351 .4806611 .141115$

$\begin{array}{lllll}\text { O } & 0.184911 & 0.518002 & 2.903964\end{array}$

C 1.3409042 .5577922 .288321

C 2.5706941 .8423101 .696130

H $1.197138 \quad 3.5221921 .788717$

O 1.6200752 .8381303 .661566

H 3.4467872 .4747301 .886221

H 2.7650540 .9199712 .258679

H -6.7991791 .0605503 .534900$

H -5.9120332 .2651824 .482477$

H -6.5775732 .6994862 .900771$

C $-4.466861-1.0481543 .305746$

H $-1.171994 \quad 1.973554 \quad 4.558837$

H $0.421443 \quad 0.7393873 .820701$

H 2.4009243 .4169053 .690824

H $-5.556365-1.1251663 .392967$

H $-4.037693-1.6754964 .097846$

H -4.1039410 .5947424 .639209$

C $2.9569583 .723741-0.675639$

O $2.0152792 .649173-0.571294$

$\begin{array}{llll}\text { C } 2.465687 & 1.526122 & 0.190612\end{array}$ 
C $3.7486720 .956985-0.429400$

C $4.8240952 .023930-0.607263$

C $4.2529773 .239596-1.336218$

H $3.1739814 .122500 \quad 0.323230$

C $2.2947704 .832105-1.500855$

H $1.6958840 .752703 \quad 0.084217$

$\begin{array}{lllll}\text { O } & 4.266849 & -0.104801 & 0.360121\end{array}$

H $3.5202430 .542283-1.417973$

H $5.2683512 .313726 \quad 0.352064$

O $5.8894891 .488084-1.395836$

H $4.0692642 .984031-2.387723$

O $5.2146494 .292245-1.311905$

C $1.2703465 .572841-0.674677$

H $3.0230235 .576905-1.841642$

H $1.8053554 .431295-2.396843$

H $3.588232-0.8007950 .385286$

H $6.2112660 .690127-0.941894$

H $6.0306363 .948769-1.714572$

O $0.0506804 .976186-0.791474$

$\begin{array}{lllll}\text { O } & 1.532325 & 6.553458 & 0.010274\end{array}$

C -1.006721 $5.581614-0.041060$

H $-1.8885704 .939673-0.115612$

H -0.729275 5.6723941 .013585

H - $1.2472366 .562080-0.462057$

SCF Energy (B3LYP/6-31G*//MMFF) $=-3245.91338778$

01530

MMFF Geometry

C $4.398836-4.0104210 .735325$

C $3.562022-3.432077-0.140171$

C $2.177056-3.943521-0.446853$

O $1.233267-2.882148-0.168074$

C $2.071786-4.289648-1.943035$

C $0.710268-4.814769-2.355330$

C $-0.119534-4.195789-3.218537$

C $0.309293-6.136258-1.751310$

C $0.099626-2.902558-3.953406$

C $-0.456657-1.656025-3.246737$

C $-1.984246-1.661284-3.071644$

C $-2.479067-0.294556-2.592441$

C $-3.992268-0.292859-2.321313$

O $-2.325949-2.655825-2.103052$

O $-4.306790 \quad 0.894560-1.577535$

C $-4.347783-1.495238-1.419783$

C $-4.802210-0.215800-3.620836$

O $-5.759954-1.631004-1.283639$

C $-3.735117-2.827015-1.899137$

C $-3.914327-3.981033-0.881986$

O $-4.383401-3.232088-3.111097$

C $0.916529-2.6510501 .134599$

O $1.318586-3.2610182 .112180$

C $0.001275-1.4906441 .168377$

C $-0.176119-0.822712 \quad 2.317702$

C -1.039986 0.3434712 .507880

C $-1.974910 \quad 0.7010641 .384151$

C $-3.279523-3.7503440 .494315$

C $-4.242885-3.2160321 .541908$

C $-4.283492-2.398323 \quad 3.897485$

O $-5.455815-3.1386391 .367825$

C $-4.325246-0.9029233 .890410$

C $-3.902142-0.1486384 .916438$

C -3.9897391 .3503124 .946098$

C -2.6608672 .0092615 .240430$

C $-2.358773 \quad 2.4357556 .478321$

C -1.723641 2.2549734 .080446

C $-0.9701621 .018502 \quad 3.673984$

$\mathrm{N}-3.586205-2.8718102 .716474$

H $4.072030-4.9209301 .234545$

H $3.852264-2.521222-0.655648$

H $\quad 1.931123-4.8206170 .164039$

H $2.823750-5.046904-2.202182$

H $2.328892-3.398681-2.525920$

H $-1.072757-4.674697-3.440441$

H - $0.611792-6.532213-2.192335$

H $1.094654-6.881861-1.912832$

H $0.138605-6.033634-0.675286$

H $1.164457-2.748557-4.157525$

H $-0.367115-2.992814-4.942826$
H $\quad 0.028471-1.555882-2.268618$

H $-0.167456-0.780626-3.841622$

H $-2.441047-1.915259-4.035336$

H $-2.2198640 .488641-3.315508$

H -1.959382 -0.025794 -1.663184

H $-4.0024601 .660926-2.093302$

H $-3.960177-1.267076-0.420871$

H $-4.5505680 .696642-4.174800$

H $-5.876305-0.152697-3.414249$

H $-4.627384-1.063934-4.285930$

H $-6.102737-0.781305-0.957354$

H $-4.973918-4.251453-0.800314$

H $-3.422164-4.866701-1.309688$

H $-4.255854-4.188603-3.224312$

H $-0.486804-1.2077150 .242984$

H $0.376043-1.1458243 .199343$

H -2.572092 -0.1689591 .093696$

H $-1.409623 \quad 1.045606 \quad 0.512560$

H $-2.690787 \quad 1.4844921 .640646$

H $-2.923315-4.716107 \quad 0.875867$

H $-2.411965-3.0878700 .422978$

H $-3.751809-2.7939774 .768771$

H $-5.303808-2.7952103 .902354$

H $-4.752039-0.4201713 .014365$

H -3.502335 -0.6357635 .803102$

H -4.7203791 .6245985 .718456$

H $-4.3929301 .747170 \quad 4.005998$

H $-1.4197632 .936517 \quad 6.690308$

H -3.041776 $2.288617 \quad 7.308431$

H $-0.9772493 .013462 \quad 4.353422$

H $-2.2848872 .705013 \quad 3.257442$

H $-0.264880 \quad 0.6726114 .430952$

H $-2.571894-2.9182762 .752090$

C $6.201955-2.1638590 .591520$

O $5.323563-1.1338261 .080441$

$\begin{array}{llll}\text { C } & 6.068721 & 0.088035 & 1.226606\end{array}$

C $7.498052-0.2254910 .788113$

C $7.591534-1.7197021 .035901$

H $6.062507 \quad 0.3370762 .294740$

C $5.405106 \quad 1.2222780 .429053$

H $7.651745-0.001487-0.274305$

H $8.236876 \quad 0.349500 \quad 1.355082$

C $8.731267-2.386818 \quad 0.286566$

H $7.714981-1.8900672 .113658$

C $4.002917 \quad 1.6097180 .955034$

O $5.3402550 .855275-0.948420$

H $6.0477012 .108791 \quad 0.488041$

H 4.1107381 .8988342 .007535

$\begin{array}{lllll}\text { O } & 3.116308 & 0.492795 & 0.933731\end{array}$

C $3.3628732 .789808 \quad 0.192363$

C 2.0465603 .2570500 .848231

H $4.0717363 .626090 \quad 0.187967$

O $3.128598 \quad 2.433420-1.166687$

H 1.3008772 .4587100 .773170

H 2.2340363 .4257251 .914925

H $8.758837-3.4613260 .491906$

H $8.632746-2.253175-0.795975$

H $9.692881-1.9610890 .591656$

C $5.764571-3.5199131 .150241$

H $4.8634390 .010361-1.008009$

H 3.0683040 .1635670 .021085

H $2.4508611 .736966-1.181324$

H $5.759372-3.4644302 .246526$

H $6.493702-4.2877880 .867482$

H $6.140169-2.163423-0.504841$

C $0.0181963 .615196-1.446103$

O $1.178142 \quad 4.406433-1.172085$

C $1.494453 \quad 4.557325 \quad 0.215337$

C 0.3022845 .1745590 .963135

C $-0.9879824 .395407 \quad 0.725421$

C $-1.2258404 .205037-0.773123$

H $0.1827822 .598813-1.067863$

C $-0.1201063 .466096-2.966059$

H $2.310593 \quad 5.2899210 .261855$

$\begin{array}{lllll} & 0 & 0.557129 & 5.244348 & 2.362406\end{array}$

$\begin{array}{llll}\mathrm{H} & 0.151478 & 6.204347 & 0.613279\end{array}$

H $-0.961646 \quad 3.437146 \quad 1.252563$

O $-2.071304 \quad 5.139611 \quad 1.285307$ 
H $-1.4867015 .173497-1.216483$ O $-2.3259283 .324646-0.980638$ C $-0.4524684 .759729-3.671937$ H $0.8090323 .077085-3.400802$ H $-0.9048562 .741600-3.212891$ H 1.3678875 .7668982 .485597 H -2.864303 4.5790561 .243886 H $-3.1212033 .757113-0.627221$ O $0.6696875 .520526-3.813350$ O $-1.5796205 .059395-4.044939$ C $0.4787296 .785443-4.453992$ H $0.1037386 .642254-5.471908$ H $-0.2099637 .404589-3.871209$ H $1.4470307 .290547-4.504668$ SCF Energy (B3LYP/6-31G**//MMFF) $=-3245.88430142$

01 531

MM̄FF Geometry

C $-0.705486-4.3786850 .410672$

C $-0.087066-3.529129-0.423094$

C $-0.703970-2.253425-0.929781$

O $-0.085542-1.161337-0.211182$

C $-0.404200-2.077021-2.427729$

C -1.131091-0.899648 -3.047148

C $-2.325573-0.991030-3.664529$

C $-0.3939740 .413537-3.009131$

C -3.163122 -2.228234 -3.822779

C $-4.673979-1.995858-3.670392$

C $-5.124294-1.679417-2.234028$

C $-6.645460-1.829265-2.128037$

C $-7.168912-1.439476-0.739904$

O $-4.747040-0.336151-1.913858$

O $-8.599920-1.346563-0.815267$

C $-6.610190-0.050810-0.367008$

C $-6.890702-2.535487 \quad 0.294907$

O $\quad-6.973975 \quad 0.2666600 .975081$

C $-5.0798110 .073653-0.578196$

C $-4.6456621 .549562-0.402843$

O $-4.414616-0.7271420 .398533$

C $-0.687293-0.7538640 .939076$

O $-1.687815-1.2226831 .457051$

C $\quad 0.082814 \quad 0.389761 \quad 1.468223$

C $-0.485101 \quad 1.2211362 .353528$

C 0.1590632 .3763912 .981943

C 1.6455272 .5320952 .788211

C $-3.2014401 .847282-0.824896$

C $-2.8410193 .309468-0.619452$

C $-0.9485834 .900409-0.923053$

O $-3.6382454 .146831-0.204754$

C -0.2242205 .0811330 .372193$

C -0.6189535 .9314381 .331491$

C $0.144406 \quad 6.1847882 .599876$

C -0.6004135 .7326173 .835322$

C - -1.5570976 .4980114 .387340$

C -0.1649454 .4375114 .481619$

C -0.6006623 .2239203 .707155$

N -1.524912 $3.571395-0.974134$ H -1.720917 -4.1676130 .736124$ H $\quad 0.939226-3.733934-0.718789$ H -1.790585 -2.247581 -0.772883 H $-0.655113-2.996394-2.971459$ H $\quad 0.673449-1.934430-2.582470$ H $-2.735816-0.093984-4.125737$ H $\quad 0.5710330 .321776-3.518412$ H $-0.9503621 .217665-3.502358$ H $-0.2118880 .726263-1.977881$ H -2.985723 -2.613562 -4.834658 H -2.860886 -3.016948 -3.126237 H $-4.986214-1.188458-4.345344$ H -5.175875 -2.911701-4.007793 H $-4.623158-2.371724-1.547407$ H -6.956466 -2.851462 -2.377947 H -7.123559 -1.181603 -2.876646 H -8.827111 -0.671737-1.477355 H $-7.1148940 .683553-1.010269$ H -7.383918 -3.4701540 .001469$ H -7.308892 -2.272204 1.272842 H $\quad-5.827515-2.7496440 .421467$
H $-6.808091 \quad 1.213957 \quad 1.114048$

H -5.303882 2.190424 -1.004214

H -4.7672391 .8489890 .645790$

H $-3.525899-0.3712720 .551730$

H 1.0923750 .5244551 .105703

H -1.5205641 .0460302 .642885$

H 1.8852042 .6591761 .727761

H 2.1757831 .6492743 .162981

H 2.0670933 .3966573 .304356

H $-2.4970891 .240864-0.250127$

H $-3.0684081 .620037-1.887866$

H -1.737837 $5.647269-1.059952$

H $-0.2456814 .989091-1.757316$

H 0.6906404 .5114290 .507469

H -1.528134 6.5102021 .184077

H $\quad 0.3414727 .2630972 .666651$

H 1.1353505 .7156842 .565828

H -2.0734296 .1945805 .291841$

H -1.846753 7.4445633 .942595

H -0.5970934 .3521175 .487928$

H 0.9155054 .4651624 .650377

H -1.671233 3.0275223 .780429

H $-0.9188722 .802021-1.241360$

C $0.838962-5.3268222 .206459$

O $1.997246-4.5654841 .804503$

C $2.150125-3.4545022 .705886$

C $1.391067-3.8527593 .964392$

C $0.189208-4.5594873 .361791$

H $1.638322-2.6006782 .244725$

C $3.629606-3.1078352 .899991$

H $1.967480-4.5494944 .583862$

H $1.118551-2.9896224 .578857$

C $-0.556702-5.4476024 .342105$

H $-0.502120-3.7945942 .987952$

C $4.417228-2.9313751 .583414$

O $4.256780-4.1636413 .637933$

H $3.712155-2.2082283 .521445$

H $4.573811-3.9132031 .120785$

O $5.723289-2.444227 \quad 1.915648$

C $3.769927-1.9830200 .559098$

C $4.647541-1.783266-0.692101$

H $2.798891-2.3891820 .260843$

O $3.542242-0.7262681 .190596$

H $5.422091-1.038540-0.478617$

H $5.150367-2.728518-0.927764$

H $-1.397262-5.9471793 .850137$

H $0.096388-6.2198414 .762321$

H $-0.953355-4.8550915 .173010$

C $-0.048022-5.6033310 .984865$

H $5.192151-3.9223103 .751033$

H $5.618245-1.5452062 .272111$

H $3.074628-0.1630640 .552647$

H $-0.832652-6.3203981 .253685$

H $0.557112-6.0785960 .201998$

H $1.228546-6.2876572 .566660$

C $3.8400961 .050893-1.663793$

O $3.070339-0.156596-1.712361$

C $3.821622-1.352795-1.927856$

C $4.630027-1.246659-3.228647$

C $5.4965020 .008557-3.261936$

C $4.6501911 .243771-2.953340$

H $4.5203161 .006992-0.805289$

C $2.8394312 .194811-1.438747$

H $3.081435-2.148165-2.083530$

O $5.454836-2.392422-3.413373$

H $3.937986-1.209886-4.080440$

H $6.345462-0.080112-2.573665$

O $6.048006 \quad 0.132521-4.574927$

H $3.9763901 .441420-3.796935$

O $5.5028042 .376325-2.809423$

C $3.4732703 .438544-0.861175$

H $2.3342482 .434280-2.382598$

H $2.0520021 .884961-0.742210$

H $4.874355-3.172667-3.417273$

H $6.6905080 .861633-4.555670$

H $5.9139902 .548091-3.673143$

O $2.8869964 .543745-1.405352$

O $4.3522833 .437759-0.009549$ 
C $3.3707115 .799765-0.919061$

H 3.2124325 .8757150 .161065

H $4.4305485 .916602-1.164315$

H $2.8057096 .595173-1.412420$

SCF Energy (B3LYP/6-31G**//MMFF) $=-3245.90227904$

01532

MMFF Geometry

C -0.246271 -3.2406560 .962972$

C $0.243157-2.846629-0.221942$

C $-0.602273-2.215729-1.292700$

O $0.023288-0.953728-1.628884$

C $-0.676963-3.066429-2.572071$

C $-1.359308-4.406119-2.408703$

C $-2.689938-4.564755-2.270113$

C $-0.448118-5.603926-2.443629$

C $-3.730483-3.480028-2.210512$

C $-4.485196-3.530660-0.876200$

C $-5.343206-2.293127-0.569972$

C $-6.290783-1.908908-1.708386$

C $-7.102138-0.646803-1.374978$

O $-4.466231-1.199572-0.264330$

O $-7.697767-0.170373-2.591905$

C $-6.135702 \quad 0.453334-0.885766$

C -8.260755 $-0.947956-0.416895$

O $-6.856521 \quad 1.594572-0.425595$

$\begin{array}{llll}\text { C } & -5.151762 & -0.028327 & 0.205671\end{array}$

C -4.1048241 .0721210 .501796$

O $-5.883486-0.2879161 .408686$

C $-0.7907310 .049503-2.053094$

O $-2.0035340 .014086-2.185292$

C $0.057254 \quad 1.229346-2.318464$

C $-0.486492 \quad 2.451522-2.228463$

C $0.2083533 .722878-2.429300$

C $1.6219313 .680359-2.947304$

C $-2.9330520 .599166 \quad 1.375044$

C $-2.445638 \quad 1.6992052 .296993$

C -1.3359893 .9088782 .338877$

O -2.5301241 .5964423 .519275$

C -1.272146 5.1014511 .441253

C $-0.1299565 .722032 \quad 1.108927$

$\begin{array}{llll}\text { C } & -0.065859 & 6.953474 & 0.249420\end{array}$

C $0.7686626 .754273-0.996192$

C $2.0754827 .066746-1.006820$

C $0.072896 \quad 6.273758-2.250253$

C $-0.4435654 .866761-2.135013$

N $-1.923786 \quad 2.7922601 .623069$

H $-1.311278-3.1316701 .150677$

H $1.301305-2.964091-0.431108$

H $-1.604578-2.021665-0.893752$

H $0.335884-3.208555-2.975060$

H $-1.188470-2.498787-3.361137$

H $-3.083031-5.576818-2.183154$

H $-0.993144-6.545536-2.318880$

H $0.078741-5.650828-3.402401$

H $0.294405-5.544240-1.641603$

H -3.295019 -2.489534-2.362390

H $-4.432498-3.640898-3.037318$

H $-5.128304-4.420090-0.868979$

H -3.761227 -3.651798 -0.058950

H $-5.919097-2.5335680 .331975$

H $-6.957386-2.743474-1.958046$

H $-5.715097-1.698388-2.619359$

H $-8.250570-0.883951-2.954072$

H $-5.5497520 .786291-1.754123$

H $-8.945588-1.679143-0.862878$

H -8.861847 $-0.051323-0.229608$

H $-7.931458-1.3500210 .543280$

H -7.451335 $1.870120-1.143945$

H -3.682488 $1.441615-0.441047$

H -4.606651 1.9211390 .983754

H -5.311990 -0.123863 2.175472

H $1.1024191 .059915-2.547986$

H -1.538804 2.532082 -1.957579

H $1.6696043 .130805-3.894022$

H $2.282616 \quad 3.192609-2.224510$

H $2.0436474 .669473-3.139473$

H $-3.212104-0.2529982 .004558$
H $-2.0963950 .264248 \quad 0.751657$

H -0.3479593 .5956032 .691669$

H $-1.949234 \quad 4.1334503 .218183$

H $-2.217218 \quad 5.4887191 .068021$

H 0.8127045 .3442631 .498789

H $\quad 0.360837 \quad 7.7626170 .856728$

H $-1.0684077 .299011-0.032269$

H $2.6771536 .955503-1.902713$

H $2.5737547 .437768-0.117274$

H $0.7219426 .373305-3.128150$

H $-0.7756986 .937662-2.461217$

H $-1.4646304 .804126-1.757553$

H -1.7162342 .6928400 .633065$

C $0.734847-2.9334273 .295552$

O $1.460326-1.7383522 .934552$

C $0.676656-0.6009093 .332300$

C $-0.191690-1.1058864 .476938$

C $-0.569981-2.4820773 .964036$

H $0.034645-0.3523172 .477974$

C 1.5558340 .6088823 .661903

H $0.376841-1.1985465 .409356$

H $-1.057492-0.4651374 .664373$

C $-1.057833-3.4287215 .047472$

H -1.373612 -2.357658 3.229994

C $2.651315 \quad 0.928780 \quad 2.623574$

$\begin{array}{llll}\text { O } & 2.216268 & 0.378222 & 4.913235\end{array}$

H $0.909461 \quad 1.4809473 .818985$

H 3.4834190 .2278632 .757639

$\begin{array}{llll}\text { O } & 3.182242 & 2.221529 & 2.944487\end{array}$

C 2.2073830 .9097261 .151891

$\begin{array}{llll}\text { C } 3.238680 & 1.466461 & 0.155168\end{array}$

H $1.961543-0.1176080 .865018$

O 1.0052031 .6651370 .994632

H $2.8264601 .316744-0.848387$

H 3.3052092 .5561420 .270646

H $-1.297042-4.4099934 .625013$

H $-0.302092-3.5713905 .827193$

H $-1.961390-3.0348465 .524183$

C $0.581470-3.8402182 .066734$

H $2.748631 \quad 1.167926 \quad 5.110484$

H 2.4640182 .8704522 .853798

H 1.2116102 .5990331 .167326

H $\quad 0.121058-4.7889612 .366002$

H $1.581255-4.0787111 .682238$

H $1.368391-3.4546194 .024434$

C $4.235931-1.239528-0.890343$

$\begin{array}{llll}\text { O } & 4.623883 & -0.572290 & 0.313359\end{array}$

$\begin{array}{llll}\text { C } 4.649081 & 0.853926 & 0.236642\end{array}$

C $5.595747 \quad 1.304572-0.886145$

C $5.2473850 .654664-2.225245$

C $5.141948-0.862549-2.071730$

H $3.202547-0.957317-1.129053$

C $4.203277-2.747136-0.596102$

H $5.087671 \quad 1.2019241 .179778$

O $5.6008862 .723061-1.012334$

H $6.6198811 .014059-0.619203$

H $4.3261231 .069964-2.650047$

$\begin{array}{lllll}\text { O } & 6.284770 & 0.939885 & -3.166798\end{array}$

H $6.153334-1.266682-1.947524$

O $4.614313-1.398250-3.283618$

C $5.559808-3.355977-0.306171$

H $3.587945-2.938027 \quad 0.290793$

H $3.753181-3.271590-1.446606$

H $4.7665912 .994975-1.430425$

H $6.3697421 .907316-3.219785$

H $4.713196-2.364446-3.247889$

O $5.545805-4.673718-0.664638$

$\begin{array}{llll}0 & 6.501128 & -2.763686 & 0.203790\end{array}$

C $6.762682-5.388672-0.427790$

H $6.994519-5.3971920 .641533$

H $6.622644-6.419189-0.764702$

H $7.582367-4.941245-0.998054$

SCF Energy (B3LYP/6-31G**//MMFF)= -3245.89589091

01_533

MM̄FF Geometry

C -1.055888 -3.111772 1.401789

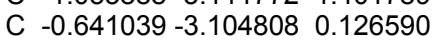


C $-1.395717-2.423122-0.981056$ O $-0.523457-1.386043-1.489580$ C $-1.751392-3.372656-2.137084$ C $-2.736861-4.463823-1.781037$ C $-4.058528-4.263377-1.610618$ C $-2.153702-5.846213-1.657937$ C $-4.794163-2.953402-1.690162$ C $-5.504331-2.648754-0.364439$ C $-5.974334-1.194231-0.202774$ C $-6.813722-0.687406-1.375974$ C $-7.2187700 .784118-1.192845$ O $-4.815232-0.369307-0.034475$ O $-7.703303 \quad 1.265827-2.456685$ C $-5.9624751 .613673-0.843812$ C $-8.3776950 .937573-0.200236$ O $-6.3420482 .952893-0.532772$ C -5.1113410 .9968420 .293362$ C -3.7716221 .7585940 .445176$ O $-5.835846 \quad 1.101478 \quad 1.522143$ C -1.108869-0.275774 -2.010876 O $-2.301575-0.069347-2.164918$ C $-0.031727 \quad 0.684919-2.325445$ C $-0.3032031 .996631-2.273954$ C $0.6488483 .085151-2.488821$ C $2.0287492 .726771-2.974076$ C -2.7541441 .0509681 .349314$ C -1.4734961 .8487881 .496554$ C -0.5365113 .8691022 .618574$ O $-0.417727 \quad 1.4739540 .992752$ C $-0.3704894 .935467 \quad 1.581264$ C 0.8196925 .2708451 .058998 C 1.0257316 .3986360 .086761 C $1.8136875 .984878-1.136181$ C $3.1570816 .025204-1.135139$ C $1.0492595 .610622-2.384541$ C $0.2512884 .347852-2.228297$ N -1.634608 2.9933792 .258472 H -1.999967 -2.633946 1.651153 H $0.294451-3.593095-0.132509$ H -2.299834 -1.957537 -0.571841 H $-0.831032-3.815443-2.543701$ H -2.149547 -2.792764 -2.980435 H -4.685956 -5.123672 -1.381780 H -2.909940 -6.595974 -1.402582 H -1.693926 -6.148881 -2.604522 H - $-1.388234-5.871058-0.876173$ H -4.127362 -2.131236 -1.959896 H -5.535444 -3.028955 -2.494592 H $-6.368941-3.317537-0.264909$ H -4.824029 -2.8775490 .467152$ H $-6.561003-1.1583250 .723120$ H -7.698983 -1.318193 -1.522233 H $-6.236310-0.756296-2.307346$ H $-8.4520330 .705660-2.723846$ H -5.349573 $1.664528-1.754978$ H $-9.2559900 .380689-0.548416$ H -8.698033 $1.982697-0.125013$ H -8.1362690 .5770200 .801634$ H -5.538106 $3.498495-0.534431$ H $-3.3081281 .884705-0.540513$ H $-3.964768 \quad 2.7642720 .837117$ H -5.2110441 .0958672 .263701$ H $\quad 0.954778 \quad 0.291731-2.535763$ H -1.314865 2.306883 -2.015807 H $1.9742152 .170856-3.916702$ H $2.5477412 .110931-2.232325$ H $2.6637403 .596205-3.158441$ H -3.1580340 .8736542 .351978$ H $-2.488437 \quad 0.071130 \quad 0.938219$ H $\quad 0.3712653 .2714082 .747551$ H $-0.792375 \quad 4.3267193 .579330$ H -1.258932 $5.485878 \quad 1.281366$ H 1.7092494 .7397001 .391138 H $1.565703 \quad 7.1975710 .612285$ H $0.0706996 .843157-0.219741$ H $3.7327875 .760014-2.015497$ H $3.7110036 .321371-0.250324$ H $1.7155965 .538669-3.252119$
H $\quad 0.3601716 .427397-2.636811$

H $-0.7619384 .512458-1.860237$

H -2.5574353 .2293232 .610842$

C $0.251266-2.7671913 .563282$

O $1.306138-1.9657262 .992828$

C $1.232515-0.6448833 .566437$

C $0.207399-0.7191004 .696160$

C $-0.741152-1.7797844 .175553$

H $\quad 0.8411000 .0113802 .783387$

C $2.614858-0.1570114 .013619$

H $0.662269-1.0448595 .638831$

H $-0.280027 \quad 0.244347 \quad 4.874759$

C $-1.635811-2.3823625 .243498$

H -1.366955 -1.316232 3.403812

C $3.687612-0.1141442 .905929$

O $3.102185-1.0164235 .052845$

H 2.5159090 .8351624 .469814

H $3.971897-1.1366612 .632730$

$\begin{array}{lllll}\text { O } & 4.866167 & 0.476987 & 3.470543\end{array}$

C 3.2884590 .6631791 .637477

C 4.4483850 .9712490 .671979

H 2.5159420 .1007361 .101963

O 2.6946581 .9127742 .000343

H $4.0248191 .505747-0.187400$

H 5.1280491 .6964341 .137930

H $-2.291970-3.1464644 .815090$

H -1.049829 -2.849180 6.042216

H -2.266644 -1.610725 5.696986

C $-0.300442-3.7550072 .529688$

H $3.142794-1.9187444 .691857$

H $4.640171 \quad 1.3765703 .761496$

H 3.3703702 .4485382 .448845

H $-0.974002-4.4632693 .026452$

H $0.534716-4.3411592 .125407$

H $\quad 0.725247-3.3547644 .361557$

C $3.748227-1.051966-1.509141$

O $4.405744-1.312364-0.266026$

C $5.249102-0.2571320 .199181$

C $6.3263100 .063513-0.848503$

C $5.7211480 .349767-2.220934$

C $4.759773-0.768433-2.626460$

H $3.096796-0.179355-1.384143$

C $2.813527-2.229346-1.801202$

H $5.771897-0.6513081 .078990$

O $7.1145241 .177307-0.441476$

H $7.005331-0.794049-0.942859$

H $5.2280311 .328726-2.239617$

O $6.7829930 .419088-3.176076$

H $5.350906-1.660740-2.865417$

O $4.083432-0.365569-3.814508$

C $3.538311-3.523584-2.083039$

H $2.151011-2.399570-0.945761$

H $2.164689-2.016152-2.658007$

H 7.5050390 .9584720 .421870

H $6.3778390 .568063-4.047742$

H $3.600296-1.136741-4.156059$

O $3.892490-4.127768-0.913495$

O $3.769636-3.940604-3.210753$

C $4.603357-5.361929-1.049203$

H $4.827818-5.736760-0.047103$

H $3.985533-6.099771-1.569931$

H $5.545089-5.199915-1.582274$

SCF Energy (B3LYP/6-31G**//MMFF) $=-3245.89015709$

01_534

MM̄FF Geometry

C $3.449738-3.031537-1.238073$

C $3.705811-3.604085-0.051658$

C $4.343614-2.9262511 .138918$

O $5.050446-1.7225130 .749951$

C $3.289027-2.4924332 .173928$

C $2.606471-3.6295722 .901247$

C $1.338560-4.0309682 .684366$

C $3.434518-4.2952013 .970184$

C $0.361885-3.5135581 .665246$

C $-0.840649-2.8056822 .302652$

C $-0.495822-1.4357962 .906067$

C $-1.733906-0.8145473 .555758$ 
C $-1.448554 \quad 0.593713 \quad 4.097748$

O $-0.024640-0.5883521 .852560$

O $-2.709132 \quad 1.213749 \quad 4.393944$

C -0.7817921 .4263582 .983688$

C -0.6670230 .5491715 .415820$

O $-0.3808792 .704122 \quad 3.474231$

C $0.400773 \quad 0.7105472 .285766$

C 0.8251031 .5215461 .039837

O 1.5011690 .6330993 .192581

C $6.330342-1.8594480 .317819$

O $6.948156-2.908826 \quad 0.194177$

C $6.941225-0.554995-0.027401$

C 6.3640660 .6366940 .194721

C $6.9387931 .945769-0.134957$

C $8.2792071 .959829-0.825068$

C 1.8778620 .8416930 .153252

C $2.0930961 .608766-1.141579$

C $3.4645571 .576664-3.218639$

O $1.4841422 .638696-1.420643$

C $4.6597262 .443575-2.978942$

C $4.5846553 .770834-2.799029$

C $5.7641594 .655941-2.514845$

C $5.7482905 .184274-1.096217$

C $5.0266326 .270703-0.772676$

C $6.6245454 .496406-0.071374$

C 6.2385953 .0601440 .168821

N $3.0433231 .003899-1.953109$

H $3.711526-1.991744-1.404629$

H $3.407552-4.6398080 .098338$

H $5.056683-3.6166211 .605472$

H $2.561303-1.8278061 .694901$

H $3.770502-1.8458992 .921495$

H $0.951065-4.8482703 .291717$

H $2.874559-5.0651844 .511713$

H $4.314221-4.7764863 .532529$

H $3.770506-3.5565144 .705208$

H $0.836385-2.8621640 .926733$

H $-0.007945-4.3796821 .101720$

H - $1.597285-2.6695411 .519826$

H -1.286944 -3.450522 3.070017

H $0.297920-1.5651823 .650091$

H -2.122162 -1.460918 4.352683

H -2.538964 -0.741233 2.810355

H -3.171835 0.6576625 .044039

H -1.556002 1.6234492 .229873

H -1.219672 -0.0251596 .169128$

H -0.5462421 .5531405 .837481$

H $0.3205620 .094745 \quad 5.313749$

H 0.4337862 .5803283 .990334

H $\quad-0.063974 \quad 1.708670 \quad 0.425430$

H 1.2153742 .5002911 .347557

H 2.3198420 .5143072 .686252

H $7.926664-0.633441-0.476048$

H $5.381730 \quad 0.6684190 .661593$

H $\quad 8.240648 \quad 1.376907-1.752274$

H $9.0506151 .535071-0.173263$

H $8.6181592 .957329-1.110131$

H 2.8375130 .7830830 .676705

H $1.563905-0.174651-0.107397$

H $2.6364622 .139662-3.662458$

H $3.7222100 .754677-3.893343$

H $5.6266151 .949374-2.941889$

H $3.6109674 .254490-2.838194$

H $5.7423585 .493575-3.224111$

H $6.7101324 .136206-2.710859$

H $5.027025 \quad 6.6723470 .235031$

H $4.4166876 .781061-1.510880$

H 6.5582495 .0135590 .895167

H $7.6681654 .606627-0.377631$

H 5.2702002 .9479180 .658392

H $3.5714630 .216612-1.590518$

C $1.240436-3.603580-2.250379$

O $0.847615-2.221086-2.291249$

C $-0.506141-2.145723-2.798980$

C $-0.896910-3.570556-3.184827$

C $0.448799-4.245768-3.381538$

H $-0.459615-1.502466-3.685631$

C $-1.384328-1.509523-1.713570$
H $-1.450148-4.059443-2.373184$

H -1.525781-3.599189-4.079922

C $0.379263-5.761031-3.317729$

H $0.859546-3.937703-4.352211$

C $-2.888199-1.487237-2.047218$

O $-0.902329-0.175132-1.533949$

H -1.218736 -2.036761 -0.767674

H -3.219322 -2.501686 -2.294063

O $-3.101521-0.691889-3.213145$

C $-3.774626-0.979269-0.887968$

C $-5.266779-0.975594-1.272466$

H $-3.617180-1.633841-0.022233$

O $-3.3694370 .332173-0.514921$

H $-5.432988-0.223093-2.050516$

H -5.513149-1.952273-1.705216

H $1.368679-6.204573-3.466178$

H $-0.002548-6.104952-2.350567$

H $-0.283586-6.148564-4.098278$

C $2.756834-3.730449-2.374963$

H $-1.3860510 .212761-0.785523$

H $-2.8229810 .215341-2.999394$

H -3.8201610 .5508010 .318279$

H $3.103271-3.275611-3.310833$

H $3.051596-4.785736-2.401427$

H $\quad 0.912567-4.007830-1.283700$

C $-6.3869261 .702171-0.250936$

$\begin{array}{lllll}\text { O } & -5.985858 & 0.572671 & 0.534653\end{array}$

C $-6.207308-0.703214-0.074077$

C -7.699839-0.872992-0.394736

C $-8.2248700 .276333-1.251951$

C $-7.8752001 .617828-0.609138$

H -5.782896 $1.746592-1.164865$

C -6.1009632 .9634640 .574001$

H -5.956609 -1.451801 0.688624

O $-7.919582-2.119218-1.045991$

H $-8.263136-0.8979780 .547313$

H $-7.8438440 .221878-2.278463$

O $-9.6486260 .190664-1.342474$

H -8.4881451 .7609720 .290170$

O $-8.1974082 .670166-1.516544$

C -4.6185013 .1759780 .763030$

H -6.5155453 .8645530 .107999$

H -6.5488262 .8828651 .572275$

H -8.879863 -2.249837-1.118056

H -9.862211 -0.606104-1.856600

H $-9.1393192 .580665-1.741804$

O $-4.0672433 .676217-0.379220$

O $-4.0217982 .915927 \quad 1.799845$

C $-2.6548563 .901941-0.331704$

H -2.4109974 .6230960 .454262$

H $-2.1273522 .958298-0.167329$

H -2.343414 4.313905-1.295227

SCF Energy (B3LYP/6-31G**//MMFF) $=-3245.91798503$

01535

MM̄FF Geometry

C $3.116240-3.4494591 .414347$

C $2.035970-4.2177131 .201959$

C $0.637909-3.9792911 .726391$

O $0.581235-2.7248972 .440439$

C $-0.345775-3.8981270 .542982$

C $-1.804720-3.9088810 .948529$

C $-2.595178-2.8182100 .972187$

C $-2.370101-5.2634651 .285402$

C $-2.196444-1.3931840 .711187$

C $-2.461586-0.902715-0.715970$

C $-3.891820-1.122637-1.231399$

C $-4.076734-0.425073-2.582328$

C $-5.516261-0.546281-3.101854$

O $-4.819581-0.601837-0.275029$

O $\quad-5.6798980 .396584-4.172922$

C -6.488809-0.125945-1.978594

C $-5.792565-1.930593-3.700218$

O $-7.840804-0.363788-2.364990$

C $-6.195898-0.808317-0.621092$

C $-7.090104-0.2535970 .518194$

O $-6.495618-2.205892-0.736452$

C $-0.085353-2.6750523 .620232$ 
O $-0.573928-3.6193914 .225398$

C $-0.220170-1.2811604 .109483$

C $0.307396-0.214522 \quad 3.487334$

C $0.143674 \quad 1.1887963 .878548$

C -0.7020201 .4813645 .090094$

C $-6.803851 \quad 1.1861980 .954001$

C $-5.6523551 .310361 \quad 1.939172$

C $-4.166468 \quad 3.0221502 .975980$

O $-5.159883 \quad 0.3514392 .527534$

C -2.8927412 .9598582 .195746$

C $-2.110954 \quad 4.0221361 .951903$

C -0.8324563 .9376821 .169271$

C 0.3859434 .3440361 .972390

C $1.2071815 .309327 \quad 1.525647$

$\begin{array}{llllll}\text { C } & 0.670975 & 3.633921 & 3.283427\end{array}$

C $0.7413412 .138850 \quad 3.128420$

N $-5.264624 \quad 2.632267 \quad 2.112034$

H $3.039969-2.5620502 .036578$

H $2.153131-5.1082310 .586368$

H $\quad 0.387022-4.8193142 .384805$

H $-0.182946-4.741772-0.140972$

H $-0.133241-2.999311-0.046710$

H -3.641862 -2.947707 1.244688

H $-3.436562-5.2197451 .530785$

H -2.255378 -5.9450490 .436233$

H $-1.851629-5.6920832 .148516$

H $-1.136754-1.2357020 .935698$

H -2.736069 -0.7568531 .423281$

H $-2.232520 \quad 0.171022-0.730823$

H $-1.755448-1.384111-1.404064$

H $-4.055981-2.200875-1.340436$

H $-3.366282-0.814735-3.322105$

H -3.839765 $0.643122-2.475156$

H $-5.0189790 .191260-4.856423$

H $-6.3888580 .959854-1.861120$

H $-5.119198-2.123845-4.544138$

H $-6.807388-1.989490-4.108611$

H -5.660377 -2.743269-2.983202

H $-7.9828990 .086895-3.215076$

H $-8.141209-0.3306870 .211305$

H -7.004572 -0.9112881 .393506$

H $-6.592503-2.5748310 .157030$

H $-0.817404-1.1781685 .009926$

H $0.898632-0.3704132 .586432$

H -0.7390002 .5401725 .353472$

H -0.3054330 .9627125 .970149$

H -1.734849 1.1550534 .925396

H $-6.624714 \quad 1.8369650 .093805$

H -7.690991 1.5705091 .473417

H -4.1120992 .3407763 .831176$

H -4.3800304 .0303113 .344373$

H -2.590350 1.9820671 .826950

H -2.405386 5.0019952 .319289

H -0.9453824 .5825280 .288404$

H -0.6781982 .9240610 .778318$

H 2.0871095 .6083832 .085572

H $1.021525 \quad 5.8212690 .587205$

H $-0.090148 \quad 3.9438274 .004052$

H 1.6335693 .9661983 .692706

H $1.351738 \quad 1.8184942 .285126$

H -5.6853283 .3517681 .531365$

C $4.956092-2.550399-0.019226$

O $5.374803-1.4828290 .859321$

C $6.625374-0.9654820 .385512$

C $6.792589-1.490562-1.032473$

C $6.165357-2.865781-0.899991$

H $7.404080-1.4326871 .004848$

C 6.7401550 .5505950 .569439

H $6.232734-0.884656-1.753735$

H $7.838767-1.526767-1.350338$

C $5.813106-3.498746-2.235031$

H $6.860770-3.523597-0.361528$

C $5.7583541 .453265-0.211274$

$\begin{array}{lllll}\text { O } & 8.076447 & 0.932535 & 0.212129\end{array}$

H $6.6417820 .779657 \quad 1.637533$

H $5.9587391 .382105-1.285530$

O 6.0602422 .8093840 .155840

C 4.2594741 .2103800 .058443
C $3.3298322 .330249-0.455118$

H $3.9580890 .255871-0.382304$

$\begin{array}{lllll} & & 4.063817 & 1.110280 & 1.471555\end{array}$

H $2.3253582 .146691-0.057481$

H $3.6327483 .282482-0.003668$

H $5.338934-4.474720-2.092391$

H $5.124116-2.869117-2.808025$

H $6.713891-3.645904-2.839793$

C $4.467598-3.7359540 .814022$

H $8.1742740 .816438-0.748245$

H $7.0034002 .957384-0.029376$

H 3.1749710 .7439601 .614897

H $5.167863-3.9335781 .634451$

H $4.420082-4.637200 \quad 0.192159$

H $4.142239-2.153116-0.635787$

C $1.3391801 .067223-2.412986$

O $2.747574 \quad 1.266857-2.600666$

C $3.2856122 .446609-1.991062$

C $2.5558593 .691166-2.514653$

C $1.0456543 .575899-2.341175$

C $0.5366582 .265136-2.935846$

H $1.1248080 .918128-1.348340$

C $0.952316-0.211663-3.172491$

H $4.3210192 .527997-2.340784$

O $3.0383654 .857716-1.857069$

H $2.7820763 .809573-3.582347$

H $0.7686493 .658388-1.286356$

O $0.4068104 .671874-2.999020$

H $0.5946712 .309974-4.030894$

O $-0.8362612 .096516-2.586372$

C $1.427638-1.461260-2.465006$

H $-0.134854-0.300410-3.270722$

H $1.403434-0.191284-4.171905$

H $2.5240905 .613753-2.188551$

H $0.6373574 .632158-3.942859$

H $-1.3142832 .885087-2.895251$

O $1.441981-2.497760-3.353241$

O $1.709928-1.527781-1.276994$

C $1.834717-3.771689-2.831926$

H $2.688687-3.682669-2.155084$

H $0.985540-4.231009-2.319935$

H $2.124566-4.407248-3.672841$

SCF Energy (B3LYP/6-31G**/MMFF) $=-3245.90455360$

01_536

MM̄FF Geometry

C $1.724726 \quad 0.7005873 .487071$

$\begin{array}{llll}\text { C } & 1.167106 & 0.233093 & 2.360784\end{array}$

C $0.011510-0.7312032 .321247$

O $-0.969876-0.2312241 .384328$

C $0.509700-2.0941981 .806430$

C $-0.561352-3.1661591 .769059$

C $-0.994450-3.7746680 .647498$

C $-1.132443-3.5683593 .104627$

C $-0.537250-3.547664-0.766273$

C $-1.350947-2.500686-1.542327$

C $-2.830375-2.870607-1.737391$

C $-3.503495-1.908057-2.717541$

C $-5.005000-2.195845-2.864680$

O $-3.487388-2.789754-0.470739$

O $-5.580461-1.069721-3.545174$

C $-5.648143-2.283569-1.462096$

C $-5.257563-3.413994-3.761482$

O $-7.003045-2.709619-1.583627$

C $-4.865590-3.188485-0.485720$

C $-5.369054-3.110342 \quad 0.976880$

O $-5.000988-4.552485-0.902960$

C $-1.7919910 .766232 \quad 1.807506$

$\begin{array}{llll}\text { O } & -1.824994 & 1.274616 & 2.916374\end{array}$

C -2.636626 1.1598870 .658355

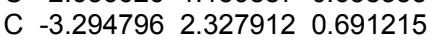

C $-4.1473192 .867415-0.370341$

C $-4.4770651 .960462-1.526385$

C $-5.327242-1.7165261 .613281$

C $-6.638756-0.9550591 .518536$

C $-7.623282 \quad 1.2821592 .003441$

O $-7.687973-1.464604 \quad 1.134489$

$\begin{array}{llll}\text { C } & -7.677978 & 2.072408 & 0.734257\end{array}$ 
C $-7.749305 \quad 3.4118940 .705106$ C $-7.8509794 .218883-0.557365$ C $-6.7910935 .293779-0.648427$ C $-7.0623186 .563042-0.300277$ C $-5.4458834 .912921-1.223662$ C $-4.6000474 .133482-0.254017$ N $-6.5062520 .357721 \quad 1.950952$ H $\quad \begin{array}{llll}1.339667 & 0.386767 & 4.453367\end{array}$ H 1.5770760 .5430101 .402950 H -0.446824 -0.8406523 .311550$ H $1.327888-2.4554022 .444241$ H $0.944866-1.9530970 .811130$ H -1.767046 -4.5366180 .746270$ H -1.783642 -4.446055 3.030532 H $-0.326970-3.818578 \quad 3.802775$ H -1.727355 -2.755203 3.531394 H $0.520746-3.265663-0.790433$ H -0.586561 -4.507495-1.296590 H -1.277344 -1.538493 -1.022516 H $-0.879200-2.374669-2.524775$ H -2.882864 -3.899054 -2.113442 H -3.010126 -1.939187 -3.696952 H -3.380810 -0.878400 -2.355272 H $-6.542458-1.209132-3.583467$ H -5.673905 -1.260414 -1.069483 H $-4.842988-3.242254-4.762174$ H $-6.329895-3.583844-3.908433$ H $-4.814143-4.332665-3.371757$ H -7.421335 -2.628863 -0.710292 H $-6.362371-3.5664681 .062694$ H $-4.707357-3.7500181 .579168$ H $-4.809323-5.127749-0.143788$ H $-2.6733120 .489509-0.192578$ H -3.1834182 .9631981 .569166$ H -4.877545 $1.008260-1.162831$ H $-3.5824711 .760503-2.125702$ H -5.235712 2.366359 -2.198494 H $-5.120156-1.8318722 .685082$ H -4.520448 -1.112528 1.187714 H -7.477105 1.9183422 .882107 H $-8.555195 \quad 0.7216482 .128874$ H -7.687003 $1.517693-0.201086$ H $-7.7691813 .961881 \quad 1.643533$ H -8.848526 $4.677146-0.579692$ H -7.800326 $3.579931-1.447760$ H $-6.3163407 .345869-0.389289$ H -8.035547 6.8463910 .086716 H -4.880783 5.817022 -1.488169 H $-5.5977284 .393696-2.173859$ H $-4.309121 \quad 4.7083330 .626432$ H $\quad \begin{array}{llll}-5.586650 & 0.710014 & 2.200501\end{array}$ C 4.1983950 .9038163 .822002 O $4.461548-0.0887822 .808365$ C $5.802906 \quad 0.0797122 .323260$

C 6.5210460 .8880303 .395982 C 5.4169101 .8292873 .837906 H 5.7369080 .6884491 .414370 C $6.432101-1.2773071 .996805$ H 6.8393350 .2606494 .236269 H 7.4006311 .4091963 .006404 C 5.6734172 .4826835 .184654 H 5.3035672 .6147603 .078520 C $5.604723-2.1817541 .057341$ O $6.608999-1.9953563 .227394$ H $7.438532-1.1242331 .589942$ H $4.705250-2.5113891 .592875$ O $6.388759-3.360103 \quad 0.821903$ C $5.202734-1.591905-0.310061$ C $6.388417-0.985062-1.084634$ H $4.779375-2.398160-0.922260$ O $4.179933-0.619233-0.141395$ H $6.716985-0.070247-0.582210$ H $7.226166-1.691006-1.056054$ H 4.8409173 .1355085 .465179 H 5.7978561 .7359475 .976085 H 6.5835243 .0904075 .150276 C 2.8996851 .6378553 .484071 H $7.025360-2.8465393 .008121$
H $5.840421-3.979710 \quad 0.310856$

H $3.442808-1.0419190 .331204$

H 2.9894012 .1126102 .498644

H 2.7116072 .4378844 .208886

H 4.1010220 .3666814 .773995

C $5.2399441 .594604-2.308438$

O $4.9677730 .248134-2.713578$

C $6.056670-0.666236-2.562436$

C $7.260768-0.177895-3.381750$

C $7.6514331 .250190-3.008008$

C $6.4328512 .169050-3.082415$

H $5.4414331 .621632-1.230846$

C $3.9764732 .420683-2.579489$

H $5.724847-1.610931-3.012314$

O $8.363891-1.058216-3.198310$

H $6.998918-0.206378-4.447587$

H $8.1172931 .296180-2.016568$

O $8.624527 \quad 1.735636-3.935547$

H $6.1594782 .324103-4.134266$

O $6.7725223 .443234-2.538634$

C $2.8475282 .002275-1.667500$

H $4.1439633 .490704-2.412810$

H $3.6549632 .305502-3.622078$

H $9.058940-0.795427-3.824603$

H $9.4386361 .221626-3.802119$

H $7.5411913 .774424-3.034024$

O $1.9190621 .293285-2.370259$

O $2.8097272 .259235-0.471271$

C $0.7955550 .817133-1.622557$

H $1.096687-0.044645-1.021105$

H $\quad 0.3789341 .605980-0.988806$

H $0.0259890 .499383-2.331153$

SCF Energy (B3LYP/6-31G**//MMFF) $=-3245.91401489$

01_537

MM̄FF Geometry

C $4.3223340 .604197-2.474807$

C $3.514527-0.031400-3.337142$

C $2.711485-1.250352-2.970667$

O $1.378449-1.028430-3.497654$

C $3.296193-2.543395-3.570853$

C $4.681594-2.914686-3.088274$

C $4.969356-3.361519-1.849687$

C $5.772330-2.787219-4.119286$

C $4.027078-3.549039-0.689433$

C $4.441230-2.6368130 .472766$

C $3.473140-2.5974341 .664118$

C $3.248918-3.9634722 .312409$

C $2.282388-3.8704183 .506686$

O $2.224378-2.0462501 .229553$

O $1.883583-5.2064323 .852450$

C $1.009784-3.1186083 .056925$

C $2.962755-3.2894234 .751529$

O $0.136243-2.8823454 .158747$

C $1.298805-1.8011782 .301641$

C $0.022138-1.1715541 .696684$

O $1.831954-0.8436293 .225950$

C $0.374285-1.800040-3.003723$

O $0.478587-2.705876-2.192727$

C $-0.893542-1.347148-3.617979$

C $-2.051335-1.866755-3.182761$

C $-3.392645-1.544478-3.672986$

C $-3.510471-0.533026-4.782407$

C $-0.723343-2.0514750 .692620$

C $-2.024144-2.5962501 .248033$

C $-4.371304-1.9177751 .754031$

O $-2.139204-3.7657091 .605937$

C $-5.207616-2.3419380 .588149$

C $-6.117806-1.553863-0.004010$

C $-7.007430-1.996886-1.130600$

C $-6.703909-1.294488-2.435156$

C $-7.207494-0.077749-2.701499$

C $-5.902266-2.048071-3.471697$

C $-4.448052-2.170298-3.110707$

N -3.024195 -1.638946 1.298249

H $4.4314090 .204473-1.471712$

H $3.4151530 .343304-4.353095$

H $2.633371-1.319508-1.879242$ 
H $3.290608-2.463290-4.667116$

H $2.626090-3.389620-3.374100$

H $6.004546-3.620128-1.628442$

H $6.755620-3.062014-3.723213$

H $5.564539-3.441172-4.972629$

H $5.838881-1.755668-4.479619$

H $2.988150-3.363159-0.972784$

H $4.082455-4.598214-0.377374$

H $5.433804-2.9382580 .832364$

H $4.540816-1.6121890 .092326$

H $3.919477-1.9109012 .393794$

H $4.201695-4.4100112 .621909$

H $2.812396-4.6562801 .580775$

H $2.685859-5.7133614 .064894$

H $\quad 0.473214-3.8031452 .389889$

H $3.830641-3.8982545 .032331$

H $2.290193-3.3136625 .616185$

H $3.310996-2.2639764 .612914$

H $0.500938-2.1412894 .671968$

H $-0.640384-0.8338122 .502617$

H $0.328465-0.2603401 .163886$

H 1.7046640 .0450972 .853985

H $-0.839483-0.602092-4.403076$

H -2.018431 -2.608359 -2.385022

H $-2.9871280 .393413-4.524703$

H $-4.541422-0.244388-4.999077$

H -3.078809 $-0.930272-5.707483$

H $-0.971831-1.455721-0.193497$

H $-0.116093-2.8906350 .340012$

H -4.754739 -1.007983 2.225531

H $-4.351824-2.7119642 .507338$

H $-5.073670-3.3614270 .234705$

H $-6.256624-0.5379040 .358223$

H -8.047175 -1.796864 -0.839492

H -6.954389 -3.083762 -1.271079

H $-7.0202300 .417498-3.648426$

H $-7.8141810 .451753-1.974175$

H $-6.034207-1.599420-4.461702$

H -6.316729 -3.059382 -3.575879

H $-4.257179-2.889591-2.313739$

H $-2.839816-0.7142590 .920888$

C $4.9833472 .957057-1.766936$

O $3.5996113 .261677-1.499974$

C $3.4391093 .440388-0.078933$

C 4.8464243 .6024930 .485970

C $5.6341002 .669654-0.412377$

H 3.0144042 .5077090 .311283

C 2.4973034 .6102130 .219536

H 5.2118434 .6305130 .379670

H 4.9030303 .3306971 .544363

C $7.1327272 .915802-0.382540$

H $5.4399651 .639502-0.091825$

C $1.0979274 .474600-0.414048$

O $3.0978165 .808403-0.287911$

H 2.4119684 .7406631 .305202

H $1.1624094 .565717-1.505354$

O $0.306200 \quad 5.580306 \quad 0.040855$

C $0.3533403 .173426-0.051371$

C - $-1.0972643 .166275-0.576955$

H $0.8841282 .318853-0.484809$

O $0.365166 \quad 3.0007291 .362471$

H -1.658230 $3.977750-0.102408$

H -1.069364 3.377290-1.652496

H $7.6539422 .228081-1.055819$

H $7.3785883 .938444-0.687858$

H 7.5270142 .7634980 .627484

C $5.0980441 .845595-2.816149$

H 2.6130046 .5617210 .088994

H $0.6569416 .383697-0.379059$

H $\quad-0.076310 \quad 3.772260 \quad 1.757174$

H $6.1518041 .578671-2.954419$

H $4.7376282 .241809-3.774189$

H $5.4179543 .870781-2.194121$

C -2.8840712 .3324371 .785680$

O $-1.967877 \quad 1.5089341 .054203$

C $-1.8307341 .826342-0.334808$

C $-3.1980011 .723280-1.025980$

C $-4.2494412 .583051-0.331637$
C -4.2845182 .2751391 .163892$

H -2.5229473 .3671001 .793464$

C -2.9118041 .8179603 .231629$

H -1.193686 $1.044274-0.767297$

O $-3.0882052 .100580-2.393545$

H -3.527902 $0.676481-1.008007$

H $-4.0727043 .648425-0.519778$

O $-5.5246852 .281279-0.900480$

H -4.7226641 .2817691 .311548$

O -5.1475073 .2068831 .813073$

C -1.6396882 .1718863 .968449$

H -3.7380362 .2521753 .804953$

H $-3.036431 \quad 0.728777 \quad 3.236312$

H -3.974932 $2.027278-2.786595$

H $-6.1893592 .797181-0.412822$

H -4.7683064 .0951161 .699033$

O -1.1216871 .0601964 .564228$

O -1.1726353 .3022024 .026779$

C 0.0839831 .2652575 .306664

H $-0.104591 \quad 1.9314996 .153787$

H 0.4170100 .2967115 .688892

H 0.8654911 .6749424 .659442

SCF Energy (B3LYP/6-31G**//MMFF) $=-3245.91408966$

01538

MM̄FF Geometry

C 3.0550591 .3557943 .045746

C 3.6064231 .9425271 .972355

C 2.8837212 .8248980 .979140

O 1.5293623 .0530291 .439065

C 3.5645484 .1950400 .792615

C 4.9433394 .1548970 .173610

C $5.1832223 .983265-1.140903$

C 6.0865624 .3981471 .124882

C $4.1844163 .772462-2.248689$

C $4.5556662 .574034-3.135693$

C $4.2288641 .212066-2.497912$

C $5.0600280 .112779-3.164538$

C $4.702763-1.276882-2.624256$

O $2.8292090 .962839-2.692306$

O $5.342877-2.256342-3.455519$

C $3.178011-1.463241-2.735206$

C $5.273729-1.500919-1.221048$

O $2.775818-2.696278-2.139589$

C $2.351649-0.282147-2.162321$

C $0.878811-0.461284-2.607360$

O $2.436649-0.315848-0.738454$

C $0.587353 \quad 3.3840960 .519233$

O $0.746943 \quad 3.525458-0.681893$

C -0.6909383 .5604671 .242555$

C -1.7599684 .0247470 .580722$

C $-3.089322 \quad 4.263477 \quad 1.142515$

C -3.3220053 .9064172 .586536$

C $-0.0922720 .650918-2.187563$

C $-1.4856640 .401854-2.749120$

C $-3.8593161 .027387-2.478179$

O $-1.709715-0.420941-3.634552$

C $-4.6294292 .273682-2.185924$

C $-5.6855322 .307673-1.358606$

C $-6.5185193 .530058-1.092305$

C -6.4312013 .9868510 .347131$

C -7.2304423 .4586531 .289192$

C -5.4682575 .1037320 .680988$

C -4.0434434 .7722650 .336593$

N $-2.451974 \quad 1.220363-2.175828$

H 1.9989831 .4989543 .259703

H 4.6573631 .7490341 .770509

H 2.8427362 .2835770 .028875

H 3.6040154 .7144081 .760458

H 2.9267794 .8464200 .180604

H $6.2199954 .019031-1.474472$

H 7.0601294 .3612930 .624610

H 5.9895135 .3869421 .585437

H 6.0983553 .6469321 .919826

H $3.1622193 .664058-1.878961$

H $4.1981474 .675182-2.872016$

H $4.0025892 .668485-4.079277$

H $5.6233002 .632552-3.382717$ 
H $4.4446551 .245615-1.425318$ H $6.1332700 .303807-3.039131$ H $4.8785130 .127443-4.248447$ H $5.025313-2.137627-4.366797$ H $2.945668-1.558556-3.804614$ H $6.365873-1.401734-1.232599$ H $5.071035-2.517719-0.866968$ H $4.885430-0.794649-0.485585$ H $2.768177-2.570229-1.175323$ H $0.855128-0.527138-3.703528$ H $0.493302-1.412310-2.221321$ H $1.6675400 .139636-0.362522$ H $-0.714215 \quad 3.3146052 .298151$ H -1.653432 $4.259368-0.477971$ H -2.718357 4.5445243 .241005 H -3.055976 2.8601942 .774747 H -4.3639424 .0178362 .896289$ H -0.164343 $0.705768-1.097118$ H $\quad 0.2507791 .618567-2.568058$ H -4.206151 $0.177425-1.881458$ H -3.968676 $0.763474-3.535465$ H -4.326729 $3.179677-2.704895$ H -5.996731 $1.391918-0.860971$ H -7.560616 $3.289746-1.341082$ H $-6.2448274 .354412-1.762664$ H -7.1910183 .7960242 .319654$ H -7.939251 2.672777 1.049997 H -5.5530965 .4026351 .732024$ H $-5.758863 \quad 5.9973430 .113192$ H -3.785943 $4.993078-0.700017$ H $-2.2090711 .740335-1.337204$ C $3.579783-1.0025643 .846599$ O $2.179246-1.2941743 .659936$ C $2.055194-2.2319372 .572216$ C $3.438073-2.8429122 .395740$ C $4.311874-1.6320962 .660629$ H $1.831773-1.6504991 .672734$ C $0.934959-3.2347922 .847851$ H $3.632170-3.6271713 .136927$ H $3.582147-3.2681911 .398025$ C $5.764378-1.9769572 .936184$ H $4.263852-0.9695721 .788865$ C -0.467471 -2.601404 2.980093 O $1.238333-3.9096894 .076197$ H $0.930344-4.0075802 .071386$ H $-0.468897-1.9433483 .858050$ O $-1.387506-3.6579893 .277828$ C $-0.984476-1.8225411 .749006$ C $-0.779088-2.5060750 .381562$ H -2.055720 -1.626792 1.875472 O $-0.352121-0.5419871 .685251$ H - $-1.078733-1.787655-0.389996$ H $0.290631-2.6627240 .202843$ H $6.348586-1.0733873 .136661$ H $5.862570-2.6415943 .801100$ H $6.209595-2.4816252 .072403$ C 3.8012850 .5009524 .030886 H $\quad 0.517637-4.5407194 .244565$ H -2.259508 -3.2552253 .429337$ H -0.521657 -0.0871692 .527842$ H 4.8731890 .7301684 .019246 H 3.4393500 .7868935 .027082 H $3.878408-1.5095424 .773637$ C -3.651729-2.895291-0.520111 O $-2.946446-3.6731490 .455083$ C -1.548194 -3.8281060 .194731$ C $-1.339235-4.506282-1.167288$ C $-2.090449-3.794745-2.288925$ C $-3.549968-3.557740-1.898552$ H $-3.231017-1.883991-0.561439$ C $-5.106810-2.782233-0.055616$ H $-1.170438-4.5309510 .944766$ O $0.043891-4.569951-1.498695$ H $-1.704027-5.540416-1.113633$ H $-1.597222-2.855551-2.558315$ O $-2.065407-4.610444-3.462341$ H $-4.080662-4.518055-1.896015$ O $-4.175887-2.738960-2.883519$
C $-5.204937-1.9626711 .209772$

H $-5.748395-2.316523-0.811950$

H -5.522845 -3.7737240 .160790$

H $0.495804-5.043034-0.779300$

H -1.131724 -4.776056-3.679032

H $-3.673238-1.908980-2.945528$

O $-4.977767-0.647150 \quad 0.932411$

O $-5.445739-2.4372512 .312137$

C -5.0083510 .2346312 .058149$

H $-4.252813-0.0594702 .792955$

H $-4.777308 \quad 1.242067 \quad 1.704248$

H -6.0056950 .2396632 .507479$

SCF Energy (B3LYP/6-31G**//MMFF) $=-3245.91835799$

01_539

MM̄FF Geometry

C $0.872508 \quad 3.458816 \quad 2.209949$

C $0.2519782 .311788 \quad 2.522710$

C $0.917874 \quad 0.965127 \quad 2.655943$

O $-0.117066-0.038312 \quad 2.493029$

C $2.079650 \quad 0.6769281 .670099$

C 1.7855480 .8709590 .189957

C $2.4930521 .661966-0.641379$

C $0.6489950 .058225-0.373829$

C $3.6413042 .571632-0.317457$

C $4.9852852 .128679-0.910141$

C $5.5813180 .885600-0.226246$

C $7.0890050 .819531-0.488783$

C $7.721599-0.4399360 .115273$

O $4.944256-0.283944-0.755485$

O $9.044943-0.572708-0.425436$

C $6.915901-1.664861-0.354269$

C $7.882071-0.3318611 .636016$

$\begin{array}{llll}\text { O } & 7.375660 & -2.851710 & 0.289868\end{array}$

C $5.385696-1.517056-0.166462$

C $4.678757-2.689126-0.891003$

O $5.080163-1.571181 \quad 1.227932$

C $0.096554-1.2699593 .027493$

O $1.055749-1.6316503 .689318$

C -1.045228 -2.122859 2.634724

C $-0.996123-3.4445522 .854251$

C -2.069914 -4.3775402 .528131$

C $-2.300975-5.4202263 .588527$

C $3.150697-2.582762-0.961977$

C $2.542193-3.752589-1.719444$

C $0.394005-4.584231-2.616352$

O $3.213219-4.663162-2.199324$

C $-1.047341-4.552265-2.221855$

C $-1.727701-5.644186-1.840390$

C $-3.198020-5.659975-1.531974$

C $-3.503410-6.170486-0.141808$

C $-3.515935-7.487464 \quad 0.125550$

C $-3.890297-5.1615700 .912659$

C $-2.735856-4.3089931 .357773$

N $1.156844-3.662509-1.796292$

H 1.9492023 .4700542 .074810

H -0.8231102 .3362642 .685376$

H 1.2917950 .8905153 .685613

H $2.394692-0.3672611 .787683$

H 2.9441521 .2703681 .981679

H $2.1986381 .689392-1.690402$

H $-0.3088970 .472396-0.048937$

H $0.6474640 .044688-1.469144$

H $0.714010-0.980661-0.038817$

H $3.3940173 .557343-0.733184$

H 3.7583812 .7342330 .756818

H $4.8711121 .948954-1.986789$

H $5.6774742 .972059-0.792361$

H 5.3894090 .9474750 .851250

H $7.5912971 .716723-0.105617$

H $7.2698640 .815149-1.572993$

H $9.5438760 .230584-0.197549$

H $7.119976-1.796679-1.426285$

H $8.515203 \quad 0.5255411 .893960$

H $8.391339-1.2126722 .042681$

H $6.932275-0.2098252 .160650$

H $8.333580-2.9137750 .133382$

H $5.051997-2.745820-1.922274$ 
H $4.938884-3.635501-0.399420$ H $4.176853-1.903617 \quad 1.343897$ H -1.900899-1.648210 2.166632 H $-0.126284-3.8861973 .335765$ H $-2.706513-4.9562534 .494280$ H -1.361672 -5.9212133 .850016$ H $-2.993489-6.2066553 .279833$ H $2.714348-2.5682950 .041676$ H $2.865827-1.663298-1.483952$ H $0.828462-5.586175-2.529887$ H $0.501505-4.261752-3.657569$ H $-1.564163-3.599416-2.300011$ H $-1.208211-6.599051-1.794502$ H $-3.691610-6.306562-2.269618$ H -3.642183 -4.667437 -1.673820 H $-3.765637-7.8625591 .112555$ H -3.273066 -8.219209-0.637997 H $-4.369219-5.6557311 .764915$ H $-4.671583-4.5058750 .509391$ H -2.456954 -3.535597 0.642416 H $0.719266-2.783975-1.533690$ C $0.247823 \quad 5.177307 \quad 0.529227$ O $-0.5709864 .287013-0.256183$ C -1.230699 $5.048816-1.285071$ C $-0.6264176 .447723-1.233978$ C $-0.287494 \quad 6.5765910 .239050$ H -2.286959 5.116633 -1.000970 C $-1.089578 \quad 4.335559-2.634223$ H $0.2824226 .519949-1.842804$ H -1.325572 $7.213540-1.583548$ C $0.696707 \quad 7.693390 \quad 0.538972$ H -1.2147616 .7490940 .801600$ C -1.650195 $2.896241-2.647333$ O $0.299945 \quad 4.264547-2.981414$ H - $1.5658624 .932444-3.420275$ H $-1.0378982 .263602-1.996268$ O $-1.4844832 .374614-3.971803$ C $-3.1333292 .785901-2.247276$ C $-3.742136 \quad 1.384072-2.429939$ H -3.257425 3.100377 -1.208506 O $-3.8875263 .697658-3.052042$ H $-4.8142251 .456065-2.209910$ H $-3.6944541 .103665-3.489631$ H $\quad 0.919657 \quad 7.741042 \quad 1.609464$ H 1.6416587 .5502760 .004137 $\begin{array}{lllll}\mathrm{H} & 0.283225 & 8.661335 & 0.237441\end{array}$ C $\quad 0.169435 \quad 4.7703202 .000062$ H $0.7554553 .782786-2.269819$ H $-2.0441052 .902738-4.566827$ H $-4.8072243 .675916-2.737013$ H -0.8790624 .6938502 .309583$ H $\quad 0.648337 \quad 5.5226792 .635901$ H 1.2763205 .0790860 .156361 C $-4.3618880 .632136 \quad 0.471312$ O $-3.0800670 .628523-0.168875$ C $-3.0942260 .288727-1.560007$ C $-3.705781-1.105600-1.751972$ C $-5.097044-1.192768-1.132342$ C $-5.051120-0.7327710 .323631$ H $-4.988301 \quad 1.4197860 .034959$ C -4.1417790 .9572561 .958870$ H -2.046290 $0.214730-1.871666$ O $-3.756239-1.427050-3.136962$ H $-3.055430-1.846677-1.269941$ H $-5.832930-0.615006-1.703391$ O $-5.546987-2.547956-1.154410$ H $-4.526464-1.4854250 .926287$ O $-6.384235-0.640686 \quad 0.823712$ C -3.8114472 .4136292 .208582$ H -5.0536550 .7347492 .524723$ H $-3.3142490 .363158 \quad 2.361541$ H -4.011687 -2.361717 -3.213958 H $-5.671176-2.801293-2.084561$ H $-6.798942-1.5125630 .705412$ O $-3.9428372 .668936 \quad 3.544779$ O $-3.462196 \quad 3.218217 \quad 1.357444$ C -3.670132 4.0125833 .955710 H -2.5911534 .1863813 .950213$
H -4.1823484 .7335593 .311088$

H -4.0400494 .1359464 .976998$

SCF Energy (B3LYP/6-31G**//MMFF) $=-3245.89497887$

01_54

MM̄FF Geometry

C $5.704052-2.1797700 .035201$

C $4.703576-2.150723-0.857108$

C $3.724438-3.279292-1.048322$

O $2.418131-2.811759-0.639609$

C $3.656363-3.664189-2.538203$

C $2.737895-4.837637-2.826566$

C $1.572751-4.759202-3.501309$

C $3.221898-6.174329-2.324116$

C $0.929416-3.538160-4.101227$

C $-0.196918-3.028449-3.204109$

C $-0.883365-1.747427-3.699623$

C $0.066197-0.549036-3.739123$

C $-0.6613310 .755110-4.095567$

O $-1.937059-1.484265-2.765836$

$\begin{array}{lllll}0 & 0.247196 & 1.823498 & -3.785594\end{array}$

C $-1.9065530 .909319-3.195630$

C $-0.9349090 .841774-5.601466$

O $-2.7191352 .002186-3.615128$

C $-2.773551-0.367991-3.094018$

C $-3.797833-0.215650-1.948103$

O $-3.528709-0.604662-4.282435$

C $2.121072-2.8668030 .688666$

O $2.841228-3.2650891 .589978$

C $0.745472-2.3499010 .859428$

C $0.209368-2.2411522 .084441$

C $-1.138030-1.7496542 .386476$

C $-2.050848-1.4873261 .219013$

C $-4.641933-1.475223-1.716779$

C $-5.395880-1.420979-0.398866$

C $-6.473802-2.943605 \quad 1.259510$

$\begin{array}{llll}\text { O } & -5.557761 & -0.386606 & 0.242698\end{array}$

C $-5.391375-3.3310732 .219679$

C $-5.271767-2.8242493 .456091$

C $-4.172297-3.2143524 .404667$

C $-3.526508-2.0217875 .077226$

C $-3.617624-1.8509946 .407212$

C $-2.786493-1.0201804 .217788$

C $-1.491566-1.5634303 .675671$

N $-5.868773-2.673656-0.032053$

H $5.842122-3.0600880 .657907$

H $4.555568-1.257407-1.459745$

H $4.008203-4.154065-0.450460$

H $4.660880-3.921319-2.900878$

H $3.351228-2.785194-3.114942$

H $0.999671-5.675075-3.642672$

H $2.584837-6.999919-2.659460$

H $4.234013-6.374185-2.690839$

H $3.235782-6.194787-1.230395$

H $1.667740-2.757033-4.288889$

H $0.524202-3.807291-5.084365$

H $-0.959447-3.812138-3.092744$

H $0.181663-2.858002-2.190548$

H $-1.318052-1.941061-4.686927$

H $0.891240-0.712439-4.441534$

H $0.533835-0.428247-2.752388$

H $-0.1696752 .661155-4.049275$

H $-1.5452561 .155753-2.187723$

H $0.0090660 .860431-6.159618$

H $-1.453114 \quad 1.771947-5.859077$

H $-1.5228170 .001484-5.977495$

H -2.178488 $2.808255-3.568717$

H -3.262363 $0.020784-1.021385$

H $-4.4766860 .621863-2.153084$

H $-3.3217650 .087473-4.929606$

H $0.206210-2.062220-0.034986$

H $0.811020-2.5255292 .946453$

H $-2.184054-2.3968980 .622413$

H $-1.635819-0.7048940 .575647$

H $-3.049291-1.1598251 .509890$

H $-5.371841-1.597368-2.524785$

H $-3.999040-2.362594-1.700722$

H $-7.170401-3.7763091 .124643$ 
H -7.028818 -2.061523 1.594557 H -4.668506 -4.069656 1.879853 H -6.008153 -2.106997 3.810975 H -3.398039 -3.799082 3.893212 H -4.608809 -3.882749 5.158091 H -3.161736 -0.999065 6.901000 H -4.149357 -2.559014 7.034264 H -2.535407 -0.1322674 .813464$ H -3.455431 -0.6466193 .438847$ H $-0.756210-1.7859634 .450276$ H $-5.624478-3.475611-0.605848$ C $6.177656-0.0312701 .310942$ $\begin{array}{llll}\text { O } & 5.039020 & 0.699658 & 0.814937\end{array}$ C 4.1356620 .9462311 .910343 C 4.8812470 .5360743 .178858 C $5.755876-0.5929432 .667357$ H 3.2830930 .2718431 .775331 C 3.6681732 .4058761 .891234 H 5.5024241 .3505643 .569240 H 4.1989460 .2246553 .975850 C $6.918664-0.9326273 .582159$ H $5.123978-1.4813782 .547534$ C 3.0119712 .8741570 .573815 O 4.8047543 .2537612 .120404 H 2.9926062 .5867972 .733662 H $3.7603242 .829838-0.227889$ O $2.665877 \quad 4.257168 \quad 0.708434$ C 1.7548032 .1146350 .111605 C 0.6671031 .8645971 .174618 H $1.3042292 .672686-0.717777$ $\begin{array}{lllll}\text { O } & 2.159473 & 0.854176 & -0.427003\end{array}$ H $-0.130405 \quad 1.2867470 .691647$ H 1.0604071 .1931181 .947249 H $7.522910-1.7409793 .158363$ H $7.572881-0.0683693 .738753$ H $6.554733-1.2599454 .561558$ C $6.652034-1.0367960 .258237$ H 5.4529963 .0703181 .418642 H 3.4689004 .7328040 .982069 H $1.3637850 .404844-0.758312$ H $7.623503-1.4508500 .552653$ H $6.810511-0.511750-0.692635$ H $6.9736380 .713309 \quad 1.446945$ C -1.475454 3.7554110 .085562 O $-0.335147 \quad 4.1064590 .876185$ C 0.0681153 .1238391 .831706 C -1.0843872 .8388072 .805806$ C -2.3704332 .4649172 .073556$ C -2.6885803 .4939200 .987879$ H -1.247520 $2.864330-0.510640$ C $-1.7458404 .919520-0.875516$ H $0.866306 \quad 3.5841702 .424434$ O $-0.734566 \quad 1.7991113 .712062$ H -1.279761 3.7365563 .406855 H -2.306455 1.4541711 .658022 O H -3.0100204 .4274371 .466491$ $\begin{array}{lllll}\text { O } & -3.788200 & 3.027258 & 0.209255\end{array}$ C $-0.7363414 .953434-1.998758$ H $-2.7339854 .828348-1.341018$ H $-1.7196075 .886669-0.359737$ H $0.0742872 .074563 \quad 4.176224$ H -4.2529332 .2205482 .533033$ H $-3.5143142 .205105-0.231011$ O $0.4529895 .445743-1.551153$ O $-0.9782524 .580047-3.139252$ C $1.5049765 .490268-2.519930$ H $2.3955485 .899779-2.035918$ H $1.7328264 .481341-2.876895$ H $\quad 1.2263716 .142382-3.353105$ SCF Energy (B3LYP/6-31G**//MMFF) $=-3245.92309769$

01_540

MM̄FF Geometry

C -2.0708271 .6327071 .981573$

C -1.4724860 .5253432 .444189$

C -1.979654 -0.871053 2.191986

O $-0.881810-1.5889351 .583227$
C $-2.430903-1.5570333 .492940$

C $-3.712240-0.9791804 .057367$

C $-4.946706-1.3068353 .625963$

C -3.541764 -0.018948 5.205729

C $-5.315114-2.2798782 .538264$

C $-6.347803-1.7171061 .549802$

C $-5.769196-0.7503720 .500220$

C $-6.913797-0.127993-0.302494$

C $-6.4035740 .750146-1.448274$

O $-4.918427-1.500953-0.371892$

O $\quad-7.5330151 .043717-2.284584$

C $-5.387443-0.056849-2.285406$

C $-5.8899092 .099172-0.930386$

O $-4.8167370 .790264-3.279619$

C $-4.296646-0.752687-1.437602$

C $-3.493782-1.809442-2.243167$

O $\quad-3.4132020 .236381-0.921657$

C $-1.153765-2.7458860 .924146$

O $-2.207931-3.3589580 .900811$

C $0.061639-3.1194550 .174997$

C $0.151436-4.312540-0.428619$

C $1.303407-4.706608-1.229674$

C $1.951343-5.983916-0.779268$

C $-2.587532-1.337440-3.383292$

C $-1.278082-0.702575-2.943638$

C $0.7243850 .451031-3.880845$

O $-0.891471-0.685314-1.778844$

C $1.787103-0.523484-4.281426$

C $2.777104-0.923561-3.468200$

C $3.865242-1.872828-3.881541$

C $3.928026-3.112210-3.016701$

C $4.944153-3.310662-2.160335$

C $2.853766-4.162137-3.201091$

C $1.692200-3.946966-2.272913$

N $-0.575820-0.177687-4.019550$

H $-2.983110 \quad 1.5368881 .400047$

H -0.5753600 .6221563 .050830$

H -2.804902 -0.846676 1.473444

H -1.629506 -1.505493 4.242332

H -2.561819 -2.634030 3.333347

H $-5.798847-0.8525104 .130399$

H $-4.5009350 .343937 \quad 5.590421$

H -3.020286 -0.5101706 .033984$

H -2.9601350 .8553834 .900778$

H -4.442932 -2.648490 1.995311

H $-5.763532-3.1517843 .031425$

H $-6.793432-2.5728201 .024776$

H -7.156700 -1.226520 2.105750

H -5.1821110 .0252101 .003694$

H -7.5816550 .4458830 .352257$

H $-7.534560-0.927799-0.731288$

H $-7.2076381 .540325-3.055200$

H $-5.951471-0.827433-2.827528$

H $-6.6860452 .629997-0.394733$

H $-5.5978122 .757918-1.755524$

H $-5.0413062 .000265-0.250755$

H $-4.1381101 .337301-2.848149$

H -2.881119 -2.386168 -1.538668

H $-4.208658-2.537109-2.651595$

H -2.576479 -0.196649 -0.685842

H $0.856036-2.3845710 .096864$

H $-0.636406-5.054450-0.328580$

H $\quad 1.263675-6.827008-0.907438$

H $2.223016-5.9209930 .280765$

H $2.867029-6.221453-1.324958$

H $-2.308260-2.222008-3.971534$

H -3.112430 -0.664971-4.064684

H $\quad 0.8544130 .801135-2.852674$

H $0.7432841 .317620-4.548797$

H $1.750935-0.900801-5.300729$

H $2.821846-0.535765-2.453393$

H $4.816216-1.326597-3.832091$

H $3.752149-2.175381-4.930121$

H $5.004604-4.210529-1.556809$

H $5.733199-2.576357-2.037884$

H $3.288493-5.160060-3.084776$

H $2.487186-4.138131-4.235069$

H $1.102258-3.059444-2.501766$ 
H $-0.948063-0.292390-4.957810$

C -1.0898613 .8134061 .063965$

O 0.2014493 .3303000 .649749

C $0.354753 \quad 3.606973-0.759387$

C $-0.9339194 .293030-1.218487$

C $-1.9439463 .801835-0.199495$

H $0.4354682 .636762-1.260586$

C $1.6193744 .442013-1.008018$

H $-0.8509185 .385434-1.180804$

H -1.197300 $4.021677-2.245903$

C $-3.1922904 .660601-0.112492$

H $-2.2244032 .778626-0.475254$

C $2.9278743 .806339-0.496334$

O $1.4782045 .711953-0.355130$

H $1.7078114 .665187-2.077507$

H 2.9173343 .7669200 .598806

O $4.0073004 .676469-0.861312$

C $3.2259212 .411721-1.071960$

C $4.6196231 .853700-0.725604$

H $2.4647191 .703602-0.729167$

O $3.1276682 .457831-2.498537$

H $4.7024220 .863301-1.190708$

H $5.3909932 .456943-1.221398$

H -3.716649 4.675143 -1.073327

H $-3.881394 \quad 4.2707900 .643229$

H -2.9505465 .6950310 .153923$

C -1.603480 3.0296562 .274529

H 1.3590245 .5416100 .594991

H $3.8095725 .553804-0.490727$

H $3.7694663 .116078-2.816267$

H -2.4486643 .5708692 .717182$

H -0.813896 3.0023403 .036314

H $-0.929844 \quad 4.8501371 .391996$

C $3.709491-0.2552341 .296289$

O $3.849771 \quad 1.1511221 .507303$

C 4.9187351 .7630930 .783037

C 6.2555241 .0874331 .125093

C $6.198020-0.4279400 .932057$

C $4.989682-1.0127311 .664028$

H $3.484847-0.4341740 .236723$

C $2.473328-0.7371222 .066448$

H 4.9859062 .7941081 .151394

O 7.2954221 .6484530 .331049

H 6.4992821 .3000022 .174379

H $6.181053-0.698915-0.129988$

O $7.375354-1.0232001 .481749$

H $5.171985-0.9747302 .744091$

O $4.840125-2.3852151 .307104$

C $2.515480-0.5054623 .562836$

H $1.595995-0.1952981 .694468$

H $2.330208-1.8083391 .890900$

H $8.140142 \quad 1.298757 \quad 0.660527$

H $\quad 8.129515-0.7355410 .940377$

H $5.678754-2.8320501 .513978$

O $1.493341-1.2049414 .141561$

O $3.311816 \quad 0.212848 \quad 4.150305$

C $1.371635-1.0590025 .559686$

H $2.285058-1.3990356 .056768$

H $1.156595-0.0167405 .814089$

H $\quad 0.537500-1.6809205 .895133$

SCF Energy (B3LYP/6-31G**//MMFF) $=-3245.92408464$

01541

MM̄FF Geometry

C $-5.636250-2.333894-0.855584$

C $-4.336626-2.616540-0.681107$

C -3.380205 -2.833947 -1.826030

O $-2.294351-1.885738-1.718417$

C $-2.776591-4.247830-1.752449$

C -1.898354 -4.580297-2.943603

C $-0.561087-4.743906-2.904709$

C $-2.631414-4.772256-4.248769$

C $0.372880-4.650223-1.729444$

C $1.393839-3.524116-1.915754$

C $2.603044-3.628084-0.973009$

C $2.221641-3.5276430 .505592$

C $3.458501-3.5249091 .419711$

O $3.491483-2.557025-1.323753$
O $3.043023-3.1171372 .731760$

C $4.448678-2.4577650 .910052$

C $4.057512-4.9273311 .577174$

O $5.672465-2.5023251 .638935$

C $4.733235-2.559878-0.603342$

C $5.584765-1.382539-1.132608$

O $5.474350-3.757752-0.865565$

C $-2.499096-0.640056-2.226753$

O $-3.524038-0.206143-2.727341$

C -1.237412 $0.115091-2.064776$

C $-1.2086251 .432294-2.315166$

C $-0.0449352 .307620-2.161836$

C $1.2890721 .661058-1.886984$

C $4.9975360 .004361-0.868379$

C $5.7253481 .101634-1.624768$

C $5.6877373 .564385-1.964075$

O $6.6804090 .892743-2.367380$

C $4.5984414 .586221-2.004342$

C $3.9260024 .906333-3.120127$

C $2.8114675 .912329-3.168991$

C $1.4619785 .248507-3.352700$

C $0.8985005 .188540-4.571622$

C $0.8225694 .715394-2.084897$

C $-0.2149163 .641626-2.279158$

N $5.1631142 .346628-1.376704$

H $-6.030636-2.265722-1.866541$

H -3.936132 -2.691696 0.326626

H -3.889604 -2.697313 -2.787817

H $-3.580447-4.994860-1.702815$

H -2.217924 -4.352133 -0.816832

H $-0.058263-4.999056-3.837887$

H -1.975759 -5.142864 -5.044174

H $-3.438679-5.502081-4.127511$

H $-3.062475-3.828010-4.594341$

H $-0.161513-4.505898-0.787905$

H $0.895194-5.611419-1.647599$

H $1.769443-3.520256-2.948357$

H $\quad 0.903938-2.550782-1.784893$

H $3.105229-4.581546-1.175574$

H $1.543135-4.3367160 .799642$

H $1.667667-2.5940810 .669780$

H $2.348352-3.7289753 .029825$

H $3.993790-1.4824301 .124212$

H $3.323104-5.6086122 .023795$

H $4.911766-4.9170182 .263252$

H $4.379237-5.3674020 .631320$

H $5.450111-2.4285602 .582899$

H $6.600662-1.436948-0.719573$

H $5.704172-1.510356-2.217802$

H $5.934665-3.655370-1.715056$

H $-0.367776-0.430184-1.716879$

H $-2.1274811 .924307-2.631369$

H $2.1262202 .359452-1.952776$

H $1.4967650 .874144-2.621045$

H $1.3078411 .219318-0.885216$

H $3.9428860 .034423-1.163961$

H 5.0687010 .2390260 .198195

H $6.088706 \quad 3.345608-2.959697$

H $6.5150403 .913755-1.338073$

H $4.3452135 .075956-1.067188$

H $4.1765394 .407855-4.053968$

H $3.0165936 .608231-3.992623$

H $2.7916306 .530699-2.262618$

H $-0.0746674 .741136-4.739098$

H $1.4023995 .596486-5.442240$

H $0.3513365 .555776-1.558510$

H $1.5971744 .355314-1.399163$

H -1.212172 4.025914 -2.495436

H $4.4856412 .429840-0.623055$

C $-7.035740-0.6651000 .473208$

$\begin{array}{llll}\text { O } & -5.935622 & 0.094426 & 1.010308\end{array}$

C -5.9469971 .4047900 .411373$

C $-7.287787 \quad 1.532777-0.307031$

C $-7.5237480 .102921-0.755159$

H $-5.1476631 .408314-0.339788$

C -5.6699162 .4880851 .461541$

H $-8.087633 \quad 1.8524130 .370682$

H -7.246178 2.243411-1.138152 
C $-8.963252-0.193645-1.135298$ H $-6.879610-0.086089-1.622277$ C $-4.416494 \quad 2.236743 \quad 2.324892$ O -6.7947412 .5583102 .347084$ H -5.6049173 .4637850 .964796$ H -4.6084301 .4254333 .038183$ O -4.1718663 .4131043 .105435$ C $-3.1407861 .915506 \quad 1.527749$ C -1.8969571 .8017652 .429122$ H $-3.2890940 .982100 \quad 0.980937$ $\begin{array}{llll}\text { O } & -2.912750 & 2.933188 & 0.551564\end{array}$ H -1.6198342 .8036542 .782399$ H -2.135824 1.2096323 .319437 H $-9.077681-1.239930-1.435868$ H $-9.646542-0.005174-0.300531$ H $-9.2770430 .435823-1.974411$ C $-6.616451-2.1232240 .263627$ H $-6.707514 \quad 3.3734342 .869152$ H $-4.894776 \quad 3.4936683 .750028$ H -2.828326 3.7798771 .022670 H $-7.504314-2.7308520 .051451$ H $-6.188954-2.5051561 .199629$ H $-7.811577-0.6498511 .250593$ C $0.911803 \quad 1.3384903 .513379$ O $\quad 0.5434501 .7540352 .196578$ C $-0.678245 \quad 1.191526 \quad 1.701787$ C $-0.594076-0.3437801 .718310$ C $-0.206271-0.8826233 .094218$ C $1.056701-0.1862503 .597429$ H $0.135092 \quad 1.6692604 .215145$ C 2.1936332 .0845153 .911790 H -0.7342491 .5081190 .656829$ O $-1.824622-0.9249041 .309992$ H $0.165211-0.6582540 .990770$ H -1.032947 -0.7785653 .806254$ $\begin{array}{lllll} & \text { O } & 0.041058 & -2.284913 & 2.977628\end{array}$ H $1.905677-0.5300182 .996078$ O $1.313090-0.5829654 .941970$ C 3.3881361 .7403343 .045107 H 2.0439663 .1668093 .821718 H 2.4311731 .8613704 .958316 H $-1.710034-1.8906171 .324778$ H $0.336138-2.5996853 .849291$ H $0.565417-0.2869285 .488874$ O $4.516973 \quad 1.7158363 .811437$ $\begin{array}{lllll}\text { O } & 3.338489 & 1.544671 & 1.838071\end{array}$ C $5.727170 \quad 1.403946 \quad 3.113957$ H 5.6859450 .3794072 .732855 H 5.8938902 .1141072 .298122 H 6.5570481 .4821383 .821258

SCF Energy (B3LYP/6-31G**//MMFF) $=-3245.91533276$

01_542

MM̄FF Geometry

C -1.538961 -4.352338 -2.332022

C $-0.749383-3.420336-1.776958$

C $-0.312959-2.175296-2.498849$

O $-0.920624-1.045449-1.828009$

C $1.204746-1.927872-2.436627$

C $2.071345-3.114518-2.782270$

C $2.797000-3.805711-1.881175$

C $2.104792-3.486977-4.240173$

C $2.873080-3.578124-0.395327$

C $4.292632-3.2638790 .089739$

C $4.903288-1.975196-0.489247$

C $6.344570-1.8324400 .009490$

C $6.994956-0.530552-0.470444$

O $4.117892-0.851302-0.069624$

$\begin{array}{lllll}\text { O } & 8.200435 & -0.336944 & 0.285298\end{array}$

C $6.0523030 .638663-0.129943$

C $7.414505-0.610451-1.942854$

O $6.5604481 .860763-0.664212$

C $4.5827440 .415237-0.570997$

C 3.7036501 .5338350 .048944

O $4.516404 \quad 0.469502-1.994419$

C $-2.155467-0.647000-2.228373$

O $-2.857835-1.147875-3.090055$

C $-2.4801080 .560307-1.441707$
C $-3.3465941 .456220-1.934331$

C $-3.6525772 .756227-1.335599$

$\begin{array}{llll}\text { C } & -3.125876 & 3.025197 & 0.048441\end{array}$

C $2.2379891 .566278-0.406379$

C $2.0025732 .454071-1.620882$

C $0.1632913 .404138-3.015847$

O $2.9134852 .995032-2.242627$

C $-0.6147714 .551179-2.446797$

C $-1.6484755 .120420-3.087316$

C $-2.4598386 .257781-2.529895$

C $-3.9544646 .049417-2.681746$

C $-4.6576516 .743033-3.592912$

C $-4.6451565 .088753-1.734106$

C $-4.3375153 .655857-2.071165$

N $0.6543242 .570098-1.930050$

H $-1.882413-4.222607-3.355251$

H $-0.426471-3.538851-0.745501$

H $-0.624117-2.191418-3.551401$

H $1.459475-1.524630-1.451962$

H $1.451165-1.098189-3.114392$

H $3.393913-4.644169-2.237904$

H $2.775906-4.329414-4.438587$

H $1.106508-3.771931-4.587132$

H $2.453045-2.639674-4.839660$

H $2.188869-2.800758-0.047313$

H $2.539365-4.5014190 .095475$

H $4.253496-3.1817901 .183967$

H $4.945748-4.114579-0.144129$

H $4.889646-2.032524-1.583261$

H $6.949398-2.695852-0.295450$

H $6.351203-1.8373091 .108859$

H $8.775748-1.1065860 .134909$

H 6.0701990 .7581740 .962192

H $8.115743-1.439721-2.096302$

H $7.9515750 .293469-2.250432$

H $6.572618-0.761504-2.621731$

H $6.3590331 .873946-1.615432$

H 3.7058161 .3932211 .137295

H $4.1542372 .515297-0.140258$

H $3.5845510 .523972-2.258574$

H $-1.9324320 .717417-0.519406$

H $-3.8207341 .251985-2.893298$

H $-2.031424 \quad 3.0482080 .044766$

H -3.4589232 .2444200 .741279$

H -3.465366 $3.971840 \quad 0.472431$

H $1.8615930 .560823-0.610868$

H 1.6373921 .9848740 .409669

H $-0.4610592 .768292-3.651486$

H $1.0054233 .781528-3.603779$

H $-0.3202114 .932985-1.472282$

H $-1.9187634 .757638-4.076909$

H $-2.155013 \quad 7.170970-3.057258$

H -2.229511 $6.429267-1.471146$

H $-5.7305496 .617489-3.694661$

H $-4.1758337 .448902-4.261361$

H $-5.7344965 .213044-1.789374$

H $-4.3769665 .359891-0.709905$

H $-4.681073 \quad 3.359555-3.063472$

H $-0.0286842 .087064-1.354361$

C $-3.273299-5.355564-0.762744$

$\begin{array}{llllllll}\text { O } & -2.955744 & -4.487481 & 0.342338\end{array}$

C $-4.008830-3.516246 \quad 0.485334$

C $-5.191693-4.076122-0.295072$

C $-4.481343-4.733764-1.465917$

H $-3.664232-2.601597-0.012301$

C $-4.261283-3.2273111 .968096$

H $-5.743178-4.8269110 .282333$

H $-5.895146-3.296250-0.601681$

C $-5.336115-5.737868-2.218294$

H $-4.169705-3.944662-2.161022$

C $-2.979180-2.8866672 .757320$

O $-4.838852-4.3937812 .567470$

H $-5.004565-2.4278082 .068005$

H $-2.343780-3.7782112 .818375$

O $-3.360234-2.5869834 .106466$

C $-2.165148-1.7078862 .191595$

C $-0.883102-1.4623373 .011337$

H $-1.879157-1.9187521 .155955$ 
O - $-2.995551-0.5503432 .188252$ H -1.156657 -1.191054 4.035683 H $-0.329508-2.4072503 .063941$ H $-4.768925-6.194816-3.035389$ H $-5.686837-6.540098-1.560522$ H -6.215802 -5.248065 -2.648469 C $-2.018093-5.580157-1.611053$ H $-4.984865-4.1938953 .508043$ H -3.879378 -1.7645104 .087294$ H -2.490792 $0.174778 \quad 1.783754$ H $-2.207718-6.364050-2.353635$ H -1.210134 -5.948423 -0.965664 H $-3.555969-6.316883-0.314027$ C -0.7264191 .5331043 .682859$ $\begin{array}{lllll}\text { O } & -0.590990 & 0.920764 & 2.395934\end{array}$ C $0.028260-0.3708452 .402761$ C $1.418345-0.2838093 .050121$ C $1.3623240 .339927 \quad 4.441481$ C $0.6255491 .677983 \quad 4.391782$ H $-1.3831510 .909415 \quad 4.301829$ C -1.478584 $2.862718 \quad 3.520732$ H $0.180525-0.6428651 .350152$ O $2.029251-1.5667623 .133147$ H 2.0687850 .3375292 .425006 H $0.901073-0.3380655 .169263$ O 2.6936730 .5815084 .902586 H 1.2588302 .4147223 .884882 $\begin{array}{llll}\text { O } & 0.410128 & 2.148040 & 5.720521\end{array}$ C $-0.794408 \quad 3.8840252 .635261$ H -2.455192 2.6606333 .065222 H -1.640966 3.3146724 .505804 H $2.081339-1.9236962 .230234$ H $3.164091-0.2696944 .885036$ H $1.278724 \quad 2.196473 \quad 6.155431$ O $-1.473506 \quad 5.0668952 .726545$ $\begin{array}{lllll}0 & 0.182409 & 3.676084 & 1.930210\end{array}$ C $-0.9713596 .128665 \quad 1.909077$ H 0.0588326 .3684322 .188847 H -1.031396 $5.852142 \quad 0.852512$ H -1.595087 7.0107782 .076187

SCF Energy (B3LYP/6-31G**//MMFF) $=-3245.92524972$

01_543

MMFF Geometry

C $4.265738-2.5793480 .809521$

C $3.566566-1.717217 \quad 1.562854$

C $2.407252-2.0167562 .489878$

O $1.251327-1.286745 \quad 2.004944$

C $1.997173-3.5003572 .593262$

C $0.938355-3.764199 \quad 3.649117$

C $-0.329618-4.1492213 .401374$

C $1.398832-3.6015565 .076803$

C $-0.997700-4.4067842 .077837$

C $-1.938993-3.258968 \quad 1.715527$

C $-2.861406-3.5354780 .518996$

C $-2.095523-3.838956-0.766953$

C $-3.029066-3.982708-1.976936$

O $-3.637278-2.3457720 .340635$

O $-2.193678-3.946590-3.145238$

C $-3.980076-2.766776-2.033406$

C $-3.720849-5.350962-1.981488$

O $-4.988200-2.945887-3.025056$

C $-4.642576-2.422566-0.677963$

C $-5.309973-1.031312-0.753535$

O $-5.672879-3.349844-0.333893$

$\begin{array}{llll}\text { C } & 1.108497 & 0.016768 & 2.366703\end{array}$

$\begin{array}{lllll}\text { O } & 1.852996 & 0.672222 & 3.077157\end{array}$

C $-0.123622 \quad 0.5193831 .718663$

C $-0.434758 \quad 1.8224851 .772179$

C -1.598929 2.4470721 .138610

C -2.6873471 .5325330 .641010$

C $-5.947992-0.5988370 .572989$

C -6.3719020 .8594560 .561584$

C -7.026715 $2.679503 \quad 2.139926$

O $-6.4007371 .546698-0.455209$

C -5.7630843 .3707582 .549692$

C -5.3606804 .5490672 .050411$

C -4.0918715 .2351602 .473152$
C $-3.252277 \quad 5.693164 \quad 1.299866$

C $-3.0216427 .000398 \quad 1.089424$

C -2.6816194 .6470550 .366111$

C $-1.6318883 .791898 \quad 1.028861$

N $-6.717916 \quad 1.295188 \quad 1.833508$

H $3.980116-3.6286260 .819121$

H $3.837990-0.6682451 .534105$

H $2.680481-1.6477983 .485963$

H $2.871620-4.1157212 .841928$

H $1.651237-3.8412591 .613205$

H $-0.987405-4.3061514 .256363$

H $0.644733-3.9356465 .797801$

H $2.302418-4.1938105 .254494$

H $1.618763-2.5525895 .296200$

H $-0.267277-4.5659321 .281286$

H $-1.566205-5.3410742 .162062$

H -2.569432 -3.002502 2.578413

H $-1.350638-2.3567901 .514896$

H $-3.530531-4.3630080 .781486$

H -1.482019-4.740939-0.661893

H $-1.397026-3.017698-0.969553$

H $-2.757312-4.091296-3.923926$

H -3.381009-1.901474-2.349906

H $-2.978080-6.152377-2.076350$

H $-4.387463-5.457168-2.844312$

H $-4.296640-5.542052-1.073276$

H $-4.543940-3.041386-3.884185$

H $-4.557006-0.286095-1.040237$

H $-6.087190-1.024655-1.528246$

H $-5.730714-4.026899-1.025820$

H $-0.732402-0.1956521 .178817$

H 0.2371972 .5011612 .295018

H $-3.0214450 .862506 \quad 1.441421$

H $-2.3305080 .924978-0.197183$

H $-3.5764342 .061646 \quad 0.295772$

H $-6.833661-1.2090170 .783387$

H $-5.236413-0.7341941 .395649$

H -7.7382392 .6780042 .971062$

H $-7.494377 \quad 3.151777 \quad 1.269962$

H $-5.1517402 .878987 \quad 3.303313$

H -5.9823735 .0572891 .317019$

H -3.485714 4.5849093 .115754

H -4.3738556 .0945163 .095196$

H $-2.4219697 .342014 \quad 0.252217$

H -3.426306 7.7602861 .749759

H -2.201574 $5.133203-0.493947$

H $-3.5023714 .066549-0.061853$

H -0.7838914 .3612911 .412518$

H -6.5976710 .6605962 .617914$

C $6.238157-0.989106 \quad 0.031500$

O $5.4501890 .169907-0.291769$

C $5.796403 \quad 1.224567 \quad 0.628818$

C $7.086468 \quad 0.783376 \quad 1.311978$

C $6.882051-0.7192141 .391228$

H 5.0021411 .2554401 .383556

C $5.8541222 .581864-0.080170$

H 7.9684531 .0130790 .703065

H $7.219363 \quad 1.2517262 .291906$

C $8.163536-1.4974481 .633922$

H $6.182966-0.9303862 .208213$

C $4.6234252 .912032-0.952482$

O $7.0033502 .628877-0.935230$

H 6.0068053 .3715490 .664785

H $4.6880712 .356075-1.894676$

O $4.6936124 .299782-1.301555$

C $3.2642912 .642702-0.287211$

C $2.0382143 .131557-1.078197$

H $3.1593011 .567694-0.107935$

$\begin{array}{llllll} & \text { O } & 3.241698 & 3.279693 & 0.993327\end{array}$

H $1.1500942 .750424-0.562348$

H $1.9586324 .223460-0.993109$

H $7.964521-2.5732421 .668716$

H $8.900486-1.3155860 .844433$

H $8.615129-1.2063882 .587942$

C $5.427349-2.285274-0.107846$

H $6.9353091 .886192-1.559677$

H $5.5548334 .448077-1.728698$

H 2.4039733 .0412051 .424971 
H $\quad 6.129348-3.122654-0.003261$

H $5.034585-2.336907-1.131572$

H $7.025293-1.022040-0.734746$

C $1.491033 \quad 0.370686-2.337494$

O $2.3989231 .381365-2.792350$

C $1.9892522 .728522-2.563793$

C $0.6382632 .991581-3.241808$

C $-0.4114181 .959614-2.836054$

C $0.1225230 .537501-3.015441$

H $1.3823360 .444802-1.249217$

C $2.166629-0.966556-2.680156$

H $2.7281033 .357972-3.075612$

O $0.1808694 .303203-2.928868$

H $0.7726282 .948570-4.330505$

H $-0.7507162 .131091-1.809593$

O $-1.5600882 .137030-3.668765$

H $\quad 0.1925150 .322718-4.089543$

O $-0.833489-0.359434-2.457736$

C $1.681318-2.146131-1.872149$

H $2.033017-1.182232-3.747106$

H $3.246594-0.885679-2.501052$

H $-0.6887074 .416178-3.349843$

H $-2.2096041 .460053-3.411525$

H -0.603765 -1.257242 -2.750247

O $2.131607-3.295108-2.457384$

O $1.012998-2.075067-0.851570$

C $1.752342-4.514033-1.811410$

H $2.016924-4.491852-0.750633$

H $0.680021-4.682307-1.939703$

H $2.295686-5.334046-2.288319$

SCF Energy $\left(\mathrm{B} 3 \mathrm{LYP} / 6-31 \mathrm{G}^{* *} / / \mathrm{MMFF}\right)=-3245.91356629$

01544

MM̄FF Geometry

C $-3.319758-1.924479-2.853966$

C $-2.479440-1.732541-1.826032$

C $-1.150715-2.431293-1.694557$

O $-0.202188-1.522219-1.084136$

C $-1.274986-3.658324-0.774309$

C $-0.020643-4.512163-0.738291$

C $0.731201-4.7288310 .358402$

C $0.359552-5.174096-2.039046$

C $0.509135-4.217173 \quad 1.752136$

C $1.217435-2.8949362 .078058$

C $2.747236-2.9359821 .938525$

C $3.372906-1.6822232 .555193$

C $4.896084-1.6416452 .357419$

O $3.076599-2.9971980 .547805$

O $5.344529-0.3120982 .663805$

C $5.218070-1.8939790 .868806$

C $5.622169-2.5786143 .329835$

O $6.622346-2.0311950 .668575$

C $4.480909-3.1127390 .276692$

C $4.646807-3.238021-1.258598$

O $5.016245-4.3106350 .853091$

C $0.260620-0.487657-1.835137$

O $-0.008565-0.241143-2.999464$

C $1.1449420 .321931-0.969976$

C $1.4306841 .585386-1.314900$

C $2.2870982 .507635-0.567618$

C 3.0792841 .9461680 .583701

C $4.144062-2.043277-2.076525$

C $5.219999-1.022438-2.410983$

C $5.5005641 .249210-3.400397$

O $6.419346-1.228441-2.246322$

C $5.5943172 .267460-2.307881$

C $5.3295203 .570130-2.491258$

C $5.4609854 .613024-1.417668$

C $4.2089055 .446726-1.256101$

C $4.0985986 .649126-1.845901$

C $3.1226514 .923691-0.343616$

C $2.3418233 .797482-0.959586$

N $4.6821110 .135831-2.956744$

H -3.067724 -2.648592 -3.624349

H -2.739735 -1.011723 -1.055870

H $-0.774892-2.722966-2.682482$

H -2.104217 -4.296057 -1.108549

H -1.539384 -3.3156660 .232071$
H $1.611354-5.3631380 .256274$

H $1.156235-5.914943-1.910349$

H $-0.502523-5.696298-2.466759$

H $0.715892-4.433743-2.761509$

H $-0.561200-4.1074951 .958231$

H $0.854533-4.9888232 .452280$

H $0.808408-2.1070991 .434721$

H $0.963425-2.6364723 .113049$

H $3.117532-3.8356102 .443922$

H $3.123919-1.6060383 .621152$

H $2.937547-0.7908042 .083920$

H $5.081502-0.1103113 .578057$

H $4.910723-0.9943850 .323184$

H $5.403467-2.2976754 .367136$

H $6.709243-2.4960673 .221228$

H $5.340847-3.626523 \quad 3.206516$

H $7.045924-1.2338491 .029929$

H $5.685389-3.488544-1.506260$

H $4.054890-4.108453-1.576911$

H $4.811742-5.0547240 .262738$

H $1.524905-0.140027-0.066421$

H $\quad 0.984557 \quad 1.990577-2.222414$

H $3.649078 \quad 1.0674530 .263639$

H 2.4149741 .6540341 .403018

H $3.8118272 .644658 \quad 0.992724$

H $3.770721-2.415096-3.039382$

H $3.309944-1.547633-1.572434$

H $5.0344751 .653977-4.304318$

H $6.5021340 .888655-3.655360$

H $5.9207451 .922477-1.329572$

H $5.0321523 .915794-3.478880$

H $6.3060295 .260885-1.685588$

H $5.7278914 .164500-0.452672$

H $3.2160807 .265977-1.712991$

H $4.8872987 .041154-2.479706$

H $2.4054565 .722982-0.112861$

H 3.5617414 .6597850 .622570

H $1.7372644 .106189-1.813688$

H $3.6729450 .236633-3.015699$

C $-5.783219-1.730377-2.282559$

O $-5.589646-1.507939-0.867353$

C $-6.524259-0.503681-0.446072$

C $-7.745580-0.765671-1.310068$

C -7.091758 -1.013747 -2.657848

H $-6.0932680 .468190-0.716078$

C $-6.757169-0.5464321 .065897$

H -8.287187 -1.661513 -0.985499

H $-8.4427370 .077082-1.321832$

C -7.961365 -1.810724 -3.616340

H $-6.871256-0.042697-3.121684$

C $-5.471051-0.5760491 .916791$

O $\quad-7.506988-1.7251281 .386515$

H -7.3892920 .3017961 .354792$

H -5.034205 -1.581177 1.866676

O $-5.855560-0.3870273 .285391$

C -4.3869730 .4554861 .550933$

C -3.1918230 .4027872 .526086$

H -4.0415220 .2770340 .528147$

O -4.9312601 .7738401 .601865$

H $-3.310478 \quad 1.1764903 .293665$

H $-3.177053-0.5642723 .043125$

H -7.439857 -1.975449 -4.564750

H -8.223622 -2.789406 -3.200570

H $-8.892092-1.274764-3.828666$

C $-4.589108-1.144592-3.044309$

H $-7.653923-1.7230292 .348050$

H -6.1746550 .5273883 .375774$

H $-5.600539 \quad 1.8544100 .902998$

H -4.416526 -0.107671 -2.728849

H $-4.806659-1.115260-4.118917$

H $-5.853040-2.813610-2.435484$

C -1.779534 2.9607821 .535752

O -1.7910991 .6720150 .915507$

C -1.8279820 .5666701 .817431$

C -0.6231840 .6126782 .768438$

C -0.4861541 .9669023 .459452$

C -0.5551963 .1061452 .444259$

H -2.6959833 .0990452 .121497$ 
C -1.764817 4.0048180 .415230 H $-1.707887-0.3298211 .195654$ O $-0.713499-0.403247 \quad 3.761889$ H 0.2942020 .4218672 .197998 H -1.243758 2.0954174 .241650 $\begin{array}{lllll}\text { O } & 0.782512 & 2.030748 & 4.115757\end{array}$ H 0.3626503 .1206431 .845090 O -0.6300144 .3491103 .138975$ C $-3.0808494 .006723-0.325553$ H -1.6103345 .0177440 .803399$ H $-0.9574943 .811434-0.301265$ H $-0.858752-1.2485463 .304554$ H $0.827316 \quad 1.278721 \quad 4.731057$ H $0.145396 \quad 4.4012933 .723754$ O $-3.0095973 .179475-1.407466$ O $-4.062478 \quad 4.644054 \quad 0.033444$ C $-4.2093803 .070832-2.178860$ H $-4.5154234 .053893-2.549269$ H $-5.0038192 .619670-1.577431$ H -4.005230 $2.422897-3.035231$

SCF Energy (B3LYP/6-31G**//MMFF) $=-3245.90142490$

\section{1_545}

MMFF Geometry

C $3.705352-4.189144-0.161868$

C $2.549903-4.272469-0.839332$

C $1.215434-3.926200-0.233009$

O $0.572356-3.027921-1.166768$

C $0.340648-5.181493-0.058112$

C $-0.795238-4.999210 \quad 0.933497$

C $-2.109141-5.024397 \quad 0.634180$

C $-0.373456-4.8299542 .373691$

C $-2.769737-5.236408-0.697310$

C $-3.294867-3.958142-1.367581$

C $-4.398956-3.219404-0.590354$

C $-5.010655-2.116820-1.461029$

C $-6.050810-1.280391-0.700115$

O $-3.831779-2.6375240 .588776$

O $-6.303290-0.089907-1.463354$

C $-5.448748-0.8298740 .648170$

C $-7.393043-2.012294-0.584230$

O $-6.433926-0.191117 \quad 1.455659$

C $-4.784974-1.9773481 .436954$

C $-4.038030-1.4955822 .707154$

O $-5.796730-2.8918341 .879178$

C $-0.052664-1.929917-0.669963$

O $-0.170812-1.6149030 .502834$

C $-0.575885-1.159717-1.822425$

C $-1.429649-0.152444-1.594194$

C -2.040053 $0.724741-2.592477$

C $-1.5849710 .590949-4.020350$

C -2.926797 -0.4676852 .475260$

$\begin{array}{llll}C & -3.377126 & 0.978159 & 2.612968\end{array}$

C -2.653616 3.3006942 .054505

O $-4.417121 \quad 1.3087193 .176104$

C -3.448765 3.6723750 .842686

C $-2.9576124 .376643-0.188301$

C $-3.7522924 .732444-1.412554$

C $-3.2305434 .058280-2.663865$

C -2.428904 $4.713133-3.520488$

C $-3.7048252 .651754-2.964113$

C -2.955958 $1.619538-2.168865$

N $-2.454794 \quad 1.8623612 .068418$

H $3.677671-3.9038150 .886029$

H $2.560503-4.572368-1.884401$

H $1.384088-3.4218470 .725094$

H $\quad 0.953764-6.0175540 .305224$

H $-0.039497-5.489636-1.037461$

H -2.814385 -4.9030391 .456109$

H -1.223836 -4.8657203 .063397$

H $0.313461-5.6313072 .665243$

H $\quad 0.126270-3.8688182 .524261$

H -2.093167 -5.740120 -1.394617

H $-3.604732-5.933831-0.552772$

H $-2.451610-3.283128-1.538561$

H $-3.683824-4.241273-2.354017$

H -5.166055 -3.948931 - 0.305357

H $-5.453434-2.541203-2.370927$
H $-4.214262-1.444700-1.805614$

H $-6.607609-0.359103-2.347024$

H $-4.690212-0.0713920 .418044$

H $-7.800021-2.224902-1.580292$

H -8.141696 -1.389109-0.082952

H -7.319238 -2.962015 -0.050537

H -6.8080620 .5387300 .933068$

H $-4.758783-1.1450163 .455880$

H $-3.560942-2.379456 \quad 3.154675$

H $-5.442108-3.4072872 .622521$

H $-0.254417-1.446203-2.816905$

H $-1.7138400 .057440-0.565258$

H $-1.910934-0.369247-4.434948$

H $-0.4933530 .646649-4.090705$

H $-1.972216 \quad 1.375892-4.673655$

H $-2.151113-0.6141463 .237593$

H $-2.458523-0.6215541 .500609$

H -1.663899 3.7672662 .061095

H -3.1888883 .6061992 .959225$

H -4.4829013 .3366950 .811485$

H $-1.9262204 .717713-0.156121$

H $-3.7171415 .823661-1.528291$

H $-4.8142414 .488398-1.283890$

H -2.072422 $4.243488-4.431301$

H -2.104893 $5.730013-3.325047$

H $-3.6483842 .452696-4.039082$

H $-4.7730202 .570307-2.725718$

H -3.225627 $1.608120-1.113534$

H -1.6508361 .4945111 .567825$

C $6.020304-3.270685-0.685752$

O $5.436723-2.111015-1.314564$

C $5.630897-0.976869-0.448266$

C $6.757301-1.3742200 .498899$

C $6.444038-2.8414990 .721697$

H $4.712054-0.8649440 .138790$

$\begin{array}{llll}\text { C } 5.887161 & 0.296541 & -1.258269\end{array}$

H $7.742157-1.2650800 .030303$

H $6.751524-0.7877681 .422531$

C $7.610397-3.6377361 .281139$

H $5.605762-2.9085481 .424558$

C $4.8276070 .624592-2.330775$

O $7.1450550 .148525-1.934095$

H $6.0099701 .144888-0.574662$

H $4.893328-0.129675-3.125299$

O $5.211504 \quad 1.872771-2.925184$

C $3.3635920 .748442-1.862052$

C $3.1735011 .714062-0.679111$

H $2.7724451 .104590-2.710382$

O $2.845425-0.529529-1.508597$

H $3.667996 \quad 1.2880720 .202850$

H $3.6795852 .662640-0.888298$

H $7.337822-4.690364 \quad 1.406066$

H $8.482908-3.5906700 .620856$

H $7.908721-3.2466612 .259335$

C $5.055859-4.460899-0.765686$

H $7.3058510 .970565-2.428605$

H $4.6035552 .045192-3.664390$

H $2.975154-1.123010-2.267753$

H $5.498365-5.329419-0.264828$

H $4.931211-4.730395-1.822257$

H $6.911074-3.517232-1.278313$

C $2.1594013 .526682 \quad 1.495807$

$\begin{array}{lllll}\text { O } & 1.583938 & 2.275289 & 1.102942\end{array}$

C $1.6963831 .976073-0.296722$

C $0.9692913 .067710-1.097226$

C $1.4715294 .466987-0.746458$

C $1.465293 \quad 4.682058 \quad 0.766317$

H $3.232813 \quad 3.5319221 .268155$

C 1.9888643 .6505113 .016977

H $1.1478471 .039366-0.435222$

O $1.0963962 .872346-2.497967$

H $-0.1023923 .023658-0.865187$

H $2.4678864 .652375-1.163997$

O $0.606043 \quad 5.439737-1.337720$

$\mathrm{H} \quad 0.430673 \quad 4.783374 \quad 1.116609$

O 2.1388645 .9017691 .068830

C 2.8495422 .6587913 .772587

H 2.2743464 .6523363 .355398 
H 0.9438853 .4567103 .287551

H $0.6984102 .010483-2.705330$

H $0.5769785 .258066-2.292862$

H $1.692144 \quad 6.6100860 .573982$

$\begin{array}{llll}\text { O } & 2.556925 & 2.746236 & 5.104522\end{array}$

O 3.6762791 .9106123 .269157

C 3.2970051 .8653775 .955649

H 3.0997290 .8232915 .686147

H 4.3667202 .0861365 .890458

H 2.9669032 .0300056 .984734

SCF Energy (B3LYP/6-31G**//MMFF) $=-3245.91205419$

01_546

MMFF Geometry

C $-0.545158 \quad 3.565280-1.656261$

C $-0.9258292 .351837-1.228703$

C $-1.2251201 .197493-2.148458$

O $-0.402840 \quad 0.103512-1.677873$

C -2.701054 $0.766791-2.071865$

C -3.687542 $1.857977-2.422872$

C $-4.4947672 .473892-1.536683$

C $-3.7490822 .222529-3.883302$

C $-4.5879342 .246501-0.051880$

C $-5.974506 \quad 1.745207 \quad 0.376936$

C $-6.3329060 .357563-0.179198$

C $-7.770888-0.0115720 .191972$

C $-8.137451-1.422613-0.289105$

O $-5.424895-0.6011990 .375062$

$\begin{array}{llll}0 & -9.360886 & -1.805831 & 0.357325\end{array}$

C $-7.048209-2.4055140 .184170$

C $-8.412858-1.462454-1.796942$

O $-7.263044-3.705494-0.361674$

C $-5.605105-1.934532-0.119579$

C $-4.599480-2.8806100 .587878$

O $-5.378201-2.000219-1.530643$

C $-0.073461-0.890970-2.538989$

O $-0.386589-0.972226-3.717807$

C $0.698390-1.951771-1.846370$

C $1.288151-1.750821-0.657998$

C $2.009953-2.7366470 .146323$

C $2.106567-4.144123-0.374341$

C $-3.151250-2.3766310 .626406$

C $-2.856393-1.4347291 .784484$

C $-0.981824-0.0721802 .719875$

O $-3.649390-1.2093272 .693938$

C $0.091692-0.7969243 .480147$

C $0.265214-2.1256723 .578026$

C $1.427694-2.7657444 .289208$

C $2.190554-3.7325513 .402566$

C $2.035485-5.0598943 .542717$

C $3.174761-3.1718012 .395169$

C $2.504397-2.3400421 .337020$

N $-1.576824-0.9074851 .693879$

H $-0.4642033 .728510-2.729708$

H -1.028429 2.154100 -0.165490

H $-0.942278 \quad 1.442747-3.179816$

H $-2.9008200 .346154-1.081367$

H $-2.865501-0.083378-2.747464$

H $-5.1695853 .243325-1.910318$

H $-4.5225392 .968986-4.093656$

H $-2.7930622 .636528-4.218149$

H -3.975655 $1.336613-4.485666$

H $-3.816363 \quad 1.568950 \quad 0.322621$

H $-4.4007113 .208590 \quad 0.441475$

H -5.9885691 .7086271 .473936$

H -6.7340102 .4745870 .067993$

H $-6.2234520 .371572-1.268825$

H $-8.4785890 .724939-0.208725$

H -7.886825 0.0241991 .284455

H -10.040487 -1.1498580 .125393$

H $-7.153921-2.5064551 .273608$

H $-9.237315-0.786300-2.053101$

H -8.735742 -2.460539 -2.113030

H -7.549557 -1.172499 -2.399338

H -8.171404 -3.968486 -0.134366

H $-4.931793-3.0737441 .615126$

H -4.601239 -3.8452200 .063561$

H -4.429996 -2.123707 -1.694791
H $\quad 0.752932-2.902415-2.365866$

H $1.220327-0.763368-0.203361$

H $2.653771-4.163926-1.323411$

H $1.108475-4.566305-0.534639$

H $2.629088-4.8206960 .306257$

H -2.491766 $-3.243566 \quad 0.763048$

H $-2.869327-1.898624-0.315926$

H -1.7455150 .3027203 .407829$

H $-0.536427 \quad 0.781342 \quad 2.197989$

H $0.812802-0.1496543 .974025$

H $-0.448334-2.8019293 .114243$

H $1.032256-3.2909645 .168253$

H $2.123936-2.0124974 .678430$

H $2.589060-5.7622802 .928537$

H $1.347797-5.4746154 .272319$

H $3.773726-3.9674451 .938276$

H $3.902081-2.5415712 .923146$

H $2.390399-1.2943531 .622364$

H $-0.991073-1.1736120 .908315$

C $-0.105170 \quad 4.555944 \quad 0.702596$

$\begin{array}{llllll}\text { O } & 0.956146 & 3.624302 & 0.989683\end{array}$

C $1.861098 \quad 4.217747 \quad 1.934970$

C 1.0566595 .3100802 .623255

C $0.2437475 .843554 \quad 1.457925$

H $2.6635334 .685574 \quad 1.350947$

C 2.4606223 .1445912 .850770

H $0.388734 \quad 4.903747 \quad 3.391360$

H $1.6930456 .069123 \quad 3.087693$

C $-0.969983 \quad 6.6534851 .880462$

H $0.8940816 .478070 \quad 0.840546$

C $2.999306 \quad 1.9101382 .096070$

$\begin{array}{lllll}\text { O } & 1.438467 & 2.691326 & 3.746526\end{array}$

H 3.2426653 .5919143 .475593

H $2.156327 \quad 1.3261361 .705410$

O $3.6751151 .067347 \quad 3.036836$

C 3.9726322 .2217800 .940058

C $4.402690 \quad 0.9407850 .208457$

H 3.4801792 .8858740 .228194

$\begin{array}{llll}\text { O } & 5.132280 & 2.884898 & 1.433568\end{array}$

H 4.8655960 .2539230 .929860

H $3.5020250 .434976-0.153333$

H -1.5316016 .9973821 .006123$

H -1.6483686 .0634472 .506026$

H $-0.663968 \quad 7.5340342 .454548$

C $-0.195693 \quad 4.765336-0.813651$

H 1.8698942 .1727454 .446194

H 3.0075090 .7072713 .643844

H 4.8545633 .7420891 .797029

H $0.7774815 .123151-1.174905$

H $-0.9294335 .550644-1.030469$

H $-1.033795 \quad 4.1219561 .092830$

C $5.088085-1.084810-1.891224$

O $5.995809-0.154369-1.287113$

C $5.4126601 .117261-0.949615$

C $4.8977181 .774486-2.239230$

C $3.9166730 .871647-2.985290$

C $4.527940-0.515232-3.200393$

H $4.265601-1.298243-1.202007$

C $5.848632-2.398306-2.124383$

H $6.2399271 .728884-0.570618$

O $4.2893193 .033305-1.992703$

H $5.7512891 .964730-2.903675$

H $2.9573660 .812929-2.457468$

O $3.6426331 .464630-4.256914$

H $5.324063-0.452559-3.953378$

O $3.528855-1.399602-3.702443$

C $6.067476-3.156114-0.830330$

H $5.271273-3.049658-2.790097$

H $6.832037-2.205349-2.567127$

H $4.9528033 .597857-1.560468$

H $2.9310050 .949958-4.673427$

H $3.261318-1.076089-4.578890$

O $6.379488-4.453818-1.120665$

O $5.992795-2.672704 \quad 0.291138$

C $6.605110-5.299067 \quad 0.011897$

H $5.703125-5.3534880 .629111$

H $6.840317-6.302046-0.353884$

H $7.453099-4.9307930 .597000$ 
C $4.7029651 .761802-1.354060$

C $5.9040480 .921560-1.415510$

C $7.2348991 .595534-1.196298$

C $1.551939-1.9373671 .312595$

C $2.561687-1.4912792 .349040$

C 4.4310480 .1299602 .673988

O $2.725922-2.1084443 .398724$

C $5.698096-0.3069091 .997499$

C $5.931654-1.5406141 .521543$

C $7.164635-1.9467170 .767394$

C $6.830375-2.443961-0.623878$

C $6.585927-3.745786-0.850366$

C $6.844543-1.444406-1.759731$

C $5.762975-0.402406-1.640882$ N $3.275009-0.3708851 .951556$ H -0.9617252 .9113680 .649167$ H 1.3833454 .8653430 .859170 H $1.1420864 .819854-1.584658$ H $-0.9727813 .392090-1.672036$ H $0.0269121 .979304-1.383306$ H $\quad 0.7388601 .830439-5.055054$ H $0.0194274 .008762-5.174259$ H -1.060105 $4.504948-3.869560$ H $\quad 0.6585914 .959042-3.832039$ H $1.720749-0.034949-4.020485$ H $1.4985910 .597643-2.405175$ H $-0.9107450 .033950-2.463874$ H $-0.802153-0.487734-4.146165$ H $0.703244-2.329159-3.523988$ H -1.786674 -2.729240 -3.335746 H - $1.758670-2.119028-1.694582$ H -2.720999 -4.596623 -2.025422 H $-0.383156-3.4645370 .121212$ H -1.218408 -5.148384 -3.718383 H $-0.192806-6.008844-2.593279$ H $\quad 0.468675-4.664127-3.547548$ H $-0.161361-5.711078-0.059754$ H $2.689255-3.6231310 .578494$ H $3.114922-2.104864-0.157559$ H $1.998838-4.561197-2.077374$ H $5.6066783 .670149-1.148577$ H $3.759011 \quad 1.227454-1.438717$ H $7.2351802 .150721-0.251320$ H $7.4512832 .291711-2.014000$ H $8.0745670 .900894-1.134610$ H $1.020032-1.0566140 .936179$ H $0.816405-2.5733461 .814603$ H $4.414528-0.1970973 .718035$ H 4.3613531 .2221322 .648093 H $\quad \begin{array}{lll}6.458076 & 0.459708 & 1.867307\end{array}$

H $7.889119-1.1258030 .704350$

H $6.365853-4.116385-1.845971$

H $6.595051-4.469549-0.041956$

H $6.693507-1.957287-2.719108$

H $7.845083-1.009494-1.830989$

H $4.757345-0.802114-1.777952$

H 3.0874520 .0313191 .037492

C -0.5153272 .9040353 .524454$

O -1.4308221 .8419383 .187602$

C -0.8118820 .5860473 .537569$

C 0.4302840 .9391014 .350809

C 0.8494082 .2405263 .694529

H -0.4899740 .1234732 .598144$

C $-1.807061-0.3274574 .258898$

H $\quad 0.189058 \quad 1.1057905 .407378$

H 1.1969800 .1624194 .303056

C 1.8390513 .0475784 .514519

H 1.2945192 .0100502 .718402

C $-3.075377-0.650773 \quad 3.440453$

O -2.2175020 .3193025 .469984$

H -1.302373 -1.253584 4.559080

H $-3.6667890 .262567 \quad 3.312467$

O $-3.888469-1.5343424 .223100$

C $-2.804581-1.2957942 .067840$

C $-4.055524-1.8219341 .340733$

H $-2.296093-0.5734231 .421151$

O $-1.904004-2.3953922 .225151$

H -3.718496 -2.298049 0.411446

H $-4.503355-2.6379731 .922140$

H 2.0991023 .9799494 .003649

H 1.4320373 .3033985 .498349

H 2.7621062 .4794934 .670325

C -0.5992744 .0410032 .505513$

H $-2.853095-0.2693365 .912113$

H -3.368484 -2.334193 4.409212

H $-2.357547-3.0853472 .738031$

H 0.0478724 .8662182 .825383

H -1.6258184 .4294722 .500558$

H -0.8555303 .2984934 .491825$

C $-4.1126980 .227040-0.919559$

O $-4.558527 \quad 0.4145290 .426170$

C $-5.120199-0.749611 \quad 1.038590$

C $-6.308925-1.2609160 .211438$

C $-5.930970-1.488951-1.252353$

C $-5.246596-0.250695-1.833494$

H $-3.315453-0.525324-0.921407$

C $-3.4479521 .524644-1.396522$

H $-5.522650-0.4234842 .005360$

O $-6.817124-2.4640450 .777624$

H -7.115696 -0.5178520 .257699$

H $-5.294001-2.374116-1.360756$

O $-7.112447-1.770000-2.006974$

H $-5.9931390 .538618-1.976444$

O $-4.711217-0.566187-3.116748$

C $-4.3642492 .729639-1.445505$

H $-2.6360561 .780887-0.707273$

H $-3.0246041 .369863-2.395320$

H -7.549421 -2.765209 0.212829

H $-7.708231-1.005334-1.928985$

H -5.443201-0.901594 -3.662502

O $-3.7879413 .688953-2.229666$

O $-5.4276902 .836511-0.851029$

C $-4.5242644 .909406-2.353062$

H $-3.9603455 .581648-3.005282$

H -5.501699 $4.718452-2.806064$

$\mathrm{H}-4.6372835 .383598-1.373448$

SCF Energy (B3LYP/6-31G**//MMFF) $=-3245.90833310$

01548

MMFF Geometry

C $-0.225210-3.2792821 .973970$

C $-0.657855-2.0918421 .524051$

C $-1.067182-0.9520442 .417043$

O -0.3123640 .1897291 .952827$

C -2.563296 -0.615626 2.277585

C $-3.489058-1.7679302 .594915$ 
C $-4.106292-2.5282551 .669052$

C -3.699602 -2.034055 4.061981

C $-4.002668-2.4218430 .171399$

C $-5.371012-2.313104-0.513413$

C $-6.171932-1.058838-0.129079$

C $-7.540442-1.085668-0.815845$

C $-8.3514350 .183524-0.527106$

O $-5.4362310 .100955-0.537172$

O $-9.4559640 .220664-1.443708$

C $-7.4697921 .409956-0.831461$

C -8.9569050 .1701810 .881306$

O $-8.1285252 .614946-0.445123$

C $-6.0616851 .343633-0.189032$

C $-5.2023902 .514235-0.733351$

O -6.1930041 .4849441 .228401$

C -0.0165371 .1829682 .829003$

O $-0.252722 \quad 1.2199594 .023923$

C 0.6246972 .2501002 .033541

C 1.0146433 .4054082 .589244

C 1.5577054 .5344131 .834312

C 1.1882425 .8750362 .409070

C $-3.7197172 .492234-0.339692$

C $-2.8204761 .768949-1.331403$

C $-0.3917951 .451433-1.836073$

O $-3.241344 \quad 1.048514-2.231055$

C $0.6945022 .446753-2.128649$

C $0.6084953 .786727-2.086266$

C $1.7573274 .706430-2.403850$

C $1.9856325 .755782-1.335082$

C $1.4548296 .984402-1.456773$

C $2.8891455 .415159-0.169482$

C 2.3056054 .3600500 .725846

N -1.481716 2.034227 -1.076443

H $-0.170044-3.4323003 .050248$

H $-0.731461-1.9054810 .456289$

H $-0.810322-1.1666163 .461739$

H $-2.754501-0.2115241 .277234$

H -2.8060440 .2275582 .938672$

H $-4.743777-3.3400572 .017453$

H -4.393912 -2.862261 4.239381

H $-2.751394-2.2893614 .545355$

H -4.113141 -1.146752 4.552374

H -3.370525 -1.590666 -0.151045

H -3.510191-3.332876 -0.191605

H -5.196040 -2.310760 -1.597278

H $-5.961088-3.209871-0.285586$

H $-6.300607-1.0388020 .958654$

H -8.107477 -1.977296 -0.519976

H -7.400771 -1.167093 -1.903191

H -9.979861 -0.588391 -1.314408

H $-7.3473221 .460869-1.922630$

H $-9.624325-0.6915641 .002539$

H -9.5786171 .0559351 .052339$

H -8.2055440 .1178561 .671853$

H -8.997393 2.622868 -0.882109

H -5.277109 2.549378-1.827339

H -5.623996 $3.453501-0.351445$

H $\quad-5.3848291 .8820901 .588459$

H $\quad 0.7306562 .0695350 .969747$

H 0.8792733 .5689523 .655922

H 1.6414646 .0045593 .398087

H 0.1004715 .9587572 .514803

H 1.5102286 .7150341 .789339

H -3.372364 $3.533052-0.296474$

H -3.564608 2.0545360 .650752

H $-0.7621061 .011960-2.767323$

H $0.0151110 .644018-1.218035$

H $1.6485902 .006279-2.409344$

H $-0.3386334 .260469-1.840698$

H $1.5345085 .196961-3.360582$

H $2.6866154 .146993-2.568418$

H $1.6321907 .754197-0.713156$

H $\quad 0.826618 \quad 7.246587-2.301661$

H 3.1460806 .3111210 .407485

H $3.8493455 .051822-0.558001$

H 2.5285723 .3461830 .397446

H -1.244364 $2.547453-0.232575$

C $0.229841-4.274330-0.371576$
O $1.256587-3.328800-0.732897$

C $1.851611-3.743412-1.977171$

C $1.056814-4.955785-2.454067$

C $0.575873-5.545791-1.143020$

H $2.872688-4.066794-1.746593$

C $1.879494-2.578281-2.971667$

H $\quad 0.203887-4.662991-3.077493$

H $1.671596-5.648390-3.037209$

C $-0.583835-6.512856-1.306165$

H $1.415704-6.063045-0.659910$

C $2.653544-1.335705-2.483488$

O $0.528617-2.173567-3.232399$

H $2.286375-2.926216-3.928819$

H $2.102323-0.859965-1.664985$

O $2.654127-0.388981-3.560951$

C $4.106811-1.603039-2.050085$

C $4.952492-0.333212-1.836046$

H $4.109666-2.206574-1.136192$

O $4.748102-2.379757-3.066839$

H $5.976321-0.651494-1.604399$

H $5.0429700 .208079-2.786364$

H $-0.900072-6.912534-0.337907$

H -1.449468 -6.027105-1.768971

H $-0.293593-7.357401-1.939828$

C $0.206071-4.4640341 .147219$

H $0.564114-1.430043-3.858544$

H $3.175998-0.771506-4.287223$

H $5.637170-2.607589-2.745271$

H $1.211703-4.7416431 .486404$

H $-0.461256-5.2957561 .400887$

H $-0.728730-3.873348-0.726174$

C $5.384478-0.5064551 .170789$

O $4.198980-0.0625010 .500086$

C $4.4287610 .613148-0.738876$

C $5.3120221 .847204-0.504957$

C $6.607104 \quad 1.4923120 .220371$

C $6.3082180 .677941 \quad 1.478611$

H $5.909259-1.2376140 .544257$

C $4.946532-1.2087702 .459968$

H $3.4518170 .980403-1.072106$

O $5.6221022 .494763-1.733668$

H 4.7679502 .5721350 .111578

H $7.3048530 .959687-0.436220$

O $7.2692162 .696168 \quad 0.615461$

H $5.849243 \quad 1.3286562 .234017$

O 7.5325790 .1888542 .021945

C $4.189776-2.4783952 .155908$

H $5.796367-1.4646203 .102524$

H $4.280539-0.5630253 .045712$

H $4.7801232 .727119-2.161493$

H $7.4148273 .222894-0.189214$

H 8.1049090 .9580492 .185430

O $5.051775-3.4527071 .748156$

O $2.975604-2.5855842 .263111$

C $4.448443-4.7068081 .415879$

H $3.758446-4.5834710 .576572$

H $5.241717-5.3978051 .118683$

H $3.929386-5.1210232 .285522$

SCF Energy (B3LYP/6-31G**//MMFF) = -3245.90079109

01_549

MM̄MF Geometry

C $1.500740-3.939801-0.385372$

C $1.240397-3.324669-1.548753$

C $-0.065485-2.646968-1.867060$

O $0.290581-1.303834-2.286312$

C $-0.816779-3.322945-3.033285$

C $-1.828805-4.359944-2.587537$

C $-3.161345-4.245472-2.752641$

C $-1.271057-5.607565-1.953579$

C $-3.925516-3.135171-3.415648$

C $-4.023952-1.823164-2.634132$

C $-4.408335-1.930725-1.151832$

C $-5.768485-2.594385-0.933440$

C $-6.177471-2.5788770 .548585$

O $-4.443535-0.587049-0.650177$

O $-7.569215-2.9232380 .625938$

C $-6.036878-1.1424711 .094598$ 
C $-5.422173-3.6393301 .359263$ O $-6.263402-1.1060542 .501919$ C $-4.679777-0.482266 \quad 0.759631$ C $-4.7198351 .022042 \quad 1.128959$ O $-3.647989-1.1194471 .515956$ C $-0.390097-0.259698-1.748228$ O $-1.283944-0.305317-0.919159$ C $0.1360130 .977543-2.362654$ C $-0.6173532 .085412-2.336848$ C $-0.2354103 .369102-2.911542$ C $-1.3109073 .989761-3.760308$ C -3.5336881 .8243220 .579463$ C $-3.604502 \quad 3.2998780 .933131$ C -2.3460595 .4210660 .599956$ O $-4.4936353 .787601 \quad 1.624741$ C $-1.6001786 .002578-0.557856$ C $-0.3236146 .411008-0.499035$ C $0.4040377 .046628-1.649303$ C $1.6432256 .283439-2.060408$ C $2.846016 \quad 6.592935-1.547275$ C $1.5031495 .237011-3.143190$ C $0.9553633 .933929-2.630369$ N -2.552136 4.0058390 .363901 H $\quad 0.707560-3.9925380 .357480$ H $2.007608-3.270993-2.316210$ H $-0.702210-2.604775-0.974509$ H $-0.108143-3.796926-3.726018$ H - $-1.307129-2.549868-3.635699$ H $-3.790683-5.057471-2.389573$ H -2.014331 -6.410548 -1.897884 H $-0.428123-5.991649-2.537315$ H $-0.934866-5.403661-0.933966$ H $-3.497273-2.935220-4.405727$ H $-4.940279-3.502239-3.617351$ H -3.069176 -1.289022 -2.704617 H -4.740065 -1.165465 -3.145536 H -3.618341 -2.478133 -0.625702 H $-5.778944-3.622523-1.312966$ H $-6.532747-2.054699-1.510018$ H -7.684192 -3.7995280 .219924$ H $-6.835126-0.5410420 .637078$ H -5.621424 -4.6406180 .958947$ H -5.765105 -3.659469 2.399614 H -4.340044 -3.4928901 .355144$ H -7.129168 -1.517275 2.666548 H $-5.636181 \quad 1.4701720 .722912$ H -4.7558751 .1376752 .219554$ H -2.905473 -0.5047881 .622612$ H $1.1128910 .948669-2.833010$ H -1.611449 2.055902 -1.899372 H $-2.2653304 .013723-3.221646$ H -1.450884 3.408071-4.678003 H -1.093412 5.019851 -4.051018 H -2.589605 1.4318420 .968133 H -3.514643 $1.750227-0.513463$ H -1.799422 5.5247221 .541911 H -3.3128495 .9213820 .718394$ H -2.153270 $6.114887-1.487326$ H $\quad 0.222178 \quad 6.3141850 .436590$ H $0.6811588 .064858-1.345238$ H $-0.2540437 .173848-2.517850$ H $3.7455726 .074163-1.861414$ H $2.9557217 .367479-0.795285$ H $2.4853445 .021895-3.585595$ H $0.9140595 .645828-3.969697$ H $1.6405943 .398607-1.973276$ H -1.775473 $3.476482-0.021358$ C $4.066568-3.982165-0.615768$

O $4.147761-2.576432-0.307615$

C $5.482203-2.2663890 .127751$

C $6.338471-3.457624-0.282377$

C $5.361433-4.601640-0.085339$

H $5.452274-2.217710 \quad 1.222961$

C $5.923377-0.912549-0.438311$

H $6.644140-3.398409-1.333222$

H $7.240786-3.5517300 .329022$

C $5.768254-5.881421-0.794424$

H $5.270852-4.8026200 .990609$
C $4.9315710 .237449-0.155602$

O $6.050471-1.038631-1.860084$

H $6.921402-0.664491-0.057597$

H $4.0200940 .091864-0.746929$

O $5.5260751 .456913-0.616460$

C $4.566366 \quad 0.415579 \quad 1.330240$

C 3.7388891 .6757171 .643304

H $4.018405-0.4623471 .682478$

O 5.7656970 .4850352 .107763

H 3.6206071 .7309402 .732409

H 4.3241782 .5661421 .381715

H $5.023247-6.667706-0.636724$

H $5.872106-5.726930-1.873688$

H $6.728503-6.243611-0.412919$

C $2.807163-4.563788 \quad 0.032176$

H $6.524534-0.253973-2.182832$

H $5.5602711 .417803-1.586970$

H 6.2839351 .2405511 .781006

H $2.887248-4.4196511 .117117$

H $2.749377-5.644095-0.142113$

H $4.025205-4.073746-1.707854$

C 1.0647910 .4907412 .580225

O $1.563211 \quad 0.572206 \quad 1.238421$

C 2.3620141 .7255670 .952046

C 1.5672973 .0075661 .241689

C 1.0203983 .0198332 .663804

C 0.2428431 .7365772 .938587

H 1.9054520 .3906703 .277694

C $0.193786-0.7744002 .675008$

H $2.545937 \quad 1.709777-0.128195$

O $2.3716404 .164773 \quad 1.042471$

H 0.7217023 .0786590 .546160

H 1.8171323 .1636433 .402850

O $\quad 0.1286014 .1245042 .818302$

H $-0.693393 \quad 1.7478712 .367932$

O $-0.100867 \begin{array}{llll}1.689892 & 4.322376\end{array}$

C $1.014921-2.0275402 .893118$

H $-0.491122-0.6919953 .526797$

H $-0.390182-0.9163001 .759177$

H 2.7128854 .1303280 .132886

H 0.6261304 .9305912 .597318

H -0.6057112 .4967374 .523076$

O $0.201976-3.0076293 .388419$

O $2.207575-2.1414682 .648503$

C $0.835317-4.2530453 .699422$

H $1.030875-4.8080972 .778734$

H $1.761831-4.0952874 .260133$

H $\quad 0.149437-4.8354854 .320419$

SCF Energy $(B 3 L Y P / 6-31 G * * / / M M F F)=-3245.89787644$

0155

MMFF Geometry

C $1.216840-2.154379-2.227296$

C $0.205716-3.031709-2.325732$

C $-0.773434-3.317455-1.217602$

O $-2.088505-2.888939-1.657847$

C $-0.904193-4.819782-0.908798$

C $0.281053-5.433329-0.199942$

C $0.390223-5.544891 \quad 1.138084$

C $1.340866-6.027086-1.090722$

C $-0.579162-5.0743922 .189632$

C $0.131839-4.3522453 .344142$

C $0.494174-2.896276 \quad 3.012467$

C $1.487815-2.3549364 .040949$

C $1.785150-0.8675103 .819731$

O $-0.717502-2.1339243 .055554$

O $2.479587-0.3810684 .978708$

C $0.450619-0.1013673 .733996$

C $2.718969-0.6406972 .626601$

O $0.703238 \quad 1.261933 \quad 3.396892$

C $-0.567782-0.7394632 .755566$

C -1.952801-0.070292 2.938139

O $-0.113098-0.5402931 .419073$

C $-2.373443-1.561077-1.593776$

O $-1.631970-0.667136-1.219971$

C $-3.765272-1.379717-2.060948$

C $-4.341173-0.172103-1.967014$

C $-5.6952690 .177189-2.401625$ 
C $-6.544578-0.922516-2.984262$

C $-3.098594-0.7355682 .163230$

C $-4.450737-0.1459912 .532053$

C -6.884174 -0.475288 2.114284

$\begin{array}{lllll}\text { O } & -4.586060 & 0.762450 & 3.347616\end{array}$

C $-7.587389-0.1618390 .832776$

C -8.1415551 .0316070 .570580$

C $-8.9162271 .349252-0.676412$

C -8.282458 $2.453914-1.491213$

C $-8.5384863 .745585-1.223696$

C $-7.4257212 .054420-2.670508$

C $-6.1104191 .453375-2.256847$

N -5.487797 $-0.761041 \quad 1.843063$

H $1.362676-1.607576-1.299112$

H $0.047959-3.556678-3.265322$

H $-0.516090-2.780755-0.296235$

H - $-1.107843-5.376808-1.834384$

H -1.814681 -4.979813 -0.315971

H $1.266282-6.0590781 .532521$

H $2.151417-6.494072-0.521166$

H $0.905629-6.798050-1.734917$

H $1.790000-5.260905-1.725245$

H -1.365766 -4.4346501 .778488$

H -1.072333 -5.9654462 .597057$

H $-0.543150-4.3613404 .210242$

H $1.029992-4.9134693 .630843$

H $0.921368-2.8447922 .004681$

H $2.417992-2.9368634 .026842$

H $1.072715-2.4794665 .051219$

H $3.293311-0.9034755 .082153$

H $0.018887-0.0993924 .744750$

H $3.672880-1.1589892 .780679$

H 2.9661710 .4209232 .525500

H $2.303818-0.9899021 .678883$

H $-0.111147 \quad 1.767025 \quad 3.556494$

H -2.213165 -0.0911054 .004723$

H -1.904733 0.9825652 .633493

H $-0.874641-0.5509130 .818697$

H -4.274546 -2.243188 -2.473396

H $-3.7653460 .646075-1.536221$

H $-6.631384-1.757037-2.279255$

H $-6.104498-1.294359-3.916143$

H -7.566156 $-0.612586-3.212587$

H -2.959022 -0.5982061 .088730$

H -3.133193 -1.809769 2.375916

H -6.9719050 .3377862 .842008$

H -7.318690 -1.374671 2.562497

H -7.675988 -0.9651510 .105859$

H -8.0652211 .8240041 .312362$

H $-9.9290481 .649446-0.376213$

H $-9.0531700 .457036-1.299619$

H -8.111874 4.543485-1.822332

H $-9.1786254 .032866-0.395967$

H -7.197854 $2.933240-3.288758$

H -8.005043 $1.398766-3.326773$

H $-5.4236152 .175866-1.813464$

H $-5.275159-1.5449131 .232165$

C $3.637456-1.907414-3.016270$

O $3.997747-0.827339-2.136463$

C $5.063448-1.277426-1.274942$

C $5.441434-2.682203-1.744026$

C $4.134236-3.177764-2.333559$

H $4.637503-1.349225-0.267326$

C $6.220786-0.269851-1.282127$

H $6.221692-2.660920-2.513273$

H $5.805273-3.306323-0.921818$

C $4.296199-4.360584-3.271287$

H $3.474456-3.458717-1.503796$

C $5.8023111 .180935-0.963916$

O $6.814972-0.265800-2.587226$

H $7.001848-0.606614-0.590299$

H $5.2235371 .578196-1.804941$

O $6.9945331 .974006-0.898479$

C 5.0157061 .3496580 .345723

C 4.7341722 .8100640 .742844

H 4.0659840 .8108900 .270312

O $\quad 5.764255 \quad 0.741763 \quad 1.402489$

H 4.1524302 .7918161 .673065
H 5.6762583 .3038361 .013749

H $3.331312-4.659557-3.692641$

H $4.967623-4.124370-4.103666$

H $4.713276-5.220405-2.736908$

C $2.148649-1.840465-3.365103$

H $7.5450270 .376624-2.571001$

H $7.5056781 .671012-0.128368$

H 5.2614690 .8562352 .226552

H $1.952756-2.512540-4.209314$

H $1.914330-0.822470-3.701686$

H $4.203282-1.737959-3.942698$

C 1.7502542 .8267740 .011570

O $2.8694552 .947867-0.869612$

C $3.9967073 .639200-0.326363$

C 3.5908975 .0457340 .138692

C 2.3999255 .0115291 .094074

C 1.2562734 .1970870 .491393

H 2.0513772 .2296280 .881392

C $0.6602942 .011226-0.700530$

H $4.6994533 .773969-1.157600$

O 4.6803565 .7056360 .774943

H $3.3142615 .650124-0.735132$

H 2.6826584 .6223222 .079079

O $1.9322836 .345108 \quad 1.310026$

H $0.8123884 .764177-0.335195$

O $0.2389654 .010849 \quad 1.472874$

C $0.1107062 .670572-1.949512$

H $1.0651741 .041179-1.011553$

H $-0.1665821 .833238-0.003843$

H 5.4176205 .7293980 .141198

H 2.6811006 .8609941 .655114

H $-0.0293674 .892621 \quad 1.783402$

O $-1.1854472 .281983-2.132748$

O $0.7462723 .407380-2.691379$

C $-1.8287632 .815449-3.294351$

H $-1.8284373 .909154-3.261449$

H $-1.3306642 .455036-4.199367$

H $-2.8645112 .466730-3.301624$

SCF Energy $\left(B 3 L Y P / 6-31 G^{* *} / / M M F F\right)=-3245.91912960$

01550

MMFF Geometry

C $-2.339566-1.249736-2.834660$

C -3.446555 -0.535133 -2.579943

C $-3.4989340 .951440-2.319636$

O $-2.154431 \quad 1.489137-2.305899$

C $-4.3105861 .727790-3.369739$

C $-5.7942101 .439394-3.387630$

C $-6.6750161 .960484-2.511351$

C $-6.2722460 .569814-4.520346$

C $-6.3727342 .840481-1.327686$

C $-7.1945402 .463311-0.086416$

C -6.9230441 .0486670 .455144$

C $-8.0167320 .669067 \quad 1.458677$

C $-7.772913-0.7116842 .076013$

O -5.6366601 .0271801 .093668$

O $-8.653391-0.8548923 .200823$

C $-6.334056-0.7527452 .619139$

C -8.118704-1.847735 1.106210

O $-6.007164-2.0625493 .081616$

C $-5.264674-0.2620231 .608570$

C $-3.910276-0.1463712 .351706$

O $-5.144663-1.2272670 .562107$

C $-1.9114422 .568516-1.518115$

O $-2.7174333 .186310-0.841837$

C $-0.4627532 .853646-1.593411$

C $0.0814983 .664853-0.675731$

C $1.4953494 .014046-0.559067$

C $2.4387423 .531013-1.628281$

C -2.7564790 .4884881 .564638$

C -1.4426320 .3343642 .319747$

C 1.0223850 .3223741 .978169

O $-1.3909820 .045023 \quad 3.513497$

C 1.6437611 .6031242 .440430

C 2.8896841 .9658862 .097924

C 3.5848263 .2089952 .579869

C 4.1379234 .0374911 .439749

C $5.3815993 .824490 \quad 0.977184$ 
C 3.2866655 .1510740 .870666 C 1.8907424 .7269420 .515505 N $-0.3341610 .557627 \quad 1.515459$ H -1.377978 -0.750449 -2.918197 H -4.392785-1.064601-2.488976 H -3.936353 $1.075545-1.322621$ H -3.882338 $1.550263-4.366287$ H $-4.1665802 .806392-3.218738$ H $-7.7311461 .734631-2.654083$ H -7.354471 $0.404472-4.490124$ H $-6.0360101 .036950-5.482002$ H -5.789564 - $0.411377-4.483759$ H -5.312384 2.836958-1.070073 H $-6.6267173 .871338-1.603395$ H -6.974549 3.1990520 .697952 H -8.259165 2.565660 -0.333933 H $-6.9206400 .336520-0.376773$ $\begin{array}{llll}\text { H } & -9.007293 & 0.703428 & 0.987620\end{array}$ H $-8.043476 \quad 1.4131462267301$ H -9.566503 -0.7550322 .881128$ H -6.299960 -0.096982 3.500770 H $-9.172522-1.7890540 .808628$ H -7.997248 -2.8258631 .584825$ H $-7.517935-1.8333430 .194555$ H -6.689181 -2.321243 3.724952 H -4.0507580 .4543303 .260500$ H -3.596525 -1.148706 2.672531 H $-4.263709-1.1557100 .165161$ H $\quad 0.1163532 .362594-2.366798$ H $-0.566577 \quad 4.094098 \quad 0.087797$ H $2.1088803 .871690-2.616051$ H $2.4889652 .436570-1.631551$ H $3.4597893 .898630-1.498009$ H -2.645704 0.0161190 .585339 H -2.9424041 .5571141 .416120$ H $1.574747-0.1244221 .145583$ H $1.002194-0.3950252 .804656$ H 1.0664852 .2361223 .109322 H 3.4660931 .3067991 .453609 H 4.4059892 .8979833 .239253 H 2.9248363 .8255913 .202293 H $5.798673 \quad 4.4230320 .174456$ H 6.0101053 .0449521 .393125 H $3.7618145 .619639-0.000018$ H 3.2176145 .9467581 .624140 H 1.1429625 .0255261 .250940 H $-0.4666910 .824790 \quad 0.544947$ C $-1.490445-3.450203-1.911888$ O $-0.122124-2.995470-1.956734$ C $0.328311-2.762144-0.608831$ $\begin{array}{llll}\text { C } & -0.669437 & -3.482717 & 0.289295\end{array}$ C $-1.960800-3.251075-0.470096$ H $0.251151-1.681655-0.434995$ C $1.785316-3.202587-0.419915$ H $-0.450661-4.5550260 .355504$ H $-0.687444-3.0733221 .303970$ C $-3.094518-4.170481-0.054416$ H $-2.269202-2.213520-0.301966$

C $2.781289-2.349837-1.240553$ O $1.887962-4.582962-0.761038$ H $2.036537-3.1068310 .643939$ H $2.607121-1.297366-0.990933$ O $2.547063-2.498539-2.639915$ C $4.258503-2.688525-0.956681$ C $5.265938-1.792452-1.702258$ H $4.440027-2.625650 \quad 0.120626$ O $4.532738-4.040035-1.333851$ H $6.266117-2.214401-1.544117$ H $5.097841-1.872513-2.783646$ H -3.993475 -3.970873 -0.646177 H $-2.827749-5.223927-0.188997$ H -3.344410 -4.018847 1.000850 C $-2.318261-2.744523-2.992034$ H $2.790732-4.873614-0.547717$ H $1.632579-2.222167-2.819136$ H $4.318432-4.129333-2.278541$ H -3.336144 -3.151645 -2.998480 H $-1.879244-2.960016-3.974101$
H $-1.460019-4.521567-2.150335$

C $6.583350-0.5113900 .720745$

O $5.319953-0.1579230 .146462$

C $5.240052-0.311338-1.272937$

C $6.3028520 .564329-1.951542$

C $7.7024070 .287870-1.403628$

C $7.7039690 .347303 \quad 0.124316$

H $6.789503-1.5727660 .537080$

C $6.471622-0.3000072 .236200$

H $4.2644780 .090332-1.571647$

$\begin{array}{llll}\text { O } & 6.271226 & 0.356273 & -3.359198\end{array}$

H $6.0559931 .619587-1.774578$

H $8.090847-0.674524-1.757189$

O $8.6053901 .287445-1.882107$

H $7.5959501 .389693 \quad 0.447928$

O $8.960607-0.120306 \quad 0.610369$

C $5.524627-1.3035522 .856167$

H $7.437715-0.4157742 .738699$

H 6.1042190 .7120872 .443016

H $6.8630131 .009687-3.768140$

H $8.6892941 .171085-2.843463$

H 9.6521520 .4263360 .199396

O $4.602842-0.659340 \quad 3.627164$

O $5.599214-2.5137262 .686385$

C $3.634530-1.5010294 .260504$

H $3.067464-2.0592753 .509311$

H $4.126840-2.1833954 .959834$

H $2.943639-0.8637264 .818912$

SCF Energy (B3LYP/6-31G**//MMFF)= -3245.91989297

01551

MMFF Geometry

C - $-1.0388924 .709188-1.758878$

C $-2.3715174 .847012-1.675911$

C $-3.3645643 .735357-1.397429$

O $-2.686662 \quad 2.484512-1.142445$

C $-4.1776874 .085030-0.135863$

C $-5.207814 \quad 3.037997 \quad 0.242210$

C -5.1976802 .3415751 .396333$

C $-6.3082792 .809364-0.761357$

C -4.2166602 .4515972 .530487$

C $-3.005213 \quad 1.5134482 .422925$

C $-3.351700 \quad 0.0219742 .560960$

C $-2.076711-0.8210062 .635823$

C -2.382405 -2.325570 2.696394

O $-4.121132-0.3805591 .425815$

O $-1.161524-3.0342772 .432636$

C $-3.367683-2.6870411 .563509$

C $-2.839285-2.7566674 .095117$

O $-3.811845-4.0354591 .686445$

C $-4.582107-1.7384361 .476923$

C $-5.455108-1.9749260 .219902$

O $-5.425637-1.9509622 .615936$

C $-2.4024951 .682654-2.202875$

O $-2.7288781 .847781-3.366541$

C $-1.5419950 .591296-1.696698$

C $-0.712383-0.036755-2.542141$

C $0.246384-1.084595-2.186637$

C $0.196676-1.626272-0.782196$

C $-4.732745-1.819620-1.123060$

C $-4.226650-3.129073-1.706335$

C $-2.855628-4.013447-3.589616$

O $-4.533205-4.230609-1.258422$

C $-1.477578-4.316759-3.092327$

C $-0.409512-4.408425-3.899334$

C $0.972804-4.755165-3.425968$

C $2.007281-3.740980-3.859597$

C $2.771661-3.952527-4.944263$

C $2.214555-2.527081-2.984208$

C $1.116172-1.508346-3.127554$

$\mathrm{N}-3.421086-2.922145-2.818295$

H $-0.5915973 .725405-1.649656$

H $-2.7990475 .839606-1.803861$

H $-4.0343243 .637751-2.260103$

H $-4.6989975 .040493-0.282046$

H $-3.477144 \quad 4.248146 \quad 0.690425$

H $-5.990426 \quad 1.612832 \quad 1.561499$

H $-7.0911772 .147416-0.375615$ 
H $-6.7848083 .759072-1.025520$ H -5.911303 2.350670 -1.672128 H -3.8606603 .4830142 .628274$ H -4.7519712 .2481703 .466738$ H -2.498159 1.6901701 .467133 H -2.2994791 .7881093 .217008$ H -3.948063 -0.1078013 .471785$ H -1.464166 -0.5208253 .494049$ H -1.460738 -0.6279771 .747117$ H $-0.510143-2.7675923 .103708$ H -2.804961 -2.628206 0.624724 H -2.063502 -2.5316244 .837212$ H $-2.993856-3.8402064 .144011$ H -3.757213 -2.261268 4.417966 H -3.020530 -4.600280 1.709925 H $-5.968666-2.9409800 .294265$ H $-6.254959-1.2202510 .236149$ H $-6.316659-1.6266682 .404453$ H - $1.5768340 .374431-0.635491$ H $-0.7096530 .266163-3.588472$ H $-0.819352-1.949727-0.533019$ H $0.508974-0.860381-0.064475$ H $\quad 0.833599-2.498579-0.625466$ H -5.445338 -1.417983-1.854924 H -3.906820 -1.106465 -1.046410 H -2.858481 -3.703969 -4.639552 H -3.488459 -4.900705 -3.485275 H - $1.357300-4.495326-2.026352$ H $-0.539522-4.257329-4.968817$ H $1.226196-5.742729-3.833270$ H $1.007494-4.868554-2.335194$ H $3.536353-3.243969-5.244599$ H $2.645754-4.837713-5.559108$ H $3.155579-2.026508-3.249089$ H $2.354235-2.856298-1.952428$ H $1.070936-1.054209-4.118290$ H -3.177590 -1.973642 -3.088296 C $0.9964495 .838077-0.880442$ O $1.8093894 .653674-1.003339$ C $3.1736884 .982032-0.687323$ C $3.156374 \quad 6.424641-0.191917$ C $1.9797037 .001216-0.957301$ H $3.7388124 .929884-1.626229$ C 3.7536063 .9752360 .317800 H 2.9733206 .4761640 .888170 H $4.0979656 .943468-0.396161$ C $1.4541148 .300058-0.372232$ H $2.2839967 .168880-1.998991$ C $3.8931242 .545974-0.257219$ O $2.950166 \quad 3.968247 \quad 1.496381$ H 4.7504174 .3191820 .618433 H $4.5368712 .606507-1.143162$ O $2.6348032 .046980-0.707577$ C $4.521117 \quad 1.5389350 .729795$ C 4.8309250 .1870130 .052507 H 5.4586491 .9612801 .110258 O $3.667363 \quad 1.3440571 .851299$ H $3.903757-0.241830-0.340410$ H $5.4832460 .383496-0.806159$ H $\quad 0.601753 \quad 8.672207-0.949030$ H $\quad 1.127844 \quad 8.1699950 .665117$ H $2.2321519 .070304-0.385775$ C $-0.0699695 .838645-1.977585$ H 2.0356103 .7736941 .230093 H 2.0236272 .0490650 .047715 H 2.8727590 .8752411 .545037 H $0.4010015 .692347-2.957031$ H $-0.5958996 .799457-1.996487$ H 0.5231735 .8010090 .110183 C $3.588464-2.1409021 .641161$ O $4.646315-1.2660672 .054814$ C 5.515911-0.818048 1.008736 C $6.154680-2.0280270 .311383$ C $5.103499-3.014521-0.183623$ C $4.151687-3.3888070 .950530$ H $2.914819-1.6110330 .958679$ C $2.795089-2.5280272 .897892$ H $6.330445-0.2719581 .501956$
O $\quad 6.968112-1.621132-0.783659$

H $6.810625-2.5499961 .020375$

H $4.556726-2.613854-1.043457$

O $5.749172-4.206359-0.636027$

H $4.681709-4.0167341 .678356$

O $3.074095-4.1616300 .426382$

C $2.003913-1.3603353 .446449$

H $2.077655-3.3297272 .692888$

H $3.491711-2.8735783 .671662$

H $7.637781-1.006773-0.437446$

H $6.379024-3.947526-1.330604$

H $3.461670-4.922620-0.039263$

O $2.002504-1.4112404 .810099$

O $1.428081-0.5272682 .759351$

C $1.282763-0.3641105 .468317$

H 1.6198630 .6166715 .118826

H $0.209520-0.4878025 .299874$

H $1.480570-0.4387116 .540881$

SCF Energy (B3LYP/6-31G**//MMFF) $=-3245.90720024$

01552

MM̄FF Geometry

C $1.734575-3.272222-0.155140$

C $0.669102-3.7577910 .498930$

C $-0.374267-2.9061871 .173311$

O $-1.625486-3.2385000 .517789$

C $-0.512517-3.2397942 .672841$

C $0.265444-2.3207633 .593607$

C $-0.305948-1.4382064 .437555$

C $1.760627-2.4895133 .585026$

C $-1.774325-1.1356394 .556029$

C $-2.082280 \quad 0.252211 \quad 5.140855$

C -1.4583541 .4306394 .376399$

C -1.891973 2.7594645 .003719

C -1.3442403 .9677194 .230699$

O $-1.874287 \quad 1.3732493 .007233$

O -2.0564345 .1228024 .700443$

C -1.6693883 .7843832 .732602$

C 0.1338744 .2182944 .550345

O $-1.0704014 .817477 \quad 1.953971$

C -1.2873252 .3911342 .183106$

C -1.7626162 .1820950 .728499$

O 0.1420242 .2781172 .167056

C -2.603291-2.294622 0.505760

O $-2.538970-1.1634230 .957065$

C $-3.790978-2.891836-0.144274$

C $-4.935102-2.194307-0.205805$

C $-6.193338-2.650869-0.799826$

C $-6.217334-4.019175-1.429193$

C -3.2765072 .2173550 .514123$

C $-3.6440331 .992993-0.944548$

C $-5.6087582 .000198-2.462965$

O $-2.8111641 .827511-1.831777$

C $-6.9948341 .447800-2.381841$

C $-7.4265350 .420951-3.129394$

C $-8.813301-0.149748-3.041131$

C $-8.812327-1.634481-2.746935$

C $-9.032456-2.527819-3.726231$

C -8.640161-2.081869-1.311357

C $-7.261333-1.826485-0.769385$

N $-5.0198012 .009855-1.135796$

H $1.888838-2.198672-0.199889$

H $0.514470-4.8344710 .527391$

H $-0.159567-1.8405971 .019012$

H $-0.194944-4.2736892 .867801$

H -1.573018 -3.239199 2.951498

H $0.344162-0.8783795 .108117$

H $2.178322-2.1224992 .643213$

H $2.246389-1.9358414 .395682$

H $2.028503-3.5441223 .705982$

H $-2.265532-1.2159393 .581809$

H -2.229702 -1.886593 5.213046

H $-3.1743710 .364757 \quad 5.148208$

H $-1.7524450 .285086 \quad 6.187208$

H $-0.367873 \quad 1.337440 \quad 4.424221$

H -1.590862 2.8100456 .057683

H -2.989688 2.8184725 .003787

H -1.771155 5.8826624 .164188 
H $-2.752645 \quad 3.9231412 .635684$ H 0.2634894 .4092725 .622482 H 0.5034615 .1157304 .042061 H 0.7800163 .3797244 .283270 H -0.1254564 .6074451 .860584$ H -1.274102 2.9156220 .073263 H $-1.399431 \quad 1.2047360 .383987$ H $0.381116 \quad 1.5291171 .596401$ H -3.693920 -3.894478 -0.544976 H $-4.958398-1.193386 \quad 0.223623$ H $-5.997717-4.790037-0.682426$ H -5.475950 -4.085938 -2.233351 H -7.181696 -4.275520 -1.873259 H -3.7651241 .4437681 .116748$ H -3.6817703 .1919500 .804081$ H $-4.9689461 .428227-3.143372$ H -5.638409 $3.036911-2.813585$ H -7.677266 $1.915055-1.675714$ H -6.749911-0.034934 -3.849030 H $-9.3150510 .044870-3.997967$ H $-9.4129540 .367798-2.282059$ H -9.064140 -3.593323 -3.524478 H $-9.183040-2.213953-4.753973$ H -8.905915 -3.138983 -1.192201 H $-9.364029-1.546036-0.683613$ H -7.152298 $-0.850839-0.295064$ H -5.604484 2.313957 -0.362015 C $4.169293-4.002694-0.386019$ O $4.656204-2.663768-0.589007$ C $5.559140-2.3367180 .488023$ C $5.745439-3.6169401 .300544$ C $4.415328-4.3144091 .086926$ H $5.043617-1.5989621 .113809$

C $6.855881-1.736290-0.069743$ H $6.564398-4.2327130 .911232$ H $5.957360-3.4092842 .353958$ C $4.439945-5.7965711 .412973$ H $3.667208-3.8225151 .717656$ C $6.634530-0.528515-1.004159$ O $7.532870-2.748439-0.826915$ H $7.524367-1.4686090 .756772$ H $6.115954-0.869419-1.906559$ O $7.916775-0.065182-1.445363$

C $5.8672550 .644264-0.366542$

C $5.7240771 .877553-1.280172$

H $4.8693020 .310545-0.066916$

O $\quad 6.527426 \quad 1.052411 \quad 0.834138$ H $5.2652302 .679525-0.688995$ H $6.7174102 .260313-1.545937$ H $3.460349-6.2507591 .233380$ H $5.176551-6.3301300 .802901$ H $4.697376-5.9545462 .465389$ C $2.728714-4.132282-0.884659$ H $8.347612-2.350743-1.179027$ H $8.4391550 .164046-0.658370$ H 7.3771621 .4547640 .587080 H $2.427198-5.185775-0.845264$ H $2.695431-3.831947-1.939637$

H $4.798856-4.650243-1.011521$

C $2.6934561 .772101-1.605971$

O $3.6634190 .953677-2.264733$

C $4.8997721 .613437-2.556375$

C $4.6493282 .854443-3.425780$

C $3.5925253 .780586-2.823318$

C $2.3317222 .997429-2.451504$

H $3.1077132 .115798-0.649420$

C $1.4876030 .902147-1.231254$

H $5.4828210 .915122-3.168700$

O $5.8665183 .565529-3.624381$

H $4.3057482 .524789-4.414944$

H $3.9908034 .314210-1.952780$

O $3.2540434 .792272-3.774989$

H $1.8052722 .701181-3.365761$

O $1.4550193 .841018-1.708479$

C $0.7994720 .214347-2.391516$

H $1.8251180 .110316-0.553444$

H $\quad 0.748571 \quad 1.516187-0.709629$

H $5.6607214 .351883-4.158465$
H $2.8944364 .353858-4.565073$

H $1.2743024 .625231-2.254650$

O $-0.351747-0.364619-1.934647$

O $1.2179380 .164146-3.539279$

C -1.120287-1.067604-2.915494

H $-2.065541-1.367713-2.456774$

H $-0.582855-1.964674-3.236505$

H -1.339738 -0.420447 -3.770328

SCF Energy (B3LYP/6-31G**//MMFF) $=-3245.91577425$

01553

MM̄MF Geometry

C $2.558884-2.624732-2.130504$

C $1.961925-1.753467-1.304013$

C $1.023343-0.666436-1.765202$

O $-0.186612-0.743713-0.975546$

C $1.6874590 .700001-1.509162$

C $1.0091781 .859461-2.208877$

C $0.2646012 .804356-1.601585$

C $1.2586621 .944341-3.694079$

C $-0.0985462 .915607-0.146021$

C $-1.5320842 .454948 \quad 0.126510$

C $-2.6311913 .314940-0.515349$

C -2.8102064 .6566330 .199393$

C $-4.0103095 .447638-0.348721$

O $-3.8421882 .554257-0.425625$

O $-4.311084 \quad 6.4971350 .584272$

C $-5.2472864 .523748-0.400887$

C $-3.6752286 .134542-1.678428$

O $-6.3411505 .171233-1.046524$

C $-4.9670963 .162063-1.073979$

C $-6.1759542 .212623-0.904021$

O $-4.733022 \quad 3.372537-2.468389$

C -1.093692 -1.701587-1.313860

O $-1.048958-2.452590-2.275289$

C $-2.130266-1.730789-0.257615$

C $-2.793708-2.874583-0.029564$

C $-3.759112-3.1136821 .046780$

C $-4.268093-1.9177621 .806546$

C $-5.8822830 .766240-1.329136$

C $-7.080806-0.146315-1.146683$

C $-7.730432-2.542867-1.372665$

O $-8.1844410 .252933-0.786353$

C -7.681640 -3.143676 -0.004027

C $-7.419033-4.4384660 .227581$

C $-7.434190-5.0571421 .594296$

C $-6.136741-5.7497101 .938223$

C $-6.034175-7.0889371 .893316$

C -4.990294-4.896626 2.431931

C $-4.116664-4.3871151 .317509$

N $-6.766357-1.463505-1.454740$

H $2.355372-2.579145-3.197310$

H $2.184583-1.794503-0.240547$

H $0.775841-0.780046-2.827345$

H $2.7319560 .678357-1.850596$

H $1.7375010 .879459-0.431649$

H $-0.1440533 .601736-2.221597$

H $0.8144152 .840372-4.140682$

H $2.3341961 .977111-3.897003$

H $0.833177 \quad 1.077565-4.208374$

H 0.5777672 .3280580 .480630

H $0.039163 \quad 3.9550160 .172137$

H -1.647456 $1.421595-0.220974$

H -1.695006 2.4097761 .211314

H $-2.4005003 .466393-1.574487$

H $-1.898396 \quad 5.2621150 .138989$

H -2.979450 4.4769281 .270311

H $-3.514537 \quad 7.0457950 .685465$

H -5.5636684 .3389630 .635345$

H -2.845183 $6.839104-1.546634$

H $-4.519766 \quad 6.732179-2.038880$

H -3.387362 $5.432334-2.463603$

H $-6.4902946 .015246-0.586797$

H -6.4777242 .1938310 .151425$

H -7.029550 2.588804-1.481888

H $-4.9557512 .566750-2.958942$

H $-2.259023-0.8453620 .353282$

H $-2.565260-3.739626-0.651447$ 
H $-4.629800-1.1479281 .116946$ H -3.473317 -1.487632 2.425062 H -5.108896 -2.147604 2.464503 H $-5.5957800 .722048-2.385066$ H $-5.0557790 .361186-0.735047$ H -7.477378 -3.266701 -2.153664 H $-8.734212-2.152899-1.569992$ H $-7.905536-2.4874150 .833737$ H -7.219967 $-5.100272-0.612345$ H -8.263190 -5.7764021 .622728$ H -7.664524 -4.314321 2.368254 H -5.115102 -7.594227 2.171578 H $-6.864346-7.7091221 .572076$ H -4.349391 -5.4966463 .092507$ H -5.380491 -4.101347 3.072528 H -3.678772 -5.1831910 .712984$ H $-5.803317-1.705767-1.669964$ C $4.967097-3.253729-2.091958$ O $5.348563-2.057284-1.386236$ C $6.748696-2.131356-1.057559$ C $7.279153-3.395334-1.727891$ C $6.043280-4.277501-1.743424$ H $6.809131-2.2629450 .028733$ C $7.445159-0.827208-1.467695$ H $7.621994-3.202430-2.750966$ H $8.114610-3.835992-1.175131$ C $6.137335-5.437146-2.718931$ H $5.876959-4.668090-0.730596$ C $6.7640450 .436893-0.898011$ O $7.411648-0.734562-2.897605$ H $8.502459-0.864348-1.180272$ H $5.7571170 .533509-1.319104$ O $7.5011081 .583445-1.339680$ C 6.7021160 .4778880 .640498 C 6.0594701 .7581381 .208519 H $6.155301-0.389771 \quad 1.020417$ O $8.0289690 .380186 \quad 1.165816$ H 6.2675591 .7908072 .284699 H 6.5672062 .6404680 .800268 H $5.222185-6.037089-2.702181$ H $6.291865-5.087133-3.745126$ H $6.974852-6.092209-2.457658$ C $3.554078-3.654907-1.670560$ H $8.031341-0.032945-3.159324$ H $7.3600611 .670009-2.297488$ H $8.537598 \quad 1.1328180 .817984$ H $3.506336-3.761700-0.579614$ H $3.279781-4.621670-2.106234$ H $4.994779-3.017778-3.164461$ C 3.7852210 .5212382 .815333 O $3.8466640 .677041 \quad 1.394369$ C 4.5397611 .8515990 .953485 C 3.8759373 .1057451 .540971 C 3.7452233 .0291993 .061042 C 3.0875271 .7134263 .478929 H 4.8084550 .4501693 .205499 C $3.145379-0.8367023 .137910$ H $4.4012431 .899893-0.132754$ O $4.605370 \quad 4.277972 \quad 1.193504$ H 2.8708663 .2160261 .114665 H 4.7156823 .1565683 .555088 O 2.9200984 .1043473 .515994 H $2.025374 \quad 1.7481363 .212536$ O 3.1655651 .5742914 .895374 C $1.701610-0.9934222 .707813$ H $3.708879-1.6254572 .624138$ H $3.201050-1.0139004 .218158$ H 4.6571874 .3124390 .222998 H 3.3288204 .9318003 .208726 H 2.7473802 .3600775 .287309 O $1.201729-2.1340243 .271582$ O $1.095864-0.2440751 .955771$ C $-0.148176-2.4580572 .925662$ H $-0.807242-1.5961043 .066879$ H $-0.184067-2.8098161 .891434$ H $-0.484014-3.2644953 .583063$

SCF Energy (B3LYP/6-31G**//MMFF) $=-3245.90915159$
$01 \_554$

MM̄FF Geometry

C $1.365329-3.471623-0.031139$

C $0.691308-3.877846-1.116691$

C $0.482505-3.086766-2.390104$

O $-0.940137-2.976117-2.666177$

C $1.193641-1.718087-2.495555$

C $1.177900-1.112299-3.885934$

C $0.5197590 .014229-4.222492$

C $2.043728-1.806504-4.906513$

C $-0.3819550 .847008-3.357337$

C $0.3794061 .960934-2.632352$

C $-0.5715542 .943984-1.932267$

C $0.2223254 .032951-1.205721$

C $-0.6877304 .983305-0.413305$

O $-1.3874742 .218051-1.004636$

O $0.138305 \quad 5.730623 \quad 0.493414$

C -1.6598104 .1480810 .447919$

C $-1.3678016 .011568-1.324833$

O -2.6252754 .9836001 .082500$

C $-2.3699513 .026165-0.340406$

C -3.2231422 .1119380 .568643$

O $-3.2716343 .621296-1.283655$

C $-1.749978-2.289835-1.817296$

O $-1.431035-1.690535-0.802910$

C $-3.130547-2.398618-2.335991$

C $-4.041985-1.493299-1.952032$

C $-5.468200-1.498206-2.284227$

C $-5.983190-2.650907-3.105814$

C $-2.448596 \quad 1.2924481 .600993$

C -3.4071650 .5148872 .481564$

C $-4.911936-1.4721272 .480944$

O -3.6834160 .8919693 .618414$

C $-5.984162-1.7780011 .483854$

C $-7.152403-1.1197201 .430536$

C $-8.233747-1.3900320 .421359$

C $-8.407498-0.242785-0.552580$

C $-9.1795640 .812992-0.244283$

C $-7.738145-0.350144-1.909124$

C $-6.243403-0.503606-1.801660$

N $-3.936515-0.6001421 .853293$

H $1.814900-2.483584-0.017298$

H $\quad 0.249637-4.872686-1.109421$

H $\quad 0.871108-3.730705-3.188415$

H $2.247795-1.833956-2.213383$

H $0.776035-1.014089-1.769274$

H $\quad 0.6229190 .384488-5.241834$

H $2.103227-1.250425-5.848276$

H $3.065454-1.915900-4.527894$

H $1.648321-2.799927-5.138032$

H $-1.1552951 .283896-4.001653$

H $-0.9167020 .223686-2.637022$

H $1.0470831 .503913-1.890393$

H $1.0097382 .510184-3.343037$

H -1.215490 $3.393013-2.698080$

H $0.8428634 .597357-1.913143$

H $0.9262973 .565368-0.504883$

H $0.8081516 .202304-0.030775$

H -1.0653683 .6989401 .252012$

H $-0.6165876 .625945-1.835899$

H -1.980582 $6.710317-0.744860$

H -1.995586 $5.555632-2.093112$

H -2.138632 5.6687461 .572095

H -4.0000102 .7065641 .067361$

H -3.776433 $1.411229-0.070309$

H $-3.9292962 .955747-1.545069$

H -3.359441 -3.230400 -2.991851

H -3.717697 -0.677939-1.308446

H $-5.831180-3.598082-2.576370$

H $-5.461924-2.698847-4.068612$

H $-7.047257-2.584478-3.338805$

H -1.7617880 .5923311 .113332$

H -1.8508391 .9318282 .257616$

H $-5.315923-1.0029103 .383838$

H $-4.398391-2.3918362 .778160$

H $-5.782175-2.5724300 .770106$

H -7.343375 -0.3257522 .149486$

H $-9.172102-1.5612380 .965016$ 
H -8.044075 -2.320277 -0.128657 H $-9.3288351 .629445-0.942792$ H $-9.678346 \quad 0.8837130 .716900$ H -7.935402 $0.549282-2.506308$ H -8.210624 -1.176388 -2.446057 H $-5.7649270 .295199-1.233298$ H $-3.539888-0.894400 \quad 0.965857$ C $1.025564-3.6405112 .479654$ O $1.595656-2.3223222 .633485$ C $0.515695-1.3874562 .806274$ C $-0.627787-2.2151163 .372189$ C $-0.496949-3.4737132 .534198$ H $\quad 0.231020-1.0388501 .805743$ C $0.953054-0.1931393 .654359$ H $-0.474428-2.4466734 .432725$ H -1.597999 -1.725621 3.262719 C -1.225969-4.673107 3.113704 H $-0.902427-3.270228 \quad 1.535623$ C 2.2063270 .5342613 .119295 O $1.244436-0.6515414 .979345$ H $\quad 0.1157750 .5087713 .749423$ H $3.089935-0.0890123 .297522$ O $2.397030 \quad 1.7116313 .913601$ C 2.1441480 .9245611 .630356 C 3.2607331 .8692311 .153699 H 2.1652510 .0167601 .018591 O $\quad 0.896461 \quad 1.5634091 .354068$ H 3.0832802 .0724770 .090087 H 3.1488112 .8447461 .644177 H -1.079079 -5.557094 2.485103 H $-0.869621-4.9119044 .121402$ H -2.301192 -4.475979 3.175685 C $1.570489-4.2994081 .207690$ H 1.5207170 .1223665 .499621 H $1.6107662 .273823 \quad 3.810124$ H 0.8701012 .3979731 .851459 H $1.134488-5.2984631 .092682$ H $2.653775-4.4329491 .321461$

H $1.370933-4.2219313 .343922$

C $4.844557-0.130291-0.542471$

O $4.856724 \quad 0.004188 \quad 0.881240$

C 4.6918361 .3397671 .364913

C $5.7852702 .251807 \quad 0.789887$

C $5.8348492 .187025-0.736134$

C $5.9209550 .736040-1.209904$

H $3.857797 \quad 0.175193-0.914157$

C $4.975579-1.622073-0.885902$

H $4.854376 \quad 1.2944032 .448654$

O 5.5762623 .5942301 .215153

H 6.7566501 .9348061 .190980

H $4.9710902 .693675-1.181110$

O $6.9823442 .901605-1.202229$

H $6.9234580 .350003-0.993706$

O $5.7469020 .689020-2.624360$

C $6.282595-2.256347-0.455158$

H $4.183149-2.183833-0.380210$

H $4.861503-1.754227-1.968010$

H $\quad 6.271698 \quad 4.139826 \quad 0.809388$

H $7.7729342 .483586-0.820150$

H $6.416006 \quad 1.274139-3.019183$

O $6.491819-3.386966-1.193197$

O $7.010329-1.8401230 .435698$

C $7.685473-4.112422-0.882288$

H $7.738686-4.979783-1.545567$

H $8.565750-3.485391-1.053217$

H $7.655517-4.4637750 .153534$

SCF Energy (B3LYP/6-31G**//MMFF) $=-3245.90036992$

\section{5}

MM̄FF Geometry

C $-0.185257-3.369200-3.710182$

C $0.230521-3.121790-2.458752$

C $1.321434-2.142422-2.127005$

O $0.792394-1.106631-1.268006$

C $2.552588-2.756408-1.427899$

C $2.292823-3.643054-0.224833$

C $2.216453-3.2127551 .049830$

C $2.215830-5.122192-0.515540$
C $2.312459-1.8064451 .572312$

C $3.574595-1.5903532 .419163$

C $4.829971-1.3801721 .559603$

C $6.087446-1.3579792 .429145$

C $7.342899-1.0452001 .601871$

O $4.680219-0.1249690 .886374$

O $8.408903-0.7431122 .514737$

C 7.0841220 .2278220 .770709

C $7.804603-2.2533620 .778724$

O $8.1660630 .482385-0.122802$

C $5.7479600 .203423-0.009248$

C $5.4664641 .608960-0.592328$

O $5.845967-0.738002-1.078900$

C $0.089617-0.102968-1.858001$

O $-0.248441-0.028784-3.028400$

C $-0.1594790 .928772-0.828286$

C $-0.8798492 .012738-1.149679$

C $-1.1361523 .167809-0.288313$

C -0.4475473 .2013881 .050841$

C $4.0581691 .795432-1.178051$

C $3.8644403 .205130-1.715606$

C $2.1661224 .658883-2.815329$

O $4.7454754 .061146-1.684585$

C $1.4510615 .465584-1.776325$

C $0.3211686 .145559-2.025743$

C $-0.4024106 .975062-1.002185$

C $-1.8790466 .649776-0.925165$

C $-2.7913637 .437753-1.518939$

C $-2.3021555 .453442-0.099358$

C $-1.9345404 .149906-0.756803$

N $2.593353 \quad 3.395396-2.240236$

H $0.288883-2.858288-4.544417$

H $-0.271265-3.613696-1.629148$

H $1.694514-1.660933-3.041522$

H $3.220450-1.939832-1.126945$

H $3.123413-3.335719-2.166167$

H $2.059907-3.9584091 .829173$

H $2.061308-5.7173220 .390896$

H $1.391832-5.349102-1.196854$

H $3.148658-5.464273-0.976274$

H $2.260184-1.059740 \quad 0.776814$

H $1.435316-1.6341242 .206286$

H $3.413098-0.6962453 .035501$

H $3.716969-2.4360233 .103254$

H $4.898598-2.1846570 .819484$

H $6.207932-2.3080522 .964583$

H $5.981899-0.5873223 .205655$

H $8.535028-1.5143073 .093790$

H 7.0578251 .0731741 .472640

H $8.028064-3.1017301 .436650$

H $8.736432-2.0349140 .245512$

H $7.061304-2.5870440 .051656$

H $8.9807260 .505147 \quad 0.408006$

H 5.5900792 .3564600 .202799

H $6.205651 \quad 1.838299-1.370756$

H $5.234247-0.480900-1.786292$

H $\quad 0.290801 \quad 0.7895320 .147889$

H -1.304425 2.076841 -2.150996

H $0.639612 \quad 3.1425370 .924996$

H $-0.7743712 .360548 \quad 1.672015$

H -0.6452614 .1085761 .624299$

H $3.8826511 .093245-1.999594$

H $3.2999351 .621395-0.406035$

H $1.5234444 .425200-3.670026$

H $3.0421895 .210124-3.171712$

H $1.8883145 .508944-0.781655$

H $-0.0969296 .126114-3.029906$

H $-0.2666108 .030108-1.273480$

H $0.044856 \quad 6.863146-0.006667$

H $-3.8532297 .226137-1.449050$

H $-2.4962498 .309991-2.092625$

H -3.391500 5.4542790 .037432

H -1.885979 5.5611420 .905227

H -2.388559 $4.020373-1.740685$

H $1.9102222 .646938-2.174026$

C $-2.690271-3.655321-4.046246$

O $-3.037658-3.254518-2.706733$

C $-3.691345-1.972078-2.762230$ 
C $-4.042135-1.744440-4.228983$

C $-2.868714-2.415248-4.921257$

H $-2.946151-1.225457-2.463709$

C $-4.873244-1.939818-1.786913$

H -4.979281 -2.240355 -4.506942

H $-4.135934-0.681838-4.473053$

C $-3.115999-2.727208-6.386338$

H $-2.001059-1.749068-4.841571$

C $-4.500522-2.366957-0.351071$

O $-5.873766-2.846585-2.266425$

H $-5.327459-0.941825-1.790332$

H $-4.224208-3.428594-0.336748$

O $-5.668411-2.240727 \quad 0.470177$

C $-3.373310-1.5338790 .289045$

C $-3.036568-2.0317611 .710160$

H $-2.467660-1.608364-0.322275$

$\begin{array}{lllll}\text { O } & -3.774062 & -0.167321 & 0.305214\end{array}$

H -3.936617 -1.9930582 .330492$

H -2.747264 -3.086529 1.633609

H -2.247669 -3.225347 -6.829069

H $-3.984084-3.381908-6.516872$

H -3.301999 -1.806867 -6.949333

C $-1.306830-4.311344-4.044311$

H $-6.692292-2.665442-1.774503$

H $-6.298118-2.926247 \quad 0.190675$

H $-3.023416 \quad 0.3588240 .628457$

H -1.103698 -4.755446 -5.025833

H -1.304795 -5.136881 -3.320675

H $-3.426090-4.419639-4.329463$

C $-3.246953 \quad 0.289827 \quad 3.697898$

$\begin{array}{llll}\text { O } & -2.272906 & 0.125623 & 2.658780\end{array}$

C -1.899956 -1.230072 2.384817

C - $1.333936-1.8789053 .657038$

C $-2.298420-1.7567194 .833397$

C $-2.738341-0.3039215 .016107$

H $-4.181409-0.2033303 .408955$

C -3.5252041 .7911453 .843372$

H $-1.079278-1.1816901 .657882$

O $-1.026210-3.2517843 .441221$

H $-0.394900-1.3788213 .927358$

H $-3.165193-2.4141514 .700339$

O $-1.651297-2.2023046 .027854$

H $-1.898406 \quad 0.2901775 .398289$

O $-3.779496-0.2495345 .988703$

C -4.2781162 .3409962 .655183$

H -4.1150932 .0173044 .738604$

H -2.582521 2.3465513 .921241

H $-0.414292-3.3000622 .687170$

H $-0.867000-1.645786 \quad 6.171147$

H -3.440443 -0.6633846 .800936$

$\begin{array}{lllll}\text { O } & -5.522202 & 1.787785 & 2.583997\end{array}$

O -3.8203423 .1812201 .892193$

C $-6.3290712 .228011 \quad 1.487349$

H -7.2716881 .6750501 .515528$

H -6.5445623 .2962421 .583694$

H -5.8287692 .0188070 .536862$

SCF Energy (B3LYP/6-31G**//MMFF)= -3245.91559048

\section{6}

MM̄FF Geometry

C $2.989764-0.6606983 .147426$

C 2.5355390 .5435043 .530426

C 2.9997251 .8871443 .009796

O 3.9966901 .6883991 .981599

C 1.8307342 .6996652 .418878

C 0.8373453 .1870673 .451936

C -0.4126542 .7076893 .600625$

C 1.3170424 .3201634 .322532

C $-1.077167 \quad 1.6041172 .825731$

C $-2.2461412 .123317 \quad 1.979465$

C -1.8046853 .0269600 .817573$

C -3.026783 3.6321330 .125917

C $-2.6283204 .483445-1.085615$

O $-1.0562602 .232455-0.109846$

O $-3.8166884 .755627-1.843670$

C $-1.6953163 .652922-1.986698$

C $-2.0559685 .843741-0.670033$

O $-1.1802084 .449794-3.052257$
C $-0.5324712 .964090-1.227692$

C $0.1501571 .954019-2.179075$

O $0.4106203 .952038-0.815714$

C 4.8627682 .7004851 .722153

O 4.8484613 .8206892 .212481

C 5.8668182 .2952020 .712006

C 6.1960821 .0109250 .504204

C $7.2067590 .509742-0.428094$

C $7.8391211 .496165-1.373088$

C $1.3231371 .160899-1.584556$

C $1.7612910 .054492-2.533434$

C $3.444318-1.721021-2.844272$

O $1.122130-0.255411-3.536979$

C $4.908198-1.893075-2.594313$

C $5.832616-1.708625-3.550629$

C $7.321780-1.920081-3.481290$

C $7.873208-2.503824-2.201095$

C $7.880747-3.831439-1.992651$

C $8.517203-1.553476-1.218019$

C $7.505935-0.805618-0.397567$

N $2.942155-0.559706-2.134299$

H $3.780703-0.7263062 .407751$

H 1.7663270 .5765754 .299451

H 3.4674662 .4202053 .846607

H 1.3490572 .1153751 .627087

H 2.2186663 .5770681 .884971

H -1.042145 3.1501864 .371953

H 0.5317344 .6948004 .987830

H 2.1529823 .9984534 .950834

H 1.6488915 .1602003 .703469

H $-0.374396 \quad 1.0491182 .198869$

H -1.466100 0.8782033 .550582

H $-2.779741 \quad 1.2537191 .576649$

H -2.950621 2.6629832 .625031

H -1.1642673 .8261411 .205413$

H -3.6209114 .2261060 .831649$

H -3.688223 $2.825305-0.216966$

H $-4.4443885 .216782-1.261360$

H -2.306823 $2.871125-2.456927$

H -2.788941 $6.401866-0.075241$

H -1.844786 $6.467199-1.545842$

H -1.142644 $5.761964-0.076876$

H -1.940692 $4.845208-3.511892$

H $-0.6114901 .237962-2.516502$

H $0.5214372 .475725-3.070658$

H $1.2502023 .515302-0.604333$

H $6.363963 \quad 3.1105480 .197794$

H 5.6997210 .2418781 .093478

H $8.4470372 .221436-0.821434$

H $7.0700042 .037746-1.935553$

H $8.4878201 .030230-2.117922$

H $2.1790771 .820416-1.405431$

H $1.0361710 .698622-0.634537$

H $3.220460-1.616574-3.911866$

H $2.896197-2.596230-2.481640$

H $5.193570-2.232035-1.603583$

H $5.485346-1.377282-4.528924$

H $7.818765-0.966575-3.697631$

H $7.597503-2.581965-4.313904$

H $8.308865-4.260747-1.092975$

H $7.453243-4.516938-2.717064$

H $9.208536-0.892339-1.748860$

H $9.153702-2.110883-0.517398$

H $6.983227-1.4391970 .320815$

H $3.297229-0.370556-1.200910$

C $1.917677-2.9207022 .650391$

O $\quad 0.831151-2.3040271 .930496$

C $0.959234-2.6422550 .536447$

C $1.947596-3.8013100 .479692$

C $2.902860-3.4244241 .594626$

H $1.401399-1.7677460 .045366$

C $-0.402416-2.942453-0.104002$

H $1.460127-4.7578250 .702772$

H $2.436344-3.889395-0.494306$

C $3.780286-4.5715972 .062976$

H $3.545045-2.6131611 .232237$

C $-1.365510-1.735904-0.086199$

O $-0.998078-4.0600770 .552291$ 
H $-0.230018-3.236857-1.146076$ H $-0.845690-0.861015-0.492565$ O $-1.748695-1.4238841 .252404$ C $-2.643656-1.965571-0.918977$ C $-3.527825-0.702890-0.972666$ H -2.356009 -2.238574 -1.940928 O $-3.384765-3.056794-0.385200$ H -3.852177 -0.4485860 .041157$ H -2.913545 $0.130396-1.333455$ H $4.438026-4.2510652 .877093$ H $3.181597-5.4144322 .424147$ H $4.409302-4.9337101 .243075$ C $2.477099-1.9607503 .705246$ H -1.170455 -3.7981501 .472960$ H $-0.938270-1.2423941 .757243$ H $-3.583343-2.8492130 .544008$ H $3.291851-2.4504224 .250989$ H $1.683611-1.7547974 .434949$ H $1.478548-3.7780993 .178140$ C $-6.375755-1.579194-0.235757$ O $-5.664501-1.882011-1.442367$ C $-4.763263-0.864185-1.890051$ C $-5.5425760 .436372-2.131646$ C $-6.3224040 .862552-0.891363$ C -7.191312 -0.290310 -0.390977 H $-5.666515-1.4733570 .593359$ C -7.291806 -2.770565 0.069378 H $-4.384104-1.198125-2.864334$ O $-4.6740491 .490695-2.527163$ H $-6.2558730 .284857-2.952399$ H $-5.6561181 .223705-0.099488$ O $-7.1788541 .956642-1.226137$ H -8.022554 -0.450788 -1.089711 $\begin{array}{llll}\text { O } & -7.755615 & 0.061062 & 0.870826\end{array}$ C $-6.495015-3.9950990 .452322$ H $-7.991700-2.5558490 .884878$ H -7.891845 -3.032086 -0.810821 H $-4.1988951 .195897-3.322703$ H $-6.6151312 .665859-1.580766$ H -8.2458300 .8920080 .747659$ O $-6.002920-3.8559341 .716718$ O $-6.329173-4.957605-0.285580$ C $-5.221118-4.9540072 .197118$ H -4.859509 -4.7015343 .197409$ H $-5.837835-5.8552122 .262881$ $\mathrm{H}-4.358475-5.1228831 .545503$

SCF Energy (B3LYP/6-31G**//MMFF) $=-3245.90964479$

\section{7}

MM̄FF Geometry

C $-4.129949-3.2726351 .668601$

C $-3.115183-2.3948831 .683776$

C $-1.952878-2.2823370 .727551$

O $-0.767754-2.2846431 .562480$

C $-1.825458-3.387185-0.342570$

C $-0.980540-2.991050-1.541513$

C $0.199674-3.540038-1.890026$

C $-1.582530-1.924260-2.425183$

C $0.971937-4.635509-1.213448$

C $2.211212-4.165531-0.435387$

C $3.291706-3.468267-1.281802$

C $4.567178-3.272294-0.454428$

C $5.642788-2.486107-1.220610$

O $2.798896-2.193181-1.707987$

O $6.660617-2.095125-0.285976$

C $5.011653-1.204659-1.810110$

C $6.359216-3.371213-2.246219$

O $5.934343-0.528597-2.659435$

C $3.693105-1.467695-2.566483$

C $2.974235-0.168468-3.012900$

O $3.975106-2.210522-3.759513$

C $0.214720-1.3908891 .277049$

O $0.266755-0.6338720 .321867$

C $1.231849-1.4829622 .348756$

C $2.417257-0.8813172 .173893$

C $3.535634-0.8639883 .116610$

C $3.301008-1.4241364 .493749$

C $2.5742110 .787466-1.886306$
C $3.6069861 .857522-1.575453$

C 4.2335983 .4839490 .211194

O $4.5042472 .173716-2.351462$

C 5.3664802 .8414590 .947874

C 5.5282022 .9090472 .278076

C 6.6711942 .2672553 .012364

C 6.2154581 .2120923 .997209

C 6.0945501 .4947335 .305065

C $5.986846-0.1919913 .481692$

C $4.704424-0.3259672 .710982$

N $3.3793782 .448091-0.339478$

H -4.178104 -4.055289 0.919129

H -3.134343 -1.621577 2.452141

H $-2.066697-1.3025830 .250159$

H -2.812509-3.644350 -0.746037

H $-1.440517-4.2965170 .129052$

H $0.671611-3.174041-2.801610$

H -1.013010 -1.777351 -3.349286

H -2.602060 -2.200774 -2.713114

H -1.615119 -0.961280-1.908123

H $0.333305-5.204745-0.531251$

H $1.284838-5.352041-1.983487$

H $1.885914-3.4958700 .365946$

H $2.650965-5.0488440 .044885$

H $3.497521-4.092330-2.159207$

H $4.966594-4.237378-0.118381$

H $4.319063-2.7198750 .461593$

H $6.247239-1.5490130 .403708$

H $4.808144-0.532264-0.967828$

H $\quad 6.862730-4.205712-1.742955$

H $7.148519-2.816612-2.765813$

H $5.686561-3.796168-2.993866$

H $6.742468-0.369925-2.142217$

H $3.5642740 .342412-3.783358$

H $2.042679-0.470712-3.512871$

H $3.233760-2.095566-4.376925$

H $0.983825-2.0486493 .239320$

H $2.597273-0.3553331 .238650$

H $3.131586-2.5052214 .444222$

H $2.427578-0.9527964 .958459$

H $4.136750-1.2576645 .177240$

H $1.6672891 .324856-2.189446$

H $2.3341890 .230592-0.978633$

H 3.6144804 .1060960 .864738

H $4.6228234 .108838-0.598982$

H 6.0937902 .2957510 .350954

H 4.8061993 .4612112 .875321

H 7.2179593 .0613983 .537710

H 7.3961331 .8259192 .316998

H 5.7973170 .7368446 .022188

H 6.2851512 .4922465 .686985

H $6.033224-0.9174624 .301108$

H $6.818775-0.4732822 .822955$

H 4.7704420 .0481051 .689418

H 2.6475832 .0771100 .259436

C $-6.472605-2.4027482 .154765$

O $-6.071598-1.0336221 .931549$

C $-6.512755-0.6394840 .619883$

C $-7.661170-1.5820330 .288467$

C -7.128835 -2.885846 0.857724

H $-5.684857-0.845494-0.070199$

C $-6.844404 \quad 0.8547730 .581523$

H -8.585122 -1.293315 0.802656

H -7.864641 -1.633259 -0.785121

C $-8.197720-3.9433881 .071120$

H $-6.378887-3.2826470 .162672$

C -5.7122771 .7699441 .098396$

O -7.9983031 .0886301 .398066$

H $-7.1281851 .137888-0.439186$

H $-5.637644 \quad 1.6744732 .188783$

O -6.1208203 .1179020 .835685$

C $-4.3369461 .509350 \quad 0.454726$

C -3.2521092 .4972790 .929278$

H -4.0194610 .4925520 .707968$

O $-4.4743721 .579009-0.961518$

H -3.4406563 .4836480 .493259$

H -3.325376 2.5945382 .018492

H $-7.764331-4.8508751 .503566$ 
H $-8.984267-3.5896411 .746154$ H -8.668320 -4.2121590 .119750$ C -5.271520 -3.217365 2.650080 H -8.1968902 .0397291 .352034$ H -5.5412643 .7078381 .346254$ H $-3.6448651 .253471-1.349753$ H -5.582982 -4.241868 2.884688 H -4.912470 -2.777190 3.589608 H -7.217013 -2.368206 2.960613 C -1.537179 $3.162356-1.553115$ O $-1.6272801 .914512-0.853336$ C -1.8266312 .0236320 .559806$ C -0.6887012 .8359321 .192240$ C -0.5116204 .1909570 .516481$ C $-0.3914964 .015676-0.996470$ H $-2.4855963 .704239-1.457294$ C -1.311549 2.845361 -3.036224 H -1.740612 1.0071630 .960292 O -0.9200223 .0075762 .586190$ H 0.2463262 .2690081 .092611 H -1.3296964 .8714320 .780259$ O $\quad 0.687383 \quad 4.787915 \quad 1.014837$ H $\quad 0.5739703 .549269-1.226883$ O $-0.3896595 .300534-1.615488$ C $-2.5929912 .377493-3.684076$ H $-0.9807203 .725292-3.599492$ H $-0.5412282 .075245-3.161197$ H -0.1855723 .5383052 .939545$ H 0.8142625 .6254530 .536739 H -1.246938 $5.721765-1.432748$ O $-2.5453351 .033904-3.906447$ O $-3.5334183 .118494-3.941292$ C $-3.7147350 .468332-4.506774$ H $-3.526019-0.593364-4.685815$ H $-4.568906 \quad 0.564396-3.830017$ $\mathrm{H}-3.9242140 .952298-5.465621$ SCF Energy (B3LYP/6-31G**//MMFF) $=-3245.90854795$

\section{8}

MM̄FF Geometry

C $-0.7872045 .636957-0.803417$

C -1.3823584 .8607990 .114583$

C $-2.5365963 .939666-0.188411$

O -2.1245082 .5768980 .066774$

C $-3.710284 \quad 4.2715280 .752253$

C -4.9717813 .4791310 .472824$

C -5.5382812 .6122391 .334990$

C $-5.624713 \quad 3.737191-0.861208$

C -5.0738242 .2327142 .713668$

C -4.1683540 .9914472 .765350$

C $-4.844228-0.3012342 .279157$

C -3.959079-1.517642 2.560201

C $-4.566053-2.8140361 .997990$

O $-5.064642-0.192770 \quad 0.871329$

O $-3.541681-3.8206282 .008253$

C $-4.963485-2.5908070 .521660$

C -5.700131-3.341536 2.885079

O $-5.684088-3.7100850 .012880$

C $-5.771406-1.2976130 .293269$

C $-5.989168-0.965352-1.202785$

O $-7.069466-1.4431570 .882387$

C -1.414282 $1.948498-0.910426$

O $-1.0731702 .413461-1.986552$

C - $-1.1065160 .579927-0.437584$

C $-0.207705-0.160324-1.103961$

C $0.216949-1.520514-0.765428$

C $-0.583672-2.258744 \quad 0.273779$

C $-4.710981-0.772607-2.026534$

C $-4.275429-2.015554-2.784332$

C $-2.416858-2.867799-4.206296$

O $-4.965461-3.026958-2.877374$

C -1.535607 -3.729020 -3.358292

C $-0.260096-4.004025-3.668988$

C $0.625395-4.901196-2.853580$

C $1.922121-4.232669-2.459117$

C $3.051589-4.447189-3.155070$

C $1.927826-3.385804-1.207633$

C $1.283295-2.042325-1.407331$
N -3.033322 -1.845981 -3.381745

H -1.133709 $5.611836-1.833298$

H $-1.0209084 .880592 \quad 1.140386$

H -2.853793 $4.038853-1.233647$

H -3.9594045 .3382650 .671671$

H $-3.3823714 .115843 \quad 1.785760$

H -6.4543372 .1109831 .023842$

H $-6.6075743 .260354-0.941900$

H -5.770994 4.811774-1.011805

H $-5.0058083 .349441-1.675997$

H -4.5566593 .0701963 .193448$

H -5.9623012 .0555943 .333316$

H -3.2686261 .1849132 .169402$

H -3.8445590 .8575693 .805101$

H $-5.806400-0.4018072 .794894$

H $-3.767089-1.6196363 .635546$

H -2.975872 -1.365219 2.095082

H $-3.227218-3.9189082 .923245$

H $-4.031457-2.532925-0.052471$

H -5.330415 -3.547109 3.896910

H $-6.085539-4.2955452 .508693$

H -6.534432 -2.642935 2.975322

H -5.132174 -4.498362 0.153473

H $-6.660346-1.702767-1.659152$

H -6.536454 -0.012324 -1.244917

H -7.670956 -0.8043120 .465142$

H -1.6151740 .2251940 .451022$

H $\quad 0.2826680 .275950-1.973188$

H - $-1.646473-2.2508250 .009898$

H $-0.463119-1.7945421 .258051$

H $-0.306974-3.3099280 .375070$

H $-4.902970-0.005099-2.787611$

H -3.886097 -0.414547 -1.402604

H $-1.859718-2.354876-4.996786$

H -3.196429 -3.481281-4.669431

H $-1.973194-4.167261-2.464357$

H $\quad 0.166020-3.586656-4.578678$

H $0.834564-5.796909-3.453023$

H $0.112514-5.262098-1.953396$

H $3.992311-3.993517-2.861051$

H $3.059152-5.077418-4.038358$

H $2.962340-3.199869-0.895349$

H $1.486435-3.957784-0.387340$

H $1.793593-1.424406-2.147638$

H -2.505495 -0.996530 -3.203182

C $1.7381445 .952375-0.683344$

O $1.947190 \quad 4.907586 \quad 0.286215$

C $2.6479923 .812106-0.353529$

C $3.1577114 .374121-1.672234$

C $2.0409465 .331966-2.044767$

H $1.8788133 .051529-0.525482$

C 3.7074293 .2942120 .622706

H $4.0948604 .925323-1.526164$

H $3.3253303 .604125-2.429782$

C $2.4283976 .342127-3.109558$

H $1.1906744 .740416-2.406037$

C 4.6884742 .2104780 .134793

O 3.0336422 .7728831 .775841

H 4.2946094 .1531420 .973511

H 5.3392321 .9913790 .989419

O $5.5272952 .711209-0.900856$

C $4.0536490 .880178-0.314285$

C $5.075982-0.239965-0.583229$

H 3.3401610 .5334650 .438211

O $3.3377181 .061116-1.535948$

H $4.520165-1.109437-0.955670$

H $5.7273750 .051708-1.417231$

H $1.5950337 .018352-3.324902$

H $3.2837666 .949460-2.795335$

H $2.7015095 .834340-4.040347$

C $0.3505796 .568022-0.492974$

H 2.4369843 .4679102 .103077

H $5.9635943 .512454-0.564676$

H $2.4015791 .188847-1.311529$

H $0.2488067 .457726-1.125557$

H $0.255956 \quad 6.9119610 .545360$

H $2.4830716 .725845-0.453406$

C $4.308298-1.9823281 .814333$ 
O $5.156415-0.828014 \quad 1.819308$

C $5.933778-0.6449800 .632490$

C $6.827982-1.8700360 .392273$

C $6.027248-3.1694190 .387850$

C $5.138294-3.2584581 .627331$

H $3.573360-1.8936211 .007202$

C $3.559110-2.0079163 .155333$

H 6.6078890 .1955280 .836039

O $7.534235-1.753045-0.838354$

H $7.579682-1.9315181 .190114$

H $5.435566-3.273638-0.528571$

O $6.930259-4.2776920 .406443$

H $5.762962-3.4358042 .512240$

O $4.259701-4.3739691 .494082$

C $2.518555-0.9117213 .246998$

H $3.033055-2.9566493 .304322$

H $4.278930-1.8732553 .972115$

H $8.045326-0.926490-0.804371$

H $7.512833-4.188349-0.367485$

H $4.811143-5.1614771 .346135$

O $2.408907-0.4953054 .542402$

$\begin{array}{llll}\text { O } & 1.855499 & -0.497962 & 2.305792\end{array}$

C 1.4394680 .5312654 .776839

H $\quad 0.4393080 .175901 \quad 4.510591$

H 1.4530710 .7748115 .842581

H 1.6945661 .4305764 .208122

SCF Energy (B3LYP/6-31G**//MMFF) $=-3245.91093325$

01_559

MMFF Geometry

C $4.156713 \quad 0.340179-1.704483$

C $3.517225-0.222335-2.740816$

C $2.674930-1.464418-2.626379$

O $1.355816-1.098404-3.103756$

C $3.219460-2.624836-3.477021$

C $4.431757-3.303325-2.874447$

C $4.367845-4.328120-2.000899$

C $5.768893-2.809964-3.362508$

C $3.132657-4.974632-1.434814$

C $3.216044-5.2053990 .080448$

C $3.127740-3.9238910 .928613$

C $3.454815-4.2610982 .388280$

C $3.278310-3.0548613 .319241$

O $1.795730-3.4004010 .824054$

O $3.341096-3.526266 \quad 4.674312$

C $1.878873-2.4521303 .078605$

C $4.440722-2.0655873 .184779$

O $1.708307-1.2556833 .836446$

C $1.562521-2.2048181 .584131$

C $0.091507-1.7621891 .381280$

O $2.392575-1.1321951 .125282$

C $0.300320-1.817906-2.637891$

O $0.340480-2.785619-1.895728$

C $-0.938495-1.214392-3.173957$

C $-2.122048-1.677159-2.744538$

C $-3.444599-1.203742-3.155107$

C $-3.507707-0.026830-4.092621$

C $-0.959580-2.8520701 .609871$

C $-2.370182-2.2850461 .659177$

C $-4.703315-2.9635102 .208501$

O $-2.625935-1.0952191 .497466$

C $-5.504778-3.003220 \quad 0.946044$

C $-6.230492-1.9637990 .505086$

C $-7.129785-2.005725-0.696961$

C $-6.722623-1.027953-1.775861$

C $-7.0841640 .264865-1.717217$

C $-5.977776-1.574739-2.971162$

C $-4.530470-1.842551-2.671415$

N $-3.312240-3.2668041 .932428$

H $4.097582-0.124164-0.724209$

H $3.5775380 .245981-3.720458$

H $2.596336-1.768230-1.576057$

H $3.452240-2.273915-4.491924$

H $2.429062-3.368111-3.641643$

H $5.304637-4.771547-1.665687$

H $6.601761-3.385066-2.943854$

H $5.828880-2.894200-4.452614$

H $5.923982-1.763849-3.085998$
H $\quad 2.227712-4.412190-1.667498$

H $3.026538-5.952941-1.919870$

H $2.386462-5.8692300 .357442$

H $4.146104-5.7412080 .309846$

H $3.836349-3.1832260 .541794$

H $4.471605-4.6641152 .476493$

H $2.786999-5.0648502 .729550$

H $2.631688-4.1784374 .803853$

H $1.154476-3.1676803 .483862$

H $5.388595-2.5558743 .438871$

H $4.337796-1.2348943 .891670$

H $4.543645-1.6532222 .179101$

H $2.186568-0.5440783 .377489$

H $-0.112876-0.8878982 .013124$

H $-0.036204-1.3962050 .354512$

H $1.945118-0.6787330 .392217$

H $-0.844266-0.409228-3.893149$

H -2.128711 -2.490369 -2.019066

H -3.103266 -0.297360 -5.073908

H $-2.9307030 .815547-3.697861$

H $-4.519644 \quad 0.351837-4.250233$

H $-0.912470-3.5932340 .803848$

H $-0.784359-3.3668572 .559494$

H $-4.765364-1.9893672 .704928$

H -5.071515 -3.725363 2.902718

H $-5.528223-3.9427890 .399941$

H -6.221035 -1.035725 1.071295

H -8.149897 -1.773738 -0.362899

H -7.187351 -3.019669-1.111970

H $-6.8183440 .962564-2.504341$

H $-7.6470450 .654337-0.875244$

H -6.099516 $-0.910013-3.831866$

H $-6.446299-2.515665-3.287877$

H $-4.375437-2.687525-1.999720$

H -3.013087 -4.234531 2.010302

C $4.2021472 .762637-1.136359$

O $4.0901322 .531305 \quad 0.284887$

C 4.3052323 .7739670 .969750

C $4.2771224 .863649-0.093461$

C $4.8748454 .126266-1.279360$

H $5.329773 \quad 3.7253341 .364403$

C 3.3556923 .9603142 .161206

H $3.2525495 .178554-0.320229$

H 4.8512345 .7487960 .195928

C $4.6147034 .809905-2.610304$

H $5.9582374 .028746-1.127654$

C 1.8464574 .1046321 .864915

O 3.7565315 .1461772 .861606

H 3.4900473 .1244842 .859506

H 1.6485795 .0550081 .355715

O $1.1679294 .186536 \quad 3.128070$

C 1.2148792 .9526191 .067395

C -0.3155753 .0558480 .951539$

H 1.6440422 .9423950 .068428

O 1.5426101 .7077321 .683864

H $-0.7575472 .884476 \quad 1.941830$

H -0.5954594 .0721830 .653466$

H $5.0497774 .237307-3.435394$

H $3.5419424 .917264-2.803539$

H $5.0597375 .810102-2.622054$

C $4.9570471 .610100-1.796586$

H 4.6800265 .0242773 .140641

H 1.5606744 .9283423 .619421

H 1.1888561 .7247112 .589797

H $5.9170461 .443201-1.293954$

H $5.1720841 .849246-2.844128$

H $3.1825392 .809730-1.538068$

C -3.2331292 .6560620 .315127$

$\begin{array}{llll}\text { O } & -2.234675 & 1.632965 & 0.400397\end{array}$

C $-0.9256412 .025590-0.029277$

C $-0.9928432 .454449-1.501931$

C $-2.0542603 .526655-1.742006$

C -3.397392 $3.109691-1.141005$

H $-2.948133 \quad 3.5099470 .942602$

C $-4.5320772 .057405 \quad 0.874337$

H $-0.317281 \quad 1.1153070 .020711$

O $\quad 0.2615542 .925730-1.973616$

H -1.253155 $1.582704-2.113090$ 
H $-1.7359634 .500544-1.352004$ O $-2.230347 \quad 3.700786-3.150907$ H -3.827227 $2.304990-1.751090$ O $-4.278373 \quad 4.228504-1.215880$ C $-4.439383 \quad 1.773347 \quad 2.359885$ H $-5.372222 \quad 2.7439110 .730892$ H $-4.743308 \quad 1.1079930 .368716$ H $0.8834372 .179027-1.933021$ H $-1.3621753 .931493-3.523737$ H $-5.1816683 .906676-1.059384$ O $-5.566072 \quad 1.1083262 .753011$ O -3.5104712 .0981053 .086434$ C $-5.621740 \quad 0.7575294 .139127$ H $-4.766994 \quad 0.129466 \quad 4.408272$ H -5.6472261 .6612434 .755331$ H -6.5401250 .1893954 .309066$ SCF Energy (B3LYP/6-31G*//MMFF) $=-3245.91128990$

0156

MMFF Geometry

C $2.489049-3.041336-1.041190$

C $1.958907-3.285316-2.249210$

C $0.747036-2.602351-2.837114$

O $-0.060539-1.940427-1.835805$

C $1.084510-1.590935-3.951630$

C $1.936205-0.401183-3.548869$

C $1.4486420 .799231-3.178309$

C $3.426600-0.576537-3.699336$

C $0.010878 \quad 1.222453-3.059019$

C $-0.243388 \quad 1.978643-1.747743$

C $-1.6046062 .688021-1.757301$

C $-1.776606 \quad 3.547730-0.505251$

C -3.144824 $4.242893-0.479831$

O $-2.6424831 .703668-1.812538$

O $-3.348902 \quad 4.765106 \quad 0.841865$

C $-4.240793 \quad 3.187052-0.724707$

C -3.199447 $5.441534-1.433962$

O $-5.5194953 .802159-0.866276$

C -3.960777 2.259498 -1.933685

C $-4.9855411 .102860-1.933766$

O $-4.1064103 .004098-3.144400$

C $-0.876239-2.732029-1.084717$

O $-0.976637-3.947750-1.146055$

C $-1.634691-1.863712-0.157838$

C $-2.343730-2.4351820 .827067$

C $-3.188868-1.7526161 .808614$

C $-3.279047-0.2529171 .733194$

C -4.677984 -0.032010 -2.917728

C $-5.678435-1.153125-2.715843$

C $-6.267186-2.960016-1.094417$

O $-6.669222-1.270772-3.432582$

C $-6.746569-2.488657 \quad 0.244133$

C $-6.690723-3.2323031 .359495$

C -7.128751 -2.739560 2.710289

C $-6.076050-2.9431233 .780904$

C $-6.258959-3.8403704 .764686$

C $-4.841858-2.0611223 .753701$

C $-3.849792-2.5045382 .713718$

N $-5.367444-1.953259-1.627313$

H $2.057805-2.269339-0.410069$

H $2.421060-4.044446-2.876984$

H $\quad 0.127173-3.381042-3.302938$

H $0.142303-1.226356-4.379877$

H $1.583915-2.116723-4.776763$

H $2.1657691 .593309-2.970819$

H $3.9795100 .342553-3.476439$

H $3.803599-1.348575-3.028081$ H $3.671995-0.862085-4.727571$ H $-0.2074401 .883248-3.907014$ H $-0.6783700 .376332-3.127997$ H $-0.193515 \quad 1.268188-0.913240$ H $0.5454232 .724674-1.592231$ H - $-1.6528653 .315335-2.655333$ H $-0.9709874 .287118-0.421383$ H $-1.6975992 .909448 \quad 0.385039$ H -2.619569 5.3782501 .037500 H $-4.296846 \quad 2.5647850 .179539$ H -2.427342 $6.174768-1.171755$
H $-4.1552545 .970405-1.348601$

H $-3.0510735 .164849-2.479752$

H -5.661678 $4.359163-0.081751$

H $-5.0401620 .673776-0.926840$

H -5.986432 $1.493896-2.159887$

H $-4.3044632 .390261-3.869154$

H $-1.600043-0.792842-0.317087$

H -2.320044 -3.5209000 .917076$

H -3.7055860 .0562110 .775969$

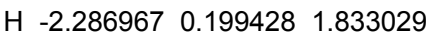

H -3.8957320 .1884682 .518628$

H $-4.7458750 .306590-3.956635$

H -3.666903 $-0.428715-2.771657$

H $-5.704132-3.895788-1.025172$

H $-7.116835-3.103673-1.768431$

H $-7.148388-1.4793310 .299903$

H $-6.310093-4.2495891 .301704$

H -8.048487 -3.2760882 .976652$

H $-7.398633-1.6765752 .677523$

H $-5.521155-3.9782395 .548180$

H $-7.147957-4.4607984 .806640$

H $-4.336568-2.0923234 .727690$

H -5.167517 -1.0265813 .614369$

H $-3.678990-3.5824722 .707452$

H $-4.518679-1.761236-1.103508$

C $4.985414-3.043671-0.742258$

O $4.933244-1.711403-0.203303$

C $6.235575-1.3617550 .300810$

C $7.161615-2.527370-0.038171$

C $6.195474-3.695204-0.078165$

H $6.139395-1.2920011 .390351$

C $6.676163-0.010381-0.274771$

H $7.642234-2.397736-1.014601$

H $7.955155-2.6520410 .705254$

C $6.736367-4.905945-0.817812$

H $5.949909-3.9807330 .953442$

C $5.6621091 .134206-0.072488$

O $6.885471-0.142690-1.687840$

H $7.648810 \quad 0.2696090 .145681$

H $4.780416 \quad 0.963015-0.700777$

O $6.2703322 .340820-0.551101$

C 5.2268561 .3575191 .386165

C 4.3955072 .6318301 .618818

H 4.6575510 .4924861 .735818

$\begin{array}{llll}\text { O } & 6.385429 & 1.443633 & 2.221489\end{array}$

H 4.1966882 .7051602 .695156

H 5.0136453 .5087331 .389049

H $6.004908-5.719981-0.822776$

H $6.979582-4.665112-1.858258$

H $7.647874-5.275010-0.336178$

C $3.671910-3.775921-0.476361$

H $6.052894-0.458071-2.079378$

H $6.5312822 .186329-1.475321$

H 6.9293972 .1806491 .894302

H $3.510669-3.8786650 .603545$

H $3.717911-4.791559-0.886530$

H $5.148956-2.947429-1.823635$

C 1.6692551 .4411722 .343282

$\begin{array}{lllll}\text { O } & 2.253322 & 1.542167 & 1.038506\end{array}$

C 3.0760902 .6942380 .824409

C $2.2580673 .971571 \quad 1.061255$

C 1.6095983 .9819652 .441695

C 0.8352722 .6882432 .674411

H 2.4595741 .3043493 .091230

C $0.765787 \quad 0.196643 \quad 2.348805$

H $3.3414542 .687876-0.239375$

$\begin{array}{lllll}\text { O } & 3.056925 & 5.136013 & 0.879296\end{array}$

H 1.4634624 .0251860 .307571

H 2.3470964 .1457613 .235863

$\begin{array}{lllll} & 0.688077 & 5.072332 & 2.518780\end{array}$

H -0.0767342 .6984602 .066123$

O 0.4292312 .6292254 .041359

C $1.531271-1.1067662 .419049$

H 0.1023020 .2220243 .220916

H $\quad 0.162156 \quad 0.169873 \quad 1.435244$

H $3.6356925 .233403 \quad 1.654104$

H 1.1847185 .8854092 .322822

H -0.0891203 .4315834 .224881$ 
O $\quad 0.644569-2.1151352 .672030$

O $2.735994-1.2321112 .256388$

C $1.185869-3.4359682 .778332$

H $2.158387-3.4372283 .279812$

H $\quad 0.491163-4.038673 \quad 3.369832$

H $1.266102-3.8739561 .780583$

SCF Energy (B3LYP/6-31G**//MMFF)= -3245.91867205

01_560

MM̄FF Geometry

C $-3.619762-1.5239182 .859470$

C $-2.586191-2.2811022 .459589$

C $-1.200491-2.3213693 .059693$

O $-0.265248-2.0748311 .977921$

C $-0.927646-1.2810094 .168991$

C $0.495813-1.2942894 .695586$

C $1.355314-0.2628954 .570415$

C $0.917665-2.5425305 .425775$

C 1.0682051 .0919223 .984623

C 1.2671751 .1428732 .469582

C 1.0813772 .5520571 .886840

C -0.3588973 .0586512 .009628$

C -0.5468054 .4252341 .331529$

O 1.4449762 .4832680 .500684

O

C $0.0343954 .363272-0.094970$

C 0.0063865 .5684572 .190795

O $0.0229365 .649063-0.710322$

C $1.4545393 .755044-0.161992$

C $1.8663283 .551261-1.642959$

O $2.374244 \quad 4.664270 \quad 0.449151$

C $0.662933-3.0327811 .711033$

O $0.724245-4.1560632 .185710$

C $1.650183-2.4615070 .768119$

C $2.895661-2.9612210 .774087$

C $4.025047-2.529391-0.053628$

C $3.761678-1.483981-1.100463$

C $2.9381172 .489765-1.904599$

C $4.2746502 .781346-1.258436$

C 5.7130482 .0916720 .659737

O $5.0632763 .590008-1.742005$

C 6.6446661 .0639030 .097431

C $7.1041310 .006000 \quad 0.783114$

C $8.091130-0.9738870 .216231$

C $7.639373-2.4115430 .327965$

C $8.273493-3.2706661 .144795$

C $6.523332-2.879796-0.581476$

C $5.229310-3.101498 \quad 0.155811$

N $4.4749762 .060303-0.092427$

H -3.509357 -0.8509103 .704613$

H $-2.741713-2.9590251 .623202$

H -1.063745 -3.328303 3.471901

H -1.605094 -1.452849 5.015443

H -1.171823 -0.2879403 .776722$

H $2.362316-0.3769844 .968976$

H $1.915505-2.4468565 .867436$

H $\quad 0.219176-2.7559076 .241623$

H $0.939359-3.4041754 .754305$

H $0.058448 \quad 1.4102104 .261746$

H $1.741473 \quad 1.8138634 .464454$

H 2.2744230 .7850822 .216327

H $\quad 0.5788290 .453517 \quad 1.966747$

H 1.7755703 .2254102 .403140

H -0.6755033 .1210523 .056732$

H $-1.0384492 .338041 \quad 1.532774$

H -2.3512484 .6295092 .088051$

H -0.637476 $3.729299-0.688496$

H -0.5045815 .5999583 .160676$

H $-0.181096 \quad 6.5411621 .722784$

H 1.0769785 .4792142 .386026

H $-0.8886255 .984769-0.662270$

H $\quad 0.986473 \quad 3.232755-2.217044$

H $2.1956074 .507818-2.068493$

H 3.2470274 .5628880 .038920

H $1.336885-1.6401680 .135043$

H $3.121291-3.7680721 .471511$

H $3.105934-1.887303-1.878449$

H $4.664775-1.119045-1.593682$
H $\quad 3.280277-0.608070-0.657117$

H $2.5764671 .503648-1.596133$

H $3.1165402 .413892-2.984329$

H 5.4657431 .8992051 .708311

H 6.1642623 .0859860 .581549

H $6.9827811 .219056-0.924886$

H $6.790446-0.1406621 .813800$

H $9.038822-0.8323010 .752159$

H $8.310439-0.753728-0.836356$

H $7.980375-4.3139701 .201876$

H $9.097102-2.9512591 .774602$

H $\quad 6.814627-3.837692-1.033943$

H $6.420965-2.194243-1.426701$

H $5.303188-3.8623920 .934744$

H $3.7619331 .405960 \quad 0.214557$

C $-5.368242-0.158493 \quad 1.699968$

$\begin{array}{llll}\text { O } & -4.569093 & 0.166472 & 0.544618\end{array}$

C $-5.4139590 .784154-0.445476$

C $-6.735966 \quad 1.083087 \quad 0.250874$

C $-6.820753-0.0683661 .233023$

H $-5.5936870 .030878-1.221176$

C $-4.7174012 .007280-1.051550$

H -6.7070252 .0390670 .786727$

H -7.575985 $1.114854-0.449421$

C $-7.8200180 .163608 \quad 2.353028$

H $-7.097190-0.9794710 .685344$

C $-3.3330201 .711445-1.665658$

O $-4.5355372 .996052-0.028859$

H -5.369634 $2.468982-1.801935$

H -2.627911 $1.468794-0.862577$

O $-2.8775542 .934038-2.258368$

C $-3.343608 \quad 0.601897-2.734702$

C $-2.0027580 .372469-3.452286$

H $-3.657853-0.340744-2.281293$

O $-4.319910 \quad 0.916002-3.732236$

H $-2.172958-0.365216-4.246176$

H $-1.7127701 .286558-3.984150$

H -7.839497 -0.6862343 .042303$

H -7.5738201 .0605702 .931222$

H -8.8288520 .2919081 .947319$

C $-4.980281-1.5435042 .218439$

H $-3.9908772 .597580 \quad 0.671788$

H -1.929698 2.836116 -2.448582

H -4.080009 $1.776708-4.116405$

H $-5.001784-2.2656491 .394533$

H $-5.700582-1.8800172 .972418$

H -5.1623540 .6101962 .456784$

C $-1.288347-2.475113-2.578395$

O $-1.204419-1.278647-1.793134$

C $-0.863720-0.094409-2.524567$

C $0.482487-0.278915-3.237139$

C $0.498918-1.531330-4.107089$

C $0.039622-2.743327-3.300291$

H -2.099912 -2.380261 -3.310061

C $-1.625815-3.639093-1.630960$

H $-0.7137810 .694256-1.777829$

O $\quad 0.777057 \quad 0.870832-4.022330$

H $\quad 1.270025-0.363893-2.479368$

H $-0.112547-1.405830-5.008329$

O $1.833059-1.781285-4.555167$

H $0.815187-3.013824-2.572090$

O $-0.121796-3.852240-4.183475$

C $-3.084425-3.667238-1.226165$

H -1.415446 -4.593426 -2.127155

H -1.032057 -3.574199 -0.713448

H $1.6857290 .777777-4.354072$

H $2.078548-1.062863-5.161956$

H $0.728533-3.988959-4.635573$

O $-3.375937-4.902215-0.718502$

O $-3.863059-2.729128-1.316638$

C $-4.728883-5.101609-0.296131$

H $-4.875747-6.171270-0.124198$

H $-4.905318-4.5682980 .641472$

H $-5.432790-4.773480-1.067066$

SCF Energy (B3LYP/6-31G**//MMFF) $=-3245.90880149$

01561

MM̄FF Geometry 
C $-0.780662-3.444682-1.415246$

C $0.501853-3.775450-1.629509$

C $1.722192-3.140958-1.000344$

O $1.414343-1.892398-0.336698$

C $2.289331-4.0903050 .072863$

C $3.802328-4.1269590 .117534$

C $4.552134-3.4436441 .003087$

C $4.450419-5.062622-0.869256$

C $4.046488-2.4361681 .997987$

C $5.139815-1.5293212 .589217$

C $6.028491-0.8200911 .554845$

C $7.013808 \quad 0.1269802 .245336$

C 7.8940490 .8708791 .229610

O $5.202477-0.0899370 .640639$

O $8.5427361 .936626 \quad 1.940585$

C 6.9820331 .5003600 .154187

C $9.014148-0.0245790 .688023$

O $7.7557542 .094384-0.885031$

C $5.9393670 .519422-0.430969$

C $4.9369431 .212040-1.381106$

O $6.623392-0.470139-1.212239$

C $1.204703-0.787577-1.100141$

O $1.252915-0.701937-2.315875$

C $\quad 0.844431 \quad 0.309203-0.178194$

C $-0.093610 \quad 1.192397-0.549758$

C $-0.610342 \quad 2.2913380 .270790$

C $0.1497042 .637578 \quad 1.524100$

C $4.1167322 .342726-0.759546$

C $3.1807712 .986292-1.769595$

C $1.7586355 .008244-2.052228$

O $3.0144742 .553521-2.906390$

C $0.6862655 .607443-1.202451$

C $-0.6083165 .623723-1.552609$

C $-1.6938256 .194703-0.687881$

C $-2.7874845 .192553-0.391457$

C $-4.0241515 .352304-0.892390$

C -2.4692104 .0496410 .550387$

C $-1.7322692 .924598-0.131977$

N $2.5529324 .105421-1.239480$

H -1.028609 -2.648570 -0.721019

H $0.716071-4.591458-2.317786$

H $2.462059-2.946374-1.786844$

H $1.948925-5.121817-0.094195$

H $1.895382-3.8151221 .059122$

H $5.626018-3.6176240 .997564$

H $5.542944-5.056520-0.792998$

H $4.111979-6.089851-0.698773$

H $4.190542-4.776296-1.893389$

H $3.286580-1.7994821 .532784$

H $3.564361-2.9684152 .826852$

H $4.636381-0.7778753 .211525$

H $5.774737-2.1223803 .259786$

H $6.587866-1.5818321 .001984$

H $7.636890-0.4150852 .967681$

H $6.453940 \quad 0.8702582 .830423$

H 9.0421882 .4621191 .291936

H 6.4594112 .3258720 .651227

H $9.650186-0.3763991 .509259$

H 9.6772270 .5319040 .016299

H $8.643697-0.9030520 .155971$

H $8.0821231 .378632-1.456646$

H $5.4685871 .582621-2.267711$

H $4.237148 \quad 0.453712-1.760167$

H $5.982613-0.880092-1.816840$

H $1.313871 \quad 0.3170530 .798742$

H $-0.5565151 .076474-1.529304$

H $\quad 1.2086762 .8066001 .298740$

H $\quad 0.073190 \quad 1.8272472 .256734$

H -0.205312 3.5459532 .014193

H 3.5182021 .9658230 .076618

H $4.7777383 .131022-0.385927$

H $1.3569904 .472479-2.918510$

H $2.4311405 .792427-2.414391$

H $0.9853916 .044767-0.252752$

H $-0.9073845 .203634-2.510187$

H $-2.1067177 .070029-1.205405$

H -1.291098 6.5698210 .261416

H $-4.8222034 .654983-0.660775$
H $-4.2668706 .182878-1.546792$

H -3.401183 3.6313050 .951545

H -1.937201 4.4517151 .416259

H -2.213683 2.577278 -1.047221

H $2.8646384 .443486-0.333160$

C $-2.814251-3.242177-2.934345$

O $-3.490319-2.264834-2.123070$

C $-3.693646-1.077047-2.918473$

C $-3.176492-1.397021-4.320677$

C $-2.116726-2.444082-4.031420$

H $-3.059742-0.296114-2.483631$

C $-5.168735-0.656389-2.863002$

H -3.962835 -1.815057 -4.960039

H $-2.778045-0.510586-4.824068$

C $-1.721809-3.267626-5.243538$

H $-1.230028-1.931285-3.638437$

C $-5.707347-0.441897-1.433058$

O $-5.970000-1.676539-3.474717$

H $-5.3219810 .247086-3.463857$

H -5.705795 -1.395195 -0.893095

O $-7.076765-0.031771-1.528555$

C $-4.9448970 .625620-0.631195$

C $\begin{array}{llll}-5.519507 & 0.904326 & 0.769812\end{array}$

H $-3.8960970 .333296-0.527539$

O $-4.9512461 .855621-1.360158$

H $-4.946147 \quad 1.731717 \quad 1.204546$

H -6.5424301 .2918450 .683059$

H $-0.964167-4.011831-4.978425$

H -2.581708 $-3.797499-5.666943$

H -1.304901 -2.624946 -6.025814

C $-1.936539-4.147777-2.069014$

H -5.813516 -2.504074 -2.988051

H -7.546132 -0.708529 -2.045898

H $-5.8795972 .116019-1.488624$

H -1.579582 -4.987639 -2.677081

H $-2.550884-4.574336-1.266086$

H -3.603370 -3.867517 -3.374346

C $-3.163302-0.2704772 .313964$

O $-4.233655-0.9871151 .692938$

C $-5.495873-0.3134641 .714747$

C $-5.907038-0.0032783 .161503$

C -4.8110460 .7406473 .925791$

C $-3.4669680 .022603 \quad 3.786572$

H -3.0238130 .6823491 .788745$

C -1.861395-1.051723 2.093127

H $-6.230650-1.027577 \quad 1.323449$

O $\quad-7.112290 \quad 0.754052 \quad 3.174637$

H $-6.116547-0.9495503 .677101$

H -4.7340231 .7802023 .587252$

O $\quad-5.1652180 .810628 \quad 5.309262$

H $-3.488458-0.9021884 .374005$

O $-2.434510 \quad 0.845434 \quad 4.324187$

C - $-1.841395-2.4450142 .685698$

H -1.703321 -1.173569 1.016063

H -1.020766 -0.486062 2.509649

H $-7.3233580 .949633 \quad 4.103695$

H -5.245491 -0.0982265 .645569$

H $-2.675751 \quad 1.050607 \quad 5.243784$

O $-0.552916-2.9016272 .689682$

O $-2.821785-3.0693763 .065820$

C $-0.370105-4.2242813 .204394$

H $0.703083-4.4272893 .248233$

H $-0.839491-4.9524762 .536542$

$\mathrm{H}-0.781179-4.3038274 .215424$

SCF Energy $(B 3 L Y P / 6-31 G * * / / M M F F)=-3245.91687206$

01562

MM̄FF Geometry

C -3.294312 -3.681308 -0.442652

C $-2.693920-3.9041380 .736346$

C $-1.207089-3.7774260 .929730$

O $-0.970574-2.7635721 .939017$

C $-0.587750-5.0917281 .439344$

C $0.727985-5.4069650 .753671$

C $1.924504-4.8960941 .103001$

C $0.622456-6.359615-0.408985$

C $2.216010-3.9078262 .196142$

C $2.547104-2.5115511 .657091$ 
C $3.801051-2.4012140 .775156$ C $5.081204-2.7546821 .535276$ C $6.341364-2.4982720 .695127$ O $3.856812-1.0384260 .327861$ O $7.474795-2.5376851 .575803$ C $6.273450-1.0725490 .111369$ C $6.563791-3.600936-0.347269$ O $7.354301-0.841546-0.789830$ C $4.928442-0.751778-0.580716$ C $4.8658150 .755090-0.927836$ O $4.826569-1.519090-1.780494$ C -1.041852 -1.463632 1.545729 O $-1.317836-1.0461170 .433552$ C $-0.696321-0.6195292 .707973$ C $-0.234961 \quad 0.6222812 .495973$ C 0.1681901 .5812723 .527349 C $-0.152768 \quad 1.2334754 .957582$ C $3.5291261 .210042-1.523003$ C $3.5079322 .714566-1.699591$ C $2.9595934 .822564-0.520521$ O $3.8020253 .245285-2.768025$ C 2.8289845 .2772670 .895859 C 1.6771555 .7052561 .434380 C 1.5354296 .1360862 .865114 C 0.7039765 .1691063 .678313 C -0.5074175 .5112034 .147590$ C 1.3195083 .8277614 .014318 C 0.7919082 .7157803 .144768 N $3.1591543 .385946-0.538180$ H -2.684926 -3.436350-1.309554 H -3.293109 -4.142044 1.611403 H - $0.720040-3.466547-0.004058$ H -1.267659 -5.9361841 .262988$ H $-0.441214-5.0610122 .526079$ H $2.798647-5.2093280 .533690$ H $1.585913-6.524703-0.902685$ H $0.251572-7.332433-0.070053$ H $-0.069688-5.964533-1.159900$ H $1.368833-3.8187902 .883218$ H $3.042819-4.2903002 .804600$ H $1.694052-2.1341741 .079392$ H $2.644842-1.8201692 .504983$ H $3.677879-3.046192-0.101493$ H $5.066912-3.7966141 .875289$ H $5.142638-2.1427562 .446206$ H 7.488311-3.408674 2.008407 H $6.409493-0.3693980 .945090$ H $6.684816-4.5737520 .144619$ H $7.489194-3.433177-0.909057$ H $5.739268-3.692858-1.057322$ H $8.177523-1.042491-0.312465$ H $5.0524591 .338370-0.016610$ H $5.6607071 .008455-1.640900$ H $4.156872-1.119531-2.356506$ H -0.802277 -1.051945 3.695999 H $-0.118567 \quad 0.9647201 .468151$ H -1.216105 0.9904585 .065705 H 0.0433542 .0448565 .660839 H $0.439007 \quad 0.3714725 .284498$ H $3.3504110 .760127-2.505176$ H $2.6909490 .917557-0.880936$ H $2.0657665 .043811-1.112915$ H $3.8117075 .309452-1.005957$ H 3.7256515 .2408661 .509769 H $\quad 0.7803375 .7390470 .820896$ H 1.0846347 .1365962 .872250 H 2.5164976 .2515383 .343091 H -1.089097 4.8252884 .754902 H -0.9443806 .4807623 .933563$ H 1.1825403 .6321055 .081520 H 2.4074953 .8765893 .878650 H 0.9807102 .8602892 .080503 H 2.7842982 .8419630 .234373 C $-5.517000-2.6591170 .207944$ O $-5.015161-1.334847-0.063244$ C $-6.120968-0.414925-0.091410$ C -7.338515 -1.192049 0.400554 C $-7.016898-2.595036-0.073714$
H $-6.278645-0.149758-1.143302$ C $\quad \begin{array}{llll}-5.800339 & 0.844184 & 0.721428\end{array}$ H -7.422339 -1.171393 1.493349 H $-8.273192-0.801388-0.013540$ C $-7.826305-3.6741470 .623799$ H -7.200245 -2.649282 -1.155243 C -4.5023941 .5735110 .313848$ O H -6.6530321 .5325300 .671392$ H -3.6367040 .9877650 .645198$ O $\quad-4.4830102 .795124 \quad 1.065183$ C $-4.3904211 .893591-1.188252$ C $-3.2345882 .831224-1.582284$ H $-4.2917740 .958764-1.750044$ O $-5.6037032 .508317-1.634951$ H $-3.2898852 .976291-2.668445$ H $-3.4146473 .829346-1.162692$ H -7.551854 -4.667491 0.255487 H -7.664918 -3.659940 1.706950 H -8.896295 -3.5302710 .441515$ C $-4.780868-3.685126-0.657455$ H -5.4793021 .2862172 .601682$ H -3.581055 3.1544381 .028083 H $-5.7342713 .316608-1.109634$ H -4.954776 -3.453079-1.716056 H $-5.171164-4.694556-0.485069$ H -5.339484 -2.853600 1.273491 C $-1.4118080 .808687-2.999609$ O $-1.5853710 .994802-1.593756$ C $-1.8307192 .339793-1.181461$ C $-0.6909703 .254776-1.651815$ C $-0.4568093 .141607-3.156504$ C $-0.2764271 .677936-3.557159$ H $-2.3478671 .078807-3.505132$ C $-1.217016-0.693530-3.262869$ H $-1.7964962 .331957-0.085923$ O $-0.9592514 .612988-1.321131$ H $0.2346792 .973243-1.134358$ H -1.267504 $3.608136-3.727959$ O $0.7338843 .850277-3.504671$ H $\quad 0.699101 \quad 1.332093-3.198802$ O $-0.2633311 .573564-4.979074$ C $0.026589-1.292343-2.634180$ H -2.070705 -1.243443 -2.848404 H -1.176951 -0.869815 -4.343755 H $-1.0968984 .657273-0.359541$ H $0.6206984 .769339-3.207241$ H $0.4429082 .157115-5.305937$ O $0.241643-2.530279-3.171386$ O $0.710976-0.763944-1.769514$ C $1.388233-3.225832-2.671229$ H $2.300625-2.742679-3.031875$ H $1.376601-3.257766-1.578258$ H $1.357496-4.250563-3.050792$ SCF Energy $\left(B 3 L Y P / 6-31 G^{* *} / / M M F F\right)=-3245.89772214$

01563

MM̄FF Geometry

C $-0.3818212 .293400 \quad 0.945506$

C $-1.2515372 .962993 \quad 0.174234$

C $-2.1078592 .291498-0.866410$

O $-3.5053312 .567623-0.597233$

C $-1.8402072 .826310-2.284784$

C $-0.4260642 .657200-2.790380$

C $0.0404371 .544796-3.388259$

C $0.4586003 .872292-2.667965$

C $-0.6861520 .255149-3.651678$

C $0.131064-0.965632-3.199770$

C $-0.054967-1.317348-1.713714$

C $0.984267-2.363753-1.296710$

C $0.737158-2.9089700 .115350$

O $-1.382328-1.836757-1.569948$

O $1.548808-4.0811820 .283806$

C $-0.734054-3.3560500 .215487$

C $1.171393-1.9196381 .201906$

O $-1.046497-3.7697081 .544460$

C -1.734208 -2.273651 -0.251097

C $-3.158149-2.878095-0.321033$ 
O $-1.715907-1.2000310 .686483$

C -4.1129521 .8692310 .398127$

O -3.5898231 .0910301 .178445$

C $-5.555140 \quad 2.1970790 .352053$

C -6.4174121 .5040441 .110876$

C -7.8689201 .6947761 .162650$

C -8.4663992 .7340260 .249729$

C $-4.234578-1.920600-0.851451$

C $-5.574823-2.611796-1.040616$

C $-7.928049-2.128899-1.683512$

O $-5.722970-3.827210-0.945478$

C $-8.915047-1.143109-1.144463$

C $-9.842628-1.459292-0.228067$

C $-10.883771-0.5071340 .287996$

C $-10.779361-0.2839331 .780187$

C $-11.361785-1.1282912 .648312$

$\begin{array}{llll}C & -10.074710 & 0.965180 & 2.258437\end{array}$

C -8.591836 0.9289072 .007243

N $-6.584409-1.704461-1.338521$

H $-0.306771 \quad 1.2151180 .843428$

H -1.3385404 .0421750 .272136$

H -1.963675 $1.204318-0.866387$

H -2.123517 $3.887500-2.337050$

H -2.541589 $2.344896-2.979928$

H $1.0619511 .565697-3.766235$

H $1.4563773 .706024-3.087538$

H $0.0151344 .717627-3.204612$

H $0.5887964 .158412-1.621040$

H $-1.6841600 .235533-3.204226$

H $-0.8277240 .186133-4.737515$

H $-0.198751-1.822401-3.802623$

H $1.193814-0.808550-3.422784$

H $0.049521-0.413074-1.104573$

H $1.998951-1.953596-1.377165$

H $\quad 0.950093-3.208054-2.000037$

H $2.478290-3.8235940 .157110$

H $-0.855872-4.242831-0.422322$

H $2.242862-1.7151221 .126731$

H $1.016914-2.3368412 .202895$

H $0.655713-0.9606201 .142343$

H $-0.400148-4.4518261 .794868$

H $-3.132850-3.759143-0.975799$

H -3.468652 -3.220554 0.674254

H $-2.541382-0.6974560 .615977$

H $-5.8691612 .989160-0.318224$

H $-6.026973 \quad 0.7179201 .756134$

H -8.198131 2.530175 -0.793196

H -8.105117 3.7330880 .517070

H -9.5571252 .7692690 .279435$

H -4.385207 -1.095546 -0.150405

H -3.935277 -1.504107 -1.819702

H -8.107806 $-3.141792-1.308334$

H -7.991184 -2.159195 -2.776070

H -8.882864 $-0.134470-1.548688$

H $-9.879125-2.4763720 .156587$

H - $11.870848-0.9242300 .048138$

H -10.841258 $0.454782-0.237456$

H -11.317223 -0.9601943 .719082$

H -11.893929 -2.009540 2.305289

H -10.216153 1.0906053 .340345

H -10.559818 1.8379151 .812383

H -8.067227 0.1947792 .620530

H $-6.328920-0.738788-1.526327$

C $1.996148 \quad 2.5896921 .667296$

$\begin{array}{lllllllllll}\text { O } & 2.395771 & 3.170957 & 0.407308\end{array}$

C 3.6026133 .9175440 .609948

C $4.2120763 .383501 \quad 1.896759$

C 2.9575963 .1508522 .719796

H 3.2900684 .9558290 .792702

C $4.4949243 .914653-0.632654$

H $4.7391072 .436717 \quad 1.737006$

H $4.905224 \quad 4.0908652 .361067$

C $3.167795 \quad 2.2361163 .913241$

H $2.592453 \quad 4.1220883 .080453$

C $5.2289462 .605655-0.992102$

O $5.490498 \quad 4.927487-0.427169$

H $3.8962594 .237902-1.493825$

H $6.0231042 .426526-0.259212$
O $5.9046522 .837305-2.238759$

C $4.3555251 .347612-1.132105$

C $5.0787750 .136461-1.753840$

H $3.9559351 .069002-0.152208$

O $3.240566 \quad 1.652899-1.971317$

H $4.357873-0.688938-1.805274$

H $5.3192750 .354921-2.802353$

H 2.2332762 .0961074 .465709

H $3.5264391 .249578 \quad 3.604741$

H 3.9062682 .6626224 .599941

C 0.5310762 .9353021 .950934

H $6.0421974 .957885-1.227700$

H $5.2234182 .950455-2.923929$

H $2.6495720 .880616-1.963481$

H $0.3971834 .024006 \quad 1.936631$

H 0.2495162 .5788932 .948168

H 2.1221821 .5036881 .569026

C $5.316537-1.5842560 .752033$

O $6.127788-0.462151 \quad 0.397157$

C $6.350570-0.310015-1.006657$

C $7.006917-1.574829-1.581364$

C $6.221553-2.839672-1.238067$

C $5.935055-2.8986820 .263508$

H $4.331116-1.4632070 .288749$

C $5.073950-1.5476742 .264465$

H $7.0822160 .499797-1.113575$

O $7.142858-1.477152-2.995591$

H $8.018939-1.675099-1.167405$

H $5.297675-2.901818-1.825349$

O $7.014153-3.969437-1.610741$

H $6.869291-3.1112250 .796950$

O $5.027588-3.963482 \quad 0.535353$

C $6.333865-1.7182333 .079838$

H $4.617430-0.5930532 .551104$

H $4.368540-2.3295772 .568536$

H $7.665880-0.679260-3.184197$

H $6.469765-4.764683-1.484982$

H $5.479902-4.7974240 .323844$

O $\quad 6.984402-0.525244 \quad 3.192589$

O $6.699876-2.7875053 .550584$

C $8.209314-0.5597883 .931032$

H 8.6277440 .4502063 .942628

H $8.021404-0.8741074 .962132$

H $8.924990-1.2304603 .446201$

SCF Energy (B3LYP/6-31G*//MMFF) $=-3245.91003039$

$01 \_564$

MMMFF Geometry

C $2.500347-1.0135653 .917224$

C $1.903009-1.5446102 .839944$

C $0.504494-1.1934382 .406739$

O $0.602263-0.3474591 .231936$

C $-0.309639-2.4306461 .992816$

C $-0.922406-3.172161 \quad 3.160134$

C -2.142375 -2.892826 3.660347

C $-0.105304-4.303399 \quad 3.722687$

C $-3.064815-1.7925713 .213181$

C $-4.285605-2.3537052 .474392$

C $-5.163050-1.2319651 .900863$

C $-6.459084-1.7988801 .320830$

C $-7.310302-0.708056 \quad 0.652496$

O $-4.415335-0.5817640 .868429$

O $-8.326281-1.359337-0.125780$

C $-6.422538 \quad 0.084017-0.330808$

C $-8.0431030 .154931 \quad 1.686401$

O $-\begin{array}{llll}-7.133056 & 1.193143 & -0.877922\end{array}$

C $-5.0727720 .542875 \quad 0.272833$

C $-4.160661 \quad 1.089307-0.854432$

$\begin{array}{lllll}\text { O } & -5.319553 & 1.574559 & 1.226877\end{array}$

C $\quad 0.7697130 .987791 \quad 1.440658$

$\begin{array}{llll}\text { O } & 0.840413 & 1.564324 & 2.514307\end{array}$

C 0.8751321 .6400800 .118468

C 1.3505332 .8909370 .034235

C $1.5166893 .671745-1.193922$

C $1.0289693 .058044-2.480173$

C $-2.7224541 .408640-0.426231$

C $-2.567362 \quad 2.821968 \quad 0.098935$

C $-2.5345915 .198347-0.654918$ 
O $-2.479463 \quad 3.0549041 .302146$ C -1.625490 $5.856288-1.643587$ C $-0.6602816 .722430-1.299597$ C $0.2513507 .388636-2.290255$ C $1.7150157 .225680-1.943415$ C $2.4358288 .262678-1.483926$ C $2.3630785 .882276-2.204340$ C $2.0860724 .892331-1.104864$ N $-2.5523693 .770026-0.910887$ H $1.949629-0.3151314 .542447$ H $2.466816-2.2214802 .203467$ H $-0.035404-0.6496043 .192818$ H $0.304257-3.1171011 .393788$ H $-1.101157-2.1236491 .296659$ H -2.517306 -3.495900 4.485704 H $-0.631371-4.8374054 .521156$ H $0.126444-5.0313022 .938304$ H $0.834239-3.9308224 .140830$ H $-3.397178-1.2413124 .101593$ H -2.542453-1.061588 2.588445 H -3.943069 -3.0010331 .656187$ H $-4.873306-2.9733943 .163188$ H -5.381374 -0.514117 2.700314 H -7.038431 -2.317470 2.094813 H $-6.218658-2.5611910 .566297$ H $-8.851139-1.9174540 .473370$ H -6.213824 $-0.584128-1.177143$ H -8.682714 -0.4695622 .321742$ H $-8.7155840 .869703 \quad 1.199360$ H -7.365742 0.7068622 .341396 H $-7.1589011 .890501-0.200875$ H $-4.0899770 .323567-1.636631$ H -4.621324 $1.976504-1.304968$ H -4.4762482 .0005081 .445776$ H $0.5684141 .069959-0.749610$ H 1.6556943 .3925130 .951890 H $-0.0318902 .795555-2.405486$ H $1.5980822 .150798-2.711630$ H $1.121413 \quad 3.718862-3.343844$ H -2.3453410 .7064600 .324313$ H -2.061205 $1.306547-1.296445$ H -2.236032 5.3914330 .380326 H -3.558006 $5.561295-0.792901$ H $-1.7735695 .617224-2.694203$ H $-0.5275746 .982178-0.251736$ H $-0.0132668 .453667-2.319467$ H $0.0812597 .010603-3.306132$ H $3.4919878 .160377-1.257457$ H $1.9854009 .235873-1.318907$ H $3.4530836 .000430-2.267357$ H $2.0576715 .529727-3.192706$ H $2.4165225 .234433-0.122559$ H -2.743898 $3.466912-1.861729$ C $4.940258-0.3387293 .593736$ O $5.024753-0.6836862 .194505$ C $4.816795 \quad 0.507821 \quad 1.417301$ C 5.2096941 .6526342 .340739 C 4.6430261 .1642353 .662521 H 3.7405290 .5678961 .218062 C 5.5630640 .4392050 .080082 H $6.297706 \quad 1.7598092 .416495$ H 4.7893372 .6108162 .021328 C 5.2441931 .8470794 .878819 H 3.5641811 .3567583 .657553

C $5.366342-0.880546-0.697562$ $\begin{array}{lllll}\text { O } & 6.965785 & 0.584803 & 0.332678\end{array}$ H $5.2704111 .297066-0.537671$ H $5.968099-1.673139-0.234755$ O $5.884104-0.702096-2.021569$ C $3.909321-1.362626-0.811155$ C $3.781255-2.632146-1.675036$ H $3.525689-1.5678590 .191518$ O $3.119481-0.319358-1.373376$ H $3.764648-2.349925-2.733532$ H $4.662615-3.263486-1.512283$ H $4.815077 \quad 1.4425505 .800971$ H $6.329710 \quad 1.7090784 .921864$ H 5.0434932 .9231484 .854302
C $3.934606-1.2668584 .292403$

H $7.4049880 .735485-0.521015$

H $6.845351-0.579556-1.948188$

H $2.190795-0.599629-1.318634$

H $4.026504-1.1549665 .379200$

H $4.185353-2.3098544 .060001$

H $5.935747-0.546124 \quad 4.006380$

C $0.886781-2.455239-2.795591$

O $1.311180-2.713922-1.454531$

C $2.522681-3.460461-1.326411$

C $2.417461-4.792919-2.083031$

C $2.020953-4.585647-3.541340$

C $0.752304-3.740438-3.622942$

H $1.625229-1.799753-3.274236$

C $-0.420199-1.647340-2.732899$

H $2.609740-3.709140-0.260492$

O $3.644985-5.511993-2.030554$

H $1.656288-5.422777-1.604200$

H $2.834104-4.136168-4.123068$

O $1.750534-5.854723-4.141165$

H $-0.096096-4.342837-3.277997$

O $0.498925-3.394419-4.982922$

C $-1.581870-2.413806-2.131559$

H $-0.281909-0.753794-2.114907$

H $-0.692020-1.325175-3.744708$

H $3.863400-5.648376-1.092763$

H $2.551543-6.397610-4.041170$

H $0.452575-4.223566-5.489393$

O $-2.747256-2.000255-2.709315$

O $-1.482889-3.255417-1.248620$

C $-3.929479-2.660199-2.244850$

H $-4.790791-2.200827-2.736771$

H $-3.896175-3.720673-2.511536$

H $-4.036733-2.538029-1.163198$

SCF Energy (B3LYP/6-31G**//MMFF)= -3245.91112853

01565

MM̄FF Geometry

C $3.5977320 .784757-2.148487$

C $3.105220-0.162222-2.960736$

C $2.762228-1.554433-2.501787$

O $1.384096-1.777807-2.891538$

C $3.643172-2.628031-3.161274$

C $5.064675-2.658429-2.643704$

C $5.454095-3.344377-1.551068$

C $6.080168-1.917173-3.473313$

C $4.590870-4.164536-0.631120$

C $4.904471-3.921715 \quad 0.851754$

C $4.433045-2.5579771 .386720$

C $4.992147-2.3473042 .798061$

C $4.461378-1.0676463 .451411$

O $2.999957-2.5586831 .411374$

O $4.818530-1.1278734 .840984$

C $2.922852-1.0705653 .355747$

C 5.1445710 .1834612 .886352

$\begin{array}{lllll}\text { O } & 2.414846 & 0.167214 & 3.851319\end{array}$

C $2.404792-1.3569341 .925426$

C $0.869153-1.5378691 .888592$

O $2.717580-0.2302141 .101413$

C $0.650026-2.649330-2.149118$

O $1.030485-3.311220-1.197384$

C $-0.729215-2.638524-2.683544$

C $-1.717109-3.189237-1.962526$

C $-3.134929-3.230092-2.325439$

C $-3.542842-2.607622-3.634933$

C $0.350393-2.8128822 .557666$

C $-0.973977-2.564623 \quad 3.249566$

C -3.336159-1.923106 2.812827

O $-1.069023-2.5869594 .475083$

C $-4.355982-2.7463502 .095079$

C $-5.304115-2.2227141 .304257$

C $-6.365901-3.029350 \quad 0.614222$

C $-6.287549-2.928789-0.892495$

C $-6.972174-1.983546-1.558328$

C $-5.488808-3.978648-1.630972$

C $-4.003879-3.805740-1.468446$

N -2.011770 -2.3123372 .366672$

H $3.7992570 .544139-1.109457$ 
H $2.913160 \quad 0.077856-4.003767$

H $2.836081-1.616352-1.409530$

H $3.643819-2.496805-4.252310$

H $3.184523-3.615954-3.024921$

H $\quad 6.512777-3.337377-1.294714$

H $7.095799-2.020034-3.076330$

H $6.087712-2.304894-4.497344$

H $5.850582-0.848946-3.508938$

H $3.525632-4.005350-0.810492$

H $4.792808-5.220439-0.850522$

H $4.408646-4.7157301 .425794$

H $5.983926-4.0344131 .014793$

H $4.780057-1.7634070 .716958$

H $6.089445-2.3417182 .784154$

H $4.708110-3.2005553 .430837$

H $4.423830-0.3543635 .279428$

H $2.567633-1.8478824 .043182$

H 6.2226380 .1499363 .085398

H 4.7779341 .0919103 .376900

H $5.0117150 .297515 \quad 1.808481$

H $1.462270 \quad 0.057394 \quad 4.007987$

H $0.384477-0.6447512 .300589$

H $0.541756-1.5681280 .840767$

H $2.059588-0.1662880 .389806$

H $-0.897276-2.153657-3.638268$

H -1.469078 -3.643443 -1.003669

H -3.069635 -3.131303 -4.472760

H -3.250054 -1.553152 -3.669227

H -4.620455 -2.630334 -3.811385

H $\quad 0.220314-3.609773 \quad 1.815805$

H $1.044375-3.2002163 .309899$

H $-3.444047-0.8537722 .603803$

H -3.433920 -2.069832 3.892932

H $-4.326332-3.8215722 .253947$

H -5.339905 -1.145294 1.167136

H $-7.342014-2.6646180 .960313$

H $-6.327111-4.0832270 .916657$

H -6.945562 -1.922134 -2.641061

H -7.569883 -1.245677 -1.033016

H -5.767060 -4.006751 -2.690276

H - $-5.766438-4.971492-1.253684$

H $-3.616817-4.224522-0.539168$

H -1.805760 -2.235092 1.374575

C $2.8833873 .171827-1.985341$

O $3.0114293 .223070-0.546334$

C $2.9438664 .594674-0.128247$

C $2.4397525 .385797-1.327921$

C $3.0661124 .609801-2.471336$

H 3.9828164 .9014250 .056924

C 2.1613524 .7823671 .180557

H $1.3478965 .351682-1.406338$

H $2.7485266 .435055-1.300150$

C $2.4251534 .894145-3.818808$

H $4.1350914 .856357-2.521725$

C $0.640348 \quad 4.5143291 .147242$

O $2.331546 \quad 6.1453421 .595611$

H $2.6075134 .156451 \quad 1.963371$

H $0.141950 \quad 5.2414860 .496212$

O $\quad 0.127528 \quad 4.7509162 .467522$

C 0.2389183 .0872290 .740388

C -1.2816012 .8723070 .717763$

H $0.6154852 .902000-0.263529$

O $0.8474992 .149806 \quad 1.621504$

H -1.711533 3.1668241 .684438

H $-1.7087203 .567291-0.012711$

H $2.8995104 .304713-4.609546$

H $1.3568124 .653134-3.813770$

H $2.5297065 .952719-4.078221$

C $3.9003682 .192904-2.574560$

H $3.2839876 .296808 \quad 1.721564$

H 0.3790695 .6569132 .716603

H $\quad 0.5269812 .3394942 .519968$

H $4.9110092 .439676-2.227489$

H $3.9035032 .265581-3.668143$

H $1.8644322 .827196-2.204713$

C $-3.8996162 .015985-0.530575$

O $-3.198272 \quad 1.3538590 .531261$

C -1.7636241 .4245190 .447975$
C $-1.3238010 .752203-0.858934$

C $-1.9549121 .444333-2.063392$

C $-3.476024 \quad 1.467907-1.901348$

H $-3.7107743 .094559-0.483299$

C $-5.4031501 .797705-0.303983$

H -1.3892000 .8100601 .275489$

$\begin{array}{lllll}\text { O } & 0.088767 & 0.712263 & -0.972649\end{array}$

H -1.663994 -0.290834 -0.838726

H $-1.5564932 .455760-2.205349$

O $-1.6482740 .717226-3.253428$

H $-3.8689470 .451585-2.035015$

O $-4.0459152 .280179-2.925652$

C -5.8975132 .4555020 .966646$

H $-5.9841332 .212840-1.134527$

H $-5.6054590 .724321-0.225493$

H $0.3097940 .190874-1.761778$

H $-0.6943830 .809892-3.416165$

H $-3.7497251 .920148-3.779346$

O $\quad-7.150879 \begin{array}{lll}1.989413 & 1.247019\end{array}$

O $-5.277633 \quad 3.2827181 .620994$

C -7.7567962 .5260922 .426984$

H -7.152082 2.2854393 .306700

H -7.8833293 .6083832 .328539$

H -8.7419712 .0665342 .542410$

SCF Energy (B3LYP/6-31G**//MMFF) $=-3245.91852193$

01566

MM̄FF Geometry

C - $-1.739516 \quad 1.8764391 .897614$

C -0.7227651 .1638372 .405326$

C -0.0134730 .0666261 .652468$

O $1.3970650 .380261 \quad 1.601065$

C $-0.183901-1.2667892 .403636$

C $0.408709-2.4606761 .680297$

C $1.437756-3.2009202 .137305$

C $-0.253666-2.8331320 .378224$

C $2.211003-3.0333823 .414853$

C $3.484710-2.1828223 .289698$

C $4.528998-2.7492982 .313594$

C $5.853330-1.9922152 .437690$

C $6.889603-2.4704031 .407754$

O $4.026048-2.6084810 .982387$

O $7.953685-1.5062981 .373260$

C $6.243347-2.4782640 .004871$

C $7.526730-3.8033421 .818433$

O $7.113977-3.073645-0.953331$

C $4.867079-3.171903-0.033280$

C $4.131278-3.000156-1.385157$

O $5.044060-4.5793220 .169331$

C 1.8013331 .2759910 .659350

O $1.1045621 .841149-0.168426$

C 3.2579421 .4735950 .825405

C 3.8447132 .5448550 .271161

C 5.2659762 .8884360 .351350

C 6.2008561 .8517820 .915692

C $3.845617-1.552823-1.801212$

C $4.891749-0.959573-2.730434$

C $5.5349241 .194363-3.805587$

O $5.776107-1.624312-3.263366$

C $6.6310251 .829016-3.009100$

C $6.9141483 .139389-3.063150$

C $8.0362083 .787107-2.303448$

C $7.5738644 .964379-1.474401$

C $7.6980676 .222142-1.930622$

C $7.0471644 .686996-0.084404$

C $5.6640534 .097925-0.095965$

N $4.6958190 .401025-2.926891$

H $-2.080676 \quad 1.6758730 .885645$

H $-0.377368 \quad 1.3724723 .415191$

H $-0.403340-0.0250750 .632911$

H - $1.251710-1.4671372 .565951$

H $0.258558-1.1648403 .400499$

H $1.768611-4.042571 \quad 1.529174$

H $\quad 0.136000-3.770408-0.033571$

H -1.330160 -2.966471 0.525701

H $-0.093827-2.056380-0.375250$

H $1.576906-2.6100704 .200965$

H $2.482626-4.0331153 .777758$ 
H $3.203765-1.1687342 .982534$ H $3.931190-2.1053314 .288947$ H $4.674445-3.8118402 .541021$ H $6.256997-2.0773813 .454266$ H $5.674190-0.9205562 .277319$ H $8.323390-1.4357062 .270079$ H $6.120791-1.430239-0.292613$ H $8.028942-3.7046342 .788377$ H $8.305049-4.1049201 .108779$ H $6.802852-4.6164481 .904014$ H $7.958504-2.592897-0.915747$ H $4.657059-3.552333-2.173450$ H $3.155067-3.496913-1.286141$ H $4.263737-5.041637-0.179047$ H 3.8024850 .7439371 .412810 H $3.2242653 .251873-0.278349$ H 6.0633280 .8939910 .402676 H 6.0155131 .7081131 .985491 H 7.2565432 .1044470 .798596 H $2.899981-1.533013-2.358083$ H $3.722867-0.908055-0.926098$ H $4.8901111 .935897-4.287763$ H $5.9664050 .550387-4.578560$ H $7.2347251 .178559-2.380524$ H $6.3261903 .781727-3.715082$ H $8.7854754 .118487-3.034468$ H $8.5530313 .066329-1.657691$ H $7.3949307 .074260-1.331266$ H $8.1014106 .427349-2.916850$ H 6.9953385 .6208530 .491346 H 7.7708884 .0687890 .453710 H $4.9084144 .777064-0.493808$ H $\quad 3.9686410 .880744-2.404267$ C $-3.936648 \quad 2.7037252 .874884$ O $-4.6469852 .673161 \quad 1.622537$ C -5.7264941 .7222411 .733766$ C -5.7590321 .2741943 .194343$ C $-4.299408 \quad 1.4026663 .586743$ H $-5.454052 \quad 0.8591741 .117216$ C $-7.035178 \quad 2.343781 \quad 1.232644$ H -6.3794601 .9352823 .810531$ H $-6.145998 \quad 0.256346 \quad 3.304672$ C $-4.067496 \quad 1.4307105 .086560$ H $-3.754873 \quad 0.5539103 .154121$ C -7.004574 2.880964 -0.215105 O $-7.375744 \quad 3.447856 \quad 2.085700$ H -7.853293 1.6237181 .344608 H -6.313844 $3.732058-0.268180$ O $-8.307183 \quad 3.396040-0.523063$ C $-6.6417301 .873968-1.323144$ C $-7.493907 \quad 0.588146-1.282968$ H -6.815582 $2.365209-2.289272$ O $-5.2530261 .581886-1.249441$ H $-7.2835640 .045595-0.356915$ H $-8.551270 \quad 0.874634-1.256783$ H -3.0018781 .5349405 .313550$ H -4.5965712 .2648735 .559543$ H -4.421989 0.5031675 .547869 C -2.447423 2.9700182 .645047 H -6.6384154 .0815562 .052390$ H -8.531363 4.0462280 .164792 H -5.020835 $1.052501-2.030864$ H -1.960940 3.1508983 .611043 H -2.337862 3.8887142 .055452 H -4.3461273 .5578993 .431531$ C $-5.571743-1.792772-1.526751$ O $-5.910372-0.843562-2.545766$ C -7.251143 -0.345789-2.492010 C $-8.242722-1.514053-2.599412$ C $-7.973570-2.582342-1.542180$ C $-6.505675-3.006072-1.580077$ H -5.635594-1.315340 -0.541938 C $-4.115541-2.212651-1.756880$ H -7.385402 $0.270558-3.390239$ O $-9.577326-1.031966-2.490230$ H $-8.145250-1.968938-3.593988$ H -8.256913 -2.239163 -0.540053 O $-8.770687-3.736163-1.817189$
H -6.316866 -3.588869 -2.490925

O $-6.234342-3.854179-0.465885$

C $-3.178626-1.085490-1.395091$

H -3.831416 -3.071996 -1.139339

H $-3.953400-2.497910-2.803345$

H $-10.175911-1.770465-2.692479$

H $-9.700546-3.490202-1.677012$

H $-6.854600-4.601870-0.512686$

O $-2.617029-0.561652-2.520770$

O $-2.987490-0.705921-0.246916$

C $-1.7184430 .532611-2.313665$

H $-0.8105740 .178728-1.817616$

H $-1.4478670 .939420-3.291612$

H $-2.1982051 .322728-1.728159$

SCF Energy (B3LYP/6-31G*//MMFF)= -3245.91493952

01_567

MM̄FF Geometry

C $0.666995-1.8496192 .794359$

C $-0.489852-1.5657362 .179496$

C $-1.833129-2.0656002 .634311$

O $-2.692061-0.9178502 .838296$

C $-2.494057-2.9320541 .549436$

C $-1.891958-4.3127561 .406939$

C $-1.044874-4.6866420 .428228$

C $-2.345768-5.3168212 .435172$

C $-0.453535-3.838311-0.663040$

C $-1.004922-4.183702-2.052445$

C $-2.441953-3.688527-2.280054$

C $-2.933957-4.095315-3.670946$

C $-4.321632-3.515361-3.981902$

O $-2.435877-2.262132-2.163036$

O $-4.558599-3.667360-5.389831$

C $-4.306613-2.000765-3.687584$

C $-5.436786-4.288608-3.268303$

O $-5.611781-1.442784-3.825264$

C $-3.722681-1.645764-2.300370$

C $-3.522325-0.115224-2.191966$

O $-4.632525-2.078898-1.288417$

C $-2.675440-0.3341974 .064029$

O $-2.014270-0.6894855 .030879$

C $-3.563346 \quad 0.849718 \quad 4.126668$

C $-4.323528 \quad 1.282180 \quad 3.108533$

C -5.1810202 .4719443 .117706$

C $-5.247803 \quad 3.2685564 .395793$

C $-2.7994230 .344965-0.916755$

C $-2.5095881 .835991-0.939141$

C -1.5556193 .6566290 .463662$

O $-2.797742 \quad 2.560854-1.888092$

C $-2.7115594 .316907 \quad 1.146634$

C $-3.643717 \quad 5.0313690 .497955$

C $-4.832848 \quad 5.672758 \quad 1.155522$

C -6.1309504 .9938500 .771173$

C $-6.7648945 .312969-0.369912$

C $-6.727335 \quad 3.997033 \quad 1.742815$

C $-5.8320832 .810384 \quad 1.983940$

$\begin{array}{llll}\mathrm{N} & -1.883858 & 2.262395 & 0.225914\end{array}$

H $0.657570-2.4475893 .700498$

H $-0.475876-0.9508371 .282271$

H $-1.760459-2.6368263 .567794$

H -2.486588 -2.3961840 .594432$

H -3.563935 -3.0391551 .776795$

H $-0.703231-5.7212270 .416357$

H $-1.918515-6.3106472 .264211$

H $-2.048000-5.0002543 .439577$

H $-3.435708-5.4190492 .410595$

H $-0.565747-2.769113-0.465320$

H $0.628530-4.022908-0.665108$

H $-0.346455-3.709445-2.791963$

H $-0.949908-5.266654-2.218569$

H $-3.093327-4.113260-1.508411$

H -2.941077 $-5.187259-3.776948$

H $-2.228775-3.726110-4.429146$

H $-4.496855-4.614185-5.603516$

H $-3.682997-1.524048-4.457054$

H $-5.434627-5.339590-3.581645$

H $-6.424002-3.898103-3.538951$

H $-5.344079-4.269928-2.180506$ 
H $-5.934845-1.677648-4.712067$ H $-2.9325930 .224554-3.053703$ H $-4.4938640 .392927-2.240985$ H -4.506696 -1.541657 -0.491374 H -3.543949 1.3677435 .080503 H -4.311113 0.7318732 .170280 H -4.2517073 .6286764 .676659$ H -5.6429202 .6522555 .211032$ H -5.8864454 .1511404 .333355$ H -3.410383 $0.134099-0.033965$ H -1.843843 $-0.178724-0.807846$ H $-1.3136684 .143279-0.487292$ H -0.6688083 .6920871 .103306$ H -2.783398 4.1915632 .223587 H -3.560330 $5.151894-0.580129$ H $-4.862064 \quad 6.7280430 .854383$ H -4.7263855 .6869422 .247151$ H $-7.7069654 .850005-0.643697$ H -6.352071 $6.044268-1.057096$ H -7.681366 3.6120391 .358850 H -6.9852664 .5259212 .664018$ H -5.712072 2.1735221 .106157 H -1.7596861 .6060440 .991580$ C $2.959706-2.5062521 .930564$ O $4.056080-1.9197381 .194756$ C $5.254189-2.0650531 .970318$ C $5.036005-3.3433332 .760790$ C $3.571822-3.2169203 .147670$ H $5.298208-1.2130232 .660531$ C $6.489375-2.0551581 .063925$ H $5.182038-4.2331872 .137678$ H $5.701209-3.4198693 .626065$ C $2.935518-4.5641133 .459080$ H $3.497240-2.5793084 .038082$ C $6.544321-0.8774690 .068341$ O $6.524942-3.2619220 .290096$ H $7.395730-2.0716401 .680109$ H $5.797067-1.033569-0.718221$ O $7.822352-0.912270-0.579558$ C $6.352747 \quad 0.5152170 .693898$ C $6.5824051 .685233-0.283582$ H 5.3441880 .5914321 .114235 O 7.2702770 .6610081 .780865 H 6.4217562 .6187420 .269935 H $7.6397781 .713579-0.575894$ H $1.884190-4.4522563 .737865$ H $2.982998-5.2379732 .596908$ H $3.453709-5.0492034 .292992$ C $1.992863-1.3707782 .287615$ H $5.696658-3.309570-0.217856$ H $7.924511-1.796376-0.971822$ H 7.1001471 .5239662 .195265 H $1.852936-0.7439681 .398392$ H $2.448267-0.7086873 .034817$ H $2.475243-3.2162881 .249627$ C $3.6646262 .582707-0.613727$ O $4.3098951 .454537-1.213055$ C $5.6887881 .650276-1.538784$ C $5.8556792 .858045-2.472494$ C $5.1780574 .110687-1.922467$ C $3.7306793 .807974-1.534278$ H 4.1675452 .8203500 .332256 C $2.2388412 .168198-0.232807$ H $5.9927010 .769409-2.116920$ O $7.232343 \quad 3.136123-2.706327$ H $5.4058462 .624696-3.446559$ H $5.7329904 .529736-1.074930$ O $5.1699825 .119637-2.935524$ H $3.1517373 .650309-2.452037$ O $3.1960464 .951813-0.873436$ C $1.4110971 .722616-1.416160$ H $2.275675 \quad 1.339576 \quad 0.483756$ H 1.7035092 .9779780 .274066 H $7.6345082 .330977-3.074782$ H $6.0941595 .274280-3.196166$ H $2.2326794 .836165-0.815706$ O $1.5111820 .371416-1.572573$ O $0.7720442 .486612-2.127566$
C $0.807213-0.179836-2.689851$

H $0.806316-1.268093-2.587077$

H $-0.2288420 .169172-2.712763$

H $1.3221230 .087180-3.617078$

SCF Energy $\left(B 3 L Y P / 6-31 G^{* *} / / M M F F\right)=-3245.90572965$

01 568

MM̄MF Geometry

C $3.396131-4.2785360 .081600$

C $2.400734-3.3808330 .124770$

C $1.304501-3.287839-0.901020$

O $0.050199-3.186322-0.188948$

C $1.463732-1.990202-1.712238$

C $0.920812-2.102094-3.119819$

C $-0.321725-1.750877-3.502374$

C $1.908101-2.615023-4.137179$

C $-1.460536-1.268594-2.644564$

C $-2.706341-2.121892-2.918148$

C $-3.917261-1.742910-2.052858$

C $-5.114571-2.618365-2.440306$

C $-6.401397-2.211711-1.711646$

O $-4.221599-0.361216-2.264793$

O $-7.506228-2.838281-2.381612$

C $-6.593245-0.688286-1.854626$

C $-6.423646-2.725500-0.267480$

O $-7.702123-0.247183-1.072958$

C $-5.3301840 .126395-1.491515$

C $-5.5416861 .617850-1.857078$

O $-5.0902990 .002438-0.092943$

C $-0.559712-4.3164610 .242337$

O $-0.221083-5.466541 \quad 0.002246$

C -1.726031 -3.995700 1.103235

C $-2.032712-2.7446821 .483432$

C $-3.139150-2.3339802 .350034$

C $-3.979049-3.4143592 .976431$

C $-4.3888812 .548809-1.453300$

C $-4.4820453 .044136-0.016963$

C -3.1063684 .1299561 .754497$

$\begin{array}{llll}\text { O } & -5.491235 & 2.926238 & 0.673045\end{array}$

C -2.6469242 .9896812 .608542$

C -3.3055782 .5556163 .694111$

C -2.8523891 .3896544 .527634$

C -3.8946660 .2926824 .623405$

C $-4.354756-0.1120035 .819263$

C $-4.415084-0.3352013 .347341$

C $-3.335823-1.0134852 .548283$

N -3.3049803 .6493600 .399088$

H $3.437048-5.002716-0.727467$

H $2.381532-2.6500270 .930290$

H $1.292840-4.166891-1.557342$

H $2.522485-1.705922-1.788445$

H $0.976548-1.158559-1.192368$

H $-0.566522-1.837005-4.560929$

H $1.472834-2.694608-5.138904$

H $2.769700-1.942445-4.202836$

H $2.264672-3.610415-3.853209$

H -1.219801 -1.306725 -1.579497

H -1.661396 -0.220462 -2.894144

H -2.975025 -2.007187 -3.977296

H -2.469023 -3.181980 -2.758470

H $-3.653641-1.893515-1.000477$

H -4.892059 -3.678575 -2.265009

H -5.293340 -2.527954 -3.521402

H -7.359724 -3.799666 -2.366562

H $-6.849492-0.480532-2.902947$

H $-6.367285-3.820497-0.249088$

H -7.363843 -2.4659180 .231066$

H $-5.596486-2.3452830 .333787$

H $-8.473572-0.775479-1.340766$

H $-5.6495011 .687938-2.947623$

H $-6.4745611 .987420-1.415946$

H -4.3458570 .5780930 .141864$

H -2.305183 -4.853129 1.429770

H -1.414722 -1.9222891 .127635$

H -3.349065 -4.118748 3.531367

H $-4.529760-3.9674642 .208150$

H -4.711990 -3.034419 3.691668

H $-3.4218402 .060828-1.616418$ 
H $-4.4224943 .442349-2.089151$ H -4.040173 4.5696522 .120148 H -2.3386324 .9087681 .721484$ H -1.7260272 .4929432 .313242$ H -4.220389 3.0575254 .000484 H -2.606421 1.7731355 .526217 H -1.9216140 .9627874 .133563$ H -5.101339 -0.8948345 .903020$ H -3.994791 $0.328372 \quad 6.743233$ H -4.8649610 .4458972 .721941$ H -5.242575 -1.019422 3.560569 H -2.665283 -0.3130422 .048465$ H $-2.5034163 .649103-0.223910$ C $5.803128-3.7526530 .523552$ O $5.609024-2.3644430 .181271$ C $6.663715-1.5848920 .767325$ C $7.769918-2.5782761 .095213$ C $6.959547-3.7858031 .525270$ H $6.268313-1.1717921 .703378$ C $7.068303-0.442407-0.168906$ H $8.372348-2.8239960 .213218$ H $8.442604-2.2107781 .875914$ C $7.745825-5.0852891 .512714$ H $6.582926-3.6089472 .541706$ C $5.8995560 .437595-0.660234$ O $7.705539-0.985488-1.333101$ H 7.8255770 .1802980 .321429 H $5.290371-0.113457-1.387464$ O $6.4659021 .545889-1.372278$ C 4.9838690 .9861030 .450668 C $3.9514861 .994388-0.087055$ H 4.4715330 .1501040 .932058 O 5.7761581 .6246331 .452395 H $4.4858452 .887531-0.437171$ H $3.4482081 .569384-0.962635$ H $7.112568-5.926306 \quad 1.812421$ H $8.145866-5.3027780 .516657$ H $8.589067-5.0312382 .209009$ C $4.502843-4.3184741 .097622$ H $7.077836-1.599934-1.751406$ H $7.0278991 .180676-2.077207$ H 6.2722162 .3422201 .022115 H $4.209744-3.7431181 .985195$ H $4.645570-5.3560061 .419669$ H $6.073393-4.272825-0.404286$ C $1.7372403 .980893-0.506570$ O 2.4977783 .8058620 .695038 C 2.9056532 .4537740 .956675 C 1.6545331 .5704341 .089722 C $0.7299451 .698689-0.120523$ C $0.4427313 .165801-0.431681$ H $2.3293763 .663899-1.374301$ C $1.4383925 .480801-0.647832$ H 3.3987622 .4816851 .935306 O $1.994426 \quad 0.202670 \quad 1.269632$ H 1.0906961 .8796171 .979967 H $1.1448611 .192746-0.999026$ $\begin{array}{llll}\text { O } & -0.513836 & 1.053092 & 0.163279\end{array}$ H -0.2157253 .5800570 .341067$ O $-0.2446933 .254763-1.677651$ C $2.6824536 .288242-0.956005$ H $0.7285985 .655364-1.463688$ H 1.0183695 .8630690 .290242 H 2.4980720 .1349672 .098796 H $-0.312243 \quad 0.136257 \quad 0.417307$ H -1.011926 2.659169-1.630934 O $2.3567987 .615363-0.960318$ O $3.7928765 .823233-1.173241$ C $3.4357998 .512445-1.241474$ H $3.0461559 .533362-1.209980$ H $4.2189738 .412331-0.483919$ H $3.8372278 .320460-2.241000$ SCF Energy (B3LYP/6-31G**//MMFF)= -3245.92365119

\section{1_569}

MM̄FF Geometry

C -3.176134 -3.329641 -2.169135

C $-1.921754-3.429792-1.706270$
C $-0.705095-3.049016-2.507069$

O $-0.096246-1.898744-1.876226$

C $0.313718-4.201902-2.485357$

C $1.435030-4.013075-3.486471$

C $2.646241-3.503303-3.191110$

C $1.129456-4.479082-4.886438$

C $3.113135-2.957187-1.870854$

C $4.210535-3.833615-1.252072$

C $4.845263-3.2373610 .016956$

C $3.857604-3.1527901 .183981$

C $4.476758-2.4694782 .411907$

O $5.353225-1.945211-0.320800$

O $3.413786-2.1609413 .327406$

C $5.089030-1.1263871 .963750$

C $5.441748-3.3931653 .162803$

O $5.781792-0.4971313 .039532$

C $6.022368-1.2513060 .738417$

C 6.4445440 .1317750 .182933

O $7.214751-1.9406801 .128884$

C $-0.514533-0.666937-2.275214$

O $-1.351654-0.414083-3.126776$

C $0.2172710 .337647-1.469284$

C $-0.0826921 .638533-1.602991$

C $0.4956332 .747864-0.839314$

C 1.6205292 .4295140 .106719

C $5.2916460 .940584-0.416047$

C $5.7460412 .247044-1.041357$

C $4.8629914 .079690-2.496394$

O $6.8266542 .772424-0.790836$

C $4.0700995 .048064-1.675793$

C $2.9740775 .682631-2.119364$

C $2.2138986 .688046-1.304337$

C $0.7404036 .370779-1.193960$

C $-0.1768177 .121512-1.828192$

C $0.3214155 .251762-0.265997$

C $-0.0251603 .982396-1.001780$

N $4.7692902 .773148-1.876451$

H $-3.350788-2.942770-3.169942$

H $-1.758628-3.797824-0.696346$

H $-0.974337-2.812100-3.544107$

H $-0.187455-5.153390-2.709899$

H $0.726304-4.321685-1.475984$

H $3.383735-3.434523-3.989827$

H $1.974923-4.334365-5.567397$

H $0.885084-5.546363-4.886295$

H $\quad 0.278061-3.927517-5.297332$

H $3.493758-1.944966-2.052094$

H $2.283702-2.847004-1.169323$

H $3.811935-4.831434-1.030480$

H $5.006933-3.965180-1.997151$

H $5.688967-3.8819770 .291120$

H $3.480492-4.1492901 .445103$

H $2.976481-2.5672850 .891896$

H $2.978542-2.9951303 .572873$

H $4.249099-0.4681841 .710264$

H $4.916585-4.2887653 .516151$

H $5.837799-2.9044994 .059848$

H $6.282589-3.7261142 .551118$

H $5.162306-0.4358253 .786813$

H 6.9595020 .7102520 .960409

H $7.192834-0.019534-0.607895$

H $7.922080-1.7147440 .502581$

H $\quad 0.958437-0.023839-0.765373$

H $-0.8526401 .922085-2.319629$

H $2.3941131 .848693-0.402068$

H 1.2555381 .8424380 .954376

H 2.1188113 .3145630 .507531

H $4.7761570 .349727-1.181655$

H 4.5680751 .2010500 .362475

H $4.4749663 .986260-3.515530$

H $5.9083924 .399133-2.547227$

H $4.4321675 .250375-0.670274$

H $2.6236265 .497370-3.132151$

H $2.3544797 .668985-1.776969$

H $2.6343346 .783526-0.295177$

H $-1.2381976 .921865-1.723048$

H $\quad 0.112067 \quad 7.948445-2.468298$

H -0.5767065 .5639920 .285085$ 
H 1.0812655 .1070470 .506655 H -0.851460 4.100479-1.704633 H $3.8965702 .267658-1.994253$ C -4.934643 -2.559537-0.553533 O $-5.375557-1.496961-1.426100$ C -6.647194-1.022709-0.961232 C $-6.831738-1.5897150 .440296$ C $-6.149440-2.9362510 .296148$ H -7.399977 -1.492277 -1.610297 C -6.796070 $0.496103-1.101245$ H $-6.313616-0.9876341 .194293$ H -7.884436-1.668980 0.727695 C $-5.806122-3.5892651 .623381$ H -6.809872 -3.606184-0.271057 C $-5.9252001 .384595-0.185676$ O $-8.1722270 .796498-0.825492$ H -6.618732 $0.776174-2.146960$ H -6.2313411 .2323010 .854358$ O $-6.264780 \quad 2.743154-0.504991$ C $-4.404276 \quad 1.202416-0.342467$ C $-3.5508292 .183846 \quad 0.484780$ H -4.125503 $0.183252-0.067083$ O $-4.064728 \quad 1.373515-1.720370$ H -2.502478 2.0382060 .202945 H $-3.773726 \quad 3.2145750 .184036$ H $-5.287566-4.540561 \quad 1.469669$ H -5.161692 -2.9477882 .232420$ H -6.716154 -3.790081 2.198306 C $-4.387191-3.725904-1.374138$ H -8.287364 $1.754979-0.943850$ H $-5.963516 \quad 3.3034490 .229919$ H -3.126185 $1.141266-1.822209$ H -5.146634 -4.084690 -2.079778 H $-4.141001-4.567482-0.715936$ H $-4.141779-2.1437210 .080920$ C $-2.143395 \quad 0.2078552 .340172$ O -3.5045190 .6591572 .421582$ C -3.715173 2.0147242 .008085 C -2.8577992 .9665732 .853006$ C -1.3904282 .5491392 .883672$ C -1.2566051 .0704743 .250275$ H -1.8018530 .2826221 .301646$ C -2.142333-1.276573 2.737951 H -4.7604942 .2473412 .242748$ O -2.9780104 .2986092 .364416$ H -3.242903 2.9673943 .881257 H -0.9031192 .7704761 .928848$ O -0.7200273 .3399973 .867690$ H $-1.5409890 .942248 \quad 4.302611$ $\begin{array}{lllll}\text { O } & 0.114042 & 0.707554 & 3.128499\end{array}$ $\begin{array}{llll}\text { C }-0.998541 & -2.075144 & 2.148519\end{array}$ H $-2.120228-1.3744173 .829451$ H -3.069155 -1.743271 2.384613 H -2.400348 4.861394 2.908041 H 0.2056873 .0425513 .894599 H $0.226182-0.1688043 .533003$ O $-1.066228-3.3558962 .619840$ O $-0.154873-1.6438351 .375736$ C $-0.032828-4.2351252 .164142$ H $0.932597-3.9150292 .565682$ H $-0.252703-5.2395212 .535533$ H $-0.004478-4.2646961 .071009$ SCF Energy (B3LYP/6-31G**//MMFF) $=-3245.90740851$

\section{1_57}

MM̄FF Geometry

C -3.647738 -2.968914 -2.508152

C $-2.411061-2.978124-1.990702$

C $-1.182523-2.612889-2.783195$

O $-0.554122-1.474464-2.144349$

C $-0.183190-3.782093-2.776505$

C $0.867608-3.646669-3.860121$

C $2.114995-3.175895-3.671812$

C $0.438796-4.116084-5.227650$

C $2.725574-2.653493-2.401774$

C $3.953965-3.484078-2.000410$

C $4.725923-2.926648-0.792349$

C $3.939154-3.0570510 .513717$
C $4.681077-2.4151231 .694919$

O $5.040965-1.561495-1.070617$

O $3.765756-2.3212252 .796228$

C $5.064580-0.9740221 .298684$

C $5.849408-3.2783452 .182875$

O $5.850506-0.3575552 .315709$

C $5.795565-0.884916-0.059105$

C $5.9786010 .578115-0.529979$

O $7.100138-1.4590900 .075720$

C -1.054914 -0.241955-2.436195

O $-1.9434680 .018116-3.232386$

C $-0.3561410 .748321-1.582801$

C $-0.7710592 .024557-1.588229$

C $-0.248522 \quad 3.126142-0.774574$

C $0.8980822 .842033 \quad 0.156824$

C $4.6718591 .304675-0.863984$

C $4.9176952 .666769-1.489539$

C $3.7329664 .436759-2.793978$

O $5.9741493 .279970-1.363551$

C $3.0576355 .382674-1.851912$

C $1.9374266 .058445-2.149161$

C $1.2899317 .037999-1.213840$

C $-0.1782186 .759393-0.987458$

C $-1.1216707 .575554-1.487844$

C $-0.5520335 .592070-0.101130$

C $-0.8381034 .336052-0.879513$

N $3.8003203 .128883-2.171466$

H $-3.780769-2.711043-3.555916$

H $-2.272991-3.241332-0.945543$

H $-1.446546-2.351583-3.815706$

H $-0.709119-4.733216-2.936775$

H $0.287396-3.871085-1.790238$

H $2.779224-3.133297-4.534616$

H $1.237381-4.019259-5.971001$

H $0.149204-5.171504-5.192321$

H $-0.415210-3.533261-5.586095$

H $3.017730-1.612587-2.584130$

H $2.002940-2.630560-1.583533$

H $3.659159-4.522491-1.803852$

H $4.644493-3.506294-2.854705$

H $5.662563-3.491989-0.717346$

H $3.710941-4.1088570 .726442$

H $2.968878-2.5543170 .418783$

H $3.428847-3.2139942 .984397$

H $4.130874-0.4016041 .239516$

H $5.486077-4.2550902 .524183$

H $6.341476-2.8222703 .049145$

H $6.602928-3.459527 \quad 1.413845$

H $5.357501-0.4364843 .150375$

H $6.554346 \quad 1.1453900 .212465$

H $6.6006400 .576439-1.436480$

H $7.670399-1.100759-0.624294$

H $0.451794 \quad 0.394147-0.953523$

H - $1.6013722 .292124-2.241416$

H 1.3997083 .7458700 .510387

H $1.6726612 .247097-0.334483$

H $0.5450372 .293743 \quad 1.034095$

H $4.0738020 .704971-1.559297$

H $4.081122 \quad 1.468403 \quad 0.043105$

H $3.1822354 .323132-3.732974$

H $4.7425764 .793662-3.020337$

H $3.5289135 .534259-0.883403$

H $1.4790635 .927598-3.126738$

H $1.4222678 .040008-1.642617$

H $1.8018917 .060752-0.243446$

H -2.175950 $7.399018-1.301375$

H $-0.862308 \quad 8.434179-2.098173$

H $-1.465214 \quad 5.8402730 .457312$

H 0.2120995 .4540930 .668808

H $-1.6724224 .443208-1.574443$

H $2.9573832 .562706-2.183417$

C $-5.767108-2.081742-1.414682$

O $-5.120503-1.227902-0.450062$

C $-5.4835110 .138172-0.735424$

C $-6.5322980 .087857-1.846889$

C $-6.141554-1.175773-2.586071$

H $-4.5817650 .624870-1.125108$

C -5.9612060 .8526720 .534583$ 
H $-7.548850-0.004952-1.447585$ H $-6.5050770 .981300-2.478513$ C $-7.239770-1.733526-3.473290$ H -5.265259 -0.942374 -3.202645 C $-4.955867 \quad 0.8373191 .703431$ $\begin{array}{lllll}\text { O } & -7.160531 & 0.208697 & 0.985760\end{array}$ H $-6.238508 \quad 1.8844300 .286050$ H -4.873492 -0.1766792 .111960$ O $-5.497194 \quad 1.6560422 .748903$ C -3.5535621 .3728841 .359022$ C -2.6490401 .6301572 .580319$ H -3.0527770 .6743670 .680396$ O -3.6742122 .6110260 .651969$ H -1.7029402 .0419802 .209659$ H -3.078578 2.437105 3.187639 H -6.907831-2.648161-3.974479 H -8.139836 -1.971949-2.896663 H $-7.517534-1.007330-4.244193$ C $-4.888836-3.296648-1.728621$ H -7.5780340 .7922031 .641353$ H -6.2651301 .1889413 .118869$ H -4.1504673 .2336441 .227711$ H -5.467067 -4.027693 -2.305956 H $-4.612328-3.785213-0.785565$ H -6.679031 -2.448538 -0.923765 C $-0.587277-0.587627 \quad 2.202090$ O $-1.919463-0.7253232 .703308$ C -2.390279 $0.392476 \quad 3.462097$ C $-1.458224 \quad 0.656578 \quad 4.655367$ C $-0.002895 \quad 0.812738 \quad 4.221618$ C $0.416792-0.3618293 .337256$ H -0.5608480 .2694991 .521001$ C $-0.262431-1.8063801 .334466$ H -3.357946 0.0914893 .880832 O $-1.862938 \quad 1.8212725 .367736$ H $-1.520984-0.1865955 .355883$ H $\quad 0.157875 \quad 1.7733493 .718594$ $\begin{array}{lllll}\text { O } & 0.818580 & 0.831068 & 5.391368\end{array}$ H $0.498838-1.2561813 .965792$ O $1.712511-0.1085192 .802062$ C $-0.188414-3.1035122 .103337$ H $-1.025324-1.9259560 .558820$ H $0.691893-1.6693530 .813634$ H -2.7834241 .6856645 .650363$ H 1.7433090 .8863675 .094782 H 1.6602920 .6883562 .248294 O $-1.443312-3.5813712 .338273$ $\begin{array}{lllll} & 0.860918 & -3.626608 & 2.455737\end{array}$ C -1.507241 -4.8075073 .072326$ H -1.057195 -4.6807494 .061711$ H $-2.560057-5.074343 \quad 3.197553$ H - $1.005731-5.6068762 .518457$ SCF Energy (B3LYP/6-31G**//MMFF) $=-3245.90303534$

01_570

MMMFF Geometry

C $4.0123541 .858777-2.125219$

C $3.6697900 .923920-3.025177$

C $3.594270-0.536653-2.679157$

O $2.316975-1.054344-3.130049$

C $4.680286-1.396315-3.354151$

C $5.990461-1.433250-2.595345$

C $6.327400-2.391510-1.707645$

C $6.968976-0.335789-2.922496$

C $5.523137-3.587104-1.269063$

C $4.225049-3.294922-0.508901$

C $4.359648-2.3293950 .679250$

C $5.038463-2.990348 \quad 1.880986$

C $5.054329-2.0704923 .111988$

O $3.030974-1.9158361 .028884$

O $5.406487-2.8870914 .239357$

C $3.628329-1.5231783 .348319$

C $6.149873-1.0037832 .998290$

O $3.622048-0.5673324 .405848$

C $2.976664-0.9379702 .076761$

C $1.497776-0.5466872 .295259$

O 3.6712110 .2604021 .707786

C $1.275143-0.985619-2.258421$
O $1.245389-0.400931-1.187955$

C $0.181113-1.814678-2.806095$

C $-0.683571-2.382565-1.952332$

C $-1.788712-3.276311-2.303716$

C $-2.106612-3.453932-3.764923$

C $0.554665-1.7149282 .588069$

C $-0.901464-1.2797842 .599428$

C $-3.189768-2.2046452 .944915$

O $-1.257008-0.1166512 .432228$

C -3.859288 -3.3143472 .200716$

C $-4.668679-3.1174531 .149046$

C $-5.341401-4.2266320 .394350$

C $-4.941204-4.270643-1.064203$

C $-5.791176-3.873263-2.025780$

C $-3.597540-4.869281-1.416563$

C $-2.466655-3.884345-1.307534$

N $-1.752728-2.3548052 .815071$

H $4.2903141 .539353-1.124516$

H $3.4103701 .216037-4.039115$

H $3.658652-0.666588-1.593498$

H $4.854982-1.057257-4.384201$

H $4.303667-2.420785-3.474324$

H $7.306183-2.323706-1.233711$

H $7.937441-0.483957-2.432575$

H $7.153091-0.299646-4.001200$

H $6.5874890 .636119-2.600909$

H $5.298432-4.209018-2.144357$

H $6.167755-4.207817-0.633871$

H $3.472244-2.913010-1.205735$

H $3.800667-4.245083-0.157369$

H $4.917252-1.4421540 .363670$

H $6.057285-3.3105911 .632474$

H $4.493418-3.9075692 .144904$

H $5.348839-2.3306875 .035241$

H $3.025676-2.3685203 .702123$

H $7.134911-1.4784222 .912334$

H $6.196642-0.3841573 .900507$

H $6.023127-0.3478792 .134594$

H 3.9778220 .2662334 .053492

H 1.4216850 .2071403 .089828

H $1.137120-0.0382651 .391236$

H $3.0990390 .777973 \quad 1.117074$

H $\quad 0.152667-1.977105-3.877075$

H $-0.554672-2.198632-0.885826$

H $-1.297388-3.991874-4.270567$

H $-2.240220-2.482325-4.253478$

H $-3.029474-4.010209-3.943197$

H $0.664366-2.4939641 .825138$

H $0.782338-2.1507143 .566588$

H -3.497068 -1.220398 2.582021

H $-3.432810-2.2670284 .010162$

H -3.669524 -4.3279012 .545880$

H $-4.864465-2.1029490 .812021$

H $-6.425451-4.0809260 .486825$

H -5.136106 -5.2024850 .852205$

H $-5.524208-3.932252-3.075664$

H $-6.771141-3.475048-1.784738$

H $-3.634473-5.323977-2.412449$

H $-3.388999-5.711974-0.744756$

H $-2.154989-3.689105-0.281770$

H $-1.347625-3.2561703 .052344$

C $3.2501684 .183529-1.430622$

O $1.8676473 .780418-1.425516$

C $1.3426863 .967903-0.093667$

$\begin{array}{llll}\text { C } 2.436687 & 4.669851 & 0.708445\end{array}$

C 3.6932944 .1590560 .030582

H $\begin{array}{llll}1.185261 & 2.968745 & 0.328111\end{array}$

C $0.0190394 .738290-0.153929$

H 2.3709345 .7605100 .618931

H 2.3908564 .4205961 .773199

C 4.9262874 .9963970 .320102

H 3.8693223 .1330990 .373573

C -1.073401 $4.055330-1.000677$

$\begin{array}{lllll}\text { O } & 0.262781 & 6.032398 & -0.723348\end{array}$

H -0.3531064 .9210890 .860511$

H $-0.7571453 .993436-2.048857$

O $-2.2327194 .900217-0.978019$

C -1.487728 2.657557 -0.499585 
C $-2.6191982 .060347-1.361582$

H $-0.6258781 .982533-0.537789$

O -1.8901092 .7676390 .862252$

H -3.466502 2.752420 -1.374444

H $-2.2510201 .990842-2.391974$

H $5.8024594 .590647-0.195071$

H $4.7932996 .033655-0.004943$

H 5.1409055 .0060791 .393820

C $4.0586533 .331214-2.413465$

H $0.6379245 .896072-1.610381$

H $-1.9568875 .779038-1.290332$

H -2.0400591 .8682951 .199983$

H $5.1051753 .656371-2.405669$

H $3.6733103 .514067-3.424693$

H $3.2642515 .218709-1.798813$

C $-4.979311 \quad 1.3773790 .463251$

O

C $-3.1001610 .669140-0.887860$

C $-4.036045-0.037991-1.877347$

C $-5.4155820 .615406-1.910027$

C $-5.9867660 .733036-0.496716$

H -4.8451172 .4355650 .210489$

C $\quad-5.4800301 .3023541 .910334$

H $-2.2030180 .046894-0.784443$

O $-3.464791-0.043924-3.180418$

H -4.147031 -1.085213 -1.571505

H $-5.3744711 .595475-2.398669$

O $-6.296550-0.177983-2.708175$

H $-6.271331-0.261896-0.132352$

O $-7.1714991 .525599-0.534712$

C -4.6349162 .1699932 .813218$

H -6.512922 1.6557812 .005211

H -5.4600370 .2696892 .277862$

H $-4.095452-0.488413-3.772408$

H -6.366464 -1.055301-2.295610

H $-7.7772791 .112649-1.173663$

O $-3.843399 \quad 1.392388 \quad 3.604282$

O $-4.661846 \quad 3.3941262 .800047$

C -2.965924 2.1003204 .485223

H -2.384903 1.3651675 .048295

H $-2.276327 \quad 2.7266683 .911274$

H -3.544264 2.7057495 .189520

SCF Energy (B3LYP/6-31G**//MMFF)= -3245.92526381

01 571

MM̄FF Geometry

C -3.719226 2.1513491 .455062

C -2.8159891 .7019822 .340197$

C $-2.533590 \quad 0.2395192 .563333$

O $-1.095370 \quad 0.0693102 .505798$

C $-3.063056-0.2435943 .927047$

C $-4.573306-0.3492253 .986323$

C $-5.277258-1.4061073 .534045$

C $-5.282444 \quad 0.799773 \quad 4.654958$

C $-4.743671-2.6624722 .902500$

C $-5.473807-3.0508991 .609939$

C $-5.118273-2.194706 \quad 0.379876$

C $-6.035798-2.591543-0.783795$

C $-5.657974-1.899253-2.097191$

O $-3.745032-2.4358640 .044175$

O $-6.378405-2.565798-3.145150$

C $-4.151812-2.118080-2.343017$

C $-6.124101-0.438672-2.118061$

O $-3.727787-1.411267-3.507285$

C $-3.274852-1.749710-1.125774$

C $-1.789808-2.122091-1.355101$

O $-3.326346-0.328891-0.957288$

C $-0.635457-1.1822342 .230975$

O $-1.307585-2.1869692 .058243$

C $0.841856-1.1414542 .170797$

C $1.503223-2.2967602 .000227$

C $2.953034-2.4771321 .938280$

C $3.813595-1.2440791 .894326$

C -1.478397 -3.620273 -1.330641

C $-0.027992-3.903812-1.691591$

C $1.494526-5.819606-2.181681$

O $0.784228-3.022505-1.958217$

C $2.441839-6.030520-1.042285$
C $3.721588-5.626548-1.075246$

C $4.732345-5.9089800 .000677$

C $5.355589-4.6533950 .569036$

C $6.363138-4.031216-0.066211$

C $4.877843-4.1737791 .918899$

C $3.440628-3.7348911 .913073$

N $0.242112-5.264819-1.703306$

H $-4.285147 \quad 1.434640 \quad 0.866566$

H -2.2683972 .4133602 .953636$

H -2.981912 -0.348166 1.755595

H -2.6990610 .4171424 .725982$

H -2.622683 -1.215996 4.180728

H $-6.359301-1.3900823 .660279$

H $-6.3671490 .652878 \quad 4.693925$

H -4.9293850 .9124675 .685334$

H $-5.099367 \quad 1.7369334 .122699$

H -3.670173 -2.611928 2.718649

H $-4.893003-3.4721663 .628175$

H $-5.211822-4.0953111 .393030$

H -6.557103 -3.022855 1.783056

H $-5.247129-1.1345550 .623996$

H -7.085834 -2.391850 -0.534690

H $-5.971488-3.678336-0.938989$

H $-6.087341-2.183600-3.991165$

H -4.020892 -3.182192 -2.573359

H $-7.216117-0.387709-2.028096$

H $-5.8817080 .043151-3.071473$

H $-5.6996140 .161004-1.310320$

H -3.642468 -0.472274 -3.269022

H -1.440187 -1.672010 -2.293491

H $-1.177506-1.646377-0.578808$

H -2.519607-0.035561 -0.503235

H $1.336931-0.1843932 .281507$

H $0.925796-3.2173061 .916242$

H $3.757932-0.7030352 .844808$

H $3.486071-0.5775821 .089358$

H $4.867133-1.4577671 .698394$

H -1.669180 -4.029593 -0.332224

H -2.106855 -4.154958 -2.049878

H $1.912137-5.157472-2.947751$

H $1.260787-6.782720-2.646594$

H $2.076360-6.581281-0.179225$

H $4.085352-5.105937-1.958640$

H $5.520399-6.537946-0.434291$

H $4.295809-6.5094520 .808314$

H $6.835488-3.1476420 .349690$

H $6.733410-4.389895-1.021035$

H $5.529939-3.3847452 .308895$

H $4.981416-4.9956132 .639791$

H $2.734444-4.5659721 .934360$

H $-0.486221-5.915957-1.424735$

C $-3.6007474 .192026-0.069271$

O $-2.1633034 .257186-0.102331$

C $-1.7252294 .012727-1.454040$

C $-2.9831144 .006938-2.319379$

C $-4.0139373 .464243-1.346600$

H -1.291124 $3.005872-1.464418$

C $-0.6632565 .042433-1.857304$

H $-3.2612135 .016303-2.643845$

H $-2.8668843 .387142-3.213715$

C $-5.4505063 .713943-1.766990$

H -3.852236 $2.384437-1.253606$

C $0.5203075 .149834-0.873497$

O $-1.2690456 .339532-1.936900$

H $-0.2965714 .820158-2.865942$

H $0.1855295 .594650 \quad 0.071507$

O $1.4756786 .061239-1.431132$

C $1.2339673 .816575-0.588867$

C 2.4339563 .9881880 .364201

H $0.5287623 .117093-0.127723$

O $1.6582293 .237816-1.820453$

H $3.1996814 .602726-0.119924$

H 2.0922464 .5333971 .251690

H $-6.1475493 .313215-1.024228$

H $-5.6558404 .783427-1.881299$

H $-5.6607493 .226667-2.724678$

C -4.0669943 .5995101 .261184$

H $-1.6601086 .534968-1.067981$ 
H $\quad 1.0102676 .897135-1.606124$

H $2.2683113 .865085-2.245052$

H -5.1558023 .6993841 .343854$

H -3.633976 4.1902932 .078618

H $-3.9485495 .233424-0.110063$

C $4.7511972 .390284-0.889906$

O $3.5471391 .881302-0.309195$

C 3.0442352 .6354590 .798485

C 4.1108242 .7418041 .900470

C 5.4430003 .2614801 .364489

C 5.8798832 .4499460 .143492

H $4.5692043 .403852-1.268305$

C $5.0875231 .552392-2.127955$

H 2.2169352 .0464401 .215111

O 3.6707353 .5816852 .962202

H $4.279517 \quad 1.7474942 .331841$

H 5.3802564 .3287621 .123265

O 6.4409993 .1495432 .382089

H $6.1682821 .443708 \quad 0.466989$

O $7.0259183 .059558-0.443659$

C $5.1742590 .068141-1.851866$

H $4.3064501 .710105-2.883517$

H $6.0272741 .869468-2.593199$

H 2.8360023 .2134523 .298923

H 6.5177462 .2115532 .626725

H $7.707876 \quad 3.1218940 .247133$

O $4.094910-0.564914-2.395020$

O $6.084868-0.464633-1.231879$

C $4.064842-1.986668-2.244650$

H $5.028750-2.430860-2.510824$

H $3.789682-2.241170-1.218138$

H $3.305994-2.383404-2.923752$

SCF Energy (B3LYP/6-31G**//MMFF) $=-3245.92185592$

01_572

MM̄FF Geometry

C $4.065992-0.124557-2.477785$

C $3.392777-1.143262-3.032616$

C $2.789473-2.266549-2.239486$

O $1.421450-2.426278-2.689596$

C $3.476220-3.618937-2.477977$

C $4.841320-3.748260-1.844663$

C $5.044018-4.110954-0.563030$

C $6.011793-3.516742-2.763427$

C $4.001547-4.3899970 .489990$

C $4.391486-3.7918391 .851177$

C $4.248643-2.2594951 .909699$

C $5.276121-1.6762742 .882619$

C $5.119179-0.1594683 .036011$

O $2.919597-1.9606652 .361196$

O $5.918490 \quad 0.255745 \quad 4.153631$

C 3.6515920 .1404843 .388321

C 5.6513560 .5973981 .815086

O 3.4192351 .5484813 .424548

C $2.617565-0.5619172 .469791$

C $1.218866-0.4177163 .120072$

O $\quad 2.627074 \quad 0.073371 \quad 1.193045$

C $0.447783-1.800231-1.982013$

O $0.581353-1.049374-1.030280$

C $-0.845247-2.233962-2.542458$

C $-1.804823-2.600124-1.682106$

C $-3.130034-3.108081-2.032742$

C $-3.595891-2.928220-3.452691$

C $0.057232-1.0736242 .364974$

C $-1.226813-1.0332243 .181298$

C $-3.609821-1.6949743 .110431$

O $-1.313302-0.4728004 .271320$

C -4.431897 -2.755102 2.453804

C $-5.506739-2.4985381 .692674$

C $-6.350396-3.5569421 .041731$

C $-6.298669-3.497069-0.469700$

C -7.220634 -2.813887-1.168246

C $-5.233531-4.307626-1.174068$

C $-3.866940-3.690768-1.065046$

N -2.271026 -1.697057 2.550215

H $4.231773-0.124401-1.404614$

H $3.231079-1.155110-4.107962$

H $2.798066-2.040453-1.166073$
H $3.539702-3.825848-3.555251$

H $2.825628-4.420234-2.100548$

H $6.073648-4.230329-0.227438$

H $6.972478-3.658136-2.256915$

H $5.977172-4.216942-3.604585$

H $5.998186-2.497627-3.159888$

H $3.008812-4.0314730 .201528$

H $3.931617-5.4786940 .603211$

H $3.753753-4.2474692 .619568$

H $5.423444-4.0900122 .077231$

H $4.390991-1.8320630 .911669$

H $6.296978-1.9208592 .562752$

H $5.151062-2.1424833 .870278$

H $6.8405680 .005043 \quad 3.972862$

H $3.496223-0.2142164 .416732$

H 6.7092540 .3618241 .648090

H 5.6079321 .6811841 .969559

H $\begin{array}{llll}5.110592 & 0.355548 & 0.899037\end{array}$

H 3.3537491 .8603922 .505862

H $1.258804-0.8605544 .124673$

H $\quad 0.978317 \quad 0.6447293 .246953$

H $1.812627-0.1624130 .721279$

H $-0.942381-2.300448-3.619043$

H $-1.585313-2.556452-0.616047$

H -3.058393 -3.609230 -4.121111

H $-3.424437-1.900253-3.791638$

H -4.665432 -3.111706 -3.580785

H $-0.135144-0.5394891 .429578$

H $0.289042-2.1192922 .135792$

H -4.026571 -0.693662 2.967149

H $-3.547483-1.8784184 .188358$

H -4.131471 -3.786559 2.621969

H -5.812074 -1.467462 1.535925

H -7.383988 -3.4204411 .386198$

H $-6.063111-4.5602651 .380018$

H -7.206283 -2.791362 -2.252818

H $-8.006485-2.256319-0.669468$

H -5.511809-4.481371 -2.218769

H $-5.196927-5.311316-0.731112$

H -3.426046 -3.781768 -0.072796

H $-2.157599-1.9532801 .573619$

C $4.0206542 .391743-2.783431$

O $2.5822222 .322260-2.684560$

C $2.1883572 .871911-1.414334$

C $3.3650373 .722184-0.956565$

C $4.5303762 .873176-1.423050$

H $2.0676612 .028193-0.723219$

C $0.8636873 .632823-1.516942$

H $3.3752374 .697143-1.458444$

H 3.3621963 .8915460 .124445

C $5.8477843 .625542-1.488031$

H $4.6421862 .031755-0.731130$

C $-0.3385192 .702790-1.798982$

O $0.9899434 .634682-2.523117$

H $0.6930094 .146360-0.563975$

H $-0.3120221 .881135-1.073989$

O $-0.2427312 .126945-3.099364$

C $-1.6942773 .421668-1.670817$

C $-2.9257802 .529620-1.905214$

H $-1.7596183 .873400-0.675481$

O $-1.7721894 .501427-2.604981$

H -3.811070 $3.169045-1.798135$

H $-2.9481142 .200742-2.952355$

H $6.6503452 .974337-1.848555$

H $5.7853644 .488333-2.159552$

H $6.1299713 .992199-0.495581$

C $4.5833751 .048977-3.262608$

H $0.1793575 .170719-2.508812$

H $0.5957181 .636915-3.144602$

H -1.657095 $4.126651-3.495282$

H $5.6780121 .061146-3.215911$

H $4.3122410 .924135-4.318945$

H $4.2383873 .148552-3.548283$

C $-3.7420992 .443768 \quad 1.027555$

O $-2.768765 \quad 1.610640 \quad 0.389483$

C $-3.0419751 .302676-0.978095$

C $-4.3854030 .566636-1.081019$

C $-5.5148461 .363976-0.428457$ 
C $-5.126725 \quad 1.787478 \quad 0.988421$ H $-3.776974 \quad 3.4204740 .529539$ C -3.278028 2.6611482 .471940 H -2.263749 $0.597676-1.294119$ O $-4.6903210 .305884-2.445976$ H -4.297817 $-0.402885-0.574419$ H $-5.7949262 .234791-1.032337$ O $-6.6850420 .548748-0.338543$ H $\quad-5.1465750 .912508 \quad 1.647490$ O $-6.0952952 .710271 \quad 1.483339$ C -2.052069 3.5419922 .521384 H -4.0515713 .1510803 .074279$ H -3.0436371 .7115042 .967248$ H $-5.457844-0.290115-2.467843$ H $-6.9832130 .362687-1.244884$ H $-6.963738 \quad 2.2753151 .430759$ O -0.9274682 .7869982 .366094$ $\begin{array}{lllll} & -2.095500 & 4.756935 & 2.665235\end{array}$ C $\quad 0.316704 \quad 3.4950642 .378611$ H 0.2450054 .4480251 .845902 H $0.629362 \quad 3.6584123 .413543$ H 1.0638482 .8748521 .876416

SCF Energy (B3LYP/6-31G**/MMFF) $=-3245.92040133$

01573

MM̄FF Geometry

C $4.194293-3.7276381 .881833$

C $2.915009-3.3827671 .671151$

C $2.083887-2.6445662 .684815$

O $1.873213-1.3163942 .153585$

C $0.718475-3.327166 \quad 2.885767$

C $-0.140251-2.6657993 .948693$

C $-1.362484-2.1361403 .742028$

C $0.427169-2.6829685 .347165$

C -2.163930 -2.074651 2.469657

C $-3.370503-3.0182822 .572527$

C $-4.328882-2.9985751 .371969$

C $-3.679366-3.5119900 .086522$

C $-4.654223-3.465407-1.100980$

O $-4.816192-1.665607 \quad 1.191341$

O $-3.893428-3.657304-2.303790$

C $-5.268195-2.050684-1.173247$

C $-5.673775-4.608911-1.052095$

O $-6.266306-1.979260-2.188353$

C $-5.821407-1.5375050 .176986$

C $-6.273110-0.0552660 .105199$

O $-6.986034-2.304500 \quad 0.512940$

C $2.357698-0.2689842 .873551$

O $2.928423-0.3100083 .952062$

C $2.070074 \quad 0.9687942 .118988$

$\begin{array}{lll}\text { C } 2.045934 & 2.136776 & 2.777815\end{array}$

C 1.7612233 .4498282 .198092

C $1.797675 \quad 3.5659790 .698224$

C $-5.1842770 .973152-0.213670$

C -4.2487331 .2532860 .949400$

C -1.9407502 .0659271 .431960$

O $-4.587053 \quad 1.122752 \quad 2.123055$

C $-1.715030 \quad 3.541867 \quad 1.356627$

C $-1.753227 \quad 4.3642382 .415557$

C -1.461852 5.8358922 .329296

C $-0.278718 \quad 6.2503753 .178709$

C $-0.450930 \quad 6.975794 \quad 4.296581$

C 1.1103605 .8908072 .689082

C 1.4820374 .4731703 .032668

N $-3.012668 \quad 1.7121690 .522772$

H $4.664224-3.4871162 .832263$

H $2.455394-3.6006770 .709940$ H $2.621750-2.6118383 .639428$ H $0.872723-4.3740933 .179731$

H $0.192426-3.3607491 .926447$ H - $1.877514-1.7028114 .599645$ H $-0.305324-2.3653356 .097288$ H $0.745397-3.6959335 .615262$ H $1.287548-2.0132525 .427995$ H $-2.503662-1.0420312 .332411$ H $-1.557949-2.3161311 .594531$ H -3.021629 -4.046331 2.734841 H -3.948598 -2.736179 3.463361
H -5.173736 -3.644508 1.640227 H $-3.288074-4.527250 \quad 0.224758$ H -2.814466 - $2.887353-0.166201$ H $-3.430686-4.509879-2.233536$ H $-4.459906-1.388032-1.498714$ H $-5.160786-5.578237-1.052140$ H $-6.309736-4.608950-1.944223$ H $-6.315353-4.577028-0.169389$ H $-7.067540-2.411205-1.846288$ H $-7.0715870 .035614-0.642691$ H -6.7418920 .2161681 .060850$ H -7.506718 -1.8039671 .162833$ H 1.8643040 .8812531 .059019 H $2.2184582 .131083 \quad 3.853655$ H $1.628077 \quad 4.581590 \quad 0.339114$ H 1.0305502 .9362690 .238223 H $2.776673 \quad 3.257333 \quad 0.314715$ H $-4.6170430 .687001-1.103428$ H $-5.669624 \quad 1.929608-0.446343$ H $-1.044963 \quad 1.5360351 .094239$ H -2.176418 1.7327222 .447521 H $-1.463993 \quad 3.9514720 .381663$ H $-1.996162 \quad 3.962513 \quad 3.396393$ H $-2.365676 \quad 6.3729502 .644566$ H $-1.279690 \quad 6.145726 \quad 1.292422$ H $\quad 0.3937407 .2979684 .896549$ H $-1.440298 \quad 7.2594234 .639993$ H 1.8552896 .5465723 .159058 H 1.1752786 .1090601 .619962 H 1.4987624 .2798394 .106678 H $-2.834792 \quad 1.809749-0.472357$ C $5.857610-3.442068-0.017640$ O $4.957498-2.686276-0.851515$ C $5.407996-1.318736-0.898580$

C $6.831277-1.329917-0.350511$

C $6.732716-2.4172560 .704722$

H $4.771849-0.754631-0.205549$

C $5.251173-0.756922-2.316276$

H $7.562551-1.612349-1.116609$

H $7.126469-0.3587720 .058162$

C $8.076510-2.9639001 .151963$

H $6.212936-1.9981441 .574989$

C $3.841258-0.959212-2.910147$

O $6.183712-1.430757-3.170731$

H $5.5287320 .303830-2.325135$

H $3.656293-2.028345-3.072538$

O $3.806013-0.341820-4.203069$

C $2.698848-0.363851-2.064495$

C $1.322558-0.632758-2.706287$

H $2.706626-0.815001-1.066564$

O $2.925394 \quad 1.032597-1.910149$

H $1.291682-0.177542-3.700381$

H $1.231523-1.716263-2.844725$

H $7.945258-3.7547931 .897222$

H $8.640374-3.3821860 .311393$

H $8.683574-2.1716491 .602092$

C $5.056151-4.4100590 .857769$

H $6.234397-0.928758-4.001522$

H $4.376761-0.863097-4.792227$

H $2.2034441 .394859-1.370186$

H $5.737426-5.0925211 .379079$

H $4.421708-5.031380 \quad 0.212489$

H $6.472021-4.037722-0.705608$

C $-0.401798 \quad 1.891643-3.104638$

O $0.0372041 .313835-1.866292$

C $0.141835-0.114462-1.851522$

C $-1.212412-0.740460-2.208741$

C $-1.735121-0.217808-3.540791$

C $-1.756192 \quad 1.308846-3.539307$

H $0.3451691 .705974-3.884743$

C $-0.5015923 .410937-2.886603$

H $0.355256-0.396088-0.812252$

O $-1.118184-2.159551-2.266790$

H $-1.944546-0.499525-1.427297$

H $-1.146848-0.603453-4.381936$

O $-3.071933-0.682629-3.738783$

H $-2.5590201 .653410-2.874876$

$\begin{array}{llll}\text { O } & -2.074929 & 1.738473 & -4.862349\end{array}$ 
C $\quad 0.852240 \quad 4.085317-2.812764$

H $-1.0380263 .891931-3.710505$

H -1.029825 $3.610434-1.947949$

H - $0.776602-2.465439-1.409171$

H $-3.052136-1.653855-3.685284$

H $-2.3512442 .668737-4.816826$

O $\quad 0.680813 \quad 5.372955-2.387944$

O $1.9181273 .569280-3.117041$

C $1.8717436 .163708-2.315131$

H $2.6302015 .668270-1.701489$

H $2.254657 \quad 6.352135-3.322349$

H $1.6165907 .119635-1.850171$

SCF Energy (B3LYP/6-31G**//MMFF)= -3245.90571415

\section{4}

MMFF Geometry

C -1.198798 3.6216231 .741371

C -2.3777113 .2673472 .271012$

C -2.7091241 .9979743 .020464$

O -3.8593151 .3930362 .360162$

C -1.6390740 .8850703 .076622$

C -0.4844491 .1011314 .033537$

C 0.8197731 .0479963 .694963

C -0.8646081 .3014425 .479124$

C $1.430074 \quad 0.8417412 .336359$

C $2.116880-0.5202292 .188399$

C $1.137022-1.7000952 .109853$

C $1.905150-3.0167201 .972352$

C $0.964884-4.2132401 .770428$

O $\quad 0.299801-1.5007320 .965827$

O $1.756587-5.327321 \quad 1.330485$

C $-0.022919-3.8839820 .632522$

C $0.299128-4.6442323 .082570$

O $-1.008733-4.9060920 .502374$

C $-0.706670-2.504618 \quad 0.784947$

C $-1.491218-2.166570-0.504668$

O $-1.613815-2.551707 \quad 1.887437$

C -5.0989621 .7735962 .763416$

O -5.3630222 .6140753 .613003$

C -6.1649481 .0644082 .019643$

C $-5.962614-0.0246231 .261283$

C $-6.992003-0.7557970 .514205$

C $-8.393116-0.1993690 .541222$

C $-2.028077-0.729007-0.566939$

C $-2.755158-0.463582-1.873479$

C $-4.3852131 .096903-2.918220$

O $-2.645255-1.190650-2.857856$

C $-5.7168620 .435817-2.749137$

C $-6.069988-0.685761-3.394674$

C $-7.376536-1.400531-3.201953$

C $-7.198116-2.717634-2.476759$

C $-6.827088-3.825729-3.140483$

C $-7.517341-2.765140-0.997967$

C $-6.636511-1.862723-0.173348$

N $-3.5230790 .693044-1.821823$

H -0.3394972 .9742231 .846424$

H -3.208699 3.9616712 .154771

H -2.9989602 .2676484 .042465$

H -1.298155 0.6688812 .059827

H -2.136147 -0.0451013 .388488$

H 1.5564091 .1827384 .486856

H $0.001357 \quad 1.2517716 .148107$

H -1.333159 2.2797925 .622531

H -1.566527 0.5245925 .799811

H $\quad 0.7110480 .983788 \quad 1.526455$

H 2.1846051 .6267462 .197090

H $2.696175-0.4880241 .258798$

H $2.828022-0.673348 \quad 3.009387$

H $\quad 0.519001-1.7136273 .014280$

H $2.555398-3.1805012 .840674$

H $2.576449-2.9566381 .104407$

H $2.433104-5.5003122 .007644$

H $0.549053-3.883876-0.306375$

H $1.057891-4.9343623 .819356$

H $-0.328231-5.5302142 .934939$

H $-0.311959-3.8587843 .531892$

H $-0.538209-5.7503640 .394818$

H $-0.834575-2.317514-1.370373$
H -2.335986 -2.858619 -0.616912

H $-2.325921-1.909327 \quad 1.745371$

H -7.1562331 .4852752 .156583$

H $-4.956576-0.4296821 .179493$

H -8.4004040 .8414640 .197957$

H -8.801688 -0.2388461 .556915$

H $-9.092308-0.733247-0.104734$

H -2.719351 -0.5447460 .259930$

H -1.207138 $-0.006957-0.495920$

H $-3.9144390 .829287-3.870175$

H $-4.4981422 .183732-2.881431$

H $-6.4103460 .895673-2.050343$

H $-5.366533-1.137873-4.090726$

H -7.820272 -1.577076-4.190434

H $-8.100590-0.772838-2.668160$

H $-6.719158-4.779805-2.635625$

H -6.620761 -3.801042-4.205602

H -7.384198 -3.784529 -0.612036

H $-8.581446-2.551749-0.866703$

H -5.593667 -2.181764 -0.142213

H $-3.6171391 .186721-0.938822$

C $-0.4191424 .653208-0.438575$

O $0.8437573 .959112-0.381482$

C $0.8336852 .904500-1.359732$

C $-0.3055613 .238991-2.314984$

C $-1.3234373 .836954-1.363548$

H $0.5919541 .978245-0.822756$

C $2.2026852 .749046-2.035959$

H $-0.0008153 .981634-3.061634$

H $-0.6780772 .356461-2.843800$

C $-2.3944494 .663774-2.052855$

H -1.804715 $3.016585-0.817949$

C $3.3288032 .376991-1.045465$

O $2.5292833 .953923-2.726874$

H $2.1206701 .960539-2.791890$

H $2.9693221 .552560-0.419648$

O $3.5850253 .490050-0.190858$

C $4.6318111 .939837-1.747929$

C $5.8071781 .605434-0.810266$

H $4.4134521 .065807-2.371236$

O $5.0848952 .961133-2.639315$

H $6.6444921 .280269-1.440885$

H $6.1656732 .525109-0.330032$

H -3.106260 $5.062604-1.323191$

H -1.960754 $5.508738-2.598205$

H $-2.9502944 .054018-2.771387$

C $-0.955983 \quad 4.893710 \quad 0.977207$

H $2.6093754 .657400-2.059904$

H 4.1387953 .1818390 .545322

H $5.1850383 .778730-2.121964$

H -1.8682745 .4990720 .925267$

H -0.2142255 .4722841 .541916$

H $-0.2030835 .629698-0.891531$

C $5.665231-1.435232-1.114219$

O $4.855419-0.608752-0.271750$

C 5.5073120 .5426210 .263033

C 6.7257140 .1144661 .091068

C $7.661566-0.7954770 .299043$

C $6.887377-1.948645-0.341190$

H $5.988987-0.862713-1.992304$

C $4.774766-2.586121-1.596203$

H $4.797050 \quad 0.9970250 .963845$

O $7.4536151 .247797 \quad 1.551582$

H $6.384944-0.4323761 .980014$

H $8.222257-0.233210-0.456983$

O $8.635504-1.3489911 .188165$

H $6.582242-2.6521530 .444249$

O $7.775151-2.636414-1.220164$

C $3.725622-2.084704-2.560459$

H $5.349176-3.354168-2.124787$

H $4.271079-3.084486-0.759398$

H 6.8411961 .7978802 .069187

H $9.100057-0.6041841 .607210$

H $7.370755-3.490980-1.444187$

O $2.635244-1.634664-1.876593$

O $3.872174-2.075440-3.776022$

C $1.582506-1.087478-2.676292$

H $1.009708-1.899539-3.132585$ 
H $\quad 0.924138-0.511143-2.021449$

H $1.976145-0.417041-3.446251$

SCF Energy (B3LYP/6-31G**//MMFF) $=-3245.89469735$

01_575

MM̄FF Geometry

C -0.691789 3.8483502 .527033

C $-1.6143983 .324302 \quad 3.346571$

C -1.731501 1.8854493 .782869

O $-2.887419 \quad 1.2807903 .133956$

C -0.4972130 .9591893 .693016$

C 0.6375461 .3185404 .632030

C 1.8928861 .6289754 .252137

C $\quad 0.307906 \quad 1.260418 \quad 6.103437$

C 2.4685051 .6998352 .865323

C 3.7079800 .8091352 .703238

C $3.411506-0.6966062 .788168$

C $4.709148-1.4959632 .647135$

C $4.453086-3.0095422 .628491$

O $2.509672-1.0373391 .730643$

O $5.654414-3.6524152 .175352$

C $3.355131-3.3133701 .588544$

C $4.172468-3.5556284 .033391$

O $2.991927-4.6923781 .624920$

C $2.102409-2.4128631 .718586$

C $1.184144-2.6304530 .490487$

O $1.396786-2.7648592 .907804$

C -2.8827521 .0384051 .795942$

O $-1.976037 \quad 1.2376191 .004526$

C $-4.210418 \quad 0.485653 \quad 1.446418$

C $-4.399528-0.0849750 .247597$

C $-5.654943-0.657178-0.241211$

C $-6.896147-0.438000 \quad 0.583600$

C $-0.066546-1.741943 \quad 0.458639$

C -1.271091 -2.444257 1.055215

C $-2.869085-4.3034660 .595553$

O $-1.666392-2.1954722 .191354$

C $-3.853344-4.435057-0.522275$

C $-5.181718-4.367921-0.347521$

C $-6.176630-4.477319-1.468230$

C $-7.191812-3.354329-1.454363$

C -8.427391-3.557404-0.965785

C $-6.811832-2.025245-2.071898$

C -5.647972 -1.349732 -1.399197

N -1.821798 -3.3812370 .194946$

H 0.0735293 .2146372 .092980

H -2.387537 3.9846153 .736373

H -2.027952 1.9225554 .838090

H -0.1550330 .8943842 .658778$

H -0.816624 -0.0647523 .931516$

H 2.6121161 .8682965 .035559

H 1.2015701 .3319556 .733188

H $-0.3570452 .082557 \quad 6.384476$

H $-0.182676 \quad 0.312046 \quad 6.346222$

H $1.741244 \quad 1.4537662 .088508$

H 2.7670172 .7398872 .684183

H 4.1425401 .0325641 .721968

H $4.458903 \quad 1.0822243 .455451$

H $2.937091-0.9135323 .751720$

H $5.414802-1.2360743 .446108$

H $5.207841-1.2186761 .707753$

H $6.372604-3.4101642 .784835$

H $3.799077-3.1459190 .597356$

H $5.013760-3.3398234 .702860$

H $4.075180-4.6468324 .021212$

H $3.273829-3.1320294 .486446$

H $2.418776-4.8268012 .398739$

H $1.765954-2.404133-0.409733$

H $0.891532-3.6855240 .431199$

H $\quad 0.516140-2.3589382 .876531$

H -4.9964650 .5612272 .189324$

H -3.555657 -0.151761 -0.438283

H -7.0480770 .6298540 .776945$

H $-6.818314-0.9623141 .542327$

H -7.806381 -0.7931810 .094850$

H $0.086291-0.7895900 .973815$

H -0.310190 -1.489534 -0.580542

H $-3.335764-3.9623761 .525197$
H $-2.388738-5.2690150 .782981$

H $-3.458125-4.596127-1.522533$

H $-5.578704-4.2344470 .656528$

H $-6.686314-5.444502-1.367418$

H -5.679856 -4.502125 -2.446212

H $-9.175942-2.772161-0.974317$

H $-8.717933-4.513590-0.543271$

H -7.667240 -1.338874 -2.103832

H $-6.549420-2.194560-3.124550$

H $-4.705357-1.463138-1.933842$

H -1.339812 -3.574791-0.678605

C - 1.0407205 .5406240 .664070

O $-0.1261604 .857530-0.215879$

C $-0.8844264 .255939-1.282995$

C $-2.2629934 .905479-1.237648$

C -2.4466765 .0971420 .255029$

H $-0.9908983 .193468-1.032854$

C $-0.1493474 .402589-2.618527$

H $-2.2738485 .875303-1.748509$

H -3.034763 4.274825-1.689223

C -3.5369016 .0898540 .617198$

H -2.695732 4.1238430 .693816

C $1.2945003 .859241-2.619361$

O $\quad-0.087478 \quad 5.789745-2.974347$

H -0.732795 $3.917437-3.409646$

H $1.9395364 .520479-2.028781$

O $1.7842553 .937830-3.964735$

C $1.4444062 .413536-2.109769$

C $2.8843961 .888601-2.278985$

H $1.1708512 .377366-1.049073$

O $\quad 0.5313371 .584918-2.822541$

H $3.1113021 .790804-3.345641$

H $3.5712642 .634641-1.862968$

H -3.6174846 .1995941 .703197$

H -3.337744 7.0788920 .191307

H $-4.506637 \quad 5.7504920 .238712$

C -0.6588475 .2972812 .127000$

H $\quad 0.3725616 .256859-2.255662$

H $1.2638823 .316452-4.502552$

H $0.5388720 .710222-2.398708$

H -1.3094715 .8931842 .777908$

H 0.3657985 .6533412 .292505

H $-0.914177 \quad 6.6111490 .454822$

C $2.669650-0.979955-3.411078$

O $2.300907-0.512923-2.107798$

C $3.1164590 .533804-1.573411$

C $4.5847600 .086340-1.522916$

C $5.072573-0.419382-2.876171$

C $4.118785-1.477650-3.423009$

H $2.546430-0.170258-4.139983$

C $1.707121-2.117492-3.781213$

H $2.7902550 .671060-0.534855$

O $5.4351261 .144064-1.093437$

H $4.688828-0.726270-0.792314$

H $5.2003010 .402152-3.590915$

O $6.360202-1.019775-2.717122$

H $4.209101-2.394647-2.826432$

O $4.492975-1.798696-4.761169$

C $0.316110-1.593092-4.054021$

H $2.027271-2.648707-4.684106$

H $1.662263-2.847714-2.963928$

H $5.1007951 .462906-0.238590$

H $\quad 6.946747-0.346484-2.331390$

H $5.421986-2.085933-4.741575$

O $-0.590912-2.251303-3.277712$

O $0.063435-0.710540-4.864062$

C -1.950395 -1.833110 -3.431134

H -2.260651 -1.905005 -4.478065

H $-2.578486-2.499956-2.836024$

H -2.070877 -0.810959-3.059994

SCF Energy (B3LYP/6-31G**//MMFF) $=-3245.92255518$

01 576

MMFF Geometry

C $4.8364591 .549276-0.179225$

C 5.7387541 .1228690 .719191

C $5.567743-0.0627461 .644588$

O $4.191256-0.4982701 .671756$ 
C $6.436057-1.2285281 .133660$

C $6.255143-2.5212191 .906843$

C $5.644876-3.6242161 .425333$

C $6.825513-2.5209743 .301088$

C $5.024086-3.8082570 .066740$

C $3.497364-3.7236950 .139669$

C $2.831984-3.341463-1.192188$

C $3.092477-1.876022-1.561305$

C $2.324156-1.444254-2.816737$

O $1.421975-3.546548-1.020104$

O $2.393037-0.010871-2.876952$

C $0.843543-1.842063-2.656248$

C $3.000548-1.961750-4.091045$

O $0.122869-1.581521-3.858601$

C $0.638493-3.305563-2.196726$

C $-0.849631-3.533967-1.839715$

O $1.005423-4.179127-3.266039$

C 3.3497160 .1110082 .551684

O $3.628048 \quad 0.975765 \quad 3.365710$

C $2.001946-0.4514222 .319222$

C $0.9381450 .002387 \quad 2.996957$

C $-0.438672-0.4614992 .814307$

C $-0.677125-1.5581561 .808259$

C $-1.143536-4.891095-1.191346$

C $-2.614454-5.022838-0.844895$

C $-4.293921-4.3752840 .860241$

O $-3.374530-5.724329-1.508699$

C -4.549194 -3.1710331 .708029$

C $-4.409369-3.1635913 .042603$

C $-4.683796-1.9724383 .915614$

C $-3.409422-1.2945984 .373774$

C $-2.829780-1.6464985 .534170$

C $-2.898484-0.1981933 .462596$

C -1.4243310 .0988963 .546143$

N $-2.976376-4.2712990 .262617$

H $3.8908411 .025724-0.287612$

H $\quad 6.688641 \quad 1.6475630 .797641$

H 5.8853290 .2215952 .655271

H $7.496251-0.9436991 .173201$

H $6.223617-1.3847620 .071506$

H $5.567255-4.4953712 .074502$

H $6.733411-3.4976733 .788179$

H $7.890110-2.2664413 .275557$

H $6.306678-1.7902083 .929007$

H $5.432040-3.101028-0.656014$

H $5.310932-4.796650-0.314160$

H $3.102810-4.6931290 .473504$

H $3.180948-3.0129410 .911205$

H $3.197833-4.017917-1.973214$

H $4.160935-1.672990-1.691989$

H $2.769500-1.234845-0.730798$

H $1.8411900 .279652-3.623415$

H $0.414301-1.174737-1.896413$

H $4.026877-1.580891-4.158621$

H $2.489331-1.597495-4.988995$

H $3.047379-3.051825-4.138229$

H $\quad 0.327769-2.294512-4.487267$

H $-1.173016-2.748754-1.145416$

H $-1.467074-3.442181-2.742160$

H $0.562478-5.033336-3.145852$

H $1.913110-1.2249931 .566647$

H 1.0816730 .7888293 .735995

H $-0.147760-2.4712692 .102466$

H $-0.328251-1.2574750 .815160$

H $-1.729400-1.8256861 .703429$

H $-0.886223-5.718388-1.860810$

H $-0.556999-5.027579-0.275615$

H $-4.317688-5.3021311 .443773$

H $-5.051350-4.4584600 .073852$

H $-4.872316-2.2670371 .197962$

H $-4.078802-4.0694043 .546128$

H -5.269176 -2.310266 4.780569

H $-5.324651-1.2442553 .402018$

H $-1.932114-1.1624645 .901837$

H $-3.249840-2.4327426 .153413$

H -3.448482 0.7212523 .697783

H -3.161671 -0.4263002 .424250$

H -1.1680950 .8749294 .267554$
H -2.229601 -3.892495 0.837845

C $4.0154993 .827529-0.940188$

O $2.6924313 .354635-1.263238$

C $1.7451204 .059120-0.432926$

C 2.5418335 .1098910 .339423

C 3.9015834 .4490670 .449150

H 1.3450823 .3304950 .280960

C $0.6185734 .646341-1.290384$

H $2.6170856 .051374-0.217587$

H $2.092805 \quad 5.3343791 .311889$

C 5.0239285 .4111820 .793677

H $3.8425093 .672171 \quad 1.221814$

C $-0.2279973 .603571-2.049451$

O $1.1882635 .536268-2.260281$

H $-0.0355365 .265519-0.665553$

H $0.3774743 .092412-2.807365$

O $-1.248993 \quad 4.317584-2.760668$

C $-0.9147982 .561471-1.144030$

C $-1.9173451 .673634-1.912521$

H $-0.1528851 .918501-0.690181$

O $-1.581373 \quad 3.236470-0.080696$

H -2.723566 $2.297172-2.312538$

H $-1.3943641 .229241-2.767565$

H $5.982710 \quad 4.886503 \quad 0.851806$

H $5.1179086 .204010 \quad 0.044120$

H $4.8394865 .885577 \quad 1.763190$

C $5.0490002 .711827-1.105078$

H $1.8139775 .020854-2.798026$

H $-0.803522 \quad 4.968899-3.329429$

H -2.236332 $3.835201-0.478575$

H $6.0561033 .126682-0.979655$

H $4.9879272 .323020-2.129259$

H $4.2459084 .607851-1.678532$

C $-4.4950281 .686492-0.217403$

O $-3.249631 \quad 1.0484800 .089631$

C $-2.5266830 .550172-1.040449$

C $-3.394290-0.457602-1.807380$

C $-4.7618490 .113842-2.170156$

C $-5.4353170 .713452-0.937082$

H $-4.3143302 .563542-0.849516$

C $-5.110712 \quad 2.166197 \quad 1.102612$

H $-1.675914-0.009144-0.635313$

O $-2.725375-0.889123-2.986299$

H -3.539665 -1.345601 -1.178152

H $-4.6768480 .849206-2.979196$

O $-5.565596-0.955017-2.674849$

H $-5.741487-0.090969-0.256146$

O $-6.6111941 .414363-1.335756$

C $-4.3406193 .331501 \quad 1.677092$

H -6.1526992 .4835880 .982101$

H -5.1057151 .3638181 .849631$

H $-3.309189-1.522585-3.437567$

H $-6.395015-0.569057-3.003303$

H $-7.2412630 .760108-1.681521$

$\begin{array}{lllll}\text { O } & -4.635687 & 4.470376 & 0.986680\end{array}$

$\begin{array}{lllll}0 & -3.567247 & 3.240785 & 2.621336\end{array}$

C $-3.9635895 .652211 \quad 1.433442$

H $-4.260323 \quad 5.8892532 .459509$

H -2.879083 5.5241711 .364691

H -4.2603306 .4784740 .782043$

SCF Energy (B3LYP/6-31G**//MMFF)= -3245.91669764

01_577

MM̄FF Geometry

C -2.524539 -2.721944 2.709751

C -1.919982 -1.569972 2.378830

C $-0.681126-1.0646163 .073818$

$\begin{array}{llll}\text { O } & 0.376459 & -1.097958 & 2.084746\end{array}$

C -0.7956570 .3807643 .603404$

$\begin{array}{llll}\text { C }-2.148819 & 0.770650 & 4.158588\end{array}$

C $-3.0380251 .540422 \quad 3.498471$

C -2.4543360 .2510335 .537167$

C $-2.8787402 .105688 \quad 2.113001$

C $-2.796107 \quad 3.6376812 .128682$

C $-1.995787 \quad 4.2372750 .959776$

C $-2.464795 \quad 3.750632-0.413521$

C $-1.585328 \quad 4.290105-1.554113$

O -0.6175183 .9025121 .169072$ 
O $-1.8742363 .525856-2.735146$

C $-0.1036204 .029490-1.202481$

C $-1.925268 \quad 5.745597-1.892551$

O $0.7622034 .613764-2.172399$

C $0.280493 \quad 4.490222 \quad 0.218910$

C 1.7182164 .0853090 .608789

$\begin{array}{llllll}\text { O } & 0.226898 & 5.921484 & 0.273378\end{array}$

C $1.651388-1.2618352 .524937$

O $2.021915-1.3808833 .681408$

C $2.530328-1.2777091 .333307$

C $3.832820-1.5643321 .475360$

C $4.827749-1.6244220 .402662$

C $4.408026-1.165231-0.968288$

C $1.9993262 .581342 \quad 0.625059$

C $3.3339892 .315804 \quad 1.295246$

C $5.7858312 .642048 \quad 0.954595$

O 3.4034551 .8991432 .449161

C 6.6155591 .7559080 .081536

C $7.541747 \quad 0.9122520 .562280$

C $8.4066780 .041499-0.303219$

C $8.415938-1.4038650 .142306$

C $9.475856-1.9222360 .785473$

C $7.232768-2.268448-0.230994$

C $6.065099-2.0759550 .696319$

N $4.4122282 .631804 \quad 0.484766$

H -2.075802 -3.3258573 .496915$

H $-2.312645-0.9558821 .574860$

H $-0.426872-1.7352113 .905371$

H -0.4704851 .0829022 .827349$

H $-0.0405600 .523355 \quad 4.389366$

H -3.983940 1.7761953 .983161

H $-3.443368 \quad 0.5634775 .888504$

H -2.430486 -0.8431455 .548764$

H $-1.715086 \quad 0.6224016 .254384$

H -2.010696 1.6772511 .606115

H -3.746610 1.7979461 .516941

H $-3.814026 \quad 4.0473672 .120675$

H -2.324015 3.9696433 .063130

H $-2.100728 \quad 5.326811 \quad 1.027967$

H -3.517052 4.013120 -0.576635

H -2.417058 $2.655839-0.457123$

H $-2.8274573 .597000-2.914211$

H $0.0476502 .945617-1.275327$

H -2.975385 $5.831455-2.197272$

H -1.338414 6.100788 -2.746566

H $-1.7706136 .429324-1.055647$

H $0.7872255 .571758-2.008117$

H $2.4370054 .595949-0.045408$

H $1.922987 \quad 4.477763 \quad 1.615570$

H $0.7602446 .221657 \quad 1.028122$

H $2.079090-1.0630400 .371127$

H $4.208828-1.7914462 .472160$

H $5.243878-1.040239-1.659960$

H $3.912253-0.189253-0.921005$

H $3.718905-1.888893-1.413308$

H 1.2284242 .0372521 .181061

H $2.0221852 .168192-0.389183$

H 5.8290852 .3462082 .007466

H $6.1386493 .674807 \quad 0.869177$

H $6.467777 \quad 1.826037-0.993591$

H 7.7112370 .8670191 .635842

H $9.4251740 .449873-0.270640$

H $8.0990110 .091665-1.355204$

H $9.501029-2.9649541 .084355$

H $10.339992-1.3144681 .032682$

H $7.514955-3.328878-0.180376$

H $6.979872-2.099692-1.281170$

H $6.273099-2.383657 \quad 1.722153$

H $4.2333173 .007699-0.442029$

C $-4.808860-2.3188311 .565026$

$\begin{array}{llll}\text { O } & -4.344440 & -1.757757 & 0.326169\end{array}$

C $-5.455221-1.622654-0.580363$

C $-6.705023-1.991396 \quad 0.210724$

C $-6.149159-2.9723751 .224708$

H -5.304932 -2.371993 -1.366491

C $-5.464857-0.219088-1.201268$

H $-7.139774-1.1255100 .722595$

H - $-7.482898-2.425142-0.425268$
C $-7.062563-3.1825562 .420447$

H $-5.980425-3.9356150 .724529$

C $-4.094198 \quad 0.224385-1.751728$

O $-5.8408760 .720696-0.186072$

H -6.236182 $-0.165498-1.978621$

H $-3.4026080 .378652-0.916248$

O $-4.256798 \quad 1.496310-2.389889$

C $-3.478959-0.740845-2.778235$

C $-2.089082-0.307107-3.281171$

H $-3.399371-1.746087-2.356049$

O $-4.352082-0.847190-3.905478$

H - $-1.839267-0.925317-4.151895$

H -2.136356 $0.716802-3.671769$

H $-6.628335-3.8977643 .125569$

H $-7.241108-2.2454042 .958342$

H -8.032708 -3.5752312 .098501$

C -3.777902 $-3.309328 \quad 2.114598$

H $-6.0842291 .550773-0.629177$

H $-4.5144512 .137112-1.706946$

H $-4.4499820 .043371-4.284499$

H $-3.475710-4.0163741 .332042$

H $-4.252618-3.8952982 .910999$

H $-4.954677-1.4869612 .266691$

C $-0.565165-2.782745-2.405558$

O $-0.976542-1.698234-1.564586$

C $-0.980729-0.417475-2.209786$

C $0.427908-0.089528-2.724300$

C $0.988527-1.201820-3.608822$

C $0.863520-2.559626-2.915043$

H $-1.246431-2.846007-3.262789$

C $-0.765793-4.093495-1.636689$

H $-1.2138650 .318022-1.430681$

$\begin{array}{llll}\text { O } & 0.412642 & 1.140765 & -3.439992\end{array}$

H $1.0955910 .047423-1.863586$

H $\quad 0.499740-1.208427-4.590106$

O $2.369702-0.925122-3.853437$

H $1.584963-2.593354-2.091330$

O $1.223104-3.573613-3.848861$

C $0.204762-4.274705-0.494726$

H $-1.773547-4.131574-1.204196$

H $-0.689208-4.954603-2.311020$

H $1.3186991 .307725-3.751345$

H $2.723023-1.662480-4.380142$

H $1.285071-4.413694-3.363678$

O $1.222630-5.099202-0.874330$

\begin{tabular}{lllll}
\hline & 0.083539 & -3.734978 & 0.596357
\end{tabular}

C $2.227340-5.3343250 .116718$

H $1.780774-5.7534901 .023445$

H $2.941397-6.056291-0.287950$

H $2.757480-4.4047520 .340659$

SCF Energy (B3LYP/6-31G**//MMFF) $=-3245.90232808$

01_578

MM̄FF Geometry

C $-0.214556-2.3524132 .044987$

C $0.834137-2.1461822 .854920$

C $1.471417-0.8363403 .249729$

O $2.835250-0.8383612 .736516$

C 0.8323520 .4706802 .736982

C $-0.4925640 .858276 \quad 3.360670$

C -1.5962881 .2030622 .668420$

C $-0.5157810 .931684 \quad 4.866603$

C $-1.797815 \quad 1.2354561 .180353$

C $-1.895502 \quad 2.6620360 .626602$

C $-0.540473 \quad 3.383177 \quad 0.573318$

C -0.7099654 .8040640 .030943$

C $0.6399685 .513665-0.151088$

$\begin{array}{lllll}\text { O } & 0.317446 & 2.629337 & -0.288426\end{array}$

O $0.4185356 .686300-0.949293$

C $1.5825034 .592635-0.953504$

C 1.2145975 .9998481 .184625

O $2.8946665 .146086-1.026967$

C $1.6474863 .146771-0.407448$

C $2.4127602 .243642-1.402935$

$\begin{array}{llllllll}\text { O } & 2.330296 & 3.154152 & 0.847160\end{array}$

C $3.798747-1.4196313 .496754$

O $3.637586-1.9817424 .572265$

C $5.133544-1.3369962 .861874$ 
C $5.436012-0.5363511 .827569$

C $6.753864-0.417817 \quad 1.192927$

C $7.838476-1.3502201 .671055$

C $2.3625800 .744475-1.069588$

C $3.148752-0.072776-2.079478$

C $4.240583-2.286277-2.372785$

O $3.4844990 .366678-3.176795$

C $5.670870-2.054332-1.999429$

C $6.515949-1.330588-2.748509$

C $7.936138-1.023842-2.370921$

C $8.1204470 .435408-2.012438$

C $8.2782341 .365886-2.969194$

C $8.1933360 .805096-0.547006$

C $6.9291330 .494725 \quad 0.212652$

N $3.413054-1.358109-1.623779$

H - $0.726047-1.5176821 .581155$

H $1.310510-3.0247283 .288530$

H $1.516814-0.7950704 .344511$

H $\quad 0.7850610 .437427 \quad 1.644311$

H 1.5339171 .2909912 .946256

H -2.481009 1.4952003 .233934

H -1.4272501 .4060105 .246552$

H -0.463485 -0.0709135 .301985$

H $0.332194 \quad 1.520108 \quad 5.232281$

H -1.0408350 .6637940 .637772$

H -2.7431490 .7253290 .974697$

H -2.300462 2.591667 -0.391132

H -2.611095 $3.246892 \quad 1.217601$

H $-0.109712 \quad 3.4109481 .580300$

H $-1.369313 \quad 5.392413 \quad 0.681124$

H $-1.2121334 .763882-0.946019$

H $-0.2336097 .242794-0.489975$

H $1.2036784 .556104-1.984716$

H $0.525936 \quad 6.7068801 .662750$

H 2.1507286 .5489821 .035525

H 1.3980105 .1896331 .893410

H $2.8094556 .046139-1.385568$

H $1.9846402 .375558-2.405499$

H $3.4639072 .555804-1.453833$

H 2.7649412 .2985070 .983878

H $5.884158-1.9664403 .329981$

H $4.661506 \quad 0.1051391 .412912$

H $7.505543-2.3926091 .607684$

H $8.105478-1.1277112 .709853$

H $8.757191-1.2947121 .084136$

H $2.7762300 .558622-0.074443$

H $1.3287670 .381144-1.084311$

H $4.069538-2.142785-3.444987$

H $3.939873-3.303532-2.108908$

H $6.013034-2.482430-1.061156$

H $6.159171-0.904591-3.683912$

H $8.581613-1.282262-3.220477$

H $8.276425-1.660604-1.545264$

H $8.4348912 .410963-2.723672$

H $8.2516851 .101220-4.021290$

H $8.3753991 .882448-0.435527$

H $9.0745670 .326184-0.111828$

H $6.081467 \quad 1.117428-0.076395$

H $3.172335-1.604019-0.667936$

C $-0.670975-4.049566 \quad 0.206031$

O $-1.617249-3.242816-0.523897$

C $-0.969332-2.738152-1.703939$

C $0.277819-3.596912-1.897197$

C $0.691233-3.847436-0.462020$

H $-0.661328-1.711418-1.471258$

C $-1.920704-2.731618-2.904464$

H $0.048486-4.547948-2.391315$

H $1.045320-3.086843-2.486409$

C $1.635826-5.022980-0.284022$

H $1.184072-2.940880-0.092586$

C $-3.290393-2.057730-2.684201$

O $-2.185790-4.082476-3.310311$

H - $1.412436-2.264811-3.756817$

H -3.945609 -2.724003 -2.110350

O $-3.896819-1.919404-3.978056$

C $-3.261434-0.676973-2.006679$

C $-4.5645620 .136159-2.141329$

H -3.012956 $-0.805057-0.948377$
O $-2.2137770 .103239-2.591991$

H $-4.4318891 .065719-1.574313$

H $-4.6636830 .460824-3.185327$

H $1.887477-5.1615840 .772306$

H $1.191686-5.954486-0.650466$

H $2.567202-4.857867-0.833869$

C $-0.733869-3.7236051 .701652$

H -2.563619 $-4.549433-2.544980$

H $-3.931808-2.805356-4.377826$

H -2.161521 $0.940802-2.101541$

H $-0.181223-4.4909472 .256900$

H $-1.776851-3.7756582 .034362$

H $-1.005225-5.0867040 .071023$

C $-5.870616 \quad 0.0401590 .643678$

O $-5.816870-1.008083-0.331625$

C $-5.854235-0.583616-1.695722$

C $-7.1339020 .222630-1.957134$

C $-7.2553801 .400713-0.998794$

C $-7.1125090 .927134 \quad 0.445442$

H -4.9627900 .6480910 .573983$

C $-5.905449-0.6239702 .031014$

H $-5.940146-1.497004-2.294988$

O $-7.1732120 .703426-3.296616$

H -8.008948 $-0.426714-1.822856$

H $-6.5277582 .187250-1.230742$

O $-8.547708 \quad 1.994649-1.149134$

H -8.0228530 .3830240 .729346$

O $-7.0263612 .085971 \quad 1.274385$

C $-4.618633-1.3351052 .392384$

H -6.0803130 .1354242 .800563$

H $-6.707897-1.3696052 .075514$

H $-7.097714-0.066543-3.885856$

H -8.637729 $2.257390-2.081340$

H $-7.208007 \quad 1.8135822 .189056$

O $-4.587494-1.5214393 .746165$

O $-3.768313-1.708322 \quad 1.597445$

C $-3.439114-2.2059494 .254803$

H -2.526867 -1.660917 3.999079

H $-3.524677-2.2504265 .343842$

H -3.406059 -3.226834 3.863803

SCF Energy (B3LYP/6-31G**//MMFF) $=-3245.90497482$

01_579

MMFF Geometry

C -2.736763 1.4376293 .222933

C -2.1270170 .5971692 .374020$

C $-1.700623-0.8252672 .635411$

$\begin{array}{lllll} & -1.700623 & -0.825267 & 0.754294 & 2.914576\end{array}$

C $-2.384127-1.6023523 .777588$

C $-3.779044-2.1006523 .453915$

C $-4.039563-3.2847942 .863671$

C $-4.919820-1.2301533 .909650$

C $-3.044018-4.2922832 .359023$

C $-3.408747-4.8685800 .982630$

C $-3.438731-3.837390-0.157799$

C $-3.905289-4.508713-1.454381$

C $-3.874194-3.546789-2.649677$

O $-2.121819-3.296157-0.327739$

O $-4.024813-4.345216-3.833585$

C $-2.485884-2.875672-2.702015$

C $-5.065799-2.582535-2.630174$

O $-2.442474-1.883451-3.724871$

C $-2.034616-2.287683-1.345739$

C $-0.584508-1.753006-1.390936$

O $-2.876905-1.169387-1.036315$

C $0.514766-1.7587852 .458502$

O $0.166869-2.7619291 .858221$

C $1.911248-1.4131952 .795270$

C $2.908935-2.0873082 .205863$

C $4.339918-1.8532412 .395898$

C $4.753201-0.7304753 .311395$

C $0.508290-2.791728-1.660462$

C $1.863603-2.120280-1.820850$

C $4.044698-2.412212-2.985841$

O $2.108252-1.000890-1.379151$

C $5.147245-2.874301-2.088128$

C $6.061161-2.043180-1.562993$

C $7.244356-2.495492-0.754371$ 
C $7.309949-1.8453420 .608802$

C $7.948643-0.6768770 .787741$

C $6.712543-2.5933761 .777462$

C $5.211987-2.6367581 .728516$

N $2.766830-2.911654-2.513681$ H -2.9508391 .1058274 .235032$ H -1.8699800 .9623511 .382769$ H -1.852394 -1.373241 1.698562 H -2.394709 -1.012549 4.702601 H -1.756881 -2.462509 4.048098 H -5.081883 -3.5715062 .728924$ H $-4.969060-0.3156753 .314498$ H -5.888682 -1.732289 3.814422 H -4.798843 -0.9596214 .963682$ H -2.034767 -3.880340 2.316855 H $-3.014676-5.1211293 .076897$ H -2.667246 -5.6427460 .745504$ H -4.383481 -5.3685721 .050655$ H -4.127440 -3.029512 0.113438 H -4.909264 -4.934885 -1.333148 H $-3.245901-5.359421-1.678400$ H $-3.929365-3.754828-4.600835$ H -1.780853 -3.656415 -3.009567 H -6.007908 -3.142329 -2.675256 H -5.064290 -1.931546-3.511394 H $-5.097443-1.954445-1.737568$ H -2.892442 -1.090003 -3.387348 H $-0.518027-0.948956-2.134677$ H $-0.359563-1.266479-0.432429$ H -2.400059 -0.580531 -0.429422 H $2.079045-0.6023803 .494588$ H $2.656657-2.8830591 .505759$ H $4.437457-0.9385244 .339462$ H $4.303603 \quad 0.2162012 .991326$ H $5.832240-0.5628693 .333379$ H $0.575745-3.508180-0.835264$ H $\quad 0.292151-3.343255-2.580092$ H $4.005658-1.320292-3.063872$ H $4.195945-2.821307-3.990009$ H $5.230932-3.943340-1.908810$ H $5.989025-0.977695-1.771556$ H $8.150874-2.246769-1.322178$ H $7.260702-3.587062-0.645576$ H $8.025581-0.2181821 .767777$ H $8.404173-0.150788-0.044694$ H $7.064764-2.1806142 .729254$ H $7.089809-3.6244121 .773132$ H $4.818154-3.4173491 .076945$ H $2.490675-3.845925-2.801301$ C $-3.847415 \quad 3.1665201 .606780$ O $-2.935538 \quad 3.1287130 .494358$ C $-3.6391992 .603461-0.649720$ C $-5.1143132 .543388-0.259645$ C -5.0198442 .2640851 .228221$ H $-3.279517 \quad 1.578777-0.789997$ C $-3.3403953 .428403-1.906566$ H $-5.6228673 .498348-0.435311$ H $-5.656221 \quad 1.767792-0.809643$ C -6.2992502 .5553691 .991304$ H $-4.760671 \quad 1.2067021 .356378$ C $-1.8383043 .596827-2.217231$ O $-3.8981534 .736560-1.730159$ H -3.860149 $2.982477-2.763418$ H -1.389238 4.293105 -1.499463 O $-1.7215964 .207845-3.508060$ C $-1.0374482 .283164-2.240003$ C $0.4108892 .410424-2.748882$ H -1.017105 $1.851509-1.233987$ O $-1.7053531 .333253-3.075922$ H $0.8765381 .421197-2.656972$ H $\quad 0.4045132 .613196-3.827774$ H -6.1713012 .3475403 .058349$ H -6.6014473 .6025341 .884686$ H -7.1182771 .9299691 .621340$ C -3.1353032 .8603902 .929921$ H $-3.8815345 .182359-2.593627$ H -2.042286 $5.122017-3.430366$ H $-1.7695781 .719463-3.966350$
H $-3.804722 \quad 3.172800 \quad 3.742116$

H -2.235202 3.4823103 .014192

H -4.1928854 .2084651 .659717$

C $1.7647752 .272402-0.000960$

O $1.1377913 .404754-0.606761$

C $1.2650593 .472813-2.028957$

C $2.7467203 .473007-2.435412$

C $3.5111942 .297936-1.824729$

C $3.2662022 .224930-0.316338$

H $1.2861641 .363097-0.383594$

C $1.4572312 .298397 \quad 1.501670$

H $0.8594254 .448773-2.322849$

O $2.8649193 .448324-3.853983$

H $3.2049644 .409447-2.091526$

H $3.2385261 .357682-2.315684$

O $4.9097892 .466761-2.069181$

H 3.7892143 .0606910 .161472

O $3.843444 \quad 1.030167 \quad 0.203238$

C 2.0541133 .4741952 .246004

H 0.3738252 .3499411 .657023

H $1.829815 \quad 1.3752381 .955075$

H $3.8135973 .421598-4.066695$

H $5.1927383 .292430-1.640494$

H $3.4006640 .274353-0.219423$

O 2.2350823 .1273753 .554894

O 2.2982824 .5679381 .755523

C 2.7806194 .1508114 .392843

H 2.8870073 .7432225 .401624

H 2.1035455 .0095444 .430572

H 3.7680154 .4518124 .030212

SCF Energy (B3LYP/6-31G**//MMFF) $=-3245.92161537$

01 58

MMFF Geometry

C $3.993523-3.531540-0.242669$

C $2.909363-3.611749-1.030027$

C $1.498426-3.454407-0.526301$

O $0.912162-2.397034-1.320235$

C $0.692815-4.752886-0.727666$

C $-0.555225-4.8458060 .133395$

C $-1.822766-4.883036-0.323539$

C $-0.308982-4.954101 \quad 1.619037$

C $-2.316343-4.852288-1.741263$

C $-2.859529-3.493082-2.206225$

C $-4.102984-2.991032-1.450644$

C $-4.684327-1.758901-2.152611$

C $-5.872537-1.156157-1.387711$

O $-3.733284-2.646766-0.110602$

O $-6.1184360 .156076-1.917911$

C $-5.480023-0.9748910 .094312$

C $-7.158542-1.960653-1.611288$

O $-6.604507-0.5724820 .871450$

C $-4.833468-2.2304080 .714753$

C $-4.292097-1.9940042 .148092$

O $-5.818487-3.267090 \quad 0.815934$

C $0.070373-1.528849-0.703428$

O $-0.297844-1.5596640 .459166$

C $-0.332143-0.514614-1.704614$

C $-1.2918590 .365881-1.389535$

C $-1.7993691 .447921-2.232811$

C $-1.1224601 .686083-3.555723$

C $-3.248598-0.8825042 .289318$

C -3.8257850 .4727562 .667816$

C -3.2102832 .8919612 .739331$

O $-4.950490 \quad 0.6188813 .138376$

C -3.8277203 .5130631 .526845$

C $-3.214806 \quad 4.428803 \quad 0.761043$

C $-3.8383115 .050088-0.456517$

C $-3.1244154 .673333-1.736905$

C $-2.2369215 .506159-2.306129$

C $-3.5017613 .366499-2.401956$

C $-2.8248402 .187695-1.763050$

N -2.913248 1.4986992 .459960

H $3.863214-3.3811190 .825042$

H $3.036430-3.770042-2.098517$

H $1.527206-3.1616760 .528349$

H $1.317958-5.621362-0.479377$

H $0.443666-4.853373-1.788993$ 
H -2.620267 -4.973433 0.413675 H -1.216595 -5.2179672 .172872$ H $0.433689-5.7311831 .827335$ H $0.053640-4.0048522 .022715$ H - $-1.530848-5.167364-2.435276$ H -3.106802 $-5.607165-1.840467$ H -2.058639 -2.752436 -2.128906 H $-3.106518-3.585992-3.271528$ H -4.841497 -3.800978 -1.429193 H -4.976207 -2.000215 -3.182379 H $-3.905221-0.990394-2.238259$ H $-6.2881640 .067128-2.871447$ H $-4.759583-0.1483450 .131960$ H -7.420472 -1.974950 -2.676154 H -8.009651 -1.496391 -1.100982 H -7.079154 -2.997723 -1.278978 $\begin{array}{llll}\mathrm{H} & -6.965837 & 0.232656 & 0.462855\end{array}$ H $-5.126538-1.8514982 .845494$ H -3.804056 -2.926767 2.465724 H -5.519712 -3.9117361 .478635$ H $\quad 0.169507-0.519478-2.665314$ H -1.752939 $0.295917-0.406995$ H $-1.2926140 .838620-4.228757$ H $-0.0427591 .815525-3.424678$ H -1.476145 2.582748 -4.069337 H $-2.558880-1.1514863 .099665$ H -2.657292 -0.7924241 .375934$ H -2.2703523 .3774883 .019518$ H -3.9012372 .9599863 .585561$ H -4.8333843 .1898861 .267869$ H -2.2110334 .7557691 .021757$ H -3.821889 $6.139461-0.320939$ H $-4.8996374 .784827-0.540613$ H -1.741588 $5.252626-3.237459$ H -1.980379 $6.456038-1.848810$ H -3.299658 $3.413668-3.476906$ H $-4.5880043 .224119-2.334644$ H $-3.2510441 .913438-0.798611$ H $-2.0334241 .291527 \quad 1.996671$ C $6.231646-2.358796-0.566285$

O $5.578251-1.242467-1.202076$

C $5.700111-0.091180-0.342898$

C $6.781725-0.4427020 .671407$

C $6.515544-1.9245780 .872029$

H 4.7549110 .0074810 .198638

C $5.9451641 .173174-1.168332$

H $7.787708-0.2880540 .264839$

H 6.6926320 .1391561 .593665

C $7.665830-2.6689011 .526523$

H $5.621038-2.0322041 .497351$

C $4.9147781 .424237-2.291163$

O $7.234801 \quad 1.055682-1.786835$

H $6.0060392 .042390-0.503521$

H $5.0295380 .635671-3.045816$

O $5.2815282 .652601-2.932899$

C $3.4309131 .515418-1.876536$

C $3.1360562 .631831-0.851992$

H $2.8458231 .745299-2.776896$

O $2.957441 \quad 0.266110-1.394850$

H 3.7442732 .4695200 .041037

H $3.4520223 .584219-1.290838$

H $7.430888-3.7326511 .632204$

H $8.586267-2.5828610 .939465$

H $7.865673-2.2659582 .524774$

C $5.404548-3.635518-0.751896$

H $7.3836791 .867795-2.301085$

H $4.6890472 .773689-3.694388$

H $3.150380-0.406080-2.070352$

H $5.899193-4.475160-0.250573$

H $5.383536-3.875919-1.822844$

H $7.182015-2.495507-1.099755$

C $1.752843 \quad 1.915317 \quad 1.828261$

O $1.281851 \quad 1.6878570 .493159$

C $1.6372882 .689756-0.468350$

C $1.1349774 .063431-0.007231$

C 1.6298964 .4043851 .390977

C 1.2803633 .2759702 .356832

H 2.8481311 .8746361 .846805
C 1.2162830 .7761602 .712930

H $1.0742302 .454030-1.378030$

O $1.5291835 .087821-0.913169$

H $0.0375884 .058150 \quad 0.007455$

H 2.7062224 .6136001 .398915

O $0.9798605 .594447 \quad 1.842758$

H 0.1959973 .2590812 .523303

O $1.9024603 .532323 \quad 3.614907$

C $2.028589-0.4923812 .566845$

H 1.2661311 .0699863 .767633

H $\quad 0.1786630 .5369222 .457287$

H $1.1794444 .852286-1.789614$

H 1.1711146 .2923131 .192720

H 1.6006364 .4070343 .914462

O $1.842039-1.2738253 .671361$

O $2.724019-0.7739721 .601530$

C $2.585198-2.4964813 .695599$

H $2.416000-2.9776964 .662609$

H $2.235673-3.1654012 .905288$

H $3.655652-2.2965703 .586629$

SCF Energy (B3LYP/6-31G**//MMFF) $=-3245.90387148$

01_580

MMFF Geometry

C $3.3380763 .121029-1.392089$

C $3.1293782 .422721-2.518456$

C $3.4228380 .953381-2.637756$

O $2.2040470 .288237-3.054951$

C $4.4927010 .628244-3.696079$

C $5.9039320 .671922-3.150364$

C $6.580650-0.413727-2.725167$

C $6.5610372 .026353-3.126669$

C $6.114246-1.843880-2.706443$

C $5.058010-2.192042-1.652070$

C $5.346810-1.712238-0.221797$

C $6.607036-2.3507990 .364762$

C $6.819738-1.9505711 .832683$

O $4.204568-2.0701370 .570935$

O $7.812395-2.8257932 .389585$

C $5.512399-2.1957452 .613746$

C $7.376329-0.5272221 .960016$

O $5.617400-1.7023143 .947850$

C $4.258035-1.6047011 .926881$

C $2.984754-2.0954952 .659873$

O $4.317601-0.1785221 .994005$

C $1.371984-0.149532-2.072426$

O $1.4915730 .027417-0.870656$

C $0.294820-0.932989-2.712817$

C $-0.290172-1.908344-2.002927$

C $-1.349836-2.806457-2.460861$

C $-2.002524-2.508584-3.783393$

C $1.669961-1.7346321 .956405$

C $0.452046-2.2535372 .703279$

C $-2.042255-2.2475912 .569388$

O $0.525177-2.9255973 .728208$

C $-2.484513-3.4963041 .874522$

C $-3.549677-3.5564441 .060743$

C $-4.001007-4.8054110 .358185$

C $-4.023711-4.650318-1.147679$

C $-5.169546-4.378389-1.794500$

C $-2.740978-4.895492-1.911851$

C -1.700023 -3.838996-1.666655

N $-0.728981-1.8716762 .080732$

H $3.7787252 .619619-0.534573$

H $2.6942912 .915326-3.384381$

H $3.7374450 .541988-1.670819$

H $4.4064461 .302353-4.558739$

H $4.290434-0.365677-4.115263$

H $7.593553-0.271114-2.350651$

H $7.6173581 .969412-2.841949$

H $6.5167882 .488421-4.118304$

H $6.0658362 .686632-2.410415$

H $5.739823-2.122172-3.699082$

H $6.994242-2.480776-2.549991$

H $4.084781-1.796119-1.965380$

H $4.915444-3.281330-1.645006$

H $5.432620-0.620518-0.227133$

H $7.495370-2.096129-0.224865$ 
H $\quad 6.517054-3.4450010 .313309$ H $8.623335-2.7349451 .860181$ H $5.380071-3.2838552 .695874$ H $8.339936-0.4454851 .442782$ H $7.576402-0.273408 \quad 3.006759$ H 6.7132710 .2324961 .540913 H $6.406535-2.1085694 .345559$ H $3.022778-3.1890412 .751060$ H $2.961557-1.6842473 .677145$ H 3.4256510 .1831901 .877727 H $0.045734-0.715223-3.744461$ H $0.057200-2.085121-0.986174$ H $-1.306853-2.705344-4.606199$ H $-2.314511-1.460213-3.834452$ H -2.902494 -3.101829 -3.962581 H $1.565542-0.6484541 .868749$ H $1.653426-2.1690340 .950849$ H -2.711714 -1.406946 2.369365 H -2.003626 -2.414605 3.650380 H $-1.900560-4.3955882 .055426$ H -4.137683 -2.6589420 .889801$ H $-5.006303-5.049430 \quad 0.725609$ H $-3.373956-5.6650240 .625582$ H $-5.202922-4.291677-2.875414$ H $-6.099389-4.228016-1.255978$ H $-2.939570-5.007710-2.983443$ H -2.326941 -5.865409-1.606895 H $-1.158963-3.974129-0.730006$ H $-0.682487-1.360866 \quad 1.203825$

C $1.9102624 .832672-0.180482$ $\begin{array}{lllll}\text { O } & 0.716847 & 4.088957 & -0.496705\end{array}$ C $0.145858 \quad 3.593046 \quad 0.729768$ C $0.875167 \quad 4.317848 \quad 1.856269$ C 2.2617914 .4748841 .262648 H $\quad 0.3829472 .522443 \quad 0.780213$ C -1.3770093 .7809340 .746051$ H $0.430823 \quad 5.3000112 .056833$ H $0.868526 \quad 3.7467832 .789722$

C 3.1141685 .5149451 .967819 H 2.7680593 .5037061 .315725 C $-2.1036892 .908715-0.303960$ $\begin{array}{llll}O & -1.661689 & 5.164618 & 0.551495\end{array}$ H $-1.745013 \quad 3.5074201 .742080$ H -1.803392 $1.867682-0.140100$ O $-1.7023743 .262586-1.626639$ C $-3.641148 \quad 3.000671-0.221738$ C $-4.3811812 .085325-1.215071$ H -3.9614192 .7686940 .799037$ O $-4.068413 \quad 4.339767-0.483098$ H -5.449602 2.328786 -1.159832 H -4.089973 2.345487 -2.240537 H 4.0968815 .5994241 .493404 H 2.6425376 .5031561 .946210 H 3.2676385 .2393873 .016303 C $2.9905154 .573935-1.234389$ H -2.619166 5.2879340 .664692 H $-0.7409243 .133730-1.689784$ H $-3.7360874 .586297-1.363619$ H $3.9002815 .130245-0.982151$ H $2.634644 \quad 4.967251-2.195317$ H $1.6311625 .893482-0.237645$ C $-5.757068 \quad 0.2862190 .815691$ $\begin{array}{lllll}\text { O } & -4.390479 & 0.211647 & 0.391337\end{array}$ C $-4.166138 \quad 0.578515-0.973208$ C $-4.981388-0.332189-1.902423$ C $-6.458294-0.372653-1.517024$ C -6.624459 -0.656780 -0.024589 H -6.1200281 .3166320 .715003$ C $-5.804598-0.0850882 .301460$ H -3.109208 $0.369932-1.177421$ O $-4.869096 \quad 0.086809-3.258534$ H -4.580677 -1.351766 -1.846900 H $-6.9696810 .556290-1.795664$ O $-7.106026-1.417994-2.245646$ H $-6.361610-1.7015490 .182239$ \begin{tabular}{llll}
\hline & -7.993466 & -0.481446 & 0.333954
\end{tabular} C $-5.248534 \quad 1.035443 \quad 3.148108$ H $-6.831220-0.2608152 .642609$
H $-5.239341-0.9999802 .511259$

H $-3.9223560 .139128-3.472379$

H $-6.956354-1.246613-3.191461$

H $-8.519399-1.071548-0.232725$

$\begin{array}{lllll}\text { O } & -3.897666 & 0.903287 & 3.273527\end{array}$

O $-5.9331321 .934346 \quad 3.619759$

C $-3.244751 \quad 1.9235814 .034845$

H -3.457826 $2.911213 \quad 3.614779$

H -3.5624901 .8717035 .080296$

H -2.166932 1.7473543 .984296

SCF Energy (B3LYP/6-31G**//MMFF) = -3245.91677762

01_581

MMFF Geometry

C -1.520393 -0.5704782 .624959$

C $-2.222247 \quad 0.297144 \quad 3.371243$

C $-2.0865151 .802423 \quad 3.364596$

$\begin{array}{lllll}\text { O } & -0.919585 & 2.172511 & 2.592032\end{array}$

C -3.3208282 .4762892 .732518$

$\begin{array}{llll}\text { C }-4.632423 & 2.235829 & 3.452473\end{array}$

C -5.6137421 .4391752 .982285$

C -4.8161093 .0000924 .735783$

C -5.5513760 .5962691 .738849$

C $-6.064021 \quad 1.351356 \quad 0.506758$

C $-5.6033800 .744302-0.827801$

C $-6.123087-0.675532-1.050685$

C $-5.590091-1.270820-2.363431$

O $-4.1726320 .753496-0.835787$

O $-5.857299-2.681751-2.347444$

C $-4.056743-1.102663-2.401037$

C $-6.317234-0.704799-3.588448$

O $-3.531329-1.510056-3.662563$

C $-3.5734090 .329078-2.065070$

C $-2.0353800 .323981-1.864969$

O $-3.9140891 .201716-3.140868$

C -0.3492773 .3822152 .818276$

$\begin{array}{lllll}\text { O } & -0.714054 & 4.225229 & 3.625131\end{array}$

C $0.781282 \quad 3.612614 \quad 1.890893$

C 1.4585562 .6129691 .304794

C $2.5457972 .780006 \quad 0.344029$

C $2.5559181 .747577-0.751515$

C $-1.4427731 .605634-1.262201$

C $-1.1028842 .691318-2.268592$

C $-0.1696215 .009662-2.354037$

O $-1.2192462 .549336-3.481985$

C $1.3039854 .886409-2.586421$

C $2.2205955 .701589-2.041867$

C $3.6945175 .597433-2.314841$

C $4.5218005 .435658-1.058176$

C $5.2153796 .467554-0.548598$

C $4.6206934 .053533-0.448137$

C $3.470172 \quad 3.752817 \quad 0.472648$

N $-0.6364463 .837550-1.638012$

H $-0.756700-0.2094591 .941961$

H -2.975162 $-0.100728 \quad 4.049217$

H -1.9426202 .1325334 .400700$

H -3.385306 2.1808641 .678120

H -3.157657 3.5611142 .679672

H $-6.540191 \quad 1.3588743 .547653$

H $-5.8019952 .830903 \quad 5.181730$

H -4.0650542 .6983985 .472402$

H $-4.715476 \quad 4.0753794 .555681$

H $-4.5314450 .231992 \quad 1.582914$

H $-6.158685-0.3025751 .901468$

H -7.1592821 .4042380 .534949$

H -5.6949552 .3850760 .542219$

H $-5.9642541 .407301-1.623109$

H $-7.219663-0.696091-1.033686$

H $-5.796663-1.327582-0.229648$

H $-6.817289-2.803579-2.250836$

H $-3.639050-1.791762-1.654166$

H $-7.392400-0.910838-3.524441$

H $-5.974416-1.188864-4.509587$

H $-6.1942060 .374524-3.699758$

H -3.832197 -2.421256-3.820514

H $-1.792706-0.477854-1.156282$

H $-1.5316590 .087561-2.809672$

H $-3.3433911 .984674-3.100869$ 
H 1.0375684 .6536041 .719422 H 1.1866101 .5799891 .509949 H $1.9032542 .055833-1.573080$ H $2.1982920 .777676-0.389468$ H $3.5556101 .570058-1.155094$ H -2.096775 $2.023993-0.490576$ H $-0.4992161 .334976-0.772900$ H $-0.4203905 .884570-1.746105$ H $-0.6921265 .089368-3.312570$ H $1.6291894 .094887-3.257696$ H $1.8950246 .506090-1.386387$ H $3.9964366 .508703-2.847790$ H $3.9159174 .770018-3.000722$ H 5.8353216 .3500470 .334185 H $5.1742927 .453851-0.998881$ H 5.5441663 .9676500 .139593 H $4.7252453 .320386-1.253098$ H $3.4066104 .424272 \quad 1.329051$ H $-0.5189783 .829147-0.629666$ C - $-1.984879-2.619607 \quad 1.258444$ O $-0.744444-2.6467920 .517375$ C $-0.626393-3.919444-0.146183$ C $-2.005249-4.563726-0.065315$ C -2.493577-4.060535 1.276411 H $0.077160-4.5185260 .443536$ C $-0.101700-3.754172-1.575796$ H -2.666233 -4.211445 -0.865860 H $-1.957217-5.655376-0.124351$ C $-3.998278-4.1785241 .453736$ H -1.990242 -4.628041 2.070812 C $1.273028-3.064308-1.689365$ O $-1.044900-2.970071-2.315807$ H $-0.071931-4.735294-2.065165$ H $1.180018-2.012789-1.395367$ O $1.623137-3.052517-3.081054$ C $2.409700-3.734717-0.892652$ C $3.824625-3.249262-1.268986$ H $2.237773-3.5867810 .179010$ O $2.365216-5.144886-1.133020$ H $4.546045-3.832150-0.683525$ H $4.034227-3.520961-2.311250$ H -4.309654 -3.788442 2.427693 H -4.538205 -3.621637 0.680266 H $-4.311394-5.2258571 .393418$ C -1.739600 -2.057483 2.660684 H $-0.697261-2.870580-3.218691$ H $1.747818-3.976088-3.359999$ H $3.038375-5.560476-0.567425$ H $-0.848148-2.5202803 .097756$ H -2.588232 -2.295012 3.312313 H $-2.677657-1.9762810 .700845$ C $4.654033-1.7947741 .281289$ O $3.745939-1.3317560 .274508$ C $4.057971-1.742443-1.058056$

C $5.461495-1.254905-1.442815$ C $6.513894-1.678011-0.422126$ C $6.076824-1.2988240 .992726$ H $4.641301-2.8907791 .311703$

C $4.134714-1.2729982 .625164$ H $3.355248-1.216744-1.714925$ O $5.835603-1.737755-2.728604$ H $5.460756-0.158601-1.500109$ H $6.731271-2.750513-0.491390$ O $7.740405-1.001183-0.708812$ H $6.139308-0.2086321 .105603$ O $6.999632-1.8850151 .908459$ C $2.811144-1.9099972 .972125$ H $4.827467-1.4708293 .449640$ H $3.977395-0.1881862 .584860$ H $5.159433-1.439293-3.360640$ H $7.987947-1.226536-1.622095$ H $6.869156-1.4630472 .773864$ O $3.002332-3.203813 \quad 3.357638$ O $1.737668-1.3274022 .898433$ C $1.815497-3.9325283 .684327$ H $1.186288-4.0410992 .796112$ H $2.111066-4.9272384 .027923$ H $1.268333-3.4318864 .488842$
SCF Energy (B3LYP/6-31G**//MMFF) $=-3245.90702721$

01582

MM̄FF Geometry

C $0.8572552 .876582-1.379431$

C $2.0920433 .393889-1.275481$

C $3.0535533 .232164-0.123383$

O 2.3788242 .8655371 .102930

C $4.0981032 .135620-0.390475$

C $5.1005782 .465786-1.473566$

C $5.0866611 .939972-2.713729$

C $6.1970493 .415865-1.067430$

C $4.0716721 .000674-3.301883$

C $4.640905-0.395986-3.575923$

C $4.944713-1.206263-2.305073$

C $5.547582-2.562039-2.682811$

C $5.779041-3.451236-1.454309$

O $3.721069-1.396243-1.583501$

O $6.032219-4.786759-1.916695$

C $4.479782-3.498772-0.626124$

C $7.021007-3.023188-0.663741$

O $4.686946-4.1997920 .598964$

C $3.869990-2.103949-0.343602$

C $2.466763-2.2791030 .286880$

O $4.717576-1.4043000 .568139$

C 1.8495723 .8720881 .845712

O 1.7593345 .0482241 .519005

C 1.3704953 .3932173 .162277

C 1.6428712 .1772503 .661549

C 1.2068141 .6604854 .962642

C 0.4410772 .5979275 .860864

C $1.699855-0.9761110 .550258$

C $0.312714-1.2477061 .110612$

C -1.674578 -0.072058 2.043411

O $-0.153189-2.378221 \quad 1.229276$

C $-1.556313-0.0132303 .531962$

C -1.437433-1.096733 4.313954

C $-1.291197-1.0471285 .808397$

C $0.090895-1.4681556 .261565$

C $0.379028-2.7610396 .487311$

C $1.113818-0.3818496 .521525$

C $1.4853990 .376266 \quad 5.274560$

N $-0.349319-0.0762281 .452674$

H $0.4630212 .240494-0.592709$

H $2.4625364 .009857-2.093285$

H 3.5671084 .1878740 .041438

H $3.5864741 .188806-0.591932$

H $4.6490751 .934140 \quad 0.539200$

H $5.8776362 .230746-3.403933$

H $6.9264313 .576383-1.868491$

H $5.7814594 .392586-0.801360$

H $6.7406233 .021044-0.202753$

H $3.1720850 .927386-2.686844$

H $3.7406951 .432630-4.254855$

H $3.900524-0.940446-4.175660$

H $5.547914-0.310120-4.187682$

H $5.646815-0.641649-1.681869$

H $6.482264-2.429983-3.242275$

H $4.863466-3.087274-3.364407$

H $6.807824-4.759022-2.502885$

H $3.747329-4.085712-1.198376$

H $7.915896-3.084334-1.294760$

H $7.203909-3.6973910 .180260$

H $6.956675-2.001827-0.282759$

H $5.054248-5.0720530 .374906$

H $1.855128-2.899241-0.382309$

H $2.555754-2.8179651 .239063$

H $4.215310-0.6867920 .983180$

H $\quad 0.796057 \quad 4.124218 \quad 3.722413$

H $2.236247 \quad 1.4846203 .067775$

H -0.4756002 .9414665 .368683$

H 1.0530413 .4712676 .112967

H $0.1317092 .148366 \quad 6.805915$

H $2.235633-0.3541651 .274023$

H $1.583726-0.407284-0.377697$

H $-2.231647-0.9574761 .722501$

H -2.1950930 .8171111 .678295$

H -1.5727230 .9746153 .983559$ 
H -1.429322 -2.082686 3.854456 H -2.047043 -1.7119786 .246388$ H $-1.522594-0.0489246 .200129$ H $1.358707-3.0708826 .835499$ H $-0.359933-3.5388226 .324730$ H $2.037903-0.8158606 .925907$ H $\quad 0.736642 \quad 0.2679337 .315633$ H $2.039166-0.2251554 .552123$ H $\quad 0.1400510 .8105921 .377083$ C -1.410199 $3.717978-2.224437$ O $-2.3181962 .680036-1.806466$ C $-3.2611633 .253056-0.879052$ C $-2.9428984 .747988-0.792210$ C -1.465865 $4.778558-1.128319$ H -3.0445302 .7995050 .094559$ C $-4.7084962 .944578-1.290217$ H -3.505675 $5.336161-1.524425$ H -3.1659025 .1573300 .198283$ C $-0.9604016 .143364-1.559400$ H $-0.9157124 .454846-0.236158$ C $-5.0658751 .450187-1.435688$ O $-4.9835953 .539875-2.566380$ H $-5.3979283 .410914-0.574565$ H $-4.6383071 .046381-2.361364$ O $-6.4916921 .378714-1.588852$ C $-4.6598840 .555378-0.254321$ C -5.359649 -0.814123-0.189195 H $-3.5751630 .412997-0.278832$ $\begin{array}{lllll}O & -4.968276 & 1.236210 & 0.967851\end{array}$ H -4.914909 -1.3609160 .651681$ H $-6.406153-0.6598920 .105905$ H $0.1064036 .105567-1.801127$ H -1.493389 $6.508210-2.443863$ H $-1.0994186 .875718-0.757579$ C $-0.0338913 .137296-2.564131$ H $-5.1768584 .480861-2.420105$ H $-6.7272241 .923909-2.359198$ H $-4.6607390 .678083 \quad 1.702342$ H $0.4673963 .827056-3.254790$ H $-0.1674142 .191080-3.099416$ H -1.836953 4.119266 -3.154303 C $-3.136748-2.689088-1.151804$ O $-3.987375-1.874553-1.966062$ C $-5.309352-1.674167-1.466654$ C $-6.011041-3.030648-1.313320$ C $-5.217011-3.963954-0.405195$ C $-3.768053-4.065890-0.876212$ H -2.928132 -2.167519-0.211291 C -1.812820 -2.865781-1.913517 H -5.849055 -1.124864 -2.246528 O $-7.323653-2.871148-0.785561$ H -6.114066 -3.504754 -2.298091 H $-5.269674-3.6504240 .643682$ O $-5.796748-5.270699-0.456708$ H $-3.740463-4.687278-1.781043$ O $-3.026858-4.750348 \quad 0.133549$ C -1.114557-1.561099-2.226874 H -1.111811 -3.480473 -1.340027 H -2.019491 -3.357730 -2.872569 H -7.808086 -2.275806 -1.382763 H $-6.727311-5.184249-0.187034$ H -2.164357 -4.994069 -0.241291 O $-0.128090-1.809931-3.139322$ O $-1.386743-0.473377-1.739759$ C $0.604842-0.668039-3.586620$ H $1.023096-0.123395-2.736924$ H $1.424410-1.016971-4.219163$ $\mathrm{H}-0.044837-0.019851-4.181373$ SCF Energy (B3LYP/6-31G**//MMFF) $=-3245.90186350$

01583

MM̄MF Geometry

C $3.0716003 .172736-1.886585$

C $2.5174573 .220630-0.665605$

C $1.0583003 .513689-0.435354$

O 0.4880402 .3811130 .266462

C 0.8611604 .7621110 .449759

C $0.0832025 .847040-0.270447$
C $-1.233766 \quad 6.083295-0.109810$

C $0.8911166 .680401-1.231766$

C -2.1885375 .3599280 .798520$

C -2.8878214 .2118740 .070999$

C -3.9850403 .5178180 .892535$

C -3.4688842 .9471082 .216336$

C -4.5453302 .1371462 .954459$

O -4.4942432 .4599170 .067092$

O $-3.9079231 .411854 \quad 4.016820$

C $-5.166287 \quad 1.118112 \quad 1.979122$

C -5.5626803 .0521503 .644843$

O $-6.2672190 .441413 \quad 2.581797$

C -5.5976751 .7388920 .629783$

C $-5.9854060 .609821-0.353495$

O -6.7281732 .5854470 .842086$

C $-0.1385111 .432177-0.480293$

O $-0.2744691 .419197-1.693530$

C -0.6548560 .3990450 .444820$

C $-1.540423-0.497750-0.013814$

C $-2.141265-1.5986970 .742466$

C $-1.659787-1.8206462 .150029$

C $-6.2296661 .070706-1.796302$

C $-6.605170-0.116900-2.661206$

C $-5.629601-2.181101-3.666873$

O $-7.772988-0.355534-2.959466$

C $-5.674966-3.229686-2.599577$

C $-4.676840-4.092355-2.355780$

C $-4.695750-5.082955-1.227305$

C $-3.659322-4.774698-0.166734$

C $-2.637608-5.6132360 .072871$

C $-3.850957-3.5274400 .672890$

C $-3.079982-2.3520650 .132446$

N $-5.509500-0.887977-3.017262$

H $2.4292453 .356376-2.746572$

H 3.1190273 .0353130 .219238

H $0.5358723 .648068-1.391250$

H 1.8244105 .1890840 .760123

H $\quad 0.3631844 .485114 \quad 1.385043$

H -1.690190 $6.878781-0.697192$

H $\quad 0.2898737 .456901-1.716553$

H $1.7104787 .179584-0.704255$

H $1.3166946 .052644-2.020901$

H -1.670666 4.9979171 .690499

H -2.9335006 .0811991 .156274$

H -3.338064 $4.580695-0.860995$

H $-2.1500503 .466259-0.247792$

H $-4.7860094 .244507 \quad 1.072713$

H -3.0921383 .7387102 .874094$

H -2.611143 2.2913332 .015082

H -3.2250870 .8401913 .627308$

H -4.4065330 .3512801 .777714$

H $-5.0624543 .687478 \quad 4.385858$

H -6.3043072 .4700184 .203118$

H -6.0914993 .7088812 .950928$

H -5.9509650 .0613213 .419332$

H $-5.180965-0.135665-0.371265$

H -6.8874990 .0977640 .005630$

H -7.2309252 .6594660 .016205$

H $-0.2988870 .408442 \quad 1.468345$

H - $1.860818-0.421749-1.052572$

H $-1.958708-0.9853442 .791857$

H -0.568532 -1.903875 2.174629

H -2.046128 -2.734074 2.606077

H $-7.0439961 .799687-1.856316$

H $-5.3374151 .548562-2.216726$

H $-4.764738-2.298408-4.327147$

H $-6.541860-2.216607-4.270211$

H $-6.564761-3.254439-1.974307$

H $-3.782588-4.065349-2.974212$

H $-4.531008-6.080936-1.652958$

H $-5.684231-5.121496-0.752218$

H -1.912561 -5.4041800 .852086$

H -2.502054 -6.522183 -0.503336

H $-3.593529-3.7504641 .712308$

H $-4.915650-3.2626610 .697517$

H -3.356256 -2.080194 -0.885880

H $-4.594250-0.632389-2.658032$

C $5.4836412 .652926-1.082918$ 
O $\quad 5.192231 \quad 1.402910-0.425066$ C $6.4340220 .777356-0.047135$ C $7.540017 \quad 1.775323-0.377136$ C $6.9365192 .531641-1.541857$ H $6.554234-0.099965-0.692438$ C $6.404843 \quad 0.353033 \quad 1.425577$ H 7.7370342 .4560420 .459333 H $8.482103 \quad 1.278359-0.628991$

C $7.6201653 .860349-1.815402$ H $6.9962871 .901373-2.439450$ C $5.292826-0.6538101 .785159$ $\begin{array}{lllll}\text { O } & 6.212489 & 1.528071 & 2.224189\end{array}$ H $7.384807-0.0538231 .704442$ H $4.310871-0.1698981 .724452$ O $5.479625-1.0300073 .156163$ C $5.292293-1.9395510 .935537$ C $4.414875-3.0799901 .486538$ H $4.974281-1.700607-0.084552$ O $6.626639-2.4483350 .834850$ H $4.533048-3.9405380 .816210$ H $4.824712-3.4226832 .445236$ H $7.1565464 .373228-2.663638$ H $7.5644274 .526354-0.947765$ H $8.6778353 .706397-2.053431$

C $4.5073092 .884645-2.239262$ H 6.4141401 .2929783 .145310 H $5.253552-0.2598233 .704334$ H $6.941570-2.6281241 .737424$ H $4.5063452 .001533-2.889878$ H $4.8596483 .729037-2.843375$ H $5.3912773 .449132-0.332791$ C $2.216864-3.105466-0.621796$ $\begin{array}{lllll}\text { O } & 2.361028 & -2.175511 & 0.458628\end{array}$ C $2.924281-2.7286411 .651215$ C $2.060091-3.8953682 .154573$ C $1.802905-4.9320251 .063697$ C $1.294833-4.257236-0.209968$ H $3.201199-3.497132-0.905403$

C $1.651177-2.340204-1.821101$ H $2.864590-1.9405402 .411390$

O $2.663932-4.5354293 .273905$

H $1.092095-3.5092852 .494863$ H $2.698938-5.529594 \quad 0.858444$ $\begin{array}{lllll}\text { O } & 0.805027 & -5.850110 & 1.514697\end{array}$ H $\quad 0.274697-3.886866-0.048055$ O $1.241539-5.218942-1.260705$ C $2.651446-1.330223-2.327839$ H $1.385357-3.005835-2.649856$ H $\quad 0.742945-1.793222-1.546132$ H $2.799302-3.8586503 .959034$ H $1.126962-6.2423592 .344425$ H $0.666525-5.943802-0.960895$ O $3.587539-1.947854-3.102984$ O $2.614830-0.139199-2.049339$

C $4.612396-1.096130-3.623692$

H $5.180458-0.643913-2.805643$

H $5.290419-1.707888-4.224444$

H $4.174980-0.324283-4.264154$

SCF Energy (B3LYP/6-31G**//MMFF) $=-3245.90303343$

\section{4}

MMFF Geometry

C $-1.4296693 .107651-1.567413$

C $-2.2116393 .075738-0.478844$

C $-3.4908913 .852600-0.325482$

O $-4.5534272 .903503-0.050995$

C $-3.441573 \quad 4.809218 \quad 0.881890$

C $-2.240613 \quad 5.732528 \quad 0.925878$

C -1.1772115 .5722051 .741287$

C $-2.2981646 .897893-0.026460$

C $-0.935637 \quad 4.460696 \quad 2.728010$

C 0.2818993 .6136102 .328059

C $0.317825 \quad 2.262456 \quad 3.058762$

C 1.5799211 .4811472 .684981

C $1.585373 \quad 0.0716903 .297231$

O $-\begin{array}{llll}0.845175 & 1.526939 & 2.662802\end{array}$

O $2.625539-0.6836242 .661465$

C $0.249742-0.6243912 .956926$
C $\quad \begin{array}{lll}1.928019 & 0.098572 & 4.791308\end{array}$

O $0.148808-1.8828573 .618190$

C $-0.986314 \quad 0.240216 \quad 3.278480$

C -2.307898 -0.3768462 .756597$

$\begin{array}{lllll}\text { O } & -1.114572 & 0.366086 & 4.699966\end{array}$

C -4.991417 $2.129070-1.079860$

O $-4.6958282 .231856-2.259349$

C $-5.8430301 .068775-0.496728$

C $-6.034318-0.066525-1.184710$

C $-6.785458-1.238654-0.731077$

C $-7.408036-1.1884830 .639403$

C $-2.393970-0.5687561 .237792$

C $-2.148990-1.9929350 .768780$

C $-2.122088-3.353605-1.322005$

O $-1.913764-2.9265851 .529691$

C $-3.480397-3.776197-1.784746$

C $-4.213338-4.719296-1.174241$

C $-5.560304-5.183271-1.652589$

C $-6.691103-4.700496-0.771407$

C $-6.933966-5.2724120 .420117$

C $-7.588519-3.607421-1.306459$

C $-6.863582-2.310444-1.547026$

N $-2.240144-2.093121-0.614505$

H $-1.725330 \quad 3.716370-2.415511$

H -1.9273932 .4480460 .360045$

H -3.739778 $4.418789-1.231877$

H $-3.534696 \quad 4.229276 \quad 1.807088$

H -4.3568805 .4180460 .880525$

H $-0.3750586 .307391 \quad 1.690030$

H $-1.420773 \quad 7.5479520 .057638$

H -2.347712 $6.544312-1.061300$

H -3.1832557 .5098850 .175742$

H $-0.7592214 .902047 \quad 3.716361$

H -1.8161413 .8245092 .837584$

H $0.251606 \quad 3.4265091 .249385$

H 1.2004154 .1774632 .533063

H 0.2799602 .4468324 .138682

H 2.4810202 .0329392 .980232

H 1.6368511 .3810581 .591427

H $3.463539-0.2064972 .787412$

H $\quad 0.264960-0.8382871 .881089$

H 2.9251320 .5278514 .946989

H $1.971223-0.9150425 .204900$

H 1.2231120 .6843195 .384448

H $0.933954-2.4020333 .373339$

H $-2.532442-1.3030503 .299053$

H $-3.1155600 .318181 \quad 3.029361$

H -2.0353940 .5953874 .908103$

H -6.2402101 .2294110 .498905$

H -5.579656 $-0.158290-2.170546$

H $-8.166262-0.3994270 .688242$

H $-6.645497-0.992323 \quad 1.401814$

H $-7.896734-2.1220940 .928056$

H $-3.411520-0.3112980 .918861$

H -1.7045690 .0969520 .709538$

H $-1.656436-4.106367-0.677549$

H -1.470151 -3.194217-2.185829

H $-3.867225-3.292569-2.678318$

H $-3.818671-5.200431-0.281900$

H $-5.557239-6.280901-1.676742$

H $-5.734558-4.875958-2.691359$

H $-7.758878-4.9488061 .046063$

H $-6.310454-6.0770950 .795829$

H $-8.456300-3.452663-0.655304$

H -8.017426 $-3.941326-2.260592$

H $-6.365906-2.263512-2.516317$

H -2.467737 -1.264635 -1.156974

C $1.1100243 .127495-1.719164$

O $2.2145252 .245684-1.410720$

C $3.3193522 .557023-2.276236$

C $2.9630413 .870122-2.960146$

C $1.4526373 .772021-3.063338$

H $\quad 3.3633631 .765512-3.033065$

C $4.6293132 .606344-1.482045$

H $3.2450924 .734910-2.347853$

H $3.4538313 .975953-3.932584$

C $0.8051495 .123428-3.317411$

H $1.2023333 .089026-3.885815$ 
C $5.0036361 .298173-0.754646$

O $4.5355843 .639417-0.491205$

H $5.4467952 .905521-2.148611$

H 4.3021291 .1230960 .069036

O $6.2825301 .500568-0.137417$

C $5.0668300 .046032-1.651857$

C $5.750457-1.177507-1.009157$

H $4.051154-0.227360-1.956658$

O $5.7776320 .348221-2.856397$

H $5.749604-1.984777-1.751937$

H $6.812502-0.955568-0.843903$

H $-0.2702515 .026151-3.482914$

H $0.9583375 .807118-2.475649$

H $1.2319375 .590876-4.211416$

C $-0.1747882 .297803-1.688330$

H $3.777993 \quad 3.4279510 .081397$

H $6.9254471 .700062-0.838728$

H $6.6950780 .562871-2.617239$

H $-0.1128731 .591935-0.850486$

H $-0.2405211 .671241-2.585411$

H $1.0933543 .883026-0.923850$

C $3.321362-2.986542-0.626523$

O $3.698419-1.8595180 .171317$

C $5.105987-1.6551730 .305093$

C $5.762362-2.8979150 .922088$

C $5.406144-4.1717770 .158125$

C $3.893575-4.279809-0.037586$

H $3.683950-2.848800-1.652558$

C $1.791138-3.025267-0.660933$

H $5.230706-0.8436691 .031880$

O $7.175519-2.7343930 .970216$

H $5.415917-2.9990651 .958972$

H $5.928185-4.210644-0.804662$

O $5.866927-5.307542 \quad 0.894168$

H $3.412580-4.5021860 .923506$

O $3.611215-5.363256-0.920055$

C $1.256651-1.843949-1.430329$

H $1.407801-3.941661-1.123592$

H $1.370905-2.9754870 .351162$

H $7.550690-3.5504821 .343307$

H $5.421880-5.3064281 .758858$

H $4.011929-6.162242-0.536649$

O $1.288870-2.104126-2.767879$

O $0.878943-0.806355-0.903368$

C $0.827739-1.043982-3.610196$

H $1.456492-0.157871-3.481615$

H $0.901180-1.377998-4.648419$

H $-0.218769-0.813143-3.389271$

SCF Energy (B3LYP/6-31G**//MMFF) $=-3245.89788518$

01585

MM̄FF Geometry

C $1.295814 \quad 4.992743-2.820514$

C $0.8373693 .768303-3.120840$

C $1.7168842 .566966-3.352557$

O $1.5082161 .657649-2.247395$

C $1.2959801 .849883-4.648201$

C $2.075990 \quad 0.578866-4.924198$

C $1.533211-0.654315-4.966128$

C $3.5498240 .758574-5.181036$

C $0.091041-1.036377-4.775892$

C $-0.304734-1.359119-3.326531$

C $0.363598-2.621550-2.758296$

C $-0.229373-2.977997-1.393996$

C $0.470096-4.192919-0.764101$

O $1.763585-2.375256-2.613920$

O $0.089335-4.249520 \quad 0.619756$

C $2.000205-3.980023-0.801442$

C $-0.001136-5.508025-1.396845$

O $2.661814-5.171003-0.381032$

C $2.524184-3.511227-2.176389$

C $4.010783-3.075954-2.159976$

O $2.414901-4.592329-3.110302$

C $2.2480341 .860568-1.123927$

O $3.1281592 .689285-0.956643$

C $1.7518550 .923162-0.094433$

C 1.9584001 .2036021 .200858

C 1.4763850 .4183872 .337800
C $0.853148-0.9183852 .040924$

C $4.352358-1.935479-1.194353$

C $4.864536-2.4028450 .158290$

C $5.505034-1.5235412 .400976$

O $5.164711-3.5689300 .398874$

C $4.372974-1.7471753 .353364$

C $4.209892-1.0396494 .481743$

C $3.108086-1.2837235 .473084$

C $2.320386-0.0323375 .791227$

C 2.5907730 .6946786 .888559

C 1.1649230 .3246794 .884376

C 1.6179260 .9244383 .580466

N $4.984227-1.3514241 .057553$

H $2.3667055 .165961-2.756436$

H $-0.2378463 .602813-3.151205$

H $2.7739012 .854033-3.411703$

H $1.4221482 .527061-5.503382$

H $\quad 0.223888 \quad 1.631769-4.593919$

H $2.192793-1.496324-5.173450$

H $4.031123-0.170617-5.504515$

H $3.7102251 .500867-5.969756$

H $4.0620081 .094066-4.274181$

H $-0.566733-0.247571-5.156704$

H $-0.117310-1.907457-5.410349$

H $-0.070953-0.497052-2.691219$

H -1.393684 -1.491696 -3.300466

H $0.207006-3.441509-3.468944$

H -1.308648 -3.156088 -1.474120

H $-0.118662-2.122784-0.714321$

H $-0.879211-4.3214360 .661288$

H $2.223452-3.215117-0.049352$

H -1.085936 -5.621200 -1.281218

H $\quad 0.443870-6.372135-0.891254$

H $0.225968-5.575731-2.462718$

H $3.606500-4.968327-0.278575$

H $4.660490-3.945682-2.006532$

H $4.250788-2.713832-3.170335$

H $3.026540-4.426524-3.846789$

H $1.1884460 .060064-0.428504$

H 2.5005682 .1132911 .456423

H $1.533381-1.5316101 .441434$

H $-0.081304-0.7860001 .487256$

H $0.624088-1.5058542 .931992$

H $5.162053-1.339937-1.635316$

H $3.496400-1.269179-1.053713$

H $6.079138-0.6238482 .644094$

H $6.180281-2.3850262 .422721$

H $3.673021-2.5460183 .119294$

H $4.928940-0.2611804 .727233$

H $3.567119-1.6767356 .389837$

H $2.423133-2.0679835 .127216$

H 2.0118291 .5772777 .139260

H 3.4012280 .4257267 .557927

H 0.5175111 .0660455 .371938

H $0.527967-0.5544744 .754372$

H 2.0872841 .9029673 .691132

H $4.662731-0.4261000 .788353$

C $0.0282806 .284493-1.029461$

O $-0.7066745 .115524-0.607667$

C -0.0971514 .6020670 .588201$

C 0.6401855 .7867401 .194205

C $1.2001836 .441277-0.056326$

H $0.6341903 .850520 \quad 0.271286$

C -1.1258683 .9312691 .506065$

H -0.0479576 .4689091 .707020$

H 1.4157385 .4799511 .902183

C 1.6353387 .8818700 .148071

H $2.0677125 .858391-0.388902$

C -1.8251492 .7212930 .845648$

O $-2.090506 \quad 4.895303 \quad 1.921679$

H -0.6053873 .5883032 .408044$

H -1.063802 2.0575240 .419976

O $-2.6574423 .153493-0.230704$

C -2.6783911 .8934431 .830358$

C $-3.329764 \quad 0.677723 \quad 1.143928$

H -2.0344051 .5651372 .650470$

O -3.7023592 .7052192 .401558$

H $-4.011147 \begin{array}{lll}1.041349 & 0.363199\end{array}$ 
H -2.5586520 .0924830 .630024$

H $2.0070788 .310879-0.787946$

H $\quad 0.8071798 .5059410 .500423$

H 2.4380827 .9395970 .890269

C $0.3923726 .156315-2.513894$

H -2.565688 5.1920851 .126526

H -2.089146 $3.596225-0.883546$

H $-4.234513 \quad 3.0639691 .670762$

H $0.8754987 .076491-2.862178$

H $-0.5326476 .048882-3.095429$

H $-0.6655427 .127973-0.920479$

C $-4.762895-1.873518 \quad 0.419620$

O $-5.204299-0.8830031 .355622$

C $-4.151681-0.2334752 .086128$

C $-3.359574-1.2854932 .879295$

C $-2.872934-2.4311671 .990141$

C $-4.024517-2.9941441 .158090$

H -4.100102 -1.416464 -0.325950

C $-6.006337-2.410042-0.304513$

H -4.6600290 .4114812 .812110$

O $-2.262960-0.684070 \quad 3.552123$

H -4.017937 -1.7034203 .652559$

H -2.048650 -2.116094 1.341322

O $-2.362380-3.4856802 .808122$

H -4.718318 -3.538396 1.811660

O $-3.507275-3.9251310 .210730$

C $-6.619153-1.381033-1.232156$

H $-5.748883-3.282382-0.914956$

H $-6.769689-2.6903750 .431175$

H -1.872303 -1.352093 4.139939

H -1.548417 -3.1618853 .229549$

H -3.076068 -4.6378190 .712425$

O $-7.774141-1.891251-1.754653$

O $-6.148245-0.278969-1.477434$

C -8.471802 -1.030981 -2.660881

H $-8.769037-0.107874-2.154343$

H -7.846020 -0.810785 -3.531171

H $-9.372028-1.551201-2.998593$

SCF Energy (B3LYP/6-31G**//MMFF) $=-3245.92199903$

\section{6}

MM̄FF Geometry

C $-2.426010-3.627524-0.909514$

C -1.405716 -4.268890 -1.500405

C $0.046225-3.843172-1.526993$

O $0.243553-2.581499-0.849709$

C $0.902823-4.900944-0.805963$

C $2.377544-4.814914-1.145738$

C $3.322877-4.241400-0.375554$

C $2.768337-5.476235-2.443893$

C $3.148788-3.5223460 .934130$

C $3.413463-2.0193820 .806715$

C $4.860897-1.6175960 .486571$

C $5.809013-1.8588031 .663310$

C $7.221065-1.3150201 .387335$

O $4.826587-0.2186350 .172015$

O $7.929327-1.2715112 .635761$

C 7.1145910 .1394400 .879328

C $8.019037-2.2500240 .470142$

O $\quad 8.387591 \quad 0.6304230 .465775$

C $6.0840320 .318778-0.257030$

C $5.8780071 .820645-0.567509$

O $6.569497-0.333723-1.433239$

C $0.072702-1.434840-1.564571$

O $-0.207008-1.336558-2.747999$

C $0.251298-0.304125-0.625075$

C $-0.0324410 .950766-1.002191$

C $0.0423232 .134519-0.143350$

C $0.584106 \quad 1.9630261 .253153$

C $4.6708262 .100220-1.475000$

C $4.5583513 .565496-1.856509$

C $3.0769805 .114084-3.136716$

O $5.3632354 .420968-1.499674$

C $2.2191625 .780188-2.109496$

C $0.9132116 .036254-2.276854$

C $0.0629316 .700896-1.233506$

C $-0.9779315 .771026-0.653351$

C $-2.2912756 .010501-0.806032$
C $-0.489378 \quad 4.600014 \quad 0.174094$

C $-0.4055363 .317479-0.614218$

N $3.4457543 .796584-2.654940$

H -2.250864 -2.703930 -0.365737

H -1.624090 -5.188351-2.041539

H $\quad 0.359248-3.753267-2.574325$

H $0.562559-5.911826-1.069967$

H $\quad 0.753470-4.8212550 .275468$

H $4.352975-4.285725-0.726679$

H $3.844448-5.412253-2.637729$

H $2.500291-6.537848-2.424603$

H $2.255138-5.003218-3.286731$

H $2.137279-3.6531341 .329001$

H $3.818447-3.9709151 .675618$

H $2.764453-1.6081000 .024866$

H $3.098141-1.5203471 .732651$

H $5.197285-2.160847-0.402052$

H $5.859390-2.9231121 .920495$

H $5.414654-1.3531262 .555942$

H $7.939931-2.1708773 .005868$

H 6.8031200 .7620241 .729726

H $8.126448-3.2389610 .931897$

H $\quad 9.038547-1.879270 \quad 0.317627$

H $7.552850-2.393386-0.506885$

H $9.0004470 .510011 \quad 1.211205$

H 5.7187102 .3702750 .369463

H $6.7835842 .227948-1.034524$

H $6.2099110 .106472-2.218765$

H $0.586324-0.5417440 .377771$

H $-0.3855411 .124671-2.017464$

H 1.6107721 .5815301 .221892

H -0.0345241 .2636341 .825143$

H 0.6111552 .8963391 .822116

H $4.7389971 .522479-2.402681$

H $3.7448391 .814293-0.962061$

H $2.5553274 .980643-4.089722$

H $3.9791555 .708803-3.312716$

H $2.7026966 .068083-1.178688$

H $0.4253445 .752221-3.206118$

H $-0.4111897 .576628-1.694581$

H $0.6789927 .093937-0.414669$

H -3.037100 $5.355899-0.367076$

H -2.649266 $6.861647-1.375212$

H -1.1770004 .4512201 .017479$

H $0.4881894 .848720 \quad 0.604513$

H $-0.7943783 .355577-1.631682$

H $2.7925733 .036196-2.821144$

C $-4.784873-3.238660-1.780153$

O $-4.857018-1.909927-1.225651$

C $-4.761008-0.961625-2.305452$

C $-5.064309-1.746590-3.575928$

C $-4.410219-3.076511-3.255552$

H $-3.715118-0.632095-2.339413$

C $-5.6717980 .244831-2.057169$

H -6.141734 -1.881434 -3.724887

H $-4.652830-1.268168-4.469591$

C $-4.877930-4.220014-4.138267$

H $-3.326628-2.958718-3.375914$

C $-5.4814350 .924430-0.685484$

O $-7.042415-0.166363-2.140630$

H $-5.5316610 .977758-2.860184$

H $-5.919630 \quad 0.2976820 .099481$

O $-6.2329372 .144417-0.694360$

C $-4.0230681 .256943-0.331976$

C -3.8354872 .1024240 .939621$

H $-3.4564980 .327160-0.217568$

O $-3.4214081 .967733-1.417319$

H -2.757532 2.1896541 .116204

H -4.1695813 .1307550 .750249$

H $-4.386768-5.156014-3.853988$

H $-5.960242-4.369555-4.062754$

H -4.639519 -4.017850 -5.187598

C $-3.848255-4.116934-0.944397$

H $-7.176305-0.876041-1.488886$

H -7.152316 $1.916375-0.915016$

H $-3.9341162 .782571-1.556295$

H -3.892041 -5.148192 -1.314402

H $-4.207529-4.1369290 .092227$ 
H $-5.799048-3.650995-1.697608$ C $-3.005739-0.2368862 .752313$

$\begin{array}{llll}\text { O } & -4.333667 & 0.155252 & 2.380386\end{array}$

C $-4.5317491 .557824 \quad 2.200234$

C $-4.175147 \quad 2.3127803 .487223$

C -2.7740281 .9625973 .981631$

C -2.5932450 .4463624 .065943$

H -2.3104690 .0374411 .950441$

C -3.043069 -1.768946 2.867001

H -5.6090151 .6931932 .043447$

$\begin{array}{llll} & -4.263805 & 3.720808 & 3.294641\end{array}$

H -4.8943382 .0494134 .273713$

H -1.999587 2.4156813 .351686

O $-2.592878 \quad 2.509131 \quad 5.290922$

H -3.193432 0.0682194 .903767

$\begin{array}{llll}\text { O } & -1.224529 & 0.185869 & 4.363483\end{array}$

C $-1.688599-2.4354222 .801106$

H -3.543214 -2.050446 3.801774

H -3.642564 -2.187706 2.048885

H -5.167576 3.9182992 .995046

H -2.740489 3.4682735 .226046

H -1.158182 -0.7345544 .667819$

O $-1.765835-3.6608763 .398506$

O $-0.695293-1.9590232 .270651$

C $-0.552768-4.4194513 .413402$

H $-0.310155-4.7485732 .399977$

H $0.267436-3.8317913 .836973$

H $-0.710778-5.3007574 .040633$

SCF Energy (B3LYP/6-31G**//MMFF) $=-3245.91410484$

\section{7}

MMFF Geometry

C $-4.156902-0.161076-3.396103$

C $-3.178726 \quad 0.082141-2.510110$

C $-1.727506-0.250271-2.722655$

O $-0.9865800 .758966-1.997205$

C $-1.437980-1.640647-2.119943$

C $-0.106426-2.226329-2.541789$

C $0.997854-2.270011-1.770982$

C $-0.078670-2.814382-3.929911$

C $1.178155-1.720934-0.383641$

C $1.509548-2.7904120 .667731$

C $2.828081-3.5385460 .411466$

C $3.168922-4.465148 \quad 1.580415$

C $4.533344-5.1479461 .389749$

O $3.865234-2.568746 \quad 0.244599$

O $4.921396-5.7181322 .649273$

C $5.588464-4.0773391 .034780$

C $4.454033-6.311275 \quad 0.393912$

O $6.835601-4.682000 \quad 0.699780$

C $5.142981-3.121074-0.095673$

C $6.144172-1.948461-0.227401$

O $5.099659-3.841017-1.328725$

C $0.257067 \quad 1.099235-2.418694$

O $\quad 0.855540 \quad 0.689224-3.398701$

C $0.7944602 .045655-1.421958$

C $2.1015202 .029749-1.128466$

C $2.6982972 .862319-0.092070$

C 3.4394432 .0811090 .956464

C $5.688979-0.841500-1.190231$

C $6.6319410 .350202-1.177712$

C $6.7891442 .704733-1.988573$

O $7.7310710 .332056-0.630160$

C $6.4275993 .571379-0.822717$

C $5.8656624 .784200-0.939836$

$\begin{array}{llll}\text { C } 5.561752 & 5.676305 & 0.229947\end{array}$

C $4.1228396 .139846 \quad 0.263200$

C $3.7842167 .373865-0.147080$

C 3.0926825 .2087350 .861743

C $2.5756724 .203574-0.130582$

N $6.103983 \quad 1.431051-1.872225$

H -3.917520 $-0.610525-4.355881$

H -3.445049 $0.497023-1.540555$

H $-1.485357-0.201038-3.790950$

H $-2.217832-2.352074-2.426084$

H $-1.509907-1.591606-1.027807$

H $1.895307-2.713109-2.200380$

H $0.892036-3.258539-4.175138$
H $-0.833025-3.602620-4.022941$

H $-0.285826-2.044506-4.679345$

H $1.982720-0.977194-0.414646$

H $0.291569-1.176713-0.049631$

H $1.574767-2.2771841 .635982$

H $0.684485-3.5096430 .741126$

H $2.725436-4.122850-0.509617$

H $2.379964-5.2132351 .727184$

H $3.204253-3.8806862 .510564$

H $4.228261-6.3439402 .921078$

H $5.766804-3.4809731 .940629$

H $3.742032-7.0678330 .745233$

H $5.417910-6.8251030 .308703$

H $4.138271-6.000901-0.604299$

H $7.084551-5.2616911 .439874$

H $6.290525-1.4918380 .760476$

H $7.119210-2.325303-0.561102$

H $5.156478-3.213757-2.065778$

H $0.0953612 .692455-0.904514$

H $2.7699041 .325692-1.617481$

H 3.9448982 .7101971 .691997

H 4.2095451 .4530040 .498772

H 2.7486181 .4286591 .502018

H $5.647953-1.215433-2.218480$

H $4.688975-0.489953-0.914389$

H $6.4925783 .149165-2.943800$

H $7.8707442 .535746-2.001732$

H $6.670423 \quad 3.1927950 .167637$

H $5.6471855 .175732-1.930608$

H 6.2315306 .5440100 .165989

H 5.8062505 .1867291 .180938

H $2.7585817 .724181-0.096323$

H $4.5238678 .058874-0.548210$

H $2.228293 \quad 5.787437 \quad 1.214784$

H 3.5021954 .7461271 .764225

H $2.0205404 .646575-0.958617$

H $5.1570291 .371221-2.234639$

C $-6.204531-1.035318-2.246342$

O $-5.574697-1.032608-0.950611$

C $-6.563543-1.2752440 .063006$

C $-7.827232-1.692887-0.678833$

C $-7.698104-0.892968-1.964501$

H $-6.751064-0.3159540 .558361$

C $-6.016967-2.2809601 .080946$

H $-7.834714-2.766592-0.899143$

H $-8.735569-1.458362-0.115631$

C $-8.589988-1.395723-3.085668$

H $-7.9421080 .156490-1.752030$

C $-4.632163-1.8949291 .651269$

O $-5.876681-3.5514410 .432008$

H $-6.744706-2.4246611 .887904$

H -3.884164 -1.956931 0.853426

O $-4.264289-2.8964952 .609036$

C $-4.575564-0.5101512 .324509$

C $-3.215195-0.1866512 .974516$

H $-4.8269560 .268501 \quad 1.598329$

O $-5.577323-0.470777 \quad 3.344790$

H -3.3490940 .6797863 .632886$

H -2.926391 -1.003013 3.647330

H -8.451105 $-0.798506-3.992175$

H $-8.372968-2.440204-3.333511$

H $-9.644073-1.330906-2.796687$

C $-5.6103010 .093115-3.092855$

H $-5.520494-4.1741071 .088795$

H $-4.887372-2.8320103 .353183$

H -5.5922410 .4318583 .706429$

H $-5.7068081 .051054-2.567907$

H $-6.1519750 .190483-4.040193$

H - -5.989722 -2.009842 -2.704584

C -2.5014152 .4467951 .628908$

\begin{tabular}{llll}
\hline & -2.430081 & 1.141177 & 1.043084
\end{tabular}

C -2.089707 0.0901191 .955096

C $-0.721153 \quad 0.379543 \quad 2.588627$

C $-0.677050 \quad 1.757470 \quad 3.242881$

$\begin{array}{lll}\text { C }-1.158994 & 2.828832 & 2.266264\end{array}$

H -3.2886842 .4670002 .390863$

C -2.878482 $3.440857 \quad 0.520741$

H $-1.974528-0.8167611 .350698$ 
O $-0.386657-0.6101993 .554927$

H 0.0540030 .3465361 .812669

H -1.2590061 .7816714 .171534$

$\begin{array}{lrrr}O & 0.671581 & 2.062482 & 3.606872\end{array}$

H -0.3999132 .9790761 .489248$

O $-1.304467 \quad 4.0655022 .961422$

C $-4.2503723 .203595-0.069233$

H -2.8553194 .4743350 .883079$

H $-2.161562 \quad 3.355567-0.304391$

H $-0.409765-1.4737903 .108350$

H $0.981754 \quad 1.348857 \quad 4.190714$

H -0.4452514 .2722663 .367799$

O $-5.186813 \quad 3.0668490 .910129$

O $-4.4691003 .203033-1.274649$

C $-6.5301572 .866503 \quad 0.458885$

H $-6.5784202 .051801-0.269351$

H -7.1423192 .5971891 .323648$

H -6.917232 3.7932910 .025715

SCF Energy (B3LYP/6-31G**/MMFF) $=-3245.90347251$

\section{8}

MM̄FF Geometry

C $3.523393 \quad 3.4577201 .934945$

C 2.9420512 .2505631 .881782

C 1.9399121 .7524742 .887788

$\begin{array}{lllll}\text { O } & 0.684205 & 1.538211 & 2.200576\end{array}$

C 2.4012090 .4069993 .472890

C $1.485569-0.1078294 .566241$

C $0.656658-1.1642984 .451902$

C $1.574257 \quad 0.6317415 .878242$

C $0.429827-2.0598163 .265053$

C $-1.027980-2.0079132 .798581$

C $-1.393362-3.1202991 .803167$

C $-0.596967-3.0374940 .499638$

C -1.047845 -4.095761 -0.519598

O $-2.795024-2.9854481 .527828$

O $-0.467799-3.736437-1.782455$

C $-2.581505-4.020405-0.666272$

C $-0.495744-5.483041-0.173466$

O $-3.065940-5.063446-1.508047$

C $-3.332842-4.013587 \quad 0.682642$

C $-4.846675-3.741150 \quad 0.518361$

O $-3.203778-5.3014381 .296749$

C -0.1118812 .6281472 .019503$

O 0.1149013 .7721542 .380970

C - $-1.3203872 .190081 \quad 1.287222$

C $-2.202052 \quad 3.1089070 .864949$

C -3.4480432 .8472340 .141415$

C $-3.8475431 .409014-0.059984$

C $-5.183319-2.444704-0.219854$

C $-6.649192-2.063211-0.108344$

C $-8.244128-0.315797-0.881869$

O $-7.467685-2.6958960 .551992$

C -8.106288 $1.117685-1.280697$

C $-8.190720 \quad 2.137932-0.414219$

C -8.012673 $3.579270-0.797036$

C $-6.7247764 .152312-0.241362$

C $-6.744815 \quad 4.8809180 .888303$

C $-5.4754193 .873709-1.057706$

C $-4.1826283 .897374-0.283592$

N $-6.920627-0.905712-0.824628$

H 3.2869224 .1296902 .756118

H 3.1602391 .5957981 .043936

H 1.7995282 .4812853 .695449

H 3.4117160 .5088883 .891457

H $2.492877-0.3278212 .666661$

H $\quad 0.067019-1.4401275 .326294$

H $0.9772740 .157715 \quad 6.664749$

H $2.6111990 .659868 \quad 6.228936$

H 1.2142481 .6591425 .769180

H $1.088316-1.8083662 .431140$

H $\quad 0.683095-3.0833893 .567283$

H $-1.702867-2.0887443 .661920$

H -1.244954 -1.027730 2.355107

H $-1.223021-4.0839642 .297562$

H $0.478420-3.134933 \quad 0.684487$

H $-0.737431-2.0423230 .054752$

H $-0.805427-4.357840-2.450295$
H $-2.787398-3.083274-1.197444$

H $0.600514-5.463500-0.154310$

H $-0.766043-6.219860-0.937911$

H $-0.836967-5.850950 \quad 0.796305$

H $-3.068197-5.885712-0.988976$

H $-5.330791-4.5877960 .014171$

H -5.295522 -3.7024581 .520778$

H $-3.924931-5.4087751 .939102$

H $-1.448800 \quad 1.1285311 .111074$

H -1.9936074 .1595331 .063762$

H -3.8686870 .8756830 .897266$

H $-3.1424720 .903150-0.728472$

H $-4.8439861 .294107-0.490669$

H -4.594295 -1.614826 0.186242

H -4.947405 -2.549401-1.284720

H $-8.734915-0.4338340 .090291$

H -8.831393 -0.865188 -1.624605

H $-7.908244 \quad 1.321382-2.330224$

H -8.3806801 .9293760 .636262$

H -8.878782 $4.140187-0.422935$

H $-8.0280563 .709783-1.886539$

H -5.8499265 .3273851 .306512$

H -7.6715045 .0555031 .425961$

H $-5.4124294 .625758-1.854963$

H -5.580226 2.918471-1.581602

H $-3.8144354 .904043-0.081362$

H $-6.212831-0.558873-1.466501$

C $3.7908494 .473439-0.396261$

O $3.2863003 .349568-1.147078$

C $1.9253173 .622969-1.521573$

C $1.7796075 .137177-1.439767$

C $2.6204805 .445323-0.214578$

H $1.290083 \quad 3.163097-0.755070$

C $1.5989113 .005618-2.884595$

H $2.2016365 .635719-2.319859$

H $0.7364895 .450655-1.335320$

C $3.0458506 .899364-0.109836$

H 2.0282115 .1918130 .672329

C $1.9797201 .517499-3.033800$

O $2.2945803 .722743-3.912972$

H $0.532423 \quad 3.142560-3.097913$

H $3.0666941 .425448-3.143834$

O $1.4098201 .047288-4.262229$

C $1.5002990 .600870-1.895782$

C $1.687948-0.905197-2.154463$

H $2.0227520 .864820-0.971903$

O $\quad 0.1086330 .824239-1.651440$

H $1.341463-1.430957-1.257390$

H $1.001539-1.225903-2.948639$

H 3.6643747 .0588530 .779062

H $3.624417 \quad 7.213077-0.985101$

H $2.1699007 .552025-0.035331$

C 4.4692763 .9772190 .887356

H $3.2446113 .682353-3.707672$

H $1.7365911 .625504-4.972886$

H $-0.3682590 .641035-2.479181$

H 5.0482114 .7957681 .331588

H 5.1865493 .1851910 .636484

H $4.5613464 .927232-1.032761$

C $4.055954-1.435222-0.278509$

O $4.092015-0.836817-1.576341$

C $3.126037-1.339878-2.502393$

C $3.296071-2.856957-2.674956$

C $3.232541-3.589139-1.335764$

C $4.207696-2.961999-0.340499$

H $3.095251-1.1863750 .190138$

C $5.133813-0.7666400 .589761$

H $3.367008-0.885990-3.471243$

O $2.299404-3.355747-3.560353$

H $4.270736-3.053810-3.140662$

H $2.215923-3.600921-0.932344$

O $3.605966-4.956029-1.521486$

H $5.225052-3.241458-0.635844$

O $3.990279-3.5213320 .954074$

C $6.554642-1.0223920 .129359$

H $\begin{array}{llll}5.004589 & 0.320298 & 0.574447\end{array}$

H $5.027914-1.1149121 .623757$

H $2.506360-4.287542-3.743431$ 
H $2.911578-5.379891-2.053417$

H $3.084925-3.296403 \quad 1.228843$

O $7.394721-0.9427931 .203169$

O $6.892013-1.227580-1.028670$

C $8.781974-1.1436040 .915134$

H $9.337903-1.0726361 .853863$

H $8.940838-2.1382680 .487725$

H $\quad 9.142789-0.3672580 .233685$

SCF Energy (B3LYP/6-31G**//MMFF) $=-3245.91503028$

\section{1_589}

MM̄FF Geometry

C $1.919791-3.1020411 .439310$

C $0.804509-3.2203112 .176825$

C $-0.232638-2.1395872 .390206$

O $-0.004136-1.054606 \quad 1.462649$

C -1.646818 -2.695445 2.138546

C $-2.747123-1.7031772 .462754$

C $-3.520661-1.0945211 .542463$

C $-2.986446-1.4554483 .930066$

C $-3.453576-1.2393680 .046757$

C $-4.441192-2.287352-0.490564$

C $-5.914859-1.932659-0.238909$

C $-6.840752-3.063700-0.688606$

C $-8.319707-2.689789-0.497606$

O $-6.217262-0.743660-0.975518$

O $-9.112586-3.643918-1.220647$

C $-8.574427-1.310079-1.140725$

C $-8.752937-2.7902370 .969783$

O $-9.891504-0.844622-0.854632$

C $-7.535537-0.231528-0.746381$

C $-7.7514711 .046481-1.604234$

O

C $0.0377820 .213857 \quad 1.946616$

O $-0.1051120 .570262 \quad 3.104718$

C $0.300749 \quad 1.1112590 .798579$

C 0.4449742 .4280531 .007870

C $0.699553 \quad 3.445287-0.014902$

C $0.8138572 .982220-1.444009$

C $-6.4884971 .842799-1.950559$

C $-5.7753572 .490089-0.777977$

C $-3.5869543 .529244-0.200362$

O -6.2688432 .6001430 .339568$

C -2.622492 $4.406672-0.931771$

C $-2.3181495 .659257-0.560611$

C $-1.347656 \quad 6.534326-1.302977$

C $-0.1381616 .902942-0.471885$

C -0.0819328 .0736960 .185156$

C $1.0397215 .951429-0.476187$

C $0.796761 \quad 4.734598 \quad 0.372987$

N $-4.5268452 .969919-1.154027$

H $2.145963-2.1573840 .951417$

H $0.616516-4.1652592 .682701$

H $-0.136812-1.8013773 .428515$

H -1.810158 -3.5948612 .747344$

H -1.725136 -3.0292001 .097331$

H -4.295014 -0.412722 1.892206

H -3.840291 -0.7922514 .105568$

H -3.194494 -2.399550 4.444276

H -2.111609 -0.9920364 .394920$

H -3.656766 $-0.261239-0.406195$

H -2.440140 -1.502093 -0.275120

H -4.272590 -2.377491 -1.571385

H -4.213353 -3.263034 -0.043616

H $-6.056206-1.7453620 .830757$

H $-6.608896-3.993925-0.155377$

H -6.669497 -3.275890 -1.753138

H -8.908041 -4.528685 -0.872090

H -8.525465 -1.450829 -2.229877

H -8.599004 -3.809076 1.345312

H $-9.824520-2.5902651 .079601$

H -8.206515 -2.110641 1.626912

H -10.511246 -1.541769 -1.129702

H $-8.1867630 .758028-2.570112$

H -8.484010 $1.704186-1.119139$

H $\quad-7.3659801 .0004130 .778161$

H $\quad 0.3703470 .662612-0.185983$

H 0.3644092 .8030002 .027428
H $-0.0940542 .452321-1.752626$

H $1.6665742 .305727-1.561557$

H $0.9529883 .795583-2.158622$

H $-5.7844181 .201762-2.494302$

H $-6.7714192 .654528-2.633198$

H $-3.0558212 .690540 \quad 0.261696$

H -4.1345414 .0637210 .582626$

H -2.135852 $3.984597-1.808074$

H -2.7980336 .0871420 .316476$

H -1.883833 $7.441920-1.609852$

H $-1.0172256 .062789-2.236956$

H $\quad 0.793048 \quad 8.3584040 .759925$

H -0.9130478 .7710960 .167150$

H $1.9309666 .452533-0.075140$

H $1.2978915 .709600-1.510573$

H $0.6965324 .963319 \quad 1.435126$

H $-4.1632252 .689416-2.060762$

C $3.094488-4.500455-0.261878$

O $3.721948-3.369617-0.896793$

C $4.692559-3.842226-1.849462$

C $4.472187-5.345519-1.965705$

C $4.005555-5.688291-0.562031$

H $5.678738-3.670473-1.402199$

C $4.572160-3.048669-3.156252$

H $3.690937-5.588829-2.695010$

H $5.382874-5.876702-2.258565$

C $3.316930-7.038267-0.464529$

H $4.873363-5.6756980 .111033$

C $4.596268-1.517801-2.951405$

O $3.322887-3.380029-3.775822$

H $5.357069-3.361475-3.854467$

H $3.687167-1.218594-2.418983$

O $4.517657-0.895776-4.239634$

C $5.842047-0.993226-2.217088$

C $5.8677490 .536169-2.036791$

H $5.923003-1.468315-1.234962$

O $7.000666-1.378362-2.961132$

H $6.8387610 .804941-1.603099$

H $5.8565561 .026702-3.018167$

H $2.988170-7.2361220 .560401$

H $2.437922-7.088458-1.115904$

H $4.000621-7.840721-0.760232$

C $2.917580-4.2090191 .229453$

H $3.260105-2.861282-4.596134$

H $5.335753-1.112644-4.719245$

H $7.781716-1.094384-2.456276$

H $3.871290-3.8860891 .664083$

H $2.601005-5.1159081 .756399$

H $2.116901-4.636300-0.743452$

C 5.6165950 .4972531 .000953

O 4.5612060 .3321560 .049369

C $4.7246511 .084514-1.157104$

C $4.8378112 .581475-0.832618$

C 5.9317752 .8659870 .195377

C 5.7673541 .9641331 .419837

H 6.5579950 .1625350 .546580

C $5.369649-0.4642402 .172933$

H $3.797900 \quad 0.951677-1.727699$

O $5.0929143 .338541-2.010685$

H $3.8844992 .936502-0.421523$

H $6.9319512 .757550-0.239989$

O $5.829004 \quad 4.2255390 .625940$

H 4.8982012 .3020241 .995131

O 6.9102932 .0962902 .261597

C $4.099487-0.1997832 .953672$

H $5.289090-1.4873071 .785589$

H $6.217641-0.4171452 .865686$

H $4.3693303 .158755-2.635072$

H $5.8906334 .784965-0.167401$

H 6.9993233 .0391742 .483386

O $4.139244-0.9153954 .116898$

O 3.1785910 .5160212 .587500

C $2.995790-0.7733804 .965319$

H $3.169582-1.3601515 .871179$

H 2.8580210 .2748925 .247134

H $2.104323-1.1587874 .463488$

SCF Energy (B3LYP/6-31G**//MMFF) $=-3245.92032165$ 
01_59

MM̄FF Geometry

C $-2.979188-2.761912-1.966358$

C $-2.449533-1.858701-2.805146$

C $-2.695477-0.381370-2.651734$

O $-1.4465230 .334455-2.789262$

C $-3.6473560 .123194-3.748381$

C $-4.3392131 .410290-3.342437$

C $-5.5350781 .458195-2.721095$

C $-3.6181822 .679517-3.711640$

C $-6.3862200 .291292-2.303120$

C $-6.9457920 .386380-0.875192$

C $\begin{array}{llll}-5.891492 & 0.207461 & 0.231309\end{array}$

C $-6.586525-0.0715721 .568216$

C $-5.594358-0.1589632 .735019$

$\begin{array}{lllll}\text { O } & -5.118827 & 1.407620 & 0.327353\end{array}$

O $-6.372481-0.1229083 .940791$

C $-4.691557 \quad 1.0911582 .706079$

C $-4.842684-1.4957142 .738329$

O $-3.653574 \quad 0.996708 \quad 3.679733$

C -4.0774061 .3709181 .314359$

C -3.3745632 .7506951 .323289$

$\begin{array}{lllll}\text { O } & -3.113667 & 0.357332 & 1.028688\end{array}$

C $-0.6151720 .371079-1.714076$

O $-0.751638-0.218442-0.654601$

C $0.4907921 .296680-2.043819$

C $1.3400101 .682809-1.080206$

C $2.4843192 .581452-1.247689$

C $2.7013133 .186678-2.609766$

C $-2.9336413 .238024-0.060812$

C -2.3675674 .6396100 .024686$

C -0.3411785 .9075640 .691678$

O $-3.0667225 .625989-0.198714$

C 1.0428335 .8570150 .129462

C 2.1499275 .9519570 .881273

C 3.5450645 .9924090 .326061

C 4.3960794 .8370010 .799910

C 5.0702174 .9006751 .960519

C $4.5324973 .645485-0.118891$

C $3.2797672 .816217-0.183378$

N $-1.032287 \quad 4.6668480 .396324$

H -3.624289 -2.413085 -1.163892

H -1.824187 -2.185195 -3.631610

H -3.121879 -0.162992-1.664981

H $-4.402377-0.635113-3.988058$

H $-3.098510 \quad 0.293499-4.684344$

H -5.961332 $2.436117-2.501743$

H $-3.4653812 .727640-4.794757$

H -4.176374 $3.574851-3.417939$

H -2.641640 $2.725485-3.220634$

H $-7.2376410 .249784-2.994081$

H -5.858234 -0.661823 -2.403511

H $-7.4585021 .348900-0.750179$

H -7.704578 $-0.400210-0.775061$

H $-5.234947-0.627918-0.037182$

H -7.187018 $-0.988327 \quad 1.511162$ H -7.2996120 .7381821 .780174$ H $-5.768357 \quad-0.2363804 .693747$ H $-5.311430 \quad 1.9525102 .992175$ H -5.546480 -2.328096 2.858996 H -4.152931 -1.560703 3.587067 H -4.276117 -1.672279 1.821774 H -4.0738040 .9331454 .553668$ H -4.0625153 .5019351 .733391$ H -2.500105 2.7199771 .985608 H -2.4191480 .7264060 .461633$ H $0.5700831 .640306-3.068957$ H $1.1945361 .295222-0.072424$ H $\quad 1.8037073 .721334-2.940588$ H $2.9371812 .407187-3.342636$ H $3.5143013 .914590-2.641465$ H -2.184841 $2.575210-0.503460$ H -3.781997 $3.272311-0.751656$ H -0.3423446 .0245671 .780509$ H $-0.8843186 .754803 \quad 0.260920$ H $1.1352755 .782669-0.951448$ H 2.0504446 .0487421 .960283 H 4.0044856 .9396090 .638773
H $3.5373946 .023711-0.770565$

H 5.7055424 .0860122 .291721

H 4.9968925 .7690782 .607004

H 5.3378772 .9880150 .233306

H $4.8672063 .990796-1.100855$

H $3.0317752 .334070 \quad 0.763016$

H -0.5851023 .7982330 .675959$

C $-2.056207-4.878094-0.888784$

O $-0.680163-4.453875-0.857081$

C $-0.261526-4.3748740 .520759$

C $-1.407981-4.9410101 .357733$

C $-2.610440-4.5877480 .504766$

H $-0.153901-3.3087010 .752676$

C $1.077682-5.091906 \quad 0.720817$

H -1.331478 -6.0281231 .476000$

H -1.445185 -4.498935 2.358291

C $-3.863336-5.3703440 .852045$

H $-2.807897-3.5175390 .637201$

C $2.213515-4.599591-0.198404$

O $0.889833-6.4895940 .460939$

H $1.377121-5.0132701 .772912$

H $2.019019-4.915309-1.230192$

O $3.409100-5.2852850 .199684$

C $2.466108-3.080825-0.164574$

C $3.727432-2.687677-0.959722$

H $1.596603-2.562515-0.584471$

O $2.598407-2.6773351 .194642$

H $4.609536-3.110144-0.467340$

H $3.660237-3.141535-1.955509$

H $-4.694783-5.0813210 .201424$

H $-3.707204-6.4486770 .742203$

H $-4.162212-5.1775921 .887577$

C $-2.784323-4.246231-2.077840$

H $1.747194-6.9275670 .599006$

H $3.640754-4.9752421 .092181$

H $2.595182-1.7054451 .208932$

H -3.772918 -4.708519 -2.184062

H -2.225083 -4.475415 -2.993816

H $-2.030302-5.963840-1.055656$

C $5.263918-0.7286420 .843966$

O $4.037029-0.4853130 .145689$

C $3.904099-1.159069-1.109301$

C $5.048969-0.742014-2.042876$

C $6.412775-0.983097-1.400200$

C $6.469148-0.334564-0.017585$

H $5.326006-1.7885211 .118435$

C 5.2365650 .0912282 .139963

H $2.974896-0.791017-1.560334$

O $4.949683-1.443006-3.277251$

H $4.9487070 .327349-2.270461$

H $6.648974-2.052164-1.341914$

O $7.430574-0.386142-2.206638$

H $6.5110390 .756194-0.130628$

O $7.667400-0.7396000 .641775$

C $4.182104-0.4230293 .089985$

H 6.1958650 .0450032 .667999

H 5.0399841 .1502091 .938239

H $5.606685-1.064866-3.885484$

H $7.469995-0.880759-3.042452$

H $8.412106-0.4957130 .065682$

O 3.0261990 .2827222 .929519

O $4.358118-1.3569263 .861685$

C $1.934207-0.1205253 .761636$

H $1.685284-1.1709973 .583869$

H $2.182257 \quad 0.0450454 .814201$

H 1.0667770 .4929583 .503744

SCF Energy (B3LYP/6-31G**//MMFF)= -3245.91302156

01_590

MM̄FF Geometry

C $0.3540523 .354361-1.583352$

C $-0.6564282 .722665-0.968178$

C $-2.0314453 .300752-0.781747$

O $-2.9401612 .283311-1.269969$

C -2.3405363 .5893450 .704255$

C -1.2459364 .3103831 .466965$

C -0.4699453 .7440782 .414176$

C -1.0663675 .7627541 .108469$ 
C -0.5221722 .3264052 .911252$

C 0.7635021 .5471112 .595570

C 0.5501440 .0420272 .807982

C $1.863317-0.7339372 .726064$

C $1.626240-2.2479462 .866717$

O $-0.332345-0.4090341 .773942$

O $2.835612-2.9209752 .486913$

C $0.531773-2.6775361 .864564$

C $1.349496-2.6498664 .320293$

O $0.177028-4.0465732 .046451$

C $-0.730105-1.7786471 .884747$

C -1.644521 -2.1257220 .678949$

O $-1.444574-2.0224273 .098067$

C $-4.2435432 .607108-1.463688$

O $-4.7614403 .704152-1.340344$

C $-4.946457 \quad 1.353162-1.816568$

C $-6.2743321 .267245-1.651911$

C $-7.1026080 .086360-1.912188$

C $-6.419042-1.138039-2.462757$

C $-2.651477-1.0396590 .277841$

C $-3.814451-0.8919591 .233684$

C $-5.946967-1.9785241 .913313$

O -3.8454110 .0026162 .075543$

C -7.111400 -2.459312 1.106682

C $-8.307416-1.8507571 .093606$

C $-9.488414-2.3558210 .314661$

C $-10.144768-1.285089-0.528544$

C $-11.325370-0.751588-0.171301$

C $-9.479011-0.897321-1.830918$

C $-8.4233580 .156845-1.643724$

N $-4.787123-1.8604301 .049505$

H $\quad 0.1955704 .341389-2.006656$

H $-0.4793861 .738731-0.538907$

H -2.154768 4.208262 -1.386315

H -2.628950 2.6533421 .192768

H -3.2542954 .1954830 .766734$

H 0.2851324 .3618982 .898217

H $-0.277816 \quad 6.242145 \quad 1.698244$

H -0.7980885 .8710920 .053214$

H $-1.994838 \quad 6.314080 \quad 1.288933$

H -0.6666622 .3504003 .998733$

H - 1.3858531 .7907542 .512851

H 1.0546431 .7239621 .553852

H 1.5754371 .9130703 .235731

H $0.077133-0.1071353 .785676$

H $2.573814-0.3837113 .484829$

H $2.342027-0.5442891 .754925$

H $3.550768-2.6007593 .062907$

H $0.984150-2.6163330 .866178$

H $2.180362-2.3426704 .966856$

H $1.280246-3.7385224 .422135$

H $0.436552-2.2060984 .722324$

H $-0.392353-4.1021382 .832634$

H -1.011812 -2.282508 -0.203505

H -2.170079 -3.0688530 .871984$

H $-2.373638-1.7756632 .972836$

H $-4.3435620 .523122-2.167031$

H $-6.8062492 .139144-1.273189$

H -5.623366 -1.473770 -1.789387

H -5.978996 -0.921889-3.442654

H -7.087011 -1.990870 -2.596436

H -2.168865 -0.0641450 .163075$

H -3.059455 -1.285442 -0.711143

H -6.145809 -1.0197602 .403253$

H -5.700134 -2.713993 2.685665

H $-6.970977-3.3654450 .521807$

H -8.457964 -0.961554 1.701673

H -10.211067 -2.758955 1.036367

H -9.210136 -3.199076 -0.329801

H -11.816390 -0.004386 -0.786143

H -11.824559-1.045098 0.746125

H -10.227612 -0.486861 -2.522005

H -9.108065 -1.797934 -2.326625

H -8.819278 $1.095436-1.251999$

H -4.586909 -2.632515 0.419692

C $2.6920613 .307738-0.641823$

O $3.7098062 .314166-0.413673$

C $4.9808142 .969219-0.253712$
C $4.6788434 .458885-0.147074$

C $3.4398884 .585396-1.018502$

H $5.5422902 .790521-1.178560$

C 5.7349602 .3570090 .934207

H 4.4475944 .7562840 .882146

H $5.5131515 .075915-0.494173$

C $2.6699295 .870604-0.768584$

H $3.7488704 .546773-2.071423$

C 5.7906690 .8141960 .910500

O 5.0834862 .7344722 .154994

H 6.7458092 .7759760 .990856

H 4.7866460 .4189621 .100412

O $6.606300 \quad 0.3756932 .002785$

C $6.3546370 .215794-0.389111$

C $6.427986-1.323148-0.394887$

H $5.7469930 .540559-1.239281$

O $7.6703060 .732953-0.599972$

H $6.866234-1.632787-1.351805$

H $7.144478-1.6634590 .363476$

H $1.8176345 .958058-1.447738$

H 2.2918115 .9192240 .258122

H $3.3136186 .741514-0.931137$

C $1.7249162 .760152-1.692672$

H 4.1605952 .4314892 .101955

H $6.242503 \quad 0.7713362 .813263$

H $7.9928360 .379828-1.446635$

H $1.6590571 .670009-1.582228$

H $2.1148922 .921038-2.704663$

H 2.1790923 .4376880 .319563

C $4.135712-1.709311-2.384825$

O $4.033942-1.433193-0.985227$

C $5.066203-2.017103-0.184985$

C $5.088082-3.541732-0.369421$

C $5.160508-3.943613-1.842571$

C $4.089104-3.216081-2.658556$

H $5.090458-1.315595-2.756542$

C $3.051684-0.907902-3.120354$

H $4.784023-1.8320290 .857770$

O $6.184223-4.1214440 .329693$

H $4.171456-3.9674540 .058953$

H $6.158631-3.751558-2.252547$

O $4.960102-5.354467-1.960649$

H $3.105066-3.630297-2.412722$

O $4.314217-3.449617-4.046558$

C $1.630840-1.203199-2.685353$

H $3.2191860 .158776-2.927143$

H $3.127382-1.083727-4.199101$

H $\quad 6.099674-3.8699081 .265288$

H $4.085331-5.566060-1.592455$

H $4.324040-4.412918-4.181101$

O $0.772516-0.501382-3.485162$

O $1.306473-1.929646-1.757278$

C $-0.616588-0.646907-3.173295$

H $-0.811732-0.314674-2.150034$

H -1.188170 -0.017207 -3.860215

H $-0.927365-1.687269-3.307085$

SCF Energy (B3LYP/6-31G**//MMFF) $=-3245.90881114$

01591

MM̄̄FF Geometry

C $-1.680924-1.1515192 .924085$

C -2.950394 -1.182593 3.359423

C $-3.988286-0.0977773 .204689$

O -3.3904961 .1810042 .887063$

C $-4.962933-0.4174532 .057080$

C $-5.887137-1.5859892 .319058$

C $-5.736656-2.8159741 .789673$

C $-7.066989-1.2835223 .205798$

C $-4.622289-3.3125800 .910650$

C $-5.085467-3.621432-0.518123$

C $-5.417999-2.364194-1.337169$

C $-5.943952-2.753813-2.720090$

C $-6.200209-1.523236-3.602178$

O $-4.219083-1.592003-1.469528$

O $-6.380316-1.979694-4.951629$

C $-4.939852-0.633566-3.587390$

C -7.496226 -0.802809 -3.212273

O $-5.171671 \quad 0.586229-4.289920$ 
C $-4.387101-0.351926-2.168126$

C $-3.001244 \quad 0.325947-2.282569$

O $-5.2889350 .523162-1.488977$

C -2.982816 1.9546323 .925642

O $-2.988173 \quad 1.645588 \quad 5.109869$

C -2.502768 3.2799743 .471999

C -2.6144003 .7335532 .213491$

C -2.1639475 .0415661 .726552$

C -1.522129 5.9703932 .725655

C $-2.261754 \quad 0.526512-0.951204$

C $-0.8976211 .161756-1.168516$

C 1.0110592 .1649590 .070915

O $-0.4084641 .330111-2.283115$

C $0.8040013 .637981 \quad 0.216379$

C $0.7830254 .485656-0.823283$

C $0.5584665 .966208-0.706907$

C $-0.7816816 .385716-1.272515$

C $-0.9321446 .624872-2.586275$

C $-1.9260236 .596817-0.304265$

C -2.3202615 .3360560 .417956$

$\begin{array}{llll}\mathrm{N} & -0.280158 & 1.503848 & 0.027277\end{array}$

H $-1.298741-0.2751272 .410944$

H -3.302209 -2.086792 3.853303

H $-4.547314-0.0017934 .143669$

H $-4.397919-0.5560201 .128696$

H -5.5769220 .4708261 .851605$

H -6.482865 -3.5722112 .030652$

H -7.728473 -2.147829 3.327762

H $-6.731398-0.9833214 .203170$

H -7.663609 -0.4703302 .779430$

H $-3.773347-2.6249740 .888526$

H $-4.240483-4.2382501 .359699$

H -4.272783 -4.166314 -1.016152

H -5.954265 -4.290742 -0.489091

H -6.170283 -1.775802 -0.800528

H $-6.854210-3.360345-2.634080$

H $-5.205450-3.392948-3.224433$

H -7.129372 -2.599840 -4.961143

H $-4.168313-1.167011-4.159497$

H -8.350960 -1.486067 -3.286835

H $-7.7133360 .020834-3.901341$

H -7.478022 -0.407684-2.194535

H -5.695214 $1.165923-3.710934$

H -2.360606 $-0.288891-2.928958$

H -3.109008 $1.304752-2.767845$

H $-4.8101600 .996912-0.791790$

H -2.061777 $3.885376 \quad 4.257700$

H -3.076763 3.0966771 .461823

H $-0.647821 \quad 5.4962363 .185827$

H -2.234718 $6.236393 \quad 3.514337$

H -1.1666756 .9069092 .292109$

H $-2.8369701 .176158-0.284274$

H - $2.106980-0.436118-0.451038$

H $1.574147 \quad 1.932288-0.838210$

H 1.5644081 .7723210 .929479

H 0.6690514 .0187981 .224752

H $0.9241154 .091960-1.827910$

H $1.366556 \quad 6.477397-1.246407$

H $0.648175 \quad 6.3033810 .333023$

H -1.881690 $6.949409-2.998378$

H $-0.1068456 .497802-3.279222$

H $-2.816207 \quad 6.956827-0.837188$

H $-1.659419 \quad 7.408798 \quad 0.377581$

H -2.786756 $4.592658-0.230136$

H $-0.791504 \quad 1.3995850 .898423$

C $-0.458927-2.9803881 .727222$

O $0.556958-2.2398641 .016571$

C $1.495454-3.175795 \quad 0.450097$

C $0.843434-4.5479290 .560807$

C $0.078826-4.4050791 .859600$

H $2.383279-3.1622841 .092934$

C $1.874187-2.776583-0.979556$

H $0.152818-4.737164-0.269454$

H $1.579119-5.3576650 .580011$

C -1.001544 -5.457939 2.042555

H $0.787338-4.4641242 .696861$

C $2.518183-1.382345-1.112197$

O $0.696999-2.792094-1.797951$
H $2.543573-3.534486-1.403086$

H $1.764374-0.610007-0.929398$

O $2.919010-1.219523-2.479167$

C $3.733194-1.138128-0.196680$

C $4.4391520 .195204-0.509100$

H $3.397333-1.1354790 .846272$

O $4.648374-2.217886-0.358088$

H $4.9322490 .122413-1.484077$

H $3.6795960 .978500-0.586041$

H $-1.543012-5.3054822 .981204$

H $-1.730774-5.4304601 .225762$

H $-0.561274-6.4601532 .065673$

C $-0.741732-2.3166053 .077093$

H $0.063599-2.161873-1.413289$

H $3.626594-1.863292-2.655875$

H $5.337270-2.1182050 .320200$

H $0.188744-1.9503543 .527210$

H -1.173523 -3.045821 3.772403

H $-1.354027-2.9745441 .092187$

C $7.467279-0.353706-0.407270$

O $6.546496-0.3330290 .691077$

C 5.4699210 .6013330 .569187

C 6.0267692 .0225290 .401284

C $7.0081702 .109003-0.763153$

C $8.0835511 .032388-0.631221$

H $6.948919-0.685392-1.315035$

C $8.554526-1.383355-0.076603$

H 4.9376890 .5794121 .528975

$\begin{array}{lllll}\text { O } & 4.979228 & 2.966009 & 0.202016\end{array}$

H 6.5525252 .3168461 .319076

H $6.4949772 .036758-1.729221$

$\begin{array}{lllll}\text { O } & 7.650647 & 3.385717 & -0.742925\end{array}$

H 8.7540001 .2861770 .199931

O $8.8694441 .008031-1.821344$

C $8.018090-2.790226-0.190848$

H $9.405541-1.313161-0.763670$

H $8.949813-1.2337870 .935607$

H 4.3748462 .8970250 .960778

H $6.9519434 .061027-0.788859$

H $9.2271801 .903102-1.951052$

O $7.624875-3.2430491 .033456$

O $7.935571-3.402249-1.248096$

C $7.084451-4.5680001 .055637$

H $6.204262-4.6325520 .408819$

H $7.845359-5.290448 \quad 0.745569$

H $6.782045-4.7945182 .081467$

SCF Energy (B3LYP/6-31G*//MMFF) $=-3245.91250849$

$01 \_592$

MM̄FF Geometry

C $-2.832880-0.5353543 .243494$

C $-2.533020 \quad 0.257826 \quad 4.284031$

C $-1.1868250 .894625 \quad 4.557979$

O $-\begin{array}{llll}0.211916 & 0.411242 & 3.606472\end{array}$

C $-1.3128742 .423448 \quad 4.407227$

C $-0.0439603 .200962 \quad 4.701021$

C $0.529194 \quad 4.077888 \quad 3.852095$

C 0.5536092 .9955236 .069693

C 0.0385114 .5201212 .501365

C 0.8892583 .9449001 .368989

C $0.4725324 .465870-0.016565$

C $-0.890825 \quad 3.923020-0.457604$

C $-1.240253 \quad 4.339289-1.894986$

O $1.4903714 .048730-0.934980$

O $-2.3556893 .547161-2.328068$

C $-0.0535393 .985430-2.813989$

C -1.697364 $5.800453-1.973332$

O $-0.2705534 .465805-4.138234$

C $1.3027664 .495628-2.284056$

C $2.4996373 .996107-3.132500$

O $1.3229325 .927670-2.349975$

C $0.840447-0.3098124 .075841$

O $1.132579-0.5227775 .241676$

C $1.575298-0.8415482 .906122$

C $2.786377-1.3913663 .083361$

C $3.612547-2.0214722 .050297$

C $2.977753-2.2446350 .703983$

C $2.6557102 .480316-3.281784$ 
C $2.8419751 .750930-1.967972$

C $4.4276421 .473913-0.070458$

O $1.9892560 .978401-1.535535$

C $5.224151 \quad 0.235942-0.332417$

C $6.353548-0.0742480 .320672$

C $7.131679-1.3334730 .072547$

C $7.129066-2.2645751 .264098$

C $8.221922-2.4092582 .032557$

C $5.888627-3.0986371 .505869$

C $4.871473-2.3927652 .366124$

N $4.0374822 .059819-1.338190$

H -2.066859 -0.7883662 .515256$

H -3.3204650 .5029124 .994298$

H -0.9030430 .6359295 .584614$

H -2.0959632 .7952505 .081777$

H -1.662304 2.6299713 .390451

H 1.4423114 .5782864 .173446

H 1.3544523 .7114236 .284772

H $-0.2128753 .126186 \quad 6.840843$

H $0.978002 \quad 1.9931506 .165253$

H -1.015397 4.2743712 .356750

H 0.0900565 .6160392 .472851

H 1.9451014 .1983011 .536693

H $\quad 0.8489632 .8472891 .379404$

H 0.4612725 .5616110 .016566

H $-1.687657 \quad 4.2410840 .224225$

H $-0.8770582 .824956-0.409577$

H -3.082896 $3.680482-1.695975$

H $-0.0237702 .890679-2.880927$

H -2.598689 $5.951869-1.367166$

H $-1.9765346 .072582-2.997222$

H -0.943683 $6.507260-1.620915$

H -1.128960 $4.118075-4.435174$

H $2.4413984 .440102-4.134928$

H $3.4227804 .397083-2.692105$

H $2.2483846 .223400-2.340535$

H $1.088539-0.7863381 .939018$

H $3.216773-1.3885784 .084242$

H $3.640858-2.718271-0.022194$

H $2.657244-1.296210 \quad 0.261115$

H $2.100903-2.8915770 .804438$

H $1.7985362 .045550-3.806432$

H $3.5304602 .267289-3.909079$

H 3.5295381 .2244130 .503777

H 5.0008942 .2279090 .477599

H $4.837454-0.458246-1.075302$

H 6.7401910 .6068931 .075132

H $8.158698-1.045519-0.186795$

H $6.749923-1.871742-0.804274$

H $\quad 8.235367-3.0992402 .869796$

H $9.124591-1.8373241 .845238$

H $6.164745-4.0250752 .027781$

H $5.484406-3.4331570 .546699$

H $5.227118-2.1929343 .378201$

H $4.7172472 .640807-1.819874$

C $-4.810654-0.4323071 .732906$

O $-4.037580-0.7700660 .565542$

C $-4.922986-0.842778-0.569283$

C $-6.318177-0.494208-0.055795$

C $-6.226435-0.8947721 .405422$

H $-4.930925-1.888610-0.894636$

C $-4.3948450 .047839-1.701241$

H $-6.5355620 .575444-0.141785$

H -7.101388 -1.032705 -0.598503

C -7.305247 -0.2697382 .272221$

H $-6.290742-1.9888341 .475677$

C $-2.954541-0.291677-2.142961$

O $-4.3716851 .412181-1.261972$

H $-5.0684940 .005726-2.565507$

H -2.250318 $-0.045808-1.339946$

O $-2.6254890 .564206-3.245349$

C -2.751909-1.749652 -2.594884

C -1.376989 -2.036106 -3.227147

H -2.903645 -2.425289-1.747804

O $-3.748776-2.085750-3.565259$

H - $-1.408938-3.050217-3.643713$

H -1.230259-1.386014 -4.098754

H -7.201462 -0.5852193 .315011$
H $-7.258484 \quad 0.8241212 .244837$

H $-8.299070-0.5726401 .926720$

C $-4.204429-1.0898552 .972572$

H $-5.2778891 .757958-1.318887$

H $-2.7280371 .481738-2.938822$

H $-3.646704-1.473397-4.314092$

H $-4.109474-2.1715342 .822210$

H $-4.856984-0.9362783 .839384$

H -4.7798980 .6615041 .833968$

C $-0.313914-4.093371-1.268414$

O $-0.357806-2.674393-1.067519$

C $-0.190091-1.891834-2.253668$

C $1.175070-2.199559-2.880530$

C $1.350962-3.694050-3.142274$

C $1.025919-4.514445-1.892271$

H $-1.141947-4.398108-1.919082$

C $-0.521789-4.7534270 .100066$

H $-0.165504-0.844351-1.931784$

O $1.335527-1.466763-4.090330$

H $1.964200-1.863921-2.196404$

H $\quad 0.737567-4.016763-3.991155$

O $2.705047-3.944203-3.530208$

H $1.841397-4.398379-1.168355$

O $0.983720-5.889316-2.270605$

C $-1.876353-4.4244880 .679851$

H $-0.433140-5.8438430 .053599$

H $0.229329-4.4001120 .816211$

H $2.199727-1.708996-4.464813$

H $3.283496-3.667242-2.799150$

H $0.986154-6.422721-1.458522$

O $-2.851729-5.0979520 .006906$

O $-2.042303-3.6659431 .626284$

C $-4.185012-4.8848500 .480333$

H $-4.307096-5.3438901 .465724$

H $-4.414881-3.8167580 .523483$

$\mathrm{H}-4.875272-5.362664-0.219896$

SCF Energy $(B 3 L Y P / 6-31 G * * / / M M F F)=-3245.91774196$

01593

MM̄FF Geometry

C $2.023875-3.6983380 .795347$

C $1.086664-3.7233231 .755123$

C $0.082936-2.6205381 .949569$

O $-1.225220-3.1917451 .703985$

C $0.103020-2.0404963 .377634$

C $1.392201-1.3605453 .784765$

C $1.705031-0.0880793 .470561$

C $2.307402-2.1730204 .661934$

C 0.8992150 .8569532 .625292

C $0.0895341 .834676 \quad 3.482725$

C -0.7336492 .8141532 .630321$

C -1.544102 3.7474583 .529443

C -2.4652444 .6698592 .721036$

O $-1.6163472 .054517 \quad 1.803030$

O -3.3722515 .2677923 .659831$

C -3.2898683 .8217561 .725165$

C -1.678875 5.8198732 .078254

O $-4.028497 \quad 4.6749640 .854238$

C -2.4317782 .8309630 .906808$

C $-3.274779 \begin{array}{lll}1.785831 & 0.138927\end{array}$

O $-1.6280843 .550223-0.030167$

C -2.204292 -2.332596 1.308487

O $-2.094671-1.1295141 .134716$

C $-3.451419-3.1086901 .129150$

C $-4.558477-2.4820520 .701387$

C $-5.875187-3.0870020 .489090$

C $-6.028814-4.549600 \quad 0.815815$

C $-4.2355402 .326346-0.919313$

C $-4.7517771 .232665-1.841537$

C $-6.3825670 .873667-3.691862$

O $-4.3234910 .082003-1.835160$

C -7.561103 $0.184181-3.079104$

C $-7.750068-1.143412-3.131590$

C $-8.960384-1.835889-2.574982$

C $-8.614332-2.995293-1.667744$

C $-8.668579-4.260368-2.118277$

C $-8.312260-2.700802-0.216623$

C $-6.876767-2.3125850 .018732$ 
N $-5.7376341 .706839-2.695163$ H $2.052127-2.8619710 .101461$ H $1.046325-4.5630992 .444279$ H $0.266995-1.8286351 .214417$ H $-0.141716-2.8331434 .098935$ H $-0.726563-1.3300923 .492361$ H 2.6382830 .3258553 .849247 H $3.237650-1.6444724 .895429$ H $1.811077-2.4075835 .609387$ H $2.578476-3.1131674 .174361$ H $1.593110 \quad 1.4129301 .984841$ H $\quad 0.236120 \quad 0.3107501 .949719$ H $-0.588094 \quad 1.2562254 .124627$ H 0.7621302 .3974694 .142064 H $-0.0463053 .385427 \quad 1.996128$ H -0.8825554 .3360314 .177521$ H -2.167600 $3.147314 \quad 4.207498$ H -3.924633 5.9085093 .181349 H -4.0250643 .2447002 .302967$ H -1.186199 6.4225282 .850659 H $-2.344328 \quad 6.5063371 .543205$ H -0.9094085 .4765281 .383318$ H -4.6504955 .1860691 .398382$ H $-2.5738091 .093279-0.346350$ H -3.8352361 .1687980 .854065$ H -1.402809 $2.953833-0.763364$ H -3.425719 -4.167528 1.358982 H $-4.501940-1.4151400 .491658$ H $-5.335877-5.1520050 .217715$ H -7.028186 -4.9398820 .613423$ H $-5.825718-4.7297651 .877244$ H $-5.0991512 .792833-0.433259$ H $-3.7378633 .074483-1.546169$ H $-5.6520090 .163933-4.094114$ H $-6.7151971 .530628-4.501339$ H $-8.3083880 .810515-2.597516$ H -7.015614 -1.764309 -3.639892 H $-9.554491-2.195383-3.425772$ H $-9.611719-1.135279-2.038029$ H -8.459945 -5.102450 -1.467168 H -8.919045 -4.482095 -3.150425 H -8.601017 -3.5496790 .413128$ H $-8.955819-1.882150 \quad 0.130949$ H $-6.665820-1.265171-0.198978$ H $-6.0743342 .658839-2.583885$ C $4.502728-4.2121610 .615881$

O $4.718065-3.365006-0.530618$ C $5.519662-2.237928-0.126119$ C $6.053090-2.5743491 .264346$ C $4.909405-3.3880211 .836950$ H $4.840836-1.382102-0.040589$ C $6.606076-1.950143-1.167082$ H $6.963828-3.1824171 .216949$ H $6.278285-1.6771761 .849035$ C $5.295095-4.2245383 .043770$ H $4.112318-2.6920822 .124027$ C $6.107154-1.726919-2.610137$ O $7.513430-3.062909-1.204722$ H $7.206643-1.092235-0.844114$ H $5.681536-2.662956-2.993504$ O $7.249140-1.437046-3.429499$ C $5.094570-0.588711-2.834056$ C $5.5228820 .750895-2.202516$ H $4.993644-0.446158-3.917670$ O $3.823618-0.994933-2.345567$ H $5.3895690 .697484-1.117479$ H $6.5897870 .905490-2.397846$ H $4.441855-4.810323 \quad 3.399813$ H $6.106883-4.9206732 .807427$ H $5.631195-3.5825993 .864597$ C $3.071281-4.7595500 .610211$ H 6.995077 -3.855679-1.426895 H $7.884304-2.163683-3.308220$ H $3.178431-0.316611-2.606352$ H $2.976657-5.5311981 .383320$ H $2.879991-5.241581-0.356466$ H $5.189430-5.060214 \quad 0.492227$ C $2.9381012 .104448-1.138119$
O $3.3331151 .886959-2.499902$

C $4.7365661 .960168-2.762053$

C $5.2827993 .324321-2.321048$

C $4.9573123 .611642-0.860616$

C $3.4593653 .450676-0.611675$

H $3.3174231 .287572-0.512882$

C $1.4012072 .075309-1.118156$

H $4.843740 \quad 1.916227-3.853725$

O $6.6891633 .385760-2.532514$

H $4.8314934 .106754-2.945152$

H $5.5486202 .976248-0.190749$

O $5.3352664 .960282-0.573624$

H $2.9298154 .285169-1.089549$

O $3.2409583 .549806 \quad 0.794922$

C $0.8569330 .683412-1.346032$

H $0.9963112 .414445-0.161464$

H $1.0175192 .736625-1.905107$

H $6.9932344 .253684-2.215972$

H 5.0875585 .1389630 .349880

H 2.2892863 .6786810 .941632

O $\quad-0.2346990 .742925-2.162023$

O $1.321371-0.330211-0.842474$

C $-0.843602-0.515930-2.465802$

H -0.124005 -1.177169 -2.957958

H -1.231454 -0.978397 -1.553970

$\mathrm{H}-1.676760-0.333964-3.149458$

SCF Energy $(B 3 L Y P / 6-31 G * * / / M M F F)=-3245.92530065$

01_594

MM̄FF Geometry

C -3.6416053 .0380720 .657439$

C $-2.7663593 .121458-0.355516$

C $-1.2727593 .192260-0.187384$

O $-0.7484752 .120239-1.015600$

C $-0.6910284 .519894-0.715495$

C -0.4820725 .5744450 .356366$

C 0.7249896 .0526240 .721525

C -1.7252696 .1168741 .009866$

C 2.0588075 .6759470 .139843

C 2.6107394 .3999240 .772848

C 3.9559943 .9392050 .192318

C $3.8631493 .598729-1.298480$

C $5.1665492 .995453-1.839890$

$\begin{array}{lllll}\text { O } & 4.331277 & 2.775696 & 0.942977\end{array}$

O $4.8883462 .432184-3.131512$

C $5.5899951 .832604-0.923165$

C $6.2456704 .062792-2.055952$

O $6.8680241 .325307-1.302518$

C $5.5917142 .201588 \quad 0.578430$

C 5.8233940 .9579601 .471322

O 6.6658693 .1149310 .828672

C $0.1405571 .243838-0.480575$

O $0.604043 \quad 1.2499790 .647506$

C $0.4486900 .245316-1.530601$

C $1.408174-0.669923-1.328576$

C $1.800029-1.728932-2.263511$

C $0.972108-1.897369-3.510916$

C $4.645616-0.0197841 .512950$

C $5.024847-1.3714942 .090874$

C $4.114761-3.6866952 .257104$

O $6.139306-1.6311052 .534826$

C $4.461948-4.3257960 .948205$

C $3.593206-5.0322180 .210097$

C $3.916262-5.607228-1.137233$

C $3.069122-5.005016-2.238092$

C $2.061391-5.700507-2.791536$

C $3.447451-3.635741-2.761107$

C $2.855812-2.511203-1.954313$

N $3.960766-2.2625722 .024380$

H -3.2588043 .0671611 .675243$

H $-3.1260813 .109848-1.380885$

H -0.9893533 .0308960 .859809$

H -1.335311 $4.942310-1.498685$

H $0.2524034 .302502-1.228133$

H 0.7703796 .8025621 .509711

H -1.5220107 .0153461 .602786$

H -2.467265 6.3914540 .252995

H $\quad-2.1621885 .3739281 .682339$ 
H $1.9713755 .585055-0.946662$ H 2.7591326 .5028880 .311136 H $2.7283324 .548603 \quad 1.855174$ H 1.8848393 .5838560 .676955 H 4.6978844 .7273500 .365757 H $3.5948504 .478521-1.893913$ H $3.0530492 .872354-1.454547$ H $4.5463003 .142487-3.701095$ H $4.8749921 .019724-1.095996$ H $5.9032324 .812053-2.779744$ H $7.1536483 .625371-2.485577$ H $6.5163444 .591378-1.139786$ H $6.8220411 .101318-2.247857$ H $6.746356 \quad 0.452715 \quad 1.159622$ H 6.0129701 .2838152 .503747 H $6.8844573 .083702 \quad 1.774643$ H $-0.1317930 .288253-2.445662$ H $1.959420-0.645930-0.390056$ H $1.070244-1.019414-4.158578$ H $-0.085623-2.031033-3.256408$ H $1.253158-2.768685-4.106134$ H 3.8325850 .3989722 .117332 H $4.259168-0.2019290 .506359$ H $3.169532-4.0587252 .663273$ H $4.908207-3.8671372 .988798$ H $5.471474-4.1665550 .576511$ H $2.583531-5.1873860 .578415$ H $3.759841-6.692339-1.084401$ H $4.976719-5.474703-1.385216$ H $1.469267-5.289189-3.602090$ H $1.802038-6.694471-2.442567$ H $3.182529-3.548397-3.820497$ H $4.540120-3.531386-2.749696$ H $3.388531-2.310746-1.025666$ H $3.100101-1.959023 \quad 1.577417$ C $-5.6353702 .207362-0.746891$ O $-5.0628870 .888006-0.844288$ C $-6.084388-0.038793-1.251011$ C $-7.2589330 .812177-1.717868$ C $-7.1501192 .002897-0.783280$ H $-6.387648-0.586943-0.351079$ C $-5.527375-1.015824-2.291138$ H -7.140111 1.134663 -2.758799 H $-8.2136530 .284404-1.634073$ C $-7.9277293 .219756-1.252771$ H -7.5147201 .7046160 .208979$ C $-4.249881-1.756794-1.836931$ O $-5.202736-0.281434-3.478296$ H $-6.307250-1.732066-2.575542$ H -3.419879-1.043296 -1.782019 O $-3.892329-2.687105-2.866714$ C $-4.381385-2.504604-0.497237$ C $-3.191295-3.412731-0.141875$ H $-4.523927-1.7803090 .308683$ O $-5.558613-3.317791-0.515522$ H -3.442316 -3.9300510 .792233$ H -3.108887 -4.211815 - 0.889512 H -7.802959 $4.054625-0.556096$ H $-7.594038 \quad 3.552697-2.241337$ H -8.996461 $2.991063-1.318472$ C -5.1362472 .8775720 .536399$ H -4.846185 $-0.916181-4.123213$ H $-4.634681-3.303721-2.983662$ H $-5.430413-4.016714-1.178739$ H $-5.454352 \quad 2.2608791 .386180$ H $-5.603428 \quad 3.8605220 .663318$ H $-5.3141702 .768363-1.633061$ C -2.041215 -1.930367 2.289697 O $-1.903595-1.5784940 .906678$ C -1.840478 -2.682991-0.002410 C $-0.672618-3.6097090 .369661$ C $-0.763810-4.0638381 .822196$ C $-0.898643-2.8520772 .739540$ H -3.004055 -2.432534 2.445186 C -2.029741 -0.6247173 .104165$ H $-1.606427-2.258244-0.985276$ O $-0.633445-4.750428-0.479969$ H $0.277020-3.0772510 .236827$
H $-1.588904-4.7686601 .976728$

$\begin{array}{lllll}\text { O } & 0.428692 & -4.763298 & 2.178599\end{array}$

H $0.049252-2.2986012 .757568$

O $-1.156896-3.3035094 .068176$

$\begin{array}{llll}\text { C } & -3.381567 & 0.056615 & 3.127548\end{array}$

H $-1.764261-0.8395904 .145801$

H -1.3064800 .0876562 .691617$

H $-0.542334-4.429603-1.393600$

H $\quad 0.498184-5.5313411 .585787$

H $-0.429158-3.8982244 .318886$

$\begin{array}{lllll}\text { O } & -3.434957 & 0.895589 & 4.204477\end{array}$

O $-4.268177 \quad-0.1086642 .302252$

C $-4.668784 \quad 1.5930344 .406977$

H $-4.705607 \quad 1.9177515 .450293$

H $-4.6982172 .476217 \quad 3.764406$

H -5.5313160 .9472094 .215512$

SCF Energy (B3LYP/6-31G**//MMFF) $=-3245.90886699$

015595

MM̄FF Geometry

C $-2.243508-0.827536-3.010185$

C $-1.266934-0.224318-3.704679$

C $-0.2423850 .667711-3.052736$

O $1.0847890 .132263-3.288456$

C $-0.2472002 .095189-3.629228$

C $-1.4970462 .894983-3.329553$

C $-1.733828 \quad 3.508516-2.152971$

C $-2.469725 \quad 3.030232-4.470268$

C $-0.844843 \quad 3.495465-0.941136$

C $-0.243824 \quad 4.881202-0.672861$

$\begin{array}{lllll}\text { C } & 0.852235 & 4.819597 & 0.400596\end{array}$

C $1.357573 \quad 6.220480 \quad 0.746568$

C 2.5370166 .1764601 .731589

$\begin{array}{lllll}\text { O } & 1.925597 & 4.037250 & -0.131937\end{array}$

O 3.1442407 .4776561 .747764

C $3.5969735 .187146 \quad 1.199398$

C 2.0684295 .8999323 .165105

$\begin{array}{lllll}\text { O } & 4.650458 & 5.013168 & 2.145037\end{array}$

C 3.0144913 .8187970 .771743

C 4.0929063 .0002770 .017678

$\begin{array}{llll}\text { O } & 2.601321 & 3.107176 & 1.936862\end{array}$

C $1.467656-0.932235-2.531947$

O $0.783773-1.544007-1.727174$

C $2.886631-1.220891-2.833976$

C $3.447093-2.330936-2.331482$

C $4.843132-2.746691-2.481133$

C $5.795410-1.765764-3.112070$

C $3.5969541 .672549-0.573026$

C $3.7366350 .517496 \quad 0.398255$

C $5.441069-0.957764 \quad 1.453308$

$\begin{array}{llll}\text { O } & 2.776175 & 0.095040 & 1.037399\end{array}$

C $6.507375-1.8174740 .853465$

C $6.481977-3.1584090 .887222$

C $7.549625-4.026758 \quad 0.285222$

C $6.992731-5.066680-0.662515$

C $6.948384-6.364163-0.315572$

C $6.558551-4.613841-2.041078$

C $5.199505-3.966576-2.026636$

$\begin{array}{lllll}\mathrm{N} & 5.034247 & 0.038786 & 0.479801\end{array}$

H -2.299656 $-0.670526-1.935747$

H $-1.187586-0.383058-4.776891$

H $-0.3995460 .716880-1.967964$

H $-0.0771142 .058617-4.714472$

H $0.6308192 .637073-3.250751$

H -2.652991 $4.081154-2.040228$

H $-3.3847993 .555556-4.176774$

H $-2.010534 \quad 3.592079-5.290327$

H $-2.7620682 .047742-4.850757$

H $-1.4395773 .179758-0.075220$

H $-0.0464292 .756066-1.043479$

H $0.1868715 .271975-1.604441$

H $-1.0385525 .570177-0.361557$

H $0.445557 \quad 4.324316 \quad 1.290204$

H $0.545006 \quad 6.8399001 .146171$

H $1.6934046 .723848-0.171080$

H 2.4657218 .1219742 .013126

H $4.057718 \quad 5.6573550 .319761$

H 1.3541286 .6665883 .488777 
H 2.9039035 .9552343 .871692 H 1.5825234 .9285433 .277102 H 4.3241134 .4239102 .846347 H $4.4499253 .605897-0.825617$ H 4.9482352 .8161180 .678594 H 2.4180842 .1876561 .688253 H $3.432327-0.507187-3.440165$ H $2.824177-3.006480-1.746884$ H $5.778074-0.811402-2.573453$ H $5.521322-1.583409-4.157040$ H $6.834160-2.100653-3.110588$ H $2.5570011 .728219-0.910968$ H $4.1936561 .431342-1.462245$ H $4.571351-1.5363491 .780718$ H $5.840454-0.4173492 .317490$ H $7.344938-1.3148820 .375175$ H $5.657833-3.6622811 .387213$ H $8.084020-4.5152341 .110303$ H $8.300942-3.427250-0.243682$ H $6.573692-7.119443-0.998495$ H $7.280903-6.7002000 .661021$ H $6.499082-5.477705-2.716374$ H $7.338634-3.973992-2.460930$ H $4.422375-4.601272-1.597237$ H 5.7680280 .5570590 .004806 C $-4.713724-1.345415-3.298209$ O $-4.942405-1.333308-1.873800$ C $-5.770468-0.196568-1.555666$ C $-6.3243730 .305218-2.885075$ C $-5.1551300 .024520-3.809280$ H $-5.1057360 .574106-1.147885$ C $-6.832102-0.575603-0.518812$ H -7.205800 -0.265082 -3.200510 H $-6.5996091 .363567-2.847444$ C $-5.5157360 .050806-5.283283$ H $-4.381951 \quad 0.776962-3.616302$ C $-6.248692-1.1317030 .798242$ O $-7.676651-1.580774-1.092146$ $\mathrm{H}-7.4726720 .291280-0.316490$ H -5.777678 -2.104195 0.614281 O $-7.338773-1.3693661 .697375$ C $-5.248399-0.1893381 .496074$ C $-4.830777-0.6376542 .909707$ H -4.354720 -0.0766320 .873099$ O H $-4.198817 \quad 0.150708 \quad 3.336931$ H -5.713234 -0.665572 3.561120 H -4.640290 -0.170543 -5.901773 H -6.291885 -0.684620 -5.520359 H -5.890402 $1.038877-5.569513$ C $-3.269022-1.749571-3.604555$ H -8.461168 -1.662630 -0.524153 H -7.849424 -2.118465 1.346846 H -5.1764081 .7055631 .984464$ H -3.133679 -1.834414 -4.689055 H -3.088172 $-2.744286-3.178053$ H -5.375355 -2.123215 -3.702330 C - $-1.880441-1.2215812 .388637$ O $-2.999382-2.0406142 .034394$ C $-4.094631-1.9907002 .954636$ C $-3.626569-2.4036634 .358525$ C $-2.425587-1.5838634 .825985$ C $-1.324363-1.5997543 .766672$ H -2.202249 -0.1727362 .403174$ C $-0.836247-1.3168831 .269346$ H -4.809147 -2.753145 2.622286 O $-4.679900-2.2698035 .306943$ H -3.337180 -3.462669 4.346462 H -2.711893 -0.556568 5.079653 O $-1.900543-2.1603356 .024219$ H $-0.870143-2.5976823 .742691$ O $-0.302717-0.6743564 .129500$ C $-0.210999-2.6900581 .153021$ H -1.294800 -1.0963630 .298955$ H $-0.050586-0.5696851 .430856$ H $-5.425037-2.8107114 .993981$ H -2.623404 -2.181924 6.674719 H $0.010455-0.9211705 .016496$
O $1.145118-2.593407 \quad 1.261780$

$\begin{array}{lllll}\text { O } & -0.846540 & -3.722300 & 0.982774\end{array}$

C $1.863206-3.8265701 .159018$

H $2.932352-3.5996271 .176118$

H $1.625392-4.4701202 .011019$

H $1.628396-4.3301960 .216300$

SCF Energy $(B 3 L Y P / 6-31 G * * / / M M F F)=-3245.90494045$

01596

MM̄FF Geometry

C $4.3259353 .920300-0.075400$

C 3.2329153 .5812070 .625518

C 1.8441053 .6237380 .039977

O 1.1701772 .4120070 .455653

C 1.0777234 .8434820 .590669

C $0.0873195 .431021-0.395771$

C $-1.2539875 .356559-0.296967$

C $0.7067446 .210415-1.531094$

C $-2.082976 \quad 4.658478 \quad 0.745277$

C -3.0578763 .6812380 .086304$

C -4.1032653 .0826481 .037821$

C -3.4848242 .3309912 .217745$

C -4.5424021 .5989643 .057913$

O -4.8839052 .1874290 .236331$

O

C -5.4420150 .7678752 .121524$

C -5.3038822 .5612653 .977225$

O -6.5237120 .1820302 .842767$

C -5.9822721 .5682200 .914728$

C $-6.6605120 .607878-0.088727$

O -6.9341122 .5289691 .370019$

C $0.3060501 .825926-0.415466$

O $0.0427942 .188014-1.550577$

C -0.2923650 .6576250 .272714$

C $-1.277278-0.038229-0.317234$

C $-1.983830-1.1919980 .250721$

C $-1.578700-1.6378801 .630754$

C $-7.1409251 .281799-1.382568$

C -7.429334 $0.235048-2.439003$

C $-6.255469-1.376901-3.935735$

O $-8.568017-0.171012-2.656904$

C $-6.035061-2.583200-3.078834$

C $-4.887092-3.275759-3.035447$

C $-4.662286-4.428650-2.099814$

C $-3.586135-4.146701-1.073467$

C $-2.509706-4.943148-0.964068$

C $-3.793463-2.985776-0.120986$

C $-2.947488-1.790229-0.482125$

N $-6.275927-0.216922-3.063929$

H $4.2042104 .245202-1.105752$

H 3.3309123 .2880051 .667735

H $1.9183933 .647955-1.053635$

H 1.7781635 .6510100 .846214

H 0.5871114 .5788931 .533291

H -1.843738 $5.866510-1.058848$

H $-0.0454616 .680044-2.173992$

H $1.3453927 .008841-1.139088$

H $1.3153335 .558224-2.164422$

H $-1.4594134 .122872 \quad 1.464288$

H $-2.6432575 .418007 \quad 1.302821$

H $-3.5960774 .182721-0.730455$

H -2.502171 $2.871132-0.401436$

H $-4.7480453 .894349 \quad 1.394881$

H -2.904462 3.0059232 .857683

H -2.7704011 .5890391 .840812$

H $-3.2312281 .174645 \quad 4.461143$

H $-4.836427-0.0665711 .741045$

H -4.6126083 .0569064 .669491$

H -6.0214662 .0230234 .606104$

H -5.8385243 .3418073 .431903$

H $-6.140382-0.3202053 .582205$

H -5.948545 -0.186310 -0.347755

H $-7.524960 \quad 0.120597 \quad 0.380092$

H -7.4978742 .7922640 .626007$

H $0.0826090 .415210 \quad 1.260811$

H $-1.6067210 .267746-1.309304$

H -1.774096 -0.8461462 .362108$

H $-0.512790-1.8798251 .655298$ 
H -2.103191 -2.528157 1.980893 H -8.056900 1.857431-1.211338 H $-6.3940211 .978839-1.778857$ H $-5.450375-1.230118-4.662062$ H -7.206012 -1.461543 -4.471473 H -6.858320 -2.878381-2.431978 H $-4.057717-2.983531-3.674947$ H $-4.404551-5.307373-2.704368$ H -5.586786 -4.687972 -1.568253 H $-1.752917-4.763113-0.208424$ H -2.362132 $-5.791996-1.623211$ H $-3.619160-3.3338330 .900883$ H $-4.846410-2.677485-0.131275$ H $-3.173331-1.375866-1.465227$ H $-5.3785210 .131704-2.738995$ C $6.6917882 .956577-0.159755$

$\begin{array}{lllll}\text { O } & 6.329701 & 1.609827 & 0.199474\end{array}$

C $6.5114760 .765366-0.952547$

C $7.3214831 .582141-1.955265$

C $6.8037802 .981203-1.684770$

H $5.5113700 .589346-1.367269$

C $7.145303-0.571370-0.554519$

H $8.3971461 .532507-1.751052$

H $7.1579881 .253545-2.986074$

C $7.7009204 .083660-2.218305$

H $5.8175553 .067106-2.155086$

C $6.449963-1.3029870 .610185$

O $8.507305-0.356771-0.160133$

H $7.190952-1.225557-1.433057$

H $6.684646-0.8087171 .561105$

O $7.017687-2.617397 \quad 0.693517$

C $4.922643-1.4394690 .479079$

C $4.326636-2.3489191 .571312$

H $4.465848-0.4462710 .546742$

O $4.604421-1.964136-0.806922$

H $4.525357-3.3963261 .317450$

H $4.832400-2.1393502 .521478$

H $7.2869605 .069241-1.982900$

H $8.7062214 .027621-1.787384$

H $7.7976264 .008187-3.306312$

C 5.7217993 .9534410 .481714

H 8.5036230 .2841220 .571570

H $7.979721-2.5116500 .791740$

H $5.054644-2.821550-0.896877$

H $\begin{array}{llll}6.110782 & 4.970137 & 0.349222\end{array}$

H 5.6887963 .7642371 .562177

H $7.682425 \quad 3.1232200 .284486$

C $1.952510-3.6091790 .056100$

$\begin{array}{llll}\text { O } & 2.055576 & -2.276547 & 0.568327\end{array}$

C $2.808760-2.1417761 .773918$

C $2.200974-3.0189332 .879091$

C $2.042241-4.4692412 .430417$

C $1.310607-4.5392321 .091248$

H $2.948775-3.980671-0.211783$

C $1.108514-3.545345-1.221577$

H $2.685013-1.0974622 .089669$

O $2.993181-2.9803484 .061394$

H $1.210738-2.6310013 .148479$

H $3.009742-4.9819422 .376064$

O $1.266178-5.1779423 .399553$

H $\quad 0.256736-4.2731591 .238729$

O $1.347763-5.879140 \quad 0.604687$

C $1.836708-2.803342-2.316274$

H $0.878118-4.545306-1.605991$

H $0.150061-3.046284-1.040901$

H $3.060410-2.0502514 .337358$

H $1.724117-5.0933654 .253648$

H $0.955056-6.4470871 .289647$

O $1.509232-1.479803-2.281247$

O $2.623445-3.333370-3.090242$

C $2.164978-0.655638-3.249423$

H $1.901638-0.978021-4.261241$

H $1.8227800 .372810-3.108549$

H $3.248735-0.686509-3.103296$

SCF Energy (B3LYP/6-31G*//MMFF) $=-3245.91441154$

01597

MM̄FF Geometry
C $-2.984623-2.6530593 .488001$

C $-2.521297-1.4973212 .985834$

C $-1.188664-0.8955893 .357970$

O $-0.379540-0.7840792 .163769$

C $-1.4096990 .521677 \quad 3.918418$

C $-0.141974 \quad 1.1976894 .401667$

C $0.3785442 .320146 \quad 3.867898$

C 0.5330230 .5526925 .585270

C $-0.146348 \quad 3.133292 \quad 2.717989$

C $0.445303 \quad 2.764047 \quad 1.348887$

C $1.9636492 .978747 \quad 1.238760$

C $2.4301272 .811368-0.207586$

C $3.9560462 .939592-0.337523$

O $2.6196282 .008867 \quad 2.057247$

O $4.3250992 .457461-1.639510$

C 4.6365952 .0157320 .697956

C $4.409898 \quad 4.403077-0.273030$

O $6.042527 \quad 2.2522230 .704738$

C 4.0461592 .1465102 .119334

C $4.555131 \quad 1.0670943 .106391$

O 4.4157903 .4197552 .662862

C $0.262219-1.9071711 .740532$

O $0.225159-3.0140462 .254390$

C $1.024061-1.5607440 .520489$

C $1.480142-2.544444-0.269413$

C $2.251404-2.374505-1.501766$

C $2.829396-1.012906-1.778712$

C $4.263633-0.3829172 .702793$

C $5.411811-1.0666291 .979324$

C $5.980157-3.2058230 .832856$

O $6.527259-0.5675561 .860179$

C $5.878996-2.998843-0.645198$

C $5.669729-3.997650-1.516261$

C $5.614939-3.812255-3.005364$

C $4.352600-4.375836-3.618001$

C $4.346383-5.589414-4.195054$

C $3.124319-3.495407-3.640326$

C $2.423809-3.441736-2.309172$

N $5.049800-2.3229261 .511184$

H -2.359104 -3.189072 4.199818

H $-3.109884-0.9363052 .266108$

H $-0.668233-1.5160364 .097677$

H -2.113357 0.4819134 .760764

H $-1.8967601 .128699 \quad 3.147435$

H 1.2917992 .7177354 .309863

H 1.3489081 .1659755 .983183

H -0.1857230 .4008856 .397160$

H $0.958514-0.4162225 .306999$

H -1.2381183 .0692412 .661596$

H 0.0645224 .1899622 .927052

H $0.200274 \quad 1.7190961 .125479$

H $-0.056603 \quad 3.3818710 .595495$

H 2.1970593 .9861891 .602702

H $1.927743 \quad 3.530249-0.866714$

H $2.1355841 .817292-0.569057$

H $3.8497422 .987830-2.301704$

H 4.4933670 .9905010 .338866

H $3.9350844 .986647-1.070995$

H $5.4890064 .490330-0.441763$

H 4.1681364 .8853160 .676307

H 6.4583911 .5645631 .250804

H $5.617711 \quad 1.222542 \quad 3.326923$

H 4.0371931 .2360204 .061893

H 4.3473063 .3738723 .630858

H $1.180081-0.5110260 .301042$

H $1.250979-3.575390-0.002562$

H $3.374058-0.645097-0.903253$

H $2.037663-0.299526-2.022747$

H $3.546114-1.002291-2.602523$

H $4.089534-0.9666963 .615940$

H $3.356636-0.4497892 .094386$

H $5.728295-4.2298671 .126197$

H $6.999743-2.9878801 .166819$

H $6.012668-1.985957-1.017823$

H $5.564769-5.013822-1.142512$

H $6.492793-4.312837-3.434775$

H $5.714374-2.755270-3.282388$

H $3.452625-5.988672-4.662811$ 
H $5.234286-6.213047-4.207363$

H $2.399164-3.882129-4.369229$

H $3.395185-2.506639-4.021312$

H $1.976171-4.391981-2.014692$

H $4.086236-2.6310201 .605412$

C $-5.269809-2.5629502 .278715$

O $-4.729704-2.4664530 .949606$

C $-5.816043-2.4099280 .007438$

C -7.101803 -2.553663 0.821364

C $-6.617990-3.2480232 .081447$

H $-5.705220-3.277131-0.654535$

C $-5.745925-1.115484-0.823700$

H -7.538404 -1.577160 1.062470

H $-7.864027-3.1270900 .284386$

C -7.573642 -3.1039273 .252818$

H $-6.469730-4.3133501 .860267$

C $-4.474230-1.018953-1.697005$

O $\quad \begin{array}{llll}-5.825321 & 0.003773 & 0.056157\end{array}$

H -6.629890 -1.079881-1.469642

H -4.446552 -1.931427 -2.307291

O $-3.310037-1.043177-0.877517$

C $-4.4155010 .178594-2.670612$

C $-4.4261581 .605826-2.088803$

H $-3.5014310 .072231-3.265174$

O $-5.4959640 .069180-3.602793$

H $-4.4221042 .296756-2.942022$

H -5.387186 $1.801139-1.598974$

H $-7.190733-3.6220584 .137359$

H $-7.727229-2.0525573 .518327$

H -8.549845 -3.5350603 .007507$

C $-4.298067-3.328972 \quad 3.178174$

H -5.059223 -0.0416550 .653637$

H -2.533784 -1.014845 -1.461988

H $-6.3149180 .326075-3.146610$

H $-4.061097-4.295848 \quad 2.716347$

H -4.786098 -3.537580 4.137321

H $-5.415817-1.5423642 .657328$

C $-1.5514592 .316922-2.792639$

O $-1.9942801 .563795-1.658664$

C $-3.2653101 .956432-1.137055$

C $-3.2175933 .429071-0.708039$

C $-2.7113184 .341180-1.824655$

C $-1.4129783 .798626-2.425873$

H -2.266065 $2.203494-3.617321$

C $-0.2131321 .720242-3.239374$

H $-3.4155781 .373968-0.221482$

O $-4.5059413 .853666-0.275178$

H $-2.545140 \quad 3.5227540 .154247$

H -3.479917 $4.480622-2.594008$

O $-2.4671795 .632120-1.260818$

H $-0.5904403 .937053-1.713975$

O $-1.0928274 .538353-3.602217$

C $-0.4285570 .415234-3.969693$

H $0.3180062 .386390-3.928515$

H $\quad 0.453179 \quad 1.540328-2.388749$

H $-4.4351264 .791575-0.027664$

H -2.247966 $6.235077-1.991004$

H $-0.8989945 .451740-3.332511$

O $-0.591272-0.603411-3.078208$

O $-0.4706460 .319800-5.189742$

C $-0.842314-1.889348-3.653626$

H -1.775337 -1.871641 -4.224772

H $-0.004944-2.191171-4.289669$

$\mathrm{H}-0.944757-2.611978-2.839613$

SCF Energy (B3LYP/6-31G $\left.{ }^{* *} / / M M F F\right)=-3245.90322983$

01598

MM̄FF Geometry

C -1.439529 $0.925722-1.137972$

C $-0.2090160 .719512-0.646721$

C $0.131123-0.4221030 .275049$

O $1.190993-1.157062-0.386214$

C 0.6419280 .0550451 .650306

C -0.2391231 .0522812 .374734$

C 0.1509692 .2930662 .731488

C -1.6222290 .5662602 .724669$

C 1.4992472 .9214812 .516434

C 1.4626063 .9866161 .413815
C 2.8714124 .2806650 .881375

C 2.8859155 .5415280 .018889

C $4.2713285 .785958-0.601178$

O 3.2602013 .1525330 .089135

O $4.1255536 .789269-1.617859$

C 4.737748 4.491926-1.302072

C 5.2642386 .3479610 .422871

O $6.0745504 .621305-1.781637$

C $4.5894343 .216079-0.435954$

C $4.8328221 .960703-1.317029$

O $5.564123 \quad 3.2547690 .607371$

C $1.470381-2.4143560 .044904$

O $0.877002-3.0536010 .897652$

C $2.674380-2.864603-0.688424$

C $3.408358-3.875262-0.200992$

C $4.645654-4.404093-0.781871$

C $5.136910-3.781475-2.062595$

C $4.2688600 .645360-0.766453$

C 5.0490030 .0797100 .399469

C $7.156165-1.1210240 .952877$

O $4.670710 \quad 0.2279341 .559050$

C $7.784950-2.3484370 .373649$

C $7.843237-3.5257231 .014974$

C $8.507002-4.7489400 .449502$

C $7.629530-5.9797220 .508505$

C $7.862246-6.9515271 .407118$

C $6.539399-6.126614-0.530635$

C $5.277663-5.409194-0.140188$

N $6.198135-0.5849220 .004169$

H $-2.2388710 .241046-0.867842$

H $0.5960621 .398168-0.918225$

H $-0.731911-1.0862210 .400644$

H 1.6610280 .4288231 .517319

H $\quad 0.767870-0.8165422 .306111$

H $-0.5651282 .931626 \quad 3.246838$

H -2.1783101 .2943053 .325008$

H $-2.208750 \quad 0.372292 \quad 1.822145$

H -1.561622 -0.3600063 .305328$

H 1.8139033 .3910983 .457039

H 2.2657792 .1725492 .304614

H 0.8365113 .6345760 .585199

H 1.0045014 .8987801 .814873

H 3.5566964 .3881751 .730444

H $2.565893 \quad 6.4154710 .599784$

H $2.1539065 .437326-0.794330$

H $3.7665137 .590328-1.199348$

H $4.1088694 .377427-2.195684$

H 4.8827367 .2816590 .853469

H $6.2170076 .606894-0.052023$

H 5.4640255 .6611591 .248009

H $6.6718254 .531898-1.019515$

H $4.3340392 .113349-2.283103$

H $5.9053801 .851480-1.518673$

H 5.7363242 .3507410 .912348

H $2.941711-2.326518-1.590673$

H $3.083173-4.3526990 .722643$

H $5.295912-2.705501-1.934287$

H $4.407442-3.933433-2.865928$

H $6.085546-4.191041-2.414020$

H $3.2210620 .749925-0.469767$

H $4.275638-0.103317-1.569050$

H $6.661659-1.3169431 .909896$

H $7.918283-0.3514251 .111813$

H $8.233321-2.259094-0.613192$

H $7.420674-3.6083052 .013829$

H $9.428354-4.9186101 .021960$

H $8.827282-4.586218-0.587325$

H $7.255421-7.8504841 .436538$

H $8.660585-6.8675662 .137069$

H $6.284792-7.188114-0.653500$

H $6.928977-5.819565-1.504595$

H $4.832945-5.7923930 .779650$

H $6.484687-0.516391-0.968486$

C $-2.8165893 .033744-1.480965$

O $-3.9970372 .345240-1.023500$

C -4.4147952 .9303390 .226501$

C -3.6339864 .2322040 .364241$

C $-2.3204603 .864302-0.298610$ 
H -4.0936492 .2463501 .020516$ C -5.9384913 .0905110 .251799$ H -4.116881 $5.054135-0.177161$ H -3.5178014 .5380151 .408499$ C -1.485139 $5.067854-0.699938$ H $-1.744672 \quad 3.2426460 .396379$ C -6.7119821 .7727110 .033840$ O $-6.3278523 .998866-0.787260$ H $-6.2500743 .558137 \quad 1.192554$ H $-6.5149101 .395694-0.975685$ O -8.1132542 .0685570 .088363$ C -6.4095350 .6800351 .076486$ C $-7.269342-0.5903220 .920341$ H -5.3503490 .4075161 .031668$ O H -7.081396 -1.235252 1.787354 H $-8.332207-0.3291230 .994513$ H $-0.5656884 .754752-1.204209$ H -2.033059 $5.728258-1.380462$ H $-1.205385 \quad 5.6529260 .182237$ C -1.810801 2.041556-2.072357 H $-6.0096813 .633474-1.630706$ H -8.287643 $2.767162-0.565315$ H -6.3897380 .5396983 .028930$ H $-0.9190472 .578425-2.415845$ H $-2.2599491 .574982-2.958281$ H $-3.1485633 .701058-2.287382$ C $-5.043349-2.5877850 .312989$ O $-5.601392-1.613698-0.574103$ C $-6.998322-1.363624-0.385610$ C $-7.799449-2.666352-0.535752$ C -7.257385 -3.7841970 .354012$ C $-5.746657-3.941306 \quad 0.168195$ H $-5.162371-2.2373711 .346211$ C $-3.527423-2.6513120 .082255$ H $-7.306295-0.710821-1.210894$ O $-9.175048-2.454604-0.236070$ H -7.746267 -2.999840 -1.580320 H -7.503218 -3.6014891 .406342$ O $-7.900506-5.0155720 .015300$ H -5.546836 -4.383700 -0.814219 O $-5.242440-4.841868 \quad 1.151312$ C -3.107249-3.002692 -1.330265 H $-3.106164-1.6624280 .294361$ H -3.077036 -3.3756530 .769874$ H $-9.497884-1.751593-0.825400$ H -7.712979 -5.205825 -0.919686 H $-5.737161-5.6746001 .063225$ O $-1.752592-3.183303-1.350926$ O $-3.852762-3.083059-2.296306$ C $-1.190238-3.485528-2.631397$ H -1.342453 -2.646117 -3.316273 H $-0.116301-3.643366-2.503347$ H $-1.633014-4.400868-3.035595$ SCF Energy (B3LYP/6-31G**//MMFF) $=-3245.90569423$

$01 \_599$

MM̄FF Geometry

C $3.392038-2.9588882 .022989$

C $2.516875-3.1220071 .018241$

C $1.035379-2.9068721 .160717$

O $\quad 0.624511-2.1031040 .033009$

C $0.219562-4.2106401 .274793$

C $-0.067991-4.958198-0.011167$

C $-1.281202-5.042658-0.594475$

C $1.090131-5.716617-0.607413$

C $-2.581170-4.408367-0.173045$

C $-3.235531-3.676757-1.352167$

C $-4.611168-3.091659-0.994022$

C $-5.329400-2.654206-2.273613$

C $-6.683926-2.003024-1.976554$

O $-4.430467-1.968553-0.119919$

O $-7.138677-1.361215-3.177603$

C $-6.472931-0.899068-0.925522$

C $-7.752248-3.037511-1.604657$

O $-7.724117-0.350980-0.513344$

C $-5.656985-1.3547020 .311484$

C $-5.318296-0.1048741 .159184$
O $-6.446819-2.254053 \quad 1.089174$

C $-0.509686-1.3718110 .168497$

O $-1.290872-1.4020591 .105235$

C $-0.602813-0.462672-0.995885$

C $-1.6552070 .363416-1.072789$

C $-1.8432771 .456886-2.022994$

C $-0.8588001 .593148-3.151956$

C $-4.471560-0.3545922 .411263$

C -4.0898380 .9794973 .027730$

C -2.5691652 .9268372 .654335$

O -4.7561131 .4884853 .926312$

C -3.1357493 .7746871 .555502$

C -2.3899824 .4220320 .646504$

C $-2.9623265 .243145-0.477427$

C $-2.4758634 .796990-1.843070$

C $-1.5064465 .473867-2.481505$

C $-3.1533513 .610015-2.500010$

C $-2.8493022 .324292-1.788365$

N -2.9827931 .5541252 .424058$

H $3.004793-2.7040633 .008064$

H $2.874406-3.3779410 .026545$

H $0.867702-2.3224422 .075849$

H $-0.724324-3.9651171 .773050$

H $\quad 0.716566-4.8942501 .976795$

H -1.363676 -5.656169-1.491881

H $0.764966-6.445141-1.358464$

H $1.787208-5.032844-1.098522$

H $1.626300-6.2737920 .167614$

H $-3.248940-5.207270 \quad 0.171321$

H -2.460631 -3.716326 0.661850

H $-2.569677-2.873353-1.691001$

H -3.348776 -4.381362 -2.186356

H $-5.194107-3.861366-0.474784$

H $-5.457821-3.501884-2.958461$

H -4.704046 -1.927262 -2.811354

H -7.205515 -2.038362 -3.872413

H -5.924655 -0.082114-1.416094

H -7.891299 -3.755023 -2.422197

H $-8.727798-2.561463-1.455526$

H -7.505135 -3.606233 -0.705794

H -8.191902 $-0.061347-1.315488$

H -4.7886030 .6147220 .522776$

H $-6.246877 \quad 0.383931 \quad 1.482311$

H $-6.069900-2.3098971 .981093$

H $0.225440-0.434476-1.694020$

H $-2.4350330 .273043-0.320364$

H $-0.8694250 .692737-3.776395$

H $\quad 0.1561191 .739298-2.770048$

H -1.071909 2.437250 -3.811816

H $-5.025015-0.9264243 .163407$

H $-3.567122-0.9219432 .177264$

H -1.475248 2.9425852 .666632

H -2.9380443 .2736463 .624461$

H -4.2197093 .8464031 .500922$

H $-1.3069674 .355304 \quad 0.707814$

H $-2.6780096 .288937-0.301732$

H $-4.0592555 .228389-0.464928$

H -1.163621 $5.176730-3.466994$

H $-1.0281286 .336572-2.029647$

H -2.890385 $3.540942-3.561338$

H $-4.2393983 .767938-2.491778$

H -3.512397 $2.134363-0.944625$

H $-2.513773 \quad 1.0492191 .679545$

C $5.505218-3.0554620 .540227$

O $5.213886-1.794315-0.095608$

C $6.395286-1.325185-0.768385$

C $7.365324-2.499997-0.782496$

C $7.029217-3.1761550 .533344$

H $6.818875-0.532087-0.141659$

C $6.030802-0.767238-2.148497$

H $7.169406-3.179429-1.620137$

H $8.408580-2.176285-0.846536$

C $7.538408-4.6034940 .631195$

H $7.458187-2.5841011 .352929$

C $4.9655830 .351551-2.123096$

O $5.520787-1.830999-2.963583$

H $6.936623-0.413260-2.653657$

H $3.998304-0.078677-1.840160$ 
O $4.804240 \quad 0.827643-3.465567$

C $5.2910921 .544617-1.204858$

C $4.3118652 .726191-1.325039$

H $5.3258281 .217953-0.160296$

O $6.5839432 .066441-1.528670$

H $4.6804563 .521252-0.664838$

H $4.3794313 .152866-2.334053$

H $7.258147-5.0530101 .588849$

H $7.130218-5.231154-0.168110$

H $8.630247-4.6282460 .553222$

C $4.894315-3.0654121 .944360$

H $4.744483-2.202273-2.509663$

H $5.6385911 .253158-3.728133$

H $7.2485001 .409289-1.263476$

H $5.296168-2.2091442 .501965$

H $5.197866-3.9718992 .480211$

H $5.067075-3.845354-0.082453$

C 2.9840992 .4311951 .413577

$\begin{array}{lllll}\text { O } & 2.705248 & 1.675139 & 0.231287\end{array}$

C $2.8416072 .400893-0.993873$

C $1.9147793 .627425-0.979404$

C $2.164785 \quad 4.516003 \quad 0.239015$

C $2.1012783 .683408 \quad 1.519156$

H $4.036673 \quad 2.741017 \quad 1.388987$

C $2.858444 \quad 1.4910562 .623387$

H $2.4803701 .734989-1.786308$

O $2.046185 \quad 4.382007-2.179156$

H $0.8737423 .283262-0.941704$

H 3.1197815 .0484660 .165109

$\begin{array}{llllll}\text { O } & 1.153411 & 5.522859 & 0.306441\end{array}$

H 1.0568373 .4156061 .711786

O $2.542126 \quad 4.4734532 .621148$

C 1.4596290 .9579112 .859633

H 3.5054720 .6182532 .474727

H 3.1842852 .0203583 .526096

H $2.8930274 .858139-2.148462$

H $1.1639926 .001505-0.540162$

H 1.9855835 .2709072 .646994

O $1.3816390 .457343 \quad 4.128109$

$\begin{array}{lllll}\text { O } & 0.555349 & 0.956903 & 2.036667\end{array}$

C $0.104998-0.0702324 .503288$

H $-0.652240 \quad 0.719124 \quad 4.475342$

H $-0.175187-0.897443 \quad 3.845124$

H $0.180761-0.4473265 .526482$

SCF Energy (B3LYP/6-31G**//MMFF) $=-3245.90935276$

01_6

MM̄FF Geometry

C $-0.5744403 .503700-1.257036$

C $0.3470554 .183989-0.559082$

$\begin{array}{llllll}\text { C } & 1.059687 & 3.608257 & 0.636211\end{array}$

$\begin{array}{llllll}\text { O } & 2.469850 & 3.614262 & 0.309626\end{array}$

C 0.8243364 .4280811 .919276

C $-0.6285794 .608960 \quad 2.304656$

C $-1.417118 \quad 3.6370182 .803675$

C $-1.172802 \quad 6.0007322 .111682$

C -1.045115 2.2098363 .100298

C -1.775016 $1.242292 \quad 2.158155$

C $-1.526663-0.2485542 .446765$

C $-2.233610-0.7098443 .723068$

C $-1.936691-2.1820464 .040380$

O $-0.117965-0.471362 \quad 2.539176$

O $-2.368986-2.4392685 .385284$

C $-0.409418-2.3929634 .008399$

C $-2.729033-3.1369493 .139623$

O $-0.086863-3.772549 \quad 4.174541$

C $0.266047-1.8413932 .730217$

C $1.805546-1.8745122 .901027$

O $-0.109771-2.654361 \quad 1.621854$

C $3.263876 \quad 2.7225250 .960305$

O 2.9438451 .9861521 .879253

C 4.5955662 .7584900 .316009

C 5.4439831 .7379670 .511824

C $6.7711651 .585470-0.086542$

C $7.2970682 .718939-0.927204$

C $2.596175-1.2913521 .721597$

C $3.009004-2.3103220 .672427$

C $4.292501-2.445758-1.468319$
O $2.761607-3.5092840 .757906$

C $5.769614-2.516636-1.240881$

C $6.674672-1.951869-2.054555$

C $8.157938-2.004957-1.820870$

C $8.780137-0.627856-1.732843$

C $9.356097-0.065836-2.809041$

C $8.8120800 .062077-0.386235$

C 7.4486590 .4378790 .126665

N $3.699361-1.707422-0.369775$

H $-0.8119392 .484681-0.966195$

H $0.5942365 .202797-0.846312$

H 0.7435522 .5690550 .787434

H 1.3055375 .4105721 .811493

H 1.3638873 .9686772 .757333

H -2.449328 3.8881473 .045515

H -2.235939 6.0720542 .364458

H -0.6315946 .7082592 .748490$

H - $1.060645 \quad 6.317417 \quad 1.070229$

H $0.0361462 .056874 \quad 3.034620$

H -1.321689 2.0083514 .140776

H $-2.854128 \quad 1.4396902 .171566$

H $-1.4337941 .447148 \quad 1.136326$

H - $1.914236-0.8101541 .588450$

H -3.315581 -0.5433453 .649138$

H $-1.896252-0.108657 \quad 4.577040$

H $-3.318932-2.2378505 .438109$

H $\quad 0.013536-1.8691354 .877153$

H -3.806287 -2.969791 3.258427

H $-2.554809-4.1811833 .421687$

H -2.493960 -3.023033 2.079842

H $-0.521005-4.0753544 .990494$

H $2.057137-1.2663463 .780412$

H 2.143499-2.896806 3.105768

H $0.514097-2.5026730 .895551$

H $4.8221723 .597773-0.331365$

H $5.1300740 .918818 \quad 1.157265$

H $7.3522403 .641129-0.338254$

H $6.6460272 .891525-1.791448$

H $8.2993892 .536880-1.321596$

H $2.040751-0.4852831 .231123$

H $3.526951-0.8647222 .116754$

H $4.041598-1.913481-2.391054$

H $3.870578-3.453972-1.520843$

H $6.108086-3.055993-0.359298$

H $6.334088-1.423550-2.942234$

H $8.607069-2.566347-2.650634$

H $8.402554-2.573733-0.915175$

H $9.8320770 .907487-2.753418$

H $9.361260-0.565993-3.771983$

H $9.4655340 .942223-0.407083$

H $9.282033-0.6102460 .343498$

H $6.985078-0.3350640 .740540$

H $3.871548-0.706933-0.332007$

C $-2.8484844 .055092-2.225667$

O $-3.3429832 .706014-2.084186$

C $-4.3177242 .688250-1.024391$

C $-4.6780644 .146960-0.757368$

C $-3.3493204 .829118-1.005556$

H $-3.8107442 .284803-0.140413$

C $-5.5164031 .809001-1.386737$

H $-5.4315174 .517085-1.462329$

H $-5.058337 \quad 4.3011910 .257077$

C $-3.4564216 .327311-1.224571$

H $-2.7105204 .643613-0.135129$

C $-5.1781830 .342554-1.722750$

O $-6.1654472 .377018-2.532486$

H $-6.248778 \quad 1.847952-0.571077$

H $-4.6690090 .297624-2.692254$

O $-6.426308-0.340528-1.911609$

C $-4.338810-0.407590-0.668216$

C $-4.355945-1.942552-0.801122$

H $-3.305216-0.048416-0.709472$

O $-4.821258-0.0967550 .643639$

H $-3.763246-2.3512240 .025394$

H $-5.375594-2.300291-0.610315$

H -2.470314 $6.764108-1.411520$

H $-4.0985706 .563757-2.079418$

H -3.879924 $6.815452-0.340733$ 
C $-1.3304314 .050903-2.433280$ H $-6.9278761 .811281-2.744123$ H $-6.913496-0.311357-1.070793$ H $-5.734076-0.423456 \quad 0.713024$ H $-0.9917135 .065853-2.671610$ H -1.091421 $3.417996-3.296825$ H $-3.3159024 .449840-3.137423$ C $-1.514206-2.774982-1.593303$ O $-2.492103-2.127285-2.416700$ C $-3.850502-2.489293-2.150448$ C $-4.032450-4.006001-2.311039$ C $-3.063082-4.780490-1.424235$ C $-1.634978-4.303301-1.673425$ H -1.638401 -2.441944 -0.557492 C $-0.126518-2.337209-2.089873$ H -4.452145 -2.020602 -2.937575 O $\quad-5.376263-4.368227-2.012665$ H $-3.850438-4.276643-3.359480$ H $-3.326530-4.698139-0.363251$ O $-3.125596-6.170516-1.750630$ $\mathrm{H}-1.303304-4.654280-2.659422$ O $-0.770694-4.890430-0.701510$ C $0.196617-0.899455-1.752701$ H $0.658357-2.951433-1.634856$ H $-0.080844-2.441744-3.180824$ H $-5.488762-5.304501-2.247876$ H -4.000655 -6.495878 -1.480083 H $-0.878712-5.855053-0.761427$ O $1.347595-0.549881-2.400640$ O $-0.455705-0.183880-1.006912$ C $1.827408 \quad 0.775233-2.156492$ H $1.2198581 .492812-2.713623$ H $1.8176201 .002519-1.087225$ H $2.8590770 .834658-2.513468$ SCF Energy (B3LYP/6-31G**//MMFF) $=-3245.90970602$

0160

MM̄FF Geometry

C $1.639990 \quad 4.2292150 .091134$

C $0.5854054 .789843-0.520838$

C $-0.6648114 .079484-0.988596$

O $-0.6253442 .667224-0.680104$

C $-1.8967594 .668558-0.276197$

C $-3.1977304 .146112-0.852541$

C $-3.8863623 .093482-0.368474$

C $-3.7084754 .896492-2.055503$

C -3.520325 2.2093390 .792486

C -4.6281172 .0758131 .850109$

C $-5.918377 \quad 1.4193231 .334354$

C $-6.895041 \quad 1.1445332 .479970$

C -8.1511570 .4011821 .994519$

$\begin{array}{lllll}\text { O } & -5.567345 & 0.189818 & 0.694941\end{array}$

O $-8.836326-0.108595 \quad 3.148849$

C -7.722452 -0.8186181 .148752$

C $-9.133688 \quad 1.344326 \quad 1.289726$

O $-8.851947-1.4464460 .546748$

C $-6.667422-0.4816950 .070821$

C $-6.127897-1.776730-0.581341$

O $\quad-7.2748630 .323895-0.942842$

C $-0.076121 \quad 1.818874-1.588994$

O $0.4335092 .109474-2.658453$

C $-0.1855390 .453103-1.027066$

C $0.547015-0.544153-1.543906$

C $0.577357-1.927981-1.062157$

C $-0.430515-2.324776-0.015262$

C $-4.855149-1.562030-1.415471$

C $-4.464406-2.800991-2.201495$

C $-2.729520-3.614443-3.795553$

O $-5.128792-3.833733-2.205615$

C -1.808013-4.502800 -3.021560

C $-0.557734-4.790928-3.413573$

C $0.344837-5.739145-2.679234$

C $1.695395-5.140576-2.362654$

C $2.792724-5.503841-3.048202$

C $1.790100-4.192965-1.189287$

C $1.505793-2.765332-1.570096$

N -3.289088 -2.605753 -2.915909

H $1.6432403 .165120 \quad 0.301010$
H $\quad 0.6182615 .858032-0.728066$

H $-0.7601074 .220271-2.072545$

H -1.895851 $5.763986-0.356932$

H -1.8448054 .4587280 .798581$

H $-4.7938392 .803482-0.895195$

H $-4.6494314 .484610-2.435529$

H -3.888195 $5.945753-1.799032$

H -2.978971 $4.857479-2.870373$

H $-3.266372 \quad 1.2182230 .398139$

H -2.6208972 .5653591 .300906$

H -4.2191891 .4625652 .663933$

H -4.8590823 .0598082 .276509$

H -6.3824762 .0906130 .603532$

H -7.170629 2.0764342 .988912

H -6.4001890 .5229873 .239458$

H -9.0489190 .6435423 .727680$

H -7.287624 -1.556874 1.837174

H $-9.4526372 .142874 \quad 1.970419$

H -10.0487280 .8165700 .999010$

H -8.7111821 .8188060 .401569$

H $-9.482077-1.652621 \quad 1.258351$

H -5.885260 -2.509969 0.198821

H $-6.908943-2.219969-1.212049$

H $-6.8174050 .178821-1.785690$

H $-0.8416720 .314055-0.174963$

H $1.212435-0.321957-2.377225$

H -1.445537 -2.072895 -0.342466

H $-0.228922-1.8073980 .928510$

H $-0.440039-3.3956240 .197393$

H -4.993409-0.746611 -2.133411

H $-4.019031-1.298375-0.757074$

H -2.216191-3.090351-4.607804

H -3.542924 -4.210767 -4.221930

H -2.200189-4.961993 -2.117008

H $-0.176092-4.358151-4.335606$

H $\quad 0.467502-6.631187-3.307405$

H $-0.118512-6.093741-1.750032$

H $3.769904-5.100194-2.804007$

H $2.737948-6.207833-3.871854$

H $2.808793-4.219684-0.780442$

H $1.155930-4.558649-0.377787$

H $2.190522-2.375896-2.324636$

H $-2.769558-1.741321-2.793018$

C $4.1285364 .592224-0.244645$

O 4.4763813 .2149600 .007981

C $4.9665472 .639816-1.218743$

C $5.1633883 .801756-2.190864$

C $4.0580574 .743917-1.764055$

H $4.1671561 .994970-1.602174$

C $6.2386221 .821388-0.980892$

H $6.1413324 .280169-2.061942$

H $5.0803323 .485295-3.235213$

C $4.2431526 .169439-2.252481$

H $3.1114754 .352595-2.156531$

C 6.0972300 .6456310 .007184

O $7.2526792 .697361-0.469446$

H $6.6119781 .454800-1.945292$

H $6.003390 \quad 1.030748 \quad 1.028994$

O $7.340953-0.071175-0.026735$

C $4.942625-0.331325-0.285363$

C $5.055527-1.6954330 .421027$

H $3.9915220 .146446-0.028141$

O $4.912846-0.592635-1.693208$

H $4.180492-2.2880590 .131396$

H $5.908133-2.240603-0.004176$

H $3.4239226 .808321-1.908065$

H $5.1825216 .599792-1.889678$

H $4.2595496 .201697-3.346883$

C 2.8642484 .9850980 .523965

H $8.0558732 .165038-0.337302$

H $7.429628-0.463332-0.912562$

H $4.130534-1.140733-1.873093$

H 2.7021856 .0654190 .429338

H 3.0146024 .7771111 .590522

H 4.9624185 .1876780 .150751

C $2.828122-1.6681242 .519636$

O $4.080982-0.9765232 .581284$

C $5.179556-1.6449001 .956197$ 
C $5.387097-3.0244542 .599845$

C $4.117219-3.8654552 .539702$

C $2.938825-3.0817363 .112669$

H $2.490623-1.7149321 .478155$

C $1.805731-0.8510803 .326138$

H $6.071106-1.0527902 .191864$

O $6.447946-3.729708 \quad 1.963677$

H $5.671442-2.8968533 .652579$

H $3.917193-4.2088301 .518711$

O $4.316489-5.043838 \quad 3.324397$

H $3.056277-3.0211144 .202181$

O $1.734098-3.8070932 .870139$

C 1.4788820 .4830102 .695262

H $0.864739-1.4051093 .416383$

H $2.206209-0.6486314 .326939$

H $7.245662-3.1774032 .028243$

H $3.484358-5.5472313 .307080$

H $1.606929-3.8594241 .907612$

$\begin{array}{lllll}\text { O } & 0.601278 & 1.144360 & 3.507847\end{array}$

$\begin{array}{lllll}\text { O } & 1.928717 & 0.894440 & 1.635832\end{array}$

C $0.194172 \quad 2.4392813 .058020$

H -0.2647702 .3700022 .068906$

H -0.5481382 .8267153 .760840$

H 1.0519293 .1178273 .042943

SCF Energy (B3LYP/6-31G**/MMFF) $=-3245.91005645$

01600

MM̄FF Geometry

C $-1.097918-2.1555651 .876313$

C $-1.881305-1.8131442 .910469$

C $-1.937509-0.4613693 .586861$

O $-0.879133 \quad 0.4029713 .102430$

C -3.2676170 .2600143 .297797$

C $-4.499959-0.438305 \quad 3.839512$

C $-5.354707-1.1690753 .094588$

C $-4.753020-0.2416875 .311021$

C $-5.234334-1.4802331 .628469$

C $-6.316164-0.7732240 .798178$

C $-5.814346-0.267377-0.564023$

C $-5.281803-1.385847-1.463509$

C $-4.658239-0.834195-2.756562$

O $-4.792208 \quad 0.695680-0.292887$

O $-3.924509-1.895698-3.384774$

C $-3.662927 \quad 0.292980-2.398766$

C $-5.733410-0.433340-3.771924$

O $-3.157641 \quad 0.920388-3.574453$

C $-4.2571791 .352593-1.445138$

C $-3.1557142 .324323-0.957080$

O $-5.2580112 .100009-2.140041$

C $0.307573 \quad 0.370297 \quad 3.761225$

O $0.554903-0.2547894 .784459$

C $1.351696 \quad 1.1648653 .073034$

C $1.184890 \quad 1.8193201 .912094$

C 2.2347932 .5374431 .178671

C 3.6206572 .5337231 .771902

C $-3.597371 \quad 3.188456 \quad 0.231447$

C $-2.553624 \quad 4.227260 \quad 0.577916$

C $-1.722536 \quad 6.468624-0.122913$

O $-1.763673 \quad 4.0695631 .505318$

C $-0.5081216 .248157-0.966132$

C $0.7437026 .372837-0.501436$

C $1.9628506 .210419-1.359223$

C $3.0053495 .311751-0.735997$

C $4.0728195 .839343-0.112275$

C $2.8798253 .820480-0.942322$

C $1.930188 \quad 3.1451810 .011280$

$\mathrm{N}-2.6070825 .329553-0.258768$

H $-0.400423-1.4340591 .460408$

H -2.559568 -2.564557 3.310136

H -1.830678 -0.6001424 .669589$

H -3.354131 $0.444946 \quad 2.220777$

H -3.221608 1.2716483 .724998

H $-6.221667-1.6117973 .582524$

H $-5.671645-0.7359775 .644590$

H $-3.926515-0.6505685 .900627$

H -4.8506120 .8244675 .540362$

H -4.234641 -1.2373081 .260534$

H -5.333933 -2.565158 1.497756
H -7.164209-1.452951 0.650665

H $-6.6962740 .094824 \quad 1.353598$

H $-6.6537630 .240828-1.053104$

H $-6.074258-2.108871-1.692987$

H $-4.508679-1.955790-0.933759$

H -3.227592 -2.183764 -2.771657

H $-2.801183-0.171885-1.900110$

H $-6.335661-1.305901-4.053210$

H $-5.283473-0.068743-4.702077$

H $-6.4172000 .330744-3.396622$

H -2.791654 $0.220392-4.141912$

H $-2.2755641 .752923-0.633566$

H -2.834550 $2.967572-1.785762$

H $-5.3096272 .993727-1.766799$

H 2.3136301 .1468553 .576775

H $0.207866 \quad 1.8077181 .432840$

H 3.9901761 .5102891 .885368

H 3.6177913 .0176992 .754916

H 4.3578023 .0644151 .167530

H -4.5435983 .7022340 .029442$

H -3.7624792 .5727351 .122249$

H -1.4737726 .6105180 .933973$

H -2.267626 $7.348840-0.477299$

H $-0.6664786 .004046-2.013982$

H $0.899706 \quad 6.6494570 .538869$

H $2.3857107 .210475-1.523907$

H $1.7055535 .832697-2.356596$

H $4.8581105 .214266 \quad 0.297971$

H 4.1864896 .9123870 .002174

H $3.8668263 .346452-0.901283$

H $2.5377373 .623773-1.966962$

H $0.8983953 .115682-0.339531$

H -3.253913 $5.326110-1.041996$

C $-1.335525-3.380996-0.290104$

O $-0.148217-2.816714-0.882523$

C $0.192288-3.570159-2.058807$

C $-1.021608-4.433640-2.374198$

C $-1.551206-4.725329-0.984637$

H $1.025469-4.227578-1.784063$

C $0.616355-2.623564-3.185309$

H $-1.767065-3.888499-2.962350$

H $-0.753655-5.337385-2.930020$

C $-2.994500-5.199380-0.972524$

H $-0.916820-5.493052-0.521346$

C $1.799269-1.697542-2.829184$

O $-0.493665-1.787659-3.539937$

H $\quad 0.853965-3.203070-4.084836$

H $1.483379-0.953329-2.089051$

O $2.146836-0.967678-4.012877$

C $3.055372-2.432258-2.326120$

C $4.317667-1.559761-2.219262$

H $2.850465-2.882966-1.351348$

O $3.361587-3.511997-3.215031$

H $5.145886-2.217821-1.929092$

H $4.590347-1.196913-3.218398$

H $-3.338472-5.3783950 .050984$

H -3.663467 -4.461655 -1.428033

H $-3.096426-6.134929-1.531939$

C $-1.133189-3.5085251 .220590$

H $-0.761188-1.302474-2.740455$

H $1.344845-0.507446-4.315096$

H $3.508172-3.132534-4.098694$

H $-0.179744-4.008268 \quad 1.425473$

H $-1.930436-4.1220741 .655023$

H -2.160858 -2.692996 -0.513195

C $4.827112-1.5236570 .795871$

O $3.835129-0.7767090 .080859$

C $4.208055-0.373740-1.240836$

C $5.4755950 .489987-1.183948$

C $6.615051-0.236320-0.478535$

C $6.153008-0.7502660 .882335$

H $4.982873-2.4907100 .302396$

C $4.286509-1.7847602 .212400$

H $3.400197 \quad 0.271877-1.604728$

$\begin{array}{llll}\text { O } & 5.896747 & 0.868146 & -2.489970\end{array}$

H $5.2654851 .415015-0.634356$

H $7.019518-1.049863-1.091536$

O $7.6942650 .677316-0.265976$ 
H $\quad \begin{array}{lll}6.047410 & 0.098148 & 1.570483\end{array}$

O $7.158482-1.6072671 .421950$

C $3.146560-2.7791962 .249656$

H $5.085991-2.1895042 .843627$

H $3.917189-0.8524942 .653787$

H $5.1568941 .339288-2.909968$

H $7.9498681 .029010-1.136104$

H $7.987264-1.0989741 .452050$

O $2.903248-3.1089103 .553480$

O $2.539628-3.1996581 .275686$

C $1.860513-4.0607583 .788048$

H $2.022232-4.5027434 .774972$

H $0.896050-3.5477773 .788533$

H $1.872061-4.8630293 .043923$

SCF Energy (B3LYP/6-31G**//MMFF) $=-3245.91438520$

$01 \_01$

MM̄FF Geometry

C $1.0957323 .805063-0.863937$

C $2.306320 \quad 3.451318-1.321648$

C $2.6645382 .102265-1.900840$

O $1.4799211 .396866-2.349185$

C $3.5995052 .203156-3.124158$

C $5.0629972 .047058-2.760728$

C $5.7546490 .896699-2.883722$

C $5.749710 \quad 3.281917-2.240169$

C $5.257984-0.428987-3.390074$

C $4.401891-1.228090-2.401903$

C $4.969189-1.386939-0.982937$

C $6.301629-2.138580-0.967736$

C $6.803487-2.3836340 .462488$

O $3.990279-2.116457-0.225733$

O $7.870400-3.3400520 .373550$

C $5.664751-3.0154041 .289760$

C $7.414760-1.1138771 .066698$

O $6.046777-3.1442262 .657894$

C $4.313673-2.2717311 .163083$

C $3.198017-3.1072581 .838352$

O $4.418562-1.0101321 .826176$

C $0.9481530 .453220-1.526446$

O $1.302057 \quad 0.182209-0.390364$

C $-0.134234-0.226034-2.273668$

C $-0.609383-1.391346-1.811618$

C $-1.639826-2.223905-2.433132$

C $-2.353610-1.677270-3.639583$

C $1.777440-2.5795661 .600970$

C $0.721974-3.4293112 .291448$

C $-1.753730-3.540636 \quad 2.623854$

O $0.985312-4.442758 \quad 2.932787$

C -2.300768 -4.4727561 .590208$

C $-3.452443-4.270068 \quad 0.932189$

C $-3.985991-5.206738-0.114321$

C $-4.110332-4.559724-1.477668$

C $-5.304658-4.154629-1.940204$

C $-2.875488-4.478686-2.349379$

C $-1.896907-3.432085-1.890157$

$\mathrm{N}-0.553147-2.9145832 .100401$

H $0.274428 \quad 3.100077-0.917415$

H $3.1186024 .165104-1.211838$

H $3.1642041 .519980-1.115841$

H $3.4533153 .154376-3.653169$

H $3.3203571 .439160-3.860077$

H $6.8019390 .894453-2.583772$

H $6.8329213 .146323-2.148591$

H $5.5849404 .125721-2.917984$

H $5.3682383 .545270-1.249671$

H $4.697872-0.288059-4.322129$

H $6.131069-1.029209-3.675983$

H $3.411105-0.766678-2.328808$

H $4.208987-2.222273-2.827194$

H $5.077632-0.392459-0.536429$

H $7.067650-1.609371-1.546359$

H $6.173400-3.111683-1.462579$

H $8.145483-3.5594721 .280374$

H $5.532428-4.0404440 .917329$

H $8.254948-0.7670020 .452913$

H $7.833305-1.3082662 .060364$

H $6.701539-0.2908071 .147669$
H $\quad 5.971312-2.2665453 .069650$

H $3.236591-4.1351561 .453956$

H $3.377901-3.1571682 .919859$

H $3.529108-0.6922602 .045598$

H $-0.4814490 .228779-3.194225$

H $-0.186833-1.791602-0.892266$

H $-1.672919-1.628020-4.496400$

H -2.736364 -0.670618 -3.442762

H $-3.216266-2.277041-3.939007$

H $1.676085-1.555513 \quad 1.974348$

H $1.557435-2.5819330 .528302$

H -2.457782 -2.740766 2.873314

H $-1.516308-4.0935783 .538084$

H $-1.711868-5.3604551 .370484$

H -4.045933 -3.3867051 .155520$

H $-4.967440-5.5612050 .226994$

H $-3.364140-6.106721-0.199049$

H $-5.411038-3.723778-2.930073$

H $-6.202430-4.240992-1.337069$

H -3.152561 -4.327302 -3.398290

H $-2.368853-5.452540-2.339709$

H $-1.307202-3.739442-1.027019$

H $-0.671659-2.0996871 .505291$

C 0.5618694 .9924591 .306790

O $\begin{array}{lllll}0.529721 & 4.088201 & 1.590020\end{array}$

C -0.0885003 .1474012 .584756$

C 1.1059463 .8044323 .268301

C 1.7750734 .4878672 .095584

H $\quad 0.2560122 .2571212 .044883$

C $-1.215787 \quad 2.768634 \quad 3.547547$

H 0.7929154 .5491544 .009283

H 1.7520783 .0764193 .768085

C 2.7455925 .5857342 .495382

H 2.3256923 .7255301 .532665

C -2.494421 2.1909172 .909624

O -1.6063693 .9283754 .296803$

H $-0.826763 \quad 2.058575 \quad 4.287444$

H -3.0744072 .9923612 .436744$

O $-3.301274 \quad 1.695391 \quad 3.989491$

C -2.287882 1.0484121 .896512

$\begin{array}{llll}\text { C } & -3.547043 & 0.207155 & 1.597861\end{array}$

H $-1.8962191 .462848 \quad 0.962405$

\begin{tabular}{llll}
\hline & -1.290982 & 0.145955 & 2.385924
\end{tabular}

H -3.274881 -0.5382120 .840388$

H -3.790308 -0.3833092 .490554$

H $3.1886686 .053347 \quad 1.610412$

H 2.2491136 .3685323 .078647

H 3.5581995 .1775473 .105185

C $0.7810485 .118806-0.206015$

H $-1.882733 \quad 4.609027 \quad 3.659091$

H -3.453612 2.4364504 .600858

H $-1.598281-0.197024 \quad 3.242707$

H $1.5723875 .851276-0.403865$

H $-0.1347795 .503998-0.671091$

H 0.2456665 .9710161 .690128

C $-4.4484280 .980605-1.243328$

O $-4.5578001 .758109-0.044812$

C $-4.787074 \quad 1.007774 \quad 1.150375$

C $-6.061707 \quad 0.163644 \quad 1.011497$

C $-6.027557-0.718178-0.231123$

C $-5.7044040 .124796-1.462045$

H $-3.5601880 .340331-1.183237$

C $-4.2631051 .957893-2.416362$

H $-4.997057 \quad 1.744144 \quad 1.934101$

O $-6.253389-0.6357992 .174220$

H -6.9258370 .8376570 .942143$

H $-5.317165-1.543193-0.109404$

O $-7.318746-1.310310-0.386853$

H $-6.5613340 .769462-1.697551$

O $-5.493742-0.733298-2.580298$

C $-2.8985812 .607577-2.412692$

H $-4.3684571 .423201-3.367037$

H $-5.0081112 .760483-2.368930$

H $-7.055522-1.1673202 .032734$

H $-7.267610-1.937819-1.127132$

H $-6.338198-1.170499-2.780659$

O $-2.661390 \quad 3.157374-3.640017$

O $-2.1453572 .649395-1.450963$ 
C $-1.4004653 .817925-3.786652$

$\mathrm{H}-0.5834923 .100802-3.665456$

H $-1.3516514 .236986-4.795053$

$\mathrm{H}-1.3117674 .634170-3.063512$

SCF Energy (B3LYP/6-31G**//MMFF) $=-3245.92350254$

01602

MM̄FF Geometry

C -3.437487 3.0084991322948

C $-2.8105264 .105808 \quad 1.775277$

C -1.3151284 .2749491 .931306$

O -0.6272613 .0390871 .638428$

C -0.8002825 .3414490 .948123$

C 0.6842685 .6189421 .090945

C 1.6233715 .3106930 .174189

C 1.0881426 .3190012 .364552

C $1.4468564 .657359-1.168498$

C $1.9633083 .215730-1.228012$

C $3.4417063 .002567-0.861903$

C $4.3898943 .807894-1.754974$

C $5.8653443 .490271-1.468492$

O $3.6998491 .596769-1.010438$

O $6.6498554 .033960-2.541625$

C $6.0576061 .961026-1.499713$

C $6.3583604 .169498-0.185414$

O $7.3802011 .603628-1.106300$

C $5.0273181 .195034-0.642507$

C $5.132882-0.336346-0.829818$

$\begin{array}{lllll}\text { O } & 5.276640 & 1.462773 & 0.742902\end{array}$

C -0.2610242 .2483742 .682445$

O -0.4059742 .4759643 .872027$

C 0.3337861 .0219532 .107366

C 0.7747170 .0427142 .910622

C $1.342560-1.2319542 .466501$

C $1.473058-1.4506250 .982795$

C $4.870536-0.840490-2.250459$

C $4.891509-2.356179-2.291899$

C $3.525824-4.353032-1.673440$

O $5.877520-2.977716-2.681029$

C $3.661359-4.794357-0.244572$

C $4.321980-4.1585050 .736440$

C $4.370218-4.6309782 .162326$

C $3.782025-3.6074183 .112480$

C $4.586492-2.8572753 .885480$

C $2.269061-3.5227223 .150108$

C $1.715806-2.1418593 .390866$

N 3.717342 -2.922232 -1.823478

H -2.8581822 .1377531 .028518$

H -3.410955 4.9660582 .064474

H -1.115866 4.5913652 .961864

H -1.342342 6.2843921 .100739

H -1.040515 $5.025770-0.072065$

H 2.6553095 .5764440 .401585

H 2.1454376 .6054072 .364966

H 0.5028917 .2350452 .496340

H 0.9243395 .6712883 .230977

H $0.3993794 .656816-1.482747$

H $1.9641615 .269382-1.916773$

H $1.3488982 .585270-0.572629$

H $1.7876702 .823599-2.239103$

H 3.5820443 .2690770 .191584

H $4.2186354 .885316-1.650914$

H $4.1822323 .571862-2.808049$

H $6.4796834 .990676-2.582689$

H $5.9509911 .652544-2.546929$

H $6.2686035 .259098-0.270543$

H $7.4221173 .969194-0.016183$

H $5.803043 \quad 3.8637300 .703617$

H $7.9953972 .101236-1.671699$

H $6.114848-0.688728-0.487192$

H $4.412292-0.814384-0.153672$

H 4.8723630 .7547521 .271547

H $\quad 0.3718910 .9489341 .026299$

H 0.6991570 .1751423 .988896

H $1.908059-2.4162180 .724065$

H $2.116108-0.6848970 .536508$

H $\quad 0.492618-1.405418 \quad 0.499320$

H $3.902594-0.488199-2.624298$
H $5.635605-0.483348-2.947385$

H $4.228042-4.903942-2.306591$

H $2.508531-4.563560-2.018955$

H $3.144653-5.720696-0.001041$

H $4.863394-3.2415110 .520860$

H $5.418568-4.8341722 .415233$

H $3.841887-5.5841122 .288931$

H $4.194771-2.1341454 .592123$

H $5.666163-2.9550873 .834299$

H $1.903664-4.1897693 .941889$

H $1.843810-3.9323062 .227803$

H $1.595825-1.8944024 .446016$

H $2.975349-2.313267-1.491434$

C $-5.3379652 .654144-0.266903$

O $-4.8451201 .379767-0.727179$

C $-5.8208710 .795525-1.611920$

C $-6.9228101 .836242-1.788013$

C $-6.8487042 .593707-0.477018$

H $-6.239132-0.073531-1.092172$

C $-5.1539240 .354931-2.919676$

H $-6.7173692 .508298-2.629315$

H -7.900097 $1.375987-1.963478$

C $-7.5274183 .951363-0.524579$

H $-7.313316 \quad 1.9828370 .308766$

C $-3.988796-0.641769-2.744059$

O $-4.632013 \quad 1.510219-3.591239$

H -5.907338 $-0.067673-3.594367$

H $-3.144846-0.143837-2.253113$

O $-3.534465-1.007340-4.054134$

C $-4.351049-1.929123-1.979648$

C $-3.264986-3.022301-2.030411$

H $-4.577211-1.683206-0.936758$

O $-5.544512-2.476960-2.547723$

H $-3.668326-3.919219-1.544793$

H -3.101815 -3.324540 -3.072464

H -7.4486524 .4630090 .439644$

H -7.078780 $4.597732-1.286415$

H $-8.5907853 .841184-0.761048$

C -4.9297602 .8808351 .189016$

H -3.999423 $1.938571-2.989005$

H $-3.307649-0.185452-4.522115$

H -5.797820 -3.248012 -2.012116

H -5.2483922 .0312481 .804457$

H -5.4251343 .7749881 .583824$

H $-4.8903843 .423323-0.910904$

C $-2.478538-3.1722270 .907746$

O $-2.101688-2.159383-0.034241$

C $-1.928399-2.618855-1.378239$

C $-0.847477-3.707161-1.425077$

C $-1.134788-4.838512-0.442912$

C $-1.422048-4.2852480 .952576$

H -3.452105 -3.5912640 .627657$

C $-2.627439-2.4920442 .273494$

H - $1.538665-1.766405-1.947036$

O $-0.728076-4.223629-2.746520$

H $\quad 0.120826-3.257739-1.171589$

H -1.956760 -5.470006 -0.800223

O $0.019575-5.680339-0.382309$

H $-0.485901-3.9123821 .384401$

O $\quad-1.872087-5.366148 \quad 1.766402$

C $-3.735200-1.4666392 .273189$

H -2.837962 -3.207804 3.074987

H -1.702355 -1.968776 2.540717

H $-0.051592-4.922120-2.725775$

H $-0.162434-6.3733260 .275408$

H -1.837291 -5.0757092 .692923$

O $-4.946988-2.0821942 .168414$

O $-3.542945-0.2617832 .370836$

C $-6.081363-1.2102002 .171427$

H $-6.012856-0.4921051 .350007$

H $-6.978454-1.8186152 .030266$

H -6.154685-0.691364 3.131890

SCF Energy (B3LYP/6-31G**//MMFF) $=-3245.91433286$

01603

MM̄FF Geometry

C 2.3428293 .8773720 .500805

C 1.3810384 .7273870 .110520 
C $-0.0573124 .380474-0.207094$

O $-0.2965042 .955316-0.182519$

C -0.9758665 .0296770 .845490$

C $-2.4559184 .856958 \quad 0.564812$

C -3.3154054 .1963441 .365373$

C $-2.9636425 .513647-0.693453$

C -3.0276143 .5064982 .669443$

C -2.7362862 .0026682 .546683$

C -3.9246591 .1716282 .035755$

C $-3.628494-0.3248742 .150020$

C $-4.761522-1.1851631 .568197$

$\begin{array}{lllll}\text { O } & -4.155525 & 1.502237 & 0.664724\end{array}$

O $-4.240678-2.5177321 .444245$

C -5.114201-0.672389 0.153104

C $-5.952576-1.2699682 .529914$

O $-6.280742-1.316973-0.352043$

C -5.2971250 .8583640 .083628$

C $-5.434474 \quad 1.390970-1.363894$

O $-6.498078 \quad 1.2178930 .778466$

C $0.0243602 .242694-1.297007$

O $0.5313332 .666168-2.323295$

C $-0.3342740 .830951-1.039332$

C $0.206114-0.138973-1.791514$

C $-0.039112-1.574689-1.642604$

C $-1.196915-1.995803-0.779078$

C $-4.237551 \quad 1.118547-2.282513$

C $-4.397894-0.109411-3.164053$

C $-3.144201-1.467871-4.837785$

O $-5.442176-0.749506-3.249175$

C $-2.632038-2.697549-4.157344$

C $-1.560029-3.384164-4.581036$

C $-1.067444-4.647388-3.935260$

C $0.394330-4.578939-3.554900$

C $1.338389-5.136570-4.331643$

C $0.750484-3.943975-2.230626$

C $0.763285-2.442053-2.293065$

N $-3.238139-0.384296-3.877304$

H 2.1170292 .8228900 .631681

H $1.6383365 .778958-0.004645$ H $-0.2928704 .771775-1.204273$ H $-0.769235 \quad 6.1065380 .908234$ H -0.7198224 .6160641 .826840$ H -4.3604514 .1494691 .060917$ H -4.056482 $5.487818-0.763704$ H -2.660478 $6.565334-0.722623$ H -2.567228 $5.008995-1.579712$ H -2.191802 3.9879003 .187590 H -3.8920933 .6541003 .329455$ H -1.8713281 .8595511 .887975$ H -2.450004 1.6396213 .541053 H -4.8078861 .4328902 .630336$ H -3.430027 -0.6065173 .191613$ H -2.705919 -0.5510661 .599296$ H -4.953413 -3.091189 1.115705 H -4.287368 -0.961169-0.504647 H $-5.642987-1.7208543 .480381$ H -6.738601 -1.921317 2.131835 H -6.393762 -0.2959952 .752210$ H $-6.091508-2.268496-0.408522$ H $-6.3750831 .042461-1.806983$ H $-5.5351112 .484170-1.296318$ H -6.790399 2.0878010 .459380 H $-1.0173840 .627337-0.222658$ H $\quad 0.918770 \quad 0.137532-2.567555$ H -2.107361 -1.470133-1.083168 H $-0.998373-1.7680110 .270594$ H -1.428647 -3.060853 -0.845657 H $-4.1276521 .970308-2.966320$ H $-3.3084301 .038106-1.710014$ H -2.481546 -1.132556 -5.641900 H -4.133976 -1.666193 -5.261579 H -3.186262 -3.055510 -3.292846 H -1.023555 -3.039964 -5.462531 H -1.229827 -5.468354 -4.646070 H -1.662247 -4.903342 -3.049638 H $2.386061-5.117130-4.050165$ H $1.084971-5.620032-5.269452$ H $1.756442-4.260384-1.922284$
H $\quad 0.091153-4.335010-1.450387$

H $1.561625-2.040543-2.917373$

H $-2.4019850 .164168-3.698175$

C $4.7848333 .745550-0.190905$

O $4.7302652 .306905-0.225733$

C $4.8008421 .872142-1.596823$

C $5.3223603 .077568-2.371606$

C $4.6396254 .215280-1.637679$

H $3.7778781 .652252-1.927701$

C $5.6794210 .624375-1.733979$

H $6.4125823 .164003-2.287148$

H $5.0638573 .033870-3.433831$

C $5.2544345 .576521-1.909642$

H $3.5851054 .235137-1.938982$

C $5.055510-0.712757-1.278941$

O $6.9189370 .824134-1.055728$

H $5.9214230 .514399-2.798905$

H $5.822210-1.487734-1.410039$

O $3.971598-1.042648-2.147636$

C $4.560812-0.8000350 .179047$

C $4.165200-2.2439650 .556940$

H $5.351622-0.4569210 .854587$

$\begin{array}{llll}\text { O } & 3.429338 & 0.044432 & 0.366855\end{array}$

H $3.260609-2.5253660 .006805$

H $4.964644-2.9229820 .238383$

H $4.7379526 .356785-1.341678$

H $6.3136685 .601965-1.632441$

H $5.1796035 .826526-2.972987$

C 3.7561014 .2936490 .801623

H $6.7159351 .082680-0.140574$

H $4.319323-1.076732-3.054884$

H $2.760279-0.208010-0.291932$

H 3.8395315 .3856640 .850784

H 3.9936253 .9131811 .803069

H 5.7853624 .0001910 .183157

C $1.594619-1.8466792 .224403$

O $2.940365-1.5414382 .601022$

C $3.931793-2.4285162 .075739$

C $3.623498-3.8747882 .495176$

C $2.200004-4.2873892 .127128$

C $1.197943-3.2625032 .657106$

H $1.510082-1.7747521 .133837$

C $0.673959-0.7508842 .780896$

H $4.874676-2.1484662 .563067$

O $4.541679-4.7880071 .903654$

H $3.736932-3.9665493 .583338$

H $2.086517-4.4254041 .045386$

O $1.906017-5.5508222 .727299$

H $1.151863-3.3403283 .749569$

O $-0.100272-3.5656172 .154583$

C $0.608979-0.7001074 .292849$

H 1.0226720 .2339382 .447976

H $-0.339266-0.9035982 .394222$

H $5.436163-4.5180432 .173421$

H $2.577008-6.1818062 .414609$

H $-0.305147-4.4809302 .411838$

O $-0.603023-0.1972264 .670675$

O $1.514964-1.0262415 .047362$

C $-0.807235-0.0717606 .081156$

H $-0.736922-1.0515456 .563186$

H $-0.078508 \quad 0.623457 \quad 6.508573$

H -1.811393 $0.328407 \quad 6.244309$

SCF Energy (B3LYP/6-31G**//MMFF)= -3245.90198409

01604

MM̄FF Geometry

C $3.626538-0.5447913 .321364$

C 2.9029240 .5750953 .166420

C 1.4736500 .8048043 .601210

O $0.752686 \quad 1.2707742 .431264$

C $0.739624-0.4394264 .149889$

C $-0.689399-0.1789114 .590052$

C $-1.778062-0.7214804 .008610$

C $-0.850750 \quad 0.721067 \quad 5.788493$

C $-1.822146-1.7139122 .880184$

C $-1.994608-1.0329581 .520071$

C $-2.033580-2.0480180 .369797$

C $-2.175997-1.330641-0.973845$ 
C $-2.264150-2.323449-2.143464$ O $-3.148222-2.9196240 .575236$ O $-2.742113-1.603782-3.293518$ C $-3.333755-3.392391-1.815672$ C $-0.882222-2.861788-2.531081$ O $-3.351431-4.430248-2.791244$ C $-3.252551-3.977566-0.384787$ C $-4.494777-4.827182-0.016034$ O $-2.163543-4.898794-0.242226$ C 0.0934242 .4578042 .519796 O 0.1164183 .2505463 .447904 C -0.7217542 .6168901 .295178$ C $-1.8166193 .390667 \quad 1.349647$ C $-2.7652303 .642552 \quad 0.263240$ C $-2.4630493 .042007-1.082588$ C $-5.862291-4.145101-0.084245$ C $-6.026755-3.0484350 .948719$ C $-6.280171-0.5990351 .195412$ O $-5.995378-3.2829732 .154826$ C $\begin{array}{llll}-6.731979 & 0.543209 & 0.345537\end{array}$ C $-5.9303071 .552803-0.025853$ C $-6.3757162 .692123-0.896867$ C $-6.2374854 .038061-0.221522$ C -7.262291 4.574295 0.462405 C $-4.9495834 .803250-0.427785$ C $-3.863721 \quad 4.3831630 .522164$ N $-6.211308-1.7973750 .381947$ H $3.191701-1.4130653 .807587$ H 3.3803011 .4316112 .694541 H 1.5040301 .5814304 .374670 H $1.283886-0.8430355 .013627$ H $0.762598-1.2177963 .380202$ H -2.758164 -0.4534834 .401510$ H -1.892833 0.7892706 .119120 H -0.2665390 .3355436 .630700$ H $-0.511693 \quad 1.7361145 .567783$ H $-0.932438-2.3516882 .874651$ H -2.664033 -2.391882 3.068996 H $-2.920955-0.4459841 .529751$ H -1.167295 $-0.336448 \quad 1.350377$ H -1.106918 $-2.633376 \quad 0.397120$ H -1.348806 -0.627031-1.130485 H -3.090959 -0.721347 -0.965646 H -2.153757 -0.843552 -3.439311 H -4.295034 -2.876471 -1.920476 H $-0.249088-2.051269-2.909496$ H $-0.951645-3.587140-3.348570$ H $-0.345947-3.320383-1.698462$ H -2.601701 -5.023097 -2.616769 H -4.521680 -5.717107 -0.659130 H -4.354436 -5.2105931 .004740$ H -1.479844 -4.696706 -0.895085 H -0.4229702 .0688700 .409407$ H -2.0560903 .8805432 .293341$ H -2.351077 $1.955107-1.005060$ H - $-1.5394213 .466514-1.486179$ H -3.244867 $3.214407-1.825073$ H -6.074493 -3.779487 -1.093162 H $-6.641658-4.8878610 .128830$ H -5.289478 -0.4309601 .628990$ H $-6.980297-0.7652182 .020779$ H $-7.771190 \quad 0.5379640 .026136$ H -4.8906331 .5383390 .289003$ H -7.417619 2.546047 -1.212086 H -5.791053 2.680109-1.824647 H $-7.184265 \quad 5.5541150 .921614$ H -8.199801 4.0412810 .582453 H $-5.1234085 .876242-0.267741$ H -4.641579 $4.735644-1.475666$ H -4.0086804 .7609061 .535258$ H $-6.025486-1.686831-0.610985$ C $5.210581-1.8717571 .909104$ O $4.596356-1.5431580 .647550$ C $5.429585-2.040455-0.417905$ C $6.498257-2.9039260 .242513$ C $6.663646-2.2061191 .578392$ H $5.919926-1.168646-0.866425$ C $4.576497-2.761922-1.467726$
H $\quad 6.155709-3.934593 \quad 0.390974$

H $7.422413-2.938776-0.342477$

C $7.360027-3.0589452 .624428$

H $7.236803-1.2817421 .425098$

C $3.437457-1.894721-2.043935$

O $3.984980-3.912071-0.850905$

H $5.221174-3.135525-2.272302$

H $2.695776-1.693905-1.262539$

O $2.767988-2.653347-3.058013$

C $3.912218-0.572521-2.674555$

C $2.8100350 .231954-3.383885$

H $4.3680510 .059406-1.909616$

O $4.935796-0.845978-3.636018$

H $3.2876441 .090628-3.871715$

H $2.398783-0.363791-4.208256$

H $7.449466-2.5199633 .572695$

H $6.811487-3.9874412 .815379$

H $8.368531-3.3267132 .292727$

C $5.055418-0.6980442 .876015$

H $3.629352-4.473852-1.559791$

H $2.296373-3.379678-2.616645$

H $4.556362-1.438096-4.308009$

H 5.4052660 .2235942 .397740

H $5.668582-0.8614933 .769253$

H $4.693833-2.7608932 .294966$

C $2.6913782 .741964-1.630391$

O $2.1584441 .446483-1.327450$

C $1.6759500 .707217-2.455482$

C $0.5764191 .498248-3.176374$

C $1.0421872 .900745-3.550759$

C $1.6416633 .609370-2.339119$

H $3.5811632 .635698-2.262987$

C $3.1137173 .397707-0.304148$

H $1.198540-0.190613-2.047119$

O $\quad 0.143706 \quad 0.825350-4.354036$

H $-0.296270 \quad 1.582538-2.518541$

H $1.7546622 .879119-4.383828$

O $-0.0789903 .663035-4.004540$

H $\quad 0.8417223 .876434-1.637563$

O $2.2518814 .825442-2.769387$

C 4.4154102 .8484040 .239406

H $3.2594764 .473744-0.454323$

H $2.3477513 .244040 \quad 0.463410$

H $-0.132917-0.069837-4.096132$

H $-0.473253 \quad 3.180233-4.751269$

H $1.5694115 .346374-3.226351$

O 4.9128823 .7265601 .160796

O $4.9207431 .781269-0.077403$

C 6.1565723 .3613571 .768057

H $6.556346 \quad 4.2425812 .276909$

H 5.9872532 .5754482 .508548

H 6.8820903 .0356521 .016225

SCF Energy (B3LYP/6-31G**//MMFF) $=-3245.89853320$

01_605

MM̄FF Geometry

C -2.467633 -1.075329 -3.020539

C $-1.778537-1.997043-3.710672$

C $-0.374173-2.468343-3.398372$

O $0.262443-1.628338-2.407970$

C $-0.448300-3.914119-2.865308$

C $0.834727-4.439434-2.253240$

C $0.908256-4.952690-1.008404$

C $2.059874-4.386726-3.126777$

C $-0.232763-5.160642-0.050725$

C $-0.642486-3.9227370 .758989$

C $\quad 0.374161-3.5203341 .836928$

C $-0.220455-2.4393592 .743677$

C $0.786149-1.9506373 .794012$

O $1.550565-3.017507 \quad 1.197847$

O $0.234988-0.7563584 .368474$

C $2.104103-1.5817373 .082307$

C $0.939882-2.9561324 .940935$

O $3.128395-1.2709164 .024179$

C $2.606280-2.6728692 .104118$

C $3.788790-2.1182171 .279231$

O $3.052267-3.8054532 .852327$

C $0.902951-0.520174-2.876177$ 
O $0.955507-0.144075-4.037252$

C $1.5410370 .168780-1.730302$

C $2.1794191 .327490-1.955148$

C $2.8992942 .150172-0.983011$

C $2.8990331 .735851 \quad 0.462274$

C $4.213504-2.9890350 .093151$

C $5.456104-2.416621-0.560417$

C $6.236626-0.519111-1.980722$

O $6.565113-2.913604-0.376590$

C $6.4796650 .724862-1.182870$

C $6.5483631 .950993-1.724272$

C $6.801268 \quad 3.201618-0.929548$

C $5.8069384 .304372-1.227797$

C $6.1683455 .387479-1.936362$

C $4.4158154 .189113-0.638713$

C $3.5460763 .248594-1.426974$

N $5.194458-1.295982-1.333603$

H -2.005365 -0.575533 -2.173399

H -2.266754 -2.497965 -4.544279

H $0.218498-2.461155-4.322177$

H $-0.742646-4.585192-3.683540$

H - $1.262929-3.967882-2.134522$

H $1.874726-5.297976-0.645112$

H $2.915327-4.896550-2.670748$

H $1.861433-4.875613-4.086275$

H $2.358187-3.351429-3.317104$

H $-1.106480-5.532356-0.600139$

H $0.035397-5.9748130 .634637$

H $-0.814810-3.0787870 .079517$

H -1.601216 -4.148011 1.243113

H $0.629223-4.4115992 .421943$

H $-1.137175-2.7957423 .230178$

H -0.524129-1.581369 2.127693

H $0.832778-0.4564375 .073795$

H $1.917826-0.6626842 .509400$

H $-0.020523-3.1026755 .449757$

H $1.629909-2.5826345 .705736$

H $1.292989-3.9348394 .609405$

H $2.827562-0.5095494 .547836$

H $3.503837-1.1424310 .875989$

H $4.656285-1.9581841 .932184$

H $3.716424-4.2875722 .335449$

H $1.481562-0.299728-0.755365$

H $2.1934781 .715719-2.973569$

H 3.6939071 .0094820 .645211

H $1.940957 \quad 1.3002240 .761598$

H 3.0537742 .5796441 .140206

H $4.437564-4.0152100 .401228$

H $3.416916-3.043270-0.656203$

H $5.892595-0.297007-2.995838$

H $7.156749-1.108523-2.039537$

H $6.6179230 .610037-0.110121$

H $6.4398642 .062200-2.800920$

H $7.8188643 .540974-1.163004$

H 6.7939832 .9965900 .148199

H $5.4690796 .193520-2.132700$

H $7.1699475 .490591-2.340574$

H $3.9232195 .170071-0.634726$

H 4.5114803 .9035520 .412707

H $3.4757473 .501054-2.486029$

H $4.242267-0.948822-1.395636$

C $-4.796240-1.164190-2.151711$

O $-4.453739-0.448428-0.949815$

C $-5.653281-0.216353-0.187876$

C $-6.782303-0.923638-0.932623$

C $-6.281244-0.888419-2.365006$

H $-5.8403240 .862914-0.219115$

C -5.448159 -0.6672791 .263826$

H $-6.906381-1.959778-0.597280$

H -7.743217 $-0.416459-0.801756$

C $-6.976943-1.885942-3.274287$

H $-6.4222660 .124882-2.763400$

C $-4.205783-0.0532951 .945286$

O $-5.284477-2.091937 \quad 1.277981$

H $-6.351993-0.454747 \quad 1.846927$

H -3.302202 -0.4715721 .486527$

O $-4.218402-0.501394 \quad 3.306155$

C -4.1676441 .4853351 .915061$
C -3.0066882 .1300672 .692695$

H $-4.119605 \quad 1.8279810 .877427$

$\begin{array}{llll} & -4.388848 & 2.001089 & 2.452640\end{array}$

H -3.126602 3.2180522 .616816

H -3.113408 1.9166963 .763849

H $-6.581036-1.829149-4.292984$

H $-6.845137-2.913290-2.918410$

H $-8.051560-1.680954-3.318812$

C $-3.897989-0.706517-3.301381$

H $-5.158829-2.3592752 .204638$

H -3.338619 -0.3307953 .682275$

H $-5.471404 \quad 1.6694353 .363425$

H $-3.9492230 .381848-3.417118$

H $-4.232720-1.153754-4.243864$

H $-4.632537-2.231205-1.946670$

C $-1.456049 \quad 3.136667 \quad 0.265857$

$\begin{array}{lllll}\text { O } & -1.462734 & 1.803429 & 0.789773\end{array}$

C $-1.604244 \quad 1.712398 \quad 2.209034$

C -0.4523612 .4569882 .897105$

$\begin{array}{llll}\text { C } & -0.336891 & 3.899356 & 2.407098\end{array}$

$\begin{array}{llll}\text { C }-0.318336 & 3.964368 & 0.878292\end{array}$

H -2.4121303 .6189510 .503557$

C $-1.3265843 .011979-1.260798$

H -1.4901230 .6518012 .458497$

$\begin{array}{lllll}\text { O } & -0.618399 & 2.453407 & 4.311086\end{array}$

H 0.4910631 .9391652 .683946

H $-1.148653 \quad 4.5146002 .811738$

O $0.869573 \quad 4.4754152 .915951$

H 0.6531793 .6117910 .516830

O $-0.446678 \quad 5.333045 \quad 0.495435$

C $-2.5288513 .598957-1.958069$

H $-0.4429443 .533878-1.645245$

H $-1.2274341 .968838-1.584828$

H -0.6718641 .5250994 .596281$

H 1.6177673 .9528972 .580470

H $-0.3466715 .387220-0.469384$

O $-3.6010782 .768091-1.818806$

O $-2.5167074 .672603-2.545909$

C $-4.8151493 .228887-2.419308$

H $-4.694973 \quad 3.300494-3.504428$

H $-5.6010212 .501915-2.200924$

H $-5.1063374 .195329-1.996848$

SCF Energy (B3LYP/6-31G**//MMFF)= -3245.89818086

01_606

MM̄FF Geometry

C $2.9307451 .846277-2.825601$

C $2.2525561 .055792-1.980768$

C $0.823014 \quad 1.263975-1.567034$

O $0.168089-0.000845-1.831673$

C $0.694373 \quad 1.590435-0.066720$

C 1.0719063 .0167530 .278560

C $0.242595 \quad 4.0710090 .143791$

C 2.4597223 .2130690 .827243

C $-1.1776054 .040008-0.345677$

C $-2.110897 \quad 4.833187 \quad 0.581049$

C $-3.582796 \quad 4.507593 \quad 0.293821$

C $-4.513855 \quad 5.486828 \quad 1.007701$

C $-5.991298 \quad 5.129928 \quad 0.777986$

O $-3.821011 \quad 3.184178 \quad 0.782421$

O $-6.773725 \quad 5.8700651 .731099$

C $-6.2050103 .632724 \quad 1.096227$

C $-6.473415 \quad 5.584714-0.604450$

$\begin{array}{lllll}\text { O } & -7.516473 & 3.206492 & 0.736642\end{array}$

C $-5.1320642 .677628 \quad 0.515124$

C -5.2318201 .3022701 .215417$

O $-5.321952 \quad 2.423263-0.876534$

C $-1.162688-0.002879-2.101714$

O $-1.9179610 .954350-2.116267$

C $-1.542046-1.406384-2.380718$

C $-2.834323-1.753885-2.461171$

C $-3.345578-3.106091-2.698619$

C $-2.344912-4.171066-3.068415$

C -4.1304770 .3179410 .797480$

C $-4.263035-1.0169361 .510147$

C $-3.054311-3.1666631 .856201$

O $-5.253688-1.3298512 .166003$

C $-3.382206-4.1503860 .778706$ 
C $-4.590290-4.714658 \quad 0.630313$

C $-4.930752-5.714107-0.438656$

C $-5.940572-5.179571-1.431237$

C -7.259579 -5.267339-1.190311

C $-5.420085-4.620920-2.738022$

C $-4.669695-3.329359-2.559461$

N $-3.150586-1.8243751 .313398$

H $2.4537692 .728757-3.242719$

H $2.7555250 .186557-1.563767$

H $0.3569552 .043569-2.182914$

H 1.3004950 .8915200 .524667

H $-0.328110 \quad 1.384674 \quad 0.273924$

H $0.616906 \quad 5.059546 \quad 0.405717$

H 2.6605664 .2575601 .087905

H 2.5938202 .6171921 .735953

H 3.2122352 .9085710 .096405

H -1.212037 $4.481137-1.349156$

H -1.541573 $3.014849-0.441896$

H -1.883387 4.5896611 .627250

H -1.9242905 .9051310 .441323$

H $-3.7487164 .535337-0.789495$

H -4.3156646 .5175930 .688872$

H -4.312619 5.4618112 .087932

H -6.5808226 .8155261 .609264$

H -6.1612173 .5501402 .191345$

H $-6.4014936 .675844-0.693281$

H -7.531366 $5.344950-0.755167$

H -5.896782 5.157083-1.426243

H $-7.5392513 .081582-0.226727$

H -5.1660891 .4386712 .302927$

H $-6.2086990 .849671 \quad 1.001393$

H -5.718239 $3.201088-1.293203$

H $-0.739081-2.125993-2.492379$

H -3.591116 $-0.984590-2.318757$

H -1.612474 -4.310514 -2.265652

H -1.811604 -3.893040 -3.984299

H -2.791956 -5.149936 -3.251000

H $-4.1853930 .128731-0.278836$

H $-3.142944 \quad 0.7347851 .026032$

H -3.722296 -3.270546 2.717612

H $-2.027258-3.3173802 .202208$

H -2.578234 -4.4169190 .098212$

H $-5.386426-4.447186 \quad 1.321799$

H -5.332385 -6.6106960 .051448$

H -4.032083 -6.053564 -0.968346

H -7.991539 -4.912512 -1.908032

H -7.635508 -5.693751 -0.266032

H $-6.252738-4.419764-3.425400$

H -4.826299 -5.390652 -3.238476

H -5.310233 -2.492056 -2.278543

H $-2.404496-1.5026340 .703407$

C $5.3760992 .183434-2.322175$

O $5.3122301 .527838-1.039300$

C 6.643068 1.195961-0.609699

C $7.5788752 .000700-1.502677$

C $6.8134701 .999368-2.811499$

H $6.7853070 .128976-0.819610$

C $6.796507 \quad 1.4540660 .892485$

H $7.7063693 .027552-1.141381$

H $8.5703201 .545053-1.583164$

C $7.2736613 .068447-3.787228$

H $6.9273841 .013358-3.281916$

C $5.7233210 .765851 \quad 1.762911$

O $\quad 6.6959972 .8666901 .116477$

H 7.8024821 .1592091 .214021

H 4.7514281 .2380751 .586181

O $\quad 6.050396 \quad 1.0137103 .136309$

C $5.603735-0.758498 \quad 1.562581$

C $4.683392-1.4547482 .586664$

H $5.250765-0.9706230 .548399$

O $6.907935-1.3335481 .683869$

H $4.803199-2.5383042 .470795$

H $5.049802-1.2433503 .598388$

H $6.6825753 .037084-4.707860$

H $7.1768564 .071606-3.358550$

H $8.3245482 .917689-4.055023$

C $4.3424181 .556103-3.256759$

H 7.0077353 .0425722 .020174
H $5.897545 \quad 1.958106 \quad 3.308173$

H $6.831071-2.2809501 .478729$

H $4.4912000 .469431-3.295672$

H $4.4713311 .930149-4.278858$

H $5.1529603 .244460-2.152059$

C $2.471750-2.8325070 .971665$

O $2.654825-1.4266251 .182203$

C $3.197065-1.0641582 .457381$

C $2.300501-1.5964033 .584202$

C $2.068013-3.0985723 .459013$

C $1.569679-3.4364462 .056154$

H $3.445471-3.3364470 .969533$

C $1.829377-3.015769-0.412360$

H 3.1423640 .0287112 .513970

O $2.874829-1.2854174 .848692$

H $1.330950-1.0829163 .535759$

H $2.970636-3.6705123 .704472$

O $1.063283-3.5039944 .391241$

H $0.542156-3.0688661 .936801$

O $1.535636-4.8543121 .902263$

C $2.785540-2.729860-1.550072$

H $1.483996-4.047506-0.541086$

H $0.977910-2.333705-0.511929$

H $2.224975-1.5159965 .533469$

H $1.430190-3.3927655 .284433$

H $0.970219-5.2092132 .609719$

O $2.104260-2.837807-2.729934$

O $3.971405-2.456454-1.429835$

C $2.874875-2.607791-3.912971$

H $3.233908-1.575443-3.933958$

H $3.712218-3.310195-3.965509$

H $2.226085-2.770687-4.777749$

SCF Energy (B3LYP/6-31G**//MMFF) $=-3245.89970733$

01_607

MM̄FF Geometry

C $1.1541651 .786212-1.186045$

C $0.9519500 .629880-0.537750$

C -0.0816820 .4426760 .539878$

O $-0.840252-0.7344540 .167282$

C 0.5452030 .2009821 .925702

C 1.1880651 .4182672 .550590

C 0.5188782 .3568783 .247639

C 2.6850981 .5031312 .421301

C -0.9625342 .4358973 .506890$

C -1.5103573 .8522023 .274764$

C -1.6297434 .2278251 .787090$

C -1.6289885 .7512511 .635995$

C $-1.840156 \quad 6.182791 \quad 0.181277$

O $-2.8612843 .681361 \quad 1.291479$

O $-2.114371 \quad 7.5920430 .174170$

C $-3.0954965 .474482-0.356398$

C $-0.5832945 .980710-0.671963$

O $-3.2662455 .735996-1.748698$

C $-3.1204093 .946181-0.094865$

C $-4.5326893 .416056-0.445885$

O $-2.1564713 .314462-0.936341$

C $-2.081953-0.8784050 .700514$

O $-2.660313-0.1002861 .441075$

C $-2.618694-2.1773070 .241787$

C $-3.781400-2.6181220 .742257$

C $-4.448160-3.8747920 .403833$

C $-3.826066-4.741597-0.658891$

C $-4.8038721 .944207-0.106593$

C $-6.2229761 .550912-0.494384$

C $-7.744641-0.380994-0.691504$

O $-7.0325012 .348863-0.963014$

C $-8.003136-1.6612010 .034363$

C $-8.233950-2.826441-0.590489$

C $-8.573452-4.1124600 .110730$

C $-7.557541-5.202134-0.150981$

C $-7.670189-6.009272-1.219329$

C $-6.453141-5.4025620 .861772$

C $-5.596158-4.1825941 .041289$

N $-6.4927760 .208668-0.251899$

H $0.5614632 .656678-0.920734$

H $1.564941-0.230585-0.792227$

H $\quad-0.759518 \quad 1.3042170 .564358$ 
H $1.275036-0.6188471 .865000$ H $-0.215279-0.1896092 .614215$ H 1.0986463 .1614913 .698965 H 3.1014352 .3750122 .937217 H 3.1547630 .6122542 .850903 H 2.9740291 .5775571 .371060 H $-1.529307 \quad 1.7189792 .909178$ H -1.125540 2.1741634 .559456 H -2.501692 3.9130913 .742195 H -0.8649544 .5689393 .798452$ H $-0.7936443 .795604 \quad 1.227297$ H -0.6978326 .1803092 .027331$ H -2.436099 6.1788912 .247678 H -1.357127 8.0464830 .581622 H -3.9618635 .9239040 .149458$ H $\quad 0.2559516 .553514-0.259537$ H $-0.7319716 .358164-1.689795$ H $-0.2713534 .937002-0.734138$ H -3.264488 $6.701683-1.864442$ H $-5.277403 \quad 4.0172460 .093256$ H $-4.7169793 .559292-1.518789$ H -2.400196 2.382929-1.051915 H $-2.039877-2.740298-0.480098$ H $-4.297949-2.0015661 .477284$ H -2.842336 -5.099417 -0.335842 H $-3.706861-4.180831-1.592876$ H $-4.424200-5.622985-0.902579$ H $-4.1129631 .289163-0.646291$ H $-4.685742 \quad 1.7745810 .968923$ H -7.675824 -0.522919-1.775691 H $-8.5588830 .325377-0.496692$ H -8.043502 -1.618912 1.119990 H -8.215172 -2.855558 -1.677937 H $-9.561421-4.437059-0.241942$ H -8.686797 $-3.962087 \quad 1.191447$ H $-6.960342-6.808908-1.402334$ H -8.474778 $-5.884870-1.936687$ H $-5.836751-6.2743530 .613824$ H -6.906818 -5.649379 1.830721 H -5.979808 -3.488104 1.789557 H -5.714181 $-0.410547-0.044474$ C $3.4514322 .606042-1.734628$ O $4.1998381 .598854-1.024727$ C $5.5992311 .750317-1.329109$

C $5.7267773 .071629-2.077123$

C $4.4001453 .125309-2.812778$

H $5.8617170 .938085-2.016725$

C $6.4286811 .636785-0.045250$

H $5.8164603 .921535-1.390791$

H $6.5917963 .087277-2.747026$

C $4.0501194 .509519-3.330657$

H $4.4337562 .421264-3.655068$

C $6.157510 \quad 0.3414810 .750104$

$\begin{array}{lllll}\text { O } & 6.099377 & 2.744309 & 0.803034\end{array}$

H $7.4949721 .731565-0.280784$

H $\quad \begin{array}{llll}5.126310 & 0.356217 & 1.113631\end{array}$

O $\quad 6.985026 \quad 0.359246 \quad 1.919886$

C $6.424584-0.956119-0.032128$

C $6.171261-2.2371240 .784804$

H $5.812371-0.981324-0.938437$

O $7.791376-0.952987-0.452909$

H $6.561355-3.0863750 .210723$

H $\quad 6.776897-2.2198491 .699307$

H $3.0810724 .503174-3.839202$

H $3.9985585 .241186-2.517351$

H $4.8041834 .854392-4.045679$

C $2.1704041 .975658-2.278467$

H 6.6203502 .6491381 .618804

H 7.9100390 .3034241 .624285

H $7.931888-1.744111-1.000578$

H $2.3952601 .010543-2.749931$

H $1.7194482 .614128-3.046433$

H $3.2194553 .402303-1.014614$

C $3.978514-3.407399-0.966578$

O $3.833631-2.352718-0.011075$

C $4.685441-2.4705331 .134236$

C $4.410093-3.7981821 .855920$

C $4.517248-4.9991770 .916212$
C $3.676642-4.775853-0.343858$

H $5.012507-3.409823-1.334191$

C $3.121928-3.076234-2.197169$

H $4.389319-1.6678721 .819743$

O $5.308229-3.9754402 .946918$

H $3.395097-3.7774442 .274197$

H $5.563570-5.2108990 .666696$

O $4.028300-6.1498191 .609955$

H $2.616451-4.865857-0.083063$

O $3.968403-5.792369-1.299694$

C $1.628925-3.013295-1.949589$

H $3.414847-2.089114-2.576166$

H $3.307299-3.821419-2.979187$

H $5.202899-3.2112043 .538873$

H $4.194938-6.9249021 .047583$

H $3.678945-6.641153-0.925295$

O $0.993647-2.913081-3.156078$

O $1.086140-3.022019-0.854340$

C $-0.432647-2.817599-3.099653$

H $-0.813558-2.810141-4.124424$

H $-0.853391-3.681553-2.576573$

H $-0.726230-1.884572-2.610094$

SCF Energy (B3LYP/6-31G $\left.{ }^{* *} / / M M F F\right)=-3245.90752521$

01608

MM̄FF Geometry

C $0.633902-3.3573010 .785266$

C $-0.044329-3.165126 \quad 1.927327$

C $0.267545-2.1410332 .994663$

O $1.618743-1.6573532 .821232$

C $-0.739124-0.9800312 .866386$

C -0.815027 -0.0765394 .078629$

C -0.3737331 .1955974 .115220$

C -1.504721 -0.6680975 .281891$

C $0.3119351 .965786 \quad 3.022025$

C -0.6674842 .9342702 .349755$

C 0.0234443 .8600911 .339875

C -0.9886764 .8232310 .715786$

C $-0.3444875 .712464-0.358264$

O 0.6198483 .0576090 .317373

O $-1.3997396 .335241-1.106672$

C $0.4417834 .820901-1.341689$

C $0.478605 \quad 6.8487860 .259792$

O $1.1820095 .613254-2.267667$

C $1.3776643 .804261-0.646415$

C $1.9400292 .813499-1.692411$

O $2.4662394 .500692-0.037646$

C $2.329875-1.3364253 .931767$

O $1.919747-1.3035005 .083691$

C $3.729956-0.9941313 .597809$

C $4.380668-1.5419892 .559317$

C $5.792409-1.3361822 .223302$

C $6.550599-0.2841982 .989735$

C $2.7075381 .631126-1.085088$

C $2.9644360 .543701-2.114545$

C $3.559844-1.876775-2.263865$

O $2.9785220 .757342-3.323838$

C $5.025561-2.181325-2.167834$

C $6.009415-1.302034-1.918854$

C $7.453694-1.681610-1.763349$

C $8.020653-1.229090-0.434447$

C $8.780645-0.123843-0.352962$

C $7.777415-2.0955550 .781421$

C $6.346213-2.0905901 .250610$

N $3.211590-0.683083-1.516245$

H $1.510754-2.7566880 .560675$

H -0.940602 -3.758727 2.101265

H $0.178825-2.6514823 .961039$

H $-1.749192-1.3810722 .704251$

H $-0.509871-0.3995881 .965052$

H $-0.514407 \quad 1.7611075 .035774$

H -1.599417 $0.051336 \quad 6.102337$

H -2.515088 -0.9962415 .015951$

H $-0.949017-1.5291395 .664538$

H 1.1411102 .5263963 .471262

H $\quad 0.766514 \quad 1.3005072 .281435$

H -1.440522 $2.346347 \quad 1.840117$

H -1.1712263 .5431403 .111494$ 
H $\quad 0.807414 \quad 4.416025 \quad 1.867531$ H -1.468543 5.4388841 .486712 H $-1.798373 \quad 4.2462770 .246772$ H -1.948829 $6.842876-0.484774$ H $-0.2955134 .260215-1.934079$ H $-0.156306 \quad 7.4809730 .892231$ H $0.8825107 .510527-0.514211$ H $1.306513 \quad 6.4925330 .876326$ H $0.5554316 .224140-2.691900$ H $\quad 1.1079812 .413579-2.285007$ H $2.6053333 .339626-2.388878$ H 3.2059513 .8847270 .083609 H $4.225775-0.3427874 .309794$ H $3.847783-2.2416611 .917292$ H $6.653827-0.5733364 .041231$ H $\quad 6.0310590 .6793672 .938574$ H $7.557025-0.1089312 .602511$ H $3.6801111 .958723-0.701810$ H $2.1468581 .197076-0.250401$ H $3.257198-1.781759-3.311076$ H $2.993019-2.697017-1.811892$ H $5.287592-3.230293-2.291067$ H $5.778497-0.245152-1.811786$ H $8.012086-1.221773-2.588996$ H $7.600844-2.763314-1.871386$ H $9.216246 \quad 0.1958310 .587704$ H $8.9812190 .489087-1.225493$ H $8.448987-1.8167111 .601987$ H $8.058001-3.1295510 .541153$ H $5.720642-2.8277730 .746868$ H $3.234542-0.743345-0.502294$ C $-0.724843-3.613947-1.275747$ O $0.014549-2.598341-1.985132$ C $-0.418919-2.588760-3.351911$ C -1.651464 -3.481111-3.422494 C $-1.325525-4.511201-2.354056$ H $0.377931-3.082508-3.925727$ C $-0.572126-1.167240-3.902493$ H $-2.561204-2.931520-3.155847$ H -1.796048 -3.921361 -4.413620 C $-2.532601-5.311012-1.896437$ H $-0.560488-5.197180-2.741606$ C $-1.651158-0.257309-3.271019$ O $-0.860585-1.292471-5.301485$ H $0.399504-0.662629-3.830730$ H -2.645967 -0.634904 -3.529902 O $-1.547713 \quad 1.021011-3.918779$ C $-1.551075-0.044848-1.751056$ C -2.448081 $1.074194-1.193905$ H -1.790295 -0.977810 -1.239216 O $-0.2034690 .278694-1.417824$ H -2.189808 $1.210399-0.137412$ H -2.188909 2.031407 -1.663396 H -2.255723 -6.022600 -1.112554 H -3.318658 -4.660515 -1.498678 H $-2.956979-5.877393-2.731986$ C $0.190846-4.323196-0.280319$ H $-0.923920-0.392879-5.665729$ H $-0.6867751 .403445-3.676151$ H $-0.1397010 .317016-0.448174$ H $1.084238-4.711169-0.783729$ H $-0.324881-5.1767330 .174341$ H -1.531565 -3.106157 -0.732226 C $-4.206511-0.690848 \quad 0.541290$ O $-4.308667-0.510385-0.874100$ C $-3.9563530 .798146-1.335417$ C $-4.8493341 .851244-0.662693$ C -4.8229341 .7359760 .861025$ C $-5.099038 \quad 0.297516 \quad 1.300402$ H -3.164435 -0.5290990 .842398$ C $-4.498981-2.1622960 .869431$ H $-4.1842070 .817688-2.408008$ O $-4.4524743 .167052-1.035804$ H -5.885082 $1.718258-1.001053$ H -3.8744772 .0962691 .275025$ O H -6.1595420 .0725491 .139352$ O
C $-5.894277-2.6254970 .503620$

H $-3.804710-2.8018710 .312326$

H $-4.343722-2.3318761 .941146$

H -4.486560 $3.218275-2.006356$

H -5.6750533 .4780291 .094642$

H -5.3953820 .8373093 .151916$

O $\quad-6.164641-3.7852141 .173904$

O $-6.640444-2.069100-0.289873$

C -7.447849 -4.3622170 .912197$

H -7.533555 -4.629970 -0.145270

H $-7.540771-5.2708411 .512908$

H -8.242060 -3.667342 1.201424

SCF Energy (B3LYP/6-31G**//MMFF)= -3245.90483979

01609

MM̄FF Geometry

C $-0.920113-4.0380330 .448216$

C $0.119926-3.2110420 .257318$

C $0.794656-3.039221-1.081450$

O $1.018716-1.630937-1.328267$

C $2.169289-3.729096-1.067008$

C $2.766735-3.879234-2.452425$

C $3.744981-3.098529-2.950905$

C $2.230723-5.031142-3.263445$

C $4.379985-1.903107-2.299491$

C $5.733820-2.256490-1.674346$

C $6.458020-1.014110-1.136597$

C $7.793912-1.398990-0.498442$

C $8.519101-0.1775130 .089255$

O $5.622798-0.395248-0.155331$

O $9.552383-0.6601750 .965652$

C 7.5328480 .6257940 .969188

C $9.2373910 .624950-1.002130$

O $8.104157 \quad 1.8597381 .395348$

C $6.1333800 .846970 \quad 0.344645$

C 5.1448871 .3414081 .425773

O $6.1402321 .859259-0.663383$

C $-0.049671-0.876378-1.696040$

O $-1.205751-1.247213-1.812772$

C $0.4355460 .502577-1.921395$

C $-0.4535291 .497759-2.053454$

C $-0.1398592 .914499-2.239289$

C $1.2930113 .293391-2.503018$

C $3.707873 \quad 1.484208 \quad 0.906547$

C 2.7718632 .0810181 .943253

C 0.3719762 .7286502 .212722

O 3.1440772 .4715343 .045735

C 0.1135484 .0934621 .658355

C -0.9878854 .4139030 .962036$

C -1.2276665 .7633420 .346630$

C $-0.4898365 .955634-0.962860$

C $0.6055296 .732630-1.018171$

C $-1.0437245 .313224-2.218628$

C -1.140171 $3.814995-2.143931$

N 1.4680292 .1334021 .472808

H $-1.279547-4.609960-0.405839$

H $0.528945-2.6364341 .082448$

H $\quad 0.173513-3.445801-1.888955$

H $2.087045-4.731836-0.626043$

H $2.856230-3.176336-0.414338$

H $4.126264-3.321510-3.946415$

H $\quad 2.727740-5.122300-4.235220$

H $2.380090-5.974443-2.728021$

H $1.160415-4.904786-3.453096$

H $4.515767-1.131802-3.067931$

H $3.717581-1.461582-1.548499$

H $5.564542-2.966687-0.854243$

H $6.370320-2.757253-2.414584$

H $6.613502-0.319746-1.970860$

H $8.437440-1.915948-1.221266$

H $7.615384-2.118790 \quad 0.312888$

H $10.131339-1.2495980 .452484$

H $7.3946490 .035075 \quad 1.885808$

H $10.0181840 .014285-1.472554$

H $9.7548951 .494123-0.582380$

H $8.5727140 .962572-1.798957$

H 8.0639852 .4842490 .651995

H 5.1328800 .6385392 .268718 
H $\quad 5.4792282 .312427 \quad 1.813154$ H $7.0166231 .904386-1.069576$ H $1.5073590 .665225-1.943036$ H -1.514355 $1.259713-1.989260$ H $1.6920732 .727695-3.352438$ H $1.9136743 .091816-1.623717$ H $1.4176874 .349757-2.753102$ H 3.6859312 .1374620 .027026 H $3.3146200 .503108 \quad 0.615193$ H $\quad 0.6181272 .791691 \quad 3.276907$ H -0.4883462 .0665102 .089471$ H 0.8805404 .8470901 .820235 H $-1.752513 \quad 3.6583480 .797966$ H -2.303948 5.9007540 .184261 H $-0.944523 \quad 6.542108 \quad 1.067474$ H $1.1337626 .901330-1.950651$ H $1.0016607 .215336-0.130749$ H $-0.4643835 .597472-3.105868$ H -2.049803 $5.715079-2.396673$ H $-2.1490313 .454723-1.946498$ H 1.2758331 .8208180 .525922 C -1.439003 -3.3334872 .889759$ O $-2.078328-2.0772542 .579039$ C $-2.845654-1.6524193 .721488$ C $-2.340687-2.4850044 .891489$ C $-2.066909-3.8076764 .203349$ H -3.887853 -1.930899 3.525062 C $-2.738180-0.1375273 .912781$ H -1.414986 -2.075539 5.312286 H -3.077087 -2.562315 5.696966 C -1.180311 -4.7397395 .011164$ H -3.024752 -4.307217 4.004661 C -3.1879350 .6818972 .684381$ O -1.3694850 .1884294 .183070$ H $-3.3074940 .161444 \quad 4.800807$ H $-2.482196 \quad 0.519273 \quad 1.862563$ O -3.0845572 .0697373 .028507$ C -4.6244710 .3925062 .214455$ C -5.1155951 .3146131 .083714$ H -4.706501 -0.6506591 .893505$ O H -6.1823081 .1120600 .928584$ H -5.0802992 .3595451 .416459$ H $-0.994979-5.6712534 .467432$ H $-0.211037-4.2804975 .232676$ H -1.656991 -4.993756 5.963675 C -1.660823 -4.310746 1.731853 H -1.3222941 .1509184 .314749$ H -3.735197 2.2472063 .729586 H -6.3970890 .3001393 .040761$ H $-2.730342-4.316631 \quad 1.483021$ H -1.397426 -5.327103 2.047661 H $-0.373041-3.1292053 .043534$ C $-5.465589-0.815546-1.059371$ O $-4.234610-0.213509-0.647404$ C $-4.3464861 .155433-0.243720$ C $-4.9152321 .995034-1.397230$ C $-6.2388941 .432452-1.914068$ C $-6.103326-0.057940-2.231311$ H -6.165500 -0.798117 -0.214062 C $-5.210811-2.305929-1.332083$ H -3.323912 $1.507524-0.062923$ O $-5.107280 \quad 3.347342-0.994897$ H -4.198450 $2.003581-2.228420$ H $-7.0567231 .609967-1.206044$ O $-6.6006392 .113575-3.118155$ H -5.512831 -0.167982 -3.146144 O $-7.393436-0.605131-2.494725$ C $-4.183043-2.606590-2.404480$ H $-4.833431-2.754372-0.403478$ H $-6.144871-2.807598-1.606934$ H $-4.2528683 .681213-0.671994$ H -6.641542 $3.063174-2.911435$ H $-7.786319-0.086274-3.217485$ O $-3.856722-3.933002-2.337587$ O $-3.731107-1.803693-3.207527$ C -2.892793 -4.379734 -3.296548 H $-1.971213-3.795802-3.215616$
H $-2.662094-5.426954-3.083960$

H $-3.308027-4.307832-4.306125$

SCF Energy (B3LYP/6-31G $\left.{ }^{* *} / / M M F F\right)=-3245.89895766$

0161

MM̄FF Geometry

C -3.955864 $1.146037-2.294041$

C $-3.0951082 .150356-2.517789$

C $-2.3380782 .875881-1.440696$

O $-0.9501302 .836737-1.856421$

C $-2.7791214 .344153-1.302480$

C $-4.1609434 .530125-0.717306$

C -4.4245664 .5681580 .603302$

C $-5.2675484 .757214-1.714604$

C $-3.4575514 .400717 \quad 1.746275$

C -4.0000763 .4478162 .822743$

C -3.8774271 .9625872 .440596$

C $-4.860803 \quad 1.1291893 .266288$

C $-4.708371-0.3713402 .993626$

O -2.5301601 .5519002 .714727$

O $-5.448835-1.081503 \quad 3.997352$

C $-3.227356-0.7487383 .170117$

C $-5.306460-0.7757171 .642973$

O $-3.002214-2.1073862 .795567$

C $-2.244296 \quad 0.1792242 .412161$

C $-0.807814-0.1381652 .896478$

O $-2.347262-0.0792121 .012846$

C $0.0044423 .052668-0.915547$

$\begin{array}{llll}\text { O } & -0.167678 & 3.282518 & 0.269972\end{array}$

C $1.3168522 .978052-1.593812$

C $2.4137553 .386764-0.941000$

C $3.7790913 .399412-1.465305$

C $4.0194402 .810483-2.829939$

C 0.3013580 .7726362 .354305

C 1.6540870 .3957622 .941051

C 4.0796470 .7570482 .683559

O $1.781911-0.4171263 .854584$

C 5.0040861 .8422902 .239027

C 6.0465311 .6264311 .421583

C 7.0393792 .6798221 .017073

C $7.0512842 .922164-0.475535$

C $7.8337912 .190748-1.286691$

C $6.2158404 .058897-1.018775$

C $4.7553963 .921561-0.695472$

N 2.7062491 .0840682 .347416

H $-4.1612080 .840007-1.273230$

H -2.901226 $2.461936-3.542137$

H $-2.4329512 .346666-0.485173$

H $-2.7162704 .845064-2.278778$

H -2.053856 $4.895223-0.690615$

H -5.4527694 .7609640 .908376$

H -6.234442 $4.931747-1.230520$

H $-5.0467255 .634821-2.331100$

H $-5.3843333 .892180-2.372922$

H -2.472169 4.0639111 .414920

H -3.3204885 .3886352 .202806$

H -3.436028 3.6267643 .747283

H $-5.0466693 .703668 \quad 3.031237$

H -4.0827701 .8383321 .372019$

H $-5.8940051 .446373 \quad 3.076669$

H -4.6837881 .3089714 .336140$

H -6.376709 -0.794822 3.944304

H -3.003371 -0.6843224 .244427$

H $-6.374981-0.5322101 .607198$

H -5.239914 -1.858291 1.487526

H $-4.825514-0.2760610 .801153$

H -3.623034 -2.655129 3.305969

H $-0.785967-0.0653073 .992241$

H $-0.556828-1.1743662 .637783$

H -1.5334010 .2171040 .577234$

H $1.3415342 .609005-2.612758$

H 2.3024373 .7630940 .075471

H $3.5217083 .410540-3.599416$

H $3.6364731 .785467-2.882853$

H $5.0780632 .755358-3.095440$

H $0.3674260 .691413 \quad 1.266030$

H 0.1005221 .8160672 .619182

H $4.312695-0.1996452 .207528$ 
H $4.1580690 .621000 \quad 3.767543$ H 4.8333582 .8385002 .639011 H $\quad 6.223208 \quad 0.621374 \quad 1.043949$ H 8.0334402 .3490851 .345797 H 6.8597643 .6244991 .545439 H $7.8685882 .372743-2.355549$ H $8.4536851 .389066-0.898776$ H $6.3648234 .182793-2.097510$ H $6.5758794 .998041-0.578340$ H 4.4889214 .3185710 .284834 H $2.5279891 .585781 \quad 1.481987$ C $-4.360816-1.100482-3.414991$ O $-2.939528-1.334703-3.331088$ C $-2.707018-2.348497-2.334783$ C $-4.037895-3.070624-2.170984$ C $-5.006401-1.909469-2.288422$ H $-2.486216-1.828633-1.396576$ C $-1.533090-3.244344-2.733951$ H -4.208597 -3.793685 -2.977022 H -4.111966 -3.597302 -1.214857 C $-6.436366-2.334054-2.573688$ H $-4.988911-1.345750-1.349488$ C $-0.188847-2.506189-2.914755$ O $-1.861238-3.873817-3.980611$ H -1.425117 -4.055753 -2.005436 H $-0.284751-1.817427-3.763952$ O $0.783786-3.482251-3.307079$ C $0.346225-1.733854-1.689674$ C $0.256374-2.459622-0.330416$ H $1.393912-1.471078-1.876638$ O $-0.364474-0.498411-1.593761$ H $0.553926-1.7472990 .447015$ H $-0.790833-2.686016-0.103337$ H -7.089513 -1.461097-2.669998 H -6.506656 -2.912619-3.500934 H $-6.821719-2.956626-1.759616$ C $-4.6528050 .403567-3.401938$ H -1.109115 -4.439985 -4.224981 H $1.611721-3.010597-3.501062$ H $0.0930750 .049833-0.934616$ H $-5.7323440 .576176-3.327251$ H $-4.3346040 .817473-4.367647$ H -4.679656 -1.502845 -4.385469

C $3.187569-2.7440690 .511614$ O $2.490952-3.471464-0.509159$ C $1.112932-3.735211-0.226108$ C $0.971170-4.502201 \quad 1.097814$ C $1.727458-3.8354102 .241815$ C $3.165526-3.531451 \quad 1.825726$ H $2.713953-1.7672240 .662201$ C $4.621042-2.5147180 .013143$ H $0.765910-4.420979-1.006058$ O $-0.397427-4.6459281 .463176$ H $1.374525-5.5151490 .968019$ H $1.218009-2.9277902 .583590$ O $1.763070-4.7229503 .361466$ H $3.724653-4.4695931 .717226$ O $3.798279-2.7708542 .850297$ C $4.662350-1.470705-1.083233$ H $5.278680-2.1601360 .813374$ H $5.022914-3.455571-0.381882$ H $-0.851840-5.0892490 .726341$ H $0.841647-4.9314373 .593530$ H $3.721454-3.2762663 .677707$

O $5.657810-1.774817-1.965832$

O $3.930027-0.491485-1.140259$

C $5.804778-0.866493-3.061783$ H $4.903885-0.877497-3.682705$ H $6.0075460 .143530-2.695364$ H $6.653314-1.196945-3.666478$

SCF Energy (B3LYP/6-31G**//MMFF) $=-3245.91407049$

01_610

MM̄FF Geometry

C $-3.067346-4.206784-0.799242$

C $-1.920382-3.540149-0.599340$

C $-0.908397-3.273201-1.681818$

O $-0.754871-1.841147-1.808258$
C $0.456792-3.874434-1.300031$

C $1.573615-3.465292-2.241785$

C $2.596777-2.653136-1.906725$

C $1.502246-4.057764-3.625378$

C $2.843016-1.981953-0.581238$

C $3.980315-2.6625430 .195856$

C $5.375057-2.251496-0.303168$

C $6.456809-3.1473580 .303329$

C $7.868304-2.686167-0.092185$

O $5.582917-0.8925100 .097196$

O $8.808493-3.3793640 .742879$

C $8.002799-1.1830510 .226969$

C $8.214001-3.060741-1.537751$

O $9.243181-0.664506-0.246664$

C $6.835286-0.329633-0.316445$

C $6.871697 \quad 1.1240450 .211187$

O $6.916735-0.268048-1.744398$

C $-1.540815-1.211167-2.718474$

O $-2.440626-1.714950-3.377991$

C $-1.1584170 .208918-2.887933$

C $-0.2590150 .847997-2.124160$

C $0.1654252 .241313-2.284241$

C $-0.4311723 .031805-3.420528$

C 6.8974681 .2405721 .737127

C 6.3924432 .5898942 .204345

C 4.2972103 .8104602 .741919

O 7.1586153 .4929432 .530610

C 2.9034133 .8124772 .203440

C 2.3587224 .8490991 .549572

C 0.9658424 .8313970 .987543

C $0.9393945 .095275-0.502948$

C $0.2933566 .163935-0.999336$

C $1.6616564 .132566-1.420668$

C $1.0599272 .753076-1.412968$

N 5.0077492 .6678812 .198560

H $-3.276164-4.578045-1.801154$

H -1.679923 -3.1469810 .384129$

H -1.229956 -3.703150 -2.639028

H $0.385229-4.970371-1.292101$

H $\quad 0.704023-3.600115-0.269181$

H $3.346029-2.435510-2.666450$

H $2.379663-3.806424-4.230893$

H $1.445858-5.149714-3.568177$

H $\quad 0.619689-3.689775-4.157515$

H $1.941250-1.9809280 .037806$

H $3.072562-0.924525-0.759245$

H $3.884120-2.3726741 .250525$

H $3.861297-3.7520430 .146319$

H $5.403892-2.315662-1.396206$

H $6.301769-4.1943890 .014304$

H $6.378193-3.1240391 .399365$

H $8.683648-4.3341550 .605989$

H $8.030095-1.0978901 .320619$

H $8.161773-4.147649-1.674331$

H $9.243522-2.777399-1.782992$

H $7.546542-2.604684-2.271603$

H $9.949862-1.2231350 .120060$

H $7.7344811 .657563-0.208486$

H $5.9904911 .651676-0.179251$

H $6.4378460 .521720-2.046281$

H -1.669930 $0.711511-3.702968$

H $0.2209630 .308725-1.309519$

H $-0.1065534 .074272-3.444332$

H -1.523244 $3.055070-3.342543$

H $-0.1559932 .587643-4.383397$

H $\quad 6.2834870 .4647602 .209837$

H 7.9174991 .1087792 .116077

H 4.8460034 .7265052 .499160

H 4.2803653 .7005693 .831059

H 2.3116212 .9127332 .357618

H 2.9404185 .7567501 .406552

H 0.3821635 .5909061 .523240

H 0.4692183 .8745601 .190609

H $0.2675286 .365038-2.065019$

H $-0.2275036 .861539-0.351986$

H $2.7121754 .052174-1.113691$

H $1.7135754 .528019-2.441519$

H $1.4288002 .117002-0.607788$ 
H $4.483793 \quad 1.8078342 .060494$

C -3.889394 -4.048420 1.652998

O $-3.899271-2.6091801 .714801$

C $-4.503390-2.1998072 .955961$

C $-4.779648-3.4761653 .744855$

C $-4.971636-4.4913872 .635613$

H $-5.460507-1.7311682 .700420$

C -3.596894-1.195965 3.677525

H $-3.928815-3.756722 \quad 4.376539$

H $-5.655388-3.3778454 .393965$

C $-4.839400-5.929813 \quad 3.105186$

H $-5.965618-4.3442032 .192216$

C -3.2537590 .0660752 .858204$

O $-2.358756-1.8381714 .012752$

H -4.052016 -0.9080374 .632196$

H -2.598849 -0.1995092 .020223$

O -2.4913190 .9356513 .705733$

C -4.475703 $0.843292 \quad 2.337332$

C $-4.152231 \quad 2.233813 \quad 1.762583$

H $-4.991594 \quad 0.2462401 .580425$

$\begin{array}{lllll} & -5.395639 & 1.028095 & 3.418556\end{array}$

H -5.1021462 .7033351 .478648$

H -3.760437 2.8707692 .565884

H $-4.983679-6.6280042 .275001$

H $-3.852144-6.1211453 .538731$

H $-5.591193-6.1553743 .868558$

C $-4.140787-4.510407 \quad 0.215452$

H -1.959929 $-2.155742 \quad 3.184361$

H -1.7228850 .4306714 .022682$

H -6.1914401 .4547103 .057639$

H $-5.067122-4.050122-0.149854$

H $-4.293337-5.5959050 .206660$

H -2.904221 -4.3870231 .999650$

C $-4.7861801 .834015-1.195292$

O $-3.6134601 .388271-0.503639$

$\begin{array}{llll}\text { C } & -3.179948 & 2.235356 & 0.566204\end{array}$

C -2.8724843 .6386580 .027707$

C $-4.0630224 .226715-0.722858$

C $-4.579888 \quad 3.243931-1.771781$

H $-5.6433281 .821681-0.511262$

C $-5.0732170 .843649-2.336579$

H -2.2299191 .8194040 .922212$

O $-2.4924554 .502741 \quad 1.092940$

H -2.017667 $3.576810-0.655111$

H -4.861622 $4.516631-0.030944$

O $-3.6586445 .435134-1.372443$

H -3.873055 $3.202770-2.609827$

O $-5.819406 \quad 3.725156-2.288978$

C $-5.491502-0.529680-1.856693$

H $-5.8866931 .230481-2.961296$

H $-4.1798270 .712039-2.955900$

H -2.326956 5.3813880 .710388

H -2.934241 5.220111-1.984620

H -5.663611 $4.623053-2.628772$

O $-5.975667-1.234090-2.923117$

O $-5.389344-0.944025-0.711418$

C $-6.413406-2.568341-2.647579$

H $-7.005820-2.913371-3.499227$

H $-5.544515-3.221796-2.536956$

H -7.040934-2.602261-1.751728

SCF Energy (B3LYP/6-31G**//MMFF) $=-3245.91030295$

01_611

MM̄FF Geometry

C $0.303411-0.8967651 .811423$

C $0.654347-0.2198502 .915046$

C 2.0689520 .0306943 .384245

O $3.006186-0.8755412 .751614$

C 2.5313841 .4543593 .029703

C 1.8320102 .5532833 .798403

$\begin{array}{llll}\text { C } & 0.874180 & 3.353608 & 3.291197\end{array}$

C 2.3129412 .7529765 .212360

C $0.252216 \quad 3.300463 \quad 1.922967$

C $0.632892 \quad 4.5079541 .057000$

C $2.090864 \quad 4.475307 \quad 0.571594$

C $2.4209475 .751012-0.205917$

C $3.8367075 .709920-0.797401$

O $2.2474953 .330916-0.274337$
O $3.9476136 .776220-1.752356$

C $4.0112184 .388609-1.572732$

C $4.9103215 .966285 \quad 0.266710$

$\begin{array}{llllll}\text { O } & 5.355917 & 4.238582 & -2.023961\end{array}$

C $3.5767823 .135205-0.774241$

C $3.5522021 .908678-1.717524$

$\begin{array}{lllll}\text { O } & 4.511452 & 2.907111 & 0.280601\end{array}$

C $3.161104-2.1043903 .308475$

O $2.578837-2.5340974 .295763$

C $4.135780-2.9335362 .562501$

C $4.856043-2.5033401 .514428$

C $5.824006-3.299050 \quad 0.751358$

C $5.998474-4.7435921 .146350$

C $3.0110860 .616615-1.088126$

C $2.892254-0.497878-2.115897$

C $2.326857-2.914237-2.312488$

O $3.160767-0.344708-3.304971$

C $3.591047-3.697531-2.154075$

C $4.600077-3.654854-3.036906$

C $5.894276-4.400516-2.881535$

C $7.055681-3.470316-2.603770$

C $7.691551-2.841977-3.607093$

C $7.525042-3.328015-1.171941$

C $6.488110-2.714230-0.269103$

N $2.445037-1.684690-1.549999$

H $1.074052-1.302792 \quad 1.166538$

H $-0.1305760 .207376 \quad 3.535625$

H $2.119261-0.114576 \quad 4.470217$

H 2.4451911 .6054351 .947795

H 3.6119991 .5355143 .213892

H 0.4621314 .1298883 .935103

H 1.8142793 .5933475 .706907

H 2.1225901 .8585105 .813350

H 3.3885562 .9573315 .222260

H 0.4803522 .3693561 .397534

H $-0.836842 \quad 3.3013072 .059632$

H $-0.0327214 .510426 \quad 0.184132$

H 0.4423605 .4355601 .610963

H $2.755775 \quad 4.379314 \quad 1.436799$

H 2.2960416 .6372410 .428783

H $1.7048755 .870694-1.031374$

H $3.7660987 .612549-1.290234$

H $3.3877344 .455460-2.475649$

H 4.7637696 .9501180 .728603

H $5.9105195 .991455-0.179486$

H 4.9078245 .2234201 .066954

H $5.5823535 .037382-2.530515$

H $2.9228662 .147393-2.585277$

H $4.5636671 .708922-2.093745$

H 4.4725041 .9752130 .545139

H $4.232760-3.9469292 .939538$

H $4.735460-1.4760801 .177198$

H $5.038254-5.2707541 .110393$

H $6.403429-4.8171362 .161703$

H $6.672392-5.3007470 .493396$

H $3.6772710 .270451-0.291560$

H $2.0182940 .785826-0.658805$

H $2.121709-2.680902-3.362589$

H $1.479910-3.480976-1.915139$

H $3.678416-4.317160-1.265747$

H $4.500793-3.030168-3.922148$

H $6.079847-4.960745-3.807209$

H $5.825497-5.160257-2.093339$

H $8.540810-2.192865-3.422152$

H $7.373355-2.962464-4.637575$

H $8.412850-2.683509-1.124703$

H $7.869057-4.304359-0.820216$

H $6.284673-1.664979-0.488120$

H $2.357328-1.746395-0.540579$

C -1.606156 -2.530892 1.421468

O $-3.006062-2.5100061 .061891$

C $-3.225735-3.434201-0.015937$

C $-2.074398-4.4209010 .069473$

C $-0.924181-3.505046 \quad 0.452203$

H $-3.137975-2.867700-0.950884$

C $-4.624351-4.0494950 .093629$

H $-2.239330-5.1675730 .854925$

H $-1.906111-4.949326-0.873777$ 
C $0.249951-4.2726151 .041452$

H $-0.588239-2.969038-0.445017$

C $-5.762316-3.008650 \quad 0.164306$

O $-4.695633-4.8460521 .283791$

H -4.794681 -4.739866 -0.740130

H $-5.690160-2.4543291 .106338$

O $-7.006984-3.717413 \quad 0.208407$

C $-5.803006-2.029120-1.023584$

C -7.004740 -1.065795-1.004509

H -4.877897 -1.448589-1.054115

O $-5.860257-2.788160-2.234391$

H -7.031596 -0.537573 -1.965129

H -7.934703 -1.646548 -0.979076

H $1.084091-3.6092841 .281328$

H $-0.035380-4.7983181 .958886$

H $\quad 0.616313-5.0184150 .327994$

C -1.110025 -1.084389 1.358971

H -4.483321 -4.267954 2.036828

H -6.960047 -4.3391430 .954865$

H -5.811464 -2.161717 -2.976516

H -1.789300 -0.4514091 .945643$

H - $1.200622-0.7132480 .331178$

H -1.555528 -2.9013712 .452667$

C $-5.6638071 .690272-0.903658$

O

C $-6.971081-0.0526850 .157784$

$\begin{array}{llll}\text { C } & -8.200371 & 0.861448 & 0.231566\end{array}$

C $-8.1913981 .908246-0.875866$

C $-6.8600072 .657157-0.895789$

H -5.616405 $1.166412-1.865986$

C $-4.3393412 .448183-0.697883$

H $-6.958882-0.6303831 .088973$

O

H $-8.210027 \quad 1.380721 \quad 1.198854$

H -8.406558 $1.461566-1.853760$

O $-9.2342922 .855744-0.630728$

H $-6.8133293 .322813-0.023947$

O $-6.8425593 .472431-2.067202$

C $-3.1285411 .643661-1.125128$

H $-4.3315763 .363770-1.298073$

H -4.2067802 .7084300 .358486$

H $-9.388720-0.5464150 .872384$

H -10.070188 2.360193 -0.587686

H -6.159698 4.153010 -1.948330

O $-2.0501392 .479723-1.205540$

O $-3.1199360 .442098-1.350249$

C $-0.8252871 .878919-1.638757$

H $-0.9801831 .273665-2.537181$

H $-0.1205542 .679524-1.878030$

H $-0.4084741 .272282-0.831541$

SCF Energy (B3LYP/6-31G**//MMFF) $=-3245.90769672$

01612

MM̄FF Geometry

C $2.231652-3.481862-0.840843$

C $1.548126-3.8292670 .261889$

C $1.557892-3.1184821 .595462$

O $2.593969-2.112516 \quad 1.596344$

C $0.185928-2.4519031 .812586$

C $-0.072267-2.0157253 .240280$

C $-0.069573-0.7340393 .654951$

C $-0.414664-3.1223734 .203419$

C $0.255005 \quad 0.479176 \quad 2.832174$

C -1.016784 1.1585052 .311449

C -0.6912042 .3995971 .470710$

C -1.9707053 .0913020 .999263$

C $-1.673553 \quad 4.2766230 .066851$

O $0.071058 \begin{array}{lll}1.973256 & 0.337785\end{array}$

O $-2.8991844 .652881-0.578619$

C $-0.7035873 .812425-1.041130$

C -1.2008685 .5093550 .846365$

O $-0.2772284 .911648-1.842188$

C $0.522144 \quad 3.042191-0.502427$

C $1.3276022 .423429-1.669825$

O $1.369350 \quad 3.950934 \quad 0.207031$

C $3.332046-1.9471192 .725159$

O $3.164337-2.4873353 .806216$

C $4.426223-1.0067922 .398219$
C $5.313940-0.6554793 .339360$

C 6.4524020 .2251283 .100979

C 6.5629481 .3513154 .089710

C 2.342298 1.367926-1.209145

C $3.3439111 .001867-2.289327$

C $5.421331-0.356452-2.577936$

O $3.3388121 .490835-3.414677$

C $6.5684350 .508114-2.158541$

C $7.5289320 .108459-1.311579$

C $8.6512920 .991086-0.849325$

C 8.3897341 .6087760 .507744

C 8.0880602 .9132990 .621806

C 8.5617970 .7462421 .738349

C $7.316116-0.0177352 .094268$

N $4.2536560 .061085-1.826601$

H $2.895303-2.622837-0.827749$

H $\quad 0.879477-4.686957 \quad 0.203447$

H $1.762544-3.8755532 .361800$

H $-0.619277-3.1463901 .534461$

H $0.082619-1.6025731 .126178$

H $-0.309564-0.5244944 .696199$

H $-0.650958-2.7441785 .203718$

H -1.288531 -3.678070 3.847635

H $\quad 0.422988-3.818742 \quad 4.305714$

H 0.8173521 .1796913 .461993

H $\quad 0.9253800 .2234242 .005135$

H -1.574030 0.4401021 .696551

H $-1.6604691 .439556 \quad 3.154117$

H -0.0872673 .0815662 .080850$

H $-2.578607 \quad 3.415021 \quad 1.853118$

H $-2.5864702 .367426 \quad 0.449917$

H -3.547365 4.8720110 .112608

H -1.264153 $3.143077-1.707300$

H -1.969869 5.8284591 .560354

H -1.041512 6.3625010 .177720

H -0.2818395 .3351131 .409634$

H -1.076608 $5.358941-2.169098$

H $0.6450421 .931986-2.375345$

H $1.8437153 .221097-2.218642$

H 2.2903153 .6545140 .137324

H $4.499144-0.6392251 .380410$

H $5.210464-1.0147824 .360561$

H 6.7839160 .9624255 .089630

H 5.6210941 .9100224 .138824

H 7.3416532 .0741383 .835776

H $2.9107501 .728732-0.344842$

H $1.8155090 .454006-0.908426$

H $5.239655-0.252351-3.652016$

H $5.595682-1.413232-2.352659$

H $6.5916681 .521079-2.553531$

H $7.500567-0.903222-0.915012$

H $8.8338341 .777035-1.594607$

H $9.5788480 .406632-0.817340$

H 7.9278043 .3732401 .591229

H $7.9892283 .549298-0.251671$

H 8.9069141 .3563122 .580529

H 9.3760280 .0295481 .571365

H $7.136600-0.8891471 .465266$

H $4.194026-0.239191-0.858345$

C $1.126723-3.490719-3.121463$

O $-0.145424-3.280126-2.472631$

C $-0.516908-1.898960-2.633765$

C $0.252481-1.428191-3.857955$

C $1.583710-2.122962-3.632199$

H $-0.147647-1.358589-1.751525$

C $-2.039589-1.745243-2.713582$

H $-0.213889-1.781524-4.785181$

H $\quad 0.339358-0.338683-3.905497$

C $2.462321-2.186285-4.868343$

H $2.124328-1.584162-2.847473$

C $-2.729198-2.083631-1.370655$

O $-2.527441-2.566458-3.770237$

H $-2.268744-0.704774-2.972436$

H $-2.270402-1.455768-0.599024$

O $-2.496157-3.442982-1.003176$

C $-4.250745-1.839557-1.368979$

C -4.915069-2.074002 0.002690

H $-4.454623-0.818270-1.704334$ 
O $-4.887488-2.707652-2.308818$

H -6.001650 -2.018198 -0.136656

H $-4.727998-3.1044940 .329705$

H $3.396885-2.714125-4.653070$

H $1.961244-2.706588-5.691285$

H $2.715035-1.178006-5.212312$

C $2.080411-4.201661-2.154329$

H -3.481882 -2.403082 -3.854701

H -1.535593 -3.569858 -0.925694

H -4.665312 -3.621545 -2.060269

H $3.067478-4.326740-2.613256$

H $1.693010-5.211386-1.965808$

H $0.930753-4.160415-3.968581$

C $-5.929162 \quad 0.7473140 .526776$

$\begin{array}{lllll}\text { O } & -4.582390 & 0.277795 & 0.667565\end{array}$

C $-4.463580-1.0829551 .094519$

C $-5.153908-1.2686292 .452335$

C $-6.595068-0.7666022 .434682$

C $-6.6654230 .651171 \quad 1.867325$

H $-6.4531550 .149155-0.228754$

C -5.8663742 .1943090 .023820$

H -3.395693 -1.260437 1.265143

O $-5.137799-2.6353162 .850155$

H $-4.602923-0.7051603 .217019$

H -7.249584 -1.444791 1.874732

O $-7.098466-0.737525 \quad 3.772405$

H -6.2333941 .3576842 .587683$

O $-8.032778 \quad 1.009944 \quad 1.679974$

C $-5.5994662 .236348-1.462724$

H -6.8160362 .7173790 .184988$

H -5.0915502 .7739070 .537893$

H -4.208953 -2.922693 2.870529

H $-7.014394-1.6374524 .131860$

H -8.4782830 .9134902 .539144$

O $-4.2758102 .030246-1.712539$

O $-6.4720422 .417134-2.302707$

C -3.903025 $2.032030-3.093921$

H $-2.8319341 .824381-3.157765$

H $-4.4430431 .249012-3.634788$

H $-4.0975813 .013862-3.535498$

SCF Energy $\left(\mathrm{B} 3 L Y P / 6-31 G^{* *} / / \mathrm{MMFF}\right)=-3245.90445005$

01613

MM̄FF Geometry

C $1.034741-1.9603641 .304001$

C $0.747744-2.6726530 .203925$

C $1.243677-2.335660-1.180511$

O $0.998498-0.931201-1.454034$

C $2.750303-2.580308-1.366092$

C $3.159863-4.035884-1.320039$

C $3.890777-4.599118-0.338332$

C $2.746124-4.859898-2.512800$

C $4.404110-3.9630830 .923358$

C $5.928086-3.778406 \quad 0.908147$

C $6.395422-2.651966-0.027320$

C $7.923286-2.584140-0.067600$

C $8.420907-1.402873-0.915020$

O $5.867825-1.4211290 .476819$

O $9.820305-1.227784-0.645134$

C $7.703447-0.119193-0.446511$

C $8.301771-1.689756-2.416422$

O $8.0347480 .984992-1.286191$

C $6.167899-0.270584-0.322270$

C $5.577894 \quad 0.969147 \quad 0.391675$

O $5.606393-0.372837-1.631909$

C $-0.265151-0.584943-1.814083$

O $-1.215827-1.344579-1.951243$

C $-0.4095230 .872914-2.021509$

C $0.5825891 .769007-1.905634$

C $0.4483353 .218123-2.092738$

C $-0.9399613 .760580-2.324351$

C $4.0923470 .841448 \quad 0.758559$

C 3.5939642 .0460731 .538598

C 1.5177922 .9944722 .533909

O $4.3220742 .976661 \quad 1.874131$

C 0.9823513 .9689361 .533637

C 1.5682795 .1470151 .273054

C 1.0858356 .1284040 .244159
C $2.0284456 .217217-0.937150$

C $3.1362716 .976035-0.880130$

C $1.6506195 .484923-2.206072$

C $1.5532653 .991840-2.024836$

N $2.238375 \quad 1.951527 \quad 1.827722$

H $1.707908-1.1125091 .223283$

H $0.081635-3.5284240 .281122$

H $0.687588-2.928614-1.916963$

H $3.310855-1.977394-0.644232$

H $3.057181-2.167298-2.337313$

H $4.147886-5.653653-0.434750$

H $3.160212-5.873452-2.482446$

H $1.656953-4.952639-2.559291$

H $3.096949-4.391714-3.438289$

H $3.906171-3.0156721 .145969$

H $4.144369-4.6323821 .753598$

H $6.240918-3.5416591 .933560$

H $6.413375-4.7225590 .631203$

H $6.000413-2.832175-1.032893$

H $8.345160-3.526889-0.437862$

H $8.310312-2.4641090 .954185$

H $10.275344-2.057677-0.869553$

H 8.1082960 .1261390 .544979

H $8.869370-2.590336-2.680122$

H $8.738834-0.878875-3.009538$

H $7.271232-1.842211-2.743521$

H $7.5256230 .893652-2.109605$

H 6.1360691 .1420141 .321396

H $5.7084481 .859199-0.237030$

H $4.684609-0.073011-1.608636$

H $-1.4168991 .179944-2.284639$

H $1.5830631 .422537-1.655560$

H -1.616982 $3.440121-1.523975$

H -1.334974 $3.407535-3.283228$

H $-0.9899814 .850842-2.338509$

H $3.4798780 .743655-0.143123$

H $3.933326-0.0440691 .383613$

H 2.1874113 .4755453 .254923

H 0.6986412 .5260263 .086640

H 0.0832463 .6818210 .995035

H 2.4683695 .4203561 .819941

H $0.997504 \quad 7.1122630 .723100$

H $0.0735965 .882400-0.098779$

H $3.8061997 .066324-1.728689$

H 3.3979697 .5228900 .019939

H $2.4009525 .665129-2.987601$

H $0.7281415 .922213-2.597652$

H $2.5144403 .511322-1.837509$

H $1.696003 \quad 1.196437 \quad 1.419174$

C $-0.179216-0.9715213 .249253$

O $-1.229547-0.4991022 .383333$

C $-2.368162-0.1343203 .183011$

C $-1.873374-0.0934194 .624086$

C $-0.831341-1.1954284 .613018$

H $-3.092038-0.9525383 .083343$

C -2.9945031 .1672982 .670213$

H -1.403824 0.8665014 .868037

H -2.678112 -0.2685165 .344511$

C $0.138471-1.1288165 .779581$

H -1.347119 -2.164941 4.629773

C $-3.2840531 .182074 \quad 1.155103$

O -2.0955852 .2498892 .941197$

H -3.9076121 .3784913 .239372$

H -2.3392091 .2533420 .603087$

$\begin{array}{lllll}\text { O } & -3.994995 & 2.392648 & 0.863802\end{array}$

C $-4.091905-0.0195570 .632997$

C $-4.453803 \quad 0.134884-0.856405$

H $-3.503423-0.9335370 .769628$

O $-5.277455-0.1427841 .412504$

H $-5.1907440 .937016-0.967342$

H $-3.5538750 .441278-1.399195$

H $0.876653-1.9346715 .719462$

H $0.679621-0.1768255 .797829$

H $-0.395697-1.2300886 .729958$

C $0.453179-2.2355172 .660902$

H -2.5120833 .0634982 .608749$

H -4.866836 2.3296431 .290725

H $-5.705672-0.9776021 .158549$ 
H $-0.301975-3.0278492 .589184$ H $1.251433-2.5953803 .319407$ H $0.566463-0.1685573 .308394$ C -7.369808 $-0.805948-1.159606$ O $-6.217844-1.610606-0.876518$ C $-4.999427-1.168808-1.482704$ C $-5.164293-1.111393-3.008248$ C $-6.360826-0.257000-3.413903$ C $-7.614645-0.712826-2.670598$ H $-7.2296290 .197041-0.739017$ C $-8.568012-1.454348-0.455684$ H $-4.255343-1.947868-1.272110$ O $-3.991573-0.602003-3.634484$ H $-5.317944-2.127456-3.394759$ H $-6.1697520 .809577-3.246581$ O $-6.593900-0.409075-4.816051$ H -7.936837 -1.686567 -3.061708 O $-8.6683540 .216201-2.917452$ C $-8.491930-1.2492591 .038387$ H $-9.519000-1.020895-0.785771$ H $-8.618573-2.529640-0.665833$ H -3.246305 -1.163758 -3.360701 H $-5.774356-0.154501-5.273965$ H $-8.7855320 .272485-3.881427$ O $-7.933935-2.3416621 .633727$ O $-8.861186-0.2280061 .603722$ C -7.795399 -2.2642233 .055999$ H -7.162461 -1.415556 3.332060 H $-8.779384-2.1813473 .526892$ H -7.316958 -3.184542 3.401244

SCF Energy (B3LYP/6-31G**//MMFF) $=-3245.93164278$

01_614

MM̄FF Geometry

C $2.403325-0.489802-2.959824$

C $2.147236-1.399726-2.007378$

C $0.901840-1.512938-1.163015$

O $-0.014289-0.424005-1.412772$

C $0.167522-2.827971-1.476292$

C $-0.927440-3.117617-0.469514$

C $-2.222403-2.777237-0.622836$

C $-0.472868-3.8322370 .777457$

C $-2.847856-2.033353-1.771560$

C $-4.063306-2.745154-2.387942$

C $-5.252179-2.910741-1.428137$

C $-6.483839-3.446469-2.161296$

C $-7.709030-3.533388-1.234335$

O $-5.548264-1.632043-0.862055$

O $-8.871230-3.712007-2.058817$

C $-7.879315-2.186511-0.495130$

C $-7.638355-4.759948-0.316800$

O $-8.915674-2.266000 \quad 0.481001$

C $-6.572303-1.6558590 .137643$

C $-6.770061-0.2115950 .654930$

O $-6.218446-2.4940001 .241302$

C $0.124510 \quad 0.705180-0.667239$

$\begin{array}{lllll}\text { O } & 0.972625 & 0.931160 & 0.180417\end{array}$

C $-0.9443951 .640824-1.083180$

C $-0.8592272 .936117-0.745869$

C $-1.811348 \quad 3.986755-1.114447$

C $-3.1224563 .559413-1.720263$

C $-5.4547280 .505498 \quad 0.997612$

C $-5.686376 \quad 1.8379501 .687865$

C $-4.429938 \quad 3.7608132 .655861$

$\begin{array}{lllll}\text { O } & -6.801691 & 2.258905 & 1.982990\end{array}$

C -4.5285874 .8863121 .675586$

C -3.6724275 .9189751 .648077$

C -3.808986 7.0860340 .713932

C -2.540261 $7.378604-0.052789$

C -1.7589298 .4218850 .274552$

C $-2.2279836 .519694-1.256820$

C -1.465001 $5.272977-0.897428$

N -4.490665 2.4936951 .952727

H $1.6612850 .259456-3.219409$

H $2.921079-2.127781-1.776927$

H $1.203142-1.504368-0.107927$

H $0.873016-3.669846-1.473558$

H $-0.243660-2.797720-2.491895$
H -2.903269 -3.016963 0.192028 H $-1.298113-4.0274181 .470521$ H -0.024157 -4.7970270 .519303$ H $0.271977-3.2345031 .312312$ H -3.145873 -1.041350 -1.411496 H $-2.128176-1.852699-2.574920$ H $-4.383832-2.142031-3.247657$ H -3.764517 -3.725685 -2.778984 H $-4.962920-3.604381-0.631210$ H $-6.272877-4.422822-2.615014$ H $-6.731567-2.775525-2.995852$ H $-8.745277-4.520939-2.583718$ H -8.223744 -1.456369 -1.240384 H -7.567049 $-5.678840-0.911195$ H $-8.552651-4.8617190 .278218$ H $-6.783027-4.7391820 .361601$ H -8.562437 -2.7461571 .249268$ H $-7.2776530 .388717-0.111321$ H $-7.420106-0.2270301 .538949$ H -5.703976 -1.979551 1.882683 H $-1.756074 \quad 1.243908-1.682449$ H $0.0060943 .268615-0.173631$ H -3.600804 $2.790173-1.103634$ H $-2.9671053 .155031-2.726147$ H $-3.8483144 .371352-1.801313$ H $-4.839481-0.1078491 .664336$ H $-4.8827840 .691566 \quad 0.080905$ H -3.489375 3.7786793 .215284 H -5.2613983 .8177513 .365958$ H $-5.365078 \quad 4.8714220 .980807$ H $-2.854905 \quad 5.9511632 .364662$ H -4.0922627 .9593591 .316145$ H -4.632602 6.9367810 .004546 H $-0.8630718 .655532-0.291389$ H -1.9937199 .0615901 .118743$ H -1.603239 $7.087006-1.960379$ H -3.150184 $6.317996-1.808532$ H $-0.4890285 .475609-0.453430$ H $-3.6204112 .101917 \quad 1.604270$ C $4.4896170 .841801-3.291834$

O $4.7564870 .813967-1.877664$

C $6.0708951 .346739-1.633748$

C $6.5748101 .873869-2.973998$

C $5.859174 \quad 0.962487-3.954285$

H $6.6977030 .499934-1.329866$

C $6.0128392 .379745-0.501463$

H $6.2801002 .915923-3.142123$

H $7.6649041 .820203-3.054794$

C $5.817107 \quad 1.505603-5.371604$

H $6.363551-0.013261-3.956091$

C $5.279413 \quad 1.8806930 .762250$

O $5.3049543 .534071-0.974377$

H $7.0263622 .718318-0.257381$

H $4.216246 \quad 1.7607220 .529208$

$\begin{array}{llll}\text { O } & 5.347473 & 2.922488 & 1.744862\end{array}$

C 5.8424200 .5812901 .367120

C $5.136916 \quad 0.144607 \quad 2.666034$

H $5.783072-0.2265120 .633238$

$\begin{array}{llll}\text { O } & 7.227877 & 0.785816 & 1.655720\end{array}$

H $5.732156-0.6516263 .128177$

H 5.1639890 .9690273 .388620

H $5.2855950 .818364-6.037283$

H $5.3106752 .475716-5.414845$

H $6.8310871 .637855-5.762959$

C $3.718100-0.416729-3.688557$

H $5.2715664 .173210-0.242027$

H 6.2797083 .0173342 .005798

H $7.590672-0.0614511 .965690$

H $4.320129-1.304835-3.461930$

H $3.524158-0.423226-4.766733$

H $3.8840401 .737051-3.486378$

C $4.130147-2.6689712 .007691$

O $3.611333-1.4243561 .521481$

C $3.685980-0.3327652 .446406$

C $2.929501-0.6824503 .733803$

C $3.409387-1.9976414 .339212$

C $3.396307-3.1032103 .284252$

H $5.202708-2.5684602 .212199$ 
C $3.946815-3.7159390 .896489$

H 3.1409360 .4979201 .985113

O 3.0624050 .3713734 .681809

H $1.859918-0.7707313 .501376$

H $4.401603-1.8839344 .791375$

O $2.517418-2.3460505 .400431$

H $2.358817-3.3732823 .048878$

O $4.035211-4.263854 \quad 3.812494$

C $4.922491-3.533605-0.247417$

H $4.109840-4.7250821 .290687$

H $2.932879-3.6461830 .485996$

H $2.582197 \quad 0.102915 \quad 5.483857$

H $2.885803-3.1253305 .849653$

H $3.487356-4.5946644 .543937$

O $4.636436-4.452501-1.218096$

O $5.823062-2.707765-0.288780$

C $5.486954-4.417572-2.368384$

H $6.512092-4.674737-2.085519$

H $5.122580-5.160000-3.083309$

H $5.452573-3.431135-2.839721$

SCF Energy (B3LYP/6-31G**//MMFF) $=-3245.91518945$

01_615

MMFF Geometry

C $-3.588179-1.578883-2.306048$

C $-3.325829-0.466269-3.007915$

C $-3.5555430 .918423-2.464883$

O $-2.3646831 .717663-2.681716$

C $-4.6940591 .657010-3.187501$

C $-6.0746301 .091962-2.945700$

C $-6.8557801 .427964-1.901168$

C $-6.5954870 .158988-4.008251$

C $-6.5285212 .364065-0.768008$

C $-6.936047 \quad 1.7793630 .592745$

C $-5.9572110 .716952 \quad 1.120577$

C $-6.616841-0.0800882 .249512$

C $-5.641265-1.0683342 .899140$

O -4.7991991 .4050371 .609492$

O $-6.251163-1.5811144 .092944$

C $-4.363009-0.3060853 .297628$

C $-5.400136-2.2912382 .009450$

O $-3.370054-1.2046903 .789006$

C -3.7748910 .5643822 .159713$

C $-2.679627 \quad 1.4862232 .752111$

O $-3.205560-0.2900101 .169658$

C $-1.2612521 .446539-1.935276$

O $-1.1215980 .549362-1.120582$

C $-0.2427902 .461490-2.283890$

C $0.9276672 .490716-1.630086$

C $2.0156253 .444135-1.862156$

C $1.7958854 .512461-2.901935$

C $-2.051767 \quad 2.4746391 .760542$

C -1.1720023 .4988152 .460696$

C 0.2388005 .4855782 .002620

O -1.0588693 .5627763 .682302$

C 1.3159075 .7905181 .012025

C 2.6231365 .7398251 .310819

C $3.725712 \quad 6.105048 \quad 0.358325$

C $4.645447 \quad 4.942571 \quad 0.059695$

C 5.6935264 .6636370 .852952

C $4.3938474 .157930-1.207097$

C $3.1434563 .326294-1.131095$

N $-0.528943 \quad 4.3374361 .557609$

H $-4.027388-1.483256-1.317549$

H -2.905854 -0.546247 -4.007420

H -3.758989 $0.901863-1.387346$

H $-4.4904191 .691613-4.267118$

H $-4.6743402 .715138-2.892343$

H -7.856304 $0.998865-1.858325$

H -7.617146 -0.177494 -3.801668

H $-6.6068890 .662231-4.980804$

H -5.968732 $-0.733196-4.087449$

H $-5.4722362 .648696-0.750645$

H -7.104109 $3.283775-0.929993$

H $-6.9829302 .607536 \quad 1.311991$

H $-7.947926 \quad 1.3611230 .518821$

H -5.6625490 .0486640 .304337$

H $-7.507487-0.6078361 .885783$
H -6.9769940 .6153023 .020851$

H $-6.448829-0.8317614 .680111$

H -4.6228570 .3546804 .136347$

H $-6.338445-2.8347841 .844813$

H $-4.721164-3.0045702 .489548$

H $-4.993274-2.0327531 .030600$

H $-3.777425-1.7254674 .502302$

H -3.123763 $2.065053 \quad 3.572929$

H -1.8722790 .8795203 .182026$

H -2.5400050 .2047340 .666889$

H $-0.4906603 .167636-3.068645$

H $1.1087021 .750664-0.851686$

H $0.8982205 .096588-2.669574$

H $1.6769634 .063492-3.894107$

H $2.6137865 .231699-2.973394$

H $-1.426731 \quad 1.9383551 .042252$

H $-2.828843 \quad 3.0187701 .212841$

H 0.6398555 .2951793 .003912

H -0.4513016 .3326452 .075158$

H 1.0050026 .0995330 .017221

H 2.9245445 .4477452 .314713

H 4.3022556 .9243770 .807906

H $3.3252986 .513205-0.578000$

H $6.372408 \quad 3.847425 \quad 0.628543$

H 5.8922755 .2443151 .747800

H $5.2300353 .474242-1.402253$

H $4.3971824 .847807-2.055121$

H $3.1987732 .525119-0.393215$

H -0.8087724 .3008070 .581175$

C -2.346024 $-3.769831-1.948674$

O $-1.063490-3.114650-1.892194$

C $-0.497666-3.331028-0.583391$

C $-1.402274-4.3466460 .110603$

C $-2.750133-4.016574-0.497056$

H $-0.555623-2.374166-0.049216$

C $0.965563-3.783927-0.677416$

H $-1.113359-5.376150-0.133122$

H -1.383134 -4.241142 1.199737

C $-3.789609-5.107157-0.314765$

H -3.111054 -3.094875 -0.028599

C $1.921114-2.679525-1.181644$

O $1.044830-4.944871-1.501501$

H $1.298068-4.0843540 .322565$

H $1.810134-1.823074-0.507954$

O $1.535638-2.231773-2.479049$

C $3.401506-3.113323-1.203753$

C $4.378536-1.994095-1.609524$

H $3.672725-3.505319-0.217285$

O $3.566926-4.183595-2.137285$

H $5.379938-2.436323-1.682026$

H $4.148234-1.659014-2.628929$

H $-4.740171-4.819360-0.774694$

H $-3.466312-6.050461-0.767425$

H $-3.971973-5.2903220 .749190$

C $-3.325977-2.968680-2.810842$

H $0.679966-4.712019-2.372257$

H $\quad 1.693190-2.962900-3.100336$

H $4.484870-4.496819-2.066394$

H $-4.281702-3.501423-2.874005$

H $-2.923279-2.915311-3.830397$

H $-2.172127-4.732262-2.449082$

C $5.780592-1.7394771 .085089$

O $4.514886-1.1776890 .717649$

C $4.405554-0.785822-0.653154$

C $5.4826790 .256268-0.983998$

C $6.882228-0.224171-0.611013$

C $6.907898-0.7321290 .830174$

H $5.959583-2.6556490 .508705$

C $5.700646-2.1164222 .568045$

H $3.436969-0.281225-0.751935$

O $5.455496 \quad 0.598337-2.364902$

H $5.2811021 .173257-0.417402$

H $7.243804-0.992026-1.304940$

$\begin{array}{llll}\text { O } & 7.796216 & 0.870638 & -0.714136\end{array}$

H 6.8187380 .1174191 .519569

O $8.163695-1.3589801 .082183$

C $4.844811-3.3457402 .760469$

H $6.688091-2.3489622 .982888$ 
H $5.286134-1.2989393 .170251$

H $4.5605150 .919851-2.568835$

H $7.736003 \quad 1.212282-1.622978$

H $8.857734-0.7052990 .889928$

O $3.543109-2.9869732 .948740$

O $5.281249-4.4890082 .723460$

C $2.625280-4.0702773 .126271$

H $1.612094-3.6595643 .134933$

H $2.705752-4.7841192 .300907$

H $2.814380-4.5649464 .083320$

SCF Energy (B3LYP/6-31G**//MMFF) $=-3245.91243340$

01_616

MM̄FF Geometry

C $4.118972 \quad 2.820492-2.313015$

C $3.2445052 .819023-1.295174$

C $1.8080243 .258696-1.418315$

O $1.0116212 .070550-1.209006$

C $1.5024964 .299910-0.324991$

C $0.2441845 .107473-0.566871$

C -0.9118374 .9596600 .109334$

C $0.3691456 .187757-1.611878$

C $-1.2430793 .945692 \quad 1.170735$

C -2.572531 $3.257860 \quad 0.854450$

C $-3.043923 \quad 2.2632291 .924941$

C $-2.089153 \quad 1.079432 \quad 2.101007$

C -2.662202 0.0036573 .036340

O -4.3255451 .8003221 .481163$

O $-1.855056-1.1766612 .905301$

C $-4.098809-0.3423332 .591460$

C -2.5364490 .4171034 .506544$

O $-4.716375-1.2336543 .517083$

C -4.9899620 .9019982 .377611$

C $-6.320700 \quad 0.489122 \quad 1.707697$

O $-5.261801 \quad 1.5122963 .638664$

C $-0.1437611 .937904-1.912319$

O $-0.6557842 .755960-2.658819$

C $-0.6820340 .589393-1.623076$

C $-1.955202 \quad 0.294458-1.928507$

C -2.613176 $-0.998203-1.715087$

C $-1.739595-2.150436-1.296226$

C -7.2560091 .6659441 .393103$

C $-8.287851 \quad 1.257377 \quad 0.362382$

C $-8.510793 \quad 0.782107-2.075640$

$\begin{array}{llll}\text { O } & -9.415840 & 0.891222 & 0.682597\end{array}$

C $-8.133823-0.657183-2.227818$

C $-7.348205-1.128055-3.207837$

C $-6.920594-2.565137-3.296979$

C $-5.427326-2.735397-3.123689$

C $-4.675498-3.288118-4.090670$

C $-4.816121-2.320684-1.800482$

C $-3.941786-1.100222-1.931621$

N $-7.7910851 .309515-0.931663$

H $3.7667033 .141167-3.291853$

H $3.5554062 .487474-0.308641$

H $1.6293603 .665118-2.421027$

H $2.3340365 .015107-0.250864$

H 1.4619123 .8051090 .652118

H $-1.7268555 .643394-0.127135$

H $-0.5446776 .783961-1.706391$

H $1.1827896 .873642-1.354291$

H $\quad 0.578444 \quad 5.752672-2.593798$

H $-0.456814 \quad 3.193742 \quad 1.262307$

H -1.318533 4.4626042 .134345

H -3.3577914 .0162870 .725555$

H - $2.5126882 .748280-0.115393$

H -3.169793 2.8039102 .870295

H - $-1.109421 \quad 1.4068872 .467524$

H -1.9025210 .6203361 .122667$

H -1.895658 -1.470859 1.979573

H $-4.029920-0.8846361 .638065$

H -1.481671 0.5531904 .775081

H -2.914996 $-0.366170 \quad 5.172520$

H -3.057810 1.3484104 .737440

H -4.126936 -2.000431 3.618925

H $-6.092607-0.044327 \quad 0.775725$

H $-6.867122-0.2114042 .351712$

H -6.0433752 .0804193 .553344$
H $-0.011248-0.124429-1.157392$

H $-2.579547 \quad 1.071835-2.366953$

H $-1.312740-1.969101-0.304401$

H $-0.922109-2.284922-2.013296$

H $-2.260987-3.107877-1.247317$

H $-7.784178 \quad 1.9994152 .293200$

H -6.7045362 .5314381 .008662$

H -8.227818 $1.379958-2.947416$

H $-9.589590 \quad 0.875922-1.917189$

H $-8.506263-1.342122-1.469230$

H $-6.969319-0.447857-3.966639$

H $-7.248188-2.954399-4.269093$

H -7.429982 -3.175067-2.540146

H $-3.608431-3.435248-3.959659$

H $-5.108963-3.607086-5.032676$

H $-4.289309-3.177882-1.372202$

H -5.606622 -2.094680 -1.073930

H $-4.485671-0.203849-2.232536$

H $-6.8118711 .544065-1.068734$

C $6.1938012 .217449-0.885803$

O $5.660073 \quad 1.033016-0.265328$

C $6.6678820 .472158 \quad 0.598523$

C 7.9100551 .3494840 .450926

C $7.701347 \quad 1.979585-0.912705$

H $6.897219-0.525754 \quad 0.209885$

$\begin{array}{llll}\text { C } & 6.137025 & 0.371616 & 2.034759\end{array}$

H $7.9596692 .120834 \quad 1.228237$

H 8.8334850 .7649490 .513879

C $8.5334663 .231904-1.127961$

H $7.9507941 .238772-1.684220$

C $4.843574-0.4584982 .176102$

$\begin{array}{lllll}\text { O } & 5.859951 & 1.697586 & 2.504364\end{array}$

H $6.922215-0.0311532 .686252$

H $4.0065390 .065528 \quad 1.700032$

O $4.530756-0.5453573 .572150$

C $4.946041-1.8911191 .621328$

C $3.764569-2.8091331 .984715$

H $5.050651-1.8555890 .532418$

O $6.134575-2.5120322 .122086$

H $3.999757-3.810344 \quad 1.603044$

H $3.718881-2.9357473 .073929$

H $8.3642163 .649897-2.124911$

H $8.2907214 .006056-0.392281$

H $9.6006473 .004235-1.036774$

C $5.5728462 .419700-2.270676$

H 5.7357831 .6475883 .467002

H 4.2504650 .3383193 .864607

H $6.075797-2.5180483 .093090$

H $5.676827 \quad 1.500071-2.859268$

H $6.1297103 .204433-2.796213$

H $5.9570663 .069607-0.234446$

C $2.604726-3.198261-0.789648$

$\begin{array}{llll}\text { O } & 2.416978-2.055697 & 0.053600\end{array}$

C $2.395591-2.3404481 .454493$

C $1.244963-3.3083891 .762818$

C $1.338954-4.5803170 .923484$

C $1.503636-4.240368-0.557089$

H $3.588574-3.641148-0.593623$

C $2.584666-2.702920-2.239643$

H 2.162978-1.394406 1.957909

O $1.228849-3.6586893 .142346$

H $\quad 0.287810-2.8185221 .543329$

H $2.149918-5.2321291 .268643$

$\begin{array}{lllll} & 0 & 0.132157 & -5.332353 & 1.075087\end{array}$

H $0.549548-3.872958-0.950038$

O $1.825424-5.427970-1.278862$

C $3.765755-1.808752-2.527522$

H $2.596680-3.529464-2.958989$

H $1.678461-2.117438-2.437615$

H $1.150894-2.8340533 .652100$

H $0.019835-5.5091042 .024975$

H $1.122476-6.075787-1.099566$

O $4.888631-2.560014-2.710123$

O $3.696066-0.587811-2.576267$

C $6.081450-1.822429-2.993062$

H $6.324964-1.161216-2.157117$

H $6.899204-2.535785-3.125306$

H $5.962765-1.248626-3.917149$ 
C $5.808091-3.199169-0.445469$

O $4.027264-4.698565-1.003594$

C $0.9386343 .280278-0.791020$

O $\quad 0.6138274 .441927-0.978583$

C $2.2888512 .821773-0.393558$

C $3.2556873 .723366-0.164393$

C $4.635103 \quad 3.4292350 .229518$

C 5.0577821 .9836620 .268309

C $6.291192-1.9216130 .243332$

C $7.731025-1.579777-0.100175$

C 9.4706250 .1340950 .373733

O $8.422924-2.246638-0.863934$

C $9.435273 \quad 1.5819270 .741382$

C $9.3819802 .573032-0.160962$

C 9.3093264 .0298980 .197180

C $7.9408784 .612390-0.092970$

C $7.7282905 .303351-1.226457$

C 6.8933604 .3847430 .981128

C 5.4622184 .4569580 .517103

N $8.139868-0.4181050 .539850$ H -2.923618 $4.459990-0.800929$ H $-1.986377 \quad 1.9724330 .721903$ H $-1.2704793 .216149-2.038210$ H $-2.7884801 .190878-2.231240$ H - $-1.6641620 .277752-1.258928$ H $0.321193-0.474331-4.362049$ H $-0.769717 \quad 1.266483-5.350686$ H $-2.3159691 .702950-4.622455$ H $-0.8527992 .641145-4.247428$ H $-0.322226-1.229397-1.436193$ H $-0.030909-2.336241-2.762932$

H $2.306587-1.341387-3.005452$ H $2.005674-0.353445-1.598910$ H $1.955268-3.409939-1.673359$ H $\quad 0.530637-2.5062790 .208583$ H $1.835354-1.4414730 .684828$ H $\quad 0.870250-3.1559892 .543967$ H $4.050853-2.5411241 .578793$ H $\quad 0.513977-4.8742821 .008055$ H $1.990887-5.6512641 .528516$ H $1.770586-5.222814-0.179692$ H $3.969660-4.5222872 .685182$ H $6.341336-4.065108-0.031726$ H $6.098789-3.157010-1.504694$ H $4.642579-4.804365-1.748040$ H $2.4499411 .754268-0.297001$ H $3.0162854 .781160-0.266044$ H $4.8348911 .488986-0.683969$ H 4.5372351 .4509591 .071527 H $\quad 6.1293841 .8529720 .430107$ H 5.668161-1.071511-0.056196 H $6.225126-2.0342441 .331223$ H $9.802394-0.025212-0.658095$ H $10.151894-0.4120641 .033999$ H 9.4363251 .8222921 .801781

H $10.0843854 .559553-0.371569$

H 9.5579114 .1951321 .253102

H $6.7705005 .754195-1.459769$

H $8.5209245 .441381-1.955296$

H 7.0377045 .1388191 .765934

H 7.0726643 .4258851 .478588

H 5.0753705 .4741850 .446354

H $7.550750-0.0394661 .276768$

C -5.2008453 .9333320 .860409$

O

C -6.8517232 .2734571 .000035$

C $-7.406098 \quad 3.573876 \quad 1.573917$

C -6.1383364 .2815402 .013555$

H $-6.714671 \quad 1.5600061 .820925$

C $-7.7033331 .640047-0.106841$

H -7.9283494 .1661620 .813728$

H $-8.106538 \quad 3.3966302 .395914$

C -6.3223305 .7721262237479$

H -5.7807293 .8171512 .942384$

C $-7.0484270 .410466-0.771851$

O $-7.9167862 .620925-1.130607$

H $-8.693443 \quad 1.385097 \quad 0.288831$

H $-6.1547780 .733739-1.316432$

O $-7.950068-0.087431-1.768303$

C $-6.688534-0.7296520 .198981$

C $-6.141865-1.996461-0.487335$

H $-5.950731-0.3749870 .925171$

O $-7.846148-1.097840 \quad 0.953290$

H $-6.049959-2.7773030 .277537$

H $-6.886092-2.385125-1.193518$

H $-5.382306 \quad 6.2403502 .545427$

H $-6.667027 \quad 6.275151 \quad 1.327747$

H -7.062894 5.9542413 .023242

C $-3.7174904 .013021 \quad 1.221765$

H $-8.4514512 .201903-1.826531$

H -8.789068 -0.307431-1.329377

H $-8.480620-1.5136940 .345434$

H -3.4896173 .2893602 .014241$

H -3.471056 5.0089051 .605960

H $-5.4166844 .562836-0.013446$

C $-3.344708-1.9918430 .718655$

O $-3.823354-1.166451-0.351717$

C $-4.792721-1.784771-1.202798$

C $-4.201930-3.052492-1.834341$

C $-3.634461-4.003594-0.783823$

C -2.688104 -3.261896 0.160219

H $-4.184097-2.2597221 .370823$

C $-2.366106-1.1311361 .529859$

H -4.974093 -1.080033 -2.023017

O $-5.179444-3.740003-2.607740$

H -3.389897 -2.770941 -2.517829

H $-4.431352-4.506753-0.223762$

O $-2.891537-5.035279-1.438274$

H -1.770047 -3.006119 -0.381853

O $-2.324233-4.1285811 .230306$

C -2.100210 -1.655338 2.922065

H -1.421172 -1.032981 0.982739

H $-2.770659-0.1187671 .646220$

H -5.511144 -3.118598 -3.278252

H $-3.495221-5.474027-2.062141$

H -1.955595 -4.937997 0.836730

O $-0.852605-1.268258 \quad 3.318828$

O $-2.901285-2.2903573 .594542$

C $-0.477479-1.6662594 .641405$

H -1.138515 -1.199319 5.377648

H $-0.504881-2.7563804 .732931$

H $\quad 0.545549-1.3254804 .821317$

SCF Energy $\left(B 3 L Y P / 6-31 G^{* *} / / M M F F\right)=-3245.91477643$

01618

MM̄FF Geometry

C $-3.197974 \quad 4.7305310 .445507$

C $-2.3423284 .210442-0.446641$

C $-0.8444094 .354604-0.369818$

O $-0.2678653 .047611-0.138589$

C $-0.3178784 .908608-1.708384$

C $1.1919054 .905242-1.847687$ 
C $1.8483974 .294713-2.855022$

C $1.9578715 .656758-0.791584$

C $1.2391393 .550765-4.013676$

C $0.8406942 .102885-3.695699$

C $2.024693 \quad 1.125756-3.626336$

C $1.522928-0.281456-3.286302$

C $2.677620-1.279462-3.121369$

O $2.9405801 .574442-2.627543$

O $2.150656-2.478962-2.533488$

C $3.722516-0.683416-2.153136$

C $3.244560-1.712153-4.477464$

O $4.884624-1.507492-2.090858$

C $4.1201540 .772810-2.494272$

C $4.9981021 .400832-1.382770$

O $4.889064 \quad 0.770182-3.701733$

C $-0.2415592 .615696 \quad 1.153900$

O $-0.696983 \quad 3.1946902 .128279$

C 0.4545391 .3093161 .193758

C $0.493498 \quad 0.6321592 .351617$

C $1.162907-0.6457642 .606049$

C $1.975763-1.2504901 .494343$

C $4.2242451 .790478-0.118698$

C $5.067611 \quad 1.7241401 .142444$

C 4.8130641 .6073913 .622584

O 6.2908211 .6197991 .131672

C $4.6936420 .156201 \quad 3.975274$

C $4.020720-0.3038515 .041212$

C $3.920845-1.7612115 .394533$

C $2.494068-2.2287095 .586993$

C $2.013664-2.4940966 .813716$

C $1.650653-2.4710364 .352635$

C $1.035130-1.2017383 .829375$

N $4.282586 \quad 1.8049522 .286329$

H -2.817725 $5.296807 \quad 1.291345$

H -2.737715 $3.627175-1.275887$

H -0.5508945 .0350970 .439814$

H $-0.6745915 .939347-1.838061$

H $-0.7710144 .333219-2.523206$

H $2.9360554 .344767-2.869160$

H $3.0262905 .724132-1.023623$

H $1.5769456 .679407-0.702761$

H $1.864206 \quad 5.161898 \quad 0.179927$

H $0.3581104 .095237-4.374049$

H $1.9463303 .566295-4.852418$

H $0.2865632 .078091-2.749499$

H $0.1505961 .758072-4.476043$

H $2.5270811 .129923-4.600852$

H $0.814246-0.636309-4.044615$

H $0.957456-0.245447-2.344163$

H $1.753606-2.249817-1.676270$

H $3.282297-0.703714-1.150510$

H $2.465739-2.197859-5.078062$

H $4.036606-2.459363-4.355408$

H $3.642465-0.880825-5.062964$

H $4.590538-2.408008-1.870552$

H $5.833106 \quad 0.725613-1.160063$

H $5.4701252 .316343-1.765386$

H $5.3974591 .596706-3.746541$

H $\quad 0.9188960 .9522720 .282478$

H $-0.013108 \quad 1.0612303 .215858$

H $2.865733-0.6464071 .304770$

H $1.391684-1.3185020 .570746$

H $2.322821-2.2635721 .706020$

H $3.8428802 .813683-0.219410$

H 3.3621101 .1351990 .019653

H 4.2397872 .2474774 .300341

H 5.8637051 .9123803 .651656

H $5.199006-0.5520063 .322312$

H 3.5337430 .4029985 .709113

H $4.495242-1.9171806 .316979$

H $4.402684-2.3895644 .635026$

H $1.000952-2.8560226 .957408$

H $2.619964-2.3502047 .701928$

H $\quad 0.828616-3.1592844 .590571$

H $2.257494-2.9967843 .611322$

H $0.410098-0.6913294 .563905$

H $3.272417 \quad 1.8399772 .187458$

C -5.2216963 .2786630 .981606$ $\begin{array}{llll}\text { O } & -4.730964 & 2.131706 & 0.259392\end{array}$

C $-4.4385451 .083764 \quad 1.204420$

C $-5.049725 \quad 1.5295752 .529708$

C -4.8722053 .0348422 .447990$

H -3.3499631 .0552741 .321212$

$\begin{array}{llll}\text { C }-4.954490 & -0.258720 & 0.676048\end{array}$

H -6.1139051 .2738462 .592938$

H -4.5434041 .0824023 .390492$

C $-5.738415 \quad 3.808517 \quad 3.425453$

H -3.8178843 .2664342 .643388$

C $-4.385934-0.680584-0.697533$

$\begin{array}{lllll}\text { O } & -6.381791 & -0.180068 & 0.543486\end{array}$

H -4.770691 -1.0448651 .415791$

H $-4.7071170 .048236-1.452629$

O $-4.985353-1.924998-1.075739$

C $-2.856510-0.845783-0.791715$

C $-2.183327-1.636396 \quad 0.349872$

H $-2.623680-1.339204-1.742608$

O $-2.2634840 .451715-0.861433$

H $-1.099073-1.5723180 .201009$

H $-2.356241-1.1262181 .304354$

H $-5.584704 \quad 4.8862393 .312234$

H -6.8026463 .6011363 .270962$

H $-5.490019 \quad 3.537882 \quad 4.456996$

$\begin{array}{llll}C & -4.688100 & 4.564550 & 0.346616\end{array}$

H $-6.5790990 .552776-0.064918$

H $-5.949804-1.810489-1.024031$

H $-1.3151640 .333014-1.039685$

H $-5.164726 \quad 5.435542 \quad 0.811454$

H $-4.9752424 .582145-0.712923$

H -6.3125683 .2683350 .853644$

C -1.142684 -4.011703-1.239507

O $-2.489147-3.797660-0.796300$

C $-2.609236-3.1147180 .454213$

C $-1.898740-3.9147601 .553572$

C $-0.461384-4.2579201 .176910$

C $-0.385771-4.873142-0.221030$

H $-0.634822-3.047545-1.363785$

C $-1.222504-4.679685-2.615676$

H -3.674442 -3.1233030 .710767$

O $-1.925251-3.1906182 .779183$

H $-2.449707-4.8494531 .721403$

H $0.176481-3.373902 \quad 1.251966$

O $0.042614-5.1940452 .132187$

H $-0.796759-5.890118-0.187315$

O $0.991874-4.969531-0.575987$

C -1.695797 -3.686993 -3.651568

H $-0.247937-5.046587-2.954241$

H $-1.904418-5.538450-2.608822$

H $-1.445224-3.7189413 .439789$

H $0.951983-5.4154381 .867276$

H $1.060489-5.550893-1.351558$

O $-3.055981-3.713763-3.739715$

O $-0.945109-2.952537-4.281010$

C $-3.632340-2.773599-4.651357$

H $-4.719119-2.885370-4.612584$

H $-3.374553-1.751933-4.355998$

H $-3.293444-2.978917-5.671078$

SCF Energy (B3LYP/6-31G**//MMFF) $=-3245.90897418$

$01 \_619$

MMFF Geometry

C $0.4783593 .822693-1.888065$

C $-0.6978494 .391436-2.194709$

C $-2.069506 \quad 3.887549-1.798281$

O $-2.0087432 .561879-1.225744$

C $-2.6716694 .833711-0.742633$

C $-4.103478 \quad 4.505216-0.367295$

C $-4.505250 \quad 4.124901 \quad 0.861511$

C $-5.120025 \quad 4.657578-1.470007$

C -3.669573 $3.943862 \quad 2.097585$

C -3.152924 2.5134162 .318157

C -4.2612841 .4688292 .530045$

C -3.6659410 .1283102 .967090$

C $-4.739964-0.9637873 .098982$

O $-4.963736 \quad 1.2955351 .296930$

O $-4.070577-2.231853 \quad 3.181979$

C $-5.596727-0.9900411 .814048$ 
C $-5.546820-0.8144454 .394357$

O $-6.708048-1.869841 \quad 1.960827$

C $-6.0850020 .404817 \quad 1.372689$

C $-6.7649640 .404421-0.018535$

O

C -1.979542 $1.508264-2.087085$

O $-2.0218231 .550550-3.306119$

C $-1.8436970 .276595-1.278900$

C -1.392539-0.845030 -1.859616

C - $1.180185-2.133983-1.197820$

C $-1.704968-2.2896080 .205076$

C $-5.890847-0.084087-1.179033$

C $-6.078232-1.554028-1.516600$

C $-5.133746-3.347375-2.965883$

O $-6.975881-2.246200-1.044778$

C $-4.206363-4.191031-2.149477$

C $-3.242390-4.953962-2.686675$

C $-2.330867-5.839531-1.886677$

C $-0.869154-5.583342-2.176868$

C $-0.191415-6.354495-3.043927$

C $-0.174782-4.489776-1.398027$

C $-0.543257-3.111406-1.876340$

N $-5.142429-1.993633-2.443846$

H $0.5021032 .911346-1.296993$

H $-0.693515 \quad 5.306798-2.783713$

H $-2.701993 \quad 3.870883-2.694068$

H -2.649168 $5.868033-1.111624$

H -2.0283764 .8164450 .143684$

H -5.5657603 .9238631 .011004$

H -6.147631 $4.554274-1.104966$

H $-5.0361445 .647331-1.930614$

H $-4.9696913 .897124-2.242124$

H -2.818056 4.6324462 .093864

H -4.273989 4.2457122 .962624

H -2.530966 2.2250151 .462664

H -2.5011872 .5276863 .200700$

H -4.949888 1.8475123 .294582

H $-3.1125040 .236127 \quad 3.908329$

H $-2.927677-0.1999852 .223147$

H -3.470141 -2.204357 3.946618

H -4.966720 -1.411970 1.022391

H -4.885177 -0.8775975 .266705$

H $-6.268252-1.6312164 .507313$

H -6.0849690 .1335224 .457600$

H -6.356816 -2.7408282 .213361$

H $-7.717620-0.1369430 .027533$

H $-7.0349371 .446110-0.245600$

H -7.5937121 .5661441 .879627$

H -2.092873 $0.334619-0.225936$

H -1.129972 -0.814031 -2.916549

H $-2.762878-2.0102120 .249892$

H -1.143767 -1.6547790 .898827$

H -1.648923 -3.3127050 .582117$

H $-6.1726850 .472412-2.082262$

H -4.832552 $0.117493-0.988161$

H -4.825792 -3.288754 -4.014634

H -6.145700 -3.762487 -2.921182

H -4.351110 -4.195259-1.071762

H -3.119635 $-4.969348-3.767417$

H -2.581435 -6.880700-2.129134

H $-2.512963-5.735673-0.809791$

H $\quad 0.864497-6.195980-3.236669$

H $-0.679280-7.158866-3.584482$

H $0.913605-4.586796-1.506770$

H $-0.360848-4.637258-0.330717$

H $-0.206169-2.901241-2.892452$

H -4.401715 -1.365163 -2.741203

C $2.6656294 .785377-1.123096$

O $2.9945223 .626374-0.331055$

C $4.3994543 .651428-0.023592$

C $4.8670025 .067598-0.331951$

C $4.0021995 .408137-1.529744$

H $4.8860402 .959928-0.722185$

C 4.6419733 .1851721 .416186

H $4.655627 \quad 5.7563260 .494129$

H $5.9387895 .114378-0.546755$

C $3.9262696 .896782-1.821687$

H $4.4051354 .890837-2.410951$
C 3.9970271 .8276311 .766760

O 4.1105134 .1574292 .325685

H 5.7200693 .1522971 .610719

H 2.9081541 .9431591 .828082

O $4.418896 \quad 1.4659593 .087959$

C $4.3254100 .679668 \quad 0.793055$

C $3.758320-0.6712971 .271819$

H $3.8967260 .911637-0.187814$

$\begin{array}{llll}\text { O } & 5.736084 & 0.588213 & 0.620735\end{array}$

H $4.314153-1.0059572 .154278$

H $2.716594-0.5200091 .578434$

H $3.2812407 .093189-2.683830$

H $3.5257987 .453221-0.967602$

H $4.9208577 .295833-2.046096$

C $1.8116474 .366399-2.321470$

H 3.1624614 .2543582 .130882

H 5.3896181 .4181663 .090208

H $6.1277670 .328401 \quad 1.471310$

H $2.3219093 .575818-2.885600$

H $1.6763195 .216763-2.999705$

H $2.1030055 .466225-0.471127$

C $5.975395-2.7209110 .649424$

O $5.130085-2.038237-0.284252$

C $3.805216-1.7644860 .179086$

C $3.106654-3.0751770 .569172$

C $3.927845-3.8810841 .570368$

C $5.357232-4.0614161 .063439$

H $6.131298-2.0923361 .534240$

C $7.332706-2.932911-0.030682$

H $3.260659-1.358564-0.683381$

O $1.816404-2.8278591 .116518$

H $2.962486-3.688164-0.329821$

H $3.918395-3.4182362 .564127$

O $3.341891-5.176031 \quad 1.723750$

H $5.359154-4.757480 \quad 0.214782$

O $6.149851-4.6437952 .096082$

C $8.073978-1.624436-0.167828$

H $7.978286-3.6052980 .545615$

H $7.212080-3.381036-1.024575$

H $1.317049-2.3109900 .461794$

H $2.419699-5.0442062 .003973$

H $5.722131-5.4782522 .354323$

O $7.912453-1.123494-1.425535$

O $8.699697-1.0992120 .744322$

C $8.5543790 .130677-1.676031$

H $8.1672130 .898819-0.999928$

H $9.6381460 .028859-1.564871$

H $8.3331800 .426091-2.705054$

SCF Energy (B3LYP/6-31G**//MMFF $)=-3245.90766108$

$01 \quad 62$

MM̄MF Geometry

C $-2.241403-3.6029720 .357738$

C $-1.374771-4.129740-0.520071$

C $-0.373152-3.278824-1.254395$

O $0.948312-3.758709-0.918796$

C $-0.572087-3.321454-2.782914$

C $-1.916907-2.813799-3.261424$

C $-2.349323-1.544570-3.121005$

C $-2.790234-3.846817-3.923171$

C $-1.593592-0.396007-2.508388$

C $-1.9142170 .932169-3.205303$

C $-1.1755112 .112152-2.554137$

C $-1.7161463 .435927-3.098128$

C $-0.9483384 .639810-2.538331$

O $0.2216751 .991322-2.849175$

O $-1.3044755 .792003-3.316969$

C $0.5602444 .401323-2.746219$

C $-1.3482694 .951895-1.091062$

O $1.3236905 .431624-2.120609$

C $1.0443903 .012253-2.261852$

C $2.5070222 .790256-2.730370$

O $0.9884912 .973750-0.838770$

C $1.951681-2.845485-1.038443$

O $1.848075-1.701558-1.453498$

C $3.208480-3.454929-0.555511$

C $4.338551-2.739321-0.657813$

C $5.669753-3.126360-0.193230$ 
C $5.800674-4.4252460 .555624$

C $3.1158081 .431642-2.360637$

C $3.7793101 .420842-0.994918$

C $5.7495042 .322823 \quad 0.245959$

O $3.2831750 .823967-0.042399$

C 7.0005601 .5075870 .142714

C 7.4075040 .6456841 .087854

C $8.693375-0.1299251 .013824$

C $8.497625-1.6210911 .182601$

C $8.715234-2.2156412 .367796$

C $8.138377-2.436693-0.039442$

C $6.696131-2.282633-0.430070$

N $4.9590872 .145226-0.958265$

H -2.190028 -2.537852 0.569600

H -1.407212 -5.191555 -0.747105

H $-0.458103-2.248311-0.895568$

H $-0.412066-4.350853-3.133273$

H $0.218836-2.748972-3.284342$

H -3.353759-1.306746 -3.468718

H -3.767290 -3.444831 -4.211291

H -2.305460 -4.225099 -4.829097

H -2.966222 $-4.689248-3.246630$

H -1.881554 -0.320091 -1.453207

H $-0.513891-0.569126-2.550453$

H -1.633369 $0.858057-4.263876$

H $-2.9964591 .108444-3.160961$

H -1.321169 $2.059883-1.469017$

H $-2.7883493 .535155-2.886934$

H -1.624983 $3.444360-4.193509$

H -2.267589 $5.911247-3.252611$

H $0.7600204 .479214-3.824201$

H -2.421335 5.169585-1.028687

H $-0.8386885 .850225-0.725454$

$\mathrm{H}-1.1389874 .132102-0.401330$

H $\quad 0.9969716 .281918-2.461499$

H $2.5235162 .856528-3.826244$

H $3.1440353 .593783-2.342796$

H $1.4542412 .180153-0.531883$

H $3.167331-4.443601-0.114880$

H $4.287339-1.755394-1.122867$

H $5.556739-5.270396-0.097320$

H $5.125873-4.4424971 .418957$

H $6.804879-4.6008460 .947589$

H $2.3690080 .634335-2.404191$

H $3.8879781 .169833-3.095589$

H 5.1546622 .0596621 .126581

H $6.0074593 .384763 \quad 0.306567$

H $7.6182301 .658170-0.739715$

H $6.8035690 .515237 \quad 1.983029$

H $\quad 9.353691 \quad 0.2495591 .804898$

H 9.2232460 .0623930 .072613

H $8.606507-3.2881472 .490818$

H $9.001787-1.6420653 .243080$

H $8.397516-3.4908420 .107993$

H $8.764762-2.118614-0.882894$

H $\quad 6.493042-1.362907-0.979461$

H $5.2978092 .580706-1.811305$

C $-4.722738-3.800864 \quad 0.787263$

O $-4.779048-2.3999531 .134078$

C $-5.392130-1.6835080 .044569$

C $-6.151215-2.735024-0.753950$

C $-5.193180-3.907612-0.665138$

H $-4.579160-1.291607-0.578524$

C $-6.244463-0.5266380 .570584$

H -7.108274 -2.989803 -0.284078

H $-6.347184-2.416801-1.782242$

C -5.823673 -5.244298 -1.012093

H $-4.362705-3.722500-1.355026$

C $-5.4652120 .472491 \quad 1.452355$

O $-7.307408-1.0741511 .360800$

H -6.720003 $-0.006756-0.269331$

H -5.151851 -0.0315472 .372559$

O -6.3829451 .4967621 .858543$

C $-4.251665 \quad 1.132670 \quad 0.766901$

C $-3.5764702 .229116 \quad 1.616151$

H -3.5131730 .3694490 .501811$

O $-4.692907 \quad 1.729447-0.455886$

H -2.8494022 .7514370 .983357$
H -4.3147732 .9963881 .879156$

H -5.094103 -6.054501-0.914890

H $-6.670838-5.471531-0.356409$

H -6.189272 -5.240264 -2.044143

C $-3.322561-4.3590151 .073627$

H -7.833418 -0.3276041 .695362$

H -6.6456991 .9872321 .060705$

H -3.908931 $2.084574-0.908465$

H -3.289083 -5.4258350 .825092$

H -3.120440 -4.268886 2.148073

H $-5.436727-4.305701 \quad 1.450652$

C -0.7659931 .0726371 .926245$

O -1.9628140 .6386222 .580583$

C -2.8874111 .6896292 .885761$

C $-2.2120682 .764487 \quad 3.752874$

C -0.8968893 .2576583 .152334$

C 0.0012432 .0732652 .795696

H -1.0367581 .5566960 .980020$

C $0.051360-0.1587091 .522070$

H $-3.670474 \quad 1.237460 \quad 3.505447$

O -3.0785993 .8760153 .954953$

H -1.997377 2.3420724 .743367

H -1.0678283 .8978822 .278969$

O -0.2136874 .0635844 .114795$

H $0.3539841 .600722 \quad 3.719780$

O 1.1475382 .5390602 .091852

C $0.593452-0.9709822 .677454$

H $-0.578170-0.8338920 .935740$

H $\quad 0.888470 \quad 0.1600370 .892772$

H -3.8968923 .5387904 .357741$

H $-0.8127584 .789315 \quad 4.360913$

H 1.5843943 .2021362 .653318

O $1.708176-1.6431562 .263295$

O $0.086124-1.0407753 .788184$

C $2.326638-2.4786663 .246025$

H $1.650129-3.2908643 .527992$

H $3.229785-2.9100402 .806333$

H $2.611623-1.8897464 .123083$

SCF Energy $(B 3 L Y P / 6-31 G * * / / M M F F)=-3245.91495390$

01620

MMFF Geometry

C $0.410401-3.8622580 .043286$

C $1.139794-2.844974-0.439148$

C $1.298049-2.546045-1.908316$

O $1.104176-1.128523-2.117984$

C $2.731237-2.884087-2.347800$

C $3.007160-2.595174-3.812597$

C $3.702689-1.524598-4.249000$

C $2.494068-3.623682-4.783850$

C $4.229455-0.405952-3.392574$

C $5.590632-0.737536-2.769718$

C $5.9126720 .155474-1.559776$

C $7.249486-0.264423-0.942620$

C $7.5503570 .478908 \quad 0.366374$

O $4.855927-0.007048-0.607749$

O $8.623645-0.2102811 .026482$

C 6.3156470 .3825661 .288406

C 8.0492471 .9046710 .103673

O 6.5087371 .1603412 .468890

C 4.9901040 .7668800 .590799

C 3.7862180 .4040091 .499378

O $5.0051262 .166642 \quad 0.320499$

C $-0.164648-0.648304-2.083399$

O $-1.181858-1.274578-1.838950$

C $-0.0957660 .793809-2.402961$

C -1.205599 $1.539824-2.314250$

C $-1.3090272 .969637-2.600761$

C $-0.1000713 .668683-3.164228$

C 2.4205280 .9147051 .007457

C 2.0699772 .2912851 .556702

C 0.2391773 .9488161 .666427

O 2.8144052 .9229682 .303355

C -0.6615124 .5854690 .657686$

C -1.9570154 .8570420 .885364$

C $-2.9311775 .574818-0.016239$

C $-2.4335535 .937448-1.398281$

C $-1.7376227 .068775-1.604805$ 
C $-2.8136625 .042498-2.556701$ C $-2.4742613 .595174-2.337348$ N $0.828303 \quad 2.739272 \quad 1.119937$ H -0.083277 -4.522699 -0.667299 H $1.665699-2.178780 \quad 0.237710$ H $0.575133-3.102725-2.516619$ H $2.941851-3.944918-2.156067$ H $3.437692-2.331314-1.717707$ H $3.887224-1.417087-5.316036$ H $2.758904-3.384740-5.819230$ H $2.915638-4.607613-4.553710$ H $1.402965-3.690986-4.730036$ H $4.3212530 .498223-4.006945$ H $3.488499-0.156912-2.626484$ H $5.596983-1.784970-2.442077$ H $\quad 6.375997-0.638897-3.529389$ H $5.9433131 .200760-1.888553$ H $8.067430-0.128800-1.661245$ H $7.224679-1.341439-0.722798$ H $9.386929-0.2242230 .424020$ H $6.241693-0.6611861 .623449$ H $8.9635901 .884497-0.501753$ H 8.3229792 .4069841 .037833 H $7.3206502 .523424-0.424093$ H 6.3611782 .0930122 .236282 H $3.724451-0.6914421 .554994$ H 3.9603370 .7621042 .520489 H 4.1218932 .4365120 .024884 H $0.8639891 .201576-2.699390$ H -2.129864 1.060079-1.996002 H $0.1949123 .217436-4.118010$ H $0.7435233 .602867-2.468113$ H $-0.2663954 .732073-3.353878$ H $2.3722870 .933335-0.086422$ H $1.644957 \quad 0.225743 \quad 1.363533$ H 1.0334964 .6514651 .940332 H -0.289186 3.6697782 .583433 H $-0.1945334 .883011-0.276201$ H -2.3758464 .5653851 .847474$ H -3.840148 $4.967316-0.105143$ H -3.248092 6.4898660 .502155 H -1.401739 $7.354493-2.595935$ H -1.486290 $7.732377-0.783755$ H -2.375034 5.395211 -3.497834 H -3.899101 5.115813 -2.706663 H -3.293797 3.015336 -1.913818 H $\quad 0.214967 \quad 2.080876 \quad 0.647497$ C $0.519652-3.1368802 .527024$ O $-0.455037-2.0775592 .418385$ C $-0.926187-1.7440703 .737519$ C $0.083300-2.3513684 .701163$ C $0.436402-3.6337693 .972366$ H $-1.883633-2.2612363 .872234$ C -1.128001 -0.2324563 .872637$ H $0.971504-1.7184024 .811175$ H $-0.339736-2.5231925 .695322$ C $1.712478-4.2863924 .475431$ H $-0.395616-4.3426844 .080388$ C -2.1005210 .3616032 .830298$ O 0.1406120 .4116663 .713868 H -1.4664870 .0029534 .888561$ H -1.6542230 .2887591 .832375$ O $-2.228481 \quad 1.762042 \quad 3.104207$ C -3.501546 -0.276958 2.836925 C -4.5237450 .4215631 .922759$ H -3.427359 -1.332672 2.557829 O $-4.010561-0.2332794 .173835$ H -5.501816 -0.0406282 .104271$ H -4.650532 1.4648452 .238630 H $1.936734-5.1943273 .907035$ H $2.570845-3.6119844 .385647$ H $1.612461-4.5636875 .529902$ C $0.199760-4.2212151 .492819$ H -0.0085851 .3696143 .790238$ H -2.659664 1.8534433 .971312 H $-4.859405-0.7075984 .180731$ H -0.857861 -4.497366 1.597707 H $\quad 0.790876-5.121241 \quad 1.698221$
H $1.506069-2.6925782 .349687$

C $-4.946540-1.877970-0.026753$

O $-3.847692-0.962083-0.009420$

$\begin{array}{llll}\text { C } & -4.180082 & 0.361572 & 0.421279\end{array}$

C $-5.2677570 .949669-0.489985$

C $-6.4939750 .041678-0.575648$

C $-6.081735-1.386983-0.934340$

H $-5.337517-1.9767170 .994085$

C $-4.410502-3.272658-0.384574$

H -3.2775210 .9677540 .279788$

O $-5.6715332 .236521-0.034027$

H $-4.8638811 .077037-1.502491$

H -7.0758560 .0603890 .353179$

O $-7.3657570 .524716-1.600830$

H -5.788534 -1.411856-1.988543

O $-7.204428-2.254188-0.789455$

C $-3.721765-3.380426-1.730447$

H $-3.662926-3.5475130 .371051$

H $-5.221209-4.008681-0.358022$

H -4.8746512 .7915050 .016321$

H -7.585885 $1.446418-1.381834$

H -7.917378 -1.901437 -1.349289

O $-3.095422-4.593907-1.804516$

O $-3.731906-2.531699-2.609979$

C -2.392783 -4.850614 -3.024535

H -1.655642 $-4.065107-3.215991$

H -1.868016 -5.803971 -2.920529

$\mathrm{H}-3.100746-4.926263-3.855007$

SCF Energy $(B 3 L Y P / 6-31 G * * / / M M F F)=-3245.91214802$

01621

MMFF Geometry

C $3.843692-2.3673412 .698218$

C $2.614513-2.5420792 .189547$

C $1.349019-2.3259762 .975518$

O $0.535538-1.3819352 .242855$

C $0.557236-3.6401283 .081448$

C $-0.660043-3.5149953 .976677$

C $-1.922055-3.3542493 .532254$

C $-0.385349-3.6228645 .454193$

C $-2.383162-3.2083642 .107396$

C $-3.097649-4.4779811 .631214$

C $-3.703691-4.383740 \quad 0.221342$

C $-2.647653-4.209230-0.872903$

C $-3.285018-4.084582-2.265846$

O $-4.633556-3.2957390 .198730$

O $-2.255126-3.628573-3.156940$

C $-4.380536-2.997222-2.208595$

C $-3.744284-5.444045-2.803606$

O $-5.084610-2.917819-3.445421$

C $-5.364885-3.170743-1.029936$

C $-6.332150-1.973721-0.878643$

O $-6.171858-4.331337-1.265718$

C $0.623551-0.0675392 .575950$

O $1.342948 \quad 0.435158 \quad 3.423108$

C $-0.3377120 .660971 \quad 1.717734$

C -0.5062111 .9826561 .870396$

C -1.4196072 .8276041 .097094$

C -2.2813182 .1502980 .063822$

C $-5.651001-0.616999-0.689076$

C $-6.6269080 .475565-0.283726$

C -6.7030942 .9209120 .166415$

O $-7.8178590 .274836-0.064972$

C -5.7227113 .9232820 .688639$

C -5.5752165 .1563220 .178575$

C -4.6208656 .2269050 .641952$

C -3.4352335 .7476661 .454624$

C -3.4455335 .8580152 .794548$

C $-2.2597945 .216000 \quad 0.655764$

C -1.4442464 .1540741 .346290$

N $-5.9902611 .704229-0.173424$

H $3.928585-2.0595583 .739154$

H $2.487551-2.8478161 .155025$

H $1.568138-1.9415333 .979311$

H $1.200310-4.4365973 .479664$

H $0.258029-3.9794392 .082182$

H -2.723292 -3.300186 4.268237

H $-1.300715-3.5652436 .052731$ 
H $\quad 0.096800-4.5793005 .681761$ H $0.273766-2.8137195 .783351$ H -3.064494-2.350334 2.061534 H -1.549745 -2.967611 1.443475 H -2.406871 -5.329736 1.671545 H -3.913584 -4.696300 2.333715 H $-4.255368-5.3172330 .056379$ H -1.924216 -5.033477 -0.851809 H -2.067528 -3.295335 -0.690706 H -2.665324 -3.471303 -4.024893 H -3.853779-2.041569-2.099033 H $-2.893281-6.132311-2.873143$ H $-4.138792-5.355186-3.821843$ H $-4.504426-5.919197-2.180355$ H $-5.702928-3.667402-3.482465$ H -7.012762 -1.927771 -1.738899 H -6.975498 -2.165970 -0.008492 H -6.982713 -4.254936 -0.735739 H -0.8867650 .0832130 .982291$ H 0.0812412 .4988242 .628426 H -2.9071571 .3820750 .531494$ H $-1.6623701 .676647-0.706176$ H -2.959708 $2.831425-0.452483$ H $-4.883282-0.6817470 .090573$ H -5.171166 $-0.305746-1.623655$ H -7.4574782 .6942720 .926905$ H -7.217821 $3.265840-0.736417$ H -5.1201213 .6092571 .536689$ H -6.218424 $5.449408-0.649467$ H $-5.1979856 .972595 \quad 1.203934$ H $-4.2398926 .758218-0.240063$ H -2.602822 5.5567523 .406036 H -4.306741 $6.263203 \quad 3.316519$ H -1.5969416 .0587290 .419969$ H -2.608992 $4.856163-0.316785$ H -0.7778174 .5472842 .115265$ H $-5.0582631 .794506-0.566965$ C $5.111959-2.9046870 .502962$ O $4.572768-1.805720-0.259022$ C $5.249023-1.747189-1.530024$

C $6.187490-2.949942-1.577526$ C $6.486825-3.168684-0.108074$ H $5.855514-0.835034-1.523422$ C $4.233385-1.700004-2.677477$ H $5.696325-3.832548-2.003716$ H $7.083536-2.749806-2.173325$ C $7.052615-4.5456820 .192779$ H $7.199946-2.4021990 .223922$ C $3.269578-0.495547-2.628041$ O $3.447715-2.897960-2.626992$ H $4.766316-1.718657-3.636031$ H $2.586637-0.593462-1.775799$ O $2.459132-0.540148-3.809612$ C $3.9675870 .877412-2.581802$ C $3.0419812 .083716-2.829240$ H $4.4671620 .999991-1.615485$ O $4.9954380 .924711-3.577173$ H $3.6679792 .984691-2.829043$ H $2.6390982 .031247-3.848877$ H $7.254360-4.6619181 .262022$ H $6.359552-5.338773-0.107443$ H $7.993591-4.700206-0.345469$ C $5.162900-2.5397151 .988898$ H $2.959133-2.963554-3.464709$ H $1.852054-1.294003-3.719400$ H $4.5740970 .777069-4.441360$ H $5.713339-1.5977762 .104642$ H $5.722809-3.3088072 .533556$ H $4.458090-3.7715580 .340558$ C $3.1181193 .325590-0.056526$ O $2.3450682 .190760-0.463301$ C $1.8890622 .232375-1.817690$ C $1.0174693 .477253-2.041072$ C $1.7282524 .759056-1.609862$ C $2.2988254 .612182-0.199260$ H $4.0275653 .390384-0.666001$ C 3.5372533 .0993641 .399029 H $1.2361501 .360863-1.948623$
O $0.632304 \quad 3.559697-3.409095$

H $0.0966753 .373396-1.456339$

H $2.5134905 .042063-2.320623$

O $0.7911965 .838463-1.593228$

H 1.4798414 .6202340 .529939

O 3.1328415 .7332230 .086628

C 4.5019061 .9443741 .505406

H 4.0129923 .9844271 .835942

H 2.6692412 .8600702 .024637

H $-0.0177814 .277430-3.490609$

H $0.5128395 .999963-2.510407$

H $2.5922716 .533617-0.028690$

O $5.755198 \quad 2.346463 \quad 1.150199$

$\begin{array}{llll}\text { O } & 4.174598 & 0.816329 & 1.847952\end{array}$

C $6.7615181 .330348 \quad 1.195129$

H 6.5149770 .5220000 .501045

H $7.710714 \quad 1.7778450 .888763$

H 6.8670530 .9461412 .214207

SCF Energy (B3LYP/6-31G*//MMFF) $=-3245.91008593$

01_622

MM̄FF Geometry

C $-0.1857394 .932734-0.689546$

C $-0.603438 \quad 4.152312 \quad 0.318467$

$\begin{array}{lllll}\text { C } & -1.709836 & 3.140320 & 0.179937\end{array}$

$\begin{array}{llll}\text { O } & -1.181180 & 1.823275 & 0.479713\end{array}$

C -2.8493723 .4012961 .183176$

C $-3.844725 \quad 4.4464140 .722167$

C $-5.0190114 .153607 \quad 0.128972$

C -3.4921855 .8787291 .025357$

C $-5.5434362 .788796-0.221858$

C $-6.6426812 .370018 \quad 0.760718$

C -7.1665400 .9535920 .486441$

C -8.3208740 .6274361 .437967$

C -8.800129 -0.8222461 .291043$

$\begin{array}{lllll}\text { O } & -6.085806 & 0.038509 & 0.690950\end{array}$

O $-9.645148-1.1006802 .417704$

C $-7.576176-1.7580821 .370945$

C $-9.673956-1.0009330 .044336$

O $-7.960684-3.1086671 .119994$

C $-6.410066-1.3368630 .445141$

C $-5.152331-2.1774090 .779679$

O $-6.797965-1.553553-0.909766$

C $-0.4633801 .201724-0.496193$

O $-0.1442571 .662062-1.580906$

C $-0.152288-0.166520-0.024266$

C $0.622436-0.961909-0.777114$

C $0.963876-2.359237-0.499937$

C $0.393618-2.988045 \quad 0.743421$

C $-3.916534-1.868956-0.079141$

C $-3.827619-2.706913-1.345142$

C $-2.346501-3.100681-3.310288$

O $-4.655351-3.562190-1.647777$

C $-1.440166-4.237523-2.954960$

C $-0.242432-4.440089-3.523954$

C $0.668601-5.577552-3.159533$

C $2.040084-5.112337-2.720918$

C $3.122671-5.330009-3.486701$

C $2.168484-4.460901-1.358276$

C $1.754489-3.013063-1.376630$

N $-2.704674-2.388977-2.096814$

H $-0.661548 \quad 4.845622-1.662986$

H $-0.1267094 .237146 \quad 1.292106$

H $-2.120707 \quad 3.121313-0.838222$

H -2.442968 $3.688132 \quad 2.163044$

H $-3.367566 \quad 2.4562751 .391398$

H $-5.6833334 .974198-0.138324$

H $-4.318586 \quad 6.5635860 .805923$

H $-3.247903 \quad 5.9952882 .086274$

H -2.6344406 .2032740 .431088$

H $-5.9515272 .825511-1.239356$

H $-4.7404232 .045701-0.248157$

H $-6.2379352 .412467 \quad 1.780944$

H -7.4722113 .0865120 .707788$

H -7.497291 $0.896140-0.557195$

H $-9.155373 \quad 1.324943 \quad 1.291746$

H -7.9882030 .7763592 .475297$

H $-9.893483-2.0402552 .374463$ 
H -7.221308 -1.7344332 .410383$ H $-10.555260-0.3507090 .101468$ H -10.062903 -2.022786 -0.025488 H $-9.149317-0.766941-0.884354$ H -8.074514 -3.2100860 .159518$ H -4.876112 -1.967224 1.821796 H $-5.384072-3.2470160 .717290$ H $-6.011885-1.478854-1.472802$ H $-0.595639-0.4913230 .909962$ H $1.029549-0.561003-1.704886$ H $-0.696322-3.0620200 .670183$ H $0.647425-2.3950311 .629127$ H $0.775781-3.9925950 .937102$ H -3.869236 $-0.806939-0.343308$ H -3.023243 -2.104075 0.512786 H -1.867081 -2.381321 -3.981561 H -3.252065 -3.483895 -3.791174 H -1.789913 -4.932454 -2.194660 H $0.101763-3.759107-4.298981$ H $\quad 0.751039-6.231299-4.037340$ H $0.233526-6.197597-2.365606$ H $4.112360-5.021145-3.166788$ H $3.043620-5.817803-4.452637$ H $3.210425-4.507474-1.021844$ H $1.594908-5.056027-0.642178$ H $2.149326-2.454330-2.226456$ H $-2.039127-1.715450-1.730424$ C $2.2358355 .547221-1.174211$ O $2.7951924 .435251-0.450762$ C $3.5474343 .622185-1.374964$ C $3.5394514 .363115-2.711624$ C $2.2254245 .118633-2.638610$ H $2.9908922 .685555-1.494130$ C $4.9448543 .339454-0.810249$ H $4.3782875 .063619-2.795782$ H $3.5940113 .677255-3.562701$ C $2.1256596 .271276-3.621044$ H $1.4143164 .406541-2.834104$ C 4.9327212 .6857310 .587484 O $5.6440564 .586474-0.705466$ H $5.5101172 .722821-1.519314$ H 4.5180333 .3807371 .327882 O 6.2920642 .4436870 .973616 C 4.1714191 .3476600 .653483 C 4.2515190 .7087402 .055811 H 3.1169861 .5144870 .407151 O $4.7125280 .473791-0.332222$ H 5.3004980 .5490552 .322641 H 3.8437901 .4276392 .776251 H $1.1660656 .787614-3.518543$ H $2.9227987 .005274-3.462054$ H $2.2052715 .907048-4.650513$ C $0.9004775 .960610-0.554969$ H $6.5839924 .383198-0.564103$ H 6.7063273 .3068061 .141533 H $4.155486-0.322524-0.359789$ H $\quad 0.550110 \quad 6.890532-1.017725$ H 1.0609136 .1813100 .508280 H $2.9386496 .380624-1.037706$ C $5.314598-2.1498721 .755539$ O $4.037451-1.6535151 .333613$ C $3.480880-0.6269942 .163397$ C $3.318178-1.1539133 .595916$ C $4.628709-1.7062194 .147560$ C $5.230025-2.7201963 .175736$ H $6.055426-1.3425161 .720573$ C $5.748969-3.2278030 .756569$ H $2.472573-0.4322811 .779344$ O $2.834490-0.1363794 .465823$ H $2.573935-1.9610363 .602173$ H $5.341893-0.9035894 .369473$ O $4.373671-2.3725835 .386148$ H $4.623624-3.6351873 .179442$ O $6.539010-3.0721773 .618939$ C $6.217310-2.604058-0.536723$ H $6.586843-3.8231901 .137249$ H $4.933742-3.9286740 .545044$ H 1.9938010 .1863164 .098848
H $3.956952-1.7257585 .981218$

H $6.457077-3.4066904 .528521$

O $5.198873-2.572879-1.440826$

O $7.351080-2.175913-0.711821$

C $5.518686-2.000100-2.712598$

H $4.607782-1.983284-3.316617$

H $5.877640-0.973646-2.591777$

H $\quad 6.267227-2.613688-3.222533$

SCF Energy (B3LYP/6-31G**//MMFF) $=-3245.92353717$

01623

MM̄FF Geometry

C -3.698934-1.866756 2.917509

C $-2.927877-0.8109202 .613013$

C $-1.636218-0.4770783 .315168$

$\begin{array}{llll}\text { O } & -0.582838 & -0.618978 & 2.333447\end{array}$

C $-1.696707 \quad 0.9813213 .804215$

C $-0.566961 \quad 1.371614 \quad 4.734137$

C 0.4995372 .1036414 .357753

C $-0.727314 \quad 0.937418 \quad 6.166614$

C 0.8206062 .5636552 .964553

C 0.4021304 .0198822 .722342

C 0.6516014 .5000991 .280553

C $-0.323816 \quad 3.8637760 .283840$

C $-0.0013564 .250239-1.167036$

O 2.0052164 .1886580 .952920

O $\quad-0.7541183 .385051-2.029937$

C $1.4915023 .958337-1.423516$

C $-0.4482595 .678868-1.493575$

O $1.8756414 .376748-2.731182$

C $2.4309014 .566459-0.359587$

C $3.8877174 .071787-0.517570$

O $2.4384945 .989999-0.505312$

C $0.584688-1.1918632 .733683$

O $0.875321-1.5626873 .860013$

C $1.451052-1.3362321 .539993$

C $2.485636-2.1901791 .589472$

C $3.391165-2.5289960 .488815$

C $3.335373-1.680808-0.751701$

C $4.0676692 .566241-0.294386$

C $5.5292662 .191820-0.127452$

C 6.9748790 .4366390 .904076

O $6.4438822 .845899-0.621550$

C $7.136293-0.7985340 .077806$

C $7.329298-2.0187300 .600258$

C $7.529586-3.253131-0.228258$

C $6.548746-4.3510530 .110465$

C $6.947129-5.4587850 .758968$

C $5.128163-4.210481-0.389073$

C $4.204907-3.5971580 .629859$

N 5.6837251 .0260190 .608330

H -3.355464 -2.545293 3.696397

H $-3.229074-0.124221 \quad 1.827442$

H -1.481834 -1.165128 4.155241

H -2.641886 1.1571154 .336488

H -1.7235551 .6572792 .940001$

H 1.2395812 .3722725 .109566

H $\quad 0.100048 \quad 1.277133 \quad 6.798524$

H -1.6523961 .3456696 .586566$

H $-0.766175-0.154168 \quad 6.234737$

H 1.9028492 .4550042 .821883

H $\quad 0.3622601 .9015872 .226365$

H -0.6539774 .1667212 .980191$

H 0.9866494 .6551903 .401463

H 0.5304035 .5899321 .270163

H $-1.358703 \quad 4.1296800 .533142$

H $-0.2764372 .769598 \quad 0.351638$

H -1.697397 $3.499180-1.823147$

H $1.5996802 .866935-1.413145$

H $-1.5293145 .784997-1.341827$

H $-0.2710975 .916774-2.548257$

H $0.0449316 .434957-0.879473$

H $1.9692515 .344529-2.717057$

H $4.2855834 .361814-1.498434$

H 4.5119264 .6016120 .216139

H $3.2575426 .336358-0.114148$

H $1.181211-0.7866680 .645442$

H $2.660086-2.7383012 .514868$ 
H $4.197501-1.818226-1.408185$ H $3.318834-0.615020-0.500573$ H $2.436292-1.917484-1.326216$ H 3.5188202 .2469940 .598343 H $3.6811712 .001848-1.149818$ H 6.9941270 .2219251 .977238 H 7.7768851 .1456970 .677108 H $7.112426-0.677768-1.002704$ H $7.375059-2.1352601 .680629$ H $8.558076-3.599557-0.062844$ H $7.461508-3.030396-1.300453$ H $6.253990-6.2650890 .975471$ H $7.972342-5.5823671 .091971$ H $4.730273-5.207382-0.624556$ H $5.126876-3.679295-1.344773$ H $4.160707-4.1562251 .565802$ H 4.8594700 .5421450 .952600 C $-5.752363-1.1232461 .540817$ $\begin{array}{llll}\text { O } & -5.084735 & -0.871190 & 0.293535\end{array}$ C -6.045886 -0.345838 -0.643698 C $-7.410814-0.4227850 .040387$ C -7.179876-1.462823 1.123699 H $-6.046464-1.023091-1.504644$ C $-5.618021 \quad 1.064457-1.081770$ H -7.7040000 .5368880 .480668$ H -8.201629 $-0.710115-0.660070$ C -8.198685-1.392846 2.247925 H -7.209305 -2.460285 0.665171 C $-4.159993 \quad 1.136000-1.584735$ $\begin{array}{llll}\text { O } & -5.727905 & 1.940427 & 0.047748\end{array}$ H $-6.312811 \quad 1.442539-1.840988$ H $-3.475590 \quad 0.925587-0.755219$ O $-3.8891082 .478457-2.003471$ C $-3.863637 \quad 0.199170-2.768067$ C -2.419207 $0.279350-3.292565$ H -4.075157 $-0.836377-2.488412$ O $-4.745257 \quad 0.510462-3.850340$ H -2.363993 $-0.322191-4.208387$ H $-2.1985401 .302481-3.621405$ H -8.006381 -2.162936 3.001088 H -8.177056 -0.4191732 .748661$ H -9.210491-1.549083 1.859512 C $-5.028944-2.2349632 .304402$ H $-5.6835282 .852851-0.284364$ H -3.902856 $3.040242-1.210379$ H $-4.5899771 .438505-4.096856$ H -4.863465 -3.0970931 .646726$ H -5.670472 -2.571084 3.127408 H -5.740104 -0.192030 2.123576 C - $1.652246-2.565641-2.541193$ O $-1.701724-1.437925-1.660689$ C -1.353906 -0.192308 -2.279757 C $0.067942-0.267934-2.858014$ C $0.260479-1.482985-3.765149$ C $-0.231746-2.760761-3.081459$ H $-2.330824-2.387278-3.384607$ C $-2.237833-3.778539-1.812603$ H $-1.3316780 .551158-1.473969$ $\begin{array}{llll}\text { O } & 0.385567 & 0.910816 & -3.591117\end{array}$ H $0.785599-0.332515-2.031377$ H $-0.237115-1.333063-4.730673$ O $1.655076-1.614080-4.052921$ H $0.468695-3.016737-2.278293$ O $-0.203748-3.817298-4.036671$ C $-1.349109-4.346501-0.732706$ H -3.204085 -3.520399 - 1.360204 H -2.443313 -4.590322 -2.520797 H $0.2439031 .670340-3.001010$ H $1.763065-2.409554-4.602019$ H $-0.372798-4.648849-3.562410$ O $-1.641827-3.7803750 .471773$ O $-0.499724-5.204722-0.938577$ C $-0.921846-4.3185811 .585201$ H -1.165728 -3.7276502 .471012$ H -1.229594 -5.3537091 .760857$ H $0.156593-4.2643191 .412036$

SCF Energy (B3LYP/6-31G**//MMFF)= -3245.92083894
01_624

MM̄FF Geometry

C $-3.796268-1.150581-2.811421$

C $-2.733612-1.702822-2.205153$

C $-1.339315-1.680633-2.769658$

O $-0.462104-1.238794-1.707209$

C $-0.849824-3.029374-3.341571$

C $-1.182643-4.273700-2.541539$

C $-0.402560-4.809590-1.584034$

C $-2.459730-4.977156-2.935880$

C $0.930506-4.333633-1.083130$

C $0.863740-3.9696510 .405566$

C $2.255569-3.9328441 .051136$

C $2.155481-3.5588842 .530937$

C $3.539770-3.4769613 .190792$

O $3.069028-2.9789220 .361566$

O $3.387033-2.8173564 .456933$

C $4.453775-2.5865292 .324349$

C $4.113555-4.8665143 .492323$

O $5.794429-2.6067642 .810440$

C $4.429576-2.9453720 .818124$

C $5.185395-1.8628950 .015046$

O $5.082078-4.2034300 .630277$

C $0.588949-0.451222-2.060992$

O $0.950380-0.164967-3.191704$

C $1.1991490 .071755-0.820337$

C $1.7694461 .284941-0.862261$

C 2.3989621 .9963260 .250004

C $2.542878 \quad 1.2674251 .558597$

C $5.047790-1.983042-1.507736$

C $5.886901-0.913603-2.177987$

C $5.9344001 .545258-2.606075$

O $7.008393-1.156533-2.617521$

C $6.1148332 .417330-1.402645$

C $5.6867593 .686800-1.335168$

C $5.8154704 .545876-0.109351$

C 4.4860295 .1100180 .347758

C 4.2064856 .4170310 .208578

C $3.505514 \quad 4.1741281 .029147$

C 2.7985883 .2703910 .053162

N $5.270603 \quad 0.326607-2.180207$

H -3.641231 -0.654300 -3.767830

H -2.852965 -2.207714 -1.250863

H -1.302812 -0.942186 -3.581290

H $\quad 0.233239-2.981619-3.506573$

H $-1.281618-3.151513-4.344565$

H $-0.746062-5.727374-1.106545$

H -2.625181 -5.894060 -2.359846

H $-3.330822-4.335735-2.780288$

H $-2.423180-5.259581-3.993407$

H $1.646392-5.151092-1.233716$

H $1.309793-3.481288-1.653752$

H $\quad 0.377917-2.990706 \quad 0.502982$

H $0.242771-4.6938160 .948233$

H $2.699923-4.9293860 .941665$

H $1.515007-4.2672393 .070989$

H $1.666869-2.5789032 .626515$

H $2.754112-3.3308554 .987852$

H $4.101579-1.5529912 .438988$

H $3.443907-5.4200144 .161622$

H $5.070155-4.7925314 .021267$

H $4.260284-5.4740162 .597092$

H $5.765310-2.3609233 .751007$

H $4.813564-0.8730350 .306029$

H $6.252162-1.8900390 .272797$

H $5.423283-4.254620-0.275966$

H $1.122806-0.5235480 .081028$

H $1.763131 \quad 1.824475-1.809457$

H $3.1866030 .392279 \quad 1.438654$

H $1.565900 \quad 0.934481 \quad 1.926621$

H $2.974373 \quad 1.8813372 .352349$

H $5.391388-2.955721-1.873776$

H $4.003522-1.872203-1.821478$

H $5.2991502 .013101-3.364555$

H $6.9064661 .313604-3.051340$

H $6.6072281 .975734-0.539272$

H $5.2088124 .133923-2.203925$

H $6.5132675 .359596-0.344821$ 
H 6.2708093 .9914900 .720680 H 3.2655206 .8334590 .552668 H $4.9096517 .099526-0.257236$ H 2.7409254 .7555571 .560044 H 4.0534233 .6100071 .790678 H $2.5889933 .729661-0.914010$ H $4.3351360 .412079-1.794151$ C $-5.502797-1.807227-0.976975$ O $-4.933949-0.996347 \quad 0.069210$ C $-5.857243-0.940651 \quad 1.173113$ C $-6.930134-1.9810420 .879301$ C $-6.988768-1.923402-0.635969$ H -6.3223750 .0516951 .145258$ C $-5.104818-1.1280892 .495655$ H $-6.629036-2.9814861 .211125$ H $-7.884499-1.7399041 .357123$ C $-7.677483-3.124251-1.260780$ H $-7.516593-1.007785-0.934817$ C $-3.933690-0.1401892 .691363$ O $-4.564477-2.4548362 .544738$ H $-5.805997-1.0569053 .334737$ H $-3.146047-0.3712131 .966003$ O $-3.360790-0.3795973 .982414$ C -4.3310001 .3427542 .586805$ C -3.179677 2.328284 2.856303 H -4.7464211 .5442251 .594893$ O $\quad-5.370863 \quad 1.6027123 .533381$ H -3.592846 3.3438222 .811109 H -2.8317372 .2155453 .890960$ H -7.685643 -3.046521 -2.352285 H $-7.174504-4.059684-0.993498$ H -8.715839 -3.191840 -0.919789 C $-5.227462-1.150730-2.332813$ H $-3.977434-2.5616181 .776417$ H $-4.032249-0.1505814 .647731$ H -5.6538872 .5252003 .413063$ H $-5.552202-0.103046-2.295268$ H -5.824749 -1.648788 -3.105510 H $-5.032332-2.797213-0.919379$ C $-2.9594903 .286851-0.035118$ O $-2.418997 \quad 2.084957 \quad 0.521342$ C -1.991296 2.1986101 .883034 C $-0.935433 \quad 3.3063442 .013673$ C $-1.397706 \quad 4.6332831 .411372$ C -1.931237 $4.422962-0.006542$ H -3.8340073 .5885060 .555603$ C $-3.5055142 .973314-1.436025$ H $-1.4902501 .253246 \quad 2.125212$ O $-0.545132 \quad 3.4867043 .370583$ H -0.0366082 .9898561 .475269$ H -2.148507 5.1230992 .042097 O $-0.285927 \quad 5.5293051 .341739$ H - $-1.0896514 .210653-0.675613$ O $-2.5457995 .625026-0.463527$ C -2.458768 $2.541197-2.440910$ H $-4.2288712 .151900-1.366857$ H $-4.0188793 .858572-1.828441$ H -1.2726853 .9286793 .839946$ H $0.080742 \quad 5.6068442 .239231$ H $-1.8802376 .331715-0.403793$ O $-2.9743922 .664183-3.699972$ O $-1.344082 \quad 2.122197-2.164912$ C $-2.094012 \quad 2.289527-4.764079$ H $-1.1883762 .903400-4.742218$ H $-1.8426301 .227809-4.691260$ H -2.612213 2.463060 -5.710884 SCF Energy (B3LYP/6-31G**//MMFF) $=-3245.90751574$

\section{5}

MMFF Geometry

C $-1.529096-4.194308 \quad 1.482174$

C $-0.982813-3.4234002 .435049$

C $0.291464-2.6366002 .284082$

O $-0.056190-1.2731752 .635207$

C $1.382057-3.1229113 .266661$

C $2.329738-4.1596412 .695473$

C $3.667704-4.0110792 .631660$

C $1.711979-5.445542 \quad 2.212287$
C $4.497015-2.8561373 .117001$

C $4.507578-1.6300752 .201315$

C $4.739527-1.9015090 .708210$

C $6.103639-2.5373600 .434579$

C $6.366879-2.697391-1.070312$

O $4.651519-0.6330090 .043737$

O $7.760754-2.993197-1.245945$

C $6.099416-1.349721-1.770542$

C $5.593808-3.882841-1.661072$

O $6.190054-1.486463-3.187275$

C $4.745509-0.707831-1.385021$

C $4.6769860 .732275-1.944816$

O $3.685436-1.483124-1.946453$

C $0.586158-0.2594931 .998527$

O $1.418547-0.352824 \quad 1.112050$

C $0.106467 \quad 1.0060612 .599392$

C $0.681166 \quad 2.1701492 .261394$

C $0.344057 \quad 3.486457 \quad 2.809841$

C -0.8075613 .5672303 .778346$

C $3.4531581 .537703-1.487497$

C $3.4750562 .954982-2.036352$

C $2.0725865 .016752-2.129647$

O $4.4185363 .414333-2.674374$

C $2.1194295 .893134-0.919982$

C $1.0465646 .522041-0.417198$

C 1.0900957 .4232780 .781457

C $0.274688 \quad 6.891723 \quad 1.938219$

C $-0.991505 \quad 7.2960392 .134549$

C $0.9604695 .973747 \quad 2.923792$

C 1.0770984 .5550452 .432401

N $2.3144443 .646051-1.720996$

H $-1.031264-4.2911950 .522329$

H -1.481723 -3.3490263 .399616$

H $0.647647-2.6699431 .247052$

H $\quad 0.920927-3.5407764 .172138$

H $1.943546-2.2530773 .625072$

H $4.251525-4.8217872 .197014$

H $2.461128-6.2261992 .039979$

H $1.008380-5.8360632 .954748$

H $1.184933-5.2871041 .267489$

H $4.171410-2.5570964 .120756$

H $5.526279-3.2131723 .249848$

H $3.560580-1.0909432 .313796$

H $5.266963-0.9244532 .564516$

H $3.929800-2.5397660 .337657$

H $6.201918-3.5069530 .936024$

H $6.892452-1.9002180 .858662$

H $7.960102-3.804415-0.747812$

H $6.903998-0.662066-1.473860$

H $5.885799-4.815895-1.164179$

H $5.832694-4.021444-2.721290$

H $4.511035-3.782311-1.561266$

H $7.058085-1.877872-3.385448$

H $5.5742091 .280215-1.627331$

H $4.6837160 .704026-3.042032$

H $2.881796-0.942148-1.984379$

H $-0.6922150 .940407 \quad 3.329805$

H 1.4899692 .1595361 .531805

H -0.5632853 .0429074 .708570$

H -1.7083913 .1164383 .348515$

H -1.0819414 .5905294 .043820$

H $2.5296051 .059719-1.828513$

H $3.4259151 .602933-0.393980$

H $1.0962605 .043068-2.621638$

H $2.826500 \quad 5.340442-2.853904$

H $3.0879646 .023725-0.442967$

H $0.0810116 .398677-0.901219$

H 0.7095368 .4065570 .474957

H $2.1214757 .602751 \quad 1.109927$

H -1.5723876 .9461072 .981258$

H -1.4775517 .9811051 .447767$

H $0.461736 \quad 6.016367 \quad 3.898363$

H $1.9709746 .352323 \quad 3.126140$

H $1.8910124 .400477 \quad 1.724056$

H $1.5959923 .183295-1.171321$

C -3.861281 -4.7949280 .623455$

O $-4.151036-3.4015820 .405163$

C $-4.580197-3.243429-0.967124$ 
C $-4.611749-4.643809-1.575882$

C $-3.554403-5.361949-0.757895$

H $-3.804159-2.663340-1.476448$

C $-5.929677-2.513868-1.007626$

H -5.591773 -5.117735-1.442851

H $-4.391524-4.632032-2.647933$

C $-3.642554-6.874842-0.849092$

H $-2.565778-5.034557-1.102632$

C $-5.847034-1.050384-0.522209$

O $-6.856095-3.219623-0.172500$

H $-6.346874-2.547217-2.020027$

H $-5.408438-1.0104510 .480538$

O $-7.178500-0.533269-0.410760$

C $-5.069002-0.139311-1.491538$

C $-4.9692281 .327127-1.036342$

H $-4.064194-0.532547-1.655490$

O $\quad-5.716452-0.155765-2.768595$

H $-4.7319391 .938996-1.914762$

H $-5.9521441 .683107-0.705484$

H -2.864329 -7.348693 -0.242839

H -4.613102 -7.242813 -0.499982

H -3.510500 -7.205631-1.884440

C $-2.793495-4.9762361 .701929$

H $-6.489980-3.2278330 .728631$

H -7.672395 -1.1295800 .177825$

H $-6.625356 \quad 0.165766-2.639535$

H -2.537667 -6.0381491 .790747$

H -3.235787 -4.679287 2.662035

H $-4.788945-5.2475661 .001632$

C $-1.9947291 .744508-1.372373$

O $-2.6260941 .053469-0.287059$

C -3.9194131 .5567090 .071304$

C -3.7881013 .0212070 .509922$

C $-3.1160973 .873598-0.563552$

C $-1.8128153 .230096-1.036174$

H $-2.5980151 .636767-2.281510$

C $-0.6370051 .075523-1.627850$

H -4.2426860 .9803730 .946189$

O $-5.0613753 .577651 \quad 0.818484$

H $-3.1831843 .074941 \quad 1.423245$

H $-3.7897354 .058654-1.408443$

O $-2.8027755 .157206-0.017195$

H -1.045192 $3.352583-0.261836$

O $-1.3548103 .911776-2.201943$

C $-0.788368-0.349865-2.113689$

H $-0.060951 \quad 1.615243-2.386723$

H $-0.0608781 .067715-0.696934$

H -5.4626093 .0235071 .509496$

H -3.632198 5.5394450 .317325

H -1.316655 $4.859549-1.988768$

O $0.219531-1.115711-1.604029$

O $-1.666499-0.735597-2.873648$

C $0.197795-2.496261-1.980024$

H $0.981157-3.013417-1.419294$

H $-0.767282-2.947627-1.730249$

H $0.405722-2.594956-3.049231$

SCF Energy (B3LYP/6-31G**//MMFF) $=-3245.91825941$

01626

MM̄FF Geometry

C $-2.954344-2.296911-1.979052$

C $-2.120396-3.018453-2.742944$

C $-0.613509-2.965382-2.704907$

O $-0.130519-2.053164-1.693668$

C $0.044948-4.338706-2.451229$

C $-0.369876-5.065543-1.184277$

C $0.272951-4.970336-0.003711$

C $-1.527518-6.022680-1.324373$

C $1.451662-4.1050940 .343716$

C $2.728448-4.9390450 .496051$

C $3.933632-4.1561851 .045273$

C $3.792711-3.8654302 .542609$

C $4.975618-3.0452913 .076986$

O $4.073750-2.938247 \quad 0.307392$

O $4.638705-2.5803914 .392995$

C $5.178671-1.8160842 .167692$

C $6.227029-3.9095483 .263176$

O $6.351053-1.0959272 .543490$
C $5.225810-2.1600450 .658431$

C $5.194287-0.850068-0.163494$

O $6.438518-2.8603870 .377030$

C $0.181065-0.783702-2.065506$

O $0.053947-0.286824-3.172793$

C $0.720517-0.096127-0.869602$

C $1.2005601 .149608-0.990607$

C 1.7830811 .9644320 .074095

C 1.8262581 .3903211 .465593

C $5.065464-1.043622-1.679771$

C $4.9263180 .294851-2.380936$

C $6.1690772 .414969-2.815292$

O $3.8733950 .640977-2.911271$

C $5.9804453 .335104-1.649112$

C $5.0958464 .343513-1.627052$

C $4.9238085 .269406-0.456313$

C 3.4899155 .3663110 .017326

C $2.7926626 .506879-0.119575$

C 2.8877734 .1664980 .719967

C $2.2439513 .195026-0.232692$

N $6.0892721 .046616-2.339459$

H -2.551752 -1.580032 -1.268627

H -2.546493 -3.714963 -3.462466

H $-0.268384-2.628340-3.691150$

H $1.134548-4.203453-2.442200$

H $-0.152589-4.994493-3.309952$

H $-0.091332-5.5730190 .827733$

H -1.696696 -6.605630 -0.412414

H -2.455756 -5.489346 -1.543786

H -1.335434 -6.735716 -2.132895

H $1.616201-3.326361-0.404800$

H $1.212668-3.5857301 .277850$

H $2.546558-5.8175441 .127892$

H $2.995917-5.318851-0.499504$

H $4.820865-4.7772920 .872665$

H $3.683429-4.7964743 .112367$

H $2.873641-3.2944632 .725896$

H $3.831921-2.0415504 .328828$

H $4.331672-1.1371022 .337805$

H $6.029772-4.715583 \quad 3.980371$

H $7.050903-3.3260663 .689013$

H $6.575362-4.3701902 .336506$

H $6.277146-0.8964233 .492433$

H $4.341529-0.2413000 .161826$

H $6.100302-0.2688590 .047510$

H $6.642778-2.773589-0.566931$

H $\quad 0.720237-0.6361710 .070296$

H $1.1748541 .621029-1.972499$

H $\quad 0.817050 \quad 1.135208 \quad 1.807929$

H 2.2403872 .0846032 .202240

H 2.4424630 .4859581 .493655

H $5.934588-1.562229-2.097735$

H $4.180492-1.642589-1.922342$

H $5.4219452 .578589-3.598484$

H $7.1669422 .554189-3.241834$

H $6.6150493 .168627-0.781430$

H $4.4772234 .528763-2.502188$

H $5.2903636 .258258-0.760870$

H 5.5547914 .9636120 .387736

H 1.7724576 .5917910 .238655

H $3.2237277 .379906-0.598356$

H 2.1285774 .5072331 .435221

H 3.6719613 .6706691 .304736

H $2.1416663 .541011-1.261356$

H $6.8992270 .675347-1.851834$

C $-5.026659-2.697282-0.642932$

O $-4.780075-1.5679730 .219003$

C $-5.975941-1.2720880 .959982$

C $-6.891809-2.4749120 .773319$

C $-6.541781-2.896700-0.640878$

H $-6.437132-0.4008590 .479798$

C $-5.624857-0.9410992 .413803$

H $-6.655765-3.2804401 .478190$

H -7.947754 -2.2161120.896916

C $-6.978576-4.311216-0.979707$

H -7.014330 -2.196935 -1.343224

C -4.5771590 .1826792 .575570$

O $-5.098951-2.1135183 .050365$ 
H $-6.538610-0.6876442 .963378$ H -3.599695 -0.1816982 .241035$ O $-4.438154 \quad 0.443152 \quad 3.978541$ C -4.9195691 .4968851 .849260$ C -3.9932522 .6761062 .197294$ H $-4.910599 \quad 1.3318890 .768216$ O $\quad-6.253648 \quad 1.8738522 .204877$ H -4.3955683 .5707351 .706854$ H -4.0744152 .8899303 .270290$ H -6.691571 -4.574007 -2.002692 H $-6.525263-5.044066-0.303763$ H -8.066343 $-4.407376-0.898824$ C $-4.452800-2.408606-2.030670$ H -4.317585 -2.396295 2.544747 H -5.2837290 .8058824 .294406$ H -6.4862412 .6591081 .680631$ H -4.847331 -1.456167 -2.402719 H -4.757216 -3.191846 -2.733979 H -4.521400 -3.560705 -0.190364 C $-2.6273863 .292406-0.469007$ O $-2.3456162 .196131 \quad 0.410600$ C -2.5148802 .4738731 .805789$ C -1.6032353 .6370072 .220819$ C -1.848900 4.8725441 .360796 C $-1.7634744 .515228-0.121453$ H $-3.6916443 .549780-0.405095$ C $-2.3297772 .834103-1.906655$ H -2.1565021 .5874802 .341800$ O -1.7985623 .9496993 .595538$ H $-0.556805 \quad 3.3274132 .108815$ H -2.8136885 .3344691 .598213$ O -0.8636495 .8630181 .663162$ H $-0.7174694 .321489-0.389720$ O $-2.1984235 .632480-0.894600$ C $-3.3278491 .827953-2.437592$ H $-2.354711 \quad 3.696145-2.583026$ H -1.341023 $2.367102-1.955910$ H -1.2273244 .7081453 .805784$ H 0.0108865 .4811321 .478931 H -1.646726 $6.392666-0.642215$ O $-3.0865041 .641293-3.769801$ O $-4.193250 \quad 1.271152-1.778178$ C $-3.9524890 .731741-4.456806$ H -3.928428 $0.981224-5.521091$ H -3.580757 -0.287469 -4.328028 $\mathrm{H}-4.9859200 .812954-4.106522$

SCF Energy (B3LYP/6-31G**//MMFF) $=-3245.90722642$

01627

MM̄FF Geometry

C $-3.3851805 .152434-0.506183$

C $-2.2747754 .759101-1.146941$

C $-0.8876905 .236345-0.803758$

O $-0.1393534 .087156-0.342688$

C $-0.2007605 .794268-2.062505$

C $1.2069826 .297651-1.808261$

C $2.3351485 .655619-2.173623$

C $1.2929777 .633664-1.115275$

C $2.4642794 .325445-2.866364$

C $3.1114353 .294408-1.941000$

C $3.2554471 .892099-2.552749$

C $1.9098341 .247924-2.897619$

C $2.069012-0.205163-3.371264$

O $3.9291501 .099100-1.566166$

O $0.770427-0.818222-3.356542$

C $2.939968-0.973402-2.358384$

C $2.569525-0.280178-4.818737$

O $3.239830-2.283846-2.834089$

C $4.244733-0.237293-1.978776$

C $4.915211-0.946632-0.778178$

O $5.135231-0.249339-3.094531$

C $-0.273116 \quad 3.742714 \quad 0.968440$

O $-0.9039804 .337405 \quad 1.827494$

C 0.4540242 .4707821 .169205

C $0.282311 \quad 1.7684252 .298819$

C 0.8797270 .4623322 .588187

C $1.947255-0.032377 \quad 1.651190$

C $6.175863-0.241655-0.262724$
C $6.500651-0.6700241 .154122$

C $5.866708-0.2268433 .527668$

O $7.347691-1.5272911 .393023$

C $4.862178-1.2457723 .965065$

C $3.948768-1.0264354 .923890$

C $3.003320-2.0801065 .424896$

C $1.559781-1.6326475 .426376$

C $0.925363-1.3555426 .578708$

C $0.827518-1.6131274 .103898$

C $0.439824-0.2285223 .659979$

N $5.738190-0.0027332 .100861$

H -3.3118705 .8813200 .296344$

H $-2.3557834 .013809-1.935573$

H $-0.9108546 .005656-0.021932$

H $-0.7954326 .622377-2.471800$

H $-0.1993045 .030184-2.846711$

H $3.2891976 .129925-1.945473$

H $2.3258427 .982794-1.010490$

H $\quad 0.7466958 .392522-1.684938$

H $0.8631727 .575275-0.110719$

H $1.4970223 .963216-3.215590$

H $3.0855784 .458650-3.759984$

H $4.1092043 .645595-1.642796$

H $2.5479793 .224027-1.003567$

H $3.8898281 .968245-3.443489$

H $1.3732161 .824297-3.659455$

H $1.2651841 .253328-2.007935$

H $\quad 0.187733-0.301229-3.938897$

H $2.339634-1.105733-1.448971$

H $1.8677670 .225034-5.493309$

H $2.622333-1.317903-5.165808$

H $3.5495830 .181363-4.957117$

H $2.398462-2.698509-3.090441$

H $4.188567-1.0196840 .039590$

H $5.190382-1.973231-1.051419$

H $6.039942-0.099565-2.778357$

H 1.0752812 .1151230 .356933

H -0.3902272 .1589563 .061117$

H $2.7353730 .719821 \quad 1.537314$

H $1.524710-0.2435740 .664102$

H $2.441241-0.9426611 .995449$

H $7.038970-0.474953-0.896282$

H $6.0643960 .848619-0.272388$

H 5.7198290 .7381314 .023047

H $6.876789-0.5839483 .752571$

H $4.918404-2.2265633 .498416$

H $3.912989-0.0556465 .413155$

H $3.315698-2.343516 \quad 6.444033$

H $3.090806-3.0053504 .841513$

H -0.122738 -1.074929 6.592215

H $1.438545-1.4029997 .533381$

H -0.095986 -2.200271 4.203821

H $1.405411-2.1469273 .344399$

H -0.3398690 .2222254 .275761$

H $5.0318010 .657576 \quad 1.790624$

C -5.2086773 .5068460 .144649$

$\begin{array}{llll}\text { O } & -4.311571 & 2.378849 & 0.067598\end{array}$

C -4.0408211 .9163321 .402966$

C -5.1257112 .5308082 .278631$

C -5.2965183 .8879121 .622827$

H -3.0681432 .3343571 .691066$

C -3.9658430 .3852861 .464120$

H $-6.059746 \quad 1.9591752 .222707$

H -4.8267222 .5949553 .329233$

C -6.5868544 .5935152 .000171$

H -4.4488844 .5183881 .917391$

C $-2.807607-0.2033490 .629523$

O $-5.209589-0.170140 \quad 1.042132$

H $-3.8234670 .093300 \quad 2.511395$

H -1.884155 0.3179670 .901651

O $-3.0222250 .019404-0.763242$

C $-2.590017-1.7131550 .864516$

C $-1.341710-2.2280670 .120170$

H -2.461293 -1.894656 1.938303

O $-3.725714-2.4531800 .431520$

H - $-1.510255-2.154581-0.959283$

H $-0.500287-1.5703750 .361526$

H -6.6681105 .5559661 .485161$ 
H $-7.4649203 .994278 \quad 1.736771$ H -6.6207974 .7823643 .078127$ C $-4.7511994 .607283-0.818137$ $\begin{array}{llll}\mathrm{H} & -5.336214 & 0.076961 & 0.109694\end{array}$ H -3.080169 $0.979635-0.903159$ H -4.486618 -2.1694930 .965502$ H $-5.4691515 .435363-0.811471$ H $-4.7515574 .200397-1.837857$ H $-6.1831233 .137864-0.201633$ C -2.174145 -4.850324 -1.241538 O $-1.991622-4.625607 \quad 0.162016$ C $-0.970263-3.681700 \quad 0.498253$ C $0.375401-4.158756-0.066870$ C $0.297222-4.417951-1.568053$ C $-0.874146-5.345397-1.887294$ H $-2.500264-3.921505-1.724047$ C -3.291835 -5.888095 -1.399051 H $-0.882731-3.7030121 .592249$ O $1.407788-3.2149980 .192540$ H $0.665242-5.094306 \quad 0.429458$ H $0.224169-3.482410-2.134307$ O $1.501440-5.056847-1.998666$ H $-0.641868-6.359627-1.537852$ O $-1.048655-5.410118-3.301196$ C $-4.629402-5.317175-0.992398$ H $-3.378636-6.249674-2.429935$ H $-3.102300-6.763344-0.765643$ H $1.456312-3.0946991 .155946$ H $2.243885-4.493527-1.720116$ H $-0.203756-5.701973-3.684493$ O $-5.108247-4.504350-1.977270$ O $-5.177038-5.559008 \quad 0.075440$ C $-6.370605-3.887533-1.706228$ H $-6.303052-3.258463-0.813613$ H $-6.630798-3.256407-2.560209$ H $-7.145476-4.650228-1.584053$ SCF Energy (B3LYP/6-31G**//MMFF) $=-3245.92136149$

0163

MM̄FF Geometry

C $3.670749-0.2767843 .325549$

C 2.9218790 .8151113 .104800

C 1.4906701 .0407453 .534988

$\begin{array}{lllll}\text { O } & 0.755821 & 1.437869 & 2.348527\end{array}$

C $0.778671-0.1875684 .145370$

C -0.6543610 .0711264 .574114$

C -1.734231 -0.5203264 .024342$

C $-0.830327 \quad 1.0312265 .722808$

C -1.761730 -1.572677 2.951164

C -1.923895 -0.9689761 .553810$

C -1.956282 -2.047941 0.462616

C -2.056047 -1.406657 -0.922917

C $-2.177034-2.458861-2.034391$

O $-3.086309-2.8935810 .697999$

O $-2.561287-1.760962-3.228762$

C $-3.313948-3.436919-1.663935$

C $-0.827625-3.118837-2.339356$

O $-3.393861-4.504065-2.605366$

C $-3.211807-3.984489-0.222529$

C $-4.441395-4.8337740 .186703$

O $-2.072278-4.851706-0.139391$

C 0.0909022 .6245282 .379437

O 0.1244053 .4698353 .259737

C -0.7473872 .7070751 .163018$

C -1.8459763 .4770541 .193523$

C -2.8167523 .6603930 .112982$

C $-2.5365062 .984339-1.201297$

C $-5.811294-4.158076 \quad 0.102467$

C $-5.970252-3.0288381 .100916$

C $-6.243256-0.5747191 .269411$

O $-5.912551-3.2217632 .313611$

C -6.7397930 .5298230 .395008$

C $-5.964331 \quad 1.533312-0.042665$

C $-6.4532502 .633086-0.940751$

C $-6.3020724 .006443-0.326353$

C -7.3098374 .5680800 .362490$

C $-5.0239004 .766562-0.601209$

C -3.9148664 .4086250 .350164$
N $-6.182368-1.8000450 .496337$

H $3.257756-1.1212503 .869342$

H 3.3780811 .6504262 .577046

H 1.5107351 .8531884 .270941

H $1.330111-0.5389325 .027074$

H $0.813940-1.0023313 .414657$

H $-2.718463-0.2481844 .403837$

H $-1.872471 \quad 1.095767 \quad 6.053977$

H -0.2354120 .7041896 .582090$

H -0.5133492 .0395615 .445720$

H $-0.867739-2.2035932 .990298$

H -2.601279 -2.245056 3.168260

H -2.849835 -0.381906 1.522923

H $-1.093901-0.2841881 .351371$

H -1.038648 -2.642834 0.542384

H $-1.200576-0.746631-1.109593$

H -2.944609-0.760141-0.959992

H -2.718144 -2.426020 -3.921033

H $-4.247016-2.871668-1.769253$

H $-0.102132-2.367136-2.668760$

H $-0.911721-3.831129-3.167418$

H $-0.397088-3.637572-1.480532$

H -2.675059-5.128249-2.407173

H $-4.465556-5.746210-0.423428$

H $-4.293377-5.176577 \quad 1.220433$

H $-2.181784-5.4284570 .635052$

H -0.4615972 .1097790 .305385$

H -2.070805 4.0197462 .111456

H -2.418461 $1.904130-1.063040$

H -1.621959 $3.387723-1.645463$

H $-3.3324703 .111326-1.937886$

H $-6.026490-3.824901-0.917209$

H $-6.588375-4.8946250 .343151$

H $-5.241013-0.3775381 .663107$

H $-6.913209-0.7207752 .123142$

H $-7.789514 \quad 0.5007160 .113122$

H -4.9141961 .5415320 .235178$

H -7.504941 2.465669 -1.209821

H $-5.9037882 .588176-1.888931$

H -7.2231415 .5667750 .777338$

H -8.2415094 .0379620 .531026$

H -5.202874 $5.845829-0.498680$

H $-4.7352174 .642107-1.649381$

H -4.0426124 .8435241 .342437$

H $-6.018473-1.721401-0.503471$

C $5.279890-1.6601571 .998163$

O $4.640634-1.4406410 .725510$

C $5.473639-1.990497-0.313836$

C $6.578058-2.7704460 .390680$

C $6.738352-1.9732651 .670514$

H $5.930138-1.140200-0.833489$

C $4.627762-2.812254-1.293429$

H $\quad 6.271225-3.7979310 .617758$

H $7.495158-2.818600-0.204385$

C $7.474180-2.7268522 .764641$

H $7.280243-1.0453151 .442555$

C $3.448908-2.026292-1.905263$

O $4.087391-3.935321-0.585972$

H $5.270634-3.220147-2.082650$

H $2.714650-1.795886-1.125023$

O $2.789084-2.876859-2.850368$

C $3.866143-0.737204-2.636593$

C $2.719925-0.013435-3.363294$

H $4.325453-0.042298-1.930628$

O $4.868705-1.047909-3.608824$

H $3.1604240 .810653-3.937776$

H $2.288055-0.681313-4.118976$

H $7.559103-2.1182063 .670086$

H $6.957304-3.6552393 .029972$

H $8.486249-2.9874482 .437790$

C $5.100485-0.4258312 .881524$

H $3.735565-4.555534-1.246531$

H $2.346792-3.582679-2.349584$

H $4.486098-1.692777-4.228423$

H 5.4239580 .4681052 .336930

H $5.723179-0.5111223 .779041$

H $4.795406-2.5343842 .453426$

C $2.6247612 .627776-1.818593$ 
O $2.124845 \quad 1.355726-1.387608$

C $1.6139720 .516495-2.429948$

C $0.471968 \quad 1.229349-3.165562$

C $0.9057332 .594906-3.686625$

C $1.5405373 .416611-2.567548$

H $3.4978982 .479805-2.465670$

C $3.0728043 .403057-0.567276$

H $1.172764-0.352841-1.930238$

O $-0.007804 \quad 0.446580-4.253163$

H $-0.3692401 .370231-2.477117$

H $1.5866022 .503025-4.540937$

O $-0.240623 \quad 3.302332-4.165345$

H $0.7624993 .740340-1.865063$

O $2.1209804 .593406-3.128410$

C $4.3888092 .910786-0.003364$

H $3.2089224 .461417-0.817838$

H 2.3237543 .3160150 .227236

H $-0.327354-0.395846-3.888219$

H $-0.6597392 .746822-4.844974$

H $1.4170525 .064110-3.606817$

$\begin{array}{llllllll} & & 1 & 4.882855 & 3.858071 & 0.848789\end{array}$

O $4.9075951 .832546-0.252917$

C 6.1391313 .5544891 .463790

H $6.529182 \quad 4.473967 \quad 1.908452$

H 5.9916522 .8168302 .256733

H 6.8616503 .1915680 .726398

SCF Energy (B3LYP/6-31G*//MMFF) $=-3245.92269290$

0164

MM̄FF Geometry

C $-3.4066072 .336757-2.548356$

C $-2.596657 \quad 1.561395-1.812777$

C $-1.3603930 .885517-2.337519$

O $-0.275329 \quad 1.166888-1.422175$

C $-1.565806-0.639091-2.363336$

C $-0.383614-1.365504-2.971590$

C $0.649692-1.873353-2.270541$

C $-0.416136-1.489932-4.472791$

C $0.867100-1.815870-0.782234$

C $1.213655-3.174194-0.151245$

C $2.552329-3.766458-0.618486$

C $2.907538-5.0201310 .183978$

C $4.285399-5.580054-0.209291$

O $3.567228-2.775501-0.437938$

O $4.679610-6.5239510 .798555$

C $5.320466-4.432577-0.186853$

C $4.224375-6.358229-1.529152$

O $6.582944-4.873401-0.682130$

C $4.854953-3.159573-0.931631$

C $5.838321-1.994383-0.670442$

O $4.826530-3.429675-2.335908$

C $0.3729112 .355035-1.542298$

O $0.157725 \quad 3.235230-2.358915$

C $1.3841962 .407975-0.462582$

C $1.9354393 .583284-0.127133$

C 2.8931413 .8075580 .958697

C 3.4180132 .5986211 .688024

C $5.299489-0.620394-1.098533$

C $6.3606290 .463525-1.018147$

C $6.6717032 .904871-1.413261$

O $7.5277550 .241263-0.707269$

C $6.7114443 .555425-0.066593$

C 6.4677254 .8597540 .132120

C $6.575116 \quad 5.5295291 .471550$

C 5.3424606 .3251481 .833866

C $5.342547 \quad 7.6676361 .772278$

C $4.141845 \quad 5.5697102 .356721$

C 3.2421555 .0770951 .254697

N $5.850067 \quad 1.710472-1.356268$

H -3.192808 $2.496155-3.601723$

H -2.835871 $1.396292-0.765670$

H $-1.0994301 .247985-3.339807$

H -2.467563 -0.888610 -2.938711

H - $1.757708-1.012981-1.351763$

H $1.453600-2.342242-2.834697$

H $0.458986-2.017616-4.866712$

H -1.305461 -2.046008 -4.787281

H $-0.441659-0.500097-4.939167$
H $1.673197-1.100320-0.582060$

H $-0.013011-1.427531-0.262924$

H $1.256914-3.0206180 .934898$

H $\quad 0.406489-3.892207-0.341322$

H $2.472020-4.016404-1.681858$

H $2.132321-5.7880820 .070318$

H $2.929785-4.7731891 .254747$

H $3.998065-7.2161620 .844343$

H $5.485290-4.1741750 .868232$

H $3.504968-7.182617-1.454385$

H $5.188646-6.825554-1.757467$

H $3.928710-5.739142-2.378598$

H $6.520955-4.919259-1.651468$

H $6.063038-1.9351620 .402479$

H $6.784309-2.194142-1.189566$

H $4.963087-2.602689-2.824114$

H $1.612007 \quad 1.4848160 .058142$

H $1.6322364 .474049-0.676129$

H $3.831677 \quad 1.8701130 .982136$

H 2.6160882 .1166902 .257919

H 4.2230212 .8281512 .389112

H $4.936242-0.648877-2.131240$

H $4.463780-0.330342-0.450804$

H $6.2428913 .559826-2.178170$

H $7.6864552 .627574-1.717251$

H 6.9908382 .9328450 .780287

H $6.2207675 .490478-0.718932$

H 7.4515616 .1900401 .440652

H $6.781097 \quad 4.8024072 .266983$

H 4.4707608 .2458852 .060477

H 6.2115018 .2181221 .427156

H 3.5384606 .2319872 .992691

H 4.4770324 .7744353 .027909

H 2.7998925 .8832610 .667145

H $4.8537751 .810670-1.528369$

C $-5.8773202 .164161-2.140444$

O $-5.7572810 .997825-1.300001$

C $-6.9605570 .847826-0.528156$

C $-8.003027 \quad 1.712574-1.223698$

C $-7.1512802 .879169-1.686257$

H $-6.756271 \quad 1.2708450 .462840$

C $-7.321423-0.633541-0.394904$

H -8.446289 $1.202641-2.086660$

H $-8.8130152 .010492-0.551226$

C $-7.8042353 .719210-2.769968$

H $-6.9349823 .519735-0.820594$

C $-6.178127-1.5122870 .157576$

O $-7.661987-1.134932-1.694621$

H $-8.222217-0.7401840 .220962$

H $-5.377055-1.564763-0.588726$

$\begin{array}{lllll} & \text { O } & -6.710424 & -2.838073 & 0.277765\end{array}$

C -5.618544-1.054989 1.520878

C -4.620691-2.036007 2.166780

H $-5.143216-0.0750511 .411636$

O $-6.715691-0.8915172 .424929$

H $-4.423070-1.6943823 .189576$

H $-5.105590-3.0109632 .294092$

H $-7.1440154 .534639-3.081602$

H -8.040134 $3.119301-3.655362$

H -8.737370 $4.160349-2.404643$

C $-4.6208773 .019722-1.982742$

H $-7.894316-2.073654-1.589884$

H $-5.959823-3.4536250 .327534$

H $-6.362623-0.519363 \quad 3.251007$

H $-4.4534353 .239106-0.920612$

H $-4.7427703 .982484-2.492103$

H $-5.9768591 .805898-3.173036$

C $-1.998083-0.4970702 .545067$

O $-2.576270-0.9470451 .312821$

C $-3.298250-2.1821011 .388471$

C $-2.381452-3.2962041 .910032$

C -1.738939 -2.922642 3.240063

C $-1.060921-1.5595873 .136409$

H -2.793616 -0.2575993 .260368$

C -1.2114620 .7898992 .248030$

H -3.554036 -2.450109 0.357305

O $-3.094872-4.5188122 .061360$

H - $-1.583897-3.4802271 .179505$ 
H $-2.467051-2.939574 \quad 4.059478$ O $-0.740569-3.8921003 .567858$ H $-0.157974-1.6480732 .519397$ O $-0.646275-1.145834 \quad 4.437422$ C $-2.102867 \quad 1.9791341 .963619$ H -0.5859581 .0615943 .105761$ H -0.5747300 .6343031 .370134$ H -3.480509 -4.7409461 .196665$ H $-1.178144-4.7605303 .590269$ H $-0.068596-1.8429124 .793109$ O -1.3122423 .0501051 .654101$ O $-3.324768 \begin{array}{lll}1.978366 & 2.007819\end{array}$ C -2.002186 4.2668791 .354964 H $-2.580503 \quad 4.1529950 .434276$ H $-2.651134 \quad 4.554645 \quad 2.187497$ H $-1.256015 \quad 5.0517561 .204954$ SCF Energy (B3LYP/6-31G*//MMFF) $=-3245.91302162$

0165

MMFF Geometry

C -3.015863 $3.638854-0.219037$

C $-2.419125 \quad 4.6711590 .395894$

C -1.0342894 .6852271 .006396$

$\begin{array}{lllll}\text { O } & -0.365354 & 3.411588 & 0.858532\end{array}$

C $-0.174236 \quad 5.747131 \quad 0.295627$

C 1.1057626 .0729641 .039325

C $2.336596 \quad 5.6493150 .691782$

C 0.9368776 .9810712 .232093

C $2.7331234 .752924-0.449212$

C 3.3698573 .4675860 .079660

C $4.0065582 .580347-0.999347$

C $3.0192742 .124918-2.075234$

C $3.6626761 .134719-3.059057$

O $4.5272441 .439697-0.306421$

O $2.6006710 .555891-3.830351$

C $4.3396050 .004098-2.256752$

C $4.5745031 .852268-4.061254$

O $5.049589-0.877256-3.124120$

C $5.256558 \quad 0.515321-1.122493$

C $5.680359-0.661449-0.213800$

O $6.419207 \quad 1.114818-1.694486$

C -0.5953182 .4559551 .800493$

$\begin{array}{lllll}\text { O } & -1.305032 & 2.549975 & 2.788599\end{array}$

C $0.152325 \quad 1.2449201 .391238$

C -0.0837180 .0703531 .993921$

C $0.552066-1.2082211 .660662$

C $1.731806-1.1754290 .726282$

C $6.529676-0.2331710 .990330$

C $6.417238-1.2253982 .128462$

C $4.885334-1.9366143 .962850$

O $7.305443-2.0398542 .367136$

C $4.122967-3.1303013 .482198$

C $2.940795-3.511198 \quad 3.989689$

C $2.221541-4.7552873 .555850$

C $0.769528-4.515243 \quad 3.217114$

C $-0.212095-5.0314773 .976758$

C $0.452697-3.7644951 .943363$

C $0.043432-2.337888 \quad 2.195224$

N $5.227369-1.0984532 .830584$

H -2.495666 $2.692655-0.320249$

H -2.974742 5.6029540 .489435

H -1.131383 4.9296442 .071096

H $-0.740715 \quad 6.6818650 .183754$

H $0.0434905 .417335-0.726112$

H $3.1768145 .981563 \quad 1.301491$

H $1.893468 \quad 7.2378342 .699735$

H $\quad 0.4582527 .9186301 .930552$

H 0.3166926 .5037872 .996843

H $1.8816934 .506667-1.086918$

H $3.4534375 .293635-1.074410$

H $4.1484493 .712140 \quad 0.816171$

H 2.6269412 .8881580 .640598

H $4.8388983 .134786-1.448436$

H $2.6048492 .977576-2.625182$

H $2.1634841 .630838-1.595201$

H $2.989551-0.128189-4.402148$

H $3.535155-0.597012-1.812263$

H $4.0023882 .592847-4.633074$
H $4.9788491 .153208-4.801631$

H $5.4089322 .372507-3.586569$

H $5.881455-0.440166-3.374149$

H $4.779048-1.1746590 .142269$

H $6.257465-1.395739-0.789849$

H $7.1250211 .132943-1.028919$

H $\quad 0.854134 \quad 1.343787 \quad 0.571953$

H $-0.8344040 .031102 \quad 2.782138$

H $2.484041-0.4635741 .084002$

H $1.419420-0.875151-0.278903$

H $2.242850-2.1357030 .633990$

H $7.585164-0.1518430 .706521$

H 6.2321550 .7498781 .373122

H $4.302632-1.3246184 .658444$

H $5.802738-2.2615784 .463983$

H $4.586348-3.7342772 .705293$

H $2.491572-2.9295084 .791245$

H $2.302964-5.4838624 .372988$

H $2.714566-5.2189992 .692157$

H $-1.256640-4.8925753 .717352$

H $0.005360-5.6009134 .874114$

H $-0.387801-4.2628471 .442769$

H $1.286879-3.8564421 .242967$

H $-0.819701-2.2445562 .855959$

H $4.530771-0.4322952 .509589$

C $-5.4041632 .786600-0.095786$

$\begin{array}{llll}\text { O } & -5.039760 & 1.399167 & -0.245685\end{array}$

C $-5.357217 \quad 0.7132840 .981442$

C -6.1663131 .6941391 .828111$

C $-5.5741573 .021581 \quad 1.404500$

H -4.4042460 .5178081 .486488$

C $-6.092680-0.6007230 .701657$

H $-7.2344531 .658427 \quad 1.583376$

H -6.0622161 .4981352 .899772$

C $-6.441590 \quad 4.2189491 .749177$

H -4.6013513 .1307011 .899403$

C $-5.322823-1.628450-0.152438$

$\begin{array}{lllll}\text { O } & -7.323388 & -0.314150 & 0.022458\end{array}$

H $-6.380964-1.0628611 .653535$

H $-5.267951-1.292137-1.194434$

O $-6.104814-2.831995-0.159886$

C $-3.908494-1.968684 \quad 0.352742$

C $-3.310846-3.266093-0.221908$

H $-3.240019-1.1263760 .146673$

O $-3.954545-2.1257681 .775858$

H $-2.314475-3.3874140 .217255$

H -3.885439 -4.1186990 .162893$

H $-5.972617 \quad 5.149048 \quad 1.412829$

H $-7.428188 \quad 4.149105 \quad 1.279082$

H $-6.589693 \quad 4.2875432 .831843$

C $-4.3977303 .694694-0.806261$

H $-7.1008770 .158598-0.798114$

H $-6.987635-2.601806-0.496917$

H -3.044423 -2.268953 2.085316

H $-4.7740624 .724590-0.804803$

H $-4.3172103 .386340-1.856157$

H $-6.3727492 .897115-0.601860$

C $-1.070348-2.284903-2.053378$

O $-2.475892-2.269725-2.330585$

C $-3.225977-3.342563-1.758743$

C $-2.684991-4.681788-2.277505$

C $-1.198355-4.832669-1.970422$

C $-0.422783-3.616631-2.473711$

H $-0.909913-2.104452-0.984716$

C $-0.443278-1.124222-2.843978$

H $-4.246871-3.244526-2.145807$

O $-3.421450-5.756079-1.703403$

H $-2.833161-4.731128-3.364241$

H -1.020502 $-4.998804-0.901888$

O $-0.691006-5.987846-2.643735$

H $-0.349328-3.677840-3.567540$

O $0.903250-3.701513-1.950356$

C $-0.8998320 .238124-2.372469$

H $\quad 0.646772-1.143024-2.753015$

H $-0.720869-1.213411-3.901417$

H $-3.140444-6.575501-2.144054$

H $-1.097597-6.769033-2.232379$

H $1.458486-3.068434-2.434853$ 
O $-\begin{array}{lll}-0.386350 & 1.183267 & -3.214974\end{array}$

O $-1.601766 \quad 0.450523-1.394993$

C $-0.705543 \quad 2.541091-2.900257$

H $-1.7860392 .697860-2.961027$

H $-0.3272632 .797944-1.907273$

H $-0.2158803 .184411-3.636093$

SCF Energy (B3LYP/6-31G*//MMFF) $=-3245.91497677$

0166

MM̄FF Geometry

C $-0.450066-3.247927-2.497145$

C $-1.432236-4.040623-2.042320$

C $-2.117562-3.826391-0.720333$

O $-3.364334-3.149162-1.009516$

C $-2.431828-5.1438230 .011353$

C $-1.248681-5.7497640 .738143$

C $-0.992405-5.5559992 .047502$

C $-0.378940-6.671992-0.074556$

C $-1.749815-4.6628472 .991231$

C $-0.852780-3.9083423 .984342$

C $0.239320-3.0411373 .338806$

C $1.040818-2.3073484 .419555$

C $2.103306-1.3767093 .817488$

O $-0.371844-2.0958652 .451513$

O $2.560362-0.5219784 .876624$

C $1.432147-0.4904942 .746856$

C $3.331159-2.1599193 .339861$

O 2.3989510 .3096782 .072900

C $0.574764-1.2858581 .738030$

C $-0.192343-0.369458 \quad 0.760434$

O $1.442606-2.098768 \quad 0.936005$

C -3.818774 -2.270522 -0.075182

O $-3.315285-2.0255831 .009311$

C $-5.043166-1.630635-0.602963$

C -5.592174 -0.6209960 .089022$

C $-6.7903290 .135106-0.274205$

C $-7.507132-0.245022-1.542364$

C -1.1524350 .6409871 .391162$

C $-1.870051 \quad 1.4655230 .331363$

C $-3.212716 \quad 3.5485670 .095168$

O $-1.769436 \quad 1.243837-0.872774$

C -4.6096953 .8186730 .555942$

C -5.642288 $3.962841-0.289161$

C $-7.048646 \quad 4.273808 \quad 0.141381$

C -8.058215 $3.298877-0.424886$

C $-8.7091693 .570435-1.568818$

C $-8.3703872 .049163 \quad 0.366890$

C -7.182834 1.1426590 .532880

N $-2.630588 \quad 2.4830760 .892554$

H - $0.147128-2.386849-1.908046$

H $-1.761999-4.880200-2.649256$

H $-1.492622-3.190227-0.082837$

H -2.849306 -5.879779 -0.689833

H $-3.254430-4.9789060 .718909$

H -0.151852 -6.087569 2.491397

H $0.392255-7.157528 \quad 0.533237$

H $-0.983053-7.463602-0.529516$

H $0.131665-6.121489-0.869110$

H $-2.355709-3.9326182 .449939$

H $-2.444647-5.2865283 .566541$

H $-1.507204-3.2713774 .593759$

H $-0.385129-4.6294204 .667039$

H $0.904589-3.6953602 .764187$

H $1.504465-3.0202085 .113046$

H $0.355571-1.7010115 .029039$

H $3.1589400 .136817 \quad 4.484649$

H $0.7871440 .209603 \quad 3.291251$

H $3.796092-2.6877074 .181305$

H $4.102448-1.4896262 .946636$

H $3.097671-2.9016252 .573167$

H $2.852780-0.2540061 .423243$

H $\quad 0.527404 \quad 0.1552260 .121601$

H $-0.770891-0.9996240 .074036$

H $0.961758-2.3613090 .134199$

H $-5.449114-1.996484-1.538671$

H -5.114659 -0.3079191 .017142$

H -7.867576 -1.277964 -1.485524

H $-6.838146-0.152676-2.405202$
H -8.375595 $0.383557-1.753161$

H -1.9050300 .1301092 .000595$

H -0.6006521 .3384342 .030010$

H $-3.1685473 .293120-0.968497$

H -2.594087 4.4373050 .257860

H -4.7764413 .9265621 .624950$

H $-5.4638543 .883200-1.359573$

H -7.284711 $5.290333-0.199697$

H -7.1383324 .3011851 .234467$

H $-9.4538432 .891863-1.971590$

H $-8.5084204 .476773-2.130491$

H $-9.2118731 .499285-0.070613$

H -8.721684 2.3435551 .364542

H $-6.594742 \quad 1.3556951 .426284$

H $-2.5776432 .627698 \quad 1.896915$

C $1.791016-3.352476-3.690167$

O $2.166348-1.990420-3.404072$

C $3.272203-2.003521-2.480591$

C $3.765702-3.446418-2.429868$

C $2.469909-4.212001-2.624583$

H $2.862961-1.740394-1.498612$

C $4.326673-0.970866-2.893604$

H $4.463345-3.667689-3.245814$

H $4.266003-3.680257-1.485141$

C $2.665317-5.660533-3.033965$

H $1.913026-4.181044-1.679834$

C $3.7820920 .460226-3.087228$

O $4.920503-1.372324-4.135253$

H $5.141533-0.965005-2.160376$

H $3.1571040 .507923-3.987397$

$\begin{array}{llll}\text { O } & 4.900038 & 1.324368 & -3.327655\end{array}$

C $2.9923791 .012413-1.887371$

C $2.5517522 .476707-2.092116$

H $2.0962720 .401170-1.739178$

$\begin{array}{llll}\text { O } & 3.780676 & 0.895246 & -0.707875\end{array}$

H $3.4358863 .119428-2.150139$

H $2.0346122 .543928-3.056213$

H $1.700442-6.156601-3.179158$

H $3.230662-5.741514-3.968348$

H $3.214064-6.207827-2.260562$

C $0.267143-3.465477-3.799255$

H $4.205735-1.451616-4.790355$

H $5.3865220 .963550-4.088766$

H $4.5815151 .432884-0.833369$

H $\quad 0.008677-4.445312-4.217839$

H $-0.091607-2.702718-4.501327$

H $2.216988-3.576114-4.677485$

C 3.2223374 .0892780 .457779

$\begin{array}{lllllllll}\text { O } & 2.260749 & 3.036966 & 0.307368\end{array}$

C $1.6146252 .975511-0.967754$

C $0.9075204 .304993-1.262998$

C $1.8613525 .488257-1.150456$

C $2.5804555 .458056 \quad 0.196511$

H $4.0521653 .934327-0.241058$

C 3.7650784 .0098491 .893996

H $0.8260702 .221973-0.873152$

$\begin{array}{lllll}\text { O } & 0.315650 & 4.297337 & -2.557266\end{array}$

H $0.0931044 .449198-0.540824$

H $2.5778075 .509408-1.979985$

O $1.1141916 .704121-1.229968$

H 1.8695755 .7014630 .996729

$\begin{array}{llllll}\text { O } & 3.595358 & 6.460299 & 0.206355\end{array}$

C 4.7544772 .8780962 .074055

H 4.2916714 .9283062 .174742

H 2.9299903 .8548922 .588118

H - $0.3011323 .545954-2.591155$

H $0.6284486 .689604-2.072576$

H 3.1645987 .3109830 .014188

$\begin{array}{lllll} & 4 & 4.732129 & 2.460986 & 3.374307\end{array}$

$\begin{array}{lllll}\text { O } & 5.490515 & 2.445568 & 1.197491\end{array}$

C $5.6599151 .421743 \quad 3.701338$

H 5.5102490 .5548143 .051221

H 6.6847991 .7950833 .616000

H 5.4809961 .1185374 .736297

SCF Energy (B3LYP/6-31G*//MMFF) $=-3245.93624827$

0167

MM̄FF Geometry 
C $-1.804884-3.298922-1.528833$ C $-0.770962-3.942459-2.092120$ C $0.699849-3.624823-1.932129$ O $0.918712-2.454158-1.114327$ C $1.392867-4.816165-1.241505$ C $2.903590-4.707096-1.180079$

C $3.622251-4.649614-0.041787$ C $3.613954-4.704252-2.509829$ C $3.124204-4.6686091 .376487$ C $2.910409-3.2780781 .995673$ C $4.193522-2.4388672 .116010$ C $3.938715-1.1684742 .929690$ C $5.175074-0.2559382 .983612$ O $4.621758-2.078626 \quad 0.801358$ O $4.725474 \quad 1.014363 \quad 3.480178$ C $5.718642-0.0478241 .550931$ C $6.210300-0.7743503 .989033$ O 6.9676540 .6381331 .569451 C $5.862094-1.3623530 .756548$ C $6.207174-1.143301-0.736309$ O $6.925840-2.1391671 .321406$ C $0.890691-1.239183-1.727224$ O $0.680123-1.013244-2.907794$ C $1.141046-0.213636-0.689361$ C $0.8728931 .074894-0.948472$ C $1.0489052 .196862-0.021928$ C 1.7808091 .9240531 .264858 C $5.187562-0.322021-1.533407$ C $5.5513151 .147114-1.668340$ C $4.6637463 .286283-2.585542$ O $6.6268611 .614055-1.304117$ C $4.1203864 .090918-1.447807$ C $3.2005095 .054783-1.603195$ C $2.6782045 .902386-0.479643$ C $1.1734105 .837733-0.355379$ C $0.3932016 .811002-0.855197$ C $0.579916 \quad 4.6857450 .423809$ C $0.5556753 .404447-0.367386$ N $4.5394531 .873228-2.282654$ H -1.629894 -2.437961 -0.890120 H $-0.986484-4.795688-2.733580$ H $1.127186-3.466286-2.929406$ H $1.149612-5.747947-1.769797$ H $0.973148-4.922045-0.235153$ H $4.706803-4.588331-0.128029$ H $4.700919-4.785846-2.400429$ H $3.283874-5.551481-3.119613$ H $3.409241-3.777945-3.055101$ H $2.192628-5.2388251 .454246$ H $3.849671-5.2255711 .983296$ H $2.168079-2.7357091 .398099$ H $2.480194-3.4200372 .994868$ H $4.963030-3.0533222 .598084$ H $3.601610-1.4160953 .944033$ H $3.112962-0.6075192 .471733$ H 5.4991721 .5966713 .564391 H 5.0082560 .6063831 .032049 H $5.778940-0.8072074 .996802$ H $7.072018-0.1009394 .054603$ H $6.574469-1.7760093 .751280$ H 6.8193021 .5115801 .968552 H $7.221710-0.738672-0.833894$ H $\quad 6.246787-2.136568-1.206853$ H $7.233682-2.7732540 .652695$ H $1.521016-0.5508950 .267981$ H $\quad 0.466246 \quad 1.334572-1.924866$ H 2.7606441 .4820021 .056723 H $\quad 1.208897 \quad 1.2344491 .894974$ H 1.9719382 .8175551 .861565 H $5.147061-0.720335-2.555489$ H $4.183407-0.412617-1.107501$ H $4.1212453 .467476-3.518704$ H $5.7187893 .533819-2.741097$ H $4.5209973 .890836-0.456890$ H $2.8199645 .270047-2.599265$ H $2.9948806 .936571-0.667639$ H 3.1348335 .6246200 .478462 H $-0.685924 \quad 6.783394-0.744875$
H $\quad 0.8161557 .654174-1.391088$ H -0.4563794 .9199830 .696601$ H $1.110308 \quad 4.597228 \quad 1.375041$ H $0.0340913 .492455-1.321466$ H $3.6581151 .416434-2.498608$ C $-3.926805-3.971510-0.384899$ $\begin{array}{lllll}\text { O } & -4.143363 & -2.716344 & 0.292842\end{array}$ C $-5.476529-2.7046520 .835167$ C $-5.976984-4.140706 \quad 0.750752$ C $-5.308617-4.609695-0.526110$ H $-6.085024-2.0836310 .167380$ C -5.470197-2.110695 2.246352 H -5.635319 -4.740833 1.602085 H -7.068965 -4.198627 0.712070 C $-5.280243-6.121048-0.675667$ H $-5.839134-4.172932-1.383135$ C $-4.894908-0.6810552 .333322$ O $-4.682160-2.946483 \quad 3.104395$ H $-6.485044-2.1298682 .660058$ H -3.816508 -0.7007862 .138690$ O $-5.052492-0.233772 \quad 3.686108$ C -5.5743140 .3382321 .400346$ C $-5.187791 \quad 1.8039531 .664659$ H $\quad-5.3523720 .0849260 .359685$ O H -5.7981432 .4318961 .004425$ H -5.504500 2.0812912 .677993 H -4.776169 -6.412825 -1.602213 H $-4.753831-6.5969570 .158517$ H $-6.298047-6.523647-0.703870$ C $-3.238975-3.711090-1.725111$ H -3.789347 -2.999816 2.722053 H $-4.617077-0.887428 \quad 4.259779$ H -7.4085420 .8225100 .913349$ H -3.754192 -2.902346 -2.255453 H $-3.292785-4.606390-2.354824$ H $-3.287452-4.5813100 .267003$ C $-3.7367332 .562556-0.907509$ $\begin{array}{llll}\text { O } & -3.214393 & 1.771656 & 0.166593\end{array}$ C -3.6917482 .1235071 .469484$ C -3.3291583 .5826151 .776940$ C $-3.875048 \quad 4.527868 \quad 0.710412$ C $-3.4602884 .057406-0.681951$ H $-4.8144502 .384053-1.004531$ C $-3.0533742 .100721-2.205770$ H $-3.133046 \quad 1.5032802 .180148$ O -3.8258183 .9445723 .060833$ H -2.2368383 .6761891 .815537$ H $-4.963857 \quad 4.632700 \quad 0.780701$ O -3.3325265 .8355320 .910443$ H $-2.3938224 .265341-0.828602$ O $-4.1713194 .815224-1.660531$ C $-3.4660030 .714111-2.650100$ H -3.304358 2.787641-3.022081 H -1.966839 2.084801-2.069205 H -3.4713084 .8230923 .277895$ H -3.687858 6.1753371 .748928 H $-3.9842535 .754765-1.491971$ O $-2.9100760 .471263-3.874890$ O $-4.167041-0.057516-2.012396$ C $-3.199271-0.799396-4.466603$ H -4.242733 -1.088996 -4.309088 H $-3.021396-0.719654-5.542385$ $\mathrm{H}-2.522750-1.552076-4.054817$ SCF Energy $\left(B 3 L Y P / 6-31 G^{* *} / / M M F F\right)=-3245.90644855$

0168

MM̄FF Geometry

C -4.638669-2.942376-1.113452

C $-3.318429-3.072071-0.919488$

C $-2.326888-3.216474-2.045787$

O $-1.383530-2.123232-1.971456$

C $-1.541416-4.531442-1.894030$

C $-0.560085-4.777389-3.024155$

C $0.780782-4.818958-2.893081$

C $-1.180904-5.020705-4.377617$

C $1.615279-4.661334-1.652040$

C $2.419238-3.359681-1.681410$ 
C $3.529076-3.298552-0.619424$

C $2.985448-3.3431940 .809721$

C $4.087164-3.1426121 .861777$

O $4.237623-2.070377-0.838309$

O $3.448951-2.8577553 .116796$

C $4.919985-1.8986841 .488982$

C $4.910271-4.4186252 .073159$

O $6.048335-1.7597682 .348804$

C $5.376326-1.8853840 .015130$

C $6.054636-0.555470-0.387696$

O $6.343383-2.923363-0.187081$

C $-1.772530-0.924429-2.486463$

O $-2.838682-0.657806-3.016671$

C $-0.665662 \quad 0.029735-2.260697$

C $-0.886321 \quad 1.347755-2.373308$

C $0.0957732 .401700-2.115387$

C $1.5135081 .982015-1.826535$

C $5.1919300 .696884-0.211621$

C $5.830816 \quad 1.925339-0.839342$

C $5.445743 \quad 4.354704-1.178744$

O $6.914769 \begin{array}{lll}1.902392 & -1.416187\end{array}$

C $4.2421005 .229799-1.312111$

C $3.6660245 .523022-2.487944$

C $2.4447706 .386263-2.632379$

C $1.216046 \quad 5.572367-2.983145$

C $0.7893415 .501587-4.255748$

C $0.5220624 .905197-1.812282$

C $-0.3028183 .690822-2.146655$

N $5.0336623 .052258-0.689236$

H -5.027594 -2.969264 -2.128243

H -2.924537 -3.0556970 .093181$

H $-2.833940-3.194803-3.018216$

H -2.239053 -5.378883 - 1.856483

H $-1.026716-4.526186-0.928345$

H $1.370268-5.008995-3.790138$

H $-0.444682-5.346137-5.120565$

H $-1.942487-5.804800-4.311061$

H -1.649269-4.108475-4.759095

H $1.011100-4.706798-0.743423$

H $2.300774-5.516609-1.604818$

H $2.884065-3.226572-2.668222$

H $1.745320-2.500928-1.562241$

H $4.214150-4.136377-0.795596$

H $2.453513-4.2816691 .003438$

H $2.243287-2.5457960 .934002$

H $2.868516-3.6069903 .334737$

H $4.283016-1.0258151 .672844$

H $4.266365-5.2365262 .418374$

H $5.662122-4.2790392 .857745$

H $5.414552-4.7586791 .166346$

H $5.718188-1.7645073 .263643$

H $6.998522-0.4343430 .160037$

H $6.341610-0.629624-1.446387$

H $6.867321-2.707744-0.976387$

H $0.294091-0.379371-1.966113$

H $-1.8843241 .690316-2.643491$

H $2.2047672 .823362-1.752713$

H $1.8986961 .342472-2.628788$

H $1.5680131 .431928-0.883159$

H $4.2114900 .551710-0.678996$

H $5.0430900 .907000 \quad 0.852982$

H $5.9764904 .231075-2.129083$

H $6.1487494 .781912-0.456343$

H $3.8220495 .634580-0.394662$

H $4.0842205 .110150-3.403226$

H $2.6475987 .136019-3.407912$

H $2.2510006 .959250-1.716603$

H $-0.0985094 .947237-4.538042$

H $1.3219256 .008412-5.054367$

H $-0.1307555 .645202-1.331026$

H $1.2549834 .638898-1.042276$

H -1.340950 3.915211-2.391223

H $4.2418343 .003602-0.053536$

C $-6.304164-1.3925830 .039951$

$\begin{array}{llll}0 & -5.366483 & -0.388516 & 0.475123\end{array}$

C $-5.7277990 .865862-0.140590$

C $-7.014580 \quad 0.613813-0.928984$

C $-6.897838-0.860092-1.261267$
H $-4.9254661 .108538-0.846696$

C $-5.859904 \quad 1.974573 \quad 0.912317$

H $-7.9092430 .797958-0.323093$

H $-7.082544 \quad 1.249929-1.817177$

C $-8.213665-1.507210-1.653126$

H $-6.186208-0.958033-2.089833$

C $-4.601337 \quad 2.2042601 .773319$

$\begin{array}{llll}\text { O } & -6.933563 & 1.624877 & 1.796729\end{array}$

H -6.1570072 .9080020 .418729$

H $-4.449793 \quad 1.3508502 .444586$

$\begin{array}{lllll} & & -4.849649 & 3.342298 & 2.609721\end{array}$

C $-3.3129352 .472643 \quad 0.974537$

C $-2.125698 \quad 2.968717 \quad 1.825233$

H $-3.023176 \quad 1.5622290 .438528$

O $-3.5843393 .470611-0.013615$

H -1.2721993 .1145811 .151518$

H -2.3463803 .9730672 .209323$

H -8.073664 -2.569828 -1.875370

H $-8.956104-1.424843-0.852211$

H -8.627866 -1.027169 -2.545765

C $-5.640437-2.772563-0.007411$

$\begin{array}{llll}H & -7.158836 & 2.417713 & 2.311755\end{array}$

H -5.5140073 .0815003 .269566$

H $-2.7743823 .593229-0.536423$

H $-6.411981-3.541442-0.134201$

H $-5.158040-2.9641800 .959586$

H -7.083077 $-1.428205 \quad 0.814294$

C $-0.4624910 .411693 \quad 1.750295$

$\begin{array}{llll}\text { O } & -1.606370 & 0.676873 & 2.567158\end{array}$

C -1.7349122 .0363942 .988812$

C $-0.4830672 .474188 \quad 3.762949$

C 0.8035222 .1990202 .984360

C $\quad 0.839010 \quad 0.7522192 .486736$

H $-0.524287 \quad 1.0295790 .846574$

C $-0.562517-1.035743 \quad 1.256858$

H -2.5672912 .0575873 .702567$

O $-0.569495 \quad 3.8567004 .091323$

H $-0.446366 \quad 1.921474 \quad 4.710749$

H 0.9133652 .9012622 .149827

$\begin{array}{lllll}\text { O } & 1.927664 & 2.434194 & 3.836356\end{array}$

H 1.0025900 .0889863 .343172

$\begin{array}{llll}\text { O } & 1.957460 & 0.612480 & 1.616659\end{array}$

C $-0.517550-2.0938892 .338615$

H $-1.525052-1.159747 \quad 0.748439$

H $0.239230-1.2372320 .539396$

H 0.2500634 .0974574 .556417

H $1.8677601 .821803 \quad 4.589317$

H $2.052297-0.3305421 .405574$

O $-0.393154-3.3181491 .743528$

O $-0.608494-1.889474 \quad 3.540468$

C $-0.355933-4.4376972 .633222$

H $-0.210113-5.3412142 .035142$

H $0.480135-4.3416653 .332224$

H $-1.304095-4.5215213 .172481$

SCF Energy (B3LYP/6-31G**//MMFF) $=-3245.90669687$

0169

MM̄FF Geometry

C -1.067724 $3.469349-1.005836$

C $0.0272634 .036993-0.478702$

C $0.945770 \begin{array}{lll}3.303885 & 0.461630\end{array}$

O $2.270483 \quad 3.288280-0.118109$

C 1.0649643 .9846561 .837053

C -0.2291094 .0813392 .616162$

C $-0.861063 \quad 3.031774 \quad 3.177956$

C $-0.792118 \quad 5.471242 \quad 2.753725$

C -0.4207561 .5930803 .167339$

$\begin{array}{llll}C & -1.332754 & 0.746414 & 2.270187\end{array}$

C $-0.887861-0.7154592 .102000$

C -1.037983 -1.5132273 .398657$

C $-0.564005-2.962828 \quad 3.232938$

O $0.469113-0.7374451 .642907$

O $-0.476605-3.527566 \quad 4.550114$

C $0.855020-2.9436912 .627256$

C -1.588013 -3.811072 2.470264

O $1.298359-4.2711042 .349949$

C $0.984888-2.0474181 .371903$

C $2.459762-1.9172720 .917380$ 
O $0.269484-2.6688620 .298699$

C $2.6850492 .127910-0.694147$

O $2.043612 \quad 1.098217-0.827065$

C $4.0854382 .321286-1.127478$

C $4.7879081 .269348-1.573326$

C $6.1783341 .292070-2.031030$

C $6.8956932 .617077-2.008650$

C $3.341275-1.0465151 .817497$

C $4.824595-1.2312101 .534111$

C $7.038640-0.5138172 .424851$

O $5.265307-2.076016 \quad 0.759476$

C $7.750461 \quad 0.028787 \quad 1.225641$

C $8.574782-0.7152770 .471743$

C $9.375545-0.196641-0.688602$

C $9.006586-0.862459-1.995472$

C $9.495736-2.071356-2.320077$

C $8.136563-0.087760-2.958400$

C $6.7437040 .134429-2.433752$

N $5.602034-0.3604962 .288242$

H -1.288220 $2.433114-0.768015$

H $0.2624685 .072834-0.708112$

H $0.5998462 .276301 \quad 0.608876$

H 1.5009054 .9857781 .710758

H 1.8129243 .4511462 .439420

H -1.790349 3.2154503 .715182

H -1.756193 5.4810793 .273058

H $-0.100882 \quad 6.1027573 .321534$

H -0.9436525 .9241231 .769018$

H 0.6225691 .5000442 .851660

H -0.4606191 .2250394 .198714$

H -2.3600510 .7750612 .653631$

H $-1.3612341 .201027 \quad 1.273216$

H -1.525102 -1.151530 1.323771

H $-2.075339-1.4863273 .754743$

H $-0.436465-1.0514334 .192714$

H $-0.100362-4.4204204 .462975$

H $1.521648-2.5649023 .410697$

H -2.548511 -3.815384 2.999581

H $-1.273695-4.8591662 .415107$

H -1.771391 -3.453657 1.455590

H $0.859972-4.5642631 .532862$

H $2.888317-2.9203560 .797169$

H $2.491645-1.480404-0.089900$

H $0.594026-2.301674-0.539913$

H $4.5010843 .320050-1.058463$

H $4.3028950 .294365-1.604349$

H $6.9014213 .033985-0.995057$

H $6.4051223 .330572-2.679921$

H $7.9405022 .556797-2.318669$

H 3.0845930 .0100231 .680250

H $3.188016-1.2968032 .871915$

H $7.258196-1.5742452 .590404$

H 7.3441990 .0433003 .316267

H $7.615376 \quad 1.0826280 .996934$

H $8.717080-1.7636050 .726576$

H $10.437438-0.378078-0.475278$

H $9.2831690 .892275-0.780695$

H $9.257819-2.543362-3.267387$

H $10.144191-2.616474-1.641995$

H $8.042495-0.628938-3.909358$

H $8.6427150 .846144-3.218389$

H $6.144232-0.776528-2.401016$

H $\begin{array}{llll}5.137929 & 0.267374 & 2.938195\end{array}$

C -3.474731 $4.186981-1.329686$

O $-3.9914052 .844182-1.192465$

C -4.6555212 .7426970 .081211$

C $-4.874863 \quad 4.1765410 .555513$

C -3.6226194 .8517370 .039984$

H $-3.952767 \quad 2.250522 \quad 0.763527$

C $-5.947851 \quad 1.928680-0.021782$

H $-5.763744 \quad 4.6256810 .097214$

H $-4.983227 \quad 4.242176 \quad 1.642354$

C $-3.7155866 .365942-0.013533$

H -2.7958984 .5715620 .701585$

C $-5.7982470 .477053-0.520248$

O $-6.8529342 .594746-0.913554$

H -6.4460341 .9252060 .955231$

H $-5.5960930 .463994-1.597771$
O $-7.076641-0.152569-0.343905$

C $-4.737890-0.3691230 .209241$

C $-4.874230-1.8932300 .027910$

H $-3.740889-0.051311-0.112927$

O $-4.808379-0.1186681 .617710$

H $-4.040305-2.3603420 .566021$

H $-5.770654-2.2313680 .563869$

H -2.788767 $6.798562-0.403399$

H $-4.5391026 .695580-0.655721$

H -3.8849476 .7762190 .987364$

C $-2.0521094 .165089-1.901232$

H $-6.4050012 .682216-1.772649$

H $-7.7303890 .378325-0.830840$

H $-5.707006-0.3449651 .913025$

H $-1.7259355 .190655-2.109748$

H $-2.0631243 .628479-2.857047$

H $-4.1274614 .683542-2.059587$

C $-2.554955-2.655494-1.831676$

O $-3.780851-2.007676-2.191629$

C $-4.924630-2.395493-1.428003$

C $-5.146568-3.910848-1.547811$

C $-3.904484-4.691406-1.133385$

C $-2.686081-4.184878-1.899537$

H $-2.264266-2.343030-0.822723$

C $-1.478483-2.183163-2.822023$

H $-5.786270-1.916429-1.906617$

O $-6.250153-4.332773-0.753455$

H $-5.385158-4.163629-2.589233$

H $-3.738188-4.645974-0.051028$

O $-4.088391-6.072836-1.452110$

H $-2.759563-4.508369-2.946016$

O $-1.509523-4.780119-1.354938$

C $-1.161512-0.711043-2.686988$

H $-0.542311-2.731742-2.674080$

H $-1.835945-2.352452-3.845454$

H $-7.028205-3.825524-1.041707$

H $-4.891848-6.370664-0.991572$

H $-1.629040-5.744696-1.390390$

O $-0.485542-0.304863-3.802598$

O $-1.453161-0.012626-1.727084$

C $-0.1367801 .081192-3.851615$

H $-1.0405951 .684641-3.970166$

H $0.4113811 .375932-2.953484$

H $\quad 0.5075011 .239333-4.720510$

SCF Energy (B3LYP/6-31G**//MMFF) $=-3245.93235117$

$01 \_7$

MM̄FF Geometry

C -3.865653 $1.391965-2.267857$

C -2.945684 $2.339201-2.506246$

C $-2.145883 \quad 3.025903-1.433504$

O $-0.770183 \quad 3.000281-1.890355$

C $-2.5600154 .495083-1.232571$

C $-3.899463 \quad 4.687333-0.558437$

C $-4.076617 \quad 4.717014 \quad 0.777037$

C $-5.0650354 .937967-1.479888$

C $-3.0398694 .524864 \quad 1.853736$

C $-3.5305803 .579648 \quad 2.961534$

C -3.5045812 .0975052 .550134$

C -4.5083711 .3029643 .388359$

C $-4.460387-0.1935193 .062507$

O $-2.176888 \quad 1.601358 \quad 2.776315$

O $-5.212152-0.890558 \quad 4.066605$

C $-3.002402-0.669860 \quad 3.171594$

C $-5.123567-0.5123901 .720743$

O $-2.877551-2.0226242 .735150$

C -1.981805 0.2252242 .420964

C $-0.559534-0.2001012 .860470$

$\begin{array}{llll}\text { O } & -2.124223 & 0.025904 & 1.014539\end{array}$

C $0.206984 \quad 3.116432-0.954069$

$\begin{array}{lllll}\text { O } & 0.061925 & 3.186669 & 0.255239\end{array}$

C $1.5019293 .175240-1.666801$

C $2.6233893 .385451-0.962992$

C $3.9816363 .483368-1.498006$

C $4.1676053 .363164-2.987335$

$\begin{array}{llll}\text { C } & 0.593493 & 0.680431 & 2.364283\end{array}$

C $1.920570 \quad 0.202586 \quad 2.936667$

$\begin{array}{llll}\text { C } 4.361546 & 0.420217 & 2.703775\end{array}$ 
O $1.998073-0.6446353 .824431$

C 5.3617031 .4127902 .210889

C 6.2881831 .1317601 .281432

C 7.3533572 .0929750 .834021

C $7.2069692 .496979-0.616050$

C $7.7767651 .777687-1.597346$

C $6.4645273 .779368-0.918228$

C $4.9955673 .667007-0.627982$

N $3.014781 \quad 0.8422352 .366983$

H -4.080468 $1.116099-1.240208$

H -2.746142 $2.637593-3.533218$

H $-2.2186512 .466918-0.492764$

H -2.549671 $5.020196-2.198061$

H -1.789235 $5.019983-0.653311$

H $-5.0803364 .919113 \quad 1.149773$

H $-5.9966015 .118176-0.932722$

H -4.873893 $5.819787-2.100454$

H $-5.2346044 .081794-2.137978$

H -2.087668 4.1648951 .456860

H -2.8492145 .5075162 .302329$

H -2.8871983 .7215873 .839394$

H -4.5448913 .8778723 .256054$

H -3.7471972 .0055851 .486211$

H -5.5258111 .6891613 .247096$

H $-4.281710 \quad 1.436580 \quad 4.455604$

H $-6.121714-0.5463884 .052684$

H -2.743041 -0.665943 4.239853

H $-6.169575-0.1838231 .719082$

H -5.147729 -1.592162 1.536166

H -4.622123 -0.0294730 .881377$

H $-3.512819-2.552212 \quad 3.246742$

H $-0.521667-0.2024793 .958114$

H $-0.368596-1.2276372 .526792$

H -1.2925840 .2670830 .577378$

H $1.4965253 .057258-2.744147$

H 2.5424773 .4968900 .117870

H $3.6783074 .198389-3.500487$

H $3.7415502 .428015-3.362288$

H $5.2153073 .364650-3.296264$

H $\quad 0.654247 \quad 0.651440 \quad 1.273057$

H $0.447508 \quad 1.7186052 .681058$

H $4.514073-0.5731852 .271914$

H 4.4401120 .3207123 .791914

H 5.3478612 .3976082 .671501

H $\quad 6.3089630 .1398160 .835909$

H $8.327806 \quad 1.6105560 .987466$

H 7.3728932 .9884691 .467880

H $7.6960962 .075695-2.637391$

H $8.3310870 .870238-1.381235$

H $6.6448934 .102658-1.948644$

H $6.8802804 .586699-0.301356$

H 4.7569263 .7552540 .432215

H 2.8723781 .3787821 .515688

C $-4.671603-0.830330-3.230456$

O $-3.339750-1.355793-3.071130$

C $-3.363050-2.358708-2.036534$

C $-4.831070-2.714573-1.830674$

C $-5.500456-1.375710-2.067548$

H -2.987240 -1.880905 -1.123964

C $-2.466350-3.541805-2.411745$

H $-5.179877-3.446918-2.568007$

H -5.023396 -3.121401-0.833290

C $-6.986690-1.481320-2.363041$

H $-5.366540-0.757340-1.175558$

C -1.012447 -3.153662 -2.745548

O $-3.019807-4.179149-3.570737$

H -2.489657 -4.288267 -1.608899

H $-0.990635-2.574443-3.676205$

O $-0.299291-4.367152-3.023938$

C $-0.272406-2.380514-1.636549$

C $1.194201-2.103418-2.025890$

H $-0.777847-1.425082-1.457607$

O $-0.340661-3.140291-0.433994$

H $1.709666-3.054435-2.191940$

H $1.191402-1.568984-2.983097$

H -7.419960 -0.491927 -2.539985

H -7.177885 -2.097031 -3.248370

H -7.514936 -1.935079 -1.518006
C $-4.6342490 .696730-3.359570$

H -2.440307 -4.927867 -3.793780

H $-0.248879-4.873314-2.194605$

H $0.030496-2.5954710 .280560$

H $-5.6573901 .088955-3.377994$

H $-4.180526 \quad 0.942377-4.328455$

H -5.050736 -1.242554-4.174878

C $2.991631-3.0887710 .250900$

O $2.113876-1.9557930 .274167$

C $1.967873-1.273997-0.975701$

C $3.339917-0.778836-1.453756$

C $4.357159-1.912645-1.528766$

C $4.396685-2.688080-0.214134$

H $2.583252-3.856530-0.416667$

C $3.032902-3.6692961 .669671$

H $1.357963-0.384828-0.774261$

O $3.242264-0.161711-2.732140$

H $3.715892-0.018747-0.756883$

H $4.150738-2.581570-2.372580$

O $5.656584-1.363017-1.760566$

H $4.890191-2.0796170 .552026$

O $5.184202-3.864751-0.388631$

C $1.728441-4.3522142 .007188$

H $3.822049-4.4208741 .783411$

H $3.229753-2.8797292 .404319$

H $2.5619230 .530053-2.670723$

H $5.603880-0.827165-2.570647$

H $6.060607-3.583246-0.703290$

O $1.076580-3.6625782 .985161$

O $1.331610-5.3714741 .456703$

C $-0.186214-4.2050303 .383013$

H -0.609446 -3.552293 4.151059

H $-0.871807-4.2349112 .530756$

H $-0.051568-5.2048113 .806528$

SCF Energy $\left(B 3 L Y P / 6-31 G^{* *} / / M M F F\right)=-3245.92658567$

0170

MM̄FF Geometry

C $-3.415740 \quad 1.8646161 .831833$

C -2.4804071 .3969562 .672113$

C $-2.103763-0.0581182 .752881$

O $-0.657534-0.1204142 .690813$

C $-2.554934-0.7129064 .071822$

C $-4.046320-0.9295004 .191794$

C $-4.694750-2.0052343 .703849$

C -4.7961050 .1119774 .980901$

C $-4.116573-3.1537332 .918094$

C $-4.986484-3.5179851 .705205$

C $-4.883383-2.5048850 .551157$

C $-6.152470-2.560343-0.302704$

C $-6.067193-1.623717-1.511783$

O $-3.739018-2.851682-0.244168$

O $-7.150352-1.945452-2.397334$

C $-4.760606-1.922478-2.266114$

C $-6.249174-0.154484-1.119312$

O $-4.559832-0.983657-3.322012$

C -3.501585 -1.981967-1.361126

C $-2.333764-2.556623-2.202092$

O $-3.171454-0.661343-0.931664$

C $-0.111568-1.2828982 .242823$

O $-0.709268-2.254961 \quad 1.809564$

C $1.354989-1.1920292 .407776$

C $2.103315-2.2637482 .108262$

C $3.557199-2.3715902 .220603$

C $4.308673-1.2005392 .791515$

C $-1.030889-2.849591-1.446603$

C $0.022608-3.420073-2.388229$

C $2.402090-4.047118-2.531801$

O $-0.196524-3.647138-3.576648$

C $3.478649-4.563917-1.634705$

C $4.696005-4.006052-1.543425$

C $5.817290-4.555985-0.707001$

C $6.228704-3.6210760 .408634$

C $7.158639-2.6717350 .210562$

C $5.612782-3.8384851 .772719$

C $4.148339-3.5053561 .792138$

N $1.238415-3.655964-1.756262$

H $\quad-3.9420981 .1590591 .195976$ 
H $-1.965628 \quad 2.084674 \quad 3.338321$ H -2.520393 -0.5972931 .893288$ H -2.193459-0.120480 4.924106 H $-2.043159-1.6759144 .199519$ H $-5.763857-2.0836703 .898448$ H $-5.862802-0.1207185 .068616$ H -4.3910430 .1817865 .995828$ H -4.7147271 .0940384 .507363$ H -3.092881 -2.961080 2.590368 H -4.080555 -4.022884 3.586278 H $-4.673943-4.5072501 .346878$ H -6.027719-3.612800 2.038889 H $-4.747400-1.4961530 .954882$ H -7.037974 -2.321051 0.299898 H -6.304633 -3.586786 -0.665336 H -7.982433 -1.830245 -1.907309 H $-4.884423-2.903413-2.746631$ H -7.220708 $-0.003637-0.633845$ H $-6.2526280 .491949-2.003503$ H -5.478882 $0.201607-0.434128$ H $-5.359807-0.994220-3.875076$ H -2.662549 -3.500051-2.659001 H -2.104082 -1.863521 -3.022128 H $-2.231788-0.633797-0.692160$ H $1.771969-0.2640042 .780720$ H $1.603468-3.1577961 .736699$ H $4.017343-1.0298583 .833683$ H $4.098209-0.2944552 .218755$ H $5.393271-1.3274382 .776454$ H - $0.628883-1.933967-1.002668$ H $-1.208493-3.580656-0.650699$ H $2.725052-3.173327-3.107499$ H $2.107943-4.824051-3.245770$ H $3.258284-5.462640-1.063968$ H $4.917477-3.118133-2.131707$ H $6.672844-4.739599-1.370429$ H $5.559217-5.537943-0.291064$ H $7.474186-2.0126241 .012441$ H $7.619296-2.528850-0.761458$ H $6.156979-3.2820942 .543265$ H $5.735783-4.8916182 .056843$ H $3.517275-4.3027581 .398868$ H $1.386822-3.250935-0.835939$ C $-3.553796 \quad 3.971423 \quad 0.394035$ O $-2.1327184 .100257 \quad 0.192986$ C $-1.8342063 .775448-1.177251$ C $-3.1682283 .798718-1.917665$ C $-4.107702 \quad 3.274550-0.850249$ H -1.451192 2.747384-1.173596 C -0.769316 4.714981-1.751180 H $-3.4590104 .814595-2.208248$ H $-3.1531003 .179475-2.819775$ C $-5.5720663 .571405-1.119971$ H $-3.9701992 .189517-0.793172$ C $0.5005674 .865421-0.890911$ O $-1.3376516 .023322-1.897153$ H $-0.508766 \quad 4.382580-2.763510$ H $0.2833655 .501139-0.023930$ O $1.4577235 .593779-1.673214$ C $1.1357483 .550312-0.404303$ $\begin{array}{llll}\text { C } 2.508452 & 3.789690 & 0.254473\end{array}$ H $0.458563 \quad 3.0706890 .311657$ O $1.2825092 .676999-1.519326$ H $3.243764 \quad 4.020082-0.524357$ H 2.4322594 .6675160 .906936 H $-6.2026113 .173531-0.318560$ H -5.757452 4.648253 -1.192595 H $-5.8908093 .112743-2.061575$ C $-3.840643 \quad 3.303798 \quad 1.743388$ H - $0.6512476 .596736-2.279503$ H $1.7218615 .024363-2.416408$ H $1.4811201 .794138-1.165331$ H -4.9167193 .3504751 .948264$ H -3.3412203 .8810392 .531980$ H -3.938270 4.9991070 .442078 C $4.1823341 .339409-0.607226$ $\begin{array}{lllll}\text { O } & 3.112820 & 1.383140 & 0.344462\end{array}$ C 3.0053402 .5929061 .096287
C 4.3155672 .8620801 .852180

C 5.5194532 .8604520 .912663

$\begin{array}{llll}\text { C } & 5.534909 & 1.586497 & 0.069472\end{array}$

H $4.0084702 .090192-1.387653$

C $4.153920-0.046848-1.262757$

H 2.2312002 .4110111 .853259

$\begin{array}{lllll}\text { O } & 4.231486 & 4.101274 & 2.547652\end{array}$

H 4.4558322 .0804682 .609461

H 5.5356883 .7524210 .275308

$\begin{array}{lllll}\text { O } & 6.723228 & 2.890575 & 1.682673\end{array}$

H $\begin{array}{llll}5.802627 & 0.732657 & 0.704399\end{array}$

O $6.5435291 .699685-0.933198$

C $2.902607-0.224407-2.086323$

H $5.026465-0.211909-1.904954$

H $4.158982-0.842873-0.509409$

H $5.018754 \quad 4.177525 \quad 3.112440$

H $6.7665583 .754928 \quad 2.125167$

H $7.3856481 .874482-0.478855$

$\begin{array}{lllll}\text { O } & 3.158634 & 0.045869 & -3.396975\end{array}$

O $1.816686-0.531441-1.611285$

C $2.031143-0.051062-4.272997$

H $\quad 1.260147 \quad 0.667686-3.978957$

H $2.366007 \quad 0.188547-5.285578$

H $1.631864-1.069580-4.267323$

SCF Energy (B3LYP/6-31G*//MMFF)= -3245.92345302

0171

MM̄FF Geometry

C -2.546707 $3.313825-0.910241$

C $-1.787612 \quad 2.247358-0.617826$

C $-0.8458741 .590331-1.592858$

O $0.453081 \quad 1.498517-0.959849$

C $-1.3286320 .162847-1.900558$

C $-0.676922-0.423354-3.135242$

C $0.374553-1.264588-3.127599$

C $-1.314582-0.024995-4.442093$

C $1.154946-1.761905-1.942874$

C $1.137202-3.296834-1.867106$

C $2.086217-3.885888-0.807797$

C $1.631676-3.5768060 .619947$

C $2.638660-4.0675321 .669275$

O $3.391929-3.364268-1.059505$

$\begin{array}{lllll}\text { O } & 2.292828 & -3.464764 & 2.926381\end{array}$

C $4.037413-3.5431101 .286467$

C $2.558213-5.5833611 .879864$

O $5.035294-4.0644962 .161116$

C $4.421588-3.832068-0.181593$

C $5.730704-3.117351-0.595210$

O $4.630745-5.238996-0.341945$

C $1.2843542 .569489-1.076071$

O $1.0899743 .582460-1.728922$

C $2.4685392 .314709-0.221666$

C $3.3626943 .296792-0.029406$

C $4.547322 \quad 3.246934 \quad 0.833777$

C $4.8244101 .959544 \quad 1.562800$

C $5.639327-1.588782-0.597554$

C $6.887185-0.933174-1.160357$

C $7.7101221 .295641-1.929230$

O $7.957206-1.522208-1.285207$

C $8.2069812 .084123-0.759126$

C $8.1761883 .423706-0.696493$

C $8.698180 \quad 4.208653 \quad 0.471447$

C $7.677936 \quad 5.168357 \quad 1.039517$

C 7.8425886 .4977190 .928662

C 6.5134204 .5912361 .816263

C 5.3009314 .3610530 .953748

N $6.6649020 .401892-1.470389$

H -2.494892 $3.754294-1.902648$

H $-1.847399 \quad 1.8115180 .375847$

H $-0.7712062 .172328-2.519742$

H $-2.4145700 .159907-2.064186$

H $-1.158099-0.486585-1.034698$

H $\quad 0.748139-1.620695-4.087321$

H $-0.820350-0.484934-5.304573$

H $-2.364717-0.334653-4.460918$

H - $1.2695811 .060032-4.577750$

H $2.184175-1.402664-2.060036$

H $0.785740-1.338500-1.006457$ 
H $\quad 0.114685-3.652092-1.688558$ H $1.445226-3.692224-2.844711$ H $2.110171-4.971723-0.959920$ H $0.644717-4.0139910 .808522$ H $1.512455-2.4939800 .752465$ H $1.378560-3.7191563 .139336$ H $4.021705-2.4579601 .446505$ H $1.559092-5.8679912 .231591$ H $3.254861-5.9109632 .659414$ H $2.761177-6.1549140 .972031$ H $4.757557-3.8623663 .070930$ H $6.560976-3.4510230 .040084$ H $5.997536-3.439431-1.611834$ H $5.197626-5.381355-1.117830$ H $2.5488651 .344408 \quad 0.255132$ H $3.2003994 .248509-0.534720$ H 4.8828241 .1228090 .860604 H 4.0278541 .7508362 .285488 H 5.7663751 .9620662 .114423 H $4.779053-1.262573-1.192964$ H $5.513941-1.2135600 .423051$ H $7.2763211 .933918-2.705342$ H $8.5306720 .718573-2.366474$ H $8.626270 \quad 1.5221220 .072405$ H $7.7781073 .988404-1.536317$ H 9.5867884 .7560340 .131306 H 9.0458293 .5455211 .273471 H 7.1292367 .1917731 .360936 H 8.6901776 .9215080 .400453 H $6.221523 \quad 5.2960942 .606487$ H 6.8451163 .6938782 .344614 H 4.9912395 .2487440 .399643 H $5.7524530 .807533-1.283994$ C $-4.9478933 .781891-0.360902$ O $-5.2990752 .385715-0.334881$ C -6.6460462 .2473360 .158948$ C -7.203996 3.6617350 .281475 C $-5.948298 \quad 4.4627130 .571050$ H $-6.565957 \quad 1.8164811 .163738$ C $-7.446977 \quad 1.303573-0.750343$ H -7.664353 $4.003596-0.652441$ H $-7.956588 \quad 3.7415401 .071873$ C -6.109407 5.9529430 .327940 H $-5.660422 \quad 4.2962591 .617904$ C -6.734876 -0.038477 -1.021616 O $-7.6378301 .954387-2.013138$ H -8.445062 $1.139254-0.327616$ H -5.818787 $0.151415-1.590796$ O $-7.574245-0.840452-1.860702$ C $-6.418656-0.8520950 .246854$ C $-5.673735-2.173655-0.026067$ H $-5.821044-0.2532170 .936476$ O $-7.637449-1.1571280 .928530$ H $-5.666721-2.7580470 .901817$ H $-6.246424-2.779182-0.738889$ H -5.1790186 .4870660 .543766$ H $-6.3852576 .162639-0.711000$ H -6.8921806 .3652380 .973156$ C $-3.491216 \quad 3.952566 \quad 0.070539$ H -8.320889 $1.459638-2.496292$ H $-7.604350-0.416618-2.734912$ H -8.193565 -1.6708390 .317968$ H -3.347257 3.5126491 .063779 H -3.2392025 .0160600 .146762$ H -5.084210 4.128617 -1.393870 C $-3.108046-1.7189431 .590926$ O $-3.477818-1.1188130 .343517$ C $-4.234261-1.960215-0.535384$ C $-3.459145-3.248633-0.842256$ C $-2.994476-3.9553530 .426342$ C $-2.267171-2.9757481 .344063$ H $-4.007971-1.9793482 .160502$ C $-2.307810-0.6767792 .388736$ H $-4.310631-1.418370-1.484547$ O $-4.245657-4.146651-1.617518$ H $-2.572619-3.001080-1.440732$ H $-3.829617-4.4368930 .948388$

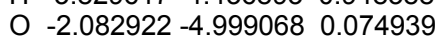

H -1.306822 -2.696768 0.893894

O $-1.992456-3.615758 \quad 2.587835$

C $-3.1822670 .428258 \quad 2.944468$

H -1.808779 -1.1406993 .246435$

H $-1.552336-0.2210071 .738209$

H -4.510601 -3.678118 -2.427479

H $-2.546174-5.591492-0.541970$

H $-1.479111-4.4187862 .393499$

O $-2.387027 \quad 1.462764 \quad 3.349305$

$\begin{array}{lllll}\text { O } & -4.399587 & 0.379789 & 3.050617\end{array}$

C -3.0672122 .5672093 .955369$

H -3.9216912.885022 3.350280

H -3.393014 2.2900124 .962053

H -2.3634073 .4006544 .028394$

SCF Energy (B3LYP/6-31G**//MMFF)= -3245.91961711

01_72

MM̄FF Geometry

C $-4.499538-2.323833-3.190216$

C $-3.296274-2.557145-2.645908$

C $-2.013100-1.983905-3.185716$

O $-1.556361-0.987285-2.238531$

C $-0.941195-3.081544-3.295072$

C $0.362843-2.560994-3.866709$

C $1.398838-2.118478-3.126076$

C $0.446924-2.558872-5.370359$

C $1.463960-2.018099-1.626944$

C $2.689471-2.707510-1.010518$

C $4.024757-2.017558-1.327663$

C $5.166872-2.658925-0.535276$

C $6.499127-1.922671-0.749061$

O $3.908223-0.636155-0.975664$

O $7.415007-2.363592 \quad 0.265119$

C $6.279191-0.409786-0.531436$

C $7.138356-2.282564-2.095490$

O $7.4503310 .330265-0.868571$

C $5.0599120 .151423-1.296753$

C $4.7792341 .609880-0.863577$

O $5.3402930 .133945-2.699092$

$\begin{array}{llll}C & -2.031376 & 0.279713 & -2.388488\end{array}$

O $-2.7770640 .688083-3.264579$

C $-1.5179921 .089881-1.261339$

C $-2.1266252 .247295-0.961143$

C -1.8050793 .1360030 .157460$

C -0.5901562 .8168950 .984593$

C $3.4152692 .143484-1.329002$

C $3.2793793 .640307-1.109660$

C $1.7329145 .534221-1.594519$

$\begin{array}{lllll}\text { O } & 4.149458 & 4.324876 & -0.578098\end{array}$

C $1.0394425 .891726-0.318663$

C $-0.1279096 .551831-0.274165$

C $-0.788573 \quad 6.9892121 .000795$

C $-2.239456 \quad 6.575687 \quad 1.086563$

C $-3.225768 \quad 7.4584550 .853656$

C -2.546669 5.1686091 .541931

$\begin{array}{llll}C & -2.615981 & 4.186896 & 0.400794\end{array}$

N $2.0709934 .123628-1.594398$

H $-4.572009-1.715275-4.088218$

H -3.233152 $-3.138798-1.728964$

H -2.164263 -1.523433 -4.170409

H $-1.306368-3.897997-3.932502$

H $-0.767734-3.541491-2.315157$

H $2.276136-1.746085-3.651706$

H $1.405230-2.172819-5.733481$

H $0.333056-3.576679-5.757539$

H $-0.343633-1.933511-5.797081$

H $1.451371-0.956830-1.352711$

H $\quad 0.576425-2.454102-1.162937$

H $2.545032-2.7062030 .076931$

H $2.728735-3.757918-1.324033$

H $4.217164-2.103349-2.402614$

H $5.267883-3.721507-0.788940$

H $4.927134-2.6289720 .537248$

H $7.507620-3.3285620 .186338$

H $6.112798-0.2544640 .543938$

H $7.336982-3.359799-2.148996$

H $8.111284-1.793107-2.214581$

H $6.512622-2.021169-2.951279$ 
H $8.187794-0.046349-0.358603$ H $4.800743 \quad 1.6806720 .231526$ H $5.5755862 .260779-1.246253$ H $4.8502330 .848327-3.134995$ H $-0.6921930 .690129-0.685136$ H -2.972624 2.560507 -1.572219 H $-0.3013773 .624817 \quad 1.660602$ H 0.2807342 .6380390 .345386 H -0.7681791 .9267041 .594549$ H $3.2668051 .950625-2.396714$ H $2.609897 \quad 1.643435-0.778763$ H $1.1062915 .718522-2.472885$ H $2.650716 \quad 6.123623-1.690793$ H 1.5410325 .6342990 .611397 H $-0.6114236 .839184-1.205151$ H -0.7097528 .0830741 .054261$ H -0.2513186 .6125391 .880182$ H $-4.269236 \quad 7.1760880 .946755$ H -3.011748 8.4804750 .559243 H -3.525645 5.1496162 .040757 H -1.841170 4.8694352 .322702 H -3.471753 4.344637 -0.257274 H $1.3819683 .469962-1.955260$ C $-6.347752-1.877685-1.496191$ O $-5.518378-1.964367-0.318413$ C $-5.250436-0.632240 \quad 0.154045$ C $-6.322150 \quad 0.248255-0.476926$ C $-6.456713-0.390974-1.846518$ H $-4.273072-0.346311-0.251735$ C $-5.194887-0.5979551 .684454$ H -7.2733650 .1837950 .063676$ H $-6.0239791 .300150-0.520342$ C -7.739583 -0.026870 -2.572820 H $-5.605764-0.062952-2.454927$ C $-4.258945-1.6452332 .323501$ O $-6.507985-0.826992 \quad 2.212750$ H -4.912554 0.4081182 .015752 H $-4.703980-2.6447152 .240871$ O $-4.179965-1.3592333 .726265$ C -2.830362 -1.682074 1.751184 C $-1.917560-2.6423352 .540337$ H -2.873365 -1.997129 0.702847 O $-2.290106-0.3651691 .788298$ H -1.657805 -2.184036 3.500863 H -2.477186 -3.5610282 .751233$ H $-7.790890-0.529530-3.543937$ H -8.624261 -0.315650 -1.995591 H $-7.790017 \quad 1.052861-2.746675$ C $-5.792570-2.799929-2.589625$ H $-6.814801-1.6856071 .873942$ H $-5.089155-1.3493154 .071556$ H -1.429365 -0.3921911 .338624$ H $-6.528017-2.897949-3.396845$ H -5.644623 -3.804812 -2.173899 H -7.329113 -2.264473 -1.192791 C $0.860640-1.2583472 .534983$ O $0.173051-1.8793131 .441641$ C $-0.627097-3.014644 \quad 1.774548$ C $0.233906-4.0798172 .466037$ C $0.977691-3.5146673 .673091$ C $1.757697-2.2632603 .271886$ H $0.117399-0.8437723 .225431$ C $1.681123-0.1042031 .932767$ H $-0.955307-3.4438310 .818796$ O $-0.571862-5.1855842 .859297$ H $0.969841-4.4612091 .745785$ H $0.289691-3.3135604 .502601$ O $1.891186-4.5124754 .134709$ H $2.601728-2.5564572 .634411$ O $2.297289-1.6442214 .435981$ C 1.8820901 .0700982 .864749 H $2.664041-0.4659081 .609875$ H $\quad 1.168850 \quad 0.2802201 .042763$ H $0.008088-5.8215523 .312237$ H $2.282863-4.1905554 .964128$ H $2.951231-2.2529614 .818584$ O $2.6874791 .984423 \quad 2.245475$ O $1.3857891 .190618 \quad 3.975564$
C 2.9449883 .1823972 .983827

H 3.4474702 .9465983 .926560

H 3.6036193 .8160742 .384038

H 2.0108243 .7217243 .166909

SCF Energy $\left(B 3 L Y P / 6-31 G^{* *} / / M M F F\right)=-3245.91485318$

0173

MM̄FF Geometry

C -1.923343 -2.892272 -0.050690

C $-1.212116-2.509736-1.120626$

C $-1.584941-1.320065-1.965090$

O $-0.463955-0.401067-2.015009$

C $-1.893391-1.702178-3.421002$

C $-3.151726-2.519077-3.602649$

C $-4.383974-1.990153-3.728912$

C $-2.953710-4.008688-3.713536$

C $-4.774226-0.536534-3.682757$

C $-6.008564-0.302400-2.798557$

C $-5.691656-0.276272-1.293379$

C $-6.987026-0.425598-0.491083$

C $-6.754847-0.2744951 .016985$

O $-5.0726740 .984268-1.008088$

O $-8.037155-0.134627 \quad 1.647349$

C -5.9841831 .0368891 .258350$

C $-6.105692-1.5231051 .624730$

O $-5.644813 \quad 1.1763132 .637481$

C -4.7284931 .1993450 .367176$

C -4.1999252 .6491280 .501874$

$\begin{array}{lllll}\text { O } & -3.734644 & 0.278867 & 0.812345\end{array}$

C $-0.1846880 .315776-0.893273$

$\begin{array}{llll}\text { O } & -0.741289 & 0.235077 & 0.189580\end{array}$

C $0.9302041 .231959-1.215125$

C $1.3841662 .075494-0.276696$

C $2.4917403 .020780-0.428383$

C $3.1759103 .082008-1.769055$

C $-2.9872162 .983420-0.376395$

C $-2.6401344 .463159-0.330259$

C $-0.9752036 .094532-1.184909$

O $-3.346844 \quad 5.301516 \quad 0.223310$

C $0.5193236 .106261-1.228223$

C $1.2761646 .747954-0.324831$

C $2.7756076 .827626-0.378005$

C 3.4352326 .2140860 .837077

C 3.6296026 .9329991 .955640

C 3.9454884 .7955840 .721608

C 2.8354133 .7860560 .628813

N -1.446496 $4.736417-0.987817$

H $-2.833151-2.3516660 .194787$

H $-0.309212-3.052144-1.385934$

H -2.443478 -0.781168 -1.545933

H $-1.036241-2.233413-3.858408$

H -1.959074 -0.786076 -4.023616

H -5.212772 -2.674658 -3.907170

H $-3.893796-4.540763-3.894690$

H -2.280886 -4.239958 -4.546042

H - $-2.521166-4.414966-2.795304$

H -3.952922 $0.109165-3.358150$

H -5.027889 $-0.237770-4.707356$

H $-6.4447330 .663936-3.084519$

H -6.761902 -1.070178 -3.015504

H $-4.995957-1.085888-1.046672$

H -7.468963 -1.387281 -0.708317

H -7.703652 $0.344909-0.809590$

H $-8.561785-0.9267331 .439307$

H -6.6772731 .8599051 .035568$

H $-6.733005-2.4052391 .448467$

H -6.017984 -1.433071 2.712995

H -5.116944 -1.735667 1.213897

H -4.8867570 .5939092 .815578$

H $-5.011614 \quad 3.340148 \quad 0.237873$

H -3.9272752 .8515331 .545627$

$\begin{array}{llll}H & -2.863297 & 0.583267 & 0.515654\end{array}$

H $\quad 1.355587 \quad 1.177466-2.210683$

H 0.9071222 .0698810 .702575

H $2.4512643 .296033-2.562691$

H $3.6690992 .129781-1.990533$

H $3.9380303 .860512-1.837582$

H $-2.1110362 .429753-0.030970$ 
H -3.181379 2.717019-1.421016 H -1.371225 $6.741513-0.394943$ H -1.378866 $6.446780-2.139783$ H $0.9965875 .590754-2.057705$ H $\quad 0.788876 \quad 7.2778720 .491073$ H $3.0538207 .887410-0.450667$ H $3.1688326 .362620-1.290327$ H 4.1277546 .5101162 .821678 H 3.2903547 .9610222 .029946 H 4.5464174 .5340681 .602945 H $4.6445824 .736826-0.117306$ H 2.2730933 .6729011 .556744 H -1.019406 $3.999056-1.541675$ C -1.098804 -3.587669 2.250345 O $0.178484-2.9209452 .183035$ C $0.198590-1.8733483 .173451$ C -1.062283 -2.051794 4.018108 C $-2.026974-2.6394083 .008351$ H $0.125931-0.9260972 .627160$ C $1.498849-1.9098313 .984675$ H $-0.904915-2.7540264 .845006$ H -1.409652 -1.105960 4.445243 C $-3.232948-3.3192613 .630558$ H -2.370577 -1.820997 2.365252 C $2.796750-1.8267773 .155859$ O $1.550086-3.1314014 .735154$ H $1.481446-1.1048694 .728723$ H $2.937932-2.7645572 .606647$ O $3.891848-1.7518164 .079557$ C $2.880128-0.6423982 .174154$ C $4.283582-0.3906461 .587043$ H $2.171718-0.8063831 .355174$ O 2.4660250 .5602472 .828747 H 4.2107990 .4704990 .911852 H $4.956705-0.0565432 .387047$ H -3.886303 -3.7357202 .857358$ H -2.934974 -4.135630 4.296889 H -3.817688 -2.603181 4.217171 C -1.549043 -4.030009 0.853663 H $1.502431-3.8672164 .100764$ H $3.777455-0.9486014 .615035$ H 3.1021620 .7503503 .538658 H -2.417930 -4.6926860 .941799$ H $-0.744919-4.6177360 .392959$ H $-0.928725-4.4940642 .847374$ C $3.831835-1.342735-1.286567$ O $4.015004-2.149900-0.120326$ C $4.902457-1.597850 \quad 0.855670$ C $6.281517-1.321844 \quad 0.234197$ C $6.182183-0.482770-1.038937$ C $5.162797-1.092779-2.002164$ H $3.404608-0.380539-0.983982$ C $2.773653-2.005310-2.172512$ H $5.051958-2.3814591 .607946$ O $7.137635-0.6650681 .162821$ H $6.756820-2.278504-0.018892$ H $5.9265110 .557357-0.806594$ O $7.459987-0.434564-1.678195$ H 5.567852 -2.027252 -2.408099 O $4.950928-0.204273-3.095265$ C $3.195402-3.349502-2.715998$ H $1.850502-2.153013-1.602040$ H $2.512918-1.364363-3.022594$ H $7.198962-1.2286851 .952967$ H $7.724740-1.345594-1.891483$ H $5.817696-0.030448-3.500787$ O $2.952522-4.324322-1.794115$ O $3.690970-3.510545-3.823763$ C $3.324413-5.648850-2.186465$ H $4.401529-5.700093-2.372125$ H $3.077002-6.328286-1.366429$ H $2.763395-5.952461-3.075360$ SCF Energy (B3LYP/6-31G**//MMFF) $=-3245.91588040$

01 74

MM̄FF Geometry

C -1.128202 -2.963760 1.581783

C $-0.695836-3.043903 \quad 0.314929$
C $-1.415210-2.407847-0.842502$

O $-0.514074-1.415614-1.388226$

C $-1.766198-3.410547-1.954083$

C $-2.769613-4.471152-1.558819$

C $-4.088334-4.243209-1.399721$

C $-2.207165-5.855779-1.378019$

C $-4.802573-2.925884-1.535142$

C $-5.485551-2.541945-0.215856$

C $-5.920930-1.071102-0.117160$

C $-6.765561-0.601050-1.302753$

C $-7.1361190 .886375-1.185052$

O $-4.741612-0.264782 \quad 0.001077$

O $-7.623948 \quad 1.322473-2.463420$

C $-5.856611 \quad 1.699381-0.890008$

C $-8.277697 \quad 1.114097-0.187232$

$\begin{array}{lllll}\text { O } & -6.171441 & 3.065489 & -0.628489\end{array}$

C $-5.0074291 .120574 \quad 0.266599$

C $-3.656908 \quad 1.8721810 .361633$

O -5.7162691 .2975631 .496225$

C -1.066355 -0.326512 -1.984653

O $-2.251363-0.105660-2.173395$

C $0.0372830 .590246-2.336303$

C $-0.2045531 .908000-2.377667$

C $0.7776032 .957640-2.645347$

C $2.1599842 .535879-3.069349$

C -2.6419401 .2102971 .302000$

C -1.352635 2.0026641 .395335

C -0.3916914 .0788742 .387796$

$\begin{array}{lllll}\text { O } & -0.302060 & 1.587426 & 0.912551\end{array}$

C $-0.213953 \quad 5.071476 \quad 1.281514$

C 0.9816165 .3657520 .747176

C $1.2005576 .421886-0.299704$

C $1.9937455 .918893-1.485245$

C $3.3375045 .941325-1.473183$

C $1.2358705 .475258-2.714607$

C $0.4041304 .244378-2.486712$

N $-1.499578 \quad 3.1933902 .086291$

H -2.060980 -2.445324 1.788468

H $\quad 0.228690-3.5725560 .099846$

H $-2.316915-1.904090-0.476530$

H $-0.845310-3.883631-2.323702$

H $-2.145101-2.869923-2.831694$

H $-4.729832-5.083173-1.136891$

H $-2.974993-6.583441-1.094451$

H $-1.749706-6.203343-2.310197$

H $-1.443594-5.859780-0.593740$

H $-4.126659-2.130901-1.858375$

H -5.558251 -3.028961 -2.322949

H $-6.363251-3.185422-0.072589$

H $-4.798545-2.7464930 .616541$

H $-6.495081-0.9796690 .812755$

H -7.666499-1.217942 -1.407370

H $-6.202661-0.727357-2.236987$

H $-8.3849410 .764831-2.699800$

H $-5.244897 \quad 1.691452-1.803236$

H $-9.1751590 .567969-0.501965$

H -8.567423 $2.170126-0.152575$

H -8.0342400 .7892090 .826386$

H $-6.6909313 .391990-1.383126$

H -3.197852 $1.928611-0.632548$

H -3.8370582 .9043440 .685912$

H $-5.083881 \quad 1.3152092 .231003$

H $1.0188380 .163659-2.499390$

H $-1.2139702 .256984-2.163511$

H $2.1148661 .915547-3.971287$

H $2.6472331 .963774-2.272776$

H $2.8189483 .376189-3.299053$

H -3.041111 1.0978722 .315650

H -2.3891810 .2047740 .948844$

H $0.509327 \quad 3.4806292 .556375$

H -0.6425734 .6022393 .315738$

H -1.0979365 .6025280 .936870$

H 1.8663574 .8563841 .123369

$\begin{array}{lllll}\text { H } & 1.742377 & 7.252556 & 0.172186\end{array}$

H $0.250276 \quad 6.849547-0.642781$

H $3.9175855 .612204-2.328739$

H $3.8873286 .286946-0.603759$

H $1.9105175 .327207-3.566091$ 
H $0.569564 \quad 6.288012-3.032177$ H -0.612955 $4.457707-2.156229$ H $-2.419153 \quad 3.4604202 .424999$ C $0.166737-2.5272583 .734000$ O $1.253857-1.7981533 .128116$ C $1.220853-0.4432773 .620351$ C $0.180248-0.4124434 .737864$ C $-0.798383-1.470867 \quad 4.270794$ H $\quad 0.8631410 .1780082 .793851$ C 2.6143230 .0210724 .056536 H $0.612457-0.6937425 .705191$ H -0.2758630 .5760914 .849559$ C -1.725123 -1.975449 5.361787 H $-1.399019-1.0356563 .463378$ C $3.702184-0.0429092 .965277$ O $3.058383-0.7916645 .151747$ H $2.550066 \quad 1.0421174 .451061$ H $3.959738-1.0877602 .755930$ $\begin{array}{lllll}\text { O } & 4.884829 & 0.562213 & 3.506278\end{array}$ C $3.347846 \quad 0.675444 \quad 1.651031$ $\begin{array}{llll}\text { C } 4.533675 & 0.871937 & 0.686568\end{array}$ H 2.5489620 .1232481 .143664 O 2.8289991 .9729041 .957179 H $4.1504241 .379705-0.207132$ H 5.2436081 .5864371 .123453 H $-2.402540-2.7421994 .972750$ H -1.164681 -2.411516 6.195582 H $-2.333960-1.1563955 .758366$ C $-0.406578-3.5593532 .756999$ H $3.059511-1.7160364 .848638$ H 5.0996220 .0921594 .330210 H 2.5197762 .3681691 .123938 H -1.108472 -4.2130573 .288137$ H $\quad 0.412622-4.1964272 .400469$ H $0.612164-3.0787934 .573372$ C $3.746578-1.221269-1.388749$ O $4.374364-1.452312-0.125883$ C $5.272207-0.4213320 .290393$ C $6.372909-0.219162-0.762712$ C $5.7958950 .028307-2.155656$ C $4.773721-1.050906-2.512564$ H $3.146972-0.305901-1.317306$ C $2.739033-2.349083-1.639091$ H $5.765987-0.794117 \quad 1.195911$ O $7.2212860 .868675-0.410066$ H $7.002347-1.117695-0.805508$ H $5.354566 \quad 1.028547-2.237033$ O $6.851655-0.021348-3.118290$ H $5.300605-1.990884-2.711040$ O $4.096759-0.682238-3.712146$ C $3.328722-3.741807-1.710400$ H $2.028692-2.362032-0.806386$ H $2.193083-2.156590-2.569148$ H 7.5848990 .6812350 .472208 H $7.5134070 .641178-2.855063$ H $4.773910-0.543253-4.396325$ O $2.360409-4.602011-2.148495$ O $4.470167-4.049599-1.398369$ C $2.752180-5.974688-2.244297$ H $1.893001-6.549014-2.600732$ H $3.571123-6.085446-2.961472$ H $3.044582-6.354568-1.260810$ SCF Energy (B3LYP/6-31G**//MMFF)= -3245.91251443

\section{75}

MM̄FF Geometry

C -3.259965 -3.549921 1.077548

C $-2.166371-2.7765741 .002662$

C -1.412252 -2.278632 2.209275

O $-0.074354-2.8380392 .269723$

C $-1.400490-0.7370792 .279577$

C -0.820686 -0.1933163 .570352$

C 0.3619950 .4432043 .682924

C $-1.697905-0.3566204 .786655$

C $1.381102 \quad 0.7290242 .615104$

C 1.5173592 .2348882 .373486

C 2.6567722 .5713781 .398511

C 2.7608064 .0888621 .226866
C 3.8253414 .4810980 .195398

$\begin{array}{lllll}\text { O } & 2.389967 & 1.947728 & 0.137247\end{array}$

O $3.6317745 .864927-0.134860$

C $3.5862293 .671749-1.093967$

C $5.244103 \quad 4.3642590 .764753$

O $4.634156 \quad 3.901312-2.034207$

C $3.4119022 .152308-0.852804$

C $2.944027 \quad 1.478948-2.168048$

O $4.6601751 .596489-0.443887$

C $0.847199-2.578362 \quad 1.307798$

$\begin{array}{llllllll}\text { O } & 0.689177 & -1.973785 & 0.260449\end{array}$

C $2.135806-3.1238501 .783323$

C $3.269624-2.4962141 .438635$

C $4.626008-2.8813241 .832228$

C $4.788743-4.1907362 .559416$

C $2.719509-0.037573-2.084956$

C $3.932067-0.863976-2.484447$

C $4.629368-3.224062-2.873468$

O $5.010153-0.369242-2.802181$

C $5.472614-3.618610-1.703089$

C $6.747410-3.229646-1.548940$

C $7.618007-3.610837-0.385214$

C $7.930499-2.4275230 .505009$

C $8.936946-1.5874640 .210230$

C $7.117800-2.2587161 .770834$

C $5.650636-2.0676181 .498970$

N $3.660567-2.226248-2.458863$

H $-3.618793-3.8793492 .049517$

H -1.827124 -2.4424780 .026716$

H $-1.911491-2.6615053 .107439$

H $-2.429681-0.3629922 .189100$

H $-0.873877-0.3208181 .416737$

H $0.6533100 .799706 \quad 4.670923$

H -1.2879920 .1502755 .666956$

H -2.6898450 .0678394 .599218$

H $-1.812832-1.4140155 .042241$

H 2.3429710 .3205882 .947878

H 1.1424300 .2309831 .674447

H $\quad 0.5666212 .609514 \quad 1.973710$

H $1.6944002 .749616 \quad 3.326490$

H 3.5901662 .1661811 .807047

H 2.9610204 .5770162 .188993

H 1.7922234 .4832730 .888773

H 3.7055296 .3820430 .685568

H $2.6654804 .054626-1.556662$

H $5.362528 \quad 5.021745 \quad 1.634487$

H $5.991308 \quad 4.6960080 .035393$

H 5.4971123 .3506361 .082244

H $4.6998554 .862692-2.166047$

H $1.9836531 .928272-2.448297$

H $3.6482001 .701432-2.978034$

H $4.6225650 .633284-0.549426$

H $2.108899-3.9929892 .429901$

H $3.200056-1.6020560 .821280$

H $4.331780-5.0072991 .988779$

H $4.313972-4.1428863 .545642$

H $5.829501-4.4788352 .718147$

H $2.391467-0.334823-1.085398$

H $1.922068-0.301946-2.790522$

H $5.232542-2.819550-3.693500$

H $4.078804-4.091695-3.249747$

H $5.012212-4.260721-0.957005$

H $7.199560-2.591675-2.305444$

H $8.550426-4.034357-0.780678$

H $7.164270-4.4155460 .206560$

H $9.182922-0.7493810 .853752$

H $9.535888-1.719511-0.684909$

H $7.461526-1.3793062 .331276$

H $7.318105-3.1108932 .425464$

H $5.426242-1.1418360 .967538$

H $2.761118-2.548728-2.113236$

C $-5.471100-3.366028-0.056749$

O $-5.381752-1.931034-0.136817$

C $-6.518671-1.433337-0.867690$

C $-7.394946-2.643447-1.182167$

C $-6.376084-3.767640-1.217104$

H $-6.132138-1.027114-1.809386$

C $-7.210483-0.326390-0.062900$ 
H -8.137517 -2.825655 -0.396786 H -7.935780 -2.525316 -2.126346 C $-6.991580-5.148688-1.078878$ H -5.822089-3.710018 -2.163693 C $-6.265854 \quad 0.8165970 .368157$ O $-7.755528-0.906751 \quad 1.129770$ H $-8.0612390 .067397-0.631056$ H $-5.539758 \quad 0.4291311 .090886$ O -7.0503751 .7823421 .080449$ C $-5.5385591 .518872-0.792442$ C $-4.7045592 .740102-0.364539$ H $-4.8963300 .805021-1.313723$ O $-6.5208331 .962610-1.733411$ H -4.379845 $3.259808-1.273934$ H -5.3523273 .4602960 .150153$ H $-6.220365-5.924642-1.103663$ H -7.541862 -5.250500 -0.137450 H -7.690343 -5.341921 -1.899364 C $-4.068558-3.969335-0.118982$ H $-8.184399-0.1912721 .629880$ H -7.6770502 .1739290 .447774$ H -6.048280 2.333495 -2.498196 H -3.570231 -3.649886-1.041630 H -4.122556 -5.063269 -0.136101 H -5.947437 -3.606442 0.903576 C $-1.9048271 .942452-1.261623$ O $-2.6041441 .455501-0.109403$ C -3.4850532 .3948980 .514760$ C -2.684039 3.6183670 .976409 C $-1.9208944 .252082-0.180303$ C $-1.0920863 .205007-0.922930$ H -2.621288 2.154227 -2.064346 C $-0.9693670 .819158-1.739067$ H -3.8651491 .9063961 .419254$ O $-3.5395224 .592421 \quad 1.564873$ H -1.9631683 .3142441 .746486$ H $-2.6005354 .780354-0.859193$ O -1.0403655 .2459610 .352404$ H $-0.2229652 .945442-0.307196$ O $-0.6024023 .815551-2.116389$ C $-1.697500-0.395154-2.274943$ H $-0.3334921 .183451-2.551611$ H $-0.3401230 .482674-0.907306$ H -4.0031854 .1649232 .305157$ H $-0.5339045 .612755-0.392650$ H $0.0979053 .248988-2.478636$ O $-0.769986-1.286988-2.736504$ O $-2.909545-0.550830-2.290476$ C -1.290455 -2.492555 -3.304502 H $-2.044557-2.269986-4.065610$ H $-0.463590-3.027783-3.778981$ H -1.709775 -3.123107 -2.516601 SCF Energy (B3LYP/6-31G**//MMFF) $=-3245.91248552$

0176

MM̄FF Geometry

C $2.674664-2.7274572 .065632$

C $2.258121-1.4576082 .188955$

C $0.840513-0.9576022 .055673$

O $-0.029520-2.0321801 .628565$

C $0.286498-0.388376 \quad 3.375477$

C $0.882540 \quad 0.942702 \quad 3.784105$

C 0.5852672 .1116753 .181796

C $1.853198 \quad 0.8986484 .933019$

C -0.3549112 .2829592 .022256$

C -0.9464013 .6965491 .941046$

C -1.8615683 .8336730 .716095$

C -2.3249665 .2775710 .529288$

C $-3.3044145 .408013-0.647751$

O -2.9938812 .9818990 .916372$

O $-3.9268006 .699010-0.562921$

C $-4.4217194 .354982-0.488197$

C $-2.5833075 .361617-2.000445$

O $-5.2724154 .336064-1.632626$

C $-3.8966132 .929193-0.195158$

C -5.0772722 .0056990 .190300$

O $-3.2612552 .411756-1.365527$

C $-1.035136-1.7220900 .765646$
O $-1.305803-0.6216270 .312502$

C - $-1.758486-2.9762410 .456211$

C $-2.785097-2.955048-0.407220$

C $-3.599472-4.102508-0.815444$

C $-3.248017-5.446143-0.231004$

C -4.6548560 .6334750 .734658$

C $-5.852156-0.202271 \quad 1.154870$

C $-6.425442-2.3000382 .372357$

O $\quad-7.009797 \quad 0.0969710 .873724$

C $-6.382749-3.5200011 .509285$

C $-7.294517-3.7931190 .564066$

C -7.278147 -5.027765 -0.291320

C $-6.995952-4.720894-1.745334$

C -7.985619-4.365784 -2.581986

C $-5.579693-4.908680-2.243445$

C $-4.620935-3.896791-1.674283$

N $-5.470861-1.3315051 .867173$

H $1.958806-3.5255791 .890712$

H $3.008249-0.6856732 .346916$

H $\quad 0.859753-0.187347 \quad 1.276076$

H $0.424300-1.1245654 .179602$

H $-0.804438-0.2857443 .305264$

H 1.0861193 .0139423 .527738

H 2.2539811 .8882975 .176358

H 1.3593440 .5116575 .830321

H 2.7014260 .2492614 .695745

H 0.1998782 .0828151 .099308

H -1.1727231 .5582722 .081458$

H -1.515219 3.9000752 .857424

H $-0.1357874 .433076 \quad 1.880846$

H -1.307776 $3.497038-0.167764$

H -1.4675195 .9478950 .391157$

H -2.8307845 .6204631 .442729$

H -3.225182 $7.372022-0.590067$

H -5.0482074 .6716720 .357652$

H -1.847346 $6.171749-2.069710$

H $-3.2839845 .522710-2.827017$

H $-2.0556484 .421453-2.174029$

H $-5.5907815 .244482-1.771805$

H -5.6784402 .4966200 .967100$

H $-5.7292601 .856443-0.679712$

H -3.256843 $1.442944-1.321991$

H -1.424279 -3.8832200 .947094$

H -3.060773 -2.005421 -0.863197

H $-3.286547-5.4124690 .863601$

H $-2.239803-5.745446-0.538024$

H -3.924212 -6.247413 -0.534482

H $-4.1121410 .067724-0.028091$

H $-4.000430 \quad 0.7575201 .604908$

H -7.427154 -1.859026 2.405634

H $-6.132984-2.5559473 .395436$

H -5.569294 -4.218434 1.687094

H $-8.109861-3.0924640 .397257$

H -8.256587 -5.517417 -0.199120

H $-6.553306-5.7617590 .082157$

H -7.797069 -4.170928 -3.632456

H -9.007132 -4.258691 -2.232009

H $-5.544404-4.806258-3.336389$

H -5.272034 -5.939955 -2.050204

H -4.799965 -2.882366 -2.033687

H $-4.482419-1.5198662 .008462$

C $4.584341-3.5917420 .720554$

O $4.445539-2.511662-0.220280$

C $5.501520-2.599281-1.195678$

C $6.305621-3.850464-0.849713$

C $6.055189-3.9858570 .642268$

H $6.137627-1.719456-1.046437$

C $4.897051-2.575437-2.607319$

H $5.935103-4.734983-1.380129$

H $7.365798-3.739013-1.097331$

C $6.358675-5.3701461 .186572$

H $6.671558-3.2452481 .169383$

C $3.910974-1.410655-2.844840$

O $4.172346-3.793357-2.829278$

H $5.695863-2.559280-3.356971$

H $3.030075-1.544484-2.207653$

O $3.443673-1.487939-4.197299$

C $4.517723-0.014125-2.626623$ 
C $3.5292051 .144202-2.860203$

H $4.9174250 .065243-1.613724$

O $5.6260180 .160751-3.512867$

H $4.1048292 .077112-2.893176$

H $3.0833491 .053958-3.858221$

H $6.157758-5.4199932 .261215$

H $5.751094-6.1369220 .694268$

H $7.412049-5.6230581 .028374$

C $4.125646-3.1257992 .101713$

H $3.485821-3.856322-2.143083$

H $3.074531-2.378587-4.325482$

H $5.2921590 .064929-4.421438$

H $4.737396-2.2783172 .430824$

H $4.253377-3.9254832 .839338$

H $3.945448-4.4122630 .366629$

C $3.6553982 .487530-0.129193$

O $2.9585401 .279450-0.459769$

C $2.4231101 .236401-1.787636$

C $1.4371792 .395198-1.990992$

C $2.0622543 .745115-1.649082$

C $2.7280693 .701377-0.274135$

H $4.5241702 .605672-0.787169$

C $4.1560202 .350597 \quad 1.317101$

H $1.8374140 .311798-1.850437$

O $0.9579212 .429016-3.331175$

H $0.5642372 .244221-1.343840$

H $2.7738344 .061738-2.420625$

O $1.0372394 .741587-1.617429$

H 1.9511653 .6755230 .497934

O $3.4658814 .905925-0.072288$

C 5.3314001 .4031761 .437872

H 4.4891683 .3160841 .712461

H 3.3455601 .9658551 .945441

H $\quad 0.5465661 .567354-3.515787$

H $0.5933224 .727515-2.482810$

H $4.1638414 .945584-0.748332$

O $5.441534 \quad 0.992613 \quad 2.735944$

O $6.084361 \quad 1.0947070 .524708$

C 6.5439590 .1248293 .019694

H $6.391951-0.3030374 .014134$

H $6.599597-0.6919242 .293683$

H 7.4736280 .7007423 .020557

SCF Energy (B3LYP/6-31G**//MMFF) $=-3245.92147806$

01 77

MM̄FF Geometry

C $2.224081-3.662850-0.101376$

C $1.448634-2.571628-0.013013$

C $0.482306-2.145134-1.087470$

O $-0.821497-2.123458-0.462766$

C $0.840091-0.736445-1.591407$

C $0.328841-0.435501-2.986402$

C $-0.6693460 .423770-3.268215$

C $1.076794-1.111583-4.107670$

C $-1.5193761 .185261-2.293084$

C $-0.9202372 .558855-1.971462$

C -1.880636 $3.440798-1.159797$

C $-1.1825654 .721519-0.696575$

C -2.076154 $5.575880 \quad 0.215784$

O $-2.3322452 .696520-0.026471$

O $-1.240716 \quad 6.541636 \quad 0.873073$

C -2.6780774 .6769691 .319092$

C $-3.108480 \quad 6.373984-0.589223$

O $-3.633646 \quad 5.3970432 .094518$

C -3.3099943 .3767020 .771740$

C -3.7049992 .4361501 .937786$

O $-4.473168 \quad 3.712098 \quad 0.016939$

C -1.880323 -2.594523 -1.172409

O $-1.896320-2.920273-2.348007$

C $-3.020126-2.708673-0.232278$

C $-4.109469-3.401604-0.597065$

C $-5.277438-3.691036 \quad 0.240331$

C $-5.291301-3.1317831 .638788$

C $-4.246457 \quad 1.066051 \quad 1.498533$

C -5.7070791 .1088941 .084039$

C $-7.3065920 .142426-0.563255$

O -6.5330991 .8177701 .654461$

C $-8.032409-0.9929010 .085161$
C $-8.570962-2.013827-0.598231$

C $-9.327734-3.1385380 .046225$

C $-8.770891-4.497989-0.309801$

C $-9.415658-5.307065-1.167396$

C $-7.514223-4.9546900 .397426$

C $-6.260593-4.461164-0.272583$

N $-5.984806 \quad 0.2612370 .022434$

H $2.162546-4.292793-0.985338$

H $1.508347-1.9460340 .874279$

H $\quad 0.500306-2.878871-1.902097$

H $1.931261-0.617652-1.623938$

H $0.4807280 .012389-0.875880$

H $-0.9211620 .598072-4.313368$

H $0.724918-0.791537-5.094298$

H $2.144417-0.875090-4.048549$

H $0.957410-2.197641-4.054370$

H -2.516764 $1.306576-2.733685$

H $-1.6658480 .604594-1.375516$

H $0.0039012 .402997-1.402070$

H -0.642582 $3.083087-2.894585$

H $-2.7397993 .679641-1.797845$

H $-0.8316565 .307169-1.555228$

H $-0.2823104 .455407-0.128040$

H -0.7914117 .0652570 .187583$

H -1.863391 4.4039072 .004470

H $-2.6059777 .055008-1.286995$

H -3.7145287 .0105710 .064843$

H $-3.7784475 .741268-1.174936$

H -3.1930326 .1983242 .425817$

H -2.809211 2.2436142 .542483

H -4.4389292 .9297702 .585522$

H -4.886180 $2.889684-0.287999$

H -2.907109 -2.268882 0.752362

H -4.138219 -3.830784 -1.598059

H $-5.139601-2.0480231 .626036$

H $-4.496795-3.5871542 .240051$

H -6.232347 -3.2982102 .166561$

H -3.6257110 .6416280 .702704$

H $-4.192807 \quad 0.3749772 .348796$

H -7.173011 -0.010640 -1.638836

H -7.861385 $1.073425-0.408934$

H -8.137132 -0.9627301 .167287$

H -8.487402 -2.031679-1.682570

H -10.373984 -3.062236 -0.277353

H $-9.347541-3.0333171 .138289$

H $-9.036373-6.295693-1.404458$

H -10.333669 -4.997356 -1.655602

H -7.468856 -6.052353 0.396953

H -7.575782 -4.6805701 .453908$

H -6.147894 -4.826580 -1.294584

H $-5.255989-0.349198-0.334831$

C $4.631609-4.078970 \quad 0.437749$

O $5.029258-2.7268350 .144890$

C $6.418794-2.5618700 .491152$

C $6.926651-3.9405870 .902225$

C $5.663687-4.5860491 .441767$

H $6.445330-1.9059291 .368918$

C $7.174171-1.907260-0.673274$

H $7.308117-4.5086900 .046095$

H $7.728981-3.8800851 .644059$

C $5.749410-6.0991541 .536659$

H $5.454916-4.1716122 .437216$

C $6.512614-0.610299-1.185747$

O $7.208131-2.832656-1.767930$

H $8.216643-1.727132-0.386496$

H $5.543343-0.861835-1.628274$

O $7.311129-0.098461-2.259937$

C $6.3486400 .485834-0.118269$

C $5.6824411 .771012-0.643719$

H 5.7681490 .1016710 .723106

O 7.6437220 .8202690 .386381

H 5.7763812 .5437430 .128676

H $6.2505862 .157002-1.498817$

H $4.816724-6.5201841 .924655$

H $5.942342-6.5523920 .558489$

H $6.559290-6.3967052 .210725$

C $3.197055-4.0875210 .963701$

H $7.681674-2.399309-2.498627$ 
H $8.1675840 .169905-1.884957$ H 7.5208901 .4604341 .108086 H $3.115979-3.4213041 .829681$ H $2.921105-5.0940131 .297114$ H $4.698571-4.645168-0.501090$ C $3.218077 \quad 1.860807 \quad 1.166745$

O 3.4406021 .0009330 .042334 C $4.2004391 .579157-1.025141$ C $3.5064582 .846251-1.541505$ C $3.2140693 .833172-0.416150$ C 2.4858203 .1356820 .731336 H 4.1768892 .1244341 .628562 C 2.3800181 .0737552 .187734 H $4.1725230 .854423-1.846845$ O $4.295096 \quad 3.490885-2.536058$ H $2.5551432 .570343-2.014833$ H $4.1294614 .320272-0.060250$ O $2.3734314 .876399-0.913762$ H 1.4627782 .8903540 .424067 O 2.3959834 .0300061 .838528 C 3.1916410 .0338542 .932782 H 1.9614681 .7440302 .946462 H 1.5617230 .5589111 .670394 H $4.4540042 .845476-3.246090$ H $2.8326125 .281854-1.669216$ H 1.9376894 .8298971 .528228 O $2.339038-0.7887813 .613308$ O $4.412719-0.0298162 .952796$ C $2.958714-1.7918184 .425518$ H $3.748038-2.3148253 .877352$ H $3.363583-1.3305405 .330771$ H $2.193193-2.5181974 .710889$ SCF Energy (B3LYP/6-31G**//MMFF)= -3245.92194864

01_78

MM̄FF Geometry

C -1.356556 -3.163468 0.815040

C $-0.332517-3.4399221 .636506$

C $0.184919-2.5853222 .771168$

O $1.468339-2.0434152 .378199$

C $-0.701480-1.3850533 .155508$

C $-0.234330-0.6918134 .420319$

C 0.4267800 .4817834 .449024

C $-0.608026-1.3796625 .708290$

C $0.894736 \quad 1.315242 \quad 3.287050$

C -0.0851112 .4566952 .998798$

C 0.5373293 .5814502 .156811

C -0.5084344 .6673501 .890622$

C 0.0455335 .7916081 .007868

O $1.012313 \quad 3.043598 \quad 0.920109$

O $-\begin{array}{llll}1.060561 & 6.581464 & 0.545044\end{array}$

C $0.705534 \quad 5.167337-0.239095$

C 0.9452006 .7421651 .807031

O $1.3775376 .164536-1.006126$

C $1.675896 \quad 4.0028120 .078177$

C $2.071413 \quad 3.277293-1.226687$

O $2.884814 \quad 4.4810190 .672092$

C $2.580380-2.8051452 .541768$

O $2.646221-3.9445312 .971185$

C $3.720876-1.9913942 .069495$

C $4.865391-2.5851991 .702131$

C $6.038537-1.8998171 .154754$

C $6.040890-0.3934171 .200614$

C $3.1565802 .212145-1.029504$

C $3.4858351 .488181-2.325298$

C $5.2322500 .015252-3.329308$

O $2.7630381 .526998-3.317287$

C $5.746693-1.289833-2.811600$

C $7.031374-1.672430-2.874131$

C $7.518798-3.006472-2.382441$

C $8.596104-2.898764-1.322422$

C $9.799193-3.466954-1.512352$

C $8.294558-2.148951-0.037677$

C $7.045837-2.6404150 .646275$

N $4.6642040 .762118-2.222613$

H -1.893529 $-2.224600 \quad 0.914035$

H $0.195011-4.3816731 .494776$

H $0.312456-3.2333973 .647285$
H -1.737838 -1.712397 3.311048

H $-0.732224-0.6648932 .330158$

H $0.6802710 .901472 \quad 5.422077$

H $-0.303770-0.8054656 .589997$

H -1.692855 -1.516680 5.764665

H $-0.128853-2.3610875 .775160$

H 1.8806101 .7206543 .546515

H 1.0463950 .7099392 .387498

H -0.9533542 .0380312 .472569$

H -0.4538032 .8850153 .939837$

H 1.3860103 .9940862 .714794

H $-0.895758 \quad 5.075607 \quad 2.832627$

H $-1.373246 \quad 4.221396 \quad 1.381224$

H - 1.5445026 .9063321 .323640

H $-0.0975724 .776018-0.877448$

H $0.373738 \quad 7.2266132 .608099$

H 1.3229097 .5545251 .176707

H 1.7952386 .2384672 .271993

H $0.7284626 .860253-1.208175$

H $1.1821342 .806462-1.664622$

H $2.4489874 .002369-1.958800$

H 2.8882965 .4509140 .635276

H $3.566142-0.9206181 .992994$

H $4.936535-3.6698821 .767880$

H $5.903611-0.0440372 .230644$

H 5.2313030 .0125730 .587490

H $6.9713910 .056702 \quad 0.849683$

H $4.0732182 .680270-0.652585$

H $2.829947 \quad 1.464929-0.298598$

H $6.0258550 .631379-3.764241$

H $4.472374-0.159020-4.097377$

H $5.012898-1.966220-2.378755$

H $7.767087-1.009009-3.322259$

H $7.894621-3.558679-3.253642$

H $6.689496-3.607028-1.988463$

H $10.581048-3.401800-0.762897$

H $10.031913-4.010252-2.422238$

H $8.240921-1.085253-0.284607$

H $9.124377-2.2605580 .671556$

H $6.968267-3.7270760 .702968$

H $5.2565480 .914110-1.412144$

C $-3.301732-4.430323-0.113059$

O $-4.093722-3.244297-0.311564$

C $-5.255347-3.575967-1.094736$

C $-5.304234-5.097523-1.148772$

C $-3.825133-5.442054-1.131153$

H $-5.067107-3.199043-2.107063$

C -6.492277-2.883509-0.509295

H $-5.802238-5.522359-0.269726$

H $-5.821450-5.465066-2.040095$

C $-3.543258-6.889156-0.767024$

H $-3.402278-5.230496-2.122465$

C $-6.281627-1.375335-0.250784$

O $-6.803619-3.4951180 .748903$

H -7.357851 -3.053460 -1.159769

H $-5.541723-1.2573090 .547601$

$\begin{array}{lllll}\text { O } & -7.503057 & -0.835060 & 0.267437\end{array}$

C $-5.863706-0.568942-1.492578$

C $-5.6130200 .923631-1.208001$

H - $-4.962231-1.000552-1.936689$

O $-6.900350-0.678648-2.470888$

H $-5.4724851 .431492-2.170038$

H $-6.5163881 .378017-0.782198$

H $-2.466693-7.086029-0.753689$

H $-3.943910-7.139808 \quad 0.220850$

H $-4.000954-7.565213-1.496591$

C $-1.822645-4.080571-0.284558$

H $-7.581557-3.0358851 .109274$

H $-8.170003-0.891589-0.438348$

H $-6.599648-0.214457-3.270802$

H $-1.669729-3.560809-1.238316$

H $-1.219343-4.995129-0.304255$

H -3.496560 -4.7785700 .909971$

C $-2.6414730 .914256-1.911106$

O $-3.242648 \quad 0.447061-0.699581$

C $-4.4000951 .185179-0.288501$

C $-4.0481902 .670213-0.112275$

C $-3.3655153 .251183-1.350068$ 
C $-2.1880542 .371028-1.774113$ H $-3.3820140 .854966-2.719208$ C - $1.530133-0.060577-2.322583$ H -4.6674950 .8046270 .704359$ O H $-3.3651352 .779546 \quad 0.740032$ H $-4.0744763 .379223-2.176417$ O $-2.8690044 .555104-1.047425$ H -1.384174 2.467123 -1.036101 O $-1.6803532 .828974-3.023917$ C $-0.404064-0.230934-1.324256$ H -1.977008 -1.054546 -2.453168 H $-1.095970 \quad 0.255782-3.277674$ H -5.6144703 .0589210 .982854$ H $-3.6208315 .085967-0.732954$ H $-1.4310083 .762354-2.910889$ O $0.515175-1.086530-1.862779$ O $-0.347377 \quad 0.288043-0.219609$ C $1.653798-1.429791-1.064715$ H $2.533565-1.428585-1.712285$ H $1.822036-0.730201-0.242363$ H $1.514627-2.439852-0.672333$

SCF Energy (B3LYP/6-31G**//MMFF) $=-3245.91959777$

0179

MM̄FF Geometry

C -3.900055 -3.576593 -2.144175

C $-3.167347-3.253483-1.066906$

C -1.756591-3.739259-0.844055

O $-0.896987-2.584755-0.692793$

C $-1.677823-4.5591070 .456364$

C $-0.303493-5.1440010 .723409$

C $0.470090-4.8340141 .782547$

C $0.181159-6.161311-0.278417$

C $0.165396-3.8905002 .911595$

C $0.662153-2.4519032 .705052$

C $2.187559-2.3158182 .577103$

C $2.598729-0.8425472 .613304$

C $4.110720-0.6633422 .404682$

O $2.593815-2.8881521 .332686$

O $4.353685 \quad 0.7258292 .130837$

C $4.552079-1.4552961 .153297$

C $4.901535-0.9978293 .675374$

O $5.973886-1.4370301 .048239$

C $4.012805-2.9024571 .118732$

C $4.254545-3.622779-0.231120$

O $4.676059-3.6718142 .129368$

C $-0.560983-1.903065-1.821105$

O $-0.888106-2.164194-2.967694$

C $0.269737-0.751530-1.412235$

C $0.4284450 .279382-2.254877$

C $1.2213451 .482033-1.997040$

C $2.1208751 .467937-0.790652$

C $3.629139-2.948908-1.457844$

C $4.579962-2.041115-2.220698$

C $4.618100-0.451486-4.142067$

O $5.783266-1.973956-1.985564$

C $4.5802390 .946932-3.611964$

C $4.1338771 .993780-4.322996$

C $4.1416793 .410158-3.823482$

C $2.7851454 .072554-3.916769$

C $2.4838904 .892096-4.938235$

C $1.8144273 .855343-2.778984$

C $1.1260412 .518207-2.855562$

N $3.926969-1.339276-3.225718$

H $-3.463950-4.254123-2.876318$

H -3.564476 -2.579300 -0.313517

H -1.408221 -4.340206 -1.692732

H -2.398379 $-5.386847 \quad 0.418030$

H -1.991078 -3.921553 1.290209

H $1.440016-5.3238631 .865949$

H $1.096146-6.6654250 .051079$

H $-0.578205-6.934831-0.432815$

H $\quad 0.398315-5.685313-1.239315$

H $-0.910309-3.8680843 .116629$

H $0.618500-4.2986723 .824184$

H $\quad 0.176491-2.0322531 .816042$

H $0.327534-1.8628823 .567256$
H $2.653877-2.8642003 .403831$

H $2.283386-0.3718523 .553024$

H $2.071598-0.2993091 .817648$

H 4.0026441 .2441592 .875316

H $4.167218-0.9047810 .287886$

H $4.580025-0.3576684 .505782$

H $5.970094-0.7968093 .540796$

H $4.782058-2.0355363 .993390$

H $6.214252-1.8089710 .183321$

H $5.324458-3.815389-0.374299$

H $3.792696-4.617798-0.151039$

H $4.600547-4.6129381 .900044$

H $0.716370-0.779060-0.425126$

H $-0.0811010 .250789-3.217115$

H $2.7734710 .589683-0.812984$

H $1.526412 \quad 1.439073 \quad 0.127647$

H $2.7824772 .333816-0.724725$

H $3.325681-3.732258-2.164400$

H $2.729903-2.389627-1.184424$

H $4.123054-0.539415-5.114357$

H $5.657982-0.776525-4.249696$

H $4.9668141 .110325-2.608601$

H $3.7747981 .831432-5.336906$

H $4.8708413 .969664-4.424216$

H $4.5053273 .468937-2.789967$

H $1.5238995 .394167-4.996114$

H $3.1891215 .074379-5.742380$

H $1.0304634 .624537-2.800027$

H $2.3337504 .017681-1.830693$

H $\quad 0.4514972 .424077-3.707732$

H $2.917652-1.416366-3.311684$

C $-5.861945-1.977275-1.609632$

O $-5.045607-0.795293-1.697996$

C $-5.8830150 .365045-1.547010$

C $-7.308690-0.151825-1.361352$

C $-7.251911-1.511323-2.031822$

H $-5.8173940 .926155-2.486893$

C $-5.3847001 .242875-0.388063$

H $-7.565653-0.257271-0.300615$

H -8.048922 $0.516658-1.812037$

C $-8.377610-2.439963-1.612337$

H -7.286084 -1.366567 -3.119903

C $-3.9708821 .829673-0.610969$

$\begin{array}{lllll}\text { O } & -5.416834 & 0.479921 & 0.818608\end{array}$

H $-6.0886622 .073590-0.258985$

H $-3.9578712 .368119-1.566415$

O $-2.9984090 .787823-0.693943$

C -3.5422342 .8039730 .507207$

C -2.1807223 .4703250 .217316$

H -4.3029773 .5872630 .604068$

O -3.4871782 .1181361 .752695$

H -1.411697 2.6975870 .119209

H $-2.2504503 .978349-0.751439$

H -8.297270 -3.406493 -2.119365

H -8.365108 -2.623767 -0.532642

H $-9.349695-2.005618-1.868113$

C $-5.277380-3.075831-2.499956$

H $-4.762974-0.2344540 .727607$

H -3.234090 $0.221827-1.448318$

H $-2.855126 \quad 1.3859461 .652853$

H $-5.216063-2.697379-3.528634$

H -5.952415 -3.939184 -2.512802

H $-5.872689-2.298331-0.559570$

C -0.3998873 .0418972 .684573$

O

C $-1.7678944 .486127 \quad 1.311352$

C $-0.553198 \quad 5.3562450 .954536$

C $0.7537104 .573074 \quad 1.046910$

C 0.8603853 .8635862 .396561

H -0.4735722 .2138001 .970707$

C -0.3609412 .4484184 .098473$

H -2.619749 5.1676961 .433708

O $-0.7123395 .903922-0.349499$

H $-0.5073806 .200667 \quad 1.654862$

H $\quad 0.842752 \quad 3.858810 \quad 0.223323$

O 1.8592365 .4699360 .930761

H 1.0180634 .6069393 .188578

O 1.9944083 .0000452 .389184 
C -1.5066611 .4939774 .346682$ H 0.5708091 .9046524 .290667 H -0.4355563 .2480554 .845749$ H $0.0079436 .540281-0.493150$ H 1.8569715 .8229880 .025149 H 2.7697673 .5401492 .158842 $\begin{array}{lllll}\text { O } & -1.400919 & 0.404841 & 3.533213\end{array}$ O -2.3799831 .6865745 .183112$ C $-2.430270-0.5808673 .673660$ H $-3.422408-0.1194653 .657415$ H $-2.356227-1.2712712 .829039$ H -2.280082 -1.139246 4.602012

SCF Energy (B3LYP/6-31G**//MMFF) $=-3245.91226160$

\section{8}

MM̄FF Geometry

C $0.245797-0.2204833 .515232$

C $-0.692791 \quad 0.6036824 .003723$

C -1.185034 1.8165183 .264780

O $-2.562612 \quad 1.5401262 .916724$

C -1.1384883 .0993504 .111540$

C 0.2531333 .5767284 .465908

C 1.0755654 .2130143 .608719

C 0.6778213 .3353955 .889855

C 0.8140684 .5137752 .157966

C 1.9004003 .8862811 .275539

C $1.5304953 .755736-0.209114$

C $1.0502185 .059410-0.846358$

C $0.6600154 .855340-2.320182$

O $0.5178192 .748630-0.322479$

O $-0.0731996 .013969-2.746880$

C $-0.3010073 .650168-2.422183$

C $1.8899734 .762182-3.231138$

O $-0.5749233 .330954-3.784735$

C $0.1983302 .391591-1.674179$

C $-0.9127161 .313077-1.650743$

O $1.334421 \quad 1.859776-2.360742$

C $-3.0407292 .097514 \quad 1.772981$

O -2.4725542 .8939561 .043605$

C -4.3792091 .5218991 .525936$

C $-4.816729 \quad 1.4340380 .261472$

C $-6.0837290 .852267-0.178639$

C -7.0854450 .4693210 .878410$

C $-0.6282940 .150283-0.692449$

C $-1.726599-0.895087-0.702123$

C $-2.750325-2.658345-2.140674$

O $-2.424729-1.106433 \quad 0.287012$

C $-3.958818-2.129305-2.848254$

C $-5.214569-2.433683-2.485754$

C $-6.438238-1.982603-3.233667$

C $-7.487359-1.373039-2.330653$

C -8.420622 -2.143064 -1.745489

C $-7.5140050 .129914-2.176229$

C $-6.2897780 .681388-1.501222$

N -1.813884 -1.576929 -1.903918

H $0.695887-0.0059662 .548991$

H -1.154087 0.3816354 .962953

H $-0.601594 \quad 1.9442762 .345460$

H -1.730509 2.9578695 .026699

H -1.670276 3.9042553 .586780

H 2.0467174 .5439703 .974354

H 1.6874233 .7085816 .092093

H -0.0057743 .8405776 .579996$

H $0.6719072 .264666 \quad 6.115682$

H $-0.1735054 .166400 \quad 1.846631$

H 0.8179605 .6021842 .025892

H 2.8191414 .4793981 .367268

H 2.1286452 .8803961 .652557

H $2.4310413 .402244-0.723739$

H $1.8120535 .842958-0.752282$

H $0.1643475 .433010-0.316019$

H $0.495114 \quad 6.792511-2.617095$

H -1.258145 $3.957239-1.977398$

H $2.4889535 .678093-3.159858$

H $1.5964704 .678388-4.283361$

H $2.5435663 .922148-2.987408$

H -0.899285 4.142104 -4.212257

H -1.860102 $1.769654-1.342176$
H $-1.0640100 .926205-2.665618$

H $1.4394720 .926066-2.120613$

H -4.9360551 .1447462 .375563$

H $-4.1732121 .800972-0.536891$

H $-7.341631 \quad 1.3359421 .498003$

H $-6.680998-0.3177351 .524401$

H -8.0233620 .0880090 .468228$

H $\quad 0.311339-0.351043-0.937347$

H -0.5327430 .5176130 .334346$

H -3.004044 -3.138597-1.190110

H $-2.241826-3.388625-2.777722$

H -3.793759 -1.504161 -3.722548

H $-5.372518-3.089446-1.632201$

H $-6.861170-2.860167-3.740670$

H $-6.182470-1.276530-4.033461$

H $-9.197989-1.715873-1.120816$

H $-8.425303-3.220230-1.876384$

H $-8.4174030 .460473-1.650001$

H $-7.5948100 .585290-3.172074$

H $-5.5003660 .970378-2.195847$

H $-1.189772-1.324115-2.664189$

C $0.207126-2.7203583 .526490$

O $0.790562-2.8409122 .213423$

C $1.113885-4.2237911 .974680$

C $0.506423-5.0128583 .129796$

C $0.583019-4.0066134 .259630$

H $2.205523-4.3052642 .030565$

C $0.621744-4.6597590 .590713$

H $-0.537096-5.2849052 .932485$

H $1.058516-5.9348533 .336220$

C $-0.322215-4.3429865 .432005$

H $1.622469-3.9498524 .610064$

C $1.176597-3.830586-0.584901$

O $-0.808647-4.5591720 .546660$

H $0.851141-5.7205810 .436803$

H $0.744541-2.824647-0.563916$

O $0.716968-4.441941-1.797535$

C $2.711396-3.731479-0.640487$

C $3.269259-3.153889-1.954322$

H $3.066170-3.1304040 .201809$

O $3.262935-5.042011-0.478135$

H $4.363170-3.144791-1.873911$

H $3.057460-3.855346-2.771393$

H $-0.238837-3.588513 \quad 6.220237$

H -1.372463 -4.395746 5.125966

H $-0.048716-5.3116315 .862970$

C $0.722234-1.4583294 .218757$

H $-1.044259-3.6363470 .745195$

H -0.251915 -4.505019-1.740640

H $4.230567-4.951482-0.445674$

H $1.818566-1.4463934 .216083$

H $\quad 0.400215-1.4448595 .266192$

H $-0.880782-2.6619883 .387524$

C $4.319629-0.423868-1.040605$

O $2.956383-0.797701-1.269591$

C $2.750207-1.749493-2.317814$

C $3.304150-1.204939-3.641528$

C $4.768323-0.801519-3.512556$

C $4.9577600 .134576-2.322253$

H $4.883872-1.292485-0.680208$

C 4.3302670 .6524810 .057382

H $1.664912-1.832943-2.447529$

O $3.174113-2.165021-4.684586$

H $2.725909-0.322272-3.943987$

H $5.424192-1.676346-3.434016$

O $5.169981-0.105542-4.695186$

H $4.5230821 .114494-2.560244$

O $6.3537750 .330901-2.102353$

C 3.9960720 .1190451 .433769

H $\quad \begin{array}{llll}5.326176 & 1.105867 & 0.120959\end{array}$

H $3.5949841 .429495-0.173527$

H $2.232377-2.398333-4.751335$

H $5.004978-0.697980-5.448802$

H $6.7333410 .668920-2.931701$

O $4.311501 \quad 1.0761212 .356865$

O $3.504356-0.9744181 .670584$

C $4.0613380 .751803 \quad 3.728517$

H $3.0157320 .963573 \quad 3.964391$ 
H $\quad 4.303739-0.2923773 .948066$ H 4.6958381 .3912754 .348025

SCF Energy (B3LYP/6-31G**//MMFF)= -3245.91981092

01 80

MM̄FF Geometry

C $2.400138-2.364288-2.534840$

C $2.171842-3.638204-2.182172$

C $1.397211-4.025798-0.952157$

O $0.043294-4.298137-1.385742$

C $1.959783-5.284560-0.266133$

C $3.193418-5.0369090 .577871$

C $3.163893-4.8321941 .910111$

C $4.512214-5.105346-0.145888$

C $1.941974-4.7250252 .780079$

C $2.052366-3.6440393 .865844$

C $2.322918-2.2261843 .338068$

C $2.388421-1.2338434 .503135$

C $2.586821 \quad 0.2107284 .023471$

O $1.275901-1.8535332 .433744$

O $2.3129791 .058456 \quad 5.150114$

C $1.543500 \quad 0.5156982 .927348$

C 4.0412360 .4791713 .619345

O $1.782113 \quad 1.7871772 .331978$

C $1.472104-0.5667811 .826984$

C $0.321740-0.3153490 .824280$

O $2.689597-0.5397401 .070173$

C $-0.950994-4.030261-0.496129$

$\begin{array}{llll}\text { O } & -0.822647 & -3.638318 & 0.652685\end{array}$

C $-2.246288-4.279345-1.164281$

C $-3.375689-3.942943-0.523467$

C $-4.741704-4.105458-1.019659$

C $-4.928569-4.711836-2.384912$

C -1.086998 -0.2804661 .422011$

C $-2.145073-0.063174 \quad 0.350175$

C -4.4546910 .8411790 .078449$

O $-1.936371-0.256296-0.844759$

C -5.6742670 .0156370 .339668$

C $-6.469081-0.442492-0.640071$

C $-7.733386-1.221417-0.406792$

C $-7.772031-2.517227-1.186891$

C $-8.333004-2.569447-2.406993$

C $-7.234620-3.765786-0.526119$

C $-5.761305-3.691842-0.238842$

N $-3.351300 \quad 0.3602640 .890273$

H $1.974094-1.563627-1.936221$

H $2.565437-4.439729-2.802544$

H $1.394319-3.188611-0.244045$

H $2.175489-6.061067-1.013187$

H $1.176334-5.7433820 .350289$

H $4.112265-4.7261382 .434974$

H $5.365010-5.0385740 .538350$

H $4.601959-6.052817-0.687230$

H $4.604182-4.283657-0.861202$

H $1.044435-4.5312362 .188481$

H $1.791987-5.691538 \quad 3.276173$

H $1.107406-3.6470974 .424795$

H $2.842570-3.9241224 .574263$

H $3.277861-2.2321062 .800845$

H $3.179645-1.5140775 .209888$

H $1.449193-1.2825725 .072290$

H 2.4842871 .9780204 .885842

H 0.5713560 .5800913 .429788

H 4.7072640 .3354334 .478703

H 4.1796621 .5192103 .303915

H $4.391969-0.1722612 .816309$

H 1.7421202 .4548703 .036534

H $\quad 0.5115830 .6157440 .275161$

H $0.348437-1.1029430 .060847$

H $2.530924-0.9771220 .218132$

H -2.235387 -4.718197 -2.154977

H -3.296697 -3.4975860 .467927$

H -4.536382 -5.734463 -2.408244

H -4.408042 -4.117862 -3.144533

H -5.974641 -4.764005 -2.695667

H $-1.314269-1.2196581 .936864$

H -1.165734 0.5370682 .144546

H $-4.1738090 .842194-0.979602$
H $-4.641384 \quad 1.875740 \quad 0.384807$

H $-5.935542-0.1843011 .375894$

H $-6.219392-0.211377-1.673610$

H $-8.576800-0.585671-0.707295$

H -7.886932 -1.4291700 .659459$

H $-8.390696-3.498321-2.964553$

H -8.743804 -1.681896 -2.877034

H -7.465312 -4.659775 -1.117252

H -7.765118 -3.9188950 .422835$

H -5.530562 -3.251382 0.731808

H -3.4138120 .5064251 .893760$

C $4.226853-0.843474-3.433126$

O $3.527358 \quad 0.391174-3.180178$

C $4.1759571 .072929-2.090164$

C $5.5200330 .377729-1.899398$

C $5.163266-1.057028-2.242775$

H $3.5604260 .895559-1.200728$

C $4.2513192 .578231-2.368106$

H $6.2774500 .756082-2.595534$

H $5.9062940 .493500-0.882209$

C $6.363090-1.933222-2.554266$

H $4.624624-1.485325-1.388599$

C $2.9036043 .227937-2.746275$

O $5.1558242 .815205-3.454940$

H $4.6849763 .090132-1.501437$

H $2.6019682 .909069-3.751526$

O $3.1039404 .645255-2.817946$

C $1.7561192 .950416-1.758965$

C $0.4601863 .693545-2.142779$

H $1.5490641 .875311-1.741605$

O $2.1617463 .325090-0.444976$

H $0.5916974 .767479-1.973621$

H $0.2803223 .549093-3.214533$

H $6.045560-2.947665-2.815472$

H $6.944969-1.535103-3.392217$

H $7.026584-1.999262-1.685807$

C $3.220269-1.961002-3.727800$

H $4.8314162 .312157-4.221779$

H $3.8267724 .803657-3.449269$

H $2.3824594 .272026-0.463805$

H $3.756032-2.828188-4.131580$

H $2.524746-1.612955-4.501583$

H $4.814189-0.668756-4.344473$

C -0.7019014 .6658280 .548905$

O -0.6136843 .3245150 .060176$

C $-0.7685913 .183896-1.354235$

C $-2.1107543 .778754-1.803556$

C $-2.2876415 .218725-1.326871$

C -2.0380915 .3192590 .178476$

H $\quad 0.1084245 .259747 \quad 0.107659$

C -0.4202024 .6518682 .058483$

H -0.821846 2.105918-1.548857

O $-2.2306143 .713260-3.220287$

H -2.921534 $3.168128-1.386617$

H -1.629469 $5.898737-1.879691$

O $-3.6206325 .650285-1.611359$

H -2.869776 4.8486020 .713697

O $-2.011525 \quad 6.6924820 .560804$

C -1.2795073 .6940032 .859759$

H 0.6199914 .3328122 .196946

H -0.5303575 .6546732 .484246$

H -3.081081 $4.119594-3.459773$

H -4.234461 $5.061625-1.139921$

H -2.851584 7.0901420 .274241

O -0.7250803 .5408694 .100191$

O -2.3046603 .1561352 .466994$

C -1.421290 2.6543504 .981744

H -0.8375572 .5622565 .901450$

H -2.4045573 .0661565 .227584$

$\mathrm{H}-1.5200211 .6626654 .529983$

SCF Energy (B3LYP/6-31G**//MMFF) $=-3245.91651402$

0181

MMFF Geometry

C -2.250306 $0.155078-3.873666$

C $-3.0200381 .215111-3.586162$

C -2.623795 $2.454792-2.824819$

O $-3.2303602 .334560-1.512068$ 
C $-1.1139572 .730781-2.653384$

C $-0.3404502 .993221-3.929211$

C $0.8335802 .411687-4.244217$

C $-0.9126874 .047217-4.843048$

C $1.6112391 .389410-3.463316$

C $2.8388952 .001078-2.775267$

C $2.4942662 .863201-1.549252$

C $3.766768 \quad 3.471770-0.957595$

C $3.480161 \quad 4.257614 \quad 0.329110$

O $1.8582892 .024299-0.579806$

$\begin{array}{lllllll}\text { O } & 4.737669 & 4.522936 & 0.969938\end{array}$

C 2.6620083 .3657331 .285394

C 2.8454555 .6226060 .036589

$\begin{array}{lllll}\text { O } & 2.274076 & 4.119982 & 2.432106\end{array}$

C $1.434868 \quad 2.698725 \quad 0.613844$

C $0.8288951 .636394 \quad 1.560697$

$\begin{array}{llllll}\text { O } & 0.452472 & 3.697808 & 0.340920\end{array}$

C $-3.5008803 .480471-0.835206$

O $-3.2698364 .621664-1.212665$

C $-4.117654 \quad 3.2120710 .483332$

$\begin{array}{llll}\text { C } & -4.620524 & 2.022549 & 0.848194\end{array}$

C $-5.288616 \quad 1.7283832 .118715$

C -5.2646712 .7880873 .189508$

C -0.2759530 .7686180 .941485$

C $-0.681295-0.3604511 .875734$

C $-2.074071-2.4242061 .927550$

O $-0.210846-0.5013013 .001703$

C $-3.530470-2.3136202 .240300$

C $-4.012404-2.2461543 .490210$

C $-5.474557-2.206144 \quad 3.829785$

C $-5.909266-0.8638754 .371143$

C $-5.676222-0.5231675 .649868$

C -6.6894560 .0461063 .451823$

C $-5.887843 \quad 0.5294942 .274291$

N $-1.621876-1.1992671 .296303$

H $-1.2238170 .114422-3.525468$

H $-4.0512211 .207918-3.936206$

H -3.092821 $3.302987-3.340324$

H $-0.675216 \quad 1.926371-2.054934$

H -0.992833 $3.623162-2.026096$

H $1.3146192 .707959-5.176144$

H $-0.2194504 .325008-5.644258$

H -1.832839 $3.690521-5.315891$

H - $1.1372114 .958209-4.278312$

H $0.9936020 .850278-2.740316$

H $1.9549470 .628239-4.175503$

H $3.4834551 .174300-2.455242$

H $3.4142392 .592608-3.498399$

H $1.8022903 .656457-1.851176$

H $4.2715224 .110655-1.693052$

H $4.4790862 .668559-0.720201$

H $5.289458 \quad 5.029278 \quad 0.349533$

H 3.3408562 .5790491 .642683

H $3.5089476 .225653-0.594967$

H $2.705156 \quad 6.1985870 .958065$

H $1.8831605 .547996-0.474181$

H $\quad 1.979414 \quad 3.4944053 .114769$

H $1.6314920 .966006 \quad 1.888104$

H 0.4201602 .1199152 .456550

H -0.4250243 .2855640 .321586$

H -4.1787834 .0790021 .133886$

H $-4.572313 \quad 1.1910760 .147055$

H -5.8638863 .6538872 .887823$

H -4.2376643 .1195333 .381430$

H -5.652973 2.4409894 .150101

H -1.1660051 .3688490 .727753$

H 0.0728230 .3179220 .005416

H -1.475689 -2.633632 2.820235

H $-1.911286-3.2407241 .217724$

H -4.219922 -2.324114 1.399994

H $-3.316975-2.2458024 .326813$

H -5.673709 -2.985998 4.576626

H $-6.088494-2.4782812 .961977$

H -6.0106410 .4270006 .052802$

H $-5.142730-1.1881336 .321171$

H -7.1199760 .8889444 .004139$

H $-7.560870-0.5023893 .070228$

H $-5.845934-0.1842351 .451001$
H -1.966853 $-0.987806 \quad 0.364462$

C $-2.795378-2.331369-3.906337$

O $-1.502838-2.684061-3.373130$

C $-1.691191-3.208076-2.043957$

C $-3.182879-3.492092-1.910944$

C $-3.765983-2.353958-2.725501$

H $-1.429261-2.401507-1.352024$

C $-0.784577-4.417390-1.802407$

H $-3.453171-4.457265-2.355326$

H $-3.517358-3.492570-0.869961$

C $-5.217067-2.562590-3.119396$

H $-3.685881-1.432658-2.134858$

C $0.720547-4.128015-1.979612$

O $-1.142234-5.460764-2.717095$

H $-0.970696-4.820509-0.800373$

H $0.940417-3.937967-3.036689$

O $1.434650-5.324078-1.640091$

C $1.256387-2.962252-1.124977$

C $2.786161-2.816100-1.239491$

H $0.780794-2.029381-1.447657$

$\begin{array}{lllll} & 0 & 0.889702 & -3.195172 & 0.232405\end{array}$

H $3.266360-3.681269-0.770305$

H $3.055877-2.826945-2.302040$

H $-5.582882-1.721422-3.716511$

H $-5.346371-3.476733-3.708350$

H $-5.848760-2.644629-2.228746$

C $-2.716261-1.019425-4.688676$

H -1.034732 -5.110664 -3.618215

H $1.296317-5.488603-0.691337$

H $1.113603-2.3956610 .737619$

H $-3.688922-0.810303-5.149664$

H $-1.998309-1.141278-5.509775$

H -3.055564 -3.130079 -4.613889

C $3.751729-2.2984521 .644985$

O $3.015213-1.4134080 .795783$

C $3.320325-1.514796-0.597707$

C $4.819457-1.270208-0.827250$

C $5.683404-2.1967120 .024985$

C $5.266399-2.114427 \quad 1.492781$

H $3.498784-3.3327101 .379245$

C $3.246895-2.1150483 .084867$

H $2.780336-0.693887-1.083684$

O $5.165216-1.445673-2.197239$

H $5.061539-0.231984-0.564815$

H $5.644012-3.230656-0.337644$

O $7.049985-1.788695-0.073677$

H $5.593149-1.1518611 .899339$

O $5.936946-3.1325252 .232784$

C $3.357810-0.7042633 .628036$

H $2.179990-2.3722203 .102258$

H $3.779467-2.7882983 .764988$

H $4.604684-0.848492-2.720839$

H $7.288922-1.806281-1.016410$

H $6.891688-3.0132712 .090668$

O $2.690266-0.6394934 .819203$

$\begin{array}{lllll}0 & 3.944581 & 0.224120 & 3.091090\end{array}$

C $2.675027 \quad 0.647753 \quad 5.444354$

H 2.1890651 .3800404 .792197

H $2.1011180 .568715 \quad 6.371428$

H 3.6942550 .9624935 .687599

SCF Energy (B3LYP/6-31G**//MMFF)= -3245.91209768

01_82

MM̄FF Geometry

C - $-1.698758-3.6750250 .676929$

C $-0.578291-4.057726 \quad 0.045645$

C $0.407800-3.069152-0.517005$

O $1.702217-3.3878840 .045502$

C $0.509595-3.133066-2.054213$

C $-0.779896-2.860929-2.800042$

C $-1.438679-1.685307-2.778131$

C $-1.309082-4.017412-3.605672$

C $-1.008704-0.436589-2.060401$

C $-1.3196040 .827837-2.870078$

C $-0.7914742 .082923-2.160215$

C $-1.3709713 .345293-2.798594$

C $-0.7926954 .616988-2.165159$

O $0.6365162 .082224-2.279941$ 
O $-1.1712055 .714705-3.008521$

C $0.7456984 .514862-2.173089$

C $-1.4126844 .894894-0.791835$

O $1.3272515 .620405-1.484475$

C $1.2919963 .173396-1.621778$

C $2.8067273 .083457-1.925515$

O $1.0896153 .134648-0.209632$

C $2.584476-2.3593650 .169070$

O $2.405977-1.194276-0.148828$

C $3.827065-2.8962070 .762832$

C $4.760266-2.0391491 .202306$

C $6.064077-2.3890921 .768022$

C $6.446733-3.8459581 .775439$

C $3.4724421 .745514-1.570078$

C $4.9219081 .709929-2.034937$

C $6.886710 \quad 0.207479-2.202665$

O $5.4588102 .648809-2.618827$

C $7.585722-0.693128-1.235548$

C $8.647571-0.309529-0.509719$

C $9.402367-1.2015150 .437043$

C $9.265268-0.7603751 .878741$

C $10.051927 \quad 0.2043862 .384639$

C $8.259757-1.4846682 .749406$

C $6.860468-1.3909392 .205837$

N $5.5500280 .510344-1.720993$

H $-1.902024-2.6127890 .791874$

H - $0.363352-5.115361-0.081348$

H $0.126785-2.062435-0.189873$

H $0.905794-4.117370-2.341670$

H $\quad 1.277577-2.431086-2.405285$

H -2.377622 -1.610648 -3.324298

H - $0.610680-4.263529-4.412324$

H -1.430201-4.902576 -2.973418

H -2.281401 -3.799438-4.059663

H -1.544117 -0.386382 -1.104826

H $0.063065-0.460434-1.841275$

H $-0.860306 \quad 0.744088-3.863656$

H $-2.4038970 .905252-3.012168$

H $-1.0670432 .032127-1.100330$

H -2.466282 $3.348995-2.732664$

H -1.135754 $3.351995-3.872312$

H $-0.7408836 .514988-2.661088$

H $1.0627404 .613833-3.220386$

H -2.500826 $4.999398-0.878761$

H -1.054608 $5.843749-0.377617$

H -1.210850 4.104759-0.066655

H $1.2386495 .453654-0.530484$

H $2.9566603 .253754-3.000225$

H $3.3382623 .882740-1.393009$

H 1.6638922 .4502770 .168180

H $3.942295-3.9726390 .812046$

H $4.559900-0.9706721 .128565$

H $6.417718-4.256306 \quad 0.759678$

H $5.757842-4.4193222 .405710$

H $7.451527-4.0359342 .156247$

H $3.4592351 .583695-0.487283$

H $2.9432020 .918002-2.055103$

H $7.4395331 .138244-2.369233$

H $6.782050-0.293813-3.170424$

H $7.218927-1.712813-1.152850$

H $9.0150020 .709468-0.611617$

H $\quad 10.460985-1.1867110 .145986$

H $9.090303-2.2481470 .334154$

H 9.9763090 .5113473 .422456

H 10.7891430 .7095571 .769206

H $8.248712-1.0576423 .760580$

H $8.597821-2.5166132 .871934$

H $6.483257-0.3681172 .158260$

H $4.980149-0.258471-1.379199$

C $-4.091392-4.513938 \quad 0.572313$

O $-4.593280-3.162288 \quad 0.637624$

C $-5.098932-2.801891-0.662394$

C $-5.306079-4.117049-1.403004$

C $-4.121509-4.918688-0.902401$

H $-4.305083-2.237477-1.166153$

C $-6.353875-1.934244-0.537272$

H -6.244090 -4.604385 -1.112672

H $\quad-5.309837-3.987434-2.489494$
C $-4.250223-6.413929-1.131127$

H -3.225435 -4.559698 -1.418469

C $-6.163087-0.6483690 .293976$

O $-7.395554-2.7004540 .081395$

H -6.723368-1.681022-1.537775

H $-6.036320-0.9153041 .348971$

$\begin{array}{lllll}\text { O } & -7.386277 & 0.096590 & 0.221878\end{array}$

C $-4.9992410 .253415-0.159563$

C -4.9638801 .6365620 .518916$

H $-4.048199-0.261506 \quad 0.014442$

O $\quad-5.110527 \quad 0.464317-1.570048$

H -4.1106342 .1885040 .104912$

H -5.8438382 .2150250 .210144$

H $-3.376268-6.943761-0.739171$

H $-5.140545-6.820463-0.639903$

H $-4.326848-6.633914-2.201061$

C $-2.722317-4.610747 \quad 1.254300$

H $-7.069170-2.9913350 .950306$

H $-7.5046510 .376780-0.702041$

H -4.362006 $1.020340-1.844173$

H $-2.366043-5.6469381 .222174$

H -2.836306 -4.345032 2.312719

H $-4.801823-5.1270121 .142043$

C $-2.493427 \quad 1.2587792 .275200$

O $-3.812406 \quad 0.7515712 .513550$

C -4.8752341 .5914932 .056420$

C -4.7845272 .9701552 .724988$

C -3.4083043 .6017812 .540862$

C -2.307976 2.6245592 .952452

H -2.3280651 .3500391 .194887$

C -1.5212880 .2053082 .820244$

H -5.8039291 .1319772 .415144$

O $\quad \begin{array}{lllll}-5.776012 & 3.853174 & 2.211026\end{array}$

H -4.9745602 .8645773 .801151$

H $-3.261083 \quad 3.947747 \quad 1.511798$

O $-3.3086554 .761879 \begin{array}{lll}3.370319\end{array}$

H -2.3167492 .5082184 .043733$

O -1.0432463 .1739062 .592868$

C $-0.109697 \quad 0.4021642 .326352$

H -1.5472410 .1994203 .917021$

H $-1.833582-0.8009102 .516418$

H -6.6420783 .4344032 .353562$

H -4.0356325 .3580403 .121227$

H -0.9740914 .0469263 .015957$

O $\quad 0.742013 \quad 0.505267 \quad 3.385855$

O $\quad 0.1993380 .459634 \quad 1.143712$

C 2.1172500 .7185063 .054389

H 2.2283581 .6027492 .419705

H 2.6691930 .8839543 .983406

H $2.521634-0.1675892 .559329$

SCF Energy (B3LYP/6-31G**//MMFF) $=-3245.92679462$

0183

MM̄MF Geometry

C $2.891305-2.7788951 .011351$

C $2.230805-2.1848952 .016066$

C $2.442930-0.7423722 .389507$

O $1.166077-0.0545412 .403388$

C $3.033227-0.5818453 .801487$

C $4.409361-1.1793573 .992769$

C $5.561892-0.5392203 .717999$

C $4.443789-2.5565464 .605898$

C 5.7423600 .8459703 .159253

C 6.7667710 .8761532 .014345

C 6.1818010 .4571790 .655264

C $7.3131340 .240795-0.353160$

C $6.790189-0.022301-1.770528$

O $5.326508 \quad 1.518708 \quad 0.219744$

O $7.9186870 .083939-2.651360$

C $5.7866291 .089579-2.136231$

C $6.250077-1.448992-1.919878$

O $5.163730 \quad 0.823335-3.391625$

C $4.7031451 .320531-1.055708$

C $3.9119372 .610147-1.388262$

O $3.8184610 .202064-1.044477$

C $0.5908250 .245702 \quad 1.207831$

O $0.982074-0.0793560 .098820$

C $-0.600326 \quad 1.0788541 .486067$ 
C -1.2290121 .6988470 .475991$

C -2.4042352 .5663960 .592633$

C $-2.9775312 .779470 \quad 1.969723$

C $2.8749963 .011863-0.330440$

C $2.3146604 .403811-0.570626$

C $0.791548 \quad 6.0775180 .471204$

O $2.6552925 .111940-1.514163$

C -0.6834565 .9443550 .674569$

C -1.589341 $6.371732-0.217811$

C $-3.0756226 .300296-0.015398$

C $-3.7601695 .435977-1.049606$

C $-4.1140765 .936636-2.245498$

C $-4.1120394 .018363-0.661554$

C -2.903839 $3.132917-0.526152$

N $1.385745 \quad 4.7559160 .400320$

H $3.617800-2.2042110 .443717$

H $1.507804-2.7524842 .596308$

H $3.096904-0.2319071 .672072$

H 2.340659-1.005096 4.543032

H 3.0541280 .4865364 .056101

H $6.493874-1.0561103 .945332$

H $5.466243-2.9129574 .770950$

H $3.939086-2.5501285 .577741$

H $3.945491-3.2861373 .962096$

H 4.7984591 .2940162 .835000

H 6.1257961 .4725773 .974231

H 7.1397421 .9058291 .932484

H 7.6264960 .2437322 .269264

H $5.593523-0.4591930 .773969$

H $7.969741-0.576934-0.029939$

H $7.9488951 .137402-0.381510$

H $7.622697-0.137821-3.550246$

H $6.3575162 .020371-2.261664$

H $7.047001-2.179400-1.735034$

H $5.902248-1.636580-2.941722$

H $5.431329-1.671200-1.233111$

H $5.861244 \quad 0.798608-4.067833$

H $4.6255573 .437688-1.496639$

H $3.3946822 .497488-2.349419$

H $2.9591630 .477152-0.689704$

H $-0.917097 \quad 1.1718402 .518677$

H $-0.8460201 .565559-0.535012$

H -2.2201433 .2010672 .640094$

H -3.330268 1.8320402 .390671

H -3.8219443 .4707371 .991851$

H $2.0345042 .313431-0.344426$

H 3.3214962 .9999150 .670087

H $1.0348546 .651905-0.428501$

H 1.2386116 .5903251 .328835

H -1.018977 5.5028111 .609583

H $-1.2444516 .828010-1.143341$

H -3.473589 $7.322225-0.069130$

H -3.3252085 .9456570 .992137$

H -4.631808 $5.331684-2.982379$

H -3.889319 $6.963556-2.514501$

H $-4.7581833 .569694-1.427151$

H $-4.7274474 .039706 \quad 0.242083$

H -2.411203 2.925066-1.477031

H $1.2353824 .127027 \quad 1.184254$

C $1.908898-4.417163-0.664400$

O $0.534228-4.055133-0.436467$

C $0.003327-3.484021-1.648860$

C $1.071048-3.681470-2.723075$

C $2.344934-3.632632-1.900072$

H $-0.113502-2.409812-1.464253$

C $-1.358444-4.109664-1.972196$

H $\quad 0.976507-4.653944-3.219856$

H $1.027799-2.907008-3.495116$

C $3.555889-4.214861-2.605770$

H $2.544353-2.583161-1.651890$

C $-2.379746-4.040092-0.817528$

O $-1.174178-5.494106-2.297790$

H -1.773015 -3.642739-2.873055

H -2.056435 $-4.692150 \quad 0.002328$

O $-3.612575-4.595537-1.295592$

C $-2.645019-2.623644-0.274194$

C $-3.718780-2.6291920 .833892$

H $-1.717509-2.2105870 .137446$
O $-3.043960-1.795461-1.361862$

H -4.654190 -3.028255 0.429852

H -3.388781 -3.318187 1.620187

H $4.438356-4.176410-1.959470$

H $3.391695-5.259516-2.890339$

H $3.778849-3.649540-3.516543$

C $2.719977-4.2164040 .616745$

H $-0.758455-5.923429-1.530326$

H -3.949295 -4.004517-1.991045

H -3.086631 -0.880699-1.035423

H $3.716642-4.6564490 .494706$

H $2.231652-4.7628071 .433744$

H $1.902355-5.492111-0.891040$

C $-5.845435-0.5559560 .077138$

O $-4.497599-0.3016710 .493449$

C $-3.972044-1.2328401 .445541$

C $-4.851733-1.2379902 .704065$

C $-6.318645-1.4938152 .371675$

C $-6.793176-0.5355651 .280321$

H -5.900332 -1.529995 -0.422531

C $-6.2257610 .520978-0.944685$

H -2.990608 -0.8459601 .745304$

O $-4.405455-2.2138253 .639140$

H -4.777226 -0.2617723 .200885$

H -6.488523 -2.536895 2.079751

O $-7.111780-1.2677243 .539153$

H -6.8644620 .4804361 .689450$

O $-8.098976-0.9197980 .855536$

C $-5.4965030 .306881-2.249810$

H -7.300512 $0.529025-1.158790$

H $-5.964273 \quad 1.518893-0.575431$

H -3.475559 -2.017484 3.844820

H $-6.774227-1.8565094 .235816$

H -8.670237 -0.9245861 .642799$

O $-6.087403-0.701536-2.953160$

O $-4.5116650 .946124-2.595344$

C -5.468217 -1.024417-4.202092

H $-4.438969-1.358356-4.039593$

H -6.033069-1.840577-4.660491

H -5.493441 -0.160016-4.872338

SCF Energy $\left(B 3 L Y P / 6-31 G^{* *} / / M M F F\right)=-3245.92573391$

0184

MM̄FF Geometry

C $0.493002-1.1652451 .768463$

C $0.590476-2.1904690 .907543$

C $0.953438-2.083643-0.552531$

O $0.499514-0.804363-1.054023$

C $2.467462-2.217594-0.782917$

C $3.036907-3.577786-0.446365$

C $3.832004-3.8340980 .610679$

C $2.702490-4.674504-1.424181$

C $4.263658-2.8860071 .695074$

C $5.750732-2.5182081 .603792$

C $6.087777-1.6024310 .415659$

C $7.592540-1.3294390 .361050$

C $7.953647-0.331162-0.748506$

O $5.375586-0.3732340 .590241$

O $9.3029700 .106690-0.526839$

C $7.0480730 .910669-0.615409$

C $7.931623-0.984853-2.135305$

O $7.2475461 .804488-1.708727$

C $5.5454660 .570707-0.475948$

C $4.7542621 .848336-0.106182$

O $5.0638170 .061134-1.719947$

C $0.235075-0.706448-2.381197$

O $0.431502-1.566980-3.228893$

C $-0.3557840 .606175-2.728579$

C $-0.8303331 .473909-1.820902$

C $-1.5145062 .736668-2.114020$

C $-1.5473133 .199438-3.546676$

C 3.2627381 .6159310 .177247

C $2.6368612 .832437 \quad 0.831124$

C $2.1231795 .236573 \quad 0.419644$

O $2.2444472 .807364 \quad 1.995628$

C 0.6728575 .3937390 .087258

C $0.2208986 .272089-0.821737$

C $-1.2031466 .578998-1.201388$ 
C $-2.2850395 .829362-0.458702$ C $-2.730154 \quad 6.2564110 .735383$ C $-2.9284364 .654796-1.157086$ C -2.093012 3.407627-1.096168 N $2.5824043 .930881-0.010378$ H $0.721882-0.1569741 .433919$ H $0.392774-3.1968381 .268893$ H $0.406201-2.870944-1.084304$ H $2.982149-1.420676-0.238676$ H $2.700908-2.000759-1.833570$ H $4.208041-4.8490730 .734026$ H $3.205933-5.616207-1.180864$ H $1.625923-4.870043-1.430521$ H $3.011492-4.389283-2.435216$ H $3.648326-1.9825361 .727740$ H $4.090497-3.3899332 .654657$ H $6.018329-2.0035192 .535925$ H $6.355573-3.4321751 .554204$ H $5.758865-2.083362-0.511930$ H $8.153180-2.2640270 .234262$ H $7.922233-0.910972 \quad 1.322407$ H $9.877546-0.677888-0.534346$ H $7.364736 \quad 1.4510720 .287879$ H $8.648843-1.813367-2.179022$ H $8.245002-0.277152-2.910735$ H $6.951916-1.383267-2.406758$ H $8.1992412 .000143-1.752849$ H 5.2015532 .2962870 .791211 H $4.8459742 .584017-0.914744$ H $4.1042610 .192016-1.767340$ H $-0.4415100 .792513-3.794423$ H $-0.7615801 .222119-0.763746$ H -2.167546 2.530112 -4.152658 H $-0.5357173 .220645-3.968173$ H -1.942509 $4.210256-3.668118$ H $2.7093641 .390602-0.740614$ H 3.1229210 .7711850 .859801 H $2.7469535 .981174-0.085610$ H $2.269195 \quad 5.3443661 .499397$ H -0.0199284 .7827520 .657964$ H $0.9575016 .869066-1.358233$ H - $-1.3169916 .415641-2.280166$ H $-1.3578467 .656898-1.056056$ H $-3.532387 \quad 5.7444121 .256442$ H -2.295693 7.1247371 .219838 H $-3.1922104 .940653-2.179498$ H -3.891445 $4.420891-0.684088$ H -2.007103 $2.996223-0.089801$ H $2.8584513 .825741-0.982280$ C $-0.919649-0.2492793 .628977$ O $-2.075131-0.2686892 .774056$ C -3.234864 0.0933393 .548563 C -2.7430570 .3779634 .966761$ C -1.471262 -0.4453565 .037918$ H -3.883363 -0.7901623 .566196$ C $-3.968219 \quad 1.2641292 .879026$ H -2.517880 1.4395755 .118596 H $-3.4814690 .090098 \quad 5.721764$ C $-0.526211-0.0097936 .143677$ H $-1.746663-1.4975745 .190410$ C -4.2591091 .0580151 .379422$ O -3.1671932 .4504402 .987128$ H -4.8959331 .4794643 .421018$ H $-3.314717 \quad 1.0692510 .825721$ O $-5.0128162 .186406 \quad 0.918516$ C $-5.047781-0.2178831 .041346$ C $-5.419230-0.351524-0.447923$ H $-4.475670-1.097895 \quad 1.342927$ O $-6.259042-0.2412031 .800519$ H -6.071281-1.226901-0.553163 H $-6.0445170 .499989-0.743473$ H $0.374463-0.6315486 .155462$ H $-0.2145091 .032628 \quad 6.017652$ H $-1.010725-0.1001507 .121372$ C $0.075481-1.3302573 .203161$ H - -2.3127872 .2688672 .558921$ H $-4.5143832 .985648 \quad 1.160855$ H -6.7649820 .5569901 .570173$
H $-0.375037-2.3197373 .345547$

H $0.968359-1.2773943 .836038$

H -0.4610070 .7435553 .526943$

C $-3.888051-2.863263-1.291357$

O $-3.343868-1.561470-1.038136$

C $-4.201833-0.469139-1.387289$

C $-4.556208-0.533899-2.878287$

C $-5.160483-1.881820-3.253576$

C $-4.260700-3.018299-2.773117$

H $-4.772447-3.022173-0.662596$

C $-2.824336-3.901915-0.897848$

H $-3.6091450 .440760-1.253652$

O $-5.4633510 .503883-3.233488$

H $-3.647082-0.383446-3.475143$

H $-6.177912-1.992164-2.860747$

O $-5.267893-1.969096-4.676361$

H -3.351577 -3.047147 -3.387794

O $-4.940630-4.258695-2.958325$

C -2.668694 -4.0525910 .600877$

H $-3.104422-4.886809-1.288684$

H $-1.848948-3.616839-1.305739$

H $-5.0467541 .349735-2.994881$

H $-5.809000-1.216597-4.971478$

H $-5.179929-4.320476-3.899103$

O $-1.891815-5.148356 \quad 0.854204$

O $-3.152858-3.3112221 .443622$

C -1.678646 -5.4474092 .237379$

H -1.019034 -6.317124 2.299115

H $-1.196067-4.6057102 .741763$

H -2.629536 -5.692169 2.720033

SCF Energy (B3LYP/6-31G**//MMFF) $=-3245.91877791$

01_85

MM̄FF Geometry

C -0.454094 -3.469363 1.610366

C $-0.621786-2.1507951 .799662$

C $-0.264182-1.436503 \quad 3.076826$

O $0.624476-0.3553432 .703673$

C $-1.505538-0.8435483 .774276$

C $-2.500640-1.8714284 .278177$

C $-3.787190-1.953098 \quad 3.883509$

C $-1.976231-2.8320865 .315139$

C $-4.510522-1.0524732 .922535$

C $-4.539712-1.6443681 .510012$

C $-5.205505-0.7013180 .499038$

C $-5.202907-1.318944-0.900868$

C $-5.752060-0.353445-1.962360$

O -4.4646350 .5220910 .487241$

O $-5.400851-0.872244-3.254377$

C $-5.0438661 .010575-1.816654$

C $-7.282955-0.279751-1.925964$

O $-5.6292691 .985616-2.676266$

C $-5.013716 \quad 1.534998-0.363817$

C $-4.1037892 .781207-0.259089$

O $\begin{array}{lllll}-6.340146 & 1.897670 & 0.029278\end{array}$

C $1.4971670 .092307 \quad 3.642052$

O $1.582288-0.2918334 .800878$

C 2.4326881 .0952413 .082964

C $2.2482351 .729173 \quad 1.914027$

C 3.1850912 .6697581 .292127

C 4.5160842 .8779391 .968865

C $-3.770633 \quad 3.1711021 .187706$

C $-3.212946 \quad 4.576167 \quad 1.261585$

C -1.205164 5.9372690 .792986

O -3.8725225 .5054861 .723100$

C $-0.0912845 .912798-0.202057$

C $1.165645 \quad 6.2759010 .093981$

C $2.2772686 .324761-0.913297$

C $3.5328515 .633392-0.434504$

C 4.5196546 .3340120 .150037

C $3.6936654 .161169-0.728610$

C 2.8377213 .2724870 .134936

N $-1.930448 \quad 4.683032 \quad 0.745945$

H $-0.026154-4.0524272 .424238$

H $-1.044775-1.5334641 .012517$

H $\quad 0.270761-2.1161453 .750470$

H $-1.970161-0.1203843 .094616$

H -1.183189-0.237015 4.630905 
H $-4.406818-2.7428314 .306284$ H -2.771179 -3.4470735 .750742$ H -1.238477 -3.5117454 .878540$ H -1.503448 -2.282733 6.136072 H -5.537762 -0.9185113 .284030$ H -4.070165 -0.0509062 .920566$ H -3.509590 -1.835586 1.192756 H -5.062722 -2.608616 1.519750 H -6.230745 -0.5029540 .833145$ H $-5.762248-2.262574-0.911366$ H $-4.172573-1.577846-1.179517$ H $-5.771736-1.768400-3.328508$ H $-4.0076580 .879982-2.157449$ H -7.718059-1.268501-2.115198 H $-7.666440 \quad 0.372037-2.718576$ H $-7.6724470 .072872-0.968667$ H $-5.6168411 .621251-3.577950$ H -3.154887 2.586959-0.775614 H $-4.5798493 .630315-0.765930$ H -6.3002052 .6272880 .667214$ H 3.3065561 .2800723 .699812 H 1.3433521 .5305231 .342384 H 4.3753913 .3000172 .970171 H 5.1746223 .5645281 .433392 H 5.0564101 .9289562 .058514 H -4.6566383 .1361401 .830369$ H -3.040855 2.4758211 .619610 H $-0.8477106 .073307 \quad 1.819340$ H -1.896403 6.7544390 .560383 H $-0.3365295 .620678-1.220476$ H $1.398561 \quad 6.6027961 .105019$ H $2.4942707 .381859-1.116468$ H $1.9672855 .898427-1.875471$ H 5.4438265 .8600300 .462453 H 4.4238587 .3995260 .331546 H $4.7462523 .862269-0.657676$ H $3.4353043 .975327-1.779474$ H $1.8509173 .072863-0.283539$ H -1.408079 3.8299780 .565669 C $-1.428483-3.520410-0.788489$ O $-0.424330-2.694863-1.407964$ C $-0.608207-2.732380-2.836389$ C -1.928261 -3.454070 -3.077804 C -1.954891-4.419042 -1.906550 H $\quad 0.199840-3.352407-3.241435$ C $-0.519953-1.314402-3.412004$ H $-2.782320-2.768770-3.028984$ H -1.955097 -3.955672 -4.049889 C -3.328564 -5.008922 -1.639696 H $-1.243186-5.232478-2.101638$ C $0.782626-0.571767-3.041933$ O $-1.616190-0.535625-2.916187$ H $-0.640173-1.345669-4.500841$ H $0.787371-0.352118-1.968383$ O $0.7829310 .692991-3.713508$ C $2.064781-1.326048-3.433337$ C $3.374148-0.568575-3.153215$ H $2.102393-2.284093-2.907111$ O $2.029038-1.636147-4.829159$ H $4.199512-1.201844-3.502544$ H $3.427810 \quad 0.328834-3.782900$ H $-3.302875-5.689460-0.783133$ H -4.066974 -4.229296-1.427368 H -3.678942 -5.574802 -2.509075 C $-0.812795-4.2797710 .388786$ H $-1.572735-0.551192-1.944383$ H $-0.0354601 .154320-3.461790$ H $1.952630-0.796101-5.313747$ H $0.104815-4.7795510 .053378$ H -1.507258 -5.0615460 .718062$ H $-2.229930-2.857573-0.445281$ C $4.186367-2.345360-0.801893$ O $3.265329-1.252194-0.781058$ C $3.587150-0.183735-1.675332$ C $4.9865790 .367892-1.364030$ C $6.047917-0.732653-1.341429$ C $5.603332-1.887122-0.440842$ H $4.203209-2.775539-1.811432$
C $3.639180-3.4565220 .106513$

H $2.8711650 .618170-1.459909$

O $5.3310631 .369988-2.314100$

H $4.965803 \quad 0.848521-0.378811$

H $6.279676-1.091303-2.351039$

O $7.266469-0.205976-0.811402$

H $5.659075-1.5641000 .604781$

O $6.503674-2.981506-0.595893$

C $3.519198-3.0783071 .568318$

H $2.630997-3.729968-0.227801$

H $4.285138-4.3384560 .028118$

H $6.1489491 .797226-2.009266$

H $7.5997030 .453977-1.442433$

H $7.396452-2.653404-0.392799$

O $3.351113-4.2157932 .306506$

O $3.542080-1.9385292 .010057$

C $3.224266-4.0197833 .718331$

H $4.129460-3.5532424 .118354$

H $2.345147-3.4084963 .939695$

H $3.097343-4.9989934 .187273$

SCF Energy (B3LYP/6-31G**//MMFF) = -3245.91010695

01 86

MMFF Geometry

C 3.8239222 .5309921 .875638

C 2.7176761 .8017441 .669003

C 1.8033871 .3249662 .764027

O 0.4621201 .7653542 .448257

C $1.784382-0.2118392 .806177$

C $1.282882-0.7473544 .130875$

C $0.050983-1.2485724 .339991$

C $2.296925-0.7502785 .247016$

C $-1.094946-1.3356903 .371659$

C $-1.156340-2.7145612 .707083$

C $-2.572568-3.0901732 .246428$

C $-2.552656-4.4492761 .541436$

C $-3.932547-4.8417820 .995764$

O $-3.075983-2.077178 \quad 1.367524$

O $-3.749630-5.9267640 .072556$

C $-4.502905-3.6563550 .189732$

C $-4.856452-5.3661912 .100971$

O $-5.843574-3.914975-0.219172$

C $-4.430026-2.3081030 .941986$

C $-4.882311-1.1164690 .065432$

O $-5.322705-2.3498372 .063220$

C 0.1328883 .0492062 .749514

O 0.8232953 .8732873 .326676

C -1.219705 3.2910012 .204985

C -1.713429 4.5373402 .186240

C -3.0166024 .9026471 .641823$

C -3.7708265 .8919792 .487213$

C $-4.044365-0.878794-1.189526$

C $-4.5278240 .317026-1.991609$

C $-3.9142621 .671390-3.997413$

O $-5.5384870 .955102-1.710696$

C $-3.4235672 .941496-3.376960$

C $-4.1884794 .034642-3.235183$

C $-3.7233505 .296033-2.565891$

C $-4.5950355 .680916-1.387430$

C $-5.2322876 .863634-1.362591$

C $-4.7399924 .702668-0.240510$

C -3.4352324 .4255350 .452614$

N $-3.6943590 .575596-3.071871$

H 4.1132222 .7982572 .888452

H 2.4493791 .5290330 .653472

H 2.1053981 .7362383 .734910

H $2.793838-0.6138622 .644009$

H $1.172604-0.6019121 .983789$

H $-0.179128-1.6407375 .330184$

H $1.900057-1.1887606 .168923$

H $3.176950-1.3342834 .958019$

H 2.6180490 .2691835 .481223

H -1.041442 -0.552513 2.608105

H -2.015773 -1.142869 3.936235

H $-0.475288-2.7064321 .846241$

H $-0.795407-3.490594 \quad 3.394430$

H -3.210554 -3.136871 3.137238

H $-2.169379-5.2296952 .210848$ 
H $-1.848441-4.4077560 .700292$ H -3.315121 -6.6553360 .548507$ H -3.905231 -3.586034 -0.727584 H -4.426096 -6.261004 2.566648 H $-5.823972-5.6778791 .692736$ H $-5.033944-4.6376502 .894678$ H $-5.844439-4.766259-0.689354$ H $-5.939862-1.236175-0.204497$ H -4.842255 -0.2017570 .674198$ H $-5.523397-1.4379862 .332025$ H -1.7703142 .4454971 .805200$ H -1.138244 5.3606782 .604333 H -4.0637195 .4323103 .437496$ H -4.678198 6.2677532 .008647 H -3.148762 6.7674492 .706357 H -3.002251 -0.704239-0.906036 H -4.088408 -1.747568 -1.853442 H $-4.9805441 .715652-4.243030$ H -3.349159 $1.453789-4.908536$ H -2.398530 $2.955370-3.013968$ H $-5.2101894 .022429-3.608078$ H $-3.7236236 .092518-3.320952$ H $-2.6838385 .203655-2.228146$ H -5.860065 $7.150594-0.525468$ H $-5.1402127 .573886-2.177631$ H $-5.1373803 .752654-0.618908$ H -5.497752 5.0469960 .471780 H $-2.7825233 .744603-0.094246$ H $-2.8578140 .013230-3.197517$ C 5.9266382 .1284020 .576591 O $5.4970230 .845301 \quad 0.076124$ C $6.3275690 .475002-1.037454$ C $7.5444191 .387649-0.966993$ C $6.9184662 .669618-0.453868$ H $5.7623480 .710793-1.947102$ C $6.636999-1.023380-0.987930$ H $8.2901191 .018400-0.253381$ H $8.0333271 .505535-1.938655$ C 7.9249513 .6533260 .117148 H $6.3775803 .150798-1.280070$ C $5.389162-1.930918-0.921224$ O $7.433276-1.2986680 .173082$ H $7.257888-1.300143-1.847776$ H $4.911585-1.8159790 .058646$ O $5.869132-3.280358-0.985537$ C $4.362431-1.693014-2.048199$ C $3.241764-2.746354-2.132488$ H $3.918327-0.698776-1.932932$ O $5.056620-1.700728-3.299863$ H $2.664447-2.550394-3.043749$ H $3.689298-3.733197-2.300971$ H 7.4236454 .5524280 .488952 H 8.4888483 .2143520 .947079 H $8.6422573 .959224-0.651426$ C 4.7033243 .0240410 .761069 H $6.927794-1.0067680 .951175$ H $5.162564-3.861425-0.657340$ H $4.416835-1.462190-3.992204$ H $4.1280393 .063426-0.172859$ H 5.0078974 .0507780 .993538 H 6.4245011 .9463871 .537813 C $0.618385-1.230393-1.692121$ O $1.623173-1.532385-0.715474$ C $2.308006-2.775867-0.905838$ C $1.301090-3.933346-0.917355$ C $0.214941-3.722513-1.965985$ C $-0.430370-2.350885-1.781760$ H $1.089889-1.084363-2.671127$ C $-0.0526970 .088788-1.273600$ H $2.936392-2.919290-0.019644$ O $1.974468-5.166269-1.148404$ H $0.830891-4.0070770 .071686$ H $\quad 0.613840-3.848589-2.979217$ O $-0.773484-4.739836-1.792459$ H -1.049312 -2.359008 -0.875718 O $-1.290127-2.086339-2.889276$ C $0.7884981 .310615-1.569900$ H $-0.9886530 .226374-1.823049$
H $-0.2602930 .077614-0.197091$

H $1.299571-5.866223-1.171427$

H -1.411795 -4.658276 -2.520812

H $-2.013578-2.734788-2.862400$

O $0.1012552 .418623-1.157834$

O $1.8926901 .304263-2.094753$

C $0.7495403 .673269-1.386323$

H $1.6742403 .731894-0.805792$

H $\quad 0.952613 \quad 3.807929-2.452945$

H $0.0759194 .468131-1.055062$

SCF Energy $(B 3 L Y P / 6-31 G * * / / M M F F)=-3245.90721029$

$01 \_87$

MM̄FF Geometry

C $-2.885658-2.793720-2.721321$

C $-1.906689-2.945260-1.816289$

C $-0.441102-2.805286-2.140354$

O $0.135409-1.840007-1.230758$

C $0.274503-4.152740-1.932367$

C $1.777578-4.096805-2.137950$

C $2.677790-4.521307-1.228966$

C $2.250195-3.549246-3.459675$

C $2.395879-5.1720990 .095157$

C $2.311307-4.2038831 .282631$

C $3.651979-3.5393571 .632563$

C $3.593959-2.9242723 .032716$

C $4.899202-2.2024453 .390706$

O $3.920925-2.5122670 .672384$

O $4.638644-1.4284874 .571525$

C $5.259108-1.2278142 .251265$

C $6.011108-3.1913263 .760969$

O $6.540947-0.6535732 .498618$

C $5.192163-1.8690350 .841413$

C $5.326610-0.772212-0.239371$

O $6.272242-2.7936260 .694926$

C $0.087251-0.534007-1.609998$

O $-0.435269-0.081085-2.616316$

C $0.8085130 .261097-0.590744$

C $0.9186991 .586630-0.763682$

C 1.6281552 .5267310 .104387

C 2.2603511 .9887861 .359283

C $4.964013-1.222832-1.658857$

C $5.275516-0.111374-2.640725$

C $4.5003732 .158784-3.329240$

O $6.291805-0.129309-3.331221$

C $4.7605623 .233875-2.319346$

C $4.0976564 .399861-2.288986$

C $4.3247755 .461517-1.249460$

C $3.0437655 .900576-0.570148$

C $2.5253967 .119657-0.796421$

C 2.3957614 .9540410 .422552

C $1.6699843 .825756-0.258098$

N $4.3316170 .903190-2.619912$

H $-2.603641-2.558681-3.746361$

H $-2.146487-3.180108-0.783247$

H $-0.299228-2.475723-3.177334$

H $-0.139312-4.897440-2.625032$

H $0.042633-4.508722-0.922707$

H $3.736341-4.427391-1.467725$

H $3.327338-3.686644-3.603851$

H $1.743320-4.060383-4.284673$

H $2.045006-2.476730-3.531094$

H $1.469243-5.7548040 .044378$

H $3.181739-5.9145120 .284827$

H $1.554850-3.4366091 .074425$

H $1.960793-4.7819322 .146833$

H $4.440046-4.3000141 .585394$

H $3.361847-3.686693 \quad 3.786829$

H $2.769613-2.1981913 .076760$

H $5.440239-0.9138934 .768952$

H $4.533410-0.4042222 .300449$

H $5.707949-3.7996524 .621658$

H $6.921327-2.6668554 .072049$

H $6.269072-3.8720222 .946984$

H 6.6558110 .0947891 .889711

H 4.6712050 .0678630 .016976

H $6.353281-0.384126-0.247224$

H $6.484831-2.885830-0.247298$ 
H $\quad 1.245348-0.2632240 .251338$ H $\quad 0.4600472 .032671-1.645429$ H 3.0862281 .3156661 .114938 H 1.5252441 .4386791 .957610 H 2.6563032 .7694472 .013001 H $5.528949-2.110555-1.961152$ H $3.900447-1.474732-1.731490$ H $3.5813182 .338287-3.895657$ H $5.3407732 .088442-4.026100$ H $5.5246903 .038622-1.570150$ H $3.3491714 .605170-3.051172$ H $4.8021346 .315859-1.746183$ H $5.0343135 .123575-0.483824$ H $1.6197007 .450104-0.298681$ H $2.9918397 .813311-1.488158$ H 1.6669555 .4963851 .037997 H 3.1733894 .5979821 .105199 H $1.1300104 .125043-1.157740$ H $3.5291380 .817302-2.003065$ C $-4.837315-3.191742-1.063777$ O $-4.560828-2.055353-0.220763$ C $-5.625203-1.922100 \quad 0.741138$ C $-6.552798-3.1150590 .528791$ C $-6.343626-3.412597-0.942522$ H $-6.171214-1.0075670 .485827$ C $-5.051335-1.8189172 .158816$ H $-6.254789-3.9754261 .139571$ H $-7.592331-2.8779380 .775922$ C $-6.808361-4.800374-1.348540$ H $-6.883263-2.660819-1.534218$ C $-4.094104-0.6291502 .385714$ O $-4.328025-3.0215262 .456526$ H $-5.872043-1.7768502 .884448$ H -3.166996 -0.7911161 .823475$ O $-3.743296-0.6555353 .775788$ C $-4.699996 \quad 0.7455652 .040798$ C $-3.868974 \quad 1.9624402 .487221$ H $-4.867967 \quad 0.8076730 .960846$ O H -4.4499772 .8622962 .249432$ H $-3.790261 \quad 1.971783 \quad 3.581654$ H $-6.639755-4.973991-2.415742$ H $-6.277353-5.580209-0.792346$ H -7.879374 -4.919108 -1.153966 C $-4.371992-2.909963-2.494687$ H $-3.620520-3.1091221 .794778$ H -2.952915 -0.102389 3.892929 H -5.8634440 .7654443 .616284$ H $-4.823719-1.971283-2.838217$ H -4.736207 -3.703543 -3.157599 H $-4.299059-4.052312-0.645347$ C -3.098983 $3.060309-0.240448$ O -2.5024641 .9439850 .430190$ C -2.4665662 .0502511 .855885$ C -1.675255 3.3004602 .266824 C -2.2051734 .5612471 .588508$ C -2.338842 4.3512800 .081018 H -4.1481153 .1558930 .064409$ C $-3.0671612 .765709-1.743112$ H -1.8989561 .1827742 .212501$ O -1.7032973 .4825653 .678551$ H -0.6240723 .1735391 .983766$ H -3.158701 4.8809182 .024934 O -1.2859435 .6340981 .808363$ H -1.342055 4.324966 -0.374610 O $-3.0352725 .459011-0.486149$ C -3.966682 $1.602690-2.080932$ H $-3.381743 \quad 3.628507-2.340938$ H -2.054548 $2.501852-2.070018$ H -1.3401952 .6766074 .084184$ H -1.1789795 .7303752 .770358$ H -2.542466 $6.263358-0.248840$ O $-5.2628652 .018254-2.155292$ O $\quad-3.564130 \quad 0.458556-2.242502$ C $-6.2175420 .997011-2.459995$ H $-6.0058900 .560999-3.441000$ H $-6.2082240 .225762-1.684863$ H $-7.2095661 .455585-2.486173$
SCF Energy (B3LYP/6-31G**//MMFF) $=-3245.90454294$

01888

MM̄FF Geometry

C $-0.918940-2.6246951 .523183$

C -1.834008 -2.469123 2.492277

C -2.064685 -1.232224 3.326761

O $-0.938329-0.3251373 .265336$

C -3.297175 -0.444882 2.845049

C $-4.614883-1.1557053 .061001$

C $-5.296460-1.8157832 .104180$

C $-5.170715-1.0624214 .458210$

C $-4.900074-2.0341000 .669770$

C $-5.909207-1.433839-0.316928$

C $-5.9371770 .103004-0.314107$

C $-7.0242610 .608870-1.265787$

C -7.020742 $2.138685-1.378969$

O $-4.6528380 .572264-0.738412$

O $-7.8354312 .500491-2.504408$

C $-5.5857752 .607648-1.690190$

C $-7.6537942 .805584-0.152105$

O $-5.5048924 .031792-1.668796$

C $-4.5148262 .000547-0.752074$

C $-3.1055222 .332355-1.300990$

$\begin{array}{llllll}\text { O } & -4.662661 & 2.558007 & 0.553517\end{array}$

C $0.096808-0.5606614 .113324$

O $0.202019-1.5017144 .889063$

C 1.1394550 .4866904 .023217

C 1.0772081 .5510343 .207865

C 2.0735842 .6233433 .115135

C 3.3051312 .5122183 .976349

C $-1.9447721 .830358-0.431353$

C $-0.6243791 .704229-1.172454$

C $0.8902562 .864580-2.780089$

O $0.1060930 .730704-0.992052$

C $1.9400253 .730727-2.148206$

C $1.8221094 .480766-1.041028$

C $2.9228885 .342883-0.492628$

C 3.5157414 .8103910 .793038

C 4.7875344 .3787200 .834662

C 2.6822024 .8798632 .052973

C 1.8406793 .6519212 .272352

N $-0.3228062 .780710-1.988189$

H $-0.245763-1.8102621 .270173$

H -2.495160 -3.304743 2.715138

H $-2.213502-1.5322144 .371714$

H -3.166817 -0.1602061 .795768$

H -3.3306150 .5213573 .367816$

H $-6.242063-2.2814502 .379861$

H $-6.139072-1.5646814 .555587$

H $-4.486385-1.5253705 .175750$

H -5.314209-0.014074 4.739997

H $-3.897503-1.6594700 .448068$

H $-4.857054-3.1178520 .502686$

H -5.634434 -1.784479-1.320499

H $-6.911365-1.825435-0.101523$

H -6.1324820 .4577340 .703804$

H $-8.012817 \quad 0.249735-0.953333$

H $-6.8564460 .188369-2.267414$

H $-8.7284982 .145812-2.354121$

H $-5.3555842 .300201-2.720107$

H -8.693438 2.477711 -0.032149

H -7.695531 $3.893867-0.271425$

H -7.1285482 .5799250 .778303$

H $-6.1831944 .369526-2.278545$

H $-3.0060961 .897378-2.304242$

H -3.003948 $3.419770-1.401894$

H -3.8584272 .3762571 .064661$

H 1.9792190 .3352324 .694648

H $0.214742 \quad 1.6645732 .553410$

H 3.8418721 .5809903 .764069

H 3.0346502 .5286465 .037805

H 4.0225833 .3200973 .819439

H -1.7849562 .4923540 .427004$

H $-2.1784120 .832735-0.044822$

H $\quad 0.613346 \quad 3.279382-3.754353$

H $1.2896891 .858617-2.938540$

H $2.8950993 .735939-2.670771$ 
H $\quad 0.876621 \quad 4.514274-0.507806$ H $2.5263036 .350604-0.316374$ H $3.7085415 .470378-1.249290$ H 5.2377564 .0296381 .757592 H $5.4082124 .361422-0.055104$ H 2.0168235 .7516222 .003261 H 3.3223755 .0844782 .918219 H $\quad 0.9190293 .6402971 .690897$ H $-0.9344533 .590319-1.994118$ C -1.049121 -3.697477 -0.745154 O $0.033523-2.959159-1.349322$ C $0.451979-3.642965-2.544562$ C $-0.678574-4.602446-2.890330$ C -1.140012 -5.019968-1.510023 H $1.342215-4.226017-2.279456$ C $0.795889-2.643131-3.652096$ H -1.492248 -4.098320 -3.424397 H $-0.338913-5.442450-3.503760$ C $-2.525641-5.643185-1.495568$ H $-0.418865-5.741146-1.102186$ C $1.845430-1.584779-3.262474$ O $-0.393137-1.939513-4.038010$ H $1.125913-3.184566-4.546292$ H $1.406644-0.882944-2.547886$ O $2.150368-0.823825-4.438679$ C $3.160459-2.145545-2.692325$ C $4.278798-1.099857-2.522044$ H $2.967849-2.621261-1.726635$ O $3.657112-3.169472-3.559065$ H $5.172633-1.623701-2.162020$ H $4.560826-0.714133-3.509731$ H -2.821738 $-5.913588-0.477168$ H $-3.279323-4.954954-1.893041$ H $-2.544917-6.551846-2.105936$ C $-0.765782-3.9015830 .743943$ H -0.745609 -1.502305 -3.243562 H $1.312235-0.461062-4.773463$ H $\quad 3.792638-2.771472-4.436204$ H $\quad 0.260623-4.2604190 .883166$ H -1.435699 -4.669809 1.147839 H -1.960435 -3.103048 -0.890434 C $4.641410-0.8881830 .520605$ O $3.560729-0.375892-0.268809$ C $3.9211620 .066674-1.581053$

C $4.9963421 .157209-1.497643$

C $6.2049400 .696548-0.692953$

C $5.759818 \quad 0.154813 \quad 0.662543$ H $5.034007-1.8022470 .058735$ C $4.076313-1.2459321 .904791$ H $3.0297320 .549065-1.993539$ O $5.421411 \quad 1.561555-2.794519$ H $4.5718562 .042655-1.010352$ H $6.795422-0.047290-1.240393$ O $7.0724171 .809731-0.466036$ H $5.4197120 .986738 \quad 1.293109$ O $6.879216-0.4437991 .313542$ C $3.248078-2.5124601 .901419$ H $4.898075-1.4036242 .612645$ H $3.435668-0.4374772 .269823$ H $4.6327741 .855391-3.281826$ H $7.3082492 .173999-1.336575$ H $7.575641 \quad 0.232762 \quad 1.373513$ O $3.040438-2.9001303 .195325$ O $2.826639-3.0804310 .904516$ C $2.300906-4.1109773 .383897$ H $1.232955-3.9062533 .281582$ H $2.615502-4.8854922 .677746$ H $2.492996-4.4693914 .398760$ SCF Energy (B3LYP/6-31G**//MMFF) $=-3245.92159039$

\section{9}

MM̄FF Geometry

C $2.548773-2.3989840 .986063$

C $1.851739-1.8804462 .007734$

C $1.995318-0.4511002 .456489$

O 0.6830690 .1617792 .528402

C $2.593413-0.3329753 .867954$

C $4.041410-0.7499053 .981528$
C $5.083623 \quad 0.0698273 .744945$

C $4.281633-2.1561444 .466844$

C 5.0439091 .5054953 .292491

C 6.0514231 .7777842 .165381

C $5.577595 \quad 1.2832730 .788175$

C $6.7647851 .221893-0.176591$

C $6.3312000 .849380-1.597950$

O $4.5982642 .214435 \quad 0.311076$

O $7.4557751 .089953-2.456585$

C $5.1947761 .798022-2.022470$

C $6.009114-0.643410-1.717323$

O $4.6785001 .424033-3.298930$

C $4.0533431 .918209-0.981854$

C $3.1309233 .094117-1.387875$

O $3.3104870 .700649-0.975235$

C 0.0536580 .4638561 .362088

$\begin{array}{lllll}\text { O } & 0.429834 & 0.203729 & 0.231059\end{array}$

C $-1.1833391 .196288 \quad 1.710959$

C -1.9670381 .6808410 .736911$

C -3.2149872 .4225680 .925241$

C -3.5954512 .7974162 .334206$

C $1.9918793 .400433-0.406638$

C $1.2696004 .690050-0.764883$

C $-0.5768226 .160460-0.004925$

O $1.6299885 .429395-1.677407$

C -1.9938045 .9146600 .402348$

C $-3.0363086 .089730-0.424068$

C $-4.4735405 .911755-0.024951$

C $-5.1706654 .834103-0.824786$

C $-5.7497145 .113269-2.004742$

C $-5.2698793 .455763-0.212686$

C -3.945026 $2.744753-0.163055$

N $0.173893 \quad 4.920172 \quad 0.058534$

H $3.281301-1.7700570 .488821$

H $1.138411-2.4986792 .543904$

H 2.6037690 .1336921 .755778

H $1.983992-0.9060514 .581152$

H $2.4815240 .703278 \quad 4.215518$

H $6.085790-0.3240323 .911567$

H $5.347681-2.3811724 .578052$

H $3.811947-2.3042115 .444962$

H $3.865702-2.8875803 .768770$

H 4.0430991 .8203332 .982502

H 5.3207972 .1251874 .154305

H 6.2138602 .8625992 .116446

H 7.0151481 .3207752 .423152

H 5.1176340 .2943140 .888570

H 7.5254620 .5192600 .186374

H $7.2580482 .203872-0.209577$

H $7.1666660 .932264-3.371842$

H $5.6456722 .790363-2.160650$

H $6.888756-1.245375-1.459216$

H $5.755184-0.914147-2.747917$

H $5.190946-0.956124-1.066365$

H $4.0972820 .655242-3.168919$

H $3.7467223 .998309-1.484161$

H $2.6864662 .897250-2.371962$

H $2.4229780 .871968-0.624101$

H $-1.414377 \quad 1.3194612 .763071$

H $-1.6748991 .512561-0.298377$

H -2.7876893 .3639022 .811521$

H -3.793989 1.9029342 .932883

H -4.4841873 .4285302 .394658$

H $1.2552022 .593271-0.421696$

H 2.3783793 .5042370 .613148

H $-0.5042146 .585078-1.011847$

H $-0.106247 \quad 6.8647190 .689051$

H $-2.1702175 .612453 \quad 1.431377$

H $-2.8511416 .411980-1.446620$

H $-4.9858576 .871265-0.176326$

H -4.5679185 .7001651 .047100$

H -6.276934 4.353008 -2.571164

H $-5.7049306 .109789-2.431807$

H $-5.9503722 .826019-0.801799$

H $-5.747111 \quad 3.5341240 .768389$

H $-3.5775772 .440644-1.144269$

H 0.0520924 .3303120 .877251

C $2.009367-3.955133-0.970528$ 
O $0.654969-3.501802-1.179789$

C $0.600543-2.809180-2.441103$

C $1.916818-3.113206-3.155728$

C $2.875614-3.215667-1.990452$

H $0.570338-1.739607-2.204253$

C $-0.632665-3.203011-3.262003$

H $1.878199-4.065236-3.697763$

H $2.189514-2.330510-3.870386$

C $4.177309-3.922114-2.324300$

H $3.098403-2.195823-1.659503$

C $-2.011457-2.987855-2.605715$

O $-0.545994-4.593691-3.605943$

H -0.602802 -2.663075-4.216689

H -2.215680 -3.783968 -1.879898

O $-2.985695-3.139262-3.650036$

C $-2.227966-1.623158-1.932820$

C $-3.696177-1.237784-1.668421$

H $-1.665709-1.600161-0.993245$

O $-1.677260-0.599429-2.770275$

H -3.681317 $-0.330709-1.053256$

H $-4.150706-0.918401-2.616103$

H $4.832158-3.964709-1.448351$

H $4.002047-4.947751-2.665762$

H $4.710732-3.390993-3.119395$

C $2.420728-3.8149160 .500319$

H $-0.486221-5.096313-2.775197$

H -2.857066 -4.020724 -4.040706

H $-1.7674520 .246261-2.299474$

H $3.388052-4.3091810 .650378$

H $1.691051-4.3501111 .118134$

H $1.997712-5.027424-1.208766$

C $-3.936619-2.0236111 .292899$

O $-4.028586-2.8883610 .157586$

C $-4.597152-2.302286-1.012094$

C $-6.015404-1.798388-0.707097$

C $-6.026808-0.8312120 .473723$

C $-5.312315-1.452867 \quad 1.671798$

H -3.229139-1.214808 1.077201

C $-3.384187-2.8555552 .461256$

H $-4.705298-3.118524-1.735623$

O $-6.572262-1.171030-1.857548$

H $-6.655481-2.657852-0.468334$

H $-5.589600 \quad 0.1344300 .199112$

O $-7.389280-0.570073 \quad 0.818613$

H -5.944435 -2.246702 2.090887

O $-5.137472-0.468846 \quad 2.687834$

C -2.033420 -3.4665382 .164658$

H $-3.283174-2.2442923 .364371$

H $-4.077995-3.6812272 .665271$

H -7.456586 -0.848322 -1.613443

H -7.392366 0.1265121 .496366

H $-6.019620-0.1963742 .991100$

O $-1.835779-4.5240433 .006265$

O $-1.249761-3.062757 \quad 1.317695$

C $-0.617068-5.2513422 .827872$

H $0.220178-4.6711613 .223903$

H $-0.693818-6.1848583 .391753$

H -0.458491 -5.495714 1.773060

SCF Energy (B3LYP/6-31G**//MMFF)= -3245.92151225

\section{9}

MM̄FF Geometry

C -3.7641940 .0563112 .512954$

C $-3.9377291 .103807 \quad 3.333851$

C $-2.9076002 .167903 \quad 3.643210$

O -1.6272381 .8364643 .062097$

C -3.3586543 .5126553 .040565$

C -2.3888804 .6500053 .302246$

C $-1.679777 \quad 5.287107 \quad 2.348593$

C -2.2530475 .0688594 .743691$

C $-1.706490 \quad 5.0501300 .862911$

C $-0.584505 \quad 4.108048 \quad 0.427436$

C $-0.477598 \quad 3.921697-1.093264$

C $-1.710967 \quad 3.236034-1.688634$

C $-1.5341922 .928845-3.183390$

O $0.686722 \quad 3.113541-1.319189$

O $-2.5736952 .020640-3.574861$

C $-0.1943892 .194864-3.386344$
C $-1.7093384 .182604-4.048504$

O $0.077565 \quad 2.005311-4.773073$

C $1.0017082 .894817-2.700756$

C $2.2492401 .981737-2.771554$

O $1.2783844 .119976-3.381232$

C -0.7404521 .1361163 .818522$

$\begin{array}{lllll}\text { O } & -0.890168 & 0.746741 & 4.964663\end{array}$

C 0.4576470 .9092552 .979601

C $1.4787650 .171983 \quad 3.439777$

C $2.700060-0.1481762 .697491$

$\begin{array}{llll}\text { C } 2.885100 & 0.498631 & 1.349327\end{array}$

C $3.4401992 .470336-1.937629$

C $4.6390801 .541557-2.048434$

C $6.9494571 .262564-1.137488$

$\begin{array}{llll}\text { O } & 4.681273 & 0.579963 & -2.810378\end{array}$

C $6.876706 \quad 0.138067-0.153957$

C $7.314370 \quad 0.229364 \quad 1.110956$

C $7.295931-0.9065282 .093663$

C $6.139566-0.8046903 .064968$

C $6.290308-0.1589364 .234106$

C $4.869263-1.5070842 .630802$

C $3.596329-0.9940133 .247903$

N $5.6673681 .936943-1.204466$

H -2.811844 -0.0822162 .008436$

H $-4.900532 \quad 1.226528 \quad 3.825916$

H -2.8161102 .2574514 .732312$

H -4.3394873 .7943213 .446366$

H -3.5089053 .3769161 .964246$

H -1.0009996 .0810332 .658356$

H $-1.639107 \quad 5.968758 \quad 4.858407$

H -3.2375415 .2896335 .168938$

H -1.7860624 .2755265 .334963$

H -2.681689 4.6726300 .545248

$\begin{array}{llll}H & -1.588094 & 6.019591 & 0.362937\end{array}$

H $0.378946 \quad 4.4804520 .802186$

H $-0.716954 \quad 3.1265940 .896926$

H $-0.3197284 .905312-1.550684$

H -2.613971 $3.838798-1.539443$

H -1.891376 2.289499-1.159500

H -3.429248 $2.438695-3.376850$

H $-0.3019561 .191395-2.952293$

H $-2.7139324 .601964-3.915540$

H -1.624387 $3.942435-5.114075$

H $-0.9913344 .970871-3.813232$

H $-0.6899051 .549353-5.159133$

H $1.9811930 .983833-2.404608$

H $2.5687661 .871695-3.815518$

H $2.2066214 .362279-3.241247$

H $0.459448 \quad 1.3434381 .986573$

H $1.408465-0.253998 \quad 4.439428$

H $2.8217601 .589217 \quad 1.436541$

H $2.116316 \quad 0.1574050 .648369$

H 3.8544190 .2873390 .893952

H $3.7646903 .462244-2.268736$

H $3.1560602 .539136-0.882273$

H $7.6969612 .008710-0.848854$

H $7.215570 \quad 0.875912-2.126785$

H $6.465383-0.802830-0.511071$

H 7.7225841 .1734091 .465146

H $8.251128-0.9060132 .634943$

H $7.261100-1.8744731 .577334$

H $5.491835-0.0880244 .963810$

H $7.231843 \quad 0.3147414 .493822$

H $4.964172-2.5732702 .874983$

H $4.788683-1.4777391 .539265$

H $3.388609-1.4063374 .235735$

H $5.5084122 .706603-0.560719$

C -5.185853 -0.967805 0.731922

O $-4.052833-1.380192-0.058093$

C $-4.523575-2.157336-1.175899$

C $-6.048198-2.123035-1.115622$

C $-6.298252-1.9478830 .369524$

H -4.189528 $-3.187170-1.008636$

C -3.928992 -1.616994 -2.481029

H $-6.459073-1.274286-1.674604$

H $-6.496940-3.034759-1.521950$

C $-7.696891-1.4524680 .692817$

H $-6.129019-2.9104250 .870658$ 
C $-2.386256-1.574837-2.515646$ O $-4.402128-0.279827-2.690573$ H -4.305018 -2.202539-3.327763 H -2.015372 -0.821474 -1.811144 O $-1.996747-1.132939-3.822744$ C -1.704557 -2.928582 -2.242572 C $-0.188053-2.949348-2.512043$ H -1.889875 -3.231582 -1.207304 O $-2.297887-3.933512-3.071243$ H $0.154669-3.985353-2.399593$ H $-0.001085-2.711598-3.566835$ H -7.832242 $-1.338278 \quad 1.772694$ H -7.897863 $-0.483506 \quad 0.223811$ H -8.448605 -2.162684 0.332892 C $-4.819472-0.9725402 .216197$ H $-4.1124540 .256169-1.932324$ H $-2.419577-0.269837-3.973756$ H $-2.173117-3.661878-3.996874$ H -4.421286 -1.953136 2.502119 H -5.712055 -0.791051 2.825226 H $\quad-5.449001 \quad 0.0488260 .409563$ C $0.847472-3.4775920 .297010$ O $0.370999-2.227718-0.216232$ C $0.634179-2.011907-1.606580$ C $2.147642-2.059108-1.867414$ C $2.779382-3.342231-1.332981$ C $2.365857-3.5883180 .117585$ H $0.346926-4.304184-0.220959$ C $\quad 0.471199-3.5473761 .780959$ H $0.306650-0.988924-1.824403$ O $2.430771-1.943298-3.257863$ H $2.626073-1.206399-1.370462$ H $2.519678-4.200340-1.963219$ O $4.204109-3.231632-1.398161$ H $2.871301-2.8653690 .767361$ O $2.794168-4.8890740 .513713$ C -1.024016 -3.575913 1.981702 H $0.894578-4.4302732 .272914$ H $0.851184-2.6680452 .312680$ H $2.026555-1.116819-3.572359$ H $4.471262-2.463775-0.864694$ H $3.754298-4.9362510 .365153$ O $-1.535140-4.7646191 .553333$ O $-1.658073-2.6529962 .475794$ C $-2.949478-4.9163221 .707679$ H $-3.479394-4.1218341 .175436$ H -3.237572 -5.8788541 .276841$ H $-3.214468-4.9120572 .769195$

SCF Energy (B3LYP/6-31G**//MMFF) $=-3245.92158686$

\section{0}

MMFF Geometry

C $-0.196494-3.5953770 .413465$

C $0.432810-2.4476210 .117951$

C $0.962063-2.127451-1.256031$

O $0.433374-0.828994-1.615036$

C $2.498390-2.046783-1.269290$

C $3.193260-3.371686-1.042218$

C $3.953770-3.6669960 .029866$

C $3.040288-4.379385-2.153345$

C $4.247817-2.8108201 .231289$

C $5.693418-2.2907841 .227166$

C $5.914635-1.1433500 .229061$

C $7.401135-0.8084790 .100089$

C $7.6307920 .393963-0.828470$

O $5.202518-0.001498 \quad 0.718055$

O $8.988436 \quad 0.828947-0.659531$

C $6.723117 \quad 1.554636-0.372440$

C $7.4760350 .016988-2.306523$

O $6.7882692 .645537-1.288500$

C $5.2490331 .142275-0.143816$

C 4.4881572 .3016080 .545292

O $4.6331020 .872594-1.405085$

C $0.292600-0.544290-2.936401$

O $0.540086-1.289463-3.874578$

C $-0.2491820 .818996-3.141041$

C $-0.2004321 .763370-2.190078$

C $-0.6818313 .140459-2.299432$
C $-1.4280193 .543840-3.541407$

C 3.1278061 .9000301 .129793

C 2.4083563 .0845051 .754873

C 0.4427043 .6038983 .204338

O $2.6968254 .252473 \quad 1.508661$

C -0.7415793 .7221952 .301444$

C -1.2619334 .8773271 .859789$

C -2.4145924 .9207420 .894175$

C $-2.1111395 .665333-0.391858$

C $-3.0056616 .538808-0.885835$

C $-0.8061655 .425577-1.125012$

C $-0.4435933 .974057-1.267612$

N 1.3790092 .6770962 .591803

H $-0.325026-4.326283-0.383242$

H $\quad 0.591902-1.695050 \quad 0.884724$

H $0.603292-2.868026-1.980095$

H $2.823490-1.285263-0.551534$

H $2.832938-1.649456-2.237232$

H $4.425924-4.6484840 .064485$

H $3.663688-5.266527-1.999029$

H $2.002551-4.717674-2.228964$

H $3.334239-3.936734-3.110778$

H $3.537963-1.9856761 .341196$

H $4.106932-3.4397272 .119621$

H $5.922144-1.9321012 .239083$

H $6.381618-3.1177021 .011676$

H $5.512887-1.432789-0.747367$

H $7.967883-1.678837-0.253636$

H $7.809575-0.5662411 .091198$

H $9.5717230 .081818-0.877533$

H 7.1279661 .9262190 .579654

H $8.187497-0.772608-2.576508$

H $7.7111890 .866545-2.957136$

H $6.475155-0.341427-2.556005$

H $7.7261072 .881788-1.391443$

H 5.0920362 .6917561 .375314

H $4.3494193 .124304-0.167596$

H $3.6783901 .030098-1.331645$

H $-0.6499981 .016541-4.129642$

H $0.2534551 .515016-1.231722$

H $-2.2702592 .868381-3.725988$

H $-0.7640233 .521208-4.412200$

H -1.849646 $4.550639-3.479042$

H $2.473743 \quad 1.491346 \quad 0.352206$

H 3.2658841 .1312341 .899053

H $0.933380 \quad 4.5672503 .374985$

H $\quad 0.141740 \quad 3.1779154 .165887$

H $-1.1794582 .788114 \quad 1.957651$

H -0.8398095 .8229792 .189468$

H -3.2626295 .3841651 .414558$

H -2.7369813 .9044640 .633042$

H -2.816713 $7.085392-1.803978$

H $-3.9496196 .727608-0.385097$

H $0.0014655 .921446-0.570133$

H $-0.8083965 .912604-2.107302$

H $0.1149353 .584316-0.417480$

H $\quad 1.1654771 .6859742 .659189$

C $-0.699076-3.0240372 .897950$

O $-1.593708-1.9272602 .622650$

C $-2.441609-1.7156243 .766014$

C $-1.743654-2.4101324 .925831$

C -1.166824 -3.625896 4.227443

H $-3.384718-2.2366263 .559827$

C $-2.714902-0.2211323 .964616$

H $-0.936751-1.7969915 .343181$

H $-2.433269-2.6651305 .735919$

C $-0.063024-4.3120565 .013875$

H -1.977622 -4.345594 4.050405

C -3.2098640 .5064762 .697767$

O

H $-3.428017-0.0816264 .784982$

H -2.386689 0.5764261 .978620

O $-3.547361 \quad 1.8510793 .063327$

C $-4.440905-0.1272732 .030712$

C -4.9118620 .6233110 .769590$

H $-4.233045-1.1694881 .771064$

O $-5.511535-0.1420682 .978115$

H $\quad \begin{array}{llll}-5.892472 & 0.223184 & 0.485276\end{array}$ 
H $-5.1016241 .676566 \quad 1.010531$ H $0.332502-5.171017 \quad 4.462808$ H $0.769392-3.6295955 .216394$ H $-0.443052-4.6728155 .975247$ C $-0.755354-4.0300361 .743348$ H -0.8349720 .2686453 .695849$ H -2.808622 2.2073303 .585363 H $-6.264458-0.5971072 .563859$ H $-1.803220-4.306871 \quad 1.568065$ H $-0.223694-4.9440762 .033490$ H $0.308591-2.6044493 .005299$ C $-4.580264-1.666156-1.197452$ O $-3.537161-0.836155-0.676249$ C $-3.929360 \quad 0.517698-0.417888$ C $-4.4349861 .168070-1.713341$ C $-5.5543880 .355882-2.363482$ C $-5.135986-1.108282-2.515082$ H $-5.393015-1.711623-0.461778$ C $-4.057409-3.106625-1.302176$ H -3.016899 $1.052055-0.128113$ O $-4.8953022 .492806-1.471098$ H -3.606332 $1.239568-2.428624$ H $-6.4916300 .442846-1.801832$ O $-5.819738 \quad 0.882920-3.665845$ H $-4.389975-1.171375-3.314425$ O $-6.255980-1.882734-2.938202$ C -2.924484 -3.302385-2.286781 H $-3.678386-3.423659-0.323104$ H $-4.882169-3.768426-1.590284$ H $-4.1497632 .997628-1.103827$ H $-6.0410271 .823860-3.557185$ H $-6.939098-1.822347-2.248808$ O $-2.764862-4.639449-2.517618$ O $-2.246946-2.408961-2.773353$ C $-1.722256-4.986241-3.434856$ H $-1.889426-4.504549-4.402999$ H $-0.749095-4.701935-3.024870$ H $-1.740200-6.070222-3.575394$

SCF Energy (B3LYP/6-31G**/MMFF) $=-3245.90138008$

\section{1}

MMFF Geometry

C $5.065788 \quad 2.867329-1.638297$

C $3.8534703 .122766-1.122545$

C $2.623283 \quad 3.338179-1.966460$

O $1.6049182 .386523-1.580744$

C $2.069504 \quad 4.754028-1.712267$

C $0.964780 \quad 5.154553-2.671038$

C $-0.3319935 .315683-2.340916$

C $1.4093705 .421389-4.088155$

C $-0.9948865 .136961-1.002316$

C $-1.8607153 .878433-0.999563$

C $-2.771500 \quad 3.730235 \quad 0.226612$

C $-2.003547 \quad 3.644177 \quad 1.545498$

C -2.946436 3.3796892 .731437

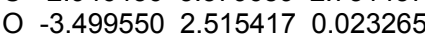

O -2.1450893 .0019753 .861221$

C -3.8547142 .1737842 .404450$

C $-3.6954054 .652597 \quad 3.145312$

O -4.8602702 .0041083 .400358$

C -4.506743 2.2365351 .004296

C -5.1143520 .8653630 .634020$

$\begin{array}{lllll}\text { O } & -5.573645 & 3.184244 & 0.951847\end{array}$

C $1.6458311 .154591-2.159917$

O $2.506387 \quad 0.721609-2.909473$

C $0.4259500 .424969-1.743744$

C $0.174576-0.802889-2.221793$

C $-1.007014-1.619454-1.923695$

C $-2.110216-0.977332-1.123026$

C $-5.7419050 .854120-0.766464$

C $-6.027060-0.549862-1.268911$

C $-6.537477-1.751665-3.397209$

O $-5.959058-1.549669-0.560199$

C $-5.205464-2.142515-3.958622$

C $-4.712748-3.389004-3.907812$

C $-3.366065-3.768290-4.456441$

C $-2.546979-4.579142-3.476090$

C $-2.213553-5.852797-3.745717$
C $-2.121249-3.925082-2.178608$

C $-1.045786-2.889293-2.380613$

N $-6.369158-0.541286-2.614994$

H $5.1802352 .786978-2.716366$

H $3.7380433 .206335-0.044422$

H $2.8564143 .213422-3.030911$

H $2.8767805 .493375-1.806139$

H $1.7260834 .821920-0.674838$

H $-1.0213845 .616762-3.129845$

H $0.6024595 .826897-4.708122$

H $2.2255776 .151105-4.099366$

H $1.7571314 .500614-4.565725$

H $-0.2668385 .096386-0.189443$

H $-1.6194246 .018439-0.812792$

H $-2.4950113 .858708-1.897070$

H $-1.2212472 .992788-1.086116$

H $-3.474644 \quad 4.571024 \quad 0.236456$

H -1.421662 4.5547631 .729014

H -1.2731212 .8255101 .489079$

H -1.5045723 .7145714 .027652$

H -3.2272591 .2736652 .445563$

H -2.987080 5.4253653 .467918

H -4.3472054 .4674124 .005988$

H -4.2962665 .0776742 .338493$

H -4.4082691 .9314414 .258589$

H -4.3274870 .1017410 .676360$

H -5.8860350 .5803751 .360060$

H -5.6723623 .5946371 .825192$

H $-0.2454350 .925438-1.056495$

H $0.899246-1.256940-2.896365$

H $-2.487693-0.086358-1.637704$

H $-1.750963-0.681561-0.131713$

H -2.969505 -1.629298 -0.959190

H $-6.6843021 .413651-0.761025$

H -5.067266 $1.331230-1.486799$

H $-7.233678-1.520775-4.208892$

H $-6.967843-2.536009-2.766197$

H $-4.615896-1.359566-4.430774$

H $-5.310289-4.179871-3.460209$

H -2.794133 - $2.880214-4.752082$

H $-3.533179-4.341714-5.377301$

H $-1.628409-6.444063-3.049128$

H $-2.516515-6.334175-4.669683$

H $-1.717050-4.685730-1.498340$

H $-3.006383-3.532165-1.673362$

H $-0.183493-3.260745-2.936663$

H $-6.3277610 .337320-3.123714$

C $6.7739531 .290570-0.585829$

$\begin{array}{lllll}\text { O } & 5.815701 & 0.601017 & 0.236790\end{array}$

C $5.727058-0.766456-0.209870$

C $6.875363-0.968660-1.193877$

C $6.9778050 .412058-1.818278$

H $4.781640-0.856070-0.758217$

C $5.718520-1.7105920 .998246$

H $7.810701-1.224877-0.683276$

H $6.662663-1.756429-1.922884$

C $8.2869220 .661757-2.544835$

H $6.1470610 .528724-2.525146$

C $4.659990-1.3512962 .063743$

O $7.000979-1.672678 \quad 1.636936$

H $5.582082-2.7435380 .658857$

H $4.939633-0.4131532 .557485$

O $4.698999-2.3566813 .083979$

C $3.224730-1.2396381 .521024$

C $2.214336-0.8746892 .625970$

H $3.186649-0.4642280 .748385$

$\begin{array}{lllll}\text { O } & 2.878570 & -2.478504 & 0.911090\end{array}$

H $2.185588-1.6732493 .373318$

H 2.5748660 .0303653 .128077

H $8.3140881 .674564-2.959123$

H $9.1463470 .547417-1.875674$

H $8.408933-0.045456-3.371738$

C $6.3162282 .730785-0.816188$

H $7.170836-0.7520181 .901272$

H $4.406125-3.1926852 .682148$

H $2.010601-2.3679300 .487324$

H $7.1137953 .295819-1.313114$

H 6.1581553 .2080290 .159842 
H $7.702309 \quad 1.3247440 .000421$ C $-0.205713-2.7787622 .514918$ O $0.202174-1.8152071 .535650$ C $0.798313-0.6247802 .061121$ C -0.1688150 .0671943 .028236$ C $-0.664264-0.8762074 .117637$ C $-1.209630-2.1608183 .496419$ H $0.672858-3.1445933 .059320$ C $-0.828575-3.9635111 .767792$ H $\quad 0.9232850 .055593 \quad 1.209977$ O $0.434420 \quad 1.206770 \quad 3.630709$ H $-1.037767 \quad 0.4258672 .463607$ H $0.123973-1.0925624 .848368$ O $-1.723564-0.2432034 .839402$ H -2.152134 -1.936993 2.980574 O $-1.513770-3.0904674 .534623$ C $0.240570-4.8730541 .210959$ H -1.446070 -4.581532 2.429913 H -1.476577 -3.6314860 .948605$ H $\quad 0.7117091 .8033442 .914628$ H -1.379819 0.6021595 .176072 H $-0.686058-3.3009134 .999770$ O $0.647180-4.429702-0.010764$ O $\quad 0.672555-5.8538381 .803286$ C $1.652198-5.220305-0.653140$ H $1.935212-4.719099-1.582656$ H $2.538601-5.305074-0.017234$ H $1.252613-6.209705-0.894050$ SCF Energy (B3LYP/6-31G**//MMFF) $=-3245.92120809$

\section{2}

MM̄MF Geometry

C $-3.7956433 .643360-0.192091$ C $-2.8989422 .654840-0.059518$ C $-1.7572422 .417235-1.012776$ O $-0.5820902 .247516-0.189575$ C $-2.0369951 .127223-1.806583$ C $-1.1840000 .954487-3.046170$ C $-0.1945600 .050350-3.185096$ C $-1.5535531 .844633-4.206229$ C $0.324506-0.921313-2.161301$ C $1.841128-0.791715-2.017775$ C $2.465483-1.769120-1.011179$ C $1.966766-1.5423290 .418011$

C $2.732117-2.3838731 .448822$

O $3.878525-1.545254-1.083649$ O $2.438403-1.8582242 .752699$ C $4.246409-2.2030211 .219488$ C $2.257801-3.8418531 .453459$ O $4.994920-3.0729182 .065943$

C $4.671442-2.401505-0.252882$

C $6.147334-1.979777-0.441432$ O $4.519105-3.775964-0.605808$ C $0.6175172 .681375-0.655564$ O $0.8574653 .175605-1.744298$

C 1.6053342 .4504200 .423846

C 2.9185112 .6057540 .198473

C 3.9859172 .4199771 .187819

C 3.5767492 .0401122 .588227

C $6.650727-2.101502-1.886531$

C $7.917591-1.294769-2.081332$

C $8.7057411 .050378-2.400918$

O $9.031178-1.807672-2.003438$

C $8.9288431 .605637-1.030577$

C $8.5932422 .851178-0.663037$

C 8.7729563 .3730040 .733675

C 7.4546143 .6762761 .409956

C 7.1549244 .9189591 .824351

C 6.5086972 .5174141 .660881

C 5.2681852 .5966220 .803952

N $7.6606360 .047403-2.315702$

H -3.705583 4.346238-1.016097 H $-3.017114 \quad 1.9415130 .753333$ H -1.635495 $3.284365-1.672426$ H $-3.0858811 .112530-2.135678$ H $-1.933506 \quad 0.263877-1.142192$ H $0.312107-0.002850-4.148374$ H $-0.9625721 .627616-5.102515$
H $-2.607776 \quad 1.708692-4.468794$ H -1.390256 $2.896573-3.954053$ H $-0.149035-0.767793-1.190492$ H $\quad 0.075000-1.937213-2.488608$ H $2.318121-0.952797-2.994787$ H $2.1086580 .235584-1.740017$ H $2.250612-2.793060-1.338671$ H $0.893968-1.7475020 .498459$ H $2.089778-0.4843930 .680778$ H $1.474226-1.8885192 .877560$ H $4.506006-1.1781491 .520299$ H $1.195837-3.8980501 .721051$ H $2.787672-4.4282322 .212087$ H $2.379179-4.3349500 .486718$ H $4.703604-2.9094222 .979374$ H $6.261097-0.939030-0.110512$ H $6.800026-2.5915280 .194210$

H $5.042039-3.958175-1.401956$

H 1.2167242 .1493971 .389423

H $3.2443352 .899676-0.798206$

H 2.9938831 .1127032 .579151

H 2.9712732 .8330323 .040770

H 4.4187161 .8568743 .258380

H $6.870640-3.144752-2.139168$

H $5.901711-1.752250-2.606402$

H $8.3706341 .814528-3.109231$

H $9.6283140 .599396-2.779608$

H $9.3726530 .935726-0.297541$

H $8.1477493 .524423-1.391389$

H $9.400244 \quad 4.271713 \quad 0.679834$

H 9.3263792 .6564891 .354027

H 6.2199255 .1326062 .331867

H 7.8363535 .7483101 .666747

H 6.2808882 .5011242 .730673

H 7.0137191 .5671401 .450541

H $5.4629032 .829225-0.243820$

H $6.7007420 .377629-2.270828$

C -6.2482173 .2839540 .030019$

O $-6.1350841 .858535-0.148483$

C -7.4256561 .2543850 .058249$

C -8.4129292 .4051410 .227990$

C -7.5292983 .4810440 .834434$

H $-7.3672260 .704521 \quad 1.004417$

C $-7.7337610 .288003-1.092681$

H $-8.8172692 .741654-0.733625$

H -9.2582622 .1328470 .867333$

C $-8.1169394 .877422 \quad 0.734233$

H -7.3519103 .2371091 .890508$

C $-6.627103-0.765179-1.323873$

O $\quad-7.8655301 .055857-2.295636$

H $-8.702488-0.195896-0.921556$

H -5.704098 -0.267061-1.640891

O $-7.029165-1.607261-2.411144$

C $-6.351605-1.665548-0.104494$

C $-5.295214-2.759462-0.354864$

H $-6.036494-1.0585340 .748980$

O $-7.567293-2.3109040 .285680$

H $-5.336770-3.4651240 .483625$

H -5.578051 -3.353997 -1.232077

H -7.441482 5.6179091 .173719

H -8.298162 $5.163044-0.307420$

H -9.0712534 .9320781 .268390$

C $-4.984868 \quad 3.803627 \quad 0.715753$

H $-8.2677190 .479584-2.967299$

H $-6.994523-1.073051-3.222442$

H -7.876127 -2.836507 -0.472284

H -4.816292 3.2577531 .652594

H -5.0882734 .8639890 .970393$

H $-6.3509283 .726318-0.970325$

C $-3.276719-2.0342851 .808031$

O $-3.481634-1.3576820 .563967$

C -3.862624 -2.210706 -0.522494

C $-2.793579-3.291582-0.740510$

C $-2.488782-4.0668770 .539593$

C $-2.187504-3.1064121 .690097$

H $-4.217120-2.5149382 .105461$

C -3.001895 -0.988361 2.897971

H $-3.865296-1.581829-1.420359$ 
O $-3.192532-4.205016-1.756651$

H -1.865530 -2.816874 -1.081928

H $-3.305796-4.749188 \quad 0.801500$

O $-1.335216-4.8849760 .329052$

H -1.205284 -2.651824 1.522594

O $-2.112045-3.8375032 .911282$

C $-1.705351-0.2238302 .734696$

H -3.806244 -0.2428602 .899061$

H -2.987814 -1.487014 3.874199

H -3.360082 -3.688959-2.563725

H -1.525427 -5.462124 -0.430464

H -1.437840 -4.5287832 .794132$

O $-1.3680840 .332317 \quad 3.936248$

O $-1.084528-0.0975601 .689162$

C $-0.1688651 .112168 \quad 3.942292$

H -0.3178632 .0257123 .359697$

H 0.0529831 .3872714 .976686

H $\quad 0.6703770 .5317203 .547845$

SCF Energy (B3LYP/6-31G**//MMFF) $=-3245.91289531$

0193

MM̄FF Geometry

C $2.052397-3.6129920 .575907$

C $1.353476-2.8984831 .470825$

C $1.723304-1.4895581 .845623$

O $0.612319-0.6135121 .543635$

C $2.012517-1.3798403 .351073$

C $2.855324-0.1595663 .671707$

C $4.199083-0.1769183 .779664$

C 2.0900151 .1153233 .909182

C $5.107762-1.3604973 .600358$

C $6.342191-1.0904902 .727452$

C $6.031424-0.9177891 .230287$

C $7.319759-1.0847700 .416974$

C $7.103840-0.827400-1.078865$

O $5.487622 \quad 0.389711 \quad 1.020044$

O $8.407411-0.723215-1.670903$

C $6.3899230 .528475-1.242920$

C $6.405311-2.009242-1.761962$

O $6.032811 \quad 0.756925-2.604867$

C $5.1397290 .678256-0.342549$

C $4.6309432 .139251-0.413977$

O $4.122159-0.199304-0.824712$

C $0.500593-0.1681950 .263581$

O $1.159726-0.520963-0.701157$

C $-0.536196 \quad 0.8853010 .246515$

C $-0.5245711 .785941-0.747501$

C -1.442895 2.914597 -0.907269

C -2.538888 3.0673210 .112780

C 3.5197882 .4805670 .586559

C 3.1063213 .9406880 .496564

C $1.6560505 .615527 \quad 1.636825$

O $3.5757294 .723076-0.325479$

C 0.3143795 .7465250 .989540

C $0.0798516 .531212-0.073077$

C -1.277509 6.752979-0.676730

C -1.376811 $6.260169-2.102074$

C $-0.9286567 .005607-3.126417$

C $-2.0857274 .947536-2.341348$

C $-1.2569403 .755677-1.946354$

N 2.1563124 .2673251 .454195

H $2.929072-3.1698280 .109087$

H $\quad 0.469590-3.3289831 .934253$

H $2.605550-1.1595361 .282972$

H $2.516755-2.2826523 .714844$

H $1.073994-1.3208133 .918675$

H $4.705200 \quad 0.7500844 .045057$

H 1.3900640 .9879204 .741302

H 2.7475841 .9554094 .156814

H 1.5220411 .3957543 .018076

H $5.460162-1.6501914 .598445$

H $4.581484-2.2288493 .193132$

H $6.868457-0.2043463 .104969$

H $7.016480-1.9468672 .855745$

H $5.293046-1.6708470 .931360$

H $7.751388-2.0819060 .571644$

H $8.073536-0.3741330 .785669$

H $8.295940-0.616595-2.630484$
H $7.1089441 .314377-0.972422$

H $7.021601-2.912876-1.682072$

H $6.277268-1.827745-2.834810$

H $5.426818-2.237045-1.333780$

H $6.8525030 .771111-3.126689$

H $5.4711502 .818707-0.217930$

H $4.2675902 .355207-1.426925$

H $3.2516380 .178761-0.626748$

H -1.2463070 .9125011 .064423$

H $0.2424261 .699285-1.516690$

H -2.1182003 .1769721 .118433$

H -3.190872 2.1898970 .101358

H -3.175139 $3.938768-0.051952$

H 2.6320681 .8690010 .405425

H 3.8672262 .2944581 .608485

H 2.3777996 .3351641 .235995

H 1.5652255 .7899322 .713618

H -0.5095585 .2062551 .448438$

H $0.9064147 .084617-0.513850$

H -1.487488 $7.830550-0.648563$

H -2.063242 $6.292898-0.065062$

H -1.026199 $6.672703-4.154361$

H $-0.4503377 .964814-2.957377$

H -2.323659 $4.835529-3.407803$

H -3.060101 4.968716-1.844595

H $-0.4228893 .567454-2.623844$

H $1.789343 \quad 3.5392112 .059357$

C $0.875119-5.057315-1.146991$

O $-0.442176-4.528347-0.900352$

C $-0.839990-3.733086-2.033260$

C $0.150380-4.061631-3.146221$

C $1.423046-4.291556-2.351757$

H $-0.705150-2.684495-1.741667$

C -2.312508 -3.986070 -2.370595

H $-0.127668-4.974165-3.685837$

H $\quad 0.238607-3.250864-3.875705$

C $2.501371-5.038104-3.116090$

H $1.814231-3.311262-2.053852$

C $-3.265460-3.853378-1.164821$

O $-2.442296-5.322712-2.872628$

H $-2.621867-3.320062-3.184734$

H $-3.090243-4.681546-0.467864$

O $-4.603005-4.030114-1.651598$

C $-3.178017-2.515030-0.407389$

C $-4.185687-2.4594320 .759548$

H $-2.167670-2.388854-0.002892$

O $-3.409003-1.457528-1.331419$

H $-5.199686-2.5846070 .368429$

H -3.984691 -3.316136 1.413072

H $3.386968-5.188207-2.490347$

H $2.151328-6.021136-3.448297$

H $2.805624-4.472571-4.002887$

C $1.697725-5.0059450 .143251$

H -3.381777 -5.463567 -3.081163

H -4.809136 -3.269394 -2.221993

H $-3.278077-0.617013-0.860917$

H $2.627707-5.5715750 .013439$

H $1.135792-5.5017290 .945220$

H $0.727192-6.112697-1.411773$

C $-5.9497040 .053587 \quad 0.574962$

$\begin{array}{llll}\text { O } & -4.540612 & -0.009672 & 0.831774\end{array}$

C $-4.100700-1.1503221 .577248$

C $-4.825543-1.1990862 .929790$

C $-6.341281-1.1719562 .756089$

C $-6.7478680 .014700 \quad 1.884255$

H $-6.247366-0.784108-0.065868$

C -6.223906 $1.362467-0.183893$

H -3.037194 -0.9857851 .793138$

O $-4.426992-2.3572463 .654384$

H $-4.521589-0.327728 \quad 3.524728$

H $-6.717947-2.1135212 .339352$

O $-6.964004-1.0128854 .032506$

H -6.5988460 .9466862 .444738$

O $-8.138312-0.0828291 .580845$

C $-5.7113011 .319486-1.607720$

H $-7.2972861 .572084-0.242030$

H -5.7298972 .1913810 .337266$

H $-4.801861-2.2900974 .548562$ 
H $-6.802055-1.8255304 .540797$

H -8.617203 -0.1274782 .426198$

O $-5.4307132 .589063-2.024896$

O $\quad-5.606891 \quad 0.305324-2.283946$

C $-4.9590802 .702136-3.371201$

H -3.991862 2.201064 -3.473533

H -5.689249 $2.280846-4.068955$

H -4.832183 3.763691-3.598939

SCF Energy (B3LYP/6-31G**//MMFF) $=-3245.91387027$

\section{4}

MM̄MF Geometry

C -1.757947 -1.046162 -2.948765

C $-1.392401-2.274593-3.346970$

C $-0.108378-2.992543-3.007818$

O $0.879972-2.032425-2.562148$

C $-0.288802-4.050546-1.903010$

C $-1.397643-5.056660-2.131525$

C $-2.508786-5.130024-1.371333$

C $-1.167795-6.037856-3.249178$

C $-2.860968-4.215322-0.230849$

C $-2.311956-4.7431881 .101304$

C $-2.195297-3.6745142 .198682$

C $-3.542264-3.0565392 .575016$

C $-3.380810-1.9446543 .622805$

O $-1.294095-2.6667551 .727492$

O $-4.616411-1.2162913 .688380$

C $-2.300095-0.959678 \quad 3.132331$

C $-3.135440-2.5065825 .027511$

O $-2.023958 \quad 0.0282334 .122483$

C $-0.986923-1.6420802 .680548$

C $-0.079413-0.5993671 .977366$

O $-0.321508-2.1721503 .824606$

C $2.191034-2.359224-2.716806$

O $2.642733-3.380783-3.208498$

C $2.993514-1.245608-2.163376$

C $4.328826-1.354444-2.100825$

C $5.254207-0.351729-1.567479$

C $4.6613490 .899659-0.975887$

C $1.203398-1.1537091 .341320$

C $2.375841-1.2745842 .299827$

C $4.695347-2.1823482 .423902$

O $2.357975-0.8282623 .443707$

C $5.672220-1.0945132 .105706$

C $6.912745-1.3256811 .649543$

C $7.913686-0.2429421 .363017$

C $8.498491-0.339304-0.028737$

C $9.711433-0.880551-0.232753$

C $7.7153800 .270781-1.169740$

C $6.579030-0.603318-1.621964$

N $3.451428-1.9327501 .719046$

H -1.113728 $-0.469852-2.290817$

H $-2.066423-2.823454-4.002828$

H $\quad 0.252140-3.461615-3.932331$

H $-0.403389-3.535863-0.941841$

H $0.652134-4.604472-1.781657$

H -3.239368 -5.908557 -1.582362

H -1.968179 -6.782201 -3.318455

H -1.115996 -5.519676 -4.211622

H $-0.228177-6.577831-3.093128$

H $-2.506799-3.199812-0.437118$

H -3.953519 -4.136220 -0.175659

H $-2.939717-5.5709911 .453849$

H - $-1.306905-5.1548640 .937356$

H -1.747817 -4.1616613 .073314$

H -4.237355 -3.826442 2.931792

H -4.013022 -2.6142201 .686881$

H $-5.322580-1.8443723 .918139$

H -2.718619 -0.4266742 .269687$

H $-3.974887-3.1402915 .338142$

H $-3.076926-1.7023155 .769179$

H -2.226474 -3.107477 5.097888

H -2.8680430 .4555994 .347937$

H $-0.658126-0.151116 \quad 1.158840$

H $\quad 0.178197 \quad 0.203643 \quad 2.677385$

H $0.595003-2.3766273 .583915$

H $2.461547-0.368739-1.813350$

H $4.793906-2.266012-2.474081$
H $3.9232500 .652528-0.204722$

H $4.1708161 .493039-1.754681$

H $5.3957321 .546366-0.491882$

H $1.020483-2.1213980 .862400$

H $1.521621-0.4527360 .559375$

H $5.054921-3.1669482 .108944$

H $4.507659-2.2069773 .502111$

H $5.351918-0.0701782 .281208$

H $7.240743-2.3520951 .501278$

H $8.713944-0.3251572 .110301$

H 7.4780070 .7538291 .505352

H $10.150351-0.927551-1.223788$

H $10.290203-1.2940690 .586415$

H $8.3721650 .420660-2.037422$

H $7.3962741 .277945-0.888113$

H $6.909353-1.543316-2.066700$

H $3.397584-2.206430 \quad 0.742395$

C $-4.031875-0.171895-2.270725$

O $-3.5286800 .797088-1.329407$

C $-4.5897691 .709096-0.983048$

C $-5.8730091 .099717-1.535718$

C $-5.3641350 .389813-2.771746$

H $-4.3838442 .644083-1.516946$

C -4.6186521 .9735010 .526397$

H $-6.3157340 .376194-0.841496$

H $-6.6301631 .858537-1.755726$

C $-6.321690-0.664408-3.301163$

H -5.185179 1.138417 -3.555499

C -3.2730702 .4323451 .119702$

O

H -5.4071752 .7003610 .754912$

H $-2.566718 \quad 1.5978001 .076376$

O -3.4835672 .7004602 .512084$

C -2.6502183 .6739010 .457974$

C $-1.3964554 .208648 \quad 1.179862$

H $-2.3992913 .444755-0.582018$

$\begin{array}{lllll}\mathrm{O} & -3.625840 & 4.718264 & 0.432471\end{array}$

H -1.0394005 .0869730 .628276$

H -1.681777 4.5959722 .166086

H -5.913415 -1.153350 -4.190960

H $-6.519382-1.438850-2.552417$

H -7.279429 -0.210233 -3.575911

C -3.028332 -0.384318 -3.405159

H -4.9934030 .9382132 .146552$

H -4.0736153 .4711292 .575784$

H $-3.2333375 .475012-0.035120$

H -2.754302 $0.579633-3.847187$

H $-3.493513-0.977102-4.201869$

H $-4.187888-1.106227-1.715687$

C $0.712063 \quad 3.357231-0.869334$

O $0.036372 \quad 2.529504 \quad 0.084047$

C -0.2661853 .1714791 .326315$

C 1.0157223 .6986591 .986807

C 1.8367874 .5702531 .038584

C $2.0434663 .853579-0.296031$

H $0.0807314 .222580-1.105511$

C $0.9101492 .523779-2.145216$

H -0.6497602 .3896251 .991079$

O $0.687371 \quad 4.4233193 .166992$

H 1.6283302 .8407872 .294718

H 1.3704595 .5513850 .890223

O $3.1225594 .814223 \quad 1.612690$

H $2.7279013 .010861-0.144277$

O $2.6695244 .742903-1.218482$

C $0.0783533 .063227-3.284167$

H $1.9526062 .542805-2.483057$

H $0.6444151 .470421-2.000166$

H $1.517198 \quad 4.6227293 .631979$

H 2.9914915 .3613122 .405343

H $2.0704315 .495230-1.362873$

O $-1.2481192 .884487-3.026164$

O $0.5548143 .586784-4.283181$

C $-2.1394723 .410062-4.014753$

H $-1.9811492 .906802-4.973251$

H $-3.1642393 .221195-3.684858$

$\mathrm{H}-1.9983064 .490238-4.116480$

SCF Energy (B3LYP/6-31G**//MMFF) $=-3245.90666084$ 
0195

MM̄FF Geometry

C $-0.289018-3.5140421 .258651$

C $-0.726831-2.272506 \quad 0.997613$

C $-0.986352-1.2377582 .059060$

O $-0.316651-0.0246951 .633242$

C $-2.488096-0.9316642 .189936$

C $-3.307641-2.0370412 .816299$

C $-4.182434-2.8142472 .148336$

C $-3.137988-2.1964324 .305009$

C $-4.471462-2.8278470 .672738$

C $-5.836388-2.2176620 .321986$

C $-5.869268-0.683751 \quad 0.423216$

C $-7.262448-0.1488490 .084923$

C $-7.299279 \quad 1.3882630 .063732$

O $-4.915903-0.166362-0.509073$

O $-8.5248461 .789573-0.567959$

C $-6.1426221 .904590-0.820377$

C -7.3313581 .9734311 .480615$

O $-6.0536933 .326888-0.766328$

C $-4.7762911 .259338-0.493855$

C $-3.7328421 .642549-1.570910$

O -4.3317351 .7395600 .776836$

C 0.0582820 .8515462 .603830

O $\begin{array}{lllll}0.055193 & 0.703002 & 3.810437\end{array}$

C 0.6210452 .0495521 .941429

C 1.2255652 .9846452 .689435

C 1.7730064 .2519642 .208739

C 1.6555185 .3622503 .220176

C $-2.4262060 .845634-1.474723$

C $-1.4886331 .212355-2.604702$

C $0.3148102 .777982-3.235806$

O $-1.4441220 .558078-3.643998$

C $0.6892834 .188405-2.920800$

C $1.8694354 .532396-2.384646$

C $2.2694385 .943198-2.070424$

C $2.0954806 .295077-0.610161$

C $1.2225437 .244724-0.233402$

C 2.9836795 .6108340 .405307

C 2.3472024 .3808050 .995528

N $-0.7432872 .350918-2.341775$

H $-0.113447-3.7877322 .297344$

H $-0.926073-1.965752-0.025146$

H $-0.564882-1.5645153 .016790$

H -2.881237 -0.6517431 .208142$

H -2.626182 -0.0226072 .790794$

H -4.745231 -3.554806 2.715649

H -3.809836 -2.954657 4.721419

H -2.113967 -2.497818 4.545923

H -3.352905 -1.251946 4.815463

H -3.675413 -2.3537170 .091253$

H $-4.472853-3.8788450 .355618$

H $-6.066080-2.507638-0.711822$

H $-6.615520-2.6525760 .960119$

H -5.585595 -0.3862611 .438671$

H $-8.010520-0.5422100 .784568$

H -7.561453 -0.513653 -0.907929

H $-9.2624121 .409124-0.060804$

H $-6.4022931 .659071-1.859229$

H -8.2043481 .5997562 .029254$

H -7.4403803 .0632151 .456008$

H -6.443862 1.7261502 .066847

H -5.6374053 .5654490 .079343$

H $-4.1571591 .460619-2.567244$

H -3.506203 2.714213-1.507519

H $-3.362557 \quad 1.7206870 .805979$

H 0.5042022 .1445850 .867539

H 1.3045372 .8394533 .765718

H 2.2720395 .1449124 .099508

H $0.615040 \quad 5.470773 \quad 3.547715$

H 1.9645956 .3368652 .837260

H -1.919051 $1.021706-0.520815$

H -2.611603 -0.232623 -1.539571

H $-0.0272442 .694109-4.272517$

H $\quad 1.1539442 .087867-3.101776$

H $-0.0415364 .959919-3.149831$

H $2.5971693 .756337-2.161702$

H $3.3193956 .085490-2.354980$
H $\quad 1.7011476 .637388-2.703816$

H 1.1090987 .5275620 .807506

H $0.5993747 .760159-0.956736$

H 3.2802056 .3226441 .183458

H $3.9303815 .319947-0.068070$

H 2.3940203 .5072280 .347836

H $-0.7380522 .712010-1.391694$

C $-0.161191-4.267602-1.215269$

O $0.846945-3.307436-1.581195$

C $1.394277-3.662494-2.863915$

C $0.487488-4.752068-3.422411$

C $0.053001-5.457705-2.150911$

H $2.382391-4.097497-2.672299$

C $1.542018-2.410470-3.735918$

H $-0.383790-4.334361-3.939935$

H $1.011540-5.409674-4.122552$

C $-1.175291-6.332538-2.331797$

H $0.885874-6.075898-1.789466$

C $2.282216-1.250074-3.034935$

O $0.233446-1.941263-4.081251$

H $2.034404-2.673259-4.679397$

H $1.651206-0.859671-2.229000$

O $2.418754-0.181702-3.979542$

C $3.673257-1.618752-2.492516$

C $4.423383-0.447672-1.832489$

H $3.586695-2.437848-1.772499$

O $4.466624-2.103655-3.579344$

H $5.436493-0.794668-1.594097$

H $4.5714930 .358152-2.562405$

H -1.457456 -6.809878 -1.388271

H $-2.033598-5.751243-2.685163$

H $-0.980021-7.122479-3.064374$

C $-0.010915-4.6164960 .267884$

H $0.343519-1.131094-4.607997$

H $3.010220-0.490295-4.687414$

H $5.319395-2.394722-3.213530$

H $1.017809-4.9531230 .450516$

H $-0.674800-5.4532730 .514730$

H -1.139064 -3.808791-1.410162

C $4.405520-1.6183050 .981886$

O $3.331264-0.9308050 .333201$

C $3.7430360 .107232-0.562727$

C $4.5827891 .148070 \quad 0.193173$

C 5.7568400 .5116190 .935475

C $5.277551-0.6563941 .797776$

H $5.030323-2.0967420 .216742$

C $3.817952-2.7746651 .804807$

H $2.8254840 .611590-0.889699$

O $5.0781502 .144792-0.693926$

H 3.9498391 .6556320 .931228

H 6.5455450 .1918240 .244742

O $6.351760 \quad 1.486495 \quad 1.795883$

H $4.728803-0.2581062 .658219$

O $6.406019-1.3639572 .306240$

C $2.921690-2.3486622 .948767$

H $3.208748-3.4091611 .149798$

H $4.636494-3.3777442 .214342$

H $4.3130152 .543413-1.141430$

H 6.6106882 .2419551 .240738

H $6.956157-0.7235502 .789326$

O $2.728450-3.4153753 .780530$

O $2.430885-1.2377953 .088739$

C $1.896964-3.1658924 .918389$

H $\quad 0.885540-2.9001884 .598076$

H $1.847305-4.0835215 .510485$

H $2.327378-2.3722865 .536617$

SCF Energy (B3LYP/6-31G**//MMFF) $=-3245.90700993$

01_96

MM̄FF Geometry

C $-5.660077-1.773445-1.319620$

C $-4.437846-2.314283-1.204433$

C $-3.490366-2.438065-2.371192$

O $-2.357539-1.574760-2.114684$

C $-2.969338-3.880470-2.508959$

C $-2.242465-4.123157-3.819682$

C $-0.923809-4.372309-3.942545$

C $-3.109885-4.106697-5.054835$ 
C $0.113044-4.499566-2.862198$

C $0.851212-3.181591-2.633003$

C $2.181947-3.325456-1.879516$

C $2.025915-3.990911-0.511234$

C $3.353069-4.0139330 .262893$

O $2.693405-1.996994-1.719348$

O $3.072840-4.3651231 .625954$

C $3.945910-2.5894570 .278958$

C $4.297086-5.098906-0.269643$

O $5.246069-2.5856260 .862952$

C $3.990371-1.914872-1.112190$

C $4.328526-0.415446-0.967278$

O $5.001266-2.484127-1.945305$

C $-2.514863-0.249686-2.385073$

O $-3.489917 \quad 0.292264-2.880968$

C -1.294425 $0.447991-1.929689$

C $-1.334017 \quad 1.774369-1.733458$

C $-0.2281462 .606874-1.257334$

C $1.1193911 .949187-1.123834$

C $4.3947390 .321802-2.310526$

C $4.5686041 .820900-2.135174$

C $4.4443283 .938082-3.456574$

O $4.8072392 .350512-1.053709$

C $3.0319984 .435267-3.450370$

C $2.6038805 .462997-2.701429$

C $1.1858775 .962415-2.720409$

C $0.6023346 .143627-1.335079$

C $0.2029327 .355091-0.912113$

C $0.4836984 .933472-0.433896$

C $-0.4738793 .901664-0.966086$

N $4.4298312 .491301-3.342129$

H -6.003899 -1.442933 -2.297077

H $-4.076730-2.624499-0.227006$

H -3.982041 -2.125885 -3.300112

H -3.805565 -4.589977 -2.452858

H $-2.318447-4.107562-1.657682$

H $-0.525699-4.518843-4.946605$

H -2.570532 -4.446663 -5.945701

H $-3.969643-4.772103-4.924158$

H -3.476507 -3.096951 -5.260747

H $-0.329516-4.858253-1.928100$

H $\quad 0.823302-5.275304-3.174976$

H $1.056428-2.686724-3.592354$

H $0.199967-2.488997-2.086446$

H $2.871800-3.896531-2.511853$

H $1.632832-5.010017-0.604947$

H $1.284364-3.4351080 .078027$

H $2.611378-5.221278 \quad 1.627632$

H $3.308663-1.9795080 .933954$

H $3.848602-6.091810-0.143254$

H $5.234841-5.1203250 .296093$

H $4.532853-4.980957-1.329343$

H $5.168800-2.9912951 .743528$

H $3.5654430 .067518-0.344847$

H $5.293513-0.292751-0.459525$

H $5.523410-3.110346-1.418731$

H $-0.410968-0.149144-1.738714$

H -2.270485 2.300513 -1.915099

H $1.9134072 .632626-0.821473$

H $\quad 1.4278741 .518780-2.082734$

H $1.0888751 .150041-0.375717$

H $5.238096-0.047822-2.904954$

H $3.4734720 .150330-2.879120$

H $4.9194434 .181508-4.411595$

H $5.0382164 .366932-2.643093$

H $2.3272043 .932372-4.109072$

H $3.3103385 .988478-2.063116$

H $0.5383885 .288889-3.295325$

H $1.1816296 .918047-3.260496$

H -0.2152737 .4994970 .078582$

H $0.2842568 .231794-1.546151$

H 0.1172105 .2328620 .557000

H $1.4817394 .531204-0.257023$

H -1.491417 4.275645 -1.088706

H $4.1806371 .960332-4.171603$

C $-6.509278-0.1170670 .411575$

O

C $-4.759626 \quad 1.356593 \quad 0.924121$
C -5.8723422 .1384510 .231190$

C $-6.5534441 .048788-0.576179$

H $-3.9188891 .244746 \quad 0.229796$

C $-4.258511 \quad 1.9635592 .241611$

H -6.5788622 .5711640 .948535$

H $-5.4817222 .952154-0.387515$

C $-7.9538441 .408622-1.038810$

H $-5.9303110 .847755-1.455228$

C -3.3417701 .0329603 .060774$

O $-5.3841912 .281567 \quad 3.071975$

H -3.7557212 .9164022 .040888$

H -3.9302870 .1857333 .433071$

O -2.9116451 .7486304 .226483$

C -2.1033610 .5071322 .312297$

C - $1.297881-0.4647623 .195689$

H -2.417286 -0.0187531 .404476$

O -1.3003631 .6142641 .922288$

H -0.9042280 .0762334 .061377$

H -1.996081 -1.220094 3.573825

H -8.400655 $0.582959-1.601650$

H $-8.6109401 .635186-0.192432$

H -7.929680 2.288586-1.689874

C $-6.596057-1.533327-0.170365$

H -5.8803601 .4586523 .222880$

H -2.345291 $2.480583 \quad 3.926769$

H -0.5172681 .2675601 .462992$

H -7.617409 -1.725618 -0.519589

H $-6.390540-2.2618450 .624324$

H -7.312645 -0.0083881 .152408$

C 1.7401340 .0650363 .343104

O $0.968221-0.2966632 .189614$

C $-0.146433-1.1631052 .436365$

C $0.334198-2.4560133 .108270$

C $1.100849-2.1655944 .391200$

C $2.228209-1.1775054 .108011$

H 1.1372630 .7015634 .000824

C 2.9530650 .8818812 .864394

H $-0.538028-1.4454391 .450521$

O $-0.754703-3.3266843 .395142$

H $0.996945-2.9943562 .423519$

H $0.438226-1.7986675 .183633$

O $1.680375-3.3783524 .877566$

H $3.008319-1.6893513 .529939$

O $2.825390-0.7837675 .343134$

C 2.6012742 .2853872 .421464

H 3.6776920 .9821103 .680803

H 3.4336950 .3803202 .017957

H -1.217351 -3.501273 2.557746

H $0.954751-4.0125625 .009448$

H $2.146949-0.3296615 .871402$

O 3.7624172 .9695732 .196938

O 1.4682972 .7220382 .282797

C 3.6468934 .3397331 .796654

H 4.5211734 .8763822 .175027

H 3.6496854 .3935260 .705282

H 2.7484514 .8153042 .201508

SCF Energy (B3LYP/6-31G**//MMFF) $=-3245.92006338$

0197

MM̄̄FF Geometry

C $0.410696-3.4339100 .067023$

C $0.846246-2.213555-0.279939$

C $1.115112-1.787774-1.697875$

O $0.509357-0.478773-1.839464$

C $2.623523-1.680897-1.977892$

C $3.285773-3.011861-2.260266$

C $4.083241-3.674455-1.400212$

C $3.045094-3.564740-3.642024$

C $4.437620-3.3012770 .011936$

C $5.903347-2.8685480 .156589$

C $6.193932-1.486919-0.450890$

C $7.678789-1.141477-0.321389$

C $7.9799230 .281747-0.815322$

O $5.407776-0.5268100 .260582$

O $9.2987520 .633934-0.370473$

C $6.9983251 .262729-0.140337$

C $8.0021820 .362768-2.346218$

O $7.1440002 .577008-0.674390$ 
C $5.5194820 .816497-0.225769$

C 4.6462031 .7144420 .681793

O $5.071863 \quad 0.933523-1.576742$

C $0.182188-0.065368-3.089323$

O $0.388839-0.670388-4.132842$

C $-0.497371 \quad 1.251671-3.074221$

C $-0.8087501 .929024-1.957494$

C $-1.5400173 .198850-1.896621$

C $-1.8580683 .887428-3.198253$

C $3.177668 \quad 1.2753530 .784350$

C $2.482072 \quad 1.976817 \quad 1.933889$

C $1.759294 \quad 4.2328212 .707905$

$\begin{array}{lllll}\text { O } & 2.124670 & 1.367075 & 2.939211\end{array}$

C 0.3075314 .4359312 .416425

C $-0.196596 \quad 5.5936341 .964323$

C $-1.658343 \quad 5.8425431 .733148$

C $-2.002626 \quad 6.0002840 .269961$

C $-1.7232707 .133976-0.395045$

C $-2.765053 \quad 4.877891-0.391043$

C $-1.9083573 .676887-0.689018$

$\begin{array}{llll}\text { N } & 2.326597 & 3.336040 & 1.721328\end{array}$

H $0.271535-4.173283-0.719611$

H $1.016910-1.4596960 .483640$

H $0.621936-2.475759-2.395788$

H $3.114056-1.157225-1.151674$

H $2.799887-1.023423-2.839418$

H $4.526397-4.612354-1.733331$

H $3.595228-4.494974-3.820071$

H $1.982644-3.778420-3.793071$

H $3.366292-2.843510-4.400619$

H $3.770678-2.5364340 .419837$

H $4.276333-4.1915230 .633542$

H $6.131974-2.8396741 .230032$

H $6.560743-3.622411-0.294130$

H $5.897963-1.489250-1.505649$

H $8.296725-1.872942-0.856872$

H $7.979046-1.2113270 .733697$

H $9.921132-0.021295-0.730012$

H $7.278394 \quad 1.327166 \quad 0.920629$

H $8.769227-0.306812-2.753717$

H $8.2733121 .368862-2.684750$

H $7.0495310 .089105-2.804507$

H $8.0817552 .821152-0.590966$

H $5.069403 \quad 1.7138761 .695284$

H 4.6785612 .7489890 .318372

H $4.1069321 .030213-1.585098$

H $-0.7643441 .625594-4.057804$

H $-0.5419101 .505226-0.990820$

H -2.590638 3.308141-3.770485

H $-0.9522214 .006851-3.803560$

H -2.262946 $4.893713-3.069474$

H $2.6293881 .489021-0.139811$

H 3.1004020 .1981790 .966529

H 2.3247895 .1690152 .657292

H 1.8822823 .8126593 .711362

H -0.3603933 .6021532 .617620$

H $\quad 0.476650 \quad 6.426973 \quad 1.775654$

H -1.9415806 .7548102 .274376$

H -2.265206 5.0447412 .179574

H -1.999894 $7.261494-1.436307$

H -1.2093367 .9583300 .088438$

H -3.279317 $5.239309-1.287423$

H -3.5789274 .5624810 .275252$

H $-1.624404 \quad 3.111467 \quad 0.198547$

H 2.5890463 .7304490 .822880

C $-0.104516-2.8379472 .530316$

O $-1.203387-1.9743412 .177343$

C $-1.941053-1.6437673 .368568$

C $-1.135116-2.1979264 .539132$

C $-0.462821-3.4004353 .905044$

H $-2.890759-2.187643 \quad 3.311530$

C -2.208757 -0.1362203 .428403$

H $-0.382233-1.4831994 .891247$

H - $1.770323-2.4597725 .390860$

C $0.726965-3.9169504 .694833$

H $-1.204840-4.2036313 .802348$

C -2.911071 $0.437867 \quad 2.179205$

O -0.9515860 .5402923 .563343$
H $-2.782856 \quad 0.0987394 .332691$

H -2.2177090 .4018901 .330907$

$\begin{array}{llll}\text { O } & -3.148808 & 1.824929 & 2.454696\end{array}$

C $-4.243233-0.2516061 .820941$

$\begin{array}{llll}\text { C }-5.038302 & 0.428280 & 0.688892\end{array}$

H $-4.052260-1.2950761 .549421$

O $-5.071704-0.268102 \quad 2.987246$

H $-6.008226-0.0770850 .607618$

H $-5.290115 \quad 1.4541110 .983869$

H $1.183573-4.7773884 .195967$

H $1.496969-3.1467474 .810353$

H $0.415522-4.2335265 .695627$

C $0.070066-3.9141091 .454913$

H $-1.140496 \quad 1.492673 \quad 3.618474$

H $-3.3621252 .262243 \quad 1.613824$

H -5.872518 -0.7758392 .771695$

H $-0.869221-4.4747671 .371062$

H $0.842647-4.6282541 .762402$

H $0.794449-2.2129592 .604729$

C $-5.060066-1.735979-1.459984$

O $-3.956555-0.900834-1.088500$

C $-4.3179520 .420844-0.673380$

C $-5.0797711 .135011-1.799149$

C $-6.274650 \quad 0.320255-2.284522$

C $-5.848137-1.108213-2.616041$

H $-5.720484-1.878979-0.597201$

C $-4.494944-3.102287-1.868261$

H $-3.3779860 .963954-0.537182$

O $-5.5321802 .417309-1.378985$

H $-4.4034051 .295053-2.649032$

H $-7.0904480 .326270-1.552039$

O $-6.7984430 .916839-3.473302$

H -5.241343 -1.105710 -3.530656

O $-7.009133-1.895104-2.874211$

C $-3.854696-3.835177-0.713027$

H $-5.268227-3.757343-2.284261$

H $-3.721959-2.969918-2.635608$

H $-4.7516302 .923827-1.099019$

H $-7.0286161 .836879-3.257216$

H -7.499789 -1.460729 -3.592908

$\begin{array}{lllll} & 0 & -4.750948 & -4.015619 & 0.297615\end{array}$

O $-2.699249-4.239013-0.729688$

C $-4.256578-4.7181911 .441809$

H $-3.395640-4.1953841 .867486$

H $-5.051839-4.7496702 .191245$

H $-3.989392-5.7431331 .168054$

SCF Energy (B3LYP/6-31G**//MMFF) $=-3245.90345030$

0198

MM̄FF Geometry

C $0.9569665 .886934 \quad 0.222700$

C $1.4397464 .937000-0.592762$

C $2.5727624 .010467-0.234211$

O $2.0323372 .675349-0.099850$

C $3.6129093 .994787-1.369478$

C $4.7783373 .057133-1.120376$

C $5.0635851 .976756-1.874560$

C $5.663068 \quad 3.4090890 .047582$

C $4.3341951 .478477-3.091450$

C $3.2291080 .450486-2.803080$

C $3.744234-0.883843-2.239062$

C $2.615297-1.915573-2.180208$

C $3.075461-3.237521-1.546539$

O $4.250708-0.659136-0.921313$

O $1.890607-3.995667-1.257198$

C $3.787960-2.936545-0.208739$

C $3.890560-4.077669-2.536570$

O $4.362021-4.121507 \quad 0.336710$

C $4.840528-1.813211-0.307886$

C $5.392686-1.3724341 .069594$

O $5.955012-2.289625-1.072938$

C $1.451407 \quad 2.360728 \quad 1.089475$

$\begin{array}{llllll}\text { O } & 1.355340 & 3.074502 & 2.075447\end{array}$

$\begin{array}{llll}\text { C } & 0.913164 & 0.988503 & 0.979602\end{array}$

$\begin{array}{llll}\text { C } & -0.049318 & 0.602851 & 1.829688\end{array}$

C $-0.704319-0.7042871 .860932$

C $-0.093142-1.8110151 .045004$

C $4.351066-0.8263562 .053068$ 
C $3.853963-1.8477353 .063809$

C $2.222495-2.0807794 .936915$

O $4.339048-2.9690143 .186948$

C $1.031629-2.8369444 .438851$

C $-0.191253-2.7303314 .980964$

C $-1.381118-3.5270684 .527382$

C $-2.575471-2.6616474 .194198$

C $-3.573528-2.4890695 .077317$

C -2.656254 -2.075128 2.804348

C -1.796871 -0.8553322 .637493$

N $2.823274-1.3387973 .843897$

H $1.4003986 .031417 \quad 1.204124$

H $0.9676404 .784721-1.561321$

H 3.0511724 .3151410 .704665

H $4.0107965 .007202-1.520290$

H $3.1055793 .729525-2.303252$

H $5.9309361 .377185-1.599885$

H 6.5569852 .7777160 .096571

H $6.0011204 .447537-0.030865$

H $5.124053 \quad 3.2857020 .991897$

H $3.9033422 .315681-3.650841$

H $5.0717971 .034744-3.772296$

H $2.5030240 .890922-2.109760$

H $2.6995320 .258319-3.744721$

H $4.553884-1.240692-2.886363$

H $2.196474-2.094862-3.178352$

H $1.789117-1.512019-1.579514$

H $2.164786-4.800920-0.785252$

H $3.006740-2.6296900 .494044$

H $3.293069-4.300002-3.429063$

H $4.156941-5.050060-2.107810$

H $4.807017-3.582879-2.865159$

H $5.184686-4.303105-0.148687$

H $5.987258-2.1798421 .513563$

H $6.106795-0.5570870 .883585$

H $6.728065-1.738664-0.865965$

H 1.3028350 .3488490 .196815

H $-0.413734 \quad 1.3223742 .562148$

H $\quad 0.964944-1.9265831 .299149$

H $-0.171069-1.596576-0.024020$

H $-0.554224-2.786248 \quad 1.214026$

H $4.817925-0.0236222 .638581$

H $3.497993-0.3910271 .524719$

H $1.957532-1.3562895 .713766$

H $2.957070-2.7800715 .349199$

H $1.190597-3.5287463 .614961$

H $-0.340718-2.0597285 .824327$

H -1.641050 -4.223627 5.335234

H -1.136047 -4.1572563 .663418$

H -4.448085 -1.8952784 .833249$

H -3.535251 -2.933545 6.066467

H -3.687262 -1.767335 2.588542

H -2.439871 -2.854506 2.069231

H $-2.153487 \quad 0.0057673 .204603$

H $2.448112-0.418413 \quad 3.633660$

C $-1.568507 \quad 6.183096 \quad 0.283229$

O $-1.7891444 .924532-0.385343$

C -2.3087893 .9801140 .568552$

C -2.820535 4.8201381 .730262

C -1.7704045 .9168951 .775168$

H -1.4603523 .3760870 .912463$

C $-3.3492913 .058625-0.081286$

H $-3.8087355 .243766 \quad 1.514548$

H -2.886422 4.2492942 .661192

C -2.1952087 .1412382 .566218$

H -0.8595565 .5029692 .225335$

C -2.740159 2.150185-1.174468

O $-4.4081873 .849169-0.615116$ H -3.784378 2.4209840 .697307

H -1.900238 $1.608095-0.728330$

O $-2.2148062 .934476-2.245817$

C $-3.7351681 .129208-1.766560$

C $-3.1025360 .191159-2.815550$

H $-4.1718670 .536803-0.956583$

O $-4.8169311 .812253-2.403176$

H -3.912694 -0.319734 -3.348736

H -2.599371 $0.793744-3.581077$

H -1.412408 7.9061042 .547453
H -3.1105717 .5846802 .160369$

H -2.385471 6.8768883 .611553

C $-0.2130376 .760169-0.136556$

H -4.030789 4.400576-1.321987

H -1.514574 $3.502301-1.882184$

H -4.435767 2.395939 -3.081534

H $-0.0671907 .747607 \quad 0.315874$

H $-0.218376 \quad 6.913393-1.223800$

H -2.352561 $6.858551-0.083085$

C $-3.683971-2.643819-1.823445$

O $-2.765550-1.696000-1.262642$

C $-2.123312-0.835577-2.211841$

C $-1.355136-1.671558-3.242994$

C $-2.251184-2.709397-3.910308$

C $-2.982606-3.538443-2.855417$

H $-4.519834-2.115209-2.296728$

C $-4.243610-3.489670-0.667918$

H $-1.368306-0.271141-1.654506$

O $-0.764815-0.824453-4.222209$

H $-0.533873-2.190697-2.732361$

H $-2.960449-2.234468-4.597768$

O $-1.449208-3.576321-4.715934$

H $-2.269427-4.203834-2.352073$

O $-3.952392-4.361406-3.500625$

C $-5.229473-2.7270200 .192049$

H $-4.776356-4.363299-1.059447$

H -3.424106 -3.823294 -0.020969

H $-0.307666-1.394990-4.863503$

H $-0.799354-4.009814-4.136685$

H -3.485148 -4.893823 -4.167083

O $-5.657484-3.5449991 .200084$

O $-5.587564-1.5724110 .007959$

C $-6.619603-2.9731102 .092049$

H -6.216545 -2.072820 2.565188

H $-7.542930-2.7445421 .551422$

$\mathrm{H}-6.840896-3.7083572 .870346$

SCF Energy $\left(B 3 L Y P / 6-31 G^{* *} / / M M F F\right)=-3245.92236980$

0199

MM̄MF Geometry

C $-1.314403-1.4140812 .051403$

C $-1.512050-0.6236373 .118632$

C $-0.6295470 .527621 \quad 3.552907$

O 0.4424230 .7053352 .594163

C -1.3880341 .8666773 .651394$

C -2.5601701 .9016354 .610455$

C -3.823734 2.210922 4.255969

C -2.2357011 .5950956 .049516$

C -4.3122912 .5958632 .888805$

C -5.0544421 .4402532 .208180$

C -5.1085121 .6336490 .686675$

C -6.0675350 .6303500 .048290$

C $-6.0410020 .707754-1.484528$

O

O $-6.707323-0.456560-1.996580$

C $-4.5769180 .636738-1.962931$

C $-6.8186251 .920162-2.009369$

O $-4.4914650 .849625-3.370460$

C $-3.6276641 .608125-1.220627$

C $-2.1569661 .271390-1.581012$

O $-3.9302892 .945564-1.610878$

C $1.605121 \quad 1.2588613 .027846$

O 1.8729611 .6194934 .162133

C 2.5054421 .3559561 .857840

C 3.7275161 .8887191 .998932

C 4.7012282 .0313410 .920712

C 6.1061591 .6834151 .329533

C $-1.0892702 .075286-0.824199$

C $-0.7001133 .382525-1.494172$

C $0.8680525 .303766-1.221740$

O $-1.1903883 .772813-2.549896$

C $2.0816204 .982559-2.036168$

C $3.3263915 .353132-1.699850$

C $4.5435465 .001895-2.506614$

C $5.5376194 .163816-1.731351$

C $6.7096434 .680834-1.325992$

C $5.2126272 .703834-1.500018$

C $4.3388752 .489062-0.294538$ 
N $0.269036 \quad 4.061549-0.767040$ H -0.461882 -1.238472 1.400465 H -2.354885 -0.8459763 .769905$ H -0.1960750 .2596394 .524461$ H -1.678241 2.156538 2.637305 H -0.6852522 .6527053 .959550$ H -4.5949702 .2021255 .024759$ H -3.090998 1.7635756 .712663 H -1.9320390 .5501456 .164159$ H -1.420143 2.2368036 .398947 H -4.994869 3.4489472 .992929 H -3.497004 2.9641962 .260882 H -4.5471990 .4917442 .428723$ H -6.0683411 .3758112 .621757$ H -5.4235542 .6607780 .467433$ H $-7.088740 \quad 0.7751180 .422494$ H $-5.781104-0.3854350 .350453$ H -7.612726 -0.464068 -1.641417 H $-4.228587-0.388580-1.783722$ H $-7.866097 \quad 1.871423-1.688154$ H -6.842048 $1.930601-3.104669$ H -6.411754 2.872631-1.663357 H $-5.0789140 .202422-3.797056$ H $-1.9844690 .218961-1.324443$ H $-1.9994021 .373358-2.661128$ H -3.186393 $3.516919-1.365324$ H 2.1462090 .9833160 .903804 H 4.0647492 .2312342 .974885 H $6.1677000 .625607 \quad 1.609269$ H 6.4176112 .2858242 .190403 H 6.8433621 .8540210 .542065 H -1.4010172 .2799080 .204605$ H $-0.1764701 .466809-0.777779$ H $1.1090225 .894578-0.332468$ H $0.1478515 .861023-1.829131$ H $1.9259344 .408660-2.946933$ H $3.4846565 .934292-0.794387$ H $5.0084155 .940965-2.833709$ H $4.2717234 .470665-3.427470$ H $7.4391514 .078012-0.795491$ H $6.9646205 .719006-1.511490$ H $6.1337432 .114497-1.434086$ H $4.6960642 .300910-2.380790$ H $3.2893422 .726846-0.461456$ H $0.671733 \quad 3.6170520 .053153$ C $-2.729755-2.505361 \quad 0.279778$ O $-1.637740-2.506439-0.659814$ C $-1.944811-3.398616-1.746961$ C $-3.430415-3.707305-1.622883$ C $-3.608296-3.693247-0.115004$ H $-1.381151-4.320786-1.560843$ C -1.495515 -2.784499-3.079393 H $-4.050178-2.930028-2.083873$ H $-3.695308-4.665905-2.078925$ C $-5.056682-3.5724920 .322736$ H $-3.187642-4.6210140 .296091$ C $-0.044887-2.255689-3.049375$ O $-2.352380-1.680126-3.387230$ H $-1.630091-3.512371-3.887533$ H $\quad 0.012847-1.420429-2.343661$ O $0.256328-1.700696-4.335519$ C $1.007914-3.323706-2.706410$ C $2.446301-2.777484-2.640971$ H $0.762938-3.801985-1.753826$ O $0.955331-4.344152-3.706680$ H $3.130936-3.632006-2.575725$ H $2.700032-2.287921-3.589316$ H -5.133542 -3.511218 1.412612 H $-5.530300-2.682321-0.101924$ H -5.630742 -4.444189-0.008362 C $-2.177112-2.5982051 .703529$ H -2.036608 -1.289249 -4.219969 H $0.246781-2.430721-4.978262$ H $1.578023-5.043849-3.445453$ H -1.550143 -3.493892 1.796919 H -3.001942 -2.697994 2.418158 H $-3.270230-1.5604570 .145566$ C $2.923383-3.4519950 .288428$
O $2.188484-2.336124-0.224772$

C $2.691112-1.812601-1.460749$

C $4.152421-1.375689-1.283425$

C $5.029526-2.486692-0.705589$

C $4.387804-3.0808600 .549578$

H $2.893441-4.264284-0.449147$

C $2.185270-4.003306 \quad 1.517469$

H $2.111412-0.904946-1.667248$

O $4.697655-0.899709-2.508847$

H $4.176979-0.531567-0.585746$

H $5.232532-3.267816-1.447403$

O $6.299324-1.939789-0.341904$

H $4.472729-2.3566951 .367192$

O $5.105995-4.248488 \quad 0.940234$

C $2.096008-3.0523612 .691659$

H $1.153422-4.244828 \quad 1.233980$

H $2.680382-4.9206801 .855531$

H $4.850626-1.663169-3.090665$

H $6.668746-1.513059-1.133978$

H $6.034711-3.988538 \quad 1.067715$

O $1.645596-3.7402803 .783089$

O $2.359431-1.8594002 .660353$

C $1.501469-2.9688874 .979726$

H $0.735148-2.2008964 .843031$

H $1.184806-3.6412885 .781404$

H $2.457480-2.5158595 .259156$

SCF Energy (B3LYP/6-31G*//MMFF) $=-3245.91481370$

021

MM̄FF Geometry

C $2.631525-3.827084-1.479504$

C $1.329400-3.601477-1.246555$

C $0.324496-3.347128-2.341101$

O $-0.490170-2.207519-1.979382$

C $-0.598685-4.570774-2.480982$

C $-1.632265-4.426729-3.581178$

C $-2.941966-4.178999-3.378136$

C $-1.110405-4.625776-4.980961$

C $-3.641258-3.943976-2.067268$

C $-4.421187-2.622343-2.084589$

C $-5.009709-2.283827-0.706339$

C $-5.848486-1.006511-0.782600$

C $-6.343478-0.5509340 .597688$

O $-3.918914-2.106168 \quad 0.203652$

O

C $-5.148415-0.521973 \quad 1.574730$

C $-7.538681-1.3832761 .076646$

O $-5.587569-0.2505622 .903248$

C $-4.307196-1.8146271 .553266$

C $-3.013084-1.7124882 .400227$

O $-5.083096-2.8853992 .106234$

C $0.018633-0.968026-2.219471$

O $1.138073-0.695795-2.622115$

C $-1.0567280 .011512-1.941146$

C $-0.8544821 .317344-2.170162$

C $-1.8462582 .385783-2.018195$

C $-3.208305 \quad 2.007213-1.500467$

C $-1.994229-0.667242 \quad 1.935020$

C -2.1374220 .6926432 .596739$

C $-1.256504 \quad 3.021600 \quad 2.440309$

$\begin{array}{lllll} & -1.2565063 & 0.898635 & 3.567022\end{array}$

C -2.096733 3.8731361 .543856

C $-1.613948 \quad 4.9195290 .857123$

C $-2.456084 \quad 5.818290 \quad 0.000248$

C $-1.9421505 .926555-1.416640$

C $-1.2007916 .977606-1.805495$

C $-2.3746124 .869586-2.405735$

C $-1.5049653 .642787-2.371580$

$\mathrm{N}-1.324948 \quad 1.643727 \quad 1.992880$

H $2.975039-3.822164-2.512789$

H $0.942026-3.611030-0.231930$

H $0.821551-3.146303-3.298531$

H $-1.085504-4.776298-1.521468$

H $0.002804-5.466029-2.690958$

H $-3.594373-4.132879-4.249485$

H $-1.904708-4.571432-5.733020$

H $-0.370993-3.857970-5.228862$

H $-0.637122-5.608623-5.073699$ 
H $-4.337333-4.772576-1.890590$ H -2.938859 -3.938866 -1.230901 H -3.747089 -1.816004 -2.399292 H -5.226727 -2.682565 -2.827103 H $-5.624852-3.127699-0.372313$ H $-6.693406-1.137628-1.470179$ H -5.240805 -0.199676 -1.211841 H $-7.519100 \quad 0.811761-0.196911$ H $-4.513517 \quad 0.3205551 .274696$ H $-8.377899-1.2851810 .377318$ H $-7.910610-1.0225352 .041983$ H -7.311533 -2.447224 1.168544 H -6.0928210 .5799892 .877514$ H -3.263414 -1.587765 3.460769 H -2.507964 -2.687045 2.335317 H -4.477869 -3.585742 2.401248 H $-2.005893-0.375754-1.590931$ H $\quad 0.120121 \quad 1.632887-2.536963$ H $-3.7333071 .386958-2.234566$ H -3.846597 $2.865982-1.281878$ H $-3.1266391 .449418-0.561734$ H -0.991650 -1.026906 2.198320 H $-2.022686-0.5493800 .848766$ H -0.2042423 .3187412 .420001$ H -1.617361 3.0982423 .470658 H -3.1557283 .6348151 .480984$ H -0.5593545 .1701620 .943465$ H -2.465662 6.8106260 .470124 H -3.503394 $5.491889-0.015003$ H $-0.8560777 .077939-2.829376$ H $-0.9150587 .756643-1.106592$ H -2.317767 $5.273511-3.426124$ H $-3.4357854 .646693-2.262869$ H $-0.4933513 .821546-2.736271$ H $-0.785671 \quad 1.386721 \quad 1.170938$ C $3.330996-3.9819681 .011732$ O $3.031230-2.6047781 .314072$ C $3.644284-2.2571622 .568453$ C 4.105101-3.569024 3.192100 C $4.466377-4.3729411 .959120$ H $4.529228-1.6562562 .329243$ C $2.675010-1.4443783 .431100$ H $4.946701-3.4295193 .877476$ H $3.298180-4.0637033 .745116$ C $4.572353-5.8658312 .218169$ H $5.425720-4.0064331 .569484$ C $2.129934-0.1712892 .753168$ O $1.551152-2.2761243 .752822$ H $3.153336-1.1939804 .385467$ H $1.454061-0.4550701 .936900$ O 1.3272210 .4928053 .738118 C 3.2046290 .8002102 .225271 C 2.5982662 .0966741 .656849 H 3.7909710 .3094831 .443101 O $4.0984121 .159242 \quad 3.278838$ H 1.8208701 .8372830 .930628 H 2.1201002 .6503262 .472561 H $5.354672-6.0732002 .955732$ H $3.631661-6.2743032 .602503$ H $4.823383-6.4052811 .299762$ C $3.709617-4.110479-0.465525$ H $0.946467-1.7462494 .300303$ H 0.7493021 .1205393 .273396 H $4.614090 \quad 0.370723 \quad 3.515746$ H $4.083248-5.122468-0.660852$ H $4.532973-3.417881-0.675922$ H $2.433281-4.5708901 .238330$ C $3.6235682 .149915-1.244638$ O $4.3727952 .376138-0.043994$ C 3.6539943 .0228271 .011614 C 3.0987014 .3725260 .531525 C $2.2851394 .229872-0.750899$ C $3.0955393 .477154-1.803268$ H $2.7878661 .470312-1.038500$ C $4.5638411 .475235-2.255192$ H 4.3952423 .2462721 .789091 O 2.2918244 .9830431 .532526 H 3.9346125 .0563220 .333893
H $1.323363 \quad 3.737028-0.567196$

O $1.9832965 .529005-1.263078$

H $3.9314934 .105212-2.137440$

O $2.2752903 .222019-2.939495$

C $4.9036900 .050685-1.873298$

H $4.105657 \quad 1.436197-3.249177$

H $5.5019362 .040996-2.310701$

H 2.8387215 .0770112 .331169

H $1.4985856 .007125-0.568382$

H $1.9421954 .079608-3.254994$

O $5.964789-0.365477-2.626727$

O $4.304929-0.620630-1.045158$

C $6.400857-1.708396-2.395203$

H $7.318604-1.870752-2.966575$

H $6.617252-1.866573-1.334238$

H $5.640008-2.411770-2.745591$

SCF Energy $(B 3 L Y P / 6-31 G * * / / M M F F)=-3245.92055433$

0210

MM̄FF Geometry

C $0.565335-0.9999712 .439999$

C $1.038487-2.1064001 .844585$

C $0.423292-2.8147190 .659744$

O $-0.522077-1.959119-0.020997$

C $-0.310054-4.0801751 .128585$

C $-0.831002-4.919215-0.021185$

C $-2.117360-4.949541-0.421670$

C $0.194140-5.792507-0.696901$

C $-3.261224-4.1434500 .128699$

C $-4.122035-3.547274-0.994532$

C $-5.270313-2.702277-0.425887$

C $-6.250869-2.298052-1.526256$

C $-7.366642-1.390024-0.987894$

O $-4.703779-1.5295790 .168259$

O $-8.036734-0.841038-2.132557$

C $-6.726858-0.228392-0.198551$

C $-8.417351-2.186266-0.205134$

O $-7.719131 \quad 0.5548380 .461313$

C $-5.657332-0.6845420 .824543$

C $-4.910744 \quad 0.553691 \quad 1.372377$

O $-6.295639-1.3585441 .910313$

C $-0.016772-1.108849-0.956475$

O $1.144790-1.022266-1.321694$

C $-1.124777-0.254463-1.435220$

C $-0.8283230 .869440-2.104579$

C $-1.7819631 .865289-2.595775$

C $-3.2477101 .536127-2.504866$

C -3.6484450 .2334192 .183235$

C $-2.919771 \quad 1.5155272 .537653$

C -1.5607263 .3375931 .496945$

O -2.9839992 .0051693 .662525$

C $-2.185910 \quad 4.227988 \quad 0.468378$

C $-1.4847294 .913589-0.447439$

C $-2.1133755 .790528-1.493762$

C $-1.6049345 .500053-2.890806$

C $-0.8167516 .377929-3.534367$

C $-2.0666614 .226085-3.570784$

C -1.297949 $3.022629-3.092940$

N -2.236346 $2.054051 \quad 1.459316$

H $-0.365282-0.5565442 .098039$

H $1.958324-2.5481302 .220657$

H $1.228520-3.092515-0.031361$

H -1.128955 -3.805933 1.803749

H $0.363684-4.7026721 .733149$

H -2.384565 -5.626984 -1.231626

H $-0.243555-6.414441-1.484989$

H $0.976134-5.181416-1.157452$

H $\quad 0.660420-6.4643340 .031286$

H $-3.881375-4.8057300 .744595$

H $-2.911633-3.3342280 .775591$

H $-3.489264-2.925170-1.640870$

H -4.529299-4.359576 -1.609292

H $-5.783522-3.2896010 .344672$

H $-6.679455-3.182830-2.013195$

H $-5.709383-1.755145-2.313978$

H $-8.781977-0.302412-1.817472$

H $-6.2435690 .433472-0.931237$

H $\quad-8.879557-2.943400-0.849839$ 
H $-9.235300-1.5393620 .131070$ H -8.005581 -2.6980140 .667232$ H $-8.3103170 .917357-0.219477$ H $-4.622517 \quad 1.1934020 .530800$ H -5.5855331 .1489002 .001306$ H $-5.724426-1.3052412 .692969$ H -2.138522 -0.545679-1.186817 H $\quad 0.220634 \quad 1.107127-2.280249$ H -3.453442 $0.555693-2.949433$ H -3.886471 $2.245570-3.035092$ H $-3.5712851 .521145-1.461177$ H $-3.894477-0.2898653 .113110$ H $-2.962916-0.4115521 .622129$ H -0.5040343 .1492191 .289047$ H -1.653127 3.7858792 .490484 H -3.2703914 .3111560 .477062$ H $-0.3991414 .851121-0.441508$ H -1.899828 $6.832836-1.223693$ H -3.206456 $5.695753-1.487432$ H $-0.4664286 .188201-4.543575$ H $-0.4994687 .303271-3.065238$ H -1.917447 $4.302728-4.655591$ H -3.146296 4.132485 -3.426103 H $-0.2149433 .147351-3.144874$ H -2.2104801 .5366870 .585987$ C 1.4515051 .1902153 .272956 O $2.207375 \quad 1.3628882 .060106$ C 3.1722252 .4138642 .250098 C 2.7995503 .0961763 .561574 C 2.2303761 .9353554 .355380 H $4.144518 \quad 1.9213822 .372894$ C 3.2180543 .3310841 .020625 H 3.6617143 .5662554 .044020 H 2.0321263 .8657423 .419999 C 1.3829232 .3648545 .540073 H $3.063244 \quad 1.3159394 .714124$ C $3.3150442 .579487-0.322993$ O 2.0183184 .1129820 .984188 H $4.0438084 .044194 \quad 1.127756$ H $2.3767442 .042341-0.507485$ O $3.4144203 .557889-1.365958$ C $4.4995321 .602632-0.430559$ C $4.5276010 .892643-1.794135$ H $4.418700 \quad 0.857097 \quad 0.361996$ O $5.7111122 .325092-0.227986$ H $3.5572390 .407406-1.944553$ H $4.6398911 .639572-2.589546$ H 1.9789332 .9513186 .246924 H $0.5339382 .980621 \quad 5.223915$ H 0.9875901 .4943396 .072640 C $1.267263-0.2997503 .570398$ H 2.0685564 .6845440 .198858 H $4.2681514 .011993-1.259792$ H $6.4225621 .676256-0.094465$ H $0.674529-0.4256134 .483176$ H $2.245225-0.7634343 .745558$ H $0.475324 \quad 1.662873 \quad 3.108418$ C $4.659672-1.994353-0.764228$

O $5.743538-1.062555-0.856262$

C $5.666624-0.140910-1.948240$

C $5.623430-0.909002-3.277585$

C $4.482510-1.923223-3.299258$

C $4.529357-2.812900-2.057739$

H $3.728798-1.453587-0.558655$

C $4.950037-2.9316200 .421021$

H $6.6072790 .423568-1.935561$

O $5.479365-0.018073-4.378422$

H $6.571256-1.444688-3.417625$

H $3.510800-1.424444-3.386185$

O $4.597918-2.743637-4.465175$

H $5.372930-3.509798-2.144015$

O $3.338520-3.595664-1.998425$

C $4.877170-2.2429631 .767026$

H $4.222739-3.7511370 .441700$

H $\quad 5.960967-3.3450710 .320777$

H $6.2223470 .608452-4.346709$

H $5.459396-3.193490-4.432775$

H $3.264055-4.077017-2.840272$
O $5.211144-3.1437802 .739346$

O $4.560606-1.0774821 .954029$

C $5.178480-2.6430984 .079606$

H $5.849144-1.7846914 .183718$

H $4.155300-2.3705094 .353827$

H $5.520989-3.4378794 .747511$

SCF Energy (B3LYP/6-31G**//MMFF)= -3245.92135907

02100

MM̄FF Geometry

C -0.9080163 .8430201 .951793$

C -0.9501072 .5125852 .124046$

C -0.7350191 .8467803 .458772$

O $\quad 0.373113 \quad 0.9213793 .343667$

C -1.9580091 .0151923 .891357$

C -3.2365911 .8152864 .031697$

C -4.2288511 .8409123 .119334$

C -3.3532102 .6167445 .301620$

C -4.2688201 .1267941 .797651$

C $-5.559090 \quad 0.315457 \quad 1.623301$

C $-5.449290-0.6295430 .418666$

C $-6.816548-1.1916340 .032880$

C $-6.704124-2.216437-1.105326$

O $-4.574227-1.6971820 .800255$

O $-7.958742-2.907644-1.202285$

C $-5.637725-3.263896-0.725441$

C $-6.471791-1.544155-2.463659$

O $-5.393439-4.158182-1.809374$

C $-4.303583-2.650089-0.233420$

C $-3.418185-3.7718470 .367546$

O $-3.630594-2.049001-1.337876$

C 1.6230951 .4058463 .557944

O 1.9264222 .5691013 .786038

C 2.6342280 .3237453 .552644

C $2.422488-0.9001333 .043140$

C $3.380473-2.0110063 .047571$

C $4.665678-1.8198623 .811354$

C $-2.152425-3.3050931 .101281$

C $-0.983732-2.9510260 .202585$

C $0.569154-3.932548-1.491274$

O $-0.487518-1.8268210 .213985$

C $1.690411-4.755764-0.943984$

C $2.932946-4.288235-0.753348$

C $4.049744-5.120600-0.195288$

C $4.688830-4.5109471 .032904$

C $5.982313-4.1475651 .022155$

C $3.867182-4.4181662 .299851$

C $3.064208-3.1466652 .389319$

N $-0.551115-4.012252-0.573008$

H -0.6710044 .4617722 .815897$

H $-1.1725321 .855127 \quad 1.289168$

H -0.5131792 .5811634 .243752$

H $-1.7335670 .534597 \quad 4.854111$

H $-2.0832520 .163380 \quad 3.212840$

H -5.0983702 .4642443 .323663$

H -4.3050343 .1543295 .368678$

H -3.2853581 .9579576 .173515$

H -2.551133 3.3588495 .362140

H -4.2197841 .8785981 .001246$

H -3.4017300 .4771551 .658142$

H $-5.751308-0.2701412 .531878$

H -6.3991921 .0078831 .488453$

H $-5.010528-0.081173-0.423409$

H $-7.506156-0.384644-0.244709$

H $-7.268390-1.6863070 .904177$

H $-8.652089-2.247186-1.372052$

H -6.057589 -3.8725520 .087881$

H -7.294186 -0.856842 -2.696075

H $-6.461638-2.282685-3.272862$

H $-5.542189-0.972811-2.506884$

H $-6.251395-4.529053-2.077930$

H $-4.016573-4.3121051 .113411$

H -3.155644 -4.489446 -0.417606

H $-2.721985-1.842468-1.070100$

H 3.5877880 .6014923 .990697

H $1.462221-1.1184362 .578921$

H $5.257788-1.0084613 .375396$

H $5.306237-2.7036143 .817663$ 
H $4.457405-1.5786814 .859796$ H -2.365106 -2.449944 1.752300 H -1.806531 -4.112387 1.760248 H $0.859676-2.888988-1.641409$ H $0.235796-4.346649-2.447542$ H $1.468926-5.791411-0.696051$ H $3.161510-3.259327-1.017966$ H $4.794855-5.249569-0.990839$ H $3.702125-6.1305080 .056773$ H $\quad 6.464949-3.7438331 .905324$ H $6.589871-4.2440840 .128409$ H $4.508798-4.5439103 .179098$ H $3.177446-5.2707222 .350625$ H $2.108655-3.1914161 .866601$ H $-0.997314-4.917956-0.462314$ C $-1.7964173 .852388-0.480843$ O $-0.8412372 .930525-1.035252$ C $-1.0506132 .843121-2.457367$ C $-2.2533073 .725903-2.776715$ C $-2.2032624 .742687-1.653100$ H $-0.1631313 .275525-2.932491$ C -1.206788 $1.377710-2.879583$ H -2.178167 4.180127 -3.769672 H $-3.1938183 .163884-2.741660$ C $-3.5157305 .480926-1.452863$ H $-1.4071645 .467514-1.869150$ C $-0.0528510 .455219-2.439162$ O $-2.412937 \quad 0.847170-2.312048$ H $-1.3371151 .321230-3.966441$ H $-0.0837080 .305000-1.353944$ O $-0.277761-0.828230-3.035055$ C $1.3503370 .940272-2.840696$ C $2.453629-0.077240-2.489073$ H $1.5591011 .885544-2.330429$ O $1.3838231 .202658-4.243068$ H $2.365904-0.342227-1.429731$ H $2.299505-0.996664-3.066703$ H $-3.7933346 .024448-2.361888$ H $-4.3311894 .790888-1.211329$ H -3.437516 $6.207473-0.638305$ C -1.1763814 .6204880 .688433$ $\mathrm{H}-2.3456730 .932100-1.345299$ H - $1.158928-1.128305-2.752840$ H $1.165616 \quad 0.373223-4.701944$ H -1.848001 5.4421020 .964457 H -0.2289725 .0727440 .373290$ H -2.657986 $3.266378-0.138090$ C $4.2480981 .687540-0.757909$ O $4.1179521 .725445-2.183880$ C $3.8712960 .456053-2.798348$ C $5.008351-0.520001-2.465248$ C $5.270455-0.610328-0.963930$ C $5.4194360 .784912-0.359038$ H $3.3174751 .316633-0.311189$ C $4.4701953 .124144-0.276216$ H $3.9099360 .630665-3.881078$ O $4.719429-1.810788-2.991585$ H $5.924916-0.172077-2.959679$ H $4.483689-1.183278-0.460219$ O $6.486729-1.333938-0.766175$ H $\quad 6.3674331 .222083-0.696724$ O $5.494206 \quad 0.6735591 .059636$ C $3.1963963 .924083-0.405544$ H 4.7645793 .1644410 .778428 H $5.2651863 .611174-0.853748$ H $5.445960-2.401738-2.729133$ H $6.669741-1.3355120 .189152$ H $4.651524 \quad 0.303570 \quad 1.372332$

O $3.3132994 .834708-1.412813$

O $2.209443 \quad 3.744480 \quad 0.296120$

C $2.1606105 .650131-1.643051$

H $\quad 1.9282076 .239246-0.750747$ H $2.3860916 .333661-2.465737$ H $1.3081575 .027822-1.928289$

SCF Energy (B3LYP/6-31G**//MMFF) $=-3245.92808624$

02101

MM̄FF Geometry
C $2.748162-4.0701700 .042842$

C $1.541130-3.526437-0.177143$

C $0.937835-3.373251-1.545309$

O $0.558412-1.981740-1.672387$

C $-0.330184-4.231227-1.685325$

C $-0.878472-4.214864-3.098501$

C $-1.891238-3.433540-3.522285$

C $-0.208943-5.168390-4.054625$

C $-2.673369-2.419030-2.731926$

C $-4.170045-2.743413-2.773915$

C $-5.050082-1.861122-1.876264$

C $-4.923839-0.365653-2.169148$

C $-5.8244930 .468139-1.241964$

O $-4.713345-2.120300-0.507821$

O $-5.418991 \quad 1.841448-1.352697$

C $-5.5712120 .039166 \quad 0.221364$

C $-7.2950990 .418657-1.672390$

O -6.4975730 .6689151 .102051$

C $-5.601701-1.4914760 .432298$

C $-5.165411-1.9329381 .851728$

O $-6.945136-1.9620540 .254728$

C $1.001781-1.285073-2.747562$

O $1.770235-1.681177-3.613035$

C $0.3819920 .058708-2.813640$

C $-0.3001160 .627352-1.806824$

C $-0.9576861 .937569-1.846257$

C $-0.9035392 .703873-3.143564$

C $-3.812924-1.3944732 .330355$

C $-3.936611-0.1309453 .168539$

C -2.6000571 .7922694 .009671$

O $-4.986923 \quad 0.2267523 .695901$

C -2.886725 2.9129373 .061019

C -1.9668633 .7975542 .649210$

C -2.2561914 .9160131 .692163$

C -1.6604354 .6680680 .325774$

C $-0.5956065 .362665-0.108275$

C $-2.3611603 .666675-0.565211$

C $-1.5823612 .386487-0.736499$

N $-2.725372 \quad 0.533523 \quad 3.297138$

H $3.300884-4.457557-0.811254$

H $0.955756-3.1364450 .650549$

H $1.671910-3.652236-2.310705$

H -1.093317 -3.893608 -0.974791

H $-0.114059-5.271643-1.407680$

H -2.188624 -3.508791-4.567917

H $-0.653546-5.136043-5.054997$

H $0.853103-4.926402-4.159468$

H $-0.297594-6.196457-3.688531$

H -2.333245 -2.348738 -1.695997

H -2.497353 -1.438726 -3.188871

H $-4.528204-2.666148-3.808660$

H -4.309514 -3.787956 -2.463786

H $-6.082432-2.188393-2.049179$

H $-5.149908-0.153077-3.221134$

H $-3.888127-0.038765-2.008428$

H $-5.4925222 .101462-2.287008$

H -4.5765650 .4169420 .489745$

H $\quad-7.4070640 .798450-2.695117$

H $-7.9126871 .069067-1.043191$

H -7.717652 -0.587638 -1.647311

H $-6.429019 \quad 1.6281230 .957217$

H -5.961960 -1.709172 2.571886

H -5.087181 -3.029688 1.839865

H $-7.029559-2.8238570 .695601$

H $0.5227260 .564860-3.763314$

H $-0.3986490 .088573-0.865933$

H $0.1334562 .867000-3.455454$

H -1.359364 $3.694132-3.088951$

H -1.425218 $2.153552-3.934485$

H -3.348621 -2.143667 2.984295

H $-3.132340-1.2294341 .488055$

H -1.587641 1.8412764 .421047

H -3.3115921 .8185484 .841304$

H -3.9069992 .9976872 .693665$

H $-0.9472993 .722133 \quad 3.017019$

H -1.859509 5.8427192 .126427

H -3.336252 5.0818011 .589744

H $-0.1864285 .206485-1.100842$ 
H $-0.1072516 .099940 \quad 0.520170$ H -2.605178 4.141883 -1.520034 H $-3.3380463 .408238-0.136947$ H $-1.565624 \quad 1.7624170 .157740$ H -1.9229270 .2069322 .766788$ C $2.873005-3.4091032 .538972$ O $2.832518-2.0038042 .229693$ C $3.119393-1.2512433 .420753$ C $3.232493-2.2653544 .556090$ C $3.697849-3.5077153 .820584$ H $4.099118-0.7843423 .266493$ C $2.051781-0.1712153 .628644$ H $3.932069-1.9384925 .331773$ H $2.265761-2.4491285 .038885$ C $3.483497-4.7919824 .601963$ H $4.766047-3.3979463 .590480$ C $1.8392710 .755406 \quad 2.414061$ $\begin{array}{lllll}\text { O } & 0.801170 & -0.818830 & 3.899745\end{array}$ H 2.2968960 .4158994 .522057 H 1.3583770 .2058841 .594749 $\begin{array}{llllll}\text { O } & 0.916797 & 1.775894 & 2.810088\end{array}$ C 3.1127401 .4388841 .881754 C 2.7848332 .5327810 .845741 H 3.7651100 .6790411 .440682 O 3.8109502 .0289102 .978040 H $2.063573 \quad 2.1314380 .126509$ H $2.300953 \quad 3.3733191 .358132$ H $4.040160-4.7658765 .544550$ H $2.425551-4.9450024 .840288$ H $3.828745-5.6592394 .030666$ C $3.474783-4.1765101 .359264$ H $0.184614-0.1405104 .223048$ H 0.0378581 .3654892 .859670 H 4.7033222 .2604402 .671010 H $3.563277-5.2390541 .612576$ H $4.491303-3.8013321 .184184$ H $1.841703-3.7355982 .725420$ C $4.0660471 .493933-1.728348$ O $4.7392802 .032465-0.582944$ C $4.030794 \quad 3.068716 \quad 0.105946$ C $3.7394154 .232645-0.852725$ C $3.0134713 .760649-2.108053$ C $3.7696052 .599420-2.750280$ H $3.1326331 .012027-1.415746$ C $4.9803500 .421668-2.339823$ H $4.717608 \quad 3.4536640 .869106$ O $2.9640775 .241643-0.215107$ H $4.6859954 .698971-1.155529$ H $1.9762963 .483348-1.890630$ O $2.9540994 .833199-3.050471$ H $4.7059272 .971723-3.185502$ O $2.9900532 .061544-3.815127$ C $5.103439-0.801871-1.457334$ H $4.5948330 .081018-3.306672$ H $5.9846170 .840508-2.478420$ H $3.4520895 .530070 \quad 0.574966$ H $2.5009935 .575229-2.614316$ H $2.7898362 .791189-4.426099$ O $6.203143-1.515714-1.840429$ O $4.324367-1.108812-0.566088$ C $6.453932-2.712638-1.097686$ H $7.418815-3.114907-1.417477$ H $6.502939-2.498439-0.025572$ H $5.677341-3.452139-1.310639$ SCF Energy (B3LYP/6-31G**//MMFF) $=-3245.90589146$

02_102

MMFF Geometry

C $-0.467335 \quad 3.539782 \quad 0.182135$

C $0.3298754 .217313-0.659281$

C $1.4030403 .655229-1.564338$

O $1.6402332 .251288-1.321475$

C $2.7347454 .391724-1.332862$

C $3.700968 \quad 4.222587-2.489399$

C $4.7694073 .402486-2.492618$

C $3.4304825 .105225-3.682699$

C $5.2176482 .458986-1.411785$

C $5.5585161 .077997-1.991419$
C $6.1925700 .159821-0.937889$

C $6.651890-1.154959-1.568935$

C $7.230075-2.118969-0.522627$

O $5.218995-0.1057290 .075574$

O $7.338614-3.400314-1.160845$

C $6.230195-2.2442160 .646405$

C $8.649164-1.715969-0.104917$

O $6.787249-2.9976771 .721463$

C $5.716998-0.8815291 .174235$

C $4.553158-1.1181152 .162273$

O $6.776018-0.2085951 .857059$

C $0.8753791 .352815-1.997545$

O $-0.0166121 .591437-2.795382$

C $1.2951380 .004295-1.560216$

C $0.432063-1.015589-1.672840$

C $0.674463-2.399079-1.259578$

C $2.084454-2.779253-0.895684$

C 3.8109970 .1543332 .588172

C $2.546049-0.2182653 .334028$

C $0.238725-1.0856362 .944226$

O $2.503521-0.2429824 .561642$

C $0.197058-2.5540752 .651048$

C $-0.830457-3.1601552 .037012$

C $-0.881586-4.6324861 .744383$

C $-1.354794-4.9378130 .338589$

C $-2.569110-5.4704690 .120000$

C $-0.393700-4.695283-0.806614$

C $-0.370525-3.251792-1.228741$

N $1.507118-0.5568822 .480922$

H -0.3724922 .4626950 .278050$

H $0.1682645 .290243-0.755601$

H $1.0732743 .801458-2.600436$

H $3.1895884 .053456-0.394711$

H $2.5613485 .468522-1.201936$

H $5.4117813 .413607-3.372900$

H $4.1846984 .987288-4.468149$

H $2.4580504 .870289-4.125683$

H $3.4313226 .158533-3.383422$

H $6.1112522 .886239-0.941192$

H $4.4626392 .343601-0.629231$

H $4.6389180 .621079-2.379659$

H $6.2518191 .191504-2.834339$

H $7.0452050 .686617-0.493374$

H $7.383582-0.972219-2.365992$

H $5.794877-1.642688-2.055299$

H $7.752813-4.016663-0.533679$

H $5.368243-2.817710 \quad 0.277105$

H $9.318420-1.723485-0.973577$

H $9.072440-2.4347230 .605414$

H $8.697939-0.7216020 .344014$

H $6.996217-3.8845491 .383551$

H $3.831201-1.8016581 .701485$

H $4.924630-1.6177703 .066440$

H 6.3933610 .4344482 .474601

H $2.282946-0.102298-1.126707$

H $-0.558558-0.814451-2.078781$

H $2.777361-2.511968-1.701831$

H $2.219313-3.847971-0.719544$

H $2.394157-2.2619100 .015478$

H 4.4281550 .7751523 .246155

H 3.5386710 .7731331 .725663

H $-0.544863-0.5360212 .416850$

H $0.131054-0.9157964 .019585$

H $1.049765-3.1496372 .969647$

H $-1.694730-2.5712231 .743717$

H $-1.559989-5.0914682 .475170$

H $\quad 0.096548-5.1034151 .902146$

H -2.907282 -5.713481 -0.882012

H $-3.255926-5.6682220 .936201$

H $-0.694917-5.287756-1.680598$

H $0.589985-5.078796-0.524279$

H $-1.348212-2.874267-1.528976$

H $1.658471-0.5147981 .477444$

C -1.8245853 .5345042 .316546$

O -2.4286002 .2400102 .099322$

C -3.626800 2.1450562 .889629

C $-3.499763 \quad 3.226403 \quad 3.954275$

C -2.8157354 .3281303 .173125$ 
H -4.4643512 .3937372 .226413$

C -3.8095020 .7306713 .445115$

H $-4.469903 \quad 3.5259124 .361923$

H -2.864286 2.9057424 .787942

C -2.1618695 .3813744 .051424$

H -3.5622084 .8154492 .531252$

C $-3.801877-0.3857362 .384225$

O $\begin{array}{llll}\text { O } & -2.737250 & 0.461164 & 4.359134\end{array}$

H -4.7327190 .6889824 .035423$

H -2.796306 -0.482752 1.964142

O $-4.063365-1.6189573 .069382$

C $-4.807489-0.2245361 .229081$

C $-4.851227-1.4847140 .340630$

H -4.5312080 .6504660 .632816$

O $\quad-6.1010820 .012417 \quad 1.781430$

H -3.825597 -1.7550290 .067326$

H $-5.260956-2.3157850 .927497$

H -2.9100785 .8782914 .677749$

H -1.408749 4.9406624 .713275

H -1.669033 6.1457213 .442658

C $-1.563693 \quad 4.2080420 .967089$

H -2.863803 -0.4421514 .696997$

H $-4.976667-1.5805683 .402166$

H $-6.674547 \quad 0.3317601 .064804$

H -1.316448 5.2647941 .123478

H -2.4783804 .1624240 .364296$

H -0.8848313 .3638482 .856534$

C $-4.019862-0.463715-2.427142$

O $-5.271202-0.247579-1.762045$

C $-5.714773-1.324511-0.930303$

C $-5.847631-2.610277-1.761512$

C $-4.555411-2.937567-2.503266$

C $-4.074584-1.725600-3.299004$

H $-3.217507-0.551607-1.684826$

C $-3.7412650 .770287-3.299711$

H $-6.726014-1.058760-0.599699$

O $-6.210130-3.716273-0.941927$

H $-6.649943-2.480898-2.499564$

H -3.781619-3.281720 -1.809758

O $-4.782862-4.026791-3.400825$

H -4.744136 -1.562254 -4.153255

O $-2.775580-1.992161-3.822873$

C -3.462906 2.012346-2.482727

H -2.868414 $0.605769-3.940607$

H $-4.6178870 .973071-3.927262$

H $-7.041487-3.487155-0.492580$

H -5.478665 -3.761451 -4.026137

H $-2.832105-2.815975-4.336766$

O $-3.556523 \quad 3.104627-3.297994$

O $-3.1774042 .024864-1.293893$

C $-3.3155894 .369454-2.674472$

H -3.948260 4.492526-1.790123

H -2.258314 $4.457463-2.411655$

H -3.564855 $5.155037-3.392825$

SCF Energy (B3LYP/6-31G**//MMFF) $=-3245.91282091$

$02 \_103$

MM̄FF Geometry

C $-0.6828330 .831343-2.295347$

C $-1.088640-0.405343-2.626370$

C $-0.625346-1.706905-2.010044$

O $0.199556-1.429299-0.857826$

C $0.178619-2.505800-3.050719$

C $0.518676-3.916067-2.613989$

C $1.743464-4.331141-2.234290$

C $-0.625164-4.897211-2.669231$

C $3.003801-3.518365-2.128664$

C $3.588096-3.581390-0.712351$

C $4.869397-2.745956-0.586137$

C $5.462136-2.8806400 .817609$

C $6.700241-1.9913551 .002079$

O $4.542208-1.376013-0.846300$

O $6.992606-1.9269152 .406218$

C $6.357464-0.5586640 .545700$

C $7.940092-2.5896910 .327198$

$\begin{array}{llll}\text { O } & 7.517606 & 0.270605 & 0.557692\end{array}$

C $5.672006-0.493388-0.841250$

C $5.1651570 .943726-1.097081$
O $6.618932-0.840423-1.852513$

C $0.002504-2.1590210 .269293$

O $-0.718797-3.1402820 .388844$

C $0.743805-1.5966561 .424069$

C $1.471067-0.4706181 .364435$

C 2.1104840 .2128982 .492495

C $1.974735-0.4025853 .858722$

C $4.2923151 .112411-2.348202$

C $3.7895322 .542068-2.413581$

C $2.1743564 .067669-1.278560$

O $4.3814353 .407480-3.054028$

C 2.7770854 .4328460 .043320

C 2.0858544 .4821001 .192320

C 2.7192724 .7375752 .530611

C 2.5743153 .5635153 .478834

C 1.7816303 .6436404 .560727

C 3.4039732 .3211343 .219370

C 2.7361841 .3833482 .251500

N $2.6643962 .746478-1.630105$

H $0.0599830 .973991-1.516349$

H $-1.833567-0.510244-3.413633$

H -1.526093 -2.254582 -1.709081

H $1.084790-1.949268-3.311358$

H $-0.391695-2.575687-3.987554$

H $1.866610-5.380998-1.969972$

H $-0.318376-5.910325-2.387873$

H -1.427361 -4.597417 -1.988467

H -1.032316 -4.947343 -3.684484

H $3.732607-3.923594-2.841126$

H $2.842320-2.474658-2.407053$

H $2.839817-3.210534-0.002590$

H $3.801780-4.624418-0.446836$

H $5.583842-3.096926-1.340261$

H $5.704755-3.9271931 .040074$

H $4.708835-2.5832531 .560952$

H $7.138858-2.8345762 .723570$

H $5.668207-0.1362351 .290495$

H $8.164180-3.5780940 .745976$

H $8.827970-1.9765440 .517101$

H $7.826085-2.707354-0.752442$

H 7.9058480 .2128091 .447568

H $4.5800571 .270544-0.229865$

H $6.0190211 .628987-1.179053$

H $6.326674-0.470319-2.700047$

H $0.600659-2.1338572 .356280$

H 1.5744890 .0362350 .406938

H $2.440256-1.3941453 .879643$

H $0.918513-0.5033634 .133010$

H $2.445597 \quad 0.1850424 .649575$

H $4.8537210 .908214-3.265413$

H $3.4358330 .428880-2.333372$

H $1.0834984 .012220-1.233390$

H $2.4614944 .796645-2.042237$

H 3.8487584 .6182750 .058250

H 1.0152924 .2912921 .180281

H 2.2515505 .6347992 .955737

H 3.7847004 .9787302 .426806

H 1.6916062 .8158195 .256308

H 1.2008744 .5352834 .772160

H $3.643217 \quad 1.8228774 .164289$

H 4.3791602 .6121612 .808228

H $2.7694691 .733203 \quad 1.222139$

H $2.293081 \quad 1.962597-1.101615$

C $-1.9262482 .988191-1.962837$

O $-3.0405672 .279217-1.392558$

C $-4.2300873 .074516-1.530209$

C $-3.7381764 .496013-1.762276$

C $-2.5085984 .244031-2.616250$

H $-4.7371592 .718063-2.435807$

C $-5.1533752 .864519-0.323822$

H $-4.4864425 .121292-2.257999$

H -3.446858 $4.986509-0.826688$

C -1.541609 $5.414743-2.657794$

H $-2.836614 \quad 4.018955-3.640081$

C -5.2882181 .3853460 .098206$

O $-4.623607 \quad 3.5808540 .798031$

H $-6.1347543 .307036-0.529998$

H -4.3311911 .0566070 .506820$ 
O $-\begin{array}{llll}-6.223525 & 1.321008 & 1.181180\end{array}$ C $-5.7388510 .431864-1.018668$ C $-5.877878-1.042363-0.587374$ H $-5.049790 \quad 0.486466-1.867667$ O $-7.0132850 .885734-1.486961$ H $-6.434421-1.1008770 .353945$ H $-6.514801-1.543117-1.327551$ H $-2.0245716 .297737-3.088927$ H -1.189185 $5.679849-1.655198$ H $-0.6661795 .174558-3.269596$ C -1.203865 2.077847 -2.958052 H -5.2112863 .4121061 .554539$ H -7.0960791 .5668650 .828825$ H $-7.2555990 .340803-2.255008$ H $-0.3604122 .612032-3.410214$ H -1.889201 $1.805477-3.770712$ H $-1.2614803 .251475-1.131263$ C $-4.108348-1.6189121 .879613$ O $-3.671421-1.3327830 .545931$ C $-4.531492-1.799293-0.496787$ C $-4.683884-3.323464-0.385611$ C $-5.211587-3.7265810 .986549$ C $-4.336565-3.1239872 .082224$ H $-5.029181-1.0622842 .088052$ C $-3.024681-1.1273992 .848831$ H $-4.003644-1.601965-1.438215$ O $-5.554325-3.830279-1.391222$ H $-3.706775-3.800810-0.537277$ H $-6.261741-3.4409521 .117037$ O $-5.163187-5.149864 \quad 1.110609$ H $-3.372928-3.6497592 .100964$ O -4.957668 -3.334839 3.348982 C $-2.824420 \quad 0.3672412 .777141$ H -3.275846 -1.369074 3.888035 H -2.067311 -1.618194 2.640120 H $-5.185490-3.572057-2.253106$ H $-5.686204-5.5211380 .379207$ H $-5.105420-4.2915693 .443752$ O O $-3.559497 \begin{array}{llll}1.180948 & 3.320717\end{array}$ C $-1.3885712 .036811 \quad 1.902500$ H -0.5605822 .1249271 .194462$ H -2.235802 2.6098941 .518946 H -1.066183 2.4260672 .872409 SCF Energy (B3LYP/6-31G**/MMFF) $=-3245.91429742$

02_104

MMFF Geometry

C $1.114322-1.7405342 .426378$

C $-0.143480-2.1530372 .215552$

C $-0.547791-2.9890131 .028908$

O $-1.352128-2.141148 \quad 0.173490$

C $-1.393686-4.1877051 .481397$

C $-1.785880-5.095800 \quad 0.332570$

C $-3.001839-5.111671-0.248368$

C $-0.715771-6.052772-0.125489$

C $-4.178126-4.2331320 .073387$

C $-4.754208-3.576971-1.189195$

C $-5.913892-2.634295-0.840634$

C $-6.620330-2.138855-2.102056$

C $-7.736446-1.137474-1.766864$

O $-5.373248-1.519990-0.122049$

O $-8.132624-0.493755-2.987480$

C -7.163894 -0.043186 -0.842730

C $-8.986931-1.833933-1.217099$

O $-8.194790 \quad 0.826117-0.379311$

C $-6.357618-0.595916 \quad 0.357257$

C -5.6262490 .5669331 .065827$

O $-7.250311-1.2143451 .285295$

C $-0.673589-1.329210-0.685326$

O $0.527485-1.332449-0.907201$

C $-1.638643-0.370086-1.264128$

C $-1.1672730 .779656-1.770417$

C $-1.9605711 .882898-2.314164$

C $-3.4373551 .659609-2.494357$

C -4.5702390 .1330782 .090409$

C -3.8458751 .3546142 .621452$

C $-2.1740593 .079361 \quad 1.938233$
O $-4.150344 \quad 1.8678263 .695632$

C $-2.6306514 .036147 \quad 0.879608$

C $-1.7939284 .782967 \quad 0.143187$

C $-2.2444895 .727091-0.935298$

C $-1.5194165 .509749-2.247184$

C $-0.5848466 .377530-2.670977$

C $-1.930394 \quad 4.329188-3.104695$

C $-1.3298493 .037164-2.616292$

N $-2.875516 \quad 1.8225041 .748603$

H $1.900318-2.0498801 .741894$

H $-0.935414-1.8068262 .875744$

H $\quad 0.330560-3.349144 \quad 0.478570$

H -2.284164 -3.837361 2.015126

H $-0.834585-4.7812072 .217510$

H $-3.180242-5.834937-1.043121$

H $-1.064969-6.716439-0.923655$

H $\quad 0.151057-5.506126-0.508974$

H $-0.389153-6.6840460 .707350$

H $-4.950903-4.8541620 .542310$

H $-3.918310-3.4531010 .793441$

H $-3.960221-3.013964-1.696692$

H $-5.102224-4.356508-1.878119$

H -6.617876 -3.175379 -0.197417

H $-7.021176-2.979626-2.681757$

H $-5.891586-1.641432-2.757604$

H $-8.431583-1.182296-3.605934$

H $-6.4908560 .574900-1.453656$

H $-9.372861-2.558121-1.944670$

H $-9.797800-1.116106-1.051104$

H $-8.804237-2.369493-0.283265$

H $-8.6641631 .162237-1.162040$

H $-5.128534 \quad 1.186306 \quad 0.311944$

H $-6.356111 \quad 1.2137531 .570119$

H $-6.841631-1.2103952 .165323$

H -2.694560 -0.605206 -1.204859

H $-0.0913220 .952237-1.749722$

H -3.932084 $2.453076-3.058376$

H -3.931779 $1.591666-1.522511$

H $-3.6259050 .733502-3.049325$

H -5.024862 -0.3916162 .937062$

H $-3.834166-0.5470581 .646757$

H $-1.1034822 .867304 \quad 1.866975$

H -2.391972 3.4861062 .930265

H $-3.701853 \quad 4.115517 \quad 0.707751$

H $-0.725634 \quad 4.7274870 .335031$

H -2.070742 $6.749316-0.574695$

H $-3.3254395 .650405-1.107090$

H $-0.0778316 .242636-3.620695$

H $-0.3014857 .239022-2.075539$

H $-1.5915424 .478813-4.138115$

H $-3.0222614 .304191-3.152510$

H $-0.248123 \quad 3.083303-2.481239$

H $-2.707051 \quad 1.3183050 .883244$

C 1.4833370 .6561522 .970386

$\begin{array}{llll}\text { O } & 2.437446 & 0.795133 & 1.902442\end{array}$

$\begin{array}{llll}\text { C } 2.957538 & 2.137830 & 1.918864\end{array}$

C $2.2579172 .863743 \quad 3.066932$

C $1.881144 \quad 1.7181493 .988547$

H 4.0248892 .0584182 .153415

C 2.7774262 .7867530 .541712

H 2.9119633 .5994853 .545532

H 1.3606723 .3948242 .729114

C $0.7862632 .073161 \quad 4.979023$

H 2.7777341 .3975734 .535521

C $3.3438391 .960779-0.631030$

$\begin{array}{lllll}\text { O } & 1.375352 & 2.957099 & 0.298335\end{array}$

H 3.2135233 .7926380 .552057

H $2.7234951 .069807-0.786957$

O $3.1958592 .749198-1.819907$

C $4.8169531 .538325-0.479154$

C $5.3437010 .834934-1.744307$

H $4.908247 \quad 0.8690730 .382446$

O $5.6006212 .700487-0.215924$

H $4.6606340 .015530-1.993978$

H $5.3249481 .538952-2.585126$

H 1.1037552 .8998565 .622901

H -0.1334782 .3782094 .468860$

H 0.5475271 .2192525 .620549 
C $1.502043-0.7746133 .509279$

H $1.2816663 .377500-0.573584$

H $3.7824463 .520392-1.734345$

H $6.473342 \quad 2.404132 \quad 0.092811$

H $0.823226-0.8685784 .364484$

H $2.505548-1.0420643 .860449$

H $0.494790 \quad 0.8846692 .548323$

C $6.291901-1.774651-0.474359$

O $6.960477-0.508394-0.437336$

C $6.786858 \quad 0.301007-1.603366$

C $7.301970-0.447991-2.840997$

C $6.659237-1.825670-2.978085$

C $6.776158-2.605190-1.669326$

H $5.207871-1.617377-0.537542$

C $6.595790-2.4921220 .845278$

H $7.4361121 .174724-1.468174$

O $7.0617140 .299306-4.028506$

H $8.388584-0.580698-2.758398$

H $5.613464-1.752417-3.298815$

O $7.339408-2.561683-3.997739$

H $7.819836-2.910036-1.518500$

O $5.992869-3.793567-1.761796$

C $5.827396-1.8730401 .987600$

H $6.308688-3.549300 \quad 0.808096$

H $7.666254-2.4618391 .082261$

H $7.4937671 .163932-3.922132$

H $7.287918-2.037062-4.815214$

H $6.304814-4.286737-2.539886$

O $6.559626-0.8770312 .561684$

O $4.702966-2.226408 \quad 2.318438$

C $5.938794-0.2143973 .667429$

H $5.885842-0.8924484 .524203$

H 6.5540280 .6481013 .936600

H $4.940647 \quad 0.139792 \quad 3.396617$

SCF Energy (B3LYP/6-31G**//MMFF) $=-3245.92193679$

02105

MMFFF Geometry

C $-3.817805-1.898447-1.188038$

C $-3.169823-1.209031-2.139908$

C $-1.698668-1.353923-2.422282$

O $-1.167314-0.005756-2.421407$

C $-1.423027-2.028862-3.777755$

C $-1.793920-3.498361-3.813363$

C $-1.042333-4.486767-3.287414$

C $-3.088629-3.822765-4.511956$

C $0.288324-4.356755-2.599038$

C $0.172191-4.586169-1.086641$

C $1.532205-4.504105-0.373580$

C $1.347896-4.5943091 .141286$

C $2.674569-4.4136591 .891972$

O $2.143246-3.256685-0.704876$

O $2.375308-4.223818 \quad 3.283450$

C $3.393923-3.1451361 .375951$

C $3.525673-5.6873571 .831052$

O $4.697740-3.0609061 .945052$

C $3.463295-3.064215-0.165029$

C $3.901576-1.675903-0.684639$

O $4.372231-4.047625-0.662375$

C $0.1548710 .149356-2.147198$

O $0.987631-0.733614-2.028645$

C $0.405126 \quad 1.595922-1.957173$

C $1.6005602 .006281-1.506650$

C $1.9742323 .382800-1.172736$

C $0.9517354 .460447-1.425571$

C $5.302913-1.208605-0.294297$

C $5.751882-0.007664-1.112594$

C $7.6576391 .581260-1.331661$

O $5.1003670 .459606-2.042872$

C $7.1978312 .866861-0.719516$

C $6.6363883 .861011-1.424790$

C $6.2334345 .180812-0.834745$

C $4.7790175 .515268-1.075048$

C $4.4201026 .392910-2.027267$

C $3.7506954 .917881-0.143113$

C $3.1935493 .612902-0.639916$

N $6.9842940 .469388-0.689165$

H $-3.257149-2.634118-0.613824$
H $-3.702478-0.480404-2.744235$

H -1.220084 -1.907463 -1.607143

H $-0.366407-1.912200-4.048188$

H -1.951254 -1.488210-4.575409

H $-1.407243-5.509934-3.366962$

H $-3.303608-4.896658-4.512185$

H -3.929915 -3.320206 -4.026084

H $-3.045553-3.495519-5.556025$

H $0.970025-5.099701-3.031153$

H $0.739105-3.380978-2.799489$

H $-0.501930-3.830004-0.666490$

H $-0.283376-5.564441-0.887872$

H $2.161663-5.322848-0.741134$

H $\quad 0.868442-5.5396391 .423922$

H $\quad 0.660339-3.8016551 .464656$

H $1.816993-3.4322423 .368624$

H $2.837037-2.2713511 .739244$

H $2.982358-6.5303122 .275131$

H $4.442754-5.5809892 .421142$

H $3.801917-5.970660 \quad 0.813157$

H $4.595879-3.0967802 .911638$

H $3.829260-1.697119-1.781553$

H $3.173111-0.919831-0.368402$

H $4.660819-3.776700-1.549687$

H $-0.4181332 .277067-2.140358$

H $2.3717091 .259653-1.324596$

H $1.3233445 .468967-1.233115$

H $0.0728014 .313381-0.788073$

H $0.6322654 .449557-2.473816$

H $5.319860-0.9200080 .762058$

H $6.034301-2.008646-0.452808$

H $7.4629861 .544364-2.408792$

H $8.7322831 .454146-1.168281$

H $7.3647012 .996146 \quad 0.347096$

H $6.4921493 .736800-2.495806$

H $6.8736525 .951941-1.283414$

H 6.4424955 .2190560 .241675

H $3.3817236 .666937-2.180468$

H $5.1560616 .853555-2.677789$

H 2.9571765 .6464000 .055051

H $4.202954 \quad 4.7450150 .842151$

H $3.8651742 .766323-0.495831$

H 7.4201700 .0601320 .132283

C $-5.992418-0.497406-1.277245$

O $-5.3101310 .666799-0.770830$

C $-6.2521001 .500405-0.078089$

C $-7.6127071 .101597-0.628119$

C $-7.434109-0.400315-0.766377$

H $-6.203110 \quad 1.2206680 .981824$

C $-5.8656502 .975458-0.232164$

H $-8.432521 \quad 1.3777760 .041557$

H -7.802759 $1.549995-1.609860$

C $-8.453631-1.055374-1.682089$

H -7.512101 $-0.854200 \quad 0.230813$

C $-4.383348 \quad 3.2763850 .084830$

O $-6.1069583 .394262-1.581081$

H -6.521459 3.5910710 .393880

H $-3.7514282 .882049-0.720382$

$\begin{array}{lllll}\text { O } & -4.198427 & 4.696233 & 0.057507\end{array}$

C $-3.8832702 .717890 \quad 1.429473$

C -2.4164903 .0892801 .715149$

H -3.9740471 .6277921 .412664$

O -4.7113453 .1876482 .491255$

H -1.8116522 .8276250 .840033$

H -2.332155 4.1738691 .854091

H $-9.467019-0.915846-1.291727$

H -8.420352 -0.628655 -2.690190

H $-8.267237-2.130714-1.764750$

C $-5.264055-1.754366-0.786336$

H $-5.5829682 .819725-2.165450$

H -4.7990345 .0887620 .713380$

H -4.575882 4.1464912 .575245

H -5.803092 -2.650925 -1.113533

H $-5.276995-1.7527710 .311886$

H $-5.970267-0.436228-2.372013$

C $-1.2839050 .263857 \quad 1.995722$

O

C -1.862552 2.3888422 .978334 
C $-0.3849542 .691578 \quad 3.270875$

$\begin{array}{lllll}\text { C } & 0.547712 & 1.963022 & 2.305641\end{array}$

C 0.2115710 .4726932 .255183 H -1.5269020 .6315720 .990504$

C $-1.711352-1.2110442 .000653$ H -2.4446822 .7681023 .827660$ O -0.1273334 .0900223 .200821$ H $-0.143790 \quad 2.369754 \quad 4.292361$ H $0.5060962 .406638 \quad 1.306158$ $\begin{array}{llll}\text { O } & 1.897498 & 2.104190 & 2.752109\end{array}$ H $0.5176290 .007643 \quad 3.199023$ O $0.959093-0.1495961 .215524$ C $-1.469007-1.9377383 .307598$ H $-2.788699-1.2749041 .806854$ H $-1.180291-1.7427381 .202711$ H -0.7186814 .5278733 .836706$ H 2.0882233 .0570862 .796290 H 1.8965370 .0595401 .368532 O $-1.514975-3.2839113 .078511$ O $-1.296272-1.4039484 .394431$ C $-1.307688-4.1133724 .226025$ H $-0.352014-3.8759844 .703337$ H -2.133912 -3.989125 4.932095 H -1.282332 -5.154130 3.892460 SCF Energy (B3LYP/6-31G*//MMFF)= -3245.90903805

02_106

MMFF Geometry

C $2.483167 \quad 1.295626 \quad 3.251102$

C 3.7925641 .1958052 .975526

C 4.5118171 .7593831 .769489

O 3.6293862 .6430151 .042684

C 4.9414320 .5942870 .858168

C $6.0677580 .911368-0.104002$

C $5.9606600 .888715-1.447048$

$\begin{array}{lll}\text { C } 7.409386 & 1.175248 & 0.532923\end{array}$

C $4.7412470 .617889-2.284545$

C $4.741334-0.803799-2.870482$

C $4.310073-1.872147-1.853124$

C $4.470073-3.281275-2.423541$

C $3.939390-4.347753-1.451321$

O $2.934881-1.630811-1.547344$

O $3.844785-5.588749-2.166811$

C $2.508290-3.963294-1.020137$

C $4.903436-4.591626-0.284208$

O $2.023018-4.840614-0.006369$

C $2.371189-2.494662-0.557367$

C $0.872014-2.134444-0.381860$

O $3.023152-2.3325290 .705335$

C $4.168447 \quad 3.7161600 .411920$

O 5.3552474 .0003690 .330028

C $3.1237674 .548254-0.226941$

C $1.841760 \quad 4.5508100 .171166$

C $0.7642155 .374175-0.386248$

C $1.0863306 .211284-1.597130$

C $0.581487-0.626080-0.363362$

C $0.468845-0.011175-1.751054$

C $0.2003802 .190224-2.877079$

O $0.521267-0.667618-2.787665$

C $-0.855034 \quad 3.248992-2.772611$

C $-1.8764743 .299779-1.902597$

C $-2.8579304 .436511-1.847891$

C $-2.8587575 .140388-0.507687$

C $-3.892145 \quad 5.007704 \quad 0.340386$

C -1.705249 $6.069208-0.187936$

C $-0.4519225 .328194 \quad 0.198737$

N $\quad 0.273568 \quad 1.360733-1.689559$

H 1.8224941 .8319312 .575872

H 4.4239530 .6431363 .669506

H 5.3710012 .3234812 .150318

H 4.0603380 .2389390 .315126

H $5.279605-0.2558501 .467025$

H $6.8568211 .092092-2.032692$

H $8.2114301 .262027-0.208087$

H 7.3929772 .1065221 .106002

H $7.6771340 .355301 \quad 1.207477$

H $3.8130530 .823438-1.742403$

H $4.7562691 .334901-3.115577$
H $4.037150-0.814172-3.712872$

H $5.733545-1.042119-3.272862$

H $4.912119-1.768650-0.944841$

H $5.517994-3.481899-2.678964$

H $3.910172-3.360951-3.365976$

H $4.729571-5.806454-2.506970$

H $1.857948-4.110844-1.894062$

H $5.881689-4.917328-0.657664$

H $4.544962-5.4011760 .361132$

H $5.064235-3.704820 \quad 0.332560$

H $2.113633-5.748875-0.342228$

H $0.282095-2.592819-1.184618$

H $0.513009-2.5645530 .562104$

H $2.609447-1.5924111 .178325$

H $3.4834325 .207076-1.010547$

H 1.5475993 .9039360 .995700

H $1.8164396 .987382-1.342702$

H $0.2187046 .717011-2.024727$

H $1.5013205 .588409-2.397634$

H $-0.388293-0.4648640 .119337$

H $1.337499-0.0847450 .215314$

H $0.0287961 .577412-3.767311$

H $1.1812222 .667072-2.978308$

H $-0.7466524 .065783-3.484210$

H $-2.0270572 .494128-1.188427$

H $-3.8530374 .027851-2.066306$

H -2.660304 5.173639 -2.636201

H $-3.9155645 .530011 \quad 1.290994$

H -4.7397624 .3748490 .101718$

H -1.9695836 .7170130 .658539$

H $-1.5615116 .750036-1.030695$

H $-0.5779504 .693053 \quad 1.077172$

H $0.3698801 .827184-0.791776$

C $0.893499-0.454674 \quad 4.067442$

O $\begin{array}{llll}0.256341 & 0.091759 & 3.394480\end{array}$

C $-1.432073-0.6454293 .800121$

C $-0.932326-1.8089614 .643211$

C $0.329274-1.2314965 .254077$

H $-2.023845 \quad 0.0484124 .409696$

C -2.201651 -1.049287 2.539842

H $-1.664511-2.1298415 .390202$

H $-0.689880-2.6734464 .012203$

C $1.268946-2.2878525 .808948$

H $0.046848-0.5381936 .057576$

C -3.407005 $-1.970358 \quad 2.784561$

O $-2.658322 \quad 0.147816 \quad 1.901863$

H $-1.508331-1.5335481 .845212$

H -3.064414 -2.934607 3.177917

O $-4.223911-1.3831513 .804460$

C $-4.292647-2.1986961 .541966$

C $-3.523229-2.711850 \quad 0.312348$

H $-4.823736-1.2717661 .303899$

O -5.303663 -3.152671 1.885228

H $-2.708930-2.0225690 .074281$

H -3.064531 -3.6783690 .554929$

H $0.777652-2.8608036 .602284$

H $1.584481-2.9922745 .031832$

H $2.167857-1.8291926 .232387$

C $1.842446 \quad 0.676424 \quad 4.464111$

H $-1.8818430 .714911 \quad 1.755457$

H $-4.987471-1.9704983 .938439$

H $-4.861613-3.9947492 .088334$

H $2.611604 \quad 0.2998975 .147938$

H $1.292291 \quad 1.4647434 .992176$

H $1.384780-1.1378553 .361475$

C $-4.410012-0.585525-1.667315$

O $-5.182204-1.709290-1.223856$

C $-4.421455-2.886477-0.933839$

C $-3.669623-3.336522-2.193560$

C $-2.834229-2.205272-2.792733$

C $-3.668134-0.933553-2.964368$

H -3.689032 $-0.300346-0.892245$

C $-5.3812200 .585011-1.861248$

H $-5.149294-3.671429-0.692658$

O $-2.838079-4.452781-1.895000$

H $-4.401048-3.670831-2.940688$

H -1.951242 -2.008547 -2.176112

O $-2.330953-2.617500-4.065849$ 
H -4.377684 -1.078625 -3.788855

O $-2.7863550 .123853-3.331769$

C $-6.0601570 .960203-0.564212$

H $-4.8839061 .479100-2.250952$

H $-6.1693290 .311372-2.573424$

H $-2.354259-4.680961-2.707282$

H $-3.090499-2.792439-4.647269$

H $-3.3267010 .875165-3.627677$

$\begin{array}{lllll}\text { O } & -5.155848 & 1.495628 & 0.304039\end{array}$

O

C $-5.6753871 .853278 \quad 1.588868$

H -6.0789220 .9703732 .093756$

H -4.8537232 .2540512 .188313$

H -6.443814 2.6251681 .485134

SCF Energy $\left(B 3 L Y P / 6-31 G^{* *} / / M M F F\right)=-3245.90523881$

$02 \_107$

MM̄FF Geometry

C $2.269771-3.5009442 .178340$

C $1.790830-2.2759981 .911719$

C $1.036060-1.4297652 .903411$

O $-0.248054-1.0690142 .345255$

C $1.827598-0.1335743 .147796$

C $1.198270 \quad 0.7831894 .174820$

C 0.5132591 .9060043 .880523

C 1.4299420 .3913075 .610996

C 0.1659962 .4469092 .518589

C -1.3281372 .7875512 .437798$

C -1.7383183 .3283991 .059257$

C -3.2167873 .7271901 .076008$

C $-3.7233084 .156150-0.307065$

O -1.5082682 .3042350 .085492$

O $-5.1587144 .181179-0.263013$

C $-3.3238443 .080256-1.337540$

C $-3.2764635 .579085-0.662006$

O $-3.646233 \quad 3.504796-2.659615$

C -1.836202 2.679524-1.261356

C $-1.4922661 .486136-2.187565$

O $-1.0337053 .785958-1.693474$

C -1.264934 -1.961743 2.491235

O $-1.188496-3.0968682 .933128$

C $-2.514610-1.2887512 .066696$

C $-3.691548-1.9179932 .204645$

C $-5.016612-1.3517711 .933289$

C $-5.0825840 .065818 \quad 1.430379$

C $-2.154857 \quad 0.152325-1.833194$

C $-3.455687-0.131105-2.562671$

C $-5.264434-1.848703-2.664188$

O $-3.9074590 .585705-3.450009$

C $-6.405598-1.450887-1.783615$

C $-7.199720-2.333797-1.159546$

C $-8.387786-1.946171-0.329217$

C $-8.327632-2.4995441 .075303$

C $-9.016904-3.6020881 .414963$

C -7.555581-1.714229 2.109582

C $-6.105559-2.1087842 .184995$

N -4.033179 -1.313995 -2.118343

H $2.075047-3.9257343 .161542$

H $1.966661-1.8217200 .942662$

H $\quad 0.889153-1.9593893 .853024$

H 1.9611560 .4006222 .200739

H $2.843226-0.3807483 .484941$

H 0.1490942 .5103564 .710676

H $\quad 0.995817 \quad 1.1101306 .314054$

H $0.980525-0.5844125 .820064$

H 2.5029590 .3331025 .820886

H $\quad 0.7566083 .3538542 .343941$

H 0.4173941 .7397431 .724005

H -1.907721 1.8831792 .661498

H -1.575474 3.5299293 .207347

H -1.1098814 .1955590 .823858$

H -3.396116 4.5202961 .812969

H -3.8184482 .8718601 .410372$

H -5.426468 4.7991540 .438535

H -3.939951 2.196280-1.131079

H $-3.672687 \quad 6.2971910 .066061$

H -3.675978 $5.888329-1.634053$

H $-2.1910375 .696449-0.679933$
H $-4.593723 \quad 3.723651-2.670636$

H -1.665109 $1.756705-3.236215$

H $-0.4097141 .322908-2.110470$

H -0.162042 3.450559-1.961636

H -2.436571 -0.2722051 .700523$

H -3.692236 -2.936301 2.591737

H -6.0882350 .3818401 .145317$

H -4.4647050 .1907040 .535639$

H -4.7282290 .7581122 .201214$

H -1.473349 -0.655651 -2.126299

H $-2.3162730 .065674-0.755150$

H $-5.147177-2.935420-2.724723$

H -5.422992 -1.460352 -3.675331

H $-6.605333-0.386858-1.681697$

H -7.011761 -3.397982 -1.283128

H $-9.284975-2.320449-0.839894$

H -8.509635 -0.856655 -0.287547

H -9.006136 -3.984035 2.430456

H $-9.602027-4.1502490 .683805$

H -7.989236 -1.894207 3.103127

H $-7.700828-0.6433021 .942305$

H -5.954187 -3.125301 2.551436

H -3.606793 -1.812536 -1.342384

C $3.534211-3.757130-0.063086$

O $2.398068-3.639835-0.945760$

C $2.790830-4.054887-2.261860$

C $4.310790-4.126888-2.251443$

C $4.565820-4.587995-0.827620$

H $2.411335-5.079237-2.383148$

C $2.151582-3.191556-3.355949$

H $4.705899-4.817253-3.002522$

H $4.762026-3.142427-2.418120$

C $5.997882-4.369490-0.370326$

H $4.320419-5.655761-0.750702$

C $2.584666-1.712236-3.461190$

O $2.416872-3.818815-4.618469$

H $1.062263-3.221874-3.229957$

H $3.622663-1.643501-3.807182$

O $1.787006-1.109993-4.492833$

C $2.417033-0.875569-2.180525$

C $2.6802320 .624658-2.415121$

H $3.113025-1.247230-1.426024$

O $1.092773-1.054482-1.687043$

H $3.6541350 .731628-2.905213$

H $1.9261651 .024986-3.103345$

H $6.692627-4.935593-0.999344$

H $6.279824-3.312532-0.425363$

H $6.133155-4.7003730 .664048$

C $3.093565-4.3774981 .265919$

H $3.372694-3.759824-4.786668$

H $1.905510-1.641236-5.298865$

H $1.044190-0.655720-0.802705$

H $3.983190-4.6769341 .832680$

H $2.509843-5.2854321 .070127$

H $3.930713-2.7479560 .102211$

C $4.8737461 .030435-0.324595$

O $3.4680960 .920955-0.088729$

C $2.6403921 .468346-1.118374$

C $2.9676342 .953536-1.325098$

C $4.4538203 .176479-1.599162$

C $5.3039062 .489558-0.528750$

H $5.1229070 .456344-1.226174$

C 5.6118900 .3280890 .824952

H $1.6131241 .421665-0.738353$

O $2.187993 \quad 3.480281-2.393564$

H $2.6875723 .507535-0.419420$

H $4.7236702 .838386-2.606291$

O $4.7099284 .582514-1.568421$

H $5.212606 \quad 3.0612300 .400701$

O $6.6773172 .545425-0.908352$

C 5.3602350 .9173832 .198872

H $5.278958-0.7160510 .870969$

H $\quad \begin{array}{llll}6.690676 & 0.345367 & 0.633951\end{array}$

H $2.4356494 .414671-2.500958$

H $5.6659784 .705469-1.698792$

H $6.7776802 .053310-1.741122$

O 6.1801330 .2954293 .098667

O $4.542898 \quad 1.7876142 .463254$ 
C $6.0447810 .729807 \quad 4.455517$

H $5.0321230 .529745 \quad 4.817920$

H 6.7537070 .1641855 .065912

H $6.281877 \quad 1.794803 \quad 4.538236$

SCF Energy (B3LYP/6-31G*//MMFF) $=-3245.91242177$

02108

MM̄FF Geometry

C $0.967902-2.584947-0.762789$

C $0.116669-1.692746-1.296557$

C $0.464101-0.622567-2.309425$

O $1.899220-0.505761-2.411814$

C $-0.0902100 .742308-1.866107$

C $-0.239024 \quad 1.726253-3.008178$

C $0.610762 \quad 2.738972-3.268985$

C -1.475492 $1.540655-3.850889$

C $1.8897433 .082265-2.556403$

C $1.8033964 .411496-1.792164$

C $0.884204 \quad 4.349259-0.562637$

C 0.8005805 .7123490 .126325

C $-0.031920 \quad 5.6528201 .416853$

$\begin{array}{lllll}\text { O } & 1.428283 & 3.385396 & 0.343922\end{array}$

O $0.216657 \quad 6.8782852 .122520$

C 0.4756854 .4823682 .288469

C $-1.5356895 .618728 \quad 1.116754$

$\begin{array}{llll}\text { O } & -0.367277 & 4.280846 & 3.420847\end{array}$

C $0.626659 \quad 3.154754 \quad 1.508877$

C 1.3484162 .1042122 .387890

O -0.6707412 .6677701 .166306$

C $2.477736-0.422153-3.633586$

O $1.910371-0.418688-4.716449$

C $3.955928-0.370493-3.514264$

C $4.590220-0.184155-2.345477$

C $6.038233-0.165954-2.129118$

C $6.929054-0.439177-3.311623$

C 1.7678920 .8327351 .635721

C $2.572417-0.1100062 .514154$

C $4.077590-2.0940962 .351805$

O $2.643472 \quad 0.0047293 .734716$

C $5.473805-1.5543072 .329022$

C $6.481703-2.1115251 .640489$

C $7.878714-1.5576251 .616751$

C $8.356815-1.2273110 .218605$

C $9.208174-2.041543-0.427726$

C $7.9202670 .085115-0.397153$

C $6.4954300 .055461-0.878664$

N $3.186302-1.1078251 .768879$

H $2.012205-2.557921-1.067195$

H $-0.935155-1.736578-1.028615$

H $0.027752-0.934039-3.266041$

H $0.5501691 .161299-1.084596$

H $-1.0766770 .624806-1.402242$

H $0.3801013 .396797-4.106286$

H -1.553495 $2.289553-4.646207$

H -1.474403 $0.555412-4.326881$

H $-2.3736931 .625419-3.230168$

H $2.2207982 .282417-1.887953$

H $2.6744723 .171708-3.318444$

H $2.8194434 .669262-1.466020$

H $1.4687475 .207432-2.469013$

H $-0.1133134 .027871-0.881682$

H $\quad 0.3965416 .470479-0.556359$

H $1.8139906 .051653 \quad 0.383879$

H -0.3407276 .8870502 .918658$

H 1.4620984 .7686042 .679722

H -1.835921 6.5235790 .574668

H -2.124444 5.6141252 .040704

H -1.8348324 .7574420 .515604$

H $-0.353435 \quad 5.0968763 .948203$

H 2.2578662 .5538272 .807831

H 0.7059041 .8201333 .231089

H -0.6249441 .7078061 .034884$

H $4.495086-0.495820-4.447445$

H $3.997504-0.039618-1.443655$

H $6.685395-1.407755-3.762471$

H $7.990399-0.475962-3.056489$

H $6.8093640 .341823-4.070460$

H $\quad \begin{array}{llll}0.889672 & 0.282774 & 1.283607\end{array}$
H $2.382161 \quad 1.0960410 .767194$

H $3.978050-3.0123811 .764629$

H $3.774459-2.3016013 .382922$

H $5.663050-0.6563062 .912677$

H $6.298656-3.0169811 .066453$

H $8.539687-2.3052592 .074510$

H $7.965715-0.6637182 .246996$

H $9.580531-1.799991-1.417681$

H $9.548942-2.9707640 .016854$

H $8.5995620 .381214-1.204409$

$\begin{array}{lllll}\text { H } & 8.021064 & 0.884041 & 0.348838\end{array}$

H $5.7682030 .243996-0.088644$

H $3.123708-1.0743410 .755562$

C $-0.705846-3.6320910 .916189$

O $-0.705088-2.5579601 .881717$

C $-1.287983-3.0422163 .099008$

C -1.993514 -4.343240 2.747425

C $-1.045001-4.9000451 .702783$

H $-0.445753-3.2925543 .759645$

C -2.137393 -1.989388 3.816812

H $-2.117683-5.0042663 .610121$

H $-2.980203-4.1579912 .307966$

C $-1.652212-6.0109990 .863838$

H $-0.146235-5.2812302 .206391$

C -3.365261 -1.419846 3.072875

O $-2.616390-2.5691655 .038607$

H -1.492891 -1.149586 4.105631

H -4.127958 -2.194282 2.933096

O $-3.956068-0.4407993 .944308$

C -3.073424 -0.7349181 .723543$

C -4.2547650 .1079551 .198402$

H -2.820227 -1.495322 0.980063

O $-1.9375340 .117267 \quad 1.865495$

H $-5.146200-0.525367 \quad 1.147275$

H -4.4636970 .9110491 .914518$

H $-1.918007-6.8657911 .494305$

H $-2.560800-5.6779020 .352093$

H $-0.945697-6.3587300 .103962$

C $0.653350-3.688694 \quad 0.216946$

H -1.840537 -2.844783 5.556142

H $-4.159374-0.8871134 .784318$

H -2.150736 0.7819902 .543010

H $0.753735-4.636755-0.324126$

H $1.439860-3.6689670 .982282$

H $-1.508200-3.4270300 .202102$

C $-4.872902-0.947967-1.628774$

O $-3.719359-0.219595-1.192217$

C $-3.9603090 .756620-0.174309$

C $-5.014304 \quad 1.768919-0.643957$

C $-6.2940471 .079083-1.104070$

C $-5.9653550 .002411-2.135653$

H $-5.263484-1.549862-0.800026$

C $-4.415641-1.888962-2.755018$

H $-3.0252351 .318908-0.064769$

$\begin{array}{llll}\text { O } & -5.295836 & 2.697593 & 0.397103\end{array}$

H $-4.6008342 .343142-1.483679$

H $-6.8533980 .658192-0.260227$

O $-\begin{array}{llll}-7.153856 & 2.036117 & -1.726046\end{array}$

H $-5.6473740 .478355-3.072419$

O $-7.145015-0.749188-2.416679$

C $-3.649737-3.083689-2.231013$

H $-5.270012-2.287438-3.312450$

H $-3.764223-1.338190-3.444898$

H -5.8582293 .3973200 .024960$

H $-7.4515532 .654036-1.037184$

H $-7.824830-0.120841-2.715304$

O $-2.865155-3.584976-3.230016$

O $-3.758561-3.545894-1.103633$

C $-2.117554-4.759684-2.900424$

H -1.378801-4.531409 -2.128190

H $-2.787277-5.559622-2.570178$

H - $1.592683-5.091760-3.800047$

SCF Energy (B3LYP/6-31G**//MMFF)= -3245.91891425

02_109

MMFF Geometry

C $0.917138 \quad 4.452555 \quad 0.119530$

C $2.1131783 .878446-0.071679$ 
C $2.6479633 .473961-1.420171$

O $2.7548802 .031018-1.418208$

C $4.0457864 .075994-1.632532$

C $4.7073063 .608379-2.913221$

C $5.6574762 .653396-2.968870$

C $4.2596054 .313819-4.165598$

C $6.1951661 .846252-1.819076$

C $6.2395010 .346886-2.145843$

C $6.634033-0.473303-0.909281$

C $6.934300-1.923975-1.284496$

C $7.262140-2.769816-0.044869$

O $5.538491-0.430258 \quad 0.013290$

O $7.229800-4.151584-0.432966$

C $6.149761-2.5674961 .003822$

C $8.676997-2.4983150 .479403$

O $6.481481-3.2120952 .231916$

C $5.793789-1.0825101 .263557$

C $4.509368-1.0073862 .121985$

O $6.863718-0.4556341 .972100$

C $1.6474041 .330518-1.788007$

O $0.5896601 .784696-2.194518$

C $1.925736-0.103219-1.554201$

C $0.897307-0.962706-1.502230$

C $0.975797-2.387110-1.175063$

C $2.341213-3.008420-1.057859$

C 3.8955960 .3929762 .249835

C 2.5824010 .3029043 .004758

C $0.236359-0.5156912 .729091$

O $\quad 2.509422 \quad 0.5566994 .204641$

C $0.117100-2.0064002 .640394$

C $-0.932799-2.6427552 .098427$

C -1.021918 -4.139090 1.978201

C $-1.386869-4.6043200 .582594$

C -2.579624 $-5.173034 \quad 0.338813$

C $-0.346444-4.484663-0.515865$

C $-0.174879-3.063932-0.979619$

N $1.537542-0.1370142 .206904$

H $0.283004 \quad 4.673908-0.735131$

H 2.7260523 .6341230 .793695

H $1.9855933 .801988-2.231178$

H $4.6869413 .850822-0.772501$

H $3.9767815 .172294-1.649464$

H $6.0962722 .420070-3.937971$

H $4.7922103 .960416-5.054801$

H $3.1908114 .151140-4.335673$

H $4.4390125 .390514-4.079886$

H $7.2108832 .195581-1.597630$

H $5.6032201 .992338-0.911934$

H $5.2537760 .022989-2.503535$

H $\quad 6.9600240 .176704-2.955345$

H $7.514842-0.008413-0.450624$

H $7.753464-1.978289-2.012265$

H $6.058264-2.361532-1.783842$

H $7.881161-4.278367-1.144025$

H $5.252572-3.0748600 .622752$

H $9.420688-2.710809-0.298106$

H $8.921813-3.1618071 .316304$

H $8.823697-1.4660600 .803712$

H $6.680779-4.1415892 .026790$

H $3.751516-1.6682011 .685864$

H $4.714422-1.3886213 .130944$

H $\quad 6.515327 \quad 0.3104512 .454827$

H $2.950741-0.398809-1.365133$

H $-0.109373-0.577783-1.661722$

H $2.950007-2.777205-1.939406$

H $2.314862-4.098648-0.990906$

H $2.856228-2.634509-0.169634$

H 4.5579561 .0735952 .794667

H $3.7088120 .841347 \quad 1.267677$

H $-0.519211-0.0041532 .124812$

H $0.140314-0.1949953 .770297$

H $0.943936-2.5929533 .035416$

H -1.772756 $-2.061446 \quad 1.724447$

H -1.773782 -4.4838412 .699861$

H $-0.079486-4.6175272 .273135$

H $-2.843207-5.533053-0.650194$

H -3.322439 -5.2878951 .121144$

H $-0.642942-5.085437-1.384903$
H $\quad 0.585170-4.921949-0.144474$

H -1.115442 -2.533817 -1.130798

H $1.720186-0.3333991 .228080$

C -0.6528273 .7024841 .900179$

O

C -2.9883053 .5124141 .868033$

C -2.4673483 .0802873 .235377$

C -1.1671043 .8554943 .327563$

H -3.5393254 .4552351 .974391$

C $-3.8830792 .469102 \quad 1.183236$

H $-3.169312 \quad 3.319313 \quad 4.040117$

H -2.2745932 .0010513 .268004$

C -0.2224403 .3308734 .393870$

H -1.3974194 .9093443 .533471$

C $-4.5575202 .981258-0.106479$

O $\quad-3.121076 \quad 1.2949000 .900769$

H -4.6658962 .1765661 .892037$

H -5.2313423 .8076110 .150146$

O $-3.5798523 .542048-0.989989$

C $-5.3361831 .918847-0.906451$

C $-6.3530691 .128167-0.070673$

H -4.635176 $1.235590-1.394455$

O $-6.0451092 .589501-1.954573$

H -5.8299650 .6014050 .732622$

H $-7.048370 \quad 1.832696 \quad 0.402428$

H -0.6850773 .3984895 .383922$

H $0.039726 \quad 2.283343 \quad 4.217169$

H 0.7054413 .9105754 .415627

C $0.363818 \quad 4.7639231 .482395$

H -2.3571531 .5605670 .361131$

H $-2.9500642 .838960-1.223231$

H -5.390120 $3.084196-2.476503$

H 1.1767284 .8198082 .215383

H $-0.1066315 .753503 \quad 1.440916$

H -0.2347622 .6970571 .753620$

C $-5.573384-1.700323-0.935683$

O $-6.336259-0.747781-1.686227$

C $-7.1698220 .114698-0.905434$

C $-8.162573-0.718478-0.083132$

C $-7.453872-1.7755780 .759409$

C $-6.499048-2.598432-0.103776$

H $-4.869903-1.176847-0.277019$

C $-4.770988-2.536933-1.944794$

H $-7.7602590 .698007-1.623057$

O -8.9429740 .1119350 .770112$

H $-8.858963-1.227784-0.761965$

H $-6.926297-1.3269571 .609277$

O $-8.427313-2.6621851 .316787$

H -7.079338 -3.256329 -0.763444

O $-5.706895-3.4311160 .740723$

C $-3.646362-1.753012-2.587823$

H -4.315162 -3.406353 -1.461692

H $-5.442243-2.881369-2.741016$

H -9.3941110 .7653520 .208443$

H -9.049971 -2.120286 1.831173

H $-6.317556-3.9727291 .269588$

O $-3.279185-2.371888-3.749031$

O $-3.128677-0.748939-2.119241$

C $-2.210720-1.748409-4.468547$

H $-2.058474-2.302583-5.398611$

H $-2.468683-0.713989-4.715370$

H -1.288672 -1.787413 -3.881991

SCF Energy (B3LYP/6-31G**//MMFF) $=-3245.92727442$

0211

MM̄FF Geometry

C $2.176276-4.064701-0.139351$

C $0.850970-3.878233-0.052105$

C $-0.026053-3.617302-1.246117$

O $-0.685492-2.346119-1.054595$

C $-1.130060-4.685449-1.347564$

C $-1.827872-4.673788-2.694716$

C $-2.929136-3.954188-2.988820$

C $-1.206314-5.566422-3.737606$

C $-3.654244-2.978313-2.103777$

C $-5.106871-3.398445-1.856178$

C $-5.891472-2.433632-0.948826$

C $-6.140342-1.073874-1.605752$ 
C $-6.888958-0.114714-0.666629$

O $\quad-5.165986-2.268340 \quad 0.273512$

O $-6.8029901 .205533-1.225915$

C $-6.156015-0.0766290 .691100$

C $-8.382128-0.448717-0.573525$

O

C $-5.839150-1.4754041 .262690$

C $-4.921243-1.4238392 .509113$

O $-7.061753-2.1036751 .668022$

C $-0.069363-1.220020-1.495365$

O $1.058352-1.121422-1.948658$

C -1.051513 -0.120314 -1.358265

C $-0.723516 \quad 1.126803-1.721829$

C $-1.6056372 .294842-1.676914$

C $-3.0549992 .066180-1.336321$

C $-3.571827-0.7218742 .306600$

C $-3.547781 \quad 0.7184202 .797054$

C -2.0400762 .7000862 .919032$

O $-4.463627 \quad 1.230214 \quad 3.435236$

C -2.4881153 .6980961 .898760$

C $-1.675961 \quad 4.6132101 .346728$

C -2.1486115 .6861240 .407385$

C $-1.3550105 .748721-0.877257$

C $-0.3701336 .648587-1.039510$

C $-1.7707604 .837260-2.008813$

C $-1.0803143 .505400-1.954954$

N $-2.357457 \quad 1.3562572 .473351$

H $2.655680-4.033314-1.114864$

H $\quad 0.369018-3.9049190 .922180$

H $\quad 0.560870-3.595515-2.173474$

H - $-1.867735-4.547858-0.546683$

H - $0.706009-5.686051-1.188005$

H -3.342676 -4.038221 -3.993169

H -1.732271 $-5.516380-4.696943$

H $-0.165903-5.276670-3.916370$

H -1.224942 -6.609278 -3.404246

H -3.148904 -2.853367-1.143951

H -3.624253 -2.003679 -2.602868

H $-5.637045-3.516610-2.809374$

H -5.095354 -4.384759-1.372924

H $-6.849692-2.918637-0.726707$

H -6.685714 -1.192099-2.550094

H -5.184332 -0.603170 -1.867573

H -7.193937 $1.179820-2.116112$

H -5.2154410 .4618950 .525557$

H -8.849435 -0.390987 -1.564001

H -8.911636 0.2803930 .049660

H -8.575393 $-1.447138-0.176246$

H -7.0524301 .5597521 .259408$

H -5.470303 -1.008031 3.362570

H $-4.698959-2.4638082 .789100$

H -6.852925 -2.789389 2.323914

H $-2.034504-0.373836-0.977548$

H $0.2841051 .313184-2.089861$

H -3.526678 $1.423328-2.087719$

H $-3.6434372 .984610-1.286611$

H -3.152615 $1.586609-0.357782$

H -2.816462 -1.254355 2.898356

H $-3.253267-0.7613761 .259812$

H -0.9613352 .7409053 .093994$

H -2.5489772 .8953473 .868726$

H -3.5447773 .7010281 .641212$

H -0.6260824 .6321651 .630429$

H -2.068794 6.6447410 .937221

H -3.214146 5.5711990 .172279

H $0.1813776 .719533-1.971190$

H $-0.0881747 .327612-0.241607$

H -1.510352 5.299443 -2.970954

H -2.860783 $4.753193-2.036090$

H $-0.0182413 .564149-2.195243$

H $-1.6718660 .871907 \quad 1.900787$

C $4.009442-3.1445111 .294615$

O $4.940977-3.014407 \quad 0.197515$

C $6.255682-2.8282340 .738507$

C $6.064768-2.4458972 .198237$

C $4.867343-3.3085992 .551398$

H $\quad 6.735983-3.8171540 .716852$

C $7.111779-1.886537-0.108386$
H $\quad 6.945122-2.6607222 .810973$

H $5.814865-1.3845862 .307523$

C $4.183159-2.9066673 .845136$

H $5.200181-4.3520922 .639165$

C $6.679149-0.409122-0.215815$

O $8.434364-1.9208950 .447326$

H $7.191558-2.305283-1.119610$

H $6.808590 \quad 0.087507 \quad 0.752484$

O $7.6263600 .224914-1.093517$

C $5.255630-0.132321-0.743196$

C $5.0465651 .355719-1.096368$

H $4.516802-0.4333100 .005533$

O $5.020832-0.894552-1.926980$

H $5.3306411 .966837-0.233441$

H $5.7199701 .619593-1.920376$

H $4.865559-3.0301204 .692553$

H $3.865428-1.8609403 .825946$

H $3.300070-3.5255004 .030803$

C $3.067578-4.3220561 .043170$

H $8.995185-1.348303-0.103821$

H $7.498284-0.150190-1.981905$

H $4.936586-1.826976-1.664580$

H $2.464911-4.5176571 .937457$

H $3.645969-5.2301000 .833668$

H $3.440650-2.2084271 .337939$

C 2.6805632 .3324820 .597625

O $2.6449791 .424126-0.509091$

C $3.6015961 .678975-1.541554$

C $3.4138093 .099419-2.093118$

C $3.4666704 .148585-0.988054$

C $2.496767 \quad 3.7833530 .132702$

H 3.6335182 .2255531 .128753

C 1.5440251 .9364201 .549683

H $3.3573500 .990494-2.360069$

O $4.3988063 .379444-3.082202$

H $2.4376283 .159020-2.591359$

H $4.4889594 .275169-0.613145$

O $3.0832425 .407799-1.545323$

H $1.4715303 .936891-0.222084$

O 2.6884364 .6768491 .228070

C 1.7991940 .6026202 .211103

H 1.4214052 .6726722 .350611

H 0.6072251 .8684250 .984690

H $4.2533224 .291230-3.387550$

H $3.0658746 .053200-0.817792$

H 3.5931114 .5504191 .561741

O $0.607352-0.0003532 .490026$

O $2.908424 \quad 0.1651982 .483581$

C $0.693635-1.2607853 .160353$

H $1.169230-1.1374344 .137814$

H $-0.321199-1.6380273 .308718$

H $1.246998-1.9783532 .549537$

SCF Energy (B3LYP/6-31G**//MMFF) $=-3245.92474675$

$02 \_110$

MM̄FF Geometry

C -0.069365 -3.115199 0.395494

C $-1.057400-2.2287090 .587164$

C $-1.252882-1.4411441 .853951$

O $-2.681267-1.3948672 .072842$

C $-0.738075-0.0052131 .668984$

C -0.6327790 .7701672 .965910$

C -1.4788231 .7450883 .351506$

C $0.554198 \quad 0.420973 \quad 3.825557$

C -2.7203952 .2263602 .654019$

C -2.5570113 .6346312 .064239$

C -1.666422 3.6722260 .812240

C -1.4907785 .1080340 .314354$

C $-0.6980145 .163812-1.000888$

O $-2.3063142 .886569-0.197093$

O $-0.8520386 .479684-1.554052$

C - $-1.3257924 .174776-2.006383$

C $0.8043474 .963787-0.769362$

O $-0.5361234 .080275-3.190008$

C $-1.5677182 .765248-1.419077$

C $-2.4186011 .920117-2.397722$

O $-0.3086262 .123437-1.216362$

C $-3.166962-1.5314193 .329979$ 
O $-2.519624-1.7125124 .351209$

C $-4.648793-1.4613753 .336635$

C $-5.372115-1.0775462 .272402$

C $-6.832680-1.0086762 .188393$

C $-7.627020-1.4636773 .383730$

C $-2.9048430 .588965-1.806651$

C $-3.827299-0.160530-2.751570$

C $-5.378131-2.115516-2.789880$

O $\quad-4.0230690 .181792-3.914318$

C $-6.744958-1.570115-2.515414$

C $-7.694246-2.234103-1.838361$

C $-9.063140-1.678382-1.564845$

C $-9.383687-1.605939-0.087147$

C -10.187193 -2.516585 0.488092

C $-8.849276-0.4318750 .704664$

C $-7.389356-0.5731601 .038437$

N -4.396702 -1.268691 -2.137049

H $\quad 0.657474-3.2906831 .184054$

H -1.768513 -2.053662 -0.216649

H $-0.750437-1.9487802 .685785$

H -1.383985 0.5234070 .961120

H $0.257922-0.0178021 .206280$

H -1.278774 2.2510114 .295567

H $\quad 0.6306611 .0603114 .711359$

H $0.489754-0.6146484 .172216$

H $1.4792700 .539653 \quad 3.254490$

H -3.0651031 .5251571 .888442$

H $-3.5218402 .256566 \quad 3.403497$

H -3.5587353 .9968451 .798279$

H -2.1594254 .3143942 .828094$

H -0.6916123 .2338921 .052051$

H -1.009523 5.7295431 .079840

H -2.4786675 .5578740 .141260$

H $-0.5276287 .120287-0.898052$

H $-2.2951204 .592300-2.313138$

H $1.1957135 .740103-0.101008$

H $1.3649175 .065436-1.705071$

H $1.0442663 .996026-0.324743$

H $-0.4176224 .983070-3.531871$

H -3.305636 $2.496495-2.691934$

H -1.845095 $1.714069-3.310367$

H $-0.4227491 .162050-1.269611$

H $-5.114027-1.7387034 .276895$

H $-4.852310-0.7866181 .360882$

H -7.369367 -2.495602 3.647816

H $-8.706731-1.448303 \quad 3.219755$

H -7.425122 -0.8191424 .246245$

H -2.055411 -0.064253 -1.587674

H -3.452401 $0.766776-0.873796$

H -5.248717 -3.128838 -2.396994

H - $-5.192904-2.130949-3.868584$

H -6.963808 $-0.576057-2.898594$

H -7.482862 -3.233863-1.465979

H $-9.790567-2.321344-2.077518$

H -9.187231 -0.682813 -2.009267

H -10.448492 -2.456773 1.539251

H -10.598394 -3.345437 -0.078603

H -9.444894 -0.2712401 .610199$

H -8.9882740 .4886090 .123000$

H $-6.727899-0.2654610 .228294$

H -4.226214 -1.425387 -1.147789

C $1.261419-3.457299-1.751335$

O $2.518307-3.683140-1.086963$

C $3.442777-2.639124-1.485039$

C $2.733373-1.848026-2.578624$

C $1.278892-1.991497-2.175182$

H $3.580696-2.018452-0.592436$

C $4.771200-3.290628-1.895644$

H $3.063318-0.807327-2.630877$

H $2.893986-2.307140-3.561785$

C $0.298636-1.649281-3.282571$

H $1.095181-1.333880-1.317474$

C $5.854054-2.343475-2.442596$

O $5.306947-3.993699-0.772195$

H $4.558768-4.053631-2.656105$

H $6.785773-2.912068-2.560859$

O $5.464702-1.942048-3.760560$

C $6.158987-1.074843-1.618737$
C $6.646471-1.347515-0.185213$

H $5.281430-0.421052-1.605987$

O $7.179062-0.345680-2.315968$

H $5.853969-1.8449370 .379924$

H $7.497609-2.038364-0.226449$

H $\quad 0.405640-0.600631-3.579433$

H $0.462734-2.268923-4.170468$

H $-0.733043-1.802504-2.951763$

C $0.090964-3.904102-0.873496$

H $4.613461-4.593083-0.446679$

H $6.159330-1.353581-4.102829$

H $7.981612-0.895003-2.326424$

H $0.237985-4.952852-0.586517$

H -0.833932 -3.867511 -1.462068

H $1.272627-4.100429-2.642064$

C 4.9288310 .6634841 .342247

$\begin{array}{lllll}\text { O } & 6.080907 & 0.945703 & 0.539047\end{array}$

C $7.091216-0.0669570 .559330$

C $7.576932-0.2995681 .996864$

C $6.420135-0.5717382 .956234$

C 5.3320990 .4931172 .810201

H $4.442196-0.2511100 .983062$

C $3.949511 \quad 1.8266701 .156933$

H 7.9439390 .3420190 .003047

O $8.507341-1.3761792 .033143$

H 8.1120710 .5974822 .334963

H $6.007702-1.5746792 .797406$

O $6.909677-0.5626914 .299479$

H 5.6899531 .4472243 .217516

O 4.1929950 .1004893 .570876

C $3.3043761 .741567-0.205328$

H 3.1399881 .8076981 .894196

H 4.4546702 .7945621 .258477

H $8.767463-1.5054922 .961348$

H 7.2831880 .3172324 .477771

H $4.486729-0.0307494 .488726$

O $3.9094732 .604061-1.069096$

O $2.3948530 .967828-0.474618$

C $3.4242652 .574261-2.415209$

H $3.8556383 .424410-2.950232$

H $3.7469421 .650302-2.903204$

H $2.3342022 .663193-2.441479$

SCF Energy (B3LYP/6-31G**//MMFF) $=-3245.91934515$

$02 \_111$

MM̄FF Geometry

C $2.382657-3.2979081 .158109$

C $1.434618-2.3512161 .092155$

C $0.831460-1.6856002 .298585$

O $-0.587146-1.9637862 .261518$

C $1.039293-0.1624092 .222215$

C 0.6470080 .5490693 .501176

C -0.5323831 .1663653 .711598$

C 1.6980170 .5406574 .581436

C -1.7015941 .2726652 .770446$

C -2.1842482 .7236832 .651552$

C -3.2454232 .9587901 .564673$

C $-4.4914562 .086651 \quad 1.733768$

C -5.5103672 .3235420 .607272$

$\begin{array}{lllll}\text { O } & -2.641359 & 2.720427 & 0.287935\end{array}$

$\begin{array}{llll}\text { O } & -6.478664 & 1.264767 & 0.658575\end{array}$

C $-4.7831102 .204721-0.750328$

C -6.2911323 .6281570 .801858$

O $-5.6514502 .552785-1.825119$

C $-3.4877403 .041083-0.828470$

C $-2.6736242 .784172-2.119674$

O $-3.8283034 .433850-0.827507$

C - $1.188111-2.3754303 .405963$

O $-0.647693-2.5715374 .486435$

C $-2.645450-2.571476 \quad 3.224670$

C $-3.311452-2.3086822 .088908$

C $-4.753187-2.4766251 .878884$

C $-5.569903-2.992393 \quad 3.035997$

C $-2.2398231 .333622-2.344301$

C $-3.1841750 .536652-3.227014$

C $-3.763312-1.813471-3.825819$

O $-3.9535561 .058499-4.029485$

C $-5.132903-1.977079-3.248174$ 
C $-5.574816-3.105762-2.674061$

C $-6.965750-3.266265-2.132914$

C $-6.992945-3.459658-0.634487$

C -7.338139-4.640453 -0.094278

C $-6.711740-2.2552490 .236307$

C $-5.273355-2.1755770 .669931$

N $-3.025427-0.831477-3.054262$

H $2.735731-3.6342722 .129809$

H $1.071174-2.0278650 .120308$

H $1.288523-2.0894923 .209900$

H $\quad 0.482602 \quad 0.245517 \quad 1.371444$

H 2.0941340 .0626922 .014555

H $-0.697012 \quad 1.6299484 .683932$

H 1.3769981 .0786515 .479752

H $1.935310-0.4852684 .879567$

H 2.6146371 .0194894 .221198

H - $1.4700820 .875311 \quad 1.779342$

H -2.509403 0.6567823 .181167

H -2.585561 3.0512743 .619321

H -1.3214143 .3651342 .429686$

H $-3.521014 \quad 4.0185931 .624829$

H -4.953179 2.2527452 .714795

H -4.2119291 .0254841 .706647$

H -6.8824961 .2696351 .543362$

H $-4.5317981 .144619-0.881069$

H -6.8433093 .6051321 .749128$

H -7.046234 3.7566390 .018638

H -5.6536864 .5143180 .815629$

H $-6.4423911 .991714-1.751851$

H -3.210939 $3.184206-2.987889$

H $-1.753323 \quad 3.381898-2.046680$

H -3.087768 4.933031-1.209920

H -3.155321 -2.9414994 .108898$

H -2.761102 -1.9302531 .229282$

H $-5.197579-3.9691773 .364700$

H $-6.626521-3.1321402 .800158$

H -5.523109 -2.294825 3.879530

H $-1.284757 \quad 1.343003-2.882273$

H -2.074989 $0.817262-1.392636$

H -3.189527 -2.745418 -3.805926

H $-3.837863-1.472885-4.863725$

H -5.804264 -1.124917 -3.326820

H -4.912639 -3.965067 -2.601004

H -7.422982 -4.125587 -2.640309

H -7.595026 -2.405250 -2.392089

H $-7.392547-4.7750920 .980657$

H -7.571567 -5.499590 -0.714280

H $-7.396450-2.244387 \quad 1.090753$

H $-6.958479-1.338699-0.314457$

H -4.600313 -1.811331 -0.106519

H $-2.397378-1.173781-2.332991$

C $4.534388-3.819363-0.019045$

O $4.926023-2.433760-0.043819$

C $6.090635-2.291221-0.879350$

C $6.535333-3.704725-1.241842$

C $5.217620-4.454598-1.228256$

H $5.759331-1.782526-1.791985$

C $7.155007-1.447216-0.166661$

H $7.041763-3.742282-2.211177$

H $7.217459-4.123670-0.493240$

C $5.377550-5.961705-1.132365$

H $4.665205-4.207752-2.144983$

C $6.652111-0.0832510 .347594$

O $7.646046-2.1715310 .970271$

H $8.019204-1.306762-0.825775$

H $5.951513-0.2366911 .177936$

$\begin{array}{lllll}\text { O } & 7.774148 & 0.620548 & 0.895622\end{array}$

C $5.9882580 .812470-0.714029$

C $5.5398922 .160496-0.121893$

H $5.1178710 .302945-1.132687$

O $6.8962581 .069970-1.783002$

H 4.9262021 .9658010 .764323

H 6.4227752 .7200000 .210435

H $5.939505-6.344133-1.990714$

H $5.914751-6.251107-0.222902$

H $4.402397-6.458328-1.119970$

C $3.009241-3.934540-0.051273$

H $6.885602-2.3668031 .544668$
H 8.1761080 .0455321 .569366

H $7.0544860 .231794-2.248225$

H $2.715273-4.989674-0.076042$

H $2.624487-3.464057-0.963799$

H $4.927939-4.245350 \quad 0.913531$

C $2.5582192 .104326-0.797247$

O $3.6386622 .360759-1.701569$

C $4.7622013 .038764-1.129089$

C $4.3165514 .392024-0.560008$

C 3.1689114 .2365940 .434612

C $2.0393113 .412128-0.181550$

H $2.8920531 .419965-0.008528$

C $1.4389151 .416209-1.594756$

H $5.4472483 .248966-1.960108$

O $5.4162465 .049810 \quad 0.060404$

H $3.9843045 .033156-1.387018$

H $3.5190303 .799015 \quad 1.376735$

O 2.6810455 .5421030 .753029

H $1.5293624 .010531-0.947729$

O $1.080128 \quad 3.098983 \quad 0.825547$

C $1.7873240 .007922-2.026757$

H $\quad 0.530266 \quad 1.346727-0.987308$

H $1.2279341 .995620-2.501686$

H 5.0857285 .8891850 .423672

H 2.0229415 .4448091 .461702

H 0.6749353 .9329831 .116507

O $0.779986-0.469841-2.816733$

O $2.795530-0.604398-1.706820$

C $0.947843-1.804164-3.305195$

H $1.922727-1.922216-3.788059$

H $0.833566-2.516474-2.483563$

H $\quad 0.166307-1.994683-4.045606$

SCF Energy (B3LYP/6-31G**//MMFF) $=-3245.90854953$

$02 \_112$

MM̄FF Geometry

C -0.872455 -2.039044 2.645325

C $-0.082986-2.9743602 .094974$

C $-0.268711-3.5206480 .695343$

O $-0.882371-2.513508-0.148732$

C $-1.163689-4.7698650 .718419$

C -1.303091 -5.428542 -0.640992

C $-2.390611-5.342808-1.433370$

C $-0.116716-6.249276-1.078904$

C $-3.662022-4.582702-1.176532$

C $-3.825510-3.427854-2.172748$

C $-5.023693-2.532146-1.824822$

C $-5.181526-1.418521-2.861600$

C $-6.290927-0.425518-2.482930$

O $-4.787459-1.965674-0.531682$

O $-6.141490 \quad 0.738810-3.310385$

C $-6.082670 \quad 0.031229-1.022599$

C $-7.684945-0.991239-2.778519$

O $-7.1782060 .827526-0.578216$

C $-5.850076-1.136177-0.042082$

C $-5.451163-0.6649201 .377893$

O $-7.064799-1.8847630 .091490$

C $-0.062326-1.522558-0.600842$

O $1.149149-1.458589-0.462720$

C $-0.894670-0.488796-1.254419$

C $-0.328610 \quad 0.680537-1.590473$

C $-0.9823171 .813444-2.248108$

C $-2.4487991 .690498-2.561854$

C -4.1460830 .1332521 .468685$

C -4.3304791 .6411151 .472135$

C $-2.9970963 .747607 \quad 1.508547$

O $\quad-5.4269792 .1887001 .539901$

C -2.9215874 .3159350 .127525$

C $-1.9040175 .076373-0.303958$

C $-1.8616245 .725577-1.656414$

C $-0.6170135 .377316-2.438830$

C $0.4487456 .195970-2.444410$

C $-0.6499114 .134640-3.297076$

C $-0.2382392 .898709-2.548056$

N -3.1108012 .3045851 .433212$

H -1.745792 -1.696534 2.096001

H $\quad 0.784820-3.3328172 .642886$

H $\quad 0.716176-3.7765170 .285005$ 
H $-2.146014-4.507071 \quad 1.123813$ H $-0.753077-5.509331 \quad 1.419341$ H -2.373996 -5.872607 -2.385349 H - $0.288496-6.750985-2.037268$ H $0.768644-5.616546-1.194154$ H $\quad 0.102650-7.024745-0.337734$ H $-4.506709-5.274835-1.279710$ H $-3.706287-4.202737-0.153833$ H -2.910155 -2.823998 -2.169042 H -3.944838 -3.830705 -3.186316 H $-5.924818-3.155617-1.788073$ H -5.365106 -1.838577 -3.858335 H $-4.237028-0.864278-2.942616$ H $-6.1942370 .455213-4.239223$ H -5.200772 $0.682100-1.015000$ H -7.787519 -1.217347 -3.846744 H -8.466039 $-0.257052-2.552241$ H $-7.901366-1.908243-2.226693$ H -7.276593 $1.562361-1.207730$ H $-6.286504-0.1321261 .848093$ H $-5.306455-1.5688281 .987458$ H -7.032486 -2.380181 0.926552 H -1.940231 $-0.709738-1.429520$ H $\quad 0.7293080 .824649-1.373557$ H -2.612522 $0.921549-3.323759$ H $-2.8949122 .616325-2.931326$ H -3.013181 $1.422561-1.662815$ H -3.667427 -0.1095712 .425990$ H -3.449639 -0.1480830 .672466$ H -2.105088 3.9740872 .100595 H -3.8685304 .1583722 .028606$ H -3.764468 4.127564 -0.533162 H -1.076166 5.2873660 .369270 H -1.910934 $6.811894-1.503742$ H $-2.7500535 .475257-2.249425$ H $1.3322205 .968746-3.031833$ H $\quad 0.4634197 .107714-1.856604$ H $0.0486274 .249613-4.137556$ H -1.630246 $4.039880-3.772851$ H $\quad 0.8172372 .895332-2.271151$ H -2.2559741 .7693911 .311180$ C $-0.453507 \quad 0.1434313 .723372$ $\begin{array}{lllll}\text { O } & 0.782478 & 0.387521 & 3.024532\end{array}$ C 1.3279531 .6421013 .476627 C 0.3050222 .2346984 .442362 C -0.3653730 .9869454 .989095$ H 2.2368931 .4046264 .040730 C 1.6705082 .5271702 .272209 H 0.7765452 .8441665 .219488 H -0.4257552 .8659573 .923583$ C -1.7063571 .2616465 .646472$ H 0.3077910 .5106135 .714203 C 2.6259201 .8636241 .257214 O $\quad 0.4538622 .8351391 .578669$ H 2.0802053 .4832892 .618732 H $2.101831 \quad 1.0349400 .769734$ O 2.8860982 .8385020 .239523 C 3.9573071 .3826841 .862076 C 4.9827040 .8480600 .848993 H $3.765421 \quad 0.6172422 .617757$ O 4.5618112 .4834602 .551638 H 5.3304951 .6777250 .217726 H 5.8827650 .5607141 .407018 H -1.585816 1.9419046 .495898 H $-2.410871 \quad 1.7213114 .944696$ H $-2.157040 \quad 0.3359896 .017331$ C $-0.619472-1.3584533 .960163$ H 0.6887193 .3838230 .810598 H $3.2930512 .379668-0.514305$ H 5.3682192 .1533412 .983250 H - $1.471720-1.5526414 .619701$ H $0.278833-1.7680924 .436962$ H -1.2610380 .5183523 .081288$ C $6.753197-0.956401-0.787784$ O $5.456146-0.469673-1.154178$ C $4.534936-0.314390-0.061365$ C $4.323876-1.6814170 .605306$ C $5.646218-2.3003181 .051606$
C $6.642109-2.326720-0.108284$

H $7.242782-0.243422-0.113618$

C $7.590059-1.044884-2.073224$

H $3.589180-0.021243-0.528247$

O $3.441139-1.6068441 .714960$

H $3.861820-2.364528-0.119888$

H $6.064153-1.7757771 .918820$

O $5.417415-3.648151 \quad 1.470666$

H $6.329836-3.084383-0.838610$

O $7.924371-2.7114820 .383456$

C $7.9269660 .321373-2.634795$

H $8.538020-1.557870-1.877988$

H $7.030170-1.590351-2.842186$

H $2.586060-1.2796771 .386406$

H $4.747626-3.6213122 .175512$

H $7.818807-3.5703880 .827402$

O $8.600354 \quad 0.159710-3.812934$

O $7.6458351 .390932-2.112084$

C $8.9926891 .370154-4.467874$

H $9.514027 \quad 1.103639-5.391055$

H $8.111167 \quad 1.966282-4.722047$

H $9.6755291 .940990-3.831359$

SCF Energy (B3LYP/6-31G**//MMFF) $=-3245.92446273$

$02 \_113$

MM̄FF Geometry

C 2.5099200 .1337663 .384166

C $3.824391-0.1217543 .294126$

C $4.814476 \quad 0.4835372 .327815$

O 4.2044451 .5605291 .584699

C $5.267543-0.5924671 .325700$

C $6.554652-0.257897 \quad 0.601672$

C $6.6355700 .123080-0.687897$

C $7.812671-0.4450081 .411137$

C $5.5136680 .391474-1.651853$

C $5.341460-0.727297-2.689969$

C $4.520388-1.915191-2.163257$

C $4.465444-3.041298-3.196516$

C $3.551418-4.190347-2.740958$

O $3.199026-1.433075-1.902688$

O $3.314700-5.038555-3.874991$

C $2.187146-3.603288-2.319352$

C $4.224963-5.065615-1.677643$

O $1.343631-4.613739-1.770248$

C $2.296700-2.399904-1.353701$

C $0.915120-1.717281-1.206847$

O $2.731141-2.867894-0.076315$

C 4.9146202 .7029841 .404586

O $6.0111902 .967037 \quad 1.878418$

C $4.1980193 .654474 \quad 0.524859$

C $3.1090773 .325841-0.186168$

C $2.4164664 .217759-1.109204$

C $2.088183 \quad 3.572242-2.428280$

C $0.951636-0.392024-0.431974$

C $-0.3794130 .337202-0.477537$

$\begin{array}{lllll}C & -1.464897 & 2.443686 & 0.297058\end{array}$

O $-1.363054-0.097809-1.069329$

C $-1.2828873 .545849-0.696939$

C $-1.2601324 .845263-0.364330$

C $-1.1281255 .961516-1.360300$

C $0.0273106 .887204-1.052866$

C $-0.1695518 .044061-0.397889$

C $1.3920776 .516036-1.587176$

C $2.0800815 .473240-0.752585$

N $-0.336063 \quad 1.536571 \quad 0.218931$

H 2.0415410 .8504832 .716083

H $4.251739-0.8520393 .980152$

H 5.6559240 .8530512 .925191

H $4.461353-0.7668450 .605966$

H $5.419037-1.5534201 .836563$

H $7.6261960 .295789-1.107491$

H $8.717580-0.2532990 .824749$

H 7.8263090 .2373932 .266367

H $7.875088-1.4726271 .784135$

H $4.5710840 .598518-1.137007$

H $5.7649291 .319231-2.182230$

H $4.817150-0.295910-3.552876$

H $6.321676-1.069457-3.043937$ 
H $4.968765-2.279285-1.232861$ H $5.472556-3.415969-3.417820$ H $4.077629-2.647069-4.146476$ H $4.175510-5.359340-4.194452$ H $1.690226-3.265291-3.239119$ H $5.165273-5.479041-2.061893$ H $3.601355-5.930237-1.424899$ H $4.454680-4.523167-0.758163$ H $1.648158-4.793205-0.864476$ H $\quad 0.516082-1.509378-2.208530$ H $\quad 0.210189-2.395062-0.708659$ H $2.446925-2.2400850 .606026$ H 4.6397334 .6440370 .450808 H $2.7073362 .318091-0.141391$ H $1.5617152 .623113-2.275959$ H $3.0075393 .366467-2.987548$ H $1.4445414 .184294-3.063560$ H $1.205107-0.5699550 .616965$ H $1.7057330 .275248-0.861709$ H -1.515252 $2.813931 \quad 1.325695$ H $-2.389176 \quad 1.9045450 .072691$ H -1.194547 $3.260754-1.742575$ H -1.3754035 .1279080 .679764$ H $-2.0698186 .526298-1.352510$ H -1.025852 $5.576931-2.382788$ H $\quad 0.648708 \quad 8.728503-0.200405$ H -1.153127 8.329381 -0.039563 H $2.0431897 .400575-1.598489$ H $1.3023756 .226554-2.638257$ H $2.344508 \quad 5.816657 \quad 0.248081$ H 0.5336521 .8257310 .656453 C $0.631167-1.5019343 .711927$ O $-0.439228-0.746448 \quad 3.107397$ C $-1.700278-1.3460413 .478462$ C $-1.356605-2.7293944 .003711$ C $-0.037646-2.4615874 .697343$ H $-2.101327-0.7205264 .286309$ C $-2.644470-1.3319752 .271458$ H $-2.120434-3.1287034 .677309$ H -1.219414 -3.438088 3.177406 C $0.766852-3.7202814 .974370$ H -0.236904 -1.946689 5.647016 C -3.962958 -2.098923 2.485714 O $-2.938522 \quad 0.032214 \quad 1.959334$ H -2.113251-1.736106 1.403031 H $-3.753976-3.1683332 .603631$ O $-4.550484-1.6764173 .723835$ C $-5.013222-1.9064731 .372236$ C $-4.505108-2.314268-0.021833$ H $-5.363428-0.8681601 .378480$ O $-6.138581-2.7240841 .711124$ H -3.589153 -1.763811 -0.251336 H -4.244410 -3.379843 -0.007173 H $\quad 0.212067-4.3927845 .636759$ H $0.988686-4.2659854 .051083$ H $1.717865-3.4779535 .458687$ C $1.618399-0.5474824 .387669$ H $-3.342256 \quad 0.4406922 .743261$ H $-4.828003-0.7503013 .623457$ H -6.889478 -2.4321301 .167162$ H $2.229971-1.0962345 .113729$ H $1.0772680 .229408 \quad 4.941375$ H $\quad 1.120837-2.0629282 .905148$ C $-5.1143010 .275034-1.483411$ O $-6.059131-0.741696-1.127781$ C $-5.538290-2.074619-1.147123$ C -5.032261 -2.412843 -2.556295 C $-4.034691-1.377633-3.071296$ C $-4.5862710 .039318-2.903074$ H $-4.2843800 .272751-0.767255$ C $-5.8280861 .626099-1.375964$ H -6.387039 -2.739899-0.947129 O $-4.441614-3.708211-2.565225$ H -5.889817 -2.446272 -3.241032 H -3.064006 -1.495075 -2.578120 O $-3.805414-1.627324-4.460226$ H $-5.3889850 .196915-3.634129$ O
C -6.1418821 .9523610 .064796$

H $-5.2277032 .444482-1.789493$

H $-6.7775741 .614316-1.924674$

H -4.116188 -3.873273 -3.466781

H $-3.196217-0.940081-4.780427$

H $-2.8432890 .860517-2.557289$

$\begin{array}{lllll}\text { O } & -5.039928 & 2.474294 & 0.674384\end{array}$

O $\quad \begin{array}{lllll}-7.229119 & 1.748010 & 0.588798\end{array}$

C -5.1955922 .7952152 .059807$

H -4.2361443 .1630972 .432912$

H -5.9463163 .5812752 .182856$

H -5.4732221 .9022022 .628279$

SCF Energy (B3LYP/6-31G**//MMFF) $=-3245.91329346$

02114

MM̄FF Geometry

C $0.1723150 .089865-1.858059$

C $0.233644-1.248742-1.781422$

C $0.477880-2.063957-0.532139$

O $0.409082-1.2083660 .628804$

C $1.882278-2.688515-0.595082$

C $2.106027-3.785314 \quad 0.424460$

C $2.805709-3.6411381 .566772$

C $1.521048-5.1257230 .060932$

C $3.432305-2.3909222 .120537$

C $4.965921-2.4299032 .074574$

C $5.535445-2.1659130 .671934$

C $7.054584-2.3418040 .665884$

C $7.657132-2.000892-0.704691$

O $5.208926-0.8188300 .319074$

O $9.079771-1.883916-0.546305$

C $7.141133-0.615285-1.150449$

C $7.428621-3.129297-1.717358$

O $7.535204-0.330242-2.490522$

C $5.611531-0.429163-1.000392$

C $5.2423821 .061337-1.171164$

O $4.888404-1.142333-2.005352$

C $-0.221719-1.6598541 .739904$

O $-0.767733-2.7449131 .883895$

C $-0.198834-0.6458232 .820707$

C 0.4320870 .5351202 .724009

C 0.4340751 .6008363 .729231

C -0.4058871 .4000014 .963113$

C $3.752021 \quad 1.349767-0.947521$

C $3.4211122 .823136-1.120964$

C $1.5489484 .438414-0.769695$

O $4.2216763 .646312-1.556237$

C 1.7408025 .0594440 .577610

C 0.7331715 .3594441 .411019

C 0.9307095 .9710562 .767894

C 0.4222625 .0882663 .886794

C -0.7360965 .3631424 .509619$

C 1.2983853 .9423154 .345259

C 1.1527402 .7166183 .483238

N $2.1135443 .102244-0.748630$

H $0.3072880 .688175-0.962934$

H $\quad 0.104510-1.827207-2.694403$

H $-0.302974-2.832668-0.488739$

H $2.633205-1.900175-0.472743$

H $2.065892-3.114480-1.590954$

H $2.922973-4.5147162 .207348$

H $1.742200-5.8936500 .809973$

H $\quad 0.432378-5.058978-0.028813$

H $1.928815-5.471411-0.894709$

H $3.054342-1.4859771 .635938$

H $3.120134-2.3134343 .169951$

H $5.335999-1.6543122 .758025$

H $5.325571-3.3946602 .453420$

H $5.069334-2.853054-0.042238$

H $7.330504-3.3612490 .963287$

H $7.503673-1.677617 \quad 1.417826$

H $9.411800-2.723526-0.184695$

H $7.6379080 .132601-0.516145$

H $7.915992-4.051657-1.378698$

H $7.881372-2.890591-2.685829$

H $6.372226-3.357702-1.874023$

H $8.501611-0.430047-2.535767$

H $5.8168381 .665876-0.457079$ 
H $5.516124 \quad 1.397478-2.179421$ H $5.520411-1.591574-2.587981$ H $-0.757771-0.9229153 .708786$ H $\quad 0.9892290 .7629251 .816955$ H -0.0324290 .5522315 .547866$ H -1.450104 1.2076024 .691030 H -0.4174282 .2659145 .628338$ H $3.1482270 .785651-1.666317$ H $3.456827 \quad 1.0471770 .063891$ H $0.4911614 .341658-1.033618$ H $2.0462725 .041825-1.535524$ H 2.7637945 .2689130 .881723 H -0.2906745 .1578021 .104850$ H 0.4065096 .9356852 .777339 H 1.9860746 .2101252 .949278 H -1.0933074 .7545145 .333490$ H -1.355922 6.2004724 .206438 H 1.1045083 .7097385 .398188 H 2.3492374 .2588374 .329504 H 1.7250772 .7670182 .557196 H $1.5661812 .373719-0.301108$ C -1.213179 $1.842851-3.000221$ O $-2.4151451 .141495-2.639568$ C $-3.4758401 .528144-3.529096$ C $-3.0154472 .832139-4.165757$ C $-1.5245782 .581668-4.303225$ H -3.528841 $0.750909-4.302002$ C $-4.8100251 .575720-2.774366$ H $-3.5096863 .027280-5.122044$ H -3.189144 $3.692309-3.509469$ C $-0.7060953 .846480-4.497386$ $\mathrm{H}-1.3613331 .917175-5.162330$ C $-5.026570 \quad 0.375040-1.827777$ O $-4.8486102 .761748-1.971393$ H $-5.6342481 .667125-3.491120$ H $-4.3190070 .459417-1.000972$ O $-6.3280790 .498047-1.243830$ C $-4.889312-1.005520-2.487882$ C $-5.120667-2.204618-1.546555$ H -3.898050 -1.112089 -2.939273 O $-5.829156-1.094928-3.564070$ H $-6.038886-2.048581-0.970559$ H -5.324172 -3.077746 -2.179595 H -1.003968 $4.360020-5.417361$ H $-0.8412814 .544026-3.663818$ H $\quad 0.360723 \quad 3.612023-4.570173$ C $-0.0639320 .842629-3.138391$ H $-5.6905612 .756486-1.484524$ H $-6.9786160 .524224-1.965564$ H -6.720178 -1.146069-3.178535 H $0.856909 \quad 1.372541-3.407580$ H $-0.2862590 .135515-3.947345$ H - $1.0059542 .552273-2.189280$ C $-4.631719-1.3759391 .386672$ O $-3.652513-1.5114530 .350890$ C $-3.921792-2.524702-0.621442$ C $-4.028943-3.887108 \quad 0.080183$ C $-5.101161-3.8648521 .165643$ C $-4.848614-2.7044082 .125496$ H $-5.574021-1.0224460 .953212$ C $-4.135529-0.3116552 .374800$ H -3.035334 -2.569854 -1.267275 O $-4.305520-4.907702-0.872090$ H -3.061202 -4.1287760 .538940$ H $-6.109819-3.811426 \quad 0.739722$ O $\quad-5.042693 \quad-5.078011 \quad 1.918929$ H -3.971717 -2.936219 2.744300 O $-5.963372-2.5712723 .005710$ C $-3.967587 \quad 1.0423621 .728326$ H -4.831772 -0.1864743 .212195$ H $-3.176355-0.6090242 .813359$ H -4.235209-5.763435 -0.416501 H $-5.315431-5.801786 \quad 1.330245$ H -6.086762 -3.4279533 .449357$ O -2.6544341 .2519041 .426455$ O $-4.8921611 .814061 \quad 1.510208$ C -2.339615 2.5188910 .841922 H $-2.9059262 .666968-0.080651$
H $-2.5450383 .322414 \quad 1.555202$

H -1.273182 2.5265020 .601156

SCF Energy (B3LYP/6-31G**//MMFF) $=-3245.91302626$

02115

MM̄FF Geometry

C -1.751691 5.3077261 .083667

C $-2.156474 \quad 4.2672350 .339565$

C -3.5909573 .8070840 .259605$

O -3.6513942 .4024760 .590838$

C $-4.0956973 .980482-1.183788$

C $-5.5121873 .483725-1.392422$

C $-5.8216782 .307391-1.974115$

C $-6.5991344 .424368-0.944909$

C $-4.8542921 .255678-2.446120$

C $-5.344954-0.159621-2.109028$

C $-4.270949-1.211881-2.422478$

C $-4.853880-2.622630-2.341264$

C $-3.778404-3.694843-2.571494$

O $-3.222534-1.064145-1.458308$

O $-4.332274-4.961970-2.185680$

C $-2.589930-3.412047-1.631072$

C $-3.401451-3.818616-4.052263$

O $-1.507319-4.302611-1.897330$

C $-2.106088-1.940732-1.651921$

C $-1.118996-1.718335-0.484353$

O $-1.442039-1.683270-2.889288$

C -3.7330052 .0735521 .907317$

$\begin{array}{llll}\text { O } & -3.698506 & 2.832176 & 2.862658\end{array}$

C $-3.8665590 .603124 \quad 1.986447$

C $-3.538345-0.0232173 .125268$

C -3.563851-1.468411 3.350099

C $-4.261397-2.3266912 .328082$

C $-0.608121-0.278921-0.329652$

C $-0.011435-0.0614461 .048563$

C $1.672596-1.0033652 .624920$

O -0.4699890 .7751541 .823334$

C $1.192647-2.2799053 .241527$

C $0.316024-2.3322504 .255503$

C $-0.194901-3.6116024 .854771$

C $-1.536494-4.0303204 .290671$

C -1.612953 -4.992592 3.355512

C $-2.790715-3.3967804 .854905$

C $-2.950738-1.9568794 .448203$

N $1.068632-0.8882431 .311612$

H -2.4961575 .8231241 .688513$

H -1.443436 $3.721983-0.272433$

H -4.2357644 .3752010 .942228$

H -3.415844 $3.474272-1.880021$

H -4.050473 $5.040975-1.467039$

H $-6.8736812 .068455-2.122844$

H -7.600156 $4.031744-1.152910$

H -6.5325754 .6012970 .133143$

H -6.507292 $5.385065-1.462048$

H $-4.7449381 .346124-3.533499$

H $-3.8632421 .401095-2.007218$

H -5.607458 -0.209607 -1.044350

H $-6.254693-0.368991-2.685368$

H $-3.872325-1.019814-3.425624$

H -5.676962 -2.746771-3.055935

H $-5.294165-2.779462-1.346270$

H -5.125849 -5.119270 -2.725536

H $-2.928500-3.650652-0.613653$

H $-4.287868-4.049760-4.655063$

H -2.706403 -4.649955 -4.214569

H $-2.953603-2.908898-4.457882$

H -1.051234 -3.983495 -2.694768

H -1.607317 -2.0072160 .454762$

H $-0.256296-2.381869-0.610967$

H $-0.865461-0.910569-2.780763$

H -4.1911270 .0764811 .097033$

H $-3.1754510 .574348 \quad 3.960872$

H -5.305234 -2.012882 2.214390

H $-4.279843-3.3863202 .592177$

H $-3.769545-2.2523941 .354048$

H $0.152521-0.040004-1.080230$

H -1.425483 $0.440290-0.451200$

H $1.420834-0.1293383 .234068$ 
H $2.756836-1.0344352 .494482$ H $1.574515-3.2057072 .817333$ H $-0.062314-1.4032904 .674124$ H $0.543153-4.4107754 .702126$ H $-0.268869-3.4963095 .942983$ H -2.567318 -5.3226812 .958981$ H $-0.721277-5.4686272 .960864$ H $-3.681905-3.9740864 .582596$ H -2.755165 -3.448268 5.950720 H -2.475546 -1.254585 5.133730 H $1.379465-1.5399230 .597940$ C 0.6790475 .3646450 .178865 O $1.176014 \quad 4.0899290 .638471$ C 2.6098914 .0510030 .444209 C $2.9420795 .256572-0.420761$ C 1.9121006 .2594440 .056109 H 3.0485284 .1729581 .442933 C $2.9990052 .689305-0.146084$ H $3.9710335 .602878-0.285992$ H $2.7911645 .029516-1.483549$ C $1.7372797 .436364-0.888651$ H 2.2089116 .6304411 .046325 C $4.4317572 .612559-0.700234$ $\begin{array}{llll}\text { O } & 2.841256 & 1.710399 & 0.885442\end{array}$ H $2.2818302 .424790-0.931239$ H $4.5312433 .288284-1.558028$ O 5.3292033 .1000590 .305820 C $4.9043151 .201171-1.103270$ C $3.9207480 .461139-2.025937$ H $5.101740 \quad 0.616007-0.198677$ O $6.1604021 .361283-1.770095$ H $2.9967610 .266572-1.471682$ H $3.6545991 .115927-2.865534$ H $2.6770847 .989118-0.990996$ H $1.4308657 .107529-1.887370$ H $0.9778688 .130195-0.515307$ C -0.3675435 .9019651 .161023$ H $3.5056941 .894176 \quad 1.571659$ H $6.2278773 .059107-0.063853$ H $6.5789530 .485130-1.822375$ H $-0.488188 \quad 6.979570 \quad 0.996820$ H -0.0086165 .7685262 .189125$ H $0.2408735 .206648-0.815000$

C $4.254882-2.229505-0.630676$

O $5.121603-1.667054-1.621884$ C $4.463652-0.865499-2.605941$ C $3.416048-1.705597-3.351015$ C $2.454348-2.403593-2.389161$ C $3.219110-3.145216-1.291383$ H $3.748974-1.424893-0.084498$ C $5.130737-3.0019970 .361178$ H $5.232870-0.591562-3.339267$ O $2.688144-0.887106-4.260181$ H $3.934839-2.467129-3.947564$ H $1.752678-1.684821-1.955226$ O $1.650157-3.335297-3.115869$ H $3.712403-4.027112-1.719154$ O $2.299768-3.604925-0.304128$ C $6.069384-2.0725571 .093979$ H $4.534530-3.5441551 .103579$ H $5.747971-3.742348-0.162359$ H $2.020819-1.449837-4.688861$ H $2.241890-3.994748-3.516542$ H $1.636104-4.153300-0.756874$ O $5.384349-1.3625202 .033938$ O $7.265083-1.9798260 .848276$ C $6.154732-0.4077832 .770070$ H 5.4841190 .1071943 .463008 H $6.934751-0.9149343 .345765$ H 6.5920690 .3312832 .091991

SCF Energy (B3LYP/6-31G**//MMFF $)=-3245.91388779$

$02 \_116$

MM̄FF Geometry

C $2.7063390 .205743 \quad 3.227053$

C 4.0099660 .5228663 .192100

C 4.6830691 .5431622 .302964

O 3.7461592 .1775891 .407609
C 5.7084300 .8243331 .408120

C $6.749083 \quad 1.745450 \quad 0.807217$

C $6.7148572 .238248-0.446257$

C 7.9212702 .0490841 .705181

C $5.6232852 .108238-1.471674$

C $5.9463611 .101825-2.585733$

C $5.538355-0.336059-2.228705$

C $5.928337-1.309326-3.341862$

C $5.424261-2.732557-3.055372$

O $4.117112-0.344258-2.052892$

O $5.553191-3.497615-4.263385$

C $3.919666-2.671156-2.717442$

C $6.279790-3.441477-1.998721$

O $3.435880-3.947809-2.305633$

C $3.564244-1.603863-1.656031$

C $2.026358-1.440947-1.571485$

O $4.061312-2.024119-0.384449$

C 3.1168983 .3118331 .804617

O 3.1924223 .8646592 .888313

C 2.2953073 .7535000 .656081

C 1.3072684 .6420510 .829647

C $0.4117105 .135865-0.219468$

C $0.6804594 .688436-1.634050$

C $1.558928-0.237610-0.738226$

C $0.043600-0.117072-0.714452$

C -1.7790531 .3020770 .227609$

O $-0.700247-0.898159-1.302297$

C $-2.1454472 .441747-0.669400$

C $-2.8727843 .492103-0.258673$

C $-3.2985904 .620262-1.154525$

C $-3.0188195 .981767-0.555597$

C -3.9861356 .6709900 .072736$

C $-1.6437626 .577348-0.759441$

C -0.6077425 .9475020 .131021$

$\begin{array}{llll}\mathrm{N} & -0.378623 & 0.966844 & 0.044061\end{array}$

H $1.997348 \quad 0.7266182 .590501$

H $4.683805-0.0162033 .856706$

H 5.1754602 .2947372 .931803

H 5.1760590 .2949610 .611705

H $6.2401860 .046798 \quad 1.973255$

H $7.5527152 .853128-0.774253$

H 8.6568922 .7025881 .224519

H 7.5843372 .5496282 .618341

H 8.4352241 .1237401 .985175

H $4.6573751 .886074-1.010666$

H $5.5034813 .099312-1.928864$

H $5.3836141 .409544-3.477051$

H $7.0112871 .144188-2.844362$

H $6.027482-0.623053-1.292352$

H $7.014657-1.309655-3.495994$

H $5.491046-0.972518-4.292313$

H $6.489095-3.484718-4.527712$

H $3.387900-2.424103-3.647189$

H $7.322157-3.508725-2.332609$

H $5.946523-4.474367-1.849018$

H $6.275744-2.933838-1.031896$

H $3.667665-4.584161-3.003550$

H $1.623464-1.314256-2.585117$

H $1.579952-2.353322-1.155531$

H $3.530784-1.6146680 .316552$

H $2.5157013 .309541-0.308526$

H 1.1251405 .0299841 .830884

H $1.6659535 .035766-1.963780$

H $-0.0466365 .061011-2.357854$

H $0.6518603 .595707-1.707977$

H $1.904598-0.3298580 .294986$

H $1.9619320 .692266-1.155520$

H $-1.9150811 .553671 \quad 1.283975$

H $-2.3990770 .433051-0.012467$

H -1.831445 2.385117 -1.708832

H -3.2178503 .5246540 .772644$

H -4.375474 4.510731-1.337525

H -2.822804 $4.550759-2.140641$

H $-3.802608 \quad 7.659307 \quad 0.481112$

H $-4.982178 \quad 6.2592920 .197744$

H $-1.6587197 .651596-0.530380$

H -1.383771 $6.527104-1.820395$

H -0.7255256 .2075291 .183910$ 
H $\quad 0.314394 \quad 1.576376 \quad 0.467902$

C $1.410527-1.9318493 .259771$

O $0.090006-1.4389712 .946030$

C $-0.871607-2.4847543 .212145$

C $-0.060802-3.7649653 .314166$

C $1.198783-3.2593093 .987205$

H $-1.319514-2.2390234 .183825$

C -1.942016 -2.465297 2.116164

H -0.569032 -4.544560 3.889102

H $\quad 0.168577-4.1626662 .317529$

C $2.372104-4.2174773 .873638$

H $0.984364-3.0766595 .048876$

C $-2.891023-3.6744532 .133844$

O $-2.718650-1.2750282 .288977$

H -1.456410 -2.384807 1.137365

H -2.329947 -4.5869941 .899804$

O $-3.390044-3.8367203 .467849$

C $-4.105279-3.556206 \quad 1.190947$

C $-3.732446-3.258348-0.271252$

H $-4.790808-2.7921531 .574132$

O $-4.824256-4.7939231 .244791$

H -3.195138 -2.307003 -0.322632

H -3.045109-4.034705 -0.629674

H $2.138866-5.1698814 .360807$

H $2.619262-4.4269622 .827354$

H $3.263923-3.8021324 .353302$

C $2.152951-0.8891984 .099288$

H -2.096975 -0.5305552 .355715$

H -3.989204 -4.602957 3.462040

H -4.246537 -5.4889940 .885804$

H $2.965903-1.3673294 .657800$

H $1.471483-0.4341904 .827904$

H $1.922243-2.1019792 .303316$

C $-5.677642-0.958665-0.744748$

O $-5.992532-2.356400-0.713830$

C $-4.958825-3.213124-1.211924$

C $-4.627066-2.848898-2.666346$

C $-4.318886-1.361898-2.832849$

C $-5.414605-0.511099-2.188713$

H $-4.789771-0.776978-0.128572$

C $-6.861504-0.216291-0.110679$

H $-5.382466-4.225372-1.224970$

O $-3.531055-3.630833-3.129229$

H -5.489485 -3.100913 -3.297477

H -3.331225 -1.117403 -2.426001

O $-4.259920-1.069815-4.230743$

H -6.330308 -0.602977 -2.785937

O $-5.0284910 .858813-2.230540$

C $-6.491346 \quad 1.161803 \quad 0.388093$

H -7.685579 $-0.149029-0.831183$

H $-7.247529-0.7755010 .750274$

H -3.340526 -3.352939-4.041601

H -4.104136 -0.113798 -4.319675

H -4.224856 $0.955958-1.693013$

O $\quad-7.5194912 .0268190 .152074$

$\begin{array}{lllll}\text { O } & -5.436014 & 1.436661 & 0.943884\end{array}$

C -7.301812 3.3726990 .587126

H -7.152443 3.4015471 .670598

H -6.4418763 .8041730 .066800$

H -8.1912123 .9582330 .339731$

SCF Energy (B3LYP/6-31G**//MMFF) $=-3245.92406606$

02

MMFF Geometry

C $-0.4046283 .187840-0.579878$

C $0.5982333 .453557-1.429551$

C $1.3780032 .383282-2.147891$

O $2.7710512 .638785-1.842675$

C $1.1532862 .410064-3.671880$

C $-0.2839242 .209529-4.109900$

C $-0.9537031 .041555-4.042031$

C $-0.9570223 .435368-4.669812$

C $-0.444813-0.283008-3.541344$

C -1.091235 $-0.640290-2.198341$

C $-0.665851-2.013098-1.655796$

C $-1.209901-2.202167-0.235540$

C $-0.716455-3.5000390 .417236$

O $0.761735-2.082908-1.650867$
O $-\begin{array}{llll}0.990997 & -3.417580 & 1.824640\end{array}$

C $0.816804-3.5847280 .260248$

C $-1.482088-4.722901-0.101231$

O $1.304189-4.8428840 .719884$

C $1.294893-3.331358-1.183897$

C $2.835749-3.254033-1.300564$

O $0.866939-4.417732-2.014986$

C $3.6515551 .613500-1.991969$

O $3.4311160 .522054-2.490029$

C $4.9356412 .041868-1.390409$

C $5.9540401 .173194-1.297150$

C $7.2582021 .429856-0.678733$

C $7.4704612 .780542-0.045218$

C $3.492924-2.083733-0.564863$

C $4.088891-2.4258560 .788687$

C $5.348683-1.3346292 .650849$

O $4.091938-3.5562401 .265150$

C $6.596841-0.5201012 .520614$

C $7.823833-1.0553812 .436642$

C $9.086872-0.2532852 .301445$

C $9.795568-0.5113120 .989712$

C $10.644457-1.5444490 .855244$

C $9.5841850 .477013-0.136479$

C $8.1849280 .447834-0.689797$

N $4.651384-1.3014511 .380082$

H $-0.6907782 .154124-0.408473$

H $0.8816304 .486438-1.616804$

H $1.1214871 .399514-1.738750$

H $1.7805891 .652066-4.157225$

H $1.5376063 .357620-4.074937$

$\mathrm{H}-1.986813 \quad 1.020917-4.387065$

H -1.996839 $3.246138-4.956928$

H $-0.9602094 .242876-3.931144$

H $-0.4256643 .783238-5.561815$

H $-0.690061-1.050653-4.284908$

H $0.645708-0.278082-3.451826$

H $-0.8184570 .129045-1.468300$

H $-2.182871-0.613866-2.291238$

H -1.058661 -2.784883 -2.328048

H -2.304555 -2.167662 -0.233102

H $-0.885442-1.3600700 .392376$

H -1.946344 -3.272765 1.936383

H $1.251190-2.8206420 .917507$

H -2.548827 -4.6361920 .137226$

H -1.140511 -5.6407520 .389458$

H -1.398433 -4.855296 -1.181842

H $0.997998-4.9547811 .636116$

H $3.287970-4.214803-1.026795$

H $3.070089-3.120021-2.366822$

H $1.427986-4.441327-2.807632$

H $4.9959953 .055343-1.009710$

H $5.8211140 .170958-1.701304$

H $7.4209223 .571034-0.802191$

H 8.4323782 .8831540 .460094

H 6.7051762 .9728930 .715266

H $4.332222-1.734803-1.179414$

H $2.799377-1.244848-0.444404$

H $5.557785-2.3694222 .940791$

H $4.689839-0.8955053 .406221$

H 6.4807490 .5605192 .492558

H $7.931756-2.1374952 .470218$

H $9.747998-0.5179763 .137182$

H 8.8928970 .8205262 .414201

H $11.179173-1.722001-0.071882$

H $10.820178-2.2381411 .671071$

H $\quad \begin{array}{llll}10.263768 & 0.252947 & -0.969417\end{array}$

H 9.8833341 .4694800 .210805

H $7.930373-0.498652-1.169011$

H $4.502890-0.3947950 .945583$

C -1.2302723 .9256861 .663423$

O

C -2.8541852 .8991353 .010915$

C -2.4129424 .1972743 .676742$

C -1.9428055 .0020402 .480965$

H -3.8614753 .0405892 .601706$

C -2.8303091 .6685373 .923101$

H -3.227172 4.6787814 .226899

H -1.5833974 .0358224 .374912$ 
C $-1.062214 \quad 6.1825722 .851987$ H $-2.823221 \quad 5.3672801 .935319$ C $-3.165048 \quad 0.340267 \quad 3.212606$ O -1.5152001 .5411524 .479661$ H -3.5082861 .8301064 .769597$ H -2.353532 0.0732192 .525104 O $-3.179399-0.6917854 .208105$ C -4.5021820 .3312692 .446458$ C $-4.874600-1.0698421 .925563$ H -4.4310901 .0239121 .603160$ O H -4.025980 -1.473225 1.364655 H -5.044689 -1.7361302 .779766$ H -1.608358 6.8833003 .491919 H $-0.166621 \quad 5.8606053 .393940$ H $-0.738015 \quad 6.7244161 .958112$ C $-1.1880124 .231325 \quad 0.164874$ H -1.518198 0.7564525 .054281 H -3.862070 -0.4667284 .862394$ H $-5.6766640 .167742 \quad 4.006691$ H $-0.7597835 .225425-0.005254$ H -2.207157 $4.234594-0.237485$ H $-0.216513 \quad 3.7544032 .048857$ C $-5.131293-0.628795-1.096818$ O $-6.038873-0.193355-0.076786$ C $-6.150399-1.0676891 .052063$ C $-6.601050-2.4622240 .594870$ C $-5.689905-3.024324-0.491732$ C $-5.533036-2.012711-1.625841$ H -4.115040 -0.662066 -0.690164 C $-5.1682060 .412114-2.226682$ H $-6.958243-0.6610401 .673250$ O $-6.647227-3.353244 \quad 1.704317$ H -7.622192 -2.391994 0.198005 H -4.719702 -3.319409-0.076774 O $-6.285734-4.216657-1.007149$ H -6.481074 -1.944525 -2.173950 O $-4.556319-2.491314-2.548188$ C $-4.4997651 .714058-1.839355$ H $-4.6473680 .041430-3.116189$ H $-6.2114410 .632768-2.483047$ H $-6.916117-4.2246091 .366127$ H -5.715288-4.537054 -1.727063 H -3.713234 -2.580548 -2.072968 O $-4.8348182 .671385-2.754651$ O $-3.7609321 .870278-0.877479$ C $-4.2728673 .966707-2.525122$ H $-4.6153824 .632521-3.321653$ H $-4.6145654 .362881-1.564214$ H $-3.1811943 .915956-2.556407$

SCF Energy $\left(\mathrm{B} 3 \mathrm{LYP} / 6-31 \mathrm{G}^{* *} / / \mathrm{MMFF}\right)=-3245.91157741$

$02 \_118$

MM̄FF Geometry

C $0.306024-0.404107-2.325914$

C $0.7840420 .801189-2.674247$

C $0.5716362 .092636-1.918152$

O $0.078026 \quad 1.801897-0.591887$

C $-0.4642222 .956597-2.661233$

C $-0.7045464 .309441-2.020676$

C $-1.7307214 .583580-1.189853$

C $0.2831235 .381862-2.394907$

C $-2.7638603 .614784-0.683519$

C $-4.1535793 .903010-1.261056$

C $-5.1455602 .738250-1.099867$

C -5.3480142 .3118860 .356142$

C $-6.2823201 .096398 \quad 0.472621$

O $-4.652250 \quad 1.641519-1.878067$

O $\quad-6.162626 \quad 0.574043 \quad 1.804988$

C $-5.784642-0.005290-0.488670$

C $-7.754331 \quad 1.4831390 .292401$

O $-6.692033-1.103594-0.520228$

C $-5.5241020 .502873-1.922904$

C $-4.849041-0.554722-2.830960$

O $\quad-6.773880 \quad 0.841626-2.536503$

C 0.6119692 .4702000 .460132

O $1.521764 \quad 3.287474 \quad 0.424244$

C $-0.0588582 .109622 \quad 1.731120$
C $-1.052827 \quad 1.2129581 .832723$

C -1.724846 $0.808953 \quad 3.070574$

C $-1.259147 \quad 1.4423774 .355892$

C $-3.506530-1.090632-2.321416$

C $-3.630680-2.395339-1.551331$

C $-2.449072-3.773070 \quad 0.155634$

O $-4.557913-3.184069-1.716727$

C $-3.217155-3.5324241 .416410$

C $-2.658917-3.4838232 .634944$

C $-3.441916-3.2559793 .895341$

C $-3.013606-2.0078374 .634087$

C $-2.269914-2.0895255 .750231$

C $-3.527293-0.6748224 .135726$

C $-2.704283-0.1174923 .004521$

N -2.558927 -2.603214 -0.695007

H $-0.301010-0.504948-1.431561$

H $1.3750300 .885201-3.583992$

H $1.5394062 .606175-1.864408$

H -1.412500 $2.410123-2.741359$

H $-0.1412093 .115476-3.699078$

H $-1.8270645 .595532-0.799781$

H $0.0617686 .338521-1.909863$

H $1.2956745 .089166-2.099579$

H $0.2706995 .548599-3.476864$

H -2.475533 $2.580877-0.889800$

H $-2.796952 \quad 3.7037190 .409068$

H -4.564669 $4.804294-0.789781$

H $-4.0554034 .115608-2.334204$

H $-6.0982143 .069457-1.530314$

H -5.7242543 .1489670 .956916$

H -4.3851542 .0305970 .802171$

H -6.3890391 .2860152 .427711$

H $-4.845775-0.387203-0.068788$

H -8.0565322 .2056001 .060174$

H $-8.409566 \quad 0.6150170 .424197$

H $-7.965725 \quad 1.930932-0.680646$

H -6.814803 -1.404395 0.396504

H $-5.554128-1.365515-3.049547$

H -4.651636 $-0.072832-3.799429$

H $\quad-6.6587640 .823858-3.501095$

H 0.3291692 .6293032 .601442

H -1.409544 0.7144450 .932998

H $-0.193710 \quad 1.2438344 .518442$

H - 1.7802631 .0708745 .240331

H $-1.4160192 .526348 \quad 4.328726$

H $-2.869846-1.316336-3.185893$

H -2.983732 -0.337922 -1.721773

H $-1.384370-3.9442390 .342822$

H $-2.856529-4.644125-0.367653$

H -4.292671 -3.4008421 .320179$

H -1.586147 -3.6281822 .737642$

H -3.308149-4.139421 4.533322

H $-4.518978-3.2021793 .693127$

H -1.986397 -1.203253 6.307454

H -1.924455 -3.045198 6.130459

H -3.6003970 .0412874 .961670$

H -4.562746 $-0.790753 \quad 3.791221$

H -2.969526 -0.522104 2.027661

H -1.886685 - $1.856546-0.550774$

C $1.143627-2.766327-2.245956$

O $2.416625-2.332085-1.736996$

C $3.408332-3.331059-2.027511$

C $2.625794-4.608391-2.297412$

C $1.410028-4.058851-3.021249$

H $3.905931-3.013090-2.952604$

C $4.443625-3.396485-0.898670$

H $3.193651-5.328202-2.894275$

H $2.314975-5.101946-1.369624$

C $0.226288-5.010870-3.040905$

H $1.695790-3.826341-4.055813$

C $4.910031-2.008750-0.408166$

O $3.865369-4.0722580 .224786$

H $5.295358-4.009432-1.215690$

H $4.077309-1.5308280 .110793$

O $5.927753-2.2130090 .578750$

C $5.447071-1.078693-1.506150$

C $5.9307760 .297106-1.005180$

H $4.687728-0.923992-2.279631$ 
O $6.550870-1.741448-2.132595$

H $6.5822220 .163966-0.135045$

H $6.5782630 .720311-1.783605$

H $0.485460-5.936413-3.565106$

H $-0.092317-5.276235-2.027122$

H $-0.628294-4.558307-3.553978$

C $0.544319-1.660412-3.116943$

H $4.531432-4.0802180 .933618$

H $6.698969-2.5977970 .127851$

H $6.830326-1.196764-2.888099$

H $-0.410782-1.995636-3.536164$

H $1.216634-1.447403-3.957459$

H $0.504144-2.958331-1.375551$

C 4.5838550 .9973691 .684172

$\begin{array}{lllll}\text { O } & 3.961786 & 0.926514 & 0.396182\end{array}$

C $4.789431 \quad 1.299394-0.708875$

C $5.2709422 .745141-0.518252$

C 6.0099742 .9123600 .804957

C $5.1451472 .401734 \quad 1.955203$

H $5.3813350 .248472 \quad 1.743475$

C 3.5283870 .6520612 .742863

H $4.1376241 .296403-1.591974$

O $6.107164 \quad 3.136502-1.601597$

H $4.4014863 .415469-0.527844$

H 6.9859802 .4141820 .779153

$\begin{array}{lllll}\text { O } & 6.273798 & 4.303329 & 1.001172\end{array}$

H $4.321604 \quad 3.1097412 .113344$

O 5.9167252 .3954413 .155007

C $2.992961-0.7506762 .584290$

H 3.9374980 .7294103 .756914

H 2.6877831 .3535172 .694488

H $6.4055324 .045118-1.424555$

H $6.699846 \quad 4.397095 \quad 1.870448$

H $6.647832 \quad 1.7654333 .036113$

O $1.788856-0.7176531 .945407$

O $3.579231-1.7527752 .971610$

C $1.142800-1.9803431 .761057$

H $0.913622-2.4312782 .730868$

H $0.206751-1.8078221 .223548$

H $1.769835-2.6460551 .163751$

SCF Energy (B3LYP/6-31G**//MMFF) $=-3245.91680632$

02_119

MMFF Geometry

C $1.0094780 .723457 \quad 4.005273$

C $-0.230114 \quad 0.2425393 .834771$

C -0.507195 -1.175153 3.404976

O $-1.023820-1.1130272 .053273$

C $-1.565726-1.8158534 .313741$

C $-1.829498-3.2686513 .970143$

C $-2.893676-3.7078903 .269701$

C $-0.819193-4.2500274 .505803$

C $-3.989657-2.8801132 .659521$

C $-4.197821-3.2244511 .177988$

C $-5.280230-2.3348240 .552174$

C $-5.630837-2.803049-0.859646$

C $-6.662921-1.876334-1.520812$

O $-4.775715-0.9957170 .504114$

O $-6.704167-2.190077-2.921102$

C $-6.175560-0.417944-1.397551$

C $-8.077891-2.114020-0.980098$

O $-7.1693440 .494419-1.859261$

C $-5.720459-0.028304 \quad 0.030313$

C $-5.0227521 .350389-0.009676$

$\begin{array}{llll}\text { O } & -6.860809 & 0.054928 & 0.886635\end{array}$

C $-0.099121-1.0043751 .058383$

O $1.114791-1.0799981 .170261$

C $-0.814422-0.700290-0.198271$

C $-0.137182-0.080225-1.176136$

C $-0.667167 \quad 0.350017-2.469774$

C $-2.064711-0.074053-2.829010$

C -4.2783801 .7323641 .276077$

C -3.5444473 .0421001 .064424$

C $-1.5697193 .996088-0.128626$

O -4.0173424 .1130901 .437322$

C $-1.7154214 .030631-1.619525$

C $-0.6817634 .173735-2.462551$

C $-0.8157664 .175355-3.959063$
C $0.127736 \quad 3.200585-4.633266$

C $1.208066 \quad 3.637317-5.302557$

C $-0.2186841 .724910-4.593989$

C $0.1225631 .098768-3.267633$

$\begin{array}{llll}\text { N }-2.350779 & 2.881400 & 0.377003\end{array}$

H 1.8626170 .0615723 .875979

H -1.0801520 .9153103 .921209$

H $0.406613-1.7824743 .425059$

H -2.494851-1.236339 4.272896

H $-1.239938-1.7609735 .361487$

H -2.996932 -4.7811063 .114399$

H $-1.079858-5.2869164 .268359$

H $0.170178-4.0548994 .080876$

H $-0.752512-4.1692235 .595679$

H $-4.916505-3.0802963 .210747$

H -3.791001 -1.809578 2.752806

H -3.250661 -3.088296 0.640126

H $-4.484125-4.2795171 .085820$

H $-6.167800-2.3667691 .195392$

H -5.996008 -3.837540 -0.849320

H $-4.722545-2.810517-1.478492$

H $-6.944806-3.128143-3.010971$

H $-5.316981-0.307188-2.074586$

H $-8.382303-3.153992-1.148394$

H $-8.811947-1.498186-1.511370$

H $-8.168116-1.9123640 .089220$

H $-7.414250 \quad 0.224161-2.760889$

H $-4.2989711 .359855-0.831706$

H $-5.7591972 .133788-0.231756$

H -6.6540360 .6433841 .629902$

H $-1.866913-0.947045-0.265200$

H $0.9095330 .167754-1.001952$

H -2.791950 $0.440965-2.197347$

H $-2.189250-1.154951-2.697228$

H $-2.3280600 .133015-3.868458$

H $-4.967773 \quad 1.8573492 .117459$

H -3.5525540 .9636841 .564701$

H $-0.532807 \quad 3.8328040 .179575$

H $-1.928702 \quad 4.9338990 .305263$

H -2.718976 $3.924660-2.025673$

H $\quad 0.3175604 .309949-2.057394$

H $-0.6155925 .196366-4.308938$

H - $-1.8439653 .949200-4.268109$

H $1.8767342 .948111-5.807633$

H $1.4516884 .693164-5.355887$

H $0.3431331 .185590-5.367437$

H $-1.2736621 .615479-4.860048$

H $1.1376381 .314416-2.930560$

H -2.062620 1.9467310 .103503

C 1.5124162 .9231172 .947629

$\begin{array}{llll}\text { O } & 2.571240 & 2.315752 & 2.185580\end{array}$

C $3.3338233 .347071 \quad 1.534326$

C 2.5907884 .6560321 .787527

C $1.9239164 .381943 \quad 3.122689$

H 4.3015883 .3865892 .048313

C 3.5502012 .9957450 .057067

H 3.2674515 .5157681 .813878

H 1.8335044 .8530641 .020262

C 0.7681345 .3185113 .428397

H 2.6790614 .4673083 .915477

C $4.0808661 .567106-0.189937$

O $2.2942043 .097441-0.625716$

H $4.2104273 .741048-0.401973$

H $3.2775540 .855094 \quad 0.023744$

O $4.3550701 .449014-1.591083$

C $5.341221 \quad 1.1936900 .605419$

C $5.964236-0.1765790 .264845$

H $\quad 5.1149641 .2141651 .677309$

$\begin{array}{lllll}\text { O } & 6.333330 & 2.197479 & 0.367201\end{array}$

H $6.307473-0.169102-0.775047$

H $6.880567-0.2646350 .862231$

H $1.1144816 .356423 \quad 3.468276$

H $-0.013846 \quad 5.2540752 .664317$

H 0.3150545 .0767414 .394935

C 1.3151452 .1702524 .265764

H $2.4549092 .885045-1.561033$

H $5.1055202 .033908-1.793241$

H 7.0964911 .9978430 .935789 
H 0.5129372 .6326894 .852133

H 2.2270602 .2155684 .873307

H 0.6027812 .8524482 .336029

C $4.345189-1.926990-1.696132$

O $3.970163-1.495494-0.385054$

C $5.042412-1.3810990 .555211$

C $5.773851-2.7283980 .670892$

C $6.246946-3.237364-0.687602$

C $5.078672-3.272757-1.671500$

H $5.010168-1.176187-2.139315$

C $3.091193-1.931124-2.583479$

H $4.576213-1.1897051 .530389$

O $6.888501-2.6353601 .551537$

H $5.091221-3.4742611 .098779$

H $7.074487-2.633341-1.078301$

O $6.749373-4.567777-0.544539$

H $4.400290-4.086511-1.391537$

O $5.569400-3.562938-2.978471$

C $1.987705-2.862499-2.122497$

H $2.663052-0.922269-2.594608$

H $3.368625-2.203034-3.608220$

H $6.555599-2.3272682 .411776$

H $7.459702-4.5380240 .119308$

H $6.058255-4.402160-2.924877$

O $1.045663-2.948704-3.109179$

O $1.935355-3.421659-1.036180$

C $-0.079741-3.782590-2.815669$

H $-0.779687-3.715443-3.652718$

H $\quad 0.242023-4.822652-2.708080$

H $-0.585992-3.439458-1.908462$

SCF Energy (B3LYP/6-31G**//MMFF) $=-3245.91617338$

$02 \_12$

MM̄FF Geometry

C $-3.301276-3.1909971 .617407$

C $-1.999726-2.9636921 .381753$

C $-0.981094-2.8704822 .487756$

O $-0.312792-1.5902262 .411533$

C $0.086323-3.9671602 .329956$

C $1.036954-4.0232893 .511719$

C $2.283366-3.5107033 .514516$

C $0.514342-4.7469454 .725656$

C $2.960560-2.7584982 .403118$

C $4.043260-3.6193001 .743549$

C $4.609890-3.0347240 .441170$

C $5.291286-1.6793980 .635734$

C $5.802343-1.105526-0.695610$

O $3.534326-2.922883-0.497594$

O $6.1312940 .276395-0.483379$

C $4.650481-1.140167-1.723054$

C $7.092482-1.789221-1.161404$

O $5.107677-0.747220-3.014030$

C $3.937408-2.506953-1.810839$

C $2.664654-2.481634-2.695648$

O $4.829785-3.464311-2.395446$

C $-0.821734-0.5802773 .162572$

O $-1.890169-0.5803493 .758931$

C 0.1412210 .5365513 .291803

C 1.1851620 .7405332 .472891

C 2.2047531 .7828432 .635386

C 2.1856192 .5791213 .915988

C $1.598586-1.457527-2.288943$

C $1.784898-0.113255-2.971723$

C $1.4635752 .324359-2.608404$

O $2.2950390 .002770-4.083809$

C $2.7282342 .831238-1.992745$

C $2.7696793 .842355-1.113466$

C $4.0347704 .336394-0.478114$

C 3.9957664 .2467651 .030723

C 3.7886905 .3452711 .776513

C 4.2964032 .9109731 .673558

C 3.1266971 .9607751 .665268

N $1.288048 \quad 0.938517-2.216470$

H -3.619448 -3.292720 2.654121

H -1.632496 -2.850194 0.366368

H -1.463272 -2.9788773 .467685$

H $\quad 0.643716-3.8156951 .397688$

H $-0.394324-4.9493422 .226135$
H $2.888784-3.6284424 .412149$

H $1.256926-4.8014685 .528949$

H $-0.367619-4.2391685 .127900$

H $\quad 0.236545-5.7734854 .465174$

H $2.240641-2.4090571 .658339$

H $3.410640-1.8563122 .833908$

H $4.861600-3.7910182 .454111$

H $3.612075-4.6018601 .508266$

H $5.333326-3.7643920 .058081$

H $6.108676-1.7552591 .363119$

H $4.578268-0.9592481 .057295$

H 6.7981020 .3206820 .223272

H $3.927985-0.381706-1.399621$

H $7.884589-1.660273-0.413948$

H $7.471875-1.331027-2.081317$

H $6.974124-2.860817-1.333108$

H $5.5263160 .125622-2.920019$

H $2.945900-2.369867-3.749867$

H $2.196423-3.473661-2.622696$

H $4.302608-4.194821-2.759379$

H $-0.057177 \quad 1.193763 \quad 4.132907$

H 1.3233110 .0812801 .617828

H 1.2677053 .1712073 .989845

H 3.0166143 .2809254 .007044

H 2.2457091 .9131974 .784154

H $0.615141-1.822903-2.608797$

H $1.562960-1.341400-1.200399$

H $0.5877772 .879691-2.263735$

H $1.5201022 .400361-3.698698$

H $3.6532362 .336804-2.280839$

H $1.8475874 .346628-0.835151$

H $4.1815925 .377773-0.792975$

H $4.9111403 .790903-0.849950$

H 3.7961795 .3047082 .860228

H 3.6043246 .3119191 .319301

H 4.6742893 .0493842 .693322

H 5.1332342 .4371261 .144701

H $3.069106 \quad 1.3388030 .771874$

H $0.9331770 .756194-1.282300$

C $-4.054618-3.332403-0.865784$

O $-3.581131-2.028973-1.254814$

C $-4.178599-1.667702-2.512852$

C $-4.842332-2.930562-3.047926$

C $-5.259749-3.617994-1.765078$

H $-4.955119-0.926583-2.289087$

C $-3.131395-1.058708-3.449859$

H -5.684011-2.707117 -3.710770

H -4.137161 -3.560838 -3.601732

C $-5.566161-5.095478-1.942344$

H $-6.151258-3.111302-1.371429$

C $-2.3385230 .122094-2.858339$

O $-2.176781-2.066767-3.811648$

H $-3.612372-0.754109-4.386627$

H -1.644192 -0.236551-2.088620

O $-1.5271960 .659315-3.912346$

C $-3.1974191 .258909-2.272863$

C $-2.3339072 .468097-1.869270$

H $-3.7448200 .882901-1.403493$

O $-4.154521 \quad 1.662201-3.250579$

H -1.514950 2.110932 -1.236379

H -1.888151 $2.899965-2.773086$

H -6.390165 -5.234747 -2.649754

H $-4.698079-5.642053-2.325948$

H $-5.857856-5.551829-0.991360$

C $-4.419879-3.3352430 .620488$

H $-1.791926-2.414354-2.988482$

H - $0.980925-0.067756-4.257175$

H $-4.8233292 .206930-2.802744$

H $-4.935077-4.2740890 .856895$

H $-5.129587-2.5221390 .815802$

H $-3.246949-4.047247-1.068702$

C -2.8993542 .7796981 .130977$

O $-3.768422 \quad 3.110546 \quad 0.039759$

C $-3.1129053 .584129-1.141188$

C $-2.2637164 .823530-0.816946$

C $-1.295673 \quad 4.5608360 .331620$

C -2.0471113 .9911821 .531161$

$\begin{array}{llll}\mathrm{H} & -2.250934 & 1.941756 & 0.847473\end{array}$ 
C -3.783195 2.334917 2.306750

H -3.907412 $3.916088-1.820066$

O $-1.527717 \quad 5.256337-1.956203$

H $-2.9252505 .651031-0.529230$

H $-0.479333 \quad 3.895016 \quad 0.029512$

$\begin{array}{lllll} & 0 & -0.686443 & 5.791701 & 0.726763\end{array}$

H -2.682332 $4.772357 \quad 1.968180$

O -1.1074643 .5994712 .527568$

C -4.441479 $0.993207 \quad 2.068304$

H -3.191069 2.240030 3.223420

H -4.5782613 .0740352 .463422$

H -2.166846 $5.428202-2.668893$

H $-0.238867 \quad 6.156054-0.056224$

H $-0.561030 \quad 4.3772892 .733382$

$\begin{array}{llllll}\text { O } & -5.393801 & 0.795302 & 3.027831\end{array}$

$\begin{array}{llll}0 & -4.147558 & 0.209452 & 1.177050\end{array}$

C -6.102467 -0.4450692 .951092$

H $-6.548709-0.5739141 .960254$

H $-5.428127-1.273653 \quad 3.184447$

H -6.903737 -0.4251983 .694501$

SCF Energy (B3LYP/6-31G**//MMFF) $=-3245.91637777$

02_120

MMFF Geometry

C -1.784061 -4.016769 0.739908

C $-1.066644-4.0307721 .875146$

C -0.828762 -2.878728 2.823953

O $-1.551506-1.6964922 .419770$

C $0.670754-2.5314972 .819729$

C $1.056106-1.5078833 .867457$

C $1.342849-0.2180423 .603688$

C $1.169758-2.0368865 .273652$

C $1.273787 \quad 0.478604 \quad 2.272210$

C 0.7089831 .8981692 .419677

C 0.6456092 .6298791 .071786

C $0.3011914 .105601 \quad 1.282059$

C $0.1450024 .851241-0.050436$

O $-0.3624472 .003281 \quad 0.270113$

$\begin{array}{lllll}\text { O } & -0.474396 & 6.117391 & 0.221354\end{array}$

C $-0.8217414 .057779-0.951230$

C $1.4998895 .160287-0.697158$

O $-0.8862724 .630523-2.255888$

C $-0.4866752 .549832-1.050031$

C $-1.6387091 .818243-1.774977$

O $0.7152052 .383423-1.800922$

C $-2.792099-1.5100292 .943861$

O $-3.386154-2.2461113 .715002$

C $-3.321225-0.2459382 .386168$

C -4.6305390 .0212802 .499481$

C -5.3243711 .1916881 .958805$

C -4.4824752 .2999901 .386440$

C -1.528822 $0.290490-1.775802$

C $-2.784113-0.304229-2.382350$

C $-5.120949-0.959559-1.802899$

O $-2.877094-0.516936-3.589226$

C $-6.0476830 .213424-1.711367$

C $-7.2231710 .183478-1.064608$

C $-8.1472581 .364798-0.962348$

C $-8.605001 \quad 1.6384780 .455570$

C $-9.877021 \quad 1.412642 \quad 0.825384$

C -7.609485 2.2433421 .425487

C $-6.673262 \quad 1.2085881 .991789$

N $-3.783990-0.519536-1.447590$

H -2.300215 -3.1139190 .428502$

H $-0.556409-4.9540352 .146443$

H $-1.136107-3.1870963 .830640$

H $0.960865-2.1809021 .823106$

H $1.269234-3.4363632 .992820$

H 1.6654000 .4103614 .432932

H $1.511780-1.2724555 .979435$

H $0.200007-2.3996085 .627923$

H $1.886584-2.8636195 .312098$

H $2.288170 \quad 0.537276 \quad 1.862692$

H $0.660799-0.0770621 .556752$

H $-0.296938 \quad 1.8391402 .855594$

H 1.3393132 .4667563 .115306

H 1.6181722 .5318520 .574970

H 1.0563844 .5967321 .908590
H $-0.644872 \quad 4.183408 \quad 1.836271$

H 0.0926096 .6003420 .846980

H $-1.8272444 .159250-0.518646$

H $2.1134595 .775053-0.027735$

H $1.3765725 .747004-1.614162$

H $2.0715994 .262576-0.936584$

H $-1.1126555 .570332-2.148840$

H $-2.5889542 .090804-1.301734$

H $-1.6968622 .158515-2.817090$

H $0.7492131 .478594-2.148811$

H $-2.6292170 .407799 \begin{array}{lll}1.867282 \\ \text { H }\end{array}$

H $-5.266422-0.7042233 .005751$

H -3.713893 2.6124152 .102755

H -5.0512293 .1996601 .143702$

H -3.9907491 .9680670 .468939$

H $-0.673325-0.050830-2.367589$

H $-1.394965-0.102864-0.761941$

H $-5.395502-1.757876-1.106306$

H $-5.123982-1.360120-2.821141$

H $-5.7372511 .132227-2.204055$

H $-7.547590-0.742619-0.595366$

H $-9.0137791 .163139-1.605325$

H -7.678779 2.271698-1.364726

H -10.219319 1.6282861 .832246

H -10.603790 1.0026250 .132059

H -8.1383512 .7044952 .269764$

H $-7.091845 \quad 3.063304 \quad 0.920931$

H $-7.187366 \quad 0.3651472 .455728$

H $-3.614378-0.272945-0.477509$

C -1.091739-4.984613 -1.503986

O $0.220610-4.447025-1.222780$

C $0.342247-3.195401-1.920471$

C $-0.547812-3.363605-3.138595$

C $-1.756392-4.024226-2.497592$

H $-0.081258-2.418184-1.270210$

C $1.803335-2.842788-2.211756$

H $-0.782848-2.414473-3.626792$

H $-0.090508-4.032151-3.877670$

C $-2.675793-4.710790-3.492470$

H -2.335011 -3.261223-1.963421

C $2.594289-2.565925-0.916986$

O $2.416553-3.880352-2.969237$

H $1.823087-1.937892-2.830695$

H $2.025531-1.832622-0.332391$

O $2.683123-3.746349-0.121349$

C $4.003307-1.987712-1.145561$

C $4.663567-1.6140060 .192634$

H $3.925946-1.103390-1.783928$

O $4.840214-2.929139-1.810556$

H $3.941916-1.0535030 .795662$

H $4.901117-2.5323330 .743683$

H $-3.088583-3.983357-4.199202$

H $-2.145105-5.475785-4.069009$

H $-3.511463-5.195084-2.977218$

C $-1.855194-5.201233-0.189737$

H $2.301259-4.710993-2.476794$

H $3.271602-4.367626-0.583159$

H $4.433303-3.128860-2.670618$

H $-1.424080-6.077458 \quad 0.311796$

H $-2.903998-5.444525-0.393151$

H $-0.929542-5.961277-1.976921$

C $4.9520571 .403293-0.137207$

O $5.765307 \quad 0.395798-0.751264$

C $5.960056-0.7909620 .026838$

C $6.632883-0.4277171 .357270$

$\begin{array}{llll}\text { C } 5.833098 & 0.628531 & 2.117293\end{array}$

C $5.5325901 .828399 \quad 1.219338$

H $3.9304781 .025785-0.010023$

C $4.9065102 .607040-1.093752$

H $6.664079-1.415855-0.536624$

O $6.793020-1.5915672 .160935$

H $7.637936-0.0369551 .152579$

H 4.9094550 .2049002 .527145

O $6.587866 \quad 1.068513 \quad 3.249515$

H 6.4525292 .4072661 .066533

O 4.5982272 .6820071 .878617

C $4.0769612 .349100-2.333594$

H $4.4658593 .473423-0.588237$ 
H $5.9219302 .856721-1.424075$

H $7.189979-1.3116063 .003547$

H 7.4245801 .4435882 .926102

H 4.9763042 .9131062 .744412

O $4.0764583 .478558-3.103311$

O $3.5001391 .304214-2.598165$

C $3.3208493 .397906-4.315992$

H $2.2621213 .238547-4.091676$

H $3.427443 \quad 4.347968-4.846186$

H $3.7067832 .595643-4.952155$

SCF Energy (B3LYP/6-31G**//MMFF) $=-3245.92818337$

02_121

MMFF Geometry

C $2.091178-0.075625 \quad 2.809174$

C 2.5963391 .0100063 .413577

C 2.3583042 .4492033 .019661

$\begin{array}{llll}\text { O } & 1.376999 & 2.575526 & 1.967617\end{array}$

C $3.681573 \quad 3.0307872 .486297$

C 3.5879074 .4691032 .016800

C 3.4537054 .8280270 .723473

C $3.707847 \quad 5.5102803 .095634$

C $3.2540033 .896855-0.440724$

C $4.5618003 .510174-1.146132$

C $4.6523441 .992425-1.373808$

C $5.9162461 .616385-2.145178$

C $5.9808290 .106104-2.419223$

O $3.496323 \quad 1.567792-2.103138$

O $7.013251-0.097569-3.395879$

C $4.641726-0.356349-3.037137$

C $6.410236-0.675197-1.170399$

O $4.637649-1.777473-3.157294$

C $3.3983590 .145042-2.260740$

C $2.106591-0.158867-3.053111$

O $3.336986-0.526300-1.001545$

C 0.0634412 .6484072 .305602

O -0.4302692 .4419153 .401519$

C $-0.665073 \quad 3.0948641 .094584$

C $-1.998822 \quad 3.2328651 .119476$

C $-2.825727 \quad 3.7952200 .046813$

C $-2.1211894 .334990-1.170982$

C $0.8380350 .487553-2.476473$

C $-0.3932630 .117517-3.286700$

C $-2.8418380 .501961-3.387001$

O $-0.361632-0.656091-4.240375$

C $-3.6650871 .747660-3.323547$

C $-4.858913 \quad 1.821037-2.716742$

C $-5.730178 \quad 3.042515-2.726049$

C $-6.1059963 .511069-1.339673$

C -7.267826 $3.139752-0.775456$

C $-5.1921514 .502895-0.660063$

C $-4.162657 \quad 3.8504410 .222081$

N $-1.5325400 .759058-2.819658$

H $1.4181900 .040068 \quad 1.964208$

H 3.2657140 .8663964 .260089

H 2.0277243 .0178343 .897730

H 4.0470402 .4040171 .664563

H 4.4587552 .9637083 .260009

H 3.4398725 .8870790 .472813

H 3.6527896 .5288832 .697312

H 2.9001265 .3970663 .825646

H 4.6653325 .4096353 .616864

H $2.7048203 .006692-0.120031$

H $2.5894354 .392396-1.160108$

H $4.5915594 .026516-2.114239$

H $5.4380813 .842343-0.576251$

H $4.6536361 .507142-0.391435$

H $6.815028 \quad 1.949367-1.611378$

H $5.9258422 .145397-3.108659$

H $7.018841-1.042968-3.625495$

H $4.6063130 .045192-4.059649$

H $7.403131-0.347190-0.839763$

H $6.505532-1.745416-1.384617$

H $5.723897-0.553806-0.329635$

H $3.891055-2.027709-3.726491$

H $2.2235480 .202723-4.083353$

H $1.949691-1.242841-3.105024$

H $2.437739-0.455906-0.645570$
H -0.0695593 .3597510 .227661$

H -2.5342452 .9476892 .024225$

H $-1.4895345 .188403-0.900728$

H -2.803312 $4.672069-1.954415$

H -1.490262 $3.566525-1.629456$

H $0.6662140 .161741-1.445497$

H $0.9345281 .579386-2.481360$

H -3.283974 -0.323478 -2.821894

H $-2.7371050 .180894-4.428516$

H $-3.2759352 .622234-3.839626$

H $-5.2622000 .937315-2.230487$

H $-6.6389862 .796061-3.291178$

H $-5.260486 \quad 3.865480-3.278994$

H -7.5615093 .5041690 .203519$

H -7.946096 2.457220 -1.276780

H $-5.7890065 .168222-0.020434$

H $-4.7515495 .175512-1.401881$

H $-4.5816763 .434306 \quad 1.139434$

H $-1.4874001 .235183-1.923037$

C $2.886808-2.3160082 .031251$

O $1.779352-2.5250971 .129301$

C $1.693663-3.9252010 .815019$

C $3.051293-4.5078961 .179543$

C $3.380957-3.7091252 .425518$

H $0.936084-4.3546721 .481635$

C $1.272996-4.120607-0.643818$

H $3.008581-5.5864251 .357834$

H $3.797876-4.3185790 .399526$

C $4.850968-3.7541612 .805519$

H $2.783710-4.0979993 .261445$

C $-0.042456-3.411546-1.030097$

O $2.302934-3.621534-1.505500$

H $1.195873-5.191880-0.862588$

H $0.100038-2.324794-1.048027$

O $-0.361313-3.795764-2.373514$

C $-1.242165-3.751702-0.124569$

C $-2.578439-3.210142-0.668128$

H $-1.064534-3.3415570 .874182$

O $-1.345673-5.1695490 .016477$

H -2.462656 - $2.145956-0.897194$

H -2.813288 $-3.723517-1.608014$

H $5.160763-4.7816883 .022364$

H $5.486043-3.3750831 .997644$

H $5.042091-3.1480873 .696629$

C $2.412480-1.4851533 .224643$

H $2.476736-2.699037-1.251054$

H $\quad 0.396624-3.551172-2.931951$

H $-1.468079-5.545060-0.872429$

H $3.176905-1.4874794 .009912$

H $1.502774-1.9287643 .644410$

H $3.660799-1.7777711 .469729$

C $-3.660877-1.3164861 .488519$

O $-3.557340-2.7444271 .558532$

C $-3.750472-3.4339390 .317488$

C $-5.134428-3.101445-0.255390$

C $-5.336720-1.596815-0.390859$

C $-5.024891-0.8928990 .927271$

H $-2.853692-0.9167000 .863095$

C $-3.485389-0.7665612 .913219$

H -3.761891 -4.5033460 .562167$

O $-5.312208-3.735637-1.517013$

H $-5.902369-3.5050890 .417129$

H $-4.723902-1.195612-1.202791$

O $-6.692230-1.330434-0.757643$

H $-5.815606-1.1155431 .655231$

$\begin{array}{llllllll} & \mathrm{O} & -5.8156066 & 0.516714 & 0.717063\end{array}$

C -2.080438 -0.9448103 .444252$

H -3.7044420 .3066152 .942688$

H $-4.165831-1.2948653 .592088$

H $-6.185186-3.472277-1.855394$

H $-7.268135-1.681846-0.057354$

H -5.8894450 .7540730 .340140$

O $-2.074932-0.6360474 .775298$

O $-1.113106-1.2917092 .782107$

C $-0.813167-0.7585065 .438083$

H $-0.111928-0.0162675 .047937$

H $-0.969069-0.5666646 .502966$

H $-0.415267-1.7707705 .320465$ 
C $1.697784-3.075102-1.067684$

O $1.318446-1.731894-1.482912$

C $0.544814-4.072943-1.314184$

C $0.170288-4.190590-2.781062$

C $-0.919222-3.638860-3.351970$

C $1.107294-5.028231-3.615297$

C $-1.974128-2.779017-2.711531$

C -3.364787 -3.404224 -2.861575

C $-4.486142-2.646860-2.131808$

C $-4.694786-1.223748-2.652581$

C $-5.815949-0.500406-1.887588$

O $-4.183173-2.617226-0.731822$

O $-5.7364290 .897782-2.206946$

C $-5.550159-0.632139-0.371639$

C $-7.207999-0.964760-2.330710$

O $-6.640961-0.1119110 .383247$

C $-5.237929-2.0761110 .080899$

C $-4.780490-2.1739531 .558416$

O $-6.425275-2.871935-0.035353$

C $0.369699-1.048205-0.787441$

O $-0.205210-1.400803 \quad 0.230038$

C $0.1154840 .218037-1.506525$

C $-0.9907020 .919048-1.218979$

C $-1.3908182 .190648-1.820758$

C $-0.4056412 .877037-2.729455$

C $-3.614378-1.2596221 .952358$

C -4.0721330 .0599802 .555491$

C -3.3067092 .3893472 .981291$

O

C -3.8932253 .2215341 .885444$

C -3.2778324 .2822881 .341647$

C $-3.877317 \quad 5.1205420 .248417$

C $-3.0410925 .121392-1.012614$

C $-2.2189916 .146410-1.293234$

C $-3.2218123 .979476-1.988595$

C $-2.6009082 .697444-1.507114$

N $-3.093188 \quad 1.0404312 .488939$

H $\quad 0.933127-4.2353451 .387662$

H $3.180594-2.4024140 .410111$

H $2.528662-3.348491-1.729734$

H $-0.331718-3.805082-0.717059$

H $\quad 0.838733-5.070107-0.959868$

H -1.072555 -3.803536 -4.418288

H $0.757938-5.141796-4.647051$

H $2.101839-4.573934-3.654668$

H $\quad 1.199282-6.032769-3.189307$

H -1.770424 -2.602454 -1.654979

H -1.949378 -1.802241 -3.207147

H -3.620089 -3.490612 -3.925260

H $-3.328000-4.424740-2.457005$

H -5.400375 -3.234148 -2.280206

H -4.903665 -1.228962 -3.729294

H $-3.774260-0.639254-2.525446$

H $-5.8209010 .990211-3.171351$

H $-4.6849600 .005241-0.152215$

H -7.349208 $-0.780551-3.402567$

H -7.994151 -0.394614-1.823525

H -7.383818 -2.027661 -2.153636

H -6.7870470 .8033810 .088543$

H -5.640924 -2.033570 2.223771

H $-4.448661-3.2083711 .728027$

H $-6.332563-3.6476640 .542444$

H $\quad 0.8330780 .529268-2.255725$

H -1.679122 $0.521130-0.474604$

H $-0.7427273 .857253-3.073938$

H $-0.2169832 .271014-3.622229$

H $\quad 0.5437773 .043584-2.208890$

H $-3.024008-1.7563412 .732755$

H -2.947455 -1.085146 1.102111

H -2.3421332 .7732703 .324394$

H -3.9918542 .3652613 .834863$

H -4.8808002 .9389961 .527731$

H -3.981376 6.1437620 .632575

H $-4.896614 \quad 4.794356 \quad 0.006579$

H -1.637458 $6.171300-2.208652$

H -2.105588 $6.982314-0.610705$

H -2.841057 $4.249012-2.980246$

H $-4.2946083 .809073-2.147449$

H $-3.2432382 .126748-0.836430$

H $-2.232627 \quad 0.8524951 .983211$

C $1.670055-2.4377143 .618348$

O $1.736858-1.1380042 .995712$

C $2.056405-0.1532763 .993300$

C $1.805099-0.8292645 .334609$

C $2.228689-2.2538135 .029441$

H 3.1290600 .0535963 .898811

C 1.2569001 .1304503 .744897

H $2.381014-0.3727596 .145152$

H $0.745274-0.8020425 .612934$

C $1.714337-3.2710316 .032863$

H $3.326076-2.2958685 .008088$

C 1.3777741 .6897652 .311818

$\begin{array}{lllll}\text { O } & -0.132796 & 0.871249 & 3.983758\end{array}$

H 1.5469521 .8949734 .474384

H 0.8574821 .0253251 .612039

O 0.6807602 .9407622 .266064

C 2.8177331 .9178241 .814487

C 2.8426152 .5159520 .395497

H $3.3595550 .966631 \quad 1.809123$

O 3.5075812 .8093312 .687708

H $2.2430201 .877941-0.260281$

H 2.3678303 .5047150 .406352

H $2.101733-3.0538147 .033527$

H $0.620503-3.263726 \quad 6.087047$

H $2.031345-4.2825255 .759849$

C $2.463110-3.4406532 .777626$

H -0.4017360 .1443743 .395825$

H -0.2331602 .7724302 .550674$

H 3.6169242 .3642893 .544326

H $2.506895-4.414578 \quad 3.277519$

H $3.496531-3.0909752 .659079$

H $0.609233-2.7183843 .653903$

C $4.6154490 .412324-0.935880$

O $5.0366361 .471568-0.072786$

C $4.2693602 .674821-0.177408$

C $4.3092333 .200404-1.620031$

C $3.8696012 .139708-2.627606$

C $4.6409020 .837870-2.409563$

H $3.5928660 .124790-0.661914$

C $5.482789-0.822194-0.648348$

H 4.7787053 .4163330 .450919

O $3.4883734 .357536-1.741378$

H $5.3361923 .508087-1.856822$

H $2.7880481 .978009-2.580198$

O $4.1401312 .629135-3.943184$

H $5.669600 \quad 0.984554-2.756844$

O $4.075386-0.188141-3.223090$

C $6.954359-0.642193-0.961739$

H $5.420921-1.0747280 .416881$

H $5.102298-1.670233-1.229478$

H $3.5264424 .644882-2.669782$

H $3.895724 \quad 1.926144-4.569454$

H $3.163401-0.340750-2.921843$

O $7.517967-1.868489-1.173337$

O $7.5452250 .428711-0.984209$

C $8.918976-1.859572-1.465127$

H $9.240157-2.892670-1.622091$

H $\quad 9.112280-1.289370-2.378788$

H $9.478425-1.442123-0.622626$

SCF Energy (B3LYP/6-31G**//MMFF) $=-3245.90629509$

$02 \_123$

MM̄FF Geometry

C $4.875642-0.5950581 .982358$

C $4.4620660 .193408 \quad 0.977712$

C 4.7782801 .6593190 .886500

O 3.5293012 .3750901 .004882

C $5.5430132 .029584-0.402456$

C $4.7878911 .917013-1.714378$ 
C $4.9349720 .912529-2.601520$

C $3.9115293 .092989-2.063128$

C $5.786951-0.315927-2.457530$

C $5.199380-1.574752-3.119832$

C $3.914925-2.108849-2.461706$

C $3.611289-3.519180-2.979039$

C $2.289917-4.068939-2.423815$

O $2.829121-1.232599-2.783059$

O $1.936525-5.229295-3.192254$

C $1.183210-3.021166-2.653308$

C $2.428535-4.537469-0.970448$

O $-0.033721-3.421221-2.025664$

C $1.574882-1.598784-2.190473$

C $0.498330-0.590228-2.657696$

O $1.642227-1.582189-0.766361$

C 3.5808613 .6479141 .472546

O $4.592745 \quad 4.2850151 .734955$

C $2.2314904 .224598 \quad 1.677647$

C 1.0720433 .5888311 .441709

C -0.2663724 .1282791 .717736$

C -0.3509035 .5446242 .229685$

C $0.868634 \quad 0.882811-2.444214$

C $-0.2166441 .820259-2.947058$

C $-0.7669274 .245338-3.097910$

O $-1.2546861 .427114-3.472309$

C $-1.4625204 .713873-1.860312$

C $-2.7254354 .383958-1.552495$

C $-3.4445874 .859198-0.323810$

C -3.6915583 .7439760 .665946$

C -4.7442262 .9203030 .529149$

C $-2.7818463 .647547 \quad 1.868671$

C -1.3477473 .3421001 .524137$

N $0.1190693 .152301-2.747601$

H $5.476796-0.1729822 .783494$

H $3.844249-0.2306010 .192328$

H 5.4139621 .9308891 .740876

H $6.4680301 .440754-0.446153$

H $5.8853763 .069557-0.315811$

H $4.3988890 .986380-3.546780$

H $3.5283463 .037516-3.087821$

H $3.0494623 .143275-1.394878$

H $4.4738384 .028657-1.979941$

H $6.750131-0.106618-2.939299$

H $5.998687-0.540638-1.407898$

H $5.009825-1.364303-4.180569$

H $5.972560-2.352566-3.078552$

H $4.060122-2.128218-1.376447$

H $4.436055-4.204728-2.746977$

H $3.542338-3.495829-4.075926$

H $2.663250-5.871274-3.116858$

H $0.979488-2.989891-3.732909$

H $3.182333-5.330361-0.893891$

H $1.493617-4.977318-0.606029$

H $2.722208-3.738197-0.287752$

H $-0.251966-4.309410-2.356185$

H $\quad 0.314833-0.733344-3.730854$

H $-0.445083-0.792833-2.135842$

H $1.454742-0.687658-0.442952$

H 2.2488795 .2320442 .083066

H 1.0944182 .5750221 .047179

H $0.0968985 .621143 \quad 3.226330$

H -1.371555 5.9251532 .302320

H $\quad 0.172150 \quad 6.230047 \quad 1.552893$

H $1.0321521 .094133-1.382521$

H $1.7866631 .117700-2.992119$

H $-1.4754803 .920672-3.867189$

H $-0.1567245 .053043-3.513814$

H $-0.8996595 .363093-1.194690$

H -3.282232 $3.736674-2.226197$

H -4.402386 $5.295223-0.637030$

H -2.9024045 .6791700 .162886$

H $-4.9510762 .138643 \quad 1.252345$

H -5.422922 $3.010369-0.312566$

H -3.124068 2.8462022 .537575

H -2.8873344 .5624102 .458466$

H -1.1979502 .3374661 .129250$

H $\quad 0.9722503 .378580-2.246497$

C $3.251142-2.3591482 .836805$
O $2.142512-1.7831992 .120286$

C $1.309477-1.0671443 .053843$

C $1.656499-1.6419364 .416731$

C $3.155427-1.8417524 .273031$

H $1.621697-0.0163392 .992914$

C $-0.149293-1.2149792 .617119$

H $1.392059-0.9720485 .239836$

H $1.152434-2.6033534 .574860$

C $3.749383-2.7854295 .303675$

H $3.642215-0.8635224 .370553$

C $-1.180651-0.6390423 .605146$

O $-0.290586-0.5640491 .348334$

H $-0.360468-2.2742782 .434935$

H -1.206272 -1.2544834 .511552$

O -0.7532430 .6609844 .028936$

C $-2.604514-0.5058943 .026520$

C $-3.203012-1.8379212 .541964$

H -2.5995880 .2318602 .217238$

O $-3.458848 \quad 0.032396 \quad 4.041893$

H -2.495032 -2.349298 1.885766

H $-3.367399-2.4900313 .408393$

H $3.604702-2.3911036 .314912$

H $3.281800-3.7748075 .259387$

H $4.824211-2.9119765 .139725$

C $4.550637-2.0594992 .083824$

H -0.0190000 .3627051 .454992$

H -0.7428811 .2406903 .249793$

H -3.466279 -0.5925984 .786932$

H $4.477080-2.4750651 .071035$

H $5.390387-2.5706762 .568769$

H $3.088857-3.4446252 .818087$

C -3.664725 -1.255069-0.417195

O $-4.411920-0.7431300 .689902$

C $-4.543573-1.6433691 .796250$

C $-5.204761-2.9487891 .333634$

C $-4.473406-3.5769880 .147936$

C $-4.279882-2.547703-0.967284$

H $-2.639818-1.464623-0.087282$

C $-3.526923-0.147083-1.471009$

H $-5.233687-1.1639382 .501755$

O $-5.267996-3.8733232 .414843$

H $-6.239198-2.7339271 .035038$

H -3.518158 -4.0138760 .461789$

O $-5.271231-4.658401-0.339690$

H $-5.246741-2.350563-1.444061$

O $-3.413065-3.082989-1.964783$

C $-4.8358770 .377198-2.024259$

H $-3.0134300 .709788-1.018165$

H -2.924291 -0.514462 -2.308464

H -5.676655 -4.688423 2.076375

H $-4.759265-5.113192-1.029361$

H -3.865704 -3.839073 -2.374478

O $-4.5645941 .198700-3.082409$

O $-5.947631 \quad 0.130634-1.578784$

C $-5.703448 \quad 1.808624-3.697385$

H $-6.3746661 .042381-4.096627$

H $-6.2265502 .444983-2.977339$

H $-5.3502752 .431373-4.523544$

SCF Energy (B3LYP/6-31G**//MMFF) $=-3245.89633511$

$02 \_124$

MMFF Geometry

C 1.8261443 .3382322 .270983

C 2.4987992 .4704581 .500535

C 3.0499112 .8102720 .143806

O $2.3297541 .961369-0.779985$

C 4.5441402 .4708080 .056304

C $5.2090713 .067628-1.167050$

C $5.4857182 .392504-2.299579$

C $5.6061174 .515797-1.036226$

C $5.1554400 .963713-2.631669$

C $6.406917 \quad 0.080711-2.734854$

C $7.050005-0.223729-1.372796$

C $8.337624-1.030589-1.552294$

C $8.944884-1.445214-0.203619$

O $6.106281-0.978252-0.606088$

O $9.943972-2.444439-0.457799$

C $7.847857-2.1104150 .653277$ 
C $9.663291-0.2779070 .483395$

O $8.324540-2.3832831 .969361$

C $6.535397-1.2947390 .724019$

C $5.426327-2.1454471 .388576$

O $6.757536-0.1181731 .503095$

C $1.7446592 .515924-1.868720$

O $1.8181763 .683218-2.225795$

C $0.9265001 .519920-2.602933$

C $0.8707870 .218845-2.275913$

C $0.042006-0.806115-2.915749$

C $-0.886445-0.370102-4.018294$

C $4.024170-1.5225301 .320670$

C $2.965897-2.4446011 .902409$

C $0.492471-2.6801492 .099221$

O $3.234015-3.4898122 .489030$

C $0.076786-3.5819590 .979241$

C $-1.101679-3.4917130 .344866$

C $-1.515946-4.397460-0.779015$

C -1.793637 -3.649025 -2.064749

C $-3.053879-3.390793-2.452521$

C $-0.620093-3.287747-2.949425$

C $0.126759-2.075248-2.463699$

N $1.683119-1.9524011 .698596$

H 1.7045684 .3660001 .939907

H $2.594091 \quad 1.4378301 .827367$

H $2.8737783 .869787-0.076912$

H 4.6774091 .3838020 .067193

H 5.0711482 .8420140 .945411

H $5.9897442 .928104-3.103383$

H $6.1101094 .892259-1.932736$

H $4.7253025 .142763-0.867197$

H $6.2930724 .645959-0.193637$

H $4.4373720 .528087-1.931106$

H $4.6513640 .965101-3.606560$

H $\quad 6.104510-0.864408-3.204624$

H $7.140790 \quad 0.552773-3.399847$

H $7.2651650 .719255-0.858608$

H $9.069221-0.469018-2.146457$

H $8.120300-1.941354-2.128070$

H $10.606326-2.060097-1.057561$

H $7.624154-3.0850390 .197113$

H $10.4732560 .100163-0.152034$

H $10.140562-0.601227 \quad 1.415069$

H 9.0017390 .5615290 .707361

H $9.138844-2.9074811 .879494$

H $5.378443-3.1223320 .889131$

H $5.679210-2.3293652 .440484$

H 5.9166250 .1756391 .885812

H $\quad 0.350013 \quad 1.927058-3.427288$

H $1.476242-0.141468-1.445802$

H $-1.5598910 .419239-3.666511$

H -1.525733 -1.172927 -4.391745

H $-0.3155030 .009342-4.872791$

H $3.992153-0.5860321 .885665$

H $3.753819-1.3092330 .280175$

H $-0.276215-1.9420772 .347950$

H $\quad 0.708408-3.2739592 .993038$

H $0.783535-4.3519790 .678132$

H -1.815517 -2.7337640 .655747$

H -2.415384 -4.937744 -0.455426

H $-0.762065-5.172094-0.967303$

H -3.265428 -2.883093 -3.387666

H -3.902897 -3.675515 -1.839882

H $-0.945148-3.162094-3.988122$

H $0.077874-4.134148-2.986753$

H $\quad 0.826795-2.289427-1.655989$

H $1.558581-1.1163821 .134663$

C $-0.3506562 .682261 \quad 3.392127$

O -0.5530421 .6294912 .423688$

C -1.454101 $2.115371 \quad 1.407411$

C $-2.261473 \quad 3.2021112 .095151$

C -1.188692 3.8787042 .928137

H -0.8215112 .5437800 .618944$

C -2.267050 $0.944142 \quad 0.851395$

H -2.744266 3.8848631 .390055

H -3.0329422 .7644982 .740705$

C -1.739486 4.7147644 .070766

H -0.6074544 .5346602 .269534$
C $-3.3521051 .366137-0.154521$

$\begin{array}{lllll}\text { O } & -1.359546 & 0.058368 & 0.189451\end{array}$

H $-2.7092980 .377796 \quad 1.678179$

H -4.0980841 .9969710 .340922$

O $-2.7553512 .194022-1.162771$

C $-4.0553360 .196400-0.870182$

C $-4.657189-0.8827160 .047652$

H -3.344674 -0.290435 -1.548447

O $-5.0769350 .725033-1.717906$

H -5.145922 -1.624960 -0.593263

H $-3.836376-1.4025930 .551996$

H -2.3658105 .5265523 .686391$

H -2.3509074 .1125454 .751016$

H -0.9257155 .1600174 .652242$

C 1.1528382 .9449313 .557643

H $-0.650991-0.1573890 .819191$

H $-2.0512641 .675170-1.588217$

H $-5.6871801 .237102-1.160802$

H 1.6254522 .0336423 .946401

H 1.3207563 .7302874 .303160

H $-0.7387872 .295743 \quad 4.342929$

C -7.714188-0.585368 -0.176244

O -6.8028570 .2748020 .518789$

C $-5.670061-0.3758051 .101234$

C $-6.136801-1.4558932 .089206$

C -7.099242 -2.441912 1.432650

C -8.239681 $-1.689400 \quad 0.749060$

H -7.211217 -1.029938 -1.043498

C $-8.8686590 .284881-0.687899$

H $\quad-5.156767 \quad 0.386771 \quad 1.697895$

O $-5.011317-2.1418822 .626582$

H -6.648436 -0.9685882 .929663$

H $-6.583259-3.1010070 .724520$

O $-7.669070-3.2883572 .433369$

H -8.900806 -1.258622 1.512139

O $-9.014665-2.610490-0.016193$

C $-8.4244941 .150584-1.842356$

H $-9.705892-0.321730-1.051603$

H -9.2646750 .9285110 .107150$

H $-5.331059-2.7243693 .335715$

H $-6.956725-3.8496912 .783016$

H -9.318416 -3.307834 0.590100

O $-8.0548112 .382225-1.389307$

O $-8.3881190 .767131-3.004593$

C $-7.6013453 .297001-2.392104$

H $-7.3066004 .225677-1.896351$

H -6.732099 $2.888900-2.916640$

$\mathrm{H}-8.4097913 .515155-3.096292$

SCF Energy (B3LYP/6-31G**//MMFF $)=-3245.90875492$

$02 \_125$

MMFF Geometry

C $0.9848240 .423158-2.595239$

C $1.981116-0.346920-3.058133$

C $2.308329-1.761274-2.642641$

O $1.340094-2.274268-1.702320$

C $3.690147-1.811582-1.971510$

C $4.255624-3.213601-1.843153$

C $4.548540-3.823386-0.678001$

C $4.576320-3.908419-3.143252$

C $4.348405-3.3159580 .722846$

C $5.661275-2.8218971 .351544$

C $6.061650-1.4182400 .871351$

C $7.450957-1.0334571 .379367$

C $7.815136 \quad 0.407875 \quad 0.988317$

O $5.089987-0.5032981 .384820$

O $\quad 8.974927 \quad 0.7898991 .743038$

C 6.6670081 .3459951 .415973

C $8.2017230 .521391-0.490996$

O 6.8896432 .6743250 .947824

C 5.2661560 .8577280 .975921

C 4.1754841 .7026251 .676877

O $5.1343141 .005701-0.438259$

C $0.346418-3.062954-2.186266$

O $0.168189-3.383061-3.353831$

C $-0.531007-3.563131-1.104303$

C $-0.555814-3.0562180 .138245$

C $-1.391543-3.5333021 .244848$ 
C $-2.268067-4.7327590 .989969$

C 2.7517591 .1447251 .529695

C 1.7810011 .8829732 .435892

C -0.3854811 .6039383 .614795$

O $1.956205 \quad 3.045732 \quad 2.791916$

C -1.021832 $0.467428 \quad 4.344898$

C $-2.261798 \quad 0.019697 \quad 4.095000$

C $-2.911855-1.0916194 .866234$

C $-3.191143-2.3128674 .020728$

C $-4.442524-2.6189563 .640187$

C $-2.036666-3.2397953 .713428$

C -1.327336 -2.8914802 .430897$

N $0.692822 \quad 1.097576 \quad 2.788277$

H $\quad 0.3220550 .051125-1.819529$

H $2.6162980 .053358-3.846957$

H $2.314394-2.369764-3.554189$

H $3.620508-1.330446-0.990903$

H $4.412624-1.219612-2.549698$

H $4.989713-4.819040-0.721821$

H $5.157580-4.824686-2.992548$

H $3.659168-4.186616-3.670769$

H $5.168301-3.252362-3.789914$

H $3.568731-2.5499950 .778703$

H $3.972872-4.1571111 .319638$

H $5.519191-2.7964152 .439913$

H $6.466646-3.5377891 .145378$

H $6.046758-1.392871-0.222654$

H $8.209421-1.7346321 .009916$

H $7.476002-1.1133612 .475373$

H $9.688140 \quad 0.162377 \quad 1.534483$

H 6.6849871 .3982872 .513881

H $9.056894-0.128021-0.713924$

H $8.5263231 .538598-0.736929$

H $7.3916340 .244857-1.169044$

H 7.7706152 .9465381 .257243

H 4.4001811 .7509482 .750860

H 4.1951092 .7312391 .294680

H $4.1940971 .096663-0.660072$

H $-1.180401-4.380976-1.400122$

H $\quad 0.095628-2.2173880 .377978$

H -2.927974 -4.5572790 .133830$

H $-2.921305-4.9834361 .828103$

H $-1.654672-5.6166650 .782572$

H $2.394523 \quad 1.249274 \quad 0.500325$

H 2.7322860 .0806391 .789854

H -1.0906102 .1185752 .959074$

H $\quad 0.005776 \quad 2.338365 \quad 4.326370$

H $-0.436308 \quad 0.0038295 .135263$

H -2.851956 0.4879183 .310554

H -3.845687 -0.6989655 .289511$

H $-2.303140-1.3850305 .730782$

H -4.655469 -3.5152803 .067431$

H $-5.282057-1.9785803 .889406$

H -2.380735 -4.279544 3.714796

H -1.307247 -3.2011974 .533088$

H - $0.661856-2.0328062 .517377$

H $\quad 0.5415750 .2257142 .288227$

C $0.6482322 .869473-2.067620$

O $-0.5303092 .697869-1.251086$

C -1.244839 $3.945314-1.199998$

C $-0.2371445 .011080-1.608287$

C $0.553216 \quad 4.275347-2.668239$

H $-2.0349313 .889811-1.958711$

C $-1.864730 \quad 4.167007 \quad 0.181674$

H $-0.7194075 .918937-1.982816$

H $0.4191675 .292162-0.776611$

C $1.9024434 .906375-2.968186$

H $-0.0405334 .250943-3.592126$

C -2.793034 3.0380390 .669582

O -0.8161294 .3110501 .149019$

H -2.405156 5.1208360 .186739

H -2.202693 2.1436530 .898383

O $-3.357491 \quad 3.4653801 .917893$

C -3.931582 $2.648490-0.293360$

C $-4.947815 \quad 1.707892 \quad 0.383737$

H -3.501530 2.174278 -1.181237

O $-4.601592 \quad 3.836400-0.713224$

H -4.3989710 .9129740 .899605$
H -5.4954672 .2739341 .146905$

H $1.7746665 .926605-3.344205$

H $2.5307454 .954526-2.072306$

H $2.442004 \quad 4.331233-3.727143$

C $0.6862081 .788763-3.149102$

H -0.2629113 .5124801 .102247$

H $-3.925944 \quad 4.2331671 .734833$

H -5.1681913.606011-1.468265

H $1.4278532 .055258-3.911360$

H $-0.2893801 .735725-3.647925$

H $1.5155732 .785927-1.400846$

C $-4.758703-0.912285-1.179070$

O $-5.3568900 .313831-1.620199$

C $-5.9765591 .095530-0.593063$

C -7.0750020 .2731500 .095379$

C $-6.530207-1.0472570 .630700$

C $-5.789981-1.802929-0.470967$

H $-3.920514-0.693993-0.506523$

C $-4.203915-1.630279-2.419908$

H $-6.4773281 .928856-1.100125$

$\begin{array}{lllll}\text { O } & -7.665439 & 1.023567 & 1.150861\end{array}$

H $-7.8696190 .063021-0.632202$

H -5.881638 $-0.881273 \quad 1.497332$

O $-7.613748-1.8500201 .105020$

H -6.515989 - $2.186698-1.199225$

$\begin{array}{lllll} & -6 & -5.125786 & -2.927510 & 0.098685\end{array}$

C $-3.061640-0.879043-3.067765$

H $-3.830340-2.626360-2.159242$

H $-5.002836-1.727206-3.165534$

H $-8.3271870 .453756 \quad 1.578834$

H $-8.217715-2.0111530 .360157$

H $-5.795141-3.4554170 .566902$

O $-2.856851-1.384791-4.320167$

O $-2.421064 \quad 0.020037-2.542344$

C $-1.817330-0.762267-5.080744$

H $-0.844097-0.995507-4.642347$

H $-1.850477-1.167255-6.095727$

H $-1.9714040 .319827-5.131794$

SCF Energy (B3LYP/6-31G**//MMFF) $=-3245.92014779$

02126

MMFF Geometry

C $-3.306636-0.675345-2.890908$

C $-2.233648-1.402462-2.541572$

C $-0.787278-1.059150-2.803182$

O $-0.6906140 .337636-3.172043$

C $-0.172987-1.931527-3.914373$

C $0.081379-3.373421-3.521315$

C $1.207925-3.788876-2.907740$

C $-0.974228-4.367384-3.926979$

C $2.343860-2.902769-2.475906$

C $3.338596-3.631770-1.562136$

C $4.479346-2.706228-1.110676$

C $5.520608-3.491942-0.311479$

C $6.636260-2.583193 \quad 0.222188$

O $3.924243-1.665438-0.297506$

O $7.371909-3.3472291 .189518$

C $5.993176-1.3844740 .950027$

C $7.634397-2.210491-0.879900$

O $6.988616-0.4467041 .354666$

C $4.875461-0.6853360 .138474$

C $4.149717 \quad 0.339966 \quad 1.043275$

$\begin{array}{lllll}\text { O } & 5.466273 & 0.003323 & -0.965228\end{array}$

C $0.4911370 .970108-2.945301$

$\begin{array}{lllll}\text { O } & 1.544416 & 0.461842 & -2.598279\end{array}$

C $0.2621452 .420294-3.128179$

C $1.1934653 .283729-2.696659$

C $1.0814344 .743227-2.678827$

C $-0.1401115 .354284-3.313472$

$\begin{array}{llll}\text { C } & 2.882599 & 0.951794 & 0.432094\end{array}$

$\begin{array}{llll}\text { C } & 2.213739 & 1.935420 & 1.378888\end{array}$

C $0.137380 \quad 3.296041 \quad 1.618640$

$\begin{array}{lllll}\text { O } & 2.736217 & 2.321972 & 2.421271\end{array}$

$\begin{array}{llll}\text { C } & 0.079799 & 4.569120 & 0.835459\end{array}$

C $0.794046 \quad 5.664276 \quad 1.137417$

C $0.7219886 .958393 \quad 0.374275$

C $2.0138667 .282831-0.345861$

$\begin{array}{llll}\text { C } 3.015039 & 7.920413 & 0.283957\end{array}$ 
C $2.1119836 .932889-1.816828$

C $2.0473735 .450073-2.054248$

N $0.971374 \quad 2.3422130 .911529$

H -3.181479 $0.251343-3.444142$

H -2.396666 -2.328105 -1.996312

H $-0.250764-1.196750-1.855467$

H $0.769746-1.483989-4.254505$

H $-0.808670-1.895931-4.810042$

H $1.322485-4.852030-2.704245$

H -1.129256 -4.333814 -5.010328

H $-0.697546-5.394765-3.666943$

H -1.925819 -4.152002 -3.433856

H $2.875811-2.545826-3.365689$

H $1.947548-2.033864-1.943082$

H $2.803529-4.013839-0.683368$

H $3.755204-4.496003-2.094478$

H $4.936555-2.262087-2.002747$

H $5.944058-4.305313-0.913945$

H $5.030034-3.9780740 .543912$

H $8.033100-2.7569061 .590130$

H $5.554304-1.7755211 .878260$

H $8.087886-3.114454-1.304103$

H $8.466405-1.620765-0.479494$

H $7.179020-1.651177-1.699817$

H 7.2504690 .0635510 .569405

H $3.858729-0.1509461 .980504$

H 4.8411621 .1514501 .304283

H $4.8589050 .696560-1.266956$

H $-0.6880642 .735848-3.543350$

H $2.1104672 .885554-2.265073$

H $-0.2206255 .048259-4.362587$

H -0.136929 $6.445677-3.311070$

H - $-1.0466615 .038799-2.785082$

H $3.1237931 .495979-0.485677$

H 2.1622210 .1631640 .190061

H $\quad 0.518916 \quad 3.4568722 .632124$

H -0.8644752 .8646021 .698175$

H $-0.6009384 .591426-0.011695$

H 1.4665395 .6370661 .992006

H $\quad 0.4853307 .7588411 .087479$

H $-0.1104996 .954017-0.340423$

H $3.9342148 .179545-0.230589$

H 2.9368308 .1946851 .330802

H $3.0619747 .291036-2.234718$

H $1.3353977 .483770-2.353569$

H $2.8906484 .907625-1.623718$

H 0.6259561 .9696110 .031809

C -5.420411 -0.002941 - 1.719842

O $-4.7364240 .157533-0.462743$

C $-5.710427 \quad 0.2946800 .588564$

C $-7.0611170 .457068-0.100339$

C $-6.860294-0.364856-1.358742$

H $-5.710263-0.6538381 .138677$

C -5.3191951 .4383751 .532076$

H -7.8878090 .0987290 .520505$

H $-7.2660251 .501779-0.360740$

C -7.869289 -0.059810 -2.451912

H $-6.926019-1.429188-1.094961$

C -3.866352 1.3703792 .044614

O -5.4616232 .6789590 .826266$

H -6.0236941 .4789562 .371330$

H -3.1790281 .5732641 .213789$

O -3.7109052 .4480342 .976887$

C -3.4916880 .0420612 .727840$

C -2.0348130 .0199303 .219628$

H -3.629792 -0.7757362 .018257$

O $-4.370576-0.1999913 .824139$

H -1.3829750 .2729342 .376552$

H -1.895925 0.7945413 .982741

H -8.886145 -0.273965 -2.107024

H $-7.830616 \quad 0.992923-2.751447$

H -7.679049 -0.669971 -3.340418

C $-4.719143-1.072247-2.558930$

H $-5.220906 \quad 3.391677 \quad 1.442897$

H -2.759231 2.5867603 .115010

H -4.2803750 .5449004 .442970$

H -5.262908 -1.229817 -3.497040

H $-4.722450-2.024366-2.015688$
H $-5.3816140 .968486-2.230164$

C $-1.044437-2.5065131 .799660$

O $-1.862354-2.4475252 .973487$

C -1.613386-1.333648 3.837149

C $-0.154228-1.3634494 .312424$

C $0.813130-1.3933773 .133758$

C $0.446127-2.5170842 .168577$

H -1.271929-1.652712 1.149352

C -1.399597 -3.800394 1.046038

H -2.245132 -1.487494 4.721059

O $0.141602-0.2356515 .129243$

H $0.013768-2.2590384 .924327$

H $0.848669-0.4285052 .617034$

O $2.135881-1.6370903 .617614$

H $0.713955-3.4808702 .620605$

O $1.215198-2.3794010 .976617$

C $-2.793098-3.7945860 .456213$

H $-0.700468-3.9545320 .216040$

H $-1.342173-4.653507 \quad 1.732807$

H -0.477285 -0.248224 5.879294

H $2.347825-0.9268524 .247678$

H $2.153093-2.3605161 .234175$

O $-2.989551-4.972387-0.209279$

O $-3.604217-2.8857190 .555313$

C $-4.267213-5.126166-0.835131$

H $-5.074859-4.940755-0.120412$

H -4.344406 -4.450436 -1.691258

H $-4.347801-6.156024-1.192883$

SCF Energy $(B 3 L Y P / 6-31 G * * / / M M F F)=-3245.93219301$

02127

MM̄FF Geometry

C $0.209900-3.672076 \quad 1.209528$

C $0.537327-2.3940221 .452410$

C $0.403885-1.7335492 .798253$

O $-0.368208-0.5326442 .560812$

C $1.770738-1.336573 \quad 3.385710$

C $2.697112-2.5106203 .627299$

C $3.704215-2.8754802 .808272$

C $2.424852-3.2886864 .888143$

C $4.099276-2.2338671 .507206$

C $5.593840-1.8861231 .476152$

C $5.927159-0.9889950 .275906$

C $7.437937-0.8560870 .091358$

C $7.7795920 .104780-1.057117$

O $5.364737 \quad 0.298246 \quad 0.543474$

O $9.179144 \quad 0.416465-0.971376$

C $7.0126181 .429535-0.850804$

C $7.569135-0.553583-2.425681$

O $7.1577642 .287052-1.980376$

C $5.5112901 .252526-0.514793$

C $4.9284712 .587463-0.000631$

O $4.7432110 .887642-1.662131$

C $-1.091419-0.0098833 .580341$

O $-1.151654-0.4354564 .725325$

C $-1.8347641 .199216 \quad 3.148042$

C -1.8741761 .6307521 .877083$

C $-2.5833982 .812183 \quad 1.377581$

C -3.3468183 .6427722 .374195$

C 3.4592832 .4892490 .433034

C 2.9643293 .7844371 .054881

C 0.9777564 .7307572 .230693

O $3.6301524 .816021 \quad 1.082164$

C -0.0126575 .4113641 .333332$

C -0.1377805 .2822610 .002723$

C $-1.2054085 .971512-0.800030$

C $-2.1058994 .992909-1.523247$

C $-2.0412284 .846666-2.857297$

C $-3.1287584 .231940-0.703841$

C $-2.511598 \quad 3.089730 \quad 0.058475$

N 1.6836923 .6483521 .571758

H -0.164265 -4.272614 2.036684

H $0.922354-1.7674350 .652779$

H - $0.143321-2.384863 \quad 3.491378$

H $1.615967-0.8091774 .336815$

H $2.237628-0.5735342 .751943$

H $4.296845-3.7478703 .080452$

H $3.129535-4.1152265 .027779$ 
H $2.506485-2.6345985 .762391$

H $1.417216-3.7153984 .865401$

H $3.882382-2.9400990 .696009$

H $3.506971-1.3396761 .299475$

H $5.867372-1.3684472 .404902$

H $6.176353-2.8144591 .428408$

H $5.462633-1.411225-0.622877$

H $7.898630-1.837037-0.079312$

H $7.888333-0.4686711 .015940$

H $9.675922-0.418542-1.015260$

H $7.4902271 .947207-0.006786$

H $8.224144-1.426501-2.534988$

H $7.8412430 .127470-3.239330$

H $6.543706-0.893425-2.585264$

H $8.1107632 .398902-2.138608$

H 5.5176702 .9306400 .859996

H $5.0092653 .356299-0.779650$

H $5.3389690 .788350-2.421025$

H -2.362870 1.7147203 .943803

H -1.3357931 .0698901 .114666$

H -4.1214943 .0409002 .862239$

H -3.8545304 .5014811 .931227$

H -2.672269 4.0372653 .142256

H $2.8235642260606-0.429857$

H 3.3349631 .6910831 .174067

H 1.6920485 .4640442 .618282

H 0.4471284 .2847973 .078383

H $-0.713024 \quad 6.0644981 .851393$

H $0.5475064 .647304-0.552371$

H $-0.7072296 .635833-1.517666$

H -1.822063 $6.623827-0.169102$

H -2.698345 $4.161733-3.383137$

H -1.325974 $5.403907-3.453215$

H $-3.8966023 .806587-1.363458$

H -3.656145 $4.941695-0.061718$

H $-1.9410282 .410828-0.577168$

H $\quad 1.1897012 .7693191 .447141$

C $0.686552-3.573930-1.336784$

O $-0.320476-2.575895-1.594271$

C $-0.566718-2.508297-3.009722$

C $0.524119-3.343270-3.670777$

C $0.773810-4.404319-2.617314$

H $-1.535320-2.991850-3.183154$

C - $0.622885-1.047695-3.470203$

H $\quad 0.203456-3.757398-4.631592$

H $1.435951-2.760111-3.844395$

C $2.094003-5.134862-2.792696$

H $-0.048036-5.132101-2.651455$

C -1.622997-0.163892 -2.698112

O $0.673561-0.453463-3.313744$

H $-0.841653-1.009477-4.543468$

H -1.264722 $0.009650-1.675787$

O $-1.6466161 .119684-3.337266$

C $-3.063100-0.707138-2.644281$

C $-4.0399910 .300329-2.010987$

H -3.074831 -1.637099 -2.068960

O $-3.510268-1.022697-3.962155$

H -3.617382 $0.641752-1.062693$

H -4.128395 $1.175454-2.665872$

H $2.122383-5.651054-3.757976$

H $2.943025-4.444019-2.755814$

H $2.234448-5.882770-2.006173$

C $0.293235-4.399607-0.107934$

H $0.923124-0.535931-2.377124$

H $-0.735097 \quad 1.459209-3.338845$

H $-3.464480-0.207793-4.491286$

H $1.000404-5.2278680 .017640$

H $-0.694114-4.843258-0.286051$

H $1.633455-3.046625-1.162806$

C $-5.100960-1.1696080 .443134$

O $-5.431406-1.432718-0.926720$

C $-5.451501-0.282989-1.780318$

C $-6.4704370 .737643-1.254087$

C $-6.2022441 .104471 \quad 0.202372$

C $-6.079117-0.156767 \quad 1.055413$

H $-4.074316-0.7906120 .509899$

C -5.171232 -2.505036 1.199586

H $-5.817331-0.629617-2.754929$
O $-6.4564081 .908863-2.063564$

H $-7.4771440 .306617-1.331040$

H $-5.314364 \quad 1.7388310 .289688$

O $\quad-7.2989001 .8869480 .679839$

H -7.072742 -0.6103491 .160484$

O $\quad \begin{array}{llll}-5.650139 & 0.206716 & 2.366320\end{array}$

C $-4.068040-3.4595310 .796365$

H $-5.079424-2.3470512 .279537$

H $-6.130735-2.9914280 .985188$

H $-7.0945962 .534869-1.680441$

H -7.1303432 .0781581 .618547$

H -4.7639390 .5994642 .291682$

O $-4.389246-4.714215 \quad 1.230907$

O $-3.046457-3.1459320 .202211$

C $-3.435297-5.7374780 .930738$

H $-3.867185-6.6995291 .218868$

H $-3.217310-5.759614-0.141402$

H $-2.521611-5.5754271 .509424$

SCF Energy $\left(B 3 L Y P / 6-31 G^{* *} / / M M F F\right)=-3245.92909728$

02128

MM̄FF Geometry

C $-0.4594363 .061671-0.407028$

C -1.392188 3.7404120 .280673

C $-2.054753 \quad 3.3087421 .568930$

O

C -3.5839203 .4641741 .447950$

C -4.3312283 .1699042 .735601$

C -4.9863402 .0183862 .987170$

C -4.3407554 .2871923 .744912$

C -5.0430600 .8091602 .096854$

C -6.404495 0.6731741 .404082

C $-6.354933-0.1506320 .106856$

C $-5.834525-1.5743780 .317208$

C $-5.696971-2.336145-1.010979$

O $-5.5200590 .563503-0.811163$

O $-4.916649-3.516216-0.764754$

C $-4.892526-1.464173-1.999300$

C -7.051766 -2.818450 -1.540573

O $-4.841054-2.068715-3.288571$

C $-5.422230-0.019402-2.116590$

C $-4.5030820 .902774-2.956470$

O $-6.700436-0.038666-2.763244$

C -1.4024571 .5575623 .088289$

O -1.2281532 .3025374 .043057$

C -1.2438390 .0892253 .208952$

C -1.544506 -0.779792 2.230971

C $-1.425809-2.2375422 .304069$

C $-0.874448-2.8344983 .572249$

C $-3.0681561 .044442-2.435299$

C $-2.1070850 .049161-3.061563$

C $-0.023023-1.224031-2.587939$

O $-2.252833-0.389531-4.200204$

C $-0.502372-2.597098-2.234777$

C $0.141163-3.414719-1.388980$

C $-0.336297-4.794881-1.039286$

C $-0.520506-4.9944200 .449550$

C $0.373689-5.697377 \quad 1.164948$

C $-1.785014-4.4688381 .092001$

C -1.799970-2.971582 1.235266

N $-1.045159-0.260878-2.224100$

H -0.103106 $2.102470-0.045613$

H -1.706819 $4.709082-0.103974$

H -1.6690933 .9538262 .367606$

H -3.9552762 .8234440 .638920$

H -3.8322654 .4881221 .136504$

H $-5.517707 \quad 1.9156193 .931701$

H -4.9148774 .0317924 .641917$

H $-3.321574 \quad 4.525750 \quad 4.063711$

H -4.7892545 .1868093 .311179$

H $-4.2343600 .828811 \quad 1.361677$

H -4.856547 -0.0788112 .713578$

H -7.1256770 .2274852 .099947$

H -6.7797851 .6734131 .148816$

H -7.373582 -0.178185 -0.297999

H $-6.479731-2.1234731 .013910$

H -4.842796 -1.546239 0.787150

H $-5.366565-4.035182-0.076132$ 
H $-3.862515-1.437454-1.623941$ H -7.522497 -3.500049-0.821833 H -6.931791 -3.394360 -2.464842 H -7.754695 -2.004599-1.729157 H -4.485495 $-2.966547-3.173538$ H $-4.5243810 .594914-4.008650$ H $-4.9466141 .908793-2.938472$ H $-6.8677410 .839800-3.143131$ H -0.878209 -0.2443924 .174927$ H $-1.920487-0.3987391 .283086$ H $\quad 0.122863-2.4336413 .785467$ H $-0.767401-3.9204433 .530655$ H -1.532142 -2.6114454 .419484$ H -2.689958 $2.036832-2.710937$ H -3.037618 $0.979703-1.342541$ H $0.891937-0.950360-2.053260$ H $\quad 0.167937-1.163339-3.664145$ H -1.421530 -2.938029 -2.705952 H $1.068112-3.078503-0.932922$ H $0.402524-5.508640-1.426683$ H -1.276029 $-5.034871-1.552486$ H $\quad 0.235295-5.8769452 .225691$ H $1.265850-6.1100980 .706145$ H -1.960883 -4.949455 2.061512 H -2.646291 -4.7701240 .482316$ H -2.184584 -2.452407 0.357163 H -1.044671 $0.086623-1.269801$ C $1.5652054 .049035-1.619810$ O $2.4879652 .979476-1.341276$ C $3.6545073 .554390-0.710535$ C $3.3749225 .050471-0.539150$ C $1.8606225 .108019-0.564150$ H 3.7358963 .0971560 .280768 C $4.9153123 .270532-1.538612$ H 3.8009005 .4406760 .390834 H $3.7974575 .639748-1.361530$ C $1.3097696 .485011-0.888317$ H 1.4946604 .7913700 .420448 C $5.2642821 .778062-1.704726$ O $4.7262483 .825314-2.848130$ H $5.7667103 .805568-1.100380$ H $4.4942751 .280282-2.306801$ O $6.4754071 .733751-2.472192$ C $5.4712321 .023449-0.375195$ C $6.057239-0.389402-0.571459$ H 4.5073930 .9592800 .140269 O $\quad 6.3543561 .782407 \quad 0.450882$ H $5.436631-0.928150-1.295681$ H $7.063020-0.308576-1.001137$ H $1.6324767 .214514-0.138469$ H $1.6540246 .834756-1.867414$ H $\quad 0.215472 \quad 6.476027-0.900310$ C $0.1240743 .543184-1.705631$ H $5.5368693 .649282-3.356117$ H $6.5551280 .839642-2.845016$ H 6.2736351 .4440931 .358416 H $0.0810242 .705335-2.412584$ H $-0.5062924 .336901-2.125749$ H $1.8360064 .430588-2.614458$ C $3.906848-1.9977500 .863659$ O $4.946608-1.2223511 .471933$ C $6.174984-1.1953360 .742078$ C $6.713476-2.6232420 .569477$ C $5.668731-3.562862-0.027799$ C $4.347973-3.4583560 .734829$ H $3.670312-1.589823-0.126934$ C $2.659562-1.8528051 .739577$ H $6.895073-0.6627881 .375540$ O $7.876758-2.636562-0.250859$ H $7.007973-3.0179691 .550725$ H $5.519054-3.368128-1.096437$ O $6.136743-4.9099790 .072191$ H $4.459098-3.9105121 .728666$ O $3.349832-4.1953590 .036032$ C $2.126469-0.4426351 .634910$ H $1.856744-2.5293681 .429203$ H $2.880076-2.0764672 .790339$ H $8.532388-2.0491130 .162430$
H $\quad 6.993415-4.952855-0.386524$

H $3.670566-5.109264-0.051508$

O 2.5692210 .2978142 .688703

O $1.441364-0.0480650 .700762$

C 2.2358101 .6916632 .719437

H 3.1632702 .2671362 .658317

H 1.5704201 .9951341 .907419

H 1.7531671 .9063953 .676304

SCF Energy (B3LYP/6-31G**//MMFF)= -3245.91572785

$02 \_129$

MM̄FF Geometry

C $2.7932190 .131067-3.229034$

C $1.7740340 .344828-2.384456$

C $0.343207-0.037125-2.635755$

O $-0.4011941 .196703-2.483653$

C $-0.189173-1.039993-1.598321$

C $0.250315-2.464785-1.840661$

C $-0.397984-3.321812-2.652335$

C $1.461143-2.916573-1.071572$

C $-1.626514-3.044294-3.473254$

C $-2.775272-4.007603-3.153609$

C $-3.362692-3.846665-1.740368$

C $-4.412237-4.935971-1.503443$

C $-5.099213-4.781776-0.143715$

O $-3.962886-2.550574-1.641986$

O $-6.255205-5.633786-0.131663$

C $-5.608718-3.333690-0.012529$

C $-4.199666-5.2575221 .003099$

O $-6.122015-3.0991421 .297918$

C $-4.554689-2.252794-0.364447$

C $-5.261199-0.879982-0.505763$

O $-3.562205-2.1380900 .653888$

C $-1.5389581 .372892-3.197939$

O $-2.0398470 .584462-3.986450$

C $-2.1704142 .673937-2.873643$

C $-1.5296503 .652132-2.214406$

C $-2.0874984 .945946-1.815992$

C $-3.5515565 .184090-2.074746$

C $-4.3234100 .335087-0.532105$

C -4.0645500 .9188460 .850994$

C -3.0122982 .8717721 .976384$

O $-4.4079520 .364487 \quad 1.892135$

C -3.1756794 .3375841 .731993$

C -2.2005745 .2377861 .930747$

C $-2.378756 \quad 6.7170141 .733659$

C -1.3475497 .3154650 .802838$

C -0.2874287 .9860571 .284481$

C $-1.5897057 .204835-0.686961$

C $-1.2691415 .833712-1.213462$

N -3.4059182 .1393750 .786434$

H $2.620326-0.391504-4.165673$

H $1.9708500 .871598-1.455735$

H $0.218461-0.416270-3.657780$

H -1.284286 -0.994561-1.567077

H $0.103701-0.727571-0.588032$

H $-0.003393-4.331815-2.755041$

H $1.700463-3.970007-1.251148$

H $2.338279-2.329171-1.353292$

H $1.291328-2.7977460 .003329$

H $-1.974126-2.013764-3.371794$

H -1.353742 -3.169935 -4.528449

H -3.568012 -3.828253 -3.891851

H $-2.431151-5.039946-3.296282$

H -2.558786 -3.925728 -1.000660

H $-3.965129-5.934119-1.594555$

H -5.180371 -4.880234 -2.287869

H $-5.958610-6.544936-0.298942$

H $-6.456605-3.220632-0.703171$

H $-3.965032-6.3227850 .888503$

H $-4.707663-5.165724 \quad 1.969233$

H -3.251223 -4.7185221 .056339$

H $-6.797934-3.7771771 .469812$

H $-5.828907-0.884993-1.446064$

H $-5.992108-0.7437030 .300296$

H $-3.811375-2.7063781 .399970$

H -3.194995 $2.783330-3.213482$

H $-0.4917023 .496794-1.923795$ 
H -3.753295 $5.194080-3.151566$ H -3.918795 $6.130344-1.673088$ H -4.158241 4.397658 -1.611654 H -3.367062 $0.089728-1.003309$ H -4.800314 $1.123266-1.128446$ H -1.975994 2.6021702 .198532 H -3.634785 2.5655422 .823073 H $-4.1544504 .678097 \quad 1.402272$ H -1.229564 4.8996532 .286179 H -2.3119807 .1929362 .720788$ H $-3.382914 \quad 6.9529861 .360011$ H 0.4427318 .4402560 .622655 H -0.1227328 .0908322 .351676$ H $-0.9527287 .918677-1.226159$ H -2.613590 $7.519597-0.904293$ H $-0.2220845 .558972-1.075953$ H $-3.0328782 .445042-0.106897$ C $5.173124-0.458503-2.606266$ O $4.834973-1.005172-1.314682$ C $6.014315-1.028793-0.490825$

C $7.189209-0.870018-1.446989$

C $6.6049320 .069595-2.480696$ H $5.961013-0.1471140 .159216$ C $6.057189-2.3004060 .362872$ H $8.077405-0.466847-0.951174$ H $7.465532-1.820697-1.917406$ C $7.3720850 .085526-3.791759$ H $6.5962531 .085036-2.061760$ C $4.788088-2.5691321 .192608$ O $6.245271-3.435698-0.494763$ H $6.935754-2.2746611 .017606$ H $3.964268-2.8458600 .525135$ O $5.041296-3.7219772 .008028$

C $4.328584-1.4171862 .107917$

C $3.079699-1.8153142 .914712$ H $4.096843-0.5350321 .505268$

O $5.365170-1.0660423 .020502$ H $2.305743-2.1342052 .208946$ H $3.319339-2.6825683 .542113$ H $8.4026850 .418394-3.630241$ H $7.407876-0.909478-4.247992$ H $6.9051730 .768148-4.508664$ C $4.182370 \quad 0.646718-2.971974$ H $5.512024-3.445114-1.133982$ H $5.311377-4.4435661 .414302$ H $6.099168-0.6892992 .507357$ H $4.5167191 .169126-3.875830$ H $4.1561261 .391712-2.166492$

H $5.119959-1.283776-3.327491$

C 1.2016490 .4873792 .213619

O 2.2767390 .5276203 .157644

C $2.535100-0.7026523 .839874$

C $1.283643-1.1224994 .621962$

C $0.072044-1.2520443 .702479$

C -0.1068010 .0196552 .874852$

H $1.473404-0.1755301 .383551$

C 1.0129641 .9084561 .652920

H $3.321088-0.4866254 .574314$

O $1.517203-2.3532055 .298523$

H $1.070361-0.3678275 .390172$

H $\quad 0.150179-2.1402883 .065192$

O $-1.084154-1.4481644 .520795$

H $-0.501153 \quad 0.814015 \quad 3.521879$

O $-1.073849-0.2170681 .853943$

C 2.1934222 .4112350 .851109

H $\quad 0.1393891 .9290390 .991031$

H 0.8610292 .6163792 .476505

H $\quad 0.694010-2.5931545 .757574$

H -1.836839 -1.6211273 .930598$

H -1.922771 -0.4066132 .287302$

O $1.845321 \quad 3.593491 \quad 0.259218$

$\begin{array}{lllll}\text { O } & 3.278204 & 1.855814 & 0.756157\end{array}$

C $2.8581804 .204455-0.545606$

H $3.0631503 .584273-1.422716$

H $2.4850075 .175392-0.881921$

H 3.7696434 .3632620 .038594

SCF Energy $\left(B 3 L Y P / 6-31 G^{* *} / / M M F F\right)=-3245.91860431$
$02 \_13$

MMFF Geometry

C $1.465312-4.420419-0.113872$

C $0.250774-3.864697-0.241267$

C $-0.391575-3.564594-1.571750$

O $-0.957579-2.231823-1.507562$

C $-1.528198-4.562318-1.847859$

C $-2.144292-4.397856-3.224660$

C $-3.352288-3.853064-3.471746$

C $-1.315621-4.931846-4.365810$

C $-4.326378-3.279578-2.481761$

C $-4.539797-1.779389-2.719310$

C $-5.421363-1.152755-1.629951$

C $-5.6802850 .324176-1.925995$

C $-6.4794961 .000309-0.802257$

O $-4.731893-1.280077-0.383284$

O $\quad-6.3892062 .421943-0.989870$

C -5.8118970 .6861990 .556446$

C -7.969934 $0.649461-0.884802$

O $-\begin{array}{llll}-6.621013 & 1.140045 & 1.637839\end{array}$

C $-5.465772-0.8066830 .754745$

C -4.591931-1.076907 2.001511

O $-6.646563-1.5915910 .965871$

C $-0.094551-1.184689-1.624757$

O $1.111112-1.244675-1.808117$

C $-0.8742310 .065569-1.474383$

C $-0.2312911 .242611-1.470350$

C $-0.8354552 .571614-1.354165$

C $-2.3336032 .653062-1.238659$

C -3.224567 -0.384509 2.020911

C -3.2139640 .9405402 .765172$

C -1.7052752 .8517353 .295039$

O $-4.171273 \quad 1.3628173 .407425$

C -1.811256 3.9511762 .287111

C -0.7891574 .7564241 .960340$

C -0.9086435 .9183631 .018628$

C $0.0527165 .836482-0.143566$

C $1.2344616 .475647-0.111709$

C $-0.4043095 .105522-1.383903$

C $-0.0249943 .650482-1.379017$

N $-1.989211 \quad 1.5838542 .651375$

H $1.993271-4.719242-1.017639$

H $-0.313243-3.5748650 .640613$

H $\quad 0.347159-3.602548-2.382107$

H -2.289018 -4.473897-1.065885

H $-1.149378-5.590431-1.766490$

H -3.691504 -3.807853 -4.506210

H $-1.835067-4.858857-5.327335$

H $-0.378431-4.374913-4.457112$

H -1.079252 -5.988306 -4.201352

H $-5.282499-3.805248-2.594431$

H -4.005786 -3.449461-1.451904

H -3.563985 -1.278318 -2.730609

H $-4.998564-1.624454-3.703768$

H $-6.364572-1.709493-1.582281$

H $-6.192440 \quad 0.445151-2.888642$

H $-4.720710 \quad 0.846837-2.030819$

H $-6.7273752 .629668-1.877813$

H -4.8844221 .2700640 .591130$

H -8.394613 $1.003878-1.831906$

H $-8.540121 \quad 1.151636-0.095746$

H -8.160905 -0.424062 -0.823477

H $-6.7868682 .088110 \quad 1.497782$

H $-5.162230-0.8658432 .914539$

H -4.399543 -2.159529 2.027899

H $-7.403147-0.992577 \quad 1.069928$

H -1.949750 -0.015605 -1.373346

H $0.8513651 .236711-1.581866$

H -2.804726 $2.309902-2.165530$

H -2.701094 $3.662357-1.040731$

H $-2.6928252 .034076-0.410033$

H -2.515873 -1.035765 2.548910

H -2.840071 -0.2367001 .007210$

H -0.7043302 .7819443 .730512$

H -2.417388 3.0274944 .107672

H -2.7870454 .1164331 .836594$

H 0.1786154 .6049252 .432927

H $\quad-0.7158976 .8330901 .595158$ 
H $-1.933514 \quad 6.0250630 .641955$ H $1.9125456 .450346-0.958341$ H 1.5518257 .0323570 .763712 H $0.0674515 .556967-2.267895$ H -1.474835 $5.268080-1.538648$ H $1.0502923 .487567-1.457693$ H -1.265734 1.1767952 .065420 C $1.862617-3.7230372 .333583$ O $2.142043-2.3582021 .961025$ C $2.704249-1.6704263 .093743$

C $2.578303-2.6236724 .277804$

C $2.688787-3.9731063 .594062$ H $3.768448-1.5239542 .877909$ C $2.021087-0.3131123 .285543$ H $3.357186-2.4563095 .028020$ H $1.606737-2.5277634 .776317$ C $2.190669-5.1279554 .444791$ H $3.740114-4.1470773 .328151$ C 2.0735320 .6075212 .048645 O $0.639887-0.5427013 .596198$ H $2.4520850 .194376 \quad 4.157006$ H $1.439616 \quad 0.193063 \quad 1.254917$ O 1.4729311 .8469562 .442152 C 3.4914300 .8766001 .504159 C 3.5029281 .9588830 .408232 H $3.897743-0.0600421 .108385$ O 4.3279801 .2929172 .582409 H $2.7494751 .698717-0.339758$ H 3.2101892 .9214470 .844975 H $2.776585-5.2068935 .366353$ H $1.140147-4.9965384 .724752$ H $2.279590-6.076633 \quad 3.906245$ C $2.217740-4.6538371 .170814$ H $\quad 0.2268880 .326677 \quad 3.735370$ H 1.2049452 .3148591 .633068 H $5.249197 \quad 1.2475892 .275987$ H $2.085561-5.7014491 .463381$ H $3.281717-4.5190940 .937782$ H $\quad 0.793455-3.7848502 .571698$ C $4.7341760 .336011-1.856993$ O $5.4271850 .918106-0.746735$ C $4.8757072 .149313-0.273102$ C $4.8731373 .189270-1.403899$

C $4.1758422 .666436-2.657511$

C $4.7269871 .296810-3.050233$

H $3.7058580 .093306-1.563043$

C $5.450712-0.971128-2.208791$

H 5.5641832 .5170550 .497441

O $4.2410934 .395531-0.989061$

H $5.9089153 .441872-1.665666$

H $3.0889052 .624973-2.526408$

O $4.4125943 .571052-3.738755$

H $5.742771 \quad 1.412246-3.449712$

O $3.917903 \quad 0.747889-4.088418$

C $5.199773-2.011711-1.144882$

H $5.095634-1.391524-3.156517$

H $6.531478-0.815886-2.311726$

H $4.7109894 .715520-0.200036$

H $4.0859474 .444091-3.461168$

H $3.9101151 .388863-4.819884$

O $6.269762-2.093456-0.304520$

O $4.162286-2.655410-1.058576$

C $6.142425-3.0383370 .762064$

H $7.060349-3.0081941 .354832$

H $5.300361-2.7705051 .405880$

H $6.016106-4.0485190 .361004$

SCF Energy (B3LYP/6-31G**//MMFF)= -3245.91211306

02 130

MM̄FF Geometry

C $-2.234586-4.0923331 .253370$

C -2.219846-3.107073 2.164124

C $-1.124128-2.8208823 .165038$

O $-0.765832-1.4120343 .140618$

C $0.144873-3.702253 \quad 3.110855$

C $1.088437-3.4655344 .276828$

C $2.262319-2.8066224 .206554$

C $0.650632-4.0709825 .587529$
C $2.887604-2.1327973 .016431$

C $4.236184-2.7691812 .665623$

C $4.922224-2.1702461 .426861$

C 5.305651-0.699591 1.603091

C $5.980624-0.1358710 .341925$

O $4.052086-2.3196840 .298899$

O $6.017624 \quad 1.2948630 .463893$

C $5.096785-0.460567-0.881985$

C $7.437254-0.5954130 .213372$

O $5.751381-0.093838-2.093328$

C $4.649135-1.937908-0.951890$

C $3.608603-2.212717-2.067637$

O $5.788146-2.759976-1.240358$

C $-0.234461-0.8505802 .023021$

O $-0.114817-1.3547690 .918871$

C 0.2459370 .4946912 .410143

C 1.1371691 .1319351 .637591

C 1.7306302 .4419571 .911348

C 1.2088093 .2164103 .092983

C $2.361347-1.321904-2.043449$

C $2.495101-0.086731-2.921235$

C $1.5933342 .216594-3.209504$

O $3.256944-0.030529-3.883597$

C $2.5522453 .144986-2.536447$

C $2.1766894 .258146-1.889208$

C $3.1364205 .182475-1.197735$

C 2.8793485 .2745160 .290321

C 2.2230126 .3242940 .812288

C 3.4550994 .1942991 .179766

C 2.6989722 .8974981 .090009

N $1.6348890 .930789-2.537378$

H -1.394602 -4.776331 1.176110

H -3.085148 -2.449177 2.226657

H -1.593346 -2.966389 4.146002

H $0.665303-3.5587362 .159104$

H $-0.139198-4.762844 \quad 3.127424$

H $2.849515-2.7167505 .120341$

H $1.402034-3.9482326 .374797$

H $-0.272795-3.6026315 .940751$

H $0.474402-5.1452455 .469509$

H $2.236008-2.1593922 .142040$

H $3.027152-1.0774243 .274527$

H $4.916797-2.6991603 .523743$

H $4.069665-3.8383612 .476344$

H $5.824241-2.7698911 .255621$

H $5.952747-0.5670962 .478695$

H $4.407880-0.0985501 .796695$

H $6.502519 \quad 1.513846 \quad 1.278064$

H $4.2084060 .177050-0.799780$

H $8.017905-0.2737561 .086500$

H $7.921577-0.133705-0.654151$

H $7.540991-1.6790760 .130070$

H $5.9903330 .846040-2.020601$

H $4.098959-2.185474-3.048341$

H $3.264503-3.249483-1.943577$

H $5.473594-3.601328-1.611014$

H -0.1157380 .9052623 .346151$

H 1.4837090 .6407810 .729997

H 1.6346404 .2184503 .178225

H 1.4352972 .6908704 .027130

H 0.1243383 .3500073 .018568

H $1.514983-1.888321-2.451723$

H $2.100348-1.042381-1.017936$

H $0.5638512 .580953-3.159567$

H $1.8680372 .094419-4.262006$

H $3.6072292 .884553-2.581786$

H $1.1226374 .522464-1.849854$

H $3.0400656 .172784-1.661598$

H $4.1773474 .879850-1.367255$

H 2.0586576 .4149131 .880729

H 1.8307147 .1151410 .181505

H 3.5183744 .5371402 .218734

H 4.4985124 .0105090 .892234

H 3.0151132 .2637020 .261876

H $1.0679000 .816978-1.702383$

C $-2.936481-4.326084-1.142724$

O $-2.636825-2.969624-1.529487$

C $-3.227500-2.713858-2.817285$ 
C $-3.625298-4.073787-3.376292$

C $-4.018192-4.807152-2.110182$

H $-4.138887-2.132602-2.635159$

C -2.264797 -1.915399-3.700597

H -4.439505 -4.002230 -4.103766

H $-2.781146-4.578144-3.860736$

C $-4.074055-6.316025-2.276148$

H $-5.002588-4.444797-1.784381$

C -1.768224 -0.595528 -3.078533

O $-1.108281-2.715902-3.980943$

H -2.733006 -1.718516 -4.671799

H -1.081218 -0.807421 -2.249960

O $-1.0001170 .086475-4.078006$

C $-2.876550 \quad 0.353851-2.587334$

C $-2.3025831 .669975-2.029470$

H $-3.460690-0.136710-1.802020$

O $\quad-3.7624650 .670735-3.659680$

H $-1.5867571 .433233-1.234419$

H -1.750486 2.188371-2.821449

H -4.819611 -6.592552 -3.028800

H -3.107784 -6.720552 -2.596086

H $-4.347917-6.803140-1.335175$

C $-3.383440-4.3550600 .319119$

H -0.712054 -2.967424 -3.128839

H $-0.287886-0.514447-4.356870$

H $-4.240805-0.139144-3.902782$

H $-3.793552-5.3408680 .566073$

H $-4.178878-3.6166340 .479226$

H -2.015012 -4.907209 -1.279009

C -3.7546071 .6858090 .678840$

O $-4.298964 \quad 1.986126-0.608943$

C $-3.3918332 .633719-1.507076$

C $-2.8444903 .929643-0.887414$

C -2.2701123 .7073150 .511177$

C $-3.2837432 .964291 \quad 1.382511$

H $-2.899678 \quad 1.0092410 .551179$

C -4.7931640 .8871651 .478518$

H $-3.9901472 .927658-2.378928$

O $-1.8542734 .500417-1.736636$

H -3.663422 4.657691-0.817416

H $-1.312512 \quad 3.1763000 .463834$

O -1.9944284 .9830551 .092516$

H -4.1262283 .6352831 .583876$

O -2.6965142 .6523212 .640817$

C -6.0643051 .6497511 .790906$

H -5.093103 -0.0004070 .908620$

H -4.3414150 .5554412 .420726$

H -1.519999 5.300995 -1.296993

H -1.673515 4.8254621 .997022

H -1.9615702 .0388572 .479057$

O

O

C -7.8844971 .7078063 .300502$

H -8.2405701 .1889714 .194431$

H -7.724184 2.7617553 .547005

H -8.635942 1.6046162 .511860

SCF Energy (B3LYP/6-31G**//MMFF) $=-3245.90850763$

02131

MM̄FF Geometry

C $2.717705-3.3814462 .517130$

C $3.201160-2.6763331 .482827$

C $4.564977-2.0446711 .478916$

O $4.382190-0.6136981 .440942$

C $5.454094-2.5517960 .322694$

C $5.053684-2.158205-1.087762$

C $4.450067-2.977953-1.971980$

C $5.487591-0.782634-1.526727$

C $3.974109-4.383808-1.739084$

C $2.688181-4.753504-2.501320$

C $1.436975-3.971141-2.065442$

C $0.174420-4.640947-2.618498$

C -1.096784 -3.847129-2.282521

O $1.532161-2.634359-2.569430$

O $-2.166290-4.361404-3.090373$

C $-0.886166-2.377372-2.694997$

C -1.525244 -4.039220 -0.822887

O $-1.978857-1.566513-2.265371$
C $0.447341-1.778332-2.188617$

C $0.667086-0.393510-2.841027$

O $0.373473-1.623790-0.772438$

C 5.3835920 .1548311 .939762

O $6.466872-0.2395702 .351907$

C $5.027811 \quad 1.5923841 .962568$

C 3.8328802 .0955891 .612849

C 3.4444003 .5090071 .699631

C 4.4990504 .4926322 .141405

C $2.0379810 .234305-2.562085$

C $2.1459931 .639774-3.128885$

C $3.7209443 .567773-3.233378$

O $1.2409812 .184025-3.755762$

C $3.5623444 .461165-2.044213$

C $2.5284945 .300269-1.882518$

C $2.3762046 .239303-0.720698$

C 1.2238795 .8656580 .183609

C $-0.0403196 .180674-0.146398$

C 1.5443465 .2239581 .514535

C 2.1761883 .8614851 .396668

N $3.3798672 .212190-2.850406$

H $3.322389-3.5008523 .412732$

H $2.587607-2.5448940 .597548$

H $5.068194-2.3020722 .421422$

H $5.544750-3.6427250 .399781$

H $6.475803-2.1818270 .482367$

H $4.290845-2.606039-2.983259$

H $5.343024-0.623644-2.600740$

H $4.917139-0.012618-1.002533$

H $6.552101-0.631067-1.320206$

H $4.767550-5.061886-2.076830$

H $3.816241-4.584391-0.674971$

H $2.857541-4.608310-3.576216$

H $2.515113-5.825613-2.343468$

H $1.402225-3.949099-0.971415$

H $\quad 0.087657-5.671205-2.250926$

H $\quad 0.254726-4.719439-3.712047$

H $-2.260326-5.309469-2.894719$

H $-0.886578-2.341378-3.793507$

H $-1.729890-5.097225-0.619717$

H $-2.459441-3.507165-0.610823$

H $-0.770043-3.707647-0.108888$

H $-2.792820-1.963617-2.619672$

H $0.564183-0.486290-3.930452$

H $-0.114606 \quad 0.295132-2.501808$

H $0.982196-0.924353-0.489973$

H 5.8229662 .2320932 .333619

H 3.0551221 .4197471 .261903

H 4.8004474 .2947963 .175776

H 4.1756165 .5342792 .094827

H 5.3825394 .4242681 .496263

H $2.2315990 .296090-1.486518$

H $2.823088-0.374644-3.022080$

H $3.0937613 .887465-4.072105$

H $4.7635003 .570031-3.566221$

H $4.3495114 .428972-1.295425$

H $1.7489335 .330406-2.640884$

H $2.2158867 .250127-1.118916$

H $3.3047746 .306395-0.141415$

H -0.8726025 .9494890 .510006$

H $-0.2708236 .674122-1.084918$

H 0.6263675 .0975252 .104477

H 2.1540335 .9178522 .100734

H 1.4835003 .0835961 .075463

H $4.0437411 .703334-2.275108$

C $0.232392-3.2124193 .072443$

O $-0.063469-2.1406942 .160932$

C $-0.503548-0.9930692 .920554$

C $-0.620233-1.4601874 .366395$

C $0.436567-2.5481694 .430394$

H $0.293669-0.2465042 .818106$

C -1.807885 -0.479971 2.301875

H $-0.448239-0.6509415 .082505$

H $-1.614637-1.8806214 .562515$

C $0.276168-3.4909255 .608909$

H $1.419420-2.0639764 .486841$

C -2.4720980 .6728513 .072214$

O $-1.516791-0.0310520 .975200$ 
H -2.509032 -1.316396 2.201857 H -2.8082010 .3197294 .054158$ O $-1.481195 \quad 1.678563 \quad 3.319783$ C -3.6441071 .3429622 .325090$ C $-4.749414 \quad 0.363821 \quad 1.894231$ H -3.2541791 .8901171 .460451$ O $-4.2165812 .331318 \quad 3.188833$ H -4.315143 $-0.443076 \quad 1.298616$ H $-5.186989-0.0973612 .788360$ H $0.357795-2.9431896 .553318$ H $-0.698012-3.9911315 .593782$ H $1.051959-4.2630395 .597181$ C $1.378606-4.0608582 .521097$ H -1.142947 -0.7859350 .488849$ H -1.918566 2.4063373 .794126 H -4.5821371 .8729443 .964731$ H $\quad 1.133297-4.3705051 .497373$ H $1.468836-4.9822023 .108903$ H - $0.667533-3.8422253 .097348$ C $-4.7938621 .006590-1.071206$ O $\quad-5.3690391 .796927-0.023885$ C -5.8763501 .0449881 .083422$ C -6.9687970 .0843850 .594750$ C $-6.485321-0.798601-0.555822$ C $-5.8392410 .048793-1.654010$ H $-3.9438250 .435351-0.680339$ C $-4.265191 \quad 1.970737-2.139389$ H $-6.356268 \quad 1.769397 \quad 1.753315$ O $-7.425923-0.7246941 .673469$ H -7.8281620 .6738290 .248844$ H $-5.800034-1.574124-0.193829$ O $-7.619939-1.483016-1.092454$ H $-6.6156900 .614762-2.184606$ O $-5.204776-0.807373-2.601290$ C $-3.1286282 .809973-1.604765$ H $-3.9075291 .444222-3.031251$ H $-5.0566042 .658727-2.461141$ H $-8.102081-1.3270511 .318606$ H -7.295142 -2.115207 -1.755350 H $-5.899127-1.324286-3.043182$ O $-2.0214882 .036326-1.420301$ O $-3.2172734 .009658-1.377153$ C $-0.8850292 .711504-0.874344$ H $-0.1059501 .969765-0.681238$ H $-0.5044213 .441886-1.593512$ $\mathrm{H}-1.1456353 .1926510 .073093$

SCF Energy $\left(B 3 L Y P / 6-31 G^{* *} / / M M F F\right)=-3245.91641156$

02132

MM̄FF Geometry

C $-1.779518 \quad 4.459794-0.256873$

C $-2.275688 \quad 3.233797-0.036057$

C -2.9353742 .8070851 .245951$

O $-2.194430 \quad 1.676573 \quad 1.762711$

C -4.3710022 .3351830 .964959$

C -5.1829432 .1231002 .225706$

C -5.3807180 .9298422 .819202$

C -5.8275993 .3640942 .786507$

C $-4.796816-0.4014652 .435941$

C $-5.849476-1.3923791 .923255$

C $-6.352832-1.0788520 .504504$

C -7.414373 $-2.098420 \quad 0.083112$

C $-7.851696-1.908050-1.376221$

O $-5.231331-1.145340-0.381985$

O $-8.582442-3.078006-1.774635$

C $-6.594675-1.836240-2.267661$

C -8.810984 -0.722502 -1.534160

O $-6.945222-1.521966-3.613913$

C $-5.528622-0.842236-1.752113$

C $-4.217366-1.013028-2.556551$

O $-6.017300 \quad 0.489693-1.909045$

C -1.1330811 .9222222 .571308$

O -0.7267593 .0191962 .929439$

C $-0.5011930 .663403 \quad 3.033480$

C $-0.733368-0.5363342 .477090$

C $-0.157336-1.8150362 .903670$

C $0.746367-1.8101884 .108507$

C $-3.083774-0.082067-2.110130$
C $-1.747112-0.557140-2.640841$

C $0.109341-2.130812-2.142957$

O $-1.256036-0.099278-3.669397$

C $0.230987-3.422528-1.402409$

C $1.173408-3.667418-0.479936$

C $1.255766-4.9496130 .297042$

C $1.263557-4.7344981 .796268$

C $2.372768-4.9789192 .514395$

C $-0.028764-4.3460042 .482690$

C $-0.464133-2.9307092 .208026$

N $-1.194416-1.560496-1.860942$

H -1.8602805 .2201020 .515068$

H -2.175412 $2.478389-0.811077$

H -2.942572 3.6208921 .981529

H -4.3504421 .4113140 .378279$

H -4.8959043 .0683740 .337925$

H -6.0088710 .8991183 .708859$

H -6.4170563 .1572283 .686068$

H -5.0668634 .1038833 .054694$

H -6.5006233 .8096612 .046553$

H $-3.983796-0.3059371 .711701$

H $-4.337144-0.8234393 .338917$

H $-5.384123-2.3868841 .920162$

H $-6.695242-1.4341152 .620955$

H $-6.769030-0.0654340 .486548$

H $-8.281869-2.0552030 .753508$

H -7.006028 -3.1135400 .189163$

H $-9.339182-3.178522-1.171819$

H -6.148976 -2.840779 -2.289899

H $-9.715279-0.879709-0.934103$

H $-9.152868-0.626016-2.570464$

H $-8.3727250 .228564-1.224535$

H -7.606800 -2.174520 -3.900695

H -3.879963 -2.053577 -2.463918

H $-4.401357-0.828732-3.622604$

H $\quad-5.2706591 .107689-1.881985$

H $\quad 0.189277 \quad 0.784587 \quad 3.862129$

H - $1.410924-0.5993831 .627261$

H $1.613066-1.1621233 .938313$

H $1.139658-2.7976404 .358959$

H $0.207129-1.4505764 .991820$

H $-3.2483610 .937420-2.475448$

H -3.020369 -0.024356 -1.018090

H $\quad 0.864916-1.398277-1.838985$

H $0.204855-2.294451-3.221236$

H $-0.507298-4.189388-1.625419$

H $1.918705-2.905404-0.266778$

H $2.171069-5.467869-0.017279$

H $0.428501-5.6238000 .041755$

H $2.385855-4.867433 \quad 3.593371$

H $3.295777-5.2957782 .040408$

H $0.034532-4.5162233 .564351$

H $-0.824305-5.0247142 .147922$

H $-1.129391-2.8399271 .349243$

H -1.606865 -1.742548 -0.949890

C $0.4418984 .825110-1.427667$

O $0.8937343 .505522-1.057441$

C $1.9105723 .637886-0.040183$

C $2.3670015 .087015-0.109693$

C $1.0613005 .793070-0.419690$

H 1.4070293 .4423490 .915293

C $2.9988882 .589480-0.288376$

H 2.8272345 .4314570 .821104

H $3.0857155 .231313-0.925938$

C $1.2395087 .204283-0.951256$

H 0.4668215 .8345010 .501097

C 4.2527052 .7680850 .584432

O $2.4340941 .300516-0.021800$

H $3.2769442 .597848-1.348485$

H $4.755003 \quad 3.7049970 .317770$

O 3.8535852 .9108951 .954032

C 5.2686391 .6108110 .515022

C $5.6799551 .225115-0.915887$

H 4.8624780 .7406651 .040198

O 6.4355622 .0012201 .247480

H $4.7954810 .896173-1.469564$

H $6.0680682 .113406-1.429847$

H $1.7442627 .833242-0.210689$ 
H $1.8403907 .216207-1.866822$ H $0.2701257 .659525-1.177527$ C -1.085146 4.841901-1.534626 H $1.6440801 .207853-0.581655$ H 3.3612302 .1090802 .201055 H 6.8299322 .7651500 .792903 H -1.385498 $4.145277-2.328282$ H $-1.4324305 .834536-1.842955$ H $0.8549865 .015379-2.426937$

C $5.323739-1.778220-0.578182$

O $6.432368-0.992525-0.126246$

C $6.7592110 .119605-0.963306$

C $7.100950-0.372439-2.377337$

C $6.000308-1.263290-2.955296$

C $5.616601-2.362352-1.962765$

H $4.420036-1.158205-0.621456$

C $5.102168-2.8821670 .461416$

H $7.6747830 .557049-0.545648$

$\begin{array}{llll}\text { O } & 7.335900 & 0.741758 & -3.231404\end{array}$

H $8.034027-0.949359-2.333567$

H $5.120420-0.677631-3.246664$

O $6.476658-1.894341-4.146235$

H $6.427328-3.099597-1.900168$

O $4.455603-3.040027-2.439091$

C $4.540345-2.3017711 .740384$

H $4.394852-3.6412040 .113167$

H $6.050989-3.3869090 .680376$

H $7.6698500 .400387-4.077886$

H $6.611776-1.199823-4.812552$

H $4.661034-3.377758-3.327734$

O $5.331137-2.6449092 .796552$

O $3.518221-1.6298091 .796317$

C $4.909109-2.1439324 .068711$

H $4.837777-1.0521094 .045373$

H $5.658112-2.429872 \quad 4.812040$

H $3.949558-2.5883864 .348214$

SCF Energy (B3LYP/6-31G*//MMFF) $=-3245.90951025$

02 133

MM̄FF Geometry

C $-2.017328-1.072503 \quad 2.988515$

C -1.8942810 .1081173 .615866$

C -2.6308801 .3922243 .308238$

O $-3.591623 \quad 1.2213612 .244397$

C -1.6408312 .4685872 .828257$

C -2.1049893 .8786983 .132031$

C $-2.757428 \quad 4.6846542 .272126$

C $-1.736194 \quad 4.3824964 .504379$

C $-3.260419 \quad 4.366971 \quad 0.891877$

C $-2.4457215 .049298-0.216065$

C $-1.168398 \quad 4.276320-0.576537$

C $-0.3453585 .035590-1.618550$

C $0.876414 \quad 4.225874-2.080154$

O $-1.572704 \quad 3.008698-1.106777$

O $1.4035404 .853867-3.258833$

C $0.4120582 .812551-2.490846$

C $2.0013204 .243810-1.038685$

O $1.5266651 .976398-2.792707$

C $-0.4939412 .129179-1.440448$

C -1.097459 $0.832139-2.030804$

O $0.291833 \quad 1.798844-0.294502$

C $-4.845048 \quad 0.8038882 .554822$

$\begin{array}{llll}\text { O } & -5.279993 & 0.507326 & 3.654292\end{array}$

C $-5.592698 \quad 0.744478 \quad 1.280327$

C $-6.685707-0.024391 \quad 1.180127$

C $-7.482725-0.222765-0.032741$

C $-7.1115610 .583081-1.251265$

C $-2.2237020 .210539-1.190918$

C $-2.705160-1.106967-1.779238$

C $-4.302995-2.969634-1.326562$

O $-2.236053-1.595720-2.803826$

C $-5.593983-2.768118-2.056120$

C $-6.733412-3.396824-1.727182$

C -8.026433 -3.232427 - 2.475567

C $-9.202812-2.947018-1.566409$

C $-10.070093-3.918347-1.234130$

C $-9.420639-1.520344-1.112181$

C $-8.486002-1.124638-0.002065$
N $-3.713623-1.679454-1.015566$

H -2.709966 -1.176311 2.159447

H -1.201252 0.1730034 .453504

H -3.1449211 .7210934 .220200$

H -1.4695402 .3570991 .752601$

H $-0.6573042 .334313 \quad 3.298264$

H -3.001532 5.6930282 .605681

H -2.089365 5.4036654 .683115

H -2.1745443 .7428675 .276955$

H -0.6483034 .3826164 .628952$

H $-3.3258683 .290306 \quad 0.716604$

H -4.2928824 .7357720 .837557$

H -3.084628 5.104074 -1.107358

H -2.2004886 .0789640 .071370$

H -0.5764834 .1219530 .331906$

H $-0.0342166 .013888-1.231572$

H $-0.9723295 .246262-2.496483$

H $1.6359325 .770573-3.031262$

H $-0.1590862 .915723-3.424211$

H $2.3321575 .272146-0.849996$

H $2.8838023 .708178-1.403256$

H $1.7073553 .811634-0.080012$

H $2.0616392 .437057-3.461551$

H - $1.5142131 .046518-3.023870$

H $-0.3030080 .088572-2.167579$

H -0.1559451 .0976030 .203180$

H $-5.193161 \quad 1.3056200 .442952$

H -7.010577 -0.5829692 .056944$

H -7.212033 $1.654582-1.045476$

H -7.731221 $0.370102-2.124210$

H $-6.0777500 .380228-1.551244$

H -1.883503 $0.016688-0.168960$

H $-3.0792130 .893741-1.144954$

H -4.443094 -3.497592 -0.377994

H -3.612494 -3.546855 -1.949597

H $-5.586047-2.094120-2.909536$

H $-6.730532-4.095882-0.893613$

H -8.205558 -4.160709 -3.033770

H -7.953317 -2.440675 -3.231629

H -10.931198 -3.717006 -0.605491

H -9.935185 -4.938125 -1.578891

H -10.443972 -1.396639-0.733039

H $-9.364643-0.856574-1.979117$

H -8.671759 -1.660690 0.929828

H -4.059759-1.184975 -0.199039

C $-0.284329-2.8388692 .343583$

O $0.704338-1.8418462 .021385$

C $0.979716-1.9118460 .603372$

C $0.326992-3.1957270 .111351$

C $-0.888258-3.2797861 .013600$

H $0.480406-1.0430370 .161667$

C $2.493722-1.8212880 .383264$

H $\quad 0.069177-3.156307-0.951209$

H $\quad 0.986673-4.0571430 .273867$

C -1.533356-4.653186 1.045773

H $-1.624169-2.5494910 .663461$

C $2.932872-2.058032-1.071677$

O $2.918512-0.513780 \quad 0.783654$

H $2.998516-2.5321371 .047247$

H $2.703489-3.089669-1.363572$

O $2.148608-1.212853-1.922275$

C $4.417894-1.757104-1.355181$

C $5.392381-2.515006-0.437901$

H $4.588090-0.677528-1.287545$

O $4.688086-2.113608-2.715836$

H $5.197756-2.2412230 .603042$

H $5.203237-3.592097-0.527058$

H -1.876962 -4.9399180 .046511$

H $-0.831422-5.4190881 .392078$

H -2.399360 -4.660669 1.715198

C $-1.258344-2.3068683 .398052$

H $2.624933-0.3812651 .701264$

H $2.441568-1.367158-2.836671$

H $4.561552-3.074075-2.802116$

H $-0.688255-2.0948624 .311954$

H - $1.983450-3.0875703 .655693$

H $\quad 0.267346-3.6768632 .790689$

C $7.107655-0.1371920 .375377$ 
O $7.167654-0.849345-0.867150$

C $6.876539-2.246333-0.776092$

C $7.876206-2.9227460 .170633$

C $7.939846-2.2238461 .528612$

C $8.130097-0.713717 \quad 1.363456$

H $6.097810-0.2112350 .797444$

C $7.377661 \quad 1.3376300 .060217$

H $7.051785-2.661234-1.776698$

O $7.538109-4.294925 \quad 0.341420$

H $8.872442-2.892268-0.289529$

H $7.043101-2.4403702 .120373$

O $9.033813-2.7585672 .278800$

H $9.153668-0.5153321 .020741$

O $7.975658-0.1113762 .646403$

C $6.210423 \quad 1.932750-0.692452$

H $7.508348 \quad 1.9377190 .966650$

H $8.2876831 .459673-0.539363$

H $8.176929-4.6764290 .967770$

H $9.853033-2.5760221 .787763$

H 8.3094580 .7993912 .590838

O $6.4254701 .843978-2.035855$

O $5.2127482 .388106-0.148205$

C $5.3586292 .319035-2.862164$

H $5.6516922 .185104-3.906800$

H $4.4504391 .738332-2.676174$

H $5.1829173 .383437-2.680480$

SCF Energy (B3LYP/6-31G**/MMFF) $=-3245.90578870$

02134

MMFF Geometry

C $0.9555850 .347929-2.586212$

C $1.970123-0.406053-3.036380$

C $2.307280-1.814991-2.598660$

O $1.315397-2.282831-1.658975$

C $3.683436-1.801477-1.912034$

C $4.314321-3.158019-1.677761$

C $4.673298-3.633468-0.468552$

C $4.648816-3.945701-2.919184$

C $4.489657-2.9937290 .881165$

C $5.772205-2.3153591 .394393$

C $6.036509-0.947607 \quad 0.743774$

C $7.380321-0.3663951 .183418$

C 7.6007901 .0428540 .608220

O $4.983338-0.0724421 .153076$

O 8.7255621 .6050821 .300435

C 6.3630181 .9124110 .922079

C $7.9895360 .992211-0.873910$

$\begin{array}{lllll}\text { O } & 6.462085 & 3.189226 & 0.295768\end{array}$

C 5.0190561 .2342160 .572203

C 3.8351352 .0651451 .135971

$\begin{array}{lllll}\text { O } & 4.879959 & 1.176743 & -0.850559\end{array}$

C $0.811806-3.531527-1.814739$

O $1.169459-4.370969-2.629380$

C $-0.274622-3.804944-0.846092$

C $-0.905940-2.852619-0.141047$

C $-2.004248-3.0663030 .806322$

C $-2.418106-4.4896631 .078015$

C $2.5425331 .253642 \quad 1.325125$

C 2.5377560 .4572612 .622606

C $1.481359-1.4745143 .741615$

O 3.2802180 .7173813 .567205

C $0.124216-2.0950953 .758932$

C $-0.900186-1.5985734 .468453$

C $-2.267856-2.2154414 .527227$

C $-3.288038-1.4251663 .739294$

C $-3.856186-0.3194564 .249334$

C -3.701747 -1.963977 2.388602

C -2.570144 -1.991608 1.396095

N $1.584506-0.5517572 .626733$

H $0.269815-0.049214-1.843800$

H $2.6357930 .018634-3.786053$

H $2.315017-2.437178-3.500780$

H $3.582150-1.261509-0.966215$

H $4.393547-1.221695-2.517803$

H $5.169052-4.603072-0.429285$

H $5.242799-3.334603-3.606766$

H $5.232744-4.845202-2.695961$

H $3.740984-4.267224-3.436225$
H $3.642247-2.3016760 .896449$

H $4.219261-3.794473 \quad 1.581780$

H $5.658741-2.1739782 .477223$

H $6.632040-2.9791341 .241111$

H $6.020684-1.060156-0.344508$

H $8.205631-1.0309510 .899454$

H $7.409785-0.3030492 .280296$

H 8.8266062 .5249321 .000426

H 6.3818562 .1055822 .003500

H $8.9027890 .399911-1.007705$

H $8.2242991 .991594-1.256894$

H $7.2137060 .555227-1.506220$

H $6.2491493 .073881-0.645994$

H 4.1149522 .5107632 .098083

H 3.6254522 .8991370 .453903

H $3.9340401 .153095-1.069435$

H $-0.559949-4.849877-0.776832$

H -0.605916 -1.814462 -0.269936

H -2.826268 -4.9493740 .171148$

H -3.178997 -4.5872721 .854193$

H $-1.560658-5.0813421 .418157$

H $1.6953351 .946748 \quad 1.384412$

H 2.3708960 .5886330 .472290

H $1.696123-0.9446084 .676533$

H $2.249960-2.2443293 .615588$

H $-0.010967-3.0037843 .179039$

H $-0.749555-0.6906475 .048734$

H -2.574802 -2.271054 5.579879

H $-2.247255-3.2560584 .180040$

H $-4.6081420 .236523 \quad 3.699372$

H -3.5793900 .0539815 .229830$

H -4.491351 -1.337564 1.959356

H -4.165274 -2.943121 2.533001

H $-2.198015-0.9998901 .134760$

H $1.134647-0.8015411 .750319$

C $0.7137812 .726436-1.866320$

O $-0.4665562 .528837-1.057587$

C -1.064412 $3.810051-0.793998$

C $0.0121614 .840424-1.108888$

C $0.6935844 .193366-2.297122$

H $-1.8823913 .930833-1.514160$

C -1.6114663 .8798200 .633690$

H $-0.4069505 .825198-1.336091$

H $0.7210014 .953636-0.280141$

C $2.0694554 .765647-2.593342$

H $0.0546394 .317731-3.181862$

C -2.656553 2.8007180 .984329

O $-0.5167293 .739854 \quad 1.548346$

H $-2.030363 \quad 4.8783180 .810196$

H -2.1666881 .8236931 .079632$

$\begin{array}{lllll} & -3.149554 & 3.135882 & 2.288871\end{array}$

C -3.836588 $2.701666-0.004110$

C -5.0057341 .8461120 .519890$

H $-3.4712752 .285356-0.948587$

$\begin{array}{llll}\text { O } & -4.335176 & 4.010809 & -0.288758\end{array}$

H -4.6080080 .9145730 .931150$

H $-5.4966542 .384246 \quad 1.339343$

H $1.9988745 .831322-2.834510$

H $2.7418124 .659031-1.735292$

H $2.5291744 .255774-3.445890$

C $0.6944101 .754446-3.046542$

H $-0.881634 \quad 3.796852 \quad 2.448094$

H -3.5888372 .3474992 .649795$

H -4.6092554 .4115010 .554021$

H $1.4391682 .053598-3.792760$

H $-0.2887531 .773506-3.532112$

H $1.5789532 .531356-1.221035$

C $-5.261302-0.623832-1.267750$

O $-5.5278530 .737259-1.628919$

C $-6.0568081 .554004-0.577094$

C $-7.3675360 .948777-0.055930$

C $-7.185022-0.4992710 .381335$

C $-6.524653-1.310958-0.730509$

H $-4.469075-0.654405-0.513183$

C $-4.751787-1.348269-2.523549$

H $-6.3156802 .515253-1.038433$

O

H $-8.1225140 .977125-0.852541$ 
H $-6.616051-0.566927 \quad 1.314810$

O $-8.465434-1.0748620 .652044$ H -7.244266 -1.463029 -1.545357 O $-6.176750-2.598843-0.224189$ C $-3.341461-0.948772-2.902067$ H -4.739443 -2.431542 -2.359456 H -5.402748 -1.115797 -3.374736 H -8.0045952 .6187910 .723839$ H -8.892182 $-0.523447 \quad 1.330257$ H $-6.988978-3.001103 \quad 0.128547$ O $-2.998255-1.609668-4.048296$ O $-2.630102-0.174339-2.278194$ C $-1.678127-1.360379-4.538793$ H -1.517426 -0.288147 -4.684093 H $-0.941430-1.776132-3.846798$ $\mathrm{H}-1.573692-1.862473-5.504350$ SCF Energy (B3LYP/6-31G**//MMFF) $=-3245.92588524$

02 135

MM̄FF Geometry

C $2.698350-0.9925523 .260387$

C $3.602597-1.8924422 .843857$

C $4.586073-1.7634791 .707186$

O $4.686042-0.3929361 .260694$

C $4.129707-2.6330910 .522011$

C $5.248141-2.985015-0.437563$

C $5.415434-2.443532-1.659536$

C $6.180911-4.0711490 .036059$

C $4.614040-1.351825-2.311420$

C $3.732903-1.875497-3.455118$

C $2.468403-2.596796-2.962342$

C $1.685080-3.185168-4.137554$

C $0.353585-3.804768-3.684818$

O $1.665716-1.629649-2.280061$

O $-0.443581-4.042251-4.854893$

C $-0.406597-2.777040-2.821209$

C $0.555815-5.167728-3.012627$

O $-1.583298-3.353075-2.257645$

C $0.459163-2.145260-1.706228$

C $-0.297182-0.955978-1.071855$

O $0.724386-3.126653-0.703520$

C 5.9293510 .0916051 .006956

O $6.991141-0.492524 \quad 1.181740$

C 5.8959321 .4701420 .469166

C 4.7864772 .1014680 .054716

C $4.7350273 .453170-0.513813$

C $6.0269854 .224502-0.604078$

C $0.541995-0.118009-0.097427$

C $-0.209781 \quad 1.1158900 .366068$

C 0.0705003 .1450161 .785979

O $-1.352746 \quad 1.3805430 .004354$

C 0.3066414 .2309310 .786252

C 1.1562385 .2512950 .972756

C $1.4015236 .316112-0.056030$

C $2.8348016 .336956-0.540228$

C $3.6750597 .311609-0.153863$

C $3.2657695 .287783-1.543226$

C $3.5459583 .946774-0.918645$

N $0.546625 \quad 1.8888841 .235255$

H $2.599219-0.0356632 .758196$

H $3.649649-2.8454393 .370192$

H $5.549638-2.1078202 .100968$

H $3.318277-2.121873-0.006442$

H $3.697341-3.5767960 .881738$

H $\quad 6.241962-2.806610-2.269662$

H $6.930207-4.334684-0.717975$

H $6.718553-3.7546870 .934863$

H $5.617271-4.9803710 .269362$

H $4.018828-0.786356-1.588520$

H $5.329848-0.631422-2.727790$

H $3.432521-1.010299-4.060765$

H $4.317471-2.538806-4.104679$

H $2.754694-3.390981-2.264494$

H $2.289631-3.925402-4.676459$

H $1.466272-2.390637-4.864785$

H $0.057115-4.632577-5.443765$

H - $0.746057-1.975290-3.492182$

H $\quad 1.038716-5.867838-3.704857$
H $-0.404557-5.623571-2.747612$

H $1.173057-5.115520-2.113321$

H -2.108071 -3.715700 -2.991725

H $-0.641227-0.288711-1.873373$

H -1.187428 -1.318903 -0.542880

H $\quad 0.890110-2.6826040 .142570$

H $\quad 6.873031 \quad 1.938370 \quad 0.398456$

H 3.8299011 .5864520 .120548

H 6.4793694 .3352680 .387817

H $5.9054915 .233892-1.001795$

H $6.7370463 .709620-1.260789$

H $0.803496-0.7072930 .786551$

H $1.4683330 .216875-0.577561$

H $\quad 0.6073013 .3168712 .724074$

H -0.9992133 .0690902 .002782$

H $-0.2484484 .167408-0.146753$

H 1.7101495 .3240081 .905557

H 1.1348397 .2815800 .393076

H $0.7336976 .200148-0.919202$

H $4.6945167 .359265-0.521006$

H 3.3628328 .0845540 .540769

H $4.1310185 .635300-2.119620$

H $2.4758905 .162987-2.295490$

H $2.6631153 .315721-0.818522$

H 1.5191141 .6426191 .394843

C $0.424849-1.7984203 .962828$

O $-0.423482-0.7065443 .554962$

C $-1.778088-0.9847863 .987991$

C -1.765047 -2.408473 4.522857

C $-0.353655-2.5208185 .060504$

H $-1.983390-0.2741864 .798390$

C -2.727461 -0.7420872 .809320$

H -2.530594 -2.577774 5.285962

H -1.923592 -3.1323373 .713566$

C $0.086349-3.9557795 .295560$

H $-0.286211-1.9598176 .002306$

C $-4.165611-1.2425293 .023335$

O -2.7757380 .6686732 .573795$

H -2.299780 -1.198736 1.910052

H $-4.169992-2.3328173 .136953$

O $-4.648477-0.7026814 .259472$

C $-5.151909-0.8370361 .907458$

C $-4.721429-1.3070760 .507306$

H -5.2965640 .2487481 .923801$

O $-6.428329-1.4106882 .212679$

H $-3.727308-0.9140470 .279972$

H -4.640796 -2.4012090.508349

H $-0.562379-4.4405286 .032578$

H $0.044907-4.544443 \quad 4.372826$

H $1.112578-3.9955275 .673406$

C $1.781503-1.2638694 .424312$

H -1.8587490 .9804172 .495387$

H $-5.562972-1.0134084 .373918$

H -6.332012 -2.378105 2.192937

H $2.262985-1.9826035 .098039$

H $1.658191-0.3317954 .988921$

H $\quad 0.541114-2.4558273 .090474$

C $-4.8647991 .350391-0.954331$

O $-5.9762470 .521843-0.590268$

C $-5.707434-0.884504-0.606531$

C $-5.278972-1.312301-2.016858$

C $-4.104684-0.479726-2.528755$

C $-4.384627 \quad 1.016553-2.373883$

H $-4.0507211 .194300-0.238221$

C $-5.3321882 .804895-0.822875$

H $-6.661456-1.382465-0.393210$

O $-4.939741-2.694744-2.028104$

H $-6.129322-1.184076-2.699065$

H $-3.178609-0.755982-2.015160$

O $-3.880741-0.777167-3.909229$

H $\quad-5.1407591 .322838-3.107723$

O $-3.1924091 .742318-2.661124$

C $-4.1799113 .766054-0.660339$

H -5.957988 $3.092682-1.676411$

H -5.9744412 .9264950 .058732$

H $-4.637177-2.913315-2.926123$

H $-4.686596-0.544678-4.401273$

H $-2.897671 \quad 1.479347-3.549792$ 
O $-4.1112804 .600689-1.736099$

O $\quad-3.425072 \quad 3.7684390 .303571$

C $-3.037147 \quad 5.545928-1.713216$

H $-2.0776525 .023642-1.769830$

H $-3.1357926 .193702-2.588409$

$\mathrm{H}-3.0891106 .164367-0.812047$

SCF Energy (B3LYP/6-31G*//MMFF) $=-3245.90884338$

02 136

MMFF Geometry

C $-2.318833 \quad 3.836272-0.576285$

C $-2.535566 \quad 4.357848 \quad 0.641069$

C -3.0855473 .6094231 .833550$

O -2.8826852 .1824551 .702072$

C -4.5919503 .8883361 .968063$

C $-5.227855 \quad 3.205308 \quad 3.162655$

C -5.9838262 .0910423 .095109$

C -4.9925433 .8815084 .488260$

C $-6.309073 \quad 1.277702 \quad 1.872403$

C $-5.894299-0.1884602 .054123$

C $-6.037024-0.986403 \quad 0.749670$

C $-5.726614-2.464607 \quad 0.985213$

C $-5.752491-3.272208-0.318965$

O $-5.117663-0.441140-0.203567$

O $-5.153348-4.545766-0.032544$

C $-4.874958-2.561094-1.373282$

C $-7.188510-3.565824-0.769809$

O $-5.014975-3.221196-2.628953$

C $-5.173556-1.050649-1.502484$

C $-4.168591-0.290527-2.406497$

O $-6.470158-0.883225-2.089972$

C -1.6495851 .7038182 .032572$

O -0.6770632 .3573452 .376715$

C $-1.6860390 .228558 \quad 1.916095$

C $-0.593847-0.4882092 .221028$

C $-0.476379-1.9491202 .198424$

C $-1.677069-2.7415351 .755650$

C $-2.702386-0.340827-1.963127$

C $-1.904858-1.480538-2.574342$

C $0.298857-2.632416-2.367359$

O $-2.319214-2.170421-3.501630$

C $0.378219-3.658487-1.281864$

C $1.508455-3.945893-0.619257$

C $1.616207-4.995840 \quad 0.446155$

C $2.079651-4.4352541 .771029$

C $3.380901-4.4399622 .107277$

C $1.023735-3.9659182 .745089$

C $0.688357-2.5076962 .589668$

N $-0.658246-1.613044-1.979036$

H -2.579594 $2.799423-0.772542$

H -2.2878095 .4024770 .816837$

H -2.567362 3.9586282 .735917

H -5.1037903 .5986671 .043935$

H -4.7624904 .9702682 .055817$

H -6.4151851 .7074524 .019003$

H $-5.508863 \quad 3.3754985 .310958$

H -3.925056 3.8941604 .728684

H -5.3553714 .9141954 .457650$

H $-7.390440 \quad 1.3290401 .697644$

H -5.8288501 .6818240 .978673$

H $-4.851562-0.2266442 .391751$

H $-6.508665-0.6459552 .839681$

H -7.060929 -0.8626110 .377665$

H -6.418806 -2.896763 1.718670

H $-4.727017-2.5580331 .429828$

H $-5.103501-5.041742-0.867926$

H -3.835129 -2.701897-1.054407

H $-7.715881-4.147726-0.004146$

H -7.202764 -4.182588 -1.675204

H -7.772040 -2.662548 -0.959674

H $-4.354174-2.850847-3.237227$

H $-4.283686-0.604533-3.450751$

H $-4.4619070 .769335-2.391752$

H $-6.5237730 .008988-2.470620$

H -2.615231 -0.2297101 .601034$

H $0.3028070 .043595 \quad 2.537926$

H -2.493673 -2.629045 2.476585

H - $1.479183-3.8106301 .651833$
H -2.025179 -2.402659 0.774098

H $-2.2093050 .583011-2.291944$

H $-2.625876-0.382593-0.873307$

H $1.257680-2.132388-2.535055$

H $-0.009857-3.106187-3.304231$

H $-0.533477-4.201569-1.044564$

H $2.423443-3.417076-0.876207$

H $2.327485-5.7553680 .095406$

H $\quad 0.666949-5.528787 \quad 0.581381$

H $3.716494-4.0688953 .070040$

H $4.139083-4.8063071 .423043$

H $1.387843-4.1050293 .772647$

H $\quad 0.145050-4.6142512 .682047$

H $1.501117-1.8461582 .892017$

H $-0.412450-1.005533-1.202653$

C $-0.4093923 .922277-2.165886$

O $0.564518 \quad 3.995162-1.107959$

C $1.8740204 .040647-1.699910$

C $1.6856354 .048193-3.215528$

C $0.2712474 .583671-3.359534$

H $2.3071685 .010569-1.421202$

C $2.7646492 .940836-1.097459$

H $2.4283694 .677110-3.717141$

H $1.7648313 .048280-3.650758$

C $-0.364378 \quad 4.258092-4.699617$

H $0.2936175 .673001-3.222043$

C $2.1073511 .551859-0.958745$

O $3.9853562 .854635-1.826403$

H $3.0244443 .275356-0.086067$

H $1.2091971 .631537-0.334116$

O $1.6673701 .109276-2.245161$

C $3.0288160 .456620-0.390184$

C 3.6746100 .8129670 .958273

H $3.7994230 .211504-1.128498$

O $2.244315-0.727521-0.230336$

H 4.3631051 .6515580 .813748

H $2.895668 \quad 1.1551001 .651441$

H $0.219927 \quad 4.691638-5.517805$

H $-0.422578 \quad 3.176825-4.863744$

H $-1.3794254 .662764-4.759883$

C $-1.7037494 .598307-1.717771$

H $3.7966382 .409487-2.669964$

H $1.2410850 .243823-2.125787$

H $1.553591-0.539567 \quad 0.426612$

H -2.424094 $4.627642-2.542347$

H $-1.4996695 .633053-1.416735$

H $-0.5904552 .858092-2.370503$

C $6.421678-0.307595 \quad 0.276871$

O $5.274432-1.055178 \quad 0.693827$

C $4.419396-0.3730501 .613112$

C $5.199999-0.0107922 .883833$

C $6.485488 \quad 0.7538342 .568907$

$\begin{array}{llll}\text { C } 7.295906 & 0.032642 & 1.489449\end{array}$

H $6.1016400 .614376-0.224521$

C $7.177099-1.162684-0.744573$

H $3.652378-1.0993361 .907227$

$\begin{array}{lllll}\text { O } & 4.399188 & 0.764352 & 3.769547\end{array}$

H $5.469979-0.9320043 .416415$

H 6.2720691 .7878752 .273804

$\begin{array}{lllll}\text { O } & 7.284422 & 0.832977 & 3.751784\end{array}$

H $7.724774-0.8830001 .915241$

$\begin{array}{llll}\text { O } & 8.387792 & 0.858073 & 1.089899\end{array}$

C $6.402693-1.232644-2.040267$

H $8.161471-0.742159-0.978984$

H $7.342091-2.182044-0.375499$

H $3.5990820 .247968 \quad 3.967355$

H $6.744231 \quad 1.262755 \quad 4.437080$

H 8.0218921 .6733420 .706473

O $5.537853-2.286808-2.013054$

O $6.526308-0.422810-2.950102$

C $4.696775-2.423418-3.162576$

H $4.081982-3.317451-3.029903$

H $4.037462-1.554623-3.251987$

H $5.302205-2.544995-4.065874$

SCF Energy (B3LYP/6-31G*//MMFF) $=-3245.92063300$

02137

MMFF Geometry 
C $-0.071772-3.2093580 .837813$

C $-0.454403-2.283914-0.054806$

C $-0.144872-2.355262-1.524266$

O $0.387693-1.049148-1.858602$

C $-1.391723-2.607967-2.385497$

C $-2.011389-3.973966-2.174119$

C $-3.109822-4.208759-1.428855$

C $-1.323368-5.107375-2.888927$

C $-3.896855-3.204887-0.635492$

C $-5.316571-3.035307-1.190366$

C $-6.001194-1.801278-0.587742$

C $-7.476548-1.742192-0.982516$

C $-8.146189-0.451223-0.487032$

O $-5.317059-0.651724-1.097047$

O $-9.411348-0.326282-1.154113$

C $-7.2861140 .755496-0.918524$

C $-8.450394-0.5042321 .014853$

O $-7.7894201 .966130-0.357444$

C $-5.783097 \quad 0.600694-0.583774$

C $-4.9764331 .724668-1.282020$

$\begin{array}{lllll}\text { O } & -5.605338 & 0.690546 & 0.828348\end{array}$

C $1.364894-0.962779-2.791695$

O $1.828764-1.883195-3.450203$

C $1.8690890 .426553-2.919426$

C $1.3347451 .482977-2.284682$

C $1.8041262 .870416-2.365238$

C $3.0902723 .127062-3.106930$

C -3.449191 $1.600345-1.171155$

C -2.8742532 .2060340 .094570$

C -2.531663 4.4021501 .238774

$\begin{array}{lllll} & 0 & -2.346311 & 1.508114 & 0.957344\end{array}$

C -1.577426 $5.413456 \quad 0.688400$

C -0.2840495 .4833911 .035243$

C $0.678058 \quad 6.4833290 .463513$

C $1.7432405 .842467-0.398605$

C $3.0287205 .813651-0.009117$

C $1.3325205 .321851-1.758435$

C $1.0771763 .837588-1.765036$

N $-3.006640 \quad 3.583478 \quad 0.138860$

H $0.439289-4.0990290 .475210$

H $-0.981742-1.3941170 .278824$

H $0.618607-3.120698-1.711500$

H $-1.122571-2.504862-3.445822$

H $-2.120832-1.803182-2.232287$

H $-3.476789-5.231452-1.355669$

H $-1.826693-6.066819-2.728945$

H $-1.305392-4.920538-3.967517$

H -0.292611 -5.214168 -2.536697

H $-3.954833-3.5541790 .403007$

H $-3.387978-2.238828-0.593437$

H $-5.269649-2.925078-2.281912$

H $-5.900294-3.938005-0.972711$

H -5.895376 -1.836299 0.502977

H $-8.015380-2.622238-0.609653$

H -7.563617 -1.779441-2.077528

H $-9.938278-1.117427-0.947765$

H $-7.3878280 .854432-2.008426$

H $-9.117128-1.3450501 .240735$

H $-8.982953 \quad 0.3956151 .341953$

H -7.555521 -0.6186431 .629961$

H -8.729282 $2.028953-0.600050$

H $-5.2115011 .693872-2.354325$

H $-5.302792 \quad 2.699668-0.903017$

H -4.6586980 .7878301 .014702$

H $2.7227540 .533053-3.581412$

H $0.4651951 .331521-1.647164$

H $3.8958992 .501259-2.707061$

H 3.443574 4.157095-3.026594

H $2.9700842 .907870-4.173322$

H -3.118268 $0.558938-1.246284$

H - $2.9853652 .128660-2.014572$

H -2.076371 3.7750452 .011394

H -3.400731 4.9074381 .671089

H $-1.971308 \quad 6.118017-0.040670$

H $\quad 0.1071194 .7871501 .771937$

H 1.1343527 .0253021 .301837

H $\quad 0.1531897 .244855-0.127331$

H $3.7991415 .383403-0.640436$
H $3.338366 \quad 6.2128150 .950985$

H $2.0784405 .615767-2.503811$

H $0.4146225 .828104-2.084527$

H $0.1649163 .546636-1.243613$

H -3.479385 $4.056478-0.626019$

C $-0.373373-1.7070602 .911526$

O $0.712549-0.8809032 .451267$

C $1.211650-0.0937893 .543980$

C $0.191837-0.2434844 .667222$

C $-0.306328-1.6588244 .437738$

H $2.156320-0.5557313 .855364$

C 1.4714491 .3424693 .075526

H $0.639524-0.0996725 .655124$

H $-0.6360460 .467575 \quad 4.566127$

C -1.627878 -1.9605195 .122082$

H $0.455727-2.3592894 .804706$

C 2.2988331 .4524291 .778236

$\begin{array}{lllll}\text { O } & 0.218391 & 1.995084 & 2.828811\end{array}$

H 1.9501721 .9118633 .880269

H 1.7075801 .0993060 .924472

$\begin{array}{llll}\text { O } & 2.552368 & 2.842254 & 1.543683\end{array}$

C 3.6494850 .7164191 .798150

C 4.4521810 .9566800 .505442

H $3.476572-0.3551431 .920526$

$\begin{array}{lllll}\text { O } & 4.416191 & 1.149357 & 2.920228\end{array}$

H $3.8217100 .691647-0.349200$

H 4.6826522 .0253910 .418886

H -1.533070 -1.850478 6.207204

H $-2.419090-1.2832144 .783401$

H -1.949506 -2.985642 4.912733

C $-0.216261-3.1192662 .335867$

H -0.2569851 .4768212 .156390$

H 1.6888113 .2811221 .468372

H 4.5457472 .1095502 .834261

H $-1.050710-3.7556892 .650859$

H $0.696338-3.5584962 .758652$

H -1.304234 -1.247266 2.556034

C $4.956216-1.900494-0.482945$

O $5.596314-1.237956 \quad 0.614822$

C 5.7816610 .1724150 .453646

C $6.6194120 .451564-0.802420$

C $6.013326-0.199905-2.041532$

C $5.743840-1.680564-1.783160$

H $3.930365-1.527944-0.590592$

C $4.895589-3.400095-0.149598$

H $\quad 6.3768150 .502165 \quad 1.314404$

O $6.753002 \quad 1.854736-1.003587$

H $7.6304780 .051687-0.650735$

H $5.1060920 .324790-2.359470$

O $6.945754-0.074492-3.117519$

H $6.703840-2.210004-1.734391$

O $5.029020-2.225802-2.890059$

C $3.955020-3.7138690 .993357$

H $4.547649-3.970818-1.017546$

H $5.893923-3.7499310 .140082$

H $7.2672531 .983839-1.818958$

H $6.564895-0.534635-3.885217$

H $4.167057-1.778833-2.939162$

O $4.075399-5.0374921 .310502$

O $3.204119-2.9141681 .532243$

C $3.243785-5.4976702 .380409$

H $3.355825-4.8602323 .262923$

H $2.200955-5.5255632 .052582$

H $3.556966-6.5121312 .641352$

SCF Energy (B3LYP/6-31G*//MMFF)= -3245.93587082

$02 \_138$

MMFF Geometry

C $3.172244-0.4227762 .988351$

C $4.336449-1.0179742 .687035$

C $5.152905-0.8438971 .424466$

$\begin{array}{lllll}\text { O } & 4.647594 & 0.213727 & 0.580267\end{array}$

C $5.056333-2.1412790 .601153$

C $6.176490-2.334035-0.398536$

C $6.080621-2.106915-1.722989$

C $7.451451-2.8943790 .179087$

C $4.938967-1.496739-2.487135$

C $4.075412-2.522066-3.241685$ 
C $2.876895-3.015318-2.416587$

C $2.030281-4.020083-3.197810$

C $0.780659-4.439472-2.403793$

O $2.079460-1.870334-2.109517$

O $-0.110416-5.115267-3.305199$

C $0.040270-3.175290-1.908795$

C $1.127392-5.449966-1.303518$

O $-1.015301-3.514261-1.013534$

C $0.961443-2.121703-1.252756$

C $0.219228-0.778251-1.079425$

O $1.373895-2.5200990 .056685$

C 4.9560141 .4989340 .890864

O $5.6135301 .895594 \quad 1.838365$

C $4.3137722 .351044-0.134293$

C $4.053247 \quad 3.637735 \quad 0.135814$

C $3.4258644 .599929-0.773953$

C $3.0569064 .122181-2.154122$

C $1.1530990 .356838-0.638801$

C $0.3996921 .559940-0.112666$

C $-1.2468513 .303822-0.780779$

$\begin{array}{llll}\text { O } & 0.520897 & 1.932703 & 1.052468\end{array}$

C $-0.6442844 .538658-1.371721$

C $-0.3864905 .650207-0.665346$

C $0.1018276 .930841-1.278800$

C $1.3294417 .490237-0.598463$

C 1.2233968 .4376450 .349048

C $2.6815797 .037984-1.097262$

C $3.2223745 .860109-0.337054$

N $-0.3979592 .164771-1.067365$

H $2.7313560 .294352 \quad 2.303829$

H $4.742867-1.7372273 .396711$

H $6.192736-0.6271261 .697035$

H $4.091279-2.1596350 .086418$

H $5.053043-3.019740 \quad 1.261196$

H $6.946552-2.339005-2.342636$

H $8.224843-3.042302-0.582127$

H $7.860297-2.2161950 .934484$

H $7.261838-3.8649950 .648936$

H $4.329589-0.851799-1.847519$

H $5.388189-0.820708-3.227030$

H $3.690421-2.022931-4.141079$

H $4.683342-3.370232-3.578354$

H $3.245860-3.471061-1.492771$

H $2.624675-4.899822-3.474186$

H $1.703558-3.567643-4.144452$

H $0.366800-5.870992-3.688477$

H $-0.431700-2.715216-2.788213$

H $1.550753-6.362067-1.741476$

H $0.230832-5.768001-0.760439$

H $1.852990-5.066649-0.582922$

H $-1.598154-4.140791-1.475804$

H $-0.241614-0.487745-2.031601$

H $-0.588118-0.888696-0.346479$

H $0.968181-3.3755220 .267748$

H $4.0576741 .886027-1.079515$

H 4.3256064 .0260391 .116545

H $3.9542123 .836197-2.713744$

H $2.5308634 .871852-2.749062$

H $2.3884063 .256346-2.098274$

H 1.8189570 .0140150 .158521

H $1.7877190 .681302-1.471253$

H -1.4026943 .3929570 .299200$

H -2.213477 $3.109642-1.253717$

H - $0.4633564 .535411-2.444060$

H $-0.600528 \quad 5.6628510 .401012$

H $-0.7172797 .659578-1.213461$

H $0.298438 \quad 6.811792-2.351554$

H $2.102646 \quad 8.8715320 .813684$

H $0.255453 \quad 8.7950860 .684530$

H $3.4058087 .856467-0.981595$

H $2.6434606 .866440-2.176752$

H 3.5166686 .1066560 .684378

H $-0.3862161 .813788-2.020423$

C $1.087780-1.4538223 .888348$

O $0.166013-0.5408023 .259897$

C $-1.170600-0.8278373 .731978$

C -1.062836 -2.110582 4.542535

C $0.342183-2.0033165 .101435$
H -1.4442190 .0133224 .381155$

C -2.108379 -0.8969992 .521624$

H $-1.829756-2.1824425 .319532$

H $-1.150039-2.9913833 .894127$

C $0.886286-3.3225655 .621618$

H $0.345511-1.2633915 .912941$

C -3.521647-1.415825 2.832233

O $-2.2174520 .422183 \quad 1.975548$

H $-1.646935-1.5209931 .748294$

H -3.470606 -2.464892 3.146441

O $-4.036317-0.6824203 .950718$

C $-4.526569-1.2690471 .671510$

C $-4.049564-1.8947910 .349664$

H $-4.761209-0.2087571 .530960$

O $-5.753074-1.8942232 .065437$

H $-3.105369-1.4330600 .049022$

H -3.849112 -2.9616160 .508823$

H $0.261014-3.7013556 .436701$

H $0.911005-4.0846124 .835262$

H $1.903843-3.2026616 .005928$

C $2.385707-0.7224434 .235500$

H $-1.3144430 .752992 \quad 1.832343$

H $-4.931982-1.0169754 .129608$

H $-5.578819-2.8439112 .180724$

H $2.987160-1.3261164 .924262$

H 2.1665410 .2282164 .736026

H $1.282553-2.258853 \quad 3.166759$

C $-4.5517550 .516377-1.430949$

O $-5.536105-0.404069-0.945558$

C $-5.078212-1.750535-0.795397$

C $-4.595521-2.284631-2.150211$

C $-3.556159-1.365271-2.789967$

C $-4.0516740 .081971-2.813402$

H $-3.7105680 .566243-0.728264$

C $-5.208326 \quad 1.899895-1.480243$

H $-5.955906-2.343106-0.508411$

O $-4.062414-3.595799-1.997662$

H $-5.457117-2.365133-2.825900$

H $-2.594828-1.445517-2.272714$

O $-3.332338-1.813318-4.128218$

H $-4.8539500 .184266-3.555288$

O $-2.983397 \quad 0.939523-3.209104$

C $-5.3731842 .453520-0.084235$

H -4.602231 2.619909-2.041105

H $-6.1913321 .863979-1.964950$

H $-3.748972-3.885473-2.871615$

H $-2.592469-1.297763-4.490690$

H $-2.756443 \quad 0.722626-4.128907$

O $-6.565614 \quad 2.052318 \quad 0.441130$

$\begin{array}{lllll}\text { O } & -4.527553 & 3.134307 & 0.481758\end{array}$

C $-6.816278 \quad 2.470778 \quad 1.786319$

H -7.7903622 .0772972 .088674$

H -6.0517212 .0674412 .457336$

H -6.8448423 .5629801 .844050$

SCF Energy (B3LYP/6-31G**//MMFF)= -3245.91175777

$02 \_139$

MMFF Geometry

C -1.676512 -1.191613 2.278625

C -1.829196 -2.4280261 .780770$

C $-2.206205-2.7492210 .350496$

O $-1.926437-1.630426-0.525467$

C $-3.709873-3.0464890 .237907$

C $-4.041773-3.930577-0.947024$

C $-4.419371-3.490155-2.162806$

C $-3.961145-5.410896-0.673139$

C $-4.517241-2.072573-2.653513$

C $-5.964016-1.635978-2.919242$

C $-6.712488-1.240196-1.637784$

C $-8.177810-0.925461-1.943979$

C $-8.926723-0.410839-0.706094$

O $-6.067030-0.078248-1.104974$

O $-10.172156 \quad 0.128246-1.174317$

C $-8.1116900 .736878-0.071984$

C $-9.277997-1.551656 \quad 0.256114$

O $-8.681781 \quad 1.1483901 .168748$

C $-6.6165680 .393883 \quad 0.129880$

$\begin{array}{llll}\text { C } & -5.839279 & 1.667546 & 0.543578\end{array}$ 
O $-6.498891-0.582326 \quad 1.167492$ C $-0.629605-1.360736-0.833399$ O $0.357021-2.008914-0.527034$ C $-0.625447-0.069503-1.553787$ C $0.4725100 .699436-1.522669$ C $0.6069812 .028245-2.124673$ C $-0.5729952 .567384-2.891704$ C -4.3108901 .5179940 .503933$ C -3.6059432 .7484341 .052834$ C -1.3280363 .6091761 .588375$ O $-4.206621 \quad 3.7430991 .451632$ C $-0.787688 \quad 4.441418 \quad 0.468815$ C 0.5074854 .7767140 .363618 C $1.0576275 .651953-0.726431$ C $2.3061575 .083435-1.364972$ C $3.5230485 .533275-1.014805$ C $2.1379284 .064231-2.469633$ C $1.7617712 .703580-1.947099$ N -2.225171 2.5993731 .056772 H $-1.899370-0.3338571 .652328$ H -1.606551 -3.2837022 .414284$ H -1.611247 -3.609205 0.017722 H $-4.272303-2.107757 \quad 0.172120$ H $-4.075134-3.5475241 .144446$ H -4.656707 -4.234880 -2.922227 H $-4.201227-6.012680-1.556010$ H -2.951203 -5.685494 -0.352233 H -4.665312 -5.6892470 .117870$ H $-4.022358-1.365772-1.982890$ H -3.961942 -2.022548 -3.598843 H $-5.928037-0.766307-3.588391$ H -6.506577 -2.429776 -3.447372 H -6.643524 -2.060031 -0.914435 H -8.685748 -1.803488 -2.362207 H $-8.227517-0.152645-2.724342$ H $-10.6889860 .411325-0.401437$ H -8.180776 $1.599923-0.749037$ H -9.929442 -2.281051 -0.240226 H $-9.843913-1.1826431 .118615$ H -8.400927 $-2.087286 \quad 0.625319$ H $-9.583641 \quad 1.4626010 .989529$ H $-6.1029872 .491238-0.133042$ H -6.1441041 .9673471 .554482$ H -5.636807 -0.4874541 .601453$ H -1.545497 $0.234655-2.040083$ H $1.3479620 .334833-0.986128$ H $-0.7927691 .929175-3.754681$ H $-0.4232993 .578735-3.274261$ H -1.461861 2.611648 -2.252209 H -3.9961740 .6582591 .102072$ H $-3.972361 \quad 1.362882-0.527082$ H -0.5349283 .0850572 .130180$ H -1.871943 4.2464292 .292805 H -1.494719 4.810253 -0.270594 H $1.2058304 .436400 \quad 1.125213$ H $1.2817436 .630999-0.282759$ H $0.3074235 .843060-1.503799$ H $4.4236015 .159809-1.489622$ H $3.6461176 .285958-0.242947$ H $3.0789953 .949022-3.023855$ H $1.4296984 .451265-3.206846$ H $2.5524612 .231795-1.362229$ H $-1.812873 \quad 1.7710200 .638102$ C $0.198041-0.1192673 .468293$

O $1.171783-0.9888992 .859518$ C $2.475633-0.6767423 .395329$ C 2.2817810 .5279744 .306706 C $0.845724 \quad 0.3464204 .766303$ H $2.774118-1.5556283 .979842$ C $3.433395-0.4638542 .217685$ H 2.9986320 .5468405 .133038 H 2.3876901 .4643173 .744467 C $0.232396 \quad 1.6063885 .350460$ H $0.811995-0.4569965 .514105$ C 4.8323090 .0354702 .613150 O $3.567335-1.7164131 .535830$ H 2.9787680 .2323881 .503084 H 4.7611021 .0516143 .018433
O $5.322521-0.7906363 .677046$

C $5.872375-0.0035781 .474981$

C 5.4349750 .7329390 .197132

H $6.120269-1.0465631 .250941$

O 7.0817560 .5914021 .959625

H $4.4884600 .317845-0.159700$

H 5.2526601 .7882700 .435176

H $0.790393 \quad 1.9308716 .234856$

H 0.2400972 .4292764 .627785

H $-0.805447 \quad 1.4323745 .651346$

C -1.119532 -0.8771913 .637970$

H $2.674455-2.0028681 .276894$

H $6.209330-0.4668263 .910856$

H $6.895127 \quad 1.5247842 .158445$

H -1.847717 -0.2696694 .185407$

H $-0.954353-1.8005364 .205634$

H 0.0620010 .7318142 .787223

C $5.911207-1.529463-1.778616$

O $6.910654-0.689788-1.187881$

C $6.4889690 .653832-0.931980$

C $6.0577861 .319046-2.246554$

C $5.0105010 .491504-2.989473$

C $5.469595-0.959671-3.130683$

H $5.047641-1.604985-1.107153$

C $6.517904-2.927914-1.931194$

H $7.3768501 .190041-0.574216$

O $5.5425672 .623802-2.011216$

H $6.9331461 .431428-2.899456$

H $4.0338660 .541394-2.495696$

O $4.824498 \quad 1.034017-4.298446$

H $6.292898-1.015845-3.854232$

O $4.393697-1.743507-3.641517$

C $6.759017-3.559177-0.580164$

H $5.873630-3.599179-2.509876$

H $7.483704-2.878740-2.448671$

H $6.233683 \quad 3.133337-1.554825$

H $4.5588981 .963454-4.190056$

H $4.114957-1.340848-4.481826$

O $5.576386-3.968378-0.038301$

O $7.864473-3.663165-0.064587$

C $5.665770-4.5469301 .267255$

H $6.075748-3.8207901 .975867$

H $4.656470-4.8178231 .588558$

H $\quad 6.280692-5.4514511 .239531$

SCF Energy (B3LYP/6-31G**//MMFF) $=-3245.91044956$

0214

MM̄FF Geometry

C $1.613637-0.3232682 .943167$

C 2.8000240 .1276173 .379848

C 3.6014221 .2817222 .827857

O 2.8432152 .0681891 .886144

C 4.8106420 .7245762 .055915

C 5.9461321 .7148811 .906059

C 6.1784992 .4683350 .813818

C 6.8917691 .7756153 .078637

C $5.3382712 .603258-0.425061$

C $5.9193501 .861901-1.638082$

C $5.4186740 .413131-1.745451$

C $6.075496-0.307195-2.923397$

C $5.499912-1.717334-3.125225$

O $4.0013120 .467813-1.939289$

O $5.964444-2.170522-4.405763$

C $3.959269-1.625211-3.175097$

C $6.045303-2.704671-2.086610$

O $3.366385-2.921794-3.208565$

C $3.350819-0.804921-2.012363$

C $1.848612-0.549018-2.287009$

O $3.492215-1.542719-0.797343$

C 2.0816313 .0882052 .356544

O $1.8804223 .388763 \quad 3.520964$

C 1.5337543 .7851201 .172035

C 0.5447634 .6788301 .310045

C -0.0878635 .4279980 .221714$

C $0.4804755 .259957-1.164399$

C $1.1796060 .425715-1.307013$

C $-0.2890320 .642176-1.633116$

C $-2.3279581 .768967-0.746452$ 
O $-0.8390700 .147917-2.614000$

C -2.528409 $3.101350-1.395832$

C $-3.3117204 .060899-0.878928$

C -3.565286 $5.384287-1.543424$

C $-3.3585616 .559552-0.612620$

C $-4.4036557 .172133-0.030964$

C $-1.9496607 .076070-0.424852$

C -1.143557 6.2168490 .511722

N $-0.913677 \quad 1.451390-0.693476$

H 1.1072900 .1664962 .117564

H $3.280576-0.4058754 .198879$

H 3.9295491 .9153033 .661114

H $4.4803400 .382803 \quad 1.069337$

H $5.213195-0.1674362 .555323$

H 7.0599023 .1092620 .816385

H 7.7033042 .4945122 .923521

H 6.3570822 .0743173 .985850

H 7.3476700 .7950723 .250379

H $4.3001572 .311993-0.245802$

H $5.3012343 .674641-0.662486$

H $5.5995672 .404862-2.537513$

H $7.0156381 .887932-1.616963$

H $5.643403-0.112226-0.811949$

H $7.164390-0.351376-2.795230$

H $5.9065240 .270558-3.843239$

H $5.658263-3.084287-4.532535$

H $3.691991-1.135102-4.121868$

H $7.136309-2.779014-2.168529$

H $5.661855-3.715935-2.261691$

H $5.807668-2.421442-1.058994$

H $3.683103-3.370903-4.010055$

H $1.736059-0.130375-3.296025$

H $1.301019-1.499043-2.268443$

H $2.823035-1.244539-0.162195$

H 1.9581253 .5313820 .206919

H 0.1526964 .8719092 .307613

H $1.5190995 .607252-1.195787$

H $-0.063508 \quad 5.813846-1.931784$

H $0.454773 \quad 4.208632-1.470921$

H $1.2409270 .041795-0.284855$

H $1.6776311 .400962-1.341720$

H $-2.701343 \quad 1.7500190 .282109$

H $-2.8487231 .001614-1.325862$

H -2.034611 $3.273282-2.349155$

H -3.831331 3.8722780 .058063

H -4.600654 5.377762 -1.908602

H -2.937640 5.511978 -2.434022

H $-4.266733 \quad 8.0325630 .615504$

H $-5.4195776 .822925-0.183925$

H -1.9722698 .0871250 .004054$

H $-1.4795267 .203421-1.403770$

H -1.483648 6.2675161 .547016

H -0.3659711 .8500140 .063066$

C $1.096039-2.7335702 .567565$

O $0.114086-2.6433941 .513918$

C $-0.369732-3.9715461 .224776$

C $0.510246-4.9311992 .016980$

C $0.856226-4.0889783 .228092$

H $-1.387820-4.0330871 .624016$

C $-0.371969-4.223529-0.286560$

H $-0.012443-5.8582162 .271787$

H $1.418019-5.1982731 .462768$

C $2.045162-4.6207274 .009973$

H $-0.019750-4.0370613 .888406$

C -1.295750 -3.281239-1.085325

O $0.961203-4.065752-0.788091$

H $-0.646803-5.265362-0.487771$

H $-0.929129-2.250687-1.028457$

O $-1.225478-3.663773-2.463911$

C $-2.769838-3.328892-0.642860$

C $-3.706608-2.510145-1.551465$

H -2.843807 -2.9477290 .379492$

O $-3.218397-4.684486-0.617732$

H $-3.325390-1.486239-1.621305$

H -3.694782 -2.929726 -2.564551

H $1.837711-5.6276444 .386655$

H $2.944194-4.6739823 .386606$

H $2.269125-3.9800914 .868413$
C $0.951499-1.5455113 .519988$

H $1.260481-3.171680-0.549302$

H $-0.290556-3.619643-2.728711$

H -3.121743 $-5.041795-1.517250$

H $1.398791-1.7811704 .492412$

H $-0.105456-1.3156983 .696762$

H $2.082547-2.7241962 .083751$

C -4.992362 $-0.745265 \quad 0.580977$

O $-5.265189-2.1288430 .330455$

C $-5.168649-2.514424-1.045067$

C $-6.146216-1.688658-1.894276$

C $-5.990197-0.187488-1.660850$

C $-6.0025790 .130318-0.165294$

H $-3.977066-0.5059300 .247607$

C $-5.058662-0.5282332 .096582$

H $-5.511649-3.555825-1.091120$

O $-5.980487-1.965239-3.280779$

H $-7.174799-1.969717-1.632311$

H $-5.0812210 .198671-2.136327$

$\begin{array}{llll}\text { O } & -7.084305 & 0.499561 & -2.272467\end{array}$

H $-7.011747-0.0270290 .236363$

$\begin{array}{lllll}\text { O } & -5.673626 & 1.503240 & 0.026137\end{array}$

C $-3.844981-1.1306352 .770277$

H -5.0722420 .5343012 .361723$

H $-5.967067-0.9878772 .504086$

H $-6.104572-2.921696-3.404692$

H $-7.0847120 .260103-3.215232$

H $-6.3042292 .028869-0.495404$

O $-4.232858-2.0106343 .736439$

O $-2.689754-0.8497962 .475802$

C $-3.168192-2.6556834 .442445$

H $-2.543944-1.9142144 .950197$

H $-3.609251-3.3162875 .193548$

H -2.569529 -3.2582933 .753501$

SCF Energy (B3LYP/6-31G**//MMFF)= -3245.92550241

02140

MM̄FF Geometry

C $2.9842350 .096223 \quad 4.237010$

C $2.907791-0.459208 \quad 3.018402$

C $4.118683-0.7369052 .163422$

$\begin{array}{lllll}\text { O } & 4.039004 & 0.095933 & 0.985999\end{array}$

C $4.095518-2.1956431 .678187$

C $5.454383-2.7033221 .242921$

C $5.912205-2.703666-0.024102$

C $6.295196-3.2865512 .349778$

C $5.266190-2.120920-1.249696$

C $4.716152-3.183468-2.209698$

C $3.321505-3.690299-1.810287$

C $2.853144-4.784313-2.770612$

C $1.413218-5.227298-2.474771$

O $2.424509-2.575412-1.868041$

O $0.956042-6.006163-3.591477$

C $0.511239-3.977413-2.393442$

C $1.338469-6.143623-1.247298$

O $-0.801442-4.357674-1.988077$

C $1.076632-2.864129-1.477164$

C $0.250867-1.564004-1.640165$

O $0.998745-3.296563-0.117604$

C 4.5095611 .3670041 .058224

O 5.0111791 .9206662 .021953

C $4.2675861 .981324-0.264995$

C $4.267803 \quad 3.315480-0.391072$

C $3.9687094 .058929-1.616873$

C $3.7168433 .263080-2.871799$

C $0.878559-0.319958-0.990350$

C $0.000927 \quad 0.909462-1.165902$

C $-0.176676 \quad 3.295078-0.462814$

O $-1.0372860 .903414-1.822254$

C $0.2520394 .144262-1.618452$

C $0.5845075 .439223-1.498827$

C $0.9789466 .313186-2.656764$

C $2.2571267 .085013-2.405086$

C $2.2225678 .379933-2.047616$

C $3.5735826 .378527-2.645705$

C $3.9039945 .405048-1.550647$

N $0.5127822 .017479-0.503910$

H 3.9699460 .3691824 .611182 
H $1.943824-0.7300162 .598152$ H $5.049363-0.5279122 .705845$ H $3.382208-2.2973740 .853515$ H $3.727212-2.8593952 .472219$ H $6.895023-3.135841-0.209904$ H $7.268916-3.6388371 .993089$ H $6.480506-2.5355533 .124394$ H $5.782383-4.1390572 .807250$ H $4.491762-1.392442-0.999297$ H $\quad 6.042929-1.550779-1.775795$ H $4.646908-2.721090-3.203295$ H $5.417835-4.022614-2.291328$ H $3.360578-4.076145-0.785944$ H $3.533781-5.644417-2.742298$ H $2.892439-4.406158-3.801969$ H $1.557735-6.763223-3.695284$ H $\quad 0.421665-3.582943-3.415611$ H $1.948956-7.041420-1.402273$ H $\quad 0.316198-6.502451-1.083707$ H $1.687080-5.662989-0.330943$ H -1.395666 -3.612529-2.175891 H $\quad 0.126975-1.349470-2.709749$ H $-0.751063-1.708052-1.217064$ H $0.928121-2.5216480 .461382$ H 4.046849 1.315231-1.091485 H 4.4715793 .9213280 .490846 H $4.6028012 .674478-3.134826$ H $3.4779963 .877853-3.741499$ H $2.8709482 .580215-2.734987$ H $1.027256-0.4789140 .082492$ H $1.850940-0.104524-1.446623$ H 0.0567643 .7593090 .500589 H -1.255700 $3.120602-0.515327$ H $\quad 0.2630463 .682720-2.602980$ H $0.5390045 .908732-0.518623$ H $0.1528037 .012819-2.839481$ H $1.0844875 .730339-3.580225$ H $3.1335178 .948077-1.890749$ H $1.2818798 .899827-1.898422$ H $4.3932107 .108072-2.690619$ H $3.5545945 .919427-3.637539$ H $4.0986115 .886191-0.590895$ H $1.4017071 .941248-0.019103$ C $0.473247-0.1672954 .862070$ O $-0.0932330 .500683 \quad 3.717282$ C - -1.4784610 .8067923 .985610$ C -1.840068 0.0382055 .247216 C -0.5275390 .0473175 .999780$ H -1.512028 $1.887733 \quad 4.170782$ C -2.3339650 .4411842 .764806$ H -2.6569490 .5056295 .805137$ H -2.135457 -0.990863 5.006279 C $-0.455018-1.0005057 .097682$ H -0.3852331 .0423686 .442864$ C -3.8442750 .6376442 .987992$ O -1.9124661 .2506591 .664421$ H -2.121134 -0.595302 2.481819 H -4.190914 -0.0134313 .799185$ O -4.0549631 .9852503 .430628$ C -4.7192210 .4091921 .740580$ C $-4.549329-0.994751 \quad 1.135994$ H -4.5074551 .1855220 .997660$ O H -3.523196 -1.101574 0.770522 H -4.685171 -1.7407391 .929163$ H -1.240157 -0.8319427 .842107$ H -0.584193 -2.011352 6.696476 H $0.509976-0.9633527 .612364$ C $1.8567350 .408375 \quad 5.185028$ H -2.121345 2.1754681 .881777 H -5.0108442 .0968253 .572001$ H $-6.6146120 .704127 \quad 1.348163$ H $2.167840 \quad 0.0404406 .170435$ H 1.7885841 .5013725 .255747 H $0.546056-1.2338384 .613251$ C $-4.412952-0.162667-1.796264$ O $-5.591379-0.281068-0.989910$ C $-5.544218-1.314260-0.002538$
C $-5.320466-2.675090-0.675646$

C $-4.099370-2.660114-1.592228$

C $-4.154092-1.471355-2.552279$

H $-3.5548210 .078741-1.157964$

C $-4.6328331 .004683-2.766410$

H $-6.542302-1.3493110 .452068$

O $\quad-5.180817-3.6928340 .309482$

H $-6.208119-2.924000-1.271516$

H $-3.174109-2.645231-1.005892$

O $-4.060668-3.872983-2.347444$

H $-4.941573-1.636551-3.298515$

O $-2.914858-1.378555-3.250458$

C $-4.4549752 .334851-2.072265$

H $-3.9090390 .980575-3.589195$

H $-5.6330230 .979727-3.214842$

H $-5.009285-4.528715-0.157241$

H -4.870994 -3.920109-2.882899

H $-2.767050-2.230319-3.696105$

O $-5.5364832 .606813-1.288416$

O $-3.4671003 .045031-2.208862$

C $-5.4660403 .827739-0.545548$

H -6.3678253 .9047470 .067592$

H $-5.4293254 .682732-1.227232$

H -4.5935243 .8210780 .115044$

SCF Energy (B3LYP/6-31G**//MMFF) $=-3245.91180767$

02141

MM̄FF Geometry

C -0.6389883 .2531351 .700351$

C -1.7062803 .6700842 .400652$

C -2.9823502 .9104122 .677704$

O -2.9156481 .5546792 .187434$

C -4.1644443 .6058111 .981852$

C -5.5096793 .0764562 .439005$

C -6.2850902 .2363091 .726567$

C -5.9900703 .6026683 .767607$

C -5.9754661 .6126440 .394017$

C -6.3821310 .1321710 .364181$

C $-6.123464-0.494809-1.012515$

C $-6.719915-1.900779-1.089623$

C $-6.415770-2.571698-2.436546$

O $-4.707936-0.557234-1.215715$

O $-6.748796-3.960785-2.296486$

C $-4.901126-2.468337-2.710015$

C $-7.310576-2.024884-3.554762$

O $-4.586680-2.962192-4.010397$

C $-4.321030-1.047337-2.506073$

C $-2.778604-1.113704-2.557207$

O $-4.785313-0.195417-3.554269$

C $-2.4733200 .602030 \quad 3.051381$

$\begin{array}{lllll}\text { O } & -2.101804 & 0.764801 & 4.202617\end{array}$

C $-2.496089-0.6919782 .336133$

C $-1.802752-1.7242862 .838034$

C -1.677339 -3.0544012 .240625$

C $-2.563811-3.3798491 .068628$

C $-2.0657060 .181886-2.151193$

C $-0.577077-0.079409-2.038277$

C $1.103897-1.129420-0.518635$

O $0.1888390 .144578-2.972309$

C $1.056575-2.624501-0.464767$

C $1.565360-3.3455260 .545754$

C $1.528867-4.8467180 .599570$

C $1.075345-5.3795451 .942521$

C $1.943245-5.9774902 .776355$

C $-0.396608-5.2830052 .288736$

C $-0.771659-3.9067812 .763629$

N $-0.225882-0.634528-0.817598$

H -0.6258652 .2656941 .248484$

H -1.6738944 .6678992 .836166$

H -3.1441502 .9092063 .762706$

H -4.0626273 .5097120 .894428$

H -4.140774 4.6859352 .181083

H $-7.256799 \quad 1.9681402 .139939$

H $-6.990003 \quad 3.2364374 .023479$

H $-5.3137393 .296326 \quad 4.571351$

H -6.0359774 .6964453 .748839$

H $-6.5364872 .156439-0.375446$

H $-4.913976 \quad 1.6961290 .144557$ 
H $-5.814591-0.4099471 .131900$ H -7.4470940 .0417850 .613121$ H $-6.5721590 .151143-1.776394$ H -7.801818 -1.877972 -0.909080 H $-6.295139-2.520752-0.287339$ H -6.487832 -4.410832 -3.118494 H $-4.406610-3.148965-2.003217$ H -8.367554 -2.179107 -3.306240 H -7.144984 -2.563139-4.494666 H -7.163704 -0.958403 -3.738421 H $-4.833984-2.276950-4.654482$ H -2.436615 -1.915336 -1.892643 H -2.450292 -1.384102 -3.569265 H $-4.175677 \quad 0.554321-3.642127$ H -3.049205 -0.7488891 .405723$ H -1.241589-1.573541 3.759772 H -3.613412 -3.173294 1.307602 H $-2.523090-4.4284040 .767492$ H -2.279887 -2.781991 0.199176 H -2.220172 $0.973957-2.891172$ H $-2.4329080 .562062-1.191294$ H $1.403174-0.6911980 .437241$ H $1.807153-0.813181-1.292856$ H $\quad 0.593000-3.138939-1.303412$ H $2.054002-2.8303751 .369469$ H $2.539456-5.2108760 .373637$ H $0.879226-5.256693-0.183842$ H $1.622507-6.3820713 .730643$ H $2.995067-6.0706192 .526476$ H $-0.642288-5.9880813 .093655$ H $-0.978847-5.6144451 .425142$ H -0.195200 -3.577934 3.629937 H $-0.947368-0.783803-0.119167$ C 0.8206654 .4237810 .023251 O $1.638723 \quad 3.388730-0.569643$ C $2.6817854 .016689-1.341400$ C $2.2519365 .466352-1.522030$ C $1.5805755 .733719-0.192760$ H $3.5870344 .000704-0.723400$ C $2.928174 \quad 3.276398-2.657865$ H $3.0988456 .130540-1.719193$ H $1.5333285 .576693-2.342846$ C $0.6938856 .967538-0.199713$ H 2.3552405 .8514190 .577138 C $3.4138201 .821956-2.499851$ O $1.7036883 .257441-3.402211$ H $3.6458563 .843939-3.262859$ H $2.6015481 .202880-2.108522$ O $3.6892451 .321308-3.815197$ C $4.6635961 .643614-1.615274$ C $5.2749940 .232410-1.728281$ H $4.3944461 .859180-0.576621$ O $5.6436862 .601563-2.019995$ H $4.474875-0.510403-1.651027$ H $5.7275250 .125412-2.721296$ H $1.2838717 .863372-0.419268$ H -0.094552 $6.890786-0.956123$ H 0.2132837 .1097750 .773283 C 0.5822964 .1111331 .502520 H $1.8724992 .769184-4.226144$ H $4.4437261 .823606-4.168186$ H $6.3004572 .673178-1.307584$ H 0.4667895 .0425392 .069487 H 1.4449733 .5812551 .923625 H $-0.1243534 .438985-0.535796$ C $4.988825-1.0789051 .037474$ $\begin{array}{llllll}\text { O } & 5.831730 & 0.021191 & 0.669547\end{array}$ C $6.361321-0.035696-0.660894$ C $7.154232-1.335838-0.865233$ C $6.340623-2.567910-0.483976$ C $5.753368-2.4032830 .916701$ H $4.105621-1.1005940 .388863$ C $4.515128-0.8427672 .480144$ H $7.0895820 .780836-0.731643$ O $7.596696-1.462588-2.212060$ H $8.053191-1.307477-0.235587$ H $5.551308-2.761947-1.219345$ O $7.185480-3.721096-0.504510$
H $\quad 6.557698-2.4432151 .662331$

O $4.871175-3.490617 \quad 1.180856$

C 3.3861880 .1648202 .540715

H $4.141643-1.7808192 .906507$

H $5.329121-0.4676583 .110087$

H $8.125770-0.673983-2.421675$

H $7.904643-3.5791330 .134405$

H $5.375789-4.3126941 .056335$

O $2.574867-0.1307883 .598655$

O $3.242654 \quad 1.1033101 .768972$

C $1.4392620 .723898 \quad 3.760657$

H $\quad 0.7945920 .6616682 .879347$

H $\quad 0.8761180 .3795124 .631940$

H 1.7608701 .7549343 .934924

SCF Energy (B3LYP/6-31G**//MMFF)= -3245.91706843

02142

MM̄FF Geometry

C -0.308705 -3.3159111 .424957$

C $0.656273-3.7071192 .271244$

C $1.686357-2.8448102 .959459$

O $1.487074-1.4341132 .715144$

C $3.099849-3.2159452 .476627$

C $4.194694-2.6099373 .336985$

C $4.945447-1.5489932 .980904$

C $4.452915-3.3126994 .644613$

C $4.813293-0.7338801 .726740$

C $5.906689-1.0873670 .710829$

C $5.619130-0.577359-0.710395$

C $5.5044080 .946762-0.781798$

C $5.1124131 .429678-2.186449$

O $4.408168-1.202048-1.149175$

O $4.7110022 .805372-2.082869$

C $3.8738930 .634571-2.651161$

C $6.2959931 .395671-3.158878$

O $3.5371530 .955147-3.997652$

C $4.023339-0.893597-2.494632$

C $2.713013-1.670752-2.785093$

O $5.004474-1.364808-3.426431$

C $0.672450-0.7660193 .573425$

O $-0.048679-1.2646674 .427963$

C $0.8025880 .702948 \quad 3.435033$

C 1.4891291 .3211482 .461660

C 1.7281472 .7649442 .367078

C 1.2497523 .6306313 .504079

C $1.524949-1.321956-1.880741$

C $0.699766-0.165538-2.414199$

C $-0.5612241 .845352-1.673989$

O $0.5083360 .011546-3.615214$

C $0.3964402 .991994-1.706023$

C $0.2394934 .099823-0.967585$

C 1.223605 5.232033-0.952589

C 1.7118615 .5447010 .444264

C 1.2396866 .6129011 .109145

C 2.8015014 .6781371 .037415

C 2.3874733 .2525031 .295040

N $0.1765920 .626167-1.402498$

H $-0.398379-2.2713741 .141589$

H $\quad 0.701083-4.7612772 .542418$

H $1.611554-3.0390744 .037036$

H $3.222004-2.9166931 .428744$

H $3.229652-4.3067222 .484236$

H $5.728576-1.2141243 .659179$

H $5.293985-2.8746265 .192679$

H $3.573800-3.2562065 .293642$

H $4.689357-4.3672264 .468903$

H $3.820337-0.8535541 .285127$

H 4.8896590 .3246792 .002698

H $6.871907-0.6973591 .056744$

H $6.001280-2.1806020 .659923$

H $6.440211-0.921626-1.350581$

H $6.4378801 .421602-0.455354$

H $4.7324711 .295984-0.084971$

H $5.4549273 .307247-1.708098$

H $3.0403430 .975251-2.027086$

H $7.1012812 .050111-2.803953$

H $6.0089411 .781051-4.143471$

H $6.7179480 .396991-3.287793$ 
H $3.4315121 .920579-4.048017$

H $2.450687-1.578061-3.846122$

H $2.923989-2.738360-2.628942$

H $4.857061-2.313129-3.577325$

H 0.3146961 .2639914 .226470

H $1.9441850 .723767 \quad 1.674383$

H 0.1572273 .5982923 .577993

H 1.5288514 .6806963 .395849

H $1.678353 \quad 3.292426 \quad 4.454067$

H $0.844014-2.180933-1.842570$

H $1.856510-1.130672-0.854825$

$\mathrm{H}-1.3020901 .956031-0.878326$

H -1.075948 $1.761124-2.635996$

H $1.2638532 .903728-2.355825$

H $-0.6372874 .197070-0.331446$

H $0.7299756 .110283-1.388425$

H $2.0866435 .024667-1.597580$

H 1.6023276 .8704512 .098564

H $0.477242 \quad 7.2543830 .679467$

H 3.1990395 .1177511 .960751

H 3.6558954 .6694870 .347504

H 2.6961692 .5587150 .513078

H $0.4324840 .437274-0.437882$

C $-1.443453-4.253619-0.637738$

O $-2.031559-3.011411-1.080341$

C $-3.123167-3.304396-1.971646$

C $-2.914106-4.744897-2.419729$

C $-2.357306-5.367440-1.158270$

H $-4.041707-3.247181-1.374822$

C $-3.188097-2.295075-3.121400$

H $-3.842747-5.214301-2.758196$

H $-2.180768-4.818923-3.230872$

C $-1.643720-6.687710-1.396097$

H $-3.186199-5.533212-0.456574$

C $-3.261295-0.822249-2.681707$

O $-2.006285-2.450488-3.918957$

H -4.036316 -2.540977 -3.771975

H -2.303619 -0.518215 -2.247462

O $-3.441717-0.026209-3.860743$

C $-4.386534-0.477191-1.685137$

C $-4.5032951 .045665-1.484104$

H $-4.182371-0.973743-0.732167$

O $-5.623126-0.982750-2.183577$

H $-3.4983471 .452084-1.347435$

H $-4.9120711 .493198-2.398448$

H $-2.332510-7.426370-1.818890$

H $-0.806739-6.571387-2.092800$

H -1.248014 -7.092177 -0.459212

C $-1.344460-4.2670950 .888222$

H -2.146885 -1.966248 -4.749820

H - $2.607065-0.047026-4.358639$

H $-6.287345-0.885032-1.480967$

H -1.129891 -5.2860301 .232117$

H $-2.311395-3.9799491 .317707$

H $-0.447449-4.318485-1.093388$

C $-3.732472 \quad 1.3597551 .452678$

$\begin{array}{lllll}\text { O } & -4.989074 & 0.895433 & 0.944471\end{array}$

C $-5.3973351 .473260-0.299049$

C $-5.5138832 .997219-0.141581$

C $-4.203088 \quad 3.6069400 .352306$

C -3.7245312 .8870691 .611681$

H -2.926261 $1.041340 \quad 0.781315$

C -3.511867 0.6934992 .821323

H $-6.4047341 .090104-0.501757$

O $-5.897137 \quad 3.584343-1.380203$

H -6.3075823 .2214580 .582936$

H -3.430654 $3.594561-0.425131$

O -4.4127274 .9811100 .686169$

H $-4.363957 \quad 3.1781742 .455220$

O $-2.397903 \quad 3.3130891 .920657$

C $-3.344429-0.8060582 .724185$

H -2.613056 1.0902463 .305484

H $-4.3826820 .887393 \quad 3.459677$

H $-6.0830974 .524203-1.216890$

H -4.604010 $5.457243-0.139356$

H -2.4133064 .2826371 .994783$

O $-3.447658-1.3429573 .975890$

O $-3.136081-1.4256061 .691340$
C $-3.330484-2.7669354 .055379$

H $-3.601324-3.0733895 .069192$

H $-4.012000-3.2532613 .350584$

H -2.296900 -3.065717 3.863383

SCF Energy (B3LYP/6-31G**//MMFF)= -3245.91029450

$02 \_143$

MM̄FF Geometry

C $2.828935-1.450573-2.924794$

C $2.090573-0.965705-1.915566$

C $0.742238-1.482919-1.499404$

O $-0.127993-0.321335-1.515907$

C $0.759191-2.055465-0.066015$

C $1.093799-3.5339600 .019581$

C $0.229642-4.4914200 .414251$

C $2.505507-3.921736-0.334462$

C $-1.193595-4.3124590 .858292$

C $-2.173647-4.543036-0.296816$

C $-3.610447-4.1646830 .087977$

C $-4.569500-4.408494-1.079586$

C $-5.994651-3.925849-0.762542$

O $-3.619310-2.7769840 .438451$

O $-6.743959-3.911405-1.987109$

C $-5.933312-2.481198-0.217688$

C $-6.730799-4.9136350 .149006$

$\begin{array}{lllll} & 0 & -7.214354 & -2.049002 & 0.233869\end{array}$

C -4.885004 -2.292550 0.901942

C $-4.725496-0.791768 \quad 1.239394$

O $-5.320667-2.9831652 .075522$

C -1.431964 -0.495702 -1.854295

O $-1.990593-1.544540-2.128698$

C $-2.069092 \quad 0.839247-1.857776$

C $-3.3437750 .969097-2.252827$

C $-4.0728412 .232473-2.322256$

C $-5.4984862 .131114-1.853023$

C $-3.480931-0.4800132 .080606$

C -3.5238390 .9186462 .663065$

C $-3.386506 \quad 3.3159432 .052241$

O $\quad-3.562166 \quad 1.105652 \quad 3.877655$

C $-4.041794 \quad 4.148600 \quad 0.999000$

C $-3.386538 \quad 5.035744 \quad 0.235126$

C $-4.0593865 .931160-0.765028$

C $-3.5153875 .767250-2.165358$

C $-2.5835036 .606183-2.648660$

C $-4.1271744 .694680-3.037278$

C $-3.500527 \quad 3.347119-2.820187$

N -3.5046561 .9148091 .699588$

H $2.464682-2.290790-3.509277$

H $2.486290-0.134696-1.338044$

H $0.375107-2.227012-2.217463$

H $-0.213855-1.8543580 .393210$

H $1.468280-1.498598 \quad 0.562039$

H $0.575202-5.5243360 .429948$

H $2.749591-4.938261-0.006679$

H $2.656377-3.883233-1.416112$

H $3.221007-3.250350 \quad 0.148962$

H $-1.399635-5.033207 \quad 1.659764$

H $-1.339172-3.3286531 .313388$

H $-1.858724-3.941250-1.157368$

H -2.136181 $-5.594446-0.607883$

H $-3.902507-4.7613840 .960183$

H $-4.576242-5.467679-1.365436$

H $-4.207066-3.863764-1.962723$

H $-6.296514-3.314056-2.610273$

H $-5.662401-1.823768-1.055659$

H $-6.813924-5.892566-0.338595$

H $-7.759228-4.5867490 .338841$

H $-6.234375-5.0649501 .109690$

H -7.840064 -2.181826 -0.498661

H $-4.643855-0.2214320 .307401$

H $-5.619787-0.4304261 .762808$

H -4.970934 -2.534185 2.861142

H -1.472992 $1.691930-1.549845$

H $-3.9185240 .090599-2.539331$

H $-6.007163 \quad 3.096080-1.798351$

H $-5.5409151 .702566-0.845934$

H $-6.0778901 .487698-2.524162$

H -3.382935 -1.179919 2.917391 
H $-2.570178-0.5761241 .477610$ H -2.319542 3.5390812 .155218 H -3.862703 3.4953503 .021702 H -5.1180824 .0409800 .887369$ H -2.313295 5.1579890 .363634 H $-3.9175976 .967169-0.429757$ H $-5.1455205 .776011-0.774570$ H -2.213239 $6.514696-3.664416$ H -2.165973 $7.399032-2.036873$ H -3.986287 4.951814-4.096029 H -5.212304 4.686509-2.900073 H -2.465085 $3.292971-3.157998$ H -3.2815851 .6518560 .744106$ C $5.309581-1.595092-2.541764$ O $5.175849-1.342871-1.127898$ C $6.430961-0.871898-0.610080$ C $7.471710-1.278505-1.643372$ C $6.695768-1.077002-2.931827$ H $6.371007 \quad 0.222824-0.579124$ C $6.654782-1.4146560 .805395$ H $8.377504-0.667706-1.585938$ H $7.761232-2.330397-1.538888$ C $7.302972-1.789356-4.127908$ H $6.648342-0.000602-3.145876$ C $5.471400-1.1840141 .768018$ O $6.874200-2.8303670 .737962$ H $7.572832-0.9880491 .224880$ H $4.618320-1.8020741 .465573$ O $5.862598-1.6499573 .065900$ C $5.016190 \quad 0.281116 \quad 1.895440$ C 3.8239010 .4303472 .856253 H $4.718497 \quad 0.6527340 .913273$ O 6.1033601 .0867392 .341953 H $3.006848-0.1977782 .483879$ H 4.1017310 .0412493 .843362 H $8.312071-1.414275-4.327506$ H $7.373140-2.869124-3.958673$ H $6.697956-1.627594-5.025649$

C $4.175894-0.888126-3.287662$ H $\quad 6.097066-3.2276820 .308376$ H $6.139995-2.5771142 .969043$ H 6.3899220 .7404243 .204449 H $4.311302-0.981052-4.371187$ H $4.1928220 .183731-3.056174$ H $5.243171-2.681869-2.678853$ C 1.9839362 .0864381 .050082 O 3.1012132 .5670621 .804610 C 3.3405091 .8872803 .040545 C 2.1068292 .0122523 .946519 C $0.849410 \quad 1.494343 \quad 3.252851$ C 0.6923482 .1445161 .880252 H $2.181787 \quad 1.0593960 .723925$ C $1.8379902 .975988-0.196914$ H 4.1590132 .4294583 .530608 O $2.292631 \quad 1.300913 \quad 5.165581$ H $1.950583 \quad 3.0667194 .208494$ H 0.8523110 .4010363 .174695 O $-0.294667 \quad 1.8334694 .040330$ H 0.3857373 .1889222 .019869 O -0.3634341 .4970751 .173818$ C $2.9793392 .835886-1.181590$ H $0.9164012 .717352-0.730766$ H 1.7992274 .0303250 .102454 H 3.0896471 .6579805 .593169 H -0.1662431 .4407314 .920903$ H -0.1105910 .5675191 .043533$ O $2.6810803 .559052-2.302644$ O $4.0025512 .193012-0.998496$ C $3.6752043 .541889-3.331948$ H $4.6402293 .877823-2.940326$ H $3.7578652 .537186-3.755601$ H $3.359414 \quad 4.230176-4.120379$ SCF Energy (B3LYP/6-31G**//MMFF) $=-3245.91256348$

\section{$02 \_144$}

MMFF Geometry

C $-0.371253-3.627348-0.332468$

C $-1.000179-2.505900-0.716180$
C $-1.198792-2.142794-2.167729$

O $-0.866573-0.743766-2.348893$

C $-2.662425-2.287993-2.623786$

C $-3.305777-3.622244-2.312076$

C $-4.241443-3.797383-1.357211$

C $-2.863955-4.773793-3.174138$

C $-4.769955-2.743079-0.423865$

C $-6.134889-2.227325-0.892709$

C $-6.495566-0.883186-0.243657$

C $-7.939344-0.507152-0.580518$

C $-8.3041760 .884663-0.050073$

O $\quad-5.5992200 .099149-0.773809$

O $-9.540377 \quad 1.282356-0.663520$

C -7.230337 $1.890987-0.513121$

C $-8.5593980 .863778 \quad 1.461490$

O

C $-5.7747861 .426604-0.257803$

C $-4.7926512 .338752-1.034403$

O $\quad-5.4342801 .4977921 .125322$

C $0.442828-0.383545-2.336782$

O $1.412881-1.114953-2.230856$

C $0.4781931 .091614-2.435782$

C $1.5616381 .759572-2.015804$

C $1.7209213 .215501-2.000065$

C $0.6106154 .045423-2.590740$

C $-3.3070692 .062895-0.755506$

C -2.7569462 .8419770 .430100$

C -0.6502453 .2065041 .708384$

O -3.4275883 .6310371 .090478$

C 0.0323034 .4209571 .159623

C 1.3264854 .7008381 .377468

C 2.0197395 .9293600 .860712

C 3.3125185 .6120940 .140891

C 4.5005685 .7748830 .747386

C $3.2292595 .180599-1.306464$

C $2.8377553 .733717-1.448517$

N -1.4157442 .5618370 .656959$

H -0.004596 -4.296012 -1.109881

H -1.401405 -1.8177250 .021651$

H $-0.556080-2.742788-2.825067$

H $-2.706857-2.120249-3.709203$

H -3.253007 -1.455531 -2.221483

H $-4.670052-4.788552-1.220428$

H $-3.384196-5.703897-2.922149$

H -3.065731 -4.557310 -4.228182

H $-1.791129-4.954254-3.055944$

H -4.868553 -3.1793820.577907

H $-4.056444-1.921742-0.310172$

H -6.122064 -2.099820 -1.983608

H $-6.899269-2.978945-0.659430$

H $-6.353536-0.9580670 .840620$

H -8.637920 -1.260338 -0.194812

H -8.072380 -0.507379-1.671638

H -10.211166 $0.611342-0.449667$

H -7.359321 $2.030162-1.595920$

H $-9.4035330 .204847 \quad 1.698128$

H -8.842770 1.8560021 .829338

H -7.6998640 .5121532 .036159$

H -8.355192 $3.435265-0.117876$

H -4.958882 2.182008 -2.108573

H $-5.0097733 .393245-0.827050$

H -6.1686591 .9088651 .608218$

H $-0.408857 \quad 1.588656-2.811768$

H $2.4023661 .189208-1.624487$

H $0.4760263 .804994-3.651230$

H $0.7871015 .120903-2.531017$

H $-0.3331813 .858728-2.066799$

H $-3.1231760 .993580-0.604285$

H $-2.7292932 .380797-1.632536$

H 0.0632882 .4694412 .084685

H -1.3222043 .4959392 .522538$

H -0.5693315 .1134300 .575522$

H 1.9189174 .0252641 .988826

H $2.222306 \quad 6.5820081 .720015$

H 1.3667506 .5080230 .195594

H 5.4322505 .5720340 .229654

H $4.5677526 .107707 \quad 1.777890$

H $4.2076495 .298738-1.791293$ 
H $2.5672105 .863234-1.845509$

H $3.5779943 .046527-1.038476$

H -0.9213651 .9462690 .018284$

C $-0.281948-3.1053462 .210268$

O $0.705585-2.0595562 .088632$

C $1.387636-1.9134173 .346813$

C $0.494364-2.5875834 .379668$

C $-0.051596-3.7514643 .580545$

H $2.325678-2.4758003 .265854$

C $1.688623-0.4409483 .639756$

H $1.048821-2.8984885 .270280$

H $-0.324945-1.9336704 .699926$

C $-1.299206-4.3731784 .184876$

H $0.729116-4.5210453 .508749$

C $2.472013 \quad 0.3012912 .539307$

O $0.453185 \quad 0.262843 \quad 3.828404$

H $2.224258-0.3619664 .593144$

H $1.8353590 .458651 \quad 1.659921$

O $2.788344 \quad 1.6048783 .047906$

C $3.786415-0.3730862 .106153$

C $4.582716 \quad 0.492613 \quad 1.110742$

H $3.561253-1.3383621 .645747$

O $4.593377-0.6327203 .253829$

H $3.930037 \quad 0.7423110 .268500$

H 4.8596181 .4364001 .595862

H -1.086707 -4.768494 5.183562

H -2.107732 -3.640408 4.278669

H $-1.663024-5.1985483 .564932$

C -0.111506-4.110685 1.067865

H $-0.080350 \quad 0.1382253 .024570$

H 1.9610432 .0007013 .370805

H 4.7725750 .2200823 .685762

H $-0.758800-4.9787851 .240276$

H $0.922363-4.4770291 .085656$

H -1.268485 -2.627904 2.173879

C $4.946836-1.429531-1.243039$

O $5.636546-1.4632380 .012153$

C $5.877793-0.1842690 .607683$

C $6.7085690 .687241-0.345068$

C $6.0587680 .801046-1.720553$

C $5.716458-0.583129-2.267007$

H $3.940424-1.021882-1.095606$

C $4.819011-2.877592-1.741569$

H $6.499730-0.3721501 .491846$

O $6.899117 \quad 1.9832250 .213405$

H $7.7036300 .237992-0.459938$

H $5.1759731 .446789-1.686197$

O $6.9840651 .438021-2.604288$

H $6.646730-1.090761-2.551560$

O $4.944795-0.435338-3.457996$

C $3.872942-3.704476-0.899084$

H $4.438094-2.903126-2.768288$

H $5.804027-3.359295-1.708183$

H $7.4057912 .506166-0.431394$

H $6.571274 \quad 1.464957-3.484641$

H $4.105305-0.006539-3.218951$

O $4.049548-5.024492-1.203379$

O $3.067011-3.255182-0.097138$

C $3.215844-5.942797-0.489646$

H $3.552264-6.956916-0.720884$

H $3.301061-5.7844970 .589886$

H $2.178199-5.833776-0.816986$

SCF Energy (B3LYP/6-31G**//MMFF) $=-3245.92500835$

02145

MM̄FF Geometry

C $0.6977923 .926037-1.003819$

C 1.1372863 .2455950 .064507

C 1.9974783 .8626941 .137031

O $3.1080012 .967731 \quad 1.397221$

C 1.2502244 .0172112 .474922

C -0.0151014 .8502462 .420536$

C -1.2458564 .3808662 .709749$

C 0.1693656 .2955842 .037767

C -1.5958612 .9978943 .182291$

C -2.0586012 .0937852 .033610$

C $-1.943997 \quad 0.5918412 .341728$

C $-2.7714470 .175897 \quad 3.559601$
C $-2.571390-1.305588 \quad 3.908912$

O $-0.557391 \quad 0.2961232 .547200$

O $-3.110188-1.5277305 .221004$

C -1.057984-1.597225 3.986486

C $-3.347585-2.2252062 .960513$

O $-0.823646-2.9915714 .177164$

C $-0.264298-1.0913452 .759562$

C $1.256792-1.2126133 .032875$

O $-0.613975-1.8789851 .626054$

C $4.1461752 .966408 \quad 0.519787$

O $4.2919843 .686570-0.454000$

C 5.0623831 .8821850 .937561

C 6.0178421 .4581610 .098050

C 6.9495630 .3537540 .340333

C $6.828283-0.3867601 .646999$

C $2.166488-0.7507401 .883579$

C $2.492067-1.8423820 .875129$

C $3.750807-2.214501-1.245517$

O $2.110402-3.0035860 .993123$

C $5.127751-2.699037-0.913464$

C $6.166770-2.608392-1.758126$

C $7.545881-3.116537-1.445157$

C $8.623929-2.083850-1.695468$

C $9.381318-2.131442-2.804595$

C $8.878595-1.047565-0.622465$

C $7.8421670 .043415-0.623045$

N $3.268788-1.364601-0.171944$

H $0.9586214 .976398-1.110427$

H 0.8727372 .1971420 .176520

H $2.396094 \quad 4.8374370 .828374$

H 1.9332184 .4718953 .205923

H 1.0633153 .0154482 .876989

H -2.093969 5.0584892 .624682

H $-0.762507 \quad 6.8663932 .111747$

H $\quad 0.897910 \quad 6.7718972 .702127$

H $\quad 0.5272826 .3850061 .008378$

H -0.7602712 .5512613 .728631$

H -2.4024793 .0886513 .920814$

H -3.0949062 .3412841 .772828$

H -1.450146 $2.297291 \quad 1.145025$

H -2.2843990 .0507391 .451105$

H -3.8339970 .3963523 .398990$

H -2.4724680 .7652894 .436182$

H -4.049260 -1.274988 5.206330

H -0.669585 -1.091587 4.881872

H $-4.422142-2.0128313 .013012$

H -3.235142 -3.276600 3.246571

H $-3.042148-2.1158391 .918971$

H -1.327049 -3.264958 4.963163

H $1.492985-0.5801413 .899163$

H $1.510759-2.2434383 .305920$

H $0.002901-1.6744180 .906242$

H 4.8968351 .4335511 .910492

H $6.1140781 .948749-0.869749$

H $7.513045-1.2318421 .738157$

H $5.819103-0.7962041 .767294$

H $7.036803 \quad 0.2840692 .487740$

H $1.7363390 .109027 \quad 1.359994$

H $3.124265-0.4338682 .314722$

H $3.735868-1.619246-2.163278$

H $3.076386-3.068006-1.367312$

H $5.272522-3.1638540 .059090$

H $6.013194-2.170430-2.741952$

H $7.723378-4.000062-2.072264$

H $7.617150-3.469066-0.408686$

H $10.174184-1.411831-2.979332$

H $9.221759-2.888329-3.565442$

H $9.852975-0.567731-0.785578$

H $8.974230-1.5515510 .342678$

H $7.8460890 .641424-1.535781$

H $3.583961-0.399391-0.155304$

C -1.600966 $3.784089-1.996858$

O $-2.4156292 .665279-1.574829$

C $-3.5983022 .623345-2.396674$

C $-3.6653613 .973274-3.099919$

C $-2.1940514 .251165-3.326040$

H -3.430247 $1.850386-3.155441$

C $-4.8383122 .285666-1.564071$ 
H $-4.2439423 .931244-4.027568$

H $-4.1063144 .744222-2.457042$ C -1.890129 $5.701895-3.656893$ H -1.837444 3.611824 -4.144916 C $-4.7897880 .907145-0.873462$ O $-4.982997 \quad 3.289754-0.552388$ H $-5.7301302 .353472-2.199264$ H $-4.0439190 .905965-0.071502$ O $-6.0600900 .689742-0.246459$ C $-4.513621-0.274369-1.824402$ C $-4.723294-1.647671-1.156576$ H $-3.485346-0.201827-2.191364$ O $-5.372684-0.185461-2.962384$ H -4.176809-1.668308 -0.209187 H $-5.787026-1.773857-0.922816$ H $-2.3860135 .996035-4.587691$ H -2.235238 $6.375113-2.865109$ H $-0.8137985 .855547-3.783588$ C $-0.1402733 .335850-2.100189$ H $-5.8692013 .191287-0.165688$ H -6.1243231 .3030790 .504986$ H $-6.290191-0.215589-2.641096$ H $0.3051953 .660210-3.048167$ H $-0.0750962 .243040-2.076894$ H -1.730785 $4.561597-1.232219$ C -1.981420 -2.995493-1.349510 O $-2.896430-2.732069-2.420824$ C $-4.282029-2.818019-2.066769$ C $-4.599967-4.214058-1.513124$ C $-3.668596-4.603132-0.369572$ C $-2.210792-4.401674-0.780305$ H -2.118821 -2.248220 -0.561152 C $-0.558564-2.857123-1.910228$ H -4.842079 -2.717527 -3.005019 O $-5.955869-4.276857-1.083911$ H -4.487232 -4.947481 -2.322571 H $-3.913023-4.0458860 .541269$ O $-3.882635-5.983077-0.064879$ H $-1.943146-5.159328-1.527415$ O $-1.372612-4.622146 \quad 0.351840$ C $-0.179798-1.412756-2.161507$ H $0.183038-3.260393-1.213101$ H $-0.489453-3.399557-2.860996$ H $-6.106570-5.168697-0.726334$ H $-3.250758-6.2259570 .633469$ H $-1.568223-3.9327441 .009420$ O $0.857307-1.374212-3.049392$ O $-0.699852-0.444722-1.623998$ C $1.348302-0.069153-3.370222$ H $\quad 0.5644950 .519786-3.855417$ H $\quad 1.7108840 .432685-2.467900$ H $2.183640-0.182002-4.066327$ SCF Energy $\left(B 3 L Y P / 6-31 G^{* *} / / M M F F\right)=-3245.92073428$

02 146

MM̄FF Geometry

C -1.952376 -3.230329 2.771922

C $-0.844694-2.5554182 .427686$

C $-0.092696-1.6420003 .362366$

O $-0.024795-0.3439102 .729593$

C $1.342482-2.1726813 .555583$

C $2.215880-1.3472694 .483214$

C $3.472246-0.9492624 .196636$

C $1.632248-1.0253035 .835683$

C $4.296368-1.2638152 .979472$

C $4.596224-0.0120062 .145812$

C $5.498054-0.3443020 .946503$

C $5.908306 \quad 0.925144 \quad 0.199074$

C $6.7279570 .605901-1.061943$

O $4.766891-1.2122320 .075953$

O $6.8303561 .807197-1.842005$

C $5.972810-0.450473-1.899297$

C $8.1715790 .225736-0.716883$

O $6.760140-0.892893-3.001697$

C $5.495397-1.664501-1.072185$

C $4.573906-2.617788-1.876597$

O $6.635736-2.435404-0.670790$

C $-0.673503 \quad 0.693356 \quad 3.324753$
O $-1.341063 \quad 0.6667414 .345923$

C -0.4115801 .9013142 .515467$

C -0.5882893 .1198103 .047816$

C -0.3667224 .3732552 .331889$

C -1.396989 5.4302122 .618077

C $3.344883-1.999374-2.549322$

C $2.410934-1.326409-1.563624$

C $1.4526950 .874377-0.951790$

O $1.854879-1.952760-0.663810$

C $1.8733742 .299850-1.098757$

C $1.0589103 .277843-1.523733$

C $1.4747694 .717388-1.634539$

C $0.6599935 .632130-0.744659$

C $-0.339926 \quad 6.372847-1.251110$

C 1.0575815 .7562790 .711439

C $0.6938514 .540915 \quad 1.516917$

N $2.296190 \quad 0.037979-1.779384$

H -2.338269 -3.100662 3.781753

H $-0.430232-2.6588681 .428903$

H $-0.603897-1.5882534 .330581$

H $1.806271-2.2471412 .566452$

H $1.298946-3.1931493 .959058$

H $4.005073-0.3649414 .946521$

H $2.379866-0.6165406 .524233$

H $0.830802-0.2871615 .749207$

H $1.228072-1.9310786 .299621$

H $5.242197-1.6995823 .326228$

H $3.831472-2.0309722 .355153$

H $3.6485360 .412844 \quad 1.790067$

H 5.0829710 .7429292 .775542

H $6.385968-0.869626 \quad 1.317632$

H $6.465867 \quad 1.603470 \quad 0.856819$

H $5.0061451 .474845-0.100158$

H $5.9312472 .108014-2.057597$

H $5.1007440 .055284-2.328052$

H $8.6692251 .053242-0.196736$

H $8.7605160 .045521-1.623102$

H $8.244500-0.656665-0.078073$

H $7.043547-0.102349-3.492081$

H $5.166876-3.131525-2.644468$

H $4.224498-3.407885-1.196964$

H $\quad 6.337619-3.335312-0.457045$

H -0.0713351 .7622991 .496105$

H -0.9435073 .2245534 .070848$

H -2.408038 5.0134102 .566671

$\mathrm{H}-1.3680406 .2603381 .907960$

H -1.249906 5.8402293 .623032

H $3.649850-1.309685-3.342715$

H $2.767019-2.788880-3.045691$

H 1.5500360 .5549320 .090730

H $0.4168380 .713742-1.261378$

H $2.8913882 .546250-0.808063$

H $0.0377963 .035314-1.808240$

H $1.3642285 .015667-2.685242$

H $2.5398314 .845232-1.404479$

H $-0.9119737 .052689-0.628546$

H $-0.6135906 .311237-2.299221$

H 0.6274796 .6610351 .155764

H 2.1428055 .9076080 .775587

H 1.4056633 .7222831 .419336

H $2.6417940 .425738-2.652572$

C -2.233641 -4.458595 0.502466

O $-2.308465-3.262068-0.293606$

C $-2.649544-3.618747-1.646504$

C $-2.698024-5.142638-1.693955$

C $-3.062763-5.488418-0.262843$

H -3.657579 -3.229291-1.827470

C -1.661154 -2.974237 -2.626420

H $-3.427517-5.508733-2.423164$

H $-1.725252-5.575396-1.954054$

C $-2.762155-6.9311420 .104490$

H $-4.133669-5.292690-0.116781$

C $-1.488658-1.452408-2.447410$

O $-0.367633-3.569366-2.453672$

H -1.960405 -3.201404 -3.655905

H $-0.979775-1.243719-1.499063$

O $-0.619971-0.984891-3.487061$

C $-2.796878-0.646199-2.510897$ 
C $-2.5581190 .871037-2.384493$

H -3.457338 $-0.969296-1.701082$

O $-3.469939-0.921735-3.737960$

H -2.018629 $1.062084-1.450612$

H -1.914324 $1.211709-3.204384$

H $-3.324925-7.616358-0.537829$

H $-1.697467-7.160730-0.010373$

H -3.041247 -7.137725 1.142240

C $-2.751940-4.1825961 .917133$

H - $0.095295-3.419221-1.531813$

H $\quad 0.196972-1.510397-3.440112$

H -2.876242 -0.660865 -4.462954

H -2.814153 -5.129935 2.465758

H $-3.770823-3.7818751 .855229$

H -1.180483 -4.767897 0.534300

C $-4.5419161 .421241-0.128128$

O $-4.8459951 .199521-1.509794$

C $-3.8644801 .694065-2.426052$

C -3.668998 $3.204842-2.229495$

C $-3.3845353 .566914-0.772732$

C -4.4193912 .9222860 .150130$

H -3.6015920 .9181760 .128594$

C $\begin{array}{llll}-5.669066 & 0.790537 & 0.696305\end{array}$

H $-4.2883151 .554945-3.428697$

O $-2.6176663 .677563-3.065054$

H $-4.5851423 .720791-2.545849$

H -2.363231 $3.286412-0.489659$

O $-3.4627734 .987670-0.642022$

H $-5.387703 \quad 3.4167220 .004138$

O $-4.040770 \quad 3.138612 \quad 1.506351$

C $-5.543208-0.7147990 .690375$

H -5.6403831 .1045621 .745336$

H $-6.647187 \quad 1.0773410 .291887$

H -2.516709 $4.629729-2.894354$

H -3.3204045 .2003540 .296092$

H $-3.1980762 .678103 \quad 1.657915$

O $-6.642746-1.2799450 .117635$

O $-4.575321-1.3155851 .139159$

C $-6.632917-2.7094410 .050623$

H -7.572664 -3.035003 -0.403219

H -5.804069 -3.052249-0.574864

H -6.561241 -3.137335 1.055135

SCF Energy (B3LYP/6-31G**//MMFF) $=-3245.92811462$

$02 \_147$

MM̄FF Geometry

C -1.083730 -2.556522 0.803422

C $-0.610628-2.118151 \quad 1.981181$

C -1.248354 -1.070567 2.866011

O $-2.583208-0.7632412 .406489$

C $-0.441551 \quad 0.2385042 .832015$

C -0.6388901 .0624874 .088779$

C -1.5290002 .0635384 .229277$

C $0.282016 \quad 0.708302 \quad 5.228931$

C -2.5460092 .5514523 .235978$

C -2.2700183 .9892382 .774981$

C -1.099481 4.0868491 .785228

C -0.8027875 .5464621 .441095$

C 0.2894245 .6718130 .368838

O

O $0.2634187 .034149-0.083806$

C $-0.0716854 .759218-0.823614$

C 1.6850485 .4334810 .955603

O $0.9877774 .711466-1.776590$

C $-0.4649913 .320241-0.408587$

C $-1.0598262 .570871-1.625517$

O 0.6958222 .6279430 .052616

C -3.644157 -1.319849 3.040702

O $-3.612721-2.0420474 .027072$

C $-4.916356-0.9640762 .367620$

C $-4.977072-0.2208581 .252107$

C -6.1958280 .1348340 .532613$

C -6.1928901 .5526370 .028083$

C -1.693664 $1.215585-1.282478$

C $-2.3836800 .592588-2.484154$

C $-3.888813-1.303987-3.085491$

O $-2.2863521 .041938-3.623051$

C $-5.300388-0.805077-3.073157$
C $-6.365255-1.594189-2.863900$

C $-7.785936-1.105369-2.892648$

C $-8.563480-1.484674-1.651511$

C $-9.383448-2.549308-1.648873$

C $-8.456931-0.580032-0.443188$

C $-7.166447-0.7722770 .304246$

N $-3.106438-0.539387-2.132937$

H -1.994193 -2.110656 0.410105

H $0.305388-2.5453172 .380924$

H -1.281170 -1.473527 3.885686

H -0.7135380 .8262531 .947455$

H $\quad 0.6323150 .0314582 .729905$

H -1.557809 2.5889305 .183520

H $0.103565 \quad 1.3243616 .116551$

H $0.145451-0.3375935 .521513$

H 1.3258750 .8527354 .931403

H -2.6360541 .8881152 .372215$

H -3.5235992 .5285123 .734052$

H -3.1807324 .3588472285722$

H -2.0851004 .6319383 .644550$

H -0.2152153 .6134442 .225774$

H $-0.524536 \quad 6.1105562 .340229$

H -1.717433 6.0232821 .060930

H $0.9804017 .152300-0.729226$

H $-0.9299815 .217349-1.334736$

H $1.8890336 .152890 \quad 1.757845$

H 2.4629785 .5995770 .202662

H 1.8117984 .4303211 .367996

H $1.1382435 .616351-2.097432$

H -1.841542 $3.194932-2.079094$

H $-0.2859352 .416705-2.387144$

H $0.5710141 .674664-0.074642$

H -5.814509 -1.363997 2.828901

H $-4.069748 \quad 0.1860030 .815004$

H -6.1256742 .2532640 .868084$

H $-7.0862561 .817299-0.540444$

H -5.334073 $1.724754-0.630890$

H $-0.9349840 .509631-0.929154$

H -2.441399 $1.341674-0.492806$

H -3.818057 -2.356618 -2.794129

H $-3.463044-1.187778-4.087172$

H $-5.4543930 .253416-3.270006$

H $-6.212308-2.657662-2.694418$

H -8.267412 -1.538396 -3.779492

H -7.833238 -0.018306 -3.032950

H $-9.966689-2.812273-0.772571$

H -9.485642 -3.185334 -2.522067

H $-9.273647 \quad-0.7928780 .259461$

H $-8.6194450 .453619-0.760139$

H -7.036236 -1.780782 0.698142

H $-3.171923-0.800517-1.153980$

C $0.645357-4.4650220 .491582$

O $1.849727-3.6731220 .521373$

C $2.962997-4.5059100 .143600$

C $2.425648-5.9298280 .058724$

C $0.990136-5.686749-0.360136$

H $3.267767-4.189451-0.860375$

C $4.122143-4.3294331 .130409$

H $2.985281-6.541239-0.655755$

H $2.459441-6.4360091 .030697$

C $0.085398-6.885143-0.129992$

H $0.973603-5.419440-1.425385$

C $4.646941-2.8850291 .255871$

O $3.696286-4.7479442 .434192$

H $4.944844-4.9993260 .854618$

H $3.899853-2.2475141 .743912$

O $5.785018-2.9122612 .127056$

C $5.072707-2.246373-0.078287$

C $5.757844-0.8791930 .105912$

H $4.190164-2.125019-0.712599$

O $5.966056-3.122551-0.766345$

H $5.117865-0.2435210 .726873$

H $\quad 6.702226-1.0174830 .645661$

H $0.436427-7.747590-0.706177$

H $\quad 0.062452-7.1738170 .926333$

H $-0.940793-6.668239-0.441694$

C $-0.511250-3.638493-0.076615$

H $2.922820-4.2099382 .676351$ 
H $5.498229-3.3136342 .965386$ H $\quad 6.736502-3.267549-0.190725$ H -1.341808 -4.311467 -0.321177 H -0.194155 -3.163415 -1.012246 H $0.430886-4.7759391 .522357$ C $3.9596310 .953417-1.558111$

O $4.891777-0.020747-2.043493$

C $6.065834-0.188497-1.241664$

C $6.8134791 .146260-1.115239$

C $5.9030582 .262807-0.613939$

C $4.6286722 .329049-1.453468$

H $3.5808210 .648984-0.575189$

C $2.7820220 .988589-2.543811$

H $6.721532-0.866833-1.802011$

O $7.931718 \quad 1.027399-0.242447$

H $7.2040901 .436514-2.099282$

H 5.6666662 .1426070 .449905

O $6.583193 \quad 3.514245-0.735557$

H $4.8679462 .711341-2.454131$

O $3.7220943 .246897-0.849188$

C $1.921372-0.253815-2.446741$

H $2.1291271 .845741-2.354454$

H $3.1710021 .057771-3.567089$

H $8.5024030 .322508-0.593452$

H $7.4102173 .443543-0.228430$

H $4.1797854 .102009-0.778057$

O $1.280165-0.449764-3.636413$

O $1.801607-0.948390-1.446654$

C $0.435494-1.602975-3.698276$

H $1.035773-2.512860-3.604309$

H $-0.325893-1.562494-2.915002$

H -0.063473 -1.608062 -4.670949

SCF Energy (B3LYP/6-31G**//MMFF) $=-3245.91486347$

$02 \_148$

MM̄FF Geometry

C -1.495125 3.9906691 .483511

C -0.9242222 .8029731 .232909$

C -0.4627811 .8612432 .313627$

O $\quad 0.850875 \quad 1.367371 \quad 1.966573$

C - $-1.417471 \quad 0.6602932 .394274$

C -1.057345 -0.3071983 .504056$

C $-0.480784-1.5086623 .304514$

C -1.447131 0.1258294 .893010

C $-0.037202-2.0880371 .990524$

C $1.275361-2.8700422 .132046$

C $1.753529-3.4084200 .776304$

C $2.906829-4.3947060 .964144$

C $3.459933-4.884656-0.381540$

O $2.187907-2.294205-0.012301$

O $4.708831-5.546818-0.131057$

C $3.766436-3.659346-1.265221$

C $2.541035-5.922341-1.036931$

O $4.148989-4.061046-2.579014$

C $2.605763-2.637686-1.339832$

C $3.101488-1.350669-2.040141$

O $1.534789-3.199886-2.098866$

C 1.9199182 .1092582 .360609

O 1.8964183 .1770862 .951625

C 3.1430811 .3908981 .936688

C 4.3303612 .0068432 .035664

C 5.6293241 .4619831 .634935

C $5.687088 \quad 0.014017 \quad 1.226414$

C $2.135430-0.162100-1.959041$

C $2.7383671 .047257-2.647737$

C $4.5255832 .770481-2.363381$

O $2.382917 \quad 1.393103-3.771903$

C $5.9671552 .450095-2.119179$

C $6.8121893 .257060-1.459721$

C $8.2532322 .916602-1.202447$

C $8.637697 \quad 3.0361180 .257454$

C 9.4742524 .0019020 .673995

C $8.112156 \quad 1.992857 \quad 1.224347$

C 6.7001652 .2831301 .655809

N $3.7281191 .653083-1.892250$

H -1.6303414 .3131442 .513048$

H -0.8100202 .4715400 .203353$

H -0.4244912 .3675723 .286485$
H $-1.4445480 .141794 \quad 1.428945$

H -2.445448 1.0100922 .561086

H $-0.317977-2.1482464 .170865$

H -1.230805 -0.641962 5.643598

H -0.9026751 .0299545 .181799$

H $-2.520791 \quad 0.335537 \quad 4.938640$

H $-0.820877-2.7661781 .632652$

H $0.095951-1.3085341 .235139$

H $2.043059-2.2118582 .559619$

H $1.124327-3.7031262 .829906$

H $\quad 0.909288-3.8992180 .277399$

H $2.595760-5.2461701 .582280$

H $3.720501-3.9040491 .516805$

H $4.540571-6.2808490 .484440$

H $4.640960-3.155582-0.829206$

H $2.417673-6.792513-0.380933$

H $2.978650-6.307180-1.964687$

H $1.544984-5.534517-1.260402$

H $4.885759-4.689090-2.486897$

H $4.052397-1.044556-1.587815$

H $3.307836-1.559663-3.097925$

H $1.007651-2.482312-2.483721$

H 3.0313690 .3894491 .536821

H 4.3548153 .0246102 .423689

H $5.155261-0.1426200 .284230$

H $5.231832-0.6224401 .993943$

H $6.704574-0.3589361 .091905$

H $1.184303-0.386469-2.449267$

H $1.9080600 .099704-0.919657$

H $4.1987033 .658468-1.813327$

H $4.3523312 .934117-3.431211$

H $6.3336001 .501264-2.505136$

H $6.4549924 .216399-1.092361$

H $8.8667673 .592201-1.812437$

H $8.4894791 .902377-1.547966$

H 9.7732064 .0803201 .714044

H $9.8751924 .739018-0.013852$

H 8.7358821 .9706422 .127351

H $8.223991 \quad 1.008658 \quad 0.761584$

H 6.5559073 .3063892 .006928

H $3.8694721 .345191-0.935354$

C -3.5315784 .8399370 .314489$

O $-3.902303 \quad 3.556177-0.230809$

C $-4.8527953 .755460-1.292004$

C $-5.3858155 .168290-1.102322$

C $-4.1351805 .884179-0.625739$

H $-4.2882573 .721223-2.231852$

C $-5.9035542 .640758-1.284021$

H $-5.7965275 .586972-2.025824$

H $-6.1636925 .209965-0.331215$

C $-4.414393 \quad 7.2215750 .037481$

H -3.471297 $6.043813-1.486122$

C $-5.3016691 .217113-1.296472$

O $-6.6937892 .766410-0.095349$

H $-6.5913022 .777827-2.126819$

H $-4.8243371 .031267-0.329349$

O $\quad-6.3861330 .286427-1.391122$

C $-4.3094230 .952334-2.441233$

C $-3.780467-0.492379-2.549399$

H $-3.4504331 .625498-2.344669$

O $-4.9583881 .275390-3.675605$

H $-4.617530-1.175062-2.727273$

H -3.179825 -0.540599 -3.466324

H $-4.8961917 .907127-0.667285$

H $-5.076178 \quad 7.1100030 .903087$

H $-3.485627 \quad 7.6875860 .381221$

C -2.0068624 .9154340 .414191$

H -7.346607 $2.045370-0.104047$

H $-6.7967950 .403216-2.265114$

H $-4.2991991 .192356-4.385631$

H -1.6863155 .9340710 .659520$

H -1.555436 $4.655016-0.551451$

H -3.9896294 .9069901 .310017$

C $-4.497488-2.361055-0.202208$

O $-3.702322-1.172011-0.169415$

C $-2.921059-0.932173-1.344701$

C -1.999272 -2.132094 -1.620281

C $-2.756856-3.455917-1.646874$ 
C $-3.620468-3.600975-0.395319$ H -5.208044 -2.295455 -1.034351 C $-5.362078-2.4063651 .062181$ H -2.264648 -0.085144 -1.106308 O $-1.299390-1.975776-2.850412$ H -1.239506 -2.186972 -0.831020 H -3.362005 -3.553410 -2.556051 O $-1.816936-4.533030-1.667358$ H $-2.967819-3.7574420 .471664$ O $-4.451803-4.751040-0.522934$ C $-4.555630-2.4952912 .334864$ H -5.963723 -1.491935 1.139049 H $-6.074081-3.2390171 .024833$ H - $0.885916-1.096253-2.842255$ H -1.236840 -4.396575 -2.436132 H $-3.869130-5.514023-0.679445$ O $-4.478393-3.7906072 .752274$ O $-4.043770-1.5282942 .883136$ C -3.715640 -4.009952 3.943012 H -3.811604 -5.063649 4.218363 H $-2.660779-3.7907183 .756068$ H $-4.099190-3.3971534 .764510$

SCF Energy (B3LYP/6-31G**//MMFF $)=-3245.92072784$

02149

MM̄FF Geometry

C $2.787280-3.5047820 .817617$

C $1.470318-3.2488510 .818551$

C $0.602176-3.440425-0.398281$

O $-0.032487-2.169316-0.674778$

C $-0.481257-4.496310-0.117978$

C -1.134199-5.034011-1.375951

C $-2.396003-4.776083-1.770923$

C $-0.278668-5.973950-2.190490$

C $-3.428522-3.905280-1.112352$

C $-3.905571-2.807676-2.070518$

C $-5.111777-2.035533-1.519840$

C $-5.535745-0.942497-2.503687$

C $-6.698071-0.101125-1.958982$

O $-4.756114-1.451017-0.262447$

O $-6.808541 \quad 1.077840-2.770992$

C $-6.3486160 .364711-0.531388$

C $-8.038873-0.835496-2.074511$

$\begin{array}{llll}\text { O } & -7.459031 & 1.024226 & 0.073408\end{array}$

C $-5.840126-0.772403 \quad 0.388198$

C $-5.313734-0.1669451 .707390$

O $-6.921313-1.6586130 .680955$

C $-0.014785-1.712493-1.953301$

O $0.472252-2.277072-2.924107$

C $-0.666030-0.387147-2.083097$

C $-1.2643720 .267933-1.076077$

C -1.909102 $1.583795-1.163029$

C $-1.9449272 .259483-2.508242$

C $-4.623246-1.1599622 .652571$

C -4.102064 -0.400200 3.856602

C $-2.344136 \quad 1.2622364 .444054$

O $-4.766936-0.2794304 .882792$

C -2.7719832 .5500013 .810482$

C -1.949322 3.3505883 .116476

C -2.4137074 .5774132 .386512$

C -2.2485074 .4593630 .885180$

C -1.417491 $5.275193 \quad 0.215234$

C $-3.107105 \quad 3.4529840 .145753$

C -2.414994 2.127729 -0.036011

N -2.867961 0.1866243 .620881

H $3.255343-3.864646-0.096134$

H $\quad 0.992391-2.887687 \quad 1.725467$

H $\quad 1.232575-3.747475-1.240749$

H -1.228598 -4.0803260 .565735$

H $-0.042139-5.3522360 .412991$

H $-2.747046-5.258268-2.683383$

H $-0.828034-6.417521-3.027953$

H $0.585558-5.449819-2.608841$

H $0.081865-6.797483-1.565498$

H -4.276294 -4.541816-0.831539

H $-3.058718-3.447689-0.191447$

H -3.074904 -2.115370 -2.247952

H $-4.174148-3.245156-3.040562$
H $-5.929132-2.750022-1.365734$

H $-5.798243-1.374347-3.477570$

H $-4.684250-0.273424-2.692788$

H $-6.9560840 .795859-3.690051$

H $-5.5529821 .117808-0.619888$

H $-8.255172-1.074538-3.122864$

H $-8.866615-0.202788-1.735597$

H -8.065810 -1.770675 -1.511519

H -7.734383 $1.737906-0.527253$

H $-4.597270 \quad 0.628524 \quad 1.469820$

H -6.1411120 .3052982 .253116$

H $-6.718749-2.1480441 .493200$

H $-0.6264510 .029671-3.084816$

H -1.281182 -0.189592 -0.088324

H $-0.9307052 .397283-2.898453$

H $-2.4017083 .250930-2.487950$

H $-2.5185301 .658976-3.222882$

H $-5.313267-1.9300603 .011083$

H -3.792182 -1.672778 2.155670

H $-1.256404 \quad 1.1588034 .475802$

H -2.7367381 .1907855 .462893$

H $-3.8265192 .805670 \quad 3.885841$

H -0.8968473 .0925973 .032284$

H -1.845587 5.4330062 .773145

H -3.466782 4.7935872 .606943

H $-1.3163765 .213975-0.863308$

H -0.8161296 .0177680 .728786$

H $-3.4348773 .876675-0.808701$

H -4.0386363 .2854480 .701546$

H -2.330224 1.5529390 .884813

H -2.4342250 .0523222 .712097$

C $4.607909-2.1223541 .817730$

O $5.439152-2.3019530 .650842$

C $6.751231-1.8049150 .944161$

C $6.644005-1.0537652 .264232$

C $5.580885-1.8780422 .970145$

H $7.377632-2.6904881 .122613$

C $7.364008-1.041726-0.232607$

H $7.592805-1.0190772 .807951$

H $6.294028-0.0262032 .114532$

C $4.981103-1.1901304 .181766$

H $6.026649-2.8308043 .287445$

C $6.6632560 .249499-0.710199$

O $8.706255-0.7063390 .145306$

H $7.445110-1.729976-1.083501$

H $\quad 6.753971 \quad 1.0280070 .055616$

O $7.4184730 .739268-1.831250$

C $5.1836050 .118474-1.125857$

C $4.6654641 .345876-1.902641$

H $4.574678-0.019399-0.229333$

O $5.006214-1.040845-1.940533$

H $4.9295872 .252649-1.349529$

H $5.1784121 .392722-2.870404$

H $5.748526-1.0214384 .944412$

H $4.552631-0.2181403 .923012$

H $4.191173-1.8013144 .628932$

C $3.696019-3.3335272 .003231$

H $9.113570-0.246097-0.608499$

H $7.3837710 .066099-2.531691$

H $5.515333-0.914524-2.758721$

H $3.111420-3.2305442 .924207$

H $4.294718-4.2474362 .098521$

H $3.999002-1.2279361 .640698$

C $2.3949922 .573876-0.254369$

O $2.3794841 .321108-0.950559$

C $3.1413071 .287693-2.162755$

C $2.6276722 .363180-3.130088$

C $2.6376543 .746072-2.488757$

C $1.8992183 .713657-1.153715$

H 3.4109552 .7859820 .098456

C 1.4802462 .4346780 .970162

H $2.9306410 .317044-2.628448$

O $3.4036922 .394577-4.322932$

H $1.5975342 .124683-3.424842$

H $3.6579614 .128007-2.366391$

O $1.9626864 .668046-3.347740$

H $\quad 0.8237403 .604413-1.339729$

O $2.0913054 .956715-0.481438$ 
C 2.0232931 .4502871 .978065

H 1.3521143 .3904121 .488988

H 0.4971502 .0824050 .644181

H $3.3685191 .506359-4.717380$

H $2.4170084 .646809-4.207556$

H $1.7784095 .659381-1.076675$

$\begin{array}{lllll}\text { O } & 0.985920 & 0.790546 & 2.570752\end{array}$

O 3.2105311 .3075712 .235588

C $1.348078-0.1584843 .577272$

H 1.8056180 .3531914 .428885

H $\quad 0.438619-0.6623173 .914767$

H $2.026724-0.9090623 .166474$

SCF Energy (B3LYP/6-31G**/MMFF) $=-3245.90994439$

02_15

MMFF Geometry

C $1.0317355 .044224-0.791709$

C $2.2226894 .425913-0.820340$

C $3.177110 \quad 4.212222 \quad 0.335872$

O $3.463522 \quad 2.7922690 .416645$

C 2.6585194 .6697601 .714314

C $3.657423 \quad 4.4763292 .841530$

C 3.5539693 .5502163 .816243

C 4.8142595 .4430592 .846032

C 2.4877812 .5036133 .984698

C $3.0813111 .093273 \quad 3.874584$

C 1.9952790 .0257103 .674236

C $2.618088-1.3719173 .658115$

C $1.600319-2.4619083 .291873$

O $1.357701 \quad 0.3002392 .422424$

$\begin{array}{llll}\text { O } & 2.330035 & -3.657481 & 2.974397\end{array}$

C $0.854055-2.0373762 .009559$

C $0.687850-2.8114004 .473250$

O $-0.193967-2.9527121 .705519$

C $0.299798-0.6002682 .072748$

C $-0.288838-0.118040 \quad 0.724343$

O $-0.759974-0.545998 \quad 3.035034$

C $4.4823232 .316587-0.352887$

O $5.1823212 .955702-1.122545$

C $4.5973890 .861792-0.108255$

C $5.4040800 .121920-0.883621$

C $5.630860-1.321050-0.772533$

C $4.873288-2.0678440 .292791$

C $0.708546-0.027261-0.436028$

C $0.755312-1.261033-1.320031$

C $1.972503-2.186145-3.289083$

O $-0.016577-2.207701-1.201598$

C $2.971573-3.179512-2.785700$

C $4.087008-3.509996-3.454491$

C $5.062391-4.553289-2.989872$

C $6.485149-4.044643-2.933442$

C $7.345200-4.284105-3.937683$

C $6.934757-3.342455-1.672722$

C $6.499550-1.905127-1.624384$

N $1.751557-1.164810-2.282985$

H 0.6530445 .4585630 .137858

H $2.5736454 .049950-1.780351$

H 4.0998464 .7611840 .109937

H $1.721234 \quad 4.1509351 .936021$

H 2.4020455 .7368971 .678765

H 4.3267803 .5254984 .583812

H $5.4570565 .318746 \quad 3.724121$

H 5.4397155 .3002291 .959647

H 4.4476096 .4747242 .852455

H $2.0284642 .626774 \quad 4.972934$

H 1.6809802 .6287203 .260424

H $3.773562 \quad 1.061473 \quad 3.023923$

H 3.6645700 .8723104 .777022

H 1.2593290 .1118294 .482217

H $3.092577-1.5958204 .621742$

H $3.427746-1.3970562 .917179$

H $2.867336-3.8954113 .749411$

H $1.574902-2.1035991 .186098$

H $1.279753-3.1936215 .313673$

H $-0.010464-3.6133974 .210042$

H $0.113104-1.9580284 .838858$

H $\quad 0.200525-3.8402791 .656958$

H $-1.166571-0.7198230 .460230$
H -0.6732710 .8997920 .884246$

H -1.3144080 .2280092 .841825$

H 4.0125340 .4381590 .698954

H $5.9585140 .618896-1.678862$

H $5.204853-1.7511621 .287176$

H $4.996179-3.151486 \quad 0.239295$

H $3.797737-1.8828860 .203319$

H $0.4076710 .803918-1.086507$

H $1.7152450 .194180-0.073077$

H $2.312890-1.679240-4.197561$

H $1.026460-2.692806-3.506117$

H $2.749053-3.675445-1.843676$

H $4.292188-3.034796-4.411254$

H $4.998664-5.399760-3.686470$

H $4.780410-4.958068-2.009940$

H $8.376238-3.949389-3.891308$

H $7.035206-4.815288-4.831658$

H $8.031746-3.349552-1.611837$

H $6.609587-3.917660-0.801306$

H $6.995549-1.279762-2.368148$

H $2.385302-0.370878-2.265812$

C $-1.2626314 .763836-1.780631$

O $-1.3005693 .322020-1.803697$

C $-2.4866072 .898479-2.516863$

C $-3.3106114 .157489-2.744431$

C $-2.232408 \quad 5.212327-2.872432$

H $-2.1304902 .498586-3.474524$

C $-3.197257 \quad 1.804860-1.708178$

H $-3.9495874 .087500-3.629916$

H -3.949296 $4.369096-1.877613$

C $-2.7526236 .629178-2.701350$

H $-1.7596725 .116550-3.859149$

C -4.597855 $1.437684-2.227377$

O $-2.371642 \quad 0.636920-1.726013$

H $-3.2557692 .123566-0.661801$

H -5.261382 2.307171-2.154041

O $-4.489828 \quad 1.125257-3.621829$

C $-5.2527120 .233153-1.522538$

C $-5.319594 \quad 0.371907 \quad 0.008030$

H $-4.723650-0.684176-1.799180$

O $-6.5809370 .093929-2.039593$

H -4.3030540 .4190750 .410415$

H $-5.807903 \quad 1.3220470 .258301$

H -3.501253 $6.858444-3.466866$

H -3.220802 $6.769263-1.721223$

H $-1.9422117 .358600-2.794453$

C $\quad 0.168218 \quad 5.256024-2.006001$

H $-2.3397130 .311687-2.642173$

H $-5.3797790 .890068-3.936185$

H $-7.0840870 .886003-1.784220$

H $0.1629496 .331174-2.218185$

H $0.6025524 .748375-2.875964$

H -1.629253 $5.077419-0.794073$

C $-4.346027-2.4296320 .723670$

O $-5.652967-2.0642950 .265186$

C $-6.097940-0.7738240 .693991$

C $-6.109835-0.699608 \quad 2.229052$

C $-4.774230-1.1232732 .837519$

C $-4.323828-2.4640172 .255620$

H -3.608651 -1.707574 0.353532

C $-4.010784-3.8012880 .128853$

H -7.140712 $-0.687277 \quad 0.363202$

$\begin{array}{lllll}\text { O } & -6.432336 & 0.614478 & 2.672518\end{array}$

H $-6.890002-1.3690832 .614728$

H $-4.012429-0.3479582 .693860$

O $-4.944917-1.2613734 .249956$

H $-4.988897-3.2540032 .626069$

O $-3.016338-2.767542 \quad 2.730240$

C $-3.958212-3.754960-1.380822$

H $-3.049881-4.1830290 .490875$

H $-4.780238-4.5328960 .404318$

H $-7.300103 \quad 0.8439842 .298265$

H $-4.098319-1.5720244 .614262$

H $-2.407060-2.0918112 .386839$

O $-2.910991-2.986845-1.794130$

O $-4.752893-4.340138-2.105701$

C -2.769151-2.848898 -3.211487

H $-1.929368-2.176594-3.406617$ 
H $-2.554221-3.821015-3.664709$

H $-3.674030-2.410997-3.643840$

SCF Energy (B3LYP/6-31G**//MMFF) $=-3245.91846040$

$02 \_150$

MM̄FF Geometry

C $-2.7148522 .065838 \quad 3.312743$

C -2.1286181 .0778992 .617654$

C -1.302678 -0.0085853 .261202$

O $0.015247-0.0234012 .659642$

C $-1.959731-1.3761663 .006279$

C -1.413669 -2.475354 3.895972

C $-0.553660-3.436902 \quad 3.507347$

C $-1.943928-2.4735155 .308523$

C $0.083542-3.6336812 .160179$

C $1.611206-3.5451092 .266221$

C $2.314022-3.8454200 .933293$

C $3.829546-3.7006011 .092223$

C $4.578929-3.849328-0.239717$

O $1.826706-2.927693-0.048716$

O $5.914091-3.353796-0.052537$

C $3.909654-2.946618-1.298256$

C $4.716744-5.319992-0.650637$

O $4.475537-3.171492-2.587043$

C $2.378882-3.112865-1.360108$

C $1.697761-2.091106-2.300678$

O $2.070951-4.416736-1.869469$

C 0.8859820 .9460483 .056946

$\begin{array}{lllll}\text { O } & 0.662149 & 1.853848 & 3.842676\end{array}$

C 2.1819780 .7079942 .380426

C 3.1687651 .6062782 .523842

C 4.5323261 .5031001 .997660

C $4.900021 \quad 0.2562281 .240870$

C $1.817405-0.622126-1.885519$

C $2.9478440 .139446-2.553908$

C $3.9860682 .408522-2.573956$

O $3.641212-0.326194-3.453041$

C $5.2918372 .307037-1.852228$

C $5.8535183 .330281-1.190478$

C $7.2087803 .263507-0.548989$

C 7.1920443 .6506530 .911062

C $7.553094 \quad 4.8838271 .304753$

C 6.8640782 .5762121 .920826

C 5.3978492 .5081822 .246853

N 3.056608 1.428828 -2.047300

H -2.5890912 .0735734 .394430$

H -2.2301301 .0275611 .538385$

H -1.199096 $0.164844 \quad 4.339544$

H -1.856070 -1.6439201 .949177$

H -3.040747 -1.3106503 .188368$

H $-0.263631-4.1853624 .244620$

H -1.566901 -3.317135 5.896603

H -1.654287 -1.556281 5.829909

H -3.036857 -2.5406625 .303731$

H $-0.202390-4.6246621 .787713$

H $-0.276534-2.907171 \quad 1.427553$

H $1.875712-2.5338782 .600161$

H $1.975689-4.2431203 .030707$

H $2.056171-4.8667770 .628899$

H $4.212019-4.4161451 .830990$

H $4.053252-2.7074471 .501427$

H $6.324433-3.8626010 .667663$

H $4.143429-1.909189-1.031041$

H $5.282709-5.877180 \quad 0.105654$

H $5.284979-5.419534-1.581937$

H $3.755167-5.822255-0.775404$

H $5.435637-3.036841-2.509398$

H $2.023585-2.249359-3.335621$

H $0.624764-2.327354-2.298309$

H $1.163799-4.407874-2.217044$

H $2.296592-0.2069251 .811618$

H 2.9697892 .5031473 .109915

H 5.8797630 .3097810 .761370

H 4.1831620 .0630820 .437079

H $4.913185-0.6036481 .917578$

H $0.899068-0.111425-2.198467$

H $1.896308-0.517135-0.798827$

H $3.522343 \quad 3.393167-2.456471$
H $\quad 4.1407142 .223592-3.641987$

H $5.8216931 .359602-1.917615$

H $5.3361404 .286143-1.151477$

H $7.8704813 .940988-1.105061$

H $7.6552662 .267284-0.657699$

H 7.5783745 .1562272 .354697

H 7.8252945 .6493910 .585695

H 7.3931982 .7851452 .861018

H 7.2740171 .6173601 .590419

H 5.0384273 .3744942 .804169

H $2.4735781 .705359-1.262532$

C -3.7007003 .2893391 .258457$

O -2.4270563 .5989610 .653999$

C $-2.6269914 .595758-0.357468$

C $-4.1280004 .666910-0.600368$

C -4.6585344 .3882320 .794335$

H -2.3178925 .5500370 .092031$

C $-1.7472584 .353684-1.588241$

H -4.445181 $5.636541-0.995397$

H $-4.4638993 .887843-1.293280$

C $-6.121878 \quad 3.9805740 .814510$

H -4.5260155 .2880931 .409747$

C $-2.063656 \quad 3.120587-2.462087$

O $-1.8626595 .519869-2.414885$

H $-0.6997314 .302035-1.265278$

H $-3.0226323 .266006-2.971771$

O $-1.0789703 .081608-3.507545$

C $-2.063878 \quad 1.763972-1.735795$

C $-2.2874170 .587287-2.703645$

H -2.860655 $1.760482-0.990972$

O $-0.8210251 .608767-1.056406$

H -3.217755 $0.769301-3.253499$

H $-1.4767690 .560891-3.441821$

H -6.7490854 .7802310 .407120$

H -6.2956573 .0794620 .216613$

H $-6.456483 \quad 3.7759791 .836220$

C -3.5437993 .2063972 .777637$

H $-1.2845075 .386968-3.185744$

H $-0.2202072 .888056-3.093301$

H $-0.9023160 .847724-0.457411$

H -4.5340373 .1357303 .242726$

H -3.0804784 .1335593 .138484$

H -4.0299492 .3239370 .858815$

C $-4.598750-0.730990-1.164830$

O $-3.202503-0.806915-0.869780$

C $-2.342715-0.794422-2.011993$

C -2.693972 -1.951472 -2.960956

C $-4.173703-1.951471-3.339166$

C -5.047634 -1.871871-2.087190

H $-4.7995620 .228275-1.659008$

C $-5.371150-0.6853470 .162629$

H -1.336171 -0.992287 -1.626925

O $-1.912774-1.890354-4.150216$

H -2.461283 -2.905562 -2.470088

H $-4.401179-1.139750-4.039892$

O $-4.462856-3.168685-4.031341$

H -4.998768 -2.836881-1.571815

O $-6.407882-1.682799-2.471929$

C $-5.196009-1.9080141 .041817$

H $-5.019857 \quad 0.1688720 .752274$

H $-6.439908-0.554735-0.041559$

H - $0.976794-1.901757-3.888681$

H $-5.415100-3.169846-4.229284$

H $-6.470264-0.834762-2.943839$

O $-6.116513-1.8595892 .050874$

O $-4.349853-2.7779850 .890494$

C $-6.064366-2.9441272 .983131$

H $-6.261117-3.8916192 .472423$

H $-5.091591-2.9691723 .483048$

H $-6.840798-2.7832063 .735691$

SCF Energy (B3LYP/6-31G**//MMFF) $=-3245.90491056$

$02 \_151$

MM̄FF Geometry

C $2.6693660 .200904-2.953304$

C $2.154107-1.007964-2.675217$

C $0.713001-1.307753-2.319198$

O $-0.019955-0.065293-2.203194$ 
C $0.625463-2.050953-0.966842$

C $0.397302-3.542799-1.106058$

C $-0.744747-4.184389-0.788376$

C $1.579913-4.334486-1.604481$

C $-2.030466-3.608501-0.262646$

C $-3.192422-3.891137-1.224081$

C $-4.535651-3.386689-0.676965$

C $-5.661676-3.670628-1.673899$

C $-7.010236-3.107645-1.200896$

O $-4.440115-1.977211-0.442730$

O $-7.890854-3.143174-2.334247$

C $-6.820581-1.632614-0.783639$

C $-7.645437-3.999330-0.127099$

O $-8.003274-1.113076-0.179894$

C $-5.612972-1.4103430 .157268$

C $-5.377042 \quad 0.1048650 .370355$

O $-5.897484-2.0064441 .427271$

C $-1.352695-0.076899-2.450823$

O $-2.028877-1.048852-2.755916$

C $-1.947052 \quad 1.272528-2.305154$

C $-1.2232962 .384146-2.102230$

C $-1.7419283 .748148-1.981014$

C $-3.2358993 .931522-1.945577$

C $-4.020566 \quad 0.434274 \quad 1.008618$

C -3.9664251 .8422961 .578655$

C -2.3349473 .4557142 .559310$

O -4.9693112 .5014791 .839282$

C -2.444888 4.6605091 .680502

C $-1.427795 \quad 5.5015351 .438933$

C -1.5718376 .7749780 .655800$

C $-0.6455706 .846635-0.534829$

C $0.5240017 .504444-0.464692$

C - $1.1238516 .239228-1.833301$

C $-0.855643 \quad 4.762937-1.908136$

N -2.662112 2.2524501 .818736

H $2.0067671 .062906-2.977210$

H $2.815149-1.870104-2.676624$

H $0.283047-1.903255-3.134687$

H $-0.153183-1.597013-0.347479$

H $1.545449-1.903667-0.384541$

H $-0.769175-5.266786-0.914552$

H $1.409311-5.415305-1.553229$

H $1.795381-4.086718-2.648147$

H $2.467014-4.117095-1.000661$

H $-2.238980-4.0736920 .708482$

H $-1.957145-2.533905-0.081622$

H -2.981537-3.407507 -2.185414

H -3.263370 -4.969428 -1.415815

H $-4.726609-3.8982800 .273773$

H -5.743951 -4.746463 -1.873591

H $-5.414181-3.206577-2.639230$

H -8.768275 -2.841191-2.044900

H -6.655470 -1.049899-1.700689

H -7.835804 $-5.001801-0.529048$

H -8.621638 -3.6113950 .184150$

H $-7.021119-4.1117860 .761623$

H -8.717776 $-1.166160-0.836449$

H $-5.4133770 .627451-0.594399$

H -6.1899730 .5084090 .986379$

H -5.422985 -1.524027 2.122270

H -3.027889 $1.303892-2.400648$

H $-0.1399702 .301878-2.037025$

H -3.542386 4.953434-1.712245

H -3.686214 3.297443 -1.174032

H -3.677107 $3.670795-2.913698$

H -3.802682 -0.2470181 .838105$

H -3.227201 0.3135860 .263172

H -1.324801 3.3276952 .961012

H -3.0307853 .5510643 .399656$

H -3.426392 4.8851791 .269878

H $-0.4517385 .295802 \quad 1.872363$

H - 1.3633697 .6087181 .339512

H $-2.606468 \quad 6.925754 \quad 0.323154$

H $1.1787037 .586090-1.326169$

H $\quad 0.8556307 .9695320 .457772$

H - $0.5983026 .706309-2.677431$

H -2.175739 $6.493297-1.991230$

H $0.2072454 .522786-1.944881$
H -1.8979121 .6295201 .575851$

C $5.160776-0.515713-2.815202$

O $4.987221-0.903284-1.440393$

C $6.270819-0.993660-0.801398$

C $7.306317-0.896474-1.916437$

C $6.598234-0.002487-2.916329$

H $6.356773-0.112765-0.153627$

C $6.346077-2.2661280 .051299$

H $8.255280-0.480750-1.564109$

H $7.516228-1.872002-2.369031$

C $7.181646-0.070706-4.316730$

H $6.655297 \quad 1.033208-2.554995$

C $5.133235-2.4980190 .974546$

O $6.432899-3.410705-0.810047$

H $7.273086-2.2624680 .635797$

H $4.255041-2.7692010 .375494$

O $5.423957-3.6384321 .794222$

C $4.769142-1.3183631 .893944$

C $3.594950-1.6688802 .825861$

H $4.498127-0.4537111 .283808$

O $5.901767-0.9448062 .674755$

H $2.773097-2.0551202 .213262$

H $3.901792-2.4766963 .501162$

H $8.2292230 .248032-4.312841$

H $7.141364-1.088476-4.719204$

H $6.6321370 .583849-5.000330$

C $4.1187700 .543464-3.191104$

H $5.651670-3.401982-1.389655$

H $5.634719-4.3772491 .197671$

H $6.173565-1.7237943 .189783$

H $4.2386820 .826562-4.243099$

H $4.3187221 .444106-2.596286$

H $5.028464-1.417737-3.425869$

C 1.5679210 .4959302 .101852

$\begin{array}{lllll}\text { O } & 2.736573 & 0.661647 & 2.913369\end{array}$

C $3.107698-0.4826263 .689135$

C $1.958559-0.8498464 .638591$

C $0.662669-1.0946493 .871254$

C 0.3556310 .0836692 .950161

H $1.765687-0.2555531 .329942$

C 1.2881281 .8382261 .404703

H $3.956684-0.169748 \quad 4.309586$

O $2.274771-2.0093285 .401456$

H $1.793868-0.0301925 .349921$

H $0.696236-2.0350703 .308995$

O $-0.417212-1.2255454 .799336$

H 0.0156590 .9338993 .555712

O $-0.717625-0.2740092 .081366$

C 2.3659532 .2317210 .419561

H $0.346194 \quad 1.7908720 .848141$

H 1.2265622 .6326652 .158391

H $3.098388-1.8252095 .884685$

H $-0.191002-1.9527925 .404386$

H -1.458579 -0.5651392 .640099$

$\begin{array}{lllll}\text { O } & 2.127836 & 3.507911 & -0.006047\end{array}$

$\begin{array}{lllll} & & 3.289165 & 1.516913 & 0.057973\end{array}$

C $3.066424 \quad 4.030385-0.951475$

H $4.0915213 .917162-0.585622$

H $2.9403353 .527378-1.914194$

H $2.8590235 .095712-1.082433$

SCF Energy (B3LYP/6-31G**//MMFF) $=-3245.91558038$

02_152

MMFF Geometry

C -0.2896602 .7842601 .395755$

C 0.4373853 .7529820 .815323

C $0.950840 \quad 3.752936-0.606294$

O $0.7330662 .465136-1.223557$

C $2.4588014 .051359-0.661590$

C $2.9417124 .283871-2.083212$

C $3.5832923 .370271-2.838734$

C $2.6772385 .662393-2.632744$

C $3.9325791 .958329-2.461609$

C $5.4542191 .754586-2.432273$

C $5.9054230 .559571-1.578350$

C $5.298058-0.768803-2.035138$

C $5.675187-1.924859-1.095260$

O $5.537267 \quad 0.853047-0.227337$ 
O $4.822707-3.039443-1.400811$

C $5.357267-1.5010440 .354464$

C $7.112210-2.406541-1.320852$

O $5.791212-2.4898941 .283785$

C $5.941472-0.1252690 .737546$

C $5.461776 \quad 0.3791352 .122118$

O $7.369215-0.2251420 .797800$

C $-0.261407 \quad 2.348657-2.138437$

O $-1.0093993 .236517-2.524340$

C $-0.3694130 .963071-2.651306$

C $0.405651-0.053703-2.242389$

C $0.329223-1.438964-2.712337$

C $-0.741319-1.782983-3.714750$

C $3.951551 \quad 0.609367 \quad 2.247379$

C $3.207135-0.6266062 .714271$

C $1.114482-1.9143882 .339270$

O $3.649128-1.3704563 .587206$

C $1.488089-3.0533551 .444345$

C $0.600421-3.7271390 .697564$

C $0.969081-4.873108-0.200517$

C $0.483271-4.680530-1.620615$

C $-0.605058-5.330077-2.067487$

C $1.300975-3.805251-2.544634$

C $1.199541-2.341415-2.213058$

N $1.986340-0.7868472 .076587$

H $-0.534651 \quad 1.8836220 .840183$

H 0.6263314 .6645901 .379328

H $0.407908 \quad 4.539515-1.144335$

H $3.0188493 .236923-0.188729$

H $2.6911024 .945771-0.068204$

H $3.8866683 .654883-3.845479$

H $3.0831375 .793144-3.641620$

H $1.6019225 .858213-2.683964$

H $3.1394686 .420871-1.992540$

H $3.4865991 .677680-1.504357$

H $3.4903501 .286482-3.207428$

H $5.8256561 .637292-3.457898$

H $5.9284122 .655765-2.020036$

H $6.9993280 .512767-1.637445$

H $5.593744-0.995516-3.066816$

H $4.202834-0.696145-2.047412$

H $4.945284-3.262258-2.339576$

H $4.265181-1.4571650 .428918$

H $7.235355-2.774217-2.346718$

H $7.351426-3.253522-0.668534$

H $7.858234-1.625489-1.161371$

H $5.388596-3.3342501 .017488$

H $5.837267-0.2819012 .912692$

H 5.9427351 .3514492 .301517

H 7.7070550 .4960091 .354059

H - $-1.1436260 .823163-3.398464$

H $1.1710030 .133528-1.491091$

H - $1.733432-1.568678-3.302822$

H $-0.746155-2.836303-4.002394$

H $-0.608179-1.205079-4.635847$

H $3.769856 \quad 1.372724 \quad 3.014555$

H 3.5320870 .9958491 .313008

H $0.092883-1.5724752 .160068$

H $1.215571-2.2146593 .386805$

H $2.535022-3.3463281 .416882$

H $-0.451055-3.4525810 .743475$

H $0.530367-5.7842790 .226638$

H $2.053095-5.042405-0.210507$

H $-0.946798-5.224605-3.091636$

H -1.181644 -5.980017-1.417601

H $1.032526-3.981141-3.592904$

H $2.354507-4.105849-2.481403$

H $1.937082-2.005578-1.483824$

H $1.730024-0.1612951 .318408$

C -0.8196411 .6232683 .586525$

$\begin{array}{llll}\text { O } & -1.612494 & 0.593260 & 2.956178\end{array}$

C $-2.5659350 .086848 \quad 3.905791$

C -2.003985 0.4531335 .273004

C $-1.401831 \quad 1.813584 \quad 4.991788$

H -3.498472 0.6400383 .737203

C $-2.820013-1.4112183 .702482$

H -2.7755090 .4758596 .048432$

H $-1.219423-0.2420445 .592673$
C $-0.375812 \quad 2.251313 \quad 6.023416$

H -2.213878 2.5529504 .966883

C $-3.148325-1.8239832 .253589$

O $-1.649929-2.1357174 .101850$

H -3.617903 -1.734530 4.381726

H -2.263615 -1.6936901 .620445$

O $-3.410678-3.234940 \quad 2.266663$

C $-4.343763-1.0924961 .612617$

C $-4.705476-1.6955330 .240068$

H $-4.100303-0.0314391 .505369$

O $-5.463002-1.1980662 .490718$

H $-3.806370-1.696006-0.385815$

H $-5.002697-2.7415920 .381317$

H -0.8340722 .3243057 .015009$

H 0.4555191 .5411606 .088636

H $\quad 0.0383503 .2320495 .768907$

C $-0.8949782 .914702 \quad 2.765824$

H -1.830503 -3.080956 3.960900

H $-4.226915-3.3758592 .776990$

H -6.120673 -0.5395332 .210382$

H -0.4125583 .7304243 .317151$

H $-1.948787 \quad 3.1871162 .627536$

H 0.2116301 .2523393 .629685

C $-4.5874330 .710930-1.669119$

O $-5.5958050 .418731-0.693335$

C $-5.861376-0.971239-0.484766$

C $-6.279892-1.633231-1.806559$

C $-5.249799-1.397908-2.905898$

C $-4.9439440 .092196-3.028466$

H $-3.6183290 .331375-1.322761$

C $-4.4967392 .240619-1.793314$

H $-6.733883-1.0193950 .177762$

O -6.475261 -3.033691 - 1.641702

H $-7.239077-1.212308-2.135452$

H $-4.334542-1.976350-2.734539$

O $-5.782004-1.847987-4.153960$

H $-5.8133270 .606104-3.458859$

O $-\begin{array}{llll}4.854757 & 0.274882 & -3.929928\end{array}$

C $-3.9258182 .897544-0.556197$

H -3.852081 2.520399-2.633316

H $-5.5002842 .653517-1.952630$

H -7.146681 -3.155229 - 0.948752

H -6.011261 -2.787288-4.047659

H $-4.097839-0.150953-4.769884$

O $-4.026505 \quad 4.253126-0.693173$

$\begin{array}{lllll}0 & -3.444743 & 2.306458 & 0.399547\end{array}$

C $-3.530491 \quad 5.028543 \quad 0.402352$

H -3.8442436 .0652340 .253567$

H -3.942946 4.6729611 .351643

H -2.438413 4.9912370 .415924

SCF Energy (B3LYP/6-31G**//MMFF) = -3245.92875311

02_153

MM̄FF Geometry

C -1.531313 -0.8928763 .009789$

C $-1.885450 \quad 0.372362 \quad 3.288018$

C $-3.234551 \quad 1.007192 \quad 3.026278$

$\begin{array}{llll}\text { O } & -4.066047 & 0.095562 & 2.276543\end{array}$

C -3.047645 2.2830852 .185312

C $-4.271807 \quad 3.1740572 .141600$

C $-5.0847113 .311522 \quad 1.075871$

C $-4.518015 \quad 3.987817 \quad 3.386120$

C $-5.0211202 .587323-0.240414$

C $-4.5465673 .490207-1.388845$

C $-3.0366173 .775208-1.354294$

C $-2.6396174 .747593-2.466840$

C -1.118385 $4.960059-2.525037$

O $-2.3585152 .528842-1.537738$

O $-0.811018 \quad 5.613838-3.765851$

C $-0.421627 \quad 3.583327-2.555272$

C $-0.6279705 .891823-1.410306$

O $0.9944003 .724425-2.460380$

C $-0.9292422 .609574-1.466958$

C $-0.3690891 .190819-1.729730$

O $-0.489036 \quad 3.067072-0.188193$

C $-5.369844-0.0406142 .619464$

O -5.9514550 .5293093 .531959$

C $-6.055964-1.0190811 .741325$ 
C $-5.494925-1.5553380 .645649$

C $-6.107891-2.536690-0.253095$

C $-7.480039-3.0485170 .098444$

C $-0.9690440 .103095-0.827309$

C $-0.518544-1.288490-1.235209$

C $-0.920240-3.689941-0.694646$

O $0.300904-1.497222-2.125118$

C - $1.888861-4.182582-1.723233$

C -2.842101-5.094091-1.477519

C $-3.811299-5.583019-2.515888$

C $-5.254196-5.324371-2.139685$

C $-6.033667-6.317156-1.678888$

C $-5.819861-3.941893-2.383524$

C $-5.406934-2.949677-1.330157$

N -1.142585 -2.272246 -0.481030

H -2.271691 -1.5615382 .576153$

H $-1.156507 \quad 1.0353743 .746958$

H -3.681452 1.2354314 .000988

H $-2.747228 \quad 1.999837 \quad 1.171680$

H -2.216402 2.8827242 .580498

H -5.9160794 .0112811 .154445$

H -5.3741314 .6626653 .279685$

H -4.7198113 .3352654 .240818$

H -3.6422774 .6033903 .616724$

H $-4.4139501 .678745-0.184706$

H $-6.0382882 .243525-0.468707$

H $-4.7885792 .978834-2.329756$

H $-5.1079664 .432730-1.378315$

H $-2.7730114 .193970-0.377139$

H -3.157210 $5.707568-2.347781$

H $-2.9696554 .347637-3.436041$

H -1.303467 $6.452126-3.792618$

H $-0.6230683 .137425-3.539454$

H -1.111258 $6.872819-1.491615$

H $0.4477526 .080262-1.497787$

H $-0.8307945 .507096-0.408761$

H $1.2731874 .329492-3.168849$

H $-0.5794000 .914255-2.771414$

H $0.7212051 .187755-1.605969$

H -0.3801762 .3084660 .406096$

H -7.066276 -1.270617 2.047718

H -4.485163 -1.254862 0.370336

H -7.481062 $-3.492071 \quad 1.100460$

H -7.842807 -3.822910 -0.580818

H $-8.211576-2.2334400 .073468$

H -0.6668120 .2605730 .212254$

H -2.063590 $0.126988-0.876252$

H $-1.048465-4.1899380 .270492$

H $0.105598-3.853263-1.040117$

H -1.795974 -3.768476 -2.724613

H $-2.929218-5.519531-0.480381$

H -3.642006 -6.659819 -2.647140

H -3.608592 -5.133104-3.495854

H -7.077962 -6.150500 -1.436647

H -5.643980 -7.318689-1.529554

H $-6.910563-3.981793-2.480143$

H -5.476234 -3.578212 -3.360561

H -4.419587 -2.519938 -1.499826

H -1.873641 -2.0037170 .171119$

C $0.989335-0.5987863 .551885$

O $1.214750 \quad 0.3157002 .460239$

C 2.6306610 .3795312 .178399

C $3.308799-0.2604893 .378649$

C 2.311657-1.341337 3.745007

H $2.776064-0.2386131 .285475$

C 3.0131431 .8364431 .890760

H $4.300559-0.6569443 .146992$

H $3.4066270 .457825 \quad 4.201974$

C $2.510374-1.9025535 .141928$

H $2.394985-2.1581773 .015561$

C 4.5091352 .1097231 .650560

O 2.2648092 .2658150 .751353

H 2.6660412 .4498532 .732433

H 4.6257353 .1473121 .312124

O 5.1808542 .0276132 .912346

C 5.2381811 .1863890 .652164

C $4.6122951 .136987-0.751417$

H 5.3089860 .1785521 .072052
O $\quad 6.588427 \quad 1.6554810 .537514$

H $3.6082510 .710004-0.686382$

H $4.5021022 .160971-1.130075$

H $3.499144-2.3641275 .233076$

H $2.433576-1.1196425 .903910$

H $1.759337-2.6662955 .366324$

C $-0.178537-1.5295453 .210341$

H 2.4410123 .2130430 .621415

H 6.1227042 .2106502 .753077

H $6.5615912 .542196 \quad 0.138807$

H $-0.282240-2.2943383 .988796$

H $0.060211-2.0527472 .275469$

H $\quad 0.761150 \quad 0.0027824 .440522$

C $4.754622-1.887912-1.123183$

O $5.829797-0.953343-1.265813$

C $5.4487990 .329203-1.770040$

C $4.7898760 .176470-3.147534$

C $3.641665-0.831602-3.125341$

C $4.090310-2.144532-2.480016$

H $4.014936-1.496425-0.414723$

C $5.340437-3.174577-0.532722$

H $6.3818760 .886390-1.922344$

O $4.3227321 .440089-3.608550$

H $5.546216-0.167566-3.865150$

H $2.767060-0.412106-2.615784$

O $3.243224-1.078312-4.475312$

H $4.786954-2.664079-3.150451$

O $2.961628-2.993238-2.288482$

C $5.665696-2.9845220 .929945$

H $4.634161-4.010182-0.595459$

H $6.250493-3.484489-1.060247$

H $3.8890031 .294361-4.466800$

H $2.435904-1.618943-4.448142$

H $2.603890-3.212437-3.164938$

O $6.929188-2.4916621 .068737$

O $4.877685-3.2195041 .836992$

C $7.344369-2.2294592 .412529$

H $7.289996-3.141783 \quad 3.014179$

H $8.382650-1.8879762 .386738$

H $\quad 6.726609-1.4398462 .850137$

SCF Energy $(B 3 L Y P / 6-31 G * * / / M M F F)=-3245.91773938$

$02 \_154$

MMFF Geometry

C $2.267543-3.6799920 .242990$

C $2.076819-3.9038651 .552880$

C $2.369580-2.9238582 .664406$

O $2.200259-1.5601312 .214090$

C $3.813319-3.1126153 .160746$

C $4.171537-2.2120824 .326497$

C $4.917376-1.0930874 .230493$

C $3.659713-2.6665545 .668860$

C $5.496865-0.4821942 .984412$

C 5.0245520 .9666602 .806029

C 5.4414141 .5378961 .442725

C 5.0533813 .0133651 .335555

C $5.3499593 .588575-0.057122$

O $4.7741340 .780395 \quad 0.426366$

O $4.6649584 .846029-0.169114$

C $4.7474752 .651096-1.125804$

C $6.8414983 .889916-0.243939$

O $5.1325143 .062710-2.434816$

C $5.1061351 .164877-0.915499$

C $4.3479710 .211787-1.874949$

O $6.5052270 .981801-1.167809$

C $0.924557-1.0813202 .212560$

O $-0.087600-1.7008392 .500343$

C 0.9637210 .3436021 .817096

C -0.1832091 .0353841 .747765$

C -0.3305142 .4567001 .429242$

C 0.9175463 .2363901 .113399

C $2.8197350 .234314-1.764328$

C $2.1392401 .203597-2.715846$

C $-0.0949912 .205517-3.186920$

O $2.7182851 .746674-3.652341$

C $-0.459748 \quad 3.407104-2.376568$

C -1.716276 $3.691023-2.001842$

C $-2.1055474 .936174-1.260514$ 
C $-2.815243 \quad 4.6478240 .041329$

C -4.1563534 .6750070 .120159$

C -1.9664724 .4312001 .271764$

C -1.5678912 .9947131 .458555$

N $0.7935641 .360638-2.413660$

H $2.688458-2.732777-0.084066$

H $1.696715-4.8727311 .869997$

H $1.674311-3.1159953 .491698$

H $4.511569-2.9608042 .330635$

H $3.966067-4.1558243 .469817$

H $5.134653-0.5419225 .144594$

H $3.984626-2.0093946 .482516$

H $2.565475-2.6833485 .677378$

H $4.025714-3.6736945 .893624$

H $6.590334-0.4997283 .063983$

H $5.240462-1.0602312 .094012$

H 3.9319371 .0005962 .895528

H 5.4375201 .5866473 .611722

H 6.5254611 .4168021 .331957

H 5.5561573 .6051532 .110558

H 3.9781553 .1203671 .530605

H 4.9808035 .4241840 .546378

H $3.6583342 .765507-1.067203$

H 7.1801304 .6209130 .500186

H $7.0332044 .347498-1.220774$

H $7.4737513 .005086-0.146072$

H $4.8674083 .993010-2.535870$

H $4.678800 \quad 0.375479-2.907774$

H $4.663658-0.812402-1.628092$

H $6.6625810 .044515-1.369172$

H 1.9299800 .7896951 .618139

H -1.113658 $0.517048 \quad 1.972323$

H 1.5563643 .3068782 .000194

H $0.7200904 .254768 \quad 0.771932$

H $1.4840542 .753518 \quad 0.310052$

H $\quad 2.438623-0.761033-2.025717$

H $2.5058910 .444831-0.738632$

H $-0.9710941 .606132-3.447360$

H $0.3904682 .519091-4.116399$

H $0.3437404 .093603-2.120513$

H $-2.5201573 .013099-2.280626$

H -2.760011 5.522768 -1.918852

H -1.235978 $5.577475-1.069642$

H -4.670996 4.5016551 .059360

H $-4.7686904 .864095-0.755226$

H -2.5333944 .7266352 .165575$

H -1.112081 $5.113810 \quad 1.256241$

H -2.409991 2.3430051 .696554

H $0.4000350 .860014-1.621974$

C $1.379184-4.077603-2.080605$

O $0.096981-3.483868-1.802535$

C $-0.753732-3.664912-2.952823$

C $0.092683-4.354370-4.021226$

C $1.114985-5.096730-3.186780$

H -1.554914 -4.346414 -2.645510

C $-1.343326-2.324018-3.405686$

H $-0.506032-5.016331-4.654863$

H $0.589530-3.631770-4.678986$

C $2.350585-5.505980-3.969636$

H $0.638939-5.992804-2.766428$

C $-2.143585-1.571355-2.323486$

O $-0.262057-1.474408-3.813759$

H -1.963232 -2.480003 -4.296829

H -1.454467 -1.227018 -1.543893

O $-2.668909-0.397234-2.956255$

C $-3.304503-2.377430-1.709325$

C $-4.267800-1.559387-0.830163$

H -2.902315 -3.220352 -1.141929

O $-4.077472-2.938805-2.777572$

H $-4.768057-0.807729-1.456353$

H -5.089171 -2.217183 -0.518456

H $2.078310-6.166384-4.799551$

H $2.866969-4.635520-4.388098$

H $3.059028-6.043086-3.331578$

C $1.968261-4.704361-0.815645$

H $-0.653923-0.646604-4.140475$

H -2.930978 $0.224628-2.256551$

H $-4.760246-3.505734-2.379869$
H $2.905344-5.214774-1.064910$

H $1.272205-5.454364-0.419601$

H $2.033169-3.274860-2.446598$

C $-5.744119-0.0776121 .327443$

$\begin{array}{lllll}\text { O } & -4.458068 & 0.258406 & 0.793920\end{array}$

C $-3.654163-0.8659630 .403052$

C $-3.410260-1.7582811 .628254$

C $-4.715454-2.1699262 .306462$

C $-5.587965-0.9450962 .582240$

H $-6.332647-0.6154340 .573632$

C -6.4658591 .2400931 .648323$

H -2.692172 -0.4373230 .106581$

O $-2.670495-2.923078 \quad 1.291310$

H $-2.815422-1.2023022 .363715$

H $\quad-5.259837-2.9153651 .715174$

O $-4.417714-2.7942023 .558073$

H $-5.144521-0.3557953 .395141$

O $-6.874788-1.3771103 .019190$

C $-6.900171 \quad 1.975651 \quad 0.396740$

H -7.3686621 .0423482 .236696$

H -5.8009481 .9068162 .209597$

H -1.810431 -2.630868 0.943109

H $-3.824846-3.5429703 .373160$

H $-6.741346-1.9422013 .799582$

O $-7.6192103 .079060 \quad 0.761525$

O $-6.6448741 .635168-0.750043$

C $-8.1216623 .871847-0.318789$

H -8.6323594 .7401760 .105805$

H -7.300702 4.222596-0.950941

$\mathrm{H}-8.8408343 .293287-0.906253$

SCF Energy (B3LYP/6-31G**//MMFF) $=-3245.91803865$

$02 \_155$

MMFF Geometry

C $-0.266300 \quad 3.164117-0.367420$

C -1.2003263 .8867850 .270216$

C -1.8844233 .5065161 .563874$

O -1.6097142 .1216061 .874693$

C -3.4099943 .6678061 .423311$

C -4.1660633 .3874732 .709192$

C -4.8215032 .2379792 .968506$

C -4.1817254 .5146213 .707101$

C -4.8704331 .0185232 .091270$

C -6.2231580 .8793541 .382258$

C -6.1658230 .0178950 .109931$

C $-5.669235-1.4062510 .370003$

C $-5.523386-2.208953-0.932833$

O $-5.3079180 .693057-0.816462$

O $-4.764176-3.392244-0.640398$

C $-4.691367-1.377924-1.933369$

C $-6.877004-2.687055-1.469202$

O $-4.627345-2.021457-3.202944$

C $-5.1984100 .069776-2.102159$

C $-4.2534870 .952544-2.956034$

O $-6.4667270 .048339-2.768149$

C -1.1301331 .8086263 .103280$

O -0.8304932 .5922233 .993728$

C -1.0215150 .3410973 .288066$

C - $-1.353116-0.5623022 .351714$

C -1.301046-2.019598 2.497544

C $-0.823339-2.578433 \quad 3.811673$

C $-2.8277081 .099668-2.412064$

C $-1.8684100 .063243-2.970380$

C $0.157880-1.251657-2.372198$

O $-1.979219-0.402667-4.102016$

C $-0.369698-2.600179-1.994888$

C $0.240685-3.420579-1.127330$

C $-0.296099-4.765820-0.732624$

C $-0.490233-4.8998720 .762477$

C $0.375205-5.6097541 .506219$

C $-1.729310-4.2912141 .381908$

C $-1.679156-2.7886371 .454806$

N $-0.851690-0.251330-2.080478$

H $\quad 0.0641242 .2156630 .047778$

H -1.493322 $4.843249-0.158671$

H -1.5065284 .1742962 .347046$

H -3.7767363 .0189080 .618562$

H -3.6504964 .6889781 .097966$ 
H -5.3590932 .1447643 .910503$ H -4.7671564 .2706114 .599957$ H -3.164811 4.7511584 .034864 H -4.621282 5.4119573 .259459 H -4.0521391 .0268091 .366115$ H $-4.694673 \quad 0.137798 \quad 2.721222$ H -6.9602320 .4630262 .079812$ H -6.5812031 .8765681 .093067$ H -7.178740 -0.007606 -0.309189 H $-6.332299-1.9254101 .073017$ H -4.684103 -1.377943 0.853386 H $-5.232376-3.8848420 .055227$ H $-3.667480-1.354183-1.541420$ H $-7.368350-3.340783-0.738320$ H $-6.751608-3.291325-2.374449$ H -7.564984 -1.869162 -1.691788 H $-4.287602-2.920720-3.055121$ H $-4.2566440 .607000-3.996764$ H -4.686744 $1.962718-2.983171$ H $-6.6160150 .917034-3.176676$ H -0.6642180 .0396364 .267745$ H - $-1.709365-0.212657 \quad 1.384078$ H $\quad 0.170923-2.1928984 .058562$ H - $0.738981-3.6672613 .816170$ H -1.514280 -2.305742 4.617016 H -2.429532 $2.072706-2.725912$ H -2.821490 $1.087673-1.317147$ H $1.052565-0.982708-1.803913$ H $0.395137-1.229719-3.440550$ H -1.301464 -2.917053 -2.458257 H $\quad 1.179606-3.112139-0.676639$ H $0.411808-5.524237-1.091562$ H -1.244989 -4.983072 -1.238842 H $0.231544-5.7389622 .573572$ H $1.251525-6.0729781 .064957$ H -1.922606 -4.7201642 .371895$ H -2.603963 -4.5821620 .786777$ H -2.017016 -2.295671 0.543011 H $-0.8803890 .125083-1.137645$ C $1.8269544 .002790-1.543986$ O $2.6085412 .982903-0.892397$ C 3.5356983 .6247680 .006674 C $3.5248505 .103867-0.362034$ C $2.0737955 .292910-0.762022$ H 3.1207123 .5192441 .015894 C $4.9077042 .949664-0.067415$ H 3.8284985 .7426120 .472746 H $4.1852155 .316516-1.210770$ C $1.819996 \quad 6.559397-1.560039$ H 1.4649285 .3178620 .150155 C 4.8681951 .4356650 .233399 O $5.4278173 .125457-1.390531$ H 5.6067713 .4574740 .607350 H $4.2922280 .932340-0.546374$ O $\quad 6.2083250 .9398360 .127939$ C 4.3087461 .0795271 .620847 C $4.378476-0.4151161 .991108$ H 3.2712251 .4196721 .706371 O 5.0681601 .8070072 .593976 H $5.369989-0.8127841 .750720$ H $4.301248-0.4859703 .083391$ H $2.0862937 .443905-0.972215$ H $2.4105556 .578757-2.482172$ H $\quad 0.7635586 .641847-1.834030$ C $0.367044 \quad 3.564237-1.670527$ H $6.2830162 .663689-1.429929$ H $6.728093 \quad 1.3453260 .843312$ H 4.6432191 .6736313 .458494 H $0.3204412 .686377-2.327563$ H $-0.2160134 .351760-2.162182$ H $2.2399724 .100922-2.556819$ C $4.435071-2.177030-0.557054$ O $3.355505-1.374815-0.064640$ C $3.259711-1.2700751 .357495$ C $3.126880-2.6695771 .977144$ C $4.268038-3.5831131 .546823$ C $4.388430-3.5962800 .026238$ H $5.388416-1.697191-0.307850$
C $4.308495-2.238261-2.085701$

H $2.316447-0.7460991 .559649$

O $3.092986-2.6075393 .398988$

H $2.179347-3.1184211 .656497$

H $5.215924-3.2932682 .014965$

O $3.994454-4.9165811 .983268$

H $3.542036-4.151250-0.398439$

O $5.578079-4.291423-0.343491$

C $4.568807-0.895595-2.725926$

H $5.023676-2.943020-2.525071$

H $3.307496-2.581315-2.375053$

H $2.388119-1.9861723 .648075$

H $3.870419-4.8851012 .947514$

H $5.531617-5.1778260 .054348$

O $3.400584-0.365983-3.187236$

O $5.675681-0.378093-2.799698$

C $3.5035130 .907939-3.829554$

H $2.4940141 .244162-4.080892$

H $3.9579011 .640556-3.157977$

H $4.0851760 .816791-4.751495$

SCF Energy (B3LYP/6-31G**//MMFF) $=-3245.92280865$

$02 \_156$

MMFF Geometry

C -1.942097 -0.1288683 .197787$

C -3.1292950 .4147303 .503308$

C -3.6857591 .7100352 .958736$

O $-4.709807 \quad 1.3258152 .009992$

C -2.6656072 .6092042 .231510$

C -3.1753184 .0051801 .938662$

C -3.5329204 .4501490 .718387$

C -3.2078554 .9401633 .120483$

C $-3.5858803 .682241-0.573483$

C $-2.4748494 .099224-1.548060$

C $-1.1050053 .487779-1.209239$

C $-0.0217504 .057349-2.128749$

C $1.3393793 .381208-1.901579$

O $-1.2099972 .072764-1.394900$

O $2.2014853 .749685-2.988753$

C $1.1465831 .852202-1.973704$

C $2.0156313 .883777-0.620159$

O $2.3564771 .170369-1.651839$

C $-0.0180411 .333474-1.100848$

C $-0.306679-0.150986-1.434823$

$\begin{array}{lllll}\text { O } & 0.354376 & 1.450150 & 0.273932\end{array}$

C -5.9109381 .9520382 .047718$

O -6.2578442 .8242252 .832042$

C -6.8195971 .4420260 .992626$

C -6.4478510 .5691950 .042319$

C $-7.3010940 .027319-1.019520$

C $-8.7492740 .442198-1.033419$

C $-1.568377-0.701798-0.757248$

C $-1.837028-2.151342-1.118308$

C $-3.538681-3.940672-0.752669$

O $-1.106429-2.812047-1.850903$

C $-4.482865-3.872332-1.911315$

C $-5.805066-4.075424-1.810518$

C $-6.748215-3.999008-2.976207$

C $-7.806849-2.931571-2.804023$

C $-9.072272-3.260116-2.493714$

C $-7.423791-1.494766-3.085517$

C $-6.756754-0.824351-1.914575$

N $-2.999336-2.614806-0.515577$

H -1.295012 0.3757162 .488997

H -3.781587 $-0.119606 \quad 4.191504$

H -4.1235072 .2472553 .808749$

H -2.351306 2.1232411 .301597

H -1.756682 2.714014 2.838893

H -3.8522705 .4879110 .627315$

H -3.518543 5.9527672 .841579

H -3.9092564 .5800883 .879059$

H -2.213585 5.0154443 .572773

H -3.581339 $2.599638-0.416050$

H -4.555005 $3.905292-1.038465$

H $-2.7724143 .761636-2.549715$

H $-2.4021015 .193330-1.583699$

H $-0.8621573 .699984-0.162494$

H $0.0639415 .143792-2.001681$ 
H $-0.314042 \quad 3.901517-3.176853$ H $2.2750894 .719348-2.998355$ H $0.9327821 .606218-3.022847$ H $2.1687784 .968514-0.669372$ H $3.0129853 .448250-0.499223$ H 1.4364993 .6741140 .281447 H $2.4574321 .184813-0.684701$ H $-0.437425-0.258203-2.519726$ H $0.549576-0.772335-1.148140$ H $\quad 0.078891 \quad 0.655046 \quad 0.754833$ H -7.8282621 .8406481 .037397$ H -5.4200290 .2101750 .029200$ H $-9.336409-0.049174-1.811924$ H -8.837798 $1.521381-1.199694$ H -9.230856 $0.191901-0.081354$ H $-1.473407-0.6474360 .329959$ H $-2.445017-0.114747-1.055154$ H $-4.034707-4.2615180 .168713$ H -2.725039 -4.638735 -0.973675 H -4.052272 -3.643503-2.883478 H $-6.236071-4.313718-0.840815$ H -7.217958 -4.984851-3.088543 H $-6.209522-3.822869-3.915692$ H $-9.845903-2.505303-2.401275$ H $-9.362470-4.291137-2.321120$ H $-8.297231-0.925352-3.421655$ H -6.737785 -1.462519-3.941846 H -5.697789-1.064689-1.820073 H $-3.572316-1.9633510 .013538$ C $0.078589-1.3993823 .950201$ O $0.733501-1.3221512 .664564$ C $1.777183-2.3152662 .607674$ C $2.029135-2.7175514 .051236$ C $0.626626-2.6573974 .623869$ H $1.356659-3.1582802 .044458$ C $2.986774-1.7301391 .869284$ H $2.485215-3.7077104 .141748$ H $2.679925-1.9894634 .551224$ C $0.591046-2.6085536 .141466$ H $0.069359-3.5399174 .281678$ C $4.234842-2.6286061 .892220$ O $2.598180-1.4934810 .512225$ H $3.224298-0.7480872 .292953$ H $4.609310-2.7127202 .919250$ O $3.843723-3.949527 \quad 1.494397$ C $5.373553-2.1810340 .955199$ C $5.819402-0.7230731 .157716$ H $5.072069-2.346408-0.084619$ O $6.492391-3.0462321 .182551$ H $4.975643-0.0540750 .963330$ H $6.107625-0.5793372 .206475$ H $1.045409-3.5097636 .565944$ H $1.138562-1.7424276 .528136$ H -0.439319 -2.545775 6.505548 C -1.438222 -1.4369763 .739978$ H $2.407913-2.3563270 .105721$ H $4.641063-4.5063211 .513751$ H $6.797101-2.9046022 .095157$ H -1.944920 -1.678251 4.681042 H -1.683721 -2.216555 3.007971 H $0.369206-0.5008164 .509920$ C $5.8038020 .114222-1.776185$ O $6.798483-0.674343-1.112235$ C $7.014748-0.3280140 .259904$ C $7.436314 \quad 1.1452240 .373879$ C $6.4752302 .082261-0.357280$ C $6.2251481 .587373-1.782196$ H $4.8442410 .003102-1.258391$ C $5.646104-0.434286-3.196830$ H $7.869470-0.9300910 .593103$ O 7.5318641 .5450971 .737029 H $8.4342891 .267900-0.067009$ H 5.5317992 .1953960 .189941 O $7.0565153 .386039-0.429171$ H $7.1402011 .718233-2.373297$ O $5.2180572 .390006-2.391789$ C $4.967228-1.783446-3.174367$ H $5.0529740 .229835-3.835514$
H $\quad 6.622759-0.561969-3.678881$

H 8.1703560 .9533122 .170473

H $7.241748 \quad 3.6704290 .482291$

H $4.3808952 .224785-1.926129$

O $3.629247-1.627702-2.959149$

O $5.557705-2.846311-3.316056$

C $2.872294-2.839172-2.881835$

H $2.926341-3.379882-3.831449$

H $3.236999-3.462700-2.059828$

H $1.829469-2.576344-2.686118$

SCF Energy (B3LYP/6-31G**//MMFF) $=-3245.92260780$

$02 \_157$

MMFF Geometry

C 4.2221622 .0090472 .608430

C 3.8815921 .8603851 .319841

C 4.3332782 .7933050 .227286

O $3.1196313 .379018-0.301161$

C $5.0824462 .048826-0.895806$

C $6.3874881 .402916-0.466866$

C $6.6586930 .084858-0.555698$

C 7.4366552 .3457850 .065493

C $5.773898-0.991404-1.118641$

C $5.022214-1.743640-0.013078$

C $3.806673-2.538388-0.516273$

C $4.193366-3.664564-1.475618$

C $2.957519-4.386501-2.036125$

O $2.928351-1.610520-1.156759$

O $3.387127-5.201275-3.137664$

C $1.986686-3.329829-2.604465$

C $2.336623-5.339638-1.008305$

O $0.769351-3.937248-3.034791$

C $1.697192-2.162623-1.631901$

C $0.951301-1.031961-2.378168$

O $0.889450-2.648346-0.560740$

C $3.2068264 .570315-0.944679$

O $4.2294295 .167272-1.249106$

C $1.8592935 .087407-1.275646$

C $0.7863854 .799876-0.521300$

C $-0.5819085 .278190-0.731166$

C $-0.8732966 .014916-2.011110$

C $0.7061940 .223377-1.531651$

C $0.1819851 .352172-2.393930$

C $-1.8921832 .166837-3.513387$

O $0.9125902 .265026-2.771253$

C $-2.9348942 .906732-2.728588$

C $-3.2958122 .716298-1.449088$

C $-4.3663603 .521065-0.767630$

C $-3.866744 \quad 4.2382860 .465867$

C -4.2806413 .8817381 .693395$

C -2.9494725 .4266550 .271754$

C -1.499740 $5.026987 \quad 0.226144$

N $-1.1590081 .211350-2.705957$

H 4.8693412 .8437932 .873119

H 3.2301681 .0459131 .016699

H 4.9541293 .5973640 .641872

H $5.3050372 .750622-1.710116$

H $4.393393 \quad 1.326581-1.347124$

H $7.627228-0.268813-0.204990$

H $8.401719 \quad 1.8501990 .216978$

H $7.6000833 .167663-0.639371$

H 7.1292532 .7657291 .027889

H $5.085167-0.577066-1.860069$

H $6.407040-1.694009-1.674416$

H $5.712116-2.4120450 .516506$

H $4.659589-1.0164980 .722438$

H $3.299382-2.9506380 .364203$

H $4.868327-4.377046-0.985510$

H $4.755453-3.256200-2.325853$

H $4.056689-5.824194-2.806884$

H $2.454874-2.920242-3.509982$

H $3.072448-6.086426-0.686064$

H $1.508333-5.907665-1.446050$

H $1.972296-4.829316-0.114269$

H $0.242368-4.136043-2.242118$

H $\quad 1.543450-0.733760-3.254157$

H $-0.008930-1.399754-2.755773$

H $0.399194-1.907543-0.174425$ 
H $\quad 1.8104415 .759645-2.125136$ H $0.920204 \quad 4.1658760 .354320$ H -1.932557 $6.235753-2.156042$ H $-0.5634795 .419258-2.877379$ H $-0.3384596 .970598-2.033945$ H $-0.0135610 .033612-0.728007$ H $1.6306160 .571142-1.057706$ H $-2.3722531 .607111-4.322195$ H -1.1969592.885054 -3.959700 H $-3.4440583 .687920-3.290711$ H $-2.8234841 .938619-0.854917$ H $-5.1821992 .835575-0.506173$ H $-4.8067574 .257821-1.451069$ H -3.944399 4.4122312 .578246 H -4.959976 3.0503961 .843163 H -3.0693836 .1235901 .112352$ H -3.270702 $5.989110-0.608647$ H -1.1741554 .4923021 .120280$ H -1.665537 $0.401661-2.359418$ C $2.903234-0.0521013 .489487$ O 1.5912330 .3953683 .090451 C $0.595325-0.3846343 .786592$ C $1.344234-1.5532654 .409322$ C $2.684586-0.9264504 .725118$ H $\quad 0.191650 \quad 0.274638 \quad 4.565075$ C $-0.511737-0.7971322 .806550$ H $0.840391-1.9533145 .294160$ H $1.465250-2.3690353 .685332$ C $3.785782-1.9454814 .964950$ H $2.575578-0.2969765 .618629$ C -1.550885 -1.747793 3.426733 $\begin{array}{lllll}0 & -1.181668 & 0.382501 & 2.354267\end{array}$ H $-0.056255-1.2583831 .924669$ H -1.057839 -2.672860 3.746688 O $-2.075825-1.1503304 .619136$ C $-2.742264-2.1132292 .518698$ C $-2.318170-2.6742301 .151802$ H -3.387365 -1.240732 2.382487 O $-3.532276-3.0873173 .209619$ H -1.867364 -1.865881 0.567239 H -1.538177 -3.429795 1.309281 H $3.537691-2.5859795 .817733$ H $3.932791-2.5903664 .091912$ H $4.737427-1.4501875 .181205$ C 3.8089991 .1510253 .775105 H $-0.507195 \quad 0.972376 \quad 1.975487$ H -2.493162 -0.3089454 .365293$ H $-2.985204-3.8820633 .331473$ H 4.7310640 .8002044 .253680 H 3.3044801 .8124814 .491262 H $3.304071-0.6562432 .667164$ C $-4.431409-1.350442-0.654894$ O $-4.605818-2.469497 \quad 0.222070$ C -3.468322 -3.3269890 .352987$ C $-3.045017-3.868625-1.020376$ C $-2.829899-2.743142-2.025765$ C $-4.047238-1.824186-2.062729$ H $-3.656218-0.685089-0.254878$ C $-5.763490-0.584243-0.697202$ H -3.807093 -4.190935 0.938564 O $-1.852643-4.640781-0.922853$ H -3.828498 -4.533277 -1.407106 H -1.921969 -2.175489-1.800098 O $-2.647328-3.299592-3.328701$ H $-4.889129-2.354247-2.526625$ O $-3.756266-0.691947-2.877412$ C $-6.0927750 .098076 \quad 0.612793$ H $-5.7392620 .195448-1.466083$ H $-6.576425-1.286388-0.919771$ H -2.023120 -5.362367 -0.293408 H $-1.886709-3.904053-3.281459$ H -3.489162 -1.023549 -3.751853 O $-7.3994450 .496448 \quad 0.586538$ $\begin{array}{lllll}\text { O } & -5.306894 & 0.283757 & 1.530761\end{array}$ C -7.8639261 .1694331 .760893$ H -8.9352661 .3542071 .645161$ H -7.7102180 .5455072 .646659$ H $-7.3518642 .129581 \quad 1.869843$
SCF Energy (B3LYP/6-31G**//MMFF) $=-3245.92069648$

$02 \_158$

MM̄FF Geometry

C $2.512577-3.808377-1.378850$

C $3.849049-3.807426-1.504863$

C $4.851812-3.155304-0.577889$

O $4.218854-2.5321020 .559187$

C $5.613895-2.060691-1.349927$

C $6.608802-1.288909-0.505492$

C $6.5583120 .039031-0.277001$

C $7.749982-2.1013750 .052513$

C $5.5403891 .033952-0.767710$

C 5.1363612 .0241740 .334796

C $4.2470503 .153201-0.211401$

C 4.0953254 .2661550 .827304

C $3.174877 \quad 5.387647 \quad 0.326050$

O $2.9595632 .614436-0.537680$

O 2.8412906 .2195461 .447739

C $1.8615764 .759872-0.178822$

C $3.8737276 .294131-0.693897$

O $1.0287845 .742558-0.791524$

C $2.0657713 .561804-1.140292$

C $0.6925682 .887198-1.396710$

O $2.577924 \quad 4.040952-2.386378$

C $3.997853-3.3030721 .657904$

O $4.227617-4.4935201 .791900$

C $3.375870-2.4340232 .675975$

C $2.425446-2.9449073 .471692$

C $1.626578-2.1827804 .432625$

C $2.139693-0.8271754 .842855$

C $0.7407031 .517332-2.085853$

C $1.0997220 .382041-1.146224$

C $0.333800-0.8285660 .873396$

O $2.106387-0.301631-1.317725$

C $-0.395659-0.4404742 .117749$

C -1.372447-1.174282 2.672581

C $-2.035649-0.8276453 .976274$

C $-1.909415-1.9310645 .008159$

C $-2.960180-2.7104615 .314694$

C $-0.586195-2.0833175 .735287$

C $0.464474-2.7166074 .863860$

N $0.1866210 .215762-0.116857$

H $2.044052-3.319790-0.529509$

H $4.283725-4.309845-2.367612$

H $5.558828-3.921402-0.236055$

H $4.889441-1.381374-1.810428$

H $\quad 6.158445-2.516164-2.188468$

H 7.3629550 .4779530 .312542

H $8.509988-1.4764740 .533504$

H $7.387422-2.8113010 .802212$

H $8.245887-2.659751-0.748175$

H $5.9920321 .587007-1.600248$

H $4.6409380 .549784-1.152057$

H 4.6060831 .4798981 .126954

H 6.0407352 .4593150 .778695

H $4.7142063 .548994-1.120910$

H 5.0738934 .6744491 .108958

H 3.6672583 .8485191 .749453

H 3.6701556 .5653351 .821053

H 1.3150794 .4008000 .704970

H $4.767266 \quad 6.750538-0.251391$

H $3.2271757 .128109-0.988871$

H $4.1862285 .764566-1.596199$

H $0.9113616 .463641-0.149494$

H $0.1681762 .783426-0.440829$

H $0.0863373 .551553-2.025869$

H $2.329648 \quad 3.420151-3.089410$

H $3.651672-1.3856992 .678681$

H $2.155612-3.9938553 .355622$

H $3.182589-0.8971465 .173529$

H $1.588309-0.3884135 .676947$

H $2.086888-0.1203974 .008300$

H $-0.2483591 .288276-2.502742$

H $1.442611 \quad 1.513645-2.925770$

H $1.397491-0.9607921 .089779$

H $-0.038047-1.7605360 .439772$

H -0.0641600 .4668232 .617543$ 
H -1.701946 -2.084983 2.177899 H -3.093006 -0.6237523 .769017$ H -1.630596 $0.101544 \quad 4.396260$ H -2.886938 -3.487674 6.068075 H -3.915986 -2.592434 4.814963 H $-0.709182-2.7201796 .620977$ H $-0.290287-1.1043176 .121116$ H $0.193118-3.7139144 .513913$ H $-0.7140250 .679894-0.189435$ C $0.611509-3.408832-2.953431$ O $-0.417238-3.130455-1.981323$ C -1.688077 -3.018098 -2.662804 C $-1.362289-2.997784-4.147852$ C $-0.131731-3.881305-4.200457$ H $-2.246018-3.927510-2.406407$ C $-2.416248-1.777990-2.132646$ H $-2.185300-3.370624-4.764681$ H $-1.117587-1.981218-4.480691$ C $0.660261-3.735573-5.488194$ H $-0.441062-4.928428-4.080748$ C $-3.667125-1.376762-2.929998$ O $-2.807620-2.039908-0.780190$ H -1.712850 -0.939565 -2.099779 H -3.380806 -1.075985 -3.944498 O $-4.502483-2.532623-3.069153$ C $-4.508646-0.261883-2.276576$ C $-3.7110361 .014624-1.957289$ H $-4.985584-0.652024-1.371521$ O $\quad-5.575608 \quad 0.068977-3.172538$ H $-2.8866750 .770314-1.282301$ H -3.265648 1.398679 -2.883324 H $0.047083-4.018297-6.350229$ H $0.995831-2.703836-5.638507$ H $1.545464-4.379036-5.479162$ C $1.586713-4.441015-2.383977$ H $-2.027620-2.376945-0.308802$ H $-5.293204-2.260892-3.566016$ H $-5.1810370 .436594-3.981814$ H $2.168279-4.896364-3.193565$ H $1.035340-5.243522-1.879493$ H $1.126677-2.460187-3.156303$ C $-4.546194 \quad 1.340077 \quad 0.956317$ O $-5.3282851 .652836-0.203119$ C $-4.5783932 .126930-1.326163$ C $-3.8028123 .395088-0.941841$ C $-2.966378 \quad 3.1944270 .320930$ C -3.8073012 .5893881 .445346$ H -3.8237250 .5503960 .715339$ C $-5.503794 \quad 0.7971142 .021495$ H -5.315532 $2.420122-2.084273$ O $-2.9687363 .805990-2.019855$ H -4.521045 4.206019-0.764420 H -2.095832 2.5649980 .113509 O H $-4.5233383 .333972 \quad 1.815120$ O -2.9516642 .2381852 .529792$ C -5.895851 -0.6245891 .694444$ H -5.0433800 .7791393 .015384$ H -6.411429 1.4072422 .100588 H -2.471676 $4.587420-1.722786$ H -3.1917815 .0399890 .944653$ H -2.467604 3.0403502 .790512 O $-6.998965-0.6339220 .893249$ O $\quad-5.274072-1.6065372 .079254$ C $-7.438987-1.9277240 .469402$ H $-7.727765-2.5314521 .334951$ H -8.312751-1.795528 -0.174141 H $-6.652497-2.426372-0.105484$ SCF Energy $(B 3 L Y P / 6-31 G * * / / M M F F)=-3245.90676132$

\section{9}

MM̄FF Geometry

C $2.206130-2.9497211 .399320$

C $1.916129-2.1950672 .469593$

C $2.749283-1.0819603 .056302$

$\begin{array}{llll}\text { O } & 2.077600 & 0.150725 & 2.703337\end{array}$

C $4.204664-0.9941402 .555617$

C $5.093005-0.1584493 .457366$
C 5.4936321 .1014713 .198951

C $5.577022-0.8573414 .703258$

C 5.1249241 .9768982 .034180

C 6.3219532 .2546641 .113459

C 6.7003941 .0508510 .237022

C $7.9643731 .344870-0.572851$

C $8.2983930 .202399-1.544617$

O $5.6041640 .804676-0.647935$

O $9.2865230 .683311-2.468499$

C $7.037903-0.137683-2.367214$

C $8.924988-0.996987-0.823816$

O $7.254123-1.288053-3.181516$

C $5.768993-0.331253-1.504996$

C $4.521473-0.415245-2.417653$

O $5.888904-1.543223-0.758428$

C 1.8484781 .0729753 .670089

O $2.107706 \quad 0.966546 \quad 4.860655$

C 1.2118072 .2913363 .114799

C 1.0728472 .5263461 .800149

C 0.4666023 .7131521 .190760

C -0.1043164 .7597902 .111217$

C $3.188840-0.367718-1.657960$

C $1.994340-0.335872-2.594489$

C $-0.4687730 .050963-2.563285$

O $2.083717-0.499886-3.807897$

C $-0.6502411 .495255-2.907965$

C $-1.5616372 .290580-2.328163$

C $-1.7321413 .743441-2.666727$

C -1.534616 $4.653513-1.473269$

C $-2.5786725 .280591-0.906070$

C $-0.1220594 .917612-0.997960$

C $0.4348913 .802902-0.155679$

N $0.811688-0.112347-1.902573$

H $3.119074-2.7758350 .838616$

H $\quad 0.982925-2.3915042 .995599$

H $2.737792-1.2321364 .142712$

H $4.221028-0.5999581 .533837$

H $4.650435-1.9959352 .500108$

H 6.1537541 .5838013 .919398

H $6.254043-0.2322325 .295342$

H $4.734597-1.1279225 .346981$

H $6.120899-1.7702744 .439106$

H $4.2835991 .576431 \quad 1.461486$

H 4.7748632 .9325382 .444930

H 6.0492323 .0959700 .463079

H 7.1854992 .5729811 .710611

H $\quad \begin{array}{llll}6.856633 & 0.174858 & 0.875869\end{array}$

H $8.814419 \quad 1.5452380 .091319$

H $7.8180432 .264034-1.157440$

H $10.061748 \quad 0.970273-1.955835$

H $6.8629350 .700689-3.056083$

H $9.851032-0.698277-0.317884$

H $9.212104-1.779389-1.535132$

H $8.266931-1.438137-0.072276$

H $8.050355-1.120205-3.714238$

H $4.5331350 .429891-3.118676$

H $4.556429-1.335192-3.014949$

H $5.003305-1.878805-0.550530$

H 0.8800303 .0063853 .860937

H 1.4422201 .7873561 .091117

H -0.8727214 .3243012 .759816$

H -0.5793875 .5900281 .584456$

H 0.6836925 .1908342 .738579

H $3.080653-1.247224-1.017934$

H $3.1449440 .526492-1.025586$

H -1.238086 $-0.306457-1.874199$

H -0.499416 -0.559359-3.471304

H $\quad 0.004790 \quad 1.904851-3.673355$

H $-2.2189771 .878182-1.566836$

H -2.741255 $3.870869-3.079785$

H -1.045432 $4.049934-3.465690$

H -2.445717 $5.962135-0.072766$

H $-3.5912675 .128172-1.262314$

H $-0.0654425 .873981-0.465636$

H $0.5316485 .056221-1.869074$

H $0.8761372 .993920-0.737874$

H $\quad 0.8524870 .077991-0.905293$

C $0.439400-3.657782-0.251496$ 
$\begin{array}{llll}\text { O } & -0.672029 & -2.890657 & 0.265939\end{array}$

C -1.895625 -3.579243 -0.063171

C $-1.560264-4.374277-1.311353$

C $-0.168030-4.865768-0.973963$

H $-2.102190-4.2494330 .781684$

C -3.036310 -2.568801-0.226591

H $-2.263251-5.190698-1.500200$

H $-1.536158-3.722268-2.193358$

C $0.630467-5.310693-2.187644$

H $-0.255729-5.714058-0.281406$

C $-4.352570-3.200146-0.712548$

O $-3.263379-1.9475841 .042043$

H -2.727886 -1.775042 -0.913523

H -4.218246 -3.599615 - 1.724819

$\begin{array}{llll}\text { O } & -4.650388 & -4.322194 & 0.129277\end{array}$

C $-5.573971-2.261577-0.685829$

C $-5.383061-0.962498-1.487274$

H $-5.837352-2.0365840 .352937$

O $-6.688956-2.980021-1.228173$

H -4.541761 - $0.398796-1.073401$

H $-5.121229-1.216183-2.522028$

H $0.135480-6.149494-2.687851$

H $0.737396-4.500155-2.916531$

H $1.634135-5.634968-1.894246$

C $1.342078-4.078628 \quad 0.911782$

H -2.419015 -1.560698 1.331030

H -5.484969 -4.708781 -0.186914

H -6.491438 -3.176104 -2.159996

H $2.009417-4.8914710 .600824$

H $0.732274-4.4633061 .739361$

H $0.977403-3.010198-0.953680$

C -6.3432550 .9993590 .631729$

O $-7.1624890 .156163-0.186416$

C $-6.651479-0.077529-1.502586$

C $-6.493598 \quad 1.255406-2.246463$

C $-5.6834912 .270780-1.442341$

C $-6.2062592 .383338-0.009367$

H -5.3522200 .5467830 .755575$

C $-7.003426 \quad 1.0837542 .011722$

H -7.425235 -0.646542 -2.033239

O $-5.885102 \quad 1.040323-3.515364$

H $-7.4919311 .670931-2.435766$

H -4.620576 $2.009347-1.445944$

O $-5.770714 \quad 3.548184-2.077580$

H -7.175233 $2.898271-0.008691$

$\begin{array}{llll}\text { O } & -5.299882 & 3.170448 & 0.759020\end{array}$

C $-6.951011-0.2515822 .716239$

H -6.522548 1.8271342 .657076

H $-8.059258 \quad 1.3663831 .920560$

H $-5.778163 \quad 1.909527-3.938447$

H $-6.705323 \quad 3.816859-2.086235$

H -5.2199464 .0338020 .318581$

O $-5.683044-0.4999973 .152704$

O $-7.919310-0.9882192 .851993$

C $-5.493085-1.759008 \quad 3.805607$

H $-5.732609-2.579788 \quad 3.122642$

H $-4.441054-1.8378994 .092128$

H $-6.108577-1.8140414 .708488$

SCF Energy (B3LYP/6-31G**//MMFF) $=-3245.91161416$

0216

MMFF Geometry

C $2.616846 \quad 0.0256823 .372683$

C $3.519497-0.9644293 .296098$

C $4.561066-1.1885982 .228036$

O $4.734297-0.003341 \quad 1.420197$

C $4.130619-2.3492341 .313786$

C $5.279731-2.9797740 .553860$

C $5.520097-2.811476-0.760894$

C $6.154641-3.9025111 .364324$

C $4.783358-1.938485-1.737988$

C $3.942802-2.754795-2.730855$

C $2.636542-3.286132-2.120020$

C $1.900904-4.191077-3.109917$

C $0.536908-4.641844-2.564797$

O $1.823123-2.153323-1.802899$

O $-0.206015-5.214015-3.651826$

C $-0.244655-3.400648-2.087087$
C $0.678356-5.743337-1.507721$

O $-1.455647-3.780143-1.436516$

C $0.577092-2.465227-1.169176$

C $-0.184751-1.134932-0.966043$

$\begin{array}{llllllll}\text { O } & 0.769577 & -3.099584 & 0.095739\end{array}$

C $6.0052760 .356372 \quad 1.103226$

O $7.034918-0.1896971 .478461$

C $6.047876 \quad 1.5394390 .215171$

C $4.9790142 .073995-0.395579$

C $5.0008483 .228668-1.300202$

C $6.3243283 .911874-1.531683$

C $0.623266-0.046846-0.245774$

C $-0.122678 \quad 1.274718-0.208866$

C $0.103914 \quad 3.608106 \quad 0.639075$

O $-1.221218 \quad 1.442972-0.731014$

C $0.467796 \quad 4.397966-0.576712$

C $1.3447465 .412480-0.575680$

C $1.7160596 .182850-1.809113$

C $3.1811526 .047143-2.161185$

C $4.0335217 .065682-1.957284$

C $3.6376364 .771094-2.835880$

C $3.8463053 .632584-1.870555$

N 0.5861862 .2485270 .480076

H 2.5648020 .7865522 .600515

H $3.518538-1.7150254 .086066$

H $5.490857-1.4269232 .757925$

H $3.361675-1.9933870 .619997$

H $3.651010-3.1435831 .901924$

H $6.362065-3.347483-1.198197$

H $6.928563-4.3832930 .756396$

H $6.660963-3.3530262 .163548$

H $5.552059-4.6975691 .815768$

H $4.171005-1.181740-1.238793$

H $5.539164-1.377664-2.302756$

H $3.696600-2.095758-3.573860$

H $4.538404-3.584765-3.130900$

H $2.865764-3.839208-1.202946$

H $2.515685-5.060755-3.373227$

H $1.734820-3.645825-4.049671$

H $0.311367-5.955153-4.010967$

H $-0.538409-2.835739-2.982970$

H $1.185648-6.618125-1.932164$

H $-0.302942-6.099264-1.174664$

H $1.246542-5.426127-0.630791$

H $-1.952751-4.342193-2.055336$

H $-0.474392-0.739995-1.948874$

H $-1.107627-1.315429-0.400739$

H $0.899416-2.4217130 .776879$

H 7.0455811 .9398520 .062807

H $3.9998511 .626777-0.234122$

H $6.7481834 .261555-0.583491$

H $6.2561294 .788068-2.179495$

H $7.0345993 .224107-2.003732$

H $0.832950-0.3471220 .785419$

H $1.5773490 .125885-0.756544$

H 0.5558344 .0123681 .550267

H $-0.983138 \quad 3.599120 \quad 0.761856$

H $-0.0156034 .115078-1.509240$

H 1.8258915 .7036940 .355099

H $1.4607437 .236034-1.633661$

H $1.1107865 .874302-2.670803$

H $5.0787566 .994342-2.237912$

H $3.7056057 .994619-1.502563$

H $4.5419164 .950113-3.428708$

H $2.8880554 .466497-3.577846$

H $2.9370813 .075169-1.646844$

H 1.5310612 .0437410 .791723

C $0.299083-0.522903 \quad 4.174997$

$\begin{array}{lllll}\text { O } & -0.503187 & 0.379818 & 3.387990\end{array}$

$\begin{array}{llll}\text { C } & -1.883218 & 0.265272 & 3.813917\end{array}$

C -1.929793 -0.9081074 .780274$

C $-0.550311-0.8472325 .401906$

H $-2.111368 \quad 1.201647 \quad 4.338319$

C $-2.768593 \quad 0.1177802 .571283$

H $-2.736353-0.8168595 .513889$

H $-2.063730-1.8544794 .241083$

C $-0.154693-2.1305156 .112287$

H $-0.518168-0.0116616 .114136$ 
C $-4.228498-0.2670942 .861894$ O $-2.766147 \quad 1.3727461 .884291$ H -2.312621 -0.6139361 .895249$ H $-4.269102-1.2588273 .326925$ O $\begin{array}{llll}-4.750972 & 0.656129 & 3.825309\end{array}$ C $-5.151904-0.2388241 .626151$ C $-4.675098-1.1471490 .479330$ H -5.2666050 .7938611 .279930$ O $-6.456133-0.6642972 .037786$ H -3.663171 -0.8602620 .182097$ H $-4.621838-2.181007 \quad 0.842552$ H $-0.850382-2.3471196 .929676$ H $-0.162586-2.9862565 .428835$ H $0.849960-2.0503306 .538591$ C $1.640523 \quad 0.128473 \quad 4.514991$ H $-1.839103 \quad 1.6426361 .775869$ H -5.678372 0.4120203 .987456 H -6.386907 -1.586363 2.339048 H $2.078005-0.3449985 .401978$ H 1.5046941 .1898134 .755529 H $0.447456-1.4282123 .570577$ C $-4.6979790 .883321-1.777443$ O $-5.8412580 .234291-1.207465$ C $-5.605702-1.103240-0.754693$ C $-5.136702-1.973128-1.929631$ C $-3.935607-1.360053-2.648592$ C $-4.1972320 .105278-2.999474$ H -3.902830 $0.952507-1.026770$ C $-5.1155692 .307781-2.155117$ H -6.578874 -1.494394 -0.432542 O $-4.817494-3.283093-1.473380$ H -5.963375 -2.074509-2.644669 H -3.029322 -1.456161-2.042867 O $-3.676864-2.093785-3.847706$ H -4.932851 $0.164019-3.811693$ O $-2.988680 \quad 0.696152-3.471284$ C $-5.385878 \quad 3.132205-0.918888$ H -4.349033 $2.821850-2.745822$ H $-6.0345122 .300383-2.753649$ H $-4.492389-3.787105-2.239036$ H $-4.464206-2.029777-4.414766$ H -2.672709 $0.157202-4.216570$ O $-4.2089873 .527441-0.354139$ O $-6.5086543 .381199-0.499024$ C $-4.333392 \quad 4.293533 \quad 0.847865$ H -4.8316083 .7028991 .622736$ H -3.328888 4.5496581 .194305 H -4.883509 5.2190410 .652724

SCF Energy (B3LYP/6-31G**/MMFF)= -3245.91087096

02160

MMFF Geometry

C -2.140764 -2.910970 2.159040

C $-3.480995-2.992145 \quad 2.153017$

C $-4.384422-2.9463200 .941962$

O $-3.608839-2.791751-0.266465$

C $-5.346056-1.7481121 .020915$

C $-6.602928-1.9609180 .200032$

C $-6.793834-1.513426-1.056146$

C $-7.704573-2.706560 \quad 0.909436$

C $-5.821163-0.784998-1.939782$

C $-6.2576430 .654962-2.241440$

C $-6.0960811 .603001-1.043000$

C $-6.5722853 .011933-1.403923$

C -6.311366 $4.014515-0.270425$

O $-4.707962 \quad 1.631173-0.699777$

O $-6.5018265 .326960-0.820118$

C -4.8373313 .8928260 .172686$

C -7.340302 3.8673570 .856396

O -4.5805144 .7187651 .306416$

$\begin{array}{lllll}C & -4.388076 & 2.437417 & 0.440141\end{array}$

C $-2.8545312 .384750 \quad 0.627948$

O -5.0303841 .9660621 .624995$

C $-3.339270-3.888121-1.016227$

O $-3.719254-5.031502-0.806415$

C $-2.473665-3.548105-2.169209$

C $-2.003308-2.314555-2.410765$

C $-1.146918-1.963807-3.537801$
C $-1.595745-0.730795-4.272615$

C $-2.2902660 .968348 \quad 0.794222$

$\begin{array}{llll}\text { C } & -0.779264 & 0.996349 & 0.713792\end{array}$

C $1.1029301 .021858-0.885147$

O -0.0823451 .1439461 .715025$

C $1.3674550 .635784-2.303321$

C $2.209859-0.345422-2.660740$

C $2.567501-0.658230-4.085397$

C $2.314812-2.100581-4.458082$

C $3.310073-3.003387-4.444129$

C $0.932577-2.471437-4.945041$

C $-0.039718-2.679541-3.818280$

N $-0.3066560 .878585-0.585198$

H $-1.597079-2.7826021 .227388$

H $-3.992423-3.1402843 .102744$

H $-4.952539-3.8842630 .915614$

H $-4.835034-0.8358010 .696337$

H -5.651032 -1.561283 2.059244

H $-7.754598-1.714835-1.529004$

H -8.592834 -2.834955 0.281941

H $-7.364044-3.7037161 .205495$

H -8.012117 -2.1628401 .808775$

H -4.806206 -0.794486 -1.535103

H -5.767252 -1.335941 -2.887328

H $-5.6326351 .018505-3.067760$

H $-7.2968210 .666427-2.592706$

H $-6.671608 \quad 1.212568-0.196437$

H -7.635195 $3.006386-1.676024$

H $-6.0372493 .357738-2.299933$

H $-6.2671315 .971881-0.130672$

H $-4.2244694 .301930-0.642150$

H -8.3535114 .0323480 .470188$

H -7.191113 4.6262021 .632435

H $-7.321492 \quad 2.8827851 .328335$

H $-4.943017 \quad 4.268707 \quad 2.088466$

H -2.374386 $2.847674-0.244271$

H -2.562754 2.9743121 .506463

H -4.524032 1.2227461 .987497

H -2.226591 -4.379657 -2.823227

H $-2.267884-1.480064-1.766916$

H $-1.7629880 .098116-3.575369$

H -2.535208 $-0.926697-4.800964$

H $-0.869822-0.375765-5.007238$

H $-2.5666840 .537242 \quad 1.762315$

$\begin{array}{llll}\text { H } & -2.673477 & 0.291676 & 0.023037\end{array}$

H $1.6769940 .421804-0.173259$

H $1.3707422 .071935-0.728177$

H $\quad 0.8842351 .230321-3.074984$

H $2.714980-0.919028-1.887121$

H $3.631733-0.421908-4.217245$

H $2.035275-0.001891-4.785098$

H $3.143554-4.033154-4.742224$

H $4.312873-2.733355-4.130658$

H $0.975760-3.410700-5.513040$

H $0.591112-1.726331-5.669648$

H $0.197224-3.536245-3.186359$

H $-0.9730780 .952470-1.348931$

C $-0.383492-1.8350423 .612947$

O $0.655193-1.8399722 .609541$

C $1.938084-1.7615953 .263750$

C $1.646722-1.2070284 .647998$

C $0.329750-1.8817944 .966689$

H $2.302567-2.7946853 .331598$

C $2.900942-0.9078782 .428015$

H $2.431525-1.4420185 .373050$

H $1.521765-0.1175334 .612880$

C $-0.441452-1.2091116 .089774$

H $0.531338-2.9238725 .249774$

C $4.267675-0.6874923 .099174$

O $3.094547-1.5568931 .167550$

H 2.4270590 .0543122 .210445

H $4.137283-0.1530064 .047550$

O $4.809339-1.9740723 .428608$

$\begin{array}{llll}\text { C } 5.305249 & 0.053206 & 2.234335\end{array}$

$\begin{array}{llll}\text { C } & 4.841208 & 1.453925 & 1.794644\end{array}$

H $5.571682-0.5637691 .368937$

$\begin{array}{lllll}\text { O } & 6.487560 & 0.179058 & 3.033308\end{array}$

H 3.9665851 .3557921 .144003 
H 4.5198562 .0141632 .681612 H $\quad 0.136657-1.2299677 .019495$ H $-0.661427-0.1623835 .854328$ H -1.392111 -1.720577 6.270877 C $-1.308860-3.0367653 .405686$ H $3.541217-2.4037001 .338880$ H $5.669375-1.8239173 .857486$ H 7.2262230 .3804002 .434133 H $-1.957108-3.160384 \quad 4.281235$ H $-0.709411-3.950766 \quad 3.309094$ H $-0.938398-0.8961573 .496570$

C $5.7179321 .335293-1.133763$ $\begin{array}{lllll}\text { O } & 6.550546 & 1.528273 & 0.016206\end{array}$ C 5.9399002 .2705541 .076208 C $5.495385 \quad 3.6535290 .577435$ C $4.6555513 .572460-0.695173$

C $5.3483222 .693553-1.737454$ H $4.8086170 .794576-0.845140$ C $6.4953450 .468643-2.128617$ H 6.7297202 .4506651 .816351 $\begin{array}{lllll}\text { O } & 4.773537 & 4.334935 & 1.597856\end{array}$ H $6.391100 \quad 4.252504 \quad 0.365901$ H $3.6446733 .211917-0.473520$ O $4.5059434 .893251-1.219465$ H $6.248453 \quad 3.206361-2.098592$ O $4.4879812 .524695-2.859540$ C $6.690201-0.928951-1.589195$ H $5.9871670 .389961-3.096038$ H $7.4899740 .891048-2.315801$ H 4.5009465 .1957051 .236036 H $4.0078484 .819411-2.051687$ H $3.6693462 .106878-2.545392$ O $5.508901-1.609138-1.616328$ O $7.762553-1.360053-1.185917$ C $5.551859-2.941667-1.097542$ H $4.541358-3.356475-1.143674$ H $6.215240-3.562502-1.706906$ H $5.879347-2.932977-0.053536$ SCF Energy (B3LYP/6-31G**//MMFF)= -3245.92470034

02161

MMFF Geometry

C -3.059134 -2.772821 -2.266087

C $-4.372681-2.996508-2.097337$

C $-5.388790-2.047636-1.495098$

O $-4.758328-0.837774-1.017978$

C $-6.081350-2.739340-0.303987$

C $-7.068879-1.8580490 .436410$

C $-6.832378-1.2850831 .634453$

C -8.400672 -1.670604 -0.243029

C $-5.561235-1.3417772 .437823$

C -5.0327210 .0665182 .740236$

C $-3.590614 \quad 0.0404793 .274755$

C $-3.1336021 .456533 \quad 3.628302$

C $-1.647116 \quad 1.5224064 .005455$

O $-2.759971-0.5064512 .245564$

O -1.2417262 .8998443 .969633$

C -0.8229960 .7909082 .930805$

C -1.3961531 .0304965 .433285$

O $0.5452120 .693583 \quad 3.317756$

C $-1.368907-0.6048882 .577487$

C $-0.645758-1.2245111 .357447$

O $-1.166242-1.4859083 .687278$

C $-4.7842240 .254062-1.833282$

O $\quad-5.2692120 .331089-2.949104$

C $-4.0918601 .354126-1.124292$

C -3.814404 2.506176-1.752691

C $-3.1126173 .655135-1.169038$

C -2.6396683 .5264880 .253915$

C $-0.816878-0.454866 \quad 0.045423$

C $0.2932200 .524539-0.279373$

C $1.0711612 .012096-2.117605$

O $1.245661 \quad 0.7422090 .463915$

C $0.8039073 .415026-1.674903$

C $0.442574 \quad 4.397844-2.513752$

C $0.2619935 .829382-2.097950$

C -1.098325 $6.384111-2.454809$

C $-1.2581717 .211155-3.502597$
C $-2.2604386 .051708-1.545269$

C $-2.9277844 .757383-1.923941$

N $0.1011101 .116829-1.520540$

H -2.634701 -1.814560 -1.986504

H $-4.771071-3.960867-2.407735$

H $-6.141796-1.812978-2.257401$

H $-5.324231-3.1282800 .384870$

H $-6.614996-3.631878-0.658585$

H -7.629259-0.698842 2.090150

H -9.081385 -1.042910 0.342233

H $-8.270324-1.191168-1.218375$

H -8.891083 -2.638030 -0.392663

H $-5.762279-1.8633983 .380886$

H $-4.788502-1.9166491 .924229$

H -5.0646290 .6649651 .821284$

H -5.6931990 .5566913 .466724$

H -3.550541 -0.6098674 .156162$

H -3.7532351 .8757614 .430346$

H -3.2940352 .1095902 .761719$

H -1.848676 3.4069454 .534994

H -0.8517421 .4189052 .033513$

H $-1.943514 \quad 1.6508026 .152500$

H $-0.338090 \quad 1.1230945 .702520$

H -1.703742 -0.0059385 .587801$

H 0.8729451 .5996593 .450303

H $0.412785-1.3989001 .586542$

H -1.076598 -2.223637 1.194466

H -1.155324 -2.400980 3.360292

H $-3.8110441 .178439-0.093088$

H $-4.1215972 .621049-2.791131$

H -3.5007883 .4499760 .925633$

H -2.035335 4.3661270 .601370

H -2.0153542 .6358100 .373069$

H $-0.817300-1.179235-0.769598$

H -1.7767430 .0637040 .008885$

H $\quad 0.996974 \quad 1.898114-3.203925$

H $2.0779561 .712647-1.808219$

H $0.9623353 .640088-0.623015$

H $0.3097594 .171168-3.569350$

H $1.0461886 .415267-2.595766$

H $0.4363505 .959871-1.022886$

H -2.228610 $7.632899-3.745305$

H $-0.4263557 .476476-4.146228$

H -3.019231 $6.843662-1.608224$

H -1.920682 $6.076680-0.506506$

H $-3.3232464 .757316-2.940745$

H $-0.7242610 .880714-2.065234$

C $-0.945022-4.076704-1.866501$

O $0.043173-3.029899-1.975772$

C $1.356158-3.620551-2.041015$

C $1.173502-5.063794-1.602884$

C $-0.196751-5.373959-2.171435$

H $1.644494-3.575672-3.099010$

C $2.345552-2.812051-1.187272$

H $1.957120-5.725325-1.982728$

H $1.159688-5.137076-0.508021$

C $-0.833623-6.610094-1.561347$

H $-0.107809-5.509802-3.257363$

C $3.751418-3.437758-1.119778$

O $2.436414-1.497113-1.744444$

H $1.939063-2.688072-0.177778$

H $3.707578-4.387576-0.574070$

O $4.155543-3.759927-2.459227$

C $4.846632-2.537217-0.518324$

C $4.549846-2.0246410 .899752$

H $5.034003-1.698483-1.194136$

O $6.063862-3.293951-0.488084$

H $3.605011-1.4737640 .900879$

H $4.425285-2.8850061 .568011$

H $-0.224981-7.496442-1.767571$

H $-0.930542-6.515750-0.474430$

H -1.832543 -6.780911-1.974495

C $-2.102589-3.791590-2.831496$

H $2.839173-1.575514-2.625924$

H $5.037783-4.165366-2.405996$

H $5.930298-4.0493310 .109677$

H -2.641443 -4.719099-3.055905

H $-1.711866-3.397213-3.777536$ 
H $-1.296615-4.075376-0.826422$

C $4.9515120 .986361 \quad 0.534817$

O $5.972130-0.0174400 .590169$

C $5.686271-1.1270061 .450106$

C $5.467212-0.630236 \quad 2.884707$

C $4.385775 \quad 0.4454182 .947713$

C 4.6767591 .5525051 .934494

H 4.0353540 .5564550 .113782

C $5.4370972 .094395-0.415521$

H $6.595954-1.7407881 .466046$

O $5.132045-1.7240143 .731523$

H $6.408980-0.2082703 .259993$

H 3.3866490 .0222702 .791673

$\begin{array}{lllll}\text { O } & 4.377409 & 1.033618 & 4.250674\end{array}$

H 5.5381142 .1406242 .276093

O $3.556722 \quad 2.433821 \quad 1.873675$

C $5.3267521 .682012-1.868925$

H $4.8288592 .995682-0.277088$

H $6.4858702 .350081-0.226004$

H $5.140537-1.4008444 .648187$

H $4.0938880 .349546 \quad 4.880312$

H 3.4015382 .7687352 .773617

O $4.9899452 .764346-2.629591$

O $5.536646 \quad 0.552423-2.290712$

C $4.847365 \quad 2.513058-4.031529$

H $4.7362783 .474206-4.540607$

H $5.7343172 .009282-4.428888$

H $3.9499391 .913204-4.210625$

SCF Energy (B3LYP/6-31G**//MMFF) $=-3245.91233678$

02 162

MMFF Geometry

C $-2.419977-3.034847-2.604568$

C $-3.451501-3.814826-2.244527$

C $-4.626186-3.428965-1.371307$

O $-4.513383-2.072963-0.884341$

C $-4.684131-4.368420-0.151291$

C $-5.894252-4.1426420 .736087$

C $-5.862440-3.5728091 .957750$

C $-7.200482-4.6485550 .178436$

C $-4.672262-3.0085292 .682717$

C $-4.871015-1.5190702 .993185$

C -3.581036 -0.8612943 .506210$

C $-3.834638 \quad 0.6031503 .869091$

C -2.5419411 .3449834 .240044$

O $-2.609961-0.9505572 .459334$

O -2.8246742 .7530624 .233166$

C -1.486797 1.0930293 .141632

C -2.0771131 .0029865 .660277$

O $-0.2241951 .638847 \quad 3.514994$

C $-1.327027-0.3967812 .776984$

C $-0.413688-0.623367 \quad 1.547470$

O $-0.727677-1.0875063 .879718$

C $-5.043408-1.083796-1.655605$

O $-5.575953-1.207216-2.746948$

C $-4.8565380 .195649-0.934714$

C $-5.1639791 .353226-1.538521$

C $-5.0654912 .691068-0.948710$

C $-4.5520332 .797663 \quad 0.462718$

C $-0.924718-0.0312390 .230993$

C $-0.370091 \quad 1.343573-0.096905$

C $-0.614114 \quad 3.168985-1.779715$

O 0.5395681 .8746340 .533180

C -1.525044 4.231474 -1.252322

C $-2.2521705 .038213-2.040116$

C $-3.1204226 .149055-1.524295$

C $-4.540976 \quad 6.069568-2.034531$

C $-4.945056 \quad 6.818310-3.074806$

C $-5.5159735 .198746-1.276392$

C $-5.4494183 .753060-1.688124$

N $-0.9912051 .888308-1.213756$

H -2.376771 -2.000817 -2.274802

H $-3.468695-4.840975-2.608729$

H $-5.544715-3.540036-1.960509$

H $-3.756641-4.2713690 .422234$

H $-4.702967-5.414354-0.487489$

H $-6.798967-3.4916882 .508717$

H -8.027574 -4.5360120 .887615$
H -7.470385 -4.100175 -0.729267

H $-7.122330-5.712818-0.066602$

H $-4.541677-3.5597763 .621656$

H -3.750610 -3.147569 2.114549

H -5.197615 -1.006156 2.079894

H $-5.670380-1.4030333 .735599$

H -3.223736 -1.419342 4.379591

H -4.5691590 .6800514 .680518$

H -4.288242 1.1147183 .010320

H -3.543076 2.9178054 .867760

H -1.8158011 .6438392 .252186$

H $-2.841614 \quad 1.289724 \quad 6.392595$

H -1.1786151 .5690395 .929360$

H $-1.871927-0.0603155 .800050$

H -0.3630222 .5805273 .714298$

H $0.608124-0.2956591 .770460$

H $-0.342882-1.7098071 .392630$

H $-0.314846-1.9020183 .548128$

H -4.4785330 .1493540 .079440$

H $-5.5386261 .321551-2.560983$

H -5.2637422 .3432751 .159874$

H -4.3856803 .8257990 .790935$

H -3.586609 2.291024 0.564206

H $-0.604806-0.688818-0.586049$

H -2.0174630 .0032010 .209460$

H $-0.6755253 .072815-2.868352$

H $0.4209503 .401366-1.509511$

H -1.562180 $4.363369-0.173590$

H -2.190369 4.921831-3.119805

H -2.665541 $7.097821-1.838191$

H $-3.1275096 .176466-0.427461$

H -5.971345 $6.791095-3.425826$

H $-4.2567747 .470503-3.602193$

H -6.543060 $5.538802-1.467695$

H $-5.3779835 .346156-0.201594$

H -5.803462 $3.575153-2.704630$

H -1.767071 $1.392199-1.642899$

C $0.068184-3.221346-2.795039$

O $0.361890-1.814305-2.914207$

C $1.768341-1.650110-3.205729$

C $2.392021-3.026517-3.034384$

C $1.250023-3.933664-3.447760$

H $1.822370-1.334182-4.255175$

C $2.327600-0.556003-2.290083$

H $3.289406-3.159998-3.645888$

H $2.661848-3.205686-1.985871$

C $1.431678-5.373544-2.999988$

H $1.151167-3.903029-4.541173$

C $3.859718-0.409339-2.344965$

O $1.7183350 .678426-2.678705$

H $2.016683-0.748129-1.256182$

H $4.315470-1.374848-2.096054$

O $4.245895-0.086859-3.679476$

C $4.3998380 .643793-1.354377$

C $5.9381780 .718821-1.344537$

H $4.0367940 .380064-0.355213$

O $3.886517 \quad 1.939527-1.654224$

H $6.345351-0.296331-1.294698$

H $6.2886261 .163600-2.283627$

H $2.335646-5.802858-3.444214$

H $1.525250-5.446370-1.911125$

H $0.580532-5.989510-3.306336$

C - $1.277461-3.515087-3.460350$

H $0.7548080 .550899-2.645409$

H $5.211420-0.177825-3.740200$

H $4.0996252 .132303-2.583298$

H -1.376826 -4.589574 -3.650515

H -1.338042 -2.997704 -4.425438

H $0.028885-3.452112-1.721771$

C $6.445214-0.1450081 .523977$

O 5.9608521 .1320741 .092106

C $6.4754621 .569961-0.170340$

C $8.0062861 .667900-0.101451$

C $8.644551 \quad 0.3726680 .398632$

C $7.968357-0.1023241 .686283$

H $6.172104-0.9116670 .789309$

C $5.742367-0.4824242 .842525$

H $6.0933712 .587958-0.318583$ 
O $8.5548131 .998043-1.372970$

H 8.2848412 .4756010 .588049

H $8.616067-0.407961-0.370861$

$\begin{array}{lllll}O & 10.026406 & 0.608658 & 0.678374\end{array}$

H 8.2432080 .5722602 .506661

O $8.461100-1.3964232 .026852$

C $4.291134-0.8230662 .594312$

H $6.205573-1.3327813 .355254$

H $5.771015 \quad 0.3684253 .533733$

H $8.1502922 .835857-1.656485$

H $10.4332040 .946534-0.138021$

H $8.222208-2.0063801 .308210$

O $4.190889-2.0915962 .102003$

O $3.364058-0.0492292 .793405$

C $2.866825-2.5264271 .778900$

H $2.470601-1.931805 \quad 0.950884$

H $2.919172-3.5722281 .464823$

H $2.215010-2.4556452 .654914$

SCF Energy (B3LYP/6-31G**/MMFF) $=-3245.89649533$

02163

MM̄FF Geometry

C $-0.641147-1.933323 \quad 3.110280$

C $0.461164-1.7745053 .857253$

C $1.345729-0.5614773 .775885$

O $2.591067-1.0169393 .194170$

C $1.637571 \quad 0.0529845 .155942$

C 0.4507360 .7312435 .804444

C 0.0214551 .9672765 .481589

C $-0.224071-0.0473886 .901850$

C 0.5779202 .8658304 .411841

C -0.5206413 .2959493 .431843$

C 0.0587873 .9942842 .191915

C $-1.048773 \quad 4.735527 \quad 1.440607$

C $-0.544574 \quad 5.352508 \quad 0.129894$

O 0.6378162 .9834991 .355616

O $-1.6882845 .752642-0.640391$

C $0.1857754 .263110-0.679298$

C $\quad 0.2787996 .622634 \quad 0.373271$

O $0.7998334 .820699-1.840240$

C $1.231122 \quad 3.473687 \quad 0.145751$

C $1.7785902 .265300-0.654311$

O 2.3387324 .3353680 .431436

C $3.326565-0.1076432 .501015$

O 3.1001391 .0848782 .378439

C $4.453968-0.8306371 .874988$

C $5.170088-0.2218430 .918384$

C $6.276754-0.8085680 .161304$

C $6.724859-2.1944840 .546768$

C $0.794626 \quad 1.104158-0.815542$

C $1.1449280 .257689-2.021815$

C $2.662550-1.513318-2.835601$

O $0.490971 \quad 0.327079-3.061132$

C $4.104178-1.865454-2.658859$

C $5.061589-1.512665-3.530871$

C $6.505161-1.917351-3.420243$

C $7.427695-0.739409-3.194731$

C $7.8501480 .016220-4.222392$

C $7.921184-0.490892-1.786121$

C $6.811781-0.094207-0.851447$

N $2.257221-0.548643-1.830297$

H -0.934345 -1.149532 2.415914

H $0.766076-2.5694484 .533826$

H $\quad 0.892451 \quad 0.1821443 .107999$

H $2.461793 \quad 0.7737125 .073395$

H $2.042526-0.7171825 .827251$

H $-0.8207112 .381756 \quad 6.033752$

H -1.062081 0.5004497 .345782

H -0.617089 -0.9933176 .517105$

H $0.488468-0.2670577 .703760$

H 1.0026303 .7561304 .891152

H 1.3948082 .3886883 .867414

H -1.094261 2.4144453 .115342

H -1.211105 3.9692333 .955308

H 0.8405104 .6950042 .507786

H -1.499199 5.5080152 .076567

H -1.860099 4.033194 1.208048

H $-2.2042786 .380975-0.106814$
H $-0.5798343 .569668-1.045626$

H $-0.325801 \quad 7.3772910 .890670$

H $0.5848887 .082503-0.572979$

H 1.1717556 .4467400 .976542

H $\quad 0.1095715 .296210-2.333377$

H $2.1456202 .607350-1.630761$

H $2.6698661 .871673-0.149146$

H $3.107623 \quad 3.7846500 .653539$

H $4.638766-1.8507492 .191311$

H 4.9061450 .7978580 .640290

H $7.060235-2.2123551 .589726$

H $7.553136-2.572180-0.055276$

H $5.904333-2.9112200 .429275$

H $0.7911840 .473103 \quad 0.081158$

H $-0.2341811 .450691-0.954828$

H $2.456303-1.107461-3.832136$

H $2.042459-2.406697-2.707597$

H $4.361856-2.473276-1.795684$

H $4.789249-0.919254-4.401180$

H $\quad 6.785368-2.428652-4.350769$

H $6.649392-2.663437-2.629204$

H $8.5328790 .845950-4.072616$

H $7.518854-0.178512-5.237166$

H $8.6616420 .319807-1.776473$

H $8.467051-1.374377-1.444378$

H $6.4083280 .898175-1.057522$

H $2.617170-0.658556-0.886359$

C $-1.224566-4.0551581 .930895$

$\begin{array}{llll}0 & -1.598098 & -3.375206 & 0.717979\end{array}$

C $-2.108680-4.344850-0.217590$

C $-1.958751-5.7112390 .446265$

C $-2.026920-5.3525831 .918474$

H $-3.176721-4.132542-0.338224$

C -1.396397-4.205583 -1.567790

H -2.744907 -6.407239 0.137630

H $-0.994534-6.1761350 .210485$

C -1.477982 -6.435733 2.830395

H $-3.073293-5.1503442 .183516$

C -1.445093 -2.784412 -2.164943

O $-0.015769-4.550640-1.392906$

H $-1.809651-4.934097-2.275463$

H -0.826739 -2.110925 -1.560024

O $-0.818125-2.831743-3.452972$

C $-2.858313-2.188177-2.303579$

C $-2.854556-0.830668-3.030312$

H $-3.286817-2.057736-1.304930$

O $-3.702440-3.100972-3.003180$

H -2.163560 -0.159787 -2.509915

H -2.472011 -0.963131-4.049451

H -2.045608 -7.364116 2.709108

H $-0.427468-6.6523682 .609146$

H -1.545736 -6.135055 3.880601

C -1.501952 -3.164753 3.141958

H $0.418496-4.455327-2.257938$

H -1.316905 -3.456399-4.005971

H $-3.390698-3.153434-3.922442$

H -1.328536 -3.723319 4.068859

H -2.550165 -2.843709 3.149320

H $-0.149570-4.2705661 .859310$

C $-4.3086660 .866269-0.953049$

O $-4.893358-0.091317-1.843518$

C $-4.257409-0.190101-3.122190$

C $-4.281728 \quad 1.172395-3.830061$

C $-3.6974992 .279830-2.956171$

C $-4.3523112 .266265-1.575102$

H $-3.2713460 .582816-0.736180$

C $-5.099496 \quad 0.8206230 .357675$

H -4.876604 $-0.871836-3.718765$

O $\quad-3.5753851 .097775-5.063815$

H $-5.322213 \quad 1.426341-4.071489$

H $-2.6068862 .192107-2.885730$

O $-3.9589393 .535861-3.585745$

H $-5.3908462 .607434-1.668245$

O $-3.6816853 .198244-0.732136$

C $-4.771280-0.4347881 .129501$

H -4.8582161 .6655491 .012564$

H -6.1795420 .8577030 .170920$

H $\quad-3.594654 \quad 1.983998$-5.464049 
H -3.615288 $4.230629-2.997917$

H $-2.7662032 .890696-0.621297$

O $-5.763873-1.3557240 .975031$

O $-3.741836-0.5831051 .775567$

C $-5.563030-2.5956361 .660209$

H $-6.406920-3.2516741 .431447$

H $-4.642994-3.0754411 .316425$

H -5.529808 -2.428550 2.741066

SCF Energy (B3LYP/6-31G**//MMFF) $=-3245.91619660$

\section{4}

MM̄FF Geometry

C 0.3864593 .4240321 .111728

C 0.8773952 .1805861 .003832

C 1.2732451 .3351672 .185612

O 2.6995551 .1304622 .059878

C $0.579411-0.0359582 .138618$

C $0.683721-0.8008703 .443219$

C $1.553557-1.8013373 .684482$

C $-0.310975-0.4023164 .502834$

C $2.631452-2.3345512 .782576$

C $2.337196-3.7596172 .292936$

C $1.227604-3.8161181 .232173$

C $0.931722-5.2619560 .831599$

C $-0.127599-5.333587-0.278772$

O $1.687567-3.0930940 .088496$

O $-0.105097-6.662652-0.825763$

C $0.271545-4.376563-1.425465$

C $-1.540765-5.1295460 .279826$

O $-0.771591-4.262044-2.389212$

C $0.724180-2.969677-0.964321$

C $1.427324-2.233161-2.127082$

O $-0.374348-2.142460-0.580927$

C 3.4813861 .2627093 .159133

O $3.112246 \quad 1.5333914 .293346$

C 4.9124851 .0614392 .827256

C 5.3421190 .6229101 .633237

C 6.7366060 .4318231 .227250

C $7.812273 \quad 0.8062512 .212379$

C $1.980908-0.858627-1.726167$

C $2.741814-0.195245-2.860266$

C $4.3201631 .687073-3.294114$

O $2.704117-0.597071-4.020156$

C $5.6733461 .048156-3.327131$

C $6.7931191 .637693-2.881358$

C $8.1452740 .983276-2.916302$

C $8.7702170 .852166-1.543880$

C $9.7534261 .680717-1.153581$

C $8.321498-0.291407-0.659300$

C $6.986361-0.037048-0.013505$

N $3.4548790 .913326-2.422168$

H $\quad 0.2459383 .8564742 .099497$

H 1.0421951 .7596590 .015392

H 1.0357261 .8638593 .116526

H $0.999382-0.6300391 .320390$

H -0.4852220 .0839361 .899146$

H $1.511529-2.2904654 .657218$

H $-0.164838-0.9514785 .439182$

H $-0.225027 \quad 0.663754 \quad 4.733878$

H -1.329528 -0.6050784 .158925$

H $2.829249-1.6724081 .934989$

H $3.561749-2.3537563 .364542$

H $3.264510-4.1564101 .859314$

H $2.076987-4.4005193 .144373$

H $\quad 0.325709-3.337574 \quad 1.628737$

H $0.616295-5.8515631 .701368$

H $1.852646-5.7360940 .464323$

H $-0.262661-7.289482-0.098983$

H $1.119079-4.844968-1.945793$

H -1.793938 -5.9327390 .982659$

H $-2.292897-5.181987-0.514398$

H -1.660678 -4.1853120 .814271$

H -1.002843 -5.162507 -2.674939

H $2.262093-2.843975-2.495418$

H $\quad 0.726902-2.098050-2.961144$

H -1.145581 -2.699445 -0.404789

H $5.601307 \quad 1.2959073 .632369$

H 4.6082100 .3833750 .865458
H $7.748468 \quad 0.177440 \quad 3.107289$

H 7.7127051 .8556982 .511584

H 8.8237490 .6933841 .816047

H $1.160785-0.191608-1.440288$

H $2.658901-0.959470-0.870835$

H $4.3520542 .708312-2.901985$

H $3.8958421 .711428-4.302939$

H $5.7329510 .045459-3.744428$

H $6.7406472 .645028-2.474937$

H $8.7893311 .587059-3.569005$

H $8.098607-0.007300-3.385801$

H $10.2298921 .576263-0.184633$

H $10.1014902 .485163-1.793068$

H $9.083965-0.5226560 .092849$

H $8.249733-1.206516-1.261273$

H $6.138720-0.280738-0.653989$

H $3.4811221 .122192-1.428113$

C $-1.3801174 .802595-0.025345$

O $-2.2727953 .732012-0.394199$

C $-3.3123334 .265366-1.239166$

C $-3.1555755 .781352-1.207393$

C -1.658946 $5.925899-1.023471$

H $-3.101123 \quad 3.915359-2.256182$

C $-4.6868833 .764332-0.784970$

H -3.525034 $6.254889-2.122256$

H $-3.6883936 .228580-0.360195$

C -1.240895 $7.304698-0.542320$

H -1.163634 $5.710735-1.979950$

C $-4.8201612 .227108-0.759588$

O -4.9293034 .2489960 .541829$

H $-5.4655414 .204597-1.418965$

H $-4.1470891 .822416-0.000144$

O $-6.1488831 .917463-0.321616$

C $-4.5586471 .539432-2.110771$

C $-4.8648950 .029099-2.158256$

H $-3.5175841 .696378-2.411009$

O $\quad-5.3631172 .173645-3.112957$

H $-5.841026-0.166408-1.701739$

H $-4.985120-0.243763-3.214787$

H $-1.5381208 .070607-1.266093$

H -1.7047357 .5522780 .418419$

H $-0.1553087 .363892-0.416991$

C $0.0656564 .307146-0.062286$

H $\quad-5.791574 \quad 3.899890 \quad 0.826011$

H $-6.7734922 .330199-0.941533$

H $-6.2928311 .954238-2.932760$

H $0.7543735 .158833-0.026299$

H $0.2515623 .772319-1.001981$

H -1.6578485 .1167350 .989654$

C $-4.657636-1.1645790 .696233$

O $-3.599040-0.668966-0.131724$

C -3.769263 -0.863551-1.537245

C $-3.950598-2.359166-1.840061$

C $-5.099207-2.958873-1.038179$

C $-4.914188-2.6562510 .445718$

H $-5.571196-0.5912240 .503281$

C $-4.239517-0.9426152 .156310$

H -2.822816 -0.558737 -2.002058

O $-4.177723-2.584421-3.226949$

H $-3.033394-2.897348-1.578219$

H $-6.072537-2.603923-1.396359$

O $-5.100974-4.378447-1.206335$

H $-4.079703-3.2519880 .836611$

O $-6.086978-3.0535881 .154148$

C -4.2846350 .5185642 .535690$

H $-4.900966-1.4672302 .855224$

H -3.226048 -1.326513 2.327631

H -3.419402 -2.214091 -3.710432

H $-5.168991-4.555822-2.160304$

H $-6.236593-3.9953830 .962360$

O -3.0193651 .0002402 .689826$

O $\quad-5.321260 \quad 1.154887 \quad 2.674137$

C $-2.9151902 .365796 \quad 3.103373$

H -1.872798 2.5614443 .367551

H -3.1995993 .0254732 .280804$

H -3.5375572 .5546083 .983431$

SCF Energy (B3LYP/6-31G**//MMFF) $=-3245.91975827$ 
$02 \quad 165$

MM̄FF Geometry

C -3.620970 -1.900668 -2.409510

C $-2.544113-2.525589-1.908427$

C $-1.110325-2.134863-2.158094$

O $-1.057053-0.697979-2.319480$

C $-0.492348-2.817734-3.388356$

C $-0.282553-4.306884-3.219552$

C $0.784395-4.859493-2.609634$

C $-1.353263-5.186452-3.811486$

C $1.965659-4.158846-1.997161$

C $1.926658-4.226629-0.465856$

C $3.249481-3.7789550 .173452$

C $3.144110-3.8053741 .699991$

C $4.425639-3.2975012 .377292$

O $3.558297-2.453491-0.268746$

O $4.096576-3.0634443 .755136$

C $4.831228-1.9490481 .742986$

C $5.526916-4.3645982 .366521$

O $6.108084-1.5221832 .213895$

C $4.826743-1.9696910 .196235$

C $5.028428-0.537701-0.355221$

O $5.906666-2.793596-0.254949$

C $0.056446-0.061356-1.871870$

O $1.097929-0.575873-1.500047$

C $-0.2441911 .386861-1.847130$

C $0.5899572 .217521-1.204881$

C $0.3987173 .655580-1.011132$

C $-0.8182624 .292431-1.628479$

C $4.778929-0.414691-1.864297$

C $5.4105180 .840818-2.430268$

C $5.2212093 .306501-2.482896$

O $6.369406 \quad 0.786316-3.198043$

C $4.7745564 .369674-1.533053$

C $4.0152725 .414618-1.895641$

C $3.5802206 .500599-0.952994$

C $2.0764426 .656141-0.900861$

C $1.4548527 .585715-1.645877$

C 1.3029705 .7963820 .073306

C $1.3128974 .336852-0.289686$

N $4.8081352 .007457-1.985391$

H -3.489225 -1.081933 -3.112279

H -2.691489 -3.357335 -1.223809

H $-0.551768-2.396546-1.251172$

H $\quad 0.460858-2.335310-3.637947$

H - $-1.113919-2.624889-4.273693$

H $\quad 0.831468-5.946074-2.545310$

H -1.128510 -6.251907 -3.693273

H -2.318940 -5.001033 -3.332896

H -1.453563 -4.989973 -4.884035

H $2.873530-4.651150-2.367343$

H $2.031627-3.116399-2.321402$

H $1.112250-3.584097-0.110293$

H $1.698399-5.248781-0.137817$

H $4.035196-4.463497-0.167674$

H $2.895409-4.8128462 .056356$

H $2.309617-3.1640272 .016372$

H $4.907107-2.7917654 .217543$

H $4.108976-1.1960472 .083674$

H $5.197916-5.2566952 .913032$

H $6.426317-4.0119942 .883325$

H $5.810089-4.6781331 .359559$

H $6.047032-1.4164593 .178097$

H $4.337908 \quad 0.149566 \quad 0.150536$

H $6.047231-0.198179-0.127822$

H $\quad 6.236705-2.460004-1.103655$

H -1.166807 $1.720141-2.308390$

H $1.4872681 .801884-0.747618$

H $-0.8342944 .123599-2.711076$

H $-0.8610735 .374498-1.484831$

H -1.732862 $3.874946-1.193354$

H $5.195930-1.265134-2.413934$

H $3.705498-0.397899-2.080920$

H $4.7894513 .428441-3.481985$

H $6.3119913 .322830-2.579808$

H $5.1054484 .283711-0.500688$

H $3.7026695 .509210-2.933253$

H $4.0414487 .438650-1.289047$
H $3.964826 \quad 6.3312750 .060433$

H $0.3803497 .726734-1.595230$

H $2.0072218 .224690-2.327083$

H $0.273826 \quad 6.1564590 .187678$

H 1.7432725 .9086121 .072878

H 2.1648573 .7925610 .119095

H $3.9109991 .937038-1.513105$

C $-5.817173-0.961762-1.669478$

O $-5.125142-0.193883-0.669684$

C -6.0936790 .4744640 .159939$

C $-7.4703850 .099515-0.387875$

C -7.195614 -1.226466 -1.072770

H $-5.9847840 .050893 \quad 1.165117$

C $-5.805248 \quad 1.9820410 .198331$

H -8.2198260 .0228170 .406093$

H $-7.8377960 .833611-1.113747$

C $-8.251693-1.616447-2.091369$

H -7.128133 -2.007349 -0.303543

C -4.3461182 .3451490 .543316$

O $-6.0803212 .533168-1.097194$

H -6.4999282 .4681480 .893466$

H $-3.6921482 .055951-0.288344$

O -4.2563093 .7738230 .622808$

C -3.8098711 .7266921 .846452$

C -2.3819502 .2024662 .169139$

H $-3.8106800 .639072 \quad 1.748749$

O -4.6786302 .0457322 .931324$

H -1.7536022 .0403071 .287024$

H -2.3957173 .2819072 .362445$

H -9.231131 -1.713758 -1.611748

H $-8.338942-0.867744-2.885771$

H -8.008281 -2.576093 -2.558018

C $-5.030779-2.231710-2.005703$

H $-5.8961453 .487092-1.051821$

H $-4.871774 \quad 4.0739531 .312811$

H -4.6190033 .0024123 .092544$

H -5.521785 -2.765788 -2.826553

H $-5.020025-2.898620-1.134896$

H $-5.896980-0.326079-2.561924$

C $-0.885293-0.4575662 .286322$

O $\begin{array}{lllll}-1.773317 & 0.070278 & 3.279151\end{array}$

C -1.7712701 .4968533 .401343$

C $-0.360361 \quad 1.983423 \quad 3.760245$

C 0.6722971 .4920292 .751700

C $0.560140-0.0186712 .559513$

H $-1.202757-0.1227891 .291389$

C $-0.991658-1.9908112 .334979$

H $-2.420048 \quad 1.730814 \quad 4.254688$

O -0.3092423 .4046593 .824384$

H -0.0843741 .6050894 .753039$

H $0.5773652 .016501 \quad 1.796205$

O 1.9847491 .7841593 .236792

H $0.946151-0.5254003 .453474$

O $1.379981-0.4125031 .462396$

C $-2.344049-2.5056681 .891193$

H $-0.242547-2.4481481 .680226$

H $-0.830629-2.3323513 .364838$

H $-0.966606 \quad 3.6909264 .481407$

H $2.0302212 .744516 \quad 3.384418$

H $2.260816-0.0266831 .603333$

O $-2.420916-3.8410132 .170462$

O $-3.216892-1.8366801 .357467$

C $-3.656587-4.4736401 .822278$

H $-4.494592-3.9761092 .319976$

H $-3.791794-4.4653420 .737201$

$\mathrm{H}-3.614200-5.5116642 .162566$

SCF Energy (B3LYP/6-31G**//MMFF) $=-3245.90479580$

$02 \_166$

MM̄FF Geometry

C -2.403338 -3.495305 -1.299879

C -1.624951 -2.785062 -2.129952

C $-0.134924-2.661893-1.966658$

O $0.156898-1.241735-1.994617$

C $0.655926-3.329294-3.103641$

C $0.998997-4.775354-2.802507$

C $2.088657-5.164938-2.109207$

C $0.030727-5.798054-3.333984$ 
C $3.159323-4.280671-1.530704$

C $2.973480-4.066464-0.022941$

C $3.991183-3.0705120 .554553$

C $3.698129-2.7871792 .030187$

C $4.621574-1.7033552 .610522$

O $3.902340-1.854463-0.193935$

O $4.042090-1.2952983 .859320$

C $4.630832-0.4822641 .662383$

C $6.008933-2.2683252 .937714$

O $5.599196 \quad 0.4813132 .069617$

C $4.863681-0.8618220 .182475$

C $4.6646620 .369517-0.732622$

O $6.205126-1.3327000 .023950$

C $0.491313-0.637308-0.823456$

O $0.686987-1.1706550 .256763$

C $0.5344440 .819607-1.072044$

C $0.895621 \quad 1.641686-0.076986$

C $0.9225923 .097485-0.154311$

C 2.1337673 .7138740 .489298

C $4.5194980 .005739-2.216088$

C $4.6688001 .210724-3.122650$

C $3.5291493 .284996-3.853144$

O $5.6020731 .301092-3.918108$

C $2.8811214 .417373-3.125259$

C $1.6876864 .931248-3.459631$

C $1.0698366 .119624-2.781231$

C $-0.2739795 .811610-2.162592$

C $-1.4171726 .088166-2.812326$

C $-0.2897895 .275155-0.750330$

C $-0.1031973 .784662-0.694782$

N $3.6607282 .148160-2.962272$

H -1.924408 -4.066972 -0.507139

H -2.069973 -2.215421 -2.940690

H $0.170711-3.084008-1.004194$

H $1.573418-2.761180-3.298541$

H $0.104244-3.266823-4.051506$

H $2.238607-6.229111-1.933843$

H $0.345302-6.823073-3.110732$

H - $0.961036-5.656258-2.895758$

H $-0.053388-5.710204-4.422107$

H $4.132962-4.751009-1.715689$

H $3.192190-3.320472-2.052472$

H $1.962025-3.6888550 .161590$

H $3.056162-5.0252720 .504030$

H $4.993385-3.4980780 .432605$

H $3.763464-3.7077592 .623578$

H $2.659604-2.4412112 .132422$

H $4.638099-0.6477244 .271879$

H 3.6494970 .0057961 .744843

H $5.929380-3.0591643 .693357$

H $6.657073-1.5010213 .375068$

H $6.515360-2.6914972 .067576$

H 5.3616640 .7784742 .963860

H $3.7562280 .901772-0.431102$

H $5.5027151 .066810-0.607174$

H $6.505135-1.149111-0.880035$

H 0.242878 1.187511 -2.049904

H 1.2046401 .2320950 .881867

H 2.1165393 .5539451 .572823

H $2.217227 \quad 4.788370 \quad 0.312117$

H 3.0497903 .2626730 .091681

H $5.276482-0.725760-2.519411$

H $3.541991-0.452538-2.408995$

H $2.9415682 .954795-4.716382$

H $4.5166323 .589582-4.214959$

H $3.4417494 .859436-2.305199$

H $1.1383144 .500625-4.293673$

H $\quad 0.960724 \quad 6.911861-3.533891$

H $1.7378116 .536503-2.017117$

H -2.384542 $5.898244-2.359170$

H -1.410318 $6.504167-3.814385$

H -1.257441 $5.495992-0.279138$

H $0.4402125 .818585-0.143371$

H $-0.9426933 .224050-1.106483$

H $2.8510301 .891238-2.405836$

C $-4.625822-2.342661-1.911805$

O $-4.238865-1.148115-1.201784$

C $-5.407595-0.347216-0.955281$
C $-6.516788-0.948487-1.810826$

C $-6.147967-2.419202-1.789899$

H $-5.665300-0.4825650 .101772$

C $-5.1091621 .129793-1.234457$

H -7.511388 -0.747889-1.401187

H $-6.491768-0.566549-2.837925$

C $-6.816052-3.231192-2.885564$

H $-6.428201-2.835649-0.813015$

C $-3.9015251 .704618-0.465418$

O $-4.840187 \quad 1.299290-2.633180$

H $-6.0027651 .729729-1.027130$

H $-2.9670141 .279732-0.852161$

O $-3.846796 \quad 3.111251-0.740381$

C -3.9539261 .5103301 .061479$

C -2.8179852 .2554061 .788503$

H -3.8920730 .4425921 .289191$

O

H $-1.867857 \quad 1.990343 \quad 1.316085$

H -2.9570503 .3353231 .659074$

H -7.905382 -3.194300 -2.781624

H $-6.560358-2.850547-3.880146$

H $-6.507413-4.280141-2.836812$

C $-3.908409-3.553320-1.305900$

H $-4.0906730 .721865-2.859341$

H -3.788251 3.215215 -1.705736

H -5.2889412 .9140361 .325558$

H -4.220942 -4.476084-1.807285

H $-4.217756-3.639609-0.256336$

H $-4.338578-2.203978-2.961408$

C $-1.3056630 .024315 \quad 3.234993$

O -2.5945660 .5532603 .571586$

C -2.7685671 .9487443 .302518$

C -1.7214542 .7629714 .076247$

C -0.3034032 .2828363 .785504$

C $-0.1971660 .773000 \quad 3.986828$

H -1.1462760 .1008872 .153351$

C -1.301539-1.463537 3.618544

H -3.7493512 .2186003 .713455$

O -1.8173454 .1490373 .765525$

H -1.9064252 .6616845 .153703$

H 0.0153212 .5690412 .778531

O $0.6049622 .918780 \quad 4.687520$

H -0.2499180 .5459835 .059398$

O 1.0727890 .3235293 .518906

C $-2.234963-2.2859582 .757081$

H $-0.300263-1.8938943 .509361$

H -1.627666 -1.567194 4.660699

H -2.724602 4.4323083 .971432

H 0.4996023 .8790084 .573472

H 1.7539070 .8292723 .994362

O $-2.479944-3.475848 \quad 3.381664$

O $-2.673625-1.943751 \quad 1.668178$

C $-3.355279-4.3720002 .690163$

H -3.584185 -5.206200 3.358594

H $-4.291450-3.8714472 .424190$

$\mathrm{H}-2.857353-4.7630081 .798700$

SCF Energy (B3LYP/6-31G**//MMFF) $=-3245.91330452$

$02 \_167$

MM̄FF Geometry

C $1.760187-3.804407-0.059912$

C $1.958491-4.1576201 .219704$

C $2.656598-3.3215242 .268098$

O $2.656528-1.9195111 .913339$

C $4.113353-3.7967842 .411319$

C $4.899632-3.0422423 .465068$

C $5.822899-2.0950213 .203279$

C $4.612712-3.4450154 .888514$

C $6.229517-1.5528191 .859963$

C $6.014991-0.0351981 .792323$

C 6.2058730 .5148360 .370294

C 6.0537902 .0369500 .365701

C $6.0895982 .622233-1.051803$

O $5.210669-0.083142-0.466902$

O $5.6222483 .977064-0.955566$

C $5.1008421 .842329-1.946346$

C $7.5232872 .700540-1.589776$

O $5.2298942 .285259-3.295338$ 
C $5.2664410 .310829-1.845738$

C $4.164350-0.482856-2.592499$

O $6.519003-0.059493-2.435420$

C $1.525192-1.2135802 .196756$

O $0.489984-1.6474672 .677051$

C 1.7675540 .1985251 .827383

C 0.7992081 .1108421 .999448

C 0.9052462 .5456451 .723182

C 2.1905043 .0484321 .121146

C $2.736307-0.287668-2.074434$

C $1.9516960 .810849-2.768675$

C $-0.2243302 .021700-2.614489$

O $2.3421421 .382660-3.782253$

C $-0.0062883 .322085-1.907544$

C $-0.9722063 .966759-1.235964$

C $-0.7861275 .299247-0.570266$

C $-1.222635 \quad 5.298971 \quad 0.877354$

C -2.4263425 .7766521 .236114$

C -0.2257544 .8422091 .916785$

C -0.1390183 .3439772 .028682$

N $0.737109 \quad 1.046634-2.136743$

H $2.137407-2.851401-0.418608$

H $1.601270-5.1282181 .557455$

H $2.133948-3.4477693 .224637$

H $4.615898-3.7335951 .440166$

H $4.130554-4.8653622 .667103$

H $6.345185-1.6426844 .045668$

H $5.239986-2.9077555 .607626$

H $3.568984-3.2368865 .143518$

H $4.798604-4.5152175 .025257$

H $7.290765-1.7768161 .699058$

H $5.683396-2.0359051 .047309$

H 4.9986970 .1954012 .134609

H $\quad 6.710011 \quad 0.4627142 .480144$

H 7.2007610 .2247660 .012619

H $\quad 6.8197832 .5062670 .995830$

H 5.0923712 .3037260 .822878

H $5.5776064 .337325-1.858203$

H $4.0937362 .123496-1.615537$

H $8.1329473 .353560-0.953498$

H $7.5492553 .148879-2.588994$

H $8.0182691 .728230-1.635277$

H $4.4917711 .910912-3.804303$

H $4.230210-0.305830-3.672609$

H $4.395249-1.550463-2.463343$

H $6.483849-1.001139-2.671808$

H 2.7459270 .4545941 .439392

H -0.1585240 .7835442 .401375$

H 3.0074852 .9608631 .844804

H 2.1454384 .0921460 .803194

H 2.4492082 .4747650 .224797

H $2.177662-1.213412-2.261469$

H $2.730783-0.114744-0.994910$

H $-1.222257 \quad 1.611227-2.429448$

H $-0.0992002 .162211-3.692899$

H $0.9831163 .767974-1.977682$

H -1.966646 $3.531070-1.194164$

H -1.373262 $6.035739-1.134584$

H $0.2548215 .639175-0.637381$

H -2.7349015 .8094522 .275797$

H $-3.133420 \quad 6.1384380 .497234$

H -0.5183355 .2209272 .905891$

H $\quad 0.7425445 .312411 \quad 1.722599$

H -1.031758 2.8884862 .458742

H $0.5252440 .560476-1.270012$

C $-0.112742-3.927019-1.723996$

O $-1.116277-3.617228-0.737810$

C $-2.418262-3.926036-1.277388$

C $-2.209372-4.155217-2.766748$

C $-0.820978-4.759735-2.791911$

H $-2.730515-4.855459-0.784926$

C $-3.387381-2.784830-0.944220$

H $-2.970069-4.809929-3.202348$

H -2.222662 -3.204055 -3.313723

C $-0.159068-4.704295-4.157875$

H $-0.890030-5.807143-2.468753$

C $-4.796022-2.970832-1.538653$

O $-3.484222-2.6849590 .480505$
H $-2.949348-1.841372-1.286327$

H $-4.743751-2.985673-2.633506$

O $-5.277911-4.259330-1.135213$

C $-5.824473-1.917959-1.081778$

C $-5.446695-0.479741-1.473359$

H $-5.977086-2.001140-0.000458$

O $-7.085240-2.234093-1.684878$

H -4.442099-0.249275-1.110501

H -5.416921 -0.404739-2.567387

H $-0.746686-5.263980-4.892902$

H $-0.065280-3.673818-4.516740$

H $0.843718-5.141315-4.124012$

C $1.052006-4.668335-1.065007$

H -3.892579 -3.5057390 .805635$

H $-6.170718-4.364302-1.506725$

H $-6.977523-2.174524-2.649455$

H $1.778744-4.972160-1.826795$

H $\quad 0.681613-5.579953-0.579838$

H $0.227390-2.970730-2.142046$

C -5.4628730 .8425301 .256011$

O $-\begin{array}{llll}6.614189 & 0.467339 & 0.489166\end{array}$

C $-6.4478490 .565762-0.929629$

C $-6.1150022 .014298-1.310864$

C $-4.9067052 .541081-0.540981$

C -5.0692822 .2974740 .960319$

H -4.6302210 .1749761 .007335$

C -5.8213150 .6211672 .730403$

H $-7.4252650 .332685-1.370041$

O $\quad-5.8639482 .128981-2.707554$

H -6.975873 $2.656492-1.084078$

H $-3.9753712 .097416-0.911403$

O $-4.7947493 .949582-0.757258$

H $-5.8345362 .980846 \quad 1.348928$

O -3.8482162 .6241231 .618505$

C $-4.612722 \quad 0.525327 \quad 3.628174$

H -6.4733931 .4230003 .097294$

H -6.402009 -0.3021932 .854359$

H $\quad-6.655007 \quad 1.808040-3.173365$

H -4.713032 $4.089360-1.716388$

H -3.1556192 .0335931 .275956$

O $-3.920645-0.618650 \quad 3.360315$

O $-4.324636 \quad 1.362907 \quad 4.473238$

C $-2.741896-0.8235834 .144644$

H -2.268099-1.749965 3.809357

H $-2.0399410 .002822 \quad 3.999212$

$\mathrm{H}-3.005112-0.9256805 .201588$

SCF Energy $\left(B 3 L Y P / 6-31 G^{* *} / / M M F F\right)=-3245.91784845$

02168

MM̄FF Geometry

C $-1.641709-3.153884-2.414698$

C $-0.795134-2.411302-1.686759$

C $0.244907-1.494312-2.267065$

O $1.509286-1.932063-1.710379$

C $0.015938-0.027258-1.853998$

C $-0.8316650 .752401-2.834949$

C $-0.334411 \quad 1.548424-3.802518$

C $-2.3199500 .636883-2.644981$

C $1.1106931 .815882-4.123077$

C $1.4692453 .307335-4.074988$

C $1.2540533 .964466-2.702088$

C $1.6250205 .448561-2.763782$

C $1.4878936 .133364-1.397538$

O $2.0699983 .296142-1.731559$

O $2.1466217 .404320-1.507010$

C $2.2355705 .291039-0.343020$

C $0.022096 \quad 6.428987-1.057435$

O $2.0042395 .794468 \quad 0.971386$

C $1.8883473 .782249-0.393570$

C 2.8362393 .0076940 .555133

O $0.540360 \quad 3.6038290 .051402$

C $2.632210-1.702422-2.440948$

O $2.704705-1.201347-3.550934$

C $3.782947-2.144429-1.628426$

C $5.008689-1.688574-1.921035$

C $6.204830-2.011411-1.153927$

C $7.024691-0.805475-0.787788$

C 2.7758481 .4805800 .418539 
C $3.447508 \quad 0.7779681 .587523$

C $3.669893-1.4822322 .616156$

O 4.0770701 .3720062 .458869

C $5.114558-1.8408612 .460785$

C $5.567113-3.1040552 .438416$

C $7.023708-3.4693662 .378516$

C $7.352298-4.4031521 .235751$

C $7.398993-5.7333441 .420709$

C $7.716196-3.786809-0.095652$

C $6.512590-3.286845-0.846392$

N $3.253606-0.5961691 .545040$

H -1.604804 -3.101469 -3.499806

H $-0.836351-2.473319-0.602914$

H $\quad 0.277800-1.598301-3.359233$

H $\quad 0.9815230 .470414-1.725221$

H $-0.4356740 .030426-0.853628$

H -1.039312 2.068331 -4.450286

H -2.878001 $1.303007-3.311555$

H -2.659851 -0.382870 -2.842469

H $-2.5874770 .904996-1.617998$

H $1.7927271 .265668-3.471998$

H $1.3013301 .447529-5.138755$

H $2.5259563 .399472-4.357899$

H $\quad 0.886140 \quad 3.845007-4.833397$

H $\quad 0.2000713 .855597-2.423505$

H $1.0203505 .971194-3.515790$

H $2.6676975 .547561-3.097674$

H $2.0272327 .880142-0.667889$

H $3.3116955 .404109-0.535874$

H $-0.4138517 .101759-1.805673$

H $-0.0648656 .951717-0.098399$

H -0.598886 $5.531818-1.016034$

H 2.3389836 .7062981 .001520

H 3.8747833 .3082090 .363508

H 2.6056303 .2860841 .591388

H $0.439507 \quad 2.7133150 .421977$

H $3.596422-2.801996-0.785788$

H $5.157232-0.992663-2.743674$

H $7.492744-0.376608-1.680482$

H $7.815879-1.022955-0.066786$

H $6.391393-0.036680-0.329551$

H $\quad 1.7389001 .135902 \quad 0.383317$

H $3.2726751 .166354-0.506232$

H $3.018368-2.3613562 .584170$

H $3.519924-0.9726313 .573903$

H $5.828213-1.0217932 .413223$

H $4.856509-3.9236312 .518584$

H $7.284725-3.9472113 .332119$

H $7.658586-2.5767962 .316255$

H $7.663737-6.4078390 .613260$

H $7.167867-6.1772062 .383410$

H $8.208542-4.531778-0.735090$

H $8.472028-3.0116430 .060726$

H $5.843206-4.078447-1.185065$

H $2.717163-0.9969650 .781606$

C $-4.085073-3.613838-2.063938$

O $-4.335701-2.408845-1.311811$

C $-5.632758-2.504864-0.693265$

C $-6.318605-3.705498-1.333859$

C $-5.138330-4.618514-1.596892$

H $-5.460880-2.7238690 .367097$

C $-6.394941-1.182916-0.838202$

H -7.071200 -4.152287 -0.677160

H $-6.809482-3.439983-2.277245$

C $-5.434046-5.718437-2.601770$

H $-4.828553-5.073868-0.646538$

C $-5.6445230 .054286-0.305896$

O $-6.668910-0.952148-2.227465$

H -7.372794 -1.266798 -0.349919

H $-4.7858010 .266234-0.951758$

O $-6.539810 \quad 1.165685-0.440714$

C -5.184962 -0.057586 1.160512

C -4.5150231 .2282391 .678101$

H $-4.475954-0.8834501 .250828$

O $-6.303900-0.372786 \quad 1.988133$

H -3.6963561 .4924531 .000120$

H -5.241513 2.0484871 .649132

H $-6.241905-6.362753-2.239493$
H $-5.740926-5.306529-3.569044$

H $-4.551853-6.344603-2.767190$

C $-2.653895-4.088110-1.812526$

H $-5.816085-0.931794-2.695028$

H $-6.0082331 .978692-0.405646$

H $-6.9583260 .338772 \quad 1.880633$

H -2.499768 -5.079874 -2.252578

H $-2.482436-4.182496-0.733534$

H $-4.230217-3.365314-3.123444$

C -1.7996120 .1924982 .688933$

$\begin{array}{lllll}\text { O } & -3.082305 & 0.017737 & 3.302762\end{array}$

C $-3.9976151 .105116 \quad 3.129947$

C -3.3883412 .4014303 .684333$

C -2.0208602 .6867843 .072370$

C -1.1181091 .4644873 .212749$

H -1.9156720 .2426991 .599246$

C $-0.945050-1.0403303 .029851$

H -4.8677210 .8701243 .755681$

O -4.2457673 .5136243 .451532$

H -3.268682 2.310449 4.771793

H -2.1016512 .9990782 .024601$

O -1.4103833 .7748113 .770009$

H $-0.844396 \quad 1.3359544 .268185$

O $\quad 0.0867581 .6868132486655$

C - $-1.425329-2.3061812 .353811$

H $0.090952-0.8793832 .710457$

H $-0.968737-1.2195814 .111443$

H $-5.1019543 .314053 \quad 3.867311$

H -2.013657 4.5349523 .703739

H 0.4885302 .5018732 .833645

O $-0.571410-3.3238362 .675281$

O $-2.413346-2.3996051 .640120$

C $-0.887929-4.6017272 .114158$

H -1.929574 -4.869335 2.316820

H $-0.692667-4.5920941 .038377$

$\mathrm{H}-0.239473-5.3474612 .581801$

SCF Energy $(B 3 L Y P / 6-31 G * * / / M M F F)=-3245.91472055$

02169

MM̄FF Geometry

C 0.3857182 .7582221 .998985

C -0.7084572 .7361052 .774643$

C -1.5316261 .5010853 .018440$

O -2.8611151 .7933542 .528566$

C -1.6147511 .1476944 .515553$

C -0.2997240 .7269075 .133781$

C $0.273505-0.4770444 .941790$

C 0.3502231 .7453356 .032394

C $-0.246663-1.6052884 .095741$

C $0.703179-1.9024922 .929082$

C $0.173128-3.0386972 .042257$

C $1.251206-3.4908391 .054769$

C $0.734261-4.5785610 .103166$

O $-0.979615-2.5620421 .332885$

O $1.685401-4.721567-0.963125$

C $-0.583202-4.088234-0.530337$

C $0.640883-5.9473850 .788612$

O $-1.180043-5.115872-1.318872$

C $-1.604093-3.5505650 .500804$

C $-2.838142-2.918110-0.189280$

O $-2.086734-4.648951 \quad 1.286090$

C -3.6439950 .7358262 .184572$

O $-3.371292-0.4486452 .290902$

C -4.9033431 .2764401 .630094$

C -5.7751360 .4447951 .041345$

C -7.0496440 .8245520 .429448$

C -7.4480502 .2764090 .498254$

C $-2.573639-1.619259-0.956828$

C $-3.741509-1.230101-1.851711$

C $-4.3518660 .486417-3.551006$

O $-4.708157-1.959870-2.054832$

C $-5.6950940 .955690-3.086967$

C $-6.8445330 .410668-3.515348$

C $-8.2159870 .892257-3.134567$

C $-9.005449-0.146618-2.368829$

C $-9.648670-1.138166-3.008490$

C $-9.1117190 .013325-0.869073$

C $-7.784872-0.132316-0.175368$ 
N $-3.550027 \quad 0.016930-2.435343$ H $\quad 0.7115331 .845017 \quad 1.507369$ H -1.031840 3.6502193 .267283 $\mathrm{H}-1.1220180 .6631972 .441262$ H -2.3554650 .3530814 .672583$ H -2.034940 1.9983725 .070357 H $1.216850-0.6855455 .444764$ H 1.2911021 .3826806 .459818 H 0.5726532 .6621485 .477894 H $-0.315646 \quad 1.9952596 .864838$ H $-0.333858-2.4948804 .731518$ H -1.251592 -1.401087 3.718075 H $0.831095-0.9944462 .325730$ H $1.688548-2.1736963 .329086$ H $-0.121561-3.8735382 .689011$ H $2.142973-3.8434591 .587723$ H $1.579906-2.6298520 .455504$ H $2.514203-5.058416-0.581844$ H -0.316884 -3.283096 -1.225105 H $1.626639-6.2609221 .152933$ H $0.323022-6.721646 \quad 0.081630$ H $-0.040897-5.9573821 .641188$ H $-0.510634-5.417872-1.956554$ H $-3.304291-3.667810-0.841659$ H -3.599800 -2.697185 0.571411 H $-2.929539-4.3892751 .693595$ H -5.0697722 .3447841 .707548$ H $-5.530545-0.6155460 .989927$ H -7.5554642 .5956581 .540895$ H -8.3946702 .4969970 .001861$ H -6.6932552 .9056180 .013322$ H $-2.373751-0.802450-0.255321$ H -1.702829 -1.721959-1.611197 H $-4.428566-0.324890-4.283288$ H -3.812981 $1.318058-4.016135$ H $-5.7207811 .800124-2.403614$ H $-6.802450-0.421958-4.214891$ H $-8.7524191 .150777-4.057308$ H -8.165263 $1.825576-2.561090$ H -10.238541 -1.871649-2.469189 H $-9.596280-1.238242-4.087695$ H -9.784084 -0.746506 -0.449066 H $-9.5979210 .968202-0.650994$ H -7.403908 -1.154505 -0.182990 H -2.678261 $0.506250-2.254670$

C 1.1944404 .4461910 .326012

O $1.9013093 .501933-0.505750$

C $2.7518264 .227728-1.412235$

C $2.3057545 .682920-1.336680$

C 1.8914925 .7909620 .115737

H $3.7706674 .153895-1.013706$

C $2.6957693 .625877-2.819404$

H $3.1064116 .377342-1.609110$

H $1.4492555 .881239-1.991458$

C $1.014106 \quad 6.9964140 .407364$

H 2.7963435 .8467190 .736090

C $3.0731442 .133139-2.899839$

O $1.3595273 .762416-3.320425$

H $3.3355764 .213937-3.488584$

H $2.2840991 .525320-2.441282$

O $3.0812381 .767147-4.286634$

C $4.4276391 .765541-2.263281$

C $4.8760050 .332139-2.607363$

H $4.3473201 .875179-1.177555$

O $5.4340832 .681019-2.698035$

H $4.041979-0.353803-2.428383$

H $5.1151690 .282144-3.676294$

H 1.5459227 .9243400 .172990

H $0.0946036 .976742-0.187455$

H 0.7306347 .0278351 .463844

C 1.2268583 .9842151 .783044

H $\quad 1.3448843 .380649-4.214847$

H $3.7553922 .305707-4.734349$

H $5.5553672 .562540-3.655133$

H 0.8866874 .7931942 .439923

H 2.2551493 .7389662 .074975

H $0.1612474 .485798-0.043513$

C $4.996745-1.1564820 .061220$
O $5.878724-0.121806-0.392868$

C $6.122677-0.106068-1.804761$

C $6.719980-1.448310-2.248169$

C $5.845187-2.623047-1.824926$

C $5.536535-2.539279-0.331077$

H $3.998265-1.005991-0.365960$

C $4.885191-1.0383031 .589102$

H $6.8948430 .654881-1.973122$

O $6.910224-1.461210-3.658841$

H $7.711401-1.563183-1.790705$

H $4.928897-2.668687-2.425095$

O $6.558897-3.833105-2.087589$

H $6.450263-2.7623540 .234082$

O $4.586257-3.5479440 .001731$

C 4.1370220 .2032242 .025152

H $4.348506-1.8978652 .005064$

H $5.890667-0.9913742 .024494$

H $7.269030-2.333641-3.895639$

H $6.007097-4.568927-1.771000$

H $3.769383-3.352371-0.487998$

O $4.283363 \quad 0.3515193 .375676$

O 3.4957950 .9390701 .288373

C 3.6335731 .4917503 .944112

H $3.786367 \quad 1.4669205 .026297$

H 4.0723562 .4118533 .547253

H $2.5604061 .456723 \quad 3.741430$

SCF Energy $(B 3 L Y P / 6-31 G * * / / M M F F)=-3245.92275959$

0217

MM̄FF Geometry

C $-0.0914262 .263894 \quad 1.733382$

C -1.105737 2.0994902 .596423

C -1.6503030 .7940903 .125366$

O $-0.968142-0.3279232 .521276$

C -3.1509940 .6631822 .809743$

C $-3.856617-0.3320253 .710032$

C $-4.204655-1.5842693 .355867$

C -4.2133290 .1869155 .080149$

C $-3.937440-2.2908902 .056640$

C $-5.223421-2.5558631 .261296$

C $-5.819874-1.2867860 .633188$

C $-7.141077-1.601496-0.070850$

C $-7.702952-0.374882-0.805443$

O $-4.871151-0.787642-0.313669$

O $-8.747262-0.825117-1.681764$

C $-6.5935380 .224545-1.694502$

C $-8.347997 \quad 0.625312 \quad 0.161113$

O $-7.0216261 .448510-2.287905$

C $-5.2521010 .436218-0.954397$

C $-4.1431260 .790878-1.972416$

O $-5.3958041 .503612-0.017043$

C $-0.502323-1.3096003 .333653$

O $-0.526173-1.3196484 .557280$

C $0.052010-2.4475742 .563287$

C $0.024878-2.5420321 .224490$

C $0.519931-3.6714840 .430285$

C $1.181742-4.808597 \quad 1.164947$

C $-2.7364470 .878133-1.366527$

C $-1.6940740 .981312-2.460384$

C $-0.479965-0.370065-4.149348$

O $-1.2146962 .061076-2.796946$

C $-0.050194-1.793903-4.282122$

C $1.181773-2.229055-3.978390$

C $1.617033-3.658362-4.111169$

C $1.905820-4.303022-2.774548$

C $3.162939-4.591280-2.399028$

C $0.726619-4.699370-1.914815$

C $0.355345-3.641972-0.909320$

N $-1.382136-0.247911-3.020819$

H $\quad 0.420565 \quad 1.398799 \quad 1.321386$

H -1.5743192 .9910393 .010803$

H -1.4851030 .8108894 .209219$

H -3.2817710 .3885291 .757949$

H -3.6560961 .6315692 .925331$

H -4.730784 -2.194441 4.089573

H -4.761175 -0.5510425 .675757$

H -3.3115040 .4544935 .639065$

H -4.8470181 .0759274 .995757$ 
H -3.207209 -1.762423 1.437586 H -3.475068 -3.2561302 .299601$ H -4.974941 -3.2660360 .461736$ H -5.966494 -3.040963 1.906393 H $-5.979557-0.5381901 .416914$ H $-7.877851-1.9937500 .641294$ H $-6.980864-2.401800-0.807115$ H -9.421655 -1.270052 -1.140306 H -6.424116 -0.478191 -2.522571 H $-9.170607 \quad 0.1510430 .709868$ H $-8.795528 \quad 1.465786-0.380791$ H -7.6473321 .0234000 .898039$ H $-7.8582861 .269237-2.750305$ H -4.133469 $0.029165-2.762761$ H -4.368193 $1.750445-2.455202$ H -4.5225921 .8789570 .174633$ H $0.468999-3.2386973 .178908$ H $-0.417707-1.7316490 .647717$ H $2.007792-4.4423221 .782888$ H $1.614490-5.5633230 .505292$ H $\quad 0.459443-5.3191921 .811073$ H -2.634792 $1.755811-0.719372$ H $-2.5071470 .000501-0.751384$ H $\quad 0.366567 \quad 0.307544-4.006484$ H -1.015933 -0.049188 -5.048439 H $-0.791288-2.500230-4.648322$ H $1.922180-1.523676-3.610165$ H $2.510032-3.678143-4.749046$ H $0.865106-4.255591-4.642404$ H $3.370935-5.081912-1.454028$ H $4.011825-4.337895-3.025110$ H $0.923330-5.665688-1.439523$ H $-0.146403-4.889020-2.552631$ H $-0.137543-2.774074-1.347151$ H -1.960231 -1.048487 -2.779660 C $0.4399903 .849633-0.167418$ O $1.3389462 .919568-0.803868$ C $2.2900823 .653009-1.594959$

C $1.6365545 .001710-1.857386$ C $0.9423925 .250689-0.533304$ H $3.1761323 .799462-0.964455$ C $2.6836662 .859918-2.847045$ H $2.3651645 .776267-2.114338$ H $0.8951844 .947729-2.662788$ C $-0.1590956 .294341-0.609396$ H 1.6939925 .5833580 .195484 C $3.0564731 .387982-2.576088$ O $1.5925912 .860211-3.775276$ H $3.5059773 .372167-3.359297$ H $2.1608820 .834573-2.269906$ O $3.4721780 .807273-3.819023$ C $4.1662681 .184462-1.528859$ C $4.470402-0.307471-1.304212$ H $3.8500221 .629340-0.583522$ O $5.3373361 .869132-1.964940$ H $3.548401-0.794064-0.965721$ H $4.742838-0.770496-2.260546$ H $0.2483447 .261998-0.919941$ H $-0.9332186 .008667-1.329679$ H $-0.638493 \quad 6.4260480 .365673$ C 0.4408333 .6166011 .344784 H $0.8149932 .503052-3.313178$ H $4.2969891 .248171-4.086308$ H $5.9480961 .918218-1.210261$ H -0.1334964 .4098261 .837992$ H 1.4672403 .6801291 .722372 H -0.558556 $3.667702-0.582557$ C $4.413771-0.3017301 .776109$ $\begin{array}{lllll}\text { O } & 5.476820 & 0.139291 & 0.923518\end{array}$ C $5.617074-0.580992-0.304894$ C $5.846147-2.071764-0.019680$ C $4.746644-2.6469610 .870247$ C $4.577696-1.7891202 .122652$ H $3.449384-0.1270871 .282850$ C 4.4657170 .5401223 .063276 H $\quad 6.534917-0.204345-0.773689$ O $5.924057-2.800158-1.239290$ H $6.812613-2.1907410 .487227$
H $3.800630-2.735907 \quad 0.325428$

O $5.097507-3.9784411 .254711$

H $5.449661-1.9259762 .775425$

O $3.433419-2.2312822 .847146$

C 4.1209671 .9977762 .848408

H 3.7540670 .1458903 .797532

H 5.4777580 .5065273 .484460

H $6.029206-3.739802-1.011763$

H $5.939035-3.9395751 .740588$

H $3.569190-3.1707783 .058499$

O 4.1450052 .6360294 .056795

O 3.8712832 .5176101 .771157

C 3.8528474 .0368114 .024036

H 2.8014634 .1921903 .766758

H 4.0338564 .4445645 .022064

H 4.5061204 .5517013 .312816

SCF Energy (B3LYP/6-31G**//MMFF) $=-3245.92963794$

02170

MM̄FF Geometry

C $0.0867683 .732238-2.155270$

C $1.4183733 .853589-2.039797$

C $2.2027714 .225187-0.800644$

O $3.0293423 .090132-0.435210$

C 1.3520834 .5989040 .430102

C 2.1744835 .0135841 .636885

C 2.3543414 .2614392 .741716

C 2.7763656 .3933551 .559929

C 1.8438322 .8708503 .002984

C 2.9919471 .9146443 .353857

C 2.5247480 .4517103 .402649

C $3.672052-0.4632503 .835240$

C $3.291923-1.9492943 .758705$

O 2.0786350 .0974482 .089926

O $4.500947-2.718666 \quad 3.853567$

C $2.681893-2.2396172 .370579$

C $2.413868-2.3740134 .941510$

O $2.177378-3.5711012 .306582$

C $1.579516-1.2406891 .964371$

C $1.109348-1.4208040 .499692$

O $0.433362-1.4412652 .800442$

C $4.2380822 .963397-1.048641$

O $4.6944013 .675111-1.929316$

C $4.9292301 .800598-0.446651$

C $6.1100401 .396163-0.938324$

C $6.9354090 .300621-0.421484$

C $6.407679-0.4786350 .753843$

C $2.175588-1.170490-0.570807$

C $2.893905-2.415601-1.058392$

C $4.746744-3.090717-2.588661$

O $2.584574-3.553992-0.720524$

C $6.021006-3.230262-1.818205$

C $7.234343-3.027130-2.353406$

C $8.520535-3.224775-1.605510$

C $9.412982-2.005473-1.639759$

C $10.434660-1.920513-2.508525$

C $9.183247-0.933308-0.599851$

C $8.1277260 .058823-1.006183$

N $3.910131-2.094210-1.949542$

H $-0.5567643 .907138-1.298393$

H $2.0223713 .667625-2.926537$

H $2.8442275 .078791-1.053983$

H $\quad 0.695465 \quad 3.762608 \quad 0.689281$

H $0.680427 \quad 5.430438 \quad 0.177293$

H 2.9397494 .6836923 .557811

H 3.2950656 .6733832 .483085

H 3.5041386 .4513890 .744912

H 1.9947597 .1396641 .384081

H 1.1377322 .9101793 .840950

H 1.2907732 .4771382 .147914

H 3.7829912 .0161482 .600481

H 3.4237322 .2009834 .321028

H 1.6848840 .3742854 .103168

H $4.014530-0.2051124 .845152$

H $4.535476-0.2963043 .178298$

H $4.937219-2.4892464 .692008$

H $3.501683-2.1793111 .644289$

H $2.945220-2.2176505 .888064$ 
H $2.185893-3.4447404 .900359$ H $1.473777-1.8214864 .997980$ H $2.905266-4.1686612 .549189$ H $0.625414-2.3964450 .371385$ H $\quad 0.317077-0.6790050 .325678$ H $-0.347757-1.0828442 .347023$ H $4.455711 \quad 1.3202060 .400890$ H $6.5275061 .930920-1.790702$ H $7.011804-1.3528491 .005330$ H $5.401883-0.8592320 .547577$ H $\quad \begin{array}{llll}6.367743 & 0.158288 & 1.643775\end{array}$ H $1.683414-0.746090-1.453341$ H $2.913225-0.439901-0.228757$ H $4.918803-2.758418-3.617312$ H $4.223366-4.051673-2.616741$ H $5.941064-3.542421-0.779541$ H $7.311887-2.739940-3.399741$ H $9.040028-4.078941-2.059768$ H $8.337897-3.514562-0.563149$ H $11.099889-1.063405-2.516282$ H $10.624605-2.703373-3.235351$ H $10.112140-0.368129-0.441447$ H $8.982400-1.4000490 .368537$ H $8.4219710 .677147-1.855522$ H $4.123566-1.116748-2.126372$ C $-1.2901251 .985583-3.388033$ O $\quad-2.2776591 .942782-2.337575$ C $-2.1433980 .686830-1.634704$ C -1.324844 -0.207872 -2.552810 C $-0.3604250 .794642-3.154144$ H $-1.5814240 .916987-0.720287$ C $-3.534730 \quad 0.150637-1.282533$ H $-0.823612-1.019948-2.019122$ H -1.957092 $-0.648412-3.333857$ C $\quad 0.339213 \quad 0.297961-4.406397$ H $\quad 0.396650 \quad 1.037183-2.399093$ C -3.520099-1.257103-0.661028 O $-4.1344181 .057647-0.351766$ H $-4.1642620 .159535-2.179532$ H -3.152766 -1.982034 -1.396987 O $-2.577037-1.2628790 .419096$ C $-4.874882-1.729916-0.098890$ C $-6.023405-1.686292-1.120717$ H $-5.125676-1.1374370 .787291$ O $-4.715580-3.0770590 .360795$ H $-6.145775-0.663262-1.487615$ H -5.757450 -2.307370 -1.985100 H $0.936974-0.592375-4.185016$ H $-0.3787700 .034070-5.190046$ H $1.0102111 .064534-4.806743$ C $-0.6138353 .359063-3.433675$ H $-4.1268541 .940200-0.760413$ H $-2.574097-2.1591530 .797025$ H $-4.508227-3.632106-0.410362$ H -1.378224 4.125023 -3.615559 H $\quad 0.0799173 .396573-4.281845$ H -1.843385 $1.847712-4.326265$ C $-8.031046-0.1668050 .580156$ O $-7.696597-1.5549050 .694576$ C $-7.365149-2.193290-0.542851$ C $-8.550510-2.095147-1.513325$ C $-9.033546-0.655962-1.687123$ C $-9.2542450 .008394-0.326786$ H -7.1787780 .3856830 .167252$ C $-8.300151 \quad 0.361498 \quad 1.992670$ H $-7.227159-3.256815-0.311076$ O $-8.195792-2.647919-2.776360$ H -9.378884 -2.698833 -1.119949 H -8.334662 -0.080447 -2.305361 O $-10.271281-0.681647-2.401842$ H $-10.141896-0.4305470 .145478$ O $-9.5278021 .394076-0.521614$ C -7.0340490 .3638352 .816986$ H -8.7020651 .3806821 .984435$ H -9.029583 -0.2721242 .511729$ H $-8.966125-2.551718-3.362263$ H -10.587648 $0.235861-2.465416$ H $\quad-8.742218 \quad 1.799970-0.926657$
O $\quad-6.212419 \quad 1.3728792 .408689$

O $-6.800554-0.4420143 .708475$

C $-4.9551191 .454748 \quad 3.087110$

H -4.3813580 .5356542 .932916$

H -4.3944492 .2922972 .663734$

$\mathrm{H}-5.1112961 .6395634 .154221$

SCF Energy $(B 3 L Y P / 6-31 G * * / / M M F F)=-3245.91730008$

02171

MM̄FF Geometry

C 3.2770342 .0297873 .108339

C 4.0537992 .7149152 .255463

C 3.6572623 .9087291 .412990

O $2.238097 \quad 4.2287311 .491259$

C $4.1057603 .842190-0.069003$

C $5.5908983 .628171-0.292956$

C $6.1142322 .560033-0.929779$

C $6.496573 \quad 4.7112270 .232098$

C 5.362574 1.425837-1.573859

C $4.9316500 .361270-0.559037$

C $4.078153-0.774306-1.142461$

C $4.885657-1.721560-2.029223$

C $3.999980-2.828989-2.619540$

O $2.999074-0.194833-1.880499$

O $4.768977-3.478725-3.642561$

C $2.773289-2.173388-3.287573$

C $3.669854-3.906176-1.579348$

O $1.859245-3.165662-3.750750$

C $2.051186-1.137595-2.391981$

C $1.012666-0.362802-3.236643$

O $1.380211-1.830127-1.338304$

C 1.2936273 .3809400 .995921

O 1.4710012 .2988350 .460688

C $-0.0275103 .994374 \quad 1.259712$

C $-1.1488983 .279751 \quad 1.078698$

C -2.5170323 .7580601 .299117$

C -2.6863275 .1962321 .719188$

C $0.3880810 .847599-2.527930$

C $-0.6775331 .513247-3.383194$

C $-2.2831893 .405568-3.365320$

O $-0.961403 \quad 1.136522-4.517309$

C $-3.1681264 .006465-2.321892$

C $-4.4441823 .636955-2.135439$

C $-5.3554754 .228312-1.097485$

C $-5.7726523 .213526-0.054961$

C $-6.8336762 .415149-0.262057$

C -5.0185603 .1819821 .257381$

C -3.5470832 .9091181 .093172$

N -1.283193 $2.573610-2.722121$

H 2.2498832 .3339913 .284258

H 5.0827322 .3795172 .140566

H $4.1516274 .776373 \quad 1.867333$

H $3.8142634 .780886-0.560166$

H $3.5250753 .078305-0.592806$

H $7.1969702 .494028-1.024775$

H $7.5393414 .561047-0.067507$

H $6.1834245 .686875-0.154004$

H 6.4703744 .7429591 .325423

H $4.5011611 .819176-2.124851$

H $6.0163180 .976514-2.330563$

H $5.807444-0.055081-0.046157$

H $4.3224180 .851060 \quad 0.206753$

H $3.664196-1.328751-0.291590$

H $5.725850-2.156008-1.473579$

H $5.332936-1.163257-2.862063$

H $4.190559-4.128398-4.078104$

H $3.139761-1.659785-4.186924$

H $4.592038-4.356545-1.192700$

H $3.101912-4.729111-2.027429$

H $3.104592-3.523095-0.727051$

H $1.362444-3.492676-2.981330$

H $1.4967630 .009340-4.149564$

H $0.214621-1.046086-3.551631$

H $0.657175-1.275836-1.006778$

H -0.0380065 .0207451 .609224$

H -1.0622572 .2484720 .738477$

H -2.234182 5.3640052 .702934

H $\quad-3.7275595 .5158841 .788559$ 
H $-2.212030 \quad 5.867314 \quad 0.993842$ H $-0.078006 \quad 0.550725-1.582829$ H $1.1604381 .594473-2.311967$ H -2.845637 2.806952 -4.090187 H -1.759505 4.193449 -3.916108 H -2.739139 4.787418 -1.699450 H $-4.8675122 .861768-2.770580$ H -6.244179 $4.618602-1.610791$ H $-4.8990605 .098644-0.610477$ H -7.1650981 .7060120 .489138$ H -7.394630 $2.453334-1.190011$ H -5.4215682 .3962101 .910213$ H $-5.212824 \quad 4.116779 \quad 1.790242$ H -3.3256991 .8887470 .778841$ H $-0.8589102 .912327-1.863100$ C $3.139565-0.4519823 .159495$ O $1.706920-0.4537623 .298764$ C $1.257773-1.8135923 .482310$ C $2.489224-2.6950563 .319886$ C $3.603451-1.7699863 .769230$ H $0.878716-1.8639614 .510186$ C $0.135114-2.0980532 .477153$ H $2.427504-3.6130373 .912149$ H $2.630146-2.9792392 .269226$ C $4.980149-2.2158293 .307398$ H $3.588849-1.7047504 .865293$ C $-0.365054-3.5523342 .495597$ O $-0.962420-1.2300662 .778216$ H $0.478225-1.8297891 .471951$ H $0.441919-4.2274692 .187600$ O $-0.688711-3.8978053 .848463$ C - $-1.615045-3.8117181 .631793$ C $-1.434316-3.427640 \quad 0.153633$ H -2.473672 -3.2876512 .065146$ O $-1.923428-5.208310 \quad 1.710533$ H -1.177211 -2.3677150 .082187$ H -0.590976 -3.993622 -0.261181 H $5.219647-3.2046983 .711853$ H $5.037062-2.2764872 .215161$ H $5.751677-1.5174003 .646002$ C $3.727870 \quad 0.790016 \quad 3.830024$ H $-0.623590-0.3183872 .764783$ H -1.012154 -4.815039 3.844309 H -1.181930 -5.699614 1.317394 H $4.8220250 .731487 \quad 3.843314$ H 3.3907320 .8615034 .870956 H $3.352415-0.4380102 .083025$ C $-3.898911-1.683353-0.207184$ O $-3.865378-3.114109-0.140519$ C -2.691127 -3.712624 -0.699696 C $-2.555687-3.321695-2.177993$ C $-2.625284-1.810196-2.380561$ C $-3.841031-1.220748-1.666913$ H -3.051492 -1.266654 0.350091 C $-5.189280-1.2210930 .473541$ H $-2.855965-4.796962-0.669923$ O $-1.335532-3.829122-2.708844$ H -3.371660 -3.790285 -2.743480 H -1.699801-1.332709-2.043461 O $-2.729753-1.548248-3.780845$ H $-4.751499-1.524416-2.198179$ O $-3.7752820 .201807-1.730663$ C $-5.108176-1.4196391 .967929$ H $-5.395456-0.162948 \quad 0.286794$ H $-6.053390-1.7893670 .108971$ H -1.276504 -3.535723 -3.634464 H -2.801296 -0.584369 -3.891118 H $-2.9677090 .486604-1.269715$ O $-4.350379-0.4329642 .526892$ O $-5.642514-2.3462462 .562957$ C $-4.174766-0.5190193 .943961$ H -3.5279280 .3051834 .256368$ H $-5.139640-0.4207224 .450333$ H -3.692009-1.464155 4.210712 SCF Energy (B3LYP/6-31G**//MMFF) $=-3245.92549894$

02172

MM̄MF Geometry
C $-0.8524914 .025031 \quad 1.331053$

C -0.8388012 .7371381 .705883$

C -0.5038802 .3009543 .108529$

O $0.652518 \quad 1.4325833 .072083$

C -1.6446551 .4845223 .742425$

C -2.9237272 .2674893 .938875$

C -4.0299182 .1321213 .182464$

C -2.9118763 .2393285 .090848$

C -4.2393481 .2308311 .997040$

C -5.3840460 .2463332 .262019$

C $-5.675939-0.6349751 .040349$

C $-6.964159-1.4307991 .258161$

C $-7.264059-2.3529000 .070003$

O $-4.577643-1.5352690 .854841$

O $-8.286418-3.2774390 .473339$

C $-6.004603-3.186385-0.243651$

C $-7.834588-1.573813-1.121146$

O $-6.174661-3.930687-1.447930$

C $-4.692563-2.362705-0.311566$

C $-3.486257-3.326441-0.341613$

O $-4.616596-1.588793-1.511049$

C 1.8767872 .0134883 .157731

O 2.1182153 .2129913 .147589

C 2.9463831 .0061583 .344562

C $2.757481-0.3178373 .232377$

C $3.762067-1.3558843 .478081$

C $5.108296-0.9190443 .996823$

C $-2.117822-2.632025-0.304218$

C $-0.995804-3.596609-0.657350$

C $1.445252-3.658589-1.125953$

O $-1.188191-4.781036-0.922982$

C $2.079845-4.417549-0.003641$

C $3.399577-4.3981440 .237379$

C $4.072901-5.2226861 .295711$

C $4.920110-4.3939392 .233337$

C $6.229900-4.2071951 .997450$

C $4.272256-3.8663883 .491383$

C $3.431237-2.6443103 .248432$

N $0.248098-2.979907-0.663890$

H -0.5745314 .7734902 .071787$

H $-1.094267 \quad 1.9538760 .999095$

H -0.2839693 .1605813 .754428$

H $-1.309864 \quad 1.1079714 .719229$

H -1.8103090 .5691313 .162347$

H $-4.8909432 .754666 \quad 3.424373$

H -3.8794813 .7338145 .228344$

H -2.674587 2.7185496 .024277

H -2.1640484 .0214584 .928008$

H -4.4923221 .8617501 .135893$

H -3.3337590 .6849411 .721134$

H $-5.119491-0.3865243 .119275$

H -6.2874550 .8079952 .532007$

H $\quad \begin{array}{llll}-5.767700 & 0.010698 & 0.159123\end{array}$

H $-7.812341-0.7598831 .443381$

H -6.865245 -2.047242 2.162845

H $-9.061903-2.7631110 .756255$

H $-5.898587-3.9249170 .563821$

H -8.780812 -1.092503 -0.845517

H $-8.072066-2.242978-1.955247$

H -7.164212 -0.791546-1.483248

H $-6.986828-4.456973-1.351192$

H -3.535861 -4.0111780 .515159$

H -3.547306 -3.938505 -1.250907

H $-5.404531-1.764881-2.048864$

H 3.9138901 .4247073 .603516

H $1.773810-0.6882902 .949558$

H $5.580807-0.2112203 .307648$

H $5.816346-1.7410364 .120272$

H $5.002763-0.4369354 .974971$

H -2.087427 -1.809276 -1.025542

H $-1.929166-2.2224370 .694383$

H $2.114297-2.899178-1.539716$

H $1.170059-4.353094-1.926824$

H $1.437348-5.0485240 .605873$

H $4.045429-3.798106-0.398715$

H $4.704299-5.9640080 .787734$

H $3.345401-5.8068841 .872655$

H $6.853350-3.6420152 .682047$ 
H $\quad 6.706349-4.6114671 .110319$ H $5.025687-3.6814724 .265002$ H $3.633117-4.6466583 .924793$ H $2.433939-2.8667312 .868065$ H $\quad 0.324656-2.003605-0.394035$ C $-1.9560323 .676530-0.991611$ O $-1.0548402 .677594-1.506690$ C $-1.2659972 .541011-2.923546$ C $-2.5381073 .316429-3.243592$ C $-2.4808294 .427564-2.217246$ H $-0.418573 \quad 3.033333-3.414835$ C $-1.3154071 .063281-3.323900$ H $-2.5515453 .682322-4.274821$ H -3.437431 $2.708427-3.091977$ C $-3.8180635 .115490-1.998735$ H -1.742821 5.171304-2.546911 C $-0.098613 \quad 0.226607-2.885822$ O $-2.477670 \quad 0.455571-2.744329$ H -1.448869 $0.984682-4.409204$ H $-0.1156530 .069234-1.800504$ O $-0.234904-1.071053-3.481645$ C $1.2682840 .807185-3.291888$ C $2.422559-0.174085-3.017794$ H $1.4402271 .738398-2.743863$ O $1.2605091 .132593-4.681988$ H $2.332587-0.541254-1.990971$ H $2.321732-1.038326-3.685053$ H $-4.1737675 .568522-2.930004$ H -4.581242 $4.408620-1.655844$ H $-3.7334165 .908783-1.249727$ C $-1.2232734 .596677-0.010874$ H -2.425235 $0.580021-1.780948$ H -1.101580 -1.423405 -3.215804 H $1.0612950 .317629-5.174021$ H -1.857137 5.4692770 .189061 H $-0.3016214 .968161-0.474652$ H $-2.7753313 .147268-0.488484$ C $4.2191521 .234405-0.987825$ O $4.0513691 .569711-2.371422$ C $3.8140350 .458507-3.243584$ C $4.977590-0.539673-3.149953$ C $5.224849-0.977853-1.711399$ C $5.3764860 .242467-0.805769$ H $3.2897030 .805820-0.594719$ C $4.5053992 .537793-0.226602$ H $3.8215280 .862779-4.263347$ O $4.731749-1.687951-3.955078$ H $5.892766-0.068969-3.532126$ H $4.425088-1.638870-1.361450$ O $6.429691-1.744610-1.667740$ H $6.3321580 .733321-1.028484$ O $5.440037-0.1925370 .549722$ C $3.3029083 .455678-0.170985$ H 4.7969342 .3281160 .808051 H $5.3163053 .079346-0.728938$ H $4.595360-1.380900-4.867590$ H $\quad 6.583273-1.988792-0.738797$ H $4.605788-0.6479190 .755195$ O $3.713176 \quad 4.691271 \quad 0.243791$ O $2.1531563 .131593-0.432752$ C 2.6865305 .6819240 .349720 H 3.1585956 .6342880 .605152 H $2.1642725 .795896-0.605021$ H 1.9872405 .4128291 .145752 SCF Energy (B3LYP/6-31G**//MMFF) $=-3245.92323915$

$02 \_173$

MMFF Geometry

C 3.0437220 .2179003 .125676

C $4.162005-0.4465472 .796463$

C $4.932569-0.3261101 .500553$

$\begin{array}{lllll}\text { O } & 4.493576 & 0.853832 & 0.788446\end{array}$

C $4.667098-1.5820230 .647072$

C $5.848673-2.081525-0.158531$

C $5.886478-2.147444-1.503786$

C $7.001422-2.6230430 .649304$

C $4.852989-1.677032-2.488480$

C $3.991210-2.821638-3.044331$
C $2.881066-3.274723-2.081460$

C $2.089822-4.444401-2.669740$

C $0.895072-4.829950-1.782662$

O $2.013479-2.156781-1.879052$

O $0.043742-5.701258-2.542461$

C $0.075996-3.560897-1.467276$

C $1.333442-5.621235-0.544760$

O $-0.961439-3.843060-0.530021$

C $0.935821-2.380061-0.961914$

C $0.092824-1.084812-0.938108$

O $1.401913-2.6698560 .355970$

C $5.3520161 .437781-0.082092$

O $6.456006 \quad 1.023827-0.408522$

C $4.8086572 .707643-0.619800$

C $3.7486653 .349431-0.101686$

C $3.2520274 .665152-0.518847$

C $3.8657325 .285644-1.747057$

C $0.8861770 .176746-0.567797$

C $0.1020481 .418604-0.934569$

C $-0.6552372 .735913-2.905823$

O $-0.5182842 .064891-0.094345$

C $-0.0216824 .071952-2.687337$

C $-0.6121475 .047244-1.981147$

C $-0.0330856 .414862-1.774974$

C $0.2948676 .691190-0.326421$

C -0.6643887 .0381440 .548520$

C $1.7487696 .660198 \quad 0.079044$

C 2.2988945 .2655530 .225711

N $0.149357 \quad 1.699703-2.291553$

H 2.6155300 .9412902 .437631

H $4.554058-1.1791053 .500123$

H $5.989581-0.2089391 .765532$

H $3.813109-1.381192-0.004528$

H $4.360168-2.4229151 .284987$

H $\quad 6.773756-2.575823-1.969035$

H $7.762033-3.0988030 .020969$

H $7.494342-1.8225031 .208379$

H $6.647177-3.3776561 .359145$

H $4.231348-0.875395-2.077541$

H $5.395545-1.217878-3.325126$

H $3.524463-2.457752-3.969295$

H $4.626361-3.673728-3.316240$

H $3.329737-3.568003-1.126856$

H $2.741961-5.311017-2.835026$

H $1.706773-4.164576-3.661311$

H $0.569244-6.474306-2.811153$

H -0.420476 -3.254036 -2.398605

H $1.848322-6.542629-0.842188$

H $0.468162-5.9397220 .047014$

H $2.009281-5.0614460 .105286$

H -1.499246 -4.563059 -0.901930

H $-0.351827-0.941135-1.930937$

H $-0.736631-1.186191-0.228461$

H $1.567566-1.8384210 .827119$

H $5.388343 \quad 3.132376-1.433491$

H $3.2241352 .906573 \quad 0.742921$

H $4.9124395 .550808-1.563276$

H $3.3526376 .190808-2.078863$

H $3.8213674 .589780-2.592776$

H 1.0927230 .2080710 .506682

H $1.8535760 .216833-1.080721$

H -1.664762 $2.686153-2.485667$

H $-0.7164222 .520731-3.977012$

H $0.9477434 .248548-3.145535$

H $-1.5884744 .861374-1.538448$

H $-0.7640887 .152667-2.131180$

H $\quad 0.855576 \quad 6.566509-2.399327$

H -0.4311757 .2690321 .582594$

H $-1.705897 \begin{array}{lll}7.094186 & 0.248893\end{array}$

H 1.8762157 .1562101 .051202

H $2.3310777 .273144-0.615570$

H 1.9045814 .7334121 .092548

H $\quad 0.700398 \quad 1.104525-2.902915$

C $0.997775-0.7857884 .143875$

$\begin{array}{lllll}\text { O } & 0.080560 & 0.032051 & 3.392087\end{array}$

C $-1.258659-0.1911013 .886782$

C $-1.159424-1.3512884 .865953$

C $0.243501-1.1748885 .412431$ 
H -1.5387180 .7313904 .410219$ C $-2.186225-0.4336202 .691607$ H $-1.930680-1.3135865 .640923$ H -1.248162 -2.312163 4.343544 C $0.783735-2.4155886 .101822$ H $0.243181-0.3365206 .121938$ C -3.619872 -0.8393323 .069405$ O -2.2447650 .7765151 .931219$ H -1.741336 -1.194174 2.040645 H -3.612197-1.818600 3.562201 O -4.1119860 .0974784 .036131$ C -4.609207 -0.8570151 .886361$ C $-4.175146-1.7676920 .724660$ H -4.7719540 .1669891 .533049$ O $-5.876414-1.3103752 .377272$ H -3.186778 -1.4653670 .369779$ H -4.084339 -2.796592 1.093862 H $0.154196-2.6852416 .956218$ H $0.811463-3.2729615 .420936$ H $1.799521-2.2468506 .472228$ C $2.289753-0.0108284 .406663$ H -1.3320821 .0182971 .697343$ H -5.022492 -0.1642104 .256244$ H -5.764111 -2.223775 2.691668 H $2.916402-0.5538245 .123227$ H 2.0650670 .9687204 .845697 H $1.204257-1.6764203 .534727$ C $-4.3352600 .217219-1.586670$ O $-5.433202-0.418116-0.917489$ C $-5.174006-1.747196-0.454446$ C $-4.782219-2.645369-1.636105$ C $-3.638056-2.047721-2.451474$ C $-3.928969-0.592925-2.824604$ H $-3.4828010 .302941-0.902042$ C $-4.7965151 .631310-1.954820$ H -6.128161 -2.127088 -0.068003 O $-4.410345-3.944555-1.188374$ H $-5.650245-2.769005-2.296552$ H $-2.693058-2.122801-1.905016$ O $-3.455769-2.814176-3.644520$ H $-4.721899-0.567405-3.582969$ O $-2.748360-0.048944-3.409911$ C $-4.9151322 .493073-0.719706$ H $-4.1114812 .132791-2.645757$ H $-5.7806131 .609885-2.438620$ H $-5.162135-4.308106-0.690173$ H $-4.279673-2.770170-4.158926$ H -2.989399 $0.782292-3.851227$ O $-3.6815582 .934683-0.341611$ O $-5.9740492 .732339-0.154138$ C -3.651495 3.7516950 .832601 H -4.069996 3.2088881 .685533 H -2.608318 3.9956361 .050228 H -4.2007424 .6815740 .657995$ SCF Energy (B3LYP/6-31G**//MMFF) $=-3245.90716019$

$02 \_174$

MMFF Geometry

C - 0.4385103 .0835132 .567267

C -0.6335001 .7915392 .262405$

C -0.4028490 .6623413 .230568$

$\begin{array}{lllll}\text { O } & 0.528390 & -0.244447 & 2.591739\end{array}$

C $-1.705631-0.108728 \quad 3.516335$

C -2.7623130 .6870594 .249685$

C $-3.940803 \quad 1.0570593 .712155$

C -2.4519631 .0071005 .688503$

C $-4.412568 \quad 0.8291632 .303535$

C $-5.204304-0.4770962 .179449$

C $-5.898251-0.6078150 .816295$

C $-6.662674-1.9302580 .726804$

C $-7.321653-2.114511-0.648254$

O $-4.908030-0.550660-0.214632$

O $-7.723248-3.488810-0.765906$

C $-6.266463-1.860527-1.748965$

C -8.602693 -1.281512 -0.776183

O $-6.866971-1.854061-3.041349$

C -5.446784 -0.563506 -1.542762

C $-4.284555-0.413195-2.551867$
O $-6.2594210 .597155-1.774541$

C $1.217525-1.1012903 .392740$

O $1.232351-1.1253964 .612611$

C $1.894524-2.0719212 .509121$

C $2.203791-3.2867422 .984556$

C $2.720196-4.3793092 .167414$

C $1.998222-5.6792812 .399476$

C $-3.197292-1.489925-2.547205$

C $-2.141965-1.338130-1.464278$

C $-0.031284-2.369129-0.676514$

O $-2.118437-0.414511-0.656721$

C $0.725531-3.643764-0.867997$

C $1.938644-3.707587-1.438770$

C $2.712172-4.983263-1.623754$

C $4.123771-4.898110-1.081676$

C $5.155998-4.625974-1.897586$

C $4.352081-5.217178 \quad 0.380708$

C $3.736929-4.1985681 .301613$

$\mathrm{N}-1.204370-2.363591-1.527117$

H $-0.0979753 .326447 \quad 3.572506$

H $-0.974991 \quad 1.5081301 .271214$

H $\quad 0.046227 \quad 1.0382394 .158168$

H - $1.477679-1.0001344 .116080$

H -2.090356 -0.5249362 .576864$

H $-4.6492651 .594226 \quad 4.341180$

H -3.291674 1.4933436 .196357

H $-2.2215390 .090213 \quad 6.240799$

H - 1.5926421 .6810215 .756849

H $-5.047147 \quad 1.6782782 .020020$

H -3.5727990 .8389391 .600885$

H $-4.514262-1.3188632 .322402$

H $-5.958250-0.5357932 .974690$

H -6.5843240 .2400410 .704326$

H -7.412354 -2.002901 1.524508

H $-5.967011-2.7651530 .891864$

H $-8.321368-3.691238-0.026028$

H -5.584269-2.718579-1.727709

H $-9.342151-1.600135-0.031286$

H $-9.077622-1.432186-1.751704$

H $-8.435383-0.212095-0.631894$

H $-7.357195-2.688572-3.137384$

H $-4.710186-0.361784-3.563360$

H $-3.8141980 .565713-2.390969$

H $-7.0853570 .315892-2.200642$

H $2.047044-1.7991611 .470622$

H $1.986793-3.5425514 .019552$

H $2.218498-6.0636203 .401525$

H $2.259744-6.4586251 .680966$

H $\quad 0.914469-5.5345892 .318017$

H -3.622957 - $2.495480-2.494714$

H $-2.661487-1.420557-3.503429$

H $-0.356854-2.2682510 .363878$

H $0.570662-1.489185-0.924521$

H $0.259143-4.554054-0.499410$

H $2.396377-2.794600-1.813842$

H $2.740629-5.201033-2.699565$

H $2.197217-5.835672-1.163484$

H $6.176095-4.593497-1.529679$

H $5.003403-4.424599-2.952861$

H $5.427388-5.2454220 .601482$

H $3.995997-6.2325910 .575904$

H $4.193267-3.2115311 .237667$

H -1.220779 -2.983582 -2.332608

C $-1.084523 \quad 4.0134590 .231561$

O $-0.090000 \quad 3.248472-0.472411$

C $-0.0340923 .692169-1.840578$

C $-1.1329794 .738329-2.002961$

C $-1.256516 \quad 5.287051-0.594623$

H $0.9399514 .177073-1.971365$

C $-0.1441312 .491962-2.788743$

H $-0.8670885 .503113-2.739248$

H -2.082124 $4.291465-2.320219$

C $-2.562278 \quad 6.021209-0.344122$

H $-0.4162495 .970170-0.411424$

C $0.8318811 .339328-2.480428$

O $-1.468962 \quad 1.950572-2.714610$

H $-0.0169872 .827887-3.824216$

H $0.5506520 .850186-1.540351$ 
O $0.678328 \quad 0.352103-3.508402$

C $2.3131121 .749175-2.412202$

C $3.2467510 .535876-2.239944$

H $2.4563362 .437711-1.573421$

O $2.6727092 .452687-3.600277$

H $2.906408-0.047952-1.377846$

H $3.166941-0.112262-3.121021$

H $-2.6593406 .874884-1.022815$

H $-3.4265775 .367014-0.500577$

H -2.6081856 .4002280 .681523$

C $-0.622730 \quad 4.278852 \quad 1.667388$

H -1.630861 $1.695026-1.789832$

H $-0.259516 \quad 0.094497-3.523605$

H $2.515281 \quad 1.857243-4.353144$

H -1.339262 4.9479972 .158002

H 0.3398084 .8043821 .640023

H $-2.013447 \quad 3.4278670 .229352$

C $4.716376 \quad 1.3269040 .312375$

O $4.9241841 .864824-0.998928$

C $4.7292490 .937105-2.070756$

C $5.688477-0.249730-1.912414$

C $5.555770-0.904981-0.540548$

C 5.6604300 .1446890 .565179

H 3.6721201 .0077340 .417584

C $4.974403 \quad 2.453977 \quad 1.316675$

H $5.020722 \quad 1.464441-2.987951$

O $5.456192-1.208128-2.938580$

H $6.7190280 .108541-2.034852$

H $4.621887-1.473555-0.468828$

O $6.617072-1.849424-0.387507$

H 6.6981810 .4968650 .622913

O $5.359332-0.4645191 .818846$

C 3.8593163 .4695501 .272273

H 5.0240062 .0803362 .345824

H 5.9262942 .9598121 .114175

H $6.085227-1.937058-2.801718$

H $6.533253-2.2373430 .500547$

H $4.432472-0.7561981 .793583$

$\begin{array}{llllll}\text { O } & 4.201686 & 4.508822 & 0.459751\end{array}$

$\begin{array}{lllll}\text { O } & 2.800346 & 3.337903 & 1.871387\end{array}$

C $3.213614 \quad 5.534396 \quad 0.323628$

H $3.6130716 .306254-0.339512$

H $2.3036635 .125219-0.123194$

H 2.9971105 .9847361 .296959

SCF Energy (B3LYP/6-31G*//MMFF)= -3245.91929685

02_175

MMFF Geometry

C $3.1955963 .868364-1.226250$

C $3.4673092 .935096-2.151387$

C $4.6240181 .958756-2.159993$

O $4.121952 \quad 0.656379-1.777343$

C $5.7917212 .314540-1.216205$

C $7.0361321 .467589-1.434277$

C $7.5325680 .576274-0.552329$

C $7.7621701 .706759-2.734753$

C $6.986218 \quad 0.221290 \quad 0.800775$

C $6.296233-1.1480040 .776775$

C $5.347988-1.3303451 .970010$

C $4.743717-2.7354131 .967048$

C $3.656484-2.9050923 .038697$

O $4.317849-0.3476741 .832425$

O $2.931534-4.1095372 .744215$

C $2.653322-1.7371902 .923387$

C $4.258586-3.0921184 .435826$

O $1.705392-1.7722543 .986789$

C $3.329890-0.3530672 .868469$

C 2.3400500 .7957292 .553402

O $3.919136-0.0669684 .141986$

C $3.546821-0.108456-2.744547$

O $3.4142040 .172642-3.924912$

C $3.076291-1.357432-2.105200$

C $2.260126-2.179390-2.781076$

C $1.690109-3.434428-2.286088$

C $2.086150-3.885062-0.904960$

C $1.656206 \quad 0.708232 \quad 1.184731$

C $0.3090320 .010760 \quad 1.208206$

C $-1.409235-0.935085-0.319252$
O $\begin{array}{llll}-0.341984 & -0.157780 & 2.235945\end{array}$

C $-1.318163-2.426781-0.338744$

C $-1.874120-3.175609-1.303417$

C $-1.859401-4.676857-1.318876$

C $-1.359303-5.241295-2.630201$

C $-2.219253-5.643163-3.581545$

C $0.133228-5.413035-2.802265$

C $0.834776-4.111191-3.080957$

N $-0.106850-0.354657-0.065481$

H $3.8176313 .957058-0.340349$

H $2.8168172 .877113-3.022834$

H $5.0051011 .920058-3.187946$

H $5.4451692 .233423-0.182143$

H $6.0802233 .364424-1.359567$

H $8.4360950 .031519-0.823756$

H $8.7257811 .187325-2.774045$

H $7.1668181 .355282-3.582607$

H $7.9639842 .774852-2.866937$

H $7.8168750 .194273 \quad 1.517104$

H 6.3125100 .9958631 .173678

H $5.718886-1.245834-0.151452$

H $7.056239-1.9389260 .771962$

H $5.902508-1.1485002 .898080$

H $5.526628-3.4945502 .087920$

H $4.291419-2.9312030 .986213$

H $3.566063-4.8464432 .734319$

H 2.089671-1.895160 1.996329

H $4.888035-3.9896274 .464173$

H $3.475977-3.2520685 .185532$

H $4.875558-2.2488754 .753239$

H $1.290551-2.6518833 .976216$

H 1.6134620 .9041093 .367426

H 2.9177221 .7314822 .553719

H $4.0296570 .894715 \quad 4.223043$

H $3.396463-1.554885-1.089092$

H $1.968312-1.903427-3.793844$

H $3.157977-4.107481-0.870616$

H $1.561652-4.781333-0.568197$

H $1.863232-3.108411-0.165795$

H $1.457651 \quad 1.7277450 .833019$

H 2.3089700 .2338860 .446736

H - $1.765772-0.536284-1.273431$

H -2.092172 -0.6192100 .473232$

H $-0.813173-2.9127850 .492262$

H -2.407042 -2.682610 -2.113379

H $-2.886075-5.020155-1.134724$

H $-1.260236-5.081355-0.493908$

H $-1.870340-6.073596-4.514281$

H $-3.291864-5.544175-3.450594$

H $0.341416-6.079752-3.649984$

H $0.538009-5.938842-1.933115$

H $0.602874-3.697994-4.063693$

H $0.506124-0.195023-0.859474$

C $0.9877914 .721390-0.317856$

O $0.0874353 .664934-0.724468$

C $-1.2436224 .211416-0.836146$

C $-1.2420125 .428107 \quad 0.072427$

C $0.1361035 .990475-0.196830$

H $-1.3552204 .514056-1.885489$

C $-2.2952383 .152418-0.473781$

H $-2.0410346 .137344-0.162445$

H $-1.3382695 .129452 \quad 1.123592$

C $0.621104 \quad 6.950808 \quad 0.876351$

H $0.1128716 .521203-1.158563$

C $-3.7342153 .701553-0.477622$

O $-2.1991772 .090820-1.426278$

H -2.0503282 .7178880 .501239$

H -3.828116 4.5028610 .264793

O $-3.9709544 .304866-1.757371$

C $-4.842198 \quad 2.656315-0.237592$

C -4.7257421 .9392691 .118603$

H $-4.8574601 .930362-1.055612$

O $-6.098416 \quad 3.344958-0.294012$

H $-3.814521 \quad 1.333395 \quad 1.127865$

H -4.6143422 .6995311 .901548$

H -0.0443657 .8174250 .946578$

H $0.652216 \quad 6.470378 \quad 1.860060$

H 1.6277087 .3144740 .646910 
C $2.0961224 .885467-1.362897$

H $-2.4547872 .448236-2.293958$

H $-4.8813454 .647373-1.752633$

H -6.1186743 .9868420 .436415$

H $2.5647425 .871634-1.256694$

H $1.6636984 .841871-2.370456$

H 1.4011974 .4321070 .656105

C $-5.273599-1.0207280 .444421$

$\begin{array}{llll}\text { O } & -6.209984 & 0.063275 & 0.465925\end{array}$

C -5.9572851 .0650991 .457736$

C -5.9213020 .4368382 .858801$

C $-4.943268-0.7296612 .935718$

C $-5.221454-1.7222191 .809144$

H -4.284945 -0.6324230 .186252$

C - $-5.710790-2.001605-0.655735$

H -6.8308651 .7286441 .443820$

O $\quad-5.576278 \quad 1.4025543 .846360$

H -6.9222600 .0636593 .112618$

H -3.902900 -0.384454 2.912103

O $-5.116250-1.4057094 .183090$

H $-6.174069-2.2299122 .006221$

O $-4.209480-2.7270421 .809488$

C $-5.325125-1.528558-2.041590$

H $-5.223385-2.971982-0.507338$

H $-6.798196-2.136116-0.640462$

H -6.2302862 .1203593 .793575$

H $-4.972208-0.7516784 .888389$

H $-3.350797-2.2864751 .694802$

O $-5.491902-2.560674-2.921812$

O $-4.934314-0.404943-2.323991$

C $-5.140287-2.274776-4.279159$

H -5.296431 -3.182012 -4.868759

H $-5.780979-1.482039-4.676823$

H $-4.085687-1.990833-4.346001$

SCF Energy (B3LYP/6-31G**//MMFF)= -3245.91195572

02176

MM̄FF Geometry

C 3.5389351 .4912322 .251292

C 4.7465971 .7158711 .710126

C 5.0564792 .6554180 .567254

O 3.9346743 .5462620 .367921

C $5.3159681 .895109-0.748703$

C $6.5193100 .976358-0.713009$

C $6.464671-0.350752-0.481267$

C $7.8431181 .651106-0.959145$

C $5.239876-1.170451-0.184311$

C $5.199673-2.464993-1.006508$

C $3.859662-3.188751-0.814754$

C $3.914078-4.609585-1.373172$

C $2.553142-5.313210-1.266187$

O $2.860531-2.433222-1.509096$

O $2.641643-6.502067-2.065662$

C $1.470699-4.398206-1.877316$

C $2.262193-5.7700580 .168691$

O $0.165814-4.931827-1.660197$

C $1.528678-2.938492-1.363466$

C $0.587071-2.057489-2.216118$

O $1.103659-2.900825-0.003323$

C $4.1716234 .771624-0.160712$

O $5.2509355 .206180-0.539130$

C $2.9322105 .582235-0.230469$

C 1.7354475 .1625730 .211448

C $0.485300 \quad 5.927920 \quad 0.188378$

C $0.5284247 .309976-0.410628$

C $0.613943-0.558942-1.880068$

C $-0.2007090 .248055-2.879795$

C $-0.8893932 .553868-3.438644$

O $-0.749565-0.254072-3.858092$

C $-1.1975923 .813596-2.697538$

C -2.433624 4.147747 -2.296965

C -2.787956 5.440599-1.619479

C $-3.0068105 .285249-0.131388$

C -4.0820714 .6405990 .352108$

C $-2.0126645 .949566 \quad 0.794739$

C $-0.631656 \quad 5.3577420 .689803$

N $-0.2477751 .598389-2.555183$

H 2.6665182 .0179941 .874893
H $5.598980 \quad 1.1697412 .109877$

H 5.9335763 .2481280 .856155

H $5.4548262 .618022-1.564200$

H $4.407277 \quad 1.358878-1.047117$

H $7.400795-0.907239-0.462529$

H $8.6815960 .947388-0.927838$

H $7.8484882 .127191-1.945107$

H $8.0283712 .418641-0.201042$

H $5.255052-1.4312850 .881430$

H $4.321056-0.603796-0.353618$

H $5.342684-2.227762-2.068683$

H $6.027109-3.113800-0.694117$

H $3.622620-3.2094570 .255310$

H $4.689809-5.198849-0.868122$

H $4.206790-4.574155-2.432193$

H $1.806679-6.991184-1.974089$

H $1.626984-4.388262-2.965202$

H $3.032780-6.4725140 .507903$

H $1.313505-6.3152710 .226068$

H $2.226077-4.9451640 .883483$

H $\quad 0.124876-5.799345-2.096237$

H $0.865083-2.172674-3.272283$

H $-0.445978-2.411743-2.111514$

H $0.990299-1.971843 \quad 0.251332$

H $3.0667506 .569457-0.661287$

H 1.6512494 .1662300 .641363

H $1.192927 \quad 7.9595310 .169764$

H $-0.4446107 .803296-0.448452$

H $0.8898507 .269288-1.444520$

H $\quad 0.197133-0.383678-0.882564$

H $1.641740-0.179782-1.900780$

H -1.789117 $2.099685-3.868497$

H $-0.2011992 .762575-4.264655$

H $-0.3716494 .496982-2.520053$

H $-3.2548903 .461265-2.487982$

H $-3.7022815 .835110-2.081816$

H -2.022351 $6.200744-1.815849$

H -4.2563194 .5510431 .419071$

H -4.813471 $4.189048-0.309995$

H -2.3323855 .8418081 .839723$

H -2.0247337 .0269900 .607727$

H -0.5634194 .3462411 .092905$

H $0.3818941 .947448-1.837585$

C $2.361098-0.6150912 .922951$

O $1.027710-0.0945672 .747497$

C $0.084141-0.9931123 .369473$

C $0.856585-2.2713673 .655162$

C $2.239700-1.7334453 .956891$

H $-0.215693-0.5097254 .307707$

C $-1.125070-1.1656112 .442782$

H $0.430426-2.8436994 .484547$

H $\quad 0.882547-2.9199972 .771467$

C $3.332112-2.7845603 .856572$

H $2.238742-1.3101454 .970340$

C $-2.125461-2.2387202 .903408$

O $-1.792898 \quad 0.096522 \quad 2.343125$

H -0.766028 -1.394935 1.436339

H -1.635403 -3.218970 2.932165

O $-2.513549-1.9364664 .249121$

C $-3.404259-2.3291172 .048133$

C $-3.129232-2.5865690 .556573$

H -3.997163 -1.418240 2.179865

O $-4.204009-3.3998472 .563045$

H -2.517836 -1.774259 0.153697

H -2.543096 -3.508688 0.455535

H $3.160206-3.5878354 .580453$

H $3.365912-3.2340492 .858543$

H $4.314616-2.3487174 .062575$

C 3.2982600 .5110203 .366044

H -2.1543210 .3066803 .221281$

H $-3.152099-2.6159464 .526079$

H $-3.709127-4.2277402 .438007$

H 4.2493620 .0912333 .713031

H $2.854975 \quad 1.0607854 .205357$

H $2.675780-1.0201721 .952359$

C $-4.867708-0.378072-0.605379$

O $-5.318767-1.633063-0.079307$

C $-4.420252-2.727990-0.281776$ 
C $-4.177786-2.931160-1.782907$

C $-3.724450-1.642600-2.463855$

C $-4.662375-0.484549-2.121721$

H -3.926138 -0.090491-0.122622

C $-5.9259510 .669602-0.243363$

H $-4.939604-3.6227290 .083688$

O $-3.214080-3.958855-1.987930$

H -5.111841 -3.267863 -2.251242

H -2.690083 -1.405322 -2.194834

O $-3.734039-1.853242-3.877213$

H $-5.619976-0.636844-2.636048$

O $\quad-4.0801090 .712398-2.630272$

C $-6.0095780 .865341 \quad 1.252570$

H $-5.7216611 .645024-0.696821$

H $-6.917106 \quad 0.350789-0.588239$

H $-3.061347-4.027872-2.945980$

H -3.448028 -1.022025 -4.293884

H $-4.7598441 .406465-2.609494$

O -4.9014041 .5206541 .701621$

O

C $-4.853779 \begin{array}{lll}1.754592 & 3.112364\end{array}$

H -5.7036102 .3690203 .424325$

H $-4.8435790 .803223 \quad 3.652796$

H -3.9300792 .2948803 .335864$

SCF Energy (B3LYP/6-31G $\left.{ }^{* *} / / \mathrm{MMFF}\right)=-3245.91492335$

$02 \_177$

MM̄FF Geometry

C $0.360428-3.762841-0.209151$

C $0.445403-3.1922771 .002210$

C $-0.698796-2.4548811 .644613$

O $-0.226353-1.1166321 .923828$

C $-1.144846-3.1171182 .961341$

C $-1.764873-4.4859842 .788033$

C $-3.010898-4.6965142 .320792$

C $-0.899109-5.6472903 .199073$

C $-4.007808-3.6596961 .880909$

C $-4.284911-3.7710520 .376973$

C $-5.349064-2.767658-0.090182$

C $-5.745078-3.054548-1.540985$

C $-6.765895-2.036912-2.068204$

O $-4.817258-1.4392490 .022052$

O $-6.838223-2.183318-3.495191$

C $-6.234371-0.617016-1.786337$

C $-8.177386-2.307279-1.534647$

O $-7.207788 \quad 0.368093-2.125344$

C $-5.756219-0.410719-0.328875$

C $-5.0890890 .972162-0.125503$

O $-6.897832-0.444707 \quad 0.539249$

C -1.168916 -0.1431302 .044473$

O $-2.379240-0.277211 \quad 1.963758$

C $-0.467774 \quad 1.1327172 .300197$

C - -1.1842242 .2578492 .440173$

C -0.6515563 .5935232 .713408$

C 0.8438013 .7342632 .828903

C $-3.7927741 .212616-0.904367$

C $-3.3732782 .675571-0.880393$

C $-1.7512744 .211241-1.983748$

O $-4.0171363 .554661-0.313931$

C $-1.0520644 .882270-0.845000$

C $-1.4183926 .079857-0.363318$

C $-0.689441 \quad 6.8182960 .723210$

C $-1.519717 \quad 6.977251 \quad 1.976571$

C -2.447242 7.9444962 .076230

C -1.2160326 .0621043 .140838$

C $-1.518974 \quad 4.6201202 .838488$

N -2.205502 2.886503-1.602669

H $-0.581881-3.679654-0.748444$

H $\quad 1.366027-3.2499581 .574998$

H -1.536716 -2.387779 0.940709

H - $-1.853623-2.4656493 .488549$

H $-0.291275-3.1754273 .651296$

H $-3.365227 \quad-5.7236282 .244349$

H -1.399087 -6.6108583 .054084$

H $0.025434-5.6651612 .614116$

H $-0.637797-5.5671754 .259413$

H -4.937170 -3.825361 2.439506

H -3.679498 -2.647101 2.128043
H $-3.351915-3.599316-0.174671$

H $-4.618699-4.7909410 .146933$

H $-6.221721-2.8638140 .566731$

H -6.135989-4.074509-1.644504

H $-4.849958-3.010642-2.177611$

H -7.098725 -3.099985 -3.689638

H $-5.388921-0.462105-2.466684$

H -8.515949 -3.305549-1.837275

H $-8.901868-1.604278-1.960645$

H -8.243946 -2.254393 -0.446262

H $-7.4738870 .207990-3.047032$

H $-5.8232831 .756161-0.353337$

H $-4.858495 \quad 1.1015620 .941419$

H $-6.662539-0.0072141 .374144$

H $\quad 0.613420 \quad 1.108803 \quad 2.374377$

H -2.2686282 .1993092 .352695$

H 1.2119813 .1939903 .707919

H 1.1816684 .7683692 .919891

H 1.3389473 .3314781 .939050

H $-2.9829100 .605971-0.487983$

H $-3.9132340 .940799-1.956984$

H -2.615682 $4.786197-2.333838$

H -1.056135 $4.089426-2.820594$

H $-0.1870414 .379434-0.423531$

H $-2.277507 \quad 6.584172-0.800732$

H $-0.417574 \quad 7.809414 \quad 0.336431$

H $\quad 0.2647356 .3328810 .962188$

H -3.0283718 .0813792 .981958$

H -2.646611 8.6197781 .250586

H -1.817120 6.3440174 .015505

H -0.1790436 .2192623 .450543$

H -2.585166 4.4161352 .729379

H -1.769560 $2.094598-2.066237$

C $2.740151-4.791920-0.205999$

O $3.471889-3.557946-0.056383$

C $4.854753-3.795217-0.373214$

C $5.022352-5.308609-0.437486$

C $3.671317-5.735080-0.971361$

H $5.020644-3.383162-1.375494$

C $5.776120-3.1042450 .636004$

H $5.854452-5.606856-1.082585$

H $5.192368-5.7427560 .554615$

C $3.374532-7.209700-0.757618$

H $3.632039-5.512635-2.046283$

C $5.566993-1.5829780 .774415$

O $5.559586-3.6892121 .927015$

H $6.819408-3.319168 \quad 0.374187$

H $4.627124-1.3821251 .302257$

O $6.601576-1.0882611 .636372$

C $5.577884-0.794557-0.549577$

C $5.6984980 .727706-0.339624$

H 4.659461-1.021103-1.101320

O $6.668967-1.228454-1.364368$

H 4.9916401 .0395580 .435073

H 6.7067960 .9521610 .027567

H $4.116625-7.829405-1.271707$

H $3.395122-7.4731820 .305319$

H $2.387059-7.471561-1.150093$

C $1.424174-4.532695-0.944743$

H $6.156851-3.2431532 .551566$

H $7.456426-1.2593341 .206320$

H $7.495476-1.006547-0.903474$

H $0.979506-5.493577-1.229854$

H $1.633012-3.983984-1.872121$

H $2.550742-5.1834020 .801766$

C $3.1313042 .096029-1.317987$

O $4.1056321 .395906-2.103031$

C $5.4590431 .515356-1.647825$

C $5.8677782 .992662-1.587330$

C $4.8980203 .816089-0.748214$

C $3.4673813 .592541-1.233955$

H $3.0873211 .667721-0.309884$

C $1.7639271 .888457-1.988252$

H $6.0831451 .050552-2.421325$

O $7.1903403 .117940-1.075415$

H $5.8822123 .397139-2.608013$

H 5.0038533 .5847390 .318364

O $5.2398995 .195128-0.902148$ 
H $\quad 3.3357274 .063437-2.216741$

O $2.5641614 .223497-0.330233$

C $1.2009700 .500052-1.768675$

H $1.0322892 .590166-1.576447$

H $1.8504232 .045410-3.069704$

H $7.3978384 .067305-1.035399$

H $4.6886425 .704775-0.284765$

H $2.7016785 .183368-0.396000$

O $-0.0152460 .424489-2.388631$

O $1.742309-0.395733-1.136602$

C $-0.701252-0.824157-2.261997$

H $-1.618135-0.770060-2.855043$

H $-0.083114-1.641138-2.645773$

H $-0.971305-0.996197-1.216845$

SCF Energy (B3LYP/6-31G**//MMFF)= -3245.91202367

02178

MMFFF Geometry

C $1.8749103 .862642-1.266382$

C $2.6293614 .665088-0.499146$

C $3.7152294 .241145 \quad 0.465549$

$\begin{array}{lllll}\text { O } & 3.959957 & 2.817698 & 0.412636\end{array}$

C 3.2859234 .6012731 .900413

C $4.340745 \quad 4.2965252 .947058$

C $4.283798 \quad 3.2747193 .825040$

C $5.497455 \quad 5.2621252 .990580$

C 3.2262602 .2109963 .933776

C 3.8355650 .8100423 .788611

C $2.756747-0.2770123 .667439$

C $3.400154-1.6635083 .599946$

C $2.370827-2.7708133 .330253$

O $2.015981-0.0169212 .471275$

O $3.088108-3.9580672 .957570$

C $1.507899-2.3670132 .115629$

C $1.571622-3.1246214 .589812$

O $0.442431-3.2933481 .919553$

C $0.945599-0.9344912 .212464$

C $0.248569-0.471306 \quad 0.908885$

O $-0.034399-0.887186 \quad 3.256081$

C $4.8594652 .374660-0.509275$

O $5.4519633 .045262-1.339691$

C $5.0042440 .912334-0.330587$

C $5.7633580 .207304-1.182663$

C $6.031797-1.232001-1.116894$

C $5.365379-2.018652-0.019397$

C $1.158712-0.367017-0.318537$

C $1.145247-1.587847-1.221272$

C $2.222581-2.460242-3.295473$

O $0.399893-2.548233-1.052837$

C $3.344924-3.374081-2.917768$

C $4.422321-3.580231-3.690174$

C $5.523002-4.542063-3.348589$

C $6.891147-3.899830-3.367263$

C $7.701403-4.028804-4.431649$

C $7.356997-3.194731-2.114327$

C $6.867097-1.775134-2.027520$

N $2.053851-1.455730-2.263798$

H $2.0230482 .787022-1.239732$

H $2.4561305 .738854-0.551314$

H 4.6346904 .7836420 .212732

H 2.3466574 .0902492 .136796

H 3.0478205 .6724581 .959166

H 5.0908763 .1783714 .550542

H 6.1946605 .0403413 .805552

H 6.0644395 .2258562 .055323

H $5.1339306 .284183 \quad 3.139082$

H 2.7445562 .2962294 .915218

H 2.4392182 .3471353 .189426

H 4.4701700 .7894822 .893914

H 4.4809540 .6005954 .650848

H $2.085561-0.2047144 .531243$

H $3.961923-1.8756904 .518229$

H $4.140590-1.6794852 .789462$

H $3.694657-4.1822843 .684052$

H $2.151860-2.4376351 .230589$

H $2.242121-3.4836895 .379908$

H $0.871416-3.9448434 .396711$

H $1.011677-2.2790984 .994260$
H $\quad 0.838644-4.1780301 .841402$

H $-0.633440-1.0906910 .708694$

H $-0.1436530 .539612 \quad 1.092640$

H $-0.626449-0.134433 \quad 3.092232$

H 4.4917180 .4561380 .507709

H $6.2517010 .735045-2.000945$

H $5.758914-1.7163190 .956804$

H $5.506532-3.097887-0.107661$

H $4.282398-1.856417-0.028144$

H $0.8088320 .469437-0.934284$

H $2.189666-0.146106-0.029714$

H $2.416212-1.931852-4.234451$

H $1.297607-3.035518-3.404315$

H $3.250361-3.914740-1.978938$

H $4.501394-3.058568-4.641449$

H $5.486361-5.360658-4.079605$

H $5.354977-5.013815-2.372451$

H $8.697326-3.598329-4.439009$

H $7.384094-4.562441-5.321465$

H $8.455007-3.156653-2.097442$

H $7.093037-3.794306-1.238659$

H $7.298571-1.122342-2.787766$

H $2.668117-0.646790-2.287241$

C $-0.570867 \quad 3.798466-1.793227$

O $-0.6287312 .400844-2.141404$

C $-1.9357512 .103380-2.680533$

C $-2.780263 \quad 3.344537-2.434197$

C $-1.742128 \quad 4.444944-2.529219$

H $-1.7866691 .951771-3.756933$

C $-2.4597380 .820784-2.023959$

H $-3.5894273 .453578-3.162345$

H $-3.2236353 .322615-1.430699$

C $-2.2022115 .762328-1.929571$

H $-1.4845394 .597742-3.585895$

C $-3.9266810 .492796-2.349925$

O $-1.629208-0.262646-2.455094$

H $-2.3197330 .896390-0.940278$

H $-4.582587 \quad 1.289089-1.981084$

O $-4.0756380 .482984-3.775631$

C $-4.404807-0.871335-1.814997$

C $-4.184105-1.114390-0.309887$

H $-3.899469-1.671522-2.368952$

O $-5.791519-1.020486-2.125706$

H $-4.602270-2.098375-0.069245$

H $-3.106018-1.183968-0.130025$

H -3.088642 6.134174 -2.453971

H $-2.4595015 .653419-0.870608$

H - $1.4199816 .523433-2.009121$

C $0.7938784 .367603-2.184486$

H $-1.766382-0.376784-3.411285$

H $-5.0038080 .265423-3.968292$

H $-6.267916-0.282245-1.709510$

H $0.7641505 .462830-2.165208$

H $1.0503294 .062028-3.206057$

H $-0.7188303 .865029-0.706875$

C $-6.989771-1.0337420 .971033$

$\begin{array}{lllll}\text { O } & -6.217735 & 0.069656 & 0.479421\end{array}$

C $-4.800212-0.0552290 .634135$

C $-4.456232-0.2476062 .119228$

C $-5.215337-1.4199312 .734118$

C $-6.710795-1.2813322 .458768$

H $-6.754779-1.933768 \quad 0.390100$

C -8.469216 -0.6941150 .750944$

H $-4.3783380 .913957 \quad 0.344452$

O $-3.054816-0.4066212 .308112$

H -4.7364600 .6640782 .662596$

H $-4.837400-2.3829082 .371497$

O $-5.018925-1.4167454 .149552$

H -7.117552 $-0.458713 \quad 3.061091$

O $-7.373893-2.476672 \quad 2.866297$

C $-8.832643-0.771030-0.712339$

H $-9.130268-1.3918711 .277596$

H -8.7061330 .3099691 .123604$

H $-2.812104-1.3121842 .051537$

H $-4.060368-1.4608274 .308830$

H -7.162304 -2.620427 3.804758

O $-8.7566630 .466491-1.279702$

O $-9.124870-1.812256-1.286241$ 
C $-9.0713410 .525146-2.674544$

H $-8.948201 \quad 1.559067-3.007275$

H $-8.389089-0.110510-3.246845$

H -10.110099 $0.223903-2.839742$

SCF Energy (B3LYP/6-31G**//MMFF)= -3245.92418931

02179

MM̄FF Geometry

C $4.024549-0.223970-2.636717$

C $2.932575-0.564031-1.935976$

C $1.525692-0.502970-2.466423$

O $0.8922120 .589345-1.758132$

C $0.750639-1.806430-2.190382$

C $0.912467-2.858398-3.268810$

C $-0.075311-3.274592-4.085738$

C $2.266385-3.513816-3.357022$

C $-1.501706-2.801689-4.147202$

C $-2.474141-3.854762-3.595759$

C -2.406787 -3.993459-2.066412

C $-3.218366-5.200720-1.594589$

C $-3.232653-5.308231-0.062924$

O $-2.939578-2.792760-1.496505$

O $-4.238668-6.2644880 .303363$

C -3.664572 -3.9492390 .522047$

C $-1.904959-5.8397590 .490822$

O $-3.558146-3.9503151 .944571$

C $-2.892253-2.743003-0.064951$

C $-3.576500-1.4331720 .387165$

O $-1.550089-2.7692120 .417555$

C $-0.161306 \quad 1.202403-2.362242$

$\begin{array}{lllll}\text { O } & -0.715377 & 0.868518 & -3.396614\end{array}$

C $-0.4944522 .395935-1.554084$

C $-1.4531623 .240946-1.959101$

C -1.813389 $4.502172-1.308162$

C $-1.0584114 .896195-0.065101$

C $-3.012305-0.156837-0.250348$

C $-3.7914301 .047248 \quad 0.241900$

C -6.0359782 .1185530 .053893$

O -3.3557521 .7842251 .123375$

C $-5.8545563 .416069-0.666428$

C $-5.7347824 .589583-0.027779$

C $-5.6167945 .921903-0.710277$

C $-4.3199716 .623282-0.379461$

C -4.168635 7.2809200 .782957

C $-3.2283186 .633287-1.422383$

C $-2.7818705 .263407-1.858042$

N $-5.0286121 .167537-0.371665$

H $3.9181390 .122475-3.661308$

H $3.052357-0.886041-0.905648$

H $1.532609-0.272242-3.539885$

H $-0.307011-1.569641-2.039385$

H $1.057034-2.238640-1.228426$

H $\quad 0.155814-4.059656-4.805403$

H $2.259968-4.394689-4.008283$

H $3.008256-2.818507-3.758592$

H $2.591796-3.849151-2.367612$

H -1.648964 -1.841155 -3.646209

H -1.742393 -2.623516 -5.202953

H -3.490155 -3.555624 -3.884164

H -2.272761 -4.822829 -4.071302

H - $-1.362339-4.107665-1.758724$

H -2.833743 -6.127213 -2.039025

H $-4.254935-5.105958-1.947216$

H -4.014781 -7.111457 -0.119323

H -4.731784 -3.8210200 .292136$

H -1.688231 -6.8321780 .077705$

H -1.955549 -5.9723261 .577296$

H -1.053953 $-5.194716 \quad 0.262414$

H -4.065107 -4.712722 2.272416

H -4.644633 -1.491630 0.140066

H -3.508224 -1.335030 1.478033

H -1.181132 -1.8729430 .391148$

H $\quad 0.093114 \quad 2.571627-0.662425$

H -2.003186 $3.008284-2.869859$

H -1.172057 4.1320130 .712283

H $-1.406107 \quad 5.8354450 .371581$

H $0.0077235 .020855-0.284048$

H -1.960914 -0.0071760 .011944$
H -3.070668 -0.196829-1.343761

H $-5.975793 \quad 2.236709 \quad 1.141137$

H -7.012747 $1.691364-0.194259$

H $-5.8565703 .393323-1.753188$

H -5.7561874 .6010311 .060151$

H $-6.4611256 .545653-0.387995$

H $-5.7271845 .825297-1.797425$

H -3.2519757 .8109451 .019211$

H -4.9601127 .3018801 .525105$

H -2.363442 $7.220161-1.089579$

H $-3.6000497 .165879-2.307937$

H -3.291557 $4.898206-2.750130$

H $-5.2735340 .527861-1.121783$

C $5.8961110 .962814-1.363165$

O $5.1324771 .149432-0.152850$

C $4.7018032 .519835-0.096208$

C 5.638104 3.278439-1.028690

C $5.8013862 .270812-2.151694$

H $3.6906162 .547632-0.520893$

C 4.6630113 .0288821 .347484

H $5.2141864 .230118-1.362839$

H $6.6070603 .479466-0.557365$

C $7.0001602 .535056-3.045539$

H $4.8932372 .295588-2.766202$

C 3.8479932 .1520892 .323107

O $6.0032183 .100014 \quad 1.848896$

H 4.2811384 .0571551 .351711

H 4.4185911 .2493322 .572506

O 3.7212152 .8757033 .553939

C 2.4537451 .7362001 .822093

C 1.5685241 .1110732 .917807

H 2.5767251 .0261560 .997763

O 1.7721162 .8746321 .292705

H 2.1853770 .4850633 .567953

H $1.152178 \quad 1.9146213 .536193$

H $6.9051243 .508028-3.538590$

H $7.9346762 .538169-2.474650$

H $7.0812351 .767848-3.822190$

C $5.422150-0.301888-2.084939$

H 5.9546703 .4324922 .761612

H 3.2342043 .6965873 .370219

H 1.6383063 .5110882 .014930

H $5.481392-1.146383-1.387281$

H $6.103806-0.533351-2.911899$

H $6.930890 \quad 0.798106-1.035647$

C $1.316267-1.9376522 .657407$

O $0.895163-0.9375411 .718126$

C 0.4157480 .2839752 .300122

C $-0.752468-0.0072133 .250236$

C $-0.387989-1.0507874 .297918$

C $0.180032-2.2972663 .623334$

H $2.175797-1.5721983 .230304$

C $1.768369-3.1713791 .859402$

H $0.0043360 .875267 \quad 1.472498$

O -1.1955451 .1799373 .898199$

$\mathrm{H}-1.601862-0.3882032 .670660$

H $\quad 0.312200-0.6494455 .040045$

O $-1.565384-1.4294435 .014339$

H $-0.620567-2.8245573 .089616$

O $0.678375-3.1810954 .626316$

C $3.138687-2.9913571 .240593$

H $1.833942-4.0474982 .513968$

H $1.060128-3.3762921 .049545$

H -1.4527601 .8114573 .204536$

H -1.942395 -0.6193385 .398707$

H $-0.056287-3.3730235 .234088$

O $3.444848-4.1138150 .523690$

O $3.854139-2.0083051 .373674$

C $4.731052-4.118846-0.103309$

H $5.517923-3.8888020 .621692$

H $4.908741-5.119911-0.505030$

H $4.743677-3.402764-0.928838$

SCF Energy (B3LYP/6-31G**//MMFF)= -3245.92326205

$02 \_18$

MM̄MF Geometry

C -1.185821 -2.4859770 .835129$

C $-0.675928-1.9755771 .967299$ 
C -1.344864 -0.957366 2.864651 O $-2.727606-0.7966912 .475193$ C $-0.647426 \quad 0.4082852 .744943$ C $-0.9116831 .307843 \quad 3.935362$ C -1.8288912 .2939153 .971937$ C $-0.025868 \quad 1.058678 \quad 5.129433$ C -2.8176642 .6832802 .908483$ C -2.5518284 .0856772 .344913$ C -1.322185 4.1442211 .426302 C -1.0581145 .5798330 .970856$ C $0.1005755 .659119-0.033591$ O $\quad-1.5904893 .3184550 .289189$ O $0.0529846 .973378-0.610017$ C $-0.1404004 .628930-1.158264$ C 1.4602205 .5375320 .663147 O $0.9840784 .545396-2.030889$ C $-0.5045313 .216295-0.639421$ C $-0.9739922 .332963-1.820266$ O $0.6483162 .624520-0.037813$ C $-3.688827-0.819217 \quad 3.430524$ O $-3.522000-0.9452034 .635625$ C $-5.041964-0.6929532 .836420$ C $-5.264905-0.4294791 .538837$ C $-6.572743-0.3189870 .886660$ C $-7.797795-0.5710111 .725749$ C $-1.5959080 .994505-1.399483$ C $-2.0573810 .185640-2.600048$ C $-3.448631-1.806329-3.168382$ O $-1.770737 \quad 0.475604-3.758383$ C $-4.819134-1.284462-3.465518$ C $-5.951325-1.910135-3.110132$ C $-7.322625-1.362856-3.384768$ C $-8.119927-1.121128-2.120738$ C $-9.119490-1.947443-1.769163$ C $-7.821934 \quad 0.124513-1.313225$ C $-6.613463-0.026560-0.430556$ N $-2.813828-0.913116-2.217280$ H $-2.155831-2.1299040 .497372$ H $0.298654-2.3105682 .312417$ H -1.279504 -1.347755 3.887430 H -0.9587380 .9032881 .818210$ H $\quad 0.4398190 .2791792 .657118$ H -1.907027 2.8841984 .884455 H -0.2401981 .7423405 .957765$ H -0.1607800 .0386655 .502079$ H 1.0258461 .1927984 .855692 H -2.867362 1.9517812 .097334 H -3.8120792 .6801203 .372850$ H -3.439346 $4.383473 \quad 1.771315$ H -2.441938 $4.802963 \quad 3.167745$ H -0.4525383 .7519861 .964807$ H -0.8653956 .2328981 .831213$ H $-1.961586 \quad 5.9799910 .489117$ H $0.8082777 .066312-1.214732$ H $-0.9785075 .000006-1.764812$ H $1.5811406 .336351 \quad 1.404630$ H $2.2818475 .666474-0.049584$ H 1.5954834 .5835561 .177051 H $1.1203145 .423996-2.422939$ H -1.733083 2.877979-2.397139 H $-0.1331272 .139057-2.496770$ H $0.5861931 .659120-0.106049$ H $-5.857398-0.8295883 .539621$ H -4.412446 -0.2836010 .877551$ H -7.755851 -1.567637 2.179352 H $-8.730047-0.5299801 .158575$ H $-7.876697 \quad 0.1759482 .523211$ H $-0.874920 \quad 0.384371-0.847047$ H -2.462754 $1.171298-0.752199$ H -3.472335 -2.802473 -2.715540 H $-2.856923-1.852753-4.087978$ H -4.879273 -0.334640 -3.992124 H -5.893965 -2.863135 -2.589196 H -7.843666 -2.080643 -4.031654 H -7.273146 -0.430267 -3.960873 H $-9.717936-1.765983-0.882751$ H -9.359791 -2.826527 -2.357968 H $-8.6997410 .425063-0.730403$
H $-7.6516740 .966569-1.996687$

H $-5.6703320 .140710-0.950562$

H -3.077711 -1.008766 -1.241445

C $0.582184-4.3517350 .506584$

O $1.781219-3.5534790 .549825$

C $2.909065-4.3930950 .232585$

C $2.369617-5.8137100 .103056$

C $0.946679-5.562792-0.351814$

H $3.276448-4.070284-0.747916$

C $4.007473-4.2376671 .291052$

H $2.949070-6.411810-0.607185$

H $2.376905-6.3410451 .064283$

C $0.036093-6.763975-0.162178$

H $0.959864-5.280903-1.413241$

C $4.544633-2.8023971 .459278$

O $3.496283-4.6578712 .563355$

H $4.836359-4.9176001 .062371$

H $3.777643-2.1561321 .903168$

O $5.625146-2.8514622 .400026$

C $5.063271-2.1644860 .158750$

C $5.756579-0.8084740 .391136$

H $4.222241-2.027648-0.527226$

O $5.983801-3.050983-0.478153$

H $5.088294-0.1641630 .972085$

H $6.663266-0.9594770 .989174$

H $0.406379-7.620573-0.735024$

H $-0.020461-7.0624190 .890056$

H $-0.979813-6.545472-0.504911$

C $-0.580985-3.535073-0.061708$

H $2.719818-4.1072392 .763482$

H $5.280549-3.2537393 .215851$

H $\quad 6.715615-3.2097740 .142636$

H $-1.395387-4.220438-0.325444$

H $-0.268304-3.035870-0.986198$

H $\quad 0.362848-4.6710621 .534079$

C $4.0620870 .969920-1.415791$

O $5.0493720 .016341-1.826034$

C $6.159138-0.120725-0.932906$

C $6.8670331 .230728-0.761744$

C $5.8972602 .338690-0.359426$

C $4.6893002 .362383-1.295359$

H $3.6358730 .673155-0.450086$

C $2.9441790 .944289-2.465340$

H $6.869969-0.791050-1.432575$

O $7.906846 \quad 1.145310 \quad 0.206827$

H $7.337007 \quad 1.514507-1.712522$

H 5.5809342 .2351070 .685332

O $6.5607763 .601299-0.454475$

H $4.9957222 .728339-2.283634$

O $3.7205713 .271310-0.781226$

C $2.119943-0.320141-2.346820$

H $2.2564821 .787157-2.351119$

H $3.3807450 .994870-3.470239$

H $8.5185110 .446884-0.082359$

H 7.3462823 .5583290 .117607

H $4.1542164 .137121-0.691342$

O $1.920398-0.861415-3.582491$

O $1.690065-0.766029-1.290626$

C $1.167880-2.078326-3.607058$

H $1.654502-2.840740-2.990735$

H $\quad 0.146424-1.896772-3.261819$

H $1.130830-2.434551-4.639821$

SCF Energy (B3LYP/6-31G**//MMFF) $=-3245.92083209$

$02 \_180$

MM̄FF Geometry

C -0.752394 -2.839343 -0.020941

C $-1.556627-3.334377-0.974697$

C $-2.187648-2.563908-2.109859$

O $-1.554943-1.276358-2.287794$

C $-3.678657-2.320810-1.822415$

C $-4.457683-1.913403-3.056379$

C $-4.772183-0.645274-3.384838$

C $-4.925910-3.055694-3.920836$

C $-4.3751660 .620162-2.677478$

C $-5.569871 \quad 1.355140-2.055734$

C $-6.1201200 .674742-0.791781$

C $-7.323743 \quad 1.450997-0.251348$ 
C $-7.823574 \quad 0.884140 \quad 1.085405$ $\begin{array}{lllll}\text { O } & -5.073211 & 0.656579 & 0.182889\end{array}$ O C -6.6249950 .7434642 .046898$ C $-8.635766-0.4018650 .891641$ $\begin{array}{lllll}\text { O } & -7.015863 & 0.091008 & 3.253250\end{array}$ C -5.4135910 .0160621 .420148$ C $-4.184348 \quad 0.1385742 .352194$ O $-5.738513-1.3615511 .234774$ C $-0.472556-1.212376-3.104753$ O $0.052301-2.147436-3.694562$ C $0.0242590 .175548-3.249984$ C $-0.3835691 .204643-2.490591$ C $0.0671862 .594424-2.608487$ C $1.0430942 .924572-3.707097$ C $-2.919298-0.5314941 .803512$ C -1.678589 -0.0154502 .501249$ C $-0.030577 \quad 1.8383872 .444658$ O $-1.159258-0.6206363 .435469$ C $-0.034643 \quad 3.2609041 .986773$ C 0.9193783 .7933531 .209025 C 0.9022595 .2110620 .717185 C $1.0856305 .307029-0.782502$ C $2.2451255 .743798-1.302954$ C $-0.0932624 .980270-1.674147$ C $-0.4054653 .508012-1.734772$ N $-1.2452351 .195276 \quad 1.983124$ H $-0.523145-1.7775110 .000048$ H $-1.783330-4.398916-0.960748$ H -2.076881 -3.156117 -3.026847 H $-3.786761-1.558798-1.044505$ H -4.142890 -3.227188-1.410775 H $-5.360192-0.482028-4.287394$ H -5.498934 -2.713585 -4.789207 H -4.072244 -3.628906 -4.295527 H $-5.570438-3.727772-3.344710$ H -3.602046 $0.450013-1.923831$ H -3.916752 $1.278749-3.426374$ H $-5.2286792 .365248-1.792878$ H $-6.368410 \quad 1.467775-2.799559$ H -6.408146 - $0.354358-1.033756$ H -8.136092 $1.470920-0.988600$ H -7.038586 2.500879-0.093567 H -9.442389 2.0001751 .036392 H $-6.310413 \quad 1.7575742 .330871$ H $-9.512959-0.209244 \quad 0.262212$ H -9.027880 -0.7691331 .846356$ H $-8.063617-1.2038840 .420837$ H -7.7708430 .5842623 .617737$ H $-3.978231 \quad 1.2028412 .525448$ H $-4.404146-0.3094323 .329459$ H -4.919055 -1.871590 1.141674 H $\quad 0.7700800 .303149-4.028302$ H -1.114193 $1.022752-1.704502$ H $1.9627322 .342102-3.592452$ H $1.3403503 .975095-3.723991$ H $\quad 0.6066872 .703565-4.687425$ H -2.960814 -1.617304 1.942845 H -2.805278 -0.3523800 .729091$ H $\quad 0.814491 \quad 1.2701892 .042391$ H 0.0099581 .7858533 .537446 H -0.8693193 .8807632 .305412$ H $1.759123 \quad 3.1769410 .900244$ H 1.7076495 .7507351 .232010 H -0.0276375 .7211240 .997826$ H $2.3825795 .847060-2.373943$ H $3.0866466 .004057-0.669445$ H $\quad 0.0556675 .379863-2.684324$ H -0.979773 $5.509697-1.301065$ H -1.111560 $3.180254-0.971529$ H -1.6507561 .5155021 .107633$ C $1.392758-3.4816581 .099448$ O $1.974319-3.791726-0.185042$ C $3.149631-4.5851960 .023963$ C $3.496665-4.4534021 .499173$ C $2.104061-4.3921252 .102713$ H $2.846921-5.627522-0.152233$ C $4.253368-4.252022-0.980023$
H $4.090074-5.2941721 .870397$ H $4.042693-3.5260211 .704496$

C $2.075426-3.8992413 .536067$

H $1.664630-5.3985352 .068371$

C $4.922465-2.864162-0.896344$

O $5.276673-5.243903-0.814547$

H $3.851726-4.388656-1.992210$

H $5.557105-2.814505-0.003920$

O $5.831534-2.785250-2.008133$

C $3.997666-1.630583-0.924500$

C $4.780603-0.322260-1.158843$

H $3.443993-1.5617180 .016299$

O $3.052325-1.750086-1.988229$

H $5.656864-0.307061-0.504037$

H $5.144297-0.318600-2.193263$

H $2.622996-4.5862624 .189332$

H $2.538385-2.9145493 .627155$

H $1.048104-3.8270113 .906045$

C $-0.121270-3.6888921 .048390$

H $5.967893-5.060024-1.473602$

H $5.298027-2.764473-2.821324$

H $2.430282-2.459093-1.753379$

H $-0.565715-3.4260522 .014797$

H $-0.341471-4.7472720 .861384$

H $1.618888-2.4278361 .307461$

C $4.639127 \quad 1.5013251 .303726$

O 3.5599841 .1199150 .439820

C $3.9120570 .938366-0.937757$

C $4.5245712 .230932-1.496477$

C $5.7042202 .704393-0.655015$

C 5.3059292 .7948510 .816140

H 5.3758800 .6911541 .353452

C $4.069778 \quad 1.7057762 .716452$

H $2.9687710 .779636-1.474402$

O $4.9508962 .060771-2.844041$

H $3.7594553 .017196-1.500455$

H $6.5713952 .047238-0.786818$

O $6.1217823 .995722-1.105266$

H 4.6249993 .6441580 .957409

O 6.4697803 .0475871 .600756

C 3.5277120 .4309833 .320933

H 4.8456822 .0803043 .393077

H 3.2482642 .4290742 .678779

H $4.1913381 .731913-3.353846$

H $5.3668844 .602836-1.020987$

H 6.8862013 .8542281 .251370

O $2.841958 \quad 0.745764 \quad 4.460101$

O $3.692525-0.6915172 .865322$

C $2.252713-0.3557895 .156408$

H $1.493242-0.8320334 .530025$

H $3.021940-1.0757665 .451326$

H $1.7704350 .030717 \quad 6.058012$

SCF Energy (B3LYP/6-31G**//MMFF) $=-3245.92693329$

$02 \_181$

MM̄FF Geometry

C $1.045556-0.6061342 .849681$

C $2.298799-0.5799593 .329749$

C 3.3698330 .4545483 .089010

O $2.815167 \quad 1.6462742 .489840$

C $4.450065-0.1111962 .150756$

C 5.7797030 .6076892 .260340

C 6.2675591 .4721091 .349744

C 6.6050620 .2478533 .470161

C 5.6107291 .9668610 .091768

C $6.2666021 .394498-1.173504$

C $5.894811-0.073424-1.436842$

C $6.650295-0.616786-2.650920$

C $6.206754-2.044133-3.007418$

O $4.486851-0.119810-1.682016$

O $6.717749-2.351797-4.313351$

C $4.667027-2.078913-3.104869$

C $6.802964-3.084060-2.051495$

O $4.197100-3.412009-3.292523$

C $3.955348-1.433271-1.894040$

C $2.444694-1.279430-2.192392$

O $4.128440-2.267849-0.748059$

C 3.2217752 .8423902 .989608 
O 3.9696563 .0209803 .942593

C 2.6442083 .9851482 .248165

C 2.0234933 .8865271 .062550

C 1.4691385 .0052510 .292903

C $1.547996 \quad 6.3819780 .901225$

C $1.680102-0.458874-1.143657$

C $0.250605-0.178576-1.571684$

C $-1.7446201 .156178-0.891586$

O $-0.262295-0.676192-2.570208$

C $-1.7245692 .367629-1.765840$

C $-2.1401083 .579504-1.369649$

C -2.097806 $4.795204-2.248845$

C $-1.2076535 .884534-1.691929$

C -1.738475 $6.985503-1.133936$

C $0.2898605 .741936-1.864036$

C $0.9062724 .755294-0.907869$

N $-0.3856750 .689016-0.694455$

H $\quad 0.690841 \quad 0.1725342 .182702$

H $2.606742-1.3939153 .985730$

H 3.7895600 .6846834 .075620

H $4.081951-0.0824891 .120098$

H $4.631562-1.1725402 .368767$

H 7.2535731 .8997421 .529002

H 7.5824500 .7422153 .469378

H 6.0910030 .5406054 .390419

H $6.784309-0.8319693 .499814$

H 4.5323461 .7833210 .087160

H 5.7219743 .0586000 .076423

H $5.9291282 .003266-2.022560$

H $7.3566151 .501918-1.110564$

H $6.130066-0.669855-0.548806$

H $7.733966-0.581590-2.482798$

H $\quad 6.460914 \quad 0.030658-3.518786$

H $7.685779-2.263095-4.284963$

H $4.385368-1.521290-4.009162$

H $7.898550-3.049778-2.083863$

H $6.526695-4.100822-2.351844$

H $\quad 6.499676-2.936246-1.013028$

H $4.658062-3.775020-4.068209$

H $2.324168-0.777686-3.161583$

H $1.975254-2.267946-2.275871$

H $3.394861-2.120626-0.131208$

H 2.7957184 .9461602 .730082

H 1.9190412 .9060330 .601516

H $1.042198 \quad 6.4032201 .873077$

H 1.0766877 .1555790 .291590

H 2.5925416 .6816631 .039909

H $1.643080-0.995962-0.190831$

H $2.1749620 .504820-0.978114$

H $-2.163296 \quad 1.3649690 .097107$

H $-2.3367170 .371755-1.368721$

H -1.346621 2.239956 -2.777635

H -2.527685 $3.709351-0.361889$

H -3.126589 $5.161867-2.359882$

H -1.764475 $4.544183-3.263708$

H $-1.1128907 .789755-0.761628$

H -2.812006 7.107303 -1.034330

H $\quad 0.7850996 .716738-1.785586$

H $0.5035845 .413552-2.889643$

H $0.8966963 .727759-1.271135$

H $0.152936 \quad 1.1161270 .053289$

C $0.028566-2.8213432 .190738$

O $-0.985011-2.5487121 .197145$

C $-1.724746-3.7634870 .952318$

C $-0.927383-4.8851741 .605135$

C $-0.346584-4.1713842 .805104$

H -2.680108 -3.666406 1.479762

C - $-1.969975-3.978765-0.543707$

H $-1.555700-5.7397491 .873822$

H $-0.125426-5.2468140 .950618$

C $0.821577-4.908756 \quad 3.438243$

H -1.138659-4.037176 3.554309

C $-2.808568-2.884680-1.230024$

O $-0.705975-4.050665-1.216912$

H -2.446657 -4.954201-0.696655

H $-2.232453-1.957279-1.277256$

O $-3.010432-3.289445-2.590717$

C $-4.175635-2.597825-0.577818$
C $-5.037232-1.650721-1.435688$

H $-4.008530-2.1611060 .412322$

O $-4.867745-3.832539-0.399902$

H $-4.437836-0.772208-1.694701$

H $-5.297366-2.151472-2.376310$

H $0.504792-5.8933773 .797678$

H $1.636692-5.0589562 .722359$

H $1.222033-4.3516144 .290851$

C $0.076763-1.6952123 .227148$

H $-0.242881-3.209423-1.061990$

H $-3.561289-4.091053-2.579457$

H $-5.622828-3.6658550 .188872$

H $0.365924-2.0948274 .207108$

H $-0.913371-1.2449873 .356050$

H $0.982356-2.9055831 .652936$

C $\begin{array}{llll}-5.461988 & 0.575072 & 0.599548\end{array}$

O $-6.138616-0.6867420 .561061$

C $-6.352606-1.212310-0.752180$

C -7.189881 -0.224599-1.577688

C $-6.5729721 .173507-1.588576$

C $-6.2571871 .637401-0.166173$

H $-4.463900 \quad 0.4669580 .161580$

C -5.2955940 .9642242 .071302$

H $-6.960543-2.116607-0.625417$

O $-7.341948-0.702678-2.909748$

H $-8.195419-0.164988-1.141221$

H -5.675052 $1.201891-2.216215$

O $-7.4919662 .090075-2.188073$

H -7.1914781 .8633110 .363309$

O $-5.4962312 .841601-0.225070$

C -4.2592660 .0889602 .734199$

H -4.9571962 .0001042 .186273$

H -6.2439650 .8741082 .614646$

H -7.849902 $-0.035713-3.402791$

H -8.306716 $2.089982-1.657094$

H $-6.0062413 .483196-0.748709$

O $-4.867095-0.8829993 .471498$

O $-3.052760 \quad 0.2394902 .590693$

C $-3.985627-1.7889784 .141660$

H $-3.345586-1.2464734 .844021$

H $-4.593970-2.5042044 .701531$

H -3.381878 -2.336815 3.413492

SCF Energy $\left(B 3 L Y P / 6-31 G^{* *} / / M M F F\right)=-3245.90873919$

$02 \_182$

MM̄FF Geometry

C $-0.2735843 .049257-0.685880$

C $1.0111793 .156607-1.058471$

C $1.9625262 .008044-1.298588$

O $1.2528420 .752382-1.172950$

C $3.1154231 .979181-0.277551$

C $4.1683293 .044028-0.482790$

C $5.1428582 .964811-1.409740$

C 4.1033494 .2177040 .457365

C $5.3608111 .857516-2.404078$

C $6.7874261 .295213-2.366200$

C $7.1452320 .560833-1.062969$

C $8.6003660 .089283-1.122741$

C $8.992530-0.7258540 .114318$

O $6.273921-0.565011-0.921865$

O $10.245585-1.358520-0.189420$

C $7.939430-1.8321680 .335983$

C 9.2476820 .1833591 .322180

O $8.163702-2.5074961 .572092$

C $6.473898-1.3300330 .278736$

C $5.512435-2.5432990 .209212$

O $6.133619-0.5749261 .440906$

C $1.490290-0.232502-2.075012$

O $2.242469-0.182272-3.037537$

C $0.758576-1.469269-1.711397$

C $-0.272000-1.480047-0.851729$

C $-0.971104-2.661404-0.344303$

C $-0.499439-4.011735-0.812453$

C $4.025057-2.1771070 .305250$

C $3.528421-2.0188231 .734996$

C $1.563426-1.1945203 .031857$

O $4.140606-2.4490262 .709352$

C $0.601890-2.3298943 .203544$ 
C $-0.699895-2.1664683 .485702$

C $-1.666025-3.3063403 .653322$

C $-2.887971-3.1779922 .767665$

C $-4.079962-2.8314643 .281803$

C $-2.745737-3.5231321 .298034$

C -1.971081 -2.4779730 .542680$

N $2.297324-1.3812451 .792678$

H $-0.7076112 .067773-0.518777$

H $1.4150154 .150102-1.240063$

H $2.3488242 .112329-2.319583$

H $3.5994860 .995783-0.299592$

H 2.7107882 .0405680 .742401

H $5.8563023 .784708-1.478201$

H 4.8978034 .9472610 .266916

H 3.1475024 .7404090 .360851

H 4.2095423 .8778331 .492959

H $4.6456071 .040160-2.280470$

H $5.1819332 .272068-3.403967$

H $6.8838760 .596047-3.207312$

H $7.5041182 .107801-2.541010$

H $6.9976831 .238903-0.215412$

H $9.2811740 .940264-1.251659$

H $8.741323-0.542188-2.011716$

H $10.542790-1.8362730 .603173$

H $8.075717-2.579198-0.458756$

H $10.091106 \quad 0.8549291 .121230$

H $9.532453-0.3989422 .205204$

H $8.3869540 .804221 \quad 1.580631$

H $9.044061-2.9170671 .529557$

H $5.666373-3.050044-0.752618$

H $5.756250-3.2659930 .996563$

H $6.878117-0.6023272 .062404$

H $\quad 1.135610-2.375929-2.172485$

H $-0.618548-0.534423-0.437984$

H -1.115532 -4.837166 -0.449443

H $0.526901-4.197903-0.477659$

H - $0.529516-4.071561-1.906305$

H $3.812163-1.273215-0.271588$

H $3.436574-2.993261-0.132524$

H $\quad 1.055149-0.2273702 .967007$

H $2.263864-1.1712413 .872557$

H $0.998032-3.337393 \quad 3.098142$

H -1.093802 -1.160916 3.615293

H -1.966828 -3.3332974 .708659$

H -1.182604 -4.271452 3.456575

H -4.965845 -2.761403 2.659285

H -4.196624 -2.604296 4.336218

H -3.734392 -3.606733 0.832666

H -2.291463 -4.515585 1.229964

H -2.262928 -1.4544230 .784184$

H $1.842402-1.1034980 .929244$

C -1.844725 4.2183790 .879598

O $-2.7365563 .088953 \quad 1.001034$

C -3.9895693 .5413201 .541685$

C -3.7191934 .9258522 .117028$

C -2.7007095 .4607531 .132153$

H -4.6810323 .6394800 .696372$

C -4.541183 2.5342052 .554307

H -4.625195 5.5365192 .174475

H -3.2824394 .8713213 .120984$

C -1.9183186 .6536051 .654004$

H -3.2234005 .7514340 .210740$

C -4.7339481 .1005622 .021062$

O

H -5.4903332 .9080332 .956127$

H $-3.763493 \quad 0.607547 \quad 1.885629$

O

C $-5.542747 \quad 0.9909620 .714314$

C $-5.943322-0.4607030 .386904$

H $-4.9513101 .406245-0.107813$

$\begin{array}{lllll}\text { O } & -6.731140 & 1.777137 & 0.815614\end{array}$

H -5.063439 -1.102622 0.488701

H $-6.680490-0.7983341 .125078$

H -2.5913617 .4888581 .872986$

H -1.377747 6.4076222 .574187

$\mathrm{H}-1.1866616 .9945220 .914825$

C -1.199113 4.218762 -0.507113

H -2.7758402 .1931193 .335282$
H -4.8788720 .4146593 .849492$

H $-7.238976 \quad 1.446451 \quad 1.576465$

H $-0.6614225 .159637-0.670323$

H -1.977432 $4.144292-1.276441$

H -1.0816344 .1067251 .660733$

C $-4.553286-1.215191-2.226802$

O $-5.628969-0.282392-2.062057$

C $-6.561383-0.604910-1.023480$

C -7.186324 -1.979721-1.297816

C $-6.120498-3.057468-1.455135$

C $-5.082177-2.632292-2.490327$

H $-3.923770-1.209252-1.331111$

C $-3.700743-0.738230-3.413155$

H -7.369995 $0.132656-1.098004$

O $-8.079933-2.354741-0.255139$

H $-7.774135-1.934836-2.223898$

H -5.648769 -3.296605 -0.496328

O $-6.734639-4.265453-1.910500$

H -5.527108 -2.686033 -3.492372

O $-3.990310-3.550430-2.460460$

C $-2.9110440 .515103-3.103948$

H -2.975534 -1.508995 -3.696587

H $-4.351304-0.517522-4.268028$

H $-8.753116-1.656678-0.182425$

H -7.421236 -4.501819-1.263455

H $-4.355077-4.440211-2.606017$

O $-2.2647460 .911988-4.240895$

O $-2.8546831 .066936-2.014540$

C - $-1.4549082 .085314-4.123421$

H $-2.0105032 .898971-3.647678$

H $-0.5488901 .849777-3.559819$

H -1.168742 2.402237 -5.129887

SCF Energy $\left(B 3 L Y P / 6-31 G^{* *} / / M M F F\right)=-3245.92304274$

$02 \_183$

MM̄FF Geometry

C -0.3419450 .3444952 .585253$

C -1.1464401 .4045082 .409379$

C -0.9614352 .4459011 .333951$

O $-0.454415 \quad 1.7775290 .152752$

C 0.0272403 .5383701 .769333

C $0.1319394 .667013 \quad 0.762023$

C $1.0833414 .755605-0.189097$

C -0.9194485 .7378380 .888330$

C $2.1771243 .763848-0.476102$

C $3.5642684 .384618-0.276216$

C $4.7038843 .356345-0.193059$

C $4.7939792 .446566-1.420253$

C $5.9002981 .389468-1.267186$

O 4.4972282 .5770790 .990679

O $5.7078160 .398563-2.288957$

C $5.720140 \quad 0.6756390 .090673$

C $7.2958721 .979747-1.496985$

O $6.799414-0.2192220 .345097$

C 5.5509501 .6466361 .279073

C 5.1809320 .9351162 .604066

O $\quad 6.7886182 .3322331 .506907$

C -1.073691 $1.993923-1.032868$

O $-2.0120902 .750261-1.241037$

C $-0.4829251 .178249-2.118564$

C $0.5846640 .377206-1.973105$

C $1.198817-0.431411-3.030790$

C $0.530712-0.432544-4.382156$

C 3.9128110 .0761562 .550029

C $4.200076-1.3885982 .264611$

C $3.164052-3.4281951 .283127$

O $5.253067-1.9361162 .582632$

C $3.737226-3.546045-0.093246$

C $3.076601-4.085198-1.128089$

C $3.655211-4.222535-2.505169$

C $2.865899-3.460876-3.545263$

C $2.021040-4.098493-4.373024$

C $3.133098-1.980004-3.691774$

C $2.315998-1.135386-2.750029$

N $3.127471-2.0280971 .661475$

H $\quad 0.555390 \quad 0.2502381 .978161$

H -2.038349 1.5029803 .023223

H -1.9482092 .8795431 .129892$ 
H 1.0165433 .1010661 .949212 H $-0.280626 \quad 3.9583752 .736327$ H $1.0657555 .614854-0.858068$ $\mathrm{H}-0.796460 \quad 6.5315600 .143935$ H $-1.918498 \quad 5.3114880 .753144$ H -0.8700796 .2029831 .878174$ H 2.0756922 .8623340 .133156 H $2.0696533 .440488-1.518417$ H $3.7674345 .090683-1.091104$ H 3.5628714 .9623050 .657999 H $5.6347003 .924320-0.077745$ H $4.9460633 .037488-2.331775$ H $3.8451961 .912647-1.562113$ H $5.7315980 .848193-3.151058$ H $4.817137 \quad 0.0594290 .000885$ H $7.3728862 .396414-2.508523$ H $8.0675371 .204621-1.433340$ H $7.5474062 .776248-0.794044$ H $6.860009-0.823455-0.414628$ H $\quad 6.041378 \quad 0.365340 \quad 2.974904$ H $5.004323 \quad 1.7175013 .355972$ H 6.7944752 .6624292 .420659 H $-0.9862791 .285075-3.074581$ H $1.0677480 .309289-0.999931$ H $-0.514423-0.750555-4.293956$ H $0.995066-1.111960-5.099497$ H $\quad 0.5592580 .568663-4.826125$ H 3.4278280 .0975543 .534325 H $3.194417 \quad 0.479626 \quad 1.828513$ H $2.140575-3.8110911 .341245$ H $3.785644-3.9830911 .993116$ H $4.750628-3.179903-0.241730$ H $2.070790-4.468405-0.977649$ H $3.675049-5.291965-2.752777$ H $4.702768-3.897537-2.534281$ H $1.477440-3.566974-5.146411$ H $1.850727-5.167148-4.292956$ H $2.987725-1.669009-4.732201$ H $4.195867-1.781295-3.503242$ H $2.725078-1.079434-1.741040$ H $2.332926-1.4777121 .350001$ C $-0.656072-2.1064412 .715225$ O $-1.612755-2.017727 \quad 1.646110$ C $-2.142480-3.3296771 .383621$ C $-1.455243-4.2779682 .365378$ C -1.070831 -3.3428383 .500884$ H -3.211022 -3.2873701 .623254$ C $-1.954856-3.665275-0.103376$ H -2.118186 -5.087975 2.685262 H $-0.560783-4.7390001 .931090$ C $0.015223-3.8973014 .404807$ H -1.967591 -3.126664 4.096631 C $-2.445478-2.562338-1.066540$ O $-0.560023-3.862649-0.371405$ H -2.440956 -4.619536 -0.334529 H $-1.794283-1.683829-0.980774$ O $-2.292889-3.041418-2.408010$ C $-3.907226-2.125610-0.864614$ C $-4.311404-1.006584-1.841118$ H $-4.046649-1.7649600 .156424$ O $-4.783791-3.233986-1.055207$ H -3.584154 -0.191492 -1.761025 H -4.261582 -1.388509-2.867852 H $-0.315350-4.8284344 .876525$ H $0.932835-4.110418 \quad 3.846210$ H $0.262398-3.1858715 .198975$ C $-0.646540-0.8006113 .511033$ H $-0.095617-3.039039-0.142639$ H -1.359358 -3.289641 -2.522254 H -4.617816 -3.871163 -0.340467 H $0.113131-0.8402114 .298660$ H -1.620294 -0.648896 3.991075 H $\quad 0.324639-2.2639132 .250767$ C -5.2441341 .1552170 .131105$ O $-5.938052-0.043232-0.236473$ C $-5.740636-0.473018-1.588220$ C $-6.1781320 .631618-2.559021$ C $-5.4898991 .960443-2.256740$
C $-5.6572042 .319657-0.780603$

H -4.1616150 .9904560 .068048$

C $-5.604267 \quad 1.469943 \quad 1.593270$

H -6.422712 -1.319096-1.739846

O $\quad-5.9152180 .238390-3.901948$

H $-7.2640580 .769042-2.472511$

H $-4.4340561 .931895-2.548934$

O $-6.1051552 .968850-3.061575$

H $-6.7034502 .592500-0.590047$

O $-4.8559913 .457868-0.472594$

C -4.9974790 .4931862 .577514$

H -5.2417212 .4659421 .870553$

H -6.6932551 .4311261 .718044$

H $-6.1871770 .972880-4.478419$

H $-5.5918763 .786600-2.950137$

H $-5.2037974 .210241-0.979901$

$\begin{array}{lllll}\text { O } & -5.472922 & 0.773643 & 3.827562\end{array}$

O $-4.199890-0.3903172 .300379$

C -4.974364 -0.063056 4.876231

H -3.8970070 .0846014 .994124$

H -5.4721060 .2245945 .806185$

H -5.200698 -1.113244 4.667974

SCF Energy (B3LYP/6-31G**//MMFF) $=-3245.90671444$

$02 \_184$

MM̄FF Geometry

C -0.426843 -0.155434 -1.783777

C $-1.018936-1.344871-1.594314$

C $-0.743265-2.288443-0.448113$

O $-0.045874-1.5989360 .614090$

C $0.135334-3.458647-0.918279$

C $0.393278-4.472500 \quad 0.180429$

C $1.517137-4.5159110 .924048$

C $-0.699957-5.4861810 .390475$

C $2.678225-3.5622490 .859874$

C $3.919433-4.2258930 .252112$

C $4.986602-3.228848-0.228470$

C $5.496553-2.3086300 .882820$

C $6.498887-1.2712380 .351083$

O $4.409239-2.462081-1.291581$

O $6.676683-0.2688651 .363726$

C $5.876360-0.566485-0.873547$

C $7.882261-1.8818060 .102008$

O $6.8122310 .317110-1.484894$

C $5.311099-1.544463-1.925491$

C $4.523115-0.836127-3.056243$

O $6.395963-2.243513-2.547836$

C $-0.779674-1.0909001 .635756$

O $-1.993046-1.1621881 .773104$

C $0.081358-0.4303032 .643083$

C $1.352632-0.0640502 .414403$

C 2.2426550 .5957233 .373700

C 1.7304010 .8006124 .776078

C $3.323568-0.002136-2.592868$

C $3.670518 \quad 1.456890-2.350928$

C $3.0588723 .403167-0.925439$

O $4.5411242 .046816-2.986524$

C 4.0893023 .4132650 .159745

C 3.8671523 .8798551 .397388

C 4.9099543 .8854612 .478099

C 4.4546033 .1780523 .735515

C 4.0680783 .8802284 .814101

C $4.536271 \quad 1.6677623 .778230$

C 3.4665370 .9903132 .963371

N $2.8708802 .037171-1.376757$

H $0.347271 \quad 0.173293-1.096853$

H -1.776301-1.673234 -2.301831

H -1.701664 -2.674081 -0.079703

H $1.086110-3.078045-1.311161$

H $-0.340663-3.970120-1.765808$

H $1.612915-5.2982141 .675401$

H -0.452024 -6.208082 1.175874

H -1.631615 -4.990770 0.680807

H $-0.877758-6.049632-0.531325$

H $2.418920-2.6562460 .305989$

H $2.900372-3.2322651 .882103$

H $4.360638-4.9140810 .983648$

H $3.612183-4.828948-0.612991$ 
H $5.815294-3.819822-0.636578$ H $5.940068-2.8932851 .698088$ H $4.657444-1.7594011 .329372$ H $6.986906-0.7111142 .172507$ H $5.0596120 .057258-0.490927$ H $8.294532-2.2872381 .033775$ H $8.594824-1.120558-0.234288$ H $7.870151-2.691269-0.630555$ H $7.1292690 .925416-0.795541$ H $5.208579-0.248675-3.678972$ H $4.127197-1.619861-3.718156$ H $6.094061-2.578923-3.408166$ H $-0.408935-0.2317703 .590751$ H $1.788298-0.2513661 .434304$ H $\quad 0.8516151 .4550454 .772584$ H 2.4610681 .2612565 .443881 H $1.454648-0.1583465 .228999$ H $2.5674240 .000376-3.387770$ H $2.858500-0.445218-1.706191$ H $2.0870823 .771646-0.581933$ H $3.3945314 .021987-1.763618$ H $5.0727753 .020508-0.089354$ H 2.8914544 .2910301 .645591 H 5.1504854 .9331182 .701089 H 5.8486253 .4344672 .132316 H 3.7728683 .3885255 .734664 H 4.0361244 .9647734 .800553 H 4.5181401 .3034534 .812176 H 5.5169031 .3525443 .399991 H 3.7539650 .8057461 .927961 H $2.2425431 .450604-0.836607$ C -1.070102 2.191992 -2.329525 O $-2.1620862 .124962-1.396140$ C $-2.9946943 .287473-1.559327$ C $-2.2631284 .204264-2.534910$ C $-1.500643 \quad 3.205385-3.385950$ H -3.922717 $2.940783-2.028851$ C $-3.3081733 .901221-0.188070$ H $-2.9533104 .824552-3.115085$ H -1.563792 $4.874498-2.022356$ C $-0.3459803 .818711-4.158630$ H -2.201785 $2.739437-4.091326$ C -3.8003862 .8862300 .864575$ $\begin{array}{lllll}\text { O } & -2.120816 & 4.507939 & 0.341766\end{array}$ H $-4.0326334 .715115-0.302413$ H -2.9713342 .2272581 .152176$ O -4.1609533 .6121112 .046344$ C -5.0019852 .0251100 .435535$ C $-5.3825181 .007978 \quad 1.524749$ H $-4.7580441 .481319-0.478643$ O H -4.4884680 .4316001 .785797$ H -5.6963791 .5451402 .428152$ H $-0.7095254 .589025-4.846566$ H $\quad 0.3839694 .284131-3.487467$ H $\quad 0.1764373 .058827-4.748195$ C $-0.7806030 .798216-2.889767$ H -1.436621 3.8183970 .394199 H -3.388033 4.1410522 .308578 H $-5.919123 \quad 3.398921-0.610190$ H $\quad 0.0556900 .846047-3.595619$ H -1.656079 $0.428703-3.436260$ H $-0.1998442 .563785-1.772726$ C $-5.226889-1.559781-0.136608$ O $-6.287493-0.597639-0.141632$ C $-6.5265000 .054808 \quad 1.109691$ C $-6.874750-0.9900762 .177443$ C $-5.791467-2.0602282 .291467$ C $-5.477668-2.6502980 .916928$ H -4.273368 -1.0542890 .057268$ C $-5.165906-2.190327-1.538853$ H -7.4175510 .6780710 .962588$ O $-7.071367-0.3569773 .437496$ H -7.823646 -1.472390 1.908456 H $-4.891415-1.6622762 .774143$ O $-6.282582-3.094547 \quad 3.147807$ H $-6.311927-3.2902490 .600923$ O $-4.318156-3.4759331 .008980$
C $-4.719173-1.222904-2.613798$

H -4.459932 -3.028226 -1.548109

H $-6.162852-2.552016-1.818976$

H -7.265080 -1.055144 4.086332

H -5.553994 -3.719302 3.301505

H $-4.532117-4.2225841 .593042$

O $-4.804206-1.844877-3.827795$

O $-4.332288-0.079084-2.424675$

C $-4.403805-1.056192-4.952703$

H $-3.336038-0.827527-4.889587$

H $-4.584504-1.640441-5.858962$

H -4.994349-0.136419-5.005322

SCF Energy (B3LYP/6-31G**//MMFF) $=-3245.90880150$

$02 \_185$

MM̄FF Geometry

C $2.919484-0.3240393 .185487$

C $3.821531-1.3131133 .280412$

C $4.872597-1.6894992 .261330$

O $4.904813-0.7628051 .155686$

C $4.522472-3.0633601 .663920$

C $5.697543-3.7621571 .013002$

C $5.958772-3.770808-0.308600$

C $6.574444-4.5414281 .959912$

C $5.255310-3.021369-1.405730$

C $4.340988-3.909770-2.262142$

C $2.923753-4.038760-1.683330$

C $2.069124-4.983690-2.529189$

C $0.616323-5.035900-2.030795$

O $2.343576-2.729336-1.682929$

O $-0.173470-5.682968-3.040134$

C $0.083109-3.594485-1.894617$

C $0.476371-5.882594-0.760273$

O $-1.211425-3.583355-1.296915$

C $1.031264-2.651585-1.116880$

C $0.540889-1.189976-1.256647$

$\begin{array}{lllll}\text { O } & 1.027186 & -3.018431 & 0.263707\end{array}$

C $5.6753310 .349306 \quad 1.256417$

O $\quad 6.3478850 .7038802 .209516$

C $5.5273171 .077255-0.022845$

C $5.8932892 .363262-0.111446$

C $5.7686633 .207301-1.301998$

C $5.2601582 .561917-2.565784$

C $1.526719-0.131746-0.740253$

C $0.9812211 .277516-0.907678$

C $1.4723113 .641961-0.280578$

O $-0.0593851 .525389-1.511755$

C $2.1882804 .347313-1.389351$

C $2.8726865 .488795-1.215834$

C $3.5476656 .238628-2.329908$

C $4.9809126 .605160-2.011261$

C $5.3000467 .838833-1.584329$

C $6.0520515 .569552-2.270543$

C $6.0978834 .511951-1.202793$

N $1.7972172 .226776-0.306392$

H 2.9076320 .3256762 .315981

H $3.800295-1.9437094 .168003$

H $5.851832-1.7172682 .754360$

H $3.713028-2.9415320 .937059$

H $4.124104-3.7334152 .437891$

H $6.813000-4.352512-0.654400$

H $7.402269-5.0416191 .446124$

H $7.008998-3.8771042 .713270$

H $5.989603-5.3126742 .471933$

H $4.711768-2.154028-1.022417$

H $6.038348-2.607968-2.055003$

H $4.268666-3.444712-3.254268$

H $4.789399-4.901081-2.400711$

H $2.990554-4.412722-0.656657$

H $2.505051-5.990266-2.549210$

H $2.064947-4.638007-3.572504$

H $\quad 0.196853-6.570307-3.187224$

H $-0.041908-3.196718-2.911668$

H $\quad 0.813303-6.909625-0.945839$

H $-0.572166-5.962960-0.452942$

H $1.053808-5.4932880 .080840$

H - $1.781459-4.165213-1.828378$

H $0.354024-0.974892-2.317036$ 
H $-0.411776-1.070238-0.725253$

H $1.255539-2.2438450 .800902$

H $5.0909570 .535817-0.855056$

H 6.3039662 .8494690 .772344

H $5.9470551 .774564-2.895641$

H $5.1494153 .257470-3.399771$

H $4.2713812 .118284-2.405007$

H $1.733645-0.2923020 .320903$

H $2.472653-0.190394-1.290096$

H $1.759366 \quad 4.0220140 .704972$

H $\quad 0.3929803 .771338-0.405398$

H $2.1117103 .919513-2.386128$

H $2.9172935 .933388-0.223930$

H $2.9649387 .150760-2.515022$

H $3.5175335 .672751-3.269325$

H $6.3282738 .118658-1.380720$

H $4.5388318 .595186-1.423324$

H $7.0409626 .046944-2.295733$

H $5.9196175 .158728-3.275137$

H $6.4498554 .885913-0.240105$

H 2.6662031 .9315500 .128754

C $0.469815-0.3679083 .689669$

O $0.061284 \quad 0.671507 \quad 2.775587$

C -1.303427 1.0446423 .072394

C $-1.838496-0.0482843 .983226$

C $-0.600824-0.4066714 .778923$

H -1.2403391 .9952653 .617831$

C $-2.0723421 .238244 \quad 1.760265$

H $-2.666250 \quad 0.2929834 .611873$

H $-2.181518-0.9108973 .398349$

C $-0.707865-1.7424985 .494307$

H -0.4145920 .3842145 .518194$

C $-3.5893691 .436637 \quad 1.955882$

O -1.5039322 .3868321 .127372$

H $-1.886751 \quad 0.384317 \quad 1.097276$

H $-3.996931 \quad 0.5510872 .456573$

O -3.8193222 .5421742 .829680$

C -4.3787851 .6337750 .644732$

C -5.8961341 .7287640 .896960$

H $-4.167070 \quad 0.783665-0.012498$

O $-3.9445222 .806533-0.038407$

H -6.2135740 .8424511 .457141$

H $-6.1062412 .599470 \quad 1.530139$

H -1.533368 -1.7280546 .213585$

H $-0.890340-2.5614384 .790231$

H $0.212422-1.9684466 .041784$

C $1.868949-0.0635414 .229916$

H -1.8793122 .4452790 .232363$

H -3.357026 3.3122082 .456999

H -4.1736473 .5745090 .511768$

H $2.068252-0.6711135 .120303$

H 1.9409790 .9891354 .528608

H $0.469885-1.3079973 .121976$

C $-6.780678-0.460514-1.026347$

O $-6.383907 \quad 0.871625-1.371564$

C $-6.7210101 .868092-0.402601$

C $-8.2408881 .893064-0.188412$

C -8.7942820 .5105650 .153092$

C $-8.301741-0.531529-0.852755$

H $-6.281296-0.765501-0.098441$

C $-6.301368-1.387949-2.146891$

H -6.443862 $2.831995-0.847986$

O

H -8.721277 2.246033 -1.110380

H $-8.5370880 .228716 \quad 1.180743$

$\begin{array}{llll}0 & -10.221357 & 0.573188 & 0.100937\end{array}$

H -8.800829 -0.362615 -1.815162

O $-8.685909-1.830166-0.406494$

C $-4.796434-1.510266-2.111980$

H $-6.709830-2.399376-2.043295$

H $-6.606447-1.017421-3.132876$

H -9.541769 $2.792867 \quad 0.950934$

H $-10.556836-0.322666 \quad 0.277129$

H -8.239623 -1.999391 0.440824

O $-4.233867-0.601966-2.958957$

O $-4.195088-2.292131-1.386735$

C -2.804021 -0.565933 -2.958905

H $-2.397666-1.537840-3.254414$
H $-2.4815400 .184729-3.685265$

H $-2.438078-0.275859-1.969904$

SCF Energy (B3LYP/6-31G**//MMFF) $=-3245.92296402$

02186

MM̄FF Geometry

C $-0.654867-4.296086-0.150348$

C $-1.909698-3.870335-0.351694$

C $-2.464999-3.481339-1.695998$

O $-2.732798-2.061266-1.651127$

C $-3.784604-4.227891-1.950752$

C $-4.501341-3.765342-3.202897$

C $-5.549022-2.916493-3.201753$

C $-3.988832-4.347493-4.492765$

C $-6.153968-2.235054-2.004463$

C $-6.392733-0.739940-2.257760$

C $-6.833167-0.026886-0.970805$

C $-7.339678 \quad 1.383470-1.271893$

C -7.7154202 .1324340 .015536$

O $-5.6991340 .043239-0.096586$

O $\quad-7.9215403 .513743-0.316367$

C -6.5385292 .0433381 .009281$

C $-9.053874 \quad 1.6483940 .583423$

O -6.8971532 .5916212 .275871$

C $-5.9779590 .610840 \quad 1.189997$

C -4.6553830 .6803941 .989474$

O $-6.920479-0.1786141 .916753$

C $-1.733078-1.228465-2.050282$

O $-0.641485-1.544817-2.495490$

C $-2.1841890 .157403-1.799847$

C $-1.2815691 .149529-1.793001$

C -1.545226 $2.546834-1.447222$

C $-2.9799912 .969876-1.276003$

C $-3.861380-0.6306172 .052450$

C $-2.533213-0.3803192 .743132$

C -0.3196540 .7165372 .354461$

O $-2.376223-0.6075153 .940293$

C -0.4215342 .2116882 .316459$

C 0.4793853 .0140641 .727752

C 0.3327664 .5100021 .658511

C 0.5340555 .0728380 .264458

C $1.5835605 .865744-0.010076$

C $-0.5220084 .802165-0.791278$

C $-0.4956853 .378621-1.281463$

N $-1.5853150 .170507 \quad 1.894494$

H $0.025340-4.386253-0.993284$

H -2.571822 -3.7593390 .504596$

H -1.759827 -3.710960 -2.504746

H $-4.446777-4.130325-1.082489$

H -3.588579 -5.305969-2.029773

H $-6.020549-2.678740-4.154034$

H -4.559583 -4.001288 -5.360883

H -2.943514 -4.064205 -4.651537

H $-4.053921-5.440097-4.468920$

H -7.112522 -2.718008 -1.779342

H $-5.524655-2.345888-1.117463$

H $-5.469912-0.281528-2.635313$

H $-7.161389-0.626817-3.032356$

H $-7.622030-0.622060-0.496107$

H $-8.1931791 .355621-1.960652$

H $-6.5528101 .948030-1.791424$

H -7.101035 $3.858553-0.707832$

H -5.7331892 .6790810 .616621$

H $-9.8551451 .795538-0.150937$

H -9.3448632 .2330861 .463331$

H -9.0475530 .5921670 .860175$

H -7.234697 3.4900552 .118288

H -4.0090191 .4436911 .540869$

H -4.8608471 .0081853 .017064$

H $-6.452178-0.9128372 .344419$

H $-3.2288280 .313300-1.558638$

H $-0.2399840 .908150-2.001879$

H -3.585389 2.646537 -2.130626

H $-3.1063934 .053630-1.216905$

H $-3.4004182 .536041-0.365617$

H -4.399544 -1.400709 2.614522

H -3.672094 -1.034209 1.051858

H $\quad 0.4612280 .3386891 .687090$ 
H $-0.113508 \quad 0.378690 \quad 3.374363$ H -1.2942502 .6574942 .789306$ H 1.3668032 .5774461 .275043 H 1.0663854 .9444912 .350022 H $-0.651984 \quad 4.8272102 .024178$ H $1.7235676 .299070-0.994886$ H 2.3268056 .1025640 .743632 H $-0.3670705 .455023-1.659642$ H -1.491490 $5.080546-0.367154$ H $\quad 0.5065282 .993467-1.476252$ H -1.8412650 .3464000 .928488$ C $0.743645-3.5018481 .765860$ O $1.930913-3.3460150 .966221$ C $3.053039-3.0882561 .837400$ C $2.468176-2.8678323 .224924$ C $1.239400-3.7532403 .187711$ H $3.660800-4.0012901 .817290$ C $3.844782-1.8972741 .285596$ H $3.167286-3.1361584 .022610$ H $2.178564-1.8184583 .364374$ C $0.224071-3.4211454 .267188$ H $1.555180-4.7995123 .296621$ C $5.003311-1.4384482 .188137$ O $4.361773-2.2660060 .002763$ H $3.150887-1.0684301 .108313$ H $4.611383-1.0833573 .148323$ O $5.820229-2.5796202 .478711$ C $5.907798-0.3575051 .564629$ C $5.147272 \quad 0.9099411 .141443$ H $6.453498-0.7834370 .715950$ O $\quad \begin{array}{llll}6.900426 & 0.002155 & 2.532374\end{array}$ H $4.3628390 .644305 \quad 0.427715$ H 4.6491061 .3370672 .020784 H $0.660858-3.5613225 .261357$ H $-0.113371-2.3822784 .193202$ H $-0.655529-4.0676674 .190985$ C $-0.111743-4.6395731 .208453$ H $4.999524-2.9873750 .140068$ H $6.552765-2.2758923 .041992$ H 6.4436510 .3980093 .294222 H $-0.934744-4.8641371 .897013$ H $0.485548-5.5541891 .110006$ H $\quad 0.196372-2.551270 \quad 1.713744$

C $6.1354941 .104129-1.722647$

O $6.8662881 .468273-0.545497$

C 6.0654481 .9870280 .520816

C 5.3261973 .2456030 .048629

C $4.5279702 .991968-1.230090$

C $5.4010652 .322258-2.293292$

H $5.4159620 .312717-1.481745$

C $7.1415350 .539656-2.731208$

H 6.7650602 .2993341 .306358

O 4.4658563 .7195141 .078265

H $6.0635554 .035613-0.144073$

H $3.6350932 .391174-1.022650$

O $4.0442944 .236816-1.739874$

H $6.1245863 .048330-2.685239$

O $4.5786461 .907596-3.381549$

C $7.722956-0.765429-2.241299$

H $6.6893070 .364200-3.713575$

H $7.9763151 .235749-2.878807$

H 4.0210824 .5142960 .739306

H $4.8116814 .802335-1.931884$

H $4.1005512 .692410-3.700354$

O $6.812859-1.769304-2.394154$

O $8.847006-0.875074-1.768907$

C $7.237604-3.057249-1.937796$

H $6.419607-3.763390-2.103779$

H $8.111104-3.391232-2.505888$

H $7.460894-3.025190-0.866999$

SCF Energy (B3LYP/6-31G**//MMFF $)=-3245.91550973$

$02 \_187$

MM̄FF Geometry

C $0.096232-1.207293-1.135766$

C $0.4780890 .013102-0.726296$

C $\quad 0.206721 \quad 0.633420 \quad 0.625520$

O $-0.668735-0.2279921 .385296$
C $-0.484181 \quad 1.9959470 .446846$

C $-0.4281482 .870577 \quad 1.682118$

C -1.4642243 .0919962 .514426$

C 0.8926003 .5570081 .920852

C -2.8393732 .4854182 .465011$

C -3.9189823 .4974882 .056358$

$\begin{array}{llll}\text { C }-3.891727 & 3.845448 & 0.559717\end{array}$

C -4.9384654 .9117280 .233448$

C $-4.9876575 .213137-1.271885$

O $-4.1857572 .651071-0.168624$

O $-6.1833755 .964463-1.534042$

C $-5.1150003 .883997-2.049185$

C $-3.8168456 .103033-1.706168$

O $-4.9902314 .098599-3.452905$

C $-4.1223142 .787061-1.594248$

C $-4.5193841 .423454-2.200948$

O $-2.7925383 .055905-2.042772$

C $-0.440340-0.3993982 .710069$

O $\quad 0.446092 \quad 0.117433 \quad 3.375173$

C $-1.408723-1.3533213 .301489$

C $-2.502765-1.7917042 .658476$

C $-3.486233-2.7493363 .170006$

C $-3.229247-3.3660064 .519603$

C $-3.6402370 .263391-1.713842$

C $-4.069659-1.067425-2.307298$

C $-3.675581-3.518702-2.048536$

O $-4.882617-1.165367-3.222444$

C $-4.787627-4.030751-1.188261$

C $-4.645331-5.016248-0.288980$

C $-5.764781-5.5157710 .579475$

C $-5.470938-5.3680772 .057119$

C $-5.117452-6.4318642 .798020$

C $-5.673099-4.0101632 .694922$

C $-4.551017-3.0521772 .397858$

N -3.417255 -2.133065 -1.702418

H $-0.465484-1.857577-0.471359$

H $1.0681550 .621909-1.409421$

H $\quad \begin{array}{llll}1.173887 & 0.741754 & 1.129574\end{array}$

H -1.5224481 .8349660 .139868$

H $-0.0187032 .556862-0.374985$

H -1.3093083 .7676643 .354952$

H 0.8670514 .2169232 .794535

H 1.6849092 .8213902 .089332

H 1.1644184 .1690911 .054668

H -2.8795101 .5994321 .824501$

H -3.0690542 .1245613 .475830$

H -4.8934333 .0538252 .299243$

H -3.8199454 .4103722 .656744$

H $-2.890673 \quad 4.1994680 .290937$

H -4.750484 5.8303660 .803005

H -5.9305954 .5601550 .549480$

H -6.159555 $6.767639-0.985877$

H $-6.1348393 .509344-1.882440$

H $-3.8585677 .069534-1.189347$

H -3.869591 $6.334001-2.775641$

H $-2.8408265 .661522-1.493197$

H $-5.6473084 .770740-3.702759$

H $-5.560676 \quad 1.194709-1.938705$

H $-4.4602951 .472497-3.295658$

H $-2.7978313 .870303-2.569860$

H $-1.172019-1.6718284 .311423$

H -2.706924 -1.421511 1.655209

H -3.242396 -2.598427 5.301121

H $-2.255115-3.8676074 .535116$

H -3.967868 -4.119608 4.801035

H $-2.5963550 .434012-1.999007$

H -3.691217 $0.185166-0.621626$

H -2.743605 -4.072292 -1.896518

H $-3.957066-3.589268-3.103864$

H -5.761444 -3.562655 -1.313399

H $-3.675527-5.493524-0.169215$

H $-5.932491-6.5718220 .330448$

H $-6.708688-5.0064250 .348269$

H $-4.934969-6.3435243 .863706$

H $-4.998423-7.4149552 .354636$

H $-5.828694-4.1085023 .775152$

H $-6.610487-3.5742972 .325776$

H $-4.649648-2.5510181 .434827$ 
H $-2.828710-1.954052-0.894326$

C $1.574219-2.772413-2.396710$

O $2.770557-2.062455-2.022843$

C $3.846581-2.458631-2.890137$

C $3.382257-3.748531-3.551231$

C $1.898642-3.468266-3.718575$

H $3.932278-1.682392-3.660253$

C $5.153250-2.534862-2.094004$

H $3.894200-3.939605-4.498986$

H $3.531545-4.617464-2.900167$

C $1.066859-4.712139-3.976952$

H $1.765085-2.768741-4.554823$

C $5.423098-1.272307-1.243562$

O $5.080388-3.652048-1.200335$

H $5.990169-2.736616-2.772457$

H $4.666952-1.212465-0.457988$

O $6.674913-1.446938-0.570656$

C $5.4508220 .041926-2.041208$

C $5.7513151 .306940-1.212786$

H $4.4971110 .189045-2.557235$

O $6.446105-0.064819-3.065239$

H $6.5881221 .115100-0.533097$

H $6.1148042 .073579-1.908951$

H $1.379288-5.197177-4.907507$

H $1.172177-5.441153-3.166451$

H $0.005546-4.459254-4.066566$

C $0.423827-1.771197-2.491796$

H $5.903323-3.663881-0.682080$

H $7.357385-1.605152-1.244295$

H $7.318076-0.058554-2.635355$

H $-0.474269-2.252319-2.894641$

H $0.691580-0.957408-3.177611$

H $1.380551-3.510874-1.607829$

C $4.900763 \quad 0.8961651 .734274$

O 4.0524661 .0025050 .585239

C $4.524730 \quad 1.863207-0.453537$

C $4.731415 \quad 3.279377 \quad 0.104173$

C 5.6798653 .2726891 .297058

C $5.196527 \quad 2.2736792 .344017$

H $5.835150 \quad 0.397513 \quad 1.451957$

C 4.1759290 .0204962 .765056

H $3.7097211 .928939-1.185921$

O $5.2388684 .158936-0.893291$

H $3.766214 \quad 3.6881140 .429483$

H 6.7115833 .0602450 .993609

$\begin{array}{lllll}\text { O } & 5.694738 & 4.571122 & 1.894582\end{array}$

H 4.2958762 .6700562 .830718

O 6.1947742 .1318933 .353268

C $4.038808-1.4081052 .296557$

H $4.710458-0.0085653 .721314$

H 3.1777070 .4240202 .972501

H 4.610104 4.152841-1.635164

H 5.9607015 .2031121 .204657

H 6.3741343 .0186783 .710461

O $2.741149-1.6693041 .970298$

$\begin{array}{llll}\text { O } & 4.974312 & -2.194399 & 2.224173\end{array}$

C $2.465770-3.0053411 .540930$

H $3.026870-3.2350080 .631737$

H $2.710709-3.7163062 .335517$

H $1.397202-3.0789281 .322219$

SCF Energy (B3LYP/6-31G**//MMFF) $=-3245.91875196$

02_188

MM̄FF Geometry

C -1.246195 3.2638002 .051396

C -1.5922701 .9682891 .998047$

C $-1.755168 \quad 1.1151813 .230577$

O $-1.076018-0.1502743 .039026$

C $-3.231096 \quad 0.754647 \quad 3.487534$

C -4.1798261 .9306293 .590919$

$\begin{array}{llll}C & -5.061684 & 2.268492 & 2.628164\end{array}$

C -4.1182202 .7022404 .880896$

C -5.2037421 .6073091 .285455$

C -6.3241680 .5611831 .278399$

C $-6.181726-0.4067650 .094433$

C $-7.389945-1.3394580 .011309$

C $-7.221364-2.388129-1.097162$

O $-4.995780-1.175788 \quad 0.317813$
O $-8.224394-3.398432-0.906964$

C $-5.850984-3.080796-0.930575$

C $-7.473699-1.786489-2.484737$

O $-5.579214-3.944277-2.032141$

C $-4.668669-2.103562-0.725267$

C $-3.415108-2.877995-0.253720$

O $-4.316868-1.434296-1.935494$

C $0.278454-0.185732 \quad 3.130185$

$\begin{array}{llllll}\text { O } & 1.033637 & 0.753509 & 3.316481\end{array}$

C $0.680527-1.6007892 .959843$

C $1.966523-1.9150952 .749848$

C $2.504012-3.2652312 .559526$

C $1.550182-4.4235112 .701816$

C $-2.180783-1.988895-0.035320$

C $-1.163367-2.187566-1.143973$

C $1.100930-3.075163-1.621040$

O $-1.385016-1.823420-2.296990$

C $1.958975-4.175087-1.084609$

C $3.292438-4.080660-0.965486$

C $4.165325-5.197401-0.467626$

C $5.123499-4.7612490 .619039$

C $6.422181-4.5486990 .347419$

C $4.597516-4.6627962 .034249$

C $3.814758-3.4014292 .268758$

N $-0.014332-2.840670-0.722997$

H -1.0429423 .6970843 .029595$

H -1.8109581 .4943051 .046184$

H -1.3399541 .6038894 .121233$

H -3.2914540 .1713524 .417246$

H -3.5671790 .0406752 .724489$

H -5.7311513 .1089362 .801603$

H -4.849940 3.5163794 .912646

H -4.322494 2.0409565 .729166

H -3.1264003 .1450195 .015693$

H -5.4198102 .3789700 .535840$

H $-4.254042 \quad 1.1642280 .971820$

H $-6.293275-0.0147912 .212809$

H $-7.294179 \begin{array}{lll}1.071768 & 1.238764\end{array}$

H $-6.0713400 .173762-0.828972$

H -8.314366 $-0.767529-0.137656$

H $-7.513630-1.8646820 .968838$

H $-9.094770-2.964829-0.926562$

H $-5.919083-3.724120-0.041904$

H $-8.501632-1.410979-2.557650$

H -7.376261 - $2.544672-3.269317$

H $-6.805426-0.955113-2.719064$

H $-6.330965-4.556060-2.113037$

H -3.648459 -3.359051 0.705423

H $-3.175336-3.674637-0.968604$

H $-4.871904-1.773846-2.655167$

H $-0.102670-2.3496163 .002081$

H $2.694346-1.1075242 .702058$

H $1.130249-4.4494673 .713518$

H $2.011915-5.3967832 .526606$

H $0.726832-4.3420091 .984851$

H $-2.426725-0.9227760 .013317$

H -1.728476 -2.246191 0.928816

H $1.650285-2.134057-1.711983$

H $0.712898-3.345521-2.608534$

H $1.463566-5.101990-0.804799$

H $3.789181-3.164387-1.276233$

H $4.728323-5.584053-1.327357$

H $3.566068-6.040627-0.101963$

H $7.123434-4.2658271 .125387$

H $6.812243-4.645219-0.660431$

H $5.433554-4.6634472 .746654$

H $4.033382-5.5692392 .268267$

H $4.423849-2.4999322 .196078$

H $0.155579-2.9288800 .273887$

C -1.493854 $3.730308-0.488060$

O $-0.4586722 .841416-0.953953$

C $-0.1725313 .142646-2.332610$

C $-1.2758704 .083097-2.800849$

C $-1.5742014 .848563-1.528540$

H $0.7785253 .686759-2.347122$

C $-0.048792 \quad 1.855034-3.151015$

H $-0.9511594 .725944-3.624744$

H - $-2.1655713 .534863-3.131798$ 
C $-2.9089395 .573492-1.558875$ H $-0.7710555 .578670-1.360064$ C $1.0059130 .858355-2.630965$ O $-1.3137921 .180331-3.157751$ H $0.1617052 .105305-4.197403$ H $0.6782080 .415725-1.682693$

O $1.079515-0.214863-3.578893$ C $2.417698 \quad 1.447634-2.458703$ C $3.4617860 .382910-2.067052$ H $2.3911842 .221610-1.685418$ O $2.8220872 .079038-3.673075$ H $3.128591-0.113444-1.149046$ H $3.511885-0.384075-2.849060$ H -2.928924 $6.307853-2.370873$ H $-3.7402884 .877557-1.714489$ H -3.087438 $6.105612-0.619472$ C $-1.146570 \quad 4.2430120 .911679$ H - $-1.5467620 .982521-2.234205$ H $0.187678-0.596615-3.652557$ H $2.8197391 .401221-4.370577$ H -1.809897 5.0802461 .159808 H -0.1231144 .6351180 .909077$ H -2.432789 $3.162191-0.475815$

C 4.6702241 .7385220 .395736

O $4.8930402 .078839-0.977797$

C $4.8796370 .969588-1.881758$

C $5.946438-0.058468-1.477137$

C $5.828998-0.463026-0.009914$

C $5.757213 \quad 0.775056 \quad 0.882714$

H $3.681288 \quad 1.2766870 .505842$

C 4.6834763 .0408341 .200767

H $5.1817151 .370541-2.857715$

O $5.862160-1.210456-2.309619$

H $6.9387880 .382066-1.640767$

H $4.966976-1.1214650 .145499$

O $6.987399-1.2222750 .340151$

H $6.734403 \quad 1.2737810 .881605$

O $5.497944 \quad 0.3717702 .224640$

C 3.4352943 .8424570 .919803

H 4.7074262 .8580202 .280741

H 5.5658653 .6438210 .954261

H $6.530959-1.844263-1.998256$

H $6.912145-1.4456311 .283864$

H $4.613303-0.0295052 .248903$

O $3.740186 \quad 4.941767 \quad 0.174295$

O 2.3196093 .5151601 .302934

C $2.6319915 .778073-0.171247$

H $3.0111796 .621726-0.753958$

H $1.9164005 .222703-0.783632$

H 2.1505896 .1635410 .732480

SCF Energy $\left(B 3 L Y P / 6-31 G^{* *} / / M M F F\right)=-3245.92085413$

02189

MM̄FF Geometry

C $0.311039-3.1150920 .037503$

C $0.822159-2.9179481 .263363$

C $0.224511-2.0612772 .357111$

O $-1.095366-1.5845182 .009729$

C $1.115413-0.8280092 .599581$

C $0.6141780 .032648 \quad 3.744897$

C -0.1142371 .1574213 .593174$

C $0.974240-0.4580845 .122749$

C $-0.5498841 .774342 \quad 2.292892$

C -0.7243873 .2959402 .405415$

C -0.8612203 .9559441 .025988$

C -1.1385055 .4542361 .155163$

C -1.333299 $6.113650-0.220023$

O $-1.926610 \quad 3.321524 \quad 0.311694$

O $-1.8732947 .427494-0.012946$

C $-2.3574575 .292939-1.036175$

C $0.0071196 .335788-0.930185$

O $-2.4507765 .778716-2.373311$

C $-2.0663253 .773520-1.042229$

C $-3.2419913 .009077-1.694935$

O $-0.8841913 .529239-1.809088$

C $-2.152872-2.3772802 .329945$

O $-2.112917-3.4987172 .809949$

C $-3.386740-1.6284821 .999725$
C $-4.571439-2.2535072 .063855$

C $-5.884198-1.6600251 .799124$

C $-5.944912-0.1730261 .569820$

C $-3.2033671 .489597-1.471538$

C $-4.2540950 .766786-2.297979$

C $-5.299606-1.477041-2.589870$

O $-4.896058 \quad 1.311318-3.192594$

C $-6.663612-1.323664-1.991647$

C $-7.405227-2.349835-1.547340$

C $-8.796553-2.189868-1.002886$

C $-8.981384-2.8295810 .354816$

C $-9.701343-3.9554130 .497041$

C $-8.405656-2.1163921 .558965$

C $-6.962349-2.4717911 .792270$

N $-4.372673-0.569565-1.937449$

H $-0.624700-2.625226-0.222753$

H $1.758163-3.3993921 .534400$

H $0.169414-2.6595153 .275219$

H $1.180856-0.2418291 .676655$

H $2.145643-1.1398352 .817876$

H $-0.451144 \quad 1.673910 \quad 4.490757$

H 0.6141110 .2122565 .910273

H $0.539291-1.4453135 .306301$

H $2.061787-0.5317135 .225834$

H $\quad 0.173518 \quad 1.564471 \quad 1.501150$

H -1.5056431 .3264431 .997347$

H -1.621908 3.4973263 .004636

H $0.127713 \quad 3.7411002 .934193$

H 0.0774813 .7940640 .486135

H -0.3364045 .9551071 .711589$

H $-2.0521775 .602937 \quad 1.747339$

H $-2.717728 \quad 7.3382860 .460904$

H $-3.3447345 .450473-0.580051$

H $0.6506436 .992247-0.331747$

H $-0.1329446 .848374-1.888525$

H $0.5580035 .410598-1.111886$

H -2.640048 $6.731314-2.323415$

H $-4.1923943 .372135-1.281987$

H -3.255372 $3.220292-2.771836$

H -0.915495 2.628957 -2.169097

H $-3.287116-0.5859091 .720519$

H $-4.585674-3.3088452 .334570$

H $-6.954903 \quad 0.203790 \quad 1.398645$

H -5.3553950 .1087230 .692741$

H -5.5516290 .3642182 .440151$

H $-2.2293001 .076365-1.750296$

H -3.375867 $1.267988-0.412232$

H -4.904918 -2.490769 -2.470341

H $-5.338161-1.237049-3.657448$

H -7.077698 $-0.318528-1.952362$

H -7.010166 -3.360918 -1.612876

H -9.487367 -2.639913 -1.727883

H $-9.080749-1.131881-0.939929$

H $-9.861578-4.4033901 .472165$

H -10.141831 -4.457138 -0.358218

H -8.961092 -2.404015 2.461754

H -8.577461 -1.042247 1.454533

H $-6.806858-3.5343271 .987189$

H $-3.850205-0.917586-1.138724$

C $2.253264-4.603515-0.842521$

O $3.259389-3.601479-0.607526$

C $4.472266-3.979731-1.281737$

C $4.247871-5.393496-1.808188$

C $2.751510-5.398636-2.050112$

H $4.581234-3.297317-2.132801$

C $5.671827-3.829339-0.338307$

H $4.830512-5.594355-2.712565$

H $4.512856-6.155015-1.065983$

C $2.160342-6.793180-2.163354$

H $2.546432-4.844454-2.976022$

C $5.783394-2.456490 \quad 0.355171$

O $5.582824-4.8181070 .698136$

H $6.595955-4.052302-0.883934$

H $4.989060-2.3575961 .105718$

O $7.023537-2.4647401 .074926$

C $5.751225-1.246297-0.597800$

C 5.9980840 .0868220 .131524

H $4.778239-1.209164-1.095016$ 
O $6.736526-1.409427-1.617064$

H $\quad \begin{array}{llll}5.331710 & 0.139702 & 0.998804\end{array}$

H 7.0278300 .1045110 .507561

H $2.611046-7.334826-3.001513$

H $2.333785-7.377581-1.253488$

H $1.080344-6.747679-2.333725$

C $0.896705-3.935421-1.084157$

H $4.737461-4.6837081 .160134$

H $6.983960-1.7566711 .739648$

H $7.600759-1.496931-1.179578$

H $0.158901-4.702044-1.348784$

H $0.985487-3.265481-1.948407$

H $2.214184-5.2434730 .048351$

C $3.4293081 .651863-0.418749$

O $4.487104 \quad 1.380873-1.346097$

C $5.798998 \quad 1.321398-0.776874$

C $6.1274352 .655037-0.091483$

C 5.0740853 .0228260 .948430

$\begin{array}{llll}C & 3.677380 & 2.967611 & 0.334443\end{array}$

H 3.3391920 .8209340 .291467

C $2.1249871 .746197-1.226158$

H $6.4930221 .212412-1.619537$

O 7.4052712 .6088590 .534329

H $6.166008 \quad 3.452947-0.844342$

H 5.1400032 .3813571 .835022

$\begin{array}{lllll}\text { O } & 5.307540 & 4.358072 & 1.403342\end{array}$

H $3.5463673 .819519-0.345449$

O 2.7085403 .1001771 .372900

C $1.6633230 .413979-1.773348$

H $1.3152792 .123668-0.595753$

H $2.2686292 .422596-2.077394$

H $8.0580012 .386655-0.151473$

H $6.214528 \quad 4.389274 \quad 1.753407$

H $28.890825 \quad 3.9359211 .835777$

O $0.5808800 .626363-2.580073$

O $2.168244-0.669770-1.519260$

C $0.007222-0.540643-3.177138$

H $0.774431-1.142833-3.673153$

H $-0.514272-1.126116-2.415330$

H - $0.719525-0.215396-3.926522$

SCF Energy (B3LYP/6-31G**//MMFF) $=-3245.92024391$

02_19

MMFFF Geometry

C $1.671036-3.810531-0.283243$

C $1.763128-4.260010 \quad 0.978101$

C $2.452557-3.5463582 .118418$

O $2.560232-2.1262771 .865731$

C $3.866129-4.1264782 .301413$

C $4.646218-3.4863683 .432506$

C $5.636757-2.5862093 .268192$

C $4.268264-3.9518364 .814655$

C $6.135299-1.9936361 .977945$

C $6.037635-0.4626332 .001787$

C 6.3237790 .1587180 .625633

$\begin{array}{llll}\text { C } & 6.290587 & 1.685888 & 0.715974\end{array}$

C $6.4242592 .356815-0.658485$

O $5.318489-0.306061-0.282109$

O $6.0434893 .734341-0.515675$

C $5.4156281 .711072-1.632650$

C $7.8760352 .357704-1.151483$

O $5.6087812 .198678-2.957947$

C $5.4602230 .169646-1.627780$

C $4.331555-0.472317-2.474238$

O $6.703414-0.261786-2.195838$

C $1.460361-1.3664742 .133763$

O $0.370015-1.7604782 .516200$

C 1.8195640 .0484821 .891519

C $0.913274 \quad 1.0169352 .093081$

C 1.1431062 .4563231 .941327

C 2.4930842 .8989251 .441930

C $2.902657-0.212475-1.987264$

C $2.2214990 .988608-2.618897$

C $0.133742 \quad 2.342879-2.432505$

O $2.683028 \quad 1.601642-3.576988$

C $0.4269953 .570579-1.629342$

C $-0.5082774 .245716-0.944059$

C $-0.2363995 .508606-0.178948$
C $-0.718603 \quad 5.446194 \quad 1.253056$

C $-1.876364 \quad 6.0222651 .618107$

C $0.191273 \quad 4.814303 \quad 2.280508$

C 0.1502953 .3113302 .265749

N $1.007077 \quad 1.267040-2.003557$

H $2.138473-2.868086-0.552865$

H $1.314625-5.2190851 .228194$

H $1.870716-3.7013983 .035746$

H $4.420935-4.0442391 .360494$

H $3.800324-5.2062852 .493890$

H $6.146887-2.2182634 .157672$

H $4.890645-3.4961055 .592096$

H $3.227369-3.6953205 .034768$

H $4.384349-5.0376104 .894804$

H $7.182398-2.2882971 .839062$

H $5.585902-2.3805561 .117305$

H $5.029248-0.1759412 .324177$

H $6.741233-0.0636692 .743302$

H $7.306618-0.1854280 .282900$

H 7.0675302 .0509541 .399299

H $5.3369032 .000244 \quad 1.158889$

H 6.6218104 .1372490 .154519

H $4.4166102 .038191-1.320070$

H $8.5165612 .910824-0.453804$

H $7.9670332 .873792-2.113508$

H $8.2943811 .354479-1.255268$

H $5.5513053 .168880-2.921113$

H $4.452351-0.205498-3.531105$

H $4.478187-1.561400-2.432542$

H $6.603350-1.179154-2.499542$

H $2.8328550 .258676 \quad 1.571548$

H $-0.085543 \quad 0.735617 \quad 2.424112$

H 3.2621992 .6896982 .192737

H 2.5453683 .9641821 .207502

H 2.7529152 .3776560 .514326

H $2.287634-1.078717-2.261924$

H $2.869859-0.120785-0.898309$

H $-0.8966321 .997312-2.300218$

H $0.2998862 .548033-3.494897$

H $1.4508793 .937009-1.636080$

H $-1.5360823 .892797-0.964504$

H $-0.7412076 .329047-0.705872$

H $0.8307055 .763062-0.191910$

H $-2.213027 \quad 6.012462 \quad 2.649402$

H -2.5163796 .5127230 .892120$

H $-0.108727 \quad 5.1302793 .289122$

H $1.202453 \quad 5.213962 \quad 2.161397$

H -0.7983902 .8988392 .611716$

H $\quad 0.733610 \quad 0.736772-1.181190$

C $-0.135013-3.680343-2.011753$

O $-1.155816-3.401954-1.034109$

C $-2.451603-3.551111-1.651310$

C $-2.193291-3.663372-3.146209$

C $-0.851221-4.365503-3.174185$

H $-2.860891-4.489925-1.257941$

C $-3.335050-2.365728-1.245751$

H $-2.978629-4.217937-3.668261$

H -2.114618 -2.669375 -3.604539

C $-0.129103-4.242477-4.504546$

H $-1.007603-5.428600-2.946476$

C $-4.719941-2.366179-1.916100$

O $-3.512436-2.4082760 .173473$

H $-2.806715-1.431675-1.467527$

H $-4.608696-2.260918-3.001650$

O $-5.324817-3.646498-1.692236$

C $-5.688038-1.289301-1.387862$

C $-5.1527240 .144946-1.533477$

H $-5.940967-1.505018-0.344332$

O $-6.913445-1.397685-2.122206$

H $-4.1955490 .233985-1.012716$

H $-4.9610350 .347453-2.594446$

H $-0.723070-4.691280-5.307406$

H $0.053319-3.194870-4.766663$

H $0.837561-4.754829-4.473152$

C $0.959199-4.545128-1.383907$

H -2.627416 -2.4141730 .577020$

H $-6.202814-3.629739-2.110315$

H $-6.719447-1.205592-3.055494$ 
H $1.697888-4.825206-2.143153$ H $0.519514-5.471313-0.993411$ H $0.274674-2.712096-2.328044$ C $-5.502143 \quad 1.101477 \quad 1.328281$ O -6.5381090 .9430990 .351388$ C $-6.1365491 .209060-0.996332$ C -5.629161 2.652729-1.110641 C $-4.5361912 .956501-0.089200$ C $-4.971507 \quad 2.5390341 .315269$ H -4.6857390 .4000121 .119131$ C -6.0987490 .7409872 .692739$ H -7.045895 $1.139151-1.606112$ O $-5.131305 \quad 2.917571-2.418007$ H $-6.4640253 .344341-0.937897$ H $-3.5877432 .478724-0.361722$ O $-4.2897214 .363945-0.078736$ H -5.7418973 .2302551 .680259$ O -3.8560442 .6348632 .198627$ C $-6.428007-0.7313782 .763724$ H $-5.4186390 .978081 \quad 3.518447$ H -7.028733 1.2947792 .869981 H -5.851389 2.737402 -3.046318 H $-4.0401564 .620960-0.983062$ H -3.521254 3.5464932 .145735 O $-5.286681-1.4571302 .937904$ O $-7.562198-1.1810182 .663221$ C -5.462974 -2.876206 2.989089 H $-4.479355-3.3365293 .114320$ H $-6.090378-3.1491293 .842864$ H -5.902368 -3.2374312.054240 SCF Energy (B3LYP/6-31G**//MMFF) $=-3245.91586982$

02_190

MMFF Geometry

C -0.6325202 .8904362 .305914$

C $-0.280740 \quad 3.742201 \quad 1.329945$

C $-0.7723483 .743739-0.099056$

O $-1.8440992 .798586-0.310281$

C $0.398075 \quad 3.351172-1.021381$

C $0.0528993 .384879-2.496971$

C $-0.1512582 .294761-3.263526$

C $-0.0086254 .760416-3.108982$

C $-0.1451090 .853576-2.832752$

C - $1.4416070 .148623-3.253986$

C $-1.548773-1.263681-2.659673$

C $-2.807726-1.964568-3.174455$

C $-3.033164-3.325106-2.499498$

O $-1.602690-1.131768-1.235786$

O $-4.376404-3.743261-2.788505$

C $-2.937426-3.145850-0.969435$

C $-2.109925-4.406154-3.073398$

O $-2.993003-4.406936-0.307364$

C $-1.681242-2.371809-0.520107$

C $-1.687769-2.0334820 .991274$

O $-0.522363-3.178326-0.763923$

C $-3.113790 \quad 3.234618-0.081672$

$\begin{array}{llllll}\text { O } & -3.448583 & 4.312897 & 0.382107\end{array}$

C $-4.0402142 .168365-0.525208$

C $-5.3650682 .332053-0.393599$

C $-6.401806 \quad 1.397612-0.842424$

C $-5.9535600 .103617-1.468831$

C -2.813332 -1.104938 1.456140

C $-4.042761-1.8130081 .996015$

C $-6.334396-1.3052982 .846691$

O $-4.109210-3.0270382 .161815$

C $-7.335538-1.3816741 .738223$

C -8.461070 -0.652373 1.707882

C $-9.499047-0.7630270 .629569$

C $-9.8092590 .563972-0.023786$

C $-10.891362 \quad 1.2755380 .335094$

C $-8.922978 \quad 1.014251-1.161977$

C -7.696589 $1.749667-0.694692$

N -5.051765 -0.9078572 .300594$

H -1.3697022 .1170642 .113596$

H $0.4709704 .497742 \quad 1.554064$

H $-1.1159484 .754588-0.351548$

H $0.7701092 .360595-0.736938$

H $1.2471494 .028621-0.854239$
H $-0.3543432 .446155-4.323068$

H $-0.201723 \quad 4.728357-4.186548$

H $-0.807935 \quad 5.350133-2.649818$

H $0.9412085 .284814-2.961165$

H $\quad 0.7077570 .351194-3.304033$

H $-0.0115930 .755512-1.753087$

H $-2.2976780 .747821-2.919395$

H $-1.4890610 .095540-4.348873$

H $-0.651760-1.830127-2.936439$

H -2.770243 -2.077880 -4.265144

H $-3.682520-1.332695-2.972324$

H $-4.474999-3.788012-3.755059$

H -3.829233 - $2.588133-0.658625$

H $-2.293983-4.536201-4.146693$

H -2.310106 -5.381136 -2.615733

H $-1.049901-4.175976-2.948446$

H $-3.805756-4.852934-0.601090$

H -1.646952 -2.952402 1.588310

H $-0.746736-1.5075561 .203406$

H $0.202019-2.857471-0.201491$

H $-3.605607 \quad 1.285683-0.978320$

H $-5.731487 \quad 3.2506750 .063199$

H $-5.4507430 .297173-2.422222$

H $-6.771538-0.592146-1.667051$

H $-5.261513-0.428679-0.807956$

H -2.435639 -0.4916732 .282656$

H $-3.113488-0.4220640 .657379$

H $-6.609185-0.5625393 .602448$

H $-6.245538-2.2809913 .334826$

H -7.136789 $-2.091048 \quad 0.938258$

H -8.668534 0.0383232 .522123

H $-10.408610-1.1752351 .086301$

H $-9.203430-1.487983-0.138986$

H $-11.1386792 .212396-0.153123$

H -11.550123 $0.939697 \quad 1.129114$

H $-9.4801231 .702507-1.812726$

H $-8.6925710 .160551-1.805460$

H $-7.9211752 .709235-0.226642$

H -4.9180550 .0752042 .081579$

C $0.9676591 .742120 \quad 3.912473$

$\begin{array}{lllll}\text { O } & 1.947838 & 1.698969 & 2.851891\end{array}$

C $1.886719 \quad 0.395504 \quad 2.237737$

C $1.384952-0.5188163 .341409$

C 0.3194860 .3555093 .974910

$\begin{array}{lllll}\mathrm{H} & 1.144071 & 0.470631 & 1.432347\end{array}$

$\begin{array}{llll}\text { C } & 3.256637 & 0.032946 & 1.659280\end{array}$

H $0.989733-1.4672282 .967458$

H $2.184224-0.7348174 .061073$

C $-0.068475-0.0752805 .378929$

H -0.5754980 .3201583 .346049$

C $3.330531-1.3946371 .085634$

$\begin{array}{lllllllll}\text { O } & 3.567612 & 0.978250 & 0.630258\end{array}$

H 4.0195440 .1664802 .433905

H $3.218885-2.1214331 .898492$

O $2.212295-1.6101100 .213990$

C $4.613659-1.7106120 .291932$

C $5.908887-1.4756371 .087690$

H $4.622590-1.126539-0.634364$

O $4.561436-3.084534-0.108717$

H $5.950781-0.4336781 .417124$

H $5.891034-2.0976961 .991212$

H $-0.489668-1.0859785 .368479$

H $0.795820-0.0786136 .051570$

H -0.8201610 .6013565 .797855$

C -0.0013392 .9090253 .674239$

H $2.8591140 .943056-0.034254$

H $2.311538-1.021055-0.552915$

H $4.552548-3.6294540 .696727$

H 0.5544303 .8470373 .801518

H -0.7907162 .9067884 .434564$

H 1.5261221 .9396024 .835917

C $7.3666330 .252939-0.946131$

O $7.200638-1.169079-0.997627$

C $7.181909-1.8160760 .278844$

C $8.498194-1.5427111 .021783$

C $8.810949-0.0488601 .101138$

C $8.698151 \quad 0.606933-0.276804$

H $6.5376300 .702622-0.386605$ 
C $7.3067250 .774578-2.385342$

H $7.147366-2.8934370 .074033$

O $8.462976-2.0849862 .337369$

H $9.319671-2.0406470 .490370$

H $8.1552190 .450442 \quad 1.823738$

O $10.139772 \quad 0.1320291 .597535$

H $9.5357850 .284909-0.908433$

O $8.7896802 .022065-0.130658$

C $5.9111880 .635830-2.946713$

H $7.5981361 .828436-2.457743$

H $7.9823820 .206146-3.035990$

H $8.285496-3.0376982 .256143$

H $10.752766-0.3059880 .982766$

H 9.6262062 .2133520 .327147

O $5.1067961 .614178-2.441005$

O $5.574770-0.244835-3.727528$

C $3.7423621 .571693-2.869277$

H $3.6803781 .706794-3.953132$

H $3.2805920 .626089-2.569486$

H $3.2096502 .392005-2.381696$

SCF Energy (B3LYP/6-31G**//MMFF) $=-3245.89958093$

$02 \_191$

MMFF Geometry

C $-0.416207-0.7491421 .880777$

C $-0.311899-1.9290051 .250112$

C $-0.805563-2.227342-0.147433$

O $-0.971641-1.008600-0.907127$

C $-2.180543-2.912481-0.098610$

C $-2.455234-3.763220-1.321725$

C $-3.108712-3.350278-2.424959$

C $-1.966658-5.185978-1.222165$

C $-3.616066-1.973435-2.751770$

C $-5.147565-1.880817-2.748454$

C $-5.727897-1.661505-1.343098$

C $-7.256976-1.679368-1.382865$

C $-7.872951-1.341045-0.017403$

O $-5.268439-0.384973-0.882981$

O $-9.267919-1.090753-0.246865$

C $-7.230007-0.0406160 .511512$

C $-7.794948-2.5304140 .946973$

O

C $-5.683994-0.0449280 .444215$

C $-5.140832 \quad 1.368714 \quad 0.770733$

O $-5.179588-0.9719871 .408673$

C $0.122163-0.461593-1.496119$

O $1.258213-0.903454-1.506569$

C $-0.3101180 .821096-2.089938$

C $0.6036091 .768844-2.340964$

C $0.3313553 .112193-2.854206$

C $-1.0878443 .437044-3.242526$

C -3.6479811 .5584140 .461993$

C $-3.1225532 .892540 \quad 0.969112$

C $-0.9969794 .173623 \quad 1.233268$

O -3.8430853 .7457121 .480873$

C -0.8444845 .1442820 .104517$

C $0.3263425 .710124-0.228287$

C $0.4847116 .722880-1.327219$

C $1.6135756 .390066-2.278521$

C $2.8069616 .999312-2.174999$

C $1.3319395 .414262-3.399570$

C $1.3557053 .987044-2.931352$

N $-1.751145 \quad 3.0160680 .785868$

H $-0.925666 \quad 0.074447 \quad 1.391735$

H $\quad 0.183571-2.7533181 .757942$

H $-0.068387-2.867898-0.647758$

H -2.968976 -2.158971 0.014040

H -2.261015 -3.558885 0.785669

H -3.267203 -4.075569 -3.222766

H -2.176293 -5.763952 -2.128389

H $-0.883923-5.206912-1.061437$

H -2.452852 -5.696643 -0.384524

H -3.184938 -1.208708 -2.101816

H $-3.258671-1.736373-3.762223$

H -5.426379 -1.025744 -3.378483

H $-5.583645-2.777017-3.206547$

H $-5.355500-2.446735-0.676124$

H $-7.625977-2.648747-1.740928$
H -7.609409 -0.937946 -2.114141

H $-9.691536-0.9286380 .612817$

H -7.601223 $0.784914-0.111787$

H -8.349663 -3.3854890 .542411$

H $-8.267257-2.2940411 .906865$

H $-6.771832-2.8600451 .139997$

H -8.6179090 .3443011 .832734$

H -5.6914172 .1164750 .184803$

H -5.3195611 .5905091 .830727$

H -4.296786 -0.6898181 .694587$

H $-1.3717330 .967847-2.252708$

H $1.6484841 .553451-2.122417$

H -1.422668 $2.778109-4.051387$

H -1.222818 $4.461932-3.592895$

H -1.762546 $3.312235-2.388524$

H $-3.059754 \quad 0.765817 \quad 0.931023$

H -3.478669 $1.516575-0.620042$

H -0.0306853 .8110931 .597055$

H -1.526336 4.6530812 .063019

H -1.742065 $5.417396-0.445405$

H 1.2163525 .4619040 .345423

H $0.6724687 .696265-0.855124$

H $-0.4451416 .844423-1.896632$

H $3.6125706 .786168-2.869672$

H $3.0065287 .720411-1.389242$

H $2.0945085 .510624-4.184086$

H $0.3947035 .690953-3.889586$

H $2.3463703 .652773-2.619000$

H -1.246806 2.2825130 .297009

C 0.9730680 .8416953 .203356

$\begin{array}{llll}\text { O } & 2.038957 & 0.731676 & 2.243487\end{array}$

C 3.2129851 .3806492 .767576

C 2.7686592 .0984994 .037572

C 1.6537951 .1893534 .524692

H 3.9075660 .5804443 .050384

C 3.8587302 .2532311 .682434

H 3.5837342 .2056434 .759684

H 2.3696163 .0971493 .830387

C 0.7353691 .8430495 .541753

H 2.1017750 .2890014 .966465

C $4.063303 \quad 1.5211280 .339551$

O $3.0104303 .372896 \quad 1.397175$

H 4.8118412 .6619092 .038627

H $3.0885521 .318698-0.120247$

O $4.7484562 .409598-0.552055$

C 4.8618120 .2087290 .435079

C $5.022825-0.459752-0.940546$

H $4.341308-0.4751761 .106951$

O 6.1408320 .4826191 .000262

H $4.031157-0.542759-1.397414$

H $5.6239760 .188320-1.590016$

H 1.2973092 .1369726 .434299

H $\quad 0.2587272 .7406605 .133624$

H -0.0555831 .1536355 .852903$

C $0.164230-0.4564153 .236030$

H 3.1462494 .0319282 .098473

H $4.2179233 .222556-0.613531$

H $6.556364-0.368617 \quad 1.217794$

H $-0.654481-0.3668283 .958241$

H $0.803705-1.2862683 .557266$

H 0.3343351 .6715592 .873949

C $3.774177-3.174568-0.248082$

O $5.102917-2.7349740 .061965$

C $5.697162-1.850646-0.893336$

C $5.771280-2.544517-2.259091$

C $4.396860-3.031887-2.711710$

C $3.728278-3.868667-1.620546$

H $3.094150-2.315951-0.232523$

C $3.347473-4.151728 \quad 0.862111$

H $6.729328-1.689060-0.558774$

O $6.321175-1.660443-3.229853$

H $6.449543-3.404451-2.185494$

H $3.758473-2.191673-3.006772$

O $4.547247-3.828780-3.890495$

H $4.225464-4.846034-1.570488$

O $2.375355-4.094656-2.014791$

C $3.180167-3.4898862 .213245$

H $2.386545-4.6170120 .620253$ 
H $\quad 4.111293-4.931480 \quad 0.970535$

H $6.314152-2.125136-4.084231$

H $5.113546-4.587916-3.670527$

H $2.011927-4.798532-1.452567$

O $2.982126-4.4584053 .156826$

O $3.198480-2.2858802 .421375$

C $2.793885-3.9847794 .494203$

H $2.709340-4.8527945 .153359$

H $3.652736-3.3857814 .812117$

H $1.870176-3.4024884 .559890$

SCF Energy $\left(B 3 L Y P / 6-31 G^{* *} / / M M F F\right)=-3245.90709442$

02 192

MMFF Geometry

C $2.819995-1.8209432 .456249$

C $3.824104-2.6060192 .034671$

C $4.813991-2.3059480 .933491$

O $4.777203-0.8940230 .618512$

C $4.497587-3.107120-0.348136$

C $4.503944-4.613171-0.176621$

C $3.397610-5.368807-0.020739$

C $5.866093-5.253797-0.207938$

C $1.979321-4.8810100 .072091$

C $1.099007-5.436590-1.054101$

C $-0.180278-4.601199-1.207784$

C $-1.177024-5.281512-2.145207$

C $-2.406396-4.397973-2.404628$

O $0.215557-3.337610-1.752758$

O $-3.120976-4.986451-3.501512$

C -1.927577 -2.997502 -2.843640

C $-3.368061-4.404803-1.210151$

O $-3.032689-2.107349-2.982419$

C $-0.845600-2.391282-1.918897$

C $-0.239815-1.128678-2.581375$

O $-1.440655-2.047079-0.670903$

C $5.879524-0.3488610 .037984$

O $6.914330-0.922216-0.262118$

C $5.6031641 .087817-0.178867$

C $6.4626211 .830328-0.891625$

C $6.3089043 .257844-1.153751$

C $7.5867364 .035486-0.994576$

C $0.905849-0.468456-1.798231$

C $0.4513370 .602673-0.821311$

C $1.3770192 .313675 \quad 0.738379$

O $-0.7267690 .895552-0.642247$

C $1.6348153 .585627-0.005124$

C 2.5231054 .5091100 .392046

C $2.7821875 .786821-0.353027$

C $4.2451805 .985512-0.685522$

C $4.9883156 .876412-0.007204$

C $4.8246435 .222717-1.856777$

C $5.1262133 .785309-1.529164$

N $1.5269421 .196362-0.175439$

H $2.664477-0.8425922 .010988$

H $3.940739-3.5814892 .504307$

H $5.813341-2.5564581 .312000$

H $5.224920-2.846410-1.128700$

H $3.548426-2.757043-0.772079$

H $3.516105-6.4459980 .085663$

H $6.361630-5.045841-1.161879$

H $6.490809-4.8630210 .601491$

H $5.816888-6.341561-0.091355$

H $1.567361-5.2011831 .037299$

H $1.932491-3.789740 \quad 0.079696$

H $1.656540-5.419727-1.999753$

H $0.849998-6.481641-0.833123$

H -0.626064 -4.448506 -0.217697

H -1.482025 -6.258070 -1.748776

H $-0.688860-5.489028-3.107950$

H -3.859505 -4.393570 -3.723788

H - $1.502584-3.107657-3.850699$

H -3.706709 -5.426695 -1.000853

H -4.274947 -3.829662 -1.427199

H -2.920447 -4.009489 -0.295912

H -3.303715 -1.829406 -2.090763

H $\quad 0.176868-1.428568-3.552417$

H -1.024729 $-0.390773-2.781288$

H $-0.820988-1.483754-0.181435$
H $\quad 4.702440 \quad 1.507824 \quad 0.255717$

H $7.3679581 .382287-1.296082$

H 8.0088043 .8748620 .004170

H $7.4554605 .113388-1.111089$

H $8.3259883 .713385-1.736262$

H $1.503648-1.211218-1.259746$

H $1.5670010 .029417-2.519456$

H 2.0824652 .1603761 .560909

H 0.3615282 .3299401 .140688

H $1.0542793 .759588-0.908224$

H $3.086226 \quad 4.3468281 .308251$

H $2.421577 \quad 6.6137520 .272522$

H $2.1943175 .837089-1.278083$

H $6.0294977 .051443-0.256392$

H $4.573327 \quad 7.4490190 .815676$

H $5.7178475 .727039-2.243429$

H $4.1144105 .250462-2.693284$

H $4.2711413 .118946-1.638896$

H $2.4697540 .919250-0.432268$

C $0.415528-2.3167942 .945311$

O $-0.126877-0.9832812 .863979$

C $-1.482750-0.9967043 .367468$

C $-1.875341-2.4617823 .455170$

C $-0.554144-3.1050913 .824116$

H -1.434177 -0.5467234 .366751$

C $-2.354539-0.1504602 .436722$

H -2.662077 -2.645277 4.192580

H -2.223406 -2.831606 2.482356

C $-0.525272-4.6044193 .580282$

H $-0.347736-2.9066714 .884440$

C $-3.863180-0.1911602 .734566$

O

H -2.168214 -0.4666511 .407605$

H -4.249655 -1.212343 2.636100

$\begin{array}{lllll}\text { O } & -4.086310 & 0.208151 & 4.092071\end{array}$

C -4.6972820 .7539751 .843498$

C -4.5789550 .4425550 .340987$

H $-4.417797 \quad 1.7932092 .047901$

O -6.0735150 .6320012 .222623$

H -3.5282200 .4387300 .044026$

H $-4.967521-0.5668470 .157272$

H -1.279978 -5.1079124 .193242$

H $-0.730272-4.8444112 .531340$

H $\quad 0.452671-5.0241013 .836065$

C $1.836520-2.2531993 .511267$

H $-0.946775 \quad 1.2039842 .425848$

H -3.730117 -0.4875874 .669203$

H $-6.346175-0.2876002 .062775$

H $2.124649-3.2326993 .910286$

H $1.884858-1.5336434 .337362$

H $0.417962-2.721947 \quad 1.925013$

C $-3.6303983 .124602-0.734520$

O $-4.9571612 .799293-0.301995$

C $-5.3548241 .445021-0.543733$

C $-5.2787961 .143185-2.046129$

C $-3.9015621 .464724-2.622214$

C $-3.4774672 .883577-2.241233$

H $-2.9052712 .516710-0.181462$

C -3.379345 4.595054 -0.384690

H $-6.4108041 .377392-0.253975$

O $-5.604634-0.221815-2.287159$

H -6.030407 $1.749546-2.568424$

H $-3.1607930 .726185-2.297740$

O $-3.981071 \quad 1.358897-4.045070$

H $-4.0800163 .609251-2.802532$

O $-2.112817 \quad 3.082797-2.604674$

C -3.1896124 .7670851 .103330$

H $-2.4903094 .994877-0.885661$

H $-4.2263475 .223299-0.685401$

H -5.510898 $-0.378912-3.242400$

H $-3.0794201 .448772-4.396824$

H -2.055266 $3.031798-3.573427$

O -1.9016014 .4745051 .443802$

O -4.0813435 .1071521 .869715$

C -1.5898464 .5961932 .834913$

H -2.2569553 .9696423 .434641$

H -0.5618094 .2552372 .983890$

H -1.6620665 .6429833 .144156$ 
02 193

MM̄FF Geometry

C $-1.0563812 .424717-1.191162$

C $-1.6140383 .563644-0.753266$

C $-1.430304 \quad 4.1935510 .606053$

O $-0.294623 \quad 3.622968 \quad 1.299024$

C -2.6578373 .9695681 .511159$

C -3.9458934 .5683980 .983478$

C $-4.878758 \quad 3.8867070 .287739$

C $-4.141102 \quad 6.030570 \quad 1.286267$

C $-4.8228742 .442373-0.125405$

C $-6.113996 \quad 1.6922920 .225197$

C -5.9149930 .1765000 .089609$

C $-7.249107-0.5626970 .154689$

C -7.051143 -2.085233 0.127062

O $-5.080673-0.2345501 .176870$

O $-8.299901-2.6956850 .487684$

C $-6.023176-2.477124 \quad 1.210125$

C $-6.709203-2.593039-1.279373$

O $-5.724222-3.868048 \quad 1.104557$

C $-4.728693-1.6232361 .178810$

C -3.893929-1.881260 2.459685

O $-3.963008-1.972670 \quad 0.027309$

C 0.9221874 .1881751 .087788

O $1.1709995 .137846 \quad 0.357770$

C 1.9688433 .5438851 .912647

C 1.8490762 .3167902 .443871

C 2.8368711 .6486353 .297537

C 4.0607082 .4359953 .690656

C $-2.648936-0.9940202 .618332$

C $-1.412375-1.5863421 .972018$

C $0.163926-3.5129722 .160116$

O $-0.905474-1.081797 \quad 0.973047$

C $1.288523-3.3870463 .135698$

C $2.518326-2.9638332 .805694$

C $3.655753-2.895813 \quad 3.782747$

C $4.242652-1.5096873 .920001$

C $5.494682-1.2483643 .509083$

C $3.425431-0.4595374 .637338$

C 2.5972370 .3832183 .703916

N $-0.958744-2.7220322 .622774$

H $-0.3824911 .865575-0.548293$

H -2.280734 4.100466-1.425702

H $-1.270577 \quad 5.2717400 .479308$

H -2.448126 4.3984942 .501363

H $-2.7701172 .899507 \quad 1.724070$

H -5.761126 $4.425231-0.055127$

H $-5.085260 \quad 6.4160320 .887233$

H -4.1482876 .1959652 .368579$

H $-3.331550 \quad 6.6233170 .848746$

H -4.675879 2.400456-1.212007

H $-3.967887 \quad 1.928498 \quad 0.320113$

H -6.4094131 .9313651 .255225$

H $-6.9173282 .032089-0.439923$

H -5.406998 -0.030901-0.859380

H -7.910644 -0.246662 -0.661471

H -7.771138 $-0.296421 \quad 1.084647$

H -8.971538 -2.405399 -0.153088

H $-6.516984-2.3352982 .181979$

H -7.502267 -2.323287 -1.987056

H $-6.649640-3.686814-1.300521$

H -5.771126 -2.188978 -1.665366

H $-5.279148-4.1415961 .923519$

H $-4.535387-1.6781793 .327379$

H -3.603007 -2.936125 2.505364

H -3.078555 -1.5845900 .115861$

H 2.8650464 .1411612 .045842

H $0.943894 \quad 1.7444792 .246868$

H 4.6111032 .7658102 .803103

H 4.7710221 .8690264 .295478

H 3.7766523 .3185544 .274574

H -2.807744 0.0147852 .222937

H -2.426470 -0.8736593 .686595$

H $0.452664-3.1977821 .155905$

H -0.169729 -4.5541862 .112577$

H $1.087329-3.6884124 .161160$
H $4.423695-3.6036003 .444092$

H $3.347568-3.2479804 .775474$

H $5.940464-0.2685783 .641960$

H $6.103174-2.0073813 .028609$

H 4.0775550 .1567365 .265703

H $2.750493-0.9456675 .353941$

H $1.683957-0.1054983 .363576$

H -1.445161 -3.041193 3.455716

C $-1.7704670 .391392-2.476954$

O $-0.632293-0.433278-2.144568$

C $-0.513668-1.476508-3.126260$

C $-1.889156-1.583174-3.769492$

C $-2.300726-0.126637-3.816546$

H $0.206386-1.126705-3.876307$

C $-0.008791-2.773033-2.489606$

H -1.853289 -2.052624-4.756998

H -2.587975 -2.151847 -3.145058

C $-3.7951630 .077597-4.000470$

H $-1.7703720 .360894-4.645847$

C $1.310490-2.646703-1.704710$

O $-0.998593-3.267807-1.576763$

H $0.092687-3.542723-3.263656$

H $1.146334-2.090209-0.775116$

O $1.703210-3.971736-1.318335$

C $2.479627-1.998434-2.468609$

C $3.803826-2.106837-1.687556$

H $2.248033-0.945898-2.657256$

O $2.635780-2.630095-3.738403$

H $3.637071-1.754554-0.664270$

H $4.092156-3.162976-1.624568$

H $-4.126983-0.347794-4.953111$

H $-4.367129-0.404131-3.200584$

H -4.046454 $1.142960-3.998716$

C $-1.3288911 .854664-2.555233$

H $-1.157931-2.573658-0.914110$

H $0.950526-4.374683-0.852655$

H $2.802112-3.574970-3.578729$

H -2.098166 $2.447537-3.063967$

H $-0.4081821 .932294-3.144897$

H $-2.5127760 .248183-1.681357$

C $4.6812450 .779381-1.188339$

O $4.7009710 .076282-2.437298$

C $4.965608-1.328087-2.344413$

C $6.325025-1.556740-1.668961$

C $6.389979-0.878906-0.304653$

C $6.0042000 .593158-0.430877$

H $3.8410350 .424083-0.579781$

C $4.4571512 .268501-1.495527$

H $5.054329-1.693168-3.375176$

O $6.581466-2.950733-1.536077$

H $7.115865-1.144739-2.309268$

H $5.759720-1.4013690 .423307$

O $7.729761-0.9716900 .183333$

H $6.8119221 .125158-0.949383$

O $5.905675 \quad 1.163154 \quad 0.871984$

C $3.0867672 .545714-2.071677$

H $4.5531082 .871627-0.586168$

H $5.2003342 .600402-2.231006$

H $7.436385-3.046739-1.082373$

H $7.761102-0.4994331 .033119$

H $5.168244 \quad 0.726881 \quad 1.332004$

O $3.0778123 .805840-2.598895$

O $2.1428931 .769151-2.048034$

C $1.8492114 .218709-3.204449$

H $2.0166985 .185400-3.686676$

H $1.5337373 .498996-3.965870$

H $1.0778214 .338165-2.439662$

SCF Energy (B3LYP/6-31G**//MMFF) = -3245.92590797

$02 \_194$

MM̄FF Geometry

C $-4.0425610 .163155-3.156048$

C $-3.5275340 .798295-2.091192$

C $-3.5894122 .291524-1.907968$

O $-2.2199412 .769103-1.960295$

C $-4.2030562 .707930-0.552924$

C $-5.6437442 .306707-0.302113$ 
C -6.1309591 .9859890 .913262$

C $-6.5897362 .361735-1.475072$

C -5.4352962 .0599182 .242958$

C -5.3259910 .7067522 .955056$

C $-4.164012-0.1729742 .465056$

C $-4.123812-1.4706733 .280382$

C $-2.882980-2.3229412 .979031$

O $-2.954898 \quad 0.5659312 .655822$

O $-2.763169-3.3087054 .016479$

C $-1.630636-1.4254083 .060325$

C $-3.033222-3.0964681 .665800$

O $-0.466970-2.1474002 .667413$

C - $1.759095-0.1120572 .255989$

C $-0.566686 \quad 0.8233772 .564363$

O $-1.757432-0.425130 \quad 0.863654$

C $-2.0275534 .041031-2.394501$

O $-2.8995784 .843758-2.702077$

C $-0.5923564 .382461-2.515450$

C $0.4229063 .645606-2.036776$

C $1.8485523 .971732-2.174047$

C $2.2022475 .178059-3.009127$

C -0.7097412 .2350531 .975362$

C 0.4268013 .1467462 .402710

C 1.1719215 .5178322 .245103

O 1.3899692 .7588893 .058534

C 2.1760265 .5913251 .139934

C $3.4365145 .149217 \quad 1.260464$

C 4.4657325 .2165950 .169698

C $4.8751213 .843850-0.317274$

C 5.8160663 .1417850 .336787

C $4.2695273 .330246-1.605741$

C $2.7672383 .196033-1.557784$

N $0.2330894 .449641 \quad 1.961645$

H $-4.5298830 .763632-3.922630$

H $-3.0317690 .235744-1.309478$

H $-4.1536592 .743041-2.733828$

H -4.145138 $3.801104-0.467951$

H -3.5486782 .3242730 .239406$

H $-7.184872 \quad 1.717846 \quad 0.985706$

H -7.637799 2.275374 -1.167039

H $-6.4892843 .318399-1.998473$

H $-6.3933191 .550616-2.179789$

H -4.460349 2.5513012 .179855

H -6.0470932 .7189712 .873701$

H -5.1700390 .9216334 .020986$

H $-6.272457 \quad 0.157567 \quad 2.876147$

H -4.294364 -0.3868121 .397949$

H $-5.040088-2.0538383 .123399$

H $-4.111404-1.2237214 .351718$

H -3.581888 -3.833605 4.027872

H $-1.484715-1.1721034 .119421$

H -3.909752 -3.754293 1.704435

H -2.174618 -3.754404 1.492483

H -3.151387 -2.442391 0.801523

H -0.471397 -2.206505 1.697322

H -0.4624160 .9229393 .652756$

H $\quad 0.361948 \quad 0.3810282 .185101$

H -1.4056190 .3284460 .365376$

H $-0.4132115 .318677-3.035837$

H $0.2028132 .725600-1.498883$

H $3.2740525 .371408-3.076719$

H $1.7562646 .084094-2.583713$

H $1.8397795 .054000-4.035667$

H -0.7158412 .1994350 .881259$

H -1.647958 2.6919882 .309961

H 1.6446075 .3378023 .216617

H $0.609218 \quad 6.4544102 .305761$

H 1.8490026 .0371420 .204402

H 3.7542984 .7114242 .204442

H 5.3436415 .7454150 .563970

H $4.1190475 .827366-0.672241$

H $6.1531292 .174374-0.017251$

H $6.2738273 .523236 \quad 1.243765$

H $4.6736572 .338775-1.848740$

H $4.6039673 .971590-2.425978$

H $2.4214532 .351080-0.961398$

H -0.5534644 .6566891 .352777$

C $-3.336688-2.221525-2.427408$
O $-1.919759-1.962710-2.453037$

C -1.211107 -3.217835 -2.502163

C $-2.230356-4.281833-2.132838$

C $-3.495110-3.710348-2.740812$

H $-0.890788-3.337810-3.544895$

C $0.006187-3.135603-1.575467$

H $-1.973231-5.268496-2.529345$

H -2.330800 -4.365899-1.044290

C $-4.766465-4.325830-2.181793$

H $-3.466009-3.871541-3.826829$

C $0.774175-4.460629-1.428891$

O $0.892573-2.144098-2.104751$

H $-0.311770-2.775970-0.590851$

H $0.147402-5.195922-0.910631$

O $1.016044-4.987463-2.740383$

C $2.136822-4.340938-0.718630$

C $2.061352-3.7052440 .679373$

H $2.828854-3.783872-1.359294$

O $2.689406-5.656863-0.594793$

H $1.584608-2.7244510 .608863$

H $1.427365-4.3270631 .323261$

H -4.794389 -5.401340 -2.385029

H $-4.836476-4.187228-1.097657$

H -5.652628 -3.872933 -2.637106

C $-4.040469-1.316528-3.441678$

H $0.386535-1.317840-2.190594$

H $1.505212-5.821153-2.632556$

H $2.097945-6.175394-0.023076$

H $-5.086713-1.629184-3.540226$

H -3.568454 -1.448828 -4.423729

H $-3.691327-2.008881-1.412405$

C $4.087215-1.4848570 .284117$

O $4.379321-2.8714240 .494263$

C $3.450606-3.5559291 .341691$

C $3.427048-2.8911372 .725398$

C $3.159933-1.3899552 .633970$

C $4.094468-0.7307131 .619138$

H $3.107648-1.381426-0.197158$

C $5.145190-0.935014-0.675462$

H $3.848972-4.5691921 .477521$

O $2.443741-3.4907703 .561999$

H $4.398926-3.0380603 .214082$

H $2.113087-1.1895472 .385215$

O $3.388446-0.7920943 .911618$

H $5.110397-0.7030022 .032355$

O 3.6890320 .6219961 .418101

C $4.942977-1.484735-2.066904$

H $5.1143830 .156525-0.738631$

H $\quad 6.155448-1.212552-0.351942$

H $2.647277-4.4399713 .618150$

H $2.803593-1.2384644 .547893$

H $2.7862130 .614927 \quad 1.056951$

O $3.943837-0.800979-2.695366$

O $5.583306-2.418958-2.530998$

C $3.631021-1.248051-4.017930$

H $3.321050-2.297399-4.001173$

H $2.800845-0.643620-4.393131$

H $4.493127-1.109670-4.677064$

SCF Energy (B3LYP/6-31G**//MMFF)= -3245.90244967

$02 \_195$

MM̄FF Geometry

C $1.399789-0.2792723 .084017$

C $2.623881-0.8030033 .255314$

C $3.928663-0.3255842 .668815$

O 3.7988041 .0120552 .138705

C $4.359148-1.2650241 .528622$

C $5.834458-1.1780011 .195742$

C $6.344446-0.5664130 .109211$

C $6.750893-1.8955362 .153942$

C $5.6131470 .210634-0.949282$

C $5.556509-0.534922-2.290438$

C $4.517521-1.667515-2.310784$

C $4.576825-2.432649-3.634051$

C $3.467106-3.489615-3.738694$

O $3.228667-1.067730-2.154906$

O $3.435800-3.917875-5.108570$

C $2.112466-2.825405-3.409561$ 
C $3.789356-4.731335-2.899058$

O $1.067290-3.793392-3.341337$

C $2.136057-1.992558-2.106115$

C $0.833568-1.165127-1.990667$

O $2.237436-2.877411-0.989514$

C 4.7940201 .8939062 .416435

O $\quad 5.767194 \quad 1.696403 \quad 3.132732$

C 4.5931033 .1959151 .742042

C $3.6857173 .422516 \quad 0.779615$

C 3.4893054 .6918560 .071306

C 4.3464435 .8625450 .479663

C $0.821520-0.174094-0.818517$

C $-0.3997150 .729064-0.842052$

C -1.299635 2.7922410 .231719

O $-1.3554360 .550811-1.592093$

C $-1.0017563 .888207-0.740008$

C $-0.6807195 .139504-0.380186$

C $-0.3571646 .223914-1.366272$

C $1.0477496 .760880-1.199955$

C $1.2597087 .967538-0.647825$

C $2.1959955 .945634-1.755925$

C $2.5526864 .759989-0.897985$

N $-0.297540 \quad 1.7532030 .090101$

H 1.2583920 .5969792 .460507

H 2.712820-1.686509 3.887114

H $4.655845-0.3435743 .489167$

H $3.754111-1.0525160 .641043$

H $4.141120-2.3098901 .789125$

H $7.423618-0.601832-0.036945$

H $7.798728-1.854773 \quad 1.837615$

H $6.692694-1.4499283 .151489$

H $6.471769-2.9518162 .227351$

H $4.6108910 .508085-0.627375$

H $6.1630741 .148867-1.097688$

H $5.2930280 .199421-3.063045$

H $6.549225-0.928277-2.542266$

H $4.707467-2.346413-1.472512$

H $5.561888-2.895366-3.772512$

H $4.459909-1.725585-4.467761$

H $2.778090-4.629119-5.188158$

H $1.864198-2.155857-4.244947$

H $4.718244-5.197153-3.249676$

H $3.011621-5.495569-3.006917$

H $3.908975-4.511617-1.836100$

H $1.005803-4.223124-4.210789$

H $0.693765-0.590250-2.915759$

H - $0.026207-1.837821-1.893271$

H $1.890884-2.435555-0.198955$

H 5.2798623 .9717132 .066790

H 3.0339852 .6089850 .466524

H 4.2047546 .0914811 .541873

H $4.1200006 .780184-0.066863$

H 5.4054345 .6437890 .303530

H $0.814160-0.7110510 .134499$

H $1.7139800 .461131-0.850158$

H $-1.274232 \quad 3.1351411 .270597$

H -2.286980 2.3761520 .022891

H -1.048679 $3.635110-1.796811$

H $-0.6415205 .399744 \quad 0.674989$

H -1.092060 7.027913-1.230414

H $-0.4880225 .877977-2.399361$

H $2.2584648 .380170-0.554030$

H $\quad 0.4384208 .570434-0.274694$

H $3.0750256 .577741-1.927395$

H $\quad 1.9270315 .582715-2.756481$

H $1.9806613 .861609-1.129466$

H $\quad 0.573794 \quad 1.8713070 .598306$

C $-0.223219-2.2175053 .150583$

O $-1.103007-2.0268692 .021528$

C -2.095506 -3.076309 2.035797

C -1.688565 -4.040241 3.144275

C -1.002312 -3.105729 4.118365

H -3.045201 -2.611875 2.320730

C $-2.209267-3.735067 \quad 0.656237$

H -2.548893 -4.559022 3.578342

H $-0.986970-4.8004732 .780485$

C $-0.136350-3.8302535 .134951$

H -1.764578 -2.514304 4.642969
C $-2.724589-2.800157-0.455099$

O $-0.917882-4.2191360 .265179$

H -2.855836 -4.6179180 .719140$

H -2.010739 -1.988668 -0.627192

O $-2.788978-3.554124-1.671305$

C $-4.117293-2.206143-0.179898$

C $-4.666961-1.386516-1.362001$

H $-4.057916-1.5643480 .702590$

O $-5.038032-3.252970 \quad 0.126476$

H -3.915858 -0.647387 -1.658850

H $-4.829547-2.044970-2.223834$

H $-0.743100-4.5130775 .738797$

H $0.647503-4.4194494 .646992$

H $0.349136-3.1225255 .813699$

C $0.179333-0.8646283 .746052$

H $-0.314523-3.4565840 .240332$

H -1.899761 -3.912298 -1.836666

H -5.065872 -3.852278 -0.639161

H $\quad 0.392873-0.959138 \quad 4.817397$

H -0.643501 -0.146092 3.650230

H $0.652666-2.7627222 .774568$

C $-5.114173 \quad 1.2182280 .139952$

$\begin{array}{llll}\text { O } & -5.941513 & 0.050318 & 0.197082\end{array}$

C $-6.006843-0.690308-1.026425$

C $-6.5587430 .202323-2.147445$

C $-5.7888851 .516691-2.268022$

C $-5.6599512 .196736-0.904324$

H $-4.0912720 .929609-0.125140$

C -5.0905001 .8355571 .543180$

H $-6.741273-1.488251-0.859191$

O $-6.528945-0.472736-3.400346$

H -7.609503 $0.439055-1.934738$

H $-4.8024961 .363084-2.721866$

O $-6.4988362 .398123-3.141244$

H -6.638812 $2.580912-0.590085$

O $-4.7770683 .310048-1.017553$

C -4.2625290 .9885312 .485998$

H -4.6474832 .8371531 .545468$

H -6.1123091 .9170661 .932786$

H -7.038888 -1.294881 -3.301392

H $-6.6032391 .938747-3.992255$

H $-5.1307313 .891609-1.712290$

O -4.9798990 .6990513 .608570$

O $-3.112973 \quad 0.6331942 .258716$

C $-4.302462-0.1079214 .577329$

H $-3.3826580 .383978 \quad 4.908550$

H $-4.963525-0.2315885 .439181$

$\mathrm{H}-4.083576-1.0943674 .158245$

SCF Energy (B3LYP/6-31G**//MMFF) $=-3245.92306975$

02196

MM̄FF Geometry

C $-2.591875 \quad 0.372292 \quad 3.132017$

C -3.0468001 .6114383 .373384$

C -3.8933412 .4576402 .450542$

O -4.4080511 .6887431 .342196$

C -3.0265923 .5747411 .843557$

C -3.8298074 .7744471 .386771$

C $-4.2500564 .991706 \quad 0.125688$

C -4.1063535 .7962142 .460257$

C $-4.1324014 .085357-1.067374$

C -3.094582 $4.567084-2.090051$

C $-1.6716604 .080011-1.773645$

C $-0.6701344 .657314-2.776230$

C $0.7408174 .085558-2.573001$

O $-1.6826722 .650068-1.858501$

O $1.5255954 .427089-3.725700$

C $0.6498362 .546698-2.528145$

C $1.4459874 .714313-1.365989$

O $1.9125731 .967851-2.209357$

C $-0.4333462 .018065-1.558819$

C $-0.6125290 .494026-1.762544$

O $-0.0203492 .269419-0.214410$

C -5.5454620 .9703521 .521649$

$\begin{array}{lllll}\text { O } & -6.212222 & 0.877017 & 2.538218\end{array}$

$\begin{array}{llll}\text { C } & -5.814061 & 0.281437 & 0.240523\end{array}$

C $-6.643251-0.7708320 .208211$

C $-6.963245-1.574047-0.974073$ 
C $-6.368785-1.145859-2.291238$

C $-1.812665-0.112219-1.020371$

C $-1.902634-1.615560-1.226713$

C -3.092636 -3.630616 -0.368228

O $-1.197480-2.225852-2.026562$

C $-4.128615-4.015783-1.377586$

C $-5.178158-4.803959-1.096504$

C $-6.192228-5.240834-2.116216$

C $-7.619033-5.011510-1.667089$

C $-8.359728-6.020084-1.177244$

C $-8.212736-3.634681-1.866632$

C $-7.752677-2.657403-0.819973$

N -2.866871 -2.196909-0.414179

H -2.833699 -0.1263762 .198540$

H $-2.773272 \quad 2.0878974 .313297$

H -4.7281962 .8788103 .024164$

H -2.446315 3.1730981 .005262

H -2.2838383 .9295202 .570984$

H $-4.7866105 .917776-0.079131$

H -4.687082 6.6466802 .087532

H $-4.673707 \quad 5.3448353 .280327$

H -3.166405 6.1887992 .862001

H -3.942085 $3.048881-0.778805$

H $-5.1166204 .076273-1.553804$

H -3.388904 4.165041 -3.068712

H $-3.1153305 .660878-2.169890$

H -1.405247 4.384146 -0.755809

H $-0.6525775 .752974-2.720819$

H $-0.9960204 .415416-3.797761$

H $1.5320265 .396220-3.807513$

H $0.4015962 .205704-3.543094$

H $1.5343275 .799469-1.497005$

H $2.4710574 .340875-1.269873$

H $0.9239214 .538620-0.423214$

H $2.5642022 .326558-2.835983$

H $\quad-0.749470 \quad 0.288198-2.832350$

H $0.299309-0.027108-1.445715$

H -0.4196821 .6102430 .373931$

H $-5.2906200 .641029-0.638345$

H -7.115426 -1.089151 1.136694

H $-6.730709-0.149280-2.567643$

H -6.611913 -1.813375 -3.120033

H -5.275131 -1.116810 -2.232782

H - 1.7328620 .0779460 .053506

H -2.747200 $0.331472-1.381095$

H $-3.397378-3.8788920 .653360$

H -2.153717 -4.148591-0.589451

H $-3.991886-3.657538-2.395348$

H $-5.295124-5.189983-0.086357$

H $-6.030353-6.310174-2.305717$

H $-6.031203-4.742045-3.080129$

H -9.392263 -5.870694 -0.879283

H -7.948163 -7.016864 -1.057796

H -9.308213 -3.683897 -1.805227

H -8.008487 -3.299904 -2.887177

H $-8.129047-2.8858620 .178461$

H -3.449451 -1.604601 0.169958

C $-0.267808-0.4115373 .606177$

O $-0.153264-1.2112562 .413304$

C $1.100099-1.9322802 .452882$

C $1.841051-1.4230803 .682205$

C $0.700022-1.0421854 .603017$

H $0.833156-2.9891632 .577734$

C $1.833952-1.7182751 .123836$

H $2.506038-2.1779404 .112615$

H $2.443272-0.5389613 .437357$

C $1.120752-0.1197525 .734018$

H $0.264284-1.9583955 .023415$

C $3.265303-2.2891911 .107086$

O $1.042306-2.3504930 .115480$

H $\quad 1.851275-0.6469270 .890504$

H $3.828878-1.8635631 .944549$

O $3.223800-3.6995471 .318381$

C $4.051216-1.966306-0.179951$

C $5.487137-2.522174-0.135517$

H $4.075321-0.877558-0.294282$

O $3.389962-2.487029-1.329951$

H $5.938612-2.2642940 .828170$
H $5.455311-3.617121-0.192696$

H $1.876829-0.6023546 .362084$

H 1.5469850 .8143805 .352698

H $0.2676930 .134808 \quad 6.370400$

C -1.718462 -0.3912484 .087928$

H $1.431789-2.129529-0.747338$

H $2.635518-4.0805390 .644196$

H $3.387825-3.456645-1.260533$

H -1.7784590 .0528465 .088017$

H -2.111172 -1.412572 4.161595

H $\quad 0.0616120 .603303 \quad 3.344025$

C $7.0307620 .087173-0.273074$

O $6.363314-0.562082-1.361799$

C $6.363221-1.992891-1.294300$

C $7.808372-2.510233-1.283362$

C $8.642171-1.858131-0.181674$

C $8.503697-0.335157-0.229384$

H $6.540677-0.1765820 .671101$

C $6.8893101 .600088-0.474457$

H $5.900542-2.343484-2.225589$

O $7.820846-3.925804-1.133845$

H $\quad 8.270615-2.285987-2.253522$

H $8.368683-2.2549160 .803029$

O $10.012127-2.209917-0.388194$

H $9.0326810 .043733-1.112700$

O $9.143090 \quad 0.2293600 .913158$

C $5.4486612 .045904-0.369503$

H 7.4705022 .1692500 .259146

H $7.2468901 .884140-1.471773$

H $\quad 8.751429-4.208005-1.115735$

H $10.534303-1.7523290 .293049$

H $8.677575-0.0903981 .704870$

O 4.9741361 .8234840 .889587

O $4.8261722 .547920-1.296408$

C 3.6066222 .1845931 .108307

H $2.954376 \quad 1.6363290 .422784$

H 3.3425141 .9112242 .133509

H 3.4772513 .2638740 .986591

SCF Energy $\left(B 3 L Y P / 6-31 G^{* *} / / M M F F\right)=-3245.92656950$

02197

MMFF Geometry

C $0.3897523 .867990-2.407882$

C $1.7306373 .922442-2.413057$

C $2.6424924 .248021-1.250827$

O $3.2528713 .018138-0.785893$

C $1.9532344 .919743-0.045799$

C 2.9278545 .4341350 .999899

C 3.1183324 .8901362 .218901

C 3.6843556 .6810790 .614269

C 2.4615843 .6711892 .803330

C 3.4983222 .5880483 .128991

C 2.8357241 .2678393 .550070

C 3.8961440 .2261963 .911701

C $3.285581-1.1558894 .189123$

O $2.052570 \quad 0.8123762 .444057$

O $4.352309-2.1175264 .185132$

C $2.339374-1.5290903 .027773$

C $2.643790-1.2255025 .579572$

O $1.635272-2.735315 \quad 3.311923$

C $1.340886-0.4101732 .669746$

C $0.537887-0.7112691 .381611$

O $0.394656-0.265728 \quad 3.735235$

C $4.3289982 .547271-1.473914$

O $4.8329113 .033849-2.473747$

C $4.7997601 .320010-0.792671$

C $5.7443700 .562317-1.369936$

C $6.342705-0.654964-0.815038$

C $5.854890-1.1249180 .529250$

C $1.376182-0.8162330 .102778$

C $1.701964-2.236227-0.323609$

C $2.970812-3.475469-2.078695$

O $1.261132-3.2326940 .240962$

C $4.284635-3.894188-1.498558$

C $5.389720-4.090895-2.233577$

C $6.692173-4.577871-1.667564$

C $7.858083-3.681525-2.016635$

C $8.689028-3.989640-3.026914$ 
C $8.118466-2.484818-1.131130$

C $7.305968-1.281297-1.523410$

N $2.529902-2.251049-1.439533$

H - $0.1625474 .045113-1.489856$

H $2.242193 \quad 3.727206-3.354278$

H $3.4230774 .921534-1.626505$

H 1.2423474 .2199550 .403148

H $\quad 1.3535105 .773702-0.388680$

H 3.8316035 .3717732 .887107

H 4.2928077 .0702741 .437674

H $4.3582796 .481651-0.224118$

H $2.987346 \quad 7.4732650 .321618$

H 1.9422273 .9641573 .723759

H 1.6974893 .2654972 .137461

H 4.1180702 .4132802 .240242

H 4.1635082 .9421293 .926489

H 2.1766641 .4609644 .404726

H 4.4939840 .5622934 .768131

H $4.603496 \quad 0.1269243 .078057$

H $4.997205-1.8525854 .863122$

H $2.970719-1.7355872 .155251$

H $3.393217-1.0337046 .356954$

H $2.250891-2.2274715 .784045$

H $1.836977-0.5024035 .716115$

H $2.298518-3.4173943 .513970$

H $-0.098768-1.5913271 .530075$

H $-0.1584090 .126707 \quad 1.231517$

H $-0.406094 \quad 0.157097 \quad 3.383337$

H 4.3652411 .0808720 .170421

H $\quad 6.131934 \quad 0.868247-2.341127$

H $6.251027-2.0995040 .822032$

H $4.765397-1.2315560 .530711$

H $6.140397-0.4091401 .307037$

H $0.804600-0.375998-0.721605$

H $2.305770-0.2463560 .188142$

H $3.036293-3.276049-3.152982$

H $2.227225-4.262129-1.914889$

H $4.318022-4.077363-0.427014$

H $5.347009-3.934651-3.309149$

H $6.867751-5.587108-2.063087$

H $6.636815-4.696134-0.578162$

H $9.547294-3.368629-3.261790$

H $8.525944-4.866338-3.644999$

H $9.175734-2.194693-1.202923$

H $7.986398-2.769360-0.083488$

H $7.589920-0.865158-2.491129$

H $2.894361-1.373176-1.798654$

C -1.116668 2.226905 -3.652969

O $-1.9869512 .071217-2.514422$

C $-1.850114 \quad 0.718617-2.020346$

C $-1.125325-0.050154-3.115585$

C $-0.1868751 .014348-3.648312$

H -1.218836 $0.797916-1.127065$

C $-3.2313460 .176686-1.638056$

H $-0.601177-0.933976-2.740717$

H -1.825984 -0.371211 -3.896512

C $0.408390 \quad 0.681754-5.004544$

H $0.627206 \quad 1.148441-2.925333$

C $-3.254288-1.329861-1.324022$

O $-3.6720190 .897850-0.482048$

H $-3.9474970 .399561-2.437362$

H -3.066069 -1.898094 -2.242596

O $-2.166932-1.624127-0.437949$

C $-4.544027-1.836235-0.650746$

C $-5.837320-1.464187-1.394099$

H $-4.574973-1.4678470 .379536$

O $-4.460973-3.262836-0.552361$

H -5.895653 -0.377949-1.506551

H -5.808211 -1.889550 -2.404963

H $1.001967-0.236690-4.948401$

H $\quad-0.3702870 .532699-5.759984$

H $1.0643851 .487019-5.349722$

C $-0.4369953 .597439-3.635282$

H -3.652477 $1.844410-0.705179$

H $-2.188660-2.579463-0.256805$

H -4.448937 -3.623049-1.455503

H -1.210539 4.375023 -3.673993

H $0.1686563 .715732-4.541773$
H $-1.7686682 .187521-4.535923$

C -7.261730 -0.2489830 .995039$

O $-7.115865-1.6462750 .715631$

C -7.108615 -1.977499-0.677209

C $-8.426825-1.529180-1.323633$

C $-8.731171-0.056343-1.049684$

$\begin{array}{llll}\text { C } & -8.597780 & 0.258091 & 0.442217\end{array}$

H -6.4354830 .3085540 .538834$

C -7.172454 -0.068056 2.513595

H -7.078999 -3.073207 -0.730440

O $-8.389481-1.768512-2.726474$

H $-9.244153-2.138185-0.915623$

H $-8.0887750 .594434-1.654407$

O $-10.0726360 .209205-1.465786$

H -9.432464 -0.2066710 .981593$

O $\quad-8.7079401 .6661870 .635775$

C -5.791014 -0.414470 3.020407

H -7.4004600 .9581172 .822337$

H -7.884231 -0.7282813 .023935$

H -9.233686 -1.458102 -3.096756

H $-10.266042 \quad 1.136381-1.244676$

H -7.964056 2.0905100 .174967

O -4.8990390 .5481422 .648580$

O $-5.535918-1.4251183 .662420$

C -3.5397650 .3089373 .028510$

H $-3.178653-0.6229192 .582469$

H -2.9308341 .1350472 .651326$

H $-3.4515950 .276148 \quad 4.118559$

SCF Energy (B3LYP/6-31G**//MMFF) $=-3245.91390374$

02198

MMFF Geometry

C -1.798210-1.883099 2.825314

C $-1.487168-0.7092373 .396275$

C $-2.2680690 .581155 \quad 3.340881$

O $-3.583421 \quad 0.3655392 .780279$

C -1.5372731 .6283422 .480641$

C -1.8610023 .0512442 .892608$

C -2.7061403 .8759142 .244064$

C -1.1150323 .5477134 .106123$

C -3.5411393 .5890871 .027464$

C $-3.0876264 .409337-0.189801$

C $-1.7646983 .908529-0.787653$

C $-1.2819574 .829849-1.908218$

C $-0.0041654 .290897-2.572524$

O $-2.0007832 .599213-1.311646$

O $\quad 0.2033115 .026548-3.787845$

C $-0.2307182 .815283-2.966442$

C $1.2335374 .533663-1.701704$

O $0.9716432 .226537-3.458995$

C $-0.8381471 .950168-1.836384$

C $-1.2763790 .580174-2.405296$

O $0.1511901 .745575-0.826309$

C -4.6395630 .9362423 .415337$

O $-4.605827 \quad 1.5852684 .452900$

C -5.9174570 .6824152 .712384$

C -6.0159950 .1958091 .465385$

C -7.266651 -0.0412850 .735581$

C $-8.5612260 .215211 \quad 1.463550$

C $-2.146536-0.248219-1.450525$

C $-2.577499-1.564224-2.075806$

C $-4.083645-3.506401-1.642801$

O $-2.170802-1.958782-3.165145$

C $-5.396723-3.205216-2.290289$

C $-6.582803-3.521745-1.750065$

C $-7.898709-3.169926-2.379917$

C $-8.725601-2.242824-1.515561$

C $-9.814291-2.694515-0.870380$

C $-8.347579-0.777265-1.473938$

C $-7.204877-0.486366-0.537230$

N $-3.465768-2.250718-1.260076$

H -2.691147 -1.975352 2.213950

H $-0.579526-0.660463 \quad 3.996956$

H $-2.352593 \quad 0.9191694 .380762$

H -1.7757791 .4689971 .423463$

H -0.4486581 .5026452 .558351$

H -2.8340974 .8844312 .636450$

H -1.3492764 .5913544 .341505$ 
H -1.3665252 .9493804 .986920$ H -0.0347663 .4836903 .938936$ H -3.569655 2.5234690 .782872 H -4.5751223 .8672321 .267882$ H -3.874918 4.336167 -0.951367 H -3.000007 5.4673310 .086966 H -1.010606 3.8543120 .004867 H -1.119427 5.848123 -1.533318 H -2.063939 $4.913692-2.676003$ H $0.2796555 .968745-3.558964$ H $-0.9310732 .819588-3.812850$ H $1.3543255 .604098-1.496495$ H $2.1482754 .224886-2.219421$ H $1.1864244 .016990-0.741163$ H $1.5365052 .023248-2.693906$ H $-1.8609790 .742022-3.320769$ H $-0.393159-0.006825-2.685165$ H $-0.0705610 .944384-0.327460$ H -6.7983150 .9596523 .283514$ H -5.105206 -0.0404990 .918160$ H $-9.448056-0.038000 \quad 0.879218$ H $-8.648847 \quad 1.2742791 .730078$ H -8.614025 -0.3851882 .378706$ H -1.604356 -0.474744 -0.527604 H $-3.0523600 .309897-1.186621$ H $-4.195840-4.105827-0.733938$ H -3.435667 -4.045817 -2.340558 H $-5.367462-2.682193-3.243537$ H -6.613948 -4.046580 -0.798101 H -8.440811 -4.106212 -2.565495 H -7.759744 -2.709911-3.366397 H -10.434993 -2.033203 -0.275426 H -10.108968 -3.737650 -0.920150 H $-9.220377-0.161258-1.229038$ H -8.063458 $-0.448927-2.482221$ H $-6.222472-0.639834-0.982581$ H $-3.785112-1.813120-0.400551$ C $0.035548-3.421791 \quad 1.913419$ O $1.004862-2.3579891 .831711$ C $1.221172-2.0385810 .440400$ C $0.654128-3.212050-0.343737$ C $-0.538466-3.5932350 .510525$ H $0.632341-1.1340730 .250294$ C $2.710740-1.7618920 .210443$ H $0.377991-2.942082-1.367311$ H $1.375330-4.037666-0.386805$ C -1.074852 -4.984654 0.227220 H -1.333918 -2.8634130 .334416$ C $3.093812-1.562022-1.266554$ O $3.056821-0.5878210 .953109$ H $3.294973-2.5809740 .643661$ H $2.923014-2.491168-1.822980$ O $2.210326-0.583014-1.827599$ C $4.536237-1.072487-1.498566$ C $5.610009-1.966079-0.855653$ H $4.629015-0.041605-1.141522$ O $4.762825-1.020613-2.911878$ H $5.438996-2.0240790 .223037$ H $5.510530-2.983917-1.252537$ H -1.402311 -5.066364 -0.814155 H $-0.312596-5.7518970 .399756$ H -1.931673 -5.2089560 .870411$ C $-0.973667-3.128748 \quad 3.026414$ H $2.566067 \quad 0.157970 \quad 0.567224$ H $2.457537-0.469302-2.761462$ H $4.701914-1.927879-3.256717$ H -0.427004 -3.043290 3.974929 H -1.656994 -3.978391 3.141575 H $0.600361-4.3199502 .196086$ C $7.136227 \quad 0.238114 \quad 0.574917$ O $7.205827-0.079995-0.820081$ C $7.049676-1.470368-1.124271$ C $8.149602-2.279864-0.421295$ C $8.214229-1.9910861 .079407$ C $8.253074-0.4838151 .338087$ H $6.159509-0.0602880 .973755$ C $7.258473 \quad 1.7594520 .707617$ H $7.219980-1.567987-2.203871$
O $7.983687-3.675556-0.649349$

H $9.117244-2.008379-0.862531$

H $7.377903-2.4574101 .613238$

O $9.405019-2.5704241 .616994$

H $9.232326-0.0935401 .033786$

O $8.124848-0.2425902 .737674$

C 6.0474332 .4488620 .124257

H 7.3609602 .0778191 .751024

H $8.1389432 .127478 \quad 0.167172$

H $7.229781-3.983121-0.118305$

H $9.386247-3.5196231 .406223$

H $7.256952-0.5774223 .020878$

O 4.9793792 .3042980 .960235

O $6.0488843 .033727-0.951308$

C 3.7550962 .8812610 .496704

H 3.8681223 .9623790 .372351

H $3.4490342 .413088-0.443962$

H 2.9834062 .6931531 .247978

SCF Energy (B3LYP/6-31G**//MMFF) $=-3245.90773963$

$02 \_199$

MM̄FF Geometry

C 2.5371353 .6160620 .846342

C $2.5616464 .263275-0.329249$

C $3.1680383 .704886-1.597972$

O $3.0734512 .257484-1.603440$

C $4.6445024 .115043-1.717109$

C $5.2751673 .709732-3.036269$

C $6.1469602 .693917-3.197550$

C $4.8854384 .558255-4.220025$

C $6.6625221 .745102-2.151961$

C $6.1494790 .321332-2.399522$

C $6.494028-0.624674-1.239763$

C $6.008573-2.043387-1.542369$

C $6.209536-2.993264-0.351882$

O $5.847900-0.124977-0.064659$

O $5.434340-4.177297-0.597020$

C $5.634076-2.3349970 .920823$

C $7.668409-3.448315-0.228107$

O $5.938456-3.1116962 .076492$

C $6.105346-0.8812001 .125863$

C $5.379575-0.1659072 .292090$

O $7.503206-0.8826061 .440802$

C $1.8377201 .729248-1.841954$

O $0.8194002 .345118-2.117254$

C $1.9210830 .261101-1.674637$

C $0.793559-0.466562-1.695362$

C $0.693869-1.923220-1.577684$

C $1.969578-2.696512-1.377148$

C $3.863443-0.0111432 .131960$

C $3.047123-1.1043192 .797933$

C $0.699187-1.9102823 .008158$

O $3.527328-1.9339513 .564981$

C $0.532490-3.0833982 .094906$

C $-0.662940-3.5158831 .668075$

C $-0.859277-4.7176680 .791510$

C - $-1.637869-4.402932-0.465340$

C $-2.965695-4.600449-0.523603$

C $-0.861965-3.961607-1.683870$

C $-0.524268-2.497671-1.669217$

N $1.701393-1.0142732 .464681$

H 3.0539732 .6651930 .940016

H $2.0489815 .217450-0.423841$

H $2.6029224 .093144-2.454563$

H $5.2047833 .698623-0.874133$

H $4.7376315 .204855-1.614349$

H $6.5396492 .509360-4.196922$

H $5.4197814 .271135-5.132013$

H $3.8145614 .466704-4.425244$

H $5.1143955 .610893-4.024223$

H $7.7587561 .748930-2.189982$

H $6.3963352 .071112-1.144544$

H $5.0604320 .353917-2.526423$

H $6.574296-0.065233-3.334373$

H $7.579996-0.611977-1.089417$

H $6.501765-2.439913-2.438682$

H $4.937456-2.014556-1.781663$

H $5.733654-4.562678-1.438355$ 
H $4.542686-2.3430470 .817499$

H $7.981615-3.980141-1.134767$

H $7.792124-4.1616310 .594082$

H $8.364359-2.620587-0.076498$

H $5.606345-4.0117391 .916571$

H $5.640821-0.6381683 .246685$

H $5.791450 \quad 0.8520152 .351532$

H $7.719506-0.0499301 .892109$

H $2.901790-0.178909-1.540750$

H $-0.1554430 .050677-1.831135$

H $2.600886-2.629244-2.269185$

H $1.809536-3.756764-1.169412$

H $2.526353-2.304174-0.519770$

H 3.5637120 .9229112 .623885

H 3.5809420 .0674431 .077264

H $-0.225583-1.3346773 .117997$

H $1.018574-2.2549163 .996999$

H $1.430825-3.6167151 .792875$

H -1.559639 -3.002289 2.004009

H $-1.395301-5.4744481 .379545$

H $\quad 0.098797 \quad-5.180478 \quad 0.524470$

H -3.526019 -4.410987 -1.433200

H -3.522896 $-4.949400 \quad 0.339522$

H -1.457630 -4.143144 -2.589181

H $0.017853-4.599746-1.807629$

H - $-1.393578-1.852502-1.803012$

H $1.403956-0.3352261 .769781$

C 0.6960323 .0083242 .385206

O -0.1960332 .8383891 .267000$

C -1.5411362 .6628281 .756416$

C -1.4206092 .5187263 .267181$

C -0.1991403 .3686353 .565645$

H -2.0740853 .5867311 .499945$

C -2.1694351 .4631031 .033198$

H -2.3176772 .8581813 .793578$

H -1.2368651 .4731783 .545309$

C 0.4222073 .0844854 .921418

H -0.4858004 .4274223 .512372$

C $-3.599544 \quad 1.146017 \quad 1.509726$

O $-2.191201 \quad 1.774344-0.362453$

H $-1.5241150 .585073 \quad 1.152694$

H -3.5629900 .9911972 .595130$

O -4.4303562 .2834001 .275181$

C $-4.261245-0.1068170 .898151$

C $-4.425446-0.098193-0.632141$

H $-5.255173-0.1944801 .351868$

O $-3.532033-1.267151 \quad 1.291210$

H $-4.8221640 .870746-0.950017$

H -3.444746 -0.225343 -1.103734

H $-0.292532 \quad 3.2947895 .723879$

H 0.7290592 .0367455 .010760

H 1.3060813 .7091725 .084093

C 1.7491684 .0656282 .043674

H -1.280398 $1.990384-0.627593$

H -5.3132182 .0823821 .629184$

H $-2.700539-1.2845750 .787929$

H 2.4315734 .2133792 .887319

H 1.2611715 .0254451 .835444

H 1.1780852 .0368302 .555462

C -7.454942 -0.119937-0.875029

O $-6.623984-1.226935-0.507250$

C $-5.337369-1.242535-1.133460$

C $-5.505218-1.297048-2.658181$

C $-6.404266-0.173855-3.171414$

C $-7.715323-0.129143-2.385159$

H $-6.9690610 .819496-0.586482$

C $-8.759843-0.241033-0.080982$

H $-4.861887-2.181628-0.827037$

O $-4.244001-1.226665-3.314482$

H -5.959616 -2.256514 -2.938056

H $-5.8940520 .795970-3.136570$

O $-6.716333-0.412959-4.545791$

H $-8.334825-0.993339-2.657386$

O $-8.438158 \quad 1.046337-2.743854$

C -8.513835 -0.0512201 .397520$

H $-9.5076690 .494408-0.397563$

H $-9.202831-1.235371-0.215233$

H $-3.701090-1.962246-2.982934$
H $-5.873095-0.474450-5.026743$

H $-8.5630931 .026853-3.708301$

O $-8.2332371 .256973 \quad 1.661804$

O $\quad-8.560160-0.9594252 .217232$

C $-7.949746 \quad 1.560323 \quad 3.031142$

H -7.724624 2.6277793 .102995

H $-8.821187 \quad 1.3416813 .655515$

H -7.077166 $0.994690 \quad 3.371957$

SCF Energy (B3LYP/6-31G**//MMFF) $=-3245.91193036$

$02 \_2$

MMFF Geometry

C $2.7814383 .322705-1.819938$

C $4.0577783 .598098-1.506954$

C $5.0977342 .635509-0.974502$

O $4.5654251 .301589-0.815348$

C 5.5879033 .1200920 .403756

C $6.7401592 .305130 \quad 0.963015$

C 6.6540751 .4523842 .004105

C $8.0710052 .532988 \quad 0.291455$

C $5.434521 \quad 1.0916772 .805472$

C $5.102295-0.3992512 .661120$

C $3.715651-0.7382033 .228781$

C $3.445781-2.240373 \quad 3.121280$

C $2.009322-2.6066823 .523018$

O $2.754699-0.0069482 .462981$

O $1.755227-3.9479553 .076498$

C $1.027134-1.6874832 .765792$

C $1.828447-2.6148975 .045187$

O $-0.309465-1.8913073 .216518$

C $1.391483-0.1927772 .861936$

C $0.524242 \quad 0.702427 \quad 1.943047$

O $1.183510 \quad 0.254198 \quad 4.206720$

C $4.6389250 .468148-1.889812$

O $5.0839610 .730828-2.995728$

C $4.064696-0.835145-1.487206$

C $3.857696-1.784652-2.411690$

C $3.314356-3.123992-2.171961$

C $2.933291-3.482047-0.759970$

C 0.6853410 .4467320 .440743

C $-0.339462-0.504082-0.146794$

C $-0.938378-1.627765-2.286708$

O $-1.309661-0.9191880 .478857$

C $-0.505627-3.056510-2.201045$

C $-0.239799-3.813065-3.276739$

C $0.132700-5.265959-3.205012$

C $1.411253-5.582146-3.947624$

C $1.380743-6.094191-5.189670$

C $2.719075-5.394248-3.213918$

C $3.187493-3.965905-3.218974$

N $-0.064865-0.802564-1.474634$

H $2.3966762 .313488-1.707609$

H $4.4100614 .619057-1.646452$

H $5.9401382 .619897-1.676699$

H 4.7431213 .1324851 .099701

H 5.9183364 .1656770 .335142

H 7.5619680 .9419932 .323857

H 8.8855241 .9949290 .788178

H $8.0415502 .194509-0.748660$

H 8.3265053 .5975650 .305609

H 5.6300461 .3184013 .860619

H 4.5718771 .6967702 .520728

H $5.129096-0.6678341 .597535$

H $5.870681-0.9967263 .167316$

H $3.670709-0.4107274 .274063$

H $4.169983-2.8080793 .718849$

H $3.602784-2.5612742 .083235$

H $2.416841-4.5309303 .486575$

H $1.052884-1.9933581 .713134$

H $2.495260-3.3540385 .505623$

H $0.812002-2.9165275 .321228$

H $2.040882-1.6488215 .507587$

H $-0.509216-2.8375653 .114287$

H -0.5267450 .6637592 .253985$

H 0.8374691 .7423962 .115364

H 1.0744201 .2195254 .199202

H $3.828163-0.978471-0.439702$

H $4.116982-1.566276-3.447004$ 
H $3.825796-3.530470-0.127422$

H $2.422142-4.443273-0.674913$

H $2.245449-2.738702-0.343371$

H $\quad 0.546516 \quad 1.399971-0.081388$

H 1.6909660 .0898970 .202185

H $-0.895824-1.239731-3.309229$

H -1.965224 -1.534545 -1.920713

H $-0.442489-3.499080-1.209682$

H $-0.333822-3.376582-4.268682$

H $-0.698074-5.842362-3.633518$

H $0.220804-5.606719-2.165902$

H $2.292987-6.352857-5.717048$

H $\quad 0.442716-6.260539-5.708957$

H $3.505766-5.991849-3.694681$

H $2.635311-5.814311-2.207692$

H $3.485339-3.612232-4.207257$

H $0.791711-0.456979-1.897601$

C $0.5655394 .438717-1.457498$

O $-0.2917983 .310350-1.730079$

C -1.664034 $3.762460-1.771859$

C $-1.6466515 .190504-1.249445$

C $-0.2926255 .672657-1.728683$

H $-1.9497293 .746792-2.831302$

C -2.534798 $2.785669-0.970781$

H -2.476469 $5.790332-1.635014$

H -1.692330 5.206114 -0.153207

C $0.1854646 .928942-1.021195$

H $-0.3470895 .863583-2.808889$

C $-3.9795553 .264520-0.754037$

O $-2.5701791 .543607-1.681167$

H $-2.0568052 .582618-0.005748$

H $-3.9801284 .175636-0.144332$

O $-4.5272603 .629174-2.027715$

C $-4.9205712 .221289-0.118973$

C -4.4134301 .6575811 .220036$

H $-5.1008411 .410046-0.831851$

O -6.1901142 .8505780 .092206$

H -3.462309 1.1414421 .058711

H -4.2136222 .4907291 .905128$

H $-0.5104647 .756263-1.195115$

H 0.2615896 .7760640 .060708

H $1.1702427 .234529-1.387694$

C $1.8155914 .356514-2.336091$

H -1.650057 $1.282014-1.855826$

H $-5.4423343 .922502-1.876907$

H -6.0638883 .5645000 .740387$

H $2.3064275 .335216-2.383214$

H $1.5366194 .076207-3.359005$

H $0.8348484 .387253-0.393973$

C $-4.896523-1.2962020 .648903$

O $-5.882861-0.319396 \quad 1.001707$

C -5.4217320 .6985251 .895072$

C $-4.9209410 .063374 \quad 3.200745$

C $-3.894110-1.0384072 .947691$

C -4.409052 -2.028900 1.902749

H $-4.052978-0.8046660 .150867$

C -5.541095 -2.261126 -0.351486

H $-6.303488 \quad 1.2991732 .152005$

O

H $-5.771791-0.3775983 .736452$

H $-2.926930-0.6195452 .649332$

O $-3.661602-1.7526904 .163919$

H $-5.223709-2.6244492 .334213$

O $-3.362625-2.9295021 .548650$

C $-5.842048-1.561273-1.656151$

H $-4.900152-3.122857-0.568676$

H $-6.488940-2.6502890 .039530$

H -5.0311981 .7194304 .224737$

H $-3.354691-1.1069454 .823290$

H $-3.041730-3.3392302 .370295$

O $-4.686867-1.347978-2.348192$

O $-6.967169-1.233833-2.010136$

C $-4.829090-0.647803-3.587605$

H $-3.834723-0.529836-4.026742$

H $-5.452775-1.223154-4.278129$

$\mathrm{H}-5.2555440 .345007-3.415154$

SCF Energy (B3LYP/6-31G**//MMFF) $=-3245.91941782$
$02 \_20$

MMFF Geometry

C $1.044210-4.2275730 .227903$

C $2.240168-3.7078350 .537243$

C $2.636977-3.2549141 .918057$

O $2.754466-1.8125371 .874819$

C $4.003743-3.8586482 .286807$

C $4.572223-3.3422423 .594332$

C $5.616497-2.4940103 .694655$

C $3.896307-3.8589874 .837576$

C $6.405790-1.8767372 .572510$

C $6.095208-0.3811602 .433478$

C 6.4363820 .1510441 .033161

C 6.2459321 .6679570 .978485

C $6.4237922 .227974-0.440035$

O $5.553564-0.4916200 .104895$

O $5.9042963 .566855-0.453876$

C $5.5614681 .401431-1.417906$

C $7.9031452 .333505-0.828333$

O $5.810498 \quad 1.789956-2.766323$

C $5.750498-0.121229-1.266585$

C $4.751878-0.943281-2.121411$

O $7.067239-0.475188-1.707161$

C $1.609236-1.0930102 .046829$

O $0.497672-1.5287372 .302103$

C 1.9343530 .3343031 .828940

C $0.952143 \quad 1.2478831 .796358$

C 1.1184412 .6837681 .555571

C 2.5075713 .1812311 .253103

C $3.270342-0.753336-1.779036$

C $2.5598620 .306237-2.603034$

C $0.4038581 .556212-2.709061$

O $3.036800 \quad 0.800980-3.620386$

C $0.6556442 .920100-2.145927$

C $-0.3178853 .729805-1.701119$

C $-0.0729335 .118904-1.182073$

C -0.7506545 .3848380 .144710$

C $-1.9145996 .054246 \quad 0.198790$

C -0.0327094 .9724761 .410359$

C 0.0298993 .4812691 .595180

N $1.3032330 .601384-2.087943$

H $0.300954-4.3728881 .007521$

H $2.961933-3.538997-0.259046$

H $1.892581-3.5478402 .668854$

H $4.706737-3.6832721 .466991$

H $3.915112-4.9516962 .352483$

H $5.941530-2.1918514 .689346$

H $4.385292-3.5090615 .752829$

H $2.853924-3.5279844 .874557$

H $3.915736-4.9536064 .853029$

H $7.474322-2.0050302 .785605$

H $6.237008-2.3947141 .626570$

H $5.029209-0.2106142 .626638$

H 6.6553130 .1742093 .196024

H $7.472308-0.117130 \quad 0.794807$

H $6.9261652 .169527 \quad 1.678170$

H 5.2336111 .9165051 .321902

H 6.3860974 .0824210 .215461

H $4.5145541 .651307-1.207990$

H $8.4321023 .004493-0.140778$

H $8.0204232 .773733-1.824609$

H $8.4208031 .372155-0.811049$

H $5.6593212 .748884-2.824293$

H $4.943564-0.778877-3.188641$

H $4.977231-2.005381-1.946770$

H $7.074137-1.420005-1.933184$

H $2.9747240 .592748 \quad 1.673516$

H -0.0734610 .9169951 .955179$

H 3.1570393 .0548762 .125976

H 2.5470804 .2352360 .971849

H 2.9361802 .6303420 .409138

H $2.746346-1.693539-1.991861$

H $3.140345-0.539682-0.715239$

H $-0.6180431 .211708-2.520901$

H $0.5791681 .565532-3.789592$

H $1.6851643 .271037-2.130438$

H -1.350683 $3.392247-1.748634$

H $-0.4474215 .823512-1.936466$ 
H $0.9998565 .328713-1.088623$ H $-2.392513 \quad 6.285317 \quad 1.144831$ H -2.419018 $6.383378-0.703804$ H $-0.548634 \quad 5.3827702 .289098$ H 0.9541845 .4431591 .425858 H -0.9390683 .0284401 .808387$ H $1.0123750 .178450-1.211256$ C $-0.242565-3.447210-1.753483$ O $-1.513462-3.437543-1.077763$ C $-2.544179-3.089562-2.028845$ C $-1.828010-2.758201-3.331118$ C $-0.579815-3.613333-3.231499$ H $-3.158602-3.990722-2.144457$ C $-3.372876-1.941879-1.441261$ H $-2.435673-2.985152-4.212193$ H -1.559648 -1.694730 -3.370099 C $0.526977-3.175276-4.175150$ H $-0.847498-4.657409-3.441961$ C $-4.422540-1.360064-2.402463$ O $-4.041305-2.434076-0.274766$ H -2.694966 -1.149942 -1.102572 H -3.923484 -0.892042 -3.259068 O $-5.200559-2.442543-2.928215$ C $-5.393632-0.353629-1.752976$ C $-4.6898800 .822344-1.055595$ H $-6.048516-0.882434-1.052606$ O $-6.249294 \quad 0.163594-2.778133$ H $-4.0005260 .439213-0.298570$ H $-4.0860091 .366170-1.792781$ H $0.195417-3.249042-5.216136$ H $0.824481-2.137600-3.990858$ H $1.414203-3.805184-4.058790$ C $0.637106-4.558798-1.181236$ H $-3.361066-2.7958230 .319001$ H -5.866487 -2.058621 -3.524276 H $-5.6943570 .647930-3.413130$ H $1.525923-4.699165-1.806950$ H $0.094947-5.511749-1.165096$ H $\quad 0.220042-2.466052-1.578934$ C -6.046502 0.5919711 .650383 $\begin{array}{lllll}\text { O } & -6.614055 & 1.155446 & 0.461923\end{array}$ C $-5.675775 \quad 1.812947-0.395448$ C $-4.994868 \quad 2.9604200 .363834$ C -4.3742702 .4886311 .678358$ C $-5.388317 \quad 1.6878742 .496813$ H $-5.303577-0.1676641 .379996$ C -7.177239 -0.1021422 .416524$ H -6.263690 $2.263278-1.205020$ O $-3.987568 \quad 3.567641-0.436607$ H -5.7375553 .7359370 .592209$ H -3.462267 1.9059191 .503515 $\begin{array}{llll}\text { O } & -3.978548 & 3.627233 & 2.446401\end{array}$ H -6.151493 2.371992 2.888467 O $-4.732127 \quad 1.107893 \quad 3.622334$ C $-7.681171-1.3062881 .656461$ H $-6.860437-0.4355423 .410919$ H -8.026449 0.5775252 .556682 H $-4.4125043 .884575-1.251803$ H -3.349567 4.1344221 .905740 H -4.0528840 .4963773 .290313$ O $-6.785317-2.329751 \quad 1.756064$ O $-8.737673-1.328308 \quad 1.038412$ C $-7.129770-3.5197121 .039950$ H -7.208823 -3.307564 -0.030572 H -6.332204 -4.251531 1.193682 H -8.066744 -3.934852 1.423086

SCF Energy (B3LYP/6-31G*//MMFF) $=-3245.90865659$

\section{0}

MMFF Geometry

C $-1.2648193 .514381-0.662575$

C $-0.313624 \quad 4.139712-1.374449$

C $0.9610363 .534216-1.919315$

O $1.0684302 .158339-1.491338$

C $2.1859864 .329140-1.424291$

C $3.3611504 .297763-2.384512$

C $4.526378 \quad 3.655320-2.173020$

C $3.1856295 .122645-3.636813$
C $4.9238052 .810781-0.996752$

C $5.4570781 .442910-1.447222$

C $5.9701500 .615502-0.261376$

C $6.494406-0.743534-0.726482$

C $6.931213-1.6207920 .457573$

$\begin{array}{lllll}\text { O } & 4.886829 & 0.426870 & 0.651400\end{array}$

O $7.091430-2.963865-0.023799$

C $5.800061-1.6491531 .507521$

C $8.294983-1.1924611 .012256$

O $6.218985-2.3240692 .691418$

C $5.249116-0.2482751 .862948$

C $3.970826-0.3856892 .717589$

O 6.2305520 .4771602 .603796

C $1.6127051 .257172-2.349933$

O $2.1110151 .479459-3.441225$

C $1.476445-0.078577-1.729492$

C $2.156259-1.121249-2.228460$

C $2.059019-2.493138-1.736712$

C $2.074280-3.522948-2.833014$

C 3.2586570 .9459713 .004247

C 1.8132910 .6894063 .375589

C $-0.301539-0.1603642 .370123$

$\begin{array}{lllll}\text { O } & 1.429047 & 0.695903 & 4.542118\end{array}$

C $-0.160389-1.6475252 .474704$

C $-0.567319-2.5152611 .536197$

C $-0.330545-3.995577 \quad 1.633704$

C $0.432667-4.5608260 .452199$

C $-0.124275-5.500173-0.330898$

C $1.867799-4.125826 \quad 0.231244$

C $1.984120-2.774233-0.420372$

$\begin{array}{llll}\mathrm{N} & 1.030678 & 0.402856 & 2.265532\end{array}$

H $-1.1539072 .464019-0.408637$

H $-0.4781855 .182225-1.643601$

H $0.8715153 .573039-3.011565$

H $2.4754633 .972388-0.430331$

H $1.9237245 .387362-1.286571$

H $5.3032863 .753608-2.930923$

H $4.0941735 .151241-4.247888$

H $2.385306 \quad 4.716157-4.262008$

H $2.9345596 .156652-3.378322$

H $5.7088743 .342867-0.446222$

H $4.0917692 .663036-0.303860$

H $4.6501600 .900376-1.954244$

H $6.2678461 .576333-2.174340$

H 6.7659181 .1849740 .233364

H $7.319230-0.620725-1.439372$

H $5.701083-1.272030-1.273813$

H $7.751563-2.949224-0.738051$

H $4.979010-2.2456291 .085488$

H $9.063343-1.2590840 .232694$

H $8.623947-1.8632061 .813644$

H $8.300520-0.1694071 .393800$

H $6.542833-3.2016812 .425205$

H $3.277430-1.0593892 .200035$

H $4.208400-0.8562843 .680192$

H $5.789644 \quad 1.1814313 .104880$

H $\quad 0.801100-0.179512-0.886703$

H $2.810593-0.982578-3.086828$

H $1.244005-3.352401-3.527674$

H $1.979625-4.547206-2.466707$

H $3.012037-3.468212-3.397472$

H $3.742177 \quad 1.478628 \quad 3.830446$

H 3.2755781 .6167042 .137997

H -0.8561820 .1441091 .477408$

H -0.8038490 .2348313 .258133$

H $0.336923-2.0272543 .364777$

H $-1.068985-2.1458010 .645345$

H $-1.310655-4.4812871 .725447$

H $0.217398-4.2470412 .550781$

H $0.418407-5.943782-1.158584$

H $-1.136023-5.852137-0.161057$

H $2.416357-4.876882-0.349729$

H $2.387994-4.0957051 .197502$

H $2.015631-1.9472450 .286685$

H 1.4810630 .3545401 .356319

C $-2.9103883 .922441 \quad 1.215993$

O -3.2630562 .5327451 .392739$

C -4.5508102 .4558712 .029410$ 
C $-4.766906 \quad 3.8166632 .677140$

C $-4.135376 \quad 4.733440 \quad 1.651627$ H -5.2904292 .3169751 .231373$ C -4.6149581 .2769423 .004702$ H -5.8252544 .0311092 .853117$ H -4.2382503 .9022073 .633509$ C -3.797832 6.1121972 .193539 H -4.8317404 .8449930 .809407$ C $-4.242659-0.0911272 .405347$ O -3.7148121 .5175354 .095541$ H -5.6154751 .2270313 .450065$ H $-3.168699-0.1308512 .199132$ O $-4.491328-1.0822003 .412045$ C $-5.008249-0.488127 \quad 1.129952$ C $-4.699345-1.9369410 .708005$ H $-4.738516 \quad 0.1957090 .320411$ O $-6.411954-0.354053 \quad 1.345017$ H -3.613561 -2.070959 0.695101 H $-5.101822-2.6217021 .464368$ H $-4.703665 \quad 6.6231322 .535912$ H -3.105577 6.0519423 .040070 H -3.3319316 .7315741 .420727$ C $-2.5474584 .182657-0.248128$ H -2.827171 1.6486483 .719717 H -3.977038 -0.8309434 .198630$ H $-6.650467-0.9239582 .096196$ H -2.482709 $5.262781-0.424857$ H -3.344868 $3.792605-0.891967$ H $-2.052555 \quad 4.1206501 .870732$ C $-3.451972-1.639253-2.062944$ O $-4.836898-1.488774-1.726622$ C $-5.302236-2.323371-0.660876$ C $-5.087557-3.797152-1.033330$ C $-3.625731-4.077739-1.366308$ C $-3.120519-3.095696-2.419992$ H $-2.831322-1.301975-1.224024$ C $-3.167109-0.729565-3.269527$ H -6.384764 -2.159494 -0.592420 $\begin{array}{llll}0 & -5.498866 & -4.658149 & 0.023148\end{array}$ H -5.699558 -4.046888 -1.909704 H -2.997411 $-4.050375-0.469320$ O $-3.509259-5.399640-1.897798$ H $-3.561838-3.352981-3.391808$ O $-1.705897-3.230011-2.548684$ C -3.302096 $0.740479-2.942580$ H $-2.148019-0.887067-3.639350$ H $-3.882770-0.956065-4.069487$ H -6.433386 -4.4646520 .210199$ H -3.865556 -6.011091 - 1.230472 H $-1.519953-4.161714-2.757250$ O $-3.268878 \quad 1.453943-4.107095$ O $-3.4086051 .205449-1.817202$ C -3.398706 $2.872820-3.976238$ H $-4.2490463 .131623-3.337761$ H -2.471754 3.291946 -3.576834 H -3.572065 3.290677-4.971441

SCF Energy (B3LYP/6-31G**//MMFF) $=-3245.91539502$

02201

MMFF Geometry

C $-1.194281-3.379249-1.729384$

C $-1.005274-2.241675-2.415408$

C $0.295765-1.486432-2.436044$

O $0.013819-0.119488-2.045606$

C $0.916424-1.416519-3.840198$

C $1.555841-2.707671-4.297701$

C $2.778312-3.118862-3.907518$

C $0.751232-3.522609-5.274331$

C $3.704118-2.427659-2.943077$

C $4.026578-3.338041-1.751279$

C $4.932095-2.642592-0.724263$

C $5.406159-3.6449150 .330311$

C $6.240996-2.9706421 .426467$

O $4.177101-1.597143-0.100732$

O $6.373924-3.919848 \quad 2.495110$

C $5.460081-1.7538781 .962874$

C $7.663509-2.6649040 .944012$

O $6.250123-1.0223702 .898627$
C $4.914558-0.8198900 .854168$

C 3.9453930 .2096921 .489132

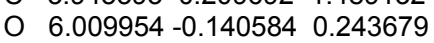

C $-0.0367800 .158580-0.718775$

O $0.152768-0.6300940 .197070$

C $-0.3590801 .582659-0.469029$

C $-0.4909362 .503800-1.436023$

C $-0.775128 \quad 3.928468-1.241514$

C $-0.911091 \quad 4.427566 \quad 0.173072$

C $3.232314 \quad 1.143216 \quad 0.502308$

C 3.9838862 .4245680 .183163

C $3.7473744 .516686-1.152930$

O 5.1024882 .6778090 .620602

C $2.6594405 .540480-1.079685$

C $2.1901916 .206525-2.145681$

C $1.1356887 .273850-2.067928$

C $-0.0492656 .994863-2.965372$

C $-0.1180497 .518604-4.201103$

C $-1.1932716 .186317-2.395676$

C $-0.8863334 .716088-2.332041$

N $3.2416893 .254937-0.646869$

H $-0.347449-3.804285-1.193409$

H -1.825621 -1.793190 -2.968330

H $1.020603-1.914603-1.732703$

H $1.664194-0.612116-3.866627$

H $\quad 0.163182-1.088553-4.570174$

H $3.162465-4.052495-4.316313$

H $1.269891-4.437845-5.578599$

H $-0.205500-3.818255-4.833274$

H $0.551405-2.939841-6.179459$

H $4.630909-2.180013-3.474566$

H $3.288466-1.481510-2.584701$

H $3.088030-3.638252-1.266465$

H $4.515498-4.249907-2.116988$

H $5.788853-2.206553-1.251245$

H $5.972404-4.462229-0.133835$

H $4.532094-4.1176530 .800641$

H $6.835245-3.4758993 .227658$

H $4.608562-2.1492782 .533588$

H $8.167462-3.5888820 .635359$

H $8.275828-2.2481471 .751303$

H $7.690618-1.9737170 .099033$

H $6.888937-0.4905592 .393993$

H $3.157030-0.3511112 .009618$

H 4.4708860 .8077022 .243157

H $5.6659180 .617624-0.253780$

H $-0.461776 \quad 1.8409220 .579459$

H $-0.3714552 .198060-2.473848$

H $-1.7900603 .981857 \quad 0.651254$

H -1.0203105 .5112910 .246693$

H -0.0219384 .1728750 .761082$

H $2.995848 \quad 0.626713-0.433819$

H $2.286664 \quad 1.4524090 .964189$

H $4.0847184 .341582-2.179636$

H $4.6076864 .843322-0.560392$

H $2.2575695 .763021-0.093861$

H $2.6105115 .999720-3.127451$

H $1.6029398 .224680-2.356757$

H $0.7880747 .420142-1.037582$

H $-0.9778827 .348959-4.840568$

H $0.6875878 .124779-4.602283$

H -2.085667 $6.301805-3.025577$

H $-1.4813706 .606954-1.428419$

H $-0.7657724 .258587-3.315228$

H $2.3812602 .896369-1.050780$

C $-3.769074-3.402706-1.970873$

O $-3.920749-2.228369-1.149124$

C $-5.301953-2.093615-0.769685$

C $-6.070418-3.121305-1.593662$

C $-5.038531-4.222749-1.743356$

H $-5.365277-2.3750120 .288095$

C $-5.764618-0.643896-0.951671$

H $-6.984755-3.453331-1.092642$

H $-6.351060-2.728149-2.577697$

C $-5.352635-5.202764-2.860072$

H $-4.969184-4.769062-0.793095$

C $-4.8925340 .399184-0.221022$

O $-5.730920-0.328977-2.350053$ 
H $-6.812100-0.553500-0.640347$

H $-3.9056030 .452164-0.696323$

O $-5.4882221 .686964-0.428019$

C -4.7159330 .1548961 .289698$

C -3.9668211 .3048851 .988898$

H $-4.161667-0.775662 \quad 1.434834$

$\begin{array}{lllll} & -5.991107 & -0.023000 & 1.904782\end{array}$

H -3.034225 1.4954761 .448242

H -4.5700002 .2184881 .925379$

H -6.307333 -5.705391 -2.673141

H $-5.422745-4.697949-3.829458$

H $-4.576560-5.970693-2.935598$

C $-2.486073-4.141549-1.577632$

H $-6.0246650 .593051-2.446675$

H -6.387693 $1.662635-0.060400$

H -6.4661210 .8239321 .861747$

H -2.399673 -5.073590 -2.147381

H $-2.561057-4.418157-0.518419$

H -3.719599 -3.068070 -3.014836

C $-1.584675-0.1575383 .226032$

O $-2.943705-0.1946193 .676748$

C -3.678702 1.0183153 .480953

C -2.984326 2.1710014 .218204

C -1.520457 2.3075603 .808658

C -0.8042580 .9629643 .927862$

H $-1.565810-0.0033562 .142307$

C $-0.954543-1.5254593 .534279$

H -4.6507670 .8676003 .967204$

O $-3.674712 \quad 3.3931153 .979434$

H -3.0328131 .9812145 .298488$

H -1.4330802 .7206092 .798213$

O $-0.902243 \quad 3.2527184 .684826$

H $-0.6647160 .716527 \quad 4.988400$

$\begin{array}{lllll}\text { O } & 0.489584 & 1.064346 & 3.335748\end{array}$

C $-1.476003-2.6288372 .638271$

H $0.131413-1.4916153 .393230$

H $-1.177500-1.8034244 .571476$

H -3.190871 4.0962164 .445800

H -0.0015493 .4124834 .356179$

H 1.0097441 .6898973 .867269

O $-1.041915-3.8304253 .123116$

O $-2.152598-2.4653941 .633277$

C -1.435599-4.980416 2.368126

H $-1.132979-5.8717722 .923997$

H -2.521742 -5.0031062 .235882$

H $-0.926946-4.9809511 .399792$

SCF Energy (B3LYP/6-31G*//MMFF) $=-3245.92425100$

02202

MMFF Geometry

C $2.019509-3.9189930 .801883$

C $0.756183-3.5753240 .507156$

C $0.189712-3.589972-0.890111$

O $-0.554446-2.361127-1.085275$

C $-0.776660-4.773911-1.056563$

C $-1.302475-4.918551-2.472258$

C $-2.548031-4.592525-2.871509$

C $-0.327526-5.506563-3.461086$

C $-3.658523-4.007497-2.045278$

C $-4.044523-2.609972-2.545749$

C $-5.075490-1.942228-1.624845$

C $-5.499906-0.583980-2.182553$

C $-6.4604950 .147627-1.233500$

O $-4.468059-1.766893-0.341863$

O $-6.5411181 .518120-1.657006$

C $-5.859613 \quad 0.1554750 .191037$

C -7.882837 -0.416812 -1.330244

O $\begin{array}{llll}-6.798370 & 0.661503 & 1.135879\end{array}$

C $-5.338575-1.2213540 .659469$

C -4.531639-1.159662 1.977430

O $-6.420336-2.1186860 .941956$

C $0.167235-1.236606-1.346639$

O $1.379627-1.155856-1.465727$

C $-0.774195-0.098476-1.447878$

C $-0.280096 \quad 1.135812-1.623802$

C -1.039756 2.379079-1.760717

C -2.541236 $2.291761-1.722606$

C $-3.272465-0.2860511 .949862$
C $-3.492124 \quad 1.1303252 .455552$

C $-2.290747 \quad 3.296154 \quad 2.731134$

$\begin{array}{llll}\text { O } & -4.543383 & 1.511613 & 2.962228\end{array}$

C -2.4379424 .1913331 .542475$

C $-1.4910395 .054187 \quad 1.143707$

C -1.6654926 .0229240 .011049$

C $-0.6137255 .871630-1.062641$

C $0.4905156 .637605-1.064393$

C $-0.896062 \quad 4.909623-2.192570$

C $-0.356216 \quad 3.529878-1.932719$

N $-2.356476 \quad 1.9132242 .300007$

H $2.657103-4.276725-0.004279$

H $\quad 0.084503-3.2357001 .290172$

H $0.989951-3.650370-1.638174$

H -1.600109 -4.676753-0.341819

H $-0.269228-5.709707-0.785048$

H -2.811235 -4.760165 -3.915362

H $-0.776580-5.657707-4.448602$

H $0.535960-4.847435-3.591461$

H $0.027411-6.481251-3.110334$

H $-4.528247-4.671983-2.116053$

H $-3.396092-3.961876-0.986470$

H -3.142642 -1.987527 -2.594346

H $-4.446992-2.682585-3.563882$

H $-5.940283-2.609241-1.529443$

H $-5.950948-0.693885-3.176701$

H $-4.6104800 .043309-2.326061$

H -6.837646 $1.529185-2.583234$

H -5.0208070 .8614210 .172127$

H -8.278969 $-0.284224-2.344272$

H $-8.5695680 .122297-0.668683$

H $-7.937582-1.482038-1.095767$

H -7.0744221 .5414950 .827389$

H $-5.190874-0.8869862 .810645$

H -4.201263 - 2.1853812 .197732

H $-7.253847-1.6230290 .901369$

H -1.835162 $-0.303219-1.374956$

H $0.7999331 .250586-1.691212$

H $-2.9115141 .743106-2.594648$

H $-3.0338453 .266551-1.717939$

H $-2.8770811 .778982-0.815373$

H -2.526203 -0.7354742 .617902$

H -2.835527 -0.2517060 .947384$

H $-1.332933 \quad 3.4360563 .240465$

H -3.0897553 .5042563 .449764$

H -3.386563 4.155986 1.012090

H $-0.553674 \quad 5.1050691 .693116$

H -1.6208247 .0361450 .432274$

H -2.663785 $5.940336-0.436409$

H $1.2315026 .557407-1.852893$

H $0.6804067 .355963-0.273880$

H $-0.4171075 .275710-3.111473$

H - $1.9643594 .912631-2.427371$

H $\quad 0.7341263 .487230-1.939922$

H -1.5401011 .5203661 .839949$

C $2.058317-2.866508 \quad 3.155044$

O $2.141706-1.5279482 .626080$

C $2.551450-0.6329063 .675576$

C $2.417904-1.4207924 .972552$

C $2.776891-2.8193754 .503807$

$\mathrm{H} 3.613960-0.4183133 .510244$

C $1.742906 \quad 0.6665713 .605238$

H $3.081865-1.0456465 .757076$

H $1.391955-1.4007115 .357858$

C $2.353267-3.9123895 .469214$

H $3.863566-2.8725244 .354294$

C $1.782751 \quad 1.3539092 .223688$

$\begin{array}{lllll}\text { O } & 0.372487 & 0.363335 & 3.898122\end{array}$

H $2.081461 \quad 1.3553564 .388213$

H 1.2381670 .7393501 .495806

O 1.0524542 .5787112 .350201

C 3.2016031 .6575511 .705003

C $3.1849572 .401006 \quad 0.357592$

H $3.743770 \quad 0.7137401 .591042$

$\begin{array}{llll}\text { O } & 3.891014 & 2.440303 & 2.677071\end{array}$

H $2.565722 \quad 1.828488-0.338478$

H $2.7119583 .382614 \quad 0.481970$

H $2.852544-3.7862056 .435447$ 
H $\quad 1.272387-3.8961415 .644992$

H $2.616435-4.9005855 .079443$

C $2.685554-3.8400222 .152740$

H -0.1234791 .1988333 .856666$

H $0.8086192 .870614 \quad 1.455598$

H 4.8312062 .4559282 .431338

H $2.727151-4.8501602 .575432$

H $3.720997-3.5231861 .969666$

H $0.996006-3.091273 \quad 3.309790$

C $4.9548670 .408720-1.128238$

O $5.3901481 .445755-0.238445$

C $4.5857052 .627702-0.252563$

C $4.5612263 .227713-1.665985$

C $4.1430322 .199726-2.715854$

C $4.9640080 .916767-2.576627$

H $3.9430810 .095351-0.846238$

C $5.907297-0.778122-0.913676$

H 5.0963283 .3514910 .394926

O $3.6811384 .345090-1.732344$

H $5.5646543 .592327-1.921631$

H $3.0698931 .983476-2.662706$

O $4.3761142 .740086-4.018932$

H $5.9940341 .111171-2.901832$

O $4.424611-0.078089-3.441299$

C $5.329016-2.118148-1.311020$

H $6.839880-0.610188-1.465399$

H $6.167601-0.849158 \quad 0.150035$

H $3.9758604 .989593-1.066523$

H $3.8730533 .570283-4.080968$

H $4.4175770 .290975-4.341129$

O $6.331220-3.046888-1.319763$

O $4.151378-2.338091-1.554534$

C $5.930418-4.379364-1.654297$

H $6.820656-5.013814-1.648272$

H $5.223589-4.758955-0.910270$

H $5.489096-4.403799-2.655252$

SCF Energy (B3LYP/6-31G**//MMFF) $=-3245.90962211$

02203

MM̄̄FF Geometry

C -2.2829753 .8547651 .064231$

C -3.3613924 .4470220 .527060$

C $-4.617818 \quad 3.771570 \quad 0.020654$

O -4.5394232 .3330880 .135669$

C $-4.8237374 .120491-1.465925$

C $-6.1313893 .604070-2.037827$

C $-6.2454992 .582963-2.911040$

C $-7.3641294 .349580-1.592486$

C $-5.1489691 .722846-3.473919$

C $-5.3368920 .255028-3.068614$

C $-4.095971-0.591248-3.389423$

C $-4.343791-2.060465-3.043054$

C $-3.079383-2.918115-3.201185$

O $-3.014671-0.075274-2.607278$

O $-3.314047-4.173915-2.545006$

C $-1.911842-2.233797-2.457680$

C $-2.793502-3.244300-4.671652$

O $-0.684239-2.915652-2.702283$

C $-1.759780-0.738746-2.802138$

C $-0.716932-0.014217-1.916097$

O $-1.317908-0.613656-4.159136$

C -4.9601241 .7756701 .304886$

O -5.3740452 .3621732 .292326$

C $-4.819120 \quad 0.307561 \quad 1.179328$

C $-5.017085-0.4747532 .250719$

C $-4.940836-1.9377382 .281798$

C $-4.593198-2.6478131 .000482$

C -1.040465 $0.030674-0.419732$

C $-0.415341-1.0872210 .395972$

C $-0.415327-2.0205232 .709083$

O $0.431924-1.856591-0.047839$

C -1.333742 -3.198787 2.779980

C $-1.929213-3.6027573 .912402$

C $-2.800011-4.8218944 .010465$

C $-4.152098-4.5309074 .620979$

C $-4.390169-4.7725165 .921369$

C $-5.253345-4.0538653 .702770$

C $-5.194067-2.573543 \quad 3.445120$
N $-0.893093-1.0948221 .700202$

H -2.2548632 .7755261 .181917$

H -3.360455 5.5328320 .443769

H -5.4672934 .1473350 .604076$

H -3.970572 $3.749613-2.042544$

H $-4.8060035 .211113-1.598552$

H -7.242098 $2.321579-3.265576$

H -8.269204 $3.997749-2.099180$

H -7.522239 $4.226077-0.516837$

H -7.261709 $5.417800-1.810291$

H $-5.1696301 .801918-4.567669$

H $-4.1625442 .071833-3.163200$

H $-5.5365440 .205549-1.990867$

H $-6.214535-0.156997-3.582040$

H -3.858697 -0.480001-4.453941

H $-5.164001-2.468392-3.646775$

H -4.679059-2.133572 -2.000473

H -4.096732 -4.583353 -2.952087

H -2.116911 -2.332923-1.385098

H -3.628521 -3.805624 -5.108262

H $-1.914782-3.891257-4.769819$

H -2.639479 -2.354978 -5.286168

H $-0.815697-3.846664-2.453904$

H $0.283633-0.418730-2.102970$

H $-0.674127 \quad 1.030704-2.256165$

H $-0.9007270 .256544-4.270174$

H -4.560452 -0.0908580 .205730$

H $-5.271723-0.0018773 .198412$

H $-5.397941-2.5232820 .268426$

H -4.429525 -3.720262 1.125098

H $-3.665043-2.2505650 .576259$

H $-0.6326210 .962997-0.012459$

H $-2.1205510 .047441-0.249160$

H $-0.369805-1.4730673 .655851$

H $\quad 0.594941-2.3550672 .452126$

H - $-1.488285-3.7652351 .864606$

H -1.748745 -3.050954 4.832323

H -2.267478 -5.5606774 .623826$

H -2.936066 -5.2975633 .031252$

H -5.367166 -4.596536 6.359243

H $-3.611207-5.1459716 .578021$

H $-6.232080-4.2629104 .156112$

H $-5.247342-4.6493712 .785308$

H -5.431458 -1.971639 4.323425

H $-1.626461-0.4437211 .965329$

C 0.2180204 .0435130 .950156

O $\quad 0.5309292 .7957151 .604751$

C 1.9270792 .7938401 .971037

C 2.5573023 .9302731 .183111

C 1.4323424 .9449661 .173753

H 1.9536073 .0148893 .045718

C 2.5196691 .4071051 .694996

H 3.4722224 .3100441 .647364

H 2.7956073 .6112460 .160722

C 1.6008656 .0260690 .120103

H 1.3739185 .4154512 .164678

C 4.0440791 .3260001 .887055

O 1.8845590 .4773392 .578781

H $2.246524 \quad 1.0972830 .680558$

H $4.549171 \quad 1.9922031 .178809$

O 4.3553441 .8255563 .194802

C $4.624343-0.0972861 .777853$

C $4.291963-0.8592760 .481395$

H $4.272046-0.6954592 .626609$

O $6.042276-0.0228751 .938114$

H $4.801287-1.8288170 .528647$

H $3.217677-1.0722390 .476925$

H $2.517536 \quad 6.5976570 .299066$

H $1.6624605 .599096-0.886556$

H $\quad 0.758370 \quad 6.724490 \quad 0.138563$

C -1.0737724 .6150081 .540034$

H $2.1449240 .709123 \quad 3.486872$

H 5.3197841 .7650893 .305907

H $\quad 6.3850680 .5729451 .250749$

H -1.168832 5.6744681 .275565

H -1.0440714 .5458262 .634347$

H $0.0937743 .822218-0.118008$

C $6.952464-0.954334-1.086672$ 
O $6.078075 \quad 0.167238-0.905762$

C $4.683006-0.145607-0.834331$

C $4.251770-0.882339-2.112106$

C $5.114312-2.111396-2.383787$

C $6.592963-1.733488-2.357785$

H $6.889557-1.613233-0.212233$

C $8.384270-0.411361-1.177606$

H $4.1569520 .816097-0.841108$

O $2.876755-1.243407-2.062415$

H $4.357353-0.195852-2.962254$

H $4.903255-2.919695-1.673871$

O $4.806778-2.624417-3.681803$

H $6.832530-1.136094-3.247107$

O $7.379972-2.921642-2.417130$

C 8.8740440 .0628950 .169278

H $9.089706-1.178124-1.517679$

H $8.4500440 .418809-1.891526$

H $2.780130-2.006146-1.467707$

H $3.853000-2.814550-3.699170$

H $7.115851-3.404740-3.218882$

$\begin{array}{lllll} & \text { O } & 8.663163 & 1.403751 & 0.298767\end{array}$

O $9.362931-0.6765751 .013576$

C 9.0793501 .9758061 .542788

H 8.8290653 .0398661 .527925

H $8.5507351 .503393 \quad 2.376254$

H $10.1616591 .870551 \quad 1.662637$

SCF Energy (B3LYP/6-31G*//MMFF)= -3245.92461854

02204

MMFF Geometry

C $0.970293 \quad 0.888405 \quad 1.838275$

C $0.844352-0.1392020 .984858$

C $-0.097794-0.143144-0.188372$

O $-0.931451-1.317206-0.031983$

C $0.641803-0.234579-1.535250$

C $1.4384300 .999221-1.893947$

C $0.895317 \quad 2.155637-2.321355$

C $2.9307930 .860359-1.775286$

C $-0.5640662 .461919-2.515016$

C -1.024552 $3.556464-1.545158$

C $-2.5071163 .909370-1.733654$

C $-2.8619125 .151846-0.912849$

C $-4.3525605 .501212-1.015821$

O $-3.302763 \quad 2.793137-1.309499$

O

C $-5.1758304 .235744-0.701844$

C $-4.696497 \quad 6.171083-2.351022$

O $-6.5659454 .465527-0.922162$

C $-4.7144802 .984372-1.486786$

C $-5.455281 \quad 1.705367-1.022694$

O $-5.0350393 .166727-2.872654$

C $-2.141572-1.313946-0.652405$

O $-2.602869-0.436301-1.363454$

C $-2.822720-2.579816-0.306612$

C $-4.088595-2.778064-0.702939$

C $-4.898522-3.968393-0.437550$

C $-4.254107-5.088367 \quad 0.337973$

C $-5.158453 \quad 1.2479830 .408561$

C -6.1290490 .1761190 .884108$

C -6.718764-1.074293 2.956614

O $-7.101394-0.1923740 .230665$

C -6.741721 -2.500990 2.506144

C $-7.856307-3.1085902 .070231$

C $-7.945383-4.556247 \quad 1.678364$

C $-8.300194-4.7426280 .219723$

C $-9.574960-4.665436-0.198702$

C $-7.184290-5.097006-0.736683$

C $-6.172650-3.994177-0.882706$

N $-5.799733-0.2892092 .152050$

H $\quad 0.3769651 .786463 \quad 1.684404$

H $1.430176-1.040306 \quad 1.145969$

H $-0.7394260 .745203-0.150157$

H $-0.071254-0.445138-2.342815$

H $1.295544-1.118367-1.535857$

H $1.5650112 .979068-2.566401$

H $3.4565411 .787024-2.027646$

H $3.2096970 .589079-0.752810$

H $3.2926140 .081246-2.453518$
H -0.705712 $2.803548-3.547689$

H $-1.1857961 .570958-2.400136$

H $-0.8571703 .217834-0.514605$

H $-0.4115584 .453752-1.699558$

H -2.684924 $4.095043-2.799488$

H $-2.2479006 .008732-1.217485$

H -2.6197364 .9708780 .144063$

H $-4.0819087 .237865-0.132425$

H $-5.061386 \quad 4.0481550 .372106$

H $-4.127527 \quad 7.101081-2.469885$

H $-5.7525406 .460172-2.387342$

H $-4.4808095 .541573-3.216599$

H -6.815122 $5.252007-0.407079$

H $-6.5342461 .847467-1.166938$

H $-5.1884170 .875920-1.692472$

H $-5.0530502 .295261-3.301648$

H $-2.265847-3.3100880 .269280$

H $-4.585241-1.993538-1.272531$

H $-3.394180-5.489233-0.209906$

H -4.922737 -5.9281280 .535730$

H $-3.913006-4.7318881 .316528$

H $-4.1372920 .858840 \quad 0.476423$

H -5.2496052 .0811551 .111507$

H $-7.708608-0.6079802 .900925$

H $-6.373510-1.0224183 .994042$

H $-5.815061-3.0637282 .580595$

H -8.781152 -2.537311 2.020410

H -8.713403 -5.0297462 .304102$

H -7.016161 -5.0900021 .911609$

H $-9.840876-4.824269-1.238343$

H $-10.380793-4.4387440 .491722$

H -7.587734 $-5.300857-1.737543$

H $-6.734938-6.042150-0.419277$

H $-6.552186-3.127092-1.425089$

H $-5.0090120 .131568 \quad 2.631170$

C 2.9257901 .9643533 .012305

O 3.9130051 .6596552 .005068

C 5.2262261 .8585582 .563452

C 5.0190202 .6400793 .854472

C 3.6951852 .0793454 .330018

H 5.6134840 .8637362 .815106

C $6.1585462 .535822 \quad 1.552724$

H 5.8338262 .4864614 .568605

H 4.9287523 .7168603 .670543

C 3.0097912 .9451035 .373409

H 3.8694281 .0801594 .752144

C 6.2518931 .8204420 .191148

$\begin{array}{lllll}\text { O } & 5.675464 & 3.863314 & 1.307409\end{array}$

H 7.1553272 .6474791 .995750

H $5.2992551 .926087-0.337707$

O $7.2133332 .521107-0.608617$

C $6.635161 \quad 0.330774 \quad 0.261723$

C $6.769079-0.302327-1.134178$

H $5.865733-0.2062470 .819557$

$\begin{array}{lllll}\text { O } & 7.855946 & 0.176398 & 0.981774\end{array}$

H $5.836968-0.130399-1.682079$

H $7.566376 \quad 0.208035-1.687892$

H 3.6376273 .0414456 .265231

H 2.8116443 .9522224 .991711

H 2.0546292 .5070655 .679187

C 1.8611110 .8665343 .048760

H $\quad 6.278462 \quad 4.2777790 .666657$

H $8.0640192 .501292-0.138803$

H 8.5712970 .5557530 .444051

H $1.2217950 .995977 \quad 3.929767$

H $2.346214-0.1118723 .141563$

H 2.4791262 .9293482 .739869

C $4.860131-2.679491-0.817138$

O $6.188751-2.566709-0.294411$

C $7.100443-1.811943-1.099605$

C $7.216479-2.450038-2.490641$

C $5.851248-2.592776-3.157537$

C $4.874517-3.302516-2.221355$

H $4.393156-1.688652-0.844186$

C $4.054164-3.5695990 .143727$

H $8.081998-1.907585-0.618263$

O $8.084930-1.679181-3.314175$

H $7.668518-3.445366-2.389904$ 
H $5.465211-1.619252-3.481051$

O $6.010667-3.373349-4.344571$

H $5.155869-4.361332-2.157823$

O $3.566581-3.257915-2.789499$

C $3.797530-2.9200941 .486247$

H $3.076059-3.805995-0.289849$

H $4.604563-4.4997140 .329998$

H $8.109962-2.104730-4.188267$

H $5.126941-3.492897-4.733007$

H $3.296749-2.325515-2.845963$

O $3.138295-3.8111032 .285689$

O $4.118245-1.7824521 .797187$

C $2.811622-3.3428043 .598059$

H $2.432532-4.1898234 .175931$

H $3.698787-2.9461134 .101118$

H $2.028858-2.5816443 .535788$

SCF Energy (B3LYP/6-31G**//MMFF) $=-3245.91570347$

$02 \_205$

MM̄FF Geometry

C $1.158798 \quad 3.3307630 .768159$

C 0.0967173 .6568091 .519309

C -0.8626622 .6282402 .057522$

O -2.1874103 .0481941 .651913$

C -0.8035682 .4984743 .590204$

C 0.5323962 .0274604 .130205

C 1.0055320 .7701374 .014173

C 1.3385093 .0770754 .848902

C $0.328172-0.4026763 .359258$

C $1.016356-0.7712292 .039452$

C $0.340921-1.9459371 .315953$

C $0.988066-2.168056-0.052203$

C $0.277195-3.266695-0.852902$

O $-1.044153-1.6399741 .143582$

O $0.754568-3.209412-2.205914$

C $-1.245552-2.994633-0.864104$

C $0.665026-4.661210-0.346712$

O $-1.930427-4.090944-1.463594$

C -1.822400 -2.688009 0.536146

C $-3.267697-2.1389010 .492833$

O $-1.806716-3.870614 \quad 1.337812$

C -3.1206252 .0706781 .494416$

$\begin{array}{llll}\text { O } & -3.003897 & 0.890768 & 1.783065\end{array}$

C -4.3047052 .6766530 .847281$

C $-5.192712 \quad 1.8766150 .237301$

C $-6.3817372 .302748-0.503710$

C $-6.7194203 .770859-0.490922$

C $-4.335115-3.077183-0.071431$

C $-5.744477-2.6464330 .306067$

C $-8.123105-3.312724-0.026711$

O $-5.987994-1.707378 \quad 1.058969$

C -8.711330 -2.331904 -0.991542

C $-9.393032-1.240633-0.609911$

C $-10.048830-0.286793-1.565483$

C $-9.6007811 .144236-1.372475$

C $-10.3712812 .027364-0.715252$

C -8.312568 $1.579028-2.031705$

C $-7.1067801 .368995-1.156395$

N $-6.703238-3.459536-0.282072$

H 1.3498562 .2819330 .557001

H $-0.0998294 .700073 \quad 1.752376$

H -0.6559231 .6615121 .585953$

H -1.591667 1.8179123 .937278

H -1.074709 3.4609354 .046222

H 1.9832970 .5451694 .438110

H 2.3006002 .6929225 .204249

H 1.5451453 .9233024 .186563

H 0.7869843 .4452055 .720295

H $\quad 0.371995-1.2558304 .046977$

H $-0.733891-0.1994443 .194582$

H 1.0093360 .1042281 .380934

H $2.067591-1.0168782 .227441$

H $\quad 0.438597-2.837888 \quad 1.945665$

H $2.052885-2.4009500 .051364$

H $0.943764-1.233695-0.629668$

H $0.554885-2.326727-2.561660$

H -1.427603 -2.122040 -1.506429

H $1.747708-4.811778-0.436704$
H $\quad 0.203958-5.446970-0.955267$

H $\quad 0.393752-4.8298690 .697524$

H -1.542368 -4.229671 -2.344457

H -3.543490 -1.866564 1.521430

H $-3.285873-1.194423-0.066841$

H -2.451321 -3.7614522 .056679$

H -4.3867623 .7573390 .849782$

H -5.0179810 .8019470 .253461$

H $-7.6742404 .002569-0.967464$

H $-5.9478024 .347123-1.013077$

H $-6.797366 \quad 4.1391150 .538412$

H -4.268858 -3.100738 -1.164672

H -4.193905 -4.094710 0.309695

H -8.269841 -3.005932 1.014351

H $-8.584500-4.295041-0.167707$

H $-8.600029-2.549208-2.051076$

H -9.523311-1.040261 0.451309

H -11.133819-0.361784-1.413831

H $-9.879420-0.584086-2.607858$

H -10.073169 $3.064543-0.604895$

H $-11.3148841 .731653-0.268716$

H -8.393142 $2.619501-2.363950$

H -8.167622 $1.013854-2.961497$

H $-6.7837860 .329156-1.100146$

H -6.410756 -4.177917 -0.938302

C $2.2612494 .130698-1.326288$

O $2.8026632 .825256-1.615250$

C $3.7691822 .940697-2.674263$

C $3.6279834 .355776-3.223827$

C $3.2189865 .123602-1.981799$

H $4.7576722 .840125-2.210964$

C $3.5583971 .827563-3.706428$

H $4.5579764 .721484-3.669522$

H $2.8423514 .420537-3.985564$

C $2.5995336 .477438-2.281049$

H $4.1055565 .264691-1.348893$

C $3.5799660 .396893-3.128341$

O $2.2857212 .010098-4.341965$

H $4.3060161 .919128-4.502742$

H $2.6900350 .220822-2.511550$

O $3.489211-0.514763-4.231389$

C $4.8402790 .050580-2.313921$

C $4.899923-1.437993-1.919872$

H $4.8631550 .666329-1.410555$

O $6.0050760 .376466-3.072642$

H $3.956203-1.714063-1.439135$

H $4.991816-2.044783-2.828537$

H $3.3117807 .116949-2.812591$

H $1.7046166 .380320-2.904871$

H $2.3119356 .988428-1.356988$

C 2.1354694 .3128280 .187586

H $1.6067302 .003665-3.645353$

H $2.685517-0.287166-4.729809$

H $5.974242-0.141553-3.895352$

H 1.8337635 .3399480 .421158

H 3.1073974 .1359410 .661995

H $1.2716244 .177383-1.799876$

C $5.040715-1.3626721 .142783$

O $6.071466-1.0002290 .215596$

C $6.095552-1.764437-0.995201$

C $6.260331-3.257789-0.675759$

C $5.202239-3.7485050 .309512$

C $5.166480-2.8401381 .538293$

H $4.060406-1.1765940 .691062$

C $5.185744-0.4571612 .375854$

H $7.001838-1.455359-1.531302$

O $6.211436-4.020274-1.876595$

H $7.253307-3.415747-0.234566$

H $4.214910-3.815288-0.161591$

O $5.534129-5.0685210 .744767$

H $6.083374-2.9946542 .121145$

O $4.076964-3.2211372 .376054$

C 4.7771190 .9737362 .097004

H $4.553790-0.8126023 .196815$

H $6.233150-0.4502342 .700881$

H $6.445902-4.936949-1.654511$

H $5.439018-5.661966-0.019145$

H $3.257166-3.1323651 .862001$ 
O $\quad 5.1898501 .7600693 .135324$

O $4.158558 \quad 1.3539121 .113089$

C $4.8729313 .150158 \quad 3.018943$

H $5.362152 \quad 3.5773292 .138340$

H 3.7897423 .2880352 .964202

H 5.2463563 .6603533 .910639

SCF Energy (B3LYP/6-31G**//MMFF) $=-3245.91526978$

02_206

MMTFF Geometry

C -2.6090640 .3341453 .185670$

C $-1.418370 \quad 0.4454103 .794189$

C $-0.366948 \quad 1.429990 \quad 3.354014$

$\begin{array}{lllll}\text { O } & 0.905585 & 0.739143 & 3.389397\end{array}$

C -0.2964342 .6610234 .277946$

C $-1.522817 \quad 3.5469724 .239738$

C $-1.847936 \quad 4.348947 \quad 3.206704$

C $-2.389076 \quad 3.5034445 .470646$

C $-1.094897 \quad 4.530949 \quad 1.917120$

C $-1.8944693 .975080 \quad 0.732374$

C $-1.1753994 .186751-0.607736$

C $-2.0866813 .769153-1.764947$

C $-1.3887103 .900799-3.125279$

O $0.027750 \quad 3.403680-0.621102$

O $-2.178762 \quad 3.196423-4.096318$

C $-0.0249903 .185037-3.049681$

C $-1.3202705 .357248-3.598090$

$\begin{array}{lllll}\text { O } & 0.730387 & 3.399849 & -4.239589\end{array}$

C $0.8143203 .578038-1.809920$

C $2.1080082 .734695-1.689327$

O $1.2313644 .943753-1.949738$

C $1.906657 \quad 1.253508 \quad 2.623615$

$\begin{array}{lllll}\text { O } & 1.847079 & 2.233575 & 1.898505\end{array}$

C 3.1115270 .4220012 .830612

C $4.231646 \quad 0.713388 \quad 2.152541$

$\begin{array}{lll}\text { C } 5.514023 & 0.014752 & 2.254618\end{array}$

C $5.600517-1.1432033 .214097$

C $1.9122731 .231564-1.474993$

C $3.1997210 .447870-1.688764$

C $3.981185-1.909026-1.900700$

O $4.256888 \quad 0.965781-2.038263$

C $5.060917-2.058148-0.877047$

C $6.359616-1.864888-1.155012$

C $7.483125-2.080162-0.181365$

C $8.240748-0.8070090 .122183$

C $9.196783-0.354727-0.706814$

C $7.941360-0.1050271 .426990$

C 6.5400980 .4362261 .485535

N $3.012040-0.912760-1.485310$

H -2.836008 $0.993746 \quad 2.351226$

H $-1.187674-0.1903714 .645019$

H $-0.562686 \quad 1.7256682 .317068$

H $0.584900 \quad 3.2675524 .032794$

H -0.1020462 .3314765 .308302$

H -2.752377 $4.950623 \quad 3.287614$

H -3.2658004 .1543755 .386352$

H -2.750169 2.4862255 .651164

H $-1.817758 \quad 3.829613 \quad 6.345870$

H $-0.923813 \quad 5.6047601 .772724$

H $-0.1056674 .068531 \quad 1.956945$

H -2.0680062 .9028870 .885599$

H -2.877207 4.4626030 .699898

H $-0.916125 \quad 5.248800-0.692231$

H -3.018888 4.347744 -1.756542

H -2.384215 2.720316 -1.630345

H -3.070885 $3.583552-4.094398$

H $-0.2417662 .111364-3.011601$

H -2.329762 $5.773275-3.702103$

H $-0.8637595 .429156-4.591340$

H $-0.7670936 .005614-2.915709$

H $0.170927 \quad 3.135512-4.989976$

H $2.7304782 .917030-2.575143$

H $2.7007543 .111840-0.843824$

H $1.9953475 .093025-1.368314$

H $3.047437-0.3947473 .539994$

H 4.2146821 .5501551 .455014

H $5.411826-0.8076834 .239739$

H $6.572087-1.6406103 .217166$
H $4.865161-1.9115822 .954418$

H $1.5369831 .034734-0.466187$

H $1.1874510 .831824-2.188166$

H $4.378350-1.619790-2.879843$

H $3.448826-2.858423-2.014508$

H $4.765935-2.3772000 .118672$

H $6.643092-1.564867-2.161784$

H $8.169981-2.819285-0.614665$

H $7.122205-2.5346100 .749326$

H $9.7630230 .543094-0.483022$

H $9.431869-0.873514-1.630531$

H $8.623140 \quad 0.743791 \quad 1.570193$

H $8.165874-0.7863132 .252198$

H $6.374624 \quad 1.2801290 .814173$

H $2.097743-1.242711-1.190278$

C $-4.142959-1.4459782 .365941$

O $-4.876397-0.584793 \quad 1.466799$

C -6.031477 -1.2908790 .990621$

C $-5.827268-2.7500671 .372931$

C -5.085856 -2.602629 2.690709

H $-6.882086-0.9108131 .573559$

C $-6.313230-0.996964-0.488256$

H $-6.770211-3.2960771 .470750$

H $-5.201452-3.2742870 .642255$

C $-4.377654-3.872094 \quad 3.131124$

H $-5.799790-2.2982843 .467842$

C $-5.300189-1.511386-1.534634$

O $-7.597119-1.549496-0.809160$

H $-6.4153930 .087884-0.614188$

H $-5.355713-2.604221-1.603339$

O $-5.717781-1.016283-2.815595$

C $-3.836734-1.092518-1.316988$

C $-2.912884-1.536492-2.465393$

H $-3.471621-1.540612-0.392157$

O $-3.7375920 .323918-1.190773$

H $-3.068770-2.604967-2.649678$

H $-3.186010-1.004581-3.384873$

H $-5.099517-4.6805293 .286530$

H $-3.653159-4.2086222 .381765$

H -3.840466 -3.713762 4.071375

C $-3.682311-0.6422983 .581543$

H $-7.528383-2.517887-0.753880$

H $-6.638958-1.298188-2.949438$

H $-4.1510830 .575486-0.347848$

H $-3.314870-1.3151384 .364240$

H $-4.523224-0.0717903 .993486$

H $-3.271378-1.8173861 .815323$

C $-0.959759-3.177817-0.790894$

O $-1.015889-1.751381-0.887426$

C $-1.419995-1.254855-2.168468$

C $-0.458044-1.757533-3.254337$

C $-0.294372-3.276278-3.221123$

C $0.031907-3.758737-1.806425$

H $-1.958165-3.586159-0.991617$

C $-0.653944-3.554080 \quad 0.665094$

H $-1.311345-0.164110-2.118097$

O $-0.898930-1.361684-4.548882$

H $0.529312-1.306006-3.102989$

H $-1.184173-3.783546-3.612307$

O $\quad 0.787351-3.646924-4.080174$

H $1.061182-3.469250-1.566948$

O $-0.027070-5.182975-1.795513$

C $0.718965-3.130595 \quad 1.139063$

H -1.372152 -3.074342 1.338511

H $-0.760346-4.6378670 .788786$

H $-0.973434-0.391942-4.547733$

H $\quad 0.582129-3.304401-4.967139$

H $\quad 0.357600-5.488878-0.957037$

O $1.244290-4.1081151 .933523$

$\begin{array}{llll}\text { O } & 1.247736 & -2.059528 & 0.874629\end{array}$

C $2.534734-3.8284262 .482809$

H $2.851032-4.6959133 .068051$

H $3.260803-3.6628541 .681953$

H $2.480908-2.9595483 .145043$

SCF Energy (B3LYP/6-31G**//MMFF) = -3245.90773073

$02 \quad 207$

MMFF Geometry 
C $-2.404604 \quad 3.8497510 .478760$

C -2.4508513 .9807391 .813954$

C -2.9662982 .9408792 .781646$

O $-2.803540 \quad 1.606428 \quad 2.246333$

C -4.4568373 .1896343 .067755$

C -5.0433452 .2336514 .087735$

C -5.8382601 .1861013 .790424$

C -4.7088822 .5440425 .523890$

C -6.2672140 .7169432 .427485$

C $-5.842231-0.7370072 .184828$

C $-6.086411-1.1688840 .731476$

C $-5.738174-2.6452960 .545358$

C $-5.876298-3.084609-0.917676$

O $-5.252879-0.367023-0.110462$

O $-5.233274-4.364417-1.031721$

C $-5.111740-2.091289-1.823794$

C -7.346055 -3.313302 -1.290275

O $-5.375865-2.392014-3.191534$

C $-5.413371-0.605157-1.516945$

C $-4.4791880 .388961-2.248188$

O $-6.730673-0.242930-1.951360$

C -1.574155 1.0355792 .396075

O $-0.588111 \quad 1.5444662 .906295$

C -1.631649 -0.3348281 .839062$

C $-0.523648-1.0913591 .818543$

C $-0.426781-2.4797491 .359865$

C $-1.673663-3.1259880 .817748$

C $-2.9862230 .267268-1.926831$

C $-2.216354-0.643531-2.867470$

C $0.039959-1.662614-3.160847$

O $-2.701388-1.124678-3.887633$

C $0.152713-2.984655-2.470694$

C $1.308132-3.474072-1.996246$

C $1.446075-4.822745-1.353866$

C $2.026662-4.7511050 .039407$

C $3.338471-4.9488160 .253571$

C $1.069312-4.5608921 .192059$

C $0.758331-3.116148 \quad 1.471521$

N $-0.907976-0.832144-2.442938$

H -2.789716 2.9460280 .013654

H $-2.088884 \quad 4.9021712 .265030$

H -2.397468 3.0219813 .716718

H -5.0227353 .1468252 .130948$

H -4.5978164 .2154523 .435429$

H -6.2233740 .5852194 .613479$

H $-5.197142 \quad 1.8567326 .222939$

H -3.629898 2.4711985 .691811

H -5.0339693 .5579585 .779036$

H -7.359340 0.7916452 .360913

H $-5.864140 \quad 1.351722 \quad 1.635620$

H $-4.775972-0.8414212 .420155$

H -6.392583 -1.396857 2.867146

H $-7.138095-0.9832740 .483719$

H $-6.354548-3.2774631 .196905$

H $-4.700726-2.8156450 .861344$

H -5.259482 -4.624308 -1.968796

H -4.045517 -2.285562 -1.655934

H -7.770396 -4.122649-0.683577

H -7.447728 -3.636962 -2.331921

H -7.968907 -2.428996-1.139475

H $-4.769658-1.862215-3.735796$

H -4.659305 $0.356037-3.329698$

H -4.785834 $1.399406-1.939659$

H -7.120035 $-0.993251-2.427697$

H -2.589192 -0.6929001 .481895$

H $0.402567-0.6650372 .201885$

H -2.414829 -3.249876 1.614209

H -1.498345 -4.1102230 .377924$

H -2.111415 -2.5157040 .020497$

H -2.530692 $1.260369-2.027283$

H $-2.832605-0.061043-0.895473$

H $0.992704-1.126285-3.185160$

H $-0.297523-1.811384-4.191440$

H $-0.754453-3.577857-2.383509$

H $2.218870-2.890888-2.109783$

H $2.095857-5.431798-1.996254$

H $\quad 0.486234-5.353320-1.325158$

H $3.756020-4.9328841 .254990$
H $4.025278-5.121241-0.568495$

H $1.511216-4.9750862 .109100$

H $0.172788-5.1661921 .029346$

H $1.608153-2.5612731 .870675$

H $-0.603382-0.413784-1.568375$

C $-0.7809224 .301109-1.369600$

O $0.306073 \quad 3.767901-0.594842$

C $1.5300933 .924708-1.335049$

C $1.1555784 .595925-2.655998$

C $-0.1302525 .318154-2.301015$

H $2.1632564 .601909-0.748796$

C $2.2458402 .573623-1.498750$

H $1.9412275 .271062-3.009686$

H $\quad 0.9751153 .860638-3.448879$

C $-0.9728885 .686081-3.509375$

H $0.1238816 .229840-1.743980$

C $2.6132121 .908453-0.152154$

O $1.4092961 .709195-2.266586$

H $3.1623792 .742230-2.077487$

H 3.1713872 .6296920 .457309

O 1.4361701 .5561210 .571860

C $3.4760150 .640401-0.310495$

C 3.8909280 .0422721 .047387

H $4.3697290 .899224-0.887637$

O $2.769342-0.350821-1.051635$

H 4.4076740 .8143801 .627666

H $2.994550-0.2375591 .613524$

H $-0.4167616 .351526-4.177741$

H -1.261861 $4.798449-4.082337$

H -1.888176 $6.202964-3.204760$

C $-1.8385474 .896918-0.440498$

H $1.9209510 .906768-2.463842$

H 0.9378112 .3723270 .745730

H $1.955024-0.559072-0.561485$

H -2.658186 $5.321082-1.030750$

H -1.3971015 .7113800 .147029$

H -1.207677 $3.468407-1.944709$

C $6.890235-0.1069780 .550559$

O $5.893078-0.9947170 .033487$

C $4.779070-1.2139260 .902216$

C $5.264717-1.8087872 .229816$

C $6.367585-0.9650272 .867417$

C $7.474059-0.6652931 .853364$

H 6.4491170 .8810590 .731740

C $7.9673240 .048447-0.528032$

H $4.160540-1.9778970 .416108$

O $4.172717-1.9507553 .131889$

H $5.657862-2.8165562 .041550$

H $5.956856-0.0429523 .295404$

O $6.914886-1.7079793 .959052$

H $8.044886-1.5805721 .650515$

O 8.3739650 .2929662 .405205

C $7.4499520 .896838-1.665913$

H $8.8641710 .544892-0.140750$

H $8.284667-0.923129-0.925352$

H $4.526019-2.3161753 .960986$

H $7.540620-1.1281714 .424745$

H $8.818321-0.1222443 .163320$

O $6.8485780 .109188-2.602443$

O $7.5399802 .117484-1.698684$

C $6.2661950 .804747-3.709015$

H $5.8140020 .065308-4.375228$

H $5.4842101 .485658-3.359226$

H $7.0368271 .350851-4.261239$

SCF Energy (B3LYP/6-31G**//MMFF) $=-3245.93772488$

02208

MM̄FF Geometry

C $2.808553-3.673297-0.959426$

C $1.606040-3.201139-0.596116$

C $0.520622-2.858128-1.586261$

O $0.000608-1.567500-1.187725$

C $-0.590465-3.924591-1.520216$

C $-1.484319-3.976042-2.744825$

C $-2.801118-3.689253-2.754260$

C $-0.821886-4.470966-4.007449$

C $-3.651714-3.227021-1.604548$

C $-4.693064-2.189294-2.052283$ 
C $-5.666046-1.838634-0.917472$

C $-6.739517-0.862072-1.398639$

C $-7.670257-0.424127-0.257389$

O $-4.915230-1.2477260 .144467$

$\begin{array}{lllll}\text { O } & -8.414145 & 0.702977 & -0.744437\end{array}$

C $-6.814514 \quad 0.0434950 .940452$

C -8.696299 -1.5124780 .080773$

O -7.6270740 .3001072 .083980$

C $-5.685412-0.9458111 .315678$

C $-4.728306-0.2922602 .337970$

O $-6.260657-2.1201431 .889705$

C $-0.514550-0.757278-2.148664$

O $-0.609922-0.987530-3.343243$

C $-0.9693480 .483042-1.487691$

C $-1.9830061 .173184-2.027878$

C $-2.5435792 .387471-1.450860$

C $-4.0163482 .295649-1.171172$

C $-3.524264-1.1675932 .710482$

C -2.421661 -0.323943 3.313899

C -0.6109661 .2665982 .673893$

O $-2.269431-0.2303284 .529230$

C - -1.1490082 .6529432 .515529$

C -0.5392723 .6064121 .795477$

C -1.081497 4.9971361 .638700

C -1.1663665 .4401810 .194932$

C $-0.3839626 .429981-0.267313$

C $-2.2192454 .805981-0.691108$

C $-1.7823823 .483138-1.262236$

N -1.662288 0.3220362 .350339

H $2.998672-3.822811-2.021227$

H $1.368946-3.0557640 .453876$

H $0.957156-2.782714-2.589179$

H -1.180367 -3.776935 -0.608990

H $-0.140884-4.922615-1.419887$

H -3.340344 -3.824517 -3.691365

H - $-1.538630-4.620306-4.822235$

H $-0.072294-3.756741-4.360145$

H $-0.329879-5.432418-3.826991$

H $-4.169305-4.101452-1.192768$

H -3.043170 -2.795151 -0.804822

H $-4.167569-1.285031-2.385282$

H -5.260934 -2.574861 -2.908268

H -6.127586 -2.767506 -0.561817

H -7.320409 -1.295616 -2.222321

H $-6.2561140 .032757-1.814266$

H $-9.0508050 .964770-0.058207$

H -6.3584481 .0033140 .662545$

H $-9.320891-1.731609-0.793526$

H $-9.385100-1.1785650 .864606$

H -8.236825 -2.4488050 .404253$

H -8.2557161 .0026491 .848100$

H -4.3648450 .6568951 .924365$

H $-5.268824-0.0509413 .262033$

H -5.581837 -2.586073 2.402364

H $-0.4845150 .785810-0.566528$

H $-2.480210 \quad 0.812011-2.925458$

H -4.559592 $1.965473-2.063846$

H -4.461565 $3.246043-0.868434$

H -4.200082 $1.573134-0.370653$

H -3.807862 -1.934319 3.439895

H $-3.117598-1.6939881 .839422$

H $\quad 0.220341 \quad 1.0696401 .991340$

H $-0.2735891 .110403 \quad 3.702783$

H -2.0815632 .8859713 .024363$

H $0.4071473 .383801 \quad 1.308696$

H -0.4269445 .6723952 .204855$

H -2.075010 5.0896852 .095245

H $-0.4586686 .776469-1.292889$

H $\quad 0.353565 \quad 6.9121150 .365624$

H -2.442126 $5.472798-1.534224$

H $-3.1457254 .723952-0.114190$

H $-0.7406463 .440762-1.577775$

H $-1.917993 \quad 0.219850 \quad 1.372575$

C $3.865626-3.6528431 .414478$

O $3.739518-2.2240201 .543856$

C $4.639933-1.7580872 .563521$

C $5.097721-3.0003003 .317234$

C $5.118811-4.0327772 .207777$
H $5.499958-1.3189332 .043242$

C $3.961022-0.6896393 .427444$

H $6.072465-2.8605703 .794333$

H $4.382032-3.2967294 .092421$

C $5.121066-5.4662222 .710059$

H $6.016399-3.8675971 .596423$

C 3.2876200 .4466062 .633618

O $2.938043-1.3035554 .224483$

H $4.685473-0.2763574 .138488$

H 2.3978040 .0635562 .119778

O 2.8209891 .4195893 .577424

C 4.1914241 .1569491 .611105

C 3.4688502 .3316000 .926670

H 4.5107190 .4394800 .851602

O $5.368481 \quad 1.6365552 .257848$

H $2.513453 \quad 1.9688430 .532580$

H 3.2401973 .0989301 .676118

H $6.010108-5.6585193 .319512$

H $4.239903-5.6771603 .325298$

H $5.125447-6.172757 \quad 1.874295$

C $3.967854-4.031580-0.066230$

H $2.314054-1.7379713 .617638$

H 2.2475690 .9560884 .211853

H 5.0889972 .2439082 .964221

H $4.135386-5.111376-0.156043$

H $4.851406-3.537591-0.488463$

H $2.972832-4.1007741 .868489$

C $3.6232291 .546128-2.019992$

O $4.6912152 .060011-1.215033$

C $4.2986542 .988889-0.199307$

C 3.611784 4.197917 -0.848268

C $2.4288823 .769290-1.709859$

C $2.8458092 .683211-2.697577$

H $2.9517550 .940724-1.398866$

C $4.2463670 .633394-3.089743$

H $5.227141 \quad 3.3512910 .259175$

O 3.1590095 .1204750 .137171

H $4.3310764 .730885-1.483491$

H $1.5864323 .438181-1.093508$

O $1.9613654 .891343-2.461929$

H $3.4576073 .134547-3.489514$

O $1.6803702 .143433-3.319216$

C $4.889249-0.607081-2.510713$

H $3.4817810 .300876-3.800503$

H $5.0271911 .185253-3.627290$

H $3.930675 \quad 5.3842130 .666828$

H $1.7976715 .613838-1.832343$

H $1.1915122 .885394-3.715015$

O $5.543427-1.269475-3.510743$

O $4.823326-0.957216-1.341702$

C $6.222634-2.469210-3.126493$

H $6.880920-2.764010-3.948112$

H $\quad 6.833369-2.306648-2.233023$

H $5.493907-3.266363-2.957406$

SCF Energy (B3LYP/6-31G**//MMFF) $=-3245.90875315$

02209

MM̄FF Geometry

C -1.381784 -2.928122 0.823626

C $-0.865347-2.786672-0.406159$

C $-1.562445-2.271471-1.643307$

O $-1.254579-0.863877-1.787845$

C $-3.099583-2.385350-1.631007$

C $-3.692511-2.511746-3.021491$

C $-4.141049-1.488863-3.774439$

C $-3.800550-3.925849-3.533744$

C $-4.075554-0.017014-3.482249$

C $-5.4509910 .606702-3.211073$

C $-5.9684110 .336219-1.789825$

C $-7.3402340 .983389-1.584119$

C $-7.8267910 .856666-0.132106$

O $-5.0226650 .899943-0.876215$

$\begin{array}{lllll}\text { O } & -8.932500 & 1.755786 & 0.043399\end{array}$

C $-6.704551 \quad 1.3318660 .815728$

C $-8.359073-0.5493100 .169416$

O -7.0545661 .0994292 .178214$

C -5.3302030 .6971730 .507387$

C -4.2216141 .3900181 .334928$ 
O $-5.373041-0.690304 \quad 0.847090$

C $-0.021447-0.497680-2.217138$

O $0.904048-1.227510-2.528993$

C $0.008163 \quad 0.980503-2.209356$

C $1.1779831 .614343-2.050918$

C $1.373744 \quad 3.064647-2.004269$

C $0.1840703 .940107-2.300556$

C -2.798850 0.9900150 .922839

C $-1.788248 \quad 1.6292081 .849979$

C $-0.4715793 .691788 \quad 2.209642$

O $-1.475793 \quad 1.1013902 .915092$

C $0.224621 \quad 4.706316 \quad 1.360487$

C 1.5533174 .8934601 .376023

C 2.2614445 .9607310 .592447

C $3.3909285 .419881-0.254481$

C 4.6571465 .4226560 .195799

C $3.0646984 .967919-1.658696$

C $2.6018283 .538658-1.708979$

N $-1.313466 \quad 2.846378 \quad 1.384607$ H -2.409256 -2.637302 1.021414 H $\quad 0.171827-3.077217-0.559065$ H - $1.162632-2.817105-2.508072$ H $-3.537261-1.521581-1.119339$ H -3.420354 -3.267874-1.062498 H $-4.574893-1.724111-4.745977$ H $-4.220975-3.973395-4.543915$ H -2.812632 -4.396318 -3.566071 H $-4.447052-4.518690-2.878472$ H $-3.3853850 .210853-2.666858$ H -3.648624 $0.465838-4.370803$ H $-5.3470881 .691619-3.345175$ H $-6.1802060 .258109-3.952638$ H -6.028456 $-0.746264-1.630445$ H $-8.077800 \quad 0.558446-2.276253$ H -7.279035 $2.052417-1.832907$ H -9.620082 $1.514662-0.600838$ H -6.6166362 .4212760 .699635$ H -9.205810 $-0.786401-0.486005$ H -8.745452 -0.6147201 .192535$ H -7.608919-1.330735 0.031976 H -7.9176951 .5207422 .331362$ H -4.3063582 .4786481 .219571$ H -4.3600911 .1669252 .400658$ H $-4.489845-0.9855321 .116787$ H $-0.9378871 .501376-2.301661$ H $2.0778371 .013445-1.932525$ H $-0.1742443 .765859-3.321030$ H $0.3948465 .007870-2.212794$ H $-0.6342243 .732225-1.602957$ H -2.647863 -0.0917140 .973734$ H -2.591774 $1.290330-0.110248$ H 0.2298243 .0645952 .768741 H - $1.123493 \quad 4.1949622 .931493$ H $-0.395715 \quad 5.3433830 .734748$

H 2.1660914 .2775292 .029582 H 2.6575056 .6909431 .310756 H $1.5635046 .523032-0.040093$ H $5.4761305 .064125-0.419150$ H 4.9009675 .7759851 .192260 H $3.961325 \quad 5.039820-2.289594$ H $2.3569235 .665173-2.115699$ H $3.3965892 .822466-1.498276$ H $-1.789348 \quad 3.2748040 .595525$ C $-0.312690-2.4621023 .059997$ O $0.720312-1.5624412 .598806$ C $1.730917-1.4530423 .620314$ C $1.085527-1.9796484 .895099$ C $0.224430-3.0970464 .345435$ H $2.538942-2.1369453 .333360$ C $2.279400-0.0237523 .715951$ H $1.824488-2.3234235 .625038$ H $0.452718-1.2274965 .378320$ C $-0.859641-3.5567305 .305515$ H $0.871195-3.9509394 .101996$ C 2.8484990 .5289152 .393662 $\begin{array}{llll}\text { O } & 1.231535 & 0.865966 & 4.115417\end{array}$ H $3.045214 \quad 0.030944 \quad 4.498938$ H 2.0370590 .6550101 .666890
O 3.3458241 .8485912 .657808

C $3.980220-0.3084911 .765577$

$\begin{array}{llll}\text { C } & 4.565917 & 0.365017 & 0.510854\end{array}$

H $3.602688-1.2977931 .497491$

O $5.035982-0.4849432 .709271$

H $3.7503590 .557744-0.192393$

H $4.991127 \quad 1.3368370 .789266$

H $-0.416396-3.9445396 .228523$

H $-1.531799-2.7349775 .574972$

H $-1.463849-4.3533614 .860517$

C $-0.615834-3.5044711 .981924$

H $1.0966330 .757528 \quad 5.071476$

H 4.1042811 .7610243 .260324

H $4.716120-1.0832573 .404907$

H - $-1.220157-4.3171112 .401785$

H $0.321501-3.9507651 .629337$

H -1.198779-1.849695 3.270495

C $4.293927-1.861648-1.576012$

O $5.248576-1.789563-0.510143$

C $5.678789-0.467674-0.167247$

C $6.3119090 .211222-1.389737$

C $5.3755120 .192743-2.594264$

C $4.855872-1.220079-2.852837$

H $3.368476-1.358266-1.273748$

C $3.980908-3.347064-1.821753$

H $6.477435-0.5913340 .575007$

O $6.6777591 .550910-1.076190$

H $7.236096-0.320982-1.649703$

H $4.5465180 .894040-2.458454$

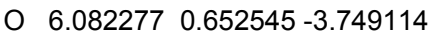

H $5.667524-1.838866-3.256357$

O $3.830536-1.164806-3.842624$

C $3.182687-3.979746-0.701866$

H $3.391430-3.462558-2.738124$

H $4.916679-3.910635-1.916792$

H $7.0408781 .950920-1.884827$

H $6.8327490 .053127-3.900939$

H $4.205299-0.727177-4.626195$

O $2.966036-5.294422-1.006096$

O $2.793056-3.406083 \quad 0.304671$

C $2.211733-6.036668-0.042545$

H $2.653538-5.9402250 .954059$

H $1.173107-5.693984-0.040823$

H $2.233303-7.090420-0.333143$

SCF Energy (B3LYP/6-31G**//MMFF) $=-3245.90036842$

$02 \_21$

MMFF Geometry

C $1.403890-0.8996392 .591564$

C $2.454702-0.4049533 .264306$

C 3.1152680 .9420683 .087959

$\begin{array}{lllll}\text { O } & 2.430914 & 1.764004 & 2.120030\end{array}$

$\begin{array}{lllll}\text { C } & 4.543526 & 0.738533 & 2.554084\end{array}$

C $5.462274 \quad 1.9143262 .813747$

C 5.7845082 .8593021 .909389

C 6.0836961 .9428634 .187130

C $5.247653 \quad 3.041660 \quad 0.517217$

C $6.2333542 .597908-0.574149$

C $6.1355071 .097635-0.890103$

C $7.1839900 .681752-1.921476$

C $7.022857-0.789823-2.329606$

O $4.824373 \quad 0.859735-1.412274$

O $7.846090-0.994491-3.487895$

C $5.554647-1.041559-2.736852$

C $7.556333-1.738071-1.248934$

O $5.357895-2.431205-2.989702$

C $4.527339-0.509422-1.708108$

C $3.098322-0.557909-2.302851$

O $4.571091-1.326277-0.537021$

C 1.3957362 .5347752 .540569

$\begin{array}{lllll}\text { O } & 0.901820 & 2.571354 & 3.654861\end{array}$

C $0.9656543 .336271 \quad 1.374347$

C $-0.227448 \quad 3.946678 \quad 1.381206$

$\begin{array}{llll}\text { C }-0.778239 & 4.760011 & 0.294984\end{array}$

C $0.1141965 .043217-0.886503$

C $2.0472370 .221634-1.496305$

C $0.673205 \quad 0.149587-2.142721$

C $-1.6600660 .978091-1.846972$ 
O $0.436735-0.533976-3.135591$

C $-2.0158822 .354636-2.309080$

C $-3.1233752 .993420-1.901840$

C $-3.5431934 .351725-2.384811$

C $-3.9264315 .281462-1.253973$

C $-5.2146525 .467725-0.919923$

C $-2.822456 \quad 6.053119-0.567223$

C $-2.047737 \quad 5.2042760 .402759$

N $-0.2590010 .928959-1.470782$

H $0.912968-0.3095011 .823648$

H $2.910779-1.0314674 .029987$

H 3.1352391 .4517594 .059044

H 4.4962130 .5305391 .480775

H $5.006076-0.1491993 .006501$

H 6.4951243 .6290522 .208950

H 6.7575982 .7953864 .322421

H 5.3090532 .0113544 .957161

H 6.6659701 .0318114 .359606

H 4.2749912 .5584050 .389333

H 5.0560534 .1154000 .391690

H $5.9900473 .161405-1.484696$

H $7.2599472 .863200-0.293227$

H $\quad 6.2738570 .5285670 .034651$

H $8.1975440 .868240-1.545231$

H $7.0794301 .304907-2.820912$

H $7.700541-1.905871-3.795057$

H $5.398661-0.524289-3.694109$

H $8.621178-1.547929-1.068093$

H $7.490004-2.783439-1.570439$

H $7.034292-1.638832-0.294949$

H $4.492241-2.537351-3.417933$

H $3.115746-0.132391-3.315005$

H $2.769241-1.600805-2.393084$

H $3.711861-1.285313-0.088318$

H $1.643873 \quad 3.3877440 .529652$

H $-0.866876 \quad 3.8300602 .255338$

H $0.9982165 .608164-0.570429$

H $-0.3707105 .624993-1.672767$

H $0.4454374 .110704-1.356418$

H $1.962833-0.183742-0.483477$

H $2.3304091 .277940-1.423856$

H $-2.2342570 .681611-0.963855$

H -1.858418 $0.262972-2.650909$

H -1.358920 $2.830737-3.032623$

H -3.790518 2.492739-1.205465

H -4.400768 4.214022 -3.056530

H -2.760537 4.820218 -2.994035

H $-5.5004886 .151781-0.127853$

H -6.012045 $4.933939-1.426772$

H -3.245254 6.8918090 .002272

H -2.188835 $6.526670-1.322217$

H $-2.6161384 .938413 \quad 1.295115$

H $0.026896 \quad 1.437954-0.639618$

C $0.923728-3.1532071 .593569$

O $-0.177023-2.8352370 .713405$

C $-0.755230-4.066306 \quad 0.234935$

C $0.238027-5.164430 \quad 0.590581$

C $0.791280-4.6456651 .900574$

H -1.674645 -4.2303340 .808127$

C -1.076334 -3.975289-1.259038

H $-0.240563-6.1441360 .682240$

H $1.037198-5.245898-0.155596$

C $2.093797-5.3111402 .311506$

H $0.041580-4.8026112 .687988$

C $-2.076587-2.865883-1.635262$

O $0.137184-3.731576-1.983146$

H -1.448941 -4.942665 -1.615413

H -1.624197 -1.883463 -1.470848

O $-2.326018-2.967433-3.042829$

C $-3.425609-2.930990-0.894289$

C $-4.462005-1.937662-1.456447$

H -3.256999 -2.720181 0.166399

O $-3.959856-4.251850-0.980205$

H -4.011331 -0.941865 -1.497424

H -4.714682 -2.220514 -2.485476

H $1.948026-6.3874872 .450261$

H $2.871817-5.1725471 .553258$

H $2.465281-4.8970613 .253976$
C $0.856028-2.2777482 .846683$

H $\quad 0.516747-2.900919-1.648063$

H -1.467608 -2.894227 -3.494401

H $-4.076774-4.460574-1.923128$

H $1.413028-2.7510483 .663624$

H $-0.181947-2.1733793 .179458$

H $1.847169-2.9606181 .031049$

C $-5.080626-0.3177081 .051921$

O $-5.530948-1.6448310 .754290$

C $-5.769810-1.902138-0.633077$

C $-6.830480-0.929447-1.168725$

C $-6.4696820 .526572-0.876786$

C -6.1215330 .7122190 .600845$

H $-4.124490-0.1300940 .549232$

C $-4.842558-0.2419582 .562805$

H $-6.202765-2.908989-0.686639$

O $-7.009765-1.118171-2.568019$

H $-7.790967-1.158349-0.688898$

H $-5.6465320 .859420-1.518128$

O $\quad-7.579750 \quad 1.364747-1.206887$

H -7.0314960 .6187381 .207076$

$\begin{array}{lllll}\text { O } & -5.605837 & 2.025139 & 0.803848\end{array}$

C -3.645049-1.078745 2.945907

H -4.6396440 .7815232 .896948$

H $-5.721257-0.5943663 .116382$

H $-7.666689-0.466792-2.868198$

H $-8.3343051 .095200-0.655931$

H -6.2661642 .6535280 .464662$

O $-4.053085-2.2261023 .558601$

O $-2.488281-0.7594852 .703276$

C $-3.009286-3.1231753 .948567$

H -2.333106 -2.634766 4.656588

H $-3.467723-3.9855144 .439782$

H -2.462798 -3.4714433 .067770$

SCF Energy $\left(B 3 L Y P / 6-31 G^{* *} / / M M F F\right)=-3245.92949871$

02210

MM̄FF Geometry

C $3.026436-0.7918132 .796753$

C $3.705388-1.9388762 .637666$

C $4.240648-2.5047461 .340687$

O $4.126349-1.5554250 .258757$

C $3.420781-3.7389710 .928063$

C $4.187895-4.6958470 .038428$

C $4.127655-4.723647-1.307238$

C $5.029011-5.7066710 .775937$

C $3.404718-3.783023-2.230385$

C $2.162223-4.408007-2.880101$

C $0.925492-4.360036-1.970435$

C $-0.268788-5.047592-2.634010$

C $-1.545437-4.913461-1.789067$

O $0.619708-2.980864-1.737129$

O $-2.660068-5.296386-2.608945$

C $-1.747632-3.429243-1.417184$

C $-1.539397-5.869534-0.590104$

O $-2.829987-3.276681-0.501219$

C $-0.478416-2.754665-0.847744$

C $-0.694180-1.225806-0.743853$

$\begin{array}{llll}\text { O } & -0.221715 & -3.279168 & 0.457372\end{array}$

C $5.112847-0.6388920 .088306$

O $6.127563-0.5059500 .750971$

C $4.6966040 .216518-1.043889$

C $5.2032681 .451045-1.166785$

C $4.8183222 .436998-2.179381$

C $3.8248442 .008270-3.228593$

C $0.585111-0.425424-0.456252$

C $0.2933231 .044063-0.201987$

C 1.4140723 .1690360 .465810

O $-0.832781 \quad 1.525908-0.282438$

C $1.7246373 .978081-0.754357$

C $2.6007254 .994885-0.763412$

C $2.8951335 .834260-1.975180$

C $4.3776705 .980759-2.245587$

C $5.0341597 .102268-1.903085$

C $5.0798564 .862576-2.985374$

C $5.3457973 .676544-2.100798$

N $1.435923 \quad 1.756307 \quad 0.132342$

H $2.807332-0.1656941 .938912$ 
H $3.910815-2.5427723 .520046$ H $5.295003-2.7708791 .486073$ H $2.504574-3.416600 \quad 0.422428$ H $3.088174-4.2992851 .812316$ H $4.701137-5.491365-1.826057$ H $5.565209-6.3784570 .097220$ H $5.775869-5.2017861 .396954$ H $4.398755-6.325021 \quad 1.423413$ H $3.151181-2.840483-1.738909$ H $4.111433-3.515503-3.026721$ H $1.947032-3.836058-3.792456$ H $2.370909-5.440407-3.185996$ H $1.163363-4.849076-1.019793$ H $-0.048804-6.103921-2.833070$ H $-0.454817-4.590138-3.615921$ H -2.511883 -6.209689-2.908728 H $-2.040024-2.898991-2.334442$ H - $1.457885-6.908938-0.929996$ H -2.480855 -5.810453 -0.032874 H $-0.715919-5.683910 \quad 0.102638$ H -3.608540 -3.703455 -0.898295 H -1.111147 -0.848167 -1.686674 H -1.428502 -1.0204900 .042327$ H $\quad 0.209687-2.6001880 .999206$ H $3.937685-0.175052-1.712038$ H $5.9399891 .785446-0.437556$ H $4.2254131 .174434-3.815808$ H $3.5667322 .796906-3.937679$ H $2.8843931 .690199-2.765211$ H $1.092941-0.8241420 .425667$ H $1.271151-0.492386-1.308099$ H 2.1475553 .3249891 .263062 H 0.4227293 .4369410 .843619 H $1.1889203 .732006-1.668082$ H 3.1126685 .2609960 .158764 H $2.4482046 .823170-1.808759$ H $2.4028145 .432437-2.869490$ H $6.0905377 .226993-2.116940$ H $4.5307997 .918932-1.396218$ H $6.0503225 .210072-3.364170$ H $4.5044644 .612798-3.880584$ H $6.0550583 .887190-1.298877$ H 2.3366521 .2886680 .111620 C $1.034333-0.3214854 .276953$ O 0.4120930 .6457753 .401176 C -0.4840941 .4551314 .179386$ C -0.7612990 .6748095 .454960$ C 0.5922150 .0400315 .700481 H 0.0847522 .3521544 .464175 C $-1.697776 \quad 1.9298803 .364928$ H $-1.089397 \quad 1.315911 \quad 6.278607$ H -1.509300 -0.108072 5.311804 C $0.543984-1.1461376 .649092$ H $1.263277 \quad 0.801776 \quad 6.120150$ C $-2.523123 \quad 0.8323902 .656435$ O $-2.5436652 .703574 \quad 4.213574$ H -1.324504 2.5988312 .579002 H -1.8431170 .2208752 .054764$ O $-3.127918-0.0381093 .608842$ C -3.6290541 .3945141 .730397$ C -4.3434890 .2769770 .947315$ H -3.183093 2.103709 1.025905 O -4.6099092 .0990842 .489048$ H -3.599515 -0.2655220 .356339$ H $-4.776397-0.441107 \quad 1.654363$ H $\quad 0.170393-0.8381857 .631131$ H -0.114712 -1.935312 6.271224 H $1.540634-1.5770476 .786699$ C $2.558773-0.2767844 .128272$ H -2.009292 3.4279484 .581656 H $-3.736747 \quad 0.4975914 .146143$ H -4.165932 2.8479752 .921287 H $3.026620-0.8532034 .935224$ H $2.912715 \quad 0.757424 \quad 4.224222$ H $0.646477-1.3062063 .986820$ C $-4.1542631 .487963-1.861380$ O $-5.062577 \quad 1.859375-0.818754$ C $\begin{array}{llll}-5.479819 & 0.790118 & 0.033677\end{array}$
C $-6.164227-0.306834-0.795711$

C $-5.287169-0.775251-1.953044$

C $-4.7817210 .417662-2.763711$

H $-3.2258221 .108333-1.420183$

C $-3.8273752 .755605-2.661763$

H $-6.246363 \quad 1.2128100 .695348$

O $-6.502782-1.4250510 .018033$

H $-7.1022000 .085372-1.210158$

H $-4.456933-1.390541-1.591196$

O $-6.069148-1.618399-2.802039$

H $-5.6190150 .844699-3.329774$

O $-3.825264-0.041099-3.717606$

C $-2.8375093 .632849-1.932231$

H $-3.3737292 .508248-3.628549$

H $-4.7257253 .348928-2.869195$

H $-7.075803-1.102088 \quad 0.734205$

H $-5.504383-1.879410-3.549742$

H $-3.072439-0.415967-3.229257$

O $-3.445621 \quad 4.266471-0.890013$

O $-1.660715 \quad 3.738702-2.253333$

C $-2.597538 \quad 5.104112-0.098621$

H $-2.1478165 .886326-0.717624$

H -3.211085 5.5761850 .673256

H $-1.824318 \quad 4.502877 \quad 0.388030$

SCF Energy (B3LYP/6-31G**//MMFF)= -3245.91870383

02211

MMFF Geometry

C -1.936136 -0.7570222 .957507$

C $-1.9860420 .547914 \quad 3.270178$

C -3.0912461 .5141932 .896750$

$\begin{array}{llll}\text { O } & -4.041014 & 0.855626 & 2.030424\end{array}$

C -2.489177 2.7010142 .122981

C $-3.4407513 .864621 \quad 1.937954$

C $-4.047240 \quad 4.183648 \quad 0.777601$

C $-3.641815 \quad 4.7285203 .156397$

C $-3.9946393 .445766-0.532125$

C $-3.166154 \quad 4.181036-1.596503$

C $-1.6506404 .080323-1.363005$

C $-0.8794794 .895415-2.402530$

C $0.6405794 .723353-2.248498$

O $-1.2870152 .700946-1.471222$

$\begin{array}{lllll} & \text { O } & 1.266227 & 5.247647 & -3.429685\end{array}$

C $0.9739963 .216866-2.191347$

C $1.1903815 .535758-1.069796$

O $2.352203 \quad 3.012310-1.888682$

C $0.0929762 .425132-1.198323$

C $0.3130110 .903196-1.387065$

$\begin{array}{lllll}\text { O } & 0.448417 & 2.792879 & 0.135054\end{array}$

C $-5.366506 \quad 1.0491102 .232531$

\begin{tabular}{llll}
\hline & -5.882916 & 1.732694 & 3.105322
\end{tabular}

C $-6.1749550 .293317 \quad 1.244774$

C $-5.647914-0.3534910 .192643$

C $-6.383337-1.129219-0.809752$

C $-7.872972-1.267115-0.635656$

C $-0.6937730 .035275-0.618390$

C $-0.538630-1.443883-0.923277$

C $-1.728470-3.616757-0.616825$

O $0.417855-1.908860-1.535209$

C -2.625839 -3.843830 -1.792063

C $-3.819026-4.452253-1.712783$

C $-4.726927-4.659236-2.891289$

C $-6.086275-4.020626-2.701703$

C $-7.160456-4.768112-2.396859$

C $-6.220898-2.534094-2.953467$

C $-5.691202-1.698400-1.819312$

$\mathrm{N}-1.593294-2.186529-0.411813$

H -2.770624 -1.197911 2.416422

H -1.1659050 .9878573 .832165$

H $-3.568877 \quad 1.845277 \quad 3.826216$

H -2.1342902 .3460191 .150374$

H -1.596930 3.0795212 .640234

H $-4.683966 \quad 5.0675520 .760173$

H -4.2908795 .5875392 .955186$

H -4.1014944 .1531433 .965527$

H -2.680937 5.1181693 .508097

H $-3.6455022 .415757-0.412791$

H $-5.0269563 .361887-0.895760$ 
H -3.405265 $3.729610-2.568319$ H -3.470616 $5.233852-1.644343$ H $-1.4205244 .441820-0.355013$ H -1.153356 $5.956346-2.347346$ H $-1.1644474 .564187-3.411154$ H $1.0055716 .180513-3.517835$ H $0.8128322 .807661-3.198510$ H $0.9762016 .602512-1.207324$ H $2.2814635 .455515-1.009313$ H $0.7683965 .236670-0.108058$ H $2.8702033 .505044-2.548107$ H $0.2193470 .655905-2.452644$ H $1.3293340 .629365-1.076598$ H 0.3072112 .0379140 .727333 H -7.2440840 .3065381 .430957$ H - $-4.570337-0.3203460 .040273$ H -8.107689-1.710735 0.338452 H -8.341021 -1.907412 -1.386411 H -8.359438 $-0.287755-0.703068$ H -0.5618020 .1656120 .458756$ H -1.718473 $0.325922-0.876710$ H -2.136404 $-4.040907 \quad 0.305887$ H $-0.747094-4.063311-0.803863$ H -2.275229 -3.482518 -2.756230 H $-4.165271-4.823346-0.750983$ H $-4.835141-5.741427-3.041418$ H $-4.276260-4.277918-3.816137$ H -8.145486 -4.326203 -2.289902 H $-7.078618-5.839109-2.243589$ H -7.260162 -2.272070 -3.181613 H $-5.670405-2.271963-3.866380$ H -4.613221 -1.541436 -1.859791 H -2.368928 -1.7012490 .029485$ C $0.507495-1.1574583 .752466$ O $1.066420-0.240362 \quad 2.790827$ C $2.486305-0.4824012 .667936$ C $2.840666-1.4257553 .808548$ C $1.571242-2.2372083 .950611$ H $2.611703-0.9785961 .698883$ C 3.2310580 .8603712 .676121 H $3.718216-2.0417133 .592491$ H $3.030152-0.8674384 .733635$ C $1.468047-2.9777905 .272480$ H $1.529742-2.9635563 .128904$ C $4.769214 \quad 0.7729692 .630922$ $\begin{array}{llll}\text { O } & 2.760625 & 1.631976 & 1.571874\end{array}$ H 2.9298781 .4061203 .579910 H 5.1768351 .7869932 .529302 $\begin{array}{lllll}\text { O } & 5.219210 & 0.292754 & 3.902177\end{array}$ C $5.382409-0.1112411 .522513$ $\begin{array}{llll}\text { C } 5.126713 & 0.400415 & 0.092786\end{array}$ H $5.041130-1.1448741 .629886$ O $6.801687-0.1428271 .738729$ H $4.0529090 .442481-0.098957$ H $5.511727 \quad 1.4248040 .024207$ H $2.297325-3.6841345 .382848$ H $1.498721-2.2879816 .122461$ H $\quad 0.533399-3.5438675 .331490$ C $-0.817087-1.7270703 .234781$ H 3.1810772 .5072361 .621945 H $6.189493 \quad 0.2391203 .866994$ H 7.1385750 .7633251 .632217 H -1.194779 -2.477846 3.938428 H $-0.618337-2.2484252 .290040$ H $0.350468-0.5982554 .683434$ C $3.952746-1.898381-1.588496$ O $5.271291-1.794137-1.033451$ C $5.814898-0.468959-0.986877$ C $5.833306 \quad 0.151505-2.390877$ C $4.4644640 .090639-3.057382$ C $3.918783-1.334398-3.016119$ H $3.241601-1.354285-0.955610$ C $3.560710-3.385139-1.579899$ H $6.864813-0.582344-0.690951$ O $6.2913391 .497892-2.331592$ H $6.552205-0.402119-3.008996$ H $3.7661220 .789981-2.586397$ O $4.5814550 .511725-4.418226$
H $4.505938-1.974198-3.687246$

O $2.577258-1.328387-3.497234$

C $3.130076-3.848522-0.204059$

H $2.718682-3.551339-2.261342$

H $4.398430-4.017229-1.894584$

H $6.2563491 .857686-3.234410$

H $5.194410-0.093786-4.869007$

H $2.587283-0.931647-4.385071$

O $2.223369-4.861437-0.333471$

O $3.553124-3.3972310 .851206$

C $1.710671-5.394948 \quad 0.891267$

H $2.526484-5.7048551 .551328$

H $1.075259-4.6519521 .381560$

H $1.103077-6.2716530 .651429$

SCF Energy (B3LYP/6-31G**//MMFF)= -3245.92083550

$02 \_212$

MM̄FF Geometry

C $1.278022-2.8042701 .741574$

C $0.588895-2.9497700 .600780$

C $1.090985-2.444270-0.723073$

O $0.159803-1.416731-1.132926$

C $1.139258-3.535123-1.805485$

C $2.099944-4.670989-1.533562$

C $3.441941-4.558885-1.571325$

C $1.456074-5.998197-1.232263$

C $4.244254-3.316722-1.844708$

C $5.042505-2.919198-0.597276$

C $5.742254-1.562134-0.754265$

C $6.719515-1.3453740 .405081$

C 7.3711170 .0423280 .357769

O $4.736941-0.538977-0.754753$

O $8.048650 \quad 0.2603001 .604864$

C $6.2613651 .104390 \quad 0.218222$

C $8.4646230 .118538-0.712780$

O 6.8234852 .4011310 .027092

C $5.247200 \quad 0.792774-0.909236$

C $4.0580331 .785672-0.890095$

$\begin{array}{llll}\text { O } & 5.903627 & 0.946206 & -2.173658\end{array}$

C $0.624127-0.435944-1.949895$

O $1.728325-0.359700-2.462581$

C $-0.4518990 .568145-2.074406$

C $-0.1536751 .808982-2.484028$

C $-1.1006202 .917609-2.608472$

C $-2.5640642 .603031-2.433187$

$\begin{array}{llll}\text { C } 3.084018 & 1.617282 & 0.280883\end{array}$

C 2.1610432 .8157450 .443559

C 0.3123353 .6176891 .907713

$\begin{array}{lllll}\text { O } & 2.341403 & 3.886954 & -0.129767\end{array}$

C $-0.695994 \quad 4.0950910 .910580$

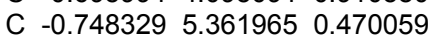

C -1.790137 $5.898163-0.471130$

C $-1.2083686 .351182-1.792643$

C $-0.6210787 .553606-1.915378$

C -1.382721 $5.440957-2.987819$

C $-0.6161624 .154207-2.848357$

N $1.138086 \quad 2.561386 \quad 1.349628$

H $2.257778-2.3334701 .712165$

H $-0.394775-3.4120380 .622893$

H $2.076042-1.984363-0.587918$

H $1.387193-3.084862-2.775741$

H $\quad 0.127597-3.935211-1.957713$

H $4.039295-5.448328-1.375324$

H $2.192900-6.785389-1.040793$

H $0.818520-5.920323-0.345793$

H $0.839268-6.320424-2.077688$

H $4.935736-3.523775-2.670512$

H $3.612790-2.489522-2.179164$

H $4.366715-2.8761130 .266794$

H $5.786813-3.699036-0.390918$

H $6.273810-1.548168-1.712861$

H $7.489052-2.1274800 .418861$

H $6.177306-1.4447201 .356186$

$\begin{array}{llll}\text { H } & 7.396223 & 0.191530 & 2.322484\end{array}$

H 5.7294781 .1393951 .176098

H $9.258093-0.607927-0.498669$

H $8.9509791 .100381-0.712686$

H $8.096910-0.083317-1.720890$ 
H 7.4517072 .5553820 .753380 H $4.4479112 .810369-0.935466$ H $3.4766141 .669954-1.814781$ H $5.2267211 .070707-2.859304$ H -1.452299 $0.271416-1.783389$ H $0.8826332 .045836-2.721352$ H -2.882011 $1.838139-3.150410$ H $-3.2201973 .462251-2.583530$ H -2.757703 2.236423-1.419606 H 2.4795340 .7153610 .135839 H 3.6220881 .5102761 .227616 H 0.9715964 .4244932 .246507 H $-0.203098 \quad 3.2056772 .781179$ H -1.434341 3.3769920 .566866 H $-0.012848 \quad 6.0763390 .834637$ H -2.284196 6.7474600 .019201 H $-2.5867135 .163941-0.641823$ H $-0.2259947 .897107-2.865487$ H $-0.5208278 .220015-1.064913$ H -1.027709 $5.934962-3.902012$ H -2.451088 $5.279035-3.155494$ H $0.4629824 .280345-2.946333$ H $1.133071 \quad 1.667703 \quad 1.832280$ C $0.301691-2.0740593 .970182$ O $-0.951495-1.5586013 .477328$ C $-0.988183-0.1378703 .719558$ C $\quad 0.245132 \quad 0.191138 \quad 4.560937$ C $1.227239-0.8646154 .094468$ H $-0.887918 \quad 0.3465102 .741306$ C $-2.313900 \quad 0.270887 \quad 4.370908$ H $0.599211 \quad 1.2126554 .392401$ H $0.051028 \quad 0.0801165 .633985$ C $2.398367-1.0763885 .036289$ H $1.607656-0.5526353 .114314$ C -3.579362 -0.154437 3.599840 O $-2.385192-0.3298855 .671592$ H -2.314982 1.3553864 .534712 H -3.707035 -1.241383 3.667375 O $-4.702471 \quad 0.4110794 .291661$ C $-3.627310 \quad 0.273648 \quad 2.120479$ C -5.0155090 .0256461 .494923$ H -2.856382 -0.2737101 .567137$ O $-3.319691 \quad 1.6645512 .039743$ H -5.325949 -0.9986301 .726723$ H $\quad-5.7430380 .700143 \quad 1.962370$ H $2.978526-0.1538605 .141941$ H $2.062358-1.3781286 .033824$ H $3.067338-1.8546254 .655535$ C $0.782524-3.2335273 .091618$ H -3.225422 $-0.050614 \quad 6.074050$ H $-4.647589 \quad 1.3778084 .198049$ H -3.159564 1.8774001 .105469 H $-0.036639-3.9538392 .971571$ H $1.598896-3.7626873 .597207$ H $0.083698-2.479778 \quad 4.967685$ C $-4.372679-1.931653-0.769429$ O $-4.083950-0.531624-0.721341$ C $-5.0549520 .260639-0.031969$ C $-6.4419810 .074892-0.666624$ C $-6.844227-1.397086-0.741799$ C $-5.737730-2.212451-1.411638$ H $-4.373432-2.3248540 .255002$ C $-3.213560-2.645231-1.479421$ H $-4.7734211 .306907-0.199773$ O $\quad-7.4114740 .8162640 .066045$ H $-6.4215710 .487595-1.683907$ H -7.086250 -1.799316 0.248913 O $-8.028151-1.526397-1.531692$ H -5.724741 -1.969220 -2.479716 O $-6.046423-3.601496-1.312242$ C $-2.941448-2.169573-2.892698$ H -2.296440 -2.464991 -0.908766 H -3.401374 -3.724399-1.505519 H $-8.2447390 .787275-0.433179$ H -8.754784 -1.104819-1.042755 H $-6.078734-3.834203-0.368650$ O $-1.989873-2.976576-3.449744$ O $-3.463562-1.205298-3.433817$
C $-1.578922-2.622897-4.773728$

H $-0.805438-3.328431-5.088766$

H $-2.424379-2.695706-5.464190$ H -1.156554 -1.613544 -4.783019 SCF Energy (B3LYP/6-31G**//MMFF) $=-3245.90616691$

02213

MM̄FF Geometry

C $1.126747-1.431792-0.301431$

C $-0.091632-0.957942-0.003047$

C $-0.425001-0.2622461 .289896$

O $-1.625131-0.8957131 .787187$

C -0.7246391 .2235841 .029772$

C -0.7206402 .0685012287158$

C -1.8237382 .5213942 .914064$

C 0.6447422 .4466072 .801669

C -3.262462 2.2551322 .569410

C -3.9808363 .5050432 .040804$

C -3.5468713 .9036980 .621256$

C -4.2440515 .1935290 .183667$

C $-3.9159665 .554651-1.273310$

O $-3.9083352 .835312-0.258319$

O $-4.838614 \quad 6.570415-1.695421$

C $-4.1666544 .318777-2.162999$

C $-2.5132236 .158281-1.409854$

O $-3.7390984 .558741-3.502062$

C $-3.5143683 .024828-1.623108$

C $-4.0189191 .804073-2.430641$

O $-2.0957343 .125823-1.756868$

C $-1.733522-1.1492413 .114246$

O $-0.900719-0.9100273 .977206$

C -3.024482 -1.807651 3.428750

C $-4.037406-1.9032952 .552730$

C -5.327655 -2.557529 2.782039

C $-5.538012-3.2520404 .101560$

C $-3.6038050 .448434-1.841256$

C $-4.223936-0.717868-2.591282$

C $-4.554106-3.183708-2.402480$

O $-4.825451-0.590872-3.654313$

C $-5.925481-3.360683-1.829401$

C $-6.266031-4.353190-0.993270$

C -7.644638 -4.518996 -0.419024

C $-7.657567-4.4903971 .094350$

C $-7.780351-5.6255831 .802732$

C $-7.619393-3.1441811 .785388$

C $-6.245966-2.5321111 .793227$

N -4.008688 -1.922646 -1.934185

H $1.935459-1.3053100 .414408$

H $-0.894592-1.067069-0.727557$

H $0.400894-0.3856732 .000513$

H $-1.685217 \quad 1.316316 \quad 0.512995$

H $0.019761 \quad 1.6463000 .342339$

H $-1.6895753 .146528 \quad 3.796354$

H 0.5935703 .0648203 .704253

H $1.225847 \quad 1.5534523 .049953$

H 1.1895133 .0179032 .043096

H -3.375344 1.4227951 .868632

H $-3.763067 \quad 1.9318403 .491029$

H -5.0569363 .2874092 .035448$

H -3.8236104 .3437032 .730570$

H -2.4597914 .0379950 .602780$

H -3.983869 6.0229660 .852971

H $-5.332414 \quad 5.067888 \quad 0.271419$

H $-4.7434157 .329979-1.095299$

H $-5.2539404 .164489-2.208003$

H $-2.4301547 .073012-0.810772$

H -2.310688 $6.458703-2.443811$

H -1.721434 $5.480086-1.085585$

H $-4.1803345 .370524-3.805822$

H $-5.1161591 .828110-2.466826$

H -3.657048 1.864929 -3.464872

H -1.715295 2.235961-1.816332

H -3.098617 -2.2043104 .436028$

H $-3.919582-1.4650911 .562883$

H $-4.762061-4.0079344 .267454$

H $-6.495163-3.7733414 .170778$

H $-5.507267-2.5296254 .924537$

H $-2.5172430 .328088-1.887882$ 
H $-3.9151830 .379946-0.792652$

H -3.866806 -3.974863 -2.087195

H -4.605541 -3.178459-3.495858

H -6.678410 -2.632373 -2.122239

H $-5.519287-5.090892-0.708592$

H $-8.042528-5.475481-0.782324$

H $-8.331451-3.752318-0.798947$

H -7.822205 -5.614238 2.886765

H -7.837814 -6.592891 1.314635

H -8.021711 -3.218076 2.801979

H $-8.304594-2.4563411 .273215$

H -5.999669 -2.0022190.872853

H -3.562961 -1.914069-1.021217

C $2.172019-3.442162-1.398759$

O $3.477140-3.205780-0.828878$

C $4.472098-3.741209-1.716603$

C $3.755192-4.841424-2.482090$

C $2.407036-4.186438-2.719313$

H $4.741198-2.938488-2.414981$

C $5.718592-4.173172-0.937899$

H $4.270186-5.112935-3.408200$

H $3.632655-5.745880-1.875508$

C $1.303247-5.170113-3.068601$

H $2.508956-3.467049-3.543336$

C $6.265129-3.1064950 .033877$

O $5.418123-5.339690-0.161543$

H $6.501585-4.477425-1.642022$

H $5.604556-3.0241490 .905938$

O $7.527898-3.566378 \quad 0.529838$

C $6.460223-1.710638-0.582972$

C $7.059494-0.7064590 .418614$

H $5.491237-1.337780-0.927125$

O $7.306890-1.796796-1.727178$

H $6.500393-0.772572 \quad 1.358144$

H $8.097471-0.9826530 .640628$

H $1.539702-5.700185-3.996997$

H $1.167597-5.917643-2.279766$

H $0.349902-4.650418-3.207952$

C $1.463794-2.099732-1.605681$

H $4.675791-5.1179150 .426759$

H $7.382749-4.4445350 .921913$

H $8.156817-2.169851-1.436322$

H $\quad 0.557092-2.247819-2.203875$

H $2.121212-1.419661-2.161697$

H $1.620108-4.066519-0.685330$

C $4.756507 \quad 1.2824210 .431356$

O $5.7498671 .138404-0.587591$

C $7.0426820 .747643-0.109507$

C 7.5600731 .7867880 .895755

C 6.5597152 .0365432 .026020

C 5.1674202 .3366631 .465501

H 4.6278760 .3173950 .937780

C $3.4057241 .564288-0.240159$

H $7.7106780 .773058-0.979807$

O 8.8096391 .3677351 .433388

H 7.7384082 .7311590 .365374

H $6.526974 \quad 1.1856002 .715659$

O 7.0002713 .1493252 .808478

H 5.1694353 .3390881 .023123

O 4.2195702 .3443052 .529927

C $3.3180132 .891710-0.962550$

H $3.2013760 .791147-0.989780$

H $2.618219 \quad 1.5275530 .520215$

H 9.0875082 .0378262 .081094

H 7.0482703 .9264562 .225961

H 4.5255242 .9926663 .187172

O $2.0056833 .261236-1.052084$

O $4.2683833 .508703-1.423082$

C $1.7556444 .493623-1.734438$

H $2.1106894 .434043-2.767717$

H $0.6760224 .663184-1.744118$

H $2.2370835 .322202-1.206491$

SCF Energy (B3LYP/6-31G**//MMFF)= -3245.92061441

$02 \_214$

MM̄MF Geometry

C $\quad 0.454016 \quad 3.376143 \quad 0.695423$

C $0.9027884 .171416-0.288539$
C $1.8331843 .778360-1.413175$

O $2.1278032 .364578-1.386535$

C $3.1596704 .543163-1.271636$

C $4.1303704 .264352-2.401836$

C $5.1982843 .448508-2.300576$

C $3.8607565 .009474-3.682632$

C $5.5972852 .625804-1.106309$

C $6.0725091 .224782-1.517102$

C $6.4207210 .374720-0.287193$

C $7.139118-0.910669-0.697437$

C $7.434472-1.8035800 .516585$

O $5.1995590 .051923 \quad 0.388543$

O $7.820337-3.0975830 .029129$

C $6.133519-1.9921411 .321879$

C $8.615365-1.2802511 .342612$

O $6.383635-2.6904502 .539896$

C $5.378566-0.6719571 .613181$

C $3.983720-1.0023812 .192533$

O 6.1099110 .0933952 .571743

C $1.3049621 .537509-2.086234$

O $0.3198781 .851369-2.734676$

C $1.8056830 .157615-1.900001$

C $1.053356-0.877255-2.301661$

C $1.377709-2.295877-2.135370$

C $2.741742-2.644037-1.601207$

C 3.0257630 .1917362 .297860

C $1.649789-0.2961132 .711258$

C $-0.294367-1.5951291 .822261$

O $1.252907-0.2071823 .870371$

C $-0.067387-3.0163121 .402714$

C $-0.828331-3.6570450 .502986$

C $-0.593146-5.069770 \quad 0.050658$

C $-0.668908-5.221978-1.455375$

C $-1.707349-5.848905-2.033648$

C $0.488370-4.704102-2.286682$

C $0.436956-3.209197-2.453867$

N $0.956659-0.8770671 .661520$

H $\quad 0.7624912 .3358960 .743824$

H $0.5700445 .207872-0.306074$

H $1.3584424 .042793-2.366235$

H $3.6220164 .306136-0.305931$

H $2.9680425 .624398-1.237978$

H $5.8585083 .359771-3.162184$

H $4.6167734 .806686-4.448646$

H $2.8889104 .722754-4.096176$

H $3.8574376 .089083-3.500915$

H $6.4137043 .144267-0.589405$

H $4.7733732 .524704-0.394334$

H $\quad 5.2827770 .730494-2.097575$

H $6.9527291 .319141-2.165127$

H $7.0577360 .970250 \quad 0.377374$

H $8.065265-0.684962-1.240315$

H $6.508366-1.473916-1.399523$

H $8.601887-2.982954-0.538314$

H $5.475739-2.6396440 .724412$

H $9.518680-1.2203560 .723769$

H $8.858957-1.9662532 .161394$

H $8.437873-0.2889451 .764835$

H $6.842036-3.5170392 .311040$

H $3.511504-1.7642281 .561457$

H $4.091141-1.4464363 .190861$

H 5.4995380 .6980243 .022687

H $2.7613580 .030427-1.404676$

H $0.089680-0.670015-2.765527$

H $2.840080-2.318792-0.562365$

H $3.525161-2.159731-2.195461$

H $2.961590-3.713039-1.627874$

H 3.3716490 .9172453 .041368

H 2.9376430 .7235151 .343919

H -1.038413 -1.092085 1.197443

H $-0.623198-1.5580412 .865012$

H $\quad 0.769287-3.5409841 .858551$

H -1.676262 -3.139621 0.065766

H - $-1.351822-5.7018490 .530065$

H $\quad 0.377731-5.4428670 .399379$

H - $1.761292-5.978910-3.109479$

H -2.531838 -6.238739-1.445693

H $0.461593-5.149064-3.289796$ 
H $\quad 1.419726-5.055074-1.834728$ H $-0.508217-2.851104-2.864331$ H $1.369704-0.8612540 .734279$ C -1.8711983 .2323541 .603280$ O $-2.480346 \quad 3.7714410 .411281$ C $-3.805430 \quad 4.217244 \quad 0.730291$ C -4.1696253 .5466672 .045206$ C -2.8254973 .5675642 .751411$ H -3.7260145 .2975720 .917494$ C $-4.7668354 .020451-0.445232$ H -4.9488434 .0852612 .592338$ H -4.5054342 .5164541 .893718$ C -2.7287942 .6097583 .924338$ H -2.6372734 .5884633 .110937$ C $-5.1823042 .581957-0.822542$ O $-5.9621834 .761016-0.161006$ H -4.325865 4.495327-1.330605 H $-5.8791532 .189028-0.073099$ O $-5.9454162 .674218-2.036793$ C $-4.0436411 .565966-1.027202$ C $-4.5333230 .262762-1.692342$ H $-3.5803231 .350333-0.059899$ O $-3.0518212 .151807-1.872514$ H -5.524857 $0.010895-1.304708$ H $-4.6367920 .439713-2.769512$ H -3.4989172 .8307654 .670283$ H -2.856149 1.5726473 .601627 H -1.7518482 .6890354 .411618$ C -0.4798883 .8407841 .778496$ H -6.3985154 .3424710 .600569$ H -5.341165 2.967025 -2.740699 H $-2.2636271 .583935-1.846683$ H -0.0542743 .5495522 .745354$ H -0.5493304 .9358481 .769634$ H -1.8005232 .1458581 .462937$ C $-4.731981-2.0216150 .353286$ O $-3.554522-1.334492-0.090178$ C $-3.559938-0.914838-1.460343$ C $-3.789187-2.120953-2.380565$ C $-5.051774-2.885895-2.006043$ C $-5.024669-3.246945-0.523721$ H $-5.587851-1.3370470 .336339$ C $-4.498413-2.4557891 .809915$ H $-2.547729-0.549435-1.670641$ O $-3.867468-1.720268-3.743952$ H $-2.936521-2.805953-2.298681$ H $-5.957017-2.321893-2.259639$ O $-5.106851-4.099871-2.758648$ H -4.267986 -4.023294 -0.351507 O $-6.288657-3.795831-0.154219$ C $-4.497796-1.2910912 .775024$ H $-5.292019-3.1383612 .133711$ H $-3.528998-2.9583641 .899319$ H $-3.044151-1.248735-3.957404$ H $-5.076427-3.857415-3.700052$ H $-6.456907-4.555067-0.738345$ O $-4.286350-1.7706824 .036575$ O $-4.663258-0.1195432 .467842$ C -4.273344 -0.801795 5.090274 H $-4.445551-1.3261626 .034037$ H $-3.291831-0.3222525 .129506$ H -5.063433 -0.055849 4.959757 SCF Energy (B3LYP/6-31G $\left.{ }^{* *} / / M M F F\right)=-3245.91209642$

\section{$02 \_215$}

MM̄FF Geometry

C -0.7988063 .6746340 .107942$

C -0.6786722 .6654190 .982776$

C -0.2690452 .8509992 .418599$

O 0.8597621 .9729632 .639180

C -1.3972482 .4288113 .379478$

C -2.6317573 .3033403 .329738$

C -3.8103582 .9146392 .803765$

C -2.4930844 .6569343 .974474$

C -4.0826571 .6053522 .115334$

C -5.3137671 .6840011 .200700$

C $-5.433090 \quad 0.460642 \quad 0.280253$

C $-6.7603750 .477665-0.480417$
C $-6.936989-0.775723-1.352309$

O $-5.350749-0.724421 \quad 1.074716$

O $-8.317554-0.854706-1.740217$

C $-6.636143-2.034235-0.505189$

C $-6.130500-0.670839-2.652385$

O $-6.615783-3.204029-1.319396$

C $-5.323012-1.9367380 .308168$

C $-5.198221-3.1231201 .294692$

O $-4.185188-1.989733-0.553403$

C 1.6997422 .2626353 .666027

O 1.6682003 .2557294 .379119

C 2.6436171 .1474093 .910200

C 2.9052750 .1978462 .999291

C $3.726061-0.9978313 .203544$

C $4.305817-1.2280934 .573614$

C $-4.051028-2.9895732 .309439$

C $-2.675258-3.0488281 .671110$

C $-0.475653-1.8893391 .668839$

O $-2.399037-3.843556 \quad 0.774984$

C $0.517513-2.8373462 .261398$

C $1.250511-3.6827741 .520138$

C $2.330578-4.5762072 .063133$

C $3.687699-4.2486461 .470679$

C $4.143196-4.8975450 .385667$

C $4.537274-3.2019062 .167141$

C $3.879733-1.8495292 .168621$

N -1.792922 -2.131088 2.220560

H -0.5676024 .6812660 .452184$

H $-0.897286 \quad 1.6468300 .675697$

H 0.0384103 .8881982 .600534

H -1.017452 2.4327104 .410226

H -1.647434 1.3739503 .204843

H -4.6527173 .6012632 .868924$

H -3.434362 5.2167973 .971232

H -2.176655 4.5509555 .017282

H -1.7499835 .2606463 .444338$

H -3.2183501 .3021461 .516192$

H -4.2495410 .8363542 .878946$

H -6.2064611 .7585961 .835630$

H -5.2774152 .5913470 .584511$

H $-4.5882480 .483564-0.418339$

H $-6.8495041 .386481-1.088478$

H $-7.590730 \quad 0.5154330 .238682$

H -8.545998 -0.031800 -2.205820

H -7.468638 -2.160533 0.201226

H $-6.4705660 .185737-3.246845$

H -6.280797 -1.552211 -3.285232

H -5.060216 -0.544336 -2.480215

H -7.462816 -3.239823 -1.796056

H $-6.126078-3.2030021 .875198$

H $-5.076023-4.0593400 .736151$

H -4.467269-2.324858 -1.420096

H 3.0931661 .1391644 .897543

H 2.4444270 .2750092 .015299

H $4.922974-0.3754524 .878406$

H $4.955979-2.1039454 .629056$

H $3.507747-1.3667005 .310876$

H -4.171196 -2.068891 2.890649

H $-4.103528-3.8220233 .021492$

H $-0.199520-0.859257 \quad 1.914144$

H $-0.528904-1.9810520 .579992$

H $\quad 0.682330-2.782263 \quad 3.334417$

H $1.084213-3.7231510 .446187$

H $2.060021-5.6142491 .830769$

H $2.385309-4.5223133 .157441$

H $5.118622-4.679741-0.036626$

H $3.546296-5.657236-0.108099$

H $5.509224-3.1033921 .668124$

H $4.742529-3.5664723 .177656$

H $3.454903-1.5659761 .204907$

H $-2.094457-1.5435702 .992144$

C $-1.7399072 .236403-1.837975$

O $-0.6392451 .310484-1.928939$

C $-0.7372150 .583560-3.166731$

C $-2.1134400 .900680-3.739457$

C $-2.3302852 .315823-3.246970$

H $\quad 0.028500 \quad 0.996268-3.833984$

C $-0.482811-0.910160-2.938647$ 
H -2.137571 $0.815667-4.830183$

H $-2.885650 \quad 0.237296-3.335087$

C $-3.7854212 .752218-3.283355$

H -1.733653 $2.997821-3.867592$

C $0.849208-1.249221-2.242129$

O $-1.534591-1.449849-2.127128$

H $-0.545372-1.440219-3.896246$

H $0.819359-0.942046-1.189958$

O $0.978323-2.677496-2.240330$

C $2.101175-0.648435-2.905571$

C $3.407111-1.202898-2.303832$

H $2.0770310 .439570-2.793773$

O $2.085500-0.926298-4.305985$

H $3.349192-1.136780-1.213269$

H $3.496714-2.264884-2.561650$

H $-4.1742922 .709740-4.305882$

H $-4.4131672 .109132-2.657912$

H $-3.8948553 .779920-2.923404$

C $-1.2437403 .593799-1.328664$

H -1.571203 -0.927847 -1.307177

H $0.183482-3.039409-1.812087$

H $2.064386-1.892749-4.414022$

H -2.044197 4.332995-1.452709

H $-0.4005293 .923863-1.947198$

H -2.472228 $1.805154-1.143859$

C $4.8038991 .247022-1.122103$

O $4.6372730 .931370-2.510352$

C $4.661911-0.465967-2.824378$

C $5.991479-1.082533-2.368632$

C $6.265118-0.810957-0.893375$

C $6.1295140 .680824-0.593772$

H $3.9636930 .841316-0.546298$

C $4.7846062 .777207-0.989983$

H $4.639274-0.533732-3.919350$

O $5.994956-2.484450-2.616701$

H $6.805698-0.649827-2.964617$

H $5.606533-1.411869-0.258990$

O $7.598675-1.229742-0.596726$

H $6.9742331 .211362-1.050988$

O 6.2261920 .8866270 .813983

C $3.4203243 .368907-1.273209$

H 5.0623853 .0855910 .023634

H $5.4936903 .209749-1.706431$

H $6.841478-2.835520-2.290969$

H $7.768069-1.0149600 .336719$

H 5.4702190 .4420081 .233032

O $3.5606454 .709391-1.497236$

O $2.3667462 .747859-1.272027$

C $2.3511755 .421265-1.774786$

H $2.6123556 .456894-2.008374$

H $1.8408774 .986156-2.639268$

H $1.7022705 .413403-0.894795$

SCF Energy (B3LYP/6-31G**//MMFF) $=-3245.92160119$

$02 \_216$

MM̄FF Geometry

C 1.3981113 .1715802 .364589

C 1.7386721 .9423001 .950212

C 2.1234430 .8258572 .884363

O $3.309171 \quad 0.1854162 .362359$

C $1.017564-0.2416822 .903711$

C $1.079703-1.1342064 .125573$

C $1.676687-2.3406904 .171913$

C $0.361214-0.5993135 .337975$

C $2.480043-3.022973 \quad 3.100271$

C $1.755215-4.2246082 .480959$

C $0.716778-3.8223711 .421899$

C $-0.016009-5.0573670 .896005$

C $-0.970526-4.711004-0.255283$

O $1.418904-3.1795630 .352515$

O $-1.364773-5.941153-0.882956$

C $-0.203274-3.887862-1.311764$

C $-2.258353-4.0496650 .249832$

O $-1.103616-3.449267-2.326335$

C $0.588174-2.699384-0.711684$

C $1.514814-2.074129-1.783391$

O $-0.334660-1.706342-0.259387$

C 4.5114150 .7863722 .545538
O $4.743279 \quad 1.814397 \quad 3.158604$

C $5.498675-0.0262831 .802336$

C 6.6738620 .5121491 .448932

C $7.703065-0.1396560 .636127$

C $7.453659-1.5617910 .203367$

C $2.552804-1.084095-1.229375$

C $3.377732-0.451792-2.339838$

C $5.176471 \quad 1.235060-2.712806$

O $3.226613-0.728043-3.527401$

C $6.4978290 .542632-2.842282$

C $7.6742171 .186218-2.779475$

C $9.0083780 .514458-2.951425$

C $10.0046030 .895712-1.876583$

C $10.949196 \quad 1.822889-2.108714$

C $9.9560570 .145536-0.563322$

C 8.7948600 .5736300 .290371

N $4.298808 \quad 0.464547-1.848936$

H 1.3897673 .3970083 .428026

H 1.7280631 .7177490 .886303

H 2.3251871 .2008363 .895569

H $1.074836-0.8502291 .994705$

H $\quad 0.0253480 .2294482 .882086$

H $1.620462-2.9018995 .104175$

H $0.430698-1.2757006 .196413$

H $\quad 0.7870490 .3624615 .641329$

H $-0.701265-0.4548595 .116442$

H $2.805061-2.3283352 .321776$

H $3.402322-3.3831833 .574184$

H $2.516865-4.8545082 .002483$

H $1.283185-4.8275703 .266473$

H $0.004312-3.1186431 .865813$

H $-0.557187-5.5626481 .705736$

H $\quad 0.718200-5.7875760 .526964$

H - $-1.792634-6.497582-0.209845$

H $\quad 0.504425-4.574379-1.797663$

H $-2.786918-4.7171970 .940883$

H -2.952939 -3.863865 -0.575635

H $-2.082547-3.1073450 .772680$

H $-0.576028-3.112043-3.069259$

H $2.066123-2.873745-2.295520$

H $0.907881-1.560406-2.539557$

H $\quad 0.083797-0.832748-0.308725$

H $5.203955-1.0295521 .515083$

H 6.8884841 .5366251 .750168

H $8.243792-1.974654-0.426226$

H $6.527970-1.632676-0.378555$

H $7.369703-2.2178781 .076900$

H $2.060047-0.278072-0.676701$

H $3.240994-1.598370-0.548837$

H $5.2750142 .230870-2.269185$

H $4.7148321 .333534-3.700622$

H $6.488178-0.529295-3.024816$

H $7.6794442 .264238-2.633283$

H $9.3990050 .802181-3.936316$

H $8.905885-0.577479-2.979657$

H $11.6837442 .083745-1.353984$

H $11.0058282 .346000-3.057594$

H $10.871350 \quad 0.3313150 .014404$

H $9.968515-0.928443-0.766500$

H $8.878181 \quad 1.6005450 .649540$

H $4.3886130 .591089-0.845260$

C -0.5138354 .4679511 .403137$

O $-1.115913 \quad 3.3305120 .749136$

C $-2.0334623 .800952-0.259195$

C -2.3101365 .2524080 .093496$

C -0.9530465 .6947810 .603358$

H -1.491900 $3.732760-1.211564$

C $-3.2619962 .885893-0.279044$

H -2.656404 $5.838183-0.762999$

H -3.0621445 .3242350 .889080$

C -0.9972506 .9761041 .417612$

H $-0.2858905 .841611-0.257030$

C $-4.3909943 .373142-1.202838$

O $-2.835281 \quad 1.590924-0.715777$

H $-3.6407002 .762388 \quad 0.742007$

H $-4.8077474 .309410-0.813205$

O $-3.8238173 .684808-2.482386$

C $-5.5234412 .353509-1.432842$ 
C $-6.1676341 .828167-0.138708$ H $-5.143408 \quad 1.523167-2.037465$ O $-\begin{array}{llll}-6.533108 & 2.987789 & -2.226947\end{array}$ H $-5.402183 \quad 1.3612640 .487254$ H -6.5745332 .6743090 .428643$ H $-1.369477 \quad 7.8062070 .808364$ H $-1.655314 \quad 6.8764712 .287375$ H $0.000927 \quad 7.242876 \quad 1.779032$ C $1.003789 \quad 4.282044 \quad 1.432127$ H -2.125986 $1.300914-0.116823$ H $-4.5493283 .986122-3.055792$ H $-6.8997493 .724509-1.708893$ H 1.4978965 .2011341 .766984 H $1.3723094 .066504 \quad 0.421067$ H -0.9212124 .5021272 .421765$ C $-5.946902-1.144565-0.743483$ O $-6.902803-0.229564-1.292452$ C -7.307072 $0.817151-0.404355$ C $-7.930230 \quad 0.2140790 .862943$ C -7.015977 -0.8206561 .516885$ C $-6.529886-1.8428160 .488606$ H $-5.034735-0.604258-0.463994$ C $-5.591305-2.147557-1.845564$ H -8.102613 $1.366918-0.922946$ O $-8.240196 \quad 1.2291321 .811744$ H $-8.874835-0.280248 \quad 0.600329$ H $-6.168594-0.3433992 .023324$ O $-7.745324-1.5183752 .529307$ H -7.362191 -2.496085 0.197033 O $-5.526394-2.6633811 .082392$ C $-4.764602-1.488472-2.924770$ H $-5.025722-3.003707-1.463844$ H $-6.498137-2.543149-2.318759$ H $-8.840478 \quad 1.8598831 .378648$ H -8.078162 -0.8528253 .155731$ H $-5.916757-3.0718731 .874037$ O $-3.500597-1.255401-2.468190$ O $-5.194727-1.207260-4.035886$ C $-2.624760-0.595833-3.387777$ H -2.461142 -1.223371-4.268710 H $-3.0372300 .376187-3.675073$ H -1.665907-0.434722 -2.887829 SCF Energy (B3LYP/6-31G**//MMFF) $=-3245.90704076$

02_217

MM̄FF Geometry

C $0.1243135 .035950-0.963400$

C $0.867244 \quad 3.919584-1.022984$

C $1.3211863 .293413-2.317088$

O $0.971973 \quad 1.890340-2.244485$

C $2.8497613 .429542-2.456018$

C $3.3934763 .002111-3.807202$

C $4.260476 \quad 1.987886-4.000337$

C $2.9474763 .832704-4.984430$

C $4.9024311 .114066-2.960800$

C $4.184309-0.231682-2.827242$

C $4.827376-1.116261-1.750111$

C $4.135496-2.478928-1.685967$

C $4.674976-3.347312-0.539462$

O $4.699083-0.440619-0.494740$

O $3.764583-4.441335-0.348690$

C $4.663252-2.5188490 .763755$

C $6.032159-3.970458-0.885630$

O $5.294960-3.2314571 .823696$

C $5.306163-1.1239320 .611108$

C $5.129707-0.2253951 .860961$

O $6.716304-1.276910 \quad 0.405771$

C $0.345571 \quad 1.330644-3.309465$

O $0.042187 \quad 1.887597-4.356101$

C $0.049968-0.104389-3.092089$

C $0.205562-0.752627-1.926575$

C $-0.092268-2.170145-1.691626$

C $-0.686757-2.952733-2.835394$

C $3.680541 \quad 0.051388 \quad 2.276266$

C $3.145453-0.9277803 .307816$

C $1.003440-1.7680914 .258481$

O $3.868174-1.6432123 .996338$

C $0.705861-3.0271203 .508472$
C $-0.518763-3.3650523 .080016$

C $-0.821698-4.6292242 .333423$

C $-1.139444-4.372491 \quad 0.879210$

C $-2.401567-4.4316330 .423727$

C $0.023827-4.142523-0.058098$

C $0.174098-2.705335-0.480535$

N $1.761335-0.8818623 .396242$

H $-0.1605275 .511818-1.900363$

H $1.1818353 .419974-0.111253$

H $0.8095563 .781571-3.154305$

H $3.3201912 .857604-1.649551$

H $3.1442214 .475970-2.297955$

H $4.5774681 .769741-5.019513$

H $3.4828523 .570962-5.903541$

H $1.8804043 .699030-5.179718$

H $3.1356364 .894016-4.790895$

H $5.9446350 .943089-3.258901$

H $4.9584601 .623239-1.994250$

H $3.137695-0.045119-2.561590$

H $4.192549-0.754198-3.791772$

H $5.890444-1.237184-1.989606$

H $4.224601-3.006764-2.643609$

H $3.059767-2.330599-1.526868$

H $3.695286-4.924057-1.190194$

H $3.610751-2.3973661 .048938$

H $5.944509-4.609534-1.772564$

H $6.383976-4.624266-0.079963$

H $6.805748-3.228039-1.091542$

H $4.847773-4.0915191 .901629$

H $5.719841-0.6229992 .695600$

H 5.5813340 .7491141 .625406

H $7.155382-0.4441940 .645786$

H $-0.336774-0.609063-3.972257$

H $0.588814-0.208561-1.065341$

H -1.606689-2.474158 -3.190845

H $-0.964323-3.974959-2.571162$

H $\quad 0.023135-3.017195-3.667270$

H 3.6354521 .0405482 .749592

H 3.0194550 .0772351 .403101

H $\quad 0.094175-1.2397864 .559364$

H $1.583564-1.9977845 .158083$

H $1.542533-3.6934023 .311181$

H $-1.357308-2.7037413 .279997$

H -1.667540 -5.1199792 .832295$

H $0.008814-5.3429182 .403839$

H $-2.631483-4.282932-0.625742$

H $-3.233242-4.6269781 .092269$

H $-0.058490-4.819882-0.914378$

H $0.958860-4.4460470 .430300$

H $\quad 0.591098-2.060570 \quad 0.293650$

H $1.241517-0.3084182 .738057$

C $-0.124358 \quad 5.0639591 .616693$

$\begin{array}{lllll}\text { O } & -0.898731 & 3.850907 & 1.685669\end{array}$

C -1.553752 3.7840112 .964972

C -0.8716094 .8291303 .837163$

$\begin{array}{llll}C & -0.567827 & 5.907290 & 2.814288\end{array}$

H $-2.593538 \quad 4.092306 \quad 2.802703$

C $-1.5178802 .351374 \quad 3.504833$

H -1.5148355 .1761254 .651356$

H 0.0587634 .4508494 .276193

$\begin{array}{lllll}\text { C } & 0.470661 & 6.912441 & 3.281483\end{array}$

H $-1.499214 \quad 6.4386942 .576364$

C $-2.052352 \quad 1.2965992 .512930$

$\begin{array}{llll}\text { O } & -0.157040 & 2.012802 & 3.802167\end{array}$

H -2.063275 2.3009414 .454619

H $-1.344037 \quad 1.2015121 .682383$

O $-2.0447180 .041405 \quad 3.201548$

C $-3.473354 \quad 1.5782091 .991866$

$\begin{array}{llll}C & -4.073178 & 0.487853 & 1.089811\end{array}$

H -3.4927472 .5280551 .453664$

O $-4.356542 \quad 1.7470313 .107261$

H $-4.250168-0.4192711 .684455$

H $-5.0852320 .808906 \quad 0.812795$

H $0.121303 \quad 7.4384024 .175978$

H $1.4194616 .424125 \quad 3.528038$

H 0.6676967 .6593082 .506050

C $\quad-0.375458 \quad 5.746311 \quad 0.269231$

H -0.1577241 .1124674 .169534$ 
H -2.176369 -0.6579792 .539789$ H $-4.323240 \quad 0.9301213 .634228$ H $0.075776 \quad 6.7454180 .276112$ H -1.4572245 .8798790 .138443$ H 0.9314554 .7856181 .724553 C $-5.090789-1.016541-1.317459$ O $-3.788326-1.120515-0.728284$ C $-3.2738070 .101575-0.172373$ C -3.181208 1.152481-1.287520 C $-4.525727 \quad 1.363157-1.977191$ C $-5.0939680 .023176-2.445583$ H $-5.824589-0.736051-0.552349$ C $-5.464355-2.408314-1.850320$ H -2.254037 -0.1379510 .145536$ O $-2.686598 \quad 2.396807-0.817865$ H -2.475413 $0.802359-2.048551$ H -5.234914 1.890249-1.328605 O $-4.339752 \quad 2.192019-3.127290$ H -4.506348 -0.343816 -3.297032 O $-6.431435 \quad 0.215651-2.901541$ C $-5.774284-3.386414-0.735786$ H $-6.356931-2.349718-2.482772$ H $-4.629782-2.818792-2.431101$ H $-1.7970402 .241446-0.457158$ H -3.929651 $3.019930-2.823108$ H $-6.4055400 .894555-3.597647$ O $-6.015794-4.615074-1.283201$ O $-5.809264-3.1144610 .456184$ C $-6.340841-5.651566-0.351495$ H $-7.253196-5.3964280 .196032$ H $-6.515913-6.572045-0.914854$ H $-5.508569-5.8160630 .339070$

SCF Energy (B3LYP/6-31G**/MMFF) $=-3245.92145138$

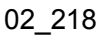

MMFF Geometry

C $0.844068-1.6153481 .233579$

C $-0.359254-1.4297720 .672998$

C $-0.545810-1.212035-0.804640$

O $-0.7968170 .200788-0.971363$

C $-1.763973-1.992319-1.322096$

C $-1.803511-2.094071-2.832957$

C $-2.561933-1.324040-3.637193$

C $-0.944171-3.183116-3.422166$

C $-3.455238-0.177879-3.251403$

C $-4.942994-0.489921-3.464559$

C $-5.517695-1.458767-2.419330$

C $-6.981927-1.778644-2.727903$

C $-7.620275-2.651596-1.636718$

O $-5.418512-0.825106-1.140336$

O $-9.042518-2.633668-1.832741$

C $-7.345790-2.010352-0.260041$

C $-7.184916-4.117303-1.750163$

O $-7.793167-2.857988 \quad 0.795821$

C $-5.863977-1.627529-0.039937$

C $-5.724682-0.7804801 .248364$

O $-5.088845-2.8199630 .094861$

C $-0.0860430 .879290-1.906090$

O $\quad 0.7321020 .412793-2.686783$

C $-0.3844502 .330426-1.863923$

C -1.328341 2.874399-1.079746

C -1.628444 $4.301863-0.948064$

C $-0.7705965 .272798-1.715996$

C $-4.335205-0.1558971 .441538$

C $-4.269900 \quad 0.7237602 .678064$

C -2.7775772 .3731173 .805951$

O $-5.175140 \quad 0.7901553 .505326$

C -3.3044983 .7140843 .401382$

C -2.5343814 .7947923 .204881$

C -3.074094 6.1340902 .790277

C $-2.502623 \quad 6.6141251 .473473$

C -1.5467837 .5580051 .441984$

C -3.098987 6.0686290 .193601

C -2.627970 $4.676039-0.122013$

N -3.0682951 .4166592 .753139$

H $1.724911-1.6027660 .596258$

H -1.241845 -1.398284 1.305352

H $0.362636-1.517365-1.337792$
H $-2.683535-1.527229-0.951501$

H $-1.763740-3.011925-0.913847$

H $-2.522851-1.512220-4.709709$

H $-1.028204-3.236192-4.512847$

H $0.109959-3.013834-3.182071$

H $-1.240022-4.158318-3.021568$

H $-3.2788060 .160398-2.226512$

H -3.185361 $0.671972-3.891439$

H $-5.4888020 .460801-3.404509$

H $-5.099550-0.889888-4.473978$

H $-4.922114-2.378341-2.416901$

H -7.076568 -2.259934 -3.709356

H -7.552784 -0.841922 -2.798005

H $-9.225859-2.967882-2.727633$

H $-7.951351-1.094976-0.199857$

H $-7.475321-4.528210-2.724622$

H -7.689166 -4.738341-1.001576

H $-6.106802-4.254391-1.643666$

H $-8.728648-3.0628990 .626005$

H $-6.4544210 .039568 \quad 1.219650$

H $-5.960744-1.3962992 .125479$

H $-4.296042-2.629590 \quad 0.619410$

H $0.2399012 .937655-2.511484$

H $-1.9359112 .221294-0.455759$

H $0.2855495 .146121-1.451941$

H $-1.0142786 .318957-1.518882$

H $-0.8852095 .116653-2.794237$

H -3.576999 $-0.936161 \quad 1.554280$

H -4.0727460 .4592280 .573233$

H -1.693294 2.3790603 .953557

$\mathrm{H}-3.2563332 .0507124 .736120$

H -4.3803213 .7982463 .264159$

H $-1.459947 \quad 4.719146 \quad 3.353513$

H -2.8374696 .8492143 .588882$

H $-4.169196 \quad 6.121426 \quad 2.723654$

H -1.1519527 .9323830 .503506$

H -1.1342547 .9752172 .354660$

H -2.904805 $6.746586-0.645246$

H $-4.193012 \quad 6.055598 \quad 0.282401$

H $-3.191790 \quad 3.8980530 .392714$

H -2.412330 1.3534761 .979851

C $2.071307-2.897113 \quad 3.052970$

O $3.359019-2.5806802 .481555$

C $3.814372-3.7244281 .739134$

C $3.073939-4.9103262 .341970$

C $1.701644-4.3014992 .566601$

H $3.472650-3.5894370 .705493$

C $5.340182-3.8205561 .761553$

H $3.055628-5.7764451 .673883$

H $3.512668-5.2194063 .297816$

C $0.837582-5.0786803 .544426$

H $1.182135-4.2575351 .601414$

C $6.069151-2.5467531 .281682$

O $5.764975-4.0856983 .103788$

H $5.654060-4.6855891 .164938$

H $5.994504-1.7664022 .048500$

O $7.465436-2.8603101 .191461$

C $5.578961-1.974178-0.063660$

C $6.511036-0.884009-0.627905$

H $4.573985-1.5607700 .072652$

O $5.469029-3.025364-1.025262$

H $6.743312-0.166822 \quad 0.165305$

H $7.453929-1.352863-0.932871$

H $0.648441-6.0902363 .170546$

H $1.319162-5.1674384 .523939$

H $-0.128155-4.5833883 .686510$

C $1.070547-1.7886012 .709995$

H $6.735713-4.1451373 .094562$

H $7.571403-3.5675040 .532957$

H $6.359786-3.378821-1.187757$

H $1.466946-0.8378003 .087451$

H $\quad 0.123357-1.9780903 .228243$

H $2.220093-2.9014224 .140229$

C $5.0003911 .796872-0.741813$

$\begin{array}{lllll}\text { O } & 4.738959 & 0.605797 & -1.496555\end{array}$

C $5.894692-0.164652-1.850572$

C $6.8841840 .699387-2.644848$

C $7.2469521 .974678-1.895067$ 
C $5.9797342 .718094-1.482553$

H $5.4149351 .529704 \quad 0.237294$

C $3.6547612 .510048-0.522140$

H $5.540698-0.943963-2.536859$

O $8.073557-0.023470-2.944025$

H $6.4308020 .980058-3.604678$

H $7.8833961 .766188-1.026987$

O $8.0044942 .827954-2.756016$

H $5.4982203 .143169-2.372845$

O $\quad 6.3364193 .804849-0.629451$

C 2.8406681 .8875050 .591853

H $3.8257153 .555114-0.239603$

H $3.0503302 .476043-1.435772$

H $7.813958-0.821630-3.435254$

H $8.7860762 .325902-3.044830$

H $6.9722164 .358059-1.115112$

O $1.782490 \quad 2.706140 \quad 0.869115$

O 3.0938340 .8269191 .144857

C 0.9226712 .2865341 .933259

H 1.5009061 .9944872 .814966

H 0.2824793 .1306962 .201542

H $\quad 0.2907951 .462642 \quad 1.594386$

SCF Energy (B3LYP/6-31G**//MMFF) $=-3245.91191186$

02_219

MMFF Geometry

C -0.6244174 .0443072 .153105$

C -1.0771412 .8109391 .878613$

C -1.481422 1.8118402 .936041

O -0.5555240 .6930493 .003536$

C -2.934222 $1.333507 \quad 2.735579$

C $-3.5080550 .608770 \quad 3.936374$

C $-3.798559-0.7060153 .982902$

C -3.8308911 .4837925 .122499$

C $-3.580111-1.7621872 .934523$

C $-4.896373-2.1964312 .273033$

C $-5.263051-1.3173061 .067031$

C $-6.708109-1.5634880 .634003$

C $-7.061941-0.765881-0.628992$

O $-4.368450-1.6629520 .002140$

O $-8.311595-1.264428-1.129755$

C $-5.996123-1.049944-1.705393$

C $-7.275040 \quad 0.722718-0.330568$

O $-6.200323-0.227932-2.853093$

C $-4.538920-0.903321-1.201293$

C $-3.575805-1.479932-2.269557$

O $-4.2469430 .477966-0.991573$

C $-0.354461-0.1188491 .933441$

O $-0.8044930 .011195 \quad 0.806852$

C $0.481904-1.2544982 .382318$

C $0.467186-2.3813411 .655300$

C $1.204252-3.6150391 .928258$

C $2.111137-3.643976 \quad 3.127857$

C $-2.102802-1.538643-1.845325$

C $-1.250492-2.307342-2.843531$

C $1.090700-3.112110-3.176202$

O $-1.697575-2.786276-3.882265$

C $1.273779-4.460694-2.555301$

C $2.407334-4.854433-1.954701$

C $2.594569-6.199239-1.311781$

C $2.884355-6.0976320 .170851$

C $4.144204-6.1596240 .633959$

C $1.710186-5.9999751 .120995$

C $1.047824-4.6521441 .079721$

N $0.072817-2.391150-2.433197$

H $-0.516316 \quad 4.3314393 .197575$

H -1.203280 2.5022240 .846241

H $-1.3998672 .298057 \quad 3.914897$

H $-3.005864 \quad 0.7072401 .841924$

H -3.5807432 .1981532 .531228$

H $-4.243215-1.0930194 .899868$

H -4.3599440 .9378545 .911141$

H -2.916817 1.8910165 .564624

H $-4.4727632 .316587 \quad 4.817117$

H -2.847411 -1.459641 2.182889

H $-3.142103-2.6304193 .443491$

H $-4.774521-3.2320561 .929497$

H $-5.706785-2.1925243 .012376$
H $-5.126901-0.263868 \quad 1.331384$

H $-7.405189-1.3221581 .446350$

H $-6.851057-2.6326700 .423203$

H $-8.979070-1.155370-0.430941$

H -6.142398 $-2.087114-2.039073$

H -8.0793950 .8547370 .402995$

H $-7.5969541 .262610-1.228036$

H -6.3834801 .2134870 .065325$

H $-7.118557-0.360747-3.144910$

H $-3.895441-2.502433-2.511605$

H -3.644629 $-0.890265-3.192510$

H $-3.2843460 .598292-0.986739$

H $1.053759-1.1428083 .295579$

H $-0.158070-2.4098960 .764265$

H $2.846331-2.8366953 .074246$

H $2.680720-4.5712343 .220953$

H $1.530780-3.5281874 .049757$

H $-1.690890-0.527505-1.761940$

H $-2.008757-2.037204-0.874842$

H $2.006054-2.514434-3.140007$

H $0.787854-3.219769-4.222340$

H $0.428507-5.143512-2.599243$

H $3.253595-4.173457-1.919378$

H $3.425236-6.702193-1.824045$

H $1.720662-6.843685-1.468636$

H $4.359310-6.1113351 .696224$

H $4.989305-6.252538-0.040129$

H $2.011524-6.2607922 .141217$

H $\quad 0.967226-6.762340 \quad 0.853121$

H $\quad 0.355838-4.535800 \quad 0.245623$

H $0.341004-1.984505-1.541700$

C $-0.4472174 .818720-0.316882$

O $0.4252773 .753780-0.737038$

C $0.8592994 .009251-2.085349$

C $0.134826 \quad 5.271724-2.544247$

C $-0.1005245 .992306-1.231030$

H $1.9327234 .223270-2.033054$

C $0.6200062 .777501-2.966564$

H $\quad 0.732160 \quad 5.854462-3.252287$

H $-0.8209635 .042831-3.029315$

C $-1.1773167 .060351-1.313828$

H $0.8431596 .453170-0.909920$

C $1.2196591 .468546-2.414183$

O $-0.794848 \quad 2.581079-3.092913$

H $0.9981412 .976406-3.976832$

H $0.6388401 .119122-1.551778$

$\begin{array}{lllll}\text { O } & 1.082123 & 0.468678 & -3.431733\end{array}$

C $2.7071021 .551687-2.025633$

C $3.2767280 .185346-1.598196$

H $2.8251342 .261583-1.201117$

O $3.4630442 .053635-3.126704$

H $2.653238-0.217375-0.792333$

H $3.210655-0.514327-2.439082$

H $-0.9040887 .825261-2.048188$

H -2.141286 $6.635788-1.613414$

H $-1.3133547 .555806-0.347645$

C $-0.234313 \quad 5.119302 \quad 1.169502$

H $-0.940537 \quad 1.939038-3.807859$

H $0.1358960 .264669-3.517929$

H $3.3356381 .442825-3.872911$

H $-0.802394 \quad 6.0182451 .436481$

H $\quad 0.824477 \quad 5.345538 \quad 1.343602$

H -1.479217 $4.489519-0.496952$

C $4.4263280 .946900 \quad 1.130079$

O $4.9798651 .250574-0.154889$

C $4.7556820 .255805-1.156996$

C $5.342523-1.089403-0.703862$

C $4.826137-1.5031540 .672767$

C $5.015498-0.3603441 .671975$

H 3.3349850 .8635121 .052076

$\begin{array}{llll}C & 4.752867 & 2.122247 & 2.056548\end{array}$

H $5.3299980 .575746-2.035680$

O $5.049673-2.115310-1.645499$

H $6.436033-1.005973-0.650186$

H $3.778766-1.8227460 .620335$

O $5.574962-2.6399821 .107750$

H $6.085117-0.2330581 .883301$

O $4.370349-0.6842542 .899776$ 
C 3.9157723 .3248961 .695592

H 4.5397221 .8912833 .106311

H 5.8146712 .3904291 .996386

H $5.407944-1.834828-2.504878$

H $5.160440-2.9696241 .922671$

H $4.842612-1.4393573 .289098$

O $44.659250 \quad 4.2208490 .987454$

O $2.733313 \quad 3.438941 \quad 1.990898$

C 3.9684485 .4040040 .575685

H 3.6086085 .9567671 .448750

H $4.671604 \quad 6.0348790 .025743$

H $3.1377955 .145665-0.086406$

SCF Energy (B3LYP/6-31G**//MMFF)= -3245.90919421

02 22

MMFF Geometry

C -0.4470073 .0156362 .577483$

C -0.6457921 .7221542 .280693$

C -0.3694880 .5988353 .244977$

O $0.575905-0.2855482 .595392$

C -1.639821 -0.2120363 .558327$

C $-2.687580 \quad 0.538306 \quad 4.350254$

C $-3.896017 \quad 0.8880293 .868537$

C $-2.331118 \quad 0.8325835 .784087$

C -4.4174440 .6802132 .474718$

C $-5.160900-0.6535782 .342646$

C $-5.908846-0.7722431 .006886$

C $-6.604212-2.1319130 .897204$

C -7.288873 -2.319914-0.466090

O $-4.970851-0.611779-0.063046$

O $-7.645397-3.704756-0.599414$

C $-6.283119-1.970624-1.586783$

C $-8.615558-1.556542-0.539716$

O $-6.914543-1.986587-2.864041$

C $-5.565630-0.620198-1.366645$

C $-4.467929-0.335803-2.422345$

O $-6.5248080 .440081-1.494888$

C $1.379471-1.0390503 .394665$

O $1.448101-1.0086544 .612654$

C $2.145238-1.9488242 .517785$

C $2.980344-2.8555303 .046780$

C $3.741211-3.8098822 .248698$

C $5.208036-3.8524432 .567158$

C $-3.336451-1.355651-2.566824$

C $-2.261103-1.291000-1.494940$

C $-0.126737-2.372195-0.842331$

O $-2.230824-0.445178-0.606572$

C $0.601455-3.643817-1.133447$

C $1.862270-3.692901-1.591678$

C $2.607477-4.973030-1.848283$

C $3.863424-5.099946-1.010960$

C $5.072427-4.850015-1.540902$

C $3.730439-5.6014080 .411698$

C $3.123489-4.5787891 .330865$

N -1.311764 -2.291246-1.673137

H -0.0712183 .2598243 .569883$

H -1.019342 1.4352041 .302387

H 0.0812670 .9917794 .164545

H -1.364397 -1.110507 4.127374

H -2.048961 -0.6161142 .623890$

H $-4.592977 \quad 1.3904754 .537815$

H -3.165675 1.2769546 .336819

H -2.048835 $-0.089397 \quad 6.302811$

H -1.492659 1.5334695 .837002

H -5.0963651 .5101802 .242190$

H $-3.6076880 .744524 \quad 1.740277$

H -4.431230 -1.469204 2.427989

H -5.875821 -0.7696773 .167082$

$\begin{array}{llll}\text { H } & -6.646500 & 0.037822 & 0.962438\end{array}$

H -7.326454 -2.268761 1.711617

H $-5.857425-2.9282631 .024306$

H -6.834624 -4.236551 -0.527240

H -5.535332 -2.772078 -1.598710

H $-9.311573-1.9291500 .221805$

H -9.112458 -1.718028 -1.502707

H -8.504594 $-0.481494-0.384840$

H -7.355481 -2.848232 -2.959396

H $-4.952209-0.223650-3.401823$
H $-4.0268640 .645589-2.204911$

H $-6.0464791 .269121-1.661922$

H $1.995113-1.8687011 .445807$

H $3.142224-2.9061354 .120669$

H $5.622895-2.8406792 .624163$

H $5.793310-4.3864801 .814370$

H $5.372593-4.3419523 .533182$

H -3.721489 -2.376917 -2.632290

H $-2.827173-1.149277-3.518006$

H $-0.433735-2.3387340 .208429$

H $0.486795-1.488329-1.040308$

H $0.074141-4.571697-0.925081$

H $2.392255-2.765285-1.795437$

H $2.861183-5.001341-2.915989$

H $1.969362-5.848673-1.675344$

H $5.980364-4.964570-0.958206$

H $5.180919-4.520076-2.568599$

H $4.698306-5.9452550 .793221$

H $3.096209-6.4971980 .417796$

H $2.042703-4.4852611 .231125$

H -1.332057 -2.825604 -2.537733

C -1.1849203 .9564770 .270653$

O $-0.2166093 .199735-0.476818$

C $-0.2052313 .660920-1.840499$

C $-1.3106674 .706432-1.954425$

C $-1.3900985 .237750-0.536591$

H $0.7634924 .148529-1.996612$

C $-0.3419192 .472357-2.799640$

H -1.069825 $5.480610-2.689663$

H -2.268991 $4.262270-2.246166$

C $-2.6902535 .962806-0.235017$

H $-0.5477455 .923436-0.373680$

C $0.6311321 .310362-2.520481$

O $-1.6683691 .938602-2.704113$

H $-0.2334212 .817939-3.833950$

H $0.3679670 .821512-1.575386$

O $\quad 0.4445920 .328850-3.548469$

C $2.1171701 .705415-2.487789$

C $3.0379250 .483622-2.303925$

H $2.2861862 .408465-1.666288$

O $2.4596422 .380059-3.696998$

H $2.734755-0.048621-1.395671$

H $2.898723-0.207167-3.144272$

H -2.814037 $6.823082-0.901070$

H -3.556258 $5.306014-0.369631$

H -2.7037496 .3307730 .795474$

C $-0.6686614 .213183 \quad 1.689392$

H $-1.8139321 .679328-1.777659$

H $-0.496870 \quad 0.084720-3.547857$

H $2.2856531 .768890-4.433439$

H $-1.367121 \quad 4.8784802 .210570$

H $\quad 0.290870 \quad 4.7414831 .627529$

H -2.1117023 .3685760 .299498$

C 4.6688721 .4223890 .106536

O $4.8011941 .860172-1.250468$

C $4.5345940 .858144-2.236396$

C $5.475380-0.339334-2.041956$

C $5.431451-0.877389-0.613887$

C 5.6286330 .2604220 .387521

H $3.633646 \quad 1.1111540 .293801$

C 4.9814572 .6198721 .008654

H $4.7889061 .308828-3.204009$

O $5.152002-1.373713-2.964907$

H $6.501826-0.020118-2.265820$

H $4.499534-1.423286-0.425737$

O $6.490821-1.824218-0.460345$

H 6.6681900 .6070830 .332799

O $5.425374-0.234804 \quad 1.707934$

C 3.8411083 .6081660 .992478

H 5.1234422 .3202422 .053297

H 5.9014843 .1272410 .694203

H $5.770865-2.106815-2.806375$

H $\quad 6.479878-2.1233470 .464733$

H $4.498525-0.5186571 .779774$

O 4.1113154 .6249520 .126403

O 2.8224163 .4763921 .658303

C 3.0984165 .6300380 .024160

H $3.4100456 .351495-0.735756$ 
H $2.1486535 .185160-0.283902$ H 2.9873696 .1505510 .980083

SCF Energy (B3LYP/6-31G**//MMFF) $=-3245.92362235$

$02 \_220$

MM̄FF Geometry

C 0.5357772 .5437561 .133520

C 1.4280763 .2981400 .475704

C $1.7217903 .095681-0.984966$

O $3.1592902 .966752-1.094220$

C $1.2598994 .251338-1.883486$

C $-0.2301174 .517485-1.881497$

C $-1.1535183 .710411-2.440779$

C $-0.6469675 .810476-1.231770$

C $-0.9187312 .390368-3.121790$

C $-1.8103501 .295890-2.516964$

C -1.296606 -0.115154 -2.848864

C $-2.451363-1.117639-2.845367$

C -1.970894 -2.550420 -3.096986

O $-0.344229-0.471169-1.836784$

O $-3.064865-3.420054-2.758579$

C $-0.811060-2.862548-2.130923$

C $-1.688176-2.793762-4.583189$

O $-0.220793-4.125365-2.428170$

C $0.267432-1.755059-2.016840$

C $1.135577-2.040963-0.767609$

O $1.165103-1.751416-3.126378$

C $3.6431742 .121324-2.042273$

O $3.0146451 .530785-2.905183$

C $5.0895171 .971863-1.785171$

C $5.6492430 .769514-1.982169$

C $7.0248680 .399476-1.649294$

C $7.9832771 .513068-1.317629$

C $2.191981-0.970911-0.474833$

C $3.021399-1.3170690 .750742$

C $4.711642-0.3359932 .297231$

O $2.985137-2.4186991 .293472$

C $6.127888-0.3564021 .815104$

C $6.889971-1.4606421 .781692$

C $8.330215-1.4824231 .348632$

C $8.553275-2.2926490 .089278$

C $8.731177-3.6232370 .145395$

C $8.650328-1.544752-1.224208$

C $7.343599-0.911684-1.618296$

N $3.821026-0.2571811 .154750$

H $-0.039654 \quad 1.806640 \quad 0.577193$

H $2.0072864 .045027 \quad 1.011777$

H $1.2650722 .154144-1.305661$

H $1.5838004 .060706-2.915798$

H $1.8040225 .164807-1.605449$

$\mathrm{H}-2.1964114 .023546-2.407432$

H -0.202953 $6.659903-1.761044$

H - $1.7334215 .947786-1.234441$

H $-0.3135355 .838207-0.189455$

H -1.166041 2.500374 -4.184977

H $\quad 0.1305532 .091285-3.087959$

H -1.864989 $1.414856-1.426998$

H $-2.8239371 .437113-2.912345$

H $-0.802864-0.104785-3.827407$

H -3.210180 -0.839777 -3.587109

H $-2.954889-1.085025-1.870849$

H -2.750861 -4.336494 -2.847343

H -1.270541 -2.980930 -1.139408

H -2.611242 -2.686557 -5.166356

H -1.342744 -3.817757 -4.761493

H $-0.957068-2.101300-5.003800$

H $\quad 0.364450-4.010520-3.195563$

H $0.490495-2.1235550 .115702$

H $1.643573-3.006628-0.889327$

H $\quad 0.692667-2.036384-3.921023$

H $5.6189142 .813084-1.353827$

H $5.025020-0.038823-2.361286$

H $8.0075032 .251123-2.127508$

H $9.0126001 .177730-1.177510$

H $7.6840172 .018162-0.392964$

H $2.875829-0.872554-1.323590$

H $1.706261-0.006320-0.301519$

H $4.474135-1.2172332 .902055$
H 4.5407980 .5524622 .913356

H 6.5519880 .5952721 .505578

H $6.462184-2.4071072 .105309$

H $8.922484-1.9082172 .169450$

H $8.719739-0.4661221 .210684$

H $8.918854-4.208099-0.748876$

H $8.689504-4.1571531 .089247$

H $8.941921-2.226482-2.034036$

H $9.467216-0.821835-1.153841$

H $\quad 6.570682-1.639718-1.869424$

H $3.7890080 .615610 \quad 0.636040$

C 0.4944491 .2167713 .231187

O $-0.534946 \quad 0.3260162 .754301$

C -1.002162 -0.479081 3.850503

C $-0.019006-0.2473464 .992345$

C $0.382476 \quad 1.1958094 .753385$

H -1.977982 -0.075473 4.145035

C $-1.150731-1.9387083 .409201$

H $-0.476402-0.4103755 .972898$

H $0.856249-0.9028474 .915518$

C 1.6590051 .5972245 .471780

H -0.4375941 .8493035 .080024$

C $-2.065125-2.1370892 .181383$

O $0.149650-2.4388903 .074491$

H -1.508227 -2.540606 4.253267

H $-1.592170-1.7258681 .281770$

O $-2.201464-3.5463771 .960457$

C $-3.474778-1.5325722 .328024$

C $-4.412341-1.9263011 .170433$

H -3.394379 -0.4428222 .374504$

O $-4.055825-1.9634263 .559257$

H -3.908421 -1.721403 0.221497

H -4.598685 -3.005845 1.214626

H 1.5392001 .4935296 .555154

H 2.5052010 .9727165 .165797

H 1.9160232 .6394885 .258850

C 0.2921362 .6016672 .613786

H $0.079941-3.4041482 .986097$

H -1.346327 -3.8756511 .636262$

H -4.091509-2.935236 3.543053

H 0.9612553 .3295113 .085637

H -0.7378222 .9363932 .777576$

H 1.4555490 .7917932 .912588

C $-5.2298010 .741981-0.117604$

O -5.6256840 .2304851 .161569$

C $-5.771668-1.1932421 .231509$

C $-6.795446-1.6738370 .192329$

C $-6.457187-1.181240-1.211049$

C $-6.2407200 .330380-1.197590$

H $-4.2322240 .366589-0.371534$

C $-5.1545482 .272686-0.007548$

H -6.201792 -1.405223 2.218375

O $-6.887950-3.0944710 .179532$

H -7.788895 -1.291471 0.461106

H -5.585264 -1.703569-1.621899

O $-7.549713-1.470886-2.085914$

H -7.203514 $0.825772-1.018983$

O $-5.7911330 .754831-2.482021$

C -3.9793932 .7450940 .821385$

H $-5.0425232 .723523-0.999896$

H -6.0685502 .6521660 .464582$

H -7.121824 -3.379766 1.079347

H -7.700426 -2.431215 -2.051273

H $-4.9450850 .313018-2.661559$

$\begin{array}{lllll}\text { O } & -4.070150 & 4.100084 & 0.974225\end{array}$

O -3.0918822 .0311871 .265971$

C -3.0230774 .7070971 .736414$

H -3.2066035 .7841651 .769736$

H -3.0252684 .3179852 .758873$

H -2.0563404 .5316231 .257127$

SCF Energy (B3LYP/6-31G**//MMFF) $=-3245.90570520$

$02 \_221$

MM̄FF Geometry

C -0.1390074 .8326881 .790508$

C -1.0138913 .8747681 .446317$

C -1.769114 3.0431132 .450075

O -1.7753011 .6687322 .001324$ 
C -3.2300473 .5182332 .514487$ C $-4.0553262 .759488 \quad 3.534271$ C -4.9545241 .8065853 .218920$ C -3.8550143 .1738844 .968212$ C $-5.2584861 .280651 \quad 1.842504$ C $-5.648740-0.2037481 .875696$ C $-5.948372-0.7337910 .466291$ C $-6.639359-2.0959710 .538056$ C -6.895942 -2.673135 -0.860641 O $-4.707630-0.853174-0.241280$ O $-7.258996-4.053711-0.708499$ C -5.578164 -2.635980 -1.658035 C $-8.075496-1.987939-1.560105$ O $-5.791862-3.025543-3.013360$ C $-4.849201-1.269482-1.606828$ C $-3.438634-1.431563-2.220019$ O $-5.583249-0.312841-2.371996$ C $-0.803266 \quad 0.8433982 .469245$ O $\quad 0.150015 \quad 1.149173 \quad 3.166494$ C -1.126642 -0.5248262 .003360$ C $-0.245553-1.5199332 .182975$ C $-0.443538-2.9249391 .815505$ C $-1.823338-3.341473 \quad 1.374707$ C $-2.498180-0.233468-2.028587$ C $-1.138858-0.544391-2.630774$ C $0.821380-2.062661-2.330222$ O $-0.754851-0.019342-3.672687$ C $0.852682-3.500187-1.917602$ C $1.797752-4.030395-1.127154$ C $1.811248-5.468799-0.696674$ C $1.861782-5.6357210 .806945$ C $2.928792-6.1913871 .405488$ C $0.638796-5.2455061 .612600$ C $0.599268-3.774784 \quad 1.928727$ N $-0.443892-1.497117-1.904250$ H $0.045667 \quad 4.9986542 .850722$ H -1.242067 3.6875670 .401295 H -1.311801 3.1137013 .444927 H -3.6904043 .4392471 .521894$ H -3.2664234 .5870892 .765337$ H $-5.533806 \quad 1.3627074 .027206$ H -4.516856 2.6332805 .653175 H -2.8256132 .9793765 .284427$ H -4.0602724 .2427585 .087314$ H $-6.093101 \quad 1.8602501 .430176$ H $-4.403473 \quad 1.4060581 .171311$ H -4.831868 -0.7833412 .325091$ H -6.533952 -0.3256922 .512561$ H -6.591222 $-0.010307-0.048886$ H -7.578861 -2.028722 1.100782 H $-6.004507-2.7990991 .095562$ H -8.051810 -4.094668 -0.146438 H -4.915087 -3.395529 - 1.219749 H -8.989420 -2.096126 -0.963809 H -8.290539 -2.460302 -2.525085 H $-7.914655-0.920978-1.727349$ H -6.233558 -3.891955 -2.999737 H -2.962505 -2.312114 -1.771309 H -3.523735 -1.633814 -3.295871 H $-4.9789550 .387661-2.664253$ H $-2.092842-0.6791221 .535841$ H $\quad 0.714744-1.2880242 .641674$ H -2.571576 -3.040079 2.116971 H -1.936140 -4.4208071 .254954$ H -2.081424 -2.881684 0.416954 H $-2.8904350 .663623-2.518434$ H -2.365598 $0.006660-0.968293$ H $1.617208-1.481594-1.859139$ H $0.921165-1.974749-3.416512$ H $0.050302-4.135483-2.285947$ H $2.608628-3.400446-0.772932$ H $2.683067-5.945761-1.162684$ H $0.934368-6.005083-1.080795$ H $2.959866-6.3403432 .479664$ H $3.795121-6.5103360 .836377$ H $\quad 0.635727-5.7878492 .567189$ H $-0.248077-5.5885371 .072869$ H $1.545535-3.3801182 .302562$
H $-0.773235-1.728110-0.972261$

C $0.4675885 .529950-0.631665$

O $1.1821554 .329478-0.988508$

C $1.9960174 .600090-2.144480$

C $1.4452485 .885513-2.743646$

C $1.0749836 .646711-1.484769$

H $3.0095694 .801219-1.777941$

C $2.0131713 .388864-3.079931$

H $2.1816126 .407252-3.362025$

H $0.5535425 .701037-3.353780$

C $0.1347677 .813107-1.735521$

H $1.9942687 .022157-1.015027$

C $2.4541802 .078857-2.392166$

O $0.6870663 .189675-3.587142$

H $2.6458673 .600022-3.949852$

H $1.6658691 .768976-1.697428$

O $2.5132991 .079147-3.416582$

C $3.8151902 .158518-1.672559$

C $4.2813350 .852158-1.012690$

H $3.8031202 .942266-0.912258$

O $4.8331652 .522771-2.612296$

H $4.4743610 .101113-1.791313$

H $5.2762891 .035873-0.587458$

H $0.6026418 .548265-2.398424$

H $-0.7980397 .483199-2.205325$

H $-0.1203528 .317375-0.798323$

C 0.6114375 .7670110 .874295

H $0.7118822 .406387-4.163496$

H $2.6288160 .216791-2.985492$

H $4.6614983 .434898-2.899967$

H $\quad 0.282673 \quad 6.784727 \quad 1.115669$

H 1.6727795 .6953631 .145156

H $-0.5824965 .382672-0.911637$

C $5.013378-1.2598720 .996826$

O $3.755261-1.1215610 .324888$

C 3.3582860 .2338030 .057464

C 3.2302400 .9793181 .391993

C 4.5193110 .9083522 .206336

C $4.977609-0.5436572 .352310$

H $5.816642-0.8443440 .376636$

C $5.281581-2.7624351 .168177$

H $2.3552520 .159894-0.372741$

O $2.8512122 .335818 \quad 1.216898$

H $2.4389580 .504987 \quad 1.984240$

H $5.308417 \quad 1.5296571 .767080$

O 4.2785691 .4310343 .515219

H $4.302815-1.0715603 .038543$

O $6.280425-0.5677322 .931345$

C $5.598931-3.438865-0.150105$

H $6.140197-2.9203561 .829797$

H $4.398233-3.2557841 .589506$

H $1.9946532 .343876 \quad 0.756254$

H 3.9366972 .3351703 .406557

H $6.231251-0.0875893 .775851$

O $5.916401-4.7457540 .089084$

O $5.565827-2.900886-1.248260$

C $6.222377-5.525989-1.070819$

H $6.438370-6.547199-0.745912$

H $5.364720-5.548492-1.750091$

H $7.105681-5.123131-1.575332$

SCF Energy (B3LYP/6-31G**//MMFF) $=-3245.91396670$

$02 \_222$

MMFF Geometry

C 0.1820585 .0875561 .374633

C 1.4276014 .5971111 .274009

C 2.2218953 .8882482 .347814

O 2.7553022 .6709621 .765748

C 1.4273143 .4776673 .601598

C 2.2874982 .8138574 .660099

C 2.3113191 .4876824 .898819

C 3.1092943 .7521825 .505109

C 1.5704650 .4177554 .143097

C $2.399344-0.8713654 .039499$

C $1.656543-1.9597363 .251369$

C $2.358273-3.3101083 .404469$

C $1.673864-4.4040032 .571423$

O $1.637867-1.5761991 .871502$ 
O $2.553635-5.5377272 .527727$

C $1.515676-3.8943641 .125126$

C $0.372782-4.8891763 .221654$

O $0.767968-4.8196850 .337606$

C $0.909481-2.4725341 .021904$

C $1.036671-1.973624-0.434783$

O $-0.471153-2.5273941 .380511$

C 4.0100162 .7159031 .243254

O $4.7362413 .692767 \quad 1.152706$

C 4.3542381 .3501470 .789022

C $5.442823 \quad 1.1678180 .026905$

C $5.908182-0.104348-0.528896$

C $5.244164-1.364875-0.043015$

C $0.632700-0.511768-0.648999$

C $0.936134-0.099981-2.076312$

C $2.8468360 .525381-3.561114$

O $0.074012-0.118027-2.951316$

C $3.898193-0.502393-3.844901$

C $5.155868-0.203021-4.204518$

C $6.213762-1.237549-4.467807$

C $7.495066-0.981639-3.701789$

C $8.617117-0.607067-4.339569$

C $7.497791-1.231301-2.206288$

C $6.903632-0.079577-1.439877$

N $2.267681 \quad 0.233291-2.262563$

H $\quad-0.3652515 .0036102 .308639$

H 1.9496294 .7286560 .327266

H 3.0436204 .5504572 .647607

H 0.6029132 .8132253 .315887

H 0.9556594 .3579634 .056952

H 2.9264401 .1295375 .723267

H 3.6698623 .2263696 .285471

H 3.8340374 .2908554 .886990

H 2.4620384 .4831516 .000860

H $\quad 0.6379410 .201303 \quad 4.677754$

H $\quad 1.304094 \quad 0.752067 \quad 3.135954$

H $3.355770-0.6470093 .549520$

H $2.621506-1.2355495 .050696$

H $0.627858-2.0215283 .625645$

H $2.406547-3.6079464 .459367$

H $3.401452-3.219043 \quad 3.070215$

H $2.716174-5.8266233 .442096$

H $2.522736-3.8725520 .685881$

H $\quad 0.567826-5.2663514 .232707$

H $-0.055273-5.7317192 .667369$

H $-0.384944-4.1069123 .301515$

H $-0.170120-4.7169270 .571699$

H $2.074897-2.095449-0.764119$

H $0.423400-2.598995-1.094588$

H $-0.932507-1.7730230 .982280$

H $3.693821 \quad 0.5394101 .073941$

H $6.0387052 .038824-0.243807$

H $5.754762-2.276036-0.361667$

H $4.216415-1.420766-0.409001$

H $5.227386-1.3970871 .052483$

H $-0.436939-0.369274-0.472667$

H 1.1639530 .1581940 .036027

H $3.261044 \quad 1.537267-3.511633$

H $2.0748710 .496060-4.335741$

H $3.608864-1.546418-3.746864$

H $5.4399340 .839700-4.326639$

H $\quad 6.412945-1.239727-5.547283$

H $5.852675-2.245343-4.226903$

H $9.545173-0.444893-3.801390$

H $8.632223-0.449479-5.412857$

H $8.527216-1.368317-1.850371$

H $\quad 6.990395-2.180706-2.015851$

H $7.3421690 .886214-1.696098$

H $2.8884760 .244673-1.459499$

C -1.910991 $5.174605-0.003635$

O $-1.7598363 .836708-0.514588$

C $-2.7517473 .612702-1.541636$

C $-3.6719284 .824078-1.513419$

C $-2.7280355 .918782-1.057310$

H $-2.1951403 .572895-2.486216$

C $-3.4456972 .273268-1.268803$

H -4.127817 $5.027226-2.487047$

H -4.478482 $4.685369-0.782463$
C $-3.4410797 .153489-0.534323$

H -2.084857 $6.202586-1.901135$

C $-4.6141291 .959953-2.217430$

O $-2.4654441 .239639-1.394910$

H $-3.7899882 .252052-0.228501$

H $-5.4215252 .685343-2.063176$

O $-4.1601372 .140811-3.564953$

C $-5.1719980 .526620-2.098003$

C $-5.6272220 .152880-0.677360$

H $-4.428358-0.187675-2.463539$

O $\quad-6.291401 \quad 0.416583-2.985875$

H -4.7906960 .2654460 .017521$

H $-6.4069160 .857694-0.363857$

H $-4.0677877 .595342-1.315866$

H -4.0859896 .9138240 .317929$

H $-2.7224067 .912419-0.209908$

C -0.5344315 .7903380 .252595$

H $-1.7207391 .478440-0.817026$

H $-4.9046401 .927432-4.153286$

H $-6.979017 \quad 1.030042-2.675055$

H $-0.643671 \quad 6.8449200 .529543$

H $0.0664525 .747997-0.664104$

H -2.4765275 .0969370 .934661$

C $-4.118388-2.464229-0.142495$

O $-5.237150-2.266286-1.016794$

C $-6.186985-1.285262-0.583827$

C $-6.737534-1.6582420 .799427$

C $-5.621175-1.8835451 .813930$

C $-4.593674-2.8694731 .260432$

H $-3.525215-1.543603-0.086609$

C $-3.243945-3.570648-0.755194$

H -7.028798 -1.345899-1.284757

O $-7.625074-0.6467931 .264364$

H -7.324111 -2.581922 0.709280

H -5.154615 -0.935084 2.104446

O $-6.198160-2.427738 \quad 3.003101$

H -5.041997 -3.870585 1.231332

O $-3.486257-2.9381622 .156351$

C $-2.485525-3.105516-1.980079$

H -2.498433 -3.916453 -0.031704

H $-3.876028-4.414597-1.056445$

H -7.934498 -0.9149112 .146589$

H -5.469362 -2.610827 3.620582

H -3.066660 -2.061193 2.181240

O $-1.822933-4.171586-2.520002$

O $-2.461441-1.959786-2.405789$

C -1.054568 -3.888154 -3.693720

H $-0.572848-4.814959-4.016311$

H -1.707986 -3.533920 -4.496601

H $-0.279110-3.149055-3.472327$

SCF Energy (B3LYP/6-31G**//MMFF $)=-3245.91377224$

$02 \_223$

MMFF Geometry

C $0.090674-2.575515-3.361160$

C $-0.873207-2.787976-2.453523$

C $-2.333371-2.938943-2.796141$

O $-3.057875-1.887739-2.118679$

C $-2.843966-4.299231-2.287249$

C $-4.318920-4.537097-2.551493$

C $-5.280477-4.538573-1.605767$

C $-4.678067-4.812290-3.989177$

C $-5.118214-4.289982-0.131866$

C $-5.744134-2.950308 \quad 0.275615$

C $-5.199041-2.4512891 .621806$

C $-5.937108-1.1879872 .064804$

C $-5.324900-0.5738603 .330555$

O $-3.807708-2.1672591 .437030$

$\begin{array}{llll}\text { O } & -5.882929 & 0.742332 & 3.467038\end{array}$

C $-3.799978-0.4246913 .133661$

C $-5.738729-1.3481284 .587518$

O -3.2030090 .0115774 .352410$

C $-3.122091-1.7102262 .611148$

C $-1.635910-1.5124662 .216311$

O $-3.153778-2.7046873 .642008$

C $-3.214037-0.714131-2.791755$

O $-2.829196-0.456812-3.921480$

C $-3.9236650 .228228-1.898668$ 
C $-4.0429991 .516111-2.253538$

C $-4.7010122 .570129-1.478031$

C $-5.2768662 .196027-0.137929$

C - $-1.379847-0.4977831 .096755$

C $-1.1052900 .914054 \quad 1.583764$

C $-0.829828 \quad 3.2390930 .727400$

O $-0.852065 \quad 1.1843162 .754480$

C -2.1038354 .0113680 .858998$

C $-2.3929705 .088357 \quad 0.112545$

C $-3.638516 \quad 5.9118370 .276250$

C $-4.3932946 .099345-1.020864$

C $-4.2341857 .211256-1.758520$

C $-5.3984805 .040647-1.412902$

C $-4.7504263 .815879-1.994573$

N -1.130765 1.8322350 .542236

H $-0.172269-2.497999-4.413026$

H $-0.604281-2.864040-1.402133$

H -2.493869 -2.873394 -3.879456

H -2.621852 -4.387960 -1.219510

H -2.274412 -5.108123 -2.765207

H $-6.307121-4.729488-1.915811$

H -5.737976 -5.059028 -4.113258

H $-4.469740-3.937577-4.612851$

H -4.098112 -5.659229 -4.370166

H $-5.613512-5.101210 \quad 0.415855$

H $-4.070600-4.3314860 .172064$

H -5.529635 -2.199625 -0.494875

H $-6.834249-3.0620270 .326379$

H $-5.312229-3.2476512 .366702$

H -7.004827 -1.3926702 .213624$

H $-5.888516-0.4415311 .261170$

H -5.4503311 .1678394 .227394$

H $-3.660523 \quad 0.3833872 .406037$

H $-6.828908-1.3275604 .705151$

H $-5.332378-0.8834075 .492523$

H -5.427691 -2.3947214 .568466$

H $-2.278478 \quad 0.2449264 .165348$

H -1.029964 -1.297096 3.104308

H -1.269362 -2.481564 1.847161

H -2.461882 -3.3617113 .459217$

H $-4.315257-0.159011-0.965917$

H -3.617422 $1.834484-3.204378$

H $-6.1140291 .501184-0.263685$

H -5.6476333 .0492950 .433467$

H $-4.515656 \quad 1.7215990 .490514$

H $-0.480459-0.8067890 .548319$

H -2.207853 -0.4864010 .383518$

H $-0.2366953 .559863-0.134835$

H -0.2282663 .3694961 .632291$

H -2.804301 3.6909551 .626683

H $-1.6754775 .424034-0.632996$

H $-3.339218 \quad 6.8902860 .674852$

H -4.308695 5.4804021 .029996

H -4.794271 $7.367242-2.674597$

H -3.535731 $7.988089-1.465037$

H $-6.0833495 .436676-2.175016$

H -6.041474 4.818036 -0.556768

H -4.295541 $3.990963-2.970640$

H -1.382301 $1.520508-0.391364$

C $2.178587-1.128261-3.375773$

O $3.613005-1.280969-3.278497$

C $4.143338-0.229003-2.444174$

C $3.0839960 .856598-2.473492$

C $1.8093240 .032144-2.446622$

H $4.218848-0.660854-1.440112$

C $5.5307710 .165560-2.963839$

H $3.1570451 .550673-1.632709$

H $3.1427301 .432686-3.405319$

C $0.5891230 .830136-2.873219$

H $1.652928-0.338436-1.424843$

C $6.2109181 .349453-2.251952$

O $6.373033-0.987194-2.875547$

H $5.4389190 .394262-4.033616$

H $7.2463901 .424562-2.608466$

O $5.5579442 .551059-2.677353$

C $6.2264331 .328933-0.708967$

C $6.8497270 .069335-0.084670$

H $5.2089841 .473797-0.334522$
O $6.9681682 .474612-0.268962$

H $6.251391-0.804363-0.354998$

H $7.850010-0.083943-0.508684$

H $\quad 0.465848 \quad 1.712779-2.237004$

H $0.6749021 .172903-3.909644$

H $-0.3208740 .232266-2.788723$

C $1.545834-2.475110-3.017751$

H $7.225560-0.760259-3.284521$

H $5.998643 \quad 3.295270-2.232511$

H $7.8875742 .365093-0.566376$

H $1.723205-2.695467-1.957022$

H $2.073419-3.269791-3.561772$

H $1.967740-0.876333-4.422741$

C $4.692148-0.3505792 .028339$

O $5.775977 \quad 0.5834572 .085173$

C $6.9813620 .144151 \quad 1.454449$

C $7.469845-1.1548582 .111970$

C $6.377960-2.2231672 .151868$

C $5.081355-1.6546172 .730342$

H $4.432266-0.5538630 .982763$

C 3.4815090 .3111522 .694059

H 7.7337400 .9140961 .667522

O $8.607090-1.6744561 .431495$

H $7.780515-0.9430003 .143360$

H $6.205011-2.6569521 .159894$

O $6.805754-3.2999362 .989314$

H $5.203503-1.4836523 .807541$

O $4.035332-2.6087662 .561997$

C 2.9466671 .4180481 .817061

H $2.662693-0.4001962 .850651$

H 3.7371540 .7287453 .675168

H $9.292798-0.984898 \quad 1.439874$

H $7.647256-3.6289562 .629084$

H $4.322172-3.4324182 .992654$

O 3.5774532 .5914122 .106270

O $2.097851 \quad 1.248150 \quad 0.951999$

C 3.2149793 .7077751 .288019

H 2.1876404 .0113611 .505450

H 3.8851524 .5371081 .529640

H 3.3310693 .4641470 .227519

SCF Energy (B3LYP/6-31G**//MMFF) $=-3245.91984218$

$02 \_224$

MMFF Geometry

C 2.0645354 .6897740 .670593

C 3.1527933 .9215860 .824202

C $4.1458803 .662426-0.279276$

O $3.9431152 .301160-0.723826$

C 5.5817693 .8031160 .248883

C $6.6273943 .611408-0.831796$

C $7.3463892 .485210-1.007923$

C 6.866414 4.808189-1.715385

C $7.2369291 .202601-0.231686$

C $7.121623-0.011965-1.163716$

C $6.941566-1.309179-0.363258$

C $7.030289-2.533840-1.273389$

C $6.774983-3.834572-0.496574$

O $5.653754-1.2644020 .260682$

O $6.567311-4.885484-1.452294$

C $5.466094-3.6872180 .306173$

C $7.987045-4.2471750 .346852$

O $5.261728-4.8095281 .161820$

C $5.377089-2.3748591 .122805$

C $3.943655-2.2091541 .676879$

O $6.293652-2.4317902 .216617$

C $3.0383482 .118687-1.725261$

O $2.4586702 .985210-2.361219$

C $2.8139590 .665273-1.875618$

C $1.6808620 .256058-2.465751$

C $1.231656-1.127140-2.624037$

C $2.198958-2.223642-2.271010$

C $3.645351-0.8328622 .285150$

C $2.169441-0.7291412 .616958$

C $-0.060352-0.4711541 .521755$

O $1.749703-0.9131893 .756872$

C $-0.530983-1.6871150 .784631$

C -1.479385 -1.659547 -0.164016

C $-1.962720-2.875954-0.902154$ 
C $-2.047891-2.662348-2.399296$ C $-3.238057-2.547527-3.012582$ C $-0.757183-2.649318-3.194189$ C $-0.028696-1.342202-3.055480$ N $1.390395-0.4573831 .502679$ H $1.8779865 .168392-0.287597$ H 3.3166663 .4173921 .773952 H $3.9980814 .362191-1.111473$ H 5.7486483 .0986631 .071619 H 5.7193344 .7985830 .692781 H $8.0996922 .476570-1.794849$ H $7.6614894 .632697-2.447861$ H $5.9589795 .063651-2.271077$ H $7.1605065 .673072-1.111818$ H $\quad 8.136277 \quad 1.0983270 .387225$ H 6.3816821 .2119350 .448838 H $6.2644750 .129379-1.834734$ H $8.022994-0.080447-1.785382$ H $7.718935-1.3505920 .408802$ H $8.002391-2.574803-1.780092$ H $6.278534-2.451643-2.070966$ H $7.360789-4.942555-2.012009$ H $4.640004-3.697545-0.418884$ H $8.867415-4.388239-0.291741$ H $7.816400-5.2115790 .837916$ H $8.247866-3.5123461 .111211$ H $5.309577-5.6080750 .608662$ H $3.228411-2.3838550 .865961$ H $3.746811-2.9744992 .439037$ H $5.991936-1.8255942 .911582$ H $3.540267-0.014518-1.446530$ H $0.9841061 .011168-2.829073$ H $3.167174-2.060996-2.758166$ H $1.867001-3.214227-2.588831$ H $\quad 2.352675-2.259717-1.189879$ H $4.216720-0.6702363 .204996$ H $3.906318-0.0214791 .596115$ H -0.3981430 .4544481 .047518$ H $-0.423162-0.4950842 .553671$ H -0.077541 -2.638672 1.052593 H -1.952841 $-0.712900-0.409336$ H -2.953122 $-3.132987-0.504959$ H - $1.322890-3.743926-0.700536$ H -3.309844 -2.414565 -4.086908 H -4.167215 -2.577611 -2.453713 H $-0.967695-2.799459-4.260898$ H -0.156661 -3.509893 -2.887804 H $-0.641410-0.475917-3.310988$ H $1.844514-0.3389850 .602350$ C -0.0775003 .8679331 .691813$ O $-\begin{array}{llll}0.748160 & 3.916252 & 0.419684\end{array}$ C -2.164295 3.7225690 .622955 C -2.3393923 .3798332 .096936$ C -1.168166 4.0981902 .734753 H $-2.634357 \quad 4.6854150 .389031$ C $-2.6645252 .631937-0.333432$ H -3.306779 3.7069792 .490169 H -2.257589 2.297603 2.258658 C -0.8209213 .5785244 .118994$ H $-1.407717 \quad 5.1680152 .799044$ C $-4.1756522 .360317-0.205087$ O $-2.3741373 .047800-1.671097$ H $-2.1033201 .707664-0.160827$ H -4.4303352 .1958680 .847942$ O $-4.8994673 .520174-0.617754$ C $-4.6620561 .119631-0.984463$ C $-6.1962890 .983503-0.973413$ H $-4.2059050 .235341-0.528516$ O $-4.2197121 .151826-2.339528$ H $-6.567040 \quad 1.1778350 .037770$ H -6.624482 $1.749554-1.630777$ H -1.668632 3.7039524 .800667 H -0.5633782 .5142064 .094886$ H 0.0317384 .1216574 .538149 C 1.0398334 .9119311 .745505 H -1.416269 3.209718 -1.722205 H -4.624397 $3.729920-1.526440$ H $-4.5783701 .955740-2.751683$
H 1.5203554 .8998772 .730922

H 0.6260615 .9182801 .605209

H 0.3441452 .8589961 .791131

C -6.810769-1.509542 0.691223

O $-6.193623-1.458255-0.601834$

C $-6.659638-0.408598-1.459220$

C $-8.174826-0.535984-1.668731$

C $-8.927444-0.589268-0.343686$

C $-8.331865-1.6649230 .561842$

H $-6.578793-0.5957661 .250949$

C $-6.203729-2.7027041 .446566$

H $-6.188850-0.579959-2.435356$

O $-8.6776430 .542876-2.449675$

H $-8.387348-1.459231-2.223558$

H $-8.933526 \quad 0.3868450 .155793$

O $-10.295004-0.922867-0.592610$

H -8.577728 -2.657406 0.162501

O $-8.925280-1.5649881 .855200$

C $-4.800638-2.4243261 .944719$

H $-6.808824-2.9433422 .327766$

H $-6.155824-3.5771570 .786923$

H $-8.1921560 .544468-3.292208$

H $-10.648964-0.252821-1.202600$

H $-9.887814-1.6441911 .739442$

O $-4.344831-3.5316102 .602700$

O $-4.186660-1.3790251 .785432$

C -3.031508 -3.441961 3.165649

H $-2.950144-4.1942503 .954931$

H -2.291944 -3.664224 2.392721

H $-2.846293-2.4575583 .606260$

SCF Energy $\left(B 3 L Y P / 6-31 G^{* *} / / M M F F\right)=-3245.92048449$

$02 \_225$

MMFF Geometry

C -0.3392680 .0046224 .034386$

C -0.9999460 .5182632 .986550$

C -2.0377581 .6033673 .092009$

O -3.1615821 .2358802 .260651$

C -1.466432 2.9072642 .513846

C -2.2598964 .1368732 .901235$

C -3.1807104 .7368512 .122086$

C -1.9097874 .7217144 .245496$

C -3.7047884 .2875810 .786530$

C $-3.1713265 .123524-0.384891$

C $-1.7810814 .674855-0.862682$

C $-1.2766525 .584455-1.985042$

C $0.0538245 .088341-2.570967$

O $-1.9053443 .330119-1.340191$

O $0.2884295 .798078-3.796602$

C $-0.0889623 .595718-2.933325$

C $1.2390055 .405833-1.651261$

O $1.1604963 .050828-3.353185$

C $-0.6835002 .736591-1.793134$

C $-1.0168951 .322391-2.326006$

O $0.2799962 .632055-0.743269$

C -4.0304040 .2991832 .717438$

O $-4.029074-0.2460823 .807911$

C -4.9613350 .0153591 .603241$

C $-5.688778-1.1101881 .613662$

C $-6.582594-1.5627670 .545337$

C $-6.724867-0.678558-0.666803$

C $-1.8036160 .436108-1.347890$

C $-2.006161-0.966829-1.898041$

C $-2.867359-3.211857-1.241354$

O $-1.659210-1.300398-3.028337$

C $-4.253358-3.388237-1.779132$

C $-5.089756-4.349288-1.356217$

C $-6.465761-4.570851-1.919627$

C $-7.526943-4.708318-0.848840$

C $-7.973316-5.918071-0.470625$

C $-8.127816-3.442894-0.277421$

C -7.200953 -2.753743 0.687070

N -2.617261 -1.806235 -0.975847

H $-0.544677 \quad 0.3707945 .036596$

H $-0.7676160 .156348 \quad 1.987702$

H $-2.374214 \quad 1.7433724 .126603$

H -1.413424 2.8272871 .423359

H -0.4310603 .0560882 .849669$ 
H -3.653706 5.6436812 .497805 H -2.496546 5.6172194 .476251 H -2.096224 3.9929115 .040491 H -0.8522015 .0037574 .273247$ H -3.5309993 .2218310 .614779$ H $-4.796353 \quad 4.401146 \quad 0.821074$ H -3.878880 $5.008574-1.216743$ H -3.156961 $6.187018-0.117041$ H -1.085940 $4.699942-0.016873$ H -1.179429 $6.618944-1.632894$ H -2.020438 $5.612816-2.793880$ H $0.307816 \quad 6.748965-3.593158$ H $-0.7611083 .532981-3.800680$ H $1.3243416 .488208-1.497272$ H $2.1859405 .092571-2.104845$ H $1.1565354 .939479-0.667412$ H $1.4939543 .612156-4.074065$ H -1.617757 $1.413663-3.240587$ H $-0.0879820 .809947-2.600014$ H $0.157149 \quad 1.793343-0.272736$ H $-4.980508 \quad 0.717340 \quad 0.776937$ H -5.599645 -1.779170 2.468701 H -7.363896 -1.099692 -1.445185 H -5.749344 -0.502022 -1.133674 H $-7.159410 \quad 0.287329-0.386493$ H - $-1.2732630 .346755-0.395451$ H -2.790922 $0.870060-1.153187$ H $-2.718095-3.751187-0.300497$ H -2.139584 -3.575548 -1.974074 H -4.573597 -2.717195 -2.572735 H -4.753566 $-5.042008-0.587685$ H $-6.431350-5.485673-2.525862$ H $-6.749484-3.767430-2.610746$ H $-8.752686-6.0253580 .276423$ H -7.565126 -6.828905 -0.896052 H $-9.049383-3.6757600 .272806$ H -8.450613 -2.798587 -1.099465 H -7.025832 -3.3229001 .601341$ H -2.924852 -1.427977 -0.084692 C $2.121868-0.4650243 .977125$ O 2.3285020 .4309292 .864482 C 3.5247660 .0403882 .160356 C $4.321094-0.7722953 .165965$ C $3.215951-1.5295323 .875334$ H $3.184772-0.5958901 .336198$ C 4.2132851 .2984321 .618450 H $5.053421-1.4343772 .696931$ H $4.845403-0.1156213 .870996$ C $3.635928-2.1093335 .214586$ H $2.881096-2.3488903 .225789$ C 5.5391001 .0683990 .872400 $\begin{array}{lllll}\text { O } & 3.311718 & 1.978374 & 0.740930\end{array}$ H 4.3939591 .9835882 .456819 H 5.8457322 .0138550 .406294 O $\quad 6.542905 \quad 0.758958 \quad 1.845519$ C $5.553209-0.036525-0.205969$ C $4.5045940 .139542-1.317070$ H $5.441112-1.0148130 .271626$ O $6.857077-0.041971-0.802358$ H $3.5029270 .084867-0.883360$ H $4.6122031 .140699-1.752905$ H $4.450129-2.8295015 .083284$ H $3.984767-1.3283455 .898408$ H $2.799165-2.6278085 .693520$ C $0.713656-1.0580713 .891418$ H 2.4822622 .1210521 .228569 H 7.3799780 .6276191 .368016 H $\quad 6.978046 \quad 0.808187-1.258818$ H $0.563779-1.8059724 .678297$ H $0.587041-1.5735112 .930338$ H 2.2357420 .1317004 .890919 C $3.479833-2.733895-1.380158$ O $4.693915-2.242768-1.959808$ C $4.628408-0.902012-2.453320$ C $3.538186-0.793350-3.527989$ C $2.192849-1.316398-3.030081$ C $2.346536-2.706440-2.412510$ H $3.211470-2.120141-0.512562$
C $3.748866-4.158775-0.886447$

H $5.587759-0.712610-2.951155$

O $3.4088250 .557488-3.958927$

H $3.843074-1.381950-4.403065$

H $1.741259-0.614785-2.320359$

O $1.302438-1.393996-4.144680$

H $2.543646-3.432886-3.210666$

O $1.114770-3.083592-1.800034$

C $4.611496-4.1371460 .353073$

H $2.820705-4.677448-0.620944$

H $4.247379-4.765254-1.652083$

H $2.6835810 .588778-4.606224$

H $\quad 0.457515-1.746863-3.816153$

H $0.924753-2.445196-1.091444$

O $5.929805-4.1097840 .006809$

O $4.163254-4.1194961 .492122$

C $6.852280-4.0464491 .098697$

H $7.865578-4.0588620 .688626$

H $6.711850-3.1165971 .657706$

H $\quad 6.727999-4.9143441 .753361$

SCF Energy $(B 3 L Y P / 6-31 G * * / / M M F F)=-3245.92128881$

$02 \_226$

MMFF Geometry

C $0.766033-2.9273631 .394478$

C $0.706587-1.6229901 .700024$

C $0.304201-1.0898543 .047677$

O $-0.756509-0.1425972 .785373$

C $1.463735-0.3416803 .730154$

C $2.516545-1.2625244 .305999$

C $3.656499-1.6185873 .682348$

C $2.214114-1.7861685 .686464$

C $4.112481-1.2327972 .302689$

C $5.559597-0.7226652 .304304$

C $6.063480-0.4780020 .875263$

C $7.554784-0.1438160 .869515$

C $8.0593170 .130371-0.554897$

O $5.3285270 .615406 \quad 0.318689$

O $9.3571860 .737795-0.455816$

C $7.1310041 .164836-1.228393$

C $8.253293-1.169424-1.345362$

O $7.4470651 .313476-2.610443$

C $5.6178650 .871995-1.061038$

C $4.7952432 .101461-1.513029$

O $5.190079-0.211289-1.891939$

C $-1.697093 \quad 0.068780 \quad 3.739118$

O $-1.775046-0.4839844 .826722$

C -2.6270691 .1483473 .328060$

C -2.7092931 .5870152 .062236$

C -3.4966512 .7215911 .578146$

C -4.3638033 .4526882 .566004$

C $3.2952772 .003883-1.199924$

C $2.9064142 .637406 \quad 0.127628$

C 0.8884173 .3009081 .434910

O 3.7246513 .0287430 .955176

C -0.1950204 .2778581 .079615$

C $-0.5713934 .690188-0.142510$

C $-1.7028565 .654296-0.388339$

C $-2.8337005 .031551-1.183285$

C $-2.9047945 .181376-2.516511$

C $-3.9131084 .281820-0.423012$

C $-3.360577 \quad 3.0760290 .283570$

N $1.5275162 .720147 \quad 0.269064$

H $0.524544-3.6495522 .172268$

H $0.949358-0.8759730 .949206$

H $-0.077970-1.8976633 .683916$

H 1.0675370 .2857194 .540289

H 1.9034560 .3878143 .038697

H $4.327742-2.3013914 .202387$

H $3.001222-2.4477256 .063357$

H $2.114592-0.9557456 .393230$

H $1.278907-2.3547745 .684162$

H $4.047803-2.1266641 .669881$

H $3.464174-0.4780631 .849982$

H 5.6084090 .2087002 .883200

H $6.207915-1.4563892 .799241$

H $5.872329-1.3817600 .284506$

H $8.138797-0.9482201 .333491$ 
H $7.732776 \quad 0.750810 \quad 1.482331$ H $9.934127 \quad 0.133750 \quad 0.042401$ H $7.3406392 .135704-0.757298$ H $9.001270-1.805853-0.857040$ H $8.642403-0.968537-2.349423$ H $7.337012-1.756077-1.438805$ H $8.3937011 .529169-2.669611$ H $5.2034223 .014238-1.062740$ H $4.8986182 .206191-2.601124$ H $5.950385-0.537205-2.398319$ H -3.2165881 .5801684 .129666$ H -2.1017131 .1009301 .300052$ H -5.0509572 .7574933 .061726$ H -4.9912954 .2191972 .105745$ H -3.7496183 .9440893 .328227$ H $2.7479812 .543428-1.983419$ H $2.9540960 .963413-1.206376$ H 1.6331493 .7954532 .066361 H 0.4578442 .4718772 .004829 H $-0.7365584 .672628 \quad 1.937994$ H $-0.0540134 .324821-1.025540$ H -1.295034 $6.518417-0.928635$ H $-2.099081 \quad 6.0572530 .552129$ H $-3.7196104 .754215-3.091693$ H -2.145463 5.730611-3.063303 H -4.697994 3.938282 -1.108420 H $-4.3883504 .988107 \quad 0.263476$ H -2.728776 $2.456573-0.353841$ H $\quad 0.9366462 .252520-0.412445$ C $1.325209-2.569643-1.112943$ O $0.095197-1.873446-1.395301$ C $-0.070464-1.767442-2.821000$ C $1.232805-2.261586-3.440557$ C $1.694900-3.278290-2.415652$ H -0.877004 -2.457028 -3.095190 C $-0.459180-0.336299-3.209351$ H $1.080162-2.691944-4.435034$ H $1.971658-1.457472-3.535069$ C $3.167346-3.632030-2.533248$ H $1.093926-4.190300-2.532063$ C $-1.679651 \quad 0.232103-2.459855$ O $0.6421660 .540366-2.936810$ H $-0.624508-0.281386-4.291518$ H -1.428138 $0.409639-1.406836$ O $-1.9684891 .518767-3.024531$ C $-2.952791-0.630798-2.538058$ C $-4.166500 \quad 0.076556-1.906570$ H $-2.775282-1.580208-2.023024$ O $-3.245855-0.937720-3.899869$ H $-3.8935480 .387260-0.894402$ H $-4.3946320 .985259-2.476629$ H $3.381075-4.066146-3.515423$ H $3.803340-2.749259-2.410639$ H $3.456188-4.362918-1.771759$ C $1.122873-3.5330100 .061359$ H $0.8460090 .461862-1.989160$ H -1.165768 2.060774 -2.936107 H -3.371281 $-0.095763-4.370530$ H $2.030876-4.1304680 .203596$ H $\quad 0.315349-4.231037-0.191768$ H $2.081242-1.812827-0.865902$ C $-4.982281-1.952118 \quad 0.205085$ O $-5.218199-2.047081-1.204762$ C $-5.438397-0.797765-1.865644$ C $-6.658329-0.095218-1.252494$ C $-6.5226410 .059857 \quad 0.260438$ C $-6.167627-1.2786950 .907869$ H $-4.061519-1.3843310 .386757$ C $-4.771561-3.3750310 .730831$ H -5.692207 -1.039685-2.905309 O $-6.847121 \quad 1.179122-1.858813$ H -7.555685 -0.689774 -1.467946 H $\quad \begin{array}{llll}-5.789876 & 0.834509 & 0.510742\end{array}$ $\begin{array}{lllll}\text { O } & -7.774271 & 0.513504 & 0.780559\end{array}$ H -7.047191 -1.9337180 .873808$ O $-5.862655-1.0643722 .284704$ C -3.446903 -3.920283 0.256425 H $-4.758233-3.4079191 .826156$
H $-5.572908-4.0467760 .400147$

H -7.611385 $1.596692-1.425816$

H $-7.6826380 .568703 \quad 1.747359$

H $-5.078608-0.4912482 .331255$

O $-3.621121-4.659388-0.875450$

O $-2.381482-3.6886270 .812433$

C $-2.427617-5.206134-1.443733$

H -1.924741 -5.857047 -0.721984

H -2.708011 -5.801164 -2.316891

H -1.762387 -4.400920 -1.767188

SCF Energy $(B 3 L Y P / 6-31 G * * / / M M F F)=-3245.92072472$

$02 \_227$

MMFF Geometry

C $1.018841-0.9046632 .849790$

C $2.154233-0.3948163 .352492$

C 2.8292400 .8944872 .944949

O $1.943807 \quad 1.6496692 .088871$

C 4.1328800 .5708752 .185169

C $5.277451 \quad 1.5244172 .463475$

C 5.7892842 .3984411 .574732

C 5.9066221 .3891233 .827701

C 5.3150822 .6942140 .179612

C $6.2526412 .132271-0.899366$

C $6.1344200 .610100-1.079115$

C $7.1088890 .109867-2.147040$

C $6.921809-1.386526-2.444506$

O $4.7927120 .330256-1.483879$

O $7.650957-1.659459-3.650775$

C $5.425178-1.663238-2.712434$

C $7.545715-2.259723-1.349390$

O $5.173251-3.060948-2.838315$

C $4.488838-1.060691-1.640116$

C $3.013788-1.161485-2.098442$

O $4.652497-1.783386-0.418479$

C 2.0902702 .9976012 .044257

O 2.9008333 .6805852 .647939

C 1.0822983 .5131721 .098221

C 1.3509084 .6268000 .404387

C $0.4444825 .216454-0.571664$

C $1.0800905 .455198-1.912452$

C $2.035951-0.382466-1.206625$

C $0.625871-0.371554-1.767306$

C $-1.6001160 .676248-1.353640$

O $0.277632-1.053281-2.726983$

C $-1.8016262 .019837-1.979165$

C $-2.7186002 .897287-1.543656$

C $-3.0276174 .199024-2.226073$

C $-3.0999705 .365129-1.267116$

C $-4.2552095 .692429-0.663044$

C $-1.8595936 .208064-1.085072$

C $-0.8204565 .535171-0.233865$

N $-0.1975740 .483500-1.048025$

H $0.477854-0.3779652 .068924$

H $2.672999-0.9568894 .127654$

H 3.0110371 .4643013 .863956

H 3.9200140 .5264891 .113609

H $4.496124-0.4319352 .450175$

H 6.6428393 .0004281 .885025

H 6.7799502 .0388153 .949683

H 5.1914841 .6522674 .612526

H $6.2399360 .359023 \quad 3.991467$

H 4.2867422 .3603360 .013300

H 5.2846773 .7865940 .074986

H $5.9884962 .620808-1.846553$

H $7.2911562 .404685-0.673742$

H $6.3369630 .119235-0.121029$

H $8.1461290 .317464-1.855691$

H $6.9437150 .669211-3.078991$

H $7.593480-2.613112-3.829732$

H $5.176744-1.211961-3.683223$

H $8.624468-2.073656-1.282886$

H $7.439318-3.324860-1.582844$

H $7.116494-2.080299-0.361442$

H $5.693777-3.388053-3.590943$

H $2.927804-0.760844-3.117117$

H $2.701020-2.212697-2.133021$

H $3.831770-1.7355280 .095754$ 
H $\quad 0.1583792 .959267 \quad 0.974649$ H 2.3089155 .1300320 .516215 H $1.8495616 .231430-1.838295$ H $\quad 0.3687905 .764209-2.681093$ H $1.5534804 .536613-2.278780$ H $1.990379-0.827131-0.207452$ H $2.3619830 .659005-1.105353$ H -2.146055 $0.572991-0.412746$ H -1.943653 -0.099106 -2.044599 H -1.212654 2.255164 -2.862192 H $-3.3279682 .635845-0.681696$ H -3.995964 $4.087048-2.732065$ H -2.303520 4.416206 -3.021009 H $-4.324366 \quad 6.5467910 .002006$ H $-5.1569235 .109369-0.817837$ H -2.117100 7.154409-0.590720 H -1.481065 $6.498667-2.068946$ H $-1.149797 \quad 5.3315210 .785893$ H $\quad 0.1890660 .995316-0.260884$ C $0.486012-3.2546782 .179698$ O $-0.574856-3.000266 \quad 1.232754$ C - $-1.222883-4.2490160 .923823$ C $-0.296542-5.342148 \quad 1.439748$ C $0.268204-4.6831752 .679829$ H -2.150749 -4.277208 1.506853 C -1.534061 -4.351226 -0.571424 H $-0.829625-6.2740301 .651153$ H $0.505937-5.5639520 .726206$ C $1.526923-5.3548873 .201746$ H $-0.498397-4.693753 \quad 3.466399$ C $-2.475482-3.259667-1.116316$ O $-0.308792-4.277852-1.312448$ H -1.956265 -5.339319 -0.789387 H -1.961760 -2.293480 -1.138699 O $-2.770769-3.589728-2.479584$ C $-3.802906-3.108677-0.350121$ C $-4.830507-2.226794-1.087323$ H $-3.595913-2.6853190 .637621$ O $-4.385068-4.396991-0.139124$ H -4.338628 -1.311194 -1.429060 H -5.180440 -2.762600 -1.977577 H $1.320706-6.3933603 .480768$ H $2.320669-5.3612132 .447166$ H $1.908922-4.8373334 .087142$ C $0.439214-2.2162043 .301569$ H $\quad 0.115838-3.430198-1.094030$ H -1.922587 -3.655370 -2.951408 H -4.525533 -4.802951 - 1.011828 H $0.988034-2.5824454 .176840$ H -0.596889 -2.040776 3.615361 H $1.430716-3.1865241 .623684$ C -5.3428990 .2697550 .624364$ O $-5.686208-1.0878950 .931180$ C $-6.050812-1.893152-0.197462$ C $-7.233327-1.257138-0.942381$ C $-6.9608650 .196646-1.310018$ C $-6.5097040 .976505-0.077381$ H $-4.4605210 .289847-0.023226$ C $-4.9817230 .971840 \quad 1.942725$ H $-6.415841-2.8429410 .213029$ O $-7.551798-1.987150-2.122095$ H -8.122409 -1.287664 -0.298792 H $-6.224200 \quad 0.276813-2.118231$ O $\quad-8.164515 \quad 0.791985-1.799314$ H $-7.353519 \quad 1.0923160 .614926$ O $-6.1025682 .282479-0.478637$ C $-3.595026 \quad 0.6012752 .428719$ H -4.9919552 .0583841 .799428$ H $-5.692560 \quad 0.7054552 .732857$ H -7.736054 -2.904488 -1.856958 H $-8.461496 \quad 0.260521-2.557927$ H $-6.8520262 .690127-0.945634$ O $-3.177400 \quad 1.538868 \quad 3.330692$ O $-2.963366-0.3860022 .078705$ C $-1.8644901 .341567 \quad 3.864104$ H -1.1206421 .4476073 .069493$ H -1.683923 2.1125554 .617801 H -1.7874290 .3601864 .341606$
SCF Energy (B3LYP/6-31G**//MMFF) $=-3245.91224445$

$02 \_228$

MMFF Geometry

C $2.030381-3.0773931 .167370$

C $1.380640-2.0286911 .698133$

C $1.918266-0.9994792 .666704$

O $1.413647 \quad 0.2733002 .182982$

C $3.452183-0.8942292 .736345$

C 3.9570890 .0335113 .822715

C 4.4164051 .2828273 .614254

C $3.975356-0.5564855 .209409$

C 4.4384122 .0610332 .328010

C 5.8495892 .2174241 .743830

C 6.3471910 .9624661 .008673

C 7.7729361 .1679490 .491805

C $8.251846-0.017141-0.361402$

O $5.462100 \quad 0.730655-0.091356$

O $9.439510 \quad 0.391214-1.057263$

C $7.184633-0.326696-1.432962$

C $8.648020-1.2195330 .503646$

O $7.516782-1.508393-2.158538$

C $5.753182-0.441325-0.862306$

C $4.722732-0.510770-2.016620$

O $5.658730-1.631693-0.077541$

C 0.7701251 .0965273 .047067

O $\quad 0.5900090 .9066794 .242150$

C 0.2610462 .3105402 .364206

C 0.4557812 .5735751 .062208

C -0.0418593 .7402590 .328316$

C -0.8768774 .7412371 .082122$

C $3.265020-0.369286-1.555300$

C $2.283361-0.397710-2.713480$

C $-0.138895-0.029827-3.196540$

O $2.612098-0.639598-3.871247$

C $-0.2326401 .381509-3.682813$

C -1.243901 2.211335 -3.386009

C $-1.3090353 .635855-3.858351$

C -1.396654 $4.632994-2.722200$

C $-2.5414955 .292100-2.476885$

C $-0.1405394 .946067-1.936924$

C $0.2528943 .850312-0.984201$

N $0.988468-0.130070-2.288434$

H $3.056663-3.2714121 .466675$

H $\quad 0.343009-1.8644461 .417837$

H $1.498160-1.2300493 .652717$

H $3.831893-0.5706641 .762262$

H $3.899433-1.8785022 .921128$

H $4.789751 \quad 1.8395754 .473121$

H 4.3809910 .1382335 .952424

H $2.962207-0.8217385 .527027$

H $4.594525-1.4592575 .230383$

H $3.753071 \quad 1.6456091 .583464$

H 4.0473563 .0615752 .554670

H 5.8156893 .0509611 .029763

H 6.5564122 .4976902 .534565

H 6.3110320 .1079451 .693061

H $8.465651 \quad 1.348347 \quad 1.323340$

H $7.8083132 .074700-0.128510$

H $10.097913 \quad 0.660495-0.393980$

H $7.2021820 .498107-2.159345$

H $9.461755-0.9478371 .186850$

H $9.035536-2.038703-0.112089$

H $7.824339-1.6023441 .109659$

H $8.416420-1.389946-2.508641$

H $4.9232360 .300200-2.729172$

H $4.834768-1.457688-2.559632$

H $4.744353-1.953977-0.090617$

H -0.2999822 .9843113 .004198$

H 1.0305921 .8696750 .463266

H -1.7461364 .2545311 .537540$

H -1.267131 5.5431240 .451756

H -0.2854625 .2146801 .873589$

H $2.996521-1.184789-0.879065$

H $3.1287660 .578756-1.022097$

H $-1.032389-0.340849-2.646011$

H $\quad 0.006372-0.709011-4.042563$

H $\quad 0.582750 \quad 1.740390-4.306861$ 
H $-2.0634921 .852699-2.768404$ H $-2.1857003 .727255-4.512941$ H $-0.4447083 .887901-4.485629$ H -2.609405 $6.035302-1.689455$ H -3.439580 $5.101543-3.054933$ H $-0.2392835 .900599-1.406837$ H $0.6874485 .110711-2.638860$ H $0.8675503 .075465-1.442360$ H $0.8315110 .123347-1.317146$ C $0.293149-3.595062-0.674405$ O $-0.893775-3.6281750 .135500$ C -2.031071 -3.878179 -0.720117 C $-1.477767-4.073782-2.127024$ C $-0.043188-4.491777-1.861744$ H $-2.484626-4.804069-0.347143$ C $-3.004920-2.700746-0.579932$ H -2.043757 -4.817939-2.695688 H - $1.501981-3.133745-2.692543$ C $0.864863-4.309321-3.065702$ H $-0.033653-5.545180-1.551785$ C $-4.227683-2.776767-1.510057$ O $-3.462355-2.6672200 .775889$ H -2.461063 -1.764509-0.748625 H -3.906969 -2.713138 -2.556254 O $-4.832227-4.066159-1.348637$ C $-5.307439-1.711511-1.231243$ C $-4.792681-0.264764-1.325739$ H $-5.758275-1.899683-0.251039$ O $-6.359625-1.884559-2.188107$ H -3.935738 -0.134036 -0.660164 H -4.437580 -0.077609-2.346663 H $\quad 0.511446-4.913397-3.907850$ H $0.894174-3.264606-3.391736$ H $1.889048-4.619331-2.837203$ C $1.502293-4.054810 \quad 0.143212$ H -2.676297 -2.619107 1.346803 H -5.610419 -4.096852 -1.931407 H -5.988055 -1.721847 -3.071971 H $2.331678-4.259536-0.544189$ H $1.272052-4.9921120 .663985$ H $0.434070-2.561122-1.011511$ C $-5.603450 \quad 0.738831 \quad 1.420950$ $\begin{array}{lllll}\text { O } & -6.474607 & 0.535313 & 0.300738\end{array}$ C $-5.8803280 .777899-0.977778$ C $-5.4086382 .235524-1.053197$ C -4.4794862 .5910800 .106166$ C $-5.0943502 .186911 \quad 1.448871$ H $-4.756196 \quad 0.045611 \quad 1.361947$ C -6.4069350 .3965052 .680715$ H $-6.6829630 .659928-1.716350$ O $-4.7463432 .491226-2.285987$ H $-6.2822202 .899214-1.014006$ H -3.493571 2.130900 -0.026658 O -4.2480024 .0024220 .107590$ H $-5.9129502 .877414 \quad 1.689341$ O -4.0909482 .3392402 .451166$ C -6.766056 -1.070477 2.710856 H $-5.8616610 .629223 \quad 3.601455$ H -7.3452740 .9636722 .709195$ H -5.367046 2.275313 -3.002560 H -5.1051784 .4490130 .213293$ H -4.5252722 .2878553 .318766$ O $-5.681727-1.8092023 .081866$ O $-7.874267-1.5059932 .426067$ C $-5.887500-3.2248043 .120435$ H $-6.164570-3.5944712 .128321$ H -4.947989 -3.696744 3.420265 H $-6.657394-3.4758333 .856168$ SCF Energy (B3LYP/6-31G**//MMFF $)=-3245.90709343$

\section{$02 \_229$}

MMFF Geometry

C 5.1019123 .4457270 .502349

C $3.7961513 .386076 \quad 0.196184$

C $2.733017 \quad 4.179676 \quad 0.913338$

O 1.6396793 .2880831 .245218

C $2.1877455 .284627-0.005763$

C 1.2027166 .2016740 .693609
C $-0.130075 \quad 6.2003750 .493704$

C 1.8170117 .1993071 .643207

C $-0.9309945 .308977-0.413792$

C -2.0298454 .5741800 .357988$

C $-2.8023543 .561051-0.500687$

C $-3.5852204 .225997-1.633029$

C $-4.436243 \quad 3.212217-2.409367$

O -3.7097612 .8839620 .376444$

O $\quad-5.3588553 .947269-3.228434$

C $-5.2774482 .382292-1.415308$

C $-3.5798802 .371429-3.366736$

O $-5.950727 \quad 1.338287-2.112816$

C $-4.455191 \quad 1.813572-0.236230$

C -5.3405391 .2442590 .900459$

O

C 1.8043062 .4894552 .335152

O 2.7810012 .4434513 .066947

C 0.5847401 .6624872 .487888

C 0.5765970 .6890423 .411051

C $-0.528809-0.2200813 .724713$

C $-1.8434500 .010443 \quad 3.031248$

C $-6.2417750 .047350 \quad 0.588518$

C $-5.508947-1.1920370 .114352$

C $-3.791969-2.8480460 .796251$

O $-5.670382-1.643585-1.017341$

C $-3.447709-3.5311002 .078775$

C $-2.192700-3.7176432 .514409$

C $-1.869132-4.4105723 .806677$

C $-0.871994-3.6542634 .655656$

C $0.283743-4.2328145 .026828$

C $-1.247512-2.2645525 .129396$

C $-0.307034-1.2033234 .622645$

N $-4.678682-1.7358811 .080764$

H $5.414324 \quad 4.1308301 .288669$

H $3.4483142 .727584-0.593705$

H $3.1301634 .622327 \quad 1.835272$

H $1.7356594 .833071-0.895122$

H $3.0166705 .899354-0.382775$

H $-0.7270996 .931603 \quad 1.038251$

H 1.0755267 .8911472 .057282

H 2.2942196 .6891692 .485150

H 2.5718367 .8006811 .126104

H -1.376222 $5.943639-1.187806$

H $-0.3070504 .573238-0.925928$

H $-1.5895784 .041878 \quad 1.212108$

H -2.7329125 .2946350 .797697$

H -2.085980 $2.833010-0.896773$

H $-2.9187164 .754776-2.323956$

H -4.252586 $4.991809-1.213558$

H $-4.8430004 .523153-3.818521$

H $-6.0564593 .039921-1.005396$

H -3.059360 $3.018749-4.082979$

H -4.201862 $1.700372-3.969365$

H $-2.8236051 .773669-2.853923$

H $-6.471003 \quad 1.751118-2.823196$

H $-4.6825500 .975597 \quad 1.738059$

H $-5.9710362 .054773 \quad 1.291207$

H $-3.2664140 .284187 \quad 0.057166$

H $-0.2612991 .872599 \quad 1.843921$

H 1.4787930 .5335774 .002269

H -2.1881751 .0391163 .185433$

H -2.643075 -0.6384033 .392544$

H - $1.746312-0.1682491 .956320$

H $-6.794372-0.2271821 .496501$

H $-7.0050270 .311264-0.149770$

H $-2.910497-2.4424050 .289683$

H $-4.289980-3.5447580 .114383$

H -4.277813 -3.900594 2.676864

H $-1.360315-3.3680831 .909725$

H -1.481697 -5.4072493 .559182$

H $-2.776492-4.5727274 .402235$

H $\quad 0.995918-3.7172555 .662448$

H $0.543664-5.2364824 .707295$

H - $-1.216635-2.2474036 .227128$

H $-2.289922-2.0469924 .880814$

H $0.685625-1.2663845 .071871$

H -4.524159-1.213515 1.938028

C $5.8043341 .402513-0.908630$ 
O $5.1711790 .465101-0.018386$

C $5.508286-0.874885-0.424198$

C $6.324247-0.735061-1.706481$

C $6.9661150 .627964-1.524056$

H $6.140649-1.3006860 .364471$

C $4.235253-1.719677-0.581375$

H $7.054587-1.542077-1.820675$

H $5.679671-0.741071-2.593868$

C $7.4993961 .224229-2.814644$

H $7.7859610 .537464-0.799152$

C $3.506666-2.0131920 .748587$

O $3.347050-1.061475-1.486230$

H $4.507833-2.674837-1.044136$

H $4.127671-2.6834181 .354593$

O $3.372754-0.8136691 .520494$

C $2.093848-2.6083390 .601443$

C $2.020565-3.864167-0.281674$

H $1.413613-1.8358540 .227555$

O $1.622596-2.9641681 .906133$

H $2.458448-3.657094-1.261596$

H $2.617752-4.6621470 .176588$

H $8.2726670 .579602-3.245125$

H $6.7062001 .341521-3.560663$

H $7.9423212 .209027-2.636596$

C $6.2207132 .643436-0.116198$

H $3.177956-0.171031-1.134810$

H $2.864654-0.1744940 .992799$

H $1.672170-2.1661302 .459872$

H $6.8096733 .316718-0.749125$

H 6.8701542 .3243410 .709640

H $5.0782351 .663485-1.688990$

C $-0.093588-2.855038-2.217014$

O $-0.313650-3.322986-0.880676$

C $0.569984-4.369671-0.463226$

C $0.422380-5.578923-1.397279$

C $0.569238-5.194412-2.868694$

C $-0.318629-3.996524-3.211261$

H $0.931193-2.477442-2.315886$

C $-1.048671-1.682663-2.458333$

H $0.222721-4.6879510 .527268$

O $1.375899-6.587407-1.080425$

H $-0.572518-6.021811-1.257164$

H $1.615384-4.989561-3.125455$

O $\quad 0.165661-6.296888-3.684257$

H -1.372236 -4.303407 -3.206528

O $-0.006064-3.544684-4.526629$

C $-0.553139-0.458566-1.723856$

H - $1.136955-1.433129-3.521685$

H $-2.059493-1.902413-2.095260$

H $1.247910-6.826923-0.146612$

H $0.714467-7.059111-3.431325$

H $-0.131213-4.297907-5.129076$

O $0.251440 \quad 0.281394-2.538700$

O $-0.819437-0.210812-0.555357$

C $0.825478 \quad 1.452537-1.949188$

H $1.6459401 .787807-2.589127$

H $\quad 0.0724432 .242725-1.898830$

H $1.2270711 .239201-0.954067$

SCF Energy (B3LYP/6-31G**//MMFF)= -3245.91264702

02_23

MMFF Geometry

C $0.544546 \quad 2.761410 \quad 0.504068$

C $1.432143 \quad 3.330068-0.324511$

C $1.7146712 .777154-1.693919$

O $3.1510882 .621208-1.777954$

C $1.2468643 .681136-2.842784$

C $-0.243740 \quad 3.937629-2.897509$

C $-1.165807 \quad 3.020101-3.250735$

C $-0.6624785 .345481-2.565779$

C $-0.9282341 .577762-3.604619$

C $-1.8284350 .653721-2.771273$

C $-1.320031-0.797499-2.768737$

C $-2.478925-1.769224-2.542530$

C $-2.002418-3.219925-2.418315$

O $-0.366515-0.914165-1.702033$

O $-3.114495-3.983055-1.926516$

C $-0.881052-3.276508-1.364743$
C $-1.639406-3.815858-3.782622$

O $-0.320236-4.586081-1.295192$

C $0.226412-2.212467-1.566593$

C $1.147508-2.213940-0.321457$

O $0.997429-2.554308-2.719293$

C $3.6270361 .576399-2.505465$

$\begin{array}{llll}\text { O } & 2.992298 & 0.804972 & -3.205757\end{array}$

C $5.0732391 .481611-2.222871$

C $5.623346 \quad 0.262403-2.131265$

C $6.995949-0.029281-1.718504$

C $7.9648371 .122580-1.662714$

C $2.150525-1.057597-0.256974$

C $2.994476-1.1124501 .006134$

C $4.665194 \quad 0.2185452 .289381$

O $2.983868-2.0689351 .776841$

$\begin{array}{llll}\text { C } & 6.080053 & 0.074378 & 1.823974\end{array}$

C $6.837930-1.0076562 .060739$

C $8.277858-1.1403341 .646465$

C $8.496811-2.2333730 .621855$

C $8.662605-3.5121920 .999209$

C $8.603419-1.826570-0.833191$

C $7.302361-1.297364-1.372817$

N $3.7705340 .027412 \quad 1.163347$

H -0.0353391 .9118610 .149157$

H $2.014838 \quad 4.184800 \quad 0.008379$

H $1.2547451 .786874-1.772551$

H $1.5665023 .247351-3.800053$

H $1.7900114 .635526-2.796385$

H $-2.209453 \quad 3.330136-3.289132$

H $-0.2215676 .049357-3.279347$

H $-1.7492455 .476390-2.598095$

H $-0.3276525 .616023-1.559375$

H - $1.1649891 .438064-4.666721$

H $\quad 0.1197451 .293942-3.490622$

H $-1.8874261 .018972-1.737698$

H -2.839343 $0.704279-3.194518$

H $-0.826537-1.013087-3.723650$

H -3.221248 -1.682770 -3.345581

H $-3.002690-1.496905-1.617316$

H -2.801045 -4.889582 -1.764348

H $-1.356089-3.102107-0.389404$

H $-2.505398-3.783733-4.454602$

H $-1.370688-4.874338-3.694587$

H $-0.817233-3.291398-4.274207$

H $0.275512-4.693365-2.056202$

H $0.527759-2.1580590 .582344$

H $1.699651-3.161562-0.275696$

H $1.867816-2.131061-2.651632$

H $5.6094392 .396672-2.001229$

H $4.992508-0.607764-2.309152$

H $7.9900211 .648728-2.623946$

H $8.9922900 .820235-1.452241$

H $7.675057 \quad 1.834115-0.881914$

H $2.831820-1.093377-1.111294$

H $1.618913-0.101659-0.270712$

H $4.424175-0.4916183 .087255$

H $4.501516 \quad 1.2293852 .675829$

H 6.5072330 .9198381 .291200

H $6.407277-1.8444942 .606419$

H $8.868998-1.3565802 .546250$

H $8.671766-0.1896671 .266073$

H $8.846923-4.2976340 .274020$

H $8.613934-3.8013172 .043948$

H $8.889166-2.686404-1.453028$

H $9.427270-1.115471-0.936601$

H $6.522628-2.057367-1.442888$

H $3.7242840 .761467 \quad 0.462578$

C $\quad 0.527453 \quad 1.9952722 .866484$

O $-0.489184 \quad 1.0012132 .624564$

$\begin{array}{llll}\text { C } & -0.962071 & 0.497986 & 3.885792\end{array}$

C 0.0167551 .0095384 .935785

C 0.4117692 .3506804 .347066

H -1.9400890 .9603764 .064034$

C $-1.106394-1.0254483 .821623$

H -0.4421091 .0920545 .925689$

H 0.8952450 .3597855 .023711

C $1.6842842 .924744 \quad 4.945068$

H -0.4122673 .0602834 .500986$ 
C $-2.008295-1.5343572 .676945$ O $0.188314-1.6142963 .642185$ H -1.475122 -1.401533 4.782776 H -1.528251 -1.361491 1.706498 O $-2.126702-2.9556762 .819003$ C -3.422626 -0.925385 2.656410 C $-4.347822-1.6013581 .626145$ H $-3.349463 \quad 0.1424242 .431442$ O $-4.011539-1.0408443 .952406$ H -3.843034 -1.620540 0.655642 H -4.516012 -2.6420351 .927470$ H 1.5622523 .0926326 .020014 H 2.5344722 .2484454 .804713 H 1.9364483 .8828794 .480199 C 0.3096273 .1821781 .926028 H $0.575967-1.2312672 .836601$ H -1.225988 -3.322833 2.811474 H $-4.048597-1.9869984 .175035$ H 0.9742774 .0094542 .198013 H -0.7224763 .5395762 .009279$ H 1.4943731 .5173022 .662095 C $-5.2106800 .670960-0.260259$ O $\begin{array}{lllll}-5.595173 & 0.471004 & 1.106056\end{array}$ C $-5.719784-0.8981251 .511048$ C $-6.734840-1.6276870 .619331$ C $-6.410681-1.470892-0.862444$ C $-6.2221490 .005944-1.203564$ H $-4.2102770 .255798-0.425766$ C $-5.1521322 .185074-0.514332$ H -6.149105 -0.8772092 .520659$ O $-6.794893-3.0072340 .965158$ H -7.732485 -1.208842 0.806670 H -5.533046 -2.066146-1.139292 O $-7.505651-1.990216-1.619665$ H $-7.1923990 .513633-1.133325$ O $-5.792248 \quad 0.124102-2.557127$ C -3.9765322 .8498650 .169487$ H $-5.0518182 .391115-1.585697$ H $-6.0674002 .656237-0.136414$ H $-7.430939-3.4306130 .363533$ H -7.302642 -1.843381 -2.559440 H -4.934572 -0.324737 -2.636859 O $\quad-4.087840 \quad 4.202791 \quad 0.012014$ $\begin{array}{lllll}\text { O } & -3.072050 & 2.268088 & 0.751036\end{array}$ C $-3.042656 \quad 4.9810570 .601877$ H $-3.243113 \quad 6.0350430 .392596$ H -3.0278364 .8341931 .685873$ $\mathrm{H}-2.078227 \quad 4.714140 \quad 0.161495$

SCF Energy (B3LYP/6-31G**//MMFF) $=-3245.93263523$

$02 \_230$

MMFF Geometry

C $-0.753046 \quad 1.6456252 .603102$

C $-1.780387 \quad 1.1261623 .293780$

C $-2.195853-0.3246943 .349559$

O $-1.176941-1.1972452 .817299$

C $-3.477062-0.5551742 .531646$

C $-4.063573-1.9419962 .717720$

C $-4.052247-2.9174331 .787109$

C $-4.747538-2.1781124 .040559$

C $-3.440167-2.8959260 .412255$

C $-4.500364-2.886948-0.700521$

C $-5.232931-1.542926-0.813959$

C $-6.386831-1.615049-1.813925$

C $-7.076205-0.249325-1.974951$

O $-4.284002-0.565528-1.253427$

O $-7.920824-0.315313-3.133920$

C $-6.004056 \quad 0.825467-2.255924$

C $-7.9926330 .073622-0.788575$

O $-6.5727702 .132710-2.251903$

C $-4.7978130 .770513-1.289920$

C $-3.6805091 .727262-1.776647$

O $-5.209797 \quad 1.2031620 .010955$

C $-0.221309-1.6504013 .669587$

O $-0.053199-1.3006394 .829914$

C $0.631927-2.680913 \quad 3.033057$

C $0.370382-3.1999591 .824226$

C $1.117061-4.2586241 .145978$
C $2.347708-4.8039451 .819150$

C $-2.2878711 .390558-1.222204$

C $-1.4806650 .473862-2.131437$

C $0.787485-0.522863-2.309736$

O $-1.8224120 .190552-3.277038$

C $0.562818-1.976171-2.586026$

C $1.582996-2.841078-2.704414$

C $1.435194-4.294112-3.058752$

C $2.092225-5.207003-2.046470$

C $3.381374-5.564024-2.172537$

C $1.238052-5.761048-0.929848$

C $0.664892-4.686459-0.050584$

N $-0.2983930 .051185-1.533422$

H -0.1445071 .0069811 .969520$

H -2.3717391 .7925313 .919672$

H $-2.378423-0.5799554 .400814$

H $-3.264729-0.3744911 .474079$

H -4.2426160 .1808622 .811327$

H -4.532286 -3.863482 2.036543

H -5.244454 -3.153352 4.083939

H $-4.025250-2.1420064 .861432$

H -5.512727 -1.414475 4.214198

H -2.739101 -2.066531 0.280999

H $-2.839750-3.8087080 .309226$

H -3.990284 -3.099415-1.648888

H $-5.221394-3.695793-0.529361$

H -5.615694 -1.264346 0.173528

H -7.115262 -2.379241 -1.515920

H $-6.003152-1.929868-2.794476$

H -8.557991 -1.037709 -2.999330

H $-5.6337760 .652061-3.276249$

H -8.762357 -0.699559-0.677905

H $-8.5336391 .012339-0.951921$

H -7.457609 $0.145107 \quad 0.160574$

H -7.312956 2.128400 -2.882820

H $-3.6425611 .734530-2.872066$

H $-3.9347232 .750249-1.468918$

H -4.4354441 .5522330 .482070$

H $1.466160-3.0231173 .636708$

H $-0.490416-2.8308071 .270800$

H $3.041017-3.9966952 .077827$

H $2.910478-5.4988871 .191012$

H $2.076761-5.3378902 .736304$

H -1.717083 2.322644-1.147803

H $-2.3461470 .961248-0.218083$

H $1.706254-0.363559-1.738382$

H $\quad 0.8573470 .025622-3.255349$

H $-0.454615-2.325711-2.738837$

H $2.599441-2.472105-2.585152$

H $1.895465-4.447135-4.043921$

H $0.381203-4.572509-3.181254$

H $3.854829-6.235759-1.464439$

H $3.993676-5.186003-2.984712$

H $1.788591-6.500691-0.338092$

H $\quad 0.404511-6.323299-1.370834$

H $-0.236766-4.228601-0.459516$

H $-0.0719800 .400284-0.606533$

C -0.4625073 .8633381 .403400$

O $0.528988 \quad 3.4113370 .455132$

C $1.2454214 .554472-0.048707$

C 0.3725575 .7610370 .270415

C -0.2075455 .3602551 .608403$

H 2.1749844 .6218380 .529708

C $1.5742184 .404286-1.537966$

H 0.9469596 .6914540 .311153

H $-0.4310755 .889685-0.463792$

C -1.4486556 .1485081 .992442$

H 0.5606095 .5093652 .379524

C $2.3327553 .119576-1.917709$

O $0.3569584 .421344-2.295631$

H $2.1401855 .282195-1.870877$

H $1.6679162 .256808-1.810237$

O $2.636708 \quad 3.202365-3.317498$

C $3.6321182 .855919-1.131937$

C $4.4215391 .675527-1.729379$

H $3.3813122 .658137-0.085812$

O $4.4401094 .030839-1.172138$

H $3.7314420 .842005-1.889454$ 
H $4.8080051 .969705-2.712889$ H -1.217669 7.2161062 .069274 H -2.244933 6.0296191 .249967 H -1.838362 5.8169282 .959972 C -0.3373703 .0866582 .715582$ H $-0.1801323 .665886-2.003717$ H $3.2656783 .934669-3.437786$ H $5.1590943 .917740-0.528193$ H -0.9296023 .5833633 .493590$ H 0.7062583 .1018153 .052273 H -1.445169 3.6937160 .946221 C $4.430214-0.3380120 .567984$ $\begin{array}{lllll}\text { O } & 5.228508 & 0.847556 & 0.463423\end{array}$ C $5.6152211 .209190-0.866133$ C $6.4226340 .065990-1.497749$ C $5.644316-1.247026-1.470945$ C $5.144887-1.544392-0.058358$ H $3.462336-0.1738790 .079757$ C $4.179658-0.5955222 .063120$ H $6.2946182 .064469-0.767801$ O $\quad 6.780100 \quad 0.395811-2.835365$ H $7.357732-0.060957-0.937016$ H $4.814009-1.227748-2.185324$ O $6.496210-2.311862-1.900746$ H $5.993240-1.8437460 .570458$ O $4.242227-2.646811-0.105396$ C 3.3101230 .4619112 .706082 H $3.679299-1.5579522 .210554$ H $5.139584-0.6009702 .594083$ H $7.257121-0.366342-3.205948$ H $7.253906-2.355406-1.292829$ H $4.710857-3.389844-0.522580$ O 3.3473150 .2963614 .061704 O 2.6719711 .3132382 .104195 C $2.5901671 .241244 \quad 4.823824$ H 2.8018101 .0707485 .882704 H 2.8826142 .2645894 .569401 H 1.5218021 .0902184 .651254 SCF Energy (B3LYP/6-31G**//MMFF) $=-3245.91452424$

\section{1}

MMFF Geometry

C $-3.211606 \quad 1.359973 \quad 3.172823$

C -4.0451300 .7013992 .351628$

C $-4.508887 \quad 1.159398 \quad 0.989878$

O -3.8623112 .4063790 .644843$

C $-6.0307151 .369546 \quad 0.891755$

C $-6.896195 \quad 0.158180 \quad 1.160274$

C $-6.956969-0.9321170 .370772$

C $-7.750613 \quad 0.2462182 .397308$

C $-6.171185-1.197706-0.883948$

C $-5.215292-2.375802-0.659020$

C $-4.191762-2.541104-1.791773$

C $-3.462973-3.878350-1.630122$

C -2.328331-4.050504-2.645905

O $-3.265148-1.451604-1.704723$

O $-1.528387-5.166357-2.226151$

C $-1.428983-2.800746-2.588087$

C $-2.854094-4.396008-4.043535$

O $-0.429033-2.849074-3.604289$

C $-2.215018-1.471138-2.680457$

C $-1.290058-0.253540-2.429135$

O $-2.743799-1.339789-4.004862$

C $-3.6081892 .639697-0.669433$

O $-3.9290251 .949761-1.622957$

C $-2.8083463 .877957-0.741850$

C $-1.9151834 .012040-1.732282$

C $-0.9841395 .127736-1.897463$

C $-1.2197866 .357966-1.059936$

C $-0.866637-0.059653-0.969947$

C $0.2305630 .980468-0.817693$

C 1.4584652 .2175840 .961741

O $0.9804261 .297514-1.737158$

C 1.4833843 .6037070 .398487

C $2.4695774 .052881-0.394129$

C $2.5740685 .452719-0.931010$

C $2.4727195 .506469-2.440557$

C $3.5342885 .229128-3.216413$
C $1.1578665 .952011-3.042827$

C $0.0445824 .982015-2.758495$

N $0.310307 \quad 1.4696890 .481244$

H -2.841562 2.3450282 .902640

H -4.386007 -0.2880022 .651161$

H $-4.1749560 .391457 \quad 0.285273$

H $-6.2829851 .760339-0.103515$

H $-6.3165582 .187126 \quad 1.568909$

H -7.632725 -1.7371580 .656845$

H -8.355044 -0.6536732 .552562$

H $-7.1224440 .379179 \quad 3.284174$

H -8.4358631 .0971842 .324412$

H -6.871952 -1.445534 -1.690334

H $-5.628751-0.310575-1.219742$

H -4.675785 -2.228109 0.286518

H $-5.808608-3.293562-0.555888$

H -4.711772 -2.498459 -2.755841

H $-4.168738-4.715745-1.700492$

H $-3.032226-3.937292-0.620230$

H -2.104392 -5.948810 -2.182140

H $-0.896031-2.834397-1.630183$

H -3.414674 -5.338295 -4.019135

H -2.029796 -4.556443 -4.747037

H -3.516213 -3.631449-4.454986

H $0.041616-3.694454-3.508433$

H $-0.416317-0.316801-3.089924$

H $-1.8066400 .662823-2.742769$

H -2.949778 $-0.403757-4.163085$

H -2.9278154 .6046420 .053173$

H -1.819102 $3.208042-2.460783$

H $-2.2381356 .734412-1.209835$

H $-0.5482027 .184940-1.297094$

H $-1.082268 \quad 6.133540 \quad 0.003474$

H -1.732256 $0.237601-0.369172$

H $-0.472185-0.990367-0.551861$

H $2.358953 \quad 1.6524520 .704125$

H 1.3857662 .2676572 .052976

H 0.6775324 .2724070 .687813

H $3.2793363 .377116-0.659250$

H $3.5419185 .864965-0.616138$

H $1.8217726 .109760-0.478372$

H $3.4764835 .288192-4.298060$

H $4.484724 \quad 4.933037-2.784371$

H $1.2437096 .041559-4.133697$

H $0.9368476 .964306-2.693638$

H $\quad 0.1327824 .044875-3.310102$

H $-0.283600 \quad 1.044558 \quad 1.187786$

C -1.1816470 .6928564 .484032$

O $-0.739053-0.2093453 .450420$

C $0.277819-1.0811343 .987536$

C $0.677883-0.4789035 .325936$

C $-0.6294790 .128345 \quad 5.792395$

H $-0.211479-2.0521444 .132922$

C $1.428405-1.2010002 .979789$

H $1.076391-1.2245286 .020406$

H 1.4361010 .3027185 .190880

C $-0.4610301 .164028 \quad 6.890318$

H -1.278363 -0.6791536 .157604$

C $2.604133-2.0646203 .471291$

O $0.904257-1.7609831 .772541$

H $1.777849-0.1961662 .725632$

H $3.051573-1.6144624 .365298$

O $2.074296-3.3346943 .870884$

C $3.697607-2.3126092 .412278$

C $4.369902-1.0192951 .919374$

H $3.289256-2.8809021 .569418$

O $4.715919-3.1267733 .005289$

H $3.614364-0.3420821 .514550$

H $4.830939-0.5162552 .778202$

H $-0.0032730 .713436 \quad 7.777015$

H $\quad 0.178427 \quad 1.991973 \quad 6.566179$

H -1.4289001 .5814307 .185163$

C -2.7098120 .7929424 .472205$

H $0.617806-2.6694971 .968754$

H $2.769107-3.7972294 .369159$

H $4.347873-4.0196293 .116321$

H -3.050065 1.4411895 .287412

H $-3.144583-0.2005704 .639462$ 
H $-0.733273 \quad 1.6705744 .263505$

C $4.059702-1.209581-1.118057$

O $4.937925-1.978399-0.285507$

C 5.458971-1.281369 0.852097

C $6.225099-0.0301250 .399785$

C $5.3866670 .856064-0.516269$

C $4.7872490 .030472-1.653759$

H $3.173215-0.910222-0.546359$

C $3.604158-2.116420-2.269328$

H $6.197269-1.9523361 .308307$

O $\quad 6.664724 \quad 0.7130131 .531648$

H $7.124928-0.345691-0.144577$

H 4.6130821 .3808120 .053253

O $6.2335851 .867129-1.066544$

H $5.592529-0.270027-2.335721$

O $3.893091 \quad 0.846223-2.405292$

C $2.740644-3.255379-1.772141$

H $3.017025-1.565219-3.011417$

H $4.486376-2.537682-2.766742$

H 7.1265481 .5020431 .199833

H $5.6917332 .397012-1.676589$

H $3.1563611 .098712-1.822854$

O $3.045221-4.393713-2.460299$

O $1.887268-3.150371-0.901415$

C $2.312894-5.561438-2.076499$

H $1.252969-5.433756-2.310821$

H $2.699314-6.408619-2.649256$

H $2.451552-5.766762-1.010615$

SCF Energy (B3LYP/6-31G**//MMFF) $=-3245.92989543$

$02 \_232$

MM̄FF Geometry

C $1.153148 \quad 0.065693-2.674012$

C $1.063675-1.271192-2.740012$

C $1.342518-2.215407-1.590760$

O $1.185096-1.542460-0.319245$

C $2.790556-2.728049-1.648789$

C $2.969043-4.056855-0.943351$

C $3.342452-4.2106450 .341897$

C $2.730602-5.267598-1.809263$

C $3.576446-3.1498901 .381541$

C $5.049454-3.0380261 .795302$

C $5.878422-2.1906120 .818417$

C $7.358195-2.2198361 .204149$

C $8.201490-1.2891750 .321047$

O $5.383330-0.847806 \quad 0.886762$

O $9.483189-1.1700390 .956658$

C $7.5422480 .106376 \quad 0.297841$

C $8.454202-1.898954-1.062791$

O $8.197427 \quad 0.968817-0.629896$

C $6.0248080 .069172-0.006384$

C $5.413936 \quad 1.473280 \quad 0.225537$

O $5.833949-0.305017-1.372670$

C $-0.073880-1.2602680 .105083$

O $-1.123484-1.579396-0.427292$

C $0.063679-0.4183141 .311766$

C -0.9340780 .4116651 .645257$

C $-0.942668 \quad 1.3403102 .776224$

C 0.2744861 .3708303 .663098

C 3.8782521 .5129640 .207692

C 3.3478402 .9355950 .300040

C 1.2234024 .2355630 .182198

O $4.076772 \quad 3.9137170 .446304$

C 0.7047034 .5271241 .554881

C $-0.558374 \quad 4.9123591 .794595$

C -1.0840885 .2599993 .158294$

C -2.3746034 .5439163 .490497$

C -3.5601945 .1660003 .375670$

C -2.2828193 .1360134 .034123$

C -2.0273812 .1223492 .954780$

N 1.9641882 .9863620 .191905

H $1.5027190 .528441-1.756302$

H $\quad 0.730418-1.734400-3.665773$

H $0.629366-3.048148-1.638129$

H $3.468549-1.983509-1.214602$

H $3.115785-2.852366-2.690439$

H $3.460982-5.2264780 .718323$

H $2.860335-6.206225-1.260072$
H $1.710809-5.257113-2.207108$

H $3.432132-5.276826-2.649974$

H $3.192371-2.1745371 .074116$

H $2.990048-3.4349362 .264497$

H $5.078962-2.5623722 .784487$

H $5.489221-4.0372121 .902171$

H $5.742436-2.578436-0.197074$

H $7.750595-3.2438751 .166281$

H $7.467264-1.8973662 .249413$

H $10.054612-0.628657 \quad 0.386426$

H $7.684898 \quad 0.551820 \quad 1.292452$

H $9.001540-2.844541-0.967723$

H $9.087519-1.246283-1.673929$

H $7.535332-2.101669-1.616912$

H $9.122007 \quad 1.061177-0.345262$

H $5.737713 \quad 1.8535021 .203394$

H $5.7968332 .165875-0.535151$

H $4.9971070 .065594-1.693077$

H $0.996140-0.4737051 .861572$

H -1.8280170 .4226951 .023602$

H 1.1737831 .5934673 .077885

H $\quad 0.4104400 .4041074 .160173$

H 0.2264522 .1290914 .447025

H $3.4977671 .073639-0.717983$

H 3.4738960 .9431881 .051995

H $0.4148024 .129870-0.547220$

H $1.8825035 .047001-0.142643$

H 1.4044194 .4493372 .383606

H -1.245796 5.0222740 .958725

H $-1.244631 \quad 6.3458873 .184324$

H -0.3419115 .0516053 .938917$

H -4.4878414 .6678603 .637493$

H -3.6298986 .1855343 .011391$

H -3.226977 2.8581604 .521817

H -1.5388703 .1079204 .834782$

H -2.8545472 .0283212 .249631$

H 1.4362152 .1203120 .136658

C $-0.2089862 .061765-3.233185$

O $-1.4570001 .441741-2.865640$

C $-2.5314552 .349977-3.175451$

C $-1.8784843 .651352-3.633652$

C $-0.5799433 .149190-4.235823$

H $-3.0697561 .916208-4.025531$

C $-3.4725902 .496889-1.973532$

H $-2.5018644 .196378-4.349079$

$\mathrm{H}-1.6741064 .322587-2.791176$

C $0.4715324 .233294-4.394771$

H $-0.7935692 .701542-5.215629$

C $-4.0660001 .166415-1.462025$

O $-2.7358183 .085169-0.892250$

H $-4.2738193 .203991-2.218752$

H -3.264796 $0.581448-0.999861$

O $-4.9860181 .484526-0.410835$

C $-4.7894840 .317311-2.521977$

C $-5.512408-0.939203-1.994265$

H $-4.078431-0.002311-3.292341$

O $\quad-5.7909381 .108704-3.170257$

H $-6.337657-0.633333-1.342612$

H -5.998983 -1.405039-2.860849

H $0.1102815 .024546-5.059771$

H $0.7240604 .691814-3.432539$

H $1.3917173 .825882-4.824872$

C $0.759977 \quad 1.008919-3.774396$

H $-3.3411123 .160290-0.134542$

H $-5.738427 \quad 1.953260-0.811227$

H $-5.340727 \quad 1.783976-3.704414$

H $1.666523 \quad 1.490077-4.156657$

H $0.2937640 .458032-4.599915$

H $0.1989932 .515711-2.321290$

C $-5.213249-1.5938081 .004950$

O $-4.190779-1.5463610 .005776$

C $-4.587997-1.963662-1.304015$

C $-5.154157-3.391368-1.242403$

C $-6.290750-3.508325-0.231496$

C $-5.835941-2.9886841 .131106$

H $-6.003300-0.8823230 .736769$

C -4.627142 -1.0677812 .323921$

H $-3.670634-2.013552-1.904216$ 
O $-5.615890-3.817647-2.519793$

H $-4.355890-4.084037-0.944772$

H $-7.190823-2.984253-0.574003$

O $-6.653617-4.882976-0.085643$

H $-5.124505-3.7002951 .564980$

O $-6.956401-2.9236202 .010772$

C $-3.448508-1.8570762 .857946$

H -4.269492 -0.044295 2.164734

H $-5.407974-1.0490853 .092368$

H $-4.869558-3.744502-3.138982$

H $-6.895803-5.212576-0.968171$

H $-7.351084-3.8119392 .043400$

O $-3.146906-1.3993184 .110327$

O $-2.849799-2.7383372 .257809$

C $-2.032531-2.033300 \quad 4.745933$

H $-2.258103-3.0870804 .934351$

H $-1.132845-1.9351434 .131128$

H -1.857935-1.533869 5.702693

SCF Energy (B3LYP/6-31G**/MMFF) $=-3245.90751591$

02233

MMFF Geometry

C 2.3893551 .9997312 .454956

C $1.834437 \quad 1.152058 \quad 3.335764$

C $2.035417-0.344453 \quad 3.414533$

O $3.018153-0.8057222 .462682$

C $0.725675-1.0788563 .073180$

C $0.598212-2.4126303 .781374$

C $0.964604-3.6037493 .269837$

C $-0.032642-2.3418055 .148915$

C $1.652301-3.8881831 .964011$

C $0.748423-4.6092280 .954852$

C $-0.204833-3.6554290 .219833$

C $-1.136310-4.430900-0.713428$

C $-2.019981-3.492281-1.548911$

O $0.599486-2.749265-0.543800$

O $-2.613162-4.265995-2.603098$

C $-1.127016-2.426581-2.218511$

C $-3.180511-2.918944-0.728725$

O $-1.915646-1.442813-2.883363$

C $-0.139677-1.743101-1.243722$

C $0.861936-0.868688-2.036292$

O $-0.872467-0.907956-0.346333$

C $4.329598-0.7837542 .810645$

O $4.816960-0.3855923 .854748$

C $5.076697-1.3099221 .648340$

C $6.364777-0.9825841 .477957$

C $7.201286-1.3714120 .340364$

C $6.604616-2.311252-0.675973$

C $2.073769-0.389922-1.221996$

C $2.9852000 .510666-2.041926$

C $5.106407 \quad 1.812700-1.862464$

O $2.7313040 .850978-3.194615$

C $6.2634201 .028580-2.397802$

C $7.5441901 .323793-2.124829$

C $8.7158450 .569928-2.689209$

C $9.7300390 .178268-1.635545$

C $10.8643190 .880739-1.474262$

C $9.469210-1.074430-0.828152$

C $8.443541-0.8528050 .249866$

N $4.1082270 .904529-1.326460$

H 3.0635681 .6242151 .691585

H $1.168103 \quad 1.5625104 .093028$

H $2.364123-0.5941704 .431224$

H $0.652066-1.2261121 .989817$

H $-0.147590-0.4713453 .346712$

H $0.788740-4.4926793 .875261$

H $-0.097691-3.3232245 .630400$

H $0.553166-1.6910255 .805940$

H -1.048836 -1.940478 5.077553

H $2.080222-2.9874191 .518090$

H $2.506312-4.5399612 .188903$

H $1.401461-5.091304 \quad 0.215279$

H $\quad 0.181400-5.4043591 .454292$

H $-0.788144-3.0942180 .958162$

H - $-1.754802-5.138019-0.146596$

H $-0.536119-5.043101-1.401339$

H $-3.121550-4.987904-2.195250$
H $-0.544020-2.935023-2.999294$

H $-3.806068-3.727989-0.333205$

H $-3.840110-2.311714-1.356873$

H $-2.852541-2.3120190 .117763$

H $-2.514301-1.912045-3.489324$

H $1.250623-1.444330-2.886940$

H $\quad 0.339150 \quad 0.004638-2.447316$

H $-0.269600-0.2467530 .027146$

H $4.526220-1.913550 \quad 0.935377$

H $6.841762-0.3442662 .220515$

H $6.338657-3.264190-0.205089$

H $7.275279-2.545771-1.504577$

H $5.702729-1.878413-1.122729$

H $1.7500850 .172860-0.340840$

H $2.662955-1.250004-0.884190$

H $5.4031272 .483091-1.049505$

H $4.6627322 .410128-2.665198$

H $6.0398890 .196600-3.061504$

H $7.7662422 .177550-1.488355$

H $9.1918651 .213605-3.440667$

H $8.390965-0.327659-3.229967$

H $11.6092670 .591401-0.740542$

H $11.0707141 .764530-2.068854$

H $10.392369-1.404709-0.333442$

H $9.212121-1.890747-1.508152$

H $8.780680-0.1764321 .036858$

H $4.2500590 .545510-0.387405$

C 1.3094934 .0042191 .301852

$\begin{array}{lllll}\text { O } & 0.001291 & 3.401217 & 1.331852\end{array}$

C $-0.365982 \quad 3.033672-0.009744$

C $0.5928453 .787043-0.924222$

C $1.8651593 .759300-0.100019$

H $-0.1731521 .958724-0.102046$

C $-1.8515813 .299595-0.283840$

H $0.7020193 .304735-1.900302$

H $0.2668984 .820797-1.087826$

C $2.9055394 .772764-0.540719$

H $2.2958082 .756312-0.174940$

C -2.784095 2.4866660 .641346

O $-2.0996204 .696925-0.140439$

H $-2.0590503 .032811-1.327484$

H -2.4786691 .4341780 .599687$

O -2.6315312 .9041561 .996933$

$\begin{array}{llll}\text { C } & -4.272103 & 2.562050 & 0.243577\end{array}$

C $\quad-5.161014 \quad 1.702768 \quad 1.161767$

H $-4.367726 \quad 2.221011-0.791077$

$\begin{array}{lllll}\text { O } & -4.737461 & 3.909445 & 0.277642\end{array}$

H -4.7180410 .705459 \begin{tabular}{lll}
1.252179 \\
\hline
\end{tabular}

H -5.1725282 .1441762 .165539$

H $3.2127164 .583507-1.574465$

H $2.5181475 .795678-0.487630$

H $3.796394 \quad 4.7152860 .092703$

C 2.1518213 .4865902 .471154

H -3.017656 $4.862256-0.414032$

H -2.733541 3.8708122 .020100

H -4.6896464 .2182231 .198263$

H 1.6485613 .7621413 .406968

H 3.1246363 .9917942 .478447

H $1.147808 \quad 5.079908 \quad 1.452105$

C $-6.348715-0.322219-0.789104$

O $-6.691782 \quad 1.064410-0.674851$

C $-6.616481 \quad 1.599890 \quad 0.651774$

C $-7.5558930 .821223 \quad 1.584110$

C $-7.279517-0.6785631 .542736$

C $-7.262352-1.178345 \quad 0.098144$

H $-5.301993-0.467367-0.498757$

C $-6.500000-0.719958-2.266334$

H -7.0042642 .6244770 .589223$

O -7.4409601 .2833332 .925813$

H -8.5954940 .9900491 .274093$

H $-6.345865-0.9210692 .063656$

O $-8.321772-1.3500032 .254515$

H -8.286863 $-1.165397-0.294638$

O $-6.828998-2.5372180 .081243$

C $-5.410967-0.138541-3.143643$

H $-6.448279-1.808664-2.376635$

H $-7.462721-0.359742-2.648070$

H -7.6435672 .2343922 .925538$ 
H -8.151610 -2.305082 2.183701

H $-5.924258-2.5657810 .436205$

O $-5.682706-0.447941-4.446621$

O $\quad-4.440678 \quad 0.490712-2.746686$

C $-4.729757 \quad 0.027160-5.402617$

H -3.749009 -0.419555 -5.213843

H $-5.066889-0.275296-6.397688$

H -4.670572 1.119237 -5.368859

SCF Energy (B3LYP/6-31G**//MMFF)= -3245.91409625

\section{4}

MM̄̄F Geometry

C $2.251623-2.9925431 .206341$

C $1.640913-1.9444961 .782778$

C $2.270313-0.8935082 .672087$

O $1.705040 \quad 0.369014 \quad 2.231375$

C $3.802931-0.7792622 .566399$

C 4.4303900 .1817703 .555000

C $4.864217 \quad 1.4206513 .250937$

C $4.608981-0.3614224 .949093$

C 4.7332442 .1550351 .945088

C 6.0591722 .2633621 .179118

C 6.4162360 .9867490 .401215

C $7.7737381 .135453-0.287363$

C $8.107490-0.089806-1.151628$

O $5.4005290 .786583-0.585134$

O $9.216740 \quad 0.253660-1.996941$

C $6.912244-0.391641-2.082922$

C $8.570125-1.273602-0.293954$

O $7.106505-1.617993-2.782985$

C $5.541173-0.407335-1.365418$

C $4.395091-0.410539-2.401522$

O $5.371642-1.583876-0.572831$

C 1.2489451 .2416783 .163669

O 1.2616341 .0890134 .377439

C 0.6925632 .4649592 .538893

C 0.6655752 .6859731 .215188

C 0.0811023 .8503840 .543980

C $-0.5643824 .905870 \quad 1.402838$

C $3.001627-0.328940-1.763151$

C $1.892379-0.314426-2.800216$

C $-0.5751990 .010203-2.990455$

O $2.089106-0.493357-3.999076$

C $-0.7629261 .433072-3.411195$

C $-1.7616172 .217599-2.979040$

C $-1.9269163 .651061-3.396014$

C $-1.8251684 .616938-2.234973$

C $-2.9216115 .215347-1.740018$

C $-0.4474274 .962362-1.709404$

C $0.1160023 .901301-0.804108$

N $0.650428-0.092340-2.219936$

H $3.306935-3.1627131 .401429$

H $\quad 0.576851-1.8017181 .608827$

H $1.963472-1.1114903 .701857$

H $4.064945-0.4861551 .545160$

H $4.274661-1.7559772 .729090$

H 5.3369602 .0043614 .040118

H 5.0933270 .3582785 .617602

H $3.639782-0.6166855 .388395$

H $5.230409-1.2626674 .929282$

H 3.9503761 .7291321 .310440

H 4.3909563 .1702292 .185100

H 5.9566223 .0907330 .464594

H 6.8716622 .5281451 .866825

H $6.429190 \quad 0.1365561 .091481$

H 8.5670281 .3142610 .449079

H $7.7604832 .023090-0.935565$

H $9.9551590 .528154-1.426555$

H $6.8886020 .400072-2.845071$

H $9.485032-1.0153780 .253071$

H $8.828191-2.137246-0.916387$

H $7.826893-1.5853210 .443013$

H $7.962972-1.557932-3.240153$

H $4.5107700 .445418-3.079107$

H $4.447718-1.321365-3.011549$

H $6.152476-2.149487-0.679056$

H 0.2810313 .1803923 .243798

H $1.091916 \quad 1.9395100 .547625$
H -1.378105 4.4753121 .996635

H -1.0002415 .7269610 .830244$

H 0.1710355 .3502822 .082543

H $2.835939-1.192864-1.112416$

H $2.9157750 .581091-1.158067$

H $-1.392249-0.344646-2.355876$

H $-0.511452-0.635381-3.872105$

H $-0.031796 \quad 1.839429-4.106475$

H -2.499769 $1.813601-2.290305$

H -2.906012 $3.743857-3.884016$

H $-1.1912083 .931824-4.160195$

H -2.857369 $5.933477-0.929625$

H -3.909882 $4.996726-2.130850$

H $-0.4570295 .937397-1.209411$

H $0.2382395 .100395-2.555296$

H $0.6067223 .088204-1.338646$

H $0.5994040 .112330-1.225971$

C $0.341264-3.604318-0.427313$

O $\quad-0.756868-3.718000 \quad 0.495829$

C $-1.937535-4.107951-0.230904$

C $-1.525244-4.222548-1.696614$

C $-0.041965-4.512885-1.592627$

H $-2.230730-5.0961780 .143522$

C $-3.080573-3.108372-0.000048$

H -2.080056 -5.007847 -2.219509

H $-1.696225-3.283384-2.236713$

C $0.712757-4.235592-2.881725$

H $0.093481-5.565204-1.309216$

C -3.634034 -3.083917 1.438996

O $-2.642368-1.797166-0.360067$

H $-3.894069-3.373919-0.684028$

H -4.059619 -4.0629101 .690831$

O $-2.574115-2.8468392 .373184$

C $-4.682112-1.9820791 .695642$

C $-5.850337-1.9750060 .696242$

H $-4.184993-1.0063011 .709510$

O $\quad-5.224627-2.1784193 .006054$

H -5.463041 -1.878919 -0.321635

H $-6.377271-2.9356920 .751477$

H $0.331541-4.865572-3.692172$

H $0.606970-3.191078-3.191834$

H $1.780418-4.445968-2.767201$

C $1.650125-4.0011240 .258848$

H -1.917708 -1.556863 0.242811

H -1.946515 -3.586049 2.303480

H $-4.479458-2.1738893 .631077$

H $2.407751-4.184409-0.512476$

H $1.516204-4.9391230 .811292$

H $\quad 0.383840-2.560656-0.762195$

C $-5.6214320 .949526-0.074919$

$\begin{array}{llll}\text { O } & -6.224636 & 0.432477 & 1.117080\end{array}$

C $-6.864887-0.8376220 .959956$

C $-7.979559-0.726967-0.090147$

C -7.475635 -0.131401-1.405254

C $-6.6887451 .157267-1.153893$

H $-4.8550690 .253220-0.436298$

C $-4.9229832 .261077 \quad 0.292564$

H -7.345427 -1.059826 1.921087

O $-8.560202-2.005869-0.322922$

H $-8.773789-0.0807520 .306074$

H $-6.877757-0.862719-1.961661$

O $-8.6079150 .164950-2.225978$

H $-7.3852711 .948809-0.851038$

O $-6.0802521 .580119-2.372256$

C $-3.696691 \quad 1.9757121 .127061$

H $-4.5891372 .806345-0.596399$

H -5.5871172 .9308570 .851503$

H -9.248632 -1.893191-1.000652

H -8.274492 $0.580855-3.039610$

H $-5.4416770 .897405-2.640723$

O $-4.019526 \quad 1.9965722 .451318$

O $\quad-2.5968741 .7229570 .653301$

C $-2.964906 \quad 1.662372 \quad 3.358979$

H $-2.416050 \quad 0.777500 \quad 3.021478$

H -2.2931902 .5174103 .468157$

$\mathrm{H}-3.4128251 .4411304 .331463$

SCF Energy (B3LYP/6-31G**//MMFF)= -3245.92789257 
02235

MM̄FF Geometry

C $1.804746-0.2383003 .171693$

C $3.001335-0.8396243 .264541$

C $4.161306-0.7026082 .310413$

O $4.054350 \quad 0.5388251 .580065$

C $4.155848-1.8638101 .301326$

C $5.521680-2.1443550 .710646$

C $5.932366-1.748070-0.509037$

C $6.432024-2.9768151 .577504$

C $5.199937-0.895982-1.506835$

C $4.771706-1.687196-2.749931$

C $3.479418-2.491995-2.536428$

C $3.176027-3.351069-3.765592$

C $1.819734-4.061499-3.654735$

O $2.424284-1.549362-2.325562$

O $1.519024-4.578738-4.959610$

C $0.742159-3.019091-3.288688$

C $1.888958-5.267515-2.710577$

O $-0.511947-3.647047-3.029418$

C $1.134029-2.124484-2.089004$

C $0.128119-0.955217-1.963648$

O $1.113333-2.909828-0.897010$

C $5.154057 \quad 1.327621 \quad 1.477905$

O $6.2531831 .122426 \quad 1.974172$

C 4.8787322 .5372750 .669204

C $3.7618692 .700743-0.055168$

C $3.4733113 .860217-0.890004$

C $2.9879143 .500375-2.267618$

C $0.4616610 .046057-0.849833$

C $-0.4510151 .259801-0.874366$

C -0.8358113 .4192250 .314267$

O $-1.3689061 .394250-1.678357$

C $-0.2420504 .485808-0.550870$

C $0.2360945 .646498-0.076944$

C $0.776976 \quad 6.744478-0.948031$

C $2.1718237 .175882-0.553892$

C 2.3681248 .2590780 .216965

C $3.3394296 .406789-1.129510$

C $3.5906265 .112871-0.407178$

N -0.1183192 .1743880 .115975$

H 1.5844710 .4197392 .336833

H $3.174266-1.5121744 .103405$

H $5.072349-0.7083602 .920559$

H $3.431583-1.6561990 .505228$

H $3.807306-2.7891301 .780099$

H $6.931495-2.041299-0.830292$

H $7.395794-3.1777631 .097679$

H $6.636694-2.4655952 .523155$

H $5.966623-3.9431341 .798082$

H $4.345270-0.378259-1.063199$

H $5.893200-0.105247-1.821587$

H $4.603944-0.962890-3.558052$

H $5.583714-2.350343-3.073207$

H $\quad 3.587227-3.122916-1.647487$

H $3.978027-4.079834-3.938120$

H $3.157986-2.711492-4.659503$

H $0.690296-5.083130-4.901398$

H $0.594199-2.376192-4.167784$

H $2.608778-6.004246-3.086899$

H $0.925938-5.787513-2.660233$

H $2.187837-5.000589-1.694544$

H $-0.786211-4.105457-3.841109$

H $0.105491-0.407120-2.915141$

H $-0.881980-1.343898-1.793453$

H $1.063399-2.320640-0.128831$

H 5.6633983 .2882160 .669399

H $3.0054921 .922956-0.083758$

H $3.7957453 .035200-2.843258$

H $2.6266694 .358164-2.838726$

H $2.1551502 .790034-2.213600$

H $0.357283-0.4243520 .133227$

H $\quad 1.492410 \quad 0.396680-0.958884$

H -0.7725183 .6659761 .378710$

H -1.8874803 .2756650 .050519$

H $-0.2363984 .307257-1.623597$

H $\quad 0.2040025 .8364440 .993561$

H $0.0890227 .596957-0.871733$
H $\quad 0.770300 \quad 6.456587-2.006663$

H 3.3664928 .5932270 .478906

H 1.5340278 .8325450 .607661

H $4.2577747 .004300-1.051375$

H $3.1885156 .271422-2.204460$

H 3.9215175 .2421150 .623946

H 0.7035062 .0078030 .688769

C $-0.060376-1.7297753 .963575$

O $-0.925557-1.5995682 .817962$

C $-2.102949-2.4061423 .044263$

C -1.922911 -3.069772 4.407570

C $-0.993717-2.1018155 .111480$

H -2.954125 -1.719629 3.101036

C $-2.288313-3.4038701 .894834$

H $-2.876417-3.2043704 .928011$

H $-1.455100-4.0575944 .317506$

C $-0.297107-2.7095646 .316775$

H -1.571129-1.222711 5.427415

C -2.542091-2.746815 0.524567

O $-1.106186-4.2089191 .786718$

H $-3.104194-4.0970442 .129493$

H -1.685947 -2.127272 0.239761

O $-2.634578-3.793126-0.450493$

C $-3.834506-1.9098650 .455405$

C $-4.121503-1.375760-0.961515$

H $-3.755494-1.0627461 .143300$

O $-4.937584-2.7039820 .891700$

H $-3.289187-0.731621-1.263409$

H $-4.151768-2.211877-1.670250$

H -1.031689-3.042322 7.057508

H $0.311346-3.5753206 .034436$

H $0.359716-1.9803466 .800613$

C $0.719575-0.4361134 .194822$

H $-0.361818-3.6127611 .595767$

H -1.800846 -4.293290 -0.417832

H $-4.998371-3.4722620 .298239$

H $1.171640-0.4346355 .193576$

H $\quad 0.0483680 .4287404 .149355$

H $0.627993-2.5603493 .755160$

C $-4.7544851 .527824-0.228500$

O $-5.6100250 .390782-0.069638$

C $-5.464239-0.618354-1.071281$

C $-5.743788-0.026460-2.460154$

C $-4.8950661 .212380-2.732612$

C $-5.0114212 .207386-1.578567$

H $-3.7073351 .211368-0.156357$

C -5.0472022 .4827270 .932916$

H $-6.254610-1.354213-0.876060$

O $-5.508887-0.987491-3.483812$

H $-6.801580 \quad 0.259984-2.524889$

H $-3.8481650 .948417-2.920544$

O $\quad-5.3653851 .851397-3.921939$

H $-6.0110232 .660631-1.588071$

O $-4.0655873 .257265-1.766945$

C -4.5379731 .9116892 .234723$

H -4.5859443 .4656110 .783258$

H -6.1247832 .6508161 .048316$

H -6.072902 -1.757171 -3.296620

H $-5.3197311 .195820-4.638937$

H -4.231395 $3.643567-2.644113$

O -3.2560322 .3209982 .451201$

O $\quad-5.192936 \quad 1.1736372 .959102$

C $-2.653177 \quad 1.846236 \quad 3.657877$

H -2.585252 0.7552603 .642255

H -1.643992 2.2615963 .718450

H $-3.2244432 .185214 \quad 4.527373$

SCF Energy $\left(B 3 L Y P / 6-31 G^{* *} / / M M F F\right)=-3245.91093211$

$02 \_236$

MM̄FF Geometry

C $-0.629138-0.849245-2.175932$

C $0.411060-0.545625-2.967619$

C $1.1500330 .768405-3.061714$

O $\quad 2.541346 \quad 0.496212-2.759221$

C $0.6662751 .895784-2.129645$

C $1.1354513 .271686-2.563650$

C $2.1156763 .979022-1.970214$

C $0.3785053 .870797-3.723079$ 
C $3.0040563 .567921-0.830617$

C $2.699727 \quad 4.349570 \quad 0.455290$

C 1.4640273 .8229841 .201541

C 1.1444624 .7058992 .408264

C -0.0027094 .1308543 .252795$

O $1.7703342 .497451 \quad 1.639759$

O $\quad-0.0212404 .8356554 .503913$

C 0.2978722 .6500533 .568849

C -1.369085 4.369612 2.600267

O -0.8203332 .0630664 .231289$

C 0.7072931 .8246752 .324029

C 1.2452080 .4383522 .750238

O -0.4340241 .6469391 .484222$

C $3.4820460 .972346-3.614792$

O $3.2807111 .581298-4.657532$

C $4.8576120 .670166-3.157006$

C $5.1665390 .154834-1.956798$

C $6.520297-0.139517-1.473408$

C $7.670618 \quad 0.120953-2.411794$

C $1.939333-0.3353811 .619930$

C $2.440328-1.6910332 .085495$

C $3.720539-3.6649051 .257592$

O $2.289161-2.1100093 .229762$

C 5.136931-3.426742 1.673204

C $6.198449-3.7251980 .909888$

C $7.614272-3.4344691 .314019$

C $8.301389-2.4712570 .370210$

C $9.250426-2.901574-0.478133$

C $7.958594-0.9999460 .474317$

C $6.674447-0.638168-0.228493$

N $3.079270-2.3779971 .062569$

H -1.005570 -0.124403 -1.461396

H $\quad 0.744124-1.299257-3.680288$

H $1.0427571 .084726-4.106521$

H $0.983861 \quad 1.685032-1.102910$

H $-0.4293101 .931575-2.107163$

H $2.3365904 .971293-2.362694$

H $0.6916894 .897971-3.938745$

H $\quad 0.5359303 .282813-4.632226$

H $-0.6942403 .895323-3.507844$

H $2.9757402 .489541-0.649199$

H $4.0360163 .787895-1.132932$

H 3.5747084 .2550421 .111854

H 2.5793455 .4160160 .227629

H $\quad 0.6106593 .7937170 .515227$

H 0.9117835 .7298852 .090206

H 2.0353364 .7867723 .047219

H -0.1482375 .7809214 .313403$

H 1.1282982 .6405364 .288795

H -1.539603 5.4420562 .447561

H -2.1828684 .0264253 .248755$

H -1.469821 3.8779531 .631441

H -0.5458311 .1952514 .570932$

H 1.9776430 .5683253 .557858

H $\quad 0.427903-0.1743023 .148687$

H -0.3392350 .8238510 .980184$

H $5.6280580 .925448-3.878260$

H $4.364180-0.067946-1.255784$

H $7.551338-0.455100-3.336301$

H $8.642373-0.157023-1.998940$

H $7.7272681 .185635-2.663950$

H $1.254349-0.4956640 .781457$

H $2.803746 \quad 0.229071 \quad 1.251071$

H $3.651892-4.2118450 .312268$

H $3.191312-4.2310352 .030473$

H $5.293331-2.9667712 .646261$

H $6.045413-4.187914-0.062290$

H $8.154470-4.389471 \quad 1.347227$

H $7.664652-3.0322252 .333690$

H $9.775192-2.216630-1.135435$

H $9.523296-3.950069-0.536665$

H $8.788903-0.3838770 .110638$

H $7.865371-0.7249991 .533032$

H $5.783620-0.7904510 .379986$

H $3.217795-1.9142170 .169275$

C - $-1.493407-2.928755-0.965187$

O $-2.129984-2.1451320 .061606$

C $-1.522658-2.4659941 .325978$
C $-0.608008-3.6613321 .062605$

C $-0.176720-3.409562-0.368038$

H $-0.908337-1.6038311 .606598$

C -2.572197-2.7331192.413932

H $\quad 0.227843-3.7087411 .766059$

H -1.157127 -4.608194 1.134094

C $0.408996-4.630612-1.053598$

H $\quad 0.565228-2.603049-0.374777$

C -3.362906-1.500657 2.893070

O $-3.454477-3.766914 \quad 1.988930$

H $-2.026735-3.1228923 .284133$

H $-4.042456-1.8229963 .692879$

O $-2.421450-0.6085243 .504720$

C -4.182494 -0.697409 1.863102

C $-5.204676-1.5228301 .068574$

H $-3.502488-0.1794651 .182587$

$\begin{array}{llll}\text { O } & -4.874088 & 0.333742 & 2.581185\end{array}$

H $-4.670092-2.1739170 .371873$

H -5.745132 -2.179152 1.762588

H $1.299058-4.981136-0.521552$

H $-0.309213-5.456680-1.085724$

H $\quad 0.700715-4.396934-2.082298$

C -1.393941 -2.141481-2.270923

H $-4.061508-3.9568132 .724335$

H -2.9159650 .1684743 .817569$

H $-5.506895-0.0960263 .181694$

H -2.408853 -1.880720 -2.592812

H $-0.973743-2.785385-3.052393$

H -2.155236 -3.787644 -1.142483

C $-4.9114870 .018138-1.582824$

O $-5.6868340 .396912-0.441278$

C $-6.254526-0.6864290 .299087$

C $-7.165661-1.524438-0.608546$

C $-6.431220-1.992294-1.863644$

C $-5.754612-0.812746-2.560533$

H $-4.034591-0.550772-1.255597$

C $-4.4289781 .308266-2.267524$

H $-6.899711-0.2260351 .058362$

O $-7.676004-2.6448420 .105815$

H -8.027385 -0.913387 -0.906863

H $-5.703568-2.776771-1.627682$

O $-7.368058-2.593587-2.761241$

H $-6.521223-0.179026-3.024916$

O $-4.918899-1.301248-3.606955$

C $-3.5185162 .143919-1.393262$

H $-3.878227 \quad 1.078022-3.185742$

H $-5.3012981 .925390-2.516650$

H -8.218091 -3.162870 -0.513494

H -8.034895 -1.924792 -2.992764

H $-5.473144-1.846110-4.191668$

O $-3.4509303 .408407-1.906368$

O $-2.9295391 .743427-0.399919$

C $-2.6518984 .336736-1.166040$

H $-1.6552313 .929291-0.974147$

H $-2.5469835 .246678-1.762952$

$\mathrm{H}-3.1531074 .587248-0.226894$

SCF Energy $\left(B 3 L Y P / 6-31 G^{* *} / / M M F F\right)=-3245.91246630$

$02 \_237$

MM̄FF Geometry

C $3.2017504 .947797 \quad 0.822264$

C 2.0702524 .3420460 .426416

C $0.714344 \quad 4.6811830 .993131$

O 0.0592553 .4578221 .407642

C $-0.1754345 .346611-0.073218$

C -1.5010955 .8406530 .480145$

C -2.714895 5.4056920 .087637

C -1.4090086 .9225621 .527892$

C $-3.0516844 .405280-0.981314$

C $-3.3804413 .033734-0.389320$

C $-3.8606322 .027049-1.447576$

C $-5.3000562 .308157-1.891055$

C $-5.8326751 .231367-2.848526$

$\begin{array}{llll}\text { O } & -3.781319 & 0.723483 & -0.854824\end{array}$

O $-7.2574231 .386258-2.939069$

C $-5.569371-0.159723-2.236279$

C $-5.286316 \quad 1.414432-4.269652$

O $-5.915479-1.192449-3.156848$ 
C $-4.115730-0.351032-1.746030$

C $-3.995800-1.681557-0.963966$

O $-3.247397-0.393435-2.879147$

C 0.4133042 .9350632 .612995

O $1.2864613 .342416 \quad 3.362507$

C -0.4807841 .7856582 .882333$

C $-0.365790 \quad 1.1170034 .039845$

C -1.2187080 .0160934 .496796$

C $-2.320053-0.4391823 .578491$

C $-2.624777-1.908167-0.314747$

C $-2.581907-3.1767530 .519283$

C -1.061343 -4.421316 2.058264

O $-3.484053-4.0094150 .526592$

C -1.619318-4.234098 3.433195

C $-0.862043-4.1304874 .536022$

C $-1.423743-4.0694625 .926622$

C $-0.999279-2.835510 \quad 6.686221$

C $-0.031524-2.8960657 .617166$

C -1.779818 -1.562767 6.449322

C $-0.991203-0.5101015 .718480$

N $-1.394522 \quad-3.2788891 .230975$

H 3.1240225 .7455561 .559313

H $2.1001773 .548704-0.314016$

H $\quad 0.818903 \quad 5.343783 \quad 1.860887$

H $-0.3377534 .632947-0.887822$

H $0.348190 \quad 6.204891-0.514554$

H -3.5908475 .8340710 .574545$

H -2.3828607 .3751191 .744009$

H -1.0189546 .5211302 .467863$

H -0.7481247 .7264991 .187066$

H $-3.9118784 .798724-1.534726$

H -2.247135 4.310808 -1.717023

H -2.4877722 .6339510 .107834$

H -4.1357843 .1233710 .402808$

H -3.175983 2.065713 -2.302291

H $-5.3891623 .297670-2.353808$

H -5.952353 2.333198 -1.006802

H -7.441667 2.287810 -3.254042

H $-6.242656-0.273551-1.374902$

H $-5.5841282 .390699-4.671126$

H $-5.7064800 .669249-4.954126$

H $-4.1971501 .357373-4.321669$

H -6.837020 -1.041218 -3.428589

H $-4.751437-1.698013-0.167370$

H -4.206919 -2.525941 -1.632345

H $-2.403816-0.791463-2.616276$

H -1.233467 1.5467262 .139892

H 0.4109751 .4229584 .739872

H -3.052829 0.3610043 .428010

H -2.864445 -1.311303 3.945712

H -1.906860 -0.7229192 .606296$

H -1.850638 -2.009137-1.081278

H $-2.366852-1.0595770 .326751$

H $0.028905-4.5150922 .065804$

H -1.481508 -5.3276391 .610107$

H $-2.702502-4.2399373 .529800$

H $0.221312-4.1509604 .442232$

H -1.086379 -4.9700416 .456892$

H -2.519183 -4.132907 5.919288

H $0.247092-2.0230018 .197990$

H $\quad 0.504024-3.8181947 .817395$

H -2.068938 -1.141391 7.422170

H -2.731658 -1.788556 5.960466

H $-0.166000-0.1047346 .306218$

H $-0.726835-2.5140991 .194332$

C $4.8191253 .336718-0.387280$

O 4.4386922 .2250810 .441108

C $5.258907 \quad 1.0879980 .116167$

C $6.2117181 .550480-0.985632$

C $6.2703033 .050274-0.759342$

H 5.8312840 .8384601 .017515

C $4.377789-0.106130-0.284673$

H $7.1913241 .068315-0.909353$

H $5.8133951 .327512-1.982596$

C $6.7703983 .825870-1.964991$

H 6.9258953 .2491060 .098847

C $3.476666-0.6463870 .849169$

O $3.5694690 .265022-1.402984$
H $5.032491-0.915782-0.625508$

H $4.105559-1.1098971 .618134$

O $2.797107 \quad 0.430594 \quad 1.506067$

C $2.391564-1.6404940 .393710$

C $2.927327-2.862215-0.369050$

H $1.642578-1.111040-0.203474$

O $1.709076-2.1214851 .558211$

H $3.601078-2.543281-1.167734$

H $3.512225-3.4843750 .319268$

H $7.7859053 .513062-2.229000$

H $6.1308003 .664801-2.839436$

H $6.7923114 .900131-1.757159$

C 4.6135024 .6381730 .389173

H $3.0771321 .065770-1.156403$

H 2.2718090 .9036580 .838796

H $1.378496-1.3452322 .042451$

H $4.9807465 .487127-0.198539$

H 5.2167474 .5944111 .305570

H $4.1874073 .318927-1.284810$

C $1.508914-2.501117-3.042548$

O $0.921941-2.933524-1.808399$

C $1.785601-3.713395-0.972281$

C $2.248446-4.968482-1.724501$

C $2.854194-4.632884-3.085110$

C $1.925343-3.714282-3.879620$

H $2.386830-1.877317-2.838117$

C $0.474329-1.633824-3.769911$

H $1.170068-4.055765-0.132828$

O $3.197169-5.702586-0.958101$

H $1.386571-5.629166-1.886590$

H $3.849943-4.185801-2.980651$

O $3.032791-5.839909-3.830059$

H $1.039097-4.274897-4.203327$

O $2.604599-3.267017-5.050961$

C $0.503852-0.216936-3.243799$

H $0.683066-1.570658-4.843995$

H $-0.542272-2.029250-3.662223$

H $2.779779-5.919512-0.106934$

H $3.595366-6.427575-3.296859$

H $2.879251-4.057586-5.546459$

O $-0.160215-0.141758-2.057036$

O $1.068890 \quad 0.707976-3.813383$

C $-0.1678041 .144223-1.431203$

H $-0.6917951 .050889-0.477232$

H $\quad 0.8533001 .480195-1.235084$

$\mathrm{H}-0.6952411 .867610-2.059108$

SCF Energy $\left(B 3 L Y P / 6-31 G^{* *} / / M M F F\right)=-3245.91814595$

$02 \_238$

MM̄FF Geometry

C $1.9841762 .624493-2.057071$

C $1.1920051 .995374-1.176300$

C $0.1395760 .982643-1.540577$

O $\quad-1.1155351 .547623-1.082927$

C $0.368795-0.361214-0.813149$

C $1.202657-1.372991-1.579986$

C $0.802805-2.632558-1.850429$

C $2.568822-0.923872-2.021691$

C $-0.485437-3.278725-1.427810$

C $-1.576398-3.104003-2.489516$

C $-2.968030-3.455106-1.946584$

C $-4.029466-3.296933-3.037946$

C $-5.451102-3.507457-2.493252$

O $-3.247268-2.559662-0.865461$

O $-6.382023-3.054772-3.488087$

C $-5.641236-2.643923-1.226781$

C $-5.761767-4.994983-2.296539$

O $-6.894065-2.917901-0.604819$

C $-4.494062-2.796011-0.201934$

C $-4.638524-1.7369270 .914882$

O $-4.551230-4.100610 \quad 0.375939$

C $-2.2505151 .194023-1.742699$

O $-2.3468960 .433498-2.691500$

C $-3.3758341 .905227-1.099189$

C $-4.6355041 .650307-1.481627$

C $-5.8178572 .275894-0.896826$

C $-6.9374661 .310908-0.616117$

C $-3.441738-1.6614591 .870562$ 
C $-3.649651-0.5723112 .903649$

C $-3.5613991 .903073 \quad 3.194718$

O $-4.010498-0.8312864 .049475$

C -4.9131512 .4945772 .940756$

C -5.1064193 .7821672 .615191$

C -6.4615154 .4043932 .429028$

C -6.5859685 .1713551 .131013$

C -6.4109656 .5032381 .095512$

C -7.002931 4.406634 -0.105881

C $-5.8725703 .607083-0.691121$

N -3.407271 0.6971522 .403770

H $1.8769762 .418066-3.118688$

H $1.2956452 .224726-0.118530$

H $0.0967950 .842249-2.628119$

H $-0.610837-0.782784-0.565789$

H $0.846533-0.1889640 .160124$

H $1.469714-3.280683-2.416883$

H $3.192545-1.761027-2.353417$

H $2.493821-0.220750-2.855930$

H $3.094984-0.438916-1.194027$

H $-0.304043-4.348490-1.264879$

H - $0.802506-2.892026-0.454004$

H -1.580460 -2.062836 -2.832777

H -1.343923 -3.729643 -3.360113

H -2.947046 -4.484569-1.570236

H $-3.833016-3.977693-3.875563$

H $-3.965890-2.282949-3.457511$

H $-6.205222-2.115229-3.665196$

H $-5.678068-1.593883-1.547003$

H -5.694162 -5.527947 -3.252869

H $-6.788953-5.142091-1.944988$

H -5.083717 -5.487304 -1.596301

H -7.584294 -2.805248 -1.280578

H $-4.759216-0.7509350 .452723$

H -5.544724 -1.931487 1.502449

H $-4.115352-4.086897 \quad 1.241934$

H -3.147492 2.617006 -0.312356

H $-4.8340590 .909267-2.252965$

H -7.883332 $1.799785-0.374980$

H -6.6778970 .6646990 .228646$

H $-7.132540 \quad 0.675928-1.488001$

H -3.294277 -2.6044362 .406675$

H -2.512175 -1.4546101 .327626$

H -2.752159 2.5832442 .910732

H -3.4587451 .6598024 .256994$

H -5.7745901 .8427623 .066984$

H -4.244902 4.4398132 .522464

H -6.6332915 .0823613 .275595$

H -7.258810 3.6530072 .487064

H -6.5279897 .0649120 .174614$

H -6.1410977 .0608951 .986251$

H -7.347136 $5.102568-0.882285$

H $-7.876148 \quad 3.7970510 .141515$

H -5.011717 $4.211155-0.980355$

H -3.148381 0.8023051 .428095

C $4.4214623 .247189-2.174225$

O $4.8530062 .000867-1.593269$

C $6.2438832 .107900-1.233238$

C $6.7433453 .414000-1.841482$

C $5.4935624 .268968-1.796828$

H $6.2778862 .192034-0.140599$

C $7.0184360 .862530-1.683332$

H $7.5774683 .840964-1.276299$

H $7.0741113 .282867-2.878012$

C $5.5513545 .476766-2.716035$

H $5.3411924 .610161-0.763942$

C $6.423889-0.478166-1.210658$

O $7.054658 \quad 0.823170-3.117034$

H $8.0627020 .943406-1.360219$

H $5.481861-0.672923-1.734009$

O $7.323850-1.520620-1.610302$

C $6.200776-0.5875620 .307439$

C $5.652443-1.9666970 .716934$

H 5.4936810 .1815630 .623406

O $7.426845-0.3387320 .991981$

H $4.737515-2.1597500 .146374$

H $\quad 6.376796-2.741100 \quad 0.437390$

H $6.3830626 .132300-2.437537$
H $5.6928205 .179602-3.760509$

H $4.6273826 .059963-2.653837$

C $3.0323863 .624075-1.653455$

H $6.1347200 .821953-3.433652$

H $7.449825-1.441474-2.571557$

H $8.071935-1.0004720 .688494$

H $2.7395494 .602724-2.050303$

H $3.0640353 .711449-0.560599$

H $4.3937133 .098493-3.261756$

C $3.161232-1.1273782 .298848$

O $4.524812-1.0577432 .731943$

C $5.377408-2.0933082 .232638$

C $4.833597-3.4666272 .652554$

C $3.381432-3.6543442 .221774$

C $2.532565-2.4732342 .688969$

H $3.115606-0.9900281 .212849$

C 2.4013610 .0289872 .969621

H $6.341885-1.9620362 .739213$

O $5.621720-4.5189632 .106578$

H $4.885377-3.5595693 .745287$

H $3.297938-3.7969891 .138006$

O $2.863713-4.8435112 .822874$

H $2.416950-2.5302073 .778773$

O $1.224867-2.5824492 .129338$

C 2.8026041 .3881912 .437493

H $1.323324-0.0737462 .803427$

H 2.6055330 .0230114 .047132

H $6.536445-4.3852812 .408509$

H $3.437544-5.5793992 .548407$

H $1.312090-2.5576241 .161578$

O 2.1880732 .3524863 .185696

O 3.5414691 .5870591 .484188

C 2.4642693 .7025242 .799484

H 3.5429103 .8872532 .788460

H 2.0265713 .9073461 .818338

H 2.0036364 .3672193 .535068

SCF Energy $\left(B 3 L Y P / 6-31 G^{* *} / / M M F F\right)=-3245.91375935$

$02 \_239$

MM̄FF Geometry

C $-2.4191151 .995926 \quad 3.376629$

C -3.6067362 .1872782 .781504$

C -3.8884822 .9895321 .531969$

$\begin{array}{llll}\text { O } & -4.061653 & 2.065784 & 0.431079\end{array}$

C $-2.7748793 .965818 \quad 1.108194$

C -3.2776475 .1005860 .236643$

C $-3.2180185 .135901-1.108573$

C -3.8296126 .2819240 .994762$

C $-2.7585294 .062426-2.054580$

C $-1.3895164 .362625-2.682321$

C $-0.2220143 .904712-1.796310$

C $1.1223384 .305356-2.404621$

C $2.3003703 .758681-1.583137$

O $-0.3023292 .478946-1.696638$

O $3.4937743 .909597-2.366508$

C $2.0943422 .245746-1.359429$

C $2.5226624 .561665-0.295727$

O $3.079696 \quad 1.726071-0.470491$

C $0.6818941 .879509-0.848431$

C $0.4788490 .346249-0.908185$

O 0.5515552 .3175650 .505933

C -5.2603931 .4537240 .262261$

O $-6.257541 \quad 1.5670470 .954576$

C $-5.1242980 .586697-0.928443$

C $-5.989642-0.416627-1.129414$

C $-5.944288-1.377949-2.233829$

C $-4.894833-1.172254-3.296038$

C $-0.973318-0.107327-0.685960$

C $-1.081606-1.619270-0.567982$

C $-2.731909-3.413300-0.032282$

O $-0.129938-2.375757-0.744107$

C $-3.367970-3.953959-1.274307$

C $-4.523924-4.636068-1.275166$

C $-5.152033-5.216055-2.511208$

C $-6.617094-4.861955-2.650834$

C $-7.575189-5.749536-2.334522$

C $-6.969120-3.512073-3.236295$

C $-6.826501-2.398885-2.233456$ 
N $-2.368969-2.022678-0.239515$ H -1.516232 2.4159982 .943342 H -4.4852871 .7380323 .241969$ H -4.820322 3.5467601 .692894 H -1.9837983 .4170030 .587060$ H -2.3015294 .4211701 .987581$ H -3.579655 $6.033571-1.609643$ H -4.1759857 .0801810 .329724$ H -4.680379 5.9772361 .612427 H $-3.060304 \quad 6.707136 \quad 1.647743$ H $-2.7662263 .074550-1.586701$ H -3.503090 $4.010246-2.859608$ H $-1.3381393 .819794-3.635368$ H -1.300048 $5.431409-2.911816$ H $-0.3323944 .350177-0.802236$ H $1.1959475 .395495-2.503648$ H $1.1965513 .905499-3.425605$ H $3.5973804 .853685-2.576064$ H $2.2539111 .748259-2.326303$ H $2.7220895 .613999-0.530999$ H $3.405527 \quad 4.2037380 .245564$ H 1.6671044 .5321140 .382219 H $3.9490221 .991254-0.815352$ H $0.786835-0.024316-1.895033$ H $1.126221-0.137334-0.167582$ H $-0.063132 \quad 1.7331130 .975720$ H -4.287261 $0.778421-1.590661$ H $-6.790733-0.564904-0.406487$ H -5.043375 -0.209658 -3.798155 H -4.900809 -1.938396-4.073306 H -3.890792 -1.186703 -2.858322 H -1.3852640 .3344320 .227132$ H $-1.5981690 .210142-1.528699$ H $-3.409367-3.4457490 .826872$ H -1.835662 $-3.994646 \quad 0.206311$ H -2.841366 $-3.799222-2.213136$ H -5.032218 -4.817932 -0.330661 H $-5.036707-6.306777-2.459917$ H $-4.616942-4.903112-3.416522$ H -8.627040 -5.513987 -2.457542 H $-7.329170-6.729890-1.940055$ H -8.013479 -3.509349 -3.576574 H $-6.382180-3.351793-4.144413$ H -7.545887 -2.460377-1.415408 H -3.100778 -1.324203 -0.151031 C - $-1.479923-0.0738854 .539217$ $\begin{array}{llll}\text { O } & -0.148448 & 0.155778 & 4.035439\end{array}$ C $0.159880-0.8824813 .087528$ C $-0.883268-1.9704623 .310003$ C $-2.105050-1.1331533 .631728$ H $0.015720-0.4485442 .092569$ C $1.607308-1.3704273 .211697$ H -1.013770 -2.610954 2.433995 H - $0.620565-2.6089844 .162076$ C -3.233227 -1.917475 4.276297 H $-2.470742-0.6835982 .700171$ C $2.647140-0.3005212 .826439$ O $1.856685-1.8351994 .536995$ H $1.719283-2.2352482 .550112$ H $2.431850 \quad 0.073497 \quad 1.820611$ O $2.5209000 .835553 \quad 3.689174$ C $4.110796-0.7777662 .887303$ C 4.428562 -2.062506 2.098296 H $4.403720-0.9363303 .932408$ O $4.952295 \quad 0.2733392 .403314$ H $5.509826-2.2313222 .163445$ H $3.953083-2.9086182 .607229$ H -3.585348 -2.706587 3.603898 H -2.911949 -2.390419 5.210175 H -4.081336 -1.263584 4.502766 C $-2.247037 \quad 1.2429404 .667605$ H $1.595685-1.1282105 .151335$ H 2.7384440 .5496074 .592439 H 4.7326411 .0767032 .905426 H -1.697895 1.9047825 .349452 H $-3.220576 \quad 1.0510495 .134436$ H -1.349199 -0.485046 5.549336 C $5.969267-1.064719-0.391597$
O $4.569360-0.961767-0.120640$

C 4.005771-2.053468 0.608762

C $4.266255-3.376105-0.128746$

C $5.747687-3.579976-0.434840$

C $6.311953-2.352905-1.150957$

H $6.513118-1.0607760 .561209$

C $6.4134430 .209366-1.126402$

H $2.921200-1.9001330 .585671$

O $3.767940-4.4699010 .634471$

H $3.709600-3.367424-1.075141$

H $6.310440-3.8103020 .477133$

O $5.878614-4.720019-1.286930$

H $5.907972-2.328844-2.168857$

O $7.726294-2.487411-1.274689$

C $5.722190 \quad 0.446747-2.455044$

H $6.1873571 .079764-0.498849$

H $7.4951110 .174245-1.298269$

H $3.964649-5.2833050 .138860$

H $6.820872-4.805872-1.512161$

H $\quad 8.103323-2.517926-0.378769$

O $6.375101 \quad 1.447420-3.118337$

O $4.725649-0.140047-2.853230$

C $5.8246101 .802072-4.390826$

H $5.9156220 .962096-5.085960$

H $4.7783432 .104085-4.285150$

H $6.3949392 .647663-4.784256$

SCF Energy $(B 3 L Y P / 6-31 G * * / / M M F F)=-3245.90985760$

0224

MM̄FF Geometry

C $2.410453 \quad 0.329115 \quad 3.277331$

C 3.7332460 .5534133 .314866

C 4.5370631 .4693942 .419879

O $3.7136792 .097017 \quad 1.414561$

C 5.5878610 .6421151 .657928

C 6.7514541 .4650341 .145220

C $6.8809181 .921431-0.115688$

C 7.8466911 .7100652 .151835

C $5.8936121 .834783-1.245745$

C $6.2756050 .793730-2.307843$

C $5.813901-0.626910-1.947631$

C $6.265270-1.633420-3.007002$

C $5.710043-3.039669-2.731426$

O $4.384091-0.604461-1.877616$

O $5.910233-3.832287-3.911767$

C $4.186306-2.940642-2.506703$

C $6.470452-3.743691-1.601455$

O $3.645693-4.198113-2.107084$

C $3.779073-1.844422-1.495236$

C $2.243357-1.648063-1.518995$

O $4.177343-2.247461-0.183593$

C 3.1226123 .2856761 .694499

O 3.1582243 .9064872 .742851

C 2.3910373 .6833890 .471938

C 1.3968854 .5792670 .542182

C $0.5646905 .019866-0.580245$

C $0.9182464 .508376-1.953647$

C $1.754926-0.401992-0.764091$

C $0.241443-0.269391-0.803849$

C $-1.5969851 .225045-0.027389$

O $-0.490584-1.098304-1.338644$

C -1.952055 2.307369-0.995648

C $-2.7088103 .364719-0.663539$

C $-3.1251814 .436016-1.630628$

C $-2.8588065 .832135-1.110164$

C $-3.8438386 .563824-0.562556$

C -1.473055 $6.408505-1.300044$

C $-0.4782465 .837693-0.327155$

N $-0.193798 \quad 0.881979-0.160887$

H 1.7860400 .8497012 .557416

H 4.3188030 .0227664 .064606

H 5.0215032 .2323293 .041167

H 5.1023870 .1240330 .824829

H $5.999282-0.1505392 .297564$

H $7.7906672 .464944-0.369451$

H 8.6738882 .2940221 .734460

H 7.4582162 .2607193 .014272

H 8.2590500 .7585772 .503482 
H $4.8748541 .669666-0.885557$ H $5.8748012 .822759-1.724216$ H $5.7873391 .090098-3.245822$ H $7.3574570 .809619-2.486989$ H $6.225744-0.899324-0.970287$ H $7.359767-1.658956-3.078073$ H $5.908576-1.309025-3.994817$ H $6.862842-3.842635-4.107671$ H $3.729243-2.701806-3.477420$ H $7.532206-3.841878-1.857951$ H $6.102702-4.765218-1.454551$ H $6.409772-3.214307-0.648285$ H $3.914053-4.853572-2.773503$ H $1.907688-1.551587-2.560073$ H $1.751703-2.535124-1.099585$ H $3.586766-1.8407050 .469606$ H $2.6705213 .192964-0.453964$ H 1.1519145 .0099831 .512033 H $1.924494 \quad 4.837548-2.236291$ H $0.241027 \quad 4.851418-2.737910$ H $0.888066 \quad 3.413617-1.980339$ H $2.059113-0.4446340 .285799$ H $2.1838660 .502401-1.210521$ H -1.7542301 .5367691 .009720$ H -2.208305 $0.342095-0.231776$ H - $1.6044502 .200977-2.020272$ H $-3.084633 \quad 3.4467860 .354080$ H $-4.1991104 .309612-1.821732$ H -2.634369 $4.310579-2.603766$ H -3.668935 $7.575354-0.211214$ H $-4.8465036 .165072-0.448101$ H -1.491142 $7.495791-1.145949$ $\mathrm{H}-1.1731406 .282997-2.343884$ H -0.6560796 .1468500 .703902$ H $0.490199 \begin{array}{llll}1.517373 & 0.238691\end{array}$ C $0.889060-1.6595873 .403751$ O $-0.348549-1.0438162 .986622$ C -1.443049-1.932419 3.304971 C $-0.804633-3.2880603 .552793$ C $0.488996-2.8831364 .228942$ H $-1.884360-1.5443264 .232103$ C $-2.466874-1.8904182 .166362$ H $-1.425209-3.9414744 .172915$ H $-0.599007-3.8021672 .605439$ C $1.535536-3.9842804 .243257$ H $\quad 0.268528-2.5839205 .262551$ C -3.579512 -2.948243 2.261775 O $-3.064618-0.5899242 .172470$ H -1.935496 -1.9829701 .213651$ H -3.147547 -3.953586 2.192611 O $-4.173896-2.8423463 .560942$ C -4.694462 -2.788166 1.207355 C $-4.173908-2.858402-0.238912$ H $-5.242557-1.8539601 .374234$ O $-5.632447-3.855481 \quad 1.382823$ H $-3.422423-2.080741-0.398225$ H -3.675553 -3.824660 -0.386680 H $1.170316-4.8544254 .798477$ H $1.785291-4.3136843 .228961$ H $2.457857-3.6406514 .721977$ C $1.717800-0.6434084 .194371$ H -3.569641 -0.5020872 .999021$ H $-4.728938-3.6292103 .693448$ H $-6.113260-3.6888542 .210976$ H $2.458229-1.1647084 .812161$ H $1.067234-0.0708464 .866611$ H $1.415141-1.9693612 .491007$ C $-5.376293-0.303485-1.399285$ O $-6.067443-1.523019-1.100768$ C -5.296301-2.714461-1.293130 C $-4.813474-2.799859-2.748346$ C $-4.088688-1.528621-3.187452$ C $-4.926321-0.291327-2.863871$ H $-4.506934-0.204170-0.739974$ C $-6.3328850 .855470-1.102356$ H -5.986495 -3.553237 -1.138424 O $-3.966261-3.930710-2.920025$ H $-5.685188-2.951561-3.398165$
H $-3.097938-1.461489-2.724111$

O $-3.854344-1.582274-4.596693$

H $-5.800284-0.253866-3.526662$

O $-4.1506660 .877940-3.111892$

$\begin{array}{llll}\text { C } & -6.539575 & 1.012119 & 0.385104\end{array}$

H $-5.9629441 .810263-1.492882$

H $-7.3155260 .679760-1.556550$

H -3.659402 -3.927791 -3.842838

H $-4.716274-1.641555-5.042924$

H $-3.8442590 .834307-4.033829$

O $-5.466694 \quad 1.646157 \quad 0.938819$

$\begin{array}{llll}\text { O } & -7.529536 & 0.609822 & 0.982227\end{array}$

C $-5.531476 \quad 1.846636 \quad 2.353719$

H -4.5808052 .2773992 .679252$

H -5.6790180 .8932532 .870230$

H -6.337069 2.5458692 .596020

SCF Energy (B3LYP/6-31G**//MMFF)= -3245.91551337

02240

MMFF Geometry

C -2.4463943 .9443312 .064618$

C -2.4544982 .6311501 .786181$

C -2.922384 1.5689782 .744722

O $\begin{array}{llll}\text { C. } & -1.958724 & 0.488538 & 2.701458\end{array}$

C $-4.274912 \quad 1.001549 \quad 2.289337$

$\begin{array}{llll}\text { C }-4.913378 & 0.103484 & 3.328934\end{array}$

C $-4.897679-1.2432493 .293129$

C $-5.644653 \quad 0.8213824 .434018$

C $-4.214579-2.134747 \quad 2.293571$

C $-5.209494-2.8947461 .405789$

C $-5.905671-2.003922 \quad 0.363979$

C $-6.932012-2.813215-0.431940$

C $-7.563153-1.986016-1.561444$

O $-4.898724-1.494217-0.515318$

O $-8.255905-2.889205-2.436417$

C $-6.437240-1.331060-2.387862$

C $-8.613742-1.002748-1.032155$

O $-6.974915-0.439943-3.362984$

C $-5.381925-0.599214-1.526464$

C $-4.172942-0.202584-2.406380$

O $-5.9673310 .574275-0.962566$

C $-0.8832430 .557002 \quad 3.524330$

$\begin{array}{llllll}\text { O } & -0.648137 & 1.431382 & 4.347064\end{array}$

C $0.018031-0.6044703 .337213$

C $-0.035541-1.4336082 .281951$

C $0.835196-2.5909532 .047956$

C $1.867104-2.9092733 .098232$

C $-3.0298110 .473774-1.638351$

C $-1.7495700 .444579-2.447143$

C $0.112208-1.060161-3.108817$

O $-1.375353 \quad 1.409865-3.108317$

C $0.570417-2.446159-2.790400$

C $1.761754-2.734213-2.246075$

C $2.212160-4.128732-1.924127$

C $2.544040-4.314509-0.459919$

C $3.822433-4.360010-0.048286$

C $1.406859-4.5626460 .506733$

C $0.677018-3.3105340 .916248$

$\mathrm{N}-1.099005-0.776517-2.363686$

H -2.831054 4.2692463 .029543

H $-2.093928 \quad 2.2741320 .826238$

H $-2.9979821 .963668 \quad 3.765249$

H $-4.148726 \quad 0.4576641 .348189$

H -4.9750261 .8178512 .065790$

H -5.416258 -1.7820644 .085444$

H -6.1198980 .1291035 .137278$

H $-4.956167 \quad 1.4498815 .007139$

H -6.4317901 .4578554 .016737$

H -3.490454 -1.5932881 .678406$

H -3.626784 -2.867703 2.860827

H $-4.647790-3.6798970 .882691$

H $-5.958500-3.3955492 .031739$

H $-6.397494-1.1690690 .875106$

H $-7.708942-3.215070 \quad 0.230434$

H $-6.438990-3.687900-0.879261$

H $-8.922868-3.363338-1.910766$

H $-5.932354-2.132303-2.945841$

H $-9.418424-1.542444-0.518379$ 
H $-9.094905-0.459159-1.852589$ H -8.204547 $-0.274950-0.328511$ H -7.622405 -0.941352 -3.887670 H -3.785771 -1.105102 -2.896793 H $-4.4936850 .481387-3.202505$ H $-5.2634901 .197233-0.723728$ H $0.760717-0.7237064 .119705$ H $-0.779798-1.2537651 .507856$ H $2.524463-2.0489433 .262416$ H $2.518389-3.7441832 .831776$ H $1.383131-3.1681164 .046330$ H -3.270019 $1.519454-1.417051$ H $-2.839367-0.020085-0.678979$ H $0.857973-0.299159-2.857226$ H $-0.114915-0.970481-4.176243$ H $-0.117510-3.256512-3.020999$ H $2.460099-1.929526-2.031767$ H $3.094221-4.342831-2.541796$ H $1.460375-4.871025-2.220212$ H $4.076742-4.5320940 .992044$ H $4.645068-4.217768-0.741610$ H $1.763477-5.1100591 .386845$ H $0.684517-5.2473120 .043225$ H $-0.090969-2.9995320 .208029$ H $-1.535628-1.527570-1.835713$ C $-1.042927 \quad 4.6209550 .012465$ $\begin{array}{lllll}\text { O } & 0.155428 & 4.025870 & 0.541582\end{array}$ C $1.2515494 .308441-0.347544$ C $0.6640705 .102586-1.511988$ C $-0.5451705 .760595-0.870019$ H 1.9499304 .9516750 .202131 C $1.9588793 .002379-0.740498$ H $1.3788095 .825575-1.917406$ H $\quad 0.353198 \quad 4.450755-2.335757$ C $-1.566254 \quad 6.262757-1.875083$ H $-0.202536 \quad 6.598235-0.247923$ C 2.7248142 .3582890 .439992 O $1.0000762 .074210-1.242028$ H $2.6787153 .201899-1.543889$ H 3.5074893 .0596720 .753413 O $1.8684552 .173257 \quad 1.566756$ C 3.3830931 .0101420 .086320 C 4.3318610 .5205301 .197397 H $3.9476991 .131811-0.843736$ O $2.3879450 .019088-0.152378$ H 5.1325531 .2586691 .319143 H 3.7919620 .4784502 .151528 H -1.120717 $7.017901-2.531040$ H -1.942574 $5.449816-2.505315$ H -2.421899 $6.719294-1.367978$ C $-1.937685 \quad 5.0541231 .175470$ H $0.6889132 .395350-2.104276$ H 1.0593261 .7352631 .251750 H $1.907952-0.1240770 .680159$ H -2.802717 5.6122650 .800587 H - 1.3656735 .7348501 .819348 H $-1.5464003 .867941-0.607559$ C $6.590607-0.264155-0.701415$ O $5.407663-1.017686-0.414015$ C $4.922317-0.8800540 .923294$ C $5.999192-1.3270691 .921817$ C $7.334106-0.6251431 .679190$ C $7.736947-0.7174180 .207205$ H $6.3961450 .805008-0.549285$ C $6.921622-0.482292-2.180718$ H $4.088014-1.5853561 .017100$ O $5.586141-1.0875493 .262532$ H $6.157452-2.4088761 .821648$ H 7.3032870 .4197512 .010185 O $8.348050-1.2622712 .460232$ H $8.026372-1.748846-0.031324$ O $8.8738490 .112237-0.019650$ C $5.9333270 .258476-3.050632$ H $7.918204-0.106241-2.437560$ H $6.902210-1.546589-2.444207$ H $4.762266-1.582786 \quad 3.406594$ H $8.062156-1.2260023 .389261$ H $\quad 9.572744-0.180578 \quad 0.590227$
O $4.915599-0.571072-3.417975$

O $6.037517 \quad 1.444420-3.337130$

C $3.8868040 .031584-4.209451$

H $4.3116310 .496960-5.104124$

H $3.193699-0.753811-4.521905$

H $3.3397080 .767324-3.612436$

SCF Energy (B3LYP/6-31G*//MMFF) $=-3245.90897240$

$02 \quad 241$

MMFF Geometry

C $2.788145-3.881364-1.239782$

C $3.255094-2.868542-0.495901$

C $4.001934-3.0628660 .798167$

O $3.292617-2.3467331 .841505$

C $5.418063-2.4616720 .753748$

C $6.319241-3.005568-0.335042$

C $6.647547-2.322136-1.450446$

C $6.889159-4.374459-0.079203$

C $6.118781-0.974540-1.853185$

C $7.0889200 .163456-1.512551$

C $6.3579291 .514050-1.483509$

C $7.3412482 .676041-1.351826$

C $6.6124574 .021255-1.202676$

O $5.484537 \quad 1.491823-0.348531$

O $7.5738864 .998557-0.776330$

C $5.5608323 .900972-0.078873$

C $6.0508084 .519028-2.539755$

O $4.7591965 .077810-0.002001$

C $4.6564612 .651106-0.202705$

C 3.8241732 .4762261 .093977

O $3.7764802 .813219-1.313547$

C $2.132060-2.8820552 .305826$

O $1.641630-3.9582862 .005670$

C $1.526121-1.8843563 .214866$

C $0.210690-1.9399463 .467903$

C $-0.554524-0.9966664 .286984$

C $0.197596 \quad 0.127334 \quad 4.949309$

C 3.0263191 .1670661 .177531

C $1.620202 \quad 1.291668 \quad 0.626947$

$\begin{array}{llll}\text { C } & -0.598779 & 2.288091 & 1.130947\end{array}$

$\begin{array}{lllll}\text { O } & 1.323814 & 0.862513 & -0.485675\end{array}$

C $-1.4588502 .309807 \quad 2.353947$

C -2.6006591 .6149182 .470395$

C -3.5032191 .6899253 .668252$

C -3.8760740 .3299014 .213373$

C $-5.072346-0.2190383 .941958$

C $-2.895700-0.3534435 .141478$

C $-1.887656-1.1753604 .390086$

N $0.7567001 .931928 \quad 1.501993$

H $2.964591-4.904644-0.917653$

H $3.065571-1.845758-0.812127$

H $4.065314-4.1219611 .078502$

H $5.900168-2.6329621 .726618$

H $5.349732-1.3680760 .700952$

H $7.334310-2.777968-2.161212$

H $7.565446-4.698315-0.877461$

H $7.456470-4.3813890 .857236$

H $6.086876-5.115351-0.006061$

H $5.935076-0.981898-2.934973$

H $5.139302-0.795398-1.401220$

H $7.542044-0.019762-0.529336$

H $7.8982550 .181441-2.252271$

H $5.7646141 .613443-2.400128$

H $8.0288942 .703633-2.206104$

H $7.9713352 .523050-0.464231$

H $8.2810475 .033871-1.443133$

H 6.1081543 .8372630 .872242

H $6.8579934 .642507-3.271846$

H $5.5917345 .508010-2.431481$

H $5.3127903 .841441-2.973864$

H 5.3636975 .8346770 .086743

H 4.5196012 .4709411 .943657

H 3.1520413 .3329571 .221824

H $3.0593272 .164389-1.238641$

H $2.169846-1.1085563 .612286$

H $-0.370608-2.7396873 .009935$

H -0.4456330 .8258095 .488213$

H 0.7425360 .7211314 .208342 
H $0.916846-0.2702075 .673863$ H $3.529300 \quad 0.3398720 .668669$ H 2.9412850 .8700802 .230449 H -0.9692751 .5951580 .369755$ H -0.5586353 .2881050 .687159$ H -1.144949 $2.956633 \quad 3.170007$ H -2.930434 0.9944951 .640609 H -4.4096992 .2302313 .365715$ H -3.056130 2.2916534 .469291 H -5.356858 -1.182849 4.351038 H $-5.789056 \quad 0.2782553 .296615$ H -3.435049 -1.032387 5.815896 H -2.4401810 .3878345 .803367$ H -2.332734 -2.031233 3.882010 H 1.1519562 .3777712 .325316 C $0.498629-3.823493-2.348725$ O $-0.015819-2.701968-1.603635$ C -1.082464 -3.167570 -0.747184 C $-1.381761-4.596257-1.184147$ C $-0.016563-5.073232-1.637768$ H $-0.672259-3.1534940 .269922$ C $-2.273896-2.207750-0.858492$ H -1.800756 -5.202994 -0.375835 H $-2.089583-4.608461-2.022542$ C $-0.058327-6.309869-2.517148$ H $0.576852-5.291642-0.741717$ C -3.507424 -2.664203 -0.060922 O $-1.864644-0.929042-0.365651$ H -2.531603 -2.071383 -1.914584 H -3.868728 -3.624589-0.445778 O $-3.115386-2.904577 \quad 1.296076$ C $-4.679742-1.663902-0.034967$ C $-5.182808-1.286599-1.437113$ H $-4.403005-0.7645950 .526284$ O $\quad-5.759928-2.275648 \quad 0.679090$ H -4.386628 $-0.762716-1.974826$ H -5.396526 -2.206662 -1.995571 H $-0.501887-7.151959-1.975702$ H $-0.653555-6.140808-3.420677$ H $0.950544-6.600411-2.826847$ C $2.015759-3.695313-2.514462$ $\mathrm{H}-1.069031-0.667700-0.860666$ H -2.740070 -2.074657 1.637705 H -5.426256 -2.510048 1.562189 H $2.244242-2.708393-2.936967$ H $2.372172-4.437201-3.238799$ H $\quad 0.045327-3.749908-3.346515$ C $-5.4386721 .737593-1.057921$ O $-6.3329220 .726709-0.574263$ C $-6.462267-0.420179-1.419226$ C $-6.9574380 .000814-2.810167$ C $-6.0859981 .095960-3.419885$ C $-5.9038492 .248010-2.431073$ H $-4.4292811 .315876-1.131257$ C -5.4223982 .8503890 .001154$ H $-7.254210-1.036281-0.975275$ O $-6.997465-1.111903-3.697398$ H $-7.9832990 .383263-2.728143$ H $-5.117017 \quad 0.702004-3.748133$ O $-6.7280831 .606420-4.590867$ H $-6.8587342 .779016-2.330694$ O $-4.9687653 .178849-2.967797$ C $-4.1925203 .729503-0.045907$ H $-6.3163483 .476536-0.104225$ H -5.4580612 .4014991 .001362$ H -7.573939 -1.784495 -3.296084 H -6.870139 $0.855863-5.192893$ H $-4.1093052 .729864-3.041939$ $\begin{array}{lllll}\text { O } & -4.388924 & 4.807334 & 0.770141\end{array}$ O $-3.1796013 .495935-0.690243$ C -3.3000735 .7332110 .840246$ H -3.596707 6.5575021 .494356 H $-2.4175325 .247616 \quad 1.266530$ H -3.078430 $6.135847-0.152745$ SCF Energy $\left(B 3 L Y P / 6-31 G^{* *} / / M M F F\right)=-3245.92445496$

02242

MM̄MF Geometry
C $1.0856641 .019824 \quad 3.213421$

C $1.940308-0.0058283 .363795$

C 3.4321110 .0465693 .103777

O 3.7293661 .2096202 .300147

C $3.895055-1.1955552 .321310$

C $5.388966-1.4342682 .415630$

C $6.282990-1.1268671 .455712$

C $5.848037-2.1112273 .682057$

C $6.035514-0.4379690 .143011$

C $6.258855-1.363982-1.060796$

C $5.163931-2.430135-1.221752$

C $5.469017-3.343127-2.411239$

C $4.333616-4.345386-2.670419$

O $3.925058-1.749170-1.437777$

O $4.545521-4.927369-3.965762$

C $2.995258-3.579445-2.732914$

C $4.353665-5.503433-1.665815$

O $1.894858-4.481574-2.826504$

C $2.783474-2.607501-1.549114$

C $1.549657-1.712783-1.815579$

O $2.568598-3.364284-0.356515$

C 4.7696952 .0098042 .633324

O 5.5554091 .8466963 .555580

C 4.8349433 .1900351 .735415

C 4.0668793 .3242740 .642054

C $4.0254794 .474170-0.263917$

C 4.8880275 .6621460 .069180

C $1.376116-0.568091-0.806651$

C $0.1868100 .313383-1.143079$

C $-0.8524592 .486447-0.498679$

O $-0.6397680 .028014-2.005095$

C $-0.4071843 .446114-1.557113$

C $-0.1199224 .736038-1.325303$

C $0.3472295 .687996-2.389894$

C $1.6981316 .298597-2.080155$

C $1.7959367 .563058-1.636192$

C $2.9389705 .479011-2.365990$

C $3.1902934 .424059-1.323121$

N $0.153191 \quad 1.452079-0.349769$

H 1.4765001 .9822422 .889444

H $1.565369-0.956583 \quad 3.732829$

H 3.9294330 .1045604 .079287

H $3.590291-1.1035541 .274025$

H $3.391101-2.0969262 .695241$

H $7.329149-1.3730261 .634502$

H $6.927711-2.2954773 .688224$

H $5.615925-1.4938044 .555216$

H $5.348870-3.0788823 .797307$

H 5.0429350 .0189250 .093682

H $\quad \begin{array}{llll}6.746232 & 0.395506 & 0.074420\end{array}$

H $6.275918-0.733640-1.959552$

H $7.242017-1.844780-0.984285$

H $5.099173-3.019209-0.300266$

H $6.420184-3.869569-2.263697$

H $5.602122-2.732014-3.315155$

H $5.420845-5.351347-3.964145$

H $2.996504-2.995026-3.663760$

H $5.304241-6.046917-1.727310$

H $3.574034-6.238431-1.894488$

H $4.228096-5.176888-0.631482$

H $2.061721-5.063548-3.587568$

H $1.641352-1.261929-2.812648$

H $\quad 0.641226-2.326821-1.820360$

H $2.038978-2.8416410 .265153$

H 5.5477683 .9519532 .033415

H 3.3806812 .5203440 .380265

H 4.6541046 .0399081 .070746

H $4.7546496 .501788-0.616466$

H 5.9481445 .3876340 .035481

H $1.217104-0.9600350 .202817$

H $2.2732810 .061501-0.794655$

H -0.9741752 .9702530 .475175$

H -1.803247 $2.027441-0.782232$

H $-0.3135103 .054959-2.567606$

H $-0.2244235 .132961-0.318126$

H $-0.4107956 .476365-2.486117$

H $\quad 0.3902485 .200383-3.371846$

H $2.7598388 .020413-1.439395$ 
H $0.9139058 .168360-1.454928$ H $3.8134716 .128111-2.485391$ H $2.8303314 .988635-3.341928$ H $2.6076503 .515850-1.478046$ H $\quad 0.928338 \quad 1.637303 \quad 0.280063$ C -1.113428 $-0.327458 \quad 3.507502$ O $-1.055057-0.9454322 .205053$ C $-2.384487-1.3659091 .822754$ C $-3.176985-1.3937423 .118453$ C $-2.600807-0.1945623 .842121$ H $-2.761620-0.5770971 .161543$ C $-2.290158-2.6971681 .068676$ H $-4.255702-1.3161902 .963118$ H $-2.971685-2.3113463 .683295$ C -2.901641 -0.1810495 .330768$ H -3.008759 0.7185493 .388418 C $-3.627780-3.3529520 .681570$ O $-1.514965-2.476526-0.110491$ H $-1.712404-3.3962171 .687301$ H -3.421819-4.204239 0.019878 O $-4.205400-3.9072831 .869118$ C $-4.683464-2.4541600 .003951$ C $-4.209926-1.753557-1.281671$ H $-5.051185-1.7154040 .722183$ O $-5.810714-3.283203-0.311486$ H $-3.413975-1.044705-1.038165$ H $-3.778393-2.502247-1.957593$ H -3.982166 - 0.1410645 .503656 H $-2.515060-1.0776285 .826695$ H $-2.449757 \quad 0.6917705 .812122$ C $-0.4005401 .026547 \quad 3.461005$ H $-1.384648-3.337249-0.543551$ H $-5.044133-4.3291791 .615187$ H $-5.523411-3.934386-0.974335$ H $-0.577948 \quad 1.574108 \quad 4.394053$ H -0.8470491 .6198892 .652736$ H $-0.627272-1.0093404 .214852$ C $-5.4242050 .999453-0.711821$ O $-6.109062-0.173790-1.165327$ C $-5.346768-1.019538-2.031158$ C $-4.895624-0.238431-3.273653$ C $-4.199648 \quad 1.072547-2.910277$ C $-5.0332111 .872346-1.908152$ H $-4.5281450 .712335-0.148058$ C $-6.366618 \quad 1.7428790 .239642$ H -6.039147 - $1.795172-2.382910$ O $-4.037095-1.042934-4.074759$ H -5.779972 $-0.009811-3.882608$ H $-3.1955200 .883884-2.517489$ O $-4.0114301 .851465-4.094056$ H -5.932389 $2.260652-2.402889$ O $-4.2777812 .991413-1.452791$ C $-6.477516 \quad 1.0084801 .554696$ H $-6.003302 \quad 2.7513720 .467548$ H $-7.3695411 .853586-0.189801$ H $-3.748120-0.500356-4.828452$ H $-4.8872942 .041728-4.471181$ H -3.998189 $3.493197-2.237642$ O $-7.492290 \quad 0.099746 \quad 1.499703$ O $-5.7308791 .201472 \quad 2.505588$ C $-7.676993-0.6900292 .678280$ H $-8.549364-1.3306712 .524550$ H $-6.801776-1.3240332 .846896$ H -7.862406 -0.047266 3.544289 SCF Energy (B3LYP/6-31G*//MMFF) $=-3245.91344460$

02243

MMFF Geometry

C $-2.500984-1.669472-4.086712$

C $-2.817784-1.046273-2.941890$

C $-3.8595940 .042708-2.863565$

O $-3.277256 \quad 1.193095-2.212579$

C $-5.031676-0.419383-1.981443$

C $-6.311603 \quad 0.348231-2.240040$

C $-6.744197 \quad 1.398567-1.515930$

C $-7.149596-0.181532-3.375679$

C $-6.0436212 .105316-0.389694$

C -6.6762551 .8308720 .981685$
C $-6.242906 \quad 0.487245 \quad 1.588326$

$\begin{array}{llll}\text { C } & -6.933968 & 0.251720 & 2.932097\end{array}$

C -6.424982 -1.0228243 .622967$

O $-4.825752 \quad 0.5403941 .777409$

O $-6.878502-0.993853 \quad 4.985373$

C $-4.881022-0.9955703 .658120$

C -7.021871-2.287925 2.994339

O $-4.387734-2.2325804 .168532$

C $-4.239807-0.6624242 .288945$

C -2.720457 -0.4076682 .446538$

O $-4.435421-1.7676911 .404224$

C -2.525780 $2.047024-2.952536$

O $-2.269057 \quad 1.972730-4.142186$

C $-2.0345253 .085298-2.021137$

C $-0.955563 \quad 3.812801-2.341215$

C $-0.3056834 .801400-1.477690$

C $-0.9593815 .108393-0.154668$

C $-2.059404 \quad 0.2213851 .209615$

C $-0.5590890 .386111 \quad 1.383368$

C $1.457146 \quad 1.1843610 .152256$

$\begin{array}{lllll}\text { O } & 0.042128 & 0.041142 & 2.397034\end{array}$

C $1.7919112 .577467 \quad 0.581567$

C $2.6994053 .335235-0.054264$

$\begin{array}{lllll}\text { C } 3.083788 & 4.723860 & 0.372441\end{array}$

C $3.0658935 .715107-0.772733$

C $4.2054046 .088214-1.379227$

C $1.7375806 .327518-1.163931$

C $0.8549555 .351142-1.891916$

$\begin{array}{llll}N & 0.028600 & 0.958516 & 0.263142\end{array}$

H $-3.012263-1.353593-4.995221$

H $-2.337135-1.329991-2.010758$

H $-4.2163930 .327182-3.861371$

H $-4.753868-0.333243-0.925882$

H $-5.242290-1.484799-2.146465$

H -7.704264 $1.840093-1.781957$

H -8.066068 $0.399351-3.524392$

H $-6.585748-0.152026-4.313418$

H -7.443740 -1.217792 -3.179038

H $-4.972391 \quad 1.888537-0.371226$

H $-6.1191803 .181289-0.593992$

H -6.3569882 .6374771 .655109$

H $-7.769798 \quad 1.8811710 .912239$

H $-6.489227-0.3179390 .887523$

H -8.0234440 .2134292 .807905$

H -6.7390811 .1050493 .596995$

H $-7.849761-0.9434574 .977135$

H $-4.592408-0.2203374 .382120$

H -8.116086 -2.2712263 .066220$

H $-6.701051-3.1862663 .533225$

H -6.763451-2.406144 1.939964

H $-3.442050-2.1198684 .361351$

H -2.551857 $0.273666 \quad 3.290779$

H -2.209514 -1.3500922 .680252$

H $-3.709695-1.7970880 .761295$

H -2.542043 $3.178946-1.067305$

H $-0.4781113 .639656-3.304758$

H $-1.9705285 .501032-0.310210$

H -0.4237795 .8514750 .438725$

H $-1.025330 \quad 4.206247 \quad 0.463493$

H -2.225744 $-0.402120 \quad 0.325836$

H -2.485970 1.2125201 .016929

H $1.7285531 .009694-0.892317$

H 1.9819320 .4639920 .785307

H 1.2938222 .9709821 .464086

H $3.2215882 .922065-0.914452$

H 4.0926114 .6703710 .802323

H $2.4350765 .089199 \quad 1.178250$

H $4.2060926 .815473-2.184276$

H $5.1631385 .669831-1.087807$

H $1.8954427 .184709-1.831858$

H $1.2673756 .749899-0.272219$

H $1.2482355 .057878-2.866619$

H $-0.5578111 .241181-0.515866$

C $-0.773339-3.333946-3.068357$

O $0.268931-2.405339-2.695789$

C $1.523326-3.118047-2.626272$

C $1.142102-4.579083-2.463514$

C $-0.071982-4.664932-3.362291$ 
H $2.012744-2.956291-3.595648$

C $2.388696-2.551658-1.492546$

H $1.941321-5.263274-2.763097$

H $0.868262-4.797520-1.423839$

C $-0.936138-5.886656-3.098656$

H $0.267101-4.689226-4.406970$

C $3.671827-3.360050-1.230051$

O $2.746272-1.211226-1.841007$

H $1.786190-2.483586-0.580267$

H $3.413845-4.376239-0.909272$

O $4.372846-3.492609-2.474440$

C $4.641559-2.733457-0.209725$

C $4.002612-2.4990231 .170604$

H $5.050569-1.801566-0.615471$

O $5.739008-3.643501-0.075371$

H $3.219066-1.7407351 .076981$

H $3.512379-3.4254421 .495731$

H $-0.367949-6.805359-3.277410$

H -1.294059 -5.909215 -2.063733

H -1.810203 -5.896158 -3.757329

C $-1.530613-2.802456-4.292045$

H $3.318701-1.252846-2.626334$

H $5.176868-4.011281-2.299298$

H $6.472839-3.1582740 .338885$

H -2.104537 -3.623354 -4.739423

H $-0.803676-2.469135-5.043832$

H -1.438866 -3.441419 -2.203582

C 5.2062960 .2632601 .721924

O $5.857661-1.0078681 .832835$

C $5.012883-2.0791902 .262226$

C $4.387828-1.7546763 .626688$

C $3.716947-0.3823843 .643913$

C $4.6576540 .687306 \quad 3.087777$

H 4.3904740 .1961090 .992679

C $6.234348 \quad 1.266074 \quad 1.189693$

H $5.673034-2.9410352 .422577$

O $3.450872-2.7624523 .990787$

H $5.181433-1.7671924 .385251$

H $2.775000-0.4015503 .085495$

O $3.364053-0.0471454 .987993$

H $5.483193 \quad 0.855372 \quad 3.790846$

O 3.9482291 .9151962 .959357

C $6.4802681 .033433-0.282282$

H 5.8869792 .3002161 .294003

H $7.188701 \quad 1.1921301 .724534$

H $3.059372-2.5025264 .842325$

H $4.181148-0.0136965 .514110$

H 3.5717572 .1274953 .830425

O $7.4579330 .098321-0.452196$

O $5.8546881 .594233-1.172823$

C $7.748625-0.247256-1.809864$

H $8.526461-1.015539-1.805723$

H $6.857333-0.654152-2.297456$

H $8.1209330 .627532-2.351333$

SCF Energy (B3LYP/6-31G**//MMFF)= -3245.91245565

$02 \_244$

MM̄FF Geometry

C $3.737404-0.5498022 .212750$

C $4.855322-1.1730391 .806614$

C $5.752580-0.7891650 .654500$

$\begin{array}{lllll}\text { O } & 5.460849 & 0.561833 & 0.222088\end{array}$

C $5.613124-1.732398-0.557878$

C $5.958674-3.186476-0.300335$

C $5.132249-4.218085-0.565248$

C $7.333734-3.4518810 .255467$

C $3.773416-4.144406-1.203332$

C $2.663094-3.988147-0.159254$

C $1.286570-3.641936-0.748645$

C $0.737221-4.749969-1.648560$

C $-0.599978-4.353503-2.290323$

O $1.416023-2.417431-1.478140$

O $-0.878655-5.289468-3.343267$

C $-0.438405-2.968221-2.949491$

C $-1.766400-4.454858-1.301359$

O $-1.685246-2.500042-3.457389$

C $\quad 0.190927-1.905335-2.015684$

C $0.517196-0.628197-2.831520$
O $-0.743336-1.585759-0.986988$

C $6.4940281 .279559-0.291475$

O $7.6435330 .888902-0.451698$

C $6.1005832 .665872-0.626526$

C $4.8347403 .108598-0.677495$

C $4.4234094 .478048-1.006710$

C $5.5083025 .515508-1.148649$

C $1.3547870 .433249-2.103196$

C $0.5830181 .268486-1.101754$

C $-1.2754422 .917771-0.921308$

O $0.860742 \quad 1.249800 \quad 0.095515$

C $-0.8235714 .324221-1.139607$

C $-0.2817945 .089667-0.180481$

C $0.1656296 .504185-0.406424$

C $1.6691256 .650015-0.362131$

C $2.274277 \quad 7.3415470 .617817$

C $2.4636886 .059965-1.506543$

C $3.1080594 .747218-1.147783$

N $-0.4211412 .025929-1.680122$

H 3.3601770 .3124041 .670611

H $5.193647-2.0353142 .379463$

H $6.779591-0.8130821 .040743$

H $6.264131-1.373068-1.366033$

H $4.598239-1.619005-0.956106$

H $5.468429-5.226962-0.331037$

H $7.577496-4.5196650 .265357$

H $8.092200-2.950796-0.355178$

H $7.415065-3.0875131 .283795$

H $3.742521-3.336370-1.941526$

H $3.621280-5.068024-1.774190$

H $2.588636-4.9003700 .445489$

H $2.944768-3.1769720 .521344$

H $\quad 0.607717-3.4747540 .096446$

H $0.635392-5.688951-1.090768$

H $1.445535-4.958674-2.460902$

H $-0.908204-6.179712-2.952701$

H $\quad 0.220680-3.095867-3.819867$

H -1.861515 -5.480713 -0.925425

H -2.717295 -4.228436 -1.794430

H -1.659052 -3.796840 -0.436643

H -2.039372 $-3.192744-4.040926$

H $1.104035-0.926190-3.710522$

H -0.413665 -0.180729 -3.198541

H $-0.423944-0.805162-0.509895$

H $6.9378353 .317882-0.856898$

H $4.0225372 .413159-0.473190$

H $6.1526775 .523730-0.261999$

H $5.1302776 .534499-1.251419$

H $6.1246895 .308170-2.030238$

H $2.213377-0.019501-1.594864$

H $1.7707341 .129215-2.843320$

H -1.259655 2.6414280 .136581

H -2.294529 2.796659-1.295941

H - $0.9556624 .733842-2.138207$

H $-0.148768 \quad 4.682380 \quad 0.819058$

H $-0.308992 \quad 7.1327260 .357892$

H -0.199532 $6.885349-1.368650$

H 3.3514897 .4712850 .632749

H $1.707647 \quad 7.790448 \quad 1.426649$

H $3.1889286 .797635-1.863423$

H $1.8051855 .892271-2.368632$

H $2.3986533 .929757-1.018941$

H $-0.5232972 .024834-2.690771$

C $1.650256-1.6425173 .140549$

O $0.685310-0.6659002 .700879$

C $-0.543619-0.8617343 .434338$

C $-0.436068-2.2447094 .054573$

C $1.041219-2.3156254 .372152$

H $-0.551777-0.0879554 .212468$

C -1.739077 -0.684660 2.491307

$\mathrm{H}-1.069241-2.3625604 .938903$

H -0.709981 -3.0204033 .328392$

C $1.541087-3.7307784 .610178$

H $1.237403-1.7106745 .267590$

C $-3.095902-0.9956193 .146528$

$\begin{array}{lllll}\text { O } & -1.749560 & 0.670251 & 2.035075\end{array}$

H -1.596040 -1.315099 1.607873

H $-3.135777-2.0526663 .435272$ 
O - $-3.189306-0.2399964 .361709$ C $-4.327065-0.6531352 .285711$ C $-4.322148-1.3486210 .913954$ H $-4.404814 \quad 0.434403 \quad 2.178213$ O $-5.478789-1.0788623 .022343$ H -3.440690 -1.026545 0.351890 H $-4.228532-2.431703 \quad 1.063079$ H $1.026293-4.1817625 .464817$ H $1.366933-4.3695923 .737545$ H $2.614845-3.7361154 .821662$ C $2.984906-0.9627073 .449717$ H -0.8751190 .8515091 .649280$ H $-4.049088-0.4479524 .766276$ H $-6.254913-0.6438842 .630427$ H $3.610391-1.6333874 .051320$ H $2.816111-0.0607994 .051495$ H $1.760958-2.3674022 .326800$ C $-4.993016 \quad 1.097133-0.764680$ $\begin{array}{lllll}\text { O } & -5.911060 & 0.313511 & 0.007362\end{array}$ C -5.594392 -1.0792980 .077998$ C $-5.562704-1.679422-1.334472$ C $-4.634548-0.900027-2.263177$ C $-4.9528100 .594310-2.212290$ H $-3.991948 \quad 1.035856-0.321241$ C $-5.4606782 .554246-0.688853$ H $-6.428356-1.5572900 .606616$ O $-5.156992-3.042605-1.299763$ H $-6.574218-1.655588-1.760426$ H $-3.581223-1.080843-2.021579$ O $-4.823426-1.353153-3.605313$ H $-5.9148800 .781334-2.705980$ O $-3.9540331 .309976-2.934806$ C $-5.312573 \quad 3.0939640 .714568$ H $-4.8987813 .206805-1.366185$ H $-6.5193862 .637278-0.962705$ H $-5.772238-3.516322-0.714420$ H $-4.634510-2.307271-3.616047$ H -3.927038 $0.941848-3.834641$ O $-3.999974 \quad 3.3475620 .980672$ O $-6.250212 \quad 3.2563781 .484552$ C -3.715979 3.8235092 .299550 H -4.2154504 .7815632 .471937$ H -4.0269913 .0844073 .044045$ H -2.635717 3.9702682 .382017

SCF Energy (B3LYP/6-31G**/MMFF)= -3245.92723403

02_245

MMFF Geometry

C -1.869331-4.244983 -0.252697

C $-3.059346-3.628072-0.230469$

C $-3.852762-3.260936-1.456984$

O $-3.863022-1.816443-1.538018$

C $-5.299523-3.764791-1.308157$

C $-6.223817-3.313927-2.422685$

C $-7.184198-2.375899-2.290117$

C $-6.021912-4.007769-3.744562$

C $-7.524050-1.581580-1.058786$

C $-7.100047-0.115039-1.209232$

C $-6.941067 \quad 0.5795620 .151824$

C $-6.6593542 .070242-0.038027$

C $-6.3503142 .775395 \quad 1.289008$

O $-5.841661-0.0453390 .825413$

$\begin{array}{lllll}\text { O } & -5.804402 & 4.062373 & 0.959862\end{array}$

C -5.2640911 .9785622 .045041$

C -7.628177 3.0439212 .092729

O -5.0742572 .5444983 .339761$

C $-5.567451 \quad 0.467457 \quad 2.137310$

C $-4.394262-0.3655382 .715599$

O $-6.6887540 .271127 \quad 3.007560$

C -2.820734-1.229411-2.190689

O $-1.910933-1.792190-2.779231$

C -2.952130 $0.235782-2.029292$

C $-1.966703 \quad 1.040016-2.454997$

C -1.921058 2.497947 -2.324176

C $-3.0515933 .170595-1.592286$

C $-3.086967-0.3153861 .917271$

C -2.1093650 .7530012374424$

C $\quad 0.0433631 .8228191 .709004$
O $-2.2375301 .378686 \quad 3.422918$

C $-0.248803 \quad 3.1173621 .016323$

C $0.621847 \quad 3.726374 \quad 0.196873$

C $0.3714825 .050380-0.466927$

C $0.5863815 .004856-1.963794$

C $1.7663045 .354809-2.503343$

C $-0.592407 \quad 4.636124-2.836288$

C $-0.863777 \quad 3.157956-2.841049$

N $-1.050190 \quad 0.895126 \quad 1.486672$

H - $-1.426853-4.532351-1.202837$

H $-3.475741-3.3246340 .727803$

H $-3.409966-3.688728-2.365034$

H $-5.691080-3.453473-0.334886$

H $-5.303183-4.862859-1.274163$

H $-7.792294-2.134258-3.160856$

H $-6.753963-3.692511-4.495679$

H $-5.026570-3.790956-4.144560$

H -6.121394 $-5.091439-3.623861$

H $-8.608701-1.629264-0.901026$

H -7.082656 -2.018394 -0.161036

H $-6.145516-0.064372-1.746871$

H $-7.8436880 .412904-1.819070$

H $-7.857494 \quad 0.429422 \quad 0.734484$

H $-7.4960622 .562499-0.549565$

H $-5.7943852 .191802-0.702857$

H -5.5404864 .4913491 .792129$

H $-4.3286922 .129852 \quad 1.493467$

H $-8.305633 \quad 3.692571 \quad 1.524154$

H -7.4084333 .5828683 .020947$

H -8.1757202 .1333442 .344745$

H -4.2883292 .1312623 .734173$

H $-4.232650-0.1157783 .770869$

H $-4.717370-1.4168052 .716097$

H $-6.673399-0.6453603 .329473$

H $-3.8398440 .611845-1.535686$

H $-1.0999600 .589424-2.937197$

H $-3.9857613 .063615-2.153649$

H $-2.8941804 .238531-1.428695$

H $-3.1836382 .729449-0.598597$

H -2.566136 -1.272458 2.049452

H $-3.286932-0.1962240 .849653$

H $\quad 0.953764 \quad 1.346953 \quad 1.331309$

H 0.1596021 .9979302 .783439

H -1.206619 3.5897311 .221534

H 1.5913613 .2671250 .021653

H $1.0565595 .781877-0.018391$

H $-0.6376615 .422805-0.251659$

H $1.9208175 .350602-3.577175$

H $2.6069005 .645120-1.881603$

H $-0.3988834 .932199-3.876178$

H $-1.4618025 .229532-2.539305$

H $-0.0923632 .580091-3.350916$

H -1.0526200 .3657430 .619373$

C $0.104554-3.5664251 .122198$

O $1.114805-3.899911 \quad 0.149817$

C $2.415068-3.8090440 .773351$

C $2.184978-3.1415092 .121322$

C $0.799704-3.6386922 .479288$

H $2.753216-4.843518 \quad 0.912517$

C $3.366424-3.055318-0.164391$

H $2.943032-3.4154922 .861001$

H $2.183948-2.0486492 .020512$

C $0.124956-2.815133 \quad 3.563422$

H $0.873287-4.6834832 .809835$

C $4.743814-2.7478140 .457395$

O $3.522731-3.843032-1.347838$

H $2.891862-2.119156-0.482421$

H $4.591018-2.1578491 .368696$

O $5.361899-3.9747830 .840978$

C $5.669319-1.951602-0.484710$

$\begin{array}{llll}C & 6.975545 & -1.508625 & 0.202027\end{array}$

H $5.118279-1.071694-0.830557$

O $5.999381-2.716450-1.641603$

H $6.737064-1.0820631 .182035$

H $7.614158-2.3830710 .376002$

H $0.704638-2.8531324 .491501$

H $0.030950-1.7642693 .269262$

H $-0.879070-3.1950123 .776853$ 
C $-1.076222-4.5298330 .992434$ H $4.009612-4.648343-1.102129$ H $6.139498-3.7591211 .382660$ H $6.406546-3.546227-1.339208$ H - $1.724126-4.4520881 .873246$ H $-0.721770-5.566257 \quad 0.940278$ H $-0.212900-2.5360090 .912036$ C 6.5627831 .4934690 .016381 O $6.955175 \quad 0.621743-1.050474$ C $7.767168-0.485513-0.645700$ C 9.0536570 .0317810 .011834 C 8.7708601 .0300381 .135205 C 7.8010452 .1180110 .665793 H 5.9971310 .9314870 .769206 C $5.6289112 .553119-0.579534$ H $8.060169-1.003125-1.567934$ O $9.821780-1.0590910 .508446$ H $9.6628480 .530164-0.753597$ H 8.3926560 .5162612 .027128 O 10.0113821 .6320351 .511885 H $8.3078802 .783865-0.044167$ O 7.3929172 .9032291 .783086 C $4.3099731 .921958-0.968778$ H 5.4051133 .3550590 .131838 H $6.0897893 .007046-1.465013$ H $10.617897-0.687757 \quad 0.926124$ H 9.8436432 .1869882 .292062 H 8.1748933 .3783632 .110729 O $4.0498482 .151750-2.286741$ O $3.6012241 .289547-0.195402$

C $2.8502611 .555228-2.789931$

H $2.6603511 .960913-3.787147$ H $2.9818080 .472053-2.867681$ H $1.9972801 .792129-2.147855$ SCF Energy (B3LYP/6-31G**//MMFF)= -3245.91653711

02246

MMFF Geometry

C 1.3684644 .7230130 .580810

C $2.534797 \quad 4.189040 \quad 0.976171$

C 2.8029463 .4055442 .240154

O 3.1186022 .0472991 .848497

C 1.6415623 .3416113 .249308

C 1.9842242 .5484614 .498042

C 1.5283121 .3045934 .748741

C 2.8492463 .2605585 .503770

C 0.6636020 .4781573 .838968

C $0.964366-1.0234153 .936503$

C $0.129715-1.8060572 .911897$

C $0.163607-3.306098 \quad 3.200355$

C $-0.614512-4.0995792 .139775$

O $0.677801-1.5487191 .612771$

O $-0.291536-5.4887502 .302718$

C $-0.115704-3.6789860 .742726$

C $-2.132128-3.9803772 .328140$

O $-0.905330-4.278798-0.282342$

C $-0.055256-2.1462030 .536010$

C $0.683642-1.838391-0.786638$

O $-1.383950-1.6303860 .458706$

C 4.4289571 .7065591 .727821

O 5.3986842 .4327911 .874763

C $4.480174 \quad 0.2736051 .362806$

C $5.623266-0.2453570 .891035$

C $5.822437-1.6207040 .427695$

C $4.692174-2.5955960 .625739$

C $1.030966-0.361491-1.001292$

C $1.771337-0.213278-2.315994$

C $4.077452-0.182813-3.273028$

O $1.174426-0.183565-3.390007$

C $4.782555-1.503837-3.288237$

C $6.117815-1.635356-3.302808$

C $6.823556-2.962124-3.297747$

C $7.876035-3.067090-2.213643$

C $9.184308-3.073610-2.520530$

C $7.412637-3.240927-0.780709$

C $6.996302-1.934825-0.159493$

N $3.146255-0.178016-2.159901$

H 0.4775874 .6050081 .190215
H $3.4039094 .330798 \quad 0.335491$

H 3.6649813 .8683742 .736895

H 0.7534952 .9152162 .769639

H 1.3528954 .3570783 .551502

H 1.7915110 .8392845 .697036

H 3.0087322 .6652526 .409229

H 3.8329533 .4804635 .078052

H 2.3818404 .2027145 .807842

H -0.3825300 .6450494 .120376$

H $\quad 0.781836 \quad 0.7891332 .796783$

H $2.033219-1.1947213 .755632$

H $0.735572-1.3697174 .951844$

H $-0.902434-1.4378782 .949644$

H $-0.228883-3.5206054 .202136$

H $1.205750-3.6549913 .203862$

H $-0.537459-5.7492523 .206828$

H $\quad 0.900415-4.0822630 .626317$

H -2.424451 -4.347306 3.319322

H -2.669817 -4.6060011 .606999$

H -2.498436 -2.955917 2.234520

H $-0.904029-5.238524-0.124625$

H $1.621403-2.407215-0.816479$

H $0.077500-2.182056-1.635090$

H $-1.376747-0.803566-0.049033$

H $3.567606-0.3038771 .456559$

H $\quad \begin{array}{llll}6.490408 & 0.407739 & 0.799801\end{array}$

H $4.375476-2.6122661 .674853$

H $4.953988-3.6250030 .373146$

H $3.834428-2.3185530 .006305$

H $\quad 0.140018 \quad 0.269307-1.044429$

H $1.6457380 .022636-0.180872$

H $4.7681390 .652525-3.121790$

H $3.541763-0.032761-4.215141$

H $4.161349-2.396809-3.284652$

H $6.739528-0.743136-3.329707$

H $7.284175-3.095617-4.285257$

H $6.112916-3.790329-3.184102$

H $9.944217-3.172797-1.752324$

H $9.525606-2.974698-3.545586$

H $8.227020-3.651270-0.169510$

H $6.623582-3.997206-0.763360$

H $7.760565-1.158860-0.228160$

H $3.538538-0.224218-1.225455$

C $0.2873954 .893127-1.724851$

O $-1.0324884 .671337-1.190271$

C $-1.5170473 .392195-1.673218$

C $-0.5774713 .011391-2.808011$

C $0.7435863 .551740-2.293492$

H -1.400325 $2.705036-0.827013$

C $-2.9977843 .535015-2.042672$

H $-0.5480671 .938294-3.003752$

H $-0.8614413 .521199-3.737153$

C $1.8175883 .662933-3.360630$

H $1.1006102 .890849-1.493876$

C $-3.7090342 .299749-2.633250$

O $-3.7170543 .914431-0.862521$

H -3.099941 $4.369811-2.747786$

H $-4.7683002 .563294-2.753841$

O $-3.2036362 .002874-3.931572$

C $-3.6535691 .030467-1.758873$

C $-4.642115-0.051835-2.235969$

H $-3.8951501 .299073-0.726454$

O $-2.3400450 .484687-1.758690$

H $-5.659606 \quad 0.345177-2.146020$

H -4.475595 -0.258593 -3.300361

H $2.0424542 .679524-3.785730$

H $1.5038144 .318315-4.179804$

H $2.7433574 .067784-2.939683$

C $1.2007635 .532680-0.675985$

H $-3.2814814 .704118-0.498080$

H $-3.3039472 .802023-4.476064$

H -2.126762 $0.230826-2.673030$

H $0.768316 \quad 6.495753-0.376009$

H $2.1752685 .753258-1.127645$

H $0.1615255 .615851-2.542487$

C $-5.639392-0.7485100 .570297$

O $-4.428907-1.193443-0.049800$

C $-4.510397-1.384968-1.463460$ 
C $-5.591183-2.423841-1.790609$

C $-6.929078-2.086369-1.132061$

C $-6.746837-1.7839610 .357156$

H $-5.944197 \quad 0.2150320 .142806$

C $-5.336175-0.5364962 .057877$

H -3.549209 -1.821536-1.764245

O $-5.753463-2.535982-3.200174$

H -5.256399 -3.404079-1.426991

H -7.414065 -1.246279-1.642210

O $-7.819853-3.194826-1.280964$

H $-6.508807-2.7093480 .896866$

O $-7.971566-1.2800470 .884566$

C $-4.5558320 .741707 \quad 2.261819$

H -6.255060 -0.445978 2.648176

H -4.761406 -1.369096 2.480642

H $-6.461081-3.183327-3.361348$

H -7.421804 -3.962190 -0.835469

H $-8.660326-1.9425290 .703797$

O $\quad-3.2350820 .534931 \quad 1.994517$

O

C -2.3995751 .6943132 .066866$

H -1.424852 1.4362911 .644462

H -2.8221532 .5167261 .482329$

H -2.2693611 .9931253 .110551$

SCF Energy (B3LYP/6-31G**//MMFF)= -3245.92637296

$02 \_247$

MMFF Geometry

C -2.258733 2.717180 -1.246807

C $-3.3539053 .293913-0.726404$

C -4.0936382 .8914780 .528655$

O $\quad-3.393348 \quad 1.863607 \quad 1.261693$

C -5.4777612 .3191690 .176145$

C -6.5067952 .5646551 .261162$

C -6.8378141 .6898382 .230317$

C -7.2139363 .8933751 .172199$

C $-6.229550 \quad 0.3468892 .521712$

C $-7.172060-0.8184212 .190122$

C -7.193999-1.162759 0.693274

C $-8.205179-2.2744030 .406295$

C $-8.159106-2.724654-1.062241$

O $-5.878219-1.5995780 .337161$

O $-8.888335-3.956719-1.169373$

C -6.695691-3.030833-1.445327

C $-8.859308-1.723837-1.988947$

O $-6.580617-3.314045-2.837970$

C $-5.705528-1.911612-1.049306$

C $-4.249451-2.398624-1.246695$

O $-5.932506-0.771984-1.881377$

C -2.3912022 .2363062 .099090$

$\begin{array}{lllll}\text { O } & -1.979816 & 3.364427 & 2.311324\end{array}$

C - -1.8517730 .9932952 .689114$

C $-0.597407 \quad 0.973168 \quad 3.160597$

C $0.091269-0.1966153 .709694$

C $-0.706990-1.4655253 .867503$

C $-3.190444-1.483096-0.613577$

C $-1.777350-1.959861-0.905197$

C $0.600704-1.324631-0.504094$

O $-1.528623-2.950994-1.586207$

C $1.168977-2.0698820 .662474$

C $2.311881-1.7168091 .271252$

C $2.924511-2.4726532 .416193$

C $3.363004-1.5681583 .548229$

C $4.651452-1.2129443 .687948$

C $2.322220-1.1421734 .560243$

C $1.400016-0.0852634 .018553$

N $-0.825562-1.134769-0.322214$

H $-1.8241691 .840396-0.777005$

H -3.760167 $4.170577-1.228947$

H $-4.196003 \quad 3.7808871 .163461$

H $-5.3954151 .245642-0.025500$

H -5.861222 $2.764238-0.751947$

H -7.6199801 .9790922 .931740$

H -7.945277 4.0316951 .975475

H -6.4935954 .7149221 .237911$

H -7.7490383 .9753520 .220373$

H -5.2639510 .2152202 .026585$

H -6.0138040 .3207923 .597583$
H $-6.817264-1.6956522 .747068$

H -8.186336 -0.597627 2.544641

H -7.447579 -0.2629710 .122134$

H -9.219380 -1.957928 0.679918

H -7.981730 -3.143702 1.040826

H $-9.796508-3.795163-0.860720$

H $-6.406396-3.950495-0.917548$

H -9.911926 -1.609484 -1.703170

H $-8.867720-2.083047-3.023953$

H -8.404315 -0.731406 -1.971432

H -7.208576 -4.028461 -3.041017

H $-4.133764-3.392244-0.793751$

H -4.039535 -2.503838 -2.318783

H -5.104496 -0.276870 -1.980235

H -2.488302 0.1157722 .672564

H $-0.008473 \quad 1.8880793 .111078$

H -1.543886 -1.306624 4.556642

H $-0.127805-2.3024434 .261821$

H -1.106629 -1.795491 2.902231

H $-3.283843-0.461913-0.996763$

H $-3.319059-1.4546840 .474580$

H $1.036820-0.327942-0.603938$

H $\quad 0.780751-1.885466-1.426578$

H $\quad 0.636426-2.9546101 .002865$

H $2.860101-0.8527370 .904346$

H $3.790531-3.0224322 .026270$

H $2.238334-3.2351352 .805027$

H $4.979686-0.5855914 .509825$

H $5.406467-1.5372932 .979753$

H $2.808316-0.7231035 .451509$

H $1.793756-2.0275654 .923714$

H 1.8902640 .8763933 .859950

H -1.129345 -0.3600330 .259626$

C $-0.1173043 .693555-2.227595$

O $0.7493742 .558578-2.047929$

C $1.7968802 .920008-1.127685$

C $1.6205644 .409897-0.833273$

C $0.1278554 .594148-1.019320$

H $1.6144792 .344782-0.211218$

C $3.1900572 .565760-1.676469$

H 1.9643554 .6741800 .171552

H $2.1724315 .032442-1.547245$

C $-0.2930556 .037498-1.228117$

H $-0.3704104 .200064-0.125228$

C $3.3882281 .067614-1.975513$

O $3.4567553 .318272-2.860679$

H $3.9248282 .886370-0.930583$

H $3.1479120 .481838-1.084457$

O $2.4602320 .643217-2.980455$

C $4.8037000 .697048-2.459362$

C $5.9520701 .093452-1.512455$

H $4.9912501 .153369-3.438936$

O $4.857552-0.715194-2.672165$

H $6.8841030 .710063-1.943711$

H $6.0398282 .185730-1.510550$

H $-0.0233746 .646257-0.358928$

H $0.1919866 .474218-2.107591$

H -1.376054 $6.110800-1.369148$

C $-1.5604693 .239641-2.472647$

H $2.7146903 .177170-3.473044$

H $2.6769991 .111520-3.804160$

H $4.127387-0.948480-3.270819$

H -1.564932 $2.439257-3.222684$

H $-2.1249014 .079256-2.896502$

H $0.2342124 .191472-3.141617$

C $6.884407-1.576209-0.280405$

O $5.695175-0.8397050 .028376$

C $5.8188720 .582808-0.056467$

C $6.928791 \quad 1.0703010 .886786$

C $8.2481830 .346703 \quad 0.637484$

C $8.033477-1.1659960 .648593$

H $7.166417-1.397691-1.324768$

C $6.559877-3.065719-0.119714$

H 4.8822020 .9920330 .339284

O $7.1063082 .476420 \quad 0.752801$

H 6.6170710 .8859601 .923239

H $\quad 8.7101370 .682208-0.298298$

O $9.157244 \quad 0.697470 \quad 1.683174$ 
H $7.822878-1.4886311 .675921$

O $9.241180-1.8114690 .250015$

C $5.668032-3.544959-1.240270$

H $7.462538-3.686562-0.147162$

H $6.067942-3.260050 \quad 0.840779$

H 7.8281442 .7365431 .350497

H 9.9759780 .1936621 .535521

H $9.433531-1.546547-0.665615$

O $4.384721-3.670757-0.799544$

O $6.064885-3.759136-2.378814$

C $3.434945-4.105827-1.777502$

H $3.282991-3.320862-2.523919$

H $3.767231-5.033560-2.253536$

H $2.486297-4.297257-1.269495$

SCF Energy (B3LYP/6-31G**//MMFF) = -3245.91219071

02248

MMFF Geometry

C $1.963021-3.6727352 .595991$

C $2.291503-2.4510162 .152470$

C $3.253888-1.5366662 .864312$

O $2.633435-0.2348042 .956560$

C $4.539085-1.3863682 .033714$

C $5.636176-0.6407062 .764114$

C 5.9299050 .6613282 .579948

C $6.452586-1.4744833 .716917$

C $5.214721 \quad 1.6552361 .707497$

C 6.0605392 .1258580 .517299

C $6.2127261 .070423-0.590868$

C $7.0905101 .614310-1.721333$

C $7.1648680 .647816-2.911924$

O $4.9063910 .766369-1.089380$

O $7.721072 \quad 1.361916-4.026602$

C $5.732307 \quad 0.235772-3.309693$

C $8.109628-0.527515-2.634375$

O $5.756339-0.768810-4.321762$

C $4.872224-0.236454-2.115247$

C $3.396962-0.391979-2.557646$

O $5.367153-1.491858-1.649364$

C $1.939802 \quad 0.0690704 .081041$

O $1.780299-0.6495355 .057967$

C 1.3770311 .4390634 .016125

C $1.374232 \quad 2.192636 \quad 2.904957$

C $0.827422 \quad 3.5458952 .776506$

C 0.2292884 .1808354 .004400

C $2.456522-0.850298-1.437107$

C $1.010933-0.549780-1.772006$

C $-0.6783451 .264735-1.681204$

O $0.262626-1.399324-2.248484$

C $-0.6529502 .758535-1.632796$

C $-1.345190 \quad 3.482797-0.740980$

C $-1.3268564 .982999-0.689407$

C -1.0543635 .5168370 .700358$

C -2.0469656 .0419991 .438426$

C $0.373474 \quad 5.5201661 .202006$

C $0.872046 \quad 4.149951 \quad 1.570124$

N $0.6715370 .767790-1.505169$

H $2.408399-4.0151663 .528656$

H $1.869930-2.0767721 .225409$

H $3.496371-1.9168763 .864228$

H $4.313724-0.8817031 .089711$

H $4.924865-2.3750601 .750618$

H $6.762074 \quad 1.0828693 .142807$

H $7.249072-0.8970594 .198221$

H $5.818322-1.8836244 .509535$

H $6.924600-2.3065823 .184357$

H 4.2468381 .2837561 .360353

H 4.9856562 .5272602 .333461

H 5.5638823 .0100370 .096132

H 7.0496932 .4475190 .866522

H $6.6578790 .164131-0.165389$

H $8.0970041 .848997-1.352905$

H $6.6744402 .567159-2.078605$

H $8.5970051 .692984-3.763387$

H $5.2490041 .115597-3.757539$

H $9.125234-0.163556-2.437323$

H $8.191407-1.183688-3.507798$

H $7.804525-1.129946-1.776206$
H $6.297003-0.428180-5.055109$

H $3.0438410 .570335-2.950611$

H $3.321700-1.119858-3.375323$

H $4.678294-1.931156-1.127052$

H 0.9407321 .7909524 .945592

H 1.8036451 .7856331 .991172

H -0.6112813 .5839874 .375581$

H $-0.1563735 .187463 \quad 3.829330$

H 0.9797814 .2649504 .798278

H $2.549201-1.928436-1.266374$

H $2.694473-0.355384-0.489876$

H $-1.2989800 .828102-0.891226$

H $-1.0633190 .918029-2.645888$

H $-0.039792 \quad 3.267608-2.372606$

H $-1.9727022 .969977-0.015491$

H -2.303328 $5.338178-1.043689$

H $-0.5875825 .398600-1.385222$

H -1.862013 6.4562312 .423817

H $-3.0691926 .066768 \quad 1.075572$

H $0.491608 \quad 6.2106022 .045472$

H 1.0261665 .9318200 .421314

H 1.3222793 .6119330 .735379

H $1.3210801 .331524-0.963159$

C $0.383746-4.2714500 .613616$

O $-0.680078-3.3291680 .878463$

C $-1.909814-3.863457 \quad 0.346127$

C $-1.476219-4.834201-0.737794$

C $-0.266936-5.470186-0.087461$

H $-2.385260-4.4010451 .177255$

C $-2.822022-2.726779-0.134328$

H $-2.250593-5.562823-0.994411$

H $-1.186715-4.296526-1.649233$

C $0.650943-6.175425-1.071846$

H $-0.613321-6.1998020 .657106$

C $-4.085392-3.230031-0.856773$

O $-3.217420-1.9629131 .009070$

H $-2.259785-2.048381-0.785988$

H -3.799027 -3.723854-1.792961

O $-4.698364-4.238416-0.040287$

C $-5.156087-2.162089-1.145168$

C $-4.641476-0.946673-1.933741$

H $-5.610988-1.840090-0.202615$

O $-6.204757-2.791263-1.892683$

H -3.879416 $-0.428708-1.343057$

H $-4.152621-1.295280-2.851958$

H $0.121952-6.992136-1.573801$

H $1.016496-5.488159-1.842520$

H $1.519951-6.599470-0.558854$

C $1.057381-4.6767511 .932358$

H -2.405190 -1.664247 1.452953

H $-5.489613-4.551686-0.511177$

H $-5.834822-3.066716-2.748968$

H $1.660468-5.5773161 .763014$

H $0.281089-4.9391102 .662757$

H $1.093433-3.780383-0.062129$

C $-5.961043 \quad 1.204684-0.222661$

O $-6.5977790 .379114-1.204742$

C $-5.7722270 .035825-2.322307$

C $-5.2996521 .308609-3.037632$

C $-4.6555852 .301062-2.071628$

C $-5.5453632 .537610-0.850522$

H -5.0778970 .6916450 .177105$

C $-6.955523 \quad 1.4072290 .924816$

H $-6.422413-0.498368-3.026865$

$\begin{array}{llll}\text { O } & -4.386440 & 0.976198 & -4.077776\end{array}$

H $-6.165907 \quad 1.788338-3.511349$

H -3.667477 $1.946394-1.764624$

O $-4.4350313 .542363-2.743936$

H $-6.4327613 .111889-1.145134$

$\begin{array}{lllll}\text { O } & -4.832166 & 3.310791 & 0.110665\end{array}$

C -7.200202 $0.110198 \quad 1.659907$

H -6.6053592 .1477851 .652346$

H $-7.922343 \quad 1.7571060 .542960$

H $-4.088764 \quad 1.809408-4.481634$

H $-5.2985543 .887667-3.027934$

H $-4.5468124 .130185-0.328786$

O $-6.108656-0.2144882 .410460$

O $-8.233704-0.5394621 .569158$ 
C $-6.198348-1.4512573 .124486$

H $-6.334478-2.2828582 .426346$

H $-5.260500-1.5996443 .666251$

H -7.019545 -1.413065 3.846298

SCF Energy (B3LYP/6-31G $\left.{ }^{* *} / / M M F F\right)=-3245.91394765$

$02 \_249$

MM̄FF Geometry

C 2.3819131 .4098152 .858602

C 3.7092801 .5873332 .764304

C 4.4539652 .3010901 .656340

O 3.5020652 .9060240 .753991

C 5.3226541 .2790230 .898575

C 6.5376421 .8534530 .198598

C $6.7490251 .812073-1.131576$

C 7.6024152 .4193191 .104866

C $5.8432021 .273535-2.203664$

C $6.285680-0.110340-2.705440$

C $5.941955-1.247626-1.730033$

C $6.492714-2.584808-2.227083$

C $6.063512-3.748150-1.318564$

O $4.516639-1.316480-1.640255$

O $6.349585-4.974824-2.007526$

C $4.534423-3.687780-1.117753$

C $6.869126-3.784255-0.014459$

O $4.103973-4.659457-0.167246$

C $4.016466-2.287842-0.713943$

C $2.471888-2.255347-0.792189$

O $4.420515-2.004401 \quad 0.626267$

C 3.8637124 .0276310 .084021

O 4.9691954 .5486610 .057623

C $2.7166334 .581491-0.667766$

C $1.4629344 .508879-0.192571$

C $0.2756295 .119028-0.794157$

C $0.4053285 .663265-2.192485$

C $1.861902-0.849046-0.674570$

C $0.370440-0.874301-0.957117$

C $-1.4868420 .519412-1.763848$

O $-0.302448-1.901215-0.897092$

C $-1.828398 \quad 1.953917-1.997479$

C $-2.8793272 .558035-1.423538$

C $-3.3307123 .952564-1.746866$

C $-3.3098994 .869965-0.547809$

C -4.3265624 .8858880 .330639$

C $-2.1591645 .840190-0.428346$

C $-0.8606725 .172440-0.067198$

N $-0.1316610 .384245-1.270616$

H 1.7211611 .8053342 .092573

H 4.3401671 .1791873 .552543

H 5.0546163 .0848532 .131893

H 4.6842340 .7444190 .189647

H 5.7019800 .5168591 .593570

H $7.6854342 .219043-1.512313$

H 8.5179082 .6759070 .560865

H 7.2502613 .3297901 .598030

H 7.8752321 .6886541 .873451

H $4.7945911 .263033-1.890938$

H $5.8862551 .976806-3.045389$

H $5.771687-0.294380-3.657976$

H $7.362579-0.104739-2.913878$

H $6.360143-1.015790-0.745280$

H $7.585873-2.547953-2.313649$

H $6.117676-2.780756-3.241465$

H $7.301993-4.993086-2.203834$

H $4.070784-3.965079-2.075033$

H $7.939364-3.890624-0.228489$

H $6.597845-4.6546120 .593179$

H $\quad 6.742329-2.8860470 .593496$

H $4.438555-5.523154-0.464276$

H $2.156922-2.670643-1.758930$

H $2.044107-2.894371-0.009146$

H $3.782407-1.3942941 .028605$

H $2.9679865 .137319-1.564315$

H 1.2915714 .0034940 .756713

H $1.0356536 .558861-2.200292$

H $-0.5524415 .930559-2.644296$

H $0.8505094 .914311-2.857719$

H $2.006982-0.4432650 .330653$
H $2.336926-0.169392-1.390491$

H $-2.1636450 .035683-1.052445$

H -1.550947 -0.030365 -2.709351

H -1.227455 $2.498161-2.721670$

H -3.492096 $1.996647-0.722566$

H $-4.3541843 .891374-2.140597$

H $-2.7353774 .385160-2.560270$

H $-4.3354785 .565976 \quad 1.175738$

H $-5.173910 \quad 4.2173680 .221671$

H -2.368693 6.5779220 .357994

H $-2.0928926 .428261-1.348345$

H $-0.856803 \quad 4.7427840 .935519$

H $0.5260651 .139632-1.443992$

C $0.945592-0.5502393 .482500$

O $-0.253145-0.1109282 .807623$

C $-1.380642-0.8388583 .339458$

C $-0.785434-2.0534494 .031170$

C $0.482621-1.4678974 .615171$

H $-1.849687-0.1696414 .072323$

C $-2.363011-1.1574892 .206806$

H $-1.448798-2.4768994 .791087$

H $-0.546485-2.8387003 .302917$

C $1.507314-2.5163095 .013676$

H $0.219555-0.8711185 .499113$

C -3.518998 -2.080611 2.628963

O -2.9082800 .0783021 .734298$

H -1.817559-1.602181 1.368166

H $-3.122308-3.0636382 .909333$

O $-4.121230-1.5352733 .810363$

C $-4.631158-2.2550041 .576941$

C $-4.127725-2.7396550 .207312$

H -5.181038 -1.314746 1.471874

O $-5.572864-3.2061312 .087996$

H -3.417597 -2.012722 -0.196457

H $-3.581327-3.6818940 .339530$

H $1.101214-3.1764385 .787057$

H $1.798181-3.1379214 .160050$

H $2.412305-2.0464235 .411329$

C $1.7257390 .665783 \quad 3.988896$

H -2.1613810 .6435281 .471616$

H $-4.849547-2.1276414 .064537$

H -5.116620 -4.061026 2.170172

H 2.4799360 .3478854 .718280

H $1.047317 \quad 1.3609104 .498536$

H $1.536503-1.1134282 .748764$

C $-5.576232-0.682136-1.504351$

O $-6.151718-1.847710-0.900528$

C $-5.271177-2.971658-0.807924$

C $-4.803159-3.382416-2.210067$

C $-4.213336-2.204315-2.984789$

C $-5.151244-0.995011-2.945193$

H $-4.706986-0.355276-0.922482$

C $-6.6302590 .429262-1.444304$

H -5.874932 -3.800568 -0.417469

O $-3.849829-4.435685-2.119853$

H $-5.663882-3.775434-2.766520$

H -3.222689-1.943535 -2.597112

O $-4.003331-2.595656-4.344072$

H $-6.029229-1.202008-3.570334$

O $-4.464611 \quad 0.114818-3.516916$

C $-6.9816920 .779894-0.016548$

H -6.297851 $1.346419-1.941650$

H $-7.5547990 .104902-1.937241$

H $-3.556315-4.641157-3.023947$

H $-4.866416-2.828167-4.726936$

H $-5.1134830 .821737-3.669354$

$\begin{array}{llll}\text { O } & -5.923031 & 1.383850 & 0.593793\end{array}$

O $-8.0704120 .546036 \quad 0.492525$

C -6.1206551 .7372081 .966220$

H -6.3656860 .8494972 .557153$

H -5.1873732 .1623482 .345094$

H -6.9103012.489592 2.051154

SCF Energy (B3LYP/6-31G**//MMFF)= -3245.90960095

$02 \_25$

MM̄MF Geometry

C $2.899779-1.2624492 .934300$

C $3.834393-2.1457872 .550667$ 
C $4.606438-2.1507001 .251223$

O $4.352246-0.9647800 .468672$

C $4.142754-3.3428630 .395318$

C $5.156448-3.775605-0.642930$

C $5.134316-3.421760-1.942539$

C $6.215179-4.722456-0.137540$

C $4.213458-2.455008-2.632554$

C $3.154377-3.142089-3.506990$

C $1.883892-3.512961-2.726113$

C $0.885046-4.246384-3.623497$

C $-0.443054-4.514190-2.897476$

O $1.305801-2.291443-2.252782$

O $-1.405313-4.945607-3.871583$

C $-0.949057-3.197585-2.269426$

C $-0.320002-5.677595-1.907730$

O $-2.100906-3.426963-1.461010$

C $0.129923-2.448868-1.452064$

C $-0.381591-1.034818-1.089139$

O $0.396928-3.177714-0.252973$

C $5.096346 \quad 0.1452880 .704859$

O $5.965930 \quad 0.2914401 .546829$

C $4.6317321 .173158-0.252345$

C $4.9268262 .464583-0.049175$

C $4.4992913 .582918-0.893430$

C $3.7206623 .257210-2.142512$

C $0.677142-0.110984-0.469718$

C $0.0970991 .251043-0.130329$

C 0.6096193 .3249251 .152793

O $-1.0155501 .614546-0.502618$

C 1.0008924 .3834970 .170476

C 1.6730295 .4942380 .510255

C $2.0298266 .585333-0.459598$

C $3.4922926 .970282-0.401388$

C 3.8920868 .0648730 .267817

C $4.4776666 .135830-1.189224$

C $4.8076424 .837610-0.504139$

$\begin{array}{llll}\mathrm{N} & 0.965896 & 2.015678 & 0.635513\end{array}$

H $2.640214-0.4258592 .293167$

H $4.061735-2.9750113 .218966$

H $5.677432-2.2248171 .475832$

H $3.194969-3.090176-0.091384$

H $3.924356-4.2141061 .027899$

H $5.899040-3.840279-2.596529$

H $6.919668-5.018081-0.922247$

H $6.794466-4.2554550 .665123$

H $5.753397-5.6355340 .252422$

H $3.749778-1.760119-1.927729$

H $4.842564-1.831656-3.281601$

H $2.878257-2.435650-4.301184$

H $3.575923-4.029267-3.995032$

H $2.159481-4.143864-1.874879$

H $1.313207-5.183652-4.000108$

H $\quad 0.679278-3.633689-4.512353$

H $-1.498843-4.244384-4.538450$

H -1.273292 -2.544062 -3.091363

H $-0.032135-6.596113-2.433494$

H -1.282480 -5.895538 -1.431603

H $\quad 0.419168-5.500038-1.123789$

H -2.758422 -3.882111-2.014601

H $-0.752771-0.545547-1.999448$

H - $1.226506-1.115673-0.394008$

H $0.720620-2.5683580 .428227$

H $4.0296750 .836816-1.089199$

H $5.5185542 .731620 \quad 0.825289$

H $4.3291702 .654048-2.825500$

H $3.396174 \quad 4.138043-2.699675$

H $2.8108732 .697912-1.898399$

H $1.076072-0.5508100 .448276$

H $1.5061510 .044346-1.168660$

H 1.1186233 .4437232 .114423

H -0.4711013 .3620101 .321901$

H $0.6925334 .245442-0.863002$

H $1.9522195 .647051 \quad 1.550347$

H $1.4063637 .457029-0.220757$

H $1.7686326 .307870-1.488394$

H $4.934736 \quad 8.3641370 .291221$

H 3.1860728 .6834400 .812008

H $5.4211746 .684134-1.314277$
H $4.1005546 .000850-2.206510$

H 5.3708374 .9689650 .420858

H 1.8935521 .6583990 .843968

C $0.676344-1.7488873 .959350$

O $-0.030109-0.6055973 .436963$

C $-1.360996-0.5820754 .004919$

C -1.517555 -1.891060 4.763930

C $-0.094665-2.1588555 .210922$

H -1.3728430 .2669124 .700008$

C $-2.369721-0.3610112 .872052$

H $-2.222116-1.8118555 .597090$

H - $1.865112-2.6905814 .097568$

C $0.139879-3.5956555 .644462$

H $0.149228-1.4835966 .041936$

C $-3.841280-0.5443213 .278784$

O $-2.196737 \quad 0.9748732 .387906$

H -2.124478-1.031274 2.040691

H -4.018848 -1.585895 3.570755

$\begin{array}{llll}\text { O } & -4.088317 & 0.251350 & 4.444582\end{array}$

C $-4.861076-0.1298842 .197674$

C $-4.650626-0.8363550 .847839$

H -4.8361280 .9580602 .070808$

O $-6.171721-0.4409302 .683559$

H -3.646562 -0.6147690 .476274$

H $-4.703750-1.9214801 .000513$

H $-0.501765-3.8498686 .494576$

H -0.080157 -4.299949 4.835131

H $1.179496-3.7485635 .949926$

C $2.136258-1.3793814 .225611$

H -1.2513801 .0937412 .195165$

H -5.0236390 .1328964 .683520$

H -6.229267 -1.406194 2.787050

H $2.604816-2.1316374 .870251$

H $2.198648-0.4166154 .746880$

H $\quad 0.611938-2.5392213 .198847$

C $-4.6647431 .626546-0.931208$

O $-5.8123460 .982496-0.363773$

C $-5.694472-0.436835-0.220557$

C $-5.459554-1.078089-1.595157$

C $-4.261910-0.459116-2.312483$

C $-4.3707261 .066698-2.330863$

H $-3.8021301 .464574-0.274961$

C $-4.964333 \quad 3.129888-0.955830$

H $-6.668585-0.7908600 .139468$

O $-5.273828-2.482500-1.454542$

H $-6.355271-0.932313-2.212899$

H -3.322207 -0.786256 -1.856076

O $-4.240727-0.945099-3.656352$

H $-5.1685431 .353895-3.027020$

O $-3.1572761 .609607-2.844101$

C $-3.7064503 .962899-0.955215$

H -5.600703 3.393319-1.809158

H $-5.5391193 .424981-0.068550$

H $-5.103817-2.843166-2.341653$

H -3.496086 -0.511909-4.108234

H $-2.4448951 .393174-2.218305$

O $-3.5484614 .576342-2.161955$

O -2.9466204 .0455380 .001333$

C $-2.3644475 .368492-2.297490$

H $-2.3329036 .145943-1.528031$

$\mathrm{H}-1.4782834 .729794-2.239218$

H -2.387001 $5.848381-3.279470$

SCF Energy (B3LYP/6-31G**//MMFF) $=-3245.92562275$

$02 \_250$

MM̄MF Geometry

C 3.4790152 .4892272 .591762

C 3.2169372 .5827791 .279237

C 3.4856213 .8472020 .497987

O $2.2901104 .241279-0.217666$

C $4.5673403 .646319-0.580570$

C $5.9110423 .168857-0.072848$

C $6.4335781 .948762-0.310560$

C 6.6968954 .1862370 .713550

C $5.8063820 .808365-1.066699$

C $5.812110-0.481762-0.233683$

C $4.932273-1.605953-0.805982$

C $5.358935-2.057362-2.203680$ 
C $4.417468-3.135067-2.769451$

O $3.583545-1.132571-0.830440$

O $4.698703-3.269907-4.171786$

C $2.958153-2.641446-2.645718$

C $4.678422-4.510130-2.143263$

O $2.057166-3.678002-3.029823$

C $2.614175-2.108202-1.237481$

C $1.240509-1.408097-1.166927$

O $2.576040-3.205236-0.317091$

C 1.2956874 .8686400 .463022

O 1.2563145 .1240621 .654619

C $0.2378775 .178019-0.524614$

C $-1.0210155 .406379-0.123297$

C $-2.1646715 .639713-1.010624$

C -1.872465 $5.869622-2.471656$

C $1.029601-0.271832-2.171261$

C $0.237397 \quad 0.872537-1.570357$

C $-1.8716461 .410627-0.369690$

O $0.7036622 .008752-1.517522$

C $-2.7506472 .175276-1.306224$

C $-4.0534272 .390755-1.068547$

C $-4.9478593 .227714-1.938430$

C $-5.5142424 .419954-1.192756$

C $-6.7109554 .346872-0.586071$

C $-4.7249665 .714290-1.209977$

C $-3.4089575 .598887-0.488525$

N -1.015968 $0.504431-1.110741$

H 3.8769193 .3728103 .089939

H 2.8265351 .7320820 .729105

H 3.7831554 .6745281 .155050

H $4.7159804 .602256-1.103266$

H $4.1729522 .993299-1.367209$

H 7.4279541 .7344330 .079683

H 7.6877493 .8170290 .998781

H 6.8431695 .0945260 .119900

H $6.168774 \quad 4.4529101 .634130$

H $4.7832911 .038968-1.370597$

H $6.3848560 .654318-1.984769$

H $6.842121-0.843650-0.123311$

H $5.445511-0.2473740 .774529$

H $4.995282-2.446726-0.104952$

H $6.395853-2.415049-2.196506$

H $5.339298-1.204543-2.894837$

H $5.635542-3.512617-4.267603$

H $2.838432-1.837573-3.383106$

H $5.711869-4.825791-2.330605$

H $4.044108-5.278410-2.599279$

H $4.520265-4.528059-1.063128$

H $1.166110-3.293944-3.081978$

H $0.433902-2.144688-1.268060$

H $1.141007-0.998635-0.153868$

H $1.996740-2.9670220 .424871$

H $0.5200185 .156398-1.571650$

H -1.245244 5.3683580 .941588

H -2.752581 $6.133733-3.060364$

H $-1.4440284 .969937-2.926528$

H -1.163811 $6.696691-2.594949$

H $\quad 1.9885340 .146588-2.498410$

H $0.509330-0.634345-3.064705$

H -1.2444442 .1058240 .198064$

H -2.4516450 .8079770 .334695$

H -2.289657 $2.615775-2.186452$

H $-4.5063231 .960340-0.178809$

H $-5.7641032 .587361-2.297398$

H -4.425802 $3.572295-2.839613$

H -7.137096 $5.200182-0.069085$

H -7.289309 $3.428756-0.589651$

H $-5.2944416 .516924-0.723294$

H -4.613452 $6.033749-2.249074$

H $-3.516000 \quad 5.424970 \quad 0.583089$

H - $-1.342038-0.445962-1.251612$

C $2.785638-0.0062512 .920543$

O $1.355324 \quad 0.0965632 .762381$

C $0.727572-1.0373703 .401699$

C $1.830098-2.0677953 .576715$

C $3.025851-1.1891713 .863986$

H $\quad 0.376623-0.6776594 .377153$

C $-0.451701-1.5200932 .549059$
H $1.620299-2.7779734 .382126$

H $1.985740-2.6350442 .651934$

C $4.356465-1.8889973 .639458$

H $2.970229-0.8552544 .909026$

C -1.097313 -2.824485 3.045622

O $-1.437648-0.4854562 .518977$

H $-0.107740-1.6447361 .520910$

H $-0.351168-3.6271113 .072351$

O $-1.526085-2.6199614 .396741$

C $-2.311433-3.2910532 .217749$

C $-1.981808-3.5240850 .731909$

H $-3.130127-2.5716232 .323510$

O $-2.783386-4.5180502 .786022$

H - $1.662357-2.5808130 .280321$

H -1.133365 -4.216329 0.661132

H $4.454232-2.7518784 .306304$

H $4.451340-2.2484622 .609184$

H $5.193027-1.2116503 .837692$

C 3.3405281 .2993753 .501372

H -1.784823 -0.383723 3.421790

H $-1.945609-3.4448514 .695916$

H -2.080633 -5.182618 2.684808

H $4.341310 \quad 1.1102003 .909411$

H 2.7053701 .6118274 .340487

H $3.208120-0.2170781 .932018$

C $-4.331676-2.053434-0.514745$

O $-4.361416-3.3501520 .091863$

C $-3.162888-4.112646-0.072553$

C $-2.874398-4.317407-1.566127$

C $-2.864585-3.000222-2.339747$

C $-4.116296-2.177796-2.027612$

H -3.525334 -1.457471 -0.072188

C $-5.663163-1.366634-0.192724$

H $-3.370331-5.1039280 .349813$

O $-1.631534-4.991542-1.736110$

H $-3.652190-4.966952-1.988899$

H -1.950240 -2.434305 -2.132487

O $-2.835770-3.308598-3.735291$

H $-4.990330-2.648126-2.495972$

O $-3.976363-0.873727-2.584789$

C $-5.787951-1.0888331 .287165$

H $-5.780914-0.417716-0.727130$

H $-6.505971-2.008009-0.478211$

H -1.481917 -5.089041 -2.692174

H $-2.729445-2.472215-4.218968$

H $-3.959862-0.965588-3.552197$

O $-4.931619-0.0923371 .650116$

O $-6.553600-1.6891072 .030211$

C -4.9355040 .2510093 .038794$

H -4.2155341 .0597293 .190187$

H -5.9267910 .6020473 .340390$

H -4.628668 -0.609131 3.641524

SCF Energy (B3LYP/6-31G**//MMFF) $=-3245.89304757$

$02 \_251$

MM̄MF Geometry

C -0.362529 -3.624967 1.405213

C $0.371500-2.6017390 .941927$

C $0.967563-1.5357671 .823090$

O $0.468981-0.2749351 .314738$

C $2.509080-1.5556081 .729210$

C $3.192297-1.6393803 .079372$

C $3.898582-0.6454153 .652915$

C $3.091365-2.9806153 .761919$

C 4.1209890 .7532583 .147580

C 5.5797371 .0168582 .750250

C 6.0072090 .2851101 .468353

C 7.4781020 .5659881 .154052

C $7.912141-0.058885-0.180164$

O $5.184010 \quad 0.757513 \quad 0.397534$

O $9.1828870 .521455-0.511290$

C $6.8984420 .342967-1.273687$

C $8.142818-1.568835-0.046567$

O $7.165236-0.339053-2.497266$

C $5.4243040 .116516-0.861268$

C $4.4811900 .756752-1.908616$

O $5.170367-1.288493-0.797405$

C 0.4787620 .7903282 .156605 
O $0.820905 \quad 0.810875 \quad 3.327466$

C $0.027068 \quad 1.9636311 .386036$

C $0.553250 \quad 3.163004 \quad 1.665970$

C $0.214866 \quad 4.3864780 .952644$

C 1.4092915 .1009880 .385870

C $3.0062690 .797362-1.485312$

C $2.1210011 .423266-2.548936$

C $-0.2760381 .949603-2.995819$

O $2.5483391 .852244-3.617044$

C $-0.460432 \quad 3.413028-2.747817$

C $-1.6388713 .962505-2.416076$

C -1.853141 $5.440452-2.258386$

C $-2.4891505 .808267-0.937922$

C $-3.8171925 .986797-0.838103$

C $-1.575758 \quad 6.0710940 .236966$

C -1.0625854 .8064110 .869010$

N $0.789776 \quad 1.438309-2.157369$

H $-0.533191-3.6881232 .478699$

H $0.565706-2.504702-0.122458$

H $0.613260-1.6623072 .853878$

H $2.859651-0.6826361 .171750$

H $2.855201-2.4198301 .145155$

H $4.366603-0.8435974 .616827$

H $3.649212-3.0117724 .703885$

H $2.048561-3.2209743 .990563$

H $3.495431-3.7663753 .115130$

H 3.4534391 .0101092 .321110

H 3.8558181 .4393063 .962289

H 5.6856202 .0985302 .594819

H $6.2460080 .742545 \quad 3.577573$

H $5.848066-0.7905051 .601943$

H 8.1243730 .2173461 .969301

H 7.6347031 .6523841 .093393

H $9.5048850 .099918-1.325789$

H $7.0409851 .413908-1.475100$

H $8.938122-1.7692880 .681485$

H $8.486090-2.002745-0.992202$

H $7.252307-2.1120790 .276789$

H $8.058050-0.087617-2.786858$

H $4.7988341 .791206-2.095245$

H $4.5617820 .214624-2.859347$

H $4.227605-1.452121-0.953468$

H $-0.696386 \quad 1.8137260 .592405$

H 1.3172553 .2657502 .433465

H 2.0273745 .5118041 .191540

H $1.1441145 .921835-0.284212$

H $2.0254424 .409191-0.200719$

H $2.630427-0.213546-1.297176$

H $2.892877 \quad 1.384809-0.566779$

H -1.176193 $1.378536-2.755827$

H $-0.022326 \quad 1.780904-4.047265$

H $0.4046354 .055586-2.893476$

H -2.510758 $3.322035-2.302407$

H -2.498580 5.770795 -3.083204

H -0.915585 $5.997112-2.380723$

H -4.278883 6.2863720 .096832

H -4.473159 $5.831120-1.688220$

H -2.116932 6.6240701 .016461

H $-0.7734996 .745735-0.075856$

H -1.836339 4.2027421 .343800

H $0.5399891 .077322-1.242208$

C $-0.839872-4.688718-0.916575$

O $-1.508373-3.526069-1.443949$

C -2.373522 -3.927417 -2.519115

C $-1.873916-5.297659-2.952466$

C $-1.476529-5.894385-1.615570$

H -3.377186 -4.038196 -2.089890

C $-2.404240-2.854255-3.613264$

H -2.642571 -5.877562 -3.471810

H $-0.998076-5.225140-3.607173$

C $-0.553460-7.094803-1.735150$

H -2.388288 -6.199068 -1.084001

C -2.656733-1.420123-3.100595

O $-1.147522-2.841634-4.303396$

H -3.154312 -3.123658 -4.365760

H $-1.752134-1.048064-2.604145$

O $-2.857867-0.599420-4.257335$

C $-3.861232-1.283891-2.152201$
C $-4.0941870 .160365-1.670460$

H $-3.693919-1.919923-1.278456$

O $-5.039955-1.761373-2.798363$

H $-3.1465430 .567836-1.302492$

H $-4.4134080 .784047-2.513663$

H $-1.042916-7.902564-2.289010$

H $0.371883-6.838688-2.262036$

H $-0.282939-7.478499-0.746340$

C $-1.007991-4.728890 \quad 0.606721$

H $-0.452161-2.669905-3.645124$

H $-2.8259460 .329492-3.975328$

H -5.167421 -1.232921 -3.604863

H $-0.615670-5.6797730 .986770$

H $-2.079351-4.7020300 .845153$

H $\quad 0.219655-4.605207-1.188307$

C $-3.811139-0.3147351 .340266$

O $-4.919604-0.6669410 .507103$

C $-5.1748920 .244911-0.567796$

C $-5.4139441 .660586-0.020205$

C -4.2973302 .1150590 .918631$

C -4.0313001 .0556411 .987968$

H $-2.901783-0.279717 \quad 0.726612$

C -3.575172 -1.447593 2.349123

H $-6.115254-0.085078-1.027266$

O $-5.5429212 .603517-1.079191$

H -6.3585421 .6746440 .539188$

H -3.3818062 .3542910 .365439$

O $-4.697721 \quad 3.318761 \quad 1.577179$

H -4.8798461 .0301372 .681333$

O $-2.878927 \quad 1.4154642 .742421$

C $-4.709133-1.6608763 .330273$

H -3.445554 -2.394989 1.812715

H $-2.657471-1.2404722 .912166$

H $-6.2761832 .307847-1.645461$

H -4.9364553 .9601490 .886531$

H -3.0289572 .3094253 .094947$

O $-4.220841-2.2756404 .448246$

O $-5.878948-1.3630163 .132089$

C $-5.186716-2.5619275 .464698$

H -5.646706 -1.635619 5.821962

H $-5.947083-3.2491835 .081561$

H $-4.669400-3.041367 \quad 6.299927$

SCF Energy (B3LYP/6-31G**//MMFF) $=-3245.89751923$

02_252

MMFF Geometry

C $0.130280 \quad 4.321657 \quad 0.620827$

$\begin{array}{llll}\text { C } & 1.366496 & 3.895705 & 0.324947\end{array}$

C $1.8242653 .607033-1.082168$

O $2.1286042 .192216-1.148526$

C $3.0952264 .405781-1.405781$

C $3.5661284 .208224-2.833715$

C $4.6014043 .425829-3.197894$

C $2.8123194 .995842-3.874282$

C $5.4600212 .574414-2.306056$

C $5.5315161 .124764-2.806298$

C $6.3289750 .246879-1.832768$

C $6.562606-1.149177-2.408558$

C $7.291454-2.057817-1.405954$

O $5.5742220 .146352-0.620802$

O $7.195768-3.407972-1.884362$

C $6.552485-2.003404-0.052509$

C $8.787165-1.732271-1.318953$

O $7.275115-2.708512 \quad 0.954235$

C $6.236425-0.567198 \quad 0.429825$

C $5.280405-0.6249411 .642908$

$\begin{array}{lllll}\text { O } & 7.447951 & 0.078986 & 0.822409\end{array}$

C $1.0740611 .350078-1.339606$

O $-0.0818421 .660802-1.582326$

C $1.552845-0.032473-1.124050$

C $0.658198-0.976229-0.792141$

C $0.953800-2.366956-0.443993$

C $2.354061-2.864779-0.679710$

C 4.6887930 .7294432 .056359

C $3.6426780 .520493 \quad 3.133221$

C $1.331633-0.3621413 .454250$

$\begin{array}{llllll}\text { O } & 3.903838 & 0.670820 & 4.324397\end{array}$

C $1.329253-1.8594773 .426246$ 
C $0.226272-2.5993603 .235366$

C $0.239905-4.1010723 .177212$

C $-0.537871-4.6603572 .003030$

C $-1.688220-5.3260512 .202069$

C $0.056286-4.5291690 .612642$

C $-0.033812-3.1231210 .080218$

$\begin{array}{llll}\text { N } & 2.422674 & 0.106358 & 2.619286\end{array}$

H $-0.5731324 .518931-0.184748$

H $2.060348 \quad 3.667272 \quad 1.130715$

H $1.0424193 .851232-1.812341$

H $3.8894394 .145917-0.697391$

H $2.9111735 .477211-1.248089$

H $4.8695153 .389040-4.253042$

H $3.2253014 .859385-4.879413$

H $1.7630984 .686975-3.906098$

H $2.8541636 .065700-3.644765$

H $6.4698903 .001886-2.292517$

H $5.1023032 .579591-1.273792$

H $4.5129950 .728757-2.909923$

H $5.999512 \quad 1.103494-3.798344$

H $7.2863330 .740049-1.626466$

H $7.119455-1.094959-3.352279$

H $5.595444-1.609311-2.655771$

H $7.598582-3.441605-2.768986$

H $5.602364-2.540000-0.182572$

H $9.261133-1.844578-2.301436$

H $9.305765-2.432976-0.655157$

H $8.985697-0.716596-0.970765$

H $7.439838-3.6060390 .617691$

H $4.449178-1.2990251 .408360$

H $5.800362-1.0557082 .508500$

H 7.2380080 .7880831 .450416

H $2.619381-0.217514-1.173545$

H $-0.391437-0.693368-0.716625$

H $3.043250-2.4159900 .039734$

H $2.691251-2.614451-1.692062$

H $2.447536-3.949802-0.595405$

H 5.4591871 .3993152 .451771

H $4.220806 \quad 1.2415431 .207984$

H $0.4064830 .057803 \quad 3.048025$

H $1.469108-0.0070264 .479944$

H $2.284365-2.3614653 .565156$

H $-0.735240-2.1029173 .125234$

H $-0.183525-4.4712534 .119994$

H $1.266224-4.4870923 .135657$

H -2.237853 -5.7668941 .377761$

H -2.106579 -5.4493073 .195661$

H $-0.475774-5.183647-0.089553$

H $1.084052-4.9005150 .651915$

H $-1.025753-2.6793730 .177279$

H $2.334309-0.0305611 .617149$

C $-1.255575 \quad 3.2441812 .370091$

O -2.4058813 .1995031 .506655$

C $-3.496357 \quad 2.575792 \quad 2.221719$

C -2.9588582 .2332343 .606474$

C - $-1.8407513 .247387 \quad 3.776752$

H -4.2813003 .3386512 .289992$

C -3.9760191 .3790291 .392215$

H -3.727774 2.306826 4.381334

H -2.555512 1.2128973 .629059

C -0.8535892 .8829994 .870437$

H -2.283733 $4.227796 \quad 3.997222$

C -5.0427520 .5071002 .073981$

$\begin{array}{llll}\text { O } & -4.516080 & 1.889669 & 0.167735\end{array}$

H -3.111299 0.7634841 .118320

H -4.6110890 .0071352 .948827$

O -6.0777501 .3663912 .568119$

C $-5.701192-0.5350171 .145587$

C $-4.700573-1.4736450 .449367$

H $-6.322321-0.0167750 .407138$

O $-6.601822-1.3248811 .930556$

H -3.955496 $-0.882781-0.089308$

H $-4.161919-2.0516841 .210661$

H -1.3585342 .8277065 .840375$

H $-0.384931 \quad 1.9124304 .681101$

H -0.0592503 .6318034 .946653$

C -0.3949894 .4585842 .023131$

H -3.823486 2.427576 -0.253115
H -6.7501290 .8006742 .985271$

H $-6.076455-1.8047172 .593507$

H 0.4323604 .5556832 .735060

H -0.9860695 .3803122 .077141$

H -0.6863842 .3199862 .197152$

C $-5.531429-0.968318-2.425814$

O $-6.224859-1.784753-1.474686$

C $-5.381240-2.455898-0.533301$

C $-4.405890-3.374826-1.281959$

C $-3.609071-2.615452-2.343218$

C $-4.550946-1.815842-3.245995$

H $-4.989541-0.172296-1.902020$

C $-6.585136-0.311896-3.323498$

H $-6.038716-3.0990050 .065020$

O $-3.527546-3.998838-0.355020$

H $-4.977995-4.172607-1.773513$

H $-2.853450-1.963694-1.888969$

O $-2.896967-3.548066-3.159154$

H $-5.102608-2.511613-3.890696$

O $-3.780596-0.976479-4.104111$

C $-7.4198470 .673914-2.540717$

H $-6.1354890 .218520-4.170277$

H -7.267589-1.064000 -3.737705

H $-3.001705-4.655471-0.841559$

H -2.214116 $-3.961368-2.604466$

H $-3.286939-0.351409-3.546300$

O $-6.712519 \quad 1.820441-2.329609$

O $-8.5579260 .444875-2.152152$

C $-7.3837012 .823148-1.560288$

H $-6.7099053 .677394-1.453761$

H $-8.2904773 .152580-2.076106$

H -7.622351 $2.439082-0.563668$

SCF Energy (B3LYP/6-31G**//MMFF) $=-3245.90888288$

$02 \_253$

MMFF Geometry

C $-0.6497582 .499063 \quad 1.206822$

C $-1.161334 \quad 1.7126532 .168371$

C -0.4903050 .5063562 .791002$

$\begin{array}{llll}\text { O } & 0.841340 & 0.346737 & 2.254510\end{array}$

C $-1.292050-0.7625152 .446369$

C $-0.952247-1.9344313 .344687$

C $-0.104252-2.932474 \quad 3.028834$

C $-1.675752-1.9517484 .667222$

C $0.717141-3.0874831 .779716$

C $2.210079-3.1711032 .122652$

C $3.086760-3.303762 \quad 0.870000$

C $4.559935-3.4319121 .262724$

C $5.484087-3.462934 \quad 0.037612$

O $2.897369-2.1359660 .066171$

O $6.820194-3.269494 \quad 0.526114$

C $5.130925-2.276276-0.883073$

C $5.462790-4.832749-0.650537$

O $5.851758-2.345864-2.111500$

C $3.615862-2.147894-1.172939$

C $3.342156-0.810737-1.897754$

O $3.203816-3.228271-2.011417$

C $1.8998490 .712673 \quad 3.023917$

$\begin{array}{lllll}\text { O } & 1.889017 & 1.032906 & 4.200570\end{array}$

C 3.0863140 .7186412 .140740

C 4.2762741 .1290122 .601170

C $5.4748411 .261226 \quad 1.775906$

C 6.7214710 .7327882 .426038

C $1.856654-0.458468-2.054257$

C $1.7236370 .895662-2.721624$

C $2.182904 \quad 3.306909-2.292055$

O $1.493557 \quad 1.004875-3.923536$

C $3.6583683 .458503-2.503315$

C $4.4588344 .190898-1.713835$

C $5.9532704 .233270-1.864408$

C $6.676678 \quad 3.643705-0.669564$

C $7.481620 \quad 4.399597 \quad 0.095914$

C $6.5248742 .158635-0.400293$

C 5.4140941 .8708550 .574609

N $1.9472681 .945950-1.844229$

H 0.3525512 .2924650 .838922

H $-2.160251 \quad 1.9154652 .545996$

H -0.4601970 .6582423 .876312$ 
H -1.137516 -1.028304 1.394271 H $-2.370137-0.5716872 .539515$ H $\quad 0.029760-3.7353773 .753137$ H -1.413317 -2.824275 5.274912 H -1.430588 -1.059204 5.251150 H -2.758658 -1.975868 4.506729 H $0.408034-4.0104431 .275265$ H $0.544684-2.2702731 .074836$ H $2.497291-2.2682092 .675554$ H $2.388330-4.0273812 .785451$ H $2.758244-4.1866130 .309175$ H $4.721389-4.3192891 .887673$ H $4.844037-2.5724421 .886997$ H $7.430710-3.339774-0.226957$ H $5.465527-1.359878-0.377050$ H $5.809505-5.6110580 .039952$ H $6.151652-4.859479-1.502191$ H $4.469903-5.119702-1.003094$ H $6.799543-2.308531-1.900066$ H $3.825642-0.000097-1.338921$ H $3.801319-0.826567-2.894681$ H $2.396958-2.972407-2.484936$ H 2.9455250 .4312151 .104542 H $4.397401 \quad 1.4034263 .646588$ H $6.607233-0.3333922 .652808$ H 6.9211911 .2639393 .363465 H 7.6126100 .8322891 .803143 H $1.332555-1.192600-2.674000$ H $1.348489-0.429322-1.083959$ H $1.8014313 .979228-1.517345$ H $1.6440423 .496150-3.225376$ H $4.0951862 .892162-3.323055$ H $4.0280364 .752652-0.888303$ H $6.2458525 .279763-2.017102$ H $6.2744263 .697321-2.766575$ H 8.0187913 .9750820 .937743 H $7.6187355 .457998-0.099009$ H $7.4828901 .752952-0.061324$ H $6.3113651 .634905-1.340605$ H 4.4374592 .2152510 .237716 H $2.1895231 .730369-0.881689$ C $-2.7995293 .901494 \quad 0.817712$ O -3.5875742 .7380170 .509228$ C -4.882202 3.1570050 .041444 C -4.8914264 .6837130 .089736$ C $-3.4165615 .018834-0.020894$ H -4.955644 2.833025-1.002894 C $-5.9851542 .487558 \quad 0.872678$ H $-5.4895095 .115274-0.719226$ H -5.2980725 .0629821 .033967$ C -3.0758526 .4193480 .456935$ H -3.114019 4.911065 -1.071188 C -5.8699940 .9543870 .995648$ O H -6.9653442 .7598160 .464318$ H $\quad-5.0202880 .6989401 .641429$ O C $-5.747271 \quad 0.204826-0.344590$ C $-5.785160-1.325705-0.172868$ H $-4.8072250 .491477-0.826330$ O $-6.8044640 .601271-1.217068$ H -5.052453 $-1.610470 \quad 0.589845$ H $-6.773201-1.6251430 .196784$ H -3.613765 $7.168401-0.133484$ H $-3.347314 \quad 6.561470 \quad 1.508397$ H $-2.004315 \quad 6.6176120 .355883$ C $-1.3260193 .642434 \quad 0.493708$ H -5.0772722 .8352922 .576933$ H -6.870938 -0.3736652 .036273$ H $-7.6450710 .396621-0.772496$ H -0.7432084 .5496890 .690318$ H -1.236164 $3.431980-0.579978$ H $-2.923520 \quad 4.1102281 .888329$ C -3.108283 -2.084739-1.414449 O $-4.295828-1.695602-2.113891$ C $-5.519784-2.091284-1.488491$ C $-5.557857-3.619615-1.343138$ C $-4.325147-4.151155-0.615598$
C $-3.048309-3.607789-1.255303$

H $-3.086467-1.603286-0.429098$

C $-1.913745-1.569959-2.223266$

H -6.321744 -1.812721 -2.183710

O $-6.727217-4.038834-0.648007$

H $-5.594398-4.077963-2.340116$

H $-4.357436-3.9170170 .454921$

O $-4.300123-5.577235-0.712688$

H -2.897652 -4.084766 -2.232299

O $-1.932123-3.948315-0.436111$

C $-1.829097-0.066409-2.130258$

H $-0.964550-1.968860-1.849358$

H -1.996039-1.863251 -3.276708

H -7.495516 -3.704006 -1.141326

H $-5.134887-5.906744-0.337538$

H $-1.934135-4.915028-0.329280$

O $\quad-2.220351 \quad 0.505541-3.303025$

O $-1.4775760 .528557-1.120362$

C $-2.2099581 .936406-3.319786$

H $-2.9147422 .327765-2.580371$

H $-1.2007232 .312733-3.127749$

H -2.524124 2.267228 -4.313126

SCF Energy $($ B3LYP/6-31G**//MMFF $)=-3245.90318360$

02_254

MM̄MF Geometry

C $-0.563529-2.3601970 .556192$

C $0.049308-2.2041891 .740874$

C $-0.299559-1.1677212 .788603$

O $-1.571988-0.5470142 .500341$

C $0.771827-0.0638862 .781720$

C 0.6464430 .8983183 .945583

C 0.0827362 .1192343 .859821

C 1.2563360 .4291195 .239690

C -0.5977692 .7159332 .659123$

C 0.3478513 .6181871 .860088

C -0.2535454 .1592680 .552037$

C -1.4428535 .0895950 .797650$

C -2.052097 $5.589021-0.519314$

O $-0.6466053 .045396-0.253999$

O $-3.3214786 .191225-0.219099$

C $-2.3383424 .370249-1.423312$

C $-1.1971336 .687204-1.161341$

O $-2.7638604 .787925-2.718672$

C $-1.1476763 .387226-1.552735$

C $-1.5581562 .075627-2.259252$

O $-0.1115473 .937829-2.375389$

C $-2.697117-1.1625702 .950451$

O $-2.765343-2.1554513 .656095$

C $-3.847930-0.4433242 .359681$

C $-5.034294-1.0634122 .281942$

C $-6.240141-0.5467701 .630287$

C $-6.2003360 .861151 \quad 1.097173$

C $-2.4397441 .140551-1.426965$

C $-2.934967-0.050665-2.230994$

C $-4.398562-2.061705-1.983792$

O $-2.706032-0.198192-3.428206$

C $-5.856144-1.721895-2.039386$

C $-6.821268-2.486221-1.505750$

C $-8.280938-2.129161-1.544250$

C $-8.942816-2.195387-0.183312$

C $-9.843781-3.1516060 .099483$

C $-8.617505-1.1112250 .825765$

C $-7.308561-1.3644631 .524098$

N $-3.684537-0.916968-1.447811$

H - $1.389294-1.6981550 .307561$

H $0.885938-2.8470702 .001011$

H $-0.337881-1.6547023 .770616$

H $0.7244260 .488593 \quad 1.835068$

H $1.776508-0.5074472 .809831$

H $\quad 0.079793 \quad 2.753437 \quad 4.744843$

H 1.1668781 .1756596 .036018

H $\quad 0.763097-0.4838335 .587461$

H $2.3220860 .218620 \quad 5.103037$

H -1.011785 1.9342942 .015842

H -1.4577433 .2910233 .020361$

H 0.6852604 .4550332 .483989

H 1.2384223 .0319911 .601038 
H $0.5460924 .699790 \quad 0.031933$ H $-1.151277 \quad 5.9348041 .433270$ H -2.230654 4.5573581 .346321 H $-3.169607 \quad 6.9088120 .419766$ H -3.187986 $3.839138-0.976977$ H -1.131005 $7.557712-0.497403$ H $-1.6526587 .052307-2.088335$ H $-0.176576 \quad 6.365367-1.378939$ H $-3.521340 \quad 5.385417-2.596265$ H -2.038822 $2.309708-3.217955$ H $-0.6495411 .521643-2.530883$ H $-0.4229394 .776892-2.750467$ H -3.668831 0.5409831 .941886 H -5.120716 -2.068706 2.692949 H $-5.897717 \quad 1.5618561 .883577$ H -7.166353 1.2164440 .733022 H $-5.492000 \quad 0.9406290 .266455$ H $-1.8765940 .763953-0.565170$ H $-3.322426 \quad 1.669909-1.055287$ H $-4.196382-2.909705-1.322015$ H -4.030610 -2.298124 -2.986565 H -6.130066 -0.797394 -2.542946 H $-6.551894-3.422877-1.023061$ H $-8.772953-2.823362-2.237757$ H -8.433658 -1.126795 -1.963521 H - $10.340391-3.1926831 .063285$ H -10.101861 -3.917902 -0.623839 H $-9.398490-1.068088 \quad 1.595925$ H $-8.653090-0.1450420 .315988$ H -7.241436 -2.358280 1.970165 H $-3.828214-0.692503-0.468425$ C $0.954566-4.278907-0.313746$ O $2.176744-3.522627-0.218490$ C $3.232294-4.254854-0.868189$ C $2.637738-5.597366-1.285030$

C $1.176297-5.246976-1.475662$

H $3.491285-3.690043-1.770669$

C $4.455164-4.3688800 .049574$

H $3.106629-5.991075-2.192139$

H $2.747455-6.355259-0.500814$

C $0.258284-6.457018-1.465362$

H $1.066031-4.717943-2.431929$

C $4.995183-3.0260040 .579355$

O $4.093322-5.1616551 .189411$

H $5.248002-4.922127-0.467977$

H $4.283526-2.6040401 .299093$

O $6.182627-3.3141121 .331622$

C $5.322086-1.967469-0.491200$

C $6.007882-0.7293940 .119347$

H $4.401899-1.663878-1.001428$

O $6.206300-2.515894-1.468373$

H $5.418155-0.3830910 .974520$

H $6.995293-1.0171860 .500353$

H $0.523355-7.147204-2.272943$

H $\quad 0.326489-7.004332-0.519181$

H $-0.784984-6.158774-1.607263$

C $-0.233392-3.340511-0.537941$

H $4.880184-5.2292671 .757063$

H $6.856688-3.6296170 .705328$

H $5.716213-3.184353-1.974925$

H $-1.135314-3.935949-0.721960$

H $-0.051214-2.748392-1.443267$

H $0.836023-4.8319590 .627129$

C $4.0660611 .503884-0.608383$

$\begin{array}{llll}\text { O } & 4.962540 & 0.822939 & -1.494698\end{array}$

C $6.1999240 .413810-0.902993$

C $6.9450351 .637725-0.352199$

C $6.0752902 .452943 \quad 0.600822$

C $4.7260852 .768096-0.045126$

H 3.7755930 .8332650 .209326

C $2.805794 \quad 1.848747-1.406354$

H $6.8107210 .009671-1.720102$

$\begin{array}{lllll}\text { O } & 8.141823 & 1.231190 & 0.302830\end{array}$

H $7.2421512 .278182-1.193115$

H 5.9514191 .9354491 .559326

$\begin{array}{lllll}\text { O } & 6.752629 & 3.677703 & 0.890775\end{array}$

H $4.8770083 .503621-0.845172$

$\begin{array}{llllllll}\text { O } & 3.877662 & 3.377782 & 0.924527\end{array}$
C $2.0191520 .596881-1.710189$

H $2.1413622 .518780-0.851220$

H $3.0580152 .353268-2.346882$

H 8.5695382 .0324660 .650355

H 6.1672624 .2036511 .462330

H 3.7236462 .7318151 .635130

$\begin{array}{llll}\text { O } & 2.218222 & 0.219885 & -3.004799\end{array}$

$\begin{array}{llll}\text { O } & 1.330551 & 0.010931 & -0.885164\end{array}$

C $1.540888-0.973669-3.408858$

H $1.731746-1.129135-4.473982$

H $1.930934-1.831656-2.853968$

H $0.461984-0.871636-3.257765$

SCF Energy (B3LYP/6-31G**//MMFF) $=-3245.92065670$

$02 \quad 255$

MMFF Geometry

C $0.2544872 .801593 \quad 1.268056$

$\begin{array}{llll}C & -0.935864 & 2.692052 & 1.875354\end{array}$

C -1.606850 1.3619762 .095372

O $-2.9458001 .417042 \quad 1.548691$

C -1.7314680 .9962853 .584161$

C $-0.409342 \quad 0.7515804 .283550$

C $0.371662-0.3283824 .078864$

C $0.001850 \quad 1.811947 \quad 5.269843$

C $0.094887-1.4788603 .150201$

C $1.016268-1.4324371 .924992$

C $0.646960-2.4833070 .868670$

C $1.555805-2.350869-0.355247$

C $1.161714-3.314458-1.483123$

O $-0.716210-2.280594 \quad 0.477865$

O $1.854109-2.871395-2.660569$

C $-0.356819-3.194846-1.740936$

C $1.653504-4.738490-1.198021$

O $-0.802460-4.190367-2.659066$

C $-1.205315-3.258214-0.449784$

C $-2.688045-2.940729-0.766192$

O $-1.133340-4.581854 \quad 0.090311$

C $-3.069941 \quad 1.1842220 .212390$

O $-2.1696810 .981779-0.587228$

C $-4.5110781 .198397-0.113079$

C $-4.8983011 .022576-1.384437$

C $-6.283240 \quad 0.982207-1.840319$

C $-6.528451-0.047195-2.910051$

C $-3.533270-2.6428780 .481030$

C $-5.026753-2.7814660 .230369$

C $-7.206016-2.2963291 .344809$

O $-5.493077-3.480922-0.665189$

C $-7.989375-1.603622 \quad 0.274706$

C $-8.888797-0.637616 \quad 0.517820$

C $-9.780307-0.039001-0.533359$

C $-9.600846 \quad 1.453422-0.686943$

C -10.3236592 .3174110 .045584$

C $-8.655492 \quad 1.938447-1.761409$

C $-7.2144521 .826060-1.352885$

N -5.779277 -2.089893 1.172016

H $\quad 0.763024 \quad 1.896517 \quad 0.945207$

H $-1.456806 \quad 3.5837932 .213167$

H $-1.0676390 .562487 \quad 1.578320$

H -2.3666290 .1059313 .686833$

H -2.296161 1.7797234 .108707

H $1.307344-0.4050954 .630711$

H $0.9706631 .596693 \quad 5.733017$

H 0.0805912 .7854924 .775941

H -0.7390551 .8888246 .072311$

H $0.259088-2.4157013 .696404$

H $-0.955428-1.4900212 .844341$

H $0.964615-0.436551 \quad 1.471006$

H $2.054481-1.5811912 .243456$

H $0.750059-3.4752611 .324182$

H $2.604034-2.502220-0.077155$

H $1.495354-1.323476-0.742172$

H $1.663049-3.499169-3.377633$

H $-0.533255-2.222673-2.222589$

H $2.747601-4.755328-1.122938$

H $1.399150-5.418043-2.018905$

H $1.251512-5.153068-0.271203$

H $-0.326756-4.054811-3.495445$

H $-2.750274-2.057028-1.414548$ 
H $-3.120084-3.779976-1.324741$ H -1.949832 -4.7745640 .577444$ H $-5.220221 \quad 1.3352900 .696479$ H -4.155356 $0.865051-2.163934$ H -7.588342 -0.212375 -3.116123 H $-6.116675-1.017974-2.609850$ H $-6.0471850 .253841-3.846886$ H $-3.291943-3.3371051 .293660$ H -3.311215 -1.632400 0.839099 H -7.467776 -1.933502 2.344131 H $-7.408547-3.3721981 .307560$ H $-7.863600-1.965020-0.742713$ H -9.039921 -0.2981221 .540071$ H -10.819176 -0.255780 -0.250478 H -9.639641 -0.530938-1.503729 H $-10.2240503 .390024-0.083205$ H -11.0279571 .9694970 .794157$ H -8.847657 2.996603-1.984705 H -8.870593 $1.416569-2.699005$ H $-6.9191582 .547309-0.590562$ H $-5.293326-1.5890901 .910188$ C $1.2357414 .255297-0.508428$ O $2.1124963 .202697-0.958328$ C $3.0779973 .752830-1.871491$ C $2.6186415 .176121-2.168556$ C $1.9488395 .558334-0.862779$ H $4.0315963 .795873-1.332352$ C $3.2133622 .850982-3.103242$ H $3.4526165 .833426-2.432995$ H $1.8936635 .207721-2.990044$ C $1.0257596 .758302-0.981716$ H $2.7279495 .775010-0.119462$ C $3.5461031 .376639-2.791694$ O $1.9785442 .861959-3.833042$ H $3.9655983 .268155-3.782612$ H $2.6860370 .882161-2.323520$ O $3.759003 \quad 0.709862-4.043269$ C $4.7926431 .169129-1.912062$ C $5.183569-0.315709-1.782426$ H $4.6031331 .582215-0.917592$ O $5.8934751 .892346-2.463445$ H $4.303517-0.886558-1.469014$ H $5.483084-0.693668-2.767185$ H $1.5843027 .642134-1.306796$ H $0.2266826 .579496-1.709018$ H $\quad 0.5592736 .989404-0.019067$ C 0.9626664 .0954820 .988355 H $1.2790332 .556904-3.229908$ H $2.957500 \quad 0.838239-4.579234$ H $\quad 6.041897 \quad 1.558808-3.364930$ H $\quad 0.3767304 .944343 \quad 1.358343$ H 1.9104984 .0823481 .538698 H $0.3020664 .157545-1.078286$ C $5.085671-0.8442901 .236894$ O $6.058133-0.0726090 .520732$ C $6.355309-0.541158-0.799517$ C $6.859998-1.990223-0.742408$ C $5.884988-2.900465-0.003605$ C $5.535106-2.3066771 .359237$ H $4.121419-0.7898840 .719807$ C $4.923774-0.2104892 .627962$ H $7.1922610 .071255-1.157718$ O $7.084277-2.508352-2.049157$ H $7.823587-2.017659-0.216928$ H $4.982550-3.089197-0.596228$ O $6.498578-4.1738540 .208932$ H $6.407326-2.3777482 .021903$ O $4.489044-3.0755831 .949406$ C 4.1815951 .1084362 .585484 H $4.355020-0.8725113 .289888$ H $5.912623-0.0231133 .063140$ H $7.717557-1.918379-2.492603$ H $6.752089-4.521112-0.663587$ H $4.791731-3.9992141 .984581$ O 4.2147471 .6705853 .830449 O 3.6319741 .5812921 .600944 C 3.5483012 .9302563 .954580 H 4.0213153 .6742523 .306505
H $2.4882752 .822833 \quad 3.709850$

H 3.6361103 .2613114 .992636

SCF Energy (B3LYP/6-31G*//MMFF)= -3245.91720703

$02 \_256$

MM̄FF Geometry

C $-1.415152-4.102305-0.518806$

C $-0.846493-3.155468-1.280395$

C $0.568323-2.674221-1.106560$

O $0.463457-1.251560-0.846118$

C $1.408275-2.853406-2.385431$

C $2.126211-4.185537-2.461750$

C $3.435927-4.361140-2.194443$

C $1.287420-5.359968-2.893819$

C $4.436837-3.318225-1.784182$

C $4.703180-3.362368-0.275853$

C $5.664454-2.249970 \quad 0.166144$

C $5.941297-2.3373021 .668353$

C $6.832881-1.1849082 .154838$

O $5.062977-0.986651-0.141879$

O $6.766525-1.1807573 .588997$

C 6.2467850 .1504751 .646798

C $8.303818-1.4235451 .794570$

O $7.114868 \quad 1.237376 \quad 1.960276$

C 5.8977810 .1425090 .140736

C $5.1304101 .433862-0.232820$

O $7.1117450 .103531-0.615922$

C $1.250896-0.7026470 .111251$

O $2.064187-1.2847560 .814774$

C $1.0301550 .757767 \quad 0.225328$

C $0.0811221 .421826-0.452011$

C $-0.1965032 .856574-0.369316$

C $0.756315 \quad 3.7123390 .423237$

C $4.4303911 .365782-1.597774$

C $4.0206042 .737205-2.109908$

C $2.6102313 .797914-3.872231$

O $4.5666183 .776532-1.748133$

C $1.6879584 .688087-3.100854$

C $0.4462945 .000180-3.502468$

C $-0.4396935 .981726-2.789585$

C $-1.7599895 .384315-2.362461$

C $-2.8564735 .503346-3.130154$

C $-1.8280084 .734408-1.000623$

C $-1.2866213 .334090-1.005322$

N $3.0187442 .660272-3.068473$

H $-0.809083-4.5729970 .253179$

H -1.422521 -2.665693 -2.060509

H $1.044592-3.175872-0.255136$

H $2.122624-2.026708-2.458245$

H $\quad 0.783310-2.730390-3.280779$

H $3.847909-5.366326-2.276065$

H $1.892487-6.252990-3.084936$

H $0.557655-5.619448-2.122611$

H $0.752726-5.127395-3.820426$

H $\quad 5.371499-3.508597-2.326615$

H $4.121208-2.317278-2.088998$

H $3.750740-3.255557 \quad 0.257045$

H $5.119465-4.340374-0.003310$

H $6.595395-2.356251-0.403387$

H $6.388072-3.3052381 .927852$

H $4.988797-2.2920232 .215505$

H $7.267129-0.4085943 .904740$

H 5.3236230 .3288942 .215007

H $8.659476-2.3575082 .246201$

H $8.945704-0.6342662 .201217$

H $8.476737-1.4885680 .718412$

H $7.851144 \quad 1.2203101 .325322$

H 4.3533031 .6352140 .515850

H $5.8264392 .281271-0.207725$

H $6.9606390 .515756-1.481320$

H $1.692937 \quad 1.2618890 .921514$

H $-0.5770740 .866877-1.118565$

H 0.6941923 .4745571 .489993

H $0.568676 \quad 4.7829840 .316408$

H 1.7880263 .5523020 .090132

H $5.0918210 .933707-2.356683$

H $3.5466790 .722639-1.524836$

H $2.1413343 .404438-4.779920$ 
H $3.5040004 .360925-4.161846$

H $2.0817685 .145296-2.196368$

H $0.0611704 .568823-4.423600$

H -0.617655 $6.823834-3.471661$

H $\quad 0.0650886 .417124-1.918108$

H -3.814139 $5.103256-2.813884$

H -2.818419 $5.998072-4.095050$

H $-2.8727164 .674634-0.665729$

H -1.341824 $5.378739-0.262475$

H -1.909559 2.633521 -1.563578

H $2.6346721 .751985-3.312098$

C $-3.795536-3.834089-1.509951$

O $-3.938251-2.476008-1.048870$

C $-5.318748-2.085347-1.154607$

C $-6.016013-3.194038-1.935599$

C $-5.211569-4.409859-1.519052$

H $-5.718570-2.071165-0.133806$

C $-5.434983-0.690312-1.778490$

H $-7.077853-3.273229-1.683177$

H -5.939252 -3.041445 -3.018308

C $-5.391445-5.601991-2.442587$

H -5.509027 -4.694706 -0.500895

C $-4.6139330 .401732-1.061868$

O $-4.969775-0.757010-3.133081$

H -6.491866 $-0.402427-1.829989$

H $-3.543967 \quad 0.228234-1.229327$

O $\quad-4.903521 \quad 1.652322-1.701247$

C -4.8748840 .5264870 .451236$

C -4.1742181 .7509601 .068258$

H $-4.521673-0.382250 \quad 0.947056$

O -6.2778360 .6139980 .697571$

H -3.1236401 .7404940 .765078$

H -4.6231072 .6648940 .661128$

H -6.438966 $-5.920501-2.459200$

H -5.096932 -5.362045 -3.469686

H $-4.786414-6.450405-2.108159$

C $-2.838109-4.595559-0.587748$

H $-5.0512820 .135122-3.512029$

H $-5.8555621 .823452-1.605774$

H $-6.596472 \quad 1.4539450 .326433$

H $-2.809335-5.652059-0.878290$

H $-3.234209-4.5536250 .434385$

H $-3.397877-3.793124-2.532054$

C -2.2986250 .5269793 .143540$

O $-3.725290 \quad 0.6320713 .227630$

C -4.2865581 .7946432 .608854$

C -3.696017 3.0590083 .246359

C -2.1710693 .0566833 .201824$

C -1.622340 1.7509683 .776023

H -1.9948380 .4304622 .094497$

C $-1.887438-0.755283 \quad 3.883929$

H -5.3567111 .7843682 .850754$

O -4.2024804 .2195482 .594885$

H -4.019315 3.1127634 .294201

H $-1.811943 \quad 3.2314942 .182912$

O -1.7024924 .1541683 .988138$

H -1.770145 1.7418754 .863689

O $-0.219076 \quad 1.6778393 .533166$

C -2.326093 -2.009596 3.159385

H $-0.799121-0.8136543 .992742$

H $-2.350739-0.7614104 .878275$

H -3.783687 4.9925223 .010908

H $-0.7404264 .214638 \quad 3.863413$

H 0.2047942 .3968014 .030984

O $-2.274513-3.0624364 .028348$

O $-2.645554-2.067611 \quad 1.980479$

C -2.655255 -4.331162 3.487726

H $-2.678109-5.0562024 .305578$

H -3.653110 -4.2761183 .041914$

H $-1.917429-4.6580542 .749679$

SCF Energy (B3LYP/6-31G**//MMFF) = -3245.91546505

$02 \_257$

MMFF Geometry

C -0.1345674 .0978861 .152648$

C -0.1583982 .7684820 .979065$

C $-0.374101 \quad 1.7774172 .092167$

O 0.7891420 .9236112 .177908
C $-1.581661 \quad 0.885314 \quad 1.757492$

C -2.0468450 .0250502 .916903$

C $-3.1593090 .255438 \quad 3.640240$

C $-1.213150-1.1918173 .221573$

C -4.0915001 .4262423 .550024$

C $-5.4936581 .066748 \quad 3.043724$

C -5.5662650 .8535101 .522310$

C -7.0254430 .9271321 .060773$

C $-7.1624720 .680413-0.445924$

O $-5.014102-0.4285541 .189234$

O $-8.5519810 .463269-0.734124$

C $-6.418692-0.620873-0.796920$

C $-6.7330381 .899563-1.271081$

O $-6.393362-0.821787-2.209276$

C $-4.981596-0.696102-0.221756$

C $-4.426816-2.121094-0.464983$

O $-4.160537 \quad 0.246588-0.910925$

C 1.8637151 .3741842 .873802

O 1.9982522 .4823383 .373916

C 2.9216370 .3400372 .959631

C $2.695459-0.9609192 .718539$

C $3.673718-2.0462562 .801525$

C $5.124506-1.6651372 .933421$

C $-3.073064-2.4338840 .184444$

C $-2.547517-3.790544-0.262840$

C $-0.508875-5.190385-0.296002$

O $-3.223154-4.583718-0.915195$

C $0.507587-5.5975720 .722147$

C $1.801678-5.8002350 .429307$

C $2.811344-6.3400081 .401379$

C $3.993549-5.4213331 .609617$

C $5.032932-5.4221640 .758024$

C $4.022684-4.5882742 .869289$

C $3.226197-3.3187502 .744476$

N -1.243752 -4.024998 0.161226

H -0.2724804 .5086542 .149771$

H $-0.0049132 .361428-0.015996$

H -0.5383082 .2792683 .054636$

H -1.327257 0.2194790 .921905

H -2.4091831 .5039801 .392176$

H -3.412191 -0.4517654 .429633$

H -1.761978 -1.9311133 .814978$

H $-0.898175-1.6898632 .300204$

H $-0.322871-0.9071373 .789115$

H -4.1921611 .8271614 .567352$

H -3.6748772 .2476642 .958552$

H -5.8592840 .1743113 .567327$

H $-6.155344 \quad 1.898376 \quad 3.317719$

H -4.978385 1.6392621 .033572

H $-7.471844 \quad 1.893423 \quad 1.327782$

H $-7.6143550 .167370 \quad 1.594071$

H -9.044326 $1.251748-0.447693$

H -7.000687 -1.453469 -0.377032

H -7.347095 $2.770987-1.013586$

H $-6.8920231 .727916-2.341408$

H $-5.6880622 .176780-1.118038$

H -7.313228 -0.786892 -2.523250

H $-5.148108-2.856327-0.083627$

H -4.336144 -2.287861 -1.546451

H $-3.2301990 .008563-0.780659$

H $3.8939420 .704223 \quad 3.274963$

H $1.691322-1.2784942 .446608$

H $5.401976-0.9313242 .167939$

H $5.808699-2.5059692 .800895$

H $5.320099-1.2331193 .920623$

H $-2.328065-1.682127-0.086468$

H -3.176295 -2.451423 1.274124

H $-0.054278-4.930871-1.257781$

H -1.206897 -6.017133 -0.465460

H $\quad 0.150273-5.7968561 .729537$

H $2.145572-5.634072-0.589239$

H $3.167983-7.3033841 .012538$

H $2.344658-6.5755722 .365695$

H $5.900033-4.7915770 .924524$

H $5.034783-6.048822-0.127830$

H $5.058235-4.3941693 .165379$

H $3.610147-5.1741173 .700882$

H $2.152881-3.4852642 .645014$ 
H $-0.711738-3.2478130 .542277$

C $1.5302565 .460291-0.225843$

O $2.2281614 .331700-0.792309$

C $3.4798684 .179091-0.102340$

C 3.7474445 .5246420 .561570

C $2.345074 \quad 5.9206190 .984727$

H 3.3134833 .4304030 .681722

C $4.5784983 .687464-1.049340$

H $4.443870 \quad 5.4443601 .401440$

H $4.1480646 .257149-0.148468$

C $2.1909027 .397923 \quad 1.299913$

H 2.0859975 .3419741 .879601

C $4.2158502 .431873-1.870916$

O $4.8901194 .731978-1.979593$

H $5.4937403 .512718-0.470560$

H $3.5384382 .709382-2.687660$

O $5.4136151 .970835-2.509450$

C $3.5811771 .281701-1.070503$

C $3.492937-0.037694-1.862348$

H $2.5793131 .588486-0.752076$

$\begin{array}{lllll} & \text { O } & 4.338636 & 1.041472 & 0.116817\end{array}$

H $3.2392080 .180355-2.903204$

H $4.478044-0.518076-1.858844$

H $2.8382597 .682536 \quad 2.135814$

H 2.4580918 .0223940 .440780

H 1.1574607 .6291781 .577196

C $0.068887 \quad 5.085203 \quad 0.036483$

H $5.5961184 .401557-2.561429$

H $6.0504311 .732066-1.815015$

H $5.2289600 .752815-0.145079$

H $-0.3606734 .675320-0.885831$

H $-0.505813 \quad 5.9882080 .273958$

H $1.5460146 .234935-1.003560$

C $0.645152-0.666955-2.816731$

O $1.109162-0.537899-1.465286$

C $2.450239-0.991773-1.236287$

C $2.584567-2.462028-1.645722$

C $2.128963-2.696725-3.079795$

C $0.728399-2.124866-3.286725$

H $1.242316-0.031010-3.479866$

C $-0.805436-0.165084-2.861325$

H $2.599410-0.958763-0.152200$

O $3.923749-2.917796-1.493318$

H $1.961174-3.073849-0.982542$

H $2.838256-2.275419-3.801943$

O $2.078976-4.102431-3.332989$

H $-0.002775-2.738312-2.744699$

O $0.398438-2.191514-4.672732$

C $-0.904933 \quad 1.340429-2.734716$

H -1.276465 -0.435444 -3.812838

H $-1.372251-0.608461-2.036132$

H $4.177545-2.771478-0.565782$

H $2.963668-4.461221-3.146615$

H $\quad 0.480077-3.121431-4.945753$

O $-2.2256141 .687019-2.706212$

O $\quad 0.0426302 .111143-2.674641$

C -2.512823 $3.082582-2.574346$

H $-1.8797703 .683865-3.234040$

H -3.557340 $3.240986-2.855764$

H -2.381837 $3.383077-1.531660$

SCF Energy (B3LYP/6-31G**/MMFF) $=-3245.90747003$

02_258

MMFF Geometry

C $-1.738257-3.597171 \quad 1.466247$

C $-2.023483-2.2983661 .640319$

C $-2.403816-1.7067322 .974139$

O $-3.595329-0.9032862 .782585$

C $-1.332789-0.7527323 .529324$

C $0.046995-1.3552693 .689462$

C $1.104549-1.0178842 .924528$

C $0.199531-2.3447734 .812733$

C $1.089315-0.0634601 .764464$

C $1.525199 \quad 1.3454742 .179483$

C $1.1075672 .398111 \quad 1.141924$

C $1.713503 \quad 3.7591491 .488472$

C $1.197440 \quad 4.875146 \quad 0.569196$

O $\begin{array}{llll}-0.322776 & 2.473858 & 1.173727\end{array}$ $\begin{array}{llll}\text { O } & 1.550696 & 6.133686 & 1.163658\end{array}$

C $-0.342827 \quad 4.812698 \quad 0.523812$

C $1.8691404 .844020-0.807459$

O $-0.8596925 .739213-0.429622$

$\begin{array}{llll}\text { C } & -0.900526 & 3.397010 & 0.242339\end{array}$

C -2.4345203 .3932090 .471355$

O $-0.608593 \quad 3.034817-1.105119$

C $-4.784024-1.5488562 .650684$

O $-4.998789-2.7417952 .787376$

C -5.773508 -0.5366762 .222593$

C $-6.860980-0.9330011 .546176$

C $-7.871023-0.057397 \quad 0.948180$

C $-7.708394 \quad 1.4278901 .139792$

C $-3.1267252 .031958 \quad 0.295765$

C $-3.6405611 .777271-1.112651$

C $-5.0309420 .162946-2.409697$

$\begin{array}{llll}\text { O } & -3.410860 & 2.524988 & -2.059168\end{array}$

C $-6.4200870 .720745-2.465735$

C $-7.507859-0.017551-2.736239$

C $-8.8978550 .548174-2.827782$

C $-9.907998-0.225755-2.007698$

C $-10.764566-1.079084-2.594143$

C $-9.9794690 .050036-0.520847$

C $-8.864331-0.6241780 .231954$

$\mathrm{N}-4.3834230 .605664-1.187813$

H $-1.755730-4.2656852 .323571$

H $-1.998690-1.6261330 .785834$

H -2.617056 -2.485199 3.717795

H $-1.668722-0.3842614 .508925$

H -1.3060410 .1568512 .916664$

H $2.069985-1.4755193 .132179$

H $1.230235-2.7008344 .914385$

H $-0.086158-1.8856825 .764796$

H $-0.435579-3.2196994 .643527$

H $1.777742-0.4398661 .001080$

H $0.105658-0.0514011 .284670$

H 1.0687781 .6000113 .145368

H 2.6126741 .3574062 .317787

H $1.4336582 .070924 \quad 0.147698$

H 2.8093413 .7143151 .460945

H 1.4524604 .0193882 .524132

H $2.517976 \quad 6.1569291 .262546$

H $-0.712875 \quad 5.1403591 .505619$

H $2.9562154 .945221-0.706862$

H $1.5463325 .690026-1.424232$

H $1.6709733 .922713-1.356396$

H $-0.4972816 .614400-0.209041$

H -2.616566 3.7040861 .509102

H $-2.9148474 .135163-0.177232$

H $-1.1711652 .285242-1.354831$

H -5.5412130 .5055852 .407943$

H $-7.004789-1.9992811 .374785$

H -7.7304151 .6808842 .205656$

H -8.490436 2.0206050 .662033

H $-6.756843 \quad 1.7698090 .717913$

H $-2.470536 \quad 1.2061520 .589848$

H -4.002076 2.0127610 .957173

H -5.024106 $-0.931521-2.404668$

H $-4.4563900 .516313-3.272024$

H $-6.5343891 .789448-2.298699$

H $-7.391275-1.080532-2.935159$

H $-9.1898710 .536363-3.886069$

H $-8.9209651 .602483-2.524884$

H -11.509286 -1.618062 -2.017844

H -10.736760 -1.262257 -3.663309

H -10.925405 $-0.328901-0.111390$

H -10.016952 $1.130944-0.363796$

H -8.890264 -1.7125920 .157007$

H $-4.5701240 .082226-0.337874$

C $0.096503-4.450085-0.012004$

O $0.732913-3.197214-0.343447$

C $1.632649-3.408964-1.454151$

C $1.828730-4.913028-1.536019$

C $0.450309-5.409253-1.148524$

H $1.101630-3.045953-2.343515$

C $2.906904-2.591875-1.220828$

H $2.139352-5.246155-2.530583$

H $2.577386-5.249943-0.808261$ 
C $0.423639-6.874507-0.749305$

H - $0.231196-5.255721-1.996145$

C $4.054354-2.890302-2.200179$

O $2.556305-1.207733-1.324310$

H $3.241112-2.748292-0.189557$

H $4.400072-3.922131-2.067209$

O $3.536709-2.801560-3.533575$

C $5.248925-1.920317-2.093243$

C $5.858988-1.841069-0.683310$

H $4.941653-0.926929-2.437083$

O $6.259285-2.358752-3.008745$

H $5.086575-1.5549330 .035281$

H $6.215341-2.837141-0.393034$

H $\quad 0.741508-7.506466-1.585135$

H $1.092617-7.0727720 .094865$

H $-0.585736-7.181783-0.458316$

C -1.407201-4.220420 0.139458

H $2.280061-1.043020-2.242087$

H $4.272684-2.983984-4.142842$

H $\quad 6.562027-3.236602-2.719813$

H -1.950216 -5.1705610 .077787$

H -1.774814 -3.584284 -0.675562

H $\quad 0.539467-4.7938090 .931935$

C $5.7515441 .172191-0.281111$

O $6.6901870 .450954-1.088733$

C $7.038096-0.845998-0.592105$

C $7.650217-0.719750 \quad 0.810220$

C 6.7489580 .0709801 .758587

C $6.321073 \quad 1.3951201 .123812$

H $4.8100350 .613702-0.218152$

C $5.4682892 .504919-0.981779$

H $7.826083-1.226586-1.253978$

O $7.914191-2.0107121 .348553$

H $8.615244-0.2033120 .726803$

H $5.874413-0.5204502 .052863$

O $7.459257 \quad 0.338777 \quad 2.970116$

H 7.1804142 .0759811 .078777

O 5.3299752 .0102001 .942364

C $4.7413922 .293996-2.288737$

H $4.8631353 .176249-0.362905$

H $6.4054363 .027829-1.208735$

H $8.274842-1.8851802 .243050$

H 8.2454680 .8659682 .747623

H 5.7061642 .1038692 .834361

O $3.4554791 .908348-2.052632$

O $5.2565002 .455636-3.387464$

C $2.6558501 .684631-3.217972$

H $2.5900722 .599292-3.814732$

H $3.0726070 .864330-3.810167$

H $1.6501461 .407202-2.891020$

SCF Energy $\left(\mathrm{B} 3 \mathrm{LYP} / 6-31 \mathrm{G}^{* *} / / \mathrm{MMFF}\right)=-3245.91617798$

$02 \_259$

MM̄FF Geometry

C $-3.162697 \quad 4.3906620 .292626$

C $-3.6237713 .692751 \quad 1.342180$

C -4.8720092 .8391231 .396889$

O $-4.474100 \quad 1.448197 \quad 1.321216$

C -5.8923673 .1006860 .269915$

C -7.1927602 .3306140 .428509$

C $-7.5967661 .321153-0.369969$

C -8.082289 2.7917631 .555382

C $-6.873898 \quad 0.731587-1.548350$

C $-6.403196-0.698063-1.252731$

C $-5.313708-1.152122-2.234876$

C $-4.955709-2.620652-2.003125$

C -3.760914 -3.069673 -2.857788

O $-4.169617-0.323302-2.004000$

O $-3.297222-4.326759-2.340408$

C $-2.608668-2.057783-2.679669$

C $-4.165313-3.322926-4.314638$

O $-1.531610-2.351548-3.565583$

C $-3.048466-0.590304-2.855262$

C $-1.940170 \quad 0.425724-2.481088$

O $-3.382823-0.362041-4.229261$

C $-4.083953 \quad 0.8344102 .471477$

O -4.0063421 .3325343 .582993$

C $-3.757060-0.5703612 .138237$
C $-3.184088-1.3620913 .056906$

C $-2.811965-2.7675132 .873300$

C $-3.073531-3.3895961 .526959$

C $-1.469140 \quad 0.373961-1.023586$

C $-0.257961-0.511430-0.792384$

C $1.091506-1.5109061 .051802$

O $0.450390-0.928315-1.703817$

C $0.652829-2.9110501 .340038$

C $0.859173-3.5148842 .520505$

C $0.468230-4.9338462 .818020$

C $-0.353697-5.0607184 .081467$

C $0.220128-5.4049505 .246926$

C $-1.850351-4.8791273 .973373$

C -2.256817 $-3.431433 \quad 3.909017$

N $-0.035046-0.7418370 .558085$

H $-3.685714 \quad 4.360891-0.658555$

H -3.0579833 .7373072 .271482$

H -5.3604363 .0308762 .360485$

H -5.427721 $2.868816-0.691962$

H -6.1378914 .1705710 .233505$

H $-8.5580800 .853543-0.159668$

H -9.0552132 .2884861 .549770$

H -7.6156612 .5906202 .524214$

H -8.2715793 .8671331 .474429$

H $-7.5593390 .715136-2.404813$

H $-6.033797 \quad 1.354772-1.860188$

H $-6.003825-0.743990-0.231721$

H -7.262025 -1.379055 -1.298233

H -5.672999 -0.999176 -3.259381

H $-5.824253-3.265541-2.186422$

H $-4.696509-2.766418-0.946577$

H -4.037424 -4.956697 -2.375469

H $-2.223380-2.194606-1.662248$

H $-4.925756-4.111296-4.368954$

H -3.315868 -3.685784 -4.903432

H $-4.574689-2.438021-4.806168$

H -1.268945 -3.274299 -3.406549

H -1.101202 $0.346412-3.182781$

H $-2.355977 \quad 1.431530-2.639262$

H -3.321803 $0.590892-4.408168$

H $-3.995219-0.9160311 .139355$

H -2.969413 -0.946260 4.040448

H $-4.150949-3.4544101 .341404$

H -2.665401 -4.396851 1.422577

H -2.612689 -2.795703 0.730343

H $-1.1643301 .383392-0.723928$

H $-2.2816230 .069513-0.359046$

H $1.458633-1.0052961 .949441$

H $1.890383-1.5185820 .304572$

H $\quad 0.169718-3.4600680 .535424$

H $1.370458-2.9700673 .311257$

H $1.392146-5.5189182 .916376$

H $-0.077694-5.3840931 .979776$

H $-0.365976-5.5276786 .151596$

H $1.290509-5.5659335 .323153$

H $-2.344931-5.3126744 .853469$

H -2.226692 -5.4682203 .132233$

H -2.097189 -2.893233 4.844390

H $-0.695430-0.380617 \quad 1.240336$

C $-0.7344244 .583948-0.326494$

$\begin{array}{llll}\text { O } & -0.197090 & 3.590417 & 0.573762\end{array}$

C 1.2199683 .8167740 .726230

C $1.6204734 .656827-0.473286$

C $0.4105165 .557687-0.613572$

H 1.3323754 .3948561 .652939

C 1.9394742 .4695480 .855721

H $2.5488255 .212502-0.312027$

H $1.7399664 .030303-1.365932$

C $0.313086 \quad 6.236917-1.968865$

H $0.453714 \quad 6.327944 \quad 0.168438$

C 3.4771142 .5876020 .866156

O 1.4943251 .8655842 .072848

H $1.633450 \quad 1.8011780 .042526$

H $3.791753 \quad 3.006979-0.097221$

O $3.8570163 .518817 \quad 1.880051$

C 4.2122181 .2441621 .078068

C 5.7353321 .3893520 .902131

$\begin{array}{llll}\mathrm{H} & 3.830424 & 0.511498 & 0.359569\end{array}$ 
O $3.974281 \quad 0.7341402 .388421$

H $5.9343321 .860137-0.066375$

H $6.1213232 .061647 \quad 1.677311$

H $1.1820186 .880632-2.139672$

H $0.2722245 .504924-2.782486$

H $-0.585876 \quad 6.858488-2.029409$

C -1.9362015 .2605910 .335628$

H $\quad 0.525574 \quad 1.7921972 .023099$

H 4.7837473 .7663341 .725419

H 3.0137270 .6441472 .501805

H $-2.1794986 .197244-0.179504$

H -1.695637 5.5175221 .375052

H -1.033772 $4.057483-1.241771$

C $6.354136-0.658456-1.294399$

O $6.030781-0.9279580 .074859$

C 6.4942800 .0464191 .014664

C $8.021030 \quad 0.1817910 .920992$

C $8.4694410 .498629-0.503134$

C $7.870356-0.509444-1.481825$

H $5.8427830 .255092-1.621046$

C $5.830372-1.838106-2.130051$

H $6.272854-0.3609112 .009209$

O 8.4756251 .1881051 .819182

H 8.480645 -0.7642561 .235848$

H $8.2124831 .525021-0.790126$

O $9.8928330 .399597-0.587066$

H $8.362917-1.481364-1.347144$

O $8.135563-0.079401-2.816066$

C $4.318952-1.889131-2.190769$

H $6.188495-1.775210-3.163237$

H $6.179804-2.777583-1.684059$

H 9.4468821 .1553951 .832529

H $10.268748 \quad 1.116932-0.049570$

H $9.099073 \quad 0.021742-2.901911$

O $3.936720-3.130572-2.614765$

O $3.571028-0.957468-1.930280$

C $2.526592-3.337292-2.742361$

H $2.050216-3.283445-1.759444$

H $2.091948-2.603096-3.427266$

H $2.365603-4.336976-3.154696$

SCF Energy $\left(B 3 L Y P / 6-31 G^{* *} / / M M F F\right)=-3245.91284768$

$02 \_26$

MMFF Geometry

C $-1.862548 \quad 4.237411-0.317375$

C $-2.3515353 .016823-0.055077$

C -2.994596 $2.631023 \quad 1.248513$

O -2.2652371 .4966701 .771622$

C -4.4434332 .1807291 .008928$

C -5.2403172 .0540642 .291175$

C -5.5215290 .8907492 .909694$

C -5.7774473 .3503242 .842046$

C $-5.069790-0.4930452 .533852$

C $-6.217304-1.3618921 .999278$

C $-6.641451-0.9958940 .567908$

C $-7.841643-1.8365680 .128135$

C $-8.214097-1.577977-1.339835$

O $-5.527217-1.254565-0.291106$

O $-9.118848-2.612673-1.754640$

C $-6.945474-1.712370-2.207989$

C $-8.962175-0.251154-1.514828$

O $-7.210611-1.341515-3.559047$

C $-5.737310-0.913238-1.667122$

C $-4.457988-1.304256-2.445861$

O $-5.9853340 .482949-1.836277$

C -1.1934521 .7373212 .567862$

O -0.7912242 .8305472 .941403$

C -0.5334090 .4762032 .979753$

C $-0.752103-0.7035292 .377498$

C $-0.126838-1.9820272 .724090$

C $0.773911-2.0196953 .930185$

C -3.164036 -0.709502 -1.870315

C $-1.929220-1.298383-2.530473$

C $0.515507-1.600262-2.151345$

O $-1.967551-1.919592-3.589528$

C $0.608209-2.971978-1.559052$

C $1.513014-3.335762-0.638288$

C $1.568514-4.711625-0.036598$
C $1.479562-4.7035511 .475166$

C $2.554594-5.0010332 .224392$

C $0.132647-4.4616522 .123557$

C $-0.383093-3.0591001 .951945$

N $-0.775526-1.033643-1.802727$

H -1.9428855 .0199810 .432033$

H $-2.2575792 .237216-0.806566$

H $-2.966743 \quad 3.4612381 .964709$

H -4.4463761 .2296120 .466550$

H -4.9620142 .8956340 .355884$

H -6.1317870 .9262203 .811667$

H -6.3813353 .2013103 .743477$

H $-4.9583784 .026883 \quad 3.104093$

H -6.412550 3.8440232 .099127

H -4.234869 -0.479907 1.827360

H $-4.675375-0.962744 \quad 3.444097$

H -5.872197 -2.404187 2.010498

H -7.079087 -1.298915 2.675290

H -6.8923480 .0695450 .528257$

H $-8.704441-1.6547800 .781113$

H $-7.600927-2.9030790 .240522$

H $-9.893319-2.586787-1.166868$

H -6.674474 -2.777474 -2.226799

H $-9.886903-0.252611-0.925228$

H -9.271636 -0.106520 -2.555920

H $-8.3758310 .616322-1.205099$

H -7.965175 -1.874669-3.862905

H $-4.357373-2.397779-2.434603$

H -4.548669 -0.995724 -3.495085

H $-5.1390690 .954481-1.878842$

H $\quad 0.1761390 .580933 \quad 3.794144$

H -1.447636 -0.7463331 .541166$

H $1.633498-1.3552223 .790597$

H $1.174581-3.0137184 .140099$

H $\quad 0.228934-1.7053944 .827155$

H $-3.1366420 .372735-2.028643$

H -3.103421 -0.903427 -0.793516

H $1.287070-0.927101-1.765860$

H $0.608274-1.657320-3.240514$

H $-0.116548-3.705455-1.905531$

H $2.248351-2.611144-0.298669$

H $2.510249-5.174591-0.359030$

H $0.771523-5.352810-0.433781$

H $2.498883-5.0370123 .307162$

H $3.517206-5.2171601 .773328$

H $\quad 0.153151-4.7248343 .188321$

H $-0.597968-5.1541431 .685080$

H $-1.048483-2.9397001 .096391$

H $-0.856715-0.574980-0.899904$

C $0.3470294 .647013-1.493737$

O $0.8573213 .372017-1.051403$

C $1.8722203 .606278-0.049984$

C $2.2527965 .072373-0.191685$

C $0.9135935 .694474-0.536202$

H 1.3854803 .4293810 .917754

C $3.0136602 .606807-0.260406$

H 2.6931165 .4853760 .720546

H $2.9650125 .213205-1.014341$

C $1.0211317 .084204-1.138386$

H $0.316475 \quad 5.7515140 .381919$

C 4.2615382 .8886730 .592568

O $2.519708 \quad 1.3017660 .061760$

H $3.2840642 .585983-1.322321$

H 4.7068973 .8430230 .287669

O $3.850303 \quad 3.0515801 .955908$

C 5.3350621 .7833230 .555781

C $5.768128 \quad 1.382937-0.864096$

H 4.9734230 .9089191 .105642

O 6.4778482 .2564081 .277683

H $4.9025960 .995457-1.409577$

H $6.1106152 .277745-1.398764$

H $1.4927697 .774273-0.431160$

H $1.6216397 .080402-2.054268$

H $0.0303957 .478272-1.385978$

C $-1.1783074 .586475-1.609730$

H $1.7204661 .153700-0.472138$

H 4.6499343 .2261022 .481344

H 6.8377383 .0209750 .796360 
H $-1.4389763 .837688-2.368884$ H -1.568848 $5.545243-1.969227$ H $0.7582264 .803680-2.499708$ C $5.561887-1.636849-0.509793$ O $6.617904-0.788767-0.044502$ C $6.9027640 .333585-0.883597$

C $7.293171-0.147415-2.288582$ C $6.242972-1.086483-2.881753$ C $5.899113-2.201746-1.893778$ H $4.623874-1.070735-0.558767$ C $5.400232-2.7607110 .521689$ H $7.7880620 .819157-0.453767$ O $7.4950480 .973054-3.143068$ H $8.249700-0.682782-2.225033$ H $\quad 5.340847-0.542158-3.185025$ O $6.764168-1.694250-4.065881$ H $6.742981-2.899961-1.822303$ O $4.777840-2.933415-2.385448$ C $4.792494-2.2435921 .807974$ H $4.747319-3.5593210 .155823$ H $6.382100-3.1968470 .743346$ H $7.8608030 .643505-3.981048$ H $6.874179-0.994191-4.731163$ H $5.009957-3.259093-3.271908$ O $5.449046-2.7804602 .876503$ O $3.836033-1.4818261 .863393$ C $4.965655-2.3770934 .161567$ H $4.999565-1.2875234 .258512$ H $3.947804-2.7474204 .313277$ H $5.615955-2.8165594 .922531$ SCF Energy (B3LYP/6-31G**//MMFF) $=-3245.91173784$

$02 \_260$

MM̄FF Geometry

C $1.592459-0.9229112 .555273$

C $0.448784-1.5951502 .368460$

C $0.230643-2.5142611 .193895$

O $-0.721208-1.8597050 .321163$

C $-0.353837-3.8548841 .659760$

C $-0.544000-4.8368360 .520521$

C $-1.727428-5.109070-0.063784$

C $0.703940-5.5551810 .075575$

C $-3.060493-4.4875430 .245489$

C $-3.749448-3.966960-1.023831$

C $-5.066453-3.256526-0.683119$

C $-5.856793-2.922327-1.947783$

C -7.141205 -2.144938 -1.620536

O $-4.744136-2.049451 \quad 0.016468$

O $-7.655541-1.606287-2.847952$

C $-6.783083-0.947971-0.715583$

C $-8.235743-3.056738-1.053424$

O $-7.958492-0.282123-0.259234$

C $-5.883313-1.3200330 .487934$

C $-5.381141-0.0292401 .174356$

O $-6.640272-2.0806881 .430515$

C $-0.209039-0.963620-0.568899$

O $0.970579-0.752312-0.805359$

C $-1.338535-0.215304-1.161445$

C -1.092722 $0.993864-1.688369$

C $-2.0789771 .919810-2.246197$

C $-3.4844371 .416123-2.431940$

C $-4.258936-0.2400772 .199039$

C -3.7792681 .1045552 .710562$

C -2.4726213 .1085541 .993507$

O -4.1749471 .5660383 .778467$

C -3.1225143 .9424450 .931683$

C -2.4573064 .8310820 .178395$

C $-3.0922835 .651658-0.908052$

C -2.341275 $5.566762-2.220720$

C $-1.5955146 .596110-2.656702$

C $-2.5131844 .318679-3.063923$

C -1.679024 $3.170926-2.556740$

N -2.916272 1.7357951 .827707

H 2.412281-1.070284 1.857510

H $-0.391354-1.4188033 .035598$

H $1.169048-2.6970150 .654913$

H -1.299694 -3.688327 2.186972

H $0.311540-4.3121462 .404667$
H $-1.749303-5.860815-0.851666$

H $0.504072-6.284125-0.716851$

H $1.441037-4.844399-0.310673$

H $1.149927-6.0971170 .915936$

H -3.694540 -5.250751 0.712895

H $-2.970274-3.6685910 .963395$

H -3.076706 -3.269316 -1.539251

H -3.943022 -4.806554 -1.702978

H $-5.653836-3.911298-0.028636$

H -6.093031 -3.832166 -2.513287

H $-5.237013-2.305904-2.614053$

H -7.823298 -2.348443 -3.453768

H $-6.240859-0.223602-1.339525$

H -8.479185 -3.852435 -1.767661

H $-9.167454-2.502773-0.894127$

H $-7.952676-3.532839-0.112326$

H -8.486667 -0.056199-1.044053

H -5.0104660 .6601620 .408193$

H -6.2179970 .4772431 .672884$

H $-6.236210-1.9868272 .307784$

H $-2.331145-0.643686-1.092495$

H $-0.0689641 .366069-1.675151$

H -4.112171 2.087208 -3.022090

H $-3.9696301 .280356-1.462553$

H $-3.487697 \quad 0.457631-2.963445$

H $-4.602613-0.8301523 .054775$

H -3.408199 -0.7734651 .760017$

H -1.382002 3.1106851 .906336

H -2.752814 3.4777552 .984365

H $-4.189353 \quad 3.8045210 .770675$

$\mathrm{H}-1.3978324 .9905580 .360047$

H -3.126148 $6.692550-0.560835$

H -4.136116 5.356919-1.072889

H -1.073025 $6.553204-3.606643$

H -1.485263 $7.502696-2.070708$

H $-2.2047654 .518152-4.098348$

H -3.579805 $4.083774-3.113360$

H $-0.6274423 .425828-2.414074$

H -2.6599901 .2621090 .966788$

C 1.4617291 .5107823 .088257

O 2.1811041 .7645391 .868671

C 2.7145843 .0984021 .898483

C 2.0338763 .7949303 .071466

C 1.8442712 .6420464 .040763

H 3.7847342 .9962152 .117493

C 2.5410533 .7713700 .531058

H 2.6422364 .6066353 .481717

H 1.0597674 .2121022 .792043

C 0.8097362 .9145765 .118543

H 2.8099132 .4237814 .516093

C $2.9345082 .886132-0.668912$

O $1.160473 \quad 4.113861 \quad 0.357314$

H 3.0949464 .7173630 .517849

H $2.1783292 .102940-0.799737$

O $2.8610643 .695300-1.849676$

C $4.3252472 .233986-0.582114$

C $4.6352111 .405076-1.842565$

H 4.3640251 .5910180 .302399

O $5.3055223 .255781-0.421163$

H $3.776251 \quad 0.757087-2.049585$

H $4.7480032 .076763-2.702394$

H 1.1074513 .7734125 .728931

H -0.1723893 .1337514 .686020$

H 0.7005052 .0501355 .780918

C 1.8035210 .1153363 .618098

H $1.0727544 .537449-0.514017$

H $3.5649164 .364107-1.789433$

H $6.1498562 .824106-0.208213$

H $1.204171-0.1096364 .507695$

H 2.8570580 .0685023 .919853

H 0.3943951 .5612152 .837299

C $5.008799-1.295443-0.468729$

O $5.951648-0.220871-0.520322$

C $5.9179730 .551002-1.724592$

C $6.174012-0.358131-2.936387$

C $5.208638-1.542303-2.974999$

C $5.201827-2.274668-1.633165$

H $3.995506-0.876654-0.517313$ 
C $5.111238-1.9636930 .910601$

H $6.7597091 .251543-1.662055$

O $6.0635160 .372402-4.153598$

H $7.198501-0.749164-2.886776$

H $4.196447-1.229008-3.256014$

O $5.634425-2.463121-3.982534$

H $6.141422-2.828933-1.527488$

O $4.143055-3.229807-1.619422$

C $6.442643-2.6314231 .187039$

H $4.973455-1.2103521 .695474$

H $4.316633-2.7130361 .005272$

H $6.6980951 .108111-4.111180$

H $5.671128-1.974738-4.822938$

H $4.270018-3.814836-2.385955$

O $6.265155-3.6085492 .124952$

O $7.508813-2.3165190 .676330$

C $7.447296-4.3206712 .503352$

H $8.170692-3.6377042 .958905$

H $7.166700-5.0776203 .240496$

H $7.882792-4.8222421 .633845$

SCF Energy (B3LYP/6-31G**//MMFF) $=-3245.92053150$

02_261

MMFF Geometry

C -2.699855-1.127559 3.385337

C $-2.252328 \quad 0.081744 \quad 3.757185$

C -2.718069 1.4324703 .268281

O -3.8091741 .3009592 .328387$

C -1.5731952 .1973742 .578510$

C -1.7307723 .7023832 .683880$

C -2.1323984 .5144531 .687061$

C $-1.345546 \quad 4.2922644 .018204$

C $-2.548275 \quad 4.1450870 .290772$

C $-1.5434444 .647035-0.757652$

C $-0.238598 \quad 3.836932-0.767535$

C $0.7921294 .460191-1.709662$

C $2.0646903 .603074-1.802485$

O $-0.5603282 .515449-1.210174$

O $2.8352614 .075356-2.917885$

C $1.6627442 .146680-2.114383$

C $2.9538693 .759196-0.563503$

O $2.7928441 .281360-2.064969$

C $0.5414201 .601374-1.200381$

C $0.0405880 .241012-1.739410$

O $1.055391 \quad 1.4125230 .119656$

C -4.8760112 .1268532 .485665$

O

C -5.8894451 .9412351 .422752$

C $-5.6822691 .265262 \quad 0.281740$

C $-6.6586821 .095511-0.800824$

C $-8.0379191 .667562-0.594873$

C $-1.294227-0.215114-1.135333$

C $-1.727617-1.566724-1.675773$

C $-3.647897-3.157445-1.572593$

O $-1.061713-2.220182-2.473932$

C $-4.606351-2.789769-2.659151$

C $-5.939405-2.820945-2.514885$

C $-6.897682-2.388864-3.585497$

C $-7.745499-1.209078-3.160885$

C $-9.049885-1.367483-2.879787$

C $-7.107966 \quad 0.164347-3.153347$

C $-6.2882070 .433464-1.917248$

N $-2.954292-1.952665-1.156076$

H -3.467167 -1.215033 2.621950

H $-1.494457 \quad 0.1248534 .538711$

H -3.0550611 .9648474 .165801$

H -1.502050 $1.887321 \quad 1.530526$

H $-0.605658 \quad 1.937242 \quad 3.028717$

H -2.182119 $5.583881 \quad 1.890813$

H -1.420938 5.3849194 .028322

H -1.994554 3.9124624 .812794

H -0.3097134 .0337564 .261614$

H -2.719576 $3.070820 \quad 0.176734$

H -3.517710 4.6227350 .100730

H -2.022058 4.566845 - 1.742255

H - $-1.3274415 .708892-0.586094$

H $\quad 0.164825 \quad 3.7927620 .249805$

H $1.0396305 .481992-1.396144$
H $\quad 0.3602674 .550796-2.716184$

H $3.0362945 .014878-2.766772$

H $1.3024002 .125728-3.152628$

H $3.2501294 .807058-0.434097$

H $3.8866313 .196902-0.675690$

H $2.4664793 .437667 \quad 0.359284$

H $3.4715161 .653508-2.653918$

H $-0.1013970 .310012-2.825901$

H $0.800718-0.529817-1.559655$

H $\quad 0.5322630 .7292530 .567726$

H -6.8328132 .4408101 .621354$

H -4.7105270 .8060710 .110054$

H $-8.497523 \quad 1.2494190 .307733$

H $-8.7256381 .454856-1.415743$

H $-7.9911742 .757582-0.494816$

H $-1.225827-0.285794-0.045361$

H $-2.0823990 .507035-1.379463$

H $-4.156430-3.562391-0.692430$

H $-2.930280-3.899459-1.936118$

H $-4.178847-2.457340-3.602340$

H $-6.367776-3.154063-1.572333$

H $-7.533126-3.249165-3.832112$

H $-6.371687-2.134958-4.514437$

H $-9.676861-0.525255-2.607923$

H $-9.520921-2.344337-2.913038$

H $-7.8675290 .941858-3.293903$

H $-6.457236 \quad 0.264940-4.031754$

H $-5.2671760 .058039-1.978728$

H $-3.451838-1.313643-0.542432$

C -1.341941 -3.2951193 .183913$

O $-0.126240-2.6045862 .838353$

C $0.219912-2.9414061 .481871$

C $-0.671347-4.1171911 .097293$

C $-1.935819-3.7969781 .871410$

H $-0.056868-2.0763010 .869948$

C $1.727072-3.1867961 .327398$

H $-0.830987-4.1825020 .017077$

H -0.255366 -5.0724571 .435416$

C -2.874576 -4.979760 2.026605

H -2.463554 -2.987940 1.356104

C $2.567180-1.9221171 .613458$

$\begin{array}{llll}\text { O } & 2.141552 & -4.237929 & 2.196686\end{array}$

H $1.925316-3.5069030 .296932$

H $2.161345-1.0898901 .025896$

O $2.479107-1.5541722 .988871$

C $4.054057-2.0844381 .237655$

C $4.856110-0.7906011 .474604$

H $4.111305-2.3676790 .181369$

O $4.653372-3.1392321 .986174$

H 4.3340270 .0415590 .989775

H $4.884089-0.5701812 .548766$

H $-3.202219-5.3421751 .046864$

H -2.389629 -5.8123952 .546979$

H -3.764663 -4.6974442 .597545$

C -2.237226 -2.4021414 .043644$

H $1.825636-5.0771861 .822978$

H $1.541719-1.3991183 .194399$

H $4.523810-2.9397452 .929238$

H $-1.689550-2.1545064 .962520$

H -3.124642 -2.964787 4.357067

H $-1.035013-4.1519643 .798984$

C $5.876309-0.381742-1.355034$

O $6.379670-1.323915-0.400724$

C $6.311491-0.8863630 .960770$

C 7.1295570 .4012051 .131534

C 6.7142181 .4783460 .129443

C $6.6838010 .918522-1.294349$

H $4.822022-0.175087-1.142348$

C $5.960788-1.034202-2.737909$

H $6.805155-1.6641161 .556978$

$\begin{array}{lllll}\text { O } & 6.998989 & 0.893556 & 2.460673\end{array}$

$\begin{array}{llll}\text { H } & 8.190900 & 0.166250 & 0.978917\end{array}$

H $5.743163 \quad 1.9074180 .400163$

$\begin{array}{lllll}\text { O } & 7.644722 & 2.562218 & 0.187962\end{array}$

H $7.7089630 .742414-1.643769$

O $6.0930561 .881243-2.164133$

C $4.938162-2.138965-2.864199$

H $5.786627-0.316208-3.547187$ 
H $\quad 6.949671-1.477187-2.906456$

H 7.5092141 .7195452 .516960

H $8.5233192 .216897-0.045237$

H $6.6003042 .706014-2.072458$

O $3.716069-1.609313-3.157297$

O $5.191341-3.324889-2.697880$

C $2.644557-2.551018-3.267938$

H $2.884644-3.323095-4.005330$

H $2.438824-2.998563-2.291099$

H $1.753860-2.013542-3.603565$

SCF Energy (B3LYP/6-31G**//MMFF) $=-3245.91073712$

\section{$02 \_262$}

MMFF Geometry

C 2.6504924 .4106470 .786168

C 3.6499463 .5774870 .462208

C $4.1400033 .363229-0.945938$

O $3.7638512 .014246-1.319453$

C $5.6719603 .487372-0.996191$

C $6.2613293 .193845-2.362967$

C $6.9598562 .083413-2.676434$

C $6.0367964 .261160-3.402761$

C $7.2860720 .923605-1.777189$

C $6.469235-0.319880-2.151335$

C $6.410120-1.329269-0.995606$

C $5.723569-2.621271-1.439698$

C $5.527795-3.601948-0.273702$

O $5.665433-0.7196320 .066260$

O $4.602661-4.614333-0.700505$

C $4.866606-2.8565040 .905869$

C $6.829760-4.3279960 .084687$

O $4.810473-3.6866542 .063098$

C $5.552258-1.5187021 .251600$

C $4.769058-0.6855892 .298329$

O $6.844054-1.7857331 .811719$

C $2.4861911 .831573-1.759786$

O $1.6471392 .696154-1.958725$

C $2.2603850 .377358-1.917793$

C $1.023477-0.076272-2.171636$

C $0.628461-1.478609-2.326341$

C $1.682922-2.529781-2.104134$

C $3.346352-0.2795171 .895430$

C $2.264764-1.2307832 .380253$

C $-0.174019-1.6869522 .140875$

O $2.471238-2.1265613 .194310$

C $-0.388837-2.7442831 .103564$

C -1.567065 -2.946177 0.494542

C -1.818420 -4.016787 -0.527474

C $-2.313981-3.462008-1.844631$

C $-3.628745-3.373292-2.107922$

C $-1.284315-3.099604-2.890859$

C -0.652786 -1.757405-2.645450

N $1.026721-0.9351551 .823469$

H 2.1812535 .0174370 .016262

H $4.0779452 .941413 \quad 1.234341$

H $3.6893814 .081130-1.642492$

H $6.1097372 .832000-0.237170$

H $5.9685564 .503054-0.700649$

H $7.3373551 .981447-3.693023$

H $6.5437794 .035261-4.346901$

H $4.9698364 .368654-3.620783$

H $6.4195635 .223589-3.047758$

H $8.3548310 .695979-1.876130$

H $7.1421071 .176398-0.724964$

H $5.447816-0.020643-2.415325$

H $6.911268-0.787044-3.040027$

H $7.431761-1.533181-0.653902$

H $6.283096-3.098810-2.253783$

H $4.737364-2.381642-1.858461$

H $4.972106-5.043926-1.491019$

H $3.829078-2.6575750 .612892$

H $7.199738-4.897595-0.776373$

H $\quad 6.666823-5.062614 \quad 0.880871$

H $7.626821-3.650240 \quad 0.397074$

H $4.350861-4.5056261 .810130$

H $4.783646-1.1925953 .270689$

H $5.329111 \quad 0.2485272 .450341$

H $7.122446-1.0117022 .328619$
H $3.111299-0.281919-1.796878$

H $0.2154830 .647216-2.275180$

H $2.450756-2.471320-2.882759$

H $1.292588-3.549515-2.109538$

H $2.159378-2.395324-1.127438$

H 3.1222960 .6906452 .356571

H $3.266176-0.1534670 .812384$

H -1.002550 -0.973474 2.180116

H $-0.064764-2.1522293 .125513$

H $0.454885-3.3841780 .856367$

H $-2.417389-2.3280250 .771747$

H -2.565084 -4.706095 -0.111601

H $-0.922274-4.625947-0.698513$

H -3.991453 -3.002885 -3.060944

H $-4.371964-3.661972-1.372783$

H $-1.757429-3.055778-3.881508$

H $-0.552107-3.907607-2.974315$

H -1.344403 -0.925416 -2.783603

H $0.956928-0.2037801 .121703$

C $0.910343 \quad 3.4295942 .245821$

O $-0.175808 \quad 3.823728 \quad 1.388118$

C -1.399819 3.2648441 .920605

C -1.0214262 .5778393 .230202$

C 0.2657813 .2848273 .617133

H -2.0614904 .1184582 .109936$

C -1.9986572 .3458160 .846109$

H -1.805591 2.6685423 .987917

H -0.8309511 .5092303 .073532$

C 1.0980572 .5096384 .623084

H $\quad 0.019633 \quad 4.2736784 .025751$

C -3.1895551 .4959481 .323931$

O $-2.4105513 .170289-0.245966$

H $-1.211777 \quad 1.687308 \quad 0.457477$

H -2.806678 0.8153652 .094114

O $-4.156917 \quad 2.3272501 .958913$

C -3.8608590 .6151360 .247218$

C $-4.8899641 .319075-0.657522$

H $-4.387397-0.1789220 .787506$

O $-2.869073-0.026840-0.546692$

H $-5.6504841 .798780-0.033135$

H -4.402005 2.112097 -1.232988

H 0.5379812 .3619305 .552225

H $\quad 1.376607 \quad 1.5226534 .238724$

H 2.0190153 .0477794 .867487

C 2.0411054 .4535912 .160401

H $-1.6346093 .681862-0.533276$

H -4.8545181 .7483222 .310981$

H $-2.5000290 .633693-1.157323$

H 2.8064274 .2480302 .917095

H 1.6641755 .4670502 .340016

H 1.2614982 .4498661 .891555

C -7.168024 -0.639164 -0.165065

O $-6.048306-0.838428-1.035044$

C $-5.5617480 .351765-1.662423$

C $-6.6769150 .987015-2.505407$

C $-7.9540171 .205610-1.695275$

C $-8.352406-0.071121-0.953448$

H -6.8940190 .0527710 .640205$

C -7.508099 -1.994500 0.464926

H $-4.7766670 .028884-2.358054$

O $-6.2556372 .229809-3.057078$

H $\quad-6.9142290 .324477-3.348088$

H -7.847271 $2.044832-0.997847$

O $-9.0185041 .556710-2.582604$

H -8.721108 -0.813521 -1.672758

O $-9.4186090 .221939-0.053635$

C -6.393618 -2.479239 1.363925

H -8.428120 -1.955339 1.058346

H -7.652154 -2.752683 -0.314559

H $-5.4536842 .060289-3.580239$

H $-8.7366612 .348519-3.072445$

H -10.142204 $0.606741-0.577360$

O $-6.295433-1.6806132 .465064$

O $-5.695717-3.4543681 .117010$

C $-5.246426-2.0184323 .377876$

H $-4.275201-1.9677412 .876235$

H $-5.257350-1.2895304 .192669$

H -5.415186 -3.015482 3.795304 
H $-2.8481263 .723036-1.435064$

H $-3.1014546 .249823-1.901264$

$02 \_263$

MMFF Geometry

C $0.832109-3.9432621 .253176$

C $0.334241-2.9864832 .051715$

C $1.106199-2.0843162 .988318$

O $1.060079-0.7299382 .475350$

C 2.592411-2.446968 3.170261

C $3.275635-1.6593394 .272966$

C $4.132663-0.6420464 .056997$

C $2.991454-2.1268275 .676403$

C $4.502298-0.0397892 .730246$

C $5.726503-0.7261872 .113586$

C $6.144879-0.1589010 .746622$

C 6.7714681 .2311820 .866731

C $7.1680111 .789541-0.506917$

O $4.994636-0.117572-0.100635$

O $7.4622303 .186752-0.347728$

C $5.9484981 .693802-1.449020$

C $8.4504791 .139657-1.038620$

O $6.3051122 .050226-2.782804$

C $5.2493110 .309774-1.444298$

C $3.8927320 .346073-2.185736$

O $6.028107-0.663211-2.151795$

C $-0.027147 \quad 0.0303382 .768119$

O $-1.043934-0.3281023 .340208$

C $0.249488 \quad 1.4037432 .286071$

C -0.7357152 .3128862 .303742$

C -0.6388573 .7166401 .899166$

C 0.7356874 .2829761 .660370

C $2.7842091 .125710-1.470908$

C $1.5803831 .369313-2.366544$

C $-0.5518422 .657692-2.419140$

O $1.4493250 .860528-3.475922$

C $-0.8614104 .065031-2.020909$

C $-2.0517434 .455597-1.540571$

C $-2.3733395 .875055-1.170277$

C -2.9267846 .0096790 .230985$

C $-4.218260 \quad 6.318144 \quad 0.437715$

C -1.9646675 .8657881 .391864$

C $-1.780568 \quad 4.428612 \quad 1.796829$

N $0.6779002 .241874-1.773637$

H $1.903362-4.1164141 .207916$

H $-0.744387-2.8437892 .064480$

H $0.611107-2.1202793 .967142$

H $3.121831-2.2979592 .221787$

H $2.695634-3.5134573 .410158$

H $4.605810-0.1699644 .916506$

H $3.561569-1.5655756 .424500$

H $1.930287-2.0072595 .914943$

H $3.256523-3.1835505 .786396$

H $3.650967-0.0795582 .043048$

H 4.7032481 .0252572 .892396

H $6.576447-0.6853652 .806403$

H $5.487798-1.7888381 .973526$

H $6.871311-0.8596940 .318255$

H 7.6391961 .2109281 .537750

H 6.0559351 .9287391 .321015

H 8.1734393 .2705550 .310382

H $5.2348312 .453795-1.110281$

H $9.2900281 .340692-0.362076$

H $8.7379341 .565385-2.006218$

H $8.3710860 .055869-1.146165$

H $6.7196992 .929320-2.747280$

H $4.0440910 .731487-3.202452$

H $3.537415-0.685352-2.323694$

H $6.812022-0.228181-2.522363$

H 1.2516721 .6300431 .940112

H -1.720391 1.9978052 .643325

H 1.3706734 .1260902 .539925

H 0.7369895 .3581291 .470448

H 1.2143613 .8068630 .800560

H $2.4452550 .571369-0.587784$

H $3.1469042 .103008-1.138979$

H -1.332670 $1.962046-2.103696$

H $-0.4388572 .589735-3.505569$

H $-0.0735464 .802941-2.153703$

\begin{tabular}{rrr}
-1.492397 & 6.521647 & -1.268798 \\
\hline
\end{tabular}

H $-4.9087076 .447445-0.389069$

H $-2.352360 \quad 6.407372 \quad 2.264908$

H -1.0268046 .3647761 .135243$

H -2.7196483 .9264522 .033529$

H $0.7828442 .440735-0.784061$

C $0.156675-4.558093-1.084852$

O $-0.578298-3.360834-1.418370$

C $-1.335821-3.602097-2.619016$

C $-0.760844-4.874549-3.227126$

C $-0.411601-5.660186-1.980082$

H -2.367371 -3.803081 -2.306950

C $-1.295069-2.382532-3.542829$

H $-1.478814-5.388316-3.873358$

H $0.142218-4.672848-3.814876$

C $0.550924-6.807118-2.235916$

H -1.337608 -6.058961-1.543970

C $-1.821353-1.082009-2.904169$

O $0.066344-2.157352-3.932596$

H -1.845139-2.603128 -4.465246

H -1.123499-0.747672 -2.127437

O $-1.794160-0.071767-3.922095$

C $-3.244729-1.161612-2.318014$

C $-3.7199050 .195714-1.766291$

H -3.274614 -1.900153 -1.513379

O $-4.161409-1.579683-3.328863$

H -2.989826 $0.549542-1.030890$

H $-3.7423800 .926086-2.583826$

H $0.112117-7.529016-2.932264$

H $1.492068-6.452042-2.669080$

H $\quad 0.786319-7.334890-1.306531$

C $-0.009007-4.8559370 .404611$

H $0.080717-1.363520-4.494355$

H -2.457927 -0.311742 -4.591257

H -3.965437 -2.506969 -3.543641

H $\quad 0.300299-5.8842350 .624540$

H -1.064719 -4.769218 0.685977

H $1.209029-4.366620-1.332390$

C $-4.522448-0.4888081 .122300$

O $-5.216315-0.832520-0.083596$

C $-5.1294620 .135387-1.134965$

C $-5.6614451 .492000-0.652449$

C -4.9791271 .9479440 .633754$

C -5.0452460 .8408191 .684358$

H $-3.446171-0.4161650 .923454$

C $-4.754254-1.6265132 .130474$

H $-5.812801-0.208802-1.921368$

O $-5.4988512 .475874-1.668698$

H $-6.7397051 .401922-0.465781$

H -3.9462352 .2599410 .442521$

O $\quad-5.6715283 .1013511 .115407$

H -6.0818130 .7235622 .026009$

O $-4.260869 \begin{array}{lll}1.210407 & 2.814686\end{array}$

C -4.018377 -2.897308 1.763251

H $-4.402677-1.3322893 .125544$

H -5.823422 -1.865254 2.177344

H $-5.8138803 .322286-1.308006$

H -5.1671913 .4543021 .867510$

H -4.6746811 .9910273 .219391$

O $-4.336075-3.8670982 .672040$

O $-3.257237-3.027426 \quad 0.815624$

C $-3.704222-5.1352512 .469847$

H $-4.144894-5.8497623 .170110$

H -3.874164 -5.495172 1.450392

H -2.633746 -5.053486 2.678598

SCF Energy (B3LYP/6-31G**//MMFF) $=-3245.91520631$

$02 \_264$

MM̄FF Geometry

C -2.2172493 .5608531 .605737$

C -1.411942 2.5547541 .233449

C -0.7510481 .6099392 .201319$

O 0.6199841 .4729481 .766304

C -1.446340 0.2412052 .119256

C $-1.078346-0.7020283 .244502$ 
C $-0.245892-1.7540553 .122279$

C $-1.764757-0.4314344 .558758$

C $0.537840-2.1682661 .907904$

C $2.003046-2.4349402 .273945$

C $2.852018-2.7906981 .045943$

C $4.275185-3.1496721 .479369$

C $5.194176-3.4120260 .279588$

O $2.874625-1.6569640 .171110$

O $6.549378-3.4152450 .753562$

C $5.064902-2.237242-0.709276$

C $4.946584-4.792505-0.339581$

O $5.792387-2.500764-1.907463$

C $3.600532-1.866548-1.047108$

C $3.584305-0.548149-1.853799$

O $3.021894-2.908619-1.833343$

C 1.6032621 .4610542 .703901

O 1.4773651 .5314963 .914838

C 2.8874191 .3623121 .976830

C 4.0536361 .4777242 .627849

C 5.3670891 .4227641 .987882

C 6.3897840 .6518052 .774473

C $2.1899250 .021066-2.145099$

C $2.3310021 .315890-2.921824$

C $3.1119683 .665082-2.623607$

O $2.2474341 .346049-4.147308$

C $4.6085443 .613758-2.583230$

C $5.3574184 .320936-1.723592$

C $6.8469834 .168906-1.603641$

C $7.2645173 .594109-0.264414$

C 8.0023924 .3113960 .599473

C 6.8872942 .1574980 .045016

C 5.6008462 .0618810 .823576

N $2.6225992 .398738-2.107496$

H -2.4043043 .7386472 .661555$

H -1.254322 2.3658090 .173535

H -0.7979072 .0230273 .215950$

H - $1.231511-0.218277 \quad 1.148817$

H $-2.536880 \quad 0.3729672 .141661$

H $-0.100041-2.3914063 .993784$

H -1.508942 -1.173487 5.322550

H -1.4788750 .5508624 .947163$

H -2.851844 -0.4522464 .432429$

H $0.094223-3.0848271 .503601$

H $0.493688-1.4124201 .119756$

H $2.418633-1.5429592 .759006$

H $2.052881-3.2538403 .002935$

H $2.381287-3.6359150 .529869$

H $4.270172-4.0142982 .154828$

H $4.697455-2.3190702 .061924$

H $6.626768-4.1043441 .435547$

H $5.542860-1.363731-0.243449$

H $5.127099-5.5816630 .400281$

H $5.644308-4.987861-1.161382$

H $3.928262-4.918693-0.713159$

H $\quad 6.706006-2.713357-1.650832$

H $4.1520180 .211326-1.301561$

H $4.099104-0.696010-2.812184$

H $2.247831-2.560079-2.301820$

H 2.8469141 .2055630 .904887

H 4.0670661 .6159023 .706754

H $6.000669-0.3376333 .041003$

H 6.6395501 .1823713 .699685

H 7.3193030 .4816632 .227197

H $1.595040-0.666262-2.752370$

H $\quad 1.6308420 .207699-1.221555$

H $2.7062854 .458070-1.987931$

H $2.7645623 .818297-3.649604$

H $5.0936412 .917831-3.264167$

H $4.8743905 .010185-1.034758$

H $7.2997355 .156058-1.760532$

H $7.244323 \quad 3.527114-2.400066$

H 8.3223433 .8931101 .548329

H $8.299405 \quad 5.3314900 .379929$

H 7.7199211 .6785920 .569301

H $6.7737371 .598677-0.892242$

H 4.7655232 .5873400 .364035

H $2.7197912 .242887-1.109050$

C -4.4026094 .0081570 .493005$
O $-4.4485392 .705410-0.124222$

C $-5.4701292 .699561-1.136800$

C $-6.3011713 .952537-0.894632$

C $-5.2386504 .919217-0.404121$

H $-4.9597282 .800446-2.102007$

C $-6.2400651 .375073-1.090950$

H $-6.8105014 .295220-1.800284$

H $-7.0585013 .795534-0.117808$

C -5.8040326 .1356220 .307598$

H $-4.6413895 .251455-1.263993$

C $-5.3393810 .120407-1.167980$

O $\quad-6.9759351 .3025440 .136186$

H $\quad-6.9890501 .352369-1.890639$

H $-4.7427760 .039242-0.253616$

O $-6.190900-1.030016-1.206640$

C $-4.4250480 .081816-2.402714$

C $-3.594911-1.203796-2.587068$

H $-3.7336750 .930605-2.378516$

O $\quad-5.226237 \quad 0.246925-3.578313$

H -4.261683 -2.071525 -2.619469

H $-3.152928-1.149131-3.589928$

H $-6.4423366 .714135-0.368196$

H $-6.4069305 .848745 \quad 1.175744$

H $-5.000567 \quad 6.7914020 .657352$

C $-2.9428614 .443896 \quad 0.628117$

H $-6.342818 \quad 1.4275870 .863972$

H -6.761533 -0.995264 -0.419798

H $-5.864597-0.486876-3.602142$

H -2.8781295 .4794880 .979823$

H -2.448041 $4.400642-0.350325$

H -4.8684613 .9082751 .482363$

C -3.451642 -3.133006 -0.179535

O $-2.965671-1.788547-0.258990$

C $-2.475828-1.379093-1.538673$

C $-1.341743-2.315754-1.981528$

C $-1.788994-3.772016-1.985152$

C $-2.388108-4.142121-0.632033$

H $-4.353799-3.233129-0.794144$

C -3.834655 -3.398202 1.281595

H -2.024244 -0.389760 -1.391033

O $-0.864779-1.972857-3.278499$

H -0.493664 -2.212410 -1.291987

H -2.495126 -3.975714 -2.798772

O $-0.654690-4.611360-2.213900$

H -1.585925 -4.203735 0.113846

O $-2.981757-5.436520-0.720750$

C $-5.067716-2.6249351 .679967$

H $-4.055183-4.4572911 .458660$

H -3.013220 -3.1301211 .956968$

H -0.640281 -1.026890 -3.267875

H $-0.256542-4.334988-3.057252$

H -2.293187 -6.050979-1.027772

O $-4.713275-1.5171842 .389084$

O $-6.206513-2.9663121 .387955$

C $-5.784390-0.6943882 .862426$

H $-5.3905590 .312246 \quad 3.027076$

H -6.601345 -0.634053 2.137923

H -6.149587 -1.091707 3.813369

SCF Energy (B3LYP/6-31G**//MMFF) $=-3245.91966962$

$02 \_265$

MMFF Geometry

C $-1.827776-1.362028-3.058925$

C $-3.134003-1.132986-3.267771$

C $-3.8580730 .190205-3.183494$

O $-2.9904191 .213312-2.647490$

C $-5.0830840 .072726-2.258670$

C $-6.1061491 .171475-2.469975$

C $-6.3222132 .198451-1.625236$

C $-6.9617681 .023323-3.702870$

C $-5.5881882 .529331-0.356207$

C $-6.4372882 .269972 \quad 0.897211$

C $-6.5992770 .776604 \quad 1.223228$

C -7.5086350 .5788602 .437291$

C $-7.590018-0.8978742 .855624$

O $-5.297798 \quad 0.2561001 .504775$

O $-8.186204-0.9549164 .160439$

C $-6.158702-1.4605492 .987665$ 
C $-8.502889-1.705758 \quad 1.925570$ O $-6.182353-2.8650353 .232716$ C $-5.257673-1.1512891 .770409$ C -3.789429-1.520280 2.091128 O $-5.702146-1.9171790 .650123$ C -2.960783 $2.417828-3.271540$ O $-3.5456972 .723296-4.302078$ C $-2.0995903 .386746-2.554952$ C -1.642923 $3.205649-1.305674$ C $-0.8193464 .150486-0.545111$ C $-0.3670365 .406855-1.243250$ C $-2.781600-1.0575641 .028073$ C $-1.347712-1.2293441 .497431$ C $0.958382-0.4248191 .003227$ O $-1.034528-1.9280062 .457649$ C $1.1791880 .758418 \quad 1.890423$ C 1.9631921 .7963981 .564105 C 2.1498573 .0002872 .441265 C $1.772197 \quad 4.287551 \quad 1.741625$ C 2.7175185 .1003141 .240045 C 0.3097304 .6746691 .700275 C -0.4953713 .8506550 .730256$ N $-0.457583-0.5203050 .702077$ H -1.165221 -0.545498 -2.787054 H -3.754865 -1.973511 -3.575378 H $-4.1672070 .436461-4.206180$ H $-4.7459540 .057141-1.217818$ H -5.594452 $-0.886305-2.417555$ H -7.111454 2.906995-1.874383 H -7.735683 $1.795689-3.767597$ H $-6.352076 \quad 1.094630-4.608423$ H -7.468270 $0.052629-3.699119$ H -4.626869 2.012019-0.286411 H -5.342381 $3.598421-0.392967$ H -5.9372522 .7649461 .740033$ H -7.4226002 .7390310 .784191$ H -7.0172880 .2616450 .351582$ H -8.5113120 .9789502 .241855$ H -7.1157151 .1567553 .285756$ H -9.062662 -0.5363344 .107939$ H $-5.707880-0.9986703 .877422$ H -9.518961 -1.293376 1.933894 H -8.598061 -2.7420872 .268067$ H -8.159714 -1.714592 0.888933 H -6.747083 -3.013611 4.010380 H $-3.507343-1.0535443 .044233$ H -3.693127 -2.606038 2.218180 H -4.958214 -2.057593 0.044019 H -1.891398 $4.295720-3.110785$ H -1.905275 2.291662 -0.775928 H $0.1858355 .162156-2.157162$ H $0.2998696 .024641-0.637874$ H -1.227896 $6.030688-1.507997$ H -2.901213 -1.6345140 .105355$ H $-2.937191 \quad 0.0015160 .793727$ H $1.486664-0.3278770 .050494$ H $1.292048-1.3374911 .506406$ H $\quad 0.658251 \quad 0.7654862 .845122$ H 2.4971901 .7850930 .616462 H 3.2013913 .0317952 .751863 H 1.5734852 .9126983 .370884 H 2.4611576 .0404600 .763623 H 3.7700834 .8418951 .290556 H 0.1950085 .7437911 .487037 H -0.1209184 .5522132 .702738$ H -0.8656352 .9167461 .153639$ H $-0.8211050 .075152-0.036391$ C $-0.348834-3.137734-2.038986$ O $0.888121-2.393269-2.055544$ C $1.991438-3.322919-2.024272$ C $1.413398-4.600817-1.444130$ C $0.042203-4.617673-2.086741$ H $2.289990-3.471165-3.070137$ C $3.146151-2.715571-1.222238$ H $2.008928-5.485651-1.687090$ H $1.326343-4.528955-0.352753$ C $-0.946754-5.532626-1.384316$ H $0.148758-4.944985-3.130022$
C $4.332808-3.669835-1.007628$

O $3.608458-1.555472-1.921363$

H $2.771083-2.372029-0.253662$

H $4.018796-4.516722-0.386121$

O $4.707132-4.216578-2.278574$

C $5.583427-3.009320-0.393773$

C $5.318229-2.3005360 .945504$

H $6.019419-2.312666-1.118137$

O $6.566006-4.031270-0.188321$

H $4.550524-1.5337090 .808443$

H $4.919867-3.0295511 .662074$

H $-0.595697-6.569439-1.408445$

H -1.081704 -5.247537 -0.335415

H -1.926015 -5.494890 -1.871937

C -1.204947 -2.721721 -3.237686

H $2.843649-0.967844-2.047481$

H $5.461293-4.812013-2.127428$

H $\quad 6.210707-4.6583380 .464599$

H -1.987083 -3.470960 -3.408615

H $-0.580841-2.683599-4.139288$

H $-0.855519-2.900775-1.096237$

C $6.561466 \quad 0.400914 \quad 0.289510$

O $7.260326-0.8084130 .610287$

C $6.589524-1.6585271 .547432$

C $6.355377-0.9051932 .864436$

C 5.6479890 .4309002 .641160

C 6.3527491 .2439041 .553956

H $5.5915050 .153938-0.158496$

C $7.3973451 .131027-0.768436$

H $7.284172-2.4789911 .767367$

O $5.603625-1.7122143 .764244$

H $7.327240-0.7158533 .338565$

H 4.5931380 .2761512 .390112

O $5.649304 \quad 1.175688 \quad 3.861425$

H 7.3195161 .5994551 .932415

O 5.5664372 .3902071 .246348

C $6.5963842 .167524-1.518587$

H $8.2836181 .585510-0.309160$

H $7.7833680 .422945-1.512380$

H $5.453400-1.1882714 .569767$

H 6.5744241 .3349174 .115504

H 5.4629652 .8995222 .068208

O $7.1766493 .393781-1.381771$

O $5.5744551 .919714-2.145808$

C 6.491748 4.472447 -2.025871

H $5.4850394 .582950-1.611360$

H $7.0517065 .392000-1.836249$

H $6.4494024 .303392-3.106102$

SCF Energy (B3LYP/6-31G**//MMFF) $=-3245.91658831$

$02 \_266$

MMFF Geometry

C $-2.789565-3.341223-2.160112$

C $-4.068223-3.736565-2.057602$

C $-5.252445-2.892199-1.637775$

O $-4.856242-1.550810-1.273249$

C $-5.926981-3.536384-0.411110$

C $-7.192348-2.8266800 .033150$

C -7.305063 -2.068622 1.142576

C $-8.395486-3.051903-0.847130$

C $-6.232267-1.7241122 .138335$

C $-6.039458-0.2049222 .237753$

C $-4.7958330 .165003 \quad 3.060414$

C -4.6748741 .6844343 .193075$

C -3.3652072 .1088323 .873992$

$\begin{array}{lllll}\text { O } & -3.656317 & -0.364267 & 2.376104\end{array}$

O -3.1890693 .5167603 .651765$

C $-2.1854471 .393656 \quad 3.180943$

C -3.4246411 .9139045 .393386$

O -0.9639201 .6421093 .871839$

C $-2.399214-0.1252913 .021584$

C - $-1.305287-0.8036562 .159873$

O $-2.359797-0.7418544 .313894$

C $-4.825983-0.614383-2.261386$

O $-5.055451-0.788444-3.447520$

C $-4.4569270 .678755-1.641895$

C $-4.2252541 .749381-2.416130$

C $-3.8885563 .097930-1.951335$ 
C $-3.765263 \quad 3.316089-0.466634$ C $-1.238263-0.3368840 .702568$ C -0.2250410 .7629570 .439377$ C $0.5537342 .264753-1.394919$ $\begin{array}{lllll}\text { O } & 0.601886 & 1.134428 & 1.266849\end{array}$ C $-0.1011943 .603980-1.280182$ C $-0.3138864 .413461-2.328701$ C $-0.9158825 .784099-2.218004$ C $-2.1038155 .979037-3.132198$ C $-1.9714186 .605376-4.313823$ C $-3.4599425 .537914-2.632143$ C $-3.721794 \quad 4.074747-2.867691$ N $-0.3312931 .250025-0.857293$ H -2.519094 -2.311250 -1.946542 H $-4.304569-4.772197-2.296994$ H $-5.965638-2.859293-2.470519$ H $-5.204110-3.5933720 .409265$ H -6.186201 -4.580617 -0.634635 H -8.278423 -1.6340331 .368254$ H $-9.300168-2.584942-0.443174$ H $-8.227767-2.632832-1.843886$ H -8.597335 -4.123294 -0.948042 H $-6.526950-2.1165933 .118973$ H -5.282312 -2.200373 1.888629 H $-5.937110 \quad 0.2070821 .226118$ H -6.9321240 .2491992 .685824$ H $-4.874354-0.3038114 .048412$ H -5.538935 2.0969723 .728854 H -4.7030732 .1374492 .193611$ H $-3.964643 \quad 3.9770244 .015897$ H -2.080458 1.8447592 .186733 H -4.2298822 .5200615 .825718$ H -2.501653 2.257964 5.872799 H -3.6016910 .8770555 .686376$ H -0.8578852 .6068203 .935408$ H $-0.330067-0.7317802 .656392$ H $-1.536351-1.8785412 .131739$ H -2.151702 -1.684120 4.201451 H $-4.396707 \quad 0.717417-0.561061$ H $-4.3042801 .635142-3.496537$ H -4.7406813 .1959060 .015986$ H -3.389261 4.306022 -0.199952 H -3.063056 $2.600550-0.025756$ H $-0.928216-1.1882010 .084958$ H -2.220939 -0.0188020 .343219$ H $0.7643651 .993509-2.434251$ H $1.4959952 .264331-0.838863$ H $-0.385263 \quad 3.934692-0.283894$ H $-0.0029154 .090569-3.319945$ H $-0.1318216 .511423-2.467145$ H -1.208816 $6.011434-1.185465$ H -2.825201 $6.778024-4.960717$ H - $1.0049876 .953832-4.662842$ H $-4.2477736 .092768-3.159970$ H -3.576878 $5.832178-1.585284$ H $-3.8269253 .818193-3.922873$ H $-1.0777740 .909607-1.456743$ C $-0.519502-4.192106-1.547078$

O $0.194296-2.951746-1.723359$

C $1.611110-3.198499-1.574733$

C $1.733619-4.627918-1.070771$

C $0.531259-5.285302-1.720582$

H $2.037147-3.112108-2.582361$

C $2.192868-2.117322-0.655409$

H 2.682449 -5.093208-1.354109

H $1.646146-4.6658550 .022278$

C $0.155117-6.614239-1.089003$

H $0.744030-5.437479-2.787172$

C $3.661278-2.353086-0.258770$

O $2.087377-0.876401-1.357771$

H $1.570617-2.0305720 .242398$

H $3.725942-3.3421810 .210785$

O $4.463275-2.394816-1.438583$

C $4.261653-1.342376 \quad 0.742823$

$\begin{array}{llll}\text { C } & 4.320146 & 0.119549 & 0.263211\end{array}$

H $5.283962-1.6768290 .953158$

O $3.549456-1.4147081 .977160$

H $4.7192790 .151724-0.755195$
H 3.3073960 .5348940 .234034

H $0.978477-7.330192-1.181283$

H $-0.074610-6.501822-0.024085$

H $-0.723195-7.045992-1.578768$

C $-1.665322-4.261446-2.557867$

H $1.155539-0.762820-1.612187$

H $5.376600-2.589267-1.167671$

H $2.687869-0.9813541 .855299$

H $-2.032710-5.290351-2.641173$

H $-1.309477-3.951196-3.547751$

H $-0.908798-4.196370-0.519881$

C $7.329396 \quad 0.540668 \quad 0.256728$

$\begin{array}{lllll}\text { O } & 6.480143 & 0.501450 & 1.409426\end{array}$

C 5.1627211 .0190401 .198359

C 5.2494362 .4868660 .754908

C $6.1650782 .664202-0.455059$

C $7.5148381 .985971-0.216498$

H $6.889672-0.060442-0.547886$

C $8.666279-0.097384 \quad 0.648615$

H 4.6684391 .0054482 .178047

$\begin{array}{lllll} & & 3.960901 & 3.006284 & 0.445151\end{array}$

H 5.6492403 .0903361 .580223

H $5.6953812 .288172-1.371617$

$\begin{array}{llll}\text { O } & 6.397794 & 4.058630 & -0.667592\end{array}$

H 8.0859112 .5576390 .526184

O $8.2633301 .990480-1.429906$

C $8.498682-1.570658 \quad 0.938775$

H $9.4227870 .009566-0.136621$

H $9.0673090 .372374 \quad 1.554967$

H 3.3993112 .8839611 .229508

H $5.5293554 .482229-0.779496$

H $8.3402312 .915737-1.719622$

O $8.265167-2.252593-0.219094$

O $8.562148-2.0547912 .061275$

C $8.053192-3.659911-0.071748$

H $7.178186-3.8468640 .558257$

H $7.866642-4.080960-1.063323$

H $8.943598-4.1351800 .350569$

SCF Energy (B3LYP/6-31G**//MMFF)= -3245.91997372

02267

MMFF Geometry

$\begin{array}{llll}C & 1.085551 & 0.263106 & -3.709837\end{array}$

C $1.607413 \quad 1.134505-2.832747$

C $0.965725 \quad 2.375800-2.273775$

O $0.553342 \quad 2.021836-0.930012$

C $-0.2642302 .904233-3.035070$

C $-0.6873224 .280909-2.557624$

C $-1.6833684 .523103-1.682832$

C $0.0958935 .428659-3.142778$

C $-2.5714243 .524870-0.990946$

C $-4.0482333 .875907-1.205900$

C $-5.039323 \quad 2.799515-0.739867$

C -4.8760552 .4068620 .729121$

C $-5.8752011 .310327 \quad 1.135789$

\begin{tabular}{lllll}
\hline & -4.875537 & 1.648804 & -1.576839
\end{tabular}

$\begin{array}{lllll}\text { O } & -5.453793 & 0.778190 & 2.401457\end{array}$

C $\begin{array}{llll}-5.794295 & 0.155707 & 0.111793\end{array}$

C -7.2859151 .8696461 .352903$

O $-6.809078-0.8140040 .357180$

C $-5.8617210 .626931-1.357985$

C $-5.590669-0.501451-2.383385$

O \begin{tabular}{llll}
\hline & -7.178928 & 1.123032 & -1.632219
\end{tabular}

$\begin{array}{llll}\text { C } & 0.929812 & 2.813948 & 0.102349\end{array}$

$\begin{array}{lllll}\text { O } & 1.614133 & 3.825855 & 0.037448\end{array}$

$\begin{array}{llll}\text { C } & 0.399822 & 2.313258 & 1.392944\end{array}$

C $-0.404177 \quad 1.244851 \quad 1.518408$

C $-0.958328 \quad 0.7271562 .773376$

C $-0.538548 \quad 1.4105104 .049843$

C $-4.276489-1.265499-2.190750$

C $-4.442818-2.540311-1.379084$

C $-3.146184-4.180073-0.031831$

O $-5.520723-3.114251-1.244275$

C $-3.386881-3.8031551 .395550$

C $-2.454130-3.8832992 .355811$

C $-2.711472-3.5513393 .795907$

C $-1.960850-2.3235524 .255274$

C $-0.880301-2.4267915 .047117$ 
C $-2.528845-0.9738943 .879316$ C $-1.802495-0.3260682 .729211$ N $-3.238877-2.992206-0.859119$ H $0.0889080 .413534-4.113075$ H $2.5954030 .920459-2.428070$ H $1.7452583 .147343-2.252376$ H -1.096093 2.197536-2.944157 H $-0.0444932 .968770-4.109022$ H -1.878980 5.559561-1.409086 H $-0.2616076 .401017-2.787565$ H $1.1540415 .350501-2.875407$ H $0.0115925 .426737-4.234426$ H -2.377676 2.501761 -1.321171 H $-2.341586 \quad 3.5615450 .079954$ H -4.273753 4.817641-0.689176 H $-4.2160714 .049451-2.277644$ H -6.041665 $3.211910-0.907178$ H -4.976742 3.2834201 .380642 H -3.8652282 .0147610 .901951$ H $-5.417788 \quad 1.5121093 .038499$ H $-4.833453-0.3455200 .282622$ H -7.277455 2.633165 2.140125 H -7.970864 1.0887881 .701393 H -7.7105692 .3262780 .456698$ H -6.717935 -1.101328 1.281890 H $-6.449698-1.182154-2.424392$ H -5.545245 $-0.033007-3.377064$ H $-7.322417 \quad 1.100319-2.592998$ H 0.7093732 .8959222 .255039 H -0.6998650 .6972670 .625351$ H $0.553614 \quad 1.4116974 .143842$ H -0.9179780 .9266284 .951896$ H -0.8954702 .4460974 .066759$ H $-3.906747-1.583047-3.173980$ H -3.507937 -0.624339-1.745618 H -2.155378 -4.615916 -0.187736 H -3.896019 -4.910795 -0.352139 H $-4.388700-3.4673841 .653829$ H -1.455891 -4.2314112 .103444$ H -2.421665 -4.423905 4.395746 H -3.783083 -3.412673 3.987504 H $-0.359564-1.5468225 .409442$ H $-0.489053-3.3934485 .346294$ H -2.558256 -0.3289384 .763608$ H -3.583367 -1.082779 3.595354 H $-2.038015-0.7639661 .758774$ H -2.406023 -2.419538 -0.960393 C $1.395085-2.176956-3.258409$

O $1.799569-1.926801-1.898047$

C $2.318385-3.140287-1.325464$

C $2.005622-4.246394-2.326715$

C $2.069532-3.491941-3.642521$

H $3.405364-3.019067-1.262993$

C $1.734252-3.3389980 .077886$

H $2.721982-5.071590-2.268648$

H $1.002461-4.661740-2.176417$

C $1.397519-4.219066-4.793706$

H $3.123977-3.314293-3.893654$

C $1.898223-2.1194011 .007942$

O $0.328889-3.593568-0.037661$

H $2.164518-4.2337320 .540727$

H $1.295245-1.2850660 .629720$

O $1.352634-2.4609062 .287693$

C $3.348166-1.6449171 .211312$

C $3.411635-0.3974262 .111885$

H $3.796382-1.4004640 .243973$

O $4.128753-2.6805121 .802190$

H 2.8166140 .3926431 .642029

H $2.944810-0.6154293 .080255$

H $1.882940-5.183466-4.975054$

H $0.339327-4.409381-4.585202$

H $1.458767-3.631762-5.715136$

C $1.803499-0.990650-4.133128$

H - $0.072082-2.831777-0.490478$

H $0.425701-2.7183822 .146733$

H $4.204063-3.4015731 .155047$

H $1.572067-1.188100-5.185645$

H $2.886817-0.832934-4.069259$
H $\quad 0.302612-2.288759-3.251662$

C $5.239345 \quad 1.199506 \quad 0.268972$

$\begin{array}{lllll}\text { O } & 5.638653 & 0.182042 & 1.192907\end{array}$

$\begin{array}{llll}\text { C } 4.847819 & 0.105420 & 2.380417\end{array}$

C 4.9130841 .4409133 .133338

C $4.5374882 .621806 \quad 2.238330$

C 5.3244502 .5814220 .926858

H $4.2152111 .007858-0.075247$

C $6.1719961 .105903-0.942940$

H $5.327862-0.6490103 .016736$

$\begin{array}{llll}\text { O } & 4.060642 & 1.401923 & 4.273213\end{array}$

H 5.9365291 .5896663 .501873

H 3.4578942 .6451502 .056187

$\begin{array}{lllll}\text { O } & 4.851512 & 3.825779 & 2.941909\end{array}$

H $6.3724182 .842568 \quad 1.122541$

$\begin{array}{lllll} & & 4.799438 & 3.552915 & 0.025407\end{array}$

C $5.962856-0.197408-1.677278$

H $5.9930881 .915869-1.658988$

H $7.2234081 .175391-0.638155$

H 4.1161502 .2701924 .707704

H 4.5124264 .5699392 .416338

H 4.9510284 .4302510 .414891

O $6.912840-1.102222-1.306197$

O $5.045997-0.396799-2.463118$

C $6.801271-2.394704-1.909714$

H $5.876639-2.881295-1.586166$

H $6.834343-2.312321-3.000307$

H $7.649965-2.998866-1.578363$

SCF Energy (B3LYP/6-31G**//MMFF) $=-3245.90743140$

02_268

MMFF Geometry

C $1.797250 \quad 4.083217-0.698611$

C 2.4330834 .7442690 .281438

C $3.103898 \quad 4.1321601 .491103$

O 3.2883052 .7060641 .337026

C 2.2411754 .3854182 .740015

C 2.8882663 .9145464 .028751

C 2.5267282 .8125694 .716392

C 3.9885454 .8009824 .553987

C 1.4665101 .8093544 .356658

C $2.0700580 .410863 \quad 4.173020$

C $1.057085-0.574373 \quad 3.571457$

C $1.661290-1.9753063 .475308$

C $0.731824-2.9592582 .752170$

$\begin{array}{lllll}\text { O } & 0.714850 & -0.098747 & 2.265924\end{array}$

O $1.516440-4.121332 \quad 2.440851$

C $0.264563-2.3316071 .419858$

C $-0.399188-3.4391903 .669161$

O $-0.712715-3.173114 \quad 0.813708$

C $-0.262829-0.8896021 .575855$

C $-0.546787-0.180907 \quad 0.228647$

$\begin{array}{llll}\text { O } & -1.497703 & -0.920015 & 2.300671\end{array}$

C $4.3887252 .301440 \quad 0.641170$

$\begin{array}{llllll}\text { O } & 5.221514 & 3.016385 & 0.105777\end{array}$

$\begin{array}{llll}\text { C } & 4.409418 & 0.821388 & 0.637825\end{array}$

C $5.3474690 .172498-0.068688$

C $5.524099-1.279696-0.150813$

C $4.538951-2.1471260 .585852$

$\begin{array}{llll}\text { C } & 0.660686 & 0.006228 & -0.693492\end{array}$

C $0.859344-1.102431-1.710754$

C $2.451678-1.828887-3.487343$

O $0.051866-2.009093-1.889538$

C $3.358012-2.880585-2.930651$

C $4.597291-3.107098-3.392211$

C $5.490785-4.202067-2.885939$

C $6.847051-3.698178-2.448595$

C $7.910339-3.775505-3.266830$

C $6.990497-3.198099-1.029875$

C $6.559317-1.765534-0.867735$

N $2.039740-0.934527-2.422960$

H $1.7170693 .001262-0.660236$

H 2.4918605 .8296640 .222170

H 4.0832094 .6099111 .620607

H 1.2604783 .9178862 .604172

H 2.0325995 .4600222 .836471

H 3.0533272 .5922255 .644375

H 4.3775644 .4550745 .517788 
H 4.8282114 .8294093 .852969 H 3.6184615 .8214374 .697216 H 0.7246251 .7811355 .163851 H 0.9254152 .0994743 .453844 H 2.9412850 .4824923 .510432 H 2.4263520 .0363465 .140821 H $\quad 0.160043-0.5829284 .201804$ H $1.925162-2.3547604 .470413$ H $2.606154-1.9225342 .919275$ H $0.956275-4.7238121 .921647$ H $1.136742-2.3308070 .755316$ H $0.014282-3.9483964 .548208$ H -1.030843 -4.179673 3.166351 H -1.035792 -2.627165 4.026366 H $-0.872500-2.843411-0.086165$ H - $-1.380032-0.663985-0.293538$ H -0.9066250 .8288040 .472942$ H -1.984614 -0.0996002 .117764$ H $3.659010 \quad 0.3055481 .224257$ H $6.0710930 .760137-0.632655$ H $4.658192-2.0219621 .667019$ H $4.644746-3.2116730 .366738$ H $3.511890-1.8860810 .310520$ H $\quad 0.5038430 .920959-1.277991$ H $1.5795240 .140028-0.116499$ H $2.944321-1.219064-4.251260$ H $1.569857-2.299963-3.933513$ H $2.963013-3.506041-2.133507$ H $4.978765-2.498218-4.208811$ H $5.610190-4.933319-3.696393$ H $5.022103-4.753224-2.061202$ H $8.893798-3.446139-2.948159$ H $7.819236-4.164517-4.275589$ H $8.043771-3.252422-0.721971$ H $6.473698-3.879841-0.348460$ H $7.217291-1.057101-1.372963$ H $2.673591-0.183616-2.164454$ C $-0.3344404 .644214-1.922387$ O $-0.7239923 .278847-2.184266$ C -1.713239 $3.275171-3.224615$ C $-2.2210314 .705105-3.345154$ C $-0.9598585 .486873-3.038378$ H $-1.1787173 .043990-4.157097$ C -2.762427 2.172175 -3.019176 H -2.626166 $4.923939-4.337973$ H $-2.9931304 .937528-2.607554$ C -1.221999 $6.931454-2.647170$ H $-0.3145945 .470391-3.927358$ C -3.364979 $2.064164-1.597575$ O $-3.7711042 .347687-4.010908$ H $-2.2661311 .214845-3.226617$ H $-2.5428271 .923791-0.887461$ O $-4.0197283 .281880-1.255788$ C $-4.359210 \quad 0.889286-1.448534$ C -4.8244120 .6749560 .003685$ H $-3.874107-0.023166-1.804869$ O $-5.5013151 .107205-2.275150$ H -3.9447490 .4814730 .626759$ H $-5.287146 \quad 1.5968960 .373686$ H -1.721724 $7.465704-3.461957$ H -1.861321 $6.997091-1.760365$ H $-0.2855577 .452894-2.426064$ C $1.1917074 .759230-1.896791$ H -4.365866 $1.580042-3.968905$ H $-4.1379193 .296953-0.291420$ H $-5.9048961 .948714-2.001051$ H $1.4850015 .815461-1.917933$ H $1.6168074 .287819-2.791727$ H $-0.7596384 .915191-0.947450$ C $-4.363716-2.355658 \quad 0.346089$ O $-5.402016-1.705215-0.395181$ C $-5.859231-0.4651840 .152967$ C $-6.363694-0.6729381 .587207$ C $-5.318407-1.3537612 .465277$ C $-4.811907-2.6268221 .789526$ H $-3.465173-1.7289720 .343539$ C -4.040730 -3.677822 -0.370799 H $-6.732104-0.174857-0.445622$
O -6.7476330 .5729982 .159680$

H -7.263301 -1.301778 1.557702

H $-4.496795-0.6685142 .704116$

O $-5.938140-1.6921743 .707979$

H $-5.604946-3.385717 \quad 1.800104$

O $-3.709992-3.1475372 .528333$

C $-3.297340-3.478445-1.674453$

H $-3.406726-4.3067890 .263849$

H $-4.969487-4.213288-0.600540$

H -7.0430880 .3960313 .069369$

H $-5.244452-2.0284504 .300008$

H -4.041489 -3.425059 3.398670

O $-2.998717-4.702971-2.202616$

O $-3.011574-2.398655-2.171095$

C $-2.283495-4.680323-3.441967$

H $-1.305997-4.208420-3.305437$

H $-2.131953-5.713503-3.765817$

H $-2.863748-4.154754-4.206400$

SCF Energy $\left(B 3 L Y P / 6-31 G^{* *} / / M M F F\right)=-3245.92731816$

02269

MM̄FF Geometry

C $-1.505813-2.0258081 .605465$

C -1.422808 -1.0075290 .736228$

C $-0.379368-0.882309-0.337997$

O $0.150490 \quad 0.460828-0.212678$

C $-0.968306-1.054823-1.749784$

C $-1.378919-2.471211-2.086236$

C $-0.515697-3.456885-2.400104$

C $-2.860167-2.735262-2.087520$

C $0.984335-3.374229-2.462423$

C $1.623731-4.277657-1.401291$

C $3.157817-4.246801-1.462088$

C $3.742848-5.329668-0.551540$

C $5.276744-5.291158-0.525520$

O $3.609069-2.951220-1.042318$

O $5.720123-6.109460 \quad 0.568311$

C $5.725842-3.847872-0.221069$

C $5.890002-5.896076-1.793741$

O $7.142480-3.723315-0.325052$

C $5.033466-2.782677-1.104934$

C $5.387674-1.342362-0.658011$

O $5.505519-2.924453-2.451673$

C $1.3876360 .705391-0.718638$

O $2.114086-0.080301-1.303774$

C $1.7117462 .118480-0.429668$

C $2.9199582 .602196-0.752552$

C $3.3846603 .972548-0.529446$

C 2.4123204 .9428880 .091238

C $4.868206-0.9282180 .721890$

C 5.4823200 .3797961 .199851

C 5.5206441 .8420083 .216666

O 6.3918760 .9543710 .607566

C 5.2358883 .1996662 .656047

C 6.2038234 .0431692 .264419

C 5.9714395 .4392061 .760451

C 6.4104235 .6185130 .323785

C 7.6995085 .8401220 .015653

C $5.3409455 .618640-0.744745$

C $4.6509584 .288643-0.873732$

N 4.9193580 .8047302 .397833

H -0.801861 -2.850965 1.540149

H -2.146164 -0.2002000 .807108$

H $0.432581-1.595922-0.154126$

H $-0.250076-0.704700-2.502302$

H $-1.822773-0.375425-1.878413$

H -0.922281 -4.438094 -2.642407

H -3.101627 -3.762461 -2.380815

H $-3.275021-2.572655-1.090017$

H $-3.365512-2.064768-2.790435$

H $1.301451-3.699381-3.460873$

H $1.340551-2.348501-2.346421$

H $1.289979-3.954873-0.406622$

H $1.272219-5.307083-1.547736$

H $3.465819-4.417446-2.500513$

H $3.392037-6.324460-0.853480$

H $3.374418-5.1808050 .473429$

H $5.377771-7.0088710 .427576$ 
H $5.476312-3.659026 \quad 0.829619$ H $5.585407-6.943887-1.903582$ H $6.984050-5.907344-1.736551$ H $5.597238-5.372152-2.705783$ H $7.536228-4.4018660 .249655$ H $6.475765-1.209064-0.716786$ H $4.976849-0.631347-1.388560$ H $5.340712-2.092111-2.924809$ H 0.9470272 .7222930 .044924 H $3.6415711 .933761-1.220572$ H $1.5347055 .071098-0.552123$ H 2.8296645 .9373330 .258740 H 2.0804144 .5809451 .070949 H $3.778685-0.8232560 .699201$ H $5.119497-1.6801741 .475714$ H 6.5956001 .6424263 .288743 H 5.0890151 .7609714 .219520 H $4.196273 \quad 3.5122222 .606790$ H 7.2412143 .7227562 .337450 H 6.5340566 .1294042 .403035 H 4.9211575 .7337211 .873145 H $8.0183845 .995954-1.009445$ H 8.4649315 .8679360 .784287 H $5.7780555 .852691-1.724530$ H $4.6453796 .438872-0.546330$ H $5.2821523 .511045-1.306419$ H $4.218914 \quad 0.215617 \quad 2.838716$ C $-3.790052-2.8323662 .314903$ O $-4.490023-2.0827631 .300819$ C -5.862837-1.920675 1.695171 C $-6.107057-2.9834412 .757092$ C $-4.773034-2.9862023 .478515$ H -5.942314 -0.9300292 .159855$ C $-6.785548-1.9874920 .473788$ H $-6.949146-2.7350663 .409803$ H $-6.296675-3.9678772 .314208$ C $-4.537468-4.2285114 .320082$ H $-4.726991-2.1025804 .129509$ C $-6.366291-1.063440-0.687429$ O $-6.780734-3.330386-0.028601$ H $-7.816335-1.7779060 .783054$ H -5.439897-1.440901-1.133031 O $-7.358250-1.173315-1.716582$ C $-6.1787360 .419022-0.314140$ C $-5.7604891 .265646-1.529385$ H -5.4024650 .4997290 .449636$ $\begin{array}{lllll}\text { O } & -7.380590 & 0.932321 & 0.252919\end{array}$ H $-4.8289490 .848947-1.928875$ H $-6.5179141 .167678-2.316444$ H -5.299109 -4.313317 5.102078 H -4.577208 -5.1385193 .711996$ H -3.557103 -4.190162 4.805431 C $-2.522130-2.0760832 .712843$ H $-7.370848-3.353241-0.801384$ H -8.212590 $-0.906332-1.337371$ H -8.047876 $0.980926-0.452307$ H -2.053021 -2.547533 3.584236 H -2.782962 -1.052893 3.009525 H $-3.540338-3.8072451 .877752$ C $-3.3487982 .717754-0.312269$ O $-4.731653 \quad 3.026410-0.106129$ C -5.589271 2.770808-1.222272 C $-5.1248043 .593524-2.432079$ C $-3.6628413 .313721-2.769216$ C $-2.7903553 .478806-1.526328$ H $-3.2339991 .636457-0.451095$ C $-2.581670 \quad 3.1321160 .955449$ H $-6.5795893 .147524-0.936927$ O $\quad-5.948375 \quad 3.317155-3.559904$ H $-5.2395754 .661134-2.203605$ H $-3.5424172 .321306-3.218222$ O $-3.2432714 .253293-3.761793$ H -2.714071 $4.548467-1.292852$ O $-1.469148 \quad 3.028612-1.821158$ C $-2.931700 \quad 2.308515 \quad 2.176413$ H - 1.5044813 .0224780 .787511 H -2.804871 4.1775731 .199851 H $-5.604246 \quad 3.838192-4.305589$
H -2.299633 $4.091031-3.933150$

H $-1.5141702 .076996-2.015999$

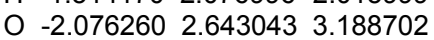

O $-3.838386 \quad 1.4920672 .247855$

C $-2.286308 \quad 1.9608794 .428982$

H -1.6270462 .4078225 .177928$

H -3.3220612 .0755224 .762668$

H -2.0287700 .9036234 .321159$

SCF Energy (B3LYP/6-31G**//MMFF)= -3245.91603684

0227

MMFF Geometry

C $4.022573-2.983634-2.269641$

C $2.733797-2.856004-1.923655$

C $1.674802-2.354821-2.871492$

O $1.058976-1.181004-2.293462$

C $0.578469-3.420709-3.042450$

C $-0.410466-3.078094-4.139209$

C $-1.621701-2.523262-3.935824$

C $0.031219-3.440015-5.533833$

C $-2.218521-2.069266-2.633479$

C $-3.419586-2.933908-2.229373$

C $-4.021073-2.472320-0.894692$

C $-5.162672-3.387328-0.451827$

C $-5.816133-2.888722 \quad 0.848105$

O $-4.508615-1.137904-1.060213$

O $-7.055063-3.5939611 .018181$

C $-6.165368-1.3915570 .694621$

C $-4.958105-3.2152682 .077302$

O $-6.632162-0.845601 \quad 1.925915$

C $-4.998301-0.5397530 .147824$

C $-5.4800830 .894579-0.184739$

O $-3.975925-0.4542991 .145711$

C $1.7252730 .001995-2.368428$

$\begin{array}{llll}\text { O } & 2.809117 & 0.210173 & -2.887668\end{array}$

C $0.8871381 .010729-1.682151$

C $1.3389012 .259916-1.493468$

C $0.607507 \quad 3.340846-0.824024$

C $-0.7879823 .032351-0.349498$

C $-4.4904241 .661396-1.070802$

C $-4.7630813 .154267-1.141251$

C $-3.826423 \quad 5.213009-2.215154$

O $-5.6600013 .713139-0.518559$

C $-2.4155215 .612711-2.515161$

C $-1.7338616 .551948-1.841465$

C $-0.3029616 .900321-2.147210$

C $0.5767486 .971608-0.914986$

C $1.2694718 .090090-0.639291$

C 0.6606825 .7711300 .005850

C $1.2056704 .541505-0.671973$

N $-3.8456643 .782380-1.972299$

H $4.324853-2.737398-3.284744$

H $2.424985-3.097125-0.909505$

H $2.102326-2.092797-3.847409$

H $0.050162-3.570619-2.093382$

H $1.031355-4.392798-3.279977$

H $-2.255638-2.342846-4.803215$

H $-0.724996-3.195046-6.287075$

H $0.946035-2.901067-5.799086$

H $0.228244-4.514858-5.602388$

H $-1.481015-2.067477-1.826393$

H -2.532628 -1.025592 -2.757054

H $-4.182423-2.874383-3.016584$

H $-3.107476-3.982611-2.149146$

H -3.225324 -2.472922 -0.143655

H $-4.811462-4.420043-0.333511$

H $-5.931181-3.418884-1.236811$

H $-6.857074-4.5458571 .043588$

H -7.003276 -1.322311 -0.013133

H $-4.810301-4.2985212 .163713$

H -5.459097 -2.907062 3.001616

H -3.970062 -2.750895 2.048824

H -7.369977 -1.4024002 .228495$

H $-6.4355600 .854021-0.723389$

H -5.6548591 .4473740 .746658$

H -3.4895400 .3780941 .037801$

H $-0.0893130 .687220-1.337655$

H $2.3341982 .515972-1.853358$ 
H -1.392603 2.665648 -1.184243 H $-1.319297 \quad 3.891848 \quad 0.061371$ H -0.7740682 .2660720 .431321$ H -3.469108 $1.535064-0.696956$ H -4.526172 $1.260492-2.091188$ H $-4.2397505 .739085-1.348682$ H $-4.4674205 .409324-3.080195$ H -1.921693 5.088331 -3.330844 H -2.224956 $7.098399-1.039942$ H $-0.3051497 .867053-2.667181$ H $0.133886 \quad 6.178664-2.848797$ H 1.9027808 .1627560 .238699 H $1.2209698 .960627-1.285074$ H -0.3193995 .6091950 .460815$ H 1.3288925 .9896400 .849789 H $2.2221774 .664545-1.048031$ H -3.260014 3.198008 -2.563061 C $5.997682-2.321532-0.807244$ O $5.279454-1.534548 \quad 0.163400$ C $5.698984-0.1615570 .040590$ C $6.878956-0.153453-0.930647$ C $6.546811-1.327463-1.829602$ H $4.8611970 .378041-0.417098$ C 6.0156390 .4389861 .414836 H $\quad 6.960031 \quad 0.793977-1.472417$ H $7.832910-0.327091-0.419514$ C $7.730465-1.849818-2.623600$ H $5.764891-1.000672-2.525547$ C $4.891697 \quad 0.3055342 .460750$ O $7.170921-0.2239821 .947365$ H $6.297510 \quad 1.491514 \quad 1.290400$ H $4.806548-0.7402822 .779419$ O 5.3068771 .0277443 .628703 C 3.5064910 .8077162 .012504 C $2.5004180 .864313 \quad 3.179713$ H $3.1267180 .141725 \quad 1.230536$ O $3.6220032 .106276 \quad 1.431484$ H $2.517258-0.0943563 .708577$ H $2.819037 \quad 1.6341243 .892738$ H $8.125270-1.070479-3.283573$ H $8.542942-2.176331-1.965941$ H $7.436101-2.701777-3.244797$ C $5.106634-3.447854-1.339903$ H 7.3634350 .1779832 .811950 H 5.4216801 .9601173 .378746 H 3.9036972 .7234312 .127443 H $4.659441-3.980373-0.490946$ H $5.722629-4.176293-1.880697$ H $\quad 6.827422-2.784738-0.256177$ C $0.300437-1.0473972 .260038$ O $\quad 0.5819190 .2622541 .749362$ C 1.0674411 .1997912 .714271 C $0.048006 \quad 1.3556983 .850823$ C -0.3297330 .0112504 .466005$ C $-0.749125-0.976186 \quad 3.377529$ H $1.226668-1.4949092 .638252$ C $-0.181554-1.8807511 .066633$ H 1.1222932 .1680202 .201715 O 0.5457472 .2140864 .871498 H -0.8643811 .8221443 .456106$ H $0.486487-0.3975065 .073291$ O H $-1.718507-0.6685442 .965821$ O $-0.923766-2.2646953 .959535$ C $-0.115223-3.3731631 .290728$ H $-1.203708-1.5849080 .804992$ H $0.438658-1.6663430 .191178$ H 0.7587503 .0675584 .456803 H -1.168989 0.8540816 .015410 H -1.578901 -2.174950 4.672763 O $-1.102091-3.9805480 .570286$ O $\quad 0.722108-3.9393561 .979937$ C $-1.150458-5.4068340 .664323$ H -1.314727 -5.7131291 .701749$ H -1.989421 -5.7580690 .057627$ H $-0.226547-5.8427630 .272929$ SCF Energy $\left(B 3 L Y P / 6-31 G^{* *} / / M M F F\right)=-3245.91397867$
02_270

MMFF Geometry

C 0.8253934 .7381351 .893406

C -0.0997873 .8055881 .616936$

C -0.9242113 .1373602 .687474$

O -0.7438411 .7059332 .586150$

C -2.4195713 .4397142 .487279$

C -3.2737052 .9104113 .625226$

C -4.0122831 .7846403 .566606$

C -3.2884323 .7601924 .869453$

C -4.1110290 .8222832 .416463$

C -5.4778740 .9276651 .730374$

C $\begin{array}{llll}-5.547366 & 0.241110 & 0.358222\end{array}$

C $-5.247361-1.2569520 .418639$

C $-5.283323-1.894883-0.979039$

O $-4.6137420 .898390-0.503387$

O $-4.687672-3.198955-0.885740$

C $-4.390012-1.064285-1.928240$

C -6.719432 -2.113861-1.467408

O $-4.506706-1.520985-3.272172$

C $-4.6494130 .459084-1.868603$

C $-3.5999901 .296908-2.637503$

O $-5.8998930 .799088-2.481482$

C 0.0642551 .1138963 .501530

O $0.776298 \quad 1.679381 \quad 4.321218$

C $-0.045029-0.3627093 .464781$

C $-0.655827-1.0640452 .496609$

C $-0.812916-2.5224932 .471437$

C $-0.267187-3.3022333 .640773$

C $-2.146618 \quad 1.108695-2.187642$

C $-1.4378820 .001149-2.948167$

C $0.261342-1.790685-2.661521$

O $-1.694317-0.268506-4.119267$

C $-0.473783-3.021651-2.238039$

C $0.054135-3.956504-1.435360$

C $-0.676981-5.193321-1.006814$

C $-0.735077-5.3417700 .496658$

C $0.060445-6.2214381 .128426$

C $-1.782877-4.5560001 .252586$

C $-1.444369-3.0994321 .426842$

N $-0.467788-0.630545-2.184923$

H 0.9918994 .9937572 .939115

H -0.3008813 .5147090 .590228$

H -0.6200303 .4932913 .679541$

H -2.7595403 .0288271 .529204$

H -2.5776194 .5239152 .411185$

H $-4.605865 \quad 1.509020 \quad 4.437142$

H -3.959855 3.3611825 .637349

H -2.2876823 .8202245 .307826$

H -3.6266344 .7742264 .632474$

H $-3.3039640 .976625 \quad 1.695414$

H -3.970937 -0.1912852 .810904$

H -6.2516280 .5126072 .388477$

H -5.7170901 .9898511 .583893$

H $-6.5588690 .408365-0.030621$

H $-5.943821-1.7672511 .095196$

H -4.245606 -1.422309 0.835614

H -5.180773 -3.707250 -0.219078

H $-3.356894-1.258213-1.617607$

H $-7.250406-2.801558-0.797998$

H $-6.733718-2.587247-2.455134$

H -7.302028 -1.191514 -1.515585

H -4.303629 -2.472146 -3.273938

H $-3.7088791 .132278-3.716599$

H $-3.8504322 .355923-2.479754$

H $-6.2592550 .007926-2.913900$

H $0.424118-0.8562864 .310632$

H -1.094957 -0.5327141 .654310$

H $0.815384-3.1556513 .727667$

H $-0.427699-4.3790103 .560918$

H $-0.743905-2.9827074 .574038$

H -1.586477 $2.026877-2.402476$

H -2.089184 $0.941798-1.106758$

H $1.268636-1.744786-2.238442$

H $\quad 0.339307-1.759771-3.752803$

H -1.485942 -3.150700 -2.614543

H $1.071021-3.832275-1.073431$

H $-0.161920-6.054676-1.451971$ 
H -1.696559 -5.217636-1.411434 H $\quad 0.003128-6.3696722 .201318$ H $0.788175-6.8158370 .586510$ H -1.988592 -5.0187102 .225270$ H -2.738574 -4.6279530 .717822$ H -1.801423 -2.4628460 .617022$ H $-0.367465-0.384193-1.204672$ C $1.4338845 .320241-0.565922$ O $1.9112254 .019257-0.959510$ C $2.7119734 .152698-2.149000$ C $2.3972995 .532199-2.708937$ C $2.1999996 .324988-1.432260$ H $3.7599104 .135311-1.825743$ C $2.4473752 .984135-3.103871$ H $3.2039775 .920686-3.337747$ H $1.4744045 .533050-3.300105$ C $1.4710257 .641171-1.643286$ H $3.1848526 .528699-0.990369$ C $2.5601131 .596593-2.440945$ O $1.1180133 .094944-3.630060$ H $3.1183223 .049359-3.967788$ H $1.7314651 .471350-1.736499$ O $2.3820730 .606099-3.459975$ C $3.9013091 .328353-1.737170$ C $4.017118-0.075440-1.117359$ H $4.0815942 .082493-0.969388$ O $4.9509681 .478171-2.699367$ H $3.943307-0.827138-1.915534$ H $5.040289-0.198534-0.741063$ H $2.0380618 .290675-2.318135$ H $0.4795957 .484920-2.081595$ H $1.3401188 .171955-0.695212$ C 1.6784285 .5246040 .932187 H $0.5052353 .121160-2.875231$ H $1.5324200 .788514-3.896965$ H $5.7961821 .358199-2.233770$ H $1.5292976 .583857 \quad 1.174497$ H 2.7244355 .2841151 .162357 H $0.3621455 .358984-0.797232$ C $4.122936-2.4211730 .777337$ O $2.949225-1.8462520 .191621$ C $3.003483-0.424340-0.005970$ C 3.2042090 .2573381 .353959 C $4.436866-0.2731942 .080434$ C $4.395339-1.8001132 .152856$ H $4.984769-2.2623480 .117801$ C $3.880604-3.9336960 .893369$ H $2.006602-0.152115-0.363858$ O 3.2869671 .6697621 .244165 H 2.3345420 .0440381 .983334 H 5.3636890 .0757601 .610916 O $4.448620 \quad 0.2332573 .417696$ H $3.619941-2.1094992 .865740$ O $5.644225-2.2803362 .645665$ C $3.841721-4.612378-0.460328$ H $4.679351-4.4095421 .472194$ H $2.915988-4.1139661 .383254$ H 2.4601731 .9807560 .836932 H $4.420348 \quad 1.2037843 .359181$ H $5.800229-1.8531053 .505367$ O $3.462179-5.913743-0.291137$ O $4.112053-4.079032-1.527418$ C $3.360751-6.685776-1.491736$ H $2.626280-6.240196-2.169279$ H $4.338875-6.760815-1.976192$ H $3.023777-7.690205-1.222188$ SCF Energy (B3LYP/6-31G**//MMFF) $=-3245.91774341$

\section{1}

MM̄FF Geometry

C 1.1372401 .0113643 .189718

C 2.0192621 .9797223 .484951

C 2.2331023 .2659942 .721474

O 1.0463303 .5501841 .944089

C 3.4529423 .1849151 .777437

C 4.7347152 .6908272 .416076

C $5.311151 \quad 1.5005802 .153502$

C 5.3802933 .6494733 .382549
C $4.8135240 .429777 \quad 1.222932$

C 5.7642730 .2412890 .033293

C $5.087961-0.525243-1.112209$

C $6.113856-0.948074-2.164447$

C $5.443970-1.598901-3.383987$

O $4.1261130 .360228-1.696759$

O $6.426850-1.715474-4.423707$

C $4.314466-0.675047-3.887697$

C $4.999082-3.034804-3.084942$

O $3.572360-1.301302-4.931859$

C $3.357414-0.200697-2.767650$

C $2.4295400 .914423-3.316147$

O $2.573524-1.306358-2.327587$

C $0.8685804 .810196 \quad 1.470674$

O 1.6013935 .7737131 .618325

C -0.3879874 .8003910 .697723$

C $-0.5355865 .633975-0.340487$

C $-1.7053805 .630867-1.210068$

C -1.365926 $5.491400-2.667576$

C $1.5504381 .610978-2.266433$

C $0.2298550 .914800-1.988517$

C $-1.7857561 .116046-0.534606$

O $-0.161120-0.067666-2.612136$

C $-2.8203701 .876020-1.304686$

C $-3.8406112 .531964-0.730864$

C $-4.9075973 .252681-1.505988$

C $-5.1739064 .648646-0.987117$

C $-6.2386584 .903587-0.207773$

C $-4.2548525 .762517-1.438894$

C $-2.9457845 .754792-0.698671$

N $-0.4671601 .539098-0.963350$

H $\quad 0.484651 \quad 1.1079862 .326426$

H $2.670378 \quad 1.8371244 .345881$

H 2.3631524 .0718143 .455128

H 3.6484154 .1794841 .355267

H 3.1814782 .5911690 .897601

H $\quad 6.240417 \quad 1.2540742 .665415$

H 6.3293953 .2687413 .774608

H 5.5877264 .6041042 .888122

H 4.7225173 .8357214 .237157

H $4.752590-0.512114 \quad 1.781983$

H 3.7968200 .6312350 .876927

H $6.0892861 .223112-0.336075$

H $6.657779-0.2958890 .374609$

H $4.575520-1.402086-0.700181$

H $6.861780-1.624022-1.731627$

H $6.671490-0.063378-2.502321$

H $6.758291-0.825133-4.630606$

H $4.7879340 .210810-4.333417$

H $5.863284-3.651651-2.809941$

H $4.565079-3.507596-3.972888$

H $4.274007-3.101139-2.271239$

H $4.208318-1.580382-5.612757$

H $3.065496 \quad 1.696905-3.751515$

H $1.8007060 .519251-4.122683$

H $1.803761-0.969568-1.844084$

H $-1.1439644 .068280 \quad 0.962620$

H $0.2618106 .318042-0.620937$

H $-2.2421685 .419607-3.314765$

H $-0.7740774 .584072-2.835279$

H $-0.778706 \quad 6.351794-3.007363$

H $2.091007 \quad 1.748819-1.324080$

H $1.2951582 .609128-2.645792$

H $-1.853777 \quad 1.2947020 .542968$

H $-1.8975510 .045003-0.722319$

H $-2.7426451 .862604-2.389422$

H -3.9388562 .5154250 .352263$

H -5.825309 2.653504 -1.443097

H -4.660300 $3.304606-2.573591$

H -6.4528925 .9062790 .146797$

H -6.9167834 .1135770 .097962$

H -4.726187 6.737211-1.255484

H -4.135365 $5.703160-2.524022$

H -3.0475095 .8769550 .380685$

H $-0.0849462 .385166-0.553100$

C $1.634223-1.4345483 .228933$

O $0.767327-1.8343092 .148571$

C $0.821776-3.2699062 .024228$ 
C $1.896374-3.7477792 .995053$

C $1.822844-2.6891644 .077027$

H $-0.145476-3.6548092 .364748$

C $1.069969-3.6681830 .565347$

H $\quad 1.697957-4.757703 \quad 3.366774$

H $2.889589-3.7525062 .530260$

C $3.049783-2.6583164 .972200$

H $0.929175-2.8682624 .689484$

C $-0.012732-3.182977-0.421154$

$\begin{array}{llll}\text { O } & 2.326718 & -3.125528 & 0.142235\end{array}$

H $1.174488-4.7568720 .492775$

H $0.000339-2.089443-0.496426$

O $0.324090-3.684525-1.720352$

C -1.434716 -3.658029-0.070933

C $-2.478130-3.305453-1.148164$

H $-1.734767-3.2035440 .877651$

O $-1.431358-5.0726680 .124656$

H -2.440203 -2.228609-1.340253

H -2.220926 -3.810278 -2.087062

H $3.175991-3.6180265 .483897$

H $3.961039-2.4612474 .397345$

H $2.959061-1.8797585 .735816$

C $1.020079-0.2589433 .987473$

H $2.277288-2.1589790 .240617$

H $1.219282-3.366785-1.930145$

H -1.130093 -5.482212 -0.704596

H $1.514509-0.1343944 .957537$

H $-0.042460-0.4414704 .185838$

H $2.590737-1.1406462 .777903$

C $-4.498804-1.8434050 .627031$

O $-4.270203-3.2557490 .553756$

C $-3.908126-3.735686-0.745711$

C $-4.997812-3.379144-1.768295$

C $-5.368042-1.897924-1.728747$

C $-5.658648-1.456879-0.294298$

H $-3.593196-1.3064220 .324666$

C $-4.791979-1.4986052 .090966$

H -3.893714 -4.830312 -0.669078

O $-4.595013-3.724960-3.088989$

H -5.901331 -3.962257-1.546401

H $-4.584653-1.275895-2.178094$

O $-6.546793-1.687286-2.509180$

H $-6.591635-1.9190550 .052423$

O $-5.841233-0.045280-0.263120$

C -3.526188 -1.575816 2.916549

H $-5.182333-0.4818492 .206266$

H -5.536462 -2.192001 2.500208

H -4.387241 -4.675002 -3.091686

H -6.357799 -2.005870 -3.408586

H $-6.552950 \quad 0.169818-0.889975$

O $-3.699432-2.434177 \quad 3.961437$

O $-2.506787-0.9454212 .665415$

C $-2.563616-2.5973454 .816625$

H -1.722783 -3.011625 4.253107

H $-2.290148-1.6406395 .271820$

H $-2.833562-3.2990695 .610223$

SCF Energy (B3LYP/6-31G**//MMFF)= -3245.92147796

0228

MMFF Geometry

C $-0.470779-0.7563381 .887050$

C $-0.385183-1.9441411 .268589$

C $-0.860974-2.244768-0.134868$

O $-0.995428-1.029434-0.906000$

C $-2.248247-2.906321-0.101551$

C $-2.524220-3.754024-1.326362$

C -3.163829 -3.333287 -2.434888

C $-2.056360-5.183488-1.221965$

C -3.650824 -1.950298 -2.766229

C $-5.181052-1.837293-2.769880$

C $-5.764594-1.607408-1.367545$

C $-7.293805-1.604381-1.413344$

C $-7.906871-1.257048-0.048610$

O $-5.288588-0.336447-0.907896$

O $-9.298478-0.967274-0.250344$

C -7.2485130 .0345830 .479107$

C -7.846483 -2.441158 0.923470

O $\quad \begin{array}{llll}-7.679973 & 0.321264 & 1.807690\end{array}$
C $-5.703754 \quad 0.012857 \quad 0.417340$

C -5.1457431 .4215310 .739785$

O $-5.212490-0.9162801 .386275$

C $0.115714-0.506502-1.484308$

O $1.243443-0.969264-1.478178$

C $-0.2859300 .780350-2.090506$

C $0.6480531 .709144-2.337255$

C $0.4061553 .054139-2.861558$

C $-1.0023183 .402719-3.267563$

C -3.6495961 .5921630 .435405$

C -3.1120582 .9247360 .933227$

C -0.9727914 .1813051 .203581$

O -3.8260743 .7920401 .430150$

C -0.7858335 .1334670 .064307$

C $0.3988205 .677713-0.254900$

C $0.5920696 .674065-1.363071$

C $1.7332116 .312995-2.289248$

C $2.9354246 .900693-2.166398$

C $1.4551565 .334749-3.408926$

C $1.4472553 .909370-2.932493$

N -1.7378413 .0295910 .759639$

H -0.9479120 .0763971 .381180$

H $\quad 0.077623-2.776626 \quad 1.793823$

H $-0.127117-2.901617-0.618903$

H -3.024289 -2.138828 0.001340

H $-2.349914-3.5499060 .782602$

H -3.325845 -4.057189 -3.233249

H -2.268354 -5.759624 -2.128811

H $-0.975052-5.219661-1.054483$

H -2.554978 -5.686122 -0.386904

H $-3.211690-1.190428-2.115770$

H -3.285944 -1.719976 -3.775674

H $-5.445679-0.979873-3.402832$

H -5.627014 -2.728623 -3.227961

H -5.406154 -2.396389-0.697357

H -7.674263 -2.569332 -1.771072

H -7.635327 -0.858632 -2.145096

H $-9.712417-1.750102-0.652571$

H $-7.6070980 .862971-0.148134$

H $-8.394775-3.2991010 .515963$

H $-8.335345-2.1979031 .873417$

H -6.827455 -2.772201 1.134312

H $-8.6523820 .338468 \quad 1.798968$

H -5.6856192 .1729080 .148727$

H $\quad-5.3255001 .6500011 .798187$

H $-4.328524-0.6420131 .675585$

H -1.342834 $0.945866-2.265475$

H $1.6862961 .475495-2.106211$

H $-1.3399432 .745860-4.077083$

H -1.114672 $4.428171-3.624212$

H $-1.6889713 .294348-2.420874$

H $-3.0723510 .797176 \quad 0.913712$

H $-3.4761971 .539503-0.645408$

H -0.0189513 .8083311 .588750$

H -1.507720 4.6809242 .017584

H $-1.6694195 .412239-0.505096$

H 1.2744295 .4247040 .338540

H $0.7859347 .650324-0.899692$

H $-0.3254916 .802213-1.950686$

H $3.750526 \quad 6.667634-2.843377$

H $3.1328697 .624051-1.382044$

H $2.2328885 .413741-4.180532$

H $0.5314005 .623163-3.917431$

H $2.4280583 .558666-2.607638$

H -1.2382402 .2839280 .284211$

C 0.9205160 .8123303 .238570

O $2.0063790 .679147 \quad 2.304983$

C 3.1737701 .3250322 .846330

C 2.7190902 .0350074 .118049

C 1.5768831 .1455164 .574994

H 3.8706610 .5253883 .123827

C 3.8177492 .2241561 .781862

H 3.5230762 .1154384 .856039

H 2.3514753 .0470363 .913959

C 0.6476161 .8132075 .572935

H 1.9985550 .2362765 .024145

C 4.0352391 .5228490 .425105

O 2.9512823 .3424581 .552302 
H 4.7599992 .6321892 .165980 H $3.0659331 .321585-0.046563$ O $4.7293692 .425679-0.443552$ C $4.842248 \quad 0.2145410 .503848$ C $5.015707-0.440123-0.876677$ H $4.327331-0.4877201 .160926$ $\begin{array}{lllll}\text { O } & 6.121442 & 0.467441 & 1.079582\end{array}$ H $4.026397-0.548579-1.333087$ H $5.5982640 .226114-1.524370$ H 1.1946232 .0955606 .478400 H $\quad 0.1959642 .7197595 .156238$ H -0.1621891 .1372565 .864886$ C $0.085707-0.469603 \quad 3.253694$ H 3.4497283 .9995201 .038283 H $4.1181423 .148743-0.663472$ H $\quad 6.572776 \quad 1.1194720 .516462$ H $-0.747125-0.362776 \quad 3.957327$ H $\quad 0.702020-1.3105283 .591010$ H $\quad 0.306529 \quad 1.6548272 .894182$ C $3.834037-3.195572-0.249922$ O $5.137386-2.720686 \quad 0.106798$ C $5.732132-1.809380-0.824020$ C $5.865578-2.485462-2.196195$ C $4.520541-3.012817-2.690973$ C $3.855869-3.881988-1.623703$ H $3.129079-2.357169-0.253928$ C $3.389992-4.1965230 .830917$ H $6.747714-1.618898-0.455024$ O $6.399232-1.584239-3.160231$ H $6.565509-3.327424-2.119236$ H $3.860562-2.191843-2.992615$ O $4.721927-3.795992-3.870856$ H $4.387951-4.839428-1.553549$ O $2.516403-4.169963-2.022383$ C $3.104340-3.5461972 .167277$ H $2.471084-4.7076050 .522758$ H $4.183069-4.9375660 .988565$ H $7.257103-1.272341-2.825061$ H $5.320409-4.529040-3.647200$ H $2.556497-4.574111-2.906019$

O $2.855798-4.5246223 .088570$

O $3.083500-2.3430982 .381501$ C $2.557342-4.0646904 .410475$ H $1.601598-3.5327714 .414234$ H $2.475775-4.9378295 .063375$ H $3.360012-3.4218984 .784683$

SCF Energy (B3LYP/6-31G**//MMFF) $=-3245.91558184$

0229

MM̄FF Geometry

C -0.1098583 .5046801 .096461$

C -0.9684293 .8608552 .064843$

C -1.9864742 .9898702 .762395$

O -2.0200331 .6485532 .228656$

C -3.3902923 .5956182 .589295$

C -4.4426902 .9121373 .440991$

C -5.3850552 .0780312 .959356$

C -4.4262323 .2717204 .904164$

C -5.5376651 .6200911 .535553$

C -6.0843930 .1872501 .457317$

C $-6.229861-0.2743150 .001128$

C $-6.987799-1.598505-0.077766$

C $-7.103661-2.094091-1.525830$

O $-4.917744-0.445351-0.540295$

O $-7.549002-3.458056-1.458619$

C $-5.702685-2.080585-2.177065$

C -8.184876 -1.325055 -2.294513

O $-5.780501-2.339741-3.576750$

C $-4.895150-0.779032-1.934132$

C $-3.420869-1.008700-2.326536$

O $\quad \begin{array}{llll}-5.362770 & 0.302766 & -2.741208\end{array}$

C $-1.209793 \quad 0.7217682 .807445$

O $-0.372140 \quad 0.914615 \quad 3.674055$

C -1.533247 -0.5916062 .205773$

C $-0.746756-1.6459242 .468321$

C $-0.931615-3.0115081 .971685$

C $-2.222587-3.3302071 .264041$

C $-2.5017640 .183908-2.029492$
C $-1.073583-0.152563-2.412714$

C $0.820437-1.639842-1.751883$

$\begin{array}{lllll}\text { O } & -0.538717 & 0.330720 & -3.407237\end{array}$

C $0.735928-3.118029-1.539893$

C $1.543406-3.792705-0.708585$

C $1.461395-5.274962-0.483443$

C $1.400173-5.6458410 .983151$

C $2.424450-6.2807421 .577756$

C $0.122981-5.3473711 .742785$

C $0.050897-3.9107802 .190640$

N $-0.497502-1.071339-1.551199$

H -0.0935202 .4846210 .724449$

H -0.9281984 .8873452 .427463$

H -1.731054 2.9607163 .828950

H -3.674816 3.5663541 .530550

H -3.3788444 .6609632 .857308$

H -6.1270901 .6892943 .655349$

H -5.2531622 .8105685 .454807$

H -3.4950202 .9390805 .372638$

H -4.5124774 .3559125 .030243$

H -6.2353312 .2972751 .028605$

H -4.5832831 .6650651 .001846$

H -5.404571 -0.4868951 .994677$

H -7.0602220 .1445801 .957320$

H $-6.7573940 .504984-0.561060$

H -7.983006 -1.5089690 .375681$

H -6.456276 -2.359143 0.511618

H -7.676509 -3.779578 -2.367103

H -5.139132 -2.915585-1.736767

H -9.166982 -1.487568 -1.834048

H $-8.274139-1.687066-3.324610$

H -8.008546 -0.247722 -2.321459

H $-6.184742-3.215772-3.693170$

H -3.041822 -1.886936 -1.790088

H -3.350673 -1.235964 -3.398360

H $-6.1623590 .022107-3.212184$

H -2.407143 -0.654939 1.567193

H $0.129640-1.4953173 .098157$

H -3.081421 -3.0269471 .874034$

H -2.357517 -4.394891 1.063366

H $-2.278237-2.8103920 .304060$

H -2.811861 $1.067994-2.597446$

H $-2.5253770 .454993-0.968015$

H $1.491019-1.157607-1.034371$

H $1.170654-1.427724-2.766265$

H - $0.022136-3.658904-2.101542$

H $2.312812-3.250179-0.166619$

H $2.343581-5.730058-0.952038$

H $\quad 0.591729-5.706584-0.994550$

H $2.379565-6.5701802 .622370$

H $3.334041-6.5184221 .036112$

H $0.066402-5.9747252 .641890$

H $-0.724817-5.647197 \quad 1.121150$

H $0.929892-3.5851412 .749444$

H $-0.978836-1.294713-0.685956$

C $0.9400234 .401290-1.015858$

O $1.6580463 .213213-1.418828$

C $2.7250363 .598186-2.303969$

C $2.3243314 .961138-2.848445$

C $1.7003235 .586782-1.619310$

H $3.6251153 .712195-1.687665$

C $2.9608312 .533208-3.376445$

H $3.1791305 .525763-3.232595$

H $1.5804684 .876764-3.649334$

C $0.8200046 .786205-1.927033$

H $2.5047425 .902553-0.940987$

C $3.2679901 .125118-2.832599$

O $1.7940952 .430671-4.203313$

H $3.7694912 .857317-4.041948$

H $2.3713990 .699278-2.371626$

O $3.5794030 .290499-3.955828$

C $4.4333091 .049325-1.827437$

C $4.857747-0.400987-1.520498$

H $4.1390261 .550573-0.900033$

O $5.5615931 .754787-2.346417$

H $3.968089-0.986850-1.270843$

H $5.294297-0.840714-2.425018$

H $1.4055477 .581944-2.398993$ 
H $\quad 0.0038886 .520860-2.607642$

H $0.3769947 .189713-1.011143$

C 0.8898094 .4663210 .512048

H $1.0465292 .191310-3.628353$

H $2.8204080 .328292-4.563148$

H $5.8003341 .344262-3.195309$

H $0.6514425 .488140 \quad 0.830450$

H $1.875714 \quad 4.2136000 .919475$

H $-0.0688474 .326202-1.440842$

C $4.391031-0.8059211 .482289$

O 5.3941130 .0075990 .860249

C $5.901167-0.490282-0.383217$

C $6.496519-1.891447-0.182314$

C $5.492344-2.8443220 .456247$

C $4.913791-2.2277721 .727330$

H $3.494697-0.8359400 .851593$

C $4.019601-0.1425432 .817736$

H $6.7324830 .170173-0.659516$

O $6.943687-2.441876-1.416699$

H $7.373661-1.8224780 .474382$

H $4.700253-3.121498-0.247322$

O $\quad 6.154906-4.059996 \quad 0.812084$

H $5.687294-2.2142352 .506223$

O $3.846801-3.0438022 .204484$

C 3.3070981 .1798232 .637310

H $3.354907-0.787170 \quad 3.403192$

H 4.9356620 .0465173 .391073

H $7.589887-1.821734-1.795653$

H $6.552987-4.4195350 .000660$

H $4.201196-3.9409062 .329221$

O $3.354049 \quad 1.8661213 .817920$

O 2.7603881 .5527761 .609081

C 2.7222823 .1492193 .818635

H 2.8999653 .6147684 .791773

H 3.1534523 .7883633 .042616

H 1.6446483 .0357053 .677096

SCF Energy (B3LYP/6-31G**//MMFF) $=-3245.93242280$

023

MM̄FF Geometry

C $1.8808113 .861335-1.474402$

C $2.6948564 .638164-0.742712$

C 3.6120164 .1849610 .372276

$\begin{array}{llll}\text { O } & 3.717645 & 2.744169 & 0.429712\end{array}$

C 3.0600734 .6863641 .719357

C 3.9712234 .3953462 .896426

C 3.7475263 .4499373 .831346

C 5.1836845 .2848403 .002715

C 2.6102722 .4684663 .898277

C 3.1295141 .0253983 .860751

C 1.9907870 .0034813 .721069

C $2.550307-1.4207263 .747435$

C $1.476399-2.4776603 .451850$

O $1.333236 \quad 0.2566252 .475554$

O $2.144620-3.7158943 .163381$

C $0.713257-2.0706702 .173789$

C $0.583925-2.7401414 .670327$

O $-0.381821-2.9499241 .936412$

C $0.224841-0.6081972 .193400$

C $-0.382235-0.1543840 .842965$

O $-0.800701-0.466746 \quad 3.183363$

C $4.6282732 .159197-0.398412$

O $5.3499122 .719844-1.208197$

C $4.5946570 .699678-0.153539$

C $5.323898-0.119028-0.926900$

C $5.425440-1.575002-0.797904$

C $4.609746-2.2404510 .277944$

C $0.584605-0.160898-0.345524$

C $0.529327-1.418177-1.194537$

C $1.624202-2.454201-3.180977$

O $-0.283824-2.320110-1.018256$

C $2.613586-3.464154-2.692582$

C $3.705069-3.824825-3.384493$

C $4.665366-4.887429-2.934379$

C $6.098316-4.408216-2.897792$

C $6.942656-4.674669-3.908812$

C $6.576912-3.709161-1.646516$

C $6.247353-2.241607-1.635469$
N $1.488452-1.395878-2.199117$

H $1.8369012 .792126-1.288140$

H $2.7109845 .707466-0.946672$

H 4.6060004 .6162160 .199467

H 2.0651724 .2597831 .884363

H 2.8997255 .7725901 .674814

H 4.4651883 .3565064 .645743

H 5.7711785 .0821413 .904627

H 5.8439675 .1407162 .142175

H 4.8814796 .3366073 .038275

H 2.0617752 .6330314 .833478

H 1.8940072 .6237273 .089162

H 3.8156280 .9182773 .011269

H 3.7049910 .8186514 .771756

H 1.2797340 .1538154 .541848

H $3.039748-1.6272264 .707463$

H $3.338286-1.5123772 .988585$

H $2.692712-3.9472423 .932812$

H $1.405980-2.2021931 .334057$

H $1.182604-3.1153945 .509219$

H $-0.155679-3.5201404 .458370$

H $0.057445-1.8486015 .017056$

H $-0.027443-3.8551941 .910761$

H -1.295934 -0.721485 0.628300

H -0.7125020 .8868420 .969901$

H $-1.337087 \quad 0.3132782 .965486$

H 3.9790550 .3392520 .661599

H $5.9199760 .318136-1.727132$

H $4.977267-1.9490401 .267294$

H $4.630250-3.3313260 .230455$

H $3.555545-1.9568940 .195225$

H $\quad 0.3144560 .666441-1.012489$

H $1.6146830 .009454-0.020131$

H $1.936555-1.987996-4.120785$

H $\quad 0.652935-2.934763-3.336821$

H $2.402398-3.946335-1.740974$

H $3.898830-3.361846-4.349479$

H $4.573956-5.732230-3.629921$

H $4.388848-5.287304-1.950770$

H $7.981292-4.362733-3.874825$

H $6.612197-5.205689-4.795401$

H $7.669872-3.792652-1.570165$

H $6.202597-4.238953-0.766078$

H $6.794101-1.670796-2.387481$

H $2.161002-0.634863-2.227208$

C $-0.4858184 .242425-2.203353$

O $-0.8538832 .849073-2.190939$

C $-2.1610782 .701926-2.787497$

C $-2.7001644 .113754-2.966387$

C $-1.4282304 .900787-3.208447$

H $-1.9913572 .231352-3.763911$

C $-3.0161031 .795188-1.893289$

H -3.415116 4.189061 -3.791013

H -3.196047 4.461515 -2.051384

C -1.597463 $6.397594-3.014325$

H $-1.0824954 .705373-4.232431$

C $-4.4929471 .703467-2.312401$

O $-2.4393860 .485578-1.912430$

H $-2.9386012 .148103-0.859197$

H $-4.9659782 .688652-2.228210$

O $-4.5415271 .346355-3.699650$

C $-5.3197370 .664959-1.528926$

C $-5.2776880 .863384-0.004258$

H $-4.985078-0.343332-1.794215$

O $\quad-6.678051 \quad 0.759748-1.972994$

H -4.2447090 .7761610 .344975$

H $-5.6116581 .881300 \quad 0.232257$

H -2.339986 $6.794241-3.714422$

H $-1.9323036 .635352-1.999033$

H $-0.6543286 .924373-3.189572$

C $0.9920204 .385456-2.569193$

H $-2.5258100 .138844-2.817006$

H $-5.4792131 .284390-3.950360$

H $-7.0090751 .641853-1.732019$

H $1.2272935 .435643-2.776483$

H $1.2122973 .815288-3.480004$

H $-0.6699174 .631320-1.192951$

C $-4.648820-2.0071800 .793733$ 
O $-5.919151-1.492522 \quad 0.376926$

C $-6.170796-0.137756 \quad 0.762988$

C $-6.100224-0.0048042 .291696$

C -4.799139 -0.5706602 .858134$

C $-4.548713-1.9801092 .322077$

H $-3.843238-1.4056120 .356163$

C $-4.525697-3.4334040 .248070$

H -7.2069690 .0747840 .471032$

O $-6.232008 \quad 1.3546552 .693119$

H -6.937621 -0.5550342 .740470$

H -3.947882 0.0861592 .644068

O $-4.899905-0.644844 \quad 4.281946$

H -5.276299 -2.673922 2.762247

O $-3.251377-2.4115002 .719246$

C $-4.477865-3.436029-1.262071$

H $-3.628099-3.9418830 .617294$

H $-5.390192-4.0374340 .549343$

H $-7.077371 \quad 1.6821772 .341201$

H $-5.094828 \quad 0.2518444 .604591$

H -3.202523 -2.338536 3.687774

O $-3.279820-2.949789-1.694644$

O $-5.399389-3.820033-1.970763$

C -3.121915 -2.876330-3.114907

H $-2.133488-2.460327-3.327133$

H $-3.183957-3.876061-3.554820$

H -3.879569-2.215159-3.546538

SCF Energy (B3LYP/6-31G**/MMFF) $=-3245.91232233$

02_30

MMFF Geometry

C $2.203462-3.6203100 .478994$

C $2.483959-2.892610-0.612887$

C $1.677149-2.806512-1.885297$

O $1.200365-1.442629-2.067808$

C $0.528661-3.821826-2.074771$

C $-0.059044-3.789856-3.474456$

C $-1.231982-3.217999-3.812937$

C $0.759879-4.493865-4.527837$

C $-2.198070-2.475728-2.930708$

C $-3.591779-3.110314-2.980823$

C $-4.616035-2.479041-2.024070$

C $-4.901743-1.006825-2.325621$

C $-5.924878-0.416568-1.340459$

O $-4.137937-2.619384-0.680914$

O $-5.8990101 .012633-1.481734$

C $-5.470306-0.7349130 .101217$

C $-7.357011-0.855848-1.665743$

O $-6.464083-0.3463301 .045542$

C $-5.088258-2.2158720 .319179$

C $-4.448617-2.4910371 .703745$

O $-6.272059-3.0222380 .246248$

C $0.349721-0.893238-1.158919$

O $-0.054497-1.393422-0.121358$

C $-0.0434180 .439230-1.664526$

C -1.091763 $1.063557-1.108469$

C -1.605468 $2.383773-1.472250$

C $-0.7986303 .206959-2.441172$

C -3.260557-1.595785 2.074879

C $-3.665459-0.3874172 .906244$

C -2.8916821 .8833253 .568535$

O $-4.700625-0.3466673 .567092$

C -3.616687 2.8419492 .678127

$\begin{array}{llll}\text { C }-3.081999 & 3.980578 & 2.212023\end{array}$

C $-3.820328 \quad 4.9442801 .327289$

C -3.158773 $5.124588-0.021415$

C $-2.3855286 .194485-0.272298$

C $-3.4636774 .114007-1.105217$

C $-2.7509542 .807605-0.899511$

$\begin{array}{llll}N & -2.717566 & 0.624498 & 2.866588\end{array}$

H $1.317990-4.2481900 .508741$

H $3.366274-2.257022-0.592361$

H $2.400569-2.961003-2.695362$

H $-0.255179-3.663152-1.328570$

H $0.900907-4.838033-1.887640$

H - $1.537492-3.266880-4.858000$

H $0.263870-4.502517-5.504465$

H $1.729049-4.002725-4.657309$

H $0.932676-5.536080-4.240200$
H -1.857851 -2.436014 -1.895294

H $-2.246725-1.442277-3.290891$

H $-3.981984-3.068194-4.005642$

H $-3.494903-4.172641-2.718566$

H $-5.536777-3.065081-2.131503$

H $-5.244242-0.879671-3.359892$

H $-3.978154-0.420100-2.237971$

H $-6.1040021 .226033-2.408333$

H $-4.591861-0.1073070 .294645$

H -7.632883 -0.540052 -2.679113

H $-8.078924-0.375202-0.996132$

H -7.499607 -1.936630 -1.607552

H -6.6562690 .5948440 .893843$

H $-5.222438-2.4701562 .480608$

H -4.079133 -3.526517 1.690989

H $-6.098710-3.8633660 .700841$

H $0.5288790 .861339-2.481475$

H $-1.6348540 .558015-0.311076$

H $-1.1946124 .213737-2.590432$

H $-0.767412 \quad 2.724692-3.424089$

H $0.2270153 .331520-2.076950$

H -2.567627 -2.171152 2.701846

H -2.708482 -1.283885 1.182523

H -1.8992092 .2459113 .850475$

H -3.4703051 .7179404 .483100$

H $-4.639622 \quad 2.5866682 .410277$

H -2.063022 4.2432502 .486912

H -3.872687 5.9063311 .853694

H -4.8629124 .6351701 .180536$

H $-1.9309636 .348516-1.245259$

H $-2.185463 \quad 6.9382450 .491915$

H -3.243801 $4.527190-2.096031$

H -4.544546 $3.923055-1.125792$

H $-3.2616402 .137807-0.207808$

H -1.9200040 .5367442 .243656$

C $2.354568-2.7062862 .798957$

O $2.309064-1.3381552 .344989$

C $2.677132-0.4677983 .430485$

C $2.653836-1.3322884 .684502$

C $3.104267-2.6713514 .129759$

H $3.710001-0.1511143 .244029$

C 1.7558350 .7579523 .456498

H $3.313620-0.9463775 .467325$

H $1.644659-1.4164645 .103657$

C $2.795514-3.8438455 .043965$

H $4.187115-2.6306253 .950136$

C $1.6529631 .497937 \quad 2.106314$

$\begin{array}{lllll}\text { O } & 0.431705 & 0.340596 & 3.814252\end{array}$

H $2.075174 \quad 1.4484974 .244858$

H 1.1213540 .8671891 .384370

$\begin{array}{llll}\text { O } & 0.840250 & 2.662107 & 2.293712\end{array}$

C $2.9975361 .937967 \quad 1.499225$

C 2.8063832 .6301710 .139959

H 3.6378281 .0658591 .357967

$\begin{array}{lllll}\text { O } & 3.670795 & 2.835012 & 2.378073\end{array}$

H $2.2270051 .962816-0.504862$

H $2.2146693 .543664 \quad 0.275518$

H $3.309921-3.7287986 .003630$

H $1.722007-3.9237925 .245592$

H $3.124814-4.7860224 .594994$

C $3.032049-3.5706051 .733902$

H $0.148510-0.3271043 .165933$

H -0.0194532 .3611282 .632413$

H 3.9108472 .3417073 .180087

H $3.172106-4.5941242 .098775$

H $4.026940-3.1694591 .507263$

H $1.314954-3.0263532 .949237$

C $4.6615110 .859967-1.520421$

O $5.056221 \quad 1.922423-0.645198$

C $4.133896 \quad 3.013533-0.554242$

C $3.9421323 .640701-1.941312$

C $3.4897042 .603572-2.965648$

C $4.4115331 .385422-2.943002$

H $3.7598000 .375909-1.126269$

C $5.799822-0.175422-1.541158$

H 4.6156953 .7675750 .080690

O $2.9973354 .703493-1.872382$

H $4.8940104 .076061-2.271971$ 
H $2.4452672 .320023-2.802969$

O $3.5380913 .202202-4.263085$

H $5.3649111 .660493-3.411950$

O $3.840278 \quad 0.354183-3.747614$

C $5.996388-0.888044-0.220013$

H $5.593958-0.944292-2.294453$

H $6.7453090 .325738-1.782163$

H $2.8856285 .052241-2.773408$

H $3.2865002 .517811-4.907019$

H $3.0025110 .082678-3.334604$

O $7.043077-1.758427-0.342560$

O $5.326727-0.7161840 .787730$

C $7.358378-2.5125070 .832173$

H $8.247029-3.1134060 .621681$

H $7.578585-1.8430461 .669194$

H $6.531391-3.1838811 .080664$

SCF Energy $\left(B 3 L Y P / 6-31 G^{* *} / / M M F F\right)=-3245.91313284$

02331

MM̄FF Geometry

C -1.622824 $3.947759-0.895924$

C -2.188042 $2.822024-0.434834$

C -2.7477352 .6609180 .951384$

O -2.0463681 .5320491 .522691$

C -4.2455652 .3248660 .887376$

C -4.9426112 .4510262 .226115$

C -5.2658851 .4164303 .026316$

C -5.3192383 .8561582 .620718$

C $-4.958413-0.0417222 .826902$

C $-6.215427-0.8775052 .549427$

C $-6.784090-0.6708681 .136742$

C $-8.075930-1.4696090 .953846$

C $-8.610085-1.379473-0.483247$

O $-5.799313-1.121750 \quad 0.201958$

O $-9.621536-2.390397-0.609523$

C $-7.465655-1.716286-1.463752$

C $-9.299908-0.035404-0.745226$

O $-7.864669-1.495560-2.814879$

C $-6.157206-0.942927-1.173851$

C $-5.007746-1.514898-2.039017$

O $-6.3490020 .435558-1.496616$

C - -1.5965341 .6075342 .798294$

O $-1.7253312 .550633 \quad 3.566056$

C -0.8759820 .3699463 .187062$

C $-0.790350-0.7154012 .401230$

C $-0.071709-1.9566752 .700572$

C $0.673884-2.0402084 .006040$

C $-3.617544-0.970747-1.679855$

C $-2.518931-1.627532-2.498549$

C $-0.033682-1.792360-2.628561$

O $-2.742385-2.382228-3.441405$

C $0.314040-3.065672-1.923382$

C $1.415638-3.235324-1.176842$

C $1.747965-4.517404-0.467803$

C $1.855028-4.351271 \quad 1.033241$

C $3.054257-4.3401251 .639478$

C $0.575817-4.2929321 .841712$

C $-0.090855-2.945021 \quad 1.781461$

N -1.255695 -1.256921 -2.056229

H -1.582231 $4.827910-0.260353$

H $-2.2116131 .944524-1.076442$

H -2.566596 3.5699111 .536870

H -4.3782231 .3144230 .486016$

H -4.754041 2.9921190 .178202

H $-5.7920661 .634053 \quad 3.955178$

H -5.8513833 .8918283 .577280$

H -4.4261364 .4808882 .718561$

H -5.9736254 .3003001 .863538$

H -4.209520 -0.2063422 .047005$

H -4.499740 -0.405507 3.755345

H $-5.940296-1.9335262 .669873$

H -6.983755 -0.655359 3.300394

H $-6.973807 \quad 0.3964280 .979301$

H -8.841106 -1.142090 1.668901

H -7.884544 -2.527037 1.185532

H -10.014656 -2.315912 -1.495242

H -7.260378 -2.791990 -1.370255

H $-10.1548180 .091401-0.070259$
H $-9.7102090 .008370-1.760153$

H -8.637246 $0.821434-0.605980$

H -8.619850 -2.078881 -2.998814

H $-4.977179-2.605834-1.917576$

H -5.203300 -1.310307-3.099295

H $-5.4920210 .837329-1.707064$

H -0.4120180 .4043524 .167367$

H -1.283606 -0.7013291 .430671$

H $1.382932-1.2107504 .102842$

H $1.258760-2.9562584 .114083$

H $-0.025485-2.0024694 .848275$

H -3.567458 $0.105952-1.867646$

H -3.403452 -1.148059 -0.619798

H $\quad 0.741565-1.028987-2.517055$

H $-0.181694-1.986488-3.695694$

H $-0.382027-3.893911-2.035644$

H $2.120546-2.414822-1.070867$

H $2.697821-4.885544-0.877229$

H $1.010625-5.299054-0.689613$

H $3.141995-4.2518272 .717113$

H $3.975870-4.4086001 .070810$

H $\quad 0.761399-4.5962542 .877895$

H $-0.125582-5.0467051 .460882$

H $-0.666156-2.7866380 .869536$

H -1.172556 -0.683805-1.221313

C $0.5374544 .063792-2.220530$

O $1.0203752 .926824-1.473681$

C $1.9711093 .392514-0.499465$

C $2.4655064 .725701-1.041737$

C $1.1754695 .304757-1.592193$

H 1.4207843 .5655130 .435018

C $3.0753102 .359330-0.248362$

H $2.9206385 .350498-0.267580$

H $3.1970524 .582754-1.846198$

C $1.3867636 .447061-2.570585$

H $0.5732985 .670330-0.751660$

C 2.5738821 .0946150 .475127

O $3.6875722 .000381-1.486007$

H 3.8475272 .8340380 .366646

H $2.162636 \quad 1.379871 \quad 1.451413$

O $1.4886600 .527109-0.266683$

C $3.634109-0.0074160 .656065$

C $4.9288630 .464716 \quad 1.337237$

H $3.856596-0.458696-0.316382$

O $3.048396-1.0437351 .449357$

H $5.406330 \quad 1.2286950 .716595$

H 4.6784520 .9387462 .294430

H $1.8944977 .284095-2.080516$

H $1.9985796 .137172-3.424466$

H $0.4287816 .810427-2.955794$

C $\quad-0.9945794 .046089-2.259766$

H $3.0016391 .589341-2.039975$

H $1.199904-0.2729960 .204912$

H $2.870373-0.6770522 .331980$

H -1.311951 $3.186077-2.863950$

H $-1.3727464 .940739-2.767081$

H $0.9085093 .933520-3.245368$

C $6.875120-0.838339-0.603759$

O $6.148886-1.4915270 .443952$

C $5.925306-0.6874991 .606042$

C $7.268753-0.2413012 .199317$

C $8.163210 \quad 0.4275961 .155551$

C $8.265588-0.429928-0.107532$

H $6.3249110 .049284-0.938644$

C $6.961046-1.816553-1.779528$

H $5.452595-1.3458292 .345929$

O 7.0520270 .6405013 .295457

H $7.787573-1.1240512 .595226$

H $7.798240 \quad 1.4325920 .914727$

O 9.4702590 .6133291 .704600

H $8.870950-1.3216450 .099021$

O $8.9294460 .314064-1.126125$

C $5.598537-2.023243-2.397962$

H $7.641265-1.465636-2.563427$

H $7.325396-2.796428-1.448101$

H 7.9247380 .9173303 .623622

H $9.829263-0.2620621 .929029$

H $9.7924850 .587371-0.770574$ 
O $5.270238-0.947021-3.168799$

O $4.903849-3.011890-2.201988$

C $3.974318-0.993591-3.772745$

H $3.866452-1.894631-4.384108$

H $3.202648-0.954995-2.999220$

H $3.867786-0.117194-4.417467$

SCF Energy $(B 3 L Y P / 6-31 G * * / / M M F F)=-3245.91209437$

$02 \_32$

MM̄FF Geometry

C $3.416198-1.5360141 .950605$

C $4.307220-2.4875631 .631440$

C $4.963268-2.7035490 .286864$

O $4.860345-1.527201-0.550660$

C $4.311537-3.845851-0.516877$

C $4.057311-5.1284360 .244978$

C $2.833887-5.5265830 .649249$

C $5.269742-5.9797820 .505438$

C $1.549544-4.7620850 .479181$

C $0.744009-5.279411-0.715834$

C $-0.352664-4.290486-1.140660$

C -1.242458 -4.926456 -2.210142

C $-2.262825-3.935929-2.783480$

O $0.299177-3.127204-1.665414$

O $-2.811069-4.548275-3.960757$

C $-1.518440-2.653507-3.208443$

C $-3.434545-3.710559-1.821636$

O $-2.433896-1.645469-3.628812$

C $-0.582791-2.087871-2.111659$

C $0.293561-0.961100-2.718540$

O $-1.369965-1.570928-1.041129$

C $5.728620-0.505246-0.330230$

O $6.631013-0.4627040 .489100$

C $5.3411030 .589059-1.246457$

C $5.7011081 .848346-0.961382$

C $5.3294463 .042876-1.723357$

C $4.5302862 .844977-2.985349$

C $1.289755-0.305254-1.749883$

C $0.749293 \quad 0.941882-1.066579$

C 1.4861082 .8319080 .386668

O $-0.417038 \quad 1.313173-1.166800$

C $1.8942253 .983276-0.478665$

C $2.6727844 .990524-0.053778$

C $3.0527406 .168162-0.906897$

C $4.5412106 .442829-0.906754$

C $5.0583987 .444827-0.175785$

C $5.4176485 .610596-1.817758$

C $5.6990364 .249091-1.244474$

N $1.727148 \quad 1.587052-0.320151$

H $3.093858-0.8167711 .203779$

H $4.598124-3.1978572 .403814$

H 6.024797 -2.934929 0.442891

H $4.951352-4.069950-1.382253$

H $3.389078-3.476873-0.983407$

H $2.737584-6.4795041 .166240$

H $5.020420-6.9056341 .034477$

H $5.749038-6.257021-0.439136$

H $5.995510-5.434972 \quad 1.117298$

H $0.954707-4.8698951 .394700$

H $1.741686-3.6895070 .384016$

H $1.420100-5.444037-1.565705$

H $0.300829-6.248971-0.456657$

H $-0.943252-4.009497-0.260817$

H -1.750581 -5.815244 -1.814978

H - $0.612965-5.288327-3.035919$

H $-3.505647-3.964873-4.310024$

H -0.907661 -2.904319-4.087239

H -3.954377 -4.656260 -1.626816

H -4.183375 -3.044600 -2.262475

H -3.128112 -3.296135 -0.858810

H $-2.933058-1.994551-4.385770$

H $0.887395-1.403101-3.530142$

H $-0.341586-0.189133-3.168574$

H $-0.815960-0.988321-0.498586$

H $4.7251350 .332913-2.100873$

H $6.2973212 .027764-0.067441$

H $4.2750653 .776172-3.494164$

H $3.5821332 .341356-2.768432$
H $\quad 5.0932472 .237498-3.702860$

H $1.630912-1.011751-0.986155$

H $2.1668530 .008863-2.329622$

H 2.0540552 .7919441 .321190

H 0.4222142 .9162920 .622123

H $1.5095614 .003109-1.495776$

H 3.0307144 .9872040 .973338

H $2.5124937 .042975-0.521617$

H $2.7101826 .039604-1.941292$

H $6.1203907 .666072-0.191033$

H $4.431928 \quad 8.0629530 .458786$

H $6.3855776 .107729-1.966869$

H $4.9655215 .576283-2.812269$

H $6.2773204 .282372-0.319587$

H $2.6781661 .232001-0.338499$

C $1.308663-1.7298793 .296444$

O $0.588950-0.6409092 .682705$

C $-0.540058-0.3009533 .517007$

C $-0.724684-1.4797764 .456872$

C $0.712264-1.8944944 .694773$

H $-0.2365690 .593173 \quad 4.076614$

C $-1.747440 \quad 0.0118932 .628445$

H -1.250006 -1.209635 5.377566

H -1.281626 -2.287326 3.965420

C $0.851775-3.2968775 .262071$

H $1.175434-1.1789245 .387690$

C -3.0580680 .2433313 .400441$

O $-1.4413351 .193497 \quad 1.883838$

H -1.880939 -0.7941581 .898063$

H $-3.380973-0.6933313 .870004$

O $-2.795834 \quad 1.1624824 .469302$

C -4.2059940 .8313082 .556183$

C -4.5427310 .0137821 .298464$

H $-3.962806 \quad 1.8653732 .290487$

O $-5.3733830 .903966 \quad 3.383214$

H $-3.643700-0.1111920 .689193$

H $-4.868414-0.9897911 .598768$

H $\quad 0.354472-3.3691876 .234946$

H $0.403183-4.0444034 .599009$

H $1.905102-3.5594575 .400872$

C $2.805186-1.4124933 .319445$

H -0.6304291 .0108841 .379791$

H $-3.633420 \quad 1.302593 \quad 4.943401$

H -5.621670 -0.003956 3.627725

H $3.318429-2.0822064 .019402$

H $2.969394-0.3863343 .670165$

H $1.110059-2.6224582 .690417$

C $-4.2677402 .238164-0.742684$

$\begin{array}{llll}\text { O } & -5.377523 & 2.034883 & 0.140478\end{array}$

C -5.6569880 .6640680 .445606$

C $-5.985075-0.092040-0.847917$

C $-4.8876080 .076758-1.897465$

C $-4.525658 \quad 1.550565-2.087875$

H $-3.3532221 .840510-0.287270$

C $-4.0862643 .749333-0.918082$

H -6.5665030 .6653821 .059305$

O $-6.188404-1.473039-0.569410$

H $-6.9276770 .296594-1.254355$

H $-4.001235-0.506743-1.627878$

O $-5.342714-0.458871-3.142693$

H $-5.3366512 .060987-2.622707$

O $\quad-3.3543601 .645450-2.895319$

C -3.7130554 .4074640 .389047$

H -3.313939 $3.989632-1.657222$

H $-5.0185634 .213950-1.261796$

H $-6.364818-1.917731-1.416100$

H -6.132136 $0.040138-3.413909$

H $-3.525127 \quad 1.152330-3.715945$

O $-2.403906 \quad 4.164247 \quad 0.682487$

O -4.4987715 .0504031 .073650$

C -1.9451934 .7120901 .922446$

H -2.4912504 .2627212 .757409$

H -0.8839294 .4745202 .030655$

$\mathrm{H}-2.0613815 .8001021 .924095$

SCF Energy (B3LYP/6-31G**//MMFF)= -3245.91739812

0233

MM̄MF Geometry 
C $-2.690605-1.9301932 .512915$

C -2.169665 -0.8431373 .105694$

C $-2.8519780 .503313 \quad 3.236266$

O -4.0039480 .5392972 .365656$

C -1.9025001 .6349292 .805170$

C -2.3587913 .0042123 .267668$

C -2.974522 3.9157692 .489577

C -2.0342713 .3348074 .702013$

C -3.4027633 .7732981 .055624$

C -2.5979424 .6717650 .106284$

C $-1.1504784 .198887-0.098816$

C $-0.3914695 .161481-1.013356$

C $1.0273734 .657601-1.317653$

O $-1.1972712 .901418-0.698218$

O $1.5453295 .431980-2.410165$

C $0.9475953 .193112-1.798909$

C $1.9776134 .884946-0.136752$

O $2.2498702 .632644-1.945320$

C $0.0823782 .286714-0.892744$

C $-0.1540020 .921279-1.582682$

$\begin{array}{llll}\text { O } & 0.763662 & 2.075128 & 0.345335\end{array}$

C -5.1850591 .0084402 .833352$

O $-5.408341 \quad 1.4519553 .950576$

C -6.2454860 .8915081 .801522$

C $-5.998951 \quad 0.532708 \quad 0.531037$

C $-6.9870260 .352104-0.534588$

C $-8.4401260 .528354-0.182072$

C $-1.1948490 .036551-0.880626$

C - $-1.476346-1.238982-1.655758$

C $-3.150952-3.084244-1.797970$

O $-0.767374-1.637479-2.575570$

C $-4.152891-2.677358-2.832992$

C $-5.458166-2.982738-2.772197$

C $-6.459891-2.563975-3.811086$

C $-7.593119-1.740277-3.236526$

C $-8.799773-2.288896-3.016837$

C $-7.357605-0.263756-2.999863$

C $-6.5479770 .004436-1.762726$

N $-2.608247-1.887900-1.181036$

H -3.691377 -1.866235 2.090628

H - $-1.186908-0.9100513 .564637$

H -3.148183 0.6173164 .285826

H -1.7894611 .6209811 .716612$

H -0.8945721 .4651203 .207052$

H -3.2308514 .8770412 .933719$

H -2.3494354 .3466874 .978115$

H -2.535837 2.6384345 .380975

H -0.9544283 .2694664 .871411$

H -3.3726042 .7348280 .713788$

H -4.457995 4.0698651 .000486

H -3.113862 $4.669580-0.862842$

H -2.606295 5.7050080 .475020

H -0.6539614 .1325670 .875449$

H $-0.3570176 .167694-0.577492$

H $-0.9327015 .265400-1.964297$

H $1.5362196 .367778-2.144940$

H $0.5004033 .204623-2.802855$

H 2.0169215 .9495900 .123516

H $3.0031334 .605361-0.398497$

H $1.686630 \quad 4.3365490 .761487$

H $2.7558373 .222647-2.529828$

H $-0.5059141 .093771-2.608463$

H $\quad 0.792361 \quad 0.372083-1.652497$

H $\quad 0.4831621 .227726 \quad 0.724212$

H -7.248402 1.1146662 .150523

H -4.9708800 .3379330 .230011$

H -8.723459 -0.1460080 .633854$

H $-9.1176610 .311168-1.010732$

H -8.636629 $1.559848 \quad 0.129871$

H $-0.849071-0.2534320 .116399$

H $-2.1388350 .582778-0.771257$

H -3.598646 -3.688086 -1.002436

H $-2.344555-3.659383-2.263662$

H -3.781191 -2.100955 -3.677201

H -5.829632 -3.570190 -1.935663

H $-6.855363-3.476038-4.277055$

H -5.982698 -2.002256 -4.623862

H $-9.627245-1.700793-2.634173$
H $-8.986393-3.340034-3.210505$

H $-8.3078460 .281096-2.972734$

H $-6.8274050 .159715-3.862711$

H -5.473682 -0.098660 -1.916989

H $-3.165142-1.440980-0.458413$

C $-0.557333-3.4114082 .615259$

O $0.165309-2.6779031 .604315$

C $1.182829-3.5333041 .042382$

C $1.355716-4.6660342 .040184$

C $-0.067032-4.8589662 .519301$

H $\quad 0.768574-3.9093250 .098070$

C $2.450268-2.7117590 .779592$

H $1.779917-5.5676151 .588475$

H $2.002423-4.3586102 .871514$

C $-0.169699-5.632326 \quad 3.822772$

H $-0.627373-5.3962631 .741965$

C $3.654371-3.5567570 .329270$

O $2.153973-1.753885-0.240711$

H $2.706451-2.1422211 .680265$

H $3.950937-4.2358851 .137173$

O $3.239318-4.385878-0.764249$

C $4.878528-2.745637-0.137526$

C $5.376991-1.7226700 .896536$

H $4.646438-2.250316-1.086228$

O $5.934143-3.670645-0.423547$

H $4.584415-0.9957411 .097119$

H $5.583679-2.2405641 .841477$

H $0.244795-6.6389343 .706484$

H $0.379155-5.1342634 .629093$

H -1.213575 -5.730941 4.136667

C $-2.062081-3.2902952 .357934$

H $1.398983-1.2261580 .070826$

H $4.011422-4.908164-1.041779$

H $\quad 6.177932-4.1102170 .408979$

H -2.606023 -3.978863 3.015272

H $-2.260696-3.6090061 .326469$

H $-0.284689-2.9834833 .587184$

C $5.6304280 .651605-0.990050$

O $6.543545-0.445530-0.869350$

C $6.660291-0.9849210 .450624$

C 7.1348490 .1053061 .421252

C $6.2603481 .356911 \quad 1.348112$

C $6.0823951 .813763-0.100888$

H $4.6227290 .330102-0.699967$

C $5.5947911 .056064-2.467162$

H $7.456376-1.7387450 .402824$

O $7.157862-0.3987032 .752234$

H $\quad 8.166287 \quad 0.379378 \quad 1.163984$

H $5.288272 \quad 1.183627 \quad 1.823546$

O 6.8758092 .4060002 .099743

H $7.0260462 .230845-0.474857$

O $5.1063482 .850113-0.148054$

C $4.954762-0.026307-3.304450$

H $5.0406911 .986745-2.630581$

H $6.609671 \quad 1.212412-2.852578$

H 7.4388600 .3282663 .334048

H 7.7438722 .5912411 .702367

H 5.4008533 .5556580 .452970

O $3.602022-0.011211-3.134159$

O $5.585346-0.800910-4.012278$

C $2.879884-1.015174-3.853517$

H $3.133201-2.006954-3.467462$

H $1.811862-0.840766-3.701451$

H $3.094525-0.949791-4.924566$

SCF Energy (B3LYP/6-31G**//MMFF) $=-3245.91073915$

$02 \_34$

MM̄FF Geometry

C - $1.584890 \quad 4.2928840 .744596$

C -2.8594984 .6995730 .636212$

C $-4.1070763 .851840 \quad 0.733839$

O -3.8027922 .4572210 .954611$

C $-4.905698 \quad 3.949097-0.577661$

C $-6.2902633 .339769-0.471976$

C $-6.6421382 .147840-0.992529$

C -7.3193744 .1939270 .223220$

C $-5.7531161 .171131-1.711204$

C $-6.102019-0.279075-1.344928$ 
C $-5.218275-1.277285-2.105110$ C $-5.727799-2.706966-1.918426$ C $-4.819799-3.727140-2.619583$ O $-3.884387-1.168214-1.596571$ O $-5.205639-5.025757-2.145443$ C $-3.362128-3.472668-2.184341$ C $-5.046240-3.737392-4.135631$ O $-2.463397-4.310717-2.908024$ C -2.927210 -1.989069 -2.278219 C $-1.556373-1.815411-1.587303$ O $-2.803953-1.622490-3.653370$ C -3.7365622 .0244542 .242216$ O $-3.8372432 .701346 \quad 3.252931$ C -3.5141230 .5613062 .210470$ C $-3.154859-0.0717963 .337057$ C $-2.884979-1.5042983 .479027$ C $-3.246441-2.4015162 .325391$ C -1.122067-0.358909-1.379606 C $0.164499-0.324653-0.579789$ C $1.033066-0.7097131 .729179$ O $1.255155-0.166695-1.121549$ C $0.836945-2.0121212 .438393$ C $0.827690-2.1393343 .773816$ C $0.621186-3.4486854 .480823$ C $-0.494506-3.3981205 .503013$ C $-0.229202-3.468671 \quad 6.818575$ C -1.923541-3.343477 4.999895 C $-2.341642-1.9422914 .634292$ N $-0.043195-0.5432730 .772190$ H $-1.357615 \quad 3.2462140 .924710$ H -3.0442395 .7606900 .474166$ H -4.7139794 .2384921 .561968$ H $-4.3401083 .477297-1.390092$ H -5.020626 $5.000199-0.875818$ H $-7.6829191 .839824-0.900463$ H $-8.314003 \quad 3.7352850 .216647$ H -7.041540 4.3584101 .268595 H -7.403371 $5.166756-0.272333$ H $-5.8916441 .312245-2.789725$ H -4.697183 $1.350144-1.488545$ H $-5.973151-0.417211-0.263404$ H -7.156557 -0.468729-1.581212 H -5.225347 -1.007268 -3.167614 H $-6.760458-2.804864-2.276027$ H -5.758093 -2.945502 -0.845746 H $-4.586486-5.673579-2.524012$ H -3.287885 -3.789301-1.134668 H -6.093364 -3.971472 -4.362542 H -4.454631 -4.522068 -4.620010 H -4.807709 -2.783795 -4.611256 H - $-2.353547-3.929203-3.795685$ H -1.589632 -2.304589 -0.606757 H $-0.778574-2.329525-2.167234$ H -2.201839 -0.864871 -3.723764 H $-3.636576 \quad 0.050247 \quad 1.262378$ H -3.0209990 .5182544 .243110$ H -2.590811 -2.2134911 .471827$ H -4.283098 -2.2308812 .012531$ H -3.175195 -3.464992 2.562431 H $-0.9457640 .146897-2.334417$ H $-1.8848290 .217820-0.844689$ H $\quad 0.9996220 .1449252 .411958$ H $1.994634-0.7136371 .211440$ H $0.699980-2.8963791 .819993$ H $0.989592-1.2602524 .393569$ H $1.568724-3.7153824 .966611$ H $0.412388-4.2567403 .768420$ H -1.024864 -3.457940 7.556308 H $0.788781-3.5335477 .188048$ H -2.608601-3.699594 5.780445 H -2.024993 -4.051371 4.173046 H -2.153673 -1.211663 5.422826 H $-0.995017-0.5828721 .123044$ C $0.6045444 .774761-0.397078$ $\begin{array}{lllll}\text { O } & 1.346306 & 3.653220 & 0.126548\end{array}$ C $2.7578003 .894221-0.065183$

C $2.8432594 .996425-1.105601$ C $1.6453185 .845405-0.731029$
H 3.1353824 .2477800 .902884

C $3.4344642 .576991-0.452576$

H $3.7878735 .546456-1.063330$

H $2.7249574 .588951-2.117223$

C $1.2133796 .802339-1.828923$

H $1.892013 \quad 6.4232590 .170042$

C $4.9161222 .709244-0.842877$

O $3.336694 \quad 1.695143 \quad 0.670164$

H $2.8721432 .112357-1.269640$

H $5.0070703 .299265-1.762375$

O 5.5860843 .4513040 .184180

C $5.6543651 .364394-1.008814$

C $5.0058140 .423207-2.037888$

H $5.7364170 .873709-0.033130$

O $6.9967791 .641690-1.423816$

H $3.9584920 .258795-1.774821$

H $5.0171090 .908036-3.021982$

H $2.0170827 .508538-2.061625$

H $0.9590626 .267323-2.750087$

H $\quad 0.3355567 .379069-1.521127$

C -0.4149005 .2367110 .646843$

H 2.3976501 .6489620 .918407

H $6.5226113 .517665-0.070100$

H $6.9560262 .073267-2.294243$

H $-0.766734 \quad 6.245596 \quad 0.401411$

H 0.0598175 .2828191 .634622

H $0.1004974 .428212-1.308432$

C $4.653866-2.023247-0.277499$

O $5.877644-1.579195-0.875676$

C $5.726216-0.940493-2.148146$

C $5.078647-1.917111-3.139504$

C $3.768022-2.490479-2.600585$

C $3.950701-3.039230-1.184326$

H $3.998671-1.163056-0.100441$

C $5.002378-2.6404331 .080904$

H $6.740865-0.735339-2.511804$

O $4.853071-1.270918-4.387549$

H $5.776674-2.743420-3.326950$

H $2.972636-1.738265-2.628494$

O $3.326634-3.545877-3.458779$

H $4.528692-3.971140-1.223530$

O $2.672093-3.351372-0.634984$

C $5.469196-1.5775702 .047035$

H $4.148543-3.1600121 .530348$

H $5.811093-3.3746680 .983895$

H $4.417457-1.913405-4.973553$

H $4.011068-4.236601-3.462357$

H $2.233218-3.964754-1.249071$

O $4.394312-0.9482912 .600816$

O $6.646748-1.3245432 .265598$

C 4.7003870 .1143993 .508196

H 5.2776820 .8929513 .000180

H $3.7577870 .546923 \quad 3.854222$

H $5.248407-0.2727364 .372277$

SCF Energy (B3LYP/6-31G**//MMFF $)=-3245.93075259$

0235

MM̄FF Geometry

C $1.644042-3.7948001 .256916$

C $0.981536-2.6393551 .099279$

C $0.518516-1.7822852 .247791$

O $-0.831805-1.3437391 .977767$

C $1.417373-0.5405542 .341827$

C $1.0890360 .349543 \quad 3.522349$

C 0.4378501 .5254373 .428982

C $1.596156-0.1332674 .856015$

C -0.1291022 .1498662 .184931$

C -1.5159442 .7568312 .435836$

C -2.0976053 .3598301 .149526$

C -3.3621424 .1650661 .449146$

C -4.0046924 .7091110 .164810$

O -2.4071562 .2819050 .259248$

O $\quad-5.322262 \quad 5.174914 \quad 0.493417$

C $-4.1715083 .548185-0.836129$

C $-3.2421525 .917361-0.391672$

O $-4.629824 \quad 4.024901-2.099523$

C -2.892459 2.697406-1.023679

C $-3.2334721 .431680-1.843858$ 
O $-1.9165653 .462479-1.732434$ C -1.841099-2.179453 2.341036 O $-1.735860-3.2687202 .881897$ C -3.114805 -1.542467 1.938143 C $-4.241947-2.2691021 .957330$ C $-5.572462-1.8204511 .541858$ C $-5.750833-0.361427 \quad 1.217728$ C $-2.1282370 .368372-1.869972$ C -2.599487 -0.847812 -2.643659 C $-4.156934-2.794933-2.461781$ O $-2.251240-1.047993-3.804740$ C $-5.629063-2.597103-2.272291$ C $-6.437517-3.504139-1.702980$ C -7.911429-3.291301-1.499038 C $-8.356123-3.555315-0.075359$ C $-9.121373-4.619244 \quad 0.222154$ C $-7.976819-2.5476680 .992321$ C $-6.561236-2.7359431 .466883$ N -3.470593 -1.639543 -1.913321 H $1.855449-4.1603572 .258537$ H $\quad 0.794361-2.2645070 .095297$ H $0.548840-2.3369043 .193892$ H 1.3586260 .0271501 .407096 H $2.467219-0.8496722 .425465$ H $\quad 0.3016292 .1084954 .338721$ H 1.3851890 .5766775 .662820 H $1.126951-1.0846085 .124884$ H $2.680861-0.2774014 .820980$ H 0.5536822 .9425961 .859777 H $-0.202187 \quad 1.4272201 .367774$ H $-2.187614 \quad 1.9757592 .815244$ H -1.437682 3.5331093 .207273 H -1.3364654 .0023350 .690849$ H -3.1474844 .9844022 .146454$ H -4.093072 3.5209911 .958213 H -5.2356035 .8605631 .177761$ H $-4.9651802 .895689-0.445521$ H -3.2140096 .7260190 .348472$ H $-3.7485236 .333278-1.269829$ H -2.210785 $5.685462-0.665877$ H $-5.4384294 .540833-1.938513$ H -4.136185 $0.970635-1.426746$ H $-3.4726601 .711928-2.877983$ H -1.322538 $2.858736-2.205219$ H -3.084469 -0.5113841 .604648$ H $-4.182323-3.3090882 .276866$ H -5.209116 -0.1028620 .304307$ H $-5.377061 \quad 0.2655612 .035282$ H -6.793802 $-0.073011 \quad 1.071114$ H $-1.2214460 .745520-2.351291$ H -1.848178 $0.055009-0.857970$ H -3.786176 -3.676167 -1.929168 H -3.926608 -2.902026 -3.525885 H $-6.050549-1.656907-2.621443$ H -6.022789-4.454715 -1.375114 H -8.440790 -3.959761 -2.190330 H -8.208358 -2.272952 -1.779477 H $-9.463965-4.803008 \quad 1.235114$ H -9.418496 -5.333295 -0.538673 H $-8.635977-2.6571851 .863344$ H $-8.167231-1.5448600 .600347$ H -6.334399 -3.7627001 .759233$ H $-3.638873-1.412998-0.938030$

C $3.674402-4.423206-0.052191$

O $3.934231-3.073878-0.493688$

C $4.858310-3.111364-1.595223$

C $5.476450-4.502549-1.566633$

C $4.293486-5.336221-1.111129$

H $4.262013-3.014164-2.510501$

C $5.850657-1.948398-1.501090$

H $5.866549-4.805402-2.542931$

H $6.291400-4.571345-0.836866$

C $4.680567-6.709549-0.590947$

H $3.601481-5.455662-1.955727$

C $5.182693-0.561110-1.364142$

O $6.679878-2.147937-0.350039$

H $\quad 6.521459-1.969058-2.368091$

H $4.707119-0.488585-0.381480$
O $6.227257 \quad 0.419217-1.377863$

C $4.171136-0.227422-2.472330$

C $3.5956341 .202767-2.434511$

H $3.341453-0.942144-2.445868$

O $4.830924-0.398900-3.732338$

H $4.414126 \quad 1.929511-2.403877$

H $3.1023911 .374152-3.399604$

H $5.169947-7.294262-1.376711$

H $5.372353-6.6379890 .255088$

H $3.797229-7.263348-0.257856$

C $2.165502-4.6152050 .110476$

H $7.288068-1.390757-0.295904$

H $6.6329670 .404076-2.261673$

H $4.163456-0.292003-4.431214$

H $1.928713-5.6673550 .303479$

H $1.650983-4.330277-0.816009$

H $4.186620-4.5464270 .910937$

C 3.9692742 .6642340 .265968

O $3.1774331 .501590-0.001585$

C $2.5781591 .442083-1.298152$

C $1.6856542 .674299-1.510978$

C $2.4616323 .969280-1.305459$

C 3.1670773 .9545770 .046937

H $4.8568822 .657085-0.376759$

C 4.4295152 .5812811 .727995

H $1.9113260 .570231-1.285772$

O $1.1111232 .680585-2.813699$

H $0.8539092 .648019-0.795732$

H $3.1733824 .146905-2.120159$

O $1.5493455 .070139-1.318574$

H 2.4227114 .0752980 .843652

O 4.0518635 .0712050 .126574

C 5.4053601 .4492761 .944084

H 4.9322073 .5002672 .050118

H 3.5664782 .4393382 .390300

H $0.6822561 .819210-2.950432$

H $1.0692425 .037681-2.163909$

H $3.5235365 .871960-0.034071$

O 4.8367640 .4882712 .725185

O 6.5348241 .4218721 .473196

C $5.655825-0.6500513 .007482$

H $5.116835-1.2896613 .711537$

H $5.837856-1.2152282 .089948$

H $6.599568-0.3408853 .466634$

SCF Energy $\left(B 3 L Y P / 6-31 G^{* *} / / M M F F\right)=-3245.92530043$

0236

MM̄FF Geometry

C -1.916211 4.4282761 .877894

C -2.4540243 .2435271 .549124$

C -2.9085662 .2232802 .562164$

O -2.4267330 .9226562 .152289$

C -4.4471572 .1820472 .590263$

C -5.0101181 .1690183 .568487$

C $-5.577269-0.003913 \quad 3.220599$

C -4.9374061 .5700875 .019420$

C $-5.733887-0.5755731 .838430$

C $-5.062796-1.9511491 .726952$

C $-4.997614-2.4384370 .271439$

C $-4.417274-3.8521550 .203694$

C $-4.201749-4.323952-1.241971$

O $-4.163642-1.527046-0.452576$

O $-3.361318-5.488094-1.204498$

C $-3.426848-3.234795-2.014560$

C $-5.515659-4.759388-1.900852$

O $-3.333927-3.563351-3.398152$

C $-4.024030-1.822230-1.848801$

C $-3.141924-0.710457-2.472225$

O $-5.292730-1.769667-2.512973$

C -1.1830550 .5573362 .567173$

O -0.3942901 .2326693 .208192$

C $-0.956551-0.8370522 .122828$

C $0.204326-1.4535862 .390920$

C $0.536002-2.8455482 .073111$

C $-0.508552-3.6681931 .365653$

C $-1.742760-0.560196-1.866345$

C $-0.665073-1.361763-2.576095$

C $1.695682-2.114422-2.278003$ 
O $-0.814677-1.852835-3.691314$

C $1.812074-3.414547-1.548380$

C $2.876910-3.742245-0.802044$

C $3.043813-5.056983-0.100112$

C $3.384434-4.8959051 .363878$

C $4.656429-4.9763841 .790045$

C $2.243080-4.7444002 .341305$

C $1.738595-3.3295512 .449596$

N $0.502264-1.424468-1.828177$

H -1.789789 4.6565552 .934961

H -2.6109232 .9816260 .506817$

H -2.528822 2.4617273 .563556

$\mathrm{H}-4.8270441 .9958611 .580290$

H -4.8372233 .1732682 .859879$

H $-5.972697-0.6368604 .014203$

H -5.4157380 .8374865 .678340$

H -3.8957371 .6685435 .339927$

H -5.442305 2.5292215 .174161

H $-6.804132-0.6771921 .622143$

H $-5.3268150 .092108 \quad 1.076798$

H $-4.045441-1.8865002 .132190$

H -5.612818 -2.676093 2.339782

H $-6.009057-2.417284-0.151204$

H $-5.054609-4.5599250 .748406$

H -3.447879 -3.8702710 .718369$

H $-3.802739-6.158924-0.655786$

H -2.403004 -3.236650 -1.621507

H $-5.964291-5.592265-1.346058$

H $-5.343973-5.134751-2.915725$

H -6.257617 -3.959695 -1.948133

H -2.935067 -4.448259-3.459801

H -3.100397 -0.827201 -3.561903

H -3.658895 $0.245265-2.302882$

H $-5.503669-0.840721-2.703699$

H -1.770566 -1.332850 1.607526

H $\quad 0.977652-0.8997302 .920934$

H -1.374446 -3.824612 2.017577

H $-0.152425-4.652571 \quad 1.054436$

H $-0.842196-3.1682240 .450013$

H -1.440021 $0.491093-1.949955$

H $-1.749220-0.811668-0.802173$

H $2.546037-1.454261-2.080482$

H $1.642163-2.295885-3.355824$

H $0.997159-4.125446-1.661326$

H $3.696585-3.035402-0.715623$

H $3.847310-5.605345-0.609321$

H $2.149013-5.683325-0.202273$

H $4.906358-4.8942512 .842565$

H $5.477125-5.1168241 .094217$

H $2.573836-5.0419893 .345995$

H $1.453663-5.4607792 .095871$

H $2.436388-2.6556332 .948796$

H $0.514688-1.021250-0.896025$

C - $-1.5439295 .238266-0.556628$

O $-0.4955094 .308669-0.897260$

C $0.1644894 .769242-2.091480$

C $-0.7511225 .827485-2.690030$

C -1.304469 $6.470107-1.432201$

H $1.0904495 .261028-1.771480$

C $0.4912713 .587359-3.008508$

H $-0.211967 \quad 6.530517-3.331989$

H -1.561041 $5.379818-3.277822$

C $-2.5478257 .307917-1.676127$

H $-0.5258757 .103655-0.986228$

C $1.3341332 .489035-2.325438$

O $-0.7419212 .992059-3.433182$

H $0.9880463 .946453-3.917310$

H $0.7189852 .005400-1.558892$

O $1.6026411 .495419-3.321488$

C $2.6649622 .983532-1.724947$

C $3.5331551 .893984-1.077856$

H $2.4863703 .765957-0.984420$

O $3.4635023 .578925-2.754460$

H $3.8842591 .200157-1.854471$

H $4.4574542 .373549-0.731480$

H $-2.3261408 .134110-2.359550$

H -3.353046 6.712311-2.119225

H $-2.9209007 .735232-0.740217$
C -1.4850195 .5329910 .945073$

H $-0.5170492 .237134-4.004024$

H $1.9496890 .709160-2.868750$

H $3.0237504 .398285-3.036362$

H -2.1103446 .4048681 .170665$

H -0.4534825 .7941061 .214694$

H $-2.4982884 .772663-0.831587$

C $4.9813800 .222252 \quad 0.970951$

$\begin{array}{lllll}\text { O } & 3.716987 & -0.071043 & 0.361089\end{array}$

C 2.9024691 .0762200 .067525

C 2.6017681 .8140021 .378408

C 3.8811002 .1963272 .116167

C 4.7759930 .9693072 .295164

H 5.5935300 .8301180 .293970

C $5.703987-1.1165201 .193978$

H $1.9576780 .669285-0.303306$

O 1.8034732 .9701541 .177991

H 2.0298831 .1479482 .034560

H 4.4154043 .0052871 .604759

O 3.5455942 .6933203 .414151

H $4.3298330 .297437 \quad 3.039944$

O 6.0430991 .3865772 .800086

C $6.378173-1.677739-0.042699$

H $6.499399-0.9895631 .937226$

H $5.002974-1.8761111 .556312$

H 0.9626332 .6814040 .783579

H 2.9333683 .4387803 .288417

H 5.8797971 .8730903 .626275

O $5.725572-1.320594-1.182383$

O $7.359374-2.4105580 .014722$

C $6.260612-1.849482-2.399545$

H $5.645804-1.484595-3.226561$

H $7.287834-1.501894-2.544641$

H $6.219063-2.942722-2.387112$

SCF Energy $\left(B 3 L Y P / 6-31 G^{* *} / / M M F F\right)=-3245.91290087$

0237

MM̄FF Geometry

C $1.685234-1.9051782 .701264$

C $2.997475-1.8250312 .973122$

C $3.818055-0.5734653 .179874$

O 3.0448810 .6032412 .856710

C $5.059972-0.5914352 .270101$

C 6.1561120 .3517512 .725554

C 6.4770031 .5120222 .120782

C $6.957298-0.1211403 .912373$

C 5.8118992 .1604840 .939452

C $6.6779472 .100290-0.327684$

C $6.7315000 .700747-0.960999$

C $7.6606240 .687116-2.176249$

C $7.637749-0.669860-2.897679$

O $5.4025290 .362513-1.365322$

O $8.268655-0.500856-4.176205$

C $6.171184-1.069614-3.166741$

C $8.453603-1.729544-2.147706$

O $6.091252-2.387958-3.704115$

C $5.259557-0.948983-1.924040$

C $3.777463-1.118087-2.335815$

O $5.606916-1.968051-0.985638$

C 3.0939311 .6548053 .713014

O 3.6724851 .6954284 .790542

C 2.3340222 .8179083 .199224

C 1.9006942 .9326031 .933874

C 1.1776334 .0775241 .371924

C 0.8183165 .2060712 .303277

C $2.777189-0.801791-1.213621$

C $1.347679-0.763247-1.724362$

C $-0.9098300 .081116-1.090117$

O $1.011067-1.218231-2.814276$

C $-1.0336461 .436315-1.709573$

C $-1.7388152 .439778-1.167034$

C $-1.8333203 .808987-1.775259$

C $-1.3483894 .892776-0.837489$

C $-2.2226705 .674368-0.181536$

C $0.1425925 .129290-0.727843$

C 0.8582304 .0650960 .060517

N $0.489639-0.173818-0.805821$

H $1.090962-1.0018432 .598030$ 
H $3.546691-2.7563863 .105462$ H $4.111241-0.5700834 .236342$ H $4.756025-0.3602131 .244710$ H $5.492427-1.6002872 .229091$ H 7.3094852 .0860752 .526840 H $7.784907 \quad 0.555078 \quad 4.152112$ H $6.325430-0.1927684 .802691$ H $7.388709-1.1071673 .710730$ H 4.8161861 .7501340 .747656 H 5.6483423 .2146921 .197559 H $6.2460212 .801699-1.053527$ H $7.6930352 .450427-0.103160$ H $7.079445-0.019394-0.212659$ H $8.6851290 .951152-1.885897$ H $7.3420611 .461616-2.888069$ H $9.173616-0.178791-4.023659$ H $5.786951-0.394541-3.944482$ H $\quad 9.498977-1.411649-2.053444$ H $8.477318-2.674628-2.701414$ H $8.078100-1.927809-1.141648$ H $6.667305-2.416848-4.487236$ H $3.563149-0.441925-3.174086$ H $3.599238-2.141800-2.688398$ H $4.835011-2.172135-0.435162$ H 2.1831773 .6096673 .926322 H 2.1037342 .1255761 .232222 H 0.2185164 .8366083 .142761 H $0.2286035 .992171 \quad 1.826866$ H 1.7229915 .6813372 .698277 H $2.828194-1.559932-0.425453$ H $3.0036860 .173282-0.767853$ H -1.451030 $0.011971-0.142109$ H -1.292479 $-0.679981-1.776997$ H $-0.5087551 .601536-2.647691$ H -2.276282 2.272689-0.236398 H -2.881831 $3.983787-2.047136$ H -1.272404 $3.869793-2.716282$ H $-1.888508 \quad 6.4753990 .469048$ H $-3.2935165 .528573-0.277755$ H $0.3508126 .122154-0.312487$ H $0.5744485 .163206-1.736671$ H $1.1588313 .205040-0.538032$ H $\quad 0.8749470 .2235280 .046182$ C $0.115769-3.308687 \quad 1.306236$ O $-1.074887-2.5088291 .470930$ C $-2.230096-3.3306941 .199412$ C -1.706753 -4.4847270 .363744$ C $-0.364804-4.7343101 .019708$ H -2.582242 -3.687206 2.176058 C $-3.309438-2.4811130 .522571$ H $-2.363011-5.3596760 .388754$ H -1.573155 -4.180011 -0.681688 C $0.592549-5.5391980 .157034$ H $-0.530758-5.272117 \quad 1.963067$ C $-4.538007-3.277918 \quad 0.053273$ O $-3.735517-1.4829501 .455096$ H -2.869897 -1.951826 -0.327885 H -4.243852 -3.979203 -0.736515 O $-5.005013-4.074271 \quad 1.149535$ C $-5.717122-2.411926-0.433128$ C $-5.343472-1.415261-1.543857$ H -6.156984 -1.887687 0.421693 O $-6.736942-3.289976-0.923223$ H -4.551647 -0.750479-1.187126 H -4.936388 -1.968520 -2.399319 H $0.178460-6.530270-0.055367$ H $\quad 0.783726-5.042536-0.800285$ H $\quad 1.553360-5.6750270 .663534$ C $0.963300-3.2216722 .577984$ H -2.944552 -0.9924321 .737783$ H $-5.785318-4.5653000 .839438$ H -6.379869-3.757716 -1.697480 H $1.686295-4.0458572 .595595$ H $0.317714-3.3290833 .458501$ H $0.660121-2.9036360 .445140$ C $-6.5209861 .081772-0.272882$ O $-7.2465510 .042855-0.941124$ C $-6.546328-0.572190-2.027415$
C $-6.1962120 .483970-3.085286$

C $-5.4568291 .677211-2.479713$

C $-6.1963372 .213479-1.252590$

H $-5.592900 \quad 0.675546 \quad 0.146844$

C -7.3902051 .5772240 .887427$

H $-7.254311-1.269995-2.492060$

O $-5.415000-0.100605-4.121648$

H $-7.1261310 .841323-3.546258$

H $-4.4266881 .406265-2.225467$

O $\quad-5.353247 \quad 2.714875-3.457462$

H $-7.1191402 .716314-1.568625$

O $-5.381322 \quad 3.182459-0.598573$

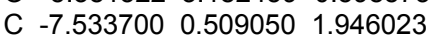

H -6.9761102 .4731001 .363107$

H $-8.398011 \quad 1.8311760 .537303$

H $-5.1928480 .605453-4.752585$

H $-6.2537852 .982579-3.708315$

H -5.180230 $3.877759-1.248093$

O $-6.373104 \quad 0.3929022 .652760$

O $-8.551536-0.1498042 .115103$

C $-6.367337-0.6199903 .663308$

H $-6.531972-1.6040723 .213897$

H $-5.385453-0.6147584 .144121$

H $-7.129799-0.4064574 .418364$

SCF Energy (B3LYP/6-31G**//MMFF)= -3245.91749204

0238

MMFF Geometry

C $-0.0989623 .033530-0.371822$

C $0.8654863 .314720-1.260108$

C $1.4647372 .268253-2.161071$

O $2.8980692 .333350-1.971331$

C $1.1491452 .499720-3.649474$

C $-0.3168192 .431275-4.017900$

C $-1.054371 \quad 1.303448-4.010825$

C $-0.9323243 .738937-4.439450$

C $-0.604618-0.079693-3.625516$

C $-1.330491-0.535243-2.355269$

C $-0.821389-1.881431-1.823314$

C $-1.707907-2.343992-0.664364$

C $-1.204470-3.643672-0.028192$

O $0.530205-1.715466-1.371318$

$\begin{array}{lllll}0 & -1.896927 & -3.821103 & 1.217592\end{array}$

C $0.290960-3.4773530 .304533$

C $-1.537149-4.869012-0.886700$

$\begin{array}{llllll}\text { O } & 0.848388 & -4.707090 & 0.763645\end{array}$

C $1.130145-2.919845-0.871115$

C $2.586225-2.612571-0.440494$

O $1.202655-3.918759-1.897265$

C $3.6099051 .203099-2.227107$

O $3.1834150 .141688-2.652130$

C $5.0168501 .476004-1.867069$

C $5.8663320 .449435-1.716907$

C $7.2759760 .547735-1.336024$

C $7.8628941 .928596-1.195316$

C $2.754125-1.4532820 .546868$

C $4.134241-1.4362441 .189278$

C $5.374645-0.2548252 .997105$

O $4.973479-2.3146711 .010312$

C $6.665304 \quad 0.1253712 .342228$

C $7.762486-0.6464982 .391700$

C $9.101417-0.2705121 .822486$

C $9.553947-1.209167 \quad 0.725529$

C $10.135353-2.3853221 .016088$

C $9.403227-0.744647-0.705537$

C $7.963866-0.592002-1.114148$

N $4.298630-0.3379612 .025749$

H $-0.4833892 .018488-0.320766$

H $1.2540374 .327038-1.333416$

H $1.1225341 .278291-1.839125$

H $1.699521 \quad 1.774277-4.263007$

H $1.5680153 .467854-3.958511$

H -2.100301 $1.370667-4.307987$

H $-1.998612 \quad 3.643103-4.670336$

H $-0.8322864 .482453-3.642377$

H $-0.4315474 .120761-5.335128$

H $-0.843798-0.764127-4.448506$

H $0.478821-0.132499-3.492912$ 
H - $1.2062120 .225301-1.576087$ H $-2.403705-0.606760-2.569387$ H $-0.843497-2.610978-2.641327$ H -2.745910 -2.463742 -0.992497 H -1.726729-1.564029 0.109655 H $-2.851138-3.844247 \quad 1.030269$ H $\quad 0.347780-2.7801771 .148684$ H $-2.622202-4.960228-1.018269$ H $-1.216672-5.794225-0.395379$ H - $1.087829-4.831877-1.881064$ H $0.299643-5.0151301 .505225$ H $3.039572-3.530495-0.044468$ H $3.180084-2.369430-1.332444$ H $1.965462-3.721457-2.465775$ H $5.3059172 .508723-1.710369$ H $5.492030-0.562645-1.867250$ H $7.7888522 .474889-2.142112$ H $8.9172731 .932013-0.913103$ H $7.3339142 .494622-0.420492$ H $2.585769-0.4987090 .037700$ H $2.032457-1.5284131 .365506$ H $5.443444-1.2173043 .516199$ H 5.0964580 .5098293 .729419 H 6.7088531 .0922391 .848295 H $7.709399-1.6022632 .909266$ H $9.833370-0.2873822 .641023$ H 9.1030490 .7637801 .457813 H $10.486924-3.051894 \quad 0.235787$ H $10.269851-2.7076742 .043541$ H $9.863218-1.466158-1.393763$ H $9.9788210 .175435-0.840080$ H $7.444701-1.544368-1.230466$ H 3.5111040 .2905332 .155562 C -0.686095 3.4760222 .013025 O -1.5463442 .3202562 .098412$ C $-2.3153212 .395732 \quad 3.311423$ C $-1.690273 \quad 3.5144504 .136817$ C -1.2209484 .4621923 .049945$ H -3.3287392 .6992513 .023025$ C $-2.356898 \quad 1.029604 \quad 4.003515$ H -2.406791 3.9709864 .826505 H -0.8361703 .1584534 .724736$ C -0.1952845 .4749413 .528277$ H -2.0930134 .9951012 .647462$ C -2.889591 -0.1184633 .119667$ O $-1.0250090 .683467 \quad 4.405706$ H -2.946396 1.1066984 .924970 H -2.165583 -0.3414912 .327237$ O $-2.945699-1.2992343 .930918$ C -4.2719690 .1355692 .487372$ C $-4.848792-1.1131141 .792349$ H -4.1835250 .9466181 .758980$ O -5.1869350 .5782043 .490131$ H -4.102516 -1.507068 1.096296 H $-5.032233-1.8884002 .545591$ $\mathrm{H}-0.6174226 .1058704 .317424$ H 0.6962814 .9833123 .931857 H $\quad 0.121963 \quad 6.1269312 .708402$ C $-0.704158 \quad 4.0218290 .583377$ H $-1.070117-0.1852184 .840794$ H $-3.552130-1.1254434 .670525$ H -5.335062 -0.1577764 .107527$ H $-0.171374 \quad 4.9779740 .535753$ H -1.739522 4.1989610 .271600 H $0.324501 \quad 3.1417082 .282752$ C $-5.293640-0.199373-1.107662$ $\begin{array}{lllll}\text { O } & -6.034929 & 0.189991 & 0.055871\end{array}$ C $-6.177018-0.8262871 .055538$ C $-6.852218-2.069290 \quad 0.457947$ C $-6.138588-2.561223-0.797395$ C $-5.954296-1.408611-1.783296$ H -4.264472 -0.442835 -0.822285 C $-5.2598741 .002969-2.063086$ H $-6.870825-0.4212261 .802950$ O $-6.914170-3.1111021 .426302$ H $-7.888014-1.8153440 .196296$ H -5.183369 $-3.036660-0.546494$ O $-6.944442-3.573049-1.405194$
H $-6.935305-1.125092-2.184925$

O $-5.170193-1.853545-2.887371$

C $-4.3855832 .127674-1.551284$

H $-4.8625580 .712507-3.041693$

H $-6.2761481 .396579-2.186498$

H $-7.332498-3.8790821 .000675$

H $-6.500315-3.841388-2.227810$

H $-4.300750-2.124910-2.549374$

O $-4.646868 \quad 3.254195-2.278920$

O $-3.5647992 .031214-0.650035$

C $-3.8941574 .413638-1.911665$

H $-4.1778325 .229958-2.581169$

H $-4.1273324 .704586-0.883057$

H $-2.8237314 .222903-2.026180$

SCF Energy (B3LYP/6-31G**//MMFF)= -3245.91434845

0239

MMFF Geometry

C $4.802157-0.6969942 .080572$

C $4.5089190 .034548 \quad 0.994376$

C $4.9118721 .471378 \quad 0.824520$

$\begin{array}{lllll}\text { O } & 3.703120 & 2.257203 & 0.899792\end{array}$

C $5.6965781 .730244-0.479411$

C $4.9350051 .607021-1.787218$

C $5.0273640 .563169-2.635602$

C $4.1222432 .813719-2.183527$

C $5.810493-0.704240-2.445634$

C $5.146149-1.954967-3.049791$

C $3.833930-2.375074-2.365491$

C $3.442622-3.792248-2.796566$

C $2.096078-4.226136-2.199384$

O $2.800071-1.458119-2.742072$

O $1.664632-5.406342-2.893793$

C $1.050836-3.128630-2.480763$

C $2.221708-4.614728-0.721369$

O $-0.177794-3.414671-1.814337$

C $1.534497-1.705883-2.115172$

C $0.510765-0.666765-2.627951$

O $1.625749-1.596292-0.697225$

C 3.8150433 .5453251 .309382

$\begin{array}{lllll}\text { O } & 4.855539 & 4.148337 & 1.537249\end{array}$

C 2.4920354 .1874821 .491080

C 1.3088923 .5689401 .342640

C $-0.006577 \quad 4.169681 \quad 1.597874$

C $-0.0399965 .626978 \quad 1.984958$

C $0.9512200 .793969-2.477450$

C $-0.0977321 .759392-3.002740$

C -0.565272 $4.197561-3.193382$

O $-1.150151 \quad 1.393282-3.518842$

C -1.241702 $4.703347-1.959467$

C $-2.501124 \quad 4.386872-1.624032$

C $-3.2035724 .906794-0.403807$

C $-3.448262 \quad 3.8291150 .627537$

C $-4.5059743 .006550 \quad 0.528073$

C -2.5322503 .7764651 .828319$

C $-1.112398 \quad 3.401043 \quad 1.494207$

N $0.2874863 .082175-2.832587$

H $5.372747-0.2497532 .890493$

H $3.919384-0.4118780 .200961$

H $5.5609211 .752127 \quad 1.665649$

H $6.5823721 .082754-0.493316$

H $6.1039842 .749540-0.440184$

H $4.4947230 .630206-3.583543$

H $3.7497302 .745534-3.211382$

H $3.2550512 .926958-1.529602$

H $4.7289203 .723047-2.121306$

H $6.779842-0.570585-2.941746$

H $6.017857-0.896070-1.388423$

H $4.966687-1.783320-4.119032$

H $5.870386-2.775858-2.971529$

H $3.980225-2.338949-1.280707$

H $4.226825-4.511426-2.528878$

H $3.363819-3.829561-3.892302$

H $2.352022-6.085828-2.785423$

H $0.831893-3.152335-3.557676$

H $2.934527-5.439213-0.601265$

H $1.268225-4.983789-0.327385$

H $2.560210-3.792357-0.089040$ 
H $-0.451428-4.308039-2.084464$ H $\quad 0.318127-0.848578-3.693520$ H $-0.440494-0.798118-2.097195$ H $1.738318-0.660406-0.468433$ H 2.5511985 .2253031 .805095 H 1.2911482 .5223171 .043781 H 0.4401715 .7778802 .957876 H -1.048369 6.0397512 .054453 H $\quad 0.4792496 .2357981 .235998$ H $1.1339961 .039169-1.426130$ H $1.8728830 .964533-3.042583$ H -1.287509 $3.885498-3.955257$ H $0.0674114 .980493-3.622338$ H $-0.6681255 .371700-1.322718$ H -3.068822 $3.720439-2.269369$ H $-4.1621445 .338194-0.721386$ H -2.651454 5.7399980 .047754 H $-4.712721 \quad 2.254827 \quad 1.282367$ H $-5.1889263 .067473-0.312942$ H $-2.897253 \quad 3.0327662 .549392$ H $-2.598957 \quad 4.7291032 .361637$ H -0.9965012 .3600411 .191493$ H $1.1484323 .287589-2.335387$ C $3.038900-2.2780603 .012336$ O $1.978394-1.6948432 .231167$ C $1.140917-0.9045563 .100671$ C $1.457956-1.3819074 .508407$ C $2.949962-1.6430574 .400876$ H 1.4658790 .1352882 .964627 C $-0.314803-1.0679202 .658977$ H $1.207590-0.6411325 .273239$ H $0.919730-2.3103774 .736188$ C $3.500668-2.5195785 .511582$ H $3.467622-0.6765104 .424647$ C $-1.347024-0.3956813 .580044$ O $-0.432272-0.4961801 .351112$ H $-0.546198-2.1342742 .556210$ H -1.392227 -0.9260324 .538344$ O $-0.893756 \quad 0.9319413 .872860$ C $-2.757616-0.2833942 .965847$ C $-3.367093-1.6304012 .540298$ H $-2.724477 \quad 0.4105642 .119344$ O $-3.623166 \quad 0.322190 \quad 3.933097$ H -2.653038 -2.189456 1.931266 H -3.566596 -2.231837 3.435532 H $3.357645-2.0410976 .485957$ H $3.001473-3.4938735 .539832$ H $4.572559-2.6931625 .373500$ C $4.368783-2.1241582 .267660$ H $\quad 0.127062-1.0251870 .757912$ H -1.5678531 .3522824 .433982$ H -3.667963 -0.268856 4.703954 H $4.280877-2.5999401 .282901$ H $5.160727-2.6606122 .802896$ H $2.808266-3.3494543 .076264$ C $-3.731987-1.155866-0.451530$ O $-4.508088-0.5967940 .612025$ C $-4.682789-1.4537851 .747035$ C $-5.343544-2.7706571 .314943$ C $-4.588708-3.4463620 .170943$ C $-4.351839-2.457814-0.972278$ H -2.722527 -1.369483 -0.079901 C $-3.540664-0.089791-1.538813$ H -5.388262 -0.9409992 .412774$ O $-5.441016-3.6555612 .426219$ H $-6.368421-2.5587880 .983095$ H -3.648160 -3.883951 0.525259 O $-5.380865-4.535191-0.308767$ H -5.307990 -2.265141-1.470830 O $-3.495351-3.056933-1.942309$ C $-4.8217430 .459640-2.130989$ H -3.007472 $0.763803-1.102861$ H -2.934943 -0.501659-2.353151 H -5.849505 -4.4784582 .106958$ H -4.909935 -4.923723 -1.065880 H -2.633177 -3.217744 -1.521896 O $-4.5014561 .281693-3.175049$ O $\quad \begin{array}{llll}-5.951836 & 0.228480 & -1.725464\end{array}$
C $-5.6086291 .916114-3.821806$

H $-5.2176702 .542195-4.628193$

H $-6.2773121 .164762-4.252627$

H -6.146515 $2.552863-3.112931$

SCF Energy $(B 3 L Y P / 6-31 G * * / / M M F F)=-3245.92589734$

024

MM̄FF Geometry

C $2.9075173 .084232-2.027275$

C $4.2226443 .249711-1.813031$

C $5.2126562 .207069-1.337765$

O $4.5718360 .941121-1.064363$

C $5.8840552 .688914-0.036626$

C 7.0097031 .7884480 .440783

C 6.9622580 .9968081 .531676

C $8.2720791 .849677-0.382214$

C 5.8148440 .8002052 .482358

C $5.299081-0.6441762 .432936$

C $3.941698-0.7943033 .135979$

C $3.488683-2.2555323 .122605$

C $2.060675-2.4304413 .661510$

$\begin{array}{llll}\text { O } & 3.007631 & 0.019623 & 2.422277\end{array}$

O $1.612048-3.7465373 .301934$

C $1.129063-1.4275782 .947589$

C $2.017627-2.3607395 .192219$

O $-0.177514-1.4516533 .517085$

C 1.6735880 .0142432 .942506

C 0.8403940 .9735212 .058093

O $1.639672 \quad 0.531917 \quad 4.277444$

C $4.4736760 .053582-2.092270$

O $4.845613 \quad 0.216323-3.243375$

C $3.812335-1.164121-1.571582$

C $3.418460-2.123851-2.421797$

C $2.761412-3.383107-2.062112$

C $2.490777-3.638765-0.603061$

C 0.8390000 .6455410 .561000

C $-0.330708-0.2059640 .108255$

C $-1.223581-1.362774-1.909329$

O $-1.292238-0.4590380 .826901$

C $-0.974577-2.829667-1.765637$

C $-0.918283-3.670736-2.809344$

C $-0.726451-5.154076-2.679707$

C $0.422123-5.671263-3.516393$

C $0.203038-6.241892-4.713132$

C $1.811881-5.610211-2.924460$

C $2.435189-4.246049-3.047302$

N -0.193820 -0.617016 -1.210607

H $2.4463502 .114202-1.868121$

H $4.6514014 .232511-2.003844$

H $5.9745042 .081545-2.116740$

H 5.1183202 .8037120 .736797

H $6.2996493 .695493-0.182302$

H $7.8473040 .411077 \quad 1.777897$

H $9.085625 \quad 1.2637380 .059056$

H $8.0963161 .458970-1.388947$

H $8.6222652 .883790-0.464584$

H 6.1603201 .0260423 .498534

H 5.0009311 .4987422 .280501

H $5.193262-0.9489151 .384021$

H $6.036963-1.3126432 .893470$

H $4.031548-0.4261534 .164700$

H $4.191860-2.8845313 .682740$

H $3.513263-2.6309522 .091285$

H $2.235783-4.3896703 .680464$

H $1.025865-1.7725521 .912004$

H $2.631437-3.1577635 .628959$

H $1.001553-2.5263605 .567056$

H $2.382908-1.4112405 .588788$

H $-0.495493-2.3696633 .473445$

H $-0.176727 \quad 1.0727552 .456049$

H $1.286840 \quad 1.9737932 .155474$

H 1.6451941 .5023234 .233855

H $3.664184-1.236166-0.500780$

H $3.595874-1.978540-3.486684$

H $3.433192-3.757149-0.058008$

H $1.894564-4.534571-0.418429$

H $1.929325-2.808567-0.161467$

H $\quad 0.7556021 .5883990 .009669$ 
H $1.775923 \quad 0.172498 \quad 0.255235$ H -1.207649 -1.038528 -2.954681 H -2.200200 -1.115870 -1.483891 H $-0.870208-3.222442-0.756929$ H -1.055468 -3.278071 -3.814482 H $-1.661600-5.637563-2.991703$ H $-0.577599-5.449646-1.633710$ H $1.018334-6.642586-5.306213$ H - $0.796396-6.315275-5.129031$ H $2.472253-6.314940-3.448090$ H $1.783506-5.976934-1.894572$ H $2.666702-3.968444-4.076574$ H $0.658304-0.394619-1.716842$ C $0.7963434 .367977-1.572747$ O $-0.1468533 .298604-1.796863$ C $-1.4807973 .850534-1.850110$ C -1.358998 $5.273179-1.328622$ C $0.0189855 .658708-1.827586$ $\mathrm{H}-1.7563623 .857366-2.912327$ C $-2.4338462 .947616-1.056255$ H -2.149412 $5.929565-1.704150$ H $-1.3889915 .290835-0.231974$ C $0.5956236 .877405-1.127852$ H $-0.0388105 .855899-2.906492$ C $-3.8513663 .519374-0.881885$ O $-2.5168521 .694268-1.741605$ H -1.989492 2.733842 -0.078119 H $-3.8122324 .434118-0.278904$ O $-4.3361643 .905204-2.174900$ C $-4.8729562 .537981-0.272041$ C $-4.462409 \quad 1.9841571 .102572$ H -5.059942 $1.720566-0.974215$ O $-6.1184793 .233714-0.136375$ H $-3.5037351 .465607 \quad 1.016813$ H -4.3134122 .8279551 .787404$ H $\quad-0.0408877 .752686-1.293817$ H $0.6745576 .719530-0.046970$ H $1.5951397 .110551-1.507750$ C $2.0007534 .189897-2.500799$ H $-2.9558501 .852551-2.594939$ H $-5.2341074 .258851-2.054139$ H -5.9873143 .9566640 .500724$ H $2.5615105 .129070-2.569513$ H $1.6577673 .933445-3.510533$ H $1.1048334 .307997-0.520578$ C $-4.756999-1.0469760 .737083$ O $-5.808603-0.0802950 .851734$ C -5.5204661 .0311991 .708916$ C -5.1880390 .5362063 .124245$ C $-4.072111-0.5048943 .114128$ C $-4.394606-1.6157422 .115883$ H -3.879740 -0.5763140 .281101$ C $-5.241420-2.163065-0.202910$ H -6.4536611 .6027261 .788607$ O $-4.831248 \quad 1.637705 \quad 3.952175$ H $-6.088671 \quad 0.083873 \quad 3.559976$ H -3.098432 -0.050590 2.897862 O $-3.962164-1.0969924 .410365$ H -5.224710 -2.222164 2.501038 O $-3.262602-2.4747841 .991316$ C $-5.147475-1.754508-1.657609$ H -4.620774 -3.056754 -0.069593 H -6.284546 -2.430706 -0.001426 H $-4.744926 \quad 1.3074434 .862049$ H -3.632843 -0.413187 5.017607 H $-3.035613-2.7875252 .883851$ O $-4.782983-2.833242-2.411131$ O $-5.385729-0.632864-2.084056$ C $-4.640207-2.585870-3.813337$ H $-4.300008-3.509857-4.288309$ H $-5.604182-2.301724-4.245597$ H -3.892433 -1.806374 -3.988591 SCF Energy (B3LYP/6-31G**//MMFF) $=-3245.91622011$

0240

MM̄FF Geometry

C -1.303705 3.3776380 .581687

C -0.8762322 .1132260 .713392$
C -0.3819721 .5317252 .013555$

$\begin{array}{lllll}\text { O } & 0.994940 & 1.125590 & 1.834367\end{array}$

C -1.2204600 .2933092 .377423$

C $-0.821307-0.3437323 .695052$

C $-0.145341-1.5041693 .818250$

C -1.2659560 .4011654 .927678$

C $0.373912-2.3868122 .718238$

C $1.906262-2.4479692 .727825$

C $2.456516-3.0784561 .439921$

C $3.978134-3.2116401 .511248$

C $4.581562-3.7024590 .186529$

O $2.088836-2.2217690 .353470$

O $5.998783-3.4746210 .236852$

C $4.028658-2.840585-0.969470$

C $4.391573-5.2124390 .000609$

O $4.440027-3.359652-2.231542$

C $2.495077-2.686271-0.939968$

C $1.964906-1.671609-1.983563$

O $1.891827-3.949670-1.241809$

C 1.9432692 .0926361 .986894

O 1.7551313 .2696372 .252602

C 3.2694801 .4729931 .768025

C 4.3671202 .2445221 .758617

C 5.7468621 .7902861 .569108

C 5.9718770 .3232361 .319191

C $2.444214-0.227240-1.803804$

C $3.6741840 .131947-2.619784$

C $5.3136911 .985625-2.932714$

O $4.134004-0.588057-3.501352$

C $6.5591981 .679641-2.162776$

C $7.4119832 .622965-1.734611$

C $8.6959732 .326164-1.014624$

C $8.8278763 .084967 \quad 0.286632$

C $9.532764 \quad 4.2271570 .352450$

C 8.2234412 .4696861 .527646

C $6.7410812 .701161 \quad 1.628487$

N $4.1737171 .380315-2.270614$

H -1.2903064 .0381231 .445284$

H $-0.8765861 .456250-0.153484$

H -0.4474152 .2667012 .825574$

H $-1.162379-0.4343651 .562993$

H -2.282282 0.5695132 .437475

H $0.069958-1.8688644 .821853$

H - $-1.020033-0.1360835 .849941$

H -0.7836651 .3817874 .980873$

H -2.3512360 .5459814 .912778$

H $-0.028975-3.3967612 .861959$

H $\quad 0.021262-2.059477 \quad 1.738847$

H $2.305063-1.4311942 .829392$

H $2.243893-3.0196543 .600963$

H $1.989181-4.0606441 .302087$

H $4.271898-3.8723832 .336457$

H $4.414985-2.2325791 .746000$

H $6.351844-3.9514051 .007550$

H $4.487024-1.849257-0.874370$

H $4.881550-5.7624690 .813100$

H $4.865788-5.560001-0.923750$

H $3.342287-5.514170-0.013690$

H $5.410684-3.419519-2.217719$

H $2.153333-2.039074-2.999435$

H $0.870905-1.648331-1.883657$

H $\quad 0.973455-3.790872-1.514947$

H 3.3077780 .4004521 .621362

H 4.2491743 .3164301 .913194

H $5.718080-0.2570532 .212430$

H 7.0020420 .0773181 .053202

H $5.355801-0.0250950 .483643$

H $1.6473440 .445102-2.147488$

H $2.626360-0.002048-0.749463$

H $5.1188363 .060928-2.995186$

H $5.4011501 .587629-3.948745$

H $6.7860970 .632158-1.977928$

H $7.1969313 .667786-1.947705$

H $9.5222082 .595155-1.685880$

H $8.810281 \quad 1.251779-0.824206$

H 9.6582314 .7612431 .288524

H $9.9951774 .656580-0.530158$

H 8.6795792 .9116832 .424149 
H 8.4952941 .4115001 .577821 H 6.4781983 .7448081 .806689 H $3.7577311 .882500-1.491498$ C $-3.2315294 .489807-0.562027$ O $-4.131586 \quad 3.393745-0.307587$ C $-5.3357153 .591592-1.071379$ C $-5.2563795 .006629-1.633595$ C $-3.761155 \quad 5.171462-1.822526$ H $-5.2941752 .883059-1.906920$ C $-6.5694353 .307748-0.207710$ H -5.822471 5.115061-2.563796 H $-5.6328895 .750897-0.922595$ C -3.324259 6.617821-1.976206 H $-3.4554054 .606020-2.713063$ C $-6.556517 \quad 1.930567 \quad 0.487142$ O H $-7.476538 \quad 3.425358-0.812214$ H -5.7883501 .9289101 .270713$ O $-7.8182221 .799596 \quad 1.154876$ C $-6.3436300 .729406-0.455093$ C $-6.404865-0.619160 \quad 0.285793$ H $-5.3686260 .818591-0.945213$ O $-7.3447600 .720012-1.471941$ H $-5.719036-0.5839941 .138942$ H -7.415563 -0.7700420 .683057$ H $-3.7936857 .069684-2.856129$ H -3.602703 $7.216306-1.102155$ H -2.239118 $6.687379-2.100070$ C $-1.8049723 .960144-0.710222$ H -7.434143 4.1151451 .352224 H -7.7312251 .0921111 .815547$ H -7.196344 $1.489996-2.045616$ H -1.130405 4.772398 -1.003072 H -1.772214 3.201691-1.502386 H $-3.301870 \quad 5.1671210 .299437$ C $-3.660276-1.783529-0.442900$ O $-4.807920-1.660929-1.288689$ C $-6.067528-1.820113-0.627881$ C $-6.131749-3.183927 \quad 0.076029$ C $-4.940844-3.4029391 .005536$ C $-3.632834-3.145746 \quad 0.259123$ H $-3.687902-0.9879980 .312516$ C -2.409241 -1.512583-1.287729 H $-6.825615-1.834555-1.420941$ O $-7.336842-3.3193350 .821528$ H $-6.130116-3.980856-0.679144$ H $-5.009879-2.7803051 .905222$ O $-4.938445-4.7598791 .455179$ H $-3.474513-3.953861-0.464740$ O $-2.548131-3.1765581 .181521$ C $-2.262299-2.471038-2.446822$ H -2.461401-0.506408 -1.722674 H $-1.500441-1.535533-0.676080$ H $-8.077684-3.1946640 .203822$ H $-5.793273-4.9210341 .890429$ H $-2.575234-4.0367261 .634437$ O $-1.396054-3.472990-2.123446$ O $-2.863664-2.352501-3.506873$ C $-1.206153-4.468323-3.134322$ H $-2.165343-4.905867-3.427582$ H $-0.699325-4.030129-3.999035$ H $-0.575646-5.259116-2.719623$

SCF Energy (B3LYP/6-31G*//MMFF) $=-3245.91166162$

0241

MMFF Geometry

C $0.984097-3.4082620 .878902$

C $1.080566-2.1488191 .328914$

C $0.614815-1.7200192 .694833$

O $-0.245483-0.5735952 .476178$

C $1.782971-1.3010913 .607211$

C $2.769573-2.4099203 .919938$

C $4.050906-2.4489693 .501150$

C $2.233544-3.5167424 .792509$

C $4.770702-1.4367902 .655993$

C $5.109389-1.9907161 .262968$

C $5.261189-0.8989470 .190526$

C 6.3575450 .1155560 .520326
C $6.3972051 .270347-0.493209$

O $3.990710-0.2551850 .083308$

$\begin{array}{lllll}\text { O } & 7.220681 & 2.309939 & 0.059279\end{array}$

C $4.9735921 .852512-0.642751$

C $7.0573800 .850756-1.811967$

O $4.9626632 .842400-1.669002$

C $3.900650 \quad 0.774616-0.906217$

C $2.4553451 .318697-0.830033$

$\begin{array}{llll}\text { O } & 4.4587869 & 0.239002 & -2.220578\end{array}$

C $-1.179278-0.2863403 .416666$

O $-1.368837-0.8919074 .462875$

C -1.982333 0.9039003 .047559

C -1.9337761 .5019851 .846513$

C -2.7298972 .6572761 .420105$

C -3.669275 3.2651492 .429166

C 2.0380521 .8771300 .534280

C $2.015710 \quad 3.392365 \quad 0.601724$

C 1.2347295 .2353292 .080805

O $2.384054 \quad 4.117502-0.317653$

C $-0.201787 \quad 5.490694 \quad 1.747156$

$\begin{array}{llll}\text { C } & -0.604568 & 6.037774 & 0.590744\end{array}$

C $-2.039896 \quad 6.2880390 .229495$

C $-2.5142525 .392373-0.893461$

C $-2.2493115 .690939-2.176712$

C $-3.3692104 .199996-0.530256$

C $-2.595422 \quad 3.105840 \quad 0.153501$

N $1.520403 \quad 3.8372551 .822363$

H $\quad 0.576476-4.1607661 .551844$

H $1.491387-1.3724810 .691928$

H $\quad 0.024471-2.5189443 .160966$

H $1.380773-0.9272254 .558497$

H $2.268356-0.4209273 .173781$

H $4.663691-3.2996963 .796670$

H $3.007965-4.2398205 .070199$

H $1.824404-3.1036625 .720407$

H $1.441208-4.0671854 .276496$

H $4.199277-0.5102242 .573784$

H $5.700331-1.1630973 .170298$

H $6.028071-2.5871601 .319964$

H $4.307606-2.6673750 .938436$

H $5.480535-1.398626-0.760421$

H $7.334744-0.3788740 .583805$

H $6.1807490 .552981 \quad 1.511682$

H $8.103323 \quad 1.9369510 .225345$

H 4.7474232 .3718250 .297402

H $8.0902500 .526231-1.636372$

H $7.1233951 .695115-2.506977$

H $6.5369000 .031078-2.311439$

H $4.1096693 .305999-1.625734$

H $2.2661872 .027616-1.644201$

H $1.7851750 .471382-1.029658$

H $3.243095-0.128104-2.529134$

H $-2.6519111 .250103 \quad 3.828659$

H -1.270342 $1.097927 \quad 1.084715$

H $-4.167617 \quad 4.1692102 .074008$

H -3.1248483 .5567633 .334461$

H -4.4529292 .5508562 .704072$

H 1.0145441 .5414130 .736079

H 2.6703251 .4972871 .343586

H 1.9093965 .8663711 .492712

H 1.4168555 .4291263 .142091

H -0.9398325 .2037852 .491685$

H $0.1421436 .320225-0.148437$

H $-2.1390207 .339547-0.069955$

H -2.6946216 .1740501 .102153$

H $-2.609945 \quad 5.068979-2.989187$

H -1.661618 $6.563843-2.441469$

H $-3.8086743 .760493-1.436263$

H -4.2282344 .5430040 .053169$

H - $-1.8804312 .605377-0.501012$

H 1.1701763 .1548982 .488838

C $1.697773-2.906679-1.573266$

O $0.566141-2.049378-1.820095$

C $0.305736-1.999242-3.233126$

C $1.565833-2.530249-3.905551$

C $2.022403-3.577819-2.911187$

H $-0.520891-2.695915-3.420111$

C $-0.109993-0.587176-3.668399$ 
H $1.363772-2.940310-4.899681$

H $2.333571-1.756221-4.011102$

C $3.484816-3.962458-3.061473$

H $1.402925-4.474923-3.044286$

C $-1.2109640 .051748-2.797756$

O $1.0392930 .265156-3.608545$

H $-0.416609-0.610664-4.721103$

H $-0.8262090 .259159-1.791379$

O $-1.543657 \quad 1.322403-3.373175$

C $-2.504609-0.777974-2.687729$

C $-3.607620-0.028456-1.916942$

H -2.286933 -1.721523 -2.181657

O $-2.980319-1.101704-3.993355$

H $-3.2020890 .280809-0.949091$

H $-3.8719160 .883393-2.465569$

H $3.671650-4.384630-4.054171$

H $4.142470-3.095464-2.935241$

H $3.769510-4.711697-2.316140$

C $1.355578-3.930106-0.483526$

H $0.821737 \quad 1.085832-4.081773$

H $-0.796248 \quad 1.922114-3.209164$

H -3.147262 -0.265454 -4.461079

H $2.196079-4.622535-0.356275$

H $0.501489-4.526622-0.828473$

H $2.526692-2.265223-1.253571$

C $-4.294942-1.9578450 .351633$

O $-4.653647-2.091291-1.029001$

C $-4.894550-0.860373-1.717889$

C $-6.032060-0.092842-1.028662$

$\begin{array}{llll}\text { C } & -5.740049 & 0.127536 & 0.452837\end{array}$

C $-5.376874-1.1936071 .127394$

H -3.330717 -1.4418090 .434979$

C $-4.134664-3.3748480 .926368$

H $-5.253802-1.134361-2.717637$

O $-6.2418121 .154221-1.682042$

H $-6.960847-0.670065-1.126400$

H -4.9510180 .8712230 .599174$

O $-6.905237 \quad 0.6459801 .097723$

H -6.279732 -1.8112921 .219460$

O $-4.906225-0.9313132 .448225$

C $-2.964297-4.122866 \quad 0.326721$

H -3.973330 -3.335622 2.009111

H $-5.040553-3.9559820 .714616$

H -7.041210 $1.556083-1.302490$

H -7.0582521 .5411080 .751145$

H $-5.597473-0.4244642 .907824$

O $-3.028647-5.429714 \quad 0.719452$

O $-2.098958-3.628887-0.381399$

C - $-1.990257-6.280440 \quad 0.223123$

H $-2.260319-7.3146420 .452901$

H $-1.888622-6.179870-0.861897$

H -1.047583 -6.044308 0.723816

SCF Energy $\left(B 3 L Y P / 6-31 G^{* *} / / M M F F\right)=-3245.91472461$

$02 \_42$

MM̄FF Geometry

C $0.112753-1.0243222 .182618$

C $0.493189-2.1529401 .563323$

C $-0.040539-2.6730040 .249213$

O $-0.697262-1.620697-0.492385$

C $-1.064491-3.7894130 .504892$

C -1.402372 -4.578259-0.744955

C $-2.513539-4.390189-1.483301$

C $-0.423419-5.664397-1.106334$

C $-3.562380-3.333049-1.281316$

C $-4.772190-3.871036-0.509441$

C $-5.823112-2.800223-0.169897$

C $-6.506621-2.236166-1.418097$

C -7.506531-1.124059-1.070901

O $-5.177324-1.7594570 .571600$

O $-7.860340-0.452683-2.290340$

C $-6.793581-0.088480-0.176749$

C $-8.811063-1.680581-0.490089$

$\begin{array}{llll}\text { O } & -7.710681 & 0.902792 & 0.281896\end{array}$

C $-6.049751-0.7170961 .024981$

C $\begin{array}{llll}-5.194390 & 0.325948 & 1.785013\end{array}$

O $-7.018336-1.2254151 .950540$

C $0.082077-0.811285-1.258598$
O $1.283775-0.906032-1.447246$

C $-0.7862040 .259689-1.794172$

C $-0.222617 \quad 1.389528-2.246556$

C $-0.9296132 .574013-2.739591$

C $-2.4265722 .485845-2.880934$

C -3.9688340 .8391591 .022353$

C -3.2948882 .0037241 .730525$

C -1.3951633 .5980831 .424640$

O -3.7430352 .5243642 .748045$

C $-1.6765554 .657778 \quad 0.404654$

C $-0.7181255 .333017-0.247742$

C $-0.9943046 .377491-1.291488$

C $-0.2285486 .139767-2.576328$

C $0.7938566 .937645-2.928294$

C $-0.6940095 .023362-3.489415$

C $-0.2063313 .676334-3.028459$

N $-2.1457282 .408471 \quad 1.066204$

H $-0.679186-0.4133081 .758382$

H $1.269400-2.7625882 .020397$

H $\quad 0.801415-3.061169-0.336427$

H $-1.976757-3.3618760 .939943$

H $-0.682519-4.4925721 .257320$

H -2.683341 -5.039330 -2.340906

H $-0.731034-6.221369-1.997748$

H $0.564564-5.239707-1.308868$

H $-0.331478-6.381989-0.284498$

H -3.143626 -2.459787 -0.773359

H -3.869672 -2.981334 -2.272711

H -5.244774 -4.685995 -1.071699

H -4.413960 -4.301272 0.435457

H $-6.568388-3.2781060 .477360$

H -6.999833 -3.035302 -1.985135

H $-5.757011-1.806680-2.095113$

H -8.240628 -1.111992 -2.895851

H $-6.0697620 .433648-0.813930$

H $-9.297478-2.347230-1.212551$

H $-9.529750-0.877270-0.293269$

H $-8.665315-2.2468190 .431951$

H -8.151666 $1.274373-0.501185$

H $-5.833688 \quad 1.1597082 .102609$

H $-4.830219-0.1242172 .719460$

H -6.599508 -1.307000 2.823205

H -1.859163 $0.110446-1.752231$

H $\quad 0.8635321 .472597-2.221898$

H -2.703134 $1.633909-3.512547$

H $-2.8742393 .366183-3.345897$

H -2.901918 $2.363769-1.902633$

H $-3.2367690 .031648 \quad 0.905472$

H -4.2503651 .1926640 .025945$

H -0.3380003 .3192881 .445161$

H -1.6910303 .9452082 .419025$

H -2.720011 4.8765320 .189665

H $0.3245635 .131724-0.016149$

H $-0.7231547 .352019-0.865202$

H -2.065371 $6.437472-1.521370$

H $1.3325526 .788627-3.858137$

H $1.1190897 .754333-2.292316$

H $-0.3076225 .183065-4.504592$

H -1.781585 $5.081477-3.580133$

H $0.8777103 .625094-2.911831$

H $-1.864979 \quad 1.9144210 .225302$

C 1.2216580 .9246043 .292808

O $2.163596 \quad 1.0186872 .208875$

C 3.2234261 .9191272 .581306

C 2.7899892 .5680213 .892114

C 1.9543821 .4668874 .517784

H 4.1057121 .2966072 .772542

C 3.5218402 .8935041 .434169

H 3.6439352 .8632524 .509276

H 2.1742283 .4590693 .725176

C 1.0346011 .9540655 .623316

H 2.6307740 .7015014 .921443

C 3.6994712 .2164040 .059318

O 2.4254103 .8088121 .316579

H 4.4008333 .4988221 .685275

H $2.7372401 .799847-0.264191$

O $4.0269243 .252923-0.874434$

C $4.785881 \quad 1.1249210 .024520$ 
C $4.9218510 .484296-1.366751$

H $4.530451 \quad 0.3513950 .750857$

O $6.0296231 .697361 \quad 0.420624$

H $3.9278170 .158554-1.691176$

H $5.2656541 .240090-2.082913$

H 1.6149432 .3967126 .439448

H $\quad 0.3350072 .7132735 .257755$

H $0.448515 \quad 1.1269336 .035974$

C $0.745046-0.5205213 .450610$

H $2.638697 \quad 4.4232600 .593318$

H $3.8708792 .905129-1.768458$

H $\quad 6.650166 \quad 0.968514 \quad 0.589421$

H $0.009455-0.5844034 .260032$

H $1.592781-1.1596383 .724411$

H $\quad 0.3738201 .5715643 .035007$

C $4.467802-2.433808-0.550849$

O $5.678458-1.682242-0.408123$

C $5.911774-0.702064-1.424140$

C $5.971655-1.384156-2.798410$

C $4.709428-2.196607-3.075185$

C $4.419142-3.144342-1.912819$

H $3.608615-1.763653-0.436697$

C $4.426338-3.4731740 .582585$

H $6.909137-0.290675-1.226111$

O $6.146735-0.426559-3.837430$

H $6.835467-2.060569-2.833622$

H $3.849907-1.548637-3.281525$

O $4.906807-2.981643-4.253901$

H $5.153222-3.959805-1.933655$

O $3.137041-3.740958-2.103368$

C $4.254675-2.8593711 .955341$

H $3.588287-4.1632460 .433426$

H $5.366028-4.0387310 .592577$

H $\quad 6.9628360 .066361-3.645690$

H $5.127653-2.368097-4.975658$

H $2.474547-3.029020-2.104745$

O $4.337656-3.8502192 .893145$

O $4.059189-1.6750982 .185147$

C $4.183616-3.4265314 .251382$

H $4.342007-4.2939174 .897802$

H $4.927025-2.6634444 .501447$

H $3.170442-3.0479444 .413648$

SCF Energy (B3LYP/6-31G**//MMFF) $=-3245.92350068$

$02 \_43$

MM̄FF Geometry

C -2.561113 $3.984608-0.663258$

C $-1.3748023 .703679-0.103227$

C $-0.0750993 .773855-0.863161$

O $0.6389912 .535683-0.635185$

C $0.7959154 .926105-0.332827$

C $1.7348315 .464762-1.394070$

C $3.0183075 .092206-1.558246$

C $1.1343846 .512847-2.297371$

C $3.7929504 .066565-0.781078$

C $4.2367822 .918735-1.696693$

C $5.1478651 .918962-0.972499$

C $5.5743580 .798586-1.922841$

C $6.417298-0.270262-1.213014$

O $4.429680 \quad 1.370060 \quad 0.135570$

O $6.482543-1.397425-2.099690$

C $5.686798-0.7102030 .073057$

C $7.8599150 .201463-0.996848$

O $6.500749-1.5820360 .854454$

C $5.2006280 .473308 \quad 0.945044$

C $4.289641-0.0575162 .073676$

O $\quad 6.3322861 .123951 \quad 1.525733$

C $0.388776 \quad 1.497214-1.476792$

O $-0.339944 \quad 1.499042-2.455740$

C $1.1521460 .331609-0.979544$

C $1.179599-0.799209-1.698874$

C $1.873346-2.023653-1.309468$

C $2.629375-2.667632-2.438016$

C 3.5610961 .0280552 .878409

C 2.6994830 .3711353 .938327

C $0.689776-1.0852154 .149762$

O 3.0699890 .2755425 .105823

C $1.176495-2.4615323 .815157$
C $0.524500-3.3131313 .009530$

C $1.082697-4.6400002 .583224$

C $1.348995-4.7055541 .092948$

C $0.736214-5.6161380 .318660$

C $2.386533-3.7662970 .509703$

C $1.772042-2.513312-0.057532$

N $1.520173-0.1432143 .421017$

H -2.572866 $4.283438-1.710285$

H -1.3123793 .4122450 .940866$

H $-0.2601963 .901335-1.937300$

H 1.3561474 .6067890 .553521

H 0.1671435 .7617200 .004450

H $3.5904345 .573350-2.351100$

H $1.8423536 .867011-3.054214$

H $0.2630456 .111683-2.824613$

H $0.816307 \quad 7.381172-1.710972$

H $4.6743334 .558222-0.351906$

H $3.217283 \quad 3.6679280 .058435$

H $3.3431482 .401339-2.067890$

H $4.7655103 .320684-2.570415$

H $6.0248272 .462319-0.601295$

H $6.1170791 .204266-2.785943$

H $4.6780450 .314037-2.335509$

H $7.056039-2.068844-1.693627$

H $4.810201-1.296082-0.234494$

H $8.3397210 .412706-1.960131$

H $8.466168-0.579852-0.525314$

H $7.9287321 .104308-0.386261$

H $6.692534-2.3682170 .316370$

H $3.531261-0.7166111 .634625$

H $4.878305-0.6696812 .769301$

H $6.054893 \quad 1.5840682 .333109$

H $1.6675020 .423639-0.029766$

H $0.676519-0.841181-2.661649$

H $3.224789-3.528960-2.129140$

H $3.325363-1.950456-2.887988$

H $1.937998-3.009240-3.216105$

H 4.2625601 .7009083 .381548

H $2.926117 \quad 1.6451122 .232623$

H $-0.344400-0.9208013 .836207$

H $0.767437-0.9062045 .226320$

H $2.143648-2.7492574 .221529$

H $-0.435673-3.0261562 .590033$

H $0.370463-5.4178062 .885857$

H $2.017014-4.8626633 .114035$

H $0.945541-5.682083-0.744014$

H $0.011132-6.3119040 .726998$

H $2.977672-4.308780-0.234006$

H $3.103695-3.4810001 .289812$

H $1.179845-1.9443990 .657856$

H $1.349652-0.0572702 .423225$

C -4.0113793 .3814771 .403416$

O

C $-4.696181 \quad 1.2655672 .145152$

C -5.4364552 .3242762 .952164$

C -5.4221393 .4917611 .987862$

H -5.3914030 .8258621 .419992$

C -4.0563830 .1608012 .990566$

H -6.4460992 .0058563 .228753$

H -4.8991472 .5856783 .871079$

C -5.7154204 .8281462 .647958$

H -6.1702013 .3083111 .204771$

C $-3.219386-0.8659542 .201083$

O -3.1920820 .7557513 .968145$

H $-4.838731-0.3556673 .559149$

H $-2.287587-0.4021131 .855925$

O $-2.825139-1.8909953 .123000$

C $-3.935802-1.5134201 .001315$

C $-3.113734-2.6534870 .366869$

H $-4.129347-0.746306 \quad 0.246017$

O $-5.205376-2.0178931 .415718$

H -2.122065 -2.2699790.103819

H -2.968785 -3.446231 1.110266

H -6.7127124 .8226873 .100069$

H -4.990206 5.0538303 .437005

H -5.6810265 .6412901 .916298$

C -3.920152 $3.946126-0.016679$

H $-2.5213831 .273333 \quad 3.490013$ 
H -3.630881 -2.292239 3.489566 H $-5.049422-2.7591492 .024659$ H -4.315043 $4.968866-0.029223$ H -4.563381 $3.344942-0.671250$ H -3.2874213 .8691202 .067704$ C $-2.896475-1.840130-2.595588$ O $-4.057044-2.301630-1.892012$ C $-3.804955-3.271508-0.869348$ C $-3.081558-4.492570-1.456194$ C $-1.830274-4.095853-2.232224$ C -2.170193 -3.018011 -3.258041 H -2.222199-1.324207-1.901537 C $-3.368535-0.832786-3.654843$ H -4.790032 -3.622762 -0.537219 O $-2.727195-5.420223-0.436692$ H -3.759202 -5.016788 -2.142925 H -1.028549 -3.762606 -1.563836 O $-1.327884-5.236575-2.931080$ H -2.796323 -3.451709-4.048493 O $-0.970825-2.556369-3.871838$ C $-3.8778120 .456361-3.046548$ H -2.550261 -0.563882 -4.331452 H -4.186644 -1.276512 -4.235355 H -3.544517 -5.671452 0.026584 H -1.163517 -5.930804 -2.269888 H $-0.518313-3.331660-4.245889$ O $-4.590874 \quad 1.137296-3.992550$ O $-3.6599190 .833148-1.903727$ C $-5.1356942 .391891-3.573290$ H $-5.7810442 .256197-2.700144$ H $-4.3283743 .096253-3.354544$ H $-5.7375252 .792183-4.393577$

SCF Energy (B3LYP/6-31G**//MMFF) $=-3245.90965165$

0244

MM̄FF Geometry

C $4.119703 \quad 3.444342-1.359617$

C $4.4663922 .253376-1.872362$

C $5.4055351 .230894-1.266423$

O $4.6383290 .039440-0.965002$

C 6.1172461 .6712000 .029660

C 7.1197370 .6583600 .557250

C $6.977204-0.0524201 .694794$

C $8.3698210 .498291-0.270657$

C $5.825543-0.0258562 .660186$

C $5.048325-1.3485852 .633668$

C $3.631370-1.1875063 .203193$

C $2.924626-2.5412323 .283130$

C $1.451774-2.4031933 .696590$

O $2.924942-0.3140312 .316922$

O $0.800830-3.6556293 .431364$

C $0.776589-1.3461112 .796922$

C $1.302514-2.1463735 .200435$

O $-0.551798-1.0766533 .237674$

C $1.572609-0.0289862 .692991$

C 1.0030150 .9411531 .627176

O 1.5234540 .6488203 .953576

C $4.488967-0.884733-1.953437$

O $4.917765-0.820344-3.094317$

C $3.686795-2.000438-1.404317$

C $3.194052-2.938343-2.226561$

C $2.368304-4.083048-1.833366$

C $2.001702-4.212552-0.378213$

C 1.0409080 .4194580 .186173

C $-0.240589-0.252180-0.266512$

C $-1.224050-1.447709-2.217947$

O $-1.266095-0.263247 \quad 0.406545$

C $-1.224988-2.912654-1.915700$

C -1.241929-3.860879 -2.864915

C $-1.288565-5.334342-2.576753$

C $-0.201453-6.105565-3.292270$

C $-0.455378-6.749982-4.443811$

C $1.152638-6.203535-2.626699$

C $1.970337-4.950423-2.787674$

N $-0.116352-0.810411-1.531002$

H $4.5072513 .756924-0.394364$

H $4.0620911 .982325-2.846413$

H $6.1680610 .999905-2.020919$
H $\quad 5.362288 \quad 1.895475 \quad 0.788121$

H $6.6555952 .612435-0.144406$

H $7.773711-0.7415881 .973033$

H $\quad 9.109037-0.149187 \quad 0.213319$

H $8.1352520 .056814-1.243863$

H $8.8453331 .471103-0.432931$

H 6.2206150 .1388723 .670340

H 5.1580570 .8162842 .468785

H $4.976109-1.7067491 .598984$

H $5.602213-2.1053763 .202584$

H $3.696190-0.7249744 .195028$

H $3.454888-3.2162223 .966547$

H $2.961090-3.0272912 .299249$

H $1.264009-4.3467613 .935102$

H $\quad 0.691486-1.7905601 .798375$

H $1.732819-2.9757465 .774846$

H $0.247176-2.0977305 .490288$

H $1.794650-1.2290135 .529823$

H -1.027053 -1.924716 3.263687

H $-0.001207 \quad 1.2757371 .914131$

H $1.626327 \quad 1.8470531 .646839$

H 1.7028851 .5919653 .804933

H $3.508679-2.011283-0.335714$

H $3.409458-2.860841-3.291535$

H $2.895947-4.405403 \quad 0.223949$

H $1.294540-5.018903-0.173892$

H $1.524247-3.295408-0.016875$

H $1.1952811 .270512-0.486622$

H $1.877475-0.266506 \quad 0.038340$

H -1.103654 -1.244257 -3.286714

H -2.165234 -1.001151-1.882581

H -1.240611 -3.206258 -0.868701

H -1.256782 -3.562974 -3.911022

H $-2.273799-5.702971-2.891445$

H -1.229198 -5.534564 -1.499814

H $0.310388-7.329784-4.948446$

H -1.433952 -6.707235 -4.910492

H $1.731363-7.024460-3.071763$

H $1.019731-6.496967-1.581723$

H $2.280771-4.766209-3.817226$

H $0.787067-0.797123-1.995449$

C $1.957164 \quad 4.735177-1.288557$

O $0.990783 \quad 3.695622-1.554205$

C $-0.2813924 .306781-1.865105$

C $-0.1637575 .748468-1.399071$

C $1.2876576 .042880-1.713097$

H $-0.3706124 .267697-2.958475$

C -1.413592 $3.503896-1.209807$

H $-0.8566976 .418751-1.915957$

H $-0.3467015 .826432-0.320043$

C $1.8239907 .271524-0.998216$

H $1.3921076 .184254-2.797442$

C $-2.7921354 .183699-1.287323$

O $-1.4817622 .232047-1.861464$

H -1.150149 $3.301140-0.165964$

H $-2.7716335 .125195-0.725844$

O $-3.0405794 .532794-2.655498$

C $-3.974743 \quad 3.321823-0.801143$

C -3.8044012 .7849790 .630019$

H $-4.1407142 .495142-1.500708$

O $-5.1543604 .132822-0.859578$

H -2.949749 2.1022430 .656770

H -3.5657703 .6220991 .297951$

H $1.2661658 .164706-1.297986$

H 1.7389447 .1718940 .089122

H $2.8788027 .435364-1.239544$

C $3.2463054 .448418-2.062705$

H -1.760312 2.385819-2.780653

H $-3.9118484 .963817-2.690928$

H $-5.0455554 .861704-0.225032$

H $3.8360315 .367012-2.164108$

H $3.0053724 .102136-3.075349$

H $2.1383364 .732096-0.205916$

C $-4.760406-0.0851020 .146043$

$\begin{array}{lllll}\text { O } & -5.568541 & 1.088514 & 0.263816\end{array}$

C -5.0657422 .0728931 .172792$

C $-4.895473 \quad 1.4674842 .574691$

C -4.0494740 .1957122 .553709$ 
C $-4.579459-0.780057 \quad 1.502308$

H -3.779485 $0.209286-0.243991$

C $-5.369429-0.995786-0.929689$

H -5.8480512 .8384171 .251862$

O $-4.303106 \quad 2.406651 \quad 3.466071$

H $-5.883438 \quad 1.2162172 .982067$

H -2.991292 0.4240472 .386986

O $-4.123361-0.438654 \quad 3.832612$

H $-5.531019-1.1890461 .859826$

O $-3.682768-1.8811131 .374865$

C $-6.744797-1.532135-0.589222$

H $-5.475222-0.437952-1.867857$

H $-4.695607-1.842474-1.105633$

H $-4.873833 \quad 3.1937823 .482078$

H -3.810826 0.2043684 .492001

H $-2.824897-1.5344361 .075485$

O $-6.955144-2.691577-1.280365$

O $-7.548191-0.9896910 .157116$

C -8.229145 -3.309361-1.072250

H $-8.263907-4.225741-1.667334$

H $-8.356018-3.570916-0.017373$

H -9.031290 -2.643843 -1.405300

SCF Energy (B3LYP/6-31G**//MMFF) $=-3245.92169472$

0245

MMFF Geometry

C $0.252468-2.8220462 .451537$

C $-0.472823-2.0838481 .597462$

C $-0.914651-2.583094 \quad 0.248290$

O $-0.532888-1.566280-0.708582$

C $-2.439927-2.747526 \quad 0.176344$

C $-2.845516-3.630040-0.988318$

C $-3.280371-3.185879-2.182982$

C $-2.760661-5.112797-0.724686$

C $-3.451743-1.764814-2.643762$

C $-4.918240-1.451740-2.976429$

C $-5.812495-1.375441-1.729551$

C $-7.287020-1.282463-2.123994$

C $-8.191402-1.118345-0.893986$

O $-5.430400-0.209180-0.993423$

O $-9.497253-0.738397-1.353702$

C $-7.6555090 .046435-0.038045$

C -8.366294 -2.435037 -0.127994

$\begin{array}{llll}\text { O } & -8.373248 & 0.148728 & 1.190137\end{array}$

C $-6.134290-0.0292490 .241634$

C $-5.664664 \quad 1.3050460 .866998$

O $-5.873987-1.0964841 .154014$

C $0.620582-1.730420-1.402147$

O $1.337003-2.721720-1.408405$

C $0.951011-0.520114-2.191007$

C $0.3681510 .672818-1.990264$

C $0.679796 \quad 1.922105-2.690367$

C $1.7659721 .886110-3.733664$

C $-4.143073 \quad 1.4502501 .008071$

C $-3.7629162 .840803 \quad 1.491248$

C -1.7738694 .2884391 .874193$

O $-4.590386 \quad 3.6893201 .814930$

C -1.484787 5.0488500 .618477

C $-0.249105 \quad 5.3831390 .217945$

C $0.042697 \quad 6.162217-1.031290$

C $0.8545935 .374560-2.034869$

C $2.1744955 .583237-2.174777$

C $0.119147 \quad 4.415549-2.944304$

C $-0.0061693 .036209-2.356806$

N -2.387450 $3.021162 \quad 1.522815$

H $0.475842-3.8521462 .179422$

H $-0.726106-1.0556881 .839914$

H $-0.418770-3.5326820 .013482$

H $-2.926327-1.7676340 .108057$

H -2.821450 -3.205517 1.098574

H $-3.544123-3.928274-2.935942$

H -3.086147 -5.708287 - 1.584287

H $-1.732600-5.406882-0.492570$

H $-3.399307-5.3833190 .122519$

H $-3.062746-1.038670-1.924487$

H $-2.853091-1.638833-3.554582$

H $-4.944447-0.486496-3.498504$

H $-5.305002-2.207594-3.671532$
H $-5.648185-2.264159-1.111144$

H $-7.590982-2.160214-2.707765$

H $-7.435770-0.416288-2.784019$

H $-9.817843-1.434074-1.953040$

H -7.857858 $0.974268-0.591798$

H -8.800464 -3.202312 -0.780158

H $-9.069447-2.3170010 .703861$

H -7.428195 -2.830006 0.267245

H -9.3183620 .2107340 .969080$

H -6.0184522 .1326390 .237390$

H -6.1207721 .4324541 .857179$

H $-5.027500-0.9324731 .598380$

H $1.745263-0.655437-2.917695$

H $-0.4044420 .758659-1.227603$

H $2.7109391 .551193-3.293331$

H $1.9693162 .857678-4.188103$

H $1.4954361 .204292-4.547332$

H $-3.7489380 .723703 \quad 1.726424$

H $-3.653124 \quad 1.2833340 .042107$

H -0.8685394 .0679382 .445511$

H -2.4502574 .8675972 .510937$

H -2.3389285 .3470240 .014635$

H 0.6038045 .1000540 .829127

H $0.5833367 .071538-0.737641$

H $-0.8806506 .515216-1.507389$

H $2.7557535 .044299-2.915514$

H $2.7040976 .292432-1.547423$

H $0.5952494 .393225-3.930131$

H $-0.8894634 .799650-3.144598$

H $-0.7706062 .959522-1.583313$

H -1.7822032 .2809981 .178582$

C $1.029017-0.8594453 .939995$

$\begin{array}{lllll}\text { O } & 1.855555 & -0.328405 & 2.885807\end{array}$

C $2.7201570 .685177 \quad 3.430510$

C $2.283986 \quad 0.889015 \quad 4.878781$

C $1.744004-0.4822685 .236237$

H 3.7341740 .2698313 .424443

C 2.6727841 .9475882 .562438

H $3.113914 \quad 1.2040595 .518841$

H 1.4927831 .6425564 .966018

C $0.848765-0.4823156 .462649$

H $2.592946-1.1573725 .408846$

C 2.9631481 .7046901 .067835

$\begin{array}{llll}\text { O } & 1.358096 & 2.507418 & 2.659300\end{array}$

H 3.3611962 .6971572 .971077

H $2.148467 \quad 1.1287260 .612520$

$\begin{array}{lllll}\text { O } & 2.974716 & 2.979073 & 0.411967\end{array}$

C 4.3032721 .0032550 .778599

C $4.6134580 .942401-0.729122$

H $4.270963-0.0126121 .184677$

$\begin{array}{lllll}\text { O } & 5.361338 & 1.690371 & 1.445026\end{array}$

H $3.7569160 .494779-1.241984$

H $4.7271991 .961208-1.119077$

H $1.400305-0.1322747 .341331$

H -0.0181690 .1729976 .326799$

H $0.478990-1.4900566 .676318$

C $0.861115-2.3702713 .753571$

H 1.3989903 .4053252 .290123

H 2.0541793 .2854560 .355970

H 5.3720512 .6049611 .114033

H $\quad 0.270217-2.7907054 .575064$

H $1.855053-2.8332443 .805440$

H $0.057702-0.3517753 .882333$

C $4.959120-2.058833-1.085240$

O $5.900867-1.156898-0.493663$

C $5.9044990 .158505-1.054302$

C $6.231850 \quad 0.083314-2.552617$

C $5.299496-0.872226-3.293825$

C $5.237442-2.225391-2.583569$

H $3.941807-1.680553-0.930797$

C $5.083754-3.397217-0.350870$

H $6.7305240 .694693-0.570305$

O $6.1573901 .371331-3.155314$

H $7.262790-0.272192-2.679746$

H $4.301107-0.435696-3.409747$

O $5.813118-1.057489-4.615155$

H $6.183650-2.761035-2.733570$

O $4.199174-3.014014-3.160860$ 
C $4.511653-3.2899091 .041671$

H $4.534143-4.196918-0.859971$

H $\quad 6.129948-3.719522-0.284914$

H $6.7752871 .952306-2.679471$

H $5.160321-1.576821-5.113893$

H $4.450433-3.206080-4.079759$

O $\quad 5.510416-3.0870561 .946709$

O $3.314586-3.3536741 .288935$

C $5.088135-2.9518643 .306967$

H $5.974910-2.7831823 .923444$

H $4.419430-2.0935383 .412789$

H $4.594965-3.8689423 .643158$

SCF Energy (B3LYP/6-31G**//MMFF) $=-3245.90070003$

02466

MM̄FF Geometry

C $0.129015-2.6399220 .624828$

C $0.703170-2.327771 \quad 1.798210$

C $0.211041-1.2907742 .784991$

O $-1.071038-0.7580062 .386593$

C $1.215815-0.1266362 .835006$

C 0.8363520 .9429353 .838323

C 0.2685332 .1211653 .513287

C 1.1776710 .6319215 .271678

C -0.1479572 .5817482 .143354$

C - -1.5292623 .2507732 .168367$

C -1.957697 3.6959490 .762984

C -3.2002764 .5835590 .829761$

C $-3.6893854 .983247-0.569365$

O $-2.2428772 .523034-0.007634$

O $-4.9968805 .551090-0.400034$

C -3.822485 $3.710481-1.431098$

C $-2.8119136 .078976-1.185905$

O $-4.1192664 .036376-2.787476$

C $-2.5803902 .786526-1.376110$

C $-2.9186811 .441464-2.059560$

O $-1.4974993 .404765-2.072650$

C $-2.183464-1.3706822 .872869$

O $-2.228798-2.3075953 .653584$

C $-3.354328-0.7253622 .239182$

C $-4.542849-1.3448532 .283117$

C $-5.777612-0.8935801 .639131$

C $-5.783221 \quad 0.477317 \quad 1.018134$

C - $1.8530360 .352313-1.896137$

C $-2.380941-0.968065-2.421660$

C $-3.972847-2.803942-1.840373$

O $-2.098682-1.373390-3.546713$

C $-5.425704-2.445461-1.908931$

C $-6.392575-3.132438-1.281088$

C $-7.848234-2.760236-1.336160$

C $-8.499562-2.7090090 .030836$

C $-9.400235-3.635426 \quad 0.400440$

C $-8.163222-1.5452520 .942984$

C $-6.833553-1.7335721 .622320$

N -3.221722 -1.603402 -1.521267

H $-0.789660-2.1321840 .340791$

H $1.623073-2.8269262 .091424$

H $\quad 0.136640-1.755723 \quad 3.775686$

H $1.325220 \quad 0.313481 \quad 1.837126$

H $2.215845-0.5040393 .089267$

H $\quad 0.0813202 .8362384 .313410$

H 0.9191581 .4532765 .948442

H $0.635991-0.2563245 .610936$

H $2.251727 \quad 0.4458825 .374636$

H 0.5918123 .3074951 .788173

H $-0.164757 \quad 1.7576241 .424980$

H -2.265158 2.5451572 .574656

H -1.496529 4.1195592 .837684

H -1.125069 4.2402490 .301940

H $-3.0107875 .477180 \quad 1.437710$

H -4.0082474 .0398241 .339873$

H -5.307155 $5.860792-1.267559$

H -4.687002 3.149217 -1.049217

H -2.823573 $6.976013-0.555253$

H -3.198306 $6.393782-2.161690$

H $-1.7709445 .774326-1.312534$

H -4.962928 4.518580 -2.797859

H $-3.8616631 .062835-1.649435$
H $-3.0846351 .599791-3.133234$

H $-0.8643502 .720134-2.340402$

H -3.1953540 .2066201 .709315$

H -4.611361 -2.299586 2.803292

H -5.1352280 .4994550 .138336$

H -5.4288621 .2286361 .733246$

H -6.7749960 .8080360 .702744$

H $-0.9465020 .602075-2.455681$

H -1.562921 $0.223551-0.847627$

H -3.765305 -3.535391-1.053074

H $-3.642015-3.210789-2.800509$

H -5.695354 -1.576487-2.505337

H -6.127679-4.017663 -0.707053

H -8.355121 -3.501790 -1.967291

H -7.992269 -1.792948 -1.833610

H $-9.888895-3.593790 \quad 1.368274$

H -9.665790 -4.459245 -0.253798

H -8.925355 -1.447663 1.726933

H $-8.220628-0.6238700 .357454$

H -6.735442 -2.693907 2.131305

H $-3.386601-1.174594-0.615854$

C $2.005765-4.236213-0.190162$

O $3.020865-3.215721-0.141747$

C $4.218568-3.714894-0.764579$

C $3.941313-5.169336-1.136380$

C $2.437109-5.166552-1.322683$

H $4.358101-3.135400-1.684206$

C $5.423041-3.5189100 .164767$

H $4.484425-5.474062-2.036436$

H $4.223291-5.858798-0.332423$

C $1.816985-6.551605-1.263321$

H $\quad 2.206897-4.708465-2.294095$

C $5.634830-2.0759210 .666689$

O $5.262066-4.3502821 .323396$

H $6.330642-3.877319-0.334909$

H $4.848910-1.8145991 .386486$

O $6.869283-2.0804951 .396584$

C $5.699358-1.008657-0.443164$

C 6.1010780 .3818650 .085750

H $4.719943-0.944166-0.927660$

O $6.636369-1.409232-1.442675$

H 5.4671210 .6289280 .943830

H 7.1366110 .3469570 .444894

H $2.228628-7.190942-2.051245$

H $2.010786-7.037055-0.300906$

H $0.732695-6.501584-1.403055$

C $0.631252-3.602513-0.420123$

H $4.430265-4.0921481 .756547$

H $6.881442-1.2892371 .960983$

H $7.499797-1.520669-1.008833$

H $-0.121536-4.391450-0.532118$

H $\quad 0.655550-3.047158-1.366314$

H $2.027326-4.767780 \quad 0.770117$

C $3.6759212 .042629-0.716172$

O $4.7078041 .559160-1.584729$

C $6.0077901 .485927-0.992034$

C $6.4367672 .878671-0.507661$

C 5.3950813 .5061160 .415386

C $4.0088553 .455587-0.226114$

H $3.563348 \quad 1.364080 \quad 0.138280$

C $2.3645062 .022808-1.507312$

H $6.6950951 .200295-1.798183$

O 7.6878092 .8229930 .169205

H $6.5699593 .538273-1.375169$

H $5.3886943 .024377 \quad 1.400262$

O $5.732214 \quad 4.8767290 .641731$

H $3.9666794 .165568-1.061970$

O 3.0355953 .8629710 .731414

C $1.9569830 .598522-1.794916$

H $1.5423812 .490601-0.954088$

H $2.4671652 .566034-2.454360$

H $8.3358112 .439372-0.446327$

H 6.6307634 .8954501 .013760

H 3.2866734 .7497501 .042114

O $2.2225090 .285662-3.094469$

O $1.487114-0.154787-0.952781$

C $1.919792-1.059228-3.477272$

H $2.166786-1.175590-4.535725$ 
H $\quad 2.524021-1.762197-2.896823$

H $0.853742-1.262491-3.341623$

SCF Energy (B3LYP/6-31G**//MMFF) $=-3245.91523746$

02447

MM̄FF Geometry

C -3.202843 -3.1881221 .666833$

C $-1.904377-2.9471541 .428893$

C $-0.891439-2.8144012 .536086$

O $-0.238141-1.5285342 .430063$

C $0.188949-3.9025122 .410562$

C $1.133298-3.9208273 .598792$

C $2.373933-3.3944153 .595545$

C $0.612437-4.6228424 .825993$

C $3.044651-2.6593882 .469098$

C $4.120456-3.5305251 .811822$

C $4.654327-2.9769490 .482455$

C $5.326274-1.6105120 .626355$

C $5.799200-1.065336-0.730888$

O $3.556732-2.900772-0.433962$

O $6.1185780 .324558-0.560995$

C $4.623514-1.137436-1.728982$

C $7.084745-1.748108-1.210533$

O $5.044966-0.771592-3.039824$

C $3.923169-2.513284-1.766179$

C $2.628714-2.523346-2.619241$

O $4.811635-3.475408-2.348916$

C $-0.760708-0.5063073 .154669$

O $-1.829079-0.505313 \quad 3.751347$

C $0.1857860 .627682 \quad 3.252169$

C 1.2286790 .8220062 .429582

C 2.2315611 .8845922 .560834

C 2.1894742 .7301623 .808633

C $1.561116-1.501857-2.209603$

C $1.715456-0.172119-2.927895$

C $1.3755652 .270222-2.615397$

O $2.193177-0.077730-4.056198$

C $2.6514982 .807460-2.051132$

C $2.7059373 .842401-1.200596$

C $3.9831384 .366993-0.615737$

C 3.9865554 .3227390 .895712

C 3.7945125 .4424411 .613476

C 4.3110493 .0079951 .570036

C 3.1592012 .0389261 .592106

N $1.2285530 .892538-2.184494$

H $-3.521882-3.2708852 .704967$

H -1.536246 -2.851865 0.411965

H -1.376249 -2.9036603 .516759$

H $\quad 0.750202-3.7667641 .478189$

H $-0.280195-4.8921822 .327259$

H $2.977608-3.4854094 .497452$

H $1.350908-4.6498675 .634456$

H $-0.277859-4.1167515 .211658$

H $\quad 0.348649-5.6584004 .587581$

H $2.318197-2.318780 \quad 1.726577$

H $3.499674-1.7518512 .882866$

H $4.954174-3.6765852 .510139$

H $3.692746-4.5224371 .611579$

H $5.376108-3.7099240 .102862$

H $6.161687-1.6600041 .335456$

H $4.616451-0.8867821 .047179$

H 6.8014340 .3931210 .128036

H $3.900649-0.378902-1.406543$

H $7.893518-1.592080-0.486452$

H $7.436843-1.310179-2.151056$

H $6.973327-2.825004-1.351628$

H $5.4572230 .107078-2.977103$

H $2.882464-2.433145-3.682568$

H $2.174174-3.518577-2.511588$

H $4.283793-4.220292-2.681390$

H -0.0240791 .3069414 .072722$

H 1.3789180 .1382851 .595912

H 1.2634823 .3127853 .849588

H 3.0110413 .4452343 .879845

H 2.2491372 .0999654 .703222

H $0.573832-1.885220-2.495843$

H $\quad 1.551317-1.360603-1.123494$

H $\quad 0.5043012 .824059-2.257407$
H $1.3979622 .319801-3.708444$

H $3.5735732 .315772-2.352980$

H $1.7859374 .343128-0.908516$

H $4.1110495 .399439-0.965935$

H $4.8542643 .818109-0.995123$

H 3.8324215 .4350192 .697288

H 3.5931056 .3939951 .132412

H 4.6895663 .1759642 .585223

H 5.1533522 .5353921 .048598

H $3.119156 \quad 1.381848 \quad 0.723220$

H $0.9010570 .729409-1.236766$

C -3.951317 -3.399507-0.814219

O $-3.505561-2.096299-1.237474$

C $-4.134879-1.768308-2.489083$

C $-4.765600-3.058817-2.998649$

C $-5.153326-3.735533-1.701339$

H $-4.933700-1.054183-2.257169$

C $-3.130729-1.132586-3.455971$

H $-5.618186-2.870966-3.658422$

H $-4.046657-3.678457-3.546721$

C $-5.417641-5.224278-1.850844$

H $-6.057225-3.248815-1.310477$

C $-2.3709720 .080101-2.886972$

O $-2.155937-2.125050-3.805682$

H -3.643814 -0.861980 -4.386706

H -1.657503 $-0.253192-2.122808$

O $-1.5928550 .640731-3.953483$

C $-3.2504201 .200478-2.296917$

C $-2.4000312 .410898-1.868755$

H $-3.8060770 .823964-1.433020$

O $-4.1969151 .647305-3.266435$

H -1.592496 $2.057298-1.219738$

H -1.938646 $2.849512-2.761411$

H $-6.242771-5.399758-2.548822$

H $-4.536877-5.751613-2.232180$

H $-5.688452-5.672433-0.889839$

C $-4.316826-3.3731410 .672081$

H -1.662640 -1.794405 -4.575046

H $-0.8873650 .007684-4.167798$

H $-4.8238040 .923460-3.429765$

H $-4.816919-4.3144490 .930147$

H $-5.040235-2.5675280 .847740$

H -3.129017 -4.101904 -1.000035

C $-2.9723182 .748587 \quad 1.124808$

O -3.8517263 .0405860 .031185$

C $-3.2115383 .515323-1.157681$

C $-2.3997114 .782203-0.850054$

C -1.4163234 .5602440 .295263$

C -2.1477673 .9856821 .506420$

H $-2.304775 \quad 1.921770 \quad 0.853274$

C -3.8432312 .2989972 .308503$

H $-4.0165973 .811064-1.841819$

O $-1.7044775 .217795-2.013719$

H $-3.0918005 .587527-0.571036$

H $-0.5858893 .916045-0.015548$

O $-0.843513 \quad 5.822700 \quad 0.641038$

H -2.800914 4.7555071 .937620

O -1.1973793 .6219452 .502816$

C -4.4718510 .9401822 .087504$

H -3.2462032 .2286073 .224201$

H -4.6546733 .0214852 .458650$

H -1.186718 $6.003127-1.766475$

H -0.1316755 .6556501 .281631$

H -0.7673264 .4367482 .811919$

O

\begin{tabular}{lllll}
\hline & -4.182086 & 0.164334 & 1.188009
\end{tabular}

C -6.073489-0.539005 3.012622

H $-6.862682-0.5339513 .769229$

H $-6.532770-0.6850012 .030156$

H $-5.375145-1.3492773 .239593$

SCF Energy (B3LYP/6-31G**//MMFF) $=-3245.90564005$

02488

MM̄FF Geometry

C -1.650472 $3.885753-0.259351$

C -2.7288744 .5899080 .118782$

C $-4.1075054 .039630 \quad 0.404277$

O -4.0943022 .6015000 .546434$ 
C $-5.0510874 .410306-0.753774$

C $-6.4820903 .956083-0.542176$

C $-7.0548002 .904990-1.162973$

C $-7.286875 \quad 4.7982530 .413921$

C $-6.4132111 .947626-2.129636$

C $-6.5272060 .501197-1.630714$

C $-5.685651-0.467058-2.475804$

C $-5.893475-1.906844-2.002075$

C $-4.966725-2.896763-2.722460$

O $-4.311502-0.091847-2.329962$

O $-4.988363-4.132721-1.990897$

C $-3.518989-2.363875-2.661483$

C $-5.467764-3.220890-4.134656$

O $-2.648515-3.173912-3.447174$

C -3.390008 -0.887507-3.088055

C $-1.970993-0.308081-2.856145$

O $-3.657756-0.783048-4.492244$

C $-3.7563202 .106584 \quad 1.770494$

O -3.3956432 .7447532 .746838$

C -3.9183920 .6365311 .723691$

C $-3.647107-0.1006472 .811039$

C $-3.807676-1.5517962 .934310$

C $-4.271240-2.309751 \quad 1.718684$

C $-1.503600-0.250383-1.398325$

C $-0.730674-1.471699-0.933343$

C $0.241305-2.4698391 .134872$

O $-0.329843-2.347288-1.694388$

C $-0.705698-3.456541 \quad 1.739213$

C $-0.711057-3.7666193 .044476$

C $-1.612083-4.7999293 .655561$

C $-2.414106-4.2631024 .818966$

C $-2.017365-4.4691326 .086331$

C $-3.723908-3.5742994 .514355$

C $-3.549246-2.1323864 .124918$

N $-0.508591-1.4440240 .437002$

H $-1.7247862 .811990-0.403184$

H -2.630103 $5.667450 \quad 0.237017$

H -4.4769854 .4898351 .334377$

H $-4.6539184 .006960-1.691458$

H $-5.0543905 .499948-0.895302$

H $-8.1024482 .694211-0.951833$

H -8.326688 4.4629910 .490414

H -6.8546724 .7584561 .418575$

H -7.301700 5.8414960 .081583

H $-6.9187372 .037594-3.098482$

H $-5.3631162 .191996-2.302978$

H $-6.1905910 .456397-0.587597$

H -7.579677 $0.191516-1.646559$

H -5.979673 -0.361336 -3.526740

H -6.942388 -2.206570 -2.119522

H $-5.687814-1.966069-0.925645$

H -5.909430 -4.442876 -1.953605

H -3.188176 -2.463355 -1.620609

H -6.464889 -3.675497 -4.091937

H -4.823474 -3.960117 -4.623075

H $-5.535917-2.341523-4.778328$

H -2.741792 -4.089347 -3.132477

H - $-1.242476-0.825331-3.492293$

H -1.984250 $0.730571-3.217209$

H $-3.2624030 .040700-4.822315$

H -4.2791750 .2006220 .800152$

H -3.294333 0.4067913 .708051

H -4.237588 -3.3944521 .841055$

H -3.634775 -2.0862630 .855754$

H -5.304138 -2.039802 1.474773

H $-0.813370 \quad 0.596582-1.293644$

H -2.345180 -0.070817 -0.724096

H $\quad 0.850596-1.9675351 .891454$

H $\quad 0.906974-2.9821380 .434434$

H -1.394723 -3.962068 1.066810 H $-0.000383-3.2789123 .708261$ H -0.980780 -5.632909 3.992020 H -2.292358 -5.2305322 .910286$ H -2.604590 -4.114718 6.927035 H -1.093362 -4.9917236 .310698$ H -4.368016 -3.5899715 .404310$ H -4.276618 -4.1544613 .769827$ H -3.211708 -1.500075 4.947434
H $-0.916603-0.697410 \quad 0.992111$

C $0.825004 \quad 3.951878 \quad 0.338054$

O $1.1459232 .604531-0.052316$

C 1.6670301 .9078211 .105408

C 1.6852202 .9213552 .247000

C 0.5861663 .8845751 .842709

H 0.9520771 .1034551 .314478

C 3.0452941 .3308870 .751436

H 1.5103482 .4537073 .220765

H $2.649333 \quad 3.4433172 .292924$

C 0.6594935 .2283802 .544298

H -0.3775823 .4107092 .066919$

C $3.7789740 .686974 \quad 1.940325$

O $2.8676800 .340971-0.266964$

H 3.6605572 .1245490 .313132

H 3.9600821 .4371722 .718822

O $2.914191-0.2962862 .520205$

C $5.111281-0.0004651 .577858$

C $6.1308690 .951470 \quad 0.927642$

H $4.919362-0.8624410 .930239$

O $5.675368-0.5275882 .784508$

H $5.7183601 .339075-0.008188$

H $6.284303 \quad 1.811717 \quad 1.591179$

H 0.5644945 .1021803 .627743

H 1.6109425 .7333952 .346942

H -0.1478345 .8872102 .209165$

C $-0.3093844 .510340-0.523516$

H $2.3832040 .760046-0.998818$

H $3.394909-0.7068203 .259210$

H 5.8744330 .2214243 .371969

H $-0.0713064 .339196-1.580709$

H $-0.3623615 .596221-0.378514$

H 1.7244034 .5474890 .128511

C $6.974610-0.756022-1.448779$

O $7.378742-0.926283-0.085265$

C 7.5017770 .2889590 .659685

C $8.5308761 .211316-0.008934$

C $8.2190571 .443763-1.485677$

C $7.9891260 .112313-2.201795$

H $5.983625-0.287906-1.482374$

C $6.866571-2.150703-2.073452$

H 7.9143630 .0083081 .636964

O 8.5939692 .4559820 .679567

H 9.5236330 .7495330 .072144

H $7.3619452 .116942-1.604270$

O $9.3355812 .108815-2.080878$

H $8.946902-0.415229-2.291203$

O $7.5289040 .367618-3.527121$

C $5.711129-2.916496-1.475111$

H $6.720383-2.109479-3.158574$

H $7.778429-2.731850-1.889733$

H 9.2430893 .0120090 .215634

H $9.1415052 .207933-3.028853$

H $6.6714780 .822088-3.463615$

O $4.532627-2.433801-1.962496$

O $5.841428-3.824025-0.664041$

C $3.353504-3.047291-1.435052$

H $3.316795-2.924804-0.348349$

H $2.487013-2.546459-1.873632$

H $3.323736-4.106426-1.707017$

SCF Energy (B3LYP/6-31G**//MMFF) $=-3245.92172621$

02449

MMFF Geometry

C -1.631408 -2.182599 2.077154

C $-1.481167-1.1889492 .968676$

C $-2.439855-0.0444613 .216558$

O $-3.533077-0.1020112 .275069$

C -1.7258971 .3074283 .045287$

C -2.4350332 .4458693 .751279$

C -3.1979363 .3757593 .144457$

C -2.1945242 .5251655 .237660$

C -3.5661743 .4685501 .690222$

C -2.9012774 .6646100 .994602$

C $-1.392534 \quad 4.475116 \quad 0.773410$

C $-0.774508 \quad 5.7281430 .151537$

C $0.7105545 .528325-0.185134$

O $-1.2216143 .361581-0.107668$ 
O $1.0936646 .629943-1.022160$ C $0.8668714 .225813-0.999359$ C 1.5896705 .6047161 .068913 O $2.2418043 .911649-1.204430$ C $0.1424003 .011644-0.369377$ C $0.1390961 .828780-1.366233$ $\begin{array}{llllll}\text { O } & 0.829619 & 2.621827 & 0.820983\end{array}$ C $-4.796918 \quad 0.0913182 .726205$ O $\quad-5.137364 \quad 0.3243793 .877725$ C $-5.785432-0.0447881 .629928$ C $-5.448376-0.1291150 .333271$ C $-6.368998-0.296443-0.793844$ C $-7.835023-0.451421-0.485965$ C $-0.7393430 .642334-0.942348$ C $-0.817201-0.417368-2.027717$ C -1.923584 -2.591414 -2.542969 O $-0.197830-0.344098-3.085195$ C $-3.011004-2.229251-3.504723$ C $-4.214153-2.821703-3.537198$ C $-5.295479-2.447546-4.510823$ C $-6.555130-1.956239-3.830691$ C $-7.639258-2.744556-3.737137$ C $-6.590741-0.524297-3.340622$ C -5.856655 -0.336147 -2.041781 N -1.661645 -1.459900 -1.672712 H $-2.509264-2.1802931 .434927$ H -0.614965 -1.191772 3.625044 H $-2.809115-0.1664184 .241996$ H $-1.614620 \quad 1.527712 \quad 1.978872$ H -0.7033581 .2576123 .443302$ H -3.6332244 .1613863 .761160$ H -2.689009 3.3891665 .694224 H -2.5726401 .6288845 .738640$ H -1.1229142 .6135845 .444154$ H $-3.3541212 .543018 \quad 1.147447$ H -4.6547853 .5974641 .637168$ H -3.390976 4.7923000 .020408 H -3.0843425 .5794861 .571765$ H $-0.913490 \quad 4.2561401 .733747$ H -0.9053526 .5957310 .810504$ H -1.309692 $5.975015-0.776414$ H $2.0453846 .554281-1.204613$ H $0.4362254 .408203-1.993884$ H 1.4887326 .5874021 .545012 H 2.6508115 .5018640 .816009 H 1.3401994 .8470471 .814679 H $2.6400564 .641334-1.707769$ H $-0.2273512 .184758-2.338252$ H $1.1636161 .467839-1.515142$ H $0.673711 \quad 1.6789330 .985776$ H $-6.820760-0.0673941 .954677$ H -4.395972 -0.0790060 .058644$ H -7.999924 -1.2998140 .187693$ H -8.448416 -0.636885 -1.370169 H $-8.225244 \quad 0.455717-0.011678$ H $-0.3372460 .169291-0.040243$ H -1.760607 $0.979153-0.731233$ H -2.195654 -3.438892 -1.906031 H -1.011858 -2.845494 -3.093229 H -2.791452 -1.437887 -4.217907 H $-4.435193-3.621308-2.834041$ H -5.514788 -3.334230 -5.119986 H -4.949130 -1.684499 -5.219250 H -8.557515 -2.391670 -3.279900 H -7.631808 -3.762605 -4.112299 H -7.623604 -0.166613 -3.264362 H -6.134414 $0.128024-4.096537$ H -4.780499 -0.212359-2.161979 H -2.208091-1.383410 -0.820075 C $0.499842-3.4816002 .772604$ O $1.508564-2.5503952 .329071$ C $2.802802-3.1697382 .477965$ C $2.567833-4.4713643 .234417$ C $1.181880-4.8486502 .747106$ H $3.152740-3.4236131 .471707$ C $3.775112-2.2011963 .160168$ H $3.325826-5.2266613 .005841$ H $2.565371-4.3126814 .319472$
C $0.507428-5.9014103 .609585$

H $1.253688-5.2152301 .714282$

C $4.059954-0.9205572 .345116$

O $3.228834-1.8107204 .426105$

H $4.717592-2.7139683 .383309$

H $3.143158-0.3313402 .224201$

O $4.971359-0.1102403 .096637$

C $4.689184-1.1981890 .968005$

C 5.0497700 .0795120 .186991

H $3.989814-1.7844470 .368480$

O $5.863370-1.994071 \quad 1.134178$

H 4.1476250 .6907420 .074517

H $\begin{array}{llll}5.773480 & 0.670623 & 0.760894\end{array}$

H $1.092327-6.8271023 .613018$

H $0.404751-5.5652284 .646842$

H $-0.492056-6.1369633 .231671$

C $-0.728960-3.3753991 .866382$

H $2.365599-1.3952314 .256609$

H 4.5675040 .0475823 .967237

H $6.483496-1.4883141 .687168$

H $-1.354390-4.2659191 .999366$

H -0.406039 -3.358786 0.817806

H $0.246828-3.2266253 .809550$

C $3.642565-0.627676-2.445198$

O $4.872866-1.161635-1.941621$

C $5.669648-0.237120-1.194704$

C $6.0084540 .991259-2.050937$

C $4.7653701 .611228-2.686502$

C $3.9307450 .541188-3.391824$

H $3.021280-0.276304-1.613214$

C $2.912294-1.775109-3.158704$

H $6.619254-0.750823-0.996452$

O $6.6964611 .955994-1.262448$

H $6.6932300 .681635-2.851449$

H $4.1639082 .146219-1.943436$

O $5.1605232 .577324-3.662199$

H $4.4616320 .187107-4.284613$

O $2.7001551 .117024-3.822567$

C $2.239466-2.709635-2.172384$

H $2.129395-1.404262-3.826819$

H $3.632891-2.353379-3.749731$

H $7.0072642 .657836-1.858447$

H $5.5894213 .311500-3.191225$

H $2.917441 \quad 1.876209-4.390177$

O $2.147041-3.957520-2.718288$

O $1.812778-2.380271-1.073683$

C $1.518084-4.941185-1.890205$

H $2.086316-5.076039-0.964658$

H $0.486583-4.649742-1.670341$

H $1.506308-5.888156-2.436075$

SCF Energy (B3LYP/6-31G**//MMFF) $=-3245.92056770$

$02 \_5$

MM̄FF Geometry

C $-1.3917630 .994802 \quad 2.442239$

C $-2.3216190 .341574 \quad 3.155744$

C -2.783182 -1.086388 2.984006

O $-2.066174-1.7744251 .937978$

C $-4.263014-1.0830002 .563575$

C $-4.973858-2.3984872 .802314$

C $-5.236977-3.3183301 .853838$

C $-5.460397-2.6103674 .213321$

C $-4.801237-3.3279250 .414897$

C $-5.918187-2.934446-0.564411$

C $-6.003810-1.417424-0.791415$

C $-7.160825-1.058965-1.723363$

C $-7.1865970 .443620-2.047193$

O $-4.766127-1.007817-1.381920$

O $-8.1050780 .611984-3.137471$

C $-5.7832660 .878171-2.525580$

C $-7.7406651 .264676-0.876641$

O $-5.7082572 .292499-2.689757$

C $-4.6384080 .400458-1.600836$

C $-3.2705410 .652335-2.282215$

O $-4.687676 \quad 1.133275-0.375034$

C $-0.895194-2.3897262 .242520$

O $-0.294223-2.3621543 .302840$

C $-0.478737-3.122478 \quad 1.026803$ 
C $0.770729-3.5929510 .908932$

C $1.293609-4.342089-0.236836$

C $0.323255-4.708775-1.331377$

C $-2.086562-0.052180-1.601553$

C $-0.7768180 .212219-2.326166$

C $1.613023-0.501871-2.342446$

O $-0.6605441 .059404-3.207913$

C $2.051563-1.823950-2.885692$

C $3.246830-2.365522-2.604626$

C $3.733237-3.672114-3.162596$

C $4.309118-4.585003-2.101474$

C $5.637666-4.686776-1.927117$

C $3.351518-5.443474-1.306000$

C $2.604093-4.663547-0.257986$

N $0.248508-0.598229-1.857326$

H $-0.855198 \quad 0.489675 \quad 1.644938$

H -2.8233280 .8838153 .956267$

H -2.651661 -1.616781 3.934983

H $-4.327478-0.8107401 .506164$

H -4.818889-0.304071 3.102995

H $-5.801346-4.2035652 .146259$

H -5.993899-3.559378 4.332555

H $-4.618253-2.6172814 .912374$

H $-6.146728-1.8080974 .503353$

H -3.905871 -2.719423 0.259253

H $-4.492933-4.3570800 .187714$

H -5.694951 -3.416001-1.525824

H $-6.884184-3.325354-0.222663$

H $-6.136383-0.9206120 .174666$

H -8.119795 -1.376379 -1.294770

H $-7.055610-1.614984-2.665707$

H $-8.179468 \quad 1.562528-3.326460$

H -5.629399 $0.439673-3.521604$

H $-8.7670880 .954582-0.646317$

H -7.799338 2.328667 -1.131669

H $-7.149828 \quad 1.1635330 .036127$

H $-6.3648872 .547463-3.359253$

H -3.311505 $0.290587-3.318209$

H $-3.070938 \quad 1.730777-2.322566$

H -3.799545 1.1615330 .013972

H $-1.224674-3.2512980 .249913$

H $1.476229-3.4098191 .718308$

H $-0.460033-5.368678-0.942088$

H $0.786464-5.226108-2.173762$

H $-0.149796-3.813053-1.748539$

H $-1.9715660 .296073-0.571071$

H -2.252834 -1.135432 -1.582713

H $2.226186-0.191514-1.491757$

H $1.6825170 .259927-3.124679$

H $1.373276-2.344529-3.557204$

H $3.931845-1.820238-1.960599$

H $4.501905-3.445924-3.913178$

H $2.936391-4.198841-3.702042$

H $6.061647-5.359180-1.188793$

H $6.331225-4.093177-2.513496$

H $3.901263-6.238496-0.784269$

H $2.689923-5.976082-1.994899$

H $3.229846-4.3471350 .577706$

H $0.066422-1.224379-1.078852$

C -1.151726 $3.281146 \quad 1.426766$

O -0.0012543 .0405300 .585690$

C $0.600758 \quad 4.305820 \quad 0.258059$

C -0.4862215 .3445740 .492013$

C $-1.1555114 .782828 \quad 1.728047$

H 1.4089554 .4708060 .980796

C $1.1670654 .294456-1.163017$

H $-0.079230 \quad 6.349140 \quad 0.641211$

H $-1.1983935 .382720-0.340592$

C $-2.540788 \quad 5.3528991 .981619$

H -0.5191584 .9939132 .598346$

C $2.2076093 .192216-1.436057$

O $0.0938304 .114634-2.096619$

H $1.5999175 .275419-1.391542$

H $1.7181752 .213792-1.456435$

O $2.726938 \quad 3.409702-2.754359$

C $3.3868753 .143414-0.445596$

C $4.5141982 .201501-0.912349$

H 3.0179412 .8196460 .532552
O $3.9289364 .453860-0.277159$

H $4.0790371 .241735-1.203909$

H $4.9865022 .629229-1.804823$

H -2.4880456 .4350382 .139728$

H -3.2113405 .1691261 .135421$

H -2.9911064 .9024002 .871795$

C -1.0380892 .4346752 .696401$

H $-0.3464683 .274681-1.879928$

H $1.9710863 .408979-3.366594$

H $4.2139064 .769142-1.152108$

H -1.6900282 .8457353 .476207$

H -0.0102482 .4707643 .074288$

H $-2.037892 \quad 3.0002080 .843234$

C $4.695766 \quad 0.020688 \quad 1.234825$

$\begin{array}{llll}\text { O } & 5.084836 & 1.394764 & 1.356845\end{array}$

C $5.604386 \quad 1.992187 \quad 0.163143$

C $6.8268781 .202898-0.327994$

C $6.504770-0.275950-0.513934$

C $5.871411-0.8407420 .754830$

H $3.854406-0.0654830 .537285$

C $4.226658-0.4540132 .619148$

H 5.9634042 .9878360 .451964

O $7.321193 \quad 1.731449-1.553932$

H $7.636073 \quad 1.2909440 .408482$

H $5.861999-0.440909-1.385574$

O $7.714435-0.994646-0.766237$

H $6.634570-0.9066821 .541217$

O $5.408200-2.164827 \quad 0.501283$

C 2.9323690 .1956413 .056559

H $4.057229-1.5363232 .623655$

H $4.993319-0.2053303 .363323$

H $7.5291472 .669646-1.404611$

H $8.127633-0.597084-1.552082$

H $6.169637-2.6826610 .188524$

O $2.769753-0.015748 \quad 4.396882$

\begin{tabular}{llll}
\hline & 2.155264 & 0.788258 & 2.321774
\end{tabular}

C $1.5860410 .539597 \quad 4.976442$

H 1.6021030 .3291066 .049065

H 1.5655501 .6238164 .833447

H $\quad 0.6990180 .071696 \quad 4.541807$

SCF Energy (B3LYP/6-31G**//MMFF) $=-3245.93232276$

02_50

MMFF Geometry

C $1.683619-4.0196441 .057086$

C $0.423042-3.703198 \quad 0.723566$

C $-0.106040-3.754321-0.687433$

O $-0.768709-2.494113-0.956408$

C $-1.136587-4.886251-0.828855$

C $-1.605711-5.084756-2.258092$

C $-2.804693-4.700854-2.739648$

C $-0.630883-5.800333-3.159050$

C $-3.910062-3.992537-2.008722$

C $-4.165177-2.601619-2.602697$

C $-5.222767-1.827955-1.802638$

C $-5.505972-0.470862-2.447806$

C $-6.4789840 .373524-1.610494$

O $-4.720871-1.641053-0.475207$

O $-6.424991 \quad 1.722535-2.100314$

C $-5.9882480 .406354-0.146396$

C $-7.929785-0.092093-1.782808$

O $-6.9482341 .039571 \quad 0.695592$

C $-5.634306-0.986048 \quad 0.415919$

C $-4.958989-0.9311221 .808766$

O $-6.838189-1.7493630 .566425$

C $0.030715-1.435910-1.265205$

O $1.244922-1.449974-1.393045$

C $-0.826388-0.235986-1.398369$

C $-0.2442140 .961701-1.557862$

C $-0.9157522 .251973-1.723780$

C $-2.4199602 .261365-1.751906$

C $-3.641284-0.1504431 .878742$

C -3.8006991 .2910792 .333719$

C $-2.470776 \quad 3.3675252 .690435$

$\begin{array}{llll}\text { O } & -4.864272 & 1.763010 & 2.725620\end{array}$

C -2.448146 4.2425401 .477853

C $-1.4055675 .017441 \quad 1.142520$

C $-1.4122375 .974046-0.013751$ 
C $-0.3034405 .711319-1.004988$

C $0.8646216 .371920-0.930320$

C $-0.5915854 .765910-2.147260$

C $-0.1541723 .357204-1.863827$

N -2.598192 1.9827852 .279909

H $2.350123-4.3775790 .274470$

H $-0.274283-3.3604791 .482443$

H $\quad 0.708464-3.898497-1.408144$

H -1.983764 -4.695743 -0.162248

H $-0.701454-5.832662-0.479733$

H $-3.029462-4.916060-3.783867$

H -1.042432 $-5.981156-4.157856$

H $\quad 0.283907-5.213197-3.283491$

H $-0.365266-6.773781-2.733761$

H -4.821204 -4.597415 -2.093171

H -3.700195 -3.905839 -0.940591

H -3.224283 -2.037980 -2.603877

H $-4.488627-2.699838-3.646527$

H $-6.136525-2.432607-1.763447$

H -5.886940 -0.597019-3.468873

H $-4.5646260 .085220-2.547923$

H $-6.660067 \quad 1.708420-3.043973$

H $-5.0907901 .036052-0.127385$

H -8.233280 -0.017533 -2.834037

H -8.620293 $0.551283-1.226585$

H $-8.089209-1.125324-1.467400$

H -7.124469 1.9203660 .322901

H $-5.675954-0.5817062 .561799$

H -4.724329 -1.9672582 .093223$

H $-6.676035-2.4577601 .211166$

H $-1.900655-0.368215-1.358730$

H $0.8431491 .003503-1.584843$

H $-2.8265501 .780313-0.856562$

H -2.786873 $1.729077-2.635563$

H -2.849010 $3.265470-1.776176$

H -2.993705 -0.6337932 .621978$

H -3.113366 -0.1747850 .920286$

H -1.554791 3.4526713 .282145

H -3.3131743 .6483913 .330629$

H -3.3517804 .2716290 .873489$

H -0.5132185 .0059241 .764175$

H -1.309276 6.9880360 .395231

H -2.379489 $5.969514-0.531470$

H $1.6501666 .213070-1.661696$

H $1.0626757 .079893-0.132475$

H $-0.0458965 .099602-3.040946$

H -1.644463 $4.835258-2.435421$

H $0.9302953 .246496-1.822138$

H -1.774935 1.5201321 .904864

C $1.677858-2.8870303 .375400$

O $1.831321-1.5651752 .816851$

C $2.317016-0.6792043 .841064$

C $2.081908-1.4048215 .159064$

C $2.350564-2.8406824 .749160$

H $3.398461-0.5838813 .687821$

C $1.6523390 .696340 \quad 3.722632$

H $2.746299-1.0519105 .953340$

H $1.047431-1.2957605 .504925$

C $1.814717-3.8655265 .733700$

H $3.434949-2.9790404 .643199$

C $1.802614 \quad 1.3476772 .332271$

O $\quad 0.251705 \quad 0.542131 \quad 3.984917$

H 2.0426451 .3609754 .502385

H 1.2003770 .7977301 .597849

O 1.2531822 .6678842 .400499

C 3.2489751 .4602561 .814098

C 3.3132252 .1957790 .463081

H $3.6743070 .458301 \quad 1.699600$

O 4.0589042 .1765102 .744174

H $2.5832001 .743733-0.213692$

H 3.0209823 .2441310 .600778

H $2.290654-3.7439166 .712219$

H $\quad 0.733030-3.763145 \quad 5.871307$

H $2.014952-4.8833035 .384464$

C $2.307865-3.9103802 .424797$

H -0.1276431 .4314884 .083546$

H 0.2877392 .5756072 .445808

H 4.1412981 .6365683 .547595
H $2.309910-4.9064942 .881689$

H $3.356695-3.6267402 .264967$

H $\quad 0.602757-3.0663503 .495049$

C $4.598445-0.023406-1.177445$

O $5.2558920 .839549-0.245488$

C $4.7188252 .164994-0.178262$

C $4.7883692 .829853-1.561610$

C $4.1249741 .976205-2.641556$

C $4.6559890 .543066-2.600674$

H $3.549325-0.128282-0.877077$

C $5.198641-1.431113-1.050185$

H 5.3824712 .7293280 .488782

O $4.1727934 .113886-1.541989$

H $5.8399362 .980419-1.838560$

H $3.0332931 .990346-2.551254$

O $4.4243432 .527240-3.926272$

H $5.6821230 .532981-2.985595$

O $3.870753-0.276694-3.463239$

C $6.662953-1.524210-1.427878$

H $5.122440-1.769658-0.009939$

H $4.628508-2.122598-1.681589$

H $4.6168894 .640274-0.855367$

H $4.1136113 .448970-3.924311$

H $3.8957950 .126048-4.348182$

O $6.954461-2.815548-1.765473$

O $7.459979-0.596678-1.397921$

C $8.315693-3.063872-2.130754$

H $8.588391-2.466352-3.005900$

H $8.979546-2.843486-1.289195$

H $8.411682-4.122687-2.385280$

SCF Energy $(B 3 L Y P / 6-31 G * * / / M M F F)=-3245.89955595$

02 51

MM̄MF Geometry

C $-4.805905-0.7514692 .508092$

C $-3.556286-1.0259022 .105989$

C $-2.321491-0.6134652 .858711$

O $-1.487698 \quad 0.177148 \quad 1.981345$

C -1.516299-1.861094 3.261671

C $-0.402292-1.5416734 .236383$

C $0.897112-1.4099403 .907649$

C $-0.832688-1.4021885 .674912$

C $1.536187-1.5007512 .548768$

C $2.533978-2.6663632 .494837$

C $3.389061-2.6575811 .217562$

C $4.347300-3.8525061 .211348$

C $5.339460-3.8036770 .038750$

O $4.132889-1.4365661 .204504$

O $6.387758-4.7474490 .307403$

C $5.993674-2.405626-0.009424$

C $4.686247-4.245285-1.275813$

O $6.813673-2.264898-1.167065$

C $4.969912-1.2525550 .056142$

C 5.6870220 .1113150 .213314

O $4.215193-1.236640-1.156960$

C -1.7360681 .5121051 .905185$

O -2.6239212 .1319622 .467664$

C -0.7178162 .0941741 .002381$

C -0.7679433 .3942540 .675012$

C $0.1593664 .092131-0.221964$

C $1.2503103 .270271-0.858940$

C $4.726427 \quad 1.245474 \quad 0.596133$

C 5.3834172 .6138370 .612619

C 4.8544694 .9788841 .198123

O $6.5438012 .816806 \quad 0.270771$

C 3.6021015 .7856591 .323472

C 3.2666406 .7929280 .503739

C 1.9863307 .5681930 .641868

C $1.1798117 .621601-0.639380$

C $0.8147828 .803493-1.164950$

C $0.7940646 .323911-1.323399$

C $-0.0209645 .411679-0.445244$

N 4.4871873 .5848031 .039804

H $-4.962088-0.1872563 .423973$

H -3.407495 -1.577153 1.182791

H -2.581046 -0.0279083 .749555$

H -1.110101 -2.347940 2.368058

H $-2.176238-2.605327 \quad 3.727825$ 
H $1.601140-1.1852254 .708865$ H $0.011599-1.2116626 .345989$ H -1.535979 -0.571621 5.789072 H -1.321097 -2.321255 6.015202 H $\quad 0.802276-1.6092201 .745159$ H $2.051443-0.5499732 .366934$ H $3.199668-2.6024083 .366312$ H $1.989135-3.6158522 .570223$ H $2.723905-2.6868240 .346719$ H $3.788395-4.7966601 .204586$ H $4.925848-3.8568712 .146198$ H $5.981065-5.6241770 .416482$ H $6.663636-2.3246010 .857979$ H $4.317703-5.274978-1.194216$ H $5.411924-4.250915-2.096473$ H $3.842033-3.617282-1.564628$ H $7.445562-3.004267-1.165930$ H 6.4466990 .0421531 .002511 H $6.205913 \quad 0.368724-0.718417$ H $3.968583-0.324539-1.375070$ H $\quad 0.046291 \quad 1.426186 \quad 0.621039$ H -1.564441 4.0068941 .095275 H $1.8727142 .799822-0.091760$ H $1.9231783 .844582-1.497452$ H $0.8180912 .483866-1.487853$ H $3.8892491 .295036-0.108083$ H 4.3164421 .0590531 .596198 H 5.4740295 .2841270 .348289 H 5.4549035 .0651172 .109392 H 2.9290795 .5205302 .136179 H $3.9411207 .077926-0.300048$ H 2.2494418 .5820630 .969841 H 1.3569567 .1478901 .436274 H $\quad 0.2387798 .862035-2.082683$ H $1.0825549 .741628-0.690160$ H $1.7086225 .852345-1.690800$ H $0.1891136 .534213-2.215011$ H $-0.864413 \quad 5.9058230 .039211$ H 3.6164503 .2732401 .461965 C $-6.501138-0.244020 \quad 0.667017$ O $-5.552820-0.261144-0.419285$ C $-5.2865641 .095835-0.811866$ C $-6.4455961 .917487-0.260406$ C -6.6864491 .2214641 .066261$ H $-4.367811 \quad 1.396762-0.293461$ C $-5.0762651 .195877-2.324747$ H $-6.1916252 .976082-0.151180$ H $-7.3368141 .841901-0.894009$ C -8.0416201 .5213301 .681996$ H -5.9040711 .5431351 .764546$ C $-4.0251000 .220058-2.895568$ O $\quad-6.318977 \quad 0.921721-2.983312$ H $-4.8165552 .230033-2.581733$ H -4.439760 -0.794706 -2.924597 O $-3.8016860 .579934-4.265266$ C $-2.682506 \quad 0.188502-2.143107$ C -1.581273 -0.577261 -2.901368 H $-2.837626-0.270544-1.160927$ O $\quad-2.2283901 .523446-1.912517$ H -1.999934 -1.502804 -3.306673 H -1.251863 0.034327 -3.749109 H -8.140067 2.5912921 .892461 H $-8.859716 \quad 1.2343121 .012814$ H -8.1685720 .9774382 .623269$ C $-6.037200-1.1987111 .769645$ H $-6.1634930 .998354-3.940442$ H -3.452887 $1.487010-4.283601$ H $-2.0535691 .937073-2.774457$ H -5.847166 -2.185978 1.328786 H -6.841996-1.334632 2.501711 H $-7.435952-0.6388800 .247979$ C $-0.762565-3.233932-1.561188$ O $-0.668145-1.904333-1.029975$ C $-0.371306-0.878627-1.987593$ C $0.929697-1.203779-2.729776$ C $0.895523-2.589985-3.357271$ C $0.520995-3.628095-2.303792$ $\mathrm{H}-1.613836-3.298246-2.248036$
C $-1.013479-4.187227-0.380892$

H $-0.1732670 .028975-1.405997$

O $1.207423-0.235608-3.735125$

H $1.761009-1.170299-2.016785$

H $\quad 0.211289-2.629485-4.212921$

O $2.193684-2.912291-3.860483$

H $1.346972-3.745052-1.591408$

O $0.326121-4.888714-2.942359$

C -2.453294-4.171504 0.086954

H $-0.793854-5.222363-0.665175$

H $-0.377362-3.9023890 .465462$

H $1.2392060 .634569-3.302070$

H $2.440924-2.213764-4.490624$

H $1.146049-5.100806-3.420775$

O $-2.547568-4.902396 \quad 1.237825$

O $-3.379491-3.617448-0.488294$

C -3.862658 -5.030565 1.787515

H $-4.198833-4.0674642 .179477$

H -4.561861 -5.4074631 .034816$

H $-3.817397-5.7476862 .611393$

SCF Energy $(B 3 L Y P / 6-31 G * * / / M M F F)=-3245.91384754$

02 52

MMFF Geometry

C $2.707556 \quad 1.4684253 .458857$

C 1.4239111 .2911613 .111405

C 0.6120850 .0899993 .517952

O $-0.011789-0.4090432 .309693$

C -0.4945710 .4889064 .507326$

C $-1.053355-0.7043795 .258263$

C $-2.237521-1.2966205 .010993$

C -0.188251-1.211064 6.386603

C $-3.260539-0.9449993 .969552$

C -3.361212 -2.044331 2.906444

C $-4.550662-1.8296551 .961352$

C $-4.579656-2.9125300 .880728$

C $-5.714529-2.684439-0.128436$

O $-4.430652-0.5393241 .355333$

O $-5.467677-3.523443-1.267270$

C $-5.659372-1.224365-0.623166$

C $-7.074909-3.1089860 .437098$

O $-6.784734-0.922164-1.445009$

C $-5.540194-0.1852880 .518199$

C $-5.2648391 .211583-0.079183$

O $-6.766664-0.146146 \quad 1.249144$

C $0.555952-1.4723881 .685809$

O $1.464169-2.1715032 .113123$

C $-0.062121-1.7192590 .360767$

C $-0.766297-0.790574-0.306160$

C $-1.353984-0.935051-1.642623$

C $-1.235813-2.275538-2.316981$

C $-4.9469672 .304613 \quad 0.951502$

C -4.5870103 .5834490 .221460$

C $-2.7931044 .511509-1.235488$

O -5.4168504 .4644390 .010081$

C $-3.0240763 .864862-2.566842$

C $-2.0451183 .359897-3.332002$

C $-2.2975162 .599112-4.601649$

C $-1.8872641 .143692-4.500723$

C $-0.9313680 .636102-5.296886$

C $-2.6411550 .251323-3.535758$

C -1.957934 $0.137009-2.197745$

N $-3.2726683 .588318-0.222482$

H 3.1727520 .7265854 .105631

H 0.9229342 .0180092 .478347

H $1.262063-0.674423 \quad 3.959114$

H -1.287990 1.0286513 .979892

H $-0.101974 \quad 1.1945075 .252107$

H -2.522688 -2.142199 5.636739

H $-0.660170-2.0316896 .937557$

H $\quad 0.769049-1.5804456 .007167$

H $0.008308-0.4070197 .103472$

H $-4.227528-0.8287814 .474364$

H -3.0476850 .0141553 .490630$

H -2.429134 -2.054577 2.329384

H $-3.455898-3.0273843 .384909$

H -5.468385 -1.861470 2.560772

H -4.659782 -3.910213 1.329992 
H $-3.625984-2.9065080 .334180$ H -5.426062 -4.444440 -0.957458 H $-4.773865-1.132336-1.267022$ H -7.063786 -4.1730520 .702413$ H $-7.866826-2.996333-0.311578$ H $-7.361365-2.5498191 .330260$ H $-6.811504-1.586173-2.155169$ H $-4.4165431 .137991-0.769568$ H $-6.1285971 .539781-0.671950$ H -6.8306950 .7015991 .715771$ H $0.150656-2.693760-0.066452$ H -0.9113550 .1854670 .154281$ H -1.703895 -3.056031-1.706827 H - $0.183356-2.533133-2.473016$ H -1.712236 -2.316771 -3.298150 H -5.8043572 .5133301 .599166$ H -4.1129512 .0138271 .599900$ H -1.732052 4.694367 -1.039977 H -3.336592 $5.459164-1.173490$ H -4.058234 $3.759504-2.887350$ H -1.012658 $3.458869-3.009078$ H -1.750620 $3.102095-5.409102$ H -3.357176 2.644015 -4.883413 H $-0.653600-0.411580-5.245427$ H $-0.4013941 .256259-6.011956$ H $-2.808253-0.726839-3.996791$ H $-3.6504260 .652242-3.376501$ H -1.984186 $1.058345-1.617393$ H -2.697906 $2.770880-0.038908$ C 3.1768053 .3971371 .800744 O 3.0286672 .5233040 .663909 C $3.6090563 .150437-0.492431$ C $3.8754984 .598360-0.097439$ C 4.1985114 .4530631 .376811 H $4.5682052 .650915-0.672994$ C $2.6925552 .972847-1.707137$ H $4.6915525 .042273-0.675600$ H $2.9879745 .227619-0.229923$ C 4.1014995 .7550022 .152692 H 5.2167874 .0529651 .473244 C $2.2958041 .513717-2.009282$ O $1.4776893 .704704-1.491348$ H $3.1605403 .424198-2.589457$ H $1.6102311 .140656-1.238991$ O $1.5587451 .515014-3.237730$ C $3.4738250 .535011-2.158627$ C $3.014093-0.864973-2.608569$ H $3.9984110 .454616-1.203119$ O $4.4072251 .048501-3.108209$ H $2.223872-1.208285-1.933412$ H $2.575502-0.794803-3.611188$ H 4.8115336 .4902031 .760089 H 3.0977926 .1881282 .086082 H 4.3304195 .5963163 .211156 C 3.6221602 .5823093 .018961 H $1.0700073 .363117-0.676822$ H $0.8064622 .119428-3.122211$ H $3.9363621 .160077-3.952001$ H 3.7899013 .2460743 .874692 H 4.5866072 .1152662 .782782 H 2.2009443 .8649541 .981552 C $4.074058-2.651833-0.366216$ O $4.839539-2.024538-1.401740$ C $4.166412-1.893437-2.657715$ C $3.748302-3.278139-3.172430$ C $2.918883-4.040411-2.143319$ C $3.629128-4.058506-0.790540$ H $3.198589-2.035730-0.132700$ C $4.963895-2.7261250 .884677$ H $4.910566-1.501911-3.362549$ O $3.007386-3.169744-4.383774$ H $4.647052-3.867294-3.397217$ H $1.911450-3.619052-2.059662$ O $2.757654-5.382645-2.608260$ H $4.497747-4.727534-0.843901$ O $2.744640-4.5807130 .199062$ C $5.216460-1.3672181 .500841$ H $4.498398-3.3451821 .659120$
H $5.935038-3.1570180 .612312$

H $3.565265-2.698295-5.025851$

H $2.137615-5.829074-2.007559$

H $2.569280-5.510533-0.022911$

O $6.256510-1.4677982 .381001$

O $4.572814-0.3548691 .265003$

C $6.613009-0.2593573 .059275$

H $7.529917-0.445133 \quad 3.624830$

H 6.8022120 .5453182 .342203

H 5.8202930 .0222063 .758048

SCF Energy $\left(B 3 L Y P / 6-31 G^{* *} / / M M F F\right)=-3245.91066753$

$02 \_53$

MMFF Geometry

C -0.8459503 .6958321 .839949$

C -0.7872312 .3583191 .923401$

C -0.3083941 .6264363 .147567$

O 0.8223690 .8251132 .733995

C $-1.381817 \quad 0.672417 \quad 3.701415$

C -2.6239931 .3719094 .205254$

C -3.7979031 .4182763 .545911$

C -2.4879752 .0242045 .556607$

C -4.1243420 .8517562 .191138$

C $-5.170316-0.2626582 .301901$

C $-5.642894-0.7521810 .925050$

C $-6.784954-1.7590311 .086973$

C -7.236131-2.335852 -0.261852

O $-4.535361-1.3615840 .251427$

O $-8.100242-3.453017-0.003444$

C $-5.995289-2.850072-1.022098$

C $-8.094096-1.337272-1.046062$

O $-6.343497-3.259325-2.343200$

C $-4.834016-1.827449-1.071607$

C $-3.548556-2.444254-1.679336$

O $-5.219570-0.739903-1.922709$

C 1.8166770 .6170653 .637192

O 1.9162641 .0959294 .754746

C $2.754405-0.3501313 .031612$

C $3.799259-0.8141913 .731709$

C $4.712120-1.8279603 .218616$

C $6.160411-1.4361263 .265737$

C $-2.872270-3.532669-0.842791$

C $-1.843665-2.9768200 .123796$

C $0.505854-2.1437490 .212045$

O $-2.061609-2.904451 \quad 1.330845$

C $1.396638-3.3258320 .439396$

C $2.614683-3.462624-0.106820$

C $3.505678-4.6525270 .122470$

C $4.856679-4.2787350 .696133$

C $5.925732-4.133049-0.104425$

C $4.994140-4.1651242 .199594$

C $4.232271-3.0036192 .767399$

N $-0.672936-2.585060-0.508632$

H -0.5232254 .2766332 .702506$

H -1.0895511 .7408041 .083150$

H 0.0054092 .3411753 .918336

H $-0.950890 \quad 0.0859534 .524521$

H -1.623809 -0.0886672 .949206$

H -4.6268201 .9456534 .017309$

H -3.4254182 .4771465 .896472$

H -2.1895371 .2845616 .306720$

H -1.732434 2.8154155 .527366

H -4.5196381 .6679351 .573707$

H $-3.2372570 .479673 \quad 1.671790$

H -4.733283 -1.100647 2.861007

H -6.0340510 .0998692 .873776$

H -5.9805800 .1167720 .348004$

H -7.635213 -1.305445 1.611535

H $-6.447354-2.5869151 .726407$

H -7.607310 -4.104220 0.524118

H $-5.655196-3.752988-0.502621$

H -8.996888 -1.082616 -0.477655

H -8.447314 -1.772783-1.987405

H -7.573472 -0.404645 -1.272303

H -7.073745 -3.897171 -2.266768

H $-3.786110-2.849345-2.671291$

H -2.826375 -1.640024 -1.863672

H $-4.417525-0.279458-2.219914$ 
H $2.548362-0.6955562 .023700$ H $4.014958-0.4448484 .730805$ H $6.305399-0.4429962 .825162$ H $6.806755-2.1213882 .711783$ H $6.513250-1.4050484 .302183$ H -3.583065 -4.143853 -0.280375 H $-2.347557-4.224831-1.514336$ H $0.212712-1.7059181 .171255$ H $\quad 0.991379-1.370897-0.391115$ H $1.021759-4.101708 \quad 1.102989$ H $2.985567-2.688154-0.773147$ H $3.638119-5.158169-0.843133$ H $3.026071-5.3917330 .776228$ H $6.905084-3.8938800 .296418$ H $5.843465-4.242691-1.180833$ H $6.049244-4.1232022 .491345$ H $4.613670-5.0858232 .660335$ H $3.157124-3.1730872 .827887$ H $-0.575564-2.747320-1.506854$ C $-1.9755413 .790130-0.491392$ O $-1.0161412 .958458-1.170286$ C -1.266536 $3.012583-2.585486$ C $-2.5486973 .818795-2.769458$ C -2.515786 $4.735753-1.564294$ H $-0.4325783 .568569-3.028646$ C $-1.327606 \quad 1.597933-3.171653$ H -2.562237 4.359709 -3.720741 H $-3.4408783 .182833-2.737818$ C $-3.8672835 .346181-1.234442$ H -1.792630 $5.539716-1.755953$ C $-0.0973990 .712704-2.888619$ O $-2.4693120 .916656-2.634589$ H -1.499323 $1.659763-4.252756$ H $-0.0870780 .398812-1.838058$ O $-0.247230-0.486355-3.660736$ C $1.2584881 .353354-3.234981$ C $2.4212290 .340769-3.222313$ H $1.4614092 .160700-2.523924$ O $1.1929781 .946086-4.533822$ H $2.351174-0.276257-2.321642$ H $2.315803-0.326864-4.085461$ H -4.234181 $5.944163-2.075043$ H -4.614156 4.574529-1.019511 H $-3.7991416 .001249-0.360523$ C -1.3056874 .5265500 .671454$ H $-2.3778570 .910862-1.665914$ H -1.103537 -0.880458 -3.421213 H $\quad 0.964788 \quad 1.243919-5.167097$ H -2.005078 5.2722001 .067903 H $-0.428226 \quad 5.071050 \quad 0.300147$ H $-2.7747803 .134064-0.122368$ C $4.3311231 .060704-0.936643$ O $4.0670041 .818362-2.124789$ C $3.7975801 .040355-3.298385$ C $4.9766250 .103643-3.592490$ C $5.329764-0.761366-2.388991$ C $5.5180760 .111628-1.150629$ H $3.4391710 .489634-0.653492$ C 4.6410042 .0555290 .192164 H $3.7491561 .752462-4.131695$ O $4.686141-0.716908-4.718967$ H $5.8516220 .711301-3.858452$ H $4.574212-1.537726-2.227472$ O $6.554162-1.442680-2.671556$ H $6.4453840 .687857-1.260754$ O $5.686135-0.727502-0.011600$ C 3.4155672 .8083210 .663641 H 5.0446451 .5290081 .064178 H $5.3708732 .795457-0.157279$ H $5.449917-1.303413-4.856093$ H $6.808085-1.928051-1.868005$ H $4.857259-1.2172660 .124284$ O 3.8004353 .7042241 .621153 O 2.2750052 .6316390 .260340 C 2.75590944989692 .189325 H 2.1510694 .9663651 .406533 H 2.1371293 .8788402 .843097 H 3.2176025 .2879372 .789053
SCF Energy (B3LYP/6-31G**//MMFF) $=-3245.92118617$

$02 \_54$

MMFF Geometry

C 2.4310832 .7738152 .200062

C 3.6150683 .2105221 .743226

C 3.8595794 .0109160 .484569

O $2.5987004 .454990-0.066438$

C $4.5869953 .159236-0.577166$

C $5.9979042 .763277-0.199501$

C 6.3739111 .5267500 .181426

C $7.0216463 .864209-0.306975$

C 5.5135880 .3051150 .356557

C $5.882210-0.766454-0.675296$

C $5.127536-2.084356-0.447315$

C $5.615341-3.145964-1.436913$

C $4.825781-4.455834-1.314799$

O $3.729263-1.841468-0.628261$

O $5.129373-5.263658-2.462557$

C $3.319577-4.130540-1.377379$

C $5.251815-5.270383-0.087852$

O $2.538164-5.296443-1.121378$

C $2.895447-2.989098-0.423213$

C $1.422754-2.566238-0.653441$

O $2.994940-3.4612820 .925670$

C $2.6090965 .529268-0.898397$

O $3.5823996 .185695-1.239772$

C $1.2557705 .776757-1.441804$

C $0.1483035 .513730-0.731716$

C $-1.2345245 .651015-1.194313$

C $-1.4516896 .000038-2.642917$

C $1.151859-1.846399-1.978267$

C $1.178482-0.338522-1.815222$

C $-0.1881981 .552837-0.944570$

O $2.1684490 .327505-2.107349$

C $-1.5558791 .988375-1.363708$

C $-2.5472342 .252240-0.499138$

C $-3.9029842 .761211-0.900381$

C $-4.3026314 .008644-0.137993$

C $-5.197421 \quad 3.9457170 .862601$

C $-3.7097065 .335751-0.572875$

C $-2.2289185 .401496-0.316469$

N $-0.0077880 .160595-1.300788$

H 1.5170043 .0069511 .661805

H 4.5075922 .9566322 .312618

H 4.4498004 .8934520 .760575

H $4.6313533 .710768-1.525675$

H $3.9730232 .284795-0.823991$

H 7.4254371 .3640290 .416504

H $8.0350153 .513937-0.083199$

H $7.0329574 .274170-1.322272$

H 6.7921224 .6734940 .392776

H $5.682328-0.0839151 .368393$

H $4.4469420 .537294 \quad 0.294245$

H $5.651554-0.380678-1.677193$

H $6.962470-0.957159-0.637938$

H $5.304927-2.4123290 .583732$

H $6.688888-3.333278-1.308552$

H $5.499081-2.767491-2.462332$

H $\quad 6.090515-5.411577-2.479235$

H $3.099366-3.836761-2.410690$

H $6.315836-5.528492-0.151001$

H $4.715289-6.224367-0.041213$

H $5.098098-4.7408630 .854490$

H $2.830984-5.983914-1.743946$

H $0.781930-3.454324-0.584786$

H $1.110108-1.9211330 .177714$

H $2.453987-2.8877221 .491936$

H $1.2237556 .198384-2.440259$

H $\quad 0.259777 \quad 5.136706 \quad 0.284373$

H - $1.0037896 .972967-2.874372$

H -2.504041 $6.073357-2.923580$

H -1.000723 $5.241998-3.292967$

H $1.854568-2.120595-2.769810$

H $\quad 0.157748-2.128380-2.345668$

H $0.5718482 .173907-1.429081$

H $-0.046310 \quad 1.6298500 .137655$

H - $-1.7224592 .131692-2.428469$ 
H -2.3725582 .1084430 .564158$ H -4.627409 $1.960386-0.712437$ H -3.952459 2.965574-1.977041 H -5.5058074 .8345091 .402928$ H $-5.6387403 .002477 \quad 1.167169$ H $-4.1782616 .162134-0.023281$ H -3.968142 5.491221 -1.623337 H $-1.958873 \quad 5.1791990 .717471$ H -0.710806 -0.497613 -0.978658 C 1.7204710 .5460473 .079598 O 0.3092030 .6630342 .809162 C $-0.376444-0.4687483 .391157$ C $0.712094-1.4256283 .852905$ C $1.832203-0.4709164 .212218$ H $-0.927692-0.0732304 .253317$ C -1.343114 -1.042289 2.349302 H $0.393721-2.0494474 .693336$ H $1.020750-2.0879163 .035739$ C $3.191681-1.1425584 .307030$ H 1.5950010 .0064845 .172357 C -1.977124 -2.390121 2.729255 O $-2.378873-0.0786532 .134629$ H $-0.812651-1.1352201 .397152$ H -1.198841 -3.157522 2.813073 O $-2.561099-2.2580434 .030854$ C $-3.077294-2.8726551 .762216$ C $-2.618352-2.955451 \quad 0.296833$ H $-3.954843-2.2258381 .853720$ O $-3.500194-4.1721202 .191204$ H -2.279972 -1.970856 -0.035255 H -1.757238 -3.632017 0.235916 H $3.183939-1.9185965 .079372$ H $3.474638-1.6133623 .359769$ H $3.969421-0.4173694 .565743$ C 2.2889941 .9220083 .432401 H $-2.898706-0.0164252 .954295$ H -2.965727 -3.1137014 .255066$ H -2.743934 -4.776716 2.099090 H 3.2609801 .8123493 .926583 H 1.6210572 .4418794 .129629 H $2.190596 \quad 0.1534262 .167473$ C -4.822115 -1.360633-1.110135 O $-4.917484-2.684319-0.569536$ C $-3.721518-3.465947-0.656875$ C $-3.277751-3.589111-2.120635$ C $-3.149966-2.226723-2.796998$ C $-4.422246-1.406296-2.591146$ H $-4.082187-0.781963-0.544487$ C $-6.199092-0.697908-0.940006$ H -3.990250 -4.474943 -0.320217 O $-2.041958-4.292333-2.200356$ H -4.021098 -4.184897-2.666399 H $-2.266521-1.689438-2.436010$ O $-2.946638-2.444877-4.194636$ H -5.236403 -1.835095 -3.189482 O $-4.202832-0.074522-3.051578$ C $-6.478123-0.2945280 .492976$ H $-6.2724050 .213484-1.542877$ H $-6.982143-1.399781-1.251387$ H $-1.785965-4.325538-3.138178$ H $-2.757424-1.582886-4.602010$ H -4.066839 -0.113120 -4.013042 O $-7.804645 \quad 0.0094640 .616470$ O $-5.643465-0.2227441 .383861$ C -8.2188540 .4343451 .918758$ H $-9.2928040 .635276 \quad 1.885209$ H -8.032213 -0.3555382 .652599$ $\mathrm{H}-7.6981121 .3547042 .199900$ SCF Energy (B3LYP/6-31G**//MMFF)= -3245.91026115

\section{$02 \_55$}

MM̄FF Geometry

C -1.727189 2.9072692 .123763

C -1.8917291 .5757072 .156966$ C $-1.804841 \quad 0.7724683 .430278$ O $-0.954639-0.379853 \quad 3.207803$ C -3.172624 0.1973503 .853299 C -4.2831371 .2087554 .054699$
C $-5.425767 \quad 1.2334493 .337861$

C -4.0609052 .2114225 .156612$

C -5.8365920 .2678522 .259870$

C $-5.4519800 .771066 \quad 0.863278$

C $-5.367823-0.333335-0.202937$

C $-6.712025-1.027992-0.420171$

C $-6.600156-2.164423-1.443710$

O $-4.374440-1.2744600 .216359$

O $-7.800339-2.949581-1.365961$

C $-5.434263-3.087078-1.031471$

C $-6.525893-1.630967-2.878979$

O $-5.197174-4.076867-2.030974$

C $-4.114507-2.342030-0.708053$

C $-3.133136-3.3095430 .000091$

O $-3.476895-1.863648-1.890577$

C $0.391624-0.2033353 .201751$

O $1.0025140 .844978 \quad 3.325352$

C $0.994872-1.5422833 .015741$

C $2.310417-1.6632972 .789096$

C $3.036907-2.9191452 .586632$

C $2.253154-4.2028282 .685843$

C $-1.711101-2.7591700 .192363$

C $-0.770186-3.106852-0.953774$

C $1.634647-2.972134-1.604277$

O $-1.142441-3.653779-1.988673$

C $2.598852-3.943147-1.001728$

C $3.918652-3.711821-0.923509$

C $4.910190-4.690898-0.363721$

C $5.817407-4.0761460 .679160$

C $7.062506-3.6811340 .364160$

C 5.313521-4.004495 2.102926

C $4.358546-2.8645522 .319350$

N $0.539787-2.736706-0.680613$

H -1.496949 3.4140723 .059744

H $-2.123842 \quad 1.0251281 .250592$

H $-1.3860801 .361304 \quad 4.256231$

H -3.039849 -0.3604134 .790887$

H -3.454491 -0.5737653 .127400$

H -6.1570652 .0109283 .552334$

H -4.9330642 .8549085 .313862$

H $-3.8603161 .695566 \quad 6.101431$

H -3.2112542 .8609644 .928249$

H -5.423202 -0.7259252 .456268$

H -6.9248460 .1399902 .314487$

H -6.1615891 .5439330 .543060$

H $-4.470498 \quad 1.2502870 .918073$

H -5.027742 $0.137763-1.132960$

H -7.478150 -0.305202 -0.727182

H -7.068891 -1.460696 0.523883

H -8.555244 -2.361911-1.541212

H $-5.750190-3.627736-0.127956$

H -7.436304 -1.071858 -3.127008

H -6.471666 -2.450562 -3.603703

H -5.677672 $-0.963337-3.045386$

H -6.039208 -4.537926 -2.187656

H $-3.537565-3.5334700 .996472$

H -3.080698 -4.261205 -0.542036

H -3.983748 -2.159626 -2.663222

H $\quad 0.331269-2.3985173 .064915$

H $2.912751-0.7583792 .743686$

H $1.857383-4.3312813 .699354$

H $2.840877-5.0937122 .455769$

H $1.414680-4.2021751 .980803$

H - $-1.717673-1.6732310 .335871$

H -1.289657 -3.215183 1.097197

H $2.105884-2.005837-1.794054$

H $1.253764-3.366280-2.551299$

H $2.198737-4.886969-0.639636$

H $4.316419-2.778966-1.316770$

H $5.510540-5.069675-1.201404$

H $4.409542-5.5692510 .062020$

H $7.730310-3.2662831 .111931$

H $7.439261-3.756027-0.650567$

H $6.155510-3.8559092 .792622$

H $4.895236-4.9740852 .387344$

H $4.836284-1.8858532 .263645$

H $\quad 0.749581-2.2623850 .192337$

C $-2.1787173 .209696-0.416046$ 
O $-1.0253852 .501320-0.910524$

C $-0.877505 \quad 2.780682-2.315404$

C $-2.1543703 .491067-2.747413$

C $-2.514308 \quad 4.249271-1.486987$

H $-0.0398703 .480477-2.412529$

C $-0.576294 \quad 1.496826-3.091610$

H -1.994253 4.142671-3.611859

H -2.949118 $2.780942-3.003835$

C $-3.9573834 .723705-1.460142$

H -1.846481 $5.116193-1.393624$

C $0.6634520 .722991-2.604427$

O $-1.6998840 .611179-2.992512$

H $-0.4724601 .730038-4.157774$

H $0.4668580 .263850-1.627948$

O $0.875836-0.354477-3.526240$

C $1.956397 \quad 1.556629-2.525854$

C $3.1873140 .703510-2.162084$

H $1.8323912 .341822-1.773508$

O $2.1847932 .203485-3.777036$

H $2.9855820 .186176-1.217969$

H $3.335387-0.065783-2.929413$

H $-4.1538205 .403793-2.295556$

H -4.657271 $3.884782-1.537902$

H $-4.176707 \quad 5.259422-0.531371$

C -1.863175 $3.834196 \quad 0.944632$

H $-1.8504680 .430454-2.048479$

H $0.062092-0.887344-3.540839$

H $2.2676611 .510571-4.454641$

H -2.650631 $4.552871 \quad 1.201444$

H $-0.927015 \quad 4.3993510 .869533$

H $-2.9959742 .484401-0.337566$

C $4.254846 \quad 2.301327 \quad 0.217449$

O $4.3567172 .642314-1.170245$

C $4.4904021 .526566-2.056083$

C $5.731536 \quad 0.700685-1.685385$

C $5.7416310 .309304-0.209532$

C 5.5050501 .5378850 .667971

H 3.3597631 .6888020 .381914

C 4.0916653 .6064861 .001157

H $4.6759311 .949381-3.051620$

O $5.810058-0.464181-2.500325$

H $6.627653 \quad 1.298875-1.895879$

H $5.005973-0.477507-0.007167$

$\begin{array}{llll}\text { O } & 7.021456 & -0.245620 & 0.099161\end{array}$

H 6.3863752 .1891900 .616056

O $5.372404 \quad 1.1263352 .025785$

C $2.719753 \quad 4.190774 \quad 0.765233$

H 4.1907303 .4532972 .081563

H 4.8528564 .3394870 .707817

H $6.588493-0.970361-2.210557$

H $7.021691-0.4608621 .047747$

H $4.569360 \quad 0.583915 \quad 2.094437$

O $2.8037235 .265408-0.068797$

O 1.6943823 .7282261 .248275

C $1.5603635 .898681-0.384451$

H $0.905248 \quad 5.200997-0.913003$

H $1.078786 \quad 6.266704 \quad 0.526427$

H $1.7680686 .748826-1.039583$

SCF Energy (B3LYP/6-31G**//MMFF)= -3245.92388781

\section{2_56}

MMFF Geometry

C $-1.987651 \quad 4.325822 \quad 0.448605$

C $-3.080704 \quad 3.551774 \quad 0.501122$

C $-3.677503 \quad 3.0074961 .772551$

O $\begin{array}{llll}\text { O } & -3.527489 & 1.567576 & 1.727973\end{array}$

C $-5.176012 \quad 3.3526751 .829376$

C -5.9074982 .7234742 .999336$

C -6.7853041 .7043082 .896579$

C $-5.608357 \quad 3.330043 \quad 4.345602$

C -7.203362 0.9788801 .646940

C $-6.610099-0.4348881 .600582$

C $-6.560085-0.990528 \quad 0.169263$

C $-6.102747-2.4501310 .178007$

C $-5.892785-3.003210-1.239063$

O $-5.630597-0.186911-0.568708$

O $-5.159092-4.232972-1.127987$

C $-5.006513-2.021284-2.035091$
C $-7.223018-3.351985-1.916729$

O $-4.909896-2.418251-3.400581$

C $-5.478877-0.556387-1.946065$

C $-4.4823490 .441753-2.590035$

O $-6.719518-0.421574-2.650425$

C $-2.3314301 .055925 \quad 2.135705$

O $-1.389487 \quad 1.6733752 .606974$

C $-2.346581-0.3997391 .866639$

C $-1.232103-1.1252562 .045500$

C -1.084386 -2.559364 1.782435

C $-2.264831-3.2834881 .190633$

C $-3.0887450 .492175-1.954759$

C $-2.060807-0.411605-2.612537$

C $0.259704-1.265321-2.287058$

O $-2.239782-0.959343-3.696603$

C $0.158677-2.658179-1.749235$

C $1.166259-3.282787-1.120689$

C $1.086817-4.695882-0.616649$

C $1.508680-4.8302670 .829912$

C $2.751366-5.2298891 .148179$

C $0.465695-4.6027691 .901116$

C $0.095419-3.1533242 .060345$

N $-0.895216-0.497093-1.860451$

H $-1.496665 \quad 4.6303351 .369233$

H $-3.5444913 .232325-0.429556$

H -3.1731343 .4084972 .660491$

H $-5.645616 \quad 3.0666910 .883353$

H -5.2987204 .4428691 .887174$

H $-7.253471 \quad 1.333325 \quad 3.807374$

H $-6.2126562 .887611 \quad 5.144688$

H -4.5564063 .1826174 .608995$

H $-5.817904 \quad 4.4046274 .332701$

H -8.2982790 .9111951 .632626$

H $-6.938343 \quad 1.537722 \quad 0.747346$

H $-5.592634-0.4175502 .009501$

H $-7.206021-1.0955012 .242529$

H $-7.555841-0.900501-0.280446$

H -6.812472 $-3.075800 \quad 0.733595$

H $-5.152479-2.5272100 .721859$

H $-5.670866-4.837397-0.563445$

H $-3.996203-2.093894-1.615455$

H $-7.757558-4.116037-1.339457$

H $-7.059516-3.787223-2.908785$

H $-7.889507-2.493365-2.020697$

H $-4.587984-3.335943-3.413420$

H $-4.4265230 .274263-3.672432$

H $-4.9136291 .446816-2.474250$

H $-6.8408630 .513020-2.886270$

H -3.269534 -0.8317721 .499155$

H $-0.337984-0.6242442 .413844$

H $-2.599570-2.7860820 .274119$

H $-3.095083-3.3075651 .904318$

H $-2.051500-4.3162400 .908669$

H $-2.698403 \quad 1.512077-2.063017$

H $-3.1394430 .273064-0.885352$

H $1.150577-0.743531-1.922824$

H $0.290504-1.293814-3.380954$

H $-0.777849-3.188189-1.906972$

H $2.114383-2.765094-0.994477$

H $1.738615-5.310635-1.251521$

H $0.078669-5.110341-0.741106$

H $3.056007-5.3656042 .180442$

H $3.494902-5.4260910 .382607$

H $0.842311-4.9465312 .873983$

H $-0.397434-5.2430461 .698591$

H $0.897152-2.5442802 .478987$

H $-0.855434-0.047601-0.950382$

C $-0.0772863 .906926-1.077633$

O $0.9609264 .350200-0.182352$

C $2.2362694 .219938-0.852996$

C $1.9419953 .570062-2.198691$

C $0.5259494 .041096-2.471146$

H $2.6044025 .243369-0.996090$

$\begin{array}{llll}\text { C } 3.177310 & 3.434662 & 0.067305\end{array}$

H $2.6525803 .872136-2.973852$

H $1.9701242 .475854-2.119916$

C $-0.1818293 .228303-3.541130$

H $0.5524935 .097178-2.770828$ 
C $4.4841792 .969690-0.595174$

O $3.498716 \quad 4.275605 \quad 1.181561$

H 2.6397402 .5727730 .478066

H $4.2624912 .255906-1.396650$

O $5.0980464 .099313-1.228336$

C 5.5113482 .3584780 .378415

C 4.9751931 .2693781 .325718

H 5.9425683 .1595420 .990867

O $6.6052131 .838768-0.381078$

H 5.8168620 .9230801 .936003

H 4.2662021 .7339322 .019722

H $0.3453223 .311140-4.497307$

H $-0.2268602 .167418-3.274925$

H -1.206655 $3.581864-3.690193$

C -1.341035 4.734521 -0.846799

H 2.6594164 .5496381 .590000

H $5.9229473 .788397-1.639450$

H $6.2535761 .160868-0.982722$

H -2.043239 4.597133 -1.676746

H -1.101469 $5.802787-0.791387$

H $-0.2666872 .847868-0.851838$

C $6.233338-1.3972010 .453829$

O $5.200670-0.663698-0.216718$

C $4.3072750 .054966 \quad 0.638341$

C $3.628784-0.9146251 .617701$

C $4.652214-1.7237752 .407895$

C $5.637883-2.3973571 .454203$

H $6.899188-0.6982150 .973172$

C $7.049298-2.126360-0.620061$

H $3.5159770 .443443-0.012254$

O $2.778651-0.2188072 .522785$

H $2.995472-1.6128981 .057075$

H $5.175377-1.1013853 .143141$

O $3.977062-2.7393093 .152043$

H $5.120555-3.2008420 .916119$

O $6.680879-3.0104952 .209552$

C $7.846130-1.151440-1.452815$

H $7.746794-2.852433-0.187263$

H $6.392155-2.679855-1.301967$

H $2.1254810 .269812 \quad 1.993279$

H $3.316559-2.2970643 .712495$

H $7.149966-2.3103632 .694864$

O $8.979205-0.787416-0.786798$

O $7.493721-0.749265-2.553944$

C $9.8157230 .153893-1.466245$

H $9.2710721 .085714-1.646075$

H $10.6753740 .368523-0.826087$

H $10.176184-0.271194-2.407745$

SCF Energy $\left(B 3 L Y P / 6-31 G^{* *} / / M M F F\right)=-3245.91179003$

0257

MMFF Geometry

C $-2.6197701 .484642-2.410329$

C $-2.9101110 .458203-3.223478$

C $-2.938615-0.972383-2.761826$

O $-1.759408-1.604592-3.309924$

C -4.186582 -1.727987 -3.254706

C $-5.502202-1.192299-2.734664$

C $-5.941294-1.360835-1.472549$

C $-6.346028-0.457222-3.741863$

C $-5.231457-2.066836-0.351388$

C $-4.880078-1.0796250 .767556$

C $-4.088959-1.7496231 .898758$

C $-3.988937-0.8074363 .100592$

C $-3.135038-1.4030454 .226799$

O $-2.779498-2.0647621 .405588$

O $-2.842831-0.3533985 .162033$

C -1.791495 -1.864498 3.629609

C $-3.887711-2.4838755 .010868$

O $-1.011518-2.5519784 .605415$

C -1.939757 -2.7324592 .357399$

C $-0.565871-3.0118421 .698777$

O $-2.493614-4.0011332 .728575$

C $-1.265480-2.677427-2.635033$

O $-1.756903-3.227337-1.662676$

C $0.016154-3.049757-3.270065$

C $0.847968-3.883482-2.628554$

C $2.161563-4.323818-3.101083$
C $2.611498-3.837601-4.454671$

C $0.109894-1.8015931 .044001$

C $1.591890-2.0355130 .791155$

C $3.606338-0.844441-0.057936$

O $2.198803-3.0215561 .200852$

C $4.129315-1.760297-1.119358$

C $5.045372-2.710397-0.875120$

C $5.655249-3.601022-1.920416$

C $5.337494-5.063272-1.697434$

C $6.032528-5.796317-0.811070$

C $4.267378-5.691077-2.561468$

C $2.901656-5.118283-2.299179$

N $2.169090-0.9868800 .085915$

H -2.376561 $1.289914-1.369145$

H $-3.1289310 .653375-4.270600$

H $-2.877369-1.002088-1.667241$

H $-4.104497-2.790979-2.992609$

H $-4.195168-1.737617-4.353931$

H $-6.912845-0.947690-1.204676$

H -7.287342 -0.097291 -3.313097

H $-5.8082460 .412291-4.132031$

H $-6.595458-1.116697-4.579660$

H $-5.897717-2.8444640 .041781$

H $-4.332389-2.582720-0.698386$

H $-4.288297-0.2553620 .348831$

H $-5.806891-0.6492211 .167903$

H -4.601193 -2.676521 2.182604

H $-4.986507-0.5459153 .475463$

H -3.5315850 .1394002 .782391$

H $-3.687703 \quad 0.0015145 .487883$

H -1.233556 -0.9578473 .374977$

H -4.794986 -2.065933 5.463800

H -3.283596 -2.859876 5.843807

H $-4.189808-3.3319674 .393029$

H $-0.926533-1.9637195 .375273$

H $\quad 0.093658-3.4783342 .441537$

H $-0.689595-3.7750570 .917896$

H -2.290196 -4.642766 2.028046

H $\quad 0.250221-2.607154-4.231395$

H $\quad 0.548419-4.271152-1.655530$

H $1.924598-4.184307-5.234748$

H $3.608287-4.181491-4.736673$

H $2.646593-2.742610-4.477926$

H $-0.381189-1.5689590 .093117$

H $0.036022-0.9154761 .680290$

H $4.070800-1.0324370 .916482$

H $3.8097680 .192335-0.339424$

H $3.769473-1.606712-2.133291$

H $5.411278-2.8384580 .141685$

H $6.743541-3.456794-1.895076$

H $5.349049-3.298157-2.929012$

H $5.828768-6.851714-0.664745$

H $\quad 6.820377-5.353855-0.210087$

H $4.208406-6.771571-2.374121$

H $4.566200-5.605296-3.609946$

H $2.491910-5.405707-1.329871$

H $1.592309-0.188939-0.164417$

C -1.266806 $3.347081-3.427229$

O $-0.2347653 .262350-2.425710$

C $0.6789994 .361072-2.610203$

C $0.2342945 .091592-3.875114$

C $-1.2511884 .799112-3.899619$

H $0.5403725 .029524-1.752782$

C $2.1276183 .862048-2.660878$

H $0.4608336 .161580-3.833450$

H $0.7169514 .686487-4.772102$

C $-1.8886615 .020260-5.260419$

H $-1.7468795 .441025-3.159010$

C $2.5813033 .058328-1.426826$

O $2.2757823 .011062-3.805905$

H $2.7972604 .715107-2.824032$

H $2.0770882 .084857-1.412145$

O $3.9754602 .766268-1.595740$

C $2.3648293 .761034-0.073361$

C 3.0618733 .0311171 .090994

H 1.2902383 .8232810 .122685

O $2.8516995 .101798-0.138216$

H 2.7996671 .9700691 .050929 
$\begin{array}{llll}\text { H } & 4.147939 & 3.105088 & 0.960297\end{array}$ H -1.768756 $6.061815-5.576351$ H $-1.4330834 .382423-6.025322$ H $-2.9599974 .799046-5.232222$ C $-2.6147452 .920245-2.847845$ H $3.2000052 .709086-3.825359$ H $4.4550323 .610668-1.640225$ H $3.8160835 .066562-0.253589$ H -3.411374 $3.081374-3.583721$ H $-2.8620273 .535164-1.973822$ H $-0.9743482 .674514-4.244776$ C 0.7897142 .2142232 .970308 $\begin{array}{lllll}\text { O } & 1.285572 & 3.537349 & 2.726903\end{array}$ C 2.6913583 .6238932 .468256 C 3.4821783 .0366373 .645394 C 3.0306121 .6198183 .983986 C 1.5136971 .5781724 .164620 H 0.9311991 .5939982 .077584 C $-0.717125 \quad 2.336316 \quad 3.242584$ H 2.9301324 .6939912 .429467 $\begin{array}{lllll}\text { O } & 4.876569 & 3.051772 & 3.358399\end{array}$ H 3.3306303 .6746784 .525918 H 3.3664480 .9069993 .221480 $\begin{array}{lllll}\text { O } & 3.658409 & 1.227852 & 5.206391\end{array}$ H 1.2495402 .1052985 .089934 $\begin{array}{lllll}\text { O } & 1.103228 & 0.222799 & 4.327500\end{array}$ C -1.511512 2.6184051 .984257 H -1.110970 1.4069533 .663973 H -0.8983243 .1582323 .945544$ H 5.3341962 .6539464 .118835 H 3.3257310 .3417555 .431106 H $1.362309-0.2667993 .528536$ O -2.8006792 .9072012 .333101$ O $-1.071944 \quad 2.572558 \quad 0.844010$ C -3.6881413 .1781191 .243840$ H $-4.665243 \quad 3.439241 \quad 1.658515$ H $-3.3206874 .022603 \quad 0.652996$ $\mathrm{H}-3.7992762 .2872640 .619350$

SCF Energy (B3LYP/6-31G**//MMFF) $=-3245.91993952$

\section{$02 \_58$}

MM̄FF Geometry

C $1.109616-3.4091432 .519204$

C $1.006183-2.1136992 .185205$

C $0.586981-1.0252053 .138932$

O $-0.712576-0.5467742 .720021$

C 1.5771520 .1481723 .040296

C 1.4057951 .1649304 .148505

C 0.7204302 .3179844 .025415

C 2.1121460 .8338955 .437196

C -0.0705232 .7899742 .837083$

C 0.6423133 .9294222 .099159

C -0.1440614 .4930630 .901539$

C -1.4143615 .2291851 .339480$

C $-2.237095 \quad 5.725608 \quad 0.142443$

O $-0.456503 \quad 3.4131910 .016738$

O

C $-2.4497234 .543468-0.825359$

C $-1.6276516 .977979-0.497094$

O $-3.1055474 .974279-2.016061$

C $-1.1433693 .797938-1.181581$

C -1.411704 2.517496-2.008715

O $-0.3314064 .660082-1.987129$

C -1.802417 -1.234999 3.153519

O $-1.817028-2.1885073 .915566$

C $-2.991142-0.6513132 .492505$

C $-4.113508-1.3824322 .419135$

C $-5.358944-1.0023921 .748897$

C $-5.486047 \quad 0.4127501 .252061$

C $-2.1245351 .397789-1.246772$

C $-2.4427770 .207219-2.136528$

C $-3.429778-2.082820-2.002140$

O $-2.329847 \quad 0.230409-3.359004$

C $-4.924054-1.984320-2.017693$

C $-5.740531-2.901691-1.477364$

C $-7.238573-2.782246-1.480769$

C -7.849262 -2.938924 -0.103846

C $-8.608044-4.0065510 .196971$
C $-7.644901-1.8228410 .902167$

C $-6.321896-1.9381331 .611774$

N -2.901574 -0.874299-1.396797

H $0.869048-3.6967983 .541072$

H $1.223234-1.7931421 .171940$

H $0.538058-1.3936294 .171162$

H 1.4757880 .6400992 .065143

H $2.610339-0.2235873 .075510$

H 0.6908182 .9932294 .879537

H 1.9742821 .6091426 .198268

H $1.733157-0.1054165 .851653$

H $3.1882600 .728910 \quad 5.264047$

H $-0.281646 \quad 1.9720102 .143061$

H -1.043603 3.1246553 .212297

H 0.8782494 .7439622 .795367

H $1.5997513 .541767 \quad 1.725930$

H 0.5240205 .1882960 .378768

H -1.167763 6.0619452 .009801

H $-2.0569314 .557319 \quad 1.922187$

H -3.410134 6.7993561 .300404

H $-3.136623 \quad 3.847333-0.329057$

H -1.5909497 .7988510 .229249$

H $-2.2466237 .339117-1.325691$

H $-0.6119856 .822604-0.866296$

H -3.920326 $5.433148-1.748618$

H -1.962954 2.777192 -2.921506

H $-0.4527042 .113835-2.361588$

H $0.2908964 .113142-2.494484$

H $-2.8949150 .331297 \quad 2.044987$

H -4.111253 -2.378379 2.861077

H -6.4975260 .6696780 .930221$

H -4.8192520 .5849920 .402801$

H -5.2336841 .1254752 .045504$

H $-1.5014191 .056317-0.412302$

H -3.076371 $1.753151-0.841649$

H $-3.075509-2.928812-1.405062$

H -3.050070 -2.185592 -3.023399

H $-5.355444-1.108790-2.497965$

H $-5.313486-3.789112-1.015856$

H -7.630240 -3.551587 -2.158749

H -7.559013 -1.820610 -1.901057

H $-9.069084-4.1144111 .173316$

H $-8.781590-4.799015-0.523521$

H -8.433255 -1.858199 1.665327

H -7.774164 -0.8668390 .387507$

H $-6.143190-2.9258302 .040345$

H -2.995976 -0.776349 -0.390854

C $2.057760-4.1766650 .239000$

O $0.981627-3.693287-0.593717$

C $1.130931-4.254243-1.905083$

C $2.525855-4.860388-1.956580$

C $2.673326-5.348604-0.527135$

H $0.410061-5.081898-1.964856$

C $0.776408-3.256985-3.011454$

H $2.610973-5.663516-2.694534$

H $3.284885-4.102487-2.181202$

C $4.106009-5.672001-0.142337$

H $2.055616-6.247050-0.394049$

C $1.655502-1.996997-3.174693$

O $0.787992-3.969506-4.257353$

H $-0.261468-2.932890-2.866654$

H $2.656657-2.272288-3.525890$

O $1.067804-1.226747-4.237588$

C $1.786945-1.078898-1.941164$

C $2.4304060 .281936-2.285158$

H $2.391802-1.571194-1.174655$

O $0.497844-0.824052-1.388090$

H $3.4153380 .102778-2.729313$

H $1.8158350 .780867-3.043318$

H $4.494045-6.486875-0.762364$

H $4.764451-4.808598-0.277481$

H $4.170574-5.9852460 .904182$

C $1.524483-4.5513361 .624405$

H $1.709074-4.208885-4.456535$

H $1.008482-1.804995-5.017371$

H $0.168234-1.654171-1.005133$

H $2.295631-5.1135492 .163960$

H $0.655812-5.2127161 .515612$ 
H $\quad 2.790184-3.367070 \quad 0.323478$

C $4.7545100 .651835-0.277552$

O $3.3455330 .688294-0.021600$

C $2.5420481 .234474-1.071703$

C $3.0105152 .654513-1.424189$

C $4.5011212 .703532-1.735227$

C $5.2932152 .050239-0.607248$

H $4.955763-0.030037-1.112071$

C 5.4339980 .1098860 .989985

H $1.532506 \quad 1.337527-0.655297$

O $2.2713623 .161680-2.529600$

H $2.8053003 .316103-0.572643$

H $4.7175762 .238770-2.704256$

O $4.8918604 .073624-1.848291$

H 5.2463162 .6932970 .280763

O $6.665306 \quad 1.968793-0.989242$

C $5.259007-1.3838181 .141168$

H $\begin{array}{llll}6.513376 & 0.295327 & 0.974581\end{array}$

H 5.0007620 .5996361 .870814

H $2.6201744 .047145-2.730667$

H $5.8524794 .088849-2.000356$

H $6.7243151 .394314-1.771581$

O $5.389030-1.7198872 .458056$

O $5.079497-2.162770 \quad 0.215565$

C $5.305555-3.1179082 .749924$

H $4.314814-3.4982272 .490411$

H $6.083437-3.6670202 .210806$

H $5.461988-3.2511913 .823632$

SCF Energy (B3LYP/6-31G**//MMFF) $=-3245.93032397$

\section{9}

MMFF Geometry

C $-0.808752-3.605566-0.342542$

C $-0.143356-2.469448-0.077713$

C $0.351353-2.1256331 .303519$

O $0.153322-0.7059461 .502663$

C $1.856635-2.4253001 .424735$

C $2.321462-2.5251092 .864260$

C $3.041827-1.5890493 .511565$

C $1.965313-3.8125863 .564853$

C $3.508271-0.2525933 .004485$

C $5.035458-0.2042762 .852406$

C $5.536889-0.9747881 .621264$

C $7.060825-1.0985691 .642367$

C $7.590940-1.7808950 .372928$

O $5.122779-0.2459090 .461026$

O $9.013014-1.5890550 .329912$

C $6.999413-1.064214-0.857236$

C $7.356921-3.2958920 .393292$

O $7.346833-1.746490-2.060619$

C $5.465985-0.861755-0.786545$

C $5.0237150 .091043-1.922776$

O $4.815794-2.122907-0.945794$

C $-0.286783-0.2860582 .716032$

O $-0.658363-0.9923583 .643801$

C -0.2623701 .1909952 .832005$

C $\quad 0.3037572 .005770 \quad 1.928046$

C 0.3748753 .4680512 .004403

C -0.2041714 .1336183 .225701$

C $3.5558280 .534299-1.853859$

C $3.2444001 .600658-2.892622$

C $1.4172003 .155681-3.578744$

O $4.0526621 .974172-3.738452$

C $1.5394224 .437561-2.818239$

C $0.4902875 .112410-2.324492$

C $0.6109306 .399668-1.563168$

C $0.1787816 .264514-0.120558$

C $-0.992772 \quad 6.7684930 .302526$

C 1.1481555 .6269530 .850367

C $0.946140 \quad 4.1415180 .983193$

N $1.9502912 .081163-2.762023$

H $-0.976366-4.3024950 .473696$

H $0.077714-1.772111-0.880903$

H -0.227767 -2.696899 2.038617

H $2.428959-1.6633440 .883767$

H $2.097358-3.3781650 .934020$

H $3.330801-1.7924954 .542471$

H $2.381948-3.8653434 .576443$
H $\quad 0.880114-3.9199103 .652386$

H $2.355776-4.6690693 .005388$

H 3.0187870 .0363922 .070219

H 3.2075610 .4998183 .744430

H 5.3291320 .8493762 .758929

H $5.508644-0.5942653 .762269$

H $5.081436-1.9706751 .605929$

H $7.395794-1.6397662 .536094$

H $7.508633-0.0971611 .712299$

H $9.391979-1.9751641 .138154$

H $7.475124-0.074981-0.915186$

H $7.850565-3.7476751 .262176$

H $7.801095-3.775627-0.485857$

H $6.299953-3.5668360 .436563$

H $\quad 8.315008-1.838437-2.072162$

H $5.6453190 .995708-1.884680$

H $5.199046-0.383130-2.896904$

H $3.910218-1.974252-1.259707$

H -0.7231081 .5764483 .736139$

H $\quad 0.7677551 .5692901 .045300$

H -1.2640103 .8830103 .337607$

H -0.1511575 .2236753 .197891$

H $0.329823 \quad 3.8116574 .126552$

H $2.886777-0.314494-2.031014$

H $3.3301680 .952082-0.865947$

H $0.3761602 .910621-3.808526$

H $1.9708353 .224845-4.520356$

H $2.5433444 .828120-2.669776$

H $-0.5141174 .725258-2.477103$

H $-0.0030217 .150331-2.077691$

H $1.6361496 .788998-1.597741$

H -1.293467 6.7006741 .342609

H -1.680572 $7.252625-0.382873$

H $1.093697 \quad 6.1340081 .819251$

H 2.1776345 .8057270 .513976

H 1.3352093 .5680600 .141972

H $1.3744871 .741820-1.997074$

C $-2.758283-4.473691-1.761747$

O $-3.635877-3.423818-1.307454$

C $-4.522384-3.974164-0.318557$

C $-4.544568-5.473937-0.586039$

C $-3.093014-5.726071-0.943355$

H $-4.049069-3.7961550 .655165$

C $-5.894821-3.294747-0.343189$

H $-4.879655-6.0486430 .282446$

H $-5.188868-5.727449-1.435642$

C $-2.864628-7.026505-1.695413$

H $-2.515664-5.760224-0.013085$

C $-5.870195-1.753519-0.319912$

O $-6.587694-3.688616-1.534828$

H $-6.491385-3.6783290 .493571$

H -5.619809-1.377198 -1.319571

O $-7.209456-1.305253-0.070754$

C $-4.912286-1.1121550 .698480$

C $-5.1388830 .404506 \quad 0.857329$

H $-3.883940-1.3048700 .376406$

O $-5.061524-1.7275321 .978746$

H $-5.4144040 .835212-0.109614$

H -5.9811300 .5704341 .539209$

H -3.171844 -7.882485 -1.085543

H -3.436742 -7.057242 -2.628796

H -1.805820 -7.150148 -1.943982

C $-1.295358-4.012909-1.707097$

H -7.461966 -3.262623 -1.516838

H -7.474368 -1.627369 0.807335

H $-5.971578-1.5737282 .283271$

H -1.178606 -3.169549 -2.396150

H $-0.651923-4.820562-2.075284$

H -3.018296 -4.641981-2.814950

C $-2.9429481 .868156-0.682457$

O -2.7908521 .0483770 .483750$

C -3.8807921 .1058571 .414620$

C $-4.1019472 .551547 \quad 1.878127$

C -4.2624713 .5125500 .705736$

C $-3.1154373 .339081-0.286753$

H $-3.8118531 .537663-1.262970$

C -1.696197 $1.677189-1.557311$

H -3.5625930 .5346722 .295973$ 
O $-5.241156 \quad 2.651287 \quad 2.725834$ H -3.2372092 .8742252 .470090$ H -5.2328603 .3846540 .211469$ O $-4.230473 \quad 4.856348 \quad 1.192205$ H $-2.187648 \quad 3.722763 \quad 0.153801$ O $-3.3876294 .114288-1.452520$ C $-1.7035740 .355878-2.297033$ H $-1.6527692 .464538-2.317205$ H $-0.788921 \quad 1.709952-0.946175$ H -5.1035592 .0433743 .472205$ H -4.9393324 .9410721 .852876$ H $-3.4993975 .037212-1.166740$ O $-0.7051290 .374383-3.227618$ O $-2.471195-0.570749-2.081668$ C $-0.561558-0.784319-4.055691$ H $-1.523227-1.264091-4.260792$ H $-0.124137-0.467080-5.006241$ H $0.123261-1.485711-3.572697$

SCF Energy (B3LYP/6-31G**/MMFF) $=-3245.90661335$

\section{6}

MM̄FF Geometry

C $-2.418118 \quad 4.232090 \quad 1.893071$

C -2.819485 2.9921361 .573098

C $-3.136574 \quad 1.9272192 .592050$

O $-2.515990 \quad 0.6901862 .172041$

C -4.6596451 .7092432 .644989$

C -5.0857250 .6306023 .622109$

C $-5.514554-0.5988663 .271446$

C -5.0422241 .0284465 .074995$

C $-5.617683-1.1760401 .886219$

C $-4.809833-2.4750451 .763898$

C $-4.708074-2.9500290 .306311$

C $-3.983959-4.2954240 .229781$

C $-3.733509-4.740030-1.218763$

O $-3.981420-1.955306-0.423451$

O $-2.775524-5.809841-1.191807$

C $-3.083967-3.574579-1.995780$

C $-5.000682-5.309774-1.866883$

O $-2.970055-3.889614-3.381050$

C $-3.824593-2.232760-1.821689$

C $-3.069487-1.034208-2.451052$

O $-5.097939-2.312989-2.474103$

C -1.227194 0.4748622 .552421

O $-0.503324 \quad 1.241853 \quad 3.166119$

C $-0.855973-0.8883572 .109697$

C $0.370465-1.3722862 .356713$

C $0.844183-2.7221012 .037546$

C $-0.114358-3.652431 \quad 1.341613$

C $-1.690443-0.735717-1.854256$

C $-0.536482-1.414315-2.572692$

C $1.899452-1.886441-2.298220$

O $-0.641056-1.925445-3.683802$

C $2.176670-3.161114-1.567275$

C $3.279620-3.359694-0.830103$

C $3.605412-4.647840-0.133798$

C $3.905811-4.4610101 .336030$

C $5.172277-4.4251871 .783891$

C $2.741386-4.4302692 .297527$

C $2.094199-3.0759142 .403931$

N $0.637978-1.339764-1.836737$

H -2.303758 4.4764592 .947913

H -2.9599832 .7114740 .533441$

H -2.769821 2.2068683 .587609

H -5.0337101 .4878921 .639732$

H -5.1561882 .6471822 .929728$

H -5.823406 -1.278857 4.064551

H $-5.418610 \quad 0.238925 \quad 5.734291$

H -4.0157891 .2508235 .382358$

H $-5.657600 \quad 1.9181425 .243988$

H $-6.672747-1.385177 \quad 1.673115$

H -5.282677 -0.4660401 .127470$

H -3.800871 -2.307041 2.160355

H -5.276824 -3.254365 2.379062

H -5.720296 -3.034158 -0.106512

H $-4.538927-5.0664850 .778510$

H -3.013386 -4.213083 0.735680

H $-3.139045-6.523898-0.640513$
H -2.061963 -3.469617-1.612059

H $-5.354200-6.186009-1.310117$

H $-4.800037-5.663368-2.884259$

H -5.822906 -4.592341-1.905405

H -2.480483 -4.727185 -3.448513

H -3.022952 -1.146557 -3.540989

H -3.683724 -0.138712 -2.278077

H -5.406995 -1.411194 -2.661355

H $-1.620548-1.4743081 .613871$

H $1.088552-0.7344502 .869762$

H $-0.948007-3.9046082 .005450$

H $0.342786-4.5904481 .019732$

H $-0.514530-3.1894390 .433218$

H $-1.5039000 .342383-1.936416$

H -1.662229 -0.989051 -0.791086

H $2.668295-1.130015-2.112131$

H $1.855539-2.076915-3.375023$

H $1.451191-3.964074-1.672903$

H $4.011384-2.560652-0.751999$

H $4.476922-5.085201-0.638566$

H $2.798666-5.381914-0.250868$

H $5.395970-4.3274552 .840971$

H $6.014026-4.4897841 .102659$

H $3.086759-4.6983643 .305705$

H $2.033330-5.2223822 .037501$

H $2.721372-2.3317412 .896803$

H $0.613785-0.931634-0.907066$

C $-2.1726405 .067887-0.547743$

O $-1.0281724 .262882-0.896758$

C $-0.4456464 .782981-2.106737$

C $-1.4837245 .727661-2.695861$

C $-2.0866336 .313012-1.432747$

H $0.4256105 .377392-1.808003$

C $-0.0074463 .635922-3.021343$

H -1.036922 $6.482132-3.350060$

H $-2.2467995 .187704-3.268606$

C $-3.4199617 .003764-1.661785$

H $-1.3776187 .033003-1.002427$

C $0.9705382 .647248-2.350517$

O $-1.1742082 .900378-3.412670$

H $\quad 0.4247704 .039456-3.944384$

H $0.4333062 .102795-1.566390$

O $1.328714 \quad 1.682477-3.346714$

C $2.2486583 .296624-1.784545$

C $3.2582852 .319611-1.163991$

H $1.9976864 .053173-1.037853$

O $2.9432173 .981384-2.833847$

H $3.6696671 .673262-1.951899$

H $4.1266412 .908163-0.842072$

H $-3.303227 \quad 7.844622-2.353419$

H $-4.1595246 .318088-2.088818$

H -3.824955 $7.393154-0.722601$

C -2.1310295 .3759740 .951855$

H $-0.8778772 .169234-3.981671$

H $1.7723990 .944315-2.897406$

H $2.4044364 .745213-3.099329$

H -2.851927 6.1692341 .181893

H $-1.1343385 .757716 \quad 1.207991$

H $-3.0703004 .494285-0.809235$

C 4.9564910 .8450590 .837240

$\begin{array}{lllll}\text { O } & 3.722536 & 0.392911 & 0.264167\end{array}$

C $2.7642151 .431317-0.003702$

C 2.4126112 .1285561 .317137

C 3.6557682 .6678722 .018832

C 4.7010681 .5616992 .168788

H $5.465547 \quad 1.5227890 .141643$

C $5.852531-0.3865071 .034826$

H $1.8662900 .911585-0.349492$

O $1.4721293 .176861 \quad 1.141177$

H 1.9460831 .3982361 .988433

H $4.0703663 .536417 \quad 1.494263$

O 3.2988903 .1206673 .327135

H 4.3633480 .8412432 .924985

O 5.9216842 .1315152 .636943

C $6.349750-0.948106-0.281228$

H $6.734806-0.1255921 .629314$

H $5.288986-1.1775841 .542811$

H $0.6624022 .785543 \quad 0.771084$ 
H 2.5959163 .7845273 .221009

H 5.7242742 .5966903 .468079

O $7.117045-2.049799-0.028722$

O $6.097876-0.490447-1.387269$

C $7.637527-2.714625-1.184086$

H $8.219235-3.576535-0.846723$

H $6.818712-3.070509-1.816843$

H $8.295829-2.043111-1.743142$

SCF Energy (B3LYP/6-31G**//MMFF) $=-3245.91505256$

0260

MMFF Geometry

C - $-1.327212 \quad 0.667413 \quad 3.579255$

C -2.6657620 .7219383 .660152$

C -3.5829961 .7720303 .077043$

O $-4.259262 \quad 1.1889251 .937404$

C -2.8933473 .0504002 .563322$

C -3.8252324 .2451732 .501040$

C $-4.490488 \quad 4.6592791 .405553$

C $-3.9375495 .025498 \quad 3.786904$

C $-4.564234 \quad 4.007960 \quad 0.053795$

C $-3.7698634 .762104-1.021311$

C $-2.2847284 .370176-1.047331$

C $-1.5225605 .197788-2.083885$

C $-0.0638564 .736493-2.225005$

O $-2.2193642 .980547-1.387285$

O $0.4749695 .333670-3.414241$

C $-0.0439453 .208644-2.437617$

C $0.8069955 .224694-1.061398$

O $1.2911112 .710112-2.437466$

C $-0.8988822 .429660-1.412016$

C $-1.0102010 .947427-1.841818$

O $-0.2708082 .499302-0.130554$

C $-5.355261 \quad 0.4156142 .139566$

O $-5.868791 \quad 0.115993 \quad 3.203859$

C $-5.805824-0.0283290 .802538$

C $-6.601534-1.1000280 .683486$

C $-7.073685-1.678437-0.576967$

C $-6.711113-0.962206-1.852941$

C $-2.0478280 .131978-1.055313$

C $-2.037520-1.333484-1.460947$

C $-3.093964-3.519128-0.887870$

O $-1.307539-1.780770-2.341873$

C $-4.230130-3.793129-1.822836$

C $-5.200234-4.683722-1.563007$

C $-6.325615-4.996331-2.508947$

C $-7.684177-4.958572-1.842258$

C -8.305036 -6.092607 -1.475793

C $-8.358012-3.613948-1.677631$

C $-7.784982-2.824140-0.531881$

N $-2.936948-2.085635-0.717154$

H $-0.779966 \quad 1.422597 \quad 3.023215$

H -3.172560 -0.0621794 .220721$

H -4.3209022 .0383543 .844840$

H -2.458726 2.8647261 .575459

H -2.057003 3.3307913 .216160

H $-5.097900 \quad 5.5601351 .490241$

H -4.6199185 .8777493 .700279$

H -4.3125914 .3850024 .591428$

H -2.957661 5.4157824 .081052

H $-4.2688072 .956640 \quad 0.088769$

H -5.622456 $4.004676-0.238303$

H $-4.2133404 .509487-1.993708$

H -3.877377 $5.845310-0.887682$

H -1.856049 4.524501-0.051399

H $-1.5661016 .265869-1.837033$

H -2.012274 $5.097253-3.062820$

H $0.4010156 .299313-3.324712$

H $-0.4466003 .014379-3.441794$

H $0.8040316 .320506-1.014952$

H $1.8538864 .934285-1.202896$

H $\quad 0.4735624 .853664-0.090108$

H $1.7901133 .216201-3.101410$

H -1.293152 $0.897890-2.901680$

H $-0.0296630 .466054-1.746226$

H $-0.524761 \quad 1.7237360 .393019$

H $-5.4338050 .521093-0.055042$

H $-6.914761-1.6185311 .588694$
H -7.139388 $0.046305-1.859559$

H $-7.068152-1.463187-2.754558$

H $-5.623721-0.881105-1.957363$

H -1.8511570 .1865510 .019785$

H -3.053645 $0.525727-1.240488$

H $-3.266941-3.9465260 .104442$

H -2.169504 -3.940360 -1.295676

H $-4.242744-3.254034-2.767112$

H $-5.166938-5.246165-0.632375$

H $-6.142542-5.997196-2.921425$

H $-6.325756-4.315769-3.369449$

H $-9.290614-6.076737-1.022352$

H -7.838931 -7.062328 -1.615835

H $-9.428681-3.750279-1.474302$

H -8.323539 -3.077611 -2.629597

H $-7.990900-3.2675060 .443580$

H $-3.531844-1.620526-0.038175$

C $0.286172-1.288210 \quad 3.296183$

O $1.228271-0.5138822530937$

C $1.293727-1.062448 \quad 1.194526$

C $0.526122-2.377178 \quad 1.240379$

C $-0.535471-2.0766542 .280072$

H $0.777785-0.3388440 .553107$

C $2.764325-1.1904100 .780936$

H $\quad 0.110868-2.6569420 .268136$

H $1.173630-3.1949681 .580996$

C -1.200490 -3.315355 2.852253

H $-1.299862-1.4401971 .819568$

C $2.976360-1.895308-0.569814$

$\begin{array}{llll}\text { O } & 3.317205 & 0.127453 & 0.702310\end{array}$

H $3.315239-1.7149281 .569702$

H $2.634883-2.934962-0.503583$

O $2.143437-1.256539-1.545219$

C $4.425817-1.858989-1.094125$

C $5.453639-2.452304-0.115544$

H $4.692760-0.829878-1.356646$

O $4.470973-2.602667-2.317628$

H $5.417742-1.9022450 .828854$

H $5.176208-3.4902500 .106555$

H $-1.696965-3.8864212 .061753$

H $-0.472415-3.9756833 .335076$

H $-1.955005-3.0428193 .596953$

C $-0.497765-0.390502 \quad 4.254794$

H 3.1652890 .5582951 .560941

H $2.287077-1.710971-2.392931$

H $4.250128-3.527188-2.112090$

H $\quad 0.212987 \quad 0.131647 \quad 4.907849$

H $-1.122933-1.0143554 .904628$

H $0.888245-1.9793853 .901564$

C $7.450339-0.158003-0.100642$

O $7.282311-1.136518-1.133552$

C $6.896597-2.435099-0.671457$

C $7.955824-2.9792220 .297463$

C $8.255710-1.9962311 .428882$

C $8.545650-0.5996420 .875927$

H $6.504603-0.0218020 .437470$

C $7.807077 \quad 1.169109-0.778246$

H $6.903864-3.088397-1.553056$

$\begin{array}{llllll} & & 7.534772 & -4.228708 & 0.833718\end{array}$

H $8.881122-3.165963-0.262996$

H $7.430860-1.9658832 .149783$

O $9.393116-2.4558952 .163283$

H $9.522163-0.5963900 .375228$

$\begin{array}{lllll}\text { O } & 8.614025 & 0.326952 & 1.957037\end{array}$

C $6.6329291 .702192-1.564505$

H $8.1082841 .936356-0.056328$

H $8.6405261 .037199-1.478912$

H $8.219737-4.523414 \quad 1.458156$

H $10.149496-2.4942521 .553223$

H $9.2919890 .003366 \quad 2.574781$

O $5.697813 \quad 2.215797-0.714964$

O $6.552577 \quad 1.654778-2.785145$

C $4.5074722 .708107-1.337097$

H $4.7429093 .557266-1.985429$

H $4.0198741 .910312-1.905468$

H $3.8262213 .043824-0.550653$

SCF Energy (B3LYP/6-31G**//MMFF) = -3245.91638686 
$02 \_61$

MM̄FF Geometry

C $4.781906-0.0645182 .840583$

C 3.6845920 .5800162 .414939

C 2.5102830 .8878023 .305330

O $1.306357 \quad 0.3673402 .699896$

C 2.3326152 .4111603 .433142

C 1.2048522 .7976994 .370755

C -0.0313713 .1523673 .966272$

C 1.5590912 .8062575 .834269

C $-0.559376 \quad 3.1665392 .558072$

C -0.6026384 .5878451 .990640$

C -1.0671744 .6053010 .526988$

C $-0.9669516 .020490-0.044738$

C $-1.5016196 .095896-1.480886$

O $-2.422101 \quad 4.146244 \quad 0.475707$

O $-1.6651607 .481615-1.818928$

C $-2.9014825 .451510-1.521870$

C $-0.5081045 .515701-2.494298$

O $-3.3810245 .371143-2.862945$

C $-2.9668114 .057560-0.850333$

C $-4.4486643 .621964-0.722857$

O $-2.2507313 .119211-1.650283$

C $0.955883-0.9152342 .976184$

O $1.640971-1.7694933 .513366$

C $-0.461907-1.0653792 .576803$

C -1.113999 -2.208849 2.831749

C $-2.540445-2.4541852 .601433$

C $-3.378178-1.2875302 .150824$

C $-4.6838812 .260730-0.051055$

C $-4.8007591 .108511-1.038123$

C $-5.682754-1.197825-1.239137$

O $-4.409231 \quad 1.173927-2.200300$

C $-6.071113-2.316685-0.327621$

C $-5.422412-3.489353-0.269410$

C $-5.832536-4.6286410 .617790$

C $-4.736335-5.0563401 .567613$

C $-4.098910-6.2279611 .399075$

C $-4.459684-4.1636862 .757997$

C -3.031669 -3.688723 2.837577

N $-5.369718-0.017186-0.456152$

H $4.812142-0.3909543 .879066$

H $3.6128940 .922641 \quad 1.387534$

H 2.6393530 .4472134 .302300

H 2.1584792 .8516512 .444115

H 3.2627852 .8695083 .795032

H -0.7590613 .4433544 .722390$

H 0.7188103 .1182366 .463602

H 1.8617951 .8065566 .161223

H 2.3861253 .4990786 .020872

H 0.0348502 .5250411 .900810

H -1.566486 2.7319962 .567544

H -1.284228 5.1939532 .601747

H $0.394715 \quad 5.0398782 .061493$

H $-0.4346683 .918964-0.046214$

H $0.0665996 .386181-0.001248$

H -1.554026 6.7083470 .580110

H $-0.7998727 .916307-1.727985$

H -3.587856 $6.123921-0.987815$

H $0.4438326 .058561-2.451016$

H $-0.8745565 .635732-3.519796$

H $-0.2921884 .458751-2.326827$

H -3.335502 $6.265951-3.241358$

H -4.962812 4.371215 -0.105623

H $-4.9308403 .629561-1.707621$

H -2.521142 2.223038 -1.396782

H $-0.949123-0.2032952 .134569$

H $-0.561951-3.0356153 .276788$

H -3.050364 $-0.939178 \quad 1.165718$

H -3.298785 -0.4565582 .860725$

H -4.440414 -1.521066 2.068713

H -3.8969532 .0236300 .672818$

H -5.6331242 .3176570 .497054$

H -4.818704 -1.443546 -1.864937

H $-6.517375-0.947075-1.902293$

H $-6.942727-2.1595020 .303441$

H -4.565166 -3.653455 -0.915627

H $-6.123403-5.465477-0.030321$
H $-6.729645-4.3789891 .198441$

H -3.339363 -6.565232 2.096303

H -4.323253 -6.877732 0.559615

H -4.681144 -4.727432 3.674429

H -5.166118 -3.328739 2.778783

H -2.336546 -4.462359 3.167079

H -5.7971710 .0787810 .460876$

C 6.0789370 .0891200 .598697

O $5.116740-0.636313-0.190948$

C $5.737604-1.056284-1.418724$

C $7.035260-0.266562-1.523804$

C $7.434820-0.164689-0.064855$

H $5.983966-2.117998-1.296989$

C $4.772389-0.884171-2.595257$

H $7.782502-0.774332-2.140938$

H $6.8728570 .733668-1.941658$

C $8.469116 \quad 0.914317 \quad 0.206515$

H $7.835848-1.1349330 .258077$

C $3.389908-1.536864-2.377083$

O $4.5599160 .515782-2.814778$

H $5.240706-1.267879-3.509456$

H $2.842595-0.969965-1.618629$

O $2.654884-1.388311-3.597979$

C $3.435833-3.024394-1.995013$

C $2.068107-3.730668-1.927433$

H $3.945907-3.146579-1.033463$

O $4.227006-3.702576-2.978535$

H $1.522853-3.562469-2.862127$

H $2.265255-4.810233-1.907486$

H $9.3917530 .710203-0.346753$

H $8.107140 \quad 1.902527-0.096959$

H $8.717303 \quad 0.9592391 .271454$

C $6.021876-0.3959382 .050464$

H $3.9300100 .601792-3.550829$

H $3.097079-1.928971-4.274955$

H $4.344994-4.620340-2.679622$

H 6.8732810 .0216242 .600976

H $6.128493-1.4882072 .067987$

H $5.819793 \quad 1.1535620 .533023$

C $-0.293645-1.754619-1.711886$

O $0.710812-2.018947-0.725786$

C $1.212474-3.357512-0.695701$

C $0.050307-4.330333-0.444297$

C $-1.031274-4.176724-1.506631$

C -1.480546 -2.720282 -1.589255

H $0.149330-1.833673-2.710816$

C $-0.770729-0.309227-1.512470$

H $1.874638-3.4169820 .177516$

O $0.501159-5.680404-0.424449$

H $-0.393831-4.1286000 .537943$

H $-0.694298-4.542095-2.483670$

O $-2.160161-4.975002-1.149086$

H $-2.076447-2.475031-0.701346$

O $-2.326242-2.554392-2.726373$

C $0.3134250 .683216-1.856793$

H $-1.631710-0.074722-2.148643$

H -1.088461 -0.143729 -0.476145

H $1.179493-5.7508310 .268846$

H - $1.847138-5.890062-1.045978$

H $-3.039390-3.211801-2.658555$

O $0.8309261 .224939-0.717915$

O $0.6671020 .932164-3.002045$

C $1.8766492 .185175-0.898863$

H $2.8245401 .664500-1.052220$

H $1.6661572 .857500-1.735958$

H 1.9494452 .7842110 .011858

SCF Energy (B3LYP/6-31G**//MMFF) $=-3245.91360464$

$02 \_62$

MM̄FF Geometry

C -2.2912352 .6404091 .925115$

C -1.510796 2.0678962 .855122

C -1.7743930 .7674463 .581488$

O $-0.622433-0.1133903 .482604$

C $-3.0729570 .012926 \quad 3.222773$

C $-3.401274-1.1401544 .151421$

C $-3.464413-2.4352953 .785211$

C $-3.757069-0.7513975 .565194$ 
C $-3.170920-3.0573802 .447718$

C $-4.453306-3.3841181 .665401$

C $-4.861973-2.2459220 .718570$

C $-6.267915-2.4628120 .161657$

C $-6.642737-1.369223-0.850319$

O $-3.912389-2.228213-0.353559$

O $-7.833554-1.789729-1.532806$

C $-5.524569-1.269167-1.907270$

C $-6.979699-0.039208-0.165605$

O $-5.753109-0.173268-2.790912$

C $-4.098977-1.177521-1.308920$

C $-3.059782-1.372326-2.441102$

O $-3.914973 \quad 0.107474-0.713844$

C $-0.227777-0.5886622 .272244$

O $-0.684978-0.304522 \quad 1.177312$

C $0.857310-1.5646692 .515584$

C $1.170319-2.4438801 .553040$

C $2.207402-3.4751821 .619854$

C $3.096594-3.5011642 .834553$

C $-1.611162-1.532219-1.961506$

C $-0.670908-1.882260-3.105207$

C $1.705400-2.527424-3.529969$

O $-1.018546-1.886550-4.282965$

C $2.322245-3.800248-3.047007$

C $3.551507-3.875306-2.514869$

C $4.168745-5.147917-2.011204$

C $4.440519-5.112052-0.522736$

C $5.671844-4.854196-0.051205$

C $3.305543-5.4609640 .415920$

C $2.326803-4.3320230 .584525$

N $0.603520-2.185510-2.648011$

H -3.2108442 .1558751 .610563$

H -0.6045152 .5878853 .160242$

H -1.8220071 .0434474 .641718$

H -3.018090 -0.3333512 .187845$

H $-3.924618 \quad 0.7050813 .260446$

H -3.755596 -3.161633 4.544133

H -4.147400 -1.595843 6.143515

H -2.878303 -0.3714196 .094556$

H -4.5289430 .0252845 .564139$

H -2.483294 -2.450391 1.852521

H -2.635783 -3.995150 2.646164

H -4.261547 -4.284712 1.067267

H -5.270839 -3.6215662 .356942$

H $-4.823373-1.2965611 .260036$

H $-7.006819-2.5042980 .971551$

H $-6.317856-3.438229-0.342198$

H $-8.529330-1.922817-0.866518$

H -5.581729 -2.178273 -2.522672

H -7.810436 -0.1708050 .538020$

H $-7.3201100 .704338-0.894863$

H $\quad-6.139747 \quad 0.3832130 .389829$

H $-6.647580-0.278608-3.157764$

H -3.320155 -2.276542 -3.007549

H -3.106471 -0.526684 -3.139110

H -2.964892 $0.296896-0.661362$

H $1.350292-1.5461153 .480707$

H $0.604071-2.4138810 .623318$

H $3.569547-2.5264152 .989825$

H $3.911210-4.2248822 .761648$

H $2.516557-3.7554193 .728316$

H -1.255859 -0.602744 -1.507375

H -1.543488 -2.330902 -1.214016

H $2.414304-1.694477-3.503329$

H $1.353987-2.645241-4.559378$

H $1.721843-4.702610-3.134753$

H $4.151138-2.972343-2.433201$

H $5.104453-5.306947-2.563059$

H $3.541235-6.016908-2.245116$

H $5.880935-4.8543071 .013356$

H $6.496698-4.629944-0.719450$

H $3.695080-5.7983521 .382301$

H $2.767255-6.3329550 .022447$

H $1.625286-4.236822-0.243750$

H $\quad 0.814780-2.050402-1.663857$

C $-1.9655643 .880706-0.235501$

O $-0.8008143 .144980-0.660430$

C $-0.2385593 .795723-1.816792$
C $-1.2444654 .858698-2.243447$

C -1.856602 $5.246056-0.913769$

H $0.6771004 .296548-1.482845$

C $0.0909162 .770688-2.906782$

H $-0.7665845 .697656-2.758492$

H -2.012101 $4.449382-2.910581$

C $-3.1785015 .982141-1.048390$

H -1.143362 $5.880468-0.369956$

C $1.0451961 .644143-2.465978$

O

H $0.5027903 .288004-3.781081$

H $0.5382480 .988876-1.749062$

O $1.3140560 .839971-3.621188$

C $2.3803012 .118777-1.865876$

C $3.3016380 .936650-1.504983$

H $2.1759482 .708037-0.967559$

O $3.0274272 .970907-2.808067$

H $2.7646870 .279453-0.811288$

H $3.5125620 .347369-2.405422$

H -3.044701 $6.912526-1.610089$

H $-3.9234775 .375969-1.574725$

H -3.586426 $6.238182-0.065774$

C -2.0021453 .9722541 .290191$

H -1.542212 $1.734602-2.580123$

H $1.8149191 .387083-4.250280$

H $3.7511863 .426234-2.345650$

H -2.792856 4.6642781 .601792

H -1.0527274 .3737351 .662527$

H -2.842058 $3.335397-0.610252$

C $3.915491 \quad 1.8628981 .341413$

O 4.5041292 .3488310 .130040

C $4.6556731 .365414-0.896311$

C $5.5245760 .201473-0.394311$

C $5.012667-0.3693280 .928903$

C $4.786808 \quad 0.7550151 .941389$

H $2.910696 \quad 1.4740381 .134523$

C 3.7853883 .0547692 .295593

H $5.2171641 .853096-1.703269$

O $5.585378-0.818479-1.384586$

H $6.5490460 .567254-0.245081$

H $4.098572-0.9559040 .783516$

O $5.987043-1.2637401 .469354$

H $5.758534 \quad 1.1608792 .249044$

O 4.1683330 .2246213 .110368

C 2.7090923 .9987551 .811647

H 3.5066062 .7448353 .308462

H 4.7382303 .5936562 .362247

H $6.222418-1.487525-1.083002$

H $6.033500-2.0364790 .881893$

H $3.287893-0.1008502 .858793$

O 3.2301375 .2383281 .591083

O 1.5421303 .6694541 .643042

C 2.3018946 .2153661 .109022

H 1.4871286 .3532421 .826404

H 2.8342347 .1635020 .996154

H 1.9101755 .9142210 .132732

SCF Energy $(B 3 L Y P / 6-31 G * * / / M M F F)=-3245.91233744$

0263

MM̄FF Geometry

C $-2.102676 \quad 3.0764951 .874892$

C -1.3694131 .9740931 .660138$

C $-0.668423 \quad 1.2107262 .755284$

O $\quad 0.7195321 .0461162 .376500$

C $-1.308418-0.1796662 .906840$

C $-0.812808-0.9395634 .122535$

C $-0.015036-2.0251244 .084963$

C $-1.328370-0.4382745 .448725$

C $0.584097-2.6897732 .878237$

C $2.099098-2.8677453 .033502$

C $2.721551-3.5839641 .824517$

C $4.227981-3.7501052 .031255$

C $4.907558-4.3700760 .804169$

O $2.467775-2.7891760 .666584$

O $6.326767-4.2075720 .954786$

C $4.496356-3.569222-0.449970$

C $4.653289-5.8795170 .720368$

O $4.973066-4.202823-1.635442$ 
C $2.972412-3.317814-0.565695$

C $2.650647-2.287409-1.674936$

O $2.276589-4.509729-0.944888$

C 1.5383462 .1134112 .593534

O 1.2391603 .1672563 .134094

C 2.8669161 .8045742 .017345

C 3.7144022 .8195471 .786916

C 5.0502292 .7336561 .193314

C $5.647647 \quad 1.367474 \quad 0.994429$

C $2.905831-0.837809-1.258009$

C $2.8647410 .145132-2.412488$

C $3.1790152 .590387-2.831962$

O $2.777461-0.193851-3.588394$

C $4.6306812 .954790-2.783145$

C $5.0889194 .151126-2.384452$

C $6.5491084 .497769-2.314517$

C $6.9678885 .023989-0.958084$

C $7.347206 \quad 6.303710-0.801560$

C 7.0247024 .0500430 .202808

C 5.6831803 .8810070 .868153

N $2.976392 \quad 1.452527-1.955973$

H -2.2125183 .4563182 .887647$

H -1.2592451 .5937490 .648473$

H -0.7259581 .7533113 .706852$

H -1.149919 $-0.757707 \quad 1.990331$

H $-2.398946-0.0788462 .999596$

H $0.235443-2.5056965 .030234$

H -1.031666 -1.0844816 .281963$

H -0.9441680 .5639865 .660145$

H -2.422623 -0.3986055 .440889$

H $\quad 0.111395-3.6712582 .754417$

H $0.387554-2.1195111 .968542$

H $2.556083-1.8754183 .146260$

H $2.316994-3.4353543 .946962$

H $2.234740-4.5586681 .703604$

H $4.434442-4.3478582 .927867$

H $4.681959-2.7662772 .216856$

H $6.591312-4.6345391 .787769$

H $5.010019-2.601292-0.392283$

H $5.070980-6.3873041 .598363$

H $5.157841-6.319060-0.147014$

H $3.592236-6.1334020 .671455$

H $5.932750-4.323663-1.533680$

H $3.200816-2.541229-2.589669$

H $1.592133-2.375548-1.953117$

H $2.924285-5.215464-1.098889$

H $3.0928390 .774046 \quad 1.770040$

H 3.3877163 .8298992 .032130

H 5.5269120 .7517701 .893065

H $6.721848 \quad 1.3931040 .795861$

H $\begin{array}{llll}5.168297 & 0.860388 & 0.154197\end{array}$

H $2.158262-0.528873-0.517435$

H $3.894633-0.748192-0.800008$

H $2.5371583 .399412-2.470037$

H $2.8878252 .336606-3.855612$

H $5.3405802 .188240-3.085696$

H $4.3803414 .925087-2.098486$

H $6.7486425 .247693-3.090885$

H $7.1750183 .632335-2.565731$

H 7.6731836 .6819370 .161833

H $7.3359317 .003247-1.630733$

H 7.7288634 .4162850 .961076

H $7.4409863 .105901-0.160098$

H 5.1836584 .8249911 .091958

H $3.0683951 .615003-0.957786$

C $-4.3160903 .740200 \quad 0.908032$

O $-4.7271032 .388590 \quad 0.618925$

C $-5.8797712 .424154-0.243116$

C $-6.3729603 .865293-0.225061$

C $-5.0663374 .622607-0.089495$

H $-5.5252182 .189277-1.253777$

C $-6.9094541 .380493 \quad 0.203414$

H $-6.9299704 .125073-1.130339$

H -7.018139 4.0648850 .638272

C $-5.242065 \quad 6.0605930 .366319$

H $-4.5526774 .613526-1.060460$

C $-6.350675-0.0520210 .325788$

$\begin{array}{llll}\text { O } & -7.425018 & 1.748452 & 1.489831\end{array}$
H $-7.7673901 .392106-0.478515$

H -5.646531 -0.1112011 .164757$

O $-7.441361-0.9222950 .653033$

C $-5.673004-0.583335-0.949747$

C $-5.184067-2.033318-0.792087$

H $-4.8190170 .051530-1.192245$

O $-6.580853-0.504135-2.046826$

H $-4.544338-2.0888700 .095237$

H $-6.045228-2.688110-0.612092$

H $-5.8422096 .622400-0.356873$

H -5.7470706 .1152411 .336601$

H $-4.2734596 .561147 \quad 0.462121$

C $-2.795895 \quad 3.847114 \quad 0.786497$

H -6.6707641 .8110702 .100992$

H -7.856866 -0.574439 1.460806

H $-7.361253-1.039018-1.820946$

H -2.4806804 .8940970 .858913$

H -2.476689 $3.479202-0.195544$

H -4.6379973 .9590151 .934618$

C $-2.230362-1.696235-1.517800$

O $-3.339106-1.717313-2.424133$

C $-4.429041-2.560541-2.034363$

C $-3.941503-4.009735-1.893286$

C $-2.761276-4.110394-0.930566$

C $-1.670040-3.111955-1.312913$

H $-2.544923-1.266552-0.558835$

C -1.146115 -0.786900 -2.120519

H -5.141365 -2.540436 -2.868783

O $-4.989364-4.860761-1.441518$

H -3.624509-4.382581 -2.875858

H $-3.082723-3.9597020 .106132$

O $-2.220439-5.433568-0.977242$

H $-1.171563-3.451959-2.230019$

O $-0.686860-3.081156-0.282431$

C $-1.5445930 .670693-2.190512$

H $-0.238697-0.836499-1.510191$

H -0.918586 -1.113043 -3.142475

H $-5.721096-4.785987-2.077552$

H $-1.932075-5.608848-1.889210$

H $-0.344282-3.985407-0.177934$

O $-0.525598 \quad 1.374123-2.769047$

O $-2.5981461 .139441-1.786056$

C $-0.7374642 .783200-2.901947$

H $-1.7208212 .994812-3.333154$

H $-0.6315403 .263734-1.925530$

H $0.0282133 .179308-3.574200$

SCF Energy $\left(B 3 L Y P / 6-31 G^{* *} / / M M F F\right)=-3245.92535032$

0264

MM̄FF Geometry

C $-0.470584-3.692612-0.409801$

C $-0.733763-2.510329-0.986020$

C $-0.601807-2.278714-2.469155$

O $0.216898-1.096676-2.634035$

C $-1.965940-2.029740-3.139045$

C $-2.908029-3.214373-3.070917$

C $-3.940732-3.323221-2.211237$

C $-2.635126-4.307631-4.070542$

C $-4.339585-2.344855-1.142956$

C $-5.735615-1.765119-1.404035$

C $-6.004918-0.548156-0.508956$

C -7.464332 -0.104728 -0.606382

C -7.7213581 .1822250 .192693$

O $-5.1480120 .504647-0.963389$

O $-9.0039791 .695660-0.197824$

C $-6.6702942 .238332-0.209400$

C -7.8018710 .9141151 .700120$

O -6.7762923 .4009250 .609861$

C $-5.2156211 .708360-0.191946$

C $-4.2764552 .750224-0.852187$

O $-4.8140221 .491151 \quad 1.159408$

C $0.957627-0.997301-3.767044$

O $1.016673-1.817502-4.673529$

C $1.7482160 .254945-3.800215$

C $1.5908231 .265851-2.931425$

C $2.3453992 .523303-2.924260$

C $3.4910392 .659732-3.893145$

C $-2.8397402 .273159-1.110322$ 
C -1.9326402 .3632890 .099873$ C -0.8332724 .0164951 .613087$ $\begin{array}{lllll} & \mathrm{O} & -1.496357 & 1.354439 & 0.649934\end{array}$ C 0.2447824 .9381401 .140442 C 1.5493604 .6255761 .141761 C 2.6240065 .5430490 .635718 C $3.3533054 .978147-0.563396$ C $4.6586794 .667318-0.499293$ C $2.5880424 .851578-1.862443$ C $1.9867713 .483927-2.046452$ N -1.667498 3.6684780 .478785 H $-0.138507-4.508223-1.050615$ H $-1.075616-1.670640-0.388144$ H $-0.097406-3.134196-2.936547$ H -1.808162 -1.769883 -4.194745 H -2.421944 -1.122865 -2.724375 H $-4.558715-4.218842-2.257057$ H -3.362670 -5.123533 -4.003862 H $-2.679340-3.909743-5.089628$ H $-1.642214-4.738075-3.907588$ H $-4.341534-2.868166-0.178596$ H -3.608585-1.539289-1.040599 H $-5.813655-1.463556-2.457028$ H $-6.488305-2.542749-1.224710$ H $-5.749678-0.8073330 .525342$ H -8.137806 $-0.904282-0.273885$ H -7.719539 $0.083815-1.658790$ H $-9.669347 \quad 1.009955-0.015506$ H $-6.9080342 .559217-1.233379$ H $-8.6103610 .206564 \quad 1.920273$ H -8.0466451 .8286292 .251365$ H $-6.879617 \quad 0.499102 \quad 2.112035$ H -7.698205 3.7068520 .560510 H $-4.6931103 .007955-1.834937$ H $-4.2647543 .666603-0.251082$ H $-3.854863 \quad 1.351216 \quad 1.178443$ H $2.4706460 .307268-4.608712$ H $0.8336711 .178788-2.153848$ H $4.1929191 .826084-3.784248$ H $4.0801453 .567498-3.748431$ H $3.1213392 .674548-4.924166$ H -2.815206 $1.247624-1.493909$ H -2.391096 $2.899692-1.892485$ H $-0.433548 \quad 3.1115412 .077297$ H $-1.4666594 .527412 \quad 2.344714$ H -0.0683705 .9116650 .770228$ H 1.8669723 .6536851 .509230 H 3.3225775 .7276761 .461672 H 2.2126626 .5245200 .367766 H $5.1910614 .287026-1.364840$ H $5.222948 \quad 4.7818120 .420099$ H $3.2331935 .132816-2.700664$ H $1.7778165 .591653-1.885872$ H $1.1405583 .284660-1.389110$ H -2.109007 $4.428628-0.030623$ C -1.042286 -2.9855882 .031580$ O $-0.045487-1.9470162 .125677$ C $0.280858-1.7348463 .509576$ C $-0.863718-2.3518184 .302751$ C -1.204450 -3.5519913 .446799$ H $1.199741-2.3013293 .704919$ C $0.521000-0.2512193 .805021$ H $-0.567521-2.6190445 .321638$ H $-1.727628-1.6802974 .365480$ C -2.583971 -4.125474 3.724691 H $-0.453144-4.3324503 .629404$ C 1.5239690 .4599232 .878029 $\begin{array}{lllllll}\text { O } & -0.719960 & 0.461831 & 3.698355\end{array}$ H $0.836504-0.1396154 .849202$ H $1.074466 \quad 0.6259231 .891438$ $\begin{array}{llll}\text { O } & 1.764998 & 1.759513 & 3.437544\end{array}$ C $2.882469-0.2411582 .706766$ C $3.8460490 .588814 \quad 1.835982$ H $2.731084-1.2186392 .242431$ O $3.469124-0.4682443 .986622$ H 3.3621670 .7810280 .871891 H 4.0122051 .5626472 .312208 H $-2.658050-4.4627164 .763828$
H -3.369365 -3.381042 3.556051

H $-2.788235-4.9830443 .076015$

C $-0.590391-4.065578 \quad 1.042287$

H -1.0562070 .3319822 .794582$

H 0.9003192 .1706973 .606159

H 3.5820670 .3975684 .415294

H $-1.286289-4.9116701 .097446$

H $0.391379-4.4408631 .355401$

H $-1.973078-2.5123631 .694958$

C $4.695632-1.454920-0.291246$

O $5.124566-1.394257 \quad 1.074286$

C $5.223928-0.077897 \quad 1.626374$

C $6.2003180 .767560 \quad 0.795938$

C $5.8077120 .791668-0.678392$

C $5.629410-0.634831-1.193804$

H $3.666767-1.082764-0.369512$

C $4.714313-2.931674-0.718385$

H $5.671425-0.1974692 .620897$

O $\quad 6.2644492 .092894 \quad 1.310323$

H 7.2073400 .3398310 .887252

H $4.9070451 .394885-0.839406$

O $6.8539861 .428843-1.414165$

H $6.614690-1.114609-1.251452$

O $5.113314-0.592608-2.521713$

C $3.664641-3.760652-0.012241$

H $4.527091-3.024453-1.793773$

H $5.693359-3.365585-0.480941$

H 6.8712582 .5965880 .741090

H $6.6043431 .400567-2.353859$

H $4.225272-0.200096-2.480353$

O $3.891780-5.080851-0.280028$

$\begin{array}{lllllll}\text { O } & 2.754787 & -3.313847 & 0.670803\end{array}$

C $2.976826-6.0027840 .320761$

H $2.896593-5.8246831 .397688$

H $1.998156-5.919831-0.159705$

H $3.360933-7.0139850 .162581$

SCF Energy (B3LYP/6-31G**//MMFF) = -3245.93788985

0265

MM̄FF Geometry

C $-3.829234 \quad 0.5487832 .813911$

C $-4.336537-0.305015 \quad 1.910744$

$\begin{array}{llll}C & -4.720624 & 0.028088 & 0.488908\end{array}$

$\begin{array}{lllll}\text { O } & -4.440216 & 1.421583 & 0.218608\end{array}$

C $-6.202887-0.231962 \quad 0.166741$

C $-6.692386-1.652564 \quad 0.344930$

C $-6.287639-2.704403-0.393699$

C $-7.725290-1.8391241 .425042$

C $-5.265988-2.708890-1.497295$

C $-4.035297-3.516999-1.066580$

C $-2.850279-3.354893-2.029341$

C $-1.770277-4.387882-1.695561$

C $-0.507215-4.199544-2.543492$

O $-2.335260-2.027200-1.869731$

O $0.536842-4.996958-1.964431$

C $-0.062288-2.728241-2.436862$

C $-0.695898-4.707690-3.977250$

O $1.028582-2.465888-3.317328$

C $-1.204613-1.716387-2.694499$

C $-0.752908-0.267916-2.379615$

O $-1.555798-1.762744-4.082407$

C $-4.065426 \quad 1.754602-1.044205$

O $-4.021853 \quad 1.027315-2.022628$

C $-3.6525473 .171958-1.012921$

C $-2.7038113 .587159-1.864066$

C $-2.1195554 .927658-1.909660$

C $-2.7930186 .006191-1.101353$

C $-0.6079840 .048141-0.887799$

C $0.0989441 .369124-0.633946$

C $0.737176 \quad 2.848648 \quad 1.271229$

$\begin{array}{lllll}\text { O } & 0.786637 & 1.932192 & -1.481159\end{array}$

C 0.4338234 .1924820 .688303

C $1.3319644 .905062-0.010180$

C $1.0903716 .281014-0.563802$

C $1.1209236 .314765-2.076817$

C $2.2856886 .354949-2.745575$

C $-0.2031916 .378796-2.806265$

C -1.003097 5.116409-2.644039 
N $-0.086679 \quad 1.8128790 .671026$ H -3.7119031 .6005262 .566958$ H -4.421212 -1.354403 2.186447 H $-4.071112-0.582161-0.145918$ H $-6.4129190 .078577-0.865806$ H -6.8171280 .4502950 .771468$ H $-6.723805-3.679921-0.181766$ H -8.045851 -2.881825 1.521273 H -7.321986 -1.526759 2.393845 H -8.613946 -1.237827 1.207008 H -5.713153 -3.171871 -2.385361 H $-4.986712-1.695814-1.797117$ H -3.723926 -3.196459-0.063193 H $-4.317564-4.575449-0.997776$ H -3.204671 -3.485284 -3.058340 H -2.159097 -5.407470 -1.811000 H $-1.491546-4.293660-0.636159$ H $\quad 0.236513-5.921843-1.952279$ H $0.323155-2.584196-1.420174$ H -0.939152 -5.777172 -3.974920 H $0.230251-4.613213-4.555074$ H -1.493233 -4.190834 -4.515174 H $1.706885-3.141334-3.146333$ H $\quad 0.174392-0.050463-2.923490$ H -1.486189 $0.436908-2.792492$ H -2.016505 -0.938744 -4.311300 H -4.088916 $3.808968-0.252665$ H $-2.2805172 .867409-2.563450$ H $-3.8468916 .097825-1.387623$ H -2.349611 $6.994846-1.232971$ H -2.739091 $5.777420-0.031364$ H -1.595892 $0.072532-0.416135$ H $-0.014569-0.720270-0.384228$ H $1.7868372 .569017 \quad 1.137250$ H 0.5227782 .8574362 .344794 H -0.5523564 .6063660 .879133$ H $2.3211194 .483505-0.178286$ H $1.8685126 .947121-0.168326$ H $\quad 0.1438406 .696217-0.196847$ H $2.3155256 .404809-3.828818$ H $3.2360456 .335197-2.222040$ H $-0.0424736 .528125-3.882227$ H $-0.7440287 .270412-2.477239$ H $-0.5868124 .265299-3.185167$ H $-0.585518 \quad 1.202780 \quad 1.312429$ C $-1.837321 \quad 0.3682704 .301947$ O $-1.130432-0.5058193 .398819$ C $0.059768-0.9963414 .054541$ C $0.175695-0.2125655 .354290$ C -1.2791250 .0534335 .687001$ H $-0.130159-2.0567474 .262137$ C $1.249244-0.8377043 .100083$ H $0.700172-0.7707406 .135443$ H $\quad 0.709470 \quad 0.732790 \quad 5.194051$ C -1.4749891 .1647046 .703298$ H -1.727129 -0.8725356 .071889$ C $2.615690-1.1650653 .722756$ O $1.027059-1.6892921 .971598$ H 1.2530290 .1863972 .715324 H $2.828040-0.4675494 .541312$ O $2.538224-2.469544 \quad 4.310335$ C $3.791211-1.1630592 .724494$ C $3.930410 \quad 0.150191 \quad 1.934814$ H $3.694222-2.0119282 .040589$ O $4.994466-1.3911073 .467422$ H $3.024356 \quad 0.3159371 .345461$ H 4.0114020 .9816572 .645809 H $-0.988750 \quad 0.9093267 .650598$ H -1.049129 2.1102906 .351199 H -2.538382 1.3282666 .903685 C $-3.343976 \quad 0.132473 \quad 4.175133$ H $1.068375-2.6093562 .284209$ H $3.410480-2.6676044 .692550$ H $5.122771-0.6348684 .065249$ H $-3.886657 \quad 0.714400 \quad 4.927968$ H $-3.570317-0.9265794 .350024$ H -1.585019 1.3971284 .011643 C $4.192311-0.909739-0.928857$
O $5.180749-0.9449440 .108495$

C 5.1634200 .1714871 .003985

C 5.3479601 .4818870 .224969

C $4.3481681 .613305-0.919712$

C $4.3570590 .353349-1.783422$

H $3.188994-0.932137-0.486586$

C $4.382937-2.172673-1.781881$

H 6.0463140 .0606301 .645900

O $5.2328262 .596068 \quad 1.104079$

H $6.3638421 .505459-0.190740$

H $3.3462531 .830363-0.537474$

O $4.7226682 .735844-1.720593$

H $5.2998870 .311505-2.343237$

O $3.3069150 .436112-2.744282$

C $3.912584-3.422929-1.068415$

H $3.820188-2.109408-2.718376$

H $5.448107-2.292270-2.015375$

H 5.3277623 .4013350 .566793

H $4.0892572 .789029-2.456872$

H $2.4637260 .478924-2.261829$

O $4.549310-4.507743-1.600551$

O $3.069693-3.452184-0.182195$

C $4.199665-5.770726-1.025512$

H $4.418093-5.774400 \quad 0.046658$

H $3.143039-5.988278-1.205488$

H $4.803988-6.543040-1.508639$

SCF Energy $\left(B 3 L Y P / 6-31 G^{* *} / / M M F F\right)=-3245.93204206$

0266

MM̄FF Geometry

C -1.3973853 .0717861 .169103$

C -0.6277261 .9769711 .077679$

C -0.2239251 .1324202 .255601$

O 1.2146981 .2204142 .361540

C $-0.622051-0.3333392 .001344$

C $-0.327529-1.2550963 .168598$

C $0.752909-2.0579233 .248010$

C $-1.365324-1.2622684 .258807$

C $1.882812-2.1423212 .259878$

C $1.844594-3.4483401 .457658$

C $2.632295-3.3858800 .138745$

C $4.112550-3.0523330 .337271$

C $4.850738-2.904449-1.003065$

O $1.999162-2.402040-0.686636$

O $6.123054-2.292622-0.741533$

C $4.057341-1.927960-1.898010$

C $5.150277-4.262056-1.648079$

O $4.628218-1.850818-3.201380$

C $2.556109-2.270900-2.000993$

C $1.734918-1.182309-2.735005$

O $2.400490-3.486030-2.743487$

C 1.7484661 .4206603 .593200

O 1.1336291 .5926614 .637619

C 3.2291801 .3990353 .567189

C 3.9682181 .1291282 .479526

C 5.4326671 .0781092 .429218

C 6.1879421 .3561043 .703316

C $1.7841660 .210351-2.095409$

C $2.8299801 .117180-2.720702$

C $4.2528153 .097395-2.219305$

O $3.2211630 .986435-3.878106$

C $5.6084962 .503041-2.002767$

C $6.5201413 .004905-1.156686$

C $7.8760232 .394626-0.947055$

C 8.1182231 .9874570 .489543

C 8.9207342 .7163551 .283404

C 7.5090010 .6906970 .977026

C 6.0347300 .7994041 .254061

N $3.2431302 .120180-1.854928$

H -1.7329373 .4099212 .146159$

H -0.2792671 .6543040 .099132$

H $-0.712099 \quad 1.5039243 .164799$

H $-0.122973-0.7034351 .097265$

H $-1.695975-0.3912371 .778640$

H $0.858108-2.7048264 .117331$

H -1.114836 -1.958284 5.066446

H -1.463685 -0.265886 4.700936

H $-2.337882-1.5618223 .854549$ 
H $\quad 1.882841-1.2798981 .588405$ H $2.825685-2.0829512 .817243$ H $2.224006-4.2697552 .077751$ H $\quad 0.801818-3.6865341 .211471$ H $2.528607-4.364647-0.344778$ H $4.599220-3.8076970 .966370$ H $4.214137-2.1020470 .877457$ H $\quad 6.601202-2.853621-0.107021$ H $4.166609-0.932734-1.449931$ H $5.785788-4.866221-0.989564$ H $5.714519-4.140572-2.579322$ H $4.252091-4.844784-1.861893$ H $5.566224-1.617472-3.093470$ H $2.011488-1.155384-3.795711$ H $0.684991-1.503623-2.719672$ H $1.498008-3.515472-3.102186$ H 3.6904361 .6052894 .528150 H 3.4657300 .9188981 .537114 H 5.9526262 .3580124 .079593 H 7.2726871 .3167263 .584896 H $5.928706 \quad 0.6200024 .472270$ H $\quad 0.8221100 .709706-2.260286$ H $1.9294710 .144045-1.011917$ H $4.0847343 .987833-1.605644$ H $4.1307483 .368359-3.272996$ H $5.8543951 .613058-2.577895$ H $6.2861703 .902376-0.588830$ H $8.6248163 .132094-1.264254$ H $8.0280351 .523576-1.596848$ H 9.1308192 .4172632 .304669 H $9.385073 \quad 3.6312750 .930508$ H $8.0421120 .316071 \quad 1.858436$ H $7.666496-0.0861190 .218197$ H $5.408581 \quad 0.6104640 .382212$ H $2.9226492 .110002-0.891061$ C -3.3552254 .1405520 .017273$ $\begin{array}{lllll}\text { O } & -4.092731 & 2.904890 & 0.050397\end{array}$ C $-5.3002613 .059871-0.720346$ C $-5.3462764 .519641-1.165401$ C $-3.8745264 .880947-1.213049$ H $-5.1890502 .424317-1.606427$ C $-6.5153302 .607166 \quad 0.100985$ H -5.848899 4.639769-2.130103 H $-5.8696515 .152537-0.439634$ C $-3.6157436 .377138-1.196097$ H $-3.4386564 .448577-2.123728$ C -6.4047241 .1842710 .685644$ O -6.6959043 .5039041 .206383$ H -7.423554 $2.694112-0.506291$ H $\quad-5.6297851 .157083 \quad 1.461761$ O $-7.642297 \quad 0.8848161 .344609$ C $-6.1257630 .081845-0.351202$ C $-6.112540-1.3182880 .292091$ H $-5.1607560 .278331-0.825105$ O $\quad-7.125611 \quad 0.137488-1.366288$ H -5.427771 $-1.299371 \quad 1.147119$ H -7.111131 -1.548337 0.683351 H -4.081338 $6.857592-2.062937$ H $-4.0230006 .844726-0.293343$ H -2.542522 $6.588677-1.230154$ C - $-1.8503023 .864804-0.025762$ H -5.8778253 .4895301 .732392$ H -7.8018191 .5895831 .995614$ H -6.831258 $-0.424517-2.102560$ H -1.301695 $4.813257-0.041410$ H $-1.6015203 .324320-0.947388$ H -3.610594 4.6890060 .933923 C $-3.317570-2.159602-0.591152$ O $-4.504041-2.155852-1.393497$ C $-5.710218-2.447955-0.682864$ C $-5.606520-3.833766-0.031180$ C $-4.357362-3.966014 \quad 0.838644$ C $-3.113269-3.531596 \quad 0.062385$ H $-3.392808-1.3862490 .182383$ C $-2.142364-1.808128-1.514130$ H $-6.503444-2.509659-1.438254$ O $-6.771951-4.099456 \quad 0.742190$ H -5.566454 -4.593971 -0.822437
H $-4.465893-3.3996091 .771113$

O $-4.224688-5.3389411 .214063$

H -2.877182 $-4.283292-0.701776$

O $-2.003071-3.4571350 .952633$

C $-2.231754-0.376535-1.995190$

H -1.179358 -1.919503-1.005404

H -2.152818 -2.473952 -2.385772

H $-6.656655-4.9745311 .150726$

H -3.491648 -5.4002241 .849268$

H -1.816634 -4.356673 1.269600

O $-1.875913-0.304299-3.309662$

O $-2.555796 \quad 0.569160-1.289949$

C $-1.9249261 .004889-3.886316$

H $-2.9525211 .381028-3.878515$

H $-1.2612101 .686515-3.345612$

H -1.584103 $0.932780-4.922418$

SCF Energy $\left(B 3 L Y P / 6-31 G^{* *} / / M M F F\right)=-3245.91404705$

0267

MM̄FF Geometry

C -2.261952 $5.023501 \quad 1.324720$

C $-2.9071293 .947148 \quad 0.848296$

C -3.8777093 .1261381 .658154$

O -3.4917021 .7317181 .552697$

C -5.2975853 .2807301 .088710$

C -6.3488022 .5349231 .888143$

C -6.9400231 .3858871 .503673$

C -6.7519483 .1903293 .184590$

C -6.6801340 .5854050 .257922$

C $-6.215736-0.8364160 .599734$

C $-5.819121-1.625833-0.656774$

C $-5.431777-3.059884-0.292310$

C $-4.909381-3.845392-1.504614$

O $-4.708424-0.956206-1.262699$

O $-4.267517-5.033822-1.016145$

C $-3.824347-3.009009-2.216940$

C $-6.051321-4.312725-2.414711$

O $-3.424952-3.629838-3.436176$

C $-4.249887-1.550645-2.484947$

C $-3.096301-0.668555-3.024556$

O $-5.282687-1.540291-3.478566$

C $-2.419377 \quad 1.3215952 .287145$

O $-1.7289912 .007707 \quad 3.024175$

C -2.229531-0.127261 2.044803

C $-1.212358-0.7746612 .634265$

C $-0.929237-2.2104212 .545469$

C -1.812226 -3.0442501 .656263$

C $-1.887891-0.515733-2.094531$

C $-0.762323-1.500427-2.365769$

C $1.431086-2.231321-1.425414$

O $-0.731432-2.240040-3.344939$

C $1.262078-3.397408-0.505086$

C $2.093375-3.6542840 .515195$

C $1.968753-4.8403931 .424103$

C $1.866239-4.4499592 .880600$

C $2.949950-4.4538883 .675346$

C $0.492235-4.1580593 .437403$

C $0.085516-2.717118 \quad 3.277428$

N $0.226039-1.425653-1.394552$

H -2.4927645 .3577732 .334473$

H $-2.7091243 .591343-0.158909$

H -3.863109 3.4211142 .714755

H -5.3093272 .9621720 .041137$

H -5.5746814 .3436961 .067175$

H -7.7012710 .9596702 .156246$

H -7.5587182 .6497543 .691130$

H -5.903856 3.2348343 .874542

H -7.1059184 .2099613 .000573$

H $-7.6103450 .534097-0.320720$

H $-5.9415941 .066628-0.386630$

H $-5.355300-0.775306 \quad 1.277631$

H -7.015022 -1.3657841 .133363$

H -6.667206 -1.621935 -1.351791

H -6.276437 -3.584867 0.170908

H -4.642042 -3.038295 0.469791

H $-4.920600-5.536475-0.499455$

H -2.944289 -3.008019-1.562701

H $-6.737041-4.967013-1.862945$ 
H $-5.672024-4.913565-3.248656$ H $-6.640490-3.488380-2.821628$ H $-3.142723-4.535377-3.221460$ H -2.797446 -1.007244 -4.024029 H $-3.5056520 .341353-3.172398$ H $-5.309910-0.659204-3.887162$ H $-2.947637-0.6280761 .406802$ H $-0.533320-0.2060423 .268765$ H -1.456345 -4.068427 1.525526 H -1.864697 -2.6154140 .650205$ H -2.824549 -3.0983902 .069879$ H -1.459769 $0.482990-2.249416$ H -2.186820 -0.582030 -1.044150 H $2.261219-1.584362-1.124627$ H $1.617436-2.585097-2.444260$ H $0.431804-4.071627-0.701332$ H $2.936284-2.9911930 .689878$ H $2.853661-5.4719181 .269388$ H $1.111404-5.4676501 .150407$ H $2.878148-4.2075594 .729468$ H $3.933874-4.6955293 .286828$ H $0.479296-4.3769604 .514202$ H -0.234035 -4.857041 3.012721 H $0.672340-2.0322093 .891283$ H $0.096101-0.804527-0.600967$ C $-0.5242375 .135886-0.581430$ O $0.0067323 .860970-0.179009$ C $1.3018423 .664094-0.783898$ C $1.4521694 .781069-1.804678$ C $0.6653305 .904368-1.152814$ H 2.0193983 .7826230 .035628 C $1.3429262 .238331-1.339652$ H $2.4961575 .046486-1.992787$ H $0.9921864 .501469-2.760528$ C $0.2800887 .015878-2.112694$ H $\quad 1.269023 \quad 6.325918-0.337882$ C $2.6531311 .777012-2.003666$ O $1.0660831 .333028-0.263087$ H $0.5234472 .121915-2.060200$ H $2.506057 \quad 0.732298-2.307157$ O $2.9191202 .520422-3.187043$ C $3.8848151 .815018-1.078527$ C $5.0630571 .005899-1.651828$ H $3.6064331 .401822-0.105236$ O $4.3095563 .157322-0.859087$ H $4.711547-0.003001-1.894975$ H $5.4031101 .462134-2.589709$ H $1.1740507 .485435-2.535960$ H $-0.3257456 .637484-2.942903$ H $-0.2977777 .790894-1.599514$ C -1.1785295 .8104300 .628571$ H $\quad 0.231354 \quad 1.6165200 .148253$ H $2.1459092 .430756-3.769852$ H $4.5190793 .544198-1.726597$ H -1.584245 6.7875200 .343146 H -0.4010865 .9925381 .382535$ H -1.267896 $4.947515-1.366143$ C $5.389090-0.7671130 .793602$ $\begin{array}{lllll}\text { O } & 5.863096 & 0.576801 & 0.649743\end{array}$ C $6.2626820 .928938-0.678879$ C $7.3979420 .004746-1.140369$ C $7.040353-1.473041-0.978765$ C $6.501412-1.7535060 .426417$ H $4.521637-0.9266620 .141819$ C $4.927168-0.9384132 .243275$ H $6.6761531 .943446-0.613142$ O $7.7328690 .281729-2.496107$ H $8.2914810 .217051-0.538788$ H $6.327844-1.788496-1.749928$ O $8.224111-2.246298-1.188466$ H $7.325038-1.6821861 .147653$ O $6.011971-3.0915150 .481976$ C $3.634786-0.1869432 .466830$ H $4.735117-1.9876582 .491598$ H $5.683095-0.5733762 .948708$ H $8.441014-0.333890-2.752025$ H $7.990187-3.178951-1.041550$ H $5.280555-3.169204-0.154072$
O $3.8819901 .007625 \quad 3.074831$

O $2.538895-0.5975352 .107180$

C 2.7328531 .8325373 .293060

H 3.0577632 .7368563 .814333

H 2.2878732 .1200742 .335902

H 2.0016291 .3107373 .917984

SCF Energy (B3LYP/6-31G**//MMFF)= -3245.92063562

02 68

MM̄FF Geometry

C $0.1859575 .122508-0.125296$

C $1.4418264 .654340-0.044947$

C 2.2381514 .3807361 .213667

O 2.8207483 .0570951 .093095

C 1.4266614 .4194472 .524767

C 2.2372624 .0718203 .760245

C 2.1489902 .9075124 .435415

C 3.1649335 .1563664 .243912

C 1.3001221 .7129404 .096240

C 2.1544170 .4458863 .947223

C $1.380658-0.6856063 .253286$

C $2.215916-1.9666413 .213500$

C $1.538784-3.0771352 .395874$

O $1.087471-0.2474621 .921968$

O $2.519473-4.0939802 .137134$

C $1.113701-2.5039321 .026911$

C $0.408175-3.7544943 .179796$

O $0.350160-3.4577420 .294094$

C $0.334569-1.1789421 .135221$

C $0.065249-0.514725-0.236402$

O $-0.943732-1.434091 \quad 1.729035$

C 4.0459902 .9632090 .504328

O 4.6927223 .8690420 .002529

C 4.4790981 .5504670 .582353

C 5.612748 1.161203-0.019864

C $6.176029-0.191683-0.009725$

C $5.390154-1.2705180 .688024$

C $1.301549-0.080816-1.026649$

C $1.807867-1.106402-2.025173$

C $3.715192-1.497572-3.586780$

O $1.200577-2.134916-2.306914$

C $4.788053-2.295183-2.915809$

C $6.085049-2.217045-3.250256$

C $7.163156-3.050040-2.619617$

C $8.302111-2.218815-2.074011$

C $9.432096-2.046342-2.780541$

C $8.168571-1.671249-0.671631$

C $7.363787-0.402235-0.615189$

N $3.013599-0.711208-2.590779$

H -0.3677175 .3571490 .778898$

H $1.9719694 .465602-0.977281$

H 3.0386175 .1288661 .275659

H 0.5608863 .7553922 .437510

H 1.0058495 .4234832 .670000

H 2.7603362 .7849595 .328740

H 3.6804394 .8807945 .170074

H 3.9313065 .3694563 .492409

H 2.6043026 .0758824 .441372

H $\quad 0.5691831 .5619034 .899774$

H $0.7244991 .876098 \quad 3.183088$

H 3.0495450 .6819393 .359009

H 2.4932020 .1177514 .937768

H $\quad 0.442256-0.853787 \quad 3.794775$

H $2.437523-2.3170144 .229255$

H $3.190005-1.7495632 .755886$

H $2.852869-4.4135302 .992991$

H $2.032781-2.3298180 .454245$

H $\quad 0.799879-4.2154794 .094570$

H $-0.040270-4.5704432 .602332$

H $-0.384214-3.0634643 .474487$

H $0.883431-4.268496 \quad 0.228897$

H $-0.595721-1.147728-0.839066$

H $-0.5060890 .402719-0.036125$

H $-1.545431 \quad-0.7103261 .488335$

H $3.8549570 .865837 \quad 1.143068$

H $6.194331 \quad 1.904941-0.563178$

H $5.357261-1.0802651 .766071$

H $5.801550-2.2721010 .546429$ 
H $4.364526-1.3113590 .305790$ H $1.0397500 .809741-1.612953$ H $2.1147690 .198828-0.353480$ H $4.119519-0.798393-4.325575$ H $3.014060-2.169951-4.091229$ H $4.478355-2.988332-2.137164$ H $6.387610-1.541536-4.047410$ H $7.540283-3.739913-3.386073$ H $6.762544-3.688339-1.822438$ H $10.266426-1.478190-2.382644$ H $9.545404-2.464884-3.775131$ H $9.163189-1.438200-0.267200$ H $7.783411-2.453266-0.011281$ H $7.8545000 .442720-1.100506$ H $3.462050 \quad 0.138035-2.259216$ C -1.927752 $4.778490-1.442715$ O $-1.8370013 .341993-1.519372$ C $-2.8568902 .857090-2.423283$ C -3.743355 $4.052821-2.738512$ C $-2.7555925 .198034-2.655904$ H $-2.3224252 .530227-3.324036$ C $-3.5865031 .675612-1.771181$ H $-4.2260753 .971775-3.717015$ H $-4.5275054 .168925-1.979765$ C $-3.4183116 .557853-2.517861$ H $-2.1307365 .190236-3.559218$ C $-4.8130041 .171736-2.551429$ O $-2.649240 \quad 0.604817-1.634463$ H $-3.8776411 .959179-0.753964$ H $-5.5845281 .950049-2.576643$ O $-4.415130 \quad 0.949491-3.910555$ C -5.416704 -0.143836 -2.018109 C $-5.824790-0.086510-0.536449$ H $-4.716013-0.965615-2.192258$ O $-6.576679-0.444592-2.803917$ H $-4.960591 \quad 0.1903560 .072954$ H -6.576242 $0.702604-0.411589$ H $-4.0570656 .763507-3.383024$ H $-4.0424256 .610864-1.619454$ H -2.670012 $7.353849-2.455161$ C $-0.5263985 .394231-1.423235$ H -2.406656 $0.307137-2.528154$ H $-5.1953020 .623859-4.391638$ H -7.225386 0.265675 -2.660892 H $-0.5983906 .481537-1.539769$ H $0.0600065 .006588-2.265288$ H -2.464993 $5.015334-0.514643$ C $-4.355979-2.4856240 .683733$ O $-5.497553-2.513747-0.183331$ C $-6.411924-1.422233-0.024735$ C $-6.926401-1.3706581 .421091$ C $-5.783647-1.3353692 .431352$ C $-4.797626-2.4689472 .154532$ H -3.745997 -1.601801 0.462663 C $-3.516021-3.7387750 .388664$ H $-7.276127-1.655138-0.659193$ O $-7.766445-0.2395951 .625895$ H -7.535069 -2.262357 1.620539 H $-5.277852-0.3627212 .432580$ O $-6.314925-1.5140193 .746380$ H $-5.272451-3.4235332 .414522$ O $-3.665098-2.3257293 .008967$ C $-2.831628-3.682294-0.960525$ H -2.728407 -3.8600831 .140520$ H $-4.164688-4.623048 \quad 0.395153$ H $-8.497102-0.2994740 .986904$ H -6.969457 -0.8094513 .891715$ H -3.232293 -1.482237 2.793168 O $-2.254892-4.895409-1.212709$ O $-2.789252-2.705127-1.694245$ C $-1.552675-4.995455-2.455431$ H $-0.732860-4.272081-2.487565$ H $-1.134117-6.002647-2.529312$ H -2.240059 -4.838361 -3.292150 SCF Energy (B3LYP/6-31G**//MMFF)= -3245.91869299

0269

MM̄FF Geometry
C $-0.6347180 .730218-2.593896$

C $-1.315798-0.419840-2.720663$

C $-0.910759-1.743537-2.114947$

O $-0.082094-1.476969-0.959190$

C $-0.135146-2.597851-3.126358$

C $0.027359-4.036912-2.679434$

C $1.159199-4.570477-2.179701$

C $-1.193356-4.903780-2.861263$

C $2.474660-3.889416-1.925281$

C $2.835600-3.937545-0.435950$

C $4.202300-3.301794-0.148475$

C $4.516154-3.3662341 .347908$

C $5.836847-2.6609571 .688213$

O $4.169832-1.937761-0.581215$

O $5.886427-2.4788173 .111550$

C $5.830107-1.2553701 .053171$

C $7.053285-3.5172411 .317472$

O $7.095400-0.6177901 .215301$

C $5.420000-1.250095-0.438855$

C $5.2269520 .205919-0.917240$

O $6.453508-1.861851-1.212121$

C $-0.472160-1.961307 \quad 0.245913$

O $-1.324920-2.8141450 .449483$

C $0.237083-1.2887131 .362005$

C $1.059302-0.2408501 .194742$

C 1.7168930 .5319092 .252979

C 1.4474780 .1395713 .680427

C $4.6324930 .350171-2.324760$

C $4.4549021 .822016-2.641123$

C $3.0294143 .769237-2.018725$

O $5.3095902 .459901-3.251299$

C $3.4838654 .220672-0.665142$

C 2.6525044 .5473280 .336272

C 3.1245494 .9060721 .716737

C 2.6717153 .9158832 .771018

C 1.8190994 .2827743 .742460

C 3.2735742 .5251862 .758179

C 2.4993221 .5696901 .890078

N $3.2843782 .343065-2.112299$

H $0.3293970 .733349-2.092290$

H -2.270736 -0.412202 -3.240526

H -1.836447 -2.245647 -1.809496

H $\quad 0.839089-2.138743-3.325229$

H $-0.656387-2.601758-4.093309$

H $1.150079-5.627833-1.916336$

H - $-1.012485-5.944665-2.572255$

H -2.023728 -4.534964 -2.252106

H - $-1.505165-4.904535-3.910929$

H $3.246437-4.408191-2.506644$

H $2.471419-2.851922-2.267877$

H $2.061469-3.407200 \quad 0.130136$

H $2.837721-4.977619-0.085910$

H $4.960336-3.845485-0.724751$

H $4.534433-4.4057851 .698091$

H $3.708763-2.8754811 .910208$

H $5.815664-3.354748 \quad 3.528310$

H $5.104168-0.6480921 .612355$

H $7.029860-4.4703921 .859620$

H $7.987010-3.0273611 .614986$

H $7.107698-3.7465880 .251318$

H $7.305674-0.6289302 .164731$

H $4.5626060 .723858-0.215785$

H $6.188770 \quad 0.734581-0.893011$

H $6.389964-1.552591-2.129090$

H -0.003805 -1.673141 2.348049

H $\begin{array}{llll}1.257851 & 0.113827 & 0.185306\end{array}$

H $1.817210-0.8731663 .875364$

H $\quad 0.3726150 .168733 \quad 3.891610$

H 1.9216200 .7991714 .410209

H $5.285509-0.082218-3.089455$

H $3.663746-0.156292-2.402355$

H $1.9581763 .922889-2.169827$

H $3.5757154 .306123-2.800122$

H $4.5576714 .238281-0.492111$

H 1.5782644 .5246940 .169135

H 2.7502145 .9119421 .946095

H 4.2188134 .9805561 .753157

H 1.5133903 .5863654 .516410 
H $1.402305 \quad 5.2835303 .781964$ H 3.3737782 .1515063 .782236 H 4.3046592 .5775152 .384963 H 2.6178321 .7616750 .825422 H $2.6769391 .729524-1.576722$ C -1.193816 $3.023420-1.831650$ O $-1.9951772 .456611-0.781159$ C -2.626965 $3.521839-0.048063$ C $-2.1764674 .826955-0.703135$ C $-1.8322984 .378389-2.113423$ H $-3.7062933 .405093-0.199244$ C $-2.295043 \quad 3.386521 \quad 1.444499$ H -2.960632 $5.590085-0.677677$ H $-1.2931315 .249821-0.211303$ C $-0.9349555 .350429-2.857486$ H $-2.7663304 .242997-2.675131$ C -2.5281791 .9717002 .015747$ O $-0.906584 \quad 3.6901841 .632057$ H -2.8552574 .1356012 .015879$ H -1.784859 1.2844871 .595452 O -2.2614622 .0185533 .423233$ C -3.9385671 .4029231 .784091$ C -4.0959400 .0006082 .398313$ H $-4.131442 \quad 1.3523530 .711060$ $\begin{array}{llll}\text { O } & -4.891226 & 2.296212 & 2.355764\end{array}$ H $-3.278057-0.6304892 .033152$ H -3.9902240 .0710493 .487731$ H - $-1.4318926 .319043-2.974307$ H $0.0053125 .519275-2.322310$ H $-0.6923414 .973297-3.855795$ C - $-1.1603852 .070156-3.028352$ H -0.7124883 .5787982 .578356$ H -2.945306 2.5770443 .831626 H -5.7694722 .0421832 .025707$ H $-0.5146882 .472104-3.816372$ H $-2.1683181 .961193-3.445573$ H $-0.1821643 .150206-1.424917$ C $-4.946513-1.566699-0.099315$ O $-5.756030-0.6840240 .687122$ C -5.455991-0.665404 2.086906 C $-5.609351-2.0746592 .677543$ C $-4.772348-3.0989791 .914904$ C $-5.060899-3.0077090 .417481$ H $-3.901808-1.233933-0.070527$ C $-5.444451-1.485614-1.552747$ H $-6.227171-0.0414252 .555244$ O $-5.248239-2.065404 \quad 4.054241$ H -6.665782 -2.368406 2.622307 H -3.701759 -2.9791712 .117190$ O $-5.120445-4.4152422 .350180$ H $-6.068123-3.3974370 .220007$ O $-4.140873-3.835638-0.290327$ C $-5.144512-0.158495-2.215676$ H $-4.965533-2.260613-2.161495$ H $-6.532007-1.625071-1.575641$ H -5.473051 -2.935246 4.424686 H $-4.825718-4.5066713 .271791$ H $-4.212671-4.7325440 .078936$ O $-5.688013-0.160550-3.469767$ O $-4.509930 \quad 0.758528-1.715774$ C $-5.4773741 .031566-4.233009$ H $-5.8796481 .900661-3.703742$ H $-4.411005 \quad 1.162635-4.438137$ H $-6.0069950 .924634-5.183352$ SCF Energy (B3LYP/6-31G**/MMFF) $=-3245.91486785$

027

MMFF Geometry

C $-0.898267 \quad 2.6484640 .691571$

C $-1.8199372 .759745 \quad 1.660645$

C -2.372445 1.6508202 .528912

O $-1.861996 \quad 0.353227 \quad 2.150190$

C $-3.9032011 .570702 \quad 2.374593$

C -4.6005271 .0595293 .619131$

C $-4.945298-0.2227453 .845659$

C $-4.950992 \quad 2.123946 \quad 4.627620$

C $-4.639475-1.4308473 .006690$

C $-5.876311-2.0220362 .317849$
C $-6.300608-1.2482831 .059296$

C $-7.530897-1.8972240 .420785$

C $-7.903866-1.244865-0.918964$

O $-5.202821-1.2824620 .142456$

O $-8.844909-2.122641-1.555503$

C $-6.641986-1.166245-1.806941$

C $-8.6248830 .092202-0.710648$

O $-6.898606-0.430725-3.001239$

C $-5.418314-0.565288-1.078255$

C $-4.146296-0.730814-1.944607$

$\begin{array}{lllll}\text { O } & -5.654483 & 0.824956 & -0.846987\end{array}$

C $-0.650888-0.046900 \quad 2.612522$

$\begin{array}{llllll}\text { O } & 0.124285 & 0.580588 & 3.313644\end{array}$

C $-0.424910-1.4034412 .068850$

C $0.824103-1.828508 \quad 1.835148$

C $1.197336-3.1380341 .294176$

C $0.100858-4.1521641 .096392$

C $-2.846409-0.352177-1.222588$

C $-1.662921-0.516426-2.152087$

C $-0.079731-2.186848-3.051004$

O $-1.287828 \quad 0.399075-2.881199$

C $0.619631-3.411760-2.558542$

C $1.952157-3.498402-2.426017$

C $2.670085-4.752627-2.019689$

C $3.651704-4.534370-0.891096$

C $4.958821-4.348187-1.141792$

C $3.134915-4.6423910 .524773$

C $2.495682-3.3721661 .013567$

N $-1.104708-1.785324-2.106628$

H $-0.469561 \quad 1.6788190 .458059$

H -2.2028653 .7536201 .888058$

H $-2.100347 \quad 1.859827 \quad 3.571296$

H -4.1577090 .9344801 .520116$

H -4.3262712 .5568872 .140213$

H $-5.476741-0.4520064 .768825$

H -5.4457101 .7128445 .514025$

H -4.0473952 .6415284 .965242$

H -5.6282372 .8602764 .182691$

H $-3.852351-1.2350592 .275251$

H -4.226770 -2.191179 3.682511

H $-5.623503-3.0503652 .026866$

H $-6.712876-2.083023 \quad 3.024693$

H $-6.514282-0.2092631 .333377$

H -8.384071 -1.8752351 .110479$

H -7.324374 -2.961436 0.237807

H $-9.141673-1.701298-2.379630$

H $-6.393235-2.190668-2.117555$

H -9.560882 -0.061958 -0.160293

H $-8.9107740 .542119-1.667821$

H $-8.0291760 .818635-0.153772$

H $-7.599353-0.894948-3.488935$

H $-4.059262-1.777859-2.263553$

H $-4.233519-0.122696-2.854053$

H $-4.808878 \quad 1.298283-0.825615$

H -1.301232 -2.002551 1.848848

H $1.649993-1.1528452 .053147$

H $-0.368351-4.4006322 .054634$

H $0.447419-5.0894110 .656268$

H $-0.668570-3.7643640 .420011$

H $-2.8569080 .688804-0.885657$

H $-2.696700-0.973453-0.332676$

H $0.608076-1.351043-3.211763$

H $-0.578197-2.400096-4.002477$

H $0.005539-4.283069-2.343714$

H $2.567104-2.636674-2.673543$

H $3.202740-5.128824-2.903316$

H $1.965175-5.546586-1.743356$

H $5.678331-4.232170-0.338276$

H $5.338566-4.304377-2.157329$

H $3.965914-4.8652121 .208097$

H $2.475599-5.5110360 .608069$

H $3.209028-2.5657561 .179137$

H -1.615230 -2.515285 -1.617353

C $-0.1754213 .486874-1.566477$

O $0.9095722 .542285-1.700600$

C $1.8391703 .036102-2.679726$

C $1.0682354 .072307-3.485162$

C $0.2186714 .707117-2.402714$ 
H $2.6423893 .540939-2.129319$

C $2.4192751 .884220-3.504901$

H $1.7282074 .785149-3.988282$

H $0.4281563 .604913-4.242457$

C $-0.9658205 .491692-2.940099$

H $0.8523025 .380838-1.809996$

C $3.0931130 .769963-2.677120$

O $1.3723641 .277233-4.272387$

H $3.1304752 .282509-4.237851$

H $2.342804 \quad 0.206573-2.109529$

O $3.676545-0.156140-3.603717$

C $4.1962141 .258156-1.718849$

C $4.9864860 .099648-1.077684$

H $3.7437411 .868364-0.931607$

O $5.1062762 .098643-2.429972$

H $4.280273-0.597646-0.615007$

H $5.516449-0.448280-1.865585$

H $-0.6247756 .317919-3.572431$

H -1.626923 $4.858721-3.541740$

H -1.557362 $5.914147-2.121711$

C $-0.3713303 .823031-0.086856$

H $0.6728491 .008925-3.651880$

H $2.965024-0.463830-4.191429$

H $5.4843071 .573761-3.156293$

H $-1.048207 \quad 4.6791360 .014917$

H $\quad 0.5942594 .1043230 .349227$

H -1.072968 $3.007355-1.976210$

C 4.6634170 .4898631 .950167

O 5.4238441 .2985811 .043800

C $6.0278700 .590497-0.044631$

C $6.956550-0.5080770 .492157$

C $6.235613-1.4323471 .465779$

C $5.535317-0.6205862 .552018$

H $3.8071190 .052644 \quad 1.424040$

C 4.1319441 .4102243 .059810

H $6.668516 \quad 1.316969-0.559840$

O $7.503678-1.283073-0.569290$

H $7.800921-0.0436501 .018208$

H $5.527381-2.0844710 .945975$

O $7.187825-2.2934552 .093337$

H $6.287638-0.187263 \quad 3.223725$

O $4.717350-1.4894953 .333026$

C 3.0597542 .3598692 .571592

H $3.689190 \quad 0.822033 \quad 3.871055$

H 4.9566822 .0144723 .457153

H $7.968388-0.672687-1.166976$

H $7.649208-2.7769041 .386412$

H $5.289540-2.1984173 .673867$

$\begin{array}{lllll}\text { O } & 2.811241 & 3.278377 & 3.552361\end{array}$

O $2.4980482 .296588 \quad 1.487511$

C 1.8047104 .2496853 .253194

H 0.8297563 .7626093 .167706

H 1.7673324 .9653734 .078712

H 2.0533524 .7891362 .334378

SCF Energy (B3LYP/6-31G**/MMFF)= -3245.93004037

0270

MM̄FF Geometry

C -0.563909 -1.988321 0.888068

C $-1.235582-2.761998 \quad 0.021634$

C $-1.663406-2.368409-1.374253$

O $-1.171141-1.047852-1.696063$

C $-3.195989-2.330264-1.475544$

C -3.684604 -2.300094 -2.910009

C $-4.132214-1.201717-3.548885$

C $-3.694646-3.634473-3.611356$

C $-4.1955280 .210189-3.036320$

C $-5.6387310 .687535-2.817259$

C $-6.3084940 .052782-1.588332$

C -7.769305 $0.493301-1.477602$

C $-8.430099-0.037575-0.195938$

O $-5.5843420 .482638-0.432347$

O $-9.6674520 .667124-0.012663$

C $-7.530387 \quad 0.307328 \quad 1.009478$

C $-8.789963-1.523596-0.311040$

O $-8.027354-0.2902772 .205040$

C -6.046233 -0.0745290 .804468$

C $-5.1869450 .529214 \quad 1.941346$
O $-5.920729-1.4971640 .824486$

C $-0.102411-0.934470-2.522595$

O $0.460823-1.840514-3.121190$

C $0.3549890 .471984-2.626393$

C $-0.1011861 .463702-1.844744$

C $0.3478132 .858287-1.863229$

C $1.4367903 .232270-2.833951$

C $-3.6729820 .403218 \quad 1.718184$

C -2.8797561 .1445292 .780688$

C -0.5788081 .9952343 .231766$

O $-\begin{array}{llll}-3.379089 & 1.568898 & 3.819344\end{array}$

C -0.5408993 .4089342 .741130$

C 0.5510283 .9822642 .214565

C 0.5949245 .3933421 .704140

C 1.0747405 .4791590 .270855

C $2.3288265 .871318-0.011187$

C $0.0819965 .195762-0.835454$

C $-0.215293 \quad 3.729452-0.999548$

N -1.540140 1.2570932 .434884

H $-0.300515-0.9708980 .613652$

H $-1.482669-3.7798010 .317304$

H -1.255917 -3.113665 -2.067376

H $-3.577487-1.464501-0.924238$

H $-3.636008-3.209347-0.985789$

H -4.482155 -1.314728 -4.574555

H $-4.102972-3.568865-4.625579$

H -2.680017 -4.036106 -3.691927

H -4.309595 -4.350317 -3.055992

H $-3.6016080 .350948-2.128541$

H $-3.7294110 .852313-3.794553$

H $-5.6080151 .776720-2.682666$

H $-6.2361730 .488613-3.715659$

H $-6.249543-1.037977-1.670006$

H $-8.3387280 .179633-2.361468$

H -7.819474 $1.591262-1.463248$

H $-10.2171900 .517197-0.800864$

H -7.5896441 .3946381 .159428$

H $-9.483715-1.685825-1.144790$

H $-9.313038-1.8738450 .585530$

H -7.921171 -2.164142 -0.476463

H $-8.955040-0.0151422 .302275$

H -5.4202241 .5982382 .035215$

H -5.4438090 .0543322 .896635$

H -5.020867 -1.734302 1.097480

H $1.1309610 .639811-3.366378$

H $-0.8680151 .240924-1.104736$

H $2.3318202 .625556-2.660564$

H $1.7519194 .274776-2.754337$

H $1.1018403 .077888-3.865501$

H -3.364480 -0.646592 1.747754

H -3.3993450 .8154440 .740110$

H $0.386900 \quad 1.4912223 .125419$

H -0.8734751 .9722514 .285500$

H -1.4587043 .9863612 .822679$

H 1.4685863 .4059622 .145953

H 1.2655475 .9633532 .360278

H -0.3846575 .8802121 .786535$

H $2.6760145 .965168-1.034634$

H $3.039356 \quad 6.1012940 .775885$

H $0.4191765 .632099-1.782626$

H $-0.8581365 .718783-0.616898$

H $-0.9950843 .365052-0.330138$

H $-1.243980 \quad 0.950680 \quad 1.513235$

C $1.420277-2.4077472 .318425$

O $1.955297-3.4527761 .476507$

C $3.027109-4.0983202 .176292$

C $3.375138-3.1941193 .349693$

C $1.995603-2.6677653 .709728$

H $2.605180-5.0248422 .591277$

C $4.173501-4.4976001 .245023$

H $3.856915-3.7331284 .170787$

H $4.031272-2.3721223 .041432$

C $2.019933-1.4505384 .615081$

H $1.429555-3.4686724 .204540$

C $4.936266-3.3791350 .502622$

O $5.117914-5.2307422 .037001$

H $3.781047-5.2038090 .502073$

H $5.538271-2.8050201 .216203$ 
O $5.887691-4.033752-0.353845$

C $4.095451-2.406913-0.347738$

C $4.954775-1.553250-1.303115$

H $3.521884-1.7478390 .309988$

O $3.171957-3.137146-1.157299$

H $5.816890-1.158700-0.757491$

H $5.337467-2.200158-2.101332$

H $2.491150-1.6934465 .573077$

H $2.582517-0.6283194 .165280$

H $1.005738-1.0930274 .818322$

C $-0.105444-2.4355032 .248786$

H $5.834324-5.5156351 .443927$

H $5.386537-4.508126-1.039664$

H $2.488002-3.501075-0.570154$

H $-0.535697-1.7590842 .995418$

H $-0.473949-3.4460782 .462545$

H $1.799693-1.4549321 .927385$

C $4.8114051 .399424-0.477101$

O $3.7476450 .576577-0.974789$

C $4.138413-0.405377-1.942696$

C $4.8123130 .274470-3.143034$

C $5.9676861 .170701-2.712126$

C $5.5124912 .140426-1.622672$

H 5.5394880 .7798860 .059910

C 4.2009782 .3908180 .525263

H $3.208664-0.847840-2.319665$

O $5.285461-0.687426-4.079340$

H $4.0713510 .891561-3.667552$

H $\quad 6.8224110 .574019-2.373230$

O $6.4353251 .918881-3.837107$

H $4.8397192 .892528-2.053702$

O $6.6515602 .829192-1.109813$

C 3.9635411 .7440851 .872644

H 4.8841933 .2332710 .681750

H 3.2396172 .7728140 .165948

H $4.527750-1.237914-4.341244$

H $5.6944652 .451197-4.173750$

H $7.0925913 .260911-1.861518$

O 4.0039062 .7023542 .844591

O 3.7446690 .5534822 .047378

C 3.7782592 .2423284 .180498

H 2.7417611 .9113104 .290309

H 4.4723451 .4347404 .433003

H 3.9528783 .0791484 .861913

SCF Energy (B3LYP/6-31G**//MMFF)= -3245.92299807

0271

MMFF Geometry

C -2.498515 -0.072389 3.280447

C $-2.1746361 .200276 \quad 3.559733$

C -2.5501712 .4367092 .773884$

O -3.3446222 .1170381 .611465$

C -1.2833363 .1355472 .249802$

C -1.468333 4.624917 2.040067

C -1.7588835 .2147520 .864216$

C -1.2462945 .4678533 .270481$

C -2.065741 $4.566919-0.457056$

C $-0.9236664 .705516-1.474322$

C $0.1619963 .634761-1.292963$

C $1.3286673 .861878-2.254644$

C $2.3718742 .737281-2.162733$

O $-0.4434352 .364673-1.560943$

O $3.2410562 .890697-3.294704$

C $1.6543371 .375400-2.285111$

C $3.2500712 .880056-0.914222$

O $2.5535720 .297273-2.037990$

C $0.4201641 .241474-1.362141$

C $-0.366911-0.041017-1.724651$

O $0.8614831 .151580-0.006814$

C -4.694195 2.0503451 .736573

O -5.3556352 .1897552 .751101$

C $-5.2234541 .742990 \quad 0.390148$

C $-6.442901 \quad 1.2041610 .255189$

C -7.065123 $0.802971-1.008975$

C $-6.322300 \quad 1.115567-2.282848$

C $-1.755200-0.141343-1.073943$

C $-2.432607-1.465664-1.390368$

C $-4.497469-2.765664-0.865617$
O $-1.922433-2.326551-2.102802$

C $-5.577686-2.546567-1.877567$

C $-6.872137-2.814782-1.644702$

C $-7.961249-2.634144-2.664316$

C $-9.150603-1.866499-2.127894$

C $-10.269492-2.505700-1.746346$

C $-9.073821-0.355612-2.114100$

C $-8.252706 \quad 0.163640-0.966761$

N $-3.671460-1.575313-0.773443$

H -3.099200 -0.296506 2.404886

H $-1.5910051 .394068 \quad 4.458598$

H -3.1132183 .1070443 .435305$

H -0.9679012 .6645091 .313558$

H -0.4441713 .0002372 .945328$

H $-1.825521 \quad 6.3022230 .843902$

H -1.3854456 .5364143 .075396$

H -1.948432 5.1838794 .060602

H $-0.2267015 .330768 \quad 3.645532$

H -2.357931 $3.520053-0.341528$

H -2.951083 $5.074700-0.861399$

H -1.359439 $4.595938-2.476060$

H $-0.4840135 .708804-1.418751$

H $0.5183343 .662181-0.257928$

H $1.7985344 .836784-2.074609$

H $0.9482513 .898756-3.285364$

H $3.9235392 .200597-3.248017$

H $1.3207561 .273406-3.327282$

H $3.8043803 .825680-0.943368$

H $4.0065422 .089769-0.871984$

H 2.6801672 .8562610 .016964

H $3.2474480 .328285-2.717673$

H $-0.515079-0.079435-2.812030$

H $0.224494-0.923313-1.448426$

H 0.1849420 .6976640 .519086

H $-4.5715701 .934035-0.455116$

H -7.0294361 .0138001 .153017$

H $-6.1895872 .197768-2.391421$

H $-6.8300090 .767425-3.184060$

H $-5.3351880 .640689-2.283854$

H -1.681389 -0.0529640 .014483$

H -2.401355 $0.664423-1.440607$

H $-4.901487-2.9558140 .133873$

H -3.880431 -3.619932 -1.161286

H $-5.279140-2.173605-2.854514$

H $-7.163200-3.218820-0.677570$

H $-8.279867-3.633630-2.988189$

H -7.585830 -2.135247 -3.566419

H -11.136819-1.962034 -1.386931

H -10.341052 -3.587824 -1.779667

H $-10.0802060 .072396-2.011722$

H $-8.723029-0.006023-3.088575$

H -8.707615 -0.0191290 .007997$

H $-4.021953-0.797899-0.222245$

C -1.255851-2.304025 3.465674

O $-0.014193-1.7414693 .000614$

C $0.316275-2.3458651 .735605$

C $-0.639381-3.5224031 .563469$

C -1.880481 -3.006047 2.263101

H $\quad 0.101305-1.5967830 .966392$

C $1.799746-2.7313001 .661820$

H $-0.808941-3.7683660 .510737$

H -0.264889 -4.422594 2.064972

C -2.877212 -4.092583 2.623635

H $-2.367416-2.2823611 .602626$

C $2.752150-1.5192681 .737185$

O $2.101123-3.6643562 .697772$

H $1.972491-3.2467910 .709706$

H $2.442182-0.7971360 .973782$

O $2.629773-0.8642392 .997574$

C $4.230395-1.8764401 .478064$

C $5.129760-0.6260981 .480000$

H $4.304336-2.3860140 .511004$

O $4.719933-2.7685442 .476625$

H $4.693634 \quad 0.1260370 .814168$

H $5.147663-0.1937262 .487930$

H $-3.226774-4.6077811 .722856$

H -2.432926 -4.841484 3.287715

H $-3.749929-3.6676623 .129341$ 
C $-2.108735-1.2349124 .155061$

H $1.847990-3.2559263 .543268$

H $3.004903-1.4546893 .672862$

H $4.198539-3.5871762 .422158$

H -1.551711 -0.8638825 .024997$

H -3.025914 -1.694544 4.541577

H $-0.981904-3.0512094 .222887$

C $6.265489-0.849549-1.330459$

O $6.646452-1.632388-0.193132$

C $6.583994-0.938648 \quad 1.057475$

C 7.5139360 .2820601 .021359

C $7.2354001 .181578-0.181236$

C $7.1934800 .361672-1.472320$

H $5.228299-0.514191-1.216499$

C $6.336644-1.758248-2.561438$

H $6.988771-1.6280141 .809284$

O $7.392033 \quad 1.0272472 .228085$

H $8.552897-0.0682500 .964549$

H $6.3103581 .750534-0.035822$

O $8.2861722 .146051-0.273804$

H $8.2103860 .032069-1.719085$

O $6.7546521 .194951-2.542559$

C $5.263372-2.819864-2.504246$

H $6.214076-1.199733-3.496219$

H $7.304761-2.270562-2.614502$

H 7.9843541 .7953682 .156679

H $8.1162812 .683994-1.066205$

H $5.8551181 .498502-2.334040$

O $4.058229-2.282749-2.848547$

O $5.469452-3.980766-2.175220$

C $2.943249-3.177557-2.786113$

H $3.075479-3.995899-3.499870$

H $2.824471-3.565160-1.769782$

H $2.042888-2.618207-3.054443$

SCF Energy (B3LYP/6-31G**//MMFF) $=-3245.91989437$

02 72

MM̄FF Geometry

C $2.416121-3.905950-1.021543$

C $3.648192-3.946831-1.553430$

C $4.749821-2.927722-1.371585$

O $4.355915-1.882713-0.456515$

C $5.068409-2.269852-2.724785$

C $6.235776-1.304926-2.658233$

C $6.1090550 .036261-2.696382$

C $7.600484-1.939327-2.602182$

C $4.8201850 .810563-2.746295$

C $4.9599942 .192359-2.091660$

C $3.6397152 .973824-2.146557$

C $3.8581904 .434445-1.748780$

C $2.5403485 .221012-1.734026$

O $2.7177192 .354997-1.241394$

O $2.8053796 .469704-1.077815$

C $1.5125284 .444581-0.886925$

C $2.0740225 .570389-3.151969$

O $0.2310765 .068519-0.948309$

C $1.4096162 .940833-1.244850$

C $0.5494372 .227103-0.178217$

O $0.7765792 .808458-2.519015$

C $4.700446-2.0285750 .850490$

O $5.305049-2.9606891 .355461$

C $4.179175-0.8500511 .577316$

C $4.123964-0.8808222 .916626$

C 3.5858110 .1660803 .787581

C 3.1445441 .4529063 .142450

C $0.5359770 .696054-0.270713$

C $-0.342396 \quad 0.136446 \quad 0.832691$

C $-0.338281-1.3769672 .805568$

$\begin{array}{llllll}0 & -1.508345 & 0.501177 & 0.973038\end{array}$

C $-0.178389-0.4526423 .973627$

C $0.294551-0.8314865 .170692$

C $\quad 0.431416 \quad 0.102964 \quad 6.340429$

C 1.8158390 .0901506 .952638

C $2.032053-0.4585678 .160098$

C 2.9343860 .7824036 .200662

C $3.501203-0.0868565 .110360$

N $0.298365-0.7895401 .639384$

H $2.125607-3.082990-0.374715$
H $3.903819-4.787995-2.195926$

H $5.640525-3.446349-0.996563$

H $4.174623-1.758946-3.103640$

H $5.300521-3.040076-3.472836$

H $7.0177420 .636071-2.697342$

H $8.405176-1.196654-2.626480$

H $7.717559-2.519742-1.681908$

H $7.744548-2.607895-3.457079$

H $4.5365390 .941058-3.797507$

H $4.0143480 .262520-2.247652$

H $5.2738132 .066177-1.047265$

H $5.7450592 .757421-2.609564$

H $3.2422232 .914047-3.166616$

H $4.5838404 .917429-2.415117$

H $4.3008434 .475657-0.743385$

H $1.9600066 .943562-0.993295$

H 1.8374304 .5300190 .159258

H $2.8394416 .160993-3.669725$

H $1.1772026 .199372-3.131163$

H $1.8626594 .690405-3.763048$

H $-0.1699854 .835953-1.803075$

H $\quad 0.9208682 .4907600 .820737$

H $-0.4812842 .595684-0.242046$

H $\quad 0.374487 \quad 1.927858-2.581947$

H $3.823509-0.0099380 .991852$

H $4.479563-1.7742303 .428858$

H 3.9679431 .9002672 .574225

H 2.8166522 .2109333 .856070

H $2.307741 \quad 1.2736392 .461098$

H $\quad 0.1196330 .351718-1.222794$

H $1.5527120 .298629-0.193163$

H $\quad 0.128260-2.3515632 .978759$

H -1.402558 -1.525054 2.597973

H $-0.485177 \quad 0.581383 \quad 3.832273$

H $\quad 0.576737-1.8699185 .327814$

H $-0.314808-0.1940537 .088824$

H $\quad 0.177702 \quad 1.132728 \quad 6.058858$

H $3.016452-0.4492748 .616513$

H $1.231625-0.9339308 .717191$

H 3.7554811 .0245536 .888246

H 2.5678081 .7461425 .838367

H $3.869698-1.0475045 .474694$

H $1.287135-0.9729911 .502634$

C $0.147559-4.346554-1.979142$

O $-0.636466-3.607167-1.019227$

C $-2.037939-3.877442-1.254147$

C $-2.102107-4.605823-2.587401$

C $-0.804161-5.387126-2.566651$

H -2.352611 -4.545691 -0.442401

C $-2.813452-2.556643-1.192092$

H $-2.985732-5.244612-2.676793$

H -2.106732 -3.893880 -3.422376

C $-0.390790-5.906083-3.933095$

H $-0.910655-6.233440-1.874788$

C $-4.269931-2.640578-1.678674$

O $-2.798598-2.1104510 .167486$

H -2.268827 -1.801038 -1.768967

H $-4.290815-2.894750-2.744796$

O $-4.910054-3.722127-0.990001$

C $-5.104100-1.366798-1.433670$

C $-4.464803-0.086786-1.998407$

H -5.293882 -1.258368 -0.360502

O $-6.385641-1.556463-2.043602$

H $-3.4955570 .075627-1.517534$

H -4.270941 -0.226376 -3.069285

H - $-1.148913-6.589045-4.330106$

H -0.265608 -5.089136 -4.651697

H $0.556437-6.451182-3.875308$

C $1.368569-4.953998-1.286418$

H $-3.316389-2.7451920 .691840$

H $-5.830221-3.760721-1.302968$

H $-6.250176-1.627803-3.003974$

H $1.790062-5.756408-1.902614$

H $1.078445-5.394321-0.324808$

H $0.457222-3.634144-2.755573$

C -4.8153591 .6400650 .482462$

O $-5.8259631 .312256-0.478113$

C $-5.3473151 .170362-1.819843$ 
C $-4.6823932 .476244-2.281113$

C -3.610063 2.952826-1.301524

C -4.1613812 .9792760 .125019$

H -4.0587440 .8486240 .495856$

C $-5.479557 \quad 1.685472 \quad 1.861358$

H -6.235869 $1.022693-2.446703$

O $-4.119342 \quad 2.307405-3.577907$

H $-5.4528043 .254168-2.364209$

H $-2.7112902 .329670-1.372939$

O $-3.2109744 .273057-1.675368$

H $-4.891488 \quad 3.7934270 .211677$

O $-3.1013143 .271391 \quad 1.031885$

C -5.8451320 .2936292 .320748$

H -4.8282742 .1294882 .622580$

H $-6.400708 \quad 2.279640 \quad 1.833843$

H -3.695496 $3.148038-3.822125$

H -2.561641 $4.575562-1.017191$

H -2.4812482 .5223491 .017365$

O $-4.724208-0.3728152 .722298$

O $-6.985607-0.1506462 .310216$

C -4.929437 $-1.724262 \quad 3.144304$

H $-5.353482-2.3164082 .327578$

H $-3.958208-2.1469063 .415212$

H $-5.582156-1.751573 \quad 4.021828$

SCF Energy (B3LYP/6-31G**//MMFF) $=-3245.93783166$

0273

MMFF Geometry

C -3.148767 1.6971272 .516323

C -2.219110 0.8619913 .006551

C $-2.361133-0.6307563 .209802$

O $-1.493475-1.294622 \quad 2.260067$

C $-3.773525-1.1897732 .956937$

C $-3.976720-2.6151213 .429410$

C -3.939104 -3.706943 2.640229

C $-4.310872-2.7551754 .892825$

C $-3.561062-3.8008521 .187756$

C $-4.763517-3.9182390 .237332$

C $-5.178217-2.561368-0.350552$

C $-6.409766-2.686577-1.245804$

C $-6.768643-1.339029-1.893757$

O $-4.072190-2.071645-1.118532$

O $-7.725515-1.591515-2.933753$

C $-5.508596-0.756177-2.568492$

C $-7.446227-0.386247-0.901432$

O $-5.7525450 .561642-3.055937$

C $-4.253344-0.760469-1.662897$

C $-3.002878-0.413757-2.508236$

O $-4.406780 \quad 0.218669-0.635242$

C $-0.214521-1.5754602 .613178$

O $0.368330-1.2316403 .627218$

C $0.343262-2.4213401 .533437$

C $1.652211-2.7069601 .494998$

C $2.307571-3.5803620 .518021$

C $1.425812-4.340313-0.440330$

C $-1.657112-0.629449-1.796475$

C $-0.487777-0.377023-2.737928$

C $1.983478-0.654948-2.905895$

O $-0.6184710 .172312-3.829303$

C $2.716759-1.957910-2.928494$

C $3.977566-2.108902-2.493983$

C $4.743070-3.397597-2.590290$

C $5.377629-3.814084-1.282498$

C $6.684948-3.605813-1.052712$

C $4.528386-4.575863-0.292197$

C $3.652779-3.6838990 .542039$

N $0.717649-0.823657-2.213143$

H -4.1158491 .3139022 .203372$

H -1.259892 1.2799293 .305200

H -2.050961 -0.8701404 .234661$

H $-3.990761-1.1231601 .887713$

H $-4.526514-0.5697573 .460675$

H -4.156555 -4.671862 3.097494

H $-4.470854-3.7980855 .186434$

H -3.497292 -2.361658 5.510209

H $-5.225962-2.2022705 .128859$

H -2.902305 -2.9798460 .893412$

H -2.951712 -4.7085391 .083636$
H $-4.465715-4.574955-0.590968$

H $-5.613178-4.3951360 .740279$

H $-5.392743-1.8735800 .471648$

H $-7.263929-3.081990-0.682365$

H -6.211617 $-3.415470-2.043947$

H -8.498946 -2.022345 - 2.531085

H $-5.298616-1.377634-3.450501$

H $-8.359898-0.839639-0.498725$

H $-7.7639840 .538181-1.396429$

H $-6.807909-0.121855-0.055660$

H $-6.5352750 .517214-3.631363$

H $-2.999010-1.042127-3.409012$

H -3.061574 $0.630210-2.841696$

H $-3.5283420 .483444-0.319758$

H $-0.355161-2.8080970 .799579$

H $2.303194-2.2742362 .253214$

H $\quad 0.804215-3.655088-1.026084$

H $\quad 0.768873-5.0257090 .106482$

H $1.980660-4.938166-1.166101$

H $-1.5538700 .050607-0.944355$

H $-1.584718-1.659176-1.428779$

H $2.545140 \quad 0.121269-2.378861$

H $\quad 1.809090-0.313472-3.930929$

H $2.197448-2.809640-3.361537$

H $4.506884-1.248123-2.093148$

H $5.521551-3.260928-3.352597$

H $4.108911-4.212568-2.961117$

H $7.157524-3.933994-0.132825$

H $7.311448-3.099719-1.779159$

H $5.173288-5.1311770 .402849$

H $3.967707-5.353961-0.817918$

H $4.206425-3.0890551 .269992$

H $0.741665-1.165148-1.256767$

C -2.928942 $3.564695 \quad 0.876083$

O $-1.6111753 .284806 \quad 0.359253$

C $-1.1710214 .414604-0.418290$

C $-2.417518 \quad 5.245782-0.684672$

C $-3.171038 \quad 5.0518750 .617245$

H -0.5006954 .9993110 .222705$

C $-0.419453 \quad 3.942363-1.664866$

H $-2.1832486 .293525-0.895402$

H $-2.9989644 .849141-1.525190$

C -4.6390965 .4318100 .530582$

H -2.6887535 .6535971 .399431$

C $0.7819313 .026826-1.348254$

O $-1.329627 \quad 3.204310-2.489178$

H $-0.0987804 .809082-2.254646$

H $0.4084442 .082544-0.939344$

O $1.4222462 .693892-2.584980$

C $1.819753 \quad 3.641275-0.390986$

C $3.0747372 .784502-0.162074$

H 1.3556843 .8559320 .572893

O $2.2433794 .910015-0.903162$

H $3.6175182 .680664-1.112141$

H 3.7628303 .3639050 .466152

H -4.7473496 .4925780 .281871$

H $-5.159143 \quad 4.851503-0.239001$

H $-5.145303 \quad 5.254962 \quad 1.484622$

C $-2.972847 \quad 3.181715 \quad 2.355501$

H $-0.8300792 .882991-3.259470$

H $1.6876453 .522190-3.018656$

H $2.746564 \quad 4.751696-1.719571$

H -3.819595 3.6685462 .852503

H -2.0579673 .5149612 .860823$

H -3.6350612 .9606060 .291399$

C $5.1772450 .992376 \quad 1.022607$

$\begin{array}{lllll}\text { O } & 4.042133 & 0.579805 & 0.251192\end{array}$

C $2.863164 \quad 1.3798880 .440220$

C $2.447142 \quad 1.305658 \quad 1.914967$

C 3.5827101 .7158822 .848338

$\begin{array}{lll}\text { C } 4.853545 & 0.930825 & 2.520117\end{array}$

H $5.4668152 .013361 \quad 0.746038$

C $6.343415 \quad 0.0598720 .664065$

H $2.0825890 .883583-0.143172$

$\begin{array}{llll}\text { O } & 1.298430 & 2.091980 & 2.189623\end{array}$

H 2.1818360 .2698032 .153165

H 3.7650042 .7961422 .810118

O 3.2132541 .4181614 .197113 
H $\quad 4.729732-0.1133262 .835686$

O 5.9438041 .4754513 .260325

C $6.8749640 .326182-0.729622$

H $7.173121 \quad 0.2102821 .363567$

H $\quad 6.018545-0.985790 \quad 0.701361$

H 0.5809061 .7717341 .616428

H $2.376457 \quad 1.8806334 .376098$

H 5.6995331 .4445624 .201244

O $8.027221-0.385541-0.907692$

O $6.347117 \quad 1.051477-1.561507$

C $8.642371-0.246852-2.192484$

H $8.9370180 .793266-2.360334$

H $9.538288-0.873056-2.209456$

H $7.961064-0.588189-2.977851$

SCF Energy (B3LYP/6-31G**//MMFF) $=-3245.92178562$

0274

MM̄FF Geometry

C -0.191357 -2.923086 1.731706

C $0.313570-3.6557520 .726710$

C $-0.126490-3.588412-0.719103$

O $-0.828267-2.353916-0.994062$

C -1.066186-4.769023-1.021333

C -1.546814 -4.810307 -2.458535

C $-2.780347-4.449919-2.867153$

C $-0.542446-5.329211-3.454742$

C $-3.903046-3.898986-2.030588$

C $-4.321454-2.507138-2.521352$

C $-5.309336-1.826266-1.561330$

C $-5.753136-0.475398-2.126758$

C $-6.6272050 .313100-1.140996$

O $-4.644219-1.642549-0.306543$

O $-6.7025201 .669789-1.606488$

C $-5.926708 \quad 0.3484990 .234275$

C $-8.066318-0.214533-1.110138$

O $-6.7726090 .938599 \quad 1.218144$

C -5.449101-1.036365 0.714344

C $-4.595319-0.9690512 .005892$

O $-6.593313-1.8459741 .013302$

C $-0.063629-1.268055-1.298877$

O $1.154574-1.224583-1.365539$

C $-0.970942-0.122737-1.533682$

C $-0.4542421 .084764-1.805803$

C -1.205711 2.311962 -2.077907

C $-2.7082542 .237707-2.031259$

C $-3.269147-0.2112221 .890245$

C -3.3349091 .2534342 .285212$

C -1.9167883 .3053622 .330542$

O $-4.313417 \quad 1.7665362 .820005$

C $-2.2597724 .156744 \quad 1.149218$

C -1.4120405 .0451360 .608847$

C -1.763390 $5.951116-0.535424$

C $-0.7936935 .842028-1.689991$

C $0.2242106 .709348-1.823019$

C $-1.0634614 .790203-2.740853$

C $-0.5208493 .439285-2.362963$

N -2.143085 1.9105392 .004081

H $-0.995910-2.2220371 .531784$

H $1.099781-4.3753980 .945177$

H $\quad 0.761494-3.652991-1.360494$

H $-1.917165-4.744281-0.332517$

H -0.553802 -5.715782 -0.801173

H -3.017968 -4.547911 -3.925899

H - $0.953309-5.388038-4.468248$

H $0.333904-4.674804-3.493139$

H $-0.214419-6.335504-3.174422$

H $-4.758525-4.581415-2.101147$

H -3.633743 -3.849636 -0.973777

H -3.425852 -1.881601 -2.620295

H -4.770434 -2.591391 -3.519043

H $-6.172559-2.487224-1.419648$

H $-6.274858-0.607487-3.082900$

H $-4.8655380 .127998-2.355598$

H -7.071659 $1.658082-2.506346$

H $-5.056519 \quad 1.007944 \quad 0.132123$

H -8.527496 $-0.125912-2.101204$

H $-8.691617 \quad 0.380368-0.435443$

H $-8.132628-1.262541-0.810561$
H -7.0321541 .8160350 .888458$

H -5.200960 -0.5974302 .841264$

H $-4.337158-2.0038162 .274546$

H -6.319487 -2.559959 1.612517

H $-2.037423-0.303141-1.479268$

H $0.6289001 .185775-1.846421$

H -3.081468 $1.620624-2.854975$

H -3.196301 $3.211665-2.106616$

H $-3.0466131 .805190-1.083912$

H -2.555359 -0.671291 2.585478

H $-2.850206-0.3002820 .883897$

H -0.8662233 .4041012 .621971$

H -2.543106 3.5876683 .182831

H -3.2631364 .0609090 .740888$

H -0.4206425 .1611251 .040510$

H $-1.7699676 .980609-0.153423$

H $-2.7834275 .766553-0.894409$

H $0.9051576 .656475-2.665945$

H $0.4030647 .486671-1.087488$

H $-0.5824705 .077715-3.686023$

H $-2.1315154 .771448-2.976030$

H $0.5687643 .396024-2.370863$

H -1.4055691 .4147191 .511649$

C $0.700151-1.6626223 .708068$

O $1.887228-1.2216563 .020196$

C $2.856182-0.7878133 .991158$

C $2.083376-0.5913725 .288620$

C $1.063539-1.7088885 .193535$

H $3.558722-1.6197564 .124266$

C 3.6144050 .4449083 .487731

H $2.725221-0.667467 \quad 6.171399$

H $1.5751600 .379173 \quad 5.319832$

C $-0.124640-1.5296096 .122919$

H $1.561840-2.6587515 .430050$

C 4.1812900 .3050262 .058608

O 2.7165351 .5622473 .483433

H 4.4120270 .6960014 .197310

H $3.3494300 .323391 \quad 1.346320$

O 4.9642541 .4790951 .815986

C $5.056340-0.9408491 .845593$

C $5.667525-1.1095850 .439762$

H $4.470370-1.8416462 .056942$

O $6.129850-0.9267202 .791105$

H $6.337229-0.2710390 .225171$

H $6.323291-1.9879320 .490213$

H $0.203501-1.5109007 .167323$

H -0.654898 -0.5929765 .920413$

H $-0.837798-2.3517946 .007393$

C $0.264554-3.0234443 .159950$

H 3.2335522 .3441443 .224800

H 5.1678231 .5083820 .867528

H $6.643478-0.1148472 .638173$

H $-0.567855-3.4156473 .755153$

H $1.093881-3.7372333 .239641$

H $-0.073119-0.9057113 .522855$

C $4.8738740 .779605-1.884288$

O $4.028782-0.069068-1.098029$

C $4.609330-1.304748-0.667620$

C $5.098843-2.101707-1.885277$

C $6.060227-1.285344-2.739165$

C $5.4165070 .045543-3.116562$

H $5.7075681 .140005-1.271048$

C $4.0354451 .989995-2.313508$

H $3.791928-1.888579-0.225217$

O $5.731564-3.313588-1.488589$

H $4.237032-2.381280-2.505715$

H $7.020621-1.131655-2.233085$

O $6.345083-2.000086-3.943460$

H $4.607426-0.131838-3.837040$

O $\quad 6.387450 \quad 0.868813-3.760522$

C $3.8014702 .936085-1.161313$

H $4.5332182 .570547-3.098896$

H $3.0649801 .679369-2.719315$

H $5.092811-3.814789-0.953287$

H $\quad 6.711236-2.863832-3.686515$

H $6.7295420 .371230-4.523103$

O $2.6289582 .633512-0.537193$

O $4.5767663 .830836-0.849861$ 
C $2.262925 \quad 3.492012 \quad 0.546801$

H 3.0897623 .6066341 .253314

H 1.9575524 .4666830 .156279

H 1.4163663 .0377021 .068153

SCF Energy (B3LYP/6-31G**//MMFF) $=-3245.91593276$

0275

MMFF Geometry

C -0.7207882 .4562210 .833095$

C $-1.6663802 .463811 \quad 1.786604$

C $-2.2852101 .269992 \quad 2.477315$

O $-1.852660 \quad 0.0236121 .891543$

C $-3.820001 \quad 1.2990792 .357954$

C $-4.502053 \quad 0.7362213 .589317$

C $-4.921313-0.5357123 .731718$

C $-4.7329691 .734487 \quad 4.695483$

C $-4.755415-1.6827682 .775242$

C $-6.086551-2.1280212 .154404$

C $-6.564701-1.192787 \quad 1.033824$

C $-7.941426-1.6183890 .521723$

C $-8.394090-0.772779-0.677872$

O $-5.606708-1.271439-0.026552$

O $-9.526787-1.444390-1.249609$

C $-7.263139-0.752789-1.728963$

C $-8.8822480 .613109-0.240013$

O $-7.566348 \quad 0.144884-2.794666$

C $-5.875021-0.408917-1.136815$

C $-4.777634-0.651138-2.200252$

O $-5.859246 \quad 0.967808-0.753790$

C $-0.702501-0.5409902 .339149$

O $0.052675-0.1172783 .197560$

C $-0.503289-1.7688011 .542890$

C $0.743193-2.1889601 .288832$

C $1.105406-3.3484810 .472834$

C $-0.008536-4.244993-0.002081$

C $-3.341011-0.608082-1.655616$

C $-2.318904-0.806891-2.762668$

C $0.138881-0.858536-3.156031$

O $-2.629547-0.973901-3.939661$

C $0.682286-2.253084-3.137151$

C $1.992213-2.526053-3.031892$

C $2.576359-3.909452-3.067744$

C $3.567314-4.154470-1.950176$

C $4.887838-4.006103-2.150853$

C $3.030209-4.646557-0.625789$

C $2.407359-3.5436200 .179865$

N - $1.016647-0.764484-2.283972$

H $-0.327013 \quad 1.5167170 .458149$

H -2.000134 3.4299162 .163198

H -1.9859901 .3030353 .532929$

H $-4.1386290 .748101 \quad 1.466192$

H $-4.187007 \quad 2.3242972 .215554$

H -5.421975 -0.8061994 .661029$

H -5.2187791 .2855885 .568250$

H -3.781869 2.160242 5.030909

H -5.3738072 .5493414 .343142$

H -4.020711 -1.4681661 .994702$

H $-4.341926-2.522873 \quad 3.347744$

H $-5.935907-3.1321321 .736728$

H $-6.854971-2.2118902 .932802$

H $-6.603800-0.166576 \quad 1.414959$

H -8.687265 -1.5741781 .325364$

H -7.902840 -2.671361 0.207971

H $-9.865133-0.899128-1.979674$

H -7.211472 -1.757304 -2.171774

H -9.7388280 .5182440 .438074$

H $-9.2408131 .195760-1.095880$

H $-8.114904 \quad 1.195756 \quad 0.273961$

H -8.385144 $-0.164131-3.217132$

H -4.925151-1.641509-2.651302

H $-4.8763520 .089611-3.004163$

H $-4.9518381 .305542-0.810825$

H - $1.385706-2.257758 \quad 1.146531$

H $1.576191-1.6148781 .692172$

H $-0.544350-4.674008 \quad 0.851843$

H $0.333463-5.081492-0.614547$

H $-0.721738-3.685119-0.616902$

H $-3.1355700 .355353-1.177253$
H -3.193354 -1.400448 -0.912938

H $0.866355-0.128087-2.795603$

H $-0.149221-0.589481-4.177185$

H $-0.024742-3.071337-3.250265$

H $2.697074-1.702768-2.956819$

H $3.077719-4.033127-4.036736$

H $1.792926-4.676508-3.038979$

H $5.605724-4.203136-1.361522$

H $5.279561-3.676193-3.107377$

H $3.843706-5.071262-0.022010$

H $2.350090-5.484945-0.799820$

H $3.134768-2.826480 \quad 0.559957$

H $-0.854264-0.659003-1.286696$

C $0.2485043 .634463-1.188360$

O $1.3679082 .738213-1.372770$

C $2.3959013 .422941-2.110689$

C $1.6941034 .584451-2.801554$

C $0.6907464 .991860-1.744641$

H $3.1035953 .821038-1.373209$

C $3.1240812 .473518-3.066961$

H $2.3857095 .388305-3.071319$

H $1.1725504 .264224-3.710879$

C $-0.4524335 .835143-2.283707$

H $1.2165055 .563768-0.967980$

C $3.7274001 .221239-2.403390$

O $2.1873522 .033011-4.059067$

H $3.9026383 .030253-3.602971$

H $2.9314850 .548756-2.067100$

$\begin{array}{lllll}\text { O } & 4.463407 & 0.513517 & -3.410827\end{array}$

C $4.6759661 .491016-1.220578$

$\begin{array}{llll}\text { C } 5.385470 & 0.205193 & -0.748477\end{array}$

H $4.1067781 .921637-0.391599$

O $5.6588112 .452888-1.601560$

H $4.630438-0.568186-0.571632$

H $6.037429-0.156123-1.552525$

H $-0.0730506 .768294-2.712779$

H $-1.0065145 .306219-3.066457$

H $-1.157556 \quad 6.091373-1.486651$

C $-0.101963 \quad 3.7193990 .299564$

H $2.6943321 .633081-4.785306$

H $3.8212960 .153086-4.045154$

H $6.1431992 .092252-2.364092$

H -0.7786684 .5648220 .471099$

H $0.815567 \quad 3.9038170 .871107$

H $-0.589614 \quad 3.224258-1.765540$

C $4.585798-0.088667 \quad 2.192832$

$\begin{array}{lllll}\text { O } & 5.486487 & 0.874910 & 1.632170\end{array}$

C 6.2518970 .4158270 .513110

C $7.082082-0.8132790 .914263$

C $6.207981-1.9116061 .512080$

C $5.340032-1.3497602 .637012$

H $3.817854-0.3481201 .454861$

C 3.8964440 .5697343 .397877

H 6.9635291 .2179450 .280653

O $7.795879-1.337030-0.200425$

H $7.828402-0.515476 \quad 1.662250$

H $5.592457-2.386928 \quad 0.740937$

O $7.038971-2.949846 \quad 2.037014$

H $5.971941-1.1219583 .504942$

O $4.399735-2.340867 \quad 3.045351$

C 2.9549791 .6834232 .995505

H $3.304382-0.1632403 .956547$

H 4.6588340 .9992514 .059288

H $8.352239-0.621878-0.553388$

H $7.613440-2.5603512 .718216$

H $4.902710-3.1382753 .284319$

$\begin{array}{llllll}\text { O } & 2.640321 & 2.414274 & 4.106068\end{array}$

$\begin{array}{lllll}\text { O } & 2.529948 & 1.874511 & 1.865094\end{array}$

C 1.7436063 .5090293 .896911

H $\quad 0.7475493 .1310013 .652751$

H 1.6830674 .0809204 .826739

H $2.1152154 .167726 \quad 3.106153$

SCF Energy (B3LYP/6-31G**//MMFF)= -3245.91089372

02_76

MMFF Geometry

C $2.147896-3.241507 \quad 0.972910$

C $1.605584-2.6129492 .027191$ 
C $2.235470-1.5337582 .872450$

O $1.587137-0.2870112 .512148$

C $3.748494-1.3262872 .675728$

C $4.371640-0.4954073 .780899$

C 4.6846030 .8111323 .682475

C $4.690124-1.2506525 .046575$

C 4.4425531 .7427052 .527834

C 5.7477662 .1692671 .840424

C 6.3378581 .0758990 .936109

C 7.6943891 .5083560 .376563

C $8.2511110 .485867-0.625985$

O $5.415307 \quad 0.865739-0.136634$

O $9.343078 \quad 1.102432-1.325050$

C $7.1629600 .171909-1.674086$

C $8.827027-0.7493030 .076423$

O $7.579811-0.878340-2.543964$

C $5.787071-0.167853-1.056320$

C $4.709575-0.214575-2.166920$

O $5.862073-1.442768-0.417454$

C 0.5106870 .1228203 .226952

O $0.008901-0.4489554 .184816$

C -0.0146691 .4146832 .722294$

C 0.3568521 .9699141 .557246

C -0.1166373 .2474471 .016066$

C -1.0677744 .0566901 .857791$

C $3.271594-0.328615-1.640646$

C $2.246347-0.186371-2.751991$

C $-0.174478 \quad 0.296111-3.096772$

O $2.534979-0.242364-3.944377$

C $-0.2149621 .773799-3.325940$

C $-1.1826662 .581823-2.867683$

C -1.176615 $4.068262-3.081142$

C $-1.2323734 .855488-1.789338$

C $-2.3271605 .567202-1.472851$

C $0.0075394 .923485-0.923026$

C $0.3210773 .641946-0.198654$

N $0.964908-0.001517-2.248660$

H $3.153411-2.9964250 .646514$

H $0.585652-2.8731672 .306769$

H $2.036724-1.7765443 .923414$

H $3.935655-0.8602281 .702137$

H $4.268628-2.2927112 .654976$

H 5.1551981 .2885144 .541598

H $5.174900-0.6186105 .798371$

H $3.777377-1.6530535 .496287$

H $5.368187-2.0829194 .831245$

H $3.7397351 .329117 \quad 1.799169$

H 3.9549912 .6389502 .932497

H 5.5240903 .0525961 .227984

H 6.4842912 .4748722 .593926

H 6.4445180 .1500531 .511725

H 8.4126781 .6814541 .187566

H $7.5861002 .473482-0.138265$

H $10.0063281 .372010-0.666588$

H $7.0480281 .066655-2.302146$

H $9.639659-0.4601800 .753762$

H $9.272130-1.442523-0.645844$

H $8.085484-1.2930780 .665209$

H $8.439053-0.619840-2.919239$

H $4.7732720 .705466-2.762984$

H $4.904920-1.056036-2.843729$

H $4.978438-1.841124-0.396721$

H $-0.7498391 .883715 \quad 3.368535$

H 1.0793241 .4477190 .932254

H -1.991963 3.4975882 .038133

H -1.3591315 .0020461 .395558$

H -0.6124174 .3055742 .822812$

H $3.112495-1.301420-1.168199$

H $3.0773920 .452028-0.896563$

H -1.070400 -0.069161-2.587221

H $-0.072479-0.229973-4.050996$

H $0.6086182 .202791-3.892693$

H -2.013619 2.159054 -2.308409

H -2.038742 4.313711 -3.714906

H $-0.2920264 .385462-3.647864$

H -2.370022 $6.172301-0.573513$

H -3.207294 $5.564900-2.106833$

H $-0.0585985 .752163-0.207255$
H $\quad 0.8667835 .181168-1.556195$

H $1.0100472 .992023-0.738521$

H $\quad 0.8402700 .099538-1.245411$

C $0.494436-3.581012-0.872980$

O $-0.672514-3.084599-0.184677$

C $-1.851762-3.484663-0.911786$

C $-1.358397-3.862643-2.296941$

C $-0.029165-4.514294-1.963322$

H -2.240705 -4.369041-0.390478

C $-2.869352-2.343226-0.848046$

H $-2.043164-4.533849-2.823225$

H -1.205412 -2.968685 -2.914422

C $0.900173-4.641385-3.158119$

H $-0.217952-5.512397-1.545957$

C $-4.093565-2.527802-1.758871$

O $-3.314264-2.2386960 .509698$

H -2.370998 -1.396869-1.083197

H $-3.783796-2.466796-2.808830$

O $-4.606032-3.852427-1.564055$

C $-5.247365-1.541042-1.492478$

C $-4.833567-0.059755-1.522886$

H -5.714522 -1.790006 -0.533819

O $-6.255500-1.758649-2.486357$

H $-4.0198660 .110841-0.812736$

H $-4.4456210 .185949-2.519367$

H $\quad 0.449857-5.274093-3.929936$

H $1.113556-3.664961-3.606409$

H $1.853271-5.092178-2.864317$

C $1.415685-4.2606180 .141702$

H -2.525596-2.115395 1.065351

H $-5.380294-3.952271-2.144142$

H -5.873978 -1.531856 -3.351667

H $2.162547-4.878930-0.369067$

H $\quad 0.839876-4.9237920 .799178$

H $0.990392-2.713836-1.324053$

C $\begin{array}{rlll}-5.919813 & 0.704532 & 1.205636\end{array}$

$\begin{array}{llll}\text { O } & -6.685874 & 0.523715 & 0.008880\end{array}$

C $-6.0074730 .893241-1.196400$

C $-5.6220632 .377251-1.136753$

C -4.8293522 .7151520 .125440$

C -5.5367032 .1783811 .371160$

H -5.0155470 .0865451 .162855$

C $-6.774314 \quad 0.2148812 .379132$

H $-6.7394240 .781568-2.006243$

O $-4.8767352 .733553-2.295646$

H $-6.5394742 .980267-1.147409$

H -3.8006182 .3427010 .054164$

O $-4.734756 \quad 4.1373650 .227436$

H -6.4329902 .7823981 .560055$

O -4.6810872 .3393432 .500535$

C $-6.971596-1.2812092 .312765$

H -6.3240310 .4559003 .348520$

H $\quad-7.7677540 .6792072 .357953$

H $-4.6522293 .676240-2.217467$

H -4.2527624 .3375441 .048143$

H $-3.889151 \quad 1.7936552 .355581$

O $-5.829136-1.9200142 .695734$

O $-8.011783-1.8155571 .950620$

C $-5.873477-3.3488062 .634290$

H $-6.060762-3.6779511 .607606$

H $-4.900724-3.7330122 .952789$

H -6.642452 -3.733437 3.310892

SCF Energy (B3LYP/6-31G**//MMFF) $=-3245.91441179$

02777

MM̄FF Geometry

C 4.0230762 .0715512 .565080

C 3.7913951 .9486071 .249266

C 4.2973642 .9384720 .228704

O $3.1637883 .466437-0.505494$

C $5.2088012 .283380-0.829625$

C $6.4360601 .566531-0.304753$

C $6.6852800 .252738-0.485644$

C 7.4253432 .4275550 .436120

C $5.833427-0.739376-1.232093$

C $4.903709-1.494519-0.276893$

C $3.673034-2.126071-0.942789$

C $4.036011-3.234924-1.927888$ 
C $2.782867-3.821932-2.596094$

O $2.943017-1.091041-1.610715$

O $3.211813-4.619016-3.711014$

C $1.933321-2.666220-3.166175$

C $2.019166-4.764804-1.659489$

O $0.688228-3.146195-3.668444$

C $1.689533-1.508868-2.164893$

C $1.047445-0.306472-2.911346$

O $0.796604-1.958480-1.146492$

C 2.4114724 .4366800 .076263

O 2.5852834 .9537261 .167190

C $1.3037554 .739874-0.857562$

C $0.2347115 .424061-0.425694$

C $-0.9568175 .748562-1.214961$

C $-0.9540315 .364011-2.671757$

C $1.1534671 .049593-2.204284$

C $0.1914081 .219296-1.049598$

C $-2.1739911 .742424-0.512410$

O $\quad 0.5385411 .0119540 .110348$

C $-3.1866452 .699880-1.052999$

C $-3.7854563 .635607-0.300313$

C $-4.7919784 .616529-0.830982$

C $-4.4755936 .047933-0.450195$

C $-5.229332 \quad 6.7061420 .446709$

C $-3.3316476 .740535-1.162175$

C $-1.9916436 .354005-0.595071$

N -1.073955 $1.614362-1.450503$

H 4.5924412 .9375792 .900007

H 3.2172521 .1094960 .870194

H 4.8318083 .7734330 .699104

H $5.5405113 .063251-1.529476$

H $4.5912831 .623601-1.448177$

H $7.595535-0.165859-0.058873$

H $8.3360261 .879700 \quad 0.700649$

H $7.7242473 .279422-0.183560$

H $6.9898242 .807813 \quad 1.364863$

H $5.275355-0.247196-2.033377$

H $6.495835-1.452089-1.738932$

H $5.472055-2.2635310 .260910$

H $4.537152-0.7941430 .479378$

H $3.050311-2.530523-0.135431$

H $4.613725-4.024225-1.431461$

H $4.687473-2.835434-2.716729$

H $3.806047-5.310913-3.373204$

H $2.479870-2.263075-4.030593$

H $2.672310-5.577121-1.318468$

H $1.189644-5.251130-2.183952$

H $1.623563-4.265002-0.772955$

H $0.885572-3.860292-4.298580$

H $\quad 1.566287-0.180580-3.870689$

H $-0.003494-0.527042-3.135099$

H $\quad 0.357730-1.189689-0.751413$

H $1.3838814 .357939-1.869093$

H $0.2148965 .754607 \quad 0.612054$

H $-0.1222665 .850736-3.193548$

H -1.863161 $5.649778-3.203504$

H $-0.8516474 .279936-2.787190$

H $2.1688831 .239830-1.844927$

H $0.9457881 .844507-2.932547$

H -1.786454 2.0550250 .462844

H $-2.6170840 .751350-0.404146$

H -3.444964 $2.621098-2.106329$

H -3.5496473 .6934230 .760148$

H $-5.7748794 .329430-0.435478$

H $-4.8756104 .549294-1.922922$

H $-5.024075 \quad 7.7389290 .707917$

H $-6.065476 \quad 6.2258970 .944157$

H -3.427853 $7.829488-1.057444$

H -3.419070 $6.549326-2.234559$

H $-1.885026 \quad 6.6006840 .462624$

H $-1.2958231 .581373-2.441741$

C $2.930242-0.1766963 .300100$

O $1.562248 \quad 0.0623502 .909899$

C $0.712087-0.9078103 .562414$

C $1.646860-1.9805224 .096532$

C $2.862123-1.1601494 .470560$

H $0.230874-0.3709814 .389913$

C $-0.344883-1.4176522 .573385$
H $1.222982-2.5273224 .943983$

H $1.899783-2.7038743 .310989$

C $4.118951-1.9948954 .651572$

H $2.649949-0.6210735 .403760$

C -1.189463 -2.585625 3.113156

O $-1.219317-0.3305112 .257896$

H $\quad 0.145925-1.7099531 .638970$

H $-0.547580-3.4576153 .286161$

O $-1.710754-2.2070974 .394523$

C $-2.381237-2.9975422 .226465$

C $-1.977446-3.3410790 .782727$

H $-3.137804-2.2055782 .243826$

O $-2.974026-4.1478792 .838678$

H -1.578496 -2.441277 0.305201

H -1.164950 -4.078273 0.804236

H $3.983027-2.7243215 .456859$

H $4.370006-2.5453403 .738520$

H $4.974934-1.3637504 .909759$

C 3.6124891 .1426383 .678042

H $-0.6658690 .398146 \quad 1.927601$

H $-2.235781-2.9557164 .725984$

H -3.858270 -4.2628162 .451284$

H 4.5264150 .9187394 .241585

H $2.952807 \quad 1.7107304 .346157$

H $3.432045-0.6491852 .449234$

C $-4.245520-1.844990-0.587896$

$\begin{array}{llll}\text { O } & -4.325015 & -3.135646 & 0.027569\end{array}$

C $-3.129815-3.914942-0.073621$

C $-2.772073-4.135371-1.550818$

C $-2.706815-2.822309-2.327762$

C $-3.964587-1.988332-2.087163$

H -3.444761 -1.269230 -0.112635

C $-5.569544-1.120822-0.327057$

H $-3.371287-4.9003740 .344067$

O $-1.529263-4.815822-1.678100$

H $-3.536388-4.771467-2.015974$

H -1.808435 -2.248946 -2.074210

O $-2.618098-3.106858-3.725434$

H $-4.819855-2.457071-2.590458$

O $-3.782817-0.695861-2.661084$

C $-5.621026-0.6214541 .097368$

H $-5.688806-0.243870-0.973375$

H $-6.432628-1.772721-0.506520$

H -1.602134 -5.653345 -1.189423

H -1.818858 -3.645314 -3.858911

H $-3.573134-0.823306-3.602222$

O $-6.099015-1.5982651 .919532$

O $-5.2497350 .494048 \quad 1.439710$

C $-6.147222-1.2622473 .309583$

H -5.143703 -1.028404 3.678334

H $-6.825372-0.4192353 .471693$

H -6.527346 -2.129534 3.855849

SCF Energy $\left(B 3 L Y P / 6-31 G^{* *} / / M M F F\right)=-3245.91924498$

02 78

MM̄FF Geometry

C $1.123733-4.080897-0.339691$

C $-0.101077-3.535387-0.372504$

C $-0.774997-3.088845-1.643122$

O $-1.160713-1.701449-1.515045$

C $-2.059184-3.907765-1.853947$

C $-2.708752-3.666497-3.202207$

C $-3.797835-2.895651-3.390490$

C $-2.091140-4.406363-4.359570$

C $-4.512444-2.072170-2.355249$

C $-5.654817-2.853242-1.697565$

C $-6.436780-2.057132-0.639467$

C $-7.311994-0.969176-1.266189$

C $-8.063254-0.158748-0.200230$

O $-5.508211-1.4880750 .290349$

O $\quad-8.6055201 .009561-0.835809$

C -7.0446790 .3302010 .849737$

C $-9.255965-0.928566 \quad 0.377953$

$\begin{array}{llll}\text { O } & -7.705680 & 0.974877 & 1.936844\end{array}$

C $-6.116634-0.7879221 .383164$

C $-4.989897-0.2207942 .282007$

O $-6.893231-1.6801092 .192816$

C $-0.221148-0.755909-1.780232$ 
O $0.961494-0.939797-2.018295$

C $-0.9025230 .559680-1.760492$

C $-0.181276 \quad 1.677660-1.926021$

C $-0.6948003 .049195-1.959432$

C $-2.1868593 .236765-2.038871$

C $-3.9301970 .613096 \quad 1.553633$

C -3.0933931 .4430012 .515438$

C $-1.458478 \quad 3.3241142 .520223$

O -3.1369941 .3119483 .735344$

C $-1.383166 \quad 4.567283 \quad 1.691624$

C $-0.237406 \quad 5.122831 \quad 1.268826$

C $-0.177767 \quad 6.395530 \quad 0.472844$

C $0.6043106 .256142-0.814003$

C $1.8192556 .814588-0.948594$

C $-0.0580755 .545346-1.975650$

C $0.1967134 .061988-1.951303$

N -2.304285 2.3624901 .839418

H $1.677905-4.202835-1.267303$

H $-0.659493-3.4167330 .552868$

H -0.109876 -3.204846 -2.507996

H -2.770295 -3.686168 -1.048575

H -1.842092 -4.981177 -1.766885

H -4.214192 -2.821602 -4.393918

H -2.628755 -4.233005 -5.297856

H $-1.055230-4.088079-4.510763$

H -2.100011 -5.484577 -4.169355

H $-3.810047-1.711820-1.597293$

H -4.898035 -1.175553-2.853201

H $-6.347494-3.231134-2.460102$

H -5.224219 -3.733370 -1.201797

H $-7.069813-2.776880-0.106361$

H -8.017290 -1.403599 -1.985385

H -6.689159 -0.271635-1.841043

H $-9.1933430 .715080-1.552670$

H -6.4368191 .1006590 .361540$

H -9.968915 -1.181493 -0.416075

H $-9.812069-0.3140601 .094675$

H -8.969805 -1.859637 0.871152

H $-8.271913 \quad 1.670387 \quad 1.560809$

H -5.440038 0.3506963 .104027

H -4.466911 -1.055138 2.770470

H -6.292718 -2.162371 2.784783

H $-1.9777800 .571563-1.621250$

H $0.8976341 .584468-2.041806$

H -2.589754 2.714221-2.914119

H -2.491005 $4.280622-2.136078$

H -2.682288 $2.845055-1.146448$

H $-3.257458-0.0443790 .990781$

H $-4.394974 \quad 1.3057120 .845902$

H -0.4806342 .8596412 .671007$

H -1.8833513 .5536203 .502769$

H -2.327031 5.0483591 .444214

H 0.7098454 .6619521 .536514

H 0.2811057 .1618991 .111201

H -1.183482 6.7679220 .240620

H $2.3737366 .741175-1.878434$

H $2.2905797 .349283-0.130693$

H $0.3439675 .931666-2.921780$

H -1.120301 5.802507-1.989566

H $1.2572693 .807805-1.935847$

H $-2.176308 \quad 2.242590 \quad 0.839682$

C $3.004544-3.7228421 .279848$

$\begin{array}{lllll}\text { O } & 4.028576 & -3.863728 & 0.270414\end{array}$

C $5.286199-4.0725660 .926580$

C $5.083063-3.6612052 .377053$

C $3.660321-4.1396692 .598586$

H $5.459586-5.1583200 .912601$

C $6.450557-3.414557 \quad 0.186368$

H $5.804225-4.1277963 .054427$

H $5.142463-2.5740802 .500295$

C $3.007155-3.5684463 .843651$

H $3.667881-5.2355562 .678191$

C $6.478145-1.8741850 .100682$

O $7.651420-3.8422820 .845090$

H $6.493292-3.825941-0.830000$

H $6.662414-1.4502171 .094198$

O $7.641380-1.535154-0.674890$

C $5.248045-1.185008-0.526499$
C $5.5120150 .305505-0.826245$

H $4.389780-1.2708640 .146602$

O $4.910340-1.822658$ - 4.757747

H 5.8719820 .7888770 .087976

H $6.3123340 .379028-1.572018$

H $3.543867-3.8950124 .740490$

H $3.009776-2.4754723 .836607$

H $1.969201-3.9037683 .931417$

C $1.789157-4.5739160 .914443$

H $8.400909-3.4520200 .363315$

H $7.486782-1.843924-1.584341$

H $4.539428-2.695722-1.544775$

H $1.074381-4.581374 \quad 1.745391$

H $2.096152-5.6124470 .740096$

H $2.729752-2.6622361 .304379$

$\begin{array}{llll}\text { C } 3.358720 & 1.867846 & 0.698537\end{array}$

O $3.180407 \quad 1.052921-0.465390$

C $4.2757531 .047603-1.384677$

C $4.5654592 .478643-1.859054$

C $4.8221303 .418137-0.686076$

C 3.6820623 .3216910 .323988

H 4.1615111 .4498251 .317131

C 2.0467241 .8151771 .492823

H $3.9342440 .486958-2.263572$

O $5.6782492 .487903-2.746888$

H $3.7007362 .846911-2.426520$

H $5.7936623 .214252-0.221327$

O $4.8893534 .755170-1.187084$

H $2.7949133 .804197-0.100340$

$\begin{array}{lllll}\text { O } & 4.027551 & 4.062549 & 1.493278\end{array}$

C 1.7994720 .4545132 .098093

H 2.0506602 .5342812 .318595

H 1.2117662 .0528040 .823470

H $5.8376983 .412391-3.003496$

H $4.9970225 .345986-0.422039$

H 4.8111183 .6452301 .890051

$\begin{array}{lllll}\text { O } & 0.457211 & 0.212794 & 2.140159\end{array}$

O $2.678451-0.2825692 .522161$

C $0.065262-1.0194672 .751207$

H $\quad 0.288170-0.9920643 .821702$

H $-1.013234-1.1376762 .618014$

H $0.570182-1.8633632 .275107$

SCF Energy (B3LYP/6-31G**/MMFF)= -3245.92584518

02_79

MMFF Geometry

C $1.750173-0.5083802 .990456$

C $3.003702-0.9750493 .104133$

C $4.140492-0.7754682 .131579$

$\begin{array}{lllll}\text { O } & 3.889294 & 0.321937 & 1.229047\end{array}$

C $4.297887-2.035828 \quad 1.262357$

C $5.701600-2.221014 \quad 0.725328$

C $6.122258-1.837716-0.495327$

C $6.643754-2.9429421 .655020$

C $5.371234-1.062924-1.541161$

C $4.951979-1.922469-2.741840$

C $3.600370-2.623376-2.532948$

C $3.270396-3.525021-3.724060$

C $1.864523-4.133724-3.615966$

O $2.607430-1.599884-2.405286$

O $1.560906-4.696725-4.901188$

C $0.853031-3.002082-3.333851$

C $1.825070-5.290625-2.610617$

O $-0.447638-3.527167-3.078573$

C $1.277035-2.073780-2.171483$

C $0.343804-0.839252-2.120837$

O $1.179193-2.795604-0.942077$

C 4.2056341 .5775361 .637065

$\begin{array}{lllll}\text { O } & 4.642226 & 1.917010 & 2.723840\end{array}$

C 3.9058922 .4790010 .503637

$\begin{array}{llll}\text { C } 3.805429 & 3.799958 & 0.705744\end{array}$

C $3.4748864 .798984-0.312952$

C $3.3252584 .322966-1.735158$

C $0.7957490 .253838-1.141725$

C $-0.1952721 .403280-1.071504$

C $-0.656394 \quad 3.4914360 .213602$

O $-1.180877 \quad 1.484152-1.799534$

C $-0.2328064 .652984-0.629010$ 
C $-0.0182535 .882807-0.136056$

C $0.3659677 .066547-0.978756$

C $1.5683067 .810360-0.438006$

C 1.4202348 .9551030 .250062

C $2.9433837 .271334-0.766310$

C 3.3044076 .0797840 .075762

N $0.1559332 .329960-0.098099$

H 1.4624540 .0944432 .135569

H $3.242698-1.5986393 .964456$

H $5.057686-0.5776182 .700313$

H $3.581951-2.0015430 .433347$

H $4.040581-2.9377701 .834385$

H $7.150347-2.067269-0.774227$

H $7.646171-3.0558951 .228565$

H $6.746923-2.3937292 .596344$

H $6.264205-3.9456661 .877044$

H $4.512730-0.532987-1.121481$

H $6.050903-0.278835-1.899823$

H $4.864800-1.253263-3.608216$

H $5.731365-2.657344-2.977861$

H $3.640069-3.212776-1.610845$

H $4.023744-4.315217-3.834170$

H $3.320497-2.935218-4.650387$

H $0.697246-5.138844-4.841191$

H $\quad 0.772445-2.398451-4.248694$

H $2.498939-6.094855-2.929435$

H $\quad 0.826352-5.738510-2.560397$

H $2.118226-4.992997-1.601578$

H $-0.733119-4.009061-3.872600$

H $\quad 0.292705-0.384664-3.119117$

H $-0.672977-1.154007-1.858234$

H $1.023455-2.174459-0.214167$

H $3.7472492 .022741-0.467007$

H $3.9553564 .184927 \quad 1.713553$

H $4.2670493 .893587-2.094940$

H $3.0499165 .113156-2.435985$

H $2.5426173 .560201-1.809748$

H $0.902485-0.155627-0.133741$

H $1.7634260 .662582-1.452612$

H -0.5424543 .6914591 .283620$

H -1.7035753 .2560730 .010780$

H $-0.1254534 .483563-1.697854$

H $-0.159344 \quad 6.0587920 .928101$

H $-0.5006257 .739474-1.018419$

H $\quad 0.5604696 .771947-2.017615$

H 2.2776939 .5088960 .617895

H $\quad 0.437558 \quad 9.3613190 .465843$

H $3.7050268 .041058-0.582766$

H $3.0027927 .073115-1.839746$

H 3.4322176 .3215181 .132042

H 1.0201252 .2037190 .419851

C $-0.009642-2.1483813 .672694$

O $-0.884188-1.9961752 .536438$

C -2.019940 -2.868236 2.718046

C $-1.771567-3.6441384 .008261$

C $-0.905167-2.6760714 .788995$

H -2.893377 -2.2253172 .868281$

C -2.210939-3.756795 1.483016

H -2.703618 -3.8979854 .522610$

H -1.231063 -4.579441 3.819592

C $-0.157054-3.3317795 .936788$

H -1.537369 -1.867376 5.179067

C $-2.521232-2.9820740 .186371$

O $-1.014973-4.5171461 .266158$

H -3.003153 -4.490010 1.672776

H -1.666933 -2.358262 -0.096959

O $-2.696179-3.940275-0.863873$

C $-3.790682-2.1157820 .265426$

C -4.164262 -1.459175 -1.077699

H -3.639622 -1.331127 1.011764

O $-4.885075-2.915737 \quad 0.714698$

H -3.313979 -0.864240 -1.428373

H $-4.347048-2.236473-1.829317$

H $-0.860229-3.7700636 .652663$

H $0.502591-4.1306525 .581635$

H $0.457001-2.6022336 .473418$

C $0.675695-0.8208933 .996266$

H $-0.288542-3.8865091 .122138$
H $-1.874886-4.458200-0.921993$

H $-5.009828-3.6310360 .067450$

H $1.118976-0.8512004 .998273$

H -0.0513690 .0002713 .990151$

H $0.736001-2.9088363 .402349$

C $-4.4352181 .427818-0.101689$

$\begin{array}{llll}\text { O } & -5.355455 & 0.350415 & 0.108396\end{array}$

C $-5.435497-0.585299-0.970926$

C $-5.8381010 .131095-2.266834$

C $-4.958821 \quad 1.345601-2.559076$

C $-4.8598562 .245739-1.325598$

H $-3.4274881 .027259-0.259200$

C -4.4252862 .2705921 .179546$

H -6.259233 -1.265791-0.719699

O $-5.798958-0.777310-3.362496$

H $-6.878367 \quad 0.469221-2.170175$

H $\quad-3.967174 \quad 1.034822-2.908884$

O $-5.5575042 .075427-3.632176$

H $-5.8274382 .729966-1.141704$

O $-3.900763 \quad 3.271114-1.564541$

C $-3.652568 \quad 1.5650732 .274609$

H -3.9477103 .2446291 .032773$

H -5.4532512 .4473011 .518060$

H $-6.044520-0.280057-4.161489$

H -4.937953 $2.778642-3.889916$

H $-4.2459133 .839123-2.273506$

O -4.3960421 .5200233 .416810$

O -2.5205521 .1179922 .138422$

C $-3.772196 \quad 0.869887 \quad 4.528777$

H $-2.841484 \quad 1.3800834 .795177$

H -4.4561000 .9208625 .380107$

H -3.582472 -0.181503 4.293362

SCF Energy (B3LYP/6-31G**//MMFF) $=-3245.92340679$

$02 \_8$

MM̄FF Geometry

C $-0.044987-3.4262361 .344536$

C $-0.670174-2.4808460 .627274$

C $-0.990272-2.607884-0.837485$

O $-0.517646-1.392111-1.464772$

C $-2.507761-2.688781-1.053358$

C $-2.872599-3.042890-2.480815$

C $-3.285507-2.159009-3.409905$

C $-2.791512-4.509711-2.818179$

C $-3.419984-0.667496-3.274189$

C $-4.882600-0.203744-3.324479$

C $-5.688159-0.580085-2.070516$

C $-7.150508-0.156307-2.223683$

C $-7.958848-0.418288-0.944253$

O $-5.0998780 .095364-0.954901$

O $-9.2054430 .285013-1.057787$

C $-7.2038860 .191110 \quad 0.255287$

C $-8.310428-1.901904-0.783076$

O $-7.841911-0.1489551 .484531$

C $-5.707805-0.1989090 .309856$

C $-4.9858790 .649791 \quad 1.383192$

O $-5.595763-1.5826130 .643659$

C $0.731517-1.381973-1.991985$

O $1.480807-2.340153-2.119201$

C $1.120113-0.018197-2.421916$

C $0.504691 \quad 1.092737-1.984882$

C $0.8623042 .474501-2.319540$

C $2.0207782 .686665-3.258776$

C -3.4665650 .4427331 .433985$

C -2.8104591 .4849472 .316678$

C -2.1952673 .8903162 .375947$

O -2.4613961 .2324803 .467275$

C -1.7121224 .8830811 .369010$

C -0.4285345 .2508101 .242539$

C $0.056326 \quad 6.2602760 .243694$

C $0.9605595 .655689-0.806391$

C $2.2939565 .810252-0.745626$

C $0.3116654 .958506-1.981478$

C $0.1498733 .478940-1.765352$

N -2.6762172 .7111541 .682513$

H $0.204196-4.3658690 .855034$

H -0.940371 -1.536494 1.090746

H $-0.494839-3.483268-1.274268$ 
H $-2.973625-1.740334-0.767757$ H $-2.947040-3.444864-0.388800$ H -3.542472 -2.538655 -4.398372 H -3.103804 -4.718122 -3.847014 H - $1.766156-4.875189-2.706088$ H -3.441965 -5.088052 -2.153921 H -2.928895 $-0.283904-2.375252$ H -2.883845 -0.213755 -4.117504 H -4.876896 $0.889094-3.429635$ H -5.369837 -0.612407 -4.218594 H $-5.624579-1.662511-1.914209$ H -7.614117 -0.660423 -3.080749 H $-7.197160 \quad 0.918831-2.447609$ H -9.661199 -0.039590 -1.853441 H $-7.272637 \quad 1.2842810 .163139$ H $-8.904679-2.249261-1.636867$ H -8.934889 -2.0659080 .102073$ H -7.431755 -2.545993 -0.710075 H -8.7733670 .1213681 .411962$ H $-5.187619 \quad 1.710615 \quad 1.186524$ H $-5.393866 \quad 0.4257522 .377103$ H -4.718412 $-1.748977 \quad 1.021420$ H $1.9838330 .023214-3.077112$ H $-0.3345690 .991976-1.298129$ H $2.9359092 .252387-2.842887$ H $2.2428643 .737883-3.451670$ H $1.8195222 .221438-4.230064$ H -3.216113 -0.5446551 .835959$ H -3.017672 0.5065420 .436278 H -1.418047 3.6017913 .090519 H -3.0330104 .3078312 .943990$ H -2.460009 5.3260690 .715494 H $\quad 0.3178534 .816779$\begin{tabular}{lll}
1.902747 \\
\hline
\end{tabular} H 0.5879937 .0463310 .795811 H $-0.7817856 .769742-0.248101$ H $2.9443965 .406791-1.514492$ H $2.764644 \quad 6.3371680 .077672$ H $0.8689805 .178255-2.897947$ H $-0.6808795 .390253-2.164227$ H $-0.6664163 .221960-1.089949$ H -3.1427902 .8427960 .789177$ C $0.462341-1.9048173 .369841$ O $1.380677-1.0989872 .605976$ C $2.100498-0.2307803 .499890$ C $1.475297-0.4211104 .878977$ C $0.988361-1.8561264 .802676$ H $3.132847-0.5969873 .530323$ C 2.0772791 .2082762 .972580 H $2.196205-0.2543465 .685186$ H $0.6306990 .258325 \quad 5.042308$ C $-0.048287-2.2018815 .856883$ H $1.852210-2.5255544 .910731$ C 2.5678381 .3584621 .518321 $\begin{array}{llll}\text { O } & 0.726439 & 1.681549 & 3.021967\end{array}$ H $2.657777 \quad 1.8529493 .643364$ H 1.8535550 .8927770 .828937 O $2.5833492 .756478 \quad 1.204453$ C 3.9739320 .7905541 .250606 C $4.4713471 .115177-0.170888$ H $3.954501-0.2949651 .389178$ O $4.897188 \quad 1.320702 \quad 2.200501$ H $3.7137810 .785326-0.887915$ H $4.5708842 .201606-0.282756$ H $\quad 0.367473-2.0730356 .861547$ H $-0.933422-1.5619735 .774810$ H $-0.374508-3.2419335 .757871$ C $0.405522-3.3176182 .779985$ H 0.7493892 .6413672 .872871 H 1.6600773 .0386331 .092438 H 4.8903572 .2889502 .107799 H $-0.242861-3.955463 \quad 3.390977$ H $1.413441-3.7497752 .822938$ H $-0.522261-1.4226043 .315312$ C $5.039919-1.694383-1.193494$

O $5.843251-0.940746-0.278758$

C $5.8373160 .472212-0.498791$

C $6.352874 \quad 0.779939-1.912037$

C $5.5819250 .010601-2.982310$
C $5.514240-1.477170-2.635309$

H $3.990136-1.394757-1.093227$

C $5.152779-3.169021-0.794600$

H 6.5630640 .8943650 .207710

O $6.2744832 .174301-2.190220$

H $7.4116880 .497558-1.979561$

H $4.5809530 .432607-3.124807$

O $6.2675670 .173006-4.226321$

H $6.502777-1.931929-2.779234$

O $4.606589-2.127440-3.522324$

C $4.404941-3.4262980 .490909$

H $4.721331-3.832896-1.552308$

H $6.200343-3.467289-0.666552$

H $6.7902502 .637523-1.508188$

H $5.715591-0.224976-4.920239$

H $4.982735-2.079100-4.417180$

O $5.270257-3.4416801 .543757$

O $3.191000-3.5709890 .550808$

C $4.674182-3.6626032 .825359$

H $5.468088-3.6495753 .576777$

H $3.962043-2.8653013 .053946$

H $4.181569-4.6393062 .851030$

SCF Energy (B3LYP/6-31G**//MMFF) $=-3245.89547900$

02880

MM̄FF Geometry

C -2.051803 $3.973938-1.353094$

C $-2.6421582 .903518-0.800793$

C -3.2067322 .8851430 .592560$

O $-2.432948 \quad 1.9161831 .338337$

C -4.6668132 .4103410 .579233$

C -5.3599792 .6357591 .907707$

C -5.5688321 .6837672 .837637$

C -5.8720134 .0354372 .133490$

C $-5.1313190 .245328 \quad 2.807788$

C $-6.316166-0.7206172 .666627$

C $-6.928921-0.7256241 .257279$

C $-8.176368-1.6098961 .213448$

C $-8.749035-1.717005-0.207667$

O $-5.940115-1.234527 \quad 0.356542$

O $-9.703832-2.789135-0.219278$

C -7.612955-2.112481-1.173187

C $-9.512234-0.451242-0.615185$

O $-8.061650-2.085152-2.526585$

C $-6.337647-1.249653-1.020235$

C $-5.184312-1.878518-1.838222$

O $-6.6000230 .066198-1.509573$

C -1.4281982 .3685402 .129160$

O -1.1469303 .5368112 .359341$

C -0.6558291 .2503062 .719193$

C $-0.730595-0.0153052 .277973$

C $0.048742-1.1529722 .773995$

C $0.982761-0.9118913 .930900$

C $-3.810756-1.232678-1.603880$

C $-2.705826-1.990585-2.321377$

C $-0.223290-2.177565-2.414277$

O $-2.923993-2.882135-3.137776$

C $0.139760-3.273383-1.461812$

C $1.263558-3.289505-0.729391$

C $1.616270-4.3941750 .224915$

C $1.817785-3.9074201 .643768$

C $3.052731-3.7957672 .161687$

C $0.592975-3.6468782 .494385$

C $-0.092607-2.3483362 .162965$

N -1.444581 -1.543946 -1.949539

$\mathrm{H}-1.9955414 .904190-0.794378$

H -2.672850 $1.969809-1.357480$

H $-3.136313 \quad 3.8774401 .053755$

H $-4.708847 \quad 1.3509130 .304014$

H $-5.2336732 .940905-0.197436$

H -6.1024921 .9644293 .745076$

H -6.3980614 .1374353 .088652$

H -5.0458514 .7528802 .135741$

H $-6.5734794 .314913 \quad 1.340481$

H -4.3846300 .0520412 .032228$

H $-4.6267410 .039173 \quad 3.760368$

H -5.949522 -1.728962 2.898956

H -7.084667 -0.4753293 .410326$ 
H -7.1858860 .3003910 .972772$ H -8.940744 -1.239719 1.908103 H -7.920156 -2.620723 1.560732 H -10.389962 -2.586401 0.439567 H -7.350466 -3.157464 -0.955588 H -10.343368 -0.2663540 .076034$ H -9.966882 $-0.565991-1.605371$ H $-8.8863300 .443413-0.624244$ H -8.852161 -2.648955 -2.581135 H -5.100001 -2.940838 -1.572871 H $-5.417100-1.828082-2.909563$ H $-5.7654080 .475648-1.785410$ H $0.026407 \quad 1.5406453 .511604$ H -1.405859 -0.2467401 .455816$ H $1.718901-0.1402183 .680422$ H $1.551511-1.7973084 .222486$ H $0.421966-0.5881644 .814546$ H -3.800632 -0.202282 -1.973112 H -3.575035 -1.219463 -0.533712 H $0.549008-1.405294-2.475684$ H $-0.380275-2.592504-3.414942$ H -0.561996 -4.099877 -1.373751 H $1.974540-2.472983-0.826169$ H $2.533654-4.870257-0.145407$ H $\quad 0.854123-5.183600 \quad 0.222034$ H $3.210186-3.4803303 .187495$ H $3.935959-4.0091471 .568543$ H $0.843664-3.6941623 .560179$ H $-0.124729-4.4650272 .350518$ H $-0.792232-2.4242221 .330317$ H $-1.365366-0.856164-1.205942$ C $0.1133563 .708863-2.647761$ O $0.3777552 .450711-1.990939$ C $1.3393352 .678909-0.938944$ C $2.0734273 .942967-1.350170$ C $0.9304474 .771775-1.907571$ H $0.7542372 .854702-0.027568$ C $2.2011501 .425402-0.775880$ H $2.5830694 .430949-0.514515$ H $2.8119513 .728014-2.132337$ C $1.3830335 .919703-2.793283$ H $0.3643285 .185403-1.064391$ C 3.3802681 .5889850 .203322 O $1.342857 \quad 0.378559-0.315685$ H $2.582017 \quad 1.108444-1.754485$ H $4.0373502 .389712-0.156084$ O 2.9033871 .9730621 .493160 C 4.2263990 .3079870 .353029 C $5.475451 \quad 0.534816 \quad 1.224995$ H $4.532018-0.022248-0.645034$ O $\quad 3.453040 \quad-0.7424820 .924424$ H $6.062440 \quad 1.350777 \quad 0.789114$ H 5.1693460 .8606772 .226755 H $2.0026746 .621997-2.226155$ H $1.9733205 .562560-3.643726$ H $0.5213606 .468698-3.186615$ C -1.400781 $3.945070-2.708918$ H $0.5832260 .336762-0.920823$ H 2.5214242 .8633861 .418144 H $3.110139-0.4244971 .776760$ H - $1.8557113 .144240-3.306491$ H -1.617032 $4.885882-3.227882$ H $0.485008 \quad 3.596719-3.674204$ C $7.489107-0.627073-0.740852$ O $6.614782-1.3635530 .121408$ C $6.344377-0.7349881 .377298$ C $7.655058-0.5193452 .146208$ C $8.696171 \quad 0.2224971 .309408$ C $8.850999-0.430767-0.065539$ H $7.0432620 .347510-0.975454$ C $7.615857-1.415624-2.047375$ H $5.748811-1.4536091 .954698$ O 7.4028720 .1894643 .354773 H $8.063675-1.4988102 .427783$ H $8.442327 \quad 1.2852641 .221404$ O 9.9478250 .1662021 .997640 H $9.358313-1.3963890 .052081$ O $9.6908230 .385579-0.878846$
C $6.331514-1.325224-2.837631$

H $8.414211-1.021798-2.686195$

H $7.845676-2.471336-1.859790$

H $8.257806 \quad 0.3175443 .800457$

H $\quad 10.608342 \quad 0.602871 \quad 1.432849$

H $9.246086 \quad 1.241417-1.003372$

O $5.522216-2.381593-2.541018$

O $6.066505-0.403796-3.599301$

C $4.248478-2.383137-3.193207$

H $3.652250-1.530389-2.854670$

H $4.374737-2.360124-4.279854$

H $3.729114-3.305916-2.921499$

SCF Energy (B3LYP/6-31G**//MMFF) $=-3245.90746881$

02 81

MM̄MF Geometry

C -1.191390 3.6289811 .803335

C -1.2582662 .2889631 .779350$

C -0.9370441 .4200782 .965977$

O $0.0678580 .471222 \quad 2.527737$

C -2.1749490 .6379273 .445771$

C -3.3155481 .5003893 .947096$

C -4.4920921 .6429183 .304389$

C -3.0871182 .1712115 .275383$

C -4.8556291 .0507061 .972461$

C $-5.517610-0.3233442 .125922$

C $-5.675065-1.0369670 .775473$

C $-6.375346-2.3860840 .960039$

C $-6.422538-3.197373-0.344315$

O $-4.362951-1.2310360 .236000$

O $-6.820970-4.538658-0.021799$

C $-5.003914-3.249221-0.954609$

C $-7.505198-2.673702-1.293658$

O $-5.020653-3.871264-2.236675$

C $-4.331316-1.863244-1.048610$

C $-2.853133-1.950672-1.488766$

O $-5.014599-1.073742-2.029066$

C $0.829110-0.1124663 .492357$

O $0.796675 \quad 0.118817 \quad 4.691017$

C $1.695576-1.1219162 .843783$

C $2.320962-2.0226803 .614967$

C $3.202744-3.0944803 .160574$

C $4.338201-3.3568084 .115596$

C $-1.929828-2.699743-0.526120$

C $-0.485363-2.531563-0.950930$

C $1.150805-3.277623-2.673701$

O $\quad 0.271047-1.752365-0.376555$

C $2.032572-4.310465-2.048840$

C $3.242516-4.031338-1.541418$

C $4.171185-5.067428-0.982588$

C $4.647963-4.741400 \quad 0.413552$

C $5.905222-4.3192520 .628844$

C $3.712911-5.0229431 .565688$

C $2.961817-3.8080572 .041032$

N $-0.155140-3.310644-2.047131$

H -0.8799654 .1091192 .729718$

H $-1.567081 \quad 1.7702080 .876619$

H -0.5215862 .0285143 .779296$

H -1.883850 -0.0442464 .255810$

H -2.500653 -0.041511 2.648056

H $-5.2681592 .253157 \quad 3.762583$

H -3.971763 2.7153295 .622947

H -2.837562 1.4272286 .038939

H -2.2649362 .8899645 .206942$

H -5.5437811 .7349681 .460738$

H -3.9718670 .9937041 .329309$

H -4.901647 -0.946852 2.787308

H -6.497302 -0.207902 2.606032

H $-6.250711-0.3906140 .102619$

H -7.386077 -2.248566 1.364216

H $-5.831676-2.9731811 .713538$

H $-6.175319-4.9057020 .605719$

H -4.398499 -3.893458 -0.305117

H -8.494339 -2.760122 -0.827481

H $-7.552985-3.273610-2.209292$

H -7.364174 -1.627719-1.573439

H -5.456833 -4.734479 -2.134881

H $-2.788334-2.381664-2.496125$ 
H -2.463312 -0.928885 -1.591108 H $-4.418403-0.367425-2.328521$ H $1.790846-1.1098341 .764648$ H $2.191975-1.9817404 .695727$ H $4.894101-2.4315934 .308069$ H $5.064072-4.0819543 .742657$ H $3.956869-3.7340625 .071014$ H -2.025220 -2.316359 0.495983 H -2.165092 -3.768840 -0.495687 H $1.570310-2.271856-2.579636$ H $1.009797-3.500297-3.735683$ H $1.675811-5.337463-2.048386$ H $3.606816-3.007772-1.572256$ H $5.028447-5.142448-1.664394$ H $3.708205-6.062163-0.982836$ H $6.274147-4.124177 \quad 1.629617$ H $\quad 6.594624-4.155049-0.192865$ H $4.266906-5.4982972 .383212$ H $2.978575-5.7828161 .268186$ H $2.100797-3.547640 \quad 1.426347$ H $-0.837388-3.968993-2.411772$ C $-1.9728743 .989923-0.646215$ O $-0.9315953 .175711-1.213228$ C $-0.8859483 .384396-2.635273$ C $-2.0929544 .249824-2.981385$ C $-2.2763315 .049029-1.706158$ H $0.0292623 .953193-2.837098$ C $-0.8302072 .035547-3.361627$ H -1.912392 $4.877119-3.859674$ H -2.986527 $3.647675-3.181237$ C $-3.6527425 .678721-1.578325$ H -1.514366 $5.839502-1.677452$ C $0.258903 \quad 1.071538-2.848580$ O $-2.088357 \quad 1.363495-3.209329$ H $-0.7099262 .202175-4.438086$ H $-0.0070250 .697323-1.852590$ O $0.267218-0.068131-3.717738$ C $1.679051 \quad 1.663234-2.804945$ C $2.7391980 .619234-2.403905$ H $1.7026082 .491029-2.091455$ O $2.0113152 .202944-4.083700$ H $2.4263060 .138038-1.471160$ H $2.782798-0.158759-3.175073$ H -3.837634 $6.369951-2.407073$ H -4.443249 $4.920667-1.589990$ H $-3.7401906 .241771-0.644077$ C - 1.5003574 .5873970 .682029 H $-2.2711921 .299844-2.255988$ H - $0.628246-0.447778-3.705236$ H $1.9609301 .478129-4.730422$ H -2.2606275 .2856261 .051569$ H -0.5890125 .1707750 .503506$ H $-2.8458533 .343094-0.488404$ C 4.0467341 .8724020 .079867

O $4.1906082 .283776-1.285571$

C $4.1523441 .226016-2.250242$

C $5.2519960 .197091-1.951538$

C $5.186527-0.307267-0.513527$

C $5.143916 \quad 0.869016 \quad 0.458610$

H 3.0567531 .4247870 .229298

C 4.1511943 .1304300 .956166

H $4.4039681 .685621-3.214242$

O $5.165209-0.896213-2.859325$

H $6.2297240 .669335-2.114073$

H $4.333781-0.979788-0.367525$

O $6.360638-1.080005-0.256991$

H $6.123019 \quad 1.3644190 .459679$

O $4.929237 \quad 0.376006 \quad 1.779125$

C 2.9577104 .0483680 .806306

H 4.2144342 .8604212 .015882

H 5.0460193 .6973930 .671894

H $5.855200-1.535438-2.611827$

H $6.331791-1.3467840 .677965$

H $4.070282-0.0783821 .790250$

O $3.2455505 .244921 \quad 1.399758$

O $1.903197 \quad 3.752736 \quad 0.263291$

C $2.204930 \quad 6.225871 \quad 1.354644$

H 2.6097707 .1684191 .732814
H $\quad \begin{array}{lll}1.862626 & 6.380758 & 0.326758\end{array}$

H 1.3757775 .9167381 .997389

SCF Energy (B3LYP/6-31G**//MMFF) $=-3245.92638239$

$02 \_82$

MMFF Geometry

C $-3.485177 \quad 0.001126-2.855574$

C $-4.7456030 .090993-2.403675$

C $-5.2910461 .124398-1.445716$

O $-4.3053952 .164630-1.230540$

C $-5.6522380 .499377-0.083578$

C $-6.770644-0.525005-0.121027$

C $-6.592441-1.8472800 .076691$

C $-8.1522730 .032163-0.338967$

C $-5.276823-2.5414210 .290530$

C $-4.904472-2.6044571 .777111$

C $-3.407700-2.8787811 .987658$

C $-3.080701-2.9273383 .482541$

C -1.570305 -3.010238 3.748082

O $-2.698654-1.809108 \quad 1.358411$

O $-1.349430-2.7218795 .137590$

C $-0.861847-1.9011932 .940781$

C $-1.025317-4.4228233 .507273$

O $0.553421-2.0216033 .050332$

C -1.273135 -1.872669 1.456460

C $-0.712824-0.6454810 .698293$

O $\quad-0.763982-3.042548 \quad 0.806033$

C $-4.755158 \quad 3.392256-0.865972$

O $-5.9200153 .705995-0.657199$

C $-3.6560204 .380137-0.752375$

C $-2.3609474 .113492-0.985892$

C $-1.2567885 .079017-0.928241$

C -1.597665 $6.504615-0.576493$

C $-1.274877 \quad 0.7116701 .132629$

C $-0.426138 \quad 1.4492292 .150036$

C -0.3972623 .5777383 .439317$

O 0.6939101 .0819222 .491140

C 0.1413894 .6551402 .551976

C 1.4005064 .6689622 .090767

C 1.9528835 .7241711 .178619

C $2.2502155 .188723-0.204219$

C $3.4039444 .551032-0.464586$

C $1.2538185 .479243-1.303323$

C $-0.0041364 .655586-1.203361$

N -1.064294 2.5976252 .604569

H $-2.7374910 .722234-2.537401$

H -5.466213 -0.648862 -2.747749

H $-6.1743661 .571953-1.918019$

H -5.9460621 .2935940 .615815$

H $-4.742361 \quad 0.0871880 .369647$

H -7.462793 -2.500786 0.071071

H -8.925780 -0.740718 -0.277289

H $\quad-8.3813770 .7880740 .419265$

H $-8.2288840 .495498-1.327372$

H $-5.342337-3.559288-0.113283$

H $-4.497643-2.048796-0.299680$

H -5.153697 -1.646143 2.252278

H -5.504538 -3.376642 2.274162

H $-3.144581-3.8239971 .498792$

H $-3.604580-3.7609873 .966491$

H $-3.461430-2.0169213 .967171$

H -1.856290 -3.365679 5.661580

H -1.132974 -0.944237 3.404001

H $-1.508202-5.1393504 .182671$

H $\quad 0.045945-4.476678 \quad 3.730021$

H -1.185477 -4.776532 2.486741

H $0.772066-2.0204463 .997831$

H $0.383471-0.657503 \quad 0.707510$

H $-0.993430-0.764447-0.356455$

H $-0.752176-2.882500-0.151816$

H $-3.9870385 .376709-0.476273$

H -2.074425 $3.102847-1.268786$

H $-2.2418596 .946174-1.344712$

H $-0.7245197 .152397-0.477480$

H $-2.116848 \quad 6.5503180 .387538$

H $-1.309834 \quad 1.3587630 .247497$

H -2.2991920 .6236141 .509522$

H 0.3945423 .0962884 .022649 
H -1.134742 $3.992124 \quad 4.133191$ H -0.5435035 .4502662 .270202$ H 2.0769503 .8673692 .378427 H 2.8736216 .1170841 .629445 H 1.2761456 .5846851 .109832 H $3.6408304 .191947-1.460336$ H 4.1358024 .3718680 .316264 H $1.7008345 .259754-2.282681$ H $1.0553826 .554543-1.330725$ H $0.1482703 .600582-1.435313$ H -1.9576712 .8580592 .196261$ C -1.947796 -1.952156 -3.143938 O $-0.718886-1.205797-3.037977$ C $0.385291-2.062776-3.401028$ C $-0.187400-3.469430-3.479654$ C $-1.600380-3.198395-3.954901$ H $0.712018-1.723815-4.392089$ C $1.509266-1.882654-2.375121$ H $0.376792-4.116122-4.158118$ H $-0.200798-3.941394-2.489131$ C $-2.547166-4.364932-3.730283$ H -1.572225 -2.955450 -5.025624 C $2.688183-2.852939-2.553987$ O $1.995190-0.540591-2.480385$ H $1.094929-1.987162-1.367701$ H $2.348797-3.882173-2.388554$ O $3.125643-2.784948-3.916887$ C $3.903752-2.558400-1.652953$ C $3.562328-2.483166-0.154909$ H $4.384636-1.632134-1.984653$ O $4.867010-3.598200-1.858254$ H $2.828536-1.6894500 .011001$ H $3.090441-3.4247740 .152572$ H -2.205862 $-5.248261-4.279873$ H -2.609781 -4.632233 -2.669948 H $-3.556723-4.121904-4.075888$ C $-3.020973-1.078923-3.795272$ H $1.2365830 .053632-2.347877$ H $3.877441-3.394786-4.010955$ H $4.474249-4.432516-1.549366$ H -3.869497 -1.698139-4.107644 H -2.619441 -0.593652 -4.693067 H -2.244240 -2.230584 -2.123927 C $4.959475 \quad 0.1503240 .440965$ O $5.583274-1.1291050 .282431$ C $4.800144-2.2363600 .738059$ C $4.478958-2.0710622 .230774$ C $3.840589-0.7142742 .528835$ C 4.6685630 .4183621 .920267 H $4.0241050 .179998-0.130833$ C $5.9116591 .198034-0.144156$ H $5.439628-3.1230010 .643219$ O $3.626208-3.1270052 .659575$ H $5.411352-2.1550962 .804137$ H $2.807032-0.6718572 .169080$ O $3.787234-0.5124973 .942208$ H 5.6065810 .5292932 .479125 O 3.9487081 .6430642 .040820 C $5.9807451 .078744-1.648353$ H 5.6061542 .2209820 .099297 H $6.927674 \quad 1.0614050 .245453$ H $3.523798-3.0521923 .622963$ H $3.152952-1.1530994 .305663$ H 3.7316411 .7618212 .981318 O $4.8317631 .556254-2.206721$ O $6.9363470 .608602-2.251651$ C $4.7546991 .464789-3.632413$ H $3.7895161 .870863-3.946785$ H $5.5517442 .055622-4.093597$ H $4.8147680 .418887-3.948252$ SCF Energy (B3LYP/6-31G**//MMFF) $=-3245.90849210$

$02 \_83$

MM̄FF Geometry

C $-2.482604-3.310531-1.833496$

C $-1.243439-3.526137-1.364720$

C $0.031214-3.015722-1.993320$

O $-0.215942-1.770202-2.690483$
C $0.626813-4.010145-3.003719$

C $1.253032-5.229464-2.360123$

C $2.492438-5.249310-1.830678$

C $0.405868-6.474351-2.358444$

C $3.474989-4.112744-1.775694$

C $3.714622-3.640058-0.335612$

C $4.750854-2.508029-0.277182$

C $5.005687-2.0733521 .166622$

C $5.969404-0.878347 \quad 1.245339$

O $4.250108-1.402599-1.036437$

O $5.885574-0.3317572 .570814$

C 5.4950540 .2245220 .272712

C $7.427155-1.3114821 .047131$

O $6.457402 \quad 1.2726230 .186566$

C $5.163870-0.300704-1.139703$

C $4.5029320 .764198-2.048417$

O $6.378513-0.702447-1.786806$

C $-0.203870-0.641283-1.928081$

O $0.078029-0.560489-0.742716$

C $-0.6456630 .489761-2.771598$

C $-1.031811 \quad 1.621590-2.164415$

C $-1.5358662 .833605-2.808601$

C $-1.4805782 .909520-4.310267$

C $3.1909921 .363075-1.527973$

C $3.3539792 .722727-0.866679$

C 2.0342374 .5290090 .208474

O $4.4348133 .290736-0.739537$

C 0.6475525 .0614810 .046579

C $0.3352226 .056075-0.797888$

C -1.048135 $6.615425-0.975533$

C $-1.6282196 .291155-2.335325$

C $-1.3346747 .043879-3.408995$

C $-2.6035585 .140037-2.437258$

C $-2.0083713 .823966-2.023919$

N $2.1334473 .244399-0.458114$

H -2.616706 -2.761465 -2.761996

H -1.119386 -4.102657 -0.451114

H $\quad 0.762866-2.827939-1.198641$

H $1.371046-3.495664-3.625484$

H $-0.140345-4.318788-3.727452$

H $2.853294-6.179934-1.394948$

H $0.921643-7.327198-1.904470$

H $-0.519078-6.313762-1.797120$

H $\quad 0.145553-6.755717-3.384161$

H $4.420587-4.460569-2.209595$

H $3.148376-3.270356-2.392044$

H $2.764498-3.2880900 .086156$

H $4.054615-4.4818520 .280534$

H $5.678408-2.871474-0.735531$

H $5.384828-2.9120071 .763912$

H $4.054496-1.7838171 .632702$

H $6.114268-1.0367493 .200604$

H 4.5913640 .6671050 .708094

H $7.717566-2.0396541 .813916$

H $8.111459-0.4643371 .166264$

H $7.609704-1.7693870 .072727$

H 6.6201681 .5919611 .090713

H $5.2303561 .544120-2.304436$

H $4.2659660 .269118-3.001582$

H $\quad 6.230080-0.705439-2.746895$

H $-0.6743700 .351481-3.845754$

H $-0.9973901 .661612-1.076195$

H $-0.4650832 .707447-4.669645$

H -1.752840 $3.892722-4.701928$

H $-2.1630782 .178669-4.757427$

H $2.5219571 .515965-2.384689$

H $2.6869190 .683120-0.833754$

H $2.7885055 .208770-0.203041$

H 2.2634154 .3818781 .268137

H -0.1303224 .6101190 .657292$

H $1.1221306 .502413-1.402291$

H $-0.9948947 .704380-0.845358$

H -1.724586 $6.263794-0.186472$

H $-1.764004 \quad 6.833524-4.382871$

H $-0.654627 \quad 7.886349-3.336727$

H $-3.0348795 .074208-3.442339$

H -3.459216 5.348508 -1.781352

H $-2.0000063 .676543-0.943361$ 
H $1.3206772 .634097-0.455682$

C -4.786492 -2.687317-1.062931

O $-4.293421-1.576574-0.290820$

C $-5.377048-1.032793 \quad 0.487984$

C $-6.635858-1.7836350 .061922$

C $-6.069893-3.124829-0.360965$

H -5.161481-1.277185 1.534625

C -5.4536430 .4889830 .312273$

H $-7.364037-1.8618330 .875293$

H -7.135403 -1.298126 -0.784346

C $-7.013724-3.933519-1.233344$

H -5.826962 -3.7021800 .541329$

C $-4.134798 \quad 1.2313580 .605629$

O $-5.809337 \quad 0.772443-1.047792$

H -6.2650460 .8876100 .932784$

H -3.398274 $0.983397-0.167057$

O -4.3818362 .6365710 .467853$

C -3.527754 0.9540061 .992275

C -2.268371 1.7980262 .257633

H -3.268744 -0.104932 2.059109

O $-4.496058 \quad 1.207737 \quad 3.008315$

H -1.5768741 .6671901 .418741$

H -2.5423402 .8595472 .287045$

H $-7.945610-4.144612-0.698504$

H -7.268250 -3.397282 -2.153740

H $-6.563075-4.890422-1.514738$

C $-3.729388-3.791003-1.144592$

H $-5.8466561 .739691-1.142609$

H $-5.0785462 .881138 \quad 1.099965$

H -4.671393 2.1636653 .023918

H $-4.127377-4.642557-1.707636$

H -3.486447 -4.143422 -0.135091

H $-5.009789-2.303175-2.067448$

C $-0.233766-0.4088912 .800612$

$\begin{array}{lllll}\text { O } & -1.256791 & 0.046706 & 3.693387\end{array}$

C $-1.571176 \quad 1.4399713 .590437$

C $-0.317346 \quad 2.2769393 .880144$

C 0.8470281 .8780152 .978560

C 1.0708290 .3674113 .025070

H $-0.571428-0.2844851 .766536$

C $-0.017570-1.9082313 .062981$

H -2.291635 1.6496604 .390947

O $-0.582933 \quad 3.6661753 .714167$

H -0.0143882 .1289444 .924882$

H $0.6834922 .224973 \quad 1.952618$

$\begin{array}{llll}\text { O } & 2.022785 & 2.540942 & 3.448994\end{array}$

H 1.5095890 .0932763 .993204

O 2.0034120 .0018772 .010809

C -1.181934-2.757652 2.600022

H $0.870381-2.2722752 .534648$

H $0.109438-2.0718554 .140045$

H $-1.312547 \quad 3.8975894 .313894$

H 2.7334182 .3683142 .808695

H $2.859356 \quad 0.3984752 .243770$

O $-1.065963-4.0042963 .146521$

O $-2.064691-2.3911381 .838006$

C $-2.096525-4.9324372 .792805$

H $-2.005398-5.204961 \quad 1.737737$

H -1.966254 -5.831837 3.400420

H -3.085269 -4.511240 2.999948

SCF Energy (B3LYP/6-31G**//MMFF) $=-3245.90635327$

02_84

MM̄FF Geometry

C $0.109858-1.8572641 .640771$

C $-1.111860-1.7946911 .092657$

C $-1.424858-2.281543-0.298007$

O $-1.615488-1.108453-1.122558$

C $-2.750920-3.056877-0.302633$

C $-2.955354-3.887432-1.552229$

C $-3.646477-3.491650-2.638932$

C $-2.359749-5.270773-1.497734$

C $-4.259753-2.146952-2.914964$

C $-5.792327-2.152035-2.849047$

C $-6.335408-2.061487-1.414258$

C $-7.864519-2.116485-1.414548$

C $-8.450827-1.903720-0.011213$

O $-5.893397-0.816015-0.861037$
O $-9.856481-1.669228-0.184698$

C $-7.822661-0.6349640 .605407$

C $-8.321249-3.1648950 .850964$

O $-8.216191-0.476121 \quad 1.966900$

C $-6.279162-0.5982580 .500723$

C $-5.7556350 .794746 \quad 0.929234$

O $-5.732687-1.5901961 .372852$

C $-0.513189-0.471215-1.596252$

O $0.648344-0.834475-1.515305$

C $-0.9797760 .809403-2.168171$

C $-0.117812 \quad 1.830367-2.270728$

C $-0.442944 \quad 3.188850-2.708027$

C $-1.8615303 .464125-3.134620$

C $-4.269662 \quad 1.039754 \quad 0.621772$

C -3.7840172 .3623951 .193605$

C $-1.708778 \quad 3.707878 \quad 1.504432$

O $-4.526253 \quad 3.159448 \quad 1.762156$

C $-1.669624 \quad 4.7768680 .457037$

C $-0.569881 \quad 5.494105 \quad 0.177924$

C $-0.5258856 .602680-0.836491$

C $0.6282506 .469008-1.806901$

C $1.7603377 .172339-1.635139$

C $0.4379935 .582242-3.018066$

C $0.5298914 .122623-2.669794$

N -2.420921 2.5470360 .999299

H $0.925268-2.310851 \quad 1.083545$

H $-1.910730-1.3015331 .640933$

H $-0.613132-2.897805-0.704146$

H $-3.585530-2.357284-0.186625$

H -2.804152 $-3.728490 \quad 0.564884$

H $-3.754352-4.200168-3.459660$

H -2.532299 $-5.836073-2.419757$

H $-1.277452-5.214606-1.344021$

H $-2.799363-5.841412-0.673164$

H $-3.852034-1.367147-2.267489$

H $-3.960345-1.865632-3.932991$

H $-6.145534-1.277505-3.411422$

H -6.190791-3.042274-3.350933

H $-5.925613-2.888941-0.824945$

H $-8.219745-3.063653-1.839698$

H $-8.255420-1.328835-2.074460$

H $-10.260369-1.5873160 .695614$

H $-8.2271580 .229340 \quad 0.060281$

H $-8.870615-3.996500 \quad 0.393623$

H -8.769809 -3.0172611 .839456$

H $-7.286640-3.4864880 .988243$

H $-9.183849-0.3885761 .986134$

H -6.3283731 .5739200 .408962$

H -5.9244370 .9313162 .005143$

H $-4.856067-1.3060241 .674682$

H -2.030939 $0.896598-2.418624$

H $0.9164611 .669590-1.968696$

H -2.124705 $2.851699-4.004362$

H $-2.0418244 .503620-3.414472$

H $-2.561028 \quad 3.237607-2.322112$

H -3.6520180 .2450961 .049295$

H $-4.1058561 .057392-0.461775$

H -0.7072283 .3744141 .791343$

H -2.2224014 .0844632 .394915$

H -2.592827 $4.993324-0.075205$

H 0.3427465 .3023120 .737762

H $-0.4401257 .549866-0.287777$

H -1.466296 $6.669554-1.397743$

H $2.5822197 .102425-2.339859$

H $1.8926147 .829619-0.782079$

H $1.2164725 .787880-3.764878$

H $-0.4997635 .849620-3.512019$

H $1.5243863 .820497-2.339150$

H $-1.894914 \quad 1.859012 \quad 0.468844$

C 0.8666980 .2241392 .783799

$\begin{array}{llll}\text { O } & 2.047729 & 0.306918 & 1.965502\end{array}$

C 2.8545611 .4075142 .425503

C 2.0726062 .0808873 .550501

C 1.2329840 .9344874 .082193

H 3.7686040 .9733752 .845490

C 3.2093852 .3266131 .250613

H 2.7332842 .5148054 .307569

H 1.4286522 .8847563 .176181 
C 0.0373411 .3892934 .900565

H 1.8711540 .2871564 .698428

C 3.8618281 .6118300 .050127

$\begin{array}{lllll}\text { O } & 2.017089 & 2.958824 & 0.766338\end{array}$

H 3.8572393 .1381521 .601504

H $3.1255250 .978452-0.459658$

O $4.2475232 .618380-0.895172$

C 5.1027320 .7703550 .398727

C $5.7830970 .186147-0.853602$

H $4.806287-0.0487591 .061210$

O 6.0417551 .5719421 .113487

H $5.034731-0.368373-1.430106$

H $6.1393141 .005085-1.490156$

H $\quad 0.364763 \quad 1.964383 \quad 5.772845$

H $-0.6339812 .023873 \quad 4.312265$

H -0.5397450 .5316335 .259873$

C $0.451474-1.2369412 .965574$

H 1.3955322 .2565870 .508121

H $3.4546843 .143694-1.098170$

H 6.2817502 .3222140 .542838

H $-0.404214-1.3088153 .646799$

H $1.268312-1.8165683 .411310$

H $\quad 0.076147 \quad 0.7774082 .258381$

C $5.787015-2.769916-0.097633$

O $6.658848-1.7538380 .410701$

C $6.989731-0.719919-0.520888$

C $7.676142-1.327847-1.752314$

C $6.835324-2.436438-2.380938$

C $6.411013-3.453265-1.321534$

H $4.820405-2.324799-0.363573$

C $5.553438-3.7805141 .030096$

H $7.731785-0.085515-0.019933$

O $7.943454-0.331628-2.733501$

H $8.643152-1.754407-1.455124$

H $\quad 5.964410-2.028928-2.907548$

O $7.616728-3.115656-3.366751$

H $7.288605-4.037952-1.018152$

O $5.480730-4.369754-1.895574$

C $4.648870-3.2018082 .090966$

H $5.069356-4.6934530 .664524$

H $6.497795-4.085978 \quad 1.496606$

H $8.4927160 .352088-2.313262$

H $7.910130-2.449481-4.011942$

H $4.694199-3.866372-2.167468$

O $5.390739-2.625143 \quad 3.078314$

O $3.426721-3.2429662 .035460$

C $4.641954-2.0377354 .146572$

H $4.115734-2.8163014 .706718$

H $5.343028-1.5336214 .816819$

H $3.935388-1.2988383 .759425$

SCF Energy (B3LYP/6-31G**//MMFF) $=-3245.91470163$

02 85

MM̄FF Geometry

C $-0.464132 \quad 3.442367 \quad 1.167471$

C -1.071805 3.5746862 .355879

C -1.402893 $2.451893 \quad 3.313043$

O $-1.130903 \quad 1.1555912 .731181$

C -2.905144 2.4943083 .657412

C -3.3430491 .3429014 .542561$

C -3.8854250 .1963624 .083717$

C -3.1317611 .5516816 .018263$

C $-4.126467-0.1801082 .647163$

C $-5.590005-0.5570072 .393684$

C $-5.938149-0.5349270 .897308$

C $-7.345363-1.0853170 .659619$

C $-7.694428-1.140274-0.834155$

O $-4.978581-1.3177520 .180247$

O $-8.868646-1.957144-0.959635$

C $-6.546137-1.836755-1.597939$

C $-8.0763220 .244252-1.372188$

O $-6.793302-1.764650-3.000605$

C $-5.145156-1.277875-1.244997$

C $-4.037322-2.149430-1.882418$

O $-5.036353 \quad 0.052433-1.758169$

C $0.076463 \quad 0.5773932 .968234$

O $1.046767 \quad 1.0872793 .503660$

C $-0.013869-0.8225002 .490256$
C $1.043320-1.6405142 .596170$

C $1.069900-3.0670862 .257337$

C $-0.227871-3.7116051 .845482$

C $-2.616865-1.834066-1.387534$

C $-1.567134-2.632557-2.143702$

C $0.900237-2.935929-2.308385$

O $-1.845199-3.449497-3.018059$

C $1.243943-4.179453-1.551661$

C $2.463911-4.425587-1.050407$

C $2.834747-5.701340-0.352098$

C $3.439523-5.4746861 .014027$

C $4.761758-5.6043111 .215743$

C $2.500282-5.2022552 .166389$

C $2.236632-3.7357382 .369236$

N $-0.279070-2.320047-1.729986$

H -0.1403742 .4588750 .839212$

H $-1.359627 \quad 4.5726212 .681298$

H -0.8177152 .5813194 .232219$

H -3.4998292 .5078822 .736403$

H -3.146343 3.4416094 .158556

H $-4.157859-0.5712944 .806643$

H $-3.465967 \quad 0.6940296 .611730$

H -2.070110 1.7087716 .233599

H -3.6903312 .4286526 .361061$

H -3.8296510 .6167541 .960460$

H $-3.488729-1.0403822 .409657$

H $-5.760607-1.5618182 .801895$

H -6.2639410 .1268782 .925095$

H -5.8726930 .5061800 .558190$

H $-8.092354-0.4988281 .208859$

H $-7.408653-2.1047521 .066074$

H $-9.062478-2.049828-1.908412$

H -6.581472 -2.901420 -1.327271

H $-8.9551980 .629393-0.840950$

H $-8.3641580 .194968-2.428153$

H $-7.2771410 .980991-1.267359$

H -6.185796 -2.380631 -3.442771

H $-4.235719-3.206842-1.663050$

H $-4.061929-2.031597-2.972994$

H $-4.1027790 .262553-1.913788$

H $-0.969209-1.1554932 .100750$

H $1.973093-1.2366762 .994113$

H -0.951572 -3.672859 2.667124

H $-0.124653-4.7593951 .556879$

H - $0.657982-3.1997700 .980040$

H $-2.384206-0.772208-1.518429$

H -2.528598 $-2.075776-0.322479$

H $1.705257-2.198064-2.269764$

H $0.701937-3.183850-3.356144$

H $0.462946-4.928211-1.441833$

H $3.253544-3.691569-1.190765$

H $3.549393-6.232596-0.994479$

H $1.970938-6.371077-0.257683$

H $5.197452-5.4756772 .201170$

H $5.438693-5.8333740 .399340$

H $2.944260-5.5802173 .097728$

H $1.587584-5.7913792 .043016$

H $3.119889-3.1810902 .686662$

H $-0.147650-1.681640-0.950744$

C $-0.5039884 .210855-1.214723$

$\begin{array}{lllll}\text { O } & 0.352867 & 3.127558 & -1.631438\end{array}$

C $0.917167 \quad 3.438624-2.915313$

C $0.0711664 .576009-3.471146$

C $-0.250575 \quad 5.348058-2.205319$

H $1.9331413 .809830-2.733726$

C $0.9733732 .178041-3.782513$

H $0.6104705 .171710-4.213673$

H $-0.8518424 .208669-3.934582$

C - $1.4198026 .305526-2.355694$

H $0.6403355 .916460-1.905851$

C $1.6806160 .976143-3.120967$

O

H $1.4483052 .411968-4.742166$

H $1.0682640 .579209-2.302164$

O $1.763444-0.065009-4.103418$

C $3.100648 \quad 1.266781-2.599556$

C $3.7991230 .003815-2.058009$

H $3.0491712 .013617-1.802893$ 
O $3.8865291 .827035-3.650939$

H $3.164406-0.443835-1.286006$

H $3.891460-0.727261-2.869719$

H -1.202602 $7.060920-3.117791$

H $-2.3331505 .779981-2.654124$

H $-1.622276 \quad 6.824167-1.413385$

C $-0.173799 \quad 4.584076 \quad 0.232472$

H $-0.8289051 .611196-3.257633$

H $0.857391-0.251965-4.403853$

H $3.9097281 .179996-4.376716$

H -0.7354095 .4774350 .527905$

H $0.893413 \quad 4.8179330 .311657$

H -1.536897 $3.847787-1.292222$

C 4.6469340 .7765530 .804954

O $5.1979161 .242277-0.433656$

C $5.211675 \quad 0.283972-1.497651$

C $5.981608-0.975393-1.072077$

C $5.460590-1.5458300 .242169$

C $5.411481-0.4548321 .306851$

H 3.5861480 .5326060 .670075

C $4.759213 \quad 1.922198 \quad 1.823716$

H $5.7879250 .743376-2.310505$

O $5.917487-1.983168-2.075671$

H $7.041811-0.722181-0.940781$

H $4.479018-2.0170240 .119493$

O $6.348515-2.570506 \quad 0.693466$

H $6.434483-0.1753221 .590200$

O $4.771465-0.9654362 .473286$

C 3.8023243 .0580711 .537689

H 4.5299471 .5616502 .832568

H 5.7760452 .3323791 .805436

H $6.272838-1.600894-2.896131$

H $6.399296-3.238795-0.011486$

H $5.261470-1.7589302 .749352$

O 4.0415724 .0661612 .428664

O 2.9503863 .0617620 .661355

C 3.2053075 .2199032 .300431

H 2.1792504 .9684552 .582783

H 3.5786525 .9876782 .983556

H 3.2428215 .6128421 .279909

SCF Energy (B3LYP/6-31G**//MMFF)= -3245.93134260

02 86

MMFF Geometry

C 2.6947832 .6607431 .914790

C $3.7889603 .132401 \quad 1.296444$

C $3.815027 \quad 4.095558 \quad 0.131201$

O $2.5025604 .653522-0.114163$

C $4.2442263 .405273-1.176104$

C $5.6663482 .889401-1.179220$

C $6.0189581 .589365-1.202030$

C $6.7354663 .953374-1.194050$

C $5.1354570 .372076-1.225530$

C $5.378954-0.5118550 .004569$

C $4.791192-1.930505-0.093234$

C $5.545960-2.796042-1.108592$

C $4.947978-4.205473-1.220189$

O $3.399563-1.853850-0.424216$

O $5.492942-4.824441-2.396380$

C $3.427257-4.074306-1.444080$

C $5.348891-5.097441-0.039748$

O $2.799430-5.354678-1.421109$

C $2.734128-3.126300-0.437055$

C $1.237299-2.906406-0.773712$

O $2.775883-3.7383720 .860081$

$\begin{array}{llll}\text { C } 2.152801 & 5.788191 & 0.545927\end{array}$

$\begin{array}{lllll}\text { O } & 2.853691 & 6.435650 & 1.311281\end{array}$

C $0.736501 \quad 6.1406990 .289869$

C $0.0147505 .594738-0.700667$

C -1.407446 $5.815923-0.964086$

C $-2.1766256 .690544-0.010340$

C $0.933481-2.192921-2.093322$

C $0.972519-0.679957-1.992842$

C $-0.2696801 .252333-1.008870$

O $1.830566-0.017959-2.571397$

C $-1.473738 \quad 1.673771-1.790886$

C $-2.6122972 .115714-1.233503$

C $-3.8011942 .589695-2.024507$
C $-4.1815184 .022704-1.711987$

C $-5.209634 \quad 4.297118-0.891497$

C $-3.4196665 .136862-2.401171$

C $-1.9698185 .171609-2.007220$

N $-0.053976-0.165731-1.218178$

H 1.7068472 .9589041 .574841

H 4.7643422 .8190341 .663636

H 4.5102744 .9115630 .361117

H $4.1374564 .121514-2.003050$

H $3.5201522 .620407-1.421583$

H $7.0835791 .356006-1.223084$

H $7.7403093 .532844-1.309577$

H $6.5730834 .643441-2.028464$

H $6.7254014 .524771-0.261066$

H $4.0757240 .629606-1.272797$

H $5.365632-0.171740-2.147058$

H $6.452859-0.5892200 .218316$

H $4.923816-0.0101880 .869117$

H $4.888225-2.3746840 .904565$

H $6.612007-2.851192-0.854918$

H $5.498994-2.336652-2.103772$

H $6.461332-4.837167-2.306881$

H $3.291828-3.682928-2.458929$

H $6.439023-5.2101330 .001046$

H $4.948034-6.110384-0.156367$

H $5.024322-4.7066230 .926547$

H $3.259991-5.914851-2.069214$

H $\quad 0.731338-3.880769-0.776078$

H $\quad 0.777173-2.348780 \quad 0.051361$

H $2.067236-3.3599841 .404790$

H 0.3139136 .8666280 .976809

H $0.498126 \quad 4.888799-1.374402$

H $-2.1123396 .298603 \quad 1.010839$

H $-3.2396136 .763406-0.251802$

H -1.779049 $7.711512-0.021147$

H $1.596493-2.507141-2.903463$

H $-0.078506-2.464833-2.417568$

H $0.606297 \quad 1.823151-1.332877$

H $-0.401867 \quad 1.4078170 .065315$

H $-1.3877941 .646761-2.874748$

H -2.698707 2.144574 -0.149725

H $-4.6410881 .922916-1.794866$

H -3.630381 $2.489181-3.103659$

H $-5.5066925 .318611-0.678620$

H $-5.7764863 .506297-0.411612$

H $-3.8891656 .111435-2.224501$

H $-3.4828154 .989289-3.486971$

H $-1.3333294 .557701-2.644992$

H $-0.743727-0.802893-0.832302$

C $2.025890 \quad 0.4155832 .831627$

$\begin{array}{llll}\text { O } & 0.611148 & 0.646177 & 2.681995\end{array}$

C $-0.114308-0.3882013 .381629$

C $0.921107-1.4249593 .790035$

C $2.157780-0.5748673 .986669$

H -0.5485480 .0956354 .265103$

C $-1.217844-0.9252422 .461996$

H $0.631140-1.9753444 .690185$

H $1.081251-2.1516152 .985472$

C $3.449665-1.3733723 .973450$

H $2.070432-0.0472964 .945993$

C $-1.972020-2.137246 \quad 3.031494$

$\begin{array}{lllll}\text { O } & -2.158845 & 0.125342 & 2.221798\end{array}$

H $-0.775821-1.1772311 .494081$

H -1.276924 -2.968631 3.198025

O $-2.487980-1.775116 \quad 4.318515$

C $-3.148231-2.6255902 .163008$

C -2.716747 -3.0260910 .740625$

H $-3.931781-1.8600312 .140058$

O $-3.702675-3.7688902 .822722$

H -2.299772 -2.1529810 .232020$

H $-1.913945-3.7706800 .810936$

H $3.455214-2.1091874 .784222$

H $3.577174-1.9130863 .029850$

H $4.316799-0.7189684 .105912$

C $2.7412851 .740348 \quad 3.102613$

H -1.6583900 .9049951 .927079$

H -2.967843 -2.546676 4.665469

H -4.586072 -3.9240412 .447608$ 
H 3.7805211 .5500393 .394138 H 2.2565532 .2590923 .939015 H $2.379970-0.0306691 .893079$ C $-4.877842-1.555214-0.835076$ O $-5.019962-2.782510-0.108709$ C $-3.859087-3.618381-0.113106$ C $-3.459396-3.967982-1.554930$ C $-3.335597-2.728232-2.436914$ C $-4.577257-1.847952-2.307905$ H $-4.063340-0.961690-0.402310$ C $-6.184066-0.772498-0.669116$ H $-4.157117-4.5592160 .365869$ O $-2.230127-4.685542-1.585368$ H $-4.224761-4.623268-1.991053$ H -2.431846 -2.156239-2.200820 O $-3.210401-3.132277-3.801874$ H $-5.433808-2.344660-2.780842$ O $-4.353305-0.622917-2.999934$ C $-6.309051-0.2384460 .738285$ H -6.254071 $0.076032-1.358157$ H $-7.051849-1.415664-0.858382$ H -2.342465 -5.480827 -1.037045 H $-2.433242-3.714288-3.860459$ H $-4.129929-0.845256-3.919947$ O $\begin{array}{llll}-5.468518 & 0.821932 & 0.909531\end{array}$ O $-7.054657-0.714061 \quad 1.584476$ C $-5.466351 \quad 1.403287 \quad 2.216571$ H $-6.452326 \quad 1.8171972 .447112$ H -5.1742530 .6585412 .963246$ H $-4.733378 \quad 2.214442 \quad 2.225714$

SCF Energy (B3LYP/6-31G**//MMFF) $=-3245.91466797$

\section{2_87}

MMFFF Geometry

C $2.1333154 .970097-0.308727$

C $3.1819214 .138263-0.409959$

C $4.054012 \quad 3.611790 \quad 0.710751$

$\begin{array}{lllll}\text { O } & 4.035108 & 2.162532 & 0.646592\end{array}$

C $3.626753 \quad 4.0291822 .132586$

C $4.558783 \quad 3.5338443 .224701$

C 4.2630862 .5641214 .114039

C $5.889251 \quad 4.2393413 .301450$

C $3.003716 \quad 1.7483554 .204166$

C $3.290552 \quad 0.2648703 .938144$

C $2.007111-0.5253013 .642510$

C $2.323303-2.0123393 .471239$

C $1.103502-2.8203083 .004714$

$\begin{array}{llllll}\text { O } & 1.453093 & 0.007349 & 2.435047\end{array}$

O $1.570225-4.1013412 .552961$

C $0.474559-2.1153521 .784495$

C $0.126993-3.0945404 .154065$

O $-0.738170-2.7536601 .398781$

C $0.232658-0.6089062 .006463$

C -0.2255210 .1281090 .724354$

O $-0.801132-0.4349992 .982141$

C $4.9339701 .561947-0.182717$

O $5.7542732 .112751-0.900043$

C $4.7426960 .098185-0.079647$

C $5.372672-0.715252-0.940503$

C $5.292878-2.177892-0.969420$

C $4.399187-2.8490930 .039062$

C $0.7714420 .096436-0.438833$

C $0.509335-1.001933-1.455277$

C $1.525369-2.036544-3.485248$

O $-0.504060-1.694951-1.457122$

C $2.293137-3.259339-3.093303$

C $3.317947-3.748483-3.808097$

C $4.058313-5.006812-3.456571$

C $5.555537-4.807106-3.386683$

C $6.344439-5.124244-4.427416$

C $6.145086-4.331283-2.078762$

C $6.017725-2.846602-1.890893$

N $1.535869-1.089235-2.386903$

H $1.818983 \quad 5.3394210 .662856$

H $3.4747443 .814612-1.407778$

H 5.0745843 .9734050 .532611

H 2.6032293 .6882942 .313726

H 3.5892085 .1245122 .202472
H 5.0110882 .3116754 .864888

H 6.4848733 .9120964 .160419

H 6.4801714 .0473092 .400778

H 5.7415835 .3199353 .399102

H $2.584388 \quad 1.8606825 .211398$

H $2.2367402 .112723 \quad 3.518500$

H 3.9671970 .1788923 .078721

H $3.807841-0.1668704 .803962$

H $1.298295-0.3726004 .464785$

H $2.730896-2.4314374 .399718$

H $3.117259-2.1279272 .721801$

H $2.037979-4.5273203 .291676$

H $1.172521-2.2434450 .948843$

H $\quad 0.617733-3.6792494 .941631$

H $-0.721348-3.6999253 .816505$

H $-0.259540-2.1833734 .615401$

H $-0.538611-3.6942871 .253338$

H $-1.218042-0.2245750 .421484$

H $-0.363483 \quad 1.186302 \quad 0.989938$

H -1.1822750 .4521092 .874531$

H $4.085916-0.2705500 .698811$

H $6.015265-0.270174-1.699299$

H $4.792632-2.7053101 .050770$

H $4.291570-3.924293-0.117811$

H $3.386160-2.436381-0.008390$

H $0.6992321 .044603-0.986404$

H $1.7980180 .013311-0.073533$

H $1.963697-1.532510-4.352409$

H $0.492824-2.309710-3.725697$

H $1.970621-3.783237-2.196427$

H $3.619014-3.238380-4.720490$

H $3.819696-5.756573-4.222563$

H $3.701703-5.430900-2.509677$

H $7.422373-5.013678-4.374115$

H $5.928602-5.496009-5.358059$

H $7.216851-4.569999-2.043290$

H $5.711131-4.905202-1.255196$

H $6.630988-2.270514-2.585380$

H $2.360372-0.506463-2.273639$

C $-0.1433845 .248923-1.347582$

O $-0.4410393 .850530-1.538409$

C $-1.6193603 .732406-2.368705$

C $-2.2115855 .130938-2.453098$

C $-0.968098 \quad 5.992145-2.397884$

H $-1.2576763 .410943-3.353435$

C $-2.5589732 .677722-1.770180$

H $-2.7995845 .285122-3.362789$

H $-2.8565025 .335147-1.589126$

C $-1.2538767 .443320-2.051350$

H $-0.464845 \quad 5.946646-3.373153$

C $-3.9358102 .596595-2.451434$

O $-1.915483 \quad 1.404835-1.871640$

H $-2.6772222 .877857-0.699730$

H $-4.4728993 .543348-2.321638$

O $-3.726537 \quad 2.437505-3.860319$

C $-4.8228191 .430722-1.970381$

C $-5.0731851 .433612-0.452493$

H $-4.3828700 .477748-2.284426$

O $-6.080348 \quad 1.529982-2.649432$

H $-4.119281 \quad 1.327900 \quad 0.072397$

H $-5.4932052 .405527-0.165104$

H $-1.9069107 .896708-2.804351$

H $-1.7495347 .533488-1.078901$

H $-0.3288058 .026634-2.012863$

C $1.3611495 .489200-1.491654$

H $-1.8322611 .189651-2.816582$

H $-4.6027062 .381360-4.279032$

H -6.504179 $2.359730-2.370788$

H $1.5600386 .564658-1.564238$

H $1.7235575 .022925-2.416131$

H $-0.4836385 .512837-0.337542$

C $-4.481954-1.5049120 .191123$

O $-5.639174-0.970080-0.458481$

$\begin{array}{llll}C & -6.045417 & 0.322021 & 0.004185\end{array}$

C $-6.305105 \quad 0.2942691 .518530$

C $-5.121300-0.2764352 .297288$

C $-4.699934-1.6211641 .705544$

H $-3.632530-0.837770-0.000732$ 
C $-4.115910-2.836375-0.479968$

H $-7.0092900 .527664-0.478005$

O -6.6015691 .5975952 .009389$

H -7.183412 -0.3323691 .721720$

H -4.2793370 .4252312 .322561$

O $-5.511361-0.4853913 .656364$

H -5.478249 -2.358417 1.932399

O $-3.510199-2.0709352 .345866$

C $-5.157119-3.924640-0.318156$

H $-3.988303-2.683426-1.558122$

H $-3.165857-3.195661-0.068495$

H $-7.374796 \quad 1.9248411 .518887$

H $-5.816343 \quad 0.370243 \quad 4.004247$

H -2.801985 -1.434853 2.146892

O $-4.549959-5.143493-0.426378$

O $-6.354733-3.735193-0.154508$

C $-5.417644-6.275689-0.311041$

H $-6.157114-6.269104-1.117387$

H $-4.809829-7.180112-0.399835$

H $-5.910110-6.2783480 .666069$

SCF Energy (B3LYP/6-31G**//MMFF) $=-3245.91541323$

02_88

MMFF Geometry

C $1.803313-0.8754082 .776874$

C $2.847900-0.259833 \quad 3.350919$

C 3.3703151 .1296253 .067164

O 2.4887921 .9038432 .225529

C $4.6980090 .995285 \quad 2.299543$

C $5.601300 \quad 2.206357 \quad 2.389262$

C $5.741628 \quad 3.1409891 .429059$

C $6.439082 \quad 2.2802703 .640468$

C $4.964718 \quad 3.2913010 .151473$

C $5.7062112 .781659-1.095760$

C $5.3545271 .327516-1.448116$

C $6.090346 \quad 0.860072-2.704021$

C $5.649197-0.549967-3.133519$

O $3.9434281 .279668-1.675486$

O $6.164107-0.754669-4.457872$

C $4.105486-0.597263-3.203053$

C $6.278000-1.631802-2.247204$

O $3.637018-1.922500-3.441598$

C $3.416042-0.022437-1.945145$

C $1.8951380 .136818-2.191276$

O $3.619924-0.916040-0.848421$

C 1.3992262 .4900482 .784841

$\begin{array}{lllll}\text { O } & 0.997143 & 2.368001 & 3.929661\end{array}$

C $0.781826 \quad 3.3319281 .735814$

C $-0.486440 \quad 3.747979 \quad 1.859690$

C $-1.196486 \quad 4.6259740 .924906$

C $-0.4322465 .146914-0.264761$

C $1.1923830 .993988-1.128659$

C $-0.3126961 .008999-1.302853$

C $-2.1019601 .769487-2.864816$

O $-1.0613650 .498210-0.472922$

C $-2.571174 \quad 3.171151-2.632883$

C $-3.6690783 .474616-1.924455$

C $-4.2103484 .864848-1.764827$

C $-4.4749255 .234976-0.323636$

C $-5.690602 \quad 5.0611160 .222722$

C -3.3664095 .9123600 .445479$

C -2.481298 $4.945517 \quad 1.185734$

N $-0.7150521 .652585-2.460640$

H $1.201950-0.3614242 .034014$

H $3.428378-0.8170104 .085353$

H $3.517017 \quad 1.6572654 .017335$

H 4.4748890 .7727851 .252217

H 5.2723930 .1329362 .665627

H 6.4696353 .9347641 .594510

H $7.0949983 .157252 \quad 3.652344$

H 5.7993072 .3369704 .526698

H 7.0741831 .3923823 .726057

H 3.9691082 .8470250 .237152

H $4.785074 \quad 4.3672930 .024065$

H $5.4026713 .419800-1.936535$

H $6.7905872 .895589-0.979744$

H $5.6173690 .685285-0.602498$

H $7.1768450 .895145-2.555229$
H $5.8791031 .554512-3.529339$

H $5.945841-1.661795-4.730390$

H $3.7999960 .003278-4.071286$

H $7.371736-1.591404-2.315333$

H $5.993344-2.634834-2.584038$

H $6.006766-1.534501-1.193807$

H $4.005542-2.217929-4.290895$

H $1.7380890 .615487-3.166098$

H $1.415268-0.847849-2.236325$

H $2.879964-0.835335-0.226452$

H $1.402667 \quad 3.5976120 .887478$

H -1.053632 3.4315542 .734061

H $0.397467 \quad 5.7838870 .060757$

H $-1.0433885 .737620-0.950512$

H $-0.0271804 .319332-0.857154$

H $1.4036200 .619907-0.123409$

H $1.5488862 .029792-1.166756$

H -2.708459 $1.034895-2.327292$

H $-2.146803 \quad 1.539246-3.933782$

H -2.006875 $3.969563-3.108849$

H $-4.2484792 .670904-1.480023$

H $-5.1476224 .921781-2.334512$

H -3.546483 $5.608466-2.222785$

H $-5.898008 \quad 5.359794 \quad 1.244975$

H $-6.5018774 .608861-0.338294$

H -3.8011736 .5866401 .196563$

H -2.805979 $6.580190-0.215562$

H -2.955375 4.5091732 .066152

H $-0.0159422 .097065-3.048287$

C $1.622986-3.1923181 .857140$

O $0.554949-2.9656360 .913044$

C $0.129921-4.2368290 .383715$

C $1.172280-5.2533520 .831610$

C $1.578636-4.6859332 .176280$

H $-0.821033-4.4815760 .869869$

C $-0.061894-4.152405-1.133479$

H $0.763493-6.2663380 .896764$

H $2.032295-5.2789320 .152042$

C $2.889943-5.2489222 .697030$

H $0.782065-4.8920492 .903778$

C $-1.096488-3.103746-1.590227$

O $1.190916-3.825644-1.748333$

H $-0.338980-5.138216-1.524698$

H $-0.722135-2.092392-1.399215$

O $-1.233749-3.220061-3.011989$

C $-2.488235-3.265771-0.950213$

C $-3.545568-2.324301-1.558027$

H -2.409282 -3.070449 0.123616

O $-2.930258-4.615161-1.100745$

H -3.169627 -1.297513 -1.512355

H $-3.688166-2.570585-2.616952$

H $2.814874-6.3325392 .834671$

H $3.713941-5.0550342 .002122$

H $3.151981-4.8042233 .661950$

C $1.429590-2.3001213 .084520$

H $1.514462-3.005816-1.337054$

H $-0.348134-3.105916-3.397737$

H -2.967012 $-4.807176-2.053494$

H $2.034409-2.6733753 .918842$

H $0.383788-2.3166703 .408293$

H 2.560906-2.943785 1.342552

C $-4.499778-0.9023100 .967633$

O $-4.812071-2.2410690 .565181$

C $-4.915955-2.433217-0.849252$

C $-6.006273-1.516553-1.422838$

C $-5.792177-0.058339-1.026143$

C $-5.583090 \quad 0.0653950 .482620$

H $-3.525740-0.6104800 .557271$

C $-4.393459-0.8946702 .495419$

H -5.261556 -3.465155 -0.990497

O $-6.060244-1.611175-2.842164$

H $-6.984887-1.836465-1.041872$

H $-4.9533520 .381062-1.575584$

O $-6.947935 \quad 0.703159-1.381651$

H $-6.529793-0.1349811 .000624$

$\begin{array}{lllll}\text { O } & -5.196058 & 1.400203 & 0.798807\end{array}$

C -3.151738 -1.6315462 .937625$

H -4.3225400 .1214362 .898951$ 
H $-5.274426-1.3633662 .950359$ H $-6.214084-2.544805-3.066719$ H $-7.0873630 .587000-2.337272$ H -5.8868931 .9915040 .454037$ O $-3.491298-2.8228543 .506419$ O $-2.015542-1.2050862 .775313$ C -2.395969 -3.628070 3.951952 H $-1.819557-3.0964934 .714992$ H -2.802657 -4.541919 4.393053 $\mathrm{H}-1.759415-3.9008023 .105820$

SCF Energy (B3LYP/6-31G**//MMFF) $=-3245.91413648$

$02 \_89$

MM̄FF Geometry

C $0.184669-0.171160-2.521919$

C $1.182603-1.042758-2.740610$

C $1.656249-2.111026-1.781284$

O $1.051933-1.916951-0.483703$

C $3.181669-2.075129-1.594766$

C $3.722722-3.407666-1.111231$

C $4.060398-3.6939130 .160951$

C $3.914950-4.446467-2.188318$

C $3.960582-2.8100281 .372352$

C $5.342032-2.4750431 .955894$

C $6.168424-1.5432941 .056373$

C $7.565172-1.3173591 .636725$

C $8.369933-0.3084090 .802011$

O $5.472694-0.297468 \quad 0.972337$

O $9.519090 \quad 0.081821 \quad 1.569080$

C 7.5156000 .9591230 .590012

C $8.903656-0.932870-0.492670$

O $8.1586191 .865515-0.303392$

C 6.0776420 .6649760 .100675

C 5.2276501 .9555550 .165483

O $6.1237590 .199979-1.249026$

C $0.108722-2.794692-0.062952$

O $-0.242007-3.821417-0.627575$

C $-0.519309-2.3448561 .202250$

C $-0.193028-1.2068991 .835629$

C $-0.811222-0.6884483 .058442$

C $-1.980434-1.4473863 .627993$

C $3.7267601 .730702-0.067375$

C 2.9241002 .9960750 .175299

C 0.5784973 .7642970 .508573

O 3.4372324 .1050360 .297306

C 0.3230583 .7947021 .982493

C -0.8820243 .5811052 .531591$

C -1.1471143 .6081914 .008952$

C - $1.7435902 .312976 \quad 4.515617$

C -3.0622532 .2084014 .752239$

C -0.8034541 .1693304 .827202$

C $-0.328330 \quad 0.453112 \quad 3.592620$

N $1.561566 \quad 2.7377420 .222189$

H $-0.336955-0.173757-1.569283$

H $\quad 1.652057-1.053437-3.722613$

H $1.354108-3.073231-2.211869$

H $3.455030-1.272983-0.901483$

H $3.683491-1.832977-2.540885$

H $4.451721-4.6885740 .372991$

H $4.343077-5.376297-1.798978$

H $2.960059-4.697778-2.659572$

H $4.595097-4.070274-2.959619$

H $3.397897-1.8931731 .175566$

H $3.385429-3.3549812 .131491$

H $5.181327-1.9879152 .926371$

H $5.898007-3.4016442 .146026$

H $6.246121-1.9850870 .057032$

H $8.109801-2.2658341 .722757$

H $7.477010-0.9278072 .660795$

H $10.030594-0.7202531 .771543$

H $7.4517201 .473477 \quad 1.559342$

H $9.550924-1.788610-0.266210$

H $9.529216-0.222819-1.044793$

H $8.112263-1.285017-1.157452$

H 9.0501842 .0338340 .046937

H 5.3441572 .4087521 .159113

H $5.5946312 .683074-0.569439$

H $5.2720960 .380543-1.676319$
H -1.295247 -3.0037451 .577474$

H $0.595226-0.5797191 .422630$

H -2.777679 -1.533552 2.882338

H -2.427697 -0.9689554 .501792$

H -1.674586-2.453074 3.936132

H $3.5370531 .408816-1.096765$

H 3.3501780 .9565280 .610901

H $-0.3187783 .514042-0.061473$

H $0.949064 \quad 4.7377510 .172746$

H $\quad 1.1715074 .0083412 .628364$

H -1.7331283 .3841101 .885607$

H -1.8330014 .4418484 .208852$

H -0.2358473 .8346514 .576347$

H $-3.496322 \quad 1.294070 \quad 5.142592$

H -3.7385603 .0334954 .554618$

H -1.2638310 .4708335 .534968$

H $\quad 0.072241 \quad 1.557475 \quad 5.363344$

H 0.5186040 .9358873 .104596

H $1.236618 \quad 1.7765530 .167560$

C $-0.6951912 .146698-3.038091$

O $-1.7593302 .049421-2.065378$

C $-2.8374582 .914515-2.462094$

C $-2.2030323 .936433-3.395635$

C -1.209265 $3.073155-4.145166$

H -3.539415 2.293968 -3.033465

C $-3.5632993 .501702-1.244665$

H -2.936359 $4.417152-4.049668$

H $-1.6667684 .718258-2.846936$

C $-0.1126103 .865381-4.836979$

H $-1.7549282 .492232-4.900986$

C $-3.9873122 .463515-0.185276$

O $-2.7110504 .438765-0.576248$

H $-4.4398894 .070622-1.577997$

H -3.099109 2.0407390 .298469

O $-4.689872 \quad 3.1736320 .845926$

C -4.878188 1.316507 -0.704019

C -5.4302580 .4569140 .451940$

H $-4.2985700 .695658-1.393688$

O $\quad-5.9651341 .883000-1.434552$

H $-4.588250 \quad 0.1186801 .065732$

H -6.0630831 .0873131 .087898$

H $-0.5398774 .544510-5.581837$

H $0.4594074 .466749-4.122406$

H $0.586198 \quad 3.196858-5.349709$

C $-0.3519930 .755210-3.577259$

H -2.730672 $5.269140-1.081101$

H -5.5099003 .5214810 .455156$

H $-6.3669511 .173385-1.963120$

H $0.3625240 .844961-4.403870$

H -1.263934 $0.293499-3.976325$

H $0.1693902 .584212-2.524659$

C $-4.520099-2.398928-0.217054$

O $-5.552596-1.640320-0.860006$

C $-6.279099-0.750058-0.007640$

C $-6.926660-1.5348911 .142610$

C $-5.898513-2.3609771 .909190$

C $-5.087977-3.2252280 .945632$

H -3.736844 -1.722170 0.145246

C -3.906669-3.331079-1.274597

H -7.102086 -0.350688 -0.612395

O $-7.601359-0.6481572 .028359$

H -7.685789 -2.209516 0.726016

H -5.244689 -1.718226 2.508595

O $-6.573078-3.2061432 .844443$

H $-5.724663-4.0299140 .555970$

O $-4.015488-3.8401631 .655532$

C -3.146735 -2.582863 -2.347131

H $-3.204550-4.031147-0.809412$

H $-4.706688-3.894475-1.770101$

H -7.966443 -1.182793 2.754189

H $-7.170669-3.7898752 .346547$

H -4.402504 -4.327855 2.402874

O $-2.797426-3.462723-3.332047$

O $-2.882694-1.389760-2.323448$

C $-2.074833-2.909739-4.436377$

H $-1.047816-2.693189-4.132509$

H -2.054151 -3.655359 -5.235569

H $-2.565926-2.007988-4.815039$ 


\section{$02 \_9$}

MM̄FF Geometry

C $-2.2667792 .777298 \quad 1.648397$

C -1.4175172 .3132032 .578669$

C -1.5993491 .0754643 .429437$

O $-0.4220520 .225248 \quad 3.361320$

$\begin{array}{llll}\text { C } & -2.881416 & 0.245754 & 3.197098\end{array}$

C $-3.126558-0.8254464 .242186$

C $-3.128344-2.1519334 .006290$

C $-3.468960-0.3169215 .620823$

C $-2.830297-2.8877132 .728984$

C $-4.108054-3.3777412 .029724$

C $-4.625961-2.3705100 .991857$

C $-6.026155-2.7443840 .509863$

C $-6.509997-1.793560-0.593237$

O $-3.712022-2.393185-0.110075$

O $-7.693035-2.378100-1.158386$

C $-5.435546-1.724363-1.698364$

C $-6.929197-0.431302-0.027421$

O $-5.808413-0.745921-2.666992$

C $-4.003345-1.465314-1.162809$

C $-2.972290-1.705760-2.294481$

O $-3.904994-0.114496-0.710891$

C $-0.058938-0.3433812 .181852$

O $-0.545027-0.1438161 .080700$

C $1.018510-1.3144422 .475033$

C $1.258947-2.2994001 .597655$

C $2.265318-3.3548181 .724223$

C $3.172375-3.3227242 .924866$

C $-1.507195-1.700185-1.838241$

C $-0.559335-2.117762-2.952624$

C $1.871838-2.528031-3.371643$

O $-0.936713-2.394380-4.087880$

C $2.434249-3.816956-2.864897$

C $3.646671-3.925548-2.300389$

C $4.209068-5.212411-1.768604$

C $4.457645-5.163057-0.276270$

C $5.690051-4.9431510 .211661$

C $3.293799-5.4481200 .648494$

C $2.341473-4.2880530 .752964$

N $0.759278-2.143900-2.521658$

H -3.184060 2.2389421 .428931

H -0.5156602 .8857852 .787039$

H -1.6142401 .4445184 .461963$

H -2.854912 -0.1944012 .196993$

H -3.7577450 .9072273 .207353$

H -3.362349 -2.813452 4.840528

H -3.791309 -1.1197286 .292755$

H -2.6020330 .1658706 .081459$

H $-4.286861 \quad 0.4091425 .567005$

H -2.205684 -2.301164 2.050406

H -2.225182 -3.762169 3.001612

H -3.869437 -4.318115 1.515348

H -4.885682 -3.601044 2.770413

H -4.641147 -1.371188 1.436294

H $-6.737730-2.7557401 .344956$

H $-6.015805-3.7682130 .110255$

H -7.968697 -1.820097-1.906038

H $-5.450903-2.695371-2.213289$

H -7.734476 -0.5556540 .706468$

H $-7.3374110 .215275-0.812188$

$\begin{array}{llll}\mathrm{H} & -6.110987 & 0.100852 & 0.462727\end{array}$

H $-5.238127-0.862724-3.445023$

H $-3.175046-2.682862-2.752725$

H -3.089324 -0.946525 -3.077739

H $-2.9704740 .145827-0.696972$

H $1.556938-1.2103493 .409618$

H $0.649853-2.3460950 .696373$

H $3.715008-2.3743212 .972405$

H $3.930449-4.1088412 .919731$

H $2.593265-3.4430193 .846973$

H -1.219337 -0.697816 -1.508073

H -1.363944 -2.395899-1.004094

H $2.607003-1.719287-3.331063$

H $1.542667-2.642435-4.408760$

H $1.807878-4.700459-2.961346$
H $5.147353-5.413216-2.302160$

H $3.554119-6.061688-1.999687$

H $5.881724-4.9319751 .279437$

H $6.533770-4.762825-0.446248$

H $3.650677-5.7580891 .636764$

H $\quad 2.741797-6.3202830 .274902$

H $1.631026-4.232161-0.071538$

H $0.971770-1.867421-1.567691$

C $-2.1089123 .836895-0.625038$

O $-0.9418193 .104998-1.049472$

C $-0.4194183 .715963-2.244580$

C $-1.4916704 .683611-2.729645$

C $-2.0903995 .144632-1.417015$

H $0.4625964 .293641-1.943859$

C $-0.0201802 .646536-3.265574$

H -1.074066 $5.501830-3.324119$

H $-2.2523054 .179291-3.336892$

C -3.453724 5.796830-1.568802

H -1.400193 $5.858988-0.947960$

C $0.9648001 .588017-2.736107$

O $-1.1960231 .949418-3.701546$

H $0.3927173 .127554-4.159762$

H $\quad 0.4673550 .945653-2.000787$

O $1.3241430 .747831-3.840071$

C $2.2563682 .153638-2.123559$

C $3.2035281 .041831-1.636587$

H $2.0036632 .793879-1.276701$

O $2.9255732 .969835-3.082445$

H $2.6618860 .409456-0.924040$

H $3.4833410 .407449-2.485528$

H -3.385467 $6.687180-2.202555$

H $-4.1767215 .113351-2.026616$

H $-3.8507016 .104992-0.596610$

C -2.0631974 .0575870 .887262$

H $-1.619796 \quad 1.567378-2.913637$

H $\quad 0.5000500 .387368-4.210317$

H $3.1244332 .412224-3.854242$

H -2.8586494 .7500851 .185822$

H -1.108351 4.5167061 .166611

H $-2.9823283 .231814-0.901851$

C 3.6997562 .0128701 .242163

O 4.2506522 .5443220 .031206

C $4.5019611 .582493-0.998665$

C $5.4498860 .486467-0.487485$

C $4.947622-0.1386320 .811079$

C 4.6365300 .9525551 .833120

H 2.7149221 .5735441 .040363

C 3.5248173 .1835392 .223562

H $5.0371112 .122051-1.790358$

O $5.624830-0.537299-1.460957$

H $6.4394530 .923051-0.299559$

H $4.074259-0.7779880 .639444$

O $5.965085-0.9844001 .351239$

H $5.573343 \quad 1.4195182 .164018$

O 4.0238680 .3685982 .978788

C 2.4056444 .1207211 .824539

H 3.2860062 .8167553 .227615

H 4.4525623 .7673272 .261651

H $5.960154-0.115931-2.270750$

H $6.165931-1.6583830 .679118$

H $4.652373-0.2777953 .343362$

O 2.4873615 .2560382 .580106

O $1.552397 \quad 3.883876 \quad 0.981844$

C 1.4753886 .2366432 .329791

H 0.5020115 .8592082 .656436

H 1.7179257 .1309632 .909836

H 1.4508556 .5040591 .268849

SCF Energy (B3LYP/6-31G**//MMFF) $=-3245.93067317$

$02 \_90$

MM̄FF Geometry

C $0.305755-2.719877-2.032642$

C $1.306952-2.122088-1.369815$

C $1.614560-2.3582870 .085494$

O $1.551663-1.0808490 .760971$

C $3.054229-2.8712540 .242798$

C $3.314625-3.5146151 .588900$ 
C $3.847182-2.8879452 .656212$

C $2.976610-4.9814481 .665047$

C $4.196274-1.4332792 .805517$

C $5.707772-1.1716432 .825073$

C $6.331260-1.1395451 .421017$

C $7.845799-0.9371731 .510196$

C $8.484349-0.7761130 .122200$

O $5.734081-0.0501600 .706799$

O $9.831581-0.3137520 .301459$

C $7.7059500 .298128-0.667956$

C $8.612069-2.122303-0.598497$

O $8.1724380 .379369-2.012833$

C $6.1736040 .088335-0.649555$

C $5.4677441 .324810-1.258190$

O $5.853168-1.063779-1.432038$

C $0.331037-0.5446001 .013333$

O $-0.758092-1.0557810 .815382$

C $0.5696720 .826377 \quad 1.514249$

C $-0.418360 \quad 1.727670 \quad 1.438597$

C $-0.3311913 .142807 \quad 1.796116$

C 0.9915283 .6542372 .302662

C $3.9434541 .352575-1.061337$

C $3.3020602 .522216-1.791878$

C $1.0720763 .483479-2.355524$

O $3.9513793 .370674-2.398477$

C $0.8029154 .632842-1.434749$

C $-0.4136665 .175273-1.268207$

C $-0.6870346 .362051-0.386198$

C $-1.846326 \quad 6.1395220 .561491$

C $-3.061171 \quad 6.6504670 .299546$

C -1.5770745 .3897401 .848235$

C -1.424126 3.9124791 .614739

N $1.9166522 .505586-1.692045$

H $-0.312635-3.451117-1.519874$

H $\quad 1.921197-1.389007-1.887035$

H $\quad 0.898658-3.0514210 .544492$

H $3.758145-2.0468030 .086387$

H $3.285506-3.610553-0.536078$

H $4.019305-3.4752523 .557816$

H $3.179995-5.4057772 .653897$

H $1.914903-5.1401851 .450693$

H $3.567076-5.5453300 .935531$

H $3.710893-0.8126782 .048671$

H $3.777661-1.1035943 .765328$

H $5.863946-0.1935253 .299118$

H $6.213771-1.9185443 .449006$

H $6.104039-2.0803020 .908155$

H $8.318650-1.7623042 .057186$

H $8.055378-0.0318482 .097124$

H 9.8019040 .5321640 .780109

H $7.9249271 .270525-0.205274$

H $9.245612-2.805510-0.019859$

H $9.107019-2.006328-1.569020$

H $7.652501-2.618439-0.758311$

H $9.1346840 .515916-1.978275$

H $5.8718172 .236240-0.797687$

H $5.6893511 .378002-2.331971$

H $4.963271-0.963186-1.803641$

H 1.5641981 .0701271 .870003

H -1.379559 1.4067721 .042812

H 1.2646623 .1491793 .235867

H 0.9968954 .7257392 .510574

H $1.7821663 .477907 \quad 1.564559$

H $3.4888450 .432418-1.439544$

H 3.7015531 .4435620 .003755

H $0.1536552 .970170-2.656007$

H $1.5813493 .845944-3.254450$

H $1.6510895 .060444-0.905208$

H -1.255318 4.768681-1.824831

H $-0.9012047 .217487-1.040388$

H $\quad 0.202233 \quad 6.6450800 .190818$

H -3.891049 6.5162370 .985326

H -3.254417 $7.209661-0.609950$

H -2.412022 5.5246522 .548580

H -0.7149945 .8399502 .347010$

H -2.3327593 .4399051 .238205$

H $1.4661441 .793253-1.125417$

C $-1.392457-1.744636-3.654464$
O $-1.500350-0.556636-2.842514$

C $-2.843701-0.491624-2.299520$

C $-3.648218-1.509094-3.094417$

C $-2.614635-2.595668-3.319883$

H -2.753236 -0.814837 -1.256071

C $-3.3286490 .961854-2.363986$

H $-4.531284-1.868121-2.561727$

H $-3.970515-1.090064-4.055525$

C $-2.999826-3.586481-4.403787$

H $-2.468631-3.136345-2.376961$

C $-4.8040841 .214637-2.010949$

O $-2.5160751 .754299-1.494128$

H $-3.143468 \quad 1.340881-3.377608$

H $-4.9608222 .298619-1.934788$

O $\quad-5.5991430 .776821-3.119474$

C $-5.3570610 .552480-0.731888$

C -4.6038360 .9050140 .561635$

H $-5.391018-0.532671-0.863245$

O $-6.723587 \quad 0.968126-0.594833$

H -3.5861660 .5110680 .505547$

H -4.5250961 .9960880 .642550$

H -3.925622 -4.106736 -4.136839

H -3.160353 -3.088147 -5.365741

H -2.216987 -4.339042 -4.539904

C $-0.025746-2.408555-3.465295$

H $-1.5870441 .574258-1.718275$

H $-6.5293450 .956244-2.899289$

H $-6.7275611 .929453-0.447232$

H $\quad 0.738583-1.734888-3.874389$

H $\quad 0.027081-3.333163-4.051032$

H -1.458251 -1.405358 -4.697183

C $-4.486430-1.9010181 .776659$

O $-5.616486-1.0222021 .737096$

$\begin{array}{llll}\text { C } & -5.301807 & 0.369810 & 1.835127\end{array}$

C $-4.5531340 .650596 \quad 3.145187$

C $-3.348808-0.2702773 .328560$

C $-3.733055-1.7319813 .100332$

H $-3.819410-1.6857970 .933015$

C $-5.010299-3.3317721 .615922$

H $-6.2635580 .893143 \quad 1.907410$

O -4.1408132 .0121393 .192728$

H $-5.2449120 .496214 \quad 3.983372$

H -2.5332790 .0215492 .662256$

O $-2.835425-0.1184374 .654023$

H $-4.351819-2.0851613 .935006$

O $-2.550770-2.528173 \quad 3.075508$

C $-5.455708-3.580630 \quad 0.194520$

H $-4.237271-4.0751081 .841964$

H $-5.852228-3.5312772 .289632$

H -3.6363052 .1362334 .014934$

H $-3.535691-0.3738915 .278624$

H $-2.072994-2.365693 \quad 3.906942$

O $-6.764698-3.2298170 .045979$

O $-4.718466-4.011928-0.682475$

C $-7.302967-3.394367-1.269568$

H $-7.185958-4.429262-1.605417$

H -8.369485 -3.157263 -1.232064

H $-6.814859-2.704402-1.963585$

SCF Energy (B3LYP/6-31G**//MMFF) $=-3245.91670505$

02 91

MMFF Geometry

C -0.081585 -4.191309 0.070295

C $0.600157-3.0443180 .204162$

C $1.186363-2.5947511 .516980$

O $0.721411-1.2533511 .798819$

C $2.720287-2.5499231 .438206$

C $3.366795-2.4992992 .808838$

C $3.890466-1.3952123 .375556$

C $3.450389-3.8226963 .527377$

C 3.9194730 .0053082 .830569

C 5.3419250 .4463962 .454987

C $5.863910-0.226677 \quad 1.175944$

C 7.3278110 .1393500 .927394

C $7.839220-0.434847-0.402519$

O 5.0568520 .2342970 .089046

O $9.091377 \quad 0.198945-0.704302$

C $6.853500-0.046662-1.523668$ 
C $8.124626-1.938171-0.306276$

O $7.207163-0.674967-2.753965$

C $5.374613-0.345501-1.182649$

C $4.454346 \quad 0.318978-2.232264$

O $5.161844-1.756834-1.190023$

C $-0.485883-1.1215362 .405567$

O $-1.252157-2.0261852 .709885$

C $-0.788551 \quad 0.2930232 .723490$

C $-0.111430 \quad 1.3410762 .227843$

C -0.3666702 .7530162 .533882$

C -1.4394083 .0619993 .546361$

C $2.9548860 .184258-1.932263$

C $2.1698241 .243769-2.678145$

C $1.6683993 .673811-2.660280$

O $1.5819420 .998958-3.728470$

C $1.559314 \quad 4.745483-1.625072$

C $0.3953985 .261831-1.203443$

C $0.2925836 .358095-0.184064$

C -0.4449265 .9334071 .065803$

C -1.7219716 .2987001 .269560$

C 0.3300195 .1894502 .129963

C $0.3688193 .700014 \quad 1.912730$

N $2.2227942 .480550-2.051469$

H $-0.171962-4.8388720 .937912$

H $\quad 0.742387-2.398889-0.657448$

H $0.873384-3.2636482 .328306$

H $3.034445-1.6932230 .833081$

H $3.103749-3.4379570 .917999$

H $4.350065-1.4952474 .358515$

H $3.973345-3.7435654 .486511$

H $2.450585-4.2173203 .731632$

H $3.994065-4.5514122 .917120$

H $3.2387680 .138593 \quad 1.984830$

H $3.545171 \quad 0.6707443 .618706$

H 5.3220601 .5336152 .303517

H 6.0242370 .2472843 .290710

H $5.760407-1.3128581 .270605$

H $7.959975-0.1967171 .758691$

H 7.4310331 .2331230 .895358

H 9.7023820 .0206410 .031243

H $\quad 6.957851 \quad 1.035157-1.688047$

H $8.876859-2.136106 \quad 0.466847$

H $8.549331-2.319835-1.241315$

H $7.238867-2.528568-0.063157$

H $8.136221-0.452401-2.936357$

H $4.7107601 .384358-2.295064$

H $4.640128-0.111786-3.224451$

H $4.220670-1.934000-1.343984$

H -1.630584 0.4259083 .395526

H $\quad 0.707811 \quad 1.1592831 .534256$

H -2.401982 2.6487823 .227412

H -1.602934 4.1305133 .699154

H -1.180329 2.6357204 .521811

H $2.581181-0.800553-2.234010$

H $2.739510 \quad 0.290488-0.862897$

H $0.7059813 .429465-3.120926$

H $2.3492273 .989091-3.457836$

H $2.4904665 .124351-1.209995$

H - $0.5358624 .894690-1.627411$

H $-0.2230047 .203802-0.657936$

H 1.2828596 .7414400 .092065

H -2.248309 6.0336952 .180107

H $-2.273106 \quad 6.8646040 .525767$

H -0.0611745 .4338843 .123833$

H 1.3627755 .5611542 .155161

H 1.1184793 .3816451 .188183

H $2.8740212 .597727-1.279687$

C $-2.205802-4.981775-1.115335$

O $-2.980517-3.781036-0.915328$

C $-4.070581-4.087923-0.024389$

C $-4.095674-5.609810 \quad 0.121816$

C -2.629204 -5.955336 -0.014718

H -3.808664 -3.645384 0.943709

C $-5.401789-3.511079-0.518973$

H -4.525582 -5.9251571 .077674$

H -4.671351 -6.090383 -0.677610

C -2.369420 -7.414271 -0.346175

H $-2.147467-5.7164570 .939624$
C $-5.437217-1.995859-0.788995$

O $-5.753691-4.164903-1.747291$

H $-6.185768-3.7785770 .200673$

H $-4.918813-1.774473-1.729854$

O $-6.811220-1.649486-1.025560$

C $-4.862393-1.0876210 .314554$

C $-5.317413 \quad 0.380577 \quad 0.169719$

H $-3.769468-1.1575860 .283574$

O $-5.266792-1.5501681 .604287$

H $-5.3784070 .638057-0.891489$

H -6.3279700 .4810280 .583768$

H -2.763623 -8.0639470 .442070$

H -2.846027 -7.703487 -1.288864

H -1.295943 -7.607315 -0.437345

C $-0.705978-4.668905-1.210503$

H $-6.614777-3.810647-2.028679$

H -7.306848 -1.818056 -0.206348

H $-6.238025-1.5306311 .640393$

H $-0.554010-3.918447-1.995659$

H $-0.172732-5.574045-1.524992$

H $-2.522197-5.373621-2.091804$

C $-3.0146201 .983985-0.995498$

O $-3.071380 \quad 1.380044 \quad 0.303331$

C -4.3727001 .3585150 .902512$

C $-4.9079012 .792211 \quad 1.030770$

C $-4.8771883 .536370-0.300901$

C $-3.4914063 .437386-0.935055$

H -3.635295 $1.416541-1.698183$

C $-1.5699671 .896308-1.488270$

H $-4.238506 \quad 0.972691 \quad 1.920942$

O $\quad-6.2365122 .7999261 .541458$

H -4.2880493 .3473571 .746384$

H $-5.6499723 .167182-0.985443$

O $-5.1652944 .918044-0.074091$

H -2.780859 $4.041053-0.358926$

O $-3.539424 \quad 3.974707-2.254537$

C -1.208382 $0.463725-1.780847$

H $-1.4108802 .483780-2.398660$

H $-0.8660462 .268118-0.736129$

H -6.2232972 .3353022 .395686$

H -6.0310834 .9637180 .366895$

H $-3.8610514 .889902-2.182994$

O $-1.5640130 .141110-3.056150$

O $-0.698963-0.285548-0.959378$

C $-1.302348-1.211016-3.442952$

H $-1.957146-1.888548-2.888034$

H -1.519898 -1.307590 -4.509925

$\mathrm{H}-0.250921-1.464730-3.276095$

SCF Energy (B3LYP/6-31G**//MMFF) $=-3245.90337707$

$02 \_92$

MMFF Geometry

C $2.503136-2.4245931 .377412$

C $1.561633-2.2797692 .321702$

C $1.436223-1.1497413 .315994$

O $0.151683-0.4745513 .136823$

C $2.547978-0.0753203 .404222$

C $3.901788-0.4803923 .958694$

C $4.9921120 .305893 \quad 3.844584$

C $4.014719-1.7767544 .717795$

C 5.0800521 .6852613 .248821

C 6.0652031 .7615272 .074788

C $5.4738701 .249157 \quad 0.753242$

C $6.5559591 .200663-0.328460$

C $5.9808200 .805767-1.695531$

O $4.4387392 .157896 \quad 0.363882$

O $6.9752641 .082345-2.693152$

C $4.7687051 .707700-2.004344$

C $5.691490-0.696176-1.774622$

O $4.108710 \quad 1.284697-3.195901$

C $3.7510851 .807018-0.843747$

C $2.7290992 .930199-1.149885$

O $3.0654370 .562631-0.716124$

C $-0.146190 \quad 0.104177 \quad 1.942123$

O 0.5208550 .0718520 .921141

C -1.439682 0.8073052 .087425

C -1.9566091 .4489851 .028941$

C -3.2264112 .1749230 .989300$ 
C $-3.949416 \quad 2.3846212 .293765$

C $1.772796 \quad 3.2537920 .005870$

C $0.9545654 .506679-0.259196$

C $-0.549688 \quad 6.1160020 .911202$

O $0.933898 \quad 5.077541-1.346357$

C -1.999735 5.7566610 .968342

C $-2.8689206 .034583-0.014968$

C -4.3420285 .7503540 .054832$

C $-4.788902 \quad 4.732860-0.969540$

C $-5.082598 \quad 5.099718-2.228384$

C $-4.9793293 .307505-0.505619$

C -3.679781 2.613422 -0.204297

N 0.2459054 .9045700 .866723

H $3.296456-1.6894151 .281923$

H $0.778653-3.0326342 .387403$

H $1.343681-1.6191624 .302489$

H $\quad 2.166570 \quad 0.733855 \quad 4.043317$

H 2.6805100 .3752142 .412606

H $5.926810-0.0471614 .279235$

H $5.012718-1.9209255 .146991$

H $3.305299-1.7938615 .551472$

H $3.821949-2.6335464 .067272$

H 4.1009832 .0793512 .959670

H 5.4463032 .3490584 .042798

H 6.3461152 .8155601 .948582

H 6.9815131 .2101782 .319778

H $\begin{array}{llll}5.044338 & 0.254159 & 0.912561\end{array}$

H $7.3636180 .516234-0.040201$

H $7.0227842 .191323-0.422835$

H $7.7761210 .579587-2.465956$

H $5.1581742 .715157-2.208269$

H $\quad 6.616242-1.273032-1.653781$

H $5.290996-0.973270-2.755696$

H $4.994042-1.031735-1.005980$

H $4.7778941 .248753-3.900779$

H $3.2793643 .848520-1.394707$

H $2.1316872 .663177-2.030966$

H $2.2255950 .710391-0.253603$

H -1.9325330 .7712423 .052120$

H $-1.410164 \begin{array}{lll}1.424224 & 0.086828\end{array}$

H -4.3071181 .4306172 .694677$

H $-4.813825 \quad 3.0465752 .210945$

H -3.284373 $2.847613 \quad 3.031717$

H 1.0690182 .4324980 .157053

H $2.334017 \quad 3.405771 \quad 0.934574$

H -0.3158626 .7511040 .050391$

H $-0.268836 \quad 6.6595641 .818843$

H -2.355311 5.2793601 .878032

H -2.508849 $6.526674-0.916044$

H $-4.8768246 .695582-0.108202$

H $-4.637315 \quad 5.423573 \quad 1.059644$

H -5.432162 4.380048-2.961111

H $-4.975566 \quad 6.129256-2.554016$

H $-5.4845732 .719579-1.283934$

H $-5.671430 \quad 3.2967810 .341304$

H -3.077245 2.417944 -1.092687

H $\quad 0.308168 \quad 4.347609 \quad 1.714294$

C $2.367277-3.029735-1.040616$

O $1.034370-2.517017-1.235102$

C $0.622599-2.807938-2.585559$

C $1.831723-3.425364-3.281884$

C $2.546270-4.090912-2.121478$

H $-0.162926-3.568892-2.518789$

C $0.080472-1.541647-3.259296$

H $1.540860-4.128321-4.068486$

H $2.471720-2.660680-3.737688$

C $3.992906-4.440273-2.423197$

H $2.001119-5.003664-1.846274$

C -1.118902 $-0.896091-2.535159$

O $1.124858-0.561220-3.325442$

H $-0.189610-1.763268-4.298226$

H $-0.801858-0.483360-1.570725$

O $-1.561154 \quad 0.215979-3.323324$

C $-2.312788-1.843614-2.320032$

C $-3.543153-1.131564-1.729062$

H $-2.008886-2.650547-1.649493$

O $-2.678864-2.447796-3.560709$

H $-3.229607-0.559909-0.851449$
H $-3.936250-0.416784-2.461780$

H $4.051280-5.144497-3.259669$

H $4.573225-3.551591-2.692046$

H $4.472632-4.906348-1.557096$

C $2.524677-3.5553290 .385500$

H $1.427463-0.391500-2.416509$

H $-0.7979620 .807154-3.442466$

H -2.912229 -1.732931 -4.177681

H $3.478954-4.0821030 .494978$

H $1.725173-4.2718220 .607053$

H $3.054805-2.195207-1.217273$

C $-3.931131-2.660850 \quad 0.884533$

O $-4.226699-3.127432-0.437424$

C $-4.672180-2.117832-1.350045$

C $-5.942822-1.450667-0.808272$

C $-5.750835-0.9244660 .611209$

C $-5.169067-2.0100181 .516266$

H $-3.107292-1.9382700 .849045$

C $-3.473266-3.8746611 .708599$

H $-4.955192-2.642704-2.271175$

O $-6.346911-0.392310-1.671040$

H $-6.755647-2.188690-0.803465$

H $-5.124593-0.0279280 .609922$

O $-7.027495-0.5226701 .111950$

H -5.935567 -2.770154 1.714750

O $-4.798418-1.4343392 .767256$

C $-2.106182-4.373912 \quad 1.290711$

H -3.408324 -3.624158 2.772889

H $-4.188270-4.6952331 .572951$

H $-7.1354760 .019021-1.277436$

H $-6.882150-0.0813001 .965792$

H $-5.610495-1.1230683 .200886$

O $-1.919716-5.6308121 .791823$

O $-1.292207-3.7369610 .637087$

C $-0.655929-6.232871 \quad 1.493995$

H $-0.688388-7.2735041 .827663$

H $-0.463230-6.217225 \quad 0.416807$

H $0.139271-5.7142552 .037018$

SCF Energy (B3LYP/6-31G**//MMFF)= -3245.92467146

0293

MMFF Geometry

C $-1.701768 \quad 3.6113801 .437264$

C $-1.0605542 .779892 \quad 2.272346$

C $-1.6725491 .723968 \quad 3.163363$

$\begin{array}{llll}\text { O } & -1.357208 & 0.424239 & 2.611028\end{array}$

C -3.2055301 .7863693 .307433$

C -3.7485720 .8694784 .391463$

C $-4.338618-0.319915 \quad 4.155010$

C $-3.650028 \quad 1.402253 \quad 5.796624$

C $-4.478319-0.996132 \quad 2.820348$

C $-5.767241-0.5919112 .094464$

C $-5.759827-0.9248160 .593456$

C $-5.740712-2.4274330 .317313$

C $-5.694325-2.718326-1.191094$

$\begin{array}{lllll}0 & -4.607518 & -0.304709 & 0.019268\end{array}$

O $-5.377402-4.109236-1.362336$

C $-4.532787-1.914925-1.819730$

C $-7.060156-2.505611-1.853927$

O $-4.549341-2.015590-3.240974$

C $-4.494696-0.425347-1.403677$

C $-3.175017 \quad 0.280951-1.794176$

$\begin{array}{lllll}\text { O } & -5.522326 & 0.328697 & -2.057694\end{array}$

C $-0.152576-0.1305942 .898678$

$\begin{array}{lllll}\text { O } & 0.772251 & 0.381784 & 3.507216\end{array}$

C $-0.172766-1.5057812 .351461$

C $0.943608-2.2460832 .378398$

C $1.078580-3.6282581 .917288$

C $-0.172048-4.345023 \quad 1.479774$

C $-1.896719-0.369740-1.245326$

C $-1.174353-1.238879-2.265517$

C $0.855835-2.647165-2.526867$

O $-1.506473-1.304280-3.446358$

C $1.173586-3.960111-1.889413$

C $2.417734-4.343112-1.563269$

C $2.757986-5.703363-1.025914$

C $3.507528-5.652663 \quad 0.285092$

C $4.847306-5.7487180 .321140$ 
C $2.694416-5.5924561 .557116$

C $2.305964-4.1882011 .925282$

N -0.093484 -1.913612 -1.709037

H -2.7804993 .5511131 .326270$

H $\quad 0.0208762 .8715822 .351597$

H -1.218282 1.8192394 .157883

H -3.6731151 .5418032 .346027$

H -3.522565 2.8101763 .545534

H $-4.736884-0.8826784 .997337$

H $-4.096373 \quad 0.722848 \quad 6.530551$

H $-2.603371 \quad 1.5496696 .079558$

H -4.171006 2.361936 5.876603

H -3.598698 -0.7865652 .205457$

H -4.468736 -2.080837 2.981822

H $-6.632487-1.0603952 .579296$

H $-5.901751 \quad 0.4937992 .189149$

H $-6.658088-0.4715280 .158105$

H $-6.605693-2.9196340 .778545$

H $-4.853343-2.882580 \quad 0.777160$

H $-6.054743-4.630659-0.898356$

H $-3.608240-2.400638-1.484668$

H -7.804587 -3.185770 -1.422445

H -7.022660 -2.742877 -2.922664

H -7.441125 -1.489019-1.737095

H -4.539293 -2.962022 -3.464840

H $-3.1285190 .415534-2.881493$

H $-3.2193181 .299993-1.382628$

H $-6.016646-0.261799-2.647656$

H -1.111042 -1.8759491 .954113$

H $1.847918-1.8001872 .786431$

H $-0.870955-4.4392072 .318223$

H $0.012954-5.3522111 .100549$

H $-0.671253-3.800726 \quad 0.670938$

H $-1.1975660 .427201-0.964186$

H -2.099118 $-0.955809-0.342783$

H $1.744936-2.018549-2.630502$

H $0.443572-2.815251-3.526725$

H $0.346186-4.649649-1.740528$

H $3.247525-3.663031-1.743448$

H $3.369314-6.213730-1.782119$

H $1.860905-6.324318-0.910636$

H $5.388715-5.7453261 .261401$

H $5.432683-5.824315-0.589269$

H $3.281997-5.9947302 .393816$

H $1.837324-6.267521 \quad 1.479147$

H $3.148867-3.5821602 .260055$

H $0.174026-1.700502-0.752335$

C -1.099705 $4.426739-0.865444$

O $-0.1600883 .393009-1.231827$

C $0.541693 \quad 3.804647-2.421412$

C $-0.2285934 .997542-2.973697$

C $-0.7158435 .650952-1.698185$

H $1.5322174 .147534-2.100900$

C $0.6806732 .641533-3.407217$

H $0.4021225 .654118-3.580639$

H - $-1.0797214 .683081-3.588978$

C $-1.8546116 .632182-1.918108$

H $\quad 0.126330 \quad 6.176717-1.228061$

C $1.4206581 .412899-2.847806$

O $-0.6354282 .218912-3.790699$

H $\quad 1.1690802 .993580-4.323522$

H $\quad 0.8123530 .947813-2.066743$

O $1.5076590 .452023-3.908839$

C $2.8324141 .674172-2.292162$

C $3.5135400 .369851-1.834117$

H $2.7771332 .365617-1.447734$

O $3.6468332 .276561-3.296670$

H $2.851543-0.145657-1.130062$

H $3.644457-0.287641-2.701802$

H -1.537020 7.448246 -2.575527

H $-2.7198016 .145560-2.380825$

H -2.182234 $7.070926-0.970659$

C -1.0189374 .6872450 .638908$

H $-0.533927 \quad 1.465030-4.396921$

H $2.1026710 .816632-4.586457$

H $3.3076543 .173184-3.456107$

H -1.510265 5.6354070 .885932

H $0.030451 \quad 4.7781290 .942881$
H $-2.0975614 .066628-1.148263$

C 4.2232390 .9743991 .097222

O $4.8404981 .502968-0.083073$

C $4.8999800 .602895-1.194872$

C $5.637662-0.685470-0.802040$

C $5.058987-1.3180050 .460745$

C $4.973036-0.2764471 .575307$

H 3.1748580 .7263800 .890357

C 4.2595942 .0743162 .170319

H $5.5167361 .099783-1.954333$

O $5.605330-1.620407-1.875511$

H $6.693553-0.444548-0.621337$

H $4.083648-1.7771890 .260852$

O $5.931795-2.3744640 .865186$

H $5.985238-0.0069581 .903587$

O $4.292551-0.8322442 .695680$

C 3.3026343 .2098921 .875034

H $3.977626 \quad 1.6697203 .148429$

H 5.2702492 .4959202 .228271

H $\quad 6.054762-2.427964-1.572184$

H $5.500023-2.8483141 .595685$

H $4.842609-1.551713 \quad 3.048371$

O $3.529234 \quad 4.2201402 .766718$

O $2.453596 \quad 3.2099540 .995327$

C 2.6844965 .3670712 .628822

H $3.050546 \quad 6.1420443 .307432$

H 2.7202125 .7534811 .605531

H 1.6594845 .1093332 .910068

SCF Energy $(B 3 L Y P / 6-31 G * * / / M M F F)=-3245.91767829$

02 94

MM̄FF Geometry

C $-0.1810824 .167026 \quad 1.531003$

C -0.7362902 .9746231 .263255$

C -1.3009402 .0885682 .343032$

$\begin{array}{llll}\text { O } & -0.706406 & 0.774479 & 2.224341\end{array}$

C $-2.821027 \quad 1.9236562 .176947$

C -3.4851971 .4813403 .466406$

C -3.8354040 .2153753 .763540$

C -3.7867092 .5923164 .440978$

C $-3.630039-1.0249322 .939663$

C $-4.962515-1.6406352 .488569$

C $-5.664246-0.8209681 .394699$

C -7.047652 -1.397532 1.088524

C $-7.723298-0.661199-0.078323$

O $-4.843781-0.8756660 .224154$

O $-8.873409-1.420900-0.479271$

C $-6.742930-0.602184-1.269032$

$\begin{array}{llll}C & -8.270589 & 0.702951 & 0.355055\end{array}$

O $-7.2707780 .198080-2.324563$

C $-5.328438-0.106589-0.883987$

C $-4.359072-0.337491-2.066251$

O $-5.3819051 .286514-0.577593$

C 0.4597560 .5431822 .879401

O 1.1155601 .3575883 .514516

C $0.846702-0.8849272 .804440$

C $0.340728-1.7485451 .909905$

C $0.651157-3.1786941 .820193$

C $1.493921-3.7796112 .915087$

C $-2.8947740 .000841-1.758312$

C $-1.986198-0.580514-2.821860$

C $-0.954525-2.706075-3.572909$

O $-1.5664330 .095279-3.757583$

C $-0.637464-4.036185-2.973967$

C $0.590750-4.405275-2.580412$

C $0.896835-5.748067-1.984070$

C $1.347067-5.662580-0.543193$

C $2.615741-5.938619-0.198239$

C $0.304034-5.3604630 .510073$

C $0.156270-3.8862980 .782463$

N $-1.722835-1.926810-2.621977$

H -0.1577584 .4954792 .569343$

H -0.7917952 .6042130 .244206$

H -1.076654 2.4999473 .335378

H -3.0376691 .2146811 .370115$

H -3.2808692 .8726151 .870246$

H -4.3207630 .0326984 .721925$

H $-4.2843842 .229270 \quad 5.346505$ 
H -2.8655043 .0945984 .751763$ H -4.4469303 .3331063 .977747$ H -2.979266 -0.8521852 .078039$ H -3.105188 -1.754058 3.569547 H -4.749039-2.645453 2.101355 H $-5.626565-1.7603173 .353763$ H $\quad-5.755765 \quad 0.2191451 .725564$ H -7.687696 -1.373192 1.979242 H $-6.946900-2.4588670 .821654$ H $-8.575825-2.308940-0.740412$ H $-6.651943-1.621305-1.670173$ H -9.0122370 .5795631 .153619$ H -8.797939 $1.197289-0.468408$ H -7.4973331 .3800890 .723572$ H -8.154200 -0.148260 -2.537569 H -4.418995 -1.391354 -2.367458 H $-4.6710830 .260374-2.932111$ H $-4.4946341 .666124-0.675296$ H $1.588539-1.1882863 .536089$ H $-0.371546-1.3839801 .171352$ H $2.480174-3.3077002 .946750$ H $1.670119-4.8503062 .796100$ H $1.009670-3.6454423 .888769$ H -2.735706 $1.084306-1.727954$ H -2.583141 -0.393613 -0.784399 H $-0.055847-2.145654-3.848226$ H -1.558887 -2.831263 -4.477397 H -1.465083 -4.732693-2.861804 H $1.420992-3.711646-2.692969$ H $1.673082-6.217356-2.602433$ H $\quad 0.029288-6.417257-2.045976$ H $2.937476-5.9145640 .837368$ H $3.362276-6.192113-0.943513$ H $\quad 0.522542-5.919048 \quad 1.426544$ H $-0.668916-5.7526260 .186435$ H $-0.448051-3.3614840 .042848$ H -2.249256 -2.420728 -1.906297 C $0.5807704 .709816-0.891032$ O $1.3996593 .531498-0.995163$ C $2.1084813 .567841-2.248445$ C $1.7488924 .892924-2.921269$ C $1.2947915 .746754-1.755082$ H $3.1771413 .564305-2.007007$ C $1.7498492 .338049-3.091426$ H $2.6024635 .322689-3.455409$ H $0.9391174 .776782-3.650647$ C $0.4269006 .922469-2.169231$ H $2.1857676 .120893-1.233120$ C $2.0005580 .979012-2.409180$ O $0.355652 \quad 2.394957-3.425964$ H $2.2863262 .379523-4.046480$ H $\quad 1.319576 \quad 0.842517-1.560297$ O $1.664352-0.038257-3.363378$ C $3.4519320 .742149-1.950994$ C $3.674395-0.697364-1.442824$ H $3.7023471 .449388-1.153691$ O $4.3396111 .001481-3.038172$ H $3.007776-0.871028-0.590885$ H $3.383843-1.407550-2.226103$ H $0.9708997 .578725-2.856558$ H $-0.4858256 .588702-2.674202$ H $0.1321867 .517744-1.299460$ C 0.4470865 .1404980 .570387 H $-0.1483262 .364771-2.594789$ H $\quad 0.7547160 .134179-3.661335$ H $4.1074440 .390298-3.758380$ H -0.1310456 .0706640 .621849$ H 1.4449935 .3689710 .962631 H $-0.4051914 .469082-1.309736$ C $5.125229-0.1705971 .206815$ O $5.664468-0.063564-0.115452$ C $5.141246-0.997382-1.062465$ C $5.384993-2.433484-0.578293$ C $4.840334-2.6536740 .830523$ C $5.359866-1.5734111 .778101$ H 4.0516630 .0548631 .185093 C 5.8256510 .8849482 .068180 H $5.735323-0.868844-1.976159$
O $4.799837-3.364376-1.481756$

H $6.465839-2.625647-0.575378$

H $3.745444-2.6794640 .826895$

O $5.258241-3.9353751 .305385$

H $6.430768-1.7306901 .960192$

O $4.693415-1.6853913 .033118$

C 5.3699172 .2716851 .689587

H 5.6320850 .7402033 .137299

H $6.913623 \quad 0.848848 \quad 1.934071$

H $4.972076-4.255328-1.132328$

H $6.230478-3.9505251 .314772$

H $4.843092-2.5884193 .361591$

O 4.2561262 .6023762 .402436

O 5.9254202 .9670440 .849163

C 3.7241563 .9008722 .132333

H 3.4663893 .9913931 .073201

H 2.8182614 .0273592 .730303

H 4.4450894 .6717412 .420555

SCF Energy (B3LYP/6-31G**//MMFF) $=-3245.92155817$

$02 \_95$

MM̄FF Geometry

C 1.1545023 .6654220 .962022

C 0.6893183 .0214212 .043485

C 1.2474321 .7074382 .517693

O $0.132327 \quad 0.8103502 .756807$

C 2.0070111 .8117143 .849695

C 3.2108632 .7260253 .845827

C 4.3895162 .4350153 .262304

C 3.0348644 .0221014 .592922

C 4.7445751 .2057092 .471699

C 5.1793441 .5968861 .053129

C 5.4811090 .3754270 .173986

C $6.1459510 .820876-1.130618$

C $6.379014-0.355145-2.087919$

O $4.241793-0.283449-0.108405$

O $\quad 6.7073820 .186098-3.376732$

C $5.056230-1.130489-2.244066$

C $7.576017-1.211314-1.659036$

O $5.249485-2.307206-3.027304$

C $4.366014-1.473933-0.900311$

C $2.940136-2.005499-1.187490$

O $5.128986-2.474095-0.228514$

C $-0.369506 \quad 0.1322201 .691485$

O $0.009197 \quad 0.1954150 .533579$

C - $-1.466122-0.7262112 .188559$

C $-1.836203-1.7959531 .470297$

C $-2.881469-2.7564911 .822826$

C $-3.767763-2.4315442 .995739$

C $2.090673-2.3130530 .051022$

C $2.203062-3.7414600 .554784$

C $1.221072-5.2531512 .281920$

O $2.919350-4.5894090 .030929$

C $-0.019309-5.8859251 .732318$

C $-1.077393-6.2223682 .485414$

C $-2.325898-6.8584341 .941663$

C $-3.567579-6.0492392 .247949$

C $-4.305876-6.3125323 .339458$

C $-3.999750-4.9923761 .255562$

C $-2.999603-3.8821881 .088531$

N $1.391101-3.9526341 .661532$

H 2.0101963 .2405820 .442032

H -0.1516763 .4197762 .602953$

H $1.9011861 .261707 \quad 1.758587$

H 2.3199790 .8048774 .159743

H 1.3105832 .1188844 .642850

H 5.1970583 .1610903 .351298

H 3.9319074 .6492304 .557317

H 2.2107784 .6019544 .165370

H 2.8110393 .8243035 .646394

H 5.5733380 .6969822 .978749

H 3.9192680 .4896842 .433207

H 4.3838192 .1919760 .585806

H 6.0711482 .2334991 .117440

H $6.137878-0.3059750 .727159$

H $7.0901401 .341906-0.928423$

H $5.5022221 .555560-1.634913$

H $7.5026560 .737054-3.277044$ 
H $4.379488-0.490527-2.826826$ H $8.485226-0.600108-1.608871$ H $7.785124-1.995226-2.395307$ H $7.439590-1.680131-0.682387$ H $5.669555-2.975025-2.458866$ H $2.404645-1.227545-1.745563$ H $2.987826-2.891576-1.831281$ H $4.575092-2.8791670 .456923$ H $-1.916462-0.4703503 .140584$ H -1.309453 -2.003158 0.540665 H -3.182665 -2.392321 3.921073 H $-4.561012-3.1657933 .154455$ H -4.260237 -1.463671 2.849223 H $2.313707-1.6231860 .871609$ H $1.039187-2.174729-0.227466$ H $1.160583-5.0926403 .362895$ H $2.088628-5.8856142 .069156$ H $-0.043807-6.0795100 .662366$ H -1.044051 -6.0420183 .557641$ H -2.413472 -7.8582342 .387013$ H -2.254888 $-7.024548 \quad 0.859539$ H $-5.213309-5.7590063 .556675$ H $-4.016585-7.0864504 .042881$ H $-4.981298-4.578111 \quad 1.515330$ H $-4.151574-5.4698520 .278429$ H -2.305151 -4.042837 0.263496 H $0.792708-3.197566 \quad 1.984107$ C $-0.820616 \quad 5.3085540 .744828$ $\begin{array}{lllll}0 & -1.728597 & 4.257391 & 0.359783\end{array}$ C -2.924748 $4.845146-0.180227$ C $-2.8313196 .339846 \quad 0.107996$ C $-1.333047 \quad 6.566163 \quad 0.044452$ H -2.885932 4.696279 -1.265679 C $-4.162890 \quad 4.153799 \quad 0.399752$ H $-3.3882486 .937326-0.620248$ H -3.2108636 .5875461 .106007$ C -0.8856397 .8637310 .694055$ H $-1.0227206 .564656-1.009094$ C -4.1889832 .6233050 .199194$ $\begin{array}{lllll}\text { O } & -4.200860 & 4.410668 & 1.809437\end{array}$ H $-5.0659294 .610055-0.023712$ H $-3.422757 \quad 2.1417780 .819403$ $\begin{array}{lllll}\text { O } & -5.454249 & 2.146078 & 0.675785\end{array}$ C $-4.0214612 .164945-1.263335$ C $-4.274644 \quad 0.655340-1.444728$ H -3.009854 $2.405683-1.603556$ O $-4.9288632 .889104-2.095355$ H -3.644132 $0.104745-0.739116$ H $-5.3181330 .437712-1.188523$ H $-1.341568 \quad 8.7227170 .190769$ H -1.170877 7.9030231 .750729 H $0.201473 \quad 7.975742 \quad 0.634286$ C 0.6032024 .9211630 .339753 H $-5.0832594 .158897 \quad 2.129706$ H -5.4547542 .2349301 .643716$ H -5.829902 2.700469-1.781204 H $1.2952265 .742870 \quad 0.555296$ H $0.6202764 .761109-0.746486$ H $-0.898147 \quad 5.426795 \quad 1.832980$ C -1.702555 -0.484486 -2.686452 O $-2.6658530 .377462-3.304762$ C $-4.0222410 .175659-2.893012$ C $-4.444233-1.275526-3.158863$ C $-3.490357-2.267950-2.504339$ C $-2.052630-1.962046-2.918341$ H $-1.661731-0.276537-1.611441$ C $-0.331545-0.158586-3.301791$ H $-4.6364040 .805320-3.548892$ O $-5.773059-1.494739-2.697248$ H $-4.449251-1.451192-4.242607$ H $-3.607897-2.261491-1.415584$ O $-3.841714-3.581071-2.944162$ H -1.915032 -2.219019-3.976630 O $-1.159342-2.775014-2.159945$ C $0.2859951 .103336-2.736724$ H $0.371060-0.973834-3.097251$ H $-0.421901-0.025428-4.386014$ H $-5.988337-2.428468-2.863847$
H $-3.309861-4.213643-2.432527$

H $-1.318693-3.700686-2.409210$

O $1.5898021 .166822-3.142276$

O $-0.290583 \quad 1.925685-2.039549$

C $2.3460822 .282280-2.661670$

H $3.2661462 .345974-3.248808$

H $1.7915073 .218103-2.779803$

H $2.6106192 .116386-1.614325$

SCF Energy (B3LYP/6-31G**//MMFF)= -3245.91461142

0296

MMFF Geometry

C $-0.439218 \quad 0.021654-2.447815$

C $-0.901551-1.213842-2.197000$

C $-0.410079-2.150986-1.116738$

O $0.661877-1.524884-0.378364$

C $0.115414-3.449273-1.748272$

C $0.554820-4.476291-0.724264$

C $1.839953-4.729474-0.405775$

C $-0.558577-5.261890-0.082062$

C $3.058087-4.023263-0.934672$

C $4.058506-3.7018450 .185251$

C $5.230458-2.861124-0.341216$

C $6.334783-2.743845 \quad 0.709034$

C $7.477982-1.8370340 .229362$

O $4.726037-1.559189-0.659706$

O $8.305464-1.533323 \quad 1.362617$

C $6.881020-0.503933-0.265470$

C $8.379877-2.540422-0.791534$

O $7.8851930 .316909-0.858202$

C $5.691689-0.674789-1.241808$

C $5.0144590 .697346-1.470112$

O $6.170835-1.171324-2.492030$

C $0.519238-1.3913640 .965609$

O $-0.374608-1.834162 \quad 1.667664$

C $1.629321-0.5385021 .438205$

C $1.406005 \quad 0.2847212 .472051$

$\begin{array}{llll}\text { C } & 2.345127 & 1.270447 & 3.005637\end{array}$

C 3.7933381 .1412092 .618591

C $3.6698330 .634325-2.205960$

C $3.0243962 .007265-2.249118$

C $1.8908283 .685713-0.785103$

O $3.0100562 .681297-3.276217$

C $2.510795 \quad 4.2867340 .437894$

C 1.8140334 .7091351 .504352

C 2.4476835 .3476852 .708783

C 2.0040024 .7300994 .017492

C 1.1597475 .3802434 .836566

C 2.6053103 .3989504 .419229

C $1.8628772 .241086 \quad 3.808983$

N $2.5050562 .393154-1.023405$

H $\quad 0.3658210 .435090-1.848585$

H $-1.720693-1.592869-2.805601$

H $-1.263428-2.369477-0.463601$

H $0.937361-3.219578-2.436558$

H $-0.663521-3.900881-2.377742$

H $2.044016-5.522504 \quad 0.312278$

H $-0.184614-6.0164780 .617933$

H -1.225189 -4.5973050 .476403$

H $-1.146003-5.782464-0.845436$

H $3.542759-4.674283-1.671951$

H $2.791763-3.095824-1.448707$

H $3.543506-3.1546500 .985163$

H $4.433800-4.6398440 .612966$

H $5.617916-3.334136-1.251408$

H $6.720380-3.7338230 .982688$

H $5.917032-2.3182751 .632406$

H $8.633193-2.3730371 .727891$

H $6.522903 \quad 0.038161 \quad 0.621292$

H $8.815040-3.448129-0.356525$

H $9.228708-1.906550-1.071266$

H $7.851955-2.831013-1.702084$

H $8.6037780 .409765-0.209535$

H $4.8487681 .173608-0.497568$

H $5.6873751 .357094-2.033212$

H $5.542731-0.926614-3.189806$

H $2.557780-0.5518110 .880821$

H 0.4216420 .2776272 .938478 
H $3.942917 \quad 1.4675871 .586556$ H 4.1315710 .1026332 .710200 H 4.4623121 .7286883 .251411 H $3.7974940 .285443-3.236012$ H $2.976444-0.057976-1.716234$ H $0.8160653 .520575-0.666734$ H $2.0482874 .342625-1.645886$ H 3.5929564 .3982900 .434736 H $\quad 0.729726 \quad 4.626073 \quad 1.498379$ H 2.1859216 .4137772 .691074 H 3.5426435 .3099242 .649758 H 0.8592864 .9565565 .789161 H $\quad 0.745767 \quad 6.3471564 .570657$ H 2.5611043 .2820545 .510094 H 3.6702083 .4076354 .171801 H 0.7980452 .2305804 .047642 H $2.4771761 .714530-0.268601$ C $-1.7211062 .113650-2.935995$ O $-2.8714411 .637296-2.211723$ C $-4.0467122 .296548-2.710240$ C $-3.5365933 .573592-3.360145$ C $-2.2532803 .075959-4.001480$ H $-4.467306 \quad 1.644687-3.486426$ C $-5.0742252 .469331-1.586988$ H $-4.2459373 .988510-4.082150$ H $-3.3085374 .346553-2.617354$ C -1.278273 $4.184349-4.360368$ H $-2.5109642 .522459-4.914562$ C $-5.2916161 .189042-0.749953$ O $-4.6187643 .494364-0.696129$ H $-6.0209992 .829317-2.006134$ H $-4.3859140 .994045-0.172657$ $\begin{array}{lllll}\text { O } & -6.321188 & 1.463547 & 0.207184\end{array}$ C -5.674209-0.057008-1.564039 C $-5.938825-1.324187-0.726184$ H $-4.897565-0.279562-2.303173$ O $-6.8676720 .252519-2.292564$ H $-6.603590-1.0813710 .109503$ H $-6.512481-2.018219-1.353675$ H -1.724273 $4.867882-5.090062$ H $-0.9950924 .770644-3.479602$ H $-0.3638473 .770738-4.797541$ C $-0.9804240 .914291-3.529993$ H -5.2712223 .5667820 .021510$ H -7.150332 $1.594392-0.284288$ H $-7.054231-0.496774-2.883594$ H $-0.1440501 .254431-4.151105$ H -1.657453 $0.343751-4.178209$ H -1.086277 2.640701 -2.212919 C $-4.508280-1.1453602 .004126$ O $-3.901307-1.291326 \quad 0.715255$ C $-4.656440-2.039976-0.240133$ C $-4.906720-3.4532370 .306714$ C $-5.615589-3.4057251 .655659$ C $-4.844577-2.5103852 .621836$ H $-5.413960-0.5351231 .912733$ C -3.521863 -0.397416 2.911076 H -4.010341 -2.150246 -1.120294 O $-5.678804-4.229902-0.602386$ H $-3.947010-3.970718 \quad 0.435379$ H $-6.656130-3.075721 \quad 1.555529$ O $-5.662531-4.7225152 .210033$ H -3.921437 -3.021788 2.924597 O $-5.622824-2.3098913 .800410$ C -3.2091260 .9866892 .396551$ H $-3.918112-0.284546 \quad 3.927010$ H -2.580987 -0.9524223 .006119$ H -5.197528 -4.254093 -1.446987 H $-6.116883-5.2911221 .564745$ H $-5.834920-3.1879214 .160736$ O O -3.9177291 .9649112 .592524$ C -1.6018022 .2187591 .167649$ H -0.7197922 .0348550 .549577$ H -2.3745522 .6752350 .545069$ $\mathrm{H}-1.3247362 .8849021 .989772$

SCF Energy (B3LYP/6-31G**//MMFF) $=-3245.91768406$
02 97

MMFF Geometry

C $-2.096488-2.8529202 .956259$

C $-3.384160-2.4763802 .999697$

C $-4.318787-2.3255461 .818849$

O $-3.740047-2.8640620 .607835$

C $-4.615432-0.8324751 .584418$

C $-5.687787-0.5909730 .543155$

C $-5.458931-0.172666-0.717056$

C $-7.101227-0.8209431 .014493$

C $-4.1414930 .102680-1.390530$

C $-4.0794911 .507370-2.001593$

C $-3.8547682 .627348-0.971443$

C $-4.1585823 .978605-1.624886$

C $-3.8320315 .153873-0.700473$

O $-2.4849262 .571925-0.551631$

O $-3.861136 \quad 6.355466-1.485689$

C $-2.3907404 .982478-0.189686$

C -4.8825365 .3282050 .402173$

O -2.0806325 .9909490 .772010$

C -2.0941083 .5785680 .396170$

C $-0.5629783 .454390 \quad 0.626304$

O -2.7911493 .4333331 .631347$

C $-3.893752-4.1945350 .371003$

O $-4.474219-5.0000351 .086142$

C $-3.203857-4.611340-0.872876$

C $-2.835394-3.747342-1.830387$

C $-2.124339-4.064482-3.070429$

C $-1.626658-5.472057-3.259561$

C -0.0766482 .2873861 .488335$

C -0.4897610 .9185691 .002457$

C $-0.136555-0.744946-0.787081$

O $-1.227900 \quad 0.203883 \quad 1.676493$

C $-0.090597-0.667549-2.276464$

C $0.721635-1.428174-3.025339$

C $0.714038-1.440582-4.527869$

C $0.333082-2.799123-5.081843$

C $1.279424-3.673719-5.462694$

C -1.137911 -3.125062 -5.248864

C $-1.904683-3.070069-3.955587$

N $0.0493760 .576821-0.225435$

H -1.629321 -3.079043 2.001626

H -3.823209 -2.245655 3.968744

H $-5.250524-2.8577772 .046415$

H -3.694816 -0.3151281 .301360$

H $-4.936520-0.3599002 .522649$

H -6.322664 -0.036346 -1.367643

H -7.842039 -0.5744430 .246444$

H -7.250519 -1.8694501 .289478$

H -7.315919 -0.1971221 .888438$

H $-3.284144-0.061544-0.734945$

H -4.046583 -0.619987 -2.209844

H $-3.251774 \quad 1.519557-2.723091$

H $-5.0012601 .693212-2.567554$

H $-4.5041312 .468167-0.103750$

H $-5.2063794 .027048-1.947470$

H -3.560218 $4.082585-2.541427$

H -4.748231 $6.436902-1.876298$

H -1.715773 $5.149027-1.041367$

H $-5.8705265 .513307-0.036419$

H -4.6622156 .2036551 .022968$

H -4.9702214 .4562421 .053508$

H $-2.265366 \quad 6.851770 \quad 0.358717$

H $-0.0665653 .404247-0.351002$

H $-0.195376 \quad 4.361322 \quad 1.122701$

H -2.5002052 .6092342 .053116$

H -3.031641 -5.678579 -0.966598

H -3.066123 -2.693867 -1.695084

H $-0.985827-5.770696-2.422477$

H $-1.032107-5.600684-4.167402$

H $-2.467691-6.170948-3.325401$

H 1.0187962 .3075221 .541129

H -0.4251212 .4064362 .520937$

H -1.100536 -1.150172 -0.463536

H $\quad 0.650015-1.386904-0.381763$

H $\quad-0.7977100 .000402-2.762542$

H $1.413848-2.107602-2.533754$

H $1.717292-1.158336-4.872491$ 
H $\quad 0.037239-0.678453-4.933739$ H $\quad 1.021230-4.641974-5.878417$ H $2.334757-3.442126-5.362297$ H -1.278522 $-4.097083-5.735750$ H -1.580286 -2.397701 -5.941984 H -2.303043 -2.079654 -3.732927 H $0.786041 \quad 1.158931-0.612627$ C $-0.112947-1.9282544 .177568$ O $0.791283-2.1605443 .080287$ C $2.142875-1.9048883 .523177$ C $2.021964-1.2948584 .911667$ C $0.759318-1.9552015 .429868$ H $2.629235-2.8868173 .574926$ C 2.835595-1.015545 2.483990 H $2.898488-1.4942925 .535355$ H $1.887306-0.2074884 .850185$ C $0.152426-1.2434816 .626484$ H $0.991370-2.9928925 .704844$ C $4.238355-0.5387732 .893794$ O $2.950563-1.7632571 .268830$ H $2.192169-0.1572202 .264206$ H $4.1734190 .091836 \quad 3.788092$ O $5.009683-1.6891793 .262139$ C 5.0046410 .2116531 .785203 C 4.2793891 .4713741 .280516 H $5.215545-0.4722480 .956058$ O 6.2791090 .5981202 .313683 H 3.2851261 .2008290 .916270 H 4.1378142 .1592952 .123209 H $\quad 0.857078-1.231647 \quad 7.464417$ H $-0.104101-0.2056106 .388365$ H $-0.759697-1.7476846 .960433$ C -1.219344 -2.983593 4.172223 H $2.083305-2.1634281 .088521$ H $5.898246-1.3770043 .504943$ H $6.121753 \quad 1.2142263 .049489$ H -1.820145 -2.902348 5.085150 H $-0.780505-3.9887954 .158826$ H $-0.541970-0.9277894 .036309$ C $4.2192460 .945006-1.716752$

O $5.3554861 .343026-0.939374$

C 5.0585572 .2049070 .164600 C $4.3860903 .488293-0.341984$ C $3.1705443 .187106-1.215397$ C $3.5184822 .174415-2.306275$ H $3.5202040 .379791-1.088911$ C $4.7237870 .012840-2.823161$ H 6.0250892 .4997560 .592066 $\begin{array}{llllll}\text { O } & 4.006250 & 4.310157 & 0.756477\end{array}$ H $5.1147374 .058148-0.932987$ H $2.3363042 .829475-0.605035$ O $2.7124124 .396694-1.823601$ H $4.1609052 .650002-3.058204$ O $2.3214741 .764903-2.962744$ C $5.215635-1.293285-2.246513$ H $3.946551-0.212835-3.561764$ H $5.5612920 .468534-3.365286$ H 3.5598605 .0942140 .393239 H $3.4324544 .747380-2.375072$ H $1.8819992 .566652-3.294425$ O $4.156261-2.087362-1.921732$ O $6.398751-1.568190-2.094574$ C $4.489122-3.354400-1.346900$ H $5.062268-3.215313-0.425134$ H $3.557191-3.872537-1.105355$ H $5.051524-3.958381-2.065056$ SCF Energy (B3LYP/6-31G**//MMFF)= -3245.91656439

\section{8}

MMFF Geometry

C 0.9324205 .0013870 .667205

C 1.9315324 .1067850 .670519

C $2.8854393 .909431-0.477368$

O $2.9688542 .492454-0.754335$

C $4.2864804 .398309-0.077573$

C $5.3061314 .243404-1.188005$

C $6.2304233 .263813-1.239470$

C $5.2661585 .307842-2.253047$
C $6.4012272 .124433-0.272072$

C $6.6620160 .798641-1.001684$

C $6.814658-0.363091-0.009448$

C $7.323539-1.619766-0.716523$

C $7.418904-2.8102230 .249403$

O $5.532791-0.6225630 .575708$

O $7.635532-3.998059-0.526799$

C $6.077386-2.9577080 .996733$

C 8.640441-2.698500 1.167888

O $6.166721-3.9527522 .014130$

C $5.545661-1.6315461 .595023$

C $4.097311-1.8441932 .092816$

O $6.363274-1.2474792 .701517$

C $2.0112691 .947119-1.550843$

O $1.0685862 .520880-2.071453$

C $2.3062860 .501208-1.659565$

C $1.401920-0.323306-2.208060$

C $1.526490-1.777799-2.326323$

C $2.849338-2.399527-1.965270$

C $3.349459-0.5614532 .476515$

C $1.903058-0.8961432 .788278$

C $-0.227099-1.5534051 .655610$

O $1.512118-1.0575273 .941786$

C $-0.221594-2.8991650 .996973$

C $-1.051968-3.2452940 .001567$

C $-1.017690-4.582523-0.684621$

C $-0.968216-4.476804-2.196020$

C $-2.003394-4.883558-2.950359$

C $0.310159-3.978911-2.843204$

C $0.455086-2.483916-2.743255$

N $1.132666-1.0439201 .645384$

H $0.8015425 .646237-0.197137$

H 2.0749163 .4710321 .541602

H $2.5466994 .441561-1.375232$

H 4.6233313 .8705950 .822628

H 4.2439115 .4573420 .211113

H $6.9453553 .278459-2.061063$

H $6.0507485 .173290-3.005228$

H $4.3045235 .292817-2.775295$

H $5.4045386 .297753-1.806131$

H 7.2557152 .3519180 .376488

H 5.5263732 .0068590 .373165

H $5.8295880 .594069-1.687206$

H $7.5739830 .892261-1.604510$

H $7.517094-0.0576870 .775029$

H $8.294357-1.436433-1.193523$

H $\quad 6.633367-1.881254-1.530960$

H $6.893379-4.096111-1.147251$

H $\quad 5.338852-3.3250670 .270499$

H $9.561762-2.6607880 .573805$

H $8.735004-3.5819651 .809234$

H $8.620558-1.8122201 .805350$

H $6.493993-4.7674071 .595582$

H $3.522391-2.3505341 .309182$

H $4.098206-2.5155382 .961665$

H $5.848480-0.6699213 .286923$

H $3.2454910 .148084-1.248850$

H $0.4650540 .098193-2.570269$

H $3.669121-1.902590-2.496726$

H $2.919925-3.456829-2.229903$

H $3.029158-2.316954-0.890636$

H $3.791148-0.0885983 .359762$

H 3.3749140 .1775671 .667826

H $-0.849158-0.8324361 .116002$

H $-0.591248-1.6397172 .683608$

H $0.510663-3.6213261 .351364$

H -1.799606 -2.531449 -0.334636

H $-1.912658-5.136580-0.373525$

H $-0.160977-5.178565-0.346059$

H - $1.967470-4.834739-4.033664$

H $-2.912250-5.271703-2.503260$

H $\quad 0.320418-4.241327-3.908924$

H $1.147712-4.518168-2.391908$

H $-0.444431-1.935543-3.023777$

H $1.564512-0.8872020 .740078$

C $-1.4825734 .902574 \quad 1.470799$

O -1.6387973 .5667360 .952652$

C $-2.6388573 .595897-0.093014$ 
C $-3.289568 \quad 4.970844-0.010305$ C -2.127603 5.8310530 .444583 H $-2.0864403 .494805-1.035947$ C -3.5941102 .4112690 .098534$ H -3.713531 $5.294445-0.965487$ H -4.0891874 .9807300 .741080$ C -2.548699 7.1781551 .004795 H $-1.4637445 .991654-0.413212$ C $-4.8611742 .466988-0.772520$ O $-2.8677891 .211195-0.189897$ H -3.8736372 .3514331 .156391$ H -5.469957 $3.330956-0.481288$ O $-4.4632182 .690963-2.131233$ C $-5.7285561 .193726-0.743815$ C -6.0717890 .6989170 .670519$ H $-5.2249990 .404996-1.310148$ O $-6.941623 \quad 1.471025-1.451867$ H $-5.1462300 .448038 \quad 1.197259$ H $-6.547993 \quad 1.5127991 .231477$ H -3.075191 7.7631570 .243622 H -3.218598 $7.064793 \quad 1.863775$ H -1.6769727 .7544061 .330304$ C -0.017931 5.1782661 .818491 H -2.644842 $1.222703-1.136529$ H $-5.2733262 .728386-2.668147$ H $-7.4288352 .148597-0.952590$ H $0.271608 \quad 4.5027492 .633836$ H $0.085808 \quad 6.198872 \quad 2.204261$ H -2.059298 4.9340112 .405199 C $-5.392655-2.2148870 .132427$ O $-6.605191-1.544712-0.228661$ C $-7.018667-0.523166 \quad 0.682748$ C $-7.244530-1.1306782 .074422$ C $-6.026029-1.9184902 .558768$ C $-5.551606-2.9061771 .490635$ H $-4.568177-1.4935210 .179119$ C $-5.083018-3.227712-0.975352$ H -7.992621 -0.1685180 .322601$ O $-7.567966-0.1067503 .008780$ H -8.107595 -1.807299 2.027015 H $-5.214097-1.2430352 .851215$ O $-6.372049-2.6393313 .744089$ H -6.267317 -3.7341711 .411571$ O $-4.297889-3.4588391 .884813$ C $-4.815158-2.547837-2.298818$ H $-4.216844-3.850367-0.730907$ H $-5.938475-3.898482-1.123300$ H -7.674919 -0.5292103 .878285$ H $-7.092657-3.2537423 .522777$ H $-4.419328-3.8580002 .763266$ O $-3.695572-1.774735-2.217532$ O $-5.514503-2.699997-3.292380$ C $-3.356567-1.051313-3.404513$ H $-2.514168-0.394426-3.172713$ H $-3.056054-1.746748-4.193475$ H $-4.198704-0.433836-3.731987$

SCF Energy (B3LYP/6-31G**/MMFF) $=-3245.91508793$

0299

MMFF Geometry

C $-2.863940-2.254770-2.625901$

C $-4.134841-2.673480-2.521050$

C $-5.211292-2.072736-1.651779$

O $-4.702854-0.944834-0.904445$

C -5.864169-3.066609-0.663183

C $-4.949759-4.0754290 .007755$

C $-4.247815-3.849651 \quad 1.135251$

C $-4.918177-5.444485-0.626572$

C $-4.191725-2.5699621 .923647$

C -3.301562 -2.677051 3.175127

C $-1.805520-2.8068832 .842793$

C $-0.991721-3.1141594 .099990$

C $0.510690-3.2010873 .790091$

O $-1.363951-1.5677572 .276167$

O $1.218455-3.1849315 .039337$

C $0.932417-1.9377613 .011385$

C $0.876868-4.5219833 .102063$

O $2.283780-2.0455862 .568075$
C $-0.002042-1.582622 \quad 1.828740$

C $0.352800-0.173506 \quad 1.297123$

O $0.194255-2.5380430 .784247$

C $-5.5179780 .134742-0.758428$

$\begin{array}{lllll}\text { O } & -6.630860 & 0.291217 & -1.234996\end{array}$

C $-4.818056 \quad 1.1128820 .102093$

C $-5.2171652 .393008 \quad 0.100795$

$\begin{array}{llll}\text { C }-4.642932 & 3.482057 & 0.894438\end{array}$

C $-3.623213 \quad 3.1201161 .941915$

$\begin{array}{llll}\text { C } & -0.663716 & 0.404593 & 0.304632\end{array}$

C $-0.2209991 .752299-0.239113$

C $-1.023895 \quad 3.643053-1.649247$

O $0.8869312 .233840-0.018675$

C $-1.357307 \quad 4.727997-0.672856$

C -2.239162 $5.709363-0.917696$

C $-2.527634 \quad 6.824580 \quad 0.047126$

C $-4.001953 \quad 6.9867650 .343840$

C $-4.7047598 .005794-0.179076$

C -4.6398336 .0198051 .317738$

C $-5.065597 \quad 4.740621 \quad 0.652526$

$\mathrm{N}-1.2034122 .348135-1.018357$

H -2.527805 -1.392685-2.056996

H $-4.449663-3.520483-3.127573$

H $-5.992883-1.720216-2.337511$

H $-6.639492-3.625610-1.204971$

H $-6.405283-2.5079520 .111442$

H -3.664227 -4.6775571 .534601$

H $-4.270916-6.141240-0.083185$

H -5.924238 $-5.876755-0.641661$

H $-4.548534-5.392735-1.654080$

H $-3.815349-1.7601121 .293984$

H -5.205021 -2.306670 2.248552

H -3.457890 -1.770714 3.774159

H $-3.628719-3.5306453 .781736$

H $-1.669723-3.6069812 .107804$

H $-1.338278-4.0413004 .573210$

H -1.149991 -2.319486 4.842598

H $0.911023-3.9412015 .568008$

H $0.916026-1.1045953 .727267$

H $0.570382-5.373853 \quad 3.721042$

H $1.961828-4.6168732 .981995$

H $0.409205-4.6393432 .122276$

H $2.294793-2.6238841 .786527$

H 0.4169490 .5270542 .140415

H $1.341404-0.1986870 .824172$

H $-0.052262-2.134731-0.062560$

H -3.9942260 .7542830 .706383$

H $-6.0380872 .678143-0.556317$

H -3.2812753 .9709732 .533756$

H -2.7332272 .6818331 .481230$

H -4.0418472 .3960002 .650010$

H $-0.803764-0.265516-0.548188$

H -1.6300610 .5339150 .801568$

H $-1.6667113 .668412-2.534604$

H $0.0186893 .743805-1.968111$

H -0.8221214 .7230950 .274172$

H -2.754888 $5.735327-1.874780$

H -2.128973 $7.749632-0.389855$

H $-1.985595 \quad 6.6861730 .990993$

H $-5.755493 \quad 8.1448150 .052994$

H -4.247262 $8.720476-0.855046$

H $-5.5384556 .472408 \quad 1.758070$

H -3.9624915 .8648832 .161324$

H $-5.8206304 .890047-0.121122$

H -2.117886 $1.910877-1.084631$

C $-0.560527-3.243237-2.745521$

O $0.170891-2.020605-2.509062$

C $1.532211-2.196703-2.954409$

C $1.739907-3.699202-3.012537$

C $0.388118-4.158836-3.521808$

H $1.576075-1.763299-3.962081$

C $2.471626-1.439251-2.011241$

H $2.564656-3.989218-3.669864$

H $1.933213-4.105179-2.011705$

C $0.127649-5.639175-3.301765$

H $0.324558-3.941320-4.596577$

C $3.966394-1.679808-2.281878$

O $2.203389-0.041305-2.159037$ 
H $\quad 2.234938-1.698572-0.974477$

H $4.221652-2.722621-2.061919$

O $4.221032-1.497640-3.681487$

C $4.924820-0.746738-1.515101$

C $4.729575-0.7711660 .009453$

H $4.832815 \quad 0.275743-1.897735$

O $6.266135-1.165462-1.792639$

H $3.696852-0.5087790 .251136$

H $4.897330-1.7917530 .375060$

H $\quad 0.865356-6.243462-3.839551$

H $\quad 0.186341-5.903314-2.240414$

H $-0.867018-5.918044-3.663428$

C - $1.845475-2.920152-3.512594$

H $1.2503690 .088892-2.017706$

H $3.964396-0.587590-3.910149$

H $6.381065-1.146620-2.758241$

H -2.263511 -3.837940 -3.942460

H -1.622095 -2.235039-4.339319

H $-0.793486-3.673774-1.763352$

C 4.4284452 .2211230 .473912

O $5.6490201 .515108 \quad 0.220201$

C $5.6912860 .186336 \quad 0.752369$

C 5.4969920 .2277982 .275467

C 4.2445241 .0056662 .676753

C 4.2079072 .3676061 .983647

H $3.590275 \quad 1.6760730 .025149$

C $4.5298233 .585839-0.213220$

H $6.708399-0.1825330 .569385$

O $5.425189-1.0866132 .816979$

H 6.3654250 .7173602 .735400

H 3.3340290 .4370992 .461027

O 4.2571661 .2184044 .090075

H 4.9814783 .0127432 .418658

O 2.9517292 .9915392 .239830

C $4.4785433 .431282-1.714716$

H 3.7205564 .2618390 .084922

H 5.4753374 .0820460 .036409

H $6.240863-1.5516662 .564071$

H 4.3053830 .3437304 .512652

H 2.2574912 .4432681 .835622

O $3.1924193 .221938-2.117559$

O $5.4647163 .474666-2.438765$

C $3.0119903 .021241-3.522575$

H $3.5917672 .157091-3.861094$

H $1.9521002 .823823-3.704138$

H $3.3017763 .921499-4.072281$

SCF Energy (B3LYP/6-31G**//MMFF) $=-3245.92434541$

031

MM̄̄FF Geometry

C $2.523360-3.9580480 .950499$

C $1.430614-3.8256300 .183342$

C $0.049668-3.5889890 .742953$

O $-0.579592-2.534749-0.023393$

C $-0.806707-4.858410 \quad 0.588961$

C $-2.165975-4.7555341 .254617$

C -3.345019 -4.792996 0.603511

C $-2.151124-4.6347582 .757599$

C $-3.588539-4.947397-0.870756$

C $-3.722011-3.627420-1.645592$

C $-4.870670-2.725164-1.164928$

C $-5.118688-1.590220-2.161332$

C $-6.200012-0.615905-1.667552$

O $-4.511165-2.1716420 .104543$

O $-6.1343450 .564770-2.482462$

C $-5.869611-0.184680-0.222064$

C -7.610012 -1.190024-1.850017

O $-6.932604 \quad 0.5845150 .334333$

C $-5.531723-1.3660010 .710745$

C $-5.004669-0.9184012 .097180$

O $-6.718536-2.1343940 .944883$

C $-0.210114-1.2538990 .245382$

O $0.576527-0.8767201 .098649$

C $-0.919317-0.379093-0.714594$

C $-0.4984110 .881112-0.891779$

C $-1.0775181 .865784-1.806914$

C $-2.4181131 .547057-2.412343$

C $-3.734511-0.0602832 .076681$
C -3.9965811 .4366392 .116079$

C -2.8095283 .6213961 .946362$

O $\begin{array}{lllll}-5.104126 & 1.922360 & 2.329158\end{array}$

C -2.9258804 .1467280 .550259$

C -2.0800095 .0506600 .033249$

C $-2.2027415 .622764-1.349876$

C $-0.9406765 .448166-2.164591$

C $-0.0336726 .435264-2.256840$

C $-0.7703584 .156312-2.932353$

C $-0.3974953 .007634-2.038901$

N -2.8336812 .1705521 .920941$

H $2.398336-3.9260682 .031523$

H $1.511649-3.871984-0.898638$

H $\quad 0.102955-3.2883751 .796301$

H $-0.282374-5.7182341 .026935$

H $-0.915353-5.080568-0.478158$

H -4.256495 -4.712399 1.195255

H -1.756546 -3.661494 3.064389

H -3.151947 -4.733055 3.191871

H -1.528051 $-5.420893 \quad 3.196437$

H $-2.802254-5.553609-1.333063$

H $-4.508986-5.531441-0.999654$

H -2.772857 -3.082878-1.582213

H -3.879054 -3.880061-2.701697

H $-5.771801-3.339979-1.056119$

H $-5.385884-1.989283-3.147842$

H -4.186850 -1.028341 -2.309696

H $-6.2673530 .297806-3.408199$

H $-4.9999520 .480326-0.281677$

H -7.801013-1.406600 -2.908017

H $-8.374313-0.464082-1.551365$

H -7.772992 -2.113506 -1.290674

H $-7.096067 \quad 1.330867-0.267327$

H -5.807754 -0.437348 2.668296

H -4.757723 -1.831732 2.657898

H $-6.597317-2.6476611 .760891$

H -1.752957 -0.800719-1.263454

H $\quad 0.363881 \quad 1.222629-0.323336$

H -2.332850 $0.715553-3.120011$

H $-2.8660772 .385926-2.948678$

H $-3.1343961 .272095-1.630668$

H -3.147469 -0.289392 2.975478

H $-3.113175-0.300727 \quad 1.209829$

H -1.872902 3.9230922 .424886

H -3.644601 3.9910482 .550000

H $-3.7566473 .787603-0.052479$

H -1.267408 5.4280710 .649430

H -2.435902 $6.690906-1.249411$

H -3.051034 5.186041-1.891447

H $0.862627 \quad 6.325813-2.858236$

H $-0.1642857 .372621-1.726248$

H $0.0312884 .262602-3.675393$

H -1.673563 $3.968349-3.519035$

H $0.5675863 .142454-1.551386$

H -1.967106 1.6851461 .708153

C $4.261599-3.722513-0.952748$

O $4.025888-2.307888-1.107932$

C $5.122246-1.726664-1.836954$

C $5.910795-2.896872-2.412874$

C $5.728252-3.940715-1.329168$

H $5.750462-1.209538-1.101946$

C $4.607096-0.727998-2.877962$

H $5.482420-3.252088-3.357316$

H $6.959525-2.641876-2.592922$

C $6.046649-5.353714-1.786125$

H $6.377881-3.682825-0.481845$

C $3.7149170 .391140-2.303838$

O $3.829909-1.445968-3.846171$

H $5.455247-0.296686-3.423069$

H $2.764736-0.036196-1.959763$

O $3.396931 \quad 1.259297-3.400119$

C $4.3643371 .212718-1.171877$

C $3.5036012 .413549-0.742426$

H $4.5310010 .561254-0.310085$

O $5.6433311 .687324-1.591189$

H $3.4583423 .137566-1.564523$

H $2.4867912 .059504-0.560782$

H $5.887918-6.071422-0.975181$ 
H $5.416761-5.654345-2.630119$

H $7.091812-5.428630-2.103658$

C $3.945334-4.1393740 .486542$

H $3.517865-0.799992-4.502901$

H $2.6197861 .784340-3.144098$

H $5.5098042 .226826-2.389349$

H $4.571296-3.5397741 .158897$

H $4.221227-5.1881640 .645097$

H $3.609348-4.242414-1.665110$

C 2.9503201 .7972912 .197680

$\begin{array}{lllll}\text { O } & 4.181407 & 2.267360 & 1.634953\end{array}$

C $4.041302 \quad 3.1408320 .509644$

$\begin{array}{lllll}\text { C } 3.220577 & 4.376226 & 0.909798\end{array}$

C 1.8758653 .9904311 .519378

C 2.0668952 .9714822 .639712

H $2.419151 \quad 1.184398 \quad 1.460943$

C 3.3020560 .9114373 .403336

H 5.0524003 .4892390 .264857

O $2.996300 \quad 5.228142-0.208876$

H $3.7816384 .960031 \quad 1.651111$

H $1.1817593 .612120 \quad 0.761525$

O $1.261226 \quad 5.1550762 .075774$

H 2.5112263 .4691113 .511297

O $0.791356 \quad 2.471636 \quad 3.037636$

C $3.955668-0.3923132 .999473$

H 4.0016411 .4495214 .054670

H $2.402960 \quad 0.6537853 .973549$

H $3.8660935 .473203-0.567996$

H $1.178823 \quad 5.8078251 .359207$

H $\quad 0.255892 \quad 3.2346803 .314759$

O $4.530931-0.9569894 .102645$

O $3.943220-0.8692581 .873918$

C $5.185432-2.211108 \quad 3.888161$

H $4.442770-2.9856623 .677257$

H $5.910376-2.1353323 .071678$

H $5.719129-2.4767764 .804499$

SCF Energy (B3LYP/6-31G*//MMFF) $=-3245.91321006$

0310

MM̄FF Geometry

C $4.217606-3.897415-0.533652$

C $3.115821-3.762631-1.288063$

C $1.727642-3.651009-0.712261$

O $1.104296-2.531309-1.384859$

C $0.920445-4.933236-0.993346$

C $-0.273195-5.129216-0.075714$

C $-1.562691-5.164126-0.465941$

C $0.060277-5.360294 \quad 1.378866$

C $-2.133997-5.030373-1.848266$

C $-2.765408-3.664032-2.153915$

C $-3.986553-3.305982-1.288216$

C $-4.686776-2.064531-1.850191$

C $-5.850790-1.597651-0.963058$

O $-3.546090-3.0448140 .048670$

O $-6.211438-0.268533-1.371255$

C $-5.362745-1.5014630 .498301$

C $-7.099778-2.464149-1.161297$

O $-6.447670-1.223308 \quad 1.378995$

C $-4.604649-2.7570280 .975191$

C $-3.972760-2.5930602 .381562$

O $-5.522186-3.8549091 .062973$

C $0.288518-1.719957-0.663486$

O $-0.020731-1.8385850 .510587$

C - $0.174576-0.642503-1.568737$

C $-1.2262980 .108968-1.212483$

C $-1.829926 \quad 1.187017-1.996505$

C $-1.102558 \quad 1.650451-3.229504$

C $-2.955009-1.4578842 .522360$

C -3.559163 -0.1354082 .964562$

C -3.1134712 .3185712 .915813$

O $-4.625727-0.0537013 .567882$

C -3.9402282 .8310741 .778964$

C -3.5522423 .8119220 .949770$

C $-4.3888274 .310271-0.194205$

C $-3.7155344 .127946-1.537604$

C $-3.1098655 .159757-2.148770$

C $-3.8318032 .783271-2.221343$

C $-2.9972441 .713623-1.571458$
N -2.743643 0.9416072 .643782

H $4.107962-3.9685670 .544856$

H $3.210960-3.708616-2.369946$

H $1.805121-3.4464790 .361490$

H $1.564258-5.814649-0.866947$

H $\quad 0.612620-4.932922-2.043875$

H $-2.315310-5.3417630 .301962$

H $0.454436-4.4499921 .839245$

H $-0.814577-5.6672521 .962413$

H $\quad 0.808402-6.153951 \quad 1.476038$

H $-1.375475-5.238975-2.608939$

H $-2.892720-5.812677-1.977203$

H -1.997801 -2.891484 -2.044923

H -3.067098 $-3.671001-3.208904$

H $-4.672251-4.161370-1.286444$

H $-5.036128-2.245692-2.874389$

H -3.961175 -1.244620 -1.926051

H $-6.440980-0.296842-2.315956$

H $-4.687315-0.6388810 .548278$

H -7.438323 -2.414424 -2.203250

H $-7.936704-2.095867-0.557755$

H -6.935085 -3.516223 -0.919922

H $-6.880917-0.4137421 .058291$

H -4.760796 -2.521975 3.141113

H -3.435663 -3.525962 2.605438

H $-5.143222-4.5269411 .653536$

H $0.344095-0.516937-2.512278$

H -1.714402 -0.101014 -0.263289

H -1.516146 2.568598 -3.653447

H $-1.1396750 .881343-4.008323$

H $-0.0528051 .866096-3.001788$

H -2.230222 -1.730330 3.300013

H -2.397586 -1.325799 1.592214

H -2.1878672 .8873303 .046370$

H -3.688452 2.3673413 .846074

H -4.9119952 .3652681 .630627$

H $-2.5862814 .287347 \quad 1.099633$

H $-4.5948005 .374060-0.016634$

H $-5.3711603 .821930-0.214071$

H -2.657486 $5.049369-3.128435$

H $-3.0486276 .136972-1.681304$

H $-3.5984082 .869295-3.288434$

H $-4.8815982 .462713-2.197743$

H -3.447656 $1.299792-0.669737$

H -1.9133980 .7830282 .080583$

C $6.540671-2.853718-0.561320$

O $5.958663-1.556096-0.803266$

C $6.004636-0.8035990 .422910$

C $7.103343-1.4556951 .252586$

C $6.871235-2.9205800 .933002$

H $5.047448-0.9658090 .931748$

C 6.1956850 .6894550 .147591

H $8.101640-1.1499570 .919202$

H $7.012387-1.2286562 .318895$

C $8.057009-3.8127021 .258620$

H $6.007438-3.2655551 .512900$

C $5.1796191 .317129-0.829386$

O $7.5017770 .876639-0.417108$

H $\quad 6.195958 \quad 1.237458 \quad 1.097331$

H $5.3732600 .920496-1.834111$

O $5.4695962 .720983-0.885805$

C $3.6853541 .150021-0.492769$

C 3.3172831 .5817100 .938824

H $3.1092751 .759965-1.196578$

O $3.276393-0.198642-0.691875$

H 3.7743350 .8882231 .653176

H 3.7358902 .5734051 .136914

H $7.845392-4.8526410 .990896$

H $8.955984-3.5003930 .716614$

H $8.281991-3.7775662 .329580$

C $5.614012-3.955287-1.091437$

H $7.6160241 .830183-0.571531$

H $4.8876553 .114152-1.558499$

H $3.503405-0.445573-1.604485$

H $6.043615-4.938571-0.868256$

H $5.569204-3.867450-2.184498$

H $7.469293-2.880557-1.146031$

C $1.315376 \quad 3.8435340 .460763$ 
$\begin{array}{llll}\text { O } & 1.100326 & 2.441049 & 0.260068\end{array}$

C 1.7898121 .5859321 .178300

C 1.3706031 .9140902 .617276

C 1.5488283 .3943632 .938148

C $0.878442 \quad 4.257812 \quad 1.871096$

H 2.3758104 .0821750 .316391

C $0.5008824 .590308-0.606544$

H 1.4367060 .5690710 .973149

$\begin{array}{lllll}\text { O } & 2.108645 & 1.139087 & 3.555598\end{array}$

H $0.312398 \quad 1.657782 \quad 2.752760$

H 2.6073003 .6577263 .048373

$\begin{array}{llllll} & 0 & 0.930264 & 3.679811 & 4.194991\end{array}$

H $-0.2111534 .177701 \quad 1.967713$

O 1.2276325 .6233392 .088923

C $1.123324 \quad 4.496704-1.984157$

H $-0.5051724 .160121-0.664440$

H $0.4286315 .655989-0.364086$

H $1.9635050 .202184 \quad 3.338575$

H $1.338217 \quad 3.095247 \quad 4.856810$

H $0.960167 \quad 5.8497762 .996301$

O $\quad 0.260546 \quad 5.036740-2.895162$

O $2.225426 \quad 4.029895-2.235046$

C $0.7102135 .025225-4.253628$

H $1.6052585 .645337-4.359408$

H $0.9087224 .000071-4.580624$

H $-0.0839045 .443968-4.877422$

SCF Energy (B3LYP/6-31G*//MMFF) $=-3245.91855439$

03_100

MM̄FF Geometry

C $-1.7885592 .974569-0.469174$

C $-1.344412 \quad 1.8247710 .060277$

C $-0.463803 \quad 0.847672-0.676789$

$\begin{array}{lllll}\text { O } & 0.802297 & 0.753266 & 0.015512\end{array}$

C $-1.122223-0.544711-0.660083$

C $-0.378877-1.584451-1.474590$

C $0.218833-2.678390-0.962928$

C $-0.360621-1.353908-2.964346$

C $0.289497-3.118020 \quad 0.472390$

C $1.553995-2.6765451 .224554$

C $2.866354-3.2031710 .621760$

C $4.038022-2.9440531 .571752$

C $5.382720-3.3667300 .958972$

O $3.098140-2.523920-0.615088$

O $6.430178-2.7949111 .758230$

C $5.509304-2.755544-0.453576$

C $5.579118-4.8866021 .011229$

O $6.663257-3.256291-1.123105$

C $4.258361-2.970167-1.330005$

C $4.304182-2.186452-2.664982$

O $4.151791-4.359391-1.664948$

C $1.724387 \quad 1.721729-0.238305$

O $1.6087752 .669119-0.999353$

C 2.9127051 .4408290 .596576

C 3.8101162 .4139370 .812909

C 5.0291072 .3004201 .616428

C $5.447507 \quad 0.9215192 .053125$

C $4.420422-0.663877-2.531837$

C $5.846028-0.143355-2.612455$

C $7.1646681 .952968-2.335540$

O $6.799943-0.837666-2.952625$

C $7.775387 \quad 1.975383-0.969841$

C $8.1795603 .102477-0.364701$

C $8.832346 \quad 3.1411180 .987364$

C 8.1403704 .0898701 .940143

$\begin{array}{llll}\text { C } 8.601470 & 5.337845 & 2.129208\end{array}$

C 6.9670543 .5628622 .734960

C 5.7185583 .4240391 .905960

N $5.9207331 .206795-2.296224$

H -1.493345 $3.249046-1.478776$

H -1.629387 1.5613211 .076599

H -0.296172 $1.172738-1.710705$

H -2.146691 $-0.478187-1.050906$

H $-1.216616-0.8712770 .380605$

H $0.715291-3.357052-1.656190$

H $0.056238-2.206430-3.511662$

H $-1.377740-1.194216-3.336870$

H $0.246048-0.478049-3.213101$
H $-0.586500-2.7725401 .029657$

H $0.225154-4.2136520 .492265$

H $1.577345-1.5813091 .261263$

H $1.464436-3.0319932 .258606$

H $2.756323-4.2781030 .435994$

H $3.879817-3.4501272 .532329$

H $4.084941-1.8718811 .804753$

H $6.318952-3.1132992 .670378$

H $5.667909-1.678952-0.319021$

H $5.551458-5.2405432 .048876$

H $6.563975-5.1714450 .624884$

H $4.817037-5.4369160 .455682$

H $7.429630-3.080582-0.550611$

H $5.084635-2.596990-3.316813$

H $3.356968-2.384231-3.187696$

H $3.594522-4.444008-2.456215$

$\begin{array}{llll}\text { H } & 3.008043 & 0.449144 & 1.022538\end{array}$

H 3.6279223 .3960420 .378091

H $5.4817780 .242724 \quad 1.194076$

H 4.7437530 .5211652 .790627

H 6.4430780 .8876512 .500025

H $3.881478-0.201492-3.368775$

H $3.952171-0.312277-1.607741$

H $6.9287202 .957510-2.700741$

H $7.8546441 .477819-3.040191$

H $7.9148661 .020974-0.467756$

H $8.0643514 .052227-0.882608$

H 9.8754973 .4523090 .844605

H $8.8819532 .142346 \quad 1.438367$

H 8.1281236 .0158512 .831531

H 9.4604155 .7108931 .581039

H 6.7304694 .2513253 .557607

H 7.2602502 .6318213 .227629

H 5.3353054 .3762051 .536124

H $5.0865661 .690789-1.976899$

C $-3.9496574 .250785-0.543666$

O $-4.6834893 .042079-0.818029$

C $-6.078193 \quad 3.269409-0.553806$

C $-6.2415814 .778865-0.424134$

C $-4.918053 \quad 5.168838 \quad 0.203677$

H -6.2878232 .8082670 .418222$

C $-6.9517242 .608144-1.624788$

H $-6.3516895 .264698-1.400268$

H $-7.1061095 .051799 \quad 0.188543$

C $-4.5932386 .646741 \quad 0.074261$

H -4.9455944 .9007121 .268596$

C $-6.6043701 .134883-1.921174$

O $-6.8036013 .331950-2.853471$

H $-8.0060212 .705631-1.338945$

H $-5.675645 \quad 1.088697-2.502817$

O $-7.6272680 .619887-2.784366$

C $-6.4720770 .224290-0.687171$

C $-6.291240-1.253682-1.084519$

H $-5.6192250 .558790-0.086992$

\begin{tabular}{llllll}
\hline & -7.648284 & 0.359229 & 0.109125
\end{tabular}

H $-7.234526-1.641883-1.487882$

H $-5.529285-1.307751-1.871863$

H -3.6254326 .8749490 .531648$

H $-4.552027 \quad 6.958098-0.974939$

H $-5.354967 \quad 7.253142 \quad 0.575114$

C -2.690153 3.9278420 .263499

H -7.373896 $2.901973-3.513796$

H -8.453239 $0.587080-2.271685$

H $-7.465927 \quad-0.0376800 .977117$

H $-2.973883 \quad 3.498916 \quad 1.232256$

H -2.132676 4.8495970 .464951

H -3.677267 $4.682064-1.515760$

C -3.981985 -2.423647 1.605691

O $-4.541018-1.7380060 .478363$

C $-5.859504-2.1443220 .095168$

C $-5.869531-3.634104-0.280708$

C $-5.311548-4.4918550 .860113$

C $-3.979556-3.9453831 .382573$

H -2.937528 -2.091447 1.644461

C $-4.652747-1.9953542 .914529$

H $-6.548288-1.9810170 .929674$

O $-5.087520-3.834713-1.456525$

H $-6.898414-3.933798-0.512864$ 
H $-5.161847 \quad-5.5200620 .508587$

O $-6.247504-4.530041 \quad 1.933299$

H -3.705046 -4.450461 2.315599

O $-2.954723-4.2601370 .434879$

C $-4.394330-0.5303413 .178110$

H -5.732303 -2.170605 2.919582

H $-4.245047-2.5539103 .765216$

H $-5.137320-4.778658-1.684668$

H -7.073378 -4.911055 1.589319

H -3.126201 -3.744869 -0.372115

O $\begin{array}{lllll}-5.517804 & 0.196774 & 2.917402\end{array}$

O $-3.314611-0.0848203 .545362$

C -5.3917371 .6094343 .104026$

H -4.659960 2.0169232 .400723

H -6.3643172 .0667632 .903964$

H -5.1044261 .8344674 .135572$

SCF Energy (B3LYP/6-31G**//MMFF) $=-3245.91495101$

03101

MM̄FF Geometry

C $2.718628-0.6841453 .266088$

C $1.994583-1.3353552 .344298$

C $0.552788-1.7256932 .547076$

O $-0.236554-1.0984341 .510403$

C $0.400568-3.2513592 .412913$

C $-1.011479-3.7500442 .653359$

C $-1.765746-4.3816081 .732363$

C $-1.558755-3.5284024 .040196$

C $-1.396900-4.7319430 .318176$

C $-1.746890-3.655938-0.720433$

C $-3.253246-3.389414-0.869511$

C $-3.523488-2.480211-2.070783$

C $-5.013124-2.126264-2.200572$

O $-3.723119-2.7532120 .321501$

O $-5.104366-1.044816-3.141066$

C $-5.531805-1.616445-0.837231$

C $-5.813879-3.286574-2.802957$

O $-6.942612-1.418718-0.875133$

C $-5.137518-2.519320 \quad 0.350443$

C $-5.473631-1.8986291 .728150$

O $-5.864269-3.751183 \quad 0.258754$

C $-0.546406 \quad 0.2169341 .673693$

O -0.2603190 .9314622 .621041$

$\begin{array}{llll}\text { C }-1.274917 & 0.654978 & 0.464247\end{array}$

C -1.3630581 .9637490 .186588$

C $-2.0074062 .554479-0.987784$

C $-2.7886681 .636114-1.889486$

C $-4.807986-0.5493652 .021727$

C $-5.6850680 .652151 \quad 1.709014$

C -5.6058123 .1414141 .617635$

O $-6.8793020 .563491 \quad 1.437115$

C -5.4364853 .5716960 .194785$

C $-4.9530094 .772859-0.157188$

C $-4.8193915 .239541-1.578369$

C $-3.4247405 .725726-1.903831$

C $-3.1258047 .035488-1.877555$

C -2.403426 $4.700929-2.342295$

C $-1.8757683 .881389-1.196118$

N -4.9821341 .8455451 .811288$

H $2.265607-0.4391134 .223438$

H $2.442134-1.5715691 .382059$

H $\quad 0.190986-1.3983663 .529494$

H $1.064379-3.7541863 .128848$

H $\quad 0.747005-3.5500181 .418131$

H -2.767490 -4.697788 2.021413

H -2.509250 -4.049056 4.199505

H -0.854185 -3.900514 4.791144

H -1.735127 -2.4637214 .220809$

H $-0.328614-4.9634130 .246845$

H -1.906904 -5.6674130 .054930$

H -1.232724 -2.725272 -0.454517

H -1.344329 -3.983839-1.686898

H $-3.762566-4.351871-0.996568$

H -3.156431 -2.938019-2.997891

H $-2.953848-1.548089-1.954242$

H $-6.033906-0.759689-3.172671$

H -5.091065 -0.623886 -0.692618

H $-5.421752-3.545462-3.793898$
H $-6.860880-3.006371-2.962317$

H -5.787732 -4.189411-2.189404

H -7.366310 -2.290655 -0.799190

H -6.560716 -1.853226 1.865759

H -5.114176 -2.600563 2.494584

H $-5.878534-4.1659471 .137371$

H $-1.688741-0.111980-0.179736$

H -0.8943052 .6723460 .868463$

H -3.520431 $1.063595-1.310218$

H $-2.1181130 .936365-2.399770$

H $-3.3597182 .159572-2.658850$

H -4.594039 -0.4958593 .097045$

H -3.854222 -0.4566891 .494127$

H -5.1341883 .8365312 .319598$

H -6.6717713 .0728201 .857202$

H $-5.7513962 .875999-0.579484$

H -4.6664605 .4770380 .620842$

H $-5.5415306 .052504-1.730721$

H $-5.1066974 .453686-2.287958$

H -2.135656 $7.395313-2.137092$

H $-3.8640337 .777002-1.590503$

H -1.541225 $5.202141-2.802597$

H $-2.8293974 .091661-3.144220$

H -1.291803 $4.462661-0.481004$

H -3.9825191 .8217261 .990891$

C 4.2848691 .2446602 .741537

O $3.704186 \quad 1.5340301 .457430$

C 3.1431812 .8581361 .484334

C 3.5470013 .4620012 .830081

C 3.6408212 .2308783 .711285

H 2.0538322 .7358801 .462757

C $3.5969393 .690807 \quad 0.273464$

H 4.5199593 .9641672 .772869

H 2.8159964 .1929793 .189572

C 4.4320022 .4480694 .988107

H 2.6201961 .9227033 .968390

C $3.1460633 .176119-1.110082$

O 5.0139743 .8453820 .300637

H 3.1719394 .6946050 .406905

H $3.5726213 .834133-1.877918$

O $1.7251933 .347939-1.202469$

C $3.4742231 .721538-1.504030$

C $4.9683101 .365010-1.458901$

H $2.8875331 .025197-0.895272$

O $3.0341131 .531527-2.856911$

H $5.5159662 .000848-2.165437$

H $5.3586571 .558243-0.457088$

H $4.478770 \quad 1.5270895 .577818$

H 5.4584912 .7655514 .776002

H 3.9622003 .2211985 .605019

C $4.139842-0.2461393 .057754$

H $5.2615744 .429783-0.435802$

H $1.3060112 .683807-0.630096$

H $2.0888051 .758622-2.887991$

H $4.710956-0.4915793 .960662$

H $4.583038-0.8255152 .237482$

H 5.3570121 .4636352 .644174

C $4.674718-2.335575-1.018179$

O $4.573305-0.918145-0.831986$

C $5.241372-0.112279-1.808584$

C $6.746196-0.426952-1.817761$

C $7.004560-1.925410-2.010223$

C $6.146839-2.779239-1.070744$

H $4.224537-2.766477-0.114760$

C $3.852584-2.814351-2.219606$

H $4.821897-0.319647-2.798456$

O $7.3535930 .001733-0.599719$

H $7.2208810 .139987-2.626890$

H $8.063611-2.139604-1.820064$

O $6.720144-2.292856-3.356597$

H $6.208760-3.834955-1.360095$

O $6.699028-2.6711730 .244995$

C $2.381741-2.563130-1.988075$

H $4.146971-2.336890-3.158396$

H $3.975371-3.893185-2.371703$

H $6.890777-0.4381650 .134063$

H $7.298437-1.765387-3.933585$

H $6.193181-3.2647030 .826027$ 
O $1.972724-1.483754-2.712935$

O $1.687722-3.232622-1.234060$

C $0.600594-1.115911-2.548202$

H $0.417809-0.207727-3.128426$

H $-0.050739-1.910500-2.923425$

H $\quad 0.390959-0.906918-1.496362$

SCF Energy (B3LYP/6-31G**//MMFF) $=-3245.91833016$

03102

MM̄FF Geometry

C $-2.526147-0.020164-3.156194$

C $-2.052556-1.180851-3.636076$

C $-0.655470-1.729199-3.420840$

O $0.030471-1.007939-2.371805$

C $-0.731053-3.206279-2.986749$

C $0.626280-3.866632-2.828652$

C $1.098888-4.376411-1.673952$

C $1.460524-3.959357-4.080608$

C $0.413064-4.425088-0.337770$

C $0.680831-3.2113870 .564571$

C $2.136139-3.0941651 .043929$

C $2.269780-1.9879642 .093162$

C $3.727957-1.7884082 .536184$

O $2.967954-2.789141-0.077779$

O $3.805164-0.5425823 .246556$

C $4.624257-1.6512651 .285733$

C $4.177288-2.8759933 .519330$

O $6.002790-1.6375051 .646798$

C $4.369964-2.7399400 .223693$

C $5.118638-2.482431-1.107106$

O $4.831679-4.0013210 .723779$

C $0.7879150 .065550-2.721288$

O $1.0155050 .477606-3.846816$

C $1.2439520 .702747-1.467151$

C $1.5152132 .015916-1.463067$

C $1.9379882 .803760-0.304069$

C 2.3371902 .0537510 .938651

C $4.783268-1.161398-1.808225$

C $5.756155-0.034160-1.501901$

C $6.0942412 .401051-1.911783$

O $6.813497-0.199492-0.900082$

C $5.6571383 .166294-0.702791$

C $5.3054214 .460669-0.739070$

C $4.907218 \quad 5.2558840 .471207$

C $3.5704835 .943106 \quad 0.303227$

C $3.4982197 .233641-0.064328$

C 2.3241955 .1583720 .644987

C $1.9656084 .148617-0.412445$

N $5.3196201 .178648-2.018911$

H -1.876850 $0.625055-2.571998$

H $-2.713239-1.793290-4.246840$

H $-0.106823-1.649118-4.366824$

H - $1.304076-3.783672-3.724520$

H -1.297283 -3.264219-2.051658

H $2.090181-4.828388-1.681997$

H $1.800059-2.967341-4.393488$

H $2.351312-4.581367-3.940966$

H $0.877568-4.404228-4.893663$

H $-0.667429-4.542969-0.469855$

H $0.734634-5.3386410 .178485$

H $\quad 0.388550-2.2980790 .033651$

H $\quad 0.027831-3.2987201 .441084$

H $2.435123-4.0596031 .468936$

H $1.632730-2.1972552 .961364$

H $1.901183-1.0420761 .673742$

H $3.188324-0.5870573 .997339$

H $4.407024-0.6709130 .846737$

H $3.542037-2.8698144 .413292$

H $5.196232-2.6900403 .876116$

H $4.137576-3.8810263 .094388$

H $6.125614-0.9226332 .294735$

H $\quad 6.199079-2.600241-0.960410$

H $4.831480-3.287464-1.799220$

H $4.985203-4.594204-0.030365$

H $1.3015580 .085078-0.578816$

H $1.3881952 .575707-2.389086$

H $1.465357 \quad 1.5782321 .397828$

H 2.8040892 .6837931 .698453
H 3.0731321 .2798020 .697863

H $4.837660-1.322217-2.892717$

H $3.763930-0.840177-1.576433$

H $5.9397602 .964944-2.837152$

H $7.1572562 .153760-1.826843$

H 5.6560402 .6400140 .248769

H $5.3354634 .991163-1.688270$

H 5.6903996 .0045250 .649444

H 4.8859544 .6314601 .373022

H $2.5448187 .742493-0.159747$

H $4.3917107 .808929-0.283285$

H 1.4666125 .8378130 .743037

H 2.4404604 .7158431 .638160

H $1.6657754 .598568-1.359919$

H $4.4057941 .232662-2.459430$

C $-4.5093851 .004102-2.049747$

O $-3.8085232 .208231-1.659381$

C $-4.7669053 .170419-1.202012$

C $-6.0716572 .411472-1.002949$

C $-5.9844011 .394265-2.127012$

H $-4.9175653 .869759-2.036688$

C -4.259612 $3.985257-0.005926$

H $-6.0981191 .901807-0.033474$

H $-6.9512513 .057822-1.076309$

C $-6.9451510 .229812-1.966904$

H -6.1888791 .900754 -3.079861

C -3.9899783 .2236381 .310048$

O -5.2490734 .9908030 .248569$

H $-3.3440684 .511434-0.304594$

H $-4.9217162 .801221 \quad 1.703299$

O -3.5443584 .1814742 .282562$

C -2.9185622 .1211801 .226255$

C -2.6413951 .4607032 .590331$

H -3.2535891 .3467960 .535561$

O

H -2.2884952 .2153253 .303604$

H $-3.586721 \quad 1.0687902 .981614$

H $-6.830092-0.486413-2.786084$

H $-6.776678-0.302802-1.025465$

H $-7.9813780 .583400-1.970827$

C $-3.9280510 .483535-3.365648$

H -4.8390385 .6676620 .812770$

H -4.3084324 .7340532 .518837$

H -1.4140203 .3653801 .306744$

H -3.887262 $1.297440-4.099424$

H $-4.562860-0.311073-3.773023$

H $-4.3546540 .268838-1.249914$

C $-2.964285-1.4072651 .604318$

O $-1.806315-0.5766721 .449862$

C -1.5789900 .3391942 .523500$

C $-1.399738-0.4345963 .836789$

C $-2.566296-1.3854624 .100950$

C $-2.842406-2.2578932 .875514$

H -3.854201 -0.7702961 .663773$

C $-3.060533-2.2661860 .334680$

H -0.6232290 .8298422 .304691$

O $-1.263048 \quad 0.4553944 .939195$

H $-0.477649-1.0273793 .785337$

H $-3.468327-0.8415304 .404483$

O $-2.231527-2.2448635 .193640$

H $-2.031746-2.9890362 .766479$

O $-4.047967-2.9885953 .079505$

C $-4.472447-2.633716-0.063026$

H -2.628062 -1.711082 -0.506105

H -2.483388 -3.189062 0.453963

H $-0.5061551 .036896 \quad 4.752850$

H -2.025724 -1.676080 5.955398

H -3.946684 -3.492051 3.905357

O $-4.422710-3.363646-1.217763$

O $-5.487219-2.3237390 .544348$

C $-5.687289-3.790425-1.732574$

H -5.506913 -4.355700 -2.650852

H -6.189022 -4.442453-1.011341

H -6.311686 -2.924676 -1.970873

SCF Energy (B3LYP/6-31G**//MMFF) $=-3245.89941643$

03103

MM̄MF Geometry 
C $-3.295998-2.660826-2.828351$

C $-2.634098-2.647471-1.661475$

C $-1.197370-3.090393-1.522083$

O $-0.412963-1.986786-1.017324$

C -1.112301-4.242337-0.502804

C $0.268834-4.857302-0.382978$

C $1.018582-4.847105 \quad 0.736634$

C $0.786669-5.549752-1.618097$

C $0.683117-4.2508542 .074451$

C $1.169744-2.8074682 .270698$

C $2.696240-2.6385882 .215528$

C $3.098828-1.2277142 .651525$

C $4.611965-0.9928762 .517565$

O $3.125705-2.8585520 .870032$

O 4.8497390 .4192352 .627459

C $5.066137-1.4176231 .103668$

C $5.393970-1.6537923 .658775$

O $6.486027-1.3754290 .990730$

C $4.546301-2.808048 \quad 0.683348$

C $4.823077-3.141661-0.803033$

O $5.202774-3.812371 \quad 1.466844$

C $-0.005990-1.049731-1.915498$

O $-0.229492-1.032402-3.115336$

C $0.758898-0.022558-1.175698$

C $1.0085591 .158472-1.760211$

C $1.7341522 .280298-1.162105$

C $2.4697212 .023838 \quad 0.126187$

C $4.210601-2.172152-1.820389$

C $5.167828-1.090610-2.293441$

C $5.2267110 .975205-3.684848$

O $6.368934-1.093289-2.038825$

C $5.1588522 .166255-2.782178$

C $4.764203 \quad 3.379795-3.196257$

C $4.7289434 .595544-2.315453$

C $3.3789405 .276442-2.319084$

C $3.1453486 .344130-3.100753$

C $2.3304844 .771334-1.354595$

C $1.7133803 .472320-1.794617$

N $4.525232-0.137131-3.072002$

H -2.757428 -2.987843 -3.716565

H $-3.131549-2.319470-0.753775$

H $-0.793165-3.415390-2.488502$

H -1.810896 -5.041205 -0.786102

H -1.453529 -3.8729660 .470351$

H $\quad 1.993933-5.3315040 .700929$

H $1.705163-6.114232-1.423546$

H $0.043514-6.259057-1.996796$

H $1.010852-4.822480-2.404145$

H $-0.396099-4.2898112 .256165$

H $1.124909-4.8886292 .850846$

H $0.704750-2.1731361 .507690$

H $0.805917-2.4642263 .247029$

H $\quad 3.151589-3.3872882 .874134$

H $2.769389-1.0277103 .678704$

H $2.578866-0.4922862 .022996$

H 4.4998820 .7162443 .484863

H $4.674967-0.6668380 .407117$

H $5.069860-1.2532534 .626904$

H $6.464023-1.4300603 .586187$

H $5.268927-2.7378733 .692823$

H $6.770038-0.4791071 .239127$

H $5.899422-3.274288-0.965585$

H $4.377330-4.127464-1.000676$

H $5.142727-4.6595890 .995014$

H $1.077445-0.258027-0.167099$

H $\quad 0.624944 \quad 1.333914-2.764627$

H 3.1287561 .1554380 .022271

H $1.764591 \quad 1.8337360 .941675$

H 3.1106782 .8513640 .436322

H $3.930687-2.741955-2.715888$

H $3.298770-1.710805-1.428682$

H $4.7543821 .162269-4.654415$

H $\quad 6.2726640 .698855-3.852779$

H $5.4725492 .031097-1.749714$

H $4.4782233 .517361-4.236738$

H $5.4978465 .290900-2.676912$

H $5.0123814 .352577-1.283824$

H $2.1887226 .855728-3.088854$
H $3.9047756 .727331-3.774452$

H $1.5148115 .502104-1.270115$

H $2.7631584 .721397-0.351688$

H $1.1521083 .553797-2.726613$

H $3.516878-0.183678-3.186494$

C $-5.608067-1.986890-1.858340$

O $-5.270722-0.692141-1.318439$

C $-6.4822900 .029490-1.030950$

C -7.600953-1.004410-1.103534

C $-7.096651-1.915600-2.200793$

H $-6.6231780 .761695-1.835312$

C $-6.419016 \quad 0.7388820 .327130$

H -7.696309 -1.556217 -0.160346

H -8.571102 -0.552026 -1.330807

C $-7.793157-3.265484-2.231826$

H $-7.237768-1.414972-3.168119$

C -5.5761092 .0285040 .398350$

O $-5.980061-0.1850821 .321800$

H $-7.449077 \quad 1.0227260 .583153$

H -5.7146662 .4289261 .411734$

O $-6.1348852 .979354-0.507337$

C $-4.059424 \quad 1.8742450 .164773$

C $-3.2979373 .147330 \quad 0.588231$

H -3.7003941 .0167840 .742139$

O $-3.8059811 .621593-1.213216$

H $-3.6108553 .984446-0.046915$

H -3.583624 3.3976551 .615878

H -7.390594 -3.896052 -3.030599

H -7.670851 -3.802325 -1.285092

H -8.866225 -3.139919-2.410102

C $-4.734111-2.290425-3.079896$

H -6.0137770 .2679942 .181311$

H $-5.7456643 .847039-0.309720$

H -2.891182 $1.301987-1.291751$

H $-4.733356-1.422471-3.751254$

H $-5.176811-3.127409-3.633272$

H $-5.441660-2.725437-1.063895$

C $-1.368447 \quad 1.8004002 .526514$

O -1.2659921 .8345921 .098392$

C -1.7614163 .0277260 .486465$

C -0.9930834 .2433501 .026680$

C -1.007197 4.3027442 .553360

C $-0.5816752 .960253 \quad 3.147208$

H -2.4230481 .8579662 .823753$

C $-0.817130 \quad 0.4486902 .991881$

H $-1.5242482 .947361-0.581366$

O -1.4970805 .4563520 .478339$

H $\quad 0.0515914 .170904 \quad 0.703561$

H -1.988282 4.6055542 .937045

O -0.0808845 .2989022 .992448$

H 0.4944892 .8145402 .988636

O -0.8074512 .9802374 .555181$

C - $-1.763066-0.6695432 .620558$

H $\quad 0.1485220 .2276492 .523442$

H -0.6610890 .4330924 .076503$

H $-2.3552545 .644920 \quad 0.893755$

H -0.3343676 .1369332 .568660$

H $-0.313971 \quad 3.7361314 .917192$

O $-2.356669-1.1763323 .737708$

O $-1.960558-1.0411171 .471164$

C $-3.276483-2.2482223 .507400$

H $-2.762687-3.0951273 .042364$

H -3.675420 -2.567469 4.473846

H -4.105716 -1.907899 2.880193

SCF Energy $\left(B 3 L Y P / 6-31 G^{* *} / / M M F F\right)=-3245.89318059$

03 104

MM̄MF Geometry

C $3.033517-3.877788-1.452084$

C $2.347219-2.752444-1.202111$

C $1.215582-2.242555-2.055192$

O $0.185792-1.707980-1.190737$

C $1.724023-1.091346-2.942255$

C $0.680398-0.546476-3.897991$

C $0.2221720 .720575-3.887997$

C $0.169603-1.515198-4.933810$

C $0.6258701 .844231-2.975730$

C $-0.2339051 .984253-1.711358$ 
C $-1.7105782 .312204-1.981020$ C $-2.4285192 .669399-0.677527$ C $-3.9293132 .917812-0.896549$ O $-2.3317341 .164716-2.565139$ $\begin{array}{llll}\text { O } & -4.567267 & 2.918513 & 0.390074\end{array}$ C -4.525996 $1.738362-1.695130$ C $-4.1937854 .296879-1.512371$ O $-5.8752292 .006041-2.067724$ C $-3.701522 \quad 1.359719-2.942740$ C $-4.1815190 .051148-3.617578$ O $-3.8210192 .402054-3.918828$ C $-0.625364-2.589072-0.547986$ O $-0.608007-3.806158-0.633352$ C $-1.533070-1.800963 \quad 0.314667$ C $-2.194967-2.4085761 .310012$ C $-3.107449-1.7637752 .256779$ C $-3.504129-0.3372601 .982125$ C $-4.139948-1.199854-2.732504$ C $-5.463067-1.525279-2.058591$ C $-6.457850-3.089075-0.391856$ O $-6.518858-0.963669-2.337224$ C $-6.575933-2.3832490 .922100$ C $-6.659335-3.0252082 .097413$ C $-6.826098-2.3334093 .420027$ C $-5.783968-2.7515564 .433175$ C $-6.056863-3.6854335 .360106$ C $-4.458516-2.0244714 .425206$ C -3.557194 -2.479222 3.308799 N $-5.331659-2.551776-1.132415$ H $2.780206-4.480559-2.320080$ H $2.625621-2.145299-0.345423$ H $0.794314-3.047446-2.669371$ H $2.581511-1.431515-3.538294$ H $2.103335-0.294476-2.292887$ H $-0.5271780 .998370-4.628773$ H $-0.451390-1.024193-5.690898$ H $1.006595-1.988897-5.457125$ H $-0.439600-2.295044-4.466921$ H $1.6765821 .745682-2.682142$ H $0.5787412 .779496-3.548446$ H $-0.1612511 .059272-1.127964$ H $0.2020472 .786321-1.105396$ H -1.755136 $3.151859-2.684488$ H - $-1.9624763 .541055-0.201451$ H -2.314476 1.8430230 .036869 H -4.1507103 .6115020 .930626$ H $-4.555100 \quad 0.879227-1.015559$ H $-3.804798 \quad 5.088215-0.860108$ H $-5.2684294 .488546-1.605880$ H -3.733202 $4.423650-2.494225$ H -6.364921 $2.214242-1.253596$ H $-5.1716220 .198977-4.065209$ H -3.506986 $-0.140777-4.464768$ H -3.602886 $2.034009-4.791163$ H -1.611862 -0.7370310 .122586$ H -2.039183 -3.4758001 .462783$ H -3.872385 -0.2343220 .956271$ H -2.6507180 .3348182 .117656$ H -4.3090910 .0240962 .625493$ H -3.902861 -2.063897 -3.366668 H -3.351865 - $1.123221-1.977450$ H $-6.284295-4.163087-0.271332$ H -7.376278 -2.946304 -0.970382 H $-6.623501-1.2969480 .903595$ H $-6.641001-4.1128852 .109600$ H -7.828752 -2.5744103 .796842$ H $-6.808130-1.2423383 .307548$ H $-5.324922-3.970928 \quad 6.108424$ H -7.017132 -4.189779 5.387390 H -3.923323 -2.208592 5.366678 H $-4.640112-0.9460764 .418220$ H -3.219252 -3.510974 3.416336 H $-4.408593-2.923331-0.927600$

C $5.479943-3.632196-0.923492$

O $5.384049-2.267269-0.466783$

C $6.553452-1.9430580 .303609$

C $7.585717-2.997165-0.068214$

C $6.701402-4.222651-0.216583$
H $\quad 6.285616-2.0745701 .359042$

C $6.960484-0.4878630 .053549$

H $8.077524-2.767442-1.020353$

H $8.358439-3.1135360 .697419$

C $7.360534-5.360632-0.976385$

H $6.421086-4.5772620 .784495$

C $5.809250 \quad 0.521540 \quad 0.266301$

O $7.398491-0.366064-1.305528$

H $7.822685-0.2314930 .680079$

H $5.0560160 .369343-0.513483$

$\begin{array}{lllll}\text { O } & 6.336167 & 1.836357 & 0.047300\end{array}$

C $5.156264 \quad 0.4658751 .661776$

C 4.0694561 .5326101 .891626

H $4.743279-0.5292151 .837978$

$\begin{array}{lllll}\text { O } & 6.184023 & 0.664738 & 2.638323\end{array}$

H 3.8295841 .5663312 .961856

H 4.4940042 .5243391 .686015

H $6.677675-6.210441-1.074754$

H $7.660016-5.050654-1.983206$

H $8.256770-5.705637-0.450725$

C $4.173271-4.357619-0.594151$

H $7.640740 \quad 0.564893-1.449004$

H 6.9766092 .0165660 .756880

H $5.780296 \quad 0.552875 \quad 3.515850$

H $3.914124-4.2089880 .462114$

H $4.285098-5.436979-0.748074$

H $5.640069-3.595292-2.008721$

C 1.5679053 .2168892 .019642

$\begin{array}{lllll}\text { O } & 2.137241 & 2.614357 & 0.852224\end{array}$

C 2.7733591 .3467311 .074542

C $1.7473660 .357887 \quad 1.650912$

C 1.0309780 .9106672 .882050

C 0.4798222 .3085842 .603754

H 2.3508103 .3924072 .768188

C 0.9888424 .5768701 .602358

H 3.0498780 .9870660 .078495

$\begin{array}{llll}\text { O } & 2.358775 & -0.883126 & 1.974106\end{array}$

H 0.9969900 .1504030 .878206

H $1.6940100 .908907 \quad 3.755125$

$\begin{array}{llll}\text { O } & -0.048476 & 0.028348 & 3.196091\end{array}$

H -0.3682312 .2383181 .910663$

O $0.0000532 .878145 \quad 3.819032$

C 2.0677365 .5665361 .214335

H $0.330957 \quad 4.446987 \quad 0.735574$

H 0.4212195 .0230472 .425883

H $1.663594-1.4709152 .316420$

H $-0.4407740 .328172 \quad 4.033228$

H $-0.765946 \quad 2.3542514 .107770$

$\begin{array}{lllll}\text { O } & 1.475080 & 6.687794 & 0.705551\end{array}$

$\begin{array}{lllll}\text { O } & 3.270939 & 5.390641 & 1.347854\end{array}$

$\begin{array}{llll}\text { C } 2.371724 & 7.724517 & 0.294113\end{array}$

H $3.0339307 .360931-0.497444$

H $1.7761928 .552389-0.099658$

H 2.9516548 .0845341 .149171

SCF Energy (B3LYP/6-31G**//MMFF) $=-3245.90643053$

03105

MM̄FF Geometry

C $0.524450-4.316798-0.903169$

C $1.518385-4.477526-1.791182$

C $2.862618-3.786325-1.713963$

O $2.742741-2.529959-1.006367$

C $3.877615-4.669125-0.968383$

C $5.269046-4.068594-0.905417$

C $5.886336-3.683777 \quad 0.229234$

C $5.981283-3.929827-2.226673$

C $5.372789-3.7703711 .639121$

C $4.643555-2.5142362 .140601$

C $5.523707-1.2547212 .196896$

C $4.812011-0.1321132 .957239$

C $5.631367 \quad 1.1687532 .957876$

O $5.792777 \quad-0.8261780 .858775$

$\begin{array}{lllll}\text { O } & 4.786492 & 2.228163 & 3.432897\end{array}$

C 6.0562541 .5009811 .509651

C 6.7910241 .1023123 .957851

O $6.9549712 .606306 \quad 1.484748$

C $6.676292 \quad 0.300797 \quad 0.763247$

C $6.9139620 .575359-0.742379$ 
O $7.954381-0.000508 \quad 1.337500$

C $2.211840-1.482825-1.695616$

O $1.908348-1.456102-2.877788$

C $2.002393-0.378948-0.735524$

C $1.1460220 .603369-1.050842$

C $0.7987381 .749360-0.209803$

C $1.583527 \quad 1.944443 \quad 1.059714$

C $5.6640300 .933964-1.553510$

C $5.4395742 .428164-1.720822$

C $3.7905214 .047735-2.656054$

O $6.2612193 .276687-1.385194$

C $2.9080234 .570241-1.566651$

C $1.6933085 .093691-1.792842$

C $0.8119645 .666991-0.720058$

C $-0.5672035 .046704-0.706173$

C -1.603915 5.647808 -1.314202

C -0.7590523 .7722470 .083503$

C $-0.1927512 .567895-0.618485$

N $4.2209672 .701107-2.328495$

H $\quad 0.691350-3.700147-0.023211$

H $1.357973-5.113270-2.658670$

H $3.211945-3.589511-2.734954$

H $3.947865-5.650591-1.455838$

H $3.497758-4.8608940 .040773$

H $6.888395-3.2639340 .147868$

H $7.026274-3.624669-2.105122$

H $5.980887-4.885688-2.760654$

H $5.491917-3.177785-2.852953$

H $4.711655-4.6354991 .757099$

H $\quad 6.226725-3.9763902 .297372$

H $3.771968-2.3290711 .502256$

H $4.265610-2.7325933 .147242$

H $6.465141-1.5146532 .694684$

H $4.583179-0.4393693 .985275$

H 3.8412640 .0654262 .482085

H 4.0159102 .2915232 .843688

H 5.1526541 .8199040 .977043

H 6.4089780 .9455984 .974056

H 7.3415922 .0490053 .989258

H 7.4992450 .2990703 .744615

H 6.5218873 .3433391 .948403

H $7.7066021 .322525-0.868854$

H $7.316752-0.350329-1.178751$

H $8.474056-0.5018570 .687578$

H $2.530853-0.421300 \quad 0.209415$

H $0.6290800 .554382-2.008643$

H 1.3673922 .8865621 .567165

H 2.6579571 .9564200 .846224

H $1.3745651 .136577 \quad 1.769354$

H $5.7822690 .529545-2.567123$

H $4.7702590 .476048-1.121063$

H $3.2728033 .996654-3.619240$

H $4.6654354 .696485-2.764675$

H $3.2966164 .542823-0.551426$

H $1.3202475 .144541-2.813511$

H $\quad 0.7320656 .747578-0.897067$

H 1.2686625 .5641660 .272042

H $-2.6013205 .221696-1.285276$

H -1.476520 $6.577095-1.859565$

H $-1.830123 \quad 3.5824100 .235983$

H -0.3552923 .9114541 .090043$

H $-0.6949282 .339843-1.559462$

H $3.5834851 .936775-2.532128$

C -1.966247 -3.864047 -0.933938

O $-1.991426-3.3169990 .400354$

C $-2.173974-1.8872830 .312470$

C $-2.641849-1.616768-1.110022$

C $-1.878406-2.670583-1.886986$

H -1.182923 $-1.448140 \quad 0.482878$

C -3.152696 -1.440769 1.403789

H -3.723246 -1.777730 -1.203505

H -2.416357 -0.598251-1.439255

C $-2.444871-2.943656-3.268715$

H $-0.843199-2.326045-1.993261$

C $-3.5115110 .052522 \quad 1.333550$

O $-2.554039-1.7036282 .676914$

H -4.057455 -2.056055 1.351484

H $\quad-3.980057 \quad 0.2853510 .372660$
O $\quad-2.302792 \quad 0.8220581 .367611$

C $-4.415610 \quad 0.5524782 .475453$

C $-5.725756-0.2314182 .677770$

H -3.8597490 .5452913 .420935$

O $-4.741662 \quad 1.9244172 .228569$

H $-5.492088-1.2267413 .073227$

H -6.3032370 .2986173 .446510$

H -1.866523 -3.720666 -3.778536

H -3.486962 -3.276293 -3.217305

H $-2.411779-2.038163-3.883543$

C $-0.848611-4.909248-1.048810$

H -2.323604 -2.648483 2.699045

H -1.8334130 .5965412 .189177$

H -3.905233 2.4035862 .099206

H -0.942093 -5.436048 -2.005688

H $-0.972712-5.651445-0.250867$

H -2.927790 -4.377968 -1.063184

C $-7.7802160 .979052-0.211646$

O -7.0185810 .9257951 .001495$

C $-6.604692-0.3774611 .417914$

C $-7.833431-1.2643611 .675965$

C $-8.745534-1.3120380 .445251$

C $-9.0302370 .088291-0.111206$

H -8.119256 $2.020566-0.285570$

C $-6.9122110 .679509-1.443710$

H $-6.002652-0.8359790 .628716$

O $-8.562108-0.7561472 .791541$

H -7.493342 -2.274806 1.933474

H -9.691674 -1.795637 0.717542

O $-8.118430-2.114047-0.551390$

H $-9.5058170 .015525-1.095628$

$\begin{array}{lllll}\text { O } & -9.974676 & 0.737068 & 0.746549\end{array}$

C $-5.7888241 .682883-1.603575$

H $-6.461475-0.314613-1.379818$

H $\quad-7.5232360 .724669-2.352097$

H $-9.310057-1.3566332 .951385$

H $-8.720084-2.157906-1.314051$

H -9.5454290 .8784301 .607891$

O $-4.8547881 .173400-2.460608$

O $-5.7404712 .779224-1.062829$

C $-3.7159972 .008339-2.691304$

H $-3.0020011 .449606-3.302135$

H $-4.0157352 .910141-3.233085$

H -3.236553 2.268791-1.743308

SCF Energy $(B 3 L Y P / 6-31 G * * / / M M F F)=-3245.90492000$

03_106

MM̄FF Geometry

C $2.3166000 .595850-2.234744$

C $1.1636890 .032462-2.623159$

C $0.307937-0.806835-1.707110$

O $-1.029087-0.257859-1.685591$

C $0.229373-2.244704-2.254377$

C $-0.492270-3.212784-1.337197$

C $-1.643953-3.842721-1.641879$

C $0.181046-3.490686-0.017017$

C $-2.448969-3.748733-2.907392$

C $-3.564704-2.692702-2.885948$

C $-4.634689-2.927071-1.807009$

C $-5.829507-1.994242-2.018360$

C $-6.876073-2.135549-0.901210$

O $-4.050703-2.667253-0.527920$

O $-7.774525-1.019057-0.995543$

C $-6.170966-2.0319240 .468921$

C $-7.731353-3.395799-1.078920$

O $-7.075542-2.3249091 .530435$

C $-4.918426-2.9241890 .584463$

C $-4.099785-2.6666901 .873526$

O $\quad-5.321515-4.2991170 .613914$

C $-1.2442840 .827248-0.892776$

O $-0.4298821 .394616-0.182176$

C $-2.6594631 .226273-1.055182$

C $-3.0494052 .448370-0.663378$

C $-4.4045882 .994530-0.763119$

C $-5.5120482 .043067-1.131066$

C $-3.575014-1.2369142 .046241$

C $-4.471398-0.3485472 .893054$

C -4.7261462 .0211173 .620283$ 
O $-5.418306-0.774233 \quad 3.548904$

C -5.751653 2.6881432 .759047

C -5.8310094 .0188962 .608412$

C -6.8784454 .7084841 .781944$

C -6.2852355 .6587590 .766006$

C -6.1977796 .9754051 .020159$

C -5.873336 $5.093898-0.574672$

C $-4.593038 \quad 4.305795-0.505264$

N $-4.061842 \quad 0.9779132 .861754$

H $2.6558710 .450213-1.211835$

H $0.8206080 .162161-3.646729$

H $0.715401-0.813911-0.688938$

H $1.242106-2.634859-2.422940$

H $-0.251025-2.217077-3.238427$

H $-2.063390-4.523630-0.901688$

H $0.181758-2.5980350 .615446$

H $-0.318909-4.2870870 .544850$

H $1.216036-3.808554-0.178641$

H -1.798299 -3.561064 -3.768131

H -2.892773 -4.734402 -3.098281

H -3.112824 -1.703284 -2.749891

H $-4.042913-2.695801-3.873337$

H -4.954834 -3.974302 -1.858859

H -6.290647 -2.168037 -2.998662

H -5.478336 -0.953833 -2.034834

H -8.171282 -1.027781 -1.883495

H $-5.872856-0.9841850 .592399$

H -8.258745 -3.367916 -2.040082

H $-8.511450-3.455450-0.312102$

H -7.147368 -4.317979 -1.050770

H $-7.835861-1.7256511 .436868$

H $-4.665453-2.9997542 .752069$

H -3.217792 -3.322189 1.831699

H $-4.606678-4.8214501 .014142$

H -3.336529 $0.510969-1.506969$

H -2.303274 $3.124239-0.247106$

H -5.404865 $1.710491-2.168924$

H -6.510468 $2.472817-1.027542$

H $-5.4946231 .165401-0.476191$

H -2.611806 -1.282925 2.570703

H -3.396595 -0.7626771 .076652$

H -3.9533272 .7179563 .959544$

H -5.211159 1.5820874 .497997

H -6.4765202 .0506082 .258058$

H -5.121767 4.6545973 .134136

H -7.5342665 .2599852 .468334$

H -7.527138 3.9861521 .271003

H -5.7995347 .6681630 .286337$

H -6.5180297 .3889831 .970748$

H -5.708605 5.909321-1.291911

H $-6.7067944 .521874-0.991397$

H -3.725024 $4.911006-0.239646$

H $-3.297317 \quad 1.2495042 .250369$

C $3.4941202 .793265-2.586346$

O $4.1840282 .684597-1.324423$

C $5.4346633 .385282-1.414957$

C $5.226404 \quad 4.413870-2.515900$

C $4.4080923 .609285-3.508477$

H $6.1831682 .654164-1.746520$

C $5.8466973 .943720-0.048376$

H $4.6472175 .275701-2.165179$

H $6.1697534 .779274-2.932325$

C $3.6545844 .463625-4.513613$

H $5.0856852 .939710-4.055504$

C 5.7526572 .9242021 .106403

O 4.9829175 .0403290 .274941

H $6.8598094 .357925-0.117572$

H 4.6999892 .7512491 .363819

O $6.362353 \quad 3.506105 \quad 2.264976$

C 6.4324691 .5697160 .833992

C 6.3497950 .6259542 .048109

H $5.948987 \quad 1.092127-0.023195$

$\begin{array}{lllllll}\text { O } & 7.800362 & 1.772854 & 0.486028\end{array}$

H 6.9543901 .0306752 .868996

H 5.3118210 .5965812 .396575

H $3.0674153 .837355-5.192706$

H $2.9683365 .157161-4.016085$

H $4.3521275 .054887-5.115634$
C $3.2162931 .391204-3.137112$

H 5.3720965 .5083211 .032515

H 5.8035084 .2485062 .549795

H 8.2261342 .2369951 .227006

H $4.1591750 .836155-3.223133$

H $2.7864331 .466252-4.142826$

H $2.5534173 .322609-2.390130$

C $4.835739-1.6688240 .731199$

O $6.224048-1.3618790 .575828$

C $6.848079-0.8047731 .737962$

C $6.714873-1.7798032 .917835$

C $5.259987-2.1751133 .168916$

C $4.604380-2.6622081 .876338$

H $4.292555-0.7398350 .946178$

C $4.291101-2.153608-0.619571$

H $7.916063-0.7218191 .500527$

O $7.256625-1.2168364 .108001$

H $7.287931-2.6911142 .702196$

H $4.688944-1.3509183 .611938$

O $5.220125-3.2431144 .118562$

H $5.008375-3.6500821 .626244$

O $3.202394-2.8177482 .083373$

C $4.846923-3.485219-1.077243$

H $3.200116-2.230967-0.553799$

H $4.538294-1.428937-1.403627$

H $8.184873-0.9900413 .927237$

H $5.676653-2.9338024 .919845$

H $3.085672-3.4260552 .833232$

O $3.902184-4.134493-1.819570$

O $5.977424-3.890620-0.843641$

C $4.292689-5.411271-2.333897$

H $3.447597-5.826649-2.889206$

H $5.141131-5.300843-3.015811$

H $4.541407-6.090830-1.513159$

SCF Energy (B3LYP/6-31G*//MMFF)= -3245.90534764

03_107

MM̄FF Geometry

C $2.242867 \quad 4.602161-1.029687$

C $3.0109493 .537408-0.757483$

C $3.757805 \quad 3.358423 \quad 0.538786$

$\begin{array}{llll}\text { O } & 3.352588 & 2.090643 & 1.106284\end{array}$

C 5.2692463 .2925050 .266986

C $6.1060613 .362214 \quad 1.530022$

C 6.6759322 .2949992 .123819

C 6.3296004 .7464922 .080243

C $6.524220 \quad 0.855508 \quad 1.724255$

$\begin{array}{lll}\text { C } 7.694828 & 0.370798 & 0.861756\end{array}$

C $7.501596-1.0885540 .425400$

C $8.682776-1.573565-0.416409$

C $8.458248-2.997921-0.950559$

O $6.298342-1.158835-0.345963$

O $9.430825-3.242565-1.978203$

C $7.067530-3.073681-1.618124$

C $8.697292-4.056596 \quad 0.132669$

O $6.750692-4.412820-1.990871$

C $5.933463-2.483609-0.749382$

C $4.622675-2.389915-1.566011$

O $5.711790-3.3376440 .376083$

$\begin{array}{llll}\text { C } 2.150475 & 2.034358 & 1.740026\end{array}$

O $1.3922102 .961112 \quad 1.973087$

C $1.893486 \quad 0.6121342 .053681$

C $0.641066 \quad 0.2013852 .302308$

C $0.230001-1.1812502 .550828$

C $1.313702-2.2145642 .726075$

C $3.549122-1.518274-0.901098$

C $2.220345-1.651196-1.615304$

C $0.251508-3.168206-1.831843$

O $1.831043-0.806324-2.418164$

C $-0.832757-2.558010-1.001513$

C $-1.771332-3.265748-0.354096$

C -2.888181-2.634083 0.427667

C $-2.921234-3.056701 \quad 1.881735$

C $-4.022692-3.6251212 .400858$

C $-1.697887-2.8428272 .753271$

C $-1.086841-1.4716962 .616702$

N $1.542744-2.812128-1.275078$

H $2.1775905 .407843-0.303522$ 
H $3.0789942 .728343-1.481426$ H 3.5315194 .1646961 .247396 H $5.5719684 .122479-0.386025$ H $5.5061652 .378339-0.291992$ H 7.2947402 .4587773 .004580 H 6.9702164 .7421812 .968531 H 6.8120605 .3804641 .329165 H 5.3777415 .2058182 .363931 H $6.464674 \quad 0.2507312 .637790$ H $5.5744720 .696127 \quad 1.204996$ H $7.7686511 .009851-0.027809$ H $8.6347840 .471678 \quad 1.418475$ H $7.392312-1.7047671 .325761$ H $9.617221-1.5198190 .155722$ H $8.818249-0.900786-1.274917$ H $10.314258-3.125833-1.588496$ H $7.121770-2.496599-2.551963$ H $9.725271-3.9933640 .509222$ H $8.589622-5.068595-0.273045$ H $8.026789-3.9537720 .988297$ H $7.480707-4.739599-2.544264$ H $4.830665-1.950159-2.550257$ H $4.227447-3.397902-1.743170$ H $4.787770-3.2601930 .660844$ H $2.737964-0.0669412 .018995$ H $-0.163461 \quad 0.9353132 .281242$ H $2.075626-1.8648943 .432063$ H $1.794751-2.4338501 .769095$ H $\quad 0.947612-3.1583243 .138054$ H $3.408597-1.7836390 .150886$ H $3.840495-0.461381-0.920901$ H $0.193616-4.261002-1.851241$ H $\quad 0.183892-2.796553-2.859514$ H $-0.862840-1.472041-0.956935$ H -1.759139-4.351416 -0.412384 H $-3.825752-2.910603-0.070455$ H -2.837115 -1.5398750 .374266$ H $-4.064124-3.9366443 .439348$ H -4.908465 -3.798242 1.800268 H $-0.982591-3.6289872 .491636$ H -1.955360 -2.985014 3.810417 H -1.806369 -0.6563872 .535942$ H $1.923150-3.412409-0.549405$ C - $0.0749224 .643216-2.068089$ O $-0.4148733 .357679-1.507922$ C -1.423584 $3.551607-0.491453$ C -1.948407 $4.966275-0.694631$ C $-0.6969625 .691670-1.146097$ H -0.8996873 .4725350 .468885$ C $-2.4826982 .449151-0.610548$ H $-2.7110854 .990402-1.483178$ H -2.382102 5.3901370 .215910 C $-0.9716887 .022974-1.822852$ H $-0.0692485 .864940-0.264152$ C $-3.6921102 .657830 \quad 0.315730$ O $-1.868351 \quad 1.200217-0.282025$ H -2.811585 2.375754 -1.653248 H $-4.201818 \quad 3.5916780 .051985$ O $\quad-3.2265632 .8302751 .660730$ C -4.7294881 .5180710 .325475$ C $-5.2221051 .131517-1.077861$ H $-4.330080 \quad 0.6381580 .843047$ O H -5.536630 $2.036668-1.612299$ H $-4.3831070 .689243-1.626726$ H $-0.0380527 .497111-2.141704$ H -1.607849 $6.901392-2.706017$ H -1.480309 $7.706363-1.134878$ C $1.4377154 .743826-2.290905$ H -1.094153 $1.095896-0.861576$ H -2.7224342 .0332961 .899922$ H -5.5247552 .2111451 .972467$ H $1.6833865 .704636-2.757494$ H $1.7352733 .961951-3.001436$ H $-0.5632264 .680313-3.051057$ C $-6.971446-2.080890-0.279705$ O $-5.976288-1.056187-0.414971$ C $-6.403431 \quad 0.140977-1.071449$
C $-6.891308-0.170695-2.495681$

C $-7.975321-1.252808-2.488415$

C $-7.560775-2.463972-1.647744$

H $-6.423600-2.953344 \quad 0.097254$

C $-8.035452-1.713300 \quad 0.761194$

H $-7.2261630 .589449-0.505259$

O $-5.803859-0.596371-3.315098$

H $-7.2871800 .748397-2.943128$

H $-8.172929-1.577948-3.517106$

O $-9.185137-0.718667-1.959040$

H $-8.410765-3.140857-1.502082$

O $-6.556461-3.199573-2.353236$

C $-7.460001-1.7671292 .156639$

H $-8.478519-0.7251500 .610281$

H $-8.864441-2.430797 \quad 0.734226$

H $-5.388437-1.362665-2.883625$

H $-9.4470860 .029672-2.522008$

H $-6.943594-3.487532-3.197493$

O $-6.859260-0.5827372 .461161$

O $-7.523585-2.7523292 .880689$

C $-6.260300-0.507600 \quad 3.758274$

H $-5.455701-1.2428793 .847420$

H -5.8350410 .4922093 .879199$

H -7.015064 -0.664112 4.534735

SCF Energy (B3LYP/6-31G**//MMFF) = -3245.91647553

03108

MM̄FF Geometry

C $-3.986752-2.468870-2.826565$

C $-3.283065-2.527193-1.685344$

C $-1.948670-3.214484-1.540131$

O $-0.952627-2.209778-1.245553$

C $-2.011574-4.192235-0.350708$

C $-0.694003-4.870292-0.032924$

C $-0.025499-4.7257191 .128905$

C $-0.146213-5.772100-1.108196$

C $-0.420170-3.9043212 .326512$

C $0.136077-2.4714012 .329239$

C $1.658780-2.3943262524460$

C $2.113950-0.9413562 .679789$

C $3.642931-0.8284762 .796787$

O $2.296003-2.9717261 .384023$

$\begin{array}{lllll}\text { O } & 3.998548 & 0.549861 & 2.607524\end{array}$

C $4.301018-1.6266371 .648827$

C $4.136498-1.2147494 .196142$

O $5.714736-1.6922341 .817031$

C $3.724822-3.0471501 .478128$

C $4.228267-3.7616500 .200620$

O $4.124655-3.8525932 .593872$

C $-0.368520-1.583593-2.303011$

O $-0.591554-1.772489-3.488101$

C $0.588347-0.594566-1.761642$

C $1.0401860 .387658-2.555807$

C $1.9415371 .469608-2.154766$

C $2.6283421 .339351-0.822530$

C $3.892308-3.061246-1.121223$

C $5.030493-2.227068-1.685826$

C $5.562680-0.731001-3.607574$

O $6.157322-2.200876-1.199189$

C $5.541448 \quad 0.678516-3.107944$

C $5.3023291 .737514-3.896129$

C $5.335733 \quad 3.159847-3.416799$

C $4.0612393 .911531-3.725452$

C $3.9852934 .742207-4.778894$

C $2.9084023 .771312-2.758795$

C $2.1064212 .517528-2.988443$

N $4.643490-1.538769-2.828661$

H $-3.561357-2.929234-3.716473$

H $-3.669454-2.059482-0.784717$

H -1.678336 -3.759203 -2.453153

H $-2.760603-4.969610-0.552961$

H -2.376782 -3.647948 0.526695

H $0.911921-5.2673391 .249180$

H $0.736172-6.328319-0.773984$

H $-0.900836-6.506220-1.408890$

H $0.147221-5.190293-1.987338$

H - $-1.510386-3.8618012 .420661$

H $-0.080392-4.429223 \quad 3.228595$ 
H $-0.145008-1.9752361 .392690$ H $-0.353113-1.9228663 .143886$ H $1.917866-2.9722073 .419458$ H $1.627650-0.4720413 .543633$ H $\quad 1.789037-0.363178 \quad 1.804218$ H 3.5385381 .0736703 .285876 H $4.125033-1.0615120 .727465$ H $3.682751-0.5664064 .954850$ H $5.218451-1.0689444 .288112$ H $3.902648-2.2471824 .463800$ H $\quad 6.039777-0.7790501 .895763$ H $\quad 5.299565-3.9788290 .287034$ H $3.737209-4.7452920 .167600$ H $4.064350-4.7875902 .337052$ H $0.864299-0.679784-0.716824$ H $\quad 0.685710 \quad 0.431551-3.584776$ H $3.1534790 .381497-0.760872$ H $1.9002011 .391864-0.007736$ H $3.3802682 .109361-0.638898$ H $3.678026-3.831550-1.873473$ H $2.996598-2.439283-1.027430$ H $5.252923-0.804897-4.654827$ H $6.574109-1.139728-3.513982$ H $5.764766 \quad 0.837174-2.055512$ H $5.1066381 .578681-4.954328$ H $6.1874143 .653724-3.902937$ H $5.5395183 .215730-2.340241$ H $3.0836555 .308224-4.988414$ H $4.8211474 .870069-5.458764$ H $2.2197884 .619301-2.875619$ H $3.2801443 .861532-1.734280$ H $1.5660032 .514944-3.936189$ H $3.671764-1.569950-3.123676$ C $-6.117044-1.460637-1.764461$ O $-5.492122-0.348423-1.100913$ C $-6.4915020 .335870-0.318940$ C $-7.837871-0.289370-0.688193$ C -7.540462 -0.973558-2.011828 H $-6.4851611 .381756-0.643816$ C $-6.131744 \quad 0.241774 \quad 1.172296$ H -8.170250 -1.017184 0.060970 H $-8.6265410 .465450-0.772168$ C $-8.532393-2.071145-2.354641$ H -7.544327 -0.215854 -2.806896 C $-4.708460 \quad 0.740996 \quad 1.504277$ O $-6.207488-1.133908 \quad 1.571375$ H -6.8796690 .7779191 .767957$ H -3.9765840 .0829501 .025006$ $\begin{array}{llll}\text { O } & -4.509221 & 0.582532 & 2.915500\end{array}$ C -4.4443282 .2096291 .123148$ C -3.0487702 .7159121 .530770$ H -4.5968902 .3483050 .051500$ O $\quad-5.4200373 .0205001 .786656$ H -3.042716 3.8117321 .478138 H -2.886816 2.5043992 .596067 H -8.293426 $-2.529402-3.319094$ H -8.533397 -2.861802 -1.596800 H -9.547382 -1.665333 -2.418964 C $-5.337482-1.824250-3.031278$ H $-5.968730-1.1755482 .513294$ H $-5.118978 \quad 1.1912203 .366987$ H -5.299155 3.9351141 .479363 H -5.184268 $-0.927378-3.643924$ H -5.934730 -2.526373 -3.624551 H -6.123316 -2.310339-1.067963 C -0.1538293 .4586801 .789617$ O -0.6628482 .1551691 .483325$ C -1.8719212 .1471550 .708245$ C -1.603253 $2.837436-0.637806$ C $-1.0280454 .241983-0.459532$ C $0.163556 \quad 4.2247660 .498239$ H -0.8887514 .0134422 .385704$ C 1.1109833 .2726462 .640870 H -2.0785901 .0914180 .504494$ O $-2.7820692 .892915-1.428640$ H $-0.8789922 .235219-1.197182$ H -1.801435 $4.943089-0.125565$ O $-0.5917794 .712963-1.737212$
H $1.0195323 .767414-0.010164$

O $\quad 0.5383525 .566773 \quad 0.802528$

C 0.8083712 .6887254 .005007

H 1.7975882 .5856962 .133474

H 1.6107114 .2340242 .801200

H $-2.5536553 .338707-2.262335$

H -0.181186 $5.583959-1.600356$

H -0.2065005 .9873801 .265173$

O 1.98770924063614 .634893

O -0.3102772 .5103714 .466299$

C 1.8658291 .8463975 .946131

H 2.8722161 .6735526 .336512

H 1.3469002 .5442946 .609989

H 1.3376420 .8891775 .901176

SCF Energy (B3LYP/6-31G**//MMFF) $=-3245.91661828$

03109

MM̄FF Geometry

C $3.278722-2.190312-2.566153$

C $2.432004-1.417100-1.869082$

C $1.181427-0.815920-2.456523$

O $0.117243-0.903739-1.481625$

C $1.4242910 .670729-2.780492$

C $0.2480181 .398376-3.407920$

C $-0.1104292 .655917-3.080079$

C $-0.5146830 .667694-4.482273$

C $0.5790173 .576507-2.112977$

C $0.0042113 .550775-0.691082$

C -1.416035 4.128717 -0.575299

C -1.8009764 .2935960 .898253$

C -3.2506884 .7670521 .072608$

O $-2.3264083 .234195-1.221128$

O $-3.614068 \quad 4.5778472 .449135$

C $-4.172678 \quad 3.857558 \quad 0.233797$

C $-3.400818 \quad 6.265927 \quad 0.788138$

$\begin{array}{lllll}\text { O } & -5.513456 & 4.342371 & 0.247079\end{array}$

C $-3.6901303 .672730-1.222926$

C $-4.5329902 .625201-1.988092$

O $-3.833903 \quad 4.916825-1.918968$

C $-0.514376-2.103874-1.363855$

O $-0.265938-3.128061-1.980030$

C -1.573270 -1.957949-0.340065

C $-2.250681-3.0430050 .063153$

C $-3.339811-3.0760401 .042552$

C $-3.705172-1.7854381 .726870$

C $-4.3395911 .176996-1.530976$

C $-5.3298590 .231440-2.191967$

C $-5.885295-2.201675-2.305744$

O $-6.2976250 .617273-2.841813$

C $-6.557125-2.707933-1.067094$

C $-6.540995-3.993730-0.684527$

C $-7.183480-4.4930830 .578551$

C $-6.232017-5.289747 \quad 1.446915$

C $-6.399512-6.6116551 .620994$

C $-5.128324-4.5435872 .171191$

C $-3.955692-4.254767 \quad 1.273321$

N -5.021409 -1.096176 -1.932793

H $3.074613-2.404035-3.612000$

H $2.653491-1.186236-0.829602$

H $\quad 0.888607-1.353527-3.367184$

H $2.2719980 .754388-3.473627$

H $1.7203921 .172492-1.851348$

H $-0.9684393 .096188-3.586376$

H $-1.2314371 .316543-4.997422$

H $0.1743360 .280345-5.239913$

H - $-1.079197-0.167674-4.056603$

H $1.6496783 .345926-2.066382$

H $0.5251554 .597514-2.511638$

H $0.0164262 .517647-0.321833$

H $0.6788864 .134261-0.052515$

H -1.439305 5.099493 -1.084146

H -1.110705 4.9783771 .406632

H -1.694470 3.3286821 .410428

H -2.9907465 .0857142 .996393$

H -4.1929632 .8799540 .730857$

H $-2.7812886 .850287 \quad 1.479120$

H $-4.430986 \quad 6.5989230 .956090$

H $-3.1088946 .542227-0.226981$ 
H $-5.7782974 .437410 \quad 1.178088$ H -5.592009 2.910597-1.948926 H -4.268462 $2.665355-3.054438$ H -3.841580 $4.738041-2.873743$ H -1.769473 -0.9647040 .046987$ H -1.995252 -4.005779 -0.378244 H -4.079149 -1.0540951 .003538$ H -2.831564 -1.361126 2.233711 H -4.474915 -1.899603 2.492554 H $-3.3224020 .844421-1.768873$ H $-4.486614 \quad 1.085762-0.450544$ H $-5.254633-2.963262-2.774744$ H $-6.635359-1.867325-3.028703$ H -7.075420 -1.977250 -0.450195 H -6.041295 -4.729042 -1.311017 H $-8.043400-5.1116030 .291363$ H -7.592903 -3.664879 1.170596 H $-5.736079-7.1858272 .259280$ H -7.200276 -7.150885 1.125784 H $-4.759358-5.1449123 .012284$ H $-5.557982-3.6433602 .618240$ H $-3.588106-5.1372420 .746754$ H $-4.204129-1.309897-1.369938$ C $5.747023-1.883422-2.252535$ O $5.596743-0.666367-1.489519$ C $6.680928-0.578728-0.553371$

C $7.817264-1.333923-1.221495$ C $7.062915-2.522922-1.790430$ H $6.373676-1.1257520 .347354$ C $6.9640920 .879400-0.187769$ H $8.271003-0.751293-2.031493$ H $8.603340-1.621440-0.517352$ C $7.809262-3.245760-2.898643$ H $6.869600-3.235980-0.977350$ C 5.7182051 .6663570 .275773 O $7.497719 \quad 1.549933-1.335298$ H 7.7475520 .9139710 .578734 H $5.0846271 .893076-0.590211$ O 6.1673622 .9340060 .771136 C 4.8703640 .9668091 .354526 C 3.7404391 .8677551 .888969 H 4.4463720 .0484790 .934569 O 5.7271380 .5990062 .435855 H 4.1689382 .6517922 .525085 H 3.2410292 .3445191 .036247 H $7.213773-4.077754-3.288214$ H $8.037559-2.574266-3.733042$ H $8.755157-3.651122-2.524783$ C $4.533815-2.778338-1.985572$ H $7.6687922 .472258-1.078388$ H $\quad 6.6792742 .7667861 .581154$ H $5.245853-0.0327402 .995558$ H $4.404740-2.919883-0.905122$ H $4.685373-3.771534-2.424033$ H $5.794699-1.594619-3.309658$ C $1.000092-0.6237722 .369444$ O $1.993296 \quad 0.2321341 .791862$ C 2.6989181 .0783222 .707194 C 1.7208282 .0051383 .444102 C 0.6179161 .1984644 .136176 C $-0.022200 \quad 0.179770 \quad 3.188544$ H $0.471456-1.0474101 .508899$ C $1.633273-1.7819413 .145722$ H $3.222770 \quad 0.4602273 .442296$ O 1.1401832 .9198632 .518646 H 2.2793362 .5914604 .183877 H $-0.151357 \quad 1.8860684 .508477$ O $1.184896 \quad 0.5260715 .257103$ H $-0.663757-0.5087373 .749722$ O $-0.878763 \quad 0.8729982 .277152$ C $2.285474-2.7520802 .189284$ H $2.398070-1.4616233 .858468$ H $0.880210-2.3306173 .723979$ H 0.5529693 .5114803 .019378 H $\quad 0.4683300 .0425635 .702427$ H -0.3165521 .4220751 .704613$ O $1.355758-3.6332871 .720089$ O $3.468983-2.7133131 .879481$
C $1.837492-4.6032420 .785504$

H $2.645831-5.1924191 .228803$

H $1.010728-5.2733660 .535539$

H $2.175697-4.109031-0.128838$

SCF Energy (B3LYP/6-31G**//MMFF)= -3245.91960665

03_11

MM̄FF Geometry

C $2.400112-2.449699-2.411361$

C $1.998413-3.038046-1.273943$

C $0.589756-3.066921-0.724762$

O $-0.239830-2.044568-1.324606$

C $-0.057941-4.437153-0.992104$

C -1.292825 -4.694374 -0.149738

C $-2.546876-4.803386-0.629198$

C $-1.047682-4.8725701 .328107$

C $-3.016090-4.697239-2.051651$

C $-3.453790-3.290584-2.488901$

C $-4.540745-2.654056-1.606053$

C $-5.108571-1.398261-2.272541$

C $-6.117217-0.668378-1.370938$

O $-3.950614-2.301535-0.350627$

O $-6.3343040 .640391-1.921090$

C $-5.494302-0.4709240 .028736$

C $-7.482663-1.366118-1.365291$

O $-\begin{array}{llll}-6.452950 & 0.055478 & 0.942100\end{array}$

C $-4.868914-1.7562350 .606459$

C $-4.083699-1.5198921 .920959$

O $-5.910752-2.6925680 .909163$

C $-0.075481-0.775904-0.858892$

O $0.689106-0.4128250 .020426$

C $-0.9771390 .121224-1.611071$

C $-0.7592311 .443264-1.553848$

C $-1.5610242 .489219-2.188354$

C $-2.8948782 .088057-2.757850$

C $-2.917449-0.530878 \quad 1.825873$

C -3.2729070 .8893902 .232903$

C -2.3338923 .1984402 .242443$

O -4.3206191 .1862492 .800275$

C -2.8924323 .9452731 .071681$

C -2.3312385 .0575750 .573411$

C $-2.8953405 .839228-0.579895$

C $-1.8803506 .069467-1.678605$

C -1.188703 $7.219273-1.750037$

C $-1.7224564 .996903-2.735140$

C $-1.0792963 .749806-2.191061$

N -2.2576051 .7850881 .923177$

H $1.671698-1.978087-3.065345$

H $2.745323-3.519495-0.645668$

H $0.651484-2.8816250 .354407$

H $\quad 0.658431-5.242451-0.780467$

H -0.293222 -4.514238 -2.059157

H $-3.348330-4.9962870 .083784$

H $-1.941172-5.2194271 .858660$

H $-0.261457-5.6155951 .497142$

H $-0.742368-3.9282181 .787393$

H $-2.250030-5.063507-2.743361$

H -3.862854 -5.384941 -2.175029

H -2.571582 -2.641413 -2.509078

H $-3.820833-3.364738-3.520118$

H $-5.334798-3.392543-1.444839$

H -5.567157 -1.644367 -3.238449

H -4.286654 -0.707660 -2.502080

H $-6.6527810 .532846-2.833755$

H $-4.7105140 .289514-0.077376$

H -7.900272 -1.397597 -2.378880

H -8.208195 $-0.810845-0.760812$

H -7.438520 $-2.392823-0.996567$

H -6.8053420 .8740830 .552670$

H -4.774901 -1.253922 2.729817

H -3.651390 -2.487089 2.216254

H -5.570108 -3.335187 1.553224

H - $1.785750-0.321899-2.178166$

H $\quad 0.093699 \quad 1.804367-0.980913$

H -3.507851 $1.610390-1.985983$

H $-2.7648241 .386585-3.589070$

H -3.480179 $2.925927-3.140382$

H $-2.130303-0.8548942 .518161$ 
H $-2.488924-0.529022 \quad 0.820817$ H -1.3221173 .5275442 .500067$ H -2.981739 3.3396953 .113483 H $-3.811327 \quad 3.5683760 .628448$ H -1.427741 5.4477011 .037116 H -3.245295 $6.803709-0.189135$ H -3.783100 5.350541-1.000508 H $-0.4767997 .403365-2.547728$ H -1.315836 $8.001000-1.008295$ H -1.086757 $5.360788-3.552978$ H -2.699835 4.813130-3.188096 H - $0.0941363 .926026-1.755675$ H -1.4457711 .4589001 .407061$ C $4.249372-0.917446-3.120201$ O $4.019474-0.095555-1.961970$

C $5.0622530 .894238-1.872741$

C $5.9298030 .722095-3.117389$

C $5.732624-0.747830-3.438238$

H $5.6583870 .637294-0.989295$

C $4.4517312 .291347-1.695054$

H $5.5803641 .341824-3.950950$

H $6.9757220 .983178-2.928710$

C $6.109929-1.115879-4.862111$

H $6.337175-1.340939-2.738951$

C $3.3966892 .381119-0.574214$

O $3.7991452 .662265-2.917487$

H $5.2509273 .024905-1.536775$

H $2.5109581 .808050-0.869869$

O $2.965892 \quad 3.745641-0.486459$

C 3.8714071 .9098640 .811818

$\begin{array}{lll}\text { C } 2.756252 & 2.043842 & 1.862787\end{array}$

H 4.1862200 .8670490 .745737

O $5.0040282 .682928 \quad 1.198965$

H 2.4938413 .1028551 .977942

H 1.8651571 .5336081 .483051

H $5.947105-2.182381-5.045888$

H $5.518002-0.553780-5.592268$

H $7.167011-0.899309-5.047805$

C $3.842140-2.364076-2.830693$

H $3.4156373 .546230-2.784545$

H $3.7205984 .269848-0.167443$

H 5.3957542 .2604661 .981818

H $4.484505-2.775293-2.042367$

H $3.986112-2.978515-3.726280$

H $3.630411-0.507686-3.929810$

C $2.752790-0.8641732 .847930$

O 3.6747220 .1690793 .213090

C 3.1239451 .4896043 .258375

C 1.9611321 .5396124 .261469

C $0.903173 \quad 0.485034 \quad 3.942821$

C $1.551452-0.8907103 .802430$

H $2.414153-0.6995511 .820769$

C $3.504887-2.2052402 .901501$

H 3.9169792 .1364213 .653791

O $1.378425 \quad 2.838490 \quad 4.263820$

H 2.3530461 .3576525 .270657

H $0.334036 \quad 0.7408813 .042407$

O $-0.043408 \quad 0.4202175 .011382$

H $1.865957-1.2462834 .792177$

O $0.582560-1.8131103 .306548$

C $4.487397-2.3841971 .763588$

H $4.068198-2.2730243 .839913$

H $2.798662-3.0409752 .839930$

H 0.7276292 .8690534 .984879

H $-0.544876 \quad 1.2529865 .009240$

H $-0.172705-1.7982313 .919168$

O $5.172145-3.5518851 .952709$

O $4.631888-1.6190190 .821700$

C $6.130972-3.8720180 .939546$

H $5.628024-4.025450-0.019796$

H $6.881546-3.0795870 .860905$

H $6.630993-4.8010471 .226234$

SCF Energy (B3LYP/6-31G**//MMFF) $=-3245.91923629$

03_110

MM̄FF Geometry

C $1.722616-3.974219-0.537471$

C $1.275957-3.8769440 .724911$
C $-0.098140-3.4126361 .149530$

O $-0.684222-2.5363540 .160829$

C $-1.028674-4.6228841 .341891$

C $-2.349676-4.2741541 .999543$

C $-3.554097-4.366138 \quad 1.402780$

C $-2.264847-3.826518 \quad 3.437072$

C $-3.869763-4.8114380 .003149$

C $-3.947335-3.679772-1.034004$

C $-4.989403-2.595182-0.713711$

C $-5.187027-1.662849-1.911390$

C $-6.149707-0.507780-1.590978$

O $-4.521652-1.836258 \quad 0.405154$

O $-6.0184730 .476176-2.629037$

C $-5.7107950 .167136-0.272550$

C $-7.613157-0.965173-1.611455$

$\begin{array}{lllll}\text { O } & -6.670845 & 1.132473 & 0.148567\end{array}$

C $-5.437756-0.8346820 .868015$

C $-4.809132-0.180292 \quad 2.123283$

O $-6.676073-1.4247301 .282972$

C $-0.223222-1.255707 \quad 0.137156$

$\begin{array}{llllll}\text { O } & 0.618248 & -0.766986 & 0.874169\end{array}$

C $-0.901410-0.543909-0.966290$

C $-0.3874660 .618149-1.393714$

C $-0.9188711 .466860-2.459918$

C $-2.2925231 .144497-2.983261$

C $-3.4697080 .530244 \quad 1.898435$

C -3.596069 2.0256831 .659307

C -2.2303254 .0167651 .046591$

O -4.6440992 .6473341 .812319$

C -2.397220 $4.295219-0.413965$

C $-1.5139775 .003112-1.134332$

C $-1.692780 \quad 5.334833-2.588807$

C $-0.5080224 .919791-3.432278$

C $0.4526795 .801401-3.756848$

C $-0.480007 \quad 3.503873-3.963646$

C $-0.1649062 .499102-2.892230$

N -2.384461 2.5949001 .289864

H $1.057726-3.747461-1.366448$

H $1.948372-4.139321 \quad 1.539413$

H $\quad 0.005817-2.8625702 .092944$

H $-0.534686-5.3814481 .964015$

H $-1.196003-5.0942540 .367359$

H $-4.432824-4.0918601 .986112$

H $-3.252061-3.7394823 .903928$

H $-1.688814-4.5470314 .026864$

H $-1.782145-2.8473923 .510328$

H $-3.147444-5.559656-0.339880$

H $-4.832847-5.3375440 .028152$

H -2.956395 -3.221590 -1.129416

H $-4.185941-4.134917-2.003301$

H $-5.933674-3.088491-0.454689$

H -5.539396 - $2.222689-2.786755$

H $-4.218024-1.235538-2.202046$

H $-6.2178570 .045016-3.477625$

H $-4.7878880 .717717-0.490299$

H $-7.875628-1.363967-2.598786$

H $-8.292955-0.123568-1.439127$

H $-7.830350-1.741744-0.875241$

H $-6.794898 \quad 1.757379-0.586317$

H -5.5391310 .4769492 .610796$

H $-4.618915-0.9872442 .845921$

H $-6.562983-1.7798192 .180262$

H $-1.787424-0.993029-1.397499$

H $0.5231850 .983565-0.922989$

H $-3.0116741 .091857-2.158935$

H $-2.2888130 .183445-3.508663$

H $-2.6829301 .890355-3.678149$

H -2.8611650 .4182472 .805120$

H -2.9158790 .0725821 .074345$

H -1.2409564 .3047501 .413784$

H -2.9860854 .5685391 .614886$

H $-3.2996953 .923456-0.893535$

H -0.626091 $5.397499-0.645021$

H $-1.8422666 .419831-2.666506$

H -2.606069 $4.882023-2.994657$

H $1.2926475 .518290-4.382571$

H $0.4232966 .825153-3.398668$

H $0.2925933 .406595-4.738030$ 
H -1.423002 $3.301362-4.478055$ H $\quad 0.8191372 .643287-2.443974$ H -1.5757451 .9976691 .143066$ C $3.741945-3.546188-1.990986$ O $3.722282-2.146209-1.651021$ C $4.964743-1.548520-2.064459$ C $5.708188-2.607985-2.874172$ C $5.210651-3.889458-2.241192$ H $5.528846-1.346317-1.147028$ C $4.732129-0.236334-2.823399$ H $\quad 5.434870-2.581539-3.935112$ H $6.794285-2.489468-2.808769$ C $5.423870-5.118902-3.107077$ H $5.733123-4.028644-1.285089$ C $3.8401540 .797730-2.110927$ O $4.104190-0.536795-4.078131$ H $\quad 5.704570 \quad 0.207526-3.069437$ H $2.7923780 .481080-2.172339$ O $3.9185182 .013974-2.869204$ C $4.1892391 .091013-0.641014$ C $3.4384512 .328121-0.109011$ H $3.9529560 .205507-0.041786$ O $5.593001 \quad 1.326419-0.535081$ H $3.9338303 .238414-0.469002$ H $2.4168532 .313359-0.504283$ H $5.049166-6.018105-2.608337$ H $4.907648-5.026886-4.068592$ H $6.489628-5.265806-3.310513$ C $3.124673-4.394571-0.874877$ H $3.9830160 .304708-4.550715$ H $4.8237942 .357918-2.777973$ H $5.813673 \quad 1.3816680 .409718$ H $3.752870-4.3308570 .022062$ H $3.105673-5.445158-1.186873$ H $3.154990-3.654648-2.912573$ C 2.3384061 .1415723 .250672 O $2.5215121 .296503 \quad 1.839105$ C 3.3694732 .3734811 .428548 C 2.8108093 .7136761 .932062 C 2.5834563 .6867803 .446657

C 1.8106642 .4386113 .885759 H 1.5562410 .3777913 .347434 C 3.6015890 .5965613 .924505 H 4.3742212 .2210631 .834970 O $1.5818493 .997876 \quad 1.266528$ H 3.5184934 .5098611 .671250 H 2.0254834 .5840253 .741288 O $3.850373 \quad 3.7307624 .096416$ H 1.8245582 .3498854 .978374 O 0.4440632 .6123333 .498485 C $3.912127-0.788343 \quad 3.406115$ H $4.476137 \quad 1.2352823 .771597$ H 3.4637280 .5140415 .008967 H 1.2704014 .8633901 .581572 H 3.6881803 .7419775 .055027 H $-0.055150 \quad 1.844073 \quad 3.824251$ O $4.970447-0.7390582 .547825$ O $3.271574-1.7876963 .706350$ C $5.336149-1.9868291 .951692$ H $6.212033-1.8183571 .319781$ H $5.595435-2.7178972 .723290$ H $4.517912-2.3560801 .327460$ SCF Energy (B3LYP/6-31G**//MMFF) $=-3245.90031307$

\section{111}

MM̄FF Geometry

C $2.6643243 .719833-1.399186$

C $1.4016374 .087692-1.136794$

C $0.2037943 .324645-1.642550$

O $-0.4861282 .760155-0.500202$

C $-0.7631794 .275408-2.368548$

C -1.934645 $3.566939-3.020861$

C $-3.2246693 .736050-2.670923$

C $-1.5877192 .637968-4.156826$

C $-3.7898004 .626182-1.600223$

C $-3.9639453 .957454-0.227214$

C $-4.8839122 .725339-0.235865$

C $-5.2176572 .286799 \quad 1.192082$
C $-6.0569280 .998826 \quad 1.217734$

O $-4.2047061 .663164-0.910671$

O -6.0428920 .4909122 .561050$

C $-5.378377-0.0646840 .326670$

C -7.5261601 .2693010 .872466$

O $-6.205543-1.2166960 .187782$

C $-4.9779060 .465537-1.065334$

C $-4.114738-0.533294-1.874927$

O $\quad-6.1612420 .714566-1.833664$

C 0.0855171 .6775450 .096869

O $1.1019661 .096811-0.249091$

C $-0.727231 \quad 1.326801 \quad 1.280341$

C $-0.2083380 .505787 \quad 2.205420$

C -0.8722820 .0498923 .427374$

C $-2.3392770 .355863 \quad 3.575843$

C $-2.796756-0.945471-1.210635$

C $-2.875637-2.257250-0.447019$

C $-1.527463-3.7038701 .069483$

O $-3.836843-3.018672-0.510723$

C $-1.916721-3.4441012 .490403$

C $-1.123192-3.7315253 .533407$

C $-1.509864-3.5115774 .968228$

C $-0.487374-2.6953495 .727536$

C $0.441153-3.291576 \quad 6.494619$

C $-0.594250-1.1881705 .661639$

C $-0.147053-0.6350174 .336419$

N -1.722494 -2.497208 0.287868

H $2.8506152 .859895-2.038123$

H $1.2165924 .922111-0.464527$

H $0.5102462 .513254-2.313403$

H $-0.2251634 .828092-3.150292$

H -1.117863 $5.026935-1.654891$

H $-3.9789693 .172234-3.219184$

H -2.476702 $2.285888-4.691494$

H $-0.9515153 .149790-4.886270$

H $-1.0580831 .754665-3.787320$

H -3.179484 $5.527937-1.482690$

H $-4.7665784 .988543-1.946292$

H -2.9759773 .6771730 .156498$

H -4.3742214 .7098540 .457766$

H $-5.8003962 .980873-0.780708$

H -5.7318823 .0890991 .735695$

H -4.2850742 .1036281 .742735$

H -6.4003191 .1806953 .146030$

H $-4.475456-0.3905400 .856428$

H -7.9634821 .9764381 .587476$

H -8.1255230 .3556120 .951689$

H $-7.6622261 .686210-0.127493$

H $-6.414586-1.5331411 .083391$

H -4.715165 -1.404440 -2.163237

H $-3.850024-0.038861-2.820867$

H $\quad-5.9224260 .716386-2.775455$

H $-1.718225 \quad 1.756557 \quad 1.361355$

H 0.8073090 .1377112 .064033

H -2.892541 0.0122292 .695221

H -2.4976051 .4333783 .692355$

H -2.804439 -0.134891 4.432928

H -2.043052 -1.089861-1.994390

H $-2.427908-0.158268-0.547802$

H $-0.474027-3.985148 \quad 0.975311$

H $-2.142675-4.5104780 .658329$

H -2.903121 -3.023180 2.670672

H $-0.148568-4.1783803 .349729$

H -1.621943 -4.497688 5.437705

H -2.493218 -3.032093 5.050319

H $1.161199-2.7132837 .063933$

H $\quad 0.504737-4.3724576 .566006$

H $\quad 0.040277-0.7311106 .432687$

H $-1.613002-0.8932575 .927110$

H $0.910306-0.8090034 .129887$

H $-0.991897-1.7919380 .313779$

C 4.1637053 .7006620 .583787

O $4.4163472 .295440 \quad 0.403419$

C 5.3539741 .8635081 .407499

C 5.6861733 .0927522 .252664

C 5.4078704 .2302991 .286346

H 6.2585891 .5524640 .873400

C 4.7719800 .6800832 .192610 
H 5.0378953 .1704723 .133079

H 6.7210213 .0777982 .608748

C $5.216506 \quad 5.5728761 .968841$

H $6.241251 \quad 4.298474 \quad 0.574552$

C $4.305981-0.4968331 .308849$

$\begin{array}{lllll}\text { O } & 3.629756 & 1.139806 & 2.928659\end{array}$

H 5.4998810 .3390122 .938065

H $3.416262-0.1908310 .748681$

O $3.872558-1.5487832 .181061$

C $5.366985-1.0509020 .341163$

C $4.926432-2.307234-0.422483$

H $5.662191-0.283468-0.381517$

O $6.543111-1.4149651 .070705$

H $5.764718-2.653242-1.044315$

H $4.783793-3.1383120 .282956$

H $5.016206 \quad 6.3594101 .234705$

H 4.3789745 .5490642 .674134

H 6.1169775 .8529652 .525372

C $3.8660224 .358997-0.764053$

H 3.2688230 .3761173 .410798

H $4.656103-1.8789812 .653472$

H $6.957280-0.5990621 .397170$

H $4.7147634 .236909-1.447461$

H $3.7027855 .435364-0.638415$

H 3.2890973 .7860721 .243763

C $2.323839-3.586156-2.735761$

O $3.505415-3.446442-1.936570$

C $3.664131-2.174069-1.290285$

C $3.743609-1.055736-2.341073$

C $2.552300-1.093571-3.307213$

C $2.274841-2.506088-3.830878$

H $2.441451-4.559406-3.229398$

C $1.082436-3.666598-1.843172$

H $2.803581-2.008802-0.632820$

O $4.955608-1.168878-3.085971$

H $3.769321-0.084415-1.835113$

H $2.755896-0.431433-4.158078$

O $1.383130-0.609568-2.655409$

H $1.309340-2.532078-4.347843$

O $3.277915-2.809671-4.806298$

C $-0.065260-4.347557-2.545207$

H $1.301711-4.252866-0.941544$

H $0.761482-2.690529-1.468980$

H $4.966678-2.047365-3.503211$

H $1.5729570 .289879-2.338661$

H $3.058703-3.674312-5.193470$

O $-0.928204-3.417272-3.040949$

O $-0.170179-5.562214-2.655960$

C -2.068241 -3.938372 -3.730640

H -2.655587 -4.578099 -3.065022

H $-2.690001-3.095551-4.044286$

H -1.750966 -4.491761-4.619545

SCF Energy (B3LYP/6-31G*//MMFF) $=-3245.91321480$

03_112

MM̄FF Geometry

C -1.253763 2.184495 3.457677

C -2.3752971 .5303093 .798226$

C -3.6764971 .4999443 .029139$

O $-3.6229082 .473161 \quad 1.960200$

C -3.935611 0.1185862 .388679

C $-3.816028-1.065873 \quad 3.322449$

C $-2.782008-1.9314403 .329360$

C $-4.968758-1.2485494 .273593$

C -1.552181-1.899929 2.462783

C $-1.398962-3.1456581 .578051$

C -2.612367 $-3.445847 \quad 0.684022$

C $-2.324175-4.632024-0.239212$

C $-3.497454-4.913646-1.190978$

O $-2.914184-2.282687-0.092239$

O $-3.029019-5.802151-2.217525$

C -3.901802 -3.595236 -1.885188

C $-4.648576-5.639144-0.484603$

O $-5.079959-3.767610-2.668404$

C $-4.087405-2.413794-0.907548$

C $-4.335550-1.067267-1.631902$

O $-5.243791-2.654542-0.093721$

C $-4.783520 \quad 2.938242 \quad 1.435916$ $\begin{array}{llll}\text { O } & -5.914554 & 2.688305 & 1.827748\end{array}$

C $-4.526717 \quad 3.765860 \quad 0.231686$

C $-3.3017814 .194703-0.110552$

C $-2.9369864 .926175-1.324153$

C $-4.0154625 .202680-2.336505$

C $-3.306490-0.644509-2.681221$

C $-1.907808-0.451295-2.135096$

C $-0.5522751 .072452-0.703212$

O $-0.988149-1.206486-2.441066$

C $0.0713542 .209548-1.458128$

C $-0.2121452 .625733-2.703361$

C $0.4373343 .819140-3.352044$

C $-0.5711174 .879592-3.748312$

C $-1.031275 \quad 4.951766-5.008592$

C -1.002967 $5.892306-2.706407$

C $-1.6409255 .255567-1.503227$

N $-1.8047080 .642666-1.292637$

H -1.2243122 .7914732 .557377$

H -2.3605380 .9352974 .709823$

H -4.4845941 .7682043 .720822$

H -3.2837620 .0064971 .515256$

H -4.9421790 .1113351 .948576$

H -2.804137 -2.752209 4.045046

H $-4.849303-2.1338994 .906942$

H $-5.062113-0.3805854 .933951$

H $-5.904349-1.3651063 .716917$

H $-1.506590-1.0054801 .835562$

H $-0.678096-1.8363873 .123090$

H $-0.512694-2.9903880 .949619$

H $-1.191145-4.0185652 .209820$

H $-3.465991-3.6794311 .330197$

H $-2.078538-5.528847 \quad 0.343213$

H $-1.432963-4.413765-0.844657$

H $-2.698979-6.609539-1.787235$

H $-3.093952-3.353820-2.586820$

H $-4.305695-6.600608-0.083642$

H $-5.454441-5.878717-1.187117$

H $-5.071741-5.0697380 .345167$

H $-4.919252-4.511782-3.273498$

H $-5.322854-1.095778-2.111507$

H $-4.404643-0.275900-0.873175$

H $-5.537230-1.8070890 .280335$

H $-5.401557 \quad 3.982122-0.372380$

H -2.463361 $3.962780 \quad 0.544070$

H $-4.7920505 .843151-1.904270$

H $-4.4773934 .268017-2.673526$

H -3.647379 $5.711174-3.230900$

H $-3.265826-1.361501-3.507837$

$\begin{array}{llll}H & -3.622296 & 0.306250 & -3.130097\end{array}$

H $\quad 0.147247 \quad 0.233300-0.640082$

H -0.7875851 .4022950 .313422$

H $0.8215892 .761975-0.898182$

H $-0.9495102 .096448-3.300731$

H $0.979276 \quad 3.464252-4.238214$

H $1.1984394 .267649-2.701180$

H $-1.7376355 .718009-5.310504$

H $-0.7142644 .243005-5.766619$

H $-1.6687546 .650304-3.135150$

H $-0.1182746 .450498-2.373384$

H $-0.9266405 .009224-0.716628$

H $-2.6136151 .243070-1.162119$

C 1.1169511 .4287303 .389464

O 1.6418222 .3926092 .453072

C 3.0667152 .1795312 .316450

C 3.3766590 .9093863 .095064

C 2.3260030 .9476064 .185605

H 3.5467963 .0449232 .789996

C 3.4055052 .1287100 .822219

H 3.2372220 .0213022 .465833

H 4.3989660 .8955713 .484054

C $2.126940-0.3932774 .871444$

H 2.6124301 .7014044 .930972

C 4.8157451 .6074750 .506864

$\begin{array}{lllll}\text { O } & 3.276267 & 3.452333 & 0.291774\end{array}$

H 2.6554601 .5172200 .311118

H 4.9286850 .5838870 .877595

$\begin{array}{llll}\text { O } & 5.762297 & 2.398609 & 1.235800\end{array}$

C $5.1965681 .657789-0.984665$ 
C $4.1987701 .007010-1.962196$ H $5.3427722 .701890-1.285977$

O $6.4793591 .040613-1.142854$ H $3.3055751 .638315-2.035082$ H $4.6755841 .000790-2.951021$ H $1.350661-0.3295985 .640098$ H $1.830728-1.1692944 .157484$ H $3.054068-0.7186715 .354516$ C $0.0274092 .082071 \quad 4.240706$ H 2.3960393 .7822490 .540993 H 6.6486282 .0605111 .020734 H $6.3922090 .104502-0.893754$ H 0.3291053 .0926004 .540179 H $-0.138187 \quad 1.5011495 .154973$ H 0.7022490 .6009572 .797893 C $4.706512-2.635279-1.231425$

O $4.927476-1.269965-1.613565$ C $3.769235-0.432995-1.613959$ C $2.726564-0.973437-2.606107$ C $2.384187-2.436657-2.309158$ C $3.644408-3.292312-2.130463$

H $5.663134-3.138139-1.424227$

C $4.400994-2.7705530 .269227$

H $3.330574-0.430023-0.613178$

O $3.205704-0.859516-3.944573$

H $1.820955-0.360974-2.537819$

H $1.780229-2.834421-3.133772$

O $1.592958-2.488007-1.125481$

H $3.388416-4.279692-1.729235$

O $4.244650-3.504548-3.411923$

C $5.551013-2.3002861 .135262$

H $3.520420-2.1888220 .553914$

H $4.218244-3.8232590 .513415$

H $4.052075-1.335379-3.999042$

H $1.339933-3.415874-0.981947$

H $3.591948-3.961747-3.969159$

O $5.140791-2.2759012 .438402$

O $6.664923-1.9983660 .730267$

C $6.118456-1.8289163 .382779$

H $6.466387-0.8238413 .125366$

H $5.646981-1.7936684 .368565$

H $6.956365-2.5314533 .417235$

SCF Energy (B3LYP/6-31G*//MMFF)= -3245.91301242

03_113

MM̄FF Geometry

C -2.179471 -2.974059-1.935124

C $-1.450427-4.088943-1.774017$

C $-0.158497-4.184850-0.991843$

O $0.445544-2.883857-0.805772$

C $-0.453011-4.8029710 .388242$

C $0.737551-4.8569941 .325690$

C $0.745578-4.3118432 .558840$

C $1.950151-5.5910550 .817567$

C $-0.388567-3.596454 \quad 3.241744$

C $-0.502596-2.1072552 .887472$

C $0.577261-1.2269703 .536132$

C $0.3362840 .245264 \quad 3.189352$

C 1.4322331 .1564723 .761702

O $1.858775-1.6361833 .055929$

O 1.3040172 .4435873 .138931

C $2.809143 \quad 0.5901513 .354147$

C $1.252744 \quad 1.386660 \quad 5.266656$

$\begin{array}{lllll}\text { O } & 3.863238 & 1.324528 & 3.974438\end{array}$

C $2.963467-0.9264053 .627327$

C $4.266601-1.4891303 .008680$

O $3.038033-1.1297265 .042857$

C $1.173340-2.399074-1.851010$

O $1.356599-2.944162-2.928673$

C $1.711212-1.074385-1.466600$

C $2.343608-0.342436-2.396666$

C $2.9832650 .961691-2.213942$

C $2.9740461 .575422-0.840564$

C $4.223122-1.6397261 .485423$

C $5.599247-1.6103150 .844366$

C $6.661193-1.345535-1.399219$

O $6.645362-1.6219501 .486444$

C $6.7676060 .122158-1.678432$
C $6.7338350 .654688-2.909460$

C $6.8370442 .129359-3.179277$

C $5.6886932 .659816-4.010839$

C $5.8761973 .035897-5.287242$

C $4.3404702 .831321-3.339398$

C $3.5634701 .544216-3.284702$

N $5.511553-1.564733-0.541414$

H $-1.847428-2.043463-1.482494$

H $-1.808059-5.018441-2.211807$

H $0.538527-4.831611-1.539898$

H $-0.832735-5.8249740 .256472$

H $-1.270470-4.2364150 .847715$

H $1.651220-4.400218 \quad 3.157289$

H $2.717304-5.7087921 .590416$

H $1.671511-6.5939270 .477840$

H $2.407017-5.051862-0.017919$

H $-1.335931-4.0950533 .005115$

H $-0.271232-3.7114324 .326677$

H $-0.470292-1.9900361 .797131$

H $-1.487647-1.7546643 .218261$

H $0.534535-1.3764634 .621452$

H $-0.6525450 .571303 \quad 3.535738$

H 0.3239390 .3648072 .096440

H $0.4086132 .777297 \quad 3.320149$

H $2.914047 \quad 0.774586 \quad 2.279086$

H $0.271097 \quad 1.8308085 .471232$

H 1.9883632 .1038825 .647177

H 1.3275300 .4687635 .853458

H 3.9197431 .0353564 .901020

H $5.114092-0.8721243 .331560$

H $4.467675-2.4856323 .425975$

H $3.441057-1.9978815 .209264$

H $1.585310-0.752031-0.440170$

H $2.416586-0.747172-3.405995$

H $1.9867421 .496055-0.374176$

H $3.2097822 .642265-0.847543$

H $3.7071271 .080061-0.198461$

H $3.739984-2.5876621 .220267$

H $3.636569-0.8345651 .038547$

H $6.505815-1.931081-2.310648$

H $7.569876-1.698296-0.901878$

H $6.8810550 .779723-0.819463$

H $6.639971-0.001832-3.771587$

H $7.7902452 .305297-3.694657$

H $6.8938272 .702126-2.245049$

H $5.0640823 .441584-5.881474$

H $6.844105 \quad 2.941847-5.768421$

H $3.7349163 .558288-3.896164$

H $4.5021613 .273983-2.352550$

H $3.4916331 .038213-4.248818$

H $4.593770-1.514482-0.972672$

C $-4.661026-2.748284-1.755094$

O $-4.563383-1.497745-1.046001$

C $-5.881800-0.936160-0.909771$

C $-6.855325-1.984597-1.440532$

C $-6.007376-2.699932-2.473160$

H $-5.918637-0.058918-1.565914$

C $-6.133806-0.5192210 .543441$

H $-7.169778-2.682943-0.656258$

H -7.757812 -1.532712 -1.863648

C $-6.553165-4.061199-2.867518$

H $-5.934317-2.066396-3.367217$

C $-5.1024750 .470012 \quad 1.124558$

O $-6.120462-1.6863091 .377418$

H -7.142660 -0.0998190 .633941$

H $-4.137654-0.029281 \quad 1.276271$

$\begin{array}{llll}\text { O } & -5.563110 & 0.851448 & 2.428222\end{array}$

$\begin{array}{llll}\text { C } & -4.887616 & 1.744460 & 0.286435\end{array}$

C $-4.041692 \quad 2.796414 \quad 1.030972$

H $-4.4049361 .470920-0.657030$

O $-6.1634862 .305779-0.024285$

H $-4.6221313 .178157 \quad 1.879438$

H -3.1488872 .3093961 .436853$

H $-5.908460-4.541296-3.610197$

H $-6.627092-4.729761-2.003213$

H $-7.553044-3.962979-3.302973$

C $-3.470662-2.911868-2.699559$

H $-5.254394-2.1153241 .266909$ 
H $-5.686800 \quad 0.0350262 .942136$ H $-6.0390582 .943622-0.746693$ H -3.411298 -2.057948 -3.384999 H -3.595540 -3.812054 -3.311986 H -4.657075 -3.545257-0.999105 C $-1.5168463 .165196-0.690460$ O $-2.8563683 .584265-0.988788$ C $-3.638242 \quad 3.9925910 .139167$ C -2.9370785 .1341320 .887871$ C $-1.503173 \quad 4.7742151 .256702$ C -0.7484194 .2883420 .021387$ H -1.539912 2.270871 -0.056282 C $-0.8505982 .796074-2.025923$ H $-4.5640904 .415161-0.268672$ O -3.6719145 .4837152 .055843$ H -2.9222116 .0226890 .243007$ H -1.476366 4.0325332 .063723 O $-0.8630205 .946745 \quad 1.765472$ H $-0.5883415 .138534-0.654581$ O 0.5296093 .8259740 .448406 C $-1.3609381 .487720-2.593176$ H -1.047417 $3.579269-2.767517$ H $0.2297012 .680114-1.899810$ H -3.181348 6.1918032 .507189 H 0.0602865 .7098691 .959730 H $1.1146123 .816649-0.327157$ O $\quad-0.746151 \quad 1.261787-3.792814$ O $-2.179811 \quad 0.753651-2.059005$ C -1.117098 $0.050732-4.458131$ H $-2.1928340 .039826-4.657453$ H $-0.5831250 .009290-5.411148$ H -0.826247 -0.814455 -3.856151

SCF Energy $\left(B 3 L Y P / 6-31 G^{* *} / / M M F F\right)=-3245.91872140$

03_114

MM̄FF Geometry

C $3.907547-3.321839-0.452993$

C $3.062778-2.301302-0.237035$

C $1.770109-2.117225-0.985465$

O $0.768491-1.772085-0.001313$

C $1.913047-0.958022-1.993611$

C $1.104534-1.155022-3.261273$

C $0.006976-0.454778-3.608031$

C $1.656583-2.190576-4.211522$

C $-0.6972950 .627944-2.838860$

C $-2.1802080 .293161-2.662753$

C $-3.0262591 .435757-2.080873$

C $-2.5038681 .938678-0.735896$

C -3.444323 $2.959639-0.082245$

O $-4.3476050 .904336-1.926953$

O -3.0126593 .0992901 .280412$

C $-4.8771982 .390425-0.078898$

C $-3.3069424 .342727-0.728994$

$\begin{array}{llll}\text { O } & -5.814830 & 3.363523 & 0.376519\end{array}$

C $-5.3235761 .839585-1.453679$

C $-6.6578661 .075477-1.296557$

O $-5.5016262 .924293-2.363957$

C $-0.505851-2.197233-0.204039$

O $-0.925013-2.860632-1.138172$

C -1.317897 -1.698735 0.929866

C $-2.653043-1.8311970 .913553$

C $-3.577180-1.3788361 .958523$

C $-2.977916-0.7296073 .179479$

C $-7.1463420 .380838-2.575865$

C $-8.229071-0.623457-2.237314$

C $-8.534869-2.832353-1.121827$

O $-9.422183-0.358991-2.361847$

C $-8.635476-2.5118100 .336371$

C -8.014797 -3.2052131 .302309$

C $-8.066888-2.8200472 .752943$

C $-6.710623-2.4302453 .299456$

C $-6.151744-3.1080814 .316177$

C $-6.031156-1.2099502 .712835$

C $-4.901567-1.5755911 .782832$

N -7.710980 -1.810976 -1.741069

H $3.657041-4.084590-1.185519$

H $3.337740-1.5267980 .475143$

H $1.499068-3.060006-1.474928$
H $\quad 2.961227-0.850197-2.306904$

H $1.660554-0.010441-1.506412$

H $-0.447620-0.675839-4.573731$

H $1.088187-2.243933-5.146274$

H $2.692862-1.951321-4.472531$

H $1.633607-3.185994-3.758497$

H $-0.2419990 .781514-1.858589$

H $-0.5956201 .566482-3.396248$

H $-2.619150 \quad 0.007613-3.628980$

H -2.288595 -0.597338 -2.031522

H -3.052832 2.251508 -2.812950

H -1.504049 2.375005 -0.839620

H $-2.3930011 .086214-0.053928$

H -3.5591513 .7839031 .701643$

H $-4.902061 \quad 1.5681840 .649719$

H $-2.2842094 .719798-0.607324$

H $-3.9569075 .076146-0.239338$

H $-3.5350284 .339907-1.797007$

H -5.5724863 .6039441 .286388$

H $-6.5360600 .319676-0.510011$

H $-7.447607 \quad 1.759841-0.960878$

H -6.079546 $2.636770-3.087867$

H $-0.789326-1.2235501 .748585$

H -3.117436 -2.310686 0.052864

H -2.4636640 .1984962 .905715$

H $-2.258633-1.4024833 .659966$

H -3.711461 -0.4715263 .945286$

H $-7.559471 \quad 1.105150-3.286216$

H $-6.332974-0.144024-3.089197$

H -8.054040 -3.798607 -1.302518

H -9.529932 -2.840921 -1.577096

H -9.228694 -1.640336 0.604578

H -7.417332 -4.074852 1.039660

H -8.482680 -3.6682653 .311253$

H -8.760079 -1.984710 2.914141

H $-5.191201-2.8152724 .727347$

H $-6.640459-3.9693284 .759369$

H $-5.710408-0.5515553 .525396$

H $-6.757800-0.6110262 .149110$

H -5.232733 -2.052156 0.859456

H $-6.706364-1.884827-1.607067$

C $6.368757-2.993232-0.721264$

O $6.157057-1.610286-1.072616$

C $7.306228-0.848717-0.675395$

C $8.448732-1.850165-0.683013$

C $7.759488-3.064188-0.080760$

H $7.132398-0.5214230 .356474$

C $7.4613960 .383772-1.569445$

H $8.785918-2.074207-1.701613$

H $\quad 9.309037-1.511098-0.098798$

C $8.485275-4.372169-0.343766$

H $7.681594-2.9186531 .005165$

C $6.160370 \quad 1.201531-1.736148$

O $7.874133-0.041035-2.873652$

H $8.2691191 .016377-1.182526$

H $5.4701390 .654195-2.390588$

O $6.5119212 .403363-2.431074$

C $5.4554711 .554393-0.412846$

C $4.1843102 .402463-0.606369$

H 5.1798660 .6290150 .101715

O $6.3605992 .253657 \quad 0.440610$

H $4.4544563 .407370-0.951415$

H $3.5640411 .925738-1.374830$

H $7.936345-5.2141310 .089988$

H $8.598130-4.559649-1.416882$

H $9.485479-4.3535580 .101265$

C $5.246477-3.4374420 .222000$

H $7.9689840 .757031-3.421415$

H $5.6889862 .814626-2.744800$

H $6.6447283 .057118-0.028083$

H $5.253683-2.8188331 .127915$

H $5.398528-4.4753560 .538155$

H $6.334193-3.566162-1.656360$

C 2.2004191 .1400482 .297301

O 2.9152601 .2298391 .058812

C 3.3766792 .5363610 .699648

C 2.1825343 .4898370 .550135

C 1.3272953 .5092491 .821528 
C 0.9960982 .0950112 .313321

H 1.8130050 .1130252 .320087

C 3.1299061 .3152893 .502571

H 4.0462782 .9131131 .479993

O $1.375705 \quad 3.104859-0.560982$

H $2.557166 \quad 4.498690 \quad 0.341520$

H $0.3983794 .055468 \quad 1.616175$

O $2.036116 \quad 4.2186382 .833512$

H 0.5685202 .1242933 .322268

O $\quad 0.0005451 .5338091 .455436$

C $4.147302 \quad 0.199387 \quad 3.555382$

H 3.6545712 .2748973 .494950

H 2.5648601 .2795014 .441355

H $1.0878202 .187673-0.413849$

H 1.4682844 .2501283 .622145

H -0.7980902 .0818521 .542534$

$\begin{array}{llll}\text { O } & 5.391936 & 0.688242 & 3.290905\end{array}$

O $3.865961-0.9684963 .792652$

C $6.450583-0.2737873 .316754$

H $6.595812-0.6416374 .336662$

H $6.235098-1.1019652 .635837$

H 7.3677740 .2207382 .986160

SCF Energy (B3LYP/6-31G**/MMFF) $=-3245.91878208$

03_115

MM̄FF Geometry

C $3.0195174 .911569-1.126764$

C $3.7175603 .792256-0.886167$

C 4.3810183 .4780440 .426506

$\begin{array}{lllll}\text { O } & 3.760663 & 2.275020 & 0.933817\end{array}$

$\begin{array}{llll}\text { C } 5.878298 & 3.183136 & 0.219674\end{array}$

C $6.5860892 .777804 \quad 1.499339$

C 7.1158301 .5584771 .724127

C 6.6867223 .8470642 .556508

C $7.1674650 .386008 \quad 0.782602$

C $5.886512-0.447963 \quad 0.817075$

C $6.008188-1.8173660 .131177$

C $6.402597-1.709802-1.344128$

C $6.389773-3.076270-2.047451$

O $4.723016-2.445366 \quad 0.254396$

O $6.452120-2.843775-3.463335$

C $5.041233-3.768348-1.763653$

C $7.622947-3.915108-1.692448$

O $5.027243-5.097207-2.278842$

C $4.659209-3.780254-0.268042$

C $3.225911-4.311121-0.025340$

O $5.551346-4.6482170 .441032$

C $2.6471452 .399629 \quad 1.702862$

O $2.106468 \quad 3.4314902 .063909$

C 2.1876411 .0275012 .010669

$\begin{array}{llll}\text { C } & 1.085310 & 0.826831 & 2.747290\end{array}$

C $0.514574-0.4803483 .085530$

C $1.222918-1.6984452 .552587$

C $2.121952-3.552956-0.766419$

C $0.730063-3.946207-0.297141$

C $-1.663988-3.305484-0.618850$

O $0.519322-4.8450330 .512146$

C $-2.069609-2.2426770 .353018$

C $-2.747956-2.4846901 .485377$

C $-3.209917-1.3998262 .416112$

C $-2.854075-1.6564023 .864742$

C $-3.816299-1.7939394 .792791$

C $-1.391975-1.743176 \quad 4.252379$

C $-0.607575-0.5272503 .833322$

$\mathrm{N}-0.248035-3.171197-0.904784$

H $2.9477465 .674746-0.356910$

H $3.7713953 .025774-1.656461$

H $4.255168 \quad 4.2993301 .142328$

H $6.3790794 .068573-0.193103$

H $5.9848842 .396978-0.536508$

H 7.5852541 .3733892 .689620

H 7.3183603 .5409733 .397485

H 7.1251184 .7574102 .134744

H 5.6985414 .0881912 .959512

H $7.3822670 .727558-0.235567$

H $8.021787-0.238342 \quad 1.073477$

H $5.568545-0.6098181 .856229$

H $\begin{array}{llll}5.067817 & 0.109637 & 0.347294\end{array}$
H $\quad 6.746291-2.4102870 .684115$

H $7.388448-1.244287-1.459313$

H $5.694688-1.046259-1.860457$

H $7.263126-2.340022-3.648948$

H $4.280257-3.209575-2.322701$

H $8.539421-3.396776-1.998943$

H $7.622318-4.867243-2.234462$

H $7.706994-4.125295-0.624372$

H $5.267622-5.045829-3.219765$

H $3.172887-5.378565-0.276608$

H $3.020673-4.2552881 .053187$

H $5.121136-4.9234561 .267573$

H 2.7694500 .2084041 .602356

H $0.5428801 .692366 \quad 3.124836$

H $2.249508-1.7447052 .933336$

H $1.255493-1.6642841 .459706$

H $0.750991-2.6438032 .823562$

H $2.240556-2.473505-0.620549$

H $2.175069-3.763985-1.840190$

H -1.872423 $-4.312425-0.243360$

H -2.198810 -3.164871-1.563142

H $-1.824126-1.2159190 .091937$

H -3.026918 -3.5060671 .732743$

H $-4.297064-1.3181182 .304615$

H $-2.807340-0.4251232 .116512$

H -3.576072 -1.974465 5.835349

H $-4.867731-1.7318354 .532707$

H $-0.990475-2.671222 \quad 3.839019$

H $-1.291351-1.8342785 .341654$

H $-1.0314770 .412754 \quad 4.189359$

H $0.035309-2.417985-1.524981$

C $0.7603495 .036362-2.273909$

O $0.4003043 .744502-1.739368$

C $-0.6703453 .925510-0.783091$

C -1.193292 $5.335521-1.017350$

C $0.0817986 .071813-1.379127$

H $-0.202798 \quad 3.8544540 .207403$

C -1.696006 $2.799701-0.965069$

H $-1.8990005 .356171-1.857229$

H -1.692013 $5.752930-0.137767$

C $-0.1571807 .410696-2.054497$

H $0.657926 \quad 6.233994-0.460147$

C -3.019782 $3.026768-0.216762$

O $-1.104848 \quad 1.585277-0.490530$

H -1.892349 $2.658030-2.034196$

H -3.521872 $3.911599-0.624971$

O $-2.738182 \quad 3.333450 \quad 1.155150$

C $-4.0114951 .845683-0.232057$

C $-4.241100 \quad 1.244610-1.626684$

H $-3.679217 \quad 1.063947 \quad 0.459119$

$\begin{array}{llll} & -5.262633 & 2.329089 & 0.266675\end{array}$

H $-4.4806642 .046453-2.336420$

H $-3.3042580 .779236-1.952402$

H $\quad 0.7917207 .892401-2.310385$

H $-0.7382267 .298194-2.976013$

H $-0.7092088 .083164-1.389774$

C $2.2813615 .151610-2.414054$

H $-0.2688711 .461330-0.972116$

H -2.239346 2.5848151 .525875

H $-5.1007982 .687171 \quad 1.156468$

H $2.5527186 .140142-2.801285$

H $2.613444 \quad 4.420476-3.162606$

H $0.3245835 .078694-3.281172$

C $-6.083963-1.855982-0.600590$

O $-5.103786-0.813942-0.709089$

C $-5.3754420 .198749-1.682369$

C $-5.505684-0.418132-3.085750$

C $-6.539448-1.548628-3.104054$

C $-6.313332-2.540615-1.958557$

H $-5.630866-2.5955670 .072049$

C $-7.381344-1.3620630 .050921$

H $-6.3130550 .701700-1.422782$

$\begin{array}{lllll} & -4.240947 & -0.929107 & -3.504544\end{array}$

H $-5.7918130 .370594-3.791692$

H $-6.480689-2.084510-4.059223$

O $-7.850919-1.004018-2.994173$

H -7.159486 -3.233052 -1.878382

O $-5.153892-3.328885-2.241559$ 
C $-7.161292-1.0383231 .509197$ $\mathrm{H}-7.804323-0.483167-0.443642$ H $-8.155858-2.1375310 .014754$ H -4.349469-1.281245 -4.404315 H $-7.981355-0.397645-3.743079$ H $-5.316152-3.799720-3.076636$ O O $-7.058035-1.8864502 .386222$ C $-6.855377 \quad 0.754973 \quad 3.022614$ H $-5.8426690 .491373 \quad 3.339223$ H -6.9556931 .8435183 .040276$ H $-7.598180 \quad 0.324966 \quad 3.701297$

SCF Energy (B3LYP/6-31G**//MMFF) $=-3245.90947290$

03_116

MM̄FF Geometry

C -3.218487 -3.903472 -0.657770

C $-2.480998-2.961850-1.266393$

C -1.036244 -3.067791-1.711590

O $-0.302524-1.967290-1.113029$

C $-0.320566-4.381698-1.335589$

C $1.143764-4.446250-1.730809$

C $2.139365-4.737500-0.869493$

C $1.456267-4.195126-3.182468$

C $2.004733-5.0769490 .590016$

C $1.952691-3.8554501 .516508$

C $3.310568-3.1663041 .720898$

C $3.164379-2.0044982 .707202$

C $4.477266-1.2300842 .885845$

O $3.779195-2.680963 \quad 0.461754$

O 4.1735470 .0029893 .556633

C $5.030156-0.8619791 .493461$

C $5.466652-1.9806593 .784475$

O $6.325898-0.2755351 .600558$

C $5.066831-2.0551670 .508042$

C $5.430277-1.602508-0.926988$

O $6.077695-2.974968 \quad 0.935861$

C $-0.298249-0.787945-1.795129$

O $-0.872658-0.550310-2.846975$

C $0.5274570 .184802-1.043635$

C $0.5292701 .465993-1.443358$

C $1.2730492 .580109-0.851498$

C 2.1586812 .2966320 .331092

C $4.309280-0.857077-1.657189$

C $4.8225670 .022622-2.783200$

C $4.0548671 .878210-4.263220$

O $5.9930420 .034001-3.153477$

C $4.1964003 .153857-3.491339$

C $3.4159834 .229557-3.674213$

C $3.5462075 .494465-2.874054$

C $2.2541355 .894682-2.193928$

C $1.5638496 .972774-2.602608$

C $1.7996065 .090657-0.991131$

C $1.1153223 .809332-1.387382$

N $3.8071460 .804386-3.319005$

H $-2.754520-4.859121-0.425119$

H -2.941048 -2.003040 -1.483991

H -1.022519 -2.969367 -2.804582

H $-0.832818-5.226617-1.813998$

H - $0.419268-4.526122-0.255665$

H $3.160414-4.759746-1.247496$

H $1.268862-3.149221-3.443686$

H $2.502502-4.410815-3.424446$

H $0.835744-4.833514-3.819821$

H $1.105827-5.684903 \quad 0.747091$

H $2.840687 \quad-5.7268710 .877470$

H $\quad 1.227457-3.132521 \quad 1.122480$

H $1.578093-4.1862572 .493283$

H 4.017911-3.909816 2.106550

H $2.792846-2.3592283 .676726$

H $2.403107-1.3062992 .332573$

H $3.752600-0.2137424 .406210$

H $4.375502-0.0822591 .087653$

H $5.032725-2.1442424 .778305$

H $6.376110-1.3924233 .948743$

H $5.753233-2.9561873 .386403$

H $\quad 6.255521 \quad 0.472543 \quad 2.217958$

H $6.353957-1.011595-0.894653$
H $5.680113-2.483625-1.534455$

H $6.325193-3.5350220 .181867$

H $1.100056-0.174451-0.196980$

H $-0.0840321 .736300-2.302821$

H 1.6246701 .7235011 .095906

H $2.5089553 .201767 \quad 0.833119$

H 3.0411191 .7316550 .019338

H $3.597580-1.576982-2.078533$

H $3.759964-0.213935-0.966001$

H $3.2099391 .904756-4.958276$

H $4.9716091 .676928-4.825858$

H $4.9794143 .188990-2.737043$

H $2.6427944 .203454-4.438654$

H $3.8773936 .286851-3.557711$

H $4.3337675 .406327-2.115220$

H $0.6500087 .280444-2.105156$

H $1.8937447 .569463-3.446492$

H $1.0873075 .677787-0.396931$

H $2.6676094 .922927-0.346715$

H $0.4100223 .923846-2.212098$

H $2.8799370 .750455-2.908624$

C $-5.593020-2.745106-0.772491$

O $-5.212751-1.430611-0.331819$

C $-5.520254-0.494514-1.383864$

C $-6.294395-1.271182-2.449671$

C $-5.736273-2.671928-2.290695$

H $-4.560675-0.179308-1.809643$

C $-6.2670020 .725383-0.833334$

H -7.372826 -1.269500 -2.253663

H $-6.140389-0.859100-3.451870$

C $-6.623758-3.753770-2.881753$

H $-4.760917-2.709235-2.786994$

C $-5.573016 \quad 1.452993 \quad 0.334947$

O $-7.553656 \quad 0.298998-0.363142$

H $-6.459908 \quad 1.427351-1.654099$

H -5.6629530 .8542501 .249970$

O -6.3300092 .6509520 .559682$

C $-4.095151 \quad 1.8137180 .094094$

C -3.5262952 .7768201 .155363$

H -3.5046780 .8919600 .077050$

O $-3.9511652 .424524-1.189961$

H -3.9624983 .7704810 .999677$

H -3.8302282 .4357452 .149520$

H $-6.180733-4.743743-2.734741$

H -7.616931 -3.756298 -2.420165

H $-6.754091-3.598634-3.957742$

C $-4.656783-3.822130-0.212967$

H $-8.0209931 .089376-0.042137$

H -6.1005022 .9843491 .443509$

H -4.520661 $3.212769-1.207092$

H $-5.109258-4.797192-0.434882$

H -4.639174 -3.7295840 .880783$

H $-6.583235-2.922089-0.329080$

C -1.3933041 .2914762 .786366$

O -1.3433881 .6567571 .401811$

C -1.9885212 .8910641 .067488$

C -1.381895 4.0405371 .882837

C $-1.4150113 .751166 \quad 3.378237$

C -0.7829392 .3913973 .665162$

H -2.4330491 .1122933 .084923$

C $-0.617230-0.0254312 .947095$

H -1.7466013 .0906770 .016802$

O -2.0581435 .2660941 .624995$

H $-0.3355664 .185106 \quad 1.584842$

H -2.4347533 .8048183 .777414$

O $-0.6581054 .748944 \quad 4.067232$

H 0.3008202 .4486263 .499765

O $-0.9897882 .063746 \quad 5.038132$

C $-1.426143-1.2219392 .494643$

H $0.307108-0.0104852 .360986$

H $-0.367048-0.1822964 .002669$

H -2.000905 5.4348570 .668861

H -1.044282 5.6122873 .839961

H -0.6135302 .7870195 .568652$

O $-0.962852-2.336721 \quad 3.133631$

O $-2.333268-1.1880841 .674916$

C -1.651247 -3.555667 2.839816

H $-2.732915-3.4297282 .947649$ 
H $-1.398434-3.8866401 .830574$ H $-1.315899-4.3147173 .551484$

SCF Energy (B3LYP/6-31G**//MMFF)= -3245.90736403

03_117

MM̄FF Geometry

C $0.938850-4.511416-2.495945$

C $2.159994-3.979374-2.656876$

C $3.334935-4.067726-1.714281$

O $3.553243-2.727407-1.208537$

C $3.151558-4.987223-0.489452$

C $4.377537-5.0393130 .405373$

C $4.413509-4.5947821 .677671$

C $5.610955-5.651195-0.208252$

C $3.285999-4.0152612 .487297$

C $3.147142-2.5054842 .291026$

C $2.243322-1.8214863 .328225$

C $0.810533-2.3597053 .315050$

C $-0.111046-1.5809874 .266915$

O $2.245371-0.4221313 .008581$

O $-1.456258-1.9632113 .938961$

C $0.043639-0.0723083 .984281$

C $0.113500-1.9847325 .728436$

O $-0.676424 \quad 0.711104 \quad 4.931819$

C 1.5132550 .3976103 .931372

C 1.6503441 .8662863 .461795

O 2.0824720 .3225375 .243561

C $4.450091-1.924517-1.837831$

O $5.141539-2.198211-2.804230$

C $4.420348-0.622300-1.134739$

C $5.1493290 .411401-1.580383$

C $5.1777541 .752100-0.987541$

C 4.3445541 .9748700 .247526

C 0.9863222 .1724302 .117827

C 1.3817633 .5290801 .559521

C $1.1014424 .911967-0.505192$

O $2.074643 \quad 4.3363192 .171925$

C $2.1583494 .602735-1.519112$

C $3.2361565 .372844-1.734742$

C $4.2687195 .073051-2.785384$

C $5.6881875 .114580-2.258123$

C $6.5776405 .990487-2.756116$

C $6.0892194 .147828-1.162498$

C $5.9281362 .708223-1.574206$

N $0.852418 \quad 3.7254810 .291545$

H $0.705785-5.105700-1.617860$

H $2.338347-3.377211-3.546912$

H $4.202536-4.404704-2.293959$

H $2.921895-6.008754-0.818446$

H $2.285067-4.6426740 .085143$

H $5.356022-4.6664562 .219264$

H $6.408364-5.8031850 .527234$

H $5.375293-6.629991-0.638633$

H $\quad 6.011519-5.008287-0.997729$

H $2.345790-4.5257102 .254373$

H $3.488185-4.2334993 .543602$

H $4.135681-2.0272732 .326347$

H $2.759440-2.2953621 .287110$

H $2.697416-1.9592084 .316620$

H $0.783050-3.4265903 .565358$

H $0.405461-2.2804082 .296183$

H -2.056431 -1.527545 4.567423

H $-0.4196770 .109048 \quad 3.007723$

H $-0.110719-3.0491725 .867875$

H $-0.565794-1.4451396 .397569$

H $1.137166-1.8145106 .068432$

H -1.612137 0.4556514 .877060

H 1.2561952 .5455054 .229057

H 2.7214172 .1012003 .385253

H 2.8474930 .9204715 .281228

H $3.775630-0.544405-0.266296$

H $5.7697640 .271660-2.464259$

H 4.6577281 .2997361 .051959

H 3.2885521 .7908830 .025810

H 4.4098072 .9860620 .651356

H 1.2535381 .4074201 .380173

H -0.1028502 .1762172 .234409$

H 1.3827545 .7451630 .146573
H $\quad 0.1643905 .161127-1.012700$

H $2.0158583 .709993-2.124284$

H $3.3672396 .282132-1.152789$

H $4.1422115 .811789-3.587660$

H $4.0869464 .095538-3.248887$

H $7.5982586 .031493-2.390217$

H $6.3068646 .688206-3.541668$

H $5.5285984 .399887-0.259564$

H $7.1448194 .291413-0.896610$

H $6.5065872 .440756-2.459720$

H $0.3349922 .969009-0.146717$

C $-1.218860-3.315924-2.871591$

O $-0.619523-2.012025-2.721253$

C $-1.570849-1.005869-3.127660$

C $-2.896853-1.731915-3.299511$

C $-2.441670-3.102022-3.760000$

H -1.211819 -0.626939-4.092729

C $-1.5794420 .114366-2.081196$

H -3.428714 -1.806713 -2.342528

H -3.555395 -1.236224 -4.018698

C $-3.508672-4.173064-3.616747$

H -2.135554 -3.035391 -4.812567

C $-2.6793571 .164518-2.308310$

O $-0.3073810 .770658-2.115754$

H -1.672521 $-0.326671-1.082945$

H $-3.6665600 .695217-2.254475$

O $-2.5694291 .674279-3.643916$

C $-2.6350532 .363294-1.343410$

C -2.6039802 .0090160 .154038$

H -1.763116 $2.989268-1.568425$

O $-3.7777323 .189018-1.589983$

H -1.6340061 .5601470 .394885$

H -2.6755262 .9531740 .709764$

H $-3.132490-5.145758-3.948509$

H -3.837592 -4.275164 -2.577213

H $-4.385843-3.925087-4.223462$

C $-0.191494-4.283108-3.462858$

H $\quad 0.3737190 .089974-1.977505$

H -1.680261 $2.056964-3.739195$

H $-3.7824143 .403947-2.538424$

H $\quad 0.197191-3.881454-4.407022$

H $-0.656087-5.249568-3.686977$

H -1.523527 -3.644335 -1.868910

C -6.1409610 .9227210 .752977$

O

C -3.7256631 .0665450 .636566$

C -3.5604620 .7006672 .120006$

C $-4.734900-0.1498272 .616519$

C -6.0860220 .4530342 .218193$

H -6.9860521 .6154780 .651996$

C $-6.358581-0.225785-0.245454$

H $-3.6822430 .149128 \quad 0.043467$

O -3.4649761 .8911672 .898421$

H -2.6215620 .1506612 .240612$

H -4.690162 -0.2352003 .709176$

O $-4.635497-1.4642012 .076126$

H $-6.889461-0.2682942 .408049$

O -6.3261181 .5805243 .065725$

C $-6.5799600 .276386-1.657130$

H $-5.499359-0.901279-0.268503$

H $-7.245638-0.8028250 .039032$

H $-3.3529251 .628523 \quad 3.827636$

H -3.788366 -1.839917 2.371020

H -7.2111031 .9239862 .855021$

O $-6.434726-0.767371-2.526760$

O $-6.8587341 .427813-1.961629$

C $-6.602016-0.442832-3.910293$

H $-7.623705-0.098795-4.096372$

H $-5.8757240 .317926-4.212715$

$\mathrm{H}-6.423507-1.349000-4.495355$

SCF Energy (B3LYP/6-31G**//MMFF) $=-3245.89980038$

03_118

MM̄FF Geometry

C $2.649424-4.6146020 .856917$

C $1.728222-4.202358-0.024887$

C $0.246397-4.1735410 .247900$

$\begin{array}{llll}\text { O } & -0.190642 & -2.794357 & 0.222259\end{array}$ 
C $-0.494334-4.930327-0.869532$

C $-2.002580-4.931508-0.717395$

C $-2.857276-4.363644-1.591231$

C $-2.537370-5.6478060 .495603$

C $-2.530270-3.637538-2.866986$

C $-2.346525-2.119687-2.712675$

C $-3.621647-1.373495-2.287398$

C $-3.410305 \quad 0.139311-2.355195$

C $-4.6521520 .908780-1.879530$

O $-3.934989-1.737486-0.943226$

O $-4.2644442 .276107-1.664548$

C $-5.1095720 .351867-0.510088$

C $-5.7401730 .937185-2.960104$

O $-6.3659370 .919890-0.149801$

C $-5.166337-1.193118-0.447145$

C $-5.348173-1.7547690 .982133$

O $-6.287096-1.704743-1.179403$

C $0.042909-2.0413181 .331509$

O $0.543074-2.4104282 .381965$

C $-0.388600-0.658496 \quad 1.030637$

C 0.0436250 .3492051 .802822

C $-0.2655791 .768777 \quad 1.622530$

C -1.3110392 .1314890 .601607$

C $-4.255393-1.3730241 .986605$

C $-4.595606-0.1610622 .838190$

C -3.6060321 .3251824 .577264$

$\begin{array}{llll}\text { O } & -5.699538 & 0.376819 & 2.836483\end{array}$

C -3.1500102 .5896683 .920656$

C $-2.204306 \quad 3.388514 \quad 4.438004$

C -1.771991 4.6853793 .816677

C -0.2766814 .7615613 .605278$

C $0.513887 \quad 5.401374 \quad 4.483442$

C 0.2874104 .1739092 .332528

C $0.388937 \quad 2.673076 \quad 2.380997$

$\begin{array}{llll}\text { N } & -3.523867 & 0.228932 & 3.630507\end{array}$

H $2.332760-4.9808321 .829807$

H $2.057637-3.828338-0.990666$

H $0.011241-4.6165111 .223436$

H $-0.151024-5.973027-0.900463$

H $-0.210767-4.492490-1.832978$

H $-3.923743-4.430633-1.379208$

H $-3.629246-5.7340510 .479179$

H -2.130794 -6.663099 0.547396

H -2.265286 -5.1136621 .410991$

H -1.631705 $-4.058908-3.330356$

H $-3.336409-3.832866-3.585661$

H -1.546509 -1.929833 -1.987702

H -2.007063 -1.725085-3.678431

H $-4.438878-1.679323-2.950916$

H -3.130756 $0.451103-3.369229$

H - $2.5646710 .417240-1.712681$

H -3.895965 2.618301-2.497060

H -4.3801520 .7136010 .223460$

H $-5.3716191 .443193-3.860833$

H -6.611811 $1.513134-2.630817$

H $-6.073037-0.058997-3.259278$

H -6.5524590 .6711010 .770910$

H -6.348267 -1.514010 1.362105

H $-5.332931-2.8515640 .898287$

H $-6.802686-0.958748-1.524007$

H $-1.023880-0.5053500 .166256$

H 0.7095550 .1183812 .633514

H -2.228413 1.5596780 .775568

H $-0.9534631 .920290-0.410829$

H -1.6038993 .1829800 .630665$

H -4.122442 -2.2085882 .685963$

H -3.296766 -1.210310 1.485431

H -2.985096 1.0578645 .438279

H -4.6411891 .4380704 .915336$

H -3.640335 2.8757362 .993077

H $-1.736303 \quad 3.1140785 .380854$

H -2.095426 5.4966464 .482138

H -2.2847604 .8648252 .863642$

H 1.5838215 .4859354 .324338

H $0.1089595 .850248 \quad 5.384425$

H 1.3015124 .5592522 .159239

H $-0.292400 \quad 4.536707 \quad 1.479078$

H 1.1242702 .3108993 .100993
H -2.628590 -0.239239 3.523035

C $4.847494-3.395298 \quad 1.242451$

O $4.412083-2.1496360 .658012$

C $4.321265-1.1616231 .701513$

C $4.999512-1.7730062 .925034$

C $4.669732-3.2416442 .752183$

H $3.252983-1.0281131 .913686$

C $4.923866 \quad 0.184168 \quad 1.272811$

H $6.085166-1.6214042 .903478$

H $4.619478-1.3538743 .861728$

C $5.546300-4.1692353 .574273$

H $3.622844-3.3879463 .044369$

C 4.1712700 .8434760 .094504

$\begin{array}{llllllll} & \text { O } & 6.298081 & -0.015486 & 0.945045\end{array}$

H 4.8864150 .8584252 .137452

H 3.1024250 .8236630 .330188

O $4.3565640 .085202-1.097086$

C $4.5968182 .304082-0.155870$

C $3.8414063 .028797-1.289716$

H 4.4652412 .8704560 .774354

O $5.9905502 .342299-0.472695$

H $4.2701024 .036241-1.359741$

H $4.0846292 .550153-2.243368$

H $5.275042-5.2154163 .400853$

H $6.605511-4.0489333 .323149$

H $5.429399-3.9621014 .643027$

C $4.126823-4.575988 \quad 0.583953$

H 6.6835410 .8541780 .745814

H $5.3109560 .062926-1.282549$

H $6.2515693 .276196-0.545278$

H $4.565095-5.5192800 .930353$

H $4.295773-4.529770-0.499784$

H $5.915824-3.4785631 .001617$

C $1.5420321 .717611-2.875960$

O $1.635238 \quad 1.928692-1.461254$

C $2.3190093 .119304-1.056876$

C $1.6383424 .346060-1.680009$

C $1.5402154 .227516-3.196019$

C $0.8701042 .907856-3.571347$

H $2.5443461 .559819-3.290308$

C $0.7294370 .437468-3.096325$

H $2.173472 \quad 3.2062420 .025731$

O $2.3276555 .544549-1.341573$

H $0.6218624 .439370-1.275182$

H $2.5235404 .323907-3.671280$

O $0.7377605 .296894-3.700536$

H $-0.1912032 .954772-3.295785$

O $0.9235792 .738547-4.986855$

C $1.551845-0.777035-2.749080$

H $-0.1657070 .419991-2.464337$

H $0.3889340 .336415-4.133283$

H $2.3577665 .599536-0.371096$

H $1.1523096 .128949-3.414556$

H $1.8593572 .696782-5.247886$

O $2.198516-1.237209-3.857030$

O $1.629461-1.242549-1.620698$

C $3.025194-2.387813-3.655847$

H $2.400063-3.261813-3.451559$

H $3.588548-2.566050-4.575603$

H $3.734327-2.220001-2.839342$

SCF Energy (B3LYP/6-31G**//MMFF)= -3245.90785714

03_119

MM̄FF Geometry

C $2.160744-2.186209-2.725602$

C $2.854564-3.211516-2.205274$

C $4.023433-3.119101-1.255546$

O $4.574866-1.781476-1.222811$

C $3.590108-3.4126190 .190876$

C $3.139105-4.825196 \quad 0.480774$

C $1.853251-5.1999250 .632409$

C $4.249402-5.826180 \quad 0.662443$

C $0.620617-4.3633260 .429362$

C $-0.107950-3.9963941 .727771$

C $0.615191-2.9367412 .576816$

C $-0.230924-2.5728563 .800780$

C $0.376073-1.4162694 .609529$

O $0.821075-1.7787091 .761398$ 
O $-0.632736-0.9304095 .509085$

C $0.718196-0.2558453 .649400$

C $1.544748-1.8867145 .483537$

O $1.420052 \quad 0.7803394 .332502$

C $1.519457-0.7072802 .409096$

C 1.7032330 .4297961 .375037

O $2.828357-1.1097242 .831701$

C $5.556298-1.465380-2.102266$

O $5.996533-2.179436-2.992510$

C $6.072278-0.095777-1.864877$

C $5.6909190 .677645-0.835168$

C $6.1766062 .028428-0.537918$

C $7.1878642 .625890-1.481922$

C $0.4275510 .832301 \quad 0.636104$

C $0.609705 \quad 2.136717-0.110970$

C $1.7014153 .146529-2.103241$

$\begin{array}{lllll}\text { O } & 0.087766 & 3.177346 & 0.284740\end{array}$

C $3.0202473 .732376-1.713892$

C $3.1489824 .770526-0.874073$

C $4.4599985 .397078-0.493713$

C $4.8934315 .028216 \quad 0.907835$

C 4.3307275 .6051621 .983021

C 6.0322544 .0433231 .054922

C 5.6850792 .6684310 .544970

N $1.4005572 .018019-1.242619$

H $2.437166-1.161913-2.492713$

H $2.536444-4.224340-2.446180$

H $4.803491-3.825810-1.563480$

H $2.818922-2.6913960 .475604$

H $4.423615-3.1718840 .866475$

H $1.648135-6.2396430 .884810$

H $3.873304-6.8251100 .907701$

H $4.839645-5.912975-0.255049$

H $4.913116-5.5146791 .475620$

H $0.830449-3.461696-0.150801$

H $-0.065746-4.951589-0.193724$

H -1.092234 -3.602581 1.440405

H $-0.285981-4.8975002 .327539$

H $1.586722-3.3357072 .889437$

H $-0.384130-3.4510464 .440434$

H $-1.233589-2.268953 \quad 3.469951$

H $-0.912728-1.6711496 .073761$

H $-0.233274 \quad 0.180856 \quad 3.324023$

H $1.205175-2.6424436 .202088$

H $1.946351-1.0630816 .083924$

H $2.361450-2.3265794 .907738$

H $0.879701 \quad 1.0401255 .098324$

H 2.1670671 .3009421 .855565

H 2.4312650 .1038710 .619177

H $3.440521-1.0268242 .082422$

H $6.8106730 .237516-2.587072$

H $4.9557720 .293464-0.130331$

H $6.7908582 .653322-2.503122$

H $8.1111472 .036263-1.478742$

H $7.4656983 .652462-1.236298$

H $0.1272520 .054602-0.075543$

H $-0.409297 \quad 0.969242 \quad 1.325568$

H $0.896635 \quad 3.886843-2.037024$

H $1.7431282 .782084-3.134333$

H $3.9051053 .293285-2.166098$

H $2.2553685 .205907-0.432005$

H $4.3563056 .487391-0.571624$

H $5.2438545 .134102-1.214395$

H 4.6536785 .3635402 .990053

H 3.5319966 .3322251 .877819

H 6.3111193 .9367232 .111763

H $6.920290 \quad 4.4597170 .571446$

H 4.9461362 .1547791 .161741

H $1.8279781 .123204-1.462308$

C $-0.313967-1.868049-2.897591$

O $-0.428736-0.440588-3.082067$

C $-1.803220-0.122785-3.401232$

C $-2.599093-1.382605-3.101499$

C $-1.602828-2.463106-3.461285$

H $-1.8179730 .090729-4.477661$

C $-2.2321501 .117603-2.607314$

H -2.855261 -1.438063 -2.035858

H -3.523816 -1.444454 -3.682036
C -1.959364 -3.823336 -2.885230

H $-1.537499-2.537071-4.555042$

C -3.743374 $1.396551-2.651923$

O $-1.5411362 .248679-3.145629$

H -1.896893 $1.015497-1.570652$

H $-4.2891840 .564066-2.196478$

O $-4.153272 \quad 1.433737-4.025484$

C $-4.1772632 .718993-1.994405$

C $-3.6985502 .951791-0.549650$

H $-3.8339013 .558872-2.610228$

O $-5.6076442 .791800-2.031026$

H $-2.6228563 .161088-0.558215$

H $-4.2036783 .856705-0.187820$

H $-1.202255-4.569876-3.143988$

H -2.037799 $-3.788411-1.793300$

H $-2.921374-4.166859-3.279357$

C $0.948418-2.381750-3.595345$

H $-0.5935622 .031343-3.152084$

H $-5.109628 \quad 1.610886-4.037594$

H $-5.9566882 .062984-1.489961$

H $1.110990-1.845145-4.537650$

H $\quad 0.838385-3.444881-3.838860$

H $-0.263161-2.042307-1.815002$

C $-5.8018780 .464951 \quad 1.311949$

O $\begin{array}{llll}-5.384960 & 1.561738 & 0.486010\end{array}$

C $-3.977092 \quad 1.8029500 .440702$

C $-3.452842 \quad 2.1262101 .849451$

C -3.8160601 .0189912 .843093$

C $-5.299605 \quad 0.6406362 .756127$

H -6.8975810 .5300291 .331041$

C $-5.423791-0.8930350 .698085$

H -3.4716970 .9056090 .074341$

$\begin{array}{lllll} & -3.4784089 & 3.367968 & 2.307868\end{array}$

H -2.364699 2.2420011 .807252

H -3.579751 $1.360573 \quad 3.858203$

$\begin{array}{lllll} & -3.008408 & -0.121651 & 2.568233\end{array}$

H $-5.499905-0.274560 \quad 3.325294$

$\begin{array}{lllll} & \text { O } & -6.078345 & 1.682176 & 3.352980\end{array}$

C $-6.122966-1.141226-0.622357$

H $-4.348534-0.9685620 .516468$

H -5.721857 -1.6981891 .379335$

H -4.9540653 .3084462 .271001$

H $-3.224500-0.7996333 .230813$

H $-5.7968591 .764146 \quad 4.280235$

O $-5.636633-2.295309-1.168818$

O $-6.985629-0.425774-1.112419$

C $-6.198408-2.661842-2.432869$

H $-6.066090-1.854417-3.159688$

H -5.670386 $-3.548006-2.795100$

H -7.258286 -2.906080 -2.315210

SCF Energy (B3LYP/6-31G**//MMFF) $=-3245.91395285$

03_12

MM̄FF Geometry

C $-3.303229-0.028288-3.586812$

C $-2.546506-0.522428-2.594358$

C $-1.199392-1.165317-2.806104$

O $-0.269120-0.603377-1.851547$

C $-1.301764-2.676630-2.531304$

C $-0.007529-3.431035-2.769024$

C $0.662052-4.115419-1.820705$

C $0.515046-3.411333-4.182593$

C $0.289435-4.295548-0.376331$

C $0.845410-3.2217250 .570331$

C $2.379497-3.2072840 .670168$

C $2.839130-2.2684811 .787162$

C $4.371321-2.1871281 .868426$

O $2.913898-2.750830-0.573371$

$\begin{array}{lllll}O & 4.710121 & -1.060881 & 2.693759\end{array}$

C $4.942787-1.8972760 .461249$

C $4.962383-3.4206672 .561493$

O $6.363368-2.003963 \quad 0.453938$

C $4.345933-2.785229-0.653737$

C $4.729127-2.323718-2.078617$

O $4.833042-4.130479-0.567850$

C $0.2335610 .632342-2.115439$

O $\quad 0.0133451 .326373-3.094578$

C $1.0860511 .013562-0.968800$ 
C $1.3927722 .304216-0.774173$

C 2.1902082 .8422390 .329422

C 2.8818811 .8566361 .233307

C $4.295931-0.902208-2.453465$

C $5.3769670 .147472-2.251363$

C $5.7355532 .611026-2.379321$

O $6.542233-0.126753-1.978064$

C $5.6979073 .171881-0.992837$

C $5.4240134 .458004-0.725821$

C 5.4311115 .0468380 .655818

C 4.1466585 .7727960 .988264

C 4.0577937 .1086690 .872745

C 2.9968664 .9592621 .537242

C 2.2757074 .1813560 .471081

N $4.8912931 .433218-2.451418$

H $-2.929890-0.110109-4.606219$

H -2.889387 -0.476220 -1.565366

H $-0.831961-0.985225-3.823499$

H -2.075025 -3.119586 -3.173269

H -1.644138 -2.819553 -1.500541

H $1.583024-4.621259-2.108708$

H $0.869406-2.411298-4.450235$

H $1.351409-4.104099-4.325681$

H $-0.273373-3.704359-4.883547$

H $-0.799008-4.341257-0.266748$

H $0.646630-5.281756-0.053021$

H $0.485210-2.2380770 .246626$

H $\quad 0.425335-3.4089941 .565930$

H $2.722432-4.2304520 .862986$

H $2.420922-2.5768612 .753522$

H $2.446758-1.2601311 .599232$

H $4.286888-1.1857143 .560501$

H $4.707484-0.8502750 .238947$

H $4.591529-3.4956493 .590966$

H $6.052315-3.3465672 .641856$

H $4.713270-4.3562922 .056290$

H $6.702725-1.4048251 .140689$

H $5.802021-2.476012-2.248608$

H $4.227733-3.005306-2.781342$

H $5.517271-4.1705470 .119242$

H $1.4082060 .225321-0.298180$

H $1.0021853 .042454-1.473473$

H 2.1489351 .2870331 .813386

H 3.5691712 .3206661 .943473

H 3.4844401 .1566940 .645065

H $4.053858-0.886086-3.524019$

H $3.393449-0.608283-1.910036$

H $5.364883 \quad 3.322110-3.124379$

H $6.7637102 .338276-2.638336$

H $5.9354922 .496663-0.174124$

H $5.2158275 .137460-1.549415$

H $6.2779845 .743120 \quad 0.715822$

H 5.6247804 .2814031 .417546

H 3.1496667 .6403281 .136662

H 4.8905217 .6999230 .506105

H 2.2569525 .6223662 .005774

H 3.3594794 .3304362 .355197

H $1.7438844 .812928-0.242055$

H $3.8993481 .570315-2.622004$

C $-5.0844211 .041118-2.051170$

O $-4.2648862 .140793-1.598951$

C $-5.1120513 .110910-0.970721$

C $-6.4453552 .419354-0.725926$

C $-6.5293161 .533072-1.955717$

H $-5.2840513 .897152-1.719667$

C $-4.455578 \quad 3.7728840 .242556$

H -6.418906 1.8093600 .184252

H -7.279413 $3.122869-0.646866$

C -7.558752 $0.423261-1.834370$

H $-6.7728372 .155612-2.827155$

C -4.0484552 .8716161 .429175$

O $-5.376858 \quad 4.760752 \quad 0.724521$

H -3.568688 4.320000 -0.101504

H -4.9409002 .4469401 .901734$

O -3.4721923 .7352012 .424010$

C -3.0490501 .7385491 .114826$

C -2.4350031 .1094292 .382653$

H -3.5604860 .9599380 .542929$
$\begin{array}{llll}\mathrm{O} & -1.993862 & 2.240972 & 0.294953\end{array}$

H -1.862122 1.8771712 .915603

H -3.2450140 .7881823 .045593$

H -7.560364 -0.208402 -2.728077

H -7.359924 -0.217524 -0.969367

H $-8.5629670 .843063-1.715530$

C $-4.6532540 .634695-3.462028$

H -4.9564705 .2055691 .480406$

H -2.686601 4.1557982 .035271

H $-1.4933152 .893248 \quad 0.813574$

H -4.626163 $1.533097-4.092000$

H -5.393916 -0.045349 -3.898120

H $-4.9400670 .217099-1.346505$

C -3.047306 -1.903170 2.244140

O $-2.150702-1.151186 \quad 1.416857$

C $-1.482244-0.0671812 .069874$

C $-0.694122-0.5806323 .283542$

C $-1.574275-1.3828904 .233682$

C -2.320602 -2.472565 3.469500

H $-3.873928-1.2607262 .569128$

C $-3.617010-3.0428191 .385200$

H -0.7403460 .3052561 .354573$

O -0.0916990 .4916024 .000507$

H $0.120041-1.2288132 .937810$

H -2.271150 -0.737101 4.780871

O $-0.750630-2.0130245 .217702$

H -1.613269 -3.2530003 .161104$

O $-3.274590-3.0809044 .338417$

C $-4.628931-2.5513610 .374788$

H -2.798055 -3.530301 0.844007

H $-4.128478-3.7899012 .001423$

H 0.4592790 .9899853 .373596

H $-0.252233-1.3104435 .669503$

H -2.790545 -3.411409 5.114698

O $-4.673910-3.423414-0.675017$

O $-5.324238-1.5542100 .510639$

C $-5.633395-3.120009-1.692131$

H $-6.640202-3.073184-1.266196$

H $-5.377705-2.177526-2.182154$

H $-5.604530-3.920362-2.436086$

SCF Energy $(B 3 L Y P / 6-31 G * * / / M M F F)=-3245.90529518$

03_120

MM̄FF Geometry

C 1.6938202 .9801410 .221295

C $1.1139451 .962568-0.432445$

C 0.3275030 .8702460 .246226

O $-1.0012520 .833568-0.322458$

C $0.997495-0.486079-0.032809$

C $0.363911-1.6498360 .703531$

C $-0.219145-2.7065410 .104218$

C $0.440861-1.5971562 .207943$

C $-0.369574-2.971348-1.367297$

C $-1.676892-2.448807-1.983184$

C $-2.947846-3.087710-1.399496$

C $-4.177252-2.699267-2.224180$

C $-5.477049-3.248093-1.613762$

O $-3.112784-2.623906-0.057000$

O $-6.578207-2.575877-2.244829$

C $-5.525961-2.882585-0.113931$

C $-5.656847-4.742474-1.906700$

O $-6.629428-3.5152740 .528479$

C $-4.221164-3.2082040 .640663$

C $-4.195845-2.6543742 .086513$

O $-4.075174-4.6305550 .736031$

C $-1.904673 \quad 1.7409670 .136750$

$\begin{array}{llll}\text { O } & -1.727060 & 2.589513 & 0.995845\end{array}$

C $-3.1619321 .535676-0.615434$

C $-4.0947772 .499323-0.617362$

C $-5.3782692 .455741-1.321078$

C $-5.797121 \quad 1.140297-1.922662$

C $-4.352865-1.1347872 .212936$

C $-5.779078-0.6819302 .479838$

C -7.1548461 .3871322 .667756$

O $-6.691281-1.4542492 .761168$

C -7.8842881 .6059651 .379989$

C -8.3648182 .8002211 .001832$

C $-9.1367813 .030556-0.265734$ 
C $-8.5534694 .142538-1.108345$

C $-9.0594185 .386317-1.057761$

C $-7.4411513 .791267-2.070374$

C $-6.1204833 .581373-1.380175$

N -5.9045790 .6989902 .405538$

H 1.5967623 .0472191 .301873

H $1.2005461 .906112-1.515322$

H $\quad 0.261771 \quad 1.0471551 .326464$

H $2.054213-0.4372080 .254616$

H $0.987434-0.664782-1.113487$

H $-0.635673-3.4876300 .739539$

H $\quad 0.123737-2.5369562 .672818$

H $1.468719-1.4064312 .531402$

H $-0.203148-0.8047392 .600579$

H $0.477572-2.555835-1.923708$

H $-0.306176-4.055818-1.524242$

H -1.718535 -1.360575 -1.856255

H -1.638302 -2.647134 -3.061519

H $-2.817699-4.176257-1.398434$

H -4.071300 -3.034547 -3.263405

H $-4.251364-1.604244-2.270383$

H -6.518311 -2.736176 -3.202257

H $-5.708569-1.803069-0.057604$

H -5.684387 -4.919701-2.988635

H -6.614181 -5.108759-1.519807

H $-4.857357-5.360152-1.492531$

H -7.432024 -3.2647960 .039551$

H $-4.923111-3.1901262 .708273$

H -3.212576 -2.905036 2.510672

H -3.471885 -4.830115 1.471031

H $-3.2758260 .607025-1.162479$

H -3.893626 $3.419317-0.069694$

H $-5.7383540 .342205-1.174960$

H $-5.1501760 .879757-2.767081$

H $-6.8266541 .133708-2.286044$

H $-3.761637-0.7965193 .073732$

H -3.963609 -0.6227111 .327886$

H $-6.9082172 .328083 \quad 3.169467$

H $-7.7702010 .782373 \quad 3.341693$

H -8.0478370 .7396470 .743449$

H -8.224149 $3.658713 \quad 1.654910$

H -10.169269 3.2779590 .013812

H $-9.2054472 .114997-0.866287$

H -8.665266 $6.183452-1.679234$

H $-9.8764345 .636308-0.388858$

H $-7.2986794 .605523-2.793681$

H -7.749856 $2.934228-2.674932$

H $-5.7312384 .483542-0.905750$

H $-5.107021 \quad 1.2575182 .115709$

C 3.8788454 .2266960 .096321

O $4.6603173 .049976-0.198921$

C $5.9110363 .457029-0.781162$

C $6.0618014 .936036-0.448663$

C $4.6251205 .405101-0.535360$

H $5.7975343 .348623-1.866775$

C $7.0686932 .577629-0.297618$

H 6.4463285 .0903570 .566108

H $6.7269655 .453984-1.146202$

C $4.375846 \quad 6.7346320 .156144$

H $4.3525955 .499375-1.595335$

C $6.8488771 .059552-0.454394$

O $7.2891842 .832477 \quad 1.096326$

H $7.9881872 .884377-0.810851$

H $\quad 6.1339580 .7258500 .302756$

O $8.0932320 .421260-0.138702$

C $6.3780400 .594893-1.840426$

C $6.393053-0.929371-2.072480$

H $5.3630670 .968384-2.018668$

O $7.2252061 .189332-2.829149$

H $6.020618-1.104160-3.091030$

H $7.434559-1.277664-2.073729$

H 3.3234417 .0246430 .076977

H 4.6334456 .6882601 .219608

H $4.9794727 .525290-0.301590$

C $2.4629814 .072727-0.464746$

H $8.026521 \quad 2.2660301 .382013$

H $8.7279030 .653522-0.838128$

H $\quad 6.8717490 .948495-3.702434$
H $2.5158963 .872112-1.542252$

H $1.9086355 .009045-0.332664$

H $3.852574 \quad 4.3234931 .189228$

C $5.699789-2.6288691 .193858$

O $6.312758-1.8843600 .132705$

C $5.556980-1.754664-1.075847$

C $5.236798-3.147618-1.647074$

C $4.558767-4.047698-0.610395$

C $5.308629-4.0403590 .725064$

H $6.486889-2.7376001 .950929$

C $4.532506-1.8633571 .831385$

H $4.624494-1.217743-0.870695$

O $6.430200-3.779163-2.110430$

H $4.581589-3.029066-2.517878$

H $4.516098-5.074745-0.993204$

O $3.221661-3.609513-0.392801$

H $4.713340-4.5313881 .503777$

O $6.517227-4.7929280 .584230$

C $4.980774-0.5885202 .513393$

H $3.791644-1.5956811 .077144$

H $4.042804-2.4799902 .593156$

H $7.054319-3.817818-1.365361$

H $2.755720-3.650114-1.245220$

H $6.269746-5.7014290 .341051$

O $3.926673 \quad 0.2777702 .579484$

O $6.103460-0.3856662 .955411$

C 4.1983891 .5332903 .209134

H 4.9749792 .0728292 .659801

H 3.2809962 .1272533 .193415

H 4.4979581 .3781914 .249930

SCF Energy (B3LYP/6-31G**//MMFF) $=-3245.90774465$

03_121

MMFF Geometry

C -2.829685 -0.209507 -3.742841

C $-2.179878-0.679703-2.666909$

C $-0.823477-1.335217-2.732033$

O $0.009851-0.761442-1.696850$

C $-0.952708-2.841727-2.448477$

C $0.346691-3.606128-2.611942$

C $0.979565-4.261427-1.619334$

C $0.919251-3.634850-4.006182$

C $0.563395-4.393596-0.181996$

C $1.129761-3.3177630 .756514$

C $2.665145-3.2731190 .821344$

C $3.128076-2.3703701 .967815$

C $4.657807-2.2278402 .008675$

O $3.158885-2.757372-0.418235$

O $4.974744-1.1292782 .877637$

C $5.167892-1.8499570 .600883$

C $5.328849-3.4664692 .613823$

O $6.592002-1.8729780 .553018$

C $4.589772-2.735271-0.521796$

C $4.942595-2.228570-1.942528$

O $5.132288-4.056775-0.404955$

C $0.5056760 .487373-1.907132$

O $0.3662401 .179389-2.902645$

C $1.2289750 .886849-0.680349$

C $1.5024652 .182186-0.468097$

C 2.1927972 .7368050 .697848

C 2.8173311 .7653161 .664234

C $4.467134-0.809760-2.274315$

C $5.5130100 .266249-2.031308$

C $5.7755192 .744588-1.986792$

O $6.6943100 .019475-1.804654$

C $5.6893583 .226454-0.572828$

C $5.3932954 .493561-0.245392$

C 5.3395515 .0051641 .166089

C 4.0395685 .7119051 .480451

C 3.9484067 .0510791 .416401

C 2.8729764 .8721951 .949916

C 2.2499384 .0779070 .835908

N $4.9768001 .542665-2.138314$

H $-2.370919-0.345647-4.720619$

H $-2.612981-0.577839-1.676122$

H $-0.353540-1.166053-3.708176$

H -1.692798 -3.287331 -3.126361

H $-1.347598-2.972183-1.435526$ 
H $1.908120-4.778402-1.860100$ H $1.755349-4.336520-4.097181$ H $\quad 0.154389-3.945588-4.725372$ H $1.290038-2.646111-4.292861$ H $-0.528570-4.404466-0.093488$ H $\quad 0.885450-5.3816970 .171701$ H $0.742734-2.3391570 .448890$ H $0.737658-3.5206321 .760213$ H $3.034839-4.2948680 .967796$ H $2.752923-2.7397732 .930441$ H $2.689776-1.3714191 .841194$ H $4.591174-1.3179603 .751598$ H $4.865843-0.8109790 .424344$ H $4.973878-3.6314563 .638287$ H $6.413090-3.3305252 .691672$ H $5.134471-4.3793422 .047144$ H $6.915086-1.2799581 .252526$ H $6.016587-2.348531-2.129347$ H $4.447206-2.901433-2.657500$ H $5.046682-4.501926-1.264326$ H 1.4948870 .1071920 .023649 H $1.1739742 .911209-1.207675$ H 3.4726421 .0660631 .134068 H $2.043746 \quad 1.1947692 .189119$ H 3.4408422 .2419242 .423106 H $4.230548-0.765187-3.345289$ H $3.552429-0.565301-1.727434$ H $5.3908503 .481133-2.699154$ H $6.8180572 .525124-2.238425$ H 5.9044132 .5085770 .215246 H $5.2077695 .215585-1.037723$ H 6.1809695 .6975011 .301326 H 5.5013064 .1987331 .892128 H 3.0284507 .5688271 .667083 H 4.7919137 .6595431 .107033 H 2.0874235 .5168282 .366591 H 3.1967154 .2511592 .789448 H 1.7710844 .6981730 .076940 H $3.9773581 .652673-2.284154$ C -4.599938 1.134121-2.429247 O $-3.6402622 .128571-2.014108$ C $-4.3418003 .320845-1.640956$ C $-5.7901222 .910283-1.427124$ C $-5.9470711 .856876-2.510625$ H -4.308095 $3.980026-2.519799$ C -3.647907 $4.043500-0.483531$ H -5.943585 $2.462998-0.439053$ H -6.485983 $3.747170-1.537728$ C -7.145784 $0.946766-2.306815$ H -6.044095 $2.362376-3.480728$ C -3.682394 3.3772350 .907005 O $-4.2596925 .332698-0.336977$ H -2.599215 $4.224926-0.752371$ H -4.6895653 .4587781 .334048$ O $-2.832370 \quad 4.155565 \quad 1.765445$ C $-3.224087 \quad 1.9095930 .986691$ C -3.0770291 .4263842 .441716$ H -3.942357 1.2693210 .466557 $\begin{array}{lllll}\text { O } & -1.953607 & 1.750424 & 0.357165\end{array}$ H -2.189434 1.8930062 .887011 H -3.9600381 .7411893 .011454$ H -7.199376 $0.190485-3.096180$ H $-7.0962800 .427017-1.344072$ H -8.075154 $1.525042-2.328144$ C $-4.1463510 .526482-3.759654$ H $-4.1478095 .808427-1.177416$ H -3.154152 5.0731781 .737466 H -2.073297 $1.901097-0.595496$ H -4.037972 $1.339532-4.489742$ H -4.913809 $-0.153802-4.145661$ H $-4.6364870 .369533-1.646103$ C -4.308129 -2.101692 2.332351 O $-4.221613-0.6708802 .285215$ C -2.929138 -0.1053852 .537209$ C $-2.412393-0.5337543 .918903$ C $-2.422852-2.0570274 .068124$ C $-3.770627-2.6554173 .664056$ H $-5.384449-2.3157472 .302672$
C $-3.666347-2.7480261 .093693$

H $-2.227913-0.4457671 .768010$

O -3.2003500 .0547824 .951898$

H $-1.390628-0.1572664 .045029$

H -2.209302 -2.323594 5.110596

O $-1.405751-2.626105 \quad 3.250458$

H -3.696132 -3.747942 3.611950

O $-4.712899-2.3518294 .697499$

C $-4.546450-2.592971-0.130654$

H $-2.690867-2.3194000 .851388$

H $-3.549723-3.8236591 .271191$

H $-4.124599-0.2125974 .810375$

H $-0.554295-2.258257 \quad 3.542473$

H $-5.547895-2.7971964 .474344$

O $-4.130454-3.467487-1.093338$

O $-5.474332-1.802803-0.236169$

C $-4.885408-3.453407-2.309260$

H $-4.972514-2.436794-2.703352$

H $-4.358259-4.068463-3.043511$

H $-5.875859-3.883222-2.134148$

SCF Energy (B3LYP/6-31G**//MMFF) $=-3245.90646245$

03_122

MMFF Geometry

C $-4.253744 \quad 0.324058 \quad 2.643316$

C $-5.025479-0.6677012 .173499$

C $-5.487088-0.8363440 .745288$

O $\begin{array}{llll}-5.299001 & 0.403041 & 0.021945\end{array}$

C $-4.686206-1.9227590 .001253$

C $-4.746763-3.3007150 .619478$

C $-3.734106-3.880801 \quad 1.293311$

C $-6.039823-4.042876 \quad 0.405397$

C $-2.386917-3.2969531 .622896$

C $-1.235228-4.0392850 .933177$

C $-1.198507-3.843887-0.591111$

C $-0.102612-4.716564-1.208696$

C $0.041759-4.478797-2.718281$

O $-0.941074-2.459766-0.858367$

O $1.274963-5.079273-3.143746$

C $0.168354-2.962631-2.969853$

C $-1.072320-5.165453-3.516667$

O $0.163590-2.674952-4.366321$

C $-0.913999-2.123789-2.252808$

C $-0.649661-0.603890-2.381825$

O $-2.183540-2.380541-2.866560$

C $-6.1654860 .702602-0.977292$

O $\begin{array}{llll}\text { C } 7.159240 & 0.066041 & -1.298947\end{array}$

C $-5.773406 \quad 1.949748-1.674882$

C $-4.5505932 .494994-1.574832$

C $-4.0752593 .690321-2.275467$

C $-5.0788384 .486430-3.067727$

C $0.611536-0.100949-1.674439$

C $0.8440691 .381698-1.912271$

C $2.0690423 .334179-0.945699$

O $\quad 0.3554901 .993807-2.858493$

C $0.8951904 .152722-0.506643$

C $0.4852545 .259604-1.144336$

C $-0.7568476 .016754-0.766290$

C $-1.6743106 .278619-1.945686$

C $-2.1428217 .516506-2.178499$

C $-2.0397465 .135860-2.869953$

C $-2.769815 \quad 4.018190-2.176784$

N $1.6923511 .931498-0.961853$

H -3.9030861 .1046301 .973493$

H $-5.345045-1.4443402 .865831$

H $-6.555080-1.0847800 .770627$

H $-3.650899-1.583474-0.122587$

H $-5.051914-1.991231-1.032745$

H $-3.887826-4.8878381 .679650$

H $-6.015343-5.0523430 .829473$

H $-6.871461-3.5087480 .875288$

H $-6.248625-4.140563-0.665062$

H $-2.327461-2.2268231 .406034$

H $-2.251480-3.3857822 .708278$

H $-0.297083-3.6651201 .363860$

H $-1.296520-5.1086811 .170838$

H $-2.172786-4.116821-1.010858$

H $-0.291192-5.778175-1.005552$ 
H $\quad 0.858585-4.488276-0.728395$ H $1.241054-6.024865-2.918675$ H $1.155159-2.664966-2.598142$ H -1.051560 -6.248930 -3.348154 H $-0.928585-5.027735-4.594031$ H -2.070951 -4.811442 -3.253152 H $\quad 0.861861-3.214019-4.775687$ H $-0.635192-0.323639-3.443049$ H -1.504420 -0.059375 -1.955199 H -2.780905 -1.642715 -2.660209 H $-6.5360812 .368022-2.323751$ H $-3.8072992 .008033-0.945304$ H $-5.9504634 .732887-2.450505$ H -5.416073 $3.918933-3.941742$ H $-4.6874475 .441303-3.426624$ H $\quad 0.531220-0.287008-0.597541$ H $1.500857-0.615645-2.046471$ H $2.4158263 .611350-1.946560$ H $2.8934383 .443254-0.235617$ H $\quad 0.332748 \quad 3.7984580 .354061$ H $1.0544505 .627186-1.995213$ H $-0.4425356 .963480-0.309141$ H -1.323692 5.4754790 .001679 H -2.795167 $7.726369-3.019834$ H -1.888051 8.349519-1.531655 $\mathrm{H}-1.1247784 .721741-3.312249$ H -2.607548 $5.501392-3.732443$ H $-2.1219783 .385491-1.568995$ H $2.0186301 .350628-0.194789$ C $-2.337472-0.0455194 .196329$ O $-1.482616 \quad 0.810761 \quad 3.419436$ C $-0.206141 \quad 0.9231094 .085546$ C -0.2857490 .0332895 .319742$ C -1.774789 $0.022627 \quad 5.611546$ H -0.1138321 .9774404 .372595$ C 0.8890280 .5342073 .086436 H $0.066211-0.9813295 .093995$ H 0.3129460 .4188126 .150663 C -2.206734 -1.122541 6.510184 H $-2.0484190 .977412 \quad 6.080114$ C 2.3091240 .5265993 .674610 O 0.8489401 .4734992 .008264 H $0.651117-0.4455932 .657288$ H $2.388498-0.2407974 .453223$ O $2.5316831 .786367 \quad 4.320985$ C 3.4262670 .3280972 .630116 C $3.309741-0.9874201 .839953$ H 3.4512681 .1864771 .949749 O $4.681784 \quad 0.334313 \quad 3.321067$ H $3.410480-1.8322762 .532239$ H $2.322472-1.0435151 .369889$ H -3.285803 -1.092801 6.690287 H -1.966337 -2.0942946 .065675$ H -1.701780 -1.061963 7.479801 C $-3.788391 \quad 0.4159104 .070771$ H -0.0515751 .4564221 .640940$ H 3.4372781 .7744524 .675534 H $4.690089-0.4233073 .930738$ H -3.886525 1.4610664 .388215 H -4.433681 -0.1833654 .723217$ H $-2.229051-1.0604143 .792105$ C $4.985959-0.049303-1.356567$ O $4.070467-0.134714-0.258098$ C $4.385052-1.1071040 .743671$ C $4.422893-2.5119940 .124866$ C $5.382896-2.569993-1.067504$ C $5.152742-1.414243-2.047681$ H $4.5022980 .633107-2.067281$ C $6.3144340 .587089-0.935273$ H $5.363562-0.8791001 .178800$ O $3.118068-2.905568-0.292621$ H $4.742950-3.2275330 .891313$ H $5.244877-3.525592-1.588474$ O $6.719065-2.529856-0.575033$ H $5.971818-1.365451-2.774734$ O $3.959792-1.701460-2.783009$ C $6.0925192 .005026-0.463018$ H $6.8236620 .028091-0.145090$
H $7.0122140 .631021-1.779694$

H $2.800119-2.245431-0.931300$

H $7.315619-2.603992-1.339356$

H $3.848002-1.001673-3.448863$

O $6.1651562 .059714 \quad 0.897147$

O $5.8560552 .943756-1.212539$

C 5.9397553 .3477861 .477637

H 6.0521403 .2559702 .561262

H 6.6769424 .0665731 .107560

H 4.9234333 .6878341 .258881

SCF Energy (B3LYP/6-31G**//MMFF) $=-3245.92686924$

$03 \_123$

MM̄FF Geometry

C $2.1189012 .479991-0.308861$

C $1.329811 \quad 1.756397-1.115515$

C $0.5654000 .543591-0.647069$

O $-0.8339120 .726467-0.957726$

C $1.071286-0.699337-1.402997$

C $0.458534-2.001519-0.926751$

C $-0.324176-2.800711-1.677666$

C $0.803915-2.4104710 .482300$

C $-0.759943-2.604373-3.102435$

C $-2.108917-1.887462-3.269948$

C $-3.298200-2.622318-2.629546$

C $-4.622476-1.984307-3.055409$

C $-5.827028-2.635563-2.356689$

O $-3.167808-2.538983-1.207944$

O $-6.964453-1.780597-2.551545$

C $-5.561219-2.691931-0.836177$

C $-6.188044-3.986342-2.986276$

O $-6.575602-3.438298-0.169403$

C $-4.171471-3.255424-0.476205$

C $-3.825964-3.1212951 .027390$

O $-4.135274-4.653320-0.789360$

C $-1.5615971 .506491-0.112529$

$\begin{array}{lllll}\text { O } & -1.166811 & 2.068383 & 0.896694\end{array}$

C $-2.9393681 .579469-0.645871$

C $-3.7645812 .542934-0.210159$

C $-5.1476282 .755172-0.642134$

C $-5.7847271 .682261-1.484986$

C $-3.817853-1.6904541 .577029$

C $-5.124574-1.2735922 .231626$

C -6.2561340 .7194003 .209950$

O $-6.034144-2.0591012 .483415$

C -7.1814941 .3040002 .190015$

C -7.6187982 .5716122 .235681$

C -8.5801153 .1636741 .245209$

C -8.0634724 .4434910 .626740$

C -8.4443225 .6422591 .099715$

C $-7.1741844 .331848-0.590982$

C $-5.7803623 .881333-0.251542$

N -5.1388090 .0804822 .539921$

H 2.2011692 .2035590 .738660

H $1.2468652 .024721-2.165916$

H 0.6842010 .3984350 .433471

H $2.161081-0.782363-1.292748$

H $0.886001-0.556841-2.473167$

H $-0.698967-3.717944-1.224168$

H $\quad 0.343237-1.7314661 .205725$

H $0.457688-3.4225760 .718248$

H $1.888830-2.3972040 .626227$

H $\quad 0.003608-2.063328-3.671208$

H $-0.818710-3.592848-3.576154$

H -2.027834 -0.878673 -2.848469

H $-2.292841-1.774152-4.345569$

H -3.263496 -3.673064 -2.940709

H -4.743101 -2.027462 -4.145003

H $-4.608956-0.916446-2.799411$

H -7.099759-1.675310 -3.508813

H $-5.634418-1.663206-0.464073$

H -6.435778 -3.860041-4.047137

H -7.082570 -4.413631 -2.519815

H $-5.380963-4.718955-2.921906$

H -7.430637 -3.037350 -0.401592

H $-4.469599-3.7803841 .622441$

H -2.807523 -3.514492 1.160561

H $-3.422771-5.066524-0.273989$ 
H $-3.230590 \quad 0.850282-1.392785$ H $-3.388623 \quad 3.2594370 .519274$ H $-5.3170751 .641548-2.474374$ H $-6.8567341 .823769-1.636717$ H $-5.676746 \quad 0.704365-1.003818$ H -3.051882 -1.6250182 .360720$ H $-3.550093-0.9704670 .798081$ H -5.8413551 .4807603 .878110$ H $-6.792201-0.0206153 .812758$ H $-7.527648 \quad 0.649028 \quad 1.393735$ H -7.2929573 .2152203 .050004$ H -9.5229773 .3594061 .772548$ H -8.8293872 .4491100 .450785$ H $-8.100752 \quad 6.5652770 .644736$ H $-9.1045795 .726857 \quad 1.956528$ H -7.084845 5.309931-1.082768 H -7.662731 $3.692295-1.331129$ H -5.2323804 .5953860 .365001$ H $-4.362037 \quad 0.666498 \quad 2.248273$ C $4.4198343 .263947-0.804830$ O $4.6544612 .435475-1.965888$ C $5.9080442 .812982-2.550034$ C $6.5884233 .740663-1.551568$ C $5.3861994 .437781-0.939583$ H $5.6627113 .412550-3.438048$ C $6.7277901 .606784-3.022143$ H $7.1327213 .176095-0.786347$ H $7.2882314 .433187-2.028783$ C $5.695990 \quad 5.139348 \quad 0.371134$ H $4.9931365 .168730-1.658943$ C $7.1307910 .554466-1.964269$ O $7.9225332 .125631-3.620973$ H $6.1726351 .103220-3.823438$ H $7.8033131 .005567-1.226771$

O $7.917922-0.442871-2.634550$ C $5.965709-0.144685-1.237958$ C $6.421563-1.314019-0.344307$ H $5.4531620 .584355-0.608786$ O $5.015860-0.622023-2.189488$ H $\quad 6.899388-2.084310-0.961451$ H $7.178564-0.9437220 .355500$ H 4.7987655 .6110710 .783659 H 6.0828964 .4394931 .119591 H $6.448700 \quad 5.920068 \quad 0.220432$ C $2.9439853 .654276-0.755627$ H $8.438806 \quad 1.367143-3.943635$ H $7.369519-0.835578-3.334559$ H $5.433510-1.337368-2.697858$ H $2.6106614 .001243-1.741152$ H $2.7810734 .471795-0.044823$ H 4.6866282 .6663180 .076547 C $5.100010-0.4475822 .284774$ O $4.463400-1.0131691 .136224$ C $5.248734-1.9713210 .421013$

C $5.665421-3.1179451 .353256$ C $6.364669-2.6062232 .610603$ C $5.522593-1.5260803 .289439$ H 5.9942430 .1000221 .959747

C $4.163278 \quad 0.6132732 .882551$ H $4.582383-2.402490-0.336994$ O $6.523739-4.034306 \quad 0.682068$ H $4.772395-3.6783311 .659617$ H $7.372722-2.2367142 .388872$ O $6.528479-3.6878253 .530962$ H $4.649975-1.9955493 .756150$ O $6.284269-0.9209294 .332225$ C 2.7692190 .1195323 .216740 H 4.6026431 .0490803 .786030 H 4.0415881 .4087942 .138096 H $6.047452-4.360539-0.100542$ H $7.029171-4.3840603 .071931$ H $6.566363-1.6307034 .934544$ O 1.9895301 .1943813 .541923 O $2.401663-1.0463913 .200109$ C $0.628017 \quad 0.892075 \quad 3.863215$ H $\quad 0.145906 \quad 0.3667853 .033076$ H $\quad 0.5823140 .2932054 .777601$ H $\quad 0.1012971 .8349664 .032999$
SCF Energy (B3LYP/6-31G**//MMFF)= -3245.90430146

03 124

MMFF Geometry

C $3.553583-3.966333-0.763687$

C $2.474829-3.697351-1.515467$

C $1.096005-3.513945-0.936892$

O $0.532005-2.359202-1.599756$

C $0.216012-4.749809-1.215936$

C $-0.955947-4.906386-0.261696$

C $-2.258164-4.882804-0.609228$

C $-0.586909-5.1647971 .179950$

C $-2.867907-4.719264-1.971679$

C $-3.419769-3.317484-2.268701$

C $-4.589861-2.871745-1.373183$

C $-5.204047-1.577266-1.917208$

C $-6.309397-1.023808-1.005516$

O $-4.104992-2.651957-0.044138$

O $-6.5709710 .331943-1.402174$

C $-5.787277-0.9734170 .446119$

C $-7.626834-1.787661-1.182381$

O $-6.830734-0.6168091 .348543$

C $-5.121437-2.2873420 .903189$

C $-4.456065-2.1804552 .299246$

O $-6.120456-3.310598 \quad 1.002339$

C $-0.288375-1.553534-0.878927$

O $-0.628412-1.6999720 .283226$

C $-0.715436-0.446872-1.763645$

C $-1.6894290 .376163-1.350580$

C $-2.2292801 .525278-2.076730$

C $-1.5940841 .888015-3.391900$

C $-3.355451-1.1246232 .430454$

C -3.8404410 .2284472 .924195$

C -3.1347882 .6208323 .031715$

O $-4.906083 \quad 0.387817 \quad 3.513046$

C -3.8872823 .3030801 .933259$

C $-3.367477 \quad 4.260817 \quad 1.150336$

C -4.1328094 .9463460 .053648$

C $-3.5502894 .688952-1.319563$

C $-2.7552745 .594571-1.913758$

C $-3.9607363 .423136-2.041040$

C $-3.2488282 .208290-1.516776$

N -2.916376 1.2329192 .668016

H $3.420497-4.0934280 .306608$

H $2.581746-3.603254-2.593499$

H $1.191201-3.3151050 .136708$

H $0.817581-5.664921-1.127478$

H $-0.126443-4.711254-2.254977$

H $-2.992597-5.0314030 .182093$

H -1.453390 -5.451434 1.785895

H $\quad 0.137959-5.9828641 .245960$

H $-0.150649-4.2729781 .638770$

H $-2.149945-4.982520-2.754722$

H $-3.679337-5.451825-2.068846$

H -2.601172 -2.596329 -2.189760

H $-3.752016-3.311025-3.314578$

H $-5.338768-3.672273-1.360271$

H -5.587457 -1.727022 -2.934379

H $-4.419357-0.815068-2.006886$

H $-6.8220550 .326378-2.341686$

H $-5.045558-0.1662300 .487478$

H -7.980705 -1.705399 -2.217262

H -8.420116 -1.357855 -0.560843

H -7.541115 -2.850730 -0.948791

H $\quad-7.206090 \quad 0.225379 \quad 1.039377$

H $-5.223669-2.0582543 .073081$

H -3.985524 -3.152398 2.507234

H $-5.784138-4.0142461 .581801$

H $-0.225223-0.344620-2.724821$

H $-2.1400350 .195135-0.376964$

H $-1.8029701 .116286-4.140701$

H $-0.5082351 .985750-3.287771$

H -1.947609 2.839418 -3.795869

H $-2.627067-1.4709973 .174610$

H -2.823096 -1.005788 1.484556

H -2.1531933 .0727983 .204659$

H -3.7091452 .6703343 .962206$

H -4.9182472 .9925181 .779139$ 
H -2.3395374 .5782621 .307782$ H $-4.129925 \quad 6.0229700 .268718$ H -5.1897674 .6519840 .059881$ H -2.357080 $5.429655-2.909374$ H -2.478865 6.517206-1.414406 H -3.820305 $3.533113-3.121516$ H $-5.0414103 .272812-1.920658$ H -3.644861 $1.849039-0.567510$ H $-2.087387 \quad 1.0160942 .122288$ C $6.039645-3.389568-0.573059$ O $5.818212-1.966590-0.614488$ C $6.336656-1.3934290 .604809$ C $7.019324-2.5294501 .367614$ C $6.232321-3.7370300 .901788$ H $5.470870-1.0533541 .184446$ C $7.270541-0.2159210 .304477$ H $8.074835-2.6312831 .089010$ H $6.976736-2.3805942 .451209$ C $6.941432-5.0578241 .143042$ H $5.276029-3.7407401 .437035$ C $6.6253910 .947607-0.473323$ O $8.372346-0.701735-0.474955$ H $7.699656 \quad 0.153297 \quad 1.243896$ H $6.4010020 .632269-1.498928$ O $7.6204871 .975490-0.591226$ C $5.357544 \quad 1.5433560 .171440$ C $4.9257202 .856113-0.513086$ H $4.550590 \quad 0.8043190 .118824$ O 5.6260671 .8020971 .549375 H $5.682473 \quad 3.628872-0.329845$ H $4.8727182 .675307-1.593886$ H $6.334059-5.8965750 .789056$ H $7.905672-5.0967920 .625176$ H $7.128264-5.2043272 .211891$ C $4.937309-4.146962-1.321705$ H $8.9572120 .054890-0.652707$ H 7.7995752 .3136860 .303140 H 4.7730471 .9205721 .999614 H $5.171399-5.217857-1.315243$ H $4.949806-3.821250-2.369789$ H $6.981110-3.562938-1.113066$ C $1.2245212 .765985-0.021421$ O $2.5651702 .456719-0.420617$ C $3.5686663 .391518-0.016653$ C $3.2646944 .787717-0.584766$ C $1.8532685 .244540-0.203991$ C $0.8092644 .165193-0.507452$ H $0.6047202 .034084-0.549809$ C 1.0077002 .5570381 .484571 H 3.5908353 .4501471 .075850 O $3.3955574 .765575-2.004677$ H $4.0069625 .495298-0.195746$ H $1.6100476 .158463-0.759273$ O $1.8354895 .563748 \quad 1.184067$ H $-0.1590714 .430050-0.068682$ O $0.6185524 .091208-1.922240$ C 1.1173981 .0990191 .880507 H $1.727513 \quad 3.1085792 .094214$ H $-0.0023712 .895963 \quad 1.742954$ H $3.2295435 .667036-2.328985$ H 0.9389285 .8698511 .403248 H $0.3046154 .960981-2.222426$ O $0.477486 \quad 0.913353 \quad 3.073096$ $\begin{array}{lllll}\text { O } & 1.714019 & 0.239346 & 1.247084\end{array}$ C $0.518944-0.4181313 .595502$ H $-0.038560-0.4311764 .535771$ H $0.045365-1.1156332 .898958$ H $1.553148-0.7137343 .795195$ SCF Energy $\left(B 3 L Y P / 6-31 G^{* *} / / M M F F\right)=-3245.89511689$

$03 \quad 125$

MM̄̄FF Geometry

C $0.9547925 .499454-0.369409$ C $0.208038 \quad 4.7465860 .452279$ C $-1.156376 \quad 4.2158600 .091496$ O $-1.1603952 .777048 \quad 0.238423$ C -2.192262 4.8001951 .071066 C -3.6291244 .4443550 .746655$
C $-4.4422593 .734544 \quad 1.553338$

C $-4.1563514 .975265-0.562055$

C -4.1315203 .1406482 .899273$

C -3.6390241 .6848942 .856539$

C -4.6659340 .6899472 .290802$

C $-4.181084-0.7512762 .462887$

C $-5.139029-1.7642841 .813106$

O $-4.840617 \quad 0.9669390 .900139$

O $-4.455438-3.0243971 .726555$

C $-5.446830-1.3184920 .365767$

C $-6.381927-2.0053232 .678397$

O $-6.461437-2.133617-0.214551$

C $\begin{array}{llll}-5.839810 & 0.168241 & 0.254461\end{array}$

C $-5.9460990 .669517-1.205844$

O

C $-0.6245042 .040683-0.773639$

O $-0.1046152 .461181-1.794796$

C $-0.7774860 .611352-0.417728$

C $-0.133282-0.326762-1.129000$

C $-0.178258-1.773069-0.893158$

C $-1.162855-2.2770380 .128172$

C $-4.6638550 .548622-2.036934$

C $-4.609890-0.698736-2.902471$

C $-3.076024-1.941687-4.419189$

O $-5.568930-1.446908-3.070455$

C $-2.533813-3.108356-3.656293$

C $-1.403626-3.745930-3.995796$

C $-0.871052-4.946299-3.269036$

C $0.561627-4.766034-2.823325$

C $1.576992-5.281807-3.536635$

C $0.808233-4.066858-1.505798$

C $0.651304-2.571876-1.597405$

N $-3.367321-0.856688-3.501642$

H $0.5811895 .741458-1.361087$

H 0.5849034 .5066721 .443997

H -1.420252 $4.478447-0.939983$

H -2.1153975 .8960581 .080869$

H -1.9359934 .4708002 .083848$

H -5.4630943 .5560241 .216481$

H -3.674963 $4.471337-1.405487$

H $-5.2367374 .826405-0.665629$

H -3.967194 $6.050843-0.640516$

H -3.394092 3.7504943 .431750

H -5.040585 3.1952093 .511931

H -2.7171581 .6413222 .264259$

H -3.3793901 .3886773 .880490$

H -5.6161980 .8385712 .817113$

H -4.034372 -0.9887113 .523890$

H -3.193677 -0.8590291 .994657$

H $-4.187578-3.2824402 .625230$

H $-4.536246-1.489948-0.219975$

H -6.093360 -2.391662 3.663395

H -7.029732 -2.770025 2.236016

H $-6.973651-1.1018832 .840019$

H -6.165413 -3.057418 -0.145853

H $-6.8016510 .198870-1.704896$

H $-6.1916281 .740767-1.162023$

H $-7.511273 \quad 1.1811100 .504276$

H -1.4090530 .3711010 .429635$

H $\quad 0.505167-0.008463-1.952190$

H $-0.896586-1.919397 \quad 1.128610$

H -1.224115 -3.365231 0.183111

H -2.172072 -1.928587 -0.114457

H $-4.618754 \quad 1.399539-2.728767$

H $-3.7723850 .595882-1.403187$

H $-2.363796-1.561038-5.157950$

H -3.994705 -2.235329 -4.937251

H -3.109894 -3.462177 -2.804346

H $-0.844288-3.407841-4.865363$

H $-0.945930-5.806262-3.947447$

H - $-1.493507-5.200041-2.401975$

H $2.606367-5.184191-3.208536$

H $1.401292-5.809893-4.468021$

H $1.833172-4.263516-1.169112$

H $\quad 0.171978-4.521709-0.742437$

H $1.335212-2.106350-2.308780$

H -2.611003 -0.223902 -3.257475

C $3.4883625 .316042-0.555401$ 
O $3.5300503 .983473-0.011230$

C $4.0061983 .087019-1.034178$

C $4.497040 \quad 3.968032-2.180347$

C $3.5526745 .151562-2.071928$

H $3.1363942 .521700-1.387833$

C $5.0533572 .131492-0.451430$

H $5.5332274 .293031-2.031593$

H $4.4402473 .459057-3.147297$

C $4.0330226 .388884-2.808351$

H $2.5784274 .849214-2.475460$

C 4.5741391 .3541540 .796300

$\begin{array}{lllll}\text { O } & 6.199041 & 2.904787 & -0.072257\end{array}$

H $5.3887551 .436934-1.231076$

H 4.4129592 .0418121 .635155

$\begin{array}{lllll}\text { O } & 5.636107 & 0.472957 & 1.175461\end{array}$

C 3.3012970 .5221160 .556203

C $2.687978-0.1684731 .791361$

H $2.514266 \quad 1.1830990 .179595$

O $3.537323-0.431817-0.473816$

H $2.277187 \quad 0.6183892 .436640$

H $1.834531-0.7588861 .438548$

H $3.3249337 .214245-2.684572$

H $5.0087926 .722747-2.439743$

H $4.1309556 .184977-3.879629$

C $2.2893456 .073786 \quad 0.013889$

H 6.9319502 .2850790 .081333

H $6.352251 \quad 1.0176921 .543138$

H $4.323969-0.940609-0.215901$

H $2.3210017 .119649-0.313287$

H 2.3735646 .0874851 .108468

H $4.3998105 .815254-0.199378$

C $3.496859-3.122787 \quad 1.363871$

O $4.297345-2.0619421 .895332$

C $3.592652-1.0767812 .650917$

C $2.860470-1.7459563 .826314$

C $1.959555-2.8854653 .359149$

C $2.742936-3.8562582 .478985$

H $2.784626-2.7133780 .639597$

C $4.438745-4.084066 \quad 0.627074$

H $4.353292-0.4258773 .097465$

O $2.079381-0.8010464 .550169$

H $3.599497-2.1558034 .527206$

H $1.071095-2.5112362 .837798$

O $1.481121-3.6042164 .498559$

H $3.447310-4.4241813 .100488$

O $1.838761-4.792123 \quad 1.894827$

C $5.005913-3.452148-0.621927$

H $5.272192-4.3945301 .269248$

H $3.920826-4.9989750 .317367$

H $2.679010-0.0979994 .853361$

H $1.019992-2.9658555 .069525$

H $1.357431-5.2231632 .621791$

O $6.162297-2.791592-0.327998$

O $4.471399-3.514907-1.721259$

C $6.787870-2.122981-1.427845$

H $7.096989-2.850539-2.184257$

H $6.109879-1.380739-1.859656$

H $7.675736-1.607890-1.051439$

SCF Energy (B3LYP/6-31G**//MMFF) $=-3245.90864919$

03 126

MMFF Geometry

C $-3.357636-2.730522-2.458412$

C $-2.151486-2.197574-2.710884$

C $-0.802824-2.768646-2.319423$

O $-0.037899-1.720289-1.672184$

C $-0.850773-3.970651-1.353603$

C $0.506933-4.546198-0.998550$

C $1.011819-4.5968590 .250193$

C $1.294877-5.122547-2.146730$

C $0.380633-4.1106861 .526068$

C $0.767226-2.6745901 .912078$

C $2.233769-2.5168252 .345269$

C $2.508226-1.0930102 .833768$

C $3.988459-0.8861463 .196903$

O $3.074173-2.8014701 .226649$

O $4.218141 \quad 0.5262403 .319724$

C $4.874226-1.3876222 .034196$
C $4.330218-1.5006534 .559696$

O $6.251774-1.3774442 .399507$

C $4.477496-2.7847411 .518265$

C $5.215017-3.1892720 .219114$

O $4.808740-3.7631612 .511328$

C $0.734697-0.927220-2.464535$

O $0.831014-0.971067-3.680839$

C $1.4459300 .031679-1.591212$

C $1.9737741 .141268-2.130305$

C $2.6680302 .205043-1.402106$

C $3.123157 \quad 1.903543-0.000044$

C $4.980189-2.267871-0.983752$

C $6.083292-1.245802-1.202207$

C $6.6912070 .655184-2.694272$

O $7.128905-1.229785-0.558835$

C $6.4423521 .923910-1.943053$

C $6.2073863 .100802-2.542501$

C $6.0099314 .390976-1.801242$

C $4.7147445 .079784-2.164645$

C $4.6948466 .098949-3.040262$

C $3.4598114 .646683-1.442849$

C $2.8600043 .390918-2.016921$

N $5.773472-0.369893-2.234602$

H $-3.434033-3.665425-1.911330$

H $-2.113777-1.279641-3.295232$

H $-0.298781-3.077826-3.243532$

H $-1.442526-4.782019-1.796589$

H $-1.378322-3.660627-0.447698$

H $1.992983-5.0508910 .384225$

H $2.191302-5.654531-1.810105$

H $0.683148-5.837015-2.707201$

H $\quad 1.621347-4.330247-2.827135$

H $-0.709913-4.1822931 .466803$

H $0.665629-4.7963242 .334131$

H $\quad 0.556176-2.009778 \quad 1.066031$

H $0.119063-2.3612652 .739931$

H $2.432174-3.2399953 .145115$

H $1.865091-0.8421563 .686585$

H $2.243308-0.3801852 .041049$

H 3.6148230 .8699234 .000782

H $4.765784-0.6654971 .217962$

H $3.713746-1.0511855 .347535$

H $5.369079-1.2920574 .838254$

H $4.173141-2.5806404 .594354$

H $6.467000-0.4760112 .694214$

H $6.283121-3.3344320 .420644$

H $4.839073-4.181934-0.068717$

H $4.883402-4.6296642 .078419$

H $1.487630-0.183830-0.529771$

H $1.8510411 .311940-3.199127$

H 3.6726500 .9573940 .023847

H 2.2674501 .8280390 .677701

H 3.8033522 .6542670 .407914

H $4.954965-2.886062-1.890597$

H $4.015870-1.754247-0.912439$

H $6.5345350 .772476-3.771233$

H $7.7208130 .323345-2.525810$

H $6.4878431 .878298-0.857363$

H $6.1892713 .149230-3.629072$

H $6.8601275 .043970-2.038663$

H $6.0498964 .240327-0.715270$

H $3.7733746 .617811-3.282951$

H $5.599016 \quad 6.433377-3.538116$

H $2.6948155 .431221-1.522992$

H $3.6658574 .572995-0.371366$

H $2.4962173 .508858-3.038699$

H $4.857201-0.417741-2.670733$

C $-5.698003-2.040829-1.829208$

O $-5.463183-0.838552-1.064288$

C $-6.726253-0.176641-0.847389$

C $-7.801233-1.192059-1.210651$

C $-7.131314-1.927265-2.352352$

H -6.776162 $0.648938-1.567125$

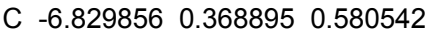

H $-8.006837-1.880589-0.382684$

H -8.743559 -0.715886 - 1.497975

C $-7.781976-3.262052-2.674632$

H $-7.155600-1.289602-3.246499$ 
C $-5.710197 \quad 1.358374 \quad 0.962624$ O $-6.773824-0.733647 \quad 1.493562$ H -7.8123180 .8348410 .722649$ H -4.7613240 .8178131 .018978$ $\begin{array}{lllll}\text { O } & -5.981466 & 1.829451 & 2.288191\end{array}$ C -5.5744132 .5642420 .018511$ C -4.5827163 .6512520 .473326$ H $-5.2721652 .214530-0.975142$ O $-6.8594783 .176902-0.123470$ H -4.616047 $4.451660-0.277859$ H $-4.9375814 .103367 \quad 1.408414$ H -7.259433 -3.765186 -3.494092 H -7.773373 -3.932133 -1.808387 H -8.824276 -3.117983 -2.977387 C $-4.660710-2.160211-2.950111$ H $-6.824198-0.3679302 .393375$ H -6.7978472 .3569552 .250672$ H $-6.7749453 .890518-0.778579$ H -4.499206 -1.180879 -3.416797 H -5.030428 -2.840939-3.725722 H -5.618411 -2.882551 - 1.128812 C - -1.7206612 .0153692 .247273$ O -3.0049372 .5714951 .930122$ C -3.1382783 .1469810 .625645$ C $-2.1259334 .288640 \quad 0.451133$ C $-0.700375 \quad 3.797570 \quad 0.709824$ C -0.5920183 .0405342 .034177$ H -1.7680931 .8060063 .323268$ C -1.4878620 .6823341 .516548$ H -2.953743 2.380829 -0.135183 O $-2.4359915 .369467 \quad 1.327497$ H -2.201142 $4.674952-0.572195$ H -0.0132844 .6524680 .725499$ O $-0.3059652 .930369-0.346947$ H 0.3818162 .5432632 .107426 O -0.6466384 .0036723 .091397$ C -2.393044 -0.4074542 .055393$ H -1.6714660 .7780820 .443138$ H $-0.457387 \quad 0.3414431 .664251$ H -2.4443565 .0230532 .236168$ H $-0.3321143 .442110-1.173384$ H -0.4981803 .5308713 .928046$ O $-2.558606-1.3765271 .107723$ O $-2.875227-0.4230593 .180416$ C $-3.393313-2.4699441 .502740$ H $-4.381182-2.1093881 .802865$ H -3.514021 -3.1386000 .647748$ H $-2.921898-3.0257602 .318761$

SCF Energy (B3LYP/6-31G**//MMFF) $=-3245.90403769$

$03 \_127$

MMFF Geometry

C $-0.936466 \quad 4.569718 \quad 0.958692$

C $0.2983495 .033772 \quad 1.204822$

C $1.583251 \quad 4.3780190 .749212$

O 1.4110222 .9504010 .586021

C $2.0366534 .966923-0.598202$

C $3.3986854 .473589-1.049185$

C $3.6218563 .731776-2.152123$

C $4.5626354 .890637-0.186020$

C $2.6230423 .244020-3.163198$

C $2.0596151 .841992-2.888334$

C $3.1047860 .715977-2.911554$

C $2.418687-0.651504-2.857534$

C $3.433386-1.803247-2.782506$

O $3.9567580 .864830-1.773750$

O $2.721005-2.992589-2.407093$

C $4.449265-1.510942-1.656438$

C $4.068857-2.091073-4.147803$

O $5.506695-2.466303-1.662556$

C $5.030470-0.083224-1.707359$

C $5.8790340 .273999-0.462295$

O $5.889660 \quad 0.034073-2.847950$

C 1.3761812 .2006291 .722179

O 1.4893882 .5993432 .870679

C 1.1471970 .7940111 .329663

C $0.714283-0.0776392 .252501$

C $0.459512-1.5035882 .043014$
C $0.928229-2.1080440 .746881$

C $5.138135 \quad 0.212462 \quad 0.878480$

C $5.329172-1.0930811 .633310$

C $4.564414-2.2679043 .694738$

O $6.133730-1.9572601 .296236$

C $3.544751-3.2937613 .312741$

C $2.630338-3.7764534 .167893$

C $1.638630-4.8483943 .817378$

C $0.210765-4.4405684 .102554$

C $-0.409099-4.8310425 .228977$

C $-0.523959-3.6574523 .038531$

C $-0.146229-2.2019273 .026010$

N 4.518048-1.160296 2.758868

H -1.058393 3.6843660 .340995

H 0.4139635 .9341421 .804037

H 2.3480684 .5544821 .515296

H $2.0771236 .062432-0.532884$

H $1.2764384 .736716-1.352364$

H $4.6492463 .442513-2.371765$

H $5.5274464 .639784-0.640258$

H $4.5496495 .973954-0.027707$

H 4.5208224 .3928850 .787401

H $1.7898103 .949116-3.253998$

H $3.1076773 .249792-4.147993$

H $1.5512961 .849931-1.917486$

H $1.2998861 .635889-3.652076$

H $3.6969890 .811645-3.829168$

H $1.749326-0.786228-3.716529$

H $1.774531-0.699066-1.968993$

H $2.027236-3.148299-3.070712$

H $3.917265-1.642532-0.707755$

H $3.297687-2.359262-4.879857$

H $4.743867-2.952486-4.096141$

H $4.623318-1.240764-4.550308$

H $5.100953-3.348081-1.604228$

H $6.797690-0.324646-0.447268$

H $6.2139811 .313942-0.588346$

H $6.4988680 .776256-2.700156$

H $1.335136 \quad 0.517887 \quad 0.298932$

H $\quad 0.5165270 .287042 \quad 3.260037$

H $0.339026-1.725080-0.092101$

H $0.862766-3.1976220 .723190$

H $1.982119-1.8678290 .573730$

H 5.5385421 .0000631 .530035

H 4.0693900 .4080430 .749693

H $4.384777-1.8567134 .693137$

H $5.562570-2.7173173 .677421$

H $3.581814-3.6807622 .297107$

H $2.615023-3.4081105 .191423$

H $1.894481-5.7415684 .402514$

H $1.728860-5.1500532 .766431$

H -1.442961 -4.567338 5.425990

H $\quad 0.105797-5.4187605 .981768$

H -1.607086 -3.707740 3.214626

H $-0.376533-4.1475552 .072760$

H $-0.437861-1.6707303 .933237$

H $3.843219-0.4208602 .931768$

C $-3.142146 \quad 4.1488682 .136672$

O -3.6738223 .2953021 .103457$

C -3.5910571 .9296341 .542854$

C -3.4234521 .9980973 .055659$

C -2.541322 3.2244243 .198739

H -2.6792211 .5129351 .094322$

C -4.7953441 .1052601 .065185$

H -4.3825072 .1603643 .561167$

H -2.9690091 .0914013 .466071$

C -2.5337333 .8194834 .595442$

H -1.5170412 .9343562 .937275$

C $-4.9779751 .115770-0.465623$

O $-5.9919791 .578244 \quad 1.682409$

H $-4.6452910 .079007 \quad 1.415098$

H -4.033992 $0.869270-0.960346$

O $-5.3293402 .433966-0.905551$

C $-6.0856200 .176265-0.975123$

C $-5.935272-1.307276-0.589880$

H -7.063095 $0.529967-0.625256$

O $-6.1313010 .259810-2.404656$

H $-6.148995-1.4283880 .478440$ 
H $-6.706399-1.861989-1.140371$ H -1.8978474 .7096144 .633710$ H -3.5405864 .1089984 .914647$ H -2.147274 3.0951755 .319895 C -2.1928405 .1805481 .513419$ H -6.1246052 .4971241 .391875$ H $-4.5911633 .025283-0.681448$ H $-6.2320261 .198707-2.637215$ H -1.949552 5.9441922 .261438 H -2.7090285 .6806090 .684891$ H -4.0056754 .6858982 .550025$ C -3.034325 -2.284455 -2.757571 O $-4.316001-1.823672-2.308857$ C $-4.559297-1.932588-0.904634$ C $-4.483595-3.404465-0.468847$ C $-3.144581-4.028132-0.875178$ C $-2.800893-3.746874-2.342859$ H $-3.095019-2.256903-3.853345$ C $-1.904649-1.334021-2.327780$ H $-3.797008-1.367524-0.360358$ O $-5.552915-4.135813-1.064085$ H -4.609895 -3.4578490 .619348$ H -3.188244 -5.111203-0.707781 O $-2.127142-3.501296-0.030926$ H -1.761801 -4.023004-2.557129 O $-3.629570-4.563447-3.175913$ C $-2.0843980 .052607-2.910769$ H $-1.865813-1.234942-1.239872$ H $-0.936889-1.712993-2.674738$ H -5.494837 -5.052666 -0.745692 H -1.290033 -3.927861 -0.281241 H $-3.448279-5.491718-2.950492$ O $-1.3888380 .953638-2.155408$ O $-2.742457 \quad 0.314115-3.908528$ C $-1.5057052 .318736-2.569497$ H $-1.2311922 .428778-3.623179$ H $-2.5254542 .674601-2.396662$ H $-0.8173792 .917259-1.968221$

SCF Energy (B3LYP/6-31G**//MMFF) $=-3245.89464256$

\section{8}

MM̄FF Geometry

C $2.118102-3.3999400 .893989$

C $1.096675-4.1238771 .375884$

C $-0.371363-3.761048 \quad 1.327015$

O $-0.569745-2.4640650 .719235$

C - $-1.125848-4.8077120 .484625$

C $-2.634847-4.746900 \quad 0.621346$

C $-3.490942-4.528314-0.395511$

C $-3.180396-5.0049212 .003653$

C $-3.176573-4.288244-1.844698$

C $-3.098689-2.805341-2.238202$

C $-4.410095-2.027926-2.033505$

C $-4.320059-0.641673-2.676343$

C $-5.5655630 .212636-2.391845$

O $-4.635303-1.891263-0.627674$

O $\quad-5.255908 \quad 1.573020-2.731792$

C $-5.8587320 .184468-0.875686$

C $-6.749461-0.193913-3.276999$

O $-7.0901070 .838524-0.582946$

C $-5.862861-1.238221-0.279841$

C $-5.978684-1.2629451 .265427$

O $-6.992086-1.957971-0.790598$

C $-0.647875-1.3795871 .534850$

O $-0.639910-1.3833842 .758418$

C $-0.751395-0.1191150 .763078$

C $-0.514430-0.033842-0.553858$

C $-0.583110 \quad 1.193668-1.339654$

C $0.5826481 .373117-2.272348$

C $-4.848993-0.5602672 .024671$

C -5.1194550 .9108952 .287318$

C -3.9292223 .0636262 .675501$

O -6.2503641 .3886302 .326486$

C $-3.887066 \quad 3.7027191 .322986$

C -2.8523124 .4257270 .870113$

C $-2.8185435 .066423-0.486415$

C -1.688899 $4.553802-1.351735$

C $-0.5789195 .286846-1.539657$
C -1.892602 $3.253907-2.095651$

C $-1.6355002 .032622-1.255481$

N $-3.949377 \quad 1.6233082 .507006$

H $1.925945-2.4468120 .410009$

H $\quad 1.322578-5.0662871 .872187$

H $-0.746276-3.7606552 .356683$

H $-0.815785-5.8196370 .779227$

H $-0.824913-4.689994-0.561923$

H $-4.557205-4.528180-0.170239$

H $-4.267299-5.1418892 .002513$

H $-2.739613-5.9149552 .423599$

H -2.958204 -4.166059 2.670147

H -2.240897 -4.781009 -2.128420

H $-3.951728-4.780348-2.445925$

H -2.296297 -2.331596-1.660615

H $-2.811163-2.755543-3.295678$

H -5.228936 -2.602975 -2.481491

H $-4.150227-0.723897-3.757196$

H $-3.443174-0.116370-2.277074$

H $-4.9839231 .594399-3.665343$

H $-5.0671170 .770476-0.393306$

H $-6.496780-0.064765-4.336453$

H -7.617463 $0.449611-3.096530$

H -7.053682 -1.233212 -3.137166

H $-7.037321 \quad 1.735459-0.955071$

H $-6.963392-0.8917141 .574114$

H $-5.955024-2.3176381 .575917$

H -7.181913 -2.699658 -0.192536

H $-0.998306 \quad 0.7643331 .344226$

H $-0.213408-0.912799-1.119274$

H $0.6746612 .394733-2.649530$

H $0.4934650 .697656-3.129822$

H $1.5236681 .149234-1.758076$

H -4.745006 -1.027467 3.012192

H -3.893904 -0.686279 1.504562

H -3.0517953 .3115463 .280785$

H -4.8280893 .3844023 .211698$

H -4.7568383 .5628110 .684840$

H $-1.9886114 .578074 \quad 1.512113$

H -2.720349 $6.150046-0.339027$

H $-3.7696284 .930312-1.016490$

H $0.2193804 .947185-2.190917$

H $-0.4404676 .240636-1.041319$

H -1.283619 $3.239670-3.006899$

H -2.925390 $3.208364-2.463539$

H $-2.439021 \quad 1.786566-0.562383$

H $-3.056337 \quad 1.1528872 .397380$

C $4.281247-3.753582-0.335815$

O $4.374443-2.380610-0.771984$

C $5.734462-2.100943-1.145737$

C $6.391506-3.459898-1.346114$

C $5.719900-4.270580-0.255800$

H $6.202500-1.611967-0.282457$

C $5.784719-1.160140-2.353914$

H $6.157425-3.890791-2.325511$

H $7.479761-3.416261-1.242993$

C $5.846182-5.772240-0.447464$

H $6.165636-3.9960380 .709937$

C $4.9927120 .151682-2.174805$

O $5.223668-1.816456-3.498076$

H $6.828066-0.932386-2.604843$

H $3.916878-0.059837-2.194067$

O $5.2479710 .962779-3.330991$

C $5.3287310 .960423-0.905149$

C $4.6631962 .349542-0.920726$

H $5.0051130 .395602-0.025647$

O $6.7446621 .118778-0.826997$

H $5.1184992 .949487-1.718056$

H $3.6053472 .226086-1.171261$

H $5.333091-6.3123540 .354374$

H $5.412774-6.093073-1.400715$

H $6.898739-6.073656-0.438610$

C $3.558649-3.8157491 .010784$

H $5.892345-2.431458-3.843378$

H $6.188083 \quad 1.212102-3.311850$

H $6.958521 \quad 1.4298330 .068494$

H $4.045050-3.1341171 .718361$

H $3.631425-4.8275991 .425873$ 
H $3.713821-4.294314-1.104023$

C 2.8246782 .3030091 .526295

$\begin{array}{lllll}\text { O } & 4.252611 & 2.423371 & 1.523001\end{array}$

C $4.8044243 .128780 \quad 0.406525$

C $4.227364 \quad 4.550724 \quad 0.353249$

C $2.7024294 .531096 \quad 0.309086$

C $2.1528753 .681146 \quad 1.451926$

H 2.5066001 .6777800 .683545

C 2.4197621 .5995572 .832445

H $5.877610 \quad 3.2307720 .607521$

O $4.7471515 .244251-0.775618$

H 4.5509965 .0997821 .247218

H $2.3291784 .176885-0.658723$

$\begin{array}{lllll}\text { O } & 2.206505 & 5.861289 & 0.474185\end{array}$

H 2.2939094 .2180262 .399160

$\begin{array}{lllll}\text { O } & 0.749534 & 3.503727 & 1.271281\end{array}$

C $2.860227 \quad 0.1545232 .888168$

H 2.8814992 .1165523 .682565

H 1.3305841 .6082542 .950578

H $4.4605216 .170658-0.710783$

H $2.4510976 .365604-0.319934$

H $\quad 0.353372 \quad 4.389407 \quad 1.206569$

O $2.580083-0.3388094 .131006$

O $3.372525-0.4639881 .967014$

C $2.944674-1.7031064 .361122$

H $3.976521-1.8909314 .048862$

H $2.865602-1.8987415 .433764$

H $2.252290-2.3632013 .832831$

SCF Energy (B3LYP/6-31G**/MMFF) $=-3245.89906606$

$03 \_129$

MMFF Geometry

C $3.007627-3.207183-2.989076$

C $1.990892-2.338211-3.090188$

C $0.554647-2.708124-2.817929$

O $0.037045-1.829799-1.791631$

C $-0.286439-2.519680-4.093637$

C $-1.756060-2.861770-3.926852$

C $-2.765092-2.013918-4.209401$

C $-2.064928-4.254035-3.441009$

C $-2.653822-0.618830-4.756628$

C $-2.472116 \quad 0.465210-3.686726$

C -3.731806 $0.736818-2.849370$

C $-3.4993811 .933684-1.923879$

C $-4.7105192 .206282-1.021922$

O $-4.030446-0.423332-2.070145$

O $-4.299626 \quad 3.125261 \quad 0.002159$

C $-5.1080470 .893368-0.317874$

C $-5.8522382 .888110-1.784197$

O $-6.324542 \quad 1.0543440 .409343$

C $-5.219605-0.316304-1.278084$

C $-5.394905-1.643031-0.499233$

O $-6.377630-0.144381-2.102732$

C $0.332769-2.155935-0.503063$

O $1.012161-3.097156-0.123131$

C $-0.316496-1.1631420 .382434$

C $-0.046746-1.1960431 .696121$

C -0.594302 -0.3173962 .731375$

C -1.5706890 .7475082 .310577$

C $-4.126166-2.1294120 .209708$

C $-4.416756-2.9655711 .443499$

C $-3.263613-3.8381413 .476946$

O $-5.542634-3.3389021 .759376$

C $-3.228568-2.7360324 .490618$

C $-2.272035-2.6070515 .422301$

C $-2.230541-1.4839076 .419614$

C $-0.918267-0.7288946 .399850$

C $-0.041130-0.8434357 .411438$

C $-0.654026 \quad 0.2178735 .244911$

C $-0.191804-0.5043794 .006339$

N $-3.255608-3.2374412 .156029$

H $2.796083-4.238395-2.718115$

H $2.193781-1.306728-3.367465$

H $0.478583-3.749784-2.481113$

H $0.118614-3.150822-4.895905$

H $-0.171273-1.483488-4.430360$

H -3.786916 -2.356695 -4.052819

H $-1.770565-4.372249-2.393695$
H -3.132407-4.489914 -3.509936

H $-1.528499-4.994340-4.043503$

H $-1.822520-0.562071-5.469081$

H $-3.551340-0.405001-5.350601$

H - $1.6424710 .184647-3.026133$

H $-2.1808501 .392179-4.196372$

H $-4.5639440 .937672-3.534262$

H -3.242256 $2.830890-2.500540$

H $-2.6314251 .729658-1.281022$

H $-3.965605 \quad 3.928579-0.432652$

H -4.3338000 .6878510 .429550$

H $-5.5144953 .842416-2.205815$

H $-6.6827523 .134963-1.113638$

H $-6.2406832 .283535-2.606427$

H $-6.207362 \quad 1.8135251 .005886$

H $-6.237514-1.5408620 .195418$

H $-5.698755-2.436256-1.196407$

H $-6.637671-1.011617-2.454724$

H $-0.993409-0.444960-0.065236$

H $0.647736-1.9530552 .059543$

H -1.1644691 .3489611 .490125$

H -1.8095281 .4550413 .107742$

H -2.510336 0.2940401 .983540

H $-3.524789-2.731839-0.481797$

H $-3.511046-1.2840660 .528218$

H $-2.388915-4.4923903 .544566$

H $-4.168281-4.4395413 .608804$

H -4.031667 -2.003280 4.450233

H $-1.477749-3.3478875 .476408$

H -2.404375 -1.9164027 .413432$

H $-3.051203-0.7742546 .255380$

H $\quad 0.890609-0.287507 \quad 7.416997$

H $-0.232074-1.4994308 .254311$

H $\quad 0.1305890 .934057 \quad 5.520929$

H -1.5589720 .8089445 .076606$

H $0.557564-1.2734064 .200668$

H -2.377561 -2.846391 1.828692

C $5.316856-2.818379-1.985039$

O $4.911108-1.732901-1.128710$

C $5.019829-2.166030 \quad 0.241874$

C $5.783273-3.4876990 .212663$

C $5.321427-4.070656-1.109283$

H $3.997783-2.3620570 .587600$

C $5.658924-1.0739681 .108233$

H $6.868371-3.3340320 .199821$

H $5.543731-4.1230251 .070843$

C $6.208094-5.186337-1.632159$

H $4.305526-4.458336-0.968001$

C 4.9747610 .3043450 .995475

$\begin{array}{llllll} & \text { O } & 7.023300 & -0.915043 & 0.698878\end{array}$

H $5.684758-1.4070112 .152821$

H $5.189248 \quad 0.7375190 .012379$

O $\quad 5.569710 \quad 1.1764101 .964214$

C $3.4565440 .286791 \quad 1.243232$

C 2.7920751 .6720261 .316423

H $2.960784-0.3071710 .472020$

$\begin{array}{lllll}\text { O } & 3.191571 & -0.380377 & 2.482998\end{array}$

H 1.7183161 .5178651 .476385

H 3.1168362 .1765312 .237544

H $5.837038-5.560149-2.591707$

H $7.238790-4.845959-1.778010$

H $6.227976-6.024091-0.927631$

C $4.445671-2.853926-3.244120$

H $7.474031-0.3864091 .378567$

H 6.4838961 .3482961 .682251

H 3.6599710 .1044813 .184044

H $4.854819-3.583306-3.952975$

H $4.498629-1.873007-3.733732$

H $6.343626-2.583077-2.296614$

C $1.2282064 .176598 \quad 0.650715$

$\begin{array}{lllll}\text { O } & 2.636681 & 3.967985 & 0.502830\end{array}$

C 3.0179112 .6322770 .130433

C $2.3506742 .267916-1.203772$

C $0.8369342 .458642-1.157799$

C $0.485318 \quad 3.858699-0.652556$

H $0.8541183 .523998 \quad 1.449202$

C 1.0100345 .6078941 .157312

H $4.0973552 .687522-0.044991$ 
O $2.6430110 .935957-1.599782$

H $2.7484082 .924775-1.989016$

H $0.3594441 .682387-0.551334$

$\begin{array}{llll}\text { O } & 0.326489 & 2.303294 & -2.484421\end{array}$

H $0.736388 \quad 4.579522-1.440039$

O $-0.923302 \quad 3.918789-0.445667$

C $1.344098 \quad 6.668501 \quad 0.134556$

H -0.0325715 .7559901 .462134$

H 1.6212805 .7986642 .048162

H $3.6060400 .867995-1.714364$

H $-0.6244992 .502391-2.453406$

H $-1.1592724 .847134-0.281094$

O $2.6932306 .850794 \quad 0.068381$

O $\quad 0.5022307 .256965-0.531871$

C $3.1369707 .821305-0.884715$

H $2.7361078 .808200-0.634910$

H $4.2285007 .864718-0.842722$

H $2.8362907 .525580-1.894375$

SCF Energy (B3LYP/6-31G*//MMFF) $=-3245.90672517$

0313

MM̄FF Geometry

C $1.965272-0.2143573 .510639$

C 2.4066281 .0193393 .800285

C 3.4881991 .7816513 .072626

$\begin{array}{llll}\text { O } & 4.285476 & 0.915528 & 2.230345\end{array}$

C 2.8885932 .8497742 .140573

C 2.2084384 .0002702 .848244

C $0.873794 \quad 4.1618412 .938288$

C 3.1365135 .0323313 .434223

C -0.2045443 .2390372 .440962$

C $-0.9917493 .830812 \quad 1.265229$

C $-0.1969413 .854270-0.050103$

C $-1.0021124 .553131-1.148157$

C $-0.2905714 .498105-2.507053$

O $0.0759142 .498774-0.425352$

O $-1.240154 \quad 4.870558-3.517544$

C $0.1247023 .040932-2.793339$

C $0.8557225 .512664-2.593009$

O $0.9158742 .962088-3.977469$

C $0.8643332 .362258-1.614996$

C $1.0326260 .852880-1.911477$

O $2.1533502 .960685-1.469324$

C $5.3505990 .293993 \quad 2.797089$

O $5.672042 \quad 0.3307203 .977452$

C $6.131149-0.4783091 .802835$

C $5.906899-0.4504690 .479388$

C $6.663666-1.186796-0.538761$

C $7.791553-2.066846-0.063430$

C $1.5935620 .020331-0.748932$

C $1.724399-1.444837-1.134147$

C $2.716459-3.589793-0.349559$

O $1.252905-1.908434-2.169750$

C $4.080545-3.721872-0.947676$

C $4.299133-3.858636-2.264163$

C $5.659313-3.971219-2.890772$

C $6.022472-2.738133-3.690087$

C $5.607583-2.591351-4.959780$

C $6.926481-1.712884-3.040231$

C $6.303704-1.059482-1.834268$

N 2.410796 $-2.180523-0.176864$

H $2.404702-0.7715612 .689217$

H 1.9329021 .5563864 .620039

H 4.1449342 .2620013 .808257

H 2.2154752 .3671451 .422953

H 3.6875803 .2579101 .505353

H 0.4984715 .0471143 .450573

H 2.5951035 .8692793 .887938

H $3.7646314 .587403 \quad 4.212040$

H 3.7859255 .4449082 .655218

H 0.1821142 .2512202 .177594

H -0.8987713 .0705603 .274131$

H -1.8885663 .2136661 .128489$

H -1.3304354 .8432011 .518187$

H $0.749210 \quad 4.381674 \quad 0.112821$

H -1.217830 $5.592309-0.869909$

H -1.979713 $4.064682-1.252456$

H -1.565324 $5.763126-3.308888$
H $-0.7942022 .473718-2.997505$

H $0.4758146 .531886-2.453768$

H $1.3211205 .500196-3.584769$

H $1.6330165 .346654-1.844198$

H $0.4084553 .382131-4.693039$

H $0.0546860 .431471-2.177923$

H $1.6902220 .720674-2.780404$

H $2.7534112 .330539-1.042031$

H $6.934744-1.0693682 .231102$

H $\begin{array}{llll}5.098934 & 0.168131 & 0.093764\end{array}$

H $7.424394-2.8088020 .654686$

H $8.570210-1.4653280 .418224$

H $8.275889-2.632099-0.861729$

H $2.5822150 .385067-0.453931$

H 0.9285500 .0804910 .119928

H $1.954773-4.060035-0.980393$

H $2.686962-4.064967 \quad 0.635527$

H $4.922540-3.703427-0.261245$

H $3.447628-3.878666-2.941129$

H $5.662041-4.852691-3.545283$

H $6.430873-4.170099-2.136895$

H $5.882545-1.723239-5.549330$

H $4.979898-3.337340-5.436178$

H $7.168153-0.911854-3.751561$

H $7.885909-2.186725-2.815516$

H $5.464517-0.406819-2.078928$

H $2.835971-1.6966530 .608207$

C $-0.410819-0.9704023 .370668$

O $-0.222128-1.9753332 .355149$

C -1.475141 -2.667449 2.147072

C $-2.510168-1.9308302 .985100$

C $-1.667918-1.3948224 .124234$

H -1.322262 -3.683784 2.530610

C -1.770718 -2.6983700 .643257$

H -2.955706 -1.1031192 .418622$

H $-3.318832-2.5863683 .321179$

C $-2.343305-0.2711094 .891558$

H -1.435502 -2.218739 4.812120

C $-3.173580-3.2142380 .287322$

O $-0.803900-3.5481450 .019407$

H $-1.614969-1.696307 \quad 0.228851$

H $-3.937130-2.574700 \quad 0.740857$

O $-3.342025-4.5085040 .878680$

C $-3.434664-3.339704-1.225700$

C $-3.166088-2.070793-2.058925$

H $-2.826574-4.156151-1.632804$

O $-4.792033-3.752826-1.418611$

H $-2.085175-1.893436-2.106524$

H $-3.509524-2.277280-3.080971$

H $-1.7021730 .092215 \quad 5.700532$

H -2.575309 0.5774594 .239033

H -3.281662 -0.6195035 .335801$

C $0.836686-0.8795394 .250265$

H $0.073584-3.2370440 .298730$

H $-4.228702-4.8251500 .634375$

H $-5.369522-3.052210-1.070464$

H $1.168948-1.8813524 .547760$

H $0.612077-0.3236495 .167736$

H $-0.585584-0.0194372 .850356$

C $-6.0877580 .072568-1.109638$

O $-5.280215-0.997464-1.618815$

C $-3.867192-0.793602-1.552346$

C $-3.4721800 .443809-2.373459$

C $-4.262337 \quad 1.679112-1.931333$

C $-5.7656691 .391983-1.832406$

H $-7.117734-0.206523-1.367364$

$\begin{array}{llll}\text { C } & -6.000778 & 0.178080 & 0.421367\end{array}$

H -3.575789 -0.628459 -0.511722

$\begin{array}{llll}\text { O } & -3.689467 & 0.212367 & -3.763366\end{array}$

H -2.400152 $0.626946-2.241788$

H $-4.0855302 .490073-2.648460$

O $-3.7682092 .101668-0.664423$

H $-6.2859692 .216920-1.331987$

O $-6.305994 \quad 1.296649-3.153717$

C $-6.481457-1.0813541 .111426$

H -4.9740850 .3587590 .750523$

H $-6.628786 \quad 1.0044620 .772761$

H - $-4.623834-0.029748-3.882089$ 
H $-4.2507752 .908100-0.415096$

H $-6.1460392 .146908-3.597822$

O $-6.105472-1.0379732 .424258$

O $-7.112365-1.9828350 .577099$

C $-6.471697-2.1786023 .206766$

H $-7.560918-2.2656273 .260256$

H $-6.030720-3.0867732 .784301$

H $-6.079489-2.0370874 .217327$

SCF Energy (B3LYP/6-31G**//MMFF) $=-3245.91606591$

03130

MM̄̄FF Geometry

C $0.5895994 .624866-0.430496$

C $1.6671233 .827379-0.472609$

C 2.6789313 .7417840 .639711

O $2.7252542 .375527 \quad 1.113754$

C 4.0749214 .0942700 .098392

C 5.1020674 .3078351 .192749

C 6.0160903 .3921091 .568904

C 5.0901705 .6736061 .828652

C 6.1460581 .9809621 .070832

C $7.2353231 .846108 \quad 0.001311$

C $7.4325440 .382918-0.421243$

C $8.5107680 .263025-1.499687$

C $8.661524-1.182751-2.000600$

O $6.187447-0.110633-0.925698$

O $9.428706-1.152197-3.213986$

C $7.267335-1.739752-2.361906$

C $9.450854-2.048594-1.011209$

O $7.337968-3.127335-2.682109$

C $6.204827-1.503066-1.264655$

C $4.799943-1.891399-1.783621$

O $6.511977-2.319990-0.130864$

C 1.7518741 .9608351 .968091

O 0.8109662 .6127442 .390118

C 2.0346240 .5454782 .295979

C $1.107109-0.2016022 .913400$

C $1.251622-1.6124043 .279595$

C $2.618876-2.2331563 .150624$

C $3.658799-1.371441-0.900806$

C $2.349948-2.050548-1.246399$

C $1.042902-4.132144-0.811541$

O $1.530216-1.528101-1.997926$

C $0.023218-3.7629350 .219112$

C $-0.484902-4.6173691 .121068$

C $-1.555890-4.2331312 .103390$

C -1.155396 -4.427813 3.550316

C $-1.910504-5.1796044 .369600$

C $0.101202-3.7546814 .069982$

C $0.172625-2.2868273 .730916$

N $2.216955-3.294827-0.648192$

H $0.428875 \quad 5.2595830 .437185$

H $1.8214613 .183640-1.335416$

H 2.4151344 .4060121 .472082

H $4.0268155 .011567-0.504248$

H $4.416163 \quad 3.309377-0.588472$

H 6.7360393 .6658202 .338759

H 5.8702595 .7850922 .589241

H 5.2553246 .4467551 .071175

H 4.1281145 .8626812 .314647

H $\quad 6.3878791 .3409361 .928583$

H 5.1913081 .6108800 .685699

H $6.9453212 .444720-0.872032$

H 8.1834032 .2509540 .376639

H $7.715321-0.1922840 .468415$

H $9.4717860 .645848-1.134283$

H $8.2413380 .897946-2.355434$

H $\quad 10.284897-0.734787-3.016882$

H $\quad 6.937725-1.227066-3.276526$

H $10.455529-1.636134-0.858709$

H $9.599933-3.060747-1.403206$

H $8.974232-2.123803-0.031708$

H $8.005573-3.229369-3.381996$

H $4.646325-1.473399-2.787146$

H $4.732514-2.982530-1.878239$

H $5.692046-2.5359380 .340490$

H 3.0008710 .1503842 .002394

H $\quad 0.1446990 .250078 \quad 3.150275$
H $2.870611-2.3926872 .099560$

H $2.707481-3.1942233 .662964$

H $3.383276-1.5903753 .602002$

H $3.866713-1.5299550 .161597$

H $3.528750-0.290742-1.033047$

H $1.367159-5.173357-0.719650$

H $0.627136-3.980705-1.812937$

H $-0.339818-2.7382980 .201009$

H $-0.143986-5.6495401 .134696$

H -2.445701 -4.831448 1.867980

H $-1.857322-3.1893721 .963364$

H -1.641643 -5.328342 5.410184

H -2.815510 -5.665168 4.019718

H $0.952966-4.3088893 .663972$

H $\quad 0.157536-3.8452515 .161985$

H $-0.766917-1.7463593 .849769$

H $2.931174-3.6101540 .001348$

C $-1.6762483 .822439-1.252187$

O $-1.3031902 .429815-1.266781$

C $-1.9959941 .754907-0.195504$

C -3.0832852 .7147370 .265696$

C -2.4079784 .0569110 .069065$

H $-1.251474 \quad 1.6073480 .596396$

C $-2.5076180 .404763-0.705936$

H $-3.9728382 .634294-0.371713$

H -3.3857282 .5381801 .301706$

C -3.3733105 .2283150 .039416$

H -1.6993794 .2020690 .893291$

C -3.382992 $-0.343098 \quad 0.312747$

O $-1.375421-0.412312-1.018058$

H $-3.0519040 .554435-1.644453$

H $-4.268407 \quad 0.2502540 .559788$

O $-2.656165-0.475643 \quad 1.541658$

C $-3.823476-1.749965-0.130134$

C $-4.538979-1.826675-1.491327$

H $-2.956524-2.419400-0.162224$

O $-4.694421-2.290540 \quad 0.868935$

H -3.812713 -1.638812 -2.290913

H $-4.894627-2.858605-1.609733$

H -2.837426 $6.169177-0.121072$

H $-4.1127445 .119659-0.761279$

H -3.9150705 .3042180 .987858$

C $-0.4436854 .690666-1.519579$

H $-0.8231270 .082445-1.647608$

H -1.813356 -0.9163211 .337168$

H $-4.229196-2.2354031 .721270$

H $-0.7448315 .736920-1.647324$

H $0.0114174 .376405-2.467655$

H -2.371754 $3.953758-2.091609$

C $-7.875432-0.338473-0.650145$

O $-6.721654-1.189792-0.676496$

C $-5.737644-0.871028-1.662330$

C $-6.349739-0.969557-3.069072$

C $-7.603492-0.096281-3.192671$

C $-8.570164-0.313921-2.022811$

H $-8.560918-0.8175730 .060853$

C $-7.5429781 .061197-0.108780$

H $-5.3867470 .152467-1.504681$

O $-6.684191-2.322107-3.374163$

H -5.601649 -0.651311-3.804271

H $-8.117474-0.329088-4.133200$

O $\quad-7.2297031 .277980-3.230865$

H $-9.3527830 .453789-2.021186$

O $-9.221595-1.577002-2.191066$

C -7.0453681 .0192191 .321233$

H $-6.7711871 .549042-0.709973$

H -8.442654 $1.686815-0.122329$

H -7.286190 -2.642981 -2.680919

H $-6.6440561 .401843-3.997053$

H -9.709702 -1.545182 -3.031598

O -6.5202642 .2388301 .643089$

$\begin{array}{llll}\text { O } & -7.116676 & 0.052067 & 2.066719\end{array}$

C -5.9948162 .3512342 .969373$

H -6.7962462 .2283013 .703686$

H $-5.205117 \quad 1.6107903 .130087$

H -5.5650293 .3503293 .080710$

SCF Energy (B3LYP/6-31G**//MMFF) $=-3245.91087779$ 
03_131

MM̄FF Geometry

C $2.5603935 .012108-1.003004$

C $3.1806363 .823709-0.967154$

C 4.1770223 .4416880 .095820

O 3.6766072 .2796450 .797376

C $5.5118393 .051063-0.560778$

C 6.6498202 .9224350 .432555

C 7.1097651 .7521880 .916765

C 7.3098614 .2183120 .826118

C $6.551972 \quad 0.3813480 .660507$

C $7.337250-0.366067-0.422668$

C $6.827836-1.804217-0.598460$

C $7.607773-2.532745-1.694328$

C $7.055084-3.944908-1.948384$

O $5.440786-1.750195-0.947038$

O $7.605675-4.418611-3.187039$

C $5.524567-3.861574-2.137447$

C $7.505384-4.935315-0.867810$

O $4.947563-5.162725-2.219247$

C $4.811811-3.034502-1.043443$

C $3.325886-2.817830-1.416146$

O $4.870328-3.7468180 .195945$

C 2.7233982 .4695551 .748652

O 2.2168673 .5264282 .087541

C 2.3924571 .1391382 .306209

C 1.3127800 .9882493 .087800

C $0.860655-0.2685223 .688281$

C $1.781965-1.4577853 .601159$

C $2.639297-1.732502-0.578123$

C $1.135349-1.784536-0.748832$

C $-0.897783-2.9586620 .098115$

O $0.551170-1.057421-1.548670$

C -1.519583 -2.021692 1.084760

C $-2.234046-2.4121252 .151815$

C $-2.910021-1.4474593 .085026$

C $-2.492338-1.6043394 .531476$

C $-3.414285-1.8099005 .487560$

C -1.021482 -1.512989 4.892215

C $-0.341164-0.3051174 .301674$

N $0.535663-2.7363060 .062342$

H $2.7850715 .754898-0.241709$

H $2.9459593 .075650-1.720949$

H 4.3306824 .2590000 .811528

H $5.7994403 .801733-1.309578$

H $5.3914772 .112880-1.117207$

H 7.9618481 .7719651 .594618

H 8.1442384 .0662491 .519050

H $7.7050034 .727489-0.058985$

H 6.5914994 .8818101 .317482

H $6.592196-0.1811161 .601827$

H $5.492674 \quad 0.432458 \quad 0.391566$

H $7.2332820 .178610-1.370013$

H $8.404567-0.383365-0.169362$

H $\quad 6.939504-2.3236390 .360729$

H $8.676252-2.576048-1.449165$

H $7.538314-1.961785-2.630918$

H $8.575002-4.405788-3.108356$

H $5.341065-3.379611-3.108158$

H $8.599856-4.999161-0.839434$

H $7.152932-5.948836-1.088924$

H $7.165862-4.661807 \quad 0.133271$

H $5.413873-5.642397-2.925236$

H $3.248057-2.510507-2.467175$

H $2.781092-3.765261-1.319079$

H $4.101913-3.5096520 .738696$

H 3.0418370 .3121312 .041499

H 0.6858941 .8565623 .286299

H $2.800622-1.1843703 .899517$

H $1.808378-1.8494612 .581626$

H $\quad 1.494507-2.2773104 .264123$

H $2.876777-1.8336370 .485094$

H $2.982624-0.735497-0.878821$

H -1.061035 -4.0079530 .362785$

H -1.317390 -2.778175 -0.896953

H -1.402486 -0.9589350 .887680$

H -2.377868 -3.4727262 .342722$

H $-3.992427-1.5924892 .981231$
H $-2.724213-0.4130972 .778176$

H -3.134272 -1.9212976 .529869$

H -4.471400 -1.872276 5.251251

H $-0.552231-2.4459334 .565913$

H $-0.898714-1.4627505 .981266$

H $-0.9193980 .617154 \quad 4.368537$

H $1.105919-3.2480490 .729105$

C $0.0928305 .183759-1.583371$

O $-0.1755063 .775897-1.431789$

C $-1.0149423 .587466-0.271436$

C -1.5034854 .9750530 .121688$

C $-0.3179845 .837471-0.265203$

H -0.3597713 .1747750 .505781$

C -2.126225 $2.591822-0.620709$

H $-2.3891975 .257185-0.461392$

H $-1.7566955 .046013 \quad 1.183613$

C $-0.6465177 .314500-0.386757$

H 0.4571585 .7066520 .499514

C -3.161812 2.396241 0.499723

O $-1.5174801 .327126-0.898489$

H $-2.6207782 .913411-1.543930$

H -3.6904773 .3375560 .688548$

O -2.4747772 .0814081 .716889$

C -4.2000681 .2844100 .237299$

C $-4.9917301 .506550-1.061748$

H -3.7180230 .3005910 .228686$

O $\quad-5.126001 \quad 1.2795231 .329003$

H $-5.4520972 .502176-1.032445$

H -4.293572 $1.478754-1.905503$

H $0.2386257 .886081-0.682981$

H $-1.4286127 .492238-1.132545$

H -1.000165 7.7100250 .571000

C $1.5399095 .391192-2.037998$

H $-0.8575021 .468636-1.598882$

H -1.8985821 .3187191 .537833$

H $-4.611084 \quad 1.1533842 .144417$

H $1.6982126 .441850-2.308055$

H $1.7132814 .803179-2.948443$

H $-0.5669065 .527862-2.391075$

C $-6.358591-1.920632-1.698255$

O $-5.475742-0.812328-1.470766$

C $-6.1019660 .463339-1.300074$

C $-6.9492890 .821927-2.533011$

C $-7.949937-0.286934-2.868845$

C $-7.277139-1.661685-2.905764$

H $-5.697015-2.755648-1.963681$

C -7.128031-2.312720 -0.426195

H $\quad-6.7498870 .432428-0.418251$

O $\quad-6.1127491 .060436-3.664099$

H -7.485592 $1.756615-2.332032$

H $-8.406807-0.082546-3.844788$

O $-8.990080-0.308681-1.896086$

H $-8.029784-2.456299-2.968928$

O $-6.471087-1.755838-4.083912$

C $-6.229567-2.9545610 .610100$

H $-7.608327-1.4539950 .048863$

H -7.895718 -3.051812 -0.684480

H $-5.5681790 .266894-3.803710$

H $-9.4209790 .562853-1.907124$

H -7.058504 -1.642568 -4.850510

O $-7.002147-3.417487 \quad 1.637654$

O $-5.012115-3.0392790 .535062$

C $-6.297597-4.0761122 .695082$

H $-5.701669-3.3500083 .254109$

H -7.035010 -4.518666 3.369955

H $-5.663000-4.8750322 .299196$

SCF Energy (B3LYP/6-31G**//MMFF)= -3245.91928269

03_132

MM̄FF Geometry

C 1.3884001 .5042083 .766346

C 0.2568391 .5838974 .482663

C -1.1014351 .0760824 .049538$

O -1.1378770 .7848912 .633017$

C $-1.437048-0.2131024 .820780$

C $-2.799018-0.7962774 .495309$

C -2.996562 -2.044496 4.026810

C -3.9812970 .1017734 .751952$ 
C - $-1.957715-3.0972513 .758831$ C -1.396621 -3.083904 2.330011 C $-2.409124-3.5240211 .259801$ C $-1.710950-3.693830-0.091663$ C $-2.697761-4.061945-1.208044$ O $-3.429710-2.528508 \quad 1.154381$ O $-2.023227-3.895053-2.464839$ C $-3.868102-3.058148-1.186662$ C $-3.122399-5.533287-1.135726$ O $-4.890012-3.449508-2.101623$ C $-4.471519-2.8423040 .221981$ C $-5.489335-1.6754610 .230418$ O $-5.174323-4.0283790 .609170$ C -1.3139571 .8451191 .796058$ O C -1.3201571 .3373530 .405689$ C -1.297735 $2.221428-0.603171$ C $-1.3436051 .923044-2.035605$ C -1.404457 $0.478990-2.455164$ C $-4.854604-0.2846170 .127401$ C $-5.7630640 .740760-0.527689$ C $-5.6572763 .029507-1.516863$ O $-6.9255140 .511036-0.848148$ C $-5.1159902 .907875-2.908128$ C $-4.4090223 .868497-3.522149$ C $-3.8428903 .726502-4.906540$ C $-2.3502103 .975475-4.958720$ C $-1.8552215 .082580-5.537832$ C $-1.4255712 .906533-4.408185$ C -1.324454 $2.955676-2.904975$ N $-5.1039331 .947800-0.723133$ H 1.3775951 .0129962 .797062 H 0.2890962 .0599565 .460563 H -1.846122 $1.848664 \quad 4.278458$ H -1.400795 -0.0179625 .900895$ H $-0.648506-0.9456634 .617170$ H -4.019058 -2.362214 3.826482 H $-4.934680-0.4284134 .651537$ H -3.938295 0.5050525 .768969 H $-3.994320 \quad 0.935513 \quad 4.043291$ H -1.128659 -3.0043334 .468868$ H -2.400310 -4.079170 3.969669 H -1.022825 -2.078594 2.098289 H -0.535378 -3.763070 2.305077 H -2.858569 -4.471919 1.578176 H $-0.914608-4.444896-0.026318$ H -1.216290 -2.751745 -0.365798 H -1.237174 -4.467989-2.461580 H -3.476324 -2.104786 -1.559552 H -2.247355 -6.188622 -1.222816 H $-3.777533-5.797048-1.973193$ H -3.632844 -5.787525 -0.204451 H -4.471678 -3.563594 -2.972216 H $-6.233654-1.845217-0.556934$ H -6.061338 -1.699462 1.168404 H $-5.807995-3.797756 \quad 1.308378$ H -1.357871 0.2650990 .253905 H -1.258332 $3.282405-0.357769$ H -2.372404 $0.044639-2.190289$ H -0.612942 -0.102741 -1.970628 H $-1.263511 \quad 0.335208-3.528950$ H -4.591886 0.0752181 .129523 H -3.933922 $-0.320750-0.460366$ H $-5.3652943 .969339-1.038370$ H $-6.7494302 .962739-1.532174$ H $-5.3108791 .975324-3.433178$ H -4.226756 $4.808484-3.006110$ H -4.369363 4.438819-5.554903 H $-4.0520262 .733149-5.322904$ H $-0.7862575 .257268-5.601278$ H -2.507204 5.841713 -5.957188 H $-0.4140573 .043343-4.812000$ H -1.771835 $1.936307-4.776125$ H -1.229642 $3.965168-2.501673$ H -4.127234 $2.020503-0.455535$ C 3.2969113 .0961783 .268432 O 3.4621492 .5326531 .952851 C 3.0438403 .5093130 .978054
C 2.9734694 .8354811 .724836

C 2.4789014 .3745373 .084868

H $2.0279333 .234306 \quad 0.670811$

C $3.9811143 .477013-0.233476$

H 3.9603465 .3036251 .817405

H 2.3006575 .5502591 .241647

C 2.6788985 .3974704 .188744

H 1.4091444 .1449143 .002763

C $4.1227542 .069616-0.852432$

O $5.2817903 .901203 \quad 0.192226$

H $3.6471024 .201483-0.985005$

H $4.595278 \quad 1.408467-0.118816$

O $5.0383922 .152778-1.951213$

C $2.7993931 .463192-1.352840$

C $2.9438820 .053361-1.943824$

H $2.0637951 .433173-0.544842$

O $2.2667782 .324449-2.362947$

H $1.976792-0.264889-2.357121$

H $3.5971690 .083886-2.826800$

H 2.3238665 .0083345 .148262

H 3.7348045 .6649994 .302553

H 2.1222936 .3142763 .968600

C 2.7147542 .0460744 .218753

H $5.8678763 .858823-0.582795$

H $4.6240882 .706260-2.635574$

H $1.4430951 .923938-2.687803$

H 2.6443572 .4634205 .229951

H 3.4065221 .1957854 .269078

H 4.3067423 .3483583 .618390

C $3.973442-3.395373-0.997357$

O $3.456209-2.255079-1.696119$

C $3.463465-1.018654-0.969985$

C $2.626316-1.1491380 .312765$

C $3.073723-2.3424841 .162386$

C $3.221387-3.6167440 .325939$

H $3.751577-4.248809-1.651427$

C $5.499114-3.321423-0.826405$

H $4.498737-0.773993-0.714663$

O $1.245004-1.291414-0.011156$

H $2.723710-0.2328560 .904344$

H $2.339575-2.5168321 .958790$

O $4.323420-2.0488811 .779845$

H $3.724310-4.4010310 .903915$

O $1.918695-4.108265-0.002288$

C $6.229643-3.367040-2.152970$

H $5.807320-2.404203-0.318324$

H $5.839594-4.181808-0.238642$

H $1.155573-2.062559-0.596898$

H $4.200576-1.2567482 .330649$

H $1.463419-4.3085630 .833159$

O $7.573730-3.291146-1.917886$

O $5.699845-3.458088-3.251605$

C $8.404414-3.319118-3.083011$

H $8.178268-2.466366-3.730102$

H $9.446251-3.247301-2.759403$

H $8.268346-4.261273-3.622348$

SCF Energy $(B 3 L Y P / 6-31 G * * / / M M F F)=-3245.91998925$

03133

MM̄FF Geometry

C $-2.117981-2.846296-2.028047$

C -1.192521 -3.772640 -2.322395

C $0.230554-3.812557-1.811294$

O $0.631859-2.545524-1.244444$

C $0.343345-4.886497-0.713468$

C $1.756477-5.110581-0.212669$

C $2.168386-4.8766641 .048932$

C $2.727735-5.664260-1.223784$

C $1.364851-4.3597992 .209576$

C $1.435789-2.8375522 .409825$

C $2.842417-2.3122592 .741464$

C $2.789925-0.8382633 .146750$

C $4.194490-0.2752813 .417930$

O $3.660145-2.4446561 .578779$

O 4.0894551 .1548023 .510190

C $5.127567-0.6153352 .229309$

C $4.711541-0.7225324 .790273$

O $6.470653-0.2657922 .552234$ 
C $5.037201-2.0900461 .769388$

C $5.751362-2.3754010 .428246$

O $5.671203-2.9688572 .707209$

C $1.057700-1.583959-2.109003$

O $1.141645-1.669198-3.323726$

C $1.384182-0.380858-1.313141$

C $1.5045720 .805137-1.927965$

C $1.802764 \quad 2.083722-1.279524$

C 2.2363832 .0456810 .161363

C $5.240811-1.577678-0.776295$

C $6.044719-0.322212-1.069225$

C $6.0864541 .635195-2.610615$

O $7.075538-0.025029-0.472076$

C $5.4903882 .812778-1.906966$

C $4.951633 \quad 3.858722-2.551247$

C $4.3900465 .068051-1.861287$

C $2.9645515 .360814-2.270172$

C $2.6884366 .290025-3.200687$

C $1.8579074 .639267-1.536185$

C $1.6802193 .218170-2.000239$

N $5.4859200 .415684-2.104340$

H - $1.873284-2.026310-1.358547$

H - $1.469736-4.592201-2.982928$

H $0.891501-4.060652-2.650680$

H $-0.036734-5.845954-1.089586$

H $-0.317182-4.605220 \quad 0.113738$

H $3.209633-5.087797 \quad 1.291159$

H $3.684684-5.944904-0.770343$

H $2.315368-6.561977-1.695636$

H $2.939405-4.924419-2.001668$

H $0.314920-4.6567932 .118774$

H $1.723097-4.8596493 .118837$

H $1.058806-2.3432751 .506560$

H $0.754077-2.5778973 .229262$

H $3.256345-2.9214453 .553246$

H $2.144839-0.6943814 .022417$

H $2.328399-0.2570802 .337131$

H $3.732174 \quad 1.4893552 .670538$

H 4.8234370 .0361891 .402122

H $4.058106-0.3429405 .585335$

H $5.703536-0.3060294 .996651$

H $4.761308-1.8080884 .898780$

H $7.004691-0.3568021 .745441$

H $6.837431-2.2790380 .546232$

H $5.584660-3.438190 \quad 0.198226$

H $6.089345-2.4356883 .401738$

H $1.495824-0.500738-0.241793$

H $1.3434800 .852895-3.004330$

H 1.4095821 .7276140 .804992

H 2.5915803 .0074850 .536881

H $3.070287 \quad 1.3470190 .289333$

H $5.325327-2.210184-1.669458$

H $4.185213-1.314294-0.659613$

H $5.9065351 .661808-3.689897$

H $7.1671521 .609981-2.437285$

H $5.5276892 .817249-0.820115$

H $4.9402323 .863219-3.638978$

H $5.0331425 .922326-2.110778$

H $4.4433794 .968693-0.769897$

H $1.6665676 .526478-3.478351$

H $3.4790456 .831502-3.709786$

H $0.9011805 .152454-1.704213$

H $2.0298084 .721441-0.459615$

H $1.3626313 .137669-3.040882$

H $4.5978800 .123297-2.501764$

C $-4.543147-3.178436-1.488326$

O $-4.615450-2.085117-0.550888$

C $-5.995660-1.856495-0.209361$

C $-6.781438-3.023484-0.797848$

C $-5.962141-3.348552-2.029799$

H $-6.298940-0.938437-0.725933$

C $-6.158584-1.6867761 .305175$

H $-6.804599-3.883625-0.118837$

H -7.816046 -2.751183 -1.028391

C $-6.249379-4.726390-2.601128$

H $-6.165123-2.589922-2.797758$

C $-5.307383-0.5552731 .917613$

O $-5.775555-2.9149141 .937315$
H -7.218983 -1.533916 1.540499

H $-4.244114-0.8249561 .898653$

O $-5.670568-0.438056 \quad 3.299691$

C $-5.493727 \quad 0.8212351 .249238$

$\begin{array}{lll}\text { C }-4.802786 & 1.950149 & 2.039022\end{array}$

H -5.0947620 .7776390 .230976$

$\begin{array}{llll}\text { O } & -6.891029 & 1.100376 & 1.158464\end{array}$

H -5.319119 2.0799382 .997712

H -3.7743091 .6457292259946$

H $-5.632642-4.920626-3.484089$

H $-6.046451-5.514863-1.868453$

H $-7.299698-4.807165-2.899644$

C $-3.517076-2.866675-2.577618$

H $-6.084942-2.8797582 .857985$

H $-5.346631-1.2324613 .756391$

H $-7.006878 \quad 1.8320440 .529622$

H $-3.719878-1.881809-3.014657$

H $-3.593398-3.601945-3.386932$

H $-4.240116-4.067609-0.919479$

C -2.750506 3.0663300 .051020

$\begin{array}{lllll}\text { O } & -4.174083 & 3.227980 & 0.018245\end{array}$

C -4.8098913 .3066151 .298608$

C $-4.225228 \quad 4.471657 \quad 2.109586$

C $-2.707246 \quad 4.381166 \quad 2.218894$

C $-2.088295 \quad 4.2104850 .833325$

H -2.4986262 .1040590 .512434$

C $-2.2574373 .057709-1.404297$

H $-5.8589823 .559576 \quad 1.103803$

$\begin{array}{llll}\text { O } & -4.808600 & 4.509850 & 3.407606\end{array}$

H $-4.487708 \quad 5.4151191 .613231$

H -2.408074 3.5742872 .897935

$\begin{array}{llll}\text { O } & -2.224984 & 5.594943 & 2.799714\end{array}$

H $-2.188944 \quad 5.1524780 .279820$

O $-0.690933 \quad 3.962246 \quad 0.978042$

C -2.491801 $1.734300-2.100899$

H $-2.7731023 .833480-1.982348$

H -1.179863 $3.241692-1.435034$

H $-4.396220 \quad 5.2467013 .890247$

H $-1.254008 \quad 5.5404512 .819238$

H -0.5850593 .1226891 .457099$

$\begin{array}{llll}\text { O } & -1.927447 & 1.791579 & -3.344649\end{array}$

O $-3.086462 \quad 0.776999-1.627128$

C $-2.0508140 .605941-4.135274$

H $-1.524753-0.223041-3.654283$

H $-3.1054380 .359401-4.290328$

H $-1.5886790 .796041-5.107580$

SCF Energy (B3LYP/6-31G**//MMFF) $=-3245.91529257$

03134

MM̄FF Geometry

C $-5.732968-2.601977-0.098812$

C $-5.165102-1.623687-0.820177$

C $-5.039879-0.193894-0.360764$

$\begin{array}{llll}\text { O } & -3.632880 & 0.089051 & -0.181658\end{array}$

C $-5.5817740 .755285-1.445380$

C $-5.4151422 .225309-1.109888$

C $-4.657904 \quad 3.087593-1.817489$

C $-6.1800862 .712444 \quad 0.093443$

C $-3.8586592 .809320-3.060665$

C $-2.4004612 .395937-2.809073$

C $-1.5207153 .516236-2.230916$

C $-0.052018 \quad 3.085737-2.200108$

C $0.846844 \quad 4.152846-1.555365$

O $-1.958797 \quad 3.806343-0.901698$

O $2.1158863 .544874-1.269014$

C $0.2369254 .580832-0.202704$

C $1.1293115 .313965-2.515947$

$\begin{array}{llllllll}\text { O } & 0.941817 & 5.691149 & 0.346402\end{array}$

C $-1.2701594 .899525-0.277694$

C $-1.9154505 .115312 \quad 1.113642$

O $-1.4564876 .112188-1.018306$

C -3.079167 -0.225673 1.020100

O $-3.643795-0.6924861 .996142$

C $-1.631060 \quad 0.055466 \quad 0.925121$

C $-0.784285-0.5791421 .749010$

C $0.674314-0.458643 \quad 1.739347$

C $1.273587 \quad 0.635075 \quad 0.896924$

C -1.8200843 .9257442 .076369$ 
C -0.6643434 .0134103 .060646$

C 0.4472472 .6976454 .864852

O $0.034300 \quad 5.0140353 .195335$

C 1.6964332 .0845224 .315643

C 2.2632730 .9804684 .826254

C 3.5463250 .3863134 .319537

C $3.414299-1.0773033 .962958$

C $3.825546-2.0362344 .809473$

C 2.895115-1.422995 2.586927

C $1.399588-1.3184092 .484412$

N -0.5314462 .8474983 .804007$

H $-6.154942-2.3748620 .876521$

H $-4.723850-1.869556-1.784036$

H -5.580155 -0.030707 0.579918

H $-6.6486130 .555596-1.612121$

H $-5.0819400 .523883-2.391996$

H -4.611943 4.122716 -1.481037

H $-6.127313 \quad 3.8007420 .206239$

H -7.238000 24445450.004823

H -5.7813422 .2686331 .010673$

H $-4.3473822 .036462-3.663775$

H -3.878062 $3.709711-3.688078$

H $-2.3837201 .527374-2.140350$

H $-1.9764652 .072125-3.767948$

H -1.643405 $4.406715-2.858680$

H $0.3048332 .844241-3.209160$

H $0.0416182 .157109-1.621190$

H $2.4771323 .196935-2.102315$

H $\quad 0.386617 \quad 3.7458410 .489688$

H $1.6222524 .947625-3.424547$

H $1.8229276 .035775-2.070720$

H $0.2262865 .845910-2.822256$

H 1.8796745 .4400720 .402450

H -1.5326836 .0369751 .568131$

H -2.9868375 .2989490 .946406$

H $-2.321276 \quad 6.487227-0.783149$

H $-1.2996790 .736150 \quad 0.149710$

H -1.191548 -1.283555 2.473244

H $1.1104310 .436676-0.166801$

H 2.3472630 .7652791 .045502

H $\quad 0.817174 \quad 1.5977311 .146432$

H -2.733748 3.9006522 .684622

H $-1.7657202 .979241 \quad 1.530865$

H -0.0103842 .0785655 .642967$

H 0.6757863 .6798395 .291058

H 2.1733902 .5901133 .479385

H 1.7992970 .4930685 .680932

H 4.3028620 .5114335 .105413

H 3.9293540 .9390063 .452423

H $3.763651-3.0869484 .546457$

H $4.229093-1.7918115 .786526$

H $3.164694-2.4566962 .331013$

H $3.415496-0.8125891 .845637$

H $\quad 0.870367-2.0724043 .068775$

H -1.1200172 .0486343 .586171$

C $-4.611760-4.890859-0.081723$

O $-3.382935-4.360576-0.623605$

C $-2.464559-4.153204 \quad 0.460947$

C $-2.908929-5.1277561 .539880$

C $-4.418241-4.9922661 .435098$

H $-2.623206-3.1284520 .817049$

C - $1.007031-4.2881780 .007466$

H -2.600801 -6.1537091 .306749$

H -2.522216 -4.8645772 .528721$

C $-5.182140-6.1404272 .071683$

H $-4.714533-4.0604081 .931689$

C $-0.637496-3.292234-1.113151$

O $-0.750453-5.623526-0.419057$

H $-0.365995-4.100616 \quad 0.876940$

H -1.015496 -2.300195 -0.842383

O $-1.266587-3.659920-2.340207$

C $0.881114-3.167907-1.346795$

C $1.201037-2.112615-2.422360$

H $1.364956-2.892177-0.403054$

O $1.436937-4.411297-1.767959$

H $0.873561-2.477163-3.403601$

H $\quad 0.640810-1.198654-2.189955$

H $-6.260982-6.0047521 .945818$
H $-4.908048-7.1022301 .625284$

H $-4.969922-6.1957803 .144393$

C $-5.793022-4.034904-0.556023$

H -1.311960 -5.793684-1.195005

H -2.227212 -3.658972 -2.190635

H $1.270581-5.062652-1.065929$

H $-6.736251-4.473569-0.210269$

H -5.822248 -4.055429-1.653342

H $-4.720670-5.893138-0.514903$

C $4.458780-0.848867-1.119890$

O $3.066270-1.152321-1.262425$

C $2.704272-1.784487-2.494357$

C $3.028680-0.849369-3.669055$

C $4.504596-0.439867-3.659789$

C $4.9606880 .035699-2.274187$

H $4.514008-0.249074-0.202804$

C $5.260101-2.133754-0.892226$

H $3.267306-2.718931-2.601417$

O $2.2115210 .319199-3.614014$

H $2.793864-1.362922-4.608735$

H $4.654306 \quad 0.362063-4.393059$

O $5.286301-1.555816-4.074447$

H $6.0526640 .114268-2.229748$

O $4.4384141 .350485-2.051108$

C $6.516471-1.862703-0.103667$

H $4.663249-2.873169-0.342701$

H $5.539761-2.629567-1.826227$

H $2.349006 \quad 0.737397-2.746631$

H $6.216353-1.272508-4.091785$

H $4.8031221 .931205-2.740538$

O $6.226090-1.8150071 .228314$

O $7.616963-1.692601-0.611519$

C $7.331737-1.5339192 .091369$

H $6.964568-1.5216813 .120992$

H $8.092789-2.3146131 .998682$

H $7.753970-0.5518751 .857741$

SCF Energy $\left(B 3 L Y P / 6-31 G^{* *} / / M M F F\right)=-3245.92167465$

03135

MM̄FF Geometry

C $-0.163566 \quad 4.3404390 .250986$

C $-1.0737434 .637666-0.689760$

C $-2.4197653 .967643-0.853920$

O $-2.4182842 .639803-0.279655$

C $-3.5085904 .800104-0.154367$

C $-4.9167594 .295231-0.402011$

C -5.7357173 .8081990 .550808$

C $-5.4046974 .391443-1.825218$

C -5.4647583 .6553932 .021060$

C -4.9059792 .2846262 .433999$

C -5.8396951 .1024602 .125489$

C $-5.325655-0.1790272 .784622$

C $-6.185436-1.3979582 .419018$

$\begin{array}{lllll}\text { O } & -5.883977 & 0.918786 & 0.708732\end{array}$

O $-5.449918-2.5633412 .823805$

C $-6.362577-1.4567790 .884730$

C -7.495687 -1.419642 3.215699

O $-7.289107-2.4870820 .549963$

C $-6.784545-0.1067240 .266387$

C $-6.752455-0.092562-1.282016$

O $-8.130691 \quad 0.1867760 .659662$

C $-1.7909041 .659944-0.988651$

O $-1.2484531 .770138-2.076861$

C $-1.8479080 .418499-0.187329$

C $-1.017864-0.593093-0.481938$

C $-0.950086-1.8828210 .208333$

C $-2.021671-2.1848981 .222330$

C $-5.391560-0.403682-1.914738$

C $-5.208139-1.861877-2.301016$

C $-3.486631-3.433556-3.182093$

O $-6.119473-2.684683-2.279944$

C $-2.913601-4.216874-2.043513$

C $-1.715477-4.818517-2.095331$

C $-1.144965-5.650061-0.982473$

C $0.235140-5.195228-0.563923$

C $1.336706-5.801772-1.037407$

C $0.332035-4.0936220 .466909$

C $0.046518-2.734867-0.111385$ 
N -3.908097 -2.127845 -2.710113 H -0.3952433 .5966421 .009050$ H $-0.8356995 .397724-1.430823$ H -2.634739 $3.886914-1.926413$ H $-3.4588415 .842892-0.495513$ H $-3.292308 \quad 4.8233580 .919013$ H -6.7304743 .4814260 .248941$ H -6.471371 4.159021-1.915156 H $-5.2595215 .406424-2.209330$ H -4.862773 $3.691636-2.468370$ H -4.7842004 .4376112 .373508$ H -6.4052723 .8352322 .557750$ H -3.9405632 .1319881 .937684$ H -4.7120752 .3173113 .513435$ H $-6.842028 \quad 1.3476272 .495752$ H $-5.269917-0.0627013 .874213$ H $-4.295848-0.3683502 .452753$ H $-5.953520-3.3439772 .535133$ H $-5.392817-1.7592770 .472764$ H -7.286076 -1.4608784 .291391$ H -8.082310 -2.317486 2.991895 H -8.123141 -0.5447123 .033318$ H -7.281436 -2.593838 -0.415825 H $-7.544560-0.734477-1.685534$ H $-7.0198960 .925984-1.599537$ H -8.5019520 .8296570 .032864$ H $-2.560456 \quad 0.376917 \quad 0.627985$ H $-0.308562-0.463412-1.298691$ H $-3.014691-2.0465240 .781619$ H -1.926378 -1.524158 2.090549 H -1.998679 -3.2116981 .592415$ H $-5.3042110 .170354-2.846177$ H $-4.572462-0.091670-1.260593$ H -2.756802 -3.269433 -3.981195 H -4.347380 -3.966200 -3.599212 H -3.522982 -4.318253 -1.148508 H -1.121404 -4.738646 -3.003199 H -1.103867 -6.690218-1.331741 H -1.807374 -5.659404 -0.107970 H $2.329524-5.499293-0.721795$ H $1.269955-6.611192-1.756857$ H $1.345580-4.0606640 .886347$ H $-0.309926-4.3429331 .315917$ H $0.771922-2.423364-0.864403$ H -3.205618 -1.396839 -2.645251 C 2.2819043 .8642060 .272286

O $2.1885673 .124663-0.960122$

C $3.5173672 .748715-1.381443$

C $4.4635283 .225562-0.286011$

C 3.7091504 .4004580 .303289

H $3.6996393 .294113-2.315172$

C $3.5429261 .234370-1.625509$

H 4.6080592 .4466280 .473370

H $5.4468813 .501318-0.677920$

C 4.1970194 .7996381 .684657

H $3.8053915 .256604-0.377735$

C $4.9419550 .686116-1.950677$

O $2.667041 \quad 0.939002-2.718174$

H $3.1256740 .724460-0.750412$

H $5.6300160 .897480-1.126426$

O $5.4536591 .400717-3.082740$

C $4.975282-0.817846-2.278797$

C $4.349439-1.749469-1.222824$

H $4.471869-0.992402-3.236910$

O $6.337407-1.203515-2.496420$

H $3.260110-1.628740-1.237729$

H $4.568543-2.779738-1.532185$

H 3.6217435 .6459882 .072833

H 4.1041303 .9733462 .397451

H 5.2505845 .0955391 .649319

C 1.2080994 .9526400 .308924

H $1.7927961 .302954-2.495523$

H $6.3341541 .037721-3.280496$

H $6.823642-1.061540-1.666372$

H $1.3487855 .640972-0.533451$

H 1.2929845 .5361531 .232194

H 2.1223853 .1468111 .089011

C $6.912989-1.5747101 .506150$
O $6.265895-1.7942650 .244973$

C $4.858474-1.5487790 .219346$

C $4.143229-2.4767441 .213243$

C $4.731706-2.3440202 .621905$

C $6.262667-2.4243222 .611691$

H $7.937305-1.9427581 .363036$

C $6.997882-0.0803091 .855771$

H $4.668402-0.5121600 .510205$

O $4.241520-3.8338940 .786676$

H $3.077936-2.2210371 .231208$

H $4.332842-3.1423773 .259481$

O $4.339706-1.0978593 .190151$

H $6.671081-2.1292833 .585356$

O $6.652644-3.7826582 .387375$

C 7.8185110 .6991820 .849304

H $\quad \begin{array}{llll}6.006909 & 0.379433 & 1.894187\end{array}$

H 7.4784670 .0424462 .832995

H $5.185301-4.0480060 .689806$

H $3.367861-1.0805373 .218057$

H $6.290971-4.3154153 .116154$

O 7.6839082 .0339781 .108634

O $8.494994 \quad 0.208687-0.044006$

C 8.3933852 .9064860 .223686

H 8.1613593 .9371350 .505166

H 9.4712972 .7479840 .322771

H $8.0715302 .745111-0.809687$

SCF Energy $(B 3 L Y P / 6-31 G * * / / M M F F)=-3245.92735288$

$03 \_136$

MM̄FF Geometry

C $-4.2389471 .871421 \quad 1.243450$

C -4.2621313 .2129731 .201751$

C -3.1090544 .1469240 .917201$

O -1.8368503 .4631200 .903066$

C $-3.3126434 .808035-0.459812$

C $-2.3136175 .910777-0.759185$

C $-1.2966445 .820965-1.640179$

C $-2.5457527 .202299-0.015510$

C $-0.8943894 .638809-2.479535$

C $0.5155694 .171308-2.113817$

C $0.9936162 .925852-2.877957$

C $0.1198661 .700818-2.607054$

C $0.6892100 .433218-3.255399$

O $2.3292762 .676063-2.424763$

O $-0.014582-0.692316-2.709038$

C $2.1727630 .282943-2.856415$

C $0.4290900 .409768-4.767509$

O $2.744856-0.817505-3.556261$

C $3.0042031 .570960-3.053099$

C $4.3870311 .512936-2.355747$

O $3.1959041 .813290-4.447064$

C -1.1780943 .3529162 .090857$

O -1.5996013 .6524003 .196526$

C 0.1822542 .8453511 .803064

C 1.0942722 .7667102 .783844

C 2.4970002 .3682962 .629086

C 2.9899092 .0893891 .234065

C $5.3860600 .471228-2.864578$

C $5.507688-0.728337-1.940503$

C $6.295701-1.3854240 .337385$

O $5.131370-1.847956-2.278581$

C $5.334258-1.0939391 .445573$

C $5.702456-0.8758512 .717197$

C $4.730953-0.6015193 .830584$

C 5.0226850 .6966294 .549442

C 5.5135300 .6987145 .800280

C 4.7484401 .9918653 .817722

C 3.2777202 .3038323 .728550

N $6.092736-0.414410-0.722745$

H $-3.314717 \quad 1.3315751 .057944$

H -5.2112813 .7141641 .386892$

H -3.099455 4.9196171 .695970

H -4.320248 $5.242086-0.520595$

H $-3.2858834 .036917-1.236502$

H $-0.6645476 .697544-1.779853$

H - $1.8549647 .992869-0.327750$

H -3.562341 $7.566985-0.195391$

H -2.4121847 .0576041 .060825$ 
H -1.602536 $3.817563-2.369154$ H $-0.9202084 .935099-3.534722$ H $1.2307804 .985465-2.296640$ H $0.5792863 .979408-1.036325$ H $1.0182933 .161092-3.948232$ H $-0.9083631 .856118-2.951971$ H $0.050043 \quad 1.537323-1.523644$ H $-0.962505-0.572471-2.890241$ H $2.1977930 .016725-1.791471$ H $-0.6452950 .490912-4.972650$ H $\quad 0.742897-0.542503-5.209148$ H $0.929144 \quad 1.221353-5.300181$ H $2.915165-0.533535-4.470228$ H $4.8568832 .498422-2.487638$ H $4.2322711 .416207-1.275454$ H $3.9817652 .374193-4.555775$ H 0.4101162 .5823680 .777804 H 0.7966153 .0445813 .794064 H 2.8674292 .9739530 .598988 H $2.437721 \quad 1.2575890 .785594$ H 4.0452921 .8164091 .190143 H $\quad 5.154931 \quad 0.127547-3.877364$ H $6.3836550 .926446-2.922936$ H $7.337773-1.2987630 .660999$ H $6.129981-2.398498-0.041707$ H 4.279019-1.072089 1.189013 H $6.756949-0.9163782 .980403$ H $4.781958-1.4449354 .531136$ H $3.699546-0.5843053 .462664$ H 5.7248351 .6254796 .323427 H $5.714858-0.2262916 .330429$ H 5.2417571 .9715922 .842818 H 5.2221242 .8294534 .347602 H 2.8269142 .5306524 .695818 H $\quad 6.3752800 .544674-0.542237$ C $\begin{array}{llll}-5.898425 & 0.212277 & 0.336464\end{array}$ $\begin{array}{llll}\text { O } & -5.030628 & -0.932644 & 0.205393\end{array}$ C $-5.839676-2.1103540 .016219$ C $-7.220946-1.604148-0.372909$ C $-7.314973-0.3503760 .473948$ H $-5.914327-2.6013680 .994015$ C $-5.185729-3.062909-0.990075$ H $-7.276673-1.348040-1.437211$ H $-8.008617-2.331587-0.155051$ C -8.4052410 .6059320 .021443$ H $-7.503665-0.6419581 .516098$ C $-3.728340-3.427015-0.641039$ O $-5.178586-2.430806-2.276109$ H $-5.796458-3.967491-1.093108$ H $-3.106763-2.533168-0.759038$ O $-3.258481-4.362107-1.619833$ C $-3.537027-4.032076 \quad 0.761478$ C $-2.089057-4.4357821 .077718$ H -3.881815 -3.3266141 .523383$ O $-4.362287-5.1943120 .873205$ H -2.050812 -4.880107 2.082165 H -1.780521 -5.2629330 .423342$ H -8.4242681 .5023650 .649118$ H -8.254762 $0.924132-1.015652$ H $-9.387674 \quad 0.1269900 .086607$ C $-5.464693 \quad 1.0482101 .542971$ H -4.753374 -3.045653 -2.898446 H -3.764458 -5.184860 -1.504902 H $-4.263254-5.5386751 .777287$ H $-5.231867 \quad 0.3941812 .392222$ H $-6.2850601 .705191 \quad 1.854817$ H $-5.8057770 .791010-0.591904$ C $1.333157-2.9762271 .273459$ O $\quad 0.204433-3.8571351 .372364$ C $-1.057199-3.302158 \quad 0.974415$ C -1.397006 -2.0781581 .839349$ C $-0.271667-1.0442041 .800950$ C $1.095019-1.6807242 .067933$ H $2.155551-3.5161961 .760558$ C $1.734219-2.725353-0.188299$ H $-0.987402-3.006863-0.076767$ O $-1.639913-2.4758173 .186748$ H $-2.316989-1.6119301 .470643$
H $-0.472188-0.2702872 .551387$

O $-0.282211-0.4252980 .518744$

H $1.897724-0.9692511 .850217$

O $1.190858-2.0060593 .457646$

C $2.251483-3.974763-0.868964$

H $0.891256-2.360802-0.778489$

H $2.538016-1.982498-0.227550$

H $-0.852059-2.9497603 .503641$

H $0.463422 \quad 0.196020 \quad 0.483735$

H $1.104453-1.1768853 .958600$

O $2.489036-3.691250-2.183934$

O $2.434920-5.054752-0.324952$

C $3.004850-4.772487-2.965722$

H $3.154767-4.411805-3.986761$

H $3.968062-5.103305-2.565652$

H $2.289190-5.599800-2.986618$

SCF Energy $(B 3 L Y P / 6-31 G * * / / M M F F)=-3245.90455913$

03137

MM̄FF Geometry

C $-2.510787-4.3613080 .628181$

C $-1.878110-3.519076-0.204087$

C $-0.416422-3.551361-0.603801$

O $0.205824-2.319541-0.161521$

C $0.399549-4.718595-0.010256$

C $1.835050-4.786757-0.494781$

C $2.917448-4.6682620 .299367$

C $2.016907-5.043821-1.968925$

C $2.962884-4.4425371 .785334$

C $3.110924-2.9699902 .200133$

C $4.442063-2.3293481 .772962$

C $4.604271-0.9425002 .398699$

C $5.883624-0.2385891 .915781$

O $4.450500-2.2090520 .349160$

O $5.786574 \quad 1.147898 \quad 2.277588$

C $5.946582-0.2983410 .373221$

C $7.131956-0.7836642 .620185$

O $7.197960 \quad 0.187645-0.105387$

C $5.676095-1.704904-0.197615$

C $5.528349-1.722505-1.738448$

O $6.776137-2.5636110 .127548$

C $0.097551-1.223937-0.960145$

O $-0.495104-1.140302-2.023582$

C $0.824549-0.119548-0.296279$

C $0.6062671 .146186-0.682400$

C $1.2174202 .339912-0.093206$

C 2.3887052 .1376310 .829939

C $4.389789-0.863309-2.300191$

C $4.8305440 .510813-2.776647$

C $3.9010442 .627807-3.705859$

O $6.0059410 .859623-2.841973$

C $3.8116103 .633468-2.602471$

C $2.9666354 .675192-2.623694$

C $2.8964375 .718155-1.546242$

C $1.5091535 .858301-0.963032$

C $0.691316 \quad 6.852289-1.348595$

C 1.1046304 .8957510 .129757

C $0.7031743 .548315-0.401612$

N $3.7487271 .293406-3.157999$

H - $1.935976-5.1396331 .123970$

H $-2.453099-2.726777-0.675395$

H $-0.377098-3.609641-1.698546$

H $-0.078908-5.673482-0.264529$

H $\quad 0.373638-4.6396401 .080884$

H $3.899427-4.752758-0.165180$

H $1.689469-4.179741-2.554949$

H $3.062566-5.239172-2.230754$

H $1.435283-5.918528-2.277453$

H $2.074381-4.8588422 .270741$

H $3.804017-5.0191802 .191171$

H $2.273251-2.3982991 .783518$

H $3.024860-2.9203803 .292827$

H $5.258584-2.9884972 .090277$

H $4.592142-1.0054173 .494026$

H $3.741778-0.3196572 .126676$

H $5.6735391 .198573 \quad 3.242268$

H $5.1815170 .390174-0.002812$

H $7.051093-0.6440183 .704948$ 
H $8.029878-0.2348932 .314989$ H $7.297683-1.8473352 .436874$ H $7.318498 \quad 1.0826390 .255464$ H $6.488643-1.486728-2.212553$ H $5.310406-2.760570-2.029107$ H $6.777013-3.312247-0.491766$ H $1.497175-0.3754420 .514100$ H $-0.1158361 .332492-1.476259$ H $2.910497 \quad 3.0625591 .083452$ H $3.137076 \quad 1.4927730 .358041$ H 2.0659781 .6739421 .767950 H $3.976628-1.371934-3.180840$ H $3.577517-0.755005-1.574647$ H $3.1146212 .763055-4.455122$ H $4.8733892 .710111-4.201983$ H $4.4908193 .514496-1.761532$ H $2.3060014 .805550-3.478023$ H $3.2167436 .672204-1.985080$ H $3.6109435 .510766-0.739955$ H -0.295067 $6.973908-0.913049$ H $0.9879917 .563037-2.112824$ H $0.241290 \quad 5.296127 \quad 0.677832$ H 1.9050394 .8438070 .872544 H $-0.1467353 .586899-1.084262$ H $2.8056720 .943587-3.015430$ C $-5.015686-3.849442-0.000818$ O $-4.984254-2.415691-0.083890$ C $-5.346132-2.031641-1.424797$ C $-5.744211-3.312933-2.160761$ C $-4.933505-4.367684-1.434815$ H $-4.437547-1.627119-1.885762$ C $-6.441630-0.959943-1.410289$ H -6.814731 -3.526980 -2.063881 H $-5.516923-3.258624-3.230125$ C $-5.464250-5.779435-1.613768$ H $-3.908749-4.326639-1.818501$ C $-6.1489160 .276004-0.539277$ O $-7.650707-1.548945-0.908710$ H $-6.657466-0.654829-2.441662$ H -6.2467160 .0125980 .521336$ O $-7.201272 \quad 1.210895-0.820679$ C -4.783172 $0.946779-0.781905$ C $-4.6718802 .325934-0.104186$ H $-3.9909420 .286860-0.413848$ O $-4.569884 \quad 1.114612-2.184488$ H $-5.3209933 .029745-0.638205$ H -5.0449142 .2500820 .921770$ H -4.847733 -6.499035 - 1.065834 H $-6.493475-5.871290-1.250834$ H $-5.454744-6.062645-2.671466$ C $-3.973350-4.3773000 .991093$ H -8.339584 $-0.862881-0.935472$ H -7.226453 $1.855301-0.093297$ H -5.302959 $1.650743-2.532505$ H $-4.228705-5.4249961 .197807$ H $-4.076858-3.8383801 .941428$ H $-6.004293-4.0921360 .413642$ C -2.553756 2.1898592 .107243 O $-2.342962 \quad 2.1013940 .691785$ C -3.231066 $2.882477-0.115915$ C $-3.130846 \quad 4.362400 \quad 0.275944$ C -3.377843 4.5658351 .765051 C -2.4736373 .6489772 .585729$ H $-3.530951 \quad 1.7632782 .362177$ C $-1.472158 \quad 1.3356382 .789358$ H -2.860962 $2.800268-1.145426$ O $-4.0525105 .155268-0.465070$ H -2.129015 4.7377570 .037452 H -4.4326254 .4138302 .022776$ O -3.0709865 .9199922 .107932$ $\mathrm{H}-1.4420384 .0195552 .521870$ O $-2.884390 \quad 3.7439783 .949029$ C -1.673443 -0.1520252 .588471$ H $-0.483002 \quad 1.5902362 .395227$ H -1.483206 1.5102633 .870288 H $-3.8611275 .019145-1.408786$ H $-3.6275816 .491778 \quad 1.551687$ H -2.1628703 .4030034 .503066$
O $-0.813632-0.819303 \quad 3.415443$

O $-2.465911-0.6627951 .810417$

C $-0.891506-2.2478013 .392722$

H $-0.359968-2.6272972 .518056$

H $-0.399480-2.6278654 .292036$

H $-1.930718-2.5899803 .395533$

SCF Energy (B3LYP/6-31G**//MMFF)= -3245.89845888

03138

MM̄FF Geometry

C $-2.310575-2.864534-2.439585$

C $-1.389260-3.837720-2.517837$

C $0.113158-3.641030-2.455980$

O $0.438290-2.397079-1.794102$

C $0.758911-4.772879-1.631924$

C $2.270945-4.682016-1.540800$

C $2.958346-4.540729-0.389995$

C $3.014600-4.784603-2.847515$

C $2.410095-4.4653821 .007522$

C $2.106580-3.0427701 .498016$

C $3.351970-2.1666521 .708015$

C $2.969897-0.841203 \quad 2.371757$

C 4.1779800 .0951632 .527568

O $3.952777-1.9055110 .437573$

O 3.6810031 .4057672 .841535

$\begin{array}{llll}\text { C } & 4.912263 & 0.203056 & 1.173411\end{array}$

C $5.072734-0.320707 \quad 3.700967$

O 6.1346340 .9222441 .313006

C $5.178295-1.1618730 .505535$

C $5.722302-1.037525-0.939240$

O $6.166309-1.870947 \quad 1.263893$

C $0.652717-1.292883-2.556050$

O $0.657585-1.218890-3.773494$

C $0.812690-0.154796-1.626244$

C $0.4386641 .070647-2.020707$

C $0.4800282 .283944-1.202837$

C 1.2213602 .2151310 .105106

C $4.816416-0.288333-1.923042$

C $5.163298 \quad 1.182746-2.088007$

C $4.3416853 .255713-3.203491$

O $6.1811041 .692261-1.627350$

C $3.6052544 .092355-2.205486$

C $2.6904015 .012324-2.547727$

C $1.9753895 .891680-1.561778$

C $0.4712355 .830913-1.708350$

C $-0.1944576 .771230-2.399862$

C $-0.2662274 .729889-0.980936$

C $-0.133023 \quad 3.394712-1.661864$

N $4.2158681 .853986-2.849964$

H $-1.990242-1.830328-2.358650$

H $-1.734133-4.861842-2.646807$

H $\quad 0.507663-3.657494-3.478813$

H $0.503569-5.746147-2.071666$

H $0.314963-4.766654-0.631414$

H $4.045079-4.487237-0.446208$

H $4.097637-4.863657-2.703703$

H $2.693269-5.674359-3.398575$

H $2.830604-3.901794-3.467423$

H $1.502227-5.0724451 .094647$

H $3.132083-4.9384561 .685343$

H $1.427711-2.5571830 .787539$

H $1.571426-3.1295852 .451453$

H $4.057354-2.7198562 .339277$

H $2.492769-1.0145913 .344338$

H $2.216536-0.3320311 .755937$

H 3.1412481 .3366683 .647662

H 4.2727870 .8006530 .513906

H $4.504042-0.3091894 .638628$

H 5.8953140 .3890883 .842002

H $5.495312-1.3208693 .584489$

H 5.9203251 .7837901 .709953

H $6.736709-0.621211-0.925441$

H $5.834297-2.059329-1.330306$

H $6.573244-2.5408560 .689926$

H $1.182565-0.367415-0.630055$

H $0.0299961 .192402-3.022965$

H $2.234121 \quad 1.829988-0.050935$

H $0.701073 \quad 1.5569630 .806432$ 
H 1.3398803 .1839960 .594269 H $4.936213-0.740734-2.916092$ H $3.763634-0.388488-1.643727$ H $3.9427513 .371688-4.216186$ H $5.3997373 .536885-3.210869$ H $3.8559503 .954595-1.156281$ H $2.4633765 .167616-3.600177$ H $2.3231936 .920845-1.721330$ H $2.2513425 .644264-0.529177$ H -1.275736 $6.750791-2.486035$ H $\quad 0.3282837 .579839-2.900199$ H $-1.3393444 .960674-0.935324$ H 0.0548924 .7110860 .064391 H $-0.6383703 .346597-2.627457$ H $3.3775481 .363513-3.147800$ C $-4.508778-2.143654-1.487619$ O $-4.401139-0.791498-1.988044$ C $-5.714824-0.229162-2.069458$ C $-6.566853-1.037123-1.105816$ C $-6.006730-2.429270-1.343429$ H $-6.073307-0.434830-3.088218$ C $-5.6992411 .288244-1.878126$ H $-6.399578-0.733819-0.066515$ H $-7.636635-0.962967-1.321373$ C $-6.335770-3.415194-0.235708$ H $-6.408112-2.813362-2.290978$ C $-5.3566031 .837659-0.479652$ O $-7.0055331 .784247-2.205817$ H $-5.0062631 .734535-2.602924$ H $\quad-6.182907 \quad 1.636300 \quad 0.212914$ O $-5.293356 \quad 3.269137-0.602129$ C $-4.0389191 .356600 \quad 0.160223$ C $-3.6966502 .166399 \quad 1.427411$ H $-4.116431 \quad 0.2949320 .415607$ O $-2.9571661 .510829-0.756020$ H -3.4360793 .1931121 .140999$ H -4.5881422 .2002252 .066117$ H -5.888372 -4.393090 -0.439922 H $-5.961129-3.069106 \quad 0.733596$ H -7.418884 -3.548964 -0.148777 C $-3.797409-3.092233-2.459535$ H -7.189549 $1.540072-3.128898$ H $-6.1447583 .566315-0.966747$ H $-3.0825470 .866787-1.473149$ H -4.151559-2.902759-3.480039 H -4.026084 -4.133020 -2.204098 H -4.021528 -2.157539 -0.506205 C $-1.999026-0.3613563 .593388$ $\begin{array}{lllll}\text { O } & -2.963112 & 0.346594 & 2.803099\end{array}$ C -2.5177641 .5794772 .229143$ C -2.0534822 .5500993 .329392$ C -1.015870 1.9102024 .257414 C $-1.454560 \quad 0.5212794 .729339$ H -2.565755 -1.184014 4.047392 C $-0.892092-0.9750012 .731583$ H -1.6876461 .3796301 .544223$ O -3.1676522 .9899014 .105894$ H -1.626898 3.4413692 .854485 H -0.8657932 .5535265 .133336$ O $0.2333161 .797003 \quad 3.583250$ H -0.6208520 .0140025 .229251$ O -2.4864750 .6942375 .704822$ C -1.455849-1.976962 1.757571 H $-0.350987-0.2300692 .142760$ H $-0.145386-1.4817363 .353393$ H -3.5950332 .2027604 .484722$ H $\quad 0.5044162 .6910923 .313315$ H $-2.712053-0.1851116 .053280$ O $-1.737485-3.1471002 .396427$ O $-1.632269-1.7376610 .570845$ C $-2.305870-4.1746561 .579308$ H -2.411094 -5.075217 2.190049 H -3.296341 -3.870409 1.229193 H -1.649641-4.400287 0.734853 SCF Energy (B3LYP/6-31G**//MMFF) $=-3245.90514809$

03139

MM̄MF Geometry
C $2.004128-3.025743-2.826839$

C $0.928364-3.754614-3.162961$

C $-0.509328-3.378998-2.876021$

O $-0.585063-2.378072-1.835469$

C $-1.173770-2.807224-4.140124$

C $-2.661059-2.557005-3.976871$

C $-3.245198-1.343182-4.011075$

C $-3.513217-3.786895-3.789894$

C $-2.598303-0.004381-4.224243$

C $-2.2086800 .741686-2.939259$

C $-3.3826561 .040381-1.992926$

C $-2.9548722 .020921-0.897428$

C $-4.0786932 .274480 \quad 0.119368$

O $-3.808428-0.186824-1.392793$

O

C -4.6325940 .9183760 .606384$

C $-5.1413173 .232100-0.433279$

O $-5.784170 \quad 1.105496 \quad 1.424603$

C $-4.956190-0.061215-0.540515$

C $-5.325054-1.482079-0.045121$

O $-6.086417 \quad 0.428974-1.273078$

C $-0.429163-2.805798-0.552443$

O $-0.311526-3.957582-0.166526$

C $-0.366938-1.6064210 .307811$

C $0.198844-1.6993811 .519721$

C $0.358788-0.6053812 .478125$

C $-0.323460 \quad 0.6973892 .157731$

C $-4.252932-2.1902160 .789584$

C -4.418354-2.013655 2.290407

C $-3.236716-2.4600284 .439833$

O $-5.426614-1.5395602 .806532$

C $-2.461918-1.2550654 .872576$

C $-1.435513-1.3129785 .734878$

C $-0.668398-0.1122446 .210983$

C $0.825098-0.2633496 .022559$

C $1.609592-0.6829057 .029704$

C 1.4164740 .1498944 .693668

C $1.091722-0.8224243 .589899$

N -3.316890 -2.487120 2.990990

H $1.870115-2.053635-2.359274$

H $1.075499-4.717106-3.648006$

H -1.038455 -4.280504 -2.544263

H -1.035590 -3.498255 -4.982399

H -0.656099 -1.882873 -4.418896

H -4.326120 -1.294599-3.881872

H $-4.584269-3.560640-3.832027$

H -3.304263 -4.517655 -4.578015

H -3.315633 -4.251244 -2.819123

H - $1.713298-0.096917-4.862731$

H $-3.2971350 .617524-4.798727$

H -1.443886 $0.161696-2.409979$

H -1.746535 $1.688650-3.241477$

H $-4.2030631 .464222-2.583828$

H -2.615532 $2.968623-1.333455$

H -2.087732 $1.610926-0.361804$

H $-3.116876 \quad 3.7666150 .965007$

H -3.860477 0.4742721 .246366

H $-4.689424 \quad 4.197738-0.690777$

H -5.9057193 .4517610 .320231$

H $-5.6375242 .852631-1.328838$

H -5.5315451 .7043772 .147983$

H -6.290994 -1.460963 0.473787

H -5.489094 -2.102291 -0.938299

H $-6.487109-0.313663-1.754684$

H $-0.761403-0.679084-0.090203$

H $0.603636-2.660647 \quad 1.833862$

H -1.3949870 .5390361 .993125$

H $\quad 0.109420 \quad 1.138586 \quad 1.254222$

H -0.2445851 .4431452 .950688$

H -4.323616 -3.269330 0.601851

H $-3.251374-1.8708560 .489623$

H -2.759960 -3.393517 4.755190

H -4.245175 -2.421755 4.863737

H $-2.773862-0.2906174 .478639$

H -1.146158 -2.276675 6.148508

H $-0.8956510 .027368 \quad 7.276097$

H -1.0084510 .8048745 .714106$

H $2.684688-0.7685776 .912072$ 
H $\quad 1.192374-0.9549697 .993733$ H 2.5112110 .2007624 .765954 H 1.1045791 .1736334 .469605 H $1.543879-1.8055823 .728586$ H -2.509324 -2.828319 2.477891 C $4.237193-3.482507-1.726038$

O $4.364261-2.141331-1.214023$ C $4.210165-2.1763340 .218890$

C $4.318810-3.644510 \quad 0.617117$ C $3.677594-4.324608-0.578466$ H $3.192042-1.8312220 .432290$ C $5.222829-1.2403290 .887900$ H $5.362086-3.9643610 .719518$ H $3.805558-3.8571841 .559781$ C $3.997195-5.804481-0.688710$ H $2.591260-4.202505-0.494435$ C $5.210151 \quad 0.195050 \quad 0.323246$ O $6.536879-1.7738520 .679218$ H $5.059056-1.2324521 .971768$ H $5.5424560 .174518-0.721486$ O $6.196793 \quad 0.9605381 .027154$ C 3.8516230 .9166040 .416781 C $3.9092842 .313435-0.222376$ H $3.0904310 .333647-0.111740$ O 3.4481601 .0000821 .778204 H 4.6483982 .9406090 .289501 H $4.2798342 .201458-1.250731$ H $3.522748-6.239362-1.574231$ H $5.075805-5.978389-0.763552$ H $3.630409-6.3436380 .190826$ C $3.424290-3.473241-3.026319$ H $7.167312-1.1657651 .101989$ H 5.9822730 .9244151 .974601 H 4.0405921 .6252452 .228953 H $3.456560-4.469474-3.482533$ H $3.890674-2.775317-3.732575$ H $5.257684-3.809098-1.966370$ C $1.4683954 .718327-1.656274$ O $2.6602083 .988351-1.345661$ C $2.5513913 .044556-0.269524$

C 2.1710483 .7778451 .028756

C 0.9186724 .6465170 .844902 C $0.9848585 .501279-0.425477$ H $1.7763655 .440791-2.422894$ C $0.387733 \quad 3.822510-2.271534$ H $1.7790362 .311606-0.524587$ O 3.2573194 .6048551 .443278 H 1.9929553 .0453661 .822560 H 0.8078265 .3020251 .717628 O $\quad-0.223750 \quad 3.798697 \quad 0.798636$ H $\quad 0.0061225 .950134-0.631582$ O $1.8960396 .576625-0.181706$ C $0.8804073 .178076-3.545853$ H $0.0518393 .034128-1.592291$ H $-0.5051674 .406695-2.523626$ H 3.0025465 .0276902 .280934 H -1.006742 4.367863 0.709464 H $1.8952917 .147589-0.968705$ O $1.1628181 .862419-3.321507$ O $1.0127203 .775551-4.605897$ C $1.6344831 .127939-4.454696$ H $2.5825231 .544717-4.807022$ H $1.7992190 .092919-4.143758$ H $0.885436 \quad 1.138809-5.252245$ SCF Energy (B3LYP/6-31G**//MMFF) $=-3245.89548533$

0314

MM̄FF Geometry

C -1.808299 -4.286640 0.659604

C $-0.673398-3.9961091 .312604$

C $0.637072-3.7686080 .599656$

O $1.181672-2.4911741 .007396$

C $1.648886-4.8627790 .986567$

C $2.885104-4.8803060 .106989$

C $4.135729-4.6073350 .526955$

C $2.657964-5.274903-1.331370$

C $4.597774-4.2370131 .906766$

C $4.675701-2.7274982 .182115$
C $5.563927-1.9440171 .200494$

C $5.823893-0.5288691 .722810$

C $6.6161990 .323308 \quad 0.718611$

O $4.885563-1.870660-0.057476$

O $\quad 6.5250581 .694638 \quad 1.135527$

C $5.9423160 .228164-0.667640$

C $8.107863-0.0319710 .723957$

O $6.7348090 .869355-1.663501$

C $5.626870-1.218800-1.097895$

C $4.777264-1.298331-2.391021$

O $6.854432-1.909020-1.362266$

C $0.619737-1.3730800 .472216$

O $-0.312141-1.321093-0.314710$

C $1.341180-0.2022601 .018033$

C 0.8001901 .0172210 .889192

C 1.3841692 .2797921 .342562

C 2.8292152 .2679851 .760078

C $3.403317-0.622451-2.320786$

C $3.3758900 .796481-2.865382$

C $1.8496412 .756388-3.093104$

O $4.3139161 .305222-3.472657$

C $2.2018403 .752724-2.033631$

C $1.3585254 .706753-1.609825$

C $1.7124895 .740759-0.578491$

C 0.7398515 .7665330 .579641

C -0.2533216 .6700440 .630028$

C 0.9662914 .7910891 .714238

C $0.607813 \quad 3.382447 \quad 1.330459$

N $2.1561881 .417209-2.625103$

H -1.780632 -4.386176 -0.423380

H $-0.682674-3.8946622 .394719$

H $0.487111-3.757766-0.486486$

H $1.177998-5.8524770 .914144$

H $1.924032-4.7332382 .039009$

H $4.940690-4.660349-0.205773$

H $2.107650-4.495211-1.866226$

H $3.597568-5.439074-1.870177$

H $2.086904-6.207573-1.384512$

H $3.966218-4.7059892 .668922$

H $5.593303-4.6756782 .053940$

H $3.659901-2.3165062 .166876$

H $5.059804-2.5980563 .201701$

H $6.509675-2.4846381 .077356$

H $6.341588-0.5601712 .689579$

H $4.863586-0.0331331 .916303$

H $\quad 6.877321 \quad 1.7570082 .039944$

H $5.0040100 .791949-0.604080$

H $8.536737 \quad 0.132187 \quad 1.719884$

H $8.6715620 .617787 \quad 0.045593$

H $8.302525-1.0707850 .449697$

H $6.8903891 .782286-1.366671$

H $5.360649-0.942282-3.248589$

H $4.592319-2.364103-2.589177$

H $6.663040-2.670621-1.934403$

H $2.292576-0.3734761 .506759$

H -0.1734351 .1035170 .410194$

H 2.9641101 .6631252 .663200

H 3.2316573 .2590231 .976957

H 3.4516801 .8526480 .960667

H $2.702948-1.198244-2.939391$

H $3.016827-0.628138-1.298522$

H $\quad 0.7840012 .776995-3.342813$

H $2.4264652 .963999-4.000044$

H $3.2046613 .704899-1.615638$

H $\quad 0.3649914 .769689-2.048770$

H $1.7238306 .718367-1.078028$

H $2.7299825 .593528-0.195530$

H $-0.941244 \quad 6.708887 \quad 1.468038$

H $-0.4058337 .386248-0.170607$

H 0.3420005 .0625442 .575735

H 1.9971544 .8933292 .062831

H -0.4309993 .2726041 .020921$

H $\quad 1.452800 \quad 0.935730-2.072587$

C $-4.134263-3.4001400 .862125$

O $-4.350446-3.502146-0.562053$

C $-5.757549-3.414593-0.824801$

C $-6.393754-2.8692600 .444808$

C $-5.518611-3.5213251 .502076$ 
H $-6.106910-4.448821-0.954339$

C $-6.044691-2.660283-2.126243$

H $-6.311243-1.7786260 .504892$

H -7.450176 -3.1390700 .535522$

C -5.629702 -2.8732122 .870373$

H $-5.799728-4.5798431 .585707$

C $-5.777880-1.140475-2.153994$

O $-7.430943-2.866326-2.430370$

H -5.475610 -3.135083 -2.935698

H -6.546256 -0.626872 -1.564346

O $-5.981899-0.702010-3.506217$

C $-4.388587-0.674494-1.686247$

C $-4.1672040 .832667-1.915589$

H $-4.275806-0.894197-0.622334$

O $-3.365761-1.374198-2.392747$

H $-4.0421451 .022302-2.988710$

H $-5.0584811 .379034-1.590481$

H -4.963185 -3.360654 3.588765

H -5.367953 -1.811403 2.834009

H $-6.653249-2.9527163 .250948$

C $-3.148207-4.4797801 .312790$

H -7.612126 -2.418785 -3.274894

H -5.270171 -1.084174 -4.048190

H $-3.387759-2.301681-2.102455$

H $-3.518422-5.4711801 .024629$

H $-3.051086-4.4684932 .404219$

H -3.710286 -2.405630 1.052226

C -3.9504921 .8266350 .954204$

$\begin{array}{lllll}\text { O } & -2.968430 & 1.077467 & 0.232260\end{array}$

C $-2.9163211 .358200-1.172720$

C $-2.6257092 .848736-1.399215$

C $-3.6047523 .746089-0.644368$

C $-3.696983 \quad 3.3318290 .825176$

H -4.9435141 .5939700 .550517$

C $-3.981104 \quad 1.3247502 .400914$

H -2.057622 $0.798719-1.564467$

O $-2.666428 \quad 3.173612-2.784755$

H -1.611004 3.075460-1.050959

H $-4.5952813 .742711-1.114404$

O $-3.1403825 .096995-0.698064$

H -2.7695373 .6154871 .334804$

O

C $-2.690026 \quad 1.549792 \quad 3.150561$

H -4.8081361 .7805202 .957839$

H -4.1606740 .2429632 .416453$

H $-2.0196552 .604895-3.236636$

H $-3.0388355 .330661-1.636701$

H -4.5896604 .9914841 .326791$

O $-2.7936052 .657762 \quad 3.938128$

O $-1.707274 \quad 0.827714 \quad 3.045783$

C -1.6371532 .9677854 .721514$

H -0.7656883 .1135904 .077623$

$\mathrm{H}-1.8320063 .8977815 .262092$

H -1.450868 2.1709225 .447561

SCF Energy (B3LYP/6-31G**//MMFF) $=-3245.91281451$

$03 \quad 140$

MM̄FF Geometry

C $4.451577-3.729777-0.592925$

C $3.325146-3.666494-1.320007$

C $1.949552-3.592459-0.707537$

O $1.238089-2.564428-1.436482$

C $1.215968-4.939201-0.867523$

C $0.068081-5.1435590 .105326$

C $-1.227370-5.295720-0.234112$

C $0.461654-5.238197 \quad 1.560057$

C -1.847950 -5.313144 -1.601475

C $-2.581526-4.021764-1.992480$

C $-3.800593-3.678521-1.118427$

C $-4.602782-2.538768-1.754415$

C $-5.776601-2.087811-0.872151$

O $-3.345595-3.2780420 .178741$

O $-6.244339-0.824917-1.371717$

C $-5.262565-1.8407360 .562220$

C -6.962311 -3.055363 -0.965098

O $-6.344617-1.5756571 .450609$

C -4.399635 -2.994759 1.112394

C $-3.747685-2.6723702 .481728$
O $-5.228607-4.146886 \quad 1.313933$

C $0.355803-1.786774-0.757321$

O $0.065754-1.8592270 .425275$

C $-0.208658-0.814746-1.722559$

C $-1.288376-0.098192-1.379094$

C $-1.9901340 .874575-2.217251$

C $-1.3592601 .256547-3.529281$

C $-2.828259-1.4479702 .507957$

C $-3.532049-0.1523192 .877238$

C -3.2841362 .3213642 .676090$

O $-4.597176-0.117417 \quad 3.487579$

C -4.1344642 .7078601 .507127$

C -3.8017523 .6537910 .615830$

C $-4.6613874 .028789-0.557886$

C $-3.9929383 .753530-1.887810$

C $-3.4189454 .745019-2.589574$

C $-4.0787272 .353320-2.454306$

C $-3.1569431 .382330-1.768829$

N -2.807134 0.9641132481908

H $4.370572-3.7641830 .489904$

H $3.388386-3.655863-2.405615$

H $2.046231-3.301850 \quad 0.344697$

H $1.918238-5.768255-0.704342$

H $\quad 0.874307-5.032500-1.903347$

H -1.941958 -5.4618130 .571631$

H $\quad 0.804077-4.2702351 .936855$

H $-0.370749-5.5586582 .196084$

H $1.266995-5.9688351 .688971$

H -1.100209-5.532292 -2.369675

H -2.554011 -6.152442 -1.640997

H $-1.867468-3.192959-1.970645$

H -2.910953 -4.134798 -3.033147

H -4.422865 -4.576368 -1.026114

H -4.962201 -2.825186 -2.750833

H -3.941485 -1.677515-1.914586

H $-6.494810-0.945272-2.303773$

H $-4.654522-0.9287720 .525387$

H -7.326554 -3.115840 -1.997775

H -7.810560 -2.699948 -0.369889

H $-6.715028-4.069352-0.644624$

H $-6.846382-0.8288281 .081509$

H -4.518927 -2.610496 3.259113

H -3.127003 -3.537729 2.755641

H $-4.784519-4.7396061 .942648$

H $0.261836-0.736502-2.695879$

H - $1.720572-0.254115-0.393201$

H -1.847951 $2.106720-4.011113$

H -1.394342 $0.415326-4.229850$

H $-0.313067 \quad 1.549153-3.387146$

H -2.061158-1.601752 3.277641

H -2.310962 -1.336387 1.552409

H -2.4065202 .9654692 .783507$

H -3.8717892 .3770763 .598005$

H -5.0762392 .1760961 .389597$

H -2.865609 4.1939290 .734606

H $-4.8990485 .096899-0.468202$

H -5.628740 $3.511805-0.525926$

H -2.965991 $4.563941-3.558509$

H -3.385114 $5.759989-2.207870$

H $-3.9060912 .363170-3.536175$

H $-5.1083371 .987239-2.348136$

H -3.533184 $1.031392-0.808466$

H $-1.969120 \quad 0.837677 \quad 1.921910$

C $6.766786-2.658457-0.681511$

O $6.179611-1.364458-0.929700$

C $6.205348-0.6143140 .297687$

C $7.321814-1.2407861 .123206$

C $7.119808-2.7109820 .809034$

H $5.252087-0.8038270 .804671$

C 6.3572480 .8856590 .033019

H $8.311717-0.915396 \quad 0.783135$

H $7.231968-1.0129772 .189548$

C $8.330534-3.5746771 .120078$

H $6.273927-3.0748921 .403216$

C $5.3363281 .499080-0.948258$

O $7.6638311 .114301-0.515557$

H $\quad 6.333844 \quad 1.4249070 .987699$

H $5.5684341 .138390-1.958691$ 
O $5.5721322 .914235-0.958058$

C $3.842048 \quad 1.262545-0.657882$

C $3.420308 \quad 1.6273840 .778787$

H $3.2684711 .881186-1.359254$

O $3.522535-0.093578-0.944843$

H 3.9616570 .9975291 .492710

H 3.7145092 .6628850 .980400

H $8.139596-4.6197380 .855960$

H $9.214771-3.2422790 .566109$

H $8.568419-3.5330482 .188012$

C $5.835007-3.768954-1.185223$

H $7.7538862 .072120-0.659148$

H $5.0027173 .301956-1.644353$

H $2.557026-0.159082-1.024473$

H $6.281503-4.746441-0.969874$

H $5.761572-3.683945-2.276959$

H $7.686264-2.690013-1.280267$

C 1.1289513 .5234650 .108187

$\begin{array}{lllll}\text { O } & 1.101441 & 2.091900 & 0.055443\end{array}$

C $1.907898 \quad 1.4396761 .038870$

C $1.446946 \quad 1.8451552 .444667$

C 1.4137993 .3606872 .617363

C 0.6410104 .0208971 .475503

H $2.151093 \quad 3.876447-0.075112$

C $0.2454924 .015266-1.042884$

H $1.705107 \quad 0.3671450 .941152$

$\begin{array}{llll}\text { O } & 2.290761 & 1.279292 & 3.441727\end{array}$

H 0.4356461 .4558932 .620482

H $2.424478 \quad 3.7777892 .697535$

$\begin{array}{llllll}\text { O } & 0.751641 & 3.677889 & 3.844368\end{array}$

H -0.4286813 .8095911 .591364$

O $0.809144 \quad 5.4333051 .563501$

C $0.4737855 .468241-1.373949$

H $\quad 0.452682 \quad 3.444311-1.956149$

H $-0.812048 \quad 3.830106-0.823064$

H 2.2781810 .3143293 .321544

H 1.2318603 .2229014 .557475

H 0.5049435 .7078832 .445665

O $-0.6535816 .188505-1.113114$

O $1.5311715 .911507-1.803035$

C $-0.5627367 .591857-1.375184$

H $0.210598 \quad 8.044880-0.747491$

H $-0.3531847 .766641-2.434812$

H -1.525941 8.046504-1.128529

SCF Energy (B3LYP/6-31G*//MMFF) $=-3245.90347853$

03 141

MM̄FF Geometry

C 5.6440112 .9126091 .123486

C 5.2591821 .7749450 .525110

C 5.4330520 .3992831 .116870

O $4.112774-0.1964451 .148438$

C $6.330343-0.4649780 .215856$

C $6.629893-1.8288960 .809828$

C $6.094637-2.9927840 .389699$

C $7.628828-1.8302921 .939914$

C $5.105941-3.215585-0.721813$

C $3.719356-3.532129-0.162180$

C $2.660555-3.856477-1.227052$

C $2.446552-2.715684-2.224970$

C $1.280680-2.998895-3.184528$

O $1.446137-4.109105-0.507920$

O $0.957429-1.773890-3.858820$

C $0.043774-3.404343-2.358945$

C $1.670164-4.001936-4.277015$

O $-1.028740-3.801923-3.209814$

C $0.333622-4.509692-1.318505$

C $-0.886786-4.684038-0.383440$

O $0.589096-5.740640-1.994713$

C $3.314133 \quad 0.1131202 .206621$

$\begin{array}{lllll}\text { O } & 3.643711 & 0.677324 & 3.237481\end{array}$

C $1.930819-0.2686001 .840837$

C $\begin{array}{llll}0.906519 & 0.237000 & 2.544067\end{array}$

C $-0.515656 \quad 0.0905852 .225289$

C $-0.888418-0.8449651 .107427$

C $-0.652247-5.6726890 .764449$

C -1.694874 -5.5074101 .850694$

C $-2.243601-4.120895 \quad 3.849447$
O $-2.675980-6.2437381 .921082$

C -3.175991 -3.011979 3.476655

C $-3.324357-1.8960914 .207232$

C $-4.320272-0.819895 \quad 3.885898$

C $-3.7155360 .564475 \quad 3.880431$

C -3.9867821 .4436904 .859959$

C -2.8860020 .9657432 .684584$

C -1.406787 0.8317552 .916645

N -1.410852 -4.4571162 .710756$

H 6.1808492 .8465872 .067630

H $4.7281711 .814109-0.421979$

H 5.8473910 .4512692 .130632

H $\begin{array}{llll}7.285296 & 0.047766 & 0.037977\end{array}$

H $5.864672-0.565135-0.769895$

H $6.394470-3.906868 \quad 0.901557$

H $7.872757-2.8429132 .278705$

H $8.564183-1.3590711 .620058$

H $7.238876-1.2815752 .802412$

H $5.060592-2.353911-1.389942$

H $5.458911-4.055917-1.331917$

H $3.784108-4.3846910 .528288$

H $3.365094-2.6979280 .454296$

H $2.964452-4.773025-1.746146$

H $3.355915-2.514850-2.802656$

H $2.227951-1.789176-1.675592$

H $1.756051-1.465164-4.320167$

H $-0.299455-2.509044-1.822908$

H $2.502748-3.612375-4.875174$

H $0.845078-4.159997-4.980440$

H $1.976991-4.971869-3.880361$

H $-1.189001-3.074005-3.834325$

H $-1.153248-3.7083910 .039109$

H - $-1.756181-5.029255-0.957255$

H $0.445253-6.475800-1.378475$

H $1.789676-0.8754610 .954034$

H 1.1281910 .8760513 .398166

H $-0.405666-1.8176411 .246850$

H $-0.575531-0.4346890 .141271$

H $-1.959877-1.0480711 .050973$

H $-0.698071-6.7069250 .405540$

H $0.336876-5.5404651 .217381$

H -1.574664 -3.840631 4.669279

H -2.820001 -5.0005174 .153414$

H -3.788091 -3.1526472 .588725$

H -2.737527 -1.772817 5.114596

H $-5.119632-0.8764984 .636514$

H $-4.807705-1.0024342 .919898$

H -3.585014 2.4515594 .838952

H $-4.612088 \quad 1.1752145 .704769$

H -3.0806102 .0202252 .446974$

H -3.226040 $0.419271 \quad 1.804157$

H $-1.031292 \quad 1.4881153 .703077$

H $-0.597719-3.8751282 .532567$

C $4.1129064 .463658-0.250863$

O $2.946904 \quad 4.0058210 .461189$

C 1.8230284 .8463790 .118791

C $2.3136515 .776473-0.981526$

C $3.7900395 .901486-0.648209$

H $1.5890725 .414979 \quad 1.027167$

C $0.649793 \quad 3.937198-0.263810$

H $2.1817455 .317034-1.969131$

H $1.7897636 .736878-0.981909$

C $4.6291086 .432369-1.796712$

H $3.8997696 .566188 \quad 0.219070$

C $-0.5932824 .676345-0.782418$

$\begin{array}{llllllllll}\text { O } & 0.274632 & 3.197497 & 0.902320\end{array}$

H $0.9911773 .205385-1.004394$

H $-0.3554735 .197752-1.716419$

$\begin{array}{lllll} & 0 & -0.955180 & 5.695586 & 0.158075\end{array}$

C -1.832141 $3.780635-1.003142$

C $-1.5657212 .600734-1.952065$

H $-2.2195143 .418511-0.043620$

O $-2.8595954 .592802-1.581988$

H $-1.1983912 .990496-2.909667$

H $-0.7849531 .967114-1.517523$

H $5.6848466 .491272-1.514085$

H $4.5520655 .790507-2.680760$

H $4.2991847 .436966-2.081246$ 
C 5.3459404 .3149840 .648295 H 1.0755742 .7563831 .233479 H -1.131946 5.2588511 .009166 H -3.018309 $5.335068-0.973874$ H 5.1849744 .9199891 .550449 H $6.236714 \quad 4.7116650 .149010$ H $4.2122643 .845043-1.151580$ C $-4.3451970 .268783-1.034158$ O $-3.1752741 .097310-0.985907$ C $-2.8149411 .735947-2.215802$ C $-2.5451700 .685033-3.304458$ C $-3.736822-0.261408-3.470490$ C $-4.219864-0.807849-2.123690$ H $-4.366099-0.248063-0.066768$ C $-5.6249351 .103463-1.140825$ H $-3.6327482 .387434-2.540030$ O $-1.385017-0.066471-2.956941$ H -2.336962 $1.200236-4.249956$ H -3.445785 -1.095863 -4.120237 O $\quad-4.791408 \quad 0.447199-4.114415$ H -5.182158 -1.317547 -2.245165 O $-3.293907-1.800086-1.669693$ C $-5.835916 \quad 1.9028410 .123571$ H $-5.6275361 .788097-1.993604$ H $-6.5028450 .457768-1.261920$ H $-1.203119-0.681488-3.687717$ H $-5.530140-0.173147-4.236120$ H $-2.447128-1.356234-1.491604$ O $-5.3529563 .167127-0.037138$ O -6.3452041 .4474791 .139654$ C $-5.4769784 .025098 \quad 1.101022$ H -6.5332174 .1964151 .329199$ H $-4.960813 \quad 3.594327 \quad 1.963686$ H -5.0100564 .9830250 .856759$

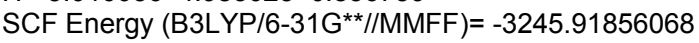

03142

MM̄FF Geometry

C -1.639340 $5.543197-0.335073$

C -2.2115194 .7154630 .551654$

C $-3.388923 \quad 3.8289570 .238167$

O $-2.9623122 .456236 \quad 0.400314$

C -4.5268104 .0999281 .238475$

C $-5.7719093 .271240 \quad 0.988524$

C -6.2699272 .3617901 .849595$

C $-6.4842213 .535070-0.313266$

C -5.7376391 .9780143 .201999$

C $-4.744400 \quad 0.8056023 .193960$

C $-5.345480-0.5257202 .714178$

C $-4.365436-1.6778932 .949468$

C $-4.901599-3.0105812 .403650$

O $-5.626371-0.4204071 .316304$

O $-3.798442-3.9299522 .390083$

C $-5.369399-2.807130 \quad 0.944872$

C $-5.952513-3.6169303 .340836$

O $-6.009765-3.9809290 .451883$

C -6.272062 -1.570302 0.753625

C $-6.568992-1.250783-0.731547$

O $-7.534223-1.813267 \quad 1.387871$

C $-2.2893521 .887542-0.636875$

O $-2.0558832 .385805-1.726524$

C -1.842383 $0.548089-0.196960$

C $-0.871097-0.076116-0.879250$

C $-0.293723-1.383254-0.560015$

C $-0.956072-2.1917090 .523740$

C $-5.338837-0.985691-1.606366$

C $-4.853850-2.206459-2.370963$

C $-2.982822-2.966910-3.829232$

O $-5.491179-3.253405-2.444818$

C -1.993055 -3.730369-3.007157

C $-0.719728-3.921475-3.383993$

C $0.279965-4.716074-2.593657$

C $1.546719-3.938706-2.315308$

C $2.629033-4.079075-3.099160$

C $1.579993-3.061217-1.084803$

C $0.786968-1.795814-1.254646$

N -3.637452 -1.973380 -2.998865

H $-2.0461065 .616707-1.339980$
H -1.7881744 .6345661 .550643$

H -3.743062 $3.989912-0.787333$

H -4.8096095 .1603821 .199670$

H -4.149289 3.9249782 .251773

H -7.1753911 .8285221 .561519$

H $-7.4509483 .022973-0.368912$

H $-6.6759204 .606462-0.431410$

H $-5.8827023 .190453-1.159841$

H -5.2679512 .8392953 .689329$

H $-6.591497 \quad 1.722123 \quad 3.842446$

H -3.8853001 .0711472 .566993$

H -4.3699050 .6805524 .217661$

H -6.278057 -0.699793 3.263607

H $-4.120813-1.7713144 .014963$

H -3.415587-1.453435 2.445160

H $-4.106218-4.753071 \quad 1.972843$

H $-4.460444-2.6827910 .345243$

H $-5.522034-3.7888114 .334765$

H $-6.281473-4.5999372 .986122$

H $-6.832626-2.9826303 .465445$

H $-6.909558-4.0044970 .819822$

H -7.213121 -2.026821-1.162122

H $-7.172328-0.331537-0.749214$

H $-8.192530-1.2106041 .003644$

H $-2.302986 \quad 0.1275570 .689269$

H $-0.4362420 .427393-1.742031$

H -2.025804 -2.304338 0.316641

H $-0.835610-1.7034151 .496746$

H $-0.560010-3.2048060 .617535$

H -5.607977 -0.239919-2.365533

H $-4.516876-0.569443-1.016305$

H -2.508537 -2.433892 -4.659350

H $-3.731331-3.653886-4.236866$

H -2.344533 -4.165594 -2.074697

H $-0.380453-3.508105-4.331266$

H $0.517346-5.620495-3.169224$

H $-0.146386-5.072131-1.647688$

H $3.549652-3.543394-2.894556$

H $2.615662-4.728160-3.968490$

H $2.612984-2.761673-0.863882$

H $1.268632-3.650549-0.218174$

H $1.184774-1.139672-2.029090$

H $-3.154819-1.093579-2.840535$

C $0.8782965 .784336-0.570594$

O $1.114696 \quad 4.482780 \quad 0.004871$

C $1.6095623 .604624-1.031189$

C $2.0157114 .514494-2.181034$

C $0.9636915 .603097-2.085209$

H $0.7575712 .978764-1.324137$

C $2.7300862 .735058-0.450813$

H $3.0168174 .931270-2.014561$

H $2.0127984 .001397-3.147130$

C $1.3247626 .871072-2.838272$

H $0.0235865 .206044-2.487107$

C $3.4868091 .897958-1.495986$

O 2.1425191 .8487320 .508555

H 3.4330483 .3709610 .099357

H $4.0316852 .563597-2.176270$

O $2.5254321 .203214-2.298836$

C $4.4601940 .853251-0.910688$

C 5.4984881 .4697470 .042816

H $3.8925270 .057452-0.413929$

O $5.135377 \quad 0.251417-2.020116$

H $6.0269362 .280051-0.474867$

H $4.966740 \quad 1.9045290 .895982$

H $0.5428367 .628151-2.721769$

H $2.2658927 .298988-2.477155$

H $1.438706 \quad 6.663908-3.907340$

C $-0.4252586 .372323-0.022240$

H 1.6618682 .3959101 .153165

H $3.0186670 .675348-2.950160$

H $5.518295-0.586869-1.710563$

H $-0.5784087 .382966-0.417941$

H $-0.329916 \quad 6.473728 \quad 1.066701$

H $1.7057996 .419363-0.227049$

C $6.664111-1.6161851 .807731$

O $5.847768-0.5630801 .276640$

C 6.5440150 .4666710 .568495 
C 7.5754491 .1472201 .484832

C $8.521230 \quad 0.1241702 .120726$

C $7.757694-1.0548302 .733029$

H $5.978152-2.2151962 .420408$

C $7.216337-2.5237960 .702988$

H $7.0665490 .029184-0.288075$

O $\quad 6.9199701 .8872082 .513400$

H 8.1534661 .8664850 .892863

H 9.1124550 .6219032 .899265

O $9.420921-0.3454471 .121261$

H $8.455575-1.8526293 .012550$

O $7.141178-0.5975683 .940391$

C $6.095322-3.2720220 .023672$

H $7.757981-1.980783-0.076295$

H $7.922024-3.2562691 .113426$

H $\quad 6.351628 \quad 1.2713603 .006831$

H $10.035598-0.9661871 .548363$

H $6.714359-1.3632574 .361001$

O $5.858806-4.447403 \quad 0.672615$

O $5.488711-2.847487-0.950866$

C $4.803006-5.2498100 .134852$

H $4.808091-6.2111730 .655487$

H $3.840375-4.7616130 .308955$

H $4.959439-5.430418-0.933044$

SCF Energy (B3LYP/6-31G $\left.{ }^{* *} / / \mathrm{MMFF}\right)=-3245.92609705$

03143

MM̄FF Geometry

C -1.613298 5.4404561 .547163

C -0.6179934 .6815292 .031549$

C 0.7747934 .5083051 .467548

$\begin{array}{llll}\text { O } & 0.938586 & 3.099823 & 1.155047\end{array}$

C 1.0562165 .2960630 .172171

C $2.4562095 .100344-0.378009$

C $2.7363994 .542078-1.572440$

C 3.5778255 .6037090 .493744

C $1.7737214 .010908-2.598660$

C $1.4849122 .505095-2.488659$

C $2.706578 \quad 1.611767-2.760157$

C $2.295062 \quad 0.140347-2.853007$

C $3.509615-0.783869-3.041457$

O $3.637667 \quad 1.776162-1.688134$

O $3.081776-2.131869-2.793149$

C $4.574229-0.445764-1.974798$

C $4.028421-0.754195-4.484120$

O $5.783285-1.160482-2.216783$

C $4.8701631 .062844-1.857762$

C $5.7738651 .415644-0.650821$

O $5.5577751 .499318-3.036816$

C 1.2695982 .2543082 .167671

O $1.4973672 .546702 \quad 3.330533$

C 1.2653850 .8819351 .617369

C $1.118293-0.1606602 .448300$

C $1.069915-1.5708712 .056972$

C $1.451688-1.9079030 .641069$

C 5.2147651 .0340880 .724424

C $5.718337-0.3000001 .250465$

C $5.402146-1.8738773 .157684$

O $6.617401-0.9420680 .714810$

C $4.512238-2.9941642 .720949$

C $3.791670-3.7372553 .574463$

C $2.933508-4.8968053 .157564$

C $1.507226-4.7721053 .643996$

C $1.092831-5.4179354 .747043$

C $0.542278-3.9722432 .799352$

C $0.704462-2.4874012 .977313$

N $5.066536-0.6709142 .419491$

H -1.464201 6.0554300 .665061

H -0.8292784 .0818872 .915815$

H $1.496816 \quad 4.8018132 .239239$

H 0.9119796 .3696190 .350604

H $0.3140815 .004952-0.578068$

H $3.7832494 .461947-1.863835$

H $4.5477305 .565728-0.013939$

H $3.399564 \quad 6.645728 \quad 0.778745$

H 3.6610415 .0003821 .402779

H $0.8255524 .557287-2.560309$

H $2.1842004 .228025-3.593191$
H $1.0818462 .291417-1.491788$

H $0.6975792 .263171-3.213523$

H $3.1681981 .937927-3.699519$

H $1.569125-0.012020-3.661356$

H $1.777386-0.150389-1.929141$

H $2.358604-2.334115-3.411267$

H $4.186599-0.806208-1.015319$

H $3.242575-1.071998-5.179964$

H $4.853358-1.461836-4.622182$

H $4.3659120 .235455-4.798665$

H $5.557909-2.105586-2.258505$

H $6.7828571 .014646-0.804663$

H $5.8949152 .508657-0.647738$

H $6.0289992 .323857-2.831657$

H $1.353096 \quad 0.7700790 .543032$

H 0.9958320 .0345113 .512953

H $2.423258-1.4689180 .392650$

H $\quad 0.702929-1.521393-0.057273$

H $1.551743-2.9792010 .456355$

H 5.5415531 .7891181 .451118

H $4.120773 \quad 1.0365280 .721085$

H $5.287373-1.6440404 .221710$

H $6.447551-2.1386082 .968920$

H $4.482027-3.2227671 .658177$

H $3.845986-3.5236084 .639794$

H $3.387604-5.8096693 .564824$

H $2.936979-5.0272862 .068156$

H $0.063634-5.3570965 .084773$

H $1.775680-6.0175605 .340008$

H -0.492140 -4.218515 3.074691

H $0.634548-4.2906831 .757952$

H $0.453207-2.1411303 .980931$

H $4.296259-0.1036922 .761818$

C $-3.9808884 .776747 \quad 1.155232$

O $-3.570778 \quad 3.4178150 .908798$

C -4.7326922 .5755760 .846882$

C $-5.9363983 .512650 \quad 0.867790$

C -5.4083214 .6770041 .684495$

H $-4.729473 \quad 1.969863 \quad 1.761851$

C $-4.6700681 .647366-0.375707$

H $-6.2060643 .847019-0.140957$

H -6.8193243 .0417661 .310736$

C -6.2190715 .9508231 .525415$

H -5.3979524 .3855522 .743368$

C $-3.4474770 .700697-0.364176$

O $-4.6755682 .431117-1.568078$

H $-5.5829161 .040789-0.390082$

H -3.4175360 .1933810 .607895$

O $-2.2343831 .443937-0.476466$

C $-3.496704-0.379189-1.465910$

C $-2.279422-1.321472-1.397346$

H $-4.428323-0.942698-1.346641$

O $-3.5272390 .242662-2.748259$

H -1.378765 -0.773814 -1.702230

H -2.131857-1.630281 -0.356893

H $-5.792307 \quad 6.7620932 .123448$

H $-6.247850 \quad 6.280747 \quad 0.481592$

H $-7.2508025 .793693 \quad 1.856875$

C -3.001836 5.4362332 .127914

H $-3.9248743 .047154-1.517893$

H $-2.1973511 .811493-1.375854$

H -3.764639 -0.436308 -3.401738

H -3.007935 4.8960623 .082800

H -3.3030826 .4687012 .335666$

H -3.9624165 .2953880 .187279$

C $-3.739457-3.916017-0.793791$

O $-3.650188-3.268011-2.069571$

C $-2.424630-2.567012-2.303359$

C - $-1.250367-3.550167-2.223104$

C $-1.248680-4.321021-0.905536$

C $-2.617066-4.951561-0.642267$

H -3.665239 -3.169713 0.005831

C $-5.127055-4.562516-0.711935$

H -2.468934 -2.205374 -3.337876

O $-0.019712-2.856816-2.392893$

H $-1.330325-4.267644-3.050278$

H $-0.946063-3.674204-0.075299$

O $-0.263720-5.353176-0.989926$ 
H $-2.763074-5.789046-1.336390$

O $-2.604884-5.4808440 .681813$

C $-6.221595-3.520538-0.736285$

H $-5.289773-5.227950-1.568620$

H $-5.256536-5.1606940 .195932$

H $0.696956-3.504129-2.277462$

H $-0.294015-5.850206-0.154465$

H $-3.381473-6.0564780 .779601$

O $-6.205263-2.7950010 .418274$

O $-7.000056-3.373984-1.669790$

C $-7.185320-1.7562030 .506952$

H $-6.996318-1.1878461 .421585$

H $-7.105275-1.078076-0.348167$

H -8.187298 -2.191630 0.562275

SCF Energy (B3LYP/6-31G**//MMFF) $=-3245.90316506$

03_144

MM̄FF Geometry

C $\quad 0.884968 \quad 5.732113 \quad 0.215417$

C $1.4013374 .839761-0.642735$

C $2.5756763 .950276-0.326207$

O $2.1251412 .576000-0.369851$

C $3.6644574 .133811-1.399453$

C $4.9329813 .348317-1.131498$

C $5.4071942 .366467-1.923406$

C 5.7034833 .7490460 .100694

C $4.8185951 .836064-3.200956$

C $3.8610760 .646831-3.023302$

C $4.512747-0.602525-2.407708$

C $3.554073-1.794048-2.456944$

C $4.148409-3.035574-1.772348$

O $4.835252-0.318109-1.045883$

O $3.084551-3.978892-1.566279$

C $4.676076-2.644429-0.371933$

C $5.173015-3.734994-2.673800$

O $5.400898-3.7186010 .219904$

C $5.541974-1.365374-0.368183$

C $5.868910-0.841978 \quad 1.048219$

O $6.821653-1.597749-0.970251$

C 1.4853192 .0953770 .731529

O 1.2750462 .6875201 .778310

C 1.0556570 .7109550 .433915

C $0.171090 \quad 0.106599 \quad 1.241494$

C $-0.354786-1.2528151 .091320$

C $0.216243-2.099338-0.015096$

C $4.657651-0.4834931 .915737$

C $4.247205-1.5824742 .881793$

C $2.514595-2.1107184 .591648$

O $4.900200-2.6074793 .057713$

C $1.551459-3.0787173 .981594$

C $0.298066-3.2482944 .428664$

C $-0.663656-4.2524343 .862629$

C $-1.955116-3.6214493 .395062$

C -3.063036 -3.664459 4.154137

C -1.982108 -3.018468 2.008107

C $-1.309988-1.6725881 .947673$

N $3.076287-1.2636163 .556899$

H 1.3289375 .8508871 .200151

H $0.9377334 .714165-1.618973$

H $2.983116 \quad 4.1743490 .667037$

H $3.9352995 .195622-1.474853$

H $3.2442363 .859389-2.373222$

H $6.3366331 .879313-1.629686$

H 6.6821193 .2600380 .155870

H $5.879116 \quad 4.8297590 .104794$

H 5.1520603 .4793291 .006366

H $4.3010132 .631721-3.747309$

H $5.6467671 .535165-3.855416$

H $3.0141540 .964601-2.403756$

H $3.4595520 .391557-4.011857$

H $5.430894-0.823897-2.964173$

H $3.272254-2.025568-3.491750$

H $2.619194-1.529490-1.945462$

H $2.681996-4.171193-2.430525$

H $3.795442-2.4753080 .259788$

H $4.695393-4.082662-3.597907$

H $5.578652-4.631396-2.192320$

H $6.003998-3.086918-2.960415$
H $4.813190-4.493473 \quad 0.231043$

H $6.543165-1.5383551 .561455$

H 6.4539180 .0808320 .920990

H $6.920920-2.550007-1.130278$

H $1.4715400 .232820-0.444948$

H -0.2149420 .6623302 .095399$

H $0.050523-1.624425-0.988632$

H $-0.224300-3.095526-0.081924$

H $1.292206-2.2391500 .131741$

H 4.9211270 .3836562 .535074

H $3.798810-0.1975631 .301350$

H $2.035524-1.4541545 .324835$

H $3.321739-2.6586135 .088775$

H $1.910129-3.6891073 .156102$

H $-0.049848-2.6552635 .271623$

H $-0.870698-4.9932554 .646161$

H $-0.214160-4.8168763 .036199$

H $-4.000594-3.2393803 .811329$

H -3.055780 -4.121688 5.138065

H -3.019722 -2.880958 1.685415

H $-1.553679-3.7439411 .312626$

H -1.674907 -0.9683172 .697041$

H $2.570310-0.4187863 .306888$

C -1.633278 5.9984260 .411898

O $-1.874404 \quad 4.717475-0.204155$

C -2.3785333 .8121990 .794946$

C -2.8039734 .6891941 .966465$

C -1.7442895 .7748141 .919406$

H -1.5361113 .1831741 .108793$

C -3.4899072 .9204520 .226800$

H -3.8000795 .1187801 .805969$

H -2.817176 4.1413762 .913448

C -2.1104107 .0232792 .701657$

H $-0.812078 \quad 5.3617582 .324372$

C $-2.9744631 .953130-0.862776$

O $-4.5273453 .756447-0.280600$

H $-3.9151472 .331167 \quad 1.048314$

H -2.155212 $1.366798-0.431200$

O $-2.4241592 .681754-1.959017$

C $-4.0428230 .973755-1.388096$

C $-3.425571-0.071696-2.336831$

H $-4.5054240 .475173-0.529934$

O $-5.0611661 .699817-2.070431$

H $-2.943570 \quad 0.439262-3.179580$

H -2.635109 -0.603419-1.794930

H $-1.322778 \quad 7.7787992 .618144$

H -3.043515 7.4663782 .338033

H -2.2422056 .7873803 .762689$

C $-0.3141876 .580713-0.102727$

H $-5.2534873 .181353-0.575166$

H $-3.1353193 .236162-2.324015$

H $-5.8114781 .097358-2.208469$

H -0.1563167 .5826650 .312728$

H $-0.3865496 .702096-1.191393$

H -2.447275 6.6531090 .073336

C $-4.688894-2.488040-0.978307$

O $-5.319181-1.607764-1.914967$

C $-4.445634-1.078604-2.914868$

C $-3.814414-2.222621-3.722765$

C $-3.145612-3.259652-2.819590$

C $-4.099598-3.699528-1.707930$

H -3.894298 -1.951697 -0.446698

C $-5.751393-2.9099240 .042313$

H -5.082424 -0.513922 -3.607631

O $-2.873849-1.695940-4.652288$

H $-4.602172-2.718458-4.305163$

H -2.205724 -2.882284 -2.399452

O $-2.807631-4.414238-3.591340$

H -4.903687 -4.311314-2.136491

O $-3.391502-4.513841-0.776968$

C $-6.221547-1.7308210 .862310$

H -6.630063 -3.325061 -0.466401

H $-5.382308-3.6775050 .731310$

H -2.589301 -2.423907 -5.229776

H -2.118893 -4.152343 -4.225244

H $-3.005133-5.254871-1.274311$

O $-5.245421-1.3452611 .732116$

O $-7.324267-1.2134430 .739857$ 
C $-5.557548-0.2163112 .553496$

H $-6.375945-0.4637893 .235845$

H $-4.6699180 .030574 \quad 3.142109$

H -5.817816 0.6473961 .934010

SCF Energy (B3LYP/6-31G**//MMFF) $=-3245.90936790$

03145

MM̄FF Geometry

C $2.315130-3.475928-0.012988$

C $2.124065-2.793154-1.153467$

C $0.895013-1.987082-1.519935$

O $-0.004345-1.944908-0.389841$

C $1.321045-0.546920-1.876058$

C $0.2816320 .249910-2.640554$

C $-0.3242941 .367840-2.194335$

C $-0.020557-0.249066-4.032564$

C $-0.1336492 .080577-0.885799$

C -1.2561931 .8550930 .137489$

C -2.617326 $2.451052-0.261427$

C -3.6042312 .3541420 .905934$

C -5.0092202 .8458920 .523508$

O $-3.1311111 .727185-1.383199$

O -5.9233112 .4040191 .539452$

C $-5.4410052 .167746-0.795147$

C -5.0898324 .3768830 .507075$

O $-6.6674212 .715445-1.270525$

C $-4.3678412 .240089-1.900695$

C $-4.7270721 .416428-3.163274$

O $-4.2219883 .601180-2.326567$

C -1.340676 -2.031891-0.610395

O $-1.915009-2.086668-1.684903$

C -1.996891-2.048869 0.717068

C $-3.307273-1.7830740 .811531$

C $-4.094887-1.7432252 .044851$

C $-3.443406-2.2390753 .308512$

C $-4.953373-0.081104-2.935950$

C $-6.404388-0.451454-2.673827$

C $-7.800039-2.282901-1.713623$

O $\quad-7.348966 \quad 0.268987-2.984825$

C $-8.166437-1.801692-0.345228$

C $-8.199977-2.5887480 .740608$

C -8.556871-2.096050 2.113882

C -7.411081 -2.2216463 .094735$

C -7.373856 -3.223858 3.988852

C $-6.356630-1.1363463 .100284$

C $-5.362379-1.2856871 .982205$

N $-6.522592-1.705572-2.090460$

H $1.514121-3.5028260 .721630$

H $2.910473-2.788228-1.904017$

H $\quad 0.422646-2.492340-2.369982$

H $2.225597-0.566120-2.499931$

H $1.608355-0.032867-0.953371$

H -1.028063 $1.859765-2.864790$

H $-0.6695950 .436259-4.588621$

H $0.905875-0.356312-4.606365$

H $-0.524623-1.218911-3.999998$

H $0.8154811 .797590-0.422321$

H $-0.0442723 .154242-1.093940$

H -1.360229 0.7799130 .314445

H -0.9364922 .3074901 .084693$

H -2.463083 $3.499291-0.542989$

H -3.230303 2.9049611 .778072

H $-3.6815351 .307573 \quad 1.227559$

H -5.615705 2.7545262 .392845

H $-5.637191 \quad 1.114798-0.558970$

H -4.8537784 .7831391 .498026$

H $-6.105903 \quad 4.7173130 .279416$

H -4.402763 4.835144 -0.207346

H -7.320487 $2.627663-0.555158$

H -5.576076 $1.877062-3.682870$

H $-3.8792231 .504203-3.857945$

H -3.812961 $3.606318-3.207860$

H -1.377276 -2.2545371 .582545$

H -3.856330 -1.552233 -0.099079

H -2.630672 -1.569035 3.609560

H $-3.033570-3.2450673 .162810$

H -4.133573 -2.311943 4.152004

H -4.668032 -0.619657 -3.848665
H $-4.315361-0.451568-2.128832$

H -7.692830 -3.371420 -1.755830

H -8.567287 -1.979541 -2.433269

H $-8.413424-0.747233-0.245292$

H -7.957322 -3.644287 0.642565

H -9.420432 -2.677158 2.462796

H -8.898304 -1.053637 2.088440

H -6.571922 -3.303656 4.715174

H -8.142068 -3.9897424 .011106$

H -5.855939-1.091667 4.073533

H $-6.844954-0.1580773 .002941$

H -5.734705 -0.9406761 .018289$

H $-5.682231-2.201478-1.808112$

C $4.808146-4.043067-0.444681$

O $5.204238-2.657647-0.458995$

C $6.635637-2.576464-0.351816$

C $7.157470-4.002884-0.493823$

C $6.016745-4.8062910 .097292$

H $6.852671-2.2210790 .662428$

C $7.197002-1.590139-1.380475$

H $7.311419-4.279707-1.543158$

H $8.105604-4.1502170 .032321$

C $6.046520-6.275737-0.285586$

H $6.058581-4.7187351 .191397$

C $6.572546-0.180246-1.337222$

O $6.964690-2.118655-2.693456$

H $8.285781-1.528859-1.264047$

H $5.548027-0.215251-1.726668$

O $7.3098790 .632245-2.262423$

C 6.5673190 .4994980 .046303

C $6.1827301 .989874-0.044946$

H $5.873124-0.0343660 .703818$

O $7.8752380 .390513 \quad 0.608515$

H $6.9892032 .527303-0.558077$

H $5.2827652 .084410-0.661509$

H $5.201893-6.8123190 .157828$

H $5.997559-6.408444-1.371613$

H $6.969041-6.7467460 .069253$

C $3.555440-4.2150660 .418352$

H $7.335283-1.484342-3.330839$

H $8.2118010 .730213-1.911589$

H 7.8078270 .5909341 .556928

H $3.783838-3.8661991 .434093$

H $3.302076-5.2787340 .493417$

H $4.606773-4.336894-1.482660$

C 3.5697702 .3098451 .534992

O 4.8795872 .0484592 .055824

C 5.9576162 .6529111 .332952

C 5.7682644 .1762411 .276248

C 4.4050384 .5534680 .705678

C 3.3011603 .8197621 .463468

H 3.4706331 .8618120 .539125

C 2.5565791 .6347302 .472929

H 6.8614602 .4755901 .927508

O 6.8098584 .7684660 .508046

H 5.8501434 .5800202 .294021

H $4.3447294 .352057-0.370272$

O $4.1925685 .957283 \quad 0.869302$

H 3.2182924 .2350822 .476289

O $2.0553914 .047366 \quad 0.808580$

C 2.5923720 .1240082 .390361

H 2.7780501 .9161953 .509711

H 1.5351751 .9426522 .224733

H 6.7336405 .7329540 .600475

H $4.823561 \quad 6.4184250 .291223$

H 1.9241285 .0097040 .759271

O $1.824818-0.3839333 .400265$

O $3.192949-0.5254701 .546420$

C $1.738850-1.8103743 .469394$

H $1.050925-2.1705292 .700744$

H $2.724268-2.2729703 .357823$

H $1.339100-2.0786344 .450884$

SCF Energy (B3LYP/6-31G**//MMFF) $=-3245.90587354$

03_146

MM̄FF Geometry

C $2.368021-3.248018-1.461421$

C $1.123604-3.723332-1.620772$ 
C $0.012553-3.503110-0.623399$

O $-0.891302-2.505885-1.151261$

C $-0.774102-4.814649-0.431168$

C $-1.819847-4.7534360 .664562$

C $-3.149215-4.8468430 .465711$

C $-1.286681-4.6180362 .067792$

C $-3.888518-5.027297-0.829926$

C $-4.346959-3.723099-1.501235$

C $-5.287783-2.863319-0.640915$

C $-5.896111-1.730606-1.471541$

C $-6.763439-0.790490-0.618772$

$\begin{array}{llllll} & -4.530001 & -2.307011 & 0.437067\end{array}$

O $-7.0117880 .397543-1.386606$

C $-5.965155-0.3599860 .631507$

C $-8.135647-1.403142-0.313782$

$\begin{array}{llll}\text { O } & -6.788370 & 0.376438 & 1.531853\end{array}$

C $-5.300579-1.5389071 .371034$

C $-4.340286-1.0908962 .500319$

O $-6.316446-2.3433511 .982895$

C $-0.542217-1.202715-0.968791$

O $0.445737-0.778057-0.390681$

C $-1.568048-0.357257-1.613905$

C -1.256778 $0.909363-1.925890$

C $-2.1469481 .895922-2.537991$

C $-3.6105531 .554369-2.620632$

C $-3.167526-0.2086792 .058516$

C $-3.422343 \quad 1.2814852 .208347$

C -2.3967803 .4891421 .683879$

O -4.3955451 .7436682 .797416$

C -3.0284204 .0369470 .443210$

C $-2.4529744 .975418-0.323968$

C $-3.0990195 .576088-1.540121$

C -2.225977 5.483911-2.771646

C $-1.4517106 .515309-3.149174$

C $-2.3141114 .226602-3.607745$

C -1.616687 $3.062069-2.961388$

N -2.4084612 .0393241 .636713$

H $2.594420-2.640328-0.588679$

H $\quad 0.886545-4.297222-2.513704$

H $\quad 0.416935-3.1672810 .339349$

H $-0.080958-5.631446-0.188069$

H $-1.237566-5.087427-1.385586$

H $-3.798698-4.7977001 .339450$

H -2.071606 -4.728626 2.823955

H $-0.533748-5.3880142 .265359$

H $-0.830182-3.6358452 .213125$

H -3.288655 -5.602293 -1.543374

H $-4.767880-5.653313-0.629951$

H -3.461571-3.138683 - 1.776085

H $-4.857097-3.992689-2.434352$

H $-6.077631-3.509272-0.239967$

H $-6.478319-2.132098-2.310270$

H $-5.088980-1.140068-1.925247$

H $-7.4488790 .133535-2.214375$

H -5.1840860 .3282370 .287135$

H $-8.676067-1.613772-1.244552$ H $-8.765999-0.7028250 .245281$ H -8.073207 -2.3370770 .248456$ H $-7.167654 \quad 1.1213141 .034804$ H $-4.908107-0.6304583 .317832$ H -3.901455 -2.002731 2.930812 H $-5.911060-2.8609822 .698111$ H $-2.538080-0.795114-1.813885$ H $-0.2459321 .261042-1.722122$ H -3.990082 $1.259101-1.636419$ H -3.774046 $0.729660-3.322635$ H $-4.2365912 .386856-2.947846$ H $-2.307050-0.4336102 .700184$ H $-2.874211-0.4296171 .028625$ H $-1.354823 \quad 3.8021341 .791894$ H -2.955125 3.8299312 .561775 H $-4.017813 \quad 3.6686750 .181773$ H $-1.4766145 .363997-0.043222$ H -3.314437 $6.629194-1.316144$ H $-4.0726815 .114523-1.746977$ H -0.842468 $6.467343-4.045573$ H -1.405840 $7.429013-2.565851$ H $-1.8379154 .388226-4.584043$
H -3.363714 4.025031 -3.837503

H $-0.5408573 .208485-2.856524$

H -1.6623541 .5734091 .128282$

C $4.871018-3.578326-1.805976$

O $5.267031-2.275451-1.334626$

C $5.929405-2.425009-0.065126$

C $6.228531-3.9146890 .080735$

C $5.033483-4.531974-0.622499$

H $5.200710-2.1349410 .701721$

C $7.151448-1.5034140 .015128$

H $7.154730-4.198332-0.432019$

H $6.316242-4.2190561 .128085$

C $5.236965-5.981386-1.026272$

H $4.173789-4.4713550 .055735$

C $6.867390-0.033365-0.357856$

O $8.149227-1.987074-0.894037$

H $7.591313-1.5697751 .017230$

H $6.6725600 .038108-1.434640$

O $8.0711920 .713161-0.135725$

C $5.7069010 .626903 \quad 0.410152$

$\begin{array}{llll}\text { C } & 5.548769 & 2.118164 & 0.053282\end{array}$

H 4.7763960 .1030350 .169585

O $5.9138010 .487991 \quad 1.814410$

H 6.4521432 .6674630 .345098

H $5.4401662 .195538-1.035483$

H $4.352525-6.367755-1.542639$

H $6.096047-6.095446-1.695923$

H $5.413868-6.605228-0.144165$

C $3.479849-3.494312-2.443098$

H $8.915801-1.392322-0.826324$

H 8.3059640 .6285620 .803798

H 6.6797601 .0335662 .060255

H $3.287128-4.417994-3.001848$

H $3.476242-2.662837-3.158824$

H $5.588780-3.836832-2.595796$

C 1.9277852 .5786540 .873829

$\begin{array}{llllll}\text { O } & 3.154569 & 2.128042 & 0.283413\end{array}$

$\begin{array}{llll}\text { C } 4.341117 & 2.781146 & 0.744037\end{array}$

C $4.267665 \quad 4.286800 \quad 0.443045$

C 2.9917114 .9100581 .016082

C $1.7450394 .092667 \quad 0.661215$

H 1.1406792 .0591100 .312932

C 1.7997452 .1562762 .344635

H 4.4407522 .6325601 .824324

O $4.315213 \quad 4.515964-0.964225$

H 5.1461524 .7782120 .877129

H $2.8856355 .928960 \quad 0.623659$

$\begin{array}{llll}\text { O } & 3.127298 & 4.998656 & 2.431545\end{array}$

H $0.8867514 .451672 \quad 1.239453$

O $1.4448504 .324645-0.717646$

$\begin{array}{llll}\text { C } & 1.704674 & 0.652220 & 2.481952\end{array}$

H 2.6385602 .4796822 .965580

H 0.8884442 .6015412 .761791

H $3.5756614 .031450-1.369516$

H 2.3259205 .4279762 .776565

H $0.6057203 .873885-0.912694$

$\begin{array}{lllll}\text { O } & 0.718013 & 0.331584 & 3.368267\end{array}$

O $2.430873-0.1437841 .901063$

C $0.534428-1.0687613 .594925$

H $0.297256-1.5744712 .654729$

H $1.431663-1.4988154 .049983$

H $-0.304929-1.1960144 .283672$

SCF Energy (B3LYP/6-31G**//MMFF)= -3245.90211259

03_147

MM̄FF Geometry

C 3.4768180 .9592493 .502367

C 2.7967700 .0472992 .792133

C $1.393814-0.3959323 .117258$

O $0.546767-0.1198051 .979336$

C $1.379952-1.9161383 .361099$

C $0.026670-2.4643003 .770139$

C $-0.672641-3.3747723 .064426$

C $-0.521339-1.9528435 .077713$

C $-0.291000-4.0342321 .768636$

C $-0.786593-3.3067850 .509378$

C $-2.317220-3.257100 \quad 0.375192$

C $-2.724218-2.708644-0.993465$ 
C $-4.250044-2.581912-1.127094$

O $-2.835034-2.4006451 .393929$

O $-4.526095-1.763967-2.275640$

C $-4.811871-1.8302670 .102025$

C $-4.902304-3.942300-1.402464$

O $-6.236072-1.8618160 .113806$

C $-4.266801-2.3438691 .452769$

C $-4.631926-1.4380812 .650602$

O $-4.819430-3.6232601 .787595$

C $0.0630851 .145431 \quad 1.853162$

O 0.2669482 .0916492 .596621

C -0.7514831 .1864820 .619734$

C -1.0671102 .3708070 .075395$

C $-1.8118282 .573668-1.169311$

C $-2.4716961 .370619-1.789537$

C $-4.132606 \quad 0.0081742 .563528$

C -5.1657700 .9844902 .025218$

C $-5.4187293 .375072 \quad 1.370849$

O $\quad-6.339270 \quad 0.6842951 .823922$

C $-5.3334613 .471593-0.119497$

C $-5.0057364 .600913-0.765349$

C $-4.9569024 .726381-2.260901$

C $-3.6294315 .253516-2.756980$

C -3.472999 $6.552000-3.065632$

C $-2.5120044 .258991-2.976336$

C $-1.8666163 .816331-1.692960$

N $-4.6266932 .249227 \quad 1.829455$

H 3.0061691 .4279824 .362802

H $3.267942-0.4030621 .922475$

H 1.0108800 .1300834 .000106

H $2.101050-2.1726104 .148679$

H $1.731587-2.4148802 .451905$

H -1.633412 -3.700417 3.462049

H $-0.799697-0.8979544 .994047$

H -1.413651 -2.502241 5.397184

H $0.226435-2.0576805 .870587$

H $0.794999-4.1576521 .705694$

H $-0.691344-5.0561871 .776300$

H $-0.381507-2.2880820 .504508$

H $-0.368027-3.826735-0.361050$

H $-2.708998-4.2714920 .514446$

H -2.322557 -3.335572 -1.799094

H -2.277813 -1.715140 -1.135435

H -4.103763 -2.178072 -3.047592

H -4.523730 -0.779313 -0.015904

H -4.533165 -4.359433 -2.347296

H -5.986781 -3.845561 -1.522680

H -4.702621 -4.678709-0.620972

H -6.541133 -1.496747 -0.734495

H -5.711283 -1.481586 2.841092

H -4.165582 -1.882976 3.542000

H -5.507072 -3.8442811 .139392$

H -1.0359680 .2410120 .172768$

H $-0.713903 \quad 3.2785940 .562760$

H -1.718852 $0.675333-2.176170$

H $-3.143306 \quad 1.617471-2.614177$

H -3.086650 $0.848047-1.049270$

H $-3.889374 \quad 0.351199 \quad 3.577381$

H $-3.2180230 .076714 \quad 1.966775$

H -5.0295554 .2694961 .867537$

H -6.4618063 .2340031 .671975$

H -5.577496 $2.580923-0.693598$

H -4.789149 $5.497608-0.188863$

H $-5.7647125 .405150-2.564381$

H -5.175972 $3.769838-2.751696$

H $-2.5322506 .933049-3.449063$

H -4.281333 $7.264122-2.936395$

H $-1.7234574 .711000-3.593073$

H -2.888264 $3.425281-3.575402$

H -1.354495 $4.622838-1.166121$

H -3.6307162 .3935951 .968164$

C 5.0060552 .4978982 .153491

O 4.6050532 .0162090 .853824

C 3.7781833 .0164190 .233685

C 4.0245044 .3027631 .015080

C 4.1882413 .7620172 .422510

H 2.7388642 .7069440 .396696

C $4.0597453 .113337-1.269607$
H 4.9439004 .8057340 .693543

H 3.1967765 .0120700 .920088

C 4.8549374 .7326383 .380969

H 3.1920583 .5127092 .808066

C 3.938078 1.783396-2.045252

O $5.3951403 .601159-1.449156$

H $3.3941523 .867427-1.707743$

H $4.8146501 .150357-1.859480$

O $3.9536192 .094206-3.444667$

C $2.6637520 .974634-1.746956$

C $2.416447-0.176320-2.742748$

H $2.7269530 .577226-0.728146$

O $1.5285501 .840672-1.793790$

H $1.9960050 .241965-3.664540$

H $3.371296-0.641214-3.000754$

H 4.9643674 .2842754 .373514

H 5.8506385 .0239313 .030295

H 4.2559675 .6431823 .484567

C 4.8863481 .3773853 .189804

H $5.4968693 .844967-2.384358$

H $4.8408152 .425879-3.662533$

H $1.5021932 .249129-2.676071$

H 5.3625611 .6915864 .126303

H 5.4452200 .5011542 .838678

H 6.0689592 .7552012 .056465

C $3.018877-2.991893-1.671053$

O $2.077457-2.035624-1.161495$

C $1.437862-1.220208-2.153799$

C $0.722996-2.101631-3.185148$

C $1.655320-3.144965-3.788886$

C $2.348743-3.935190-2.679510$

H $3.843641-2.470936-2.167338$

C $3.589914-3.762232-0.471397$

H $0.651334-0.663654-1.629366$

O $0.148052-1.291264-4.203950$

H $-0.105043-2.620958-2.687312$

H $2.388592-2.687632-4.464096$

O $0.891329-4.062811-4.574418$

H $1.611936-4.578712-2.181795$

O $3.323249-4.778768-3.290986$

C $4.490018-2.9135430 .398947$

H $2.769557-4.123300 \quad 0.159886$

H $4.182338-4.628902-0.781343$

H $-0.405315-1.864458-4.760568$

H $\quad 0.559339-3.579341-5.349421$

H $3.576621-5.458508-2.644640$

O $5.461939-2.320599-0.350242$

O $4.372822-2.834591 \quad 1.615165$

C $6.386356-1.4960190 .364996$

H $5.879522-0.5930240 .713871$

H $6.827960-2.0427181 .203804$

H $7.185348-1.206220-0.322546$

SCF Energy $\left(B 3 L Y P / 6-31 G^{* *} / / M M F F\right)=-3245.89892279$

03_148

MM̄FF Geometry

C $0.3747494 .916543-2.062900$

C $-0.4239834 .406443-1.112748$

C $-1.6976133 .654786-1.411675$

O $-1.7336592 .459034-0.597086$

C $-2.9097864 .523096-1.028216$

C $-4.2468353 .896131-1.373161$

C $-5.1880083 .554837-0.471236$

C $-4.5127943 .686773-2.842274$

C -5.1424303 .7111851 .022815$

C -4.6190692 .4818401 .781926$

C -5.4760341 .2184081 .599562$

C $-5.047236 \quad 0.1258102 .581334$

C $-5.826669-1.1813952 .363851$

$\begin{array}{lllll}\text { O } & -5.305508 & 0.745203 & 0.260837\end{array}$

O $-5.148679-2.223297 \quad 3.082984$

C $-5.774330-1.5591820 .867182$

C $-7.242666-1.1035782 .947130$

O $-6.624800-2.6692140 .592520$

C $-6.118319-0.387365-0.075721$

C $-5.862974-0.706956-1.569315$

O $-7.511196-0.0748020 .051786$

C $-0.9705411 .403320-0.988227$ 
O $-0.2516611 .336251-1.972142$ C $-1.1490760 .332748 \quad 0.017660$ C $-0.277584-0.6852030 .062633$ C $-0.307825-1.808748 \quad 1.001824$ C $-1.502472-1.9273201 .909782$ C $-4.421846-1.089262-1.925371$ C $-4.174031-2.588373-1.966021$ C $-2.332878-4.245079-2.234457$ O $-5.072027-3.423100-1.898388$ C $-1.891879-4.723042-0.887316$ C $-0.683535-5.258384-0.656652$ C $-0.241536-5.7873190 .677165$ C $1.048730-5.1607221 .154793$ C $2.226530-5.7847580 .984804$ C $0.961173-3.853358 \quad 1.908093$ C $0.726747-2.674617 \quad 1.003232$ N -2.827006 -2.885134 -2.126227 H $0.0757604 .795177-3.102785$ H $-0.1704214 .519712-0.062753$ H -1.748126 3.373231-2.470413 H -2.850312 $5.492188-1.541623$ H $-2.854323 \quad 4.7443070 .043180$ H -6.114089 $3.116429-0.842206$ H $-5.547165 \quad 3.384045-3.038060$ H -4.334568 $4.613767-3.397003$ H -3.861934 $2.905360-3.245797$ H -4.5439134 .5841361 .303846$ H -6.158205 3.9413581 .369466 H -3.5894012 .2802411 .463751$ H -4.5833572 .7411942 .847434$ H -6.5263151 .4871221 .763257$ H $-5.157103 \quad 0.4682203 .617931$ H -3.977058 -0.0818722 .447733$ H $-5.108162-1.9637334 .019433$ H $-4.750377-1.8917990 .660932$ H $-7.202208-0.9021444 .024311$ H -7.766682 -2.059955 2.841822 H -7.853093 -0.3228472 .489020$ H $-6.359282-3.3918581 .186857$ H -6.580259 -1.457944 -1.921836 H $-6.0961170 .204723-2.138372$ H -7.794530 $0.401701-0.746132$ H -1.9834180 .4208550 .703760$ H $0.554661-0.686025-0.639867$ H $-1.524257-2.8549372 .485177$ H $-2.428566-1.9145041 .325462$ H $-1.525693-1.0987122 .625709$ H $-4.205079-0.718412-2.935425$ H -3.708097 -0.617295 -1.243561 H $-1.514309-4.237217-2.960814$ H -3.128114 -4.895051-2.613565 H -2.607817 $-4.651137-0.071891$ H $0.019253-5.354313-1.481259$ H $-0.114182-6.8734440 .578583$ H - $-1.017859-5.6506351 .440230$ H $3.153066-5.3535621 .349259$ H $2.291323-6.7370750 .468937$ H $1.903391-3.6644382 .440293$ H $\quad 0.209752-3.9455922 .696728$ H $1.540568-2.5036410 .298406$ H $-2.145941-2.131239-2.119303$ C $2.2876205 .619222-0.465028$ O $2.681896 \quad 4.266627-0.160140$ C $3.999466 \quad 4.277747 \quad 0.415319$

C 4.2766845 .7289920 .784046

C $3.5593986 .458895-0.335111$

H $4.6925893 .971814-0.378993$

C 4.1049173 .2890651 .585163 H 3.8365135 .9896361 .753792

H 5.3473495 .9507730 .826043

C $3.3045377 .926435-0.038254$

H $4.1605786 .377059-1.250736$

C $3.890747 \quad 1.8241151 .141046$

O 3.1550843 .6610852 .581890

H 5.1038583 .3857282 .027591

H $4.556718 \quad 1.6362550 .291874$

$\begin{array}{lllll}\text { O } & 2.557005 & 1.639033 & 0.668297\end{array}$

$\begin{array}{llll}\text { C } 4.191904 & 0.783292 & 2.238345\end{array}$
C $3.972265-0.6698621 .789061$

H 5.2215000 .9175612 .587665

O 3.3407001 .0188573 .362369

H $4.150240-1.3428742 .639462$

H $2.907962-0.8207611 .570208$

H $2.7704638 .404969-0.864983$

H $2.705056 \quad 8.0543320 .869341$

H 4.2502048 .4590020 .106291

C $1.6718075 .660368-1.866869$

H 3.2737163 .0642663 .339860

H 1.9564951 .8405991 .406473

H 3.5859590 .3826614 .055810

H $2.3903705 .227282-2.575028$

H $1.5066356 .700820-2.169544$

H 1.5583645 .9274770 .294151

C $5.055766-3.134569-0.787390$

$\begin{array}{llll}\text { O } & 4.432589 & -2.503276 & 0.339701\end{array}$

C $4.801717-1.1369470 .579593$

C $6.318422-1.0150730 .794536$

C $7.101371-1.652623-0.356591$

C $6.588033-3.057922-0.684336$

H $4.775436-4.192356-0.704995$

C $4.510285-2.597436-2.119826$

H $4.499192-0.544936-0.288969$

O $6.674214-1.6455282 .023467$

H 6.5870500 .0443300 .876512

H $8.162180-1.703863-0.083077$

O $6.990576-0.811700-1.501169$

H $7.033198-3.423791-1.616872$

O $6.993878-3.956870 \quad 0.351631$

C $3.067440-2.998830-2.340445$

H $4.575247-1.507323-2.167783$

H $5.081418-3.004377-2.961968$

H $7.631074-1.5266892 .148619$

H $7.508244-1.219473-2.216224$

H $7.965529-3.9437460 .387448$

O $2.409433-1.998954-2.996363$

O $2.588360-4.076354-2.013544$

C $1.037406-2.262586-3.306801$

H $\quad 0.972873-3.033687-4.079871$

H $0.490156-2.569880-2.411473$

H $0.591213-1.340477-3.688811$

SCF Energy (B3LYP/6-31G**/MMFF)= -3245.89304406

03_149

MM̄FF Geometry

C 3.6982093 .1711810 .145251

C 2.5483823 .7127390 .571658

C $1.2152993 .323051-0.013220$

O $0.4582962 .732336 \quad 1.066875$

C $0.4645574 .551707-0.561443$

C $-0.5913744 .210751-1.599098$

C $-1.9192844 .392735-1.456370$

C $-0.0609903 .673256-2.907304$

C $-2.6738244 .963349-0.290492$

C $-3.375522 \quad 3.9242190 .596362$

C $-4.468113 \quad 3.096762-0.104354$

$\begin{array}{llll}C & -5.259811 & 2.288475 & 0.929301\end{array}$

C $-6.297043 \quad 1.361268 \quad 0.278355$

O $-3.8509852 .205405-1.039601$

O $-6.7313620 .418774 \quad 1.271635$

C $-5.6132430 .549195-0.842068$

C $-7.5475562 .129637-0.164616$

O $-6.570436-0.208219-1.577219$

C $-4.7765491 .416902-1.804536$

C $-3.9611770 .582214-2.825725$

O $-5.654717 \quad 2.254532-2.567502$

$\begin{array}{llll}\text { C } & -0.291621 & 1.637106 & 0.778177\end{array}$

O $-0.452033 \quad 1.122668-0.316476$

C $-0.886277 \quad 1.1545832 .045002$

$\begin{array}{llll}C & -1.874763 & 0.250337 & 1.998371\end{array}$

C $-2.563647-0.346242 \quad 3.141985$

C $-1.995993-0.0917004 .512497$

C $-2.959585-0.406505-2.223058$

C $-3.516460-1.803747-2.005349$

C $-3.085047-3.880119-0.688315$

O $-4.494627-2.238739-2.606772$

C $-4.038955-3.8313270 .463203$ 
C $-3.735359-4.2442561 .703263$

C $-4.697558-4.1977222 .856438$

C $-4.216718-3.3092123 .983526$

C -3.608448 -3.831026 5.062066

C $-4.518621-1.8287773 .902040$

C $-3.647820-1.1137782 .906338$

N -2.760557 -2.524266 -1.090407

H $3.6662292 .442841-0.663038$

H 2.5577404 .4368001 .381944

H $1.3835452 .581079-0.802134$

H $\quad 1.1734255 .239732-1.042119$

H 0.0278665 .1043490 .276564

H -2.556843 $4.122779-2.297980$

H -0.839839 $3.603650-3.674695$

H $\quad 0.7212084 .331451-3.299414$

H $0.3576432 .671022-2.778186$

H -2.0182875 .5710060 .340964$

H -3.423135 $5.662355-0.683188$

H -2.618740 $3.253621 \quad 1.013991$

H $-3.8250554 .463788 \quad 1.439613$

H $-5.132606 \quad 3.785137-0.639782$

H -5.7447492 .9542331 .654330$

H -4.5652651 .6710221 .513701$

H -7.0896610 .9199402 .024234$

H $-4.951721-0.173353-0.348830$

H -8.0274032 .6087230 .697344$

H -8.298800 $1.453063-0.586777$

H -7.334179 2.910781-0.897098

H -7.057612 $-0.757069-0.938961$

H $-4.6383960 .086946-3.532008$

H $-3.377716 \quad 1.291832-3.429990$

H -5.186571 2.542744 -3.368492

H -0.4988251 .5565382 .973945$

H -2.226897 -0.0821801 .024002$

H $-2.108677 \quad 0.9633384 .784707$

H $-0.931347 \quad-0.3505484 .543524$

H -2.476075 -0.6822725 .296132$

H -2.119542 -0.523918 -2.919354

H $-2.554022-0.016785-1.287289$

H -2.145469 -4.377167 -0.427419

H -3.541047 -4.414078 -1.528017

H -5.033066 -3.438462 0.261978

H -2.744374 -4.645101 1.903626

H $-4.833511-5.2250083 .218882$

H -5.693269 -3.868428 2.533989

H $-3.286620-3.2054105 .887792$

H -3.413996 -4.895579 5.140418

H -4.450196 -1.363670 4.891542

H -5.567766 -1.691502 3.609441

H -3.980428 -1.2317571 .875157$

H $-2.006423-2.057923-0.594975$

C 5.6218952 .2918221 .492737

O 5.5267461 .0880400 .712273

C $6.775464 \quad 0.379658 \quad 0.764413$

C 7.5438330 .9911491 .928943

C 7.1016072 .4423851 .849440

H $7.2987190 .612297-0.171616$

C $6.521604-1.1324280 .833427$

H 7.2408330 .5623392 .890759

H 8.6257000 .8642021 .826460

C 7.3495193 .2290043 .124435

H 7.6411962 .9240651 .022947

C $5.408555-1.619499-0.121959$

O $6.117859-1.4769012 .164308$

H $7.459361-1.6705670 .653280$

H $4.452030-1.2373770 .240077$

O $5.331039-3.045698-0.013711$

C $5.604511-1.234113-1.595441$

C $4.506021-1.744653-2.551384$

H $5.680937-0.146589-1.696711$

O $6.857777-1.780490-2.021712$

H $4.916219-1.693681-3.567312$

H $4.322553-2.808897-2.369814$

H 6.9999954 .2609173 .018898

H 6.8296322 .7825983 .978778

H 8.4187593 .2558593 .358086

C 5.0618353 .4748950 .696600

H $5.941565-2.4334182 .176228$
H $\quad 6.157628-3.411603-0.372620$

H $7.031268-1.452410-2.920503$

H $5.7127853 .694133-0.158869$

H 5.0438434 .3730511 .324633

H 5.0168022 .1295322 .393864

C $1.874387-2.327513-1.026247$

O $2.483344-1.052637-1.264527$

C $3.192616-0.927868-2.501221$

C $2.230272-1.207202-3.666918$

C $1.570613-2.576734-3.534243$

C $0.915392-2.707851-2.163211$

H $2.655007-3.088381-0.913520$

C $1.096022-2.2424710 .293569$

H $3.4829110 .127859-2.579415$

O $2.888301-1.088292-4.923584$

H $1.442072-0.443086-3.661067$

H $2.283405-3.390840-3.709779$

O $0.552468-2.707913-4.528620$

H $0.025730-2.066342-2.131026$

O $0.477091-4.051935-1.976644$

C $1.996645-1.9555981 .470117$

H $0.331105-1.4594580 .240752$

H $\quad 0.566725-3.177500 \quad 0.510872$

H $3.389642-1.904978-5.085668$

H $\quad 0.973249-2.569013-5.394414$

H $-0.126465-4.264218-2.709311$

O $1.921074-0.6344531 .797917$

O $2.690696-2.7995082 .021294$

C $2.694225-0.2241692 .928759$

H $3.759510-0.3405802 .718803$

H 2.4878940 .8331593 .115275

H $2.408899-0.7987783 .815026$

SCF Energy (B3LYP/6-31G**//MMFF) $=-3245.91476837$

$03 \_15$

MM̄FF Geometry

C -3.220285 -2.444723 -3.135636

C $-2.566929-2.505325-1.964988$

C $-1.135926-2.966828-1.831436$

O $-0.349740-1.907022-1.240868$

C $-1.082934-4.184519-0.889168$

C $0.298211-4.789064-0.731318$

C $0.974989-4.8550050 .432147$

C $0.903294-5.376008-1.980726$

C $0.542758-4.3651761 .786421$

C $0.983944-2.9306412 .116438$

C $2.506269-2.7535422 .234066$

C $2.852388-1.3525222 .743148$

C $4.371124-1.1147782 .784965$

O $3.085301-2.9426550 .941947$

O 4.5912250 .2951352 .948245

C $4.986365-1.5126281 .424738$

C $5.020048-1.7976243 .994897$

O $6.409867-1.4719551 .475868$

C $4.517579-2.8911980 .918089$

C $4.957836-3.190397-0.535513$

O $5.082896-3.9136741 .747299$

C $0.087798-0.922460-2.072443$

O $-0.143731-0.809760-3.265777$

C $0.9102350 .012189-1.273285$

C $1.2036251 .221760-1.773498$

C $2.0032762 .258215-1.117377$

C $2.771217 \quad 1.865502 \quad 0.115574$

C $4.443414-2.206885-1.593381$

C $5.442580-1.124910-1.968195$

C $5.6472390 .914421-3.385695$

O $6.608932-1.118884-1.584364$

C $5.5533072 .107350-2.488525$

C $5.1782653 .322559-2.915728$

C $5.1334084 .539767-2.038064$

C $3.7904485 .232678-2.069082$

C $3.5878516 .314008-2.840928$

C $2.7137184 .726911-1.137269$

C $2.0255083 .494972-1.656901$

N $4.883265-0.181781-2.820672$

H $-2.677051-2.717755-4.038992$

H $-3.069748-2.228380-1.043692$

H $-0.716483-3.233765-2.809190$ 
H $-1.753944-4.970180-1.261947$ H -1.480933 -3.8858600 .085906$ H $1.958034-5.3248750 .423652$ H $1.823978-5.932676-1.774371$ H $0.201619-6.071271-2.453027$ H $1.152121-4.587322-2.697046$ H $-0.544594-4.4370991 .892706$ H $0.946756-5.0510362 .541993$ H $0.591691-2.2533861 .348755$ H $0.517604-2.6471023 .068038$ H $2.889140-3.5158882 .922624$ H $2.409089-1.1756333 .730986$ H $2.403169-0.6041092 .076722$ H 4.1454940 .5751953 .766072 H $4.680349-0.7481450 .702519$ H $4.584788-1.4183334 .927334$ H $6.090099-1.5695824 .050636$ H $4.897365-2.8826053 .991511$ H $6.663836-0.5822961 .775474$ H $\quad 6.046985-3.308688-0.583387$ H $4.548856-4.176631-0.799401$ H $5.078805-4.7497621 .252575$ H $1.243119-0.316741-0.295840$ H $0.8040461 .491524-2.750410$ H $3.3978680 .992783-0.093232$ H $2.090816 \quad 1.6172970 .935503$ H $3.4468772 .643170 \quad 0.477157$ H $4.243084-2.766986-2.516051$ H $3.501625-1.743539-1.282747$ H $5.242563 \quad 1.112217-4.383324$ H $\quad 6.694610 \quad 0.612059-3.487664$ H $5.8370171 .972820-1.447340$ H $4.9231263 .461151-3.963959$ H $5.9159575 .227649-2.384213$ H $5.3940974 .296528-1.000516$ H $2.6361226 .834980-2.846079$ H $4.3680386 .698959-3.489269$ H $1.9400295 .496563-1.010923$ H $3.1342264 .593132-0.136651$ H $1.4423523 .674763-2.561392$ H $3.892194-0.232231-3.038590$ C -5.549861 -1.920080 -2.130612 O $-5.200666-0.730027-1.398876$ C $-6.400726-0.152305-0.848548$ C $-7.537224-1.113625-1.181834$ C $-7.033432-1.757247-2.458745$ H $-6.5716070 .787064-1.386492$ C $-6.217747 \quad 0.1235930 .648380$ H -7.674363 -1.870624 -0.401078 H -8.492065 -0.594257 -1.310160 C -7.746203 -3.054956-2.798706 H -7.159713 -1.045816 -3.285898 C $-4.999159 \quad 1.0141290 .978085$ O $-6.039549-1.1289651 .320031$ H -7.1364290 .5618831 .055834$ H $-4.081014 \quad 0.4790930 .718121$ O $-4.967351 \quad 1.1956752 .398779$ C -5.0273112 .3921540 .297017$ C -3.907936 3.3636720 .717988 H $-4.9957812 .268277-0.790899$ O H -4.1851194 .3541790 .335428$ H -3.906382 3.4732091 .807595 H -7.355894 -3.486426 -3.725402 H -7.625792 -3.798929-2.004056 H $-8.818464-2.879965-2.935305$ C -4.657918 -2.053159 -3.368565 H $-5.955204-0.9364892 .269555$ H -5.7550221 .7094872 .646816$ H -6.3395193 .8339970 .094292$ H -4.653251 -1.106011 -3.922404 H -5.084759 -2.814788 -4.031754 H $-5.406011-2.775530-1.457308$ C -1.515483 2.0719822 .193563 O C -2.5120852 .9843650 .179086$ C $-1.5110114 .148408 \quad 0.180187$ C -1.0668934 .4972951 .595785$
C $-0.5391553 .251105 \quad 2.300895$

H -2.3930542 .2582702 .823325$

C -0.8354310 .7812432 .669825$

H $-2.6487142 .687905-0.868955$

O $-2.072023 \quad 5.290652-0.456804$

H $-0.633283 \quad 3.858175-0.407959$

H -1.8828324 .9576052 .164428$

O $-0.0288045 .478251 \quad 1.538508$

H 0.4303952 .9751341 .871001

O -0.3193863 .5504843 .678409$

C $-1.850624-0.2569773 .078549$

H $-0.1863810 .361849 \begin{array}{lll}1.891767 \\ \text { H }\end{array}$

H -0.2020100 .9522263 .548128$

H $-1.413766 \quad 6.004843-0.406010$

H $0.720503 \quad 5.094637 \quad 1.052340$

H 0.2822214 .3136893 .717623

O $-2.058248-1.1416392 .063463$

O $-2.394933-0.2724354 .175535$

C $-2.970880-2.2071912 .346367$

H $-3.311651-2.6271301 .397114$

H $-2.453607-2.9864932 .912619$

H $-3.844102-1.8523082 .901093$

SCF Energy (B3LYP/6-31G**//MMFF)= -3245.90913055

03 150

MM̄FF Geometry

C -1.281806 2.737961 -1.219173

C $-1.4382341 .619894-1.945830$

C $-0.5437370 .399755-1.944056$

O $0.6277640 .591767-1.122304$

C $-1.335090-0.790234-1.369953$

C $-0.602879-2.114960-1.434710$

C $-0.240906-2.841794-0.359455$

C $-0.309001-2.632555-2.819297$

C $-0.464599-2.5241451 .092361$

C $0.693264-1.7768041 .771597$

C $2.009341-2.5698441 .825754$

C $3.036947-1.8546772 .706091$

C $4.395206-2.5751202 .707642$

O $2.523905-2.686551 \quad 0.497086$

O $5.367366-1.6805863 .271010$

C $4.825848-2.8483171 .249558$

C $4.378966-3.8180213 .605555$

O $5.994351-3.6630331 .207752$

C $3.713457-3.4802060 .388823$

C $4.074094-3.561887-1.114674$

O $3.478049-4.8215190 .835184$

C $1.7039751 .196376-1.695247$

O $1.8036361 .591074-2.845751$

C $2.7432661 .332285-0.651486$

C $3.7240792 .232821-0.812285$

C $4.8062892 .506670 \quad 0.135655$

C $4.976484 \quad 1.5553681 .290293$

C $4.373922-2.221036-1.794157$

C $5.853362-1.879924-1.860396$

C $7.390685-0.034741-2.524099$

O $6.743478-2.674206-1.569624$

C $7.8119820 .709195-1.296465$

C $8.2764381 .967550-1.323208$

C $8.7441262 .714439-0.107261$

$\begin{array}{llll}\text { C } 8.059529 & 4.053370 & 0.051924\end{array}$

C $8.6505595 .185729-0.365503$

C $6.730713 \quad 4.086227 \quad 0.772138$

C $5.5963523 .579679-0.078525$

N $6.064473-0.587879-2.323578$

H $-0.4302782 .840608-0.554162$

H $-2.3040141 .550247-2.602879$

H $-0.2436500 .188606-2.977446$

H -2.280633 $-0.904048-1.915993$

H $-1.609920-0.553536-0.336876$

H $0.273560-3.786593-0.532394$

H $0.079155-3.656685-2.806073$

H -1.220652 -2.637696 -3.425476

H $0.438348-2.005673-3.315041$

H $-1.382418-1.9433161 .223062$

H $-0.647418-3.467308 \quad 1.622951$

H $0.855263-0.825742 \quad 1.250701$

H $0.378244-1.5347922 .794465$ 
H $\quad 1.793830-3.5679712 .224716$

H $2.661749-1.7434673 .731023$

H $3.187086-0.8332902 .330978$

H $5.071011-1.4407904 .165786$

H $5.104049-1.8810490 .815219$

H $4.137146-3.5405554 .638757$

H $5.367147-4.2891153 .646653$

H $3.651370-4.5674723 .287479$

H $6.676774-3.2186051 .739478$

H $4.883653-4.285347-1.269493$

H $3.202621-3.988543-1.632420$

H $3.050633-5.3154310 .115898$

H 2.6561430 .7090670 .230792

H $3.7230212 .848357-1.711000$

H 5.0248070 .5222740 .930257

H 4.1388301 .6455081 .990060

H 5.8953391 .7165641 .857714

H $4.028655-2.276075-2.834689$

H $3.829487-1.403435-1.312312$

H $7.3424940 .612848-3.405266$

H $8.098164-0.845689-2.724609$

H $7.7578300 .180895-0.347454$

H $8.3559892 .482865-2.278011$

H $9.8282942 .859702-0.202564$

H 8.6052192 .1226420 .806035

H $8.1791796 .153387-0.229685$

H $9.6174815 .169187-0.857673$

H 6.4832265 .1179571 .056720

H 6.8260383 .5522351 .721500

H $5.3949314 .205367-0.949286$

H $5.266614 \quad 0.018760-2.489912$

C -2.9827394 .1233710 .064852$

O -3.6101542 .9056910 .516652$

C -3.4066162 .7898611 .939103$

C -2.9678154 .1710312 .410048$

C $-2.128270 \quad 4.6242551 .229724$

H -2.569212 2.0971932 .084222

C -4.6630022 .2395962 .619521$

H -3.8230014 .8424682 .550416$

H -2.406382 4.1323303 .348318

C $-1.866786 \quad 6.1199821 .205801$

H -1.167625 4.0961461 .264837

C -5.1147590 .8680292 .073590$

O $\quad-5.7326183 .1702702 .412230$

H $-4.5037832 .186923 \quad 3.702918$

H -5.4520740 .9926811 .041442$

O $-6.264194 \quad 0.4560512 .822292$

C $-4.044175-0.2337342 .149228$

C $-4.516827-1.6389441 .731486$

H -3.1888290 .0439901 .524506$

O $-3.571074-0.3112663 .497214$

H -3.694908 -2.336613 1.938016

H $-5.345267-1.9547632 .378365$

H $-1.274290 \quad 6.3975540 .328275$

H $-2.8008596 .691068 \quad 1.176973$

H $-1.312958 \quad 6.4274782 .098844$

C -2.253687 $3.885419-1.260495$

H -6.5270832 .7962442 .830651$

H -5.9757550 .2945573 .736991$

H -2.838318 -0.9503983 .515548$

H $-1.7205994 .793214-1.564640$

H -3.006814 $3.691049-2.034360$

H $-3.7981354 .834943-0.121213$

C $-6.792189-1.259680-1.215911$

O $-6.257912-1.2684690 .115460$

C $-4.913368-1.7397810 .248976$

C $-4.804643-3.184269-0.263639$

C $-5.327341-3.310210-1.697447$

C $-6.699772-2.654449-1.861777$

H -7.857175 -1.028799-1.087207

C $-6.168327-0.146374-2.074097$

H $-4.243447-1.099818-0.333199$

O $-5.528521-4.0681160 .590595$

H -3.753656 -3.494300 -0.226233

H -5.402345 -4.371115 -1.966268

O $-4.412964-2.693564-2.598720$

H -6.962539 -2.592892 -2.924443

O $-7.667554-3.506616-1.241318$
C $-6.6074401 .232809-1.623895$

H $-5.076099-0.173464-2.039846$

H $-6.491899-0.256386-3.115344$

H $-6.451503-3.7632010 .620394$

H -3.557725 -3.146521 -2.504076

H $-8.546245-3.126683-1.411943$

O $-5.8701492 .173452-2.285783$

O $-7.4960081 .462639-0.814986$

C $-6.2016763 .532388-1.983411$

H $-6.0971243 .723017-0.911029$

H $-5.5081664 .181177-2.524690$

H -7.220319 $3.752955-2.316234$

SCF Energy (B3LYP/6-31G**//MMFF $)=-3245.90647510$

03151

MM̄FF Geometry

C -0.0218744 .9610341 .463576$

C 0.8377384 .4209250 .585748

C 1.9931003 .5403540 .995655

O 2.0245332 .3841090 .126220

C 3.3218214 .2979750 .819054

C 4.5186383 .5645451 .394642

C 5.5558063 .0977190 .672442

C 4.5243403 .3972542 .893625

C $5.7738973 .186907-0.810961$

C $5.2568451 .984037-1.615070$

C $5.8642690 .632973-1.201046$

C $5.511884-0.451355-2.222519$

C $6.011564-1.839011-1.789614$

O 5.3327460 .2730610 .077454

O $5.363400-2.818643-2.615977$

C $5.562506-2.107457-0.336529$

C $7.517621-2.000840-2.027546$

O $6.142377-3.3103030 .161286$

C $5.866528-0.9404970 .624522$

C $5.241441-1.1280512 .029441$

O $7.284023-0.8398830 .809112$

C 1.1328821 .3887550 .376706

O $\quad 0.312979 \quad 1.345373 \quad 1.279619$

C $1.3165140 .351760-0.662305$

C $0.352107-0.559178-0.854097$

C $0.373826-1.660725-1.816945$

C $1.676963-1.948604-2.512419$

C $3.714350-1.2540672 .060687$

C $3.207810-2.6871102 .075036$

C $1.089336-3.9966481 .953702$

O $3.938410-3.6598282 .240982$

C $0.943483-4.5317250 .563978$

C $-0.229951-4.9274360 .047101$

C $-0.385958-5.508057-1.329979$

C $-1.424888-4.781400-2.154751$

C $-2.676274-5.257390-2.265162$

C $-0.989988-3.546265-2.912690$

C $-0.758090-2.370694-2.004161$

N $1.828516-2.7481301 .920186$

H $\quad 0.1407864 .7691802 .522925$

H $0.7282384 .607734-0.478139$

H 1.8774203 .2082732 .034343

H $3.2626935 .277497 \quad 1.312312$

H $3.4706474 .503065-0.246736$

H 6.3598672 .5909071 .205633

H 5.4824143 .0177563 .265371

H 4.3439994 .3591403 .384602

H 3.7499522 .6914003 .208158

H $5.3296044 .103046-1.214662$

H $6.8524743 .294062-0.985191$

H $4.1656541 .941492-1.520589$

H $5.4815012 .172571-2.672346$

H $6.9519700 .748543-1.127399$

H $5.906568-0.195226-3.213605$

H $4.421035-0.497127-2.339892$

H $5.575303-2.616220-3.543378$

H $4.478622-2.270978-0.364276$

H $7.752931-1.872766-3.091020$

H $7.852863-3.010868-1.767170$

H $8.120003-1.281465-1.469082$

H $5.907953-4.022734-0.457781$

H $5.727884-1.9613212 .550562$ 
H $5.494259-0.2328572 .616186$ H $7.454037-0.3574681 .635173$ H $2.2322830 .369142-1.240756$ H $-0.549420-0.490355-0.249805$ H $2.475489-2.096657-1.777790$ H $1.953492-1.118311-3.171074$ H $1.657481-2.851281-3.125744$ H $3.348375-0.7943332 .987862$ H $3.259693-0.7133091 .226580$ H $\quad 0.121524-3.7883142 .420932$ H $1.628709-4.7209572 .572427$ H $1.846866-4.623775-0.034421$ H $-1.127689-4.8602990 .657730$ H $-0.671406-6.562063-1.216067$ H $0.567832-5.517521-1.872237$ H -3.421268-4.755383 -2.873602 H $-2.985953-6.156764-1.742988$ H - $-1.765430-3.251892-3.632315$ H $-0.117337-3.797531-3.520961$ H -1.649367 -2.075854 -1.449877 H $1.308908-1.8939101 .740275$ C $-1.7928195 .752433-0.252380$ O $-2.4140734 .461628-0.431645$ C $-3.7051504 .648138-1.042218$ C $-3.7112546 .075085-1.573918$ C $-2.8940756 .783086-0.511561$ H -4.448846 $4.575096-0.239925$ C $-3.9661923 .568959-2.096638$ H $-3.2145406 .147777-2.548528$ H $-4.7238506 .476589-1.677444$ C $-2.3779068 .143078-0.949000$ H $-3.5143096 .906658 \quad 0.386383$ C $-3.9022452 .126723-1.549944$ O $-2.9757793 .693360-3.125068$ H -4.934283 $3.751664-2.577795$ H $-2.8701091 .898924-1.261979$ O $-4.215216 \quad 1.239907-2.632120$ C $-4.8572541 .839379-0.375836$ C $-4.9611150 .356910 \quad 0.023803$ H $-4.5678342 .437696 \quad 0.491737$ O $-6.170802 \quad 2.276156-0.746385$ H $-5.671716 \quad 0.2808670 .856336$ H -5.456451 $-0.196285-0.786665$ H - $1.7805578 .607106-0.157935$ H -1.750804 8.065856-1.843563 H $-3.2115498 .814125-1.180387$ C -1.208733 $5.839321 \quad 1.160874$ H $-3.1486992 .994841-3.779377$ H $-5.1439981 .393217-2.877081$ H -6.7502852 .1495520 .024408$ H -1.995091 $5.559744 \quad 1.874527$ H -0.9213206 .8725421 .387817$ H -1.011821 5.847545 -1.015695 C $-4.660956-2.308131 \quad 1.413477$ O $-3.838836-1.7885720 .360883$ C $-3.647263-0.3652620 .381981$ C $-2.9745530 .026831 \quad 1.702865$ C $-3.764454-0.4729862 .909063$ C $-4.052298-1.9703442 .781501$ H -5.670881 -1.8871211 .340454$ C $-4.761038-3.8259541 .195202$ H -2.937298 -0.163361 -0.425226 O $-2.785479 \quad 1.431631 \quad 1.784230$ H -1.976556 -0.4291091 .735521$ H $-4.686917 \quad 0.104323 \quad 3.041914$ O $-2.979063-0.2487724 .082856$ H -3.118296 -2.525868 2.937762 O $-4.951302-2.3300243 .828489$ C $-5.581460-4.176929-0.029733$ H -3.759781 -4.250063 1.055599 H -5.245956 -4.305901 2.051155 H -2.349838 1.6192442 .633329 H -3.480400 -0.6027364 .837519$ H -4.953291 -3.298953 3.900457 O $-5.646762-5.537444-0.140771$ O $-6.101124-3.371431-0.789562$ C -6.405591-6.028686-1.250363 H $-5.994013-5.652728-2.191806$
H $-6.339863-7.119908-1.250510$

H -7.455535 -5.739574 -1.145070

SCF Energy (B3LYP/6-31G**//MMFF) $=-3245.90519132$

03_152

MM̄FF Geometry

C -4.141191-2.374039-2.378190

C $-2.989922-2.863374-1.895339$

C $-1.700280-2.859081-2.676340$

O $-0.776935-1.952080-2.031144$

C $-1.077316-4.265590-2.672041$

C $0.136331-4.383265-3.573194$

C $1.416672-4.391616-3.151579$

C $-0.172201-4.539159-5.041039$

C $1.936612-4.246821-1.746686$

C $2.863506-3.035070-1.634837$

C $3.537573-2.872173-0.263507$

C $2.540793-2.7241390 .887493$

C $3.237264-2.4173312 .222040$

O $4.344933-1.692323-0.360077$

O $2.234977-1.9777653 .151562$

C $4.215104-1.2423022 .022876$

C $3.874314-3.6719182 .831544$

O $4.993583-1.0223943 .197343$

C $5.141637-1.4091370 .797626$

C $5.894437-0.0856020 .524213$

O $6.086522-2.4474031 .056380$

C $-0.865136-0.635334-2.369571$

O $-1.657700-0.125017-3.145153$

C $0.1833790 .092063-1.622534$

C $0.2127461 .432629-1.639868$

C $1.1780872 .273872-0.930545$

C $2.4451471 .617896-0.454869$

C $6.779939-0.121167-0.728141$

C $7.0323231 .274795-1.260232$

C $5.9534543 .081658-2.602916$

O $8.0804041 .874487-1.034160$

C $5.3187764 .058809-1.664314$

C $4.2610764 .820496-1.984215$

C $3.6835795 .860471-1.067672$

C $2.1884635 .733214-0.889188$

C $1.3512326 .637842-1.425941$

C $1.6759614 .620923-0.001945$

C $0.8895143 .579712-0.755902$

N $5.9625751 .765887-1.993689$

H $-4.160708-1.944571-3.376997$

H -2.966152 -3.272019-0.888141

H -1.870346 -2.537988 -3.711841

H -1.820663 -5.004421 -3.001413

H $-0.820977-4.552874-1.647392$

H $2.197248-4.512993-3.902189$

H $\quad 0.731767-4.671035-5.645301$

H $-0.806770-5.416342-5.204378$

H $-0.694299-3.655851-5.421398$

H $1.121551-4.150777-1.027693$

H $2.487944-5.158034-1.486695$

H $3.654186-3.101678-2.395514$

H $2.313404-2.119721-1.883506$

H $4.190004-3.737805-0.098862$

H $1.926594-3.6241970 .993524$

H $1.843218-1.9060270 .664588$

H $1.578839-2.6896923 .244259$

H $3.612613-0.3354861 .880416$

H $3.111915-4.4394023 .011728$

H $4.316936-3.4549803 .809881$

H $4.642808-4.1144762 .194344$

H $4.374258-0.9101993 .938828$

H 5.1604020 .7224780 .421686

H 6.5317010 .1706011 .380100

H $6.851660-2.3313200 .471415$

H $0.893167-0.494180-1.053419$

H $-0.5561101 .967496-2.195082$

H $2.9350251 .091965-1.281632$

H 2.2342010 .8925600 .335093

H $3.1788112 .323207-0.060349$

H $7.747701-0.584377-0.504826$

H $6.327022-0.713067-1.531718$

H $5.4064142 .999685-3.547351$ 
H $\quad 6.980798 \quad 3.391365-2.819741$ H $5.7795564 .173736-0.685761$ H $3.8186234 .730093-2.973518$ H $3.9298516 .843513-1.490003$ H $4.1637025 .833601-0.081240$ H $0.2793926 .577964-1.267775$ H $1.7176947 .457908-2.034564$ H 1.0106675 .0557000 .756814 H $2.498404 \quad 4.1876770 .574307$ H -0.059366 $3.950153-1.147482$ H $5.1210721 .203556-2.076739$ C $-5.596256-1.095601-0.774727$ O $-5.4945920 .078033-1.604799$ C $-6.5520530 .985208-1.260403$ C -7.1273450 .4995530 .062690$ C $-6.953187-1.002625-0.073943$ H $-7.3249580 .851348-2.030143$ C $-6.0780322 .441938-1.315416$ H -6.5563550 .8678580 .920742$ H -8.170831 $0.799138 \quad 0.199295$ C $-7.012274-1.744378 \quad 1.250319$ H -7.743231-1.385731-0.733733 C $-5.1659332 .944633-0.175680$ O $-7.2547643 .262167-1.320862$ H -5.576646 2.611545 -2.276620 H -5.7654793 .0729560 .733259$ O $-4.727855 \quad 4.265119-0.529731$ $\begin{array}{llll}C & -3.933765 & 2.085072 & 0.149744\end{array}$ C $-3.0436862 .724596 \quad 1.231864$ H $-4.257025 \quad 1.1026650 .497736$ O $-3.1404201 .896396-1.018614$ H -2.4917613 .5720270 .807610$ H -3.689172 3.0966662 .037047 H $-6.871986-2.8196821 .101362$ H -6.236122 -1.3956621 .940165$ H $-7.983508-1.5935051 .732758$ C $-5.437625-2.360657-1.619847$ H $-6.9626554 .187530-1.389538$ $\mathrm{H}-4.1288424 .179830-1.291471$ H -3.646299 $1.339230-1.633944$ H $-6.248657-2.428259-2.355420$ H $-5.512280-3.250266-0.983361$ H $-4.781075-1.040335-0.042420$ C -2.025445 -0.3711253 .043097$ O -2.7939690 .7107122 .502274$ C $-2.035624 \quad 1.7160201 .818344$ C -1.0531192 .3836412 .792447$ C -0.1544111 .3463643 .473410$ C $-0.953100 \quad 0.153898 \quad 4.014396$ H -2.752697 -0.9495363 .627469$ C - $1.498785-1.2754361 .917798$ H $-1.481551 \quad 1.2504040 .996220$ $\begin{array}{lllll}\mathrm{O} & -1.784040 & 3.100958 & 3.785767\end{array}$ H -0.4441403 .1092612 .240636$ H $0.386142 \quad 1.826402 \quad 4.298368$ \begin{tabular}{lllll}
\hline & 0.810668 & 0.897207 & 2.529958
\end{tabular} H $-0.273787-0.6587014 .291926$ O -1.6088790 .5600145 .221016$ C -1.244602 -2.6926672 .383225$ H -2.251197 -1.3185931 .120114$ H $-0.576190-0.886477 \quad 1.479914$ H $-1.138276 \quad 3.549112 \quad 4.358225$ H $1.3871150 .256302 \quad 2.980187$ H -2.260216 1.2442604 .989257 O $-0.999772-3.4840651 .297209$ O $-1.248835-3.064176 \quad 3.548950$ C $-0.757570-4.8637241 .591801$ H $0.101524-4.9663332 .261817$ H -1.649862 -5.3153442 .035545$ H $-0.532736-5.3798690 .655182$

SCF Energy (B3LYP/6-31G**//MMFF) $=-3245.93035307$

03_153

MMFF Geometry

C -2.816363 -2.526214 -1.995547

C $-1.986586-3.561583-2.199307$

C $-0.569097-3.713866-1.696541$

O $-0.011121-2.464537-1.234425$
C $-0.558074-4.705590-0.517508$

C $0.830798-5.104842-0.060034$

C $1.350921-4.8297631 .152109$

C $1.640567-5.899756-1.053235$

C $0.718538-4.085476 \quad 2.294667$

C $1.093246-2.5964872 .374740$

C $2.596245-2.3341482 .567900$

C $2.855268-0.8615862 .891559$

C $4.356843-0.5537452 .998045$

O $3.272189-2.6751781 .355708$

$\begin{array}{lllll}\text { O } & 4.493206 & 0.875441 & 2.989906\end{array}$

C $5.082860-1.1049051 .749969$

C $4.932674-1.0313954 .336775$

O $6.491921-0.9684471 .917231$

C $4.700940-2.5624261 .414534$

C $5.243818-3.0514990 .049157$

O $5.244943-3.4293222 .417122$

C $0.533972-1.641770-2.172155$

O $0.550871-1.810161-3.381066$

C $1.112064-0.477627-1.466448$

C $1.3689570 .649385-2.146447$

C $1.9180231 .885056-1.584732$

C $2.5115911 .820129-0.202162$

C $4.773716-2.251806-1.170951$

C $5.735185-1.157306-1.602135$

C $5.9264210 .725730-3.223759$

O $6.859229-1.020743-1.127405$

C $5.5854072 .003472-2.524615$

C $5.1510613 .099754-3.164458$

C $4.8455554 .401732-2.481801$

C $3.4545024 .908146-2.789471$

C $3.2521735 .842874-3.733476$

C $2.3125394 .396073-1.942282$

C $1.8758093 .009008-2.330453$

N $5.197563-0.368289-2.610276$

H $-2.491970-1.669469-1.411762$

H $-2.355837-4.409996-2.773753$

H $0.043438-4.101209-2.519655$

H $-1.093672-5.623611-0.795133$

H $-1.125190-4.2659010 .309847$

H $2.357739-5.1886621 .364649$

H $2.566869-6.289718-0.617329$

H $1.064391-6.757964-1.413912$

H $1.919481-5.278900-1.909984$

H $-0.372203-4.1737152 .265248$

H $1.018934-4.5810203 .226828$

H $0.743756-2.0958811 .464177$

H $0.540783-2.1599523 .216208$

H $2.956534-2.9735863 .382262$

H $2.337697-0.5680423 .813167$

H $2.426736-0.2353312 .097564$

H $5.444107 \quad 1.0806872 .990092$

H $4.801061-0.4530110 .915201$

H $4.421684-0.5333895 .169751$

H $5.990503-0.7629414 .433052$

H $4.836666-2.1090524 .484767$

H $6.916051-1.1896791 .071435$

H $6.336257-3.1386730 .081890$

H $4.878859-4.079567-0.091843$

H $5.298957-4.3280642 .051950$

H $1.290260-0.571814-0.401656$

H $1.1259510 .684747-3.207604$

H $3.2119230 .981927-0.126275$

H $1.728043 \quad 1.6918530 .551182$

H 3.0837312 .7104410 .067839

H $4.692907-2.938630-2.023411$

H $3.781809-1.821289-1.002817$

H $5.6505300 .745991-4.282826$

H $7.0021590 .538192-3.146390$

H $5.7266332 .036156-1.446819$

H $5.0371503 .072264-4.245928$

H $5.5913425 .135134-2.815428$

H $4.9737834 .325234-1.394909$

H $2.2610206 .232814-3.940327$

H $4.0732526 .235071-4.324438$

H $1.4385255 .051305-2.057404$

H $2.5810934 .480004-0.885289$

H $1.4326982 .954538-3.325817$ 
H $4.236739-0.520659-2.902941$

C $-5.254860-2.658405-1.417316$

O $-5.367505-1.427976-0.674253$

C $-6.761892-1.165719-0.416825$

C $-7.505546-2.445484-0.776433$

C $-6.664267-2.959311-1.928297$

H -7.068875 -0.381942 -1.118886

C $-6.960409-0.6827941 .024410$

$\mathrm{H}-7.500536-3.1660320 .049533$

H -8.547063 -2.256920 -1.053851

C $-6.908736-4.424017-2.248511$

H -6.877928 -2.354312 -2.819940

C $-6.0955290 .539658 \quad 1.394409$

O $-6.604558-1.7519421 .910695$

H -8.021535 -0.4725331 .203001$

H $-5.041242 \quad 0.240978 \quad 1.387514$

$\begin{array}{lllll}\text { O } & -6.413936 & 0.885014 & 2.747808\end{array}$

C -6.3264851 .7658080 .491568$

C -5.4908133 .0015140 .854228$

H $-6.1172721 .507333-0.549934$

O

H -5.7615533 .8240660 .177213$

H -5.7879893 .3831651 .840611$

H $-6.273620-4.755745-3.075600$

H -6.698279 -5.064026 -1.385053

H -7.952042 -4.584764 -2.539164

C $-4.222637-2.494140-2.534346$

H $-6.735267-1.4296302 .819086$

H -5.7333041 .5029583 .063205$

H $-7.9264672 .333743 \quad 1.465127$

H $-4.366420-1.536076-3.048553$

H -4.350581 -3.285491 -3.282187

H $-4.936248-3.432710-0.706695$

C -1.950198 4.0776751 .238992

O $-3.381070 \quad 4.068650 \quad 1.129284$

C -3.9677722 .7933070 .832748$

C $-3.4385752 .264303-0.510501$

C $-1.9099092 .218562-0.531911$

C -1.293280 $3.532702-0.040627$

H $-1.691435 \quad 5.1411851 .320917$

C $-1.500537 \quad 3.3877502 .535211$

H -3.7044202 .0994361 .637729$

O $-3.9064463 .080891-1.581969$

H $-3.8247731 .253947-0.683512$

H -1.574983 2.013499-1.555779

O

H -0.2171283 .4174010 .119551$

O $-1.456524 \quad 4.526697-1.057317$

C -0.0545823 .6718332 .876043$

H -2.1145913 .7393353 .372849$

H -1.6437682 .3054512 .473110$

H -3.621929 $3.994169-1.406119$

H $-0.514270 \quad 1.1307600 .293865$

H -1.002981 4.206497 -1.855769

O 0.4207052 .6794323 .684642

O 0.5741654 .6523312 .501211

C 1.7855792 .8205634 .090093

H 2.0558931 .9456434 .686828

H 2.4393782 .8648213 .213941

H 1.9045493 .7166744 .706442

SCF Energy (B3LYP/6-31G**//MMFF) $=-3245.92568322$

03_154

MM̄FF Geometry

C $3.1091435 .075814-0.925006$

C $3.7446883 .895796-0.965682$

C 4.6441913 .4021270 .135322

O 4.0291462 .2197990 .696077

C $6.0220112 .999598-0.421134$

C 6.9704592 .4997560 .653401

C 7.4500931 .2421080 .724866

C 7.3891593 .5186511 .682673

C $7.2094140 .104664-0.228604$

C $5.940021-0.6784570 .106943$

C $5.845255-2.042230-0.594685$

C $5.890278-1.931903-2.120840$

C $5.670837-3.291087-2.804091$

O $4.602221-2.626989-0.178741$
O $5.412168-3.047865-4.195710$

C $4.402386-3.942734-2.217184$

C $6.924286-4.171854-2.744820$

O $4.226579-5.266257-2.716042$

C $4.374609-3.954620-0.673526$

C $3.017904-4.438462-0.107563$

O $5.375465-4.857810-0.189181$

C 3.1153432 .3910211 .688811

O 2.7580923 .4434152 .192038

C 2.6079311 .0485672 .047530

C 1.6719700 .9139432 .998812

C $1.070411-0.3494473 .434989$

C $1.532822-1.6048262 .743443$

C $1.802372-3.639228-0.581816$

C $0.540133-3.9764010 .195015$

C $-1.845823-3.2534530 .390110$

O $0.490777-4.8442821 .061677$

C $-2.004834-2.1166661 .350288$

C $-2.514517-2.2481022 .584648$

C $-2.735960-1.0884653 .513524$

C $-2.129047-1.2963834 .884440$

C $-2.905290-1.3115145 .981261$

C $-0.628972-1.4780684 .996671$

C $0.139039-0.3229564 .411480$

N $-0.525833-3.184515-0.208250$

H $3.2716295 .740720-0.080703$

H $3.5662003 .222369-1.800723$

H 4.7656074 .1658140 .913146

H $6.4872593 .857625-0.923627$

H $5.8829402 .233736-1.192569$

H $8.1114940 .992361 \quad 1.553833$

H 8.1871313 .1462862 .334200

H 7.7660054 .4216441 .191351

H 6.5452373 .7935142 .322438

H $7.1777980 .471152-1.260074$

H $8.079639-0.561892-0.176663$

H $5.871944-0.8442441 .190826$

H $5.056438-0.081744-0.150321$

H $6.669178-2.667403-0.230811$

H $6.836954-1.496158-2.460819$

H $5.103612-1.241835-2.457297$

H $\quad 6.174627-2.568598-4.563013$

H $3.552177-3.355566-2.585976$

H $7.762638-3.678658-3.251667$

H $6.768138-5.117280-3.275976$

H $7.243943-4.396690-1.725362$

H $4.245533-5.215108-3.687011$

H $2.872135-5.502339-0.336258$

H $3.066127-4.3797670 .989109$

H $5.136747-5.1264590 .713616$

H $3.0159310 .201606 \quad 1.507252$

H 1.3044831 .8079083 .500728

H $2.606113-1.7589922 .901042$

H $1.343238-1.5321491 .668042$

H $1.034603-2.5120093 .088204$

H $1.990935-2.565064-0.475073$

H $1.601556-3.851423-1.637848$

H -1.986403 -4.226144 0.872042

H -2.571497 -3.152905 -0.422909

H -1.717039-1.128654 0.998941

H -2.833121 -3.2290192 .928904$

H -3.819544 -0.937080 3.597171

H -2.341905 $-0.160023 \quad 3.085418$

H -2.484601 -1.456888 6.970762

H -3.980451 -1.180317 5.915996

H $-0.365983-2.4371024 .544776$

H $-0.334107-1.5613806 .050947$

H -0.1142420 .6396124 .858237$

H $-0.366141-2.464177-0.906871$

C $0.6668825 .333064-1.561837$

O $0.3715573 .926352-1.440379$

C $-0.5037183 .733255-0.306197$

C -0.9821125 .1232320 .092088$

C $0.2271265 .972477-0.245812$

H $\quad 0.1225893 .3003220 .483700$

C $-1.6216142 .758913-0.697998$

H -1.844994 $5.427890-0.513457$

H -1.2662525 .1835061 .147031$ 
C $-0.0756247 .456459-0.352463$

H $0.977073 \quad 5.817553 \quad 0.539115$

C $-2.702316 \quad 2.585676 \quad 0.383087$

O $-1.0308091 .481145-0.956052$

H -2.075096 $3.093268-1.637743$

H -3.195652 3.5456460 .573596

O -2.0719062 .2158531 .615455$

C -3.7825751 .5299990 .067261$

C $-4.480361 \quad 1.773223-1.281127$

H -3.3542230 .5223650 .092745$

$\begin{array}{llll}\text { O } & -4.765666 & 1.584060 & 1.105844\end{array}$

H $-4.8149072 .817009-1.332669$

H -3.744953 $1.616336-2.078209$

H $0.8265828 .019167-0.612336$

H $-0.8321967 .658272-1.118044$

H -0.4518867 .8416210 .600891$

C $2.1306855 .530492-1.970230$

H $-0.3448641 .610757-1.633274$

H $-1.5611891 .404507 \quad 1.450450$

H -4.3058351 .4204521 .947347$

H $2.3194566 .588595-2.185214$

H $2.313975 \quad 4.981205-2.902654$

H $0.036188 \quad 5.700939-2.382297$

C $-6.283056-1.488453-1.650975$

O $-5.261492-0.496089-1.476160$

C $-5.7006100 .864043-1.530686$

C $-6.3628321 .166667-2.885768$

C $-7.4888990 .173027-3.188548$

C $-7.038357-1.275458-2.974234$

H -5.739936 -2.439232 -1.729742

C $-7.215536-1.576949-0.431479$

H $-6.4274641 .041030-0.731208$

O $-5.3846871 .105026-3.922406$

H $-6.7552902 .190421-2.864878$

H -7.818011 $0.296454-4.227394$

O $-8.606408 \quad 0.440214-2.346874$

H $-7.898080-1.954466-3.015447$

O $-6.156809-1.653598-4.035466$

C $-6.504341-2.0923510 .801343$

H -7.644426 $-0.605060-0.175166$

H -8.030810 -2.277281 -0.647667

H $-5.827365 \quad 1.330821-4.758121$

H -8.891038 $1.354194-2.517147$

H $-6.648462-1.566774-4.869796$

O $-7.386469-2.1119001 .844664$

O $-5.333260-2.4400250 .848222$

C $-6.860719-2.5868693 .088062$

H $-6.113196-1.8858993 .469342$

H -7.684222 -2.645925 3.804609

H -6.429641 -3.585565 2.967683

SCF Energy (B3LYP/6-31G**//MMFF)= -3245.91139761

03_155

MM̄FF Geometry

C -2.306095 -3.625301-2.347052

C $-1.445578-3.621100-1.317789$

C $0.053177-3.616031-1.466267$

O $0.542593-2.376496-0.903527$

C $0.664924-4.778567-0.664711$

C $2.178426-4.839441-0.740077$

C $3.003304-4.6724990 .312668$

C $2.754327-5.130191-2.102228$

C $2.634211-4.4135451 .746866$

C $2.552417-2.9287972 .134130$

C $3.889964-2.1775792 .031437$

C $3.777686-0.7873562 .661598$

C $5.074762 \quad 0.0220572 .503095$

O $4.235384-2.045500 \quad 0.650083$

$\begin{array}{lllll}\text { O } & 4.779720 & 1.392078 & 2.817662\end{array}$

C $5.517574-0.0156471 .024228$

C $6.152267-0.4281393 .496887$

$\begin{array}{lllll}\text { O } & 6.803380 & 0.577071 & 0.862061\end{array}$

C $5.511026-1.4322820 .414815$

C $5.750169-1.437960-1.115502$

O $6.565978-2.2020031 .005053$

C $0.486396-1.270735-1.693639$

O $0.126635-1.212796-2.858576$

C $0.907465-0.116217-0.872857$
C $0.5584711 .120150-1.256362$

C $0.8769052 .361353-0.551020$

C 1.8867812 .2890450 .563725

C $4.727582-0.654047-1.945911$

C $5.1568400 .765233-2.282059$

C $4.3007852 .846262-3.357014$

O $6.2876551 .196068-2.073783$

C $3.8593423 .805034-2.296591$

C $2.9675424 .784092-2.513000$

C $2.5509895 .784220-1.472408$

C $1.0501305 .856544-1.297894$

C $0.328436 \quad 6.812648-1.906845$

C $0.4007054 .874905-0.349599$

C $0.268623 \quad 3.499788-0.943829$

N $4.132367 \quad 1.484992-2.883263$

H $-1.929107-3.657907-3.365424$

H $-1.835786-3.564854-0.303407$

H $0.348140-3.689852-2.520078$

H $\quad 0.263382-5.733044-1.030173$

H $\quad 0.338573-4.6944380 .377450$

H $4.076423-4.7390610 .135996$

H $3.835668-5.301734-2.067960$

H $2.294822-6.029797-2.524828$

H $2.577620-4.292542-2.783690$

H $1.683345-4.8976861 .993692$

H $3.377509-4.9111452 .382970$

H $1.801116-2.4366191 .505844$

H $2.191358-2.8759873 .168874$

H $4.656802-2.7683412 .546270$

H $3.499628-0.8602513 .720474$

H $2.961889-0.2340992 .176969$

H 4.4311391 .4227223 .725133

H 4.8129700 .6120130 .467330

H $5.795732-0.3101974 .527286$

H 7.0486600 .1966513 .417911

H $6.445771-1.4718823 .367647$

H $\quad 6.7576141 .474877 \quad 1.233277$

H $6.777570-1.125229-1.337760$

H $5.688344-2.484694-1.447293$

H $6.779492-2.939630 \quad 0.409964$

H $1.467523-0.3162530 .032843$

H $-0.0392941 .236354-2.159670$

H $2.800657 \quad 1.7954480 .217633$

H 1.4868741 .7273661 .413994

H $2.196936 \quad 3.2676190 .936099$

H $4.598277-1.166708-2.907890$

H $3.751716-0.635040-1.452671$

H $3.7129362 .944987-4.275075$

H $5.3543863 .021921-3.597130$

H $4.3155893 .707272-1.314278$

H $2.5350384 .896932-3.504724$

H $2.9367866 .764765-1.781109$

H $3.0209585 .574685-0.503464$

H $-0.7443306 .888914-1.764138$

H $0.7943127 .537499-2.566221$

H $-0.6124815 .212723-0.092624$

H $0.941225 \quad 4.8798150 .601302$

H $-0.442173 \quad 3.450092-1.769881$

H $3.2124441 .063019-2.971195$

C $-4.362400-2.140949-2.419051$

O $-3.681257-1.171987-1.594440$

C $-3.4368670 .001668-2.388212$

C $-4.317798-0.121116-3.622901$

C $-4.269688-1.622308-3.855067$

H $-2.395728-0.066140-2.731279$

C $-3.5510541 .275903-1.542508$

H $-5.3453190 .199878-3.429094$

H $-3.9346300 .454129-4.471035$

C $-5.370491-2.134193-4.767235$

H $-3.297131-1.873093-4.296505$

C $-4.7495541 .321546-0.575732$

O $-3.562503 \quad 2.425224-2.383844$

H -2.637374 $1.330954-0.941886$

H -4.7656550 .4220800 .047496$

O $-5.971567 \quad 1.296050-1.319136$

C $-4.787167 \quad 2.5623720 .338643$

C $-3.5074812 .842168 \quad 1.149548$

H $-5.0211153 .454617-0.254953$ 
$\begin{array}{llll}O & -5.873278 & 2.412178 & 1.260057\end{array}$ H -2.729321 3.2120520 .471664 H -3.7389253 .6527521 .852948$ H $-5.302409-3.219872-4.888048$ H $-6.363595-1.900562-4.368959$ H -5.290822 -1.677820 -5.759294 C -3.795395 -3.540285 -2.160861 H $-2.7659042 .388988-2.940331$ H -5.969036 2.066018 -1.913517 H -6.6749972 .2359450 .738387$ H $-4.281522-4.275283-2.811859$ H $-4.041086-3.827723-1.130665$ H $-5.411028-2.133410-2.093924$ C $-3.594876 \quad 0.069022 \quad 3.673537$ $\begin{array}{llll}\text { O } & -3.926517 & 1.240598 & 2.914541\end{array}$ C -2.9551471 .6420531 .946390$ C -1.628673 1.9804412 .642331 C -1.1328010 .7952693 .476531$ C $-2.237688 \quad 0.236356 \quad 4.381391$ H -4.3684310 .0095134 .450220$ C -3.686956 -1.208105 2.822254 H -2.780255 0.8183341 .250205 O $-1.8041683 .120553 \quad 3.479655$ H -0.8881052 .2418141 .880559$ H -0.284081 1.1185024 .091660 O $-0.666652-0.2191312 .590751$ H -1.927699 -0.7239804 .808264$ O $-2.416606 \quad 1.1289725 .486115$ C $-5.095123-1.4577902 .322307$ H -3.031925 -1.158870 1.948414 H $-3.398038-2.0745353 .428093$ H -0.9405483 .3298943 .874495$ H $-0.325122-0.9494813 .134358$ H -2.765051 1.9673885 .137130 O $-5.077670-2.5107261 .451634$ O $-6.086297-0.8217772 .653622$ C $-6.347342-2.8603980 .891793$ H $-7.055651-3.1189031 .684728$ H $-6.730628-2.0349610 .284323$ H -6.208441 -3.734171 0.249846

SCF Energy (B3LYP/6-31G**//MMFF) $=-3245.89765879$

03_156

MM̄FF Geometry

C $3.2270305 .261483-0.384604$

C $3.2416194 .146629-1.130060$

C $4.017598 \quad 2.904547-0.775324$

O $3.0610901 .845986-0.540443$

C $4.9122762 .486963-1.956133$

C $5.691346 \quad 1.209142-1.708724$

C $5.538895 \quad 0.073067-2.418276$

C $6.7072151 .266510-0.596937$

C $4.618120-0.173075-3.581074$

C $3.237262-0.729954-3.202072$

C $3.273429-2.147667-2.608714$

C $1.857100-2.712094-2.471659$

C $1.853994-4.100146-1.811838$

O $3.887966-2.090305-1.318992$

O $\quad 0.503992-4.404622-1.430459$

C $2.686571-4.043612-0.512778$

C $2.283855-5.198183-2.791754$

O $2.851718-5.345508 \quad 0.042833$

C $4.062389-3.368673-0.690838$

C $4.801135-3.1256110 .648432$

O $4.902565-4.217850-1.482713$

C $2.511633 \quad 1.7643180 .701377$

O 2.7785702 .4496191 .675278

C 1.4735150 .7132310 .653367

C $0.5112720 .696276 \quad 1.587365$

C $-0.624089-0.2253341 .628176$

C $-0.596945-1.4026670 .691709$

C $4.056259-2.2507441 .663099$

C $3.260386-3.0388082 .690985$

C $1.643789-2.7121474 .559665$

O $3.348156-4.2563092 .824127$

C $0.251495-2.9468454 .064942$

C $-0.836476-2.4444424 .668102$

C $-2.243610-2.7015194 .211280$
C $-3.028188-1.4262693 .999329$

C $-3.859348-0.9599414 .946699$

C -2.916042 -0.7436562 .656431$

C -1.6293710 .0208672 .494014$

N $2.465486-2.2116273 .473364$

H 3.8231035 .3072650 .522755

H $2.6258204 .102047-2.026032$

H 4.6310473 .0623190 .120209

H $5.6271243 .289648-2.180883$

H $4.2856942 .383608-2.848542$

H $6.154332-0.784502-2.148825$

H $7.3321830 .367694-0.558878$

H $7.3754202 .122524-0.736738$

H $6.2124201 .362444 \quad 0.374273$

H $4.4831490 .744400-4.163917$

H $5.114512-0.871550-4.266970$

H $2.755406-0.043711-2.495999$

H $2.623330-0.740942-4.111486$

H $3.873303-2.781549-3.272203$

H $1.356617-2.751647-3.447149$

H $1.253655-2.033102-1.853804$

H $-0.052254-4.354994-2.226749$

H $2.100374-3.4673880 .212174$

H $1.608656-5.225689-3.655526$

H $2.216956-6.189109-2.329230$

H $3.298644-5.061638-3.170971$

H $1.963996-5.7242490 .162251$

H $5.109095-4.0821101 .087775$

H $5.738140-2.6020350 .409203$

H $5.828932-3.977136-1.315657$

H $1.4993220 .017203-0.176994$

H $\quad 0.527226 \quad 1.455848 \quad 2.367987$

H $0.354998-1.9374610 .772994$

H $-0.730779-1.077464-0.345073$

H $-1.370414-2.1397560 .908963$

H $4.796977-1.6736072 .231602$

H $3.400103-1.5344611 .159877$

H $1.678609-1.9678655 .361526$

H $2.064510-3.6501894 .935749$

H $\quad 0.131669-3.5784653 .187689$

H $-0.713127-1.8366945 .561943$

H -2.734641 -3.3175404 .976140$

H $-2.264941-3.300563 \quad 3.292530$

H $-4.448185-0.0620824 .790669$

H -3.965422 -1.463385 5.901843

H $-3.735242-0.0224512 .531140$

H -3.076350 -1.480886 1.865582

H $-1.5565320 .897368 \quad 3.139216$

H $2.416830-1.2196873 .259496$

C $1.066246 \quad 6.5320870 .043679$

O $0.2424645 .400009-0.306891$

C $-0.307044 \quad 4.843015 \quad 0.899609$

C -0.1782815 .9391581 .949095$

C $1.1598376 .546163 \quad 1.570691$

H 0.3380294 .0010401 .179551

C $-1.734693 \quad 4.324567 \quad 0.685417$

H $-0.9761206 .684815 \quad 1.852549$

H -0.1986545 .5448722 .969476$

C 1.3959687 .9261182 .158486

H 1.9510055 .8732381 .922791

C -1.820461 $3.194284-0.362934$

O $-2.587076 \quad 5.405084 \quad 0.312815$

H $-2.102838 \quad 3.942138 \quad 1.644859$

H $-1.0575382 .445076-0.129341$

O $-1.5334773 .691762-1.668932$

C $-3.1916752 .485495-0.389561$

C $-3.1902321 .296173-1.364257$

H -3.4319802 .1268580 .618163$

O $-4.2096913 .399977-0.785246$

H -3.210059 $1.651672-2.402502$

H $-2.248103 \quad 0.742323-1.259244$

H 2.3637328 .3243341 .837541

H 0.6197678 .6336331 .848006

H 1.3928237 .8837253 .252561

C $2.3934876 .467630-0.719913$

H $-2.2703995 .742397-0.543030$

H $-0.636608 \quad 4.066330-1.651507$

H $-4.2393754 .113899-0.126293$ 
H $2.9855517 .368121-0.519886$ H $2.1778056 .466825-1.796419$ H $0.5183767 .417984-0.303322$ C $-4.986870-1.909942-1.984090$ O $-4.076160-0.804205-2.042808$ C $-4.3401520 .288062-1.148580$ C $-5.7453160 .858334-1.393970$ C $-6.814388-0.237253-1.343253$ C $-6.434017-1.442884-2.206811$ H -4.711464 -2.545437 -2.835959 C $-4.808696-2.737991-0.701410$ H $-4.268002-0.080416-0.120181$ O $-5.7811241 .500941-2.666243$ H $-5.9677381 .611769-0.630436$ H -7.768468 $0.177181-1.691029$ O $-6.986623-0.6417200 .011926$ H -7.118702 -2.279140 -2.024285 O $-6.565615-1.092141-3.587076$ C -3.505614 -3.511066 -0.688682 H -4.825703 -2.116382 0.196605 H -5.617736 -3.475295 -0.636994 H $-6.6647791 .891969-2.773126$ H -7.684001 -1.3191540 .029534$ H -7.490068 $-0.830617-3.736608$ O $-3.502876-4.348558 \quad 0.391729$ O $-2.604395-3.395386-1.507309$ C $-2.343207-5.1752660 .535350$ H $-2.516927-5.8609831 .368967$ H -2.175733 -5.763326 -0.372070 H $-1.468656-4.5606790 .765021$

SCF Energy (B3LYP/6-31G**//MMFF) $=-3245.90264487$

03 157

MM̄FF Geometry

C $4.776241-0.9190043 .077641$

C $3.740546-1.4813932 .435963$

C $2.464194-1.8839053 .131144$

O $1.341364-1.2587102 .468784$

C $2.276527-3.4091453 .045975$

C $1.060837-3.9031423 .807129$

C $-0.074431-4.3610493 .242690$

C $1.201016-3.9026105 .309001$

C $-0.415585-4.4699191 .781429$

C - $1.722820-3.7402401 .458159$

C $-2.236384-4.0005410 .032968$

C $-1.287352-3.472100-1.043672$

C $-1.864916-3.651373-2.455721$

O $-3.501784-3.335603-0.073121$

O $-1.079213-2.858978-3.360010$

C $-3.296686-3.075941-2.487278$

C $-1.739357-5.101665-2.936321$

O $-3.938941-3.359385-3.727127$

C $-4.187362-3.550587-1.316333$

C $-5.527787-2.787717-1.235946$

O $-4.560894-4.925780-1.464472$

C 1.0278200 .0094852 .850839

$\begin{array}{lllll}\text { O } & 1.661853 & 0.727222 & 3.607945\end{array}$

C $-0.262193 \quad 0.3631372 .217673$

C -0.8238601 .5525742 .480591$

C -2.1114162 .0316071 .975471$

C -2.9860631 .0489651 .240724$

C -5.393804 -1.269491-1.106667

C $-6.694111-0.594653-0.705602$

C -7.610792 $1.662438-0.193400$

O $-7.739351-1.203216-0.496713$

C -7.0154802 .9243620 .340908$

C -6.8589863 .1709351 .650131$

C -6.2079184 .4094242 .196263$

C $-4.836664 \quad 4.1135532 .770521$

C $-4.676907 \quad 3.9784184 .098437$

C -3.7134894 .0201551 .754222$

C -2.4681123 .3112332 .216499$

N $-6.530777 \quad 0.778623-0.588539$

H $4.675232-0.7366664 .146607$

H $3.798076-1.6812201 .370974$

H $2.481336-1.5773884 .184625$

H 3.162822 -3.915999 3.451565

H $2.225369-3.7136421 .995826$
H $-0.861251-4.7172303 .907469$

H $0.339642-4.3594115 .808050$

H $2.089169-4.4690885 .607851$

H $1.294623-2.8807135 .688499$

H $0.382818-4.0763621 .150081$

H $-0.521292-5.5338641 .538200$

H -2.506259-4.046243 2.165228

H $-1.600308-2.6608841 .615346$

H $-2.393779-5.079320-0.081570$

H $-0.304781-3.952791-0.983587$

H $-1.113913-2.401392-0.873062$

H $-0.154261-3.151983-3.291033$

H -3.191624 -1.985025 -2.433134

H $-0.684144-5.395369-2.995086$

H $-2.139150-5.219291-3.949352$

H $-2.239831-5.815973-2.279242$

H $-3.357463-3.034326-4.435767$

H $-6.153467-3.027807-2.105834$

H $-6.080838-3.170811-0.366685$

H $-4.273209-5.233574-2.338755$

H $-0.725697-0.372126 \quad 1.570404$

H -0.2916782 .2482353 .128379$

H $-3.1179790 .133025 \quad 1.828165$

H -2.5459860 .7835190 .274503$

H -3.9919201 .4294201 .049349$

H $-4.644370-1.019088-0.347585$

H -5.075645 -0.839140 -2.062769

H $-8.243184 \quad 1.1559670 .543654$

H -8.223046 $1.870753-1.076520$

H $-6.6731973 .655856-0.386939$

H -7.1907072 .4306812 .374753$

H -6.868549 4.8321682 .963817

H -6.1183285 .1864851 .426564$

H -3.710850 3.7855424 .550751

H -5.5211484 .0634144 .775447$

H -3.4344525 .0400571 .458719$

H -4.0796543 .5515730 .834658$

H -1.7843113 .9483632 .778347$

H $-5.6723791 .195112-0.939488$

C $6.353549-0.7720511 .018110$

O 5.5426730 .1191650 .225730

C $6.3631870 .711727-0.797744$

C $7.629012-0.131958-0.857618$

C $7.802129-0.5002210 .601710$

H $6.6287451 .715658-0.444797$

C $5.5965670 .816061-2.119100$

H $7.489188-1.036032-1.461567$

H $8.4794580 .421660-1.266645$

C $8.739934-1.6747610 .822953$

H $8.190826 \quad 0.374514 \quad 1.140649$

C $4.2384121 .540725-2.007915$

O $5.344095-0.507141-2.607856$

H $6.2307051 .301923-2.870324$

H $3.5459880 .914226-1.439272$

O $3.7020721 .635797-3.333354$

C $4.3067542 .947238-1.392694$

C $2.9892603 .747672-1.428445$

H $4.6571372 .883677-0.356791$

O $5.2882393 .694337-2.122090$

H $3.2429384 .794888-1.219370$

H $2.5903903 .753972-2.448302$

H $8.824192-1.9128021 .887823$

H $8.388064-2.5726610 .303891$

H $9.742404-1.4419580 .448945$

C $6.108185-0.5070932 .506855$

H $4.840442-0.419681-3.435400$

H $4.2776872 .233341-3.841049$

H $5.4088864 .544898-1.666511$

H 6.2203040 .5672752 .702072

H $6.877013-1.0243813 .093428$

H $6.069503-1.7973410 .749881$

C $0.5379051 .913577-1.849252$

O $1.3848871 .988003-0.696493$

C $1.9274303 .279059-0.408631$

C $0.7820394 .282580-0.206308$

C $-0.1451684 .318676-1.417275$

C $-0.6257182 .910100-1.758014$

H $1.1289472 .109808-2.750641$ 
C $-0.0044530 .481182-1.932562$

H 2.4421863 .1860860 .556308

O 1.2983095 .5825930 .056239

H 0.2046843 .9870950 .677684

H $0.3470624 .785527-2.277946$

O $-1.2766475 .141397-1.123413$

H -1.343713 $2.579351-0.998867$

O $-1.3165592 .938106-3.005640$

C $1.088254-0.514602-2.237512$

H $-0.4879470 .195793-0.991285$

H $-0.7602790 .376534-2.719183$

H 0.5398356 .1846690 .145565

H -1.735267 $4.758438-0.356609$

H -2.030672 $3.593804-2.927837$

O $1.398008-1.224433-1.115559$

O $1.605264-0.641098-3.339727$

C $2.382031-2.250990-1.272981$

H $3.379537-1.806906-1.247053$

H $2.283715-2.944727-0.434366$

H $2.231945-2.811032-2.201172$

SCF Energy (B3LYP/6-31G**//MMFF) $=-3245.91563329$

03_158

MM̄FF Geometry

C $-2.554046 \quad 3.331597-0.163215$

C -1.9972322 .2430390 .389198$

C $-0.8448691 .490010-0.221820$

O 0.2529381 .6104130 .710497

C $-1.2266670 .005180-0.386962$

C $-0.331250-0.773896-1.330751$

C $0.475642-1.794640-0.979221$

C $-0.430368-0.378165-2.784183$

C $0.692430-2.402598 \quad 0.377465$

C $2.025427-2.0349201 .045526$

C $3.279216-2.5401860 .311300$

C $4.521113-2.3369451 .183805$

C $5.813732-2.7299330 .455796$

O $3.427732-1.806321-0.907247$

O $\quad 6.904111-2.2040131 .228737$

C 5.843561-2.049781-0.931118

C $6.001864-4.2516330 .430140$

O $6.962489-2.529715-1.672160$

C $4.530742-2.226774-1.725328$

C $4.474025-1.397812-3.033921$

O $4.395644-3.602959-2.102819$

C 1.5060481 .7600220 .206569

O $1.8375441 .755054-0.967728$

C 2.4261841 .9318271 .354131

C 3.7430361 .7576451 .170895

C 4.7847931 .8680142 .192850

C 4.3912232 .4169603 .538210

C $4.6142940 .117763-2.864072$

C $6.0464170 .618793-2.948783$

C $7.4612942 .575318-2.322951$

O $6.951714-0.022690-3.475250$

C $8.1612532 .159665-1.067600$

C $8.3827032 .978998-0.028719$

C 9.0820612 .5551621 .231267

C 8.1928152 .6494142 .452224

C 8.2843853 .6925003 .294155

C 7.2636011 .4922082 .747026

C 6.0403491 .4919131 .872325

N $6.1859891 .886658-2.401443$

H -2.156526 $3.724640-1.095451$

H -2.3947121 .8590491 .326261$

H $-0.5827381 .946619-1.183076$

H -2.250308 -0.073864 -0.779304

H -1.249525 -0.4620110 .602332$

H $1.037298-2.285592-1.773411$

H $\quad 0.127184-1.056394-3.439363$

H -1.475179 $-0.396278-3.111729$

H $-0.0319120 .627874-2.943236$

H $-0.116361-2.1342491 .063209$

H $\quad 0.633453-3.493347 \quad 0.272228$

H $2.074640-0.9470801 .152558$

H $2.012781-2.4576012 .058180$

H $3.141546-3.6037630 .083870$

H $4.429866-2.8901492 .127048$
H $4.592868-1.2788821 .467565$

H $7.725646-2.3853420 .740133$

H $\quad 6.018157-0.982868-0.746828$

H $6.064426-4.6442041 .452258$

H $6.945415-4.527711-0.053423$

H $5.188920-4.775881-0.076565$

H $7.041458-1.992135-2.477628$

H $5.193269-1.789522-3.763005$

H $3.485310-1.571817-3.483059$

H $3.773729-3.659002-2.847190$

H 1.9931782 .1698242 .318972

H 4.0959631 .4858840 .178037

H 3.7228751 .7206554 .056256

H 3.8786563 .3792753 .427664

H 5.2430952 .6007304 .196906

H $4.0750110 .612301-3.681931$

H $4.1543970 .445570-1.927941$

H $7.2551683 .649970-2.346701$

H $8.0730942 .317841-3.193327$

H $8.502791 \quad 1.128381-1.013777$

H $8.0474364 .012088-0.083293$

H 9.9639813 .1973321 .355527

H 9.4749181 .5341911 .146833

H 7.6725923 .7527714 .188071

H 8.9699594 .5120013 .105392

H 6.9929561 .4757313 .808337

H 7.8025190 .5486042 .591007

H 6.2189371 .0996830 .871471

H $5.3943182 .311052-1.927106$

C $-4.9156704 .088816-0.534575$

O $-5.3065572 .746883-0.883506$

C $-6.7356392 .632158-0.782967$

C $-7.2650984 .059035-0.709773$

C -6.1586504 .7459230 .066837$

H -6.9397632 .1314610 .170370$

C $-7.2947321 .783200-1.929139$

H -7.363038 $4.511053-1.703184$

H $-8.2389044 .116177-0.214302$

C $-6.1762286 .259591-0.054188$

H $-6.2549194 .471236 \quad 1.126073$

C $-6.5702070 .438592-2.145975$

O $-7.1835462 .525380-3.150620$

H $-8.3671771 .621818-1.766817$

H $-5.5919910 .621640-2.607014$

O $-7.316834-0.304519-3.118365$

C $-6.372607-0.417977-0.882252$

C $-5.805161-1.813815-1.204284$

H $-5.6889220 .102515-0.203673$

O $-7.613117-0.562569-0.191714$

H -6.562711 -2.404099-1.734051

H $-4.936809-1.693304-1.863716$

H -5.3524416 .7054300 .511968$

H $-6.0808826 .580011-1.097118$

H -7.1143896 .6659710 .337621$

C -3.7311644 .0505330 .433385$

H $-7.5504301 .971784-3.861168$

H -8.207068 -0.457235 -2.759277

H -8.211737 -1.082263 -0.754170

H -4.0325253 .5577661 .365647$

H -3.425584 5.0711930 .690039

H $-4.6263014 .586069-1.469546$

C -3.713073 -2.463885 1.818059

O $-4.231917-1.9197750 .597657$

C $-5.384639-2.5793110 .062860$

C $-5.059548-4.049380-0.239777$

C $-4.513500-4.7628291 .001128$

C -3.392806 -3.961118 1.670701

H -2.764552 -1.934998 1.977155

C $-4.625706-2.1470273 .007191$

H $-6.202115-2.5313100 .789959$

O $-4.109252-4.140952-1.299709$

H -5.971597-4.550557 -0.583903

H $-4.129811-5.7494390 .714153$

O $-5.563092-4.961321 \quad 1.943398$

H -3.151777 -4.380535 2.654393

O $-2.212970-4.0663650 .869036$

C $-4.701661-0.6539533 .226465$

H $-5.639039-2.5384812 .880306$ 
H $-4.235635-2.5905443 .930658$

H $-3.322954-3.630698-1.039488$

H $-6.249441-5.4967521 .510263$

H -1.969576 $-5.006860 \quad 0.826710$

O $-5.928222-0.1889452 .854745$

O $-3.773500 \quad 0.020033 \quad 3.654736$

C -6.1175841 .2222052 .992014$

H -5.9786141 .5262984 .033882$

H $-5.426077 \quad 1.7589382 .336669$

$\mathrm{H}-7.1416461 .4587842 .691691$

SCF Energy $\left(B 3 L Y P / 6-31 G^{* *} / / M M F F\right)=-3245.92161104$

03_159

MM̄FF Geometry

C $1.7803694 .619759-0.953517$

C $2.5826155 .230434-0.067528$

C 3.6890544 .5394030 .695952

O 3.3308803 .1620300 .966669

C $5.0009744 .560361-0.104354$

C 6.1391683 .8509930 .604590

C 6.5802082 .6140670 .297197

C 6.8059794 .6435231 .698574

C $6.0354621 .682198-0.751418$

C $5.7666400 .282048-0.187489$

C $5.206914-0.688138-1.241039$

C $3.773106-0.340812-1.653684$

C $3.197167-1.366770-2.640638$

O $5.236993-2.004712-0.667977$

O $1.779249-1.157796-2.717611$

C $3.407034-2.783589-2.070454$

C $3.741942-1.163157-4.059252$

O $3.032816-3.771029-3.027893$

C $4.844936-3.047326-1.577107$

C $4.995727-4.404548-0.841285$

O $5.723963-3.093514-2.709409$

C 2.4560952 .9428181 .987727

O 1.9218943 .7857212 .691105

C 2.2597721 .4821122 .108743

C 1.3752241 .0051002 .997068

C $1.113129-0.4072663 .284738$

C $1.943471-1.4289442 .553477$

C $4.128453-4.5915410 .408285$

C $2.759823-5.1947810 .130790$

C $0.516717-5.4587061 .200753$

O $2.460512-5.730565-0.932159$

C $-0.293379-4.236772 \quad 1.498472$

C -1.140123 -4.137178 2.533809

C -1.886083 -2.879104 2.878191

C -1.647206 -2.444644 4.308708

C $-2.656243-2.4119015 .195444$

C $-0.239473-2.0647844 .716745$

C $0.169043-0.7129554 .200027$

N $1.917875-5.0772151 .226091$

H $1.9674653 .578711-1.206675$

H 2.4108816 .2759410 .175803

H 3.8355145 .0568841 .652649

H $5.2995065 .599110-0.300216$

H $4.8392934 .122665-1.095626$

H 7.4296802 .2209670 .854568

H 7.6465874 .1052102 .149196

H 7.1943315 .5869781 .301233

H 6.0936984 .8689192 .498165

H $5.1179692 .075423-1.191714$

H $6.7729111 .609341-1.559746$

H $6.699858-0.1355980 .214505$

H $\begin{array}{llll}5.081471 & 0.335537 & 0.667678\end{array}$

H $5.876576-0.667943-2.108806$

H $3.708606 \quad 0.663393-2.086734$

H $3.132988-0.323369-0.760887$

H $1.624248-0.238378-2.994018$

H $2.717054-2.883988-1.223674$

H $3.491167-0.160018-4.424731$

H $3.279281-1.862023-4.764851$

H $4.826140-1.274851-4.123019$

H $2.113617-3.588738-3.287894$

H $4.856153-5.231842-1.548047$

H $6.041805-4.478266-0.510853$

H $6.527292-3.578083-2.456699$
H 2.8635250 .8386641 .480946

H $\quad 0.798118 \quad 1.7151413 .588323$

H $3.011597-1.2586392 .729207$

H $1.753118-1.3793351 .475478$

H $1.741474-2.4542402 .864473$

H $4.637054-5.2928361 .082092$

H $4.013363-3.6443020 .946644$

H $\quad 0.379178-6.2414231 .953182$

H $\quad 0.245427 \quad-5.860678 \quad 0.220112$

H $-0.151248-3.3803830 .843119$

H $-1.292331-4.9968703 .182374$

H -2.953398 -3.066457 2.705552

H -1.610088 -2.056870 2.207972

H -2.495564 -2.105584 6.223829

H -3.666932 -2.686883 4.912778

H $\quad 0.456690-2.856708 \quad 4.429264$

H $-0.166592-2.0238905 .812262$

H -0.3735150 .1062914 .674563$

H $2.283011-4.6824332 .088111$

C $-0.7103464 .462148-1.370550$

O $-0.6109873 .182442-2.030108$

C $-0.9259832 .147546-1.077229$

C $-1.7546032 .834159-0.004177$

C -1.067657 4.1814000 .093208

H $0.0360251 .808537-0.672925$

C $-1.6427920 .999388-1.792901$

H -2.795045 $2.954121-0.330851$

H -1.7503232 .2889930 .943495$

C -1.925983 5.2608580 .729686

H -0.1667954 .0554170 .703517$

C $-2.075743-0.141728-0.855225$

O $\quad-0.7608960 .466061-2.784224$

H $-2.507526 \quad 1.400314-2.332509$

H $-2.7568800 .228289-0.082238$

O $-0.926201-0.651778-0.165991$

C $-2.727113-1.336634-1.575359$

C $-3.931490-0.999216-2.473644$

H -1.977113 -1.858673 -2.182488

O $-3.154770-2.283939-0.590588$

H -3.579295 -0.465405-3.363958

H $-4.356408-1.953150-2.812526$

H -1.3889336 .2142750 .760366$

H -2.8567495 .4141600 .173236$

H -2.190119 4.9865841 .756346

C $0.5828605 .260022-1.598455$

H -0.493688 1.202167 -3.361193

H $-0.6541180 .012250 \quad 0.489468$

H $-2.376244-2.515943-0.056506$

H $0.4464426 .283582-1.230530$

H $\quad 0.7809075 .319881-2.675462$

H $-1.5328534 .990663-1.868547$

C $-6.565845-0.2673230 .091557$

O $-5.566719-0.926649-0.697450$

C $-5.048824-0.176035-1.798023$

C $-6.1782550 .169739-2.782577$

C $-7.3268330 .893928-2.072245$

C $-7.749717 \quad 0.172947-0.786443$

H $-6.931940-1.0374070 .782853$

C $-5.9726420 .874340 \quad 0.932073$

H $-4.6119570 .754824-1.426014$

O $-6.669272-1.026480-3.384011$

H $-5.7705590 .800422-3.581947$

H $-8.1851440 .960056-2.752037$

O $-6.9071772 .221725-1.771700$

H $-8.4220100 .808665-0.199363$

O $-8.502555-0.991446-1.141065$

C -4.9347420 .3802891 .918324$

H $\quad-5.492575 \quad 1.626250 \quad 0.300035$

H $-6.7664021 .361071 \quad 1.509840$

H -7.352371 $-0.772259-4.027529$

H $-7.6543422 .676237-1.346583$

H -7.902971 -1.604221-1.600788

O $-4.251764 \quad 1.4535012 .416852$

O $-4.749964-0.7896282 .223488$

C $-3.208770 \quad 1.139546 \quad 3.344483$

H $-2.7260832 .073742 \quad 3.643443$

H -3.625002 0.6573364 .233776

H -2.4622720 .4979782 .867482$ 
C $-3.177852-3.672131-0.312636$

C $-2.160364-3.9496090 .515781$

C $-0.719479-3.8316520 .097497$

O $-0.099040-2.8537940 .963887$

C $0.032403-5.1630140 .267269$

C $1.313579-5.208980-0.547119$

C $2.561962-5.106228-0.051204$

C $1.122947-5.419583-2.030159$

C $2.997765-4.9394861 .375215$

C $3.393283-3.5098851 .776396$

C $4.481991-2.8636700 .903498$

C $5.043756-1.6141741 .587871$

C $6.071388-0.8841870 .709454$

O $3.902316-2.493467-0.354332$

$\begin{array}{llll}\text { O } & 6.285237 & 0.419749 & 1.272718\end{array}$

C $5.473895-0.673450-0.698459$

C $7.432941-1.589190 \quad 0.721774$

O $6.454369-0.153728-1.592430$

C $4.840873-1.947959-1.295110$

C $4.084499-1.693915-2.624550$

O $5.875976-2.896061-1.587913$

C $-0.029847-1.5748170 .508178$

O $-0.434347-1.149007-0.561950$

C $0.663050-0.7700591 .537307$

C $1.294141 \quad 0.3453901 .147401$

C 2.0746981 .2556031 .985272

C $2.026170 \quad 1.0627703 .475262$

C $2.994286-0.617105-2.579756$

C $3.4693000 .758755-3.027633$

C $2.7796813 .159002-3.011921$

O $4.5287540 .946569-3.619676$

C $3.5412353 .769203-1.877239$

C $3.0106964 .635135-0.999786$

C 3.7594645 .1940600 .176715

C 3.2392254 .6731431 .501608

C 2.4822955 .4424922 .301702

C 3.6695113 .2865011 .939269

C $2.7930112 .210763 \quad 1.361301$

N $2.5492721 .753026-2.722374$

H -2.961226 $-3.380723-1.338151$

H -2.363761 -4.2320561 .545444$

H $-0.657247-3.502497-0.946475$

H $-0.598611-6.000965-0.058189$

H $\quad 0.237767-5.3359241 .328952$

H $3.391412-5.166135-0.755848$

H $2.074524-5.536535-2.560200$

H $0.535831-6.325991-2.211713$

H $0.601809-4.570453-2.481592$

H $2.228001-5.2944832 .067581$

H $3.857938-5.6016511 .538267$

H $2.496484-2.8851091 .759871$

H $3.738107-3.5484512 .817394$

H $5.278379-3.5993390 .740923$

H $5.484806-1.8676272 .560026$

H $4.221621-0.9206921 .806737$

H 6.5861390 .3043792 .190272

H $4.6988210 .096686-0.599569$

H $7.830360-1.6360161 .742860$

H $8.173305-1.0300450 .139060$

H $7.390715-2.610670 \quad 0.338312$

H $6.8077300 .660040-1.193847$

H $4.802328-1.501060-3.431086$

H $3.588596-2.636883-2.897408$

H $5.534897-3.531415-2.239213$

H $\quad 0.674699-1.1350802 .557191$

H 1.2518880 .6134460 .092650

H $0.993764 \quad 1.096795 \quad 3.837683$

H $2.5745191 .826498 \quad 4.030294$

H 2.4604110 .0952553 .750375

H $2.195942-0.901062-3.277425$

H $2.549125-0.554842-1.582693$

H $1.8015223 .628576-3.154040$

H $3.3514183 .255652-3.940320$

H $4.5767543 .458837-1.757877$
$\begin{array}{llll}H & 3.677251 & 6.287946 & 0.136711\end{array}$

H 4.8330194 .9794580 .102603

H 2.1296525 .0813463 .262140

H 2.1912636 .4461422 .010160

H 3.7039553 .2420803 .031824

H 4.7024153 .1096701 .613837

H 2.7745022 .2052720 .274073

H $1.7284401 .514888-2.173398$

C $-5.216964-2.2865900 .049116$

O $-5.215908-1.784336-1.306002$

C $-6.481435-1.163377-1.562454$

C $-7.139117-0.959572-0.206001$

C $-6.671456-2.2105100 .516695$

H -7.077832 -1.908979-2.107495

C $-6.3622050 .072074-2.458271$

H $-6.756537-0.0633840 .295474$

H $-8.228356-0.882664-0.271277$

C $-6.851638-2.1493962 .022220$

H -7.231776 -3.072042 0.128176

C $-5.5794461 .289904-1.923525$

O $\quad-7.6909830 .528552-2.751638$

H -5.917884 -0.225136 -3.416786

H $-6.1162811 .753547-1.087632$

O $-5.5769342 .269931-2.976531$

C $-4.1148271 .038388-1.511575$

C $-3.3218512 .348161-1.309448$

H $-4.0958900 .455743-0.586421$

O $-3.457454 \quad 0.265031-2.516189$

H $-3.2339382 .859596-2.275376$

H $-3.8893703 .005510-0.643143$

H -6.456430 -3.050256 2.501548

H $-6.341509-1.2843202 .454580$

H -7.913519-2.069742 2.277345

C $-4.627689-3.6978710 .081593$

H $-8.163683-0.202469-3.185135$

H $-6.5048412 .449372-3.206185$

H $-3.5062620 .762834-3.350312$

H $-5.157169-4.346308-0.626722$

H $-4.745609-4.1332381 .080366$

H $-4.591325-1.6052160 .637858$

C -2.3536652 .3844581 .600361$

O -1.9096641 .5236220 .544548$

C $-1.8957792 .111731-0.759943$

C $-1.0014183 .360126-0.761294$

C -1.4235024 .3542960 .314469$

C -1.5011303 .6580081 .670361$

H -3.4069232 .6454841 .444199$

C $-2.243174 \quad 1.5974412 .913726$

H -1.414615 $1.380855-1.421208$

O $-1.0202434 .005392-2.029782$

H $0.0373723 .062175-0.569286$

H -2.3745294 .8394430 .065363$

O -0.4512915 .3973250 .402760$

H -0.4869443 .4161762 .013391$

O -2.0708824 .5514892 .624738$

C -3.1997120 .4286062 .958239$

H -1.224719 1.2084653 .012794

H -2.463412 2.2296033 .780393

H $-0.7395853 .352378-2.693528$

H $-0.3804585 .801639-0.478979$

H -1.522886 5.3551002 .632264

O $-2.662699-0.5643193 .725844$

O $-4.295204 \quad 0.4062692 .414534$

C $-3.470156-1.7349663 .879607$

H $-4.417834-1.4814644 .363837$

H -3.644855 -2.204116 2.907996

$\mathrm{H}-2.928799-2.4402734 .515831$

SCF Energy (B3LYP/6-31G**//MMFF) $=-3245.90864183$

03_160

MM̄FF Geometry

C $3.2490262 .898642-0.578822$

C $2.3599783 .746734-1.119040$

C $0.8621303 .529471-1.134307$

O $0.5158332 .573715-0.106228$

C $0.0966564 .836563-0.851041$

C $-1.3516944 .799127-1.308374$ 
C $-2.4308234 .832661-0.501745$ C -1.554263 4.771867-2.804577 C $-2.487217 \quad 4.9148050 .996245$ C -2.8129493 .5897941 .701611$ C -4.1944482 .9960541 .371469$ C $-4.508837 \quad 1.834798 \quad 2.320785$ C -5.8281151 .1308301 .969303$ $\begin{array}{llll}\text { O } & -4.187089 & 2.522847 & 0.020041\end{array}$ O $-5.860508-0.1222192 .670981$ $\begin{array}{llll}\text { C } & -5.837795 & 0.800799 & 0.461428\end{array}$ C $-7.044101 \quad 1.928962 \quad 2.454138$ $\begin{array}{lllll}\text { O } & -7.107251 & 0.291664 & 0.061569\end{array}$ C $-5.4458311 .997010-0.429427$ C $-5.2982611 .620878-1.926150$ O $-6.4731492 .994142-0.356853$ C $-0.2497561 .505285-0.440308$ O $-0.7729921 .274587-1.517652$ $\begin{array}{llll}\text { C } & -0.335279 & 0.643472 & 0.760584\end{array}$ C -1.327424 -0.2518800 .848427$ C $-1.563425-1.1928371 .942338$ C $-0.535466-1.2720193 .037759$ C $-4.2493990 .551020-2.242164$ C $-4.794257-0.868359-2.282550$ C $-4.022984-3.244910-2.231117$ O $-5.987547-1.128259-2.410019$ C $-4.330182-3.717263-0.845286$ C $-3.511118-4.489731-0.114766$ C $-3.834403-4.9599981 .275898$ C $-2.879618-4.4175852 .318044$ C $-1.889219-5.1796222 .811673$ C $-3.124163-3.0214082 .850384$ C $-2.672177-1.958597 \quad 1.890055$ N -3.779948 -1.813659-2.206422 H $2.8949461 .982279-0.113320$ H $2.7298174 .628826-1.636943$ H $0.617758 \quad 3.142645-2.130121$ H $0.576393 \quad 5.677199-1.370335$ H $0.165983 \quad 5.065200 \quad 0.217372$ H -3.412452 4.824908 -0.975040 H -2.605028 4.903712 -3.084987 H $-0.9888225 .580329-3.279646$ H - $-1.222803 \quad 3.818444-3.225907$ H -1.5479855 .3013681 .403828$ H $-3.246593 \quad 5.6599921 .265594$ H -2.033102 2.8630201 .455251 H -2.752749 3.7709242 .782284 H -4.9452393 .7878991 .477147$ H -4.5252912 .1786273 .362591$ H -3.699681 1.0954382 .266936 H -5.7691270 .0639053 .621222$ H $-5.112391-0.0076830 .309392$ H -7.0135042 .0482943 .543998$ H $-7.977719 \begin{array}{lll}1.398072 & 2.238186\end{array}$ H -7.104158 2.9275502 .016576 H -7.304304 -0.4732930 .628815$ H -6.277124 $1.359590-2.346300$ H -4.987333 $2.531617-2.458126$ H $-6.3974013 .571756-1.134433$ H 0.4069440 .7797701 .538638 H -2.044744 -0.3054920 .032809$ H $-0.519811-0.3407773 .614530$ H $0.462914-1.4409642 .621752$ H $-0.714277-2.087306 \quad 3.742353$ H $-3.8461870 .744215-3.244572$ H -3.414653 $0.612752-1.540687$ H - $3.127829-3.722456-2.641700$ H $-4.867485-3.461026-2.893460$ H $-5.283437-3.406771-0.423141$ H -2.559034 -4.805373 -0.534702 H -3.805796 -6.057514 1.270840 H $-4.861848-4.6971171 .557143$ H -1.217658 -4.8063973 .577789$ H - $1.723181-6.1907612 .455248$ H -2.657338 -2.8962843 .833065$ H $-4.197292-2.8888183 .038906$ H -3.359961 -1.804245 1.059572 H -2.825657 -1.504387 -2.045375 C $5.3941702 .876694 \quad 0.741877$ $\begin{array}{llll}\text { O } & 5.066116 & 1.565840 & 1.248129\end{array}$

C $6.2764310 .813787 \quad 1.409092$

C 7.3461891 .8659551 .654401

C $6.9258622 .939366 \quad 0.666144$

H 6.4717320 .3169260 .450648

C $6.117085-0.2501232 .498996$

H 7.3021492 .2606932 .675792

H $8.356568 \quad 1.487847 \quad 1.473333$

$\begin{array}{llll}\text { C } 7.493541 & 4.313579 & 0.977991\end{array}$

H $7.2667202 .641197-0.334552$

C $4.829159-1.0945432 .388825$

$\begin{array}{lllll}\text { O } & 6.091739 & 0.395647 & 3.778327\end{array}$

H $7.003460-0.8957692 .502519$

H $3.972923-0.4931132 .717963$

O $4.935042-2.1661553 .335513$

C $4.528062-1.6745530 .994251$

C $3.302753-2.6114331 .016357$

H $4.357409-0.8505370 .293453$

O $5.670948-2.3996770 .542849$

H $3.571779-3.5572701 .501915$

H $2.513995-2.1310101 .606315$

H $7.1457865 .051144 \quad 0.247626$

H 7.1916344 .6559231 .973539

H $8.587821 \quad 4.294346 \quad 0.946573$

C $4.7400963 .072603-0.631280$

H $5.999950-0.2995724 .452412$

H $5.645786-2.7561343 .030696$

H $5.539965-2.605017-0.397831$

H $5.1233102 .317723-1.328299$

H $5.0068364 .056936-1.032995$

H $4.996723 \quad 3.603041 \quad 1.461201$

C $1.769577-1.752806-2.275032$

O $2.250649-1.688167-0.926850$

C $2.757563-2.915793-0.391771$

C $1.645200-3.975913-0.368326$

C $1.035924-4.165959-1.762043$

C $0.678194-2.827223-2.420281$

H $1.304144-0.774948-2.453613$

C $2.921316-1.913722-3.275720$

H $3.583354-3.273420-1.014573$

$\begin{array}{llllll} & & 0.626954 & -3.577158 & 0.546436\end{array}$

H $2.064796-4.922328-0.006706$

H $0.133014-4.783312-1.678151$

O $1.971099-4.872826-2.571356$

H $0.451755-2.974441-3.482171$

O $-0.520092-2.331115-1.817641$

C $3.915821-0.780754-3.147218$

H $3.449119-2.860163-3.128640$

H $2.546954-1.909645-4.305368$

H $-0.049274-4.275700 \quad 0.557424$

H $1.559555-5.012585-3.441218$

H $-0.335457-2.181553-0.874822$

O $5.184200-1.282242-3.119565$

$\begin{array}{lllllll}0 & 3.608617 & 0.402574 & -3.087743\end{array}$

C $6.229248-0.314023-2.989020$

H $6.1626520 .178177-2.015279$

H $7.187044-0.837206-3.052211$

H $6.1764510 .419932-3.798834$

SCF Energy (B3LYP/6-31G**//MMFF) $=-3245.92022233$

$03 \_161$

MMFF Geometry

C $2.332922-4.142881-1.235429$

C $1.011935-4.030632-1.439752$

C $0.024736-3.672194-0.358450$

O $-0.787124-2.572999-0.834025$

C $-0.907686-4.864401-0.082379$

C $-1.923291-4.601337 \quad 1.013216$

C -3.258562 -4.590062 0.830955

C $-1.356014-4.3644432 .389564$

C $-4.021333-4.838581-0.440040$

C $-4.344059-3.575694-1.253360$

C $-5.278928-2.588432-0.535772$

C $-5.750588-1.494615-1.496300$

C $-6.622602-0.443653-0.791879$

O $-4.565501-1.9886710 .548760$

O

$\begin{array}{llll}\text { C } & -5.897485 & 0.043683 & 0.482864\end{array}$ 
C $-8.047204-0.960163-0.557267$

O -6.7441710 .8811851 .265749$

C $-5.355784-1.1064201 .357827$

C $-4.456097-0.6185912 .520310$

O $-6.458393-1.8053421 .950011$

C $-0.237231-1.329459-0.805297$

O $0.864742-1.019443-0.382592$

C $-1.211917-0.402208-1.419576$

C $-0.7861010 .778728-1.890265$

C $-1.6089161 .803581-2.534296$

C $-3.0980511 .583279-2.567118$

C $-3.222777 \quad 0.191752 \quad 2.105892$

C $-3.436567 \quad 1.6966792 .134194$

C $-2.302150 \quad 3.827048 \quad 1.508757$

$\begin{array}{llll}\text { O } & -4.421643 & 2.229386 & 2.637768\end{array}$

C - 2.7482914 .2957510 .160501

C -2.006641 $5.092655-0.623926$

C $-2.4680015 .627494-1.948915$

C $-1.4888405 .349176-3.066646$

C $-0.5912576 .274486-3.446047$

C $-1.6140944 .035886-3.803533$

C $-0.9959632 .888030-3.053081$

N -2.371887 2.3794201 .563689

H $2.724978-4.008647-0.231277$

H $0.612895-4.171639-2.441737$

H $0.540157-3.3728060 .562364$

H $-0.313459-5.7416110 .206481$

H -1.412409-5.135268 -1.015957

H $-3.890388-4.3903161 .695897$

H -2.134206 -4.3352483 .159932$

H -0.662116 -5.1678202 .658106$

H $-0.821323-3.4104742 .428824$

H -3.484032 -5.546281 - 1.080469

H $-4.957539-5.348842-0.179506$

H -3.406810 -3.072875 -1.519089

H $-4.815312-3.897210-2.190588$

H -6.136540 -3.149059-0.145808

H -6.288964 -1.928357 -2.348278

H $-4.874502-0.988283-1.924369$

H $-7.3170451 .330187-1.287492$

H -5.0577680 .6650960 .150635$

H -8.525938 -1.204123 -1.513326

H -8.676806 $-0.191949-0.095133$

H -8.081282 -1.8542490 .068762$

H $-6.968923 \quad 1.6590800 .728385$

H $-5.059151-0.0839183 .264085$

H $-4.084802-1.5145213 .038760$

H -6.136007-2.282586 2.732515

H -2.244194 $-0.725282-1.486147$

H $\quad 0.2744651 .014020-1.814374$

H $-3.4785301 .384556-1.559434$

H $-3.3464120 .732915-3.211059$

H -3.658973 2.445159-2.934532

H -2.418288 -0.0125862 .823909$

H -2.864167 -0.1125121 .117806$

H -1.2676314 .1119301 .723481$

H -2.946956 4.2579432 .281223

H -3.739859 $3.994860-0.169191$

H $-1.0271365 .414630-0.276898$

H -2.605855 $6.711373-1.839251$

H -3.454831 $5.232298-2.219831$

H $0.0943416 .094064-4.267273$

H $-0.5185837 .231195-2.939225$

H -1.099412 $4.099739-4.772010$

H -2.662805 $3.864348-4.061877$

H $0.0895672 .959166-2.969033$

H -1.6136261 .8537491 .138049$

C $4.344011-3.321022-2.560216$

O $3.676425-2.067271-2.815687$

C $4.309754-1.040129-2.017681$

C $5.669693-1.611897-1.645785$

C $5.322887-3.068971-1.413389$

H $3.682460-0.934917-1.124269$

C $4.3305250 .274736-2.808497$

H $6.374532-1.516853-2.481127$

H $6.111897-1.140418-0.764788$

C $6.529352-3.991557-1.418992$

H $4.821859-3.151523-0.441665$
C $5.0636271 .450520-2.131163$

$\begin{array}{llll}\text { O } & 2.984827 & 0.673833 & -3.079738\end{array}$

H $4.7890820 .083268-3.786941$

H $4.8411492 .379581-2.671400$

O $6.4751231 .236865-2.256885$

C $4.7698741 .677133-0.633429$

C $3.3043841 .997869-0.296717$

H $5.1112120 .811890-0.058845$

O $5.5741932 .777946-0.183729$

H $3.0451252 .981649-0.706243$

H $2.6485871 .250880-0.750273$

H $6.224191-5.031667-1.267401$

H $7.075202-3.934247-2.366740$

H $7.222977-3.721799-0.615922$

C $3.317965-4.436776-2.333580$

H $2.532073-0.084405-3.487216$

H $6.6811681 .188730-3.205984$

H $5.3125983 .564402-0.692465$

H $3.833990-5.375955-2.104105$

H $2.774165-4.596053-3.273717$

H $4.902614-3.556131-3.475797$

C 2.9558650 .4031743 .058256

$\begin{array}{llll}\text { O } & 3.138285 & 0.633327 & 1.656183\end{array}$

C $3.0538321 .997721 \quad 1.225647$

C 1.6825782 .5891831 .583708

C 1.3651322 .4124293 .071279

C $1.611697 \quad 0.974808 \quad 3.539759$

H $2.911243-0.6893273 .155182$

C $4.156310 \quad 0.893440 \quad 3.874847$

H 3.8379372 .5826111 .717246

$\begin{array}{lllll}\text { O } & 0.660757 & 1.973764 & 0.805944\end{array}$

H 1.6795363 .6554941 .329062

H $0.3156242 .678033 \quad 3.246607$

O 2.1746403 .3148623 .818903

H 1.5519390 .9173964 .632722

$\begin{array}{llll}\text { O } & 0.557349 & 0.158961 & 3.019383\end{array}$

C 5.3759510 .0568373 .568452

H 4.4029281 .9446743 .700874

H 3.9616290 .7960674 .949524

H $0.703703 \quad 1.0148900 .963002$

H 1.9394443 .2118754 .756793

H $0.682283-0.7408673 .366173$

O 6.1081160 .6434872 .579743

O $5.628700-1.0054664 .122113$

C $7.268444-0.0800552 .158657$

H $6.987463-1.0752961 .800832$

H $7.7307580 .469703 \quad 1.334438$

H $7.986251-0.1527702 .981034$

SCF Energy (B3LYP/6-31G**//MMFF) $=-3245.90999359$

$03 \_162$

MM̄FF Geometry

C $2.112560-3.878289-0.239504$

C $0.896670-3.777706-0.797043$

C $-0.365434-3.555240-0.001321$

O $-1.069261-2.415388-0.544574$

C $-1.279212-4.787614-0.139510$

C $-2.512613-4.7412090 .741249$

C $-3.780437-4.713663 \quad 0.285892$

C -2.257001 -4.7659612 .227018$

C $-4.259432-4.717071-1.138524$

C $-4.509347-3.322694-1.734609$

C $-5.588745-2.507883-1.002617$

C $-5.948205-1.248421-1.794047$

C $-6.954037-0.361525-1.041559$

O $-5.077971-2.131440 \quad 0.278683$

O $-6.976398 \quad 0.922601-1.684357$

C $-6.451027-0.1344560 .401402$

C $-8.380760-0.916129-1.133748$

$\begin{array}{llll}\text { O } & -7.433755 & 0.538671 & 1.183560\end{array}$

C $-6.013909-1.4323911 .109856$

C $-5.319428-1.1836312 .471010$

O $-7.169845-2.2377971 .371814$

C $-0.676298-1.180511-0.129976$

O $0.229032-0.9174730 .645300$

C $-1.541277-0.186851-0.805500$

C $-1.2042861 .110537-0.770366$

C -1.926668 2.211561 -1.409601 
C $-3.3161691 .930887-1.913614$ C $-4.047802-0.3294172 .415612$ C $-4.276331 \quad 1.1396542 .730884$ C -3.0626343 .3143662 .818231$ O C -3.3837254 .0883791 .579069$ C -2.5998565 .0656551 .099100$ C $-2.9285715 .881714-0.117903$ C -1.832324 5.839219-1.158229 C $-0.9346186 .833998-1.259908$ C $-1.8265844 .679264-2.129017$ C $-1.3166653 .411423-1.500711$ N -3.1268541 .8933132 .532924$ H $2.208554-3.7877310 .839959$ H $\quad 0.790978-3.885677-1.873794$ H $-0.132893-3.3752111 .055006$ H $-0.719388-5.6967830 .118773$ H -1.562318 -4.895469-1.192183 H -4.584385 -4.692978 1.021205 H -3.178128 -4.8987482 .804956$ H - $1.589422-5.5946422 .484765$ H -1.797606 -3.829209 2.556423 H -3.559594 -5.258915 -1.783408 H -5.190432 -5.296924 -1.180176 H -3.564919 -2.765992 -1.737838 H $-4.808717-3.457611-2.781415$ H -6.473180 -3.142892 -0.873659 H $-6.336290-1.509461-2.786567$ H -5.037238 $-0.662672-1.975327$ H $-7.2204110 .787206-2.616063$ H -5.5896690 .5404480 .334770$ H -8.699022 $-0.981288-2.181229$ H $-9.097557-0.246896-0.645210$ H -8.483151 -1.911392 -0.696469 H $-7.662789 \begin{array}{lll}1.360433 & 0.716647\end{array}$ H $-6.041632-0.790917 \quad 3.196770$ H -5.020417 -2.166184 2.864382 H $-6.956445-2.8604252 .086518$ H -2.414812 -0.550536 -1.333569 H $-0.2963521 .396920-0.242455$ H $-3.925240 \quad 1.486344-1.119751$ H $-3.2863241 .240481-2.763269$ H -3.852080 $2.823962-2.240760$ H -3.350467 -0.6988553 .178622$ H -3.549882 -0.4221991 .445694$ H -2.0548053 .5257313 .189680$ H -3.7836183 .5612283 .604251$ H -4.3150353 .8528521 .069320$ H -1.681606 5.3161081 .625786 H -3.093853 6.9165620 .209446 H -3.874461 5.561971 -0.572530 H -0.160195 $6.821766-2.019740$ H $-0.9507727 .680085-0.580864$ H -1.170403 $4.906082-2.980041$ H -2.825639 4.575101-2.560185 H $-0.3046643 .503674-1.106725$ H -2.3016611 .4498772 .139632$ C $4.438386-3.088038-0.818383$ O $4.831924-3.035376 \quad 0.569022$ C $6.258923-3.143176 \quad 0.649037$ C $6.786699-2.766798-0.725474$ C $5.717699-3.378270-1.612978$ H $6.473086-4.2090900 .810906$ C $6.816401-2.3633091 .842380$ H $6.821106-1.681918-0.868438$ H $7.785580-3.169569-0.917437$ C $5.708380-2.824159-3.026523$ H $5.886722-4.462642-1.662893$ C $6.821075-0.8237001 .760363$ O $8.180991-2.7648782 .032505$ H $6.275367-2.6598632 .750039$ H $7.601516-0.4917061 .064432$ O $7.220911-0.3365133 .051999$ C $5.494341-0.1403241 .381613$ C 5.5642261 .3890041 .561871 H $5.239776-0.3722110 .342798$ O $4.433530-0.6174572 .209128$ H 5.5264551 .6249312 .632739
H $\quad 6.516821 \quad 1.753333 \quad 1.157574$

H $4.891379-3.257975-3.611537$

H $5.587528-1.736860-3.029788$

H $6.649771-3.055993-3.535504$

C $3.371360-4.169685-1.008871$

H $8.184715-3.7226152 .201273$

H $8.080021-0.7419603 .261019$

H $4.277448-1.5494121 .982207$

H $3.755218-5.131754-0.646431$

H $3.150341-4.288088-2.076010$

H $4.032934-2.101172-1.071830$

C $3.7412802 .744020-1.403096$

O $4.7058062 .115463-0.548804$

C 4.4037182 .1128490 .852195

C 4.2349433 .5500541 .367225

C 3.1916204 .3155790 .550548

C $3.4425074 .186352-0.953810$

H $4.2315182 .800009-2.383645$

C $2.4804681 .881911-1.557880$

H 3.4772101 .5551541 .024192

O 5.4808724 .2432881 .325951

H 3.9239833 .5140342 .417882

H 3.2215085 .3736360 .838699

O $1.8996853 .809045 \quad 0.871861$

H $2.5878544 .583403-1.514177$

O $4.5694555 .005851-1.279648$

C $2.7340530 .646657-2.396119$

H $2.0906571 .547684-0.594136$

H $1.7118302 .463948-2.078235$

H 5.8025094 .2229560 .408404

H 1.2471704 .3634950 .411233

H $4.6814354 .981903-2.245282$

O $1.542546 \quad 0.026321-2.642352$

O $3.8297690 .272514-2.789392$

C $1.612782-1.144132-3.461940$

H $2.321666-1.865537-3.046509$

H $0.620251-1.601940-3.486402$

H $1.898732-0.868012-4.481061$

SCF Energy $(B 3 L Y P / 6-31 G * * / / M M F F)=-3245.90908858$

03163

MM̄FF Geometry

C $-0.8616225 .669824-0.468742$

C -1.383783 4.8069590 .415660

C -2.5579073 .9110160 .115043$

O -2.1350422 .5334530 .247726$

C $-3.6750994 .171768 \quad 1.142113$

C -4.9560663 .4086390 .869338$

C -5.4841192 .4798871 .690354$

C $-5.6759973 .771915-0.404470$

C -4.9532991 .9940513 .010128$

C $-4.042430 \quad 0.7592642 .920746$

C $-4.728833-0.4910092 .346124$

C $-3.827028-1.7186062 .488770$

C $-4.455037-2.9667861 .847962$

O $-4.991348-0.2632430 .961167$

O $-3.429069-3.9651891 .726771$

C $-4.919022-2.6270310 .412146$

C $-5.538974-3.5724152 .747745$

O $-5.674769-3.696659-0.148158$

C $-5.721178-1.3116050 .310402$

C $-5.968302-0.848805-1.143004$

O $-7.032097-1.4530250 .871890$

C $-1.4315451 .993580-0.785646$

O $-1.1124702 .544470-1.827193$

C $-1.0997840 .597646-0.423964$

C $-0.175563-0.069094-1.132083$

C $0.249860-1.454153-0.911604$

C $-0.525331-2.2642070 .093096$

C $-4.708939-0.581899-1.974645$

C $-4.290944-1.751367-2.850148$

C $-2.476994-2.457568-4.404159$

O $-4.977896-2.756644-3.009062$

C $-1.580890-3.403269-3.669269$

C $-0.313313-3.647591-4.034204$

C $0.582227-4.631842-3.339324$

C $1.888637-4.016058-2.893867$

C $3.009013-4.167276-3.620288$ 
C $1.913677-3.302740-1.561393$

C $1.290661-1.933023-1.625122$ N $-3.068480-1.521418-3.467556$ H -1.297914 $5.750679-1.461052$ H $-0.9339854 .719123 \quad 1.402207$ H -2.931616 4.080827 -0.901941 H -3.9202245 .2423241 .159606$ H -3.292765 3.9329802 .140573 H -6.4183512 .0064401 .389152$ H -5.105935 $3.442540-1.278309$ H -6.667165 $3.309877-0.467785$ H $-5.8180464 .855845-0.465488$ H -4.4174502 .7948593 .530738$ H -5.8108641 .7614063 .654476$ H -3.165225 1.0109822 .313093 H -3.6812210 .5375043 .932664$ H -5.674324 -0.6437032 .879404$ H -3.589062 -1.907720 3.542922 H -2.865345 -1.521880 1.996543 H -3.061256 -4.126992 2.612482 H $-4.011859-2.533148-0.196405$ H -5.108103 -3.8905123 .704780$ H -5.968292 -4.474938 2.299372 H -6.348989 -2.874719 2.970856 H -5.124364 -4.497038 -0.099177 H -6.648464 -1.544103 -1.649869 H $-6.5198960 .101359-1.086855$ H -7.177076 $-2.387175 \quad 1.091472$ H $-1.624116 \quad 0.1586510 .416729$ H $0.3266880 .448334-1.948664$ H $-0.412619-1.8420541 .097250$ H $-0.216312-3.3092410 .150943$ H $-1.588943-2.274267-0.167429$ H $-4.9169940 .250650-2.658969$ H -3.869768 $-0.281210-1.339924$ H -1.936153 -1.870378 -5.153057 H -3.271362 -3.018832 -4.906919 H $-2.001850-3.932891-2.817829$ H $0.096305-3.137375-4.903228$ H $0.777026-5.455638-4.038466$ H $0.082624-5.089782-2.476586$ H $3.956918-3.754622-3.291348$ H $3.002104-4.704154-4.563124$ H $2.951289-3.172371-1.233399$ H $1.460727-3.950299-0.806743$ H $1.788579-1.260986-2.325795$ H $-2.540128-0.686103-3.232727$

C $1.6557245 .954561-0.647549$

$\begin{array}{lllll}\text { O } & 1.931525 & 4.734820 & 0.068445\end{array}$

C $2.5025323 .780641-0.845066$

C $2.8757174 .572419-2.093721$

C $1.7690185 .609735-2.131065$ H $1.7091823 .065003-1.095118$

C $3.6742783 .030626-0.198810$

H $3.8524695 .058647-1.983865$

H $2.9080663 .944693-2.989370$

C $2.075123 \quad 6.802148-3.019195$

H $0.855898 \quad 5.121219-2.492362$

C $3.2642132 .145962 \quad 0.997559$

$\begin{array}{lllll}\text { O } & 4.667259 & 3.967404 & 0.219919\end{array}$

H $4.1354202 .404212-0.969226$

H $2.516535 \quad 1.4084430 .682702$

O 2.6367522 .9444062 .008916

C 4.4420881 .4311751 .685967

C $5.350494 \quad 0.5955370 .767549$

H 5.0662112 .1701012 .203950

$\begin{array}{lllll}\text { O } & 3.922563 & 0.580263 & 2.710425\end{array}$

H 5.9046261 .2779470 .113140

H 6.0964580 .1022591 .401652

H $1.2543377 .525772-2.994546$

H $2.9884817 .316370-2.701335$

H $2.2138606 .482859-4.057276$

C $0.3250806 .542566-0.173334$

H 4.2605394 .5389570 .893896

H 1.8230553 .3166001 .628472

H 3.3590401 .1267683 .284642

H $0.1612697 .522016-0.637317$

H 0.3823576 .7147250 .909369
H $2.4538476 .654724-0.366811$

C $4.665982-2.3335491 .455482$

O $3.885642-1.408240 \quad 0.688622$

C $4.629713-0.470207-0.090339$

C $5.552141-1.215862-1.064843$

C $6.447079-2.217033-0.342514$

C $5.616138-3.134604 \quad 0.553246$

H $5.242135-1.7886772 .212618$

C $3.677197-3.2583862 .176686$

H $3.8940900 .058774-0.706451$

O $6.364275-0.306702-1.800225$

H $4.943475-1.761516-1.796714$

H $7.232275-1.7120620 .232434$

O $7.117969-3.026665-1.311984$

H $5.054360-3.834131-0.078699$

O $6.518554-3.9024651 .348279$

C $2.872252-2.505043 \quad 3.208353$

H $2.965900-3.6984861 .467376$

H $4.176990-4.0907202 .683314$

H $5.7692530 .308592-2.261872$

H $7.616559-2.427040-1.893197$

H $6.017933-4.634141 \quad 1.745815$

O $3.598631-2.3652464 .354001$

O $1.739355-2.083946 \quad 3.014336$

C $2.942318-1.6667465 .416598$

H $3.636182-1.5996796 .258703$

H $2.675704-0.6538725 .100622$

H $2.053001-2.2178825 .736969$

SCF Energy (B3LYP/6-31G**//MMFF) $=-3245.91083107$

$03 \_164$

MM̄FF Geometry

C $2.341353-0.908647-3.312092$

C $2.086565-1.899616-2.443126$

C $0.741258-2.258986-1.855220$

O $-0.211621-1.180221-1.992747$

C $0.171585-3.505342-2.554762$

C $-0.973653-4.150382-1.798172$

C $-2.247359-4.211749-2.231817$

C $-0.607153-4.789631-0.481771$

C $-2.824948-3.676086-3.510433$

C $-3.397399-2.252763-3.418164$

C $-4.471271-2.065750-2.333002$

C $-5.178879-0.718991-2.499303$

C $-6.182781-0.445211-1.368247$

O $-3.829959-2.109786-1.054623$

O $-6.5307410 .945319-1.456184$

C $-5.489786-0.682692-0.006936$

C $-7.483223-1.232357-1.569666$

O $-6.427149-0.612171 \quad 1.064046$

C $-4.724122-2.0203660 .062504$

C $-3.874344-2.1756121 .348745$

O $-5.665352-3.1012740 .053960$

C $-0.065904-0.129127-1.139243$

O $0.782000-0.004160-0.270300$

C $-1.1094180 .869496-1.449954$

C $-0.966357 \quad 2.119304-0.984841$

C $-1.911573 \quad 3.223132-1.156240$

C $-3.2734072 .889337-1.703613$

C $-2.798359-1.1059841 .562726$

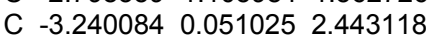

C $-2.484468 \quad 2.292462 \quad 3.227699$

$\begin{array}{lllll}\text { O } & -4.277730 & 0.041350 & 3.099761\end{array}$

C -3.168552 3.3372182 .402518

C -2.7283714 .6010502 .304209$

C $-3.420620 \quad 5.672374 \quad 1.509563$

C $-2.493952 \quad 6.363206 \quad 0.533084$

$\begin{array}{llll}\text { C } & -1.903872 & 7.528868 & 0.847464\end{array}$

C $-2.3131705 .741146-0.834444$

C $-1.5261914 .461237-0.782440$

N -2.3143351 .0862732 .439183$

H $1.528892-0.304483-3.705715$

H $2.919727-2.495700-2.075217$

H $0.888294-2.459081-0.787411$

H $0.956844-4.264167-2.674154$

H $-0.139099-3.228983-3.568173$

H $-2.977814-4.710052-1.594626$

H $-1.427907-5.383895-0.065700$ 
H $\quad 0.247949-5.461519-0.608942$ H $-0.347845-4.0283750 .259316$ H -2.085386 -3.711564 -4.317436 H -3.624001 -4.359072 -3.826991 H -2.571669 -1.554547 -3.241462 H $-3.824208-2.004291-4.397820$ H -5.191916 -2.888138 -2.411881 H $-5.677209-0.658721-3.475060$ H -4.429756 $0.083902-2.499710$ H -7.198553 $1.132975-0.775305$ H $-4.7809380 .141750 \quad 0.137282$ H -7.963798 $-0.938437-2.510511$ H $-8.208991-1.008456-0.780205$ H -7.330239 -2.313185 -1.599165 H $-6.801110 \quad 0.284878 \quad 1.070838$ H -4.528633 -2.271896 2.223609 H -3.348672 -3.1386921 .273210$ H $-5.231776-3.8901310 .419905$ H -1.959172 $0.552098-2.041251$ H $-0.0669112 .364412-0.421182$ H -3.743069 2.104120 -1.101324 H -3.197925 2.541406 -2.739541 H -3.967605 $3.731491-1.699244$ H -1.946616 -1.568521 2.076751 H -2.434110 -0.7219360 .606671$ H -1.489279 2.6090853 .555570 H -3.089793 2.0659484 .111238 H -4.0790463 .0490221 .882116$ H -1.8334274 .8933432 .849340$ H -3.825079 6.4053022 .220079 H -4.2887655 .2743510 .969426$ H -1.257881 8.0441870 .144505 H -2.0501087 .9913301 .818015$ H -1.770116 $6.429656-1.495406$ H -3.296938 $5.620628-1.295232$ H $-0.5137984 .592486-0.396732$ H -1.5003171 .0252891 .834444$ C $4.0557610 .921760-3.448286$ O $3.7995011 .220083-2.061678$ C $4.9256291 .925591-1.514021$ C $5.7316442 .408795-2.713656$ C $5.5271331 .265812-3.689279$ H $5.5174711 .180278-0.969464$ C $4.4722003 .012299-0.529448$ H $5.3243853 .331575-3.140364$ H $6.7831832 .582683-2.466505$ C $5.8483501 .624279-5.129580$ H $6.1667510 .428251-3.379661$ C 3.4177882 .5546060 .499162 O $3.8743274 .100017-1.246824$ H $5.3460863 .426058-0.011454$ H $2.4472962 .451283-0.000621$ O 3.2436663 .6143701 .449694 C 3.7321241 .2452421 .245364 C 2.7086980 .9536452 .361034 H 3.7322980 .4211480 .525086 O 5.0407031 .3017421 .810457 H 2.8576651 .6535753 .192357 H 1.7014791 .1136961 .959106 H $5.6719060 .770736-5.791759$ H $5.2322092 .458029-5.482699$ H $6.8986871 .917387-5.227990$ C $3.732394-0.545484-3.749729$ H $4.5934584 .637422-1.619686$ H 4.0982653 .7671511 .886625 H 5.0424591 .9905902 .496076 H $4.458607-1.192901-3.243334$ H $3.829221-0.733502-4.825029$ H $3.3994251 .578023-4.034364$ C $2.566922-2.7742862 .142280$ O $2.483516-1.3766361 .839030$ C $2.818937-0.4830062 .905987$ C $1.882957-0.7105844 .103572$ C $1.897116-2.1769824 .545124$ C $1.700119-3.1301713 .360633$ H $2.133825-3.2753591 .267021$ C 4.021181-3.238236 2.271115 H $3.855870-0.6573093 .212694$
O $0.554266-0.3300413 .750081$

H $2.203572-0.0645714 .929783$

H $1.100647-2.3368475 .282044$

O $3.140219-2.4463945 .186460$

H $1.901404-4.1654683 .659462$

O $0.333371-3.0759612 .942178$

C $4.736014-3.0976530 .948028$

H $4.580696-2.6894203 .033954$

H $4.071136-4.2965032 .553758$

H $-0.006401-0.4552374 .534397$

H $3.124311-3.3733235 .479905$

H $-0.216590-3.3424483 .698415$

O $5.539207-1.9963520 .966232$

O $4.577810-3.8616810 .004424$

C $6.254620-1.736461-0.244717$

H $6.894300-0.865130-0.081380$

H $6.886151-2.590511-0.507057$

H $5.553274-1.511101-1.052574$

SCF Energy (B3LYP/6-31G**//MMFF)= -3245.88763308

03_165

MM̄MF Geometry

C $-2.021108-3.5346741 .018946$

C $-0.706988-3.5131411 .287235$

C $0.357542-3.3415020 .233301$

O $1.321220-2.3678970 .701237$

C $1.099258-4.6731230 .019336$

C $2.123371-4.627745-1.098480$

C $3.449211-4.799487-0.930727$

C $1.571863-4.401852-2.483279$

C $4.196230-5.0726720 .344082$

C $4.724413-3.8214611 .062640$

C $5.723378-2.9948410 .236213$

C $6.406295-1.9404711 .110313$

C $7.345853-1.0377600 .295014$

O $5.009361-2.345239-0.819364$

O 7.6783320 .0991921 .107090

C $6.587864-0.503164-0.939701$

C $8.669676-1.739607-0.030430$

O $7.4686470 .200152-1.811754$

C $5.836333-1.598960-1.723072$

C $4.913779-1.034797-2.832132$

O $6.789761-2.452592-2.368102$

C $0.953407-1.0585820 .684515$

O $-0.105252-0.5955220 .292464$

C $2.068298-0.2779771 .263918$

C 1.8459240 .9713011 .697664

C 2.8373751 .8641382 .301508

C 4.2763441 .4201542 .284696

C $3.818498-0.073975-2.355403$

C $4.2033041 .393984-2.443286$

C $3.3926363 .657988-1.791234$

O $5.2063641 .794152-3.027644$

C $4.0467554 .073219-0.511146$

C 3.5459355 .0203390 .296173

C 4.2034105 .4768921 .566405

C 3.2811365 .3908672 .761966

C 2.5874106 .4638213 .178272

C 3.2327584 .0865353 .524800

C 2.4192303 .0359402 .824180

N $3.2687842 .212312-1.822176$

H -2.356217 -3.458850 -0.012536

H $-0.370155-3.6076992 .317135$

H $-0.079033-2.989749-0.709377$

H $0.378533-5.467539-0.216253$

H $1.572777-4.9640590 .963282$

H $4.085541-4.748814-1.813882$

H $1.183046-3.384146-2.584033$

H $2.331484-4.539847-3.260487$

H $\quad 0.762430-5.109587-2.690531$

H $3.577725-5.6485951 .040664$

H $5.040919-5.7316460 .104918$

H $3.873818-3.1929701 .351742$

H $5.210347-4.1530551 .988676$

H $6.468433-3.677228-0.189620$

H $6.952123-2.4130751 .936355$

H $5.640851-1.3093301 .581269$

H $8.091619-0.2272571 .924755$ 
H $5.861598 \quad 0.230344-0.570705$ H $9.186466-2.0287950 .892636$ H $9.354679-1.066610-0.557655$ H $8.540325-2.641841-0.631668$ H $7.9009830 .895930-1.287602$ H $5.516469-0.594124-3.635452$ H $4.401021-1.892261-3.291910$ H $\quad 6.347577-2.908401-3.103437$ H $3.036588-0.7595611 .333893$ H $\quad 0.837351 \quad 1.3750391 .622183$ H 4.4192340 .5561512 .942377 H 4.9765252 .1939172 .606073 H $4.5802031 .144248 \quad 1.269310$ H $2.942742-0.196943-3.005523$ H $3.504105-0.311770-1.335104$ H $2.3851344 .072358-1.895872$ H $3.9978383 .992336-2.639815$ H $4.9848903 .590074-0.248219$ H 2.6234775 .5225680 .013457 H 4.5254026 .5163681 .420062 H $5.1210284 .910961 \quad 1.769697$ H 1.9455336 .4186564 .051873 H 2.6415577 .4102142 .650415 H 2.7749974 .2448554 .510635 H 4.2531153 .7602863 .744790 H 1.3549353 .2712752 .782489 H $2.4915291 .791267-1.321326$ C $-4.194860-2.6296771 .885180$ O $-5.054460-3.065910 \quad 0.806148$ C $-6.396388-3.1502841 .303460$ C $-6.446606-2.2189892 .503316$ C -5.089747 -2.491994 3.122646 H $-6.526588-4.1796891 .667758$ C -7.438746 -2.908564 0.210559 H -6.515903 -1.168747 2.200211 H -7.278521 -2.441084 3.178007 C $-4.632087-1.4095934 .085888$ H $-5.136100-3.4482653 .661460$ C $-7.563884-1.476422-0.345798$ O $-8.709766-3.274891 \quad 0.766144$ H $-7.250267-3.601976-0.618335$ H -7.976506 -0.8184590 .427534$ O $-8.560489-1.516768-1.380263$ C $-6.279752-0.850031-0.920997$ C $-6.5519050 .536417-1.534366$ H $-5.530726-0.743031-0.131117$ O $-5.736290-1.694730-1.932381$ H $-7.2960690 .443035-2.334659$ H $-6.9920651 .174146-0.759692$ H -3.640932 -1.641114 4.488578 H $-4.576146-0.4325273 .593837$ H -5.328078 -1.323261 4.926691 C $-3.076912-3.6585872 .081741$ H $-9.377441-3.148650 \quad 0.070143$ H -8.196934 -2.039059 -2.116049 H - $-5.396652-2.494392-1.496080$ H -3.492043 -4.672527 2.028999 H -2.624308 -3.5314983 .072241$ H $-3.784521-1.6550241 .591990$ C $-4.3349992 .099750-0.113146$ O $-4.190251 \quad 1.220187-1.231706$ C $-5.2969151 .207398-2.136945$ C $-5.5518592 .621652-2.679899$ C $-5.7387693 .635043-1.552489$ C $-4.5787533 .546887-0.562153$ H -5.1822591 .7585940 .494990$ C $-3.090661 \quad 1.9470880 .772772$ H $-4.9862850 .586667-2.986809$ O $-6.6982352 .646508-3.523606$ H -4.695479 $2.936844-3.290171$ H $-6.7011073 .503878-1.044633$ O $-5.7548754 .954069-2.104067$ H -3.682599 $3.964215-1.036786$ $\begin{array}{lllll}O & -4.866501 & 4.347248 & 0.581571\end{array}$ C -1.8383602 .4747940 .107612$ H -3.2408922 .4604241 .729741$ H -2.906206 $0.890971 \quad 1.003733$ H $-6.5476232 .005714-4.239466$
H $-6.4699224 .982299-2.763045$

H -5.0452795 .2499340 .266385$

O -1.3829303 .5720380 .777176$

O $-1.3424601 .990394-0.901400$

C -0.2395914 .2118710 .202965$

H 0.5764203 .4965610 .067244

H $-0.5103724 .670863-0.752347$

H 0.0913684 .9958510 .889047

SCF Energy $(B 3 L Y P / 6-31 G * * / / M M F F)=-3245.90768699$

03166

MM̄FF Geometry

C $0.5714075 .260309 \quad 1.574959$

C $1.1469494 .743470 \quad 0.478943$

C 2.4830434 .0459750 .481619

O 2.2403292 .6456820 .211048

C $3.3764774 .603217-0.640642$

C $4.7439733 .950251-0.708276$

C $5.1908803 .218184-1.747947$

C 5.6389734 .1807140 .482318

C $4.4780362 .904272-3.033608$

C $3.6540861 .607623-3.015364$

C $4.4895250 .330881-2.825953$

C $3.625880-0.913956-3.042583$

C $4.417581-2.207433-2.795684$

O $5.0076560 .315799-1.494494$

O $3.476553-3.288248-2.692249$

C $5.138393-2.110995-1.431410$

C $5.328158-2.543174-3.983013$

O $6.031998-3.205037-1.247714$

C $5.891397-0.779479-1.213636$

C $6.402646-0.5902850 .233172$

O $7.071853-0.707544-2.023742$

C $1.9178761 .856279 \quad 1.271026$

O 1.8844832 .1775832 .448312

C 1.5487210 .5265010 .740575

C $0.794406-0.2859631 .495201$

C $0.299792-1.6092901 .114646$

C $0.827935-2.208300-0.161827$

C $5.326912-0.6084891 .324231$

C $5.137809-1.9652521 .983584$

C $3.693200-3.1282133 .649021$

O $5.883309-2.9212091 .787726$

C $2.653713-3.9292202 .930364$

C $1.503355-4.3193953 .500335$

C $0.468985-5.1633942 .811823$

C $-0.906945-4.5361222 .838271$

C -1.815354 -4.898494 3.759690

C -1.255671 -3.5539831 .743407$

C $-0.588127-2.2197011 .926976$

N $4.053279-1.9706912 .851069$

H 1.0918645 .2116722 .527907

H $0.6059284 .770144-0.464514$

H $2.9851134 .161315 \quad 1.449913$

H $3.5174145 .683245-0.500995$

H $2.8518384 .488206-1.595214$

H $6.1939112 .797592-1.682690$

H $5.247698 \quad 3.667115 \quad 1.365717$

H 6.6569823 .8129920 .313976

H 5.7117095 .2510650 .701597

H $3.8263743 .735556-3.323786$

H $5.2290212 .842467-3.831686$

H $2.8981051 .676509-2.224679$

H $3.1163851 .543639-3.969634$

H $5.3193050 .352034-3.541964$

H $3.192104-0.917543-4.050389$

H $2.773294-0.890952-2.350607$

H $2.952995-3.309931-3.511620$

H $4.363386-2.206617-0.661648$

H $4.731580-2.704832-4.889066$

H $5.872496-3.478532-3.813599$

H $6.050637-1.754985-4.204928$

H $5.515779-4.022237-1.355660$

H $7.204324-1.3078970 .446558$

H $\quad \begin{array}{llll}6.883349 & 0.398085 & 0.275947\end{array}$

H $7.208463-1.568235-2.450849$

H $1.8704150 .272288-0.262027$

H $\quad 0.4821990 .0618182 .479369$ 
H $\quad 0.482655-1.633099-1.027752$

H $\quad 0.521703-3.244220-0.318935$

H $1.923308-2.214201-0.157084$

H 5.6341630 .0776882 .123908

H $4.368100-0.2521080 .937690$

H $3.335820-2.7573844 .614897$

H $4.581363-3.7451603 .819057$

H $2.869019-4.2253391 .906346$

H $1.304637-4.0450724 .534050$

H $0.443636-6.1374313 .317999$

H $\quad 0.752059-5.378251 \quad 1.773876$

H -2.814011 -4.474401 3.766992

H -1.578491 -5.6246594 .530417$

H -2.338633 -3.372691 1.731200

H -1.034674 -4.0125760 .776419$

H $-0.907956-1.6971872 .829853$

H $3.461857-1.1472682 .914670$

C $-1.923770 \quad 4.8707541 .888559$

$\begin{array}{lllll}\text { O } & -1.993286 & 3.881647 & 0.840049\end{array}$

C -2.0213332 .5764971 .440928$

C -2.5163192 .8063922 .861817$

C -1.805682 4.1027203 .207449

H $-0.9853822 .222637 \quad 1.473373$

C $-2.855257 \quad 1.5859010 .618279$

H -3.603206 2.9476472 .890404

H -2.2573801 .9824483 .533473$

C -2.4054894 .8338594 .395646$

H $-0.758776 \quad 3.8672513 .433927$

C $-2.3137271 .341308-0.806801$

O -4.2039822 .0450190 .541405$

H $-2.871740 \quad 0.637471 \quad 1.165102$

H $-1.2863150 .961809-0.759054$

O $-2.2591742 .578726-1.527432$

C $-3.1709450 .387287-1.661570$

C -3.344429-1.012809-1.052405

H $-4.1458950 .844927-1.863600$

O $-2.5231150 .227736-2.930115$

H $-2.357347-1.477620-0.941183$

H $-3.776107-0.928591-0.052128$

H -1.870484 5.7699724 .584930

H -3.4603715 .0756134 .228247$

H -2.341490 4.2179995 .298535

C -0.8029825 .8713381 .579063$

H -4.192867 2.8992120 .076243

H -1.622136 3.156119-1.073590

H $-2.4164351 .115438-3.313194$

H $-0.818816 \quad 6.6883672 .309725$

H -0.9942936 .3238390 .597326$

H -2.879491 5.4091041 .852733

C $-6.375956-1.203158-1.096788$

O $-5.498977-1.347784-2.221344$

C $-4.229341-1.936004-1.923621$

C $-4.441018-3.340928-1.346821$

C $-5.371335-3.319955-0.136371$

C $-6.665435-2.570838-0.461395$

H $-5.919166-0.536383-0.355946$

C -7.655594 -0.533551-1.610236

H $-3.715068-2.057474-2.885077$

O $-3.201499-3.933293-0.979311$

H $-4.889276-3.984851-2.114670$

H $-4.877958-2.8872660 .741953$

O $-5.707125-4.6641550 .217566$

H -7.274046 -3.188897 -1.134106

O $-7.394013-2.4059540 .753527$

C $-7.3747680 .868047-2.099291$

H $-8.075917-1.095888-2.452896$

H -8.433673 $-0.472723-0.842175$

H $-2.633432-3.938321-1.768634$

H $-4.873285-5.1331190 .392878$

H $-8.297686-2.1325520 .524109$

O $-7.2105821 .709160-1.038642$

O $-7.300401 \quad 1.174087-3.282389$

C $-6.9142963 .068422-1.373878$

H $-6.8032723 .631449-0.443415$

H $-5.9744653 .124361-1.931819$

$\mathrm{H}-7.7346033 .503587-1.952293$

SCF Energy (B3LYP/6-31G**//MMFF) $=-3245.91465502$
03167

MM̄FF Geometry

C $3.117926-0.8112653 .454042$

C $2.338335-1.4517932 .569058$

C $0.859213-1.6727422 .757022$

O $0.146604-1.0333271 .674326$

C $0.560707-3.1830112 .695878$

C $-0.888034-3.5382042 .967072$

C $-1.711077-4.1310482 .079699$

C -1.391703 -3.219159 4.351661

C $-1.407644-4.5613030 .671608$

C $-1.759131-3.521884-0.404126$

C $-3.258094-3.196019-0.500516$

C $-3.545561-2.326013-1.726187$

C $-5.023263-1.906757-1.800016$

O $-3.645517-2.4912030 .680661$

O $-5.132771-0.847726-2.763930$

C $-5.454740-1.321910-0.436635$

C $-5.913024-3.047895-2.307709$

O $-6.858975-1.080780-0.406788$

C $-5.046342-2.1981540 .764465$

C $-5.287608-1.5144872 .132311$

O $-5.824645-3.4013350 .754998$

C $-0.1239120 .293971 \quad 1.805282$

O $0.201205 \quad 1.0249382 .727287$

C $-0.8853940 .715496 \quad 0.608879$

C -1.0174192 .0225810 .337856$

C $-1.6981212 .596767-0.824651$

C $-2.5456891 .674994-1.659688$

C $-4.540314-0.1935662 .348665$

C -5.3700951 .0411522 .038632$

C -5.1740463 .5196891 .882122$

O -6.5750241 .0046351 .805068$

C -5.0428763 .8942990 .439943$

C -4.5015165 .0503040 .027387$

C $-4.4007765 .460460-1.413386$

C $-2.9894865 .822028-1.817203$

C $-2.5911527 .104520-1.865601$

C $-2.0696714 .702455-2.247819$

C -1.533365 $3.910636-1.085469$

N -4.6079242 .2006492 .092216$

H $2.677770-0.3996534 .358460$

H $2.785634-1.8564441 .663922$

H $0.521601-1.2629633 .716746$

H $1.180441-3.7134173 .431610$

H $0.869410-3.5588401 .714483$

H -2.730557 -4.347299 2.397700

H $-2.386071-3.6387344 .538985$

H $-0.716038-3.6335845 .107143$

H -1.461071 -2.137008 4.497984

H $-0.352764-4.8364120 .568809$

H -1.962370 -5.4873300 .472915$

H -1.192969-2.603900 -0.207556

H -1.417476 -3.913430 -1.370238

H -3.812357 -4.139781-0.563592

H $-3.246826-2.840295-2.648206$

H -2.929059-1.418301-1.678790

H -4.796599-1.179945 -3.613971

H $-4.969109-0.343906-0.343533$

H $-5.594570-3.365379-3.307871$

H $-6.952923-2.720306-2.415573$

H -5.892769 -3.927522 -1.661109

H -7.070764 -0.506627 -1.162598

H $-6.363412-1.4079472 .316232$

H -4.929648 -2.208191 2.906894

H $-5.822180-3.7775401 .650805$

H -1.289183 -0.059458 -0.032233

H $-0.5500572 .743158 \quad 1.007869$

H -1.918748 $0.961875-2.205262$

H $-3.1648072 .196086-2.392914$

H $-3.2412001 .117560-1.023984$

H -4.271005 -0.118961 3.410095

H -3.609959 -0.1648001 .772867$

H -4.6395864 .2113892 .540948$

H -6.2312543 .5125502 .166458$

H $-5.434073 \quad 3.196008-0.296270$

H -4.136100 5.7588720 .767609

H $-5.0637976 .323031-1.560893$ 
H $-4.7820354 .678131-2.081392$ H -1.588856 7.374109-2.181749 H -3.257321 7.913505 -1.584555 H -1.202193 $5.114662-2.780989$ H $-2.5824874 .083760-2.989425$ H $-0.8990274 .494872-0.417215$ H -3.6045212 .1317422 .236058$ C 5.0499580 .6972792 .747901 O 4.5042810 .9045881 .431957 C 4.1480122 .2959061 .298446 C 4.7828463 .0069062 .488093 C 4.6712711 .9337553 .559030 H 3.0598202 .3534011 .419382

C $4.5389842 .804798-0.095419$ H 5.8355333 .2505662 .308754 H 4.2586903 .9313942 .748164 C 5.5598352 .1774424 .765302 H $3.6258501 .880163 \quad 3.887770$ C $3.9478561 .945663-1.236585$ O $5.9631332 .755328-0.238772$ H $4.2425403 .853078-0.218321$ H $4.3873430 .946492-1.184433$ O $4.3668142 .493116-2.490933$ C $2.4136931 .847666-1.218190$ C $1.7940930 .973547-2.323524$ H $2.083998 \quad 1.439657-0.258611$ O $1.855907 \quad 3.159891-1.312947$ H $\quad 0.7055221 .089560-2.256255$ H $2.0791261 .362559-3.309056$ H $5.456301 \quad 1.3675135 .494303$ H 6.6153382 .2424644 .480822 H 5.2871463 .1151895 .260355 C $4.604035-0.6606323 .289126$ H $6.3363373 .502527 \quad 0.257830$ H $4.0896423 .424472-2.517956$ H $2.0161503 .488174-2.213905$ H $5.081262-0.8512834 .257404$ H $4.965606-1.4421902 .608567$ H 6.1403260 .6644662 .621159 C $3.938189-2.041441-2.856498$ O $3.397693-0.714286-2.894540$

C $2.169495-0.513348-2.187595$ C $1.072488-1.432126-2.747014$ C $1.513222-2.900091-2.745829$ C $2.912169-3.079491-3.342189$ H $4.754247-2.029800-3.590717$ C $4.549541-2.366478-1.484285$ H $2.318982-0.737417-1.126551$

O $0.757976-1.027026-4.078562$ H $\quad 0.166018-1.311913-2.141529$ H $\quad 0.799138-3.482965-3.339674$

O $1.475690-3.439665-1.428008$ H $3.290325-4.088847-3.143106$ O $2.821583-2.943027-4.765394$

C $5.825277-1.587723-1.229531$

H $3.862701-2.133205-0.668282$

H $4.811646-3.429605-1.438895$

H $\quad 0.036030-1.595906-4.395637$

H $1.850551-2.795172-0.807831$

H $2.227880-3.641441-5.089887$

O $6.250156-1.8393040 .044316$

O $6.385907-0.860907-2.038169$

C $7.453331-1.1687360 .432587$

H $8.292628-1.520247-0.174749$

H $7.334276-0.0850170 .337243$

H $7.652732-1.4080401 .480470$

SCF Energy $\left(B 3 L Y P / 6-31 G^{* *} / / M M F F\right)=-3245.88257473$

03_168

MM̄FF Geometry

C $-1.574680-3.947599-1.896180$

C $-0.604287-3.830785-0.976731$

C $0.821163-3.472826-1.320164$

O $1.230326-2.351537-0.504123$

C $1.742711-4.661769-0.989438$

C $3.182094-4.462205-1.422822$

C $4.235436-4.407418-0.584397$

C $3.409437-4.356969-2.909664$
C $4.239801-4.5237780 .913885$

C $4.155739-3.1845931 .663269$

C $5.322743-2.2253971 .375392$

C 5.298020-1.0370972.339476

C $6.393415-0.0080192 .015415$

O $5.194205-1.7493870 .033296$

O 6.0924651 .1975612 .735608

C 6.3341860 .3392350 .511522

C $7.771214-0.4711332 .503790$

O $7.436868 \quad 1.159461 \quad 0.134385$

C $6.265210-0.901409-0.402206$

C $5.998910-0.554109-1.887471$

O $7.519679-1.592349-0.353237$

C $0.815773-1.113980-0.887549$

O $0.128370-0.833432-1.856380$

C $1.338204-0.1443380 .099968$

C 0.8320891 .0967910 .138172

C 1.2367002 .1659081 .053776

C 2.4530721 .9291561 .908186

C $4.6939610 .201203-2.163312$

C $4.8603931 .709046-2.255517$

C $3.5203003 .796695-2.490973$

O $5.9534632 .267277-2.288201$

C $3.3784224 .433744-1.144958$

C $2.4010565 .301605-0.842529$

C 2.2697005 .9862920 .487528

C 0.9108205 .7781961 .117039

C -0.0495466 .7124991 .015525$

C 0.6967934 .5221561 .931660

C 0.5107693 .3026481 .073163

N $3.6356092 .358604-2.337648$

H -1.314488 -3.769817 -2.938484

H $-0.817878-4.0021220 .074182$

H $0.914175-3.204757-2.379418$

H $1.368399-5.570233-1.480423$

H $1.689129-4.8556840 .087329$

H $5.224983-4.268775-1.018911$

H $4.473212-4.381032-3.169868$

H $2.929026-5.193189-3.428283$

H $2.999366-3.420156-3.298610$

H $3.427826-5.1742951 .255601$

H $5.161370-5.0418121 .208974$

H $3.206534-2.6977321 .410583$

H $4.129019-3.4064072 .737379$

H $6.261745-2.7817561 .479476$

H $5.387989-1.3768833 .378745$

H $4.322456-0.5371922 .273040$

H 6.0516000 .9788363 .682413

H 5.4309020 .9417440 .360805

H $7.759838-0.6272373 .589263$

H 8.5337050 .2939392 .320453

H $8.099148-1.4040152 .040716$

H 7.4298551 .9393240 .715351

H $6.865980-0.036403-2.315164$

H $5.932345-1.506105-2.434126$

H $7.597940-2.146289-1.147612$

H $2.115314-0.4819530 .775792$

H $0.0318201 .353449-0.555158$

H 3.3070701 .6459951 .283885

H 2.2651691 .1294982 .632700

H 2.7706562 .8077632 .472792

H $4.307644-0.121215-3.138889$

H $3.932625-0.038836-1.415259$

H $2.6540493 .986056-3.132768$

H $4.4152794 .183417-2.989016$

H $4.1281324 .192303-0.395093$

H $1.6683145 .561528-1.603485$

H 2.4473637 .0580880 .329178

H 3.0494225 .6587861 .186543

H -1.017792 6.5826291 .486916

H $\quad 0.1100457 .6266490 .453118$

H -0.2061494 .6245092 .548137$

H 1.5163614 .4218562 .647981

H $-0.349916 \quad 3.3706730 .408138$

H $2.7786521 .820180-2.249460$

C $-3.470207-4.543939-0.227544$

O $-3.508035-3.2815530 .471279$

C $-4.742977-3.1955391 .204928$ 
C $-5.312076-4.6073851 .233623$

C $-4.886296-5.117297-0.127967$

H $-5.416841-2.5652980 .614499$

C $-4.523217-2.5666362 .582720$

H $-4.857951-5.2129382 .026548$

H $-6.396763-4.6163161 .377312$

C $-4.953690-6.629452-0.256461$

H $-5.531368-4.662797-0.892175$

C $-3.890091-1.1599282 .553326$

O $-3.657333-3.419858 \quad 3.341500$

H $-5.477374-2.5398613 .122940$

H -2.833320 -1.233492 2.270246

O $-3.898709-0.6622543 .898382$

C $-4.596740-0.1478801 .630615$

C -4.0811841 .2903391 .831635$

H $-4.451629-0.4582150 .590060$

O $-5.997339-0.1809901 .908073$

H -4.4144271 .6629402 .808010$

H -2.9842091 .2697391 .823324$

H -4.623501 -6.953007 -1.248538

H -4.320179 -7.124305 0.487345

H -5.980131-6.982019-0.111058

C -3.017896-4.319412 -1.673401

H -3.530535 -3.003835 4.211442

H -4.827422 -0.5286954 .155274$

H $-6.457237 \quad 0.242986 \quad 1.164475$

H -3.625053 -3.519287 -2.115742

H $-3.205777-5.228603-2.256826$

H -2.767195 -5.1935870 .308516$

C $-4.3108282 .648887-1.637896$

O $-3.9395451 .886884-0.482479$

C -4.5800172 .2601890 .742349$

C $-4.268702 \quad 3.7242101 .090005$

C $-4.6282794 .658885-0.069088$

C $-4.0756834 .151268-1.405780$

H $-3.6176002 .319475-2.422949$

C $-5.7337462 .319038-2.100978$

H -5.6627692 .1455110 .634867$

O -2.8845263 .8562581 .399095$

H $-4.8352454 .001237 \quad 1.987242$

H -4.2253045 .6581700 .136414$

O $-6.0470534 .767102-0.138759$

H $-4.5098424 .720539-2.235367$

O $-2.6666254 .397038-1.442397$

C $-5.8211410 .881487-2.556410$

H $-6.4874412 .489586-1.327003$

H $-6.0221462 .944988-2.953811$

H $-2.728014 \quad 4.7806801 .656614$

H $-6.2589645 .385215-0.858892$

H $-2.2523263 .841514-0.760402$

O $-6.4288870 .123345-1.599998$

O $-5.3791950 .478313-3.624722$

C $-6.555688-1.268313-1.907243$

H -5.565987 -1.730781 -1.964097

H $-7.120773-1.743942-1.101301$

$\mathrm{H}-7.102151-1.405787-2.845194$

SCF Energy (B3LYP/6-31G**//MMFF) $=-3245.90926344$

03169

MM̄FF Geometry

C $2.2683540 .157448-3.503017$

C $3.426743-0.514585-3.416592$

C $4.618508-0.171375-2.555196$

O $4.440127 \quad 1.090873-1.870565$

C $4.824838-1.228365-1.453398$

C $5.311602-2.572417-1.949744$

C $4.540249-3.670199-2.075894$

C $6.782382-2.642005-2.269762$

C $3.062037-3.797706-1.827803$

C $2.741961-4.695836-0.625598$

C $3.114427-4.0629220 .723925$

C $2.841700-5.0405911 .868909$

C $3.108422-4.4025143 .240074$

O $2.320758-2.8831880 .889787$

O $2.535796-5.2553364 .243084$

C $2.363148-3.0541453 .314001$

C $4.607573-4.3145583 .549124$

O $2.694592-2.3543564 .511701$
C $2.600793-2.1435972 .085047$

C $1.630380-0.9399802 .138084$

O $3.946875-1.6666462 .110016$

C $4.8801372 .215450-2.489788$

O $5.3726652 .286818-3.607827$

C $4.6832853 .418431-1.647633$

C $4.2877633 .385025-0.365050$

C 4.0806534 .5479700 .504529

C 4.296852 5.912204-0.099109

C $1.627066-0.0600290 .879127$

C $0.605988 \quad 1.0594670 .987729$

C $-0.2611753 .033902-0.254152$

O $-0.123398 \quad 1.207242 \quad 1.965034$

C $0.436003 \quad 4.226247 \quad 0.317933$

C 0.2326064 .6739201 .565853

C 0.9418795 .8524552 .169418

C 1.9393745 .4351363 .228871

C 1.5451985 .1894894 .489855

C 3.4016105 .3727032 .841754

C 3.6842974 .3432451 .779433

N $0.6032331 .874089-0.136318$

H $2.1214561 .074951-2.941839$

H $3.531291-1.425215-4.004037$

H $5.513869-0.118265-3.186100$

H $3.903943-1.329262-0.868307$

H $5.551645-0.846351-0.722606$

H $5.011944-4.589358-2.421815$

H $7.094344-3.645336-2.578745$

H $7.031900-1.955194-3.084196$

H $7.375265-2.369979-1.390264$

H $2.571493-2.826814-1.715117$

H $2.618255-4.248404-2.724706$

H $1.662129-4.893574-0.641312$

H $3.249862-5.661717-0.739433$

H $4.174530-3.7874530 .709804$

H $3.435296-5.9555881 .749444$

H $1.790313-5.3590271 .834203$

H $2.946741-6.1329594 .160303$

H $1.288814-3.2786163 .374495$

H $5.058297-5.3143513 .545549$

H $4.780871-3.9121154 .553289$

H $5.158744-3.7026582 .832010$

H $2.519280-2.9551205 .256214$

H $0.608129-1.3146782 .282540$

H $1.871059-0.3092083 .003489$

H $3.999722-0.8319741 .619650$

H $4.9039644 .354516-2.150978$

H 4.0948852 .4214780 .102682

H $3.6570216 .049480-0.978305$

H $5.3425846 .036271-0.401421$

H 4.0624406 .7358490 .577348

H 2.6099890 .3954320 .723090

H $1.377199-0.658634-0.003933$

H -1.2103982 .8352550 .253884$

H -0.464392 $3.195002-1.315942$

H $1.1412534 .740162-0.329366$

H -0.4780294 .1535752 .204759$

H 0.1875846 .5172422 .610169

H $1.4411576 .454077 \quad 1.400028$

H 2.2550584 .9131885 .262360

H 0.5006965 .2573784 .775949

H $4.0165905 .113563 \quad 3.713803$

H 3.7277926 .3760652 .555472

H $3.543463 \quad 3.3168042 .121288$

H $1.3090741 .732133-0.852331$

C $-0.127172-0.538825-3.434608$

O $\quad-0.8346950 .712759-3.296944$

C $-2.2483950 .469731-3.481018$

C $-2.419995-1.037174-3.375650$

C $-1.137644-1.526259-4.015555$

H $-2.4811620 .809379-4.498458$

C $-3.0335551 .293681-2.454022$

H -2.469584 -1.352534 -2.325710

H $-3.317443-1.398236-3.886588$

C $-0.822363-2.980172-3.705849$

H -1.211095 -1.395207 -5.103431

C $-4.5202060 .916435-2.352878$

O $-2.9371182 .673102-2.823892$ 
H $-2.5527951 .199364-1.474514$ H -4.612061 -0.121508 -2.020586 O $\quad \begin{array}{llll}-5.090295 & 0.963417 & -3.667085\end{array}$ C $-5.3594591 .830379-1.440759$ C $-4.8006072 .066865-0.026555$ H $-5.4857142 .804975-1.927334$ O $-6.679876 \quad 1.285997-1.344123$ H $-3.8839592 .663293-0.101939$ H -5.5399432 .6718000 .514777$ H $\quad 0.122475-3.282844-4.168254$ H $-0.738300-3.151980-2.627277$ H -1.611812 -3.634624 -4.089605 C $1.103862-0.332003-4.320831$ H -1.996151 $2.874019-2.963123$ H $-6.0290120 .722367-3.584320$ H $-6.6137230 .419696-0.907423$ H $0.8939950 .408949-5.101240$ H $1.371428-1.270926-4.819097$ H $0.163633-0.851261-2.422641$ C $-5.680879-1.0715131 .834366$ O $\begin{array}{llll}\text { C } & -4.516613 & 0.793451 & 0.797037\end{array}$ C $-3.774786 \quad 1.1308702 .100781$ C -3.596713 -0.111168 2.975950 C $-4.917193-0.8665753 .154273$ H $-6.722351-1.3071112 .090167$ C $-5.130691-2.2271630 .989380$ H $-3.892714 \quad 0.1206840 .202345$ O -4.4761452 .1352242 .831135$ H -2.7940301 .5477451 .849074$ H -3.2093810 .1954453 .955201$ O $-2.629456-0.9624972 .368149$ H $-4.745226-1.8384153 .631356$ O $-5.767505-0.1194954 .029177$ C $-6.186594-2.7332870 .035327$ H $-4.238307-1.9686150 .412712$ H -4.854575 -3.0767221 .625760$ H -5.3797521 .8121482 .988845$ H $-2.500070-1.7258632 .956116$ H -5.304118 -0.0261504 .879068$ O $-6.164986-2.005283-1.115344$ O $-6.952390-3.652723 \quad 0.294370$ C -7.136734 -2.372100 -2.099095 H -6.988035 -3.410238 -2.410883 H -8.146922 -2.226326 -1.704982 H -7.001959 -1.722060 -2.967765

SCF Energy (B3LYP/6-31G**//MMFF) $=-3245.91219640$

0317

MMFF Geometry

C -1.165876 $3.867714 \quad 0.511074$

C $-0.166477 \quad 4.311223 \quad 1.289881$

C $1.183138 \quad 3.653157 \quad 1.489390$

O $1.376237 \quad 2.5455450 .582001$

C 2.3102144 .6689921 .220776

C 3.6886764 .1585701 .598566

C 4.7002263 .9594910 .731030

C $3.9092113 .896603 \quad 3.067274$

C $4.7123464 .193018-0.752655$

C $4.3624692 .961265-1.601168$

C $5.3257611 .774949-1.425720$

C $5.0697320 .713519-2.498785$

C $5.947663-0.532143-2.301269$

O $5.1148441 .203691-0.130753$

O $5.417716-1.580369-3.127737$

C $5.826598-1.008350-0.837434$

C $7.387111-0.294696-2.773418$

O $6.750113-2.059556-0.567256$

C $6.003334 \quad 0.124706 \quad 0.193767$

C $5.687235-0.3178081 .644702$

O 7.3676830 .5633890 .180699

C 0.9227351 .3212730 .961838

$\begin{array}{lllll}\text { O } & 0.387996 & 1.022373 & 2.016996\end{array}$

C $1.1473090 .404297-0.175744$

C $0.367793-0.677363-0.310774$

C $0.451049-1.666797-1.385776$

C $1.660700-1.624186-2.280682$

C $4.262829-0.8300451 .884585$
C $4.118132-2.3404151 .792126$

C $2.377274-4.1265951 .795138$

O $5.074892-3.1072981 .727007$

C $2.078123-4.5239560 .383712$

C $0.943991-5.1398530 .016137$

C $0.645705-5.578820-1.389295$

C $-0.676075-5.047587-1.898463$

C $-1.784342-5.806653-1.865406$

C $-0.692318-3.669162-2.521070$

C $-0.540994-2.572832-1.502711$

N $2.786589-2.734641 \quad 1.830288$

H -1.042346 $2.951520-0.057819$

H $-0.3374225 .211178 \quad 1.878392$

H 1.2357033 .3023002 .526911

H 2.1290545 .5914831 .788433

H 2.2783174 .9521870 .163200

H 5.6466043 .5928381 .127745

H 4.9604083 .6946203 .299844

H 3.6073384 .7671943 .658670

H 3.3295183 .0293673 .397293

H $4.0380755 .012970-1.021914$

H $5.7136004 .546737-1.030322$

H $3.3402902 .646373-1.361983$

H $4.3655933 .274221-2.652680$

H $6.3535052 .149603-1.498185$

H $5.219076 \quad 1.130733-3.502407$

H $4.0165890 .405127-2.458702$

H $5.416611-1.265275-4.047775$

H $4.823366-1.436303-0.725740$

H $7.402143-0.033926-3.838558$

H $7.989081-1.205384-2.680583$

H $7.8895080 .508928-2.231173$

H $6.589028-2.761935-1.220453$

H $6.438842-1.0369971 .991983$

H 5.8146010 .5666052 .285742

H 7.5555841 .0112741 .022185

H $1.9253640 .660556-0.884617$

H $-0.419280-0.8439960 .422573$

H $2.578370-1.623181-1.682716$

H $1.646025-0.724652-2.905251$

H $1.740085-2.483542-2.949600$

H $3.965257-0.5593292 .905913$

H $3.556517-0.3524851 .201013$

H $1.503311-4.2245002 .446701$

H $3.182168-4.7535312 .191956$

H $2.838860-4.317631-0.365531$

H $\quad 0.197019-5.3710350 .772232$

H $0.634270-6.676714-1.399427$

H $1.446084-5.285859-2.080089$

H -2.730962 -5.443302 -2.251837

H $-1.771451-6.806059-1.443213$

H -1.646989 -3.499009 -3.036951

H $\quad 0.063932-3.627774-3.309535$

H -1.370592 -2.516312 -0.799353

H $2.057910-2.0269831 .840094$

C $-3.1396494 .356991-0.968298$

O $-3.4809292 .965208-1.154020$

C $-4.8301752 .874496-1.642461$

C $-5.1859404 .271773-2.134187$

C $-4.4471715 .134195-1.130749$

H $-5.4631762 .633198-0.780001$

C $-4.9532351 .775119-2.701139$

H $-4.8049134 .457023-3.145096$

H $-6.2657574 .447852-2.140988$

C $-4.2574466 .570775-1.586049$

H $-5.0072595 .129906-0.185887$

C $-4.4923350 .377084-2.238960$

O $-4.1514312 .141906-3.831911$

H $-5.9885011 .733618-3.060657$

H $-3.4020210 .365350-2.122300$

O $-4.777450-0.535958-3.308675$

C $-5.149978-0.138901-0.943067$

C $-4.821554-1.622184-0.681268$

H $-4.8143530 .478442-0.104308$

O $-6.5644800 .012983-1.058402$

H $-5.314584-2.234242-1.446158$

H -3.742779 -1.765434 -0.796226

H $-3.706878 \quad 7.148321-0.836885$ 
H -3.700769 $6.623191-2.527879$ H $-5.2266357 .055903-1.741384$ C $-2.505474 \quad 4.5443000 .412463$ H $-4.2443081 .435501-4.494113$ H -5.744602 $-0.590568-3.396879$ H $-6.947714-0.102577-0.172867$ H $-3.1605984 .098101 \quad 1.169822$ H -2.404466 5.6121990 .636932 H -2.427476 $4.620072-1.760828$ C $-3.295519-1.5531011 .963006$ O $-4.704996-1.3713461 .780647$ C $-5.279750-2.125098 \quad 0.708070$ C $-5.044174-3.6248350 .942371$ C -3.562784 -3.937459 1.130811 C $-2.957417-3.0315632 .200962$ H $-2.761003-1.1793061 .082592$ C $-2.872912-0.7125163 .178150$ H $-6.362865-1.9644530 .766096$ O $\quad-5.573582-4.374510-0.145777$ H $-5.589945-3.9332441 .843583$ H $-3.014602-3.8516980 .188071$ O $-3.410146-5.2911821 .562627$ H $-3.321658-3.3456043 .187811$ O $-1.539415-3.1906672 .201501$ C $-2.968156 \quad 0.7758562 .923500$ H $-3.528785-0.9486654 .025188$ H -1.834398 -0.9237183 .455249$ H $-5.515761-5.316050 \quad 0.088207$ H $-3.678977 \quad-5.8658140 .826209$ H -1.353504 -4.135631 2.336538 O $-2.894591 \quad 1.430812 \quad 4.120214$ O C -2.9650272 .8580394 .053947$ H -2.0580073 .2530353 .589254$ H -3.8553813 .1761863 .503197$ H -3.031349 3.2426255 .075221

SCF Energy (B3LYP/6-31G**//MMFF) $=-3245.91138223$

03170

MM̄FF Geometry

C $-3.886789-1.8980231 .700381$

C $-2.971276-2.876821 \quad 1.678880$

C $-1.763676-2.7915880 .781727$

O $-0.645336-2.4477091 .628694$

C $-1.483151-4.1297690 .074605$

C $-0.658090-3.978232-1.191637$

C $0.601992-4.423093-1.364985$

C $-1.366362-3.308344-2.345680$

C $1.485012-5.156569-0.397726$

C $2.569228-4.2955200 .267611$

C $3.582063-3.658349-0.700221$

C $4.757908-3.0634020 .081484$

C $5.750701-2.325208-0.829199$

O $2.928300-2.621987-1.441669$

O $6.606921-1.5291680 .005510$

C $4.972161-1.347761-1.735555$

C $6.664820-3.299404-1.581549$

O $5.833593-0.759661-2.706187$

C $3.756265-1.992567-2.432401$

C $2.881085-0.971991-3.205360$

O $4.218234-2.948455-3.395766$

C $0.155329-1.4304731 .214131$

O $0.083471-0.8183120 .160700$

C $1.147943-1.1716822 .280137$

C $2.235559-0.4461431 .984577$

C $3.310239-0.0689722 .901069$

C $3.095460-0.3138344 .370197$

C $2.3044490 .174642-2.369950$

C $3.1750391 .420298-2.341444$

C $3.5611593 .490578-1.004140$

O $4.0319891 .660798-3.187482$

C $4.7375123 .192630-0.128564$

C $4.849403 \quad 3.6094191 .142052$

C 6.0371463 .3160432 .014752

C 5.6788272 .4998103 .238132

C 5.4768743 .0907884 .427762

C 5.6412180 .9930873 .103424

C 4.4153620 .5061872 .383678
N $2.8518512 .254611-1.279782$

H -3.718097 -1.030056 1.067611

H -3.081615 -3.744068 2.323166

H -1.941131 -2.003044 0.041212

H -2.428937 -4.609251 -0.212254

H - $-1.000211-4.8137280 .779671$

H $1.061938-4.263096-2.339928$

H -1.543375 -2.249687 -2.135181

H $-0.789895-3.362699-3.275654$

H $-2.329928-3.793437-2.533427$

H $0.893814-5.6368480 .388103$

H $1.966500-5.980404-0.939917$

H $2.080628-3.5122120 .854394$

H $3.107716-4.9377860 .975998$

H $3.936257-4.434034-1.389389$

H $5.274520-3.840900 \quad 0.658074$

H $4.375164-2.3501710 .823086$

H $7.044614-2.1231350 .639059$

H $4.621075-0.532688-1.090895$

H $7.246592-3.902089-0.873691$

H $7.400213-2.761901-2.190339$

H $6.117798-3.989118-2.227542$

H $\quad 6.570106-0.343930-2.226112$

H $3.426879-0.595715-4.079142$

H $2.023737-1.526321-3.613763$

H $3.507429-3.104623-4.039485$

H $0.960698-1.5837323 .264689$

H $2.361718-0.0963370 .961991$

H 2.1449310 .1215674 .699372

H 3.8687610 .1315615 .000249

H $3.080484-1.3884324 .581615$

H $1.3490640 .485812-2.811196$

H $2.099148-0.160760-1.351322$

H $2.8547534 .176888-0.527669$

H $3.9003683 .938148-1.943814$

H $5.5416172 .607028-0.568783$

H 4.0498254 .2023031 .580272

H 6.4728174 .2770592 .318467

H 6.8282312 .8024151 .454201

H 5.2482482 .5108025 .315656

H 5.5306044 .1688594 .538748

H 5.7404410 .5144664 .083718

H 6.5263640 .6593182 .546593

H $4.4708100 .630108 \quad 1.302266$

H $2.1675861 .944085-0.596110$

C $-6.396442-1.5654691 .739982$

O $-6.210219-0.3662020 .952016$

C $-6.400203-0.714930-0.429958$

C $-7.379825-1.876714-0.393848$

C $-6.813930-2.6762140 .767022$

H $-5.439333-1.084843-0.808510$

C $-6.8336180 .494263-1.256737$

H $-8.397650-1.539649-0.165171$

H -7.400807 -2.439812 -1.331450

C $-7.798454-3.6682121 .362777$

H $-5.936053-3.2314000 .413230$

C $-5.8566721 .690257-1.203636$

O $-8.1082420 .942482-0.782937$

H $-6.986457 \quad 0.180357-2.296484$

H $-5.9389992 .193109-0.232722$

O $-6.3045742 .650543-2.169712$

C $-4.3744921 .346603-1.464507$

C $-3.4965622 .588045-1.718655$

H $-3.9850260 .794382-0.603266$

O $-4.2683810 .479938-2.596239$

H $-3.7546773 .002977-2.699910$

H -3.729345 $3.350374-0.969320$

H -7.350115 -4.197019 2.209963

H $-8.706392-3.1691751 .717844$

H $-8.094331-4.4122370 .615991$

C $-5.124713-1.8584102 .549190$

H -8.363608 $1.711665-1.320614$

H $-6.2580652 .235880-3.047593$

H $-4.5903000 .962958-3.375882$

H $-5.247299-2.787522 \quad 3.116889$

H $-4.984614-1.0482343 .275712$

H -7.211489-1.345855 2.440608

C -1.4587322 .9904980 .546851$ 
O $-1.5259701 .903997-0.387992$

C -1.986822 2.245200 -1.700828

C $-1.080720 \quad 3.325079-2.304537$

C $-0.9874274 .547376-1.400927$

C $-0.582761 \quad 4.1339230 .011588$

H -2.4686653 .3620610 .754681$

C $-0.8746572 .427757 \quad 1.853258$

H - 1.851609 1.345295 -2.313802

O $-1.5445213 .724870-3.589498$

H $-0.0704592 .917752-2.441802$

H $-1.9255585 .115163-1.393254$

O $0.0147165 .430594-1.911632$

H $0.473047 \quad 3.8369310 .005707$

O $-0.7026595 .281925 \quad 0.850833$

C -1.8389701 .5178592 .583029$

H 0.0295621 .8500651 .630909

H -0.6255613 .2364852 .547562$

H - $1.5781122 .929571-4.148007$

H $-0.2356045 .654310-2.824560$

H -0.2064595 .1086381 .667883$

O -1.1998390 .9853083 .666854$

O -3.0006651 .3139942 .261074$

C -1.979935 0.1157784 .493197

H $-1.447297-0.0205815 .438188$

H -2.962015 0.5486954 .707235

H $-2.083315-0.8570194 .006623$

SCF Energy (B3LYP/6-31G*//MMFF) $=-3245.90051351$

03171

MM̄FF Geometry

C $1.519649-0.786048-3.697192$

C $2.161749-1.959873-3.588201$

C $3.523200-2.183319-2.969601$

O $4.169387-0.932543-2.627088$

C $3.438171-2.963074-1.647232$

C $2.977152-4.397647-1.755289$

C $1.780767-4.844759-1.327316$

C $3.985431-5.358529-2.328103$

C $0.647451-4.034333-0.764368$

C $0.441796-4.2357490 .742178$

C $1.543196-3.6125441 .615885$

C $1.254991-3.8782533 .096471$

C $2.267818-3.1826044 .017682$

O $1.571351-2.2022651 .366132$

O $1.743856-3.215523 \quad 5.354488$

C $2.369374-1.6975163 .609904$

C $3.607860-3.9259344 .060548$

O $3.399399-1.0369444 .341750$

C $2.583145-1.4908492 .093524$

C $2.506690 \quad 0.000212 \quad 1.684249$

O $3.895262-1.9530731 .747663$

C $4.946474-0.329307-3.559768$

O $5.109264-0.688972-4.717506$

C $5.6099180 .877645-3.011481$

C $5.6045821 .208367-1.709643$

C $6.2726882 .367108-1.108820$

C $6.9585573 .332600-2.040892$

C $1.1254100 .639543 \quad 1.830762$

C 1.1757692 .1379461 .611478

C $1.536215 \quad 3.875447-0.131315$

O 0.9989702 .9263102 .538509

C $2.9789114 .268359-0.144423$

C 3.5669314 .9450720 .853263

C $5.0075795 .368542 \quad 0.856310$

C 5.8669624 .4936971 .741785

C 5.7827604 .5627113 .081267

C 6.8763853 .5909311 .068637

C 6.2333102 .5153090 .233147

$\begin{array}{lllll}N & 1.424418 & 2.494116 & 0.295937\end{array}$

H $1.9868390 .118921-3.320149$

H $1.677688-2.850047-3.985280$

H $4.154487-2.729879-3.680586$

H $2.820342-2.393943-0.945430$

H $4.430024-2.960974-1.172528$

H $1.567057-5.909005-1.417130$

H $3.627511-6.393615-2.314965$

H $4.212459-5.102930-3.367631$

H $4.914406-5.324801-1.749594$
H $\quad 0.748060-2.971067-0.996732$

H $-0.263524-4.358824-1.282709$

H $-0.517950-3.7729801 .005308$

H $0.353302-5.3068310 .963086$

H $2.508353-4.0481761 .335141$

H $1.229069-4.9562473 .299754$

H $0.251546-3.5037193 .344025$

H $1.596966-4.1461325 .596027$

H $1.426559-1.2239283 .908076$

H $3.467608-4.9453454 .440156$

H $4.305804-3.4425954 .752918$

H $4.088192-4.003763 \quad 3.083179$

H $3.224547-1.1860115 .286819$

H $3.257466 \quad 0.5789312 .238148$

H 2.8059780 .0854710 .630124

H $4.177614-1.5096710 .931022$

H $6.1412811 .470859-3.748939$

H $5.0788710 .567200-1.003940$

H $6.2574183 .698365-2.799731$

H $7.8013372 .847019-2.544929$

H $7.3519164 .220359-1.542150$

H $0.4174600 .205828 \quad 1.117273$

H $\quad 0.7114700 .4768342 .830269$

H 0.9474814 .5169430 .533018

H $1.1179583 .952874-1.138910$

H $3.5568193 .993652-1.022512$

H 2.9781705 .2149051 .727503

H 5.0636836 .4085371 .203655

H $5.4084015 .387652-0.164448$

H 6.4139343 .9546603 .720891

H 5.0791015 .2304033 .567573

H $7.498903 \quad 3.0879851 .820674$

H 7.5735804 .2101110 .497237

H 5.7019081 .7675770 .823545

H $1.6209241 .769146-0.387321$

C $-0.962091-0.238567-3.401976$

O $-1.274747-1.341684-2.530616$

C $-1.600940-0.819377-1.224393$

C $-1.726722 \quad 0.690157-1.389154$

C $-0.7304130 .967619-2.494995$

H $-0.746060-1.068225-0.585619$

C $-2.876000-1.493683-0.704258$

H $-2.7399320 .966707-1.707294$

H -1.500996 $1.231169-0.466182$

C $-0.937322 \quad 2.305446-3.181823$

H $\quad 0.2720440 .941747-2.054934$

C $-3.323982-0.9629810 .668232$

O $-2.640335-2.899043-0.597175$

H $-3.674560-1.370232-1.443680$

H -3.5185250 .1123930 .609624$

O $-2.234122-1.1163231 .587653$

C $-4.549526-1.6820861 .260676$

C $-5.799102-1.7310460 .360721$

H $-4.274109-2.7093031 .527668$

$\begin{array}{llll}\text { O } & -4.888255 & -1.051942 & 2.501507\end{array}$

H $-5.616891-2.421526-0.470958$

H $-6.613293-2.1529100 .964103$

H - $0.1877152 .460347-3.964143$

H $-1.9277312 .370141-3.645058$

H $-0.8516373 .125686-2.462244$

C $0.176622-0.617398-4.354886$

H -2.361908 -3.212491 -1.474774

H $-2.532124-0.7780382 .449459$

H $-5.134826-0.1309832 .310707$

H $0.2764160 .157222-5.124508$

H $-0.096143-1.545007-4.873949$

H $-1.863584-0.076894-4.008322$

C $-6.953774 \quad 1.8294550 .538516$

O -6.5828340 .4895050 .889646$

C $-6.258741-0.371079-0.204155$

C $-7.470742-0.516566-1.138266$

C $-7.9834270 .851251-1.601720$

C $-8.148303 \quad 1.829640-0.432712$

H -7.2860552 .2834051 .480867$

$\begin{array}{llll}\text { C } & -5.753956 & 2.643706 & 0.028206\end{array}$

H -5.430593 $0.062809-0.771106$

O $-8.523111-1.221036-0.482302$

H $-7.177699-1.115181-2.008702$ 
H $-8.9484160 .716861-2.105847$

O $\quad-7.0642421 .380856-2.552563$

H $-8.3222642 .844120-0.809836$

O $-9.321073 \quad 1.446048 \quad 0.292014$

C -4.6710212 .7888321 .077401$

H $-5.2974782 .178439-0.849427$

H $-6.0831993 .653138-0.243168$

H $-8.749149-0.7319670 .327432$

H $-7.4220672 .229349-2.864945$

H -9.4648432 .1073570 .990271$

O $-3.563863 \quad 3.3400370 .496990$

O -4.7779022 .4690092 .253306$

C $-2.442317 \quad 3.525557 \quad 1.366173$

H -2.122012 2.5657351 .783137

H -1.622573 3.9425750 .775979

H -2.694875 4.229493 2.164624

SCF Energy (B3LYP/6-31G**//MMFF) $=-3245.91420994$

03172

MM̄FF Geometry

C $-2.815053-2.814422-3.073204$

C $-2.199273-2.694740-1.887407$

C $-0.753726-3.074744-1.672019$

O $-0.036167-1.940056-1.136777$

C $-0.671399-4.219299-0.644826$

C $0.729867-4.760759-0.435890$

C $1.398187-4.7227160 .733579$

C $1.368913-5.409124-1.637473$

C $0.933110-4.1641522 .048918$

C $1.310780-2.6942812 .288435$

C $2.824678-2.4455602 .387684$

C $3.110738-1.0131862 .843068$

C $4.618048-0.7103052 .871359$

O $3.405530-2.6561081 .098817$

O $4.778917 \quad 0.7123832 .984274$

C $5.246072-1.1296631 .523949$

C $5.298114-1.3223554 .101896$

O $6.666919-1.0278991 .569037$

C $4.834606-2.5432581 .066014$

C $5.285795-2.871961-0.378259$

O $5.445908-3.5119851 .927041$

C $0.387781-0.998114-2.022507$

O $0.190715-0.975882-3.226990$

C $1.1422200 .022517-1.263962$

C $1.3518291 .229418-1.809860$

C $2.0584842 .347430-1.181945$

C 2.8459392 .0601680 .068143

C $4.733180-1.945499-1.467889$

C $5.681247-0.827272-1.870065$

C $5.7892991 .187828-3.334048$

O $6.845043-0.756498-1.485276$

C $5.6026272 .402087-2.480559$

C $5.152983 \quad 3.574277-2.953793$

C $5.0096904 .814216-2.118676$

C $3.6232555 .413282-2.193967$

C $3.3631096 .455241-3.001707$

C $2.5643654 .862040-1.267029$

C $1.9815953 .565640-1.756978$

N $5.0801070 .066348-2.747058$

H -2.229668 -3.180281 -3.915717

H $-2.742861-2.328913-1.022214$

H $-0.288543-3.387494-2.614877$

H -1.309134 -5.053579-0.966591

H -1.089889 -3.8659220 .302992$

H $2.399030-5.1526720 .763524$

H $2.301005-5.925723-1.384036$

H $0.693264-6.152570-2.072895$

H $1.606956-4.661241-2.399890$

H $-0.150861-4.2765162 .152600$

H $1.358532-4.7817452 .850367$

H $\quad 0.886372-2.0818721 .484254$

H $0.834600-2.3765623 .224232$

H $3.245080-3.1637183 .101396$

H $2.662697-0.8191473 .825645$

H $2.628159-0.3098682 .151170$

H 4.3238251 .0020153 .793446

H $4.907036-0.4038430 .777153$

H $4.848299-0.9305605 .022176$
H $\quad 6.357404-1.0462024 .146423$

H $5.223050-2.4111344 .136106$

H $6.884032-0.1183751 .836216$

H $6.378841-2.947225-0.424269$

H $4.916829-3.882056-0.608762$

H $5.475498-4.363847 \quad 1.460922$

H $1.486866-0.240594-0.270786$

H $\quad 0.9418901 .431904-2.798558$

H $3.5087261 .203802-0.089480$

H 2.1777941 .8360350 .904781

H 3.4918882 .8849430 .376298

H $4.565989-2.541200-2.374604$

H $3.768534-1.519919-1.174917$

H $5.3953191 .327534-4.345685$

H $6.8551040 .947758-3.404866$

H $5.8779232 .321360-1.431583$

H $4.9052663 .660289-4.009514$

H $5.7500505 .542189-2.475784$

H $5.2672774 .623453-1.069444$

H $2.3785136 .909511-3.039601$

H $4.1280736 .873650-3.647453$

H $1.7331265 .575177-1.181016$

H $2.9703934 .792128-0.253899$

H $1.3939593 .671346-2.670099$

H $4.093693-0.038282-2.966360$

C $-5.195067-2.155653-2.252838$

O $-4.915768-0.812751-1.803062$

C $-6.155116-0.093156-1.711984$

C -7.265552 -1.131911-1.744501

C $-6.667078-2.160327-2.682757$

H $-6.2380810 .494017-2.637860$

C $-6.1520950 .903330-0.544410$

H -7.447734 -1.580586 -0.764963

H -8.212332 -0.722288 -2.109615

C -7.332921 -3.523260 -2.590272

H $-6.758385-1.788657-3.712537$

C $-\begin{array}{llll}-5.633081 & 0.374060 & 0.807608\end{array}$

O $-7.4625831 .440078-0.375384$

H $-5.4970541 .731840-0.839408$

H -4.6061540 .0136810 .693415$

O $-6.394648-0.7646451 .218159$

C -5.6980321 .4010631 .959894$

C -5.0470142 .7671851 .674976$

H -6.7452281 .5737102 .237749$

O

H $\quad-5.5930243 .2650240 .865910$

H -5.1730143 .3819292 .576352$

H $-6.863543-4.235601-3.275812$

H -7.264405 -3.935194 -1.577810

H -8.393550 -3.451435 -2.852404

C $-4.255063-2.526494-3.405771$

H $-7.7349741 .823923-1.226397$

H -7.320036 -0.4800941 .313171$

H $-5.504338-0.0270943 .281927$

H $-4.258821-1.718710-4.148722$

H $-4.643199-3.424034-3.902722$

H -5.049238 -2.823597 -1.394810

C -1.420248 2.2186812 .401664

$\begin{array}{llll}\text { O } & -2.8369502 .384998 & 2.537668\end{array}$

C -3.5451182 .7059301 .337025$

C -3.0337984 .0378980 .768340$

C -1.5211113 .9807360 .532686$

C -0.7719723 .4591181 .763521$

H -1.048889 2.1445223 .432080

C -1.0784480 .9032541 .689264$

H $-3.380497 \quad 1.9199140 .594704$

O -3.3459025 .0956211 .671276$

H -3.555046 $4.242763-0.174455$

H -1.1494024 .9816410 .281704$

O $-1.2463703 .126338-0.572761$

H 0.2708723 .2363601 .514325

O -0.7504594 .4867282 .759601$

C -1.617313 -0.2810122 .461670$

H -1.4861930 .8772470 .674962$

H $\quad 0.0057390 .773321 \quad 1.611645$

H -3.021682 5.9232151 .277395

H -1.695089 $3.497736-1.351273$

H -0.2876225 .2529922 .380141$ 
O $-2.265464-1.1320551 .616076$ O $-1.477516-0.4368813 .667934$ C $-2.880894-2.2641952 .237923$ H $-2.129170-2.8764362 .743920$ H $-3.649107-1.9352562 .944111$ H -3.357614 -2.864373 1.458570

SCF Energy (B3LYP/6-31G*//MMFF) $=-3245.88537562$

03173

MM̄FF Geometry

C -2.024226 5.4032250 .538692

C $-1.369394 \quad 4.728379-0.418353$

C $0.1155824 .470597-0.399717$

O $0.322767 \quad 3.054972-0.181181$

C $0.725612 \quad 4.842409-1.763644$

C $2.2264784 .640790-1.842498$

C $2.8381103 .785055-2.685065$

C $3.0527905 .496670-0.917278$

C $2.205606 \quad 2.876755-3.702632$

C $1.853298 \quad 1.474460-3.182748$

C $3.0696610 .628535-2.772671$

C $2.644849-0.801955-2.433927$

C $3.830868-1.651210-1.950316$

O $3.6705621 .222482-1.621938$

O $3.302600-2.841269-1.342791$

C $4.593995-0.885303-0.844146$

C $4.699113-2.118266-3.124894$

O $5.808765-1.548617-0.506752$

C $4.8840700 .591237-1.191492$

C 5.4168441 .4129810 .003848

$\begin{array}{lllll}\text { O } & 5.906099 & 0.703600 & -2.189937\end{array}$

C $\quad 0.273260 \quad 2.615237 \quad 1.106294$

$\begin{array}{lllllll}\text { O } & 0.100294 & 3.290771 & 2.108702\end{array}$

C $0.4381881 .145324 \quad 1.092635$

C 0.0770430 .4416952 .176196

C $0.171268-1.0100142 .342156$

C $0.914795-1.7819901 .285203$

C 4.4886701 .4875321 .221139

C 4.8255390 .4873112 .314836

C $4.030476-0.2801864 .547971$

O $5.822259-0.2294932 .292881$

C $3.333487-1.5911174 .366905$

C $2.429245-2.0690925 .235108$

C $1.763509-3.4078595 .098363$

C $0.255003-3.3135305 .119517$

C $-0.438664-3.558296 \quad 6.244112$

C $-0.450456-3.0246573 .814331$

C $-0.390611-1.5697703 .434367$

N 3.8935090 .5171803 .344142

H -1.472226 5.8126681 .380845

H $-1.9343414 .298848-1.242760$

H $\quad 0.604275 \quad 5.045660 \quad 0.396456$

H $0.5108835 .895424-1.990165$

H $0.2220264 .257161-2.540822$

H $3.925873 \quad 3.729133-2.655514$

H 2.9005665 .1983130 .124396

H $4.1252015 .418755-1.126479$

H $2.7756286 .550300-1.025129$

H $1.3044373 .338537-4.120428$

H $2.8953372 .784082-4.551401$

H $1.1659651 .571091-2.333896$

H $1.3105670 .952242-3.980057$

H $3.7860960 .630539-3.602484$

H $2.161435-1.278353-3.295637$

H $1.886422-0.779322-1.639585$

H $2.746540-3.292846-2.000539$

H $3.962900-0.9166470 .051250$

H $4.114099-2.747558-3.806759$

H $5.529734-2.743787-2.780689$

H $5.105162-1.291693-3.711906$

H $5.581538-2.461237-0.258877$

H $\quad 6.421026 \quad 1.0690650 .280491$

H $5.5563032 .443821-0.353813$

H $6.273841-0.178395-2.359409$

H $\quad 0.8357710 .6869390 .195102$

H -0.3441200 .9782713 .025865$

H $0.394244-1.7220960 .324282$

H $1.036290-2.8413681 .519227$
H $\quad 1.925142-1.3789341 .161384$

H 4.5943872 .4816861 .674621

H $3.440499 \begin{array}{lll}1.371021 & 0.930281\end{array}$

H $3.609866 \quad 0.3029325 .373326$

H $5.092716-0.4533424 .749138$

H $3.608496-2.1885183 .500800$

H 2.177149-1.482059 6.115533

H $2.109479-4.0376515 .928587$

H $2.083966-3.9240424 .184858$

H $-1.522843-3.5217246 .259141$

H $0.063419-3.7953097 .176411$

H - $-1.512116-3.2939793 .895623$

H $-0.052064-3.6884883 .042658$

H $-0.904125-0.917348 \quad 4.142439$

H $3.067641 \quad 1.101643 \quad 3.252508$

C $-4.298762 \quad 4.4084101 .115499$

$\begin{array}{lllll}\text { O } & -4.135863 & 3.264151 & 0.253236\end{array}$

C $-3.8099102 .120501 \quad 1.060307$

C -4.2802502 .4761262 .463444$

C $-3.916303 \quad 3.9492082 .524423$

H -2.7167942 .0307021 .058056$

$\begin{array}{llll}\text { C } & -4.411782 & 0.835171 & 0.479004\end{array}$

H -5.363772 2.3456792 .568595

H $-3.784601 \quad 1.878643 \quad 3.234418$

C $-4.625008 \quad 4.711724 \quad 3.629793$

H -2.8338374 .0270412 .683108$

C $-3.873870 \quad 0.489227-0.925981$

$\begin{array}{llll}\text { O } & -5.833655 & 0.945862 & 0.430483\end{array}$

H $-4.1824670 .018191 \quad 1.170456$

H $-2.7786050 .439316-0.911347$

O $-4.2153101 .530799-1.850125$

C $-4.443456-0.812704-1.517583$

C $-4.252843-2.080509-0.666738$

H $-5.515419-0.689222-1.715207$

O $-3.840764-1.033278-2.795588$

H $-4.924055-2.0212650 .198038$

H $-4.590718-2.934982-1.265276$

H -4.3438845 .7695713 .612573$

H $-5.713510 \quad 4.6493543 .527230$

H -4.3567364 .3054484 .610495$

C $-3.515463 \quad 5.5963310 .544786$

H $-6.0468881 .681841-0.168855$

H $-3.7693252 .343020-1.556231$

H $-3.975408-0.227757-3.323752$

H $-3.7453316 .501977 \quad 1.118231$

H $-3.8495755 .783503-0.483942$

H -5.3672814 .6572821 .079769$

C -1.901515 -3.508016-2.093815

O $-1.847663-2.369812-1.225521$

C $-2.812053-2.348362-0.173127$

C $-2.670211-3.6081870 .691403$

C $-2.735664-4.882822-0.142153$

C $-1.742034-4.812303-1.300557$

H $-2.856749-3.515741-2.632127$

C $-0.771273-3.335746-3.115523$

H $-2.535690-1.5032960 .467392$

O $-3.669884-3.653024 \quad 1.702980$

H $-1.699399-3.5883401 .199464$

H $-3.752071-5.076581-0.504435$

O $-2.383595-5.9971950 .682281$

H $-0.723059-4.901763-0.901719$

O $-1.977349-5.936247-2.146890$

C $-1.051976-2.174956-4.039411$

H $\quad 0.183567-3.131425-2.615799$

H $-0.623077-4.230433-3.729556$

H $-3.585343-2.8417502 .232734$

H -3.001202 -6.006816 1.433689

H $-1.214720-6.027105-2.741830$

O $-1.788357-2.596728-5.106644$

O $-0.681555-1.028148-3.824843$

C $-2.129958-1.582267-6.056348$

H $-2.714500-2.047117-6.854665$

H $-2.738586-0.806851-5.581629$

H $-1.223486-1.150089-6.490864$

SCF Energy (B3LYP/6-31G*//MMFF) $=-3245.91323633$

03174

MM̄FF Geometry 
C 1.2221921 .3405773 .633323

C 0.3007570 .8080494 .450020

C $-0.657278-0.2900224 .043479$

O $-1.048727-0.1315172 .657599$

C $0.007656-1.6680524 .207190$

C $-0.910023-2.8297443 .879779$

C $-0.720756-3.6904982 .860050$

C $-2.090689-3.0089994 .799316$

C $0.399136-3.7095521 .857509$

C $0.123618-2.9234720 .566147$

C $-1.050382-3.467218-0.264312$

C $-1.107017-2.782808-1.631974$

C $-2.318714-3.246161-2.456643$

O $-2.267232-3.213651 \quad 0.442507$

O $-2.476667-2.335834-3.556200$

C $-3.593184-3.133634-1.591638$

C $-2.092782-4.632738-3.070946$

O $-4.713425-3.712012-2.256270$

C -3.440302 -3.749454-0.185904

C $-4.640540-3.4466180 .744956$

O $-3.352552-5.174930-0.304616$

C - $-1.937246 \quad 0.8615932 .377434$

O -2.4775861 .6112933 .175446$

C $-2.117406 \quad 0.909074 \quad 0.910518$

C $-2.6307702 .016521 \quad 0.354788$

C $-2.8849842 .233673-1.069703$

C $-2.7847091 .044557-1.986750$

C $-4.913523-1.9611221 .007387$

C $-5.981044-1.3560270 .109681$

C $-7.0680040 .829268-0.405133$

O $-6.689770-2.021372-0.640494$

C $-6.4904831 .369038-1.675343$

C $-6.4917562 .673366-1.991083$

C $-5.9663833 .222223-3.286978$

C $-4.9549274 .329147-3.088654$

C $-5.3180995 .620253-3.172011$

C $-3.5089803 .937925-2.884397$

C $-3.2290043 .471276-1.482411$

$\begin{array}{llll}\mathrm{N} & -6.076231 & 0.019559 & 0.277908\end{array}$

H 1.3409820 .9238882 .635978

H $\quad 0.190141 \quad 1.1978895 .458554$

H -1.553526 -0.2246304 .672314$

H $0.361672-1.7901625 .239462$

H $0.902703-1.7009813 .576723$

H -1.449023 -4.489740 2.725632

H $-2.634598-3.9389714 .600728$

H -1.758629 -3.042191 5.842120

H -2.800235 -2.1843464 .682926$

H $1.326331-3.3408972 .308780$

H $\quad 0.606887-4.7567901 .602836$

H $-0.055986-1.8720840 .819524$

H $1.035495-2.957143-0.042750$

H $-0.914759-4.548557-0.384338$

H $-0.177434-2.949020-2.190631$

H -1.179534 -1.696146-1.490733

H -1.651364 -2.341539-4.070781

H $-3.811418-2.065253-1.487539$

H -1.212873 -4.622511 -3.725474

H -2.935051 -4.924943 -3.707937

H -1.937780 -5.413103 -2.323116

H $-4.790305-3.280264-3.124279$

H -5.536933 -3.966606 0.386010

H -4.413823 $-3.901111 \quad 1.720454$

H $-3.594519-5.5708180 .548928$

H $-1.812240 \quad 0.0451670 .332149$

H -2.8775212 .8566181 .003309$

H -3.370379 $0.208152-1.592886$

H -1.745081 $0.724229-2.092637$

H -3.171949 $1.233512-2.990147$

H -5.286886 -1.855621 2.034341

H $-3.995106-1.3723420 .932613$

H -7.367892 1.6247550 .284372

H -7.945946 $0.215515-0.631696$

H $-6.0818370 .650203-2.381700$

H $-6.9285903 .386504-1.295441$

H $-6.8239693 .601354-3.858301$

H -5.525650 $2.432504-3.908047$

H -4.593472 6.419931 -3.060479
H $-6.3499045 .906224-3.348198$

H -2.855821 $4.800641-3.073621$

H $-3.2207063 .205140-3.643557$

H -3.293492 $4.267209-0.739178$

H -5.4124360 .4943320 .882904$

C 2.0251233 .6449032 .959996

O 2.4088843 .1516941 .659667

C 1.3564313 .4626080 .728399

C 0.6608044 .6768381 .321820

C 0.6462964 .2960362 .793688

H $0.6548312 .619676 \quad 0.742285$

C $1.9218203 .619115-0.684337$

H 1.2447105 .5923591 .172454

H $-0.340214 \quad 4.8311250 .908569$

C 0.4107075 .4701563 .727706

H -0.1537873 .5629222 .951620$

C $2.8043492 .428783-1.122395$

O $2.7295164 .800700-0.727030$

H $1.1021133 .778160-1.394731$

H $3.7339112 .447737-0.543587$

O $3.1889932 .640807-2.485796$

C $2.1289591 .051114-0.997373$

C $2.994846-0.122596-1.474494$

H 1.8314540 .8698290 .039461

O $0.9286851 .063093-1.772586$

H $2.409399-1.050363-1.404061$

H $3.187975-0.029398-2.552268$

H 0.4337745 .1441974 .772391

H 1.1729806 .2458413 .598416

H -0.5667415 .9244493 .535305$

C 2.1088252 .5025663 .982280

H $3.0875034 .874151-1.628470$

H $2.3846872 .579481-3.029374$

H $\quad 0.4946100 .201534-1.650699$

H 1.8776232 .8868214 .982335

H 3.1391602 .1263574 .004719

H 2.7722664 .4024493 .227854

C $6.219359-1.840588-0.780784$

O $4.920307-1.496298-1.278770$

C $4.333778-0.303721-0.739097$

C $4.196122-0.4131740 .788211$

C $5.529071-0.7778901 .447608$

C $6.204270-1.9640590 .751810$

H $6.421768-2.838454-1.191489$

C $7.305327-0.888572-1.306523$

H $4.9775870 .543941-0.993438$

O $3.218214-1.4001191 .106644$

H 3.8428780 .5435881 .188964

H $5.351405-1.0225592 .501801$

O $\quad 6.381766 \quad 0.362618 \quad 1.407147$

H $7.230282-2.0923711 .115802$

O $5.493127-3.1617591 .078012$

C $7.483100-0.991802-2.807612$

H $7.0755670 .155607-1.079477$

H $8.266607-1.146920-0.847338$

H $3.114802-1.4101112 .073378$

H 7.2133510 .1234401 .850573

H $5.519460-3.2645632 .044604$

O $8.464113-0.122785-3.195289$

O $6.851163-1.732880-3.547498$

C $8.747791-0.101560-4.597729$

H $7.8593050 .202583-5.159496$

H $9.5409820 .629992-4.773132$

H $9.097272-1.084265-4.928527$

SCF Energy (B3LYP/6-31G**//MMFF) $=-3245.89596001$

03175

MM̄MF Geometry

C $2.399415-4.185795-1.800754$

C $1.326841-4.991038-1.784761$

C $-0.104842-4.504197-1.827575$

O $-0.252657-3.255729-1.107707$

C $-0.530012-4.278984-3.290171$

C $-1.968347-3.831902-3.453979$

C $-2.345282-2.636518-3.949229$

C $-3.014598-4.837037-3.046409$

C -1.471559-1.511764 -4.432815

C $-1.155561-0.442253-3.374275$ 
C $-2.3886760 .325724-2.871353$

C $-1.9754991 .504354-1.987082$

C $-3.1958032 .237474-1.405246$

O $-3.202953-0.571078-2.113049$

O $-2.7319773 .100156-0.355678$

C $-4.1454831 .206371-0.755426$

C $-3.8651873 .143236-2.446282$

O $-5.3670731 .819871-0.352276$

C $-4.440249-0.009013-1.656552$

C $-5.212957-1.136238-0.929509$

O $-5.2525630 .410761-2.760051$

C $-0.263580-3.325751 \quad 0.252448$

O $-0.144345-4.331310 \quad 0.935243$

C $-0.436178-1.9546740 .782871$

C $-0.178910-1.7216702 .078793$

C $-0.311262-0.4421042 .778131$

C - -1.0300340 .6742782 .069552$

C $-4.500097-1.7477920 .282069$

C $-4.959298-1.1908471 .619507$

C $-4.508806-1.4137524 .062130$

O $-5.890880-0.4004291 .740510$

C $-3.706665-0.2285404 .496479$

C $-2.885563-0.2411175 .557386$

C -2.1242170 .9600276 .038852$

C $-0.639587 \quad 0.703312 \quad 6.159693$

C $-0.0814080 .416907 \quad 7.348056$

C $0.208727 \quad 0.873878 \quad 4.920866$

C $0.183491-0.3379314 .029445$

N $-4.221150-1.7155612 .672793$

H $2.246649-3.112574-1.884905$

H $1.469142-6.067737-1.737265$

H $-0.748222-5.259322-1.359325$

H $-0.392393-5.205871-3.863254$

H $\quad 0.148352-3.546743-3.740606$

H -3.412739 -2.433112 -4.029158

H $-4.027477-4.511824-3.307587$

H $-2.839053-5.793545-3.549483$

H -2.992577 -5.000139-1.964658

H $-0.530329-1.896914-4.838465$

H -1.973073 -1.036507 -5.285657

H $-0.643578-0.917336-2.529134$

H $-0.4505160 .269357-3.821166$

H $-2.9496540 .681638-3.743546$

H -1.337808 $2.202789-2.542319$

H -1.360766 $1.136747-1.154082$

H $-2.1041903 .732017-0.746051$

H $-3.657220 \quad 0.857236 \quad 0.160875$

H -3.154899 $3.892145-2.816337$

H $-4.6922773 .709327-2.003786$

H $-4.2448372 .595634-3.311291$

H -5.1387242 .5641610 .230514$

H -6.226431 -0.799585-0.680376

H $-5.350828-1.951169-1.655222$

H $-5.706719-0.367671-3.122815$

H $-0.762730-1.1805680 .098538$

H $0.179116-2.5477842 .692633$

H -1.976661 0.3156721 .653872

H $-0.421741 \quad 1.076603 \quad 1.255717$

H -1.291054 1.5072502 .726335

H -4.725451 -2.822031 0.306775

H $-3.413998-1.6446930 .199523$

H $-4.273213-2.3099874 .644717$

H -5.576223 -1.196941 4.173581

H -3.8333050 .6920873 .931622$

H -2.782096 -1.155092 6.137933

H -2.5343311 .2419647 .017699$

H -2.292436 1.8285555 .389845

H 0.9879350 .2625897 .447642

H -0.6824810 .3244458 .246603$

H 1.2556461 .0407895 .208623

H -0.0826301 .7922114 .403647$

H $0.672440-1.2092684 .467602$

H -3.426699 -2.316547 2.472994

C $4.626761-3.984247-0.606791$

O $4.589189-2.551074-0.777559$

C $4.112619-1.9672880 .447270$

C $4.482964-2.9724861 .526177$

C $4.140917-4.2728350 .818593$
H $3.018454-1.9225690 .380707$

C $4.665709-0.5533810 .624102$

H $5.552589-2.9372011 .763014$

H $3.916178-2.8249002 .450194$

C $4.782917-5.4983201 .445311$

H $3.051626-4.4025420 .840237$

C $4.3859070 .386895-0.571929$

O $6.084754-0.6324580 .798451$

H $4.266066-0.1228411 .549853$

H $5.0193780 .100755-1.419828$

O $4.8172621 .694425-0.189013$

C $2.9140260 .421516-1.027630$

C $2.6034631 .406268-2.175563$

H $2.657995-0.576821-1.401322$

O $2.0779610 .663426 \quad 0.096896$

H $3.3749901 .309902-2.948861$

H $1.6607271 .060135-2.620448$

H $4.524412-6.4012260 .883096$

H $5.874661-5.4138981 .466059$

H $4.436696-5.6292792 .475741$

C $3.825509-4.655627-1.729180$

H 6.4141230 .2763420 .906824

H 4.2405811 .9928040 .534981

H $1.1608820 .678494-0.225459$

H $3.876624-5.744874-1.617988$

H $4.292822-4.403955-2.689455$

H $5.679781-4.273813-0.713408$

C $0.9774324 .302615-0.446358$

O $1.2642042 .992158-0.950010$

C $2.4127422 .888052-1.796726$

C $2.2242043 .772517-3.041378$

C $1.9189075 .222091-2.648842$

C $0.8066545 .307596-1.596605$

H 0.0067714 .2019620 .054516

C 2.0007614 .7438120 .607825

H $3.2963453 .229662-1.254584$

O $1.1578793 .257915-3.836657$

H $3.1383383 .730614-3.645823$

H $1.6212135 .780292-3.544811$

O $3.1118835 .816915-2.146370$

H $0.7391886 .321593-1.185614$

O $-0.448395 \quad 5.027418-2.223706$

C 1.9578653 .8294731 .811902

H 3.0192554 .7578050 .210337

H 1.7836095 .7571450 .964191

H $1.085953 \quad 3.817155-4.628770$

H $2.9084326 .741785-1.925495$

H $-0.5826915 .691107-2.921452$

O 3.2043383 .3423602 .072445

O $0.941503 \quad 3.5809182 .447237$

C 3.2962282 .4482243 .185669

H 2.9533182 .9404504 .100646

H 2.7129821 .5442222 .988734

H 4.3453812 .1673003 .309951

SCF Energy (B3LYP/6-31G**//MMFF)= -3245.90879335

03176

MM̄FF Geometry

C $4.0065402 .102990-1.661956$

C $3.8373083 .417937-1.873732$

C $2.5614794 .212645-1.709895$

O $1.4164723 .360115-1.492438$

C $2.7083965 .144090-0.490829$

C $1.4989676 .023775-0.238216$

C 0.6170025 .8537360 .767874

C $1.3366987 .177654-1.194346$

C 0.6004664 .7678821 .810264

C $-0.6586543 .910011 \quad 1.675936$

C -0.6679072 .6543712 .561784$

C 0.4160091 .6451632 .165139

C 0.3049170 .3306342 .952664

O -1.9645742 .0629112 .395148$

O $1.129706-0.6464592 .299128$

C $-1.148519-0.1752102 .871206$

C $0.8549720 .475658 \quad 4.376215$

O $-1.331771-1.3304303 .686727$

C $-2.2044560 .904853 \quad 3.203665$

C $-3.615351 \quad 0.3692562 .864397$ 
O -2.1365831 .2073614 .597502$

C $0.7479932 .917277-2.593751$

O $1.0551623 .088885-3.762329$

C $-0.4518182 .191770-2.122449$

C $-1.2672971 .577170-2.991515$

C $-2.4839640 .843566-2.636942$

C $-3.0327671 .025403-1.244170$

C -4.7333841 .4125872 .974677$

C $-6.068538 \quad 0.8273712 .554583$

C $-7.383502 \quad 0.192127 \quad 0.541623$

$\begin{array}{llll}\text { O } & -6.942798 & 0.556835 & 3.374451\end{array}$

C -7.032296 -0.549035 -0.708440

C -7.170954 -0.037100 -1.940749

C $-6.836362-0.779538-3.203421$

C $-5.592818-0.232431-3.872734$

C $-5.6981700 .681781-4.852662$

C $-4.280402-0.816509-3.388929$

C $-3.0737790 .065441-3.568639$

$\begin{array}{llll}\mathrm{N} & -6.161115 & 0.633208 & 1.185579\end{array}$

H $3.168847 \quad 1.485139-1.350838$

H $4.7029124 .005837-2.175434$

H $2.406654 \quad 4.814828-2.613794$

H $3.5825795 .796009-0.626427$

H $2.940365 \quad 4.5430520 .394220$

H $-0.201873 \quad 6.567100 \quad 0.856100$

H $0.4909207 .821363-0.930224$

H $2.2363667 .801675-1.193066$

H $1.1663136 .813014-2.211833$

H $1.4919104 .144877 \quad 1.748879$

H $0.619645 \quad 5.234752 \quad 2.802220$

H -1.539299 4.5199281 .921876

H $-0.807500 \quad 3.613607 \quad 0.631642$

H -0.549371 $2.964423 \quad 3.606520$

H 1.4208172 .0627612 .291135

H 0.3221181 .4146451 .095415

H $2.039327-0.3029242 .272324$

H $-1.311738-0.5072461 .837312$

H $1.9068070 .785404 \quad 4.350328$

H $0.841328-0.4836274 .905295$

H $\quad 0.3103801 .2100454 .973158$

H $-1.392275-1.0339934 .610757$

H $-3.608048-0.0169701 .837448$

H -3.863111 -0.4719513 .523983$

H -2.982144 1.5868754 .882316

H -0.635502 2.172839-1.056120

H - $1.0160611 .594016-4.050798$

H $-3.1105202 .089421-0.993100$

H -2.394011 $0.537568-0.501981$

H $-4.0380940 .616807-1.127894$

H -4.8383741 .7764814 .001986$

H -4.5255612 .2856082 .345328$

H $-7.985323 \quad 1.084430 \quad 0.338426$

H -7.954104 -0.4512211 .219034$

H $-6.651070-1.560558-0.590422$

H $-7.5506980 .975759-2.055732$

H $-7.698687-0.706659-3.878884$

H -6.707437 -1.852723 -3.013262

H $-4.8322371 .076865-5.371643$

H $-6.6673421 .056296-5.166916$

H $-4.103325-1.758566-3.924238$

H $-4.368511-1.107053-2.336341$

H -2.626342 $0.005003-4.561061$

H $-5.449862 \quad \begin{array}{lll}1.057512 & 0.597417\end{array}$

C $5.821474 \quad 0.850937-0.470142$

O $5.118365-0.376376-0.181988$

C $6.068879-1.3592740 .274473$

C $7.339740-0.5883680 .603590$

C $7.3072880 .488666-0.462948$

H $6.277263-2.016318-0.578148$

C $5.488928-2.1781521 .432242$

H $7.295280-0.1376771 .601866$

H $8.233453-1.2177140 .553862$

C $8.2281521 .660251-0.167882$

H $7.5899210 .041947-1.425892$

C $4.149502-2.8660441 .097504$

O $5.262471-1.2985292 .540864$

H $6.226684-2.9162651 .768068$

H $3.379694-2.0978330 .971665$
O $3.752302-3.6360822 .238765$

C $4.193217-3.785510-0.136361$

C $2.882357-4.537713-0.409908$

H $4.460229-3.204359-1.024191$

O $5.230622-4.7517210 .051055$

H $3.013962-5.174823-1.295916$

H $2.692299-5.2614410 .394876$

H $8.1595732 .419513-0.953077$

H 7.9769642 .1381530 .784997

H $9.2692551 .325936-0.111829$

C $5.3388391 .416050-1.808350$

H $4.884650-1.8320183 .261131$

H $4.397039-4.3567122 .344373$

H $5.277007-5.292878-0.755638$

H $5.2262760 .609177-2.542541$

H $6.0783742 .120632-2.206227$

H 5.5970591 .5463370 .349541

C $-0.730188-3.904455-1.026517$

O $0.557486-4.531688-0.929450$

C $1.639833-3.652882-0.590515$

C $1.798639-2.582408-1.680402$

C $0.496378-1.807995-1.880658$

C $-0.711226-2.740272-2.033147$

H $-1.381517-4.689261-1.432467$

C $-1.255011-3.5386050 .369911$

H $1.412152-3.1802960 .371339$

O $2.197156-3.183330-2.910204$

H $2.587474-1.878280-1.394699$

H $0.590631-1.175677-2.771625$

O $0.320925-0.953001-0.756134$

H $-1.644542-2.174087-1.956661$

O $-0.680463-3.314623-3.343902$

C $-2.761883-3.3987640 .417679$

H $-0.975103-4.3356511 .070066$

H $-0.809774-2.6108360 .735846$

H $1.531195-3.852392-3.143890$

H $-0.517854-0.479720-0.875008$

H $-0.742255-2.585031-3.984080$

O $-3.150056-3.2292041 .716447$

O $-3.507649-3.431202-0.551467$

C $-4.560531-3.1042061 .923766$

H -4.734756 -2.940895 2.990464

H $-4.952138-2.2471371 .368952$

H -5.068909-4.024496 1.621353

SCF Energy (B3LYP/6-31G**//MMFF)= -3245.91843979

$03 \_177$

MM̄FF Geometry

C -3.516402 2.7937630 .548377

C $-3.607788 \quad 3.264794 \quad 1.801884$

C $-2.447583 \quad 3.4187542 .757889$

$\begin{array}{llll}\text { O } & -1.414715 & 2.447748 & 2.470827\end{array}$

$\begin{array}{llll}\text { C } & -1.827956 & 4.822704 & 2.639051\end{array}$

C $-0.685306 \quad 5.0532713 .611114$

C $0.6058225 .213456 \quad 3.257217$

C $-1.073975 \quad 5.1110005 .066608$

C 1.1942675 .2406241 .873161

C 1.7583193 .8774181 .470329

C 2.5663253 .8995450 .163745

C $1.7175484 .302903-1.044148$

C $2.5022544 .202529-2.360523$

O $3.0795932 .571926-0.023917$

O $1.5615124 .276696-3.442663$

C $3.1719132 .817125-2.439759$

C $3.4649725 .382196-2.541834$

$\begin{array}{lllll}\text { O } & 4.029919 & 2.729747 & -3.575394\end{array}$

C $3.9447062 .425772-1.158569$

C $4.3828380 .943004-1.249236$

O $5.1062463 .250399-1.046446$

C -1.5683001 .1971162 .985268$

$\begin{array}{llll}\text { O } & -2.499136 & 0.786197 & 3.659112\end{array}$

C -0.3934540 .4009792 .568213$

C $-0.350363-0.9137062 .828521$

C $0.724885-1.8267092 .441537$

C $1.984700-1.2228931 .879660$

$\begin{array}{llll}\text { C } & 4.968815 & 0.372378 & 0.048403\end{array}$

C $5.388213-1.081770-0.104258$

C $6.323211-2.9703001 .239704$ 
O $5.365370-1.682647-1.174847$

C $5.185989-3.9228251 .430071$

C $4.810682-4.4045462 .624746$

C $3.701404-5.3985472 .820632$

C $2.425935-4.7453213 .308242$

C $2.164146-4.6635224 .624062$

C $1.494770-4.2547302 .218529$

C $0.549028-3.1534352 .614804$

N $5.814177-1.6186481 .102632$

H $-2.539527 \quad 2.5471190 .145214$

H -4.5843813 .5397292 .193738$

H -2.817371 3.2582863 .778142

H -2.595317 5.5878662 .815935

H -1.489521 4.9702011 .608056

H 1.3393875 .3556384 .050245

H -0.2372165 .4064675 .708648$

H -1.873662 5.8437155 .217124

H -1.4243104 .1342745 .413488$

H 0.4593865 .5879821 .142149

H 1.9968515 .9887151 .864944

H 2.4021483 .4866302 .269998

H 0.9427203 .1515191 .373872

H 3.4084174 .5892440 .294398

H $1.3158675 .316169-0.930384$

H $0.8449073 .638903-1.108064$

H $1.0747505 .114776-3.360022$

H $2.3740072 .080643-2.599280$

H $2.9123226 .329428-2.550874$

H $3.9797385 .327531-3.507425$

H $4.2166455 .447674-1.752427$

H $3.5016412 .967364-4.356558$

H $3.5154210 .325399-1.514228$

H $5.1223270 .826597-2.051801$

H $5.7727442 .793205-0.511017$

H $\quad 0.397754 \quad 0.9135162 .033904$

H $-1.191480-1.3725243 .346351$

H $2.388611-0.4687232 .564715$

H $1.790841-0.7486990 .912793$

H $2.782367-1.9525301 .728896$

H $5.855327 \quad 0.9372520 .355060$

H 4.2292760 .4347020 .853525

H $7.008624-2.9768852 .093502$

H $\quad 6.890087-3.2417240 .343162$

H $4.661133-4.2473470 .534721$

H $5.334626-4.0754983 .519220$

H $4.043685-6.1550353 .538930$

H $3.500264-5.9533751 .895194$

H $1.249290-4.2247835 .005642$

H $2.866292-5.0447615 .358879$

H $\quad 0.902125-5.1070541 .861791$

H $2.078434-3.9370741 .348461$

H - $0.387245-3.5076923 .046832$

H $5.726964-1.0621531 .948067$

C $-4.7338371 .119828-0.862942$

$\begin{array}{lllll}\text { O } & -4.873100 & 0.214156 & 0.251395\end{array}$

C $-5.972228-0.667217-0.009400$

C $-6.206186-0.617426-1.510866$

C $-5.9190660 .845926-1.794036$

H $-6.846171-0.2159130 .481749$

C -5.746911 -2.0467210 .610560$

H -5.500133 -1.251450 -2.056841

H -7.220949 -0.917040 -1.788938

C $-5.6287951 .137864-3.256288$

H $-6.7884521 .439191-1.479892$

C $-4.729159-2.986932-0.066386$

O $-7.018872-2.7118930 .604756$

H -5.469951 -1.917233 1.664407

H -5.145776 -3.349830 -1.013397

O $-4.627251-4.151750 \quad 0.770496$

C $-3.311942-2.434331-0.318542$

C $-2.360102-3.540947-0.816939$

H -3.350187 -1.627284 -1.056138

O $-2.773401-1.9014810 .887439$

H -2.180940 -4.259451-0.007309

H -2.855202 -4.067612 -1.642377

H $-5.4082772 .199699-3.404701$

H -4.770282 $0.561581-3.617585$

H $\quad-6.4918780 .880609-3.878779$
C $-4.6937312 .565552-0.356622$

H $-6.894745-3.5778591 .030010$

H $-4.192239-3.8800581 .597226$

H -3.277631-1.099984 1.107730

H -5.6237832 .7949790 .177957$

H -4.620378 $3.254950-1.205225$

H $-3.7905520 .863097-1.358217$

C $-0.064502-1.646203-3.078752$

O $-1.225493-2.227680-2.472085$

C $-0.992281-3.015026-1.299649$

C $-0.028547-4.176248-1.608862$

C $1.262655-3.685609-2.270116$

C $0.974811-2.723880-3.427903$

H $-0.429351-1.213063-4.018848$

C $0.522098-0.513977-2.232893$

H $-0.562383-2.378916-0.519036$

O $-0.672418-5.110066-2.475892$

H $0.201094-4.701064-0.674139$

H $1.824679-4.549117-2.646220$

O $2.065143-3.043352-1.284862$

H $1.902498-2.242623-3.757729$

O $0.489188-3.473575-4.544992$

C $-0.4710550 .601008-2.038237$

H $0.824269-0.844841-1.236621$

H $1.418363-0.099679-2.706739$

H $-0.062518-5.855650-2.608890$

H $2.890958-2.761853-1.714221$

H $-0.370927-3.853781-4.296808$

O $-0.5413871 .370966-3.159879$

O $-1.1136420 .755631-1.008495$

C $-1.4687882 .459624-3.109764$

H $-1.2998503 .089826-3.986790$

H $-2.4917222 .075662-3.145328$

H -1.314512 $3.064521-2.211479$

SCF Energy $\left(B 3 L Y P / 6-31 G^{* *} / / M M F F\right)=-3245.92577830$

03178

MM̄FF Geometry

C -3.431573 -1.704896 -2.809554

C $-2.704663-1.049846-1.890853$

C $-1.353746-1.508353-1.409890$

O $-0.524546-0.324954-1.358709$

C $-1.484763-2.1156490 .002948$

C $-0.359131-3.0560120 .396030$

C $0.445136-2.9004991 .466452$

C $-0.204333-4.285771-0.465726$

C $0.415574-1.8183232 .506896$

C $1.459436-0.7117292 .305667$

C $2.915275-1.1633312 .510681$

C 3.8501400 .0489552 .517502

C $5.326327-0.3579972 .621771$

O $3.280602-2.0436831 .444787$

O 6.1038150 .8061402 .300436

C $5.631102-1.4402301 .562097$

C $5.702559-0.7410014 .058111$

O $6.955293-1.9324271 .751899$

C $4.603131-2.5935511 .553444$

C $4.784913-3.5849900 .375945$

O $4.745277-3.3544722 .759737$

C $0.803624-0.454417-1.607727$

O $1.414658-1.479486-1.859793$

C $1.3997250 .896711-1.508672$

C $2.7242251 .023520-1.346525$

C $3.4636262 .279236-1.208061$

C $2.7044433 .565175-1.401541$

C $4.693844-2.971060-1.024268$

C $6.030495-2.514386-1.584350$

C $7.000847-0.987722-3.300960$

O $7.108358-2.941024-1.178559$

C $7.4255590 .228095-2.539563$

C $7.3139611 .480366-3.007808$

C $7.7429052 .697814-2.239216$

C $6.5937413 .637653-1.946131$

C $6.3866514 .730215-2.700241$

C $5.7411883 .361660-0.727177$

C $4.7830372 .220929-0.931718$

N $5.876576-1.603060-2.619753$

H $-3.045203-2.618638-3.253320$ 
H $-3.114386-0.152051-1.433487$ H - $0.940752-2.221488-2.131994$ H $-2.414143-2.6979460 .073538$ H -1.583321 -1.2971910 .723820$ H $1.201524-3.6638141 .646606$ H $0.478026-5.019671-0.023122$ H -1.171559-4.782443 -0.596249 H $0.191999-4.026850-1.451435$ H $-0.577150-1.3622152 .558329$ H $0.564700-2.2839963 .489340$ H $1.341531-0.2931391 .300617$ H 1.2338370 .0897923 .020172 H $2.979060-1.6993273 .464889$ H 3.5858440 .7409623 .327034 H 3.7125240 .6157281 .587569 H 7.0400710 .5417662 .292358 H $5.617547-0.9314770 .590636$ H 5.5429450 .1090614 .732403 H $6.766758-0.9889524 .137731$ H $5.123238-1.5825874 .443577$ H $7.172449-2.5071910 .999497$ H $5.709205-4.1608080 .504127$ H $3.973221-4.3234640 .447739$ H $4.352901-4.2320582 .618708$ H $\quad 0.728991 \quad 1.747491-1.545350$ H $3.3280110 .119913-1.288787$ H $1.9627393 .696783-0.606270$ H $2.1875773 .566562-2.367898$ H $3.3438804 .450736-1.396925$ H $4.323905-3.736051-1.718689$ H $3.976268-2.146326-1.038509$ H $6.676040-0.748008-4.318294$ H $7.830285-1.699876-3.357843$ H $7.8498730 .066269-1.551201$ H $6.897474 \quad 1.643858-3.999002$ H $8.510173 \quad 3.213063-2.831841$ H $8.2366932 .423917-1.298354$ H $5.5841955 .426047-2.479129$ H $7.0123224 .950595-3.558714$ H $5.2138164 .267920-0.409514$ H 6.3944103 .1180420 .120830 H $5.2397451 .239614-0.804821$ H $4.947637-1.256233-2.840742$ C $-5.896263-2.029783-2.501698$ O $-5.805118-1.726845-1.093700$ C $-7.047365-1.152298-0.662158$ C $-8.080450-1.702314-1.631543$ C $-7.308536-1.622989-2.937732$ H $-6.967558-0.068666-0.810158$ C $-7.284851-1.4388420 .823022$ H -8.334814 -2.744465 -1.406966 H $-9.002634-1.114200-1.641417$ C -7.881175 -2.497235 -4.039928 H -7.310415 -0.579610 -3.281330 C $-6.080193-1.0930801 .727696$ O $-7.561110-2.8353770 .978845$ H -8.183568 -0.9057711 .156031$ H $-5.289324-1.8427511 .597212$ O $-6.505614-1.1883303 .092142$ C -5.4842450 .3098351 .501076$ C -4.3575780 .6320312 .502745$ H $-5.093980 \quad 0.3706290 .480006$ O -6.5258941 .2765921 .627990$ H -4.782901 0.7788093 .502899 H -3.675582 -0.2265852 .538632$ H -7.276680 -2.420184 -4.949332 H -7.910282 -3.550808 -3.742273 H $-8.902800-2.188696-4.284210$ C -4.800253 -1.260291-3.243895 H $-7.890947-2.9712571 .882803$ H $-6.697413-2.1231903 .276098$ H $-6.1952512 .118253 \quad 1.272072$ H -4.908180 -0.184555-3.058601 H -4.892061 -1.409912 -4.325644 H -5.745838 -3.111669 -2.604281 C -2.1750372 .7222770 .307913$ O $-2.903573 \quad 1.6259050 .874212$ C -3.5620561 .8946512 .116651$
C $-2.5304582 .281393 \quad 3.187951$

C -1.642095 3.4397842 .719289

C $-1.1130043 .238371 \quad 1.294654$ H -1.652008 $2.294077-0.556340$ C $-3.1083853 .820241-0.212932$ H $-4.2650762 .722751 \quad 1.985172$ O $-1.7124921 .166133 \quad 3.531103$ H -3.064129 2.5715924 .100439 H -0.7926483 .5501743 .404448$ O -2.3910664 .6525992 .755539$ H -0.6933494 .1779610 .917170$ O $-0.0270022 .309028 \quad 1.340567$ C $-4.0061213 .284014-1.303921$ H -3.729034 4.2529920 .576677 H -2.534524 $4.649080-0.643472$ H -1.4155130 .7477512 .707077$ H -2.6740714 .7954983 .674911$ H $-0.379127 \quad 1.416101 \quad 1.191210$ O $-5.3027783 .282654-0.882244$ O $-3.6016162 .903081-2.394985$ C $-6.2596862 .800253-1.829844$ H $-6.3224563 .485788-2.679973$ H $-5.9946861 .793907-2.165028$ H -7.234669 2.758266 -1.337166 SCF Energy (B3LYP/6-31G**//MMFF) $=-3245.91407195$

03_179

MM̄FF Geometry

C -2.490338 -2.544995 1.568791

C $-1.278384-3.1187341 .570696$

C $-0.296024-2.9813270 .434847$

O $\quad 0.831251-2.209522 \quad 0.905278$

C $0.212712-4.3722200 .013803$

C $1.119226-4.349075-1.201710$

C $2.416680-4.712876-1.197586$

C $0.472997-3.911532-2.491594$

C $3.240037-5.207133-0.041644$

C $4.004265-4.1118280 .718427$

C $5.038690-3.356422-0.132366$

C $5.912236-2.4594620 .748215$

C $6.896096-1.618619-0.081791$

O $4.341045-2.550088-1.085115$

O $7.425455-0.5911230 .770676$

C $6.124515-0.909441-1.216616$

C $8.095504-2.448813-0.554564$

O $7.021518-0.255285-2.109975$

C $5.190220-1.850058-2.004626$

C $4.270453-1.105818-3.003619$

O $5.982221-2.766001-2.771150$

C $0.738775-0.8554650 .803798$

$\begin{array}{lllll}\text { O } & -0.180762 & -0.212477 & 0.323042\end{array}$

C $1.954861-0.2753671 .413039$

C 1.9485871 .0133101 .783920

C 3.0547451 .7372452 .411513

C 4.4087891 .0792462 .400026

C $3.339932-0.055440-2.387172$

C $3.8885241 .361046-2.439219$

C $3.3846593 .667995-1.644437$

O $4.8907271 .674076-3.076076$

C $4.2063673 .949873-0.426382$

C $3.8984334 .907600 \quad 0.461364$

C $4.7299605 .230577 \quad 1.669773$

C 3.9273915 .2003962 .951306

C 3.4356426 .3311193 .485579

C 3.7658353 .8707283 .653399

C 2.8086852 .9533072 .942612

N $3.0995732 .247210-1.717584$

H $-2.779898-1.9281540 .721382$

H $-0.971134-3.7139402 .427170$

H $-0.763002-2.482808-0.423151$

H $-0.639778-5.026509-0.213463$

H $\quad 0.726309-4.8297340 .866201$

H $2.963472-4.659636-2.138665$

H $0.225354-2.846337-2.458630$

H $1.125694-4.068954-3.357230$

H $-0.445424-4.480671-2.669380$

H $2.617814-5.7638260 .666999$

H $3.957157-5.943163-0.427577$ 
H $3.281875-3.4022451 .137785$ H $4.515235-4.5892801 .563754$ H $5.657208-4.093487-0.657972$ H $6.452826-3.0542201 .494978$ H $5.269636-1.7764141 .320047$ H $7.850946-1.0225461 .531454$ H $5.522126-0.123040-0.746495$ H $8.638479-2.8603680 .304698$ H $8.818358-1.827430-1.094459$ H $7.811920-3.285939-1.195862$ H $7.5726670 .345171-1.579280$ H $4.865809-0.688058-3.824460$ H $3.623925-1.862475-3.471628$ H $5.436018-3.114898-3.494987$ H $2.808451-0.9261431 .560112$ H 1.0397021 .5938201 .634182 H 4.4158070 .2002243 .053275 H 5.2169621 .7357002 .728585 H 4.6715090 .7648921 .384230 H $2.405637-0.040163-2.963005$ H $3.083809-0.312776-1.355388$ H $2.4234934 .191426-1.626259$ H $3.9345373 .976356-2.539498$ H $5.1066313 .357305-0.280570$ H $3.0121465 .516746 \quad 0.297158$ H 5.1583226 .2306281 .522165 H 5.5878394 .5527431 .760398 H 2.8817556 .3221964 .418390 H 3.5720137 .2911152 .998492 H 3.3709204 .0250134 .666563 H 4.7540013 .4273993 .801975 H 1.7899423 .3414692 .895592 H $2.3153801 .891569-1.178498$ C -4.896298-3.058769 2.205656 O $-5.426643-2.0020941 .377139$ C $-5.912377-2.587010 \quad 0.157281$ C $-6.179463-4.048850 \quad 0.492145$ C $-4.999901-4.3549311 .395940$ H $-5.088999-2.538214-0.566111$ C $-7.113325-1.812076-0.387584$ H -7.119094 -4.175155 1.042214 H -6.213951 -4.682721 -0.398839 C $-5.186166-5.5961052 .251244$ H -4.113919 $-4.496730 \quad 0.765147$ C $-6.888151-0.294549-0.553609$ O $-8.218674-1.9876520 .508128$ H -7.422050 -2.255697 -1.342204 H -6.9140750 .1922010 .429372$ O $-8.0235820 .198111-1.278385$ C $-5.5919530 .097509-1.290494$ C $-5.533606 \quad 1.590267-1.668987$ H $-4.734334-0.153147-0.657670$ O $-5.468404-0.673221-2.487829$ H $-6.2383521 .770871-2.488969$ H $-5.8622672 .189527-0.814631$ H -4.316761 -5.7557272 .897018$ H -6.071690 -5.514922 2.890556 H $-5.307750-6.4829081 .620685$ C -3.489612 -2.683749 2.684504 H -8.974801 -1.5045310 .132872$ H $-8.0496551 .163444-1.166884$ H -6.258861 -0.505570 -3.029143 H -3.148024 -3.422643 3.418824 H -3.543480 -1.715470 3.195999 H -5.554982 -3.107731 3.082152 C $-3.3453243 .012986-0.048201$ O $-3.1755141 .994362-1.045024$ C $-4.1192652 .021415-2.123028$ C $-4.0662103 .382144-2.829037$ C $-4.2795604 .530255-1.851048$ C $-3.3089174 .415320-0.677599$ H -4.2991042 .8660220 .471761$ C -2.2105512 .8390230 .975259$ H $-3.7730421 .276141-2.850038$ O $\quad-5.0388493 .461572-3.865522$ H $-3.0834583 .508315-3.301968$ H -5.318137 4.573901-1.501786 O $-4.027475 \quad 5.768138-2.520907$
H $-2.2967324 .656238-1.027749$ $\begin{array}{lllll}\text { O } & -3.680418 & 5.398767 & 0.287656\end{array}$ C -2.4307631 .6548161 .893008$ H -1.2550642 .6864250 .460076$ H -2.1382453 .7222301 .618147$ H $-4.8708592 .726947-4.480228$ H -4.626485 $5.809388-3.286173$ H -2.9285175 .5266910 .889398$ O -1.4875221 .6890292 .881163$ O C $-1.5502910 .633673 \quad 3.844809$ H -2.5597350 .5427814 .257429$ H $-1.231898-0.3056753 .386046$ H -0.8617010 .8765624 .658537$ SCF Energy $\left(B 3 L Y P / 6-31 G^{* *} / / M M F F\right)=-3245.90797146$

0318

MM̄FF Geometry

C $2.2005204578370-0.436301$

C $1.411473 \quad 3.9054550 .413602$

C -0.0685693 .7352290 .174906$

$\begin{array}{llll}\text { O } & -0.391422 & 2.325932 & 0.228562\end{array}$

C $-0.870974 \quad 4.4564381 .272274$

C -2.3545994 .5611570 .969106$

C -3.3308104 .0001731 .708510$

C $-2.7232125 .394190-0.232912$

C -3.189356 3.1801862 .957755

C -3.0542061 .6699812 .721079$

C $-4.253477 \quad 1.0267392 .007977$

C $-4.159459-0.4998632 .068712$

C $-5.294169-1.1783611 .285436$

O $-4.246277 \quad 1.4491920 .641716$

O $-4.950483-2.563291 \quad 1.122592$

C $-5.368062-0.562933-0.129007$

C $-6.617546-1.1489592 .059071$

O $-6.509561-1.043503-0.833747$

C $-5.3607150 .979694-0.129528$

C $-5.2279251 .591075-1.546233$

O -6.5974051 .4561020 .415822$

C $-0.0575221 .569311-0.853099$

O $0.483501 \quad 1.948777-1.879575$

C $-0.4551440 .174484-0.565159$

C $0.031498-0.818508-1.323727$

C $-0.263309-2.244487-1.172171$

C $-1.355620-2.630911-0.210889$

C $-3.9733891 .181313-2.325791$

C $-4.1891260 .005056-3.264184$

C $-2.931480-1.558490-4.740911$

O $-5.298954-0.440654-3.542770$

C $-2.685690-2.841493-4.011252$

C $-1.732454-3.716227-4.366698$

C $-1.492119-5.025683-3.671093$

C $-0.053332-5.196745-3.237173$

C $0.817132-5.892047-3.988602$

C $0.353113-4.630410-1.895454$

C $0.442756-3.130350-1.905566$

N -2.992474 -0.464622 -3.789194

H $1.7497615 .051214-1.306096$

H 1.8410483 .4307941 .291713

H $-0.3455334 .123842-0.812415$

H $-0.4888575 .477481 \quad 1.405395$

H -0.7046613 .9409762 .224398$

H $-4.3625164 .148766 \quad 1.390784$

H $-2.4377414 .887059-1.159399$

H $-3.7995585 .590915-0.286751$

H $-2.2183416 .364919-0.192961$

H -2.336719 3.5268343 .551864

H -4.0687783 .3677343 .587348$

H -2.1402311 .4837982 .145960$

H $-2.925622 \quad 1.196271 \quad 3.700347$

H -5.1728751 .3722092 .495040$

H -4.150149 -0.8491183 .108799$

H -3.201882 -0.821434 1.637803

H $-4.821970-2.9462682 .007332$

H $-4.487908-0.922779-0.675440$

H $-6.510275-1.6655483 .020518$

H -7.403945 -1.686110 1.517622

H $-6.970550-0.1368632 .267144$ 
H $-6.462856-2.014981-0.833068$ H $-6.1416981 .405703-2.123456$ H -5.179739 2.682914 -1.423244 H -6.7356522 .3693190 .114276$ H -1.1276980 .0030020 .266589$ H $\quad 0.726933-0.568649-2.124185$ H -1.050478 -2.4250700 .820181$ H -1.632020 -3.685735 -0.263400 H -2.271660 -2.070417 -0.426804 H -3.669277 $2.023275-2.960883$ H $-3.1418970 .965913-1.648413$ H -2.131491 -1.326109 -5.450958 H -3.877856 -1.620749 -5.287596 H -3.334557 -3.072639 -3.169707 H -1.104357 -3.493861 -5.226809 H $-1.768973-5.828398-4.367229$ H -2.152092 -5.146788 -2.803234 H $1.844049-6.042284-3.672566$ H $\quad 0.522649-6.322506-4.940141$ H $1.342941-5.010860-1.609130$ H $-0.326179-5.013688-1.129460$ H $\quad 1.211383-2.748222-2.578807$ H $-2.115444-0.067505-3.464894$

C $4.4990643 .902876-1.291175$

O $4.5039702 .516329-0.899553$

C $4.5218221 .703177-2.089311$

C $4.6957222 .659162-3.270247$

C $4.041483 \quad 3.922289-2.749573$

H $3.5339761 .231913-2.155502$

C $5.6072670 .623682-2.000889$

H $5.7509922 .847639-3.497535$

H $4.2255532 .275796-4.181236$

C $4.4389935 .178492-3.503405$

H $2.9568543 .784163-2.829414$

C $5.568613-0.241808-0.728055$

O $6.888067 \quad 1.269453-2.035257$

H $5.556657-0.009636-2.895303$

H $5.903667 \quad 0.3424310 .138115$

O $6.528665-1.294763-0.898640$

C $4.205703-0.883481-0.402906$

C $4.320478-1.9092320 .741090$

H $3.493492-0.098362-0.128770$

O $3.689133-1.537851-1.560428$

H $4.908701-2.7672110 .393473$

H $4.876174-1.4506751 .565682$

H $3.9447026 .058629-3.080102$

H $5.5204625 .346477-3.462554$

H $4.1505895 .102433-4.556897$

C $3.6861824 .734390-0.291854$

H $7.5559710 .587554-2.217777$

H $7.413386-0.893791-0.862946$

H 4.337172 -2.209398 -1.833970

H $3.9228295 .796475-0.427889$

H 3.9898594 .4679610 .728159

H $5.5452244 .231266-1.224751$

C $2.524327-0.7508142 .912520$

O $2.097526-1.3890831 .703414$

C $2.956432-2.4358041 .239126$

C $3.074163-3.5235492 .316061$

C $3.567062-2.9468203 .640918$

C $2.697012-1.7622184 .058665$

H $3.480333-0.2480562 .722106$

C $1.525607 \quad 0.371347 \quad 3.229957$

H $2.455445-2.8880780 .375855$

O $3.956060-4.561118 \quad 1.900915$

H $2.090873-3.9832382 .480353$

H $4.624638-2.6630023 .591751$

O $3.470277-3.9523224 .653376$

H $1.729539-2.1593204 .388571$

O $3.305466-1.1291195 .181744$

C $0.202665-0.1556103 .736385$

H $1.9464861 .060523 \quad 3.971571$

H $\quad \begin{array}{llll}1.312517 & 0.968158 & 2.335371\end{array}$

H $3.613104-4.9174871 .063458$

H $3.996732-4.7137654 .354722$

H $2.675789-0.4758585 .530371$

O $\quad 0.107496 \quad 0.0517855 .081163$

O $-0.623635-0.719938 \quad 3.032141$
C $-1.047128-0.499303 \quad 5.722627$

H $-0.860106-0.5148076 .799802$

H -1.915571 0.1338425 .526992

$\mathrm{H}-1.233010-1.5249405 .389116$

SCF Energy $\left(B 3 L Y P / 6-31 G^{* *} / / M M F F\right)=-3245.90311546$

03180

MM̄FF Geometry

C -3.613693 -3.104550-1.669322

C $-2.398491-2.716018-2.086570$

C $-1.069270-2.871668-1.382944$

O $-0.499907-1.537968-1.350996$

C $-1.127235-3.4385990 .051390$

C $0.200724-3.9383860 .591453$

C $0.855851-3.4234301 .650535$

C $0.763476-5.156351-0.101347$

C $0.463776-2.2689422 .527711$

C $1.197754-0.9576522 .214138$

C $2.705445-0.9713592 .517151$

C 3.2978990 .4293312 .331688

C 4.8221110 .4474292 .527937

O $3.351991-1.8849321 .627382$

O 5.3194731 .6896122 .005855

C 5.454435-0.680762 1.683276

C 5.2036460 .4195554 .012644

O $6.846693-0.8039531 .959341$

C $4.758428-2.0449531 .864900$

C $5.275848-3.1357390 .893926$

O $5.004660-2.5261053 .192491$

C $0.811116-1.386898-1.675458$

O $1.629968-2.267104-1.883108$

C $1.0827530 .065761-1.760793$

C $2.3473410 .505346-1.687813$

C $2.7852731 .899482-1.769822$

C $1.7855602 .922538-2.239357$

C $5.134004-2.814894-0.596753$

C $6.362099-2.153936-1.200665$

C $7.066809-0.780430-3.158702$

O $7.480386-2.227986-0.698244$

C $7.1685880 .613277-2.624627$

C $6.8122691 .706615-3.315281$

C $6.9164283 .103235-2.772781$

C $5.5731873 .793346-2.678331$

C $5.1795194 .666406-3.620839$

C $4.7286813 .543124-1.448264$

C $4.0639282 .193966-1.456577$

N $6.070523-1.508126-2.394171$

H $-3.737389-3.599569-0.712123$

H -2.329495 -2.248291-3.068695

H $-0.464177-3.519096-2.028539$

H $-1.809005-4.2971980 .091717$

H -1.553063 -2.675485 0.708545

H $1.784949-3.9069111 .950591$

H $1.644890-5.5568090 .411141$

H $0.015055-5.955087-0.131516$

H $1.063486-4.921045-1.126222$

H -0.613429 -2.086609 2.472931

H $0.654101-2.5512333 .570858$

H $1.034976-0.7079461 .160593$

H $0.730066-0.1654542 .811350$

H $2.843162-1.3095573 .550954$

H 2.8160151 .1481183 .006374

H $3.0776310 .783351 \quad 1.316043$

H 4.8677892 .4135732 .472560

H $5.367933-0.3750730 .633494$

H 4.7909551 .2940354 .530063

H 6.2895040 .4804284 .143440

H $4.841401-0.4699084 .532143$

H 7.2502000 .0680721 .809311

H $\quad 6.306547-3.4086141 .151160$

H $4.680881-4.0417981 .078831$

H $4.862830-3.4872353 .203497$

H $\quad 0.234810 \quad 0.731967-1.873275$

H $3.141829-0.222629-1.535369$

H $1.0112803 .079358-1.483289$

H $1.3066732 .597746-3.170042$

H $2.2325033 .896176-2.453427$

H $5.000411-3.755656-1.145959$ 
H $4.245355-2.204335-0.779735$

H $6.754800-0.801628-4.207573$

H $8.035071-1.283759-3.072116$

H $7.5592770 .726216-1.615816$

H $6.4292461 .597145-4.327193$

H $7.5860723 .668850-3.433872$

H $7.4003363 .115580-1.788246$

H $4.2326535 .190731-3.546429$

H $5.7940644 .873138-4.490793$

H $3.9905654 .342289-1.315085$

H $5.3645963 .611256-0.556000$

H $4.7150931 .383352-1.130820$

H $5.103854-1.442240-2.699226$

C $-6.034040-2.375771-1.693299$

O $-5.753411-1.028519-1.262910$

C $-6.892785-0.199290-1.556792$

C $-8.043935-1.154173-1.847847$

C $-7.317690-2.297403-2.523551$

H $-6.6480030 .349026-2.474736$

C $-7.1677150 .794970-0.423876$

H $-8.526324-1.506453-0.928926$

H -8.812483 -0.698275 -2.479326

C $-8.114848-3.590320-2.553071$

H -7.082113 -1.999949 -3.554331

C -5.9526321 .6363940 .011957$

$\begin{array}{lllll}\text { O } & -7.613119 & 0.066479 & 0.728695\end{array}$

H -8.001294 $1.446810-0.712356$

H -5.2641220 .9950880 .568328$

O -6.4291382 .6159710 .943626$

C $-5.1922792 .342242-1.120305$

C $-4.1163363 .349153-0.666168$

H $-4.7199131 .589198-1.761407$

O $-6.1374183 .050445-1.927987$

H -3.656504 $3.763365-1.573618$

H -4.607559 4.195497-0.168165

H -7.547463 -4.388396 -3.042028

H -8.369264 -3.927959-1.542756

H $-9.049629-3.452980-3.106558$

C $-4.857350-2.932542-2.498509$

H -7.7812530 .7144651 .434242$

H $-6.996606 \quad 3.2353290 .453197$

H -5.653217 $3.434427-2.678766$

H -4.650768 -2.269048 -3.347854

H -5.126120 -3.914785 -2.904238

H -6.195305 -2.971854 -0.785882

C -2.6726742 .1054542 .557832$

O -3.5486002 .6540761 .564510$

C -3.0188802 .7601350 .239295$

C -1.767422 $3.653606 \quad 0.248542$

C -0.7317193 .1689561 .265976$

C -1.3571192 .8997732 .636351$

H -3.202638 2.2516723 .507478

C -2.4622540 .5956912 .365029$

H $-2.7559771 .764238-0.133494$

O $-2.124905 \quad 5.003742 \quad 0.541234$

H -1.326635 $3.651354-0.754911$

H 0.0535953 .9292931 .362086

$\begin{array}{lllll}\text { O } & -0.130621 & 1.977772 & 0.772466\end{array}$

H -0.6424652 .3765863 .282429$

O $-1.625426 \quad 4.164791 \quad 3.248667$

C $-3.733487-0.1921832 .606785$

H -2.111768 0.3671601 .354852

H -1.722599 $0.227703 \quad 3.084180$

H -2.578384 5.0112281 .401612

H $\quad 0.600016 \quad 1.7517601 .372360$

H -1.958995 3.9925754 .145790

O $-3.606994-1.4293582 .042880$

O $-4.704896 \quad 0.212271 \quad 3.231071$

C -4.732901 -2.297262 2.204059

H -5.610026 -1.873724 1.706619

H $-4.492906-3.256177 \quad 1.737655$

H -4.934272 -2.466026 3.266246

SCF Energy (B3LYP/6-31G**//MMFF) $=-3245.90752872$

03_181

MM̄MF Geometry

C 1.4606162 .2009062 .424585

C 1.3016561 .1253413 .210918
C $\quad 0.317407-0.0047272 .996928$

O $-\begin{array}{llll}0.615610 & 0.289326 & 1.934241\end{array}$

C $1.101987-1.2685632 .598491$

C $0.250199-2.5138112 .459410$

C $0.087110-3.204721 \quad 1.313835$

C $-0.414563-2.9950553 .723231$

C $0.681948-2.917521-0.036459$

C $-0.198011-2.053648-0.953266$

C -1.536942 -2.705270 -1.336497

C $-2.248939-1.886825-2.416002$

C $-3.631731-2.465427-2.758522$

O $-2.362048-2.770113-0.170965$

O $-4.348820-1.475696-3.512768$

C $-4.422688-2.696388-1.452064$

C $-3.523725-3.701498-3.659338$

O $-5.641188-3.388930-1.710044$

C $-3.615237-3.438978-0.367892$

C $-4.330198-3.4858311 .004761$

O $-3.413563-4.796744-0.779688$

C $-1.717474 \quad 1.0222722 .250768$

O -2.0288771 .4499393 .350543$

C -2.4699251 .2524960 .997995$

C -3.3609312 .2532080 .938834$

C $-4.1596062 .628424-0.230090$

C $-4.1476971 .692930-1.409981$

C $-4.652214-2.1225501 .626806$

C $-6.065507-1.6363781 .350516$

C -7.5321070 .3509951 .679153$

O $-6.933116-2.3378630 .837766$

C $-7.577357 \quad 1.1342390 .405381$

C -7.9101042 .4328520 .352716$

C $-8.0029573 .223153-0.920825$

C $-7.1735814 .486932-0.882823$

C -7.733849 $5.672759-0.589596$

C $-5.7155214 .387202-1.269284$

C $-4.8678523 .776331-0.187104$

N $-6.255580-0.3306361 .783410$

H $0.8349962 .328840 \quad 1.545543$

H 1.9527841 .0152254 .076215

H $-0.228079-0.1753843 .932798$

H $1.876464-1.4741673 .348876$

H $1.634467-1.0619891 .663659$

H -0.543295 -4.093049 1.340122

H -1.188883 -2.291754 4.044273

H $-0.892587-3.9723073 .595272$

H $0.323134-3.0958564 .526006$

H $1.661699-2.4410490 .066278$

H $\quad 0.884539-3.876730-0.529796$

H $-0.380780-1.089392-0.464493$

H $\quad 0.373152-1.849261-1.867273$

H - $-1.333920-3.719795-1.698984$

H -1.631031 -1.812200 -3.319589

H $-2.382769-0.856364-2.060328$

H $-3.824802-1.264653-4.304486$

H $-4.700718-1.706762-1.071281$

H $-3.015146-3.448135-4.597254$

H -4.514930 -4.068890 -3.947218

H -2.971489-4.522305 -3.196981

H $-6.130028-2.876887-2.376829$

H $-5.219403-4.1249800 .945142$

H $-3.652800-3.9977381 .703634$

H -3.218930 -5.332117 0.007395

H $-2.2480590 .612106 \quad 0.152371$

H -3.5027802 .8762041 .821188$

H $-3.1638601 .692951-1.891156$

H -4.885398 1.944327 -2.174588

H $-4.3833510 .672253-1.090719$

H -4.570794 -2.212585 2.717773

H $-3.933100-1.3625281 .307311$

H -7.6319720 .9896682 .562484$

H $-8.341504-0.3861591 .687486$

H -7.352543 $0.603555-0.516957$

H -8.1620412 .9530201 .274152$

H -9.059686 $3.475699-1.079404$

H -7.710597 2.620745 -1.789881

H $-7.1521406 .588521-0.587972$

H -8.786494 $5.753040-0.339161$

H $-5.3100585 .388816-1.466946$ 
H $-5.6345573 .862376-2.225070$

H $-4.815404 \quad 4.3834600 .717840$

H -5.4655950 .1936252 .148276$

C 3.5528393 .2106871 .537668

O 2.9556253 .6327710 .292436

C $3.8642884 .518157-0.376195$

C 5.2039824 .3727620 .331742

C 4.7491924 .1350621 .761012

H $3.4950145 .534924-0.181102$

C $3.8650224 .307067-1.893684$

H $5.7632223 .505211-0.035009$

H 5.8362055 .2590100 .223600

C 5.8295163 .5423312 .649231

H 4.4122295 .0886632 .189221

C $4.4498142 .985447-2.437074$

O $4.6206055 .387214-2.459517$

H $2.8376294 .417905-2.263393$

H $5.5345742 .971142-2.278377$

O $4.2557173 .018889-3.859503$

C $3.8163021 .699662-1.875948$

C $4.3410670 .417572-2.547000$

H $4.0320371 .640386-0.808339$

O $2.3983651 .751993-2.022982$

H $4.0287020 .399018-3.597902$

H $5.4358650 .438462-2.532003$

H 5.4521533 .3709323 .662113

H 6.1905482 .5851582 .257877

H $6.685678 \quad 4.2213472 .717996$

C 2.5076993 .2569222 .651883

H $4.5921645 .284561-3.426367$

H $4.8543932 .362734-4.254503$

H $2.2044631 .851401-2.970859$

H 2.0079244 .2327922 .666579

H 2.9877923 .1161903 .626698

H $3.8983132 .179191 \quad 1.394445$

C $5.423188-1.024925-0.077924$

O $4.051073-0.885342-0.458724$

C $3.815570-0.870562-1.869932$

C $4.342620-2.167296-2.498732$

C $5.815267-2.398911-2.166144$

C $6.048613-2.302748-0.657712$

H $5.979365-0.155815-0.451557$

C $5.501748-0.9315211 .454222$

H $2.726053-0.868728-1.996101$

O $4.180347-2.149827-3.912946$

H $3.763175-3.018458-2.117745$

H $6.465203-1.703202-2.709492$

O $6.189976-3.710963-2.594280$

H $5.634488-3.202825-0.190056$

O $7.454972-2.310605-0.421987$

C $4.824476-2.0678082 .193745$

H $6.552858-0.8945731 .761762$

H $5.011053-0.009317 \quad 1.786835$

H $3.233949-2.021546-4.096330$

H $\quad 6.001005-3.768724-3.546595$

H $7.594337-2.4338650 .531938$

O $5.259721-2.0733563 .489065$

O $4.000796-2.834576 \quad 1.715352$

C $4.702543-3.0954364 .321674$

H $3.620090-2.9644894 .409790$

H $4.940686-4.0846753 .919222$

H $5.148649-3.0045615 .315565$

SCF Energy (B3LYP/6-31G**//MMFF) $=-3245.89689237$

03_182

MM̄FF Geometry

C $0.7287074 .788698-1.740387$

C $-0.0276914 .415104-0.697229$

C -1.482188 $4.035363-0.804863$

O $-1.6175972 .659575-0.375086$

C -2.3188774 .9186490 .137949$

C -3.8089084 .6460640 .074185$

C $-4.5467754 .187706 \quad 1.104469$

C $-4.4747694 .950567-1.243336$

C -4.0895953 .8561052 .497645$

C -3.6668592 .3930702 .704729$

C -4.7982351 .3744202 .489676$

C $-4.359874-0.0243242 .930231$
C $-5.436862-1.0831052 .642158$

O

O $-4.828144-2.3773792 .772491$

C $-5.900120-0.9488401 .174585$

C $-6.572691-1.0335503 .671115$

O $-7.015275-1.7965480 .912962$

C $-6.2347020 .500670 \quad 0.766917$

C $-6.4998730 .666604-0.749352$

O -7.4254970 .9178701 .446212$

C $-1.2838161 .690143-1.271047$

O $-0.922614 \quad 1.847614-2.426402$

C $-1.4021570 .384633-0.584428$

C $-0.847391-0.703935-1.138393$

C $-0.854710-2.058361-0.580353$

C $-1.684248-2.2981320 .652897$

C $-5.3465070 .251096-1.669282$

C $-5.467084-1.166567-2.203588$

C $-4.202044-2.857833-3.523770$

O $-6.477692-1.852339-2.076600$

C $-3.571249-3.831271-2.579031$

C $-2.527627-4.605256-2.912431$

C $-1.905981-5.616802-1.993119$

C $-0.414349-5.422385-1.841209$

C $0.455155-6.146304-2.566262$

C $0.067750-4.449139-0.789728$

C $-0.126603-3.013262-1.196344$

N $-4.320421-1.563729-2.878596$

H $0.2812014 .855408-2.728748$

H $\quad 0.4267704 .3393990 .288076$

H -1.842560 $4.135900-1.835879$

H $-2.1566995 .977239-0.105216$

H -1.9498274 .7840421 .160487$

H -5.6130894 .0353940 .940143$

H -5.566362 $4.880526-1.183010$

H -4.231119 $5.967906-1.566539$

H $-4.1444384 .248462-2.014830$

H -3.2653214 .5110612 .799420$

H -4.9083134 .0951853 .188649$

H -2.831878 2.1660132 .031582

H -3.2895042 .2978403 .730585$

H -5.6699451 .7007483 .068905$

H -4.091461 -0.0314793 .993966$

H -3.445421 -0.3046972 .390185$

H -4.464891 -2.448554 3.671790

H $-5.076950-1.3127690 .548454$

H $-6.182179-1.2087384 .680848$

H -7.303816 -1.829707 3.491816

H -7.099116 -0.077126 3.683609

H $-6.755973-2.7009021 .159260$

H $-7.4377820 .169188-1.024408$

H $-6.6767621 .736887-0.930361$

H $-7.8145551 .662086 \quad 0.957755$

H -1.9226160 .3599920 .365679$

H $-0.316601-0.592798-2.083337$

H -2.717366 -1.975137 0.486149

H $-1.274864-1.7453141 .505363$

H -1.741211 -3.3480810 .946075$

H $\quad-5.3515770 .907619-2.548747$

H $-4.3794800 .377104-1.173042$

H -3.606194 -2.714882-4.430906

H $-5.196454-3.214320-3.810885$

H -4.007377 -3.922282 -1.586980

H -2.112169-4.531245 -3.915149

H $-2.111946-6.613214-2.405848$

H -2.378643 -5.604185 -1.003140

H $1.527107-6.032112-2.443367$

H $\quad 0.113617-6.865856-3.303027$

H $1.142423-4.591009-0.614300$

H $-0.406293-4.6941910 .164264$

H $\quad 0.440102-2.736467-2.086697$

H $-3.509641-0.951687-2.888285$

C $3.0101683 .858665-1.204732$

O $2.8943842 .815753-2.191193$

C $4.1228222 .052824-2.200450$

C $5.0244482 .681496-1.143492$

C $4.5091274 .106338-1.082349$

H $4.5504482 .186120-3.201509$

C $3.7737740 .579709-1.953577$ 
H $4.8913282 .189478-0.171759$

H $6.0837292 .618698-1.410016$

C 4.9221414 .8420240 .180079

H $4.8753304 .653124-1.961408$

C $4.995770-0.323800-1.721203$

O $3.0555540 .094403-3.092801$

H $3.0842990 .513586-1.105089$

H $5.5442650 .002786-0.832514$

O $5.909116-0.165379-2.814517$

C $4.673350-1.823777-1.589279$

C $3.576976-2.182274-0.570019$

H $4.379437-2.227796-2.565902$

O $5.872393-2.516391-1.227852$

H $2.611590-1.836307-0.953609$

H $3.535134-3.277786-0.512850$

H 4.5261535 .8621480 .185753

H 4.5569174 .3336291 .078872

H 6.0132294 .9034790 .249185

C $2.2015115 .080955-1.642634$

H $2.2829770 .672629-3.215763$

H $5.434020-0.402112-3.629616$

H $6.552375-2.282015-1.882413$

H $2.5407645 .409025-2.633263$

H $2.3571705 .920226-0.955487$

H $2.6191223 .454575-0.261537$

C $5.355489-1.7020492 .692156$

O $4.978589-2.182774 \quad 1.395214$

C $3.781591-1.6228270 .852751$

C $2.585070-1.9372321 .766160$

C $2.848031-1.4729293 .202444$

C $4.222043-1.9269663 .709648$

H $6.204776-2.3308872 .988064$

C $5.848264-0.2467562 .644416$

H $3.892301-0.5374920 .784497$

O $2.331337-3.3400311 .755652$

H $1.695497-1.4360081 .366478$

H $2.065887-1.8752983 .857837$

O $2.756674-0.0517193 .242245$

H 4.462661-1.4209774.651959

O $4.138925-3.3261153 .996660$

C $7.064991-0.0820641 .757587$

H $5.072451 \quad 0.4233152 .264673$

H 6.1293080 .0843043 .650493

H $1.541646-3.4993872 .300043$

H 2.8971820 .2254454 .163656

H $4.992683-3.6011394 .371929$

O $7.2398691 .245726 \quad 1.487091$

O $7.780043-0.9928901 .363485$

C 8.3459541 .5597210 .635353

H 9.2859841 .2735691 .116285

H $8.236044 \quad 1.057402-0.330725$

H 8.3503222 .6402920 .469061

SCF Energy (B3LYP/6-31G**//MMFF) $=-3245.89143998$

03_183

MM̄FF Geometry

C -1.667249 -1.818760 1.691399

C $-0.424984-2.3168101 .595738$

C $0.468928-2.1272060 .397523$

O $1.726904-1.569194 \quad 0.840757$

C $0.754525-3.494878-0.249694$

C $1.559194-3.410534-1.531979$

C $2.784473-3.944000-1.704002$

C $0.890683-2.696741-2.679215$

C $3.610131-4.713916-0.711920$

C $4.582393-3.8582530 .115491$

C $5.648059-3.129332-0.719705$

C $6.712210-2.5044570 .185582$

C $7.742143-1.689462-0.613554$

O $5.004841-2.099530-1.474759$

O $8.485692-0.8863480 .316399$

C $6.998256-0.716362-1.554726$

C $8.761752-2.593732-1.316193$

O $7.909000-0.062555-2.434494$

C $5.872401-1.387999-2.367742$

C $4.996245-0.379370-3.150603$

O $6.453275-2.273414-3.333203$

C $1.767784-0.2303381 .079562$
O $\quad 0.856153 \quad 0.5706390 .949244$

C 3.1291160 .0955431 .558314

C 3.3460951 .2620032 .183914

C 4.6249641 .7251592 .726813

C 5.8565370 .9325372 .377368

C $4.2825430 .674956-2.296900$

C $5.0289731 .995077-2.195813$

C $4.9379124 .190542-1.020778$

O $6.0085682 .271194-2.882756$

C 5.8927814 .1647350 .130689

C 5.7978074 .9922251 .182544

C 6.7716095 .0104332 .325689

C 6.0932454 .8755553 .670449

C 5.8223455 .9578384 .419499

C 5.8077173 .4814454 .181404

C 4.6366762 .8369473 .491803

N $4.4437642 .849398-1.270528$

H -2.075903 -1.229111 0.875301

H $-0.023691-2.9068922 .416760$

H $0.007836-1.454992-0.333605$

H $-0.192433-4.001927-0.479640$

H $1.266369-4.1275340 .483561$

H $3.260759-3.823367-2.676538$

H $0.829164-1.622672-2.480652$

H $1.434545-2.824723-3.621529$

H $-0.121344-3.085072-2.832796$

H $2.967471-5.281950-0.030900$

H $4.179819-5.473131-1.263078$

H $4.005922-3.1290390 .696698$

H $5.080530-4.5219980 .833078$

H $6.106269-3.854032-1.403039$

H $7.215155-3.2739650 .784540$

H $6.225744-1.8350550 .907895$

H $8.901430-1.4829790 .962116$

H $6.5612820 .064189-0.921126$

H $9.302486-3.203232-0.582117$

H $9.525292-2.001861-1.832948$

H $8.305600-3.275028-2.037307$

H $8.5858530 .367246-1.883955$

H $5.5795900 .079249-3.958012$

H $4.209967-0.959427-3.655441$

H $5.804967-2.426908-4.040273$

H $3.908974-0.6401571 .400284$

H $2.503971 \quad 1.9367142 .333534$

H $5.831051-0.0507342 .859024$

H $\quad 6.788219 \quad 1.4177142 .675392$

H $5.925650 \quad 0.7919191 .293434$

H $3.3188560 .907317-2.768345$

H $4.0715570 .293550-1.293480$

H $4.0682304 .825451-0.824072$

H $5.4484164 .563802-1.914450$

H 6.7136113 .4528330 .084963

H 4.9918505 .7224021 .210856

H 7.3208965 .9600422 .279533

H 7.5307034 .2254862 .219556

H 5.3612705 .8682165 .397449

H 6.0509196 .9586384 .068247

H 5.5690553 .5154465 .253023

H 6.7219572 .8848954 .119633

H 3.6896663 .3443953 .681985

H $3.6651582 .517994-0.708573$

C -3.834007 -2.821928 2.583422

O $-4.775229-2.0064641 .862423$

C $-5.627929-2.8794381 .091190$

C $-5.130619-4.3090901 .327417$

C $-3.691291-4.0924791 .751292$

H -5.473174 -2.619190 0.038260

C -7.098887 -2.674164 1.479758

H $-5.693010-4.8130902 .121995$

H $-5.221474-4.9254980 .427209$

C $-3.091353-5.2654472 .505311$

H -3.101641 -3.904659 0.845387

C $-7.612719-1.2296921 .324324$

O $-7.252376-3.0434252 .857393$

H -7.725862 -3.3645060 .902057$

H $-7.120829-0.5678352 .047635$

O $-9.007806-1.2307741 .658702$

C $-7.462151-0.640930-0.092113$ 
C $-8.1769050 .717160-0.236825$

H $-6.399560-0.520156-0.322183$

O $-7.998697-1.553452-1.050105$

H $-9.2513640 .575905-0.069804$

H $-7.812934 \quad 1.3887890 .547830$

H -2.056316 -5.056133 2.793092

H -3.656125 -5.4877493 .416945$

H -3.092866 -6.165046 1.881192

C -2.552454 -2.040983 2.885189

H -8.204062 -3.1390113 .030427$

H $-9.078125-1.3694602 .618183$

H -8.936337 -1.691255 -0.831490

H -1.996254 -2.568992 3.669572

H -2.816717 -1.056103 3.289374

H -4.312865 -3.0551503 .544610$

C $-5.9521202 .582387-1.207739$

O $-6.6175091 .562876-1.963044$

C $-7.9936651 .358579-1.630629$

C $-8.7785442 .663863-1.825466$

C $-8.1605223 .814667-1.038107$

C $-6.6709023 .931156-1.351592$

H $-5.9082242 .290074-0.151729$

C $-4.5171752 .685132-1.745950$

H $-8.3814070 .641590-2.364940$

O $-10.1389002 .508384-1.435366$

H $-8.7784692 .932962-2.889877$

H $-8.3289183 .703650 \quad 0.039480$

O $-8.7933515 .038730-1.418832$

H $-6.5441994 .318877-2.370907$

O $-6.0776244 .874143-0.460855$

C $-3.6832731 .466427-1.415588$

H $-4.5498902 .786055-2.838009$

H -3.998144 $3.551345-1.321985$

H -10.511613 $1.779710-1.960604$

H -9.745942 $4.935168-1.251525$

H $-6.5647555 .710392-0.557506$

O $-2.617770 \quad 1.418045-2.269164$

O $-3.9120160 .679566-0.507568$

C -1.703431 $0.340215-2.053304$

H $-0.9618240 .356353-2.856191$

H -1.190462 $0.480011-1.098333$

H -2.224824 -0.621598 -2.076907

SCF Energy (B3LYP/6-31G**//MMFF) $=-3245.91313110$

03_184

MM̄FF Geometry

C -2.366193 -1.487995 2.089423

C $-1.189535-2.1075121 .916347$

C $-0.377418-1.9966120 .650912$

O $0.983456-1.6521910 .998606$

C $-0.356310-3.357292-0.068497$

C $0.308427-3.316501-1.430823$

C $1.428412-3.990243-1.757811$

C $-0.385868-2.476189-2.472885$

C $2.250219-4.903443-0.892301$

C $3.412082-4.219187-0.155201$

C $4.453789-3.571378-1.082407$

C $5.692196-3.144508-0.290851$

C $6.713548-2.406595-1.171717$

O $3.861925-2.421433-1.692508$

O $7.662171-1.766368-0.304188$

C $5.992140-1.287417-1.954533$

C $7.514696-3.376374-2.048647$

O $6.865847-0.688318-2.907602$

C $4.699231-1.759717-2.650321$

C $3.872244-0.600648-3.259265$

O $5.040321-2.639964-3.728427$

C $1.241530-0.3494681 .293101$

O $\quad 0.448142 \quad 0.577569 \quad 1.288138$

C $2.673160-0.2445091 .648860$

C 3.1111940 .8433452 .299435

C 4.4889111 .0978002 .725256

C 5.5583920 .1731472 .206930

C $3.4084990 .471193-2.266350$

C $4.3287331 .678060-2.186137$

C $4.6726893 .773612-0.882518$

O $5.2463401 .880843-2.976618$

C 5.7445313 .5419770 .135231
C 5.8893654 .2977191 .234460

C 6.9835894 .1067552 .245504

C 6.4548793 .9680153 .655671

C 6.4245845 .0216484 .489181

C 6.0413312 .5909474 .123439

C 4.7300092 .1491353 .536253

N $3.9770672 .527569-1.145525$

H $-2.764009-0.8704181 .288230$

H $-0.789565-2.7309502 .712318$

H $-0.789580-1.224816-0.010137$

H $-1.383339-3.721225-0.208017$

H $0.138746-4.0880480 .580206$

H $1.807297-3.885798-2.774169$

H $0.040787-2.617756-3.471796$

H -1.446261 -2.742456 -2.531837

H $-0.303877-1.412400-2.230632$

H $1.617733-5.417328-0.160477$

H $2.651324-5.701668-1.530185$

H $3.002963-3.4635810 .525487$

H $3.904804-4.9789580 .464331$

H $4.727522-4.297942-1.856389$

H $6.160902-4.0090980 .195385$

H $5.388024-2.4733470 .523572$

H $8.072831-2.4525760 .249370$

H $5.738989-0.506169-1.228132$

H $8.058146-4.096405-1.424974$

H $8.279163-2.846436-2.627521$

H $6.890128-3.945681-2.739901$

H $7.653901-0.382870-2.426533$

H $4.408168-0.160952-4.109091$

H $2.962632-1.044745-3.689316$

H $4.296792-2.664533-4.353382$

H $3.322272-1.0693191 .379545$

H 2.3910741 .6168702 .563780

H 5.5001310 .0955841 .115934

H $5.444208-0.8273062 .637649$

H 6.5730270 .5094042 .428865

H $2.4353500 .854158-2.599911$

H $3.2679970 .048372-1.267302$

H $3.9253604 .496478-0.540299$

H $5.1195964 .144960-1.810280$

H $6.4481422 .734247-0.052862$

H 5.2010615 .1227651 .404302

H 7.6506784 .9765712 .182128

H 7.6078833 .2378572 .002808

H 6.0727594 .9252025 .510907

H 6.7462746 .0061514 .165925

H 5.9229002 .5841645 .215414

H 6.8566191 .8891553 .927692

H 3.8855272 .7637453 .851507

H $3.2334412 .255543-0.509070$

C $-4.553794-2.2556213 .123904$

O $-5.335803-1.5085782 .170245$

C $-5.967919-2.4398481 .271242$

C $-5.872255-3.8026771 .947735$

C $-4.515431-3.6986982 .617508$

H $-5.357018-2.4614280 .360460$

C -7.389206-1.986221 0.927545

H $-6.657175-3.9401212 .700361$

H $-5.937259-4.6280701 .232503$

C $-4.289640-4.7289683 .709799$

H -3.743964 -3.824337 1.847935

C $-7.480568-0.5517160 .367668$

O $-8.185517-2.0381742 .117909$

H -7.838272 -2.699580 0.226125

H $-7.2767490 .169557 \quad 1.168002$

O $-8.842734-0.328541-0.020248$

C $-6.556156-0.261780-0.830184$

C $-6.805718 \quad 1.135752-1.428317$

H $-5.515039-0.344566-0.501508$

O $-6.779906-1.253772-1.830579$

H -7.812558 $1.170691-1.862185$

H $-6.777411 \quad 1.871180-0.616993$

H $-3.304768-4.5988924 .169188$

H $-5.044042-4.6494174 .499744$

H $-4.339993-5.7424473 .298544$

C $-3.194179-1.5851323 .339307$

H $-9.082753-1.748521 \quad 1.879123$ 
H $-9.031948-0.913458-0.774049$ H $-6.057683-1.189186-2.477736$ H $-2.646259-2.1147104 .127612$ H -3.357446 -0.561145 3.697362 H $-5.109458-2.2079194 .069718$ C -3.998206 2.279855-1.109269 O $-4.4374431 .355564-2.112529$ C $-5.7944571 .522398-2.531892$ C $-5.9910552 .932595-3.102189$ C $-5.5419894 .013184-2.121327$ C $-4.1287723 .726258-1.609465$ H -4.600185 2.143521 -0.203108 C $-2.5476431 .903766-0.780338$ H $-5.9475350 .820305-3.360904$ O $-7.3554423 .130489-3.457846$ H $-5.4022313 .026691-4.024163$ H -6.254796 4.112845 -1.294244 O $-5.5494125 .264901-2.812118$ H -3.405445 $3.913564-2.413343$ O $-3.831204 \quad 4.623637-0.542994$ C -2.0344162 .5259530 .493965$ H -2.442099 $0.815396-0.687614$ H -1.881093 2.186710-1.604390 H -7.439126 4.038919 -3.795043 H $-5.3654925 .961666-2.159833$ H $-3.7912855 .521002-0.914118$ O -2.7099462 .0260131 .567166$ O $-1.1276513 .347906 \quad 0.530381$ C -2.3063132 .5366912 .841099$ H -2.4303743 .6234892 .872292$ H -2.9474622 .0892843 .605194$ H -1.268254 2.2595193 .047119 SCF Energy (B3LYP/6-31G**//MMFF) $=-3245.90793656$

03 185

MM̄FF Geometry

C $5.896046-2.494006-1.403206$

C $5.260211-2.413440-0.224713$

C $5.537108-1.3684810 .819220$

O $4.449059-0.4173410 .770247$

C $5.545177-2.0048432 .222555$

C $5.696147-0.9922843 .341758$

C $4.736050-0.6919504 .240093$

C $7.035945-0.3059593 .416261$

C $3.357087-1.2835424 .363821$

C $2.302165-0.3877693 .713060$

C $0.855406-0.8444043 .957011$

C $0.546937-2.200078 \quad 3.314520$

C $-0.937387-2.5795263 .450721$

O $0.0094590 .167593 \quad 3.389522$

O $-1.192286-3.6977012 .586043$

C -1.807612 -1.396245 2.976109

C -1.263037 -3.079622 4.862141

O $-3.189343-1.6419823 .223205$

C -1.395040 -0.045571 3.598693

C -2.1456921 .1543402 .972506$

O $-1.708743-0.0507384 .996207$

C 4.6472680 .7406330 .084120

O $5.6339031 .062532-0.557233$

C 3.4429411 .5766550 .283083

C $3.4671302 .880179-0.032925$

C 2.3723193 .8326700 .177283

C 1.1068903 .2977250 .793200

C -2.0235571 .2568981 .452048$

C -2.4575182 .6082220 .908547$

C $-2.6191653 .824803-1.277167$

O -2.8408903 .5334711 .617398$

C $-1.3201564 .327504-1.825143$

C $-0.9152755 .604385-1.752561$

C $0.3983026 .081605-2.307168$

C $1.1960666 .906729-1.319165$

C $1.5332328 .176272-1.603618$

C $1.5998136 .277609-0.002351$

C $2.5542715 .124447-0.170143$

N $-2.3490072 .647194-0.473552$

H $6.683969-1.785079-1.642578$

H $4.450624-3.110464-0.018105$

H $\quad 6.497618-0.8729430 .633174$
H $\quad 6.362479-2.7346582 .294748$

H $4.622880-2.5801182 .354663$

H 4.9581170 .0663134 .990394

H $7.127808 \quad 0.3312354 .302391$

H $7.839870-1.0477943 .463076$

H 7.1953860 .3278172 .538661

H $3.319906-2.2924913 .946193$

H $3.134938-1.3968015 .432311$

H 2.4090150 .6413594 .082683

H $2.479722-0.3271452 .633340$

H $0.686329-0.8800925 .039505$

H $1.172304-2.9947123 .737237$

H $0.800441-2.1562202 .245954$

H $-0.973375-3.4350391 .675742$

H -1.692021 -1.337162 1.886817

H $-0.673775-3.9750665 .094349$

H -2.312528 $-3.384146 \quad 4.942100$

H -1.058555 -2.340256 5.639027

H -3.409164 -2.498309 2.817823

H -3.2067891 .1263693 .253385$

H -1.7474422 .0742973 .422683$

H -1.778374 0.8697915 .299485

H 2.5735101 .0997920 .720477

H $4.3779733 .297262-0.460178$

H 1.3059692 .8736281 .783688

H 0.6806862 .5169090 .154514

H 0.3284874 .0496650 .928485

H -0.9860841 .0898801 .143118$

H -2.646553 0.4909030 .979294

H -3.139732 $4.578939-0.679017$

H $-3.271073 \quad 3.511351-2.098298$

H $-0.6760693 .599560-2.313790$

H -1.562299 $6.345041-1.288635$

H $0.1798556 .672494-3.205977$

H $1.0135845 .237844-2.643181$

H $2.1026578 .781157-0.905542$

H $1.2488288 .641440-2.541679$

H $0.698640 \quad 6.0099120 .554231$

H $2.107167 \quad 7.0191510 .629638$

H $3.5124555 .413182-0.604400$

H $-2.0191231 .818930-0.959132$

C $4.323558-3.090941-3.316238$

O $3.157921-2.946726-2.476565$

C $2.558591-1.661697-2.744430$

C $3.025959-1.300619-4.143261$

C $4.465815-1.781306-4.096296$

H $2.985102-0.966777-2.009651$

C $1.046507-1.771433-2.540607$

H $2.457663-1.855245-4.900122$

H $2.942211-0.231382-4.358010$

C $5.098625-1.952102-5.466270$

H $5.054300-1.050178-3.528364$

C $0.254746-0.538017-3.003390$

O $0.813466-1.962610-1.139258$

H $\quad 0.671819-2.668290-3.046822$

H $\quad 0.312018-0.449505-4.094307$

O $0.8761720 .644156-2.479034$

C $-1.222393-0.508739-2.564641$

C $-2.015156-1.773112-2.934498$

H -1.283811 $-0.325294-1.485747$

O $-1.8507900 .607262-3.205842$

H -2.013010 -1.900097 -4.023819

H - $1.527363-2.644863-2.486262$

H $6.124655-2.322853-5.375859$

H $4.536166-2.660914-6.083269$

H $5.129125-0.995423-5.997910$

C $5.528289-3.500741-2.459456$

H $1.320481-2.746212-0.865308$

H $0.8874060 .564838-1.509538$

H $-1.3012511 .390436-3.032600$

H $6.401175-3.675581-3.098937$

H $5.304795-4.456483-1.967529$

H $4.096915-3.915433-4.004085$

C $-4.719460-1.451257-0.390014$

O $-3.441789-1.638078-1.012343$

C $-3.475338-1.716026-2.441726$

C $-4.286115-2.946601-2.873100$

C $-5.700283-2.904321-2.280744$ 
C $-5.690692-2.579365-0.780361$ H $-4.507666-1.5472080 .681652$ C $-5.244668-0.031990-0.655274$ H -3.947842 -0.812309 -2.844379 O $-3.622889-4.131294-2.435780$ H -4.331622 -2.976581 -3.968245 H $-6.190767-3.873927-2.430675$ O $-6.471071-1.920815-2.964705$ H $-6.701700-2.326547-0.442301$ O $-5.306078-3.759432-0.065921$ C -5.9428480 .5503050 .553623$ H $-4.3989140 .620950-0.900275$ H $-5.9419830 .008248-1.495587$ H -4.142500 -4.891016 -2.749449 H -6.498801 -2.169630 -3.904221 H $-4.386981-3.966573-0.307786$ O O $-6.541040-0.102357 \quad 1.398251$ C -6.4180272 .6004881 .633442$ H -7.4991592 .4334831 .643312$ H -5.9664512 .2735912 .574979$ H -6.228218 3.6697471 .507093

SCF Energy (B3LYP/6-31G**//MMFF) $=-3245.92752108$

$03 \_186$

MM̄FF Geometry

C $-0.381148 \quad 5.423707 \quad 0.335026$

C $0.6804604 .822893-0.223217$

C 1.6935694 .0153110 .547090

O $1.8403372 .732002-0.104016$

C 3.0593104 .7240980 .508234

C 4.1520783 .9877371 .259240

C 5.2658323 .4862610 .689468

C 3.9481853 .8522462 .746426

C $5.6752863 .549516-0.755508$

C $5.2455112 .338744-1.598217$

C $5.9019971 .015503-1.173088$

C $5.630913-0.081273-2.205276$

C $6.215012-1.434829-1.769733$

O $5.358475 \quad 0.6204790 .089227$

O $5.648002-2.450519-2.612078$

C $5.760175-1.740707-0.326087$

C $7.732722-1.495392-1.979984$

O $6.417327-2.899577 \quad 0.179960$

C $5.956592-0.5587440 .646051$

C $5.305667-0.7920812 .031584$

O $7.358441-0.3634040 .871273$

C 0.9005121 .7837920 .154740

$\begin{array}{lllll}\text { O } & -0.056856 & 1.878148 & 0.905849\end{array}$

C $1.2271230 .603187-0.675326$

C $0.289388-0.332764-0.879257$

C $0.426879-1.533363-1.704959$

C $1.792846-1.857515-2.246977$

C $3.796637-1.0621242 .014271$

C $3.435538-2.5386752 .041046$

C $1.461381-4.0542911 .928409$

O $4.258179-3.4315662 .224747$

C $1.215350-4.5479200 .538976$

C $0.008044-4.9330480 .098848$

C $-0.245208-5.491991-1.270877$

C $-1.283978-4.707000-2.037208$

C $-2.562234-5.117604-2.094344$

C $-0.818486-3.493647-2.808256$

C $-0.673690-2.277693-1.938463$

N $2.072200-2.739798 \quad 1.873840$

H -0.5251475 .3563241 .409629$

H $0.8300744 .898026-1.298158$

H $\quad 1.3737113 .8667091 .585637$

H 2.9672705 .7301510 .938850

H $3.3458204 .869287-0.538807$

H 5.9836252 .9777621 .332453

H 4.8320643 .4480353 .251671

H 3.7369924 .8299653 .191700

H 3.1122303 .1800712 .962374

H $5.2946024 .462416-1.225547$

H $6.7679213 .647285-0.794680$

H $4.1541502 .242703-1.555790$

H $5.5114062 .550760-2.641278$
H $6.9805651 .184334-1.071691$

H $6.0216060 .204221-3.189898$

H $4.546151-0.196883-2.335918$

H $5.864633-2.230236-3.534301$

H $4.692791-1.984633-0.377342$

H $7.979357-1.334994-3.036485$

H $8.127408-2.486030-1.728592$

H $8.276718-0.748993-1.397573$

H $6.248184-3.624962-0.445311$

H $5.850297-1.5723632 .576626$

H 5.4548620 .1270242 .616859

H 7.4716240 .1229671 .704723

H $2.2140480 .553331-1.120429$

H $-0.688841-0.189941-0.424451$

H $2.101907-1.109501-2.984825$

H $1.849152-2.835318-2.729654$

H $2.530282-1.878307-1.437521$

H $3.356678-0.6256762 .920303$

H $3.318519-0.582677 \quad 1.154731$

H $0.535934-3.9588562 .503213$

H $2.122497-4.7499242 .454878$

H $2.075009-4.623512-0.122621$

H $-0.844726-4.8796430 .771918$

H $-0.576735-6.531729-1.149892$

H $0.679295-5.546302-1.859072$

H $-3.306634-4.570682-2.663719$

H -2.895189-6.003251-1.563516

H -1.551285 -3.246132 -3.588284

H $0.095224-3.741786-3.354942$

H -1.613699-1.956775 -1.490311

H $1.469046-1.9431291 .690052$

C $-2.8021465 .605528-0.435316$

O $-2.7763374 .248920-0.922305$

C $-3.6624003 .454505-0.111149$

C -4.5076984 .4485960 .676919$

C -3.5058955 .5603810 .920206$

H -3.0334192 .8869120 .586980$

C $-4.4836592 .478008-0.963784$

H -5.3522234 .8160950 .081542$

H -4.9047814 .0167731 .600567$

C -4.1419346 .8766641 .330929$

H -2.8172685 .2359731 .709564$

C $-3.6324351 .364964-1.605778$

O $-5.1927673 .192682-1.975229$

H $-5.2398972 .022545-0.317139$

H $-3.1042250 .814105-0.820836$

O $-2.605021 \quad 1.942285-2.420135$

C $-4.4224930 .377261-2.483802$

C $-5.627291-0.311617-1.819110$

H $-4.7755430 .887441-3.388502$

O $-3.529074-0.642426-2.943403$

H $-6.4457140 .411686-1.721390$

H -5.967914 -1.093677 -2.509982

H -3.3797617 .6483151 .477824$

H $-4.845647 \quad 7.2369910 .573027$

H -4.691395 6.7612352 .270915

C -1.394206 $6.206919-0.453839$

H -4.547061 $3.731595-2.463086$

H -3.035607 $2.427514-3.144102$

H -2.769575 -0.199196 -3.358693

H $-1.4226767 .232670-0.068819$

H -1.065397 $6.272624-1.499178$

H -3.417797 $6.170148-1.148520$

C $-3.866304-2.4569960 .708858$

O $-4.287247-1.885427-0.541151$

C $-5.365241-0.952119-0.438102$

C $-6.618363-1.6594300 .106023$

C $-6.329883-2.3470771 .444170$

C $-5.038871-3.1757101 .399890$

H $-3.124768-3.2149360 .427679$

C $-3.154527-1.4016621 .569189$

H -5.080489 -0.156949 0.257052

O $-7.065130-2.630091-0.839027$

H $-7.418876-0.9195580 .226523$

H -7.174636 -2.997716 1.701134

O $-6.237360-1.3479282 .455420$

H -4.744339 -3.478673 2.410516

O $\quad-5.288591-4.3767400 .661889$ 
C $-2.256275-1.9907972 .633099$

H $-2.526720-0.7832390 .918902$

H $-3.868052-0.7344492 .059674$

H -7.876145 -3.032968 -0.485307

H $-6.081099-1.8005313 .301877$

H $-5.991481-4.8643681 .124194$

O $-1.638151-0.9686543 .297394$

O $-2.116249-3.1833082 .863448$

C $-0.773925-1.3585974 .368957$

H $-1.329797-1.9314565 .117364$

H $0.067434-1.9401733 .983842$

H $-0.386078-0.4512184 .839641$

SCF Energy (B3LYP/6-31G**//MMFF) $=-3245.89386504$

03_187

MM̄FF Geometry

C $-2.8946831 .230676-3.252242$

C $-2.2090760 .622178-2.272121$

C -1.010301 $-0.255376-2.517008$

O $-0.0002770 .024684-1.518962$

C - $-1.420792-1.732230-2.369735$

C $-0.338540-2.712208-2.779424$

C $0.240649-3.605679-1.954568$

C $0.064624-2.674158-4.231930$

C $-0.029748-3.834968-0.495110$

C $0.871288-3.0321010 .454581$

C $2.374404-3.312096 \quad 0.291919$

C $3.164933-2.6921091 .444534$

C $4.677583-2.8996321 .272333$

O $2.811985-2.729467-0.937003$

O $5.347915-2.0264692 .196493$

C $5.098934-2.456094-0.148781$

C $5.093943-4.3262621 .651290$

O $6.456681-2.818541-0.386764$

C $4.181738-2.994794-1.272138$

C $4.432908-2.342371-2.652274$

O $4.394801-4.394748-1.493031$

C $0.687568 \quad 1.193077-1.624104$

O $\quad 0.5440402 .061013-2.470387$

C $1.6550291 .240787-0.506699$

C $2.2110822 .409790-0.158209$

C 3.1859472 .6138100 .915056

C 3.7874691 .3909521 .556248

C $4.254697-0.821428-2.709032$

C $5.541431-0.039020-2.502018$

C $6.3678582 .295251-2.210910$

O $6.651879-0.562584-2.479120$

C $6.5512942 .583181-0.754078$

C $6.5528083 .823764-0.242565$

C 6.7785384 .1319131 .210259

C 5.6922495 .0052321 .797578

C 5.8562226 .3349611 .902470

C 4.4558464 .3267192 .342065

C $3.5169783 .876578 \quad 1.256691$

N $5.303206 \quad 1.323458-2.381365$

H $-2.5923161 .091373-4.286902$

H -2.530586 $0.743683-1.240995$

H $-0.588648-0.066560-3.511317$

H -2.304982 -1.937654 -2.988372

H -1.728257 -1.907386-1.332747

H $1.004445-4.262554-2.370255$

H $\quad 0.718435-3.510327-4.503395$

H $-0.820051-2.731309-4.874666$

H $0.605354-1.750536-4.459541$

H -1.077674 -3.624965 -0.255458

H $0.099524-4.905710-0.291870$

H $\quad 0.677431-1.9644280 .300600$

H $\quad 0.568952-3.274248 \quad 1.480597$

H $2.525321-4.3972870 .259981$

H $2.830686-3.0947792 .408891$

H $2.963232-1.6133511 .485070$

H $5.037867-2.2427193 .092628$

H $5.060926-1.360653-0.148089$

H $4.857671-4.5273692 .703393$

H $6.176985-4.4614821 .558362$

H $4.594345-5.0913751 .053221$

H $\quad 6.735066-2.402189-1.219559$

H $5.409871-2.647773-3.045726$
H $3.698153-2.773910-3.348068$

H $5.127717-4.691505-0.930841$

H 1.8775810 .3111690 .003874

H $1.9148113 .308122-0.698594$

H 4.1517240 .6953250 .792637

H 3.0466290 .8766602 .176048

H 4.6491291 .6116202 .190085

H $3.893758-0.552589-3.710010$

H $3.502432-0.487600-1.989337$

H $6.0852743 .188235-2.777176$

H $7.2981801 .896696-2.628071$

H $6.7166631 .734871-0.094347$

H $6.4163474 .671762-0.910088$

H 7.7488684 .6386021 .295877

H 6.8719023 .2152131 .805535

H 5.0945876 .9659972 .348488

H 6.7546156 .8229951 .538641

H 3.8968545 .0216832 .983528

H 4.7543923 .5152213 .012108

H 3.0399644 .7007010 .724598

H $4.3455041 .661717-2.360392$

C $-5.3820451 .290500-2.914501$

O $-5.3369250 .463022-1.734534$

C $-6.5803000 .582318-1.024477$

C $-7.5558451 .217553-2.006164$

C $-6.6358022 .153608-2.768424$

H $-6.4102631 .282290-0.199234$

C $-6.996039-0.776245-0.455355$

H -7.990292 $0.477062-2.687446$

H $-8.3756401 .736997-1.501022$

C $-7.2098222 .632708-4.090437$

H -6.420669 $3.025438-2.136052$

C $-5.890758-1.4766080 .366149$

O $-7.335515-1.636548-1.550074$

H -7.907182 -0.6585700 .143396$

H $-5.080868-1.806576-0.296623$

O $-6.458297-2.6646740 .933507$

C $-5.295256-0.6294601 .510749$

C -4.269856-1.429128 2.340629

H $-4.8048430 .253797 \quad 1.088662$

O $-6.358350-0.1794622 .347552$

H -4.742028 -2.345913 2.713282

H $-3.457223-1.7371941 .672677$

H $-6.5019773 .287935-4.607914$

H $-7.4401871 .794075-4.756087$

H $-8.1341523 .196120-3.926827$

C $-4.0913512 .105575-3.004765$

H $-7.816060-2.398910-1.185843$

H $-6.649570-3.2763620 .202995$

H -6.0064540 .5255462 .916860$

H $-3.9368202 .671826-2.079068$

H -4.156435 $2.838005-3.817627$

H $-5.4729720 .614953-3.774916$

C $-2.016966 \quad 0.6655752 .456669$

O -3.2208870 .6313613 .232392$

C $-3.703324-0.6715403 .562370$

C $-2.634834-1.4495264 .343924$

C $-1.304330-1.4823183 .596338$

C $-0.886256-0.0687953 .193904$

H $-2.196356 \quad 0.203870 \quad 1.477641$

C -1.660145 2.1431012 .238355

H $-4.544196-0.5185874 .250720$

O $-3.089665-2.7726774 .604502$

H $-2.482456-0.9624155 .315978$

H -1.349863 -2.141389 2.721827

O $-0.291619-2.0155974 .452951$

H $-0.592427 \quad 0.4831744 .096455$

O $0.263127-0.1749122 .359124$

C -2.5888272 .8194391 .257678$

H -1.6904942 .7076533 .177862$

H -0.6492162 .2523101 .831196$

H -2.449976 -3.192361 5.203717

H $-0.496121-2.9543414 .599674$

H $\quad 0.689727 \quad 0.6971882 .329508$

O $-3.813606 \quad 3.0149101 .822659$

O -2.2541913 .1519320 .127909$

C -4.7806183 .6639350 .992523$

H -4.9533223 .0799230 .085758$ 
H -5.7182463 .7304261 .550663$

H $-4.446306 \quad 4.6742720 .739832$

SCF Energy (B3LYP/6-31G**//MMFF) $=-3245.90010052$

03_188

MM̄FF Geometry

C -0.3213314 .6970610 .746280$

C 0.8714404 .9630851 .300317

C 2.1387064 .1578841 .121903

O $1.8670282 .798648 \quad 0.711503$

C 3.0056984 .8257370 .038296

C $4.3710654 .194185-0.142662$

C $4.7901623 .584909-1.269115$

C 5.3052334 .3103931 .034657

C $4.0371403 .381972-2.554996$

C $3.3124422 .030354-2.663846$

C $4.2508290 .811953-2.655736$

C $3.479633-0.471228-2.968901$

C $4.374709-1.716623-2.874199$

O $4.836473 \quad 0.702957-1.357810$

O $3.502138-2.857363-2.873748$

C $5.137083-1.700688-1.529224$

C $5.268134-1.859701-4.112443$

O $6.089683-2.760866-1.504240$

C $5.806661-0.344143-1.223278$

C $6.375153-0.2388620 .212810$

O $6.902839-0.150541-2.125084$

C 1.4829611 .9222531 .681046

O 1.2900842 .1688842 .861014

C 1.3311580 .5911181 .053152

C $0.670751-0.3781501 .704309$

C $0.442769-1.7422931 .220929$

C $1.194647-2.179055-0.007829$

C $5.349041-0.4110701 .338655$

C $5.284037-1.8188571 .905729$

C $4.018963-3.1794603 .566726$

O $6.074759-2.7073951 .600776$

C $3.053884-4.0418232 .817211$

C $1.932678-4.5375513 .361979$

C $0.986164-5.4518622 .639867$

C $-0.435778-4.9398992 .641060$

C -1.343084 -5.415854 3.510578

C $-0.827473-3.9458561 .572755$

C $-0.405521-2.5395361 .903540$

N $4.253489-1.9543812 .826648$

H -0.4319813 .8388200 .089271$

H 0.9689525 .8457091 .929493

H 2.6753394 .1404502 .078298

H 3.1559455 .8862190 .282687

H $2.4495634 .812015-0.905212$

H $5.8028963 .183132-1.282307$

H $\quad 6.3179433 .9669840 .797191$

H 5.3824125 .3537911 .357751

H 4.9449513 .7085591 .874459

H $3.3116724 .186519-2.713070$

H $4.7502933 .476352-3.383990$

H $2.5913981 .948181-1.841864$

H $2.7376022 .033148-3.598287$

H $5.0375490 .977952-3.400981$

H $3.007657-0.412382-3.957650$

H $2.654807-0.580776-2.252016$

H $4.053044-3.649041-2.746902$

H $4.399319-1.925868-0.751611$

H $4.653890-1.947269-5.016759$

H $5.865388-2.777116-4.067969$

H $5.945769-1.014677-4.251829$

H $6.451761-2.815673-0.604186$

H $7.232332-0.9113930 .334542$

H $\quad \begin{array}{llll}6.789638 & 0.774242 & 0.320527\end{array}$

H $7.5091210 .502801-1.738589$

H $1.759410 \quad 0.4479690 .067785$

H $0.239364-0.1502422 .678238$

H $\quad 0.824708-1.655476-0.894556$

H $1.123382-3.250073-0.207501$

H $2.262304-1.9670540 .105636$

H $\begin{array}{llll}5.641613 & 0.239156 & 2.173384\end{array}$

H $4.349830-0.1003061 .017822$

H $3.641515-2.8953894 .554140$
H $4.966280-3.7128413 .695880$

H $3.305312-4.2965891 .790306$

H $1.697549-4.3041494 .398101$

H $1.030273-6.4319683 .132869$

H $\quad 1.308777-5.628473 \quad 1.606253$

H -2.373061 -5.0747223 .495787$

H $-1.074419-6.1529034 .260112$

H -1.918336 -3.937699 1.458126

H $-0.454351-4.2913230 .605016$

H $-0.898774-2.1337752 .787917$

H $3.609109-1.1822142 .970830$

C $-2.769775 \quad 4.779148 \quad 1.432448$

O $-3.213990 \quad 3.8731180 .405270$

C -3.6658212 .6573631 .028344$

C -3.7436342 .9517712 .523651$

C -2.6030413 .9367462 .695566$

H -2.8840701 .9066910 .852384$

C -4.9838792 .1635780 .413616$

H -4.6976453 .4205712 .792731$

H -3.627729 2.0487823 .130653

C -2.6691634 .7361043 .984120$

H -1.661209 3.3745992 .671667

C $-4.847764 \quad 1.812372-1.081468$

O -5.9871373 .1632710 .588018$

H $-5.300798 \quad 1.2830190 .981933$

H $-4.006326 \quad 1.127152-1.217977$

O $-4.5101092 .987923-1.827589$

C $-6.1029901 .202025-1.730802$

C -6.693784 -0.029944-1.022251

H $-6.8875461 .963221-1.816625$

$\begin{array}{lllll}\text { O } & -5.779611 & 0.828244 & -3.075655\end{array}$

H $-7.1813670 .288768-0.093392$

H -7.475440 -0.435848 -1.677720

H -1.8338175 .4404424 .047648$

H -3.5999205 .3087774 .054697$

H $-2.6175194 .070828 \quad 4.852190$

C -1.539522 5.5532010 .954955

H -6.8407832 .7751210 .333021$

H $-5.1910303 .657496-1.642754$

H $-5.4060861 .612313-3.513452$

H $-1.3251186 .359977 \quad 1.665909$

H -1.772353 $6.024214-0.008261$

H -3.5880295 .4987961 .569672$

C $-4.076689-2.609586-1.778731$

O $-5.105196-1.619500-1.921089$

C $-5.686614-1.153508-0.701843$

C $-6.354295-2.3209290 .041233$

C $-5.357156-3.4579110 .285576$

C $-4.586232-3.828016-0.987342$

H $-3.868023-2.944193-2.803154$

C $-2.780724-2.009875-1.210233$

H $-4.900973-0.738728-0.064810$

O $-7.467337-2.814849-0.700445$

H $-6.743321-1.9565440 .999115$

H $-5.894216-4.3412970 .651864$

O $-4.428318-3.065770 \quad 1.291332$

H -3.742619-4.486705 -0.750022

O $-5.458498-4.559745-1.854884$

C $-2.204130-0.944177-2.119324$

H -2.949983 -1.562180 -0.227265

H -2.018485 -2.789304 -1.104568

H -7.148017 -3.066362 -1.584032

H $-4.932954-2.8767402 .100640$

H $-5.733753-5.364138-1.382920$

O $-1.463242-0.069174-1.377305$

O $-2.361892-0.898562-3.331853$

C $-0.8521830 .998312-2.109220$

H $-0.0740780 .601545-2.767815$

H -1.602565 $1.550542-2.683208$

H $-0.3911111 .682578-1.392697$

SCF Energy (B3LYP/6-31G**//MMFF) $=-3245.91221175$

03_189

MM̄FF Geometry

C $3.578499-0.0509553 .766088$

C $2.766866-0.6890672 .910943$

C $1.269716-0.5381662 .924823$

O $0.857378-0.0371281 .633266$ 
C $0.590181-1.8999673 .132250$

C $-0.909218-1.7809113 .332575$

C $-1.835769-1.9765302 .372529$

C -1.340045 -1.460352 4.741079

C -1.598764 -2.290545 0.920689

C $-2.366392-1.394763-0.068755$

C $-3.862011-1.734478-0.186126$

C $-4.465329-1.092661-1.438340$

C -5.977755 -1.346935 -1.541243

O $-4.531065-1.2366460 .973677$

O $-6.503986-0.468984-2.548692$

C $-6.641141-0.949963-0.205423$

C $-6.290642-2.774270-2.004820$

O $-8.021067-1.305911-0.188118$

C $-5.930688-1.5367471 .033868$

C $-6.481508-0.9753952 .369410$

O $-6.139188-2.9549241 .066769$

C $0.860325 \quad 1.309601 \quad 1.450334$

O 1.2349122 .1619632 .239037

C $0.290826 \quad 1.5668970 .109845$

C $0.0493682 .826619-0.279691$

C $-0.5617403 .234705-1.546718$

C $-0.9315292 .148138-2.523283$

C -6.5319890 .5474842 .517549$

C -5.1696541 .2027122 .397161$

C -3.9036692 .8744481 .067548$

$\begin{array}{lllll}\text { O } & -4.246347 & 0.926846 & 3.160109\end{array}$

C $-3.9288163 .309347-0.362471$

C $-3.9405844 .592570-0.754084$

C $-3.9429885 .028341-2.192941$

C $-2.7528115 .897960-2.539854$

C $-2.8614487 .236942-2.569065$

C $-1.4591615 .210582-2.926518$

C $-0.7926004 .548650-1.752174$

N $-5.0981592 .101177 \quad 1.344107$

H 3.1468740 .5921944 .529304

H $3.198016-1.3198552 .138761$

H $0.9497160 .157856 \quad 3.710597$

H $1.017347-2.4053464 .008951$

H $\quad 0.806447-2.5588082 .284276$

H $-2.879646-1.9178212 .670290$

H $-0.933025-0.4947905 .056541$

H -2.429321 -1.405753 4.841973

H $-0.983180-2.2313585 .431892$

H $-0.537876-2.1884720 .676958$

H $-1.859907 \quad-3.340430 \quad 0.742817$

H -2.242158 -0.3433420 .218837$

H -1.897268 -1.523751 -1.052519

H -3.970055 -2.824362 -0.232308

H -3.956995 -1.448882 -2.343078

H -4.296603 $-0.007315-1.408030$

H -6.039893 -0.657693 -3.382450

H $-6.6043520 .144417-0.156295$

H -5.846749 -2.962422 -2.989862

H -7.369114 -2.921232 -2.129886

H -5.914690 -3.539823 -1.323192

H -8.431199 -0.915671 -0.978976

H -7.491370 -1.371830 2.538592

H $-5.866696-1.3759153 .187842$

H -5.990043 -3.265245 1.975533

H $0.0608520 .708375-0.510552$

H $\quad 0.2985723 .6383100 .402679$

H $-0.0370251 .603420-2.844973$

H -1.415943 $2.517637-3.428683$

H -1.629781 $1.437688-2.066683$

H $-7.245020 \quad 0.976744 \quad 1.806583$

H -6.9162330 .8002153 .513707$

H -3.019452 2.2585191 .262011

H -3.889268 3.7203571 .762713

H -3.913753 2.524488-1.114795

H $-3.9564475 .377700-0.001627$

H $-4.8755585 .578703-2.373440$

H $-3.9735074 .167564-2.872631$

H -2.021396 $7.867377-2.840859$

H $-3.7928747 .732868-2.316620$

H $-0.7482025 .940192-3.336166$

H -1.662927 $4.520083-3.748783$

H $-0.4844485 .254577-0.979240$
H $\quad \begin{array}{llll}-5.960996 & 2.388628 & 0.890959\end{array}$

C 5.7498091 .0052722 .966595

O $5.503500 \quad 0.8602231 .552087$

C 5.1406802 .1446291 .016286

C 5.5925153 .1690932 .052517

C 5.3166482 .4242973 .343809

H 4.0454612 .1590180 .959216

C $5.7270292 .348861-0.384471$

H 6.6623353 .3918541 .969691

H 5.0419424 .1113231 .972860

C 6.0488282 .9871374 .549073

H 4.2380062 .4755413 .532958

C $5.4560611 .204694-1.382079$

O $7.1495012 .483534-0.265223$

H $5.3605963 .300906-0.788162$

H $6.1020690 .346577-1.157632$

O $5.8407561 .667821-2.684121$

C $3.9954550 .721706-1.456694$

C $3.757193-0.234914-2.641349$

H $3.7333600 .224479-0.517195$

O $3.1230361 .842431-1.600282$

H $3.7415090 .348532-3.569840$

H $4.598067-0.931625-2.710617$

H 5.8255702 .4014685 .446579

H 7.1338272 .9780394 .400952

H 5.7445084 .0222544 .735426

C $5.077948-0.1414363 .730417$

H $7.4856402 .821650-1.112030$

H $6.8077251 .764518-2.688123$

H $3.3906812 .325661-2.400664$

H $5.441607-0.1581574 .764615$

H $5.373601-1.0956943 .276010$

H 6.8360960 .9009993 .086975

C $3.277630-3.022372-1.482021$

O $2.409598-1.886512-1.385792$

C $2.425816-1.011691-2.520781$

C $2.044709-1.795961-3.783585$

C $2.961871-2.996688-3.996035$

C $3.001714-3.864416-2.738593$

H $4.314280-2.665883-1.517276$

C $3.174657-3.818126-0.170972$

H $1.635975-0.270750-2.349748$

O $2.091144-0.961219-4.936085$

H $1.012588-2.158574-3.690724$

H $\quad 3.970385-2.686507-4.293997$

O $2.452952-3.790412-5.071298$

H $2.049550-4.400891-2.664569$

O $4.025359-4.843871-2.905943$

C $1.809402-4.4109770 .112109$

H $3.908472-4.631395-0.174037$

H $3.403001-3.1490970 .666177$

H $1.492990-0.211045-4.777319$

H $2.396533-3.214636-5.853247$

H $3.920441-5.505151-2.201601$

O $1.899667-5.2106951 .216970$

O $0.791155-4.193301-0.528435$

C $0.679195-5.836251 \quad 1.625839$

H $\quad 0.294135-6.476306 \quad 0.826394$

H $0.891975-6.4568002 .500328$

$\mathrm{H}-0.059258-5.0789561 .904784$

SCF Energy (B3LYP/6-31G**//MMFF) $=-3245.89733407$

03_19

MM̄FF Geometry

C -3.348397 $2.516567-2.222744$

C $-2.6192471 .767784-1.381582$

C -1.291862 $1.150051-1.729257$

O $-0.436974 \quad 1.382345-0.586401$

C -1.472729 -0.367201-1.944646

C $-0.361603-1.033703-2.735145$

C $0.416850-2.038918-2.287198$

C $-0.191906-0.549212-4.154780$

C $0.374338-2.735397-0.957343$

C $1.458934-2.2895970 .032871$

C $2.893894-2.661304-0.377587$

C $3.863667-2.3817510 .774716$

C $5.326278-2.6387300 .381081$

O $3.265310-1.882199-1.518365$ 
O $6.164597-2.0352791 .379092$

C $5.623564-1.917199-0.951075$

C $5.661010-4.1348410 .382384$

O $6.920031-2.256539-1.434889$

C $4.566885-2.184714-2.042816$

C $4.771122-1.327707-3.317969$

O $4.649812-3.556168-2.451315$

C $0.892617 \quad 1.558571-0.794721$

O $1.487213 \quad 1.529891-1.859172$

C 1.5132421 .7868620 .529776

C $2.8376201 .636162 \quad 0.673028$

C 3.5984211 .7978781 .912966

$\begin{array}{llll}\text { C } 2.868112 & 2.325046 & 3.119688\end{array}$

C $4.7348800 .188964-3.108473$

C $6.0976240 .805195-2.837766$

C $7.1515342 .828527-1.830075$

O $7.1534370 .269578-3.164644$

C $7.5768992 .405665-0.459358$

C $7.499614 \quad 3.1938450 .623426$

C 7.9251302 .7638141 .998313

C 6.7817792 .7647472 .989689

C 6.6140083 .7843423 .848490

C 5.8884791 .5450893 .047279

C 4.9103401 .4829451 .907812

N $5.9942852 .047182-2.226527$

H -2.982633 2.713878 -3.226836

H $-3.0128461 .554681-0.391557$

H $-0.8814661 .650434-2.613423$

H $-2.404079-0.555869-2.496726$

H -1.599149 $-0.843571-0.966835$

H $1.161426-2.443817-2.971796$

H $0.489161-1.184280-4.731994$

H $-1.155470-0.552710-4.674933$

H $\quad 0.2138150 .465994-4.176903$

H $-0.603631-2.608318-0.484705$

H $0.467269-3.814415-1.134463$

H $1.384719-1.206146 \quad 0.173058$

H $1.235901-2.7578850 .999613$

H $2.912582-3.725531-0.640581$

H $3.596485-2.9728201 .659769$

H $3.768255-1.3318291 .080143$

H $5.929999-2.4203082 .240760$

H $5.641256-0.843139-0.729599$

H $5.507140-4.5601631 .381582$

H $6.717206-4.3041830 .145504$

H $5.052278-4.711527-0.316990$

H $7.556047-2.049931-0.728668$

H $5.685042-1.639791-3.837685$

H $3.948242-1.569883-4.005966$

H $4.241557-3.641699-3.328822$

H 0.8589712 .0442731 .355100

H $3.422957 \quad 1.345385-0.197159$

H $2.114386 \quad 1.605043 \quad 3.457077$

H $2.369313 \quad 3.2723502 .885570$

H 3.5243802 .5282813 .969009

H $4.3713070 .661234-4.030038$

H $4.0340570 .452401-2.311760$

H $6.862553 \quad 3.883613-1.867938$

H $7.9677352 .664104-2.540659$

H $7.9699781 .396672-0.355767$

H 7.1139544 .2054270 .520350

H 8.7152533 .4489252 .332546

H 8.3892511 .7698671 .981329

H $5.8156353 .779596 \quad 4.582908$

H 7.2688134 .6493293 .832615

H 5.3726021 .4883704 .012045

H $6.5112680 .641433 \quad 3.020739$

H $5.344365 \quad 1.1029280 .983337$

H $5.0806592 .380902-1.933666$

C -5.824256 2.173408-2.352639

O $-5.7526730 .912573-1.656346$

C $-7.061808 \quad 0.562090-1.176739$

C $-8.0304371 .450251-1.945265$

C -7.219986 2.728129-2.065766

H -7.095212 $0.843295-0.117230$

C $-7.286965-0.947038-1.315178$

H -8.251533 $1.047803-2.940437$

H $-8.9778191 .589397-1.415964$
C $-7.7353733 .679269-3.131389$

H $-7.228343 \quad 3.241232-1.094500$

C $-6.157638-1.804122-0.703904$

O $-7.363422-1.269495-2.709949$

H $-8.257773-1.217181-0.884169$

H $-5.248207-1.683284-1.304989$

O $-6.533202-3.180880-0.838531$

C $-5.836607-1.511597 \quad 0.774417$

C $-4.678232-2.3822941 .290874$

H $-5.558507-0.4625240 .891458$

O $-6.987359-1.7524731 .580341$

H $-4.944741-3.4405431 .182100$

H -3.803907 -2.203192 0.655062

H $-7.1081994 .574273-3.190984$

H $-7.7446693 .207668-4.119807$

H $-8.7575533 .996965-2.901124$

C $-4.695173 \quad 3.079481-1.860336$

H $-7.498006-2.230316-2.778218$

H $-7.300746-3.332383-0.260546$

H $-7.655884-1.0858781 .349860$

H $-4.761557 \quad 3.197094-0.772085$

H $-4.7841764 .080359-2.296984$

H $-5.7044851 .954184-3.421536$

C $-2.916570-0.1820232 .503502$

O $-4.102724-0.746507 \quad 3.072855$

C $-4.324437-2.1288352 .775627$

C $-3.141754-2.9673793 .281459$

C $-1.817770-2.464352 \quad 2.710973$

C -1.672371 -0.9634052 .951275$

H -2.997065 -0.1856461 .410067$

C $-2.814285 \quad 1.2776682 .980647$

H -5.202580 -2.427293 3.362206

O $-3.338144-4.3360612 .944356$

H $-3.104006-2.9051854 .376946$

H -1.721543 -2.701946 1.646195

O $-0.733388-3.1222673 .369881$

H $-1.484262-0.7868064 .018427$

O $-0.536764-0.4823712 .235996$

C -3.9026012 .1753322 .431409$

H -2.888357 1.3142404 .074232

H -1.8548411 .7051422 .667681$

H $-2.640979-4.8514513 .383365$

H $-0.761687-4.0599853 .115579$

H $0.235323-0.9878632 .543096$

O -3.6660693 .4553302 .848629$

O $-4.841177 \quad \begin{array}{lll}1.813673 & 1.737287\end{array}$

C $-4.620762 \quad 4.4312542 .419466$

H $-4.5743284 .548793 \quad 1.333092$

H $-5.629095 \quad 4.1468532 .735494$

H -4.364263 5.3856932 .886734

SCF Energy (B3LYP/6-31G**//MMFF) $=-3245.91075147$

03_190

MM̄FF Geometry

C $-3.405172 \quad 2.175344 \quad 1.838517$

$\begin{array}{llll}\text { C }-3.076289 & 3.424218 & 2.204836\end{array}$

C $-1.780777 \quad 4.134276 \quad 1.881543$

$\begin{array}{llll}\text { O } & -0.737880 & 3.190576 & 1.546679\end{array}$

C $-1.988414 \quad 5.077116 \quad 0.683790$

C $-0.8029145 .987362 \quad 0.427842$

C $0.1054295 .820470-0.554077$

C -0.6991407 .1749201 .351586$

C $0.1849774 .715253-1.572060$

C $1.4753923 .913233-1.396968$

C $1.6895092 .813673-2.448466$

C $0.5745521 .765271-2.460872$

C $0.888646 \quad 0.603316-3.416039$

$\begin{array}{lllll}\text { O } & 2.936836 & 2.189725 & -2.116261\end{array}$

O $-0.049177-0.450137-3.154834$

C $2.306308 \quad 0.071501-3.123673$

C $0.6539920 .999134-4.877931$

O $2.691368-0.909568-4.083953$

C $3.375907 \quad 1.184542-3.039628$

C $4.6982520 .599001-2.490870$

$\begin{array}{lllll}\text { O } & 3.602568 & 1.724800 & -4.341359\end{array}$

C -0.0847742 .5944322 .583772$

$\begin{array}{lllll}\text { O } & -0.260558 & 2.789567 & 3.775781\end{array}$

$\begin{array}{llll}\text { C } & 0.870109 & 1.622287 & 2.009588\end{array}$ 
C $\begin{array}{llll}1.484090 & 0.733417 & 2.804417\end{array}$

C $2.408040-0.3121782 .359331$

C $2.923814-0.2218300 .949426$

C $5.7979381 .645251-2.268353$

C $6.8228431 .154285-1.266330$

C 7.1748610 .8577331 .187067

O $7.9065120 .696130-1.619836$

C $6.823646-0.5520801 .544821$

C $6.417341-0.9323502 .766219$

C $6.153118-2.3624193 .140240$

C $4.820653-2.564596 \quad 3.823457$

C $4.755206-2.8687555 .131259$

C $3.573951-2.5199852 .969654$

C $2.735227-1.2964883 .221504$

N $6.385390 \quad 1.274186 \quad 0.044512$

H -2.719686 $1.588101 \quad 1.233217$

H $-3.785583 \quad 3.9980172 .798177$

H -1.472830 4.7113802 .762309

H -2.871742 $5.708462 \quad 0.851943$

H -2.222626 $4.487854-0.209293$

H $0.899213 \quad 6.561608-0.643899$

H $\quad 0.138903 \quad 7.8314331 .093989$

H - 1.6132747 .7756361 .301340

H $-0.553467 \quad 6.8477522 .385517$

H $-0.6766834 .049093-1.506708$

H $0.1624015 .164436-2.571969$

H $2.3399584 .591140-1.431721$

H $1.5074723 .470707-0.394354$

H $1.7809793 .292856-3.430134$

H $-0.3909752 .209639-2.728286$

H $0.447667 \quad 1.360722-1.447815$

H $0.056680-0.732695-2.230222$

H $2.271302-0.445225-2.156173$

H $-0.3929851 .287659-5.031433$

H $0.8315120 .152908-5.550944$

H $1.2779461 .834465-5.202536$

H $1.997423-1.591076-4.095435$

H $4.4914220 .090200-1.542073$

H $5.089533-0.160733-3.179340$

H $4.4725382 .153949-4.357800$

H $1.014887 \quad 1.6359730 .936800$

H $1.2634180 .744176 \quad 3.870770$

H 3.3602020 .7656880 .764885

H $2.111460-0.3795990 .233560$

H $3.705225-0.9497290 .722781$

H $6.3149761 .869868-3.208032$

H $5.3910782 .593436-1.899020$

H 6.9634511 .5569612 .002123

H 8.2389550 .9214590 .938048

H $6.950825-1.3043970 .769621$

H $6.321934-0.1868823 .552379$

H $6.967342-2.6838963 .803035$

H $6.207316-3.0202302 .263648$

H $3.804203-3.0549005 .619505$

H 5.648859-2.937193 5.742576

H $2.954880-3.3976763 .202015$

H $3.835175-2.6444421 .915029$

H $2.300176-1.2662764 .221663$

H $5.447911 \quad 1.6217960 .222682$

C -5.6619941 .3404331 .084095$

$\begin{array}{lllll}\text { O } & -5.121213 & 0.439274 & 0.095299\end{array}$

C -6.101821 $-0.571280-0.196305$

C $-7.430540-0.006956 \quad 0.288111$

C -6.9950270 .7348251 .533897$

H $-5.842850-1.4434710 .416399$

C $-6.080069-0.959230-1.678625$

H $-7.8645180 .693840-0.434221$

H $-8.167937-0.7901250 .488523$

C -8.0059821 .7611352 .016211$

H -6.8242380 .0018702 .334229$

C $-4.702140-1.361343-2.239247$

O $-6.5490860 .142311-2.467920$

H -6.798565 - $1.770256-1.846889$

H $-4.080820-0.470308-2.393023$

O $-4.915842-1.926693-3.539767$

C $-3.918501-2.374398-1.388637$

C $-2.669740-2.913756-2.112132$

H $-3.623825-1.893109-0.450493$
O $-4.756133-3.479579-1.047854$

H $-2.973743-3.645259-2.870864$

H $-2.166100-2.080803-2.615434$

H -7.6409422 .2790022 .908674$

H -8.2095252 .5152911 .248564$

H $-8.9542201 .276582 \quad 2.270884$

C -4.6818521 .4980242 .248310$

H -5.974622 $0.904209-2.278083$

H $-5.390066-1.262811-4.069406$

H $-5.077821-3.872020-1.877588$

H -4.4154580 .5121842 .649561$

H -5.1592102 .0565093 .062138$

H -5.8208152 .3033090 .581949$

C $-0.145615-2.952594 \quad 0.635559$

O $-1.078271-2.537542-0.369257$

C $-1.687044-3.586854-1.131282$

C $-0.614835-4.408660-1.860977$

C $0.431358-4.942148-0.876382$

C $0.963364-3.8355090 .038940$

H $0.318106-2.0208810 .978564$

C $-0.856446-3.5865841 .835347$

H $-2.252779-4.242952-0.460923$

O $0.020216-3.599656-2.848175$

H $-1.103205-5.241100-2.381620$

H $1.267702-5.382193-1.432834$

O $-0.142513-5.968574-0.072441$

H $1.564357-4.2621420 .850521$

O $1.828427-2.985633-0.719059$

C -1.696777-2.556211 2.552768

H $-1.521706-4.4095091 .559335$

H $-0.132722-3.9992832 .548548$

H $0.673523-4.154440-3.307063$

H $-0.452999-6.670470-0.669519$

H $2.564915-3.530939-1.044010$

O $-0.946705-1.9174283 .495650$

O $-2.870306-2.3295742 .287946$

C -1.625586 -0.8961614 .232754$

H $-2.508654-1.3063634 .731953$

H $-0.940117-0.5127304 .993148$

H -1.904663 -0.075889 3.565827

SCF Energy (B3LYP/6-31G**//MMFF) $=-3245.91363955$

03_191

MM̄FF Geometry

C $-1.937288-3.728116-0.683578$

C $-0.995705-4.209953-1.509218$

C $0.464254-3.814724-1.525694$

O $0.682601-2.537844-0.883633$

C $1.287517-4.880441-0.778437$

C $2.787181-4.690106-0.885778$

C $3.603456-4.4194230 .151614$

C $3.369035-4.860349-2.266221$

C $3.244451-4.2351121 .599632$

C $3.009175-2.7755092 .020567$

C $4.233562-1.8615981 .851370$

C $3.979695-0.4924642 .485102$

C 5.1470810 .4774382 .245662

O $4.481261-1.6948750 .454526$

$\begin{array}{lllll}\text { O } & 4.678124 & 1.788974 & 2.595837\end{array}$

C 5.5066560 .4866490 .741778

C 6.3240620 .1786103 .182493

$\begin{array}{lllll}\text { O } & 6.689295 & 1.255400 & 0.537167\end{array}$

C $5.652059-0.9285690 .141354$

C $5.792844-0.944654-1.400247$

O $6.829945-1.5447020 .675763$

C $0.427109-1.423452-1.624202$

O $0.015655-1.384329-2.773122$

C $0.722552-0.240753-0.784954$

C $0.3119420 .969720-1.192113$

C $0.512602 \quad 2.237992-0.488269$

$\begin{array}{llll}C & 1.471168 & 2.242092 & 0.671972\end{array}$

C $4.628488-0.314779-2.172911$

C $4.8612641 .135454-2.559819$

C $3.7061023 .067892-3.624419$

O $5.937113 \quad 1.709009-2.412966$

C $3.2466043 .989383-2.539599$

C $2.2554154 .878370-2.702693$

C $1.8120415 .841976-1.640391$ 
C $0.3297215 .753110-1.359039$

C $-0.5341626 .615327-1.921256$

C $-0.1381094 .720742-0.358745$

C $-0.1533153 .328370-0.923687$

N $3.7288151 .706221-3.125008$

H -1.667161 -2.993691 0.069014

H -1.279725 -4.965714 -2.238982

H $\quad 0.790215-3.751440-2.570919$

H $1.048383-5.878713-1.169549$

H $\quad 0.976127-4.8878380 .271224$

H $4.669138-4.320533-0.053830$

H $4.464407-4.860582-2.258468$

H $3.046419-5.812271-2.700467$

H $3.047618-4.047404-2.924147$

H $2.360449-4.8261551 .860429$

H $4.056923-4.6564932 .205481$

H $2.167101-2.3743621 .444489$

H $2.708198-2.7769943 .075366$

H $5.095560-2.3484082 .322381$

H $3.776678-0.5905563 .558885$

H $3.070674-0.0558242 .050026$

H 5.3865712 .4210132 .383774

H $4.6943401 .017440 \quad 0.231820$

H $\quad \begin{array}{llll}6.011238 & 0.275130 & 4.229191\end{array}$

H $7.1348040 .902743 \quad 3.045857$

H $6.733318-0.825073 \quad 3.049855$

H $6.8179271 .356607-0.420649$

H $6.758261-0.519594-1.699221$

H $5.837841-1.999697-1.707881$

H $7.100548-2.2626090 .079780$

H $1.254966-0.3984460 .145327$

H $-0.2445011 .042418-2.125858$

H $2.437787 \quad 1.8278290 .366917$

H 1.0782081 .6434211 .500345

H 1.6802523 .2389361 .064625

H $4.501492-0.864253-3.114662$

H $3.690017-0.399490-1.616109$

H $3.0405353 .084473-4.493266$

H $4.7120203 .353260-3.948890$

H $3.7698793 .943026-1.587396$

H $1.7524994 .941322-3.665201$

H $2.0670446 .854050-1.981070$

H $2.3704035 .696301-0.707193$

H -1.596075 $6.576046-1.702116$

H $-0.1987017 .376348-2.618061$

H $-1.1625594 .951501-0.040436$

H $\quad 0.465140 \quad 4.8094720 .548375$

H $-0.8281653 .214936-1.773645$

H $2.8628661 .175497-3.151869$

C $-4.327069-2.966415-0.665468$

O $-4.126628-2.121347-1.815948$

C $-5.407162-1.786443-2.367958$

C $-6.441121-2.140105-1.308842$

C $-5.804138-3.366382-0.681945$

H $-5.554023-2.466834-3.218429$

C $-5.435160-0.349891-2.898078$

H $-6.547750-1.348462-0.560018$

H -7.428399 -2.339583 -1.736353

C $-6.364076-3.7095230 .687757$

H -5.954774 -4.221567-1.354589

C $-5.452014 \quad 0.795137-1.864521$

O $-6.620913-0.198911-3.692429$

H -4.584853 -0.196052 -3.574377

H $-6.4300380 .833116-1.369328$

O $\quad-5.3419532 .025889-2.598666$

C $-4.3457650 .780128-0.792403$

C $-4.3836972 .048328 \quad 0.081131$

H -4.465279 $-0.093166-0.146597$

O $-3.0632910 .701318-1.407886$

H -4.139844 $2.923342-0.534105$

H -5.4056702 .1687430 .460946$

H -5.865124 -4.590435 1.103655

H $-6.231641-2.882711 \quad 1.393821$

H -7.435035 -3.927039 0.622041

C $-3.376112-4.162824-0.720007$

H $-6.571306-0.842787-4.419560$

H -6.065602 $2.040981-3.248473$

H $-2.984637-0.177093-1.816771$
H $-3.567237-4.744242-1.630530$

H $-3.559844-4.8179610 .138954$

H $-4.109074-2.3551220 .217718$

C -2.995639 0.7806693 .327913

O -3.8090230 .9671982 .158786$

C -3.3966232 .0095701 .266106$

C -3.3522553 .3655901 .992366$

C $-2.509383 \quad 3.2969553 .266817$

C -2.8726472 .0890574 .130854$

H $-3.5582960 .072714 \quad 3.950313$

C -1.6357610 .1443342 .991155$

H $-2.399828 \quad 1.7765690 .880170$

O $-4.665321 \quad 3.8188322 .315152$

H -2.9277574 .1118851 .312433$

H -2.6514664 .2136613 .852181$

O -1.1302513 .2056682 .919581$

H -2.1244561 .9618584 .921718$

O $-4.109321 \quad 2.352996 \quad 4.799874$

C -1.751832 -1.357249 2.831508

H -1.1920970 .5338502 .072870$

H -0.9417410 .3255093 .820771$

H $-5.162607 \quad 3.0712482 .684572$

H -0.8996784 .0090092 .423135$

H -4.8336241 .9812574 .270140$

O $-0.550473-1.934773 \quad 3.127804$

O $-2.763774-1.9464362 .477537$

C $-0.509432-3.3653233 .084285$

H $-0.357310-3.6931572 .053931$

H $0.339119-3.6981783 .687818$

$\mathrm{H}-1.420572-3.8074853 .499047$

SCF Energy $($ B3LYP/6-31G**//MMFF $)=-3245.91701563$

03_192

MMFF Geometry

C $3.135757-2.565694-2.476477$

C $2.265738-1.695784-1.939483$

C $1.282232-1.937834-0.813097$

O $-0.031192-1.514544-1.265455$

C $1.162321-3.408585-0.362139$

C $0.227382-3.6290540 .811857$

C $-0.916076-4.3397970 .763945$

C $0.666971-3.0234652 .119592$

C $-1.533355-5.042384-0.412126$

C $-2.599152-4.222671-1.157610$

C $-3.807392-3.821372-0.294375$

C $-4.919765-3.225265-1.160057$

C $-6.102380-2.722578-0.315334$

O $-3.377518-2.8462090 .659127$

O $-6.923379-1.895709-1.154928$

C $-5.569462-1.8213920 .820838$

C $-6.993065-3.8774560 .158541$

O $-6.612594-1.4731251 .727045$

C $-4.387652-2.4424161 .592824$

C $-3.723463-1.4637692 .592456$

O $-4.859288-3.5601512 .355529$

C $-0.309593-0.182069-1.239294$

O $\quad 0.425451 \quad 0.716780-0.862936$

C $-1.6695920 .008467-1.787881$

C $-2.0365901 .223617-2.221871$

C $-3.3310941 .576551-2.808776$

C $-4.4384230 .561502-2.710155$

C $-3.151018-0.1814701 .978802$

C -4.0990901 .0050422 .034842$

C -4.3304013 .3438411 .205339$

O -5.1299351 .0135872 .701943$

C $-5.1815433 .366817-0.024598$

C $-5.1389094 .357132-0.928523$

C $-6.0112014 .416343-2.149343$

C $-5.2159064 .590990-3.423609$

C $-5.0602705 .804089-3.980357$

C $-4.6754663 .349100-4.095528$

C $-3.4684812 .786867-3.390111$

N $-3.6325242 .074521 \quad 1.282943$

H $3.196448-3.585557-2.110616$

H $2.267006-0.682390-2.338718$

H $1.585679-1.3185620 .039389$

H $2.148635-3.790140-0.067579$

H $0.843435-4.009758-1.220002$ 
H -1.490061 -4.438988 1.685013 H $0.041841-3.3451322 .959618$ H $1.695513-3.3189312 .348750$ H $0.613341-1.9325712 .073129$ H $-0.766842-5.359476-1.126465$ H $-1.986909-5.973526-0.048824$ H -2.126067 -3.324379-1.571030 H -2.947483 -4.824022 -2.006404 H -4.168597 -4.7139850 .229693$ H -5.263990 -3.951875 -1.906499 H $-4.518026-2.378498-1.732259$ H -7.207008 -2.430012 -1.916522 H $-5.236224-0.8869770 .354260$ H -7.395987 -4.427228 -0.700491 H $-7.863829-3.5052020 .709483$ H $-6.464977-4.5950340 .789772$ H -7.325107 -1.064944 1.205830 H -4.410191 -1.2462863 .419413$ H -2.877836 -1.996516 3.051429 H -4.225712 -3.734614 3.071176 H $-2.319859-0.856311-1.841261$ H -1.313087 2.036359 -2.167068 H $-4.5551270 .223800-1.674983$ H -4.222084 -0.306575 -3.341900 H $-5.4144400 .948665-3.009362$ H -2.2646780 .1123632 .556061$ H -2.828010 -0.3479440 .947023$ H -3.5698304 .1310041 .197849$ H -4.9587283 .4733482 .092357$ H -5.879642 2.544994-0.165745 H $-4.4584235 .190890-0.769421$ H $-6.7030875 .259463-2.023535$ H $-6.6450973 .524918-2.233998$ H $-4.5152075 .934890-4.909113$ H -5.470372 $6.694882-3.515873$ H $-4.3671463 .582796-5.123647$ H $-5.4854672 .623174-4.207123$ H $-2.6058043 .454583-3.405087$ H -2.8029091 .9563580 .709344$ C $5.469865-1.811466-3.172949$ O $5.416698-0.598897-2.396763$ C $6.371445-0.699713-1.322024$ C $7.248874-1.903543-1.650289$ C $6.247661-2.820610-2.330291$ H $5.800194-0.927586-0.414126$ C $7.1166140 .627745-1.147321$ H $8.056758-1.641963-2.343509$ H $7.699290-2.345161-0.756212$ C $6.887012-3.935994-3.137618$ H $5.607144-3.261517-1.556502$ C $6.1868601 .846070-0.958127$ O $7.9073730 .862334-2.319016$ H $7.8199300 .546451-0.310295$ H $5.6360922 .034377-1.887260$ O $7.0077303 .002493-0.753044$ C 5.1918571 .7140350 .209177 C 4.3115802 .9670220 .385388 H 4.5385790 .8558280 .023062 O 5.9000941 .4450581 .417877 H 4.9380803 .8465970 .576115 H $3.7730633 .133857-0.555312$ H $6.122858-4.553553-3.620150$ H $7.544720-3.540715-3.918911$ H $7.486677-4.584073-2.490329$ C $4.059669-2.212180-3.610557$ H $8.3650521 .711456-2.194626$ H 7.5619202 .8406120 .028910 H 6.3940862 .2465251 .659550 H $4.110206-3.068116-4.293707$ H $3.622446-1.383848-4.183346$ H $6.043989-1.563903-4.075920$ C 1.5897301 .3255322 .349874 O 2.5118991 .6686921 .308768 C 3.3115822 .8286031 .550219 C 2.4099254 .0628391 .700763 C 1.3460473 .8455382 .783757 C 0.6303952 .4973742 .627647 H 1.0020920 .4946381 .940892
C $2.3019350 .807023 \quad 3.609107$

H 3.8867552 .6880922 .471337

O $1.7686824 .362873 \quad 0.462111$

H 3.0313734 .9280591 .959319

H 0.6113274 .6583452 .727554

O 1.9769123 .9158994 .059006

H 0.0270712 .2818743 .517026

O $-0.2759472 .614978 \quad 1.526913$

C $3.115062-0.4437803 .343114$

H 2.9855581 .5475824 .031530

H 1.5464920 .5588004 .364093

H 1.2680213 .5751840 .188195

H 1.2850453 .8129134 .734487

H -0.7319651 .7621601 .429274$

O $3.527344-0.9640574 .537428$

O $3.375106-0.8996682 .238344$

C $4.330462-2.1453174 .448203$

H $3.761907-2.9551773 .981611$

H $5.245597-1.940303 \quad 3.884298$

H $4.603961-2.4476985 .462525$

SCF Energy (B3LYP/6-31G**//MMFF) = -3245.90561192

03_193

MMFF Geometry

C $2.579956-0.0270494 .180810$

C $1.901498-0.8520203 .368686$

C $0.406621-1.0392733 .419012$

O $-0.157652-0.6443012 .147820$

C $0.090040-2.5305613 .638219$

C $-1.388114-2.8331413 .783660$

C $-2.101335-3.5964472 .932363$

C $-2.059251-2.2452634 .998663$

C $-1.628192-4.2960871 .688517$

C -1.823447 -3.4959690 .390806$

C $-3.292876-3.1930830 .053817$

C $-3.417194-2.599973-1.350510$

C $-4.869696-2.224895-1.684602$

O $-3.788701-2.2479671 .002852$

O $-4.852273-1.390883-2.854437$

C $-5.452893-1.369829-0.535647$

C $-5.698337-3.461870-2.051431$

O $-6.850926-1.160586-0.712299$

C $-5.184596-1.9463380 .871929$

C $-5.541576-0.9702882 .016079$

O $-5.987529-3.106503 \quad 1.123678$

C -0.3811560 .6835251 .950408$

O -0.1367591 .5954312 .724234$

C $-0.9703310 .842745 \quad 0.602544$

C $-0.9913792 .056624 \quad 0.032333$

C $-1.5042922 .373509-1.301868$

C $-2.2990951 .311622-2.013212$

C -4.7797300 .3596572 .013262$

C -5.5290411 .4981451 .341052$

C -5.2408143 .8821290 .675760$

O -6.6965151 .4131800 .969881$

C $-4.9182333 .934721-0.783895$

C $-4.2889864 .969372-1.361102$

C $-3.9967535 .057005-2.831280$

C -2.534769 $5.312311-3.119310$

C $-2.0887726 .551410-3.386737$

C $-1.6086984 .119285-3.182681$

C -1.246661 $3.592853-1.819793$

N $-4.7447582 .639378 \quad 1.236295$

H 2.0428070 .5623234 .919070

H $2.446135-1.4300612 .624912$

H $-0.037690-0.4392694 .222612$

H $0.598494-2.8883674 .543720$

H $0.517158-3.1025242 .807357$

H $-3.157663-3.7507973 .150976$

H -2.118934 -1.155580 4.917905

H $-3.080014-2.6198205 .131515$

H -1.498528 -2.500154 5.903885

H $-0.573531-4.5766631 .776681$

H -2.168869 -5.2478621 .609144$

H -1.259550 -2.558806 0.465762

H - $-1.379891-4.076028-0.427985$

H -3.864744 -4.125192 0.129933

H $-3.015703-3.289181-2.103787$ 
H $-2.803178-1.692465-1.416532$ H $-4.409124-1.882380-3.567179$ H $-4.978206-0.384484-0.607776$ H -5.282821 -3.950804-2.940958 H -6.725662 -3.187391-2.314426 H $-5.730257-4.207714-1.254461$ H $-6.975912-0.764626-1.591813$ H $-6.626549-0.8143042 .054436$ H -5.291444 -1.477987 2.959189 H $-6.608672-3.2208960 .386816$ H $-1.344341-0.0448410 .106279$ H -0.5615482 .8955700 .578218$ H -1.649171 $0.485702-2.319518$ H -2.811275 $1.675595-2.906330$ H -3.084383 $0.918365-1.359059$ H -4.624656 0.6706653 .054497 H $-3.792646 \quad 0.2504871 .553893$ H -4.7729984 .6995341 .233565$ H -6.3249793 .9389560 .816205$ H -5.239813 $3.096560-1.397678$ H $-3.9931375 .818566-0.749015$ H -4.605938 $5.872043-3.243579$ H -4.319822 4.151489-3.359764 H -1.046422 $6.738945-3.622453$ H $-2.7557517 .407035-3.370310$ H $-0.6694784 .399789-3.678550$ H $-2.0483643 .358494-3.833720$ H $-0.6644004 .296153-1.222615$ H -3.7696402 .6016541 .518741$ C 4.5727051 .2969843 .306963 O $4.192998 \quad 1.106167 \quad 1.931619$ C 3.8344212 .3852531 .371643 C 4.2923483 .4310002 .382903 C 4.0751642 .6887493 .690752 H 2.7396482 .4128601 .325332 C $4.4222772 .518764-0.038400$ H 5.3521053 .6818932 .258795 H 3.7145584 .3574132 .312384 C 4.8057293 .3016204 .871848 H 2.9988712 .6743063 .903430 C $4.0422221 .340190-0.964448$ O 5.8486762 .5561710 .075144 H $4.1181363 .475310-0.479050$ H $4.4651670 .414220-0.567895$ O $4.6423341 .552458-2.246299$

C $2.526511 \quad 1.189617-1.168657$ C $2.0899420 .029476-2.082654$ H $2.0268461 .067312-0.204392$ O $2.0240332 .405034-1.735139$ H $1.0771980 .258293-2.438012$ H $2.7138030 .009147-2.982205$ H 4.6309172 .7188045 .781747 H 5.8863803 .3420184 .699206 H 4.4560874 .3234145 .052106 C $4.075300 \quad 0.1207334 .147371$ H $6.2024242 .842007-0.784005$ H $5.6034181 .456465-2.139068$ H $2.4627702 .530452-2.594278$ H 4.4383330 .2161165 .177304 H $4.514428-0.8030993 .748241$ H 5.6708761 .2802923 .321931 C $4.191328-2.240916-2.141313$ O $3.378761-1.823057-1.036495$ C $2.075866-1.341310-1.370088$ C $1.302334-2.429318-2.126574$ C $2.059471-2.883672-3.367352$ C $3.479691-3.302767-2.996080$ H $4.443960-1.369139-2.755998$ C $5.485935-2.814692-1.548085$ H $1.553881-1.193158-0.416102$ O $0.009451-1.972833-2.507742$ H $1.154963-3.296641-1.469918$ H $2.065407-2.108697-4.142897$ O $1.392890-4.014681-3.934264$ H $3.436100-4.261252-2.462422$ O $4.200023-3.518264-4.209404$ C $6.358022-1.741614-0.943143$ H $5.260775-3.536966-0.753433$
H $\quad 6.088271-3.344909-2.293714$

H $-0.440595-1.669210-1.701240$

H $\quad 0.479601-3.743059-4.129890$

H $5.014472-4.001447-3.993013$

O $7.068874-1.114759-1.923523$

O $6.406130-1.4981840 .255543$

C $7.987244-0.110558-1.480875$

H $7.5151130 .569698-0.767130$

H $8.863119-0.587618-1.032059$

H $8.3064090 .466213-2.352974$

SCF Energy (B3LYP/6-31G**//MMFF) $=-3245.90391818$

03_194

MM̄MF Geometry

C $2.4802694 .654274-0.891567$

C $3.6864924 .503762-0.323177$

C $4.3741803 .178763-0.069076$

O $3.4281962 .084131-0.095615$

C $5.4321812 .928389-1.158327$

C $6.2404891 .662932-0.947564$

C $6.2340810 .603574-1.780639$

C $7.116321 \quad 1.6431790 .278887$

C $5.4648780 .436082-3.061335$

C $4.087665-0.226731-2.902894$

C $4.140641-1.675684-2.391093$

C $2.759537-2.329141-2.473252$

C $2.760752-3.749506-1.888829$

O $4.575600-1.662425-1.029857$

O $1.386619-4.136938-1.732864$

C $3.410301-3.724920-0.486559$

C $3.382052-4.756747-2.864217$

O $3.563523-5.058868-0.007856$

C $4.752419-2.962083-0.447749$

C $5.303234-2.7401760 .982577$

O $5.739046-3.715580-1.163298$

C 2.6819141 .8779991 .024564

O 2.7561602 .4852342 .080792

C 1.7087890 .8048230 .722691

C $0.635406 \quad 0.651907 \quad 1.512513$

C $-0.440555-0.3252911 .333199$

C $-0.258092-1.3716170 .265940$

C $4.379292-1.9685271 .931291$

C $3.521688-2.8578782 .816026$

C $1.689897-2.7315184 .500408$

O $3.668645-4.0745742 .893730$

C $0.392256-3.0576403 .831237$

C $-0.800197-2.6849524 .320440$

C $-2.114929-3.0433243 .689448$

C $-2.978104-1.8324663 .416329$

C $-3.955133-1.4730594 .265742$

C $-2.763946-1.0940652 .115203$

C $-1.527000-0.2392872 .129227$

N $2.592328-2.123523 \quad 3.540556$

H $1.9450673 .777383-1.247107$

H 4.2264015 .3880880 .007818

H 4.8503593 .2187490 .918043

H $6.1300383 .775350-1.202095$

H $4.9285232 .903011-2.130740$

H $6.861483-0.249941-1.525474$

H 7.7797140 .7719310 .301654

H 7.7497272 .5357840 .308190

H 6.5079831 .6145621 .187941

H $5.339403 \quad 1.400842-3.564501$

H $6.076277-0.163340-3.748073$

H $3.4751700 .382400-2.228013$

H $3.597280-0.212359-3.884303$

H $4.863149-2.229979-3.001515$

H $2.393721-2.341182-3.507602$

H $2.037792-1.722510-1.909722$

H $1.373943-5.008463-1.300865$

H $2.691826-3.2321550 .178746$

H $2.817104-4.774436-3.804043$

H $3.332259-5.776780-2.467490$

H $4.422890-4.532601-3.106866$

H $3.848486-5.0128490 .919875$

H $5.628344-3.6927091 .417407$

H $6.221333-2.1425030 .884289$

H $6.619515-3.427358-0.870206$ 
H $1.8842760 .196126-0.156445$ H 0.5146551 .3260292 .359855 H $-0.241168-0.909633-0.727019$ H -1.043511 -2.1296100 .253557$ H $0.682466-1.9112180 .418992$ H $5.001859-1.3731822 .611673$ H $3.739847-1.2728751 .380312$ H $1.564842-2.0208815 .323514$ H $2.140916-3.6475734 .895393$ H $0.433181-3.6504102 .920411$ H $-0.838537-2.1162155 .246926$ H $-2.636800-3.7264724 .372503$ H -1.973560 -3.608115 2.759581 H $-4.597675-0.6230354 .062295$ H -4.131221 -2.018856 5.186786 H -3.614255 -0.428996 1.917306 H -2.773968 -1.817249 1.296253 H -1.5634470 .5605962 .870268$ H $2.505084-1.1252893 .372705$ C $0.4334786 .051342-0.356699$ O $-0.425394 \quad 4.977409-0.797644$ C -0.8709994 .2542880 .361946$ C -0.8052115 .2639501 .496657$ C 0.5040895 .9614851 .172586 H -0.1292583 .4673210 .547284$ C -2.2326083 .5876560 .132529$ H -1.633735 5.9794871 .453141 H -0.8058174 .7891652 .482149$ C $0.6665237 .308327 \quad 1.855734$ H 1.3286405 .3108561 .488792 C $-2.1872172 .534487-0.997531$ O $-3.2213864 .567344-0.170809$ H -2.5349843 .0870041 .060960$ H $-1.3331681 .869683-0.820989$ O $-1.9819073 .155862-2.265403$ C $-3.4567381 .662734-1.071771$ C $-3.3214720 .554238-2.132360$ H $-3.6351411 .211678-0.089620$ O $-4.5984562 .450642-1.400427$ H -3.283995 $1.003564-3.132305$ H $-2.3775740 .023605-1.958475$ H $1.6138487 .776167 \quad 1.569257$ H -0.1438257 .9948691 .588828$ H 0.6616697 .1906182 .944277 C $1.7828615 .972578-1.083427$ H -3.4000205 .0720960 .639945$ H -1.148447 $3.653861-2.219300$ H -4.699958 $3.126876-0.709779$ H $2.4210126 .805400-0.766243$ H $1.6101206 .089918-2.160571$ H $-0.0665006 .980425-0.658184$ C -5.475815 -2.070374 -0.578508 O $-4.432291-1.120943-0.829761$ C $-4.489286-0.447806-2.090927$ C $-4.410647-1.466663-3.238082$ C $-5.490497-2.544268-3.099871$ C $-5.529420-3.135789-1.685800$ H -5.177464 -2.571182 0.351848 C $-6.820579-1.381950-0.324131$ H $-5.4295010 .108531-2.163659$ O $-3.124957-2.084355-3.268595$ H -4.535659 -0.938494 -4.190596 H -5.292891 -3.340479 -3.828012 O $-6.752819-1.966015-3.418252$ H $-6.421331-3.758241-1.549841$ O $-4.393781-3.988581-1.509397$ C $-6.763436-0.5720290 .949592$ H -7.134779 $-0.726381-1.141252$ H -7.619803 -2.122255 -0.199627 H -2.967576 -2.486988 -2.397238 H $-7.422025-2.668537-3.353543$ H -4.449107 -4.692145 -2.178351 O $-\begin{array}{llll}6.482016 & 0.730375 & 0.662211\end{array}$ O $-6.920460-1.0482032 .066403$ C $-6.362828 \quad 1.6012451 .791179$ H -6.1699772 .6118681 .421672$ H -7.293805 1.6091492 .366064 H $-5.522427 \quad 1.2918812 .419033$
SCF Energy (B3LYP/6-31G**//MMFF)= -3245.91880815

03_195

MM̄FF Geometry

C $0.4491964 .642169-1.986576$

C $-0.3375114 .229935-0.980801$

C -1.738218 $3.704985-1.174236$

O $-1.8116082 .394028-0.565162$

C -2.743326 $4.621292-0.453842$

C $-4.1891444 .198653-0.630086$

C $-4.999273 \quad 3.8063210 .372941$

C $-4.7235164 .263028-2.038119$

C -4.6802073 .7091381 .838363$

C -4.1720762 .3336952 .297483$

C -5.1865321 .1934022 .111704$

C $-4.707480-0.0767872 .818716$

C $-5.656325-1.2620372 .578813$

O $-5.3265640 .933143 \quad 0.712475$

O $-4.977433-2.4598052 .987629$

C $-5.928612-1.3932111 .064276$

C $-6.919487-1.1641633 .442617$

O $-6.933907-2.3709880 .810810$

C $-6.310657-0.0595640 .390165$

C $-6.380809-0.152621-1.154043$

O $\quad-7.6099680 .3409680 .843089$

C $-1.3017141 .346351-1.267739$

O $-0.8268291 .372930-2.391640$

C $-1.3884530 .149587-0.402716$

C $-0.659545-0.935809-0.701307$

C $-0.609560-2.1786460 .071562$

C $-1.590714-2.3361251 .202388$

C $-5.086005-0.590474-1.847759$

C $-5.018743-2.079429-2.146410$

C $-3.450908-3.818700-2.999576$

O $-5.979231-2.835428-2.029504$

C $-2.842721-4.551130-1.845466$

C -1.690172 -5.232552 -1.933352

C $-1.084616-6.008016-0.798600$

C $0.346894-5.608007-0.519633$

C $1.373776-6.320675-1.013005$

C $0.584849-4.4306150 .398170$

C $0.303705-3.111897-0.268042$

N $-3.762449-2.458765-2.600634$

H $0.0417604 .627626-2.995949$

H 0.0326514 .2323960 .040483

H $-1.9892603 .629262-2.239128$

H -2.641776 $5.649222-0.826790$

H -2.4822064 .6580990 .609343$

H -6.0226613 .5280280 .123025$

H -5.805591 $4.097775-2.079431$

H $-4.5278815 .248115-2.474416$

H -4.252326 $3.500823-2.666050$

H -3.9493774 .4726962 .126059$

H -5.5887713 .9623322 .399924$

H -3.2472302 .0981801 .757978$

H -3.9139072 .4144213 .360714$

H -6.149610 1.5181222 .522692

H $-4.5811270 .099793 \quad 3.894217$

H -3.711850 -0.3477052 .442143$

H $-4.732196-2.360576 \quad 3.923558$

H $-5.004936-1.7692760 .608764$

H -6.654717 -1.146972 4.506841

H -7.559388 -2.043167 3.308456

H $-7.512032-0.2704713 .236578$

H $-6.643640-3.2000511 .228129$

H $-7.232248-0.774485-1.455852$

H -6.612536 $0.855553-1.527472$

H $-7.983790 \quad 0.962470 \quad 0.196562$

H -2.0264330 .2054940 .471504$

H $-0.016189-0.905313-1.579669$

H - $1.367460-1.6271712 .006890$

H -1.595478 -3.335654 1.641643

H -2.612229 -2.158611 0.849290

H $-5.025545-0.083661-2.819577$

H $-4.207877-0.291297-1.268102$

H $-2.771838-3.757743-3.855903$

H $-4.367643-4.326304-3.316281$

H $-3.386494-4.542382-0.903717$ 
H -1.163444 -5.261749 -2.884788

H -1.129227 -7.072931 -1.061943

H -1.677407 -5.9062390 .119054$

H $2.402964-6.054931-0.795344$

H $1.207480-7.182600-1.650703$

H $1.635187-4.4100730 .719217$

H $\quad 0.014468-4.5762101 .319339$

H $0.965737-2.904463-1.108747$

H -3.011356 -1.774818 -2.618827

C $2.5815754 .937350-0.541790$

O $2.6288953 .539911-0.196263$

C 3.9492523 .2054810 .255124

C 4.6411234 .5337040 .528136

C $4.0368235 .409822-0.552707$

H $4.4454302 .704162-0.585966$

C 3.9072502 .2397101 .449039

H 4.3866934 .9255161 .519803

H 5.7305414 .4572800 .461927

C $4.2053466 .897103-0.296312$

H $4.5080475 .156485-1.511890$

C $3.1174840 .948046 \quad 1.159045$

O 3.3417762 .8855152 .589247

H 4.9428171 .9980561 .707298

H 3.4351840 .5159170 .206689

O $1.723580 \quad 1.2513961 .017861$

C $3.224670-0.1191092 .260819$

C $4.649305-0.5892622 .605516$

H 2.7440040 .2418693 .178220

O $2.471543-1.2683761 .854742$

H $5.1557230 .187344 \quad 3.190951$

H $4.546685-1.4677083 .255537$

H $3.7402557 .485363-1.093538$

H 3.7482027 .1950270 .653164

H $5.2668797 .162282-0.255887$

C $1.8815335 .104844-1.894881$

H $2.425722 \quad 3.1227882 .363665$

H $1.626901 \quad 1.8418770 .251725$

H $1.568139-0.9662901 .658879$

H $2.4394124 .533192-2.648120$

H $1.9150986 .157530-2.198821$

H 2.0210885 .4497180 .250158

C $5.546907-2.357527-0.585998$

O $4.873058-1.9558370 .615344$

C $5.540553-0.9654291 .400482$

C $6.918625-1.4795081 .850703$

C $7.756818-1.9403120 .652840$

C $6.964308-2.868510-0.274773$

H $4.962971-3.206890-0.963261$

C $5.522260-1.255335-1.658383$

H $5.683543-0.0662800 .794277$

O $6.750792-2.5669872 .758044$

H $7.435673-0.6766292 .389756$

H $8.649093-2.4679471 .011193$

O $8.196204-0.807507-0.090536$

H $7.507083-3.027839-1.213859$

O $6.828198-4.1473230 .352135$

C $4.113054-0.920604-2.098823$

H $5.985376-0.335980-1.290125$

H $6.070732-1.580019-2.549628$

H $7.635513-2.8478273 .047465$

H $8.721723-0.2491780 .507512$

H $7.723050-4.4968190 .502653$

O $4.075361 \quad 0.367522-2.551887$

O $3.167059-1.695250-2.067359$

C $2.788627 \quad 0.838561-2.965477$

H $2.893906 \quad 1.878711-3.284992$

H $2.4207090 .246151-3.808403$

H $2.0853010 .799892-2.128546$

SCF Energy $\left(B 3 L Y P / 6-31 G^{* *} / / M M F F\right)=-3245.90189671$

03_196

MM̄FF Geometry

C $2.599807-2.494428-2.316439$

C $2.272179-3.121702-1.175609$

C $0.889548-3.159954-0.568584$

O $0.149519-2.004856-1.027353$

C $0.123926-4.432890-0.960595$

C $-1.142337-4.639318-0.147189$
C $-2.394134-4.622558-0.646207$

C $-0.936757-4.9124401 .322478$

C $-2.824318-4.419727-2.070709$

C $-3.139267-2.964803-2.445179$

C $-4.341734-2.351466-1.705983$

C $-4.685847-0.986020-2.310208$

C $-5.802203-0.267017-1.539143$

O $-4.003962-2.198950-0.324118$

O $-5.8118621 .107588-1.956156$

C $-5.456988-0.276930-0.035288$

C $-7.185546-0.832086-1.880065$

$\begin{array}{lllll}\text { O } & -6.533934 & 0.247202 & 0.736417\end{array}$

C $-5.058829-1.6705960 .493307$

C $-4.553451-1.6456851 .958921$

O $-6.206540-2.5282550 .467471$

C $-0.092685-1.014109-0.134983$

O $0.081191-1.0611291 .074377$

C $-0.5746410 .217888-0.791591$

C $-0.7702970 .355743-2.110551$

C $-1.2019361 .599962-2.739170$

C $-0.4381531 .935900-3.989887$

C $-3.301246-0.8014632 .209769$

C -3.5903800 .6460702 .568538$

C -2.5193382 .9006142 .530943$

O -4.6578311 .0199633 .047060$

C -3.0979283 .5661661 .321033$

C -2.4154844 .4075930 .529221$

C $-3.0214945 .121072-0.646793$

C $-2.3130904 .824088-1.949953$

C $-1.3643905 .648957-2.424388$

C $-2.7694153 .624307-2.749922$

C $-2.2057582 .335921-2.221407$

N -2.497986 1.4657562 .324267

H $1.820798-2.039985-2.923589$

H $3.059204-3.585018-0.584460$

H $1.028401-3.1211500 .517791$

H $\quad 0.762861-5.314388-0.817250$

H $-0.103347-4.392032-2.031175$

H -3.220248 -4.7896710 .044643$

H -1.861576 -5.2232721 .820468$

H $-0.207977-5.7176901 .461255$

H -0.574281 -4.018056 1.837979

H -2.063814 -4.804999-2.758531

H $-3.711421-5.040190-2.251205$

H $-2.247578-2.355470-2.262730$

H $-3.332890-2.935519-3.524864$

H -5.193213 -3.035514 -1.799347

H -4.955626 -1.086306 -3.369089

H -3.792300 -0.349232 -2.291159

H $-5.9442901 .127987-2.919280$

H $-4.6107180 .408607 \quad 0.096148$

H -7.392521 -0.717191-2.950911

H $-7.977130-0.279456-1.362056$

H -7.287563 -1.892142 -1.638985

H $-6.735258 \quad 1.1328620 .388532$

H $-5.370234-1.3642532 .634503$

H $-4.293651-2.6791152 .229893$

H $-6.053098-3.2675001 .079328$

H $-0.7385391 .056022-0.120883$

H $-0.572053-0.464936-2.794731$

H $-0.698451 \quad 1.238180-4.793350$

H $0.640528 \quad 1.865116-3.811625$

H $-0.6273162 .948815-4.352295$

H -2.758395 -1.221134 3.066201

H $-2.632727-0.848307 \quad 1.346450$

H -1.4907973 .2190632 .726358$

H -3.129824 3.1323423 .409565

H $-4.144558 \quad 3.364981 \quad 1.103320$

H $-1.3738994 .624657 \quad 0.755221$

H -2.979531 $6.198392-0.437833$

H $-4.0886304 .887678-0.749722$

H -0.873016 $5.459074-3.372695$

H -1.053141 $6.528975-1.871486$

H -2.528370 $3.757142-3.810389$

H $-3.8648933 .564192-2.725424$

H $-2.7047041 .973834-1.322736$

H -1.665145 1.0664361 .903622

C $4.258096-0.820170-3.118655$ 
O $3.9435730 .002897-1.981716$

C $4.8218781 .143860-1.971937$

C $5.7063921 .019944-3.211597$

C $5.705558-0.479262-3.455392$

H $5.451347 \quad 1.043407-1.080122$

C $3.9972862 .436255-1.881731$

H $5.2806271 .545447-4.073901$

H $6.7088961 .425860-3.042523$

C $6.126426-0.867789-4.860965$

H $6.381468-0.951272-2.729710$

C $2.9504642 .435690-0.746651$

O $3.2866012 .610602-3.114741$

H $4.6704303 .296776-1.789686$

H $2.1682121 .703010-0.974774$

O $2.2992863 .712751-0.749143$

C 3.5218142 .1608710 .655578

C 2.4294272 .2001261 .739650

H 4.0009621 .1798380 .659954

$\begin{array}{llllll}\text { O } & 4.524078 & 3.135249 & 0.937589\end{array}$

H $1.9843173 .202143 \quad 1.768103$

H 1.6343761 .5020301 .455616

H $6.102942-1.954564-4.988638$

H $5.465685-0.425487-5.614127$

H $7.146322-0.526029-5.065459$

C $4.014815-2.294813-2.785077$

H $2.7606423 .424785-3.033388$

H $2.9606354 .378404-0.493263$

H 5.0236062 .8294011 .713165

H $4.715461-2.618386-2.005881$

H $4.191484-2.913809-3.671133$

H $3.589575-0.507885-3.932719$

C $2.930095-0.5490083 .023349$

O 3.7000290 .6468223 .196032

C 2.9483431 .8645713 .156837

C 1.8557041 .8520604 .236746

C 0.9774110 .6067314 .139353

C $1.845435-0.6502844 .104263$

H $2.470292-0.5457452 .029020$

C $3.896856-1.7416823 .111273$

H 3.6499282 .6628273 .429625

$\begin{array}{lllll}\text { O } & 1.061134 & 3.028491 & 4.135927\end{array}$

H 2.3352521 .8702095 .224212

H $0.3138590 .648236 \quad 3.269169$

$\begin{array}{lllll}\mathrm{O} & 0.131574 & 0.528842 & 5.288374\end{array}$

H $2.307186-0.8018535 .088407$

O $1.020954-1.784142 \quad 3.842813$

C $4.760813-1.888778 \quad 1.876223$

H $4.562044-1.6093173 .972978$

H $3.345260-2.6819013 .220132$

H 0.4496713 .0386414 .891179

H -0.5105241 .2564105 .228980$

H $0.323631-1.799804 \quad 4.520638$

O $5.749059-2.7970882 .130870$

O $4.583155-1.3093790 .814162$

C $6.633608-3.0617831 .037062$

H $7.129940-2.1407640 .716668$

H $7.394300-3.769041 \quad 1.377972$

H $6.081393-3.5123020 .207046$

SCF Energy (B3LYP/6-31G**/MMFF) $=-3245.91203141$

03_197

MMFF Geometry

C $-3.702188 \quad 0.136081-3.595364$

C $-2.682881-0.579070-3.098863$

C $-1.241360-0.354152-3.477522$

O $-0.480166-0.086867-2.275812$

C $-0.677437-1.617746-4.154164$

C $0.800120-1.543734-4.492482$

C $1.706866-2.460594-4.099007$

C $1.233053-0.377079-5.340906$

C $1.444274-3.712998-3.309106$

C $1.369948-3.502354-1.791861$

C $2.727692-3.249442-1.118967$

C $2.537910-3.080667 \quad 0.391125$

C $3.861672-2.7713681 .101859$

O $3.307788-2.066056-1.665633$

O $3.555504-2.3152712 .429068$

C $4.560081-1.6005450 .376207$
C $4.718450-4.0318291 .267886$

O $5.874486-1.3951740 .889108$

C $4.614115-1.756350-1.165126$

C $5.086919-0.458747-1.859072$

O $5.570299-2.750449-1.551453$

C $-0.5241841 .189208-1.800596$

O $-1.1836372 .113947-2.250357$

C $0.3863411 .283508-0.636120$

C 0.4293752 .4326930 .055607

C 1.2753302 .7500151 .208309

C 2.2633481 .7210231 .687285

C $4.0408860 .658649-1.893725$

C $4.6551882 .039353-2.041467$

C $4.0508874 .445022-1.769249$

O $5.8441582 .228222-2.282142$

C $4.2459684 .783354-0.323158$

C 3.5361515 .7151780 .330872

C 3.7350296 .0413611 .784095

C 2.4598005 .9352252 .593058

C 1.8502027 .0342953 .068980

C 1.9339474 .5512722 .919264

C 1.1385483 .9642021 .783071

N $3.7065453 .038744-1.866496$

H $-3.4987260 .919289-4.321445$

H -2.888261 -1.355653 -2.368167

H $-1.1458420 .495656-4.165902$

H -1.230584 -1.809273 -5.083351

H $-0.874350-2.475334-3.502067$

H $2.746695-2.318008-4.389430$

H $1.1529130 .559561-4.780554$

H $2.271878-0.470705-5.675536$

H $0.607048-0.306319-6.236404$

H $0.510214-4.173544-3.652879$

H $2.226652-4.445686-3.543418$

H $0.689881-2.669603-1.573543$

H $0.926242-4.403120-1.350346$

H $3.380974-4.103153-1.334080$

H $2.069475-3.9716220 .827217$

H $1.841170-2.2517990 .581413$

H $3.035293-3.0073592 .872176$

H $3.992636-0.6949060 .620091$

H $4.194526-4.7719291 .885092$

H $5.652154-3.8102561 .796094$

H $4.961384-4.5129590 .318054$

H $5.795192-1.2785331 .851427$

H $6.020696-0.121069-1.392292$

H $5.358588-0.687193-2.899489$

H $6.037363-3.059942-0.759151$

H $0.9947080 .420557-0.393750$

H $-0.2259993 .244132-0.260860$

H $1.8330250 .715241 \quad 1.695060$

H 2.5967901 .8988992 .713083

H 3.1479221 .7180401 .044599

H $3.3519970 .497385-2.731293$

H $3.4506600 .657562-0.975097$

H $3.2336555 .014526-2.222337$

H $4.9717234 .641873-2.326746$

H 5.0150394 .2306940 .212010

H $2.7792696 .282727-0.205684$

H 4.1367067 .0611541 .842441

H 4.4974475 .3948402 .236641

H $\quad 0.9503606 .964847 \quad 3.671215$

H 2.2338108 .0280882 .862960

H 1.2752724 .5981553 .796130

H 2.7797683 .9239993 .215088

H 0.3727394 .6365891 .393007

H $2.7562042 .775284-1.625091$

C $-5.6655870 .917019-2.173608$

O $-5.063991 \quad 0.661767-0.886622$

C $-4.7718801 .925291-0.262542$

C $-5.5138232 .984222-1.075832$

C $-5.4371282 .399431-2.470986$

H $-3.6940302 .087022-0.380680$

C -5.1383901 .9150851 .225759$

H $-6.5602073 .081176-0.764197$

H $-5.0459963 .970359-0.995940$

C $-6.4392962 .994026-3.444150$

H $-4.4241822 .576771-2.851804$ 
C $-4.492203 \quad 0.7947872 .066037$

O

H -4.893065 2.894272 1.655963

H -4.990304 -0.163952 1.875399

O $\quad-4.734186 \quad 1.1053493 .445868$

C $-2.9749720 .615018 \quad 1.873724$

C $-2.323819-0.2411082 .981138$

H $-2.793270 \quad 0.162851 \quad 0.892353$

O

H $-2.222600 \quad 0.3752343 .881989$

H $-2.987460-1.0737073 .228575$

H $-6.3483042 .525929-4.429419$

H -7.468484 2.853728 -3.097259

H $-6.2688904 .069038-3.562961$

C -5.139902 $-0.076461-3.213777$

H $-6.807116 \quad 1.9820562 .254102$

H $-5.686546 \quad 0.999813 \quad 3.608542$

H -2.5433482 .3295142 .717178$

H $-5.744750-0.007548-4.125765$

H -5.268771 -1.096139-2.830295

H $-6.7386420 .725828-2.039290$

C $-1.487280-3.0782732 .168321$

O $-1.025701-1.8367241 .617601$

C $-0.929760-0.7566902 .556045$

C $0.002341-1.1451243 .711199$

C $-0.421862-2.4518364 .369458$

C $-0.580227-3.5433793 .313725$

H $-2.510836-2.9611872 .539334$

C - $-1.510300-4.1157981 .039189$

H -0.4369790 .0637352 .025213$

O $0.060712-0.1165904 .693285$

H $1.021387-1.2712973 .323731$

H -1.342447 -2.329287 4.952397

O $0.590218-2.8655085 .289882$

H $\quad 0.407397-3.8242172 .926122$

O $-1.147071-4.7028863 .920348$

C -2.513502 -3.773051 -0.036225

H $-0.525513-4.1663190 .562634$

H -1.752088 -5.1184951 .408321$

H $\quad 0.345736 \quad 0.698478 \quad 4.245649$

H $0.710946-2.1429355 .929771$

H $-0.567048-4.9569424 .658575$

O $-3.764953-3.6765390 .494860$

O $-2.217914-3.640621-1.216799$

C $-4.807580-3.338770-0.424473$

H $-5.764690-3.4482060 .092084$

H -4.696008 -2.297918 -0.738493

$\mathrm{H}-4.798076-4.008908-1.289400$

SCF Energy (B3LYP/6-31G**//MMFF) $=-3245.91538168$

03198

MM̄FF Geometry

C 0.0640355 .6676140 .972805

C $0.280975 \quad 4.7082680 .059225$

C $1.6440614 .146678-0.260437$

O $1.5858352 .707360-0.112221$

C $2.0063464 .457787-1.723476$

C $3.3916463 .985804-2.121285$

C $3.6425313 .043707-3.051627$

C $4.5404734 .665555-1.420576$

C $2.6563872 .276081-3.886479$

C $2.2261490 .923855-3.296970$

C $3.377570-0.078842-3.113508$

C $2.833846-1.469386-2.782119$

C $3.959144-2.476262-2.505648$

O $4.2052520 .372204-2.038679$

O $3.346616-3.633535-1.915807$

C $4.936903-1.875991-1.470758$

C $4.616835-2.954350-3.805839$

O $6.067577-2.732612-1.329958$

C $5.370701-0.431923-1.804353$

C $6.1696220 .255800-0.669380$

O $6.218939-0.454259-2.958994$

C 1.6289772 .2045251 .151210

O 1.7510182 .8321422 .190953

C 1.4662950 .7373831 .052958

C 1.1157530 .0405772 .143889

C $0.895511-1.4050362 .210267$
C $1.300154-2.2277291 .016252$

C 5.4417810 .3661120 .675073

C $5.749813-0.7661151 .640821$

C $5.054671-1.6691833 .857755$

O $6.638004-1.5920861 .449442$

C $4.081903-2.7881003 .656850$

C $3.215759-3.1880114 .600403$

C $2.262603-4.3367014 .432148$

C $0.830511-3.9552614 .734265$

C $0.287401-4.2075595 .937251$

C $0.005554-3.361178 \quad 3.615314$

C $0.359755-1.9254653 .334099$

N $4.935646-0.7222902 .764717$

H $\quad 0.924825 \quad 6.089217 \quad 1.488888$

H $-0.5522394 .265779-0.479298$

H $2.406995 \quad 4.551970 \quad 0.415152$

H $1.9535465 .540814-1.897566$

H $1.2473404 .010094-2.374154$

H $4.6831192 .791723-3.253884$

H $5.5094744 .396693-1.854914$

H $4.4411915 .753497-1.495075$

H $4.5680204 .387175-0.362842$

H $1.7629882 .877918-4.083646$

H $3.1080752 .109233-4.873041$

H $1.7297951 .097788-2.335184$

H $1.4775400 .491685-3.972738$

H $3.962385-0.107588-4.040351$

H $2.182262-1.835484-3.585263$

H $2.199076-1.405194-1.888135$

H $4.057745-4.251172-1.672577$

H $4.412104-1.886016-0.508396$

H $3.874952-3.439775-4.451380$

H $5.382202-3.713175-3.608465$

H $5.075120-2.144657-4.377725$

H $6.598600-2.406899-0.584349$

H $7.155193-0.212101-0.559696$

H $6.3761101 .284497-0.999418$

H $6.7442960 .362962-2.971447$

H 1.6142320 .2761240 .083657

H 0.9495910 .5809803 .075096

H $2.328096-1.9938460 .719442$

H $\quad 0.635282-2.0284720 .170241$

H $1.281296-3.3038571 .200797$

H $5.7718561 .287249 \quad 1.172349$

H 4.3602420 .4416420 .531036

H $4.867291-1.1188944 .785354$

H $\quad 6.073877-2.067813 \quad 3.887778$

H $4.109209-3.3095942 .702821$

H $3.211267-2.6824215 .563671$

H $2.584572-5.1380485 .110221$

H $2.321752-4.7633503 .423030$

H $-0.747316-3.9630446 .153578$

H $0.867848-4.6596726 .734685$

H -1.060301 -3.382983 3.880230

H $\quad 0.083507-4.0023332 .732691$

H $0.106417-1.2470864 .150128$

H $4.192157-0.0316582 .814804$

C -2.5172575 .5110120 .891978$

O $-2.519762 \quad 4.1522291 .363579$

C -3.8704943 .7449011 .637191$

C -4.7601494 .9140051 .222569$

C -3.8298846 .0999511 .401045$

H -3.9399863 .6005562 .722430$

C -4.1956492 .4196130 .928385$

H -5.0755964 .8299850 .175895$

H -5.6639794 .9816981 .836115$

C -4.2839347 .3442640 .658708$

H -3.7513826 .3253242 .472898$

C -3.3036161 .2352061 .358600$

O $-4.0863482 .595239-0.485261$

H -5.2427342 .1802521 .140812$

H -3.399627 1.0657352 .437966

O $-1.926171 \quad 1.5431501 .111581$

C $-3.580643-0.0797540 .604401$

C $-5.029387-0.5781450 .724830$

H $-3.3008350 .027728-0.449919$

O $-2.724247-1.0886021 .155781$

H $-5.267995-0.7383001 .783416$ 
$\begin{array}{llll}\text { H }-5.706790 & 0.186294 & 0.330136\end{array}$ H $-3.580563 \quad 8.1687250 .811167$ H $-4.3620397 .162900-0.418543$ H $-5.2662437 .668598 \quad 1.017125$ C -1.2674356 .2269231 .411382$ H -3.159289 2.817424 -0.679001 H -1.685721 2.2971521 .676065 H $-1.809220-0.7749091 .055393$ H -1.274272 6.1892342 .508514 H -1.299657 7.2839901 .124035 H $-2.5162375 .481410-0.205422$ C $-5.236225-2.735667-2.320039$ O $-5.107455-1.612425-1.437938$ C $-5.264352-1.894407-0.042770$ C $-6.658235-2.4790300 .228104$ C $-6.919004-3.710541-0.645755$ C $-6.586043-3.444980-2.117679$ H $-5.238813-2.297889-3.327098$ C $-4.028194-3.675385-2.224057$ H -4.507595 -2.6213200 .268781$ O $-7.666754-1.500144-0.013624$ H -6.727295 -2.7540451 .286996$ H -7.973158 -4.001158 -0.560486 O $-6.126233-4.801912-0.187286$ H -6.595893 -4.380004 -2.689961 O $-7.597768-2.601677-2.676898$ C -2.803634 -3.025985-2.825097 H $-3.786836-3.984083-1.203178$ H $-4.210836-4.595792-2.791723$ H $-7.565398-1.188717-0.929425$ H $-6.370549-4.9743030 .737894$ H -8.445849 -3.071347 -2.599934 O $-2.207907-2.210744-1.909234$ O $-2.435443-3.206741-3.978646$ C $-1.059977-1.494700-2.374771$ H $-0.247392-2.194264-2.589380$ H $-1.308112-0.905193-3.262692$ H $-0.739753-0.811855-1.583263$

SCF Energy (B3LYP/6-31G**/MMFF) $=-3245.92781951$

03199

MM̄FF Geometry

C $-0.6166564 .811770-0.144990$

C $0.6351015 .284127-0.249626$

C $1.8333324 .479363-0.700897$

O $1.6736793 .079244-0.375824$

C $1.9967994 .610847-2.225936$

C $3.209686 \quad 3.891382-2.781474$

C $3.1554372 .858819-3.646173$

C $4.5469364 .418883-2.328864$

C $1.9360922 .206887-4.237696$

C $1.4024261 .004370-3.442819$

C $2.366843-0.191861-3.389576$

C $1.690357-1.405956-2.751053$

C $2.657447-2.592272-2.606174$

O $3.5043340 .176625-2.608677$

O $2.048800-3.546081-1.720789$

C $3.961209-2.112111-1.928342$

C $2.870590-3.315918-3.941632$

O $4.928242-3.159451-1.944577$

C $4.535192-0.818786-2.547808$

C $5.700177-0.202268-1.735246$

O $5.044193-1.112714-3.854389$

C $1.887387 \quad 2.718406 \quad 0.919793$

$\begin{array}{lllll}\text { O } & 2.209904 & 3.449017 & 1.843258\end{array}$

C 1.6340251 .2656301 .031130

C $1.462646 \quad 0.7213312 .245099$

C $1.202892-0.6909572 .530685$

C $1.335592-1.6649411 .390785$

C $5.3619010 .182135-0.290186$

C $5.749326-0.8692650 .736138$

C $5.609116-1.3324203 .181212$

O $6.404474-1.8710790 .462482$

C $4.520634-2.3334833 .404599$

C $3.872139-2.4685154 .571380$

C $2.819701-3.5080784 .828065$

C $1.525082-2.9163785 .337369$

C $1.233028-2.9156336 .648843$
C $0.531522-2.411798 \quad 4.316454$

C $0.879629-1.046173 \quad 3.791825$

N $5.305244-0.5339142 .008575$

H $-0.8240683 .782823-0.428278$

H $\quad 0.830250 \quad 6.3244290 .000936$

H $2.7255014 .862919-0.190888$

H $2.0736945 .671439-2.501098$

H $1.0832434 .242567-2.704963$

H $4.0969852 .426122-3.983053$

H $4.6974484 .225333-1.262488$

H $5.3804943 .954166-2.866563$

H $4.6082955 .498254-2.502071$

H $1.1295512 .937482-4.360924$

H $2.1837181 .884750-5.257213$

H $1.1597681 .330300-2.424496$

H $0.4631980 .684490-3.911195$

H $2.683583-0.424257-4.413071$

H $0.802231-1.703405-3.322546$

H $1.323996-1.133546-1.752136$

H $1.193507-3.806941-2.103382$

H $3.714972-1.930915-0.876469$

H $1.914927-3.683109-4.334862$

H $3.501048-4.202921-3.814792$

H $3.319128-2.679325-4.707013$

H $5.676743-2.883687-1.389688$

H $6.585925-0.846142-1.789735$

H $5.9911280 .727355-2.246273$

H $5.682769-0.422599-4.099273$

H 1.5824720 .6897520 .114672

H $1.498408 \quad 1.3734473 .117152$

H $0.560453-1.4870080 .638835$

H $1.258122-2.7103591 .695592$

H $2.315720-1.5600110 .915568$

H $5.9318921 .083768-0.031125$

H $4.3005220 .425016-0.181758$

H $5.718094-0.6427314 .024313$

H $6.561253-1.8515783 .030493$

H $4.277787-2.9994972 .579781$

H $4.137314-1.8202145 .403664$

H $3.223044-4.2132685 .566879$

H $2.619169-4.1062073 .930359$

H $0.292526-2.5206097 .018486$

H $1.930969-3.3052337 .382691$

H $-0.466189-2.3375824 .770070$

H $0.419684-3.1590153 .526085$

H $0.818376-0.2639744 .549910$

H 4.7182910 .2860902 .131594

C -2.6260454 .9321231 .401408$

$\begin{array}{llll}\text { O } & -3.248114 & 3.756547 & 0.847892\end{array}$

C -3.2305532 .7144821 .843161$

C -2.8406803 .3910183 .154490$

C -1.8935154 .4697922 .660163$

H -2.426541 2.0230551 .566593

C -4.5721021 .9765161 .858277$

H $-3.7049353 .844143 \quad 3.653447$

H -2.3713482 .6949543 .856270$

C -1.641272 5.5759223 .668564

H -0.9377873 .9946532 .405850$

C $-5.0304381 .429168 \quad 0.488447$

O

H -4.5433141 .1724702 .602520$

H -5.247952 $2.279232-0.170450$

$\begin{array}{lllll}\text { O } & -6.278094 & 0.754259 & 0.702093\end{array}$

C $-4.0750020 .455583-0.231250$

C $-3.679635-0.7585630 .628568$

H $-4.5868260 .086798-1.127492$

O $-2.9022401 .126012-0.677283$

H -3.150989-0.413955 1.525035

H $-4.589440-1.2652720 .968277$

H $-0.9671756 .332393 \quad 3.254445$

H -2.5718046 .0750613 .958760$

H -1.180119 5.1716044 .575455

C $-1.7838345 .629936 \quad 0.329882$

H -6.4234682 .4137002 .325538$

H $-6.6156900 .479748-0.167636$

H $-3.1828631 .882934-1.219213$

H -1.445221 6.6006090 .710682

H $-2.4195165 .824194-0.543056$ 
H -3.4493275 .6054941 .675343$

C $-4.423005-2.904563-1.412313$

O $-3.238671-2.099616-1.406105$

C $-2.755792-1.757157-0.104505$

C $-2.448463-3.0320530 .696517$

C $-3.624948-4.0065460 .707118$

C $-4.156271-4.237355-0.706816$

H $-5.235786-2.371268-0.904423$

C $-4.828013-3.114995-2.874851$

H -1.802175 -1.238404 -0.263747

O $-2.103280-2.7213792 .042027$

H -1.583003 -3.538478 0.249664

H $-4.425401-3.6593251 .371214$

O $-3.188223-5.262972 \quad 1.231243$

H -3.433653 -4.833159-1.278959

O $-5.369055-4.983300-0.635714$

C $-5.273400-1.815492-3.502969$

H -3.981240 -3.490828 -3.461959

H -5.643096 -3.839683 -2.979857

H -1.385533 -2.067011 2.016566

H -2.820626 -5.0947302 .115953$

H $-5.174681-5.807750-0.157747$

O $-6.506538-1.468615-3.034416$

O $-4.600438-1.180491-4.304416$

C -7.025505 -0.229769-3.527493

H $-7.147952-0.275836-4.613743$

H -8.004710 $-0.065442-3.070065$

H $-6.3647350 .596058-3.246629$

SCF Energy (B3LYP/6-31G**//MMFF) $=-3245.91501835$

032

MM̄FF Geometry

C $-1.3195284 .308149-0.193894$

C $-0.199775 \quad 4.967447 \quad 0.141834$

C 1.1823034 .3630510 .273622

O $1.1368722 .918208 \quad 0.247117$

C $2.0632554 .839329-0.895889$

C $3.4953354 .346870-0.828169$

C $4.0650273 .523573-1.730498$

C 4.3078804 .8603650 .332755

C $3.4458962 .926731-2.963774$

C $2.8164601 .540364-2.758051$

C $3.8210130 .439836-2.380302$

C $3.145915-0.933426-2.394799$

C $4.097574-2.048895-1.933552$

O $4.3187950 .708947-1.068006$

O $3.307588-3.217740-1.663958$

C $4.757260-1.632994-0.600274$

C $5.089846-2.441273-3.034663$

O $5.772553-2.559098-0.222888$

C $5.331306-0.201610-0.618490$

C 5.7998720 .2870200 .774072

O $6.474714-0.164593-1.481472$

C $0.8073802 .284458 \quad 1.406437$

O 0.5709662 .8004252 .487176

C 0.7406430 .8351761 .120123

C 0.0540450 .0341021 .948183

C $-0.126418-1.4115461 .799853$

C $0.666135-2.1035770 .723619$

C 4.7163040 .3227531 .857763

C $4.689218-0.9106792 .745461$

C $3.364523-1.9469784 .584436$

O $5.562203-1.7740282 .729208$

C $2.502217-3.0214904 .001966$

C $1.388712-3.4748304 .597574$

C $0.540630-4.5875264 .051894$

C $-0.915644-4.2016763 .923328$

C $-1.807284-4.5413194 .869451$

C $-1.351340-3.5014532 .656882$

C $-0.972892-2.0454332 .638196$

N $3.588891-0.9119273 .592836$

H -1.274084 $3.248099-0.421681$

H -0.2660596 .0322520 .356251$

H $1.6149164 .694291 \quad 1.225628$

H $2.0821535 .937203-0.924155$

H $1.5952844 .525400-1.834176$

H $5.1069633 .243929-1.578258$

H 3.9329534 .4532761 .276673
H $5.365198 \quad 4.5845820 .254394$

H $4.2586665 .953246 \quad 0.377430$

H $2.6908073 .600814-3.381741$

H $4.2232012 .859356-3.735774$

H $2.0416001 .612817-1.986042$

H $2.3162931 .259625-3.693277$

H $4.6482660 .466871-3.099187$

H $2.743444-1.160040-3.390043$

H $2.279914-0.917287-1.719650$

H $2.824149-3.446498-2.476304$

H $3.979894-1.6925600 .169889$

H $4.554371-2.793160-3.924591$

H $5.722684-3.277277-2.716933$

H $5.735583-1.616754-3.343595$

H $5.364955-3.441699-0.197689$

H $6.679511-0.2824251 .097333$

H 6.1544231 .3207330 .650409

H $7.0188400 .603071-1.239745$

H 1.2339200 .4744250 .225291

H -0.4429730 .4802632 .808825$

H $\quad 0.357225-1.757414-0.267350$

H $0.563311-3.1903910 .732090$

H $1.734155-1.8981770 .848170$

H $4.922262 \quad 1.1705382 .524101$

H 3.7257570 .4837571 .421930

H $2.901451-1.4690295 .453503$

H $4.325894-2.3720744 .890811$

H $2.823982-3.4673063 .063685$

H $1.085856-3.0468905 .550703$

H $0.639603-5.4445174 .730978$

H $0.912744-4.936823 \quad 3.080847$

H -2.859018 -4.293665 4.770716

H -1.503907 -5.070065 5.767011

H -2.444345 -3.5507082 .559949$

H -0.972979-4.054235 1.792581

H -1.495091 -1.453179 3.391074

H $2.886143-0.1843673 .498736$

C -3.7005374 .3062920 .670915$

$\begin{array}{lllll}\text { O } & -3.970331 & 2.940267 & 0.294863\end{array}$

C -4.1953002 .1740801 .495575$

C -4.2393413 .1766942 .648457$

C -3.3120124 .2646452 .147718$

H -3.3184131 .5292401 .625598$

C -5.4647641 .3255941 .370844$

H -5.2481713 .5772972 .802250$

H -3.9105212 .7323793 .593123$

C -3.4747485 .5906632 .868493$

H -2.2808993 .9128782 .274348$

C $-5.4684320 .318250 \quad 0.203629$

O

H -5.6446970 .8019152 .317836$

H $-5.5288880 .853373-0.751860$

O $-6.688163-0.4271040 .329840$

C $-4.272450-0.6556090 .188661$

C $-4.455414-1.828507-0.795670$

H $-3.364492-0.099634-0.065607$

O $-4.081202-1.1933851 .498723$

H -5.265324 -2.470989-0.430791

H $-4.763315-1.432251-1.768238$

H -2.7892456 .3409702 .462222$

H -4.4947095 .9778422 .772393$

H -3.257664 5.4774163 .935665

C $-2.6798294 .938259-0.279599$

H -7.3785521 .6575041 .124540$

H $-6.864184-0.851350-0.527018$

H -4.899832 -1.6538621 .751225$

H -2.618190 $6.014938-0.081976$

H $-3.0313104 .818597-1.311748$

H -4.6509524 .8434290 .549764$

C $-2.220202-1.633608-2.889970$

O $-2.090187-1.953583-1.499421$

C -3.179885-2.690204-0.936020

C $-3.379259-4.003707-1.704505$

C $-3.550097-3.762873-3.199831$

C $-2.404984-2.903621-3.730415$

H $-3.075536-0.962458-3.034641$

C $-0.948963-0.882000-3.310807$

H $-2.868868-2.9662780 .077691$ 
O $-4.506102-4.721146-1.213001$

H -2.501365 -4.646291 -1.556208

H $-4.521716-3.308584-3.426664$

O $-3.521089-5.017013-3.885014$

H -1.477706 -3.491053 -3.735388

O $-2.686076-2.536196-5.079818$

C $-0.9989700 .556869-2.850601$

H $-0.048847-1.335848-2.882188$

H $-0.843388-0.890448-4.401778$

H $-4.362607-4.874605-0.263467$

H -4.228174 -5.568243 -3.508015

H -2.820312 -3.358079 -5.582252

O $-0.714034 \quad 1.386207-3.895222$

O $-1.2549450 .903626-1.705423$

C $-0.7507362 .785335-3.600999$

H $-0.0303633 .025210-2.815316$

H $-0.4750143 .331662-4.506743$

H -1.761149 $3.081872-3.304407$

SCF Energy (B3LYP/6-31G**//MMFF) $=-3245.91362414$

0320

MM̄FF Geometry

C $-1.7828212 .883779-0.462391$

C -1.2554431 .8025290 .130559$

C $-0.414374 \quad 0.776277-0.584114$

O $0.885027 \quad 0.7226230 .048021$

C $-1.073200-0.608504-0.439703$

C $-0.362021-1.708954-1.201263$

C $0.244581-2.768620-0.631266$

C $-0.389010-1.584560-2.703379$

C $0.356274-3.1042660 .829514$

C $1.644685-2.6143771 .508138$

C $2.935505-3.1997420 .912804$

C $4.137932-2.8700201 .800605$

C $5.459925-3.3565591 .185303$

O $3.132909-2.630381-0.383803$

O $6.535738-2.7279841 .899780$

C $5.546061-2.870220-0.277990$

C $5.647684-4.8680341 .361931$

O $6.674286-3.438365-0.937708$

C $4.266797-3.146889-1.093440$

C $4.275404-2.479914-2.490910$

O $4.140011-4.558554-1.305341$

C $1.7913861 .673673-0.307035$

O $1.6368592 .574423-1.116281$

C 3.0181961 .4415640 .486494

C 3.9288292 .4204320 .594428

C 5.1822282 .3530131 .348885

C 5.6096641 .0056191 .867498

C $4.407059-0.952879-2.491373$

C $5.832349-0.455322-2.667671$

C $7.1716241 .645739-2.629636$

O $6.769546-1.186498-2.975138$

C $7.8344801 .781140-1.295244$

C $8.2635232 .953491-0.803914$

C $8.968573 \quad 3.1027970 .513552$

C 8.3149184 .1306051 .409876

C 8.7859435 .3873021 .478037

C 7.1716643 .6770252 .289124

C 5.8916743 .4874681 .525292

N $5.9263260 .917212-2.478446$

H -1.586620 $3.064534-1.516250$

H $-1.4373461 .634814 \quad 1.189231$

H $-0.2988491 .030731-1.644613$

H -2.110948 $-0.566114-0.797145$

H $-1.131337-0.857307 \quad 0.625174$

H $\quad 0.715086-3.499310-1.288823$

H $\quad 0.006170-2.476820-3.201199$

H -1.416463 -1.444106 -3.054689

H $0.214211-0.732979-3.031998$

H $-0.502443-2.7160251 .386114$

H $\quad 0.289979-4.1951020 .930450$

H $1.676573-1.5198791 .456058$

H $1.583093-2.8851892 .569606$

H $2.814149-4.2856840 .822837$

H $4.006740-3.2895942 .805779$

H $4.199067-1.7819271 .937712$

H $6.451014-2.9659992 .838993$
H $5.716991-1.787930-0.241875$

H $5.648742-5.1316652 .426472$

H $6.618492-5.1940590 .972881$

H $4.865771-5.4568280 .878008$

H $7.460038-3.220958-0.407351$

H $5.031199-2.952371-3.129730$

H $3.310364-2.711660-2.964778$

H $3.559231-4.704686-2.070421$

H 3.1295180 .4804090 .974102

H 3.7309123 .3720080 .102366

H 5.5989840 .2647151 .060882

H 4.9375690 .6689742 .664004

H 6.6246910 .9937572 .269774

H $3.840587-0.557073-3.344246$

H $3.976195-0.519408-1.583896$

H $6.9277222 .616843-3.071926$

H $7.8310271 .104595-3.315885$

H $7.9919130 .871954-0.719699$

H $8.1296823 .856626-1.395488$

H $10.005543 \quad 3.3970820 .304523$

H $9.0353322 .145045 \quad 1.044479$

H 8.3409636 .1233832 .139401

H 9.6244265 .7094760 .869452

H 6.9746954 .4280153 .066105

H 7.4775862 .7828112 .838958

H 5.4993944 .4141461 .103530

H $5.1070101 .435805-2.175068$

C $-3.9514534 .148433-0.498462$

O $-4.7275652 .939506-0.594217$

C $-6.1230053 .268147-0.450528$

C $-6.2062344 .791282-0.395474$

C -4.8615335 .1553530 .201668$

H $-6.4398352 .864370 \quad 0.518212$

C $-6.9437072 .627129-1.576485$

H $-6.3100135 .232285-1.393563$

H -7.0517155 .1358940 .207945$

C $-4.4726006 .606638-0.018899$

H -4.8908894 .9489661 .280104$

C $-6.7399601 .106363-1.728840$

O $-6.5634153 .234933-2.818612$

H $-8.0054342 .860068-1.432305$

H $-5.7274410 .907522-2.099756$

O $-7.6287490 .654259-2.759659$

C $-6.9896890 .285204-0.450797$

C $-6.866468-1.227156-0.712065$

H -6.2709660 .5888320 .313785$

$\begin{array}{lllll}\text { O } & -8.296252 & 0.583792 & 0.036834\end{array}$

H -7.665910 -1.540321 -1.394553

H -5.914253 -1.413968 -1.220501

H $-3.496530 \quad 6.8220560 .426738$

H $-4.4170696 .849494-1.085389$

H $\quad-5.207658 \quad 7.2756600 .440584$

C -2.6472943 .8827090 .255479$

H -7.094774 $2.819092-3.519101$

H $-8.5375970 .763810-2.430814$

H -8.3629490 .2298930 .939443$

H -2.874757 3.5223081 .266094

H -2.0812784 .8154430 .357511$

H $-3.7363384 .472413-1.525607$

C $-4.654487-1.9482861 .273112$

O $-6.030067-1.6856301 .575001$

C -6.961647 -2.089719 0.567145

C $-6.831821-3.5972230 .313476$

C $-5.398954-3.991357-0.034580$

C $-4.427019-3.4443411 .010173$

H $-4.350210-1.3618190 .397896$

C -3.823720 -1.486545 2.482271

H $-7.960503-1.9238930 .988760$

O $-7.716567-3.997350-0.727835$

H -7.137391 -4.1373651 .219279$

H $-5.132524-3.659538-1.044810$

O $\quad-5.324797-5.419080-0.046827$

H -4.539409-4.011109 1.943494

O $-3.090269-3.632157 \quad 0.551740$

C -3.8016960 .0188572 .639979$

H $-4.252086-1.9105743 .398727$

H $-2.782917-1.8131592 .385853$

H $-7.585187-4.950350-0.870947$ 
H $-4.440867-5.664398-0.368183$

H $-2.923582-4.5886450 .507744$

O $-3.2749490 .327921 \quad 3.862187$

$\begin{array}{lllll}\text { O } & -4.174721 & 0.824004 & 1.799089\end{array}$

C -3.1865201 .7249624 .160645$

H -2.4479932 .2004243 .509128$

H $-4.1640192 .204808 \quad 4.052310$

H -2.8587491 .8303275 .198214$

SCF Energy (B3LYP/6-31G**//MMFF) $=-3245.90958620$

03200

MM̄FF Geometry

C $-0.018421 \quad 5.5728150 .346445$

C $1.0761444 .944685-0.107941$

C 1.8888213 .9665920 .700724

O $1.9636592 .726174-0.040607$

C 3.3193654 .5060240 .876140

C 4.2304173 .5848781 .663755

C 5.3426763 .0076301 .167812

C 3.8396523 .3464383 .099442

C $5.9166253 .145292-0.214991$

C $5.4314162 .086919-1.217767$

C $5.8823570 .656347-0.882838$

C $5.556267-0.299945-2.032314$

C $5.936196-1.750456-1.695528$

O $5.199931 \quad 0.225021 \quad 0.297482$

O $5.312972-2.584995-2.684079$

C $5.343289-2.121281-0.317297$

C $7.445910-1.980025-1.831381$

O $5.802253-3.4043610 .099796$

C $5.608351-1.0640640 .775375$

C $4.840179-1.3316262 .092428$

O $7.004590-1.0722631 .100424$

C $0.899087 \quad 1.883304 \quad 0.042714$

O -0.1083402 .0336530 .715103$

C $1.1576680 .747544-0.869809$

C $0.133997-0.030832-1.249952$

C $0.198589-1.164099-2.174204$

C $1.559179-1.597055-2.651883$

C $3.315829-1.4270081 .961893$

C $2.806302-2.8505641 .806097$

C $0.734179-4.1315741 .294300$

O $3.512590-3.8409571 .975665$

C $0.623306-4.408905-0.171786$

C $-0.532121-4.737060-0.768095$

C $-0.675352-5.042637-2.229638$

C -1.673694 -4.135441-2.911945

C $-2.966718-4.485078-3.021088$

C -1.151487 -2.866797 -3.544684

C $-0.955399-1.755235-2.549837$

N $1.455269-2.8878461 .490963$

H -0.3485265 .4009041 .367102$

H $1.4000105 .117874-1.132080$

H 1.4313023 .7852151 .681013

H $3.2884985 .476811 \quad 1.388647$

H $3.7406324 .702818-0.115570$

H 5.9233132 .3650801 .828872

H 4.6083062 .7963113 .653005

H 3.6862734 .3001203 .614973

H 2.9151982 .7636843 .156659

H $5.7090524 .141402-0.620406$

H $7.0102713 .095433-0.136390$

H $4.3376192 .127034-1.279997$

H $5.8217762 .361550-2.205717$

H $6.9625760 .673578-0.696551$

H $6.0493610 .019334-2.958892$

H $4.477953-0.262227-2.240105$

H $5.480490-3.510606-2.436040$

H $4.261805-2.218633-0.466059$

H $7.775141-1.751701-2.852276$

H $7.704455-3.031654-1.664678$

H $8.035906-1.367158-1.146877$

H $\quad 6.709957-3.3009050 .432570$

H $5.257293-2.2106842 .598416$

H $5.047333-0.4861382 .764423$

H $7.118294-0.6688091 .977063$

H $2.1707590 .604594-1.226741$

H $\quad-0.8597490 .201554-0.870256$
H $2.224698-1.778180-1.800767$

H $2.006399-0.827264-3.289753$

H $1.543891-2.527374-3.223684$

H $2.864500-1.0426142 .885499$

H $2.949492-0.8092121 .135894$

H -0.244185 -4.017763 1.771106

H $1.268464-4.9508911 .785412$

H $1.532993-4.353176-0.764916$

H $-1.431436-4.824644-0.164374$

H $-0.999036-6.087476-2.324105$

H $0.290056-4.988094-2.747733$

H -3.682892 -3.851161-3.533194

H -3.338617 -5.409815 -2.592143

H $-1.867626-2.502580-4.294106$

H $-0.247799-3.090913-4.118721$

H $-1.890998-1.374058-2.138227$

H $0.956048-2.0179811 .327332$

C $-2.2055825 .949402-0.893841$

O $-2.0492524 .680767-1.561938$

C $-3.0226643 .751974-1.024238$

C $-4.0661374 .627399-0.348206$

C $-3.1949325 .717676 \quad 0.246937$

H $-2.4770053 .157124-0.282427$

C $-3.5149802 .854196-2.162609$

H -4.756781 $5.052000-1.087363$

H -4.6516754 .1014380 .409178$

C $-3.966215 \quad 6.960641 \quad 0.655118$

H -2.6874755 .3104551 .129903$

C $-4.6362561 .847128-1.843713$

O $-2.3972912 .106746-2.658169$

H $-3.8469613 .496105-2.988857$

H $-4.8171691 .266613-2.758336$

O $-5.8494542 .530420-1.541052$

C $-4.3239810 .833113-0.722709$

C $-5.384190-0.285277-0.663190$

H $-3.3432240 .382497-0.903745$

O $-4.262211 \quad 1.4848350 .541556$

H $-6.3834380 .153306-0.551130$

H -5.359754 -0.818750 -1.621198

H $-3.291087 \quad 7.7226931 .057147$

H $-4.5022137 .398071-0.193767$

H $-4.702917 \quad 6.7191961 .428421$

C $-0.8362586 .510338-0.499199$

H $-1.6901802 .742113-2.864195$

H $-6.0547663 .111677-2.292953$

H -5.1411191 .8608330 .720170$

H -0.9575657 .4584490 .037072$

H $-0.2829636 .740236-1.419078$

H $-2.6490406 .627881-1.634527$

C $-3.464371-2.7395371 .409873$

O $-3.860625-1.8415390 .365260$

C $-5.165494-1.2746070 .500283$

C $-6.231822-2.3821030 .517704$

C $-5.935716-3.4421971 .584905$

C $-4.471064-3.8928101 .560301$

H $-2.522675-3.1729301 .055922$

C -3.161870 -2.004538 2.725644

H $-5.217652-0.7083401 .435224$

O $-6.300352-3.017494-0.759311$

H -7.213029-1.931082 0.705453

H $-6.576209-4.3130571 .399593$

O $-6.274616-2.9602582 .881526$

H -4.230322 -4.476382 2.456360

O $-4.282248-4.758860 \quad 0.434846$

C $-1.910918-1.1555372 .627970$

H $-3.973481-1.3403563 .028891$

H -2.989917 -2.742687 3.517757

H -5.414205 -3.359916 -0.968669

H -5.962010 -2.046590 2.972052

H $-4.863198-5.5290440 .556810$

O $-1.612855-0.659503 \quad 3.865442$

O $-1.278778-0.943858 \quad 1.603107$

C $-0.449300 \quad 0.171624 \quad 3.930824$

H -0.6248921 .1027913 .384679$

H $-0.2608770 .410475 \quad 4.980840$

H $0.422708-0.3537333 .530005$

SCF Energy (B3LYP/6-31G**//MMFF) $=-3245.91786726$ 
03_201

MMFF Geometry

C -2.614881 $2.641081-1.842512$

C $-2.0607461 .550550-1.291786$

C $-0.9700260 .752084-1.959122$

O $0.1258540 .602523-1.025813$

C -1.483862 -0.652975-2.319242

C $-0.500992-1.459273-3.147706$

C $0.104772-2.591097-2.738426$

C $-0.235070-0.936512-4.537193$

C $-0.046399-3.304879-1.425884$

C $0.990369-2.922842-0.358756$

C $2.448386-3.191313-0.763558$

C $3.373256-3.0615360 .448330$

C $4.850297-3.2287220 .065015$

O $2.826514-2.231956-1.753715$

O $5.625741-2.786928 \quad 1.190259$

C $5.174481-2.302811-1.128818$

C $5.210525-4.703504-0.151494$

O $6.496200-2.568362-1.592057$

C $4.150151-2.406970-2.279626$

C $4.340366-1.335884-3.382629$

O $4.286222-3.685830-2.911451$

C $\quad 0.956621 \quad 1.667702-0.861504$

O $0.8972312 .744715-1.432800$

C 1.9538601 .3018330 .167333

C 2.6609932 .2670350 .772832

C 3.6724582 .0700421 .813031

C 4.1245980 .6595482 .083601

C $4.2526410 .119284-2.909712$

C $5.5965510 .740197-2.567290$

C $6.5874652 .788849-1.551821$

O $6.6712860 .210288-2.835855$

C $6.8928312 .532351-0.109541$

C 7.0432033 .5119420 .794864

C 7.3874593 .2769322 .237869

C 6.4201733 .9530033 .184043

C $6.7029915 .151963 \quad 3.720985$

C 5.1661523 .2012403 .570484

C 4.1546503 .1534202 .457185

N $5.4534181 .978601-1.956126$

H -2.288858 $2.967313-2.827146$

H $-2.3850521 .223276-0.307041$

H $-0.6094831 .265261-2.858671$

H -2.419249 -0.574565 -2.889476

H - $-1.736565-1.183629-1.395707$

H $0.789684-3.080876-3.430132$

H $\quad 0.346047-1.641307-5.141733$

H -1.178346 -0.758305 -5.063765

H $\quad 0.327770 \quad 0.000775-4.497941$

H -1.051258 -3.154543-1.017885

H $0.021842-4.383216-1.619478$

H $0.864781-1.863730-0.107622$

H $0.761936-3.4981740 .546056$

H $2.511560-4.200708-1.186389$

H $3.097119-3.7810641 .229416$

H $3.240777-2.0689270 .899372$

H $6.563417-2.8232720 .933776$

H $5.177230-1.279941-0.733797$

H $5.030932-5.2774550 .765608$

H $6.276453-4.823885-0.374306$

H $4.636446-5.172007-0.953618$

H $6.724965-1.890129-2.249075$

H $5.264144-1.525257-3.941973$

H $3.529461-1.477803-4.111911$

H $3.901363-3.632244-3.801967$

H 2.0658180 .2520420 .410561

H $2.469003 \quad 3.3034440 .497934$

H 4.4398410 .1749091 .153199

H 3.3131930 .0741202 .529223

H 4.9799700 .5956052 .758923

H $3.8380810 .724517-3.726142$

H $3.575650 \quad 0.213806-2.055939$

H $6.3226103 .834184-1.739640$

H $7.4577942 .532736-2.164261$

H 7.0200681 .4974540 .199418

H 6.9433514 .5470820 .475423

H 8.4017123 .6624422 .405370
H 7.4369262 .2058052 .469964

H 6.0278295 .6318914 .421735

H 7.6147435 .6827503 .467184

H 4.6810263 .6913374 .425306

H 5.4461632 .2107693 .938787

H 3.7636484 .1345452 .183706

H $4.5229922 .317450-1.728651$

C $-5.0222663 .368476-1.831743$

O $-5.5747312 .065700-1.539696$

C $-6.7846012 .246998-0.787546$

C $-7.3094093 .603407-1.229969$

C $-6.0193494 .398298-1.282465$

H -6.4960822 .3067170 .269458$

C -7.748978 $1.074363-0.983914$

H -7.764795 $3.558545-2.225874$

H $-8.0417274 .019142-0.531762$

C $-6.1140025 .660884-2.122402$

H $-5.7425094 .681590-0.257756$

C -7.131978 -0.319115 -0.741017

O $-8.237171 \quad 1.105075-2.330522$

H $-8.622548 \quad 1.220992-0.336779$

H -6.528547 -0.620416-1.606555

O $-8.207078-1.262892-0.651125$

C $-6.273868-0.4432850 .530861$

C $-5.877502-1.8985170 .843461$

H $-5.370503 \quad 0.1647140 .412745$

O

H $-6.738737-2.4247641 .272773$

H -5.591345 -2.400449-0.089056

H $-5.1530956 .184856-2.144319$

H $-6.3990375 .435274-3.155513$

H $-6.8633426 .343371-1.708266$

C $-3.6506223 .489797-1.163009$

H $-8.975850 \quad 0.476720-2.391544$

H -8.625682 -1.317174 -1.526465

H $-7.823162-0.4130261 .725544$

H $-3.7268573 .208604-0.104782$

H $-3.3055134 .529884-1.197818$

H $-4.9359833 .442651-2.922625$

C $-2.306471-1.5908731 .860403$

O $-3.516814-1.6420691 .094375$

C $-4.700656-1.9625731 .835705$

C $-4.561342-3.3537102 .469145$

C $-3.310963-3.4378853 .348245$

C $-2.067545-2.9062432 .624921$

H $-1.517324-1.4950841 .104441$

C $-2.279430-0.3253192 .728658$

H -4.848306-1.214539 2.623435

O $-4.504899-4.356458 \quad 1.456405$

H $-5.451572-3.5586383 .075435$

H $-3.151138-4.4840653 .636609$

O $-3.541046-2.6922154 .539504$

H -1.235542 -2.779347 3.326510

O $-1.659162-3.8852031 .664241$

C $-0.8750350 .117302 \quad 3.079871$

$\begin{array}{llll}\mathrm{H} & -2.753097 & 0.497124 & 2.178335\end{array}$

H $-2.841510-0.4663253 .655627$

H $-3.761443-4.1422770 .867040$

H $-2.750264-2.7808045 .098411$

H $-1.434780-4.6973332 .149689$

O -0.9490031 .2304333 .868696$

O $0.154156-0.4303992 .710055$

C 0.3063501 .7802434 .280819

H 0.8225352 .2117093 .419275

H 0.1078162 .5747425 .005001

H 0.9254021 .0163484 .761165

SCF Energy (B3LYP/6-31G**//MMFF) $=-3245.92080080$

03_202

MM̄FF Geometry

C -1.325400 $4.677100 \quad 0.034357$

C -2.520955 $4.930792-0.520674$

C $-3.7096813 .993831-0.524968$

O $-3.3160612 .643051-0.192548$

C -4.7411014 .4636420 .516172$

C -6.0164403 .6433570 .526731$

C -6.4367602 .8948891 .565553$

C $-6.8536993 .721061-0.724234$ 
C -5.7763912 .7094012 .903132$ C -4.8446281 .4908672 .996651$ C -5.5551840 .1403352 .808534$ C $-4.621088-1.0188413 .166364$ C $-5.276904-2.3846882 .904481$ O $\quad \begin{array}{llll}5.953380 & 0.023271 & 1.439882\end{array}$ O $-4.257988-3.3928012 .990075$ C $-5.857338-2.4038741 .472030$ C $-6.287051-2.7418954 .000462$ O $-6.618511-3.587217 \quad 1.247594$ C $-6.707216-1.1597601 .139952$

C $-7.115820-1.078091-0.351432$ O $-7.920150-1.2019751 .902044$ C $-2.7661581 .892666-1.186499$ O $-2.6080442 .215394-2.352994$ C $-2.3384480 .609531-0.588851$ C -1.440984 -0.148652 -1.235586 C $-0.890145-1.421282-0.766241$ C - $1.510132-2.0385580 .459083$ C $-5.955662-1.029774-1.351712$ C $-5.585557-2.383447-1.935396$ C $-3.901984-3.456786-3.426567$ O $-6.249152-3.400916-1.756125$ C $-2.840965-4.106025-2.595457$ C $-1.612066-4.381208-3.058027$ C $-0.547454-5.070476-2.253903$ C $0.745646-4.288047-2.211010$ C $1.756034-4.575655-3.048982$ C $0.894853-3.230171-1.141913$ C $0.129849-1.974872-1.454349$ N $-4.439352-2.311745-2.715962$ H -1.165473 3.7433050 .566882 H -2.670136 $5.879129-1.032677$ H -4.154305 $4.003839-1.527555$ H $-5.011814 \quad 5.5111760 .327362$ H -4.2673334 .4486701 .503594$ H -7.3750142 .3518641 .455864$ H -7.825344 $3.229526-0.605068$ H -7.047359 4.765959 -0.987844 H $-6.3430043 .236454-1.561992$ H -5.2166283 .6068493 .186850$ H -6.5655992 .6176973 .660407$ H -4.0452911 .5960182 .253619$ H -4.3710081 .5115043 .986038$ H $-6.4432260 .126748 \quad 3.451239$ H $-4.290438-0.9476474 .210026$ H -3.708465 -0.946182 2.559107 H $-3.574639-3.1927902 .328400$ H -5.005814 -2.444265 0.782879 H $-5.788928-2.7892404 .976588$ H $-6.714712-3.7371573 .835785$ H -7.106044 -2.024447 4.082706 H $-6.043998-4.3450881 .450872$ H -7.825892 -1.878389 -0.592412 H -7.680169 -0.142927 -0.480040 H $-8.578293-0.6356641 .466209$ $\begin{array}{llll}\text { H } & -2.748921 & 0.340912 & 0.377463\end{array}$ H - $-1.0491750 .207268-2.187740$ H - $1.158858-3.0522730 .662683$ H -2.596228 -2.1157820 .339362$ H -1.296092 -1.432962 1.346159 H -6.258545 -0.405157 -2.202042 H -5.069191 -0.568384 -0.906601 H -3.514122 -3.092025 -4.382946 H -4.704526 -4.174061 -3.626092 H -3.103163 -4.385776 -1.577896 H -1.363352 -4.124989-4.085655 H $-0.372080-6.054789-2.707749$ H - $0.885565-5.274133-1.230266$ H $2.696150-4.036107-3.008448$ H $1.662152-5.353445-3.799762$ H $1.950373-2.944069-1.041578$ H $0.631309-3.660904-0.172100$ H $\quad 0.501171-1.452802-2.337437$ H -3.925526 -1.436786 -2.767795 C $1.0800484 .997843-0.698216$ O $1.5462083 .861056 \quad 0.056235$ C $1.9276562 .818564-0.869604$
C $2.0052063 .485578-2.235238$

C $0.8912684 .509028-2.133994$

H $1.1100722 .086759-0.846694$

C $3.2322472 .175787-0.385501$

H $2.9730283 .983979-2.372220$

H $1.8632092 .778781-3.058238$

C $0.9729205 .610330-3.175819$

H $-0.0646893 .983261-2.247596$

C $3.8359591 .162898-1.373249$

O 2.9585851 .5011340 .847035

H $3.9615872 .961762-0.159485$

H $4.1407151 .675672-2.292588$

O $2.8199650 .229176-1.761262$

C $5.0273130 .348676-0.830799$

C $6.1828611 .220129-0.313155$

H $4.689740-0.342837-0.050229$

O $5.529024-0.456867-1.903986$

H $6.5419021 .862488-1.126846$

H $5.810803 \quad 1.8619270 .492396$

H $\quad 0.1564006 .327935-3.047414$

H $1.9184906 .158530-3.107868$

H $0.8994885 .191238-4.184667$

C $-0.1513195 .613241-0.025497$

H 2.5808962 .1567181 .458350

H $2.501389-0.207997-0.952727$

H $4.790899-1.003196-2.224674$

H $-0.4216186 .542499-0.540350$

H $\quad 0.1043875 .8788191 .007804$

H $1.8919705 .736151-0.659401$

C $7.856765-1.2089571 .982055$

O $6.902802-0.3333091 .367143$

C $7.367106 \quad 0.388676 \quad 0.221064$

C $8.565313 \quad 1.2752130 .595376$

C 9.6767670 .4506001 .253585

C $9.134369-0.4466472 .370624$

H $7.371644-1.5413682 .909172$

C $8.124540-2.4501271 .124125$

H $7.674628-0.320655-0.554233$

O 8.1397832 .3010591 .490464

H $8.940477 \quad 1.763555-0.311985$

H $\quad \begin{array}{llll}10.435061 & 1.122827 & 1.673193\end{array}$

O $10.311871-0.365677 \quad 0.273881$

H $9.903631-1.1604952 .686302$

O 8.8428910 .3650183 .512553

C $6.882153-3.3036971 .031399$

H $8.462735-2.2126280 .111506$

H $8.908708-3.0721031 .572185$

H 8.9107302 .8617561 .681843

H $\quad \begin{array}{lll}10.676049 & 0.223723 & -0.408427\end{array}$

H $8.1102050 .959206 \quad 3.275779$

O $6.218969-3.037087-0.129033$

O $6.530293-4.0905851 .900690$

C $4.996630-3.755023-0.320829$

H $4.585625-3.468991-1.292154$

H $5.183726-4.833006-0.322525$

H $4.276020-3.4874550 .457749$

SCF Energy (B3LYP/6-31G**//MMFF) $=-3245.91551496$

03203

MM̄̄F Geometry

C $-3.300796-4.126473-1.486252$

C $-2.255073-3.566178-2.114401$

C $-0.783959-3.803336-1.847623$

O $-0.208093-2.570853-1.348367$

C $-0.471340-4.905297-0.814242$

C $1.009920-5.192595-0.646139$

C $1.692389-5.0419140 .506291$

C $1.721846-5.712030-1.869088$

C $1.169853-4.5848471 .839466$

C $1.299773-3.0754522 .091517$

C $2.750638-2.5784402 .201905$

C $2.787163-1.1187782 .660022$

C $4.219748-0.5659122 .707516$

O $3.370382-2.6870480 .918542$

O 4.1117580 .8598972 .838725

C $4.922503-0.8694091 .365254$

C $4.967742-1.0554693 .952756$

O $6.300110-0.5082941 .422474$ 
C $4.759170-2.3303950 .897990$

C $5.274678-2.570762-0.541833$

O $5.521147-3.1814291 .763715$

C $0.177821-1.631022-2.252162$

O $0.083071-1.688391-3.467324$

C $0.722728-0.487154-1.490965$

C $\quad 0.7112390 .736234-2.039785$

C $1.1645961 .968642-1.392762$

C $1.9544231 .832836-0.119006$

C $4.583164-1.747163-1.634486$

C $5.325729-0.477404-2.017514$

C $5.0893331 .546416-3.455154$

O $6.456395-0.209833-1.620661$

C $4.6567472 .695989-2.601123$

C $4.0060363 .767215-3.080161$

C $3.6086314 .948729-2.242608$

C $2.1377325 .279738-2.358101$

C $1.7112966 .261172-3.171124$

C $1.1733314 .534023-1.465036$

C $0.8648943 .150924-1.969354$

N $4.5857310 .307193-2.892645$

H -3.142653 -4.827046 -0.671912

H -2.465411 -2.867310 -2.922647

H $-0.315731-4.067375-2.803894$

H -0.959514 -5.843072 -1.110296

H $-0.910401-4.6135700 .145293$

H $2.753782-5.287790 \quad 0.506513$

H $2.735303-6.060794-1.642882$

H $1.174835-6.558556-2.296847$

H $1.807709-4.929071-2.628612$

H $0.121488-4.8765321 .963216$

H $1.709732-5.1315592 .623139$

H $0.781638-2.534627 \quad 1.290670$

H $0.775053-2.8495303 .028137$

H $3.279298-3.2171722 .919131$

H $2.297909-1.0012553 .635043$

H $2.204208-0.5072331 .958751$

H 5.0101891 .2293872 .787354

H $4.467706-0.2052020 .622951$

H $4.435681-0.7486774 .861348$

H $5.962052-0.6008094 .023274$

H $5.081866-2.1410163 .984833$

H $6.768411-1.2035461 .915106$

H $6.364974-2.457514-0.576188$

H $5.086499-3.627964-0.779009$

H $5.693268-4.0174211 .299496$

H $1.075976-0.676284-0.483955$

H $0.2951650 .855215-3.039326$

H $2.7991341 .152835-0.267300$

H 1.3254091 .4373930 .683173

H 2.3820742 .7729730 .234948

H $4.534359-2.356129-2.546474$

H $3.555620-1.499334-1.352016$

H $4.702157 \quad 1.619986-4.476345$

H $6.1825751 .510113-3.498421$

H $4.9154292 .657771-1.545529$

H $3.7734733 .815346-4.141720$

H $4.2102045 .806137-2.571768$

H $3.8656544 .796982-1.186890$

H $0.6607196 .524174-3.237023$

H $2.4031686 .819665-3.792914$

H $0.2195065 .075131-1.412080$

H $1.5496684 .542007-0.438154$

H $0.2935943 .143993-2.898672$

H $3.6346420 .034227-3.123241$

C $-5.446851-3.037420-0.774563$

O $-4.954270-1.682605-0.813200$

C $-6.066438-0.775191-0.851800$

C -7.262530 -1.590365 -0.378107

C $-6.957142-2.940842-0.994544$

H $-6.216018-0.499898-1.903525$

C $-5.8011460 .489493-0.022554$

H -7.288755 -1.6672840 .715378$

H -8.214608 -1.169194 -0.714583

C $-7.743298-4.084187-0.376663$

H -7.179082 -2.895051 -2.069313

C $-4.6887151 .410685-0.569430$

O $\begin{array}{llll}-5.545924 & 0.136283 & 1.332290\end{array}$
H $-6.7363891 .065133-0.024089$

H $-4.6124062 .294307 \quad 0.074297$

O $-5.105787 \quad 1.891417-1.855800$

C $-3.2786340 .813708-0.748675$

C -2.6211460 .2083010 .504821$

H $-3.3101240 .047152-1.530113$

O $-2.4212601 .830353-1.282102$

H -3.214259-0.639715 0.859982

H -1.651459 -0.1964510 .191107$

H -7.472683 -5.038264 -0.839735

H $-7.554379-4.1660200 .698910$

H -8.818342 $-3.932231-0.518069$

C $-4.736522-3.860213-1.850284$

H -5.4962910 .9603561 .845723$

H $-5.9474292 .363104-1.734506$

H $-2.3604992 .544972-0.626193$

H $-4.789073-3.337263-2.813637$

H $-5.234125-4.828181-1.979302$

H $-5.224992-3.4290840 .226488$

C $-0.975313 \quad 3.1109372 .212466$

O -1.3418962 .1039491 .259903$

C -2.3694671 .1937261 .664781$

C -1.9501150 .4510032 .943764$

C -1.558600 1.4279254 .055655

C -0.5697962 .4857953 .558215$

H -0.0816613 .5841071 .784823$

C -2.0575684 .1880162 .343545$

H $-3.287068 \quad 1.753617 \quad 1.862719$

O $-0.857745-0.4251822 .675560$

H -2.785822 -0.1746613 .278447$

H -1.1056410 .8723834 .885950$

O -2.7223422 .0830734 .551321$

H -0.4453653 .2783304 .305395$

O $\quad 0.712042 \quad 1.8767943 .379185$

C -2.1080695 .0384981 .096372$

H -3.0552183 .7815972 .532485$

H -1.836316 4.8652333 .177185

H $-0.1389470 .104968 \quad 2.290817$

H -3.3226751 .3977824 .891229$

H $0.993407 \quad 1.5240604 .240564$

O $-3.094534 \quad 4.5953260 .267584$

O -1.3440225 .9695240 .877081$

C $-3.2332315 .307915-0.965599$

H -4.089163 4.891485-1.503235

H $-3.4209586 .368945-0.775210$

$\mathrm{H}-2.3367135 .176585-1.578304$

SCF Energy $\left(B 3 L Y P / 6-31 G^{* *} / / M M F F\right)=-3245.90842357$

03_204

MM̄FF Geometry

C $3.031116 \quad 1.7721552 .110391$

C 3.6827232 .8680751 .689107

C 4.8910972 .8958200 .781698

O $5.2768321 .555677 \quad 0.407434$

C $4.6923933 .794765-0.457797$

C $3.5476043 .441780-1.388468$

C $2.4883504 .234847-1.647193$

C $3.6701462 .125081-2.115357$

C $2.1452425 .585023-1.075848$

C $1.0389435 .552660-0.005892$

C $-0.3706335 .231224-0.539524$

C $-1.3896405 .244147 \quad 0.604767$

C -2.7820734 .7495260 .171441$

O $-0.3319883 .940304-1.138653$

O

C $-2.6306993 .419247-0.603577$

C $-3.5566575 .843931-0.572611$

O $-3.8809212 .989584-1.136321$

C $-1.5615413 .489276-1.710077$

C $-1.2765122 .122661-2.367792$

O $-2.0141314 .369646-2.744287$

C 6.5653601 .3254030 .048741

O $7.4561072 .156989-0.053829$

C $6.790113-0.108455-0.252507$

C $5.999664-1.0830490 .223787$

C $6.160091-2.5221930 .004284$

C $7.216738-2.976372-0.966563$

C $-0.6665311 .072313-1.433970$ 
C $0.012355-0.041317-2.213210$

C $1.782876-1.752575-1.994955$

O $-0.190691-0.244040-3.407837$

C $2.236841-2.712996-0.946308$

C $2.349921-4.032588-1.158381$

C $2.826554-5.018538-0.128949$

C $4.271098-5.418648-0.339878$

C $4.615092-6.281793-1.310675$

C $5.309896-4.8697350 .613572$

C $5.338940-3.366650 \quad 0.662064$

N $0.861309-0.794818-1.413210$

H 3.3507910 .7895541 .774878

H 3.3588713 .8382992 .061420

H $5.708298 \quad 3.3227121 .379451$

H $5.6085433 .779798-1.062272$

H $4.6003184 .826629-0.104654$

H $1.7727243 .877120-2.386144$

H $2.9401532 .027245-2.926453$

H $4.6641942 .024932-2.563049$

H $3.5045401 .289379-1.429212$

H $3.0287846 .061800-0.641757$

H $1.8364766 .240004-1.899906$

H 1.3168194 .8170050 .759682

H $1.010886 \quad 6.5329040 .486228$

H $-0.6362525 .975974-1.299055$

H -1.4572806 .2445901 .050174$

H -1.0284054 .5904531 .408534$

H -3.569271 5.2680401 .898707

H -2.3379102 .6549840 .126742$

H -3.693215 6.7210660 .071730

H $-4.5660705 .507762-0.833335$

H -3.055185 6.181667-1.481845

H $-4.072563 \quad 3.533195-1.919346$

H -2.184703 $1.731286-2.841654$

H $-0.5719902 .292973-3.194824$

H -1.521071 $4.172666-3.557742$

H $7.672846-0.322059-0.845942$

H $5.156326-0.8097590 .855910$

H $8.216211-2.743381-0.583587$

H $7.087790-2.482435-1.936303$

H $7.186629-4.050842-1.164141$

H $0.0875861 .533854-0.787973$

H $-1.4386620 .627789-0.797560$

H $2.638424-1.183960-2.374376$

H $1.300758-2.253638-2.841229$

H $2.514933-2.3030820 .020707$

H $2.092034-4.436493-2.134938$

H $2.184902-5.908005-0.179111$

H $2.687186-4.6256120 .885524$

H $5.645127-6.590439-1.455555$

H $3.872035-6.698296-1.982862$

H $\quad 6.305565-5.2690870 .390101$

H $5.082000-5.2366961 .623249$

H $4.596765-2.9437831 .340447$

H $1.075339-0.441344-0.484381$

C $0.634637 \quad 1.1225242 .557485$

O $0.877787-0.2872842 .354150$

C $-0.233102-1.0344362 .898215$

C -1.341347 -0.0189443 .109380$

C $-0.5433891 .196523 \quad 3.529962$

H $0.113546-1.4221673 .865109$

C $-0.572899-2.195701 \quad 1.957707$

H -1.876241 0.1769102 .171512

H $-2.066515-0.3349983 .864353$

C -1.338970 2.4849883 .467104

H -0.1887351 .0473734 .558989$

C -1.911668 -2.881606 2.271839

$\begin{array}{llll}\text { O } & 0.472138 & -3.1670642 .071598\end{array}$

H $-0.562806-1.8463250 .920727$

H -2.738402 -2.184183 2.105693

O $-1.951078-3.2043663 .669515$

C -2.175911-4.180852 1.493945

C -2.069465 -4.092798 -0.037437

H $-1.489369-4.9642341 .838329$

O $-3.487486-4.6515661 .826854$

H - $1.017953-3.976451-0.325705$

H -2.399515 $-5.066339-0.422158$

H $-0.712324 \quad 3.3494023 .706184$
H -1.764509 2.6338012.471952

H -2.1678752 .4601364 .182019$

C 1.8958601 .7988953 .097458

H $1.317233-2.7025061 .947630$

H -1.196599 -3.7889893 .856922$

H $-3.539247-4.7073202 .796474$

H 2.2365821 .2730413 .998143

H 1.6684462 .8311583 .387923

H $\quad 0.360786 \quad 1.546722 \quad 1.583929$

C $-5.143980-2.064540-0.792668$

O $-4.250016-3.056833-0.270890$

C $-2.897604-2.983845-0.726940$

C $-2.833613-3.110768-2.262576$

C $-3.780304-2.128024-2.960325$

C $-5.174196-2.127667-2.328027$

H $-6.136707-2.360295-0.429398$

C $-4.839593-0.656890-0.252648$

H -2.476559-2.018597-0.431278

O $-3.190532-4.438970-2.646856$

H $-1.799379-2.941940-2.586006$

H $-3.873137-2.395012-4.020282$

O $-3.245290-0.812536-2.891290$

H $-5.762487-1.288568-2.716669$

O $-5.861584-3.320024-2.717678$

C $-5.124890-0.525513 \quad 1.228208$

H $-3.794610-0.376891-0.404858$

H $-5.4774790 .072200-0.766091$

H -3.085102 -4.504025 -3.611332

H $-2.402453-0.815494-3.375840$

H $-5.394415-4.075446-2.321148$

O $-4.8244550 .749227 \quad 1.619607$

O $-5.557188-1.413567 \quad 1.949293$

C $-5.061727 \quad 1.0436362 .999436$

H -4.7112032 .0605383 .194703$

H -6.1332330 .9933853 .213834$

H $-4.5074890 .352326 \quad 3.640942$

SCF Energy (B3LYP/6-31G**//MMFF)= -3245.91194937

03205

MM̄FF Geometry

C $2.593137 \quad 3.201516-0.830876$

C $2.834773 \quad 3.959752 \quad 0.250200$

C 3.3482003 .4207491 .569246

$\begin{array}{llll}\text { O } & 2.930696 & 2.042769 & 1.734571\end{array}$

C 4.8838373 .4676221 .601976

C 5.4569643 .1981022 .979453

C $5.9455212 .008503 \quad 3.382518$

C 5.5015974 .3889923 .900882

C 5.9427950 .7182562 .613194

C 7.3302590 .3774892 .056847

C $7.311630-0.9503641 .285792$

C $8.705326-1.3111570 .770144$

C $8.684309-2.588697-0.085767$

O $6.412669-0.8054520 .182136$

O $9.933977-2.670385-0.787830$

C $7.574363-2.463326-1.152291$

C $8.589441-3.8520260 .778128$

O $7.412454-3.684945-1.869266$

C $6.215177-2.003157-0.578493$

C $5.227987-1.684355-1.726651$

O $5.662589-3.0519350 .221442$

C $1.623897 \quad 1.8174212 .045718$

$\begin{array}{lllll}\text { O } & 0.790011 & 2.645059 & 2.375907\end{array}$

C 1.3604550 .3817211 .803079

C $0.105292-0.0148621 .542857$

C $-0.308357-1.3650651 .155911$

C $0.727369-2.4575651 .210286$

C $3.994529-0.898035-1.267989$

C $2.986345-0.764932-2.389750$

C $1.097520-1.966616-3.486719$

O $2.9373510 .238601-3.097190$

C $-0.168122-1.494831-2.842451$

C $-1.284714-2.233773-2.753671$

C $-2.559923-1.731662-2.137851$

C $-3.060429-2.605960-1.006739$

C $-4.275478-3.175514-1.075974$

C -2.175612 -2.8367350 .205566$

C $-1.583440-1.5666910 .760586$ 
N $2.178127-1.883224-2.521958$

H $2.8430582 .144098-0.803319$

H 2.5946545 .0196720 .223749

H 2.9276894 .0183642 .387436

H 5.2391634 .4525801 .269857

H 5.2937932 .7509350 .879229

H $6.367487 \quad 1.9349904 .383857$

H 5.9461134 .1472064 .872061

H 6.0987335 .1914423 .455563

H 4.4920414 .7683294 .086333

H $5.612442-0.0793013 .290569$

H 5.2076020 .7417331 .803799

H 7.6503811 .1861681 .386814

H 8.0569150 .3201102 .876657

H $6.937874-1.7317831 .958104$

H $9.413438-1.4166841 .601450$

H $9.089409-0.4875260 .151975$

H $10.648549-2.676284-0.127908$

H $7.909772-1.716110-1.885223$

H $9.444736-3.9132111 .461691$

H $8.634488-4.7565870 .161621$

H $7.681230-3.8910181 .383007$

H $8.284553-3.931125-2.222521$

H $5.732722-1.077351-2.489654$

H 4.913892 -2.615653 -2.214378

H $4.695336-2.9776660 .222147$

H $2.212251-0.2874561 .761824$

H -0.6896580 .7296791 .559849$

H $1.240759-2.4573752 .178721$

H $1.470236-2.3230650 .419487$

H $0.305106-3.4594241 .101911$

H $3.508942-1.372554-0.409903$

H $4.2760670 .111471-0.947289$

H $1.032275-3.008428-3.815479$

H $1.329096-1.338313-4.352605$

H $-0.171463-0.481985-2.446762$

H -1.295087 -3.239672 -3.166322

H -3.310055 -1.675421 -2.937416

H $-2.442720-0.704632-1.773288$

H -4.649200 -3.809437 -0.278804

H $-4.926359-3.022836-1.930532$

H -1.399878 -3.547612 -0.094345

H -2.750006 $-3.314324 \quad 1.009064$

H -2.279874 -0.7292610 .807766$

H $2.268266-2.640859-1.851503$

C $0.6023292 .915157-2.259259$

O $-0.3186563 .310191-1.224476$

C $-1.6597743 .284147-1.760753$

C -1.537889 $2.753180-3.182986$

C $-0.1345113 .189738-3.564014$

H -1.997118 4.327945 -1.770432

C -2.531180 $2.449486-0.816005$

H $-1.6154001 .658955-3.200034$

H -2.308536 $3.156157-3.846889$

C $0.4250902 .445484-4.763251$

H $-0.1425924 .267703-3.773175$

C -3.953410 $2.177350-1.339929$

O $-2.6030503 .150236 \quad 0.429260$

H -2.037195 $1.494320-0.603706$

H $-3.8763631 .588780-2.261148$

O $-4.5830353 .409401-1.690371$

C $-4.8457421 .404828-0.345891$

C $-6.1825590 .986519-0.985601$

H $-4.3134490 .510095-0.004313$

O

H $-6.7369961 .878052-1.302995$

H -5.957439 $0.386914-1.875729$

H $1.4425242 .778464-4.990549$

H $\quad 0.456227 \quad 1.365069-4.587248$

H $-0.1938022 .623320-5.648776$

C $1.9171883 .677040-2.085418$

H -1.690972 3.2827180 .740864

H $-4.6670183 .933598-0.875319$

H -4.293085 2.3898751 .248287

H $1.7260454 .755307-2.031586$

H $2.5855563 .493481-2.933131$

H $\quad 0.7689191 .836145-2.140842$

C $-7.012591-1.8704301 .275579$
O $-6.372646-1.0232990 .312822$

C $-7.0725740 .179742-0.019674$

C $-8.448293-0.151049-0.619705$

C $-9.253125-1.0510520 .323628$

C $-8.430370-2.2470160 .817031$

H $-6.411640-2.7886161 .284506$

C $-6.961272-1.2687322 .683429$

H -7.2179430 .7752760 .887526$

O $-8.305834-0.822727-1.869403$

H $-9.0008090 .779156-0.800354$

H $-10.144494-1.413337-0.203060$

O $-9.687663-0.2706491 .433674$

H $-8.954351-2.756071 \quad 1.633650$

O $-8.318588-3.199413-0.245408$

C $-5.532858-1.1751193 .166220$

H $-7.388060-0.2636392 .741588$

H -7.527002 -1.883282 3.393928

H $-8.008207-0.169277-2.524392$

H $-10.222983-0.8467362 .005447$

H $-7.803482-2.787743-0.960624$

O $-5.162458-2.347698 \quad 3.755745$

O $-4.826215-0.1866543 .017032$

C $-3.821796-2.3884614 .254181$

H $-3.108348-2.2249823 .441771$

H $-3.688798-1.6400885 .041278$

$\mathrm{H}-3.647143-3.3803384 .679342$

SCF Energy $(B 3 L Y P / 6-31 G * * / / M M F F)=-3245.91978842$

03206

MM̄FF Geometry

C -3.369046 -3.102685 -2.518053

C $-2.700035-2.908973-1.372814$

C $-1.196907-2.927821-1.276954$

O $-0.821528-1.603138-0.838070$

C $-0.750285-3.970475-0.234876$

C $0.727282-4.309339-0.276573$

C $1.610279-4.0648350 .712046$

C $1.190114-5.030427-1.519194$

C $1.370640-3.4252142 .050653$

C $1.825434-1.9617592 .151358$

C $3.347643-1.7568542 .062614$

C $3.710453-0.3039592 .384440$

C $5.212517-0.0273162 .214070$

O $3.784239-2.0765420 .738195$

O 5.4001521 .3965632 .196168

C $5.672117-0.5581940 .838419$

C $6.025299-0.5547073 .402581$

O $7.087897-0.4691840 .706449$

C $5.205810-2.0005290 .551436$

C $5.517124-2.472870-0.891213$

O $5.888734-2.9002951 .434023$

C $0.289509-1.029527-1.365600$

O $1.080405-1.525716-2.150269$

C $0.3772270 .338931-0.806146$

C $1.5376621 .006188-0.875653$

C $1.7979372 .349486-0.356103$

C 0.6242313 .1482810 .143262

C $4.906317-1.627309-2.012430$

C $5.823633-0.527933-2.524117$

C $5.7673561 .611179-3.807299$

O $7.040230-0.534598-2.355824$

C $5.8533252 .680954-2.764944$

C $5.1469923 .821063-2.802431$

C $5.2282554 .884599-1.744938$

C $3.9191695 .078360-1.010271$

C $3.1102386 .111019-1.300511$

C 3.5909914 .1284470 .121118

C $3.0675022 .805469-0.365336$

N $5.1272920 .441571-3.232632$

H -2.814326 -3.307579 -3.430363

H $-3.254832-2.695107-0.462135$

H $-0.768904-3.138136-2.263765$

H -1.300888 -4.908397 -0.390695

H -1.038416 -3.6168760 .759060$

H $2.638550-4.391030 \quad 0.559519$

H $1.129667-4.378541-2.395180$

H $2.227560-5.372875-1.439599$

H $0.569185-5.914440-1.698062$ 
H $\quad 0.313752-3.4779242 .325055$ H $1.893200-4.0197542 .810600$ H $1.326943-1.3828971 .367410$ H $1.471625-1.5705953 .113583$ H $3.826574-2.4351362 .778458$ H $3.384959-0.0371213 .397702$ H 3.1585040 .3638101 .710688 H 5.0376941 .7572493 .023501 H 5.2429370 .1107670 .082598 H $5.700632-0.0719714 .332263$ H $7.087762-0.3104053 .294835$ H $5.930302-1.6334323 .542483$ H 7.3366030 .4567920 .869742 H $6.599584-2.591407-1.021523$ H $5.099464-3.484567-0.996805$ H $5.853480-3.7928681 .051824$ H $-0.5069370 .747643-0.330930$ H $2.3897430 .515347-1.341247$ H -0.174896 $3.175298-0.606326$ H $\quad 0.870001 \quad 4.1901170 .360732$ H 0.2287582 .7067001 .064170 H $4.711294-2.278439-2.874116$ H $3.946476-1.206010-1.701404$ H $5.1699011 .920518-4.670600$ H $6.7716161 .347557-4.154190$ H $6.5314172 .502960-1.933319$ H $4.4708474 .002868-3.634555$ H $5.5307505 .819530-2.234393$ H $6.0211544 .666248-1.018659$ H $2.184866 \quad 6.273759-0.758099$ H $3.3528576 .813795-2.090743$ H 2.8941094 .5957820 .825132 H $4.496193 \quad 3.9488470 .715507$ H $3.8464862 .140527-0.736295$ H $4.1127220 .399505-3.263143$ C $-5.393021-1.724581-3.207820$ O $-5.246052-0.669083-2.236385$ C $-4.9143180 .549200-2.929976$ C $-5.086826 \quad 0.258440-4.420617$ C $-4.735664-1.214003-4.489399$ H $-3.853906 \quad 0.742137-2.728449$ C $-5.7632401 .719355-2.420521$ H $-6.1193280 .416042-4.753086$ H -4.438162 $0.885109-5.040481$ C $-5.223550-1.905465-5.749867$ H $-3.643536-1.297443-4.436231$ C $-5.7140751 .948198-0.897199$ O $-7.130561 \quad 1.468179-2.773452$ H -5.469901 $2.633469-2.951330$ H $-6.2589031 .152390-0.374247$ O $-6.4215283 .166583-0.625719$ C $-4.3001562 .069616-0.295018$ C -4.3337882 .5463241 .171374$ H $-3.7995301 .097691-0.356326$ O $-3.5247672 .991743-1.060662$ H -4.7153103 .5739911 .204455$ H -5.0396591 .9217621 .728576$ H -4.953473 -2.966179 -5.740272 H $-6.311750-1.834985-5.851432$ H -4.773292 -1.449554 -6.637561 C $-4.865269-3.045053-2.637623$ H -7.621008 2.299351-2.658537 H -7.365308 $2.999842-0.787769$ H -3.986729 $3.847628-1.046658$ H -5.184340 -3.875386 -3.278586 H $-5.318318-3.214551-1.652471$ H -6.474813 -1.835162 -3.362674 C $-2.985017 \quad 0.2651802 .686367$ O $-2.347286 \quad 1.2303931 .838839$ C -2.9447112 .5304741 .846724$ C -2.9333403 .0967563 .273298$ C $-3.5981232 .145646 \quad 4.264631$ C -2.9993430 .7445504 .145919$ H -4.010874 0.1084662 .335086 C -2.209187-1.048885 2.519476 H -2.2924973 .1724051 .242330$ O -3.5859134 .3607773 .328400$ H -1.894914 3.2574853 .591450
H -4.6861722 .1211834 .131802$

O -3.3668542 .6205455 .593605$ H -1.9815410 .7636944 .557132$ O $-3.773967-0.1383814 .953129$ C $-3.046483-2.2769432 .794687$ H -1.840695 -1.143849 1.493684 H -1.324374 -1.044864 3.167427 H -3.1341234 .9502012 .700530$ H -3.7232703 .5242395 .643095$ H -3.267842 -0.9611015 .060639$ O $-2.268710-3.236188 \quad 3.374543$ O $-4.229619-2.3923822 .504332$ C $-2.924846-4.4767883 .652572$ H -3.284965 -4.930313 2.724184 H -2.196907 -5.1504124 .112379$ H $-3.750494-4.3202354 .353335$ SCF Energy (B3LYP/6-31G**//MMFF) $=-3245.90545139$

03207

MM̄FF Geometry

C -1.3483214 .8395260 .492888$

C $-1.7506814 .744159-0.783922$

C $-2.6725323 .681993-1.342827$

O $-2.7701982 .544084-0.454489$

C $-4.0839564 .260667-1.542827$

C $-5.0290863 .318709-2.264025$

C $-6.1368392 .777234-1.720475$

C $-4.6806883 .016001-3.699346$

C $-6.6828992 .972453-0.334396$

C -6.2215201 .9283480 .694100$

C -6.6243850 .4838080 .353758$

C $-6.371347-0.4418061 .546213$

C $-6.676923-1.9103401 .211413$

O $-5.843041 \quad 0.045157-0.761456$

O $-6.110619-2.7257592 .249230$

C $-5.958257-2.289312-0.101771$

C $-8.184052-2.1929641 .212575$

O $-6.348100-3.588819-0.538234$

C $-6.172346-1.267698-1.236902$

C $-5.290467-1.543409-2.480278$

O $-7.536557-1.327699-1.671763$

C -1.726495 $1.668597-0.446257$

O $-0.7205361 .713368-1.136471$

C -2.0027270 .6540080 .593670$

C $-0.991460-0.0757981 .087169$

C $-1.098374-1.1099762 .118578$

C $-2.480895-1.5497072 .521947$

C $-3.777811-1.532720-2.232823$

C $-3.181094-2.906914-1.974307$

C $-1.043713-3.999196-1.299717$

O $-3.800979-3.953847-2.139870$

C -1.090324 -4.308906 0.163540

C $0.006991-4.5258210 .904489$

C $-0.022381-4.8757112 .364975$

C $0.821095-3.943973 \quad 3.206802$

C $2.073842-4.2770553 .561033$

C $0.180727-2.6695943 .710032$

C $0.036014-1.6268262 .635471$

N $-1.854002-2.826430-1.572223$

H $-1.7378304 .148195 \quad 1.235797$

H -1.354655 $5.449132-1.512028$

H -2.262009 $3.346126-2.302845$

H -4.028788 5.192395 -2.121517

H -4.488522 $4.536965-0.563201$

H $-6.7389392 .116146-2.343278$

H $-5.4747442 .461831-4.211685$

H $-4.5215123 .945156-4.256246$

H $-3.7707382 .411324-3.757747$

H -6.4404993 .9723910 .040876$

H $-7.7785642 .952146-0.398054$

H -5.1325001 .9930560 .800322$

H -6.6568782 .2044911 .662633$

H -7.6868660 .4753640 .083690$

H $-6.952689-0.1201292 .419376$

H $-5.317396-0.3666831 .845890$

H $-6.500636-2.4474243 .095500$

H $-4.887940-2.3446900 .128465$

H $-8.614377-1.9700002 .196262$ 
H $-8.387381-3.2541861 .031455$ H -8.731481 $-1.604850 \quad 0.473192$ H $-6.173778-4.2049190 .193875$ H -5.613067 -2.468121 -2.973823 H $-5.495593-0.741828-3.204812$ H $-7.590888-0.957427-2.568506$ H $-3.0252990 .549810 \quad 0.936715$ H $0.0154650 .110796 \quad 0.714997$ H -3.069580 -1.820397 1.638975 H -2.998554 $-0.746783 \quad 3.057488$ H -2.488761 -2.430165 3.167686 H -3.282307 -1.152787 -3.135515 H $-3.519645-0.859721-1.410663$ H -0.028150 -3.779132 -1.644059 H -1.430372 -4.850312 -1.869335 H $-2.070448-4.3815710 .629086$ H $\quad 0.983487-4.484190 \quad 0.427419$ H $\quad 0.345848-5.9050192 .467626$ H -1.048224 -4.890405 2.753636 H $2.676656-3.6277154 .187290$ H $2.527970-5.2033203 .224841$ H $0.796250-2.2278794 .505470$ H $-0.767051-2.9145364 .197556$ H $\quad 0.990132-1.2412402 .274979$ H -1.435736 -1.914133-1.414680 C 0.9325315 .0634831 .429976 $\begin{array}{lllll}\text { O } & 1.555685 & 4.449284 & 0.285148\end{array}$ C 2.9872374 .4428350 .481726 C 3.2274725 .0270371 .867886 C 2.0225075 .9341302 .042464 H $3.3971685 .105320-0.290452$ C 3.4846833 .0071240 .279084 H 3.2249574 .2384872 .630796 H 4.1806285 .5599311 .937101 C 1.7670926 .3303873 .485611 H $2.1690586 .837901 \quad 1.436028$ C 4.9627792 .7837070 .635985 O $3.2933552 .669469-1.099635$ H 2.8538682 .3238540 .859488 H 5.1122982 .9352451 .711111 O $5.7590523 .780954-0.016107$ C 5.5262731 .4036360 .237192 C 4.6901300 .2240030 .758736 H $5.6352811 .340790-0.851763$ O $\quad 6.8429521 .2930110 .788706$ H 4.6279780 .2803801 .852622 H $3.675673 \quad 0.3051220 .354336$ H 0.8858736 .9746023 .564042 H 1.6005745 .4529504 .119519 H 2.6232686 .8798483 .890408 C -0.3176325 .8200890 .980938$ H $2.3495532 .794662-1.299882$ H $5.6066883 .697569-0.973276$ H 7.3610022 .0448600 .453598 H $-0.061645 \quad 6.5309370 .185869$ H -0.7448476 .3900331 .812873$ H $0.659964 \quad 4.2535462 .120838$ C $5.792063-2.443681-1.620611$ O $5.252372-1.238201-1.062383$ C $5.267537-1.1513900 .367003$ C $4.451111-2.3006490 .978890$ C $4.945789-3.6597430 .476775$ C $5.083518-3.683542-1.049338$ H $5.551758-2.386310-2.690352$ C $7.317605-2.499100-1.485672$ H $6.300845-1.2101390 .723948$ O $3.077273-2.1393300 .632035$ H $4.527608-2.2486942 .071584$ H $4.240334-4.4385220 .791459$ O $\quad 6.203143-3.937776 \quad 1.085136$ H $5.616363-4.586858-1.366944$ O $3.777033-3.763143-1.628211$ C $7.960759-1.439957-2.348964$ H $7.669707-2.373482-0.457848$ H $7.703626-3.467140-1.826873$ H $2.582728-2.8607531 .054867$ H $6.491699-4.8133370 .775483$ H $3.307657-2.937435-1.418362$
O $8.189544-0.314503-1.615134$

O $8.202730-1.588040-3.539944$

C $8.7610710 .783501-2.332523$

H $8.9048961 .609992-1.631429$

H $9.7336350 .501760-2.747004$

H $8.0818201 .110571-3.125428$

SCF Energy (B3LYP/6-31G**//MMFF) $=-3245.91735580$

03208

MM̄FF Geometry

C $2.779512-4.141516-0.193669$

C $2.323749-3.047009-0.821757$

C $1.001494-2.999286-1.549816$

O $0.115163-2.101513-0.845228$

C $1.216380-2.444452-2.971100$

C $-0.045191-2.388607-3.811520$

C $-0.575626-1.257356-4.317496$

C $-0.700649-3.714496-4.100905$

C $-0.0580230 .147657-4.179152$

C $-0.6057470 .908331-2.962829$

C $-2.1083351 .221202-3.038920$

C $-2.5265942 .135870-1.885816$

C $-4.0397632 .398539-1.883247$

O $-2.833252-0.006694-2.954690$

O $-4.3567792 .992589-0.614675$

C $-4.7931101 .052448-1.980126$

C $-4.4319953 .428270-2.949751$

O $-6.1884221 .294916-2.143370$

C $-4.2548450 .127740-3.093848$

C $-4.843147-1.304046-3.052116$

O $-4.5954680 .685429-4.368834$

C -0.598261 -2.619143 0.192052

O $-0.571004-3.7665270 .607541$

C -1.415123 $-1.527190 \quad 0.763052$

C $-1.868689-1.6351512 .020712$

C $-2.648362-0.6297472 .745186$

C $-3.1760590 .541051 \quad 1.960270$

C $-4.601945-2.075916-1.749527$

C $-5.761302-2.010433-0.769018$

C $-6.402464-2.7419411 .525729$

O $-6.850838-1.515152-1.043960$

C $-6.296320-1.5638722 .441318$

C $-6.129425-1.6746963 .768078$

C $-6.075924-0.5030114 .705839$

C $-4.842061-0.5093775 .579729$

C $-4.889771-0.9738646 .839798$

C -3.5787780 .1054235 .022261$

C $-2.863001-0.8067124 .065601$

N -5.444737 -2.6074440 .444181$

H $2.146410-5.027266-0.182985$

H $2.914348-2.135765-0.823967$

H $0.551603-3.997963-1.608409$

H $1.950878-3.064465-3.502475$

H $1.663771-1.447825-2.889271$

H -1.484449 -1.340169-4.912454

H $-1.507246-3.626984-4.836869$

H $\quad 0.032319-4.421106-4.503730$

H -1.133856 -4.138397 -3.189909

H $1.0366510 .148494-4.136631$

H $-0.3065630 .697474-5.095785$

H $-0.4001270 .323265-2.060122$

H $-0.0485171 .849372-2.875854$

H $-2.3116901 .701554-4.003058$

H $-1.9734293 .082894-1.917464$

H $-2.2538931 .664274-0.931681$

H $-5.3226153 .101416-0.573986$

H -4.672581 $0.559954-1.009022$

H $-3.9176854 .379590-2.767472$

H $-5.5030203 .655663-2.907830$

H $-4.1881103 .108089-3.964871$

H $-6.6507540 .443820-2.065355$

H -5.904668 -1.287978 -3.325593

H -4.350656 -1.876902 -3.851534

H $-4.566942-0.023145-5.033000$

H $-1.588459-0.6519270 .147836$

H -1.619851 -2.531342 2.587909

H -3.7192010 .1928221 .076153$

H -2.3559451 .1881381 .635757$ 
H -3.8801141 .1608462 .519204$ H -4.473455 -3.137714 -1.996992 H -3.684601 -1.742782 -1.254779 H $-6.184942-3.6852812 .036557$ H -7.414200 -2.795371 1.110587 H $-6.392335-0.5758631 .997161$ H $-6.063061-2.6650284 .213215$ H $-6.976487-0.5385585 .332996$ H $-6.1331150 .448678 \quad 4.162823$ H -4.016431 -0.9544867 .483157$ H -5.802625 -1.3889917 .254035$ H -2.878872 0.3288025 .838923 H $-3.812951 \quad 1.0813454 .587865$ H -2.446898 -1.694437 4.544000 H -4.497909 -2.939632 0.604264 C $5.284144-3.529615-0.065058$ O $5.214316-2.1386230 .303172$ C $6.548283-1.6541700 .556814$ C $7.497911-2.7934880 .203009$ C $6.634591-4.0097760 .467471$ H $6.613953-1.4646231 .634198$ C $6.805372-0.359580-0.223932$ H $7.799162-2.761494-0.850499$ H $8.409523-2.7733550 .808212$ C $7.158553-5.271076-0.198463$ H $6.574615-4.1700931 .552351$ C 5.7984830 .7713970 .063060 O $6.743567-0.639790-1.629542$ H $7.826058-0.008603-0.033011$ H $4.8124990 .493929-0.326612$ O $6.2156461 .926333-0.676549$ C 5.6867851 .1673681 .546151 C 4.8205902 .4094601 .807021 H 5.2882350 .3283942 .124369 O $\quad 6.991301 \quad 1.4313612 .070437$ H 4.8313622 .6336562 .882857 H $5.295773 \quad 3.2953261 .362899$ H $6.513784-6.1280670 .018593$ H $7.212143-5.157521-1.286441$ H $8.164193-5.5075990 .164538$ C $4.096321-4.2995310 .521517$ H $5.864302-1.011489-1.816302$ H $6.2792561 .664486-1.611076$ H 7.3801822 .1495891 .541987 H $3.945989-4.0288951 .573718$ H $4.330134-5.3707190 .496273$ H $5.269336-3.577734-1.162003$ C 1.3986653 .7304721 .178596 O 2.7492803 .5693091 .633657 C 3.3712192 .3190531 .306138 C 2.5802271 .1557821 .921682 C 1.1071431 .2057691 .509427 C 0.5007892 .5982101 .702827 H $1.0606834 .667174 \quad 1.640681$ C $1.3238273 .905305-0.342864$ H 3.3921652 .2205500 .216683 O $2.677368 \quad 1.187168 \quad 3.344024$ H 3.0137520 .2053621 .591716 H 0.5406420 .4801452 .104206 O $0.989208 \quad 0.841626 \quad 0.138872$ H -0.4802752 .6632291 .218573$ O $\quad 0.2951602 .8239893 .100145$ C $2.0289475 .169789-0.769682$ H $1.7814743 .080411-0.896100$ H $\quad 0.2821813 .959624-0.681646$ H 2.3553982 .0542313 .645157 H $1.337140-0.0611980 .041849$ H -0.3226122 .1435263 .418388$ O $1.184673 \quad 6.234804-0.658563$ O $3.1962705 .206851-1.136629$ C $1.7440587 .502005-1.017005$ H $0.9702788 .263705-0.889009$ H $2.5859907 .743290-0.361105$ H $2.0594007 .494666-2.064859$ SCF Energy (B3LYP/6-31G**//MMFF) $=-3245.92800194$

03209

MM̄FF Geometry
C $1.2377583 .801981-1.060593$

C $1.8168754 .668093-0.215075$

C 2.7502524 .3011140 .916018

O 2.7338052 .8769791 .165408

C 4.1963474 .7008500 .570940

C 5.1707064 .4373381 .704343

C 6.1769873 .5419511 .656971

C 4.9679075 .2683622 .945765

C 6.5856692 .6657050 .505587

C 5.8710651 .3141430 .527099

C $6.5077200 .261202-0.392961$

C $6.5235440 .686561-1.863704$

C $7.053014-0.429775-2.779282$

O $5.736230-0.939399-0.236134$

O $6.769830-0.062894-4.138543$

C $6.296824-1.738299-2.465213$

C $8.579217-0.545061-2.704028$

O $6.847825-2.836490-3.187016$

C $6.246555-2.070301-0.958336$

C $5.332688-3.278967-0.644125$

O $7.561370-2.414588-0.505172$

C 1.7813772 .3855632 .003959

O 0.9151693 .0145692 .588931

C 1.9791120 .9204752 .073366

C 1.1984480 .1651822 .860759

C $1.298653-1.2888893 .021892$

C $2.337293-2.0002062 .194913$

C $3.893604-3.147049-1.146960$

C $2.974281-4.216283-0.578719$

C $0.565347-4.890455-0.601753$

O $3.357939-5.1091590 .171171$

C $-0.248159-4.1558690 .416973$

C $-0.664778-4.693621 \quad 1.573786$

C -1.517349 -3.954287 2.566367

C $-0.958836-3.9800143 .972811$

C $-1.663823-4.5256874 .978580$

C $0.407068-3.3766764 .226389$

C $0.470080-1.9088923 .888664$

N $1.669263-4.045611-1.018931$

H $1.4728862 .743169-0.991557$

H $1.5787455 .725895-0.303816$

H 2.4187624 .8332181 .815869

H 4.2417915 .7679150 .316595

H $4.5031124 .160213-0.331371$

H 6.8015263 .4267432 .542356

H 5.7828335 .1427603 .666890

H 4.9236296 .3315002 .687726

H 4.0379364 .9910543 .450830

H $6.4192673 .176293-0.448226$

H 7.6701172 .5124140 .574588

H 5.8525370 .9114661 .549073

H 4.8183791 .4459920 .248822

H $7.5274190 .074406-0.036009$

H $7.1160021 .597099-2.010568$

H $5.5005990 .940820-2.174379$

H $5.808890 \quad 0.054771-4.228627$

H $5.272500-1.605735-2.834508$

H $9.0482830 .391721-3.028997$

H $8.951917-1.317604-3.385652$

H $8.946300-0.765544-1.699705$

H $6.865868-2.586739-4.126726$

H $5.777461-4.200651-1.041967$

H $5.310504-3.4125780 .446699$

H $7.479195-2.9380420 .309230$

H 2.7750650 .4992401 .469261

H 0.4192140 .6516693 .445605

H $3.342831-1.6463642 .448382$

H $2.157692-1.8175191 .130612$

H $2.344770-3.0829632 .326926$

H $3.485638-2.167658-0.874057$

H $3.868254-3.239483-2.238460$

H $0.946831-5.842755-0.219991$

H $-0.043692-5.083792-1.490114$

H $-0.528138-3.1322710 .179700$

H $-0.411110-5.725346 \quad 1.805377$

H -2.515767 -4.410208 2.544896

H -1.659803 -2.911723 2.262311

H -1.278097 -4.5508515 .992495$ 
H $-2.643981-4.9604614 .812788$ H $\quad 1.152644-3.9715243 .693837$ H $\quad 0.670853-3.4713575 .288225$ H $-0.241727-1.3004934 .448347$ H $\quad 1.450971-3.248846-1.610516$ C $-1.1473193 .557466-1.865873$ O $-1.0393472 .118027-1.847503$ C $-1.6855471 .626605-0.654774$ C $-2.6455592 .729088-0.239934$ C -1.830556 $3.966278-0.557202$ H -0.8906831 .5050560 .092463$ C $-2.3433190 .276688-0.953654$ H $-3.5589372 .701265-0.847234$ H -2.9277572 .6683650 .814675$ C $-2.6619805 .232393-0.670093$ H -1.0954764 .1035390 .244751$ C $-3.184388-0.2630860 .215270$ O $-1.308433-0.668411-1.244204$ H -2.952402 $0.360524-1.859873$ H -4.0004920 .4288230 .444418$ O $-2.371030-0.2993451 .395853$ C -3.761875 -1.673052 -0.002289 C $-4.586798-1.863759-1.288295$ H -2.952426 -2.411531 -0.002076 O $-4.587910-2.005021 \quad 1.118590$ H $-3.914639-1.838518-2.153997$ H -5.023702 -2.869980-1.243360 H -2.029587 $6.090822-0.917740$ H -3.428640 $5.141822-1.446800$ H -3.1680465 .4456540 .277241$ C $0.2323634 .183387-2.111269$ H $-0.778653-0.302681-1.973395$ H $-1.583187-0.832508 \quad 1.192434$ H $-4.052228-1.8824871 .921074$ H $\quad 0.1343665 .272815-2.183370$ H $\quad 0.619088 \quad 3.828647-3.074489$ H -1.792214 $3.799847-2.720068$ C $-7.731086-0.017612-0.419851$ O $-6.648909-0.958181-0.417593$ C $-5.724153-0.842969-1.501183$ C $-6.446716-1.063622-2.840143$ C -7.636537 -0.108002 -2.987857 C $-8.522117-0.099654-1.736918$ H $-8.394973-0.3505260 .388461$ C $-7.2550801 .403875-0.080949$ H $-5.2895310 .160339-1.500239$ O $-6.906262-2.411525-2.917734$ H -5.732216 $-0.910061-3.657591$ H -8.243180 -0.406241 -3.851575 O $-7.1614721 .213799-3.225850$ H $-9.2357560 .730565-1.786441$ O $-9.299358-1.301263-1.717788$ C -6.6516571 .4902651 .305678$ H -6.498972 $1.751363-0.789919$ H -8.104216 $2.096039-0.110474$ H $-7.329519-2.527864-3.785421$ H $-6.6332221 .193698-4.041886$ H -8.687982 -2.048629 -1.600275 O -6.0092932 .6892861 .435163$ O -6.7396180 .6291952 .169851$ C -5.3740732 .9128792 .697804$ H -4.8635233 .8785732 .654656$ H -6.1219032 .9450543 .495645$ H -4.631746 2.1328482 .893847

SCF Energy (B3LYP/6-31G**//MMFF) $=-3245.91469970$

0321

MM̄FF Geometry

C 2.0330241 .0488934 .424922

C 1.5438840 .0095603 .732381

C $0.075569-0.3199723 .644549$

O $-0.349187-0.1898052 .268694$

C $-0.147812-1.7818764 .074844$

C $-1.606190-2.1937744 .123153$

C $-2.160511-3.1474933 .348916$

C $-2.452481-1.4829655 .148678$

C -1.500074 -3.994389 2.297442

C $-1.600628-3.4358080 .869931$
C $-3.036409-3.3096770 .336085$

C $-3.027920-2.976293-1.157742$

C $-4.445259-2.742156-1.704838$

O $-3.691783-2.2602191 .052242$

O $-4.322701-2.123800-2.995409$

C $-5.180803-1.736699-0.791870$

C $-5.194671-4.061351-1.926455$

O $-6.551368-1.618656-1.164064$

C $-5.071277-2.0764220 .708530$

C $-5.626692-0.9634981 .630732$

O $-5.830044-3.2613350 .979560$

C -0.6167361 .0648051 .813762$

O -0.5319002 .1101012 .438476$

C - -1.0213090 .9492860 .395870$

C -1.033486 $2.043879-0.378898$

C $-1.3834272 .089365-1.800247$

C $-1.9667710 .843845-2.412800$

C -4.9485430 .4044491 .493172$

C -5.6734051 .3602540 .560002$

C $-5.4267333 .605181-0.491678$

O $-6.791204 \quad 1.1395450 .102234$

C $-4.9414253 .429875-1.895872$

C $-4.3237654 .400541-2.585746$

C $-3.8684184 .257025-4.009426$

C $-2.4064274 .598271-4.189082$

C $-2.0283715 .811365-4.626434$

C $-1.3906983 .505865-3.943760$

C $-1.1693483 .234894-2.481147$

$\begin{array}{llll}\mathrm{N} & -4.936038 & 2.514167 & 0.329217\end{array}$

H 1.3508571 .7047824 .959738

H $2.229911-0.637316 \quad 3.190957$

H $-0.5161390 .353354 \quad 4.276597$

H $0.281284-1.9447615 .072646$

H $\quad 0.411549-2.4340083 .395167$

H -3.220503 -3.360368 3.485104

H $-3.447706-1.9297205 .247872$

H -1.975909-1.528869 6.133238

H -2.591920 -0.432911 4.874421

H $-0.445041-4.1614302 .538785$

H -1.959498 -4.990771 2.327091

H -1.114935 -2.454598 0.841793

H -1.029652 -4.101033 0.210983

H -3.557572 -4.257661 0.513815

H -2.522122 -3.764106 -1.729902

H -2.438241 -2.064254 -1.321894

H $-3.794507-2.712854-3.561074$

H $-4.723524-0.756068-0.968309$

H $-4.653162-4.693797-2.640270$

H $-6.180559-3.886257-2.371098$

H $-5.326343-4.638965-1.009247$

H $-6.576302-1.382057-2.107117$

H $-6.715241-0.8902821 .520130$

H $-5.463086-1.2922342 .667422$

H $-6.039751-3.2831001 .927907$

H -1.276290 -0.035784 0.023295

H -0.7335952 .9950970 .059327$

H -2.821788 $0.493636-1.824785$

H -1.216081 $0.047247-2.452522$

H $-2.3417030 .989613-3.427683$

H -4.9436170 .8883602 .478439$

H $-3.9066070 .299845 \quad 1.175495$

H -5.068055 $4.536529-0.042134$

H $-6.5214373 .609013-0.477727$

H $-5.1296332 .472047-2.375246$

H $-4.1625155 .366707-2.112638$

H $-4.4852424 .924729-4.625123$

H $-4.0568713 .246020-4.391504$

H $-0.983086 \quad 6.055140-4.785108$

H $-2.756186 \quad 6.589728-4.830542$

H $-0.4191273 .795025-4.367007$

H $-1.6839792 .614585-4.504929$

H $-0.7422204 .083293-1.944230$

H -3.9954692 .5888140 .705793$

C 3.9614882 .4656523 .556061

O 3.8361442 .0111492 .195430

C 3.4738493 .1349701 .367007

C 3.5880124 .3731992 .252249

C 3.2311883 .8048153 .614401 
H 2.4205082 .9980901 .095694

C $4.343814 \quad 3.154575 \quad 0.103462$

H 4.6079714 .7744622 .262549

H $2.915942 \quad 5.1746181 .930811$

C 3.6501954 .6873124 .776016

H 2.1445763 .6580063 .648023

C $4.3175441 .830447-0.690609$

$\begin{array}{lllll}\text { O } & 5.704769 & 3.407798 & 0.476525\end{array}$

H $4.0469183 .990463-0.539735$

H $4.7844881 .044484-0.087724$

O $5.1509161 .984556-1.845883$

C $2.9144191 .391914-1.144042$

C $2.8966080 .097785-1.970654$

H $2.260417 \quad 1.268198-0.276323$

O $2.3530412 .439063-1.939803$

H $1.865692-0.106283-2.293191$

H $3.4302410 .253355-2.918607$

H 3.3831674 .2248915 .731532

H 4.7308924 .8643354 .777062

H 3.1506935 .6600034 .719642

C 3.4973231 .3706154 .517812

H 5.9785772 .7011861 .086340

H $4.7401592 .657892-2.415130$

H $1.4771442 .143336-2.239375$

H 3.7201871 .6636005 .550462

H 4.0781870 .4604264 .319531

H 5.0359302 .6230293 .722577

C $3.808772-3.502094-1.756788$

O $3.281870-2.238056-2.180128$

C $3.471597-1.145421-1.270529$

C $2.812672-1.4539390 .082107$

C $3.291834-2.7917190 .651401$

C $3.228430-3.911162-0.392999$

H $3.444489-4.218863-2.504401$

C $5.340843-3.523908-1.798722$

H $4.546484-0.992056-1.133385$

O $1.397660-1.485089-0.079931$

H $3.049352-0.6511680 .788975$

H $2.668111-3.057176 \quad 1.513904$

O $4.628467-2.6323861 .117476$

H $3.747002-4.806804-0.031664$

O $1.860917-4.276207-0.601007$

C $5.833964-3.388762-3.219626$

H $5.805726-2.738976-1.195442$

H $5.725489-4.475470-1.412578$

H $1.007928-1.6040660 .802860$

H $4.910317-3.4822041 .496859$

H $1.504145-4.575100 \quad 0.252658$

O $6.054280-2.075447-3.513469$

O $5.979347-4.335046-3.982565$

C $6.496297-1.808101-4.847547$

H $7.453523-2.303369-5.035919$

H $5.742048-2.136251-5.569141$

H $6.633351-0.728392-4.950164$

SCF Energy (B3LYP/6-31G**/MMFF)= -3245.90143704

03210

MMFF Geometry

C 1.8403135 .2434110 .878645

C 3.0003294 .6103160 .644586

C $3.6359114 .305297-0.690176$

O $3.8528352 .872187-0.712086$

C $2.7903374 .655328-1.932609$

C $3.4555714 .272273-3.243485$

C $2.9815773 .341843-4.097236$

C $4.7214415 .019615-3.575757$

C $1.7171392 .536035-3.970774$

C $1.9427521 .240831-3.191711$

C $0.7297490 .298748-3.159898$

C $-0.4932030 .944021-2.501721$

C $-1.648669-0.049021-2.308206$

O $1.144565-0.857772-2.416025$

O $-2.5865340 .548138-1.402575$

C $-1.103325-1.318095-1.624352$

C $-2.411589-0.304944-3.612552$

O $-2.106711-2.323900-1.529149$

C $0.152765-1.889122-2.312451$

C $0.778392-3.066598-1.527812$
O $-0.198421-2.392255-3.606340$

C $5.1022472 .391407-0.479368$

O $6.1123563 .031734-0.240953$

C $5.0139430 .916098-0.560953$

C $6.0924630 .151208-0.335513$

C $6.118184-1.314555-0.370251$

C $4.840682-2.014083-0.754035$

C $1.119249-2.761777-0.067534$

C $2.002143-3.8298120 .558583$

C $3.253021-4.298105 \quad 2.672704$

O $2.337548-4.856476-0.024912$

C $4.602043-3.6500872 .711693$

C $5.753818-4.3000592 .482669$

C $7.103349-3.6434572 .574605$

C $7.982203-3.9031511 .368331$

C $9.164002-4.5282391 .506001$

C $7.509650-3.4420010 .004562$

C $7.262909-1.955522-0.053731$

N 2.366538 -3.4910171 .854016$

H 1.2503265 .6361290 .056058

H 3.5618094 .2468301 .504603

H $4.5913704 .841169-0.735265$

H $2.5859625 .733498-1.955115$

H $1.8181904 .158577-1.845246$

H $3.5522483 .135764-5.001913$

H $5.0805854 .798243-4.586545$

H $4.5497746 .099559-3.520462$

H $5.5219984 .756062-2.878272$

H $\quad 0.927764 \quad 3.139702-3.512794$

H $1.3614272 .300475-4.981521$

H $2.7977010 .694621-3.613444$

H $2.2293401 .473773-2.160022$

H $0.499917-0.004911-4.187926$

H $-0.8434621 .808680-3.077149$

H $-0.2059391 .337538-1.516619$

H $-2.8894261 .386939-1.790424$

H $-0.847938-1.036290-0.595385$

H -2.861652 $0.624376-3.982088$

H $-3.244915-0.997696-3.453355$

H $-1.778891-0.701266-4.409201$

H -2.845859 -1.941837-1.025908

H $0.118292-3.942799-1.574128$

H $1.699535-3.366786-2.047276$

H $0.477079-3.034576-3.880794$

H $4.0440450 .492419-0.797000$

H $7.0365220 .635829-0.091833$

H $4.513982-1.702185-1.752526$

H $4.052776-1.771529-0.034863$

H $4.917858-3.101711-0.781156$

H $1.640617-1.8010570 .006837$

H $0.201020-2.7022480 .527705$

H $3.295015-5.3202592 .283644$

H $2.826560-4.3219963 .680202$

H $4.634093-2.5935112 .969305$

H $5.730726-5.3631522 .255206$

H $7.588826-4.0226003 .483301$

H $7.005190-2.5604322 .718927$

H $9.805922-4.7204340 .652641$

H $9.518280-4.8634602 .475085$

H $6.633071-4.032486-0.271218$

H $8.265440-3.672719-0.757774$

H $8.139150-1.3560650 .197099$

H $2.059473-2.5999772 .233510$

C $-0.142720 \quad 4.850826 \quad 2.370831$

$\begin{array}{lllll}\text { O } & -0.132895 & 3.444074 & 2.062869\end{array}$

C -1.0206682 .7549732 .968949$

C $-1.748707 \quad 3.8373213 .754892$

C $-0.725394 \quad 4.9542353 .778521$

H $-0.371992 \quad 2.1657773 .628358$

C -1.9495531 .8376202 .161760$

H -2.6547004 .1607743 .227254$

H -2.041666 3.5029254 .754706

C -1.316991 $6.313626 \quad 4.107485$

H $\quad 0.042844 \quad 4.7060584 .523009$

C $-2.952374 \quad 1.062593 \quad 3.034803$

O $\begin{array}{llll}\text { O } & -1.142241 & 0.889384 & 1.456880\end{array}$

H -2.471376 2.4325251 .404771

H -3.566288 1.7571523 .618786 
$\begin{array}{llll}\text { O } & -2.217589 & 0.283615 & 3.988554\end{array}$

C -3.882927 0.1097352 .256702

C $-4.827906 \quad 0.851554 \quad 1.296410$

H -3.298938 -0.6462951 .720311$

O $-4.694859-0.5879413 .208730$

H -5.4300671 .5648991 .873321$

H $-4.234327 \quad 1.4180260 .572802$

H -0.5426017 .0869504 .107580$

H -2.0834276 .6040003 .381191$

H $-1.781080 \quad 6.301046 \quad 5.099085$

C $1.271596 \quad 5.4270132 .260013$

H -0.5018421 .3927840 .925258$

H -1.644922 -0.3241673 .489562$

H $-4.093407-1.0511063 .816747$

H 1.9225424 .9419132 .998264

H 1.2569786 .4979782 .491055

H -0.8060245 .3318811 .639780$

C $-5.713383-1.873171-1.110749$

O $-4.995011-0.895258-0.346373$

C $-5.783741-0.0922970 .538295$

C $-6.8448450 .689366-0.251380$

C $-7.706703-0.255812-1.094689$

C $-6.849718-1.222333-1.915942$

H $-4.982243-2.259169-1.832363$

C -6.167602 $-3.047808-0.237729$

H $-6.285825-0.7395341 .264493$

O $-6.2043441 .640200-1.099910$

H -7.472426 1.2463540 .454622

H $-8.3376730 .328873-1.775285$

O $-8.563782-1.011036-0.243491$

H $-7.480077-2.003612-2.355542$

O $-6.270198-0.506538-3.011575$

C $-4.973450-3.8604260 .205521$

H $-6.729490-2.7463820 .650744$

H -6.820845 $-3.721460-0.805177$

H -6.900978 2.134900 - 1.563957

H $-9.119341-0.3817840 .247185$

H -5.639137 $0.136985-2.645808$

O $-4.429900-3.316298 \quad 1.330919$

O $-4.557914-4.842756-0.395332$

C -3.249059-3.959376 1.820055

H -2.457848 -3.9250401 .065019$

H -2.909798 -3.416984 2.706631

H -3.470345 -4.992812 2.102680

SCF Energy (B3LYP/6-31G**/MMFF) $=-3245.92290646$

03211

MM̄FF Geometry

C -4.020231 -3.578592 2.191545

C $-4.491543-4.1094641 .051977$

C $-5.095782-3.327916-0.092012$

O $-4.545620-1.991205-0.108846$

C $-4.726819-3.985566-1.438655$

C $-5.319629-3.280329-2.644610$

C $-4.598118-2.685199-3.615633$

C $-6.824550-3.291172-2.732070$

C $-3.101715-2.622655-3.753046$

C $-2.534018-1.325848-3.176837$

C $-1.045835-1.106763-3.490120$

C $-0.148173-2.174884-2.859089$

C $1.345055-1.867993-3.056758$

O $-0.707951 \quad 0.188277-2.971671$

O $2.097000-2.735197-2.193171$

C $1.623773-0.410338-2.634375$

C $1.805978-2.214595-4.476392$

O $2.962679-0.036814-2.943543$

C $0.637788 \quad 0.606113-3.247210$

C $0.810742 \quad 2.031083-2.667417$

O $\quad 0.866070 \quad 0.698561-4.658074$

C $-5.373320-0.9325290 .088885$

$\begin{array}{lllll} & -6.563305 & -0.957163 & 0.353499\end{array}$

C $-4.5554250 .286400-0.104598$

C $-5.1137461 .504580-0.048640$

C $-4.4134992 .772145-0.280063$

C -2.974170 $2.690654-0.715987$

C $0.7077852 .115588-1.144029$

C $0.528713 \quad 3.535504-0.634381$

$\begin{array}{llll}\text { C } & 0.112589 & 4.762431 & 1.506997\end{array}$
O $0.5008394 .519770-1.366818$

C $-1.334627 \quad 4.7487591 .889601$

C $-2.181195 \quad 5.766914 \quad 1.671936$

C -3.631002 5.7320272 .069750

C $-4.565008 \quad 6.149788 \quad 0.952732$

C $-5.3585297 .225861 \quad 1.090563$

C $-4.5842395 .335483-0.323723$

C $-5.0843193 .931090-0.111136$

N $0.3984773 .559233 \quad 0.747693$

H $-4.105885-2.5084872 .353665$

H $-4.439475-5.1889420 .924713$

H $-6.184418-3.3202720 .036319$

H $-5.070186-5.028524-1.451360$

H $-3.634828-4.025890-1.515011$

H -5.136494 -2.195918-4.426661

H $-7.187782-2.894664-3.686400$

H $-7.201950-4.315013-2.640723$

H $-7.265283-2.683787-1.936446$

H $-2.629573-3.493887-3.290921$

H -2.860690 -2.688097-4.821546

H $-3.100308-0.466059-3.560531$

H -2.678846 -1.306922 -2.090007

H $-0.924164-1.093506-4.579566$

H $-0.375899-3.172005-3.252925$

H $-0.357322-2.225214-1.782369$

H $1.825759-2.561346-1.275588$

H $1.534326-0.376023-1.542369$

H $1.662233-3.283961-4.673278$

H $2.878955-2.030134-4.600773$

H $1.268900-1.662084-5.250177$

H $3.548427-0.696693-2.534623$

H $1.7664962 .460166-2.995422$

H $0.0349842 .672118-3.109089$

H $0.5152851 .548595-4.971761$

H $-3.5008940 .143692-0.313221$

H $-6.176777 \quad \begin{array}{lll}1.584358 & 0.173402\end{array}$

H -2.881072 2.103396 -1.636680

H -2.368661 2.2189660 .065391

H $-2.5203863 .660159-0.924147$

H $-0.1438511 .523804-0.792243$

H $1.6183641 .715038-0.687350$

H $0.379653 \quad 5.647210 \quad 0.920676$

H 0.7347314 .7325482 .406619

H -1.704185 3.8515082 .381871

H -1.812685 $6.676931 \quad 1.204486$

H -3.7502646 .4001762 .932604$

H $-3.919326 \quad 4.7335342 .420877$

H -6.0318687 .5382020 .299182$

H -5.3543457 .8201901 .998297$

H -3.593471 $5.372657-0.782584$

H $-5.2499085 .803813-1.061311$

H $-6.1286343 .883866 \quad 0.200694$

H $0.4006772 .678792 \quad 1.254719$

C -2.036623 $-3.768053 \quad 3.772464$

O $-1.180794-3.4301312 .660103$

C $-0.762029-2.0567072 .812385$

C $-0.910644-1.7610584 .295213$

C $-2.196488-2.4973594 .612867$

H $-1.474056-1.4581872 .228575$

C $0.652874-1.8862362 .249961$

H $-0.073048-2.1853004 .862733$

H $-0.970549-0.6907584 .512623$

C $-2.394178-2.7650136 .095486$

H $-3.039024-1.8941464 .254286$

C $1.267416-0.5051152 .534933$

O $0.585756-2.0715930 .832706$

H $1.304109-2.6763492 .640896$

H $1.427199-0.3883203 .613016$

$\begin{array}{lllll}\text { O } & 0.326506 & 0.511764 & 2.164798\end{array}$

C $2.584337-0.200568 \quad 1.796774$

C $3.659535-1.2843751 .974772$

H $2.390890-0.043654 \quad 0.730584$

$\begin{array}{lllllll}\text { O } & 3.093425 & 1.037270 & 2.305057\end{array}$

H $3.794235-1.4967633 .042656$

H $3.307436-2.2021841 .491273$

H -3.323039 -3.3171556 .270143$

H $-1.568826-3.3522966 .511584$

H -2.449935-1.823773 6.652052 
C $-3.349292-4.385818 \quad 3.270505$ H $0.191621-2.9464830 .673426$ H $0.088647 \quad 0.361997 \quad 1.233804$ H 2.4095421 .7134192 .159877 H $-4.042360-4.5128264 .109733$ H $-3.125870-5.3876872 .881831$ H $-1.497913-4.5295074 .350810$ C $5.994118-0.071305-0.682761$ O $4.834686-0.567129-0.000157$ C $5.025112-0.8816801 .382588$ C $6.074326-1.9935691 .532442$ C $7.387917-1.6061250 .841855$ C $7.155647-1.075158-0.578023$ H $5.698804-0.013373-1.737986$ C $6.3647911 .347485-0.223659$ H 5.3724620 .0103621 .914221 O 5.575068 -3.199980 0.957799 H $6.244375-2.1801682 .599278$ H $8.048436-2.4802340 .790685$ O $8.052834-0.6046441 .606132$ H $8.072361-0.613547-0.962143$ O $6.866798-2.182249-1.438153$ C $5.2354992 .330744-0.451508$ H $\quad 6.612744 \quad 1.3658130 .840995$ H $7.2346201 .709453-0.783065$ H $6.245983-3.8902681 .095410$ H $8.222616-0.9734212 .489617$ H $6.017778-2.564070-1.156155$ O $5.398284 \quad 3.402138 \quad 0.379608$ O $4.3358212 .189955-1.268044$ C $4.387058 \quad 4.410592 \quad 0.287465$ H 4.6656195 .2333130 .951257 H $4.3180144 .793224-0.735325$ H 3.4258244 .0053920 .615465 SCF Energy (B3LYP/6-31G**//MMFF) $=-3245.93002088$

03_212

MM̄FF Geometry

C -1.704999 -4.396503 -1.985113

C $-0.854373-4.042049-1.008980$

C $0.591280-3.683742-1.255140$

O $0.908827-2.478796-0.521726$

C $1.480834-4.818735-0.711567$

C $2.959172-4.645271-0.996998$

C $3.912403-4.534581-0.051174$

C $3.350269-4.641598-2.452774$

C $3.744969-4.5443071 .443043$

C $3.580607-3.1525172 .074376$

C $4.805767-2.2386761 .906768$

C $4.642895-0.9542492 .722574$

$\begin{array}{llll}\text { C } & 5.807231 & 0.024793 & 2.496207\end{array}$

O $4.935028-1.9068900 .522945$

O 5.4150391 .3041443 .017853

C $6.031315 \quad 0.206864 \quad 0.978018$

C $7.060279-0.3938843 .274796$

$\begin{array}{lllll}\text { O } & 7.206857 & 0.971202 & 0.724584\end{array}$

C $6.093466-1.1256860 .204329$

C $6.112119-0.946854-1.333092$

O $7.296185-1.8198770 .557961$

C $0.636729-1.284969-1.114769$

O $0.134991-1.104781-2.212459$

C $1.047984-0.218166-0.172778$

C $0.6951551 .054533-0.408660$

C $0.985007 \quad 2.203264 \quad 0.452418$

C 2.0184902 .0163821 .531083

C $4.905152-0.210383-1.924709$

C $5.1403251 .275109-2.138417$

C $3.9371073 .361330-2.766962$

O $6.2442261 .804683-2.043877$

C 3.632644 4.105881-1.505866

C $2.6872795 .053595-1.422563$

C $2.3920785 .830139-0.172188$

C $0.924887 \quad 5.8063760 .191994$

C $0.119516 \quad 6.829528-0.140174$

C $0.417095 \quad 4.6443691 .015971$

$\begin{array}{lllll}\text { C } & 0.328086 & 3.361817 & 0.231711\end{array}$

N $3.971591 \quad 1.938341-2.487473$

H $-1.337260-4.389414-3.010014$

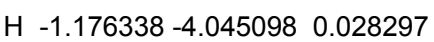

H $0.781355-3.530194-2.324068$

H $1.168647-5.776347-1.150249$

H $1.306654-4.9090880 .366217$

H $4.945907-4.429601-0.379925$

H $4.436186-4.680104-2.591350$

H $2.926045-5.512511-2.963235$

H $2.989969-3.734413-2.947436$

H $2.894461-5.169913 \quad 1.733728$

H $4.622567-5.0376951 .880211$

H $2.696674-2.6695581 .640850$

H $3.381401-3.2913663 .144266$

H $5.694121-2.7902682 .236412$

H $4.536127-1.1821083 .790529$

H $3.709061-0.4558412 .429644$

H 5.1969021 .1901113 .958688

H $5.187516 \quad 0.797183 \quad 0.601694$

H $6.848538-0.4290714 .350286$

H 7.8651920 .3393353 .152952

H $7.439049-1.3753112 .982255$

H 7.1173131 .8098911 .208871

H $7.059604-0.493590-1.648300$

H $6.112937-1.953934-1.775076$

H $7.500247-2.460637-0.143317$

H $1.604289-0.5139740 .709555$

H $\quad 0.112978 \quad 1.277922-1.300849$

H 2.9303621 .5774321 .112425

H 1.6395301 .3568842 .318925

H 2.3289172 .9502262 .004407

H $4.695807-0.629748-2.917309$

H $4.009812-0.358231-1.312081$

H $3.1779383 .523597-3.538637$

H $4.9092933 .678935-3.157562$

H $4.2301003 .871448-0.627698$

H $2.1111065 .307390-2.309649$

H $2.7181146 .865352-0.338434$

H $2.984314 \quad 5.466752 \quad 0.676961$

H $-0.926588 \quad 6.8431720 .143615$

H $0.4895357 .677979-0.706250$

H -0.5919564 .8618111 .391201$

H $1.033706 \quad 4.5521231 .914316$

H $-0.398943 \quad 3.397236-0.580838$

H $3.0902301 .433152-2.481221$

C $-3.691746-4.964250-0.418541$

O $-3.957963-3.6376050 .077643$

C $-5.237668-3.6354740 .739433$

C $-5.613422-5.098490 \quad 0.927001$

C $-5.025116-5.709357-0.331027$

H $-5.953741-3.1859870 .041525$

C -5.168595 -2.803981 2.023819

H $-5.140928-5.5288231 .817628$

H $-6.694557-5.2433171 .012016$

C $-4.888526-7.220642-0.262326$

H $-5.661743-5.443354-1.185680$

C $-4.683949-1.3564011 .791045$

O $-4.249794-3.4358892 .923334$

H -6.144572 -2.809207 2.522914

H -3.638343 -1.379869 1.466725

O $-4.689746-0.6795863 .053900$

C $-5.538063-0.5570630 .791781$

$\begin{array}{llll}C & -5.123658 & 0.912051 & 0.625247\end{array}$

H $-5.535075-1.044991-0.185255$

O $-6.897145-0.580173 \quad 1.245174$

H $-5.8247991 .372742-0.081248$

H -5.3162011 .4514391 .563335$

H $-4.445749-7.615864-1.181696$

H $-4.254956-7.5287330 .576334$

H $-5.869489-7.689339-0.131716$

C $-3.131487-4.867566-1.840487$

H $-4.205010-2.886357 \quad 3.724558$

H $-5.615598-0.6083053 .343490$

H $-7.437256-0.1238720 .577618$

H $-3.758577-4.187006-2.430304$

H $-3.185875-5.854184-2.315818$

H $-2.966421-5.430067 \quad 0.260257$

C $-4.0685993 .432517-0.641999$

$\begin{array}{lllll}\text { O } & -3.381437 & 2.594911 & 0.297917\end{array}$

$\begin{array}{llll}\text { C } & -3.669887 & 1.190982 & 0.187522\end{array}$ 
C $-3.2539540 .714799-1.209682$

C $-3.9447871 .521380-2.304681$

C $-3.7277263 .020006-2.081659$

H -5.150802 $3.366850-0.480201$

C -3.641606 $4.879261-0.353932$

H -3.0106190 .7044430 .914684$

O $-3.495486-0.673000-1.381503$

H $-2.1726760 .854557-1.315541$

H -5.010524 $1.271997-2.365250$

O $-3.3735151 .151603-3.562268$

H $-2.6832843 .263968-2.315728$

O $\quad-4.5534413 .722667-3.007908$

C $-4.1090225 .356721 \quad 1.005878$

H -2.548554 $4.945093-0.373098$

H -4.060146 5.565692 -1.096608

H $-3.210537-0.911020-2.280448$

H -3.798252 $1.699539-4.244694$

H $-4.2617854 .649426-3.023914$

O -3.5263956 .5626351 .274720$

O

C -3.8704267 .1455982 .535383$

H -3.566246 6.4858053 .353564

H -3.3323408 .0924802 .630063$

H -4.9450987 .3462372 .578745$

SCF Energy (B3LYP/6-31G $\left.{ }^{\star *} / / \mathrm{MMFF}\right)=-3245.90727330$

03_213

MM̄FF Geometry

C $-0.4881184 .712272 \quad 1.835522$

C $0.461906 \quad 4.3475120 .960669$

C 1.6976533 .5742471 .349083

O 1.8047632 .4222130 .477592

C 2.9512324 .4423561 .142867

C 4.2309483 .7805561 .617559

C 5.2595573 .4367970 .817841

C $4.3289973 .534483 \quad 3.101910$

C $5.3855713 .626639-0.667180$

C $4.9170332 .433256-1.513659$

C $5.6818221 .127059-1.242608$

C $5.3589430 .081067-2.310793$

C $6.042443-1.264584-2.021830$

O $5.287768 \quad 0.6288690 .038574$

O $5.439324-2.252890-2.872310$

C $5.749707-1.682739-0.563267$

C $7.532728-1.233857-2.383707$

O $6.514241-2.839871-0.232882$

C $5.988577-0.550560 \quad 0.460355$

C $5.486742-0.8855871 .887435$

O $7.396112-0.3010160 .560666$

C 0.9720301 .3733220 .718190

$\begin{array}{llll}\text { O } & 0.158411 & 1.271794 & 1.622257\end{array}$

C $1.2006870 .368704-0.343870$

C $0.311265-0.619786-0.516580$

C $0.376241-1.676977-1.526944$

C $1.661966-1.827490-2.294591$

C $3.994123-1.2150012 .001668$

C $3.681668-2.7014341 .949893$

C $1.752771-4.2784081 .872037$

O $4.545532-3.573247 \quad 1.987596$

C $1.490588-4.6882070 .457138$

C $0.310431-5.1709510 .039590$

C $0.035847-5.624419-1.365534$

C -1.147796 -4.914372 -1.983494

C $-2.370932-5.470631-1.976919$

C $-0.893706-3.594044-2.675753$

C $-0.704030-2.465946-1.702682$

N $2.314970-2.940817 \quad 1.892278$

H -0.3342894 .4790002 .887649$

H $0.3485674 .569837-0.096579$

H 1.6362343 .2334122 .389664

H 2.8410685 .3912881 .684361

H 3.0245824 .7052910 .082128

H 6.1289442 .9682041 .278312

H 5.3305693 .2094273 .403727

H 4.1049404 .4523043 .655272

H 3.6256182 .7560843 .412756

H $4.8489654 .524985-0.990432$

H $6.4404683 .831288-0.891361$
H $3.8456772 .276844-1.342876$

H $5.0384422 .709754-2.568485$

H $6.755418 \quad 1.348954-1.237304$

H $5.6363640 .443947-3.308392$

H $4.273161-0.080910-2.344017$

H $5.558179-1.967740-3.794543$

H $4.695564-1.982735-0.533652$

H $7.663795-0.991732-3.445285$

H $7.994321-2.217516-2.243394$

H $8.099083-0.500247-1.806382$

H $6.209450-3.163070 \quad 0.631208$

H $6.109999-1.6682662 .336076$

H 5.6588720 .0073592 .506142

H 7.5703760 .1458461 .405623

H $2.0801420 .481343-0.966814$

H $-0.562975-0.6496930 .131890$

H $2.510630-1.904162-1.606584$

H $1.819551-0.967175-2.953749$

H $1.697139-2.724177-2.916477$

H $3.635674-0.8629342 .977633$

H $3.417533-0.6929871 .233183$

H $0.837753-4.2550332 .471960$

H $2.455674-4.9786352 .334616$

H $2.313653-4.607300-0.248877$

H $-0.501565-5.2761500 .755764$

H $-0.152390-6.705669-1.336373$

H $\quad 0.915850-5.496289-2.008033$

H -3.219511 -4.976882 -2.438818

H -2.552004 -6.429272 -1.502110

H -1.750575 -3.331652 -3.310897

H $-0.056053-3.709243-3.368684$

H -1.588153 -2.274048 -1.094555

H $1.674726-2.1562951 .813160$

C -2.2387815 .4049070 .058053$

O $-2.3483764 .051500-0.424397$

C $-3.6160133 .881530-1.079732$

C $-4.1045575 .291758-1.382222$

C $-3.6175576 .036893-0.152123$

H $-4.2941913 .433140-0.344127$

C $-3.4706472 .937284-2.278247$

H $-3.6319485 .709341-2.277860$

H $-5.1895155 .338789-1.513642$

C $-3.5828227 .545300-0.325189$

H -4.2782285 .7903050 .689881$

C $-2.7466341 .607279-1.972802$

O $-2.6945893 .585892-3.296642$

H $-4.4529392 .734323-2.721216$

H $-1.6934351 .827724-1.756233$

O $-2.7549570 .834778-3.181862$

C $-3.325370 \quad 0.732813-0.843346$

C $-4.8177910 .400602-1.016301$

H -2.774057 -0.212322 -0.836427

O

H $-5.4147951 .316854-0.943608$

H -4.965877 -0.025019-2.015696

H $-3.207856 \quad 8.0322980 .580561$

H -2.935768 $7.839097-1.158535$

H $-4.5868477 .932380-0.526905$

C -1.802135 5.3843551 .527095

H $-3.2788184 .210302-3.758571$

H $-2.2157720 .040594-3.025953$

H -2.1662311 .5226950 .514603$

H -2.564268 4.8441352 .104467

H $-1.765696 \quad 6.4068061 .920137$

H -1.488866 $5.911023-0.562513$

C $-4.895834-2.8399980 .855787$

O $-4.591890-1.810430-0.094833$

C $-5.328837-0.5912950 .048936$

C $-6.835404-0.858285-0.092876$

C $-7.303354-1.9476740 .878266$

C $-6.397099-3.1819610 .834051$

H $-4.348628-3.7212560 .497686$

C $-4.370450-2.4975282 .259347$

H $-5.131033-0.1630541 .036898$

O $-7.148566-1.248957-1.428725$

H -7.3796390 .0729040 .102398$

H $-8.327631-2.2470950 .623645$

O $-7.316432-1.4334612 .206547$ 
H $-6.638698-3.8555841 .664553$

O $-6.682259-3.890053-0.376460$

C $-2.857376-2.4415962 .305140$

H -4.750681 -1.531961 2.602499

H $-4.683170-3.2662332 .975095$

H $-6.621518-2.038927-1.638769$

H -7.923640 -0.6740962 .221552$

H $-6.158398-4.709237-0.368286$

O $-2.468150-1.732348 \quad 3.405415$

O $-2.109214-2.9806901 .501645$

C $-1.054525-1.6206933 .599883$

H $-0.877652-0.9088964 .410529$

H $-0.642409-2.5921453 .887321$

H - $0.566265-1.2476412 .695473$

SCF Energy (B3LYP/6-31G**//MMFF) $=-3245.91300621$

03214

MM̄MF Geometry

C $3.785756-3.072117-3.103271$

C $3.063310-1.944814-3.179488$

C $1.593881-1.898347-3.507453$

O $0.867201-1.563703-2.302334$

C $1.337954-0.790643-4.546692$

C -0.121766 -0.607432 -4.910551

C $-0.8336530 .510511-4.664540$

C $-0.770975-1.771265-5.613520$

C $-0.3660611 .779204-4.004769$

C $-0.5849521 .823480-2.484585$

C $-2.0633581 .887797-2.068088$

C $-2.1913292 .103186-0.559552$

C $-3.6590152 .102827-0.104536$

O $-2.6873740 .652479-2.417876$

O $-3.677003 \quad 1.972696 \quad 1.326145$

C $-4.3723720 .854852-0.675736$

C $-4.3438713 .437211-0.422488$

O $\quad \begin{array}{lllll}-5.776850 & 0.914517 & -0.444108\end{array}$

C $-4.0997740 .606517-2.175395$

C $-4.593271-0.773236-2.667887$

O $-4.7942091 .553594-2.996898$

C $0.594240-2.581493-1.440830$

O $0.910823-3.754604-1.560939$

C $-0.162668-2.021182-0.301177$

C $-0.235262-2.7206620 .840989$

C $-0.950043-2.3215122 .054084$

C $-1.913656-1.1713881 .945074$

C $-3.951393-1.987964-1.986899$

C $-4.791085-2.591851-0.872514$

C $-4.725869-4.421958 \quad 0.822177$

O $-5.932561-2.224121-0.609264$

C $-4.472231-3.7884272 .153350$

C $-3.877763-4.4226783 .175485$

C -3.681759-3.814395 4.534520

C -2.245572 -3.869432 5.003753

C - $-1.833862-4.8188785 .860984$

C $-1.313400-2.7675614 .555466$

C $-0.734911-3.0165263 .190075$

$\mathrm{N}-4.119655-3.622101-0.225831$

H $3.309047-4.030452-3.291044$

H $3.550291-0.995351-2.971223$

H $\quad 1.243638-2.862010-3.897726$

H $1.897642-1.010520-5.465749$

H $1.7523190 .147844-4.162992$

H -1.876646 $0.530507-4.978482$

H - $0.848829-2.632545-4.943141$

H -1.782227 -1.534782 -5.961555

H $-0.183113-2.061230-6.490529$

H $0.6940101 .955012-4.215917$

H $-0.8901272 .622273-4.472844$

H $-0.1075440 .948885-2.027857$

H $-0.0663142 .708161-2.097557$

H $-2.5420532 .707497-2.616155$

H -1.694557 $3.030809-0.250545$

H $-1.6658631 .295387-0.034963$

H -3.156418 2.7038521 .701040

H -4.002099 -0.004760 -0.107607

H -3.8536334 .2565980 .116977$

H $-5.3856033 .439622-0.083829$

H $-4.3224253 .686633-1.485508$
$\begin{array}{llll}H & -5.908141 & 1.032818 & 0.512301\end{array}$

H $-5.688500-0.818124-2.630070$

H $-4.341386-0.838888-3.736645$

H -5.347702 2.112772 -2.429054

H -0.629732 -1.052091 -0.430350

H $\quad 0.294219-3.6707300 .904485$

H -1.383997 -0.2382491 .740013$

H -2.513619-1.014183 2.843606

H $-2.623751-1.3507101 .132666$

H -3.830982 -2.778928 -2.738739

H $-2.954871-1.749752-1.602593$

H $-4.297580-5.4269190 .753102$

H -5.804386 -4.490462 0.646977

H $-4.827576-2.7702572 .293768$

H -3.549254 -5.451229 3.043292

H $-4.323942-4.3616095 .237358$

H $-4.034383-2.7758074 .563510$

H $-0.812747-4.8456516 .226817$

H -2.507389 -5.594009 6.211352

H -0.468101 -2.688012 5.252807

H $-1.821861-1.8023804 .634775$

H $-0.029304-3.8481873 .162839$

H -3.152320 -3.815055 -0.469322

C $5.539611-2.873494-1.252917$

O $5.209804-1.522395-0.876152$

C $4.654554-1.5357400 .453943$

C $4.909261-2.9373081 .003072$

C $4.810933-3.775734-0.259034$

H $3.571932-1.4036140 .351017$

C $5.248098-0.3928881 .283035$

H $5.909118-3.0280131 .442952$

H $4.178392-3.2216961 .766254$

C $5.416203-5.161191-0.124208$

H $3.750525-3.873031-0.522205$

C $5.095124 \quad 1.0138780 .662102$

O $6.654359-0.6430621 .433076$

H $4.829036-0.4127012 .295454$

H $5.6778721 .049034-0.266885$

O 5.7159181 .9397591 .565276

C 3.6591801 .5069870 .385183

C 2.7535541 .4731361 .628807

H 3.7220482 .5460510 .040010

O $3.0562360 .762426-0.665206$

H 2.6837630 .4438391 .999389

H 3.2182852 .0679842 .422723

H $5.333449-5.714560-1.064997$

H $6.476123-5.1122730 .146753$

H $4.897384-5.7336200 .651652$

C $5.243708-3.095516-2.739627$

H 7.0209550 .0813001 .968486

H 5.7047012 .8149301 .141588

H $3.6364940 .830117-1.442436$

H $5.658673-4.060499-3.054497$

H $5.761272-2.327230-3.328718$

H $6.624903-2.965263-1.110782$

C 1.7095774 .3465121 .373901

O $1.280824 \quad 3.2002460 .633050$

C $1.3142521 .970876 \quad 1.365274$

C 0.4507942 .0750112 .633996

C 0.7816733 .3130263 .466602

C 0.8067724 .5664872 .592934

H 2.7393994 .1827641 .716188

C 1.7836895 .5403300 .416329

H $\quad 0.843543 \quad 1.221513 \quad 0.716293$

O $0.603521 \quad 0.923431 \quad 3.459149$

H -0.6066752 .1273862 .345373$

H 1.7304143 .1925154 .003164

O -0.2255013 .4828814 .467569$

H -0.2219414 .8005782 .293066$

O $1.2916965 .651593 \quad 3.378713$

C $0.4364376 .011090-0.078246$

H 2.2767006 .3948920 .894155

H $2.3966515 .287039-0.458101$

H 0.4755630 .1386252 .901184

H -0.2486272 .6662104 .995452$

H 1.1412976 .4701912 .876656

O $0.0980665 .352609-1.222419$

$\begin{array}{llll}\text { O } & -0.236086 & 6.862317 & 0.489666\end{array}$ 
C $-1.1519505 .735170-1.804543$

H $-1.9700335 .554013-1.101774$

H -1.123672 $6.788040-2.100718$

H -1.313472 $5.124364-2.696682$

SCF Energy (B3LYP/6-31G**//MMFF) $=-3245.90403511$

03_215

MM̄MF Geometry

C $2.695646 \quad 3.5560710 .707030$

C 2.0144862 .4048320 .606922

C 1.0963811 .8797561 .678889

O $\quad-0.196151 \quad 1.6271901 .079647$

C 1.6484450 .5497192 .221513

C 0.9046680 .0571003 .447228

C $-0.000692-0.9409093 .454825$

C 1.2690360 .7489884 .736760

C $-0.491346-1.7591032 .292917$

C - $1.979395-1.5164312 .044096$

C -2.586454 -2.407088 0.950070

C $-1.945930-2.200196-0.423465$

C $-2.662452-2.998899-1.522959$

O $-3.975136-2.0621930 .888370$

O $-2.178046-2.494498-2.776819$

C $-4.177890-2.718912-1.442203$

C $-2.285065-4.484431-1.474032$

O $-4.904615-3.558406-2.336963$

C $-4.753473-2.858345-0.014070$

C $-6.199849-2.3119430 .027443$

$\begin{array}{llll}\text { O } & -4.757012 & -4.235568 & 0.362948\end{array}$

C -1.0459372 .6852620 .963926$

O -0.8567813 .8269621 .352636$

C $-2.2523192 .216536 \quad 0.244821$

C $-3.175693 \quad 3.101697-0.159522$

C $-4.3928422 .794288-0.916624$

C $-4.7528131 .343831-1.096133$

C $-6.822068-2.3223071 .430202$

C -7.896714 -1.264495 1.569032

C $-8.199800 \quad 1.192767 \quad 1.868263$

O $-9.091706-1.5493161 .550884$

C -8.4777531 .7868590 .523714$

C -8.2682083 .0780170 .224220$

C $-8.6323673 .686055-1.099140$

C $-7.5167354 .503507-1.706386$

C -7.613069 $5.841078-1.800007$

C $-6.3376243 .765064-2.298962$

C $-5.1128293 .818545-1.420275$

N -7.372797 0.0123221 .711883

H 2.5928984 .1675181 .599860

H $2.1263461 .794671-0.286788$

H 0.9902752 .6033412 .496419

H 2.7073210 .6647002 .491961

H $1.634409-0.2053381 .428734$

H $-0.452130-1.2120674 .408714$

H $\quad 0.749608 \quad 0.320678 \quad 5.600594$

H 2.3442310 .6601414 .924071

H 1.0098021 .8111214 .692667

H $0.073795-1.5440851 .385168$

H $-0.324799-2.8172452 .526307$

H -2.542777 -1.683354 2.972943

H -2.152115 -0.4621921 .796108$

H -2.491883 -3.4519501 .268523$

H $-0.883289-2.462971-0.412912$

H $-1.987029-1.134620-0.686375$

H -2.576869 -3.021791 -3.489341

H -4.339548 -1.687393 -1.782936

H -1.206585 -4.608781 -1.628078

H -2.769085 -5.043378 -2.282513

H -2.542238 -4.960795 -0.525663

H $-4.596723-3.365812-3.238354$

H $-6.200762-1.286952-0.362331$

H $-6.846753-2.903176-0.632880$

H -5.409187 $-4.368121 \quad 1.068913$

H $-2.333622 \quad 1.1570810 .036490$

H -3.013403 4.1572660 .055487

H $-4.7578620 .826419-0.130308$

H $-4.0308160 .848802-1.754425$

H -5.745222 $1.191026-1.525109$

H $\quad-7.269679-3.299827 \quad 1.643590$
H $-6.075461-2.1432552 .212476$

H -7.661665 1.8843562 .524042

H -9.1426000 .9136342 .349946$

H $-8.9139621 .131908-0.227187$

H -7.8666013 .7435730 .984749$

H $-9.5162614 .317261-0.938159$

H -8.942490 $2.917739-1.818571$

H $-6.8318246 .430311-2.269177$

H -8.471604 $6.376517-1.408559$

H $-6.0722964 .232333-3.257410$

H $-6.6327362 .745714-2.561904$

H $-4.7550944 .833074-1.236719$

H $-6.3672350 .145237 \quad 1.653268$

C 5.0827364 .0209360 .098547

O 5.4597812 .6634470 .405835

C $6.6994852 .360299-0.253970$

C $7.3027203 .705735-0.634931$

C $6.0570964 .497938-0.980468$

H $6.4404071 .817465-1.170531$

C 7.5834191 .4729170 .627166

H 7.8238184 .1735390 .208178

H $8.0071303 .625477-1.468250$

C $6.2728866 .001172-0.993540$

H $5.7065634 .180355-1.971916$

C 6.8781610 .2248041 .197304

O 8.0466992 .2455691 .742201

H 8.4784561 .1846640 .062941

H 6.1875890 .5264851 .994216

O $7.876082-0.5782901 .841715$

C $6.111528-0.6303170 .171171$

C $5.609626-1.9580630 .771829$

H $5.257936-0.055180-0.202783$

O $6.947466-0.910749-0.951343$

H $6.466028-2.609790 \quad 0.983485$

H $5.096920-1.7418771 .717358$

H $5.3426136 .526053-1.232791$

H $6.6247876 .364625-0.022185$

H $7.0194626 .276112-1.745762$

C $3.6243584 .058101-0.361505$

H 8.6109641 .6662272 .282496

H $8.527203-0.8425291 .170003$

H $7.678689-1.474858-0.648228$

H $3.5062983 .452104-1.267673$

H $3.3359355 .082696-0.621879$

H 5.2077064 .6005991 .022180

C $2.416042-2.469139-1.089523$

O $3.430233-1.954996-0.216763$

C $4.650290-2.702248-0.172250$

C $4.373920-4.1356380 .305244$

C $3.316450-4.817320-0.567827$

C $2.078729-3.933171-0.757042$

H $1.528792-1.861736-0.869622$

C $2.776635-2.249326-2.562271$

H $5.095428-2.732850-1.172330$

O $3.941540-4.1319751 .664527$

H $5.307748-4.708657 \quad 0.266667$

H $3.025367-5.764712-0.097819$

O $3.896674-5.119696-1.833064$

H $1.425088-4.341176-1.536606$

O $1.328646-3.9350370 .460091$

C $2.904311-0.773599-2.860177$

H $3.706952-2.749696-2.845679$

H $1.996513-2.647916-3.221035$

H $3.156977-3.5604311 .725761$

H $3.221547-5.575639-2.363988$

H $1.053310-4.8512120 .634379$

O $4.206252-0.461927-3.118910$

O $1.9632590 .009413-2.845234$

C $4.4659150 .917545-3.392963$

H $3.9102061 .240723-4.278163$

H $4.2018391 .528489-2.525728$

H $5.5354321 .030391-3.588584$

SCF Energy (B3LYP/6-31G*//MMFF)= -3245.90838267

03 216

MM̄MF Geometry

C $2.241308-3.428796-1.800851$

C $1.704681-2.329077-1.251302$ 
C $0.631036-1.512646-1.926579$

O $-0.381364-1.168547-0.952708$

C $1.233320-0.203403-2.470044$

C $\quad 0.2673810 .607938-3.312645$

C $-0.189717 \quad 1.834779-2.994198$

C $-0.159928-0.028814-4.611229$

C $0.1309942 .663822-1.783233$

C $-0.8652612 .517162-0.623378$

C $-2.3076442 .920471-0.968248$

C -3.1562243 .0132290 .301479$

C $-4.6291353 .313106-0.011175$

O $-2.8567831 .923234-1.832626$

O $\quad-5.3685393 .073053 \quad 1.196437$

C $-5.1342522 .321408-1.083106$

C $-4.8396774 .792232-0.356835$

O $-6.4481662 .691086-1.493880$

C $-4.1864482 .197431-2.295871$

C $-4.5652181 .052956-3.268394$

O $-4.2269293 .416641-3.047214$

C -1.285278 -2.129345-0.620710

O $-1.344632-3.268110-1.055810$

C -2.195135 -1.552501 0.393065

C $-2.963409-2.3664691 .131821$

C -3.906495 -1.950668 2.172234

C $-4.222084-0.4819392 .276986$

C $-4.593750-0.350730-2.653311$

C $-5.968716-0.789547-2.178460$

C $-7.096406-2.632961-0.937410$

O $-6.998028-0.167645-2.426935$

C $-7.289208-2.222593 \quad 0.488437$

C $-7.470348-3.0997681 .487257$

C $-7.706835-2.7059072 .917170$

C $-6.745079-3.3769873 .872015$

C $-7.098082-4.4871184 .541937$

C $-5.408572-2.7123994 .112671$

C $-4.453341-2.8970822 .963865$

N $-5.914079-1.983179-1.471219$

H $1.899733-3.756113-2.779947$

H $2.060655-1.987992-0.282421$

H $\quad 0.167742-2.083018-2.740694$

H $2.111561-0.426580-3.091001$

H $1.6035440 .390951-1.628980$

H $-0.8759252 .317963-3.689408$

H $-0.7190950 .664163-5.249380$

H $0.715804-0.358797-5.179570$

H $-0.804327-0.893278-4.425472$

H $1.1384952 .442476-1.416741$

H $0.1670263 .716075-2.093732$

H $-0.8507151 .477901-0.276875$

H $-0.505606 \quad 3.1403870 .203865$

H -2.285862 $3.886106-1.486639$

H -2.749814 3.7659640 .988711

H -3.1032002 .0584640 .841937$

H -6.3121353 .1938620 .992561$

H $-5.2210451 .347830-0.586411$

H $-4.5356655 .426440 \quad 0.484656$

H $-5.8987675 .013410-0.529317$

H -4.274252 5.112340-1.234496

H $-6.7943231 .982874-2.062046$

H $-5.5021781 .288074-3.787179$

H $-3.798377 \quad 1.033704-4.056521$

H $-3.9158373 .233709-3.949303$

H -2.197419 $-0.475548 \quad 0.513782$

H -2.882894 -3.441605 0.976342

H -4.518724 -0.0839931 .300490$

H -3.3489120 .0748112 .633752$

H -5.049726 -0.2596512 .953627$

H -4.294373 -1.072431 -3.424327

H -3.877409 -0.434914 -1.830751

H -6.947945 -3.712531-1.041604

H -7.971694 -2.341690 -1.526818

H -7.306225 -1.157003 0.704966

H -7.480943 -4.1639431 .262027$

H $-8.738248-2.9828103 .172345$

H -7.652577 -1.618043 3.048455

H $-6.424958-4.9591985 .249787$

H -8.067201-4.951726 4.393031

H $-4.928827-3.1436475 .001689$
H $-5.573728-1.6622554 .368494$

H -4.164578 -3.936762 2.802739

H $-5.010728-2.401874-1.269463$

C $4.702195-3.811056-1.651059$

O $5.078666-2.604345-0.954265$

C $6.469452-2.692575-0.588037$

C $7.026799-3.890159-1.345715$

C $5.820591-4.810775-1.364007$

H $6.503130-2.9177020 .483756$

C $7.166255-1.358530-0.872455$

H $7.319860-3.622614-2.367786$

H $7.896716-4.329118-0.847887$

C $5.928482-5.929653-2.385243$

H $5.689900-5.244936-0.363664$

C $6.542806-0.162708-0.119592$

O $7.085671-1.083008-2.276918$

H $8.234275-1.434441-0.639796$

H $5.511119-0.010145-0.455276$

O $7.2676481 .018291-0.483957$

C $6.589645-0.2975191 .413881$

C 6.0547750 .9247832 .173715

H $6.034159-1.1829581 .732699$

O $7.943922-0.5073941 .826807$

H 6.2796870 .8084863 .243355

H 6.6282541 .8196761 .895059

H $5.030705-6.555449-2.375849$

H $6.054350-5.536675-3.399624$

H $6.788718-6.569910-2.164177$

C $3.323792-4.253160-1.161191$

H $6.143637-1.065191-2.518576$

H $7.2419591 .085909-1.453864$

H $8.4638300 .257726 \quad 1.526087$

H $3.262324-4.167271-0.069087$

H $3.137858-5.302755-1.415272$

H $4.677183-3.567705-2.721871$

C 2.9425082 .8900342 .701046

O 4.2856142 .3933102 .759295

C 4.5548811 .2125751 .990804

C 3.6466430 .0585932 .447829

C 2.1689470 .4593492 .456634

C 1.9421031 .8098883 .143373

H 2.9097783 .6978173 .443730

C 2.6115883 .4996071 .330568

H $4.372297 \quad 1.4362940 .935499$

O $4.020465-0.3760373 .754503$

H $3.780951-0.7950991 .774235$

H $1.593998-0.3187012 .973608$

O $1.7069160 .517922 \quad 1.112429$

H $\quad 0.9202882 .1663392 .968133$

O 2.0911831 .6424244 .556093

C 3.4503884 .7213461 .019812

H 2.7707292 .7844090 .519780

H 1.5632673 .8178641 .322041

H $3.9655020 .391178 \quad 4.349743$

H $\quad 0.7516080 .6944601 .140892$

H 1.4256630 .9956754 .846542

O $3.1022305 .189059-0.216381$

O 4.2964895 .2074221 .756920

C $3.8140666 .349092-0.658068$

H $3.446896 \quad 6.615024-1.652830$

H 3.6294397 .1877380 .020061

H $4.8848976 .133878-0.725122$

SCF Energy (B3LYP/6-31G*//MMFF)= -3245.92511146

03_217

MM̄MF Geometry

C -0.0929334 .8321190 .166836$

C $0.9764774 .459493-0.551538$

C 2.2390033 .9105040 .064095

O $2.4261232 .558081-0.415525$

C $3.4489594 .749562-0.384375$

C 4.7545004 .3331290 .265644

C $5.8164903 .834300-0.397005$

C 4.8371174 .5372511 .757176

C $5.9591143 .586159-1.872299$

C $5.5736942 .170171-2.328231$

C $6.4234521 .051641-1.702917$

C $6.150623-0.285807-2.395401$ 
C $6.913824-1.444385-1.733432$

O $6.084597 \quad 0.945871-0.317574$

O $6.355720-2.674784-2.220576$

C $6.665158-1.409029-0.209491$

C $8.394141-1.452677-2.132935$

O $7.485901-2.3609090 .462233$

C $6.863372-0.0118920 .412582$

C 6.4176020 .0666561 .893659

$\begin{array}{lllll}\text { O } & 8.255623 & 0.325609 & 0.377674\end{array}$

C 1.6931911 .5787730 .181890

O $0.909047 \quad 1.7056351 .108916$

C $1.9907520 .308061-0.514160$

C $1.159871-0.733795-0.362536$

C $1.304712-2.052016-0.983650$

C $2.595565-2.345342-1.700719$

C $4.946213-0.2714792 .157952$

C $4.707466-1.7149462 .570391$

C $2.856922-3.3151453 .046739$

O $5.612648-2.4951392 .852236$

C $2.636951-4.1809111 .846581$

C $1.489527-4.8362981 .613531$

C $1.267235-5.7465190 .439739$

C $0.037877-5.376670-0.359789$

C $-1.127868-6.018265-0.172188$

C $0.186069-4.322227-1.433538$

C $0.289137-2.932740-0.867280$

N $3.353384-2.0185132 .625841$

H $-0.0601514 .760174 \quad 1.251258$

H $0.9352014 .512233-1.636974$

H 2.1707423 .9037671 .158748

H $3.2756765 .807905-0.147425$

H $3.5288224 .692849-1.475286$

H 6.7028093 .5760810 .181966

H 5.8485804 .3710282 .143741

H 4.5542445 .5625592 .017048

H 4.1700323 .8454352 .280094

H $5.3771174 .315340-2.445917$

H $7.0040823 .778840-2.147329$

H $4.5149342 .000925-2.100593$

H $5.6787832 .133784-3.419680$

H $7.4807121 .323918-1.802617$

H $6.394438-0.228346-3.463624$

H $5.075725-0.506527-2.346835$

H $6.438272-2.678428-3.189559$

H $5.627174-1.724203-0.050967$

H $8.496477-1.553060-3.220226$

H $8.914890-2.315571-1.703313$

H $8.923850-0.545486-1.835180$

H $7.313199-3.2286410 .058535$

H $7.089968-0.5299362 .521953$

H $\quad 6.564374 \quad 1.1066832 .219454$

H 8.4229051 .0133661 .043267

H $2.8726780 .270586-1.142643$

H $0.276441-0.6075680 .262641$

H $3.449840-2.147392-1.044599$

H $2.688179-1.722730-2.596956$

H $2.694484-3.385452-2.016911$

H $4.594876 \quad 0.3448272 .995612$

H $4.324376-0.0294521 .291269$

H $1.933120-3.1422743 .607858$

H $3.586995-3.7869053 .712338$

H $3.467101-4.2963741 .153634$

H $0.671035-4.7409872 .323668$

H $1.166241-6.7691140 .826371$

H $2.140924-5.767195-0.223486$

H $-2.007261-5.779546-0.760991$

H -1.230008 -6.7942510 .579235$

H $-0.688402-4.335987-2.097734$

H $1.029555-4.587354-2.076441$

H - $0.601360-2.622132-0.320403$

H $2.674167-1.3244562 .327168$

C $-2.4453044 .220309-0.391358$

O -2.7961393 .9315120 .975064$

C -4.1972233 .5794861 .030655$

C $-4.7017833 .605815-0.407368$

C $-3.7623574 .601723-1.058911$

H -4.6793424 .3657271 .624068$

C -4.3273702 .2194791 .727342$
H $-4.5961172 .619834-0.877668$

H $-5.7538033 .898990-0.475020$

C $-3.7449484 .510729-2.574783$

H $-4.0581315 .615439-0.757345$

C -5.7522061 .6427651 .712220$

O

H -3.627115 1.5139231 .266482

H -6.0874001 .4956110 .681603$

O -6.6529552 .6057652 .274285$

C -5.9222460 .3253642 .493386$

C $-4.920022-0.7884422 .138467$

H -5.8514710 .5218143 .570228$

O $-7.247457-0.1673012 .272997$

H -3.918629-0.484625 2.464571

H -5.198627 -1.674122 2.724790

H $-3.053755 \quad 5.242910-3.003332$

H -3.435161 $3.516424-2.913680$

H $-4.7413904 .709654-2.982897$

C -1.380502 $5.315857-0.439112$

H -3.0117352 .7388143 .077501$

H $-6.3494262 .797943 \quad 3.178211$

H -7.8653310 .5486802 .499618$

H $-1.710305 \quad 6.1956230 .126567$

H -1.214289 $5.637032-1.473624$

H $-2.0519253 .290775-0.825617$

C $-6.271103-2.237784-1.042491$

O $-6.115496-1.8296330 .323048$

C $-4.880326-1.1846790 .647536$

C $-3.691708-2.1119640 .343958$

C $-3.731323-2.603034-1.105608$

C $-5.112075-3.146798-1.483801$

H -7.186460 -2.843443 -1.047424

C $-6.490224-1.039273-1.973385$

H $-4.777407-0.2789200 .043952$

O -3.716698 -3.229421 1.229313

H $-2.761547-1.5619410 .531111$

H -2.982572 -3.391677 -1.239675

O $-3.381427-1.519286-1.961166$

H $-5.169872-3.312866-2.565300$

O $-5.285330-4.428210-0.870602$

C $-7.820512-0.379113-1.698558$

H $-5.719740-0.269937-1.870154$

H $-6.480032-1.348569-3.025414$

H $-2.928487-3.7676771 .043390$

H -3.380261 -1.854760 -2.873757

H $-5.310679-4.2958840 .092721$

O $-8.827723-1.107069-2.260544$

O $-7.949287 \quad 0.654143-1.055149$

C -10.146355 -0.590546-2.055873

H -10.378898 $-0.558534-0.987148$

H $-10.2368760 .404638-2.501618$

H -10.854732 -1.261593 -2.549028

SCF Energy $\left(B 3 L Y P / 6-31 G^{* *} / / M M F F\right)=-3245.90898925$

03_218

MM̄FF Geometry

C -1.467230 $2.691010-1.431735$

C $-1.7918561 .438476-1.076105$

C $-0.9632710 .497766-0.231448$

O $0.4217850 .904168-0.181840$

C -1.5186310 .4889251 .204793$

C $-0.883750-0.5498362 .107033$

C $-0.144833-0.2705223 .198332$

C $-1.150616-1.9866321 .738999$

C 0.2240101 .0754513 .754944

C 1.5736741 .6241733 .264433

C 2.7801760 .7335003 .605754

C 4.0934781 .4597783 .305440

C 5.3176730 .5603013 .543138

O $2.703885-0.4533002 .811481$

O 6.4536281 .1919192 .932126

C $5.106655-0.7868682 .816968$

C 5.6459060 .4300335 .035321

O $6.138813-1.7114413 .149291$

C $3.726363-1.4198163 .086949$

C $3.435142-2.6595592 .205642$

O $3.667645-1.8553074 .450910$

C $1.1985470 .580216-1.252445$ 
O $0.856085-0.028063-2.253979$

C $2.5509241 .126793-1.008566$

C $3.3996741 .261776-2.038366$

C $4.7647061 .787319-1.970050$

C $5.3584602 .002538-0.603314$

C $3.453814-2.4131980 .692885$

C $4.784871-2.731754 \quad 0.032357$

C $5.961602-2.539319-2.155300$

O $5.698382-3.320096 \quad 0.604275$

C $6.861749-1.344797-2.113249$

C $7.319390-0.730610-3.214837$

C $8.2556440 .443680-3.193082$

C $7.7377841 .615487-3.996850$

C $8.1520451 .825330-5.257756$

C $6.805516 \quad 2.587809-3.309672$

C $5.4209052 .030104-3.124120$

N $4.817505-2.314425-1.291750$

H $-0.5260873 .123650-1.104111$

H $-2.7556511 .048443-1.394899$

H -1.026812 $-0.505913-0.666361$

H -2.599433 0.2968341 .185978

H -1.3997351 .4915731 .629601$

H $0.248803-1.1081303 .773579$

H $-0.645365-2.243754 \quad 0.803815$

H $-0.795305-2.6851802 .504300$

H $-2.225192-2.1562051 .618740$

H -0.5577851 .8126703 .543877$

H $0.2481670 .992314 \quad 4.849225$

H 1.5202321 .7731582 .179720

H 1.7153522 .6125243 .719095

H 2.7247100 .4689084 .668199

H 4.1739172 .3798063 .897645

H 4.0978531 .7805612 .255143

H 6.5549412 .0744253 .328194

H $5.200651-0.5860261 .743328$

H 5.8566681 .4154035 .468190

H $6.553153-0.1641215 .190255$

H $4.836123-0.0172575 .615345$

H $6.987904-1.2850462 .941957$

H $4.095720-3.4874762 .489756$

H $2.419957-3.0011182 .455234$

H $2.969747-2.5265704 .530609$

H $2.804068 \quad 1.416548 \quad 0.004103$

H $3.0594730 .977054-3.033348$

H $4.8451802 .819507-0.085079$

H $6.4223972 .246511-0.621319$

H $5.269347 \quad 1.092212-0.001142$

H $2.716487-3.0779270 .224451$

H $3.162623-1.3854400 .457685$

H $5.576056-2.732532-3.161259$

H $6.507646-3.424471-1.813673$

H $7.171544-0.984943-1.134889$

H $7.030284-1.111386-4.192055$

H $9.2186760 .109239-3.600910$

H $8.4671820 .769860-2.167174$

H $7.8071052 .678605-5.832355$

H $8.8421251 .144800-5.745499$

H $6.7057503 .502292-3.909880$

H $7.2638062 .917732-2.373379$

H $4.9033141 .834912-4.064598$

H $4.034259-1.788444-1.668476$

C $-3.4736454 .269669-1.462824$

O $-4.4464743 .295046-1.030922$

C $-4.732882 \quad 3.5219550 .359775$

C $-4.2881524 .953040 \quad 0.639072$

C -3.036237 $5.039285-0.213489$

H $-4.091277 \quad 2.8383420 .929166$

C $-6.201927 \quad 3.2244180 .673726$

H $-5.028628 \quad 5.6848220 .296106$

H -4.0981855 .1306911 .701705$

C $-2.5817426 .459879-0.498426$

H $-2.231308 \quad 4.5131850 .313824$

C $-6.691007 \quad 1.8319690 .221411$

$\begin{array}{lllll}\text { O } & -7.012583 & 4.204777 & 0.013797\end{array}$

H $-6.3718583 .356558 \quad 1.749296$

H $-6.823348 \quad 1.811011-0.867577$

O

$\begin{array}{llll}\text { C } & -5.789923 & 0.653814 & 0.639728\end{array}$
C $-6.441507-0.7174330 .372053$

H -4.8431710 .7186360 .095198$

\begin{tabular}{llll}
\hline & -5.484979 & 0.751496 & 2.031364
\end{tabular}

H -7.293611 -0.8426131 .050538$

H -6.832372 $-0.730947-0.650293$

H -1.689354 $6.461579-1.132295$

H -3.360236 $7.036254-1.009291$

H -2.336765 $6.979648 \quad 0.433578$

C -2.361471 $3.575719-2.256685$

H $-7.911048 \quad 4.137745 \quad 0.378026$

H -8.603332 $2.243003 \quad 0.355407$

H -6.3268330 .7412682 .518522$

H $-1.7350844 .330348-2.747304$

H $-2.8113672 .974224-3.057149$

H $-4.0117644 .938808-2.146655$

C $-4.663500-2.179413-1.675392$

O $-4.355723-1.849608-0.316407$

C $-5.460945-1.8924490 .592823$

C $-6.119344-3.2786660 .559078$

C $-6.529347-3.675529-0.855143$

C $-5.339452-3.552831-1.805227$

H $-5.340824-1.414654-2.075397$

C $-3.372401-2.055794-2.501408$

H $-5.034700-1.7685431 .596223$

O $-7.261795-3.3234661 .407442$

H $-5.410064-4.0269920 .936614$

H -7.381175 $-3.083129-1.209463$

O $-6.961175-5.038662-0.852471$

H $-4.640393-4.368650-1.590531$

O $-5.815453-3.741465-3.136885$

C $-2.224945-2.926985-2.029254$

H $-3.569400-2.282152-3.554281$

H $-3.021053-1.020732-2.434740$

H $-6.969678-3.0825922 .303212$

H $-7.691925-5.108630-0.214460$

H $-5.043385-3.877408-3.711189$

O $-1.137039-2.683033-2.820573$

O $-2.260143-3.706174-1.087779$

C $0.050374-3.403777-2.475324$

H $-0.124289-4.481286-2.549644$

H $0.835057-3.125315-3.183869$

H $\quad 0.377332-3.132735-1.466905$

SCF Energy (B3LYP/6-31G**//MMFF) $=-3245.89806520$

$03 \_219$

MM̄FF Geometry

C $-2.696515-3.6624410 .739465$

C $-1.947408-2.879337-0.051067$

C $-0.862867-1.960643 \quad 0.449754$

O $0.278810-2.196296-0.406636$

C $-1.327327-0.498282 \quad 0.307810$

C $-0.507305 \quad 0.5162191 .081587$

C $0.177706 \quad 1.539913 \quad 0.534436$

C -0.5319810 .3695042 .583336$

C $0.3118051 .916709-0.913856$

C $1.6461161 .504094-1.554081$

C $2.8787932 .241282-1.001567$

C $4.1186741 .908006-1.837235$

C $5.3957912 .542365-1.263978$

$\begin{array}{llll}\text { O } & 3.093812 & 1.837205 & 0.353620\end{array}$

O $6.5227531 .908562-1.889673$

C $5.4838492 .226805 \quad 0.245170$

C $5.4928674 .033364-1.608225$

$\begin{array}{llll}\text { O } & 6.565902 & 2.929590 & 0.849578\end{array}$

C 4.1755002 .5165121 .008498

C 4.2045082 .0414492 .483196

O 3.9513403 .9320831 .037404

C $1.524325-2.1115910 .126839$

O $1.823115-1.8206901 .273037$

C $2.483951-2.437898-0.952976$

C $3.775391-2.106696-0.811678$

C $4.842134-2.343561-1.785672$

C $4.523198-3.204868-2.978803$

C 4.4547240 .5452092 .692589

C 5.9199130 .1875122 .883063

C $7.479644-1.7548632 .754813$

O $6.7711941 .001963 \quad 3.229791$

C $8.176588-1.5943461 .440842$ 
C $8.474380-2.6146720 .622218$

C $9.165771-2.444708-0.700316$

C $8.304469-2.874240-1.868237$

C $8.489479-4.065838-2.460796$

C $7.291508-1.887459-2.406425$

C $6.054727-1.798462-1.555835$

N $6.157543-1.1623112 .663221$

H -2.532834 -3.655926 1.813950

H $-2.129423-2.882870-1.123931$

H $-0.618206-2.2152541 .487314$

H $-2.362148-0.406870 \quad 0.663944$

H $-1.352314-0.246892-0.757136$

H $\quad 0.6965002 .216383 \quad 1.212854$

H -0.0518051 .2126823 .091863$

H -1.562088 0.3187652 .945814

H -0.009312 -0.5382162 .897000$

H $-0.5020981 .491117-1.507667$

H $0.1893513 .003986-0.996931$

H $1.7761220 .423459-1.431575$

H $\quad 1.5714101 .699204-2.631308$

H $2.6767283 .318324-1.033571$

H $3.9767592 .211425-2.882053$

H $4.2560350 .819193-1.860957$

H $6.4372852 .026271-2.851334$

H 5.7157181 .1582430 .331100

H $5.5106224 .174458-2.695767$

H $6.4284114 .465195-1.236203$

H $4.6599584 .619683-1.214857$

H 7.3718572 .7005840 .355868

H 4.9098212 .6518823 .060150

H 3.2152262 .2575722 .911834

H 3.3418544 .1330041 .766767

H $2.096241-2.919915-1.843265$

H $4.082276-1.5944000 .097902$

H $3.804851-2.701061-3.634590$

H $4.096868-4.162947-2.660488$

H $5.399334-3.448531-3.583893$

H 3.9485540 .2320643 .614672

H $4.025068-0.0381501 .873547$

H 7.349069 -2.806259 3.029490

H $8.052194-1.2564653 .543580$

H $8.447812-0.5821391 .148980$

H $8.209000-3.6281430 .913949$

H $10.089356-3.037482-0.672518$

H $9.489842-1.407401-0.852098$

H $7.897218-4.369008-3.317723$

H $9.234271-4.766888-2.098803$

H $7.040668-2.121375-3.446954$

H $7.750145-0.891438-2.456835$

H $\quad 6.179952-1.183671-0.665127$

H $5.404223-1.7475322 .314113$

C -5.173420 -4.051362 0.580314

O $-5.427785-2.825545-0.135858$

C $-6.681522-2.928800-0.828373$

C $-7.423249-4.060086-0.126729$

C $-6.287007-5.017158 \quad 0.172276$

H -6.460448 -3.229484 -1.859724

C $-7.438758-1.596231-0.823549$

H -7.887961 $-3.717748 \quad 0.805793$

H -8.202564 -4.501131 -0.755382

C $-6.623125-6.0505461 .233354$

H $-6.013745-5.536896-0.755993$

C $-6.842414-0.441776-1.659391$

O $-7.608675-1.1616210 .525947$

H -8.440581 -1.801519 -1.223862

H $-7.5463150 .394683-1.558176$

O $-6.838966-0.797002-3.040434$

C $-5.4553980 .085158-1.240991$

C $-5.1710741 .470400-1.855330$

H $-5.3993910 .153009-0.149932$

O $-4.425070-0.789536-1.693555$

H -5.140987 $1.383963-2.948606$

H -5.996666 2.146390 -1.608110

H $-5.765716-6.7028201 .427102$

H $-6.906944-5.5769712 .179207$

H -7.459221 -6.6780380 .907773$

C -3.778665 -4.568906 0.220827

H $-8.158428-0.3597840 .511963$
H $-6.096811-1.408263-3.185786$

H $-4.462590-1.595593-1.154717$

H -3.690804 -4.665929 -0.868840

H $-3.621824-5.5661460 .647593$

H $-5.234563-3.8139651 .649982$

C -4.5949712 .9784420 .725085$

$\begin{array}{llll}\text { O } & -3.720398 & 2.063769 & 0.054921\end{array}$

C $-3.8294962 .067621-1.371809$

C $-3.5181713 .468818-1.914408$

C $-4.3609124 .546679-1.235922$

C -4.2892294 .4135380 .285884$

H -5.6386182 .7326540 .493139$

C -4.3897802 .7903802 .231080$

H -3.040394 $1.401053-1.740260$

O $-3.7254313 .529442-3.321231$

H $-2.4596153 .697989-1.735226$

H -5.401086 $4.518167-1.581663$

O $-3.8542775 .834051-1.595733$

H $-3.292871 \quad 4.7130110 .635304$

O $-5.2374365 .298844 \quad 0.877286$

C $-5.069751 \quad 1.5242492 .698281$

H -3.325602 2.7418072 .491266

H -4.8238073 .6138522 .809449$

H -3.165959 $2.845922-3.728347$

H -3.872273 $5.890238-2.566541$

H $-5.0346856 .195400 \quad 0.559129$

$\begin{array}{lllll}\text { O } & -4.196150 & 0.480192 & 2.655739\end{array}$

O $-6.246378 \quad 1.467903 \quad 3.032244$

C $-4.722317-0.7875133 .058731$

H -5.084229 -0.738773 4.089983

H $-5.523294-1.0960502 .382056$

H -3.914983 -1.522490 3.004979

SCF Energy $\left(B 3 L Y P / 6-31 G^{* *} / / M M F F\right)=-3245.92153512$

0322

MM̄̄FF Geometry

C $-2.576140-2.680891-1.948613$

C $-1.684210-3.143193-2.838239$

C $-0.216195-2.783531-2.917349$

O $0.144754-1.746247-1.977086$

C $0.638814-4.028578-2.615631$

C $2.134681-3.804112-2.742194$

C $3.021902-4.042466-1.755857$

C $2.621073-3.311168-4.080317$

C $2.735474-4.584958-0.383862$

C $2.379435-3.5125720 .653181$

C $3.565037-2.6371191 .088355$

C $3.139926-1.7067792 .227928$

C $4.272573-0.7624752 .652299$

O $4.003250-1.862461-0.029514$

O 3.7045380 .2585493 .487301

C $4.829676-0.0614831 .396906$

C $5.329649-1.4790683 .499979$

$\begin{array}{lllll}\text { O } & 5.988018 & 0.707481 & 1.715582\end{array}$

C $5.141226-1.0318330 .231769$

C $5.479060-0.263492-1.070004$

O $6.287205-1.8167700 .579536$

C $-0.103254-0.460840-2.353302$

O $-0.636522-0.089959-3.387422$

C $0.3827300 .433267-1.278415$

C $0.0198491 .724503-1.297952$

C $0.4334322 .771773-0.361504$

C 1.4227152 .4141190 .714939

C $4.2699650 .399714-1.739812$

C $4.6123411 .695292-2.454449$

C $3.4900163 .700267-3.429885$

O $5.7613122 .091489-2.626639$

C $3.2356864 .672654-2.319261$

C $2.2233855 .553208-2.313807$

C $1.9709266 .522285-1.193691$

C $0.5651146 .429713-0.639601$

C $-0.3378717 .394885-0.882213$

C 0.2290535 .2586250 .262043

C $-0.0805264 .009790-0.521694$

N $3.4673362 .358874-2.877271$

H $-2.261210-1.995265-1.167782$

H $-2.032211-3.829637-3.608135$

H $-0.009276-2.436434-3.937426$ 
H $\quad 0.361723-4.841895-3.299574$

H $\quad 0.388098-4.377269-1.608367$

H $4.075297-3.846403-1.951496$

H $2.302690-2.278154-4.250634$

H $3.713516-3.334209-4.158782$

H $2.223685-3.939386-4.884066$

H $1.923062-5.318974-0.433080$

H $3.610684-5.152464-0.043580$

H $1.581408-2.8768700 .250954$

H $1.974989-4.0222221 .536532$

H $\quad 4.378727-3.295574 \quad 1.414269$

H $2.779281-2.2830843 .089122$

H $2.288100-1.0948951 .898608$

H $3.282230-0.1768134 .247607$

H $4.0697920 .657376 \quad 1.070751$

H $4.874178-1.8989554 .404839$

H $6.097071-0.7793513 .848977$

H $5.823197-2.2962132 .969951$

H 5.7460501 .3098232 .439732

H $6.2813670 .454540-0.862529$

H $5.908558-0.962564-1.801189$

H $6.679825-2.168758-0.236290$

H $\quad \begin{array}{lll}1.028766 & 0.009413 & -0.518837\end{array}$

H -0.644833 $2.059130-2.094258$

H 2.4167662 .2741830 .281323

H 1.1338181 .4955401 .235421

H 1.5079443 .1762881 .492727

H $3.831421-0.290027-2.471186$

H $3.4954200 .627778-1.003565$

H $2.7206163 .747045-4.206627$

H $4.4652653 .899500-3.884755$

H $3.9240164 .650647-1.477224$

H $1.5452835 .590407-3.163317$

H $2.1587207 .532144-1.580813$

H $2.6866836 .380103-0.374213$

H $-1.339427 \quad 7.348476-0.467537$

H $-0.0982088 .250227-1.505251$

H -0.6542615 .4948460 .869596$

H 1.0503425 .1260520 .971573

H $-0.8156494 .162986-1.313428$

H $2.559724 \quad 1.965174-2.649073$

C $-4.960096-1.849746-2.206255$

O $-4.843155-0.898749-1.129291$

C $-4.7877380 .425799-1.692545$

C $-5.2813050 .289172-3.128556$

C $-4.720969-1.069968-3.501571$

H $-3.7287480 .712070-1.713473$

C $-5.5697831 .419522-0.827707$

H $-6.3757430 .274543-3.185200$

H -4.917768 $1.096096-3.771805$

C $-5.375637-1.691221-4.722508$

H -3.648549 -0.951477 -3.698106

C -5.1920871 .4011960 .667209$

O $-6.9657691 .106644-0.920124$

H -5.453929 2.429334 -1.239808

H $-5.573307 \quad 0.4812951 .128209$

O

C -3.6848591 .5414600 .947658$

C -3.3593871 .6170962 .450457$

H -3.1644120 .6834920 .511425$

$\begin{array}{lllll}\text { O } & -3.181092 & 2.709928 & 0.303052\end{array}$

H -3.725671 2.5671272 .857921

H $-3.894467 \quad 0.8138802 .967930$

H -4.945180 -2.674939 -4.935519

H -6.454055 -1.818088 -4.579742

H $-5.225722-1.058169-5.603176$

C -4.034698 -3.045995-1.959535

H $-7.444397 \quad 1.755547-0.376257$

H -5.8830522 .3430982 .242406$

H -3.673106 3.4736410 .650070

H -4.234149 -3.819696 -2.710188

H $-4.267542-3.481172-0.979722$

H -5.996585 -2.210324 -2.173726

C -1.640268 -0.8834352 .681036$

$\begin{array}{lllll}\text { O } & -1.242467 & 0.376549 & 2.124023\end{array}$

C -1.8433501 .5221652 .736263$

C -1.478685 1.5599214 .225913

C -1.8331050 .2525284 .932080$
C $-1.267756-0.9468514 .169689$

H $-2.723145-0.9980602 .560293$

C $-0.939885-1.9743291 .855708$

H -1.3807882 .3992142 .267444$

O -2.1326472 .6394094 .884239$

H -0.3984411 .7261024 .330027$

H $-2.915990 \quad 0.1525365 .071562$

O $-1.2602270 .260957 \quad 6.242396$

H $-0.177110-0.9592614 .295076$

O $-1.783190-2.1345184 .764524$

C $-1.732765-3.2573311 .728306$

H $-0.768003-1.6060060 .837861$

H $\quad 0.036419-2.2103242 .292035$

H -1.874671 3.4587244 .428036

H -1.6064161 .0442146 .703705$

H $-1.304868-2.8892774 .382754$

O $-1.003505-4.1510280 .995822$

O $-2.843858-3.4566232 .198148$

C -1.622566 -5.421990 0.776651

H $-2.567833-5.2974850 .240176$

H $-0.948736-6.0263810 .163488$

H $-1.783328-5.9327931 .730628$

SCF Energy (B3LYP/6-31G**//MMFF) $=-3245.90909472$

03_220

MM̄FF Geometry

C -1.970600 $5.092698-0.923019$

C $-2.9754054 .460563-0.298418$

C $-3.5975183 .195025-0.834106$

O -3.3166982 .1230250 .099310$

C $-5.1247033 .347668-0.945063$

C $-5.7871282 .214827-1.707079$

C $-6.6390521 .318890-1.171850$

C $-5.4666732 .143443-3.179522$

C $-7.126555 \quad 1.2256750 .245244$

C -6.2961180 .3141651 .161786$

C $-6.145870-1.1326000 .661432$

C $-5.565152-2.0233701 .763051$

C $-5.293097-3.4534031 .270359$

O $-5.263472-1.129805-0.465378$

O $-4.460087-4.1040522 .242686$

C -4.485065 -3.389304-0.044114

C $-6.581853-4.2797431 .183264$

O $-4.349090-4.685336-0.620687$

C $-5.077210-2.412335-1.079903$

C $-4.166240-2.207701-2.316640$

O $-6.321172-2.935086-1.562375$

C -2.0593931 .6005330 .078939$

O $-1.1454551 .910512-0.668876$

C -1.9689380 .5780391 .142472$

C -0.7579170 .1956371 .573454$

C $-0.478102-0.8362562 .572967$

C $-1.627897-1.7047463 .009808$

C $-2.771905-1.643381-2.024026$

C $-1.689316-2.699598-1.874014$

C $0.701663-2.948533-1.211224$

O $-1.853319-3.880369-2.168329$

C $0.734977-3.4689340 .191590$

C $1.812274-3.3756130 .985870$

C $1.875728-3.9271942 .382022$

C $2.352092-2.9069403 .392929$

C $3.634937-2.8801353 .791490$

C $1.326118-1.9766764 .003148$

C $0.789673-0.9809693 .012539$

N $-0.498694-2.156438-1.406994$

H $-1.6089364 .708328-1.874098$

H -3.317422 4.8193500 .669096

H -3.172161 $2.938102-1.811651$

H $-5.3714424 .285587-1.460302$

H $-5.546258 \quad 3.433757 \quad 0.062301$

H $-7.0503090 .552780-1.828983$

H $-6.0967691 .419009-3.706851$

H $-5.6283743 .117886-3.651877$

H $-4.4258671 .845394-3.336766$

H -7.194966 2.2202730 .698870

H -8.1592960 .8543090 .218797$

H $-5.304770 \quad 0.7614651 .294862$

H $\quad-6.7800080 .3085772 .146503$ 
H -7.132246 -1.499727 0.354588 H -6.226574 -2.039979 2.638319 H -4.618507 -1.592941 2.115157 H -4.924278 -4.083128 3.097110 H -3.474209-3.056251 0.220868 H $-7.058580-4.3498642 .168548$ H $-6.370639-5.3109850 .879865$ H $-7.315714-3.8590230 .492839$ H -3.951148 -5.2620960 .053662$ H -4.113564 -3.132759-2.903363 H $-4.668607-1.478891-2.969176$ H $-6.519379-2.519089-2.417637$ H -2.8918430 .1649751 .529875$ H $\quad 0.119407 \quad 0.683914 \quad 1.152038$ H -2.112946 -2.160343 2.139716 H -2.369889 -1.114229 3.557913 H -1.333326 -2.531185 3.658946 H -2.467082 -1.015762 -2.871447 H -2.788524 -1.007440 -1.135849 H $1.556515-2.302666-1.433388$ H $\quad 0.701070-3.786453-1.915679$ H $-0.157428-3.9677660 .562606$ H $2.712226-2.9028760 .601216$ H $2.561075-4.7849452 .369095$ H $0.905368-4.3283732 .699962$ H $3.981633-2.1726434 .537322$ H $4.369726-3.5619463 .375965$ H $1.774458-1.4048614 .826593$ H $0.542894-2.5795374 .470038$ H $1.550826-0.2993842 .630811$ H $-0.467700-1.175822-1.143781$ C $0.025686 \quad 5.783414 \quad 0.385599$ O $0.8980805 .081195-0.516911$ C $2.2481305 .187071-0.026088$ C $2.199506 \quad 6.1395181 .171338$ C $0.899764 \quad 6.8940170 .951895$ H $2.8423015 .639697-0.828970$ C 2.8136913 .7950600 .311619 H 2.1746925 .5928202 .121389 H 3.0722706 .7997671 .202055 C 0.3547797 .5396022 .213179 H 1.0673507 .6658830 .189031 C $2.9409122 .871488-0.923653$ O $\quad 1.9754323 .1996241 .302559$ H 3.8070373 .9244470 .758756 H $3.5615153 .384042-1.668416$ O $1.6651372 .664803-1.526513$ C $3.5834071 .501862-0.617523$ C $3.7518490 .648353-1.889826$ H $4.5564961 .674037-0.143069$ O 2.7616820 .7931010 .306702 H $2.765408 \quad 0.412857-2.308017$ H $4.3037751 .242242-2.628355$ H -0.5784238 .0730882 .007658$ H $\quad 0.152921 \quad 6.7946282 .990179$ H 1.0727698 .2610132 .617013 C -1.224315 $6.264554-0.351691$ H 2.3853382 .3609601 .572775 H $1.0892262 .257840-0.856402$ H 3.2921220 .0737930 .688055 H $-0.9511376 .928355-1.180578$ H -1.869795 6.8362640 .324535 H -0.2596525 .0777091 .178431$

C $6.636017-1.474211-0.819682$

O $5.814715-0.356296-1.181538$

C $4.495191-0.676674-1.633172$

C $4.559364-1.557575-2.889708$

C $5.398316-2.813409-2.632084$

C $6.745414-2.476035-1.981836$

H $7.632577-1.044584-0.653330$

C $6.179058-2.1133230 .496099$

H $3.965827-1.224102-0.848139$

O $5.126075-0.813582-3.965782$

H $3.539444-1.837182-3.179736$

H $5.571433-3.331784-3.583025$

O $4.655133-3.687573-1.787353$

H $7.235109-3.390721-1.629548$

O $7.607353-1.915640-2.977358$
C $6.355405-1.1460651 .642668$

H $5.138584-2.4472810 .478275$

H $\quad 6.780757-3.000350 \quad 0.727301$

H $5.120098-1.385405-4.752381$

H $5.186842-4.490913-1.655834$

H $7.226543-1.065161-3.255962$

O $5.188299-0.4818621 .877213$

O $7.412682-0.9811372 .237335$

C 5.2312850 .4958202 .921131

H 5.8934221 .3183392 .635380

H 4.2207800 .8895443 .059575

H 5.5611730 .0411183 .860117

SCF Energy (B3LYP/6-31G**//MMFF)= -3245.91517326

03_221

MM̄MF Geometry

C -3.3192562 .1217092 .285298$

C -4.4824512 .6110091 .827182$

C -4.6429233 .8129620 .924187$

O -3.3503634 .3516510 .573356$

C $-5.4616633 .494598-0.344461$

C $-4.9247412 .380177-1.224983$

C $-5.4883951 .163818-1.368788$

C $-3.7016642 .724764-2.038135$

C $-6.7101000 .604395-0.693364$

C $-6.441315-0.6074060 .216986$

C $-6.015662-1.882847-0.532153$

C $-6.090942-3.1001990 .395552$

C $-5.534691-4.376724-0.258938$

O $-4.673218-1.705679-0.990328$

O $-5.346603-5.3578590 .772889$

C $-4.157124-4.070578-0.892564$

C $-6.557711-4.996031-1.217614$

O $-3.674282-5.187196-1.634817$

C $-4.160185-2.798067-1.762784$

C $-2.753022-2.398968-2.255427$

O $-4.957884-3.028569-2.930212$

C -3.2526335 .6571220 .220285$

$\begin{array}{lllll}\text { O } & -4.168553 & 6.463647 & 0.142230\end{array}$

C -1.851523 $6.018859-0.107441$

C -0.8029565 .2394760 .201389$

C $0.6072635 .517427-0.077207$

C $0.9384926 .726815-0.909308$

C $-1.723726-2.140380-1.153486$

C $-0.588839-1.293075-1.693350$

C $-0.0369921 .071298-2.286396$

O $0.447244-1.794458-2.121863$

C $0.9881051 .528626-1.298192$

C $2.2309491 .882247-1.659740$

C $3.2999412 .346224-0.711960$

C $3.5672443 .831041-0.822101$

C $4.3152104 .326037-1.822763$

C 3.0291824 .7327770 .266483

C 1.5339204 .6627440 .403586

N $-0.8898680 .060142-1.689888$

H -2.3848692 .6023892 .009378$

H -5.4075362 .1280652 .135664$

H -5.1841774 .5720941 .504348$

H -5.528969 4.396589-0.966769

H $-6.4934543 .288270-0.042462$

H $-5.0275440 .486858-2.085999$

H -3.480000 $1.967084-2.797678$

H -3.844182 $3.675041-2.562778$

H $-2.8217972 .806399-1.394169$

H $-7.2114481 .363986-0.087579$

H $-7.4303190 .319779-1.470478$

H $-5.675349-0.3376860 .955263$

H -7.367664 -0.8130660 .768227$

H $-6.681110-2.022901-1.392186$

H $-7.120907-3.2637560 .736855$

H $-5.513809-2.8930781 .304338$

H $-4.717560-5.0002261 .422328$

H -3.450850 -3.932042 -0.064944

H $-7.476256-5.256964-0.677616$

H $-6.186901-5.935888-1.641298$

H $-6.836200-4.330889-2.037539$

H $-4.159315-5.215819-2.477011$

H $-2.364599-3.159912-2.945133$ 
H $-2.856625-1.490833-2.866180$ H -4.707263 -2.379802 -3.608664 H -1.727672 $6.980108-0.595399$ H $-0.9829134 .300763 \quad 0.722221$ H $0.6798667 .645447-0.371804$ H $0.3873416 .706661-1.856315$ H $1.9968396 .791586-1.173729$ H -2.171878 -1.624221 -0.299334 H -1.307365 -3.080525 -0.775478 H $-0.6823851 .909394-2.565501$ H $0.4224890 .657961-3.190428$ H $0.6953161 .592571-0.254050$ H $2.5077121 .830366-2.710877$ H $4.2199471 .793154-0.936577$ H 3.0539902 .0825130 .322913 H $4.5323855 .386402-1.896709$ H $4.7256853 .679129-2.591320$ H 3.3614515 .7671600 .125102 H $3.472814 \quad 4.4262201 .223059$ H $\quad \begin{array}{llll}1.194817 & 3.808213 & 0.990825\end{array}$ H $-1.7633510 .370806-1.274229$ C -2.306809 -0.1509692 .605386$ O $-\begin{array}{llll}0.950159 & 0.331050 & 2.504028\end{array}$ C $-0.052556-0.6787613 .012813$ C $-0.872591-1.9543933 .089260$ C $-2.235066-1.4115953 .467072$ H $0.232786-0.3448134 .018579$ C $1.181100-0.7569542 .106980$ H $-0.913854-2.4507052 .111487$ H $-0.478885-2.667323 \quad 3.819863$ C $-3.359695-2.398593 \quad 3.218012$ H -2.224362 -1.139252 4.530913 C $2.132037-1.9169992 .446774$ O 1.9013190 .4724032 .241190 H $0.860368-0.8243521 .062177$ H $1.626600-2.8756802 .283434$ O $2.441331-1.8666053 .846320$ C $3.466953-1.9151151 .676520$ C $3.296724-1.9480930 .148728$ H $4.076591-1.0550101 .976148$ O $4.193658-3.0885572 .061402$ H $2.740286-2.850698-0.132111$ H $2.715951-1.074542-0.164287$ H $-4.332282-1.9537483 .449700$ H -3.372511 -2.723853 2.173351 H $-3.236769-3.2891513 .842818$ C -3.202111 0.9365513 .203661 H 1.2803831 .1959182 .049059 H $2.858843-1.0058684 .022828$ H $4.294734-3.0619793 .028371$ H $-2.772146 \quad 1.2921194 .148177$ H -4.1935490 .5252623 .425263$ H $-2.637519-0.3933861 .588333$ C $6.568292-0.495692-0.920208$

O $5.271614-0.683071-0.339688$ C $4.645865-1.943826-0.598501$

C $4.469458-2.156271-2.110288$ C $5.797550-1.991512-2.855566$ C $6.528266-0.709097-2.443230$ H $6.8037070 .560233-0.734339$ C $7.634687-1.339888-0.214245$ H $5.272279-2.746968-0.197331$ O $3.514851-1.237723-2.636369$ H $4.071969-3.163483-2.281111$ H $5.599503-1.969423-3.934315$ O $6.615035-3.125887-2.583854$ H $7.547490-0.707984-2.846761$ O $5.841891 \quad 0.395812-3.039980$ C $7.833934-0.8563071 .202495$ H $7.399218-2.407532-0.188695$ H $8.601841-1.250033-0.723236$ H $3.840146-0.339178-2.458104$ H $7.438729-3.021723-3.090056$ H $6.3386421 .203222-2.823438$ O $7.067565-1.5898312 .058293$ O 8.5609850 .0811451 .505375 C $7.132003-1.1996053 .433238$ H $6.774632-0.1722823 .551343$
H $\quad 6.480660-1.8649884 .006232$

H $8.154764-1.2997773 .808710$

SCF Energy (B3LYP/6-31G $\left.{ }^{\star *} / / M M F F\right)=-3245.90898575$

03222

MMFF Geometry

C -2.183643 -3.817740 -0.878410

C $-0.953952-3.759495-0.345915$

C $0.263576-3.298779-1.106722$

O $0.887534-2.223090-0.367850$

C $1.270723-4.457494-1.226413$

C $2.547343-4.102745-1.966758$

C $3.785117-4.274439-1.461515$

C $2.379090-3.556064-3.360557$

C $4.150962-4.858630-0.125892$

C $4.219426-3.8332181 .013640$

C $5.421595-2.8796770 .922945$

C $5.507851-2.0176612 .185507$

C $6.654642-0.9997562 .108786$

O $5.262980-2.042456-0.224824$

O $6.470104-0.0467453 .166998$

C $6.542861-0.2254470 .779521$

C 8.019786-1.654949 2.348577

O $7.673308 \quad 0.6233860 .589281$

C $6.351588-1.139705-0.454800$

C $6.034955-0.320043-1.729363$

O $7.572309-1.849434-0.695412$

C $0.413127-0.967683-0.596544$

O $-0.498934-0.655428-1.346192$

C $1.200834-0.022108 \quad 0.227868$

C $0.855678 \quad 1.2743310 .230145$

C 1.5156982 .3620920 .953925

C 2.7134362 .0266861 .800357

C $4.6246630 .276459-1.766319$

C $4.5157121 .476669-2.690645$

C $2.9532163 .352364-3.205334$

O $5.4369021 .872362-3.399050$

C $3.1403484 .401707-2.153067$

C $2.1676815 .226741-1.737624$

C $2.3514546 .242138-0.645515$

C 1.3619336 .0680060 .487593

C $0.384766 \quad 6.9669290 .695316$

C 1.5428234 .8866461 .422726

C 1.0120103 .6081200 .832003

N $3.2595842 .064146-2.611048$

H -2.331117 -3.519879-1.913665

H $-0.805696-4.0593800 .688958$

H $-0.013599-2.951097-2.109787$

H $\quad 0.799149-5.299489-1.750747$

H $1.502086-4.814902-0.217031$

H $4.637133-3.986334-2.075684$

H $1.944909-2.551989-3.332048$

H $3.332011-3.485744-3.896389$

H $1.723000-4.207228-3.947360$

H $3.442292-5.6492230 .146073$

H $5.118118-5.367805-0.222933$

H $3.287208-3.2546451 .036223$

H $4.278930-4.3876391 .958703$

H $6.330605-3.4819030 .809375$

H $5.613464-2.6447613 .079621$

H $4.564870-1.4675362 .315234$

H $6.465873-0.5319054 .009941$

H 5.6762110 .4390040 .871273

H $8.048991-2.1274213 .337965$

H $8.821545-0.9082782 .350521$

H $8.263754-2.4219471 .610715$

H 7.7500141 .1854121 .379339

H $6.8036670 .451744-1.859116$

H $6.133843-0.967615-2.611788$

H $7.573390-2.159142-1.616117$

H $2.037471-0.4121530 .795781$

H $\quad 0.0023231 .580805-0.373900$

H $2.476175 \quad 1.2256632 .509319$

H 3.0643122 .8686072 .402425

H 3.5487201 .7036661 .172728

H $3.908987-0.484704-2.099440$

H $4.3177160 .608397-0.771426$

H $1.9222103 .308228-3.569437$ 
H $3.6213303 .544383-4.050335$ H $4.1262864 .473944-1.699177$ H $1.1842735 .166556-2.198232$ H $2.2447307 .237568-1.095465$ H $3.368717 \quad 6.206739-0.235647$ H -0.3159316 .8598661 .516903$ H 0.2592137 .8267040 .045843 H 1.0058365 .0702382 .362112 H 2.6042954 .8149921 .680196 H $\quad 0.1090203 .7380400 .235488$ H $2.5814481 .679907-1.960413$ C $-4.420568-3.1750440 .053340$ O $-4.979864-2.805418-1.226508$ C $-6.405762-2.713616-1.094877$ C $-6.693213-2.660000 \quad 0.398464$ C $-5.608532-3.578260 \quad 0.929367$ H $-6.806714-3.663233-1.476879$ C $-7.000415-1.584255-1.942740$ H -6.560368 -1.6499300 .801244$ H -7.703341 -2.9991240 .646400$ C $-5.360472-3.4252032 .419528$ H $-5.893107-4.6181990 .719021$ C $-6.691415-0.131932-1.520499$ O $-8.424653-1.755685-1.914293$ H -6.689787 -1.725348 -2.985655 H $-7.1868950 .087706-0.567540$ O $\quad-7.3175420 .711468-2.499503$ C $-5.1967420 .223490-1.437330$ C $-4.9399911 .707094-1.114392$ H -4.734413 -0.382047 -0.656116 O $-4.553114-0.104772-2.667210$ H -5.326702 $2.334244-1.926457$ H $-5.4964451 .969313-0.208025$ H -4.548960 -4.079963 2.751866 H -5.090285 -2.396453 2.675046 H $-6.259489-3.6857892 .987568$ C $-3.397219-4.295961-0.130512$ H -8.810162 -1.065088 -2.480511 H $-7.3978921 .600454-2.114754$ H $-4.9900070 .403102-3.372291$ H -3.840256 -5.118150 -0.705761 H -3.105483 -4.7000290 .845845$ H -3.932411 -2.2811990 .460959$ C $-3.170778 \quad 1.2785291 .338331$ O $-2.7846701 .138563-0.034243$ C $-3.4382702 .032048-0.939604$ C $-3.1507273 .485160-0.538628$ C -3.4905243 .7529580 .926113$ C -2.8604452 .6968261 .834642$ H $-4.243701 \quad 1.073593 \quad 1.429284$ C -2.4015980 .2046752 .124383$ H -2.967374 $1.869051-1.917343$ O $-3.8747934 .395878-1.359142$ H -2.085421 3.697101 -0.690861 H -4.5743333 .8061571 .083026$ O -2.9683845 .0303151 .301117$ H -1.7760442 .8543161 .871573$ O $-3.3563612 .863456 \quad 3.159381$ C -3.088408 -0.2364423 .397830$ H -2.282701 -0.6898251 .500395$ H $-1.397000 \quad 0.5663952 .371684$ H -3.623281 4.220283 -2.281999 H -3.3443535 .6869170 .689835$ H -3.162116 3.7773263 .429679 O $-2.255135-1.0811294 .075883$ O $-4.213137 \quad 0.091475 \quad 3.748805$ C -2.775041-1.604654 5.302007 H -2.987875 -0.7906326 .001539$ H -3.675697 -2.195372 5.109848 H $-2.015726-2.2563005 .742578$

SCF Energy $\left(\mathrm{B} 3 \mathrm{LYP} / 6-31 \mathrm{G}^{* *} / / \mathrm{MMFF}\right)=-3245.90744857$

03_223

MM̄FF Geometry

C $-4.029054-2.1756552 .093389$

C $-4.349531-3.1105641 .187158$

C -4.685601-2.850862 -0.261984

O $-5.086227-1.469272-0.428425$
C $-3.467395-3.073265-1.180156$

C $-2.793643-4.420685-1.070029$

C $-1.550103-4.612583-0.586966$

C $-3.592145-5.583568-1.597699$

C $-0.603226-3.592563-0.009794$

C $0.558976-3.275782-0.961839$

C $0.170501-2.281582-2.067882$

C $1.224636-2.264344-3.175561$

C $0.909175-1.213903-4.250044$

O $0.061888-0.980695-1.470037$

O $2.079915-1.051957-5.065840$

C $0.6541580 .137734-3.553267$

C $-0.213725-1.667662-5.189388$

O $\quad 0.2242841 .124457-4.487344$

C $-0.3470860 .052015-2.379699$

C $-0.4456861 .379102-1.588666$

O $-1.657111-0.219121-2.894992$

C $-5.907271-1.154559-1.460956$

O $-6.488028-1.938417-2.198531$

C $-6.0273100 .314192-1.619951$

C $-5.1349851 .173087-1.101397$

C $-5.1317062 .628747-1.257413$

C $-6.2831683 .264336-1.989799$

C $0.8805531 .933899-1.065035$

C $0.6413463 .174396-0.227602$

C -0.0286473 .8935062 .064456$

O $0.6795004 .300710-0.717910$

C -1.5214923 .9773192 .089165$

C -2.2161395.087969 1.800123

C -3.7180365 .1352701 .778330$

C -4.2769135 .5564340 .433738$

C -5.1374396 .5848720 .346499$

C $-3.8502094 .806924-0.811442$

C $-4.1027813 .326786-0.733329$

N $0.3508742 .884187 \quad 1.093982$

H -4.006535 -1.127598 1.805543

H -4.332287 -4.155155 1.491645

H -5.519412 -3.506966 -0.539541

H -2.754098 -2.257455 -1.014218

H $-3.777851-2.929856-2.224384$

H $-1.156350-5.628654-0.584830$

H -3.031800 -6.523743 -1.561256

H $-4.504672-5.721545-1.009324$

H -3.873276 $-5.408703-2.641344$

H -1.112997 -2.675834 0.299916

H $-0.189697-4.0245620 .910585$

H $1.372984-2.843514-0.366906$

H $0.936756-4.207472-1.401001$

H $-0.797876-2.570241-2.488161$

H $1.328578-3.257052-3.630924$

H $2.203322-2.030833-2.739217$

H $2.300803-1.918633-5.447981$

H $1.6258910 .473070-3.169974$

H $\quad 0.068506-2.594847-5.702637$

H $-0.389008-0.929636-5.979915$

H - $-1.158532-1.852212-4.674094$

H $0.882378 \quad 1.152976-5.202785$

H $-0.9389712 .141934-2.206290$

H -1.120102 $1.222129-0.734990$

H $-2.3114340 .060190-2.233141$

H $-6.8523650 .645625-2.241597$

H $-4.3006150 .776988-0.524273$

H -7.237433 $2.969082-1.539021$

H $-6.2830472 .961771-3.042480$

H $-6.2600954 .356607-1.964848$

H $1.4149681 .186168-0.469967$

H $1.5443942 .226048-1.885394$

H 0.4376944 .8488381 .802818

H 0.3468443 .5723913 .040645

H -2.0586263 .0658842 .342738$

H -1.6849856 .0040841 .553485$

H -4.0388305 .8313842 .563838$

H -4.145026 4.160872 2.047209

H $-5.5470316 .901165-0.607135$

H $-5.454508 \quad 7.134478 \quad 1.226504$

H $-2.7746154 .959380-0.970312$

H -4.318418 5.231771-1.706708

H $-3.3198272 .778972-0.208318$ 
H $\quad 0.273881 \quad 1.912218 \quad 1.377863$

C -2.096822 -2.659171 3.604413

O $-1.431682-1.5303613 .014692$

C $-0.277446-1.1919723 .806418$

C $-0.130935-2.2979294 .841438$

C -1.570854 -2.737727 5.035176

H $-0.524230-0.2386454 .288680$

C $0.924806-1.0344462 .870041$

H $0.472124-3.1251414 .446613$

H $0.340101-1.9471925 .764684$

C -1.709426 -4.109467 5.670831

H -2.079983 -1.995277 5.664317

C $2.216863-0.5940363 .579044$

O $0.585536-0.0558531 .884934$

H $1.091374-1.9729672 .329679$

H $2.562793-1.3874724 .251849$

O $1.910240 \quad 0.5363494 .407123$

C $3.351431-0.1716692 .624799$

C $3.799871-1.2766971 .653583$

H 3.0496340 .7263902 .074375

O $4.479330 \quad 0.2156053 .420458$

H 4.263694 -2.091176 2.223006

H $2.928857-1.6769451 .126562$

H -2.763361 -4.384832 5.777258

H -1.217982 -4.881774 5.069593

H - $-1.255308-4.1192636 .667068$

C -3.612729 -2.479053 3.505243

H $-0.212783-0.3709961 .426779$

H $2.7365150 .810896 \quad 4.840757$

H $4.768313-0.5632133 .926113$

H $-3.938853-1.6393184 .131260$

H $-4.125748-3.3727533 .878804$

H -1.788224 -3.5433203 .031131$

C $4.8512750 .759081-1.290347$

O $4.078426 \quad 0.112488-0.272314$

C $4.806082-0.7468160 .612573$

C $5.469464-1.881212-0.181097$

C $6.347933-1.328869-1.308687$

C $5.618454-0.268036-2.141270$

H $4.1078771 .245435-1.935145$

C $5.7466531 .857052-0.707174$

H $5.579389-0.1686081 .128788$

O $4.480274-2.750949-0.726438$

H $6.081158-2.4809810 .502994$

H $6.654561-2.157797-1.958507$

O $7.524065-0.767960-0.732362$

H $\quad 6.325814 \quad 0.248832-2.800165$

O $4.684620-0.947803-2.984680$

C $4.9070082 .932378-0.057488$

H $6.462331 \quad 1.4757470 .026863$

H $6.3391992 .338073-1.494182$

H $3.890341-2.214019-1.281838$

H $8.086552-0.453962-1.460635$

H $4.263331-0.283027-3.555946$

O 5.0063782 .8448101 .299262

O $4.2267653 .736302-0.681896$

C 4.2296673 .7922832 .037930

H 4.3945873 .6101013 .103152

H 4.5493204 .8115081 .801163

H 3.1663423 .6599761 .821319

SCF Energy (B3LYP/6-31G**//MMFF) $=-3245.92670636$

03_224

MM̄FF Geometry

C $-1.5370565 .533813-0.111229$

C -2.1345314 .6098780 .656053$

C $-3.247675 \quad 3.712314 \quad 0.179037$

O -2.8380042 .3406020 .386256$

C $-4.5137923 .963731 \quad 1.017596$

C -5.7120023 .1451030 .576897$

C $-6.3270372 .218174 \quad 1.337528$

C $-6.2359623 .442221-0.804928$

C -5.9917371 .7995492 .741678$

C -5.0275340 .6076432 .844166$

C $-5.576382-0.6993792 .248880$

C $-4.667026-1.8776592 .601157$

C -5.144462 -3.185974 1.950975

O $-5.637376-0.5613330 .827771$
O $-4.079483-4.1436872 .062445$

C $-5.385116-2.9493220 .442678$

C $-6.344081-3.7849932 .695918$

O $-5.988616-4.104826-0.134573$

C $-6.217484-1.6822520 .145165$

C $-6.273180-1.310793-1.357399$

O $-7.568088-1.8996360 .571899$

C $-2.0378421 .776205-0.558285$

O $-1.6510022 .286423-1.597349$

C $-1.6798030 .420970-0.084304$

C $-0.664653-0.233557-0.666837$

C $-0.155233-1.551656-0.284593$

C $-0.986459-2.3685870 .668095$

C $-4.916247-1.061413-2.025244$

C $-4.347628-2.276547-2.738901$

C $-2.300992-3.042954-3.935967$

O $-4.981851-3.313235-2.915039$

C $-1.453381-3.838532-2.994358$

C $-0.137780-4.031201-3.173890$

C $0.721049-4.858275-2.260783$

C $1.930339-4.102581-1.757499$

C $3.130787-4.250848-2.342479$

C $1.764356-3.242443-0.525488$

C $1.018226-1.965389-0.807313$

N -3.053496 -2.052381 -3.189348

$\mathrm{H}-1.8728605 .674450-1.135130$

H -1.7958654 .4694641 .680210$

H $-3.4597073 .879527-0.883796$

H -4.7898755 .0253980 .963708$

H -4.280562 3.7651702 .069299

H $-7.183913 \quad 1.6963860 .912407$

H -7.187483 2.937925 -1.005414

H $-6.4058634 .517271-0.924121$

H -5.524393 $3.112331-1.567750$

H -5.5783632 .6413163 .307376$

H $-6.9298741 .548603 \quad 3.253409$

H -4.0837850 .8708522 .352523$

H -4.8052170 .4514543 .907194$

H -6.584959 -0.8624892 .646424$

H -4.588169-1.998057 3.688876

H $-3.647733-1.6674952 .249937$

H -3.872083 -4.253025 3.006260

H $-4.394299-2.849060-0.016171$

H -6.084225 -3.9839463 .742648$

H $-6.630314-4.7534072 .270966$

H -7.220111 -3.133055 2.692335

H -6.000968 -3.985749 -1.098769

H $-6.868068-2.047819-1.909860$

H $-6.838379-0.370422-1.435069$

H $-8.144470-1.2739640 .102305$

H -2.2364370 .0141200 .751781$

H $-0.1301540 .254183-1.481082$

H $-0.982248-1.9185351 .666543$

H $-0.643572-3.4000960 .772412$

H -2.021185 -2.431560 0.314770

H $-5.046413-0.290073-2.795190$

H -4.185194 -0.683817 -1.304107

H -1.702941 -2.504429-4.677906

H -2.993329 -3.708669-4.461191

H - $1.947722-4.296851-2.140868$

H $0.347940-3.594212-4.043742$

H $1.043078-5.746222-2.820454$

H $\quad 0.147929-5.239892-1.406657$

H $4.008721-3.734907-1.969413$

H $3.261201-4.888663-3.210470$

H $2.748837-2.959287-0.129403$

H $1.306883-3.8408260 .266951$

H $1.542418-1.298494-1.493270$

H $-2.586686-1.182027-2.951387$

C $0.9541146 .038129-0.226110$

O $1.2894454 .676200 \quad 0.108221$

C $1.8425274 .040195-1.057983$

C $2.2161195 .173726-2.005189$

C $1.0898546 .155716-1.744025$

H $1.0360163 .446841-1.507890$

C $3.0065653 .108764-0.694891$

H $3.1819715 .618564-1.737269$

H $2.2683684 .846027-3.047647$ 
C $1.3812207 .564991-2.228683$

H $0.1895365 .786228-2.249216$

C 2.5885441 .9102510 .179073

O $4.0302093 .848859-0.032082$

H $3.4344292 .737384-1.632160$

H $1.8040031 .343164-0.332295$

O $2.0230252 .368977 \quad 1.411994$

C 3.7345040 .9511890 .554626

C $4.5107170 .416088-0.661290$

H $4.421448 \quad 1.439072 \quad 1.256174$

O $3.161213-0.161575 \quad 1.250947$

H $3.8044430 .017281-1.399747$

H $5.0459521 .252722-1.123788$

H $0.5474878 .235405-1.997673$

H $2.2829147 .973091-1.759786$

H $1.5332397 .574999-3.312932$

C $-0.408487 \quad 6.4042480 .369076$

H 3.6505934 .1921970 .795172

H 1.2230582 .8784231 .198239

H 2.6756600 .1942922 .014850

H -0.6486527 .4487280 .139253$

H $-0.3377526 .331911 \quad 1.462204$

H $1.714427 \quad 6.6627030 .261390$

C $7.395117-1.0907741 .172305$

O $6.436608-0.160550 \quad 0.652133$

C $5.515405-0.694782-0.301967$

C $6.271710-1.217488-1.532446$

C $7.339165-2.240171-1.126178$

C $8.206541-1.7353780 .033730$

H $8.081027-0.4797871 .772911$

C $6.741907-2.1161612 .112498$

H $4.959022-1.5215560 .151319$

O $6.887700-0.126391-2.213284$

H $5.552793-1.674228-2.222679$

H $7.977228-2.454905-1.992177$

O $6.688893-3.452831-0.757278$

H $8.819189-2.5530860 .431085$

O $9.108044-0.756605-0.492632$

C $6.067717-1.4550873 .297137$

H $5.984705-2.7075061 .590921$

H $7.503523-2.7987172 .505713$

H $7.338841-0.485719-2.996050$

H $7.379286-4.099180-0.531542$

H $9.697851-0.4809510 .229525$

O $5.238527-2.359316 \quad 3.898005$

O $6.253835-0.302378 \quad 3.662282$

C $4.518619-1.8718745 .034621$

H $3.880794-1.0305744 .747103$

H $3.884737-2.6806065 .408053$

H $5.214872-1.5774985 .825727$

SCF Energy $\left(\mathrm{B} 3 \mathrm{LYP} / 6-31 \mathrm{G}^{* *} / / \mathrm{MMFF}\right)=-3245.91443458$

03 225

MM̄FF Geometry

C 1.7291464 .6841650 .880928

C 0.5853654 .1554841 .342111

C -0.6250763 .9052200 .479119$

O $-1.1851492 .616144 \quad 0.825321$

C -1.698720 4.9724920 .757245

C $-2.9105694 .866065-0.148891$

C $-4.1619864 .597090 \quad 0.271937$

C $-2.6566445 .115863-1.614013$

C $-4.6389394 .359599 \quad 1.676792$

C -4.6712082 .8849472 .107132$

C -5.5973391 .9996781 .256598$

C -5.7977160 .6349161 .919468$

C $-6.645549-0.3081861 .051252$

O $-4.9954291 .819037-0.028472$

O $-6.509173-1.6360571 .581189$

C $-6.067237-0.332840-0.380140$

C $-8.137756 \quad 0.036608 \quad 1.125039$

O $-6.918805-1.061456-1.260564$

C $-5.7923031 .070891-0.957482$

C $-5.0227001 .037181-2.301348$

O $-7.0403861 .729245-1.207182$

C $-0.519827 \quad 1.5055800 .409325$

O $0.5026781 .463348-0.255380$

C -1.2509200 .3318920 .934112$
C $-0.630828-0.8541901 .010933$

C -1.217392 -2.104034 1.497894

C $-2.695846-2.1159001 .782260$

C $-3.6477140 .360724-2.257670$

C $-3.663442-1.103751-2.665192$

C $-2.178123-3.101668-2.782301$

O $-4.638481-1.652754-3.170793$

C $-2.380600-3.968293-1.579813$

C -1.459765 -4.838617-1.138497

C $-1.662088-5.7584480 .030896$

C $-0.585177-5.6134341 .082409$

C $0.466090-6.4494951 .119422$

C $-0.774931-4.5521082 .141754$

C $-0.414622-3.1781651 .645791$

N -2.439353 -1.717726 -2.433493

H $1.7850704 .987832-0.160251$

H 0.5124613 .8718042 .389633

H $-0.3514303 .902272-0.582889$

H -1.269928 5.9755180 .629421

H -1.9967084 .9019891 .808870$

H $-4.9523684 .549601-0.476719$

H -2.065759 4.304123 -2.048741

H -3.585645 5.188729-2.189816

H -2.114815 $6.057375-1.751061$

H -4.0345034 .9260132 .393086$

H $-5.6491804 .779870 \quad 1.764719$

H -3.650603 2.4855462 .080340

H -5.0029622 .8540763 .152414$

H -6.5593782 .5129391 .142944$

H -6.2478820 .7468442 .913787$

H -4.8185850 .1662092 .086438$

H -6.801485 -1.619956 2.508578

H $-5.122927-0.887042-0.329422$

H $-8.496061-0.0337872 .159163$

H -8.737632 -0.6789890 .551969$

H $-8.363733 \quad 1.042807 \quad 0.766231$

H -7.046204 -1.945293 -0.875139

H $-5.6573720 .613082-3.088802$

H -4.849685 $2.081436-2.599941$

H $-6.8919442 .434602-1.858675$

H $-2.2760260 .479698 \quad 1.253720$

H $0.411130-0.9158210 .701780$

H -2.924679-1.484843 2.647504

H -3.093666 -3.110920 1.991310

H -3.255546 -1.744426 0.917398

H $-2.9901160 .867522-2.975615$

H -3.191495 $0.461791-1.269039$

H -1.153107 -3.152797 -3.161490

H $-2.859233-3.414489-3.580173$

H $-3.338021-3.899258-1.068914$

H $-0.516505-4.924179-1.672093$

H -1.670355 -6.787048 -0.352899

H $-2.646334-5.6098190 .492195$

H $1.228523-6.3657031 .886962$

H $\quad 0.592533-7.2301060 .376533$

H $-0.132454-4.7663533 .005622$

H -1.793049 -4.6141392.535318

H $0.644208-3.0674891 .410916$

H -1.701148 -1.198226 -1.967418

C 4.2769394 .5908631 .043832

O $4.275128 \quad 3.2859730 .431311$

C $5.0313493 .361831-0.795673$

C $5.6978134 .735666-0.807122$

C $4.6838495 .572495-0.053644$

H $4.3070163 .324252-1.616946$

C $6.0199462 .195728-0.899707$

H $6.6565254 .725447-0.275070$

H $5.8836745 .096446-1.823491$

C 5.2451306 .8861600 .462109

H $3.8420415 .782229-0.724392$

C $5.3598370 .800368-0.938062$

O 6.8894642 .2496540 .238440

H $6.6563242 .334966-1.782217$

H 4.8691920 .5960680 .018848

O $6.409602-0.163728-1.085423$

C $4.3683320 .603096-2.101377$

C $3.865347-0.837885-2.332866$

H $3.5008681 .256706-1.962364$ 
O $4.9999991 .022583-3.318380$

H $3.407436-0.862471-3.330522$

H $4.718423-1.521591-2.399867$

H 4.4802957 .4526451 .002324

H 6.0881776 .7238781 .142108

H $5.5988317 .505442-0.368800$

C 2.9448404 .8782521 .743848

H 7.6369901 .6534000 .063886

H $6.891330-0.201678-0.241818$

H $5.7848080 .462605-3.448144$

H $2.9481125 .905627 \quad 2.124986$

H 2.8716274 .2120052 .613158

H 5.0595234 .5643421 .814547

C $4.278231-2.482706 \quad 0.249088$

O $3.359860-1.4090420 .025737$

C $2.831589-1.328283-1.299035$

C $2.141202-2.651087-1.675147$

C $3.057905-3.855201-1.472194$

C $3.638584-3.841136-0.058792$

H $5.147771-2.347190-0.403503$

C $4.814073-2.3695231 .680067$

H $2.047404-0.562484-1.268720$

O $1.687577-2.630910-3.024283$

H $1.253380-2.786264-1.045663$

H $3.853057-3.884205-2.226360$

O $2.303889-5.057197-1.635612$

H $2.835456-4.0641770 .652402$

O $4.607309-4.878708 \quad 0.070752$

C $3.745169-2.5718472 .729131$

H $5.628484-3.0851111 .842985$

H $5.236454-1.3720841 .852981$

H $1.088712-1.870561-3.119084$

H $1.905608-5.030091-2.522620$

H $5.319766-4.704784-0.567928$

O $3.947269-3.754633 \quad 3.376879$

O $2.835938-1.7797372 .940728$

C $2.998980-4.0653444 .401836$

H $3.068945-3.3341785 .212735$

H $1.986413-4.0890853 .990193$

H $3.238910-5.0553894 .798760$

SCF Energy (B3LYP/6-31G**//MMFF) $=-3245.89760576$

03_226

MMFF Geometry

C -2.161565 2.4447591 .987938

C - 2.6694813 .6242021 .596476

C $-3.596432 \quad 3.805427 \quad 0.413083$

O $-3.3503712 .770786-0.570489$

C $-5.061971 \quad 3.7057210 .863734$

C $-6.0416804 .155839-0.202156$

C $-6.7150613 .322675-1.019562$

C $-6.2739995 .642475-0.274146$

C $-6.5764731 .829618-1.108326$

C $-7.7534211 .103636-0.447102$

C -7.607239 $-0.420266-0.565909$

C $-8.804857-1.1403250 .055368$

C -8.629044 -2.6677150 .029090$

O $-6.408320-0.802740 \quad 0.115316$

$\begin{array}{lllll}0 & -9.611277 & -3.240587 & 0.905601\end{array}$

C -7.242923 -3.0258330 .608126$

C $-8.900208-3.248763-1.363810$

O $-6.970738-4.417611 \quad 0.461525$

C $-6.087640-2.194658 \quad 0.005445$

C $-4.778219-2.4491080 .789541$

O $-5.887932-2.584735-1.355962$

C $-2.2188002 .878991-1.320563$

O $-1.460698 \quad 3.833512-1.380124$

C $-2.0084771 .573526-1.983555$

C $-0.766181 \quad 1.226337-2.352405$

C $-0.361848-0.063339-2.913988$

C $-1.448495-1.045597-3.265566$

C $-3.678447-1.4229320 .491580$

C $-2.366017-1.8361991 .123672$

C $-0.373669-3.286560 \quad 0.742174$

O $-2.000477-1.3848872 .206561$

C $0.690924-2.4216530 .144769$

C $1.646299-2.872038-0.683431$

C $2.742674-1.999284-1.224047$
C $2.816740-1.979101-2.736700$

C $3.967244-2.280203-3.363240$

C $1.583342-1.604971-3.538433$

C $0.953857-0.312883-3.085504$

N - $1.674646-2.7678850 .364861$

H -2.4770561 .5353881 .482884$

H -2.3635824 .5328322 .109137$

H -3.401675 $4.782810-0.045633$

H $-5.225156 \quad 4.319915 \quad 1.759747$

H -5.2883652 .6766951 .170197$

H $-7.4230773 .752027-1.727030$

H -7.005795 $5.912500-1.042785$

H $-6.649814 \quad 6.0150710 .684364$

H $-5.3417816 .165525-0.509341$

H $-6.5254781 .556693-2.169919$

H $-5.6324861 .492825-0.670810$

H $-7.792407 \quad 1.3868210 .612932$

H -8.695910 $1.422525-0.909328$

H $-7.517260-0.674031-1.628838$

H $-9.736725-0.851683-0.446563$

H $-8.917610-0.8234241 .101734$

H -10.489598 $-2.961743 \quad 0.594557$

H -7.280819-2.829695 1.688919

H $-9.923686-3.017026-1.682467$

H $-8.830018-4.341987-1.356378$

H $-8.222284-2.864163-2.128640$

H $-7.712583-4.9014640 .863399$

H $-4.978710-2.4025691 .868077$

H $-4.410510-3.4605010 .575709$

H -4.962601 -2.427591-1.601243

H -2.860837 $0.910518-2.077058$

H $\quad 0.043745 \quad 1.934892-2.182809$

H $-2.217417-0.567934-3.883299$

H $-1.919969-1.437893-2.360513$

H $-1.089883-1.897808-3.847725$

H $-3.526675-1.293394-0.584374$

H $-3.949793-0.4380750 .888901$

H $-0.317047-4.3197950 .385903$

H $-0.281339-3.2858891 .833073$

H $0.693856-1.3737480 .437024$

H $1.665209-3.923397-0.959638$

H $3.686684-2.367248-0.804546$

H $2.637323-0.971454-0.859021$

H $4.042888-2.268501-4.445537$

H $4.862026-2.545203-2.809543$

H $0.885659-2.445145-3.470461$

H $\quad 1.839180-1.493170-4.599515$

H $1.6669910 .478876-2.853667$

H -2.042061 -3.037355 -0.542905

C $0.1300091 .625906 \quad 2.433859$

$\begin{array}{lllll}\text { O } & 0.722215 & 2.524547 & 1.476977\end{array}$

C $2.1586332 .374867 \quad 1.522093$

C $2.434131 \quad 1.2349432 .491756$

C 1.2431231 .3200333 .428111

H 2.5434113 .3208361 .922715

$\begin{array}{llll}\text { C } 2.663911 & 2.154521 & 0.091199\end{array}$

H $2.4400990 .271808 \quad 1.967060$

H 3.3921511 .3501183 .006858

C 1.0163640 .0589114 .242410

H 1.3887162 .1710084 .106850

C $4.1779961 .869234-0.009267$

O $2.3472353 .336788-0.651198$

H $2.1062541 .335639-0.376785$

H 4.3949170 .9662840 .568492

$\begin{array}{lllllllll} & \text { O } & 4.898342 & 2.937919 & 0.604551\end{array}$

C $4.6915711 .658014-1.450055$

C $6.2104001 .450936-1.584899$

H $4.1742270 .804370-1.900733$

O $4.3932462 .799238-2.260930$

H $6.4482991 .496615-2.656868$

H $6.7291402 .304592-1.129547$

H $\quad 0.1620730 .178525 \quad 4.916064$

H $0.815575-0.803713 \quad 3.598695$

H $1.896765-0.1711264 .851150$

C $-1.1116742 .274122 \quad 3.048417$

H $1.3886823 .482663-0.571001$

H 4.7148023 .7434610 .090980

H $3.4259212 .877977-2.314470$ 
H $-0.8515543 .245203 \quad 3.486198$ H -1.518935 1.6433463 .845603 H $-0.147358 \quad 0.716457 \quad 1.884101$ C $7.342833-0.9032541 .111907$ O 6.8180350 .2354680 .411294 C $6.7551390 .129079-1.013958$

C $8.150181-0.169480-1.597598$ C $8.804890-1.379148-0.931214$ C $8.741436-1.2794410 .594415$ H $7.461178-0.5631942 .148767$ C $6.367080-2.0912911 .103306$ H $6.066242-0.676881-1.286387$ O $9.0034060 .966100-1.453401$ H $8.048320-0.355495-2.673495$ H $9.851185-1.441524-1.254243$ O $8.140091-2.558532-1.373300$ H $9.060898-2.2208681 .056037$ O $9.651369-0.2696831 .038935$ C $5.197608-1.8883602 .042367$ H $5.959106-2.2788790 .107791$ H $6.889496-2.9916911 .448431$ H $9.0306181 .199590-0.509739$ H $8.584118-3.318110-0.959244$ H $10.541843-0.5277930 .745352$ O $4.419104-3.0114832 .022507$ O $4.993128-0.8875072 .713923$ C $3.279335-2.9857992 .886400$ H $3.595824-2.8850013 .928863$ H $2.611776-2.1678602 .605128$ H $2.742947-3.9313792 .771284$

SCF Energy $\left(B 3 L Y P / 6-31 G^{* *} / / M M F F\right)=-3245.91444166$

03227

MMFF Geometry

C $2.383229-4.226486-0.288919$

C $1.104470-4.180364-0.689746$

C $-0.028757-3.7330650 .199462$

O $-0.750756-2.665722-0.457256$

C -1.001792 -4.907539 0.410387

C -2.171660 -4.585395 1.319337

C $-3.463941-4.6085270 .937536$

C -1.819798 -4.251002 2.746336

C $-4.024013-4.947539-0.416053$

C $-4.248984-3.735972-1.334338$

C $-5.335052-2.768975-0.836604$

C $-5.652881-1.713807-1.897830$

C $-6.688243-0.691893-1.398363$

O $-4.861245-2.1193140 .345066$

O $-6.6791100 .420336-2.307268$

C $-6.243872-0.153716-0.019312$

C $-8.110334-1.264528-1.430971$

$\begin{array}{lllll}\text { O } & -7.253424 & 0.674578 & 0.551837\end{array}$

C $-5.822767-1.2601120 .971529$

C $-5.173460-0.7129172 .265616$

O $-6.987042-1.9957301 .368977$

C $-0.216547-1.415632-0.396655$

O $0.833518-1.0868850 .131464$

C -1.137749 -0.502175 -1.108963

C $-0.7105890 .718200-1.465762$

C $-1.4838401 .729004-2.190615$

C $-2.9454251 .453684-2.425349$

C -3.9204330 .1467892 .063333$

C -4.2016131 .6403142 .042919$

C -3.070999 3.8232731 .648576

O

C -3.2553094 .2531220 .227774$

C $-2.4148725 .086419-0.403390$

C $-2.6157665 .576164-1.807310$

C -1.431079 $5.290798-2.701240$

C $-0.4777786 .216162-2.903026$

C $-1.4067253 .972613-3.439014$

C $-0.8534572 .846472-2.609307$

N $-3.0705802 .374187 \quad 1.713582$

H $2.633523-3.927470 \quad 0.726265$

H $0.851313-4.478323-1.704530$

H $\quad 0.345883-3.3770751 .167101$

H $-0.462784-5.7617670 .841697$

H - $-1.359528-5.240835-0.569614$
H $-4.220328-4.366740 \quad 1.683477$

H -1.295614 -3.291958 2.800735

H -2.705997 -4.1764203 .385859$

H - $-1.175895-5.027843 \quad 3.171183$

H $-3.381109-5.668738-0.931389$

H $-4.976175-5.473149-0.268990$

H -3.300794 -3.199296-1.456843

H $-4.536060-4.115973-2.322678$

H $-6.231845-3.353677-0.600005$

H -5.998197 -2.185455 -2.826352

H $-4.732303-1.174960-2.160705$

H $-6.8884790 .083812-3.195340$

H $-5.3859250 .501199-0.207918$

H -8.370530 -1.585524 -2.446742

H $-8.849775-0.502028-1.162344$

H $-8.241563-2.123437-0.769684$

H -7.9455570 .0936670 .911081$

H $-5.925293-0.1961482 .874259$

H $-4.865404-1.5824562 .864417$

H -6.793582 -2.448546 2.206672

H $-2.133001-0.865955-1.336947$

H $\quad 0.314900 \quad 0.998622-1.228006$

H $-3.0723640 .624858-3.129749$

H $-3.4911272 .310990-2.825519$

H -3.442651 $1.195139-1.484284$

H $-3.243349-0.0236552 .910228$

H -3.384224 -0.1391931 .152564$

H -2.1213584 .1693102 .068539$

H -3.8853084 .2210812 .262417$

H $-4.1359073 .886179-0.294295$

H -1.5524715 .4744450 .133396$

H -2.791589 $6.659056-1.759829$

H -3.524012 $5.152997-2.253883$

H $\quad 0.3583786 .030223-3.568981$

H $-0.5090617 .177082-2.400156$

H $-0.7668364 .058375-4.328142$

H -2.401500 $3.759757-3.840627$

H $0.2075572 .957848-2.382436$

H -2.2178541 .8866351 .453564$

C $4.445730-3.520621-1.516865$

O $5.064577-3.004647-0.323624$

C $6.412162-2.609371-0.636539$

C $6.587519-2.785919-2.139479$

C $5.607148-3.908715-2.429906$

H $7.070524-3.332807-0.136856$

C $6.695314-1.222201-0.045997$

H $6.300764-1.891878-2.698848$

H $7.617967-3.033799-2.411920$

C $5.234014-4.022007-3.897792$

H $6.048776-4.856515-2.093897$

C $5.759163-0.098660-0.548313$

O $8.062632-0.899710-0.277618$

H $6.556165-1.3151621 .038973$

H $4.742642-0.495750-0.653658$

O $6.1869100 .345066-1.833467$

C $5.6978341 .097248 \quad 0.422548$

C $4.7501312 .208478-0.064683$

H $5.3634810 .724147 \quad 1.395317$

O $6.9970681 .651950 \quad 0.616903$

H $5.2330652 .768109-0.874560$

H $3.8452121 .752722-0.479405$

H $4.524648-4.839461-4.059223$

H $4.774502-3.099208-4.267300$

H $6.122528-4.221931-4.505739$

C $3.521286-4.683063-1.159250$

H $8.244629-0.0555160 .168472$

H $5.4752040 .886271-2.214211$

H $7.3213031 .951854-0.249743$

H $4.076040-5.450283-0.605344$

H $3.138662-5.153736-2.072094$

H $3.866900-2.699116-1.960225$

C 2.4523912 .0054011 .924821

O 3.7643512 .5245142 .178207

C 4.3777073 .1914781 .067794

C 3.5006144 .3685430 .619986

C 2.0667863 .9308760 .335994

C 1.5037933 .1366751 .514381

H $2.4992791 .253348 \quad 1.127972$ 
C $\quad 1.972847 \quad 1.306953 \quad 3.204594$

H $5.318313 \quad 3.6124931 .444431$

O $4.0364374 .994318-0.540898$

H $3.4789755 .127073 \quad 1.413288$

H $2.0004383 .353008-0.593156$

$\begin{array}{lllll}\text { O } & 1.251584 & 5.088973 & 0.147736\end{array}$

H 1.3276993 .8120622 .361346

$\begin{array}{lllll}\text { O } & 0.246569 & 2.577966 & 1.143474\end{array}$

C $2.503711-0.1059333 .283762$

H 2.2814321 .8458374 .107818

H 0.8796871 .2284193 .231202

H $4.9414705 .279378-0.327339$

H $1.6346545 .595481-0.588917$

H $-0.295286 \quad 3.2981450 .779176$

O $3.836362-0.095263 \quad 3.568629$

O $1.812781-1.1012783 .106828$

C $4.463039-1.3807603 .621737$

H $5.530333-1.2309923 .804874$

H $4.043863-1.9711724 .441823$

H $4.342308-1.9027722 .667348$

SCF Energy (B3LYP/6-31G**//MMFF) $=-3245.91701036$

03_228

MMFF Geometry

C $-0.140393 \quad 5.702752 \quad 0.361946$

C $0.8342745 .027630-0.265207$

C $2.142284 \quad 4.649965 \quad 0.379557$

$\begin{array}{lllllll}\text { O } & 2.135360 & 3.217753 & 0.587183\end{array}$

C $3.3175034 .991479-0.554737$

C $4.6612194 .508392-0.040766$

C $5.4106203 .549317-0.622466$

C 5.1592115 .1944501 .205782

C $5.1227122 .776686-1.880547$

C $4.6811661 .332665-1.624343$

C $5.7117310 .416636-0.941389$

C $6.9892390 .260879-1.772679$

C $7.950097-0.781720-1.182148$

O $5.067553-0.856692-0.778203$

O $8.936391-1.088281-2.180104$

C $7.166718-2.081224-0.910432$

C $8.715112-0.2315320 .027293$

O $7.981848-3.038374-0.238300$

C $5.862595-1.852738-0.119510$

C $5.010854-3.138724-0.012226$

O $6.185535-1.4492031 .216287$

C 1.5197342 .7548451 .709860

O $\quad 0.952558 \quad 3.4130142 .566890$

C 1.6513451 .2829261 .714930

C $0.895774 \quad 0.550617 \quad 2.547393$

C $0.932958-0.9077982 .678854$

C $1.988570-1.6378941 .893894$

C $4.551342-3.724660-1.348570$

C $3.430471-4.727862-1.149996$

C $0.979567-4.876077-0.746586$

O $3.625453-5.939090-1.216083$

C $-0.019909-4.051195-0.000368$

C $-0.674400-4.4708301 .093238$

C $-1.672196-3.6250891 .834816$

C $-1.405842-3.5605043 .324311$

C $-2.318504-4.0098684 .202637$

C $-0.091181-2.980973 \quad 3.803080$

C $0.044030-1.5130563 .494095$

N $2.213524-4.121456-0.875985$

H $0.011315 \quad 6.0361361 .385482$

H $0.6609404 .668444-1.277152$

H $2.2756805 .163397 \quad 1.340109$

H $3.3680506 .078560-0.701439$

H $3.1129884 .572358-1.544468$

H $6.3601743 .292320-0.153929$

H 6.1783094 .8908881 .468824

H 5.1686546 .2799981 .063290

H 4.5164004 .9590092 .059512

H $4.3534943 .266432-2.484032$

H $6.0202242 .794820-2.509149$

H $3.7602251 .334282-1.026639$

H $4.3915390 .881550-2.583266$

H $\begin{array}{llll}5.940899 & 0.817787 & 0.052039\end{array}$

H $7.5091271 .219340-1.884506$
H $\quad 6.721279-0.054856-2.790888$

H $9.388468-0.260853-2.419135$

H $\quad 6.919663-2.515160-1.887872$

H $9.3211410 .634533-0.264958$

H $9.421168-0.9720020 .418840$

H $8.0604770 .087376 \quad 0.840924$

H $8.788147-3.156105-0.769311$

H $5.552130-3.9050320 .558017$

H $4.127729-2.9109710 .595585$

H $5.427331-1.6454291 .791122$

H 2.3674320 .8428301 .030710

H $\quad 0.181027 \quad 1.0617173 .191032$

H $2.985920-1.2756432 .167473$

H $1.842510-1.4841230 .819119$

H $1.995679-2.7160212 .059534$

H $4.195292-2.942778-2.029249$

H $5.378785-4.235519-1.853249$

H $\quad 1.189537-5.831498-0.254317$

H $0.615304-5.083843-1.757888$

H $-0.219518-3.053758-0.384985$

H $-0.497378-5.4762421 .467736$

H -2.666932 -4.0481071 .644334$

H -1.697817 -2.604330 1.436559

H -2.142836 -3.9695415 .272460$

H -3.264659-4.426300 3.872764

H $0.730294-3.5794593 .402120$

H -0.005897 -3.087063 4.892912

H $-0.676109-0.8904204 .026608$

H $2.140834-3.117616-1.017722$

C $-2.4414914 .763250-0.215958$

O $-1.971383 \quad 3.752354-1.131166$

C -1.995250 $2.474260-0.463447$

C -2.9383232 .6520650 .715796$

C $-2.6286004 .075077 \quad 1.138739$

H $-0.9705142 .296109-0.115699$

C $-2.4124691 .393934-1.465144$

H $-3.9843212 .563450 \quad 0.396601$

H -2.7570681 .9245471 .511790$

C -3.7075884 .7055272 .001563$

H -1.6919604 .0611001 .707708$

C $-2.549541-0.003443-0.837945$

O $-1.4198651 .331261-2.494846$

H $-3.3477701 .692504-1.950685$

H $-3.3020710 .011740-0.044304$

O $-1.316505-0.343097-0.190946$

C $-2.885270-1.122505-1.842613$

C $-4.137830-0.886792-2.709650$

H $-2.029907-1.282680-2.509375$

O $-3.036531-2.352870-1.127511$

H -3.933869 -0.083668 -3.427164

H $-4.308034-1.805504-3.286177$

H -3.442436 5.7350642 .262552

H $-4.6737314 .725156 \quad 1.486367$

H -3.834717 4.1415432 .931501

C $-1.4909045 .968848-0.243884$

H $-1.3315982 .225600-2.866757$

H -0.616619-0.330959-0.866196

H -3.769909-2.245329 -0.498257

H -1.947689 6.8075460 .294859

H -1.353290 $6.298277-1.281691$

H -3.413088 $5.088154-0.610143$

C $-6.864361-1.489042-0.214990$

O $-5.739722-1.665950-1.085925$

C $-5.423437-0.552925-1.925336$

C $-6.604348-0.243912-2.858500$

C $-7.891093-0.014979-2.056317$

C $-8.123320-1.118590-1.017951$

H -7.032691 -2.477806 0.231301

C $-6.553080-0.5078240 .926092$

H $-5.2293720 .326237-1.304728$

O $-6.793111-1.329010-3.764263$

H $-6.3633010 .645724-3.452618$

H $-8.7492050 .017983-2.738774$

O $\quad-7.8197391 .240337-1.386560$

H $-8.922700-0.824843-0.328240$

O $-8.583125-2.293231-1.694490$

C $-5.405985-0.9873791 .791435$

H $\quad-6.2832970 .4784380 .539039$ 
H $-7.430143-0.3992661 .573808$ H $-7.524504-1.090929-4.359195$ H $-7.710177 \quad 1.929050-2.064083$ H -7.860047 -2.611638 -2.261870 O -4.9709090 .0491452 .567680$ O $-4.952641-2.1235671 .795821$ C $-3.857245-0.2469613 .415774$ H $-3.6008820 .659673 \quad 3.970192$ H -4.120994 -1.033397 4.129069 H $-2.994516-0.5434212 .812096$ SCF Energy (B3LYP/6-31G**//MMFF) $=-3245.91711035$

\section{3_229}

MMFF Geometry

C $2.415236 \quad 0.9927104 .084587$

C $1.802039-0.0384713 .484580$

C $0.314652-0.2857353 .556202$

O $-0.265954-0.0912322 .249891$

C $0.071311-1.7491963 .975981$

C -1.390304 -2.122801 4.122424

C $-2.013371-3.0662693 .388604$

C $-2.150655-1.3866455 .195266$

C -1.432589-3.927756 2.301015

C $-1.586280-3.3500310 .885476$

C $-3.039508-3.2881890 .388506$

C $-3.093430-2.865154-1.081172$

C $-4.539279-2.711192-1.582124$

O $-3.752178-2.336731 \quad 1.180799$

O $-4.499121-2.006632-2.832985$

C $-5.329533-1.826848-0.591786$

C $-5.188147-4.070093-1.871391$

O $-6.714815-1.800363-0.924825$

C $-5.151283-2.2445780 .881618$

C $-5.765162-1.2364751 .883055$

O $-5.806959-3.5004871 .096722$

C -0.7573651 .1461341 .966027$

O -0.7284052 .1351682 .680755$

C -1.3275751 .0977480 .601898$

C -1.490921 2.239750-0.082379

C $-1.9632902 .356013-1.463617$

C $-2.5355151 .122855-2.109530$

C $-5.197926 \quad 0.186221 \quad 1.815418$

C $-6.032609 \quad 1.1427350 .980213$

C $-6.0231033 .482543 \quad 0.124769$

O

C $-5.6047203 .455450-1.310968$

C $-5.0823564 .516350-1.944410$

C $-4.7004364 .519376-3.396212$

C $-3.2675544 .946475-3.619084$

C $-2.9692896 .206455-3.978650$

C $-2.1916833 .890359-3.510675$

C -1.854746 $3.549259-2.084529$

N -5.4080762 .3739250 .829816$

H 1.8188771 .7386794 .604098

H $2.404833-0.7804342 .965295$

H $-0.153378 \quad 0.3852374 .286910$

H $0.568425-1.9453534 .935723$

H $0.559250-2.4034103 .245350$

H -3.066438 -3.257279 3.592501

H -2.272388 -0.3325374 .928228$

H $-3.151987-1.8017445 .353847$

H -1.617407 -1.448162 6.149440

H $-0.372470-4.1258982 .490524$

H -1.917100 -4.911413 2.347632

H -1.143809 -2.347497 0.861385

H -1.003290 -3.9782750 .200756$

H -3.487591 -4.280603 0.516686

H -2.542979 -3.574528 -1.711586

H -2.580680 -1.901089-1.200121

H -3.942585 -2.516442 -3.446428

H -4.960273 -0.802824 -0.716604

H -4.615284 -4.613368 -2.632595

H -6.194691 -3.947607 -2.286366

H $-5.253423-4.710470-0.989404$

H -6.785016 -1.508421 -1.849916

H $-6.858766-1.2433031 .802329$

H -5.548237 -1.613275 2.893157

H $-5.988283-3.5952572 .046434$
H -1.5453380 .1233810 .180478$

H -1.207639 3.1769920 .395014

H $-1.7495740 .381092-2.287147$

H -3.025342 $1.318257-3.065633$

H $-3.3016680 .677214-1.467049$

H -5.1863490 .6017142 .831379$

H -4.1658190 .1862371 .450856$

H -5.7118094 .4020170 .630550$

H -7.112502 3.3978940 .192814

H $-5.7638692 .531151-1.861223$

H $-4.9499295 .447914-1.398554$

H -5.380167 $5.205880-3.917808$

H $-4.8639043 .537087-3.856664$

H -1.946197 $6.513449-4.168508$

H -3.741862 $6.960754-4.085363$

H $-1.2668404 .248996-3.982404$

H -2.484464 $3.018959-4.102860$

H -1.423829 4.383820 -1.529266

H $-4.4657182 .502137 \quad 1.187278$

C 4.6050281 .9868033 .141173

O 4.7382361 .2642711 .905612

C 4.9471592 .2288510 .849758

C 4.7598833 .6144351 .477410

C 3.9659403 .3071782 .737841

H 4.1521042 .0706930 .116151

C 6.3204481 .9973300 .195484

H 5.7189454 .0830781 .726429

H 4.2337454 .2978320 .802907

C 4.0546794 .3914873 .795479

H 2.9168773 .1586612 .451630

C $6.5444930 .551004-0.298211$

O 7.3348572 .2822491 .168629

H $6.4662252 .711217-0.623655$

H $6.517340-0.1324950 .559274$

O $7.8723760 .487468-0.832898$

C $5.5444790 .100281-1.377864$

C $5.699259-1.383305-1.763173$

H $4.5308430 .250526-1.003657$

O $5.6850810 .919255-2.536491$

H $6.717339-1.574551-2.122078$

H $5.561204-1.988847-0.860135$

H 3.4774054 .1171394 .684095

H 5.0902294 .5647594 .107049

H 3.6560305 .3364093 .412063

C 3.9068641 .1306854 .195552

H $8.1953892 .133370 \quad 0.740528$

H $8.115288-0.450185-0.910362$

H $6.5969950 .816844-2.858487$

H 4.1145541 .5535975 .186772

H 4.3589440 .1299674 .192493

H 5.6293332 .1636083 .499558

C $2.703151-1.918139-1.558071$

O $3.394226-1.346566-2.673955$

C $4.720149-1.839701-2.870171$

C $4.678841-3.358206-3.098293$

C $3.898284-4.086479-2.002137$

C $2.538441-3.424746-1.763183$

H $3.265247-1.730730-0.634376$

C $1.357493-1.197605-1.442382$

H $5.076275-1.381756-3.801936$

O $5.993579-3.898761-3.173232$

H $4.190519-3.564639-4.059704$

H $4.473566-4.138692-1.070248$

O $3.671238-5.438488-2.407448$

H $1.876486-3.623906-2.615368$

O $1.933159-3.993296-0.604794$

C $1.5957570 .250877-1.085106$

H $\quad 0.795469-1.248557-2.382513$

H $0.724072-1.631208-0.661771$

H $6.458216-3.437331-3.892090$

H $4.540637-5.835925-2.587263$

H $1.870910-4.952770-0.751374$

O $1.4890531 .031831-2.196838$

O 1.8984490 .6292200 .038909

C $1.8078812 .417353-2.028186$

H $1.4016032 .815804-1.093953$

H $2.8937072 .543731-2.055599$

H $1.3686562 .970214-2.862760$ 
MMFF Geometry

C $-2.9826620 .184739-3.218527$

C $-2.276740-0.133683-2.122513$

C $-1.072600-1.039334-2.146843$

O $-0.041570-0.477085-1.302494$

C -1.449532-2.418500-1.574096

C $-0.368508-3.468079-1.752051$

C $0.269015-4.093600-0.743470$

C $-0.039795-3.836014-3.177160$

C $0.067948-3.9154850 .733910$

C $0.980495-2.8657991 .383248$

C $2.483814-3.1550481 .249780$

C $3.291181-2.2218952 .154540$

C $4.803433-2.4200071 .980243$

O $2.866774-2.941722-0.110952$

O $5.446240-1.3105442 .627429$

C $5.154068-2.3618680 .476578$

C $5.292172-3.6807292 .703663$

O $6.526074-2.7027350 .293656$

C $4.239258-3.245502-0.399949$

C $4.435639-3.028676-1.921176$

O $4.526293-4.623802-0.133302$

C $0.6639080 .579372-1.787806$

O $\quad 0.533787 \quad 1.117531-2.875386$

C $1.6258350 .986856-0.741002$

C $2.1878712 .202902-0.796619$

C 3.1506572 .7510860 .160678

C 3.7142641 .8133451 .195437

C $4.199647-1.598039-2.418229$

C $5.461963-0.755642-2.498325$

C $6.2129371 .584030-2.915493$

O $6.591701-1.222055-2.378406$

C $6.4440842 .272123-1.606939$

C $6.4574273 .606978-1.471252$

C $6.7287564 .316482-0.175262$

C 5.6569425 .3259830 .171703

C $5.8169836 .629199-0.114328$

C 4.4401864 .8361430 .924038

C $3.497640 \quad 4.0508360 .053898$

N $5.1783330 .578585-2.757796$

H -2.691742 $-0.223565-4.182894$

H -2.584674 $0.258486-1.156283$

H $-0.685758-1.138528-3.167925$

H -2.357763 -2.792411 -2.065817

H - $1.703587-2.299689-0.514899$

H $1.025809-4.834401-0.999799$

H $0.604904-4.719405-3.239494$

H $-0.955729-4.063323-3.732517$

H $0.481500-3.015465-3.679161$

H $-0.975639-3.6669150 .955856$

H $0.237904-4.8862251 .217361$

H $\quad 0.753956-1.8871820 .945087$

H $0.720382-2.8165522 .447413$

H $2.661578-4.2018771 .522183$

H $3.006371-2.3533763 .205941$

H $3.047370-1.1794731 .908785$

H $6.401971-1.3879822 .463353$

H $5.046407-1.3132010 .175251$

H $5.078793-3.6100803 .777184$

H $6.379452-3.7893012 .622464$

H $4.826723-4.5961622 .332444$

H $6.755363-2.533046-0.635232$

H $5.413198-3.411374-2.237424$

H $3.699086-3.664379-2.434371$

H $4.215075-5.154444-0.885345$

H $1.832290 \quad 0.2805530 .054422$

H $1.9064642 .869027-1.611408$

H 4.1152910 .9125070 .718394

H 2.9387131 .5184341 .909981

H 4.5396852 .2396071 .769029

H $3.802558-1.647444-3.440289$

H $3.454544-1.086761-1.802116$

H $5.8761112 .276202-3.693553$

H $7.1394831 .106034-3.249261$

H $6.634858 \quad 1.646127-0.738382$
H $7.6986684 .822212-0.269550$

H 6.8450443 .6077930 .653983

H 5.0661837 .3642450 .155486

H $6.701306 \quad 6.989234-0.629875$

H $3.8737125 .689247 \quad 1.321282$

H 4.7672364 .2837631 .809158

H $3.0383064 .646247-0.736532$

H $4.2102540 .884542-2.793397$

C $-5.4857330 .261605-3.114767$

O $-5.535798-0.393965-1.829693$

C $-6.6933600 .073441-1.121609$

C $-7.6769370 .454076-2.214655$

C $-6.7437871 .135714-3.200997$

H $-6.3965560 .981708-0.582954$

C $-7.166491-0.974408-0.112427$

H -8.133984 -0.429166 -2.675315

H $-8.4741141 .110604-1.854268$

C -7.313339 $1.244747-4.604950$

H $-6.5247412 .147473-2.833189$

C $-6.048024-1.5057490 .811216$

O $-7.706555-2.092815-0.826056$

H $-7.991447-0.5617540 .480612$

H -5.395802 -2.1803320 .242348$

O $-6.686984-2.3066361 .812256$

C $-5.201827-0.4091441 .485417$

C $-4.158376-0.9644962 .472687$

H -4.6821250 .1654540 .712321$

O $\quad-6.057752 \quad 0.5005452 .177133$

H -4.663489 -1.366224 3.359133

H $-3.612502-1.7794431 .981694$

H $-6.594121 \quad 1.721272-5.278758$

H $-7.5596860 .259865-5.015784$

H $-8.2279371 .846672-4.603584$

C $-4.1923501 .076131-3.196561$

H -8.005702 -2.740676 -0.165195

H $-5.998862-2.8333462 .252758$

H $-6.559034-0.0107752 .835327$

H $-4.1245701 .758532-2.340451$

H $-4.1849511 .694163-4.101657$

H -5.498043 -0.527572 -3.876788

C -1.4302271 .5405641 .991991$

O $-2.3743880 .482242 \quad 1.785651$

C $-3.164148 \quad 0.123763 \quad 2.924997$

C $-2.252443-0.3603774 .060919$

C $-1.1897380 .688673 \quad 4.404124$

C -0.4753821 .2149713 .153025$

H -0.8373091 .5672221 .069534$

C -2.127852 2.8983972.137703

H $-3.7340210 .995507 \quad 3.263449$

O $-1.615350-1.5826393 .696100$

H -2.865418 -0.5679384 .945698$

H $\quad-0.457004 \quad 0.2409875 .086670$

O -1.8211041 .7656625 .090595$

H $0.1231662 .102043 \quad 3.390721$

O $0.431890 \quad 0.2127292 .688441$

C -2.9189193 .2358990 .893045$

H -2.8013642 .9161602 .998985$

H -1.395783 3.7003532 .285821

H -1.150427 -1.432529 2.855341

H -1.1302492 .4072225 .328674$

H 1.0766570 .0475023 .397339

O $-4.1888423 .597016 \quad 1.235660$

O $-2.4640873 .195156-0.242903$

C $-5.0461793 .948470 \quad 0.145674$

H -6.0353134 .1773800 .551016$

H -4.661181 $4.835320-0.366292$

H $-5.1392903 .110921-0.550536$

SCF Energy (B3LYP/6-31G**//MMFF) = -3245.92673347

03230

MM̄FF Geometry

C $-2.368909-2.5309172 .101366$

C -1.581411 -1.574960 1.583389

C $-0.612938-1.6975110 .425873$

O $0.695040-1.2920520 .904756$

C $-0.471369-3.114512-0.165851$

C $0.466428-3.198743-1.355786$ 
C $1.613425-3.905716-1.380558$

C $0.029910-2.450661-2.589267$

C $2.219341-4.739248-0.286163$

C $3.254252-4.0023140 .578499$

C $4.505692-3.542977-0.188543$

C $5.569655-3.0150630 .776713$

C $6.797434-2.4610110 .035231$

O $4.128470-2.501709-1.092229$

O $7.573539-1.7009110 .974841$

C $6.328193-1.478216-1.060367$

C $7.710151-3.584091-0.471374$

O $7.419632-1.072416-1.881949$

C $5.188910-2.035761-1.937467$

C $4.581383-0.982658-2.896335$

O $5.699748-3.097091-2.753625$

C 0.9850630 .0377820 .900142

$\begin{array}{lllll}\text { O } & 0.272771 & 0.947842 & 0.507027\end{array}$

C $2.3261870 .204849 \quad 1.500905$

C 2.6700581 .3940992 .016902

C 3.9411791 .7163302 .668735

C 5.0591090 .7155502 .544223

C $3.973530 \quad 0.251416-2.220280$

C $4.916121 \quad 1.441073-2.144596$

C $5.0654293 .738996-1.192847$

O $5.9992791 .482709-2.721247$

C 5.8688773 .6878550 .068054

C 5.7890674 .6213181 .028350

C 6.6179514 .6084792 .280385

C 5.7781354 .6987433 .534460

C 5.5940195 .8736914 .160079

C 5.2248973 .4140274 .108285

C 4.0490822 .8877943 .330135

N $4.3832372 .472762-1.382926$

H -2.354902 -3.535281 1.690352

H -1.632171 -0.5837322 .031751$

H $-0.941890-1.009695-0.361056$

H -1.452951 -3.481645 -0.492435

H $-0.137350-3.7898910 .628428$

H $2.194536-3.898641-2.302234$

H $\quad 0.038137-1.371353-2.411379$

H $0.683896-2.643717-3.446550$

H $-0.980819-2.753870-2.877928$

H $\quad 1.441718-5.1509840 .365605$

H $2.693677-5.614074-0.749207$

H $2.770774-3.1384241 .049474$

H $3.558750-4.6811401 .384789$

H $4.894677-4.397287-0.755055$

H $5.871321-3.7940171 .487949$

H $5.138800-2.2078061 .384319$

H $7.814846-2.2900921 .710087$

H $5.972199-0.577499-0.546916$

H $8.064505-4.1966790 .366433$

H $8.610438-3.178322-0.945901$

H $7.216063-4.251322-1.180545$

H $8.102893-0.705019-1.295327$

H $5.313028-0.705606-3.664755$

H $3.763586-1.476607-3.441114$

H $5.106804-3.214175-3.514359$

H $2.978728-0.6596691 .528663$

H 1.9428482 .2046351 .985436

H $4.829902-0.1906643 .114926$

H 6.0221111 .0899642 .897334

H 5.2113600 .4415901 .494829

H $3.1134210 .583205-2.816160$

H $3.6040890 .011751-1.218717$

H $4.2987754 .519767-1.164766$

H $5.7268503 .929078-2.044175$

H 6.5656792 .8610760 .184498

H 5.1111665 .4616140 .895453

H 7.3075775 .4615122 .231854

H 7.2553803 .7170072 .331269

H 5.0163415 .9427965 .075904

H 6.0128726 .7940333 .766687

H 4.8797593 .5809345 .137607

H 6.0361532 .6869774 .202011

H 3.1810993 .5481883 .357820

H $3.5024482 .330275-0.897168$

C $-4.762134-2.3609622 .998513$
O $-5.155181-1.2580902 .162596$

C $-6.331151-1.6519411 .420781$

C $-6.664262-3.0804131 .854931$

C $-5.308224-3.6001882 .299623$

H $-6.045298-1.6729150 .364062$

C $-7.458821-0.6343071 .644227$

H -7.375219 -3.098554 2.689117

H -7.100418 -3.665714 1.039248

C $-5.385455-4.8313513 .183752$

H $-4.722251-3.8328831 .401783$

C $-7.068500 \quad 0.818217 \quad 1.298396$

O $-7.822576-0.6678933 .031124$

H $-8.349720-0.9372161 .081716$

H $-6.2639411 .148473 \quad 1.966831$

O $-8.207648 \quad 1.637871 \quad 1.588715$

C $-6.6622221 .020806-0.173166$

C $-6.2148792 .461041-0.480468$

H $-5.8355750 .349064-0.410554$

O $\quad-7.7486640 .662329-1.024577$

H -7.024597 $3.161139-0.244092$

H -5.3744932 .7074630 .178171$

H $-4.384862-5.1599273 .481713$

H -5.961968 -4.636757 4.094252

H $-5.867253-5.6577082 .651013$

C $-3.261871-2.3127013 .291751$

H $-8.536574-0.0197683 .157973$

H -7.902648 2.5591121 .642366

H $-8.500888 \quad 1.235379-0.796718$

H -3.014115 -3.074164 4.040965

H -3.031042 -1.341113 3.747472

H $-5.285397-2.2180653 .954555$

C $-3.6349031 .664628-1.924284$

O $-4.960541 \quad 1.659751-2.465045$

C $-5.8230082 .681424-1.959838$

C $-5.2210764 .063786-2.250550$

C $-3.7875874 .180255-1.734777$

C $-2.9425123 .000625-2.215654$

H $-3.6752021 .492981-0.841545$

C $-2.8787570 .498121-2.573812$

H $-6.7515462 .605605-2.539990$

O $-6.0108755 .096886-1.671521$

H -5.211779 $4.234297-3.334886$

H $-3.7586184 .262028-0.641915$

O $-3.2032015 .382920-2.240591$

H -2.753565 $3.102324-3.292075$

O $-1.6792183 .026447-1.554887$

C $-3.454016-0.832783-2.142146$

H $-2.9502640 .577540-3.665594$

H -1.817958 $0.498102-2.303085$

H $-6.9100975 .013765-2.032419$

H $-3.7706596 .120624-1.957873$

H -1.275463 $3.892873-1.734436$

O $-3.654042-1.630635-3.229599$

O $-3.685084-1.137552-0.979644$

C $-4.202896-2.922125-2.947758$

H $-3.527982-3.488209-2.298672$

H $-5.189339-2.821258-2.484888$

H $-4.312097-3.458967-3.893782$

SCF Energy (B3LYP/6-31G**//MMFF $)=-3245.91000921$

03_231

MMFF Geometry

C $-0.026708 \quad 4.8124990 .356833$

C $1.0073374 .396497-0.389343$

C 2.3071383 .8932260 .184146

O $2.4781732 .525437-0.254333$

C $3.4840264 .720527-0.362126$

C 4.8305564 .3029950 .198335

C $5.8392083 .790308-0.533832$

C 5.0185024 .5173421 .678635

C $5.8735663 .536093-2.014576$

C $5.4262802 .128840-2.438595$

C $6.3169670 .994980-1.904793$

C $5.950169-0.334557-2.567593$

C $6.779349-1.499785-2.005868$

O $6.1189670 .884666-0.493882$

O $6.159179-2.725947-2.425420$

C $6.715108-1.472414-0.461546$ 
C $8.196564-1.513219-2.591221$

O $7.619743-2.416694 \quad 0.103910$

C $6.965578-0.0772870 .151968$

C $6.658324-0.0073141 .665613$

$\begin{array}{llll}\text { O } & 8.344288 & 0.300262 & 0.047252\end{array}$

C 1.8308671 .5595750 .451920

O $\quad 1.169502 \quad 1.705437 \quad 1.467273$

C $2.0354230 .278904-0.258010$

C $1.205559-0.745611-0.014212$

C $1.255061-2.064620-0.646812$

C $2.463900-2.390999-1.483064$

C $5.219686-0.3509742 .066580$

C $5.023921-1.7983032 .489109$

C $3.232139-3.4000943 .151552$

O $5.952367-2.5839352 .658679$

C $2.855921-4.2446951 .975107$

C $1.677594-4.8784861 .872394$

C $1.298746-5.7665380 .721680$

C $-0.007542-5.3589850 .077580$

C $-1.159864-5.9696280 .401308$

C $0.037348-4.298957-0.999530$

C $0.230863-2.917362-0.436597$

N $3.683416-2.0996812 .691551$

H 0.0691824 .8290061 .439067

H $0.9046174 .369234-1.471939$

H 2.2957023 .9338491 .280033

H $3.3314805 .782783-0.128919$

H $3.4854144 .647734-1.455104$

H $6.7622903 .527864-0.017819$

H 6.0528754 .3445951 .995073

H 4.7633125 .5471571 .949123

H 4.3831443 .8358642 .252188

H $5.2675854 .277288-2.546852$

H $6.9000683 .707430-2.363694$

H $4.3914711 .971226-2.113044$

H $5.4272072 .098225-3.535367$

H $7.3630261 .250826-2.109627$

H $6.066521-0.273087-3.656873$

H $4.887093-0.547895-2.391981$

H $\quad 6.112144-2.721536-3.396872$

H $5.706127-1.801374-0.185763$

H $8.159902-1.645441-3.679404$

H $8.776445-2.359572-2.207390$

H $8.750461-0.593062-2.393253$

H $7.406569-3.286064-0.276487$

H $7.386221-0.6063302 .226252$

H 6.8376061 .0311001 .980788

H $8.848913-0.456021-0.292247$

H $2.842807 \quad 0.223457-0.978447$

H $\quad 0.395490-0.5996770 .699277$

H $2.511393-3.437023-1.792618$

H $3.382779-2.203150-0.916942$

H $2.479533-1.780232-2.392009$

H $4.948456 \quad 0.2591502 .937734$

H $4.518222-0.1060311 .264275$

H $2.387313-3.2295033 .826162$

H $4.034685-3.8880933 .713820$

H $3.595221-4.3622721 .186405$

H $0.951537-4.7802102 .676576$

H $1.220698-6.7925211 .104631$

H $2.087897-5.795679-0.039767$

H -2.096552 -5.699547 -0.075029

H -1.192805 -6.7492871 .155290$

H - $0.908573-4.289279-1.557761$

H $\quad 0.794870-4.573296-1.738586$

H $-0.596051-2.5866960 .192391$

H $2.977918-1.4001502 .479462$

C $-2.4587964 .197386-0.049112$

O $-2.0874522 .963451-0.698984$

C -2.4197681 .8650990 .177887$

C -3.3889902 .4382751 .199947$

C -2.830865 3.8349991 .388653

H -1.4805801 .5752200 .664351$

C $-2.9639190 .700371-0.655379$

H -4.4069972 .4812020 .792656$

H -3.412022 1.8611992 .128609

C -3.809546 4.7999642 .034305

H -1.9370723 .7627132 .019875$
C $-3.526559-0.4546330 .190289$

O $-1.8963540 .199226-1.466591$

H $-3.7276841 .073404-1.346328$

H $-4.383811-0.1046020 .774024$

O $-2.532287-0.840505 \quad 1.147525$

C $-3.920274-1.705808-0.616077$

C $-4.898696-1.473307-1.783027$

H -3.015944 -2.181021-1.013865

O $-4.480103-2.6681890 .284658$

H $-4.379400-0.938662-2.586855$

H $-5.174347-2.462129-2.172145$

H -3.3663225 .7961252 .131084$

H -4.7283454 .8943651 .445782$

H -4.0870494 .4535983 .035328$

C $-1.3471435 .238117-0.223438$

H $-1.5378010 .949182-1.971524$

H -2.898775 -1.577367 1.665862

H $-5.289929-2.2859400 .663644$

H $-1.645121 \quad 6.186627 \quad 0.237813$

H -1.222625 $5.435653-1.296117$

H $-3.3484984 .560070-0.580614$

C $-8.094616-0.8475010 .070857$

O $-6.901311-1.460054-0.435970$

C $-6.189220-0.708834-1.421178$

C $-7.078616-0.470862-2.652040$

C $-8.3996900 .198360-2.255428$

C $-9.068213-0.512637-1.073076$

H $-8.564297-1.6206510 .692616$

C -7.7802690 .3539590 .976762$

H $-5.907070 \quad 0.260218-1.000900$

O $-7.347316-1.714766-3.295452$

H $-6.5334910 .158966-3.365262$

H $-9.0848950 .192841-3.111836$

O $-8.1619681 .557935-1.902139$

H $-9.8965030 .088896-0.680690$

O $-9.627649-1.747561-1.530440$

C $-6.977814-0.0438102 .198179$

H -7.2069551 .1169650 .443640$

H $-8.7142030 .804021 \quad 1.332021$

H -7.886528 -1.528136 -4.082594

H $-7.7671671 .997767-2.674180$

H -10.284195 -1.536021 -2.215831

O -6.5212541 .0866242 .815205$

O $-6.781796-1.1916972 .572220$

C $-5.7279150 .869767 \quad 3.986250$

H $-4.8575780 .250404 \quad 3.748313$

H -5.3779121 .8417004 .343924$

H $-6.3328680 .402698 \quad 4.769011$

SCF Energy (B3LYP/6-31G**//MMFF) $=-3245.90470704$

032232

MM̄FF Geometry

C $-2.9484544 .791894-0.869928$

C $-1.9289334 .148392-1.457549$

C $-0.5017734 .228683-0.979932$

O $-0.1001452 .912901-0.530066$

C $0.4126004 .657430-2.141894$

C $1.8882894 .703816-1.792919$

C $2.8422874 .032026-2.468167$

C $2.2709545 .585361-0.633011$

C $2.6550673 .158054-3.677147$

C $2.3000581 .700157-3.355431$

C $3.4643200 .891103-2.762404$

C $3.062450-0.578812-2.616808$

C $4.169969-1.415194-1.961464$

O $3.7999591 .431147-1.482438$

O $3.603719-2.678171-1.581549$

C $4.618807-0.715260-0.662968$

C $5.307103-1.727761-2.940756$

O $5.753139-1.370322-0.098595$

C $4.9128340 .794099-0.843000$

C 5.1494221 .4944510 .517429

O $6.1096640 .941754-1.615653$

C -0.4650022 .5692270 .736888$

O

C 0.0301461 .2011481 .009252

C -0.3672610 .5886582 .135257$

C $0.031924-0.7387462 .607300$ 
C $1.001676-1.5239071 .766333$

C 3.8818351 .6879321 .355768

C 4.1584041 .7516442 .847510

C 2.9464561 .6083495 .024358

O $5.288200 \quad 1.7851883 .326506$

C $2.731527 \quad 0.1599795 .339690$

C $1.704476-0.3023006 .068448$

C $1.485965-1.7589326 .365063$

C $0.104705-2.2383295 .972388$

C $-0.819170-2.5205006 .906680$

C $-0.182689-2.4664564 .501155$

C $-0.483935-1.1820093 .773566$

N $2.9788851 .757403 \quad 3.581200$

H -2.758089 $5.424906-0.007011$

H $-2.1307673 .498607-2.306285$

H $-0.4034304 .947389-0.156477$

H $0.1137165 .654198-2.492841$

H $0.2427373 .974960-2.981966$

H $3.8751504 .129067-2.136631$

H 1.9019155 .1672550 .308641

H $3.3564225 .698051-0.538355$

H $1.8492596 .587473-0.762441$

H $1.8802613 .580802-4.327235$

H $3.5745503 .190538-4.275114$

H $1.4452351 .678112-2.667944$

H $1.975841 \quad 1.220340-4.287486$

H $4.3264910 .987118-3.432878$

H $2.786981-1.005234-3.589499$

H $2.160498-0.648968-1.991939$

H $3.241574-3.097139-2.381091$

H $3.805575-0.8385070 .061379$

H $4.924979-2.283752$-3.805398

H $\quad 6.060192-2.375363-2.478362$

H $5.806176-0.833073-3.318808$

H $5.522161-2.3089810 .008735$

H $5.9243210 .949531 \quad 1.070208$

H 5.5760762 .4920820 .343420

H $6.471144 \quad 1.829605-1.458587$

H $\quad 0.7026530 .7472520 .291140$

H $-1.052103 \quad 1.1200462 .795774$

H $0.663245-1.5785630 .726064$

H $1.122897-2.5579202 .095856$

H $1.990306-1.0569891 .789970$

H 3.3794442 .6159231 .057493

H $3.1802440 .866823 \quad 1.191129$

H 2.1355092 .2397355 .400429

H 3.8918791 .9495015 .456866

H $3.459890-0.5443134 .943750$

H $0.9852720 .401454 \quad 6.481446$

H $\quad 1.644987-1.9054397 .441257$

H $2.235647-2.3848745 .865041$

H -1.803037 -2.888679 6.635251

H $-0.614665-2.3851687 .963604$

H -1.057198 -3.1203034 .390587$

H $\quad 0.659279-3.0143004 .069070$

H -1.210151 -0.5498274 .287270$

H 2.0949141 .6874833 .086895

C $-5.1553343 .560961-0.604111$

O $-4.6023222 .274070-0.945981$

C -4.5889831 .4501530 .235431$

C -5.4714002 .1642071 .254857$

C -5.1884183 .6182110 .923020$

H -3.5594621 .4530130 .611613$

C $-5.0143410 .018575-0.108387$

H -6.5337251 .9409111 .103792$

H -5.2128411 .8995992 .284616$

C $-6.2158734 .586776 \quad 1.480555$

H -4.2019613 .8734591 .328880$

C $-4.207333-0.626303-1.258868$

O $-6.391577 \quad 0.048313-0.504946$

H -4.961685 -0.6043460 .792938$

H -4.407239 -0.102908 -2.201654

O $-4.692536-1.964446-1.410992$

C $-2.688913-0.676784-1.006358$

C $-1.814150-1.154633-2.184456$

H $-2.3364660 .333908-0.779783$

O $-2.419406-1.4601260 .150651$

H $-1.863699-0.382946-2.962846$
H $-0.776661-1.175743-1.828983$

H -5.9727665 .6159671 .198038$

H $-7.2218804 .363337 \quad 1.110058$

H -6.2413654 .5330822 .573940$

C $-4.3761434 .667412-1.320611$

H $-6.717959-0.867321-0.496778$

H -5.582439-1.911282 -1.798328

H -2.812952 -2.336383 0.001407

H -4.872873 $5.632959-1.170170$

H -4.394195 4.470905 -2.400529

H $-6.1801843 .560142-0.998454$

C $-0.945185-4.017877-1.348801$

O $-2.205871-3.586760-1.874980$

C $-2.147897-2.519531-2.823507$

C $-1.234213-2.900095-4.000967$

C $0.140711-3.360389-3.530322$

C $-0.002941-4.454274-2.476790$

H $-0.488904-3.201655-0.776365$

C $-1.222417-5.189011-0.397137$

H $-3.154212-2.427113-3.247778$

O $-1.077011-1.810816-4.904275$

H -1.696383 -3.720309-4.565991

H $0.736484-2.524049-3.149175$

O $0.864428-3.897994-4.639040$

H $-0.377608-5.371466-2.949367$

O $1.280300-4.748307-1.929562$

C -1.741951-4.708275 0.935551

H -1.947676 -5.893024 -0.822972

H $-0.311965-5.761962-0.185766$

H -1.964285 -1.552797 -5.207468

H $\quad 0.911631-3.202376-5.317297$

H $1.854729-5.016964-2.666994$

O $-3.090844-4.5221630 .877706$

O $-1.026816-4.5113321 .909315$

C -3.701332 -4.063712 2.088741

H $-3.625413-4.8347642 .860967$

H -3.236044 -3.132670 2.426616

H $-4.757873-3.8714421 .884193$

SCF Energy $\left(B 3 L Y P / 6-31 G^{* *} / / M M F F\right)=-3245.91404506$

03233

MMFF Geometry

C $-2.296191-3.762877-2.730039$

C $-1.673878-3.353486-1.613154$

C $-0.190149-3.494457-1.380890$

O $0.332847-2.219914-0.938694$

C $0.061792-4.519028-0.259526$

C $1.529502-4.824120-0.029699$

C $2.198542-4.5634831 .110450$

C $2.244958-5.497076-1.173821$

C $1.676296-3.9413062 .374436$

C $1.861080-2.4191102 .465965$

C $3.325114-1.9535292 .426049$

C $3.427221-0.4688932 .781479$

C 4.8669600 .0516302 .663290

O $3.829382-2.1579631 .104485$

O 4.7968841 .4851942 .711943

C $5.444006-0.3501011 .286991$

C $5.719037-0.3774183 .864044$

O $6.828395-0.0144941 .233284$

C $5.218293-1.8377410 .939190$

C $5.590176-2.200313-0.519928$

O $6.036870-2.6464101 .793115$

C $0.539959-1.263054-1.882687$

O $0.340369-1.355409-3.083108$

C $1.042614-0.057154-1.188805$

C $1.0246061 .125411-1.820652$

C $1.4766022 .403206-1.267003$

C 2.2368652 .3677230 .032294

C $4.831632-1.426657-1.604152$

C $5.564909-0.196818-2.113058$

C $5.2475921 .772036-3.607377$

O $6.7312710 .059110-1.826982$

C $4.8904122 .961710-2.773463$

C $4.2540684 .037473-3.261273$

C $3.9250395 .256825-2.448669$

C $2.4606545 .627420-2.521373$

C $2.0300836 .580766-3.364900$ 
C $1.5120824 .953405-1.556254$

C $1.2007333 .535664-1.947141$

N $4.7696720 .565580-2.958209$

H -1.696619 -4.232755 -3.507853

H -2.236569 -2.886680 -0.810930

H $\quad 0.323763-3.799207-2.300310$

H $-0.444823-5.463992-0.497531$

H $-0.402635-4.1496530 .661338$

H $3.252683-4.8362251 .155881$

H $3.246595-5.840469-0.892885$

H $1.683308-6.374579-1.510486$

H $2.360230-4.809001-2.016626$

H $0.616909-4.1796632 .516311$

H $2.189351-4.4159243 .220874$

H $1.300748-1.9461861 .651222$

H $1.407736-2.0873403 .407972$

H $3.900208-2.5582623 .136837$

H $3.032975-0.2794933 .787732$

H 2.7903580 .1106202 .099532

H 5.6957051 .8286062 .569341

H $4.938727 \quad 0.2786230 .544956$

H 5.2892150 .0128684 .794463

H $6.7298560 .041178 \quad 3.804207$

H $5.801542-1.4615353 .965765$

H $7.134165-0.1512680 .321251$

H $6.675708-2.140333-0.662532$

H $5.341117-3.262259-0.660645$

H $6.152210-3.5163321 .376054$

H $1.398119-0.172677-0.171615$

H $\quad 0.6210921 .170059-2.831472$

H $3.0712951 .661772-0.033928$

H 1.5821212 .0613830 .854784

H 2.6785303 .3275960 .307253

H $4.703675-2.084960-2.473180$

H $3.833068-1.142136-1.259545$

H $4.789150 \quad 1.809192-4.600654$

H $6.334527 \quad 1.717499-3.725557$

H $5.1910772 .948828-1.728431$

H $3.9799294 .058930-4.313848$

H $4.5378516 .084926-2.828266$

H $4.2176705 .130741-1.398860$

H $0.9858936 .872626-3.403549$

H $2.7123677 .087041-4.039733$

H $0.5574525 .496049-1.525893$

H $1.9118405 .040603-0.542295$

H $0.6476913 .455338-2.884259$

H $3.7972090 .306056-3.096113$

C -4.627791 -2.868539-2.061410

O $-4.265443-1.475448-2.113885$

C $-5.456979-0.674713-1.940733$

C $-6.578681-1.653627-1.625474$

C -6.122915 -2.896508 -2.366703

H -5.633766 $-0.188842-2.908248$

C $-5.1698920 .371306-0.858011$

H -6.634269-1.851352 -0.547466

H -7.556754 -1.290413 -1.954873

C -6.828990 -4.162138-1.912734

H $-6.292149-2.750261-3.441885$

C $-6.3962621 .161986-0.384080$

O $-4.2108201 .291045-1.392562$

H -4.698808 $-0.126834-0.004603$

H -7.124012 $0.476310 \quad 0.066455$

O $-7.029748 \quad 1.723624-1.540505$

C -6.0987722 .3085520 .608310$

C -5.3538641 .8806611 .883790$

H $\quad-5.591864 \quad 3.1365740 .104067$

O $\quad-7.3656702 .8288971 .044248$

H -5.7159052 .4638442 .741802$

H -5.6330390 .8489812 .139286$

H $-6.463547-5.033714-2.464567$

H $-6.671245-4.348874-0.845060$

H -7.907487 -4.082908 -2.084118

C $-3.767133-3.654950-3.051869$

H $-3.4343940 .773054-1.666767$

H -7.909128 $2.037252-1.270473$

H -7.7623083 .3009610 .292837$

H -3.842863 -3.182268 -4.039464

H $-4.155626-4.675184-3.148888$
H $-4.455704-3.218994-1.036239$

C -1.8987291 .2796123 .135480$

O $-\begin{array}{llll}\text { O } 3.326449 & 1.383225 & 3.034950\end{array}$

C -3.8191351 .9880541 .830513$

C -3.2985783 .4295711 .720112$

C -1.7691373 .4740401 .811258$

C -1.238125 2.6613302 .996914

H $-1.7190070 .923151 \quad 4.158231$

C -1.3285850 .2295212 .173182$

H $-3.451361 \quad 1.4067520 .986397$

O

H -3.6123763 .8601300 .763807$

H -1.4396844 .5154561 .910679$

O -1.2094202 .9520650 .610767$

H -0.1509082 .5397372 .925867$

O -1.5022113 .3813404 .205055$

C -1.850370 -1.143602 2.524291

H $-1.5563150 .430768 \quad 1.122451$

H -0.2351830 .1904632 .245695$

H -3.6427673 .8339213 .602765$

H -1.521652 $3.504939-0.125582$

H -1.0374364 .2333884 .145395$

O $-2.930918-1.445008 \quad 1.749945$

O $-1.375292-1.8481993 .405212$

C $-3.558367-2.6999282 .029411$

H $-4.464845-2.7687301 .422605$

H -2.888470 -3.521545 1.760001

H -3.841328 -2.761564 3.084799

SCF Energy $\left(B 3 L Y P / 6-31 G^{* *} / / M M F F\right)=-3245.91562998$

03234

MMFF Geometry

C $3.980386-0.4976163 .933155$

C $2.784841-0.9698843 .551164$

C $1.482772-0.2571803 .810813$

O 0.9205940 .1359592 .537330

C $0.495522-1.2174134 .498369$

C $-0.855625-0.5986094 .799450$

C -2.020682 -1.018963 4.267933

C -0.8498940 .5429865 .783053$

C $-2.238044-2.1531013 .305438$

C $-2.157660-1.755911 \quad 1.824274$

C $-3.293461-0.8308571 .360195$

C $-3.253709-0.644478-0.157688$

C $-4.3316540 .336640-0.644084$

$\begin{array}{llll}\text { O } & -3.135994 & 0.439433 & 1.995947\end{array}$

O $-4.0207590 .694476-1.999695$

C -4.2532641 .6308630 .194787$

C $-5.720058-0.313047-0.674807$

O $-5.3420762 .500541-0.104117$

C -4.1835091 .3779591 .714707$

C -3.879546 2.6590002 .529999

O -5.4499470 .8825342 .166567$

C 1.4187391 .2638411 .960852

O 2.2858281 .9991402 .405649

C $0.736491 \quad 1.4433250 .662080$

C $1.2972132 .230848-0.267551$

C $0.7637472 .502854-1.602938$

C $-0.6428072 .054670-1.893731$

C -2.5529713 .3521362 .195796$

C -2.6863694 .5107741 .220253$

C -1.294206 $6.151633-0.040500$

O $-3.7665154 .973566 \quad 0.864445$

C $-1.2266795 .708621-1.467804$

C $-0.2342746 .054136-2.302338$

C $-0.1865705 .664626-3.752169$

C $1.1137504 .996009-4.138394$

C $2.0982045 .690671-4.733225$

C $1.2344773 .505603-3.918290$

C $1.5438683 .154526-2.490110$

N $-1.446554 \quad 4.9966370 .824784$

H 4.0381620 .4430524 .474796

H $2.737483-1.8989122 .987274$

H $1.635471 \quad 0.6313614 .435782$

H $0.925597-1.5764085 .442933$

H $0.375975-2.1039463 .866002$

H -2.933193 -0.499962 4.559057

H -0.3513861 .4183545 .356116$ 
H $-1.861864 \quad 0.849547 \quad 6.069127$ H $-0.325917 \quad 0.252415 \quad 6.699471$ H $-1.523275-2.9605713 .497218$ H $-3.222202-2.5934093 .511080$ H -1.188833 -1.281666 1.630156 H -2.186726 -2.677511 1.233839 H $-4.246685-1.2780791 .665533$ H $-3.352933-1.605755-0.674716$ H $-2.270666-0.251898-0.448299$ H -3.989998 $-0.122967-2.525990$ H $-3.3440792 .154947-0.117503$ H $-5.718997-1.187052-1.337441$ H $-6.4683530 .373102-1.086625$ H $-6.059783-0.6453240 .308262$ H $-5.3358542 .653228-1.064536$ H -4.7268893 .3527052 .472419$ H -3.820746 2.3615963 .587191 H $-5.522638 \quad 1.0474103 .121210$ H -0.1914750 .9081920 .500008$ H $2.2519052 .704312-0.041300$ H $-0.7119190 .963601-1.883858$ H -1.021929 2.394918 -2.859291 H -1.328484 2.453921-1.140551 H -2.146108 3.7816023 .120558 H -1.816186 2.6370751 .817497 H $-0.386116 \quad 6.6755810 .274145$ H -2.148556 6.8226050 .096205 H -2.049403 $5.103200-1.840670$ H $0.5720476 .685191-1.935016$ H $-0.3248206 .578205-4.345351$ H - $-1.0260875 .010938-4.019622$ H $3.0219995 .211983-5.040585$ H $2.0041886 .755190-4.920799$ H $2.0496453 .100328-4.533250$ H $0.3359003 .008870-4.295404$ H $2.5466653 .450695-2.179074$ H -0.6039854 .5148571 .124812$ C $5.895741-0.7627212 .283651$ O $5.074415-1.2335181 .196593$ C $5.061912-0.2321410 .160740$ C $6.142792 \quad 0.7790950 .533972$ C $6.087003 \quad 0.7351752 .049710$ H 4.0863860 .2648710 .215345 C $5.238607-0.886499-1.213946$ H 7.1329330 .4676380 .181763 H 5.9381161 .7730990 .124635 C $7.316662 \quad 1.3127532 .727183$ H 5.2041721 .3003332 .372134 C $4.213674-1.999893-1.516671$ O $6.546462-1.470668-1.265046$ H $5.210372-0.115410-1.993149$ H $4.373623-2.854847-0.848345$ O $4.458013-2.472289-2.847967$ C $2.741002-1.551733-1.435366$ C $1.765661-2.684119-1.817904$ H $2.511861-1.235325-0.412212$ O $2.545290-0.422788-2.279737$ H $1.979594-3.028976-2.835679$ H $1.966126-3.529007-1.147377$ H $7.229547 \quad 1.2417993 .816002$ H 8.2273120 .7840702 .426064 H 7.4394562 .3687252 .465590 C $5.280002-1.1827743 .621292$ H $6.735500-1.684504-2.194198$ H $5.301725-2.954789-2.836654$ H $2.776832-0.691039-3.185701$ H $5.983149-0.9749824 .436325$ H $5.119293-2.2686293 .615224$ H $6.857759-1.2821232 .177700$ C $-0.642191-4.517771-1.746242$ O $-0.475905-3.292132-1.025186$ C $0.272869-2.282974-1.721638$ C $-0.418218-1.963025-3.059581$ C $-0.687214-3.215690-3.894401$ C $-1.392173-4.286606-3.062980$ H $0.349022-4.926477-1.983476$ C -1.286305 $-5.546368-0.804263$ H $0.207088-1.388311-1.091286$
O $0.348156-1.057855-3.844974$

H $-1.380223-1.474997-2.855764$

H $0.236146-3.609392-4.335668$

O $-1.536840-2.870149-4.992287$

H $-2.425200-3.964995-2.896936$

O $-1.433973-5.489970-3.825826$

C $-2.644864-5.164721-0.251630$

H $-1.380430-6.518663-1.298599$

H $-0.619247-5.663508 \quad 0.060107$

H $0.539697-0.279722-3.295233$

H $-1.089130-2.166910-5.493247$

H $-2.043554-6.101257-3.379364$

O $-2.924013-5.965445 \quad 0.821077$

O $-3.379351-4.295098-0.696118$

C $-4.176852-5.714164 \quad 1.465737$

H $-4.264967-6.3965632 .315368$

H $-4.213348-4.6854461 .837085$

H -5.002580 -5.9054730 .773908$

SCF Energy (B3LYP/6-31G**//MMFF)= -3245.90745711

03_235

MM̄FF Geometry

C $1.4481495 .534735-0.246713$

C $2.1640894 .605160-0.897464$

C $3.1336713 .672969-0.216223$

O $2.8763922 .324129-0.675725$

C $4.573240 \quad 4.044732-0.615205$

$\begin{array}{llllll}\text { C } 5.638108 & 3.268841 & 0.135474\end{array}$

C $6.5261562 .433807-0.438377$

C $5.695603 \quad 3.505734 \quad 1.623438$

C $6.6679632 .084262-1.892755$

C $5.877102 \quad 0.843507-2.336054$

C $6.271179-0.450793-1.605081$

C $5.628569-1.666776-2.274722$

C $5.919947-2.966244-1.509561$

O $5.816121-0.360420-0.252962$

O $5.030669-3.965790-2.031827$

C $5.584361-2.765648-0.014021$

C $7.341547-3.471623-1.784468$

O $6.002067-3.909870 \quad 0.726470$

C $6.182500-1.4711540 .577596$

C $5.682326-1.1457952 .007060$

O $7.606787-1.6077210 .654785$

C $1.8251921 .655355-0.127716$

$\begin{array}{llll}\text { O } & 1.072170 & 2.052723 & 0.746045\end{array}$

C $1.7359680 .340981-0.802808$

C $0.620356-0.396986-0.696792$

C $0.395647-1.703971-1.320950$

C $1.586928-2.386561-1.941729$

C $4.166214-0.973492 \quad 2.151587$

C $3.439180-2.2413682 .565804$

C $1.142178-3.1723502 .815076$

O $4.016721-3.2485012 .966252$

C $0.853638-3.9907291 .596095$

C $-0.386360-4.3269101 .209729$

C $-0.681407-5.1721690 .003832$

C $-1.735961-4.565090-0.895020$

C $-3.005644-5.002708-0.851139$

C $-1.303774-3.532586-1.912422$

C $-0.843730-2.239279-1.294481$

N $2.060800-2.1035382 .469462$

H 1.5852545 .6522550 .824676

H $2.0384754 .487949-1.971408$

H 3.0143393 .7139250 .872995

H $4.7469145 .113355-0.429239$

H $4.6834493 .902794-1.695764$

H 7.2505891 .9384290 .207567

H 6.5837653 .0566872 .081309

H 5.7274894 .5791001 .837152

H 4.8201703 .0745542 .118166

H $6.3845842 .932389-2.525083$

H $7.7338471 .921225-2.097783$

H $4.8075861 .037163-2.192857$

H $6.0405040 .713301-3.412973$

H $7.363865-0.538432-1.620193$

H $5.954750-1.755826-3.318525$

H $4.540591-1.523623-2.315144$

H $5.157131-4.775194-1.507035$ 
H $4.490821-2.7268950 .054983$

H $7.473970-3.670999-2.854732$

H $7.529437-4.424995-1.278385$

H $8.114856-2.762969-1.481072$

H $5.664211-3.8192351 .632917$

H $6.077906-1.8750602 .723846$

H $6.135036-0.1858172 .295635$

H $7.943324-0.9675741 .303600$

H $2.5914240 .023297-1.387897$

H - $0.214121-0.003532-0.118205$

H $2.418492-2.423923-1.229839$

H $1.913810-1.850798-2.839386$

H $1.398248-3.422641-2.229766$

H $3.974413-0.2408662 .946066$

H $3.726495-0.5797691 .230585$

H $0.240303-2.7072883 .225549$

H $1.594618-3.8047093 .585510$

H $1.704058-4.3414651 .015885$

H -1.234804 -4.001348 1.807428

H -1.018198 -6.1538040 .362396$

H $0.225394-5.366071-0.582464$

H -3.764271 -4.607744 -1.517780

H -3.316241 -5.766183 -0.145393

H $-2.140991-3.287038-2.579862$

H $-0.549945-3.979417-2.566674$

H $-1.643220-1.677410-0.809301$

H $1.671299-1.2532702 .072743$

C - $-1.0022156 .167612-0.481054$

O $-1.3295114 .778776-0.694791$

C $-1.882608 \quad 4.2505590 .528387$

C $-2.4120825 .461972 \quad 1.276381$

C $-1.325467 \quad 6.4791650 .983338$

H -1.0408713 .8041121 .072457$

C -2.9206653 .1778860 .190845$

H -3.3715295 .7900240 .857749$

H -2.5442995 .2778302 .346432$

C - 1.7546547 .9166851 .222084

H -0.4671846 .2610431 .629595$

C -3.683620 $2.642349 \quad 1.415435$

O $-2.2342752 .088189-0.435524$

H -3.625295 $3.572287-0.550061$

H -4.3136453 .4364161 .833834$

O -2.7235622 .3064262 .425851$

C $-4.534487 \quad 1.3874341 .142193$

C $-5.558561 \quad 1.5706090 .008746$

H $-3.881725 \quad 0.5317250 .933534$

O

H -6.2585512 .3716990 .275656$

H $-5.0335001 .862885-0.906389$

H $-0.944513 \quad 8.6096190 .973761$

H $-2.625370 \quad 8.1826780 .613524$

H -2.021026 8.0689572 .273218

C $0.4434096 .432312-0.917219$

H $-1.7440702 .450420-1.193569$

H -3.208785 $2.167243 \quad 3.256496$

H $-4.618972 \quad 0.7410602 .988218$

H $\quad 0.7095237 .476524-0.717716$

H $0.5046256 .292536-2.004261$

H -1.668579 $6.740039-1.138924$

C $-5.963963-2.004564-0.964033$

O $-5.424889-0.700226-0.723480$

C $-6.357610 \quad 0.284589-0.268742$

C $-7.457990 \quad 0.492143-1.319675$

C $-8.134424-0.834559-1.682682$

C $-7.113108-1.937828-1.985908$

H $-5.138908-2.565397-1.419556$

C $-6.346471-2.7208570 .340596$

H $-6.814227-0.049966 \quad 0.668360$

O $-6.9168101 .087845-2.497213$

H -8.202642 $1.190381-0.920161$

H -8.775678 $-0.678193-2.559022$

O $-8.967448-1.230516-0.596968$

H -7.614227 -2.910788 -2.048218

O $-6.555983-1.671141-3.276852$

C $-5.146674-2.973428 \quad 1.231345$

H $-7.069752-2.1532280 .931067$

H $-6.785389-3.6943300 .091130$

H $\quad-6.2094370 .506891-2.825946$
H $-9.416704-2.052834-0.856824$

H -5.957455 -2.405404 -3.496361

O $-5.495081-3.8735582 .198379$

O $-4.051844-2.4407471 .112764$

C $-4.459140-4.2106503 .126410$

H $-4.880244-4.8874103 .874724$

H -3.643603 -4.723798 2.608534

H -4.092195 -3.312800 3.633075

SCF Energy (B3LYP/6-31G**//MMFF) $=-3245.91499218$

03236

MM̄FF Geometry

C $1.602248 \quad 1.491726 \quad 4.273165$

C 1.3421240 .3796893 .569175

C $-0.011767-0.2818023 .528393$

O $-0.530220-0.1737142 .182438$

C $0.125590-1.7768073 .868756$

C -1.201169-2.505935 3.957378

C $-1.585805-3.5029013 .136482$

C $-2.111600-2.0729655 .078589$

C $-0.828069-4.1104881 .989974$

C -1.132065 -3.493805 0.616109

C $-2.599222-3.6169940 .174574$

C $-2.756651-3.208236-1.292114$

C $-4.226057-3.233222-1.742555$

O $-3.395157-2.7571490 .994188$

O $-4.315149-2.533849-2.993798$

C $-5.081169-2.446517-0.724878$

C $-4.715037-4.661801-2.009718$

O $-6.470053-2.586954-1.011070$

C $-4.804811-2.8367650 .741692$

C $-5.507917-1.9114171 .765184$

O $-5.293610-4.1636120 .975385$

C -1.128409 1.0001621 .840308

O -1.2949951 .9784002 .551400$

C -1.5307690 .9021310 .421096$

C -1.812364 $2.022810-0.259786$

C $-2.2002172 .099527-1.669493$

C $-2.5431720 .809869-2.366626$

C $-5.134617-0.4274331 .677963$

C -6.0952920 .4028720 .842623$

C $-6.3681962 .694905-0.096353$

O $-7.173957-0.0191720 .435071$

C $-5.9252132 .699019-1.525466$

C $-5.5573583 .812532-2.177127$

C $-5.147343 \quad 3.842530-3.621665$

C $-3.8010034 .499225-3.830173$

C $-3.7104985 .788574-4.197849$

C $-2.5656693 .638020-3.694458$

C $-2.2403703 .311052-2.262575$

N -5.6203781 .6916480 .638361$

H 0.8113491 .9512554 .860616

H $2.130178-0.0630012 .964123$

H $-0.703303 \quad 0.2003324 .230030$

H $0.640492-1.8933174 .831857$

H $\quad 0.772441-2.247826 \quad 3.120674$

H -2.563426 -3.953186 3.306619

H -2.494327 -1.063857 4.898935

H -2.976692 -2.735532 5.190484

H -1.571559-2.079683 6.031016

H $0.251470-4.0637402 .168503$

H -1.064028 -5.1820321 .960948$

H $-0.835600-2.4384200 .628153$

H $-0.496726-4.000552-0.120303$

H $-2.916039-4.6569030 .317087$

H $-2.146510-3.847283-1.942481$

H -2.369254 -2.189401-1.427689

H $-3.722197-2.974620-3.626470$

H $-4.839032-1.386074-0.861647$

H $-4.108637-5.133738-2.792218$

H $-5.743469-4.663650-2.387426$

H -4.669590 -5.303000 -1.127083

H $-6.605773-2.307721-1.932786$

H $-6.593824-2.0589341 .722830$

H $-5.208489-2.2525072 .766853$

H $-5.429725-4.2785981 .930506$

H -1.559050 -0.082691 -0.029788

H -1.7245182 .9798590 .252967$ 
H -1.649857 $0.187598-2.486828$ H -2.978364 $0.950972-3.357951$ H $-3.2872410 .250181-1.789743$ H $-5.167443-0.0022392 .689436$ H $-4.115353-0.2992151 .302384$ H $-6.190406 \quad 3.6565480 .395361$ H -7.437531 $2.466896-0.042490$ H -5.930849 $1.748854-2.054438$ H -5.579888 4.765199-1.652506 H -5.919505 $4.392608-4.175546$ H $-5.1336662 .835405-4.056567$ H $-2.7497506 .260031-4.376503$ H $-4.5958446 .403107-4.323291$ H -1.694537 4.161137-4.111279 H -2.681566 $2.748903-4.320033$ H - $1.9761424 .187115-1.668272$ H $-4.692702 \quad 1.9394830 .970401$ C $3.115567 \quad 3.2147603 .169284$ O 3.1511592 .5481931 .892104 C 2.3681293 .3081430 .952455 C 2.1694224 .6779521 .590676 C $2.045124 \quad 4.3013263 .056778$ H 1.3922362 .8129440 .883723 C $3.0416653 .307392-0.424323$ H 3.0382585 .3288371 .440052 H 1.2860195 .1935091 .202276 C 2.2494795 .4650754 .009960 H 1.0430613 .8849393 .216346 C $3.4115401 .894984-0.931143$ O $4.2521974 .068511-0.333635$ H $2.4009043 .825453-1.147753$ H $4.2206021 .495054-0.312476$ O $3.9285252 .020107-2.260259$ C $2.2312840 .909310-0.969886$ C $2.530334-0.487158-1.551178$ H $1.827138 \quad 0.772408 \quad 0.038227$

O $1.1824421 .482608-1.757212$ H $1.565373-0.999727-1.658453$ H $2.909876-0.384451-2.573256$ H 2.1684775 .1333475 .050038 H 3.2346245 .9249303 .877726 H 1.4928496 .2380933 .841024 C $2.9382852 .179253 \quad 4.283939$ H $4.5646114 .230952-1.239505$ H $4.7918942 .462309-2.198327$ H $1.5343441 .630900-2.651818$ H 3.0713382 .6585015 .260920 H 3.7283481 .4221034 .196250 H 4.1041413 .6767853 .291176

C $5.553621-1.003452-1.853192$

O $4.808188-0.847748-0.641645$

C $3.469445-1.346281-0.677294$

C $3.487124-2.838300-1.043061$

C $4.234535-3.094732-2.348782$

C $5.629521-2.472131-2.291294$

H $5.064414-0.426116-2.646295$

C $6.925314-0.341099-1.654052$

H $3.088548-1.2758540 .349898$

O $2.165880-3.356587-1.147489$

H $3.988015-3.400467-0.243886$

H $3.671968-2.724268-3.213865$

O $4.380389-4.504627-2.535772$

H $6.245953-3.065107-1.605375$

O $6.216613-2.569352-3.586860$

C $7.797779-1.017299-0.616370$

H $7.461028-0.324425-2.609830$

H $6.7900970 .694560-1.319899$

H $1.724041-3.194326-0.296601$

H $3.487553-4.890330-2.530443$

H $7.157378-2.340466-3.503394$

O $9.108876-0.754205-0.894737$

O $7.385584-1.6634730 .337182$

C $10.056513-1.3166030 .018357$

H $9.970112-2.4074140 .030918$

H $11.059362-1.048479-0.324411$

H $9.905965-0.9046801 .020709$

SCF Energy (B3LYP/6-31G**//MMFF) $=-3245.90005469$

\section{7}

MMFF Geometry

C $-3.207230-2.072530-2.761006$

C $-1.958296-1.621805-2.959964$

C $-0.662579-2.256541-2.495989$

O $0.083914-1.277883-1.729519$

C $-0.810831-3.513636-1.613441$

C $0.504288-4.204532-1.298849$

C $1.026880-4.347724-0.064876$

C $1.229032-4.795914-2.482312$

C $0.468435-3.8779941 .248631$

C $1.038266-2.5376831 .737272$

C $2.542458-2.5568782 .050602$

C 2.959641-1.2581912.745603

C $4.476850-1.1900112 .980509$

O $3.255615-2.6867850 .818762$

O 4.8088420 .1650543 .321468

C $5.208100-1.5138061 .659297$

C $4.907602-2.0546984 .171085$

O $6.613255-1.6254591 .869017$

C $4.678867-2.7820140 .959904$

C $5.265167-2.986516-0.458537$

O $5.042078-3.9317711 .733888$

C $0.882516-0.414147-2.413803$

O $1.016631-0.331636-3.624304$

C $1.5689840 .442882-1.424188$

C $2.0128341 .652291-1.797207$

C $2.6756662 .628442-0.930729$

C 3.1620412 .1514300 .411817

C $4.972205-1.863665-1.460422$

C $6.082922-0.833400-1.578935$

C $6.6004831 .337626-2.688590$

O $7.184787-0.963780-1.053443$

C $6.3840902 .447128-1.708846$

C $6.1347293 .713636-2.074450$

C $5.9601154 .846204-1.104242$

C $4.6681125 .601989-1.317212$

C $4.6467396 .753145-2.010357$

C $3.4200115 .069451-0.651420$

C $2.8288943 .891859-1.379260$

N $5.7064480 .238243-2.377895$

H -3.375550 -2.978282 -2.186716

H - $1.836137-0.725720-3.566619$

H $-0.096363-2.521521-3.397428$

H -1.451824 -4.250621 -2.113928

H $-1.324316-3.231428-0.690049$

H $1.972735-4.8812370 .025496$

H $2.075918-5.421964-2.180709$

H $0.551541-5.426227-3.067563$

H $1.621605-4.005991-3.129585$

H $-0.622670-3.801657 \quad 1.203571$

H $0.669329-4.6516712 .000530$

H $0.838730-1.7748790 .976723$

H $0.486291-2.2507942 .640958$

H $2.754558-3.4205532 .691487$

H $2.419321-1.1318813 .692148$

H $2.667712-0.4033332 .120720$

H 4.2980580 .4090524 .112240

H $5.059388-0.6539890 .995946$

H $4.405644-1.7214705 .087463$

H $5.981141-1.9538554 .365212$

H $4.678142-3.1137684 .037054$

H $6.910426-0.7993412 .287318$

H $6.337897-3.205844-0.396113$

H $4.807248-3.898609-0.868095$

H $5.020974-4.7123081 .155848$

H $1.6594030 .071291-0.410744$

H $1.8447261 .974812-2.823996$

H $3.738322 \quad 1.2263840 .303869$

H 2.3187151 .9630911 .083413

H 3.8280272 .8594560 .909656

H $4.865596-2.310491-2.457491$

H $4.029381-1.359772-1.226144$

H $6.390467 \quad 1.646100-3.717612$

H $7.6379160 .992779-2.632197$

H $6.4624382 .202825-0.652043$

H $6.0844803 .960064-3.132880$

H $6.8139695 .525190-1.228807$ 
H $6.0111254 .496838-0.065508$ H $3.7292007 .316898-2.142759$ H $5.5460387 .154772-2.465528$ H $2.6473945 .850029-0.625265$ H 3.6346264 .8594100 .400351 H $2.4501844 .138598-2.372226$ H $4.7536420 .288774-2.726985$ C $-5.479764-1.060250-2.311375$ O $-5.084892 \quad 0.165476-1.658513$ C $-6.2125921 .059739-1.645410$ C $-7.4368580 .206751-1.930984$ C $-6.860071-0.788438-2.919413$ H -6.078755 $1.735913-2.501889$ C $-6.2206801 .924783-0.378244$ H -7.791652 -0.321651-1.042535 H $-8.2687470 .787018-2.340969$ C -7.712867 -2.034339-3.091609 H $-6.750173-0.293325-3.893942$ C -6.1510551 .1555490 .955908$ O $-7.3649572 .774709-0.373770$ H -5.340133 $2.571850-0.447154$ H $-5.3748540 .386044 \quad 0.911284$ O $-7.3777890 .452398 \quad 1.177991$ C -5.9070452 .0489452 .191319$ C -4.6675412 .9634642 .127090$ H -6.7890582 .6723632 .381463$ $\begin{array}{llll}\text { O } & -5.771345 & 1.203101 & 3.339568\end{array}$ H $-4.871153 \quad 3.7941361 .441393$ H -4.5189273 .3929823 .126276$ H -7.252267 -2.728357 -3.801621 H $-7.846312-2.563438-2.141910$ H $-8.705302-1.771038-3.471810$ C $-4.436076-1.444465-3.363672$ H -7.362176 $3.276756-1.206539$ H -8.0976531 .1063001 .159183$ H $-6.555714 \quad 0.628923 \quad 3.371771$ H $-4.161443-0.561008-3.952967$ H $-4.862456-2.180061-4.055909$ H $-5.552350-1.828246-1.531705$ C $-1.915351 \quad 0.4343292 .343828$ $\begin{array}{llll}\text { O } & -3.032519 & 1.271585 & 2.674005\end{array}$ C -3.3701632 .2552301 .693819$ C $-2.200704 \quad 3.2304491 .511494$ C -0.9297312 .4699701 .116420$ C $-0.661964 \quad 1.2765752 .039321$ H $-1.715524-0.1403133 .257382$ C -2.273656 -0.5666461 .233304$ H $-3.553301 \quad 1.7655640 .735095$ O $-1.974363 \quad 3.9701722 .707765$ H -2.457446 3.9534690 .728564 H -0.0708253 .1514341 .149227$ O $-1.0636541 .991615-0.218981$ H 0.1200610 .6386521 .613162 O -0.1521851 .7909253 .273785$ C $-3.313236-1.5698581 .691102$ H $-2.665619-0.0704850 .342258$ H -1.383032 -1.1328530 .948966$ H $-1.813006 \quad 3.3337543 .425185$ H -1.1742812.764719-0.798152 H $\quad 0.100662 \quad 1.031398 \quad 3.826002$ O $-3.617259-2.398630 \quad 0.649851$ O $-3.797718-1.6266032 .812831$ C $-4.595183-3.4050350 .928626$ H $-4.251492-4.055266 \quad 1.738781$ H $-5.552299-2.9399991 .183468$ H $-4.727606-4.0089210 .027032$ SCF Energy (B3LYP/6-31G**//MMFF) $=-3245.89602076$

03238

MM̄FF Geometry

C $-0.5347795 .295208-0.699726$

C -1.210281 4.6523970 .264002

C -2.5692534 .0339610 .058864$

O $-2.4508302 .609830 \quad 0.283540$

C -3.5634614 .6007661 .087847$

C -4.9775094 .0781400 .922298$

C -5.6310353 .3349181 .836929$

C $-5.6727734 .465045-0.358119$
C $-5.1406332 .875446 \quad 3.181213$

C $-4.463617 \quad 1.496033 \quad 3.183880$

C -5.3849850 .3395262 .762232$

C $-4.709292-1.0091893 .018979$

C $-5.578330-2.1832032 .541580$

$\begin{array}{lllll}\text { O } & -5.662153 & 0.465295 & 1.366329\end{array}$

O $-4.753486-3.3590942 .509637$

C $-6.036720-1.9205691 .088752$

C $-6.711385-2.4806973 .530869$

O $-6.995953-2.888317 \quad 0.672692$

C $-6.593289-0.4983800 .854368$

C $-6.815950-0.161716-0.637908$

O $-7.883965-0.335925 \quad 1.456127$

C $-1.923447 \quad 1.862483-0.724395$

O $-1.5771342 .250791-1.828656$

C $-1.8064470 .473163-0.233297$

C $-1.002555-0.385829-0.876648$

C $-0.768664-1.783886-0.511750$

C $-1.638903-2.3778720 .564168$

C $-5.569064-0.232253-1.526084$

C $-5.398037-1.560445-2.245149$

C $-3.785516-2.788187-3.695146$

O $-6.261741-2.433120-2.266086$

C $-2.998435-3.740964-2.851917$

C $-1.806091-4.234424-3.219301$

C $-1.021615-5.225034-2.407516$

C $0.395717-4.768312-2.144223$

C $1.412745-5.194928-2.911981$

C $0.641435-3.886008-0.941686$

C $0.194608-2.467556-1.163703$

N $-4.174148-1.640993-2.896375$

H $-0.9870415 .414334-1.680858$

H $-0.7495184 .521048 \quad 1.240494$

H $-2.9356424 .214963-0.958853$

H -3.5945295 .6957601 .009968$

H -3.1894984 .3798082 .093446$

H -6.6483913 .0202361 .605824$

H $-5.2099433 .967165-1.215416$

H $-6.7332414 .190650-0.353949$

H $-5.6172685 .548160-0.508411$

H -4.4525213 .6102483 .612782$

$\begin{array}{llll}H & -5.998576 & 2.855750 & 3.865677\end{array}$

H $-3.587315 \quad 1.5297132 .526146$

H $-4.096361 \quad 1.309123 \quad 4.200647$

H -6.3193660 .4159353 .330535$

H $-4.460455-1.1253514 .081154$

H -3.751852 -1.044538 2.482227

H $-4.382084-3.4897433 .398986$

H $-5.154293-2.0610660 .453397$

H $-6.301481-2.7666564 .507177$

H $-7.314064-3.3331913 .199307$

H -7.373821 -1.6276253 .692514$

H $-6.595837-3.765750 \quad 0.799057$

H $-7.634080-0.769377-1.043619$

H $-7.1797920 .875348-0.681457$

H $-8.180476-1.1962201 .793535$

H -2.367596 0.2024800 .653288

H $-0.446000-0.029372-1.742660$

H $-1.417386-1.9204151 .534171$

H -1.518866 -3.4570690 .677979$

H - $2.697474-2.2170800 .332450$

H $-5.6610150 .528596-2.311819$

H $-4.664440-0.004874-0.954812$

H -3.207058 -2.411151-4.544486

H $-4.681823-3.287695-4.076869$

H $-3.440433-4.059941-1.910997$

H -1.380894 -3.935059-4.174871

H $-1.009919-6.173514-2.960357$

H $-1.517609-5.445943-1.454197$

H $2.435990-4.893362-2.715505$

H $1.243024-5.850730-3.759659$

H $1.713929-3.850568-0.713851$

H $\quad 0.186387-4.346945-0.060782$

H $0.766089-1.949635-1.935074$

H $-3.497687-0.891767-2.780972$

C $1.9480114 .968437-1.118648$

O $2.0035543 .700086-0.435493$

C $2.3287262 .669952-1.398398$ 
C $2.7056433 .400789-2.681632$

C $1.829274 \quad 4.635726-2.603684$

H $1.4057032 .095300-1.541007$

C $3.4346621 .780316-0.816497$

H $3.7658023 .683741-2.673376$

H $2.5207532 .800850-3.577581$

C $2.2688115 .758037-3.526478$

H $0.8047094 .340581-2.860832$

C $4.030098 \quad 0.756584-1.801202$

$\begin{array}{lllll}\text { O } & 2.897403 & 1.086732 & 0.314899\end{array}$

H $4.2264212 .430301-0.428504$

H $4.4815411 .269870-2.658552$

O $2.979113-0.067219-2.321075$

C $5.077095-0.194691-1.183734$

C $6.2100970 .546627-0.452320$

H $4.576922-0.902774-0.513493$

O $5.628493-0.963008-2.258103$

H $6.614903 \quad 1.321402-1.115917$

H $5.788403 \quad 1.0615680 .416553$

H $1.6135046 .628187-3.419972$

H $3.2934666 .078436-3.310446$

H $2.2320365 .433112-4.571407$

C $0.8493545 .848106-0.514841$

H $2.2197560 .470435-0.012687$

H $2.4126250 .489304-2.880879$

H $6.099194-1.718314-1.866538$

H $0.8852016 .849340-0.959454$

H 1.0496535 .9727350 .557210

H $2.9124505 .456878-0.924317$

C $6.333047-1.3303071 .941018$

O $6.937442-1.5414240 .660208$

C $7.380589-0.3525170 .003689$

$\begin{array}{llll}\text { C } 8.420456 & 0.366724 & 0.875499\end{array}$

C 7.9001690 .6228942 .289170

C $7.325659-0.6576552 .895527$

H $5.435947-0.708787 \quad 1.828978$

C $5.895386-2.6982332 .473886$

H $7.898083-0.680490-0.906761$

O $8.808483 \quad 1.604924 \quad 0.289894$

H $9.323702-0.2530030 .947958$

H 7.1580401 .4295232 .304888

O $8.981899 \quad 1.0610943 .115106$

H $8.145942-1.3465563 .134850$

O $6.661768-0.3427624 .117293$

C $4.841912-3.3027571 .575578$

H $6.747836-3.3860922 .533777$

H $5.470012-2.6280113 .481093$

H $9.1510991 .412497-0.599680$

H $9.360428 \quad 1.8542102 .697946$

H $7.306950 \quad 0.1052554 .690854$

O $5.407655-4.2469350 .770168$

O $3.669491-2.9509751 .565610$

C $4.530010-4.856192-0.180330$

H $5.081605-5.647524-0.694744$

H $3.666524-5.3019090 .322664$

H $4.211107-4.114423-0.918218$

SCF Energy (B3LYP/6-31G**//MMFF) $=-3245.90726111$

03239

MM̄FF Geometry

C $-0.5143865 .267826-0.781087$

C -1.325212 4.6035880 .055992

C $-2.5624393 .864869-0.386861$

O $-2.4808652 .506276 \quad 0.103561$

C $-3.807866 \quad 4.5214510 .235932$

C $-5.116777 \quad 3.903786-0.216520$

C $-5.992093 \quad 3.2823310 .598121$

C $-5.435847 \quad 4.045169-1.683067$

C $-5.887843 \quad 3.0673992 .081915$

C -5.2377631 .7375332 .494215$

C $-6.011727 \quad 0.4896652 .038461$

C $-5.433431-0.7706092 .685131$

C $-6.143153-2.0414652 .192206$

O $-5.899678 \quad 0.379673 \quad 0.618516$

O $-5.342938-3.1712392 .576099$

C $-6.191311-2.0328240 .646680$

C $-7.502616-2.2258572 .877393$

O $-7.005375-3.0934390 .154866$
C $-6.660645-0.691006 \quad 0.042255$

C $-6.471719-0.604235-1.489703$

$\begin{array}{llll}\text { O } & -8.065409 & -0.489536 & 0.243246\end{array}$

C $-1.7359821 .621744-0.613556$

O $-1.140007 \quad 1.834791-1.657012$

C -1.7479560 .3361140 .119528$

C $-0.864813-0.620164-0.203595$

C $-0.725152-1.9206300 .454557$

C $-1.820443-2.3427441 .397578$

C $-5.032099-0.764763-1.989984$

C $-4.682751-2.180803-2.418342$

C $-2.750559-3.555402-3.184293$

O $-5.512196-3.080820-2.516978$

C $-2.226351-4.332704-2.018521$

C $-0.982244-4.832362-1.966578$

C $-0.455159-5.646466-0.819923$

C $0.838542-5.097870-0.260695$

C $2.022253-5.630239-0.608847$

C $0.753204-3.9960250 .770756$

C $0.362229-2.6711780 .179419$

N $-3.332085-2.312144-2.713668$

H $-0.7574875 .302843-1.839668$

H -1.0854824 .5710521 .116446$

H $-2.644917 \quad 3.857643-1.480431$

H $-3.8322335 .589113-0.020520$

H -3.7182304 .4740051 .326626$

H -6.9043232 .8824460 .156109$

H -4.756688 $3.436136-2.287299$

H $-6.4572813 .726939-1.918235$

H $-5.3412015 .090752-1.993879$

H -5.3432213 .8915642 .554781$

H -6.8999883 .1185432 .503427$

H -4.2159381 .7045132 .098141$

H -5.1601791 .7316453 .588653$

H -7.0650750 .6150872 .315231$

H $-5.481888-0.7054923 .779325$

H $-4.366484-0.8492942 .437631$

H -5.229353 -3.144676 3.541578

H $-5.170712-2.2358520 .301671$

H -7.373381 -2.333894 3.961181

H $-7.994244-3.1456942 .542575$

H $-8.182540-1.3873492 .712191$

H $-6.657428-3.9221710 .526466$

H -7.153395-1.300354-1.993613

H $-6.8058810 .397699-1.797052$

H $-8.444387-1.2986640 .622394$

H -2.4629850 .2231260 .926119$

H $-0.154134-0.423919-1.005059$

$\begin{array}{llll}H & -2.798632 & -2.243457 & 0.914908\end{array}$

H -1.809532 $-1.725702 \quad 2.302224$

H $-1.748311-3.3860581 .711488$

H $-4.903831-0.137214-2.881306$

H $-4.312647-0.420429-1.241067$

H - $-1.962140-3.295668-3.897793$

H -3.514764 -4.141422 -3.704812

H $-2.907942-4.513651-1.190664$

H $-0.311283-4.673789-2.807990$

H $-0.299728-6.670850-1.183354$

H $-1.193914-5.728905-0.012896$

H $2.952421-5.267172-0.184866$

H $2.087388-6.441328-1.326563$

H $\quad 1.729851-3.856737 \quad 1.253935$

H $\quad 0.086897-4.3159681 .576482$

H $1.096877-2.278527-0.524673$

H $-2.708000-1.527089-2.551621$

C $2.0299785 .309179-0.779096$

O $2.0985513 .974781-0.237104$

C $2.5519973 .083445-1.270564$

C $3.1759183 .976230-2.336365$

C $2.2520905 .178074-2.286449$

H $1.6575622 .601308-1.685865$

C $3.4904082 .000295-0.720939$

H $4.1980714 .268716-2.068097$

H $3.2044473 .495850-3.318951$

C $2.8313926 .423571-2.933742$

H $1.3202774 .913934-2.800304$

C $2.809246 \quad 1.069058 \quad 0.303894$

$\begin{array}{lllll}\text { O } & 4.631039 & 2.624291 & -0.133761\end{array}$ 
H $3.839377 \quad 1.415194-1.578587$

H $1.8999710 .645697-0.136679$

O $2.396037 \quad 1.821246 \quad 1.450858$

C $3.689123-0.0823810 .828292$

C $4.268673-0.965120-0.290855$

H $4.485026 \quad 0.3257801 .460279$

O $2.862551-0.8858871 .677700$

H $3.449296-1.312419-0.932271$

H $4.929470-0.360182-0.918734$

H $2.1340567 .263527-2.852724$

H $3.7741106 .720559-2.462229$

H $3.028506 \quad 6.248411-3.996414$

C $0.7297485 .987167-0.337243$

H 5.2815151 .9305820 .065863

H 1.7470242 .4808961 .153163

H $3.448222-1.4505112 .209835$

H $0.6958157 .013648-0.719866$

H 0.7331006 .0596250 .758158

H $2.8673935 .858840-0.329172$

C $7.106732-1.0993630 .790300$

O $5.982179-1.8619001 .244144$

C $5.041830-2.2004800 .220979$

C $5.732888-3.017192-0.881581$

C $6.994028-2.331148-1.404409$

C $7.897916-1.897720-0.249999$

H $6.761739-0.1570360 .348358$

C $7.961913-0.7650442 .016644$

H $4.303265-2.8626340 .689936$

O $4.849541-3.253668-1.973034$

H $6.016704-3.998126-0.478617$

H $6.747912-1.477704-2.047520$

O $7.723196-3.249651-2.221981$

H $\quad 8.352627-2.782217 \quad 0.214123$

O $8.954201-1.088291-0.761016$

C 7.2376910 .1968222 .929405

H $8.179671-1.6699822 .596661$

H $8.922252-0.3141561 .743170$

H $4.053820-3.684780-1.618417$

H $7.125316-3.542498-2.931153$

H $\quad 9.419020-1.610835-1.437061$

O 7.2133881 .4414412 .371883

O $6.744048-0.1237344 .002823$

C 6.5143542 .4390523 .122423

H 5.4656482 .1529553 .248349

H 6.9948252 .5878524 .094051

H 6.5559113 .3768222 .562113

SCF Energy (B3LYP/6-31G**//MMFF) $=-3245.90595533$

0324

MMFF Geometry

C $-2.3811962 .305996-2.075042$

C $-1.8078511 .383396-1.286975$

C $-0.7101770 .464520-1.761469$

O $\quad 0.3738330 .484783-0.804538$

C $-1.237709-0.980575-1.846126$

C $-0.288313-1.930717-2.552732$

C $0.310906-2.992376-1.979351$

C $-0.054958-1.652483-4.016974$

C $0.186960-3.482459-0.565718$

C $1.262530-2.9588900 .397298$

C $2.704297-3.308240-0.004353$

C $3.669674-2.9979981 .141109$

C $5.133463-3.2422040 .742966$

O $3.055755-2.521251-1.144664$

O $5.966006-2.6290551 .740332$

C $5.424535-2.523619-0.593655$

C $5.481353-4.7360220 .747732$

O $6.725920-2.877859-1.055443$

C $4.358766-2.793217-1.679977$

C $4.517055-1.909389-2.942318$

O $4.462508-4.156494-2.108497$

C $1.2195601 .549812-0.838405$

O $1.1613522 .514686-1.583557$

C 2.2414411 .3414420 .210583

C 2.9926792 .3758650 .615750

C 4.0456942 .3293971 .632202

C 4.5025160 .9715882 .096642

C $4.452552-0.397383-2.699736$
C $5.8114220 .256883-2.514567$

C $6.8482422 .437546-1.898712$

O $6.873001-0.322912-2.725816$

C $7.2091932 .423234-0.446671$

C 7.3934653 .5388800 .275319

C 7.7937093 .5451401 .722892

C 6.8607014 .3707792 .580312

C 7.1622525 .6392442 .905677

C 5.6217663 .6983983 .127755

C 4.5634783 .4928762 .078707

N $5.6966971 .582433-2.117820$

H -2.045893 2.411662 -3.103939

H $-2.1492351 .265215-0.261975$

H $-0.332550 \quad 0.789989-2.738085$

H -2.191663 -1.000219-2.390247

H $-1.458065-1.334718-0.833137$

H $0.967783-3.601775-2.599856$

H $0.483604-2.466966-4.513651$

H - $1.010113-1.530573-4.538357$

H $0.536699-0.741931-4.149915$

H $-0.800852-3.244438-0.159167$

H $0.228904-4.579189-0.584918$

H $1.158862-1.8707490 .481418$

H $1.053067-3.3833261 .386828$

H $2.746186-4.372578-0.263361$

H $3.414165-3.5810122 .034662$

H $3.560436-1.9444501 .431947$

H $5.746392-3.0267062 .600329$

H $5.450794-1.450880-0.367663$

H $5.312571-5.1650691 .742921$

H $6.543332-4.8956450 .530794$

H $4.891281-5.3159350 .035158$

H $6.939477-2.308879-1.813379$

H $5.420166-2.193491-3.495480$

H $3.680893-2.154828-3.613416$

H $4.051194-4.236608-2.985087$

H 2.3420020 .3455270 .625848

H $2.8115703 .355496 \quad 0.174939$

H $4.7621820 .341699 \quad 1.238649$

H 3.7132890 .4755422 .671743

H 5.3940791 .0014602 .725953

H $4.0051130 .076255-3.583020$

H $3.812979-0.163343-1.843577$

H $6.5798223 .439110-2.249069$

H $7.6930192 .073669-2.492457$

H $7.349281 \quad 1.4534140 .025237$

H $7.2801934 .506958-0.207922$

H 8.8121623 .9504681 .784923

H 7.8563192 .5270902 .126977

H 6.5125036 .2299623 .542769

H $8.064196 \quad 6.1170272 .537353$

H $5.1754774 .317673 \quad 3.917569$

H 5.9133892 .7725613 .630833

H 4.1672414 .4249541 .672841

H $4.7770721 .965292-1.918930$

C $-4.8264242 .778155-2.255134$

O $-5.2194911 .516516-1.679210$

C $-6.6116011 .577330-1.319172$

C $-7.1704592 .795472-2.040485$

C $-5.9821023 .738678-1.974798$

H $-6.6506991 .768982-0.240259$

C -7.294042 $0.239455-1.624505$

H -7.420236 $2.572946-3.084290$

H -8.064909 $3.194849-1.553132$

C $-6.0710264 .898644-2.950714$

H $-5.9021214 .134437-0.953083$

C -6.544526 -0.981417 -1.047554

O $-7.3582210 .073698-3.046136$

H $-8.3304820 .263749-1.268337$

H - $-5.597335-1.111235-1.584888$

O $-7.319316-2.152180-1.332785$

C $-6.274206-0.9088940 .466189$

C $-5.470248-2.1193850 .966612$

H $-5.708969-0.0018140 .682260$

O $-7.519746-0.8213381 .152290$

H $-6.029873-3.0398410 .759804$

H $-4.540812-2.1714820 .388121$

H $\quad-5.1855135 .537833-2.878361$ 
H $-6.1500454 .547914-3.985222$

H $-6.9506905 .514490-2.736867$

C $-3.4970573 .213888-1.638240$

H -7.785646 $-0.782862-3.218640$

H -8.142395 $-2.090261-0.817958$

H -7.332628 -0.5734162 .073325$

H $-3.5728903 .210892-0.545071$

H -3.247935 4.235998 -1.944131

H $-4.7158812 .614534-3.335232$

C -3.303002 -0.5228472 .455178$

O $-4.624490-0.8260702 .915682$

C -5.150824 -2.083635 2.479322

C $-4.234665-3.2239372 .943063$

C -2.794233 -3.009898 2.484304

C $-2.312691-1.6161432 .882831$

H $-3.308600-0.4206151 .363167$

C $-2.8876420 .824853 \quad 3.073374$

H $-6.107248-2.2096763 .002230$

O $-4.722021-4.4713282 .459926$

H -4.254242 -3.2737324 .039659$

H -2.695698 -3.177048 1.406023

O $-1.974685-3.992937 \quad 3.120377$

H -2.165211 -1.5825223 .970260$

O $-1.051411-1.3569272 .271485$

C -3.6574212 .0078062 .527354$

H -3.0561320 .8007704 .156685$

H -1.826824 1.0165342 .875534

H -4.101056 -5.159659 2.754125

H -1.080301 -3.914361 2.747709

H $-0.402199-1.9576202 .674085$

O $\quad \begin{array}{llll}-3.172670 & 3.149700 & 3.101385\end{array}$

O -4.5652531 .9508421 .711638$

C -3.8086094 .3641392 .689878$

H -3.3676615 .1877793 .257757$

H -3.632996 4.5389751 .624416

$\mathrm{H}-4.8809224 .3256062 .904201$

SCF Energy (B3LYP/6-31G**//MMFF) $=-3245.91781588$

03240

MM̄FF Geometry

C $-1.4991442 .317648 \quad 1.633716$

C -1.5154653 .3950440 .833769$

C $-2.1981053 .461149-0.514300$

O $-2.3103652 .136992-1.088870$

C $-3.6121524 .041674-0.363443$

C $-4.2504854 .389598-1.693301$

C $-5.1518483 .614988-2.327920$

C $-3.8519765 .722101-2.271457$

C $-5.6323092 .254545-1.909280$

C $-6.9879212 .327176-1.200816$

C -7.524395 $0.930576-0.857409$

C $-8.8916011 .030613-0.177967$

C $-9.419272-0.3459360 .252923$

O

O $-10.516349-0.136177 \quad 1.155111$

C $-8.316439-1.0782481 .045399$

C $-9.979619-1.139543-0.933464$

O $-8.703781-2.4195391 .336372$

C $-6.935914-1.0641710 .346162$

C $-5.858233-1.6275021 .303089$

O $-6.996048-1.892415-0.818378$

C $-1.2021291 .621268-1.686881$

O $-0.1392342 .188848-1.881117$

C - $1.4884240 .205866-2.010576$

C $-0.462403-0.623358-2.252965$

C $-0.547138-2.058548-2.533481$

C $-1.919010-2.662652-2.674396$

C $-4.412020-1.4071890 .833563$

C $-3.420846-2.2047031 .667223$

C $-1.001923-2.8006671 .760434$

O $-3.756693-2.9155282 .611098$

C $-0.703421-3.9359740 .831143$

C $0.525901-4.2106090 .369079$

C $0.834907-5.346697-0.563241$

C $1.583353-4.901172-1.800744$

C $2.893861-5.159882-1.947149$

C $0.794731-4.224454-2.902350$

C $0.603000-2.755801-2.646228$
N -2.115787 -2.038475 1.223866

H $-2.053148 \quad 1.4276661 .344462$

H -0.9743164 .2882531 .137070$

H -1.598157 4.091746-1.181997

H -3.5864364 .9505390 .252824$

H -4.2470423 .3342900 .184816$

H $-5.5788533 .976434-3.262235$

H $-4.3661155 .936703-3.214362$

H $-4.0963476 .528108-1.571856$

H $-2.7761505 .748434-2.470498$

H $-5.7163991 .633065-2.809457$

H $-4.8984401 .753753-1.270939$

H $-6.8722542 .912364-0.278981$

H -7.712599 $2.853095-1.834929$

H $-7.6009530 .356266-1.788332$

H $-9.6161981 .532065-0.831689$

H -8.8076521 .6637300 .716659$

H $-11.189656 \quad 0.3903120 .690770$

H -8.216656 -0.567911 2.013821

H $-10.805929-0.592728-1.403524$

H $-10.402386-2.095448-0.605053$

H -9.235034 -1.338376 -1.706966

H $-9.565017-2.3809951 .786853$

H $-5.955076-1.1508472 .287480$

H $-6.032723-2.7017481 .444701$

H $-6.114891-2.253190-1.002540$

H -2.522000 -0.120220 -1.995488

H $\quad 0.548407-0.218692-2.220060$

H -2.478441-2.574229-1.738067

H -1.906444 -3.722803 -2.933808

H $-2.481591-2.155845-3.466499$

H -4.291044 -1.714624 -0.209988

H $-4.151094-0.3452610 .908812$

H -1.258932 -3.1881582 .751035$

H $-0.154191-2.1177431 .858480$

H $-1.537113-4.5656840 .528988$

H $\quad 1.364228-3.5994070 .693439$

H $-0.077870-5.874708-0.866375$

H $1.431862-6.080284-0.005873$

H $3.431317-4.864602-2.842233$

H $3.460392-5.666317-1.172686$

H $-0.144941-4.763701-3.046089$

H $1.329067-4.321490-3.856872$

H $1.544630-2.214249-2.542433$

H -1.939996-1.453290 0.412633

C 0.2895251 .0802332 .813807

O $1.315964 \quad 1.424527 \quad 1.863886$

C 2.5572640 .8266172 .283922

C 2.2409870 .0283343 .546876

C 1.0403010 .7711764 .102517

H 3.2394001 .6477712 .536084

C $3.162149-0.0200311 .153512$

H $1.975549-1.0088033 .307966$

H 3.0874640 .0015654 .240037

C $0.242709-0.0387855 .109321$

H 1.3848551 .7022704 .571506

C $3.6669070 .807673-0.045866$

O $2.200447-0.9754290 .706184$

H $4.002294-0.5894241 .565105$

H 4.4817801 .4596350 .285395

O $2.6419241 .695170-0.505160$

C $4.128043-0.029294-1.254927$

C $5.159620-1.132167-0.954088$

H $3.258001-0.496307-1.731621$

O $4.6779690 .853767-2.237695$

H $4.686747-1.914562-0.349369$

H $5.434812-1.585323-1.915724$

H -0.6159600 .5310455 .477685$

H $-0.134060-0.9681644 .668888$

H $\quad 0.865745-0.3029485 .970047$

C -0.7211532 .2214742 .916209$

H $1.402623-0.4896760 .436570$

H $1.882677 \quad 1.157561-0.786080$

H $4.0131621 .540842-2.416333$

H -0.2040353 .1661453 .122359$

H -1.4263152 .0382543 .733913$

H -0.2038190 .1745022 .438910$

C $8.3171120 .850768-0.605179$ 
O $7.1329310 .241513-1.135153$

C $6.447511-0.651937-0.254413$

C $7.365495-1.8258780 .123431$

C $8.685731-1.3238960 .718544$

C $9.318573-0.220065-0.137966$

H $8.766626 \quad 1.378936-1.456014$

C $7.987257 \quad 1.894040 \quad 0.473244$

H $6.166572-0.1178600 .657375$

O $7.632841-2.613171-1.035213$

H $6.843949-2.4648650 .846381$

H $9.384398-2.1652620 .803144$

O $8.432412-0.8364822 .032980$

H $10.137949 \quad 0.2607330 .407943$

O $9.903573-0.821762-1.297429$

C $7.1069383 .003063-0.064457$

H 7.4679431 .4359451 .319274

H 8.9079452 .3562090 .846169

H $8.183085-3.365342-0.758016$

H $9.282145-0.5484952 .407917$

H $9.182938-1.193798-1.834657$

O 6.4530193 .6001570 .975519

O $7.0168663 .320140-1.242719$

C 5.5610554 .6591520 .614008

H $4.7843364 .287221-0.061334$

H 5.0842495 .0280481 .525961

H 6.1168035 .4797940 .150602

SCF Energy (B3LYP/6-31G**//MMFF) $=-3245.90720727$

03241

MM̄FF Geometry

C $-2.881132-3.659975-2.285337$

C $-2.275265-3.423227-1.111751$

C $-0.825269-3.743172-0.840759$

O $-0.121719-2.510873-0.568907$

C $-0.730765-4.6369370 .410644$

C $0.677966-5.0823590 .749357$

C $1.327608-4.7646261 .886593$

C $1.347869-5.967989-0.269880$

C $\quad 0.834617-3.9288003 .035144$

C $1.197850-2.4383442 .942056$

C $2.707763-2.1554763 .005730$

C $2.975327-0.6524153 .112229$

C $4.479225-0.3319143 .085679$

O $3.314848-2.6605941 .814667$

O 4.6250911 .0780722 .854735

C 5.132220-1.054807 1.886493

C $5.145561-0.6201714 .436316$

O $6.550783-0.9248711 .924173$

C $4.743386-2.5435241 .781963$

C $5.226025-3.2147120 .473196$

O $5.343334-3.2639292 .865738$

C $0.387673-1.836114-1.635719$

O $0.309008-2.149845-2.812685$

C $1.052309-0.618738-1.120195$

C $1.3661430 .371968-1.968665$

C $1.9698691 .657092-1.608245$

C $2.4980131 .807935-0.206840$

C $4.706712-2.581416-0.822460$

C $5.671847-1.589965-1.450731$

C $5.8309590 .039462-3.327361$

O $6.825837-1.430978-1.062126$

C $5.5992661 .412905-2.781751$

C $5.2079252 .447483-3.540601$

C $5.0063913 .840336-3.017872$

C $3.6312654 .383599-3.332723$

C $3.4336335 .200244-4.381323$

C $2.5048814 .047321-2.382561$

C $2.0125852 .634211-2.538501$

N $5.100731-0.926697-2.528486$

H -2.282067 -4.070352 -3.096631

H -2.835215 -2.996035 -0.284965

H $-0.370400-4.255545-1.697227$

H -1.344524 -5.5369690 .269419$

H -1.174443 -4.099359 1.255536

H $2.335664-5.1547522 .023103$

H $2.294367-6.3802660 .096278$

H $\quad 0.700553-6.813816-0.523149$

H $1.567521-5.407712-1.183847$
H $-0.250789-4.0236883 .145293$

H $1.250540-4.3449463 .961727$

H $\quad 0.782607-2.0287602 .013785$

H $0.702442-1.9211513 .773278$

H $3.118613-2.6786113 .877169$

H $2.511388-0.2371584 .015529$

H $2.496327-0.1382852 .268137$

H 4.1540841 .5481803 .564169

H $4.795321-0.5356380 .981597$

H $4.680670-0.0204015 .228089$

H $6.202484-0.3318384 .425418$

H $5.076123-1.6684674 .733801$

H $6.753830 \quad 0.0253301 .964748$

H $6.319318-3.2997990 .474266$

H $4.856090-4.2503380 .486906$

H $5.386629-4.2041442 .624640$

H $1.230308-0.554883-0.053122$

H $1.1222710 .250939-3.023290$

H 3.1757600 .9826950 .035367

H $1.675303 \quad 1.8128640 .516213$

H $3.0742192 .722415-0.052762$

H $4.564820-3.378309-1.563927$

H $3.735082-2.101469-0.668399$

H $5.480951-0.061835-4.359535$

H $\quad 6.899925-0.194372-3.295727$

H $5.7846761 .569040-1.721609$

H $5.0494462 .293882-4.605879$

H $5.7741944 .480817-3.471357$

H $5.1800963 .892801-1.935882$

H $2.4553785 .616564-4.597932$

H $4.2454385 .466432-5.050159$

H $1.6466354 .706477-2.571319$

H $2.8188014 .283488-1.362382$

H $1.5992622 .425391-3.526346$

H $4.119714-1.078256-2.744558$

C $-5.304305-3.274337-1.456111$

O $-5.144501-1.970615-0.866369$

C $-6.436395-1.480708-0.456773$

C -7.426964 -2.613236 -0.705329

C $-6.775610-3.342667-1.864005$

H $-6.682988-0.650503-1.128462$

C $-6.383066-0.9833900 .992866$

H -7.514540 -3.277324 0.162284

H $-8.428929-2.240932-0.940207$

C $-7.306528-4.752414-2.059978$

H $-6.938147-2.762294-2.782281$

C -5.2890950 .0728531 .253860$

O $-6.113701-2.102861 \quad 1.847272$

H -7.367342 -0.6005641 .287380$

H $-4.306860-0.4028701 .162404$

$\begin{array}{llll}\text { O } & -5.398868 & 0.486896 & 2.621867\end{array}$

C $\quad-5.367570 \quad 1.313597 \quad 0.348750$

C -4.3712262 .4281880 .703894$

H $-5.2342401 .025534-0.697038$

O

H $-4.5332183 .252653-0.001234$

H -4.6489792 .8663081 .673102$

H $-6.814205-5.244131-2.904434$

H $-7.144329-5.369622-1.169793$

H $-8.382353-4.732787-2.263591$

C $-4.333828-3.443572-2.629453$

H -6.077954 -1.768400 2.759856

H -6.2466580 .9539312 .719185$

H $-6.7433392 .608591-0.170254$

H $-4.390669-2.569452-3.289991$

H $-4.644673-4.315218-3.217790$

H -5.095825 -4.016594 -0.674041

C $-2.014510 \quad 4.304153 \quad 0.481812$

O $-2.107397 \quad 3.1325451 .304307$

C -2.8753722 .0518090 .748763$

C $-2.2218651 .616153-0.567756$

C $-2.1237092 .782732-1.545664$

C $-1.4063643 .963156-0.887004$

H -3.0093724 .7451240 .348892$

C -1.1456425 .3173051 .244019$

H $-2.767801 \quad 1.2275261 .462280$

O $-2.9125450 .525717-1.157221$

H $-1.2077121 .261202-0.347087$ 
H $-3.113653 \quad 3.070027-1.918369$ O $-1.3777102 .356109-2.686756$ H $-0.3428363 .716784-0.781275$ O $-1.5048465 .080273-1.767491$ C - -1.8242415 .8272202 .499257$ H -0.2050864 .8401311 .544701$ H -0.9289296 .1906880 .621163$ H $-2.453406 \quad 0.301457-1.984778$ H -1.276490 $3.126882-3.271234$ H $-0.8686875 .751648-1.469892$ O $-0.937725 \quad 6.5970483 .198186$ O -2.9810225 .5954192 .822660$ C -1.4381147 .1600274 .415149$ H -1.7323906 .3653565 .107373$ H $-0.636607 \quad 7.7454904 .873541$ $\mathrm{H}-2.2824267 .8237784 .206280$ SCF Energy (B3LYP/6-31G**//MMFF) $=-3245.90925963$

03242

MM̄FF Geometry

C $5.013423-2.085357-2.540320$

C $5.229733-0.770034-2.385943$

C $5.847971-0.068720-1.201069$

O $4.808475 \quad 0.762930-0.628873$

C $6.370565-0.983144-0.073839$

C $6.976722-0.2159301 .088361$

C $6.481667-0.2177662 .342486$

C 8.2219150 .5731470 .775468

C $5.295943-0.9830112 .863136$

C $3.983661-0.2295452 .648875$

C $2.785525-0.8333903 .396014$

C $2.475473-2.2661282 .959202$

C $1.215480-2.8092653 .646975$

O 1.6649140 .0108063 .105358

O $0.849412-4.0150632 .956729$

C $0.062854-1.7967473 .467953$

C $1.506622-3.2215845 .095026$

O $-1.062194-2.2048014 .242059$

C $0.454216-0.3350173 .793721$

C $-0.6150360 .689823 \quad 3.353566$

O $0.591543-0.1318445 .205074$

C $4.7283162 .066149-1.004355$

O $5.4540222 .659881-1.784107$

C $3.5641602 .643347-0.295220$

C $3.1720743 .902742-0.538692$

C 2.0187394 .5700670 .073538

C $1.2395153 .789807 \quad 1.099523$

C $-0.943666 \quad 0.679797 \quad 1.858695$

C - -1.8238531 .8511451 .453450$

C $-2.7733122 .923871-0.596376$

O -2.3102692 .6403532 .257851$

C -1.829240 $3.864133-1.277441$

C -1.889855 $5.200147-1.165085$

C $-0.9629626 .131342-1.896013$

C $-0.3113997 .160335-0.995314$

C $-0.4999378 .473662-1.210324$

C 0.5612006 .6855550 .149192

C $1.7098745 .825639-0.313541$

N -2.004943 1.8957830 .079148

H $5.309548-2.788844-1.768205$

H $4.907064-0.105038-3.186228$

H $6.6720100 .551077-1.574355$

H $7.132954-1.666317-0.469496$

H $5.546004-1.6100440 .282219$

H 6.9885790 .3841393 .095839

H $8.6936980 .977972 \quad 1.677255$

H $8.961306-0.0650670 .280485$

H $7.991663 \quad 1.4174300 .118791$

H $5.255916-1.9772242 .406492$

H $5.451839-1.1549593 .935609$

H $4.095700 \quad 0.817306 \quad 2.963379$

H $3.748457-0.1861281 .578881$

H $2.994228-0.7884374 .471020$

H $3.320585-2.9364653 .153802$

H $2.318057-2.288321 \quad 1.871660$

H $0.016920-4.3352813 .345006$

H -0.236992 -1.866116 2.415218

H $2.243998-4.0333325 .118073$
H $\quad 0.610491-3.6190125 .584026$

H $1.899552-2.4041765 .703230$

H -1.815200 -1.643906 3.992289

H $-1.5341910 .555663 \quad 3.938085$

H -0.2512901 .6926123 .620853$

H $\quad 0.386999-0.9619155 .663379$

H $3.042831 \quad 1.9988850 .404082$

H $3.7319124 .494463-1.261443$

H 1.8847753 .5012811 .937012

H $\quad 0.8280372 .8840070 .644980$

H $\quad 0.3992864 .3349321 .531610$

H -0.0220820 .7224111 .267944$

H -1.476759 -0.2379191 .591230$

H -3.428103 3.4339640 .116902

H -3.391312 2.419698-1.344166

H $-1.0707743 .421574-1.919623$

H -2.664442 $5.649712-0.548364$

H -1.551676 $6.631068-2.676304$

H -0.180553 $5.573042-2.424942$

H $-0.0393539 .223545-0.575688$

H -1.119697 $8.831185-2.025984$

H $-0.078817 \quad 6.1850490 .879404$

H 0.9931607 .5443530 .679533

H $2.3490766 .310841-1.052503$

H -1.537869 $1.205795-0.500668$

C $2.957553-3.243098-3.383865$

O $2.086656-2.177764-2.949297$

C $0.818671-2.298465-3.626388$

C $0.797802-3.704714-4.204534$

C $2.254664-3.902118-4.570748$

H $0.839398-1.552278-4.430927$

C $-0.316435-1.991248-2.640724$

H $\quad 0.491019-4.434207-3.444408$

H $\quad 0.125476-3.798949-5.062272$

C $2.636909-5.356513-4.783770$

H $2.462718-3.339834-5.490972$

C $-1.718631-2.194803-3.243549$

O $-0.163742-0.627111-2.236988$

H $-0.183928-2.606770-1.743623$

H -1.851918 -3.246482 -3.522190

O $-1.808656-1.450218-4.466482$

C $-2.901759-1.764426-2.354572$

C $-2.919941-2.455828-0.981871$

H -2.916524 -0.674834 -2.236229

O $-4.108031-2.118831-3.042173$

H $-2.917141-3.543211-1.127542$

H -2.010782 -2.182455 -0.435430

H $3.699460-5.448615-5.029599$

H $2.444696-5.957277-3.888571$

H $2.060426-5.788641-5.608366$

C $4.330320-2.670496-3.746098$

H $-0.717909-0.491379-1.450922$

H $-1.645183-0.515485-4.252715$

H -4.086859-1.679072 -3.909418

H $4.216315-1.902207-4.521212$

H $4.972883-3.455939-4.159583$

H $3.042978-3.944865-2.544120$

C $-5.160415-0.1255040 .897951$

O $-4.068012-0.6772050 .151489$

C $-4.152068-2.077135-0.133931$

C $-4.220904-2.8898261 .168114$

C $-5.367008-2.4056922 .062714$

C $-5.361697-0.8821092 .222038$

H -4.8441940 .8964381 .143199$

C $-6.435145-0.0288340 .053348$

H $-5.054465-2.272575-0.722208$

O $-2.985979-2.7707001 .869719$

H $-4.356378-3.9485380 .916504$

H $-5.281083-2.8675163 .053938$

O $-6.613745-2.8061421 .501923$

H $-6.293352-0.5501002 .693808$

O $-4.304767-0.5164993 .113979$

C $-6.2528250 .963486-1.070380$

H $-6.745113-0.987613-0.372163$

H -7.2781070 .3250050 .658760$

H $-3.044443-3.3283002 .664339$

H -6.610685 -3.776544 1.441272

H $-3.464023-0.7488112 .685296$ 
O $\quad \begin{array}{rll}-5.977970 & 0.307063 & -2.232849\end{array}$

O $-6.3227052 .177196-0.924843$

C $-5.7696281 .137830-3.378851$

H -6.647111 $1.765866-3.560149$

H $-4.8756011 .752404-3.241108$

H $-5.6182370 .488931-4.245510$

SCF Energy (B3LYP/6-31G**//MMFF) $=-3245.91424866$

03243

MM̄FF Geometry

C $-2.6592630 .323725-3.308587$

C $-2.016854-0.694198-3.902200$

C $-0.546508-1.031624-3.766030$

O $0.086819-0.171442-2.792144$

C $-0.396267-2.489258-3.285932$

C $1.027649-3.014492-3.288486$

C $1.601446-3.655755-2.251652$

C $1.806975-2.834273-4.566151$

C $0.986759-4.005936-0.926458$

C $1.272824-2.9984880 .196474$

C $2.761737-2.8718010 .561369$

C $2.928322-2.0614271 .848160$

C $4.403734-1.8004162 .186286$

O $3.429919-2.210091-0.514468$

O $4.449722-0.7743443 .190286$

C $5.117298-1.2405980 .936739$

C $5.073020-3.0364912 .798810$

O $6.521748-1.137817 \quad 1.156821$

C $4.844129-2.056905-0.341996$

C $5.393130-1.381237-1.623195$

O $5.489782-3.331057-0.228544$

C $0.742608 \quad 0.928131-3.245561$

O $0.9731821 .212175-4.413187$

C $1.1044791 .842332-2.138555$

C $0.976474 \quad 1.538732-0.838558$

C 1.2873382 .4538170 .254315

C 2.1402291 .8486691 .331754

C $4.749797-0.040197-1.993200$

C $5.5026731 .181885-1.494324$

C $5.3368353 .674775-1.496336$

O $6.5730081 .123701-0.896815$

C $4.6689934 .158802-0.248489$

C $3.8909135 .250829-0.201287$

C 3.2722065 .7855551 .057987

C 1.7688385 .9155140 .965601

C 1.1950107 .0800620 .618302

C $0.9320634 .726031 \quad 1.374814$

C 0.7900853 .7062850 .279346

N $4.8363282 .354404-1.824649$

H -2.112680 $1.014624-2.673185$

H $-2.590133-1.358543-4.546441$

H - $0.086694-0.912572-4.753762$

H $-0.988433-3.152145-3.931054$

H -0.828384 -2.560004 -2.281309

H $2.628388-4.000134-2.369141$

H $2.069822-1.782261-4.713372$

H $2.740432-3.407471-4.568025$

H $\quad 1.216287-3.174563-5.423073$

H $-0.096275-4.132186-1.027077$

H $\quad 1.361001-4.993800-0.628843$

H $\quad 0.878042-2.017606-0.098047$

H $\quad 0.713677-3.321443 \quad 1.082807$

H $\quad 3.178903-3.8777140 .687802$

H $2.424905-2.5571112 .687634$

H $2.425366-1.0937631 .729543$

H $3.939146-1.0833353 .958262$

H $4.749341-0.2190420 .789803$

H $4.562053-3.3293423 .724078$

H $6.109315-2.8246593 .084396$

H $5.067249-3.9016642 .132547$

H $6.651107-0.5977131 .955139$

H $6.486348-1.307868-1.575851$

H $5.185943-2.063830-2.460274$

H $5.608365-3.698635-1.119968$

H $1.4810222 .813402-2.448644$

H $0.6328250 .555920-0.526220$

H 1.5643371 .1228021 .913014

H 2.5475742 .5871912 .026012
H $3.000774 \quad 1.334978 \quad 0.891106$

H $4.7324290 .038593-3.088232$

H $3.7127210 .011476-1.646861$

H $5.1293224 .322314-2.354113$

H $6.4205123 .636116-1.346277$

H 4.8638123 .6013970 .664509

H $3.7183005 .818860-1.112799$

H 3.7171376 .7701171 .254198

H 3.5351285 .1696071 .926830

H $\quad 0.1162247 .1903580 .582498$

H 1.7923867 .9472230 .356359

H -0.0819215 .0556041 .640072$

H 1.3299294 .2990332 .300603

H $0.1648614 .034739-0.551428$

H $3.9391082 .296718-2.296566$

C $-4.7924060 .608998-2.062197$

O $-4.3940721 .811654-1.366817$

C $-5.5467352 .368644-0.723576$

C $-6.6163541 .286638-0.750969$

C $-6.3200210 .636613-2.090931$

H $-5.8919683 .186777-1.371937$

C -5.2226352 .9754820 .642804$

H $-6.4780330 .565440 \quad 0.062503$

H -7.630229 $1.692073-0.685422$

C $-6.971119-0.723583-2.264014$

H $-6.6660091 .303107-2.892804$

C -4.6858302 .0393941 .747401$

O -6.4314563 .5828021 .120888$

H -4.5041053 .7922120 .497299$

H $-5.468227 \quad 1.3345442 .052220$

O -4.4476932 .8859042 .884807$

C $-3.387014 \quad 1.2751611 .414071$

C -2.7293170 .6223112 .646792$

H $-3.607350 \quad 0.4995280 .675875$

O -2.4429662 .1699240 .823250$

H $-2.395497 \quad 1.414417 \quad 3.327237$

H $-3.4807100 .028396 \quad 3.176720$

H -6.696813 -1.170074-3.224689

H -6.672692 -1.416934-1.471879

H $-8.061810-0.632684-2.233429$

C $-4.1364970 .578492-3.442369$

H -6.2253704 .0058441 .972187$

H $-4.4311702 .319553 \quad 3.674837$

H -2.267647 2.8802291 .464094

H $-4.2706431 .543572-3.945298$

H $-4.601596-0.192969-4.065964$

H -4.442149 -0.236465 -1.458734

C $-2.584221-2.4023072 .125508$

O $-1.845833-1.3667851 .465014$

C $-1.501337-0.2448052 .284049$

C $-0.680221-0.7097533 .494758$

C -1.404392 -1.794323 4.283146

C $-1.827840-2.9277373 .353067$

H -3.565737 -2.018411 2.427377

C $-2.795526-3.5347841 .109413$

H -0.8378830 .3804441 .676225$

O $-0.379136 \quad 0.3777054 .362592$

H $\quad 0.277739-1.1165093 .148273$

H -2.264260 -1.390645 4.830452

O $-0.517123-2.3327275 .266174$

H $-0.939449-3.4896083 .037521$

O $-2.669186-3.8305424 .068796$

C $-3.749404-3.1419220 .004692$

H -1.832376 -3.7942340 .655995$

H $-3.212007-4.428157 \quad 1.586792$

H $\quad 0.0847341 .0519613 .837459$

H -0.222326 -1.593078 5.824887

H -2.174114 -4.130930 4.850343

O $-3.445616-3.847310-1.123985$

O $-4.670353-2.3467330 .130169$

C $-4.293840-3.608676-2.251011$

H -3.928693 -4.213504-3.085238

H $-5.320657-3.908260-2.021375$

H $-4.254586-2.555593-2.539921$

SCF Energy (B3LYP/6-31G**//MMFF) $=-3245.90081177$

03244

MM̄MF Geometry 
C $-2.443455-4.022378-0.076016$ C $-1.444887-3.721548 \quad 0.767935$ C $-0.006795-3.6105520 .326186$ O $0.421336-2.2434430 .527102$ C $0.888240-4.5341521 .174679$ C $2.272265-4.7531490 .589565$ C $3.428765-4.4237021 .196952$ C $2.317402-5.438512-0.753945$ C $3.627086-3.7888632 .542779$ C $3.726636-2.2570752 .519220$ C $4.882301-1.7028671 .667996$ C $5.062912-0.2042871 .925902$ C $6.1214110 .423768 \quad 1.006644$ O $4.569173-1.919677 \quad 0.288817$ O $5.978966 \quad 1.8511201 .079687$ C $5.8253260 .016953-0.453178$

C 7.5449250 .1072551 .479744 O $6.8684230 .448854-1.322518$ C $5.586513-1.496266-0.628998$ C $5.116628-1.881123-2.054712$ O $6.814224-2.197050-0.394227$ C $0.225045-1.378931-0.502800$ O $-0.242462-1.647892-1.602005$ C $0.656610-0.004991-0.169905$ C 1.0296800 .4069551 .049956 C 1.4205081 .7779901 .366171 C 0.8244762 .2929752 .645640 C $3.796464-1.250370-2.507950$ C $3.962013 \quad 0.053577-3.271507$ C $2.6453962 .040879-4.014243$ O $5.0250600 .415365-3.768029$ C $3.0497763 .113866-3.053103$ C $2.1927593 .977350-2.487350$ C $2.6152895 .061834-1.538421$ C $2.0652204 .869243-0.142826$ C 1.0431625 .6161200 .307543 C 2.7607603 .8758010 .762367 C 2.2596992 .4718070 .571514 N $2.7649920 .749965-3.363425$ H -2.217945 -4.190536 -1.126711 H $-1.664708-3.5362301 .816430$ H $\quad 0.082500-3.879358-0.732684$ H $\quad 0.417455-5.5218181 .272562$ H $0.955570-4.1233962 .187806$ H $4.358976-4.6433250 .673372$ H $3.329474-5.761937-1.020829$ H $1.684157-6.331691-0.747815$ H $1.971001-4.766381-1.544567$ H $2.828018-4.0837973 .231550$ H $4.546159-4.2010002 .978868$ H $2.776387-1.8550162 .151719$ H $3.844391-1.9163543 .555296$ H $5.798557-2.2471831 .925462$ H $5.310756-0.0182652 .978431$ H $4.108908 \quad 0.308641 \quad 1.751515$ H 6.0773652 .1152512 .010548 H $4.9198980 .557962-0.754705$ H 7.7091430 .5025512 .489459 H 8.2912200 .5950210 .843152 H $7.759753-0.9629331 .507865$ H $6.9715271 .407824-1.197232$ H $5.920504-1.699633-2.778312$ H $4.961108-2.969855-2.060674$ H $6.755831-3.071364-0.813956$ H $0.6245320 .693303-1.001543$ H $1.015856-0.2755741 .895076$ H -0.2629062 .1717282 .632420$ H 1.0158553 .3538132 .820170 H 1.2229831 .7389363 .502456 H $3.299328-1.942313-3.199743$ H $3.124269-1.102097-1.657395$ H $1.6065472 .148848-4.341786$ H $3.2934312 .067435-4.896124$ H $4.1093623 .186966-2.818994$ H $\quad 1.132998 \quad 3.910507-2.721605$ H $2.2733886 .018822-1.953948$ H $3.7084965 .142137-1.486124$ H 0.6635005 .5034951 .317583
H $\quad 0.557340 \quad 6.350851-0.325997$

H 2.6741574 .1906261 .807970

H 3.8399223 .8924410 .563826

H $2.6573891 .977274-0.313596$

H $1.9443000 .386845-2.888830$

C $-4.680480-2.892093-0.041179$

O $-4.612588-2.644664-1.461693$

C $-5.921330-2.287245-1.931154$

C $-6.758709-1.995430-0.693680$

C $-6.170120-2.9880010 .293185$

H $-6.328807-3.193362-2.401425$

C $-5.870976-1.189135-3.001096$

H $-6.609489-0.972054-0.332963$

H -7.828900 -2.143916 -0.866013

C $-6.508162-2.6778321 .740513$

H $-6.543972-3.9910210 .046433$

C $-5.4870770 .236768-2.547413$

O $-7.179608-1.129792-3.584710$

H -5.185551 -1.506934 -3.797150

H $-6.3032140 .672338-1.958388$

O $-5.368778 \begin{array}{lll}1.051963 & -3.722441\end{array}$

C $-4.1737960 .359649-1.757024$

C $-3.8122451 .821048-1.430320$

H $-4.268910-0.187816-0.818482$

O $-3.094146-0.210889-2.493060$

H $-3.5758412 .358067-2.356859$

H $-4.6847552 .310457-0.984599$

H $-6.032111-3.3949232 .416433$

H $-6.177226-1.6740352 .024389$

H -7.590024 -2.729669 1.900808

C $-3.886984-4.1476730 .321135$

H -7.108102 -0.631553-4.416114

H $-6.2598251 .162706-4.094409$

H -3.231885 -1.172745 -2.522040

H $-4.298629-5.020018-0.201123$

H $-3.970939-4.347606 \quad 1.395674$

H $-4.232878-2.0236780 .456636$

C -3.7381941 .5782681 .607384$

$\begin{array}{lllll}\text { O } & -2.742989 & 1.116031 & 0.689339\end{array}$

C $-2.5936171 .926709-0.482462$

C $-2.2200763 .359816-0.080763$

C -3.2063473 .9483640 .926585$

C -3.4147482 .9916342 .101968$

H -4.7114281 .5894091 .100887$

C -3.8805580 .5474862 .731770$

H $-1.7402001 .515104-1.033151$

O $-2.1515654 .208745-1.221118$

H -1.2234583 .3572930 .377425$

H -4.1622854 .1999200 .452481$

O

H -2.5124352 .9895042 .723567$

O -4.4886093 .4627372 .912132$

C -2.6293160 .3754183 .558985$

H -4.7213520 .7982763 .389070$

H -4.104132 -0.439246 2.310356

H $-1.4955113 .827048-1.829271$

H -2.504521 5.7543080 .677090

H -4.2697564 .3704513 .184361$

O $-2.758551 \quad 1.0386574 .743045$

O $-1.653093-0.2657493 .192497$

C -1.6398350 .9437385 .629820$

H -1.406217 -0.1039855 .842401$

H -0.7742871 .4530865 .197484$

$\mathrm{H}-1.9065511 .4393506 .567006$

SCF Energy (B3LYP/6-31G**//MMFF) $=-3245.89631607$

03245

MM̄MF Geometry

C -6.448709 -0.835900 -1.023967

C $-5.7360780 .247994-0.681200$

C $-5.6135590 .773010 \quad 0.724995$

O $-4.261266 \quad 0.512413 \quad 1.167764$

C $-5.8413802 .295196 \quad 0.747114$

C -5.5950062 .9195052 .107342$

C -4.5682913 .7419132 .404819$

C -6.6113892 .5750743 .166063$

C -3.470605 4.2337881 .499958

C -2.1143643 .6384701 .886681$ 
C $-0.9450854 .187221 \quad 1.052675$ C $-1.0116653 .749685-0.413693$ C $0.2223574 .200399-1.211641$ O $\quad 0.261675 \quad 3.691763 \quad 1.652245$ O $0.1929053 .480956-2.453608$ C $1.4948833 .784726-0.442739$ C $0.1482765 .688972-1.571740$ O $2.667704 \quad 4.309740-1.058757$ C 1.4656364 .1880591 .047046 C 2.6636763 .6187081 .842740 O 1.5409075 .6153691 .151428 C $-4.023743-0.687677 \quad 1.764844$ O $-4.833295-1.571348 \quad 1.995868$ C -2.576275 -0.758699 2.057680 C $-2.016753-1.9418312 .350853$ C $-0.595176-2.1760572 .606221$ C $0.306344-0.9718642 .691812$ C 2.8145902 .0971701 .787535 C 3.8214821 .5775402 .800875 C $4.839387-0.5456393 .599728$ O 4.4329442 .3015343 .581227 C $4.416941-1.9786833 .615848$ C $3.695485-2.5276684 .604655$ C $3.220652-3.9532674 .602232$ C $1.723283-4.0467474 .385206$ C $0.900610-4.2713875 .424124$ C $1.262637-3.9021642 .947613$ C $-0.158920-3.4445272 .758417$ N $3.937847 \quad 0.1962522 .738130$ H -7.004538 -1.378072 -0.263832 H $-5.1589650 .760579-1.448115$ H $-6.332922 \quad 0.285413 \quad 1.394617$ H -6.8714912 .5188030 .438677$ H -5.205235 $2.762934-0.010392$ H $-4.5022294 .125298 \quad 3.422815$ H -6.4447643 .1261504 .097853$ H -7.620075 2.8202122 .817831 H -6.5735831 .5078263 .404904$ H -3.6877204 .0261710 .451340$ H -3.4273685 .3265791 .588199$ H -1.9121143 .8406402 .947512$ H $-2.1423882 .545403 \quad 1.800724$ H $-0.9543935 .280596 \quad 1.132149$ H -1.920941 4.119137 -0.901259 H -1.073500 $2.652970-0.459229$ H $0.9313203 .796137-3.000969$ H $1.5657872 .693172-0.514409$ H $-0.7260275 .884148-2.204395$ H $1.0190935 .998010-2.160394$ H $0.0758156 .339648-0.697889$ H $2.7183603 .941242-1.956337$ H 3.5965364 .0924291 .509624 H 2.5438483 .9192962 .893230 H 1.8585655 .8427802 .041025 H $-2.002048 \quad 0.156914 \quad 1.982364$ H -2.649975 -2.827679 2.376539 H $-0.059389-0.2692153 .449122$ H $\quad 0.354937-0.453741 \quad 1.728488$ H $1.329540-1.2213912 .977719$ H 1.8531411 .6152361 .994987 H 3.1518531 .7871900 .792184 H $4.831287-0.0983114 .599704$ H $5.853016-0.4498013 .197289$ H $4.701381-2.5860122 .760259$ H $3.403623-1.9146605 .454437$ H $3.504418-4.4081865 .559736$ H $3.734143-4.5435693 .832572$ H $-0.172665-4.3650605 .302484$ H $1.287021-4.3743866 .433169$ H $1.385503-4.8710172 .446223$ H $1.929317-3.2225512 .407416$ H -0.881086 -4.259369 2.703238 H $3.522189-0.2862191 .945969$ C $-5.375111-2.406629-2.717158$ O $-4.084957-1.777749-2.561327$ C $-3.288740-2.585784-1.679398$ C $-3.876624-3.983038-1.797687$ C $-5.360699-3.660748-1.837899$
H $-3.458137-2.211408-0.663021$ C -1.793234 -2.473279-1.997997 H $-3.565138-4.471195-2.728694$ H -3.600447 -4.627404 -0.957964 C $-6.219008-4.792526-2.375051$ H $-5.689893-3.427397-0.817808$ C $-1.236278-1.047253-1.811073$ O $-1.545289-2.912690-3.331544$ H -1.262164 -3.159393 -1.329659 H -1.448715 -0.696477 -0.794255 O $-1.897230-0.138119-2.699469$ C $0.271764-0.898709-2.084440$ C $1.145249-1.858356-1.259256$ H $0.480771-1.022773-3.153560$ O $0.6374220 .443975-1.747901$ H $\quad 0.838153-1.816184-0.208943$ H $0.973672-2.879161-1.618834$ H -7.272992 -4.498385 -2.406128 H $-5.918038-5.079163-3.388322$ H $-6.132175-5.677569-1.736500$ C $-6.482492-1.386004-2.424420$ H -2.024948 -2.313077 -3.928814 H $-2.839389-0.124765-2.460438$ H $\quad 0.0856611 .038521-2.284943$ H -7.465521 -1.837121 -2.602138 H $-6.388330-0.552483-3.132771$ H -5.443556-2.690478 -3.775105 C $4.415708-1.314692-2.980661$ O $3.051320-1.656475-2.703460$ C $2.648248-1.530653-1.337643$ C $3.490802-2.449621-0.441853$ C $4.986533-2.178131-0.630251$ C $5.377247-2.145034-2.112669$ H $4.572895-1.611477-4.025829$ C $4.6538730 .201322-2.883193$ H $2.790905-0.496169-1.008889$ O $3.205336-3.812235-0.746321$ H $3.205628-2.2773250 .601542$ H $5.559811-2.957947-0.114509$ O $5.303641-0.931682-0.018104$ H $6.397561-1.763430-2.235109$ O $5.367832-3.481074-2.624345$ C $3.8717750 .970872-3.928300$ H $4.3665020 .596395-1.905448$ H $5.7160690 .413943-3.050423$ H $3.723618-4.364016-0.136007$ H $6.259872-0.788614-0.120678$ H $6.011092-3.997154-2.109324$ O $4.0841072 .308229-3.743945$ O $3.1744900 .471924-4.800830$ C $3.4270253 .169084-4.679631$ H $2.3523172 .965351-4.703084$ H $3.5800254 .202341-4.357052$ H $3.8650393 .041497-5.673856$ SCF Energy $\left(B 3 L Y P / 6-31 G^{* *} / / M M F F\right)=-3245.91325084$

03246

MM̄FF Geometry

C -1.954576 -0.614834 3.034170

C $-1.864966-1.6764882 .217202$

C $-0.681901-2.0524071 .350812$

O $0.385964-1.0949741 .524434$

C $-0.161452-3.4396771 .771689$

C $0.937913-3.9871440 .882908$

C $2.192102-4.2574141 .296187$

C $0.544326-4.270503-0.544556$

C $2.767441-4.0965432 .676122$

C $3.452319-2.7450422 .927868$

C $4.742597-2.5323832 .119439$

C $5.444908-1.2429692 .552826$

C $6.694282-0.9530951 .705284$

O $4.409043-2.4494510 .731978$

O 7.0842550 .4080841 .946265

C $6.327076-1.0581690 .208342$

C $7.882970-1.8244522 .128817$

O $7.491570-0.964184-0.607589$

C $5.541739-2.339125-0.141467$

C $5.006900-2.356282-1.594250$ 
O $6.407597-3.473258-0.005896$

C $0.803398-0.390607 \quad 0.441273$

O $0.441376-0.516045-0.716793$

C 1.7705680 .6189540 .916372

C 1.8034201 .8148800 .310819

C 2.6711822 .9345400 .676097

C 3.8808892 .6234521 .517088

C $4.071367-1.202000-1.968478$

C $4.768690-0.045520-2.665460$

C $4.3238702 .244614-3.538673$

O $5.940497-0.082979-3.030094$

C $4.7429873 .257533-2.520806$

C $4.2669354 .511473-2.495690$

C $4.696508 \quad 5.553229-1.503211$

C $3.5275686 .156189-0.757245$

C $2.9685657 .308365-1.164434$

C 3.0614215 .4653190 .504681

C $2.354796 \quad 4.168240 \quad 0.229964$

N $3.9111901 .028256-2.864537$ H $-1.128497 \quad 0.086646 \quad 3.111637$ H -2.713517 -2.356682 2.162418 H -1.032431 -2.0741580 .312922$ H $-0.986543-4.164715 \quad 1.766474$ H $0.177975-3.3781982 .811252$ H $2.893776-4.659628 \quad 0.566345$ H $1.330531-4.798333-1.095173$ H $-0.351706-4.899049-0.573913$ H $0.337121-3.341237-1.082600$ H $1.992492-4.2481003 .435033$ H $3.488581-4.9067242 .843006$ H $2.740955-1.9400742 .711616$ H $3.690911-2.6849713 .997229$ H $5.396219-3.3965852 .286846$ H $5.705358-1.2802583 .618013$ H $4.751040-0.3981342 .445569$ H 7.2465470 .5090282 .899800 H $5.706549-0.184897-0.027773$ H $8.135514-1.6419413 .180426$ H $8.782803-1.5709191 .557536$ H $7.692494-2.8937432 .017625$ H $7.942214-0.134363-0.375052$ H $5.840323-2.447366-2.301259$ H $4.425937-3.282623-1.712295$ H $6.039858-4.206081-0.527001$ H $2.387148 \quad 0.369673 \quad 1.771110$ H $1.1057822 .007348-0.503726$ H $4.437312 \quad 1.7816331 .090131$ H 3.5848162 .3659222 .539576 H 4.5919133 .4497851 .579258 H $3.323316-1.578841-2.677644$ H $3.533394-0.829438-1.091894$ H $3.4749892 .586760-4.138896$ H $5.1611092 .026980-4.209520$ H $5.4852412 .953540-1.786434$ H $3.5439444 .818454-3.248401$ H $5.2320646 .338090-2.053264$ H $5.4209485 .150227-0.784598$ H $2.1470477 .762427-0.620427$ H $3.3178437 .820820-2.054848$ H 2.3553186 .1085921 .046586 H 3.9150305 .3522401 .178380 H $1.453748 \quad 4.283295-0.374677$ H $2.9696120 .991304-2.484639$ C -3.873334 0.9419753 .522083 O -4.5653630 .7273082 .274411$ C -5.953745 1.0601032 .451898 C -5.9914611 .9873293 .656873$ C $-4.943438 \quad 1.3433124 .542787$ H -6.4705140 .1266712 .708261$ C $-6.550528 \quad 1.632501 \quad 1.162946$ H -5.6884693 .0077903 .395967$ H -6.980935 2.0301864 .121566 C $-4.428886 \quad 2.257621 \quad 5.641534$ H -5.3786030 .4463935 .004165$ C $-6.2861170 .770341-0.088235$ $\begin{array}{lllll}\text { O } & -5.976789 & 2.923596 & 0.923392\end{array}$ H $-7.6259151 .796321 \quad 1.300058$ H $-5.2280120 .854207-0.347801$
O $-7.0113451 .350960-1.178377$

C $-6.671373-0.711170 \quad 0.045342$

C $-6.521865-1.547126-1.240785$

H $-6.064959-1.1767210 .830391$

O $-8.036072-0.7870430 .466429$

H $-6.853675-2.567299-1.005856$

H -7.219885-1.171352 -2.000066

H -3.662531 1.7542706 .239437

H $-3.988577 \quad 3.1717915 .229363$

H -5.2437352 .5496896 .311998$

C $-3.139490-0.3401743 .919301$

H $-6.358417 \quad 3.261608 \quad 0.094987$

H $-7.959421 \quad 1.237848-0.992631$

H $-8.247997-1.7250970 .610316$

H $-3.834915-1.1884093 .888466$

H -2.772962 -0.2587024 .949298$

H $-3.164327 \quad 1.762862 \quad 3.358189$

C $-3.567373-0.226860-3.122006$

O $-4.868448-0.385634-2.541314$

C $-5.087361-1.589212-1.797704$

C $-4.871438-2.812689-2.702779$

C $-3.494795-2.778143-3.373653$

C $-3.214328-1.421951-4.025670$

H $-3.653936 \quad 0.659252-3.763224$

C -2.496794 $0.062290-2.059349$

H $-4.388667-1.633760-0.955371$

O $-5.889156-2.876047-3.700488$

H $-4.963095-3.721169-2.096217$

H $-3.442930-3.561403-4.139958$

O $-2.482030-3.043025-2.407260$

H $-2.163109-1.362057-4.331358$

O $-4.001639-1.338184-5.217559$

C $-2.6800341 .415788-1.404071$

H $-2.507785-0.693741-1.270357$

H $-1.5051670 .069998-2.524898$

H $-5.863723-2.044535-4.204365$

H $-2.657100-3.922483-2.031141$

H $-3.767440-0.508250-5.666917$

O $-1.988056 \quad 1.438182-0.226754$

O $-3.3212132 .345556-1.874313$

$\begin{array}{llll}\text { C }-2.032797 & 2.672938 & 0.494154\end{array}$

H $-1.4590522 .548766 \quad 1.416371$

H $-1.576332 \quad 3.472022-0.097587$

H -3.064875 2.9248790 .754000

SCF Energy (B3LYP/6-31G**//MMFF) $=-3245.90718088$

03247

MM̄FF Geometry

C -1.977910 $2.933695-0.644918$

C -1.3503181 .9410800 .002895$

C $-0.4207860 .958008-0.658600$

O $0.8783111 .111800-0.043179$

C $-0.917867-0.477009-0.401384$

C $-0.183099-1.536806-1.199140$

C $0.531406-2.545890-0.663679$

C $-0.325375-1.444897-2.697598$

C $0.764811-2.854968 \quad 0.787630$

C $2.057294-2.2628821 .367344$

C $3.346547-2.767856 \quad 0.697813$

C $4.572790-2.3239661 .500033$

C $5.891038-2.6997640 .806115$

O $3.411694-2.221689-0.622496$

O $6.949051-1.9633551 .439346$

C $5.835799-2.231622-0.664271$

C $6.227856-4.1839800 .989817$

O $6.973336-2.692674-1.387801$

C $4.542309-2.652484-1.392038$

C $4.403120-2.035636-2.806742$

O $4.538686-4.075742-1.558808$

C $1.7681411 .937026-0.653025$

O $1.6236652 .499257-1.730501$

C 2.9988342 .1119380 .150036

C 3.1361461 .6933291 .416604

C $4.332128 \quad 1.8981442 .227663$

C $4.0348862 .394253 \quad 3.615913$

C $4.365840-0.505169-2.858617$

C $5.7323350 .136175-3.031659$

C $6.9164072 .301207-2.671588$ 
O $6.705744-0.463606-3.479680$

C $7.6589692 .102068-1.387782$

C $7.7821973 .043960-0.440677$

C 8.5463442 .8426460 .835941

C 7.6724802 .9494642 .065700

C 7.6736434 .0617012 .819484

C 6.8738021 .7328092 .477060

C 5.5625481 .6233851 .749944

N $5.7259051 .472984-2.660098$

H -1.805121 $3.071351-1.709334$ H -1.5101151 .8193641 .071377$ H $-0.3652461 .149610-1.736292$ H $-1.983345-0.551867-0.657577$ H $-0.846452-0.6835480 .671689$ H $1.003131-3.252791-1.345904$ H $0.064300-2.332902-3.207011$ H $-1.380429-1.349172-2.974586$ H $0.220292-0.579892-3.086271$ H $-0.080409-2.5201431 .397614$ H $\quad 0.784743-3.9462010 .903045$ H $2.006062-1.1712351 .288343$ H $2.085577-2.5097492 .435934$ H $3.304191-3.8617970 .639742$ H $4.543361-2.7368902 .516033$ H $4.547228-1.2337881 .622203$ H $6.941863-2.1828602 .386825$ H $5.895075-1.136451-0.647669$ H $6.336607-4.422527 \quad 2.054720$ H $7.190431-4.4312780 .528695$ H $5.466273-4.8509970 .580764$ H $7.763621-2.388075-0.909864$ H $5.173497-2.443163-3.472468$ H $3.446454-2.386083-3.220382$ H $3.924361-4.300904-2.277080$ H $3.7999692 .652053-0.346237$ H 2.3153761 .2000691 .930997 H 3.5172651 .6217154 .195027 H 3.3924793 .2815683 .575403 H 4.9287452 .6834664 .173131 H $3.777864-0.198773-3.733210$ H $3.866651-0.098113-1.973595$ H $6.5938173 .338155-2.807987$ H $7.5520412 .018950-3.516969$ H $8.1278631 .132245-1.237205$ H $7.3217154 .017358-0.592693$ H $9.341647 \quad 3.5989090 .870043$ H $9.064790 \quad 1.875577 \quad 0.843571$ H 7.0754894 .1332903 .721636 H 8.2691204 .9271462 .548524 H $6.727313 \quad 1.7244503 .562891$ H 7.4604930 .8270222 .277988 H 5.6479501 .2397310 .733811 H $4.8828621 .870264-2.257257$ C $-4.257473 \quad 3.984369-0.721080$ O $-4.916382 \quad 2.704122-0.731867$ C $-6.3348312 .907730-0.582037$ C $-6.561926 \quad 4.416755-0.616229$ C $-5.2508594 .941572-0.066311$ H -6.5992442 .5355510 .414679$ C $-7.1052832 .124940-1.652351$ H -6.719710 $4.784323-1.636735$ H -7.429016 $4.716310-0.019278$ C $-5.0044236 .406811-0.380625$ H -5.2474014 .8001201 .022938$ C $-6.760016 \quad 0.623765-1.718758$ O $-6.8009452 .689378-2.935319$ H -8.182363 2.265346 -1.502358 H $-5.7387120 .498929-2.098083$ O $-7.6168330 .028490-2.702998$ C $-6.911379-0.138854-0.389898$ C $-6.651829-1.646318-0.565581$ H $-6.2119680 .275122 \quad 0.340023$ $\begin{array}{llllll} & \mathrm{O} & -8.231970 & 0.066411 & 0.107762\end{array}$ H -7.430190 -2.071704-1.210524 H $-5.695188-1.774400-1.083801$ H -4.0479206 .7402910 .033363$ H -4.985643 $6.587853-1.460696$ H -5.7940027 .0306300 .051163$
C -2.9258353 .8886690 .025883$

H $-7.2996002 .183656-3.599803$

H $-8.5271590 .072935-2.363180$ H $-8.250107-0.236153 \quad 1.031188$ H -3.107252 3.5706831 .059780 H -2.4498654 .8749990 .063061$ H $-4.0853884 .264351-1.769059$ C $-4.347852-2.041677 \quad 1.405469$ O $-5.736213-1.8898091 .724426$ C $-6.644007-2.4368530 .762961$ C $-6.381203-3.9400270 .592193$ C $-4.923713-4.217667 \quad 0.231974$ C $-3.990205-3.5229091 .221793$ H $-4.115848-1.4813930 .491802$ C -3.539788 -1.4374612 .566112$ H -7.646272 -2.337963 1.197306 O $-7.248078-4.473463-0.402815$ H $-6.617612-4.4508001 .534852$ H $-4.696516-3.918023-0.797817$ $\begin{array}{llll}\text { O } & -4.675081 & -5.623385 & 0.306879\end{array}$ H $-4.034441-4.0425822 .187675$ O $-2.649889-3.623138 \quad 0.745664$ C -3.6627150 .0690562 .647249$ H $-3.903801-1.8516343 .514344$ H $-2.474430-1.6668552 .457238$ H $-7.145889-5.439956-0.396637$ H $-5.184891-6.049611-0.402630$ H $-2.454619-4.567776 \quad 0.621246$ O $-3.136953 \quad 0.4922003 .835231$ $\begin{array}{llll}\text { O } & -4.136154 & 0.788358 & 1.779513\end{array}$ C -3.1815861 .9046444 .061819$ H $-4.2044132 .279853 \quad 3.958113$ H -2.841730 2.0954945 .083171 H -2.5098192 .4161293 .366941$ SCF Energy (B3LYP/6-31G**//MMFF) $=-3245.90388040$

03248

MM̄FF Geometry

C -2.144968 2.5691501 .715681

C $-2.204509 \quad 3.681458 \quad 0.966147$

C $-2.7151503 .726050-0.459505$

O $-2.5525442 .429195-1.083441$

C $-4.2084514 .087850-0.476618$

C $-4.726714 \quad 4.407448-1.865187$

C $-5.4114983 .543564-2.640128$

C $-4.4729315 .814893-2.338085$

C $-5.7164132 .103380-2.341463$

C $-7.1739461 .902587-1.910562$

C $-7.4688770 .425646-1.612040$

C $-8.9331650 .220724-1.224510$

C $-9.219263-1.244328-0.857003$

O $-6.631766 \quad 0.021213-0.526284$

O - $-10.494886-1.294848-0.198830$

C $-8.167647-1.728270 \quad 0.168305$

C $-9.346978-2.121752-2.108301$

$\begin{array}{llll} & -8.3469786 & -3.130207 & 0.397919\end{array}$

C $-6.709104-1.373816-0.207850$

C $-5.764262-1.6283890 .986212$

O $-6.223209-2.187472-1.278457$

C $-1.2909382 .071781-1.451378$

O $-0.3030352 .787250-1.493487$

C $-1.302240 \quad 0.611124-1.682677$

C $-0.167402-0.077761-1.489799$

C $-0.018853-1.532125-1.553086$

C $-1.199083-2.339727-2.024859$

C $-4.341036-1.1188300 .735158$

C $-3.418857-1.5212361 .866330$

C $-1.883557-3.3427192 .599212$

O $-3.247943-0.7995502 .846329$

C $-0.505151-2.9299692 .188666$

C $0.481483-3.7906721 .896033$

C $1.868876-3.3551091 .517574$

C $2.309363-3.8768780 .165186$

C $3.421351-4.6231090 .053732$

C $1.483019-3.545635-1.064596$

C $1.158011-2.079288-1.182918$

N -2.843838 -2.7679331 .675606$

H -2.5450361 .6393451 .320028$ 
H $-1.8191774 .615676 \quad 1.367094$ H -2.134787 4.466914-1.023161 H -4.3938254 .9587800 .166669$ H -4.794103 $3.270829-0.036668$ H -5.770278 $3.889050-3.608660$ H $-4.8909076 .001079-3.333119$ H $-4.9276006 .534724-1.649662$ H $-3.3981206 .014310-2.390287$ H -5.517690 $1.519392-3.249072$ H -5.038852 $1.705563-1.580652$ H -7.362721 2.503127 -1.011062 H -7.847967 2.264044 -2.696841 H $-7.218630-0.162461-2.502881$ H -9.600723 $0.552659-2.029243$ H $-9.1732480 .849557-0.355632$ H $-11.158133-0.914380-0.799911$ H -8.400126-1.237716 1.124103 H - $10.183608-1.781535-2.730437$ H $-9.575223-3.159807-1.843257$ H -8.451089 -2.110702 -2.732579 H $-9.202316-3.3071840 .657913$ H -6.148937-1.121690 1.880766 H -5.729395 -2.701892 1.211295 H -6.908217 -2.831453 -1.517916 H $-2.2502190 .140878-1.916829$ H $\quad 0.7313800 .469978-1.207244$ H -1.592784 -1.938743 -2.965830 H -1.996532 -2.327123-1.277047 H $-0.953037-3.384702-2.229067$ H -3.945984 -1.505288 -0.209919 H $-4.322688-0.0257810 .658322$ H -2.015832 -4.4288412 .575721$ H $-2.091118-2.9846203 .612539$ H $-0.302642-1.8625322 .148996$ H $\quad 0.294190-4.8602541 .952031$ H $2.549225-3.7080392 .303533$ H $1.954196-2.2614551 .524622$ H $3.752883-5.004837-0.906265$ H $4.028393-4.8690780 .918606$ H $\quad 0.577030-4.158103-1.019898$ H $2.021228-3.840253-1.974200$ H $1.973739-1.412047-0.904962$ H $-3.008638-3.2612710 .803076$ C -0.3739671 .4492993 .040419$ O 0.7024041 .9397352 .215114 C 1.9578461 .6519842 .866184 C 1.6284090 .6909963 .998045 C $0.247814 \quad 1.1679994 .405347$ H 2.3073082 .6109743 .269570 C 2.9496611 .1203491 .824706 H $1.585785-0.3425903 .632355$ H $2.3553690 .735648 \quad 4.814361$ C $-0.5209990 .158903 \quad 5.240273$ H 0.3497812 .1032354 .972093 C 4.2823070 .6356122 .421419 O 3.2210142 .1793880 .900278 H 2.4805620 .3146581 .248871 H $4.111083-0.2576263 .033173$ O $4.783106 \quad 1.6367513 .318636$ C $5.395957 \quad 0.344787 \quad 1.396328$ C $4.995888-0.6643610 .308524$ H $5.740711 \quad 1.2800900 .940491$ O $6.513477-0.1992342 .109345$ H $4.735857-1.6191880 .780756$ H $4.113969-0.288406-0.219913$ H -1.517022 0.5369335 .491195 H $-0.645513-0.7904354 .709388$ H $0.007729-0.047426 \quad 6.176468$ C - 1.5076782 .4775203 .072024 H 2.3688832 .4615160 .525030 H 4.9022582 .4554332 .806872 H 6.7614240 .4457682 .793775 H -1.119117 3.4565913 .376935 H -2.274090 2.1788073 .795257 H -0.7190150 .5141762 .581558$ C $7.3905970 .314956-2.389568$ O $6.337371 \quad 0.324171-1.416878$ C $6.124770-0.905779-0.715192$
C $5.778095-2.029482-1.703789$

C $6.839214-2.155822-2.800577$

C $7.173990-0.797976-3.428242$

H $7.2966591 .278038-2.908463$

C $8.7739150 .273345-1.731115$

H $7.036906-1.175538-0.173338$

O $4.505359-1.794858-2.301882$

H $5.706458-2.973793-1.153093$

H $6.470328-2.839759-3.574749$

O $8.012588-2.731544-2.234063$

H $8.059731-0.875125-4.069464$

O $6.086344-0.393872-4.265447$

C $9.0341641 .552872-0.972309$

H $8.906121-0.570765-1.048342$

H $9.5626160 .182926-2.487480$

H $4.530815-0.918350-2.722408$

H $8.665264-2.830237-2.948090$

H $5.978122-1.072620-4.953306$

O $8.712011 \quad 1.389960 \quad 0.342015$

O $9.4466022 .581071-1.493472$

C 8.8736712 .5491321 .165043

H $8.216022 \quad 3.352240 \quad 0.819252$

H 8.5928312 .2819492 .187318

H 9.9181632 .8748281 .161365

SCF Energy $(B 3 L Y P / 6-31 G * * / / M M F F)=-3245.91927064$

03249

MM̄FF Geometry

C $1.079398 \quad 5.487127 \quad 0.227187$

C $2.0688214 .771934-0.329320$

C 2.9182513 .7830020 .427473

O $2.7747922 .493479-0.213119$

C 4.4007994 .1876050 .352376

C 5.3175553 .2680091 .136911

C 6.2736872 .4905970 .591257

C 5.1261843 .2740232 .632303

C $6.6544812 .367135-0.856935$

C $5.945706 \quad 1.235891-1.617495$

C $6.242770-0.171589-1.074952$

C $5.742351-1.241886-2.047979$

C $5.952978-2.661711-1.498622$

O $5.577482-0.3263910 .182274$

O $5.178497-3.564540-2.303452$

C $5.386314-2.737442-0.064365$

C $7.413174-3.111717-1.626412$

O $5.711956-3.9836910 .545565$

C $5.842281-1.5736160 .840926$

C $5.110846-1.5398292 .206053$

O $7.240615-1.7164731 .121289$

C $1.6706501 .763948 \quad 0.100771$

O 0.8045022 .0519530 .910692

C $1.6779760 .554495-0.750122$

C $0.540541-0.135268-0.917295$

C $0.368412-1.329374-1.746441$

C $1.607882-1.988967-2.288084$

C $3.583767-1.4268462 .135011$

C $2.859957-2.7613782 .220726$

C $0.574384-3.7438312 .053442$

O $3.421431-3.8168732 .500754$

C $0.299876-4.2471890 .671779$

C $-0.938966-4.4142760 .184978$

C $-1.241346-4.947785-1.185144$

C $-2.067038-3.987713-2.011181$

C $-3.403398-4.107074-2.084827$

C $-1.330766-2.939682-2.814534$

C $-0.884074-1.776681-1.971665$

N $1.498340-2.6266101 .984574$

H $\quad 0.8854305 .388581 \quad 1.291579$

H $2.2589324 .866419-1.396203$

H 2.6001043 .7128831 .474772

H 4.5285165 .2077710 .738085

H $4.7016474 .222514-0.700218$

H 6.8706351 .8712161 .260023

H 5.9110952 .7153133 .153513

H 5.1489414 .2998313 .014412

H 4.1679392 .8199122 .901754

H $6.4828683 .311385-1.384574$

H $7.7394652 .208552-0.907973$ 
H $4.8652251 .419016-1.601946$ H $6.2674661 .292182-2.665048$ H $7.325661-0.264267-0.931320$ H $6.226330-1.135922-3.026838$ H $4.668648-1.094979-2.228263$ H $5.474105-3.472979-3.225506$ H $4.294111-2.709625-0.154884$ H $7.727196-3.098676-2.677007$ H $7.538650-4.146920-1.290332$ H $8.107178-2.480493-1.067697$ H $5.384716-4.687527-0.040506$ H $5.424543-2.3910752 .822272$ H $5.468544-0.6480182 .740903$ H $7.446016-1.2134541 .926763$ H $2.6072930 .282424-1.236248$ H $-0.3613480 .223298-0.422503$ H $2.319771-2.187379-1.479673$ H $2.091462-1.347872-3.032806$ H $1.416006-2.953355-2.763062$ H $3.239577-0.8391182 .995856$ H $3.270954-0.8973061 .230986$ H $-0.332241-3.3843562 .550172$ H $1.008490-4.5457282 .658968$ H $1.156242-4.5048540 .053164$ H -1.792834 -4.1803320 .817029$ H - -1.782148 -5.895400 -1.062909 H $-0.322851-5.202628-1.728129$ H -3.998958 -3.434726 -2.693499 H $-3.933091-4.873028-1.528082$ H -1.989903 -2.536543 -3.595379 H $-0.510909-3.414916-3.359780$ H -1.711926 -1.225059-1.527139 H $1.126931-1.7200701 .715698$ C $-1.2487905 .920435-0.702179$ O $-1.2644874 .624324-1.335382$ C $-2.2021993 .780424-0.630101$ C -3.0715714 .7294510 .179691$ C -2.0569725 .7807730 .588229$ H -1.5976343 .1583860 .042102$ C $-2.9428262 .894837-1.637648$ H -3.854816 $5.172885-0.447623$ H -3.5477784 .2419531 .035500$ C -2.6818797 .0793111 .066909$ H $-1.4400675 .366763 \quad 1.394799$ C $-4.1122592 .094509-1.020823$ O $-1.9621752 .010096-2.188391$ H -3.309806 $3.510130-2.467872$ H $-4.8692552 .803774-0.667725$ $\begin{array}{llll}\text { O } & -3.635144 & 1.387408 & 0.120040\end{array}$ C $-4.7819331 .119350-2.007451$ C $-6.1473840 .564287-1.558893$ H $-4.9523571 .657382-2.948834$ O $-3.8980730 .043356-2.307833$ H $-6.8656531 .393061-1.520044$ H -6.479773 -0.108722 -2.359843 H -1.9092567 .8085881 .330269$ H -3.319943 7.5262380 .297108 H -3.2991026 .9048241 .954263$ C $0.1927256 .418985-0.552600$ H -2.387448 $1.496424-2.895270$ H $-2.9775450 .742621-0.193564$ H -4.339954 -0.535951-2.951508 H $0.2008047 .402031-0.068294$ H $0.6129316 .560016-1.556832$ H -1.770047 $6.597900-1.391335$ C $-5.181995-2.0596690 .963833$ O $-5.241681-1.250933-0.221759$ C $-6.215138-0.203693-0.221299$ C -7.631121 -0.7709900 .009779$ C $-7.692182-1.6567171 .256941$ C $-6.554265-2.6811331 .273981$ H -4.499106 -2.880270 0.710171 C $-4.591760-1.3019982 .165466$ H $-5.9841790 .501200 \quad 0.583099$ O $-8.034817-1.537388-1.124867$ H -8.3325000 .0668920 .104604$ H -8.651584 -2.187520 1.284625 O $-7.612178-0.8494052 .427129$
H $-6.513041-3.1901752 .244171$

O $-6.804740-3.6850450 .286903$

C $-3.106010-1.0509542 .030978$

H $-5.071479-0.3304262 .307345$

H -4.726070 -1.899458 3.074793

H -8.944165 -1.842712 -0.965418

H -8.357382 -0.2252542 .405408$

H -7.652944 -4.106631 0.506405

O $-2.685890-0.3216233 .107626$

O $-2.401598-1.438493 \quad 1.110572$

C -1.2973520 .0217853 .116536$

H -1.127391 0.7325423 .929435

H $-0.695197-0.8722593 .298691$

H -1.012305 0.4958062 .173594

SCF Energy $(B 3 L Y P / 6-31 G * * / / M M F F)=-3245.89717095$

0325

MM̄MF Geometry

C $3.114623-0.8197103 .460669$

C $2.330863-1.4616482 .580708$

C $0.852305-1.6820262 .773717$

O $0.137604-1.0390531 .694514$

C $0.553330-3.1919962 .710052$

C $-0.896575-3.5473882 .974114$

C $-1.714691-4.1399582 .082166$

C $-1.406905-3.2281384 .356213$

C $-1.402900-4.569347 \quad 0.675637$

C $-1.741144-3.526159-0.400694$

C $-3.238188-3.195938-0.511063$

C $-3.510675-2.319261-1.735402$

C $-4.986557-1.896222-1.822804$

O $-3.635821-2.4959140 .669450$

O $-5.083475-0.832379-2.782760$

C $-5.430964-1.316650-0.461439$

C $-5.873427-3.032796-2.344982$

O $-6.834862-1.072297-0.444987$

C $-5.036754-2.1995120 .740351$

C $-5.290060-1.5209652 .108570$

O $-5.817827-3.4007250 .717926$

C $-0.1227860 .290209 \quad 1.825037$

O $0.207568 \quad 1.0188962 .746986$

C $-0.8797530 .717943 \quad 0.627972$

C -1.0042162 .0263070 .359559$

C -1.679928 $2.607129-0.802436$

C $-2.5259471 .690712-1.644907$

C $-4.542129-0.2025712 .337756$

C -5.3667391 .0354912 .026778$

C -5.1660373 .5147901 .889401$

O -6.5694531 .0025301 .781562$

C -5.0275053 .8982470 .450239$

C -4.4836185 .0563590 .047351$

C $-4.3763165 .475206-1.390444$

C $-2.9630055 .838073-1.785940$

C $-2.5638777 .120497-1.826137$

C $-2.0420004 .720047-2.218031$

C $-1.5124493 .922040-1.056166$

N -4.603312 2.1934212 .094510

H $2.679842-0.4068604 .367121$

H $2.773720-1.8656941 .673001$

H $0.517804-1.2741763 .735351$

H $1.170030-3.7231623 .447634$

H $0.865970-3.5669681 .729576$

H -2.736047 -4.356505 2.394233

H $-2.403229-3.6457614 .538012$

H $-0.736196-3.6444255 .114937$

H -1.474592 -2.145931 4.502912

H -0.348631 -4.849055 0.580320

H -1.960462 -5.4922330 .470912$

H $-1.175177-2.609858-0.195882$

H -1.390582 -3.915793 -1.364385

H $-3.794054-4.138125-0.584488$

H -3.203349 -2.829700 -2.656733

H -2.892860 -1.413078-1.677077

H $-4.737302-1.160892-3.630223$

H $-4.944186-0.340195-0.358578$

H $-5.544989-3.346965-3.343069$

H $-6.911328-2.702315-2.462810$

H $-5.862383-3.915305-1.701926$ 
H -7.037239 -0.493774-1.200045 H -6.367402 -1.413011 2.282388 H $-4.941212-2.2186942 .883838$ H -5.825761 -3.780236 1.612306 H - $-1.286491-0.053753-0.015033$ H -0.5346642 .7429761 .032389$ H $-3.2257351 .132555-1.014554$ H -1.898296 $0.978036-2.190244$ H -3.140298 2.215954 -2.379031 H -4.281612 -0.1339213 .401985$ H $-3.606725-0.1726201 .770065$ H -4.6335914 .2016202 .554921$ H -6.2245043 .5076542 .168623$ H -5.415569 $3.204683-0.292075$ H -4.1214815 .7603210 .793410$ H $-5.0380686 .339283-1.535566$ H -4.755299 $4.697277-2.064852$ H -1.560196 $7.391151-2.136589$ H -3.231153 $7.928358-1.544101$ H -1.172146 5.134451 -2.745387 H $-2.5519184 .105375-2.964961$ H $-0.8796504 .502146-0.382711$ H -3.6013772 .1221932 .246515$ C 5.0361370 .6895952 .730742 O $4.461896 \quad 0.897072 \quad 1.426926$ C 4.1245712 .2929421 .292802 C 4.7766903 .0002812 .475647 C 4.6772821 .9279103 .547854 H 3.0373912 .3669641 .412031 C $4.5346002 .798314-0.095094$ H 5.8264043 .2445962 .274934 H 4.2585343 .9260482 .743084 C 5.5867682 .1680954 .739270 H 3.6370291 .8789183 .893283 C $3.9378891 .958765-1.246129$ O $5.9621962 .728655-0.196677$ H $4.2676283 .855506-0.203948$ H $4.3711250 .956448-1.202775$ O $4.3715942 .523389-2.487789$ C $2.4028571 .873514-1.243383$ C $1.8005720 .992150-2.351279$ H $2.0453831 .482184-0.286122$ O $1.8523473 .182901-1.399856$ H $0.7113861 .114017-2.304046$ H $2.1031221 .372147-3.335216$ H 5.4911161 .3595395 .470719 H $6.6378282 .227527 \quad 4.437272$ H 5.3270723 .1076965 .237782 C $4.599153-0.6666103 .283355$ H $6.2033683 .021223-1.092328$ H $3.9555003 .398354-2.572948$ H $2.0353593 .680547-0.585335$ H $5.085640-0.8521364 .248013$ H $4.956450-1.4503442 .603041$ H $\quad 6.1235180 .6546332 .580999$ C $3.910013-2.051972-2.867331$ O $3.384469-0.718336-2.913668$ C $2.165789-0.494928-2.195663$ C $1.055428-1.413084-2.727424$ C $1.490054-2.879868-2.678629$ C $2.863325-3.082691-3.325058$ H $4.719719-2.059943-3.608599$ C $4.522973-2.371188-1.494133$ H $2.323824-0.709623-1.133095$ O $0.730491-1.042898-4.065013$ H $0.156019-1.268951-2.116476$ H $\quad 0.742524-3.492882-3.196475$ O $1.529699-3.297790-1.317077$ H $3.237994-4.093625-3.126839$ O $2.732914-2.960154-4.744422$ C $5.794726-1.586640-1.240811$ H $3.830492-2.144131-0.679941$ H $4.790044-3.433150-1.448016$ H $0.010991-1.624564-4.363606$ H $1.774866-4.238599-1.305341$ H $2.105597-3.641193-5.041443$ O $6.225753-1.840527 \quad 0.030403$ O $6.349174-0.855293-2.049627$
C $7.430124-1.1697300 .414178$

H $8.266943-1.520212-0.197337$

H $7.310448-0.0860320 .320533$

H $7.634561-1.4102731 .460807$

SCF Energy (B3LYP/6-31G**//MMFF) $=-3245.89435220$

03_250

MM̄FF Geometry

C -2.803940 -3.262474 2.017379

C $-1.911165-2.3471531 .610480$

C $-0.817199-2.5049890 .575966$

O $0.388856-1.8913501 .104924$

C $-0.472144-3.9613170 .204327$

C $0.549232-4.087520-0.910600$

C $1.785516-4.604134-0.771352$

C $0.091652-3.622635-2.269998$

C $2.445087-5.1385460 .467871$

C $3.332954-4.1245821 .207788$

C $4.475814-3.5406190 .359354$

C $5.443847-2.7378631 .231664$

C $6.539657-2.0519890 .399019$

O $3.907353-2.679573-0.631062$

O $7.179492-1.0736111 .233492$

C $5.884692-1.285677-0.772062$

C $7.632842-3.040633-0.024248$

O $6.873249-0.777376-1.663696$

C $4.854836-2.125373-1.553204$

C $4.055844-1.305844-2.596940$

O $5.538749-3.159543-2.271904$

C $0.478762-0.5343681 .025062$

$\begin{array}{llll}\text { O } & -0.341083 & 0.227080 & 0.536796\end{array}$

C $1.740485-0.1154851 .671105$

C $1.876677 \quad 1.1605482 .062019$

C 3.0504251 .7480042 .710485

C 4.3116960 .9269342 .736260

C $3.236665-0.138705-2.035434$

C $3.9487551 .202145-2.095646$

C $3.6956113 .568356-1.357044$

O $5.0010631 .381431-2.702677$

C $4.5209013 .769224-0.125191$

C 4.3089174 .7659130 .747639

C 5.1509345 .0045851 .968437

C 4.3286865 .0852553 .235226

C 3.9675636 .2728303 .750060

C 3.9961893 .7926133 .946689

C 2.9470892 .9914093 .224962

N $3.2460022 .190156-1.418549$

H -2.808410 -4.261456 1.594013

H -1.982093 -1.357479 2.060267

H -1.122682 -1.962961 -0.324852

H - $-1.374537-4.490459-0.126976$

H -0.125347 -4.478899 1.104687

H $2.415802-4.655439-1.659162$

H $-0.071148-2.541030-2.274943$

H $\quad 0.823437-3.844215-3.054544$

H $-0.841470-4.122879-2.546248$

H $1.703532-5.5370131 .167805$

H $3.056671-6.0024330 .176996$

H $2.699180-3.3117711 .580459$

H $3.756332-4.6307592 .084237$

H $5.000657-4.369101-0.130800$

H $5.892243-3.3754382 .003719$

H $4.885089-1.9634441 .773575$

H $7.533035-1.5315942 .015292$

H $5.377378-0.415790-0.338447$

H $8.106268-3.4882460 .857819$

H $8.436457-2.532661-0.568572$

H $7.256451-3.856502-0.644679$

H $7.486376-0.235445-1.138205$

H $4.717786-0.982910-3.409392$

H $3.337912-1.994633-3.065666$

H $4.966131-3.462803-2.995751$

H $2.510049-0.8630711 .817936$

H 1.0416351 .8434991 .907982

H 4.1877510 .0507163 .381506

H 5.1842901 .4745833 .097466

H 4.5658220 .5880561 .726287

H $2.329125-0.028085-2.643133$ 
H $2.919385-0.339529-1.008532$

H $2.8033334 .202313-1.366445$

H $4.2958403 .795530-2.244007$

H 5.3445323 .0795380 .043990

H 3.5017415 .4708270 .560900

H 5.6963345 .9455041 .818119

H $5.921448 \quad 4.2318222 .082418$

H 3.4008396 .3412424 .672638

H 4.2267117 .2038403 .256818

H 3.6065344 .0048114 .951380

H 4.9214553 .2354144 .116151

H 1.9861183 .5028983 .151390

H $2.4078271 .940275-0.901558$

C $-5.206359-2.5863112 .571346$

O $-5.146411-1.3188341 .890108$

C $-6.200278-1.2856940 .902619$

C $-6.960138-2.6065161 .033410$

C $-5.892793-3.5311361 .591978$

H -5.714587-1.264305 -0.078093

C -7.063762 -0.0321711 .100080$

H -7.801232 -2.5237111 .731836$

H $-7.361389-2.946110 \quad 0.073408$

C $-6.448374-4.7918092 .228487$

H $-5.214393-3.8052180 .774225$

C $-6.276872 \quad 1.292672 \quad 0.996318$

O $-7.646402-0.0984782 .408920$

H -7.897544 -0.0379130 .388287$

H -5.5020041 .3219881 .771721$

O

C $-5.660558 \quad 1.541226-0.394914$

C $-4.8512942 .848776-0.476735$

H $-4.9948070 .714110-0.644475$

O $-6.6956801 .554728-1.376799$

H $-5.4806543 .692331-0.170898$

H -4.0255652 .7876350 .241048$

H $-5.641190-5.4167222 .623289$

H $-7.129323-4.5587583 .053992$

H -7.003040 -5.382170 1.491857

C $-3.823718-2.9854213 .087430$

H -8.1868150 .7019172 .524973$

H $-6.680837 \quad 3.1373181 .524337$

H -7.310184 2.272023 -1.144383

H -3.907678 -3.878871 3.717518

H $-3.450636-2.1841033 .738985$

H -5.851527 -2.429942 3.447193

C $-2.3918341 .697954-1.932502$

O $-3.6605022 .042644-2.500969$

C -4.316276 $3.158907-1.894372$

C $-3.4201564 .404002-1.969821$

C -2.028967 4.140193 -1.398486

C -1.423998 $2.884910-2.024701$

H -2.526611 $1.401201-0.885352$

C $-1.8527010 .492645-2.718835$

H $-5.1963603 .363718-2.517453$

O $-4.0081035 .501414-1.279634$

H $-3.3089664 .706632-3.019210$

H -2.049651 4.062046 - 0.305170

O $-1.1773225 .246630-1.705489$

H -1.173082 $3.087100-3.073933$

O $-0.2095862 .562376-1.351904$

C $-2.713808-0.738243-2.531752$

H $-1.8392600 .741033-3.787363$

H $-0.8365530 .230533-2.405810$

H $-4.8799105 .663862-1.678782$

H -1.594339 $6.043251-1.334494$

H $0.3665263 .344398-1.401782$

O $-2.664763-1.505410-3.659583$

O $-3.331916-1.009326-1.511618$

C $-3.451506-2.700676-3.623793$

H -3.205177 -3.302028 -2.743345

H $-4.515179-2.444919-3.628780$

H $-3.224453-3.283950-4.520199$

SCF Energy (B3LYP/6-31G**//MMFF) $=-3245.91254303$

03_251

MMFF Geometry

C -3.265090 -2.739876 1.869234

C $-2.063668-3.2856752 .109598$
C $-0.854434-2.984208 \quad 1.260875$

O $0.032346-2.1157102 .002469$

C $-0.086720-4.276106 \quad 0.935091$

C $0.945584-4.085049-0.159821$

C $2.260667-3.8668460 .040304$

C $0.410178-4.205523-1.564094$

C $2.985210-3.7187821 .349704$

C $3.782386-2.4089401 .493689$

C $5.063430-2.3578070 .643766$

C $5.995684-1.2475541 .136218$

C $7.252209-1.1243250 .260201$

O $4.695463-2.100044-0.713195$

$\begin{array}{llllll}\text { O } & 7.893484 & 0.108415 & 0.622040\end{array}$

C $6.818157-1.019769-1.217839$

C $8.262587-2.2370610 .560918$

O $7.947821-1.037916-2.086589$

C $5.788837-2.091857-1.638680$

C $5.213841-1.855386-3.058416$

O $6.434445-3.372035-1.664962$

C $-0.257657-0.7858841 .994989$

$\begin{array}{llll}\text { O } & -1.229272 & -0.247136 & 1.489199\end{array}$

C $0.842722-0.081438 \quad 2.687004$

C 0.8408361 .2590372 .716438

C 1.8967662 .1164753 .257292

C 3.1127181 .4428963 .837285

C $4.649341-0.464006-3.357349$

C $3.508327-0.070856-2.438874$

C $2.7735191 .727194-0.883654$

O $2.510328-0.774735-2.303764$

C $3.5127902 .550467 \quad 0.121993$

C $3.3271893 .867747 \quad 0.297359$

C 4.0701404 .6872251 .316425

C 3.1495035 .3480372 .320844

C 2.7801466 .6320932 .179288

C $2.7176194 .538456 \quad 3.527244$

C 1.7446223 .4533903 .154048

N $3.7358281 .132781-1.791056$

H -3.362029 -2.040189 1.042506

H $-1.935782-3.9543852 .956714$

H $-1.148672-2.4901280 .326681$

H $-0.786138-5.0585660 .610295$

H $0.389124-4.6696361 .841071$

H $2.894324-3.808051-0.841927$

H $1.182856-4.038445-2.322062$

H $-0.003509-5.206086-1.727018$

H $-0.381796-3.471619-1.737026$

H $2.286087-3.766173 \quad 2.189964$

H $3.662655-4.5728841 .469410$

H $3.134890-1.5605421 .240391$

H $4.054664-2.3114452 .552088$

H $5.565687-3.3298140 .709911$

H $6.276649-1.4086712 .184525$

H $5.459938-0.2880021 .114384$

H 8.6487650 .2347190 .022254

H $6.370703-0.025829-1.328325$

H $8.562849-2.2031521 .615273$

H $9.184708-2.101925-0.015078$

H $7.873454-3.2368650 .358452$

H $8.257925-1.957399-2.149415$

H $5.989227-2.077888-3.803315$

H $4.417120-2.592252-3.232439$

H $5.904229-3.969507-2.218400$

H $1.642880-0.6786413 .108055$

H $\quad 0.002242 \quad 1.7869042 .263770$

H 3.6027690 .8147643 .084904

H 2.8312070 .8135664 .688904

H 3.8684582 .1407714 .202250

H $5.451103 \quad 0.281257-3.338677$

H $4.250429-0.449591-4.379254$

H $2.2010660 .938579-0.384684$

H $2.0825872 .325385-1.485545$

H $4.2293752 .027493 \quad 0.751180$

H $2.6107574 .392394-0.330609$

H 4.6451865 .4500560 .775320

H 4.8145894 .0832091 .850305

H 2.1401217 .1203052 .906628

H 3.1057837 .2221811 .329004

H $2.221355 \quad 5.1847274 .262921$ 
H 3.6111364 .1617304 .031362 H $0.818903 \quad 3.8357162 .721437$ H $4.5089271 .714556-2.101400$ C $-5.795114-2.9507511 .892157$ O $-5.971467-1.6370221 .320318$ C $-6.360426-1.787064-0.057595$ C $-6.871336-3.216557-0.182731$ C $-5.908823-3.947295 \quad 0.736744$ H $-5.449207-1.689612-0.659125$ C $-7.357690-0.694552-0.450032$ H -7.898580 -3.3140690 .187291$ H $-6.841999-3.581389-1.213688$ C $-6.389658-5.3239771 .159927$ H $-4.949040-4.0539910 .215899$ C $-6.8530620 .743994-0.201581$ O $-8.563811-0.8665610 .305163$ H -7.642470 $-0.814434-1.501576$ H -6.7804350 .9380040 .875579$ O $-7.8473111 .645754-0.705361$ C $-5.5052391 .076527-0.872588$ C $-5.1179162 .558485-0.694980$ H $-4.7236300 .441256-0.442240$ O $-5.6043610 .771165-2.262799$ H $-5.8585343 .194812-1.194742$ H -5.1401712 .7865410 .377719$ H $-5.670355-5.7946241 .837602$ H $-7.354997-5.2721001 .674605$ H $-6.509221-5.9745240 .287403$ C $-4.492412-2.9897232 .698428$ H $-8.327649-0.8263471 .247901$ H $-8.6877801 .422884-0.269594$ H $-4.7027980 .740484-2.624240$ H $-4.413600-3.9492043 .222260$ H $-4.536086-2.1971803 .456080$ H -6.632197 -3.087689 2.589081 C $-1.3930692 .301678-0.999184$ O $-2.7468512 .149526-0.548877$ C $-3.7261042 .907532-1.266788$ C $-3.4215054 .409907-1.155170$ C -1.996843 $4.718193-1.628949$ C $-0.9681013 .779888-0.990927$ H $-0.7866151 .780623-0.248687$ C $-1.163501 \quad 1.606561-2.344968$ H $-3.7063072 .620826-2.322676$ O $-3.575064 \quad 4.8286440 .199498$ H -4.150299 $4.964874-1.757817$ H -1.740177 $5.754025-1.375249$ O $-1.9240624 .585457-3.045584$ H $-0.0008143 .885246-1.494726$ O $-\begin{array}{llll}0.761610 & 4.180131 & 0.366933\end{array}$ C - $-1.3408530 .113974-2.200640$ H -1.828989 $1.965341-3.135274$ H $-0.141684 \quad 1.776007-2.704205$ H $-3.407855 \quad 5.785927 \quad 0.231387$ H -2.559995 5.211258 -3.432266 H -1.5871854 .0161110 .854445$ O $-2.570717-0.257099-2.657068$ O $-0.496422-0.627141-1.715238$ C $-2.872930-1.651715-2.554033$ H $-3.883567-1.809986-2.939583$ H $-2.171830-2.235884-3.157211$ H $-2.844179-1.969871-1.508236$ SCF Energy (B3LYP/6-31G*//MMFF) $=-3245.92166032$

03_252

MMMFF Geometry

C -2.130894 -2.759373 -2.102027

C $-1.224357-3.667408-2.495592$

C $0.206466-3.763325-2.012871$

O $0.638368-2.534818-1.385415$

C $0.327378-4.898898-0.980738$

C $1.751036-5.171327-0.534922$

C $2.207035-5.0011930 .721612$

C $2.680226-5.695483-1.600156$

C $1.448923-4.5254801 .929077$

C $1.530876-3.0122952 .184636$

C $2.951839-2.4957092 .465409$

C $2.914388-1.0432072 .944396$
C $4.324301-0.4697323 .149227$

O $3.710394-2.573721 \quad 1.256522$

$\begin{array}{lllll} & & 4.179405 & 0.952107 & 3.290822\end{array}$

C $5.173607-0.7379991 .886182$

C $4.948724-0.9696274 .457828$

O $6.525252-0.3557362 .129038$

C $5.088616-2.1975911 .390561$

C $5.751068-2.4261340 .009475$

O $5.764623-3.0478612 .324890$

C $1.020984-1.519430-2.207607$

O $1.063333-1.534760-3.427576$

C $1.351548-0.355529-1.357539$

C $1.3848290 .868347-1.904622$

C $1.6836122 .118230-1.203533$

C 2.2140202 .0152360 .201668

C $5.178960-1.590601-1.141333$

C $5.943938-0.306141-1.413643$

C $5.8500481 .743810-2.828542$

O $7.017150-0.034499-0.882449$

C $5.2935992 .872046-2.018933$

C $4.7148203 .952958-2.563975$

C $4.1947935 .116256-1.769360$

C $2.7515555 .439427-2.084594$

C $2.4342706 .421097-2.945670$

C $1.6791614 .683082-1.334361$

C $1.4856583 .284988-1.852102$

N $\begin{array}{lll}5.300097 & 0.487889 & -2.353427\end{array}$

H - $1.864978-2.015837-1.355316$

H $-1.517591-4.411468-3.233541$

H $\quad 0.848118-3.969970-2.878059$

H $-0.079862-5.829196-1.399027$

H $-0.304481-4.653949-0.120252$

H $3.251759-5.2382090 .920617$

H $3.645022-6.013559-1.190137$

H $2.236252-6.563471-2.098443$

H $2.882117-4.925166-2.350428$

H $0.395588-4.818321 \quad 1.866055$

H $1.839116-5.0592492 .805186$

H $1.108608-2.4836901 .322128$

H $\quad 0.890522-2.7866113 .046530$

H $3.408200-3.1383113 .227282$

H $2.325957-0.9503323 .865792$

H $2.394835-0.4289492 .196576$

H $5.071971 \quad 1.3340623 .352278$

H $4.792068-0.0664621 .108569$

H $4.330351-0.6708595 .313002$

H $5.931521-0.5162654 .627865$

H $5.060273-2.0553654 .492380$

H $7.005962-0.4051581 .286137$

H $6.839692-2.3199250 .086479$

H $5.584490-3.480397-0.256161$

H $\quad 5.999770-3.875888 \quad 1.874242$

H $1.538091-0.535320-0.305470$

H $1.1511750 .971609-2.963750$

H $1.443721 \quad 1.6275230 .876621$

H 2.5571882 .9677090 .610664

H $3.079193 \quad 1.3448470 .234509$

H $5.242552-2.183280-2.063089$

H $4.123119-1.359408-0.973558$

H $5.5939311 .829182-3.889327$

H $6.9405181 .724577-2.733193$

H $5.3968332 .810518-0.938094$

H $4.6387184 .023973-3.646884$

H $4.8285035 .983763-1.996178$

H $4.3035484 .946592-0.690928$

H $1.4012246 .678000-3.155015$

H $3.2014896 .986033-3.464753$

H $0.7149545 .199345-1.434563$

H $1.9022594 .716828-0.264700$

H $1.1026443 .250900-2.873091$

H $4.3872550 .208020-2.700773$

C -4.578204 -3.106436 -1.628741

O $-4.563396-2.215717-0.495080$

C $-5.916877-1.855885-0.166609$

C $-6.808918-2.829629-0.927106$

C $-6.000603-3.049388-2.189108$

H $-6.075007-0.849677-0.569923$

C $-6.132443-1.8423491 .350291$ 
H $-6.932917-3.776694-0.389604$ H -7.804172 -2.417456 -1.119084 C $-6.410879-4.288828-2.965329$ H -6.115242 -2.168332 -2.834926 C $-5.114788-1.0008622 .145396$ O $-6.040143-3.1894311 .834985$ H -7.154892 -1.510435 1.568011 H $-4.146906-1.5180132 .152160$ O $-5.576265-0.9893953 .503381$ C -4.9230330 .4466201 .652745$ C -3.9979021 .2612902 .576976$ H $-4.504466 \quad 0.4357490 .640744$ O $-6.179279 \quad 1.123122 \quad 1.599789$ H -4.520274 1.4737953 .517805 H -3.1060740 .6622452 .799277$ H -5.794448 -4.410832 -3.861341 H $-6.306577-5.194820-2.358895$ H -7.456273 -4.215204 -3.282407 C $-3.525956-2.677985-2.652919$ H -6.198074 -3.163084 2.794518 H $-4.835777 \quad-0.7055634 .065431$ H -6.7162020 .7088620 .904428$ H -3.697324 -1.637649-2.955085 H -3.614156 -3.292568 -3.556457 H -4.351637 -4.110184 -1.246222 C -2.1570593 .4146190 .143297$ O $-2.6552062 .292820 \quad 0.883408$ C -3.5686332 .5990691 .942748$ C -2.9016373 .5264712 .970306$ C -2.3427374 .7862672 .303593$ C -1.4968924 .4478181 .071545$ H -1.373256 $2.994183-0.498976$ C $-3.2336544 .013419-0.767252$ H -4.4530683 .0940361 .527989$ O -1.8524402 .8412613 .651902$ H -3.6438193 .8086293 .726234$ H - 1.7318255 .3325413 .032524 O H -1.2624785 .3533150 .500091$ O $-0.2527973 .895321 \quad 1.509579$ C $-3.6395653 .015727-1.826383$ $\mathrm{H}-4.1262084 .331808-0.221215$ H -2.856489 4.901089-1.288675 H -1.2229582 .5205432 .983439$ H $-3.057573 \quad 6.431401 \quad 1.533992$ H 0.1980684 .5725942 .042105 O $-4.8576162 .482858-1.524285$ O $-2.9440112 .727473-2.791797$ C $-5.3449941 .501068-2.443614$ H $-4.6951430 .621459-2.430857$ H $-6.3466531 .202922-2.123214$ H -5.410418 $1.919754-3.452314$ SCF Energy (B3LYP/6-31G**//MMFF) $=-3245.91759482$

03_253

MM̄FF Geometry

C -2.544811-1.591695 3.018957

C $-1.270078-1.7819523 .388917$

C $-0.206442-2.2333722 .418050$

O $0.855988-1.2511212 .389529$

C $0.393734-3.5747702 .876854$

C $1.249028-4.2474791 .820010$

C $2.576483-4.4536281 .917506$

C $0.512400-4.7382930 .598884$

C $3.499004-4.0821813 .042176$

C $4.178522-2.7114102 .898340$

C $4.955283-2.5192161 .584657$

C $5.842283-1.2742071 .659576$

C $6.553087-0.9892610 .326935$

O $4.008297-2.3656490 .521924$

$\begin{array}{llll}\text { O } & 7.081378 & 0.344952 & 0.385704\end{array}$

C 5.514001-1.012061-0.815681

C $7.752484-1.9200900 .111718$

O $6.153071-0.915908-2.085596$

C $4.592332-2.248828-0.782975$

C $3.431116-2.176339-1.806793$

O $5.359035-3.415422-1.107009$

C $0.598221-0.0779871 .749292$
O $\quad-0.4408790 .247399 \quad 1.197252$

C 1.8108350 .7630721 .836238

C $1.7057582 .081417 \quad 1.616849$

C 2.8036573 .0490781 .607772

C 4.2072042 .5065821 .652277

C $2.481882-0.983583-1.649175$

C $2.8450480 .215657-2.509601$

C $2.2547692 .613126-2.851313$

O $3.6617300 .164528-3.425377$

C $3.2206033 .483020-2.109317$

C $2.9556234 .753957-1.769903$

C $3.9311135 .656591-1.068205$

C 3.3472616 .2966280 .172336

C 2.8233207 .5333580 .130130

C 3.4443175 .5342611 .475271

C $2.5027574 .361517 \quad 1.520738$

N $2.1162371 .344855-2.159983$

H -2.829152 -1.793658 1.988267

H $-0.967350-1.5812384 .413224$

H $-0.621185-2.3298881 .407286$

H $-0.410025-4.2736603 .144786$

H $0.970310-3.4090803 .793373$

H $3.075245-4.9546641 .087868$

H $1.139228-5.372081-0.037920$

H $-0.358637-5.3335540 .891367$

H $0.174156-3.896303-0.011350$

H $2.978365-4.1233104 .004833$

H $4.274782-4.8563323 .105595$

H $3.414808-1.9310702 .991020$

H $4.863715-2.5927613 .747034$

H $5.566185-3.4120851 .407296$

H $6.574741-1.3665802 .471254$

H $5.223737-0.4039521 .915390$

H $7.6819790 .396173 \quad 1.148990$

H $4.901064-0.109699-0.703507$

H $8.483977-1.7945620 .919129$

H $8.284100-1.672582-0.813616$

H $7.476002-2.9759380 .080256$

H $6.702524-0.113591-2.073561$

H $3.824804-2.246136-2.827905$

H $2.822416-3.081943-1.668425$

H $4.750294-4.110637-1.407207$

H 2.7508930 .2760272 .063724

H 0.7177812 .4967601 .424246

H $4.358171 \quad 1.7730320 .852894$

H 4.4022752 .0234002 .615690

H 4.9770263 .2678031 .512606

H $1.478692-1.293098-1.969387$

H $2.411160-0.678509-0.602312$

H $1.2578923 .060374-2.913910$

H $2.6228512 .438000-3.867293$

H $4.1915993 .058088-1.865890$

H $1.9943225 .184736-2.041667$

H $4.2337256 .436128-1.779789$

H $4.8532315 .123783-0.804687$

H 2.4264078 .0108001 .019802

H $2.7753218 .094252-0.797521$

H 3.1873176 .1898102 .318034

H 4.4876585 .2552081 .644551

H 1.4510654 .6501411 .490369

H $1.4876301 .305867-1.362744$

C -4.1283720 .2896253 .504253$

$\begin{array}{lllll}\text { O } & -4.599047 & 0.246914 & 2.143065\end{array}$

C -5.9377080 .7653422 .090604$

C -6.0826301 .6168493 .343160$

C $-5.3066260 .785146 \quad 4.348655$

H -6.610485 -0.098318 2.167854

C $-6.187583 \quad 1.4790270 .757073$

H -5.6103702 .5989523 .226290$

H -7.1280621 .7697183 .626567$

C $-4.889526 \quad 1.5567445 .588581$

H -5.932862 -0.0636504 .655018$

C $-5.7170760 .688262-0.483159$

$\begin{array}{llll}\text { O } & -5.475666 & 2.723710 & 0.759137\end{array}$

H -7.2503281 .7355440 .674215$

H $-4.6232780 .726576-0.520337$

O $-6.1932461397263-1.632346$

C $-6.196359-0.771060-0.540978$ 
C $-5.798565-1.557416-1.805498$ H $-5.800304-1.3144260 .324280$ O $-7.621428-0.791150-0.430321$ H -6.140438 -2.590455 -1.656446 H -6.364963 -1.177554 -2.665639 H $-4.319354 \quad 0.917767 \quad 6.270316$ H -4.2650752 .4193865 .333330$ H $-5.769075 \quad 1.925726 \quad 6.126013$ C -3.637289-1.099904 3.924351 H $-5.6468183 .156436-0.095029$ H $-5.7319441 .040343-2.410152$ H -7.902654 -1.721820 -0.412250 H -4.460372 -1.822710 3.863412 H -3.299955 -1.077089 4.966896 H -3.2988191 .0076713 .527452$ C $-2.653558-0.184309-3.292799$ O $-4.018839-0.390868-2.905893$ C $-4.287004-1.545300-2.099661$ C -3.841026 -2.819554 -2.831405 C $-2.366703-2.730056-3.236949$ C $-2.065896-1.429440-3.981823$ H $-2.6958210 .615148-4.043817$ C $-1.8033110 .323564-2.117159$ H $-3.740828-1.468120-1.153165$ O $-4.655654-3.016112-3.984821$ H -3.996218 -3.678979 -2.168039 H -2.110337 -3.579726 -3.881392 O $-1.543246-2.796000-2.076199$ H $-0.983085-1.311173-4.107702$ O $-2.626277-1.533953-5.294055$ C $-2.0818381 .784486-1.823242$ H -1.980846 -0.235095 -1.195239 H $-0.7432650 .243507-2.383413$ H -4.353352 -3.829809 -4.422860 H -1.733507 -3.638353 -1.629423 H $-2.372859-0.734888-5.786839$ O $-1.0320342 .312878-1.128435$ O $-3.0983692 .384751-2.145403$ C $-1.1516033 .699877-0.796796$ H $-0.1898124 .041092-0.407092$ H -1.392533 $4.292579-1.684697$ H -1.914386 $3.831828-0.024042$ SCF Energy (B3LYP/6-31G**//MMFF) $=-3245.89830173$

03_254

MM̄FF Geometry

C -6.330450 -0.967464 -0.022074

C $-5.6131120 .047814-0.525515$

C $-5.281202 \quad 1.299794 \quad 0.245417$

O $-3.842622 \quad 1.3436420 .399759$

C $-5.7232352 .541602-0.547413$

C -5.5620773 .8330210 .231460$

C -4.5819084 .7382010 .039048$

C -6.6257254 .0900841 .269216$

C $-3.4386404 .680943-0.937107$

C $-2.1062844 .542860-0.200228$

C $-0.8695374 .565731-1.111017$

C $-0.8652763 .441998-2.149778$

C $0.447423 \quad 3.397572-2.947266$

O $0.2609364 .425384-0.240978$

O $0.4916342 .146383-3.649980$

C $1.6342903 .408336-1.963674$

C $0.5034924 .496773-4.014797$

O $2.8739163 .511228-2.660558$

C $1.5366454 .513920-0.888784$

C 2.6224954 .2923980 .190885

O $1.7322515 .789556-1.498669$

C $-3.309307 \quad 0.645741 \quad 1.440959$

$\begin{array}{llll}\text { O } & -3.916222 & 0.054180 & 2.319629\end{array}$

C $-1.8373280 .677807 \quad 1.294445$

C $-1.080849-0.1705202 .006978$

C $0.374260-0.311471 \quad 1.912024$

C $1.1187390 .711856 \quad 1.098530$

C 2.5301695 .2645761 .373760

C 3.2184044 .7029082 .601235

C 2.8868623 .0694414 .458487

O 4.3390565 .0742942 .941334

C 3.5536641 .7820664 .088818
C 3.2120560 .5922404 .607584

C $3.944199-0.6828444 .302737$

C $3.029770-1.8132923 .892711$

C $2.849546-2.8818524 .687886$

C $2.411042-1.7567742 .515687$

C $0.963042-1.3504412 .539462$

N 2.4501263 .7505693 .255207

H $-6.728748-0.8910820 .986329$

H -5.200102 -0.039416-1.528073

H $-5.762131 \quad 1.2963201 .231148$

H $-6.7796612 .445419-0.832749$

H -5.171900 $2.588846-1.492538$

H -4.5882645 .6350910 .658089$

H -6.5019105 .0608101 .761194$

H -7.6177364 .0823460 .805665$

H -6.5994243 .3223452 .048420$

H -3.560155 $3.861241-1.646757$

H -3.441412 $5.606019-1.525782$

H -2.004055 5.3533240 .535107

H -2.1022573 .6222720 .395434$

H $-0.8230915 .545348-1.601208$

H -1.711489 $3.533878-2.840210$

H $-0.9952442 .476468-1.642054$

H $-0.2926342 .095928-4.222810$

H $1.6460422 .434943-1.455766$

H $-0.3267064 .385808-4.722758$

H $1.4182564 .420419-4.612892$

H $0.4468765 .503765-3.596460$

H $2.9065572 .780594-3.301848$

H 2.5448373 .2653150 .565139

H $3.6215124 .392432-0.252112$

H $2.0123576 .423922-0.820135$

H $-1.417826 \quad 1.358447 \quad 0.563941$

H -1.574080 -0.8577332 .693140$

H $\quad 0.852623 \quad 1.723346 \quad 1.423388$

H 0.8719960 .6125380 .037150

H 2.2044130 .6395091 .188560

H $2.9992306 .223073 \quad 1.124237$

H 1.4905605 .4844181 .642270

H 2.0021472 .9130065 .083753

H 3.5917603 .7069085 .001940

H $4.387914 \quad 1.8384583 .393318$

H 2.4008570 .5387945 .330062

H $4.506807-0.9617545 .203328$

H $4.695985-0.5318793 .517680$

H $2.225284-3.7141514 .379282$

H $3.319506-2.9479345 .663589$

H $2.462351-2.7544742 .060573$

H $3.020093-1.1238981 .866178$

H $0.327261-2.041013 \quad 3.095691$

H 1.5549803 .4793492 .859227

C $-5.648854-3.389927-0.390837$

O $-4.300582-3.055304-0.782750$

C $-3.419483-3.3478830 .314994$

C $-4.183756-4.3283891 .194781$

C $-5.591513-3.7706031 .090792$

H -3.287780 -2.408340 0.866140

C $-2.041159-3.824232-0.164750$

H $-4.154856-5.346910 \quad 0.792413$

H $-3.805046-4.3520902 .220867$

C $-6.673297-4.7485131 .514205$

H -5.651987 -2.881983 1.730329

C - $-1.324145-2.778765-1.046827$

O $-2.164774-5.043160-0.894024$

H $-1.417113-4.0255920 .715072$

H - $1.366708-1.809911-0.538794$

O $-2.009160-2.620736-2.288300$

C $0.158704-3.109608-1.315028$

C $0.848844-2.006392-2.138569$

H $0.673581-3.230327-0.355171$

O $0.283387-4.334874-2.033219$

H $\quad 0.428896-1.986595-3.151704$

H $0.642063-1.041805-1.661964$

H -7.666120 -4.302103 1.399455

H $-6.643683-5.6651380 .915553$

H $-6.547505-5.0301542 .564758$

C $-6.598777-2.246421-0.765769$

H $-2.349714-5.753651-0.257471$ 
H $-1.997704-3.483744-2.736888$ H - $0.100053-5.039594-1.484469$ H -7.635996 -2.546197-0.577282 H -6.515845 -2.059795 -1.844305 H $-5.922544-4.267683-0.990672$ C $4.328152-2.312109-0.812407$ O $2.917949-2.081046-0.928306$ C $2.371692-2.213548-2.245592$ C $3.035162-1.208568-3.201483$ C $4.559366-1.356835-3.183823$ C $5.114118-1.387004-1.755145$ H $4.576573-2.0238070 .216785$ C $4.678390-3.794858-0.975903$ H $2.554147-3.229096-2.612195$ O $2.713707 \quad 0.128674-2.828171$ H $2.661465-1.371832-4.219651$ H $5.005864-0.517294-3.730832$ O $4.906462-2.558234-3.865999$ H $6.168338-1.685849-1.764633$ O $5.075722-0.061562-1.216037$ C $4.109525-4.5990940 .169177$ H $4.322989-4.225591-1.916180$ H $5.764960-3.941493-0.962667$ H $\quad 1.7835390 .287335-3.061820$ H $5.876885-2.621376-3.873049$ H $4.1425200 .203064-1.142236$ O $2.904416-5.128451-0.185135$ O $4.658243-4.7159651 .257480$ C $2.238993-5.8853240 .830514$ H $2.845033-6.7500171 .116623$ H $2.030682-5.2542391 .699522$ H $1.289538-6.2412840 .421696$ SCF Energy (B3LYP/6-31G**//MMFF) $=-3245.91734705$

03_255

MM̄FF Geometry

C $-0.8457115 .508600-1.405674$

C $-1.2154094 .659635-0.434450$

C $-2.5320553 .922198-0.437929$

O $-2.2811062 .521360-0.174573$

C -3.4337694 .4597300 .688004$

C $-4.825290 \quad 3.855913 \quad 0.695807$

C -5.3308863 .1060501 .694589$

C $-5.6812474 .168545-0.505467$

C -4.6721102 .7119342 .986291$

C $-3.958031 \quad 1.3517862 .955971$

C $-4.883394 \quad 0.1602582 .658508$

C $-4.160926-1.1627702 .919132$

C -5.029064 -2.373550 2.547091

O -5.2730120 .2223051 .284425$

O $-4.163492-3.5194452 .537375$

C $-5.583057-2.184008 \quad 1.117232$

C $-6.094922-2.6495773 .614652$

O $-6.517269-3.2206530 .825768$

C $-6.206045-0.7908030 .881222$

C $-6.559197-0.510351-0.600679$

O $-7.424702-0.6922641 .628462$

C $-1.7872451 .765984-1.192510$

O $-1.5314272 .133199-2.327890$

C -1.589105 $0.396333-0.670179$

C $-0.811339-0.465197-1.342039$

C $-0.511401-1.841336-0.942204$

C $-1.316305-2.4281770 .187169$

C $-5.384275-0.582343-1.582596$

C $-5.226135-1.932378-2.262441$

C $-3.689216-3.167707-3.788020$

O $-6.058047-2.831515-2.177508$

C $-2.787130-4.045014-2.978583$

C -1.596857 -4.481925 -3.417654

C $-0.699110-5.397745-2.635587$

C $0.702997-4.851350-2.484251$

C $1.695420-5.258902-3.293281$

C $0.970930-3.899815-1.340824$

C $0.452007-2.512819-1.606505$

N -4.056717 -2.000680 -3.008099

H - $-1.5451315 .689731-2.220357$

H $-0.5530324 .459270 \quad 0.402513$

H $-3.0339004 .020989-1.408167$
H -3.540002 5.5486740 .592201

H -2.934112 4.2870151 .647271

H -6.3486232 .7321091 .585852$

H $-6.7197133 .847323-0.370168$

H $-5.6965245 .247459-0.691255$

H $-5.2968033 .663852-1.396838$

H $-3.961123 \quad 3.4792903 .310184$

H -5.4439872 .6941383 .766569$

H -3.1536431 .3905092 .211959$

H -3.4857171 .2050363 .935263$

H -5.7710280 .2433623 .296360$

H -3.836445 -1.231729 3.964984

H -3.241309 -1.196620 2.319431

H $-4.687793-4.2815722 .236360$

H $-4.735461-2.3243160 .436871$

H $-5.620538-2.8524254 .582414$

H -6.675732 -3.5467723 .373737$

H $-6.788866-1.8177093 .752923$

H -6.746532 -3.160257 -0.116565

H -7.394498 -1.144830 -0.920085

H $-6.9457960 .518203-0.649650$

H $-7.9696530 .011076 \quad 1.238143$

H -2.0746460 .1399850 .264250$

H $-0.326817-0.126095-2.256819$

H -2.387481 -2.307802 -0.005487

H $-1.072384-1.9336401 .133077$

H -1.156127 -3.4990760 .327752$

H $-5.5610030 .143604-2.386780$

H -4.445544 -0.306277-1.093528

H -3.206368 -2.807883 -4.702095

H $-4.591574-3.722257-4.064771$

H -3.140491-4.355903-1.998205

H -1.258853 -4.191645 -4.410135

H $-0.668292-6.361370-3.161021$

H -1.114388 -5.616317 -1.643922

H $2.709705-4.893191-3.172909$

H $1.513873-5.963773-4.097907$

H $2.052765-3.809948-1.171416$

H $0.582509-4.332458-0.414590$

H $0.970986-2.003941-2.419995$

H $-3.401023-1.225310-2.977789$

C $1.5859015 .850947-0.571539$

O $1.9685974 .486818-0.837279$

C $3.3950724 .415411-0.984222$

C $3.9305745 .698193-0.363899$

C $2.853696 \quad 6.688025-0.762423$

H $3.6007474 .418869-2.062475$

C $3.9637223 .126671-0.373038$

H 3.9915615 .6229220 .728213

H $4.9214955 .963802-0.743850$

C 2.8733767 .9705420 .051101

H $2.9843576 .937954-1.824000$

C $3.4084911 .835696-1.008241$

O 3.7123493 .1036991 .031866

H $5.0502573 .159493-0.502393$

H $3.5648161 .854417-2.093784$

O $1.9930511 .757146-0.797476$

C $4.0023160 .534323-0.432961$

C $5.5312160 .441280-0.567214$

H 3.7024670 .4108810 .614076

O $3.429624-0.560128-1.158595$

H $5.8072740 .576846-1.620220$

H $5.987972 \quad 1.2493750 .013819$

H $2.0677108 .642344-0.261174$

H 2.7494147 .7694631 .120523

H $3.8241348 .496802-0.083565$

C $0.4573336 .256102-1.525531$

H 2.7472533 .0930561 .153589

H $1.5837702 .511738-1.253823$

H $2.464568-0.495495-1.057378$

H $0.8052506 .107158-2.556352$

H $\quad 0.241577 \quad 7.324657-1.409282$

H 1.2523785 .8985550 .472236

C $6.274166-2.2201411 .953640$

O $5.854686-0.9995581 .327079$

C $6.099079-0.906770-0.080146$

C $7.602419-1.033238-0.370830$

C $8.177067-2.3174150 .236305$ 
C $7.768876-2.4844131 .703347$

H $6.144740-2.0380753 .028677$

C $5.371868-3.3995601 .568151$

H $5.570352-1.715242-0.594898$

O $8.308183 \quad 0.0941680 .143854$

H $7.754023-1.036214-1.456492$

H $9.271387-2.2959920 .165775$

O $7.713697-3.446298-0.499006$

H $8.027515-3.4870142 .063584$

O $8.505578-1.5514162 .499751$

C $4.017565-3.2669232 .223341$

H $5.220143-3.5103830 .491015$

H $5.803794-4.3436811 .921456$

H 8.1173940 .1552291 .095601

H $8.004613-3.336263-1.420321$

H $9.450367-1.7509602 .385215$

O $3.246513-2.3841301 .528281$

O $3.693695-3.863567 \quad 3.242181$

C $1.957687-2.1165312 .087300$

H $1.323373-3.0033412 .002584$

H $2.047040-1.8034173 .132138$

H $1.505756-1.2994181 .519204$

SCF Energy (B3LYP/6-31G**//MMFF) $=-3245.92982845$

03_256

MM̄FF Geometry

C 3.0014995 .3246820 .476826

C 3.2473404 .3196451 .331791

C 4.1181003 .1050031 .104508

O 3.2152721 .9867210 .900812

C $5.0427793 .170635-0.126985$

C $5.8795051 .919319-0.327591$

C $5.8014011 .118378-1.409523$

C 6.8606881 .6001100 .770376

C $4.9319301 .293642-2.623902$

C $3.5464020 .637426-2.527491$

C $3.581317-0.898610-2.489617$

C $2.168242-1.472207-2.618607$

C $2.168894-3.005913-2.532886$

O $4.146198-1.316783-1.245513$

O $0.814447-3.437953-2.330621$

C $2.967596-3.445701-1.284747$

C $2.629669-3.641947-3.849876$

O $3.155164-4.857826-1.270387$

C $4.331512-2.735447-1.127192$

C $5.004772-2.9938030 .240772$

O $5.281911-3.210059-2.089841$

C 2.6702471 .3887151 .992451

O 2.9359111 .6014703 .163909

C 1.6215240 .4691361 .501123

C 0.6226810 .1151582 .322232

C $-0.529855-0.7134961 .965283$

C $-0.507263-1.4026800 .626422$

C $4.194901-2.5681601 .470200$

C $3.372804-3.6888022 .087331$

C $1.667312-4.0956513 .859848$

O $3.480868-4.8662441 .756136$

C $0.299917-4.1204513 .252782$

C $-0.820699-3.8961013 .955857$

C $-2.204022-3.9556263 .373465$

C $-3.003526-2.6997923 .641111$

C -3.881807 -2.641758 4.656386

C $-2.849431-1.5458302 .678228$

C -1.546487 -0.8152182 .846908$

N $2.526395-3.2202623 .083738$

H $3.4869855 .360398-0.493287$

H 2.7043594 .3177832 .276228

H 4.7212592 .9404512 .005548

H $5.7204684 .029387-0.037505$

H $4.4294383 .349256-1.015590$

H $6.4463590 .241161-1.445767$

H $7.535650 \quad 0.7814460 .498131$

H 7.4816842 .4743830 .990826

H 6.3363151 .3020201 .683310

H $4.8064972 .358192-2.850966$

H $5.4679860 .883571-3.489345$

H $3.0277391 .019835-1.640820$

H $2.9671070 .957511-3.402598$
H $4.213551-1.248301-3.314292$

H $1.695233-1.141920-3.551892$

H $1.538871-1.078216-1.809196$

H $0.276728-3.097863-3.066381$

H $2.344094-3.203809-0.416066$

H $1.949285-3.368264-4.665440$

H $2.602496-4.735546-3.793279$

H $3.634461-3.333686-4.146239$

H $2.275101-5.267794-1.330057$

H $5.322296-4.0412800 .312598$

H $5.936625-2.4096450 .254548$

H $4.889984-3.954851-2.572909$

H 1.6680220 .1592160 .463726

H $\quad 0.6238220 .5032143 .340066$

H $\quad 0.431287-1.9510410 .491854$

H $-0.607889-0.673034-0.184105$

H -1.301630 -2.141887 0.504763

H $4.895080-2.2409062 .249546$

H $3.544527-1.7205441 .237641$

H $1.657992-3.7137034 .885605$

H $2.085541-5.1072783 .867773$

H $0.226762-4.3583192 .194140$

H $-0.744393-3.6864685 .020739$

H -2.713803 -4.8220863 .814774$

H -2.177559-4.147732 2.293640

H $-4.481273-1.7555134 .835881$

H $-4.017948-3.4788455 .333125$

H $-3.651706-0.8128312 .834597$

H -3.001909 -1.909696 1.660415

H -1.467626 -0.2809223 .794732$

H $2.469086-2.2213383 .259576$

C 0.6549225 .9859590 .029339

O $0.1212794 .798243 \quad 0.648951$

C $-1.3207394 .873860 \quad 0.650485$

C -1.675858 $6.129411-0.133270$

C -0.4672847 .0126820 .127614$

H -1.6122544 .9831251 .702383$

C $-1.858413 \quad 3.5581520 .077713$

H -1.764909 $5.909392-1.204488$

H -2.616511 6.5781440 .199695

C $-0.3390928 .168762-0.847769$

H -0.5303917 .4048141 .151368$

C $-3.3765203 .525741-0.150455$

O -1.5198202 .5145920 .997550$

H -1.334348 $3.333343-0.858371$

H $-3.6515694 .251415-0.924381$

O -4.0393143 .9548751 .045627$

C $-3.9448332 .140541-0.527521$

C $-3.2430591 .497315-1.735502$

H -3.9082451 .4658930 .335963$

O $-5.3279872 .313364-0.855609$

H -3.308109 2.179107 -2.592437

H $-2.1848541 .351460-1.493407$

H $0.551428 \quad 8.766519-0.630118$

H $-0.2634157 .815391-1.881628$

H $-1.2117548 .826759-0.780488$

C $1.9548306 .377306 \quad 0.735502$

H -0.5574042 .5506151 .132513$

H -3.773672 3.3507921 .760506

H $-5.7596232 .718993-0.084052$

H 1.7827346 .4797361 .813965

H $2.326198 \quad 7.3402820 .369133$

H $0.8530565 .737413-1.022378$

C $-4.186047-2.087037-1.239415$

O $-3.638598-0.773675-1.058874$

C $-3.8498590 .140697-2.140369$

C $-3.214051-0.403038-3.430094$

C $-3.741036-1.804063-3.753043$

C $-3.669610-2.731581-2.536071$

H $-3.789964-2.674650-0.402016$

C $-5.713277-2.084403-1.122514$

H $-4.9249950 .273309-2.298749$

O $-1.795693-0.448119-3.281806$

H $-3.4403170 .284804-4.253762$

H $-3.150487-2.230654-4.573081$

O $-5.089090-1.689535-4.198949$

H $-4.225488-3.655439-2.731121$

O $-2.307027-3.117545-2.332841$ 
C $-6.129114-1.7388160 .288111$

H $-6.200024-1.390286-1.813339$

H $-6.123669-3.076920-1.343424$

H $-1.422922-0.761769-4.123253$

H $-5.401069-2.582868-4.422972$

H $-1.807425-2.322923-2.078038$

O $-6.450805-0.4169340 .369601$

O $-6.138523-2.543098 \quad 1.211257$

C $-6.838267 \quad 0.0454331 .666792$

H $-7.728237-0.4902792 .010334$

H $-6.014153-0.0792232 .375097$

H -7.076688 1.1096231 .591022

SCF Energy (B3LYP/6-31G**//MMFF)= -3245.91421772

03_257

MM̄FF Geometry

C $-2.445870 \quad 0.2855942 .576963$

C $-1.175795 \quad 0.0057512 .905114$

C -0.280833 -0.880885 2.075542

O $1.007543-0.2376571 .937724$

C $-0.065476-2.2212322 .801807$

C $0.715637-3.2369511 .990480$

C $1.918395-3.7346142 .338993$

C $0.044868-3.7236630 .730860$

C $2.734930-3.4221233 .561297$

C $3.760964-2.2928443 .379306$

C $4.834543-2.5815142 .317230$

C $5.948648-1.5335972 .375367$

C $6.991242-1.7385401 .264383$

O $4.216865-2.5457911 .028007$

O $7.793196-0.5495371 .188142$

C $6.265259-1.879703-0.091645$

C $7.952022-2.8882691 .590482$

O $7.179850-2.238715-1.123759$

C $5.091000-2.879611-0.058643$

C $4.239743-2.868272-1.352240$

O $5.608201-4.2079090 .088385$

C 1.1390280 .7039790 .965986

O $\quad 0.2886581 .0647480 .169044$

C 2.5118971 .2472751 .050877

C 2.7898872 .4251420 .472805

C 4.0892253 .1003420 .473144

C 5.2797362 .3154840 .956999

C $3.595382-1.523209-1.705475$

C $4.403737-0.690339-2.686794$

C $4.4472641 .566881-3.741134$

O $5.377790-1.122662-3.296959$

C $5.4182192 .432540-3.001881$

C $5.3827203 .773154-3.039091$

C $6.3723824 .658379-2.337416$

C $5.7064005 .684745-1.448545$

C $5.4998056 .941025-1.878208$

C $5.3581575 .276370-0.034897$

C $4.156013 \quad 4.372315 \quad 0.026697$

N $3.8816490 .588260-2.831082$

H $-2.866424-0.149476 \quad 1.673597$

H $-0.756157 \quad 0.4168343 .820184$

H $-0.715596-1.0520861 .083251$

H -1.036437 -2.668964 3.053311

H $0.433938-2.0245043 .756681$

H $2.378057-4.4672121 .676078$

H $0.583009-4.5589000 .269682$

H - $0.968731-4.0728070 .952408$

H $-0.013801-2.923345-0.012850$

H $2.085764-3.1792504 .409271$

H $3.259601-4.3387173 .860131$

H $3.228876-1.3685933 .125736$

H $4.248992-2.1289304 .348227$

H $5.242686-3.5823212 .501342$

H $6.433355-1.5344853 .359586$

H $5.511128-0.5328732 .259653$

H $8.199174-0.4055802 .060156$

H $5.878481-0.886364-0.348108$

H $8.485644-2.6869822 .527221$

H $8.726009-2.9869950 .821510$

H $7.448020-3.8508441 .699552$

H $7.888501-1.572570-1.127732$

H $4.821633-3.271409-2.189877$
H $3.416135-3.581429-1.202513$

H $4.935828-4.836643-0.222132$

H $3.2489040 .673713 \quad 1.600258$

H $1.9862362 .959392-0.032680$

H $5.340518 \quad 1.354066 \quad 0.435996$

H 5.2058472 .1285012 .033586

H 6.2345242 .8134590 .776918

H $2.632681-1.718633-2.195334$

H $3.390840-0.935748-0.805659$

H $3.6143002 .141288-4.158457$

H $4.9612921 .051475-4.558752$

H $6.1996641 .935494-2.432032$

H $4.6165454 .266554-3.633462$

H $6.9703635 .164674-3.106629$

H $7.0871764 .072521-1.746257$

H $5.0479717 .691267-1.237733$

H $5.7735087 .245863-2.882997$

H 5.1242906 .1651710 .566436

H 6.2440824 .8451800 .439165

H $3.2353814 .851764-0.309228$

H $3.1018580 .877307-2.247618$

C -3.992126 2.2708172 .592840

O $-4.867074 \quad 1.703811 \quad 1.596391$

C -6.1773592 .2778261 .743024$

C -5.9496873 .5987362 .460856$

C -4.8616403 .2037873 .442786$

H -6.7424241 .6082522 .403825$

C -6.8857682 .3617840 .386681$

H -5.5786114 .3738061 .780573$

H $-6.854772 \quad 3.9722772 .948837$

C -4.1037294 .3861214 .021595$

H -5.3216202 .6416534 .266877$

C $-6.8256631 .050182-0.424976$

O $-6.2579393 .381873-0.399190$

H -7.923555 2.6830460 .533116

H $-5.7962510 .901844-0.759761$

O $-7.6063721 .226606-1.611924$

C $-7.317734-0.1983540 .323465$

C -7.293372 -1.502078 -0.498123

H $-6.711584-0.3528401 .222818$

$\begin{array}{llll}\text { O } & -8.659930 & 0.035821 & 0.756727\end{array}$

H -7.719957 -2.2920310 .134019$

H -7.968264 -1.405960 -1.358233

H -3.3197164 .0478484 .706622$

H -3.6299134 .9830203 .235176$

H -4.7808985 .0416284 .578872$

C -3.3598821 .1410733 .410663$

H $-6.7101983 .404656-1.259941$

H -8.536297 $1.312527-1.340249$

H -8.939005 -0.7345371 .280395$

H $-4.1481620 .488116 \quad 3.805769$

H -2.817463 1.5604664 .265967

H -3.2201182 .8361392 .057167$

C $-4.281585-1.431300-2.723148$

O $-5.581798-1.202573-2.161202$

C $-5.881051-1.913351-0.954609$

C $-5.783389-3.426763-1.200095$

C $-4.415715-3.809569-1.771302$

C $-4.033767-2.930256-2.965629$

H $-4.313639-0.945727-3.706975$

C $-3.177100-0.753296-1.895906$

H -5.167114 -1.628953 -0.174165

O $-6.813872-3.852215-2.089557$

H $-5.942334-3.950696-0.250188$

H -4.440775 -4.861906 -2.079051

O $-3.439393-3.678104-0.742088$

H -2.984776 -3.086410 -3.242914

O $-4.824046-3.315712-4.094758$

C $-3.2523000 .758079-1.983879$

H -3.241063 -1.031012 -0.840610

H $-2.190767-1.044019-2.274884$

H -6.734264 -3.332122 -2.907553

H -2.582379 -3.946660 -1.114869

H $-4.631165-4.250181-4.282178$

O $-2.502678 \quad 1.302627-0.981366$

O $-3.867631 \quad 1.385230-2.835349$

C $-2.4546502 .732613-0.956988$

H $-3.4581923 .143753-0.816952$ 
H $-1.8303293 .038739-0.113556$

H $-2.0062603 .111887-1.880109$

SCF Energy (B3LYP/6-31G**//MMFF) $=-3245.91006229$

03_258

MM̄MF Geometry

C $1.947684-4.096684-1.275760$

C $1.453440-2.923362-0.853713$

C $0.528033-2.061035-1.672714$

O $-0.519889-1.557853-0.812723$

C $1.309044-0.853675-2.224266$

C $0.4999820 .030479-3.153331$

C $0.218341 \quad 1.327814-2.922340$

C $0.025306-0.618262-4.428624$

C $0.6238372 .168752-1.745096$

C $-0.3856852 .180475-0.587502$

C -1.757939 $2.769104-0.952770$

C -2.6153842 .9600370 .300530$

C $-4.0275173 .463790-0.039633$

O $-2.4168041 .866003-1.843872$

O $-4.846737 \quad 3.299692 \quad 1.128537$

C $-4.6325542 .568710-1.143513$

C $-4.0338384 .962020-0.366103$

O $-5.8702393 .100263-1.608806$

C $-3.6807362 .333218-2.334322$

C $-4.203306 \quad 1.274252-3.336004$

O $-3.527453 \quad 3.557351-3.063357$

C $-1.543979-2.399728-0.510163$

O $-1.698818-3.544551-0.903586$

C $-2.444464-1.683806 \quad 0.420320$

C $-3.345109-2.3765601 .132885$

C $-4.289834-1.8194152 .103523$

C $-4.410171-0.3201492 .175316$

C $-4.451975-0.120341-2.750555$

C $-5.894463-0.368899-2.341237$

C $-7.323602-2.060821-1.199513$

O $-6.8168290 .391398-2.622616$

C $-7.555908-1.6222360 .211867$

C $-7.917800-2.4633671 .192389$

C $-8.197480-2.0343422 .604168$

C $-7.398730-2.8195523 .620286$

C $-7.941150-3.8649034 .267448$

C $-6.002584-2.3391153 .946016$

C -5.008182 -2.669991 2.866534

N $-6.037285-1.567757-1.655067$

H $1.676080-4.471493-2.259394$

H $\quad 1.747987-2.5442900 .121204$

H $\quad 0.082918-2.636208-2.493567$

H $2.191338-1.202617-2.777691$

H $\quad 1.692167-0.272221-1.379450$

H $-0.3682961 .863353-3.668184$

H $-0.402470 \quad 0.107140-5.129125$

H $0.859812-1.109954-4.938943$

H $-0.747115-1.364326-4.218795$

H $\quad 1.6005011 .852390-1.365083$

H $0.7776773 .196419-2.098591$

H $-0.5096821 .157389-0.213320$

H $0.050636 \quad 2.772187 \quad 0.225613$

H -1.597906 $3.730020-1.455664$

H -2.125912 $3.641431 \quad 1.007613$

H -2.7072562 .0000970 .826627$

H -4.436632 3.7998351 .854912

H $-4.8629431 .603701-0.677324$

H -3.6589385 .5410120 .486522$

H -5.052164 5.322955 -0.547370

H -3.418068 $5.215012-1.231590$

H $-6.4483303 .208953-0.834242$

H $-5.0862171 .656565-3.862156$

H -3.432816 $1.156635-4.111794$

H -3.224859 $3.345208-3.961933$

H $-2.326527-0.6099650 .508613$

H -3.382965 -3.458485 1.012139

H -4.5950860 .0964351 .179571$

H -3.491312 0.1209102 .576373

H $\quad-5.2364840 .0231012 .800947$

H $-4.223856-0.865698-3.523439$

H -3.789470 -0.316633 -1.902256

H $-7.306607-3.150901-1.297414$
H $-8.115113-1.664192-1.843550$

H -7.448080 -0.5626510 .431167$

H $-8.052944-3.5181310 .962746$

H $-9.271215-2.1726042 .787421$

H -8.010581 -0.9621962 .742728$

H -7.384822 -4.416324 5.018076

H $-8.952783-4.1959814 .056663$

H $-5.645927-2.8177934 .868169$

H -6.039142 -1.272543 4.183489

H $-4.852129-3.7416502 .734336$

H $-5.210550-2.109165-1.419612$

C $4.347318-4.688979-0.930129$

O $4.742226-3.355210-0.549275$

C $6.072067-3.396180-0.000511$

C $6.625311-4.770768-0.353450$

C $5.369326-5.620275-0.279528$

H $5.965924-3.3346821 .089106$

C $6.886288-2.202761-0.510836$

H $7.040860-4.794807-1.367332$

H $7.406233-5.0953330 .340721$

C $5.504722-6.966785-0.968310$

H $5.113578-5.7801760 .776544$

C $6.200446-0.838839-0.271477$

O $7.071941-2.353207-1.924178$

H $7.887007-2.217926-0.063884$

H $5.296136-0.784320-0.886162$

O $7.0733570 .183106-0.769478$

C $5.867215-0.5403651 .203054$

C 5.2654090 .8559751 .444142

H $5.197305-1.3072081 .595621$

O $7.080857-0.6325281 .957700$

H 5.2530531 .0482122 .524401

H 5.9565561 .6147511 .052637

H $4.570887-7.533957-0.903503$

H $5.756524-6.852274-2.027898$

H $6.294575-7.561537-0.497751$

C $2.908631-4.929407-0.472349$

H $7.578138-1.582363-2.233235$

H $7.8693700 .185725-0.210286$

H $6.852464-0.5170232 .895917$

H $2.808857-4.6869040 .593261$

H $2.634020-5.983246-0.592142$

H $4.419575-4.743512-2.024492$

C 3.5338463 .2858041 .832402

O 3.6374612 .4973170 .640328

C 3.8581431 .0953590 .855904

C $2.6782000 .525798 \quad 1.656971$

C $2.440347 \quad 1.2972262 .953139$

C 2.3579262 .8012852 .690734

H 4.4668333 .2200032 .405652

C 3.3450104 .7441681 .387730

H $3.8290060 .644275-0.140784$

O $2.873327-0.8512201 .944571$

H $1.7723560 .594398 \quad 1.041674$

H 3.2114801 .0627863 .696137

O 1.2006490 .8552813 .513643

H 1.4022723 .0242452 .200852

O 2.3580443 .4630423 .954224

C 4.5778515 .3000750 .704208

H 2.5137554 .8044500 .674896

H 3.1374925 .3903542 .246270

H $2.102074-1.1558242 .452837$

H $1.047531 \quad 1.3747704 .321503$

H 2.0848734 .3840393 .808800

O $4.277741 \quad 6.526708 \quad 0.182331$

O $5.665782 \quad 4.743864 \quad 0.645057$

C $5.3523557 .186457-0.494626$

H 6.1773557 .3749470 .198845

H $5.6904306 .586004-1.344588$

H $4.9815088 .144868-0.867754$

SCF Energy (B3LYP/6-31G**//MMFF) $=-3245.90849675$

03_259

MM̄MF Geometry

C $3.2680074 .856321-0.948732$

C $3.9128953 .703604-0.717703$

C 4.5326163 .3316040 .601000

O 3.8651812 .1348531 .061205 
C 6.0246422 .9948310 .417746

C 6.6895152 .5175031 .695480

C $7.1687841 .271607 \quad 1.885811$

C 6.8063113 .5436712 .792651

C 7.1932510 .1314610 .903571

C $5.876844-0.6453830 .881007$

C $5.944711-1.9849660 .132331$

C $6.360496-1.825305-1.332349$

C $6.287828-3.152892-2.103055$

O $4.629638-2.555498 \quad 0.213555$

O $6.377164-2.854079-3.504982$

C $4.903664-3.789924-1.865669$

C $7.474115-4.068626-1.779714$

O $4.829551-5.088838-2.447497$

C $4.505836-3.857318-0.375887$

C $3.045549-4.327494-0.172017$

O $5.346735-4.8030120 .295386$

C 2.7259232 .2687091 .789754

O 2.1851883 .3047632 .137796

C 2.2439850 .8998002 .077919

C 1.1092860 .7039822 .765035

C $0.527645-0.6017893 .089422$

C $1.289675-1.8252282 .649816$

C $1.986549-3.473366-0.874118$

C $0.572947-3.830314-0.441509$

C $-1.788959-3.080476-0.760321$

$\begin{array}{lllll}\text { O } & 0.312718 & -4.769173 & 0.305533\end{array}$

C $-2.181694-2.0914310 .291137$

C -2.875495 -2.410840 1.394700

C $-3.327824-1.3984642 .408751$

C $-2.911111-1.7363933 .824288$

C $-3.832621-1.9099304 .786698$

C $-1.435327-1.8680294 .142361$

C $-0.643062-0.6469653 .758760$

N $-0.362886-2.975159-1.006480$

H $3.2069015 .604215-0.163397$

H $3.9553272 .953868-1.505279$

H 4.4156724 .1351501 .338149

H $\quad 6.5632263 .8781280 .050188$

H $6.1242022 .234923-0.365876$

H $7.611022 \quad 1.0343152 .852606$

H 7.4037543 .1824713 .636747

H 7.2909624 .4498462 .414517

H 5.8185153 .8105673 .180334

H $7.446140 \quad 0.499591-0.096314$

H $8.012707-0.5397651 .189956$

H $5.535972-0.8401491 .907064$

H $5.091698-0.0305030 .426381$

H $6.647575-2.6377550 .663313$

H $7.369248-1.404375-1.415373$

H $5.692301-1.102715-1.821632$

H $7.213865-2.382486-3.658218$

H $4.177084-3.166692-2.401868$

H $8.418401-3.582490-2.053112$

H $7.432101-4.992077-2.367586$

H $7.536525-4.333994-0.722392$

H $5.083384-5.002608-3.382318$

H $2.941379-5.375070-0.483655$

H $2.834031-4.3227490 .906731$

H $4.894984-5.0978601 .103465$

H 2.8410690 .0768841 .700506

H $\quad 0.5490391 .5718043 .109579$

H $2.301164-1.8215943 .071828$

H $1.365576-1.8489181 .559512$

H $0.835806-2.7677972 .960114$

H $2.153201-2.411501-0.661444$

H $2.041635-3.620766-1.958257$

H -2.040639-4.107799-0.477738

H -2.296944 -2.841060 -1.699536

H -1.914125 -1.052246 0.115241

H -3.172653 -3.443851 1.558425

H -4.421040 -1.338495 2.341873

H -2.958657 -0.3970642 .157742$

H -3.549159 -2.150348 5.806048

H -4.892751 -1.817616 4.578591

H - $1.073514-2.7741933 .650031$

H -1.290989 -2.025971 5.218803

H -1.0981040 .2945184 .069229$
H $-0.040375-2.195327-1.572258$

C $1.0487165 .088033-2.154498$

O $0.6328493 .801452-1.648500$

C $-0.4510484 .004923-0.712327$

C $-0.9262315 .432147-0.943439$

C $0.3794446 .133080-1.264487$

H -0.0067443 .9112360 .286725$

C $-1.5036282 .910597-0.926431$

H -1.609176 $5.482949-1.800674$

H -1.434340 $5.855238-0.071857$

C $0.1983637 .486233-1.929339$

H $0.9376506 .267116-0.329923$

C $-2.8395753 .172769-0.212211$

O $\quad-0.9598631 .677738-0.442844$

H -1.676067 $2.778750-2.000924$

H $-3.3049454 .073005-0.630135$

O -2.5866493 .4666621 .167646$

C $-3.8637132 .020879-0.260817$

C $-4.0585461 .422219-1.662306$

H $-3.577740 \quad 1.2307350 .442199$

O -5.1176572 .5394330 .194221$

H -4.215082 $2.229460-2.388890$

H -3.133892 $0.903173-1.937891$

H $1.1676117 .941396-2.155869$

H $-0.3625097 .402091-2.866136$

H $-0.3494048 .167932-1.270519$

C $2.5758665 .154950-2.249375$

H $-0.1156681 .531753-0.903249$

H -2.121122 2.7022181 .549020

H -4.9791292 .8837861 .093318$

H $2.8914806 .142757-2.603500$

H $2.9045784 .431874-3.007183$

H $0.6434685 .158239-3.172884$

C $-6.172571-1.513194-0.678316$

O $-5.112635-0.549067-0.751520$

C $-5.2462950 .444358-1.771674$

C $-5.318798-0.212297-3.160666$

C $-6.424587-1.271403-3.216912$

C $-6.349255-2.232931-2.026227$

H $-5.827034-2.2547110 .053601$

C $-7.475760-0.902725-0.145666$

H $-6.1643361 .015455-1.597626$

O $-4.065926-0.821406-3.469442$

H $-5.4993850 .567499-3.910224$

H $-6.336807-1.845116-4.147566$

O $-7.699503-0.636185-3.218814$

H -7.244315 -2.864765 -1.987131

O $-5.229500-3.106137-2.196065$

C $-7.354288-0.5632041 .322294$

H $-7.7761250 .014233-0.658890$

H $-8.299181-1.616543-0.270691$

H -4.134881-1.199102 -4.362618

H -7.734942 -0.050320 -3.994099

H $-5.361840-3.594796-3.026047$

O $-8.191632-1.3520862 .054550$

O $-6.606938 \quad 0.298811 \quad 1.765935$

C -8.185754 -1.113972 3.465284

H -7.192245 -1.309310 3.877699

H $-8.497574-0.0867683 .677684$

H -8.899326 -1.800141 3.929279

SCF Energy $\left(B 3 L Y P / 6-31 G^{* * / / M M F F}\right)=-3245.90528718$

03_26

MM̄FF Geometry

C $3.962633-3.1260531 .329606$

C $3.119821-3.1859390 .286735$

C $1.621064-3.195068 \quad 0.428779$

O $1.152590-2.119936-0.419849$

C $1.024685-4.527422-0.058749$

C $-0.387223-4.7826700 .438452$

C $-1.486859-4.865478-0.336124$

C $-0.517394-5.0088801 .925828$

C $-1.601468-4.736350-1.827866$

C $-2.144085-3.385040-2.316560$

C $-3.583098-3.064517-1.874593$

C $-4.103052-1.831208-2.620404$

C $-5.498442-1.403317-2.141128$

O $-3.591370-2.810884-0.465562$ 
O $-5.746410-0.076447-2.632513$

C $-5.498160-1.318087-0.599582$

C $-6.598755-2.294547-2.728848$

O $-6.812602-1.080182-0.103904$

C $-4.895352-2.5628860 .082626$

C $-4.743118-2.4064081 .617679$

O $-5.763140-3.683666-0.131199$

C $0.168685-1.3095320 .044120$

O $-0.439709-1.4122861 .096014$

C $-0.045844-0.255829-0.973944$

C $-1.1697460 .472549-0.930244$

C $-1.5558391 .539927-1.853286$

C $-0.549681 \quad 1.979963-2.882821$

C -3.863976-1.241694 2.081494

C $-4.627725 \quad 0.0457882 .345077$

C -4.2733252 .5067342 .558770$

$\begin{array}{llll}\text { O } & -5.836448 & 0.078530 & 2.559324\end{array}$

C -4.7002523 .0745801 .241653$

C $-4.089446 \quad 4.1051580 .637514$

C $-4.5148784 .667019-0.689447$

C $-3.4597054 .498521-1.762020$

C $-2.6376985 .509673-2.088930$

C $-3.4155973 .187199-2.516653$

C -2.782536 $2.083993-1.716403$

N $-3.789564 \quad 1.1529662 .356497$

H $3.542813-3.1483772 .333749$

H $3.505392-3.177391-0.728906$

H $1.357606-2.9976121 .473319$

H $1.644872-5.3640870 .290939$

H $1.067150-4.556733-1.152232$

H $-2.434390-5.0799800 .157455$

H -1.519323 -5.350162 2.208680

H $\quad 0.191039-5.7751152 .257538$

H $-0.320143-4.0868422 .480207$

H - $0.637348-4.917979-2.312835$

H $-2.259193-5.538037-2.187008$

H $-1.469723-2.593647-1.975669$

H -2.102418 -3.392071 -3.413147

H $-4.212943-3.934881-2.092848$

H -4.108102 -2.005881 -3.703599

H -3.412179 $-0.993661-2.459360$

H -5.666411 $-0.095882-3.601622$

H $-4.897130-0.440343-0.332563$

H -6.592800 -2.237576 -3.824051

H -7.592365 -1.953866 -2.417729

H -6.491662 -3.346018 -2.454647

H -7.145519 -0.276783 -0.539188

H -5.731705 -2.376787 2.091568

H -4.269275 -3.3259921 .990451$

H $-5.570136-4.355710 \quad 0.543421$

H $0.711260-0.125382-1.738688$

H -1.891126 $0.260202-0.144085$

H $-0.3787441 .182795-3.614548$

H $0.4066682 .231329-2.411998$

H $-0.8578332 .869738-3.436412$

H -3.396756 -1.5137713 .036578$

H -3.058710 -1.060643 1.366184

H -3.4595363 .0801273 .013871$

H -5.121470 2.4936723 .250759

H -5.5572442 .6091020 .760004$

H -3.2390374 .5774371 .122395$

H -4.739004 $5.731996-0.544613$

H -5.455000 4.215946 -1.031163

H -1.897777 $5.404711-2.875383$

H -2.681551 $6.462389-1.571597$

H $-2.9158973 .315565-3.482606$

H -4.438703 $2.886658-2.777182$

H $-3.4386581 .676970-0.947596$

H -2.811732 1.0359622 .107083

C $6.052886-2.500014-0.053274$

O $5.483701-1.224312-0.406551$

C $6.517923-0.372657-0.927584$

C $7.741959-1.261675-1.120750$

C $7.563401-2.271107-0.002514$

H $6.7383650 .364487-0.146343$

C $6.0286610 .343395-2.190847$

H $7.730481-1.768248-2.092701$

H $8.677724-0.699139-1.047337$
C $8.387914-3.533300-0.183874$

H $7.840936-1.7934500 .946739$

C $4.6873541 .089520-2.024982$

O $5.848345-0.631462-3.226944$

H $6.8082931 .027752-2.546011$

H $3.8707120 .363999-1.927691$

O $4.4374251 .792212-3.250242$

C $4.6303012 .082967-0.848338$

C $3.3265372 .905408-0.852292$

H $4.717871 \quad 1.5278860 .091820$

O $5.7486392 .963561-0.943526$

H $3.2953703 .525901-1.755922$

H $2.4802702 .212240-0.905331$

H $8.216146-4.2331770 .639897$

H $8.137823-4.045403-1.119111$

H $9.456312-3.294507-0.206834$

C $5.461047-2.9707151 .278821$

H $5.542514-0.158193-4.019806$

H $5.1138212 .486521-3.332483$

H $5.8347873 .424861-0.092427$

H $5.718271-2.2308652 .048562$

H $5.917241-3.9202101 .580324$

H $5.803629-3.203304-0.857837$

C 2.2340682 .2416191 .922821

O 3.3099953 .1355631 .610881

C 3.1764383 .8367270 .372102

C 1.8872434 .6696940 .379887

C 0.6650103 .8161910 .703657

C 0.8956802 .9928571 .970149

H 2.1923351 .4464661 .168381

C 2.5844091 .6007043 .271422

H 4.0096354 .5490240 .332039

O $1.7199795 .315044-0.877962$

H $1.9797925 .458151 \quad 1.138277$

H $\quad 0.396817 \quad 3.168747-0.136417$

O $\quad-0.459657 \quad 4.666787 \quad 0.932807$

H 0.8677603 .6575322 .843037

O -0.1744222 .0607902 .110186$

C $1.8223590 .325193 \quad 3.522615$

H 3.6516821 .3470533 .310600

H 2.4321342 .3082224 .095693

H $0.9641175 .921029-0.801486$

H $-0.684098 \quad 5.0902870 .087311$

H -1.0041602 .5683672 .103460$

O 0.8862820 .5189894 .494056

O $2.025822-0.7165352 .913643$

C $0.067462-0.6156104 .794498$

H $0.686560-1.4638285 .102289$

H $-0.594796-0.3469965 .621619$

H $-0.544530-0.8777303 .926525$

SCF Energy (B3LYP/6-31G**//MMFF) $=-3245.90971646$

03_260

MM̄FF Geometry

C $-1.852384-2.946045-4.043962$

C $-1.166621-3.017764-2.892404$

C $0.326387-3.176270-2.773485$

O $0.807586-2.069646-1.971343$

C $0.646975-4.483926-2.027360$

C $2.115707-4.661878-1.697954$

C $2.607457-4.738357-0.444967$

C $3.043821-4.769989-2.879290$

C $1.841554-4.6883640 .849140$

C $1.641670-3.2781401 .425728$

C $2.940699-2.6086681 .902181$

C $2.637592-1.2972262 .630028$

C $3.921798-0.5662053 .053593$

O $3.763906-2.3406760 .764900$

O 3.5632640 .7752343 .420563

C $4.871513-0.4636751 .839790$

C $4.554063-1.2014294 .297675$

O $\quad 6.142430 \quad 0.0508152 .228350$

C $5.052279-1.7959041 .083326$

C $5.826557-1.640464-0.248775$

O $5.804018-2.7016141 .900856$

C $0.933078-0.860726-2.582673$

O $0.783001-0.615696-3.768868$

C $1.2307330 .137739-1.532250$ 
C $1.0667781 .441197-1.801812$

C $1.2625212 .548756-0.865107$

C 1.8766652 .2316360 .471788

C $5.193253-0.690673-1.272263$

C $5.7730310 .714736-1.254992$

C $5.4579852 .954269-2.305125$

O $6.7710071 .025976-0.610855$

C $4.7589173 .842949-1.325550$

C $4.0491914 .923236-1.686056$

C $3.3875235 .854346-0.710744$

C $1.9185666 .062796-1.004114$

C $1.4942647 .148156-1.673262$

C $0.9370085 .059230-0.443727$

C $0.8891823 .788563-1.244721$

N $5.063568 \quad 1.575377-2.082809$

H -1.316438 -3.066719-4.982386

H -1.690092 -2.914703 -1.945802

H $0.817222-3.165817-3.754272$

H $0.318249-5.341195-2.629732$

H $0.048269-4.517630-1.110553$

H $3.682722-4.864163-0.323438$

H $4.068383-5.017355-2.580990$

H $2.702181-5.556974-3.559541$

H $3.081778-3.824133-3.428297$

H $0.863066-5.1668620 .731072$

H $2.371074-5.3090131 .583394$

H $1.151780-2.647670 \quad 0.674324$

H $0.952587-3.3598252 .275634$

H $3.458073-3.3053062 .572176$

H $1.992963-1.4734823 .500094$

H $2.066200-0.6363891 .964229$

H $2.908274 \quad 0.7252224 .137806$

H $\quad \begin{array}{llll}4.434068 & 0.272817 & 1.157381\end{array}$

H $3.849306-1.1811395 .137840$

H $5.430373-0.6336584 .629423$

H $4.853281-2.2401724 .143643$

H 5.9868580 .9005402 .675246

H $6.873218-1.381892-0.048341$

H $5.859663-2.633708-0.719635$

H $\quad 6.202287-3.376801 \quad 1.326995$

H $1.535003-0.227964-0.558441$

H $\quad 0.725307 \quad 1.727306-2.795961$

H 2.8138631 .6826200 .338619

H 1.1957881 .6228111 .074111

H 2.1284443 .1168681 .059154

H $5.389619-1.086314-2.277287$

H $4.107562-0.641633-1.147813$

H $5.2060483 .200851-3.341299$

H $6.5414223 .047084-2.178101$

H $4.8675983 .604899-0.270107$

H $3.9671485 .175683-2.740972$

H $3.9162896 .815550-0.758888$

H 3.5067005 .5045350 .322135

H $\quad 0.4397017 .319735-1.862160$

H $2.1931907 .886601-2.051977$

H $-0.0771425 .481594-0.449252$

H 1.1569594 .8898330 .614151

H $0.4585423 .919314-2.238569$

H $4.2160531 .245594-2.535593$

C -3.864274 -1.705284 -3.080601

O $-3.251246-0.410501-3.236209$

C $-4.1300780 .577260-2.643508$

C $-5.387969-0.179195-2.222903$

C $-5.355102-1.402380-3.126498$

H $-4.3652531 .290568-3.442517$

C $-3.3351721 .263041-1.523937$

H -5.328545 -0.488629-1.172858

H $-6.2944720 .421281-2.344294$

C $-6.242496-2.533485-2.639204$

H - $-5.653328-1.107217-4.141055$

C $-4.1722992 .040689-0.499047$

O $-2.4120272 .169342-2.140771$

H $-2.7234920 .512781-1.012523$

H -4.8308401 .3570380 .045211$

O $-5.0422092 .943941-1.192090$

C -3.3532792 .8599590 .518225$

C $-2.1915602 .119827 \quad 1.206855$

H $-2.943922 \quad 3.7527210 .029091$
O $\quad-4.2429593 .345973 \quad 1.528469$

H -1.4011281 .9411180 .470738$

H $-1.7825752 .798001 \quad 1.967392$

H -6.178964 -3.396206 -3.309404

H -5.956998 -2.864778 -1.635004

H $-7.288383-2.211766-2.601459$

C $-3.331125-2.649958-4.159348$

H $-1.8781531 .655047-2.770707$

H -4.485088 $3.533615-1.728971$

H -4.9632993 .8196501 .078257$

H -3.490068 -2.182805 -5.139772

H -3.878061 -3.598925 -4.157435

H -3.612379 -2.076232 -2.079315

C $-3.962258-0.1202473 .632799$

O -3.4686861 .0403482 .950122$

C -2.5526980 .7829081 .884884$

C -1.3017220 .0642422 .419264$

C $-1.677817-1.2101133 .181287$

C -2.800401-0.960266 4.195837

H -4.5354200 .2764624 .480510$

C $-4.929609-0.9345512 .758131$

H $-3.037350 \quad 0.1387201 .147678$

O -0.5789260 .9415853 .280258$

H $-0.648588-0.1833241 .573501$

H $-0.791261-1.5900963 .703818$

O $-2.079428-2.1991522 .237740$

H -3.182576 -1.913934 4.578086

O $-2.232325-0.2625625 .308351$

C $-6.140464-0.1285672 .336313$

H $-4.439191-1.2900021 .847939$

H $-5.293143-1.8044213 .316850$

H $0.2209310 .472966 \quad 3.573174$

H $-2.282455-3.0111152 .732575$

H $-2.930820-0.1548405 .976230$

O $-6.773273-0.7813971 .316440$

O -6.4964320 .9276602 .839945$

C -7.928012 -0.1233310 .786072$

H $-8.703200-0.0436901 .553910$

H $-7.658614 \quad 0.8657040 .402737$

H $-8.311934-0.726031-0.041239$

SCF Energy $\left(B 3 L Y P / 6-31 G^{* *} / / M M F F\right)=-3245.88684309$

03_261

MMFF Geometry

C $-2.063924-3.864337-0.846476$

C $-2.775001-4.6021070 .020299$

C $-3.775038-4.0455381 .010060$

O $-3.536797-2.6390131 .254203$

C $-5.200281-4.2222780 .456083$

C $-6.270337-3.5339981 .281509$

C $-6.936944-2.4243840 .901428$

C $-6.578195-4.1860492 .604232$

C $-6.767987-1.643952-0.373208$

C $-6.173087-0.256281-0.113430$

C $-5.517570 \quad 0.350497-1.365085$

C $-4.154578-0.293050-1.649604$

C $-3.4228080 .381296-2.817442$

O $-5.3501631 .753882-1.121335$

O $-2.054860-0.056078-2.791605$

C $-3.4136701 .907086-2.585144$

C $-3.989606-0.057409-4.172568$

O $-2.8817942 .584104-3.721030$

C $-4.8009832 .482184-2.231975$

C $-4.7573033 .981617-1.842523$

O $-5.6510222 .381203-3.382699$

C $-2.560654-2.3222102 .151321$

O $-1.840433-3.0984412 .758858$

C $-2.520691-0.8477492 .270382$

C $-1.653370-0.264523 \quad 3.111508$

C -1.5282221 .1755473 .358810$

C -2.4226992 .0909952 .564199$

C $-3.9565394 .329116-0.583902$

C $-2.5129424 .730705-0.841528$

C $-0.450805 \quad 5.3926140 .409660$

O $-2.0136714 .792663-1.960533$

C 0.2710714 .3485291 .199853

C 0.9181844 .5963072 .348536

C 1.6210003 .5370853 .148036 
C 1.0833323 .4132044 .557057 C 1.8718583 .6605315 .616705 C -0.3644823 .0077574 .744887$ C $-0.640981 \quad 1.599267 \quad 4.284615$ N $-1.852138 \quad 5.0210860 .343919$ H $-2.221644-2.789679-0.886288$ H $-2.630197-5.6797080 .041825$ H $-3.682390-4.592047 \quad 1.957178$ H $-5.438782-5.2928040 .392744$ H -5.226984 -3.870734 -0.579552 H -7.688856 -2.019112 1.577415 H -7.397934 -3.6878833 .132741$ H $-6.871234-5.2301882 .453537$ H $-5.701382-4.1604913 .258645$ H -6.169879 -2.188187-1.104230 H -7.754761 -1.527393 -0.838782 H -6.9686600 .4118190 .243204$ H -5.437812 -0.2871940 .700230$ H $-6.199376 \quad 0.220251-2.213513$ H $-4.249490-1.368181-1.838401$ H $-3.520000-0.204337-0.757582$ H -2.048402 -1.027007-2.849907 H $-2.7272002 .098818-1.750707$ H $-3.881974-1.141556-4.299104$ H $-3.4328970 .396362-4.999917$ H $-5.0477850 .182924-4.293986$ H $-1.9996302 .212604-3.892875$ H -4.437244 $4.585812-2.700253$ H $-5.7937584 .290797-1.643755$ H $-6.3798313 .016140-3.286211$ H $-3.222104-0.2786081 .672898$ H $-0.980037-0.8980883 .687334$ H $-3.477188 \quad 1.8638442 .756594$ H -2.229386 $1.977907 \quad 1.491326$ H -2.287175 3.1477122 .797886 H -4.434065 5.194907 -0.106433 H $-3.976433 \quad 3.5057210 .137811$ H -0.3986816 .3787230 .881910$ H $-0.0247255 .464256-0.595516$ H $0.2521093 .334570 \quad 0.806914$ H 0.9473805 .6112652 .737977 H $2.6896313 .786663 \quad 3.163634$ H 1.5500432 .5620082 .655978 H 1.4991863 .5756506 .632154 H 2.9095003 .9529645 .494207 H -1.004907 3.7489444 .261321 H $-0.632196 \quad 3.0542625 .809044$ H -0.0494420 .8492834 .812179$ H $-2.369684 \quad 4.984094 \quad 1.217438$ C $0.366659-3.829057-1.540596$ O $0.332829-2.390735-1.663969$ C $0.946018-1.818672-0.490032$ C $1.830156-2.9198940 .071304$ C $0.967198-4.141790-0.167221$ H $\quad 0.124152-1.5940350 .202081$ C $1.679208-0.531038-0.876044$ H $2.768683-2.993087-0.492306$ H $2.069638-2.772302 \quad 1.128000$

C $1.736211-5.450609-0.118597$ H $0.189520-4.1664380 .605528$ $\begin{array}{llll}\text { C } & 2.524601 & 0.054653 & 0.266974\end{array}$ O $0.7005820 .440603-1.259595$ H $2.300472-0.714367-1.759154$ H $3.309491-0.6517560 .554074$ $\begin{array}{lllll}\text { O } & 1.694800 & 0.195859 & 1.427695\end{array}$ C $3.160242 \quad 1.423814-0.033334$ C $3.9966731 .497361-1.325844$ H $2.3763332 .188610-0.078148$ O 3.9816141 .7719321 .086200 H $3.3284361 .424860-2.191913$ H $4.4598372 .492209-1.356183$ H $1.072344-6.298237-0.315968$ H $2.541285-5.471513-0.860664$ H $2.186806-5.595210 \quad 0.868864$ C -1.030484 -4.409031-1.791365 H $0.1615860 .047319-1.967515$ H $\quad 0.9447310 .765430 \quad 1.184128$ H 4.3440132 .6588530 .920655
H $-0.990220-5.503266-1.741725$ H $-1.348832-4.141727-2.806556$ H $1.035321-4.183984-2.335080$ C $7.088748-0.351132-0.295599$

O $6.0327090 .615208-0.375996$

C $5.1074990 .434391-1.450461$

C $5.8389440 .526046-2.799603$

C $7.003974-0.467537-2.863300$

C $7.886223-0.397150-1.610858$

H 7.7583090 .0283560 .486963

C $6.573126-1.7271370 .154057$

H $4.646940-0.554004-1.368593$

O $6.3320281 .851391-2.985011$

H $5.1233780 .321789-3.605206$

H $7.612252-0.251880-3.750250$

O $6.470887-1.780355-3.010995$

H $8.579346-1.245572-1.587670$

O $8.6940630 .781846-1.686338$

C $5.946900-1.6795531 .532313$

H $5.819988-2.114901-0.537121$

H $7.403575-2.4407670 .197225$

H $6.755067 \quad 1.887148-3.859837$

H $7.220835-2.395713-3.077877$

H $8.101754 \quad 1.551722-1.635285$

O $5.216078-2.8172851 .727868$

O $6.090015-0.7733852 .341536$

C $4.549114-2.9094852 .990571$

H $3.856716-2.0713753 .116537$

H $3.977003-3.8410343 .005088$

H $5.281011-2.9329423 .803351$

SCF Energy (B3LYP/6-31G**//MMFF) = -3245.90944312

03_262

MMFF Geometry

C $2.846890-3.2049771 .666075$

C $2.361479-4.3535191 .170710$

C $1.070992-4.491975 \quad 0.389045$

O $0.273391-3.2895800 .469009$

C $1.424888-4.726693-1.092808$

C $0.228716-4.766752-2.021674$

C $0.003698-3.877171-3.009427$

C $-0.733914-5.903152-1.796111$

C $0.854824-2.701100-3.407385$

C $0.472112-1.374562-2.731052$

C $-0.887171-0.815234-3.181037$

C $-1.1197150 .582706-2.602215$

C $-2.5067641 .132489-2.975409$

O $-1.917402-1.694379-2.725845$

O $-2.771445 \quad 2.270712-2.141883$

C $-3.5806890 .073474-2.641837$

C $-2.546174 \quad 1.639059-4.421863$

O $-4.859576 \quad 0.471674-3.128747$

C $-3.236640-1.337314-3.160306$

C $-4.201833-2.430105-2.639007$

O $-3.326470-1.345853-4.590463$

C $-0.528646-3.1350951 .556549$

O $-0.690472-3.9225392 .474366$

C $-1.143826-1.794278 \quad 1.456441$

C -1.627656 -1.205245 2.559723

C -2.209091 0.1363662 .630552

$\begin{array}{llll}C & -2.476266 & 0.847389 & 1.331404\end{array}$

C $-4.247457-2.596973-1.115598$

C $-5.400039-1.867663-0.444278$

C $-6.333007-1.3943351 .820134$

O $-6.303258-1.313487-1.064222$

$\begin{array}{lll}\text { C }-5.977101 & 0.012719 & 2.182412\end{array}$

C $-5.8710830 .446426 \quad 3.447752$

C $-5.572966 \quad 1.870156 \quad 3.820878$

C -4.3966921 .9971774 .762520$

C $-4.5824712 .156704 \quad 6.083821$

C -3.011015 2.034000 4.160632

C -2.4795110 .6625323 .843026$

N $-5.318460-1.9440810 .940518$

H $2.280504-2.2849701 .546228$

H $2.951031-5.2615251 .277858$

H $0.499307-5.3422310 .780186$

H $1.976566-5.670866-1.196109$

H $2.121699-3.942359-1.408077$ 
H $-0.889739-4.007243-3.619052$ H - $-1.517022-5.942260-2.560894$ H $-0.203411-6.860504-1.821229$ H -1.228842 -5.802275 -0.825357 H $\quad 1.912069-2.906790-3.210027$ H $\quad 0.789676-2.585718-4.496958$ H $0.477801-1.511151-1.643108$ H $1.252315-0.640388-2.967946$ H - $0.895333-0.779501-4.276824$ H $-0.3330061 .275840-2.925483$ H $-1.0460600 .538900-1.507363$ H -2.050915 2.911314 -2.270008 H -3.662522 $0.038297-1.550265$ H -1.806837 $2.435896-4.568158$ H -3.517879 $2.086815-4.657751$ H $-2.3384330 .857731-5.155768$ H $-5.0487751 .351623-2.760175$ H $-5.202488-2.283528-3.062962$ H -3.853028 -3.389265 -3.048771 H -3.447398 -2.263185 -4.886586 H $-1.137909-1.3099180 .486579$ H -1.561038 -1.7386913 .507125$ H -3.0663660 .2115560 .664656$ H -1.5372541 .1018260 .831049$ H -3.047054 1.7709551 .447478 H $-4.391251-3.661890-0.890519$ H $-3.303528-2.291168-0.654345$ H $-6.390066-2.0464092 .697456$ H -7.302183 -1.409699 1.311166 H $-5.8321220 .715096 \quad 1.364780$ H $-6.045374-0.2509994 .264165$ H -6.4736832 .2842684 .292920$ H -5.3963082 .4890632 .932287$ H -3.744297 2.2794896 .761768 H -5.5769712 .1621076 .517602$ H -2.3081222 .4941014 .868566$ H -3.005322 2.7061283 .297598 H $-2.259800 \quad 0.0646924 .728554$ H $-4.501704-2.3710221 .367933$ C $5.151605-2.3151851 .412442$ O $4.608649-1.0286071 .068447$

C $5.681105-0.0814190 .937804$ C $6.973422-0.8678741 .143274$ C $6.511590-2.0110762 .030154$ H 5.5614190 .6388611 .756645 C $5.5844870 .659687-0.406624$ H $7.366661-1.2544140 .195738$ H $7.757726-0.2586081 .603027$ C $7.465934-3.1914352 .047613$ H $6.383434-1.6318083 .052785$ C $4.2530891 .430451-0.575967$

O $5.759381-0.267349-1.476632$ H $6.4145131 .373656-0.464920$ H 4.1191232 .0470230 .317504 O $3.1562200 .520340-0.629906$ C $4.2096442 .332149-1.822515$ C $2.9156373 .161524-1.973689$ H $5.0744983 .006062-1.809070$ O $4.3339011 .514670-2.990072$ H $2.9382093 .605744-2.976469$ H $2.0502422 .490150-1.979117$ H $7.086769-3.9905562 .692090$ H $7.608907-3.6068871 .044407$ H $8.446450-2.8875422 .428458$ C $4.183740-3.0563282 .336031$ H $5.087526-0.963039-1.375698$ H $3.2227240 .027182-1.465214$ H $4.4081922 .106453-3.757953$ H $4.031896-2.4914493 .263483$ H $4.595241-4.0338992 .610949$ H $5.272109-2.8725590 .473200$ C 1.0091973 .3402990 .481982 O $2.368348 \quad 3.7852290 .364993$ C $2.7639964 .278445-0.918402$ C $1.8394885 .428511-1.341678$ C $0.3762595 .007714-1.313161$ C 0.0253714 .4423400 .060789 H $0.8589512 .453289-0.143826$
C $0.7982662 .957727 \quad 1.957362$

H $3.7610464 .716850-0.782685$

O $2.1937515 .900379-2.637128$

H $1.9791556 .268980-0.649067$

H $0.1529664 .293605-2.114722$

O $-0.4286636 .162242-1.562284$

H 0.0235365 .2614580 .791683

O $-1.3008043 .924204-0.010842$

C 1.4012011 .6112082 .292357

H 1.2572413 .7205052 .598564

H -0.2611192 .8889792 .220755$

H $1.5664416 .605841-2.871131$

H -1.358735 $5.884500-1.499267$

H $-1.621553 \quad 3.7941170 .896908$

O 1.9800331 .6605273 .526795

O 1.3299330 .6228331 .574902

C 2.5532780 .4325683 .986434

H $1.809864-0.3704113 .969957$

H 3.4182990 .1719323 .370891

H 2.8873340 .5781015 .017120

SCF Energy (B3LYP/6-31G**//MMFF) $=-3245.90177828$

03_263

MMFF Geometry

C $0.652374-0.8934635 .374528$

C 0.9938370 .0506214 .484434

C 2.3252910 .7524114 .458946

O $2.8947860 .522706 \quad 3.149261$

C 2.1360532 .2711994 .629447

C 3.4339483 .0483234 .514819

C 3.7101893 .9276643 .530902

C 4.4468702 .7930015 .600888

C 2.8162064 .3570262 .399547

C 2.8745743 .3971811 .210666

C $2.2549383 .958759-0.078093$

C 0.7760294 .3188280 .080014

C $0.1586984 .784032-1.247887$

O $2.4013322 .938530-1.075118$

O $-1.2692014 .796448-1.091421$

C $0.4801493 .742804-2.340864$

C $0.5654766 .222645-1.587472$

O $0.0667284 .198839-3.625731$

C $1.9659053 .318446-2.388621$

C $2.2106322 .111269-3.321448$

O $2.7923154 .361288-2.919537$

C $3.641735-0.5966072 .960736$

O $3.949971-1.4302123 .795911$

C $3.992630-0.6406181 .523402$

C $4.672411-1.6836721 .024649$

C $5.035478-1.874543-0.382590$

C $4.607932-0.806230-1.354780$

C $1.4147470 .851593-2.971858$

C $1.867962-0.362874-3.766007$

C $1.446079-2.822975-3.923877$

O $2.704717-0.310168-4.662568$

C $2.293442-3.618070-2.981002$

C $3.389084-4.297007-3.354601$

C $4.204669-5.134398-2.409822$

C $5.685410-4.818821-2.456015$

C $6.576512-5.767312-2.791632$

C $6.146300-3.416663-2.110350$

C $5.711716-2.988642-0.733166$

N $1.207650-1.510952-3.353083$

H $1.362911-1.1435526 .160153$

H $\quad 0.3062580 .322786 \quad 3.688553$

H 2.9954620 .3659705 .236303

H 1.6854422 .4826355 .607920

H 1.4156812 .6255443 .883309

H 4.6840574 .4156303 .537622

H 5.3020913 .4746665 .539006

H 3.9897622 .9324536 .586094

H 4.8356211 .7723425 .536204

H 1.7867384 .4749562 .754161

H 3.1376865 .3571072 .082097

H 3.9165313 .1202540 .999581

H 2.3711132 .4570551 .465624

H $2.8378054 .836248-0.381296$

H 0.6322545 .0908720 .844822 
H $\quad 0.222180 \quad 3.437531 \quad 0.432983$ H -1.484877 $5.386089-0.348617$ H $-0.1285692 .860481-2.112905$ H $0.2036756 .914632-0.817119$ H $0.1064286 .553145-2.525410$ H $1.6466346 .356224-1.663081$ H $-0.8708164 .448294-3.558353$ H $2.0111752 .395033-4.363436$ H $3.2823951 .868210-3.287592$ H $2.2217295 .076076-3.244159$ H $3.655147 \quad 0.1832300 .904287$ H $4.975811-2.4809291 .701751$ H $5.0516490 .159014-1.086085$ H $3.518638-0.705186-1.346698$ H $4.898494-1.002437-2.387910$ H $1.5163480 .621945-1.905220$ H $0.3525951 .003998-3.192598$ H $1.906658-2.721846-4.911808$ H $0.470341-3.305263-4.038095$ H $1.971974-3.660321-1.942542$ H $3.696536-4.281855-4.397729$ H $4.033642-6.185913-2.674832$ H $3.847351-5.025583-1.378371$ H $7.640514-5.557904-2.827334$ H $6.263972-6.776349-3.039396$ H $5.796248-2.743006-2.895836$ H $7.242178-3.359482-2.137299$ H $5.994422-3.6902030 .053073$ H $0.569409-1.453568-2.565305$ C $-1.513208-1.5926514 .147887$ O $-0.801324-2.0914792 .998470$ C - $-1.734367-2.7565112 .119829$ C $-3.112219-2.5587962 .738351$ C $-2.784095-2.4347974 .213828$ H -1.454426 -3.816896 2.133479 C $-1.569759-2.1743310 .710500$ H -3.576669 -1.634341 2.372649 H $-3.790542-3.3882482 .518115$ C $-3.903891-1.8139635 .030016$ H -2.555715 -3.433484 4.609440 C $-2.628551-2.660866-0.291804$ O $-0.275274-2.5423590 .221416$ H -1.576095 -1.0807700 .771862$ H $-3.628551-2.3684480 .042181$ O $-2.633182-4.094384-0.306417$ C $-2.416422-2.175762-1.737608$ C $-2.242347-0.655664-1.911649$ H -1.542833 -2.675874 -2.172400 O $-3.533980-2.596144-2.527069$ H -1.273106 -0.354868 -1.497752 H $-2.212842-0.456716-2.990868$ H -3.622695 -1.737429 6.084901 H $-4.151140-0.8085194 .673066$ H -4.810260 -2.425051 4.966053 C $-0.622602-1.7017965 .389643$ H $0.379198-2.2320210 .870798$ H -1.739571-4.387905 -0.554310 H -3.630213 -3.556541 -2.407370 H $-0.325770-2.7513575 .514045$ H -1.190679 -1.4177706 .282613$ H $-1.770975-0.5443373 .948489$ C $-5.7443840 .565743-1.369751$ O $-4.594292-0.103980-1.903444$ C $-3.3487430 .214404-1.279979$ C $-3.0474381 .714219-1.430832$ C $-4.2016012 .567249-0.892860$ C $-5.5590532 .092258-1.423128$ H $-6.5636190 .305291-2.052367$ C -6.1146690 .0455890 .028244$ H -3.407917 -0.024185 -0.214775 O $-2.8304682 .021355-2.806357$ H -2.121980 $1.942871-0.889225$ H $-4.0533073 .614236-1.184060$ O -4.2141342 .5104120 .530451$ H $-6.3702982 .577940-0.869061$ O $-5.6902402 .511602-2.785213$ C $-6.461891-1.4286390 .018242$ H $\quad \begin{array}{llll}\text {-5.292624 } & 0.184111 & 0.735449\end{array}$
H -6.9912070 .5848950 .404844$

H $-2.6182212 .968369-2.865366$ H -3.3597942 .8520950 .845271$ H $-5.0228232 .035340-3.308357$ O $-6.480472-1.8879121 .304905$ O $-6.699812-2.091891-0.981735$ C $-6.779901-3.2788361 .457041$ H $-6.725175-3.5234582 .521217$ H -7.792299 -3.488339 1.098971 H $-6.045252-3.8867030 .919920$ SCF Energy (B3LYP/6-31G**//MMFF) $=-3245.91456397$

03_264

MM̄̄FF Geometry

C $1.490846 \quad 1.7966264 .064837$

C 1.3202320 .7497633 .244646

C $0.092331-0.1258973 .265815$

O $-\begin{array}{llll}0.593423 & 0.018131 & 2.000137\end{array}$

C $0.498050-1.6022293 .426733$

C $-0.681797-2.5484083 .545034$

C $-0.980525-3.5172032 .657214$

C $-1.538306-2.3757854 .773754$

C $-0.244976-3.8825931 .398936$

C $-0.753530-3.182786 \quad 0.129587$

C $-2.207097-3.517817-0.240492$

C $-2.545785-2.977301-1.631847$

C $-4.019496-3.213888-1.998688$

O $-3.072004-2.9158870 .725768$

O $-4.323711-2.403489-3.144714$

C $-4.914769-2.714883-0.843416$

C $-4.276099-4.666991-2.415406$

O $-6.277502-3.067224-1.066402$

C $-4.463192-3.2134860 .544218$

C $-5.231402-2.5467691 .712042$

O $-4.702456-4.6233000 .637324$

C -1.3805391 .1172351 .841986$

O

C $-1.931598 \quad 1.081500 \quad 0.471022$

C $-2.3846112 .215524-0.083340$

C $-2.9508932 .354663-1.426196$

C $-3.2378881 .093389-2.196880$

C $-5.111496-1.0207251 .793251$

C $-6.255527-0.2733691 .127402$

C -6.9728472 .0362790 .524464$

O $-7.280689-0.8217600 .732061$

C $-6.6820212 .275041-0.923544$

C $-6.5483313 .499984-1.454644$

C $-6.3042663 .759276-2.913721$

C $-5.1027674 .645461-3.156072$

C $-5.2483415 .964821-3.364943$

C $-3.7446163 .987046-3.242397$

C $-3.2048933 .594666-1.894198$

N -6.0119641 .0924331 .063955$

H 0.7219232 .0175944 .801884

H 2.0825790 .5231522 .505138

H -0.5813250 .1659734 .080792$

H $1.119225-1.7208234 .324575$

H $1.132818-1.8858962 .580786$

H -1.854099-4.137772 2.854674

H $-2.093619-1.4340144 .729378$

H $-2.271053-3.1822674 .885874$

H $-0.915668-2.3746005 .674379$

H $0.826746-3.6865021 .508413$

H $-0.321560-4.9693121 .265138$

H $-0.646752-2.0994050 .255604$

H $-0.097345-3.479830-0.697815$

H -2.328320 -4.607068 -0.213853

H -1.887253 -3.416559 -2.391606

H $-2.351062-1.896726-1.658398$

H $-3.714808-2.655538-3.860017$

H $-4.869185-1.619935-0.858541$

H -3.659648 -4.930721 -3.283269

H $-5.314917-4.809591-2.732994$

H $-4.054441-5.385722-1.623864$

H $-6.529799-2.713203-1.936328$

H $-6.278889-2.8715931 .708723$

H -4.808046 -2.945356 2.645444

H $-4.743338-4.8676811 .576799$ 
H $-1.9337010 .130149-0.047115$ H $-2.318427 \quad 3.1377160 .493021$ H $-2.3054710 .602891-2.493798$ H -3.822852 $1.259633-3.103877$ H -3.823711 $0.396995-1.587888$ H -5.134146 -0.728102 2.850986 H $-4.158533-0.677731 \quad 1.380195$ H $-6.8965672 .952344 \quad 1.118918$ H -7.9837991 .6312210 .635240$ H $-6.6067351 .403011-1.568953$ H $-6.6535234 .370700-0.811141$ H $-7.2054464 .235540-3.321950$ H $-6.1864972 .823332-3.473870$ H $-4.3948586 .603229-3.567989$ H $-6.224746 \quad 6.437055-3.332238$ H -3.018916 4.678951 -3.690850 H -3.795891 $3.149009-3.943130$ H $-2.9721884 .447718-1.255111$ H -5.1128081 .4530841 .369737$ C 2.9534853 .4711802 .736239 O $3.6393002 .628671 \quad 1.793920$ C 3.4173893 .1658660 .470421 C 2.4140424 .3153870 .621767 C 1.7632134 .0252781 .962020 H $2.9588932 .367001-0.122081$ C $4.7547103 .596721-0.153459$ H 2.9099565 .2928000 .634763 H $1.6930234 .334164-0.202261$ C 1.1115535 .2369622 .602545 H 1.0044653 .2509111 .800484 C $5.8093512 .475360-0.239659$ O 5.3057224 .6466940 .655617 H $4.5774424 .031782-1.144221$ H 6.0534082 .1217000 .769465 O $7.001598 \quad 3.074317-0.767067$ C $5.4027951 .293590-1.141904$ C $6.4886340 .202114-1.212135$ H $4.4813120 .847632-0.757544$ O $5.1194521 .771503-2.455651$ H $7.4398130 .644060-1.530879$ H $\quad 6.644069-0.189817-0.200697$ H 0.6613014 .9740013 .564976 H 1.8377416 .0377992 .777344 H $\quad 0.3210285 .6343401 .957590$ C 2.6699862 .7266684 .045999 H 6.1463434 .9153350 .246790 H $7.7510772 .508162-0.517388$ H $5.9262342 .197487-2.792971$ H 2.4923053 .4777784 .826657 H 3.5598462 .1644954 .355059 H 3.6531614 .2848312 .974285 C $4.585146-2.156221-0.843724$ O $4.836858-1.442332-2.057887$ C $6.167485-0.944760-2.196894$ C $7.166330-2.110484-2.148047$ C $6.978028-2.968050-0.897484$ C $5.512437-3.371248-0.734071$ H $4.733133-1.4893760 .014917$ C $3.115012-2.583400-0.865835$ H $6.224876-0.513480-3.204470$ O $8.507888-1.636442-2.191176$ H $7.021524-2.749527-3.028853$ H $7.345105-2.456709-0.000022$ O $7.757093-4.160144-1.024023$ H $5.252485-4.115497-1.497775$ O $5.331796-3.9849380 .540046$ C $2.215696-1.370914-0.865534$ H $2.895254-3.188133-1.754201$ H $2.852582-3.1903510 .007334$ H $8.606650-1.113803-3.005333$ H $8.680384-3.888013-1.164109$ H $5.949595-4.7343760 .591206$ O $1.725345-1.151263-2.118489$ O $1.999615-0.683948 \quad 0.123700$ C $0.882148-0.004116-2.253612$ H $-0.025839-0.139674-1.661194$ H $1.4143410 .901433-1.946619$ H $\quad 0.6033520 .092380-3.306147$
SCF Energy (B3LYP/6-31G**//MMFF)= -3245.90965607

03_265

MMFF Geometry

C $-2.758702-3.927678-0.399139$

C $-1.496680-4.291518-0.127589$

C $-0.311649-3.683423-0.834259$

O $0.465482-2.944367 \quad 0.140592$

C $0.586268-4.777056-1.435606$

C $1.682816-4.223756-2.326642$

C $3.001489-4.296490-2.060637$

C $1.219634-3.577956-3.608936$

C $3.680260-4.923937-0.877133$

C $3.980273-3.9645890 .284835$

C $4.844422-2.752022-0.099156$

C $5.360469-2.0397761 .153618$

C $6.165898-0.7798120 .799750$

O $4.038618-1.844768-0.856906$

O $6.368826-0.0250452 .004903$

C $5.3457440 .091448-0.180634$

C $7.574686-1.1406280 .315707$

O $6.1265401 .175737-0.674184$

C $4.742306-0.700448-1.362885$

C $3.7364750 .120127-2.204596$

O $5.754481-1.094187-2.298509$

C $-0.041689-1.7552220 .569259$

O $-1.068408-1.2127210 .192570$

C $0.859396-1.2255111 .613368$

C $0.412773-0.2609292 .431248$

C 1.1728050 .3776143 .506358

C 2.6540600 .1126513 .559378

C $2.5141830 .652007-1.449322$

C $2.6806352 .065304-0.915201$

C $1.552273 \quad 3.7280800 .560105$

O $3.6064172 .803669-1.239981$

C 2.0772943 .6585571 .958903

C 1.3998544 .1211773 .020735

C 1.9263744 .1004714 .427380

C 0.9771143 .4274695 .393782

C 0.1245194 .1490936 .140868

C 1.0752811 .9268685 .551920

C $0.518348 \quad 1.1850634 .366935$

N $1.6469512 .418946-0.058087$

H -2.946604 -3.208847 -1.193674

H -1.298375 -4.978515 0.691117

H $-0.640954-2.996088-1.621322$

H $-0.017859-5.470226-2.035914$

H $1.012797-5.371991-0.620997$

H $3.691116-3.860220-2.783108$

H $2.052166-3.350625-4.283556$

H $0.539180-4.245896-4.147325$

H $\quad 0.699383-2.637470-3.404827$

H $3.099335-5.771284-0.497671$

H $4.623863-5.362286-1.227630$

H $3.031405-3.6209790 .711871$

H $4.492858-4.5433151 .063410$

H $5.681572-3.101881-0.714326$

H $5.963992-2.7178021 .769791$

H $4.507107-1.747477 \quad 1.780019$

H 5.4984550 .1991362 .375013

H $4.5266240 .532206 \quad 0.399768$

H $8.130637-1.6528001 .110558$

H $8.154980-0.2425340 .077364$

H $7.575313-1.795858-0.557995$

H $6.464326 \quad 1.6645480 .095699$

H $4.2590350 .923736-2.737825$

H $3.357740-0.548539-2.991644$

H $6.589186-0.664500-2.052133$

H $1.854656-1.6466951 .686741$

H -0.6120810 .0891802 .314322$

H 3.1117360 .3107352 .583748

H $2.849729-0.9290863 .834990$

H 3.1860600 .7445294 .273486

H $1.6669840 .690026-2.145652$

H $2.237579-0.019636-0.632733$

H 0.4996054 .0266280 .526560

H $2.1399114 .450619-0.014449$

H 3.0684903 .2346392 .101250 
H $\quad 0.420727 \quad 4.5704522 .869114$ H 2.0946385 .1408584 .735414 H 2.9083773 .6145514 .481799 H -0.5407503 .6770416 .855973$ H 0.0695125 .2289496 .049616 H $\quad 0.5061701 .6020056 .433305$ H 2.1118661 .6598795 .775907 H $-0.556576 \quad 1.3194834 .235837$ H 0.9339591 .7330750 .172732 C $-4.218157-3.3368701 .520557$ O $-4.416005-2.0272510 .957998$ C $-5.347085-1.3022621 .782751$ C $-5.715927-2.2296722 .939487$ C $-5.477859-3.6030252 .336182$ H $-6.239566-1.1331201 .169314$ C $-4.735803 \quad 0.0444042 .197255$ H -5.066375 -2.076815 3.808805 H $-6.748350-2.081348 \quad 3.271201$ C $-5.330101-4.7039563 .371098$ H -6.315119-3.842399 1.667049 C $-4.195507 \quad 0.887613 \quad 1.020644$ O $-3.638124-0.1881743 .090529$ H -5.4718550 .6195462 .770348$ H $-3.299916 \quad 0.4059300 .614807$ O $-3.7505212 .146181 \quad 1.540859$ C $-5.2065221 .133359-0.112686$ C $-4.7167732 .087121-1.212308$ H $-5.4801360 .179040-0.572540$ O $-6.415687 \quad 1.6703190 .431040$ H -5.526442 2.228092 -1.942392 H $-4.5787413 .095226-0.796481$ H -5.155579 -5.671269 2.889894 H $-4.491879-4.5083894 .048298$ H $-6.238817-4.7874043 .976142$ C -3.950973 -4.353167 0.408482 H $-2.990856-0.7431492 .622351$ H -4.5057352 .5729381 .979395$ H -6.2208442 .5573270 .777785$ H $-4.811120-4.417881-0.268476$ H -3.794314 $-5.351466 \quad 0.832315$ H $-3.342712-3.2707182 .181634$ C $-2.0516572 .522367-3.770845$ O $-3.2275262 .666091-2.964340$ C $-3.4272641 .675435-1.944847$ C $-3.4835540 .269019-2.561261$ C $-2.260928-0.005196-3.445004$ C $-2.0052141 .134219-4.432166$ H $-2.1669433 .268663-4.567523$ C $-0.7735592 .867981-2.990607$ H -2.596303 $1.735710-1.234595$ O $-4.6743640 .139173-3.335499$ H -3.531178 -0.475269-1.758425 H -2.414372 $-0.936464-4.003917$ O $-1.103306-0.170953-2.633171$ H -1.038015 $0.990171-4.928240$ O $-3.008696 \quad 1.074654-5.448928$ C $-0.6742854 .353336-2.712080$ H $-0.7092052 .356323-2.027703$ H $0.0963382 .583892-3.595027$ H $-4.694119-0.764160-3.694618$ H -1.297786 $-0.865105-1.981461$ H -2.807002 $1.765919-6.102405$ O $0.6410644 .706972-2.616606$ O $-1.6293685 .104422-2.567574$ C $0.8887176 .089140-2.340850$ H $\quad 0.489094 \quad 6.713373-3.145641$ H $\quad 0.4483846 .368401-1.378876$ H $1.9706646 .235914-2.286673$ SCF Energy (B3LYP/6-31G**//MMFF) $=-3245.90033005$

03_266

MM̄FF Geometry

C -2.282721 -2.282105 2.420654

C $-1.571928-2.1668043 .553223$

C $-0.344721-1.3013853 .732287$

O $0.391978-1.1770732 .493316$

C -0.7693320 .0963094 .223356$

C 0.3553331 .0982864 .407679
C $0.2682892 .387303 \quad 4.020920$

C 1.6010330 .6066545 .096348

C -0.9276733 .0720923 .417632$

C -0.9884043 .0167381 .885834$

C 0.0436043 .9147831 .184573

C $-0.1991293 .904867-0.327637$

C $0.8753924 .683565-1.099169$

O $1.3528383 .421791 \quad 1.477912$

O $\quad 0.7621314 .338245-2.488111$

C $2.2668634 .208607-0.630127$

C $0.6534836 .197938-1.012451$

O $3.2979955 .001348-1.213790$

C 2.4187944 .1895160 .907106

C 3.7581273 .5619381 .358081

O 2.4051115 .5379901 .391493

C $1.245530-2.2011102 .202353$

O $1.413425-3.2183682 .858312$

C $1.958813-1.8797010 .944744$

C $2.838950-2.7691390 .458916$

C $3.654946-2.640351-0.751198$

C $3.498367-1.393470-1.578053$

C 3.8877432 .0569981 .110912

C 5.2698131 .5399381 .475925

C $6.544674-0.5978561 .660543$

O 6.2269572 .2779641 .694256

C $6.989609-1.0848720 .316721$

C $7.287387-2.3659160 .051870$

C $7.702725-2.859034-1.305051$

C $6.869426-4.028558-1.784342$

C $7.398082-5.260572-1.876333$

C $5.445290-3.758722-2.226453$

C $4.500490-3.646650-1.060163$

N $5.3141810 .153213 \quad 1.489422$

H -1.989360 -1.716403 1.540283

H -1.896071 -2.715182 4.435118

H $0.299141-1.7672014 .489520$

H -1.289517 -0.0054305 .185291$

H -1.5030980 .4915393 .511138$

H 1.1250123 .0376854 .190423

H $2.138318-0.1078264 .465557$

H 2.2946721 .4216125 .330034

H $1.3432490 .117956 \quad 6.041730$

H $-1.8489842 .639013 \quad 3.825261$

H -0.9342354 .1176463 .750560$

H $-0.858931 \quad 1.9772001 .557228$

H -1.9944383 .3291511 .578295$

H -0.0562844 .9328451 .579396$

H -1.197907 $4.292791-0.564336$

H $-0.1937502 .866927-0.683305$

H $\quad-0.1430594 .546328-2.776699$

H $2.4031713 .190493-1.015474$

H -0.315147 $6.468090-1.450337$

H $1.4059986 .742110-1.593396$

H 0.6660496 .5762110 .011755

H $3.1684814 .981251-2.177519$

H $4.591543 \quad 4.1058370 .895373$

H 3.8788153 .7244002 .438794

H 2.8082835 .5539042 .275132

H $1.748702-0.9312520 .465784$

H $2.988604-3.6973821 .010143$

H $2.515923-1.385959-2.059965$

H $4.243759-1.297633-2.369758$

H $3.596108-0.494423-0.962249$

H $3.1409251 .514817 \quad 1.702297$

H 3.7280051 .8217950 .054399

H $6.330468-1.4211292 .349054$

H 7.3160480 .0414662 .100705

H $7.064525-0.347359-0.479239$

H $7.234287-3.1009460 .851871$

H $8.760279-3.146467-1.244328$

H $7.651819-2.057430-2.052651$

H $6.815136-6.099902-2.240922$

H $8.424059-5.459396-1.584676$

H $5.087670-4.583443-2.857140$

H $5.439984-2.878742-2.874556$

H $4.520709-4.519712-0.405558$

H $4.473275-0.3689601 .263935$

C $-4.796371-2.3574922 .374724$ 
O $-4.884363-1.423910 \quad 1.280574$

C $-6.202586-1.4979580 .706767$

C $-7.055390-2.259018 \quad 1.713871$

C $-6.043926-3.2374662 .277104$

H -6.110853 -2.104352 -0.202783

C $-6.719501-0.1019590 .338515$

H -7.430307 -1.605628 2.509808

H $-7.914176-2.7490391 .245214$

C $-6.468101-3.8577873 .597309$

H -5.883477 -4.037142 1.541253

C $-5.7362010 .748550-0.490281$

O

H -7.679327 $-0.197075-0.183440$

H -4.9126461 .0806270 .154521$

O $-6.4438621 .929623-0.886403$

C $-5.1714740 .042623-1.735287$

C $-4.2422010 .947058-2.564681$

H $-4.614994-0.841652-1.411919$

O $-6.238797-0.415245-2.563242$

H -4.833034 $1.719891-3.071036$

H $-3.5521561 .459408-1.885860$

H -5.703471 -4.547189 3.968490

H -6.634162 -3.094024 4.364481

H -7.399591-4.420288 3.475964

C -3.495586 -3.158289 2.282573

H -7.3346401 .4939691 .290461$

H -5.788614 2.576997-1.196793

H $-6.7756610 .358577-2.805992$

H -3.429547 -3.6619061 .310134$

H -3.483581 -3.941112 3.050127

H -4.813777 -1.764626 3.298505

C -1.693586 $-0.659048-2.204176$

O $-2.774482-0.975426-3.086648$

C $-3.4497950 .160858-3.635414$

C $-2.4539051 .014447-4.432216$

C $-1.2395121 .406100-3.591596$

C $-0.6214920 .181019-2.914067$

H $-2.085798-0.088825-1.352530$

C -1.175791-1.973179-1.601924

H $-4.186676-0.234616-4.345972$

O $-3.0779872 .194769-4.926465$

H $-2.1024760 .445677 \quad-5.302815$

H -1.497132 2.173669-2.852594

O $-0.251481 \quad 1.990524-4.444491$

H $-0.091193-0.404450-3.673531$

O $0.3485720 .639146-1.975828$

C $-0.510235-2.901957-2.595508$

H $-0.464216-1.744847-0.802538$

H -2.011825 -2.532075 -1.164142

H $-3.833047 \quad 1.918258-5.473440$

H $-0.6647402 .752554-4.885487$

H $0.784951-0.138693-1.593700$

O $0.349924-3.726305-1.927481$

O $-0.737764-2.928834-3.797162$

C $1.051856-4.673469-2.738612$

H $\quad 0.345346-5.324073-3.262857$

H $1.700004-4.152710-3.449786$

H $1.673202-5.288624-2.082411$

SCF Energy (B3LYP/6-31G**//MMFF) $=-3245.90461484$

03267

MM̄FF Geometry

C $-2.9388430 .017046-3.152261$

C $-2.258704-0.183295-2.012858$

C $-1.068024-1.100771-1.901192$

O $-0.037230-0.432140-1.134589$

C -1.473081-2.375475-1.133040

C $-0.597165-3.572560-1.450442$

C $0.316474-4.113118-0.620978$

C $-0.838585-4.201195-2.801414$

C $0.693237-3.6737020 .765228$

C $1.924029-2.7664150 .786078$

C $3.206038-3.3646990 .189453$

C $3.796546-4.4693491 .067711$

C $5.159575-4.9567520 .548949$

O $4.138762-2.2825490 .080402$

O $5.750748-5.7259561 .607555$

C $6.066941-3.7348870 .277196$
C $4.995395-5.909103-0.642107$

O $7.280933-4.126827-0.359603$

C $5.373649-2.633072-0.556301$

C $6.251411-1.360550-0.609326$

O $5.172048-3.115953-1.886692$

C $0.6435220 .583752-1.731511$

O $\quad 0.554586 \quad 0.955973-2.890144$

C $1.4742951 .225538-0.690269$

C $1.6606062 .552854-0.738634$

C 2.3510473 .3630030 .268077

C $3.1223862 .637148 \quad 1.338547$

C $5.531871-0.142209-1.207248$

C $6.4188221 .088458-1.254045$

C $6.3611043 .480996-1.955450$

O $7.5825531 .096824-0.862697$

C $6.0440744 .320782-0.759177$

C $5.4245655 .508177-0.832578$

C 5.1497016 .3791370 .358085

C $3.689026 \quad 6.7412540 .492683$

C 3.2378487 .9492030 .114048

C 2.7711945 .7393721 .155523

C 2.2425054 .7066440 .196823

N $5.7482152 .176904-1.796574$

H -2.636182 $-0.498867-4.059535$

H -2.581666 $0.320552-1.104843$

H $-0.668200-1.345035-2.892327$

H -2.505152 -2.660160 -1.381203

H -1.475573 -2.166331 -0.057793

H $\quad 0.869993-4.983572-0.972223$

H $-0.244112-5.109073-2.950611$

H $-1.892083-4.480673-2.906577$

H $-0.581019-3.506747-3.606410$

H $-0.137590-3.1543041 .251837$

H $\quad 0.871480-4.5661691 .376460$

H $1.692220-1.8396520 .249687$

H $2.120012-2.4533301 .819873$

H $2.994988-3.737675-0.817986$

H $3.103849-5.3142191 .160861$

H $3.935372-4.0836282 .087606$

H $6.588758-6.0949921 .281465$

H $6.347075-3.3091421 .250883$

H $4.429802-6.799538-0.342139$

H $5.965982-6.275642-0.994172$

H $4.472675-5.454050-1.486129$

H $7.744741-4.7363200 .238559$

H $6.565830-1.0941170 .408396$

H $7.160426-1.558905-1.191060$

H $\quad 5.129279-2.366108-2.499585$

H 1.8311340 .6081390 .125088

H $1.2177913 .108052-1.565010$

H $3.811886 \quad 1.9117350 .893274$

H 2.4402912 .1052552 .010498

H 3.7389523 .2966851 .952811

H $5.201355-0.350108-2.230158$

H $4.6494970 .104302-0.606608$

H $5.9701963 .913655-2.881694$

H $7.4456233 .369255-2.052823$

H $6.364088 \quad 3.945520 \quad 0.210175$

H $5.1268635 .893705-1.805229$

H 5.7534387 .2896140 .248916

H 5.4940635 .9084191 .287315

H $2.196321 \quad 8.2271790 .237242$

H $3.898478 \quad 8.684465-0.333129$

H $1.9000726 .260937 \quad 1.575354$

H 3.2766725 .3049442 .022165

H $1.6523745 .133033-0.615850$

H $4.7618152 .087773-2.024025$

C $-5.4447930 .116741-3.195213$

O $-5.530181-0.556165-1.921914$

C $-6.701605-0.095131-1.233118$

C $-7.6528000 .324575-2.341248$

C $-6.6882981 .009055-3.294923$

H $-6.4076350 .793687-0.661477$

C -7.212532 -1.165315 -0.265284

H $-8.114544-0.540902-2.830083$

H $-8.4471910 .986965-1.985159$

C $-7.2241571 .149908-4.709162$

H $-6.4650272 .011267-2.904511$ 
C $-6.116869-1.7573250 .648747$

O $-7.771632-2.245670-1.021597$

H $-8.033681-0.7517750 .331854$

H -5.469414 -2.416111 0.057144

O $-6.753783-2.6073621 .609761$

C $-5.243231-0.7192411 .377052$

C $-4.222370-1.3710862 .329259$

H $-4.703629-0.1247800 .633454$

O $\quad-6.0693640 .1842082 .109494$

H -4.745069 -1.836887 3.173265

H $-3.689690-2.1554131 .777650$

H $-6.4836801 .628172-5.358258$

H $-7.4737570 .175561-5.142536$

H $-8.1306791 .763866-4.718079$

C $-4.1396370 .916198-3.245150$

H -8.086091 -2.912086 -0.386809

H -7.379188 -2.065702 2.119913

H $-6.487728-0.3111382 .833640$

H -4.114171 $1.639982-2.421598$

H -4.078402 $1.490002-4.176930$

H $-5.450466-0.662126-3.967704$

C $-1.468548 \quad 1.1401002 .074357$

O $-2.4376530 .129891 \quad 1.771855$

C $-3.205632-0.3495772 .880172$

C $-2.280037-0.9688623 .937736$

C -1.1932280 .0232994 .368399$

C $-0.494610 \quad 0.6645643 .164288$

H $-0.898527 \quad 1.262328 \quad 1.146148$

C -2.130482 2.4847522 .390362

H -3.7595340 .4834443 .325721$

O $-1.672095-2.1454373 .409548$

H -2.883477 -1.269402 4.802518

H $-0.446733-0.4949054 .982549$

O $-1.771256 \quad 1.0514385 .167514$

H $\quad 0.1312751 .5058303 .484649$

O $0.374780-0.2994502 .565223$

C -2.8953992 .9938571 .190596$

H -2.8099002 .4278883 .245545$

H -1.3788643 .2428252 .639902$

H -1.110528 -2.525054 4.106336

H $-2.182348 \quad 0.6273515 .939986$

H $1.031513-0.5581263 .233775$

O $\quad-4.2332902 .9833451 .452887$

O $-2.367274 \quad 3.345913 \quad 0.143554$

C $-5.074735 \quad 3.4434620 .391745$

H $-4.9071222 .852724-0.512578$

H -6.1149563 .3170290 .703261$

$\mathrm{H}-4.8902544 .5045220 .199508$

SCF Energy $\left(B 3 L Y P / 6-31 G^{* *} / / M M F F\right)=-3245.89511750$

03268

MM̄FF Geometry

C $1.2895921 .407625-3.566457$

C $1.3591830 .086468-3.341965$

C $0.289116-0.779140-2.713836$

O $-0.7972630 .003822-2.170368$

C $-0.291867-1.730497-3.775645$

C $-1.215716-2.790800-3.208432$

C $-2.526280-2.902484-3.500264$

C $-0.573203-3.790894-2.280828$

C $-3.353802-2.039878-4.410717$

C $-4.071028-0.873799-3.711992$

C $-5.070556-1.306563-2.626410$

C $-5.911360-0.116556-2.158048$

C $-6.854729-0.495360-1.004589$

O $-4.335078-1.831686-1.518159$

O $\quad-7.346013 \quad 0.720931-0.419827$

C $-6.048228-1.2277530 .090657$

C -8.084608 -1.262751-1.504415

O $-6.911994-1.7451591 .099034$

C $-5.148546-2.353347-0.458815$

C $-4.191591-2.9478650 .603850$

O $-5.972577-3.428624-0.925978$

C $-0.6035390 .580614-0.952170$

O $0.3964120 .514110-0.255362$

C $-1.8230081 .349166-0.621461$

C -1.764389 2.2995320 .323095

C $-2.872196 \quad 3.151450 \quad 0.760329$
C $-4.2526982 .801275 \quad 0.271734$

C $-3.217810-1.9527931 .244989$

C $-3.705656-1.3742562 .563092$

C $-3.1154190 .348648 \quad 4.263473$

O $-4.684920-1.8032893 .167208$

C -3.9398681 .5728924 .018270$

C -3.5995882 .7901034 .468563$

C -4.4346734 .0221724 .267704$

C -3.6543815 .1587673 .645893$

C -3.1289626 .1337424 .406916$

C -3.5575095 .2080412 .137704$

C -2.6047954 .1854041 .585468$

N $-2.889629-0.3425523 .007950$

H $\quad 0.387263 \quad 1.957897-3.314927$

H $2.277098-0.435702-3.607022$

H $0.751715-1.356746-1.906744$

H $0.522132-2.245783-4.303252$

H $-0.810002-1.130860-4.531723$

H -3.083894 -3.714943 -3.034819

H -1.239004 -4.629061-2.048258

H $\quad 0.329214-4.210194-2.737595$

H $-0.300267-3.317947-1.332850$

H -2.744866 -1.643483 -5.230186

H $-4.100812-2.680834-4.896390$

H -3.318409 -0.206398 -3.276087

H $-4.602506-0.301434-4.482562$

H -5.715555 -2.089038 -3.043042

H $-6.4803790 .310855-2.993012$

H $-5.2443750 .684184-1.811335$

H $-7.7935451 .229588-1.117633$

H $-5.417246-0.4742790 .576242$

H -8.649005 -0.655054 -2.221989

H -8.778145 -1.476006 -0.683654

H -7.831963 -2.203712 -1.997151

H -7.441822 -1.002381 1.435954

H $-4.763452-3.4997111 .359622$

H -3.576892 -3.7055860 .096343$

H $-5.437205-4.238882-0.951901$

H -2.726821 $1.125219-1.175485$

H -0.8094672 .4884050 .812441$

H $-4.473512 \quad 1.7480440 .474687$

H $-4.3355912 .977284-0.806148$

H $-5.046914 \quad 3.3720490 .756982$

H $-2.283336-2.4811941 .473633$

H -2.973139-1.140522 0.554518

H $-2.1332710 .587563 \quad 4.683539$

H -3.640284 -0.3163304 .956790$

H $-4.870107 \quad 1.4488473 .468792$

H -2.682725 2.9053445 .042489

H -4.8183514 .3278655 .250022$

H -5.3214293 .8113073 .657107$

H -2.591830 6.9675903 .967129

H -3.2200316 .1165935 .487951$

H -3.1952466 .1937531 .815805$

H -4.5624075 .1292011 .714045$

H -1.569777 4.3519631 .887952

H -2.129177 -0.0180612 .417636$

C $3.0105273 .154106-3.099338$

O $3.7075622 .375969-2.103177$

C $4.9843732 .986871-1.845475$

C $4.9086684 .382278-2.451486$

C $4.0446994 .124138-3.670289$

H $5.7288432 .406112-2.403161$

C $5.3110612 .947973-0.349832$

H $4.4135085 .092142-1.778757$

H $5.8962324 .782214-2.700129$

C $3.4451965 .384578-4.268901$

H $4.6533923 .619491-4.432724$

C 5.2668631 .5366980 .274649

O 4.3575813 .7603720 .346960

H $6.2906053 .410603-0.179542$

H 4.2259301 .1995780 .334523

O $5.718076 \quad 1.6532501 .630601$

C $6.1190630 .480674-0.451783$

C $6.261115-0.8508570 .299007$

H $5.7024980 .294972-1.447279$

O $7.4346061 .010926-0.643615$

H $6.904652-1.522981-0.286287$ 
H $6.840822-0.6945111 .219972$

H $2.8188085 .145965-5.134136$

H $2.8253705 .918950-3.541229$

H $4.2354186 .065576-4.601462$

C $2.4152512 .215439-4.149758$

H 4.5797013 .7204861 .293119

H 6.6579811 .9029141 .606380

H $7.9406310 .360317-1.159757$

H $3.1952641 .549524-4.539326$

H $2.0211322 .788337-4.996656$

H $2.2168343 .703037-2.575572$

C $4.277838-3.6008751 .814616$

O $5.344732-2.7812611 .321489$

C $4.952546-1.5727630 .654230$

C $4.068023-1.896152-0.559547$

C $2.883032-2.787084-0.172277$

C $3.315529-3.9845140 .679677$

H $4.770630-4.5187652 .161053$

C $3.578597-2.9643733 .020889$

H $4.397759-0.9494601 .362337$

O $4.835855-2.544467-1.571841$

H $3.690802-0.962715-0.991681$

H $2.395242-3.143849-1.087324$

O $1.939829-2.0013180 .549132$

H $2.442218-4.4967631 .100157$

O $3.992602-4.927893-0.155579$

C $4.528029-2.8629544 .190543$

H $3.174682-1.9686862 .816829$

H $2.729860-3.5783883 .345178$

H $5.228133-3.344012-1.180950$

H $1.173732-2.5671740 .744756$

H $3.368082-5.208929-0.846049$

O $5.148569-1.6486454 .182770$

O $4.714117-3.7653884 .996704$

C $6.102424-1.4372325 .227797$

H $6.521739-0.4347915 .107004$

H $5.613986-1.4984446 .204903$

H $6.913173-2.1681695 .153813$

SCF Energy (B3LYP/6-31G**//MMFF) $=-3245.90393413$

03269

MMFF Geometry

C $3.112719-3.532625-1.506508$

C $2.184572-4.355868-0.992771$

C $0.796735-3.948725-0.550277$

O $0.412165-2.746807-1.255851$

C $-0.231803-5.057724-0.864343$

C $-1.473433-5.0213530 .010301$

C $-2.734046-4.827068-0.425214$

C $-1.239416-5.2915281 .477798$

C $-3.226154-4.612780-1.827395$

C $-3.594750-3.160844-2.165614$

C $-4.756056-2.574839-1.342566$

C -5.198055 -1.234251-1.937182

C $-6.296822-0.563098-1.100508$

O $-4.316808-2.374210 \quad 0.004175$

O $\quad-6.3997250 .806583-1.522147$

C $-5.863381-0.5412740 .382286$

C -7.667471 -1.197925 -1.363222

O $-6.925011-0.0869431 .216388$

C $-5.336816-1.8993210 .897259$

C $-4.721867-1.8247692 .315587$

O $-6.397913-2.8557631 .020040$

C $-0.293548-1.802794-0.583754$

O $-0.675877-1.8521240 .573213$

C $-0.534844-0.682850-1.522139$

C -1.443725 $0.251174-1.208332$

C $-1.8232851 .407216-2.021376$

C $-1.0426901 .667525-3.281628$

C $-3.523583-0.8853942 .474788$

C -3.8922830 .5306062 .886008$

C -2.9735552 .8511302 .897239$

O $-4.9602250 .819284 \quad 3.419064$

C -3.6157393 .5242891 .725458$

C $-2.985101 \quad 4.391640 \quad 0.919135$

C $-3.6393715 .059392-0.257107$

C $-2.9939704 .690926-1.575810$

C $-2.1277765 .524138-2.176191$
C $-3.4241593 .404156-2.246062$

C -2.839506 $2.183803-1.592098$

N $-2.866617 \quad 1.4294752 .624753$

H $2.888946-2.478036-1.643200$

H $2.459334-5.395667-0.826277$

H $0.861869-3.7591120 .527230$

H $\quad 0.221509-6.047527-0.718467$

H $-0.495489-5.001349-1.925315$

H $-3.531521-4.8549270 .317026$

H -2.175293 -5.4376182 .028173$

H $-0.643822-6.2013221 .606508$

H $-0.711848-4.4580311 .949939$

H -2.491695 -4.962787 -2.559363

H $-4.107127-5.249818-1.976920$

H $-2.704663-2.536844-2.041412$

H $-3.862345-3.130459-3.229455$

H $-5.585614-3.291333-1.353750$

H $-5.530989-1.359444-2.975233$

H $-4.336237-0.556527-1.982207$

H $-6.5805460 .813931-2.477660$

H -5.0599010 .2010820 .461642$

H -7.954167 -1.068312 -2.413925

H $-8.451526-0.706293-0.777358$

H -7.691224 -2.268504 -1.148796

H -7.2042170 .7826870 .882116$

H $-5.503098-1.6025123 .052792$

H -4.368655 -2.835884 2.565038

H $-7.245210-2.3896270 .933717$

H $\quad 0.021585-0.672117-2.452369$

H -1.977979 $0.155642-0.265709$

H $-1.2432240 .886352-4.023028$

H $\quad 0.0333871 .685577-3.077049$

H -1.278962 $2.627830-3.745663$

H -2.880374 -1.271754 3.275596

H -2.927230 -0.866206 1.560326

H -1.9632303 .2255193 .089042$

H -3.579979 3.0080263 .795015

H $-4.658523 \quad 3.2822281 .532283$

H -1.9452714 .6422701 .113922$

H -3.581553 $6.144220-0.098397$

H $-4.7110954 .828731-0.302395$

H -1.684062 $5.282491-3.136374$

H $-1.8363156 .462987-1.717257$

H $-3.1923393 .429833-3.316343$

H $-4.518527 \quad 3.328017-2.207237$

H $-3.3483311 .898491-0.671814$

H $-2.043174 \quad 1.1094762 .123364$

C $5.595283-3.559683-0.937419$

O $5.659045-2.125658-0.810971$

C $5.994315-1.8048120 .555269$

C $6.368006-3.123378 \quad 1.228214$

C $5.479624-4.1019370 .486226$

H $5.077930-1.4251401 .022369$

C $7.092847-0.7384080 .610337$

H $7.423808-3.3752261 .074176$

H $6.181984-3.1045822 .306525$

C $5.906891-5.5510750 .635799$

H $4.457448-3.9884760 .867346$

C $6.7466650 .574907-0.119817$

O $8.275393-1.2750820 .002833$

H $7.350131-0.5344531 .656635$

H $6.7092900 .398793-1.201233$

O 7.8386901 .4808810 .091093

C 5.4377531 .2454990 .340332

C $5.2363872 .626895-0.313440$

H 4.5957690 .5912590 .089902

O $\quad 5.4738131 .3901421 .759122$

H $6.0389053 .306312-0.001817$

H $5.3031902 .496165-1.400370$

H $5.239257-6.213790 \quad 0.076331$

H $6.926194-5.7095960 .267891$

H $5.878815-5.8535961 .687597$

C $4.490437-3.976380-1.911828$

H $8.960733-0.5862570 .045041$

H 7.8582931 .6997991 .038635

H 4.5657771 .5597992 .061564

H $4.517149-5.063927-2.050670$

H $4.694853-3.531470-2.893834$ 
H $\quad 6.556677-3.866503-1.371337$

C $1.5112482 .844735-0.112801$

O $2.8470502 .421620-0.411044$

C $3.8906593 .284974 \quad 0.047025$

C $3.7483584 .681000-0.581524$

C $2.3530195 .261109-0.328870$

C $1.2487094 .254445-0.669443$

H $0.8706222 .145206-0.660468$

C 1.1787342 .7099321 .380499

H 3.8328853 .3785181 .135989

O $3.9810194 .598178-1.986281$

H $4.5151275 .340079-0.156752$

H $2.2230716 .164593-0.936982$

$\begin{array}{lllll}\text { O } & 2.260339 & 5.640920 & 1.040827\end{array}$

H $0.2824644 .616567-0.302726$

O $1.1334404 .164459-2.092247$

C $1.166743 \quad 1.266978 \quad 1.840663$

H $1.889943 \quad 3.2426422 .016248$

H $0.176868 \quad 3.1183371 .555672$

H $3.9234645 .499431-2.346826$

H 1.3795386 .0292561 .178469

H $1.9524353 .765374-2.432731$

O 0.5518851 .1984043 .058868

$\begin{array}{lllll}\text { O } & 1.653445 & 0.325677 & 1.230088\end{array}$

C $0.479895-0.105594 \quad 3.643574$

H $1.481486-0.5326463 .751817$

H $0.031260-0.0082424 .635711$

H $-0.154515-0.7554713 .035076$

SCF Energy (B3LYP/6-31G**/MMFF) $=-3245.91238882$

0327

MMFF Geometry

C $-4.927611-1.4630552 .948786$

C $-5.195862-0.2218112 .515449$

C -5.8523590 .1834921 .218016$

$\begin{array}{lllll}\text { O } & -4.845754 & 0.886184 & 0.448606\end{array}$

C $-6.377142-0.970075 \quad 0.338352$

C $-7.042619-0.491743-0.940355$

C $-6.585777-0.754408-2.181301$

C $-8.3051170 .311981-0.762091$

C $-5.390272-1.581714-2.567242$

C $-4.086455-0.787760-2.498053$

C $-2.893326-1.473643-3.179733$

C $-2.585444-2.854251-2.594779$

C $-1.312998-3.465909-3.200310$

O $-1.772852-0.597810-2.992759$

O $-0.961012-4.592725-2.382699$

C $-0.171873-2.433113-3.102606$

C $-1.568191-4.019955-4.606614$

O $0.999675-2.906008-3.763045$

C $-0.560029-1.031543-3.621019$

C $0.524780 \quad 0.028633-3.319556$

O $-0.712995-1.087874-5.044013$

C $-4.747150 \quad 2.2352290 .576746$

$\begin{array}{llll}\text { O } & -5.445020 & 2.968727 & 1.256733\end{array}$

C $-3.5946132 .659276-0.249234$

C $-3.1437853 .921015-0.187139$

C -1.981582 $4.448384-0.908269$

C $-1.2819813 .515457-1.861603$

C $0.8391510 .216648-1.833435$

C $1.7339541 .417411-1.578171$

C $2.683164 \quad 2.738400 \quad 0.318007$

O $2.2521462 .076485-2.474750$

C 1.7673853 .8331650 .766509

C $1.917860 \quad 5.1217390 .422364$

C 1.0227916 .2203160 .923950

C $0.4581317 .087605-0.182346$

C $0.7109758 .407356-0.212850$

C $-0.4037746 .444324-1.251071$

C -1.594096 $5.720367-0.677330$

N $1.8901591 .650531-0.219752$

H -5.201471 -2.327951 2.352561

H -4.894303 0.6123293 .147956

H $-6.6826630 .856948 \quad 1.462114$

H -7.105808 -1.5677440 .900992$

H $-5.544963-1.6406050 .098000$

H -7.136584 -0.346983 -3.028043

H -8.818857 $0.493250-1.712414$
H $-9.005490-0.220686-0.110516$

H $-8.084933 \quad 1.287179-0.317611$

H -5.334622 -2.478614 -1.940844

H $-5.549029-1.942265-3.591491$

H $-4.2220960 .201915-2.955532$

H $-3.827177-0.588840-1.451384$

H $-3.109618-1.548604-4.251868$

H $-3.427803-3.542414-2.731661$

H $-2.445819-2.763554-1.508262$

H $-0.114029-4.941168-2.710644$

H $0.085852-2.365812-2.038951$

H $-2.344607-4.793875-4.575359$

H $-0.674955-4.511255-5.007978$

H $-1.890323-3.255936-5.317073$

H $0.866626-2.792489-4.719486$

H $1.444602-0.206203-3.870469$

H $\quad 0.182707 \quad 0.992343-3.722929$

H $-0.610093-0.189827-5.400286$

H $-3.123621 \quad 1.903431-0.867932$

H -3.6516324 .6259220 .469457$

H $-1.9798643 .146427-2.621861$

H $-0.8741652 .660081-1.316329$

H $-0.4537563 .972852-2.405048$

H $-0.0873070 .352239-1.264166$

H $1.356217-0.663873-1.439408$

H $3.4045403 .080066-0.430866$

H $3.2296612 .340752 \quad 1.177634$

H 0.9545993 .5542131 .433498

H $2.7461775 .409458-0.220712$

H 1.6143126 .8311351 .618492

H 0.1949435 .8134051 .517669

H $0.3122349 .042502-0.996954$

H 1.3233278 .8845990 .545101

H $0.238176 \quad 5.795357-1.851502$

H $-0.787763 \quad 7.207224-1.940623$

H $-2.186415 \quad 6.3241220 .011815$

H 1.3959051 .0585900 .442049

C -2.837899-2.356009 4.010059

O $-1.985790-1.3963823 .349650$

C $-0.703848-1.3704014 .011617$

C $-0.670647-2.6093714 .893086$

C -2.122261 -2.7259115 .308955$

H $-0.704593-0.4606474 .624964$

C $0.411560-1.3058162 .959624$

H $-0.365701-3.4910074 .315336$

H $0.010431-2.5043895 .742889$

C -2.491584 -4.0964795 .849228$

H -2.326440 -1.970570 6.079833

C $1.825642-1.3869183 .561040$

O $0.288653-0.0690712 .250520$

H $0.263647-2.1060342 .225913$

H $1.960818-2.3504994 .065477$

O $1.954143-0.3870914 .581077$

C $2.981927-1.1816802 .562798$

C $2.947685-2.1626971 .380088$

H $2.997481-0.1470492 .202445$

O $4.208399-1.3881373 .273368$

H $2.936960-3.1899641 .764818$

H $2.023403-1.9993640 .815158$

H $-3.550549-4.1356576 .123068$

H $-2.305258-4.8829335 .110003$

H $-1.902776-4.3291086 .742534$

C $-4.221175-1.7448574 .246651$

H $-0.611729-0.0336711 .883917$

H $1.7967860 .477701 \quad 4.164287$

H $4.220247-0.7622564 .017866$

H $-4.120377-0.8190704 .827206$

H $-4.843195-2.4297524 .833837$

H -2.910466 -3.226329 3.345061

C $5.139805-0.347134-1.040917$

O $4.074234-0.701939-0.151366$

C $4.152814-2.0064100 .430206$

C $4.160889-3.085537-0.663948$

C $5.273136-2.829990-1.686918$

C $5.277232-1.377707-2.174646$

H $4.8247690 .603390-1.490097$

C $6.448477-0.095258-0.285094$

H $5.074132-2.0866611 .016141$ 
O $2.898977-3.100710-1.326173$

H $4.296657-4.065873-0.191676$

H $5.140221-3.494698-2.549291$

O $6.538207-3.124163-1.101890$

H $6.192218-1.174505-2.742736$

O $4.187804-1.194060-3.083826$

C 6.3133831 .1005350 .628331

H $6.771641-0.9561230 .307068$

H $7.2663230 .123802-0.981680$

H $2.924768-3.805272-1.995721$

H $6.526770-4.057930-0.830761$

H $3.362031-1.332429-2.589537$

O 6.2141450 .6893651 .924144

O 6.2815042 .2590380 .233969

C 6.0780841 .7312092 .895349

H 6.0356561 .2691273 .885181

H 6.9421902 .4010772 .857502

H 5.1508382 .2864222 .728276

SCF Energy (B3LYP/6-31G**//MMFF) $=-3245.93832474$

03270

MM̄FF Geometry

C $-2.4904815 .278615-0.220677$

C -1.632427 $4.737772-1.098966$

C $-0.2368904 .318309-0.717960$

O $-0.0835992 .952334-1.166560$

C $0.8215375 .191804-1.416110$

C $2.1938035 .111203-0.769304$

C $3.2990504 .588468-1.336526$

C 2.2952475 .7251160 .606640

C $3.4619523 .981513-2.700187$

C $3.4956482 .446236-2.721864$

C $4.6752201 .807872-1.966928$

C $4.7437980 .306660-2.266777$

C $5.844534-0.400927-1.461843$

O $4.4942662 .005693-0.560045$

O $5.608583-1.815600-1.541055$

C $5.716836-0.0071610 .025630$

C $7.233084-0.159752-2.066062$

O $6.817174-0.5087390 .779298$

C 5.5763481 .5132380 .246789

C 5.2825341 .8930051 .720543

O $6.8091562 .156161-0.102966$

C $0.4170922 .044845-0.289100$

O 0.7725232 .2453290 .860946

C $0.4710190 .740079-0.987061$

C $1.115890-0.279584-0.404631$

C $1.286329-1.630511-0.939442$

C $0.587894-1.972577-2.226063$

C 4.0256201 .2669162 .331829

C $4.281630-0.0166653 .108397$

C $3.060564-1.9435894 .130386$

O $5.399377-0.378048 \quad 3.465864$

C $3.273311-3.0796483 .180425$

C $2.308585-3.9324992 .802034$

C $2.526705-5.0535801 .825146$

C $1.767662-4.8567140 .529458$

C $0.634891-5.5348280 .281576$

C $2.365793-3.933710-0.513156$

C $2.051780-2.490390-0.237392$

N $3.101993-0.6915673 .394280$

H -2.150132 5.4626620 .795234

H -1.948951 $4.563102-2.124193$

H $-0.1356534 .378614 \quad 0.371385$

H $0.5129816 .245704-1.392226$

H $\quad 0.870340 \quad 4.915467-2.474337$

H $4.2233204 .619609-0.759968$

H 1.7376045 .1351751 .339568

H 3.3307175 .7874530 .959140

H 1.8942156 .7440060 .598734

H $2.6709394 .320142-3.376458$

H $4.3940974 .366850-3.132769$

H $2.5525952 .071965-2.314047$

H $3.5364662 .134090-3.773038$

H $5.5984092 .303137-2.290233$

H $4.8836950 .131130-3.340773$

H $3.781295-0.156479-2.014595$

H $5.606617-2.067177-2.480552$
H $4.818774-0.5067780 .409969$

H $7.274258-0.540104-3.093869$

H $8.005309-0.706852-1.514273$

H $7.5104650 .895809-2.095705$

H $6.854906-1.469410 \quad 0.632857$

H $\quad 6.167162 \quad 1.7025592 .340327$

H 5.1378442 .9827781 .748839

H $6.836808 \quad 3.0253110 .330416$

H $0.0022880 .660692-1.961028$

H $1.570056-0.1109630 .569558$

H $0.698096-3.019810-2.511055$

H $0.990825-1.372947-3.049864$

H $-0.487170-1.782012-2.150996$

H 3.6015871 .9750053 .055536

H 3.2674921 .0942851 .563941

H $2.087262-1.9982874 .628220$

H $3.843626-1.9458234 .895498$

H $4.275810-3.1979022 .775327$

H $1.304298-3.8158013 .202863$

H $2.213385-5.9862702 .312060$

H $3.592753-5.1857991 .601744$

H $0.098944-5.414762-0.653563$

H $\quad 0.211852-6.2162101 .012168$

H $2.039264-4.237657-1.512180$

H $3.455921-4.059149-0.526293$

H $2.539169-2.1079350 .657358$

H $2.229366-0.3480543 .004193$

C $-4.9653594 .842120 \quad 0.197921$

O $-4.9463783 .478568-0.269291$

C -5.1116932 .6022410 .861847$

C -5.5896163 .4856962 .012122$

C -4.852182 4.7779431 .722402

H $-4.1145142 .219623 \quad 1.107276$

C -6.0475981 .4364680 .527068$

H -6.6718173 .6550931 .978340$

H -5.3453993 .0601702 .990272$

C -5.4297535 .9890472 .433161$

H -3.8121014 .6441432 .040373$

C $-5.6984440 .637274-0.743996$

$\begin{array}{lllll}\text { O } & -7.369067 & 1.966876 & 0.337537\end{array}$

H $-6.113407 \quad 0.7652411 .391612$

H -5.871929 $1.279069-1.617339$

O $-6.651136-0.433219-0.831499$

C $-4.2892640 .024694-0.833965$

C $-3.878041-0.7844850 .412462$

H $-4.275975-0.633016-1.711457$

O $-3.3430001 .061305-1.077909$

H -3.617862 $-0.093176 \quad 1.221891$

H $-4.728480-1.3810290 .756528$

H $-4.867960 \quad 6.8933032 .178487$

H -6.4774036 .1539542 .159851$

H -5.3833395 .8562493 .518986$

C $-3.9058565 .671126-0.538570$

H -7.9580661 .2158710 .150394$

H -6.510304 -0.882732 -1.682299

H $-2.5036110 .645199-1.334618$

H -4.030394 $6.730322-0.284664$

H -4.077142 $5.579201-1.618616$

H $-5.9515105 .236177-0.082236$

C $-3.851456-3.646310-0.654079$

O $-2.906997-2.611126-0.941695$

C $-2.664727-1.7058150 .142638$

C $-2.156213-2.4779851 .367951$

C $-3.087669-3.6261611 .749004$

C $-3.386784-4.5001690 .530235$

H $-4.817492-3.188599-0.408185$

C -4.104119-4.449059-1.934228

H -1.846272 -1.055305 -0.179888

O $-1.994349-1.6125812 .486280$

H -1.167333 -2.898613 1.144044

H $-4.014124-3.2581452 .205444$

O $-2.448747-4.4405302 .735222$

H -2.485514 -5.0667130 .270391$

O $-4.404880-5.4411220 .859520$

C $-2.891661-5.194322-2.440625$

H $-4.906280-5.181571-1.785711$

H -4.447165 -3.781086 -2.734902

H $-1.376791-0.9086322 .223178$ 
H $-2.223345-3.8629763 .484735$

H $-4.093654-5.9473921 .629442$

O $-2.182441-4.408570-3.299962$

O $-2.610950-6.340176-2.112943$

C $-1.039076-5.028179-3.897089$

H $-0.323988-5.332412-3.127497$

H $-1.347142-5.888473-4.498790$

H $-0.559110-4.296374-4.552207$

SCF Energy $\left(B 3 L Y P / 6-31 G^{* *} / / M M F F\right)=-3245.90215889$

\section{1}

MMFF Geometry

C $-2.132882-1.2815613 .735084$

C $-1.061238-1.3534034 .539852$

C $0.346919-0.9104904 .209424$

O $0.523175-0.6592002 .796684$

C $0.6766840 .386847 \quad 4.969839$

C 2.1350920 .7937024 .877647

C 2.5848481 .9184324 .287570

C $3.116931-0.1416605 .537309$

C 1.7894992 .9979813 .610150

C 1.6556722 .8398822 .087676

C 2.9926572 .8626981 .327810

C $2.7548052 .964521-0.180459$

C $4.0667192 .897154-0.978016$

O 3.6980621 .6523291 .614706

O $3.7319032 .669006-2.355987$

C $4.8851631 .676073-0.504756$

C $4.8299564 .226065-0.931458$

O $6.1823491 .672105-1.094842$

C 5.0050791 .5728641 .029380

C 5.6402490 .2416901 .502270

O 5.8458692 .6306281 .506557

C $0.705767-1.7412661 .990784$

O $0.708796-2.9160972 .322989$

C $0.891095-1.2480470 .609567$

C $0.688529-2.084430-0.418885$

C $0.848790-1.756994-1.836150$

C $1.583794-0.488456-2.176601$

C $4.883804-1.0295321 .099933$

C $5.409616-1.693847-0.162414$

C $4.907567-3.586164-1.707928$

O $6.444333-1.349731-0.726717$

C $4.210213-3.053760-2.919769$

C $3.401707-3.795652-3.692174$

C $2.735130-3.286756-4.938250$

C $1.238906-3.507332-4.934391$

C $0.692077-4.549386-5.583158$

C $0.373146-2.471002-4.255116$

C $0.363755-2.614947-2.757269$

N $4.599720-2.748612-0.563486$

H -2.043259 -0.8330012 .749456$

H -1.183152 -1.797346 5.526311

H $1.031671-1.7105144 .516093$

H 0.4277260 .2722656 .033500

H $0.028322 \quad 1.1847764 .592191$

H 3.6589362 .1020454 .295730

H 4.1270140 .2798355 .581759

H $2.808144-0.3537926 .566122$

H $3.181246-1.0858764 .988133$

H 0.7887843 .0775414 .047237

H 2.2716703 .9594603 .829272

H $1.124627 \quad 1.9046981 .874333$

H $\quad 1.0237743 .660674 \quad 1.726547$

H 3.5765423 .7207741 .680879

H $2.2049643 .881183-0.427508$

H $2.1118722 .134419-0.501663$

H $3.1533063 .394236-2.647906$

H $4.3700750 .785097-0.880213$

H $4.2160805 .034674-1.346522$

H $5.7312354 .186490-1.553125$

H 5.1197844 .5188330 .079745

H $6.0622201 .710068-2.059247$

H 6.6942080 .1987651 .202507

H 5.6530850 .2640482 .601768

H 6.1910152 .3803062 .379609

H $1.182488-0.2127810 .477271$

H $\quad 0.355817-3.100113-0.207975$
H $2.544611-0.454269-1.653121$

H $0.9994390 .388097-1.886093$

H $1.816173-0.388686-3.238811$

H $5.001080-1.7690201 .902646$

H $3.813248-0.8336310 .990696$

H $4.588858-4.603630-1.460265$

H $5.989076-3.591217-1.877283$

H $4.402535-2.018062-3.189952$

H $3.234254-4.839331-3.435268$

H $3.185744-3.809201-5.792417$

H $2.945946-2.222306-5.099902$

H $-0.382323-4.698284-5.609986$

H $1.307539-5.280236-6.097249$

H $-0.667518-2.570434-4.593158$

H $0.674102-1.473162-4.586989$

H $-0.139744-3.518338-2.409914$

H $3.732681-2.927454-0.065178$

C $-3.997240-2.9100743 .162565$

O $-4.244220-2.3548551 .855630$

C $-3.682171-3.2386070 .868524$

C $-3.482940-4.5738561 .576056$

C $-3.069736-4.1117082 .960384$

H -2.698733 -2.827572 0.607399

C $-4.560085-3.288583-0.385986$

H $-4.414104-5.1487431 .634667$

H $-2.727616-5.1958731 .086530$

C $-3.205999-5.1780774 .032684$

H -2.020746 -3.797341 2.910892

C $-4.955173-1.906973-0.945842$

O $-5.774186-3.983114-0.073045$

H $-4.055626-3.886454-1.154494$

H -5.708360 -1.451758 -0.291107

O $-5.614850-2.115496-2.201451$

C $-3.792186-0.916155-1.135868$

C $-4.2414230 .379115-1.830729$

H $-3.370098-0.669574-0.155816$

O $-2.758875-1.539507-1.894838$

H $-4.3452520 .223680-2.912396$

H $-5.2415630 .658342-1.472321$

H -2.910748 -4.784514 5.010479

H $-4.236620-5.5399334 .110946$

H -2.565319 -6.036352 3.805307

C $-3.488875-1.816340 \quad 4.109273$

H $-6.311003-4.008960-0.883788$

H -4.986658 -2.553509-2.800036

H -3.085931 -1.673201 -2.800132

H -3.479090 -2.205550 5.134433

H $-4.189650-0.9726914 .090312$

H -4.974757 -3.249155 3.529212

C $-3.281513 \quad 3.965767-2.082362$

O $-3.9213882 .695276-2.256110$

C $-3.3174991 .586619-1.574943$

C $-1.8676971 .398259-2.040094$

C $-1.0636902 .695232-1.898715$

C -1.801876 $3.895724-2.496929$

H $-3.7951074 .636325-2.783290$

C $-3.4899854 .524318-0.665592$

H $-3.3328611 .785207-0.497601$

O $-1.8557840 .969190-3.399120$

H $-1.3996460 .614077-1.435569$

H $-0.0963592 .576625-2.401880$

O $-0.8087392 .918185-0.515166$

H -1.293482 $4.827085-2.221291$

O $-1.7299273 .788056-3.921556$

C $-4.9443924 .836529-0.378456$

H -3.1521553 .8232990 .101789$

H -2.930051 $5.460732-0.559489$

H $-0.9289340 .816887-3.650743$

H $-0.2918553 .738077-0.440423$

H $-2.1509944 .579260-4.298473$

O -5.0481305 .3163650 .897030$

O $-5.8705944 .684619-1.162705$

C -6.3721755 .6566901 .320269$

H -7.0186714 .7744341 .289604$

H -6.3177506 .0158542 .351455$

$\mathrm{H}-6.776926 \quad 6.4553080 .691255$

SCF Energy (B3LYP/6-31G**//MMFF) $=-3245.89220561$ 
03272

MM̄FF Geometry

C -1.576843 4.8154470 .144807

C $-1.9720814 .651540-1.127682$

C $-2.8241143 .506663-1.632246$

O $-2.7092882 .361514-0.755998$

C $-4.3001393 .937896-1.680933$

C $-5.2348752 .862184-2.198891$

C $-6.2181222 .292989-1.473766$

C $-5.0262032 .448480-3.632785$

C $-6.6067942 .589694-0.052108$

C -5.9001031 .7221361 .000871$

C -6.2632030 .2303820 .928786$

C $-5.704561-0.5224452 .137694$

C -5.994021 -2.028402 2.055968

O $-5.705806-0.320661-0.266437$

O $-5.179420-2.6640913 .053319$

C $-5.553082-2.5590020 .672997$

C -7.449004 -2.341839 2.426543

O $-5.970899-3.9141550 .527946$

C $-6.053410-1.693001-0.504769$

C $-5.437043-2.080273-1.871957$

O $-7.472751-1.848121-0.627995$

C -1.619374 $1.561550-0.917553$

O $-0.7596891 .650026-1.779372$

C -1.6224470 .5793200 .187918$

C $-0.487853-0.0647070 .499385$

C $-0.313766-1.0186251 .596613$

C -1.548932 -1.459592 2.337865

C $-3.906379-2.034783-1.943286$

C $-3.240568-3.374135-1.674366$

C $-0.996752-4.398962-1.335802$

O $-3.862292-4.429872-1.588606$

C $-0.689338-4.4892930 .125585$

C $0.558246-4.6021120 .605054$

C $0.895151-4.7278132 .061727$

C $1.893625-3.6875372 .517384$

C $3.207426-3.9649782 .564448$

C $1.358597-2.3616163 .006056$

C $0.931429-1.4519651 .886328$

N -1.860870 -3.257744 -1.574425

H -1.9111354 .1127330 .904477$

H -1.650328 5.370089-1.878039

H -2.479490 $3.230336-2.636162$

H -4.407724 $4.824077-2.320755$

H $-4.6015094 .254506-0.676634$

H $-6.8297711 .528965-1.952248$

H $-5.8122771 .774032-3.988998$

H $-5.0293813 .327000-4.286068$

H $-4.0708371 .927796-3.749651$

H $-6.432543 \quad 3.6455920 .181593$

H -7.6925032 .4565840 .038682$

H -4.8156651 .8461000 .898392$

H -6.1770032 .1090091 .989559$

H $-7.355766 \quad 0.1456040 .897266$

H -6.098657 -0.1074153 .073796$

H -4.616056 -0.379358 2.182906

H -5.302324 -3.625124 2.964750

H $-4.456934-2.5658670 .681092$

H -7.662880 -2.003669 3.447645

H -7.635805 -3.4213952 .425015$

H -8.172837 -1.866963 1.760943

H -5.582318 -4.261511 -0.292026

H -5.828731 -3.049006 -2.204298

H $-5.807820-1.353181-2.609426$

H $-7.733838-1.590024-1.527673$

H -2.545429 0.4459800 .740139

H $0.4091920 .149268-0.080356$

H -2.309880 -1.818809 1.636565

H -1.968094 -0.629065 2.915974

H -1.369988 -2.284093 3.031306

H -3.615022 -1.749517 -2.962228

H -3.502302 -1.279166 -1.262978

H - $0.096442-4.256839-1.941569$

H -1.497061 -5.315364 -1.664839

H - $-1.528581-4.4837430 .817019$

H $1.389719-4.638051-0.093871$

H $\quad 1.306793-5.7328002 .223459$
H $-0.003562-4.6742642 .688510$

H $3.927614-3.2351502 .919278$

H $3.592901-4.9264842 .241741$

H $2.135767-1.8291923 .571452$

H $0.564096-2.5403793 .736268$

H $1.763410-1.0827141 .284697$

H -1.433871 -2.336566 -1.608406

C 0.6025115 .3035581 .227163

O $1.372741 \quad 4.6656490 .191382$

C 2.7730704 .8238760 .484496

C 2.8479695 .5230231 .839520

C 1.5539506 .3157331 .854887

H $3.1850375 .488556-0.285135$

C 3.5000683 .4703630 .434895

H 2.8656434 .7996322 .663622

H 3.7385276 .1525721 .930539

C 1.1448696 .7805873 .241177

H 1.6654357 .1873411 .196166

C $3.5374102 .858436-0.981085$

O $2.8542092 .573311 \quad 1.338969$

H 4.5239923 .6248940 .793332

H $4.0560133 .550730-1.655274$

O $2.2067702 .735496-1.496610$

C $4.1892961 .467336-1.091084$

C $5.6108701 .398174-0.510813$

H $3.5482790 .711903-0.621731$

O $4.2630801 .134586-2.483065$

H $6.2387722 .153829-0.999027$

H 5.5691841 .6289380 .558782

H $\quad 0.203826 \quad 7.3381103 .204907$

H 1.0094925 .9353063 .924285

H 1.9096417 .4381163 .667305

C -0.6614215 .9099860 .618619$

H $3.4112181 .782221 \quad 1.426894$

H $1.6885452 .222003-0.853248$

H $3.3591561 .179474-2.839510$

H $-0.3967116 .571982-0.214712$

H -1.195993 6.5081821 .364325

H $\quad 0.339715 \quad 4.5261801 .957903$

C $5.906259-2.306386-0.105527$

O $5.479075-0.9425620 .012181$

C $6.2665020 .017200-0.700765$

C $7.718184-0.006233-0.199094$

C $8.307288-1.419392-0.274727$

C $7.370644-2.4630750 .341333$

H $5.278018-2.8552290 .606243$

C $5.629353-2.877123-1.501886$

H $\quad 6.256267-0.225454-1.768081$

O $7.7899720 .468186 \quad 1.144075$

H $8.3155190 .678770-0.811790$

H $9.267786-1.4431670 .254694$

O $8.547635-1.766565-1.635416$

H $7.727107-3.4730280 .107234$

O $7.427942-2.3182371 .763731$

C $4.147157-3.077620-1.713569$

H $6.009474-2.258201-2.319045$

H $6.101068-3.861166-1.611954$

H $7.215762-0.0959781 .689925$

H $9.170102-1.114308-1.999372$

H $6.889205-3.0280512 .153197$

O $3.555454-1.893879-2.038105$

O $3.584235-4.157501-1.590482$

C $2.138221-1.938818-2.228717$

H $1.801464-0.939353-2.516759$

H $1.882516-2.640170-3.028440$

H $1.643147-2.218331-1.294982$

SCF Energy (B3LYP/6-31G**//MMFF) $=-3245.91826758$

03_273

MM̄FF Geometry

C $2.2561630 .578099-2.267426$

C $1.1029770 .018338-2.660242$

C $0.252059-0.836153-1.753956$

O $-1.085190-0.288202-1.718238$

C $0.171860-2.265088-2.322563$

C $-0.548012-3.245839-1.417533$

C $-1.700280-3.871762-1.727784$

C $0.129798-3.542288-0.103817$ 
C $-2.508399-3.761025-2.990079$

C $-3.626108-2.707586-2.951131$

C $-4.694224-2.960043-1.874422$

C $-5.889113-2.025286-2.068945$

C $-6.935120-2.189328-0.954933$

O $-4.109739-2.716909-0.593065$

O $-7.837117-1.073889-1.034338$

C $-6.234109-2.1077200 .420019$

C $-7.786319-3.449167-1.156943$

O $-7.163899-2.425228 \quad 1.453121$

C $-4.972614-2.994158 \quad 0.518985$

C $-4.139438-2.7527331 .802193$

O $-5.368263-4.3713050 .530493$

C $-1.2981670 .782658-0.905531$

O $-0.4816241 .337142-0.187260$

C $-2.7138181 .184656-1.057035$

C $-3.1036302 .397841-0.638652$

C -4.459032 $2.945689-0.725304$

C $-5.5671972 .000914-1.108848$

C $-3.627265-1.3209631 .995038$

C $-4.526245-0.4552552 .861794$

C $-4.789472 \quad 1.8964113 .642464$

O $-5.474645-0.8991083 .503480$

C -5.8118052 .5818252 .791783$

C -5.8879543 .9153462 .666038$

C $-6.932724 \quad 4.6224261 .851240$

C $-6.335908 \quad 5.586306 \quad 0.850342$

C $-6.242686 \quad 6.8983771 .125256$

C $-5.927656 \quad 5.041109-0.499560$

C $-4.647138 \quad 4.251837-0.442916$

N $-4.119807 \quad 0.8724272 .862813$

H $2.6012890 .415604-1.249025$

H $0.7536440 .166705-3.679151$

H $0.663938-0.858537-0.737676$

H $1.184438-2.652860-2.498688$

H $-0.310825-2.223220-3.304990$

H -2.117465 -4.562861 -0.995811

H $0.128083-2.6602670 .543387$

H $-0.364459-4.350700 \quad 0.445803$

H $1.165920-3.851673-0.274404$

H - $1.860116-3.559564-3.849373$

H $-2.950660-4.744718-3.194283$

H -3.175957 -1.719556 -2.800808

H $-4.105889-2.697097-3.937816$

H $-5.012678-4.006947-1.941144$

H $-6.349960-2.181252-3.052268$

H $-5.539579-0.984201-2.065760$

H -8.237071 -1.074142 -1.920898

H $-5.952711-1.0576420 .561531$

H -8.303874 -3.410635 -2.123071

H -8.574786 $-3.519663-0.399375$

H -7.200780 -4.370495 -1.133639

H $-6.741387-2.2303322 .306060$

H $-4.684517-3.1112602 .683352$

H -3.250359-3.396683 1.734018

H $-4.648061-4.8960890 .917684$

H -3.391360 $0.478778-1.522953$

H -2.357296 $3.065153-0.209402$

H $-5.4623541 .688292-2.153232$

H $-6.5654182 .428193-0.994764$

H $-5.5478291 .110789-0.471067$

H -2.659847 -1.366271 2.511752

H $-3.461099-0.8288991 .032179$

H -4.0193442 .5862824 .001810$

H -5.2780391 .4363844 .507360$

H $-6.537157 \quad 1.955506 \quad 2.277734$

H $-5.178193 \quad 4.5396173 .204603$

H -7.586042 5.1655022 .546675

H -7.584560 3.9111001 .328982

H -5.841916 7.6011760 .402288

H -6.5607817 .2982662 .082515$

H -5.764404 $5.867080-1.204970$

H $-6.7624894 .475800-0.922647$

H -3.778515 $4.852056-0.167725$

H -3.3546841 .1598972 .259488$

C $3.3828232 .802969-2.595798$

O $4.0613432 .703220-1.327211$

C $5.2862763 .450315-1.393144$
C $5.0603624 .476595-2.492898$

C $4.2853003 .649066-3.501351$

H $6.0667562 .749230-1.715380$

C $5.6529844 .015412-0.016534$

H $4.4472655 .316817-2.147739$

H $5.9973334 .875228-2.892914$

C $3.522307 \quad 4.481659-4.517221$

H $4.9928383 .002244-4.037739$

C 5.5818372 .9840931 .129639

$\begin{array}{lllll}\text { O } & 4.740333 & 5.073818 & 0.299315\end{array}$

H $6.6494474 .470748-0.066349$

H 4.5333332 .7675091 .370509

O 6.1513823 .5806112 .301139

C 6.3187841 .6599960 .856965

C 6.2636120 .7048142 .063561

H $5.8639021 .168257-0.007970$

$\begin{array}{lllll}\text { O } & 7.680695 & 1.921472 & 0.524416\end{array}$

H 6.8418151 .1293062 .893296

H 5.2244680 .6268442 .400877

H $2.9668063 .839000-5.207711$

H $2.8061695 .151879-4.029969$

H $4.2109025 .096407-5.106057$

C $3.1452341 .398485-3.158002$

H $5.0965285 .550308 \quad 1.067740$

H 5.5593994 .2979242 .583286

H 8.0794902 .3980621 .272597

H $4.1016640 .866503-3.238765$

H $2.7226311 .468950-4.167118$

H $2.4272953 .307657-2.406364$

C $4.858248-1.654693 \quad 0.730774$

O $6.230945-1.2778860 .580355$

C $6.827727-0.699307 \quad 1.746058$

C $6.743096-1.6864982 .919110$

C $5.311048-2.1613673 .162282$

C $4.686279-2.6744261 .864132$

H $4.269541-0.7573550 .958933$

C $4.346384-2.143200-0.630183$

H $7.890681-0.5649131 .509132$

O $7.250772-1.1034604 .114505$

H $7.364922-2.5643332 .699741$

H $4.695317-1.3721013 .609281$

O $\quad 5.329713-3.235878 \quad 4.105819$

H $5.157031-3.6325631 .612467$

O $3.301895-2.9205112 .097381$

C $4.905585-3.482533-1.046815$

H $3.253900-2.230179-0.618857$

H $4.593586-1.421049-1.417952$

H $8.166085-0.8264673 .938328$

H $5.758801-2.9036784 .913082$

H $2.954256-3.4016571 .327939$

O $6.192194-3.344170-1.474267$

O $4.270840-4.528256-0.992396$

C $6.838557-4.553939-1.881176$

H $7.856580-4.306901-2.194077$

H $6.889633-5.256138-1.043441$

H $6.309163-5.000099-2.728370$

SCF Energy (B3LYP/6-31G*//MMFF) $=-3245.90646188$

$03 \_274$

MM̄FF Geometry

C $2.1019642 .100774-1.274885$

C $1.2570681 .337947-1.983209$

C $0.462698 \quad 0.212836-1.368090$

$\begin{array}{llll}0 & -0.946445 & 0.461446 & -1.567981\end{array}$

C $0.825430-1.108534-2.071820$

C $0.277180-2.342268-1.382639$

C $-0.627754-3.183158-1.920177$

C $0.845285-2.635240-0.017031$

C $-1.281137-3.107049-3.270919$

C $-2.620625-2.354919-3.300967$

C $-3.698035-2.945851-2.377069$

C $-5.064197-2.320914-2.669449$

C $-6.145570-2.816685-1.695885$

O $-3.329678-2.669250-1.022767$

O $-7.284795-1.951771-1.825342$

C $-5.629403-2.668232-0.247778$

C $-6.630129-4.226791-2.053824$

O $-6.531042-3.2692660 .677657$ 
C $-4.209168-3.233645-0.040920$

C $-3.614160-2.9061121 .351121$

O $-4.250683-4.661874-0.150138$

C -1.544052 $1.352094-0.729679$

O

C $-2.9689051 .446893-1.115094$

C $-3.7007512 .483107-0.679802$

C $-5.1193242 .715396-0.958851$

C $-5.8953151 .590199-1.590437$

C $-3.493552-1.414731 \quad 1.683397$

C $-4.672583-0.8604532 .466185$

C -5.6222321 .2935953 .282466$

O $-5.534129-1.5690192 .979660$

C -6.6859941 .7506342 .334289$

C -7.109682 3.0217232 .263726

C -8.2010193 .4933811 .345670$

C -7.7759524 .6686560 .493431$

C -8.0792555 .9276180 .852345$

C $-7.0723644 .378019-0.813415$

C $-5.6585843 .903835-0.615004$

N $-4.627173 \quad 0.5243092 .558823$

H $2.1974321 .929612-0.205699$

H $1.1527131 .500535-3.052902$

H $\quad 0.6679610 .134371-0.294244$

H $1.917145-1.217078-2.123200$

H $0.477580-1.059391-3.109359$

H $-0.939000-4.043052-1.327410$

H $\quad 0.497646-1.8994120 .712894$

H $\quad 0.552166-3.6244350 .351041$

H $1.939422-2.611031-0.046447$

H $-0.603703-2.658292-4.005345$

H -1.441443 -4.133885 -3.624268

H -2.442011 -1.306467-3.035860

H -2.984506 -2.368494 -4.335875

H -3.733394 -4.030209-2.534524

H -5.366360 -2.512933 -3.706596

H -4.989971 -1.229094 -2.578656

H -7.576449 -1.977023 -2.752839

H $-5.620191-1.594698-0.024238$

H -7.050732 -4.241280 -3.066560

H $-7.440450-4.547003-1.389608$

H - $-5.836248-4.975552-2.017011$

H -7.405530 -2.869357 0.531736

H $-4.158542-3.4516912 .131340$

H -2.593563 -3.315476 1.370210

H $-3.468941-5.0279730 .295579$

H -3.373350 $0.667311-1.749470$

H -3.214780 $3.244270-0.070496$

H -5.555984 $1.417143-2.617262$

H $-6.9718221 .767972-1.629887$

H $-5.764250 \quad 0.667087-1.015470$

H -2.612238 -1.2716112 .321603$

H $-3.340421-0.8214810 .777304$

H -5.1021442 .1318203 .756848$

H -6.0682160 .6703194 .064266$

H -7.1447120 .9997381 .695236$

H -6.6685043 .7641572 .925300$

H $-9.060278 \quad 3.776578 \quad 1.967649$

H -8.5593832 .6835810 .698038$

H -7.802405 6.7750920 .234162

H $-8.6075986 .139001 \quad 1.776256$

H -7.024210 $5.288023-1.426471$

H -7.681014 3.680630-1.395222

H -5.010951 4.652234 -0.155690

H -3.9028561 .0340282 .061429$

C $4.4451122 .788782-1.686417$

O $4.7566011 .679595-2.558269$

C $6.0675441 .881766-3.102112$

C $6.6788383 .049495-2.337857$

C $5.4436633 .884629-2.047589$

H $5.9184382 .212647-4.139563$

C $6.8925460 .590634-3.144863$

H $7.1427482 .717623-1.402332$

H $7.4322143 .586622-2.921600$

C $5.6540834 .917140-0.954205$

H $5.1303794 .390723-2.970500$

C $7.205998-0.112182-1.804231$

O $8.1357450 .917060-3.781352$
H $\quad 6.385319-0.124576-3.804773$

H $7.8585440 .525160-1.196436$

O $7.981792-1.273762-2.139933$

C $5.978086-0.552553-0.982371$

C $6.342718-1.4138220 .241720$

H $5.4508440 .337933-0.637560$

O $5.095344-1.287996-1.827656$

H $6.784595-2.358944-0.095624$

H $7.106136-0.8854560 .823033$

H $4.7351855 .481520-0.767618$

H $5.9585464 .448301-0.012307$

H $6.4344855 .628784-1.242894$

C $2.9772063 .174022-1.859179$

H $8.6520210 .095155-3.845203$

H $8.445436-1.561285-1.335478$

H $4.232497-1.335688-1.383115$

H $2.747503 \quad 3.318497-2.921674$

H $2.7614204 .113646-1.339346$

H $4.6270912 .451538-0.657993$

C 5.0035780 .2884192 .408264

O $4.361194-0.5991751 .488562$

C $5.128076-1.7560711 .137901$

C $5.485671-2.5573912 .398944$

C $6.172605-1.6894463 .451148$

C $5.352843-0.4276103 .717898$

H 5.9247120 .6669101 .947002

C 4.1076961 .5170492 .606974

H $4.464046-2.3903850 .538167$

O $6.328243-3.6602032 .081903$

H $4.567718-2.9695962 .838072$

H $7.198759-1.4371483 .159586$

O $6.272474-2.4237294 .673690$

H $4.443332-0.7064614 .263392$

O $6.099157 \quad 0.455928 \quad 4.550536$

C 2.8090101 .2155523 .318267

H 4.6317572 .2868623 .185818

H 3.8618351 .9738241 .641621

H $5.860310-4.2064071 .427189$

H $6.760081-3.2425994 .479672$

H $6.328316-0.0325095 .359894$

O $1.896706 \quad 0.7039802 .443862$

O $2.631258 \quad 1.408446 \quad 4.514547$

C 0.6206090 .3841163 .007673

H -0.0325950 .0378042 .202935$

H $\quad 0.725771-0.4179883 .744155$

$\mathrm{H} \quad 0.1709831 .271803 \quad 3.462780$

SCF Energy $\left(B 3 L Y P / 6-31 G^{* *} / / M M F F\right)=-3245.89978230$

03_275

MM̄FF Geometry

C $3.797171-3.288306-0.525213$

C $2.978993-2.253648-0.277085$

C $1.703661-1.999108-1.034756$

O $0.683935-1.731628-0.045190$

C $1.871547-0.761141-1.939327$

C $1.083602-0.844959-3.231991$

C $-0.010407-0.118244-3.532401$

C $1.653290-1.791542-4.261329$

C $-0.7320360 .889844-2.681800$

C $-2.2145270 .530893-2.556087$

C $-3.0824821 .615577-1.900557$

C $-2.5790862 .030539-0.518383$

C -3.5457352 .9806940 .201410$

O $-4.3964821 .054017-1.800003$

O -3.1297153 .0243521 .575134$

C $-4.9679902 .387392 \quad 0.147127$

C $-3.4286374 .411499-0.337193$

O $-5.927148 \quad 3.306040 \quad 0.666612$

C $-5.3918021 .936504-1.270009$

C $-6.7164641 .144024-1.187869$

O $-5.5760933 .084664-2.097281$

C $-0.583926-2.149694-0.297807$

O $-0.985888-2.739980-1.286819$

C $-1.413542-1.7512870 .862780$

C $-2.747829-1.8859740 .818450$

C $-3.685588-1.5283611 .887996$

C $-3.103197-0.9716613 .161674$

C $-7.1811810 .544658-2.522994$ 
C $-8.264992-0.485283-2.278457$

C $-8.580079-2.776921-1.348316$

O $-9.457151-0.214238-2.397870$

C -8.702885 -2.576115 0.129354

C $-8.091931-3.3431881 .044460$

C $-8.163473-3.0756572 .520507$

C $-6.816971-2.7163963 .110370$

C $-6.259482-3.4680194 .074770$

C -6.146494 -1.445419 2.630469

C $-5.006161-1.7246741 .685864$

N $-7.750131-1.707154-1.870350$

H $3.537061-4.009489-1.295520$

H $3.261101-1.5233650 .477594$

H $1.438869-2.895297-1.608186$

H $2.925973-0.635369-2.224016$

H $1.6151500 .143909-1.379600$

H $-0.448666-0.253346-4.521227$

H $1.097061-1.765894-5.204532$

H $2.691365-1.526003-4.486971$

H $1.629802-2.822730-3.896725$

H $-0.291251 \quad 0.960677-1.685448$

H $-0.6297191 .873514-3.154643$

H -2.636122 $0.317865-3.548507$

H $-2.323634-0.407229-1.998616$

H -3.115452 $2.481660-2.572215$

H -1.590123 $2.497603-0.587670$

H $-2.448755 \quad 1.1345760 .102092$

H -3.689386 3.6688012 .040148

H -4.9840111 .5110720 .809990$

H -2.415591 4.798264 -0.173178

H -4.0995085 .0930480 .197170$

H -3.642960 $4.484920-1.405638$

H $-5.6960103 .481297 \quad 1.594112$

H $-6.5920010 .330445-0.461548$

H -7.519501 $1.789187-0.809183$

H $-6.1383552 .844218-2.850240$

H $-0.897493-1.3484021 .726869$

H -3.199924 -2.291935 -0.085400

H $-2.587582-0.0249002 .964742$

H -2.388406 -1.677757 3.599280

H -3.846568 -0.771807 3.935374

H -7.586335 $1.317838-3.184601$

H $-6.3566460 .062645-3.060193$

H -8.093775 -3.724518 -1.599873

H -9.568322 -2.750307 -1.817527

H $-9.303375-1.7309940 .459063$

H -7.486462 -4.186285 0.720708

H -8.575304 -3.9698183 .004985$

H -8.867654 -2.263016 2.740167

H -5.306108 -3.1988664 .517577$

H $-6.741893-4.3675154 .442164$

H -5.839765 -0.852149 3.496820

H $-6.875373-0.8102482 .111047$

H $-5.324752-2.1294810 .724707$

H $-6.747426-1.789537-1.728031$

C $6.278607-3.099534-0.748466$

O $6.157036-1.705163-1.098231$

C $7.344990-1.016401-0.680207$

C $8.422579-2.087681-0.673682$

C $7.649971-3.255685-0.081944$

H $7.174966-0.6870950 .351976$

C $7.5829590 .217245-1.557030$

H $8.756401-2.338635-1.686453$

H $9.295187-1.803468-0.078739$

C $8.298252-4.606534-0.330324$

H $7.561595-3.1030441 .002202$

C $6.3298481 .105014-1.733316$

O $7.980438-0.190150-2.871273$

H $8.4104260 .811909-1.151266$

H $5.6227100 .604389-2.406990$

O $6.7548062 .297762-2.403468$

C $5.6205761 .478386-0.417651$

C $4.3999472 .394885-0.624096$

H $\quad \begin{array}{llll}5.286960 & 0.563012 & 0.079971\end{array}$

O $\quad 6.5450142 .1188340 .460901$

H $4.7288903 .387438-0.953503$

H $3.7700291 .959367-1.409129$

H $7.690778-5.4118950 .094810$
H $\quad 8.418362-4.803535-1.400944$

H $9.289638-4.6485830 .132483$

C $5.115404-3.4817420 .171894$

H $8.902899-0.492006-2.818996$

H $5.9603052 .749412-2.734474$

H 6.8816132 .9101540 .006518

H $5.142818-2.8683061 .080935$

H $5.202309-4.5281140 .485122$

H $6.228659-3.666432-1.686538$

C 2.3029871 .2075322 .232126

O 3.0483861 .2724851 .010328

C 3.5757352 .5584430 .668938

C 2.4319223 .5695710 .502543

C 1.5452983 .6147751 .752566

C 1.1401792 .2137492 .221965

H 1.8747200 .1968652 .241402

C 3.2103991 .3388443 .459475

H 4.2470012 .8988511 .464543

O $1.6356503 .238343-0.633364$

H 2.8602594 .5606690 .314211

H $0.639576 \quad 4.194741 \quad 1.535923$

O 2.2410584 .2688992 .809697

H 0.6823922 .2677693 .216865

O $\quad 0.1397621 .727014 \quad 1.325298$

C 4.1728760 .1764113 .533693

H 3.7801312 .2722793 .467589

H 2.6216051 .3280914 .384417

H $1.2771422 .345557-0.493444$

H 2.4549745 .1682232 .508005

H -0.1951510 .8891801 .686486$

O 5.4402570 .6005493 .265054

O $3.834863-0.9729153 .786580$

C $6.450728-0.4112783 .307127$

H $6.567203-0.7811564 .329987$

H $6.202341-1.2308232 .627133$

H 7.3944230 .0367292 .984584

SCF Energy $\left(B 3 L Y P / 6-31 G^{* *} / / M M F F\right)=-3245.90416963$

03_276

MM̄FF Geometry

C $0.990683 \quad 1.797789 \quad 3.374813$

C $-0.0747861 .669247 \quad 4.181218$

C -1.3143820 .8570263 .882492$

O

C -1.149936 -0.569782 4.439001

C $-2.332508-1.4921614 .205640$

C $-2.217335-2.7496023 .733179$

C $-3.691394-0.9516984 .566148$

C $-0.946678-3.4810113 .398680$

C $-0.454120-3.2684551 .961543$

C $-1.308456-3.9685410 .893019$

C $-0.660467-3.806892-0.484972$

C $-1.521323-4.408407-1.604221$

O $-2.611845-3.3829440 .890191$

O $-1.006019-3.936058-2.858706$

C $-2.958022-3.861137-1.471743$

C $-1.422498-5.937506-1.643794$

O $-3.833126-4.501185-2.398030$

C $-3.529057-3.973282-0.038399$

C $-4.887273-3.2450290 .103981$

O $-3.754950-5.3554200 .261855$

C -2.1569641 .8928351 .907173$

O -2.4380262 .9354152 .479074$

C -2.4084451 .6205740 .474126$

C $-2.8740602 .611932-0.301357$

C $-3.2196142 .528890-1.722493$

C $-2.9590041 .229761-2.435773$

C $-4.796001-1.7173990 .076542$

C $-6.153408-1.052379-0.074656$

C $-7.1531111 .219490-0.352520$

O $-7.199928-1.677648-0.219004$

C $-7.0246131 .665505-1.776270$

C $-6.9685242 .951932-2.153257$

C $-6.8171463 .393058-3.581541$

C $-5.6525374 .338981-3.785788$

C $-5.8586655 .634497-4.077709$

C $-4.2490623 .768458-3.728163$

C $-3.7554153 .617209-2.315155$ 
N -6.044656 $0.331595-0.053607$ H $\quad 1.0186901 .2590712 .432842$ H $-0.076672 \quad 2.1900645 .136575$ H $-2.167406 \quad 1.3417024 .374819$ H $-0.973305-0.5170515 .521620$ H $-0.244468-1.0010703 .997035$ H $-3.125040-3.3360283 .597845$ H -3.987089 -0.155339 3.876156 H $-4.468190-1.723054 \quad 4.527431$ H $-3.681773-0.5503235 .584888$ H $-0.153189-3.1853534 .095139$ H $-1.101996-4.5515853 .581967$ H -0.413254 -2.192118 1.754136 H $0.572654-3.6499671 .898041$ H -1.389205 -5.0299251 .155768$ H $0.345715-4.244263-0.497295$ H $-0.524639-2.737625-0.696918$ H $-0.073412-4.205182-2.920090$ H $-2.922738-2.804223-1.762704$ H $-0.385540-6.249374-1.817594$ H $-2.002095-6.349477-2.477273$ H $-1.758033-6.413882-0.720480$ H $-3.441698-4.402032-3.282849$ H $-5.581112-3.614068-0.661686$ H -5.347787 $-3.524568 \quad 1.062003$ H $-4.389034-5.4129880 .995373$ H $-2.2276050 .618250 \quad 0.105095$ H -3.039875 3.5881460 .153809 H -1.925998 $0.900680-2.279234$ H -3.090822 $1.294271-3.517766$ H $-3.6346800 .451103-2.071824$ H $-4.333261-1.3550451 .002103$ H $-4.181447-1.384684-0.763598$ H $-7.0992792 .056567 \quad 0.350414$ H -8.102849 $0.695535-0.208497$ H -6.971598 $0.887593-2.534790$ H -7.041549 $3.731664-1.398422$ H $-7.7558393 .877557-3.879974$ H $-6.6974192533489-4.252849$ H -5.029921 6.313343 -4.249688 H $-6.8597556 .046973-4.146198$ H $-3.5524474 .435721-4.252236$ H $-4.2370142 .829324-4.287794$ H -3.871817 4.522918-1.717412 H -5.1233340 .7489810 .033483$ C $2.610086 \quad 3.5960182 .567650$ $\begin{array}{lllll}\text { O } & 3.348219 & 2.867335 & 1.569626\end{array}$ C 3.2207853 .5677380 .314228 C $2.2929394 .761028 \quad 0.564212$ C $1.516515 \quad 4.3236901 .792259$ H $2.7307392 .878758-0.381763$ C $4.6048243 .955422-0.225479$ H 2.8523795 .6804890 .771179 H $1.6478914 .962385-0.297155$ C 0.8717795 .4676532 .552832 H $\quad 0.737919 \quad 3.6252791 .461141$ C $5.5976672 .782759-0.355431$ O $5.1841674 .911694 \quad 0.673240$ H $4.4879584 .465959-1.189152$ H $5.886760 \quad 2.417192 \quad 0.637753$

O $6.7925443 .293873-0.962503$

C $5.0980971 .599237-1.207082$ C $6.1989220 .543097-1.433892$ H $4.2439191 .131123-0.707902$ O $4.638698 \quad 2.076662-2.470606$ H $7.0351531 .001885-1.975170$ H $6.5829560 .226269-0.458167$ H $0.332694 \quad 5.097128 \quad 3.430470$ H 1.6176946 .1922372 .896098 H $\quad 0.1560245 .9977181 .916016$ C 2.1738932 .6633253 .702177 H $5.937500 \quad 5.3231110 .217374$ H $7.2290093 .868248-0.310913$ H $5.3853562 .523470-2.905134$ H $1.952615 \quad 3.272532 \quad 4.587351$ H 3.0095872 .0018783 .961583 H $3.317421 \quad 4.322626 \quad 2.991346$ C $4.767925-1.964124-0.445559$
O $4.558488-1.292248-1.692922$

C $5.722082-0.678138-2.251328$

C $6.807231-1.739388-2.485598$

C $7.109068-2.531609-1.215796$

C $5.818704-3.069598-0.598854$

H $5.087764-1.2397680 .313666$

C $3.420917-2.546922-0.006310$

H $5.424228-0.300664-3.237555$

O $8.009976-1.146136-2.962894$

H $6.466679-2.441774-3.257507$

H $7.669612-1.930684-0.490155$

O $7.944343-3.644521-1.543664$

H $5.425042-3.880059-1.225853$

O $\quad 6.107310-3.622747 \quad 0.683413$

C $2.473009-1.4438770 .394860$

H $2.956068-3.135899-0.806306$

H $3.527413-3.211560 \quad 0.858509$

H $7.793475-0.662932-3.778494$

H $8.743755-3.289837-1.969440$

H $6.791243-4.302904 \quad 0.558616$

O $1.783671-1.005082-0.696280$

O $2.377159-1.0105251 .535559$

C $0.8603560 .058054-0.453408$

H $1.3821160 .933128-0.058434$

H $\quad 0.3934450 .327191-1.403962$

H $0.083851-0.2732320 .240338$

SCF Energy (B3LYP/6-31G**//MMFF) $=-3245.91020535$

03_277

MM̄FF Geometry

C $-2.070746 \quad 3.269230-1.253650$

C $-1.5520452 .121109-0.793373$

C $-0.5534861 .291583-1.557774$

O $0.577016 \quad 1.020161-0.698504$

C $-1.200138-0.052485-1.939871$

C $-0.316046-0.934248-2.799599$

C $0.155354-2.140030-2.426290$

C $0.007338-0.399632-4.171409$

C $-0.078450-2.862140-1.130066$

C $0.987450-2.611492-0.052168$

C $2.400151-3.074159-0.443967$

C $3.340371-3.023228 \quad 0.762481$

C $4.784201-3.3907920 .383612$

O $2.893419-2.204907-1.466422$

O $5.637488-3.0032421 .472035$

C $5.213238-2.557238-0.843822$

C $4.960215-4.9044130 .213378$

O $6.481680-2.985391-1.332047$

C $4.178660-2.570086-1.987151$

C $4.511850-1.573582-3.124611$

O $4.142049-3.876906-2.573981$

C $1.5065032 .004054-0.558818$

O $1.5066353 .100000-1.095961$

C $2.5280401 .519787 \quad 0.395421$

C 3.3629962 .3977700 .970816

C 4.4115272 .0813641 .943195

C 4.7341130 .6281692 .169680

C $4.607072-0.102513-2.704651$

C $6.0222590 .363752-2.405260$

C $7.2705322 .330187-1.519531$

O $7.021034-0.303312-2.660143$

C $7.6060602 .086933-0.082080$

C $7.8907293 .072360 \quad 0.782822$

C 8.2722522 .8446732 .217396

C 7.4145873 .6346493 .180255

C 7.8378704 .8081993 .679935

C 6.1070793 .0220003 .628164

C 5.0393673 .0984562 .569901

N $6.039761 \quad 1.637901-1.853239$

H $-1.7614173 .649144-2.223873$

H -1.8726901 .7400950 .173860$

H $-0.2120821 .817175-2.457866$

H $-2.1340020 .126712-2.490061$

H $-1.488508-0.574206-1.020877$

H $\quad 0.778180-2.686699-3.133574$

H $0.513063-1.142427-4.797827$

H $-0.910516-0.105978-4.691137$

H $0.6656270 .471628-4.101881$ 
H $-1.062297-2.608494-0.723079$ H $-0.128387-3.937483-1.344242$ H $1.001833-1.5425070 .190151$ H $0.675434-3.1429550 .854994$ H $2.333361-4.097279-0.832373$ H $2.978056-3.6758431 .566638$ H $3.339599-2.0074691 .179947$ H $5.331327-3.4645752 .271652$ H $5.344663-1.526205-0.495464$ H $4.706802-5.4262201 .144221$ H $6.004612-5.1599180 .002921$ H $4.335372-5.322538-0.578586$ H $7.108513-2.939236-0.589951$ H $5.410055-1.901101-3.661723$ H $3.695881-1.638247-3.859317$ H $3.768719-3.803239-3.467963$ H 2.5512280 .4584150 .613539 H 3.2572683 .4539290 .725576 H 4.9273940 .1266201 .215450 H $3.901846 \quad 0.1228712 .671146$ H 5.6270320 .4678002 .777138 H $4.2537410 .518523-3.537968$ H $3.9616840 .105466-1.846005$ H $7.1141063 .391970-1.734696$ H $8.0815741 .960441-2.155035$ H 7.6429141 .0538780 .255701 H 7.8810064 .1026320 .433889 H 9.3248003 .1355662 .331967 H 8.2306021 .7803042 .480100 H 7.2417105 .3678544 .392948 H 8.7887565 .2380203 .382423 H 5.7251243 .5545664 .509620 H 6.2922142 .0030223 .978783 H 4.7369024 .1192692 .331249 H $5.1612202 .095788-1.627938$ C $-4.4601764 .005960-1.191568$ O $-4.9339402 .643274-1.195971$ C $-6.2944932 .620066-0.734722$ C $-6.7890174 .057220-0.834062$ C $-5.5326184 .823806-0.469822$ H -6.2593982 .3352710 .322529$ C $-7.1173991 .591268-1.515287$ H $-7.1039384 .311315-1.852628$ H -7.626417 4.257044 -0.158869 C $-5.570356 \quad 6.286443-0.876907$ H -5.3871684 .7598010 .617123$ C $-6.499003 \quad 0.178714-1.568354$ O $-7.2555932 .047746-2.867545$ H $-8.1323931 .550732-1.102423$ H $-5.626790 \quad 0.188423-2.233112$ O $-7.457299-0.681999-2.199727$ C $-6.092007-0.427678-0.212082$ C $-5.630775-1.892372-0.350229$ H -5.2874150 .1662550 .234879$ O $-7.203782-0.4094130 .684232$ H $-6.493666-2.518791-0.607536$ H -4.897840 -1.957092 -1.163476 H -4.636583 $6.789952-0.607695$ H -5.714907 $6.398966-1.956679$ H $-6.3919776 .805001-0.372088$ C $-3.0957794 .070788-0.503440$ H -7.784133 $1.385248-3.344716$ H $-8.218438-0.762640-1.599468$ H -7.395490 0.5159830 .908105 H -3.180001 3.6965780 .523996 H $-2.7494495 .108547-0.441075$ H $-4.3756974 .315092-2.241551$ C $-3.008288-2.1527292 .295046$ O $-3.708350-1.8419331 .082626$ C $-5.008533-2.4276430 .952993$ C $-4.883778-3.9574640 .951569$ C $-4.177656-4.4493982 .219860$ C $-2.890143-3.6727702 .514991$ H -1.996167 -1.756071 2.141950 C $-3.621494-1.4201883 .494622$ H $-5.636947-2.1186201 .794663$ O $-4.176343-4.415087-0.198658$ H $-5.887893-4.394500 \quad 0.900327$
H -3.939837 $-5.515352 \quad 2.118013$

O $-5.055194-4.302168 \quad 3.334699$

H -2.567914 -3.863049 3.545339

O $-1.845761-4.1799881 .681389$

C -3.5576440 .0765813 .294029$

H -4.660496-1.713643 3.670625

H -3.072913 -1.647945 4.415828

H -3.549898 -3.723905 -0.470753

H $-5.846795-4.8385533 .158007$

H -2.071379 -3.981256 0.759162

O $-4.817571 \quad 0.596244 \quad 3.253213$

$\begin{array}{lllll}\text { O } & -2.514047 & 0.706246 & 3.177475\end{array}$

C -4.8922382 .0104103 .051709$

H -4.4064652 .2850682 .111320$

H -5.9472882 .2912092 .995964$

H -4.4315402 .5364753 .892916$

SCF Energy $(B 3 L Y P / 6-31 G * * / / M M F F)=-3245.91000483$

03278

MM̄FF Geometry

C $2.0700164 .258019-0.488923$

C 1.2062613 .6989290 .372338

C $-0.282873 \quad 3.6379460 .132854$

O $-0.7294782 .272641 \quad 0.276920$

C -0.9946784 .4861491 .205286$

C -2.4954854 .5935381 .019267$

C -3.4099514 .1550681 .906843$

C $-2.9520165 .279006-0.243376$

C -3.1727553 .4810793 .229828$

C -3.1827311 .9453443 .170820$

C -4.5465671 .3406692 .798620$

C $-4.525947-0.1819272 .951593$

C $-5.847657-0.8246412 .498672$

O -4.8368531 .6756181 .440098$

O $-5.625787-2.2367522 .359432$

C $-6.221875-0.2871961 .099226$

C $-6.948909-0.6617263 .553340$

O $-7.523548-0.723857 \quad 0.717278$

C -6.1280571 .2482010 .986666$

C $-6.2989681 .770395-0.460540$

O -7.1708821 .8416131 .770163$

C $-0.8531581 .528008-0.854613$

O $-0.5878141 .868368-1.996265$

C $-1.3581690 .194833-0.455992$

C $-1.243296-0.835091-1.307546$

C $-1.619152-2.222867-1.032737$

C $-2.514212-2.477720 \quad 0.149778$

C $-5.277618 \quad 1.245154-1.475799$

C $-5.7687690 .053654-2.280770$

C $-4.988587-1.593175-3.978814$

O $-6.924331-0.359385-2.241171$

C $-4.642131-2.868091-3.277832$

C $-3.810308-3.786215-3.792101$

C $-3.479315-5.086622-3.119057$

C $-1.990948-5.286396-2.945589$

C $-1.289277-6.041232-3.807802$

C $-1.334604-4.680220-1.725915$

C -1.146561 -3.191705 -1.844623

N $-4.765627-0.474836-3.082229$

H $1.6948054 .647994-1.432096$

H $1.5691773 .332976 \quad 1.329954$

H $-0.5291074 .019942-0.865023$

H $-0.5844425 .505278 \quad 1.203578$

H -0.7595124 .0675342 .189624$

H -4.4624964 .3032021 .667645$

H $-2.7285824 .663212-1.119817$

H -4.030444 $5.471075-0.245231$

H -2.449196 $6.245189-0.355035$

H -2.2268383 .8129503 .670178$

H $-3.9462893 .822063 \quad 3.929744$

H -2.4214971 .6128452 .455236$

H -2.884358 1.5707604 .157881

H $-5.307253 \quad 1.7793773 .454947$

H $-4.296806-0.4664763 .986076$

H -3.711991 -0.595979 2.341429

H $-5.316903-2.577576 \quad 3.216392$

H -5.520342 -0.7387060 .387985$

H $-6.646211-1.1294054 .498010$ 
H -7.867984 -1.174045 3.248104 H -7.1888810 .3822043 .765165$ H -7.532943 -1.694447 0.777468 H -7.329269 $1.609609-0.800215$ H $-6.1737242 .862489-0.425806$ H -7.3244642 .7446601 .446239$ H -1.7635950 .0855550 .543055$ H $-0.783626-0.659834-2.279209$ H -1.973138 -2.308693 1.086748 H -2.917687 -3.4918800 .180315$ H $-3.383048-1.8117830 .119827$ H $-5.0743042 .040846-2.204141$ H $-4.3283550 .994557-0.991806$ H -4.370526 -1.426133 -4.866647 H $-6.039957-1.613265-4.283450$ H -5.113909-3.055154-2.316016 H $-3.360218-3.607913-4.766346$ H $-3.895860-5.895558-3.733464$ H -3.974720 -5.172183 -2.143814 H $-0.226087-6.212122-3.674950$ H -1.761969 -6.500880 -4.669619 H $-0.338039-5.118597-1.580427$ H -1.900801-4.971063-0.837019 H - $0.501092-2.900774-2.674690$ H -3.821853 -0.106008 -3.007982 C $4.5550563 .818432-1.061228$ O $4.7939312 .475416-0.607738$ C $5.3920431 .742080-1.700154$ C $5.3217792 .649566-2.933694$ C $4.2637843 .669063-2.547770$ H $4.7617780 .864935-1.871455$ C $6.8197501 .304198-1.329745$ H $6.2808053 .140340-3.135795$ H $5.0554822 .087215-3.834734$ C $4.3519804 .964442-3.334050$ H $3.2805113 .208306-2.706404$ C $6.9178780 .481392-0.028720$ O $7.6219172 .480853-1.155463$ H $7.2626040 .750207-2.165979$ H 6.6170031 .1053030 .821809 O $8.303511 \quad 0.1673010 .161027$ C $6.102783-0.823731-0.050829$ C $6.216896-1.6281251 .258131$ H $5.052438-0.577523-0.220647$ O $6.522415-1.636434-1.145379$ H $7.259616-1.9228461 .426023$ H $5.928868-0.9776842 .091512$ H $3.5787635 .669876-3.014347$ H $5.3251095 .449117-3.200668$ H $4.2148434 .775085-4.403696$ C $3.5180524 .519938-0.182001$ H $8.5216002 .186492-0.932142$ H $8.421028-0.0998631 .088172$ H $7.468460-1.826191-1.023940$ H $3.6619865 .603429-0.285520$ H $3.724577 \quad 4.2794730 .868728$ H $5.5047324 .354567-0.921318$ C $3.215471-1.8953011 .649901$ O $4.031768-2.6887370 .781471$ C $5.359765-2.9151821 .258114$ C $5.306935-3.6443412 .608750$ C $4.404027-2.9288293 .614512$ C $3.043048-2.6049292 .994712$ H $3.678867-0.9128901 .804757$ C $1.878489-1.6746100 .939496$ H $5.827366-3.5991900 .538399$ O $\quad 6.609695-3.782013 \quad 3.165720$ H $4.913108-4.6577262 .455995$ H $4.880083-2.0208764 .003491$ O $4.189670-3.7812744 .741962$ H $2.467710-3.5306002 .865770$ O $2.310779-1.762573 \quad 3.881109$ C $2.088669-0.810828-0.282935$ H $1.430032-2.6274810 .633796$ H $1.150192-1.1655601 .580254$ H $7.155363-4.2589742 .517252$ H $5.063779-4.0055035 .104738$ H $2.246213-2.2256514 .733951$
O $2.108523-1.586812-1.403059$

O $2.254308 \quad 0.401018-0.236339$

C $2.326363-0.915123-2.649084$

H $1.9061720 .095174-2.652729$

H $3.399254-0.883161-2.854259$

H $1.835943-1.495423-3.435381$

SCF Energy (B3LYP/6-31G**//MMFF) $=-3245.90728366$

03279

MM̄FF Geometry

C $2.430666-5.2321111 .274486$

C $1.741867-4.4995440 .385289$

C $0.236312-4.4468410 .322166$

O $-0.167276-3.0558650 .309288$

C $-0.254701-5.090969-0.986352$

C $-1.765210-5.128508-1.116846$

C $-2.469766-4.474214-2.061052$

C $-2.486554-5.992673-0.113980$

C $-1.953463-3.598492-3.168098$

C $-1.900661-2.098762-2.836313$

C $-3.267397-1.476742-2.504723$

C $-3.1693200 .049320-2.452564$

C $-4.4946850 .702139-2.030947$

O $-3.689186-1.966920-1.229294$

O $-4.1963622 .071421-1.718551$

C $-5.0088550 .016150-0.745376$

C $-5.5009920 .728872-3.187245$

O $-6.3210040 .463389-0.414563$

C $-4.991824-1.524702-0.824408$

C $-5.308525-2.2081560 .529103$

O $-5.991906-1.957233-1.755438$

C $-0.082111-2.3632781 .476856$

O $0.261040-2.7917722 .566706$

C $-0.456003-0.9624321 .187380$

C -0.1160910 .0049112 .051665$

C -0.4134971 .4310131 .905479$

C -1.3462241 .8325250 .794563$

C $-4.345560-1.8777781 .675109$

C $-4.810135-0.7384242 .567544$

C $-4.025990 \quad 0.6917724 .453069$

O $-5.939263-0.2586172 .516621$

C $-3.5522112 .002798 \quad 3.909600$

C -2.6958352 .8039264 .561706$

C -2.2475084 .1436634 .051693$

C -0.7422144 .2878214 .032724$

C -0.0946804 .9041455 .036040$

C 0.0007353 .7972502 .811003

C 0.1368752 .3002342 .778341

N -3.821220 -0.346599 3.460609

H $1.874275-5.8647521 .963392$

H $2.265105-3.865774-0.324914$

H -0.214436 -4.945109 1.188819

H $0.118611-6.121495-1.056477$

H $\quad 0.189251-4.550950-1.829672$

H -3.554006 -4.582910-2.053635

H $-3.550811-6.101837-0.349227$

H -2.052565 -6.997733 -0.096295

H -2.416380 -5.562095 0.889490

H $-0.959419-3.925623-3.490752$

H -2.598239 -3.748161 -4.043774

H $-1.210440-1.945045-1.998554$

H -1.473046 -1.583806 -3.705563

H $-3.984717-1.787284-3.273274$

H $-2.8346300 .453020-3.416337$

H $-2.3975830 .334562-1.725472$

H $-5.0286692 .516637-1.486535$

H -4.3508450 .3379050 .070058$

H -5.099444 $1.307651-4.027796$

H $-6.429127 \quad 1.231849-2.894125$

H $-5.752678-0.266394-3.559779$

H $-6.274073 \quad 1.419735-0.248446$

H -6.352030 -2.022790 0.810649

H $-5.241151-3.2935740 .365795$

H $-6.212891-2.883455-1.562343$

H $-0.987320-0.7646320 .263691$

H $0.445755-0.2674032 .944273$

H -2.289385 1.2812340 .870511

H $-0.8951841 .619104-0.178788$ 
H -1.612096 $2.891108 \quad 0.803863$ H -4.267346 -2.758549 2.325548 H -3.342589 -1.662287 1.295909 H $-3.4776860 .393681 \quad 5.352321$ H -5.0907190 .7578054 .698750$ H -3.953116 2.3201962 .949813 H -2.320235 2.4968265 .535319 H -2.6853054 .9108594 .703880$ H -2.646255 4.3461123 .049873 H 0.9818805 .0378485 .016885 H -0.626839 5.2840825 .902006 H 1.0169714 .2128882 .800323 H -0.4729214 .2042491 .913492$ H $\quad 0.791573 \quad 1.9073493 .557491$ H -2.897898 -0.7654433 .396589$ C $4.694294-4.0808800 .841518$ O $4.275319-2.8545721 .471912$ C $5.393270-1.9535981 .505123$ C $6.563879-2.6664790 .833962$ C $6.205106-4.1266321 .052621$ H $5.654541-1.8233482 .564016$ C $4.996055-0.5757630 .949587$ H $6.625203-2.441065-0.234703$ H $7.524820-2.3937611 .281830$ C $6.937233-5.0756350 .120462$ H $6.431426-4.3921012 .093836$ C $4.124203-0.607308-0.326613$ $\begin{array}{lllll}\text { O } & 6.166617 & 0.207918 & 0.742620\end{array}$ H $4.399026-0.0692051 .719366$ H $3.236176-1.217456-0.124662$ O $4.824653-1.239808-1.394292$ C $3.641300 \quad 0.784388-0.788796$ C $2.6556370 .672293-1.968157$ H 3.1500191 .2924210 .049308 O $4.7431991 .588822-1.201675$ H $3.1493810 .187614-2.819328$ H $1.8199420 .035343-1.652662$ H $\quad 6.644387-6.1129990 .309747$ H $6.723051-4.851363-0.929915$ H $8.019629-4.9991420 .266661$

C $3.928653-5.2533701 .459343$ H $\quad 6.649490 \quad 0.2430611 .586039$ H $5.595351-0.684671-1.604037$ H $5.3255581 .706979-0.432467$ H $4.110732-5.2529362 .541939$ H $4.307054-6.2039751 .067551$ H $4.475948-3.997407-0.230798$ C $0.9116303 .953998-1.574671$ O $1.4248602 .635922-1.341877$ C $2.1224402 .039085-2.437613$ C $1.1890531 .906163-3.650474$ C $0.5607263 .254479-4.020239$ C $-0.0243383 .968684-2.796308$ H $0.3069814 .180743-0.687016$ C $2.0323525 .002575-1.651581$ H $2.9773262 .666772-2.709210$ O $\quad 0.1551720 .961520-3.381215$ H $1.7634421 .517828-4.499419$ H $-0.2345453 .094756-4.758630$ O $1.5441114 .095573-4.615819$ $\mathrm{H}-0.2853155 .004440-3.042793$ O $-1.2359323 .311729-2.413188$ C $2.7936275 .126983-0.347777$ H $2.7645844 .770397-2.428847$ H $1.5877295 .981032-1.868936$ H $-0.311157 \quad 1.253617-2.579480$ H $1.8807903 .639985-5.406166$ H -1.849529 $3.362588-3.165704$ O $3.6009646 .227586-0.410453$ O 2.7115924 .3544710 .596800 C $4.397923 \quad 6.4712720 .752834$ H 5.0839175 .6358640 .923125 H 4.9846247 .3772350 .579347 H 3.7569036 .6298231 .625318 SCF Energy (B3LYP/6-31G**//MMFF) $=-3245.91237832$

0328

MM̄FF Geometry
C $-2.4465171 .997500-3.392261$ C $-2.3010550 .961964-2.552904$ C $-1.293984-0.141453-2.751220$ O $-0.329823-0.060362-1.675827$ C $-1.986524-1.512079-2.662724$ C $-1.054130-2.682089-2.911918$ C $-0.746480-3.625049-1.999518$ C $-0.473819-2.771333-4.300273$ C $-1.243724-3.747592-0.586776$ C $-0.362776-3.0688580 .471857$ C $1.055131-3.6506120 .583866$ C $1.767195-3.0939801 .819371$ C $3.220968-3.5831611 .915857$ O $1.788885-3.291913-0.590071$ O $3.894854-2.7713862 .890315$ C $3.925839-3.3418840 .563269$ C $3.303485-5.0274942 .423895$ O $5.223306-3.9312920 .553868$ C $3.113831-3.838404-0.650543$ C $3.725692-3.415118-2.009166$ O $3.078277-5.271010-0.638199$ C $0.6814830 .839122-1.812231$ O $0.9031351 .564448-2.768470$ C $1.4634340 .820968-0.558509$ C $2.2089051 .888088-0.236730$ C 3.0111122 .0433390 .977674 C 3.1669050 .8383691 .867513 C $3.869976-1.904655-2.225624$ C $5.239902-1.355086-1.862333$ C $6.4610830 .803383-1.610780$ O $6.210802-2.067570-1.622628$ C $6.4916101 .196413-0.167343$ C 6.6958922 .4562010 .246940 C 6.7735672 .8632451 .690854 C 5.8319193 .9981312 .027892 C 6.2655715 .2696602 .058851 C 4.4113843 .6488892 .410719 C 3.5771503 .2426891 .227297 N $5.2603920 .033371-1.878330$ H -1.830551 $2.049963-4.286719$ H $-2.8970360 .929370-1.645394$ H $-0.783588-0.038670-3.716456$ H -2.804004 -1.563955 -3.393614 H -2.452197 -1.601627 -1.676286 H -0.063265 -4.419634 -2.297983 H $0.234612-1.956465-4.476825$ H $0.061732-3.712291-4.466656$ H -1.270146 -2.713354 -5.049478 H $-2.261553-3.355480-0.501168$ H -1.331546 -4.815821 -0.350347 H $-0.306468-1.9965310 .251906$ H -0.869364 -3.174692 1.439222 H $0.976814-4.7423200 .648154$ H $1.216686-3.3480082 .733842$ H $1.773253-1.996778 \quad 1.770650$ H $3.416360-2.8553073 .732902$ H $4.073044-2.2591670 .473005$ H $2.848507-5.109898 \quad 3.418357$ H $4.344980-5.3452272 .545028$ H $2.797744-5.7416251 .770833$ H $5.709390-3.5743161 .316915$ H $4.674123-3.939941-2.175212$ H $\quad 3.049960-3.782362-2.795345$ H $2.876782-5.582147-1.536308$ H $1.375492-0.0509660 .078478$ H $2.2145412 .741500-0.913819$ H 2.2104750 .5778912 .333189 H 3.8922510 .9785812 .671268 H $3.522419-0.0211131 .289082$ H $3.732912-1.693284-3.293923$ H $3.096506-1.354840-1.682110$ H $6.443747 \quad 1.672299-2.276238$ H $7.3432620 .199670-1.847162$ H $\quad 6.367051 \quad 0.406467 \quad 0.569481$ H $6.8492943 .238478-0.493355$ H $7.8086463 .166547 \quad 1.895803$ H 6.5772112 .0150882 .358464

H 5.6042246 .0869152 .326660 
H 7.2915155 .5218391 .811729 H 3.9194224 .5181302 .867748 H 4.4305802 .8935623 .201197 H 3.4086854 .0530580 .516521 H $4.3966880 .542724-2.041508$ C -2.814572 $4.247003-2.239560$ O $-2.7867803 .778989-0.874425$ C $-1.4760854 .018045-0.335468$ C $-0.8850525 .128360-1.195257$ C -1.401471 4.741278 -2.568789 H $-0.9010073 .099089-0.498136$ C $-1.5333224 .310466 \quad 1.167887$ H -1.273728 $6.113677-0.914048$ H $\quad 0.2072705 .161537-1.140425$ C - $-1.3732675 .874825-3.579249$ H $-0.7720223 .927297-2.945335$ C $-2.3806203 .318476 \quad 1.992210$ O -2.0977225 .6141901 .357142$ H -0.5098984 .3585341 .559936$ H -3.4478283 .5285241 .847248$ O -2.1078333 .5554023 .379428$ C -2.1233061 .8296181 .700664$ C -2.8748350 .9005702 .672884$ H -2.4298311 .6095040 .674556$ O $-0.7275941 .549394 \quad 1.792117$ H $-2.366521 \quad 0.9118153 .644605$ H -3.885446 1.2896502 .830286 H -1.773498 $5.544135-4.543006$ H -1.968468 $6.729653-3.241243$ H $-0.3473806 .222566-3.738740$ C $-3.3829413 .149185-3.150504$ H -1.944631 5.8704852 .281951 H -2.4590774 .4336393 .602848$ H -0.4302571 .8123552 .680032$ H -3.642066 $3.582270-4.124067$ H -4.315888 2.765893 -2.717580 H -3.519047 $5.088419-2.243965$ C $-5.044945-0.4319590 .982309$ O $-3.632645-0.659487 \quad 0.908763$ C $-2.936558-0.5561102 .157528$ C $-3.524674-1.5551213 .162601$ C $-5.029247-1.3635273 .334203$ C $-5.729833-1.3836981 .975189$ H -5.2164040 .6004351 .311207$ C -5.621143 $-0.494240-0.441454$ H -1.903002 -0.8645451 .960189$ O $-2.889454-1.4357774 .431021$ H $-3.343643-2.5780652 .807357$ H $-5.257779-0.4414643 .881621$ O -5.550508 -2.436859 4.122331 H $-5.735082-2.4156351 .608091$ O $-7.088540-0.9957252 .169419$ C $-5.451197-1.823238-1.148851$ H $-6.688637-0.249617-0.424213$ H $-5.106786 \quad 0.253464-1.056297$ H -1.936845 -1.576121 4.294650 H -5.069719 -2.434028 4.967781 H -7.574286 -1.2007791 .353087$ O $-6.019461-1.734171-2.389054$ O $-4.889588-2.808651-0.693247$ C $-5.930749-2.915587-3.191999$ H -6.477546 -3.735809 -2.717108 H $-6.388788-2.702709-4.161519$ H -4.883802 -3.190536 -3.351935 SCF Energy (B3LYP/6-31G**//MMFF)= -3245.89947493

$03 \_280$

MM̄FF Geometry

C -0.152768 $3.875935-2.526571$

C $-1.4703053 .690830-2.701290$

C $-2.5907614 .190004-1.821348$

O $-3.2891623 .007258-1.358945$

C $-2.1543374 .978420-0.568468$

C $-3.310490 \quad 5.385628 \quad 0.327313$

C -3.492595 $4.934428 \quad 1.585080$

C $-4.284007 \quad 6.369587-0.267864$

C -2.607358 3.9977122 .362604

C -2.9803802 .5349282 .124238$
C $-2.2218571 .538183 \quad 3.012268$

C -0.7131421 .5453052 .749168$

C 0.0163020 .4445113 .535246

O $\quad-2.766583 \quad 0.2435502 .714893$

O 1.3368250 .3147192 .989330

C $-0.700851-0.8989163 .294321$

C 0.1936470 .8166965 .011456

O $-0.146358-1.9314294 .104925$

C $-2.227565-0.8254743 .505650$

C $-2.951504-2.1267253 .085548$

O $-2.505717-0.6230724 .895924$

C $-4.4676412 .664398-1.940850$

O $-5.0438613 .239865-2.848320$

C $-4.9341951 .434605-1.261498$

C $-6.0787490 .839196-1.627969$

C $-6.629776-0.383139-1.033925$

C $-5.869095-0.9906610 .115913$

C $-2.690914-2.5721191 .644770$

C $-3.657896-3.6501631 .181426$

C $-4.252217-4.946462-0.874029$

O $-4.514307-4.1471041 .906937$

C $-5.168235-4.206035-1.797982$

C $-6.486389-4.439806-1.894534$

C -7.383321 -3.715903 -2.860242

C $-8.637357-3.162209-2.215699$

C $-9.851906-3.565078-2.626677$

C -8.499711 -2.143963-1.102527

C $-7.768471-0.900341-1.541168$

N -3.441285 $-3.986210-0.147641$

H $0.2145664 .474458-1.698573$

H $-1.7920283 .083686-3.546410$

H -3.249870 $4.814946-2.435446$

H -1.613881 $5.885675-0.867668$

H -1.442854 4.368418-0.001958

H -4.3678515 .2795702 .133863$

H -5.0193246 .7198430 .464469$

H -3.751845 $7.250113-0.642410$

H -4.836458 5.915851-1.096100

H -1.5552474 .1809322 .124807$

H -2.7152164 .2360473 .428263$

H -4.0583712 .3949682 .283895$

H -2.806620 2.2710001 .075196

H -2.4343931 .7796414 .060313$

H -0.2723992 .5210542 .983423$

H $-0.531048 \quad 1.3840891 .677006$

H 1.7775341 .1793853 .054704

H -0.498636 -1.177882 2.252728

H $\quad 0.787947 \quad 1.7335495 .105994$

H 0.7509640 .0423825 .549932

H -0.7530660 .9848845 .528846$

H $0.812434-1.9446043 .942152$

H -2.692677 -2.941892 3.774107

H -4.031694 -1.968295 3.213335

H -3.414654 -0.916518 5.074053

H $-4.3074331 .042409-0.468229$

H $-6.6609341 .276806-2.437317$

H -5.788672 -0.2789510 .945370$

H -4.861352 -1.268237 -0.207965

H $-6.325965-1.8923940 .525782$

H -2.781163 -1.719525 0.962658

H -1.676835 -2.978050 1.556109

H $-4.798170-5.583713-0.171336$

H -3.566998 -5.570234-1.456159

H -4.717032 -3.453169-2.440907

H $-6.938184-5.212292-1.276808$

H -7.649091 -4.426599 -3.653616

H -6.847168 -2.898965 -3.358802

H -10.759697 -3.177416 -2.176279

H $-9.968224-4.292533-3.423234$

H $-8.036174-2.633654-0.243193$

H -9.491132 -1.827505 -0.752467

H $-8.250518-0.377631-2.368625$

H -2.722037 -3.492032 -0.667956

C $1.8117712 .277156-2.654216$

O $2.6430652 .992312-1.719963$

C $2.8534632 .157728-0.557768$

C $2.2503160 .798329-0.893859$

C $1.1310471 .176141-1.842236$ 
H 2.2965282 .6357400 .257546

C $4.3532932 .121249-0.238829$

H $2.9828850 .159566-1.403164$

H $1.8967070 .267837-0.004829$

C $0.6179050 .015812-2.675326$

H $\quad 0.3055291 .579835-1.243104$

C $4.7156841 .148313 \quad 0.895044$

O 4.7660003 .4373870 .141865

H $4.9075871 .874243-1.150416$

H 4.4004340 .1319370 .640342

O $3.966431 \quad 1.5028262 .064845$

C 6.2049101 .1306681 .281686

C 7.1930580 .8819580 .128198

H $6.4749842 .074777 \quad 1.770244$

O $6.404626 \quad 0.1133902 .269699$

H $7.2338531 .773523-0.508794$

H 8.1863530 .7586450 .579355

H $-0.179722 \quad 0.343773-3.349242$

H $1.414689-0.425371-3.283566$

H $0.213384-0.771145-2.030108$

C $0.8938523 .248127-3.404440$

H $4.5167394 .039304-0.580430$

H 4.1919132 .4219502 .290033

H 5.7753830 .2816772 .991976

H $0.4247402 .721248-4.244002$

H $1.4975904 .059255-3.829603$

H $2.4990521 .830874-3.385776$

C $6.573377-2.749755-0.587111$

O $6.902557-1.5192350 .071972$

C $6.895667-0.351235-0.751989$

C $7.919419-0.493862-1.891133$

C $7.680279-1.778303-2.692694$

C $7.514304-2.997450-1.779585$

H $6.770126-3.5303410 .159308$

C $5.084774-2.823283-0.964274$

H $5.901387-0.227746-1.189554$

O $9.238349-0.512746-1.348284$

H $7.8442820 .382633-2.545841$

H $8.525700-1.953182-3.369355$

O $6.508044-1.634068-3.489278$

H $7.152019-3.854137-2.359096$

O $8.798546-3.368461-1.268867$

C $4.179343-2.8329850 .249733$

H $4.783349-1.977045-1.586909$

H $4.893882-3.750814-1.516452$

H $9.861587-0.567356-2.092698$

H $6.649065-0.879062-4.085636$

H $9.106206-2.650987-0.688589$

O $2.879728-2.786899-0.171328$

O $4.553674-2.8822971 .412993$

C $1.894516-2.7844130 .866373$

H $0.918110-2.6065320 .407795$

H $\quad 1.878127-3.756519 \quad 1.367512$

H $2.094334-1.9840711 .584195$

SCF Energy (B3LYP/6-31G**/MMFF)= -3245.91483501

$03 \_281$

MM̄FF Geometry

C -2.903644 4.315471 1.043859

C -2.0553043 .8929520 .094710$

C $-0.556014 \quad 4.003186 \quad 0.184234$

O -0.0060122 .6657770 .166108$

C $-0.0240854 .756714-1.048617$

C $1.4835404 .912046-1.072873$

C $2.2908554 .376446-2.010002$

C 2.0727825 .7519690 .030926

C $1.9039643 .542493-3.200193$

C $1.9118882 .025902-2.951298$

C $3.3022051 .448787-2.638470$

C $3.260482-0.080444-2.613724$

C $4.616447-0.687435-2.218876$

O $3.7181971 .931224-1.358976$

O $4.403412-2.072077-1.903021$

C $5.122587-0.003364-0.929823$

C $5.613685-0.657232-3.383184$

O $6.452932-0.412094-0.623640$

C $5.0431971 .536175-0.978248$

C 5.3459952 .2064940 .384297
O $6.0133052 .027250-1.911489$

C -0.0157951 .9723551 .336939$

O -0.4152292 .3627192 .422378$

C 0.5247100 .6240661 .060306

C $0.319721-0.3607701 .947226$

C $0.759644-1.7500241 .808837$

C $1.721054-2.0647330 .694937$

C 4.4139051 .8093971 .534913

C 4.9612250 .7007832 .419115

C $4.309663-0.7447664 .342241$

O 6.1114920 .2788132 .339608

C $3.938115-2.0949763 .816596$

C $3.147141-2.9515994 .480461$

C $2.805689-4.3288423 .988814$

C $1.315328-4.5808203 .946653$

C $0.697267-5.2411664 .940505$

C $0.560040-4.1454042 .712035$

C $0.295535-2.6654522 .684739$

N 4.0184990 .2638523 .340745

H -2.5164734 .7840661 .944294$

H $-2.4553623 .408266-0.792931$

H $-0.2486674 .518226 \quad 1.102636$

H $-0.4721115 .758570-1.091286$

H $-0.3669374 .236048-1.949317$

H $3.3614674 .561098-1.926812$

H $3.1410755 .943223-0.117646$

H 1.5708596 .7240140 .078110

H $1.9616065 .252150 \quad 0.997929$

H $0.9168323 .836216-3.572699$

H $2.5960893 .777724-4.019079$

H $1.2215271 .795515-2.131421$

H $1.5172651 .538195-3.851566$

H $4.0008871 .799173-3.407201$

H $2.931086-0.477445-3.581978$

H $2.508585-0.409829-1.884204$

H $4.009601-2.500328-2.682421$

H $4.490462-0.364164-0.110933$

H $5.220224-1.219438-4.238674$

H $6.554566-1.148074-3.110864$

H $5.8385710 .353933-3.728779$

H $6.454144-1.383456-0.576631$

H 6.4008432 .0646620 .648324

H 5.2266623 .2906920 .243469

H $6.2010022 .956965-1.700758$

H 1.0518540 .4732340 .125547

H $-0.250875-0.1397902 .848427$

H $1.240146-1.927116-0.278009$

H $2.109634-3.0845010 .724737$

H $2.593861-1.4064600 .750014$

H 4.2824962 .6811502 .189088

H 3.4238091 .5280191 .163684

H $3.745930-0.4806525 .242573$

H $5.378004-0.7234444 .580529$

H $4.362329-2.3930492 .860641$

H $2.747898-2.6617845 .449957$

H $3.286248-5.0506344 .662375$

H $3.235805-4.5195182 .997718$

H $-0.366455-5.4518514 .904006$

H $1.241000-5.5813875 .815622$

H $-0.416342-4.6473142 .673721$

H $1.086930-4.5019121 .822635$

H -0.393454 -2.334814 3.463221

H 3.0720990 .6303253 .297438

C -4.9432382 .9522121 .700866$

O -4.3141661 .7411131 .237033$

C -4.0897610 .8756972 .367794$

C -4.8873221 .4795213 .517519$

C $-4.7652532 .962293 \quad 3.216676$

H -3.020216 0.9306322 .606797

C $-4.463411-0.5724042 .024163$

H -5.9375941 .1652063 .484261$

H -4.4812511 .2036284 .495406$

C -5.7781343 .8206113 .952671$

H -3.7535283 .2883413 .488619$

C $-3.502403-1.2246991 .003067$

O $-5.804279-0.6081571 .539266$

H -4.440636 -1.169324 2.943544

H $-2.498510-1.2299541 .441326$ 
O - $-3.448505-0.448761-0.188547$ C $-3.887541-2.6740640 .645939$ C -2.753657 -3.5018690 .005950$ H $-4.168217-3.1991231 .567796$ O $-5.026233-2.709898-0.210386$ H -1.962133 -3.6380280 .751716$ H -3.174084 -4.493419-0.208275 H $-5.659600 \quad 4.8758483 .687838$ H $-6.805175 \quad 3.525544 \quad 3.712701$ H -5.6465873 .7269805 .035599$ C $-4.393977 \quad 4.156166 \quad 0.933231$ H $-5.838002-0.0529100 .740960$ H -3.0788210 .4215460 .036584$ H $-5.753153-2.2541190 .245955$ H -4.8758875 .0782491 .277503$ H -4.663061 4.043462 -0.125096 H -6.0093432 .8538641 .455449$ C $-2.727817-2.335754-3.560984$ O $-3.138629-2.861752-2.290861$ C -2.123132 -2.953568 -1.289388 C $-0.973354-3.854107-1.776636$ C $-0.425478-3.379573-3.125649$ C $-1.547141-3.136948-4.139798$ H $-3.590676-2.494752-4.220302$ C $-2.459074-0.823226-3.498205$ H $-1.717273-1.959328-1.083217$ O $-1.410343-5.207157-1.891520$ H - $0.172816-3.842595-1.028595$ H $0.264504-4.137858-3.515953$ O $0.312927-2.178762-2.922779$ H $-1.150926-2.629852-5.027223$ O $-2.030347-4.412864-4.570772$ C $-3.724658-0.027365-3.251150$ H $-1.752267-0.571137-2.703382$ H -2.049694 -0.482985-4.456370 H $-2.170223-5.223486-2.498210$ H $0.678091-1.909529-3.782742$ H $-2.696382-4.257227-5.262098$ O $-3.3952971 .290022-3.094861$ O $-4.855390-0.491642-3.202604$ C $-4.4946242 .169803-2.839462$ H $-4.1087713 .191321-2.789612$ H $-5.2266802 .112390-3.650678$ H $-4.9590741 .921487-1.880349$ SCF Energy (B3LYP/6-31G**//MMFF)= -3245.90915305

03_282

MMFFF Geometry

C -2.273473 -2.784900 -2.026650

C -1.545257 -3.910302 -1.960266

C $-0.240071-4.070791-1.211597$

O $0.383833-2.789307-0.966572$

C $-0.510641-4.759646 \quad 0.139481$

C $0.726744-4.9981710 .983318$

C $0.879269-4.5470312 .244500$

C $1.815561-5.8207420 .345277$

C $-0.109566-3.7609763 .060980$

C $-0.060352-2.243402 \quad 2.834974$

C $1.209994-1.5635143 .373804$

C $1.113447-0.0471953 .180787$

C 2.3970730 .6740243 .616569

O $2.336496-2.0742932 .661966$

$\begin{array}{lllll}\text { O } & 2.337189 & 2.018528 & 3.114892\end{array}$

C $3.602692-0.0090292 .939134$

C $2.4977660 .784408 \quad 5.141869$

$\begin{array}{llll}\text { O } & 4.831660 & 0.539694 & 3.410924\end{array}$

C $3.603625-1.5492713 .072104$

C $4.690216-2.1935832 .176195$

O $3.895880-1.8919614 .430274$

C $1.105206-2.255680-1.992417$

O $1.307400-2.759616-3.086373$

C $1.590389-0.923834-1.566413$

C $2.189703-0.127460-2.464916$

C $2.7244941 .215553-2.225906$

C $2.7338191 .719094-0.808628$

C $4.336713-2.2083630 .686844$

C $5.537687-1.946022-0.194518$

C $6.976383-0.095484-1.028668$
O $\quad 6.121955-2.851169-0.784069$

C $6.6940791 .323368-1.407725$

C $6.5826541 .749494-2.674963$

C $6.3360083 .182334-3.049983$

C $5.1295073 .361128-3.943759$

C $5.2729373 .641771-5.250282$

C $3.7580793 .312038-3.304960$

C $3.1796821 .922811-3.281629$

N $5.860572-0.599776-0.253927$

H -1.937518 -1.892810-1.504690

H $-1.908663-4.800733-2.469003$

H $0.432144-4.692371-1.816075$

H $-0.993521-5.730784-0.034303$

H -1.236995 -4.153905 0.692894

H $1.808846-4.7761752 .763863$

H $2.607846-6.0826811 .054906$

H $1.404483-6.757519-0.044940$

H $2.282512-5.271901-0.478266$

H -1.126061-4.120275 2.861645

H $\quad 0.066282-3.9790174 .121924$

H $-0.160555-2.0386321 .761616$

H - $0.934232-1.7998403 .328588$

H $1.312625-1.8104144 .436998$

H $\quad 0.245270 \quad 0.358934 \quad 3.715011$

H $\quad 0.938651 \quad 0.1738402 .117909$

H 1.5321092 .4349823 .467544

H 3.5513270 .2533201 .878196

H 1.6288401 .3184125 .545055

H $3.372621 \quad 1.3733595 .438691$

H $2.548215-0.1865925 .638939$

H 5.0025370 .1672044 .292748

H $5.661220-1.7197682 .366088$

H $4.827895-3.2406052 .480482$

H $4.210715-2.8105714 .455819$

H $1.432590-0.635305-0.533711$

H $2.289989-0.487098-3.488446$

H $1.7351541 .662479-0.364584$

H $3.0546782 .757673-0.711996$

H $3.4160651 .117150-0.203848$

H $3.907756-3.1780870 .408054$

H $3.578404-1.4595950 .442229$

H $7.137492-0.738613-1.900261$

H $7.869105-0.146681-0.397341$

H $6.5927752 .039196-0.595064$

H $6.7036941 .038156-3.488866$

H $7.2377523 .550540-3.556605$

H $6.2195803 .813813-2.160182$

H $4.4116253 .803072-5.890256$

H $6.2532103 .710769-5.709969$

H $3.0641743 .941476-3.878358$

H $3.8096513 .775859-2.316820$

H $3.1117631 .467225-4.270954$

H 5.4263910 .0288450 .415682

C $-4.765674-2.619731-1.845910$

O $-4.705203-1.446787-1.011374$

C $-6.037095-0.925718-0.844451$

C $-6.980953-1.934287-1.492985$

C $-6.103331-2.526812-2.576601$

H $-6.0808670 .011270-1.411332$

C $-6.324419-0.6598990 .637424$

H $-7.292667-2.713399-0.787667$

H -7.886155 -1.459655 -1.884194

C $-6.614820-3.852793-3.112238$

H $-6.031306-1.806592-3.402652$

C $-5.326157 \quad 0.286387 \quad 1.334846$

O $-6.301213-1.9036401 .352021$

H -7.343510 -0.2713100 .748442$

H $-4.353108-0.2059241 .453148$

O $-5.817092 \quad 0.5236292 .661463$

C -5.1258521 .6439130 .634965$

C -4.3079822 .6257801 .497082$

H -4.626235 $1.477824-0.324353$

O -6.4074582 .2124290 .365514$

H -4.9031642 .9052292 .374691$

H -3.4107972 .1129371 .859428$

H $-5.950020-4.244307-3.888426$

H $-6.686544-4.604950-2.319409$

H $-7.610325-3.731513-3.551631$ 
C $-3.562595-2.661265-2.787493$ H $-5.424471-2.3026601 .216920$ H -5.930326 -0.3431833 .087803$ H $-6.2843592 .930152-0.277854$ H $-3.512625-1.738324-3.377961$ H -3.666707 -3.491866 -3.494938 H $-4.752948-3.489634-1.175560$ C $-1.791303 \quad 3.197423-0.171808$ O $-3.1305863 .647820-0.420458$ C $-3.9157313 .917493 \quad 0.745494$ C -3.2204154 .9704301 .618383$ C $-1.789762 \quad 4.568916 \quad 1.955547$ C $-1.023197 \quad 4.2181320 .681980$ H $-1.815540 \quad 2.2287880 .341701$ C -1.123354 $3.006646-1.543881$ H $-4.845694 \quad 4.3758380 .388560$ O -3.9636315 .1894952 .812652$ H -3.200649 5.9243051 .074935 H $-1.770096 \quad 3.7442652 .677806$ $\begin{array}{lllll}\text { O } & -1.151745 & 5.677637 & 2.594296\end{array}$ H $-0.838228 \quad 5.1389880 .113671$ O $\quad 0.2409213 .686922 \quad 1.073510$ C $-1.598575 \quad 1.758424-2.258532$ H $-1.347173 \quad 3.864175-2.189360$ H $-0.0396382 .910679-1.435926$ H -3.476028 5.8429463 .342931 H -0.2304115 .4189942 .768631$ H 0.8403293 .7585340 .312331 O $-0.9517151 .668608-3.459218$ O $-2.4199920 .962079-1.827135$ C $-1.2859610 .528505-4.256484$ H $-2.3548330 .527729-4.490077$ H $-0.7222820 .590548-5.190981$ H $-1.002790-0.391348-3.737420$ SCF Energy (B3LYP/6-31G*//MMFF) $=-3245.91225478$

03283

MM̄FF Geometry

C $-0.9055495 .386337-1.039235$

C $-1.8283494 .797708-0.264140$

C $-2.8765923 .847925-0.785346$

O $-2.7094362 .576196-0.116456$

C $-4.2772704 .388333-0.444577$

C $-5.4114663 .542909-0.990490$

C $-6.3216342 .901395-0.231491$

C $-5.5082093 .467867-2.492910$

C -6.4265912 .8740231 .267691$

C -5.6970921 .7005821 .940462$

C $-6.247192 \quad 0.316443 \quad 1.559074$

C -5.632735 -0.7732332 .440647$

C $-6.095230-2.1792372 .024527$

$\begin{array}{lllll}\text { O } & -5.919850 & 0.059024 & 0.191261\end{array}$

O $-5.235529-3.1337692 .666753$

C $-5.897744-2.348230 \quad 0.501751$

C -7.516986 -2.478663 2.515014

O $-6.470778-3.5711090 .046972$

C $-6.451932-1.168994-0.324009$

C $-6.073823-1.238173-1.823939$

O $-7.883330-1.177368-0.254685$

C -1.780409 $1.719252-0.622117$

O $-1.076267 \quad 1.891167-1.604204$

C $-1.7475880 .527853 \quad 0.255655$

C $-0.732649-0.343513 \quad 0.154451$

C $-0.537941-1.5408230 .976603$

C -1.677229-1.965094 1.864774

C $-4.571086-1.264751-2.124859$

C $-4.009427-2.662346-2.328236$

C $-1.850336-3.855525-2.674259$

O $-4.711220-3.665999-2.418525$

C $-1.395617-4.421519-1.366360$

C $-0.121774-4.760580-1.117393$

C $0.349821-5.3599750 .175752$

C $1.470395-4.5660160 .808276$

C $2.753269-4.9352870 .655296$

C $1.096601-3.3939721 .686987$

C $0.639536-2.1959950 .897495$

N -2.625058 -2.652098 -2.436068

H $-0.9022705 .189396-2.108240$
H -1.8227854 .9887480 .806638$

H $-2.7796883 .712594-1.869342$

H $-4.3902765 .404649-0.845593$

H $-4.3592794 .482277 \quad 0.643701$

H -7.096782 $2.329006-0.739946$

H -6.429976 $2.978672-2.825979$

H $-5.4992434 .473696-2.925384$

H $-4.6693222 .900078-2.906622$

H $-6.063012 \quad 3.812852 \quad 1.698567$

H -7.4912852 .8350201 .531986$

H $-4.630776 \begin{array}{lll}1.756242 & 1.692416\end{array}$

H $-5.783807 \quad 1.833866 \quad 3.025977$

H $-7.3368980 .334146 \quad 1.678746$

H $-5.860911-0.593193 \quad 3.498546$

H $-4.538224-0.7300682 .360354$

H -5.292330 -2.989671 3.626839

H $-4.817784-2.4260250 .329336$

H -7.566778 -2.4107193 .608474$

H -7.813579 -3.5035592 .266096$

H -8.264129-1.794063 2.108656

H $-6.078051-4.2884810 .572999$

H $-6.598826-2.071362-2.306556$

H $-6.474138-0.330747-2.299214$

H $-8.231352-0.675114-1.010084$

H -2.5448800 .4158120 .981334$

H $0.049643-0.147982-0.577915$

H -2.600978 $-2.052261 \quad 1.282921$

H $-1.833480-1.2360812 .667014$

H $-1.526991-2.9389822 .334646$

H $-4.397468-0.727924-3.066463$

H $-4.001982-0.749578-1.344981$

H -1.011132 -3.578817-3.320430

H -2.469072 $-4.590383-3.199157$

H $-2.149422-4.579837-0.598656$

H $0.623902-4.626478-1.897547$

H $0.689091-6.382774-0.034920$

H $-0.473142-5.4645790 .893832$

H $3.560657-4.3852591 .127333$

H $3.025575-5.7911170 .046503$

H $1.967321-3.0748922 .275644$

H $0.364138-3.7270372 .427022$

H $1.399763-1.8127130 .215750$

H -2.120215 $-1.782006-2.294200$

C $1.5086175 .645646-0.274282$

$\begin{array}{lllll}\text { O } & 1.389038 & 4.677303 & 0.787905\end{array}$

$\begin{array}{lllll}\text { C } 2.045403 & 3.460146 & 0.376687\end{array}$

C $2.9680093 .864859-0.763341$

C $2.1283584 .914103-1.465866$

H 1.2489542 .8020380 .006163

C 2.7386042 .8189861 .584995

H $3.8943264 .307564-0.376872$

H $3.2303943 .025752-1.414409$

C $2.9231735 .816995-2.392632$

H $1.3617174 .395244-2.053540$

C 3.5481671 .5606391 .210192

$\begin{array}{lllll}\text { O } & 1.701781 & 2.490801 & 2.514308\end{array}$

H 3.3860723 .5560782 .074682

H $4.293797 \quad 1.8454740 .461157$

$\begin{array}{llll}\text { O } & 2.676773 & 0.619906 & 0.584075\end{array}$

$\begin{array}{llll}\text { C } & 4.286438 & 0.875360 & 2.378906\end{array}$

C $4.972490-0.4446001 .989206$

H 5.0204561 .5751992 .793373

O 3.3632590 .5745143 .427095

H $5.470599-0.8667202 .873233$

H $4.206517-1.1975461 .753202$

H $2.2730696 .565835-2.856047$

H $3.7177776 .345268-1.855050$

H $3.3903815 .232362-3.191837$

C $0.1530926 .317389-0.515941$

H 2.1266402 .1636503 .324898

H 2.0007550 .3773651 .240062

H 3.8699520 .1986394 .167188

H $0.2689607 .142957-1.227900$

H $-0.192945 \quad 6.761713 \quad 0.426280$

H 2.2039476 .4095120 .097455

C $7.298883-1.881055-0.525892$

O $6.433263-1.705666 \quad 0.603714$

C $5.965342-0.3678010 .820393$ 
C $7.1534540 .570178 \quad 1.075575$

C $8.1904440 .481529-0.051073$

C $8.532930-0.968652-0.411302$

H $7.639582-2.921993-0.457630$

C $6.544579-1.725586-1.855783$

H $5.419916-0.046651-0.071545$

O $7.775453 \quad 0.2495032 .318630$

H $6.791416 \quad 1.600762 \quad 1.156243$

H 9.1076740 .9981180 .257886

O $7.691661 \quad 1.139818-1.211952$

H $9.107259-0.999447-1.344615$

O $9.379624-1.4826450 .621501$

C $5.388595-2.697630-1.965027$

H $6.143984-0.714240-1.965364$

H $7.217681-1.915218-2.699177$

H $8.060504-0.6794712 .277356$

H $7.5198422 .066650-0.973565$

H $9.657019-2.3761320 .356370$

O $4.429578-2.167271-2.779642$

O $5.341755-3.792193-1.420558$

C $3.256918-2.967959-2.955578$

H $3.520186-3.957209-3.342034$

H $2.719269-3.052368-2.007246$

H $2.609685-2.469044-3.681855$

SCF Energy (B3LYP/6-31G**//MMFF) $=-3245.91008344$

$03 \quad 284$

MM̄FF Geometry

C 1.1526392 .1023932 .342140

C $1.0098560 .959701 \quad 3.034331$

C $-0.051085-0.1041172 .836510$

O $-0.8933190 .175857 \quad 1.696464$

C $0.598353-1.4823982 .609785$

C $-0.383909-2.6382052 .679133$

C $-0.662341-3.4746261 .659868$

C $-1.048826-2.8543024 .015379$

C $-0.084843-3.4737710 .273435$

C $-0.887393-2.666733-0.757275$

C $-2.304284-3.204005-1.014424$

C $-2.938094-2.498796-2.216093$

C $-4.391135-2.944611-2.445433$

O $-3.098419-2.965043 \quad 0.150297$

O $-4.998661-2.014790-3.355936$

C $-5.167382-2.843788-1.113984$

C $-4.464920-4.320455-3.118430$

O $-6.470872-3.405296-1.241446$

C $-4.430953-3.4903320 .077111$

C $-5.109536-3.2127981 .441171$

O $-4.412693-4.912660-0.097320$

C -1.9845820 .9637741 .894682$

O $-2.349678 \quad 1.4720262 .942306$

C -2.649442 1.1364100 .585099

C -3.4438902 .1985020 .387381$

C $-4.1597772 .519187-0.848846$

C $-4.2262251 .451496-1.908588$

C $-5.246824-1.7342591 .820564$

C $-6.592331-1.1270321 .458652$

C -7.7832361 .0607821 .405858$

O $-7.553318-1.7917131 .081235$

C $-7.732516 \quad 1.6140600 .016748$

C $-7.9086012 .914551-0.262317$

C $-7.8989173 .483386-1.652294$

C $-6.9252604 .630776-1.803433$

C $-7.3409255 .905598-1.716356$

C $-5.4882874 .299656-2.137970$

C $-4.7338143 .735022-0.965669$

$\begin{array}{llll}\mathrm{N} & -6.604961 & 0.248766 & 1.645607\end{array}$

H $0.4691762 .324958 \quad 1.530054$

H 1.6790750 .7848483 .875458

H $-0.659144-0.1300373 .748718$

H $1.377139-1.6618213 .362714$

H $1.105361-1.4700491 .641345$

H $-1.383640-4.2719401 .837255$

H -1.764722 -2.054192 4.226025

H $-1.596403-3.8021674 .058174$

H $-0.300204-2.8763294 .814057$

H $0.945618-3.1072880 .288893$

H $-0.009226-4.515404-0.063891$
H $-0.943009-1.623755-0.425129$

H $-0.324461-2.677989-1.698875$

H $-2.236780-4.282805-1.197477$

H -2.340949 -2.660392 -3.122147

H -2.931131 -1.413988 -2.044614

H $-4.475355-2.012603-4.175632$

H $-5.305402-1.775639-0.908797$

H -3.957112 -4.298781-4.090239

H $-5.502244-4.602028-3.330078$

H $-4.005394-5.113646-2.525011$

H $-6.909319-2.955001-1.983574$

H $-6.071635-3.7362551 .496937$

H -4.481565 -3.678706 2.214425

H $-4.265628-5.3276200 .768813$

H $-2.4468250 .401191-0.184558$

H -3.5653652 .9156891 .198221$

H -3.237186 $1.281932-2.347601$

H $-4.9096371 .688787-2.726443$

H $-4.5872650 .510396-1.480028$

H $-5.158788-1.6471762 .911257$

H $-4.443065-1.1379361 .378990$

H $-7.794444 \quad 1.8486642 .165638$

H -8.6813500 .4464331 .525583$

H $-7.5682370 .911621-0.796904$

H -8.1007213 .6096800 .552183$

H $-8.9168203 .826901-1.878693$

H -7.674205 $2.712925-2.400306$

H -6.654917 $6.735260-1.850750$

H $-8.3770216 .147762-1.503513$

H $-4.9567865 .207470-2.453980$

H $-5.4698293 .646404-3.014580$

H $-4.6323314 .436805-0.136392$

H $-5.7476650 .723574 \quad 1.913291$

C 2.9224533 .8239981 .568530

O 3.9008352 .9322281 .007986

C $4.1939373 .386985-0.333956$

C $3.2651924 .573022-0.612284$

C 2.1330744 .3365340 .370003

H $3.9236502 .569366-1.009835$

C $5.6861183 .725525-0.472915$

H $3.7569575 .532048-0.412058$

H $2.9289234 .591959-1.653885$

C $1.298685 \quad 5.572563 \quad 0.650777$

H $1.4919003 .548875-0.043809$

C $6.6385882 .550827-0.170302$

$\begin{array}{lllll}\text { O } & 5.994037 & 4.784354 & 0.444301\end{array}$

H $5.8808914 .121192-1.477038$

H $\quad \begin{array}{llll}6.550146 & 2.267594 & 0.885599\end{array}$

O $7.972695 \quad 3.040677-0.355415$

C $6.4198181 .324891-1.074934$

C $7.4182210 .182886-0.808619$

H $5.4059880 .950845-0.917075$

O $6.5104551 .714691-2.444876$

H $8.4343590 .512774-1.055496$

H $7.404934-0.0531740 .260961$

H $0.4989785 .349011 \quad 1.363815$

H $1.907126 \quad 6.3815851 .068542$

H $0.8356595 .941310-0.270330$

C 2.1448143 .1708562 .714565

H 6.9389654 .9902990 .341110

H 8.5787172 .4149330 .075821

H $7.3960862 .091647-2.584557$

H 1.5852323 .9573363 .236470

H 2.8687292 .7642103 .432264

H 3.4931914 .6541642 .009714

C $5.345527-1.978844-0.275798$

O $5.738287-1.470614-1.555779$

C $7.111741-1.081151-1.645148$

C $8.015596-2.284257-1.339125$

C $7.651967-2.956231-0.015522$

C $6.150160-3.2383540 .057071$

H $5.516215-1.2189900 .496367$

C $3.841062-2.261549-0.343654$

H $7.281971-0.809315-2.694631$

O $9.385599-1.898710-1.306254$

H $7.909836-3.027238-2.140486$

H $7.977150-2.3542070 .841167$

O $8.345687-4.2021080 .084550$ 
H $5.893233-4.049158-0.636379$

O $5.817990-3.674907 \quad 1.372797$

C $3.061628-0.965295-0.391449$

H $3.610453-2.854996-1.236622$

H $3.487602-2.8161540 .531974$

H $9.596526-1.497454-2.166638$

H $9.295795-4.0108780 .001655$

H $6.365955-4.4547701 .566441$

O $2.191703-0.975867-1.440421$

O $3.200014-0.0552720 .415931$

C $1.3894810 .200950-1.584418$

H $2.0216851 .056127-1.840131$

H $0.6800720 .031079-2.398647$

H $\quad 0.8269850 .397494-0.667038$

SCF Energy (B3LYP/6-31G**//MMFF) $=-3245.90790652$

03285

MM̄FF Geometry

C $0.542830-4.317912-0.924965$

C $1.535939-4.473671-1.814937$

C $2.879292-3.781154-1.734291$

O $2.754246-2.523546-1.029801$

C $3.892131-4.661599-0.982604$

C $5.281719-4.057929-0.909727$

C $5.891782-3.6778310 .230411$

C $6.000215-3.910007-2.226364$

C $5.369581-3.7745861 .636453$

C $4.605301-2.5364422 .129809$

C $5.456148-1.2582262 .208104$

C $4.690557-0.1465802 .929591$

C 5.4902631 .1647372 .967573

O $5.762260-0.8298540 .879741$

O 4.5926572 .2186943 .352114

C 5.9871621 .4991961 .542537

C 6.5865111 .1224504 .038912

O 6.8890292 .6017991 .562251

C $6.637726 \quad 0.3051890 .808774$

C $6.9076050 .577268-0.689182$

O $7.926093-0.0056981 .355018$

C $2.223716-1.478572-1.722604$

O $1.928084-1.453277-2.906797$

C $2.002075-0.375991-0.763299$

C $1.1504640 .607544-1.087586$

C $0.7910541 .751085-0.248135$

C 1.5504261 .9379821 .038315

C $5.6764780 .926579-1.531946$

C $5.4455992 .419702-1.701930$

C $3.8090784 .031353-2.671171$

O $6.2545393 .272785-1.347176$

C $2.9181314 .558230-1.590649$

C $1.7089995 .088817-1.829198$

C $0.8196755 .666495-0.765246$

C $-0.5622205 .051908-0.763960$

C $-1.5910795 .657499-1.380594$

C $-0.766125 \quad 3.777164 \quad 0.022415$

C $-0.1903752 .573961-0.672414$

N $4.2387012 .686634-2.335105$

H $0.711023-3.705763-0.042115$

H $1.375153-5.104742-2.685637$

H $3.232637-3.585945-2.754208$

H $3.967843-5.642593-1.469967$

H $3.506374-4.8545730 .024190$

H $6.892953-3.2541010 .157356$

H $7.044078-3.603888-2.097741$

H $6.004323-4.862572-2.766186$

H $5.512603-3.154947-2.850378$

H $4.728700-4.655878 \quad 1.748239$

H $6.222232-3.959698 \quad 2.302369$

H $3.741346-2.3669851 .476892$

H $4.213650-2.7665303 .128567$

H $6.384866-1.4932992 .740905$

H $4.417057-0.4568543 .945847$

H 3.7417720 .0398312 .408705

H $4.203782 \quad 1.983096 \quad 4.212006$

H 5.1075101 .8236490 .974175

H 6.1442630 .9911825 .033977

H 7.1379172 .0681664 .080055

H 7.3001200 .3093323 .889633
H 6.4319283 .3424501 .996354

H $7.697017 \quad 1.330872-0.799178$

H $7.331329-0.346040-1.110775$

H 8.1634400 .6790072 .000703

H $2.517459-0.4211480 .188592$

H $0.6473470 .561660-2.052872$

H $2.6289351 .954413 \quad 0.847400$

H $1.328906 \quad 1.1238651 .736917$

H 1.3211872 .8750331 .549386

H $5.8243320 .525361-2.542831$

H $4.7755850 .460804-1.123595$

H $3.2990473 .976007-3.638101$

H $4.6847364 .680000-2.775327$

H $3.2958194 .528242-0.571389$

H $1.3468355 .142241-2.853760$

H $0.7461046 .747375-0.943824$

H 1.2664585 .5625410 .231352

H -2.590336 $5.235214-1.360934$

H $-1.4552756 .586937-1.923867$

H $-1.839297 \quad 3.587703 \quad 0.159851$

H -0.3762423 .9162791 .034208$

H $-0.6750422 .350795-1.623803$

H $3.6088001 .919409-2.551077$

C $-1.947198-3.864037-0.951966$

$\begin{array}{lllll}\text { O } & -1.968023 & -3.318137 & 0.382979\end{array}$

C $-2.145504-1.8877960 .296528$

C $-2.619712-1.615500-1.123526$

C $-1.861588-2.670084-1.904643$

H $-1.151936-1.4523630 .461718$

C $-3.116588-1.4376151 .393151$

H $-3.701705-1.774621-1.211959$

H $-2.393819-0.597225-1.453232$

C $-2.434961-2.941086-3.283929$

H $-0.826247-2.327389-2.015478$

C $-3.473454 \quad 0.056114 \quad 1.322304$

O $-2.510106-1.6990882 .662854$

H $-4.022493-2.0517791 .347984$

H -3.9490260 .2875950 .364431$

$\begin{array}{lllll}\text { O } & -2.263243 & 0.823706 & 1.345583\end{array}$

C $-4.367990 \quad 0.5610122 .469283$

C $-5.676759-0.2211892 .685810$

H $-3.8044910 .557494 \quad 3.410254$

$\begin{array}{llll}\text { O } & -4.695437 & 1.932041 & 2.219214\end{array}$

H $-5.440509-1.2140723 .086113$

H -6.2484230 .3138403 .455581$

H $-1.860205-3.718665-3.797150$

H -3.477332 -3.272096 -3.227911

H $-2.403134-2.035177-3.898209$

C $-0.830429-4.909661-1.071587$

H -2.281359 -2.644241 2.685367

H $-1.787616 \quad 0.5987502 .163584$

H -3.859891 2.4102132 .080774

H $-0.924860-5.433172-2.029947$

H $-0.954179-5.654196-0.275909$

H -2.909405 -4.377302 -1.078850

C $-7.749704 \quad 0.969120-0.199294$

O

C $-6.564802-0.375252 \quad 1.433783$

C $-7.793497-1.2573691 .708503$

C $-8.714567-1.3138750 .484986$

C $-9.0006870 .082158-0.081451$

H $-8.0870072 .010579-0.280140$

C $-6.8920870 .656285-1.435447$

H $-5.969286-0.8418990 .644261$

O $-8.513136-0.737798 \quad 2.824714$

H $-7.453398-2.2661781 .972333$

H $-9.659605-1.7932730 .768309$

O $-8.096289-2.125600-0.509156$

H $-9.4840350 .001642-1.061476$

$\begin{array}{lllll}\text { O } & -9.937026 & 0.740553 & 0.777688\end{array}$

C $-5.7696841 .657241-1.616043$

H $-6.440988-0.337008-1.364986$

H -7.511109 $0.692439-2.338809$

H $-9.260941-1.3353362 .995314$

H $-8.703638-2.175119-1.266812$

H $-9.5009440 .888786 \quad 1.634429$

O $-4.8501631 .142988-2.485829$

O $-5.7106962 .755269-1.079796$ 
C $-3.713781 \quad 1.975021-2.737909$

H $-3.0093221 .411918-3.355759$

H $-4.0203892 .873994-3.280547$

H -3.220245 2.240371-1.798426

SCF Energy $\left(B 3 L Y P / 6-31 G^{* *} / / M M F F\right)=-3245.90655319$

03286

MM̄FF Geometry

C $1.541158-3.782313-2.155406$

C $0.379779-4.329839-2.545076$

C $-0.980247-3.671386-2.458761$

O $-0.966030-2.541623-1.556349$

C $-1.397294-3.165391-3.851747$

C $-2.805345-2.604895-3.903739$

C $-3.110779-1.324481-4.192315$

C $-3.912400-3.592483-3.636048$

C $-2.174179-0.196415-4.522399$

C $-1.7386050 .652670-3.318555$

C $-2.886478 \quad 1.389547-2.611067$

C $-2.3374902 .361496-1.564434$

C $-3.4649613 .063899-0.791652$

O $\quad-3.7176720 .426001-1.963307$

O $-2.886004 \quad 3.6831090 .368427$

C -4.470553 $2.006432-0.278984$

C $-4.0868694 .196856-1.617038$

O $\begin{array}{lllll}-5.627818 & 2.626986 & 0.273409\end{array}$

C $-4.892066 \quad 0.973117-1.347704$

C $-5.695660-0.216728-0.775799$

O $\quad-5.7619301 .557068-2.325954$

C $-1.055151-2.808768-0.224293$

O $-1.174575-3.904088 \quad 0.300947$

C $-0.942813-1.5227380 .497474$

C $-0.683349-1.5283551 .813325$

C $-0.517789-0.3492252 .665454$

C -0.8568590 .9915002 .069954$

C $-4.980034-1.0444010 .297088$

C $-5.329606-0.6401111 .719918$

C $-4.722483-1.1406964 .083263$

O $-6.217214 \quad 0.1623541 .995936$

C $-3.793450-0.0745074 .570969$

C $-2.962496-0.243703 \quad 5.610574$

C $-2.052416 \quad 0.827407 \quad 6.138759$

C $-0.609508 \quad 0.380875 \quad 6.207823$

C $-0.076006-0.0582757 .360235$

C 0.2344690 .5247164 .961809

C $-0.072399-0.5236183 .927558$

N -4.552398 -1.3134632 .653060$

H $1.551066-2.767967-1.766194$

H $\quad 0.387510-5.336881-2.956840$

H -1.700300 -4.414375 -2.095110

H -1.331693 -3.983522 -4.581556

H -0.672356 -2.411474 -4.177284

H -4.163355 -1.042786 -4.205601

H -3.900166 -3.912700 -2.589928

H -4.903199 -3.170621 -3.837218

H $-3.798318-4.474226-4.275047$

H -1.281085 -0.571872 -5.033375

H -2.669885 $0.450439-5.257493$

H -1.211979 $0.010502-2.602749$

H -1.017412 $1.394244-3.681627$

H $-3.4691641 .927646-3.367757$

H -1.674451 3.101615 -2.029205

H $-1.713924 \quad 1.810707-0.847454$

H -2.186276 4.2881930 .068085

H $-3.975810 \quad 1.4781240 .544420$

H $-3.3343114 .961241-1.845823$

H -4.873525 4.711852 -1.055030

H -4.503741 $3.855462-2.566959$

H -5.3254683 .2400440 .965286$

H $-6.6803470 .123607-0.432648$

H -5.903809 $-0.895664-1.615739$

H -5.990292 $2.456303-2.041519$

H -1.052400 -0.610100 -0.076347

H -0.553687 -2.487155 2.314007

H $-0.146722 \quad 1.252016 \quad 1.277656$

H -0.8487121 .8073562 .795297$

H -1.866860 0.9758161 .646734

H $-5.293602-2.0911570 .192575$
H $-3.894591-1.0131330 .163336$

H $-4.525273-2.1094504 .553128$

H $-5.757340-0.8531964 .295401$

H -3.8233310 .8866934 .063173$

H -2.955012 -1.197712 6.133389

H $-2.406619 \quad 1.1011707 .141246$

H -2.1221661 .7446445 .540897$

H $0.965788-0.3540297 .425061$

H $-0.671429-0.1369358 .263964$

H 1.2984460 .4235095 .215605

H $\quad 0.1349841 .5446854 .581215$

H $\quad 0.146869-1.5383794 .262913$

H -3.800984 -1.917757 2.333281

C $3.611952-4.528633-0.891170$

O $3.879537-3.190300-0.427291$

C $3.567237-3.121350 \quad 0.977257$

C $3.521262-4.5654811 .458184$

C $2.895503-5.2472140 .253976$

H $2.558448-2.6987411 .057866$

C $4.567615-2.2115161 .697306$

H $4.525320-4.9650741 .642156$

H $2.932606-4.6818972 .372934$

C $3.073848-6.7548430 .240613$

H $1.822454-5.0183310 .248892$

C $4.693567-0.8054731 .069942$

O $5.865896-2.8171691 .663555$

H $4.300994-2.1302542 .757168$

H $5.175847-0.8863860 .090429$

O $5.574255-0.0270301 .888591$

C $3.359089-0.0515410 .948857$

C 3.4600061 .3814370 .407916

H $2.661065-0.6112380 .322263$

$\begin{array}{lllll}\text { O } & 2.750492 & 0.021220 & 2.242498\end{array}$

H $2.443418 \quad 1.7931890 .387468$

H 3.9754722 .0128761 .145357

H $2.622917-7.191122-0.656310$

H $4.132472-7.0346450 .257086$

H $2.593615-7.2064321 .114826$

C $2.871147-4.478708-2.231388$

H $6.097031-2.9648230 .730324$

H $6.408462-0.520797 \quad 1.967597$

H 3.3712750 .4787142 .835348

H $2.757100-5.495884-2.623818$

H $3.484316-3.920565-2.950114$

H $4.594420-4.987864-1.062830$

C $3.1557403 .737308-1.450656$

O $4.3495632 .978641-1.211912$

C $4.1430671 .578185-0.961362$

C $3.4651430 .958184-2.189846$

C $2.1583891 .668464-2.527678$

C $2.3831513 .176126-2.653412$

H $2.5209663 .717517-0.557288$

C $3.5893535 .188767-1.688487$

H 5.148417 1.149349 -0.885799

O $3.215916-0.429458-2.028920$

H $4.135519 \quad 1.057856-3.054094$

H $1.3755831 .444058-1.793248$

O $1.6820831 .183407-3.786093$

H $2.9253343 .376743-3.586662$

O $1.1052633 .802106-2.753670$

C $4.1690485 .793048-0.431103$

H $4.3625565 .241509-2.464597$

H $2.7607805 .825848-2.015348$

H $4.074665-0.871495-1.918772$

H $1.5608020 .222363-3.697598$

H $1.2433644 .717091-3.049545$

O $3.165904 \quad 6.127158 \quad 0.430463$

O $5.3686585 .939955-0.236121$

C 3.5880756 .6955321 .673925

H 4.1312637 .6291001 .498797

H 2.6967106 .9142522 .267824

H 4.2100525 .9829592 .224069

SCF Energy (B3LYP/6-31G**//MMFF) $=-3245.91614514$

03_287

MM̄MF Geometry

C $-0.469325-4.728718-0.036517$

C $0.783360-4.249286-0.039871$ 
C $1.564162-3.983320-1.299292$

O $1.891743-2.573958-1.321338$

C $2.885430-4.773052-1.303344$

C $3.685076-4.577538-2.578633$

C $4.894449-3.986202-2.642144$

C $3.050800-5.112824-3.837571$

C $5.727216-3.451401-1.510995$

C $5.427388-1.982098-1.211999$

C $6.509334-1.290546-0.368027$

C $6.727434-1.9605000 .990433$

C $7.733166-1.1900211 .860625$

O $6.0820100 .066768-0.182002$

O $7.620547-1.7259503 .188318$

C 7.3181970 .2960061 .904410

C $9.177713-1.4393681 .411391$

O $8.297174 \quad 1.0889202 .570808$

C 7.0300870 .8939060 .509121

C 6.4396022 .3226350 .582856

O $8.2560870 .988075-0.226583$

C $0.978741-1.717760-1.854293$

O $-0.105651-2.000324-2.337138$

C $1.537747-0.352468-1.733950$

C $0.8417870 .703038-2.182768$

C $1.2701042 .103773-2.120028$

C $2.6060632 .386638-1.484783$

C 5.1733572 .4522301 .431632

C 4.4868033 .7978551 .262390

C 2.4587455 .0307702 .055111

O 4.8985164 .6785660 .513156

C 1.2394694 .6897751 .257613

C 0.7073945 .4900510 .320906

C $-0.5325345 .135343-0.450371$

C $-0.3848565 .326801-1.945040$

C -1.191085 6.173571-2.607845

C $0.6842804 .544478-2.678953$

C $0.4566963 .055893-2.623809$

N 3.3505973 .8855292 .054612

H -0.948554-4.980152 -0.979566

H $1.254626-3.9786290 .902100$

H $0.971811-4.241284-2.185478$

H $2.681354-5.845249-1.184472$

H $3.477773-4.475819-0.430795$

H $5.363789-3.886073-3.620368$

H $3.738438-5.091089-4.690098$

H $2.744184-6.154316-3.695068$

H $2.171332-4.522412-4.111041$

H $5.597039-4.062185-0.611660$

H $6.781011-3.564157-1.795756$

H $5.311240-1.419351-2.148346$

H $4.459940-1.896599-0.701741$

H $7.439039-1.291635-0.949370$

H 7.059083 -2.999039 0.875022

H $5.768644-2.0095941 .525738$

H $8.287982-1.2930883 .746839$

H 6.4059720 .3464452 .511806

H $9.425916-2.503676 \quad 1.501806$

H $9.889556-0.9100892 .054417$

H $9.363224-1.1427570 .377159$

H 8.3829910 .7519453 .478266

H 7.1996613 .0256810 .948427

H $6.2090292 .648157-0.441406$

H $8.1444791 .652885-0.926421$

H $2.513472-0.253007-1.271592$

H $-0.1326050 .530216-2.637794$

H $3.4096721 .881617-2.032456$

H $2.6119272 .034294-0.448036$

H $2.8670673 .445363-1.456120$

H 4.4550841 .6702081 .161698

H 5.4219212 .3421722 .493178

H 2.9791545 .9100241 .662663

H 2.1789635 .2195283 .096065

H $\quad 0.7568823 .7392241 .475111$

H $\quad 1.165387 \quad 6.4550680 .118450$

H -1.349099 $5.760772-0.066958$

H $-0.8288244 .099024-0.252805$

H -1.100211 $6.320994-3.678834$

H -1.963115 $6.738491-2.095714$

H $1.6627314 .843252-2.295933$
H $\quad 0.697248 \quad 4.826500-3.740244$

H $-0.4880972 .744157-3.071544$

H 3.0773163 .0802292 .610731

C -2.396603 -3.818023 1.232186

$\begin{array}{lllll}\text { O } & -3.395518 & -4.120957 & 0.238477\end{array}$

C $-4.674145-3.6325730 .709179$

C $-4.409170-2.9896492 .065731$

C $-3.174344-3.7316162 .539505$

H $-5.310641-4.5185970 .822327$

C $-5.244950-2.691272-0.359420$

H $-4.186540-1.9210161 .955781$

H -5.259165 -3.088763 2.747449

C $-2.441157-3.017343 \quad 3.661288$

H -3.467706 -4.7360192.872578

C $-6.453035-1.8616020 .103440$

O $-5.639055-3.484804-1.484160$

H $-4.446717-2.032037-0.715846$

H $-6.167907-1.2113100 .935833$

O $-7.451678-2.7440190 .631349$

C $-7.112305-1.006388-0.995106$

C $-6.161316-0.117176-1.817428$

H -7.662419-1.653344 -1.689376

O $-8.098379-0.167279-0.384105$

H $-5.555638-0.754125-2.472823$

H $-6.7867340 .513685-2.462773$

H $-1.556214-3.580576 \quad 3.972602$

H -2.113960 -2.018105 3.354810

H -3.092221 -2.903919 4.534497

C -1.299770 -4.881600 1.207952

H -4.860254 -3.995143 -1.765874

H $-7.691726-3.369844-0.073676$

H -8.694520 -0.7424290 .125570$

H $-1.736543-5.8871361 .208512$

H $-0.665512-4.7958702 .097168$

H -1.976700 -2.834357 0.978132

C -5.2981732 .5889170 .630334$

O $-6.0222221 .719408-0.251586$

C $-5.2265710 .795541-0.996872$

C $-4.2394781 .552498-1.900174$

C $-3.3816032 .523708-1.083155$

C $-4.2301903 .385079-0.141453$

H -6.0461733 .3030250 .998426$

C $-4.732511 \quad 1.8341391 .844138$

H $-4.6566630 .173588-0.302341$

O $-4.9610452 .275616-2.895263$

H $-3.6016580 .825019-2.416401$

H -2.826063 3.180611-1.762337

O $-2.4344401 .790608-0.312021$

H -3.5845113 .9135590 .569042$

O $-4.8917344 .392065-0.913527$

C -5.8205011 .1970672 .683081$

H -4.0493101 .0384391 .535265$

H -4.1855592 .5291872 .491076$

H $-4.3098902 .716342-3.467382$

H -1.882852 $1.281850-0.930154$

H $-5.5237683 .946531-1.503592$

$\begin{array}{lllll}\text { O } & -5.246962 & 0.314586 & 3.554476\end{array}$

O -7.0152351 .4448432 .598288$

C $-6.157029-0.3908404 .403962$

H $-6.869321-0.9650053 .803573$

H $-5.578276-1.0853465 .018788$

H -6.6804040 .3098825 .061156$

SCF Energy (B3LYP/6-31G**//MMFF)= -3245.90892441

03_288

MM̄FF Geometry

C -3.628174 -3.790665 -0.472538

C $-2.734153-3.016828-1.107678$

C $-1.265874-3.317415-1.341101$

O $-0.500588-2.170453-0.888556$

C $-0.724885-4.556530-0.598860$

C $0.748544-4.833825-0.823297$

C $1.681114-4.8310210 .150052$

C $1.144214-5.162720-2.239531$

C $1.490574-4.5542851 .616863$

C $1.712761-3.0865422 .014889$

C $3.174495-2.6228801 .905571$

C $3.334061-1.1947252 .429583$ 
C $4.777056-0.6879342 .270048$

O $3.563434-2.6607130 .533639$

O $4.768764 \quad 0.7356662 .463442$

C $5.252958-0.9387620 .819383$

C $5.694606-1.2632403 .355750$

O $6.644512-0.6660090 .681715$

C $4.944490-2.3589770 .296414$

C $5.180771-2.523574-1.221411$

O $5.798338-3.3371820 .902907$

C $-0.288855-1.162997-1.779405$

O $-0.686876-1.103629-2.931970$

C $0.504392-0.115893-1.098345$

C $0.5684861 .112619-1.633890$

C $1.2648722 .268640-1.064158$

C 2.2008972 .0197490 .087638

C $4.341368-1.611557-2.123133$

C $5.074529-0.364125-2.587105$

C $4.7199691 .700004-3.935158$

O $6.262856-0.153508-2.361249$

C $4.5473862 .839411-2.982327$

C $3.9068983 .973989-3.301487$

C $3.7701955 .145292-2.373176$

C $2.3312015 .556673-2.165375$

C $1.8034586 .593334-2.838145$

C $1.5340684 .822975-1.112100$

C $1.0237863 .489392-1.587229$

N $4.2454050 .475960-3.318747$

H -3.292358 -4.739317 -0.060033

H -3.062714 -2.064240 -1.511598

H -1.120995 -3.456213 -2.419699

H -1.279678 $-5.449975-0.913352$

H $-0.923035-4.4299030 .468749$

H $2.708328-5.062450-0.129348$

H $1.024616-4.288241-2.886429$

H $2.188231-5.485195-2.315159$

H $0.522284-5.975265-2.629121$

H $0.491483-4.8624631 .941899$

H $2.181518-5.1944462 .179960$

H $1.073493-2.4493681 .392620$

H $1.379620-2.9633693 .052904$

H $3.797620-3.3166782 .481968$

H $3.015635-1.1243173 .477092$

H $2.668057-0.525347 \quad 1.869053$

H $4.3910250 .917393 \quad 3.341075$

H $4.730444-0.2117190 .187587$

H $5.353609-0.9504784 .350170$

H $6.715747-0.8797153 .256449$

H $5.730405-2.3547463 .351813$

H $\quad \begin{array}{llll}6.792431 & 0.246323 & 0.984569\end{array}$

H $6.251105-2.445106-1.447682$

H $4.910247-3.558815-1.476549$

H $6.457798-2.8796321 .448462$

H $0.984091-0.373936-0.161599$

H $0.0260031 .306072-2.558714$

H 1.6421601 .7446340 .988155

H 2.8233352 .8818130 .336835

H $2.8960761 .209863-0.155628$

H $4.083544-2.169635-3.032540$

H $3.402438-1.323427-1.639194$

H $4.1503561 .840964-4.859182$

H $5.7789291 .590882-4.191094$

H $4.9945012 .738992-1.996202$

H $3.4835954 .082835-4.297617$

H $4.3395815 .979376-2.803860$

H $4.2355254 .943326-1.400280$

H $0.7800256 .912860-2.672327$

H $2.3818647 .141804-3.574252$

H $0.6551135 .418671-0.829739$

H $2.1266484 .754051-0.195791$

H $\quad 0.3252113 .564788-2.421979$

H $3.257708 \quad 0.252927-3.402216$

C $-5.828495-2.380827-0.901384$

O $-5.318120-1.105145-0.481210$

C $-5.444410-0.183403-1.582022$

C $-6.205656-0.917874-2.687305$

C $-5.835751-2.365607-2.427270$

H -4.426992 $0.020320-1.935280$

C $-6.108970 \quad 1.121849-1.125798$
H -7.289496 -0.781855 -2.596616

H $-5.914014-0.573771-3.684645$

C $-6.797102-3.362135-3.052088$

H $-4.835557-2.542458-2.835297$

C $-5.430848 \quad 1.8166140 .072593$

O $-7.460500 \quad 0.830258-0.745406$

H -6.169272 $1.809544-1.978352$

H -5.6353291 .2665890 .999571$

$\begin{array}{llll}\text { O } & -6.041155 & 3.105444 & 0.225474\end{array}$

C $-3.9114832 .018352-0.067623$

C -3.3307062 .9501271 .016508$

H $-3.4161211 .043262-0.020880$

O $-3.6160652 .576186-1.349983$

H -3.6742723 .9733410 .824494$

H -3.7195462 .6508371 .994406$

H $-6.491107-4.389506-2.830670$

H -7.817529 -3.225768 -2.678461

H $-6.819627-3.243937-4.140457$

C $-5.090481-3.533219-0.214213$

H $-7.9331931 .675794-0.667785$

H -6.9525152 .9643310 .532546$

H -4.093158 $3.420781-1.420631$

H $-5.623200-4.461310-0.458311$

H $-5.188315-3.3961460 .870896$

H $-6.867069-2.409872-0.542244$

C $-1.409951 \quad 1.3197702 .780170$

O $-1.266417 \quad 1.6572381 .393752$

C -1.7871472 .9355551 .014736$

C -1.124200 4.0422781 .843024

C $-1.2410963 .778653 \quad 3.338371$

C $-0.7392312 .374782 \quad 3.672212$

H -2.4743571 .2505393 .034688$

C $-0.781382-0.0700602 .970585$

H -1.477756 $3.097820-0.023459$

O $-1.6858175 .315127 \quad 1.543125$

H $-0.0579964 .096229 \quad 1.588027$

H -2.268383 3.9253953 .692883

O -0.4316564 .7230784 .043696$

H $\quad 0.3520612 .3460323 .556371$

O -1.0407242 .1253145 .044509$

C $-1.750054-1.1652722 .576903$

H $0.121910-0.1892742 .363593$

H -0.515076 -0.228965 4.020233

H -1.581030 5.4623010 .587453

H -0.7396905 .6109873 .793328$

H -0.4879551 .3844945 .343257$

O $-1.609440-2.2221463 .429075$

O $-2.525242-1.1013751 .632729$

C $-2.508617-3.3156933 .223521$

H $-3.540624-2.963023 \quad 3.133641$

H $-2.212597-3.8749542 .333910$

H -2.441836 -3.977099 4.091374

SCF Energy $\left(B 3 L Y P / 6-31 G^{* *} / / M M F F\right)=-3245.89218666$

03_289

MM̄FF Geometry

C $\quad 0.042023 \quad 4.4210840 .513021$

C $-0.8230714 .712519-0.470667$

C -2.049585 $3.912629-0.853052$

O $-2.0539452 .608964-0.228395$

C $-3.3091274 .668806-0.390486$

C $-4.6105124 .037074-0.843547$

C $-5.5486283 .537402-0.015120$

C $-4.8443714 .017538-2.332568$

C $-5.529523 \quad 3.4849541 .487144$

C -4.9676112 .1804652 .074190$

C -5.7911390 .9288081 .728692$

C $-5.295382-0.2828732 .520110$

C $-6.040238-1.5681782 .128840$

$\begin{array}{llll}\text { O } & -5.652521 & 0.666465 & 0.331040\end{array}$

O $-5.299039-2.6654472 .684983$

C $-6.029751-1.7187210 .590223$

C $-7.433616-1.6247702 .767221$

O $-6.856914-2.8161380 .211748$

C $-6.442654-0.429085-0.151918$

C $-6.227303-0.491984-1.683676$

O $-7.838915-0.1913610 .064554$

C $-1.3682681 .612508-0.853596$ 
O $-0.751888 \quad 1.689795-1.904411$

C -1.470193 $0.400712-0.009003$

C -0.660423 $-0.641059-0.250539$

C $-0.605089-1.8911640 .511302$

C $-1.701474-2.142827 \quad 1.511269$

C $-4.788706-0.767006-2.135305$

C $-4.509540-2.228662-2.442725$

C $-2.652218-3.750837-3.104481$

O $-5.381576-3.093036-2.462581$

C $-2.169034-4.468524-1.884163$

C $-0.946383-5.011864-1.787925$

C $-0.458855-5.771725-0.588413$

C $0.823507-5.203566-0.024784$

C $2.014580-5.748127-0.326221$

C $0.715476-4.0545420 .952212$

C $0.414305-2.7443610 .277676$

N -3.168530 -2.448442 -2.727408

H -0.116199 3.5415151 .131278

H $-0.6513675 .608771-1.064032$

H -2.058248 $3.797116-1.943517$

H -3.286349 $5.699264-0.770406$

H -3.2812704 .7499660 .701448$

H $-6.4500953 .118021-0.460884$

H $-5.8578793 .691077-2.589716$

H -4.709092 $5.019896-2.752094$

H -4.146195 $3.333640-2.824612$

H -4.9673814 .3304001 .897584$

H $-6.557267 \quad 3.627647 \quad 1.844758$

H -3.935434 2.0517201 .727910

H -4.9307162 .2945653 .164717$

H -6.8426901 .1338401 .961234$

H -5.379028 -0.1052873 .599607$

H $-4.224312-0.4285892 .325028$

H $-5.723359-3.4883652 .386489$

H $-5.003861-1.9873880 .311904$

H $-7.352445-1.5965813 .860650$

H -7.941545 -2.566185 2.530401

H -8.079331 -0.799024 2.461180

H $-6.731931-2.970862-0.739276$

H -6.937899-1.191569-2.139688

H $-6.4992100 .494590-2.087084$

H $-8.1643150 .395981-0.637693$

H -2.185896 0.4104900 .804680

H $0.052104-0.564186-1.070222$

H -2.681716 -2.046117 1.032483

H $-1.641226-1.4272082 .338276$

H $-1.676304-3.1432741 .947228$

H -4.609622 -0.219591-3.069880

H $-4.065037-0.403556-1.399302$

H -1.851790 -3.584497-3.832352

H -3.445582 -4.332919-3.584419

H -2.863776 -4.568088 -1.053586

H $-0.262739-4.932617-2.630467$

H $-0.303103-6.814407-0.894907$

H $-1.219827-5.8074250 .201066$

H 2.936648 -5.361278 0.094667

H $2.094266-6.589420-1.006737$

H $1.667062-3.9293451 .484074$

H $-0.009841-4.3170741 .726435$

H $1.158595-2.465775-0.468912$

H $-2.506358-1.684119-2.630497$

C 2.5353324 .4659800 .669208

O $2.7858534 .263448-0.739322$

C $4.1798104 .486814-0.993846$

C 4.8645924 .5451840 .365382

$\begin{array}{llll}\text { C } 3.775816 & 5.173742 & 1.213828\end{array}$

H $4.2479185 .488486-1.440608$

C $4.7675473 .486352-1.999378$

H $5.105023 \quad 3.5452980 .741473$

H 5.7871465 .1330560 .346501

C 3.9895384 .9860812 .705963

H 3.7263356 .2474250 .987954

C $4.7965531 .999442-1.580341$

O $6.122245 \quad 3.877880-2.261514$

H $4.2266853 .570452-2.950064$

H $5.4611861 .878236-0.719616$

O $5.4152331 .260876-2.645839$

C $3.4258171 .366216-1.291021$
C $3.474467-0.137953-0.992384$

H $2.9480951 .869043-0.450420$

O $2.5809231 .565075-2.427467$

H $2.448208-0.505819-0.876883$

H $3.837745-0.685659-1.872958$

H $3.1742815 .440183 \quad 3.277870$

H 4.0389553 .9251212 .973964

H 4.9263855 .4562503 .021947

C $1.243248 \quad 5.261186 \quad 0.851407$

H $6.1024584 .783135-2.616032$

H $4.8309711 .321499-3.421276$

H $1.6950851 .236831-2.196172$

H 1.2674286 .1610530 .224808

H 1.1416815 .5868251 .892762

H 2.4382293 .4727281 .126816

C $4.852205-2.5966511 .432225$

O $4.111922-1.967174 \quad 0.378618$

C $4.302769-0.5529590 .231476$

C 3.9042190 .1731591 .525595

C $4.638396-0.4008782 .741653$

C $4.562238-1.9286662 .788425$

H $4.461942-3.6215351 .474808$

C $6.350812-2.6825851 .103696$

H $5.357312-0.3632740 .008119$

$\begin{array}{lllll}\text { O } & 2.495523 & 0.081294 & 1.730623\end{array}$

H 4.1442121 .2375921 .433886

H $4.1972490 .007708 \quad 3.659300$

O $6.007358-0.0085302 .703041$

H $5.249428-2.3159613 .549913$

O $3.239165-2.2858183 .198607$

C $6.625496-3.574403-0.090054$

H $6.774256-1.7003830 .879185$

H $6.885740-3.1113041 .958966$

H $2.261583-0.8618651 .769097$

H 6.0349360 .9634582 .709341

H $3.216952-3.2516613 .309130$

O $7.971027-3.589201-0.328063$

O $5.779083-4.181597-0.731240$

C $8.390837-4.392367-1.435642$

H $8.121424-5.439542-1.267971$

H $7.944245-4.021328-2.363090$

H $9.478566-4.318567-1.516178$

SCF Energy (B3LYP/6-31G**//MMFF)= -3245.91343381

03_29

MMFF Geometry

C -2.972955 $1.954040-2.919795$

C $-2.2906051 .094078-2.148593$

C $-1.1093710 .291388-2.625886$

O $-0.0809690 .334813-1.609443$

C $-1.528474-1.178336-2.811975$

C $-0.442232-2.055169-3.404695$

C $0.108241-3.116814-2.783691$

C $-0.000022-1.699010-4.801284$

C $-0.212925-3.661816-1.420962$

C $0.638688-3.081406-0.282565$

C $2.144356-3.364213-0.411330$

C $2.877869-2.9732240 .871997$

C $4.393711-3.1881590 .749459$

O $2.660105-2.588786-1.494006$

O $5.022964-2.5078761 .848133$

C $4.902971-2.513329-0.545979$

C $4.762885-4.6695580 .894698$

O $6.266471-2.862314-0.770195$

C $4.043320-2.824149-1.794913$

C $4.380810-1.944514-3.021544$

O $4.248974-4.167787-2.248916$

C $0.6452251 .479071-1.496998$

O $0.5505742 .484051-2.182878$

C $1.5700581 .303835-0.356590$

$\begin{array}{llll}\text { C } 2.160388 & 2.378276 & 0.186396\end{array}$

C $3.0814552 .371246 \quad 1.324324$

C 3.5683601 .0346301 .818710

C $4.226258-0.433906-2.814829$

C $5.5141240 .270642-2.421378$

C $6.3675132 .506339-1.724280$

O $6.612544-0.278361-2.428742$

C $6.5065532 .536877-0.234968$ 
C 6.5282203 .6730610 .478316

C 6.7123353 .7248311 .967989

C 5.6280404 .5183972 .662406

C 5.8224235 .8039353 .002119

C 4.3542203 .7962453 .038462

C 3.4572883 .5527521 .857188

N $5.294517 \quad 1.601715-2.092399$

H -2.684108 $2.091298-3.958374$

H -2.598683 $0.945848-1.116757$

H $-0.7110850 .700369-3.562174$

H -2.402839-1.234880 -3.474208

H $-1.855220-1.572402-1.843208$

H $0.879834-3.674771-3.313366$

H $0.654051-2.462214-5.236977$

H $-0.867792-1.599446-5.461487$

H $0.553025-0.754981-4.803093$

H $-1.272421-3.512900-1.185566$

H $-0.079443-4.750852-1.451106$

H $0.469348-1.999480-0.233420$

H $0.271486-3.5115610 .657403$

H $2.280947-4.430739-0.624682$

H $2.480856-3.5242061 .733767$

H $2.696764-1.9113781 .084809$

H $4.654082-2.8652332 .674071$

H $4.881459-1.434086-0.353979$

H $4.462551-5.0438941 .881036$

H $5.847081-4.8159620 .838078$

H $4.285702-5.3055700 .146200$

H $6.599602-2.311051-1.497882$

H $\quad 5.374038-2.198669-3.410974$

H $3.680720-2.229058-3.820999$

H $4.936821-4.580483-1.702995$

H 1.7218260 .2985450 .018883

H $1.9329263 .360686-0.225558$

H $3.9672640 .440227 \quad 0.989577$

H $2.7511700 .478723 \quad 2.289619$

H $4.376898 \quad 1.1073132 .548936$

H $3.9122550 .014868-3.766002$

H $3.449089-0.214731-2.077015$

H $6.1193653 .489087-2.137533$

H $7.3045782 .164178-2.175060$

H 6.6220291 .5840620 .276583

H $6.4417414 .625561-0.040133$

H 7.6913314 .1807542 .166242

H 6.7626952 .7184812 .401731

H 5.0609936 .3724403 .525602

H $6.746508 \quad 6.3182012 .759141$

H 3.7811814 .3946093 .759620

H 4.6080152 .8804793 .579483

H 3.0549134 .4688301 .422242

H $4.3432521 .957380-2.063183$

C $-5.4523481 .970243-2.519188$

O $-5.4160110 .890620-1.563056$

C $-6.6147230 .922555-0.772287$

C $-7.6046001 .755180-1.573818$

C $-6.6834192 .806828-2.163190$

H -6.3720251 .4590470 .153166$

C $-7.065750-0.501163-0.440793$

H $-8.0736511 .171531-2.374305$

H -8.396891 2.177932 -0.949005

C -7.282730 $3.550301-3.344090$

H $-6.4310763 .531951-1.377417$

C $-5.973584-1.3673320 .223646$

O $-7.453087-1.151143-1.658221$

H $-7.963539-0.4643030 .187466$

H $-5.181921-1.586782-0.502595$

O $-6.570729-2.6317680 .543209$

C -5.349634 -0.762542 1.497974

C $-4.363108-1.7340342 .174017$

H $-4.8279090 .163377 \quad 1.238947$

O $-6.402887-0.434607 \quad 2.402102$

H -4.889299 -2.665670 2.413332

H -3.577023 - 1.9832171 .452535

H $-6.5734224 .281341-3.744578$

H -7.553924 $2.864947-4.154156$

H -8.187671 4.087265 -3.041504

C $-4.1446802 .756482-2.425872$

H $-7.730661-2.054544-1.428504$
H -7.236889 -2.473974 1.234347

H -6.0404370 .1731623 .068605$

H $-3.9639813 .057676-1.386834$

H $-4.2051593 .676438-3.018527$

H $-5.5625451 .515010-3.511723$

C -1.8635390 .0357692 .575631$

$\begin{array}{lllll}\text { O } & -3.093688 & 0.068763 & 3.309302\end{array}$

C $-3.740941-1.195466 \quad 3.482449$

C $-2.799402-2.188878 \quad 4.180419$

C $-1.470353-2.314502 \quad 3.442594$

C $-0.864417-0.9309063 .227504$

H $-2.061859-0.2635591 .538741$

C $-1.283791 \quad 1.4607492 .575152$

H $-4.571501-1.0185804 .176763$

O $-3.427688-3.461114 \quad 4.290735$

H -2.606089-1.835072 5.201720

H $-1.581096-2.8485872 .491729$

O $-0.556032-3.077568 \quad 4.232397$

H $-0.528262-0.5286184 .192154$

O $0.285331-1.0493142 .394198$

C $-2.007907 \quad 2.393968 \quad 1.629994$

H -1.3415381 .9020503 .576718$

H $-0.234547 \quad 1.4311192 .261227$

H $-2.862203-4.0228914 .846813$

H $-0.891076-3.9893554 .269405$

H $0.892977-1.6740442 .825973$

O

O $\begin{array}{llll}-3.156199 & 2.247153 & 1.236774\end{array}$

C $-1.716654 \quad 4.422148 \quad 0.433149$

H $-2.712425 \quad 4.741560 \quad 0.755495$

H $-1.051117 \quad 5.2893940 .449008$

H $-1.7477824 .023227-0.583975$

SCF Energy (B3LYP/6-31G**//MMFF) $=-3245.92160008$

$03 \_290$

MMFF Geometry

C -2.324994-2.138146 1.957785

C $-1.136418-2.7411121 .806773$

C $-0.242001-2.5420270 .608711$

O $1.000028-1.9524041 .053384$

C $0.075360-3.911078-0.022990$

C $0.892751-3.829026-1.297115$

C $2.141480-4.314162-1.442286$

C $0.206731-3.181506-2.472207$

C $2.991024-5.022014-0.424922$

C $3.946987-4.111136 \quad 0.361133$

C $4.969907-3.365808-0.511984$

C $6.041469-2.7023730 .356547$

C $7.026341-1.868991-0.479391$

O $4.278836-2.363034-1.261876$

O $7.773605-1.0367650 .421534$

C $6.229703-0.926320-1.408116$

C $8.050193-2.753776-1.201275$

O $7.097784-0.255330-2.317576$

C $5.102868-1.637729-2.184825$

C $4.179835-0.663309-2.957675$

O $5.684287-2.517716-3.154909$

C $1.015086-0.6047271 .244181$

$\begin{array}{lllll}\text { O } & 0.094205 & 0.176663 & 1.067336\end{array}$

C $2.360769-0.2390981 .737321$

C 2.5406290 .9403942 .349692

C 3.8006831 .4399812 .903633

C 5.0564540 .6783832 .570851

C $3.4543460 .379727-2.100405$

C $4.1701981 .717838-2.017906$

C $4.0560273 .910774-0.839958$

O $5.131206 \quad 2.013869-2.722582$

C $5.009933 \quad 3.9001780 .312546$

$\begin{array}{llll}\text { C } 4.902896 & 4.728606 & 1.362375\end{array}$

C 5.8727594 .7596212 .508590

C 5.1906444 .6213733 .851333

C 4.9027055 .7035714 .593942

C $4.922945 \quad 3.2262324 .368497$

$\begin{array}{llll}\text { C } 3.775751 & 2.555080 & 3.663647\end{array}$

N $3.5807402 .561980-1.085998$

H $-2.670979-1.441314 \quad 1.198791$

H $-0.784733-3.4242732 .576501$

H $-0.716664-1.888807-0.132527$ 
H $-0.860362-4.437248-0.255956$ H $\quad 0.590137-4.5282860 .721147$ H $2.621050-4.201196-2.414466$ H $\quad 0.780045-3.287967-3.399504$ H $-0.772004-3.641937-2.640878$ H $0.067967-2.111463-2.295353$ H $2.368757-5.5788330 .283428$ H $3.577259-5.787525-0.949580$ H $3.354697-3.3889710 .934811$ H $4.481004-4.7388241 .085483$ H $5.428921-4.086677-1.198663$ H $6.581398-3.4514630 .948789$ H $5.557453-2.0387091 .085465$ H $8.223280-1.6155441 .060725$ H $5.786956-0.152911-0.769441$ H $8.627201-3.340513-0.476325$ H $8.782989-2.147722-1.745379$ H $7.592967-3.455264-1.901909$ H $7.774600 \quad 0.201064-1.789011$ H $4.730906-0.196512-3.782874$ H $3.399600-1.271592-3.438305$ H $5.022913-2.698082-3.843287$ H $3.160049-0.9577541 .600722$ H 1.6801511 .5957412 .479658 H 5.1396030 .5324171 .488409 H $5.052781-0.3019133 .059183$ H 5.9717041 .1906042 .873765 H $2.4783830 .585841-2.558615$ H $3.268221-0.000379-1.091696$ H $3.1775384 .534068-0.645246$ H $4.5622524 .289169-1.733782$ H $5.8388483 .197488 \quad 0.270335$ H 4.0885295 .4493651 .387291 H 6.4125495 .7146492 .462202 H 6.6399533 .9819742 .407068 H 4.4398105 .6128075 .571148 H 5.1195516 .7056924 .239039 H 4.6660153 .2636805 .435768 H 5.8494132 .6468964 .326433 H 2.8144043 .0396033 .840650 H $2.8176232 .215453-0.511869$ C $-4.641657-2.8244142 .746031$ O $-5.334910-1.7633302 .060689$ C $-6.088551-2.3345920 .974077$ C $-6.103246-3.8408721 .216286$ C $-4.736917-4.0530611 .840800$ H -5.516549 -2.135431 0.060402 C -7.469548 -1.6782610 .866590$ H $-6.889851-4.1345291 .920725$ H $-6.247424-4.4084150 .292077$ C $-4.594184-5.3727302 .577089$ H -3.988716 -4.011240 1.039778 C $-7.436848-0.1363070 .816936$ O $-8.239893-2.0586602 .013729$ H -7.995296 -2.080675 -0.007836 H $-7.165486 \quad 0.263581 \quad 1.801974$ $\begin{array}{lllll}\text { O } & -8.765916 & 0.325852 & 0.544817\end{array}$ C $-6.4916220 .454837-0.246190$ C $-6.5935111 .991754-0.314399$ H $-5.4628230 .164396-0.009034$ O $-6.822614-0.103579-1.516433$ H -7.575748 $2.280623-0.707762$ H -6.5040262 .3817710 .706909$ H -3.597532 $-5.464323 \quad 3.020310$ H $-5.331453-5.4659503 .381446$ H $-4.738095-6.2135351 .890692$ C -3.231598 -2.368894 3.134534 H $-9.164889-1.8158911 .840423$ H $-9.3108950 .128648 \quad 1.324884$ H $-6.0976180 .103832-2.129423$ H -2.792373 -3.1062523 .817115$ H -3.302757 -1.421476 3.682937 H -5.206949 -2.998273 3.671375 C -3.092532 $2.800246-1.343908$ O $-4.2365492 .323515-0.624375$ C $-5.5088902 .635092-1.200422$ C $-5.6876964 .158186-1.310505$ C $-4.5265544 .796914-2.078941$
C $-3.1665004 .322062-1.552922$

H $-2.2417872 .593403-0.681296$

C $-2.8726892 .019353-2.643485$

H $-5.5721972 .194865-2.200483$

O $-5.7661854 .726526-0.004796$

H $-6.6367124 .366226-1.819267$

H -4.594552 $5.887949-1.989556$

O $-4.6584684 .467741-3.458594$

$\mathrm{H}-2.3603934 .635323-2.226666$

O $-2.9155094 .939650-0.287139$

C $-2.5449940 .575773-2.346012$

H $-3.7338502 .050901-3.316810$

H $-2.0274912 .431140-3.207546$

H -5.907936 $5.682717-0.108607$

H $-3.9280064 .898784-3.934392$

H $-2.9175465 .902251-0.424820$

O $-3.651304-0.200585-2.524714$

O $-1.4422600 .190816-1.980003$

C $-3.472046-1.597951-2.276016$

H $-3.144672-1.763733-1.246139$

H $-4.434630-2.095147-2.421264$

H $-2.751284-2.015399-2.984522$

SCF Energy (B3LYP/6-31G**//MMFF) $=-3245.89287266$

03 291

MM̄FF Geometry

C -2.951922 -3.469754 -2.411036

C $-2.174650-3.267245-1.336204$

C $-0.672329-3.416176-1.367054$

O $-0.055835-2.250349-0.775479$

C $-0.276620-4.644421-0.524731$

C $1.200862-4.978438-0.568538$

C $2.028000-4.9437940 .494664$

C $1.731931-5.413151-1.910979$

C $1.709616-4.5696411 .915528$

C $2.010694-3.1049842 .272924$

C $3.493960-2.7182332 .150994$

C $3.742763-1.3260162 .734976$

C $5.197289-0.8680082 .534910$

O $3.844037-2.7185420 .765744$

O $5.255648 \quad 0.5417392 .802473$

C $5.593429-1.0623481 .054259$

C $6.146373-1.5384313 .535608$

O $6.983123-0.8131520 .860451$

C $5.226735-2.4529280 .499129$

C $5.424292-2.573253-1.031390$

O $6.067368-3.439477 \quad 1.109844$

C $0.066153-1.141706-1.554575$

O $-0.348663-0.992850-2.692834$

C $0.812688-0.127181-0.776942$

C $0.8702131 .138314-1.217735$

C $1.5701892 .246979-0.564639$

C 2.5240531 .9088750 .549162

C $4.588648-1.607651-1.879746$

C $5.334413-0.352905-2.301673$

C $4.975061 \quad 1.800927-3.501650$

O $6.537008-0.186923-2.118743$

C $4.8372892 .885487-2.481078$

C $4.2038964 .043635-2.719532$

C $4.1004165 .160531-1.722118$

C $2.6715805 .580686-1.465358$

C $2.1502136 .665585-2.062602$

C $1.8779384 .794243-0.447829$

C $1.3403723 .502899-1.000127$

N $4.4978460 .549248-2.945694$

H $-2.464284-3.715270-3.353410$

H -2.618189-3.025949-0.375513

H $-0.310983-3.534151-2.395880$

H $-0.828303-5.527254-0.875693$

H $-0.602130-4.4804080 .507398$

H $3.068600-5.2257730 .336283$

H $2.760078-5.786153-1.850283$

H $1.116455-6.220761-2.320567$

H $1.729292-4.577221-2.616861$

H $0.662016-4.7824542 .151351$

H $2.289153-5.2271422 .576250$

H $1.407026-2.4542551 .629659$

H $1.678779-2.9381513 .305110$ 
H $4.092211-3.4679562 .681922$ H $3.474536-1.2959623 .798460$ H $3.080517-0.6028972 .240433$ H 4.9386980 .6879073 .710288 H $5.058042-0.2986690 .479182$ H $5.849116-1.2996234 .563889$ H $7.168685-1.1605343 .425192$ H $6.167701-2.6262403 .443617$ H 7.1677380 .0792791 .200208 H $\quad 6.490764-2.516944-1.280386$ H $5.114723-3.589237-1.317264$ H $6.081639-4.2275370 .541892$ H $1.291548-0.4477790 .140953$ H $\quad 0.336232 \quad 1.396692-2.131461$ H $3.216596 \quad 1.1234280 .230060$ H 1.9813211 .5597901 .432699 H 3.1493632 .7488440 .858845 H $4.307858-2.120342-2.808977$ H $3.661747-1.327756-1.368765$ H $4.3869362 .001583-4.402636$ H $6.0265821 .695266-3.787528$ H $5.3040692 .722525-1.512462$ H $3.7609734 .214949-3.698195$ H $4.6755906 .010002-2.113497$ H $4.5783254 .894990-0.770835$ H $1.1343646 .988966-1.860326$ H $2.7258807 .251138-2.771953$ H $1.0148565 .385544-0.113920$ H 2.4831224 .6577200 .452403 H $0.6404253 .641880-1.825246$ H $3.5019560 .355275-2.998916$ C $-5.223797-3.233903-1.173488$ O $-5.180552-1.836165-0.816718$ C $-6.515663-1.385544-0.523947$ C $-7.314561-2.656344-0.265754$ C $-6.707499-3.591492-1.289452$ H $-6.904395-0.902009-1.428893$ C $-6.545115-0.4047610 .653755$ H $-7.144687-3.0341200 .749932$ H -8.390106 -2.509065 -0.402015 C $-7.012956-5.056516-1.027974$ H -7.084548 -3.319229 -2.284645 C $-6.113661 \quad 1.0453160 .349980$ O $-5.770825-0.932677 \quad 1.728715$ H -7.586781 -0.3556130 .999356$ H -6.2723521 .6157221 .275030$ O $-7.0041261 .580093-0.629079$ C $-4.646214 \quad 1.253598-0.074717$ C -4.280947 $2.751732-0.120496$ H -3.9983690 .7252190 .632642$ O $-4.4345330 .701706-1.370570$ H -4.826313 $3.229593-0.943015$ H $-4.612737 \quad 3.2275130 .808724$ H -6.536394 -5.695172 -1.778119 H -6.655840 -5.371377 -0.041559 H $-8.092218-5.236677-1.067244$ C $-4.457165-3.454263-2.482125$ H $-5.849027-0.3192272 .478873$ H $-6.8836222 .543991-0.648738$ H -3.475197 $0.626666-1.507779$ H -4.741074 -2.677951 -3.203944 H -4.754918 -4.419291-2.910209 H -4.771032 -3.796014 -0.347652 C -2.046618 2.7348091 .949108 O $-1.969006 \quad 2.274994 \quad 0.594739$ C $-2.7735303 .010861-0.332546$ C $-2.3729894 .494692-0.319006$ C -2.3430735 .0828371 .091166$ C - 1.5534224 .1814172 .040281 H -3.082901 2.6749142 .303368 C -1.1969341 .7903742 .804428$ H -2.523202 $2.624179-1.328148$ O $-3.2257445 .267183-1.158128$ H -1.368698 $4.586976-0.745256$ H -3.355482 5.2565361 .474505 O $-1.7039286 .360686 \quad 1.052510$ H -0.4844024 .2351201 .799572$ O $-1.717925 \quad 4.652974 \quad 3.375644$
C $-1.905471 \quad 0.4678012 .983027$

H -0.2200491 .6192412 .340044$

H -1.0162642 .1897553 .808297$

H $-4.0836635 .365876-0.712013$

H -2.1883896 .9076590 .410432$

H -1.4210845 .5790813 .394519$

O $-1.225790-0.5241732 .341531$

O $-\begin{array}{llll}-2.950526 & 0.336879 & 3.608408\end{array}$

C -1.817482 -1.823232 2.434671

H $-2.789733-1.8303551 .933159$

H $-1.157680-2.5343871 .933725$

H -1.919925 -2.122888 3.482146

SCF Energy (B3LYP/6-31G**//MMFF) $=-3245.89562252$

03292

MM̄FF Geometry

C -2.0875264 .7480020 .392306$

C $-2.6103584 .661382-0.841165$

C $-3.5048773 .539756-1.324259$

O $-3.2967202 .344187-0.535061$

C $-4.9835413 .945938-1.211189$

C $-5.9399272 .902317-1.756596$

C $-6.8399922 .226887-1.014995$

C $-5.8567342 .650690-3.240516$

C -7.1092312 .3555170 .457782$

C -6.3019711 .3995321 .349037$

C -6.590896 -0.090161 1.100938

C $-5.960211-0.9504792 .198862$

C $-6.158359-2.4519861 .938746$

O $-6.038928-0.458344-0.166609$

O $-5.275768-3.1707382 .814587$

C $-5.720829-2.7751430 .493836$

C $-7.580210-2.9081942 .286918$

O $-6.043533-4.1210230 .154158$

C $-6.308638-1.812415-0.558093$

C $-5.708558-2.013860-1.972075$

O $-7.719120-2.041522-0.668152$

C $-2.1609601 .631667-0.775793$

O $-1.3313271 .836956-1.647386$

C -2.0686970 .5816210 .260530$

C -0.8796770 .0175200 .518022$

C $-0.614962-1.0093701 .527193$

C $-1.800402-1.6209712 .226704$

C $-4.189551-1.837329-2.076623$

C $-3.405218-3.135243-1.964984$

C $-1.067316-3.982405-1.785881$

O $-3.929664-4.244967-1.999749$

C $-0.724104-4.244361-0.353018$

C $0.537858-4.3123180 .097528$

C $0.911945-4.6111221 .520861$

C $1.822814-3.5609892 .117604$

C $3.155582-3.7308602 .137647$

C $1.181567-2.3573192 .769691$

C $0.664872-1.3631571 .766920$

N -2.039113 -2.907229 -1.862361

H -2.3652724 .0147821 .145754$

H -2.334456 $5.403079-1.587100$

H -3.251379 $3.320912-2.368786$

H $-5.1556974 .885564-1.752750$

H $-5.2066024 .158209-0.160167$

H -7.473559 $1.497951-1.519449$

H -6.663107 $2.000848-3.597405$

H $-5.9314503 .594324-3.790769$

H -4.909376 $2.167800-3.498595$

H -6.9372283 .3842660 .792432$

H -8.1804592 .1810130 .621989$

H -5.2331321 .5978911 .208783$

H -6.5403901 .6417842 .392262$

H -7.677803 -0.2322311 .083001$

H $-6.357966-0.6778973 .184427$

H $-4.881895-0.7461292 .245532$

H -5.491494 -2.920040 3.729225

H $-4.627433-2.7000160 .471538$

H -7.797016 -2.711846 3.343891

H -7.691725 -3.990046 2.155467

H -8.348704 -2.407089 1.694839

H $-5.633225-4.6953840 .823267$

H $-6.032260-2.977004-2.384775$ 
H $-6.159584-1.252558-2.625018$ H -8.020292 -1.702199-1.527316 H -2.9720820 .3263790 .801767$ H -0.010698 $0.352994-0.047082$ H -2.531080 -1.9853101 .496602$ H -2.287516 -0.8832222 .873332$ H -1.544749 -2.481194 2.848720 H -3.955972 -1.427227 -3.067685 H -3.826484 -1.122388 -1.333423 H $-0.195046-3.672379-2.370174$ H - $-1.485845-4.888068-2.236167$ H -1.547305 -4.4090880 .338100$ H $\quad 1.356028-4.178178-0.605757$ H $1.412921-5.5880681 .535152$ H $0.023172-4.7272582 .153320$ H $3.813654-2.9951352 .588064$ H $3.619344-4.6069471 .696488$ H $1.916069-1.8317113 .394772$ H $0.414254-2.6950693 .471914$ H $1.455034-0.864471 \quad 1.203875$ H -1.696621 -1.953055 -1.796752 C $0.2409905 .032773 \quad 1.171543$ O $0.7841684 .404023-0.006401$ C 2.2251384 .4825630 .044337 C 2.5668905 .0648691 .408825 C 1.3553825 .9364611 .686676 H $2.5127015 .182200-0.750436$ C $2.7921963 .087777-0.244474$ H 2.6480294 .2719852 .163025 H 3.5067465 .6249391 .401955 C 1.2133356 .3303483 .145994 H 1.4221066 .8425971 .069823 C $4.3145162 .971900-0.038912$ O $2.4632792 .765036-1.598932$ H 2.2869122 .3412270 .380809 H 4.5309443 .1415541 .022028

O $4.9820814 .003968-0.764824$ C $4.8968411 .600319-0.440705$ C $6.3663631 .458810-0.003842$ H 4.3011990 .8054390 .023244 O $4.8392201 .426372-1.854882$ H $6.9727292 .233376-0.489410$ H 6.4154001 .6189271 .080099 H 0.3208616 .9447913 .299816 H 1.1324315 .4505693 .793393 H 2.0824266 .9099153 .474002 C -1.055735 5.7585480 .808136 H $1.4962272 .827084-1.685506$ H $4.8061283 .853785-1.709616$ H $3.9040051 .457344-2.117568$ H $-0.8725786 .472207-0.004203$ H -1.438413 6.3191601 .667505 H $0.0379134 .231374 \quad 1.895491$ C $6.589927-2.2762260 .072538$ O $6.206025-0.918698 \quad 0.329624$ C $6.9668760 .082494-0.355045$ C $8.451144-0.0102000 .033186$ C $8.995893-1.422490-0.201543$ C $8.077443-2.4936710 .397203$ H $6.003414-2.8713190 .783982$ C $6.205742-2.723961-1.342608$ H $6.870213-0.066616-1.435327$ O $8.607418 \quad 0.337917 \quad 1.407599$ H $9.018050 \quad 0.717675-0.559745$ H $9.992711-1.5032840 .248786$ O $9.132738-1.627536-1.604550$ H $8.383933-3.4883930 .054933$ O $8.232424-2.491973 \quad 1.819685$ C $4.706228-2.839099-1.479004$ H $6.573603-2.060282-2.129977$ H $6.620982-3.715869-1.558631$ H 9.5578130 .3083601 .611162 H $9.503005-2.516949-1.736486$ H $7.898136-1.6420782 .154170$ O $4.177525-1.642725-1.861251$ O $4.081135-3.866971-1.252821$ C $2.754096-1.611019-2.002790$ H $2.470516-0.624831-2.379755$
H $2.422345-2.368871-2.718943$

H $2.279175-1.763744-1.030393$

SCF Energy (B3LYP/6-31G**//MMFF) $=-3245.92041959$

03_293

MMFF Geometry

C $2.0803043 .734793-2.111393$

C $1.1844004 .690704-1.821072$

C $-0.2145464 .485605-1.277747$

O $-0.5049473 .087954-1.052263$

C $-0.3380095 .206548 \quad 0.079168$

C -1.7391055 .1937830 .658503$

C $-2.0872354 .594317 \quad 1.814012$

C $-2.7790475 .945736-0.132664$

C -1.2152113 .8247002 .766312$

C -1.2203692 .3020702 .554501$

C -2.5986821 .6450892 .737416$

C -2.4748870 .1196582 .765330$

C $-3.847607-0.5676482 .851721$

O $-3.4326892 .033326 \quad 1.642477$

O $-3.667000-1.9579092 .541297$

C $-4.775596 \quad 0.016324 \quad 1.764212$

C $-4.422937-0.5112944 .272088$

O $-6.105873-0.4720161 .915202$

C -4.7828841 .5578581 .725975$

C -5.5509872 .1370170 .512277$

O -5.4303292 .0544822 .904090$

C $-0.9856232 .359632-2.095643$

O $-1.2243042 .752759-3.225743$

C $-1.1300300 .965015-1.624238$

C $-1.138024-0.039240-2.512882$

C $-1.245787-1.464565-2.193681$

C $-1.600852-1.832275-0.777613$

C $-5.0166971 .725796-0.863946$

C $-5.7178980 .518732-1.464614$

C $-5.560637-1.057355-3.388744$

O $-6.7462850 .034782-0.999908$

C $-4.852011-2.301926-2.955722$

C $-4.246523-3.138510-3.812110$

C $-3.562865-4.410501-3.399733$

C $-2.142016-4.498340-3.909852$

C $-1.850843-5.181339-5.029789$

C $-1.050486-3.867795-3.075640$

C -1.037151 -2.366742 -3.175362

N $-5.0764470 .067356-2.610480$

H $1.8198572 .688738-1.978106$

H $1.4717265 .729073-1.978293$

H $-0.9292774 .906073-1.995005$

H $-0.0283326 .255278-0.024510$

H $\quad 0.3717584 .751273 \quad 0.778195$

H -3.1275984 .6666992 .130003$

H -2.984647 $5.439165-1.080613$

H $-3.729407 \quad 6.0308410 .405364$

H $-2.4335176 .962641-0.345545$

H -0.1817564 .1839212 .727340$

H -1.5538614 .0455113 .786853$

H -0.8363982 .0840191 .551061$

H $-0.515306 \quad 1.864502 \quad 3.272322$

H -3.032424 2.0089513 .676333

H -1.831819 -0.2041193 .593134$

H $-1.974271-0.2192641 .848510$

H -3.059722 -2.335625 3.199722

H $-4.410611-0.3611300 .801598$

H -3.746711-1.007316 4.978793

H -5.372299-1.054392 4.336073

H $-4.5825090 .507600 \quad 4.630445$

H -6.060435 -1.443327 1.907911

H $-6.623221 \quad 1.9276730 .609533$

H -5.4638403 .2319360 .568129$

H -5.7298702 .9624672 .731897$

H $-1.1949980 .801746-0.554879$

H -1.029776 $0.199213-3.570132$

H $-0.779838-1.576672-0.099815$

H -1.819352 -2.893071-0.641379

H -2.501792 -1.297665 -0.457610

H $-5.1872752 .554338-1.563337$

H -3.938492 $1.544516-0.828913$

H $-5.381070-0.823596-4.442804$ 
H $-6.638105-1.173489-3.234714$ H -4.857515 -2.537732 -1.894211 H -4.267511 -2.913570 -4.876396 H $-4.152307-5.248486-3.794353$ H $-3.567485-4.534098-2.309627$ H $-0.830632-5.273314-5.387469$ H $-2.627809-5.659564-5.617029$ H $-0.068600-4.220889-3.416477$ H -1.133258 -4.229338 -2.047068 H $-0.790111-1.999167-4.172318$ H $-4.2017630 .499168-2.894597$ C $4.5071213 .312274-1.727227$ O $4.4358701 .888873-1.948771$ C $5.7659501 .349206-1.894993$ C $6.6904022 .485751-1.477014$ C $5.9541723 .691404-2.033976$ H $6.030908 \quad 1.077815-2.926043$ C $5.7984750 .064111-1.054479$ H $6.7812392 .565305-0.390202$ H $7.6986622 .374211-1.887989$ C $6.3968345 .007212-1.417978$ H $6.1098863 .728963-3.120533$ C $5.0469470 .137950 \quad 0.293973$ O $7.150011-0.336345-0.852585$ H $5.308159-0.721986-1.643754$ H 4.0376200 .5262060 .115835 O 5.6876801 .0502891 .180227 C $4.897575-1.2346560 .983924$ C $4.060052-1.1451812 .274004$ H $4.414154-1.9199030 .280440$ O $6.173393-1.7903721 .292059$ H $4.596030-0.5367483 .012440$ H $3.120401-0.6284172 .050592$ H $5.8366665 .846210-1.841990$ H $6.2440765 .014014-0.333505$ H $7.4607815 .181906-1.608519$ C $3.4732514 .012239-2.610040$ H $7.562819-0.430013-1.728016$ H $6.618151 \quad 0.7789751 .258799$ H $\quad 6.600947-1.2126161 .946265$ H $3.5439973 .638292-3.638588$ H $3.6619515 .091150-2.631573$ H $4.2855393 .484571-0.665089$ C $1.822673-3.1198651 .597321$ O $3.175304-3.4304441 .955883$ C $3.774173-2.5307382 .894846$ C $2.946122-2.4881104 .187712$ C $1.473374-2.192123 \quad 3.915286$ C $0.929481-3.1338822 .841947$ H $1.780683-2.1324321 .122427$ C $1.368587-4.1706980 .574087$ H $4.747659-2.9659893 .153591$ O $3.458343-1.5160955 .092791$ H $3.013221-3.4616214 .690860$ H $1.318446-1.1441453 .632353$ O $0.722002-2.3993025 .113613$ H $0.859799-4.1512723 .247616$ O $-0.387210-2.7242812 .484525$ C $2.054381-3.976291-0.761951$ H $1.611933-5.1714710 .951482$ H $\quad 0.290789-4.1210780 .390187$ H $4.389151-1.7389715 .264499$ H $\quad 1.104843-1.8232595 .797537$ H $-0.914606-2.7104193 .301243$ O $2.309629-5.192582-1.325769$ O $2.309520-2.888164-1.260765$ C $2.977293-5.156702-2.591182$ H $3.973931-4.719666-2.477223$ H $3.082995-6.184139-2.949047$ H $2.389431-4.590617-3.319595$

SCF Energy (B3LYP/6-31G**//MMFF) $=-3245.90599232$

03_294

MM̄FF Geometry

C $0.3207445 .308848-0.343722$

C $1.2224694 .532698-0.963484$

C $2.3895683 .888323-0.259552$

O $2.4501782 .494555-0.645547$
C $3.6998894 .561316-0.706299$

C 4.9278784 .0611510 .029877

C $5.9670093 .431805-0.553119$

C 4.9619064 .3373991 .511663

C $6.1518443 .094263-2.005603$

C $5.6463501 .701355-2.411950$

C $6.3520900 .539718-1.693102$

C $5.971225-0.798790-2.328658$

C $6.600783-1.985432-1.582344$

O $5.9434410 .541096-0.323988$

O $5.941733-3.183880-2.022209$

C $6.312674-1.847009-0.069645$

C $8.081773-2.152660-1.942926$

O $7.033894-2.8210070 .679124$

C $6.597037-0.4378760 .495376$

C $6.080047-0.2359821 .938423$

O $8.004831-0.1822560 .578055$

C $1.5462881 .642160-0.090404$

O $0.686194 \quad 1.9100450 .732766$

C $1.7735300 .308930-0.690711$

C $0.831405-0.640281-0.588318$

C $0.913416-1.993366-1.144418$

C $2.238593-2.434664-1.708422$

C $4.570772-0.4175232 .132565$

C $4.169948-1.8139512 .579134$

C $2.152869-3.2258262 .961980$

O $4.977505-2.6617432 .949383$

C $1.960266-4.1076271 .768813$

C $0.788742-4.6853501 .462781$

C $0.589221-5.6047190 .292287$

C $-0.583018-5.203089-0.575011$

C $-1.774592-5.810146-0.445033$

C $-0.347709-4.170921-1.654689$

C $-0.184762-2.777114-1.110270$

N $2.792723-1.9898942 .551125$

H 0.4463615 .5214920 .714130

H $1.1113354 .325565-2.025378$

H $2.275627 \quad 3.9550900 .829067$

H $3.6334765 .646308-0.548620$

H $3.8146014 .418156-1.786228$

H 6.7974543 .1236280 .081573

H 4.2131993 .7352142 .034985

H 5.9361734 .1050201 .955178

H 4.7604505 .3959801 .706207

H $5.6734303 .846598-2.641837$

H $7.2225633 .168148-2.235924$

H $4.5667691 .648906-2.227380$

H $5.7945491 .597723-3.494043$

H $7.4348590 .699794-1.753448$

H $6.251970-0.821316-3.388990$

H $4.879385-0.913736-2.306372$

H $\quad 6.038642-3.242543-2.988189$

H $5.248533-2.0720090 .066854$

H $8.193947-2.357406-3.014591$

H $8.520982-3.013555-1.427315$

H $8.680269-1.267862-1.715729$

H $6.800930-3.6940710 .319349$

H $6.653713-0.8592082 .635177$

H $6.314800 \quad 0.8010112 .220445$

H $8.482251-1.0011970 .369557$

H $2.7062350 .151958-1.220311$

H $-0.095385-0.401590-0.068066$

H $2.468443-1.882713-2.626018$

H $2.279228-3.499060-1.948539$

H $3.040420-2.262097-0.982491$

H 4.2398480 .2618462 .928600

H $4.021827-0.1496021 .225220$

H $1.203949-2.9581833 .437215$

H $2.782868-3.7345833 .698618$

H $2.828538-4.3000861 .142938$

H $-0.069386-4.5149992 .108564$

H $0.428809-6.6160150 .688713$

H $1.493404-5.670170-0.325610$

H $-2.612052-5.556375-1.085827$

H -1.940937 -6.5727130 .308656$

H -1.202037 -4.146282 -2.344775

H $0.497124-4.487938-2.272523$

H $-1.096973-2.370687-0.673228$ 
H $2.203111-1.2514742 .178102$ C $-2.2153275 .441309-0.458045$ O $-2.2940754 .001502-0.468927$ C -2.8575193 .5626410 .788160$ C -3.4937434 .8010331 .402801$ C -2.5208565 .8808870 .973325$ H -2.0069103 .2278721 .395352$ C -3.8210762 .3993910 .527614$ H -4.4858914 .9843440 .971656$ H -3.5987374 .7313272 .489316$ C -3.0849837 .2870521 .079232$ H -1.6284455 .8099001 .606124$ C -4.6512691 .9632481 .749658$ O $\quad-3.0524001 .2870990 .056147$ H -4.487812 2.680261 -0.295134 H -5.3036012 .7813042 .078289$ O $\quad-3.7692891 .6739992 .842729$ C -5.5039390 .6971751 .532497$ C -6.4063980 .7661290 .289506$ H $-4.846070-0.1765961 .489112$ O $\quad-6.320350 \quad 0.5389942 .698168$ H -7.032905 1.6647700 .352756 H -5.7802920.874104 -0.600971 H $-2.352108 \quad 8.0268240 .742242$ H -3.9880987 .4048630 .470984$ H -3.3469707 .5191262 .116686$ C $-0.8740345 .905776-1.034588$ H -2.4796600 .9944630 .785991$ H -3.3807322 .5119873 .143746$ H -6.662854 -0.370765 2.695302 H $-0.8044616 .998258-0.983209$ H $-0.8481605 .636567-2.098450$ H -3.011318 5.792965 -1.127791 C $-5.710056-1.989219-0.811351$ O $-6.625388-1.6980640 .251405$ C $-7.329875-0.4607280 .115552$ C -8.137389-0.458799-1.190699 C $-7.265485-0.787132-2.401168$ C $-6.450922-2.056145-2.151971$ H $-4.931035-1.218239-0.852442$ C $-5.042979-3.331635-0.484707$ H $-8.055111-0.4295140 .938161$ O $-8.768970 \quad 0.800046-1.398188$ H -8.933588 -1.211916 -1.125368 H $-6.6112550 .052033-2.665516$ O $-8.104595-1.011536-3.536890$ H -7.118234 -2.927356 -2.172283 O $-5.502248-2.219192-3.204068$ C -4.155961 -3.2238720 .736506$ H $-5.816046-4.086549-0.295287$ H $-4.416287-3.685860-1.309685$ H -9.329584 $0.976765-0.623563$ H $-8.641560-0.210131-3.661644$ H -5.996913 -2.230952 -4.041397 O $-4.390356-4.2761291 .572482$ O $-3.341486-2.3290750 .921289$ C -3.615120 -4.290309 2.774978 H $-3.888224-5.182213 \quad 3.345073$ H $-2.548507-4.3383362 .538818$ H $-3.837905-3.4052773 .378600$

SCF Energy (B3LYP/6-31G**//MMFF) $=-3245.90693289$

03_295

MM̄FF Geometry

C $1.604059-2.3550723 .323181$

C $2.722094-1.6831763 .639918$

C $3.975571-1.5624812 .803037$

O $3.889176-2.4690511 .679062$

C $4.156451-0.1361512 .238268$

C 4.0621830 .9829323 .252139

C 3.0058851 .8113353 .379290

C 5.2674591 .1438074 .140148

C 1.7243811 .7931382 .590723

C 1.4741213 .0888491 .805697

C 2.6167743 .4924210 .860428

C 2.2305614 .7273390 .042777

C $3.3271055 .113772-0.962217$

O $2.9031702 .395517-0.011988$
O $2.7627616 .052723-1.890802$

C $3.7227553 .860207-1.771756$

C $4.5010035 .829631-0.284281$

O $4.8378334 .126740-2.618371$

C $4.0120022 .623098-0.893011$

C $4.2499021 .338418-1.725305$

O $5.2142632 .849200-0.144236$

C $5.030627-2.8620041 .061697$

O $6.174719-2.5994861 .403947$

C $4.727509-3.618715-0.177891$

C $3.500924-4.080818-0.466772$

C $3.082530-4.742640-1.702733$

C $4.096584-4.895086-2.803905$

C $3.1550060 .944295-2.717898$

C $1.8172160 .645998-2.074599$

C $0.654639-1.041609-0.656866$

O $0.8464801 .381439-2.237395$

C $0.010209-2.149218-1.437708$

C $0.202407-2.467459-2.728328$

C $-0.455794-3.640101-3.405686$

C $0.553847-4.632007-3.949665$

C $0.919155-4.599643-5.242454$

C $1.097222-5.696656-3.017347$

C $1.791812-5.118889-1.817156$

N $1.830792-0.513957-1.318847$

H $1.542217-2.9099612 .391693$

H $2.740555-1.1430124 .584984$

H $4.829781-1.8460263 .430378$

H 3.4492450 .0100681 .414364

H $5.133372-0.0693171 .740191$

H 3.0496622 .5856474 .144065

H 5.1621131 .9825904 .836430

H 5.4286620 .2394004 .735120

H 6.1616891 .3275013 .535472

H 1.6673690 .9419391 .906972

H $\quad 0.897334 \quad 1.6534663 .298221$

H $\quad 0.5542802 .9438551 .224291$

H 1.2799193 .9093162 .508125

H $3.5034873 .712197 \quad 1.465768$

H 1.9975405 .5734520 .701125

H $1.3073684 .519263-0.516579$

H $2.4383306 .817047-1.384339$

H $2.8760633 .637981-2.432752$

H 4.1574126 .7494150 .204282

H $5.2498546 .143584-1.019805$

H 4.9962715 .2206310 .474571

H $4.6124924 .903595-3.158253$

H $5.1941911 .438704-2.276545$

H $4.4073390 .502383-1.030193$

H $\begin{array}{llll}5.558421 & 1.989527 & 0.149916\end{array}$

H $5.566725-3.752367-0.852325$

H $2.701977-3.9342680 .258123$

H $4.929267-5.525783-2.473783$

H $4.492264-3.917783-3.102393$

H $3.687743-5.357125-3.705892$

H $3.0145891 .715407-3.482265$

H $3.4707760 .044045-3.260837$

H $-0.068180-0.241809-0.468864$

H $\quad 0.993525-1.4324340 .307336$

H $-0.669552-2.768859-0.858640$

H $0.867845-1.868639-3.344383$

H -1.077822 -3.249245 -4.221455

H -1.147622 -4.159532 -2.730842

H $1.625394-5.315523-5.649311$

H $0.522138-3.853113-5.922376$

H $1.760383-6.392005-3.544947$

H $\quad 0.260417-6.315154-2.667828$

H $1.125785-4.962813-0.967770$

H $2.670759-1.085220-1.305906$

C $-0.786222-1.6668583 .435066$

O $-1.340621-2.5827552 .468180$

C $-2.777816-2.4152502 .439871$

C $-3.074193-1.200043 \quad 3.307178$

C $-1.957320-1.2709304 .328796$

H $-3.194382-3.3214762 .897118$

C $-3.222843-2.3000660 .977417$

H -2.997462 -0.273952 2.723248

H $-4.071074-1.2376423 .756477$ 
C - $1.750724 \quad 0.030780 \quad 5.084133$ H -2.177266 -2.076389 5.042015 C $-4.673293-1.8309010 .789327$ O $-3.074553-3.585800 \quad 0.365554$ H -2.540421 -1.623889 0.451747 H $-4.794797-0.8325531 .218350$ O $-5.531419-2.693766 \quad 1.545633$ C $-5.163856-1.829456-0.670976$ C $-4.251016-1.118181-1.687052$ H -5.305757 -2.864793 -1.003021 O $-6.467471-1.240103-0.710783$ H $-3.326485-1.696289-1.798715$ H $-4.767845-1.139911-2.655680$ H $-0.926591-0.0566095 .798738$ H -1.519536 0.8575014 .403864 H -2.6538180 .2982645 .642415$ C $0.366675-2.3418074 .179761$ H $-2.167725-3.8902480 .540856$ H $-6.443734-2.3841951 .412227$ H -6.383272 $-0.311729-0.434027$ H $0.108708-3.3782564 .427387$ H $0.568673-1.8172505 .120290$ H $-0.426834-0.7916202 .876352$ C $-4.9550742 .530968-1.169904$ O $-5.0933941 .132560-1.462644$ C $-3.901794 \quad 0.347512-1.353853$ C $-2.8136120 .887442-2.296482$ C $-2.5532612 .374882-2.050852$ C $-3.8571073 .178078-2.032037$ H -5.913792 $2.971991-1.473156$ $\begin{array}{llll}\text { C }-4.774376 & 2.784026 & 0.331739\end{array}$ H $-3.5319960 .399063-0.326284$ O $-3.1839370 .683614-3.658388$ H $-1.8909790 .321649-2.130192$ H -1.894020 2.756472 -2.839866 O $-1.8767252 .519676-0.805190$ H -3.675638 $4.201632-1.684100$ O $-4.3632493 .276692-3.366470$ C $-6.087907 \quad 2.611086 \quad 1.056815$ H -4.0290352 .1366390 .802184$ H -4.4506753 .8153480 .517061$ H -4.044914 $1.113005-3.800156$ H $-1.6762603 .464043-0.688801$ H -3.687144 $3.723676-3.903912$ O -6.2382481 .3158591 .449953$ $\begin{array}{llll}\text { O } & -6.893470 & 3.516638 & 1.229885\end{array}$ C -7.459901 1.0123892 .129931 H $-8.314228 \quad 1.1963191 .471638$ H -7.444887 -0.0475932 .397360$ H -7.541614 1.6047673 .046155

SCF Energy (B3LYP/6-31G**//MMFF) $=-3245.91140638$

03_296

MM̄FF Geometry

C -1.2654815 .4326950 .510967$

C $-0.323008 \quad 4.748870-0.154775$

C $0.936290 \quad 4.2230520 .486196$

$\begin{array}{llllll}\text { O } & 1.022495 & 2.797850 & 0.251300\end{array}$

C $2.1588924 .905357-0.156575$

C 3.4996604 .4253540 .366428

C $4.4948013 .972034-0.421968$

C 3.7022924 .5052481 .856795

C $4.4942773 .877916-1.922920$

C $3.8772722 .585469-2.474452$

C $4.7456061 .335366-2.260173$

C $4.0928490 .120917-2.926662$

C $4.881823-1.170775-2.669969$

O $4.8835571 .104231-0.857568$

O $4.048751-2.278307-3.046844$

C $5.145661-1.299804-1.155688$

C $6.139174-1.256404-3.542795$

O $6.007024-2.403126-0.882228$

C $5.714620-0.010863-0.515796$

C $5.763689-0.112158 \quad 1.028307$

O $7.0568110 .179177-0.977041$

C $0.299696 \quad 1.995997 \quad 1.082564$

O $-\begin{array}{llll}0.454290 & 2.356223 & 1.973383\end{array}$

$\begin{array}{llll}\text { C } & 0.592183 & 0.586244 & 0.733435\end{array}$
C $-0.063838-0.3920791 .376672$

C $0.114851-1.8354561 .203239$

C $1.113615-2.3110340 .183230$

C $4.396174-0.0055461 .709729$

C $4.371368-0.6426873 .087211$

C $2.738762-1.4357144 .797983$

O $5.374958-1.0591663 .658485$

C $2.315815-2.8130474 .389848$

C $1.112812-3.3393504 .663970$

C $0.691267-4.713240 \quad 4.226210$

C $-0.596746-4.7087153 .430663$

C $-1.724973-5.228327 \quad 3.943406$

C $-0.560180-4.1761482 .011224$

C $-0.623912-2.6711961 .963856$

N $3.080591-0.6948653 .598141$

H -1.1308885 .6318261 .570959$

H $-0.4572484 .550818-1.215834$

H 0.9365294 .4159521 .566509

H $2.0996145 .989376 \quad 0.010368$

H $2.1008654 .759651-1.240993$

H $5.421713 \quad 3.650050 \quad 0.050347$

H 3.0675033 .7781802 .372669

H 4.7382974 .2987272 .146149

H 3.4559005 .5082452 .220241

H $3.9652014 .738319-2.349135$

H $5.5276843 .975324-2.278797$

H $2.8910472 .433253-2.018099$

H $3.7153882 .720913-3.551159$

H $5.7344851 .525034-2.694038$

H $3.9701690 .286719-4.004199$

H $3.077082-0.010537-2.526866$

H $3.812226-2.169979-3.984006$

H $4.185455-1.539594-0.685048$

H $5.869801-1.228949-4.605647$

H $6.661207-2.207673-3.391440$

H $6.844062-0.442531-3.361165$

H $5.608120-3.190642-1.290447$

H $6.286562-1.033788 \quad 1.312230$

H $\quad \begin{array}{llll}6.386886 & 0.700284 & 1.427237\end{array}$

H $7.5159100 .769993-0.357538$

H $1.3459030 .395619-0.021675$

H $-0.804278-0.1104712 .124931$

H $0.993356-1.779665-0.766732$

H $1.007795-3.371415-0.058404$

H $2.132210-2.1552640 .548013$

H 4.1137201 .0489301 .812744

H $3.627284-0.4963751 .108884$

H $1.938192-0.8880645 .304599$

H $3.606796-1.4915465 .461980$

H $3.038974-3.405607 \quad 3.833574$

H $0.393059-2.7571665 .234649$

H $\quad 0.578811-5.3284405 .128212$

H $1.473889-5.1984953 .629470$

H -2.646031 -5.2554293 .370947$

H - $-1.754555-5.6358424 .948425$

H $-1.415740-4.5650431 .445762$

H $0.333013-4.5758911 .521646$

H - $1.359400-2.2362552 .642210$

H $2.312614-0.365353 \quad 3.021541$

C -3.7787325 .1798310 .196010$

O $-3.6815903 .848906-0.347227$

C $-4.2986892 .931995 \quad 0.577405$

C $-5.0302413 .790716 \quad 1.606115$

C $-4.1280125 .008902 \quad 1.673254$

H -3.4849942 .4045001 .088583$

C $-5.181766 \quad 1.929400-0.173284$

H $-6.0306624 .077932 \quad 1.262460$

H -5.1383893 .2815662 .568583$

$\begin{array}{llll}C & -4.785960 & 6.226414 & 2.297270\end{array}$

H -3.2404254 .7418142 .259432$

C $-4.462278 \quad 1.167396-1.309588$

O $-6.276266 \quad 2.648559-0.756456$

H -5.6191201 .2233870 .543275$

H $-4.2176821 .850722-2.131774$

$\begin{array}{llll}\text { O } & -5.394943 & 0.211239 & -1.823624\end{array}$

C $-3.1900560 .427083-0.857855$

C $-2.373753-0.270494-1.966233$

H -2.504651 1.153717 -0.409492 
O $-3.511953-0.498552 \quad 0.173898$ H $-1.9639770 .512309-2.616733$ H $-1.517290-0.749954-1.478580$ H -4.0979897 .0776082 .306999$ H $-5.684336 \quad 6.5245241 .746548$ H -5.0799896 .0171543 .330983$ C -2.512297 $5.973245-0.130407$ H $-6.9425381 .996016-1.030414$ H $-6.0783190 .700551-2.311641$ H -4.217108 $-1.073004-0.168667$ H -2.636565 7.0162150 .183673 H -2.381327 $5.987877-1.220167$ H $-4.6175675 .656948-0.328582$ C -2.927923 -3.264858 -1.417534 O $-3.766866-2.325930-2.099810$ C $-3.083057-1.320347-2.848785$ C $-2.165863-1.978266-3.893459$ C $-1.217425-2.992193-3.261345$ C $-1.994995-3.983529-2.399463$ H -2.338136 -2.742193 -0.656553 C $-3.845053-4.271491-0.711116$ H $-3.849280-0.782999-3.419010$ O $-1.403702-1.001852-4.595550$ H $-2.781619-2.500305-4.637719$ H -0.429031 -2.499610 -2.681762 O $-0.554627-3.724328-4.294995$ H -2.573495 -4.654072 -3.047873 O $-1.074891-4.788931-1.665445$ C $-4.584046-3.6292390 .438581$ H -4.580155 -4.695207-1.406300 H $-3.279578-5.114131-0.297362$ H $-2.030720-0.381998-5.005900$ H $-0.096212-3.077459-4.858348$ H $-0.478682-5.208504-2.309067$ O $-5.778473-3.1320270 .007217$ O $-4.140883-3.5537951 .577041$ C $-6.568985-2.4746351 .002550$ H $-6.858476-3.1846491 .782877$ H $-6.019892-1.6312311 .432014$ H -7.473106 -2.093475 0.520504

SCF Energy (B3LYP/6-31G**//MMFF)= -3245.91272365

03_297

MMFF Geometry

C -3.217225 -3.7557120 .241015$

C $-2.327177-2.828232-0.146505$

C $-0.830487-2.991530-0.310709$

O $-0.136456-1.9617200 .451683$

C $-0.218367-4.386752-0.043769$

C $1.100719-4.626724-0.757975$

C $2.299747-4.778866-0.163111$

C $1.006489-4.762425-2.259614$

C $2.650900-4.7193441 .295227$

C $3.212998-3.3683551 .765920$

C $4.440014-2.8566830 .992173$

C $5.020266-1.6124501 .672037$

C $6.159242-0.9769230 .858135$

O $4.019036-2.520113-0.332148$

$\begin{array}{lllll}\text { O } & 6.394179 & 0.339752 & 1.381984\end{array}$

C $5.699496-0.801448-0.606719$

C $7.473373-1.7490151 .024368$

O $6.776791-0.364051-1.430059$

C $5.066981-2.074965-1.200970$

C $4.440025-1.860631-2.600445$

O $6.077555-3.079794-1.348246$

C $-0.327098-1.8606751 .792433$

O $-1.012630-2.5945792 .491978$

C $0.415612-0.7187572 .375390$

C $1.177107 \quad 0.1378401 .675954$

C $1.9578051 .245328 \quad 2.238675$

C $1.8374351 .501876 \quad 3.719097$

C $3.314772-0.822578-2.667543$

C $3.7829520 .562622-3.080220$

C $3.0075552 .929314-3.148036$

O $4.887514 \quad 0.785767-3.567704$

C $3.6666123 .559444-1.963045$

C $3.0414224 .394860-1.121205$

C 3.6991685 .0179600 .073499
C 3.1902084 .4480821 .377890

C 2.3930885 .1665042 .186093

C 3.6920863 .0827371 .791049

C 2.7495721 .9683411 .418068

N $2.7999441 .520520-2.872310$

H -2.865228 -4.7527120 .492026$

H -2.680386 -1.829609 -0.388776

H $-0.642847-2.723776-1.356909$

H $-0.897610-5.171760-0.398451$

H $-0.110461-4.5472761 .032184$

H $3.157241-4.979228-0.805837$

H $1.949171-5.095251-2.707606$

H $0.242842-5.499550-2.528495$

H $0.747852-3.805525-2.722053$

H $1.788256-4.9772061 .918075$

H $3.395258-5.5012431 .492892$

H $2.414461-2.6263631 .688481$

H $3.466073-3.4621442 .829201$

H $5.187415-3.6574690 .949886$

H $5.359343-1.8480612 .688582$

H $4.226750-0.8633741 .791042$

H 6.6042920 .2531892 .327664

H $4.9531980 .003103-0.611491$

H $7.783176-1.7546932 .076385$

H $8.288935-1.2653590 .475682$

H $7.404196-2.7884410 .697509$

H $7.1337210 .448700-1.032582$

H $5.225630-1.657261-3.338006$

H $3.998642-2.820363-2.906477$

H $5.772254-3.731950-2.000542$

H $0.309542-0.631058 \quad 3.452432$

H 1.2638110 .0097430 .598602

H $\quad 0.7903501 .6594234 .000010$

H 2.3744752 .3910464 .054915

H 2.2318290 .6533734 .289040

H $2.596337-1.138667-3.434930$

H $2.771920-0.764293-1.717788$

H $2.0297013 .370677-3.363153$

H $3.6423153 .042785-4.032667$

H $4.7106063 .309426-1.788571$

H $1.9989684 .645394-1.296043$

H $3.520156 \quad 6.1000350 .028611$

H 4.7896624 .9023630 .031699

H 2.0540464 .7743013 .138993

H 2.0571096 .1606031 .910605

H 3.9233193 .0816042 .861253

H $4.6599372 .885370 \quad 1.311784$

H 2.7692541 .7091710 .359716

H $1.9454041 .256405-2.390376$

C $-5.288317-2.2019350 .090692$

O $-5.140115-1.894028-1.312498$

C $-6.351928-1.287329-1.778548$

C $-7.156471-0.926621-0.538686$

C $-6.786707-2.0799390 .376938$

H -6.901168 -2.079640 -2.306790

C $-6.098816-0.157258-2.780423$

H $-6.8252770 .024572-0.107039$

H $-8.230503-0.861510-0.736303$

C -7.139260 -1.837755 1.833120

H -7.305903 -2.984604 0.032064

C $-5.338098 \quad 1.093697-2.290792$

O $-7.373106 \quad 0.292265-3.264069$

H -5.564069 -0.564089-3.648076

H $-5.9438091 .652405-1.567872$

O $-5.1932201 .959457-3.430042$

C $-3.9338190 .852649-1.702473$

C $-3.1245942 .155215-1.533951$

H $-4.0294570 .361974-0.730833$

O $-3.199223-0.024343-2.557464$

H -2.917952 $2.570815-2.527263$

H $-3.7384382 .884778-0.996490$

H -6.813825 -2.673104 2.460462

H $-6.672174-0.9255622 .214822$

H $-8.222533-1.7299441 .951106$

C $-4.711780-3.5892470 .383151$

H -7.820862 $-0.471891-3.665737$

H $-6.0857502 .133580-3.775601$

H $-3.1485290 .393085-3.434356$ 
H $-5.179149-4.319868-0.289193$ H -4.972363 -3.8786121 .408069$ H $-4.739402-1.4382530 .652477$ C -2.4628802 .4757151 .445883$ O C $-1.7736301 .937620-0.811182$ C $-0.8423263 .158277-0.847955$ C -1.3469884 .2733600 .060895$ C -1.5820143 .7322091 .468014$ H -3.4852892 .7406991 .152051$ C -2.5144011 .8389842 .842083$ H $-1.2531601 .126454-1.335348$ O $-0.7044463 .659246-2.172914$ H $0.1590822 .861242-0.511118$ H $-2.2546914 .740628-0.338343$ O -0.3644635 .3069740 .136756$ H -0.6158303 .5084351 .938502$ O -2.2174164 .7362892 .256588$ C -3.5148930 .7091982 .911463$ H -1.525858 1.4400893 .092026 H -2.7997492 .5700033 .605910$ H $-0.3781382 .930491-2.727892$ H $-0.2049505 .620344-0.769947$ H -1.648181 5.5247302 .234745 O $-3.098538-0.2078893 .832336$ O -4.5496850 .6582752 .261209$ C $-3.959243-1.3356864 .016393$ H $-4.952674-1.0088454 .337494$ H $-4.021654-1.9161923 .092043$ H $-3.528646-1.9676294 .797622$

SCF Energy (B3LYP/6-31G**//MMFF) $=-3245.90278723$

03_298

MM̄FF Geometry

C $-3.0328163 .502195-2.138247$

C $-2.5539842 .662013-3.070882$

C -1.237112 $1.916255-3.010019$

O $-0.7587541 .905232-1.645797$

C - $-1.4192280 .459027-3.487055$

C $-0.133391-0.231702-3.903996$

C $0.414720-1.301968-3.294727$

C $0.5190910 .320353-5.148827$

C $-0.074409-2.059803-2.093710$

C $0.729948-1.822860-0.807028$

C $2.197802-2.277403-0.858978$

C $2.818482-2.2107810 .540401$

C $4.316313-2.5514180 .531877$

O $2.919351-1.416513-1.744574$

O $4.871199-2.132621 \quad 1.788725$

C $5.016267-1.722284-0.566689$

C $4.553872-4.0631140 .436795$

O $6.375172-2.123240-0.717244$

C $4.296115-1.778914-1.929334$

C $4.899308-0.817106-2.984305$

O $4.411658-3.102713-2.467039$

C $0.5697062 .075460-1.422555$

O $1.4515102 .201038-2.256107$

C 0.7702782 .0773040 .045001

C $2.002707 \quad 1.8919860 .539218$

C 2.3732561 .8454981 .954268

C 1.3277832 .2334952 .965095

C $4.8843530 .668346-2.612704$

C $6.1422451 .139363-1.901618$

C $6.9702532 .977228-0.432201$

O $7.2128700 .540991-1.962420$

C 6.9434832 .4086050 .951477

C 6.5674013 .1012202 .037051

C 6.5339432 .5227673 .422683

C 5.1393492 .4948144 .009541

C 4.7426023 .4276084 .891442

C 4.2368651 .3361093 .645679

C 3.6306881 .4780932 .277513

N $5.9383642 .334069-1.224707$

H -2.444278 $3.703183-1.248563$

H -3.139155 2.515264 -3.977021

H $-0.5414372 .460588-3.658998$

H -2.089318 $0.429068-4.357115$

H - $-1.927777-0.107345-2.702816$
H $\quad 1.322536-1.715820-3.732455$

H $\quad 0.9103791 .326095-4.971805$

H $1.356243-0.298538-5.489812$

H $-0.2052410 .366231-5.968743$

H -1.122171 -1.828252-1.885795

H $-0.055408-3.129677-2.336767$

H $0.681625-0.758856-0.555925$

H $\quad 0.227154-2.3676970 .000712$

H $2.227923-3.304513-1.241092$

H $2.280695-2.8673281 .236113$

H $2.698663-1.1953870 .940130$

H $4.383271-2.5853352 .497899$

H $5.031172-0.683255-0.215737$

H $4.102198-4.5759851 .294655$

H $5.622887-4.299100 \quad 0.477249$

H $4.134879-4.507218-0.468447$

H $6.797207-2.0507160 .155901$

H $5.905566-1.150840-3.265048$

H $4.294284-0.918001-3.896932$

H $4.260538-3.060451-3.425884$

H -0.1031282 .2032820 .674907$

H $2.8228821 .737856-0.159007$

H 1.7173692 .3152303 .982385

H 0.5217651 .4932702 .983376

H 0.9002863 .2131462 .723173

H $4.8241531 .258796-3.535879$

H $3.9998590 .908734-2.016726$

H $6.7684214 .052906-0.442686$

H $7.9494392 .801197-0.888902$

H 7.2483901 .3703951 .062124

H 6.2673254 .1410111 .929057

H 7.1978833 .1284614 .053147

H 6.9561601 .5101683 .443742

H 3.7554083 .3976745 .340396

H 5.3948734 .2452035 .180443

H 3.4634521 .1956464 .408570

H 4.8167550 .4044113 .674521

H 4.3103091 .2087611 .469416

H $5.0006452 .721581-1.176042$

C $-5.2706774 .078325-1.046852$

O $-5.4719682 .685924-0.732811$

C -5.2458852 .5035520 .676976$

C -5.4324943 .8824521 .296122$

C -4.7872414 .7579810 .238154$

H -4.1950222 .2147590 .796888$

C -6.1495801 .4062601 .242825$

H -6.4924984 .1394101 .405752$

H -4.9541123 .9691592 .276157$

C $-5.174106 \quad 6.223587 \quad 0.339671$

H $-3.698818 \quad 4.6770170 .340300$

C -6.0687210 .0447490 .519589$

O $\quad-7.509527 \quad 1.856057 \quad 1.158174$

H -5.9415631 .2796842 .311946$

H $-6.5442250 .147182-0.464458$

O $-6.877703-0.8715741 .269427$

C $-4.670388-0.5777540 .342900$

C $-3.869669-0.6928741 .655433$

H $-4.810220-1.585797-0.065276$

O $-3.9455520 .160992-0.632413$

H $-3.795426 \quad 0.2898232 .133426$

H $-4.423583-1.3380432 .346443$

H -4.699967 $6.806598-0.456218$

H $-6.257643 \quad 6.3601310 .258769$

H -4.8546676 .6397311 .300706$

C $-4.3504444 .221807-2.265372$

H -8.0698981 .1559981 .535065$

H -6.913107 -1.706504 0.772039

H $-3.179414-0.374744-0.896670$

H $-4.1549905 .283947-2.452113$

H $-4.8868503 .836422-3.142123$

H $-6.2587234 .471189-1.320365$

C $-2.927659-3.5652291 .202332$

O $-2.387306-2.3744570 .621830$

C $-2.431386-1.2207561 .461516$

C $-1.664539-1.4831192 .765102$

C $-2.123951-2.7663903 .453939$

C $-2.148275-3.9342452 .467812$

H $-3.986736-3.4119091 .445062$ 
C $-2.836554-4.6687330 .143702$ H $-1.881913-0.4348150 .927155$ O $-1.806980-0.393538 \quad 3.670334$ H $-0.593789-1.5767012 .540733$ H -3.103604 -2.637118 3.929210 O $-1.208673-3.0879624 .503993$ H $-1.120064-4.2197772 .210948$ O $-2.765250-5.0594383 .088793$ C $-3.813998-4.400551-0.976654$ H -1.826082 $-4.739019-0.276984$ H $-3.081017-5.6531780 .557761$ H -1.5277450 .4127303 .204359$ H $-1.178162-2.3234925 .104660$ H $-2.267258-5.2509823 .901975$ O $-3.207073-3.680978-1.962641$ O $-4.985527-4.755250-0.957341$ C $-4.046143-3.296403-3.055525$ H $-4.422519-4.183402-3.573608$ H $-4.873452-2.675819-2.698161$ H -3.444795 -2.708667 -3.754275

SCF Energy (B3LYP/6-31G**//MMFF) $=-3245.90075170$

03_299

MMFF Geometry

C $-1.230404 \quad 5.970787 \quad 0.687438$

C $-2.102246 \quad 4.995536 \quad 0.984758$

C $-3.095854 \quad 4.4226660 .008282$

O $-2.8169703 .010566-0.135102$

C $-4.526038 \quad 4.568926 \quad 0.559236$

C $-5.5831523 .923784-0.318271$

C -6.3368212 .8620370 .033777$

C $-5.7820824 .566716-1.667428$

C $-6.3333402 .105427 \quad 1.333370$

C $-5.653713 \quad 0.735860 \quad 1.242875$

C $-6.298767-0.283247 \quad 0.287403$

C $-7.742121-0.6143380 .679999$

C $-8.333845-1.750615-0.167552$

O $-5.483564-1.4639250 .353758$

O $-9.537189-2.200064 \quad 0.475035$

C -7.350757-2.937935 -0.161999

C $-8.743912-1.266899-1.563572$

O $-7.789595-3.969252-1.042826$

C $-5.902876-2.533019-0.506229$

C $-4.908058-3.701978-0.320196$

O $-5.837399-2.139191-1.881660$

C $-1.8462052 .650778-1.017784$

O $-1.1606713 .390382-1.704745$

C $-1.7612661 .174913-1.001180$

C $-0.7026110 .558399-1.547790$

C -0.500134 -0.890791-1.623052

C $-1.646471-1.763474-1.187551$

C $-4.830339-4.2646241 .100254$

C $-3.584884-5.1100621 .288310$

C $-1.138780-4.9319171 .704836$

O $-3.631078-6.337777 \quad 1.305174$

C $-0.067672-3.9658921 .310194$

C $0.949529-4.2675180 .488761$

C $2.011506-3.2790860 .095292$

C $2.237083-3.213702-1.401190$

C $3.433951-3.526161-1.926494$

C $1.086507-2.798520-2.293714$

C $0.674216-1.364701-2.090247$

N $-2.434761-4.3453021 .414212$

H - $-1.2372516 .411186-0.305781$

H -2.080024 4.5504121 .977287

H -3.022828 $4.916382-0.968835$

H $-4.770536 \quad 5.6335520 .673498$

H $-4.556948 \quad 4.149877 \quad 1.569649$

H -7.064481 2.494007 -0.688921

H $-6.6361674 .144340-2.207630$

H -5.967272 $5.639744-1.552876$

H $-4.8966754 .429121-2.295612$

H -5.8461552 .6766872 .128389$

H -7.370398 1.9931651 .669384

H -4.6023010 .8700950 .956313$

H -5.6156490 .2985732 .249976$

$\begin{array}{llll}H & -6.254506 & 0.110355 & -0.734094\end{array}$

H -8.3852940 .2704950 .610469$
H -7.767986 -0.921421 1.735011 H $-10.139716-1.4401600 .548915$ H -7.371115 -3.3636020 .849495$ H $-9.511742-0.487470-1.488226$ H $-9.196322-2.077259-2.145605$ H $-7.911767-0.851867-2.135874$ H $-8.700927-4.197257-0.790955$ H $-5.140096-4.512365-1.023627$ H $-3.912791-3.351231-0.616969$ H -4.919157 -2.227826 -2.185951 H $-2.5770600 .634505-0.534818$ H $0.0897201 .167322-1.979868$ H $-2.545796-1.530247-1.768582$ H $-1.865594-1.608501-0.125643$ H $-1.463626-2.830944-1.318471$ H $-4.817870-3.4646561 .849473$ H $-5.702191-4.8916961 .317036$ H - $1.051872-5.8926771 .186751$ H $-1.097976-5.1189782 .782765$ H $-0.130576-2.9626701 .726265$ H $1.032587-5.2754350 .089206$ H $2.937869-3.5722480 .605384$ H $1.767603-2.2755240 .463648$ H $3.612271-3.484757-2.995736$ H $4.265306-3.829085-1.298898$ H $0.261104-3.502306-2.162677$ H $1.372066-2.901231-3.349464$ H $1.440333-0.647436-2.387636$ H $-2.543044-3.3445301 .554926$ C 1.2128715 .9717801 .389712 O $1.2275114 .528722 \quad 1.403731$

C 1.9679594 .0684590 .252506

C $2.8083775 .256539-0.184234$

C 1.8428956 .4006850 .063918

H $1.2186393 .835487-0.513650$

C 2.7393682 .8001380 .627006

H 3.6989005 .3585310 .448376

H $3.1290785 .189272-1.227902$

C $2.510578 \quad 7.763817 \quad 0.114127$

H $1.0978606 .402781-0.740862$

C $3.7165822 .321331-0.465074$

$\begin{array}{llllll}\text { O } & 1.772779 & 1.778150 & 0.889698\end{array}$

H 3.2888332 .9552201 .563258

H $4.4905403 .086916-0.592763$

O $3.0316402 .217440-1.713542$

C $4.4093300 .979688-0.149309$

C $5.4890280 .636947-1.192565$

H 4.8638321 .0334940 .846557

O $3.461056-0.084371-0.143503$

H $5.024757 \quad 0.522863-2.179797$

H $6.1965811 .473424-1.241814$

H $1.775596 \quad 8.5489850 .317748$

H 3.2768837 .8074520 .895227

H $2.9930997 .990892-0.842124$

C $-0.2057496 .482378 \quad 1.663080$

H 1.1705412 .1160791 .574722

H $2.3682591 .512836-1.619591$

H 2.8098780 .1031190 .553067

H -0.2213407 .5781081 .651737$

H -0.4923896 .1760642 .677562$

H 1.8541356 .2871862 .223188

C $7.638193-1.6045370 .887816$

$\begin{array}{llllll} & 0 & 6.928994 & -0.466288 & 0.381790\end{array}$

C $6.243642-0.664962-0.858606$

C $7.237959-1.054148-1.962346$

C $8.063272-2.279456-1.552624$

C $8.651419-2.128261-0.146122$

H $8.200630-1.2199401 .748325$

C $6.682902-2.6863261 .405363$

H $5.509208-1.467459-0.739871$

O $8.1083090 .043530-2.228935$

H $6.679780-1.263460-2.882876$

H $8.882326-2.425909-2.267545$

O $7.244149-3.444243-1.587625$

H $9.066904-3.0849220 .191585$

O $9.740357-1.203635-0.224746$

C $5.893515-2.1743292 .587158$

H $5.983420-3.0428270 .644196$ 
H $7.239569-3.5670951 .746947$ H $8.711961-0.226503-2.941577$

H $6.900010-3.534992-2.492552$

H $10.153350-1.1601060 .654407$

O $4.630418-1.8426972 .196808$

O $6.354412-2.0592623 .715431$

C $3.783922-1.3150853 .222465$

H $2.794222-1.1425502 .791405$

H $3.687754-2.0313224 .044066$

H $4.181066-0.3616913 .583232$

SCF Energy (B3LYP/6-31G**//MMFF) $=-3245.91120011$

03 3

MMFF Geometry

C -3.847508 2.196819 1.370298

C $-3.031975 \quad 1.8870102 .389653$

C -2.648018 $0.475902 \quad 2.741742$

O $-1.204397 \quad 0.4557392 .857557$

C -3.257759 0.0127564 .075708

C $-4.750546-0.225798 \quad 4.016169$

C $-5.316321-1.3662783 .574625$

C $-5.611562 \quad 0.8887884 .550575$

C $-4.623182-2.5949543 .049705$

C $-5.231211-3.0970591 .732383$

C $-4.831370-2.2720190 .495530$

C $-5.715162-2.676822-0.689079$

C $-5.291349-1.991024-1.992037$

O $-3.453027-2.5439610 .210678$

O $-5.973774-2.639422-3.076194$

C $-3.784870-2.233205-2.198307$

C $-5.714955-0.518625-2.030267$

O $-3.308573-1.509107-3.332331$

C $-2.927643-1.893519-0.955810$

C $-1.461985-2.359159-1.152700$

O $-2.909663-0.471396-0.794989$

C $-0.577896-0.7255392 .609207$

O $-1.100374-1.7813702 .289671$

C $0.874383-0.5214002 .803722$

C $1.710077-1.5381482 .545542$

C $3.165419-1.5336702 .697875$

C $3.818233-0.2706993 .192817$

C $-1.254218-3.870696-1.037402$

C $-0.044127-4.324155-1.827766$

C $2.430155-4.336200-1.848738$

O $-0.166385-4.871714-2.922245$

C $3.488135-4.567293-0.818350$

C $4.622473-3.854198-0.740869$

C $5.723787-4.1321190 .243294$

C $6.009235-2.9613811 .157875$

C $6.920561-2.0320700 .825326$

C $5.317025-2.9244662 .501499$

C $3.841403-2.6610762 .392669$

N $1.162296-4.054919-1.199502$

H $-4.280770 \quad 1.3954840 .779077$

H -2.611112 2.6832732 .998800

H $-2.947921-0.1998411 .933454$

H -3.023018 $0.738076 \quad 4.867155$

H -2.757375 -0.9041684 .413394$

H $-6.402220-1.4438023 .614701$

H -5.4632361 .8070633 .975864$

H $-6.6788270 .646027 \quad 4.509408$

H $-5.360877 \quad 1.0909305 .597255$

H -3.547493 -2.450433 2.931240

H $-4.750998-3.3823433 .803051$

H $-4.893633-4.1319551 .587212$

H -6.324132 -3.127214 1.827886

H $-4.950674-1.2049590 .711429$

H $-6.770563-2.467012-0.474195$

H $-5.652072-3.764644-0.835171$

H -6.930184 -2.564723 -2.916032

H $-3.659994-3.295679-2.441132$

H -6.806562 -0.432259-1.969546

H $-5.429253-0.048143-2.977326$

H $-5.2944090 .067781-1.211740$

H $-3.866859-1.756422-4.089367$

H $-1.088427-1.985196-2.113729$

H $-0.821143-1.880207-0.400829$

H $-2.112659-0.222386-0.298802$
H 1.2112070 .4462093 .156528

H $1.287277-2.4775932 .190087$

H $4.909253-0.3007693 .146899$

H $3.538368-0.0800214 .234451$

H 3.5130470 .5900982 .589642

H $-1.130699-4.1614350 .012610$

H -2.113364 -4.434160 - 1.415217

H $2.659652-3.490068-2.503965$

H $2.326176-5.227155-2.477110$

H $3.328019-5.388987-0.124204$

H $4.793063-3.049072-1.451427$

H $6.627265-4.381472-0.328872$

H $5.507131-5.0245250 .843727$

H $7.158601-1.2092161 .491101$

H 7.445444-2.071199-0.123573

H $5.802033-2.2041293 .168513$

H $5.456990-3.8935512 .998155$

H $3.272576-3.5259712 .050084$

H $1.162902-3.444863-0.386670$

C -3.660331 $4.063779-0.344839$

O $-2.2200864 .044744-0.295970$

C $-1.7280293 .502142-1.536334$

C $-2.9011703 .559159-2.508860$

C $-4.0599903 .253883-1.578681$

H -1.490979 $2.448453-1.349885$

C $-0.4699714 .247375-1.987028$

H -3.020269 4.556086 -2.948619

H -2.8011912.836082 -3.323990

C $-5.4189293 .620996-2.146333$

H $-4.0457152 .179788-1.364674$

C $0.6601874 .314615-0.938074$

O $-0.841090 \quad 5.597400-2.302919$

H $-0.0983443 .809574-2.920890$

H $0.3286934 .950494-0.107510$

O $1.7612944 .996604-1.555181$

C $1.1786742 .973190-0.383123$

C $1.5835001 .971832-1.479725$

$\begin{array}{lllll}\text { H } & 2.057710 & 3.181851 & 0.236101\end{array}$

$\begin{array}{lllll}\text { O } & 0.212160 & 2.368846 & 0.469838\end{array}$

H $0.6910491 .662730-2.035586$

H $2.2521992 .467748-2.190862$

H $-6.2137093 .392811-1.429112$

H $-5.4785424 .687042-2.389715$

H $-5.6180583 .055488-3.062615$

C -4.2332323 .6006010 .997443$

H $-0.0363486 .056479-2.599198$

H $2.4494135 .115778-0.878246$

H -0.0256963 .0177891 .153841$

H -5.3269973 .6675870 .977286$

H $-3.8881134 .290267 \quad 1.778455$

H $-3.9421045 .115500-0.487354$

C $4.5229741 .526274-0.775409$

O $3.3814761 .007820-0.082619$

C $2.2555850 .703458-0.910564$

C $2.655657-0.329191-1.970886$

C $3.8780880 .112809-2.769289$

C $5.0097920 .526622-1.830859$

H $4.2662122 .479527-1.252582$

$\begin{array}{llll}\text { C } 5.614631 & 1.788053 & 0.272069\end{array}$

H $1.5168240 .221626-0.258445$

O $1.582704-0.587360-2.868453$

H $2.893248-1.275334-1.470868$

H $3.6319910 .921372-3.467528$

O $4.337945-0.980249-3.567732$

H $5.422616-0.365735-1.344584$

O $6.0578141 .121281-2.593050$

C $5.250866 \quad 2.928514 \quad 1.198356$

H $\begin{array}{llll}5.752618 & 0.885023 & 0.877818\end{array}$

H $6.5693682 .046850-0.197534$

H $0.819503-0.864814-2.335202$

H $3.592924-1.264449-4.124794$

H $6.3287250 .474730-3.267238$

O $5.808797 \quad 2.7093162 .425133$

$\begin{array}{lllll} & 0 & 4.586268 & 3.904609 & 0.877818\end{array}$

C 5.5432263 .7128563 .409799

H 5.9617254 .6730873 .093777

H 4.4661053 .7979703 .583170

H 6.0253013 .4094044 .342862 
H $-5.0073885 .065280-1.054314$

03_30

MMFF Geometry

C $2.7560790 .996795 \quad 3.215408$

C 1.9281610 .7824394 .249217

C $0.570747 \quad 0.120504 \quad 4.154125$

O 0.0258090 .2300782 .818361

C $0.696953-1.3728274 .504202$

C $-0.625349-2.1142734 .509697$

C $-0.947058-3.1115553 .662214$

C $-1.608185-1.6885695 .570104$

C $-0.108505-3.7042302 .564272$

C $-0.325595-3.0752751 .179754$

C $-1.742305-3.2624740 .614384$

C $-1.799851-2.814691-0.847701$

C $-3.221812-2.906963-1.422991$

O $-2.651875-2.476569 \quad 1.387277$

O $-3.245099-2.171158-2.656282$

C $-4.205526-2.206316-0.460084$

C $-3.600517-4.350939-1.773027$

O $-5.555017-2.417670-0.866777$

C $-4.028661-2.625648 \quad 1.013076$

C $-4.865877-1.7702961 .995413$

O $-4.458953-3.9835451 .169449$

C -0.5210901 .4272752 .469684$

O $-0.6242122 .421313 \quad 3.170831$

C -0.9499411 .3325721 .057605$

C -1.187820 2.4592090 .369912

C $-1.6066152 .543640-1.030052$

C $-2.0459511 .271241-1.703783$

C $-4.558478-0.268448 \quad 1.988447$

C $-5.498822 \quad 0.553374 \quad 1.121630$

C -5.8517842 .8888280 .322809$

O

C $-5.3522692 .994912-1.083124$

C $-4.9643114 .151149-1.642376$

C $-4.5104014 .280740-3.068060$

C -3.157862 $4.945077-3.193529$

C $-3.0540396 .251642-3.490021$

C $-1.9286894 .074846-3.064049$

C -1.593002 $3.748997-1.636302$

N $-5.091593 \quad 1.8784881 .034698$

H $2.473500 \quad 0.6447442 .226582$

H $2.227081 \quad 1.1107295 .242065$

H $-0.108722 \quad 0.6171654 .857574$

H $1.156321-1.4857885 .495294$

H $1.392078-1.8373643 .797073$

H -1.928590 -3.572776 3.767562

H -1.972121 -0.6751925 .375448$

H -2.482417 -2.3470585 .614967$

H -1.134703 -1.707932 6.557143

H $\quad 0.955867-3.6489372 .815866$

H $-0.330522-4.7778122 .510829$

H $-0.082771-2.007517 \quad 1.230576$

H $\quad 0.390658-3.5374290 .490833$

H -2.009499 -4.3223980 .699588$

H -1.101546 -3.398150-1.460005

H -1.462605 -1.772050 -0.921378

H -2.575192 -2.555739 -3.247274

H $-4.020471-1.130083-0.545977$

H -2.901611 -4.761684 -2.511506

H -4.590194 -4.397991 -2.240409

H -3.594619 -5.017314 -0.907941

H -5.630458 -2.116856 -1.788501

H $-5.935006-1.9669881 .850415$

H -4.640831 -2.131819 3.009422

H -4.667393 -4.134957 2.106270

H $-1.038273 \quad 0.3453710 .619632$

H $-1.0393983 .415600 \quad 0.869991$

H $-1.1971140 .596561-1.849106$

H -2.507584 $1.431167-2.680401$

H $-2.799230 \quad 0.759987-1.096229$

H $-4.678483 \quad 0.112523 \quad 3.011004$

H -3.523939 -0.0776201 .688563$

H $-5.739820 \quad 3.8277210 .874454$

H -6.9108742 .6119350 .317643$

H $-5.3375352 .084070-1.677100$

H $-5.2656684 .870171-3.604480$

H $-4.4859903 .306178-3.571529$

H -2.087280 $6.729148-3.610811$

H -3.934079 $6.874262-3.612899$

H $-1.0568714 .591781-3.487881$

H -2.048563 $3.184244-3.687457$

H -1.267721 4.615896-1.059465

H -4.209493 2.1570591 .454640

C 4.2294722 .8454242 .306144

O 4.1501582 .3292030 .958832

C 3.2195183 .1392760 .219645

C 3.1331924 .4561780 .981208

C 3.1879643 .9635732 .413790

H $2.2461442 .637780 \quad 0.281258$

C $3.6387823 .274181-1.245800$

H 3.9913405 .1046260 .770176

H 2.2184975 .0113010 .752202

C 3.5484835 .0414773 .420913

H 2.2022393 .5590292 .673484

C $3.8234161 .941700-2.002634$

O $4.8847223 .980682-1.301696$

H $2.9080423 .903431-1.768956$

H $4.7564151 .459510-1.685754$

O $3.9970362 .292154-3.382582$

C $2.6518640 .949932-1.864221$

C $2.701784-0.217887-2.869173$

H $2.6419450 .557447-0.841490$

O $1.4177231 .642295-2.060247$

H $2.4240940 .160528-3.859912$

H $3.727314-0.591409-2.936344$

H $3.592043 \quad 4.627598 \quad 4.433314$

H 4.5217895 .4911763 .197512

H 2.7998885 .8405863 .413466

C 4.0779641 .7037883 .318313

H $5.1222384 .074646-2.240053$

H $4.3698151 .519593-3.839713$

H $1.4437802 .052675-2.941643$

H 4.2339922 .0918394 .331479

H 4.8605850 .9600663 .131975

H 5.2401903 .2613362 .407099

C $3.326114-2.868864-1.460562$

O $2.165513-2.041403-1.293758$

C $1.729689-1.355754-2.474195$

C $1.434252-2.367751-3.588664$

C $2.618559-3.291405-3.846641$

C $3.088830-3.931722-2.541412$

H $4.183805-2.247027-1.740284$

C $3.632751-3.504944-0.099989$

H $0.772403-0.886613-2.217208$

O $1.077860-1.709124-4.799262$

H $\quad 0.574660-2.985334-3.298117$

H $3.439824-2.761659-4.344059$

O $2.212489-4.334250-4.736534$

H $2.339851-4.662883-2.210354$

O $4.297815-4.638040-2.813599$

C $4.153940-2.4670480 .863449$

H $2.734105-3.9492690 .341996$

H $4.382702-4.300213-0.166062$

H $\quad 0.312513-1.140370-4.607656$

H $1.885207-3.909835-5.548263$

H $4.462776-5.244005-2.072379$

O $5.465267-2.2066060 .593606$

O $3.473129-1.9421391 .733893$

C $6.081634-1.2046931 .406725$

H $5.909529-1.4021122 .469118$

H $7.158255-1.2288271 .218023$

H $5.698329-0.2198631 .126562$

SCF Energy (B3LYP/6-31G**//MMFF) $=-3245.90553862$

03_300

MM̄FF Geometry

C $-0.3965525 .263754-0.324344$

C $0.7052084 .648904-0.778913$

C 1.9571494 .4605990 .037990

O 2.1381153 .0426980 .259076

C $3.1816994 .981634-0.736198$

C $4.5007844 .724765-0.030254$ 
C $5.4755623 .912919-0.488189$

C 4.6955115 .4696571 .265924

C $5.5041963 .121889-1.766808$

C $5.2525761 .624730-1.565839$

C $6.2887710 .868252-0.716347$

C $7.6925370 .924551-1.327326$

C $8.6944720 .033345-0.578211$

O $5.831319-0.491356-0.648608$

O $9.865538-0.100625-1.398775$

C $8.088334-1.375832-0.426426$

C 9.1575850 .6748470 .735069

O $8.921223-2.2066920 .378392$

C $6.653295-1.3647810 .138514$

C $6.003652-2.7677660 .113207$

O $6.687739-0.9407061 .505952$

C 1.4307372 .4753821 .274144

O $\quad 0.6376883 .0258852 .020897$

C 1.7867591 .0414231 .312808

C 1.0275580 .1868352 .014695

C $1.253259-1.2548172 .141363$

C $2.524401-1.8034001 .552486$

C $5.871995-3.393542-1.276637$

C $4.883396-4.544292-1.270129$

C $2.443757-5.032615-1.306696$

O $5.251918-5.715931-1.295913$

C $1.226980-4.371640-0.741554$

C $0.453063-4.9130190 .211680$

C $-0.760991-4.2279890 .774459$

C $-0.780813-4.2041732 .288942$

C -1.771914 -4.804297 2.969852

C $0.338085-3.4903443 .019309$

C $0.327127-2.0058542 .773322$

N $3.565478-4.115121-1.215814$

H -0.4016635 .6821730 .678777$

H $0.6942374 .211112-1.774777$

H $1.883664 \quad 4.977657 \quad 1.002925$

H $3.0800896 .062214-0.903725$

H $3.1847454 .528215-1.732028$

H 6.3778663 .8117590 .114175

H 5.7069485 .3471371 .668000

H $4.532096 \quad 6.5418231 .115720$

H 3.9948025 .1120612 .026597

H $4.7723553 .499000-2.486640$

H $6.4744213 .283119-2.250423$

H $4.2590131 .479008-1.121518$

H $5.1909181 .146228-2.552832$

H 6.2891891 .2841500 .297353

H $8.0690611 .953359-1.362224$

H $7.6452610 .587520-2.372513$

H $\quad 10.216530 \quad 0.790460-1.568251$

H $8.074129-1.826312-1.427112$

H 9.6607121 .6293410 .538157

H 9.8973290 .0462191 .242735

H $8.337957 \quad 0.8741651 .428257$

H $9.811684-2.189939-0.012439$

H $6.553301-3.4518500 .773146$

H $5.006734-2.6879560 .561782$

H $5.886686-1.263244 \quad 1.950813$

H $2.659280 \quad 0.7273020 .751287$

H 0.1507720 .5721382 .533486

H $3.394939-1.3059661 .994641$

H $2.544637-1.6465370 .468665$

H $2.663055-2.8725371 .719823$

H $5.537118-2.659381-2.018458$

H $6.838558-3.777481-1.621464$

H $2.695456-5.964806-0.790272$

H $2.290738-5.257970-2.367068$

H $0.965238-3.394379-1.141798$

H $0.693498-5.9006820 .597880$

H -1.644707 -4.7540880 .391065$

H $-0.837437-3.1996030 .402072$

H -1.800461 -4.794645 4.054434

H -2.579780 -5.317765 2.459041

H $1.290029-3.9603882 .761166$

H $\quad 0.238540-3.6379504 .102981$

H $-0.556830-1.5078363 .174149$

H $3.381461-3.127533-1.370336$

C $-2.6813864 .266606-0.819787$
O $-2.1501672 .992987-1.240600$

C $-2.4588892 .007426-0.231394$

C -3.5259852 .6398750 .649154$

C -3.1019764 .0953420 .639751$

H -1.5320201 .8599020 .336162$

C $-2.8753190 .701986-0.916996$

H -4.5212782 .5252560 .201579$

H -3.5486752 .2101331 .654523$

C -4.193966 5.0526971 .082423

H $-2.242770 \quad 4.202189 \quad 1.312878$

C $-3.377021-0.3747740 .059429$

O $-1.7377280 .185750-1.616391$

H $-3.6361380 .916120-1.675272$

H $-4.265125-0.0194970 .590357$

O $-2.384459-0.583396 \quad 1.071322$

C $-3.680941-1.738484-0.587091$

C $-4.667411-1.708848-1.768901$

H $-2.749402-2.204769-0.929329$

O $-4.204545-2.6127760 .418217$

H -4.182218 -1.236634 -2.631025

H $-4.874033-2.752162-2.041275$

H -3.8425326 .0884201 .041165$

H -5.0818914 .9713440 .446471$

H -4.4984414 .8387672 .112255$

C -1.668651 $5.377612-1.117068$

H $-1.4249420 .884462-2.216484$

H $-1.562031-0.8462570 .623587$

H -3.567602 -2.6250281 .153267$

H $-2.1173306 .357070-0.914579$

H -1.427451 $5.357916-2.187656$

H $-3.5674684 .440857-1.444366$

C -7.910669-1.101321 0.008440

O $-6.677978-1.686069-0.432057$

C $-6.008566-0.998374-1.491237$

C $-6.901790-0.953963-2.741502$

C $-8.267485-0.335563-2.420315$

C $-8.894457-0.953307-1.165537$

H $-8.335083-1.8331040 .708105$

C $-7.6797890 .207107 \quad 0.780752$

H $-5.7949990 .027434-1.178430$

O $-7.084245-2.275951-3.244598$

H -6.393972 -0.371019-3.519209

H -8.947656 -0.484270 -3.267926

O $-8.1220021 .067464-2.220727$

H $-9.758451-0.360019-0.845349$

O $-9.395321-2.252713-1.496139$

C -6.860639 -0.007117 2.036584

H -7.1545080 .9444260 .167949$

H -8.6422170 .6320151 .087642$

H -7.624960 -2.211428 -4.050099

H $-7.757178 \quad 1.444330-3.039529$

H -8.634145 -2.818028 -1.713338

O -6.4461971 .2065212 .507856$

O $-6.623268-1.0921652 .549212$

C $-5.6440791 .164501 \quad 3.692292$

H -4.7428660 .5678523 .520432$

H -5.3450132 .1873673 .936446$

H -6.2241000 .7583794 .526243$

SCF Energy (B3LYP/6-31G**//MMFF $)=-3245.90911013$

03_301

MM̄FF Geometry

C $-2.502125-1.7322492 .014760$

C -1.323168 -2.297830 1.717789

C $-0.526314-1.9625950 .482679$

O $0.821766-1.6273570 .882386$

C $-0.459200-3.193059-0.439429$

C $0.283287-2.944672-1.738428$

C $1.400100-3.598068-2.115384$

C $-0.318572-1.906230-2.650877$

C $2.133726-4.680699-1.373416$

C $3.244993-4.176106-0.439486$

C $4.424151-3.509718-1.167099$

C $5.557658-3.200315-0.188070$

C $6.723561-2.474494-0.877712$

O $3.967677-2.289726-1.749098$

O $7.574542-1.937640 \quad 0.148525$

C $6.177573-1.270519-1.681752$ 
C $7.589168-3.449781-1.685038$

O $7.216680-0.705773-2.477058$

C $4.941880-1.605942-2.549821$

C $4.231153-0.363658-3.132529$

O $5.304903-2.384511-3.696788$

C $1.051306-0.3552191 .305935$

$\begin{array}{lllll}\text { O } & 0.242978 & 0.556285 & 1.377658\end{array}$

C $2.474821-0.2703671 .699672$

C 2.8873350 .7473542 .469668

C $4.249263 \quad 0.9549542 .966471$

C 5.3372310 .0800172 .402070

C $3.7080770 .640384-2.100091$

C $4.6478481 .805621-1.841209$

C $4.8654243 .839746-0.418863$

O $5.6740402 .001876-2.486307$

C $5.829346 \quad 3.5341750 .683497$

C $5.859216 \quad 4.208079 \quad 1.843174$

C $6.853137 \quad 3.9462382 .937872$

C 6.1943313 .6902544 .274506

C $6.084345 \quad 4.6679975 .189777$

C $5.7444702 .280025 \quad 4.581292$

C 4.4603621 .9172723 .888860

N $4.1836402 .623613-0.819636$

H $-2.918818-0.9851751 .344726$

H $-0.905778-3.0451152 .388776$

H $-0.971851-1.117264-0.054097$

H - $-1.476016-3.525225-0.688452$

H $\quad 0.002322-4.017540 \quad 0.114566$

H $1.844223-3.337892-3.075631$

H $0.156719-1.895381-3.637620$

H $-1.384110-2.105518-2.804881$

H $-0.206736-0.905234-2.223100$

H $1.432440-5.291568-0.794678$

H $2.565576-5.368371-2.111737$

H $2.811299-3.474593 \quad 0.283233$

H $3.618654-5.0361340 .130038$

H $4.770098-4.186502-1.957136$

H $5.912244-4.1160810 .301081$

H $5.177369-2.5585150 .618264$

H $7.864084-2.6737620 .714423$

H $5.901019-0.510651-0.941784$

H $8.026913-4.209079-1.025466$

H $8.436940-2.936013-2.151349$

H $7.030570-3.976370-2.461797$

H $6.8871310 .129880-2.848099$

H $4.8649230 .121120-3.884763$

H $3.356819-0.728950-3.691583$

H $6.269778-2.485613-3.713444$

H $3.136966-1.0618921 .368174$

H 2.1548001 .4867022 .791120

H $5.3128120 .097575 \quad 1.307383$

H $5.213362-0.9543362 .740054$

H $6.343320 \quad 0.3981842 .683173$

H $2.7809351 .078089-2.488851$

H $3.4659380 .148982-1.152902$

H $4.0973504 .556690-0.111939$

H $5.4058944 .252601-1.276785$

H $\begin{array}{llll}6.554560 & 2.742919 & 0.508471\end{array}$

H 5.1511955 .0182362 .004044

H 7.5092934 .8237023 .007869

H 7.5120413 .1051552 .689208

H 5.6393204 .4852046 .162170

H 6.4323805 .6750454 .984433

H 5.5718082 .1684485 .660486

H 6.5605981 .5870014 .359042

H 3.6057382 .5030104 .230634

H $3.3536352 .351397-0.300943$

C -4.670072 -2.681137 2.942044

O $-5.474069-1.7756822 .160510$

C $-6.171601-2.5365731 .154464$

C $-6.044121-3.9979231 .570294$

C $-4.654578-4.0066372 .180131$

H $-5.621047-2.3962560 .216407$

C -7.606943 $-2.026730 \quad 0.991457$

H $-6.791033-4.2742162 .323433$

H $-6.147029-4.6819980 .722647$

C $-4.375371-5.2170473 .052990$

H -3.921974 -3.980266 1.363906
C $-7.708084-0.512028 \quad 0.713567$

O $-8.325303-2.294237 \quad 2.202313$

H -8.110536 -2.5967030 .201623$

H -7.4131410 .0450191 .610796$

$\begin{array}{llll}\text { O } & -9.090193 & -0.200645 & 0.496397\end{array}$

C $-6.883077-0.023103-0.491260$

C $-7.0715951 .483827-0.747851$

H $-5.825109-0.230875-0.303520$

O $-7.274916-0.762844-1.645572$

H -8.119904 $1.685931-0.999193$

H -6.8550612 .0216570 .182003$

H $-3.368427-5.1649553 .479274$

H $-5.089131-5.2893503 .880373$

H $-4.446982-6.1388192 .466462$

C -3.304482 -2.057407 3.243134

H $-9.231219-1.9614092 .081125$

H $-9.361344-0.634669-0.330912$

H $-6.621861-0.588116-2.344119$

H $-2.740568-2.7193043 .910792$

H $-3.458030-1.1151133 .783830$

H $-5.201180-2.8076853 .894910$

C $-4.0999572 .180221-0.710512$

O $-4.8320041 .599956-1.794165$

C $-6.1913642 .032230-1.895060$

C $-6.2485783 .558207-2.060640$

C $-5.4689764 .284109-0.962791$

C $-4.056848 \quad 3.708554-0.833349$

H -4.5885651 .9080350 .233546$

C $-2.7183491 .516757-0.665971$

H $-6.5815911 .595735-2.823102$

O $-7.6047513 .990622-2.073472$

H $-5.8152303 .823174-3.034090$

H $-5.9959484 .245784-0.002101$

O $-5.352250 \quad 5.667918-1.303468$

H -3.4736814.022034-1.707899

$\begin{array}{lllll} & \mathrm{O} & -3.443797 & 4.277765 & 0.320745\end{array}$

C $-1.837868 \quad 1.889330-1.836816$

H -2.2116351 .7751830 .270871$

H $-2.818840 \quad 0.425597-0.688015$

H -7.611842 $4.934728-2.303708$

H $-6.2425626 .055945-1.263919$

H $-2.5035094 .031822 \quad 0.308358$

O $-0.6652992 .418980-1.384899$

O $-2.150509 \quad 1.729429-3.009571$

C $0.253893 \quad 2.830877-2.400513$

H $\quad 0.505742 \quad 1.988522-3.051885$

H $-0.1725743 .653985-2.981422$

H $1.1661363 .182041-1.911265$

SCF Energy (B3LYP/6-31G**//MMFF) $=-3245.91061540$

$03 \_302$

MMFF Geometry

C $-4.116768 \quad 1.740094 \quad 1.618416$

C $-5.1872301 .842787 \quad 0.814588$

C $-5.3351642 .825955-0.324950$

O $-4.144002 \quad 3.632683-0.440872$

C $-5.692978 \quad 2.165294-1.670034$

C $-4.6769951 .199526-2.247905$

C $-4.829518-0.137995-2.315793$

C $-3.4439161 .827321-2.850961$

C $-5.954782-0.992705-1.797836$

C $-5.595721-1.791096-0.533041$

C $-4.636778-2.974925-0.758616$

C $-4.366221-3.690540 \quad 0.569028$

C $-3.288634-4.7803990 .452022$

O $-3.414143-2.475070-1.296798$

O $-2.858439-5.1221541 .779083$

C $-2.059479-4.197986-0.280779$

C $-3.851271-6.066963-0.163274$

O $-1.092196-5.211007-0.542494$

C $-2.430110-3.471152-1.588298$

C $-1.228609-2.759506-2.247236$

O $-2.918398-4.427561-2.536408$

C $-4.2509964 .900728-0.905150$

O $-5.2565815 .430000-1.357525$

C $-2.9741335 .640304-0.747333$

C $-1.8374615 .086750-0.293154$

C $-0.5773745 .787181-0.027200$ 
C $-0.5367077 .271521-0.282596$

C $-0.630229-1.602653-1.442694$

C $0.441254-0.872823-2.235063$

C $1.844096 \quad 1.159857-2.222591$

O $0.871459-1.280009-3.311894$

C $2.5175502 .008132-1.194275$

C $2.7789723 .310715-1.378724$

C $3.5261764 .155305-0.388394$

C $2.8808405 .503291-0.160329$

C $3.3194836 .592305-0.814323$

C 1.8082295 .6119870 .897070

C $0.470492 \quad 5.082986 \quad 0.452882$

N $0.8859310 .273910-1.589197$

H $\quad-3.2556352 .3807301 .447625$

H -6.054449 1.2178141 .016746

H $-6.1673673 .486268-0.044259$

H $-5.8498212 .953125-2.419023$

H -6.670749 $1.683611-1.565298$

H $-4.045477-0.698029-2.822441$

H -2.864903 $1.115443-3.449553$

H -3.717852 $2.655526-3.512657$

H -2.780908 2.205504 -2.067789

H -6.836332 $-0.385081-1.574582$

H $-6.264241-1.681661-2.593163$

H $-5.158971-1.1049440 .202619$

H $-6.526573-2.176023-0.097573$

H $-5.095908-3.661560-1.479453$

H $-5.293322-4.1066870 .982468$

H $-4.020901-2.9518631 .302419$

H -3.639013 -5.4108982 .282355$

H $-1.584799-3.4827460 .403367$

H $-4.645829-6.4784890 .470819$

H -3.083305 -6.845891-0.223446

H $-4.273484-5.917403-1.159072$

H $-0.884742-5.6397350 .305507$

H $-0.449619-3.492235-2.495564$

H $-1.559543-2.354338-3.214532$

H -2.846117 -4.046707 -3.427216

H $-3.0347486 .695113-0.996359$

H -1.824295 $4.023600-0.059979$

H -1.2563287 .7927950 .358308$

H $-0.7756177 .487949-1.329906$

H $0.4410857 .719187-0.092905$

H - $-1.409051-0.880839-1.172550$

H $-0.169844-1.979627-0.525263$

H $1.3001171 .756248-2.962772$

H $2.5880710 .554313-2.750023$

H $2.8484551 .525152-0.278533$

H $2.4879023 .781674-2.315208$

H $4.5445894 .295376-0.774450$

H 3.6425403 .6383980 .571937

H $2.8932747 .572984-0.632148$

H $4.1144896 .526774-1.550074$

H 1.7170676 .6453901 .251531

H 2.1236255 .0528361 .787845

H $0.355802 \quad 4.0083900 .597253$

H $\quad 0.3531120 .623421-0.797611$

C -2.859055 -0.1376032 .751198$

O $-1.627788 \quad 0.5644432 .495118$

C $-0.575879-0.0117363 .297461$

C $-1.163952-1.2672683 .924904$

C -2.624356 -0.8870184 .062988$

H -0.3455070 .7355574 .066521$

C $0.643270-0.2624672 .403903$

H $-1.054964-2.1282573 .253260$

H $-0.692561-1.5188474 .879657$

C -3.542935 -2.072302 4.294319

H $-2.722057-0.1932984 .909362$

C $1.828556-0.9095803 .140382$

O $1.073763 \quad 0.9961891 .875080$

H $\quad 0.338403-0.8746461 .550397$

H $1.534800-1.8772843 .562063$

O $2.179896-0.0789324 .254997$

C $3.088957-1.0995472 .273121$

C $2.879294-2.0885311 .114203$

H $3.445232-0.132957 \quad 1.900735$

O $4.126898-1.6308183 .105794$

H $2.662885-3.0808981 .528671$
H $2.021753-1.7712140 .515020$

H $-4.590987-1.7590464 .321368$

H -3.432348 -2.825339 3.509734

H $-3.310345-2.5550365 .249366$

C -4.0273250 .8486602 .825709$

H $\quad 0.3078821 .4042151 .435885$

H $2.418707 \quad 0.796133 \quad 3.903473$

H $4.251391-1.0098463 .843931$

H $-3.893646 \quad 1.5075253 .693196$

H -4.9652650 .3024662 .978603$

H -3.005651 -0.8350091 .917206$

C $5.310596-0.827048-1.419635$

O $4.194867-0.957682-0.530736$

C $4.113085-2.1882730 .194819$

C $4.012556-3.372814-0.777675$

C $5.167415-3.363726-1.784275$

C $5.354419-1.986402-2.429456$

H $5.1139530 .097527-1.977308$

C $6.621621-0.630592-0.652152$

H $5.009058-2.3065420 .812745$

O $2.768645-3.342756-1.473461$

H $4.033699-4.305161-0.201345$

H $4.965754-4.109414-2.563178$

O $6.361160-3.748443-1.108665$

H $6.295107-1.955840-2.991439$

O $4.296391-1.803942-3.375374$

C $6.581245 \quad 0.666855 \quad 0.121036$

H $6.842042-1.4471100 .041312$

H $7.472933-0.572087-1.340425$

H $2.724746-2.506000-1.966891$

H $7.075863-3.773067-1.767569$

H $4.452833-0.960722-3.833705$

O $6.2777720 .421701 \quad 1.427221$

O $6.763604 \quad 1.766994-0.384284$

C $6.155287 \quad 1.578425 \quad 2.260049$

H 5.3441202 .2182941 .900309

H 5.9137351 .2437843 .272458

H 7.1003622 .1288182 .287183

SCF Energy (B3LYP/6-31G**//MMFF)= -3245.91936714

0331

MMFF Geometry

C $-2.0643112 .980377 \quad 1.471688$

C $-2.131600 \quad 3.833244 \quad 0.437607$

C $-2.5061803 .451905-0.977501$

O $-2.228182 \quad 2.049709-1.209550$

C $-4.0040463 .694409-1.216007$

C $-4.3829113 .626670-2.682132$

C $-4.9534002 .557589-3.270823$

C $-4.128594 \quad 4.883219-3.472921$

C $-5.249228 \quad 1.226691-2.640585$

C $-6.7227101 .109233-2.239518$

C $-7.043520-0.275407-1.660327$

C $-8.527075-0.381018-1.301425$

C $-8.861659-1.725843-0.638777$

O $-6.242748-0.470517-0.489048$

O $-10.164515-1.614385-0.045763$

C $-7.862711-1.9797380 .509613$

C $-8.948686-2.861964-1.664920$

O $-8.036547-3.2849391 .057067$

C $-6.384740-1.7672690 .104530$

C $-5.481494-1.8316841 .359053$

O $-5.994904-2.806741-0.797292$

C $-0.9433611 .711451-1.505203$

O $-0.0114322 .471484-1.713132$

C $-0.8490040 .235427-1.468189$

C $0.349431-0.333307-1.266390$

C $0.627214-1.768214-1.160207$

C $-0.521876-2.716210-1.382775$

C $-4.040439-1.351572 \quad 1.128020$

C $-3.139558-1.6826892 .307298$

C $-0.743054-1.6355412 .987457$

O $-3.542782-2.241013 \quad 3.324356$

C $-0.047538-2.8511602 .460731$

C $1.282899-2.9333262 .310592$

C $1.984532-4.1485051 .777627$

C $2.966160-3.8222820 .673611$

C $4.289332-3.8471930 .904518$ 
C $2.421496-3.559242-0.714318$ C $1.886242-2.160583-0.870028$ N -1.826215 -1.291505 2.083612 $\mathrm{H}-2.3487301 .940807 \quad 1.327331$ H -1.860568 4.8751780 .592545 H -1.909624 4.057270 -1.671246 H $-4.2953154 .681947-0.833367$ H $-4.5939312 .970975-0.639356$ H $-5.2205222 .627293-4.324250$ H -4.451236 $4.790805-4.515535$ H -4.672766 $5.725530-3.033134$ H -3.061083 $5.124235-3.479415$ H $-4.9989140 .443609-3.367050$ H $-4.6046971 .045452-1.775386$ H $-6.9465901 .880239-1.490498$ H -7.362402 $1.301168-3.110037$ H -6.776769 -1.029671 -2.410154 H $-9.154778-0.222739-2.187182$ H $-8.7898990 .424012-0.600646$ H -10.794581 -1.380183 -0.748697 H -8.104067 -1.2715341 .314867$ H -9.726811 -2.648117 -2.407430 H $-9.241778-3.803690-1.187857$ H -8.014104 -3.024564 -2.205626 H -8.971472 -3.3701591 .311238$ H $-5.908403-1.2044802 .152619$ H -5.464214 -2.862728 1.735052 H $-5.042275-2.966743-0.711212$ H $-1.766448-0.333325-1.565343$ H $\quad 1.2164450 .316002-1.148434$ H -1.316879 -2.539584 -0.651391 H $-0.247130-3.768506-1.291446$ H $-0.937025-2.584896-2.388244$ H $-3.611066-1.8242610 .239001$ H -4.026377 $-0.265846 \quad 0.977507$ H -1.141959 -1.836340 3.986480 H $-0.071326-0.7750893 .040702$ H $-0.665944-3.7053592 .194663$ H $1.904287-2.0906672 .604262$ H $1.268995-4.8952541 .411266$ H $2.507858-4.6236332 .617596$ H $5.002355-3.6525590 .112386$ H $4.689374-4.0628311 .889644$ H $1.688258-4.332035-0.958412$ H $3.219767-3.683055-1.458721$ H $2.648125-1.391151-0.735026$ H -1.580935 -0.871689 1.191964 C -0.3237752 .5100713 .162442$ $\begin{array}{lllll}\text { O } & 0.763894 & 2.921831 & 2.314325\end{array}$ C 2.0008262 .7738853 .036421 C 1.6341182 .1678604 .388627 C 0.2104062 .6601834 .580483 H 2.4001413 .7840503 .189796 C 3.0078331 .9447732 .222343 H 1.6610401 .0718494 .356906 H 2.3116532 .4933535 .184058 C -0.5650371 .8762175 .624188$ H 0.2367083 .7207144 .863653 C 3.4601782 .6238140 .913064 $\begin{array}{lllll}\text { O } & 2.442787 & 0.668051 & 1.923484\end{array}$ H 3.8861121 .7674022 .852475 H 3.9570633 .5742131 .143837 O 2.3244912 .9466220 .103088 C $4.3811051 .769502 \quad 0.021333$ C $5.6382691 .248376 \quad 0.734634$ H $3.8182910 .934815-0.412226$ O $4.8022062 .593102-1.073784$ H 6.1856392 .0965041 .164393 H 5.3333130 .5867101 .552620 H -1.586138 2.2578655 .721191 H $-0.625854 \quad 0.8137195 .365611$ H -0.0809501 .9565446 .603081$ C -1.5650923 .3415702 .843342$ H 1.6538270 .8202531 .375284 H 1.7801433 .5849450 .593822 H $3.9993142 .902297-1.527555$ H -1.327111 4.4105642 .895379 H -2.3618083 .1381703 .566610$
H $-0.516607 \quad 1.4533222 .942920$

C $6.603603-1.505445-1.606395$

O $5.894771-0.694435-0.659426$

C $6.5825540 .478859-0.210503$

C 7.9008720 .0967800 .478731

C $8.771807-0.759448-0.446132$

C $7.980370-1.920076-1.058356$

H $5.997258-2.414612-1.705528$

C $6.670517-0.841941-2.986304$

H $6.8069521 .118517-1.070068$

O $7.621656-0.6219161 .678191$

H 8.4341441 .0137000 .756959

H $9.620723-1.1557790 .123969$

O $9.2896860 .073204-1.479699$

H $8.562215-2.395410-1.856035$

O $7.784433-2.920408-0.054441$

C $5.302980-0.820391-3.627623$

H $7.0609750 .179280-2.966440$

H $7.326633-1.409713-3.657048$

H $8.472237-0.8193062 .106106$

H $9.859666-0.478096-2.042679$

H $7.220707-2.5381910 .639874$

O $4.6347810 .311254-3.267289$

O $4.874932-1.715091-4.345396$

C $3.3097840 .442686-3.791296$

H $2.717492-0.451892-3.577389$

H $2.835311 \quad 1.299597-3.305616$

H $3.3537570 .626376-4.868563$

SCF Energy (B3LYP/6-31G**//MMFF) $=-3245.92276532$

0332

MMFF Geometry

C $0.989598 \quad 5.3060810 .576045$

C $1.9340794 .689104-0.149107$

C $2.8764313 .663470 \quad 0.427874$

O $2.6641942 .414707-0.272286$

C 4.3359054 .0962040 .203657

C 5.3456733 .1969610 .891421

C $6.2561092 .435154 \quad 0.254261$

C 5.3137953 .2106082 .399023

C $6.4864592 .301065-1.224104$

C $5.7091731 .161408-1.900972$

C $6.005160-0.235943-1.330940$

C $5.413912-1.322041-2.233252$

C $5.600449-2.728910-1.643089$

O $5.417761-0.326215-0.029401$

O $4.745304-3.627515-2.367101$

C $5.116332-2.730003-0.176633$

C $7.031814-3.242691-1.838616$

O $5.422268-3.9681340 .459023$

C $5.677852-1.5591060 .655835$

C $5.041789-1.4483442 .064088$

O $7.084790-1.7511940 .849241$

C $1.568151 \quad 1.6860840 .075077$

O $0.760947 \quad 1.941467 \quad 0.954319$

C $1.5006640 .519405-0.830446$

C $0.345359-0.149695-0.954224$

C $0.112707-1.323864-1.796266$

C $1.312885-1.957465-2.448842$

C $3.522447-1.2493812 .087991$

C $2.731117-2.5378722 .241890$

C $0.395025-3.4030312 .159872$

O $3.242636-3.6105832 .551119$

C $0.148096-4.0152830 .817081$

C $-1.080375-4.2133290 .315288$

C $-1.350756-4.863240-1.011895$

C -2.260489-4.036402 -1.893706

C $-3.572814-4.310688-1.982197$

C $-1.635240-2.950547-2.740126$

C $-1.148603-1.786214-1.923579$

N $1.372572-2.3383522 .033772$

H 0.9154745 .0970861 .640390

H $2.0023114 .884067-1.216708$

H 2.6858213 .5153401 .497573

H 4.4843175 .1175880 .579172

H $4.5288984 .137734-0.873808$

H $\quad 6.931111 \quad 1.8327240 .861809$

H 4.4026882 .7332862 .771989 
H $\quad 6.1646312 .6775782 .837289$

H 5.3491954 .2397272 .771133

H $6.2603723 .241226-1.738771$

H $7.5609482 .142498-1.383088$

H $4.636370 \quad 1.371598-1.822684$

H $5.9631051 .177237-2.968163$

H $7.092162-0.355745-1.252410$

H $5.846589-1.269453-3.240164$

H $4.338680-1.140026-2.361985$

H $4.988438-3.580197-3.307631$

H $4.022649-2.653659-0.204595$

H $7.279864-3.287363-2.905962$

H $7.139013-4.266759-1.464275$

H $7.782815-2.616420-1.352789$

H $5.031598-4.675904-0.081708$

H $5.348182-2.2995812 .683806$

H $5.481977-0.5643102 .547884$

H $7.364297-1.2287271 .619248$

H $2.3975860 .250896-1.375398$

H $-0.5203980 .202279-0.396377$

H $1.747617-1.282303-3.193721$

H $1.091898-2.898369-2.956243$

H $2.075196-2.192143-1.698035$

H $3.266789-0.6318762 .958779$

H $3.184777-0.713503 \quad 1.197226$

H $-0.511129-2.9627382 .587607$

H $0.772627-4.1653942 .848774$

H $1.015606-4.3334920 .243647$

H -1.944549 -3.9195830 .905799$

H -1.811163 -5.840336 -0.815189

H $-0.419384-5.077824-1.550734$

H -4.227692 -3.741657 -2.633617

H -4.023579 -5.107729-1.400165

H -2.370465 -2.557370 -3.455052

H $-0.851802-3.396047-3.358691$

H $-1.948223-1.261879-1.398882$

H $1.044268-1.4274631 .726942$

C $-1.3996185 .618526-0.220164$

O $-1.3197764 .612826-1.247122$

C $-2.1703983 .492711-0.890898$

C $-3.000608 \quad 3.9850340 .289131$

C -2.043109 4.9363060 .980981

H -1.492229 2.691008 -0.576932

C -2.950303 $3.056805-2.139572$

H -3.889454 4.525801-0.057359

H $-3.320166 \quad 3.1857070 .960687$

C -2.719203 5.8940541 .945472

H -1.3133434 .3319521 .532806$

C $-3.6772241 .697183-2.074980$

O $-1.9814252 .938240-3.201386$

H $-3.6339383 .854676-2.453889$

H -2.928226 $0.897284-2.042785$

O $-4.364991 \quad 1.554452-3.327687$

C $-4.6821101 .503025-0.931787$

C $-5.4906040 .194028-1.034866$

H -4.1378841 .4992680 .012967$

O $-5.5960042 .598300-0.901336$

H $-6.2088760 .278893-1.858733$

H -4.811034 -0.630374 -1.273432

H -1.9862446 .5650192 .404521$

H -3.4710376 .5095651 .440146$

H -3.2212015 .3421492 .746849$

C $-0.022761 \quad 6.2548590 .000759$

H -2.468033 $2.704013-4.010395$

H -4.577076 $0.612756-3.442610$

H $-6.0357562 .639618-1.767859$

H -0.1134067 .1138620 .675543$

H $\quad 0.3452816 .642654-0.957712$

H -2.070301 $6.394635-0.612443$

C $-4.596067-1.4473271 .393893$

O $-5.388979-0.2528451 .393247$

C $-6.266364-0.1057290 .269203$

C -7.223209-1.303164 0.186611

C $-6.476500-2.6327770 .200073$

C $-5.502057-2.6846451 .375374$

H $-3.933754-1.4579630 .520210$

C -3.720320 -1.422543 2.656634

H -6.8832810 .7771280 .479595$
O $-8.033634-1.233363-0.981467$

H -7.902264 -1.282185 1.049066

H $-5.958809-2.809543-0.749184$

O $-7.416076-3.6990790 .351095$

H $-6.065168-2.7549722 .314888$

O $-4.701463-3.858421 \quad 1.268824$

C -2.608032 -0.3977512 .569819$

H -4.335862 -1.172844 3.528902

H -3.244482 -2.396744 2.812174

H $-8.513119-0.387735-0.953832$

H $-8.052681-3.628868-0.380991$

H -5.305378 -4.619462 1.223505

O $-1.930937-0.379575 \quad 3.756405$

$\begin{array}{lllll}0 & -2.353776 & 0.290996 & 1.592078\end{array}$

C -0.8388970 .5428293 .826780$

H $-1.183321 \quad 1.5611203 .621965$

H -0.4295690 .5084644 .839872$

H $-0.055495 \quad 0.250527 \quad 3.122093$

SCF Energy $\left(B 3 L Y P / 6-31 G^{* *} / / M M F F\right)=-3245.91149860$

0333

MMFF Geometry

C $2.592123-4.6525110 .789733$

C $1.681616-4.219354-0.093153$

C $0.197797-4.1832370 .168244$

O $-0.225740-2.7996090 .168912$

C $-0.541195-4.908235-0.971063$

C $-2.050318-4.899642-0.828145$

C $-2.894497-4.299434-1.690669$

C $-2.599022-5.6441740 .361537$

C $-2.553375-3.541024-2.943612$

C $-2.363128-2.028915-2.747476$

C $-3.635364-1.288765-2.303066$

C $-3.4246830 .226262-2.349170$

C $-4.6513960 .993934-1.831269$

O $-3.947139-1.682588-0.964613$

O $-4.2546802 .355551-1.604804$

C $-5.0707930 .412655-0.463280$

C $-5.7768731 .037730-2.871502$

O $-6.3028060 .979040-0.024803$

C $-5.165924-1.127348-0.451301$

C $-5.375524-1.7148710 .966107$

O $-6.288405-1.534716-1.243498$

C $0.007297-2.0734551 .295930$

O $0.491588-2.4712432 .343351$

C $-0.399116-0.6780881 .018982$

C $\quad 0.0577520 .3071601 .805645$

C -0.2223131 .7356651 .651863$

C -1.2603132 .1382660 .638453$

C $-4.275817-1.3885621 .983242$

C $-4.584425-0.1899662 .865757$

C -3.5434921 .2492214 .614686$

$\begin{array}{lllll}\text { O } & -5.683755 & 0.355730 & 2.903538\end{array}$

C -3.0807882 .5228303 .980541$

C -2.1250713 .3027574 .508251$

C -1.6843434 .6084913 .911157$

C -0.1885554 .6790703 .700474$

C 0.6069295 .2946574 .591421

C 0.3713374 .1138242 .415661

C 0.4494132 .6114262 .428378

N $-3.491471 \quad 0.1740193 .641651$

H $2.264331-5.0323731 .753782$

H $2.022122-3.830949-1.049133$

H $-0.049240-4.6449751 .131956$

H $-0.206415-5.952958-1.023406$

H $-0.247472-4.451465-1.922372$

H -3.962848 -4.362212 -1.486477

H $-3.691657-5.7193270 .336648$

H $-2.202371-6.6642650 .387410$

H $-2.327309-5.1381861 .293009$

H - $1.653821-3.954509-3.411902$

H -3.355340 -3.712449 -3.673276

H -1.563321 -1.862242 -2.016746

H $-2.020677-1.609772-3.701884$

H -4.454154 -1.580580 -2.971287

H $-3.1671960 .554389-3.364000$

H -2.563104 $0.490999-1.722113$

H $-3.9176092 .712944-2.444211$ 
$\begin{array}{llll}H & -4.309410 & 0.725531 & 0.260034\end{array}$

H $-5.4291731 .523376-3.791167$

H $-6.6201361 .640145-2.516361$

H $-6.1492210 .047700-3.142507$

H $-6.192547 \quad 1.945363-0.021084$

H $-6.369760-1.445673 \quad 1.342640$

H $-5.392511-2.8094960 .861950$

H -6.548797 -2.430419 -0.971619

H $-1.035074-0.4982600 .160258$

H $\quad 0.722307 \quad 0.0483112 .629148$

H -2.1926001 .5909490 .810653$

H $-0.912728 \quad 1.927024-0.377447$

H -1.5227183 .1974350 .677820$

H -4.168791 -2.244353 2.662457

H -3.311000 -1.242342 1.488689

H -2.9124280 .9536565 .458812$

H -4.5714611 .3680974 .972089$

H -3.5744792 .8320573 .062343$

H -1.654017 3.0049575 .442453

H -2.002046 5.4090984 .591948

H -2.196272 4.8090102 .961648

H 1.6773835 .3748474 .433550

H 0.2056095 .7273155 .501901

H 1.3922044 .4865782 .255014

H -0.1997824 .5063651 .569543$

H 1.1776332 .2205093 .140530

H -2.603495 -0.3006183 .505730$

C $4.791711-3.4489721 .213579$

O $4.371247-2.1937040 .638579$

C $4.277115-1.2162971 .691867$

C $4.934489-1.8474232 .916880$

C $4.595660-3.3114142 .722574$

H $3.207742-1.0748021 .892535$

C $4.897987 \quad 0.1282341 .285504$

H $6.021582-1.7042112 .911276$

H $4.545722-1.4360553 .853432$

C $5.454195-4.2553423 .545098$

H $3.544067-3.4526892 .999566$

C $4.165654 \quad 0.8090640 .106536$

O $6.273749-0.0820090 .971124$

H 4.8575370 .7929342 .157505

H 3.0944010 .8007280 .331253

O $4.3532570 .060009-1.090629$

C $4.6124612 .266288-0.125201$

C $3.8776103 .012143-1.258851$

H 4.4798412 .8252300 .809477

O $6.0094682 .289129-0.428807$

H $4.3241414 .012353-1.318856$

H $4.1195342 .536255-2.214326$

H $5.176575-5.2972373 .356603$

H $6.517347-4.1409793 .308722$

H $5.325437-4.0592284 .614569$

C $4.072196-4.6172550 .531760$

H $\quad 6.670404 \quad 0.7859270 .786896$

H $5.3090180 .029868-1.268291$

H $6.2833203 .220199-0.489431$

H $4.502532-5.5669960 .870074$

H $4.251985-4.557882-0.549487$

H $5.862598-3.5360150 .985309$

C $1.5670911 .749965-2.870364$

O $1.6551291 .950043-1.453628$

C $2.3552843 .126864-1.036072$

C $1.6990954 .368192-1.654865$

C $1.6053024 .257650-3.172255$

C $0.9142112 .952370-3.561206$

H $2.5692481 .582889-3.281973$

C $0.7392690 .481478-3.102527$

H 2.2036823 .2097060 .046227

O $2.4130085 .542563-1.285547$

H $0.6857834 .470930-1.244150$

H $2.5960544 .332243-3.634924$

O $\quad 0.8497295 .358861-3.681512$

H $-0.1501143 .006010-3.297581$

O $\quad 0.996658 \quad 2.782537-4.974755$

C $1.545396-0.746156-2.763370$

H $-0.1575180 .470332-2.472730$

H $\quad 0.399714 \quad 0.392183-4.140769$

H $1.9810506 .295854-1.723406$
H $-0.0370215 .325326-3.283636$

H $0.605473 \quad 3.572401-5.385900$

O $2.195384-1.200554-3.871640$

O $1.608272-1.226316-1.640110$

C $3.007458-2.362888-3.678569$

H $3.708734-2.215513-2.851535$

H $2.370682-3.232988-3.495208$

H $3.579690-2.533123-4.594351$

SCF Energy $\left(B 3 L Y P / 6-31 G^{* *} / / M M F F\right)=-3245.90461922$

03_34

MM̄FF Geometry

C $3.604895-3.336854-2.926804$

C $2.715641-2.366723-3.185954$

C $1.228312-2.589161-3.276605$

O $0.608251-1.928938-2.148650$

C $0.688037-1.949639-4.568570$

C $-0.812135-2.084254-4.742423$

C -1.666854 -1.043720 -4.802250

C $-1.334050-3.491286-4.876638$

C $-1.3412820 .422285-4.725141$

C $-1.3459361 .004696-3.303741$

C $-2.7328741 .029462-2.641108$

C $-2.6820011 .784835-1.311896$

C $-4.0324861 .757707-0.579160$

O $-3.154411-0.315346-2.406626$

$\begin{array}{llll}O & -3.806855 & 2.183812 & 0.773793\end{array}$

C $-4.5484980 .303114-0.516008$

C $-5.0269342 .754544-1.186119$

O $-5.8773270 .254171-0.003437$

C $-4.478691-0.434480-1.868486$

C $-4.791178-1.946643-1.756069$

O $-5.4484450 .128329-2.760849$

C $0.596288-2.604784-0.967030$

O $1.026792-3.727303-0.754969$

C $-0.009681-1.7209350 .051295$

C $0.205117-1.9799421 .349703$

C $-0.296634-1.1988652 .481331$

C -1.316362 -0.1311882 .190394$

C $-3.862758-2.743629-0.832190$

C $-4.421622-2.9667950 .563831$

C $-3.800458-3.9672582 .761052$

O $-5.571006-2.6790980 .886143$

C $-3.465073-2.855083 \quad 3.703232$

C $-2.681470-3.0131104 .780925$

C $-2.384037-1.9204245 .767167$

C $-0.901757-1.7116345 .980229$

C $-0.272258-2.2489687 .038908$

C $-0.171895-0.8048105 .016226$

C $0.161397-1.4861593 .717676$

N $-3.490432-3.5679151 .401005$

H $3.261483-4.357855-2.782721$

H $3.068974-1.344037-3.300026$

H $0.986284-3.659024-3.261625$

H $1.176862-2.406642-5.439215$

H $0.978208-0.893440-4.580618$

H -2.726830 -1.258936 -4.932348

H -1.218878 -4.037379 -3.935473

H -2.396386 -3.516395 -5.142142

H $-0.789036-4.025521-5.661723$

H $-0.3693750 .622534-5.189272$

H -2.066562 $0.966723-5.343252$

H $-0.6465580 .435558-2.680182$

H $-0.9622082 .030447-3.363393$

H $-3.4325691 .517208-3.329879$

H $-2.3575392 .819276-1.469994$

H $-1.9234201 .328517-0.661487$

H -3.3988163 .0664680 .746202$

H $-3.918243-0.2258000 .206677$

H $-4.625823 \quad 3.774112-1.135527$

H $-5.9637622 .774230-0.618398$

H -5.260398 $2.543659-2.231818$

H -5.8721540 .7046980 .858396$

H $-5.846891-2.095424-1.499425$

H $-4.676046-2.374809-2.762428$

H $-5.643990-0.522979-3.454660$

H $-0.586743-0.870985-0.293654$

H $0.829625-2.8328931 .613280$ 
H $-0.8654250 .692606 \quad 1.628107$ H -1.772173 0.2931563 .087164 H -2.141928 -0.5452781 .603671$ H -3.721131 -3.742591-1.264834 H -2.875912 -2.276294 -0.761424 H -3.227202 -4.875741 2.971254 H $-4.867037-4.2031512 .835674$ H -3.913681-1.883722 3.508645 H $-2.257728-3.9930404 .989462$ H -2.862140 -2.192062 6.717554 H $-2.846087-0.9718215 .466683$ H $0.784740-2.0784487 .214702$ H $-0.799179-2.8761427 .750409$ H $0.777154-0.4739245 .459289$ H $-0.747990 \quad 0.114312 \quad 4.881330$ H $0.894121-2.2871813 .825110$ H -2.542964 -3.718071 1.066321 C $5.499731-2.639742-1.376742$

O $4.928064-1.348559-1.083510$

C $4.432458-1.3609620 .268247$

C $5.081022-2.5690220 .934623$

C $5.107030-3.557189-0.218066$

H $3.353418-1.5419360 .209263$

C $4.695631-0.0121610 .945372$

H $6.101496-2.3511081 .270156$

H $4.507775-2.9266481 .795300$

C $6.057808-4.721918-0.007453$

H $4.091766-3.949545-0.354026$

C 4.1606321 .2066890 .157678

$\begin{array}{llll}\text { O } & 6.113435 & 0.144413 & 1.085610\end{array}$

H $4.280081-0.0253711 .960317$

H $4.7069391 .316626-0.786776$

O 4.4414762 .3705810 .942327

C $2.6468231 .154089-0.121856$

C $2.0841202 .250393-1.050141$

H $2.4120690 .209531-0.621831$

$\begin{array}{lllll}\text { O } & 1.932377 & 1.140754 & 1.108374\end{array}$

H $2.4931202 .073409-2.052650$

H $1.0016502 .087245-1.119657$

H $6.050943-5.390032-0.874474$

H $7.086259-4.3786390 .146467$

H $5.763364-5.3045350 .871562$

C $5.079596-3.085832-2.781020$

H $6.266494 \quad 0.8756151 .707462$

H 5.4017042 .5186630 .913702

H 2.2027501 .9309731 .605603

H $5.616610-4.000492-3.057909$

H $5.379530-2.316181-3.504097$

H $6.587557-2.489939-1.380891$

C $0.504354 \quad 4.100028 \quad 0.869982$

O 1.9204784 .0433090 .661578

C $2.3391073 .723732-0.666181$

C $1.7546304 .739245-1.661684$

C $0.2400494 .853507-1.532125$

C $-0.1503865 .111409-0.079532$

H $0.069803 \quad 3.1045410 .717862$

C $0.272524 \quad 4.5158192 .329191$

H $3.4246363 .870924-0.693377$

O $2.082945 \quad 4.391931-3.002873$

H $2.1925885 .727581-1.469932$

H $-0.2642923 .964392-1.924451$

O $-0.2190875 .954286-2.319949$

H $0.140738 \quad 6.1326580 .198789$

O

C $0.591827 \quad 3.397198 \quad 3.290815$

H 0.8804845 .3878562 .599606

H -0.7738504 .7922682 .504605$

H $3.0525164 .347434-3.063766$

H $0.0715645 .795886-3.234595$

H -1.956724 $5.652334-0.583316$

$\begin{array}{llllll}\text { O } & 1.931647 & 3.357447 & 3.539593\end{array}$

O $-\begin{array}{llll}0.251681 & 2.644330 & 3.759183\end{array}$

C $2.361406 \quad 2.3327504 .441770$

H $1.8873102 .463628 \quad 5.418980$

H 2.1325031 .3453834 .030542

H 3.4445962 .4187604 .561723

SCF Energy (B3LYP/6-31G**//MMFF) $=-3245.91413707$
03_35

MMFF Geometry

C $2.914593-3.832804-0.463570$

C $2.064276-4.7522910 .016907$

C $0.597529-4.5524170 .330721$

O $0.199895-3.1650500 .246178$

C $-0.231886-5.345241-0.698558$

C $-1.728677-5.300077-0.466798$

C $-2.620006-4.782721-1.335383$

C $-2.209956-5.9170320 .821140$

C $-2.351972-4.152626-2.674807$

C $-2.200031-2.623861-2.635499$

C $-3.499801-1.875472-2.297242$

C $-3.304932-0.363673-2.424382$

C $-4.5651300 .415716-2.012941$

O $-3.873228-2.190867-0.955610$

O $-4.1958611 .793356-1.844363$

C $-5.053104-0.092168-0.637594$

C $-5.6343610 .385759-3.111372$

O $-6.3162120 .475961-0.301042$

C $-5.124812-1.629172-0.539726$

C $-5.394248-2.1383180 .896995$

O $-6.199341-2.101397-1.361671$

C $0.362478-2.3934621 .356211$

O $0.871306-2.7257632 .414764$

C $-0.188325-1.0517771 .055460$

C $0.079861-0.0219011 .873014$

C $-0.363231 \quad 1.362221 \quad 1.686763$

C $-1.446181 \quad 1.6195430 .675170$

C $-4.340483-1.7486181 .940561$

C $-4.714375-0.5314862 .770005$

C -3.8019350 .9482884 .555302$

O $-5.814645 \quad 0.011148 \quad 2.718095$

C -3.3667542 .2239623 .907386$

C $-2.4349703 .035536 \quad 4.429125$

C -2.0281544 .3431643 .814625$

C -0.5391074 .4367143 .574390$

C 0.2570325 .1159564 .417356

C 0.0104483 .8225322 .308539

C 0.2160702 .3359732 .419632

N $-3.677770-0.1441473 .609327$

H $2.566768-2.821772-0.655047$

H $2.437221-5.7616190 .183201$

H $\quad 0.402940-4.9311851 .341483$

H $\quad 0.077443-6.399375-0.692338$

H $\quad 0.012227-4.967911-1.697414$

H -3.674780 -4.821352 -1.065254

H -1.880366 -5.323995 1.679726

H -3.302422 -5.9818840 .868194$

H -1.817952 -6.933896 0.925580

H - $-1.457450-4.585881-3.134075$

H -3.174432 -4.420270 -3.350243

H $-1.419590-2.360787-1.911458$

H -1.849291 -2.295487 -3.621795

H -4.281184 -2.219159-2.985292

H $-3.003496-0.094093-3.444338$

H $-2.476464-0.050618-1.775013$

H $-3.8017232 .101899-2.678274$

H -4.3408340 .2765560 .107885$

H $-5.2429050 .821794-4.038402$

H $-6.5007690 .997503-2.836698$

H -5.982588 -0.622905 -3.342848

H $-6.221396 \quad 1.442681-0.350160$

H $-6.405933-1.8578241 .213427$

H $-5.400578-3.2372280 .853205$

H $-6.461960-2.983250-1.049938$

H $-0.781225-0.942070 \quad 0.154391$

H $\quad 0.717217-0.1955412 .739155$

H -1.057962 $1.516978-0.341078$

H -1.8936692 .6123650 .761183$

H -2.2687820 .9109490 .810363$

H -4.236411-2.579041 2.651006

H -3.360252 -1.589552 1.479419

H $-3.1897770 .695193 \quad 5.426712$

H -4.8454141 .0349004 .875389$

H -3.8623472 .5076952 .981775$

H $-1.9597112 .763770 \quad 5.369115$

H -2.3485305 .1434294 .494567$ 
H $-2.560327 \quad 4.5269482 .872891$ H 1.3220875 .2130064 .234421 H -0.1377065 .5852665 .312307$ H 0.9900354 .2628792 .076452 H $-0.6224164 .105774 \quad 1.462316$ H $0.9828642 .054508 \quad 3.142275$ H $-2.778411-0.6113173 .538915$ C $5.375601-3.350187-0.006470$ O $5.141505-1.934262-0.131704$ C $5.219360-1.3458471 .180172$ C $5.972258-2.3538112 .036520$

C $5.411153-3.6555141 .492213$ H $4.191949-1.2761561 .558299$ C 5.8353930 .0528501 .099059 H $7.054005-2.3037201 .866318$ H $5.784751-2.2176623 .105595$ C $6.237954-4.8771541 .852123$ H $4.395640-3.7869131 .886590$ C 5.1139010 .9951790 .110028 O $7.201575-0.0514290 .681398$ H 5.8606390 .5038682 .097529 H $5.3293110 .685916-0.918068$ O 5.6705632 .3060120 .260871 C 3.5951231 .0853190 .329508 C $2.8622822 .127861-0.523708$ H 3.1319250 .1102560 .160765 O 3.3453431 .4039281 .703713 H $1.8003192 .068831-0.262532$ H $3.1503513 .135386-0.191486$ H $5.802396-5.7827141 .418073$ H $7.266654-4.7885351 .486904$ H $6.277747-5.0078732 .938463$ C $4.344882-4.128277-0.826463$ H $7.213092-0.518010-0.172016$ H 6.6311052 .2299730 .127744 H 3.7755302 .2559181 .891322 H $4.553757-5.202046-0.752398$ H $4.462886-3.856221-1.883244$ H $6.365775-3.531511-0.444969$ C $1.0435833 .331576-2.595847$ O $2.4744463 .237600-2.655835$ C $3.0293122 .054381-2.054192$ C $2.4665110 .822473-2.778518$ C $0.9397690 .816294-2.790332$ C $0.4000762 .145132-3.323943$ H $0.7145953 .342122-1.550547$ C $0.6541294 .675637-3.220653$ H $4.1038232 .099859-2.264275$ O $2.934547-0.394151-2.216606$ H $2.8088420 .838319-3.821984$ H $0.5369450 .594587-1.796439$ O $0.480118-0.230581-3.649210$ H $0.5932182 .201174-4.402962$ O $-1.0128082 .155555-3.134998$ C $1.0857735 .819429-2.331802$ H $1.1408054 .809096-4.194238$ H $-0.4248984 .769499-3.380266$ H $3.900778-0.412685-2.320718$ H $\quad 0.847078-1.064590-3.308747$ H -1.380484 2.877270 -3.671426 O $0.2670625 .896635-1.243069$ O $2.0401386 .547401-2.571936$ C $0.5932456 .919483-0.297249$ H $0.5290527 .905272-0.767288$ H $-0.132515 \quad 6.871208 \quad 0.519124$ H $1.593391 \quad 6.7498240 .112869$

SCF Energy (B3LYP/6-31G**//MMFF)= -3245.91285586

0336

MM̄FF Geometry

C $0.290426 \quad 5.466757 \quad 0.116799$

C $0.9735684 .686125-0.733662$

C $2.3029994 .055486-0.405845$

O $2.1516492 .619673-0.496313$

C $3.3594304 .489752-1.437444$

C $4.7491603 .955411-1.147420$

C $5.4252193 .104846-1.944609$

C 5.3927824 .4595480 .119251
C $4.9886622 .517138-3.257135$

C $4.2849961 .155456-3.151291$

C $5.1628950 .034010-2.571752$

C $4.475387-1.323872-2.728900$

C $5.299017-2.456987-2.097375$

O $5.3734060 .293176-1.182615$

O $4.452454-3.613097-1.993215$

C $5.691740-2.059656-0.655844$

C $6.473403-2.864230-2.995309$

O $6.613428-2.993777-0.101156$

C $6.260402-0.628225-0.532520$

C $6.413725-0.151610 \quad 0.930270$

O $7.582303-0.541547-1.079707$

C 1.6181201 .9793690 .579262

O 1.2845582 .4759321 .643230

C 1.4798470 .5505810 .223051

C $0.661638-0.2310760 .942652$

C $0.404288-1.6536740 .711826$

C $1.304129-2.372820-0.257062$

C $5.122167-0.1181071 .754659$

C $4.896423-1.3594662 .602821$

C $3.213386-2.3826604 .131222$

O $5.736062-2.2442902 .742770$

C $2.423656-3.4086593 .381377$

C $1.198674-3.8100153 .753882$

C $0.411054-4.8729893 .042936$

C $-0.961015-4.3976602 .621108$

C $-2.049268-4.6788863 .357413$

C $-1.078896-3.6686361 .302069$

C $-0.603568-2.2442631 .386935$

N $3.651542-1.3430183 .218488$

H 0.7135795 .6898131 .092669

H $0.5436714 .454724-1.705636$

H 2.6292604 .3298800 .604607

H $3.4169865 .585913-1.471531$

H $3.0274384 .173277-2.432193$

H $6.4209622 .793718-1.630088$

H 4.8788764 .0617450 .999426

H $6.445573 \quad 4.1666830 .195163$

H 5.3562305 .5531440 .155280

H $4.3363063 .213658-3.794493$

H $5.8769732 .413726-3.893654$

H $3.3805591 .269721-2.542529$

H $3.9608540 .871437-4.160307$

H $6.1252100 .040898-3.096687$

H $4.275046-1.540175-3.785747$

H $3.493129-1.293094-2.238734$

H $4.124793-3.826641-2.883604$

H $4.778095-2.127028-0.053875$

H $6.106638-3.237075-3.959384$

H $7.042859-3.689236-2.553736$

H $7.158930-2.040184-3.203791$

H $6.205518-3.874491-0.162065$

H $7.198984-0.7281361 .434429$

H 6.7943550 .8796320 .892322

H $7.879248-1.434042-1.318760$

H $2.037550 \quad 0.187372-0.631945$

H $\quad 0.108710 \quad 0.2159341 .767632$

H $1.147275-2.003133-1.275548$

H $1.151805-3.453664-0.276632$

H $2.354515-2.2207100 .013556$

H 5.1827080 .7231542 .457041

H 4.2506210 .0511811 .115966

H $2.623586-1.8976664 .915484$

H $4.086285-2.8550954 .593215$

H $2.891580-3.8616962 .510605$

H $\quad 0.745033-3.3735574 .641147$

H $\quad 0.316103-5.7286013 .724489$

H $0.950519-5.2534702 .166546$

H $-3.039681-4.3632073 .046172$

H $-1.972813-5.2257514 .291393$

H -2.128978 -3.639201 0.981222

H $-0.566035-4.2483800 .530511$

H -1.192466 -1.635184 2.072576

H $3.000166-0.5933853 .004923$

C -2.2223935 .3065900 .474503$

O $-2.2837483 .951832-0.017391$

C -2.4868523 .0652901 .103540$ 
C -2.9910803 .9488902 .234089$ C -2.1873235 .2138562 .000599$ H -1.496172 2.6632111 .348192 C -3.4321891 .9321910 .691155$ H -4.064264 4.1485912 .124433 H -2.820267 3.5113163 .222111 C -2.7505586 .4364372 .703165$ H -1.1668255 .0386412 .361730$ C -3.8319170 .9963391 .846610$ O $-2.7806121 .172202-0.332801$ H $-4.325825 \quad 2.3635420 .227675$ H -4.4387701 .5463622 .575661$ O -2.6389870 .5998542 .532633$ C $-4.572941-0.2830311 .409296$ C $-5.883559-0.0021150 .656937$ H $-3.906901-0.9099860 .808620$ O $-4.873648-1.0438202 .585843$ H $-6.5536180 .556176 \quad 1.321996$ H $-5.6818170 .636007-0.207083$ H -2.1457307 .3223632 .485297$ H $-3.778701 \quad 6.6432892 .387919$ H $-2.755687 \quad 6.2882333 .787976$ C -1.060364 $6.050869-0.191895$ H -2.0096760 .7364150 .069075$ H -2.902916 0.0027263 .253690 H $-5.468031-0.510883 \quad 3.141220$ H -1.064180 7.1024300 .117009 H -1.217154 $6.041599-1.278300$ H -3.1578805 .7830530 .153204$ C $-5.569950-1.517752-1.983697$ O $-5.785571-2.079779-0.682825$ C $-6.598559-1.292758 \quad 0.195804$ C $-7.975596-1.052680-0.438270$ C $-7.855554-0.430714-1.825158$ C $-6.905149-1.251193-2.693696$ H $-4.999813-0.585318-1.894031$ C $-4.730261-2.522982-2.789467$ H $-6.763709-1.9062871 .090073$ O $-8.778413-0.2119750 .383520$ H -8.504693 -2.009928 -0.532602 H $-7.5361350 .616591-1.772449$ O $-9.140753-0.426670-2.451486$ H -7.385398 -2.201664 -2.960397 O $-6.656889-0.544093-3.907718$ C -3.280774 -2.567685 -2.354512 H -5.141955 -3.532321 -2.670912 H -4.732954 -2.251680 -3.851148 H $-8.852944-0.639867 \quad 1.253640$ H -9.744432 $0.064696-1.868320$ H -7.518589 -0.373563 -4.325262 O $-2.611873-3.447076-3.159454$ O $-2.792412-1.921229-1.439032$ C - $-1.213612-3.588541-2.892652$ H $-0.789053-4.256338-3.646896$ H $-0.711447-2.618943-2.961676$ H - $1.064338-4.034641-1.905840$ SCF Energy (B3LYP/6-31G**//MMFF)= -3245.91616657

0337

MMFF Geometry

C $2.737480-4.291160-1.632022$

C $1.850400-3.396065-2.091346$

C $0.362729-3.511013-1.874359$

O $-0.106375-2.311527-1.217463$

C $-0.346424-3.619274-3.237183$

C $-1.847199-3.814852-3.139720$

C $-2.762237-2.959633-3.637585$

C $-2.302420-5.081255-2.461010$

C $-2.518818-1.673715-4.376619$

C $-2.436218-0.429291-3.480367$

C $-3.750452-0.078748-2.765275$

C $-3.6350111 .270019-2.051181$

C $-4.9072811 .609320-1.257191$

O $-4.033280-1.098912-1.805572$

O $\quad-4.5972682 .698422-0.373777$

C $-5.2898570 .403712-0.370140$

C $-6.0345512 .101816-2.172628$

$\begin{array}{llll}\text { O } & -6.556082 & 0.608240 & 0.250721\end{array}$
C $-5.285179-0.941469-1.124584$

C $-5.453294-2.166202-0.192549$

O $-6.379242-0.965649-2.049593$

C $0.058020-2.2321690 .131239$

O $0.573848-3.0629480 .861546$

C $-0.490230-0.9301490 .570655$

C $-0.186860-0.4621881 .790517$

C -0.6079090 .8237532 .350988$

C -1.6418701 .6112611 .591987$

C $-4.379620-2.3198860 .891247$

C $-4.784775-1.7555172 .243185$

C $-3.872978-1.3428244 .524716$

O $-5.922386-1.3726262 .501982$

C $-3.538526 \quad 0.1106104 .639129$

C -2.6665090 .5957895 .535834$

C -2.3541912 .0558235 .694763$

C -0.8731512 .3470055 .607721$

C -0.1334142 .5078996 .717991$

C $-0.2706002 .536896 \quad 4.234584$

C $-0.053816 \quad 1.235810 \quad 3.510328$

N $-3.728880-1.7683903 .145076$

H $2.380464-5.154504-1.076373$

H $2.203357-2.534954-2.653601$

H $0.123088-4.384956-1.256487$

H $0.067176-4.464841-3.803252$

H $-0.114698-2.721361-3.820240$

H -3.815473 -3.208811-3.513496

H -2.082907 -5.046074 -1.389632

H -3.380114 -5.245769-2.568856

H -1.795742 -5.949185 -2.895500

H $-1.604264-1.743074-4.975520$

H -3.326573 -1.541400 -5.107735

H -1.641365 -0.576869 -2.739892

H -2.139546 $0.416573-4.112577$

H -4.550779 -0.047531-3.514003

H $-3.4036522 .070788-2.764531$

H $-2.7876741 .239708-1.352752$

H $-4.2803463 .442017-0.914682$

H $-4.551556 \quad 0.358540 \quad 0.438074$

H $-5.7186222 .997727-2.720609$

H -6.913298 2.401975-1.591209

H $-6.3448601 .357703-2.909003$

H $-6.508998 \quad 1.4505450 .734522$

H $-6.464528-2.1811700 .231378$

H $-5.392092-3.063523-0.825329$

H $-6.581188-1.891447-2.264355$

H -1.101540 -0.377889 -0.133722

H $0.461466-1.0600012 .429944$

H $-1.2233501 .997794 \quad 0.657565$

H -2.0392702 .4616252 .149841$

H -2.5044910 .9798281 .356769$

H -4.201908 -3.391296 1.051448

H -3.430488 -1.875056 0.576279

H $-3.209919-1.9701645 .128824$

H -4.904706 -1.509921 4.850603

H -4.0571350 .7974493 .974496$

H -2.170709 -0.090873 6.218465

H -2.740080 2.3739626 .672250

H $-2.8891972 .664007 \quad 4.954676$

H $0.9239712 .745046 \quad 6.663908$

H -0.5681592 .4003787 .706253$

H 0.7107623 .0232874 .319869

H -0.8819123 .2458023 .669542$

H $0.6824870 .590048 \quad 3.990633$

H -2.805300 -2.048947 2.827992

C $5.030389-3.822737-0.620034$

O $4.732096-2.472237-0.218354$

C $4.770083-2.4052121 .221387$

C $5.337972-3.7396151 .699509$

C $4.833181-4.6844960 .625416$

H $3.728698-2.3314501 .556958$

C $5.555279-1.1711221 .682606$

H $6.433928-3.7356481 .716833$

H $4.988187-4.0017702 .702822$

C $5.567430-6.0122540 .581193$

H $3.768465-4.8692390 .812503$

C $5.059198 \quad 0.1562841 .071067$

O $6.925832-1.3409801 .299469$ 
H $5.542678-1.1190982 .777899$ H $5.310874 \quad 0.184706 \quad 0.005144$ O 5.7813601 .2281321 .689565 C 3.5568280 .4229661 .265553 C 3.0806851 .8177110 .827512 H $2.971908-0.3357750 .740862$ $\begin{array}{lllll}\text { O } & 3.231954 & 0.276399 & 2.653008\end{array}$ H 2.0027841 .8747311 .018074 H 3.4991382 .5709011 .510200 H $5.172708-6.648300-0.217424$ H $6.639111-5.8735520 .403698$ H $5.450592-6.5480101 .528885$ C $4.219271-4.196853-1.862695$ H $7.447731-0.6705421 .771199$ H $6.704736 \quad 1.170176 \quad 1.391895$ H 3.7716250 .9145873 .150620 H $4.564717-5.162988-2.249147$ H $4.418998-3.454942-2.646533$ H $6.091805-3.821872-0.903435$ C $1.7954574 .076137-0.720185$ O $3.1668793 .666341-0.763686$ C $3.3846162 .251554-0.621386$ C $2.6433791 .512251-1.744940$ C $1.1610591 .873075-1.779705$ C $0.9778503 .390555-1.822568$ H $1.376553 \quad 3.8111210 .258321$ C $1.7549985 .608466-0.779240$ H $4.4584622 .112640-0.785766$ O $2.7810680 .103188-1.638087$ H $3.0830591 .799753-2.709453$ H $0.6286621 .429830-0.932604$ O $0.5853581 .304526-2.957931$ H $1.2769203 .742813-2.817165$ O $-0.4093313 .676913-1.662905$ C 2.173676 6.172721-2.116604 H $0.7456325 .977503-0.563262$ H $2.4114666 .041000-0.013999$ H $3.727763-0.105863-1.709104$ H $-0.3417281 .596103-2.993318$ H $-0.5391634 .622329-1.847086$ O $3.5318666 .157916-2.230050$ O $1.3826726 .570495-2.962469$ C $4.0512526 .650915-3.468751$ H $3.7692127 .698963-3.607758$ H $5.1416906 .582866-3.431335$ H $3.6898156 .039130-4.300830$ SCF Energy (B3LYP/6-31G**//MMFF) $=-3245.90483077$

0338

MM̄FF Geometry

C $-3.276741-1.441337-3.357196$

C $-2.440563-1.883216-2.406213$

C $-0.950917-2.010751-2.603838$

O $-0.277768-1.244541-1.579361$

C $-0.529098-3.483644-2.447113$

C $0.933239-3.737341-2.760955$

C $1.842457-4.189219-1.874865$

C $1.349568-3.484932-4.187988$

C $1.636870-4.542300-0.429087$

C $1.922755-3.401525 \quad 0.559251$

C $3.381864-2.9184430 .557765$

C $3.636082-1.9615571 .724756$

C $5.061786-1.3868731 .698228$

O $3.632740-2.239939-0.675068$

O $5.105109-0.2747342 .605940$

C $5.358021-0.8299100 .288215$

C $6.093131-2.4012532 .206831$

O $6.724405-0.4429260 .170471$

C $4.988184-1.805206-0.847835$

C $5.089149-1.171559-2.256924$

O $5.891039-2.917907-0.824927$

C $-0.1312330 .091397-1.791562$

O $-0.521806 \quad 0.732774-2.753699$

C $0.5833350 .650684-0.623138$

C $0.6004821 .977908-0.431483$

C 1.2125992 .6758300 .701204

C 2.1362921 .8847351 .588101

C $4.2029710 .056930-2.492054$
C $4.9152131 .381108-2.270572$

C $4.4713983 .830701-2.175195$

O $6.128121 \quad 1.476120-2.104540$

C $4.3473814 .244034-0.742787$

C $3.7147705 .360824-0.352650$

C 3.6124635 .8104971 .076486

C 2.1842076 .0618921 .505012

C 1.6736457 .3047851 .513511

C 1.3779104 .8838042 .002842

C 0.9225343 .9804450 .888265

N $4.0355772 .454579-2.319430$

H -2.871890 -1.162402 -4.326996

H -2.837932 -2.170717 -1.437501

H $-0.657158-1.639269-3.593008$

H -1.130499-4.116205 -3.113781

H $-0.764656-3.806869-1.427876$

H $2.863449-4.339319-2.224851$

H $1.329025-2.414499-4.413876$

H $2.364084-3.842928-4.394137$

H $0.675914-4.003084-4.878288$

H $\quad 0.619699-4.908959-0.263132$

H $2.286654-5.394692-0.192585$

H $1.254709-2.561800 \quad 0.334644$

H $1.665429-3.7600821 .563775$

H $4.035740-3.7950720 .634189$

H $3.440913-2.4565282 .684318$

H $2.924221-1.1269571 .670349$

H $4.847467-0.596713 \quad 3.486682$

$\begin{array}{llll}H & 4.763887 & 0.084746 & 0.178387\end{array}$

H $5.861833-2.6983883 .236861$

H $7.096171-1.9618462 .241054$

H $6.134267-3.3103511 .603428$

H $\quad \begin{array}{llll}6.909416 & 0.188909 & 0.886231\end{array}$

H $6.137905-0.968553-2.504775$

H $4.764559-1.935982-2.978089$

H $5.884348-3.338433-1.700781$

H $1.043860-0.0458550 .067785$

H $0.0824892 .614422-1.147797$

H 1.5747051 .1449192 .168217

H 2.6954472 .5002682 .295864

H $2.888471 \quad 1.363790 \quad 0.986363$

H $3.8830020 .056790-3.541980$

H $3.2997400 .017242-1.875360$

H $3.8485224 .439698-2.837989$

H $5.5143913 .919636-2.495888$

H 4.8189363 .6098300 .004258

H $3.2677456 .007149-1.104952$

H 4.2001176 .7322561 .178504

H 4.0762435 .0878661 .759377

H $\quad 0.6589107 .497518 \quad 1.845763$

H $2.2579898 .157180 \quad 1.183073$

H 0.4748165 .2379612 .518085

H 1.9508344 .3606242 .773132

H $\quad 0.240596 \quad 4.4642380 .187263$

H $3.0389102 .278812-2.407834$

C $-5.2963850 .095569-3.040533$

O $-4.9513300 .613735-1.740505$

C $-4.5352911 .983671-1.890001$

C $-4.9989142 .413508-3.278655$

C $-4.8134281 .127278-4.060694$

H $-3.4383181 .974644-1.876018$

C $-5.0515562 .852975-0.736206$

H $-6.0543062 .708629-3.285633$

H $-4.4102713 .246957-3.673795$

C $-5.5701821 .091064-5.376347$

H $-3.7422021 .013312-4.264604$

C -4.7805212 .2921350 .675804$

O $-6.4709242 .997107-0.873934$

H $-4.6289543 .860569-0.834046$

H -5.4914301 .4877690 .903596$

O -5.0345823 .3369681 .623046$

C -3.3520761 .7738700 .905367$

C -3.0758091 .4011722 .375399$

H -3.1863310 .9030710 .263875$

O -2.4093332 .7691760 .508503$

H -2.7951492 .3028672 .932615$

H $-3.990777 \quad 1.0112372 .831052$

H $\quad-5.4126640 .136412-5.888184$ 
H $-6.647066 \quad 1.219471-5.224432$

H $-5.2271241 .891694-6.039589$

C $-4.767762-1.334468-3.195182$

H -6.757796 $3.694989-0.261671$

H -5.9865743 .5307971 .599909$

H -2.593488 3.5738591 .022822

H - $-5.231298-1.798193-4.074298$

H $-5.083431-1.923777-2.324668$

H $-6.3935910 .048182-3.053059$

C -3.300810-1.639945 2.486303

O $-2.286631-0.8709201 .829398$

C -1.9418690 .3562742 .487367$

C $-1.469595 \quad 0.0693743 .918167$

C $-2.488594-0.7531354 .702591$

C $-2.885450-2.0048803 .918364$

H $-4.224471-1.0503112 .524948$

C -3.646226 -2.850183 1.607532

H $-1.083017 \quad 0.7652831 .944040$

O -1.2018511 .2794814 .618590$

H $-0.527851-0.4939613 .881063$

H $-3.370667-0.1562294 .963284$

O $-1.902741-1.1659575 .939914$

H -2.039720 -2.702429 3.924626

O $-3.967022-2.6340404 .601515$

C -2.511839-3.833862 1.418000

H -4.501974 -3.379607 2.041585

H -3.934464 -2.505529 0.608297

H -0.5406641 .7740144 .104568$

H -1.624247 -0.3613376 .409998$

H -4.084999 -3.518870 4.217135

O $-3.033274-5.0546951 .097360$

O $-1.322613-3.5615641 .502140$

C $-2.084220-6.1010130 .867469$

H -1.323394 -6.124289 1.653797

H -2.621247 -7.0532940 .875710$

$\mathrm{H}-1.622514-5.966891-0.114328$

SCF Energy (B3LYP/6-31G**//MMFF) $=-3245.89608390$

0339

MM̄FF Geometry

C $-2.710260-0.221502-3.207831$

C $-2.127540-1.342898-3.658759$

C $-0.710802-1.799580-3.377839$

O $-0.092462-1.011608-2.335201$

C $-0.727629-3.262036-2.889582$

C $0.653119-3.874331-2.746313$

C $1.180952-4.316716-1.588106$

C $1.445587-4.007788-4.022105$

C $0.554281-4.318582-0.222975$

C $0.894147-3.0944250 .640468$

C $2.388073-2.9537860 .975599$

C $2.601006-1.8719372 .036335$

C $4.090215-1.6387332 .333651$

O $3.092052-2.595326-0.216263$

O $4.204427-0.4107353 .069619$

C $4.846199-1.4384871 .002248$

C $4.669917-2.737783 \quad 3.232007$

O $6.253816-1.3886261 .219132$

C $4.515496-2.507520-0.059868$

C $5.113990-2.190695-1.452782$

O $5.064422-3.7653120 .353293$

C $0.5818770 .112318-2.695036$

$\begin{array}{lllll}\text { O } & 0.748690 & 0.547636 & -3.822562\end{array}$

C $1.0505650 .757918-1.450011$

C $1.2624012 .081801-1.435720$

C $1.7102772 .867007-0.284385$

C 2.2412592 .1142240 .906753

C $4.655148-0.871404-2.083495$

C $5.5958850 .296120-1.833894$

C $5.763942 \quad 2.759079-2.181227$

O $6.7123100 .169931-1.338752$

C $5.3678973 .468744-0.925106$

C $4.9348084 .738528-0.902552$

C $4.573104 \quad 5.479428 \quad 0.352812$

C $3.188777 \quad 6.0858720 .293471$

C $3.0136787 .377229-0.034456$

C $2.0184335 .218156 \quad 0.697084$

C $1.6502244 .212984-0.357521$
N $5.0519951 .497768-2.268305$

H -2.145539 $0.473058-2.593132$

H $-2.713908-2.003415-4.295279$

H $-0.135746-1.721093-4.307906$

H -1.297943 -3.886294 -3.590515

H $-1.269058-3.302144-1.938190$

H $2.182850-4.744385-1.615403$

H $1.751563-3.024686-4.392163$

H $2.354943-4.603336-3.886034$

H $0.845486-4.502681-4.792668$

H $-0.533897-4.411639-0.297650$

H $0.880404-5.2277820 .298298$

H $0.540036-2.1907280 .130967$

H $\quad 0.327560-3.1815261 .575191$

H $2.752704-3.9215001 .339831$

H $2.062434-2.1210572 .959226$

H $2.167132-0.9274591 .681401$

H $3.670608-0.495148 \quad 3.878237$

H $4.554143-0.4543200 .617834$

H $4.131350-2.7753864 .186618$

H $5.714854-2.5299013 .486983$

H $4.614513-3.7318552 .783680$

H $6.421820-0.6879081 .872371$

H $6.207695-2.264445-1.419294$

H $4.790549-2.992532-2.132489$

H $5.156838-4.333544-0.429382$

H $1.1794340 .131989-0.574915$

H $1.0668162 .649993-2.344278$

H $1.4346001 .578173 \quad 1.415248$

H 2.7264552 .7538821 .646947

H 3.0005801 .3897370 .593683

H $4.618037-1.003119-3.172632$

H $3.647078-0.607179-1.751924$

H $5.5148853 .334564-3.078409$

H $6.8425042 .571920-2.174758$

H 5.4663182 .9222590 .009948

H $4.8647915 .290865-1.837190$

H 5.3198296 .2711080 .498582

H 4.6513544 .8342991 .236732

H $2.0268447 .827862-0.052115$

H $3.8536428 .011434-0.298027$

H 1.1305165 .8409870 .871405

H 2.2292294 .7606181 .667747

H $1.2599534 .665488-1.270324$

H $4.1001931 .514340-2.623059$

C $-4.8367190 .543391-2.178562$

O $-4.3479341 .833275-1.747748$

C $-5.4539952 .599283-1.256188$

C $-6.616907 \quad 1.628326-1.108993$

C $-6.352900 \quad 0.694766-2.278067$

H $-5.717412 \quad 3.304458-2.057312$

C $-5.091747 \quad 3.426464-0.020850$

H -6.562946 $1.080466-0.161283$

H -7.590795 2.123352 -1.166672

C $-7.121911-0.611465-2.196173$

H $-6.6168841 .209514-3.211785$

C $-4.6086102 .679016 \quad 1.241441$

O -6.2585524 .1855920 .325679$

H $-4.3237374 .156290-0.307626$

H -5.4300002 .0870551 .661888$

$\begin{array}{lllll}\text { O } & -4.320267 & 3.703551 & 2.207816\end{array}$

C $-3.356217 \quad 1.7942341 .065191$

C $-2.728544 \quad 1.3441352 .399345$

H -3.6233980 .9079580 .484567$

$\begin{array}{llll}O & -2.364015 & 2.506838 & 0.323534\end{array}$

H -2.3324482 .2260602 .916249$

H -3.510488 0.9145363 .033158

H -6.872029 -1.266775 -3.036186

H $-6.901696-1.150804-1.269711$

H -8.200309 $-0.423561-2.223990$

C $-4.1424250 .154025-3.482815$

H $-6.024797 \quad 4.743433 \quad 1.087337$

H -4.3274263 .2892013 .087268$

H -2.147963 $3.314808 \quad 0.819783$

H $-4.1455601 .002101-4.177689$

H -4.669275 $-0.679361-3.960328$

H $-4.583917-0.173067-1.388351$

C $-2.797321-1.7265002 .475959$ 
O $-2.005503-0.892563 \quad 1.620602$

C $-1.568322 \quad 0.340307 \quad 2.202802$

C -0.7555170 .0630623 .474846$

C $-1.532658-0.7969744 .464118$

C $-2.049121-2.0569183 .774848$

H $-3.742974-1.2229492 .707978$

C $-3.109527-3.0155181 .700604$

H -0.8771380 .7869251 .478461$

O $-0.3655891 .274278 \quad 4.113019$

H $0.166255-0.4668193 .207160$

H $-2.352366-0.2372364 .929570$

O $-0.662013-1.1952505 .525637$

H $-1.206390-2.7280933 .565478$

O $-2.931299-2.7453084 .659599$

C $-4.078092-2.781872 \quad 0.563577$

H -2.180018 -3.4170861 .282159$

H -3.560204 -3.773507 2.350070

H 0.1337741 .7993903 .464593

H $-0.307797-0.3837215 .928251$

H -2.439212 -2.923121 5.479586

O $-3.863620-3.717366-0.407451$

O $-4.940979-1.9150120 .549487$

C $-4.736824-3.648714-1.538312$

H $-5.775102-3.793982-1.225723$

H -4.618095 -2.690571-2.050246

H $-4.461960-4.450240-2.229031$

SCF Energy (B3LYP/6-31G**//MMFF) $=-3245.90819413$

03_4

MM̄FF Geometry

C -3.266248 -0.027552 -3.594601

C $-2.521299-0.522961-2.594040$

C $-1.175962-1.173891-2.792068$

O $-0.249737-0.610786-1.834116$

C $-1.288619-2.682990-2.509246$

C $0.001051-3.447134-2.739929$

C $0.663731-4.130704-1.786334$

C $0.526322-3.439451-4.152615$

C $0.286983-4.299123-0.341619$

C $0.846079-3.221480 \quad 0.599009$

C $2.380237-3.208916 \quad 0.696447$

C $2.844211-2.2706021 .812878$

C $4.376630-2.1726891 .882024$

O $2.913526-2.759540-0.551748$

O $4.708452-1.048598 \quad 2.712076$

C $4.929132-1.870540 \quad 0.471840$

C $4.996253-3.4044432 .552968$

O $6.352341-1.9402360 .456251$

C $4.345878-2.783161-0.626578$

C $4.738544-2.342493-2.058394$

O $4.846683-4.113759-0.447158$

C $0.2583550 .622034-2.101186$

O $0.048297 \quad 1.310252-3.086764$

C $1.1017551 .009162-0.949942$

C $1.4080102 .300869-0.761674$

$\begin{array}{llll}\text { C } 2.199333 & 2.846145 & 0.342762\end{array}$

C 2.8795101 .8662601 .261302

C $4.306058-0.926025-2.453814$

C $5.3847840 .127208-2.258727$

C $5.7344302 .592031-2.383017$

O $6.554057-0.143987-1.999426$

C $5.701027 \quad 3.154029-0.996779$

C $5.4361484 .442333-0.731186$

C 5.4469635 .0330710 .649725

C 4.1694605 .7728510 .978920

C 4.0934357 .1089220 .856719

C 3.0117314 .9730321 .531633

C 2.2910504 .1862940 .471092

N $4.8930651 .411782-2.450682$

H -2.883986 $-0.113800-4.610115$

H $-2.872922-0.471801-1.568237$

H -0.800252 -1.001853 -3.807832

H -2.063168 -3.124697-3.150520

H -1.634296 -2.817882 -1.478502

H $1.581745-4.644623-2.069437$

H $0.887930-2.443558-4.425546$

H $1.358391-4.138713-4.289785$

H $-0.262746-3.731483-4.853254$
H $-0.801786-4.339170-0.234078$

H $0.639190-5.284628-0.010778$

H $0.486878-2.2388950 .271331$

H $0.427097-3.4034091 .596168$

H $2.720352-4.2324500 .892851$

H $2.437019-2.5858652 .781605$

H $2.440724-1.2650701 .632360$

H $4.304386-1.1910993 .585217$

H $4.665606-0.8314310 .245203$

H $4.611332-3.5185023 .573545$

H $6.081654-3.2955732 .653820$

H $4.790168-4.3335992 .017763$

H $6.679936-1.3335561 .142091$

H $5.811985-2.498447-2.219434$

H $4.238556-3.030274-2.755752$

H $4.766939-4.589355-1.290636$

H $1.4187450 .224800-0.272668$

H $1.0232503 .034746-1.468905$

H $2.139507 \quad 1.2999121 .835390$

H 3.5574752 .3342071 .977680

H $3.489187 \quad 1.1633870 .684560$

H $4.071361-0.923046-3.526126$

H $3.398835-0.627489-1.920691$

H $5.3585403 .301602-3.126902$

H $6.7623572 .322012-2.646194$

H $5.9340012 .477949-0.177453$

H $5.2329655 .122225-1.555668$

H 6.3007055 .7208800 .710116

H $5.6316994 .266811 \quad 1.412841$

H 3.1904417 .6505901 .117882

H 4.9317467 .6902580 .487250

H 2.2737705 .6460691 .988947

H 3.3670474 .3515602 .358231

H $1.7671484 .813044-0.252090$

H $3.8989651 .546244-2.611745$

C $-5.0595221 .047763-2.077254$

O $-4.2414292 .143484-1.612366$

C $-5.0933863 .116692-0.995964$

C $-6.4315292 .429331-0.766883$

C $-6.5037751 .544275-1.998177$

H $-5.2537563 .902269-1.748328$

C -4.4520093 .7797710 .224635$

H -6.4174041 .8186470 .142986$

H $-7.2642003 .135466-0.697119$

C -7.538428 $0.437795-1.890267$

H $-6.7348612 .168459-2.871948$

C $-4.063927 \quad 2.882522 \quad 1.420255$

O -5.3795704 .7707630 .689936$

H -3.559497 $4.325614-0.106670$

H $-4.9652762 .464728 \quad 1.883582$

$\begin{array}{llll}\text { O } & -3.489554 & 3.769943 & 2.394857\end{array}$

C -3.0533821 .7560661 .115957$

C $-2.450157 \quad 1.1140272 .382138$

H -3.5490230 .9802390 .526635$

$\begin{array}{lllll}\text { O } & -1.984943 & 2.275980 & 0.323205\end{array}$

H -1.8734951 .8742992 .921474$

H -3.2648990 .7920943 .038584$

H $-7.530868-0.193541-2.784173$

H $-7.352625-0.203746-1.022932$

H $-8.5426570 .860911-1.783944$

C $-4.6137160 .642826-3.483663$

H $-4.963904 \quad 5.224464 \quad 1.443277$

H -3.5424803 .3300793 .260242$

H -1.5566912 .9851810 .832700$

H $-4.574408 \quad 1.542674-4.110922$

H $-5.352835-0.032291-3.930155$

H $-4.9266690 .221801-1.372602$

C -3.071856-1.897131 2.232731

O $-2.171997-1.1450341 .409093$

C -1.500715 -0.0652662 .066220$

C $-0.716102-0.5850163 .279609$

C $-1.600637-1.3869074 .225982$

C $-2.349202-2.471903 \quad 3.457542$

H $-3.897523-1.2536102 .558014$

C -3.642694 -3.0331311 .369898$

H -0.7567190 .3067891 .352749$

$\begin{array}{lllll}\text { O } & -0.111273 & 0.482900 & 4.000977\end{array}$

H $0.096701-1.2348612 .933483$ 
H $-2.296413-0.7400134 .773263$ O $-0.781149-2.0223645 .209905$ H -1.643944 -3.253864 3.148263 O $-3.306775-3.0794074 .323094$ C $-4.648233-2.536038 \quad 0.355878$ H $-2.823787-3.5230850 .831074$ H -4.159777 -3.778953 1.982955 H $\quad 0.442527 \quad 0.981427 \quad 3.376518$ H $-0.279949-1.3227485 .663421$ H -2.825117 -3.4143685 .098949$ O $-4.693743-3.407610-0.694173$ O $\quad-5.338510-1.5350710 .489294$ C $-5.646143-3.098376-1.716040$ H -6.653115 -3.030725 -1.293307 H -5.373135 -2.165208 -2.214257 H -5.628929 -3.906362 -2.452063 SCF Energy (B3LYP/6-31G**//MMFF) $=-3245.90838401$

0340

MM̄FF Geometry

C $-2.9555420 .050073-3.725872$

C $-2.512005-0.631989-2.658619$

C $-1.179892-1.345431-2.644386$

O $-0.301842-0.692164-1.697467$

C $-1.369155-2.804048-2.189430$

C $-0.111800-3.645635-2.297952$

C $0.528167-4.208703-1.254456$

C $0.406171-3.871914-3.696011$

C $0.164626-4.1493620 .201986$

C $0.848571-3.0231780 .992016$

C $2.381714-3.1182971 .035281$

C $2.956573-2.1076662 .030566$

C $4.493710-2.1195902 .037679$

O $2.893736-2.840057-0.269940$

O $4.939357-0.9442182 .732145$

C $5.009567-2.0083650 .585915$

C $5.049543-3.3190552 .814627$

O $6.421972-2.1900960 .531201$

C $4.316060-2.980308-0.390516$

C $4.678886-2.717614-1.873021$

O $4.726839-4.319878-0.090014$

C $0.3460510 .426794-2.123906$

O $0.2397300 .971262-3.211718$

C $1.2228790 .892092-1.028037$

C $1.6463932 .164664-1.024388$

C $2.5217122 .782454-0.027107$

C 3.2451721 .8725450 .929240

C $4.316401-1.325764-2.403537$

C $5.462867-0.328396-2.363816$

C $5.9534962 .077751-2.796002$

O $6.620697-0.639669-2.098719$

C $5.9929792 .802464-1.487855$

C $5.7766534 .121231-1.368622$

C $5.8621664 .870048-0.069521$

C 4.6170575 .6766900 .223880

C $4.5671536 .992648-0.043630$

C 3.4654174 .9762950 .908285

C $2.6619174 .124436-0.033206$

N $5.0430050 .952107-2.700490$

H -2.311283 $0.090144-4.603560$

H $-3.101407-0.679725-1.748801$

H $-0.719884-1.324926-3.639847$

H -2.150045 -3.285415 -2.793202

H -1.738883 -2.800948 -1.159274

H $1.420013-4.799129-1.462908$

H $1.205898-4.619998-3.726449$

H $-0.398332-4.230123-4.346601$

H $0.810174-2.945140-4.114251$

H $-0.919783-4.0643850 .327051$

H $\quad 0.426309-5.1145420 .654637$

H $0.546799-2.0578140 .568816$

H $0.465116-3.0616002 .018456$

H $2.654995-4.1391381 .326955$

H $2.567714-2.2895323 .040408$

H $2.618025-1.0985981 .759183$

H $4.565205-0.9662033 .629630$

H $4.815998-0.9810060 .259196$

H $4.698046-3.2985813 .853166$
H $6.143178-3.2823962 .869009$

H $4.756650-4.2800072 .386807$

H $6.821134-1.5434461 .138053$

H $5.733696-2.958351-2.051774$

H $4.111175-3.440922-2.476420$

H $4.578188-4.871696-0.875767$

H $1.4871130 .180924-0.254619$

H $1.3050132 .828276-1.818169$

H 3.7210511 .0494940 .387171

H $2.554528 \quad 1.4536531 .666317$

H 4.0487462 .3695461 .477306

H $4.035281-1.421740-3.460280$

H $3.453537-0.913204-1.872616$

H $5.5978762 .714751-3.612038$

H $6.9557351 .715948-3.047401$

H $6.2395002 .220662-0.602762$

H $5.5586274 .705469-2.259936$

H $6.7323375 .537148-0.127706$

H $6.065314 \quad 4.1945190 .770729$

H 3.6887097 .5846520 .190622

H $5.4024707 .506170-0.508370$

H 2.7772835 .7172151 .337739

H 3.8417584 .4193111 .771402

H $2.1093844 .700640-0.776684$

H $4.0541221 .128128-2.852742$

C $-5.3998300 .495360-2.931238$

O $\quad-5.072537 \quad 0.879710-1.585189$

C $-6.1654331 .623377-1.019421$

C $-7.3529141 .391517-1.944275$

C $-6.6678851 .277689-3.290416$

H $-5.8726842 .679699-1.067685$

C $-6.3945851 .231450 \quad 0.445437$

H $-7.8784770 .457814-1.715059$

H $-8.0806902 .207425-1.898131$

C -7.526242 $0.610361-4.352056$

H $-6.4024002 .287922-3.630488$

C $-5.106567 \quad 1.124828 \quad 1.285595$

O $-7.030016-0.0534150 .490082$

H $-7.1026381 .933214 \quad 0.901815$

H $-4.557300 \quad 0.2374080 .962906$

O $\begin{array}{lllll}-5.503382 & 0.882350 & 2.641145\end{array}$

C $-4.1865702 .352248 \quad 1.243920$

C -2.9544442 .2669502 .168319$

H -3.8493632 .5354350 .218154$

O -4.9597763 .4903601 .640034$

H -2.5359383 .2788502 .243392$

H -3.275537 2.0188553 .185684

H $-6.9914430 .546642-5.304758$

H -7.811256 -0.405128 -4.057076

H $-8.4450861 .182166-4.518758$

C $-4.2401870 .819266-3.879673$

H -7.152565 -0.285103 1.426804

H -5.9491631 .6842302 .963972$

H -4.4026184 .2788621 .524274$

H -3.985458 $1.883817-3.797276$

H $-4.5833000 .652485-4.908413$

H -5.596283 -0.584158 -2.920539

C $-2.378897-0.6567072 .994143$

O $-2.225028-0.0747031 .694387$

C -1.848856 1.3049031 .675195

C -0.5125841 .4707902 .411304$

C -0.5873760 .9324613 .836167$

C -1.099252 -0.5063823 .828749$

H -3.222482 -0.1840363 .508776$

C -2.708172 -2.143906 2.807554

H $-1.666971 \quad 1.5569490 .622524$

O -0.1135552 .8360522 .418722$

H $\quad 0.2572090 .9116191 .867481$

H $-1.2131861 .571386 \quad 4.469171$

O $\quad 0.717520 \quad 0.961270 \quad 4.420389$

H $-0.315289-1.1650883 .434596$

O $-1.363263-0.9191205 .167935$

C -4.010969-2.350696 2.073079

H -1.912388 -2.646799 2.247049

H -2.790781 -2.663438 3.768938

H 0.7255292 .8923562 .907311

H 1.3054120 .4138473 .872241

H $-0.545703-0.7895655 .678774$ 
O $-3.759833-2.694697 \quad 0.777808$

O $-5.113217-2.2068042 .584728$

C $-4.901336-2.931535-0.052380$

H $-5.299570-1.976857-0.403266$

H $-4.574174-3.513288-0.918435$

H -5.671176 -3.500873 0.477219

SCF Energy (B3LYP/6-31G*//MMFF) $=-3245.91320466$

0341

MM $\mathrm{MFF}$ Geometry

C $3.0809620 .218053 \quad 3.677763$

C $2.440204-0.6021882 .831285$

C $1.013994-1.0532713 .010993$

O $0.239991-0.6175501 .870836$

C $0.973968-2.592375 \quad 3.053091$

C $-0.407168-3.1659123 .299802$

C $-1.083019-3.9378322 .426234$

C -1.015742 -2.851019 4.642462

C $-0.651973-4.394561 \quad 1.060507$

C $-1.121274-3.496202-0.094549$

C $-2.648791-3.407220-0.244132$

C $-3.025524-2.693606-1.544144$

C $-4.545858-2.511717-1.681234$

O $-3.171736-2.673224 \quad 0.865613$

O $-4.782529-1.566688-2.736359$

C $-5.099633-1.885241-0.382404$

C $-5.239413-3.812995-2.101549$

O $-6.524336-1.855535-0.398168$

C $-4.602829-2.5864250 .898484$

C $-4.985666-1.8338842 .196309$

O $-5.194333-3.8890550 .980750$

C $-0.212116 \quad 0.6658351 .876028$

O $-0.029973 \quad 1.5088732 .739585$

C -0.9613340 .8786550 .618250$

C $-1.220418 \quad 2.1290180 .208353$

C $-1.9075892 .504861-1.028867$

C $-2.5743231 .408190-1.815447$

C -4.449191 -0.4025742 .310421$

C $-5.436147 \quad 0.664042 \quad 1.865403$

C -5.5910353 .1196861 .471845$

$\begin{array}{llll}\text { O } & -6.614601 & 0.430357 & 1.611679\end{array}$

C $-5.445138 \quad 3.384540 \quad 0.006723$

C $-5.0608564 .569523-0.491035$

C $-4.9503234 .865683-1.958984$

C -3.592651 $5.410034-2.341804$

C $-3.3931736 .731066-2.486313$

C $-2.4922714 .417544-2.640744$

C $-1.9109003 .803440-1.396430$

N $-4.8504651 .922363 \quad 1.822831$

H $2.559366 \quad 0.609138 \quad 4.547317$

H $2.967300-0.976998 \quad 1.956894$

H $0.582741-0.6397123 .930754$

H $1.639299-2.9594153 .846249$

H $1.385422-2.9771392 .113643$

H -2.067189-4.299591 2.722856

H $-1.261448-1.7872784 .715642$

H $-1.938568-3.4128964 .822373$

H $-0.317181-3.1063895 .446094$

H $0.437030-4.4971381 .009750$

H - $-1.039417-5.4098190 .906034$

H $-0.703026-2.492303 \quad 0.044547$

H - $0.695209-3.898902-1.021636$

H $-3.055568-4.425224-0.235600$

H $-2.623137-3.227723-2.414002$

H -2.557034 -1.700698 -1.560092

H $-4.374258-1.914773-3.547714$

H $-4.765990-0.841259-0.367576$

H $-4.846745-4.162220-3.064152$

H -6.312764 -3.656812 -2.256027

H -5.107964 -4.621768 -1.379848

H $-6.795694-1.385442-1.205097$

H $-6.070754-1.8741452 .349589$

H $-4.554134-2.398586 \quad 3.035494$

H $-5.163284-4.1840481 .905964$

H -1.2484820 .0031370 .047604$

H -0.8668202 .9600700 .817544$

H $-1.8258170 .740710-2.255282$

H -3.204206 $1.774377-2.628577$
H -3.233536 $0.820646-1.167947$

H $-4.236614-0.1964383 .367428$

H $-3.510597-0.2868811 .759832$

H $-5.194868 \quad 3.9370392 .082692$

H -6.6487022 .9803251 .717828$

H -5.691664 $2.574306-0.675481$

H $-4.842071 \quad 5.3862280 .193547$

H $-5.7296065 .597032-2.210441$

H $-5.172023 \quad 3.978802-2.565547$

H $-2.4298397 .128193-2.788447$

H -4.188462 $7.445448-2.300261$

H $-1.6681294 .918071-3.166810$

H $-2.8645153 .675222-3.352020$

H $-1.4000624 .523799-0.755798$

H -3.8538512 .0145551 .996939$

C 4.7216561 .9321692 .749746

$\begin{array}{lllll}\text { O } & 4.216824 & 1.801686 & 1.408001\end{array}$

C 3.5997683 .0472591 .025146

C 4.0033224 .0615742 .089867

C 4.0595433 .1809723 .327272

H 2.5162802 .8958941 .092809

C $3.9913893 .398348-0.415550$

H 4.9888344 .4961691 .886575

H $3.283393 \quad 4.8809782 .176779$

C 4.8173103 .8013684 .486979

H 3.0312652 .9666023 .644347

C $3.6972212 .255362-1.413512$

O $5.3996053 .658393-0.443599$

H $3.4930694 .325012-0.723161$

H $4.3079551 .387414-1.154234$

O $4.1106992 .669715-2.720187$

C $2.2100641 .866687-1.485635$

C $1.8816900 .698008-2.436813$

H $1.8363211 .612265-0.491079$

O $1.466466 \quad 3.011309-1.917687$

H $\quad 0.8513230 .826622-2.791904$

H $2.5096430 .756108-3.331858$

H $4.843753 \quad 3.1196125 .342934$

H 5.8506214 .0378124 .211612

H 4.3347254 .7299914 .808811

C 4.5190960 .6193873 .506267

H $5.6026914 .072819-1.299161$

H $5.0815002 .717897-2.717456$

H $1.7902963 .253170-2.802547$

H 4.9831690 .6824884 .497381

H $5.047027-0.1791212 .968013$

H 5.8025942 .0994882 .650551

C $4.299730-1.232310-2.262714$

O $3.315319-0.950695-1.262591$

C $2.002972-0.684470-1.757190$

C $1.508134-1.860239-2.611502$

C $2.494243-2.215033-3.720041$

C $3.890007-2.426533-3.134538$

H $4.415665-0.349222-2.902952$

C $5.648135-1.416280-1.554386$

H $1.353004-0.647869-0.874359$

O $\quad 0.235980-1.580594-3.184771$

H $\quad 1.383470-2.742477-1.970534$

H $2.507225-1.452871-4.508097$

O $2.078825-3.433234-4.342502$

H $3.884790-3.359348-2.557841$

O $4.809121-2.588008-4.211601$

C $5.696380-2.640640-0.670228$

H $6.465997-1.491880-2.279909$

H $5.875468-0.544958-0.927342$

H $-0.370370-1.365385-2.455937$

H $1.176000-3.293385-4.676118$

H $5.651566-2.896306-3.837665$

O $5.113312-2.374517 \quad 0.533130$

O $6.183209-3.709390-1.016178$

C $5.070480-3.4694411 .453182$

H $6.082116-3.8265811 .668396$

H $4.619258-3.1152752 .383801$

H $4.452446-4.2764601 .048450$

SCF Energy (B3LYP/6-31G*//MMFF)= -3245.90364097

0342

MMMFF Geometry 
C $-1.8429943 .431838-0.845772$ C $-1.2933152 .372981-0.232560$ C $-0.443518 \quad 1.352825-0.946692$ O $0.7682051 .135517-0.189653$ C -1.205741 $0.017511-1.013594$ C $-0.526926-1.038151-1.864448$ C $-0.093481-2.227900-1.404188$ C $-0.381726-0.709867-3.328382$ C $-0.187252-2.765252-0.004844$ C $1.017725-2.4447780 .892335$ C $2.344472-3.0453930 .399788$ C $3.436871-2.8905871 .460365$ C $4.798736-3.4015690 .962550$ O $2.738836-2.357435-0.789968$ O $5.805848-2.9261621 .869404$ C $5.101491-2.774464-0.415946$ C $4.877868-4.9326430 .991951$ O $6.271955-3.349182-0.990945$ C $3.925078-2.874209-1.408368$ C $4.151861-2.066741-2.709947$ O $3.748231-4.243504-1.792095$ C $1.7632412 .053735-0.319745$ O $1.7562173 .056274-1.015620$ C 2.8734681 .6308800 .561852 C 3.8319172 .5109400 .886944 C 4.9831022 .2498551 .753929 C 5.2439510 .8210122 .151546 C $4.382490-0.563163-2.520618$ C $5.848914-0.164843-2.480038$ C $7.3138751 .806466-2.060638$ O $6.764770-0.931384-2.765280$ C $7.8405551 .730125-0.662283$ C 8.3076792 .7957680 .005817 C 8.8790902 .7314691 .393066 C $8.221673 \quad 3.7112372 .339221$ C 8.7867064 .9014362 .603726 C 6.9558353 .2750173 .041508 C $5.753703 \quad 3.2902172 .135033$ N $6.0129361 .167496-2.124431$ H -1.667757 $3.585404-1.907813$ H -1.479932 2.2105670 .826426 H - $0.1867221 .697272-1.955624$ H -2.208044 $0.185818-1.426209$ H -1.355195 -0.3483300 .008035$ H $0.375361-2.910563-2.112120$ H $-0.055953-1.574342-3.916985$ H -1.340008 $-0.378872-3.741571$ H $\quad 0.3574720 .083274-3.475986$ H -1.098714 -2.403352 0.480029 H $-0.312269-3.853999-0.065900$ H $1.115128-1.3562260 .981104$ H $\quad 0.797517-2.832323 \quad 1.894805$ H $2.180112-4.1055320 .173548$ H $3.153371-3.4008552 .389280$ H $3.539716-1.8288101 .722575$ H $5.586764-3.2534012 .758711$ H $5.327548-1.716527-0.238964$ H $4.723933-5.3041922 .012141$ H $5.872128-5.2830680 .693642$ H $4.135529-5.4130570 .351341$ H $\quad 6.993242-3.243089-0.347175$ H $4.949457-2.527492-3.305178$ H $3.240853-2.171283-3.317152$ H $3.265025-4.267611-2.634558$ H 2.8611170 .6127340 .933424 H 3.7590663 .5264710 .499690 H 5.2725040 .1771001 .266094 H 4.4592220 .4592762 .824620 H 6.2003770 .6753942 .657582 H $3.949255-0.035767-3.380330$ H $3.876119-0.195879-1.622985$ H $7.1850252 .839432-2.399060$ H $\quad 8.003713 \quad 1.298536-2.742340$ H $7.8635480 .751694-0.188153$ H $8.309818 \quad 3.767841-0.482645$ H 9.9531692 .9476721 .320855 H 8.8094681 .7190251 .809885 H 8.3366295 .5987473 .302436
H $9.710103 \quad 5.2071612 .122777$

H 6.7358503 .9540953 .876338

H 7.1261692 .3029553 .512258

H 5.4841964 .2886911 .787301

H $5.2011251 .715252-1.853912$

C $-4.1795344 .281281-0.610113$

O $-4.7036783 .034044-0.109057$

C -5.9992723 .2705720 .470531$

C $-6.4329314 .641067-0.031460$

C $-5.1038485 .371358-0.066109$

H -5.8526353 .3281361 .555858$

C -6.9514992 .1176310 .139649$

H $-6.8630474 .589393-1.038427$

H -7.166995 5.1133320 .628381

C $-5.126236 \quad 6.636769-0.905745$

H -4.8171465 .6291040 .962410$

C -6.3994910 .7231950 .511359$

O $-7.2037872 .123711-1.270752$

H -7.919712 2.2913370 .624109

H $-5.5618210 .484238-0.149828$

O $-7.425925-0.2338360 .222501$

C -5.9769850 .5734351 .981330$

C $-5.542525-0.8455262 .400329$

H $-5.166224 \quad 1.2734332 .210133$

O

H $-5.550889-0.873823 \quad 3.497332$

H -6.303085 -1.571093 2.093528

H $-4.1427187 .116932-0.913948$

H -5.406829 $6.425290-1.943052$

H $-5.8488867 .353303-0.501812$

C $-2.7268304 .433433-0.156822$

H -7.789392 $1.371612-1.464051$

H $-8.162970-0.0677620 .834982$

H $-6.7952290 .955928 \quad 3.720678$

H -2.660782 4.3060670 .930957

H $-2.3546405 .436981-0.391648$

H $-4.2411264 .234960-1.705305$

C $-4.741596-2.628764-0.000457$

O $-4.075194-1.4573890 .485183$

C $-4.137814-1.2481651 .898711$

C $-3.543311-2.4613222 .630171$

C $-4.232796-3.7563182 .213606$

C $-4.222092-3.8958960 .693237$

H $-5.821124-2.5273280 .159375$

C $-4.481285-2.717505-1.511126$

H $-3.475878-0.3985722 .110275$

O $-3.633808-2.2874604 .040112$

H $-2.475497-2.5326392 .390508$

H $-5.254774-3.8036012 .606444$

O $-3.541053-4.8664832 .790726$

H $-3.201471-4.1164480 .355308$

O $-5.040451-5.002403 \quad 0.318721$

C $-5.179094-1.611414-2.266727$

H -3.404352 -2.663771-1.712416

H $-4.839923-3.663531-1.932069$

H -3.278534 -3.091492 4.456360

H -2.624097 -4.851198 2.467131

H $-4.705840-5.7842820 .790579$

O $-4.257714-0.785573-2.837689$

O $-6.395394-1.494919-2.338978$

C $-4.7829630 .322259-3.574872$

H $-5.2521371 .034076-2.891043$

H -3.949907 $0.818829-4.079590$

H $-5.496909-0.018791-4.330675$

SCF Energy (B3LYP/6-31G**//MMFF) $=-3245.91498280$

0343

MM̄FF Geometry

C $1.607951-4.047736-0.211596$

C $1.054415-3.9709111 .009061$

C $-0.349459-3.5019611 .313440$

O $-0.786819-2.5298390 .337286$

C $-1.323787-4.6928801 .288707$

C $-2.711489-4.3538541 .797445$

C $-3.831382-4.3744621 .048511$

C $-2.803086-4.005303 \quad 3.261749$

C $-3.973016-4.719147-0.407043$

C $-3.887337-3.520857-1.365596$ 
C $-4.955051-2.439697-1.128495$

C $-4.944405-1.413692-2.262497$

C $-5.948076-0.276760-2.013932$

O $-4.660108-1.776203 \quad 0.101358$

$\begin{array}{llll}0 & -5.643173 & 0.786555 & -2.931505\end{array}$

C $-5.7434180 .288722-0.587955$

C $-7.381931-0.718690-2.332690$

O $-6.7850651 .210639-0.278655$

C -5.631522 -0.8004590 .504647$

C $-5.173392-0.266157 \quad 1.881373$

O $-6.901235-1.4133370 .762080$

C $-0.378131-1.2456710 .528991$

O $0.283083-0.8173641 .461307$

C $-0.857491-0.438943-0.613795$

C $-0.3189700 .770285-0.827752$

C $-0.6768701 .704840-1.896170$

C -1.907346 $1.396301-2.705586$

C -3.8109870 .4344451 .899868$

C $-3.892808 \quad 1.9474531 .787234$

C -2.4356893 .9626871 .636532$

O -4.9513172 .5696581 .807380$

C -2.386874 4.4071110 .209115

C - $-1.3991215 .163453-0.293328$

C -1.362653 $5.659842-1.710887$

C $-0.0951165 .263609-2.433932$

C $0.9343416 .119644-2.547745$

C $-0.052858 \quad 3.899221-3.087184$

C $0.0979722 .793624-2.080487$

N -2.633852 2.5265611 .693922

H $1.015315-3.792420-1.086084$

H $1.646099-4.2675321 .872457$

H - $0.345539-3.0395362 .307873$

H -0.930559-5.511699 1.906275

H $-1.373979-5.0829990 .266363$

H $-4.772565-4.1168411 .533763$

H -3.840058 -3.9248953 .605439$

H $-2.318690-4.7778233 .867867$

H -2.317773 -3.0455323 .462664$

H $-3.228650-5.465709-0.703366$

H $-4.942611-5.215683-0.542250$

H -2.888716 -3.076505 - 1.284835

H $-3.986205-3.906376-2.387960$

H $-5.934294-2.927366-1.058383$

H $-5.144232-1.896442-3.227313$

H $-3.940344-0.977965-2.349713$

H $-5.6870080 .426782-3.833945$

H $-4.8107220 .865018-0.618454$

H -7.469751 -1.000013 -3.389257

H $-8.0928240 .101112-2.182834$

H -7.707569 -1.575924 -1.739569

H -6.5516551 .6563580 .552759$

H -5.9559520 .3599552 .326719$

H -5.091798 -1.1361792 .549836$

H $-7.583065-0.9410250 .258388$

H -1.627571 -0.862133 -1.247034

H $0.4712701 .110564-0.160344$

H -2.776163 $1.285238-2.047935$

H $-1.7736810 .468727-3.272721$

H -2.165155 $2.174191-3.426403$

H -3.3274550 .2252552 .862875$

H -3.157828 0.0464541 .113781

H -1.5075654 .1835252 .171522$

H -3.262014 4.4646172 .150186

H $-3.2163974 .119387-0.432572$

H -0.5834395 .4718870 .356935$

H $-1.4502406 .753983-1.682844$

H -2.231995 $5.309782-2.281458$

H $1.8377415 .849927-3.084724$

H $0.8989317 .107509-2.100428$

H $\quad 0.8038513 .835636-3.771004$

H $-0.9372883 .788107-3.719362$

H $0.9709922 .917112-1.440489$

H -1.8136141 .9301231 .632869$

C $3.685576-3.746946-1.603484$

O $3.757203-2.336560-1.324482$

C $4.976344-1.814190-1.889570$

C $5.657210-2.975543-2.611541$

C $5.129858-4.175387-1.851808$
H $5.605749-1.507723-1.046277$

C $4.679218-0.609329-2.791167$

H $5.361906-3.031857-3.665609$

H $6.748375-2.894525-2.580802$

C $5.273318-5.482994-2.610776$

H $5.669265-4.249143-0.897825$

C $3.9027960 .532816-2.107759$

O $3.888513-1.060272-3.900648$

H $5.616782-0.230926-3.216061$

H $2.8800380 .200859-1.889541$

O $3.7904521 .582757-3.078586$

C $4.5626541 .081564-0.827137$

C $3.8280952 .318681-0.281927$

H $4.5748210 .291524-0.071351$

O $5.9168501 .425773-1.114993$

H $3.9792743 .160023-0.968979$

H $2.7579082 .098917-0.261999$

H $4.884798-6.321135-2.024208$

H $4.730424-5.456463-3.561579$

H $6.326636-5.686087-2.830546$

C $3.024263-4.495613-0.443926$

H $3.718903-0.287947-4.466950$

H $3.0836012 .182022-2.785948$

H $6.3663991 .592022-0.269616$

H $3.612111-4.3472900 .470199$

H $3.011388-5.568837-0.664841$

H $3.087049-3.863108-2.517132$

C $2.894807 \quad 1.2733182 .418080$

O 4.2217751 .6948612 .080313

C 4.2916502 .7570821 .124283

C 3.5505043 .9878881 .664408

C 2.1135623 .6596072 .064503

C 2.0774152 .4412052 .987889

H $2.4000380 .873522 \quad 1.526425$

C $3.021354 \quad 0.141715 \quad 3.450321$

H $5.350803 \quad 3.0295501 .042387$

O 3.5598125 .0306090 .694508

H 4.0854824 .3648752 .545921

H 1.4810703 .5129101 .181153

O $1.582994 \quad 4.7935152 .755006$

H 2.4619442 .7213863 .977011

$\begin{array}{lllll}\text { O } & 0.725425 & 2.017689 & 3.155052\end{array}$

C $3.576239-1.1296472 .842996$

H $3.6986790 .460627 \quad 4.252153$

H $2.048025-0.1108043 .884037$

H 3.0583635 .7770901 .064741

H $0.647354 \quad 4.6128152 .944046$

H $0.2501832 .723308 \quad 3.624985$

O $4.134096-1.8919243 .829198$

O $3.502252-1.4337361 .660717$

C $4.696918-3.1364543 .401752$

H $3.901991-3.8117643 .073105$

H $5.424478-2.9763232 .600051$

H $5.210813-3.5876404 .254775$

SCF Energy (B3LYP/6-31G**//MMFF)= -3245.91124916

0344

MM̄FF Geometry

C $-3.180094-2.012463-3.358136$

C $-2.416489-2.274192-2.286175$

C $-0.939869-2.578355-2.381006$

O $-0.203431-1.661279-1.538131$

C $-0.681501-4.018112-1.895259$

C $0.775908-4.440704-1.908653$

C $1.422840-4.943948-0.838075$

C $1.497727-4.304394-3.223581$

C $0.852394-5.2030840 .529316$

C $0.842779-3.9749201 .448044$

C $2.224718-3.5760521 .987545$

C $2.090009-2.3698792 .921617$

C $3.452652-1.8686473 .418267$

O $3.071686-3.2479790 .886157$

O $3.257148-0.5738294 .008093$

C $4.383932-1.6751312 .203819$

C $4.024977-2.7666934 .520873$

O $5.708366-1.3554512 .625054$

C $4.410264-2.8894981 .245969$

C $5.182380-2.572397-0.058069$ 
O $5.084220-3.973773 \quad 1.893742$

C $0.056456-0.431508-2.064511$

O $-0.3484270 .005400-3.131156$

C $0.954504 \quad 0.296232-1.137027$

C $1.2990491 .558540-1.435524$

C $2.2154022 .432367-0.699469$

C 2.8913841 .8945780 .532095

C $4.423191-1.665129-1.031004$

C $5.340231-0.927906-1.989625$

C $5.3145261 .012286-3.556957$

O $6.538214-1.171605-2.101192$

C $5.6073102 .231104-2.737466$

C $5.1423513 .456230-3.024697$

C $5.4333884 .670038-2.188890$

C $4.1800075 .380899-1.725863$

C $3.8448166 .583984-2.221880$

C $3.3558484 .734979-0.629594$

C $2.4188853 .683094-1.166221$

N $4.6507320 .041131-2.707564$

H -2.699075 -2.000461 -4.335180

H $-2.853488-2.292243-1.292688$

H $-0.588239-2.480595-3.416045$

H -1.241168 -4.720248 -2.527921

H $-1.096307-4.120674-0.887180$

H $2.470523-5.219489-0.950212$

H $2.486395-4.775653-3.204818$

H $\quad 0.923440-4.783266-4.023382$

H $\quad 1.642879-3.249843-3.477247$

H $-0.169653-5.5889770 .435397$

H $1.422065-6.0142390 .999560$

H $0.404846-3.1273540 .907121$

H $0.183328-4.1902652 .298102$

H $2.646749-4.4339732 .524160$

H $1.434349-2.6043983 .769660$

H $1.597998-1.5477232 .382891$

H $2.610351-0.6671544 .728437$

H $4.017530-0.7979541 .658963$

H $3.343726-2.7996505 .379559$

H $4.970368-2.3675244 .904592$

H $4.191373-3.7949324 .193332$

H $5.647004-0.5846363 .214830$

H $6.166519-2.1595770 .195819$

H $5.397820-3.508751-0.591368$

H $5.385086-4.6004451 .215372$

H $1.324975-0.231382-0.266444$

H $\quad 0.8758752 .004997-2.335478$

H $3.7260321 .246250 \quad 0.252597$

H 2.1974921 .3257121 .158860

H 3.2843242 .6837911 .178492

H $3.718223-2.261790-1.622042$

H $3.844460-0.916409-0.485870$

H $4.644851 \quad 1.226562-4.395475$

H $6.2463050 .590581-3.946117$

H $6.2367792 .095138-1.860649$

H $4.5276313 .599353-3.910422$

H $6.0527655 .346597-2.791869$

H $6.0402414 .413405-1.311429$

H $2.9617347 .111153-1.876215$

H $4.4424327 .066734-2.988060$

H $2.7479095 .496525-0.124872$

H 4.0401274 .3450380 .129567

H $1.8559704 .003302-2.044340$

H $3.6684110 .196793-2.502489$

C $-5.442346-1.873591-2.086643$

O $-5.118285-0.771603-1.215210$

C $-6.337347-0.228536-0.674582$

C -7.417551-1.264128 -0.955688

C $-6.958294-1.809110-2.292588$

H $-6.5610740 .675521-1.253700$

C $-6.163616 \quad 0.1351810 .803129$

H -7.424224 -2.062200 -0.204374

H -8.418141 -0.822928 -0.990564

C -7.591622 -3.142634-2.652015

H -7.200867 -1.075316 -3.073093

C -4.9618951 .0617251 .087754$

O $-5.972559-1.0707961 .552053$

H -7.091164 0.5825011 .179786

H -4.0346460 .5118580 .903864$
O $\quad-4.979926 \quad 1.3632652 .488450$

C -4.9684552 .3766420 .292132$

C $-3.8660613 .383897 \quad 0.673932$

H $-4.9005002 .163526-0.780186$

O -6.2333543 .0128650 .512900$

H -4.1548124 .3539240 .249718$

H -3.8676893 .5393901 .757935$

H -7.222966 -3.503650 -3.617256

H -7.370919 $-3.907422-1.899637$

H $-8.679973-3.044606-2.722074$

C $-4.662094-1.744444-3.399170$

H $-5.846100-0.8175912 .482561$

H -5.7745351 .8959042 .664071$

H $-6.2801103 .786359-0.074537$

H $-4.794521-0.731348-3.799654$

H $-5.085796-2.440383-4.133415$

H -5.171772 -2.797090 - 1.559172

C -1.5106242 .0488742 .169474$

O -1.8962861 .8658180 .801854$

C -2.4590803 .0112590 .157219$

C -1.4573014 .1741840 .206397$

C $-1.0381904 .485681 \quad 1.639099$

C -0.5332463 .2209062 .328721$

H -2.403785 2.2208112 .780422

C -0.8452260 .7468792 .636680$

H $-2.5769042 .739647-0.899696$

O $-2.0075335 .333935-0.408424$

H $-0.569436 \quad 3.900547-0.374821$

H -1.861392 4.9398542 .201987

O 0.0076045 .4601441 .624681

H 0.4456942 .9504911 .916960

O $-0.3460133 .486773 \quad 3.717664$

C $-1.848426-0.3718692 .778031$

H $-0.065801 \quad 0.4390321 .930011$

H $-0.361770 \quad 0.862113 \quad 3.613403$

H $-1.350626 \quad 6.046455-0.326336$

H $0.7580125 .087278 \quad 1.131698$

H $\quad 0.2574594 .2461223 .789507$

O $-1.656726-1.3005021 .798501$

O $-2.702995-0.4120663 .653820$

C $-2.532668-2.4305721 .843179$

H -2.507204 -2.901822 2.830482

H $-3.548496-2.1238241 .585798$

$\mathrm{H}-2.191235-3.1579001 .103369$

SCF Energy $\left(B 3 L Y P / 6-31 G^{* *} / / M M F F\right)=-3245.91464630$

0345

MM̄FF Geometry

C $2.812691-3.542583-1.244927$

C $1.520644-3.446268-1.596558$

C $0.395917-3.295591-0.602799$

O $-0.571468-2.337146-1.097049$

C $-0.325273-4.645715-0.438142$

C $-1.395949-4.6454840 .635268$

C $-2.708285-4.8498670 .409546$

C $-0.910469-4.4269982 .045312$

C $-3.399403-5.104314-0.900194$

C $-3.953594-3.844943-1.586092$

C $-5.001162-3.086631-0.754544$

C $-5.694892-2.014079-1.596545$

C $-6.686453-1.183783-0.764903$

O $-4.336998-2.4625650 .347810$

O $-7.034791-0.018972-1.529272$

C $-5.982734-0.6870800 .517588$

C $-7.994432-1.944068-0.513733$

O $-6.912364-0.0622631 .398667$

C $-5.212971-1.7945151 .265660$

C $-4.338273-1.2603712 .426722$

O $-6.151263-2.7132371 .839788$

C $-0.201057-1.029928-1.146944$

O $0.888496-0.563066-0.857037$

C -1.360962 -0.249584-1.631608

C $-1.175031 \quad 1.017909-2.028057$

C $-2.2027511 .939329-2.515985$

C $-3.6329191 .471765-2.479524$

C $-3.274204-0.2289562 .033865$

C $-3.721890 \quad 1.212350 \quad 2.214455$

C -2.9790323 .5469831 .763642$ 
O $-4.763077 \quad 1.529836 \quad 2.782673$

C -3.563417 4.0343410 .475740

C $-3.0036254 .997880-0.271512$

C $-3.6186195 .545460-1.527076$

C $-2.6793465 .498751-2.710256$

C $-1.9430016 .569939-3.051067$

C -2.667939 $4.244377-3.553312$

C -1.817996 $3.154577-2.959332$

N $-2.798553 \quad 2.108977 \quad 1.695054$

H $3.072359-3.536034-0.190655$

H $1.249994-3.478934-2.649438$

H $\quad 0.774939-2.9412320 .362709$

H $0.402358-5.428227-0.183325$

H $-0.750829-4.932894-1.405671$

H -3.379981 -4.835986 1.267623

H $-0.580067-3.3929792 .181241$

H $-1.691307-4.6213392 .788565$

H -0.074367 -5.097319 2.269830

H -2.736028 $-5.626616-1.597289$

H $-4.224698-5.804090-0.715406$

H -3.118517 -3.176825 - 1.827211

H $-4.404085-4.156193-2.536666$

H -5.734531-3.810971 -0.380626

H -6.201657 -2.462860 -2.459791

H $-4.938608-1.335654-2.013721$

H $-7.413800-0.317044-2.373935$

H $-5.2745020 .090248 \quad 0.208035$

H -8.473917 -2.205222 -1.464861

H $-8.717454-1.3201820 .023245$

H -7.851579 -2.8694550 .047960$

H -7.3556580 .6444360 .898649$

H $-4.975763-0.8937603 .240327$

H -3.800231-2.122325 2.847562

H -5.711288 -3.1921802 .561620$

H -2.328999 $-0.735995-1.648390$

H $-0.1642651 .423550-2.003553$

H -3.784655 $0.646369-3.183200$

H -4.354336 $2.251555-2.731973$

H $-3.898138 \quad 1.130393-1.473359$

H -2.404283 -0.3614462 .690089$

H -2.934253 -0.3827631 .005143$

H -2.000517 3.9904921 .971202

H -3.652064 3.7958392 .590362

H $-4.509797 \quad 3.5990840 .163024$

H -2.071981 5.4518270 .059393

H -3.910021 $6.585173-1.327315$

H $-4.5506555 .023860-1.777816$

H -1.290386 $6.554951-3.917724$

H -1.972306 7.483718 -2.466795

H -2.256245 $4.467450-4.547128$

H -3.696671 $3.928717-3.747761$

H $-0.7554563 .401377-2.934862$

H -1.985117 1.7559961 .198884

C $5.282989-3.147327-1.805245$

O $5.201370-1.774643-1.379524$

C $6.198455-1.560052-0.355622$

C $6.992273-2.860598-0.240652$

C $5.961928-3.892372-0.658399$

H $5.658350-1.4046270 .584258$

C $7.054142-0.332709-0.692308$

H $7.848918-2.874911-0.924792$

H $7.374804-3.0213730 .772142$

C $6.567123-5.228447-1.051444$

H $5.267067-4.043703 \quad 0.176347$

C $6.2541710 .981535-0.802371$

O $7.692958-0.569626-1.954108$

H $7.857566-0.2265700 .046309$

H $5.5302460 .904826-1.622614$

O $7.1884162 .004895-1.169581$

C 5.5472951 .3903980 .505298

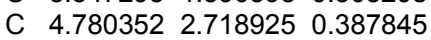

H $4.8414080 .608507 \quad 0.789290$

O $\quad 6.506745 \quad 1.489095 \quad 1.556848$

H $5.4760013 .521548 \quad 0.116693$

H $4.0566832 .627638-0.429390$

H $5.788124-5.938885-1.344956$

H $7.262132-5.123555-1.891208$

H $7.118893-5.662392-0.211046$
C $3.917597-3.675461-2.255291$

H $8.2298100 .215423-2.157677$

H $6.6804412 .753371-1.525288$

H 7.1603842 .1601061 .295218

H $4.015684-4.731214-2.532747$

H $3.627505-3.125752-3.160427$

H $5.943806-3.155925-2.683464$

C 2.0806081 .7820721 .497272

O 3.2572832 .0978942 .249621

C 4.0711013 .1370101 .697757

C 3.2536754 .4308781 .575830

C $1.974714 \quad 4.211616 \quad 0.773559$

C $1.193358 \quad 3.0221201 .327288$

H 2.3686281 .3892640 .514898

C $1.325203 \quad 0.683026 \quad 2.264574$

H 4.8614993 .3254042 .435365

O 4.0181025 .4636050 .963069

H 2.9784664 .7843092 .577974

H $2.1848234 .080622-0.294312$

$\begin{array}{lllll}\text { O } & 1.147464 & 5.373324 & 0.877039\end{array}$

H $0.742793 \quad 3.2999452 .288698$

$\begin{array}{lllll}\text { O } & 0.129913 & 2.709324 & 0.432932\end{array}$

C $2.066756-0.6378192 .273921$

H $1.193148 \quad 1.0005643 .306275$

H $\quad 0.341540 \quad 0.497264 \quad 1.820320$

H 4.8165525 .5947371 .502369

H 1.6697376 .1288050 .556688

H -0.3603303 .5309900 .261159$

O $1.589146-1.4126223 .292377$

O $2.935571-0.964644 \quad 1.477940$

C $2.213236-2.6931343 .433769$

H $2.133101-3.2658822 .505010$

H $3.261831-2.5683963 .719647$

H $1.694320-3.2383844 .226558$

SCF Energy (B3LYP/6-31G*//MMFF)= -3245.91620712

03_46

MM̄FF Geometry

C -3.308136 -1.682368 -3.256042

C $-2.422198-1.697029-2.247875$

C $-0.976287-2.095084-2.410559$

O $-0.169175-1.269102-1.537542$

C $-0.786000-3.557939-1.973016$

C $0.619252-4.085522-2.186276$

C $1.432425-4.509684-1.199062$

C $1.086069-4.151937-3.617822$

C $1.148199-4.5642280 .275709$

C $1.565162-3.3090821 .057157$

C $3.073887-3.014648 \quad 1.017404$

C $3.437581-1.9145832 .016951$

C $4.926039-1.5392571 .943621$

O $3.419143-2.585782-0.302575$

O $5.105072-0.3082462 .661616$

C $5.303718-1.2619510 .472327$

C $5.811775-2.5794902 .639385$

O $6.709523-1.0742560 .334089$

C $4.820534-2.354654-0.503753$

C $5.014461-1.971995-1.992059$

O $5.574375-3.552397-0.278756$

C $0.0858850 .007092-1.929584$

O $-0.2645540 .543692-2.967920$

C $0.8483650 .657347-0.840731$

C $0.929398 \quad 1.995215-0.801291$

C $1.6176862 .787860 \quad 0.218274$

C $2.5001222 .054681 \quad 1.192946$

C $4.291218-0.698358-2.444671$

C $5.155207 \quad 0.551680-2.404277$

C $5.0067613 .023371-2.691960$

O $6.3700470 .527008-2.226683$

C $4.9168293 .673926-1.347600$

C $4.4147124 .903803-1.157926$

C $4.3571745 .590208 \quad 0.176931$

C $2.9716716 .094358 \quad 0.514823$

C 2.6309377 .3759470 .298123

C 2.0127085 .1345031 .181613

C 1.4436904 .1258900 .221387

N $4.4106121 .701681-2.631964$

H $-2.969574-1.998334-4.241228$ 
H $-2.730293-1.416153-1.245738$ H $-0.648895-1.955634-3.447815$ H -1.477886 -4.207262 -2.525934 H -1.065889 -3.641276 -0.918610 H $2.422258-4.873067-1.473674$ H $2.035203-4.688907-3.720242$ H $\quad 0.347995-4.674873-4.234742$ H $1.234105-3.146522-4.023406$ H $\quad 0.086212-4.7613920 .458545$ H $1.672563-5.4353720 .689081$ H $1.009292-2.447800 \quad 0.668473$ H $1.254427-3.4515692 .099639$ H $3.612068-3.9396251 .255425$ H $3.166642-2.2089953 .038383$ H $2.845525-1.0166401 .796604$ H $4.802073-0.4473293 .575182$ H $4.833234-0.3100420 .199990$ H $5.532827-2.6744173 .695760$ H $6.862665-2.2700862 .634775$ H $5.741732-3.5721542 .189987$ H $6.968402-0.3621350 .943856$ H $6.082885-1.939704-2.236928$ H $4.604534-2.796761-2.593126$ H $5.521688-4.104237-1.076570$ H $1.2963650 .019745-0.087340$ H $0.4217992 .569607-1.575377$ H $3.198867 \quad 1.4008180 .660534$ H 1.8972731 .4454041 .873113 H 3.1174072 .7148781 .805600 H $3.991249-0.825057-3.492961$ H $3.379991-0.535135-1.861885$ H $4.4695263 .586594-3.461433$ H $6.0566042 .937985-2.990500$ H $5.3065973 .120094-0.496714$ H $4.0528345 .466047-2.016094$ H $5.061955 \quad 6.4316860 .148618$ H 4.7131284 .9345270 .981300 H 1.6488777 .7530210 .563416 H $3.3257418 .075657-0.154752$ H 1.1645025 .6858521 .609705 H 2.5059294 .6748642 .042674 H $0.7894984 .565345-0.533062$ H $3.4009981 .632264-2.720920$ C $-5.154467-0.366701-2.019149$ O $-4.5069690 .911296-2.192379$ C $-5.4798801 .948848-2.014709$ C $-6.6586841 .304727-1.303209$ C $-6.655120-0.071192-1.947497$ H $-5.8124752 .228931-3.024251$ C $-4.8725073 .192967-1.361084$ H $-6.4832141 .220200-0.225060$ H -7.594614 $1.849244-1.458621$ C -7.449529-1.108160 -1.172468 H $-7.0703430 .012608-2.960824$ C -4.4151873 .0885520 .108359$ O $-5.8474074 .243691-1.416331$ H $-4.0181573 .529686-1.962276$ H $-5.288426 \quad 3.0554090 .771174$ O C -3.4769801 .9200560 .459820$ C -2.8573602 .0643811 .862371$ H $-4.0273320 .975920 \quad 0.403142$ O $-2.395500 \quad 1.858569-0.468822$ H -2.0890412 .8473281 .835055$ H -3.6391072 .3658262 .570476$ H -7.388924 -2.087862 -1.656660 H -7.078264 -1.214920 -0.147983 H -8.504862 -0.821567 -1.118216 C $-4.761233-1.283165-3.181061$ H -6.066752 $4.391960-2.352098$ H -4.3510345 .0440270 .232751$ H -2.755478 $1.582564-1.328146$ H $-4.992498-0.764915-4.121028$ H -5.362297 -2.199440 -3.161611 H -4.816353 -0.776959-1.062095 C $-2.894869-1.4044073 .249920$ O $-3.277279-0.1127202 .758327$ C $-2.210803 \quad 0.7506182 .345088$
C $-1.2351740 .994530 \quad 3.505307$ C $-0.723113-0.3266624 .084346$ C $-1.867968-1.2924874 .391480$ H $-3.815323-1.8210693 .679247$ C $-2.450428-2.3337452 .109230$ H -1.6716110 .2877031 .511754$ O $-1.861749 \begin{array}{lll}1.759894 & 4.532486\end{array}$ H -0.3909351 .5885613 .136766$ H $-0.162648-0.1289545 .006409$ O $0.160096-0.946518 \quad 3.155714$ H $-1.468109-2.2841984 .633793$ O $-2.539451-0.817106 \quad 5.562328$ C $-3.636509-2.8024401 .289600$ H $-1.747128-1.8556001 .422806$ H $-1.978486-3.2258922 .537525$ H -2.655581 1.2777894 .820864 H $0.902812-0.3373443 .008488$ H $-3.216306-1.4733135 .800292$ O $-3.281249-3.9140500 .581163$ O $-4.730602-2.2556731 .258630$ C $-4.303265-4.466744-0.254507$ H $-4.704618-3.706093-0.930483$ H $-3.856968-5.264996-0.853422$ H $-5.099685-4.891418 \quad 0.363365$ SCF Energy (B3LYP/6-31G**//MMFF) $=-3245.90816436$

0347

MM̄FF Geometry

C 2.0474801 .8617834 .065823

C 1.7116520 .7033663 .478988

$\begin{array}{llll}\text { C } & 0.325269 & 0.113568 & 3.524110\end{array}$

$\begin{array}{llll}\text { O } & -0.205668 & 0.071588 & 2.179875\end{array}$

C $0.400191-1.3314994 .051440$

C $-0.952625-1.991764 \quad 4.232555$

C $-1.372904-3.078928 \quad 3.555994$

C $-1.846258-1.3747265 .278281$

C $-0.638968-3.8640742 .505188$

C $-0.931924-3.4298191 .060952$

C $-2.398526-3.5953690 .631952$

C $-2.547594-3.367141-0.874381$

C $-4.016483-3.423482-1.324018$

O $-3.184516-2.6320261 .337401$

O $-4.091689-2.881897-2.652061$

C $-4.860035-2.503597-0.414060$

C $-4.528455-4.865922-1.414906$

O $-6.250663-2.653406-0.687226$

C $-4.594047-2.7170191 .090001$

C $-5.279282-1.6595151 .989651$

O $-5.107810-3.9958571 .482206$

C $-0.725076 \quad 1.2251361 .677471$

$\begin{array}{llll}\text { O } & -0.792345 & 2.308647 & 2.235670\end{array}$

C $-1.1898110 .944403 \quad 0.301627$

C $-1.4485331 .965050-0.528970$

C $-1.8904281 .853146-1.920720$

C $-2.2756320 .485892-2.419783$

C $-4.867869-0.206252 \quad 1.727246$

$\begin{array}{llll}\text { C } & -5.810518 & 0.543511 & 0.800719\end{array}$

C $-6.0389922 .722456-0.385272$

\begin{tabular}{lllll}
\hline & -6.898049 & 0.100958 & 0.441328
\end{tabular}

C $-5.6258252 .540966-1.811481$

C $-5.2408413 .556384-2.599264$

C $-4.8636693 .399466-4.044228$

C $-3.502390 \quad 3.979171-4.355684$

C $-3.379102 \quad 5.207377-4.886822$

C $-2.292524 \quad 3.100982-4.130850$

C $-1.9318182 .970821-2.676219$

$\begin{array}{llll}\mathrm{N} & -5.308175 & 1.790457 & 0.452486\end{array}$

H 1.2937672 .4244614 .610490

H 2.4674490 .1502352 .926686

H $-0.335720 \quad 0.713453 \quad 4.161278$

H $0.914935-1.3463495 .021533$

H $1.024370-1.9190923 .369345$

H -2.365296 -3.467034 3.783502

H -2.736154-1.982315 5.475312

H $-1.307524-1.2703466 .225739$

H $-2.190041-0.3863744 .958864$

H $0.442344-3.8243732 .674456$

H $-0.906604-4.9217932 .623967$ 
H $-0.635272-2.3822660 .942239$ H -0.293868 -4.026520 0.397776 H $-2.727193-4.6062750 .900543$ H -1.946175 -4.090376 -1.439248 H $-2.143918-2.378625-1.132421$ H $-3.503910-3.405464-3.223156$ H $-4.599154-1.472045-0.678096$ H -3.930110 -5.439770 -2.132690 H -5.557006 -4.897362 -1.790980 H -4.492806 -5.394485 -0.460131 H $-6.378995-2.487749-1.636881$ H $-6.368239-1.7856161 .957703$ H -4.992853 -1.884604 3.027367 H -5.248976 -3.990123 2.443377 H -1.285257 -0.0931470 .003658$ H -1.298831 $2.981488-0.166707$ H -1.396883 $-0.166056-2.471423$ H $-2.7337110 .494587-3.410690$ H -3.014281 $0.029870-1.752022$ H -4.8865190 .3358792 .681547$ H $-3.846550-0.1479211 .338661$ H -5.822298 $3.729783-0.015833$ H $-7.1133522 .537260-0.286169$ H $-5.6702741 .534891-2.221960$ H $-5.2248284 .565516-2.193158$ H $-5.6306713 .905606-4.645174$ H $-4.8922612 .347772-4.354922$ H $-2.4078815 .619823-5.139494$ H -4.247265 $5.830281-5.075829$ H -1.418763 $3.527766-4.641827$ H $-2.4569082 .136933-4.619617$ H -1.632052 $3.915136-2.219154$ H -4.3735542 .0530700 .752110$ C 3.6216183 .5299752 .961372 O 3.4895082 .9607371 .645383 C 2.8795563 .9405910 .780137 C 2.8216465 .2405041 .578689 C 2.6641034 .7184362 .995894 H 1.8532753 .6012400 .597815 C $3.6471254 .022528-0.545256$ H $3.7487395 .817948 \quad 1.486538$ H 1.9941775 .8830361 .262557 C 2.9893525 .7436784 .066786 H 1.6271324 .3833973 .121784 C $3.8146132 .658114-1.247852$ O $4.9587684 .534817-0.276789$ H $3.1619464 .745634-1.211284$ H $4.4570322 .019125-0.632844$ O $4.5313902 .872781-2.469997$ C $2.4913421 .941906-1.566355$ C $2.6612360 .603329-2.300069$ H $1.9236451 .773334-0.646973$ O $1.7010222 .805622-2.387680$ H $1.6671540 .194483-2.530515$ H $3.0985120 .775171-3.293357$ H 2.8734085 .3122045 .065944 H 4.0173826 .1094993 .974186 H 2.3181976 .6053943 .990628

C 3.4312002 .4446474 .022329 H $5.4342734 .571043-1.124567$ H $3.9623793 .404527-3.052932$ H $0.8774642 .335928-2.601072$ H 3.6655842 .8488735 .014065 H 4.1541921 .6400703 .834387 H 4.6579643 .8876693 .031839 C $4.230910-2.742869-1.902390$ O $3.439178-1.642795-2.371659$ C $3.494688-0.458982-1.562505$ C $2.990207-0.762419-0.143802$ C $3.743183-1.9365510 .486663$ C $3.839336-3.132882-0.466442$ H $3.955151-3.578090-2.558963$ C $5.721386-2.456615-2.113305$ H $4.534130-0.115773-1.524407$ O $1.598405-1.063344-0.195480$ H $3.1157390 .127903 \quad 0.481873$ H $3.226600-2.2397191 .405706$ O $5.045964-1.4935200 .853468$
H $4.543330-3.877865-0.080059$

O $2.559261-3.770187-0.535220$

C $6.553664-3.711803-2.040659$

H $5.887431-1.987339-3.091423$

H $\quad 6.119945-1.740317-1.388953$

H $1.291891-1.1721340 .720806$

H $5.505264-2.2433171 .268689$

H $2.327282-4.0599730 .363649$

O $6.451143-4.408838-3.207917$

O $7.206886-4.038606-1.058230$

C $7.188349-5.634166-3.258643$

H $6.833407-6.321833-2.484936$

H $7.021199-6.091241-4.237535$

H $8.258344-5.437971-3.140778$

SCF Energy (B3LYP/6-31G**//MMFF) $=-3245.90588810$

0348

MM̄FF Geometry

C $4.774562-0.9334373 .075080$

C $3.738069-1.4897732 .429426$

C $2.459871-1.8919543 .121615$

O $1.338539-1.2660632 .457376$

C $2.272153-3.4169883 .034685$

C $1.055959-3.9121423 .794079$

C $-0.078494-4.370103 \quad 3.228048$

C $1.194533-3.9127915 .296101$

C $-0.418800-4.4773501 .766573$

C $-1.725158-3.7459271 .444171$

C $-2.239642-4.0030760 .018739$

C $-1.285343-3.482944-1.058429$

C $-1.868370-3.633693-2.472218$

O $-3.504708-3.334127-0.085025$

O $-1.068049-2.846002-3.366954$

C $-3.287579-3.031460-2.489852$

C $-1.783261-5.079224-2.975493$

O $-3.931149-3.267167-3.739206$

C $-4.181413-3.532868-1.335433$

C $-5.530890-2.780528-1.257301$

O $-4.485445-4.917701-1.537915$

C 1.0238050 .0017532 .840065

O 1.6566730 .7193583 .598269

C -0.2663790 .3548102 .206448$

C $-0.831194 \quad 1.5419182 .473117$

C -2.1195822 .0196161 .968566$

C -2.9910821 .0375141 .229371$

C $-5.405929-1.261673-1.126387$

C $-6.707841-0.594289-0.718492$

C $-7.6299931 .657206-0.190279$

O $-7.749806-1.208061-0.509072$

C $-7.0352762 .917947 \quad 0.347198$

C -6.8721743 .1584791 .656841$

C -6.2199574 .3952662 .205179$

C -4.8470194 .0980172 .775215$

C -4.6836243 .9618944 .102586$

C -3.7269464 .0045491 .755504$

C -2.4795193 .2974712 .214134$

N $-6.549257 \quad 0.778922-0.595487$

H $4.672600-0.7556054 .144613$

H $3.796505-1.6854941 .363826$

H $2.475075-1.5860264 .175297$

H $3.158055-3.9243483 .440500$

H $2.221864-3.7204021 .984076$

H $-0.865751-4.727156 \quad 3.891841$

H $\quad 0.333076-4.3708745 .793828$

H $2.082922-4.4786605 .595403$

H $1.286719-2.8910685 .676416$

H $0.380091-4.0837071 .135723$

H $-0.525535-5.5410671 .522351$

H -2.509129 -4.051997 2.150622

H -1.601572 -2.667016 1.602865

H $-2.400560-5.081422-0.095924$

H -0.311655-3.982636-1.005539

H -1.090513 -2.417755 -0.876719

H $-0.151159-3.165418-3.310057$

H -3.165749 -1.944254 -2.402408

H $-0.738287-5.409887-3.013469$

H $-2.161060-5.163469-4.000330$

H $-2.328170-5.786161-2.346732$ 
H $-3.339858-2.936133-4.436875$ H $-6.152774-3.025362-2.128210$ H $-6.082043-3.164832-0.387496$ H -5.291673 -5.132183 -1.039919 H $-0.727193-0.3792021 .556013$ H $-0.3013532 .236506 \quad 3.123969$ H -3.1207520 .1187151 .812834$ H -2.5496250 .7774040 .262216$ H -3.997890 1.4156791 .039030 H -4.654553 -1.008537 -0.369991 H $-5.093780-0.828174-2.083162$ H $-8.256768 \quad 1.1447860 .547610$ H $-8.2476851 .868163-1.068848$ H -6.698297 $3.653634-0.378897$ H -7.1985632 .4140732 .379627$ H -6.8789104 .8154032 .975745$ H $-6.1321605 .174798 \quad 1.437776$ H -3.716409 3.7682014 .552067 H -5.525755 4.047164 4.782185 H -3.449328 5.0244841 .458781 H $-4.095817 \quad 3.5355610 .837229$ H -1.797372 3.9340812 .778575 H $-5.6936241 .200144-0.947536$ C $6.356307-0.7849451 .019192$ O $5.548761 \quad 0.1106120 .228489$ C $6.372810 \quad 0.706550-0.790173$ C $7.636354-0.140398-0.852125$ C $7.806534-0.5155700 .605788$ H $6.6401781 .707837-0.431135$ C $5.6085090 .820230-2.111986$ H $7.495100-1.041199-1.460435$ H $8.4890730 .412589-1.257258$ C $8.740641-1.6939190 .823062$ H 8.1971320 .3555361 .149211 C $4.2511181 .545907-1.998119$ O $5.354642-0.499527-2.609546$ H $6.2445291 .309901-2.859136$ H $3.5567110 .915822-1.435894$ O $3.7179451 .652296-3.323977$ C $4.3197572 .947492-1.371541$ C $3.0030973 .749449-1.403713$ H $4.6679592 .875297-0.335469$ O $5.3034083 .699174-2.092873$ H $3.2573464 .794701-1.186200$ H $2.6061563 .763904-2.424346$ H $8.822570-1.9371021 .886952$ H $8.386961-2.5883930 .299335$ H $9.744253-1.4622110 .451444$ C $6.109033-0.5239592 .508195$ H $4.852362-0.406019-3.437145$ H $4.2955542 .253051-3.825580$ H $5.4246454 .545617-1.629841$ H 6.2238790 .5494932 .706833 H 6.875319-1.0452813.094523 H $6.070150-1.8088020 .747347$ C $0.5514531 .920151-1.843021$ O $1.3958651 .985626-0.687836$ C $1.9390253 .274181-0.389624$ C $0.7942044 .277193-0.182367$ C $-0.1300104 .323174-1.395285$ C $-0.6110632 .917456-1.747379$ H $1.1448382 .121776-2.741554$ C $0.0075290 .489056-1.937705$ H $2.451793 \quad 3.1738190 .575634$ O $1.311377 \quad 5.5747280 .090979$ H $0.214566 \quad 3.9759130 .698293$ H $0.3648624 .795777-2.251034$ O $-1.2614745 .144699-1.098040$ H -1.331618 $2.581947-0.992760$ O $-1.2982552 .955003-2.996768$ C $1.099966-0.506437-2.244569$ H $-0.4804260 .198321-1.000141$ H $-0.7453370 .390637-2.728238$ H $0.5531836 .176844 \quad 0.183444$ H -1.722667 $4.756083-0.335637$ H $-2.0113063 .611585-2.916711$ O $1.403313-1.224358-1.126018$ O $1.621826-0.626368-3.345119$
C $2.385951-2.251913-1.286465$

H $3.384135-1.809378-1.260157$

H $2.287238-2.947536-0.449330$

H $2.234147-2.809436-2.215775$

SCF Energy (B3LYP/6-31G**//MMFF) $=-3245.91651480$

03_49

MM̄FF Geometry

C -2.474588 -4.062941 0.201730

C $-1.187865-4.3059930 .490782$

C $-0.055102-3.736518-0.325495$

O $0.714158-2.8418150 .515544$

C $0.875994-4.860128-0.811930$

C $1.913388-4.389247-1.813919$

C $3.243515-4.386783-1.600134$

C $1.375249-3.927781-3.145470$

C $3.990362-4.832930-0.375848$

C $4.272800-3.7252950 .650898$

C $5.070703-2.5334170 .095999$

C $5.557766-1.6338821 .234570$

C $6.274660-0.3786630 .712507$

O $4.215221-1.781731-0.771030$

O 6.3966800 .5454091 .805152

C $5.3901630 .301017-0.355609$

C $7.702373-0.6903440 .247958$

O $6.0861691 .373821-0.984333$

C $4.855091-0.675569-1.422768$

C $3.815119-0.033548-2.375085$

O $5.944644-1.122987-2.239319$

C $0.158612-1.6351760 .817174$

O $-0.919010-1.2039310 .438550$

C $1.086097-0.9201731 .719182$

C 0.6373710 .1333542 .417425

C $1.422740 \quad 0.955563 \quad 3.339631$

C 2.9136210 .7450643 .359578

C $2.5629410 .536451-1.700252$

C $2.6559312 .018313-1.374923$

C $1.4642943 .809451-0.116597$

O $3.5231832 .755652-1.835563$

C 2.0832693 .9926371 .232858

C 1.4447794 .5808372 .255980

C 2.0537394 .8051823 .610543

C 1.1928384 .2638274 .730520

C 0.3560365 .0674565 .408761

C 1.3625142 .8111635 .115265

C 0.7797891 .8692334 .096937

N $1.6259252 .431424-0.540941$

H -2.719916 -3.470431 -0.676936

H $-0.933065-4.8676551 .385869$

H -0.440542 -3.172915 -1.182179

H $0.286503-5.654640-1.288915$

H $1.358487-5.3192250 .057617$

H $3.887517-4.025992-2.401854$

H $2.170728-3.765809-3.880915$

H $\quad 0.696087-4.678719-3.562429$

H $0.832512-2.983852-3.039310$

H $3.465370-5.6551990 .121867$

H $4.944806-5.264138-0.704876$

H $3.318983-3.3721021 .059190$

H $4.829690-4.1791261 .480211$

H $5.922921-2.921484-0.474283$

H $6.210891-2.1903091 .918269$

H $4.697304-1.3160151 .838216$

H $\quad \begin{array}{llll}\text { H } & 688543 & 0.103038 & 2.518124\end{array}$

H $4.540620 \quad 0.7467110 .175377$

H $8.293297-1.0996301 .076235$

H $8.222906 \quad 0.220236-0.068630$

H $7.739355-1.412306-0.570363$

H $6.3839201 .977394-0.282197$

H $4.3017860 .710977-3.016637$

H $3.471739-0.824318-3.057810$

H $5.585174-1.449773-3.080745$

H $2.104758-1.2819331 .788327$

H -0.4094120 .4182182 .315806$

H $3.322901 \quad 0.8174312 .345984$

H $3.157775-0.2410953 .768922$

H 3.4530251 .4852513 .953696

H $1.7184770 .433268-2.393449$ 
H $2.314945-0.027326-0.796948$

H $0.3909474 .019721-0.106036$

H $1.9455974 .475632-0.839425$

H 3.1055783 .6477711 .368469

H 0.4322144 .9496252 .105416

H $2.1930285 .886787 \quad 3.738344$

H 3.0577724 .3680613 .677419

H -0.2455444 .6941556 .230673$

H 0.2507596 .1166075 .152982

H 0.8524902 .6103536 .067008

H 2.4194662 .6198555 .319662

H -0.3042251 .9496734 .002371$

H $0.969979 \quad 1.744424-0.180638$

C -4.000349 -3.329173 2.021032

O $-4.321065-2.1450401 .269672$

C $-5.320301-1.3965221 .986599$

C $-5.615071-2.1773863 .266739$

C $-5.236507-3.5941842 .872765$

H $-6.218960-1.3988071 .359031$

C -4.8399180 .0453542 .201409$

H $-4.997855-1.8364204 .105657$

H -6.662001 -2.084163 3.571990

C $-4.992666-4.5098054 .058982$

H -6.039553 -4.010900 2.250441

C -4.3568840 .7505820 .915495$

$\begin{array}{lllll}\text { O } & -3.734005 & 0.028623 & 3.114744\end{array}$

H -5.6321650 .6261652 .688109$

H -3.4065720 .3065880 .600124$

O $-4.0566312 .111008 \quad 1.253575$

C $-5.3583490 .729860-0.251720$

C $-4.932821 \quad 1.572920-1.463112$

H -5.531764 -0.302289-0.572259

O $-6.612036 \quad 1.2348880 .216838$

H -5.722664 $1.525438-2.225944$

H -4.918851 2.636673 -1.186572

H -4.720041 -5.5161793 .726089$

H -4.184352 -4.134999 4.695859

H $-5.895043-4.5913004 .673701$

C -3.628110 -4.469695 1.072443

H $-3.441961 \quad 0.9488113 .233531$

H -4.8942672 .5457091 .489255$

H -7.248289 $1.165663-0.515249$

H -4.472351 -4.716644 0.417612

H -3.382607 -5.3737301 .641166$

H -3.141375 -3.079782 2.659428

C $-2.1834831 .987686-3.930685$

O $-3.4201882 .095959-3.214315$

C $-3.5807661 .207129-2.098584$

C $-3.483899-0.255422-2.559111$

C $-2.197154-0.508644-3.354926$

C $-1.9744260 .551478-4.436536$

H -2.310577 2.636942 -4.806869

C - $-1.0016212 .525037-3.116431$

H -2.792295 $1.418981-1.369788$

O $-4.614137-0.570051-3.370575$

H -3.513154 -0.913400-1.683333

H -2.246102 -1.497298 -3.827300

O $-1.077054-0.501552-2.476473$

H $-0.9660330 .455475-4.855037$

O $-2.8851810 .314028-5.513522$

C $-1.1254194 .015869-2.913976$

H $-0.8965432 .054659-2.134990$

H $-0.0568202 .349135-3.644788$

H -4.546043 -1.508293 -3.616803

H -1.252725 -1.144631 -1.769241

H $-3.786196 \quad 0.465821-5.179942$

O $-1.8757474 .274320-1.805096$

O $-0.6293234 .848270-3.661755$

C -2.076926 $5.658600-1.505721$

H -2.614418 $6.148257-2.323275$

H $-2.6822185 .725067-0.597818$

H -1.116441 $6.150260-1.324025$

SCF Energy (B3LYP/6-31G**//MMFF)= -3245.91695200

03

MM̄FF Geometry

C $2.946371 \quad 0.7421242 .996032$

C 2.1966510 .3320624 .030145
C $0.865484-0.3778803 .914167$

O $0.233393-0.1122442 .640032$

C $1.079231-1.8973254 .035556$

C $-0.208370-2.6976614 .045710$

C $-0.566498-3.5853223 .097117$

C $-1.107963-2.4718185 .233979$

C $0.190872-3.9823181 .861078$

C $-0.180720-3.1924640 .596608$

C -1.642085 $-3.358740 \quad 0.150708$

C $-1.856571-2.737454-1.231364$

C $-3.326999-2.808769-1.672746$

O $-2.486840-2.7048021 .100112$

O $-3.491768-1.929464-2.796415$

C $-4.224338-2.265510-0.538973$

C $-3.706175-4.211902-2.161374$

O $-5.602636-2.472815-0.836009$

C $-3.890434-2.8553590 .846198$

C $-4.643730-2.1566992 .004896$

O $-4.271626-4.2365800 .872598$

C -0.3859251 .0914042 .493889$

O $-0.481706 \quad 1.9762493 .329636$

C -0.9102441 .1625271 .112991$

C -1.2497992 .3552620 .602205$

C -1.773799 $2.599770-0.742435$

C $-2.2090861 .405091-1.548535$

C $-4.374493-0.6551372 .155242$

C $-5.4122520 .230703 \quad 1.485132$

C -5.8876252 .6286430 .996307$

O $-6.460574-0.1967491 .009949$

C $-5.5142752 .914366-0.423901$

C $-5.2139224 .142078-0.874089$

C $-4.8850104 .451820-2.306318$

C $-3.5713915 .185204-2.458974$

C $-3.5380856 .520681-2.604544$

C $-2.3063174 .361068-2.531931$

C $-1.8550713 .872825-1.182137$

N -5.0433921 .5693581 .516533$

H $2.612646 \quad 0.5329381 .983114$

H 2.5498710 .5143305 .042513

H $0.210346-0.0209054 .717979$

H $1.628907-2.1251304 .958716$

H $1.724618-2.2230083 .212835$

H -1.516605 -4.104781 3.219197

H -1.531413 -1.463114 5.212108

H -1.945437 -3.177369 5.261121

H $-0.546195-2.5970246 .165401$

H $1.270698-3.9021742 .024773$

H $\quad 0.010453-5.0502191 .682861$

H $0.039141-2.1313820 .762858$

H $0.474414-3.536591-0.212385$

H $-1.875310-4.4297030 .127957$

H -1.210364 -3.216293 -1.977233

H -1.552405 -1.682411-1.206255

H $-2.877738-2.216443-3.493954$

H -4.072734 -1.180805 -0.507534

H -3.076285 -4.504964 -3.009964

H -4.736802 -4.236437 -2.532245

H -3.597484 -4.978007 -1.390834

H $-5.777205-2.064818-1.701466$

H $-5.717088-2.372647 \quad 1.941487$

H $-4.308382-2.6307422 .939029$

H -4.381148 -4.508658 1.798768

H $-0.984826 \quad 0.2391010 .550931$

H $-1.109913 \quad 3.243997 \quad 1.216514$

H -1.345432 $0.802983-1.846963$

H $-2.754876 \quad 1.667511-2.457343$

H -2.887865 $0.777350-0.962575$

H -4.406432 $-0.404315 \quad 3.223425$

H $-3.377741-0.3936351 .788200$

H -5.7509553 .5002611 .644191$

H -6.9361562 .3177151 .046236$

H -5.519457 2.078095-1.119085

H $-5.2377704 .981025-0.181900$

H $-5.7021985 .064295-2.709657$

H $-4.8684143 .542576-2.920018$

H $-2.6020987 .051445-2.743980$

H $-4.4464337 .113651-2.581846$

$\mathrm{H}-1.4876844 .965831-2.945215$ 
H $-2.4430333 .552953-3.256112$ H $-1.5233214 .672680-0.518470$ H -4.1309641 .8261561 .882024$ C 4.3092622 .7667492 .310726 O 4.1580972 .4613670 .907506 C 3.1283413 .3092090 .370817 C 3.0606884 .5029521 .313531 C 3.2465153 .8161412 .653665 H 2.1864622 .7522530 .447334 C $3.4037423 .647847-1.094974$ H 3.8785835 .2100251 .133171 H 2.1128735 .0439401 .235855 C $3.6553134 .756143 \quad 3.774258$ H 2.2975823 .3394702 .928437 C $3.6144562 .423363-2.011416$ O $4.5877304 .451939-1.166375$ H $2.5872044 .271716-1.478983$ H $4.6033501 .986279-1.825204$ O $3.6405302 .928774-3.353038$ C $2.5320151 .333423-1.888811$ C $2.6091850 .257999-2.988265$ H $2.6165680 .857441-0.906194$ O $1.2407091 .939973-1.952809$ H $2.2708520 .698303-3.933420$ H $3.651791-0.045233-3.121081$ H 3.7961614 .2055564 .709877 H 4.5917995 .2745293 .542926 H 2.8837355 .5150343 .940264 C 4.2530861 .4731843 .131151 H $4.7353454 .667116-2.103317$ H $4.0218012 .238732-3.921597$ H $1.1794312 .419751-2.796615$ H 4.4672541 .6972354 .182517 H 5.0405380 .7986232 .773887 H 5.3131823 .1974622 .416263 C $3.434511-2.445784-1.793678$ O $2.227161-1.713168-1.545386$ C $1.721675-0.966925-2.660331$ C $1.469604-1.904519-3.848377$ C $2.717583-2.699783-4.210114$ C $3.262352-3.411125-2.974595$ H $4.254886-1.748457-1.999531$ C $3.781807-3.225228-0.514710$ H $0.742192-0.583258-2.349873$ O $1.025860-1.179412-4.990033$ H $\quad 0.671544-2.612404-3.590064$ H $3.483240-2.063941-4.669838$ O $2.378614-3.691362-5.182254$ H $2.584788-4.228920-2.696203$ O $4.525551-3.993134-3.292057$ C $4.295100-2.3343380 .595524$ H $2.894559-3.745986-0.139124$ H $4.570096-3.957719-0.722206$ H $0.224851-0.691645-4.732692$ H $1.994361-3.227349-5.945921$ H $4.389830-4.583649-4.052988$ O $4.620907-3.1194421 .665597$ O $4.404490-1.1182790 .534348$ C $5.173790-2.4443742 .799652$ H $4.374060-1.9551363 .360185$ H $5.644312-3.1930213 .442646$ H $5.935689-1.7196102 .497162$ SCF Energy (B3LYP/6-31G**//MMFF)= -3245.91339574

\section{50}

\section{MM̄FF Geometry}

C $4.001596-3.555150-0.826349$

C $2.853762-3.400445-1.504218$

C $1.510122-3.249792-0.840459$

O $0.815649-2.215470-1.575670$

C $0.721918-4.571690-0.934133$

C $-0.393284-4.7126750 .085902$

C $-1.701252-4.858717-0.204249$

C $0.048101-4.7512601 .529436$

C $-2.365974-4.922924-1.549509$

C $-3.082593-3.632062-1.972739$

C $-4.273100-3.235245-1.082504$

C $-5.064724-2.095296-1.730778$
C $-6.204499-1.591627-0.832028$

O $-3.777511-2.8103590 .191376$

O $-6.653843-0.330999-1.353985$

C $-5.649057-1.3196370 .582804$

C $-7.415425-2.531270-0.869886$

O $-6.701999-1.0049671 .489641$

C $-4.800575-2.4795561 .142616$

C $-4.105665-2.1416702 .486512$

O $-5.651638-3.6057651 .393295$

C $-0.027160-1.395930-0.897223$

O $-0.292035-1.4286290 .292817$

C $-0.592696-0.440186-1.876759$

C $-1.6931130 .255269-1.555976$

C $-2.3823461 .231564-2.400574$

C $-1.739021 \quad 1.602412-3.710321$

C $-3.155373-0.9410602 .460711$

C -3.8228110 .3792202 .807070$

C -3.5435632 .8377642 .514404$

$\begin{array}{llll}\text { O } & -4.871386 & 0.454212 & 3.442354\end{array}$

C -4.3989813 .1987121 .340866$

C -4.0687024 .1264590 .429919$

C $-4.9288804 .482288-0.748589$

C $-4.2644394 .166526-2.070816$

C $-3.6187465 .119222-2.764150$

C $-4.4254572 .770605-2.631752$

C $-3.5374581 .765974-1.952241$

N -3.088144 1.4684672 .358846

H $3.967355-3.6015540 .258219$

H $2.873935-3.386535-2.591541$

H $1.659972-2.932506 \quad 0.197325$

H $1.399600-5.421837-0.774891$

H $\quad 0.337988-4.678180-1.953646$

H -2.390949 -4.979302 0.630750

H $-0.765055-5.0382372 .205275$

H $\quad 0.851668-5.4832441 .661740$

H $\quad 0.410115-3.771817 \quad 1.854731$

H -1.648237 -5.193214 -2.330079

H -3.090990 -5.746611 -1.529569

H -2.352054 -2.817150 -1.997225

H -3.439443 -3.775802 -3.000389

H $-4.913359-4.115364-0.950849$

H -5.456296 -2.401181-2.709019

H $-4.388087-1.255221-1.932578$

H $-6.929950-0.469513-2.276121$

H $-5.020105-0.4244370 .506734$

H $-7.807211-2.609324-1.891347$

H $-8.239391-2.139680-0.263373$

H -7.184625 -3.542585 -0.528978

H -7.194233 -0.2554781 .113172$

H -4.854992 -2.041940 3.281203

H $-3.499840-3.0158032 .765774$

H -5.206529-4.193545 2.026065

H $-0.100145-0.350809-2.838135$

H $-2.1437740 .088904-0.579662$

H $-1.7352760 .744729-4.391693$

H $-0.7054381 .931432-3.553959$

H -2.244151 $2.422682-4.225007$

H -2.379223 -1.092662 3.221274

H $-2.652609-0.868271 \quad 1.493280$

H -2.6562073 .4725872 .594616$

H -4.1222812 .9311403 .438923$

H -5.3411442 .6651431 .237311$

H -3.1326334 .6689730 .535933$

H $-5.1533375 .555225-0.686871$

H $-5.9024793 .978691-0.700190$

H $-3.1648314 .908456-3.726723$

H -3.524325 $6.129849-2.380818$

H $-4.2670652 .769569-3.715639$

H $-5.4688782 .451682-2.511308$

H $-3.9204301 .429560-0.989232$

H -2.2643541 .3093581 .785862$

C $6.440797-2.807342-0.915061$

O $6.047448-1.421386-0.944764$

C $6.569760-0.7779860 .235684$

C $7.518110-1.7797830 .889318$

C $6.864044-3.0972040 .524160$

H $5.719674-0.6177560 .907193$

C $7.2248240 .559507-0.118367$ 
H $8.526401-1.7225160 .462255$

H $7.602173-1.6255101 .969422$

C $7.784916-4.2956350 .672160$

H $5.990209-3.2362511 .171425$

C $6.2754961 .581139-0.775077$

O $8.2992530 .305061-1.033205$

H $7.683037 \quad 0.9924020 .778947$

H $5.9779821 .224951-1.768188$

O $7.0346232 .777825-1.000674$

C 5.0169631 .9320740 .045393

C $4.2154643 .084078-0.590894$

H $4.3713011 .051787 \quad 0.134253$

O 5.3961742 .3342601 .361850

H $4.8374613 .986994-0.623307$

H $3.9643112 .802606-1.620780$

H $7.270788-5.2197500 .390389$

H $8.675230-4.1989020 .041726$

H $8.118281-4.3981261 .710133$

C $5.340692-3.701188-1.493962$

H $8.7006691 .162951-1.254207$

H $7.2598633 .150313-0.130698$

H 5.6985961 .5439831 .839355

H $5.651431-4.750170-1.428442$

H $5.237874-3.464330-2.560861$

H $7.316300-2.887934-1.573955$

C 0.7814462 .4453630 .759277

$\begin{array}{lllll}\text { O } & 2.027484 & 2.317231 & 0.059136\end{array}$

C $2.927398 \quad 3.4230260 .187837$

C $2.2733174 .712119-0.333863$

C $0.935334 \quad 4.9743180 .361245$

C 0.0425163 .7310090 .351208

H 0.1806631 .5956910 .414645

C 0.9597842 .3013492 .275042

H 3.1847973 .5581351 .243128

O $2.0735204 .634713-1.743977$

H $2.9527335 .553119-0.151644$

H $0.4249965 .801134-0.147579$

O $1.1893345 .376571 \quad 1.703958$

H -0.8232553 .8735511 .005465$

O $-0.4609833 .537081-0.972232$

C 1.2428230 .8633492 .638978

H 1.7565962 .9248452 .689609

H 0.0394072 .5833002 .800551

H $1.5355143 .845052-1.925460$

H 0.3313985 .5639462 .121335

H $-0.9912784 .318884-1.202738$

O $2.571101 \quad 0.594887 \quad 2.490925$

O $0.379736 \quad 0.0704432 .992940$

C $2.962978-0.7503592 .780640$

H $4.053351-0.8049632 .732514$

H $2.644330-1.0365823 .787632$

H $2.542454-1.4299282 .034110$

SCF Energy (B3LYP/6-31G**//MMFF) $=-3245.90558609$

03 51

MM̄FF Geometry

C $-0.681586 \quad 4.842310 \quad 0.020348$

C $-1.5633814 .818516-0.991271$

C $-2.7143933 .849672-1.141788$

O $-2.4370722 .595540-0.477804$

C $-3.9859244 .458658-0.523399$

C $-5.2422483 .649067-0.777302$

C $-5.976843 \quad 3.0510640 .180951$

C $-5.6832923 .561819-2.216409$

C -5.7374493 .0347491 .664775$

C -4.9393291 .8230142 .172099$

C $-5.6218490 .467957 \quad 1.921466$

C $-4.882965-0.6556722 .650920$

C -5.486285 -2.034664 2.343342

$\begin{array}{lllll}\text { O } & -5.605177 & 0.206994 & 0.516697\end{array}$

O $-4.547175-3.0145952 .812894$

C $-5.624753-2.2036680 .812959$

C $-6.780588-2.2686263 .132186$

O $-6.332121-3.4075360 .526433$

C $-6.287427-0.9917720 .122998$

C $-6.233596-1.046608-1.423394$

O $-7.670698-0.9418020 .492334$

C $-1.6651081 .697593-1.151319$
O $-1.1756681 .836122-2.261319$

C -1.499705 $0.505380-0.290289$

C $-0.559016-0.401496-0.594103$

C $-0.261569-1.6256620 .152691$

C -1.240707 -2.043062 1.216690

C -4.828071-1.121387 -2.030339

C $-4.374509-2.532952-2.362520$

C $-2.394814-3.783285-3.214017$

O $-5.111274-3.512759-2.292569$

C $-1.691502-4.410144-2.052200$

C $-0.399952-4.771446-2.083524$

C $0.309988-5.444242-0.944299$

C $1.553559-4.700100-0.514860$

C $2.770360-5.078050-0.941882$

C $1.391158-3.5695920 .475347$

C $0.855069-2.315911-0.159646$

N -3.051466 -2.563466 -2.783037

H -0.7826844 .1341660 .838810$

H -1.468219 $5.555202-1.786380$

H -2.867739 $3.662903-2.211533$

H $-4.1500705 .467676-0.925170$

H -3.8224254 .5860400 .552112$

H $-6.8685212 .506190-0.128028$

H -6.670598 $3.099025-2.321054$

H -5.744608 $4.562367-2.656806$

H $-4.9784202 .963261-2.801350$

H -5.234032 3.9520191 .987933

H -6.7142563 .0560712 .164895$

H -3.9489711 .8305611 .701984$

H -4.7858481 .9534353 .250553$

H -6.6588250 .5342342 .270668$

H $-4.869491-0.4763533 .733283$

H -3.830344 -0.659515 2.337141

H -4.886474 -3.891096 2.561972

H -4.608995 -2.335427 0.422408

H $-6.584448-2.2143984 .209841$

H -7.179682 -3.272955 2.952222

H -7.561592 -1.541575 2.900037

H $-6.288973-3.558465-0.432504$

H -6.882581 -1.846412 -1.799192

H $-6.685294-0.114462-1.793626$

H -8.147937 -0.414184 -0.169382

H -2.1448720 .4138190 .575486$

H $0.070311-0.226212-1.465392$

H -1.242127 -1.324600 2.043293

H -1.031873 -3.025965 1.643164

H -2.251685 -2.101667 0.800403

H $-4.832691-0.571844-2.980563$

H $-4.089207-0.643455-1.379837$

H -1.705045 -3.513220 -4.019890

H $-3.141412-4.478349-3.611591$

H -2.277057 -4.600880 -1.155874

H $0.176160-4.603354-2.990920$

H $0.575402-6.458636-1.269789$

H $-0.352817-5.576488-0.080106$

H $3.669077-4.564686-0.616320$

H $2.894214-5.906155-1.631902$

H $2.366163-3.3185550 .910955$

H $0.787811-3.9184331 .317328$

H $1.496262-1.926239-0.950572$

H $-2.499661-1.710951-2.755084$

C 1.7818235 .1088790 .391952

O $2.0977164 .202990-0.678554$

C $3.5287514 .122182-0.816111$

C 4.1181325 .0643330 .233461

C 2.9841046 .0432570 .469602

H $3.7703324 .493154-1.819380$

C $4.0104392 .668078-0.678262$

H 4.3657064 .5329441 .160092

H $5.0340825 .548799-0.119352$

C 3.1021206 .8033051 .778777

H $2.9598756 .757419-0.364454$

C $3.4761821 .708195-1.762506$

O 3.6448622 .1714440 .610494

H $5.1049142 .676294-0.717228$

H $3.8272142 .027602-2.751195$

O $2.0455651 .757259-1.801916$

C $3.8397610 .227183-1.536117$ 
C $5.348115-0.043814-1.441866$

H $3.325314-0.149473-0.646262$

O $3.336105-0.526010-2.647210$

H $5.8102750 .216720-2.401814$

H $5.7959660 .598883-0.680481$

H 2.2647967 .4965281 .905439

H 3.1102076 .1239592 .637780

H 4.0283977 .3866221 .802390

C 0.4597275 .8193980 .102962

H 2.6730612 .1413550 .645582

H $1.7858892 .656358-2.064543$

H $2.383211-0.342416-2.712298$

H $0.540316 \quad 6.381115-0.835977$

H $0.241515 \quad 6.537100 \quad 0.901477$

H 1.6971784 .5109791 .309586

C $5.677368-1.3627571 .299220$

O $5.083382-1.9378390 .128282$

C $5.661894-1.523740-1.116328$

C $7.156230-1.870331-1.140881$

C $7.891619-1.2886860 .061958$

C $7.179358-1.6765631 .356720$

H $5.520932-0.2776761 .297794$

C $4.960376-1.9532482 .524433$

H $5.182462-2.132476-1.892997$

O $7.748588-1.405541-2.348952$

H $7.267097-2.962555-1.129047$

H $7.999430-0.201657-0.029695$

O $9.217266-1.8230410 .079549$

H $7.332709-2.7477411 .538735$

O $7.784310-0.9807842 .445481$

C $3.569525-1.3890162 .723618$

H $4.864093-3.0394552 .409558$

H $5.523539-1.7319463 .437905$

H $8.694450-1.629276-2.313764$

H $9.656950-1.4797860 .876417$

H $7.644965-0.0285352 .305622$

O $3.002942-1.9949713 .809997$

O $3.043679-0.5316442 .028704$

C $1.679020-1.5619874 .136579$

H $1.660738-0.4817344 .309768$

H $0.987675-1.8374663 .336077$

H $1.372318-2.0691935 .055025$

SCF Energy (B3LYP/6-31G**//MMFF) $=-3245.93537555$

03_52

MM̄FF Geometry

C 2.5478340 .9555083 .558119

C $2.149482-0.0646552 .782870$

C $0.811415-0.7447942 .916041$

O $0.015917-0.4144791 .754208$

C $1.003439-2.2722312 .943231$

C $-0.281897-3.0441883 .170082$

C $-0.839567-3.8860082 .277687$

C $-0.932847-2.8482864 .515833$

C $-0.344531-4.2515830 .906775$

C $-0.922163-3.401235-0.235324$

C $-2.449710-3.490319-0.382458$

C $-2.905674-2.824755-1.683173$

C $-4.436552-2.816146-1.819944$

O $-3.053885-2.8229820 .728510$

O $-4.779522-1.902294-2.873469$

C $-5.057960-2.259044-0.520339$

C $-4.977972-4.187066-2.242743$

O $-6.476744-2.390884-0.537520$

C $-4.485785-2.9017520 .759491$

C $-4.956950-2.2028952 .058750$

$\begin{array}{lllll}\text { O } & -4.922917 & -4.264337 & 0.837307\end{array}$

C $-\begin{array}{lll}0.636053 & 0.780352 & 1.767602\end{array}$

O $\quad-0.641317 \quad 1.6088772 .664191$

C -1.3334630 .9263230 .472462$

C -1.7233132 .1456320 .072313$

C $-2.4019082 .463303-1.185187$

C $-2.9211701 .312029-2.004800$

C $-4.598519-0.7173052 .175887$

C $-5.7024920 .222838 \quad 1.720064$

C -6.1408572 .6390591 .294888$

O $-6.845980-0.1491821 .471024$

C $-6.0050802 .915593-0.169151$
C $-5.7650494 .138204-0.666912$

C $-5.6667334 .442387-2.134397$

C $-4.3868035 .162934-2.494876$

C $-4.3607506 .499780-2.628903$

C $-3.1608864 .326017-2.782171$

C $-2.5398463 .759544-1.534303$

N $-5.2674331 .540377 \quad 1.662600$

H 1.8893721 .3265034 .339095

H $2.804804-0.4124781 .987332$

H $0.294413-0.4153023 .825499$

H $1.707872-2.5446833 .740607$

H $1.475293-2.5818852 .004185$

H -1.763994 -4.388515 2.561179

H -1.763438 -3.5431974 .680509$

H $-0.206116-3.0145065 .317730$

H $-1.332391-1.8339814 .608594$

H $0.748814-4.2085720 .864673$

H $-0.593003-5.3057680 .728780$

H $-0.624015-2.357095-0.085214$

H $-0.455613-3.739508-1.168647$

H $-2.735669-4.548640-0.374946$

H -2.446956 -3.312827 -2.552202

H -2.549588 -1.785970 -1.702984

H $-4.332581-2.198824-3.684804$

H $-4.844119-1.183934-0.503472$

H $-4.549682-4.486873-3.206815$

H -6.062390 -4.154086 -2.395347

H $-4.753706-4.977258-1.523336$

H $-6.798899-1.951849-1.343263$

H $-6.029584-2.3738832 .211033$

H $-4.460887-2.7128842 .897304$

H -4.858083 -4.556597 1.761638

H $-1.4824430 .035193-0.125407$

H -1.5026342 .9991200 .712353$

H -3.536227 $0.649717-1.386149$

H $-2.0923350 .732128-2.424051$

H -3.557806 $1.619624-2.836861$

H $-4.422132-0.4862243 .234393$

H $-3.675617-0.4911891 .634112$

H $-5.8545873 .499783 \quad 1.907548$

H $-7.1779152 .375272 \quad 1.525747$

H $-6.1355112 .078471-0.850966$

H -5.6637254 .9779980 .017196$

H -6.531471 $5.062701-2.404256$

H $-5.7563493 .532051-2.740288$

H $-3.4539027 .022300-2.914574$

H -5.245509 $7.101990-2.450664$

H $-2.3931344 .939207-3.273350$

H $-3.4167903 .559696-3.518913$

H -2.133439 $4.521667-0.867757$

H $-4.2891361 .750325 \quad 1.838410$

C 3.8621312 .7597882 .333239

O $3.6254172 .181249 \quad 1.034767$

C 2.6876903 .0124810 .324706

C 2.6851724 .3459041 .059955

C 2.8326223 .8781352 .498652

H 1.7000742 .5528130 .447079

C $3.0505683 .062669-1.162335$

H 3.5395434 .9698570 .772919

H 1.7685674 .9169190 .884989

C 3.2637454 .9724033 .458517

H 1.8672623 .4758922 .830179

C $3.1918791 .662861-1.801248$

O $4.3094023 .733624-1.297828$

H $2.3177343 .668633-1.706890$

H $4.0652611 .174584-1.362676$

O $3.4899991 .833935-3.190870$

C $1.9558940 .757556-1.660714$

C $2.099279-0.645021-2.278503$

H $1.6973760 .625625-0.605294$

O $0.8370411 .376913-2.299713$

H $1.119368-1.134763-2.195743$

H $2.303263-0.555077-3.353122$

H 3.3769864 .5764434 .472687

H 4.2199105 .4153003 .160100

H 2.5177765 .7732943 .488677

C 3.8674211 .6553083 .393568

H $4.5398513 .729164-2.242722$ 
H $2.7040862 .221326-3.613262$

H $0.6208032 .184339-1.804425$

H 4.1645842 .0707144 .363510

H 4.6280030 .9099403 .127324

H 4.8698853 .1918632 .290692

C $5.573391-1.876682-1.615441$

O $4.440839-1.182607-2.157614$

C $3.168809-1.512329-1.589295$

C $2.881499-3.010103-1.768721$

C $4.009086-3.861656-1.181946$

C $5.380406-3.401408-1.683558$

H $6.403661-1.625689-2.288196$

C $5.931455-1.368134-0.209138$

H $3.171860-1.273813-0.520387$

O $2.717891-3.301777-3.154437$

H $1.937694-3.248618-1.266919$

H $3.847483-4.910657-1.457651$

O $3.953936-3.7716880 .239041$

H $6.182735-3.895229-1.123144$

O $5.530595-3.791497-3.051937$

C $6.5274010 .025240-0.240914$

H $5.057633-1.3369930 .446693$

H $6.687701-2.0241270 .237181$

H $2.513417-4.248979-3.232993$

H $4.663383-4.332608 \quad 0.596274$

H $5.454238-4.760002-3.091120$

O $\quad 6.737871 \quad 0.4459301 .041856$

O $6.7915390 .660285-1.252552$

C 7.3250101 .7444241 .172008

H $\quad 8.350741 \quad 1.7301530 .791577$

H 6.7278962 .4924870 .641583

H 7.3468402 .0025902 .234062

SCF Energy (B3LYP/6-31G**//MMFF) $=-3245.89762839$

03_53

MM̄FF Geometry

C -3.841256 -2.913549-1.346546

C $-2.894574-3.158633-0.428060$

C -1.420014 -3.094672 -0.725909

O $-0.865741-2.2070790 .272896$

C $-0.765775-4.480963-0.590332$

C $0.603442-4.576066-1.239746$

C $1.766305-4.777963-0.589144$

C $0.610386-4.483596-2.746896$

C $2.004090-4.9576630 .882784$

C $2.562083-3.7188231 .598960$

C $3.962479-3.2769751 .137992$

C $4.512645-2.2001602 .079124$

C $5.864302-1.6438711 .606067$

O $3.871180-2.751958-0.190513$

O $6.122015-0.4325912 .333825$

C $5.757392-1.2574910 .115125$

C $7.017603-2.5989361 .934917$

O $7.030926-0.887438-0.405944$

C $5.130565-2.361974-0.759937$

C $4.872069-1.912154-2.220533$

O $6.030265-3.476086-0.827050$

C $0.016170-1.255727-0.124340$

O $0.465086-1.081091-1.245005$

C $0.347780-0.4466661 .069876$

C 1.4548640 .3074821 .057475

C 1.9322691 .1757942 .133126

C $1.050910 \quad 1.350848 \quad 3.340557$

C $3.937031-0.711295-2.389420$

C $4.6498400 .630572-2.453669$

C $4.1944953 .079261-2.254701$

O $\quad 5.845840 \quad 0.746787-2.706289$

C $4.6951483 .456526-0.896601$

C $4.0536494 .292095-0.065339$

C 4.5692504 .6686971 .295189

C 3.6671114 .1985492 .415943

C 2.8212205 .0462773 .024892

C 3.8047262 .7660802 .886358

C 3.1173491 .7976861 .966312

N $3.7774101 .688910-2.240756$

H -3.526495 -2.717355 -2.370012

H -3.172461 -3.3697280 .600661$

H -1.273702 -2.680240 -1.729601
H -1.401818 -5.241467-1.063391

H $-0.715591-4.7478210 .470260$

H $2.672376-4.851135-1.190082$

H $1.592692-4.714199-3.173531$

H $-0.103413-5.195317-3.174645$

H $\quad 0.340899-3.476528-3.077609$

H $1.087178-5.2683541 .392917$

H $2.702977-5.7937161 .013469$

H $1.855200-2.8933211 .471078$

H $2.597663-3.9478622 .671603$

H $4.619061-4.1549111 .139377$

H $4.595399-2.5849273 .103309$

H $3.798607-1.3685712 .132946$

H $6.108521-0.6443563 .282995$

H $5.124848-0.3627690 .064620$

H $7.085612-2.7578463 .017910$

H $7.981513-2.1737121 .634612$

H $6.910072-3.5790821 .465879$

H $7.378278-0.1746230 .157080$

H $5.825260-1.755117-2.739683$

H $4.394824-2.756898-2.738207$

H $5.804327-4.008202-1.607954$

H $-0.316491-0.5049891 .924255$

H 2.0794690 .2905880 .166908

H 0.9593390 .4058493 .886902

H 0.0496491 .6828823 .046122

H 1.4246732 .0959374 .046280

H $3.407939-0.813866-3.345593$

H $3.180526-0.697853-1.601060$

H $3.3294843 .677821-2.557407$

H $4.9887253 .216235-2.995662$

H $5.6408393 .019088-0.584497$

H $3.1097594 .734185-0.375986$

H 4.6689645 .7616161 .322560

H 5.5821444 .2800871 .459798

H 2.1909834 .7225363 .846476

H 2.7356796 .0814032 .711170

H 3.4459702 .6626503 .915843

H 4.8687952 .5024772 .941098

H 3.6816541 .5869381 .058601

H $2.814011 \quad 1.492488-1.984755$

C $-5.799579-2.6399220 .328249$

O $-5.188581-1.4619560 .893066$

C $-6.212731-0.6081021 .424231$

C $-7.418411-1.5126551 .636148$

C $-7.313674-2.4241180 .428028$

H -6.4489280 .1218190 .640257$

C -5.7159780 .1354832 .668522$

H -7.331348 -2.100502 2.556938

H -8.358294 -0.954051 1.673816

C $-8.108638-3.7114300 .561881$

H -7.676033 -1.875843 -0.452194

C -4.3395800 .8160562 .510253$

$\begin{array}{lllll}\text { O } & -5.603780 & -0.797167 & 3.751474\end{array}$

H -6.4740070 .8664112 .974932$

H -3.5512580 .0538372 .541570$

O $-4.130137 \quad 1.6359403 .667957$

C -4.1606291 .6704381 .242643$

C -2.8282382 .4481481 .254109$

H -4.2109521 .0182390 .364378$

O $-5.242722 \quad 2.597240 \quad 1.157218$

H -2.913342 3.3128191 .923326

H $-2.042701 \quad 1.786178 \quad 1.637567$

H $-7.984958-4.338860-0.326602$

H -7.787431 -4.292849 1.432651

H $-9.175610-3.4946230 .677522$

C $-5.327070-2.820323-1.119823$

H $-5.300430-0.3009614 .531156$

H $-4.7743412 .363807 \quad 3.631130$

H -5.2014543 .0231780 .284933$

H $-5.666297-1.953020-1.700904$

H $-5.801745-3.704293-1.560841$

H $-5.489210-3.4914930 .945702$

C $-1.7596752 .007769-2.288005$

O $-2.0988731 .772938-0.916615$

C $-2.4230942 .937691-0.149059$

C -1.224466 $3.899007-0.109903$

C $-0.7409384 .242195-1.523156$ 
C $-0.5830792 .991271-2.396897$

H -1.418485 $1.034175-2.663037$

C $-2.9872312 .423679-3.108916$

H -3.278132 $3.447674-0.603523$

O $-0.158313 \quad 3.3034720 .623677$

H -1.5214794 .8115990 .420654$

H $0.2222634 .763196-1.456593$

O $-1.6774325 .136713-2.116226$

H $-0.4418283 .275823-3.445533$

O $0.6079352 .305848-1.999028$

C -4.074182 $1.373372-3.032958$

H -3.397948 $3.374444-2.758059$

H $-2.7244462 .552624-4.164585$

H 0.5685703 .9480050 .665890

H -1.342453 $5.369306-2.998979$

H $0.4788841 .993048-1.087401$

O $-5.281592 \quad 1.974441-2.827549$

O $-3.881226 \quad 0.170575-3.151195$

C $-6.4010021 .090032-2.719640$

H $-6.2933570 .456831-1.834894$

H $-7.3039841 .696180-2.608761$

H $-6.4960460 .480530-3.623259$

SCF Energy (B3LYP/6-31G**//MMFF) $=-3245.91652753$

03_54

MMFF Geometry

C 3.2146413 .1996220 .930154

C $2.6596093 .203534-0.292124$

C $1.2328552 .849145-0.645143$

$\begin{array}{lllll}\text { O } & 0.545172 & 2.372680 & 0.532297\end{array}$

C $0.498603 \quad 4.085997-1.207499$

C $-0.659613 \quad 3.762217-2.135107$

C $-1.9538324 .062735-1.908127$

C $-0.2773573 .101530-3.437978$

C -2.565811 $4.777394-0.737722$

$\begin{array}{llll}\text { C } & -3.267226 & 3.868540 & 0.282267\end{array}$

C $-4.4802503 .090661-0.258671$

C -5.2261562 .4122990 .895720$

C $-6.385713 \quad 1.531326 \quad 0.407244$

O $-4.0243842 .098740-1.184669$

O $\begin{array}{llll}0.788951 & 0.687933 & 1.497941\end{array}$

C $-5.8737760 .601288-0.713315$

C -7.6142552 .3671520 .029787$

O $-6.949612-0.122822-1.303723$

C $-5.0749361 .341264-1.805579$

C $-4.4255770 .385445-2.839405$

O $-5.9606532 .197580-2.538288$

C $-0.3737121 .386606 \quad 0.370691$

$\begin{array}{llll}\text { O } & -0.737987 & 0.886322 & -0.680467\end{array}$

C -0.8593631 .0001291 .714370$

C -1.9226460 .1900631 .815189$

C $-2.525248-0.3051513 .052221$

C $-1.793032-0.0448544 .341419$

C $-3.436585-0.633846-2.268190$

C $-4.058634-1.970304-1.900484$

C $-3.627409-3.990410-0.500398$

O $-5.125262-2.365601-2.362851$

C $-4.463837-3.8017180 .726099$

C $-4.079551-4.1700221 .957589$

C $-4.927892-3.9898123 .184761$

C $-4.282758-3.0879874 .214611$

C -3.610731-3.602744 5.258002

C $-4.490821-1.5950994 .084760$

C $-3.684344-0.9907182 .969551$

N -3.255019 -2.690019-1.025852

H 2.6193932 .9434491 .801868

H $3.2880533 .467940-1.141282$

H $1.2985232 .043333-1.385419$

H $1.1961824 .708466-1.784800$

H $0.1671664 .706801-0.368845$

H -2.675502 $3.790206-2.677922$

H -1.121204 $3.032009-4.133110$

H $0.5085323 .676342-3.938972$

H $\quad 0.087757 \quad 2.085323-3.268677$

H $-1.8167895 .376444-0.210431$

H -3.290790 $5.503466-1.127530$

H -2.5323793 .1670180 .687832$

H -3.596331 4.5031051 .114916
H -5.140142 $3.796707-0.776275$

H -5.5893893 .1576441 .614462$

H -4.5259021 .7788641 .455498$

H $-7.036131 \quad 1.2606902 .244263$

H -5.220948 $-0.139346-0.235105$

H -7.9734252 .9328190 .897985$

H $-8.449387 \quad 1.727343-0.275695$

H -7.416344 $3.084078-0.769510$

H $-7.411594-0.590912-0.587329$

H $-5.202526-0.102000-3.440653$

H $-3.8599341 .010132-3.545758$

H $-5.5537472 .399702-3.397000$

H -0.3322821 .3907232 .577191$

H $-2.412451-0.1387890 .900670$

H -1.7877551 .0263614 .570267$

H $-0.756420-0.3946044 .273996$

H -2.232711 -0.5578095 .199846$

H -2.680775 $-0.855498-3.032329$

H $-2.914758-0.217829-1.404351$

H -2.701668 -4.535919-0.291659

H $-4.195018-4.542380-1.256386$

H $-5.441131-3.344320 \quad 0.589079$

H -3.106977 -4.637085 2.094796

H $-5.106744-4.9839553 .615220$

H $-5.921450-3.5995302 .931050$

H $-3.170051-2.9652196 .017194$

H -3.482099 -4.6738945 .373396$

H $-4.287072-1.0899305 .035355$

H $-5.553431-1.3957433 .893705$

H $-4.136142-1.1226481 .986407$

H $-2.420014-2.253188-0.646380$

C $5.4149842 .401754 \quad 1.855997$

$\begin{array}{lllll}\text { O } & 5.436083 & 1.248923 & 0.990196\end{array}$

C $6.787044 \quad 0.765897 \quad 0.885927$

C $7.5378381 .388296 \quad 2.055607$

C 6.8793332 .7509162 .135464

H $7.1901401 .171308-0.050440$

C $6.821558-0.7654730 .832209$

H 7.3722220 .8369992 .988151

H 8.6160671 .4405321 .878041

C 7.1010043 .4586013 .461135

H 7.2780453 .3782201 .326761

C $5.857233-1.390526-0.196418$

O $6.458617-1.2798842 .120400$

H 7.850185-1.098809 0.650201

H $4.834697-1.2761510 .172644$

O $6.114385-2.799717-0.227556$

C $5.957309-0.826452-1.621585$

C $5.101399-1.554558-2.677834$

H $5.6732050 .231958-1.614557$

O $7.323634-0.892947-2.039308$

H $5.281877-1.057061-3.640106$

H $5.471755-2.581193-2.797166$

H 6.5950934 .4291843 .474217

H 6.7185802 .8677294 .300233

H 8.1686713 .6313453 .630942

C $4.6567713 .547017 \quad 1.179542$

H $6.479632-2.2505862 .061726$

H $7.000652-2.927319-0.607549$

H $7.384067-0.474513-2.915103$

H 5.1477543 .8012390 .231600

H 4.6899264 .4407431 .813253

H 4.9048392 .0965572 .778316

C $1.969365-2.744735-1.004219$

O $3.330979-2.599935-1.430797$

C $3.591451-1.557221-2.376968$

C $2.764744-1.790525-3.652127$

C $1.273805-1.932823-3.335100$

C $1.026366-2.936475-2.204672$

H $1.954890-3.677158-0.425337$

C $1.539614-1.601349-0.074260$

H $3.322936-0.588732-1.941524$

O $3.222907-2.957077-4.333618$

H $2.915312-0.942902-4.331066$

H $0.746706-2.258400-4.240162$

O $0.765217-0.654004-2.970181$

H $-0.014151-2.887073-1.862977$

O $1.237106-4.260034-2.705925$ 
C $2.258829-1.6403991 .258113$

H $1.737071-0.628140-0.530426$

H $\quad 0.468512-1.6778110 .143071$

H $3.171095-3.703813-3.712608$

H $-0.181944-0.756377-2.776329$

H $0.606148-4.402127-3.432188$

O $2.178004-0.4096331 .844241$

O $2.797926-2.627426 \quad 1.739375$

C $2.781657-0.2992993 .136212$

H $2.6356730 .723728 \quad 3.492849$

H $2.301271-0.9867913 .838806$

H $3.854873-0.4991793 .071275$

SCF Energy (B3LYP/6-31G**//MMFF) $=-3245.91138743$

0355

MM̄FF Geometry

C $3.032613-3.8459190 .728659$

C $2.156197-3.0577451 .368786$

C $0.653276-3.1996191 .426353$

O $0.092213-2.0978720 .669007$

C $0.086439-4.5116980 .846309$

C $-1.379206-4.7468881 .167278$

C $-2.355936-4.8892840 .250251$

C $-1.720387-4.8732072 .631352$

C $-2.243135-4.846901-1.246706$

C $-2.504240-3.465608-1.864954$

C $-3.912967-2.905911-1.601875$

C $-4.162183-1.658633-2.454199$

C $-5.511131-0.994912-2.135982$

O $-4.011448-2.566823-0.215742$

O $-5.505051 \quad 0.318450-2.716865$

C $-5.633823-0.809921-0.607517$

C $-6.680822-1.746670-2.781799$

O $-6.932359-0.341436-0.254841$

C $-5.299667-2.0859200 .190823$

C $-5.247827-1.8590361 .722644$

O $-6.314685-3.069317-0.047362$

C $-0.128013-0.9259011 .318318$

O $0.029052-0.7121442 .513025$

C $-0.600741 \quad 0.1323840 .399139$

C $-0.6092450 .030316-0.938044$

C -1.049564 $1.087235-1.845709$

C $-0.187878 \quad 1.225607-3.069989$

C $-4.213113-0.8375202 .204284$

C $-4.7595190 .573800 \quad 2.340294$

C -3.9916882 .9401922 .512516$

$\begin{array}{llll}\text { O } & -5.958896 & 0.830454 & 2.398261\end{array}$

C -4.1884963 .4827611 .132118$

C -3.305359 4.2781330 .510828

C $-3.5060514 .828184-0.871152$

C $-2.5024894 .290130-1.866498$

C -1.473312 $5.042797-2.290230$

C $-2.7459182 .912060-2.441681$

C $-2.1527191 .820617-1.594485$

N -3.7424041 .5138502 .426867$

H $2.687470-4.7034950 .158907$

H $2.548645-2.2056151 .919787$

H $0.364172-3.1183562 .480720$

H $\quad 0.642502-5.3682191 .249482$

H $0.247439-4.513252-0.236688$

H $-3.368726-5.0692280 .610171$

H -2.737984 -5.246933 2.789982

H - $-1.038253-5.5745663 .122875$

H -1.648110 -3.902453 3.131038

H -1.259954 -5.202968 -1.572335

H -2.959090 -5.567917 -1.661496

H -1.753893 -2.765758 -1.480859

H -2.345968 -3.549705 -2.947315

H -4.646880 -3.684191-1.841720

H -4.095570 -1.898206 -3.522953

H -3.366304 -0.927795 -2.264249

H $-5.341487 \quad 0.223829-3.670932$

H -4.926833 -0.019229-0.327551

H -6.569731 -1.762281 -3.872746

H -7.632629 -1.240954 -2.585750

H $-6.763277-2.782350-2.445921$

H -7.093736 $0.473213-0.760867$

H $-6.250318-1.6288682 .103150$
H $-4.976423-2.8203752 .182543$

H $-6.293169-3.7166350 .676743$

H -0.9261951 .0464390 .885856$

H $-0.238642-0.867435-1.426169$

H $-0.4148752 .113852-3.663248$

H $-0.3044750 .351088-3.719405$

H $0.866396 \quad 1.303817-2.788129$

H -3.876933 -1.127725 3.208022

H $-3.332940-0.8376771 .553726$

H -3.133211 3.3937223 .017965

H -4.8871203 .1214413 .115607$

H -5.1084663 .2076030 .621415$

H $-2.3889534 .555731 \quad 1.024057$

H -3.431608 $5.921660-0.808436$

H $-4.5208704 .626690-1.236679$

H $-0.7681284 .671670-3.026429$

H -1.308963 $6.043747-1.905399$

H $-2.3796202 .864268-3.472809$

H $-3.8255972 .737516-2.530531$

H $-2.7227161 .602189-0.692767$

H -2.7788881 .2117712 .319990$

C $5.021763-3.190350-0.642846$

O $4.539589-1.865755-0.950929$

C $5.607661-1.109789-1.553003$

C $6.709491-2.114155-1.869389$

C $6.544417-3.103417-0.732432$

H $5.980856-0.425869-0.781594$

C $5.087483-0.306067-2.750689$

H $6.547095-2.616675-2.828849$

H $7.699540-1.648985-1.895729$

C $7.228040-4.436201-0.983630$

H $\quad 6.950937-2.6569690 .185124$

C $3.9322940 .658251-2.407145$

O $4.584288-1.205525-3.746741$

H $5.9099870 .251195-3.215358$

H $3.0395360 .083030-2.134754$

O $3.5862521 .368860-3.604018$

C $4.2457391 .672294-1.289800$

C $3.1172722 .701265-1.085993$

H $4.4060711 .131614-0.354344$

O $5.4635382 .354533-1.587628$

H $2.9887173 .279402-2.008666$

H $2.1810292 .163885-0.905434$

H $7.072399-5.119914-0.143378$

H $\quad 6.843626-4.920428-1.887749$

H $8.306698-4.296931-1.109750$

C $4.518525-3.6103220 .737601$

H $5.345930-1.585955-4.214910$

H $4.3780441 .841468-3.911096$

H $5.3103062 .914412-2.367394$

H $4.774798-2.8397831 .474720$

H $5.005385-4.5389511 .055676$

H $4.628879-3.861516-1.418090$

C 2.4610662 .4181591 .899073

O $3.6237413 .019427 \quad 1.315847$

C 3.4026993 .6876620 .069105

C 2.3441624 .7872300 .236152

C 1.0589964 .2507330 .860252

C 1.3740583 .4741482 .137322

H 2.0830261 .6280431 .239940

C 2.8877611 .7752353 .228686

H $4.3452554 .193859-0.174654$

O $2.0684995 .393177-1.022097$

H 2.7519375 .5710530 .888030

H $0.491468 \quad 3.6326410 .154584$

O $\quad 0.2146645 .3483801 .213240$

H 1.6966374 .1751062 .918197

O 0.1866112 .8374062 .603317

C 3.7573550 .5506273 .038063

H 3.4616142 .4982823 .820470

H 2.0072561 .4560063 .796951

H $1.4888736 .156677-0.862418$

H $-0.083718 \quad 5.7665790 .388215$

H -0.4891843 .5280172 .714262$

O $4.1319320 .081846 \quad 4.265879$

O 4.0653570 .0594151 .961795

C $4.957866-1.0868144 .255212$

H $5.843476-0.9289473 .631927$ 
H $5.283063-1.2822835 .280562$ H $4.382358-1.9461733 .899580$

SCF Energy (B3LYP/6-31G**//MMFF) $=-3245.89978167$

03 56

MM̄FF Geometry

C -0.5898234 .8939961 .168235$

C 0.6328304 .6650820 .666166

C 1.6285223 .7190131 .286085

O 1.8057172 .6061950 .377996

C 2.9851984 .4250941 .456653

C 4.0485903 .5599792 .104446

C 5.1907593 .1745041 .501824

C 3.7803363 .1483363 .529312

C 5.6604403 .5009080 .111691

C $5.2382512 .489084-0.965356$

C $5.8056971 .075669-0.757392$

C $5.5352840 .197007-1.981143$

C $6.014976-1.248100-1.768628$

$\begin{array}{lllll}\text { O } & 5.176117 & 0.497335 & 0.388807\end{array}$

O $5.437547-2.060447-2.802716$

C $5.463916-1.771822-0.423954$

C $7.535995-1.371343-1.920062$

O $6.021379-3.044237-0.106883$

C $5.679758-0.7956740 .751302$

C $4.950825-1.2240952 .048713$

O $7.077715-0.7333501 .060506$

C 0.8316101 .6549940 .373907

$\begin{array}{lllll}\text { O } & -0.149817 & 1.604361 & 1.098194\end{array}$

C $1.1431790 .692395-0.703639$

C $0.178507-0.128694-1.144440$

C $0.308079-1.121528-2.211695$

C $1.685770-1.368678-2.767772$

C $3.430674-1.3812161 .930689$

C $2.981481-2.8059081 .651464$

C $0.949830-4.1274891 .082757$

O $3.732049-3.775397 \quad 1.721947$

C $0.901781-4.362093-0.393952$

C $-0.220146-4.696843-1.048814$

C $-0.280658-4.977364-2.522635$

C $-1.327789-4.147275-3.230388$

C $-2.552418-4.643124-3.474678$

C $-0.925938-2.780009-3.733808$

C $-0.801303-1.766448-2.630070$

N $1.627596-2.8733701 .351760$

H -0.8918964 .4046642 .090664$

H $0.9193195 .140836-0.269190$

H 1.2727043 .3494372 .255619

H 2.8589605 .3270302 .070346

H $3.323205 \quad 4.7732760 .474492$

H 5.8835992 .5544202 .069888

H 4.6446312 .6572493 .989454

H 3.5409304 .0251814 .139749

H 2.9418042 .4470733 .577972

H $5.321748 \quad 4.499542-0.184823$

H $6.755783 \quad 3.5683650 .131635$

H 4.143560 2.446335-1.003807

H $5.5800702 .873511-1.934378$

H $6.8843031 .160780-0.580024$

H $5.9983030 .625394-2.878765$

H $4.455640 \quad 0.177437-2.182856$

H $5.715705-1.696129-3.660534$

H $4.387587-1.927235-0.563434$

H $7.848097-1.050423-2.921239$

H $7.859560-2.413888-1.826548$

H $8.089266-0.771152-1.194867$

H $5.841841-3.634751-0.858426$

H $5.419636-2.1243532 .464048$

H $5.128735-0.4353622 .794238$

H $7.178004-0.4072391 .970232$

H $2.1446550 .702329-1.117326$

H -0.811978 -0.055406 -0.697311

H $2.389950-1.594755-1.959737$

H $2.045093-0.488233-3.311065$

H $1.734777-2.217241-3.453180$

H $2.977514-1.1034092 .890999$

H $3.022598-0.7090351 .170108$

H $-0.047610-4.0562321 .524729$
H $1.489239-4.9469481 .568447$

H $1.835434-4.278569-0.945289$

H -1.145502 -4.811457 -0.489722

H $-0.505047-6.044685-2.649440$

H $\quad 0.694055-4.822857-3.001665$

H $-3.301176-4.066199-4.006947$

H -2.835597 -5.635898 -3.140536

H -1.680583 -2.400194 -4.435758

H $-0.015179-2.873159-4.331974$

H -1.753317 -1.523875 -2.155384

H $1.089653-2.0153391 .271437$

C -2.105729 $5.156457-0.828530$

O $-2.7876223 .908613-0.583849$

C $-3.9422033 .839362-1.441102$

C $-3.8273075 .010324-2.412079$

C $-3.1148416 .040139-1.559201$

H $-4.8157144 .007738-0.800740$

C $-4.0433292 .468691-2.119033$

H -3.219442 $4.754451-3.287808$

H $-4.8045675 .348779-2.769714$

C $-2.4885137 .165710-2.363910$

H -3.833091 $6.463067-0.844001$

C $-4.1133331 .265943-1.154527$

O $-2.8921272 .285980-2.954149$

H $-4.9114112 .463928-2.789720$

H $-3.1268581 .096106-0.709640$

O $-4.386366 \quad 0.103318-1.949227$

C $-5.1716651 .381566-0.043461$

C -5.4744280 .0752620 .702591$

H -4.8600942 .1494830 .673174$

O $-6.400778 \begin{array}{lll}1.829073 & -0.626252\end{array}$

H -6.214188 0.2819721 .489449

H $-6.014416-0.6099150 .032885$

H -1.978417 $7.878302-1.708376$

H -1.755376 $6.785393-3.083168$

H $-3.2557447 .711460-2.922766$

C -1.615596 5.7580570 .491189

H -2.973119 $1.411902-3.373165$

H $-5.283114 \quad 0.200308-2.313344$

H -7.0367451 .9632960 .097133$

H -2.4610435 .8727401 .181054$

H -1.195372 6.7575950 .330651

H -1.258617 $4.928529-1.488540$

C -3.910029-2.738346 2.537934

O $-4.844372-1.8186301 .963699$

C $-4.278637-0.6748701 .305896$

C -3.4464440 .1500802 .296809$

C $-2.391885-0.7207142 .993838$

C $-2.987041-2.0231513 .537201$

H -4.528641 -3.448885 3.101706

C $-3.158861-3.5287241 .462366$

H -3.641084 -1.021301 0.486348

$\begin{array}{llll}\text { O } & -4.311178 & 0.726428 & 3.273592\end{array}$

H $-2.957617 \quad 0.9725151 .763171$

H -1.943450 -0.160778 3.823637

O $-1.352679-1.0459572 .076053$

H -2.185393 -2.704033 3.845381

O $-3.743791-1.7268914 .714925$

C $-4.116918-4.3827540 .667220$

H $-2.598502-2.8967760 .767200$

H $-2.429434-4.2049361 .922443$

H $-3.763273 \quad 1.2686063 .866387$

H $-0.949674-0.2105751 .785014$

H $-4.501747-1.1775574 .450946$

O $-4.546906-3.700933-0.432490$

O $-4.465136-5.5074281 .002659$

C $-5.511586-4.380950-1.240760$

H $-6.443019-4.510363-0.681530$

H $-5.712680-3.764437-2.120850$

H $-5.122652-5.348760-1.571440$

SCF Energy (B3LYP/6-31G**//MMFF $)=-3245.91859077$

$03 \_57$

MMFF Geometry

C $-0.0966385 .149100-0.016111$

C -1.2881714 .7294060 .433764$

C $-2.2374823 .895850-0.389311$

O -2.4045952 .6203090 .275131$ 
C $-3.6134554 .579823-0.465864$

C $-4.612323 \quad 3.837090-1.332636$

C $-5.7519693 .277634-0.880707$

C $-4.2794553 .766309-2.801325$

C -6.2867853 .2670670 .523454$

C -5.8793422 .0447391 .360370$

C $-6.373304 \quad 0.7022470 .797063$

C $-6.167770-0.4195691 .816207$

C $-6.580851-1.7882791 .253057$

O $-5.6295140 .403680-0.386982$

O $-6.063266-2.797757 \quad 2.134465$

C $-5.907410-1.998679-0.121774$

C $-8.104710-1.9623831 .243850$

O $-6.416552-3.184311-0.728369$

C $-6.051203-0.786622-1.069429$

C $-5.194371-0.895193-2.355777$

O $-7.417895-0.679732-1.487165$

C $-1.402248 \quad 1.709810 \quad 0.135743$

O $-0.385526 \quad 1.827309-0.530073$

C $-1.740714 \quad 0.5381320 .971904$

C $-0.763613-0.3052061 .335315$

C -0.914601-1.509957 2.153105

C $-2.313565-1.9411612 .505379$

C $-3.686268-1.057924-2.133889$

C $-3.214508-2.502646-2.137427$

C $-1.187587-3.887177-1.713459$

O $-3.932377-3.444235-2.463468$

C $-1.190859-4.397288-0.307226$

C $-0.079462-4.7865590 .335337$

C $-0.065677-5.3459921 .728444$

C $0.863961-4.5901952 .650771$

C $2.122715-5.0110072 .861137$

C $0.304058-3.3998103 .395369$

C $0.197444-2.1742122 .532328$

N $-1.881558-2.614009-1.765272$

H $0.2027014 .917418-1.035396$

H - 1.5738334 .9371121 .462419

H - $1.8422803 .730455-1.398980$

H -3.502138 $5.595461-0.868519$

H -4.0022474 .6985600 .551202$

H $-6.3921712 .770425-1.602043$

H -5.104879 $3.358768-3.394846$

H -4.061945 4.766417-3.190553

H -3.408290 $3.126403-2.970576$

H $-5.992615 \quad 4.1771701 .056765$

H $-7.382048 \quad 3.3140960 .467487$

H -4.7875672 .0269641 .456008$

H -6.289271 2.184120 2.368526

H -7.4363360 .8024450 .548473$

H -6.712954 -0.2080062 .744573$

H -5.106849 -0.4656192 .096936$

H -6.428841 -2.637628 3.021296

H $-4.844831-2.1786840 .079764$

H -8.507833 -1.8601802 .258639$

H -8.386079 -2.9677490 .911569$

H $-8.615692-1.2336180 .611358$

H -5.885130 -3.366915 -1.520871

H $-5.593017-1.677065-3.013097$

H $-5.330090 \quad 0.043749-2.912328$

H -7.449782 $-0.158666-2.306603$

H -2.7734000 .4111001 .273501$

H $0.254272-0.0834211 .017728$

H -2.781082 -1.215509 3.179574

H $-2.359989-2.9164472 .994160$

H -2.926887 -2.027514 1.602018

H $-3.159201-0.563831-2.960206$

H $-3.371130-0.571443-1.206653$

H $-0.174501-3.721861-2.092117$

H $-1.689245-4.607198-2.367721$

H -2.152377 -4.4751170 .194635$

H $0.875649-4.735377-0.182157$

H $\quad 0.249862-6.3956251 .662786$

H -1.073302 -5.3694142 .161517$

H $2.786867-4.4901723 .542783$

H $2.519312-5.8816142 .349297$

H $0.959474-3.1431074 .238717$

H $-0.645447-3.6798503 .859868$

H $1.166440-1.7838452 .217429$
H -1.375663 -1.785221 -1.466211

C 2.0035124 .8965341 .282313

$\begin{array}{lllll}\text { O } & 2.780487 & 4.478951 & 0.144477\end{array}$

C $4.130588 \quad 4.221755 \quad 0.575557$

C 4.1619844 .4963422 .077745

C 3.0244935 .4860962 .247151

H $4.7728424 .947347 \quad 0.061750$

C 4.5584852 .7952100 .196170

H 3.9688573 .5843262 .655584

H 5.1281704 .8942562 .403107

C 2.5367975 .6051503 .680162

H 3.3557996 .4704711 .890380

C $4.6958662 .561362-1.322604$

$\begin{array}{lllll}\text { O } & 3.627533 & 1.858382 & 0.740760\end{array}$

H 5.5264392 .5983760 .670387

H $5.4698743 .224984-1.727482$

O $3.4767362 .903915-1.992684$

C $5.0062421 .106479-1.726588$

C $6.1891720 .481068-0.970954$

H $4.1090490 .491315-1.597213$

O $5.3089801 .093355-3.126057$

H $7.0643021 .136133-1.066375$

H $\begin{array}{llll}5.944878 & 0.426302 & 0.094425\end{array}$

H $1.7165726 .325513 \quad 3.756319$

H 2.1776634 .6439834 .063102

H 3.3458325 .9464934 .334190

C 0.9099365 .8674820 .838492

H 2.7667792 .0256100 .319017

H $3.3121373 .850275-1.844043$

H $4.5449331 .476047-3.590525$

H 1.3367306 .6868910 .248332

H $\quad 0.4178616 .3120891 .710825$

H 1.5523073 .9921841 .714523

C $4.805772-2.178760-0.432849$

O $5.440889-1.778006-1.651425$

C $6.575661-0.925428-1.484045$

C $7.638950-1.638024-0.637872$

C $7.070789-2.1591370 .682650$

C $5.788791-2.9607540 .444681$

H $4.444731-1.2943630 .107112$

C $3.589889-3.030459-0.811752$

H $7.002144-0.785936-2.485624$

O $8.732336-0.761032-0.387677$

H $8.033326-2.487902-1.210200$

H $6.902104-1.3367961 .387911$

O $8.054325-3.006746 \quad 1.280958$

H $\quad 6.036877-3.919974-0.027629$

O $5.167741-3.2421941 .696877$

C $2.505568-2.164675-1.407882$

H $3.852397-3.813783-1.532727$

H $3.158259-3.5302510 .062082$

H $9.372430-1.2443460 .162449$

H $7.731211-3.2539952 .163709$

H $5.756120-3.8373882 .190790$

O $2.686169-2.041979-2.753484$

O $1.621704-1.633663-0.748718$

C $1.756335-1.184014-3.422113$

H $0.743267-1.588371-3.342310$

H $1.803350-0.173385-3.004824$

H $2.034592-1.139439-4.478367$

SCF Energy (B3LYP/6-31G**//MMFF)= -3245.92077303

03_58

MM̄FF Geometry

C -3.232049 -2.239876 -3.050837

C $-2.327369-2.606002-2.131898$

C $-0.842129-2.659415-2.374060$

O $-0.205699-1.687076-1.513241$

C $-0.313141-4.054349-1.992858$

C $1.173142-4.234304-2.228698$

C $2.078901-4.465996-1.258020$

C $1.619354-4.170847-3.666933$

C $1.842942-4.5963270 .221016$

C $1.999216-3.2886131 .013569$

C $3.417810-2.6970640 .973960$

C $3.550108-1.5347661 .960249$

C $4.933162-0.8682031 .881851$

O $3.675773-2.223568-0.349946$ 
$\begin{array}{llll}\text { O } & 4.858579 & 0.384019 & 2.580966\end{array}$

C $5.255730-0.5423880 .406865$

C $6.006662-1.6990832 .594765$

O $6.596982-0.0812360 .267968$

C $5.004159-1.722921-0.552678$

C $5.122314-1.332742-2.046550$

O $5.980134-2.743399-0.308575$

C $-0.204048-0.392363-1.932807$

O $-0.6755770 .047278-2.969498$

C $0.4593270 .416836-0.887686$

C $0.3236391 .750931-0.905789$

C 0.8824532 .6879420 .069995

C 1.8949732 .1533141 .046572

C $4.158702-0.238159-2.519230$

C $4.7614611 .157219-2.517905$

C $4.1370693 .539420-2.897793$

O $5.9550521 .377362-2.331846$

C $3.9345434 .202013-1.572022$

C $3.1990565 .312239-1.409701$

C $3.0215836 .015025-0.094521$

$\begin{array}{llll}\text { C } & 1.567230 & 6.219505 & 0.264488\end{array}$

C 0.9557827 .3933210 .031928

C 0.8490415 .0924870 .970652

C $0.470534 \quad 3.972576 \quad 0.039691$

N $3.8090032 .130442-2.790202$

H -2.902962 -1.967124 -4.049731

H -2.670546 -2.872963 -1.136731

H $-0.604040-2.439392-3.421750$

H $-0.844779-4.822769-2.570052$

H $-0.557914-4.244953-0.942307$

H $3.121029-4.586763-1.552320$

H $2.671021-4.452219-3.788286$

H $1.025641-4.856719-4.280032$

H $1.504572-3.157137-4.062799$

H $0.851812-5.0170130 .419184$

H $2.548697-5.3403470 .612256$

H $1.280261-2.5539240 .632796$

H $1.727265-3.4962282 .055955$

H $4.129269-3.492707 \quad 1.224263$

H $3.342449-1.8668822 .984937$

H $2.788654-0.7775441 .730842$

H 4.5815530 .2008833 .495221

H 4.6078070 .2930640 .118400

H $5.747642-1.8323263 .652076$

H $6.974202-1.1851302 .587048$

H $6.139122-2.6921022 .160471$

H 6.7065890 .6763530 .867802

H $6.163449-1.091167-2.291931$

H $4.888111-2.232440-2.634132$

H $6.042066-3.306184-1.098160$

H $1.015369-0.106573-0.118749$

H $-0.2825152 .202234-1.690591$

H $2.694579 \begin{array}{lll}1.626698 & 0.515430\end{array}$

H 1.4239531 .4589001 .748856

H 2.3876582 .9299431 .635091

H $3.882137-0.447621-3.560664$

H $3.236826-0.240522-1.929812$

H $3.4948033 .963796-3.675964$

H $5.1811313 .648370-3.208679$

H $4.4371723 .765017-0.712339$

H $2.7207945 .762658-2.276705$

H $3.5281076 .986612-0.165639$

H 3.5261775 .4786520 .718693

H $-0.079415 \quad 7.5573680 .312429$

H $1.4759228 .214971-0.449291$

H $-0.0819845 .464007 \quad 1.420192$

H 1.4521104 .7596861 .819814

H $-0.2642774 .263046-0.712613$

H $2.832841 \quad 1.862352-2.876759$

C -5.271251 $-0.775927-2.625241$

O $-4.684532-0.106367-1.491626$

C -4.665426 1.312011-1.764955

C $-5.3506821 .503502-3.118207$

C $-5.076551 \quad 0.176592-3.800224$

H $-3.6134871 .601924-1.862398$

C $-5.3337372 .079955-0.617178$

H -6.429958 $1.662428-3.006687$

H $-4.9456222 .359879-3.666364$
C $-5.985049-0.100906-4.984007$

H $-4.0322230 .175274-4.136387$

C -4.626402 1.9247650 .747147

O $-6.6815251 .610487-0.472763$

H -5.415364 $3.141218-0.877212$

H $-4.6694910 .875447 \quad 1.052880$

O -5.3928492 .6513451 .716902$

C -3.1712712 .4363600 .780243$

C -2.5050252 .4130702 .171465$

H $-2.550702 \quad 1.865506 \quad 0.082896$

$\begin{array}{lllll} & \text { O } & -3.147352 & 3.801201 & 0.346517\end{array}$

H $-1.672714 \quad 3.1287542 .170886$

H -3.212772 2.7811522 .923597

H $-5.752832-1.070994-5.434247$

H $-7.039450-0.110014-4.688003$

H $-5.8589690 .667351-5.753871$

C $-4.706948-2.189823-2.771244$

H $-6.6402420 .662393-0.259317$

H $-5.340893 \quad 3.594451 \quad 1.484531$

H $-3.2913513 .808211-0.614732$

H $-5.229092-2.717335-3.578071$

H $-4.920230-2.743959-1.847758$

H $-6.342952-0.870021-2.401221$

C -2.691745 -1.217486 3.038341

O -3.0608290 .1436622 .775416$

C -1.9611191 .0320922 .572024$

C $-1.108393 \quad 1.102413 \quad 3.849090$

C $-0.638331-0.292044 \quad 4.276978$

C $-1.786546-1.3098774 .279087$

H -3.638767 -1.718479 3.277301

C $-2.122370-1.8661891 .773236$

H -1.3438120 .6621551 .746070$

$\begin{array}{lllll}\mathrm{O} & -1.874146 & 1.693204 & 4.897247\end{array}$

H $-0.243711 \quad 1.7510183 .664838$

H $-0.200329-0.2296685 .280588$

O $0.389249-0.7167613 .386852$

H $-1.398554-2.3294514 .378248$

O $-2.612544-1.0672795 .422919$

C $-2.193525-3.3702591 .832040$

H -2.675784 -1.523205 0.890092

H $-1.083899-1.5840611 .578452$

H -1.3013391 .7550245 .680469$

H $0.689197-1.5939273 .680403$

H -2.057644 -1.175569 6.213948

O $-3.427188-3.7945251 .436235$

O $-1.266975-4.081632 \quad 2.197595$

C -3.616207 $-5.212578 \quad 1.432589$

H $-2.903997-5.688048 \quad 0.751283$

H -3.508236 -5.6110712 .445802$

H -4.630046 -5.419534 1.079905

SCF Energy (B3LYP/6-31G**//MMFF) $=-3245.89736077$

03_59

MM̄FF Geometry

C $2.291708-1.232527-3.282797$

C $2.033187-2.160836-2.348214$

C $0.690311-2.453913-1.719388$

O $-0.235041-1.356745-1.895062$

C $0.075239-3.715928-2.348907$

C $-1.091066-4.277816-1.559104$

C $-2.366155-4.320685-1.991527$

C $-0.746575-4.852198-0.207668$

C $-2.924702-3.840047-3.300119$

C $-3.450449-2.395904-3.289873$

C $-4.529624-2.117452-2.229730$

C $-5.186820-0.756512-2.472583$

C $-6.195494-0.396099-1.370012$

O $-3.905876-2.121070-0.941941$

O $-6.5037491 .001141-1.493982$

C $-5.527945-0.5891430 .009557$

C $-7.520523-1.147791-1.543988$

O $-6.476280-0.4247131 .060389$

C $-4.809331-1.945524 \quad 0.157614$

C -3.979541-2.061365 1.460707

O $-5.785300-2.9943610 .192286$

C $-0.048977-0.269614-1.096127$

O $0.812357-0.126581-0.243328$

C $-1.0658250 .743153-1.448148$ 
C $-0.885728 \quad 2.008187-1.040957$

C -1.796195 $3.131227-1.265663$

C $-3.1689572 .814420-1.795554$

C $-2.869496-1.0189081 .631978$

C -3.2783270 .1934312 .451530$

C -2.446942 $2.438207 \quad 3.144959$

$\begin{array}{llll}\text { O } & -4.324148 & 0.253416 & 3.092346\end{array}$

C -3.083203 3.4688592 .265732

C -2.5924814 .7082782 .111694$

C $-3.2368125 .767383 \quad 1.262435$

C $-2.278875 \quad 6.3710490 .258735$

C -1.644143 7.5266390 .518074

C $-2.1171615 .674054-1.074615$

C -1.370513 $4.373453-0.954506$

N -2.313816 1.1919472 .413446

H $1.483501-0.638860-3.700681$

H $2.860474-2.749953-1.956589$

H $0.851245-2.606644-0.645570$

H $0.833426-4.506979-2.426961$

H $-0.227624-3.484455-3.375842$

H $-3.113247-4.757206-1.328816$

H $-1.589441-5.3867590 .243932$

H $0.080223-5.564592-0.295453$

H $-0.454034-4.0591800 .486236$

H -2.185573 -3.944473 -4.101522

H $-3.744873-4.513733-3.580533$

H -2.604099 -1.716310 -3.139470

H -3.858289-2.185633 -4.286408

H $-5.278484-2.916819-2.277832$

H -5.670189-0.728206 -3.457165

H $-4.4114110 .020400-2.499989$

H $-6.8537641 .152809-2.388654$

H -4.7925280 .2168440 .121440$

H -7.975386 $-0.902609-2.511322$

H -8.250342 $-0.843829-0.785640$

H $-7.407421-2.232932-1.500132$

H -6.8869470 .4494650 .945860$

H -4.646237 -2.089437 2.331040

H -3.486286 -3.044179 1.442392

H $-5.383780-3.7756900 .607159$

H - $-1.9251410 .424364-2.024695$

H $0.020518 \quad 2.252239-0.488421$

H -3.659316 2.069303 -1.159747

H $-3.1068642 .420953-2.815853$

H -3.838490 $3.675970-1.824346$

H -2.037552 -1.4844092 .174937$

H -2.485452 -0.6937700 .661810$

H -1.444040 2.7323933 .470212

H -3.069191 2.274332 4.030701

H -4.001026 3.1922651 .751953

H -1.689919 4.9905962 .649473

H -3.612855 6.5499861 .934378

H $-4.118792 \quad 5.379793 \quad 0.737331$

H $-0.9750617 .979946-0.205701$

H -1.7758908 .0429251 .463344$

H $-1.5508196 .309747-1.768138$

H -3.103496 $5.562390-1.532132$

H $-0.3538014 .491161-0.575853$

H -1.494677 $1.072098 \quad 1.824633$

C $4.0338050 .551567-3.565102$

O $3.8167940 .935915-2.193680$

C $4.9444491 .697645-1.732018$

C $5.7385972 .069552-2.979061$

C $5.5028620 .858033-3.859349$

H $5.5456001 .015952-1.119005$

C $4.4922312 .882151-0.870136$

H $5.3420272 .967910-3.465560$

H $6.7964852 .243879-2.760529$

C $5.8003141 .101956-5.328420$

H $6.1388790 .038886-3.497766$

C 3.4602852 .5377020 .222803

O $3.887113 \quad 3.867242-1.719213$

H $5.3746493 .361942-0.429807$

H $2.4807122 .380286-0.242894$

O $3.3103903 .699217 \quad 1.050831$

C $3.7967811 .322573 \quad 1.105446$

C $2.794807 \quad 1.1678282 .267283$

H 3.7975160 .4218210 .483165
O $5.1109641 .488003 \quad 1.635029$

H 2.9859151 .9408853 .021783

H 1.7825521 .3175681 .872732

H $5.6037770 .202292-5.920136$

H $5.1854271 .912834-5.733208$

H $6.8512981 .375436-5.467877$

C $3.680845-0.925469-3.766968$

H $3.6206664 .612796-1.154317$

H 4.1465343 .8249311 .531668

H 5.3739490 .6459242 .042630

H $4.404780-1.552357-3.232273$

H $3.754094-1.183470-4.829441$

H $3.3733761 .181867-4.174792$

C $2.516172-2.5556232 .419792$

O $2.473393-1.1918511 .984153$

C $2.867627-0.2119252 .949747$

C $1.954846-0.2862434 .183995$

C $1.934395-1.7018544 .769099$

C $1.675425-2.7603853 .692028$

H $2.042202-3.1212971 .607332$

C $3.956985-3.0581842 .554729$

H $3.902716-0.3969963 .253506$

$\begin{array}{llll}\text { O } & 0.631236 & 0.104075 & 3.822989\end{array}$

H $2.3171590 .426646 \quad 4.934509$

H $1.151742-1.7616575 .535445$

O $3.184889-1.9484175 .405236$

H $1.853336-3.7630764 .097753$

O $0.290961-2.693714 \quad 3.339079$

C $4.637575-3.064168 \quad 1.206259$

H $4.556322-2.4625253 .249111$

H $3.979102-4.0870872 .932545$

H $\quad 0.083728 \quad 0.066515 \quad 4.625604$

H $3.144345-2.8375175 .796923$

H $0.111698-3.4157732 .713399$

O $5.467924-1.9883711 .097769$

\begin{tabular}{lllll}
\hline & 4.432918 & -3.909290 & 0.344404
\end{tabular}

C $6.152588-1.863620-0.151917$

H $6.823150-1.002639-0.087541$

H $6.749502-2.758275-0.352766$

H $5.433058-1.689507-0.956582$

SCF Energy (B3LYP/6-31G**//MMFF) $=-3245.90168285$

03_6

MM̄FF Geometry

C $3.960939-3.1479641 .178987$

C $3.043769-3.2008680 .201153$

C $1.560183-3.2203200 .453383$

O $1.017287-2.172960-0.384881$

C $0.949027-4.568415 \quad 0.034830$

C $-0.446536-4.8016980 .583824$

C $-1.571197-4.893556-0.152876$

C $-0.528292-4.9925252 .079181$

C $-1.732851-4.791451-1.642556$

C $-2.294937-3.449370-2.135291$

C $-3.725474-3.135497-1.663114$

C $-4.276084-1.916377-2.409633$

C $-5.664946-1.499581-1.901522$

O $-3.700886-2.864328-0.257849$

O $-5.940433-0.181467-2.401449$

C $-5.629487-1.397531-0.361307$

C $-6.768268-2.410127-2.453147$

O $-6.935040-1.1714590 .162871$

C $-4.993545-2.6263760 .320269$

C $-4.803070-2.4516751 .848569$

O $-5.852104-3.7603750 .141022$

C $0.131640-1.2982240 .154406$

O $-0.335452-1.313143 \quad 1.281025$

C $-0.178754-0.298038-0.892377$

C $-1.2976780 .431133-0.786948$

C $-1.7655041 .451092-1.725510$

C $-0.840207 \quad 1.859710-2.840043$

C $-3.933508-1.2664772 .278292$

$\begin{array}{llll}\text { C } & -4.717249 & 0.007687 & 2.550641\end{array}$

C -4.4088012 .4759402 .749671$

O \begin{tabular}{llll}
\hline & 5.922555 & 0.018005 & 2.786450
\end{tabular}

C $-4.872346 \quad 3.0283451 .438493$

C -4.2739014 .0462230 .801563$

C $-4.7341154 .588196-0.521831$ 
C $-3.7176004 .381918-1.624711$

C $-2.9152605 .383249-2.022931$

C $-3.6926013 .041556-2.327413$

C $-2.9885041 .984118-1.526298$

N $-3.901773 \quad 1.1312182 .543220$

H $3.616691-3.1861592 .210805$

H $3.353761-3.177335-0.839815$

H $1.369733-3.0047191 .510645$

H $1.580859-5.3921130 .394319$

H $0.957291-4.638618-1.057586$

H -2.503095 -5.0925850 .375501$

H - $1.525481-5.3085482 .404550$

H $0.177882-5.7641072 .403226$

H $-0.294934-4.0620112 .604412$

H $-0.784195-4.977678-2.155044$

H $-2.398646-5.601168-1.967152$

H -1.618837 -2.648964-1.818796

H $-2.279035-3.469244-3.232396$

H -4.352354 -4.014111 -1.855292

H -4.304852 -2.102770 -3.490502

H $-3.591158-1.069750-2.273232$

H $-5.883580-0.210846-3.371841$

H $-5.034264-0.509023-0.117928$

H -6.787388 -2.367163 -3.548838

H -7.758307 -2.076042 -2.124012

H $-6.643574-3.456750-2.168054$

H -7.288793 -0.377115 -0.272821

H $-5.779026-2.4351892 .348557$

H $-4.302836-3.3589382 .217323$

H $-5.633939-4.4219460 .818322$

H $0.506418-0.208134-1.727680$

$\begin{array}{llll}\text { H } & -1.946847 & 0.257641 & 0.068625\end{array}$

H $-0.7083201 .035364-3.549085$

H $0.1420332 .142145-2.446470$

H -1.198894 $2.722060-3.406256$

H $-3.439034-1.5209253 .224473$

H -3.147309 -1.0777611 .542667$

H -3.598432 3.0682003 .186087

H -5.2426102 .4496513 .458346$

H -5.7450982 .5599570 .989040$

H $-3.405613 \quad 4.519351 \quad 1.253000$

H $-4.9387305 .658886-0.390909$

H -5.690982 4.144168 -0.824010

H -2.204179 $5.249737-2.831488$

H -2.946361 $6.356275-1.543893$

H $-3.2575563 .140883-3.327470$

H -4.722941 $2.710689-2.510856$

H $-3.5819301 .601211-0.696959$

H -2.9264191 .0329282 .276999$

C $5.927236-2.446731-0.341465$

O $5.300458-1.178832-0.617257$

C $6.264039-0.294790-1.214238$

C $7.498674-1.143076-1.503806$

C $7.429816-2.174136-0.393466$

H $6.5193730 .449994-0.451222$

C $5.659630 \quad 0.402133-2.438105$

H $7.436906-1.634855-2.481331$

H $8.419510-0.552131-1.485457$

C $8.275435-3.408043-0.654697$

H $7.760278-1.7023670 .541677$

C $4.3215841 .124762-2.172677$

O $5.417340-0.584735-3.450332$

H $6.394696 \quad 1.098526-2.859113$

H $3.5292030 .385770-2.003046$

O $3.9613301 .808076-3.381670$

C $4.3381742 .131793-1.006493$

C $3.0352762 .953098-0.935344$

H $4.4937231 .589345-0.069152$

O $5.4434303 .016567-1.187520$

H $2.9780533 .603039-1.816602$

H $2.1853882 .264698-0.981864$

H $8.184422-4.1247010 .167392$

H $7.973265-3.913841-1.577793$

H $9.331947-3.136723-0.750574$

C $5.448929-2.9721691 .015788$

H $5.039211-0.124203-4.219145$

H $4.6166222 .512321-3.525809$

H $5.5874613 .485442-0.348588$
H $5.759955-2.2560931 .787580$

H $5.938495-3.9248371 .247145$

H $5.638944-3.135453-1.145726$

C $1.8830542 .243621 \quad 1.809834$

O 3.0171223 .0760761 .535500

C 2.9325033 .8389780 .327546

C 1.6938654 .7463580 .365289

C 0.4204123 .9567820 .652776

C 0.6019853 .0831711 .892324

H 1.7854541 .4815821 .028037

C 2.1455061 .5321613 .146048

H 3.8049044 .5033820 .323564

O $1.5712525 .452664-0.864528$

H 1.8303715 .4963191 .155561

H $0.1180603 .355138-0.210090$

O $\quad-0.6519464 .8649160 .910149$

H 0.6273653 .7219072 .784690

O -0.5223812 .2158002 .023588$

C 3.2843010 .5397513 .058073

H 2.4087552 .2789123 .905305

H $1.2600880 .978593 \quad 3.476292$

H $0.8552346 .101945-0.762835$

H -0.8437625 .3347740 .081394$

H -1.319706 2.7727732 .021887

O $3.719834 \quad 0.251690 \quad 4.320220$

O 3.7210050 .0597362 .021795

C $4.805344-0.6766774 .405533$

H $5.633842-0.3655523 .761714$

H $5.156504-0.6941505 .440650$

H $4.458999-1.6778444 .135319$

SCF Energy $\left(B 3 L Y P / 6-31 G^{* *} / / M M F F\right)=-3245.91118377$

03_60

MMFF Geometry

C $-2.672068-3.864291-0.117796$

C $-1.487527-3.8663900 .511445$

C $-0.176523-3.649581-0.200466$

O $0.435478-2.4646710 .359717$

C $0.766277-4.8441910 .034756$

C $1.993630-4.840624-0.858844$

C $3.266846-4.797926-0.420001$

C $1.728863-4.938043-2.340830$

C $3.761960-4.7506850 .996627$

C $3.994190-3.3362431 .546688$

C $5.033821-2.5111450 .769230$

C $5.393104-1.2386651 .541227$

C $6.340134-0.323998 \quad 0.749174$

O $4.473827-2.160301-0.499939$

O $6.345697 \quad 0.963207 \quad 1.386379$

C $5.772243-0.119272-0.672143$

C $7.786202-0.8319390 .789003$

O $6.6965590 .591407-1.491063$

C $5.362144-1.436342-1.362655$

C $4.628655-1.221831-2.710360$

O $6.537942-2.204037-1.649372$

C $0.103308-1.272067-0.200127$

O $-0.608624-1.099225-1.180789$

C $0.713877-0.1334420 .518898$

C $1.348196-0.2324641 .696459$

C 1.9206390 .8988812 .421726

C 1.6675240 .8537763 .903587

C $3.335806-0.403058-2.636319$

C $3.5307671 .081489-2.899222$

C $2.3650523 .268637-2.620787$

O $4.5414941 .548716-3.416782$

C $2.9834603 .866345-1.396846$

C $2.2854194 .471853-0.424502$

C 2.9137775 .0762890 .797310

C 2.5447674 .3432702 .067573

C 1.6416884 .8498222 .923793

C 3.2885833 .0647852 .387875

C 2.6291531 .8544241 .787652

N $2.4206261 .822545-2.518476$

H $-2.701389-3.716400-1.194795$

H $-1.452632-4.0043091 .589297$

H - $0.345432-3.513195-1.274851$

H $\quad 0.228862-5.783923-0.150587$

H $1.056343-4.8586081 .090955$ 
H $4.062730-4.808736-1.164390$

H $2.640601-5.142485-2.912719$

H $1.026785-5.751925-2.549892$

H $1.307812-4.002946-2.721812$

H $3.076609-5.2848841 .663580$

H $4.703049-5.3136541 .045222$

H $3.035962-2.8057411 .553599$

H $4.318287-3.4351612 .590041$

H $5.926543-3.1293870 .617845$

H $5.827459-1.4859152 .517870$

H $4.475719-0.6776101 .758601$

H 6.6213020 .8390522 .310708

H $4.8865280 .519885-0.569107$

H $8.149336-0.8714761 .823083$

H $8.460487-0.1479180 .261940$

H $7.902262-1.8302040 .362105$

H $6.9100731 .422288-1.033017$

H $5.322428-0.814158-3.455500$

H $4.349331-2.216277-3.087713$

H $\quad 6.325992-2.842340-2.350690$

H $\quad 0.586658 \quad 0.8314130 .035831$

H $1.429134-1.1900282 .203627$

H 0.6019670 .7042854 .108341

H $1.9553601 .771507 \quad 4.420891$

H 2.2226720 .0264884 .359376

H $2.651814-0.759841-3.416938$

H $2.836433-0.549148-1.673626$

H $1.3142083 .551067-2.734879$

H $2.9102903 .596445-3.511643$

H $4.0655153 .797996-1.311058$

H $1.2041994 .542893-0.508476$

H $2.591444 \quad 6.1241530 .854284$

H 4.0066735 .1136100 .706465

H $1.395048 \quad 4.3411873 .849788$

H $\quad 1.123528 \quad 5.7797172 .713958$

H 3.4102422 .9628093 .471408

H 4.3148723 .1299132 .004760

H 2.7795901 .7556730 .713623

H $1.6514201 .355322-2.048090$

C $-4.807601-2.7445560 .539616$

O $-5.156090-2.414969-0.823239$

C $-6.536630-2.028537-0.860944$

C $-6.942379-1.7632270 .581045$

C $-6.134781-2.8319641 .295124$

H -7.090890 -2.915080 -1.201147

C $-6.803441-0.909069-1.870224$

H -6.630191 -0.7654110 .909191$

H $-8.019833-1.8608300 .742860$

C $-6.029269-2.6189262 .793785$

H -6.603084 -3.808493 1.110125

C $-6.1812030 .479908-1.611015$

O $-8.225748-0.743497-1.968780$

H -6.474996 -1.250765 -2.859555

H $-6.6426010 .950648-0.735258$

O $-6.5388251 .305978-2.732296$

C $-4.6473040 .523192-1.462286$

C $-4.0847031 .959598-1.529076$

H $-4.3640330 .066055-0.510285$

O $-4.041573-0.246511-2.501848$

H -4.267293 2.362766 -2.532285

H -4.633258 $2.587783-0.820119$

H -5.397235 -3.3837203 .255431$

H -5.605745 -1.640048 3.033144

H -7.019292 -2.674221 3.258350

C $-3.995499-4.0399930 .572614$

H -8.543089 $-0.377206-1.125873$

H -7.508776 $1.293060-2.804223$

H $-4.3228850 .133017-3.351894$

H $-4.538533-4.8402690 .055484$

H -3.845171 -4.362675 1.609086

H -4.204242 -1.9124220 .921485$

C -2.6241382 .5079911 .114668$

O $-2.234710 \quad 1.6048360 .072622$

C $-2.5648442 .023566-1.255107$

C $-1.9247843 .387984-1.547033$

C $-2.3071434 .428392-0.500687$

C -2.010842 3.8972710 .898991

H -3.7175172 .5792821 .150896$
C $-2.136817 \quad 1.9149602 .443860$

H -2.086865 $1.303362-1.930245$

O $-2.2900163 .863769-2.837555$

H $-0.8330153 .280052-1.542646$

H $-3.3569404 .729927-0.594913$

O $-1.5283245 .609593-0.703424$

H -0.9244123 .8565321 .049046$

O -2.5411554 .8000871 .866917$

C -2.8238160 .6076972 .763575$

H -1.0592181 .7322152 .377456$

H $-2.3250812 .595613 \quad 3.280564$

H -2.022229 $3.188579-3.484212$

H -1.689329 5.908548 -1.615040

H -2.147864 5.6728341 .694908

O $-1.976848-0.1847163 .482316$

O $-\begin{array}{llll}-3.972928 & 0.332595 & 2.447223\end{array}$

C $-2.496413-1.4593933 .870543$

H $-2.754693-2.0482512 .986571$

H $-1.718059-1.9882404 .427132$

H $-3.367532-1.3316134 .519728$

SCF Energy $(B 3 L Y P / 6-31 G * * / / M M F F)=-3245.90737957$

03_61

MMFF Geometry

C $-2.629636-2.899126-2.609052$

C $-2.016758-2.948388-1.416641$

C $-0.548535-3.257512-1.252171$

O $0.118083-2.091759-0.718358$

C $-0.380485-4.410817-0.244652$

C $1.051287-4.879588-0.075724$

C $1.753377-4.8019861 .071914$

C $1.683358-5.502557-1.294435$

C $1.310910-4.2542102 .400099$

C $1.685858-2.7834072 .642641$

C $3.200117-2.5181492 .672148$

C $3.495002-1.0953133 .151188$

C $4.998802-0.7766333 .104655$

O $3.712629-2.683774 \quad 1.348755$

O $5.1577920 .638028 \quad 3.294727$

C $5.560806-1.1470491 .711540$

C $5.743858-1.4237894 .278019$

O $6.979878-1.0129101 .714189$

C $5.137080-2.5553531 .238139$

C $5.496622-2.853656-0.237997$

O $5.805830-3.5382302 .037916$

C $0.526713-1.148544-1.610829$

O $0.372923-1.163471-2.821812$

C $1.200691-0.072375-0.852310$

C $1.4342941 .107021-1.447126$

C $2.0742412 .269788-0.828805$

C 2.7790262 .0569100 .484051

C $4.880733-1.903163-1.270670$

C $5.801144-0.770071-1.691280$

C $5.8326831 .272727-3.116784$

O $6.974242-0.685973-1.338424$

C $5.6117152 .467807-2.244961$

C $5.1327583 .633743-2.703817$

C $4.9438834 .854764-1.851153$

C $3.5341635 .396318-1.917056$

C $3.2274826 .430641-2.718256$

C $2.5031914 .795453-0.989647$

C $2.0031963 .459967-1.461139$

N $5.1612090 .124378-2.538687$

H -2.025159-3.055957-3.500959

H -2.579798 -2.773565 -0.504719

H $-0.098537-3.536515-2.212707$

H -0.981144 -5.272965 -0.564639

H $-0.796085-4.0947870 .718086$

H $2.773126-5.1864011 .070489$

H $2.652558-5.961555-1.071111$

H $1.036671-6.288231-1.698831$

H $1.849771-4.750164-2.071138$

H $\quad 0.229969-4.3700872 .529434$

H $1.758250-4.8743353 .187537$

H $1.216459-2.1659411 .867725$

H $1.250490-2.4832993 .603900$

H $3.665241-3.2515413 .341065$

H $3.100756-0.9326874 .162005$ 
H $2.965187-0.3811542 .506396$ H 4.6819281 .0967812 .582068 H $5.176076-0.398758 \quad 1.009217$ H $5.342344-1.0595455 .231593$ H $6.803843-1.1459304 .275998$ H $5.673412-2.5133934 .284765$ H $7.292152-1.1196680 .800247$ H $6.583490-2.935414-0.356852$ H $5.108173-3.857122-0.466388$ H $5.811118-4.3800081 .552748$ H $1.471745-0.2739450 .177507$ H $1.0990551 .249495-2.473718$ H 3.3893882 .9076470 .794164 H 3.4656891 .2066210 .413596 H 2.0568751 .8598741 .283374 H $4.666303-2.476241-2.182133$ H $3.932640-1.489042-0.913830$ H $5.4275421 .412974-4.123929$ H $6.9049951 .067598-3.197009$ H $5.8813372 .378047-1.195349$ H $4.8891093 .728478-3.759840$ H $5.6536385 .616938-2.198921$ H $5.2110154 .659518-0.805032$ H $2.2247936 .843692-2.750071$ H $3.9718846 .883844-3.364719$ H $1.6303895 .459057-0.920597$ H $2.908845 \quad 4.765578 \quad 0.025280$ H $1.4719793 .508763-2.412826$ H $4.1703440 .007755-2.730097$ C $-5.063774-2.887244-1.722756$ O $-4.965731-1.816138-0.764098$ C $-6.282496-1.518080-0.262345$ C -7.220932 -2.563132 -0.857111 C $-6.528237-2.893456-2.162432$ H $-6.555623-0.535628-0.664370$ C $-6.284911-1.4804721 .269691$ H -7.290025 -3.456338 -0.225397 H $-8.235302-2.175750-0.994656$ C $-6.994240-4.204336-2.773179$ H -6.710176 -2.076934-2.874087 C $-5.300132-0.4717151 .893262$ O $-5.935181-2.7829501 .757131$ H -7.303695 -1.2835881 .624752$ H -4.269985 -0.817682 1.746923 O $-5.517972-0.487626 \quad 3.311137$ C -5.4341490 .9726331 .375166$ C -4.600912 1.9680902 .205320 H -5.1263921 .0016890 .325331$ O H -5.0551942 .0701613 .198276$ H -3.5960741 .5564642 .346352$ H -6.474919 -4.406597 -3.714811 H $-6.810607-5.048228-2.099690$ H $-8.068348-4.169739-2.983226$ C -4.091046 -2.656711 -2.883024 H -5.946216 -2.741072 2.728866 H -6.407071 -0.1284153 .474873$ H -6.9187392 .1572460 .903749$ H -4.193907 -1.631163 -3.256874 H $-4.364229-3.328504-3.705712$ H -4.822044 -3.820494 -1.196984 C -2.5400473 .0291140 .209618$ O -3.9414853 .3259310 .250218$ C -4.5162873 .3699171 .560399$ C -3.7936344 .4185412 .415748$ C -2.2865824 .1868032 .447726$ C -1.740431 4.053965 1.026969 H -2.3616382 .0211890 .602492$ C $-2.1116653 .059615-1.265954$ H -5.5449763 .7270621 .431721$ O $-4.317955 \quad 4.418713 \quad 3.739383$ H $-3.9886215 .413617 \quad 1.994550$ H -2.035516 3.3122623 .059474 O -1.6794905 .3139343 .083717$ H -1.764451 5.0329280 .530986 O -0.3797083 .6343631 .088984$ C -2.554941 $1.831235-2.032763$ H $-2.5470423 .936606-1.759560$
H -1.022016 $3.103937-1.347574$ H -3.819456 5.0798724 .249598 H -0.7339485 .1163923 .191035$ H $\quad 0.135437 \quad 4.3578841 .483614$ O $-2.2135321 .991239-3.346529$ O $-3.112597 \quad 0.855269-1.551613$ C $-2.5618180 .906332-4.211237$ H $-3.6449530 .751851-4.208465$ H -2.247445 $1.165456-5.225687$ H $-2.039457-0.003978-3.904645$ SCF Energy $(B 3 L Y P / 6-31 G * * / / M M F F)=-3245.91496586$

03 62

MM̄FF Geometry

C $-2.705737-2.6282181 .818352$

C $-1.528357-3.2702391 .829952$

C $-0.501248-3.1286520 .733145$

O $0.614697-2.356593 \quad 1.232363$

C $0.015782-4522590 \quad 0.328567$

C $0.924040-4.514194-0.885209$

C $2.229632-4.846920-0.870757$

C $0.266978-4.140131-2.189280$

C $3.064069-5.2929980 .296392$

C $3.815445-4.1670791 .022787$

C $4.804858-3.3917460 .137273$

C $5.693614-2.4823730 .989263$

C $6.629727-1.618844 \quad 0.128631$

O $4.057269-2.595065-0.786167$

O $7.170450-0.5853690 .966616$

C $5.803079-0.919342-0.972655$

C $7.826516-2.423581-0.392342$

O $6.653857-0.243233-1.894435$

C $4.859099-1.873902-1.731641$

C $3.891675-1.142084-2.694452$

O $5.640895-2.770993-2.530623$

C $0.463953-1.0031261 .259200$

O $-0.515844-0.3643280 .909157$

C $1.702329-0.4154291 .813010$

C 1.6837680 .8534572 .247985

C 2.8123591 .5873282 .823067

C 4.1769400 .9617582 .709880

C $2.957824-0.116039-2.043052$

C $3.4812261 .309870-2.080614$

C $2.9707703 .590539-1.220626$

O $4.4576391 .652448-2.741856$

C $3.8311303 .858129-0.026393$

C 3.5394814 .7903480 .893426

C 4.4135355 .1066262 .073257

C 3.6644065 .0450693 .385533

C 3.1760046 .1596333 .955390

C 3.5555093 .7037414 .075046

C 2.5756932 .7841553 .399907

N $2.7010122 .168386-1.316899$

H -2.925347 -1.947157 0.999859

H -1.285650 -3.925045 2.663344

H $-0.932243-2.624755-0.139898$

H $-0.832736-5.1840700 .106841$

H $\quad 0.528764-4.9681001 .187794$

H $2.775074-4.808783-1.813583$

H $0.926632-4.300796-3.048770$

H $-0.631118-4.745209-2.350869$

H -0.017525 -3.085222 -2.189349

H $2.454191-5.8397661 .023411$

H $3.791640-6.027936-0.071943$

H $3.083266-3.4739771 .452346$

H $4.360360-4.6199901 .860384$

H $5.417053-4.115571-0.413335$

H $6.272961-3.0703311 .712111$

H $5.060040-1.8150631 .588701$

H $7.631215-1.0120861 .709285$

H $5.202977-0.147642-0.476218$

H $8.409396-2.8274380 .444259$

H $8.516436-1.786085-0.956211$

H $7.535471-3.263779-1.026065$

H $7.2121940 .365381-1.380822$

H $4.451288-0.705234-3.530363$

H $3.246982-1.909274-3.147610$

H $5.075816-3.127961-3.235795$ 
H $2.585101-1.0414261 .859534$ H $\quad 0.747161 \quad 1.4078302 .196347$ H 4.3854810 .6878871 .670289 H 4.2410410 .0619293 .330898 H 4.9892281 .6251953 .013304 H $2.016382-0.103754-2.606225$ H $2.720432-0.396155-1.012723$ H $2.0061454 .101954-1.156351$ H $3.4841463 .926326-2.127302$ H 4.7474953 .2807160 .071218 H 2.6363325 .3854580 .776407 H 4.8208956 .1154091 .924869 H 5.2843024 .4407722 .118320 H 2.6612476 .1284764 .910000 H 3.2758347 .1283213 .476725 H 3.2111543 .8386285 .109207 H 4.5549773 .2706684 .167774 H 1.5488413 .1521713 .425206 H $1.9386531 .790212-0.761877$ C $-5.183777-2.8183562 .341562$ O $-5.510914-1.553148 \quad 1.731740$ C $-6.173583-1.8045010 .478917$ C $-6.633257-3.2562910 .541252$ C $-5.480145-3.8945301 .295055$ H -5.411423 -1.713019-0.304202 C $-7.277644-0.7709980 .239780$ H -7.563508 -3.3650351 .110868$ H $-6.788779-3.687037-0.452391$ C $-5.809647-5.2525361 .888758$ H $-4.639546-4.0087270 .599368$ C $-\begin{array}{llll}6.808038 & 0.693287 & 0.375237\end{array}$ O $-8.316663-0.981707 \quad 1.204051$ H -7.730743 $-0.941522-0.743945$ H $-6.579194 \quad 0.9074221 .425993$ O $\quad-7.914310 \quad 1.541617 \quad 0.041869$ C $-5.5937251 .070196-0.495433$ C $-5.2279632 .561939-0.373021$ H $-4.7319570 .468551-0.189250$ O $\quad-5.863058 \quad 0.752362-1.859743$ H $-6.0570703 .178056-0.741438$ H -5.0811862 .7882470 .690197$ H -4.951404 -5.655027 2.436287 H $-6.655314-5.1937782 .582040$ H -6.071942 $-5.964071 \quad 1.098995$ C -3.752565 -2.770529 2.887139 H $-9.004722 \quad-0.3142761 .039554$ H -8.185344 $1.335235-0.868584$ H $-6.553877 \quad 1.357320-2.178154$ H -3.564696 -3.666129 3.490679 H -3.662346 -1.899402 3.547916 H $-5.869165-2.923963 \quad 3.192594$ C -1.594317 $2.405342-1.258172$ O $-2.8582142 .213928-0.607899$ C $-3.9595152 .939918-1.164788$ C $-3.6861634 .452060-1.110766$ C $-2.3572494 .799596-1.787586$ C -1.218220 $3.895340-1.304694$ H $-0.8667221 .899872-0.611076$ C -1.550066 $1.727692-2.631548$ H -4.102853 $2.636080-2.207083$ O -3.6536314 .8820320 .249002$ H -4.511398 4.978472 -1.605366 H $-2.1074285 .846180-1.574932$ O $-2.5195194 .668342-3.196916$ H $-0.3374284 .028586-1.942151$ O -0.8300204 .3026450 .010934$ C -1.635068 $0.227976-2.485566$ H -2.345093 $2.059366-3.305095$ H $-0.6043541 .946090-3.142334$ H -3.5201235 .8453240 .248843$ H $-1.6770154 .917428-3.613696$ H -1.575134 4.1226830 .609735 O $-2.916079-0.191230-2.685458$ O $-0.678121-0.481378-2.204497$ C -3.131984 -1.599731-2.557339 H $-2.500999-2.148892-3.262559$ H -2.932913 -1.918639-1.530920 H $-4.179915-1.805686-2.790808$
SCF Energy (B3LYP/6-31G**//MMFF) $=-3245.91299961$

03 63

MMFF Geometry

C $-5.008597-1.6677202 .261256$

C $-5.112782-0.3297252 .240904$

C $-5.765216 \quad 0.528190 \quad 1.181927$

O $-4.700648 \quad 1.2902250 .561895$

C $-6.502293-0.2387330 .063473$

C $-7.0441380 .647521-1.046466$

C $-6.6568320 .557832-2.336392$

C $-8.0821351 .657879-0.636261$

C $-5.675929-0.430101-2.909934$

C $-4.2322850 .041149-2.736359$

C $-3.168692-1.032741-3.007644$

C $-3.237932-2.185657-1.999028$

C $-2.072960-3.171753-2.157605$

O $-1.906180-0.360294-2.894436$

O $-2.077344-4.013182-0.993464$

C $-0.752611-2.377585-2.164191$

C $-2.280113-4.099532-3.359632$

O $0.351971-3.238554-2.422991$

C $-0.756268-1.179706-3.138299$

C $0.490693-0.277446-2.974902$

O $-0.753275-1.669909-4.483353$

C -4.4219942 .5325051 .036403$

O -4.9772153 .1178751 .951004$

C -3.2916543 .0553270 .235120$

C $-2.766767 \quad 4.2592190 .508048$

C $-1.6423184 .884268-0.195697$

C $-1.0388054 .119874-1.345576$

C $0.6779140 .287609-1.565881$

C $1.7389281 .371129-1.489917$

C 2.8454402 .8476210 .201269

O $2.3704561 .770489-2.463165$

C 2.1389423 .9105140 .980973

C 2.2955165 .2258240 .765550

C 1.5877716 .2796601 .570993

C 0.9187227 .3401360 .720528

C 1.2044078 .6405250 .904862

C $-0.0767936 .915975-0.341070$

C $-1.212021 \quad 6.096446 \quad 0.214474$

N $1.8790261 .839369-0.190601$

H -5.439855 -2.268397 1.466441

H -4.6588520 .2279873 .058988$

H -6.4758621 .1996181 .679277$

H -7.337889 -0.8056250 .493749$

H $-5.816021-0.978026-0.360496$

H -7.089834 $1.245504-3.060731$

H -8.522592 2.171899-1.497344

H $-8.8981431 .165625-0.097166$

H -7.643847 $2.421547 \quad 0.013178$

H $-5.847346-1.413490-2.463989$

H $-5.889754-0.557939-3.978375$

H $-4.0515400 .898465-3.399459$

H $-4.0700800 .431597-1.726385$

H -3.288616 -1.398650 -4.033775

H -4.187142 -2.727659-2.070683

H -3.206554 -1.775082 -0.979439

H -1.293739 -4.587548 -1.042522

H $-0.616665-2.009909-1.140371$

H -3.203829 -4.678207 -3.238656

H - $1.474514-4.838223-3.435023$

H $-2.346957-3.563052-4.308413$

H $\quad 0.370509-3.418210-3.378471$

H $1.394099-0.819088-3.282387$

H $0.4002910 .561893-3.678562$

H $-0.408306-0.971600-5.064186$

H $-2.9131632 .423301-0.560762$

H -3.1913674 .8400631 .325651$

H $-1.7888403 .943397-2.124871$

H -0.659337 $3.153635-1.000926$

H $-0.2035094 .628444-1.829001$

H $-0.2624360 .714486-1.199417$

H $0.980133-0.512421-0.883962$

H $3.3597613 .245646-0.678688$

H 3.5793532 .3498720 .840740

H $1.4736463 .581641 \quad 1.776429$ 
H $2.982176 \quad 5.563147-0.007064$ H 2.3324146 .7399992 .233383 H $\quad 0.834907 \quad 5.8288532 .229473$ H $\quad 0.736214 \quad 9.4141750 .305291$ H 1.9135888 .9635411 .659955 H $0.4724936 .400589-1.132272$ H $-0.5206617 .799450-0.818392$ H -1.7341376 .5768691 .043250$ H 1.3580881 .3783520 .550586 C -3.042069-3.134475 2.797940 O $-2.055570-2.1544222 .406182$ C $-0.819389-2.4400843 .090416$ C $-0.911996-3.9051373 .480805$ C -2.374953 -4.019130 3.854387 H $-0.818358-1.8006013 .982362$ C $0.372226-2.0960452 .186879$ H $-0.681860-4.5516012 .624669$ H $-0.241497-4.1687904 .303931$ C -2.889963 -5.448291 3.869653 H -2.514133 -3.583076 4.852980 C $1.731949-2.4332602 .826440$ O $\quad 0.333939-0.6918371 .914466$ H $0.263832-2.6087411 .224956$ H $1.807769-3.5133772 .996190$ O $1.793161-1.8245604 .124501$ C $2.969417-1.9619482 .038851$ C $3.064795-2.5626640 .627650$ H $2.999126-0.8671411 .994679$ O $4.131770-2.3775642 .767238$ H $3.083169-3.657017 \quad 0.703028$ H $2.175938-2.2770820 .057377$ H -3.953612 -5.4758194 .126640$ H $-2.766053-5.9298582 .893844$ H $-2.347076-6.0449484 .609977$ C $-4.288463-2.4264063 .340800$ H $-0.530468-0.4990241 .512019$ H $1.691249-0.8648624 .002304$ H $4.065315-1.9967463 .659713$ H $-4.001769-1.7443944 .151191$ H -4.984619 -3.159951 3.763284 H -3.286268 -3.714157 1.898828 C $5.332891-0.063458-0.983884$ O $4.219884-0.687317-0.330260$ C $4.326588-2.100532-0.130120$

C $4.486931-2.824020-1.475539$

C $5.667498-2.255937-2.270779$

C $5.619441-0.727177-2.342588$

H $5.003090 \quad 0.965400-1.175013$

C $6.5630580 .007581-0.071891$

H $5.202272-2.3118570 .491882$

O $3.290101-2.687542-2.235728$

H $4.637015-3.893526-1.285370$

H $5.652644-2.661136-3.290093$

O $6.892844-2.653146-1.663009$

H $6.559307-0.341052-2.754210$

O $4.582416-0.364385-3.258403$

C $6.3186280 .957746 \quad 1.076082$

H $6.861071-0.9614490 .338145$

H $7.4316740 .388934-0.621989$

H $3.413726-3.170119-3.070482$

H $6.914105-3.625262-1.652919$

H $4.5761520 .605402-3.329369$

O $5.820957 \quad 0.277518 \quad 2.147242$

O $\quad 6.5217812 .1635901 .016256$

C 5.5188721 .0718293 .298206

H 4.7429381 .8048623 .059485

H 5.1434030 .4070464 .080621

H 6.4221251 .5694133 .663718

SCF Energy (B3LYP/6-31G**//MMFF) $=-3245.92402472$

\section{4}

MM̄FF Geometry

C $2.067503-0.043082-3.524298$

C $3.213153-0.735542-3.429700$

C $4.436355-0.369439-2.623383$

O $4.3071860 .936894-2.014598$

C $4.649191-1.361259-1.463749$

C $5.086532-2.746046-1.888682$
C $4.284327-3.828336-1.922451$

C $6.544457-2.875175-2.246773$

C $2.811866-3.900052-1.622557$

C $2.508134-4.711458-0.356286$

C $2.940628-4.005180 \quad 0.937914$

C $2.684287-4.9023172 .150488$

C $3.012935-4.1870843 .469010$

O $2.180642-2.7977171 .054732$

O $2.455259-4.9608304 .542246$

C $2.302522-2.8180913 .482074$

C $4.523487-4.1184413 .722434$

O $2.691309-2.0537634 .621552$

C $2.518521-1.9925112 .191318$

C $1.578789-0.7639322 .200802$

O $3.875369-1.5488262 .141994$

C $4.7530252 .009675-2.716088$

O $5.2116951 .999344-3.850530$

C $4.6105663 .266862-1.945548$

C $4.2562913 .322981-0.651645$

C $4.1055374 .542747 \quad 0.149129$

C $4.3314715 .860793-0.546547$

C 1.5496610 .0350680 .889297

C 0.5619851 .1869860 .963804

C $-0.3000983 .105116-0.364822$

O $-0.126646 \quad 1.415186 \quad 1.954981$

C 0.4488444 .3100320 .107287

C 0.3017434 .8398291 .330819

C 1.0627736 .0327001 .834699

C 2.0865065 .6534322 .883229

C 1.7313855 .4952524 .169568

C 3.5317955 .5282672 .451070

C 3.7499484 .4279821 .447014

N $0.5380251 .931029-0.207915$

H $1.9564370 .908662-3.014241$

H $3.281470-1.681171-3.964648$

H $5.314539-0.377902-3.280081$

H $3.744024-1.400405-0.847552$

H $5.407204-0.954072-0.779589$

H $4.721630-4.779744-2.223448$

H $\quad 6.821634-3.903967-2.500625$

H $6.785676-2.247759-3.110286$

H $7.171100-2.564604-1.404354$

H $2.349557-2.911116-1.557468$

H $2.328866-4.394448-2.475077$

H $1.423697-4.880173-0.326037$

H $2.987559-5.696058-0.423838$

H $4.005816-3.7582580 .872311$

H $3.251695-5.8379402 .069827$

H $1.624929-5.1952652 .170529$

H $2.841785-5.8523774 .501121$

H $1.225948-3.0109713 .591735$

H $4.950408-5.1275473 .766881$

H $4.740656-3.6590264 .693174$

H $5.063754-3.5663562 .950710$

H $2.527363-2.6021385 .407909$

H $0.554387-1.1032902 .403929$

H $1.864665-0.0864083 .015640$

H $3.930324-0.7470151 .599588$

H $4.8364254 .164209-2.513056$

H $4.0569292 .395495-0.118538$

H $3.6662985 .957944-1.412055$

H $5.3691775 .940680-0.888780$

H 4.1372946 .7306530 .083367

H 2.5370640 .4523020 .668704

H $1.251370-0.6106540 .055856$

H -1.2341592 .9635640 .188818$

H -0.538885 $3.207949-1.426450$

H $1.1440864 .762428-0.594467$

H -0.3993334 .3805702 .024590$

H $0.342316 \quad 6.7443322 .258593$

H 1.5495276 .5714071 .012474

H 2.4612365 .2472694 .932858

H 0.6996305 .6085474 .486275

H 4.1711125 .3075753 .316166

H 3.8720696 .5028982 .091051

H 3.5979773 .4287941 .857878

H $1.2116601 .725524-0.939506$

C $-0.340718-0.675206-3.352798$ 
$\begin{array}{llll}\text { O } & -1.008178 & 0.602394 & -3.263648\end{array}$

C -2.434159 $0.390968-3.379279$

C $-2.641680-1.104087-3.199139$

C $-1.395118-1.660162-3.853471$

H $-2.7007740 .692237-4.400386$

C -3.152723 $1.280169-2.357818$

H -2.666522 -1.369668 -2.134753

H -3.563837 -1.460772 -3.666433

C $-1.112657-3.106194-3.481460$

H $-1.499857-1.579875-4.943813$

C $-4.6394990 .940213-2.165690$

O $-3.0491542 .637915-2.799718$

H -2.623649 $1.225569-1.401017$

H $-4.743230-0.082782-1.790363$

O $-5.2745350 .955958-3.450759$

C $-5.4064291 .907474-1.245586$

C $-4.788793 \quad 2.1542660 .143600$

H -5.518887 2.874609-1.749665

O $-6.7413941 .413455-1.084751$

H -3.8855562 .7653300 .031187$

H -5.5143572 .7461100 .716699$

H - $0.192669-3.460277-3.957042$

H $-0.999123-3.227371-2.398618$

H $-1.933024-3.753975-3.807318$

C $0.870339-0.552246-4.280987$

H -2.114379 $2.809546-3.003966$

H $-6.2131860 .741162-3.312424$

H $-6.6885020 .553416-0.634087$

H $0.6565380 .146054-5.098763$

H $1.102646-1.525468-4.728545$

H $-0.030613-0.942865-2.333643$

C $-5.468370-1.1206431 .838422$

O $\begin{array}{llll}-5.636227 & 0.134224 & 1.163516\end{array}$

C -4.4402430 .8866160 .950046$

C $-3.7860701 .230862 \quad 2.297608$

C $-3.534417-0.030193 \quad 3.129967$

C $-4.778500-0.9245393 .200297$

H -6.489868 -1.473844 2.030661

C $-4.772529-2.1631300 .948300$

H -3.7395250 .2872530 .363183$

O $-4.6091332 .131150 \quad 3.036590$

H -2.837850 1.7460492 .109560

H -3.234246 0.2665654 .142292

O $-2.452131-0.7512522 .548647$

H -4.528552 -1.903276 3.626036

O $-5.733789-0.3192424 .077109$

C $-5.580489-2.492026-0.289860$

H $-3.791358-1.8154740 .614347$

H -4.640922 -3.095850 1.508595

H $-5.483314 \quad 1.717734 \quad 3.140727$

H - $2.277192-1.5237153 .112792$

H -5.318072 -0.2382964 .952431$

O $-4.822679-3.271494-1.117630$

O $-6.726849-2.123731-0.505349$

C $-5.449408-3.655252-2.345398$

H $-6.303068-4.308721-2.142715$

H -5.764404-2.770270 -2.907104

H $-4.717638-4.205802-2.942468$

SCF Energy (B3LYP/6-31G**//MMFF) $=-3245.91381919$

0365

MM̄FF Geometry

C $-0.538762 \quad 4.595341-0.698268$

C $0.656856 \quad 5.140799-0.426849$

C $1.942648 \quad 4.371488-0.204316$

O $1.6888202 .958429-0.030994$

C $2.855567 \quad 4.546723-1.431732$

C $4.1959533 .848939-1.309166$

C $4.6162772 .859388-2.122156$

C $5.0947994 .344430-0.205985$

C $3.888922 \quad 2.253504-3.290476$

C $2.9980161 .052634-2.937751$

C $3.770973-0.181390-2.445726$

C $2.831682-1.379984-2.294321$

C $3.551747-2.608982-1.716881$

O $4.3597420 .123268-1.179797$

O $2.550026-3.551521-1.303197$

C $4.330193-2.191793-0.449449$
C $4.402584-3.318634-2.776680$

O $5.148632-3.259317 \quad 0.020842$

C $5.182647-0.920885-0.642277$

C $5.793857-0.3879450 .676556$

O $6.276086-1.216591-1.520023$

C $1.2715202 .545511 \quad 1.197214$

O $1.1439823 .228156 \quad 2.200883$

C 0.9375841 .1085731 .091690

C $\quad 0.1384290 .5522482 .014009$

C $-0.306807-0.8424842 .044932$

C $0.290764-1.7829741 .032996$

C $4.782896-0.0193711 .768294$

C $4.561526-1.1141332 .799261$

C $3.161191-1.6474194 .790174$

O $5.250904-2.1280102 .866704$

C $2.097479-2.6124864 .371988$

C $0.952055-2.7829625 .049557$

C $-0.101310-3.7854414 .675040$

C $-1.472285-3.1647174 .530225$

C $-2.366148-3.2194505 .532129$

C $-1.835588-2.5610293 .193100$

C -1.209152 -1.210742 2.978517

N $3.523265-0.8025973 .667594$

H $-0.6262123 .517363-0.783856$

H $0.7357256 .223436-0.354726$

H 2.4360414 .7653000 .692592

H $3.0418175 .614702-1.607154$

H $2.3169874 .186229-2.315086$

H $5.6023272 .432932-1.941512$

H $4.677445 \quad 4.094040 \quad 0.774124$

H $6.0965773 .904303-0.257280$

H $5.2121625 .431001-0.270604$

H $3.2815653 .011363-3.797318$

H $4.6346111 .945265-4.034414$

H $2.2692821 .359266-2.178280$

H $2.4321610 .782161-3.837663$

H $4.560727-0.404593-3.172598$

H $2.360890-1.628644-3.253452$

H $2.008945-1.111876-1.617946$

H $2.002273-3.765861-2.077778$

H $3.585429-2.0030410 .331881$

H $3.774500-3.650349-3.612412$

H $4.863369-4.226160-2.371202$

H $5.190692-2.684886-3.188643$

H $4.571060-4.030600 \quad 0.153070$

H $6.556854-1.0832721 .046538$

H 6.3396010 .5342710 .429085

H $6.973130-0.554810-1.377956$

$\begin{array}{llll}\text { H } & 1.334746 & 0.559542 & 0.245976\end{array}$

H -0.2439231 .1814672 .816930$

H $0.032954-1.4692220 .016618$

H $-0.041035-2.8170971 .141960$

H $1.381133-1.7978631 .127986$

H 5.1731040 .8439202 .322939

H $3.8231130 .278012 \quad 1.335610$

H $2.828669-0.9884695 .598483$

H $4.044474-2.1979245 .129797$

H $2.291413-3.2203743 .491476$

H $0.774481-2.1944235 .946997$

H $-0.122088-4.5542285 .458709$

H $0.159157-4.3151353 .750146$

H -3.361981-2.802156 5.425329

H $-2.119348-3.6781106 .483941$

H $-2.923719-2.4274103 .126921$

H $-1.595378-3.2762622 .402008$

H $-1.577962-0.4506743 .668847$

H $2.9695120 .033213 \quad 3.503788$

C -2.9404384 .9328860 .029430$

O $-3.347940 \quad 3.579737-0.262423$

C -3.7644102 .9555310 .969303$

C -3.7278894 .0445042 .042413$

C $-2.636206 \quad 4.9584501 .525160$

H -3.0101362 .1984541 .212001$

C $-5.1443552 .309844 \quad 0.810398$

H -4.6805584 .5839532 .105243$

H -3.512393 3.6364863 .035001

C -2.656089 6.3466862 .138696

H -1.6696344 .4853721 .738103$ 
C $-5.218174 \quad 1.151095-0.205669$

O $\quad-6.066498 \quad 3.3254750 .389926$

H -5.4981801 .9651421 .789998$

H $-5.0651031 .530354-1.223256$

O $-6.5700830 .671599-0.150112$

C $-4.248590-0.0183060 .076716$

C $-4.607135-1.316371-0.680580$

H $-3.2297140 .290703-0.180784$

O $-4.255274-0.3064801 .477975$

H $-5.569337-1.679233-0.302352$

H -4.734504 -1.085876 -1.742309

H $-1.854426 \quad 6.966493 \quad 1.724784$

H -3.6074796 .8554611 .950949$

H -2.513593 6.2900373 .222703

C -1.806916 5.371416-0.901472

H -6.9398892 .9057050 .305497$

H $-6.7375940 .168899-0.965223$

H $-5.162490-0.556636 \quad 1.723601$

H -1.625992 $6.445076-0.774293$

H -2.121755 $5.215274-1.940657$

H $-3.8141875 .563081-0.185326$

C $-2.408852-2.334552-2.615678$

O $-2.344362-2.155871-1.194316$

C $-3.551908-2.428663-0.477554$

C $-4.042709-3.852218-0.777139$

C $-4.179208-4.102309-2.272640$

C $-2.873422-3.750241-2.978847$

H $-3.104428-1.604904-3.047071$

C $-0.999583-2.060303-3.170551$

H -3.279196 -2.4224120 .583028$

O $-5.288032-4.114199-0.139484$

H -3.320922 -4.574129 -0.372550

H $-5.023023-3.547925-2.700108$

O $-4.447719-5.487988-2.496521$

H -2.100862 -4.483297 -2.712906

O $-3.072804-3.828391-4.389586$

C $-0.785322-0.612826-3.551005$

H $-0.223414-2.336248-2.448680$

H $-0.831860-2.637484-4.087396$

H $-5.165080-3.9637310 .813508$

H $-5.256729-5.708463-2.003727$

H -3.388076 -4.726762 -4.588293

O $-1.3057890 .224576-2.612964$

O $-0.180886-0.270734-4.560577$

C -1.221795 $1.619099-2.915819$

H -1.509301 $1.818767-3.952941$

H $-1.9206482 .145381-2.262127$

$\mathrm{H}-0.206756 \quad 1.976307-2.722985$

SCF Energy (B3LYP/6-31G**//MMFF)= -3245.91396789

0366

MM̄FF Geometry

C $-3.419243-3.390333-2.534375$

C $-2.779348-3.138885-1.381868$

C $-1.350525-3.545215-1.116460$

O $-0.587358-2.356877-0.802356$

C -1.293335 $-4.485530 \quad 0.101412$

C $0.088001-5.0485380 .375288$

C $0.808427-4.8080001 .488340$

C $0.643149-5.964800-0.685861$

C $0.435683-3.9641682 .674433$

C $0.910034-2.5048102 .600388$

C $2.436137-2.3360562 .528206$

C $2.825007-0.8654922 .695652$

C $4.337682-0.6466242 .535455$

O $2.882306-2.8001851 .251776$

O $4.564104 \quad 0.7631732 .380934$

C $4.810663-1.3251001 .230944$

C $5.111018-1.0748143 .788175$

O $6.231430-1.2925461 .126133$

C $4.304879-2.7735811 .073882$

C $4.602059-3.378337-0.320101$

O $4.958342-3.6069992 .039226$

C $-0.179282-1.587200-1.848009$

O $-0.373747-1.794848-3.035301$

C $0.542372-0.420688-1.294653$

C $0.7751680 .639790-2.082400$

C $1.4731721 .867295-1.695983$
C $2.1869381 .869287-0.370781$

C $3.995517-2.623095-1.508166$

C $4.949316-1.638017-2.163667$

C $5.0070820 .137765-3.910045$

O $6.147881-1.583053-1.902592$

C $4.9182661 .474168-3.243596$

C $4.5215962 .586536-3.879923$

C $4.4668343 .944104-3.240537$

C $3.1133504 .601625-3.388499$

C $2.8833575 .499245-4.361593$

C $2.0560304 .281740-2.356664$

C $1.4532602 .915785-2.545826$

N $4.307472-0.849724-3.109626$

H -2.878789 -3.925669 -3.313494

H -3.281416 -2.602790 -0.582185

H $-0.914162-4.038509-1.993386$

H -1.977496 -5.331331 -0.049608

H -1.667728 -3.9466180 .978013$

H $1.787814-5.2783781 .571963$

H $1.562283-6.465525-0.362800$

H $-0.083320-6.746199-0.931758$

H $0.878926-5.405060-1.595891$

H $-0.647374-3.9785242 .835076$

H $0.861037-4.4342963 .570412$

H $\quad 0.445559-2.0266971 .730269$

H $0.535294-1.9892823 .493254$

H $2.889367-2.9442393 .319767$

H $2.481978-0.4783963 .663297$

H $2.308483-0.2665551 .933877$

H $4.202250 \quad 1.2124703 .163969$

H $4.422345-0.7215970 .402170$

H $4.772922-0.5028524 .660701$

H $6.180255-0.8588993 .685939$

H $4.993162-2.1344814 .023679$

H $6.506765-0.3631111 .204208$

H $5.681202-3.529335-0.443309$

H $4.165635-4.387751-0.333390$

H $4.909214-4.5283341 .734702$

H $0.850409-0.461833-0.256678$

H $0.4053770 .618105-3.106888$

H $2.8860391 .028153-0.314079$

H $1.470381 \quad 1.785177 \quad 0.451997$

H $2.7794012 .768343-0.191733$

H $3.733364-3.353201-2.284803$

H $3.074191-2.107499-1.220397$

H $4.5450080 .137719-4.902333$

H $6.057260-0.153544-4.013165$

H $5.2184571 .534925-2.200116$

H $4.2497052 .526835-4.931606$

H $5.2369924 .566755-3.714399$

H $4.7366073 .899131-2.177971$

H $1.9239835 .996616-4.457681$

H $3.6488095 .752830-5.087692$

H $1.2343365 .007544-2.423821$

H $2.4771834 .430289-1.358623$

H $0.9089842 .809360-3.485357$

H $3.300766-0.926163-3.222559$

C $-5.639632-2.230834-1.865398$

O $-5.060179-0.928462-1.662701$

C $-6.1130950 .027688-1.445240$

C $-7.415389-0.767920-1.414679$

C -7.079758 -1.956199 -2.293881

H $-6.1176280 .693656-2.316949$

C $-5.8607260 .852521-0.173709$

H -7.661881 -1.100889 -0.399452

H $-8.263483-0.184610-1.786429$

C $-8.023076-3.131633-2.106112$

H -7.105579 -1.634529 -3.343702

C $-4.5860561 .726504-0.253939$

$\begin{array}{llll}\text { O } & -5.785536 & -0.041207 & 0.937112\end{array}$

H $-6.728347 \quad 1.504017-0.011855$

H -4.651953 2.347215 -1.155309

O $-3.4411670 .890352-0.398807$

C -4.4185062 .6538120 .967642$

C -3.3648713 .7705150 .813772$

H -5.3804883 .1516861 .144947$

O -4.1471341 .8776382 .129406$

$\mathrm{H} \quad-3.7404164 .4787760 .064653$ 
H -3.322843 $4.315057 \quad 1.764802$ H -7.743998 -3.965972 -2.756703 H -8.013279 -3.492752 -1.072207 H -9.050254 -2.842632 -2.351447 C -4.827939-3.003805-2.908050 H -5.7000820 .4946641 .743539$ $\begin{array}{llll}H & -3.376611 & 0.343393 & 0.402708\end{array}$ H -4.1791082 .4741382 .896565$ H $-4.758700-2.401089-3.822864$ H -5.358493 -3.927209 -3.168263 H -5.625161 -2.753316 -0.899899 C -1.0435372 .7314242 .580731$ O $-1.3911902 .328366 \quad 1.250792$ C -1.933409 3.3508240 .416153 C -0.9440374 .5204060 .311365$ C -0.5116175 .0318691 .680749$ C -0.0238193 .8775712 .555242$ H -1.9438473 .0523483 .117484$ C $-0.477631 \quad 1.4915433 .282976$ H -2.003395 2.923484 -0.591109 O $-1.5085745 .579215-0.454331$ H $-0.0528654 .179174-0.226274$ H -1.320042 5.5908652 .166389 O $\quad 0.5603995 .9595101 .492465$ H $0.946424 \quad 3.5295762 .178949$ O 0.1808104 .3889843 .871102 C - -1.5712430 .5002893 .602087$ H 0.2771010 .9919072 .664948 H $\quad 0.0052541 .7403524 .233735$ H $-0.8522716 .296426-0.486276$ H $\quad 0.849654 \quad 6.2492582 .374804$ H $\quad 0.7028313 .7369414 .367428$ O $-1.769763-0.3383832 .546028$ $\begin{array}{lllll}0 & -2.186091 & 0.487235 & 4.660834\end{array}$ C $-2.785833-1.3290022 .728959$ H -2.930272 -1.8463661 .777859$ H -2.465974 -2.055592 3.481246 H -3.732852 -0.864605 3.019725

SCF Energy (B3LYP/6-31G**//MMFF) $=-3245.90210480$

\section{7}

MMFF Geometry

C $-0.005928 \quad 5.5720870 .335933$

C $1.0866214 .940918-0.119387$

C 1.8983453 .9626210 .689801

O $1.9731222 .721867-0.051170$

C 3.3289204 .5017260 .865966

C 4.2381723 .5823131 .657674

C 5.3508213 .0027471 .165319

C 3.8453633 .3487693 .093607

C $5.9266773 .135110-0.217208$

C $5.4421232 .073465-1.216837$

C $5.8857380 .642954-0.872011$

C $5.564915-0.317928-2.019006$

C $5.938051-1.767802-1.672478$

O 5.1931620 .2193720 .305196

O $5.319551-2.605243-2.661396$

C $5.334305-2.130088-0.296684$

C $7.448116-2.002488-1.796285$

O $5.786719-3.412303 \quad 0.129780$

C 5.594639-1.068259 0.792793

C $4.816583-1.3266312 .105990$

O $6.988537-1.0782851 .127694$

C $0.906661 \quad 1.880960 \quad 0.029421$

O $-0.1021042 .033387 \quad 0.699332$

C $1.1650400 .744839-0.882547$

C $0.141290-0.032885-1.263811$

C $0.206466-1.166298-2.187844$

C $1.567564-1.600393-2.663350$

C $3.293135-1.4221311 .965396$

C $2.784715-2.8463901 .812442$

C $0.718851-4.1306111 .284618$

O $3.489622-3.8358731 .992734$

C $0.616574-4.406690-0.182432$

C $-0.534003-4.740016-0.785215$

C $-0.668790-5.041485-2.248208$

C -1.666376 -4.134654 -2.932548

C $-2.958847-4.485155-3.044205$
C $-1.143926-2.865273-3.563606$

C $-0.947179-1.756681-2.565353$

N $1.436478-2.8853291 .485751$

H -0.3342125 .4026791 .357490$

H $1.4091205 .111886-1.144330$

H 1.4402663 .7813551 .669851

H 3.2978105 .4739111 .375801

H $3.7518894 .695436-0.125627$

H 5.9300022 .3619391 .829341

H 4.6129882 .8000553 .649994

H 3.6919214 .3041193 .606022

H 2.9205362 .7666913 .151681

H $5.7207564 .129921-0.626634$

H $7.0201583 .084466-0.136837$

H $4.3488912 .117412-1.284404$

H $5.8380002 .341645-2.204363$

H $6.9647390 .657585-0.678012$

H $6.065550-0.004644-2.943704$

H $4.488312-0.278017-2.234589$

H $5.481964-3.530127-2.407326$

H $4.253752-2.225070-0.452832$

H $7.785460-1.779762-2.815767$

H $7.702457-3.053947-1.622840$

H $8.035024-1.387982-1.110438$

H $6.692144-3.309580 \quad 0.469213$

H $5.229450-2.2026532 .620654$

H $5.019545-0.4768052 .773779$

H $7.096920-0.6708002 .003253$

H $2.1784370 .600888-1.238204$

H $-0.8527330 .200078-0.885484$

H $2.231318-1.782615-1.811219$

H $2.016809-0.830567-3.299926$

H $1.552558-2.530383-3.235691$

H $2.835286-1.0336122 .883935$

H $2.933123-0.8081181 .133946$

H $-0.262267-4.0188481 .755988$

H $1.251940-4.9493361 .778011$

H $1.528624-4.344275-0.771251$

H -1.435802 -4.834523 -0.186262

H -0.989199 -6.086865 -2.347715

H $\quad 0.299041-4.982689-2.761292$

H -3.674393 -3.851434 -3.557430

H -3.330902 -5.410084 -2.616062

H -1.859385 -2.500640 -4.313474

H $-0.239761-3.088819-4.137113$

H -1.883302 -1.374935 -2.155200

H $0.938314-2.0162361 .314951$

C $-2.1951975 .951654-0.899509$

O $-2.0438104 .682402-1.567572$

C $-3.0161613 .755618-1.024775$

C $-4.0560234 .633277-0.346080$

C -3.181266 $5.722953 \quad 0.244681$

H $-2.4683573 .161190-0.284356$

C -3.514411 $2.856542-2.159669$

H -4.748681 $5.057986-1.083288$

H $-4.639711 \quad 4.1091670 .414009$

C -3.9490826 .9677620 .653778$

H -2.6716245 .3159851 .126505$

C $-4.6356261 .851162-1.834332$

O $-2.3998542 .107285-2.659248$

H $-3.8494653 .497683-2.985273$

H $-4.8227501 .270997-2.747927$

O $-5.8458022 .536586-1.524943$

C $-4.3187890 .836395-0.715152$

C $-5.384403-0.276069-0.644717$

H $-3.3418520 .380833-0.904855$

$\begin{array}{lllll}\text { O } & -4.242315 & 1.488688 & 0.548127\end{array}$

H $-6.3795750 .167837-0.519140$

H -5.375334 -0.808032 -1.603948

H -3.2712017 .7290221 .052664$

H $-4.4872977 .405145-0.193856$

H -4.6835986 .7285911 .429864$

C $-0.8235016 .509938-0.509602$

H -1.693185 2.741561 -2.870103

H $-6.0548323 .117405-2.276157$

H $\quad-5.118464 \quad 1.8667370 .735738$

H -0.9411727 .4592090 .025219$

H $-0.2722586 .736684-1.431492$ 
H -2.639618 $6.630844-1.638965$

C $-3.457524-2.7510381 .388819$

O $-3.858420-1.8414830 .355210$

C $-5.157842-1.2655510 .508708$

C $-6.230654-2.3658200 .537905$

C $-5.924594-3.4135711 .612166$

C $-4.471974-3.8976661 .537075$

H -2.519398 -3.184352 1.026325

C -3.152996 -2.030399 2.712045

H $-5.195415-0.7047121 .448310$

O $-6.322656-3.006513-0.733520$

H $-7.205600-1.9059400 .737013$

H $-6.604681-4.2643401 .483155$

O $-6.179842-2.8415422 .891553$

H -4.217433 -4.498145 2.418147

O $-4.325134-4.7483790 .396186$

C $-1.929793-1.1433782 .610198$

H $-3.983799-1.4012153 .040173$

H -2.946462 -2.777106 3.487703

H $-5.439620-3.345650-0.960186$

H $-6.007379-3.5269843 .559230$

H $-4.931137-5.5001310 .511041$

O $-1.650314-0.6288853 .844466$

$\begin{array}{lllll}0 & -1.299981 & -0.923041 & 1.585727\end{array}$

C $-0.511616 \quad 0.236058 \quad 3.906593$

H -0.7161571 .1606113 .359486$

H -0.3285530 .4820034 .955919$

H $\quad 0.375161-0.263708 \quad 3.505178$

SCF Energy (B3LYP/6-31G**//MMFF) $=-3245.92084386$

03_68

MM̄FF Geometry

C $-4.342076-3.5345832 .047627$

C $-5.005140-2.4115721 .730302$

C $-5.630404-2.0492130 .405719$

O $-4.937064-0.859677-0.047099$

C $-5.494844-3.106937-0.708798$

C $-6.065008-2.654655-2.041196$

C $-5.327616-2.452293-3.151941$

C -7.555928 -2.441569-2.077165

C $-3.846767-2.662274-3.320231$

C $-3.038484-1.442872-2.877113$

C $-1.542359-1.526043-3.208773$

C $-0.842849-2.686599-2.495560$

C $0.673435-2.683178-2.740223$

O $-0.967669-0.278563-2.789702$

O $1.251712-3.598046-1.796146$

C $1.223528-1.275798-2.434919$

C $1.020839-3.226455-4.130775$

O $2.599342-1.184260-2.785797$

C $0.424690-0.135741-3.100241$

C $0.877511 \quad 1.262657-2.616626$

O $0.638847-0.178693-4.516193$

C $-5.482786 \quad 0.359721 \quad 0.197218$

O

C $-4.5209861 .362922-0.313086$

C $-4.7433672 .674555-0.143627$

C $-3.844213 \quad 3.752154-0.569324$

C $-2.5809363 .350300-1.285207$

C $0.8211001 .465626-1.101299$

C $0.9863982 .920652-0.696896$

C 0.9154034 .3701121 .338056

O $1.2036453 .824626-1.498389$

C $-0.4779734 .808787 \quad 1.664823$

C -0.9614736 .0313891 .394688$

C $-2.3450626 .474778 \quad 1.781080$

C -3.114102 7.1083220 .640107

C $-3.541314 \quad 8.3796840 .726500$

C -3.399805 $6.282480-0.597832$

C $-4.1797595 .029443-0.291670$

N 0.8608293 .0799400 .676103

H -4.234367 -4.338316 1.326167

H -5.082022 -1.635649 2.491250

H -6.691679 -1.833385 0.577260

H $-6.003682-4.032346-0.409752$

H $-4.436180-3.361900-0.825789$

H -5.836010 -2.110128 -4.052394

H -7.921675 -2.240686 -3.089863
H $-8.075608-3.334327-1.713930$

H -7.841188 -1.591206-1.450616

H -3.529750 -3.562039 -2.783508

H $-3.652934-2.866525-4.380683$

H -3.448796 -0.534829 -3.339504

H $-3.147016-1.293416-1.797398$

H $-1.437287-1.618253-4.296124$

H -1.263503 -3.652913 -2.796400

H -1.020844 -2.605834 -1.413675

H $2.219132-3.531266-1.875946$

H $1.177642-1.162169-1.345288$

H $0.649086-4.252157-4.242522$

H $2.105464-3.286609-4.274047$

H $\quad 0.597902-2.629897-4.941767$

H $2.653074-1.082648-3.751480$

H $1.892121 \quad 1.476385-2.977860$

H $\quad 0.2320872 .012724-3.095008$

H $0.4374800 .698142-4.883624$

H $-3.6295850 .986493-0.803047$

H -5.6528732 .9895720 .365555$

H $-2.8157242 .796153-2.201063$

H -1.969835 $2.714851-0.637276$

H -1.952454 $4.189647-1.585791$

H $-0.1387731 .112031-0.709208$

H $1.6228690 .898333-0.616955$

H 1.4409415 .0922520 .705227

H 1.4828414 .2335892 .263373

H -1.1133734 .0909982 .179189$

H -0.3206996 .7635240 .909069$

H -2.238908 7.1899772 .607146

H -2.9283305 .6380942 .185020$

H -4.094939 8.847476-0.080901

H -3.3474238 .9841751 .606399$

H -2.450681 $6.083073-1.100346$

H -3.992841 $6.864986-1.314881$

H -5.1262295 .2163510 .217569$

H 0.6450082 .2676861 .247354

C $-2.149128-3.8783473 .218694$

O $-1.603795-2.7091212 .572565$

C $-0.483958-2.2252233 .338248$

C $-0.084371-3.3764114 .248320$

C $-1.429505-3.9969784 .563313$

H $-0.868753-1.3822093 .925707$

C $0.629461-1.7565592 .390563$

H $0.552169-4.092583 \quad 3.713752$

H $0.450579-3.0421165 .142139$

C -1.337749 -5.4191565 .088714$

H -1.930897 -3.372824 5.315324

C $1.863304-1.2232853 .138845$

O $0.112263-0.7071751 .567729$

H $0.908212-2.5766541 .719682$

H $2.281650-2.0070873 .780435$

O $1.445016-0.1720644 .021240$

C $2.986234-0.6622362 .246222$

C $3.577663-1.7089011 .284574$

H $2.6392390 .217066 \quad 1.691768$

O $4.036878-0.2138963 .112447$

H $3.891085-2.5866081 .863198$

H $2.801906-2.0240660 .578025$

H $-2.334369-5.826195 \quad 5.286745$

H $-0.839645-6.0804084 .371705$

H $-0.768587-5.4479096 .023612$

C $-3.666686-3.7328413 .376406$

H $-0.668280-1.0593161 .106112$

H 1.0380290 .5226533 .475281

H $3.6537890 .447836 \quad 3.713534$

H -3.886135 -2.882869 4.034781

H $-4.082800-4.6272913 .853465$

H -1.914261 -4.733031 2.571501

C $5.3551450 .528124-1.114827$

O $4.333292-0.162390-0.383927$

C $4.797346-1.1780930 .509165$

C $5.519659-2.278020-0.279435$

C $6.677663-1.685826-1.092754$

C $6.248172-0.453070-1.897030$

H $4.8106451 .141638-1.844407$

C $6.1452711 .477062-0.203666$

H $5.501557-0.741973 \quad 1.224884$ 
O $4.596958-2.927312-1.149711$ H $5.897164-3.029757 \quad 0.423910$ H $7.068252-2.444110-1.782417$ O $7.735496-1.312529-0.213807$ H $7.130597 \quad 0.078883-2.270619$ O $5.529595-0.895797-3.051635$ C $5.222138 \quad 2.463642 \quad 0.477699$ H 6.6977940 .9259490 .562748 H $6.8784892 .052157-0.779858$ H $5.079950-3.628687-1.619130$ H $8.018340-2.1120350 .261752$ H $4.716185-1.331030-2.743597$ O $5.5159612 .527726 \quad 1.808031$ O $4.349625 \quad 3.101133-0.097194$ C 4.6837703 .3962702 .582623 H 3.6655692 .9983412 .618919 H 5.0827983 .4312073 .599796 H 4.6904004 .4089622 .168539 SCF Energy (B3LYP/6-31G**/MMFF) $=-3245.93779572$

0369

MM̄FF Geometry

C $3.713711-2.499219-1.822932$

C $3.173071-2.911918-0.665713$

C $1.695677-2.942128-0.356539$

O $1.042484-1.952132-1.185077$

C $1.086072-4.328037-0.622285$

C $-0.235380-4.5524520 .090479$

C $-1.439550-4.658639-0.505334$

C $-0.137587-4.7098881 .588759$

C $-1.776898-4.593989-1.967325$

C $-2.392175-3.265400-2.431965$

C $-3.743903-2.910932-1.787929$

C $-4.374962-1.715265-2.509312$

C $-5.682944-1.257546-1.846299$

O $-3.533570-2.580479-0.410545$

O $-6.008891 \quad 0.042126-2.364124$

C $-5.447572-1.090511-0.329922$

C $-6.856358-2.174692-2.210503$

O $-6.671938-0.8230180 .348100$

C $-4.738999-2.297720 \quad 0.318218$

C $-4.353479-2.0576101 .800706$

O $-5.622930-3.426146 \quad 0.302573$

C $0.174827-1.091677-0.595420$

O $-0.247707-1.1358790 .547366$

C $-0.166889-0.054899-1.594188$

C $-1.2460160 .714616-1.394850$

C -1.724607 $1.781322-2.273149$

C $-0.8100172 .243695-3.375722$

C -3.439249 -0.8576062 .061735$

$\begin{array}{llll}\text { C } & -4.182383 & 0.430456 & 2.378861\end{array}$

C -3.858716 2.9055232 .396066

O $-5.335320 \quad 0.4542632 .800942$

C $-4.453996 \quad 3.4201341 .124085$

C $-3.918877 \quad 4.4137350 .398438$

C $-4.5171424 .928558-0.879819$

C $-3.6050084 .731984-2.071479$

C $-2.8373995 .736479-2.526483$

C -3.647946 $3.400606-2.788945$

C -2.941956 2.310379-2.032399

N $-3.386745 \quad 1.5502182 .180469$

H $3.067577-2.249717-2.661073$

H $3.831430-3.1692820 .160978$

H $1.602404-2.6609720 .698983$

H $1.776535-5.113449-0.286321$

H $0.976796-4.467742-1.702521$

H $-2.302526-4.8429870 .134161$ H $-1.096649-4.9817642 .043077$ H $0.577401-5.4998391 .841334$ H $0.191711-3.7791512 .058204$ H $-0.895584-4.799422-2.582609$ H -2.477810 -5.409187 -2.187963 H $-1.674037-2.462143-2.243671$ H -2.522972 -3.328649 -3.519705 H $-4.398979-3.787323-1.857984$ H $-4.544425-1.945598-3.568593$ H $-3.670290-0.873289-2.498417$ H $-6.078682-0.029533-3.331567$
H $-4.818142-0.200806-0.205695$

H $-7.016397-2.178453-3.295457$

H $-7.792702-1.812258-1.772050$

H $-6.705105-3.209796-1.897569$

H -7.072631-0.043923 -0.074051

H -5.257264 -2.014929 2.420819

H $-3.808278-2.9497642 .141200$

H $-5.323873-4.0605940 .975062$

H $\quad 0.4687920 .037324-2.467378$

H $-1.8534940 .536691-0.510314$

H $-0.7029991 .464181-4.137828$

H $0.1827242 .484745-2.979040$

H $-1.1630063 .146338-3.879679$

H -2.822206 -1.071577 2.943643

H -2.760062 -0.7032101 .220576$

H -3.0010213 .5017452 .722548$

H $-4.6113482 .913727 \quad 3.190815$

H -5.3741042 .9492910 .785585$

H $-3.002250 \quad 4.8892840 .740110$

H $-4.7316835 .996491-0.741632$

H $-5.4881794 .460486-1.084373$

H $-2.202373 \quad 5.612988-3.397402$

H -2.822652 $6.703254-2.034190$

H $-3.254042 \quad 3.496492-3.806671$

H $-4.694767 \quad 3.101612-2.930133$

H $-3.5279371 .897729-1.211418$

H $-2.4706721 .436647 \quad 1.757205$

C $5.392196-0.807861-2.514560$

O $4.7304820 .122696-1.639477$

C $5.4790501 .351451-1.606277$

C $6.6578311 .162567-2.559837$

C $6.844272-0.345945-2.541528$

H $5.8697311 .446712-0.586390$

C $4.5440392 .533362-1.907777$

H $6.4278781 .498738-3.576054$

H $7.5467331 .707371-2.226712$

C $7.644318-0.877113-3.717031$

H $7.345410-0.624727-1.604829$

C $3.2466752 .535998-1.067522$

O $4.1263852 .477252-3.278251$

H $5.0798943 .481381-1.779591$

H $2.6070791 .702248-1.381067$

O $2.517063 \quad 3.727510-1.390511$

C 3.4594852 .4740770 .455637

C 2.1263952 .5126551 .226290

H 3.9935211 .5553850 .704563

$\begin{array}{lllll}\text { O } & 4.280566 & 3.573087 & 0.844475\end{array}$

H 1.6146493 .4588421 .012757

H $1.485977 \quad 1.7064240 .852154$

H $7.741868-1.965964-3.661927$

H $7.168132-0.629378-4.671556$

H $8.651520-0.447783-3.722046$

C $5.182933-2.238560-2.010441$

H $4.8392902 .855737-3.819642$

H $3.0389064 .484461-1.073247$

H $4.5961843 .400782 \quad 1.747407$

H $5.712272-2.377822-1.060210$

H $5.593200-2.956424-2.728491$

H $4.933013-0.693566-3.506250$

C $2.439263-0.0159072 .953937$

O $3.074958 \quad 1.254706 \quad 3.140045$

C 2.2910352 .3905182 .758669

C 0.9668282 .4119113 .537479

C 0.2105491 .0923503 .409436

C $1.129068-0.0787443 .750441$

H $2.243854-0.1786351 .887126$

C $3.412478-1.1016523 .443906$

H 2.8574513 .2720973 .083322

$\begin{array}{lllll}H & 0.155402 & 3.491938 & 3.088614\end{array}$

H 1.1854212 .5920144 .598320

H -0.2246990 .9719512 .411237$

$\begin{array}{lllll}\text { O } & -0.879357 & 1.077718 & 4.333676\end{array}$

H $1.343085-0.0697254 .827037$

O $0.457080-1.3022903 .462610$

C $4.615465-1.2762402 .541893$

H $3.784307-0.8376684 .441306$

H $2.907494-2.0728793 .489170$

H -0.6109253 .5528043 .683280$ 
H -1.5222111 .7449984 .040307$ H $-0.383373-1.2953153 .951841$ O $5.456326-2.196433 \quad 3.102311$ O $4.797670-0.6990521 .480204$ C $6.642192-2.4813522 .353395$ H $6.380619-2.9460121 .398258$ H $7.222129-1.5672792 .193283$ H $7.248408-3.1841472 .931225$ SCF Energy (B3LYP/6-31G**//MMFF)= -3245.90321605

\section{7}

MM̄FF Geometry

C $1.627192-4.029773-0.337987$

C $1.059097-4.003158 \quad 0.878100$

C -0.346965 -3.544675 1.188755

O $-0.793658-2.5671510 .222269$

C $-1.316469-4.7391231 .157896$

C $-2.697341-4.4108311 .692210$

C $-3.826458-4.4066350 .956979$

C $-2.769990-4.1026673 .166599$

C $-3.988209-4.710646-0.505217$

C $-3.922406-3.486359-1.431793$

C $-4.975146-2.405489-1.134144$

C $-5.012055-1.363864-2.255307$

C $-5.982756-0.213241-1.943703$

O $-4.629714-1.7646340 .097591$

O $-5.710187 \quad 0.855084-2.864061$

C $-5.6896210 .326947-0.526535$

C -7.442239-0.621990 - 2.176404

O $-6.6726841 .280335-0.132501$

C $-5.574854-0.7806760 .540355$

C $-5.089222-0.2598021 .915834$

O $-6.865547-1.3672450 .750386$

C $-0.388683-1.2834680 .422772$

O $0.274025-0.8611451 .356625$

C $-0.872224-0.468656-0.711841$

C $-0.3118450 .728882-0.934601$

C $-0.6703221 .672856-1.993960$

C -1.933606 $1.402462-2.765791$

C $-3.718944 \quad 0.426414 \quad 1.916749$

C -3.7842561 .9413581 .812948$

C -2.308977 3.9366031 .586852

O -4.8305872 .5791901 .892601$

C $-2.292708 \quad 4.3712280 .155471$

C $-1.3130755 .118370-0.376059$

C $-1.3069405 .604765-1.797639$

C -0.050756 $5.211128-2.542223$

C $0.9761916 .068171-2.669175$

C $-0.0184573 .849304-3.201366$

C $0.1309852 .738257-2.200430$

N -2.5235682 .5035241 .658168$

H $1.045349-3.740916-1.209282$

H $1.642410-4.3316621 .735630$ H $-0.342028-3.0899052 .186598$ H $-0.912520-5.5664241 .756962$ H $-1.379823-5.1133380 .130350$ H -4.760548 -4.1590811 .460822$ H -3.802471 -4.0275633 .525017$ H $-2.282139-4.8938463 .745255$ H -2.277726 -3.1511233 .388621$ H $-3.246467-5.446036-0.834256$ H $-4.958330-5.206720-0.639158$ H -2.918989-3.050650-1.367835 H $-4.052877-3.843420-2.460926$ H $-5.952396-2.892299-1.033691$ H $-5.269692-1.832251-3.213474$ H $-4.007354-0.942084-2.391326$ H $-5.8160230 .507739-3.766336$ H $-4.7358960 .865400-0.583347$ H -7.594031 -0.920997 -3.220581 H -8.121012 $0.220942-2.005898$ H -7.760298 -1.455281-1.546554 H -6.695733 $1.972124-0.815621$ H $-5.859354 \quad 0.3735062 .372287$ H -5.003349 -1.133472 2.578486 H -6.867726 -1.804689 1.617937 H $-1.661894-0.875460-1.331374$ H $\quad 0.498297 \quad 1.050409-0.282087$
H -2.783083 $1.303589-2.081630$

H $-1.8402000 .478845-3.347261$

H -2.197232 2.195513 -3.467891

H -3.2228850 .2083782 .871386$

H -3.0825300 .0326801 .119994$

H -1.3641664 .1493082 .095268$

H -3.114676 4.4527892 .118818

H $-3.1390094 .083498-0.464094$

H $-0.4810195 .427247 \quad 0.253071$

H -1.399825 $6.698608-1.775646$

H $-2.1851015 .245819-2.348654$

H $1.8709805 .801086-3.221468$

H $0.9470567 .054392-2.217795$

H $0.8344453 .783601-3.889730$

H $-0.9054593 .744761-3.831231$

H $1.0266322 .834821-1.587294$

H -1.7150451 .8963941 .558468$

C $3.722538-3.660360-1.687408$

O $3.775200-2.260930-1.353835$

C $5.000782-1.706650-1.872699$

C $5.704917-2.832242-2.628008$

C $5.175201-4.065926-1.926549$

H $5.610425-1.430224-1.004862$

C $4.713247-0.467394-2.729681$

H $5.428766-2.848821-3.688622$

H $6.794593-2.743379-2.574558$

C $5.344618-5.341019-2.733944$

H $5.698213-4.173084-0.966623$

C $3.9134340 .638390-2.014630$

O $3.950117-0.875259-3.873952$

H $5.657302-0.065522-3.116994$

H $2.8895430 .291639-1.831588$

O $3.7939381 .739360-2.926899$

C $4.5292041 .140968-0.695132$

C $3.7544992 .346626-0.134387$

$\begin{array}{llll}\mathrm{H} & 4.532118 & 0.322863 & 0.029976\end{array}$

O $5.8843521 .517261-0.931828$

H $3.9004933 .209056-0.796104$

H $2.6889012 .104172-0.146494$

H $4.952882-6.205071-2.188554$

H $4.819049-5.281468-3.692951$

H $6.403596-5.526571-2.941700$

C $3.048979-4.460357-0.569576$

H $3.777909-0.079300-4.405871$

H $4.6850512 .105204-3.061885$

H $6.3061621 .661945-0.068269$

H $3.623501-4.347154 \quad 0.358065$

H $3.045871-5.523472-0.835198$

H $3.140896-3.746703-2.615277$

C $2.799077 \quad 1.1865622 .508762$

$\begin{array}{llll}\text { O } & 4.118846 & 1.660510 & 2.214655\end{array}$

C $4.1738262 .755452 \quad 1.294649$

C 3.3812103 .9464791 .854772

C 1.9478103 .5547612 .210299

C $1.9374282 .310333 \quad 3.097942$

H 2.3354950 .8044851 .593026

C $2.9372230 .024566 \quad 3.504973$

H 5.2252303 .0636381 .244244

$\begin{array}{lllll}\text { O } & 3.382035 & 5.014084 & 0.913042\end{array}$

H 3.8838614 .3123132 .759577

H $1.3356173 .400561 \quad 1.313775$

$\begin{array}{lllll}\text { O } & 1.333169 & 4.619395 & 2.939456\end{array}$

H 2.2982692 .5746934 .100365

$\begin{array}{lllll}\text { O } & 0.594976 & 1.847602 & 3.235378\end{array}$

C $3.528320-1.2140632 .865046$

H $3.595596 \quad 0.3313844 .326921$

H $1.963595-0.2640943 .915080$

H 2.9761945 .7864861 .341153

H 1.2274175 .3693262 .330191

H 0.0690932 .5839873 .591940

O $4.078101-2.0019703 .835653$

O $3.485586-1.4773431 .671486$

C $4.669339-3.2217103 .376299$

H $3.894448-3.8913272 .992741$

H $5.421303-3.0196042 .607121$

H $5.160029-3.7026744 .226711$

SCF Energy (B3LYP/6-31G*//MMFF)= -3245.91042004 
03_70

MM̄FF Geometry

C $3.071414-0.9075003 .424588$

C $2.292198-1.5239482 .522491$

C $0.810681-1.7390012 .701428$

O $0.106354-1.0827081 .623423$

C $0.501952-3.2463712 .622193$

C $-0.948929-3.5939882 .892324$

C $-1.781048-4.1642671 .998649$

C -1.444262 -3.294471 4.284478

C $-1.488610-4.574090 \quad 0.582374$

C $-1.843699-3.516943-0.475032$

C $-3.341853-3.183782-0.556269$

C $-3.634471-2.301793-1.772244$

C $-5.108896-1.869619-1.828848$

O $-3.713988-2.4869620 .634648$

O $-5.218370-0.802031-2.783131$

C $-5.522015-1.291849-0.456904$

C $-6.012973-2.999099-2.337015$

O $-6.923412-1.037497-0.412001$

C $-5.111228-2.1820490 .732989$

C $-5.334603-1.5079202 .108699$

O $-5.900261-3.3781910 .720240$

C $-0.158586 \quad 0.244171 \quad 1.769188$

O $\quad 0.165051 \quad 0.9623932 .701541$

C $-0.912306 \quad 0.6835590 .574174$

C $-1.039085 \quad 1.9942970 .318737$

C $-1.7112992 .584462-0.840828$

C $-2.5567241 .675076-1.691652$

C $-4.573146-0.1959352 .329983$

C -5.3926341 .0490872 .033590$

C -5.1719233 .5260111 .886236$

O -6.5997661 .0263391 .809867$

C -5.0408153 .9069940 .445618$

C -4.4917495 .0611380 .038067$

C $-4.3895395 .477496-1.400822$

C $-2.9758795 .830997-1.803865$

C $-2.5678227 .110709-1.844188$

C -2.064895 $4.707248-2.242305$

C $-1.5408963 .900740-1.085361$

N -4.6182742 .2004732 .087319$

H $2.629020-0.5150824 .336416$

H $2.739934-1.9110721 .609816$

H $0.471659-1.3377683 .664147$

H $1.118939-3.7901993 .350245$

H $\quad 0.806137-3.612767 \quad 1.635943$

H -2.801032 -4.376837 2.317857

H -2.441201 -3.7091784 .468950$

H - $0.769070-3.7273595 .029817$

H -1.503978 -2.2144734 .449746$

H $-0.435514-4.8511030 .468096$

H -2.047971 -5.4948840 .373190$

H -1.274792 -2.603480 -0.266266

H -1.506922 $-3.893161-1.449029$

H $-3.901943-4.123878-0.622532$

H $-3.348783-2.811140-2.700993$

H -3.009959-1.399624-1.723885

H -4.893703 -1.130440 -3.639104

H -5.025564 -0.319421 -0.360187

H -5.707492 -3.310369 -3.343159

H $-7.051351-2.662548-2.431055$

H -5.992808 -3.884677 -1.698415

H -7.136263 -0.454078-1.160512

H $-6.407779-1.3929442 .302619$

H -4.976711 -2.211513 2.874487

H $-5.894746-3.7612261 .613025$

H -1.314556 -0.081824 -0.079117

H -0.5735942 .7052541 .000230$

H -1.928286 $0.965604-2.240450$

H $-3.1687182 .206124-2.423565$

H $-3.2589391 .113350-1.066846$

H $-4.296900-0.1315353 .390400$

H -3.645886 -0.172314 1.748794

H -4.6286904 .2086142 .547205$

H $-6.228353 \quad 3.5276882 .173536$

H $-5.4377313 .215128-0.293448$

H -4.1204905 .7634840 .781305$

H $-5.0464976 .345599-1.543673$
H $-4.7768634 .701264-2.072279$

H -1.563884 $7.374711-2.159499$

H $-3.2276797 .922747-1.557123$

H -1.192614 5.116710 -2.769723

H $-2.5816784 .100220-2.990620$

H $-0.9084184 .474986-0.406604$

H -3.6146262 .1206052 .222841$

C 5.0146880 .6164762 .784431

O $4.486477 \quad 0.869707 \quad 1.469615$

C 4.1274612 .2638861 .380792

C 4.7519342 .9346012 .598851

C 4.6290791 .8263263 .632559

H 3.0381482 .3138431 .495741

C 4.5245342 .8197840 .006635

H 5.8066993 .1818912 .437608

H 4.2265353 .8507662 .884600

C 5.5060642 .0284764 .854874

H 3.5804621 .7635793 .949280

C $3.9651571 .980014-1.164557$

O $5.9507352 .803631-0.121085$

H $4.2078903 .864841-0.090656$

H $4.4297080 .991272-1.136365$

O $4.3810842 .572323-2.399151$

C $2.4340931 .842814-1.162786$

C $1.8503380 .965535-2.284451$

H $2.1040631 .413315-0.212633$

O $1.8439763 .141339-1.246835$

H $\quad 0.7580381 .046104-2.222824$

H $2.1289261 .376997-3.262682$

H 5.3946941 .1946885 .555105

H 6.5644142 .1020004 .582933

H 5.2293382 .9493305 .378360

C $4.559090-0.7587653 .271606$

H 6.3024103 .5450750 .399472

H $4.0832083 .497551-2.401917$

H $2.0026533 .483869-2.142644$

H $5.030357-0.9875574 .234527$

H $4.920550-1.5154172 .563249$

H 6.1063760 .5850402 .670179

C $4.075190-1.980458-2.880622$

O $3.496643-0.668088-2.894161$

C $2.274371-0.509594-2.164886$

C $1.198159-1.459076-2.709948$

C $1.692414-2.908983-2.695842$

C $3.066689-3.041245-3.356840$

H $4.880196-1.939512-3.625910$

C $4.708197-2.305933-1.517804$

H $2.447399-0.741997-1.108396$

O $0.849918-1.073592-4.037205$

H $\quad 0.297322-1.362629-2.091757$

H $0.972968-3.546878-3.223116$

O $1.786483-3.376003-1.353047$

H $3.482023-4.040978-3.183279$

O $2.921355-2.890847-4.771968$

C $5.946968-1.475361-1.249413$

H $4.010292-2.126996-0.696012$

H $5.019634-3.356654-1.497159$

H $\quad 0.155995-1.680217-4.346479$

H $0.916548-3.258354-0.937126$

H $2.322737-3.591779-5.081354$

O $6.374285-1.7176340 .025423$

O $6.482175-0.721868-2.050675$

C $7.548042-1.0030060 .424625$

H $8.401432-1.308094-0.188142$

H 7.3821690 .0758650 .347059

H $7.758147-1.2510601 .468374$

SCF Energy (B3LYP/6-31G**//MMFF)= -3245.88613007

03 71

MM̄FF Geometry

C 4.3623473 .1818041 .798482

C 3.6572402 .3884182 .620637

C 3.9663920 .9676443 .023505

O 2.8419810 .1555952 .596248

C 5.2309890 .3484502 .393666

C $5.491342-1.0750432 .853804$

C $5.447335-2.1621562 .057799$

C $5.835962-1.2337814 .313188$ 
C $5.183940-2.2288840 .578446$

C $3.731551-2.6019210 .277656$

C $3.484210-2.992463-1.187654$

C $3.754347-1.842206-2.160781$

C $3.392272-2.214853-3.607615$

O $2.110622-3.399218-1.279680$

O $3.367115-1.004406-4.380366$

C $1.962693-2.793692-3.631114$

C $4.452919-3.116436-4.249694$

O $1.627846-3.286762-4.925481$

C $1.725155-3.892873-2.572449$

C $0.241031-4.327579-2.484088$

O $2.480824-5.058913-2.920010$

C $1.889020-0.1658593 .511298$

$\begin{array}{llllll}\text { O } & 1.826704 & 0.199596 & 4.673501\end{array}$

C $0.929593-1.0742052 .842588$

C $-0.062508-1.6438293 .543221$

C $-1.044288-2.5989833 .020220$

C $-0.894059-3.0248381 .583727$

C $-0.739294-3.179456-2.246437$

C $-2.123106-3.639382-1.818627$

C $-4.335940-2.663575-1.162730$

O $-2.413036-4.812436-1.605523$

C $-4.323975-2.2142930 .264896$

C $-4.926461-2.8715131 .266973$

C $-4.906507-2.3938472 .692438$

C $-4.483800-3.4696813 .670548$

C $-5.326298-3.8965654 .626740$

C $-3.094359-4.058114 \quad 3.545247$

C -2.010893 -3.0561353 .844185$

N $-2.986573-2.560317-1.686639$

H $\begin{array}{llll}5.285600 & 2.832228 & 1.347139\end{array}$

H 2.7326662 .7849683 .038351

H 4.0597610 .9481294 .115849

H 6.1112720 .9538882 .645208

H $5.1325440 .382873 \quad 1.303945$

H $5.638507-3.1341102 .512136$

H $6.155427-2.2529964 .556179$

H $6.657667-0.5627184 .584295$

H $4.972020-1.0025544 .943420$

H $5.450213-1.2907290 .084210$

H $5.855287-2.987507 \quad 0.156721$

H $3.417517-3.4394820 .915634$

H $3.071437-1.7685350 .546448$

H $4.122519-3.852692-1.420979$

H $4.799943-1.516514-2.112744$

H $3.154089-0.969935-1.866069$

H $4.244182-0.589083-4.315480$

H $1.285259-1.953339-3.433184$

H $5.424911-2.608933-4.270252$

H $4.206695-3.333189-5.295032$

H $4.587996-4.063140-3.722881$

H $1.783739-2.566032-5.559670$

H $-0.048462-4.875450-3.390286$

H $0.147826-5.050243-1.661500$

H $2.080156-5.827199-2.480235$

H $\quad 1.079089-1.2672941 .786554$

H $-0.159522-1.4025944 .600829$

H -1.019634 -2.1632310 .921216$

H -1.621380 -3.7731601 .266482$

H $0.095321-3.4624641 .409407$

H $-0.353902-2.524766-1.458678$

H $-0.851653-2.589302-3.163121$

H $-4.702144-3.688923-1.273493$

H -4.963481-1.992012 -1.756606

H -3.793935 -1.2909950 .486779$

H -5.479167 -3.782930 1.051862

H $-5.916360-2.0374842 .933578$

H $-4.246827-1.5252742 .809023$

H $-5.037296-4.6674355 .333313$

H $-6.325389-3.4850664 .726383$

H -2.992057 -4.520169 2.560678

H -2.969264 -4.8858964 .256310$

H -2.029053 -2.691535 4.872192

H -2.621832 -1.624305 -1.840619

C $3.3984604 .553070-0.048971$

O $2.307138 \quad 3.620217-0.156387$

C $1.2021984 .248798-0.819111$
C $1.7948185 .462044-1.521261$

C $2.8442835 .899965-0.514332$

H $0.5125544 .583047-0.032808$

C $0.4714043 .250958-1.729570$

H $2.2707405 .186123-2.469543$

H $1.0462586 .233218-1.725675$

C $3.8961496 .830410-1.091730$

H 2.3389686 .4072020 .318513

C $-0.0484612 .012962-0.970757$

O $1.3289992 .830279-2.789571$

H $-0.3693293 .776224-2.194403$

H $-0.6695712 .337732-0.130768$

O $1.0455471 .297229-0.380281$

C $-0.8304741 .007796-1.832510$

C $-2.0514451 .569187-2.582160$

H $-0.1588890 .539302-2.562356$

O $-1.277318-0.048926-0.978445$

H $-1.7137462 .247088-3.374388$

H $-2.5445190 .717941-3.069872$

H $4.6380497 .097214-0.332422$

H $4.4246896 .366796-1.931605$

H $3.4347707 .754790-1.454298$

C $3.9020264 .558421 \quad 1.396028$

H $2.1576012 .513549-2.391246$

H $1.6078240 .964040-1.099735$

H $-0.505195-0.363625-0.477899$

H 3.1004554 .8919072 .067099

H 4.7339255 .2619371 .510616

H $4.1857534 .198933-0.726986$

C $-4.4866251 .899028 \quad 0.245052$

O $-3.5788201 .364275-0.725950$

C $-3.087296 \quad 2.291538-1.696399$

C $-4.256722 \quad 2.854198-2.519756$

C $-5.323568 \quad 3.474179-1.608373$

C $-5.6987402 .545458-0.447191$

H $-4.848142 \quad 1.0272700 .805275$

C -3.7769922 .8288551 .240612$

H $-2.5833003 .118916-1.188195$

O $-4.841952 \quad 1.807310-3.291863$

H $-3.8695863 .605034-3.218814$

H $-6.2250073 .693075-2.193764$

O $-4.8413114 .705140-1.077666$

H $-6.295358 \quad 3.0898500 .293539$

O $-6.534128 \quad 1.498082-0.951496$

C $-2.6621642 .123351 \quad 1.985967$

H $-3.338626 \quad 3.6908380 .730373$

H -4.4916143 .1959951 .985503$

H $-5.5561662 .198749-3.823305$

H $-4.646524 \quad 5.292353-1.827867$

H $-5.9975480 .953928-1.553425$

O $-1.829754 \quad 3.060962 \quad 2.529132$

$\begin{array}{llll}0 & -2.537816 & 0.910723 & 2.087405\end{array}$

C -0.6918832 .5453303 .226673$

H -0.1546823 .3873363 .671359$

H $-1.0046201 .866888 \quad 4.026026$

H -0.0254782 .0375082 .523990$

SCF Energy (B3LYP/6-31G**//MMFF) $=-3245.91444882$

03_72

MM̄FF Geometry

C $-2.209174-3.814365-1.556125$

C $-1.031343-3.773194-0.915941$

C $0.244072-3.283114-1.554302$

O $0.719908-2.130038-0.822728$

C $1.312973-4.390479-1.488462$

C $2.661500-4.003534-2.067657$

C $3.828033-4.129900-1.403876$

C $2.657624-3.481643-3.480546$

C $4.029596-4.685964-0.021386$

C $3.835483-3.6605971 .103071$

C $4.972324-2.6331531 .218828$

C $4.737706-1.7246132 .429200$

C $5.806542-0.6285882 .542561$

$\begin{array}{llll}\text { O } & 5.012574 & -1.850468 & 0.024031\end{array}$

$\begin{array}{lllll}\text { O } & 5.340667 & 0.345334 & 3.489567\end{array}$

C 5.9286640 .0879551 .181835

C $7.128713-1.1729013 .095442$

O 7.0144081 .0128421 .190507 
C $6.066880-0.881659-0.016200$

C $5.981050-0.136853-1.370526$

O $7.352663-1.5095170 .045066$

C $0.173990-0.929734-1.166129$

O $-0.707015-0.736572-1.990186$

C $0.838726 \quad 0.128860-0.372419$

C $0.4239801 .395952-0.521938$

C $0.9488012 .585938 \quad 0.149128$

C 2.0669062 .4084231 .139859

C $4.576267 \quad 0.349176-1.738695$

C $4.5919791 .467794-2.765333$

C $3.073196 \quad 3.189375-3.741799$

O $5.6126691 .859855-3.323658$

C $3.028328 \quad 4.346333-2.791778$

C $1.969798 \quad 5.158210-2.651333$

C $1.9196576 .294642-1.669746$

C $0.744357 \quad 6.197282-0.720013$

C $-0.2801497 .063496-0.796551$

C $0.787831 \quad 5.1396250 .366196$

C $0.395683 \quad 3.781086-0.147369$

N $3.319217 \quad 1.983741-2.972498$

H -2.270798 $-3.469003-2.585274$

H $-0.973871-4.1077130 .117445$

H $0.073636-3.010420-2.603737$

H $0.953663-5.274794-2.031533$

H $1.423511-4.697200-0.442287$

H $4.744437-3.823565-1.906155$

H $2.186846-2.494765-3.526923$

H $3.669273-3.381345-3.888208$

H $2.108395-4.165150-4.136268$

H $3.348896-5.5299110 .141381$

H $5.036694-5.116900 \quad 0.039259$

H $2.882272-3.1398020 .952427$

H $3.757509-4.2081572 .050786$

H $5.917791-3.1776201 .326161$

H $4.691639-2.3119483 .354815$

H $3.755967-1.2392292 .334060$

H $5.186390-0.1090694 .335538$

H 5.0192800 .6865531 .055670

H $6.978392-1.6019644 .093469$

H $7.863852-0.3706073 .222859$

H $7.569893-1.9493692 .467188$

H 6.8835031 .6064671 .949861

H $6.7054910 .687036-1.374912$

H $6.311546-0.807592-2.175935$

H $7.571638-1.852807-0.836875$

H $1.642118-0.1571520 .296123$

H -0.386359 1.590748 -1.223816

H $1.827102 \quad 1.6253951 .867364$

H 2.2678193 .3082851 .726454

H $2.9934472 .138417 \quad 0.625911$

H $3.988826-0.483447-2.143620$

H $4.054620 \quad 0.726008-0.856084$

H $2.1265153 .051456-4.273290$

H $3.8734153 .336243-4.473627$

H $3.9122454 .517125-2.181003$

H $1.0902874 .999766-3.271197$

H $1.8684107 .228226-2.244656$

H $2.8449366 .355321-1.083210$

H -1.114948 $7.013190-0.105317$

H $-0.3101927 .838583-1.555067$

H $\quad 0.092507 \quad 5.405337 \quad 1.172959$

H 1.7885585 .1463830 .808660

H $-0.4462263 .799047-0.841331$

H $2.5474481 .616131-2.424712$

C $-4.414225-3.197587-0.526345$

O $-4.841655-2.448507-1.682877$

C $-6.263358-2.266493-1.613180$

C $-6.667831-2.585878-0.180879$

C $-5.691076-3.6962820 .154868$

H $-6.697192-3.039266-2.263449$

C -6.696101 -0.899614 -2.154413

H $-6.508832-1.7350430 .489647$

H -7.714358 -2.894177 -0.098498

C -5.539671 -3.9396801 .646882$

H $-6.034971-4.622349-0.325132$

C $-6.412630 \quad 0.339481-1.278282$

O $-8.115490-0.971620-2.355720$
H $-6.246839-0.750444-3.144241$

H $-7.0491840 .305714-0.385864$

O -6.853933 1.476097 -2.036679

C $-4.9414950 .542167-0.871640$

C $-4.733214 \quad 1.821613-0.038752$

H $-4.618589-0.317496-0.281284$

$\begin{array}{lllll}\text { O } & -4.136916 & 0.593443 & -2.046770\end{array}$

H $-4.9912312 .700177-0.641783$

H $-5.4264701 .795171 \quad 0.809337$

H $-4.810456-4.7321451 .841705$

H $-5.204414-3.0372012 .168781$

H $-6.495478-4.2446822 .085302$

C $-3.478511-4.332319-0.942518$

H $-8.396020-0.122421-2.738226$

H $-6.9708132 .216782-1.418363$

H -3.209050 $0.491632-1.774618$

H $-3.968240-4.973510-1.685786$

H $-3.248184-4.963340-0.075873$

H $-3.881047-2.4954020 .124374$

C -3.316412 0.4848302 .330487

$\begin{array}{llll}\text { O } & -2.750989 & 0.827148 & 1.062525\end{array}$

C -3.284512 2.0112710 .465301

C $-3.100996 \quad 3.2085191 .409872$

C -3.6896302 .9394722 .793151$

C $-3.158395 \quad 1.618945 \quad 3.351302$

H -4.3870990 .2822522 .195600$

C -2.700033 -0.8459732 .788609$

H -2.673079 $2.207863-0.423290$

$\begin{array}{lllll}\text { O } & -3.694964 & 4.382308 & 0.866545\end{array}$

H -2.031185 $3.416138 \quad 1.532061$

H -4.7855542 .9466462 .774853$

O

H -2.109497 1.7516713 .636681

O $-3.877794 \quad 1.284104 \quad 4.536132$

C -1.186123 -0.8621152 .860457$

H -3.096156 -1.137474 3.767056

H -2.980133-1.613257 2.057832

H -3.277013 4.5476220 .004026

H $-3.603373 \quad 4.8259913 .292536$

H -3.788522 2.0326755 .150801

O -0.769691 -2.153469 3.029272

O $-0.4573840 .116402 \quad 2.786568$

C $0.647478-2.3383703 .101423$

H $1.134910-1.9095942 .221479$

H $1.037913-1.8852094 .017176$

H $0.850431-3.4123273 .124072$

SCF Energy (B3LYP/6-31G**//MMFF)= -3245.90867137

0373

MM̄FF Geometry

C $-2.241174-3.908852-0.360878$

C $-1.082779-3.818177 \quad 0.309184$

C $0.249431-3.592111-0.360501$

O $0.882820-2.437097 \quad 0.234915$

C $1.154995-4.813479-0.112807$

C $2.478340-4.761888-0.850817$

C $3.686837-4.727408-0.255979$

C $2.390613-4.790146-2.355697$

C $4.001280-4.7253491 .213862$

C $4.164974-3.3275411 .831685$

C $5.321477-2.507496 \quad 1.236246$

C $5.563213-1.2359462 .052854$

C $6.648068-0.344721 \quad 1.425450$

O $4.977173-2.150108-0.104244$

$\begin{array}{llll}\text { O } & 6.573231 & 0.946438 & 2.049931\end{array}$

C $6.333477-0.139170-0.073152$

C $8.056644-0.8807601 .708100$

O $7.4018310 .537273-0.730169$

C $6.006316-1.450345-0.815663$

C $5.492236-1.227299-2.258673$

O $7.194675-2.245037-0.914438$

C $0.519338-1.211711-0.232371$

O $-0.301975-0.968072-1.101684$

C $1.297466-0.2006120 .518680$

C 0.9657601 .0955080 .424549

C $1.6153372 .207441 \quad 1.122613$

C 2.9074941 .9202071 .838119

C $4.219174-0.382439-2.381152$ 
C $4.4799201 .081758-2.693603$

C $3.2807733 .246709-2.976652$

O $5.5894181 .525963-2.975446$

C $3.4515364 .045475-1.723683$

C $2.6183705 .033131-1.363140$

C $2.7971235 .871750-0.130554$

C 1.5832955 .8434610 .770034

C $0.681228 \quad 6.8392660 .748931$

C 1.4589274 .6975141 .749317

C $1.026753 \quad 3.4206821 .084987$

N $3.311967 \quad 1.831958-2.657607$

H -2.240759 -3.801737 -1.442990

H -1.080929 -3.9306501 .390951$

H $\quad 0.122808-3.428979-1.437481$

H $0.635223-5.729894-0.423811$

H $1.320425-4.9109480 .965532$

H $4.568352-4.704352-0.896000$

H $\quad 1.964146-3.856937-2.735857$

H $3.371474-4.916975-2.826575$

H $1.761673-5.623824-2.684668$

H $3.238617-5.2750901 .775247$

H $4.928109-5.2944621 .362334$

H $3.223808-2.777473 \quad 1.715464$

H $4.333348-3.4568382 .908050$

H $\quad 6.221340-3.1338361 .230033$

H $5.823027-1.4826303 .089913$

H $4.630225-0.6593982 .109159$

H 6.6949640 .8239883 .006980

H $5.4633090 .525846-0.126760$

H $8.237347-0.9307242 .788656$

H $8.823541-0.2083161 .308067$

H $8.225393-1.8794221 .300126$

H $7.5598531 .366798-0.247808$

H $6.300065-0.839672-2.891057$

H $5.252736-2.217932-2.672008$

H $7.082892-2.878148-1.642918$

H $2.109047-0.5522261 .144867$

H $\quad 0.1213221 .371274-0.205024$

H 2.7404561 .2371382 .677880

H 3.3938492 .8106172 .240611

H $3.627702 \quad 1.4645431 .150750$

H $3.626313-0.770941-3.219451$

H $3.602113-0.460785-1.480702$

H $2.3243503 .447480-3.469697$

H $4.0902813 .480296-3.675894$

H $4.3121083 .819110-1.098691$

H $1.7725185 .274095-2.003280$

H $2.9997276 .901039-0.454095$

H 3.6816455 .5631010 .440590

H $-0.179407 \quad 6.837000 \quad 1.409797$

H 0.7800057 .6760110 .065318

H 0.7054634 .9375952 .511351

H 2.3996904 .6010712 .297125

H 0.0895193 .5178520 .537633

H $2.445907 \quad 1.392496-2.359164$

C $-4.524322-3.0099190 .182105$

O $-4.865402-2.772826-1.201485$

C $-6.287381-2.610201-1.302382$

C $-6.788665-2.3585720 .112281$

C $-5.851220-3.247046 \quad 0.907384$

H $-6.680708-3.584019-1.627087$

C $-6.683496-1.572776-2.358216$

H $-6.656606-1.3130050 .410663$

H -7.842670 -2.622163 0.239605

C $-5.815786-2.9197062 .389349$

H $-6.165873-4.2918540 .780613$

C -6.380309-0.090494 -2.053257

O $-8.100444-1.699892-2.545402$

H -6.216291 -1.843893 -3.313351

H $-7.0108840 .250166-1.223679$

O $-6.8049870 .650915-3.207280$

C $-4.9033230 .233067-1.767377$

C $-4.6449491 .734981-1.547897$

H -4.591559 -0.302564 -0.869135

O $-4.090112-0.231386-2.842891$

H $-4.8928832 .290044-2.460326$

H -5.316009 2.091458 -0.758769

H $-5.101266-3.5596342 .916288$
H $-5.527001-1.8787512 .560219$

H -6.802042 -3.071154 2.840091

C $-3.565465-4.1952680 .289682$

H -8.357603 -1.070514 -3.241030

H $-6.9111921 .579194-2.938956$

H $-4.3907850 .215493-3.652734$

H -3.995882 $-5.071110-0.211585$

H -3.420832 -4.462959 1.342810

H $-4.040695-2.0984760 .554350$

C -3.2472931 .4870641 .157479$

O $-2.6752991 .231208-0.131150$

C $-3.1746132 .051787-1.189695$

C $-2.9111483 .529654-0.871596$

C -3.4577563 .9238300 .498872$

C -2.9958742 .9421921 .576320$

H -4.3244201 .2871621 .116122$

C -2.6015690 .4828432 .126074$

H $-2.5760951 .800420-2.074623$

O $-3.4837864 .376326-1.862777$

H -1.829920 $3.714810-0.880044$

H -4.5512324 .0029040 .486595$

O -2.9673095 .2227890 .841859$

H -1.9290863 .0980471 .772406$

O $-3.689040 \quad 3.2224142 .788954$

C -3.4432460 .1680463 .342560$

H $-2.429075-0.4665341 .603583$

H -1.624402 0.8541502 .456121

H $-3.1039574 .119531-2.720528$

H -3.2331205 .8293860 .129448$

H -3.521902 4.1545123 .010786

O $-2.718163-0.6289754 .183322$

$\begin{array}{llll}\text { O } & -4.590920 & 0.546222 & 3.529817\end{array}$

C $-3.391485-1.0346715 .378949$

H -3.660872 -0.1595485 .977819$

H $-4.280194-1.6231175 .132003$

$\mathrm{H}-2.707271-1.6596685 .958876$

SCF Energy $\left(B 3 L Y P / 6-31 G^{* *} / / M M F F\right)=-3245.90380441$

0374

MM̄FF Geometry

C $-1.954983-3.710131-0.734849$

C -1.013762 -4.190505 -1.561753

C $0.448819-3.804797-1.568592$

O $0.670847-2.531171-0.920907$

C $1.261365-4.877703-0.820304$

C $2.762614-4.693248-0.915004$

C $3.571687-4.430097 \quad 0.129811$

C $3.354602-4.859808-2.291582$

C $3.201588-4.2510661 .575697$

C $2.966525-2.7927862 .000952$

C $4.194726-1.8816001 .845239$

C $3.941477-0.5155182 .485769$

C 5.1146980 .4507322 .261181

O $4.452186-1.7066290 .451171$

O $4.648758 \quad 1.7617892 .616730$

C 5.4851090 .4683120 .760108

C 6.2836300 .1408863 .204488

O 6.6727431 .2330440 .569120

C $5.628262-0.9429510 .151175$

C $5.779705-0.947996-1.389491$

O $6.799895-1.5679570 .689015$

C $0.425814-1.413076-1.659242$

O $0.022973-1.367774-2.810984$

C $0.719243-0.234726-0.813199$

C $0.3117190 .977912-1.217000$

C $0.5086722 .242518-0.505554$

C 1.4649132 .2414940 .656481

C $4.624011-0.306692-2.165551$

C $4.8660911 .145467-2.540112$

C $3.7272513 .090425-3.599721$

O $5.9430611 .713398-2.380189$

C $3.2573594 .005033-2.513391$

C $2.2683534 .895745-2.680281$

C $1.8151845 .852592-1.616004$

C $0.3301725 .762532-1.349057$

C $-0.5281586 .628901-1.913629$

C $-0.1473984 .723719-0.359950$

C $-0.1583193 .334068-0.936054$ 
N $3.740722 \quad 1.725120-3.110377$ H -1.682011 -2.985341 0.026155 H -1.299957 -4.937322 -2.299828 H $0.781434-3.739694-2.611711$ H $1.020565-5.873001-1.217922$ H $0.941903-4.8886960 .226896$ H $4.639185-4.333862-0.066745$ H $4.449876-4.863487-2.275370$ H $3.032389-5.809070-2.732156$ H $3.040652-4.043348-2.948760$ H $2.314194-4.8412331 .826775$ H $4.008015-4.6771452 .186137$ H $2.129935-2.3869301 .420390$ H $2.657501-2.7981833 .053408$ H $5.051934-2.3742352 .318693$ H $3.730201-0.6194083 .557411$ H $3.037472-0.0724152 .046547$ H 5.3614362 .3922832 .413994 H 4.6791691 .0064890 .247780 H 5.9631810 .2308494 .249461 H 7.0977950 .8632533 .079345 H $6.690514-0.8631943 .067621$ H $\quad 6.808713 \quad 1.340027-0.386952$ H $6.749192-0.525210-1.678730$ H $5.821945-2.000970-1.704867$ H $7.071282-2.2826570 .089382$ H $1.246435-0.3974590 .119187$ H $-0.2401141 .055419-2.152974$ H 2.4327091 .8304720 .350775 H 1.0714911 .6372521 .480445 H 1.6716773 .2364991 .054986 H $4.500969-0.848502-3.112138$ H $3.681533-0.390873-1.615677$ H $3.0717413 .115272-4.475985$ H $4.7373663 .375747-3.910568$ H $3.7710423 .951792-1.556337$ H $1.7750334 .965522-3.647274$ H $2.0735496 .866716-1.947945$ H $2.3645645 .700860-0.678451$ H -1.592091 $6.588901-1.704652$ H $-0.1858987 .394461-2.602106$ H -1.174837 $4.952445-0.049564$ H 0.4471594 .8057560 .553636 H - $0.8306063 .224837-1.788590$ H $2.8732821 .197772-3.149149$ C $-4.339311-2.932057-0.709410$ O $-4.134308-2.073600-1.849250$ C $-5.413373-1.721591-2.394239$ C $-6.447938-2.081446-1.337947$ C $-5.819046-3.320967-0.729439$ H $-5.566849-2.389455-3.253299$ C $-5.431429-0.277809-2.905984$ H $-6.546715-1.299130-0.578322$ H -7.437534 -2.267523 -1.765883 C $-6.379473-3.6793190 .636162$ H -5.976636 -4.165457 -1.413738 C $-5.4316040 .853472-1.856527$ O $-6.619026-0.107264-3.693228$ H -4.582442 -0.126115 -3.584421 H $\quad-6.4106390 .892162-1.364504$ O $-5.3234852 .092403-2.577276$ C $-4.3279980 .811353-0.781979$ C $-4.353247 \quad 2.0673570 .109107$ H -4.456394 $-0.070037-0.149348$ O $\quad-3.0470760 .729678-1.400314$ H -4.102366 $2.948754-0.494074$ H -5.3732552 .1921610 .493054$ H -5.886602 -4.5698261 .038662$ H $-6.239821-2.863607 \quad 1.353653$ H -7.452214 -3.887901 0.568895 C $-3.396692-4.134290-0.780418$ H -6.577737 -0.741778 -4.428925 H -4.435697 2.123269-2.973785 H -2.975489 -0.145561-1.817453 H -3.590222 -4.700638 -1.699768 H $-3.586511-4.8006930 .068624$ H $-4.116087-2.3338720 .181534$ C $-2.983303 \quad 0.7442093 .339553$
O

C -3.3646722 .0011021 .291061$

C -3.3098793 .3438872 .039178$

C -2.4532753 .2483743 .303301$

C -2.8448982 .0419104 .156530$

H $-3.558931 \quad 0.036167 \quad 3.949517$

C -1.6301770 .0929643 .003643$

H $\quad-2.3701691 .7651390 .900752$

O -4.6239523 .7775572 .387297$

H -2.8960384 .1045301 .368805$

H -2.5746874 .1624063 .897620$

O -1.0794773 .1333332 .947420$

H $-2.116247 \quad 1.9009244 .963415$

O -4.1015562 .3273874 .777532$

C -1.764857 -1.405212 2.827461

H -1.1775330 .4861202 .091071$

H -0.9382110 .2568193 .838560$

H $-5.034596 \quad 3.0796412 .925851$

H -0.8397963 .9298762 .444379$

H -4.3098621 .5878955 .373621$

O $-0.572572-2.0015043 .122774$

O $-2.782353-1.9775702 .462323$

C $-0.551344-3.4317703 .062338$

H -0.419763 -3.750407 2.026076

H $0.301563-3.7829693 .649106$

$\mathrm{H}-1.462389-3.8652443 .486377$

SCF Energy $\left(B 3 L Y P / 6-31 G^{* *} / / M M F F\right)=-3245.91997854$

0375

MM̄FF Geometry

C $-2.9275352 .093210-2.838782$

C $-2.2537371 .196401-2.102652$

C $-1.0711600 .413464-2.609168$

O $-0.0454280 .410870-1.588826$

C $-1.491683-1.045843-2.862665$

C $-0.406008-1.897044-3.492619$

C $0.133297-2.992686-2.922991$

C $0.048728-1.472719-4.865834$

C $-0.203606-3.603871-1.592632$

C $0.628898-3.074021-0.416346$

C $2.136026-3.356031-0.530105$

C $2.850284-3.0086260 .776528$

C $4.367275-3.2270080 .670391$

O $2.671052-2.547794-1.578627$

O $4.982655-2.5831021 .798453$

C $4.900504-2.517116-0.596712$

C $4.727636-4.7138570 .776930$

O $6.264743-2.868826-0.811995$

C $4.057223-2.781586-1.866888$

C $4.417393-1.864459-3.058998$

O $4.262047-4.110947-2.361770$

C $0.6794721 .549892-1.424448$

O $0.5796222 .586279-2.061279$

C $1.613536 \quad 1.322817-0.300437$

C 2.2093672 .3730150 .282730

C 3.1476972 .3184581 .404902

C 3.6640860 .9658131 .818578

C $4.271052-0.360289-2.804202$

C $5.5616810 .322708-2.382075$

C $6.4297182 .535958-1.633015$

O $6.655170-0.236018-2.391738$

C $6.5732882 .523941-0.143868$

C 6.5913583 .6386270 .602661

C 6.7832083 .6469792 .092335

C 5.6964634 .4094312 .816699

C 5.8836425 .6841393 .198643

C 4.4294943 .6662313 .174022

C 3.5174353 .4751501 .993552

N $5.3510351 .647796-2.024334$

H $-2.6300692 .276613-3.867760$

H $-2.5697491 .001429-1.081146$

H $-0.6691080 .862871-3.525092$

H -2.364711 -1.070936 -3.528754

H -1.821008 -1.482680 -1.913309

H $\quad 0.905807-3.527729-3.474323$

H $\quad 0.698740-2.218386-5.336564$

H $-0.813642-1.331035-5.525418$

H $0.610491-0.535223-4.815069$ 
H $-1.267194-3.470360-1.366151$ H $-0.064693-4.689714-1.672469$ H $\quad 0.461531-1.994478-0.324426$ H $0.244542-3.5426020 .498045$ H $2.272951-4.415472-0.775609$ H $2.437879-3.5853651 .613739$ H $2.669295-1.9534781 .020924$ H $4.598034-2.9621472 .607368$ H $4.884154-1.444147-0.372340$ H $4.410323-5.1158131 .746947$ H $5.811935-4.8637570 .732814$ H $4.259332-5.3249330 .002500$ H $6.611833-2.298603-1.518230$ H $5.413618-2.113415-3.443820$ H $3.725779-2.116959-3.876393$ H $4.933045-4.548564-1.814467$ H 1.7702630 .3017040 .027262 H $1.9746773 .372455-0.081433$ H $4.037206 \quad 0.4141180 .949040$ H 2.8706730 .3801182 .292636 H $4.499571 \quad 1.0143732 .520212$ H $3.9646410 .120656-3.742035$ H $3.491883-0.159488-2.063275$ H $6.1862113 .531313-2.017882$ H $7.3638282 .201663-2.095910$ H $\quad 6.696634 \quad 1.5572270 .338734$ H $6.497024 \quad 4.6055140 .113233$ H 7.7594374 .1051052 .298976 H 6.8447402 .6283012 .494998 H 5.1204656 .2294043 .743854 H 6.8030786 .2128092 .969320 H 3.8639294 .2288773 .929184 H 4.6908832 .7266953 .669124 H 3.0968324 .4077151 .614479 H $4.4028122 .011178-1.994671$ C $-5.4116422 .108020-2.470514$ O $-5.401617 \quad 0.987214-1.561860$ C $-6.6133440 .997893-0.790410$ C $-7.5820291 .872394-1.573013$ C $-6.6418692 .939605-2.100983$ H -6.3819221 .4936010 .160330$ C -7.084222 $-0.433355-0.525660$ H $-8.0415591 .327228-2.405649$ H $-8.3819792 .275503-0.944810$ C $-7.2139543 .737417-3.259717$ H $-6.3976183 .628550-1.280850$ C $-6.015061-1.3365350 .126334$ O $-7.452215-1.030378-1.775657$ H $-7.994561-0.4122720 .085020$ H $-5.211565-1.537649-0.592331$ O $-6.632171-2.6050870 .386055$ C $-5.409745-0.7878381 .434467$ C $-4.453936-1.7983912 .096782$ H -4.8689290 .1381551 .219448$ O $-6.477202-0.4753252 .327649$ H $-5.004058-2.7239392 .304237$ H -3.663570 -2.045573 1.379299 H -6.491514 $4.478205-3.616736$ H $-7.4765573 .089321-4.102613$ H $-8.1195654 .269349-2.950325$ C $-4.0997152 .877747-2.318777$ H -7.743063 -1.939288 -1.587931 H $-7.309813-2.4658111 .069831$ H -6.1204820 .1045053 .021570$ H $-3.9313843 .123126-1.263114$ H $-4.1453473 .827656-2.863577$ H $-5.5075981 .697530-3.483824$ C $-1.910235-0.1137202 .571797$ O $-3.147810-0.0621253 .293768$ C $-3.838252-1.3076413 .426951$ C $-2.938654-2.3423894 .117500$ C -1.607164 -2.497911 3.391099 C $-0.948690-1.1329553 .205561$ H $-2.106890-0.3760681 .524730$ C $-1.298300 \quad 1.2975432 .617471$ H $-4.674344-1.1200394 .111747$ O $-3.609609-3.595048 \quad 4.197799$ H -2.745514 -2.013422 5.147102
H -1.725921 -3.011883 2.429942

O $-0.730504-3.3083224 .177701$

H $-0.598266-0.7749624 .182734$

O $0.196743-1.3134752 .376953$

C -2.0210822 .2897081 .732274$

H -1.3241411 .6942693 .638928$

H -0.2591891 .2650272 .275560$

H $-3.068486-4.1852984 .748424$

H -1.093599 -4.209920 4.185905

H $\quad 0.766119-0.5336582 .478658$

O -1.1890193 .3455331 .486863$

O $-3.1691032 .171617 \quad 1.329329$

C -1.7204694 .3870880 .662295$

H -2.7212584 .6809050 .993335$

H -1.0591045 .2538750 .743418$

$\mathrm{H}-1.7383234 .056478-0.379456$

SCF Energy (B3LYP/6-31G**//MMFF)= -3245.91375195

03_76

MM̄FF Geometry

C $-2.071058 \quad 3.230014 \quad 0.878951$

C $-2.1404413 .863306-0.302416$

C $-2.5064863 .210677-1.616968$

O $-2.2218931 .791500-1.569771$

C $-4.0039463 .395311-1.904216$

C $-4.3754053 .043149-3.330978$

C $-4.9422601 .879124-3.702997$

C $-4.1180574 .122466-4.349844$

C $-5.2400740 .696007-2.827071$

C $-6.7138890 .659890-2.411905$

C $-7.036130-0.585139-1.574428$

C $-8.519390-0.616562-1.200869$

C $-8.855145-1.805950-0.288604$

O $-6.234799-0.549675-0.388038$

O $-10.157194-1.578998 \quad 0.272361$

C $-7.855360-1.8330180 .886625$

C $-8.944836-3.119975-1.073849$

O $-8.030460-3.0063441 .677723$

C $-6.377774-1.7057620 .446706$

C $-5.472982-1.5253071 .688726$

O $-5.990094-2.901593-0.235534$

C $-0.9355791 .407194-1.795258$

O $-0.0065692 .115814-2.147641$

C $-0.836501-0.033662-1.473801$

C $0.364320-0.552325-1.174278$

C $0.642270-1.939032-0.789489$

C $-0.505077-2.914060-0.828905$

C $-4.031712-1.1014401 .366375$

C $-3.130004-1.1904722 .587281$

C $-0.734611-0.9932063 .244738$

O $-3.530431-1.5428563 .693830$

C $-0.034725-2.2877432 .973543$

C $1.295850-2.3941382 .840867$

C $2.000740-3.6902012 .564550$

C $2.980963-3.5926471 .416417$

C $4.304465-3.5751911 .645534$

C $2.434441-3.6087610 .004484$

C $1.900144-2.266014-0.422862$

N $-1.819050-0.8422032 .291033$

H -2.3467232 .1799780 .941175$

$\mathrm{H}-1.8768434 .917242-0.353490$

H -1.908962 $3.671921-2.413113$

H $-4.3008544 .437054-1.721678$

H -4.593822 $2.794853-1.200409$

H $-5.2043521 .742107-4.751069$

H $-4.4382573 .829786-5.355465$

H $-4.6628795 .034096-4.083150$

H -3.050437 4.357311-4.400130

H $-4.989842-0.213384-3.387447$

H $-4.5960820 .685759-1.942637$

H $-6.9376281 .562164-1.827758$

H -7.352748 $0.678862-3.303728$

H -6.770740 -1.471392 -2.163202

H $-9.147614-0.633011-2.100265$

H $-8.7805240 .310079-0.670411$

H - $10.787823-1.485231-0.462122$

H -8.094940 $-0.981248 \quad 1.538797$

H $-9.724005-3.053802-1.842788$ 
H $-9.238035-3.950591-0.422350$ H -8.011258 -3.385845 - 1.573840 H $-8.965323-3.039177 \quad 1.944000$ H -5.898069 -0.7545782 .344976$ H -5.456135 -2.462999 2.258968 H - $-5.037788-3.043698-0.119642$ H -1.753584 -0.610756 -1.450485 H $1.2318940 .106819-1.191341$ H -1.299075 -2.605214 -0.141642 H $-0.228017-3.930542-0.544411$ H $-0.922070-2.973634-1.840510$ H $-3.602522-1.7423840 .589616$ H $-4.017343-0.0676681 .002009$ H -1.132998 -0.9901304 .263939$ H $-0.065933-0.1372213 .123054$ H $-0.650261-3.1802382 .887271$ H $1.914498-1.5072592 .956522$ H $1.287050-4.4975152 .357820$ H $2.525605-3.9835143 .483054$ H $5.016001-3.5443430 .829127$ H $4.706246-3.5919662 .653154$ H $1.700026-4.413554-0.080100$ H $3.231191-3.878541-0.701994$ H $2.661331-1.484149-0.437666$ H $-1.575490-0.603617 \quad 1.334267$ C $-0.328747 \quad 3.1196082 .629502$ O $\quad 0.7510053 .3561591 .707988$ C 1.9930603 .3753592 .435574 C 1.6389503 .0565943 .886340 C 0.2105523 .5627653 .982847 H 2.3796014 .4002302 .375293 C 3.0101742 .4072111 .807208 H $1.6782831 .977766 \quad 4.080125$ H $2.3163103 .545774 \quad 4.593317$ C -0.5511993 .0015225 .170272$ H 0.2259154 .6591524 .042216 C 3.4591652 .8150130 .388639 O 2.4551601 .0918491 .771802 H 3.8887262 .3673212 .460243 H 3.9553343 .7922110 .438928 O $2.3011792 .989547-0.433255$ C $4.3736421 .797319-0.320382$ C 5.6361981 .4348720 .479059 H $3.8102470 .890195-0.568988$ O $4.7919312 .378216-1.561323$ H $\quad 6.1858412 .3524410 .722262$ H $5.336810 \quad 0.9552051 .417243$ H - 1.5762293 .3842585 .193489 H $-0.6009151 .907913 \quad 5.135219$ H $-0.063926 \quad 3.2858796 .108745$ C -1.5782463 .8584772 .153151$ H $1.665424 \quad 1.1250281 .204743$ H $2.5801823 .446921-1.244153$ H $4.0263222 .370601-2.160453$ H -1.351725 4.9195501 .993955 H $-2.373726 \quad 3.7930972 .902939$ H -0.5138502 .0389542 .633521$ C $6.569919-1.727913-1.284884$ O $5.876734-0.748373-0.501082$ C $6.5752320 .484143-0.296363$ C 7.8916880 .2298280 .451988 C $8.753793-0.794032-0.294364$ C $7.951409-2.041285-0.684051$ H $5.958194-2.634664-1.203859$ C $6.622247-1.333624-2.765282$ H $6.8017230 .938839-1.266290$ O $7.608805-0.2469291 .765604$ H $8.432703 \quad 1.1786200 .551224$ H $9.597161-1.0864080 .343159$ O $9.285251-0.173476-1.461389$ H $8.523439-2.655158-1.388678$ O $7.762025-2.8430450 .486146$ C $5.234020-1.181906-3.342282$ H $7.144421-0.386579-2.929108$ H $7.156202-2.087864-3.355474$ H $8.458268-0.3654222 .223576$ H $9.850695-0.825732-1.909046$ H $7.212401-2.3357061 .107814$
O $4.642007-2.403267-3.471336$

O $4.738963-0.103925-3.645245$

C $3.315710-2.393616-4.008821$

H $3.352241-2.161125-5.077003$

H $2.889001-3.391756-3.878663$

H $2.684560-1.672994-3.480437$

SCF Energy (B3LYP/6-31G**//MMFF)= -3245.91413597

0377

MM̄FF Geometry

C $-3.1900380 .227251-3.212258$

C $-2.474466-0.075580-2.118085$

C $-1.320254-1.044793-2.126527$

O $-0.249519-0.514796-1.311945$

C $-1.766876-2.381356-1.504217$

C $-0.744923-3.493560-1.645289$

C $-0.148240-4.122915-0.614352$

C $-0.430727-3.921306-3.056698$

C $-0.345545-3.8877150 .855632$

C $0.621642-2.8693861 .475628$

C $2.105739-3.2552651 .374112$

C $2.961666-2.3294892 .241023$

C $4.460252-2.6294252 .094646$

O $2.513165-3.1337190 .009408$

O $5.165549-1.5305842 .692549$

C $4.827436-2.6704270 .594164$

C $4.862702-3.8796122 .886475$

O $6.176478-3.1059270 .445203$

C $3.865793-3.537917-0.247976$

C $4.087118-3.411923-1.775795$

O $4.062744-4.9160190 .091083$

C $0.5224700 .469352-1.845626$

O $0.4190070 .969771-2.953781$

C $1.5198830 .851617-0.822070$

C $2.1863052 .008395-0.945455$

C $3.1870522 .529519-0.011966$

C 3.6603581 .6120811 .084265

C $3.949495-1.995895-2.346235$

C $5.266705-1.247389-2.462759$

C $6.1791261 .006152-3.007380$

O $6.361298-1.781366-2.304760$

C $6.4572881 .743057-1.735291$

C $6.5646903 .078916-1.669153$

C $6.8825283 .833747-0.410220$

C $5.8772734 .925113-0.117411$

C $6.1216376 .200436-0.463292$

C 4.6293564 .5500320 .649506

C $3.6412003 .787883-0.189223$

N $5.0764570 .085365-2.801798$

H $-2.938714-0.235194-4.163336$

H $-2.7448220 .369132-1.163661$

H $-0.953438-1.199000-3.148280$

H -2.695761 -2.723513 -1.980225

H $-2.010157-2.210768-0.449470$

H $\quad 0.566675-4.912740-0.843525$

H $\quad 0.163814-4.840988-3.089023$

H -1.355068 -4.113303 -3.611258

H $0.138403-3.146352-3.578803$

H $-1.374528-3.5743921 .063339$

H $-0.232223-4.8502631 .370789$

H $0.460263-1.8970300 .996361$

H $\quad 0.353907-2.7620952 .533512$

H $2.216241-4.2961391 .699727$

H $2.660029-2.3902343 .294299$

H $2.785337-1.2876561 .941067$

H $6.116018-1.6757422 .544639$

H $4.789229-1.6339120 .238819$

H $4.644952-3.7411153 .952381$

H $5.941569-4.0597712 .823798$

H $4.343904-4.7818822 .556320$

H $6.423867-2.999863-0.488473$

H $5.039571-3.873735-2.061932$

H $3.314227-4.023381-2.264201$

H $3.723751-5.463782-0.636273$

H 1.6631660 .1789150 .015523

H $1.9658372 .647022-1.799801$

H 3.9896430 .6546360 .666470

H 2.8557901 .4250431 .803092 
H 4.5126232 .0023591 .644046 H $3.551666-2.070540-3.366581$ H $3.240064-1.403310-1.761284$ H $5.8923271 .678983-3.821747$ H $7.0698710 .447468-3.312036$ H $6.6017961 .151411-0.834330$ H $6.4478333 .665032-2.578065$ H $7.8826964 .271040-0.527790$ H 6.9540153 .1620290 .454126 H $5.4187166 .994053-0.232896$ H $7.0287356 .478293-0.989785$ H 4.1193305 .4555931 .004917 H 4.9181444 .0178351 .559820 H $3.2388904 .371117-1.018972$ H $4.1319340 .454567-2.861951$ C $-5.6861670 .386208-3.116962$ O $-5.764459-0.241259-1.818552$ C $-6.8982360 .295228-1.120828$ C $-7.8711790 .675329-2.223810$ C $-6.9173781 .295149-3.231116$ H $-6.5631021 .210946-0.616754$ C -7.408742 -0.698843 -0.074325 H -8.358391 $-0.205789-2.656790$ H $-8.6449781 .369007-1.882570$ C $-7.4841671 .376244-4.638183$ H $-6.6670112 .311231-2.897055$ C $-6.308589-1.2303640 .872589$ O $-7.984057-1.820728-0.752881$ H $-8.219096-0.2346630 .500475$ H $-5.681494-1.9612840 .346686$ O $-6.944373-1.937124 \quad 1.943520$ C $-5.408669-0.1398731 .481208$ C $-4.400098-0.6911672 .507029$ H $-4.858237 \quad 0.3597670 .677893$ O H -4.927926 -0.9952563 .418752$ H -3.907601 -1.572373 2.077944 H $-6.7501991 .806266-5.327197$ H $-7.7628490 .386815-5.016110$ H -8.378524 $2.007393-4.657218$ C $-4.367822 \quad 1.160933-3.203730$ H -8.456928 -2.354034 -0.092296 H -7.358522 $-2.731446 \quad 1.566785$ H -6.7426670 .4073182 .802029$ H -4.282332 $1.851447-2.354715$ H $-4.3378611 .765961-4.116988$ H -5.716125 -0.417693 -3.862640 C -1.5300721 .5888511 .839215$ O C -3.3385990 .3633222 .872820$ C $-2.467928-0.0687954 .059823$ C -1.3550740 .9507674 .327262$ C $-0.606648 \quad 1.3420283 .045467$ H $-0.923051 \quad 1.4941320 .931114$ C $-2.2023822 .964410 \quad 1.812434$ H $-3.845714 \quad 1.3038023 .116845$ O $-1.892687-1.3487943 .808756$ H $-3.100238-0.1672234 .949729$ H $-0.649900 \quad 0.523317 \quad 5.050592$ O H $\quad 0.0210302 .223736 \quad 3.215617$ $\begin{array}{lllll}\text { O } & 0.272175 & 0.270718 & 2.689685\end{array}$ C -1.2473434 .0454451 .373431$ H -3.058756 2.9640251 .125725 H -2.6174683 .2480332 .783969$ H -1.410823 -1.2941992 .966026$ H -1.216338 2.7326425 .113135 H $\quad 0.9031230 .1522513 .420049$ O -1.1038124 .0176470 .017590$ O -0.6927724 .8173252 .144841$ C $-0.2261345 .005093-0.532051$ H $-0.0505434 .757168-1.582187$ H $-0.6979105 .990289-0.475349$ H $0.7344055 .008944-0.008632$ SCF Energy (B3LYP/6-31G**//MMFF) $=-3245.92181895$

0378

MM̄̄FF Geometry
C 1.0603775 .2152610 .672961

C $2.0226224 .651467-0.072409$

C 3.0135853 .6531710 .470043

O $2.7881852 .391538-0.202382$

C 4.4513834 .1088640 .165106

C 5.5118433 .2330420 .805145

C 6.3983102 .4786220 .126335

C 5.5618863 .2621362 .312033

C $6.5492562 .331670-1.361122$

C $5.752207 \quad 1.174596-1.982747$

C $6.106366-0.214270-1.425013$

C $5.467216-1.314656-2.276067$

C $5.710863-2.714782-1.690702$

O $5.613942-0.301926-0.084802$

O $4.817615-3.628436-2.346457$

C $5.330783-2.709860-0.193809$

C $7.130896-3.214181-1.982447$

O $5.693966-3.9400370 .427214$

C $5.936476-1.5266280 .588468$

C $5.400440-1.4126022 .037283$

O $7.355582-1.7020180 .682623$

C 1.7430041 .6379090 .236061

O $1.0036641 .883013 \quad 1.175830$

C $1.6297570 .460116-0.650139$

C $0.485251-0.238679-0.668610$

C $0.211504-1.430495-1.473007$

C $1.368818-2.046738-2.213482$

C $3.885399-1.2223902 .166593$

C $3.114630-2.5124992 .395149$

C $0.780573-3.3798522 .527141$

O $3.653706-3.5820002 .665439$

C $0.448969-4.0391131 .225478$

C $-0.807551-4.2695350 .815001$

C -1.151049-4.968109-0.470227

C $-2.124972-4.183490-1.321172$

C $-3.435094-4.480650-1.321395$

C $-1.569884-3.116424-2.238181$

C $-1.045035-1.923004-1.488305$

N $1.742631-2.3167952 .302200$

H 0.9979464 .9791421 .732168

H $2.0716794 .871847-1.136317$

H 2.8819913 .5202531 .550675

H 4.6011095 .1360950 .523465

H $4.5863894 .143619-0.921387$

H $7.115472 \quad 1.8937730 .701916$

H 4.6793172 .7762012 .738640

H 6.4428602 .7455602 .708182

H 5.6025274 .2955162 .671589

H $6.2815693 .263177-1.871338$

H $7.6156872 .186524-1.577503$

H $4.6826371 .366814-1.840312$

H $5.9400331 .187347-3.063676$

H $7.197718-0.319336-1.422811$

H $5.827920-1.264979-3.311089$

H $4.383384-1.146410-2.331254$

H $4.993169-3.585344-3.302111$

H $4.237091-2.645232-0.144683$

H $7.303571-3.266025-3.064226$

H $7.276410-4.233339-1.607928$

H $7.906712-2.574693-1.556268$

H $5.274340-4.655795-0.080093$

H $5.755438-2.2583502 .638298$

H $5.867838-0.5232112 .484589$

H $7.683148-1.1707641 .427254$

H $2.4845490 .207122-1.265657$

H $-0.3397390 .098176-0.042811$

H $1.729905-1.370128-2.995604$

H $1.127415-2.995617-2.695672$

H $2.192290-2.259408-1.523303$

H $3.688851-0.5931983 .044335$

H $3.480441-0.7015901 .294767$

H -0.098310 -2.928526 2.998330

H $1.208585-4.116613 \quad 3.214584$

H $1.279686-4.3660720 .604285$

H $-1.637403-3.968411 \quad 1.450766$

H - $1.584042-5.943481-0.212253$

H -0.252291 -5.189799-1.059240

H $-4.135303-3.946266-1.954244$ 
H $-3.837196-5.263920-0.687338$ H -2.355200 -2.751817 -2.913905 H $-0.822966-3.573086-2.892401$ H -1.811224 -1.405499 -0.909147 H $1.387684-1.4077872 .020485$ C $-1.3262385 .447375-0.161123$ O -1.161422 4.461681-1.197452 C $-1.9726713 .299161-0.885564$ C -2.8768223 .7413570 .255860$ C -1.9870134 .7184261 .002991$ H $-1.2708232 .531950-0.538187$ C -2.649332 $2.833565-2.179951$ H $-3.7683384 .252724-0.125397$ H -3.1930122 .9174030 .898118$ C -2.7414955 .6292381 .954729$ H $-1.2521734 .137541 \quad 1.573563$ C $-3.421917 \quad 1.499600-2.138434$ O $-1.5805682 .650227-3.132749$ H -3.272699 $3.636868-2.590887$ H $-2.7213600 .687350-1.913788$ O $-3.8846341 .274424-3.480776$ C $-4.6278171 .423586-1.187196$ C $-5.4765130 .150266-1.381299$ H $-4.2700161 .445964-0.156673$ O $-5.4643382 .562939-1.380320$ H $-5.9485310 .177541-2.370626$ H -4.814161 -0.721638 -1.358130 H $-2.056837 \quad 6.3219242 .454310$ H $-3.4979886 .221677 \quad 1.429392$ H -3.250915 5.0419462 .725692 C 0.0119476 .1386790 .120695 H -1.989159 2.394651 -3.977663 H $-4.0555090 .322222-3.577141$ H $-5.7473492 .566464-2.310827$ H $-0.134135 \quad 6.9625540 .828584$ H $\quad 0.3799936 .585904-0.811645$ H -2.014805 $6.199811-0.567988$ C $-5.333292-1.1427301 .382532$ O $-6.085697 \quad 0.0191251 .013311$ C $-6.595720 \quad 0.011291-0.323478$ C $-7.508499-1.206437-0.525502$ C $-6.802619-2.508314-0.159446$ C $-6.183828-2.409406 \quad 1.233598$ H -4.435005 -1.2140960 .756915$ C $-4.890299-0.9557282 .837115$ H $-7.2288240 .902904-0.413217$ O $-7.966911-1.285685-1.870839$ H $-8.396902-1.1041080 .111408$ H -6.048324 -2.776218 -0.906782 O $-7.754207-3.574756-0.150527$ H $-6.981569-2.4190331 .987417$ O $-5.363543-3.5526581 .465695$ C -3.8323880 .1150032 .942651$ H -5.731360 -0.6498403 .471337$ H -4.486361 -1.879314 3.267533 H -8.428390 $-0.453468-2.071357$ H -8.168105 -3.599818 -1.030417 H -5.919504 -4.340171 1.335782 O $-2.598947-0.4297722 .738883$ O C $-1.505767 \quad 0.4880322 .822078$ H -1.478329 0.9609543 .808417 H -0.579621 -0.0735292 .678281$ H -1.588288 1.2422862 .034657 SCF Energy (B3LYP/6-31G**//MMFF) $=-3245.90492285$

0379

MM̄FF Geometry

C -3.416861-1.875075 -2.754672

C $-2.695866-1.159532-1.877520$

C $-1.352263-1.587954-1.351677$

O $-0.516733-0.408645-1.385867$

C $-1.500506-2.0832690 .102548$

C $-0.385211-2.9965270 .579001$

C $0.407498-2.7632421 .643907$

C $-0.228212-4.289499-0.183996$

C $0.372903-1.6036842 .597181$

C $1.427823-0.5226722 .325972$
C $2.878996-0.9705342 .569423$

C $3.824180 \quad 0.2315442 .494612$

C $5.296228-0.1787682 .637190$

O $3.243031-1.9280161 .571765$

O $6.085478 \quad 0.952477 \quad 2.236421$

C $5.597947-1.3380351 .661280$

C $5.661150-0.4588904 .100059$

O $6.916709-1.8258341 .895126$

C $4.559795-2.4799961 .729824$

C $4.740696-3.5570230 .629779$

O $4.687727-3.1513382 .989585$

C $0.812622-0.563850-1.611680$

O $1.421069-1.608622-1.772651$

C $1.4132030 .788672-1.621255$

C $2.7344730 .925946-1.441418$

C $3.4766342 .186722-1.399376$

C $2.7262763 .453477-1.715648$

C $4.664429-3.047485-0.812352$

C $6.008020-2.641336-1.393797$

C $7.001275-1.246354-3.206395$

O $7.079965-3.046120-0.951301$

C $7.4281080 .018360-2.530209$

C $7.3327021 .233934-3.089582$

C $7.7640692 .500719-2.407098$

C $6.6209943 .470464-2.200341$

C $6.4322174 .502945-3.039315$

C $5.7530253 .297328-0.973345$

C $4.7909852 .149090-1.097044$

N $5.867908-1.804519-2.492147$

H -3.030816 -2.819147 -3.129369

H $-3.105973-0.230441-1.487205$

H $-0.938080-2.357394-2.012716$

H -2.434102 -2.653297 0.208708

H -1.600875 -1.2109100 .756887$

H $1.156954-3.5143011 .891367$

H $0.444868-4.9906920 .321387$

H -1.196827 -4.789421 -0.287292

H $\quad 0.180700-4.108842-1.182052$

H $-0.616743-1.1376302 .600864$

H $\quad 0.506437-1.9936363 .614232$

H $1.316520-0.1706341 .294671$

H 1.2067450 .3261952 .984893

H $2.933477-1.4371243 .559978$

H $3.5613750 .982413 \quad 3.250388$

H 3.6966490 .7308521 .525287

H 7.0193580 .6806312 .253964

H $5.594686-0.9014690 .655223$

H 5.5056140 .4396844 .709440

H $6.722632-0.7094244 .204112$

H $5.072141-1.2648604 .542469$

H $7.133250-2.4560751 .188219$

H $5.659217-4.1293090 .806811$

H $3.922280-4.2816430 .750033$

H $4.286979-4.0329632 .911191$

H $0.7472531 .634268-1.749840$

H $3.3324310 .029423-1.289468$

H $1.9691123 .652985-0.949475$

H $2.2291603 .374762-2.689135$

H $3.3686354 .335264-1.771902$

H $4.294298-3.859176-1.451451$

H $3.952836-2.221600-0.892457$

H $6.685656-1.078009-4.240791$

H $7.826555-1.965576-3.205512$

H $7.840145-0.074579-1.527872$

H $6.9289751 .328027-4.094971$

H $8.5442202 .963638-3.025526$

H $8.2426192 .292704-1.441806$

H $5.6343615 .220944-2.882172$

H $7.0692054 .650190-3.905344$

H $5.2246614 .227711-0.737173$

H $6.3955593 .122425-0.100578$

H $5.2421711 .181269-0.879290$

H $4.943055-1.468000-2.744051$

C $-5.884682-2.163078-2.438545$

O $-5.788431-1.774786-1.052106$

C $-7.031362-1.182320-0.647962$

C $-8.066008-1.774127-1.591669$

C $-7.291944-1.766618-2.898508$ 
H $-6.950998-0.106231-0.843169$ C $-7.271899-1.4048050 .847667$ H $-8.328339-2.801761-1.315323$ H -8.984452 -1.181351-1.632832 C $-7.870273-2.689874-3.956755$ H $-7.284201-0.741566-3.293363$ C $-6.072715-1.0166731 .742374$ O $-7.544226-2.7944091 .062895$ H -8.173558 -0.8610351 .154029$ H -5.278857-1.768727 1.650233 O $-6.508013-1.054828 \quad 3.106811$ C -5.4782980 .3769361 .460394$ $\begin{array}{llll}\text { C }-4.376876 & 0.754867 & 2.471236\end{array}$ H -5.0662660 .3903740 .445853$ $\begin{array}{lllll}\text { O } & -6.527479 & 1.342602 & 1.518048\end{array}$ H -4.8276850 .9409173 .453533$ H -3.686766 -0.0931362 .560949$ H $-7.263858-2.663545-4.867763$ H -7.908904 -3.726910 -3.606717 H -8.889039 -2.385254 -4.218187 C $-4.780187-1.456948-3.229245$ H -7.878204 -2.891957 1.970275 H $-6.693727-1.9820743 .330686$ H -6.1975392 .1641491 .117790$ H -4.882412 -0.370038 -3.124704 H $-4.867385-1.685056-4.297487$ H -5.748553 -3.251144 -2.473328 C $-2.128137 \quad 2.757993 \quad 0.264177$ O $-2.873703 \quad 1.6845340 .852047$ C -3.5861842 .0082412 .050464$ C $-2.6038472 .465783 \quad 3.138885$ C -1.7350753 .6302652 .649070$ C -1.126639 3.3531461 .269875 H -1.556892 $2.287452-0.545700$ C -3.049184 $3.807433-0.367655$ H -4.2944042 .8171151 .846942$ O $-1.765674 \quad 1.3838163 .538178$ H -3.173092 2.7728144 .023829 H -0.9253293 .8073213 .367813$ O -2.5201204 .8166982 .576390$ H $-0.687517 \quad 4.2706700 .860713$ O $-0.057743 \quad 2.4206431 .449399$ C $-3.8961043 .190674-1.456857$ H -3.7078204 .2800560 .366516$ H $-2.4661174 .614530-0.825981$ H $-1.286016 \quad 1.0767542 .750137$ H -2.864690 4.9959453 .467852 H 0.3914592 .3204710 .593491 O $-5.211962 \quad 3.230317-1.101398$ O $-3.4420652 .722364-2.492929$ C $-6.1256582 .677181-2.052841$ H $-6.1227513 .276121-2.968199$ H $-5.8670881 .637909-2.270758$ H -7.127901 2.703462 -1.617061 SCF Energy (B3LYP/6-31G**/MMFF) $=-3245.91794822$

$03 \_8$

MMFFF Geometry

C -2.956127 1.970662 -2.902369

C -2.282054 $1.103676-2.131577$

C $-1.1064540 .292037-2.607644$

O $-0.077504 \quad 0.329925-1.591535$

C $-1.536097-1.175040-2.790564$

C $-0.457943-2.059459-3.386264$

C $0.092057-3.120655-2.764075$

C $-0.023895-1.711909-4.787466$

C $-0.221545-3.658005-1.396664$

C $0.644749-3.080657-0.267692$

C $2.148316-3.366274-0.414718$

C $2.897383-2.9948340 .867099$

C $4.415318-3.187145 \quad 0.723413$

O $2.653850-2.585004-1.500778$

O $5.049484-2.5231471 .827903$

C $4.892562-2.478506-0.562788$

C $4.814906-4.6642910 .818656$

O $6.262768-2.768695-0.825374$

C $4.037494-2.812487-1.802435$

C $4.387211-1.944580-3.036622$
O $4.262475-4.177839-2.174841$

C $0.653747 \quad 1.470818-1.478870$

O $0.5640212 .476113-2.164951$

C $1.5772341 .292125-0.337779$

C 2.1656262 .3657880 .209081

C 3.0862192 .3569691 .347253

C 3.5706831 .0195211 .841482

C $4.224901-0.432157-2.846043$

C $5.507715 \quad 0.283074-2.454205$

C $6.3407432 .518781-1.732585$

O $6.613860-0.249279-2.486246$

C $6.4921932 .535467-0.244163$

C 6.5211883 .6654860 .478778

C $6.7168193 .704604 \quad 1.967470$

C 5.6422274 .4989142 .675975

C $5.8456425 .781338 \quad 3.022111$

C 4.3673143 .7812103 .057208

C $3.4648063 .537771 \quad 1.879727$

N $5.2746591 .606024-2.101708$

H -2.664266 $2.107936-3.940207$

H -2.593263 $0.955500-1.100779$

H $-0.7055770 .696569-3.544759$

H -2.413243 -1.226824 -3.449666

H $-1.862264-1.565921-1.820315$

H $\quad 0.857463-3.684315-3.296477$

H $0.625524-2.479035-5.223230$

H $-0.895845-1.613806-5.442527$

H $0.531573-0.769361-4.798072$

H $-1.277689-3.500528-1.151959$

H $-0.096168-4.748077-1.424040$

H $0.478161-1.998562-0.215210$

H $\quad 0.287639-3.5114670 .675856$

H $2.277792-4.431896-0.637338$

H $2.517397-3.5681301 .721961$

H $2.706222-1.9399851 .104950$

H $4.701768-2.9098252 .649806$

H $4.832538-1.400894-0.369800$

H $4.506472-5.082587 \quad 1.784279$

H $5.903224-4.7829450 .775946$

H $4.369038-5.2809520 .035601$

H $6.768587-2.521062-0.032543$

H $5.387053-2.201078-3.406834$

H $3.696848-2.235702-3.841599$

H $4.010431-4.288624-3.106618$

H 1.7294110 .2856530 .034349

H $1.9376263 .348996-0.200665$

H $3.9723420 .426500 \quad 1.012821$

H 2.7513450 .4630702 .308230

H 4.3761631 .0902482 .575270

H $3.9147700 .005825-3.803496$

H $3.441321-0.209449-2.115961$

H $6.0780053 .503082-2.133194$

H $7.2778702 .192049-2.194781$

H 6.6103491 .5780960 .257969

H $6.4322394 .622390-0.031074$

H 7.6997414 .1535412 .162213

H 6.7648632 .6945422 .393021

H 5.0911916 .3502823 .555135

H 6.7702486 .2926292 .774872

H 3.7982104 .3818333 .779604

H 4.6207822 .8651673 .597911

H $3.0636954 .454598 \quad 1.445196$

H $4.3196081 .949098-2.056676$

C $-5.4356812 .003458-2.505047$

O \begin{tabular}{lllll}
\hline & -5.406788 & 0.921143 & -1.551709
\end{tabular}

C $-6.6104260 .951971-0.768232$

C $-7.5919731 .796295-1.567956$

C $-6.6621152 .845857-2.147595$

H -6.3701291 .4794690 .162647$

C $-7.070045-0.473066-0.453229$

H $-8.062262 \quad 1.220797-2.373602$

H -8.383782 $2.220099-0.943069$

C $-7.253076 \quad 3.600657-3.325642$

H $-6.4074353 .564002-1.356325$

C $-5.985773-1.3478550 .211684$

O $-7.447527-1.109847-1.680920$

H $-7.974201-0.4408130 .165735$

H $-5.186752-1.552564-0.511096$ 
O $-6.582940-2.6193490 .501850$ C -5.371637 -0.7721641 .504368$ C $-4.356884-1.7421492 .139076$ H $-4.8676020 .174816 \quad 1.290885$ O $-6.405560-0.515368 \quad 2.453482$ H -4.859555 -2.692646 2.352970 H -3.568382 -1.949323 1.407322 H $-6.5379364 .329751-3.719250$ H -7.525893 $2.922162-4.141054$ H -8.155569 4.141375 -3.022430 C $-4.1228472 .780705-2.409006$ H -7.728012 -2.015305 -1.463148 H -7.252451 -2.476982 1.193099 H $-6.933163 \quad 0.2313452 .124460$ H -3.941532 $3.079553-1.369504$ H $-4.1764403 .701683-3.000735$ H -5.548148 $1.551466-3.498808$ C -1.8591780 .0195542 .581825$ O $-3.095506 \quad 0.0473113 .304860$ C -3.747086 -1.217690 3.460176 C $-2.812805-2.2180684 .157287$ C -1.478121 -2.3387053 .428861$ C $-0.867474-0.9539393 .233381$ H -2.048165 -0.269764 1.540362 C $-1.278801 \quad 1.4441822 .598375$ H $-4.584800-1.0432544 .146948$ O $-3.443353-3.4905514 .252057$ H $-2.627311-1.8730725 .183149$ H $-1.582750-2.8621192 .471393$ O $-0.571542-3.112148 \quad 4.217674$ H $-0.540153-0.5616184 .205147$ O $0.290054-1.0655282 .410136$ C -1.9956412 .3842151 .654265$ H $-1.344991 \quad 1.8774383 .602927$ H -0.2269861 .4168822 .292957$ H -2.883737 -4.0570244 .809134$ H $-0.908826-4.0235314 .242160$ H $0.892370-1.6959742 .840842$ O -1.1650593 .4270261 .356090$ O -3.1413282 .2409891 .252222$ C $-1.694123 \quad 4.4182640 .470242$ H -2.691056 4.7382530 .788054 H - 1.0268215 .2839830 .493561 H -1.720125 $4.023562-0.548670$ SCF Energy (B3LYP/6-31G**/MMFF) $=-3245.91185010$

0380

MM̄FF Geometry

C -2.596490 -2.641227 -2.355153

C -1.731669 -3.659912 -2.486336

C $-0.225247-3.601102-2.348038$

O $0.199426-2.371505-1.721005$

C $0.250078-4.759133-1.446741$

C $1.756949-4.856947-1.301616$

C $2.417302-4.772731-0.129692$

C $2.525931-5.095595-2.575612$

C $1.838581-4.5814621 .244393$

C $1.725095-3.1152811 .685854$

C $3.075705-2.4199731 .919225$

C $2.868113-1.0244312 .512609$

C $4.196398-0.2700322 .685326$

O $3.756480-2.3059520 .668449$

O $3.888871 \quad 1.1123242 .924473$

C $4.992263-0.3331901 .362705$

C $4.972935-0.7601033 .913263$

O $6.302565 \quad 0.2018341 .529340$

C $5.075522-1.7505030 .760829$

C $5.681613-1.774128-0.663608$

O $5.917795-2.5627961 .588194$

C $0.521908-1.321681-2.521477$

O $0.493052-1.281527-3.740374$

C $0.883103-0.195464-1.634437$

C $0.7550141 .062165-2.081325$

C $1.0373332 .275407-1.312909$

C $1.7849182 .115545-0.016070$

C $4.924280-0.954887-1.715270$

C $5.498936 \quad 0.432779-1.949491$

C $5.0576612 .519886-3.238798$
O $6.559443 \quad 0.816327-1.463992$

C $4.4584973 .527648-2.309910$

C $3.6993224 .552886-2.725159$

C $3.1326245 .599177-1.809063$

C $1.6363295 .757087-1.957412$

C $1.1157046 .747894-2.701084$

C $0.746414 \quad 4.821853-1.171847$

C $0.6276283 .463093-1.805000$

N $4.7000681 .183148-2.802602$

H $-2.233560-1.636533-2.160644$

H $-2.137908-4.644164-2.715155$

H $\quad 0.217767-3.701868-3.345985$

H $-0.109657-5.715946-1.848441$

H $-0.222589-4.644464-0.465801$

H $3.502766-4.864795-0.147040$

H $2.485943-4.213601-3.222125$

H $3.582300-5.316250-2.387736$

H $2.106585-5.948841-3.118683$

H $\quad 0.851545-5.0513541 .312542$

H $2.463468-5.1307491 .959994$

H $1.147304-2.5613800 .936266$

H $1.151686-3.0920852 .620772$

H $3.666655-3.0414392 .602183$

H $2.332811-1.0820523 .468632$

H $2.222064-0.4389101 .844576$

H 3.3228481 .1612533 .713888

H 4.4777740 .3226190 .651990

H $4.375928-0.6238004 .822952$

H $5.885272-0.1726144 .064385$

H $5.246971-1.8153913 .853942$

H 6.2050961 .1021671 .884094

H $6.744135-1.505085-0.626001$

H $5.660547-2.819252-1.005093$

H $6.241040-3.310698 \quad 1.059073$

H $1.208332-0.432783-0.627978$

H $\quad 0.3775201 .221328-3.090543$

H 1.1705041 .5932820 .724123

H 2.0979913 .0626390 .428052

H $2.7021241 .540449-0.176409$

H $4.995716-1.478723-2.677267$

H $3.861855-0.873719-1.465956$

H $4.6949062 .634499-4.265162$

H $6.1476132 .622791-3.240560$

H $4.6833023 .425667-1.250797$

H $3.4994704 .668563-3.788065$

H $3.6323536 .549059-2.040579$

H $3.3743865 .387707-0.760035$

H $0.0430846 .885833-2.788746$

H $1.7511257 .440197-3.243669$

H $-0.2690695 .235836-1.109447$

H $1.0903134 .781944-0.134388$

H $\quad 0.1005203 .473697-2.760157$

H $3.8087430 .805738-3.111071$

C $-4.794734-1.991996-1.357294$

O $-4.979914-0.638119-1.834161$

C $-6.342295-0.259540-1.598535$

C $-6.871866-1.229962-0.554330$

C $-6.189327-2.509187-0.999421$

H $-6.881905-0.457782-2.535894$

C $-6.510784 \quad 1.228224-1.284758$

H $-6.543247-0.9523810 .453725$

H $-7.963260-1.303906-0.556906$

C -6.191956 -3.598442 0.059151

H $-6.692536-2.885137-1.900611$

C $-5.8100501 .788581-0.028581$

O $-7.9205831 .462679-1.153242$

H $-6.1837541 .807021-2.157750$

H $-6.239412 \quad 1.3385240 .873934$

$\begin{array}{lllll} & 0 & -6.157924 & 3.182839 & 0.016265\end{array}$

C $-4.272863 \quad 1.658585-0.008878$

C $-3.5999602 .504142 \quad 1.093363$

H $-4.0026860 .608317 \quad 0.132974$

O $-3.746107 \quad 2.064394-1.273971$

H $-3.817498 \quad 3.562628 \quad 0.910330$

H -4.0389092 .2406682 .061089$

H -5.654853 -4.485411 -0.291186

H $-5.716100-3.2598990 .985184$

H $-7.217447-3.8968430 .300253$ 
C $-4.090008-2.815860-2.438486$ H -8.041244 $2.413772-0.989490$ H -6.0376183 .4861890 .932059$ H -4.007358 2.990151-1.418424 H -4.420346 -2.491358 -3.432190 H -4.340844 -3.876990 -2.325619 H $-4.177105-1.923817-0.455193$ C -1.8711880 .5444472 .732959$ $\begin{array}{llll}\mathrm{O} & -1.673918 & 0.985548 & 1.383884\end{array}$ C -2.0641772 .3365121 .120804$ C -1.3323253 .3050142 .062406$ C -1.4879842 .9085203 .525253$ C $-1.1102271 .442006 \quad 3.716394$ H -2.9402070 .5629442 .974852$ C -1.366159 -0.9044612 .815130$ H -1.7070772 .5624440 .109651$ O -1.7909334 .6412051 .887408$ H -0.2621653 .3050491 .822594$ H -2.502900 3.1053523 .890649 O $-0.609024 \quad 3.703611 \quad 4.324101$ H -0.0273991 .3192683 .582966$ O $-1.426570 \quad 1.0506765 .050873$ C -2.357656-1.895424 2.244243 H $-0.430393-0.9943222 .252065$ H -1.192474 -1.204650 3.853968 H -1.6592844 .8746370 .952563$ H -0.8328714 .6357064 .159063$ H $-0.952740 \quad 1.6530575 .649845$ O $-1.725188-3.0882642 .038024$ O $-3.542936-1.6693362 .044933$ C $-2.552515-4.1539881 .561631$ H -3.389709 -4.324367 2.245262 H $-2.915202-3.9275600 .555947$ H -1.945851 -5.062443 1.519751 SCF Energy (B3LYP/6-31G**//MMFF)= -3245.90722037

03_81

MM̄FF Geometry

C $2.414952-3.5292150 .632215$

C $1.678400-4.400208-0.075339$

C $0.390167-4.101954-0.809200$

O $-0.235026-2.890190-0.330883$

C $0.685558-3.933088-2.311142$

C $-0.552612-3.757330-3.169118$

C $-0.822185-2.662053-3.906860$

C $-1.504640-4.925530-3.193651$

C $0.005840-1.415416-4.047862$

C $-0.335602-0.309702-3.038162$

C $-1.7373380 .295783-3.217394$

C $-1.9186981 .513756-2.309010$

C $-3.3393002 .093284-2.398996$

O $-2.709092-0.695886-2.879267$

O $-3.5146202 .998320-1.297574$

C $-4.3649760 .952947-2.214532$

C $-3.5363862 .920040-3.675058$

O $-5.6909131 .413355-2.461741$

C $-4.068904-0.287820-3.081129$

C $-4.965794-1.501464-2.734430$

O $-4.3095490 .032701-4.456936$

C $-0.971751-2.9851120 .810249$

O $-1.141674-3.9803531 .496702$

C -1.532494 -1.647679 1.098513

C $-1.962933-1.3719962 .338597$

C -2.535825 -0.1031972 .790984$

C -2.9296290 .8954051 .736976$

C $-4.854486-2.012310-1.292917$

C $-5.947023-1.501229-0.367296$

C $-6.669376-1.6082322 .017404$

O $-6.912939-0.850092-0.755267$

C $-6.310901-0.3096172 .667413$

C $-6.101161-0.1760393 .985971$

C -5.7993291 .1338574 .655652$

C -4.5453001 .0870875 .499258$

C -4.6136290 .9224196 .831078$

C -3.2211421 .3243824 .810106$

C $-2.704158 \quad 0.0958254 .114764$

N -5.732304 -1.8952240 .947338$

H $2.106121-2.4902760 .709210$
H $2.025117-5.429181-0.153128$

H $-0.294974-4.944635-0.654672$

H $\quad 1.232140-4.809737-2.684260$

H $1.362123-3.080508-2.434700$

H -1.741969-2.653132 -4.490749

H -2.292019-4.803885 -3.945408

H $-0.967018-5.849264-3.431775$

H -1.995205 -5.044679 -2.222866

H $1.072304-1.651982-3.969423$

H $-0.124950-1.030541-5.067201$

H $-0.235926-0.714086-2.024379$

H $0.4128120 .485172-3.146242$

H -1.856523 $0.582186-4.268889$

H -1.174758 2.285870 -2.540494

H $-1.7310881 .220297-1.267613$

H $-2.8236583 .680339-1.357297$

H $-4.3296800 .662892-1.159133$

H -2.820865 $3.750683-3.707183$

H $-4.5299903 .381085-3.698064$

H $-3.4047982 .336339-4.588535$

H $-5.8458382 .176907-1.879725$

H -6.005560 -1.294486 -3.014360

H $-4.651471-2.330114-3.385828$

H -4.458108 -0.794075 -4.944976

H -1.559175 -0.9254170 .290894$

H $-1.866772-2.1394813 .105743$

H -3.5829840 .4248830 .996024$

H $-2.046911 \quad 1.2843061 .224236$

H -3.483365 1.7513112 .127909

H -4.955679 -3.105348 -1.306750

H $-3.874845-1.781289-0.863469$

H -6.628888 -2.447895 2.718458

H -7.682181 -1.546673 1.606012

H -6.2536620 .5679502 .027683$

H -6.187873 -1.049529 4.628471

H $-6.661671 \quad 1.3906305 .284966$

H -5.7163251 .9481173 .924940$

H -3.7203460 .9184147 .446913$

H $-5.564403 \quad 0.7821357 .334639$

H -2.463059 1.6203895 .548085

H -3.3063642 .1890394 .145649$

H -2.401837 $-0.696758 \quad 4.800677$

H $-4.873772-2.3824921 .187222$

C $4.922367-3.4098160 .605340$

O $4.988879-1.9722500 .673146$

C $6.359735-1.5785410 .881422$

C $7.197792-2.8426740 .725418$

C $6.234019-3.9064501 .212111$

H $6.432418-1.2391081 .921315$

C $6.739978-0.436273-0.067322$

H $7.469541-3.026337-0.320457$

H $8.122733-2.800373 \quad 1.308673$

C $6.622378-5.3127430 .789169$

H $6.182507-3.8597662 .308309$

C 5.7938440 .7794950 .004215

O $6.702985-0.931570-1.412348$

H $7.776081-0.1309590 .120739$

H $4.8164980 .493427-0.399345$

O $6.3052411 .782819-0.882767$

C 5.6308061 .3878781 .408317

C 4.7924232 .6745411 .438463

H 5.1861930 .6507462 .083925

O $6.929137 \quad 1.692858 \quad 1.925117$

H 4.7708243 .0659282 .465182

H 5.3091783 .4676740 .880159

H $5.898108-6.0463571 .156234$

H $6.671798-5.404581-0.301054$

H $7.604662-5.5780461 .193568$

C $3.682671-3.9114661 .346143$

H $6.945309-0.194476-1.998963$

H $7.1540692 .089526-0.519511$

H 6.8129252 .0312632 .829403

H $3.642032-3.4796972 .353780$

H $3.730922-5.0002331 .464096$

H $4.867327-3.677776-0.458348$

C 1.4272913 .9672870 .521883

O 2.7603383 .8383611 .032204

C 3.3567982 .5407150 .905192 
C 2.5181541 .4910101 .649771

C 1.0578581 .5176961 .189661

C $0.4873112 .939063 \quad 1.172448$

H $1.100177 \quad 4.9657810 .839957$

C $1.3915123 .931084-1.014474$

H $3.4102172 .289172-0.158259$

O $2.582893 \quad 1.7406443 .052359$

H $2.940766 \quad 0.495734 \quad 1.471385$

H 0.4544890 .8959281 .861066

$\begin{array}{lllll}\text { O } & 0.960194 & 0.963411 & -0.117741\end{array}$

H $-0.4790572 .961575 \quad 0.656093$

O $\quad 0.2517593 .3613462 .518535$

C $2.1178245 .105351-1.637034$

H $1.8474293 .020544-1.411320$

H $0.3496753 .979930-1.351883$

H 2.0649951 .0490483 .498278

H $1.2859460 .048087-0.075040$

H -0.3894602 .7445932 .911230$

O $2.0335355 .002957-2.997284$

O $2.6844065 .996945-1.020578$

C $2.6793576 .046318-3.733735$

H $3.7514296 .056796-3.515044$

H $2.5407265 .845971-4.799543$

H $2.2263547 .013665-3.496456$

SCF Energy (B3LYP/6-31G*//MMFF) $=-3245.89705037$

0382

MMFF Geometry

C 1.7305202 .3392482 .197098

C $1.4333291 .435483 \quad 3.143876$

C $0.3642180 .367325 \quad 3.071704$

O $-0.492524 \quad 0.539627 \quad 1.921285$

C $1.034854-1.0156122 .958920$

C $0.066051-2.1777453 .064976$

C $-0.172584-3.0712472 .084806$

C $-0.634278-2.3254674 .391842$

C $0.437858-3.1307740 .713521$

C $-0.359053-2.396838-0.375458$

C $-1.762189-2.973116-0.625265$

C $-2.386942-2.351402-1.876499$

C $-3.826879-2.839220-2.104329$

O $-2.581874-2.6817010 .509173$

O $-4.435454-1.976280-3.077796$

C $-4.629555-2.677559-0.794609$

C -3.861566 -4.252994-2.697082

O $-5.919049-3.272958-0.911292$

C $-3.902312-3.2375210 .444735$

C $-4.610374-2.8933681 .778058$

O $-3.853513-4.667007 \quad 0.355503$

C -1.528829 1.4140132 .037776

O -1.8328622 .0715753 .020016$

C -2.219855 1.4610650 .730756

C -3.0052342 .5082090 .438837$

C $-3.7378882 .713885-0.812316$

C -3.810057 $1.557011-1.773496$

C $-4.779195-1.3978052 .067817$

C $-6.129360-0.8372111 .652386$

C -7.3603731 .3213911 .467696$

O $-7.071860-1.539707 \quad 1.297172$

C $-7.306468 \quad 1.7953540 .049681$

C $-7.491816 \quad 3.076116-0.304134$

C $-7.4826923 .563328-1.724678$

C $-6.5181864 .708005-1.940301$

C $-6.9455345 .982028-1.934204$

C $-5.0770874 .369844-2.247577$

C $-4.317728 \quad 3.912531-1.031825$

N -6.1701780 .5463101 .763807$

H $1.1761072 .351807 \quad 1.262896$

H 2.0132941 .4428704 .065090

H -0.2359810 .4223023 .987821$

H $1.785585-1.1336203 .751852$

H $1.585507-1.0573682 .012792$

H $-0.887410-3.8687232 .285512$

H -1.347575 -1.510212 4.545537

H $-1.191615-3.2656344 .465078$

H $0.093792-2.3147735 .209525$

H $1.459773-2.7411940 .731311$

H $0.542931-4.1867120 .433412$
H $-0.435207-1.336883-0.106478$

H $0.218449-2.452720-1.305642$

H $-1.672502-4.059378-0.743946$

H $-1.770214-2.553134-2.761258$

H $-2.404015-1.258596-1.768119$

H -3.896694 -2.010381-3.886824

H $-4.793278-1.602734-0.654607$

H -3.335754 -4.277523 -3.659199

H $-4.889102-4.567181-2.910571$

H $-3.398430-5.000886-2.050067$

H $-6.352785-2.875221-1.685523$

H $-5.564326-3.4298911 .847843$

H $-3.988920-3.3022442 .588104$

H $-3.714227-5.0271411 .247080$

H -2.043027 0.6472800 .037292

H -3.1043663 .3053801 .174693$

H $-2.8250041 .355042-2.207482$

H $-4.5044741 .718270-2.600554$

H $-4.1603190 .655211-1.260138$

H $-4.708781-1.2453083 .152590$

H -3.979678 -0.8129831 .603264$

H -7.3933352 .1509082 .181113$

H -8.248368 $0.698327 \quad 1.614753$

H $-7.133006 \quad 1.048792-0.721819$

H -7.6932323 .8151900 .468207$

H -8.502791 $3.884431-1.973026$

H -7.249858 $2.752559-2.426156$

H $-6.2657716 .807795-2.115969$

H -7.984728 $6.227591-1.741850$

H $-4.557763 \quad 5.256117-2.636556$

H $-5.0476343 .648360-3.068624$

H $-4.2127554 .685733-0.269174$

H $-5.325546 \quad 1.0511132 .016439$

C 3.9439563 .0857701 .323346

O $3.479083 \quad 3.387773-0.010871$

C $4.5245804 .074153-0.712219$

C 5.7844163 .9110350 .126048

C 5.1956913 .9398201 .524831

H $4.2544125 .139665-0.692869$

C $4.6284943 .655830-2.180872$

H $6.2708282 .947535-0.063865$

H $6.5144864 .706804-0.048838$

C 6.1459983 .4279532 .592179

H 4.9079344 .9718311 .767425

C $4.9685462 .182372-2.496124$

O $5.6385514 .484345-2.774651$

H $3.686413 \quad 3.912411-2.682028$

H $5.9906721 .956200-2.170918$

O $4.9767532 .087141-3.930543$

C $3.9887101 .132414-1.932985$

C $4.183973-0.276404-2.530243$

H $4.1091931 .075840-0.848321$

O $2.6457881 .546844-2.191534$

H $3.949797-0.241529-3.600468$

H $5.236308-0.561329-2.431360$

H 5.6691113 .4320263 .576871

H 6.4768932 .4067072 .380430

H 7.0372234 .0617812 .647015

C 2.8304753 .3577622 .333682

H $5.6853454 .251944-3.717917$

H $5.4887631 .296054-4.169709$

H $2.5455341 .636172-3.154820$

H 2.3961274 .3499812 .162930

H 3.2340573 .3453153 .352379

H 4.2083552 .0222991 .334302

C $4.779578-2.195700-0.190890$

O $3.555774-1.509598-0.482531$

C $3.265455-1.332071-1.872378$

C $3.242670-2.689517-2.589721$

C $4.529629-3.471494-2.360171$

C $4.819285-3.572612-0.865534$

H $5.628760-1.592171-0.531916$

C $4.862768-2.3493701 .336245$

H $2.242619-0.938686-1.919752$

O $3.033567-2.529085-3.988406$

H $2.405372-3.287995-2.208300$

H $5.375278-3.027029-2.898151$

O $4.374234-4.795907-2.874921$ 
H $\quad 4.089039-4.244874-0.396290$

O $6.111438-4.147558-0.678544$

C $5.185437-1.0444552 .028725$

H $3.899902-2.7107421 .717388$

H $5.648032-3.0575471 .621776$

H $2.197837-2.045385-4.104772$

H $4.146887-4.714332-3.817154$

H $6.114706-5.007341-1.133202$

O $4.788211-1.1216183 .332995$

O $5.752463-0.0954471 .505663$

C $5.0707550 .028901 \quad 4.134747$

H 6.1511010 .1906214 .196017

H $4.5713160 .909937 \quad 3.723934$

H $4.684898-0.1545635 .140889$

SCF Energy (B3LYP/6-31G**//MMFF)= -3245.91129039

0383

MMMFF Geometry

C -2.345540 $2.374286-1.993392$

C $-1.7808021 .432416-1.222085$

C $-0.6906370 .513681-1.713848$

O $0.4003310 .518065-0.764804$

C $-1.226329-0.927755-1.810596$

C $-0.288834-1.874800-2.536762$

C $0.304998-2.949681-1.982495$

C $-0.061743-1.577377-3.998215$

C $0.184924-3.459032-0.575354$

C $1.264549-2.9506760 .391110$

C $2.704258-3.299480-0.018325$

C $3.674436-2.9911751 .122929$

C $5.132531-3.2578190 .719862$

O $3.054006-2.506569-1.153418$

O $5.979220-2.6437541 .705698$

C $5.429954-2.555586-0.626102$

C $5.456054-4.7566860 .750967$

O $6.714219-2.947947-1.103203$

C $4.347307-2.789000-1.706821$

C $4.498805-1.884866-2.952313$

O $4.405167-4.124512-2.223381$

C $1.2523591 .578285-0.795514$

O $1.1932122 .551308-1.530083$

C $2.283510 \quad 1.352354 \quad 0.240831$

$\begin{array}{llll}\text { C } 3.047962 & 2.376733 & 0.647129\end{array}$

C 4.1117682 .3114721 .651090

C $4.568105 \quad 0.9453182 .090884$

C $4.454060-0.376666-2.684766$

C $5.8228420 .258570-2.504763$

C $6.891322 \quad 2.424178-1.889496$

O $6.875684-0.335719-2.720002$

C $7.261763 \quad 2.398389-0.440109$

C $7.458618 \quad 3.508196 \quad 0.287719$

C 7.8684873 .5022081 .732598

C 6.9448544 .3252772 .602375

C 7.2549815 .5892972 .936850

C $5.705968 \quad 3.6545743 .151708$

C 4.6397613 .4661762 .108586

N $5.7277551 .584692-2.104747$

H -2.006571 2.497574 -3.019228

H $-2.1251071 .296753-0.200125$

H $-0.3184810 .847581-2.689719$

H - $2.185262-0.936062-2.346396$

H -1.439418 -1.292472 -0.799698

H $0.953588-3.555014-2.615399$

H $0.464587-2.390242-4.510414$

H $-1.018703-1.437248-4.511435$

H $0.539265-0.671579-4.121321$

H $-0.801238-3.224477-0.162827$

H $0.224345-4.555480-0.609889$

H $1.163665-1.8635310 .490158$

H $1.056959-3.3877961 .375610$

H $2.744690-4.362162-0.283859$

H $3.415251-3.5648912 .021551$

H $3.579102-1.9343291 .405827$

H $5.749194-3.0169412 .573897$

H $5.490034-1.483751-0.402294$

H $5.324170-5.155727 \quad 1.764161$

H $6.504226-4.9420960 .491973$

H $4.822569-5.3455090 .084134$
H $6.935356-2.383897-1.863032$

H $5.392895-2.166863-3.521375$

H $3.653869-2.114411-3.618355$

H $5.169605-4.577278-1.833355$

H 2.3801970 .3518280 .645807

H 2.8701623 .3619590 .217838

H $4.809767 \quad 0.324562 \quad 1.220930$

H 3.7857980 .4468202 .672897

H 5.4706590 .9626472 .704987

H $4.0016800 .115994-3.555159$

H $3.828157-0.149262-1.817050$

H $6.6332443 .430674-2.233432$

H $7.7273732 .052521-2.490544$

H 7.3979061 .4248050 .024925

H $7.3489494 .480177-0.188468$

H 8.8892403 .9025651 .790754

H 7.9293512 .4810812 .128834

H 6.6121916 .1778103 .582978

H $8.157076 \quad 6.0656492 .567047$

H 5.2701604 .2672413 .952453

H 5.9949512 .7208583 .641797

H 4.2439844 .4057501 .720204

H $4.8139401 .979221-1.900925$

C $-4.7826872 .874361-2.188991$

O $-5.1926011 .599272-1.656306$

C $-6.5912961 .656946-1.322206$

C $-7.1289782 .902971-2.011706$

C $-5.9350953 .834129-1.894513$

H $-6.650977 \quad 1.811691-0.238423$

C $-7.2747130 .334795-1.687466$

H $-7.3626112 .717137-3.066267$

H $-8.0287783 .292618-1.526330$

C $-5.9993995 .027516-2.831281$

H $-5.8687914 .194031-0.858763$

C $-6.544570-0.909681-1.136141$

$\begin{array}{lllll}\text { O } & -7.308793 & 0.217648 & -3.114962\end{array}$

H $-8.3184270 .353747-1.352867$

H -5.586626 -1.027453-1.656723

O $-7.319775-2.065104-1.477311$

C $-6.307290-0.8893340 .384726$

$\begin{array}{llll}\text { C }-5.523376 & -2.122075 & 0.862747\end{array}$

H -5.7406790 .0056840 .643312$

O $-7.567289-0.814946 \quad 1.045514$

H $-6.085043-3.0304970 .613207$

H $-4.581421-2.162640 \quad 0.304114$

H $-5.1104625 .656754-2.722740$

H $-6.063890 \quad 4.712886-3.878330$

H $-6.8779895 .642471-2.610565$

C $-3.457516 \quad 3.280579-1.543553$

H $-7.736924-0.629929-3.325821$

H $-8.154184-2.014181-0.980005$

H $-7.399391-0.5955801 .977432$

H $-3.5468183 .247008-0.451914$

H -3.195505 $4.308572-1.817270$

H $-4.6600322 .742595-3.272133$

C $-3.376311-0.5947892 .451096$

O $-4.711419-0.8987832 .869918$

C $-5.238454-2.1376292 .383120$

C $-4.345076-3.3032692 .832386$

C $-2.892054-3.0868642 .414604$

C $-2.410303-1.7112852 .870381$

H $-3.353089-0.4596741 .362797$

C $-2.9629890 .728560 \quad 3.120403$

H $-6.207863-2.2697562 .879966$

O $-4.838047-4.5259852 .295715$

H $-4.392148-3.3865973 .926006$

H $-2.759450-3.2104561 .334104$

O $-2.064342-4.071472 \quad 3.037543$

H -2.293057 -1.714208 3.961999

O $-1.126727-1.4525102 .306641$

C -3.7038401 .9363602 .588955$

H -3.161901 0.6742884 .197649

H -1.8950090 .9132452 .957532$

H $-4.328345-5.2506822 .694981$

H $-2.297315-4.9343002 .655398$

H $-0.532595-2.1604652 .608592$

O $-3.2276693 .054122 \quad 3.215064$

$\begin{array}{llll}\text { O } & -4.584676 & 1.914974 & 1.742512\end{array}$ 
C -3.8381424 .2874862 .822040$

H -4.9167364 .2543423 .003176$

H -3.4059145 .0885563 .427656$

H -3.6289094 .4926801 .768230$

SCF Energy (B3LYP/6-31G**//MMFF) $=-3245.92123921$

0384

MM̄FF Geometry

C -3.286253 $4.640348-0.039352$

C $-2.4410344 .015156-0.872636$

C $-0.9402954 .119205-0.788370$

O $-0.4172122 .827429-0.400994$

C $-0.3734154 .472729-2.175689$

C $1.1354134 .611782-2.209537$

C $1.9575583 .826360-2.933060$

C $1.7087375 .735256-1.384823$

C $1.5890452 .682160-3.836452$

C $1.5761011 .304976-3.154578$

C $2.9506740 .847576-2.639561$

C $2.896902-0.607574-2.170261$

C $4.233275-1.070368-1.565799$

O $3.3277801 .684752-1.544848$

O $3.992142-2.298120-0.860303$

C $4.705824-0.033164-0.521724$

C $5.263129-1.392465-2.655421$

O $6.016033-0.342476-0.053274$

C $4.6370371 .424285-1.022976$

C 4.9038612 .4674090 .088673

O $5.6404741 .618489-2.027696$

C -0.4304502 .5272350 .926728$

O -0.8222013 .2375371 .839197$

C 0.0955251 .1541721 .084518

C -0.1457690 .4894632 .224384$

C $0.281471-0.8756472 .537153$

C $1.248570-1.5343741 .590356$

C 3.9339092 .4271981 .274865

C 4.4552471 .6524392 .474052

C 3.7579930 .9040244 .746994

O 5.6024861 .2225512 .554879

C $3.389491-0.5441214 .682168$

C $2.572639-1.1356745 .567027$

C $2.237090-2.5989845 .548739$

C $0.747617-2.8561445 .514370$

C $0.070974-3.1587876 .635005$

C $0.063439-2.8514294 .166366$

C $-0.197945-1.4604943 .654858$

N $3.491651 \quad 1.5328103 .467135$

H -2.8935305 .2717600 .753115$

H -2.846610 $3.366466-1.646235$

H $-0.6373714 .878012-0.056497$

H $-0.8108955 .418052-2.523881$

H $-0.7006183 .709046-2.889571$

H $3.0275984 .025903-2.883798$

H $1.5786275 .535773-0.316857$

H $2.7802395 .876505-1.563243$

H $1.2103516 .679017-1.629229$

H $0.6139032 .857214-4.303270$

H $2.3025062 .664792-4.670357$

H $\quad 0.857271 \quad 1.323437-2.326776$

H $1.2072670 .575335-3.886011$

H $3.6770990 .958447-3.453261$

H $2.604674-1.266909-2.995945$

H $2.113438-0.716210-1.407972$

H $3.648448-2.948057-1.496712$

H $4.043342-0.1420830 .343560$

H $4.877948-2.167472-3.329193$

H $6.181001-1.805257-2.222463$

H $5.525546-0.526054-3.265926$

H $6.648882-0.065549-0.737511$

H 5.9493002 .4118540 .415020

H $4.7975983 .461233-0.370220$

H $5.8217582 .570148-2.101777$

H $\quad 0.640377 \quad 0.7214430 .253683$

H $-0.733248 \quad 0.9818402 .998481$

H $0.771566-1.7282150 .624188$

H $1.641353-2.4849221 .956402$

H $2.119806-0.8917141 .428850$

H 3.7736683 .4553801 .624308
H 2.9597702 .0282660 .977336

H 3.1788201 .4459145 .501357

H 4.8215801 .0028734 .986990

H $3.839363-1.1392073 .890875$

H $2.146313-0.5434886 .373793$

H $2.672847-3.0509646 .449413$

H $2.714778-3.1096334 .703222$

H $-0.992141-3.3739716 .612879$

H $0.564728-3.1928237 .600549$

H $-0.908425-3.3580784 .237413$

H $0.646110-3.4619693 .472141$

H $-0.893363-0.8946494 .276544$

H $2.549826 \quad 1.8705613 .291226$

C -5.3361363 .2766960 .612672$

O $-4.8412042 .070777-0.003671$

C -4.4682031 .1443121 .033558$

C $-5.128771 \quad 1.6621282 .305841$

C $-5.009793 \quad 3.1626712 .101942$

H -3.3824911 .2252461 .157886$

C $-4.840138-0.2846750 .625286$

H $-6.183321 \quad 1.3693132 .366001$

H -4.6239971 .3093113 .210151$

C -5.9259213 .9782462 .996466$

H -3.9705503 .4542142 .298424$

C $-4.267869-0.714852-0.746538$

O $-6.268790-0.3675120 .552516$

H $-4.524813-0.9839971 .409179$

H $-4.714543-0.115158-1.548649$

O $-4.667647-2.071612-0.964971$

C $-2.732319-0.633007-0.830136$

C $-2.104596-0.872587-2.218058$

H -2.416476 $0.375500-0.548080$

O $-2.153196-1.5129320 .126566$

H $-2.386037-0.025417-2.856149$

H -1.016093 -0.821472 -2.091729

H -5.8151345 .0480972 .793256$

H $-6.977343 \quad 3.7125902 .844209$

H -5.6858353 .8071324 .050926$

C $-4.7804054 .498897-0.123678$

H $-6.505178-1.3094410 .514068$

H $-5.624264-2.071013-1.137215$

H -2.508358 -2.400489 -0.049180

H -5.2396545 .4130970 .270001$

H $-5.0680474 .436757-1.181404$

H -6.4245293 .2610510 .468588$

C $-0.851853-3.695983-1.959259$

O $-2.225940-3.353331-2.172297$

C $-2.456943-2.181194-2.955642$

C -1.782084 -2.321897 -4.330492

C $-0.301645-2.667792-4.205981$

C $-0.118079-3.878233-3.294538$

H $-0.366266-2.909758-1.369031$

C $-0.823887-5.000359-1.152238$

H $-3.534728-2.147646-3.152341$

O $-1.918713-1.128399-5.094944$

H $-2.276494-3.122801-4.895730$

H $\quad 0.281721-1.812144-3.848979$

O $0.215030-2.995191-5.497673$

H $-0.490814-4.771993-3.810609$

O $1.274269-4.084693-3.062311$

C $-1.180673-4.7650820 .294977$

H -1.515368 -5.744793 -1.565325

H $0.173307-5.455808-1.158803$

H $-2.869628-0.944082-5.180666$

H $\quad 0.053673-2.229838-6.075897$

H $1.607676-3.323872-2.557751$

O $-2.534653-4.7642860 .453250$

O $-0.350772-4.5898701 .177301$

C $-2.998763-4.5448151 .789416$

H $-2.661213-5.3541682 .443514$

H $-2.649218-3.5771072 .161162$

H -4.091805-4.538067 1.772095

SCF Energy (B3LYP/6-31G**//MMFF) $=-3245.90495834$

03 85

MM̄FF Geometry

C $-0.069273 \quad 4.7554750 .183312$

C $0.9820344 .329253-0.532537$ 
C 2.2761963 .8549970 .077282

O $2.4901242 .489933-0.351746$

C $3.4485174 .706647-0.440656$

C 4.7868014 .3290520 .165481

C $5.8304843 .835300-0.529472$

C 4.9232724 .5631791 .648423

C $5.9176883 .565432-2.005167$

C $5.5258882 .140523-2.425466$

C $6.4246091 .039269-1.839160$

C $6.130930-0.307541-2.504897$

C $6.949933-1.449004-1.881467$

O $6.1679260 .941116-0.435509$

O $6.383578-2.691527-2.326496$

C $6.790020-1.405275-0.346293$

C $8.404387-1.437734-2.367010$

O $7.663456-2.3391780 .282869$

C $7.001939-0.0004180 .254636$

C 6.6425030 .0812401 .758936

O $\quad 8.384508 \quad 0.357504 \quad 0.136809$

C $1.837366 \quad 1.5118770 .332162$

O 1.1350091 .6455341 .321329

C $2.096463 \quad 0.234317-0.365667$

C $1.282603-0.808618-0.146735$

C $1.384532-2.128451-0.771526$

C $2.630308-2.429481-1.561814$

C $5.196934-0.2854402 .112553$

C $5.012465-1.7317962 .543061$

C $3.225867-3.3697903 .125382$

O $5.948728-2.4912872 .776661$

C $2.928003-4.2328441 .939917$

C $1.774214-4.9013171 .789548$

C $1.472928-5.806978 \quad 0.629673$

C $0.180553-5.447718-0.069150$

C $-0.962366-6.0957510 .212968$

C $0.232574-4.393271-1.151545$

C $0.373920-3.004647-0.593893$

N $3.670553-2.0637582 .675590$

H 0.0071684 .8025031 .266099

H $0.9008394 .274720-1.615931$

H $2.2340793 .897418 \quad 1.172352$

H $3.2624955 .766701-0.222700$

H $3.4856664 .623893-1.532294$

H 6.7434893 .6025040 .017753

H 5.9517414 .4220751 .998053

H 4.6319835 .5883231 .899929

H 4.2893333 .8702302 .209670

H $5.3073354 .281995-2.565247$

H $6.9492993 .762897-2.324464$

H $4.4835651 .959229-2.138502$

H $5.5715412 .094902-3.520766$

H $7.4705511 .324639-2.001772$

H $6.310183-0.255042-3.586082$

H $5.064222-0.543801-2.390803$

H $6.409412-2.701370-3.298703$

H $5.767834-1.735114-0.125714$

H $8.444467-1.543855-3.457827$

H $8.962394-2.289964-1.963685$

H $8.936844-0.520924-2.105870$

H $7.480783-3.212772-0.103673$

H $7.362826-0.4966742 .350461$

H 6.7864451 .1266452 .068370

H 8.5797681 .0518600 .787769

H $2.9294040 .195728-1.057543$

H $0.446419-0.6794040 .538846$

H $3.523154-2.219258-0.963221$

H $2.665338-1.820774-2.471600$

H $2.713057-3.474875-1.866034$

H 4.8839530 .3272702 .967745

H $4.520206-0.0594881 .283731$

H $2.344394-3.2126093 .755024$

H $4.009685-3.8346563 .731926$

H $3.705128-4.3337161 .186033$

H $1.010666-4.8195252 .560152$

H $1.414485-6.8333461 .015362$

H $2.292879-5.812267-0.099218$

H -1.887905 $-5.861361-0.302458$

H -0.998772 -6.8716140 .970553$

H $-0.694219-4.412146-1.740766$
H $1.021192-4.651836-1.863254$

H $-0.483775-2.6917960 .001942$

H $2.961307-1.3844672 .416007$

C $-2.5031414 .137304-0.208323$

O $-2.1280252 .857584-0.759815$

C -2.4949501 .8266470 .182961$

C -3.4779942 .4807351 .141410$

C -2.9069613 .8811131 .243936$

H -1.5706421 .5591050 .709729$

C $-3.0367660 .614464-0.580883$

H -4.4866512 .5057140 .710323$

H -3.527161 1.9704992 .107434

C -3.8867664 .8989911 .800486$

H -2.0263983 .8442001 .896285$

C $-3.639729-0.4698340 .328568$

O $-1.9556810 .039370-1.322118$

H $-3.7748870 .947905-1.318228$

H $-4.509417-0.0721540 .860327$

O $-2.690807-0.8072221 .348966$

C $-4.034118-1.772177-0.391660$

C $-4.971743-1.606977-1.601592$

H -3.132963 -2.301973 -0.723634

O $-4.665727-2.6415730 .554226$

H $-4.418878-1.138082-2.424131$

H -5.251966 -2.616429-1.930020

H -3.4337565 .8946531 .837475$

H -4.7923754 .9621131 .187826$

H -4.1885564 .6260602 .817077$

C -1.381222 $5.156762-0.432702$

H -1.574765 $0.743882-1.874301$

H $-1.879550-1.1070040 .905590$

H -4.060346 -2.738591 1.309244

H -1.675167 $6.133275-0.031435$

H -1.246476 $5.288812-1.514169$

H $-3.3792414 .466621-0.782434$

C $-8.218342-0.8085710 .095066$

O $-7.020433-1.472960-0.328135$

C $-6.260597-0.801011-1.335126$

C $-7.102137-0.632644-2.611051$

C $-8.4265480 .076602-2.309641$

C $-9.144855-0.540950-1.103703$

H -8.723009 -1.5290780 .751667$

C $-7.9150010 .450076 \quad 0.923227$

H $-5.9761310 .189771-0.970498$

O $-7.367120-1.913579-3.179664$

H $-6.523165-0.057222-3.343617$

H $-9.0760840 .015435-3.191430$

O $-8.1564761 .453750-2.064096$

H $-9.9741370 .101418-0.787139$

O $-9.728493-1.784263-1.506038$

C $-7.1613870 .129772 \quad 2.197388$

H $-7.312637 \quad 1.164790 \quad 0.356281$

H $-8.8523290 .938388 \quad 1.212993$

H -7.865545 -1.771882 -4.002499

H $-9.0075961 .895330-1.902345$

H $-9.004071-2.396654-1.721745$

O $-6.672801 \quad 1.2908832 .726684$

O $-7.028105-0.9872262 .677772$

C -5.9225481 .1472323 .936626$

H -6.5665870 .7702214 .736434$

H -5.0696930 .4798793 .779239$

H -5.5467612 .1334134 .222172$

SCF Energy (B3LYP/6-31G**//MMFF) $=-3245.90374275$

03_86

MM̄FF Geometry

C $\quad 0.2202115 .4514580 .150136$

C $0.9183124 .680943-0.697398$

C $2.2516454 .062879-0.362027$

O $2.1197752 .626434-0.472386$

C $3.3138914 .523405-1.376117$

C $4.7066594 .001657-1.078359$

C $5.4021793 .172398-1.881402$

C $5.3296574 .492900 \quad 0.203597$

C $4.9877452 .600246-3.207899$

C $4.2998911 .228455-3.131225$

C $5.1870100 .107989-2.563960$

C $4.515476-1.254883-2.745149$ 
C $5.348069-2.388200-2.125610$

O $5.3874820 .348700-1.170026$

O $4.513509-3.554839-2.042808$

C $5.729009-2.007887-0.676240$

C $6.531381-2.769824-3.023079$

O $6.657830-2.940129-0.130360$

C $6.281357-0.572193-0.529142$

C $6.422631-0.1149210 .940808$

O $7.604808-0.463340-1.068830$

C 1.5899021 .9642090 .591534

O 1.2431442 .4416461 .659912

C 1.4750450 .5381320 .215839

C $0.669304-0.2663340 .924096$

C $0.432670-1.6893170 .673084$

C $1.340147-2.380744-0.308771$

C $5.126959-0.1072151 .759366$

C $4.912609-1.3622222 .590040$

C $3.237712-2.4234684 .100844$

O $5.761423-2.2399222 .720031$

C $2.464917-3.4507893 .335524$

C $1.244289-3.8735523 .698520$

C $0.474120-4.9383892 .971425$

C $-0.902014-4.4759562 .548510$

C $-1.990178-4.7802643 .275714$

C -1.022771 -3.7322591 .237834$

C $-0.565089-2.3043431 .341337$

N $3.666145-1.3676243 .202464$

H 0.6329635 .6748001 .130496

H $0.4996284 .450544-1.674492$

H $2.562798 \quad 4.3289260 .655505$

H $3.3589385 .620522-1.394746$

H $2.9962384 .216477-2.378511$

H $6.3982082 .868532-1.560408$

H 4.8106184 .0744801 .071235

H 6.3850564 .2116540 .286875

H 5.2790525 .5852290 .256923

H $4.3324643 .297168-3.741163$

H $5.8841782 .518249-3.836094$

H $3.3893601 .321206-2.527732$

H $3.9870710 .957735-4.147445$

H $6.1519760 .133419-3.083430$

H $4.323539-1.457652-3.806126$

H $3.530062-1.242354-2.260313$

H $4.193108-3.759133-2.938106$

H $4.813124-2.093833-0.080067$

H $6.173599-3.132296-3.994571$

H $7.107436-3.595095-2.590656$

H $7.209110-1.935482-3.216055$

H $6.259732-3.824264-0.206347$

H $7.211877-0.6897991 .440603$

H $\quad \begin{array}{llll}6.792140 & 0.920831 & 0.919357\end{array}$

H $7.912781-1.349115-1.318680$

H $2.0384570 .195805-0.644142$

H $\quad 0.1100690 .1603921 .755409$

H $1.177496-1.995970-1.320956$

H $1.201179-3.462886-0.347057$

H $2.388850-2.220027-0.036606$

H 5.1746660 .7253622 .472994

H 4.2561090 .0600801 .119199

H $2.637992-1.9555504 .888090$

H $4.114696-2.8896864 .561177$

H $2.942352-3.8869132 .461163$

H $0.781256-3.4542874 .589218$

H $0.387202-5.8029873 .642468$

H $1.022729-5.3012372 .093360$

H $-2.982967-4.4737502 .963233$

H -1.911063 -5.337302 4.203440

H -2.071509 -3.7133990 .911837$

H $-0.497538-4.2956340 .462484$

H -1.161388 -1.713735 2.036986

H $3.006483-0.6229512 .996650$

C $-2.2945015 .275363 \quad 0.490174$

O $-2.3371893 .916613 \quad 0.007400$

C -2.5258643 .0343701 .134612$

C $-3.057227 \quad 3.917244 \quad 2.253374$

C -2.2738215 .1944532 .017378$

H -1.527341 2.6570021 .385765

C -3.4445101 .8783010 .726799$
H -4.1326314 .0966752 .131152$

H -2.8894513 .4930233 .247390$

C $-2.865820 \quad 6.4125012 .704211$

H -1.254243 5.0409702 .390199

C -3.8216060 .9227421 .875846$

O $-2.781793 \quad 1.138313-0.304770$

H -4.3485422 .2939690 .269426$

H -4.4224441 .4466552 .628722$

O -2.6262530 .4867972 .534850$

C $-4.566132-0.3515511 .426644$

C $-5.892206-0.0536570 .707830$

H $-3.904573-0.9652430 .806718$

O $-4.846182-1.1115052 .608694$

H -6.5516060 .4784691 .404567$

H $-5.7086720 .614577-0.137150$

H -2.2745527 .3073612 .485131$

H -3.894295 6.5984772 .376926

H -2.8793806 .2729643 .790098$

C -1.132469 $6.025951-0.169129$

H $-2.001734 \quad 0.7141820 .091937$

H -2.2705371 .2379523 .037885$

H $-5.033368-2.0247452 .333488$

H -1.145499 7.0783110 .137144

H -1.281685 $6.012963-1.256663$

H -3.231089 5.7403430 .155539

C $-5.578991-1.502045-1.971377$

O $-5.809829-2.095581-0.686995$

C $-6.619781-1.3221760 .206086$

C $-7.989643-1.047168-0.430548$

C $-7.853801-0.394378-1.801501$

C $-6.906831-1.204231-2.683311$

H -5.001335 -0.577356-1.852993

C $-4.743539-2.494481-2.797533$

H $-6.803554-1.9572061 .080887$

O $-8.785670-0.2147650 .406333$

H $-8.531534-1.994488-0.550227$

H -7.523580 $0.648010-1.722402$

O $-9.134741-0.361606-2.435661$

H -7.395419-2.142834 -2.975664

O $-6.643068-0.470955-3.878387$

C $-3.294913-2.557058-2.362263$

H $-5.161158-3.503739-2.702208$

H -4.742555 -2.199746 -3.853055

H $-8.868141-0.6597201 .267129$

H $-9.7370320 .122345-1.844744$

H $-7.500252-0.279519-4.296399$

O $-2.628587-3.418231-3.188750$

O $-2.804667-1.937074-1.429771$

C $-1.230991-3.571264-2.924859$

H $-0.807699-4.218865-3.697209$

H $-0.725804-2.601771-2.966157$

H -1.083941-4.045139-1.950854

SCF Energy $\left(B 3 L Y P / 6-31 G^{* *} / / M M F F\right)=-3245.90864396$

03_87

MM̄FF Geometry

C $1.805109-0.473124-2.408333$

C $0.561537-0.889444-2.689921$

C $-0.335647-1.569633-1.686693$

O $-1.582301-0.840024-1.613838$

C $-0.648106-3.000591-2.161138$

C $-1.465100-3.809677-1.172518$

C $-2.702751-4.286625-1.410205$

C $-0.793543-4.1051210 .144632$

C $-3.531472-4.140797-2.655462$

C $-4.483909-2.934850-2.656559$

C -5.547042 -2.970316-1.546286

C $-6.596843-1.878643-1.766738$

C $-7.623280-1.826851-0.623615$

O $-4.897413-2.756066-0.290736$

O $-8.350654-0.593854-0.740247$

C $-6.875038-1.7823300 .726918$

C $-8.658434-2.952458-0.736671$

O $-7.784482-1.9036781 .817244$

C $-5.763610-2.8447080 .848776$

C $-4.882219-2.6667052 .109580$

O $-6.362528-4.1434740 .938194$

C $-1.6001320 .295558-0.864411$ 
O $-0.676852 \quad 0.771189-0.223357$

C -2.952654 $0.883984-0.979991$

C $-3.1473242 .164652-0.632799$

C $-4.4153202 .894169-0.703814$

C $-5.6609002 .097375-0.988566$

C $-4.148784-1.3250422 .216767$

C $-4.879370-0.2870063 .052723$

$\begin{array}{lll}\text { C }-4.752710 & 2.113178 & 3.710477\end{array}$

O $-5.862553-0.5450623 .741560$

C -5.6926412 .9026212 .855126$

C -5.5735674 .2246162 .659400$

C $-6.531628 \quad 5.0405521 .839784$

C $-5.8368595 .854250 \quad 0.771027$

C $-5.543206 \quad 7.150276 \quad 0.970735$

C $-5.5616615 .187814-0.557871$

C $-4.4057474 .227893-0.497640$

N -4.2755350 .9607712 .969514$

H $2.207665-0.634557-1.411283$

H $\quad 0.158097-0.731767-3.687433$

H $0.131958-1.593853-0.694972$

H $0.288622-3.543096-2.347444$

H -1.161874 -2.943340 -3.126884

H $-3.187160-4.862163-0.621820$

H $-0.686302-3.1918810 .737730$

H $-1.360529-4.8220870 .748278$

H $0.199578-4.534521-0.022439$

H -2.891923 -4.089860 -3.542944

H $-4.117089-5.060668-2.781306$

H -3.891965 -2.015913 -2.572707

H -4.984558 -2.908661-3.632414

H $-6.019036-3.959796-1.551368$

H -7.103903 -2.014572 -2.730201

H -6.096906 $-0.902667-1.828343$

H -8.768550 -0.574294 -1.618163

H -6.424071 -0.7863040 .805760$

H $-9.200515-2.879723-1.687254$

H $-9.418897-2.870590 \quad 0.047620$

H -8.215480 -3.949197 -0.686187

H $-8.451769-1.203307 \quad 1.717419$

H -5.466926 -2.882565 3.011950

H $-4.107179-3.4461932 .073898$

H $-5.721590-4.7515361 .342503$

H $-3.7451800 .253604-1.366187$

H $-2.2939392 .744377-0.282876$

H -5.646634 $1.714536-2.014575$

H $-6.5831742 .668946-0.866244$

H -5.739395 $1.251101-0.297826$

H -3.187389-1.495650 2.718321

H $-3.931850-0.9135151 .226494$

H -3.874132 2.6948864 .006896

H -5.2708301 .7773114 .614541$

H -6.5226592 .3686322 .398416$

H -4.7585204 .7602143 .141435$

H -7.070092 5.7087592 .524737

H -7.301490 4.4109991 .376531

H $-5.070566 \quad 7.7488310 .199146$

H -5.7646997 .6402421 .913240$

H $-5.3114745 .944883-1.313341$

H $-6.4830674 .725974-0.922891$

H $-3.4504104 .712907-0.291262$

H -3.4950301 .0955442 .333194$

C $3.0500931 .630903-2.962527$

O $3.8688341 .596413-1.776125$

C $4.8861812 .610475-1.881710$

C $4.5440003 .435197-3.118230$

C $3.8691152 .401405-3.997175$

H $5.8325382 .088840-2.063600$

C $4.9799733 .418688-0.582684$

H $3.8477194 .249539-2.886512$

H $5.432742 \quad 3.877263-3.578890$

C $3.0436303 .007545-5.118883$

H $4.6403301 .748778-4.427950$

C 5.2154142 .5669680 .681490

$\begin{array}{lllll}\text { O } & 3.747679 & 4.129284 & -0.407027\end{array}$

H $5.7653404 .177675-0.683221$

H 4.3208011 .9761910 .914469

O 5.4072013 .4649191 .782580

$\begin{array}{llll}\text { C } & 6.438018 & 1.632044 & 0.607115\end{array}$
C $\quad 6.7213040 .927499 \quad 1.948143$

H $6.2655300 .877473-0.166706$

$\begin{array}{lllll}\text { O } & 7.590985 & 2.378165 & 0.219063\end{array}$

H 6.9659741 .6784772 .709011

H $5.8072990 .421222 \quad 2.276714$

H $2.5721022 .227230-5.724080$

H $2.251793 \quad 3.655837-4.729069$

H $3.6763123 .609614-5.779376$

C $2.704200 \quad 0.207724-3.401858$

H 3.8893714 .7953630 .286407

H $4.553023 \quad 3.8915491 .964649$

H 7.7285223 .0736350 .885059

H $3.618078-0.390572-3.490549$

H $2.230557 \quad 0.226206-4.390057$

H $2.1368022 .183982-2.705534$

C $6.679536-1.9854051 .042776$

O $7.727937-1.034110 \quad 0.824458$

C $7.896917-0.072847 \quad 1.870147$

C $8.202287-0.7903573 .192905$

C $7.149021-1.8451193 .522937$

C $6.930006-2.7766752 .331582$

H $5.715743-1.4649441 .101189$

C $6.645526-2.915560-0.174056$

H $8.7894980 .508832 \quad 1.607441$

$\begin{array}{lllll}\text { O } & 8.288950 & 0.136195 & 4.270097\end{array}$

H $9.177600-1.2887033 .118200$

H $6.205373-1.3859363 .840035$

O $7.602056-2.6322154 .627018$

H $7.805420-3.4277352 .211173$

O $5.806173-3.6145912 .593063$

C $6.109358-2.185796-1.381889$

H $7.644755-3.304585-0.404645$

H $5.994937-3.781928-0.009983$

H $8.9762270 .785343 \quad 4.042033$

H 7.773513-2.022215 5.365020

H $5.982079-4.0840323 .426510$

O $7.137388-1.772986-2.175935$

O $4.917812-1.985739-1.579020$

C $6.756210-1.049042-3.349487$

H $6.236324-0.127969-3.072633$

H $7.664781-0.787214-3.897950$

H $6.125027-1.670949-3.991278$

SCF Energy (B3LYP/6-31G**//MMFF)= -3245.90783965

03_88

MMFF Geometry

C 1.2749450 .6403903 .647848

C $0.226406 \quad 0.303001 \quad 4.414813$

C $-0.972660-0.493007 \quad 3.953687$

O $-1.280931-0.2102402 .568994$

C $-0.694628-2.0000294 .095477$

C -1.896595 -2.874842 3.796398

C $-1.969792-3.7551242 .778670$

C $-3.057521-2.7388384 .748401$

C $-0.925349-4.0684921 .744577$

C $-1.031845-3.2409520 .454833$

C $-2.342556-3.445106-0.322313$

C $-2.260071-2.786863-1.701679$

C $-3.586327-2.894321-2.471207$

O $-3.408940-2.8514940 .422712$

O $-3.524536-1.987927-3.583678$

C $-4.738621-2.413867-1.562228$

C $-3.789455-4.293913-3.063567$

O $-6.003413-2.652830-2.173638$

C $-4.710073-3.036938-0.151639$

C $-5.735614-2.3993240 .818153$

O $-5.034700-4.429540-0.246793$

C -1.8846810 .9813062 .303009$

O $-2.203113 \quad 1.8426243 .107424$

C -2.0689851 .0751370 .839752$

$\begin{array}{llll}C & -2.253505 & 2.278060 & 0.276777\end{array}$

C $-2.4384812 .544723-1.150901$

C $-2.5986691 .361334-2.067995$

C $-5.566982-0.8950181 .059830$

C $-6.444123-0.022752 \quad 0.176382$

C $-6.8511092 .369774-0.394344$

O $-7.347919-0.466646-0.526363$

C $-6.1631912 .690670-1.683572$ 
C $-5.8224933 .937093-2.045581$

C $-5.1780384 .282993-3.357463$

C $-3.9120515 .093776-3.191440$

C $-3.9276716 .431974-3.311899$

C $-2.6165224 .345573-2.973866$

C $-2.4745573 .827561-1.568243$

N $-6.123145 \quad 1.3231160 .298581$

H 1.3199910 .2916242 .620784

H 0.2021550 .6331895 .450901

H -1.834100 -0.2076164 .569855$

H $-0.359573-2.2228915 .117296$

H $0.142324-2.2628003 .439624$

H -2.887243 -4.332712 2.669570

H -3.534093 -1.759960 4.640081

H $-3.827675-3.4983274 .575367$

H $-2.716439-2.8511395 .782740$

H $\quad 0.080462-3.9497072 .161346$

H -1.004779 $-5.134347 \quad 1.495274$

H $-0.913693-2.1808250 .706167$

H $-0.187163-3.518323-0.187978$

H $-2.520227-4.522198-0.423202$

H -1.440035 -3.216356 -2.290496

H -2.015667 -1.722967 -1.581530

H -2.756495 -2.233205 -4.127646

H -4.636659-1.326430 -1.471121

H -2.972497 -4.540092 -3.752410

H -4.707342 -4.341351 -3.660050

H $-3.828677-5.079042-2.305754$

H -5.989193 -2.223753 -3.046359

H $-6.756553-2.6476150 .504202$

H $-5.605488-2.8905641 .793392$

H -5.343192 -4.7342950 .622668$

H -2.0310750 .1561990 .267529$

H -2.251389 3.1576790 .919473

H -1.658869 $0.805734-2.146940$

H $-2.9032561 .632288-3.081047$

H $-3.375750 \quad 0.687413-1.692785$

H -5.865753 -0.675555 2.093037

H $-4.521169-0.5936940 .951859$

H $-6.8957093 .232806 \quad 0.277472$

H -7.872623 $2.031589-0.596153$

H $-5.9635011 .864963-2.362533$

H $-6.0512444 .763899-1.376558$

H -5.908858 $4.852544-3.946628$

H $-4.9608783 .383971-3.947573$

H -3.019413 $7.018838-3.223103$

H -4.851045 $6.971090-3.496769$

H -1.762302 $5.010905-3.159191$

H -2.522663 $3.560773-3.729815$

H $-2.3603574 .618130-0.825261$

H $\quad-5.3259391 .5957930 .865997$

C 2.7826642 .6326413 .159157

O 3.5363082 .1127392 .048742

C $3.3591663 .002657 \quad 0.926342$

C 2.3839204 .0947501 .376498

C 1.6459063 .4256032 .521350

H 2.8901612 .4146950 .130503

C 4.7187763 .5321170 .450808

H 2.9053244 .9922071 .728216

H 1.7176704 .4050230 .565062

C 0.9596394 .3967863 .463709

H 0.8954522 .7553802 .084533

C 5.7600632 .4396270 .135633

O $5.2691624 .357266 \quad 1.488114$

H $4.5744004 .185922-0.417653$

H 6.0469401 .9273321 .062393

O $6.9312403 .123583-0.331941$

C $5.3153191 .410487-0.922480$

C $6.4319890 .410067-1.279126$

H $4.4498470 .858309-0.543601$

O $4.8957902 .090220-2.104489$

H $7.2951400 .954140-1.680160$

H $6.760909-0.082830-0.357722$

H 0.4508573 .8616224 .271725

H 1.6757095 .0904863 .916776

H 0.2109104 .9887302 .927227

C 2.4067281 .5039644 .124828

H 6.1221324 .6959901 .165850
H $7.6873142 .522765-0.220818$

H $5.6505532 .612591-2.426369$

H 2.1533531 .9449095 .096712

H 3.2800710 .8575994 .275413

H $3.462728 \quad 3.3116803 .692281$

C $4.888629-2.197286-0.877078$

O $4.801808-1.312157-1.999030$

C $6.015114-0.637479-2.335646$

C $7.107838-1.666387-2.660255$

C $7.290199-2.672711-1.526868$

C $5.946538-3.279859-1.125576$

H $5.134792-1.6242330 .025497$

C $3.504337-2.826915-0.680561$

H $5.808938-0.084842-3.260812$

O $8.352453-1.026910-2.921702$

H $\quad 6.830862-2.216096-3.569248$

H $7.789598-2.219914-0.662316$

O $8.142304-3.731155-1.971131$

H $5.606961-3.964249-1.913766$

O $6.117110-4.0491720 .063287$

C $2.512276-1.818535-0.152848$

H $3.110617-3.238842-1.617722$

H $3.538750-3.6508240 .041687$

H $8.213243-0.407688-3.658546$

H $8.978711-3.326702-2.259201$

H $6.806423-4.711561-0.116246$

O $2.069285-1.026208-1.169313$

O $2.176774-1.7477771 .022041$

C $1.124393-0.012125-0.817177$

H $1.1379640 .751832-1.599156$

H $\quad 0.127588-0.454673-0.773548$

H 1.3762020 .4624350 .135027

SCF Energy (B3LYP/6-31G**//MMFF) $=-3245.90826110$

03_89

MM̄FF Geometry

C $0.7106555 .944267 \quad 1.149587$

C 1.1278414 .9999620 .291676

C 2.4271144 .2448950 .404870

O 2.1025142 .8330880 .372687

C $3.3227414 .551402-0.808058$

C $4.6124313 .753330-0.829566$

C $4.9390202 .858907-1.783883$

C 5.5684194 .0235190 .303365

C $4.1476102 .482803-3.005901$

C $3.1777981 .306786-2.812818$

C $3.867755-0.035925-2.522344$

C $2.857746-1.184457-2.576013$

C $3.502475-2.530509-2.214715$

O $4.4503670 .021154-1.218535$

O $2.430979-3.461567-1.998297$

C $4.280804-2.383641-0.887844$

C $4.326593-3.089410-3.380940$

O $5.014637-3.578450-0.629890$

C $5.203077-1.144997-0.851449$

C $5.812255-0.8653200 .545005$

O $6.298314-1.352605-1.751918$

C 1.6135422 .2726081 .511388

O 1.5147592 .7973192 .608682

C 1.1538800 .9095691 .166186

C $0.3121800 .270823 \quad 1.991751$

C $-0.283167-1.0472171 .765824$

C $0.218407-1.8449220 .592319$

C $4.798761-0.6408911 .672807$

C $4.485256-1.8886462 .482264$

C $2.993392-2.6901224 .312024$

O $5.109328-2.9402522 .368729$

C $1.846736-3.4453003 .717464$

C $0.677098-3.6258034 .349968$

C $-0.465358-4.4240563 .789709$

C $-1.762170-3.6466783 .757899$

C $-2.687893-3.8029584 .719222$

C $-2.022350-2.7584762 .563273$

C -1.239402 -1.476507 2.615650

N $3.445944-1.6758413 .378229$

H $1.376086 \quad 6.2452531 .955897$

H $\quad 0.4840334 .688943-0.526540$

H $2.9516674 .482723 \quad 1.338161$ 
H $3.5750965 .619928-0.821213$

H $2.7473214 .367551-1.721957$

H $5.8926332 .339918-1.694073$

H 6.5322073 .5225780 .161689

H $5.767623 \quad 5.0969870 .384432$

H 5.1527733 .6711521 .252347

H $3.5879693 .347328-3.379423$

H $4.8561152 .238746-3.807888$

H $2.4788241 .547900-2.003525$

H $2.5861081 .211336-3.731861$

H $4.656021-0.185565-3.269417$

H $2.381121-1.241241-3.562651$

H $2.044611-0.985990-1.864435$

H $2.824511-4.298412-1.696364$

H $3.528156-2.300334-0.095922$

H $3.688516-3.238830-4.260305$

H $4.737734-4.075994-3.140204$

H $5.150661-2.436320-3.675643$

H $5.383681-3.5149640 .266758$

H $6.542376-1.6411570 .804453$

H $\quad \begin{array}{llll}6.397727 & 0.061939 & 0.460196\end{array}$

H $7.025330-0.761803-1.494223$

H 1.4838950 .4894410 .223325

H -0.0009750 .7738062 .905936$

H $1.309445-1.9291140 .625999$

H $-0.068741-1.363367-0.348137$

H $-0.159602-2.8685950 .564121$

H 5.2248940 .0809572 .381533

H $3.870108-0.2108481 .287285$

H $2.715627-2.1772315 .238274$

H $3.815729-3.3797094 .528402$

H $1.995680-3.8887192 .735816$

H $0.541513-3.2049225 .343951$

H $-0.582985-5.3191154 .414718$

H $-0.238915-4.7959942 .782726$

H $-3.633305-3.271648 \quad 4.685569$

H -2.518663 -4.462746 5.563644

H -3.084890 -2.486406 2.519312

H -1.844016 -3.3325931 .651414$

H -1.533321 $-0.822523 \quad 3.437831$

H $2.952703-0.787786 \quad 3.367107$

C - 1.7578405 .8111680 .450236

O -1.9095944 .5368921 .101984$

C -3.2804184 .1017910 .960099$

C -4.0002195 .2081720 .197269$

C $-3.149891 \quad 6.428136 \quad 0.516752$

H -3.673980 4.0161651 .980054

C -3.2539642 .7318580 .272811$

H -3.992516 $5.009086-0.881690$

H $\quad-5.0430695 .317590 \quad 0.509592$

C $-3.3665227 .586128-0.440245$

H -3.370360 6.7538391 .542000

C $-4.6286002 .167457-0.112363$

O $-2.6220921 .810563 \quad 1.169159$

H -2.613745 2.792434 -0.614694

H $-5.0934592 .806935-0.871579$

O $\quad-5.4785492 .2228221 .039533$

C $-4.5963280 .705581-0.609714$

C $-3.6306840 .473312-1.785867$

H $-4.351050 \quad 0.0374530 .223485$

O $\quad-5.9224350 .355120-1.022495$

H -3.925000 $1.117514-2.623597$

H -2.618102 $0.754048-1.477116$

H $-2.7266038 .434254-0.177761$

H -3.141424 $7.301152-1.473484$

H $-4.4071557 .924971-0.404288$

C -0.6506116 .6032851 .151799$

H -1.7615842 .1915581 .414759$

H -6.3384741 .8472230 .783789$

H $-6.1572410 .918742-1.779432$

H $-0.934144 \quad 6.7325792 .204442$

H -0.5557557 .6037510 .715943$

H -1.491578 $5.622629-0.597964$

C $-2.992220-3.192801-1.470817$

O $-3.058215-1.782993-1.216400$

C $-3.608346-0.986456-2.271493$

C $-2.771921-1.148207-3.551149$

C $-2.638199-2.621290-3.946251$
C $-2.208500-3.490901-2.759919$

H -2.413913 -3.600564 -0.632807

C $-4.379743-3.841229-1.434149$

H $-4.636214-1.306773-2.470165$

O $-1.473636-0.583215-3.375084$

H $-3.255868-0.591783-4.362495$

H -1.901188 -2.707368 -4.753949

O $-3.891997-3.072825-4.449549$

H -2.302014 -4.555290-3.004835

O $-0.826197-3.246449-2.484057$

C $-4.954227-3.774154-0.038663$

H $-5.091626-3.385047-2.127907$

H -4.321464 -4.901721 -1.706678

H -1.058965 -1.022048 -2.612572

H -3.782924 -4.000531-4.719784

H $-0.320405-3.483524-3.280126$

O $-5.765317-2.6852920 .079687$

O $-4.689535-4.5782290 .845822$

C $-6.350869-2.4874261 .369879$

H $-6.968624-3.3482551 .643055$

H $-5.570552-2.3182932 .117473$

H -6.987191 -1.599819 1.321553

SCF Energy (B3LYP/6-31G**//MMFF) $=-3245.92057392$

03 9

MM̄FF Geometry

C $-2.230158-3.482453-1.164043$

C $-1.214376-4.182110-1.692591$

C $0.255431-3.828707-1.632240$

O $0.475954-2.498312-1.113436$

C $0.982083-4.823960-0.708300$

C $2.492093-4.682072-0.719114$

C $3.238522-4.3449470 .350782$

C $3.168434-4.981289-2.032845$

C $2.775551-4.0446181 .748268$

C $2.514339-2.5574982 .028106$

C $3.757710-1.6615861 .908298$

C $3.460548-0.2553342 .434206$

C 4.6555080 .6933062 .248404

O $4.130462-1.5828190 .530398$

O 4.1886302 .0354552 .456570

C 5.1468090 .6085770 .786687

C 5.7466830 .4464333 .297073

O 6.3564881 .3417110 .612369

C $5.330031-0.8371070 .281649$

C $5.615607-0.921186-1.238169$

O $6.450092-1.4295960 .951124$

C $0.324284-1.456639-1.976600$

O $0.003351-1.512798-3.152913$

C $0.592547-0.212974-1.223607$

C $0.1076720 .951485-1.678224$

C $0.2721132 .258889-1.040298$

C 1.2363922 .3565040 .111991

C $4.529722-0.332401-2.146065$

C $4.7910561 .102609-2.574030$

C $3.7150182 .986175-3.802188$

O $5.8547741 .683282-2.376388$

C $3.1397743 .948146-2.811067$

C $2.1492164 .803891-3.105436$

C $1.5930045 .807471-2.136335$

C $0.0898935 .714699-1.998125$

C $-0.7196956 .545421-2.676554$

C $-0.4684424 .718335-1.008250$

C $-0.4293383 .305290-1.523642$

N $3.702693 \quad 1.647434-3.242907$

H $-2.031647-2.572049-0.606036$

H -1.445098 -5.095770 -2.237806

H $0.661923-3.891774-2.648870$

H $0.741132-5.854090-1.003694$

H $\quad 0.590293-4.700850 \quad 0.307418$

H $4.318519-4.2827980 .219670$

H $2.933981-4.210431-2.773172$

H $4.258965-5.023027-1.938495$

H $2.838284-5.951335-2.418614$

H $1.872041-4.6151381 .988347$

H $3.538369-4.4143582 .445496$

H $1.735783-2.2012761 .343714$

H $2.114721-2.4776083 .046385$ 
H $4.570671-2.1204732 .483094$

H $3.158697-0.2887843 .488537$

H $2.600750 \quad 0.1600181 .891534$

H 3.8088922 .0852733 .350587

H 4.3898641 .1055810 .169597

H 5.3460570 .5915694 .307610

H $6.564574 \quad 1.1680683 .193943$

H $6.166034-0.5606673 .250816$

H 6.1912322 .2503100 .917156

H $6.602943-0.498911-1.460394$

H $5.693298-1.988146-1.492924$

H $6.769663-2.1754260 .416691$

H $1.160895-0.294213-0.304603$

H $-0.4861750 .946569-2.591522$

H $2.2120991 .949920-0.173477$

H $0.865310 \quad 1.7993540 .977781$

H 1.4233433 .3808250 .440386

H $4.495478-0.922516-3.071017$

H $3.543523-0.402178-1.677921$

H $3.1382952 .954611-4.731997$

H $4.7443823 .273338-4.039496$

H $3.5766223 .960504-1.815351$

H $1.7341974 .808403-4.111053$

H $1.8724156 .806615-2.495843$

H $2.0568395 .710084-1.147000$

H -1.797669 $6.504094-2.561349$

H -0.321916 $7.281819-3.366999$

H $-1.5195214 .952753-0.791498$

H $0.0428894 .838088-0.049114$

H -1.094661 $3.135492-2.371330$

H $2.8425401 .111460-3.314496$

C $-4.541308-2.851987-2.010058$

O $-4.607190-1.634147-1.243154$

C $-4.574948-0.518192-2.154719$

C $-4.781106-1.101268-3.549781$

C $-4.094400-2.446687-3.414281$

H -3.562435 -0.100022 -2.100669

C $-5.5987160 .544551-1.742272$

H $-5.843321-1.239374-3.782292$

H $-4.344260-0.471468-4.330812$

C $-4.474897-3.442695-4.494796$

H $-3.010723-2.279808-3.448983$

C $-5.4726931 .017697-0.279444$

O $-6.913860-0.002590-1.905146$

H -5.534103 $1.398530-2.427164$

H $-5.792161 \quad 0.2140760 .395494$

O $-6.4119252 .087451-0.113518$

C -4.0647651 .5041160 .109791$

C -3.995557 2.0811321 .535603

H -3.3671430 .6646220 .030514$

O $-3.6311452 .500420-0.815085$

H -4.576266 3.0098891 .583343

H $-4.459142 \quad 1.3698352 .227260$

H -3.962463 -4.397537 -4.341112

H -5.552927 -3.635138 -4.502641

H $-4.194326-3.063615-5.483028$

C -3.672488 -3.887367 -1.291474

H -7.548513 $0.689092-1.650318$

H -6.5402062 .2223630 .840590$

H -4.270552 $3.232513-0.781196$

H -3.758494 -4.851092 -1.807159

H -4.060686 -4.032115 -0.276258

H - $-5.568502-3.239254-2.044125$

C -1.9273280 .1930492 .754055$

O $-1.682021 \quad 1.2659721 .835899$

C $-2.5476402 .395531 \quad 1.975237$

C -2.4244882 .9736263 .390467$

C -2.6492441 .9097114 .461951$

C -1.772803 0.6833694 .201339

H -2.941301 -0.1930642 .598458$

C $-0.931629-0.9143092 .391409$

H -2.1680273 .1579421 .283997$

O -3.3465104 .0398373 .589019$

H -1.417481 3.3899363 .524659

H -3.7065121 .6278794 .531579$

O -2.2944202 .4510085 .737209$

H $-0.7279690 .944966 \quad 4.413080$

O $-2.160628-0.3344945 .120127$
C -1.307993 -2.273746 2.922949

H $-0.837348-1.0057701 .302805$

H $0.073235-0.6588702 .748216$

H -3.167245 4.7111932 .908508

H -2.8375593 .2460605 .874480$

H $-1.443167-0.9891745 .155235$

O $-2.442508-2.7213112 .315074$

O $-0.659515-2.872356 \quad 3.771509$

C $-2.898097-4.0099272 .737933$

H $-3.863647-4.2024442 .263045$

H $-2.188570-4.7793242 .419442$

H $-3.033656-4.0332273 .823554$

SCF Energy (B3LYP/6-31G**//MMFF) $=-3245.91348259$

03_90

MM̄FF Geometry

C $0.435494-4.6894190 .334763$

C $-0.652896-4.398759-0.393071$

C -1.945268 -3.9047470 .207006$

O $-2.210510-2.581148-0.314367$

C $-3.104237-4.826356-0.213557$

C $-4.431653-4.4681350 .426537$

C $-5.521525-4.053083-0.248695$

C $-4.501638-4.6316081 .923612$

C $-5.679047-3.858680-1.730519$

C $-5.378022-2.437008-2.230197$

C $-6.292269-1.351924-1.637518$

C $-6.098143-0.022091-2.370253$

C $-6.9278681 .109133-1.741618$

O $-5.960403-1.185246-0.256582$

O $-6.4426882 .355022-2.266051$

C $-6.6772231 .133676-0.217890$

C $-8.4062281 .018964-2.138651$

O -7.5518232 .0557700 .426880$

C $-6.793666-0.2531320 .446102$

C $-6.344134-0.2618281 .927996$

$\begin{array}{llll}\text { O } & -8.163991 & -0.671687 & 0.423549\end{array}$

C $-1.535863-1.5424440 .250508$

O $-0.745055-1.5944111 .179096$

C $-1.907739-0.313344-0.483494$

C $-1.1401330 .780064-0.364927$

C -1.361962 2.067848 -1.025889

C $-2.6662282 .261256-1.752536$

C -4.8948920 .1688922 .179687$

C -4.7407321 .6352892 .548894$

C -2.9873573 .3554502 .971123$

O -5.6899122 .3693312 .809620$

C $-2.8219694 .195528 \quad 1.744244$

C -1.7172384 .9128361 .488382$

C -1.5512045 .7971870 .285859$

C $-0.2997315 .480250-0.501805$

C $0.8237636 .197782-0.332373$

C $-0.3797004 .387005-1.543520$

C $-0.4013603 .011086-0.935593$

N -3.406601 2.0190572 .592015

H $\quad 0.398175-4.587577 \quad 1.416640$

H -0.608142 -4.481515 -1.476494

H -1.878106 -3.859975 1.300731

H $-2.869312-5.8652050 .054525$

H -3.187847 -4.807044 -1.305590

H $-6.421380-3.8302540 .324066$

H -5.521199-4.515357 2.306866

H $-4.157248-5.6297032 .213831$

H $-3.877408-3.8853482 .424098$

H $-5.055193-4.569440-2.282868$

H $-6.710970-4.121073-1.997407$

H -4.330958 -2.198670 -2.009956

H -5.485334 -2.440257 -3.322132

H $-7.331780-1.688679-1.727544$

H $-6.338442-0.125649-3.435795$

H $-5.0380800 .262394-2.330289$

H $-6.5253142 .325006-3.234670$

H $-5.6592641 .513089-0.070657$

H $-8.5142811 .081161-3.228241$

H -8.976632 $1.862143-1.733646$

H $-8.882040 \quad 0.091517-1.813550$

H -7.430015 $2.919677-0.002751$

H -7.0498820 .3129122 .539654$ 
H $-6.430044-1.2986662 .284572$ H -8.291200 -1.347684 1.109736 H $-2.789977-0.347238-1.111570$ H -0.2511120 .7256230 .262692$ H -3.508831 2.034235 -1.090762 H $-2.7197541 .605977-2.628564$ H $-2.8257043 .283145-2.101992$ H $-4.508168-0.4008373 .034555$ H -4.260061-0.061951 1.319396 H -2.053639 3.2551023 .533485 H -3.7427473 .8032743 .624902$ H -3.657869 4.2377551 .049721 H -0.8943184 .8903992 .199596$ H -1.515664 $6.836150 \quad 0.639265$ H -2.423451 5.741256-0.377229 H $1.7176655 .996625-0.913306$ H 0.8760757 .0006050 .395762 H $0.4954024 .433254-2.205578$ H -1.234976 $4.581400-2.196221$ H $0.5046922 .771099-0.378610$ H -2.688154 1.3575552 .312556 C $2.750948-3.965417-0.241895$ O $3.093616-3.6278141 .115807$ C $4.472420-3.1946721 .152358$ C $4.968694-3.227880-0.288086$ C $4.083103-4.288693-0.910294$ H $5.003028-3.9370931 .760830$ C $4.529424-1.8126621 .815418$ H $4.805611-2.261213-0.781065$ H $6.034406-3.465270-0.355330$ C $4.053919-4.231503-2.427537$ H $4.436401-5.277379-0.588573$ C $5.912233-1.1442511 .747123$ O $4.162536-1.9610253 .190919$ H $3.770255-1.1668261 .361524$ H $6.214306-1.0025730 .705138$ O $6.887251-2.0313452 .310696$ C 6.0141660 .2022272 .487325 C 4.9505551 .2520262 .113846 H $5.9689910 .036278 \quad 3.570657$ O $7.307606 \quad 0.7614932 .234910$ H 3.9796140 .9367602 .513812 H 5.2261682 .1849592 .622824 H $3.403272-5.010427-2.836717$ H $3.687401-3.263615-2.785755$ H $5.058336-4.382438-2.836938$ C $1.747863-5.118381-0.259111$ H $3.287569-2.3856293 .212930$ H $6.620593-2.2146203 .228002$ H 7.9674570 .0893692 .477390 H $2.125156-5.9631750 .329535$ H $\quad 1.599598-5.475917-1.284383$ H $2.305087-3.068360-0.693646$ C $6.1011512 .315027-1.291542$ O 6.0432072 .0654440 .119078 C 4.8064041 .5400410 .604607 C 3.6610132 .5247910 .317807 C $3.5992262 .880054-1.171459$ C $4.9741583 .268968-1.725967$ H $7.0576802 .830604-1.445615$ C $6.1386331 .010886-2.104813$ H 4.5911340 .5980700 .093299 O $3.8448553 .711131 \quad 1.086845$ H $2.7168002 .067170 \quad 0.636517$ H $2.9028283 .714695-1.312306$ O $3.0815281 .760366-1.883841$ H $4.9372603 .327012-2.820108$ O $5.2794034 .582823-1.248684$ C $7.3591640 .171844-1.788551$ H $5.2575470 .393801-1.909924$ H $6.1705201 .242684-3.175443$ H 3.0906394 .2975830 .906295 H $3.0111862 .015370-2.819465$ H $6.1247844 .851789-1.646868$ O $7.185320-1.077315-2.314317$ O $8.3432840 .558970-1.174048$ C $8.255953-1.997371-2.080399$ H $9.169749-1.644883-2.567806$
H $8.415900-2.129093-1.005793$

H $7.975462-2.961453-2.513038$

SCF Energy (B3LYP/6-31G**//MMFF) $=-3245.89299112$

03 91

MM̄FF Geometry

C 2.5658712 .5462603 .084304

C 2.2678271 .3671992 .519506

C 1.0973750 .5165992 .942683

O 0.1994240 .3923151 .814448

C $1.575336-0.8882163 .351186$

C $0.455876-1.8392383 .736954$

C $0.289977-3.0638253 .198218$

C $-0.489698-1.3607714 .807562$

C $1.172756-3.7326302 .182438$

C $0.870891-3.3536610 .726855$

C $-0.418829-3.9801350 .174320$

C $-0.573817-3.641243-1.310944$

C -1.887957-4.179899-1.892222

O $-1.534398-3.4718940 .909232$

O $-2.094110-3.553631-3.168025$

C $-3.051267-3.740352-0.979804$

C $-1.821438-5.688789-2.155318$

O $-4.275917-4.344075-1.391869$

C $-2.799187-4.0222980 .521204$

C $-3.893276-3.3897391 .415152$

O $-2.838551-5.4377290 .736817$

C $-0.690857 \quad 1.407501 \quad 1.634487$

O -0.8051382 .4115462 .320883$

C -1.5118431 .0981090 .442821$

C $-2.3409632 .041431-0.029828$

C $-3.2449051 .926480-1.176061$

C $-3.2435090 .637076-1.952245$

C $-3.812468-1.8651001 .532737$

C $-5.136513-1.2328411 .924271$

C -6.2405021 .0037571 .993966$

O $-6.131693-1.8819412 .232959$

C -6.7349721 .3126930 .614197$

C -6.8589312 .5556820 .124806$

C -7.331502 $2.855069-1.269665$

C $-6.3612183 .719853-2.046322$

C $-6.6572774 .997932-2.337753$

C $-5.0809313 .083253-2.552569$

C $-4.0308992 .981669-1.478650$

N -5.0705450 .1535841 .874105$

H 1.9557262 .9102183 .907527

H 2.8616701 .0123911 .682073

H 0.5689010 .9762953 .787479

H $2.262060-0.8055834 .203938$

H $2.159268-1.3057542 .524186$

H $-0.549097-3.6694253 .537196$

H -1.116079 -0.5446184 .434397$

H -1.159454 -2.155148 5.154397

H $0.071392-1.0050835 .677889$

H $2.220466-3.5042872 .406191$

H $1.087689-4.8194992 .307126$

H $\quad 0.819277-2.2614790 .638068$

H $\quad 1.714933-3.6870970 .110379$

H $-0.359644-5.0660480 .313545$

H $0.279910-4.018300-1.887284$

H $-0.563193-2.549404-1.437490$

H -1.329847 -3.763508 -3.731766

H -3.178901 -2.661475 -1.126054

H $-1.018567-5.918006-2.866380$

H -2.745009-6.049736-2.620995

H $-1.637592-6.274471-1.252311$

H -4.399542 -4.135245 -2.333603

H $-4.879057-3.715327 \quad 1.060387$

H $-3.811460-3.7962522 .432813$

H -3.002798 -5.6022031 .679998$

H $-1.4123770 .116740-0.005744$

H -2.3737493 .0023940 .483357$

H $-2.2234570 .344558-2.225566$

H $-3.7954540 .700231-2.893066$

H -3.693582 -0.165224 -1.361067

H -3.061774 -1.589704 2.283021

H $-3.510250-1.4205770 .581967$

H $\quad-5.9370511 .9036672 .537430$ 
H $-7.019643 \quad 0.4894782 .564694$ H -6.999088 $0.466210-0.015896$ H $-6.612423 \quad 3.404808 \quad 0.758124$ H -8.305186 $3.355525-1.189479$ H -7.515692 $1.932756-1.834697$ H -5.978838 $5.617575-2.914804$ H -7.581991 $5.455283-2.001661$ H $-4.6626173 .684468-3.370053$ H $-5.3335122 .112547-2.988839$ H -3.924756 $3.889495-0.882577$ H -4.2079050 .5911791 .565893$ C 3.2749774 .4851361 .585795 O 3.0745333 .8380760 .311900 C $1.8188314 .277734-0.233243$ C 1.5078875 .5904470 .476183 C 2.0035605 .2890071 .878305 H 1.0734093 .5295920 .063100 C $1.8697274 .353246-1.763837$ H $2.072876 \quad 6.4284710 .052509$ H 0.4437015 .8416560 .440981 C 2.2457646 .5256232 .725988 H 1.2454704 .6713952 .373351 C $2.4699693 .112387-2.457699$ O $2.6715955 .480558-2.139087$ H $0.8604244 .554449-2.143619$ H $3.5637623 .128682-2.369063$ O $2.1771563 .203972-3.857676$ C $1.9510231 .757658-1.947269$ C $2.4679950 .573201-2.785501$ H $2.2532571 .631235-0.903788$ O $0.5251901 .741630-1.975383$ H $1.8660530 .484570-3.697949$ H $3.4979690 .774087-3.095807$ H 2.6187726 .2485793 .717079 H $2.979927 \quad 7.1931782 .262717$ H 1.3165067 .0893232 .858379 C 3.6857103 .4424952 .634154 H $2.5370675 .635749-3.088833$ H $2.6697373 .962766-4.212903$ H $0.2440881 .933504-2.886491$ H 4.0845093 .9523173 .519350 H 4.5003152 .8274312 .230618 H 4.1232595 .1654711 .435773

C $4.617244-0.701321-1.041667$

O $3.202891-0.724767-0.815588$

C $2.400823-0.759124-2.003444$

C $2.764476-1.995884-2.834911$

C $4.256760-2.044913-3.153503$

C $5.082955-1.905766-1.873531$

H $4.8729240 .217312-1.583619$

C $5.321082-0.563851 \quad 0.317437$

H $1.362529-0.880098-1.670701$

O $2.022230-2.029453-4.049000$

H $2.503957-2.901824-2.273637$

H $4.536965-1.281066-3.888427$

O $4.565827-3.307085-3.750650$

H $5.003326-2.841965-1.310616$

O $6.451474-1.747800-2.243034$

C $5.032001-1.6755651 .304317$

H $6.405420-0.4994160 .180086$

H 4.9783880 .3669160 .785628

H $1.078479-2.013643-3.814404$

H $4.007868-3.395956-4.542316$

H $6.989276-1.861858-1.441688$

O $5.626990-1.3689782 .496126$

O $4.375653-2.6805471 .074121$

C $5.442834-2.3295583 .540700$

H $5.894060-3.2854773 .258056$

H $5.943588-1.9564134 .437849$

H $4.378398-2.4546803 .760145$

SCF Energy $\left(B 3 L Y P / 6-31 G^{* *} / / M M F F\right)=-3245.90372564$

03_92

MM̄MF Geometry

C $-3.4629002 .824903 \quad 1.255012$

C -2.8143442 .3743732 .339704$

C -2.6739130 .9156862 .684728$

O
C $-3.5268730 .535673 \quad 3.911216$

C $-5.008974 \quad 0.396470 \quad 3.605954$

C $-5.597576-0.754276 \quad 3.219429$

C -5.8501251 .6233493 .846742$

C $-4.924260-2.0802293 .017947$

C $-5.471279-2.9101281 .850583$

C $-5.263912-2.3242440 .440469$

C $-5.655871-3.388030-0.596327$

C $-5.328365-2.976051-2.036367$

O $-3.892847-1.9476470 .286109$

O $-5.401618-4.150215-2.860513$

C $-3.868086-2.483428-2.088063$

C $-6.363129-1.992108-2.594227$

O $-3.556195-1.968865-3.380789$

C $-3.549324-1.429557-1.009810$

C $-2.048632-1.045637-0.994288$

O $-4.285566-0.235845-1.302925$

C $-0.790412-0.5699582 .849950$

O $-1.446816-1.5774822 .640441$

C $0.684349-0.5427792 .989523$

C $1.375221-1.6550652 .690022$

C $2.825957-1.8456822 .760471$

C $3.660712-0.6852783 .230330$

C $-1.096877-2.126165-0.480624$

C $-0.331380-2.894646-1.539731$

C $1.573167-4.502689-1.699118$

O $-0.518853-2.776626-2.745246$

C $2.663285-4.931954-0.769498$

C $3.883482-4.375010-0.736441$

C $4.965583-4.7940790 .217363$

C $5.418691-3.6695011 .124623$

C $6.457906-2.8906580 .780098$

C $4.759898-3.5239852 .479429$

C $3.332819-3.0490682 .414231$

N $0.619500-3.714634-0.944854$

H -3.9339352 .1102300 .588072$

H -2.365894 3.0885163 .026555

H -2.9528110 .3000201 .821060$

H -3.379853 1.2691924 .716012

H -3.154598 -0.3982714 .350043$

H $-6.676771-0.7591883 .075209$

H -5.5737452 .4318343 .166560$

H $-6.918327 \quad 1.4282973 .702514$

H $-5.7201501 .975174 \quad 4.875504$

H -3.846963 -1.968925 2.894004

H $-5.072289-2.663423 \quad 3.935812$

H $-4.963257-3.8829011 .903192$

H $-6.539481-3.1091342 .005718$

H -5.889762 -1.4310990.331756

H -6.718586 -3.645094 -0.501136

H $-5.108489-4.317540-0.382492$

H -6.295103 -4.523800 -2.771778

H $-3.220373-3.356158-1.933225$

H -7.354497 -2.459842 -2.631431

H -6.126183 -1.711072 -3.626107

H $-6.455411-1.081798-1.998170$

H -3.759605 -2.666618 -4.026949

H -1.739957 -0.659444-1.973117

H -1.939382 $-0.191741-0.310793$

H $-3.8162360 .522838-0.921334$

H $1.1523180 .377570 \quad 3.317400$

H $\quad 0.819417-2.5318232 .357970$

H $4.735080-0.8648313 .147678$

H $3.445775-0.4590844 .280245$

H 3.4538440 .2091042 .633228

H $-0.332891-1.6218600 .123324$

H -1.606703 -2.846478 0.167306

H $1.945678-3.905024-2.537995$

H $1.051839-5.373226-2.109377$

H $2.425291-5.734303-0.075055$

H $4.122923-3.576314-1.434276$

H $5.814415-5.155045-0.378464$

H $4.653200-5.6518410 .825878$

H $6.826018-2.1132801 .441134$

H $6.963924-3.013123-0.171833$

H $5.358568-2.8798503 .132229$

H $4.770356-4.5016192 .979374$

H $2.638533-3.8245942 .089425$ 
H $\quad 0.593857-3.8292410 .065141$ C -2.935850 $4.714867-0.365249$ O $-1.5219904 .457569-0.269018$ C -1.069336 $3.921913-1.526820$ C $-2.1787444 .218335-2.529996$ C $-3.4089214 .048601-1.657838$ H $-1.0045542 .834917-1.404264$ C $0.3045554 .491485-1.884581$ H $-2.1216175 .244566-2.910812$ H -2.159422 $3.535983-3.385155$ C $-4.6662604 .664784-2.244909$ H $-3.5765452 .975385-1.509570$ C $1.3862054 .307188-0.798869$ O $0.1582225 .901285-2.109446$ H $0.6428334 .066876-2.837024$ H 1.1216764 .9253240 .068184 O $2.6038774 .851233-1.327691$ C $1.6681552 .863732-0.336510$ C $1.9474381 .896063-1.499921$ H 2.5464932 .8836290 .317059 $\begin{array}{lllll}\text { O } & 0.589962 & 2.359129 & 0.444931\end{array}$ H $1.0353651 .773159-2.094728$ H $2.7056812 .332828-2.158199$ H $-5.516900 \quad 4.530017-1.569336$ H $-4.542700 \quad 5.738276-2.422653$ H $-4.9153544 .192707-3.200891$ C $-3.632448 \quad 4.2819410 .926068$ H $1.0355096 .248804-2.345545$ H $3.2736394 .810821-0.623605$ H $0.4337902 .985153 \quad 1.172438$ H -4.7041884 .5022410 .860671$ H $-3.235743 \quad 4.8872081 .751296$ H $-3.038507 \quad 5.804675-0.454924$ C $4.7555220 .951397-0.764722$ O $3.5248880 .562195-0.143339$ C $2.3979560 .498540-1.022635$

C $2.672887-0.492942-2.161489$ C $3.990630-0.202995-2.874558$ C $5.128376-0.044844-1.868299$ H $4.6577871 .957838-1.188795$ C 5.8312830 .9907140 .329683 H $1.5722030 .085838-0.429814$ O $1.621155-0.480038-3.120678$ H $2.720239-1.507103-1.748100$ H $3.9122730 .681486-3.517882$ O $4.307194-1.298977-3.736497$ H $5.367819-1.022451-1.432499$ O $6.293095 \quad 0.418786-2.547549$ C 5.6293552 .1439701 .289434 H 5.7892820 .0594750 .904567 H $6.8341371 .101174-0.095886$ H $0.789109-0.648480-2.647274$ H $3.555328-1.416147-4.342397$ H $6.488053-0.218056-3.256470$ O $6.147472 \quad 1.8106322 .508052$ $\begin{array}{lllll}\text { O } & 5.112312 & 3.214462 & 1.000187\end{array}$ C $6.025124 \quad 2.811198 \quad 3.523477$ H 6.5749803 .7120703 .235130 H 4.9706843 .0417063 .703188 H 6.4590442 .4147474 .445229

SCF Energy (B3LYP/6-31G**//MMFF) $=-3245.91768558$

03_93

MM̄FF Geometry

C -2.059635 $3.278785-1.274912$

C $-1.5392392 .131948-0.813642$

C $-0.5422801 .301302-1.578914$

O $0.5859441 .024714-0.718131$

C -1.190636 -0.040132-1.966139

C $-0.305011-0.921991-2.824366$

C $0.160162-2.130671-2.452622$

C $0.027945-0.383705-4.192446$

C $-0.084084-2.856594-1.160343$

C $0.974582-2.612484-0.073992$

C $2.388598-3.078539-0.457334$

C $3.321437-3.031598 \quad 0.754991$

C $4.766583-3.4031620 .384730$

O $2.890711-2.209457-1.475620$
O $5.614177-3.0192561 .478745$

C $5.205995-2.569838-0.839185$

C $4.939109-4.917140 \quad 0.214107$

O $6.476070-3.001649-1.319885$

C $4.177771-2.577992-1.989165$

C $4.520795-1.581586-3.123784$

O $4.140607-3.884068-2.577614$

C $1.5164462 .006515-0.571626$

O $1.5208373 .104137-1.105319$

C 2.5328301 .5175990 .386049

C 3.3667652 .3924830 .967382

C 4.4100522 .0716921 .943957

C 4.7272080 .6171462 .170102

C $4.618816-0.111161-2.701852$

C $6.0342880 .350040-2.395537$

C $7.2850442 .312754-1.505072$

O $7.031952-0.320794-2.645313$

C $7.6139492 .068440-0.066271$

C 7.8974043 .0530240 .799990

C 8.2723532 .8241642 .236110

C 7.4123073 .6159963 .195314

C 7.8362694 .7880673 .697346

C 6.1010633 .0065413 .636954

C 5.0382073 .0860242 .574594

N $6.0534851 .624454-1.843934$

H $-1.7560703 .654726-2.248497$

H -1.8536581 .7550120 .157227$

H $-0.197726 \quad 1.828748-2.476597$

H -2.121995 $0.142289-2.519506$

H -1.483300 -0.562891-1.049094

H $\quad 0.784833-2.677155-3.158420$

H $\quad 0.533130-1.126589-4.819193$

H $-0.885917-0.083776-4.715823$

H $0.6899920 .484016-4.116224$

H -1.069916 -2.601560 -0.759338

H $-0.135371-3.930975-1.378474$

H $0.990732-1.5442820 .171648$

H $\quad 0.654020-3.1456540 .829302$

H $2.321357-4.100938-0.847273$

H $2.952260-3.6840321 .556169$

H $3.321115-2.0161521 .173480$

H $5.301857-3.4806832 .275923$

H $5.338408-1.539535-0.489048$

H $4.678665-5.4388381 .142951$

H $5.983899-5.1755430 .009746$

H $4.317882-5.332774-0.581728$

H $7.098407-2.957874-0.573741$

H $5.420826-1.911587-3.656301$

H $3.708799-1.642694-3.863032$

H $\quad 3.772627-3.808169-3.473517$

H 2.5527670 .4556790 .601302

H 3.2644353 .4494030 .723985

H 4.9236470 .1162001 .216430

$\begin{array}{llll}\text { H } & 3.890996 & 0.113683 & 2.666827\end{array}$

H 5.6165900 .4535002 .781859

H $4.2713360 .511794-3.536064$

H $3.9703010 .097979-1.845848$

H $7.1327453 .374982-1.720998$

H $8.0974831 .940427-2.137277$

H 7.6467341 .0354030 .271662

H 7.8918274 .0834560 .451130

H 9.3250193 .1128002 .355222

H 8.2271721 .7599352 .498378

H 7.2381525 .3491234 .407766

H 8.7895335 .2157493 .404401

H 5.7168713 .5391874 .517390

H 6.2817831 .9866363 .987177

H 4.7398184 .1078862 .335758

H $5.1756252 .085328-1.622553$

C $-4.4484024 .020931-1.185346$

O $-4.9321092 .661798-1.163943$

C $-6.2939092 .657482-0.704566$

C $-6.7721524 .100177-0.807824$

C $-5.5048184 .855332-0.459171$

H -6.2665462 .3728240 .353572$

C -7.130698 1.639879 -1.484457

H -7.093378 $4.352024-1.825086$

H $-7.6017374 .313221-0.127049$ 
C $-5.530967 \quad 6.315614-0.875150$ H $-5.350566 \quad 4.7958550 .626817$ C -6.543606 $0.213297-1.525122$ O $-7.2552162 .089337-2.840072$ H -8.147802 $1.622553-1.074372$ H -5.683513 $0.187311-2.205059$ O $-7.534480-0.634532-2.122979$ C $-6.127402-0.370910-0.161811$ C $-5.782406-1.870949-0.254676$ H -5.2686310 .1922890 .219555$ $\begin{array}{llll}0 & -7.208253 & -0.198278 & 0.755172\end{array}$ H $-6.704067-2.443623-0.415221$ H $-5.122687-2.024577-1.117693$ H $-4.590802 \quad 6.812007-0.615124$ H -5.681449 $6.422833-1.954651$ H $-6.3441956 .845305-0.368303$ C $-3.0738644 .088114-0.518585$ H $-7.7964701 .434873-3.314067$ H -8.288128 $-0.681467-1.509669$ H -6.861429 -0.3349951 .652514$ H $-3.143463 \quad 3.7245790 .513660$ H -2.723989 5.125565 -0.470837 H $-4.3780214 .315371-2.240579$ C -2.983962 -2.182554 2.196282 O $-3.770689-1.8487061 .045275$ C $-5.089596-2.4048021 .013568$ C $-5.015220-3.938657 \quad 1.025048$ C $-4.223476-4.4378952 .238877$ C $-2.884810-3.7069892 .386157$ H $-1.978585-1.8069311 .964964$ C $-3.482242-1.4478153 .445577$ H -5.649295 -2.068704 1.891661 O $-4.394353-4.393882-0.175273$ H $-6.034345-4.3430141 .045308$ H -4.033452 -5.513396 2.137006 O $-4.989247-4.2419203 .423826$ H -2.443001 -3.923198 3.365471 O $-1.975608-4.2254071 .411443$ C $-3.405197 \quad 0.048258 \quad 3.247455$ H $-4.509765-1.7179723 .706019$ H $-2.865947-1.6941114 .317904$ H $-4.390882-5.366019-0.152945$ H $-5.817754-4.741134 \quad 3.324214$ H $-2.342472-4.0324750 .531923$ O $-4.660990 \quad 0.579428 \quad 3.219290$ O $-2.356814 \quad 0.6667493 .116032$ C $-4.725861 \quad 1.9931783 .012260$ H -4.2821362 .2521362 .047306$ H -5.7784072 .2880583 .004524$ H -4.218550 2.5199563 .825879

SCF Energy (B3LYP/6-31G**//MMFF) $=-3245.91486948$

03_94

MM̄FF Geometry

C -2.802320 $0.655714-3.639343$

C $-2.181940 \quad 0.106443-2.583694$

C $-0.850740-0.595330-2.660280$

O $0.0310280 .051844-1.714464$

C $-1.022328-2.067102-2.240702$

C $0.205947-2.925267-2.471243$

C $0.868007-3.591430-1.505261$

C $0.660876-3.052523-3.903132$

C $0.566394-3.639339-0.034636$

C $1.301999-2.5817530 .800738$

C $2.835849-2.6826250 .750041$

C $3.467422-1.7551321 .791741$

C $5.001323-1.7373061 .700751$

O $3.272218-2.302382-0.557918$

O $5.475794-0.6148912 .460650$

C $5.415925-1.4938610 .233097$

C $5.623573-2.9845562 .339162$

O $6.825790-1.623855 \quad 0.074501$

C $4.686465-2.411884-0.769009$

C $4.964144-2.051983-2.250331$

O $5.131579-3.761183-0.581053$

C $0.7913351 .082105-2.167756$

O $0.8950591 .458081-3.327743$

C $1.5193501 .753985-1.068089$ $\begin{array}{llll}\text { C } & 1.274840 & 1.535481 & 0.231994\end{array}$

C 1.9498652 .2239351 .326474

C 1.0057342 .7624862 .365557

C $4.555614-0.637847-2.673557$

C $5.6673950 .388664-2.534261$

C $6.0202912 .851088-2.356867$

O $6.8562450 .089369-2.465009$

C $6.2292283 .081793-0.893400$

C $5.7186824 .123135-0.219379$

C $5.9469334 .355223 \quad 1.246308$

C 4.6693034 .3023152 .053720

C 4.0738625 .4286882 .480327

C 4.1353642 .9438392 .449707

C $3.2939062 .313857 \quad 1.374464$

N $5.1748611 .685909-2.533938$

H $-2.3145500 .594698-4.610635$

H -2.636674 $0.144671-1.598554$

H $-0.439437-0.534993-3.674167$

H $-1.849432-2.519079-2.804703$

H $-1.318289-2.095708-1.187053$

H $1.732809-4.186473-1.798017$

H $1.421459-3.830632-4.029466$

H $-0.183105-3.316296-4.549000$

H $1.094132-2.112054-4.256491$

H $-0.510332-3.5493310 .144314$

H $0.833480-4.6380510 .333801$

H $0.986161-1.587840 \quad 0.464018$

H $\quad 0.972490-2.6933041 .840379$

H $3.121526-3.7234010 .943084$

H $3.146766-2.0311772 .804078$

H $3.100959-0.7329861 .632964$

H $5.150075-0.7103493 .372103$

H $5.168003-0.4499080 .005308$

H $5.355949-3.0446753 .401006$

H $6.717768-2.9403162 .309162$

H $5.301570-3.9148461 .866805$

H 7.245679-1.014144 0.705137

H $6.012025-2.260600-2.498109$

H $4.376000-2.746280-2.867889$

H $4.940424-4.264694-1.389721$

H $2.2551342 .489450-1.380062$

H 0.5007260 .8393280 .541048

H $0.276223 \quad 3.4364441 .902315$

H 1.5042233 .3337483 .151592

H 0.4600351 .9450682 .849004

H $4.293083-0.653446-3.739080$

H $3.667399-0.308873-2.124922$

H $5.5217893 .695403-2.843239$

H $6.9837632 .683865-2.848930$

H $6.8408842 .354539-0.364390$

H $5.1126914 .855803-0.747232$

H $\quad 6.425246 \quad 5.337242 \quad 1.356941$

H 6.6648333 .6346771 .658011

H 3.1755925 .3973923 .087896

H 4.4687326 .4071352 .227455

H 3.5851293 .0129613 .394590

H 4.9747092 .2741312 .676150

H 3.8698951 .8558240 .571063

H $4.170437 \quad 1.833387-2.548707$

C $-4.849267 \quad 1.497587-2.303099$

O $-4.1536462 .466568-1.489089$

C $-5.1243013 .316996-0.866121$

C $-6.4701932 .628616-1.036591$

C $-6.2903152 .000272-2.406572$

H $-5.1487474 .238098-1.465832$

C $-4.7399993 .709788 \quad 0.562968$

H $-6.6226951 .853114-0.277422$

H -7.311324 $3.326699-0.991698$

C $-7.309270 \quad 0.917233-2.715128$

H $-6.3594482 .786646-3.170326$

C -4.597042 2.5862501 .612184

O

H $-3.798808 \quad 4.2734610 .536169$

H -5.5684752 .1201201 .812823$

$\begin{array}{llll}\text { O } & -4.206428 & 3.221708 & 2.841948\end{array}$

C -3.5573931 .4910291 .298643$

C -3.2408370 .5978892 .517164$

H -3.9283960 .8635340 .484238$ 
$\begin{array}{llll}\text { O } & -2.344278 & 2.098029 & 0.852771\end{array}$ H -2.811562 1.2216623 .309946 H -4.1773200 .1802952 .901119$ H -7.121597 $0.470807-3.696552$ H -7.283235 $0.115820-1.969857$ H -8.321727 $1.334258-2.722005$ C $-4.1397181 .354931-3.651410$ H $-5.768344 \quad 5.3762290 .445940$ H $-4.883092 \quad 3.8890413 .049631$ H -2.031247 2.6838321 .563262 H $-3.9787202 .352702-4.078727$ H $-4.7840210 .803202-4.345920$ H -4.836716 $0.547868-1.759420$ C $-3.765243-2.3098581 .636068$ O $-2.717771-1.432751 \quad 1.204326$ C -2.229144 -0.5262042 .197318$ C $-1.733984-1.2988973 .428385$ C $-2.800163-2.2415643 .972163$ C -3.328961 -3.134889 2.853811 H -4.655565 -1.721805 1.888286 C $-4.100316-3.236098 \quad 0.456163$ H -1.347252 -0.0456561 .759005$ O $-1.320084-0.4126784 .462488$ H $-0.857090-1.8968583 .152250$ H -3.615235 -1.693174 4.459009 O $-2.220667-3.078762 \quad 4.975392$ H -2.553487 -3.855896 2.564060 O $-4.445153-3.8786363 .339170$ C $-4.877117-2.524548-0.628606$ H $-3.169637-3.6186520 .019998$ H $-4.716584-4.083000 \quad 0.776559$ H $-0.6226590 .157167 \quad 4.096098$ H - $-1.850945-2.4949235 .660066$ H $-4.146214-4.3722994 .122163$ O $-4.689870-3.175014-1.814302$ O $-5.593610-1.549387-0.449954$ C $-5.409101-2.644508-2.931519$ H $-5.060742-1.633873-3.157917$ H -5.213858 -3.284230 -3.796102 H -6.484489-2.646499-2.729506

SCF Energy (B3LYP/6-31G**//MMFF) $=-3245.90508494$

03_95

MMFF Geometry

C $0.121068 \quad 6.051964 \quad 0.367680$

C $0.776128 \quad 5.188394-0.423082$

C $1.9992264 .411763-0.010270$

$\begin{array}{lllllllll}\text { O } & 1.626167 & 3.017717 & 0.085887\end{array}$

C $3.0864784 .541823-1.093265$

C $4.331558 \quad 3.722229-0.815954$

C $4.7626602 .705012-1.588828$

C $5.117886 \quad 4.121357 \quad 0.405860$

C $4.1461402 .179511-2.856610$

C $3.1449161 .030463-2.656821$

C $3.779765-0.274014-2.148296$

C $2.754403-1.409039-2.143049$

C $3.351143-2.710294-1.584129$

O $4.248029-0.064793-0.816755$

O $2.264102-3.608291-1.306281$

C $4.047965-2.420246-0.233178$

C $4.230829-3.412665-2.625939$

O $4.769569-3.571166 \quad 0.197942$

C $4.963539-1.173648-0.253606$

C $5.437470-0.721700 \quad 1.147061$

O $6.173903-1.427119-0.977863$

C 1.0793322 .5989551 .259045

$\begin{array}{lllll}\text { O } & 0.892330 & 3.262944 & 2.266027\end{array}$

C 0.7024091 .1791951 .092083

C $-0.205704 \quad 0.641601 \quad 1.919816$

C $-0.723075-0.7258221 .858754$

C $-0.029626-1.6918160 .936578$

C $4.324955-0.3559992 .135715$

C $3.935282-1.4869143 .072930$

C $2.314491-2.0267104 .888201$

O $4.538136-2.555113 \quad 3.129994$

C $1.218753-2.8626854 .306890$

C $-0.009796-2.9421104 .840041$

C $-1.102283-3.817265 \quad 4.296830$
C -2.379736 -3.056026 4.025052

C $-3.393599-3.0689454 .906933$

C $-2.516852-2.3605152 .691119$

C $-1.778622-1.0521072 .632981$

N 2.847797-1.145827 3.866355

H $0.477207 \quad 6.237628 \quad 1.377223$

H $0.3871934 .993590-1.420777$

H 2.3885224 .7653360 .952458

H $3.3804455 .594865-1.196847$

H $2.6532214 .256092-2.057801$

H $5.6747142 .188921-1.290804$

H 6.0690203 .5833360 .481879

H 5.3493215 .1910990 .375925

H 4.5492963 .9110041 .316876

H $3.6528752 .988090-3.406675$

H $4.9565441 .843734-3.516159$

H $2.3598681 .356464-1.964509$

H $2.6620170 .836319-3.622722$

H $4.625805-0.522150-2.799847$

H $2.348425-1.574658-3.148832$

H $1.897499-1.122898-1.518643$

H $1.780201-3.759622-2.136040$

H $3.245160-2.2631900 .495641$

H $3.638130-3.679771-3.509551$

H $4.628997-4.356683-2.238110$

H $5.065059-2.796357-2.967534$

H $5.066263-3.4125061 .109641$

H $6.129760-1.4588291 .571474$

H $6.0430840 .184978 \quad 1.001574$

H $6.181238-2.355452-1.259701$

H $1.1489390 .630547 \quad 0.271106$

H $-0.639671 \quad 1.2712302 .695724$

H $1.049506-1.6879831 .120499$

H $-0.207399-1.421555-0.108711$

H $-0.351286-2.7265621 .069410$

H 4.6870870 .4589222 .775800

H $3.4349780 .008577 \quad 1.614101$

H $1.962904-1.3952045 .710232$

H $3.113155-2.6748825 .263160$

H $1.455831-3.4527363 .424683$

H $-0.235501-2.3731445 .739287$

H $-1.290527-4.6092475 .033670$

H $-0.785209-4.3351973 .383135$

H $-4.325649-2.5514774 .705102$

H -3.311938 -3.5933115 .853292$

H $-3.573385-2.1352332 .498056$

H -2.230065 -3.0494421 .891686$

H -2.205807 -0.2833283 .278391$

H $2.373456-0.2616033 .707611$

C -2.421683 6.0009740 .222552

$\begin{array}{llll}\text { O } & -2.397301 & 4.733455 & -0.466478\end{array}$

C $-2.769724 \quad 3.700518 \quad 0.462372$

C $-3.534533 \quad 4.417790 \quad 1.564616$

C $-2.715858 \quad 5.6912751 .692208$

H -1.839732 3.2981660 .882392

C $-3.5307382 .565957-0.234229$

H $-4.5604454 .649924 \quad 1.254891$

H -3.5750613 .8387412 .491803$

C $-3.435280 \quad 6.811313 \quad 2.422610$

H $-1.791146 \quad 5.4551122 .233488$

C $-2.6448231 .812562-1.251219$

$\begin{array}{llll}\text { O } & -4.687183 & 3.107548 & -0.866411\end{array}$

H $-3.873353 \quad 1.8582180 .530565$

H $-1.7375841 .487150-0.730504$

O $-2.2242282 .692264-2.293255$

C $-3.3098800 .567381-1.871692$

C $-2.299547-0.227674-2.716917$

H -3.690116 $-0.060191-1.061077$

O $-4.4146690 .965406-2.675578$

H $-1.8729140 .432065-3.482657$

H $-1.473517-0.524469-2.061564$

H -2.812372 7.7105832 .463434

H -4.3764307 .0743691 .928139$

H -3.668086 6.5130353 .450052

C $-1.1278156 .770128-0.066663$

H $-5.1811962 .369384-1.261188$

H -3.025702 $3.014554-2.740372$

H $-4.9243800 .166552-2.892385$ 
H -1.1570317 .7527740 .417866$ H -1.066543 $6.957992-1.146736$ H -3.256782 $6.563805-0.213976$ C -2.923997 -3.011042-1.578215 O $-3.659301-2.304338-2.582608$ C $-2.877465-1.466208-3.436856$ C $-1.812374-2.302689-4.161971$ C $-0.943784-3.074225-3.171159$ C $-1.820797-3.873769-2.209953$ H $-2.490894-2.294139-0.870864$ C $-3.916931-3.907080-0.816824$ H -3.564936 -1.088734 -4.204296 O $-1.003672-1.459119-4.974656$ H -2.312255 -3.016170 -4.829975 H $-0.267665-2.409686-2.622186$ O $-0.112023-3.995027-3.880322$ H $-2.269520-4.717766-2.750049$ O $-1.004388-4.419053-1.175179$ C $-4.906454-3.1302550 .025006$ H $-4.498418-4.507885-1.526047$ H -3.370126 -4.574178 -0.140496 H - $0.428464-2.029408-5.511775$ H $0.521382-3.475156-4.403118$ H $-0.307324-4.946596-1.601728$ O $-5.601498-4.008351 \quad 0.808763$ O $-5.065748-1.918675-0.001790$ C $-6.587179-3.4227541 .665242$ H $-7.358210-2.9256981 .069003$ H -7.053431 -4.224238 2.244498 H -6.118801-2.717039 2.357805

SCF Energy (B3LYP/6-31G**//MMFF) $=-3245.91925556$

03_96

MM̄FF Geometry

C $1.935624-4.280160-0.958029$

C $0.875483-5.060089-0.699382$

C $-0.539307-4.596260-0.428067$

O $-0.621072-3.164394-0.244488$

C $-1.421708-4.972042-1.634106$

C $-2.893122-4.655740-1.454552$

C $-3.590145-3.802512-2.230733$

C $-3.591125-5.393827-0.341282$

C $-3.093630-2.999057-3.401052$

C $-2.598345-1.590494-3.038883$

C $-3.705374-0.641415-2.552344$

C $-3.1571240 .771941-2.350839$

C -4.230982 $1.727080-1.805100$

O $-4.208709-1.126083-1.308355$

O $-3.5693782 .912076-1.333140$

C -4.919584 $1.081620-0.579242$

C $-5.1874372 .183478-2.913346$

O $-6.0530301 .840480-0.167903$

C -5.327431 $-0.392948-0.792147$

C $-5.763401-1.1052710 .507918$

O $-6.461429-0.502600-1.661679$

C $-0.346748-2.6752690 .996260$

O $0.004398-3.3129511 .976266$

C $-0.537290-1.2073480 .955392$

C $-0.066587-0.4525721 .959971$

C $-0.159095 \quad 1.005432 \quad 2.063510$

C -1.0833531 .7189601 .114908$

C $-4.701289-1.1740521 .611498$

C $-4.848534-0.0983912 .675014$

C $-3.750730 \quad 0.732627 \quad 4.751743$

O $\begin{array}{llll}-5.794894 & 0.682440 & 2.721886\end{array}$

C $-3.015596 \quad 1.9871804 .402176$

C -1.968772 $2.447507 \quad 5.103327$

C $-1.257048 \quad 3.7327124 .794625$

C $0.229918 \quad 3.544176 \quad 4.601982$

C $1.097188 \quad 3.826796 \quad 5.588778$

C $0.7151433 .115743 \quad 3.236653$

C 0.5746631 .6354853 .004526

N $-3.807016-0.1380863 .592854$

H $1.817935-3.200859-0.987553$

H $1.016825-6.139456-0.693217$

H $-0.902121-5.1016810 .475197$

H -1.332531-6.047441 -1.839695

H $-1.025854-4.462605-2.519360$
H $-4.648785-3.664237-2.013541$

H $-3.226019-5.0569140 .633633$

H $-4.675416-5.238935-0.356205$

H $-3.414398-6.470743-0.429738$

H $-2.293479-3.534205-3.923183$

H $-3.907335-2.921744-4.133454$

H $-1.817109-1.673090-2.273782$

H -2.131342 -1.159771 -3.933196

H $-4.509328-0.640709-3.297684$

H $-2.7353631 .163261-3.284978$

H $-2.3240780 .740416-1.635784$

H $-3.0433453 .274364-2.066615$

H $-4.199808 \quad 1.1294810 .244963$

H $-4.6392262 .729384-3.690933$

H $-5.9357282 .884709-2.528331$

H $-5.7050081 .354094-3.400095$

H $-5.7495932 .750428-0.007060$

H $-6.704032-0.677438 \quad 0.875535$

H $-6.008117-2.142958 \quad 0.237140$

H -6.776402 $0.389362-1.877729$

H $-1.043375-0.7869000 .094032$

H $0.465391-0.9433282 .774188$

H $-0.666809 \begin{array}{lll}1.732874 & 0.104597\end{array}$

H -1.2866832 .7528921 .402969$

H $-2.058326 \quad 1.222638 \quad 1.086542$

H $-4.808631-2.1341762 .132847$

H -3.689124 -1.1395601 .195926$

H -3.2601210 .1737635 .554938$

H -4.7690010 .9791245 .069812$

H -3.380242 2.5589423 .552012

H $-1.622548 \quad 1.8885585 .969937$

H -1.4416814 .4230035 .628299$

H -1.679471 $4.222653 \quad 3.908417$

H $2.1678673 .722314 \quad 5.447517$

H $0.755177 \quad 4.1634976 .561859$

H $1.7816753 .356312 \quad 3.128120$

H 0.2193893 .7178132 .469894

H 1.2042201 .0320833 .659985

H $-3.031528-0.7763823 .439711$

C $4.385627-4.368910-0.274177$

O $4.460534-2.930880-0.228650$

C $4.583964-2.525543 \quad 1.148334$

C $4.998233-3.7758651 .912530$

C $4.205819-4.8373271 .170293$

H $3.581241-2.2355671 .484476$

C $5.536391-1.3319461 .264077$

H $6.073457-3.9698961 .822414$

H $4.748503-3.7167852 .976006$

C $4.690728-6.253392 \quad 1.424367$

H $3.154937-4.7612431 .476408$

C $5.143626-0.1340970 .371333$

O $6.856205-1.740805 \quad 0.885192$

H $5.608880-1.0145482 .310395$

H $5.307573-0.390975-0.680409$

$\begin{array}{llllll}\text { O } & 6.032542 & 0.949927 & 0.664452\end{array}$

$\begin{array}{llll}\text { C } 3.701226 & 0.354448 & 0.582779\end{array}$

C $3.3127821 .619932-0.193729$

H $2.992664-0.4382740 .332476$

$\begin{array}{lllll}\text { O } & 3.506791 & 0.626469 & 1.975401\end{array}$

H $2.272599 \quad 1.8531420 .063918$

H 3.8717132 .4775500 .206956

H $4.099404-6.9752760 .852206$

H $5.741144-6.3758901 .139615$

H $4.599060-6.5064162 .485679$

C $3.308785-4.810009-1.267394$

H $6.805789-2.094750-0.019529$

H $6.9397820 .622760 \quad 0.538645$

H $4.146943 \quad 1.3118242 .233548$

H $3.299736-5.904193-1.334873$

H $3.575872-4.432859-2.262848$

H $5.357782-4.708746-0.655293$

C $1.9555583 .416148-2.191720$

O $3.2994972 .918998-2.260606$

C $3.4891941 .597758-1.726182$

C $2.6215100 .619951-2.529740$

C $1.1525991 .030473-2.512098$

C $0.9939762 .487896-2.948835$

H $1.6412193 .502461-1.144899$ 
C $1.9693344 .829245-2.795643$

H $4.5380401 .353463-1.927631$

O $2.740507-0.715573-2.064916$

H $2.9586990 .619966-3.574894$

H $0.7052390 .858846-1.527942$

$\begin{array}{lllll} & \mathrm{H} & 0.425373 & 0.208827 & -3.428969\end{array}$

H $1.1676922 .553517-4.030714$

O $-0.3573812 .874135-2.704796$

C $2.7394715 .812435-1.937175$

H $2.4482674 .802601-3.781769$

H $0.9520025 .220751-2.894084$

H $3.667860-0.984287-2.177998$

H $0.555491-0.715131-3.153814$

H $-0.5219473 .703646-3.182758$

O $2.8326957 .001433-2.603990$

O $3.1906605 .578641-0.824417$

C $3.5332208 .040543-1.912931$

H $4.5678257 .738790-1.723691$

H $3.5377728 .929172-2.549644$

H $3.0230478 .282796-0.975748$

SCF Energy (B3LYP/6-31G**//MMFF) $=-3245.91817932$

03_97

MM̄FF Geometry

C -0.4385624 .7501841 .822843$

C $0.496934 \quad 4.360047 \quad 0.943295$

C 1.7274873 .5778491 .330307

$\begin{array}{lllllll}\text { O } & 1.823104 & 2.421107 & 0.463394\end{array}$

C $2.987253 \quad 4.4351541 .116734$

C 4.2627773 .7670631 .593890

C 5.2890213 .4136930 .795492

C 4.3599533 .5266353 .079324

C $5.4159743 .596114-0.690278$

C $4.9405572 .401375-1.531173$

C $5.6970201 .091891-1.252722$

C $5.3669920 .041444-2.314475$

C $6.040931-1.307212-2.017185$

O 5.3000930 .6039800 .031415

O $5.430764-2.296458-2.861524$

C $5.745660-1.714339-0.556016$

C $7.531324-1.289392-2.379486$

O $6.502250-2.874675-0.218658$

C $5.993120-0.577150 \quad 0.460551$

C $5.489334-0.8994381 .889876$

O $7.402274-0.336764 \quad 0.559043$

$\begin{array}{llll}\text { C } & 0.981222 & 1.380552 & 0.708315\end{array}$

$\begin{array}{llll}\text { O } & 0.165086 & 1.290868 & 1.611500\end{array}$

C $1.2048200 .367455-0.346885$

C $0.310409-0.617305-0.514287$

C $0.372365-1.682035-1.517185$

C $1.659296-1.844852-2.280287$

C $3.994285-1.2172052 .006644$

C $3.671201-2.7016651 .964366$

C $1.731773-4.2658051 .896223$

O $4.528860-3.5793662 .006801$

C $1.471803-4.6836590 .483273$

C $0.291160-5.1652260 .066060$

C $0.017878-5.625105-1.337234$

C $-1.162843-4.914985-1.960674$

C $-2.387550-5.467998-1.952436$

C $-0.904502-3.599186-2.659732$

C $-0.711312-2.466889-1.690361$

N $2.302890-2.931626 \quad 1.909113$

H -0.2755694 .5315992 .876846$

H $0.3746384 .568155-0.115745$

H $1.666203 \quad 3.2414252 .372226$

H 2.8849515 .3882681 .652600

H 3.0608134 .6906920 .054203

H $6.1555082 .941676 \quad 1.257926$

H 5.3597373 .1968613 .381947

H 4.1416504 .4480103 .629069

H 3.6522942 .7537453 .393832

H $4.8842674 .495953-1.017782$

H $6.4719463 .793817-0.915624$

H $3.8681122 .252387-1.359944$

H $5.0643132 .671672-2.587332$

H $6.7720601 .306713-1.249017$

H $5.6469910 .396366-3.314214$
H $4.280202-0.113145-2.346631$

H $5.551779-2.018207-3.785476$

H $4.689426-2.006672-0.524306$

H $7.664073-1.055233-3.442641$

H $7.985953-2.275243-2.232756$

H $8.103037-0.556049-1.806934$

H $6.195770-3.1903700 .647606$

H $6.106889-1.6839232 .343221$

H $5.668206-0.0038712 .502856$

H 7.5799860 .1141391 .401131

H $2.0859190 .470838-0.969111$

H $-0.565078-0.6381240 .132687$

H $2.506015-1.918421-1.589549$

H $1.822126-0.991394-2.947040$

H $1.692439-2.747648-2.893563$

H $3.638716-0.8561822 .980389$

H $3.421055-0.6958971 .235031$

H $0.814945-4.2322752 .492892$

H $2.428761-4.9672332 .365877$

H $2.296649-4.609224-0.221528$

H $-0.522691-5.2641230 .781024$

H $-0.172905-6.705764-1.303162$

H $0.899295-5.502367-1.978848$

H $-3.234410-4.974555-2.417570$

H -2.571534 -6.423501 -1.472448

H $-1.760283-3.337534-3.296473$

H $-0.067026-3.720651-3.351964$

H -1.595939-2.267023 -1.085542

H $1.668012-2.1431081 .825329$

C -2.207272 5.4223940 .056308

O $-2.3419554 .058472-0.386783$

C $-3.607768 \quad 3.894799-1.046573$

C $-4.0898175 .305300-1.360998$

C $-3.5811036 .065585-0.149844$

H $-4.2913093 .449114-0.314596$

C $-3.4600942 .959111-2.249846$

H -3.630361 $5.701306-2.273848$

H $-5.1764805 .357577-1.477123$

C $-3.5328637 .570344-0.349401$

H -4.2350565 .8401980 .703273$

C $-2.7430641 .623598-1.955707$

O $-2.689036 \quad 3.638366-3.252644$

H $-4.4441882 .777292-2.697234$

H $-1.690137 \quad 1.838337-1.732687$

O $-2.749104 \quad 0.866830-3.174218$

C $-3.3300580 .741072-0.837795$

C $-4.8228770 .415863-1.020352$

H $-2.782010-0.206100-0.836786$

$\begin{array}{lllll}\text { O } & -3.125120 & 1.333321 & 0.439769\end{array}$

H $-5.4165621 .334259-0.947530$

H $-4.966798-0.005337-2.022335$

H $-3.1435098 .069272 \quad 0.543575$

H -2.891819 $7.843011-1.194491$

H $-4.5350647 .964293-0.547399$

C $-1.7472345 .434604 \quad 1.518361$

H -2.614971 $3.039150-4.015271$

H $-2.2198850 .064835-3.023611$

H -2.174169 1.5145410 .532546

H -2.507816 4.9209222 .121609

H -1.689384 6.4660511 .884102

H $-1.4611705 .901916-0.589291$

C $-4.920219-2.8275060 .846230$

O $-4.607509-1.797499-0.101519$

C $-5.343611-0.577500 \quad 0.038202$

C $-6.849026-0.842909-0.116096$

C $-7.325772-1.9294730 .853774$

C $-6.420933-3.166106 \quad 0.815325$

H $-4.375940-3.7112640 .488909$

C $-4.400265-2.4879832 .252305$

H $-5.153111-0.1510861 .028498$

O $-7.151290-1.237658-1.453149$

H $-7.3941650 .089230 \quad 0.072570$

H -8.349985 -2.224364 0.594510

O $-7.344468-1.4147142 .181883$

H $-6.658629-3.8434491 .643927$

O $-6.669052-3.884196-0.397335$

C -2.887581 -2.428409 2.302851

H $-4.784186-1.5240912 .596374$ 
H $-4.713706-3.2587992 .965463$

H $-6.613328-2.020768-1.660737$

H $-7.945618-0.6504692 .192399$

H -7.604489-4.149733 -0.394134

O $-2.503240-1.7086943 .398035$

O $-2.135816-2.9732791 .506648$

C -1.090449-1.591245 3.595130

H $-0.917925-0.8726504 .400835$

H -0.676107 -2.559212 3.891005

H $-0.601026-1.2235932 .689018$

SCF Energy (B3LYP/6-31G**//MMFF) $=-3245.92294619$

03_98

MM̄FF Geometry

C -1.026005 5.150460-0.638508

C $-0.0589144 .427090-1.225063$

C $1.1323313 .890467-0.469247$

O $1.4784782 .587677-0.995574$

C $2.3421514 .820546-0.682928$

C 3.5040484 .5383920 .250219

C $4.7284694 .138200-0.143886$

C 3.2400424 .7774541 .715969

C $5.2200003 .876181-1.538571$

C $5.0891082 .417432-2.003060$

C $5.8186091 .399171-1.110294$

C $5.8671620 .026782-1.785807$

C $6.486331-1.046422-0.875777$

O $5.106694 \quad 1.296596 \quad 0.126343$

O $6.186548-2.331574-1.442511$

C $5.802501-0.9941450 .508549$

C $8.015384-0.939779-0.831741$

O $6.457607-1.8588791 .432351$

C 5.7137300 .4296851 .093540

C 4.8622970 .5118002 .385182

O $7.0301640 .881696 \quad 1.434385$

C $0.8154631 .506398-0.505645$

$\begin{array}{lllll}\text { O } & -0.057421 & 1.499835 & 0.346807\end{array}$

C $1.3429440 .308892-1.198406$

C $0.638839-0.832975-1.198150$

C $1.048247-2.096801-1.815926$

C $2.485789-2.217976-2.247810$

C $3.400926 \quad 0.0724662 .242901$

C $3.152365-1.3795852 .614844$

C $1.387622-3.1355382 .520588$

O $3.982406-2.081043 \quad 3.186444$

C $1.698345-4.0071491 .344722$

C $0.798860-4.8182750 .767754$

C $1.115555-5.716083-0.394636$

C $0.125304-5.576692-1.529907$

C $-0.862294-6.473773-1.690199$

C $0.335083-4.459761-2.529477$

C $0.141148-3.089674-1.932568$

N $1.872735-1.7918842 .264938$

H -0.9392435 .3625430 .423553$

H $-0.0997094 .240680-2.295183$

H 0.8972393 .8035300 .598118

H $2.0443375 .865970-0.525205$

H $2.6588204 .749543-1.729255$

H 5.4836133 .9793330 .625648

H 2.5571314 .0217902 .115269

H 4.1565914 .7380672 .314723

H $2.7966475 .767251 \quad 1.866726$

H $4.7136234 .527074-2.259254$

H 6.276566 4.170553-1.582841

H $4.0245852 .161758-2.058519$

H $5.4891352 .357037-3.022952$

H $6.8338631 .766799-0.920602$

H $6.4101980 .081025-2.737656$

H $4.846721-0.283961-2.044386$

H $\quad 6.545651-2.351815-2.346162$

H $4.787498-1.3878420 .376183$

H 8.436872 -1.065836 -1.836444

H $8.450430-1.740139-0.223234$

H $8.3676850 .018942-0.446041$

H $6.471183-2.7477521 .037798$

H $5.366492-0.0167843 .203190$

H 4.8431881 .5678272 .691779

H 6.9512951 .6002422 .083597
H $2.3015640 .402112-1.695159$

H $-0.332417-0.847931-0.708290$

H $2.771392-3.226826-2.551167$

H $3.153126-1.954481-1.420346$

H $2.691944-1.553971-3.094013$

H 2.7900980 .6702912 .931379

H 3.0324650 .2613881 .230939

H $\quad 0.312338-3.0640842 .710725$

H $1.878547-3.5331763 .414592$

H $2.716600-3.9873680 .962811$

H $-0.211791-4.8612361 .167814$

H $1.115119-6.749727-0.024549$

H $2.129087-5.538067-0.775596$

H -1.556966 -6.410422 -2.520595

H $-0.999067-7.293723-0.992763$

H $-0.379272-4.553799-3.358004$

H $1.318125-4.587979-2.989972$

H $-0.875103-2.905458-1.582586$

H $1.270461-1.1607161 .744609$

C $-3.5708015 .425059-0.806878$

O $-3.8411414 .017886-0.946577$

C -4.4834903 .5545300 .256400$

C -4.9412604 .8079150 .997181$

C -3.8209655 .7730660 .661638$

H -3.7056173 .0663820 .854415$

C $-5.5991652 .553327-0.060132$

H $-5.8938805 .187900 \quad 0.611013$

H -5.0551604 .6367142 .071886$

C -4.1746817 .2308650 .897966$

H -2.9581225 .5133241 .285013$

C $-5.2033441 .374028-0.972041$

O $-6.6599303 .258853-0.722349$

H -6.0261432 .1797420 .878301$

H -5.036788 $1.760117-1.986014$

O $-6.3454150 .508889-1.050427$

C $-3.9897340 .529355-0.545193$

C -4.0584540 .0347140 .912750$

H $-3.940651-0.336820-1.213456$

O $-2.8042001 .284171-0.762942$

H -3.9510070 .8870591 .592356$

H $-5.046496-0.4000921 .095839$

H -3.3379837 .8825300 .627010$

H -5.0448897 .5344390 .306506$

H -4.409862 7.4032681 .953321

C $-2.1870265 .765846-1.371415$

H $-7.3695752 .616873-0.896737$

H -6.139228 -0.192095 -1.692346

H $-2.0469970 .681386-0.687140$

H -2.055060 $6.854269-1.356195$

H $-2.1575905 .454815-2.423508$

H $-4.3173255 .931749-1.433336$

C $-4.069283-2.9807750 .456503$

O $-2.945363-2.0984130 .346882$

C $-2.957390-0.9946981 .257612$

C $-2.996737-1.5088412 .703624$

C $-4.143459-2.4902012 .931344$

C -4.135177 -3.583862 1.863995

H $-4.995546-2.4338010 .243977$

C $-3.899362-4.074009-0.607254$

H $-1.995397-0.4842571 .134185$

O $-3.112621-0.4316093 .626776$

H -2.054021 -2.024164 2.928734

H $-5.111893-1.9769122 .958930$

O $-3.982306-3.1119114 .208441$

H $-3.280544-4.2523802 .030219$

O $-5.321774-4.3651361 .985601$

C -4.105880 -3.536007 -2.007830

H $-2.885289-4.483582-0.538820$

H $-4.620342-4.886330-0.468155$

H $-2.3626760 .167967 \quad 3.471722$

H -3.952136 -2.402362 4.873102

H -5.349483 -4.710696 2.894428

O $-3.375285-4.278119-2.889510$

O $-4.842344-2.602947-2.298032$

C $-3.472245-3.879993-4.260275$

H $-4.503485-3.980855-4.611907$

H -3.120150 -2.851122 -4.381635

H $-2.833182-4.540944-4.851738$ 
H 5.9013045 .1650921 .869409

H 8.3221954 .8335912 .674128

03_99

MM̄FF Geometry

C $-1.969259-1.1641872 .312138$

C $-0.860810-1.8309321 .958478$

C $-0.115208-1.5880070 .670172$

O $1.269731-1.3181820 .987853$

C $-0.169650-2.853930-0.204733$

C $0.513124-2.701564-1.550347$

C $1.601285-3.394343-1.940204$

C $-0.120824-1.718022-2.500616$

C $2.364297-4.438710-1.174619$

C $3.567271-3.903698-0.382116$

C $4.661299-3.262902-1.252177$

C $5.916171-2.979744-0.422807$

C $6.996103-2.253234-1.241054$

O $4.156867-2.034448-1.781791$

O $7.975356-1.742961-0.322710$

C $6.362447-1.034342-1.947149$

C $7.739895-3.211398-2.179142$

O $7.286209-0.431333-2.849343$

C $5.048760-1.365830-2.683759$

C $4.310178-0.111654-3.212335$

O $5.341086-2.188224-3.820416$

C $1.583702-0.0565971 .390189$

$\begin{array}{llll}\text { O } & 0.825370 & 0.894025 & 1.495621\end{array}$

C $3.027557-0.0374491 .712424$

C $3.524417 \quad 0.9633112 .454512$

C 4.9190211 .1151882 .875305

C 5.9363830 .1933802 .256938

C $3.9056280 .912674-2.146236$

C $4.9002982 .048489-1.972034$

C $5.3524334 .025821-0.524520$

O $5.8425962 .243984-2.734658$

C 6.3888893 .6599990 .490344

C 6.5497294 .3204401 .647110

C 7.6128073 .9958232 .656858

C 7.0502153 .7719684 .042545

C 7.0576714 .7549434 .958790

C 6.5600932 .3846814 .390477

C 5.2211112 .0727573 .777094

N $4.5853982 .846543-0.880047$

H -2.365442 -0.3976761 .651684$

H $-0.461501-2.5922762 .624647$

H $-0.538343-0.7357290 .125092$

H -1.216180 -3.134932 -0.385052

H $\quad 0.272344-3.6844220 .356166$

H $1.996227-3.202651-2.937561$

H $-0.052915-0.698561-2.109562$

H $0.360210-1.720941-3.484739$

H -1.174876 -1.967439 -2.654243

H $\quad 1.702325-4.981316-0.491687$

H $2.713218-5.194349-1.890154$

H $3.209581-3.1773550 .356896$

H $3.998605-4.7451240 .174482$

H $4.894558-3.949213-2.074638$

H $\quad 6.318570-3.9069140 .003877$

H $5.648388-2.3483710 .435053$

H $8.331116-2.4934250 .183441$

H $6.155187-0.290534-1.169045$

H $8.224710-4.009905-1.604606$

H $8.546246-2.696079-2.712606$

H $7.086594-3.685291-2.914431$

H $8.087535-0.215412-2.342381$

H $4.8861180 .351634-4.022390$

H $3.378956-0.460039-3.682456$

H $4.604690-2.117017-4.450223$

H $3.635915-0.8577691 .350259$

H 2.8430611 .7355522 .809745

H $5.785333-0.8342712 .603554$

H 6.9688860 .4665552 .484003

H $5.8553550 .212346 \quad 1.165004$

H $2.9626371 .380962-2.457105$

H $3.727306 \quad 0.428933-1.181336$

H $4.6454904 .770320-0.145199$

H $5.8383364 .428995-1.418788$

H 7.0535222 .8330430 .251464

H 8.1993743 .1192252 .354958

H 6.6821634 .5943265 .963879

H 7.4350055 .7443744 .721965

H 6.4455792 .2890255 .478797

H 7.3326011 .6565524 .128593

H 4.4120472 .6954404 .161752

H $3.8163942 .579211-0.272413$

C $-4.100302-1.9916563 .429470$

O $-4.943682-1.1264272 .645903$

C $-5.859388-1.9483161 .889608$

C $-5.640842-3.3849862 .360217$

C $-4.175205-3.3574832 .752207$

H $-5.548954-1.8813170 .841882$

C $-7.295610-1.4343282 .054407$

H $-6.265078-3.6283863 .227823$

H $-5.862221-4.1142701 .574820$

C $-3.757920-4.5159833 .639430$

H $-3.574542-3.3713001 .834013$

C $-7.481790 \quad 0.031223 \quad 1.608354$

O $-7.641493-1.5268033 .441753$

H -7.987604 -2.090539 1.513255

H -6.8577310 .6951152 .218901$

O $-8.8425240 .407676 \quad 1.851904$

C $-7.1869610 .267706 \quad 0.114938$

C $-7.3587001 .742960-0.292543$

H $-6.159804-0.033796-0.103238$

O $-8.041520-0.553851-0.678404$

H -8.363661 $2.089259-0.022171$

H -6.6503082 .3433930 .289588$

H -2.697050 -4.445031 3.899565

H -4.333294 -4.536552 4.570969

H $-3.916049-5.4698683 .125712$

C $-2.705827-1.3861973 .601471$

H $-8.603839-1.4081493 .510962$

H -8.9614490 .4628712 .815223$

H -8.959336 -0.306265 -0.472346

H -2.116382 -2.016002 4.278386

H -2.802814 -0.407043 4.087185

H $-4.563846-2.0468394 .424177$

C $-4.7602271 .855892-1.894211$

O $-6.016077 \quad 1.329184-2.335033$

C $-7.1647681 .993776-1.805603$

C $-7.1369583 .477135-2.200986$

C $-5.8178854 .146366-1.816044$

C $-4.6267703 .324217-2.310085$

H $-4.6825551 .767199-0.803363$

C $-3.6604050 .992708-2.520327$

H $-8.0309531 .542929-2.306568$

O $-8.2138644 .186778-1.598228$

H -7.261920 $3.564144-3.288276$

H $-5.7540554 .314506-0.734597$

O $\quad-5.7516765 .438015-2.425389$

H $-4.5550793 .403973-3.402433$

O $-3.4252903 .860283-1.761073$

C $-3.711437-0.408604-1.958514$

H $-3.7705250 .944340-3.610513$

H $-2.6602161 .390368-2.315806$

H $-9.0396413 .753044-1.873405$

H $-6.5327965 .936919-2.129945$

H -3.382622 4.797729 -2.016660

O $-4.311057-1.250798-2.846886$

O $-3.298326-0.710709-0.846867$

C $-4.465176-2.603053-2.404505$

H $-5.159208-2.643615-1.560068$

H -4.882988 -3.185005 -3.230248

$\mathrm{H}-3.496544-3.031287-2.129477$

SCF Energy $\left(B 3 L Y P / 6-31 G^{* *} / / M M F F\right)=-3245.91133188$

0400001

MM̄FF Geometry

C 0.5842362 .7079122 .732683

C 0.8905301 .5521522 .125050

C 1.0434330 .2473892 .863470

O $2.368885-0.2313632 .542291$

C $0.014442-0.7832662 .369440$

C $-0.194216-1.924373 \quad 3.344050$ 
C $0.379602-3.1398903 .247735$

C $-1.159964-1.6331574 .464085$

C $1.375926-3.6186302 .228147$

C $0.819558-4.7344961 .331840$

C $-0.257997-4.2449190 .353603$

C $-0.800635-5.400608-0.488594$

C -1.817505 -4.912651-1.532887

O $0.341482-3.269628-0.503829$

O $-2.028425-5.975092-2.475208$

C $-1.209778-3.728571-2.315350$

C $-3.182015-4.617443-0.901295$

O $-2.177483-3.134416-3.178072$

C $-0.570845-2.644980-1.414458$

C $0.233117-1.647082-2.283054$

O $-1.602333-1.939827-0.722276$

C $3.150715-0.7280353 .531683$

O $2.851203-0.8548794 .710326$

C $4.495935-1.0835433 .017704$

C $4.795451-1.1271541 .709769$

C $6.106574-1.4132141 .123850$

C $7.263049-1.6343492 .062357$

C $1.129631-0.695199-1.478679$

C $1.9462940 .217558-2.376331$

C $3.8290941 .851546-2.275467$

O $1.782194 \quad 0.289138-3.591311$

C $5.0277761 .063258-2.702986$

C $6.2601581 .234649-2.200973$

C $7.4514330 .426831-2.634105$

C $8.092452-0.335329-1.493487$

C $9.2407110 .091076-0.941030$

C $7.453668-1.634843-1.050401$

C $6.218263-1.421688-0.220994$

N $2.874967 \quad 0.953103-1.651168$

H 0.4274302 .6911913 .810104

H 1.0555011 .5259361 .052378

H 0.9449850 .4174053 .942429

H $0.319789-1.1742261 .394039$

H $-0.959184-0.3022232 .203392$

H $0.129744-3.8833434 .003948$

H -1.283550 -2.485630 5.140296

H $-0.810834-0.7864435 .063163$

H -2.147050 -1.388838 4.058431

H $1.766255-2.8022301 .614244$

H $2.239690-4.0151182 .776779$

H $1.661180-5.1465270 .759941$

H $\quad 0.420928-5.5467601 .952283$

H - $1.069630-3.7802480 .924281$

H $-1.246298-6.1732070 .150237$

H $0.030042-5.889848-1.016458$

H -2.341840 -6.753823 -1.983985

H $-0.425526-4.139938-2.966477$

H -3.587599 -5.517664 -0.424184

H -3.915532 -4.325631 - 1.660846

H -3.137490 -3.834612 -0.142838

H -2.539953 -3.842256 -3.737834

H $0.882764-2.208558-2.967544$

H -0.454195 -1.054372 -2.899791

H -1.288370 -1.048001 -0.506436

H $5.232913-1.3039583 .783030$

H $4.010918-0.9186510 .984301$

H $7.395544-0.7692522 .721773$

H $8.216195-1.7818351 .550065$

H $7.091741-2.5236542 .678904$

H $0.527242-0.060963-0.821966$

H $1.827630-1.269276-0.858286$

H $4.0788162 .625442-1.543050$

H $3.3676922 .327937-3.146276$

H $4.8695250 .308642-3.470474$

H $6.4265281 .995376-1.441990$

H $8.1766171 .119096-3.081366$

H $7.187804-0.278129-3.432465$

H $9.724789-0.462860-0.143640$

H $9.7209131 .006356-1.271022$

H $8.177435-2.263241-0.519725$

H $7.181505-2.223313-1.936164$

H $5.319900-1.252267-0.814760$

H $2.9839450 .768100-0.658116$

C 0.6984734 .2133170 .608060
O $-0.275548 \quad 3.474658-0.154925$

C $-0.7534404 .297018-1.234770$

C $0.1989115 .483939-1.315491$

C 0.5795855 .6658550 .138327

H $-1.7458894 .655558-0.935665$

C $-0.8680453 .489215-2.532145$

H $1.0905645 .255812-1.910395$

H $-0.2770046 .368227-1.750269$

C 1.8438076 .4853660 .335102

H $-0.2523796 .162128 \quad 0.656256$

C $-1.6571542 .173016-2.404785$

O $\quad 0.4523353 .152721-2.976797$

H $-1.3016964 .122438-3.315412$

H -1.081507 $1.459620-1.805303$

O $-1.7447451 .591453-3.712524$

C $-3.0691842 .302689-1.804761$

C $-3.8390420 .970891-1.862942$

H $-2.9837112 .630345-0.765222$

O $-3.8065473 .307773-2.497279$

H $-4.0354190 .714982-2.911129$

H $-3.2001910 .183313-1.452809$

H 2.0833206 .5867431 .398045

H $2.7032576 .021966-0.161173$

H $1.7183527 .491073-0.079432$

C 0.4207234 .0686132 .108832

H $0.3614912 .632771-3.793831$

H -2.215105 $2.218257-4.287725$

H $-3.9562102 .998360-3.406481$

H -0.6083794 .3894142 .311331$

H 1.0809524 .7509252 .657939

H 1.6856183 .8057330 .355660

C -4.3786950 .4087451 .086368$

O

C $-5.1889091 .008500-1.112914$

C $-5.989529-0.296595-1.217972$

C $-5.342751-1.414017-0.405073$

C $-5.089848-0.9518621 .028915$

H $-3.3361080 .307498 \quad 0.761878$

C $-4.388650 \quad 0.9438182 .527403$

H $-5.7915331 .797592-1.579612$

O $-6.110278-0.690074-2.580879$

H -7.006214 -0.124360 -0.841340

H $-4.423353-1.764222-0.885564$

O $-6.233780-2.531414-0.385359$

H $-6.052472-0.8935241 .552592$

O $-4.311081-1.9357141 .708327$

C -3.5202282 .1691782 .705418$

H -5.4135091 .2212312 .802894$

H -4.0194870 .1841043 .224394$

H -6.594819-1.533210 -2.598015

H $-5.822940-3.2188680 .166693$

H -3.434869 -1.965550 1.287241

O $-3.7849802 .721687 \quad 3.926429$

$\begin{array}{llll}\text { O } & -2.704225 & 2.579651 & 1.893067\end{array}$

C $-3.0431703 .901386 \quad 4.251241$

H -2.0028443 .6375294 .459582$

H -3.102939 4.6355843 .441862

H -3.480982 4.340284 5.151793

SCF Energy (B3LYP/6-31G**//MMFF $)=-3245.92388014$

0400002

MM̄FF Geometry

C 0.2366232 .1796662 .979880

C $\quad 0.718131 \quad 1.1369382 .287288$

C $0.873844-0.2460012 .864678$

O $2.247293-0.6199192 .610806$

C -0.045614 -1.244306 2.144601

C $-0.236162-2.5372552 .911088$

C $0.378416-3.7016332 .624724$

C $-1.232944-2.4698794 .040134$

C $1.408035-3.9713461 .562531$

C $0.880173-4.8848870 .446656$

C $-0.154739-4.200672-0.459109$

C $-0.688847-5.174196-1.511523$

C $-1.653819-4.480478-2.486495$

O $0.491874-3.100881-1.106386$

O $-1.886654-5.366362-3.591782$

C $-0.990033-3.195025-3.028734$ 
C $-3.029738-4.260421-1.849509$

O $-1.909611-2.435327-3.810215$

C $-0.371631-2.303622-1.926281$

C $0.487612-1.188606-2.570529$

O $-1.421422-1.708097-1.163457$

C $2.954760-1.2498873 .579369$

O $2.554029-1.5747224 .687923$

C $4.354210-1.4826663 .145976$

C $4.770781-1.3040791 .882086$

C $6.138264-1.4613421 .382652$

C $7.215125-1.8023072 .378265$

C $1.332234-0.391089-1.566674$

C $2.2181660 .636213-2.248589$

C $4.0525132 .249818-1.740191$

O $2.1696030 .874164-3.452336$

C $5.3061191 .543485-2.153197$

C $6.4826741 .666894-1.520229$

C $7.7332360 .950158-1.946484$

C $8.2977940 .055098-0.863599$

C $9.3710440 .432931-0.148823$

C $7.676991-1.311874-0.668180$

C $6.367450-1.2539020 .068931$

N $3.064746 \quad 1.263123-1.343268$

H -0.0871432 .0071504 .005120$

H $1.047061 \quad 1.270978 \quad 1.261545$

H $0.674273-0.2230923 .942518$

H $0.349074-1.4513591 .145290$

H - $-1.036506-0.8018451 .982711$

H $\quad 0.137397-4.5702523 .236624$

H -1.360278 -3.435257 4.541425

H $-0.911153-1.7477764 .796633$

H -2.214065 -2.164072 3.663260

H $1.818559-3.0505181 .138332$

H $2.254312-4.4730142 .048773$

H $1.740719-5.190978-0.162387$

H $0.451752 \quad-5.7954730 .883596$

H $-0.977001-3.8267160 .160523$

H -1.175577 -6.035471 -1.037258

H $0.153396-5.584948-2.085727$

H -1.031600 -5.558652 -4.012823

H $-0.185734-3.503771-3.710745$

H -3.478279 -5.220906 -1.568388

H -3.727181 -3.802029 -2.559290

H -2.991264 -3.639687 -0.952524

H -2.268921 -3.027863 -4.492673

H $1.176679-1.640727-3.296048$

H $-0.157290-0.493853-3.123040$

H $-1.108479-0.872657-0.783680$

H $5.027482-1.8084453 .931977$

H $4.046569-0.9983661 .128587$

H $7.252129-1.0539553 .177876$

H $8.215623-1.8369701 .941569$

H $7.027171-2.7848902 .824723$

H $0.6873230 .144363-0.863823$

H $1.979715-1.066666-0.995885$

H $4.2117402 .916188-0.886524$

H $3.6651402 .838866-2.577516$

H $5.2399590 .897652-3.026027$

H $6.5571672 .321580-0.655074$

H $8.4714491 .711763-2.230004$

H $7.5639200 .354612-2.852302$

H $9.802330-0.2130840 .608584$

H $9.840340 \quad 1.399264-0.301801$

H $8.378755-1.989368-0.169311$

H $7.505191-1.773890-1.648981$

H $5.518879-1.012096-0.571375$

H $3.0825120 .941209-0.379885$

C 0.6868903 .9712121 .147672

O $-0.1215213 .395103 \quad 0.101345$

C $-0.5022784 .433602-0.818656$

C $0.5052725 .552335-0.603213$

C 0.6883615 .4847880 .899670

H -1.492541 $4.784317-0.501726$

C $-0.5897763 .898540-2.251726$

H $1.4568945 .348078-1.107109$

H $0.1345426 .521746-0.949098$

C 1.9440026 .1859621 .390183

H $-0.1856105 .945599 \quad 1.379805$
C $-1.4250712 .611105-2.396695$

O $\quad 0.7331203 .609232-2.716805$

H $-0.9786794 .685852-2.908222$

H $-0.8746921 .771656-1.957666$

O $-1.5380762 .309105-3.792022$

C $-2.8266772 .672477-1.765742$

C $-3.6411751 .392704-2.023968$

H $-2.7165002 .817481-0.687007$

O $-3.5430623 .800042-2.264607$

H -3.904418 $1.331027-3.086921$

H $-3.0123330 .526160-1.797316$

H 2.0419226 .0959772 .476573

H 2.8447295 .7610090 .934346

H 1.9117877 .2515431 .140574

C 0.0990483 .6072412 .517439

H $0.6545093 .253833-3.618726$

H -1.976142 $3.060221-4.226203$

H $-3.7134613 .653465-3.210262$

H -0.9721903 .8457362 .526170$

H 0.5766634 .2320263 .282250

H 1.7000383 .5655121 .035574

C $-3.9995940 .509150 \quad 0.867213$

O $-4.704180 \quad 1.5621410 .202846$

C $-4.9425891 .339266-1.191366$

C $-5.7544560 .049994-1.379748$

C $-5.082507-1.145025-0.706877$

C $-4.742531-0.8270270 .749745$

H -3.0063550 .4074870 .412253$

C -3.7383860 .9402592 .318360$

H -5.569056 2.173535 -1.531044

O $-5.939353-0.237508-2.761804$

H -6.751886 $0.179463-0.940070$

H $-4.190388-1.459830-1.257882$

O $-5.975949-2.260542-0.723613$

H $-5.669325-0.8123041 .334598$

O $-3.922533-1.8650751 .281957$

C $-4.989418 \quad 1.1400603 .148974$

H -3.1114430 .1884052 .810069$

H $-3.202537 \quad 1.8968772 .326140$

H $-6.3813580 .530075-3.163064$

H $-6.202673-2.434172-1.653448$

H $-4.397225-2.7043261 .155144$

O -4.6702811 .0122084 .471210$

O -6.0970881 .4079432 .703986$

C $-5.755591 \quad 1.1923405 .386471$

H -6.1579012 .2063165 .301181$

H -6.5363990 .4489935 .199846$

$\mathrm{H}-5.3720181 .0510986 .400486$

SCF Energy (B3LYP/6-31G**//MMFF) $=-3245.91525386$

0400003

MM̄FF Geometry

C $1.541714 \quad 1.6748942 .213893$

C 0.6788740 .9458372 .939351

C $0.707817-0.5518443 .150724$

O $1.768164-1.1723662 .391751$

C $-0.602339-1.1964582 .657821$

C $-1.004395-2.4035253 .481646$

C $-0.634023-3.6749503 .233073$

C -1.915237 $-2.095490 \quad 4.642437$

C $0.298338-4.1828892 .168831$

C $-0.398628-5.0732701 .131898$

C $-1.266552-4.2776900 .146863$

C $-1.968493-5.214283-0.837429$

C $-2.781520-4.433027-1.881495$

O $-0.407552-3.385728-0.571668$

O $-3.135724-5.341817-2.935274$

C $-1.882884-3.342736-2.505107$

C $-4.099179-3.917323-1.295042$

O $-2.635073-2.492258-3.368704$

C $-1.091531-2.506051-1.470137$

C $-0.032881-1.642630-2.198622$

O $-1.998995-1.647864-0.776858$

C $3.010562-1.2567382 .929226$

O $3.379768-0.8714784 .024674$

C $3.864664-1.8583471 .884014$

C $5.152619-1.5001001 .796677$

C $6.086045-1.9248280 .751592$ 
C $5.595772-2.935246-0.253713$

C $0.997989-0.977477-1.273244$

C $1.998167-0.140821-2.055857$

C $4.0772461 .202875-1.748543$

O $1.8871730 .083729-3.258653$

C $5.2585210 .373705-2.147635$

C $6.5221160 .684663-1.817577$

C $7.722476-0.106137-2.258691$

C $8.688734-0.396780-1.129811$

C $9.7889400 .354393-0.952743$

C $8.424491-1.606707-0.261009$

C $7.314914-1.3687830 .723903$

N $3.0184590 .343910-1.248409$

H 2.3682991 .1913941 .703360

H $-0.120581 \quad 1.4672873 .461979$

H $0.854540-0.7458694 .220926$

H $-0.509765-1.4853411 .605056$

H -1.431634 -0.4774282 .690837$

H -1.013231 -4.453869 3.893917

H -2.164603 -2.987668 5.226270

H -1.438055 -1.380242 5.320319

H -2.854164 -1.661356 4.283313

H $\quad 0.830254-3.3737991 .664817$

H $1.070251-4.7786442 .672550$

H $\quad 0.385091-5.5982570 .570154$

H - $-1.002816-5.836727 \quad 1.637150$

H -2.003589 -3.6978050 .713493$

H -2.610067 $-5.928477-0.306450$

H -1.217995 $-5.819724-1.364888$

H -3.639773 -6.075078 -2.542573

H -1.161499 -3.862638 -3.150604

H -4.708384 -4.751239-0.925982

H -4.706892 -3.420924-2.059498

H $-3.949034-3.227084-0.463856$

H -3.135530 -1.872348 -2.811195

H $0.524638-2.275192-2.902242$

H $-0.536356-0.867480-2.789996$

H - $-1.524164-0.855350-0.480966$

H $3.386407-2.5183591 .169071$

H $5.547583-0.7979892 .530050$

H $5.314229-3.8677810 .247955$

H $6.336763-3.196859-1.011251$

H $4.723740-2.551475-0.794859$

H $0.504461-0.316937-0.552881$

H $\quad 1.552802-1.742027-0.717885$

H $4.3240601 .911595-0.951982$

H $3.7094121 .761341-2.615250$

H $5.071146-0.502606-2.763772$

H $6.7096711 .582960-1.233272$

H $8.2319670 .474902-3.038843$

H $7.427245-1.048849-2.735936$

H $10.5014450 .136813-0.163933$

H $9.9988051 .207343-1.589655$

H $9.322826-1.8623800 .316822$

H $8.250984-2.475586-0.901515$

H $7.570979-0.6388891 .493439$

H $3.0634360 .052668-0.276608$

C 1.5519123 .6001400 .605509

O $\quad 0.3898893 .146496-0.114073$

C $-0.0247874 .181582-1.026828$

C $1.0734755 .239506-1.003453$

C 1.5831145 .1157340 .418514

H $-0.9381714 .620108-0.608404$

C $-0.3147763 .591200-2.411989$

H $1.8757265 .012269-1.714926$

H $0.6913796 .237734-1.238518$

C 2.9519425 .7398360 .627225

H 0.8607245 .5933801 .094154

C - $1.3293002 .430411-2.399835$

O $0.915293 \quad 3.093434-2.951899$

H $-0.6511214 .387971-3.086200$

H $-0.8964601 .556647-1.898338$

O $-1.5767542 .040498-3.755749$

C $-2.6794152 .774117-1.744296$

C $-3.6914571 .620596-1.851668$

H $-2.5150793 .009071-0.690610$

O $-3.2319433 .940475-2.352169$

H $-3.9616841 .471321-2.903916$
H $-3.2076580 .701537-1.508323$

H 3.2816535 .6226221 .664174

H $3.7060105 .279335-0.020080$

H 2.9244006 .8109320 .401835

C 1.4646433 .1700532 .070453

H $\quad 0.7661802 .896983-3.891985$

H $-0.7721781 .606666-4.086838$

H $-3.344713 \quad 3.752494-3.299846$

H 0.5340273 .5448052 .511239

H 2.2971373 .6056732 .634340

H 2.4244673 .1400650 .124836

C -4.2218331 .0722731 .099888$

O -4.7352452 .1663840 .331245$

C $-4.9877181 .876204-1.047819$

C $-6.0184220 .744307-1.154858$

C $-5.560401-0.496429-0.392228$

C $-5.172049-0.1328361 .040597$

H -3.2295270 .7931550 .725491$

C -4.0728401 .5564312 .551542$

H -5.450506 2.776999-1.470269

O $-6.2479020 .427083-2.523352$

H -6.973072 $1.088992-0.736528$

H $-4.734241-0.994122-0.909405$

O $-6.629736-1.442427-0.334214$

H -6.0863050 .0840101 .607518$

O $-4.559518-1.2615391 .663740$

C -2.987030 2.5973972 .715485

H -5.0169982 .0072902 .880908$

H -3.8200000 .7214713 .213973$

H -6.987486 -0.202377 -2.560597

H $-6.787370-1.763068-1.238056$

H -3.731053 -1.451089 1.190806

O $-3.100423 \quad 3.1628573 .953882$

$\begin{array}{llll}\text { O } & -2.135534 & 2.867390 & 1.881028\end{array}$

C -2.1364914 .1738534 .265420$

H -2.4430234 .6598045 .195461$

H -1.1550053 .7159054 .415393$

H -2.0946864 .9293963 .474637$

SCF Energy $\left(B 3 L Y P / 6-31 G^{* *} / / M M F F\right)=-3245.91734528$

0400004

MMFF Geometry

C $-0.3024292 .675601 \quad 1.384768$

C -0.9243442 .0310922 .384433$

C $-0.4992240 .738648 \quad 3.045128$

$\begin{array}{llll}\text { O } & 0.747988 & 0.243068 & 2.510859\end{array}$

C -1.585106 -0.3254012 .799649$

C $-1.338647-1.6348623 .522924$

C $-0.955712-2.7843362 .931786$

C $-1.601798-1.6050735 .006980$

C $-0.648037-3.0169131 .478805$

C $0.809465-3.459427 \quad 1.294561$

C $1.228889-3.466146-0.182738$

C $2.664660-3.974005-0.328457$

C $3.183959-3.853584-1.768986$

O $1.129688-2.122039-0.662479$

O $4.609427-4.026282-1.739217$

C $2.915326-2.422454-2.281843$

C $2.630962-4.966230-2.667100$

O $3.242835-2.308409-3.664309$

C $1.467300-1.950400-2.044628$

C $1.252755-0.456081-2.385736$

O $0.584671-2.703406-2.885722$

C 1.8948200 .7009203 .085720

O $1.989198 \quad 1.5175683 .988234$

C 3.0334650 .0338992 .415227

C 4.2883280 .4094352 .704903

C $5.516224-0.1669902 .150992$

C $5.375684-1.2716261 .137948$

C $2.0486180 .540786-1.537638$

C $3.3627720 .985351-2.154809$

C $5.3762122 .359939-1.625568$

O $3.6967260 .713825-3.304376$

C $6.4887191 .450325-1.210418$

C $7.4987101 .834100-0.414766$

C $8.6548180 .949790-0.046510$

C 8.8759360 .8622421 .446366

C 9.8096101 .6153362 .052311 
C $8.083997-0.1674742 .218338$

C 6.7041250 .3094232 .579491

N $4.1034881 .761266-1.271944$

H 0.6249822 .2863580 .975405

H -1.845776 2.4597482 .774926

H $-0.397855 \quad 0.916822 \quad 4.122804$

H -1.693248 -0.4887021 .722410$

H -2.5591450 .0598343 .128962$

H $-0.838970-3.6677193 .558697$

H -1.487125 -2.590868 5.470145

H $-0.906857-0.9243855 .507972$

H -2.624755 -1.267789 5.204569

H -1.316722 -3.799667 1.100773

H $-0.846033-2.1286700 .875786$

H $1.461684-2.7734541 .849644$

H $0.946067-4.4589851 .725921$

H $0.534786-4.103261-0.743452$

H $2.747903-5.0092500 .025668$

H $3.323179-3.3850900 .323062$

H $4.798033-4.895914-1.346637$

H $3.599310-1.756437-1.742290$

H $2.935080-5.949858-2.289001$

H $3.040126-4.896375-3.680918$

H $1.541006-4.963008-2.732146$

H $4.166487-2.594567-3.767926$

H $1.397469-0.284822-3.459068$

H $0.192939-0.239978-2.197973$

H $-0.236923-2.198817-3.004000$

H $2.808562-0.7535951 .706135$

H $4.436080 \quad 1.2102473 .428549$

H $6.320101-1.5614570 .672847$

H $4.719732-0.9635620 .317065$

H $4.955519-2.1663091 .609147$

H $1.4465311 .451492-1.426320$

H $2.2319110 .147753-0.533701$

H $5.4274333 .332431-1.125565$

H $5.4164152 .522001-2.707458$

H $6.4805400 .439443-1.611571$

H $7.5196892 .854718-0.038990$

H $9.5514551 .358261-0.531295$

H $8.534111-0.060365-0.457442$

H $9.996397 \quad 1.5347283 .118068$

H $10.403708 \quad 2.333408 \quad 1.496752$

H $8.598780-0.4030973 .159945$

H $8.080108-1.1125931 .667961$

H 6.7042681 .1170573 .312982

H $3.7709931 .885797-0.320001$

C $-0.9456653 .809223-0.761220$

O $-1.8204402 .721285-1.110746$

C $-2.5799583 .086616-2.279445$

C $-2.0459974 .441813-2.736095$

C -1.550244 $5.039355-1.433543$

H $-3.6186703 .208377-1.951288$

C $-2.4903741 .974311-3.333619$

H -1.217081 4.338217 -3.445435

H $-2.8193845 .047208-3.219106$

C $-0.5744786 .187067-1.624541$

H -2.417386 $5.393572-0.860081$

C $-2.8570040 .569338-2.812507$

O $-1.1463031 .907800-3.830738$

H -3.115206 2.231586-4.196400

H -2.108633 $0.242032-2.081436$

O $-2.759774-0.325253-3.927739$

C $-4.2644460 .467524-2.196289$

C $-4.608678-0.959743-1.733176$

H $-4.3282491 .141579-1.339653$

O $\quad-5.2370020 .904584-3.144227$

H -4.639330 -1.625603 -2.603558

H -3.807609 -1.316852 -1.076760

H $-0.2431526 .582984-0.659562$

H $0.3136015 .870161-2.181632$

H -1.046023 $7.004252-2.180112$

C $-0.839498 \quad 3.9348730 .759617$

H $-0.5568781 .789154-3.066753$

H -2.703624 -1.229966 -3.576846

H $-5.158586 \quad 0.335414-3.928987$

H -1.826624 4.1675821 .177228

H $-0.1702184 .763127 \quad 1.018147$
H $\quad 0.0386873 .579604-1.190347$

C $-5.266113-0.4541371 .217354$

O $-6.081517-0.1391890 .082462$

C $-5.974973-1.050935-1.015322$

C $-6.349427-2.468955-0.559933$

C $-5.526598-2.9086760 .649173$

C $-5.599677-1.8533281 .751615$

H $-4.207469-0.4030630 .936717$

C -5.5414750 .6130062 .289689$

H $-6.733788-0.738710-1.744020$

O $-6.178379-3.385232-1.635555$

H -7.413301-2.483940 -0.288728

H $-4.485532-3.1186730 .376416$

O $-6.061603-4.1268911 .171030$

H $-6.604251-1.8580362 .193808$

O $-4.677700-2.1930652 .784925$

C -4.9571361 .9638791 .934033$

H $-6.624506 \quad 0.7388252 .408787$

H -5.1037170 .3201143 .250035$

H -6.544862 -4.240819-1.356171

H $-5.908887-4.8217280 .508713$

H -4.894634 -3.092578 3.084536

O -5.4328862 .8969282 .811292$

O -4.1691142 .1768151 .023986$

C -4.9546154 .2306782 .609544$

H -3.8802884 .2773312 .809652$

H -5.1736064 .5676511 .591603$

$\mathrm{H}-5.4719014 .8872013 .314157$

SCF Energy $\left(B 3 L Y P / 6-31 G^{* *} / / M M F F\right)=-3245.91027918$

0400005

MM̄FF Geometry

C $-0.1145290 .528757-2.519669$

C $-0.992713-0.361326-3.004810$

C $-1.188004-1.794837-2.569234$

O $-2.570061-1.883560-2.140321$

C $-0.299308-2.278262-1.406955$

C $-0.289491-3.784803-1.239059$

C $-0.966562-4.460849-0.290647$

C $0.604874-4.527634-2.199151$

C $-1.919202-3.9174060 .737486$

C -1.352662 -3.9917402 .162711$

C $-0.288560-2.9204042 .446352$

C $0.301449-3.0966753 .846856$

C $1.286430-1.9701864 .196545$

O $-0.927782-1.6446062 .348921$

O $1.547886-2.0317595 .606487$

C $0.610784-0.6107793 .920204$

C $2.635646-2.1566503 .494992$

O $1.535003 \quad 0.4625994 .085855$

C $-0.055941-0.5222262 .528399$

C $-0.922444 \quad 0.7574792 .441679$

O $0.960427-0.4766001 .524927$

C -3.337094 -2.901129-2.603362

O $-2.989157-3.792465-3.365384$

C $-4.723611-2.813475-2.085398$

C $-5.120683-1.928266-1.157328$

C $-6.478548-1.778357-0.626286$

C $-7.555557-2.664482-1.195032$

C -1.8220490 .8179201 .200131$

C -2.6672782 .0790581 .164528$

C $-4.5299023 .116620-0.131857$

O -2.5606632 .9866601 .984518$

C -5.7858552 .7627290 .600030$

C $-6.9461442 .465569-0.004383$

C -8.1938232 .0828470 .738938$

C -8.6897730 .6992930 .376028$

C $-9.7651670 .539724-0.413652$

C -7.999042 -0.4956300 .998806$

C $-6.701696-0.8418990 .319728$

N -3.5533392 .0678490 .095426$

H $0.5653150 .246239-1.721845$

H -1.651535 -0.038898 -3.810012

H -1.021154 -2.414911 -3.458413

H $-0.620123-1.794568-0.478556$

H $0.741424-1.970251-1.562897$

H $-0.851007-5.543736-0.255575$

H $\quad 0.617519-5.605954-2.007451$ 
H $\quad 0.268749-4.381893-3.230196$ H $1.635366-4.167316-2.115110$ H $-2.244868-2.8996250 .504115$ H -2.826774 -4.5331300 .693637$ H -2.191178 -3.8504162 .857349$ H $-0.941181-4.9912332 .350077$ H $\quad 0.501707-2.9910071 .690963$ H $\quad 0.787037-4.0756253 .944265$ H - $0.509538-3.0892134 .588655$ H $1.906045-2.9135695 .806696$ H $-0.165884-0.4717224 .685365$ H $3.095830-3.1065033 .792921$ H $3.345587-1.3750893 .787370$ H $2.551582-2.1588432 .406736$ H 1.9201520 .3788594 .974901 H - 1.5757420 .8105923 .322828 H $-0.280273 \quad 1.6456552 .462045$ H 0.6383030 .0295990 .763270 H $-5.411218-3.533726-2.517537$ H $-4.389295-1.236861-0.742240$ H -7.630405 -2.532317 -2.280255 H -8.548477 -2.457528 -0.790379 H $-7.338228-3.717103-0.982673$ H -1.2212740 .7940850 .287240$ H -2.501445 -0.0423561 .185353$ H $-4.6849473 .190939-1.212768$ H -4.139752 4.0720950 .232835 H $-5.7319532 .741581 \quad 1.686187$ H -7.005150 $2.492962-1.089935$ H -8.960902 2.8338710 .509812 H -8.045186 2.1366851 .824690 H -10.148940 -0.445879 -0.654683 H $-10.2844001 .391418-0.840547$ H -8.670899-1.360955 1.023257 H -7.794141 -0.2837592 .056299$ H $-5.856064-0.2498220 .669346$ H $-3.6108491 .237958-0.488041$ C $-0.2799912 .953899-1.905370$ O $\quad 0.7119382 .824929-0.867269$ C $1.3061324 .111354-0.628492$ C $0.2868225 .118286-1.138623$ C $-0.2173324 .407505-2.381457$ H $2.2025864 .166384-1.259069$ C $1.714357 \quad 4.2546420 .841149$ H $-0.5354165 .261173-0.427875$ H $0.7321536 .094566-1.351682$ C -1.545729 $4.940186-2.890947$ H $0.5348864 .511525-3.175076$ C 2.5249633 .0605311 .387487 O $0.530447 \quad 4.3696501 .639567$ H 2.2670415 .1919650 .974711 H 1.8583002 .2004541 .509364 O 2.9716073 .3905222 .707209 C 3.7270582 .6379810 .525637 C 4.5061561 .4628001 .143961 H $3.3634772 .340148-0.462038$ O 4.6029623 .7454520 .329076 H 4.9875861 .7890062 .073899 H 3.7958900 .6720541 .409222 H -1.877054 4.380765 -3.771527 H $-2.3281044 .862620-2.128494$ H -1.454823 $5.994206-3.173054$ C $0.0118501 .943356-3.017328$ H 0.8114104 .4471252 .567472 H 3.5230274 .1885642 .645163 H 5.0105233 .9612071 .184720 H $1.0389182 .073775-3.380003$ H $-0.6610552 .116869-3.864988$ H -1.251050 $2.741129-1.445529$ C $4.187580-0.498833-1.166222$ O $5.0832220 .614686-1.108130$ C $5.594827 \quad 0.9067190 .196761$ C $6.346710-0.3130600 .748363$ C $5.481294-1.5735950 .724736$ C $4.865511-1.778216-0.660848$ H $3.311199-0.281310-0.542758$ C $3.652665-0.616200-2.598495$ H 6.3316901 .7087360 .062270
O $6.793494-0.0447072 .073023$

H $7.239782-0.4859300 .133608$

H $4.706275-1.5370761 .496283$

O $6.291607-2.7127661 .021402$

H $5.660936-2.092047-1.346910$

O $3.917422-2.841997-0.609020$

C $4.708489-0.993144-3.610947$

H $2.857541-1.368336-2.653861$

H $3.2065760 .333002-2.920439$

H $7.368569-0.7792232 .345366$

H $6.582326-2.6336081 .945523$

H $3.200297-2.575564-0.008602$

O $5.4556600 .098211-3.940679$

O $4.853797-2.126868-4.050214$

C $6.506271-0.137523-4.882929$

H $7.220544-0.862091-4.480325$

H $6.091541-0.489164-5.832466$

H $7.0264130 .808323-5.055881$

SCF Energy (B3LYP/6-31G**//MMFF)= -3245.90629673

\section{6}

MM̄FF Geometry

C 1.2441341 .8807582 .393899

C 0.4219050 .9204312 .845880

C $0.775607-0.524497 \quad 3.110258$

$\begin{array}{lllllll}\text { O } & 2.146167 & -0.777823 & 2.729685\end{array}$

C $-0.127523-1.4748342 .305341$

C $-0.275762-2.8309292 .967076$

C $0.405633-3.9410712 .622831$

C $-1.310501-2.8921704 .062352$

C $1.468154-4.0892241 .569165$

C $1.018954-4.9928290 .411675$

C $-0.083449-4.359975-0.448986$

C $-0.558848-5.326468-1.533513$

C $-1.614451-4.677471-2.441148$

O $0.459128-3.185103-1.058823$

O $-1.769574-5.535367-3.581592$

C $-1.080922-3.315430-2.936490$

C $-2.984883-4.621867-1.757270$

O $-2.086038-2.610759-3.662790$

C $-0.488891-2.425058-1.816782$

C $0.257445-1.225911-2.447596$

O $-1.551532-1.942682-0.994672$

C $2.953015-1.4419053 .593662$

O $2.653136-1.8612544 .702913$

C $4.320344-1.5982143 .042496$

C $4.640415-1.3401131 .764511$

C $5.970748-1.4609071 .162244$

C $7.115969-1.8448552 .062072$

C $1.077024-0.390371-1.453189$

C $1.8836030 .687799-2.158226$

C $3.7213822 .320125-1.743944$

$\begin{array}{lllll}\text { O } & 1.734877 & 0.970633 & -3.344334\end{array}$

C $4.9549131 .643559-2.253429$

C $6.1622361 .757672-1.679869$

C $7.391142 \quad 1.059615-2.188308$

C $7.995328 \quad 0.120166-1.166700$

C $9.1109130 .456812-0.497820$

C $7.361835-1.242816-0.985429$

C $6.108695-1.197420-0.154178$

N $2.7815701 .306560-1.299707$

H $2.275914 \quad 1.6470302 .147224$

H $-0.596572 \quad 1.1989873 .111171$

H $0.641991-0.6792514 .187935$

H $\quad 0.263670-1.5857991 .289179$

H $-1.133527-1.0507632 .188196$

H $0.192102-4.860298 \quad 3.167291$

H $-1.398344-3.8934704 .497262$

H $-1.055204-2.2030344 .873159$

H -2.294719-2.616714 3.669055

H $1.807922-3.1241771 .183180$

H $2.344848-4.5447262 .046421$

H $1.897823-5.189098-0.216029$

H $0.678974-5.9584740 .806100$

H $-0.922922-4.0813750 .197039$

H $-0.949128-6.251336-1.090627$

H $0.296493-5.628625-2.154265$

H $-2.376330-5.094797-4.201337$ 
H $-0.285021-3.534712-3.661321$ H -3.307220 -5.628944 -1.466708 H -3.754392 -4.251531-2.443278 H -2.990503 -3.998678 -0.860815 H $-2.701863-2.225061-3.016151$ H $0.951588-1.602057-3.211103$ H $-0.458844-0.568891-2.956618$ H - $-1.279380-1.111345-0.575724$ H $5.056057-1.9576223 .754836$ H $3.859089-1.0066171 .083645$ H $7.205489-1.1383892 .894940$ H $8.083998-1.8485791 .556514$ H $6.964261-2.8508412 .468394$ H $0.4218680 .104051-0.728384$ H $1.775884-1.032837-0.905423$ H $3.9309492 .972093-0.890672$ H $3.2675642 .919079-2.539841$ H $4.8451301 .023836-3.140427$ H $6.2776312 .383988-0.798354$ H $8.1186851 .831491-2.471245$ H $7.1820960 .501595-3.109590$ H $9.570658-0.2212460 .213280$ H $9.5880541 .420338-0.643354$ H $8.084103-1.952931-0.567286$ H $7.113725-1.659299-1.970390$ H $5.218367-0.929036-0.722849$ H $2.8819010 .954153-0.352186$ C 1.0558323 .8984860 .880401 O $\quad 0.1178273 .312746-0.046274$ C $-0.5661184 .370794-0.741390$ C $0.3160515 .600807-0.581284$ C 0.8188415 .4115260 .834298 H $-1.5067204 .541192-0.204226$ C $-0.8757733 .987321-2.191937$ H $1.1581525 .594310-1.282895$ H $-0.2384206 .533566-0.720418$ C $2.053414 \quad 6.2364531 .156164$ H $0.0154245 .688513 \quad 1.530518$ C -1.589704 $2.633340-2.364766$ O $0.358577 \quad 3.921192-2.916651$ H -1.463015 $4.789223-2.655930$ H $-0.8767161 .815717-2.216622$ O $-2.0348682 .539101-3.723497$ C $-2.8000802 .404971-1.443410$ C $-3.5514911 .103863-1.784826$ H $-2.4536502 .367053-0.405770$ O $-3.7072623 .501569-1.543247$ H -4.129172 $1.239146-2.707298$ H $-2.8127070 .312526-1.954417$ H 2.8839615 .9942260 .484450 H 1.8392677 .3053301 .053949 H 2.3870646 .0550532 .182664 C 0.8394103 .3246032 .281538 H $\quad 0.1436563 .852167-3.861870$ H -1.246631 $2.455600-4.286075$ H -3.973635 $3.579161-2.475436$ H -0.2121663 .4411232 .568356$ H 1.4376313 .8899673 .005973 H 2.0601213 .6542110 .516380 C $-4.407026-0.0695161 .648221$ $\begin{array}{llll}\mathrm{O} & -3.698106 & 0.330243 & 0.470103\end{array}$ C $-4.5087640 .671993-0.659793$ C $-5.383120-0.521398-1.078025$ C $-6.191126-1.0700390 .102030$ C $-5.322014-1.2674551 .350683$ H $-3.626560-0.4069352 .342459$ C $-5.136414 \quad 1.1084302 .302962$ H $-5.1502151 .521463-0.401678$ O $-4.559028-1.555977-1.608789$ H $-6.057984-0.196789-1.879372$ H $-6.640526-2.028179-0.186411$ O $-7.254372-0.164816 \quad 0.383090$ H -5.946488 -1.4737142 .227691$ O $-4.479973-2.4079551 .157994$ C -4.1585982 .1827332 .718011$ H -5.8876051 .5541001 .644729$ H -5.6687670 .7859593 .205329$ H $-5.144146-2.273059-1.906777$
H $-7.771413-0.5381401 .117160$

H $-5.055965-3.1764911 .007137$

O $-4.3482513 .300961 \quad 1.960861$

O -3.3213442 .0409623 .599811$

C -3.4790044 .3988532 .251574$

H -2.4370064 .1064772 .100023$

H -3.7172135 .2133841 .562298$

$\mathrm{H}-3.6381984 .7462533 .276663$

SCF Energy (B3LYP/6-31G**//MMFF) $=-3245.91626060$

\section{7}

MM̄FF Geometry

C 0.8509362 .5288012 .683509

C $0.838902 \quad 1.3096902 .124783$

C $0.7283840 .031102 \quad 2.916275$

O $1.815000-0.8304232 .506452$

C $-0.576433-0.7045802 .566585$

C $-1.012451-1.6764403 .643897$

C $-0.684667-2.982603 \quad 3.691377$

C $-1.912215-1.0843764 .698191$

C $0.231043-3.7473902 .776453$

C $-0.494103-4.8132071 .944239$

C $-1.318616-4.2210300 .792169$

C $-2.022456-5.3273620 .004180$

C $-2.782930-4.768148-1.208930$

O $-0.420745-3.516653-0.071883$

O $-3.130098-5.871860-2.059284$

C $-1.837670-3.852277-2.017486$

C $-4.103249-4.114142-0.790436$

O $-2.541376-3.189341-3.066262$

C $-1.059421-2.831120-1.154031$

C $0.039043-2.150887-2.007379$

O $-1.969400-1.834615-0.683970$

C $3.065643-0.5611632 .958743$

O $3.395590 \quad 0.2715853 .785709$

C $3.979220-1.4229642 .179160$

C $5.241693-1.0217881 .977119$

C $6.224100-1.6860361 .118832$

C $5.810483-2.9713510 .449779$

C $1.058108-1.335779-1.196245$

C $2.081148-0.652986-2.089150$

C $4.1339320 .758881-1.974067$

O $2.017495-0.670828-3.315870$

C $5.326181-0.121729-2.190334$

C $6.5880100 .280421-1.969186$

C $7.798324-0.572552-2.234131$

C $8.789617-0.567757-1.089131$

C $9.8808600 .215651-1.124635$

C $8.557158-1.527868 \quad 0.057530$

C $7.420857-1.0903550 .937894$

N $3.063582-0.001897-1.353204$

H 0.7491212 .5960713 .765449

H 0.9351581 .2003641 .049303

H $\quad 0.7961650 .216637 \quad 3.995650$

H - $0.463810-1.2383781 .616934$

H $-1.392940 \quad 0.0128112 .407998$

H -1.088385 -3.5793734 .508887$

H -2.182756 -1.810457 5.472192

H $-1.416686-0.2434535 .193840$

H -2.840681 -0.7230634 .244569$

H $0.804707-3.0892642 .120357$

H $0.972117-4.2512223 .410416$

H $0.272924-5.4776191 .525178$

H $-1.134692-5.4252732 .590931$

H $-2.052398-3.5204251 .206289$

H $-2.698824-5.8977870 .652848$

H $-1.276372-6.048408-0.358713$

H $-3.664663-6.495539-1.538500$

H $-1.110229-4.509877-2.513000$

H $-4.745963-4.839191-0.276866$

H $-4.673822-3.776551-1.662434$

H $-3.959125-3.268121-0.117469$

H $-3.042870-2.456972-2.669147$

H $0.600454-2.919597-2.554652$

H $-0.429817-1.498061-2.754557$

H - $-1.487232-1.005437-0.540155$

H $3.562476-2.3132131 .721935$

H $5.572551-0.0935902 .441398$ 
H $\quad 5.552755-3.7273651 .199977$ H $6.586925-3.408803-0.180242$ H $4.940938-2.807681-0.196658$ H $0.553116-0.556721-0.618116$ H $1.595493-1.989730-0.500140$ H $4.3579571 .602774-1.313911$ H $3.7859401 .145581-2.937256$ H $5.149633-1.122155-2.578171$ H $6.7649561 .294823-1.618181$ H $8.281454-0.187055-3.141523$ H $7.515729-1.607335-2.464332$ H $10.6103150 .206917-0.321624$ H $10.0666410 .887552-1.956186$ H $9.453162-1.5915850 .689147$ H $8.426176-2.534998-0.346427$ H $7.619425-0.1559911 .465391$ H $3.062374-0.081816-0.340653$ C 1.3306143 .8459690 .491985 O $\quad 0.2381923 .287094-0.260441$ C $0.0482834 .054802-1.462400$ C $1.2215395 .026103-1.541647$ C $1.5362415 .250709-0.076916$ H $-0.8763334 .627085-1.321375$ C $-0.0952703 .129639-2.677184$ H $2.0871414 .585471-2.049380$ H $0.9596355 .947161-2.071525$ C 2.9239555 .8211800 .162009 H $0.787555 \quad 5.9383430 .339150$ C -1.146098 $2.014034-2.512557$ O $1.1689992 .497246-2.911501$ H $-0.3097693 .732038-3.568293$ H $-0.793637 \quad 1.269328-1.788573$ O $-1.2608151 .331328-3.767930$ C -2.547386 $2.497612-2.091942$ C $-3.5745881 .350921-2.127270$ H $-2.4898172 .921944-1.085884$ O $-2.9655803 .534498-2.977717$ H -3.754105 $1.069619-3.171758$ H $-3.1401870 .481371-1.625259$ H 3.1088065 .9679351 .230552 H $3.7031355 .156635-0.226503$ H $3.0315146 .791176-0.334543$ C 0.9956883 .8576061 .987541 H $1.1339782 .083727-3.790233$ H $-0.4351550 .838611-3.911090$ H $-3.7602523 .945483-2.598268$ H 0.0581594 .4050732 .141271 H 1.7795904 .4102902 .519113 H 2.2121353 .2203950 .300300 C $-4.375937 \quad 1.1688610 .812818$ O C $-4.9332581 .703043-1.481231$ C $-5.9643290 .566034-1.533996$ C $-5.591341-0.573784-0.589431$ C $-5.329426-0.0365450 .815631$ H -3.3552010 .8467990 .573811$ C -4.3666671 .8262792 .202757$ H $-5.3516892 .541668-2.050578$ O $-6.1232820 .076948-2.861677$ H $-6.9422680 .961098-1.230936$ H $-4.728700-1.133890-0.963680$ O $-6.674317-1.503432-0.521083$ H $-6.288836 \quad 0.247095 \quad 1.266595$ O $-4.787233-1.079281 \quad 1.625692$ C -3.2859032 .8729832 .355765$ H -5.3310752 .3192192 .376294$ H -4.198130 1.0760622 .982509 H $-5.350142-0.469535-3.081226$ H $-6.853697-1.806421-1.427709$ H $-3.916162-1.3165951 .263935$ O -3.4947243 .5507363 .523358$ O -2.3696213 .0623941 .569585$ C -2.5518904 .5846653 .822573$ H -2.9437455 .1694874 .659076$ H -1.5994384 .1390634 .121870$ $\mathrm{H}-2.4165325 .2511602 .965151$ SCF Energy (B3LYP/6-31G**//MMFF) $=-3245.91515051$
0400008

MMFF Geometry

C $2.0286053 .756348-1.427118$

C $2.3641634 .553659-0.400342$

C 3.1524324 .1680490 .834265

O $3.414844 \quad 2.747047 \quad 0.874921$

C 2.3523424 .5398832 .097581

C 3.1386734 .3780313 .386507

C 2.9130383 .4319864 .320317

C 4.2220395 .4032423 .610625

C 1.8830782 .3384644 .295852

C 2.5467030 .9627834 .157900

C $1.528896-0.1320323 .807763$

C $2.210828-1.5001423 .750988$

C $1.269214-2.6020463 .242287$

O 0.9759350 .1983052 .530036

O $2.070540-3.7449852 .903873$

C $0.595652-2.1236681 .938500$

C $0.292013-3.0639974 .329519$

O $-0.389278-3.056108 \quad 1.504956$

C $-0.019310-0.7142242 .050472$

C $-0.526311-0.1632470 .696285$

O $-1.143210-0.7561082 .936072$

C 4.5502362 .3012530 .269629

O $5.3964182 .975380-0.295735$

C 4.5683960 .8253030 .381565

C $5.4347760 .116598-0.357676$

C $5.580238-1.341143-0.365311$

C $4.678837-2.1393150 .538364$

C $0.5478530 .011148-0.381470$

C $0.653221-1.146676-1.357826$

C $2.068844-1.954356-3.246660$

O $-0.167852-2.057237-1.418826$

C $3.008987-2.998931-2.732941$

C $4.179371-3.292451-3.320090$

C $5.095290-4.389048-2.857521$

C $6.518308-3.921659-2.651591$

C $7.447931-4.095841-3.606191$

C $6.884800-3.344626-1.303836$

C $6.509211-1.893424-1.173485$

N $1.760685-1.014347-2.186346$

H $2.3329682 .713984-1.431628$

H $2.0656905 .599882-0.447798$

H 4.0967134 .7256530 .821529

H 1.4316883 .9488092 .120386

H 2.0235115 .5865182 .037853

H 3.5407703 .4301115 .210800

H 4.6853785 .3098644 .598694

H 5.0161225 .2964822 .865583

H 3.8088426 .4147443 .538817

H 1.3138902 .3739185 .232846

H 1.1523212 .4912483 .499459

H 3.3062181 .0101903 .367358

H 3.0639660 .7108285 .091906

H $0.731719-0.1283264 .560338$

H $2.624135-1.7686504 .731258$

H $3.071860-1.4450193 .072364$

H $2.563040-4.0116083 .699049$

H $1.372342-2.1107921 .164896$

H $0.839504-3.4755335 .186049$

H $-0.349063-3.8749833 .966664$

H $-0.345140-2.2600034 .703518$

H $0.043860-3.923628 \quad 1.431718$

H $-1.373387 \quad-0.7583970 .337069$

H -0.9380930 .8373720 .890909$

H $-1.729041-0.0084782 .731956$

H $3.8602580 .362491 \quad 1.058397$

H $6.1083690 .654318-1.023951$

H $4.758384-3.2187030 .394011$

H $3.628985-1.8887040 .355085$

H $4.914204-1.9329891 .587573$

H $\quad 0.2861530 .886960-0.985279$

H $\quad \begin{array}{llll}1.528627 & 0.206082 & 0.062533\end{array}$

H $2.498650-1.381121-4.074197$

H $1.145161-2.428598-3.593779$

H $2.694218-3.565585-1.859616$

H $4.476535-2.745449-4.212283$

H $5.073228-5.181024-3.618001$ 
H $4.726929-4.857323-1.936295$ H $8.478523-3.793118-3.453727$ H $7.197063-4.539935-4.563942$ H $7.970885-3.411096-1.152435$ H $6.462585-3.969836-0.511986$ H $7.110173-1.232753-1.800095$ H $2.414289-0.253418-2.024963$ C $0.1499413 .245636-3.017239$ O $-0.836628 \quad 3.160893-1.971725$ C $-2.145370 \quad 3.044507-2.571434$ C $-1.9153492 .902159-4.070524$ C $-0.621827 \quad 3.665364-4.265534$ H $-2.6563213 .989341-2.349652$ C $-2.8925581 .862831-1.936280$ H $-1.7816501 .848620-4.347049$ H -2.744152 $3.304024-4.661171$ C $0.0812953 .339760-5.571809$ H $-0.8403754 .741111-4.229924$ C $-4.2626461 .581419-2.577097$ O $-3.0707972 .143150-0.544576$ H -2.257549 $0.972181-1.990781$ H $-4.1342891 .318082-3.633426$ O $-5.0218042 .797354-2.551054$ C $-5.093526 \quad 0.491052-1.874332$ C $-4.380752-0.870138-1.788724$ H $-5.3851500 .835581-0.876475$ O $-6.3124350 .331263-2.610491$ H $-4.069801-1.171651-2.796706$ H $-3.470809-0.766790-1.190627$ H $1.0104263 .909837-5.668966$ H $0.3304372 .275400-5.639098$ H $-0.5579583 .589866-6.424938$ C $1.2523264 .230853-2.625474$ H $-3.6555752 .917059-0.473504$ H $-5.8873412 .607187-2.952088$ H $-6.0837400 .000916-3.496112$ H $0.8112295 .214097-2.420357$ H $1.9541214 .347088-3.458991$ H $0.5683332 .238456-3.146859$ C $-4.933503-1.5195731 .142920$ O $-5.857711-1.5961320 .051510$ C $-5.276157-1.983084-1.197190$ C $-4.594155-3.353402-1.066760$ C $-3.623231-3.4044390 .112263$ C $-4.299080-2.8917231 .384465$ H $-4.152841-0.7829430 .917765$ C -5.708995-1.033254 2.371012 H $-6.113640-2.115236-1.893928$ O $-3.920268-3.675697-2.278400$ H $-5.368540-4.117068-0.915945$ H $-2.709047-2.839238-0.099198$ O $-3.221755-4.756668 \quad 0.339784$ H -5.061227 -3.611094 1.710262 O $-3.334429-2.7900632 .427005$ C -6.1412660 .4036612 .198602$ H $-6.613480-1.6342852 .523327$ H -5.116128 -1.099290 3.290091 H $-3.597734-4.589360-2.203646$ H -2.680401 -5.033032 -0.418601 H $-2.899429-3.6561432 .508436$ O $-5.0689501 .234326 \quad 2.342233$ O C $-5.343620 \quad 2.627829 \quad 2.170158$ H $-6.0437052 .972192 \quad 2.937054$ H $-4.403937 \quad 3.175522 \quad 2.280222$ H -5.7430942 .8155961 .168831$

SCF Energy (B3LYP/6-31G**//MMFF) $=-3245.91483449$

\section{9}

MM̄FF Geometry

C $-0.5019172 .438548-0.090311$ C $-1.363583 \quad 2.6785350 .909737$ C $-1.6822101 .752102 \quad 2.061144$ O $-1.0795730 .458781 \quad 1.833357$ C $-3.204937 \quad 1.5500842 .156065$ C -3.6594170 .9259573 .459226$ C $-4.063499-0.3517993 .599025$ C -3.6972271 .8621184 .639701$
C $-4.075730-1.4395952 .561498$

C $-5.492512-1.7983252 .091194$

C $-6.097795-0.7612791 .131428$

C $-7.527884-1.148420 \quad 0.748703$

C $-8.116537-0.198059-0.305546$

O $-5.276198-0.725621-0.039057$

O $-9.305328-0.806052-0.833410$

C $-7.118046-0.068114-1.475385$

C $-8.548921 \quad 1.138643 \quad 0.308437$

O $-7.5545430 .914333-2.412113$

C $-5.6722210 .238634-1.022642$

C $-4.7027720 .106666-2.222635$

O $-5.6145351 .572211-0.514424$

C $-0.436454-0.1571132 .854943$

O $-0.243094 \quad 0.292596 \quad 3.975812$

C $0.028476-1.5102912 .463506$

C $-0.277961-2.0938781 .293734$

C $0.117706-3.4364470 .860752$

C $0.985723-4.2468271 .786150$

C $-3.2172710 .193407-1.841366$

C $-2.308214-0.120256-3.017720$

C $0.049210-0.758515-3.526353$

O $-2.704127-0.163934-4.179432$

C $0.014090-2.252107-3.620991$

C $1.013975-3.054911-3.227176$

C $0.946742-4.554287-3.292411$

C $1.120253-5.211865-1.939306$

C $2.257292-5.858238-1.631724$

C $-0.053150-5.214852-0.982903$

C $-0.307062-3.878183-0.341800$

N $-0.995983-0.330232-2.613637$

H $0.055566 \quad 1.506296-0.118906$

H -1.885992 3.6336760 .917710

H -1.2888952 .2164672 .972793$

H -3.5369870 .9419301 .308950$

H -3.7260972 .5111752 .049295$

H $-4.402898-0.6757204 .582333$

H $-4.086806 \quad 1.3787525 .542069$

H $-2.6932642 .229698 \quad 4.872544$

H -4.3413972 .7205994 .423224$

H $-3.433160-1.2039371 .708288$

H -3.629779 -2.329769 3.023736

H $-5.429445-2.7639181 .572505$

H $-6.150303-1.9385262 .957834$

H -6.0885220 .2224181 .613481$

H $-8.171697-1.1847041 .636294$

H $-7.531542-2.166617 \quad 0.334840$

H $-9.916543-0.961456-0.092863$

H $-7.116041-1.027722-2.011267$

H -9.3112950 .9765391 .079967$

H -9.016102 $1.785560-0.442282$

H $-7.724451 \quad 1.6853600 .770838$

H -8.459461 $0.677878-2.679239$

H $-4.866214-0.865426-2.706750$

H $-4.9204890 .882761-2.967178$

H $-4.712547 \quad 1.913926-0.613278$

H $0.622976-2.0182523 .216116$

H $-0.892393-1.5489060 .579374$

H $1.903584-3.7020102 .029200$

H $1.299355-5.2025981 .360666$

H $0.452213-4.4680932 .716908$

H $-2.9691971 .200506-1.493448$

H $-2.991030-0.515625-1.037086$

H $1.000963-0.387922-3.132682$

H $-0.121183-0.317102-4.513293$

H -0.892689-2.696609 -4.025585

H $1.926611-2.616221-2.833311$

H $1.731386-4.891313-3.982275$

H $0.000640-4.892372-3.733362$

H $2.382525-6.363166-0.679802$

H $3.091534-5.895100-2.324476$

H $\quad 0.068617-5.991234-0.218666$

H $-0.957320-5.513512-1.529404$

H $-0.937950-3.220823-0.940279$

H $-0.784528-0.348504-1.620360$

C $1.0878304 .106219-1.080839$

O $2.1585313 .146974-1.133111$

C $3.2587383 .712150-1.870485$ 
C $2.8918865 .167057-2.144721$

C $1.3783195 .096115-2.206818$

H $3.3006483 .174698-2.825086$

C $4.5699703 .506660-1.103326$

H $3.2065175 .830235-1.330830$

H $3.3430235 .539207-3.069638$

C $0.7008056 .444905-2.039824$

H $1.0870844 .662350-3.173003$

C $4.8081932 .050534-0.650524$

$\begin{array}{lllll}\text { O } & 4.543860 & 4.322294 & 0.075468\end{array}$

H $5.4100033 .868971-1.707255$

H $4.0752251 .796251 \quad 0.118667$

O $6.0907012 .004712-0.012677$

C $4.769801 \quad 1.009567-1.779333$

C $5.122348-0.428337-1.349241$

H $3.7840241 .008213-2.256749$

O $5.7164951 .415428-2.774876$

H $5.385874-0.984588-2.257771$

H $6.036906-0.421291-0.746931$

H $-0.3880626 .344357-2.086616$

H $0.9556046 .905937-1.079622$

H $1.0099887 .131362-2.834801$

C $-0.255777 \quad 3.394272-1.224957$

H $5.372857 \quad 4.161544 \quad 0.558242$

H $6.7626742 .183216-0.692779$

H $5.6169690 .818444-3.535971$

H $-0.2785492 .814263-2.155924$

H - $1.0663074 .129685-1.285338$

H $1.1503474 .604737-0.104307$

C $4.623512-0.8159201 .680946$

$\begin{array}{lllll}\text { O } & 3.645686 & -0.625093 & 0.652044\end{array}$

C $3.977680-1.173445-0.625449$

C $4.217015-2.684088-0.490796$

C $5.292284-2.9899340 .545420$

C $4.960607-2.3023371 .867083$

H $5.528485-0.2510111 .431028$

C $4.042835-0.2489712 .983397$

H $3.084183-1.047595-1.249863$

O $4.579406-3.241850-1.749564$

H $3.280764-3.166619-0.184549$

H $6.285817-2.7063700 .179171$

O $5.327514-4.404078 \quad 0.751394$

H $4.115199-2.8228642 .332304$

O $6.066062-2.4438532 .758141$

C $3.895619 \quad 1.2522692 .924247$

H $3.062601-0.6944153 .192423$

H $4.682783-0.4723403 .844668$

H $4.775165-4.183350-1.605280$

H $5.983753-4.578691 \quad 1.447817$

H $6.825563-1.9783352 .367754$

O $2.577691 \quad 1.5882072 .830448$

$\begin{array}{lllll}\text { O } & 4.840310 & 2.030495 & 2.948517\end{array}$

C 2.2949172 .9899282 .791130

H 2.7970773 .5120833 .611302

H 1.2163323 .1227272 .905206

H 2.6003453 .4021581 .826591

SCF Energy (B3LYP/6-31G**/MMFF) $=-3245.92334070$

0400010

MM̄FF Geometry

C $-0.423358-0.6583292 .028643$

C $-0.924167-1.8913941 .857073$

C $-1.254137-2.544703 \quad 0.535513$

O $-0.781534-1.716282-0.549492$

C $-2.775252-2.707483 \quad 0.392173$

C $-3.170154-3.654337-0.722068$

C $-3.621608-3.271299-1.932088$

C $-3.074571-5.119404-0.381341$

C $-3.766486-1.873909-2.468769$

C $-5.235200-1.455163-2.620897$

C $-5.908071-1.114484-1.282006$

C $-7.399827-0.841477-1.482292$

C $-8.077370-0.393416-0.179006$

O $-5.264910 \quad 0.052673-0.759243$

O

C -7.2617750 .7641940 .432085$

C $-8.319218-1.5688290 .775255$

O $\begin{array}{llll}-7.768648 & 1.122590 & 1.716094\end{array}$
C -5.7442520 .4760350 .523243$

C $-4.9927751 .774200 \quad 0.905805$

O $-5.515482-0.514598 \quad 1.526414$

C $-0.077507-2.288132-1.556418$

O $0.210702-3.471358-1.669932$

C $0.341512-1.280972-2.560634$

C $0.0362660 .024046-2.479403$

C $0.4480931 .073625-3.415604$

C $1.3118140 .673395-4.582969$

C $-3.462578 \quad 1.6738310 .825238$

C $-2.794838 \quad 3.009178 \quad 1.113676$

C $-0.557199 \quad 4.1093491 .062394$

O $-3.413158 \quad 4.0044321 .481948$

C $-0.3847324 .747936-0.279425$

C $0.7886924 .816737-0.925926$

C $0.9623225 .445281-2.277883$

C $1.4043344 .450554-3.328889$

C $2.6839684 .395411-3.734792$

C $0.346513 \quad 3.580630-3.972569$

C $0.0525022 .341082-3.172659$

N -1.421672 $2.952273 \quad 0.920174$

H $-0.249762-0.0207121 .166588$

H - $1.093059-2.5104162 .736097$

H $-0.748820-3.5177750 .525586$

H $-3.231712-1.7242380 .234773$

H -3.209760 -3.0865151 .327181$

H $-3.901211-4.048565-2.642503$

H -3.410217 $-5.760864-1.203128$

H $-2.040703-5.394810-0.151644$

H $-3.697656-5.3479440 .489487$

H $-3.216139-1.139822-1.873075$

H $-3.296937-1.858669-3.460745$

H $-5.260427-0.567419-3.266389$

H $-5.795610-2.246012-3.134829$

H $-5.768862-1.948925-0.586297$

H $-7.905444-1.724017-1.893781$

H $-7.528481-0.046591-2.230519$

H $-9.871531-0.567401-0.965210$

H $-7.4080561 .641511-0.213792$

H $-8.958420-2.3224990 .299701$

H -8.856934 -1.244493 1.673035

H -7.398342 -2.0667391 .085605$

H -8.7176011 .3082121 .611291$

H -5.3068412 .5793560 .228376$

H -5.2711422 .0778011 .923186$

H $-4.612716-0.4205361 .867428$

H $0.937413-1.678157-3.376123$

H $-0.5647670 .373161-1.641462$

H $2.2278540 .182788-4.237398$

H $1.6316301 .516252-5.198872$

H $0.771084-0.016517-5.240214$

H -3.085492 0.9491251 .553618

H -3.1552801 .350347 -0.175833

H 0.3914283 .7594051 .477742

H $-1.001160 \quad 4.823976 \quad 1.762335$

H $-1.273023 \quad 5.171826-0.741932$

H $1.6793684 .402663-0.460534$

H $1.7042206 .248476-2.179748$

H $0.0408155 .938950-2.610996$

H $3.0016583 .704145-4.508247$

H $3.4434985 .035335-3.297942$

H $0.6313423 .336483-5.001301$

H $-0.5827194 .154723-4.080605$

H $-0.5743842 .525437-2.300482$

H -1.0125142 .1049790 .537645$

$\begin{array}{llll}\text { C } & 1.435128 & 0.288485 & 3.373968\end{array}$

O 1.6356901 .4798092 .581887

C 2.5730572 .3246703 .261732

C 3.1911031 .4801684 .366589

C $2.0067530 .611733 \quad 4.752564$

H 1.9786893 .1126453 .745988

C 3.5481893 .0117212 .303620

H 4.0118230 .8617853 .985201

H 3.5703202 .0825435 .197357

C $2.392081-0.6055225 .573992$

H 1.2900621 .2188395 .322002

C 4.4498022 .1203301 .421405

O 4.3906813 .8555083 .100411 
H 2.9754213 .6815891 .650793

H 5.1753801 .5883422 .046598

O $\quad \begin{array}{llll}4.231169 & 3.015095 & 0.610321\end{array}$

C 3.7374831 .1134810 .494535

C $4.6835420 .502998-0.560517$

H $3.3050870 .303793 \quad 1.088457$

O $2.6759701 .765855-0.202548$

H $5.0579491 .305236-1.206960$

H $5.5460800 .060860-0.051471$

H $1.514306-1.2175535 .802949$

H $3.114414-1.2355025 .045099$

H $2.846693-0.2998946 .522003$

C $-0.046418-0.0838063 .367510$

H $4.996014 \quad 4.3161192 .494309$

H $4.6216553 .463790-0.000989$

H 1.9629901 .9328490 .436787

H $-0.663394 \quad 0.8051213 .544264$

H $-0.260713-0.8050684 .163738$

H $2.025075-0.5071552 .904782$

C $4.501011-2.558382-0.223882$

O $3.493020-1.660044-0.703916$

C $3.977490-0.541864-1.454637$

C $4.803204-1.020594-2.655924$

C $5.923159-1.963162-2.234006$

C $5.357754-3.095421-1.379764$

H $5.140985-2.0375350 .497635$

C $3.779962-3.713495 \quad 0.489194$

H $3.091944-0.049213-1.869520$

O $5.3372890 .093192-3.363445$

H $4.139371-1.551181-3.351357$

H $6.720356-1.420632-1.712457$

O $6.513877-2.516018-3.411904$

H $4.759049-3.758455-2.016950$

O $6.437326-3.874869-0.867737$

C $3.294419-3.3282131 .869293$

H $2.912932-4.024164-0.106567$

H $4.445679-4.5724620 .625867$

H $5.864964-0.257141-4.101403$

H $7.191882-3.151524-3.124523$

H $6.973071-3.302139-0.292660$

$\begin{array}{llll}\text { O } & 2.298470 & -4.182947 & 2.246623\end{array}$

O $3.762629-2.4270852 .551177$

C $1.787210-3.9914453 .569061$

H $0.955246-4.6852033 .716104$

H $1.417260-2.9707423 .693619$

H $2.564494-4.2114484 .306805$

SCF Energy (B3LYP/6-31G*//MMFF) $=-3245.92802482$

0400011

MM̄FF Geometry

C 0.8309864 .6040811 .323162

C $-0.193350 \quad 4.166957 \quad 0.575849$

C $-1.641604 \quad 4.3467490 .948506$

O $-2.260308 \quad 3.039858 \quad 1.013383$

C $-2.3539805 .174704-0.135289$

C $-3.830156 \quad 5.3884300 .137457$

C $-4.830744 \quad 4.751536-0.503925$

C $-4.144795 \quad 6.422674 \quad 1.187548$

C $-4.7152743 .691820-1.565050$

C $-5.4018592 .391620-1.126692$

C $-5.0938551 .230771-2.084540$

C $-5.871238-0.021848-1.676745$

C $-5.491748-1.242632-2.527656$

O $-3.6856840 .978097-2.022617$

O $-6.005470-2.413066-1.872576$

C $-3.954169-1.379019-2.551514$

C $-6.148049-1.198218-3.912574$

O $-3.550620-2.406543-3.453239$

C $-3.224979-0.063462-2.894467$

C $-1.686608-0.160357-2.729709$

$\begin{array}{llll}\text { O } & -3.478578 & 0.267122 & -4.265558\end{array}$

C -2.079988 2.3266362 .160089

O $\begin{array}{llll}-1.419823 & 2.654565 & 3.133478\end{array}$

C -2.843599 1.0650482 .033642

C $-2.8202890 .168323 \quad 3.031121$

C $-3.554317-1.099168 \quad 3.073254$

C $-4.356490-1.4836031 .858405$

C $-1.190487-0.455382-1.310176$
C $-0.957234-1.928420-1.020309$

C $-0.350656-3.4519880 .860735$

O $-1.004312-2.802822-1.880519$

C -1.578070-4.017375 1.500356

C -1.629568 -4.404352 2.783797

C $-2.835871-5.0315893 .419728$

C $-3.283255-4.3076004 .668735$

C $-2.935966-4.7527895 .888269$

C $-4.213465-3.1281574 .505196$

C $-3.487020-1.8477444 .194202$

N $-0.653387-2.1423230 .317234$

H $\quad 0.6326915 .1335042 .251463$

H $0.0130093 .628233-0.346495$

H $-1.7451004 .847678 \quad 1.919405$

H $-2.2077004 .703353-1.112763$

H $-1.8729276 .158770-0.222251$

H $-5.8554305 .008752-0.238122$

H -5.2219106 .5822701 .305085$

H -3.7505636 .1149522 .160771$

H $-3.697927 \quad 7.3853370 .918485$

H $-5.1917744 .062100-2.480676$

H -3.673940 $3.487457-1.821308$

H $-5.0587882 .128726-0.118536$

H -6.485668 $2.552493-1.070945$

H $-5.3625661 .536284-3.102651$

H $-6.9523300 .159220-1.724717$

H -5.655920 $-0.255095-0.625842$

H $-6.968063-2.307590-1.781797$

H -3.649689-1.703199-1.549152

H -7.240589 -1.185243 -3.818019

H $-5.909357-2.096564-4.492522$

H -5.858956 -0.322138 -4.496471

H -4.013231 -3.220627 -3.190532

H -1.271317 $-0.867125-3.458245$

H -1.270082 $0.818762-3.007508$

H $-2.7813000 .870028-4.572349$

H -3.4207660 .9152851 .129335$

H $-2.2239550 .390288 \quad 3.915377$

H $-4.758891-2.4977301 .904049$

H -3.735253 -1.448701 0.957123

H $-5.202752-0.8007191 .729810$

H $-0.2197750 .038978-1.174191$

H $-1.872838-0.042647-0.562424$

H $\quad 0.468175-3.3258391 .575949$

H $-0.013644-4.1177540 .060589$

H -2.454326 -4.1436490 .868781$

H - $0.742887-4.3012303 .405291$

H $-2.579205-6.0709973 .663812$

H -3.673936 -5.095243 2.714587

H -3.280324 -4.257333 6.789906

H $-2.294629-5.6192496 .011386$

H -4.769284 -2.962140 5.438398

H $-4.985399-3.3714803 .769607$

H $-2.873793-1.4872335 .021537$

H $-0.674931-1.3580710 .962788$

C 2.9479403 .4078681 .920317

O 2.3283372 .1107901 .802376

C 3.3546021 .1098011 .635294

C 4.6414031 .7768652 .090750

C 4.4293843 .1972461 .607638

H 3.3889430 .9054070 .559782

C $2.947967-0.1500742 .408641$

H $4.725823 \quad 1.7570403 .184318$

H 5.5355461 .3131491 .666083

C 5.3498684 .2088422 .267352

H 4.5902113 .2259320 .521960

C $3.951970-1.3155712 .369917$

O $1.692594-0.6138021 .907890$

H 2.7710650 .1282793 .455727

H $3.479971-2.1979832 .821039$

O $5.053203-0.9734173 .218942$

C $4.512687-1.7130960 .987233$

C $3.445599-2.110620-0.046419$

H $5.137731-0.9032990 .598313$

O $5.395351-2.8263271 .182849$

H $2.818569-2.9038910 .378464$

H $2.793740-1.255800-0.242819$

H 5.1462305 .2193431 .899155 
H 5.2255824 .2151173 .355459

H 6.3971493 .9743322 .050019

C 2.2716634 .3849590 .956770

H $1.0703410 .132807 \quad 1.946898$

H $5.677791-1.7187233 .200000$

H $4.863744-3.5727511 .508726$

H $2.3404003 .999525-0.068413$

H 2.7846905 .3530880 .974289

H 2.8101073 .7325262 .959704

C $4.479914-0.485115-2.410153$

O $5.010661-1.722872-1.924030$

C $4.041720-2.622088-1.379413$

C $2.994881-2.968378-2.447325$

C $2.363676-1.712760-3.046640$

C $3.446936-0.739604-3.513182$

H $4.0161870 .067010-1.584724$

C $5.6575610 .344990-2.931409$

H $4.581017-3.549531-1.149029$

O $1.997159-3.811954-1.885811$

H $3.482582-3.535664-3.250754$

H $1.686224-1.224848-2.337793$

O $1.571985-2.070904-4.180722$

H $3.939541-1.141242-4.407908$

O $2.8370680 .497181-3.877540$

C $6.4920230 .862918-1.784051$

H $6.301065-0.240832-3.598677$

H $5.3180261 .218432-3.499768$

H $1.399630-4.083606-2.602442$

H $\quad 0.807041-2.575733-3.856406$

H $2.1680840 .301559-4.556022$

O $7.436294-0.057421-1.437763$

O $6.3072251 .946023-1.244236$

C $8.2706210 .301770-0.332291$

H $8.8047391 .232970-0.544086$

H 7.6700960 .3978900 .576966

H $9.002718-0.496764-0.185782$

SCF Energy (B3LYP/6-31G**//MMFF) $=-3245.92337150$

04_00012

MM̄FF Geometry

C $-1.1705411 .609300-1.767681$

C $-0.3407600 .816740-2.464544$

C $-0.567346-0.635625-2.826743$

O $-1.821435-1.121158-2.299941$

C $0.530096-1.513033-2.199795$

C $0.733017-2.825291-2.929613$

C $0.167334-3.999624-2.587996$

C $1.691439-2.758366-4.091294$

C $-0.847055-4.271149-1.511286$

C $-0.271185-5.044305-0.316530$

C $0.510909-4.1449590 .651437$

C $1.129543-4.9632621 .786185$

C $1.838184-4.0652572 .812425$

O $\quad-0.409177-3.190193 \quad 1.194637$

O $2.112164-4.8779523 .963066$

C $0.873861-2.9380663 .241824$

C $3.196832-3.5847412 .291142$

O $1.534487-2.0002934 .089460$

C $0.191102-2.2175222 .055979$

C $-0.921701-1.2793372 .581561$

O $1.169810-1.4397181 .366222$

C $-2.954348-0.953170-3.027026$

O $-3.064310-0.449956-4.131633$

C $-4.062125-1.445411-2.179869$

C $-5.295760-0.949024-2.345231$

C $-6.462246-1.265675-1.517953$

C $-6.287470-2.293361-0.429391$

C -1.843492 -0.7160971 .489750$

C -2.8518120 .2740112 .050905$

C -4.7759361 .6986121 .342546$

$\begin{array}{llll}\text { O } & -2.844236 & 0.648239 & 3.220784\end{array}$

C -6.0995151 .0159051 .492413$

C $-7.218851 \quad 1.4361520 .882069$

C $-8.5632990 .787003 \quad 1.058064$

C $-9.2737440 .533135-0.254573$

C $-10.239747 \quad 1.360881-0.687555$

C -8.924014 -0.723337-1.021473

C $-7.614321-0.603274-1.751531$
N $-3.7466540 .707906 \quad 1.081923$

H -2.116130 $1.221004-1.403636$

H $0.591406 \quad 1.243568-2.830813$

H -0.559884 -0.728740 -3.919759

H $\quad 0.285323-1.709392-1.151453$

H $1.492946-0.984863-2.184951$

H $0.427492-4.879443-3.176128$

H $1.814221-3.726900-4.587610$

H $1.332853-2.046746-4.841736$

H $2.679857-2.434584-3.750203$

H $-1.340677-3.357124-1.172334$

H $-1.639103-4.879883-1.966070$

H -1.115716 $-5.491746 \quad 0.223793$

H $0.362927-5.867195-0.668274$

H $1.294481-3.6226420 .092077$

H $1.822273-5.7170661 .391809$

H $0.338926-5.5247902 .303730$

H $2.497044-4.3017564 .645857$

H $0.094793-3.4053653 .859566$

H $3.844642-4.4409772 .068126$

H $3.729255-2.9973773 .047217$

H $3.114879-2.9846351 .383106$

H $2.086451-1.4303653 .527078$

H -1.555400 -1.828585 3.290487

H $-0.466560-0.4465763 .132400$

H $0.732348-0.6994220 .917781$

H -3.813771 -2.152908 -1.396484

H $-5.459293-0.214781-3.133087$

H $-6.006866-3.261445-0.859081$

H -7.187378 -2.461203 0.165056

H $-5.507185-1.9829190 .274413$

H $-1.260500-0.1945470 .725221$

H -2.394625 -1.530979 1.006598

H -4.7669852 .4005950 .502602$

H -4.5363012 .2466092 .259173$

H -6.1463590 .1539222 .153811$

H -7.1758642 .3199890 .249211$

H -9.1715841 .4521871 .685060$

H -8.482849 -0.1548561 .614893$

H -10.773557 $1.172029-1.613085$

H -10.514725 2.249126 -0.128419

H -9.694683 -0.933336 -1.775286

H $-8.961153-1.578356-0.341449$

H -7.635849 $0.128304-2.560746$

H $-3.697953 \quad 0.310847 \quad 0.148644$

C -0.9488573 .3399420 .041578$

$\begin{array}{lllll}\text { O } & 0.165973 & 2.705377 & 0.701996\end{array}$

C 0.8442293 .6854711 .507396

C -0.1597544 .8104701 .711511$

C -0.8425084 .8341120 .358950$

H $1.678306 \quad 4.0638680 .904898$

C 1.3934403 .0635792 .795041

H -0.8865294 .5710042 .496298$

H $\quad 0.3214095 .7588681 .967714$

C -2.1796885 .5553530 .364841$

H $-0.1757165 .326236-0.361941$

C 2.2436551 .7942122 .592972

O 0.3044922 .7089363 .657546

H 1.9727103 .8167423 .341911

H 1.5899820 .9458092 .368435

O $2.877707 \quad 1.486856 \quad 3.840425$

C 3.3243661 .8959791 .503831

C 4.2321830 .6518651 .451580

H 2.8370102 .0307200 .533018

O $4.134973 \quad 3.048976 \quad 1.728997$

H 4.9055950 .6469722 .317169

H $3.602331-0.2435571 .499352$

H -2.639757 $5.531477-0.627982$

H -2.8797625 .0968831 .071512$

H -2.0498206 .6037640 .652545$

C $-0.8961073 .054503-1.461769$

H -0.2768582 .1046813 .164542$

H 2.1760121 .4029334 .508800

H 4.5286352 .9634022 .614352

H $0.0834663 .342123-1.860701$

H -1.652009 $3.655783-1.980159$

H -1.859212 2.9179300 .483452

C $4.7640630 .402509-2.233439$ 
$\begin{array}{llll}\text { O } & 4.172126 & 0.418784 & -0.928842\end{array}$

C $5.077907 \quad 0.609366 \quad 0.164061$

C $6.118893-0.5202630 .192522$

C $6.838529-0.654226-1.153060$

C $5.853443-0.677656-2.328996$

H $3.9458680 .114104-2.906317$

C $5.2473881 .794640-2.654791$

H 5.5878621 .5719860 .050083

O $5.498235-1.761520 \quad 0.519586$

H $6.848649-0.3091160 .982931$

H $7.429087-1.578572-1.149053$

O $7.7425650 .436952-1.295868$

H $6.384542-0.575307-3.282356$

O $5.192401-1.946621-2.351617$

C $4.0943402 .770568-2.703528$

H $6.0152032 .189372-1.983354$

H $5.6936621 .767898-3.655680$

H $4.798844-1.925713-0.136038$

H $8.2112550 .321297-2.139974$

H $5.874400-2.630339-2.465502$

O $4.2716513 .746955-1.768309$

O $3.1477352 .672039-3.473685$

C $3.2431204 .739278-1.709468$

H $3.2208665 .313303-2.640391$

H $2.2732464 .271778-1.521220$

H $3.4706845 .416703-0.882185$

SCF Energy (B3LYP/6-31G**/MMFF) $=-3245.94071504$

0400013

MM̄FF Geometry

C $1.474161 \quad 3.754387 \quad 1.394437$

C 1.5042804 .4730580 .260780

C $2.1847774 .071396-1.028303$

O $2.3692372 .638053-1.105059$

C $3.5576164 .755964-1.135580$

C $4.2098464 .581522-2.494279$

C $5.2366523 .749443-2.759639$

C $3.6528025 .457099-3.588414$

C $5.9290062 .805603-1.816707$

C $5.7772401 .352495-2.284256$

C $6.3609760 .357185-1.270550$

C $6.226505-1.075961-1.788526$

C $6.669695-2.115835-0.748555$

O $5.637565 \quad 0.507948-0.045458$

O $6.196172-3.401060-1.180907$

C $5.975705-1.8104240 .596565$

C $8.197006-2.222239-0.666702$

O $6.476640-2.6552431 .629437$

C $6.090575-0.333124 \quad 1.023595$

C $5.238020 \quad 0.0071862 .270420$

O $7.454453-0.0482311 .358499$

C $1.2718371 .901996-1.442528$

O $0.1479602 .324590-1.667517$

C $1.6679900 .476982-1.489498$

C $0.729895-0.455197-1.715134$

C $0.943888-1.898966-1.835532$

C $2.348144-2.415766-1.678032$

C $3.724520-0.1677062 .105235$

C $3.190371-1.5066352 .584102$

C $1.078233-2.8294612 .665034$

O $3.867954-2.3220653 .203137$

C $1.065532-3.8230501 .547652$

C $-0.061870-4.314037 \quad 1.011443$

C $-0.091015-5.363006-0.061084$

C $-0.877713-4.935596-1.278273$

C $-2.160099-5.304550-1.437731$

C $-0.146574-4.168294-2.354934$

C $-0.119652-2.687023-2.097695$

N $1.846595-1.6643012 .269889$

H 2.0195052 .8169251 .446677

H $0.973617 \quad 5.4225300 .227920$

H $1.5483274 .388222-1.864138$

H $4.2122204 .386132-0.339637$

H $3.4544565 .833145-0.945369$

H $5.6322943 .733282-3.774635$

H $4.1995265 .344623-4.530817$

H $2.6054945 .209254-3.785880$

H $3.7133126 .511334-3.298671$
H $6.9933653 .067790-1.782798$

H $5.5544492 .906207-0.796005$

H $4.7111731 .138235-2.429929$

H $6.2708051 .223825-3.255804$

H $7.4147270 .608594-1.101746$

H $6.785359-1.204891-2.724007$

H $5.176366-1.270183-2.041929$

H $6.565426-3.575306-2.063653$

H $4.915908-2.0626890 .472934$

H $8.502047-2.9977560 .044539$

H $8.677780-1.283711-0.383389$

H $8.611872-2.525526-1.635431$

H $6.365185-3.5743551 .331507$

H $5.615882-0.5328413 .146847$

H 5.4050121 .0710942 .493521

H 7.4752010 .7362761 .931152

H $2.7148190 .235668-1.352366$

H $-0.302120-0.129782-1.843573$

H $2.787395-2.063751-0.739026$

H $2.972712-2.074512-2.510057$

H $2.410876-3.505662-1.648483$

H 3.2286130 .5913702 .722543

H 3.4146320 .0006081 .069631

H $0.071801-2.4856552 .922261$

H $1.527852-3.2811543 .555082$

H $2.027654-4.1806591 .188356$

H $-1.022560-3.9760801 .393762$

H $-0.539186-6.2673430 .371769$

H $0.921943-5.656174-0.363369$

H $-2.720500-5.030547-2.325113$

H $-2.679130-5.883660-0.681117$

H $-0.648718-4.318461-3.320678$

H $0.850218-4.594832-2.499402$

H $-1.099245-2.216896-2.189068$

H $1.380050-0.9580251 .707778$

C -0.3546843 .1397813 .049228$

O $-1.200196 \quad 2.7838551 .934206$

C $-1.295737 \quad 1.3432851 .878725$

C $-0.9247330 .868755 \quad 3.273628$

C $\quad 0.1949901 .829795 \quad 3.616473$

H -0.5417991 .0190091 .149776$

C $-2.6956680 .941670 \quad 1.405349$

H $-1.7665930 .995144 \quad 3.965673$

H $-0.611419-0.1773303 .299316$

C $0.519698 \quad 1.8855405 .098802$

H 1.0934021 .5182673 .073288

C -2.960260 $-0.573341 \quad 1.469997$

O $-2.841662 \quad 1.3713280 .047572$

H -3.4473011 .4830051 .990450$

H $-2.940115-0.9086052 .513160$

O $-1.886543-1.2595320 .814216$

C $-4.276934-1.0340860 .813787$

C $-5.523359-0.323397 \quad 1.367951$

H $-4.210889-0.900195-0.271089$

O $-4.411485-2.443557 \quad 1.028726$

H $-5.570318-0.4780892 .453176$

H $-5.4294620 .753755 \quad 1.202648$

H 1.3192262 .6068185 .294870

H -0.3534752 .1791805 .690908$

H $0.8532660 .905515 \quad 5.455606$

$\begin{array}{llll}\text { C } 0.697116 & 4.168274 & 2.614938\end{array}$

H -2.652000 2.3250230 .022277

H $-1.873413-0.963253-0.111960$

H $-4.476846-2.5933491 .987395$

H $1.3956174 .358716 \quad 3.437365$

H $0.181644 \quad 5.1153542 .410401$

H -1.0119583 .6150603 .789001$

C $-6.7610380 .454956-1.296870$

O $-6.795218-0.841512-0.688310$

C $-6.842704-0.8309510 .741563$

C $-8.096687-0.082183 \quad 1.213895$

C $-8.199613 \quad 1.3107790 .592124$

C $-8.018799 \quad 1.247015-0.926017$

H $-5.8660380 .996625-0.966750$

C $-6.6531340 .248877-2.810701$

H -6.957068 - 1.8771511 .051779

$\begin{array}{llll}\text { O } & -8.104442 & 0.014211 & 2.634106\end{array}$

H -8.982144 -0.6639560 .926752$ 
H $\quad-7.471087 \quad 1.994743 \quad 1.042058$ O H $\quad-8.9033550 .786079-1.383604$ O $-7.9099102 .571783-1.440977$ C $-5.297440-0.317017-3.162510$ H -7.431480 -0.432329 -3.175238 H $-6.7648391 .189114-3.361895$ H -8.8980680 .5158092 .887705$ H -10.159621 1.2828720 .502891 H $-8.7048303 .058234-1.162385$ O $-5.385998-1.666311-3.337710$ O $\quad-4.2767880 .355633-3.232991$ C $-4.150356-2.333750-3.609291$ H -3.496615 -2.272326 -2.734882 H $-4.369126-3.385625-3.811243$ H -3.663692 -1.901815 -4.488987 SCF Energy (B3LYP/6-31G**//MMFF) $=-3245.90934875$

0400014

MM̄FF Geometry

C $1.199990-1.2381533 .202909$

C $0.140222-1.9187132 .743046$

C $0.156176-2.7001861 .453664$

O $-0.639132-1.9701010 .489896$

C $-0.469817-4.0895161 .666950$

C $-0.422903-4.9703010 .431743$

C $-1.486099-5.284565-0.335918$

C $0.934186-5.5353330 .098121$

C $-2.910343-4.838262-0.161412$

C $-3.327546-3.866164-1.272047$

C $-4.638950-3.143616-0.932050$

C $-5.082073-2.251538-2.092480$

C $-6.314977-1.407691-1.736742$

O $-4.396090-2.347203 \quad 0.232151$

O $-6.441255-0.403215-2.755020$

C $-6.062361-0.691212-0.391428$

C $-7.599645-2.241933-1.795480$

O $-7.233471-0.0045410 .042698$

C $-5.535846-1.626668 \quad 0.715724$

C $-5.091573-0.8696631 .991183$

O $-6.584523-2.5233621 .101899$

C $-0.007084-0.968256-0.185349$

O $1.170606-0.655820-0.103135$

C -1.005064 -0.283284 -1.034878

C $-0.6691870 .867233-1.637474$

C $-1.5218041 .671143-2.515343$

C $-2.9286761 .192387-2.754360$

C $-3.943747 \quad 0.127972 \quad 1.797233$

C -4.3964901 .5608471 .572578$

C -3.4883353 .8111931 .001834$

O $-5.553701 \quad 1.9364611 .737710$

C -3.681239 4.091202 -0.454600

C $-2.9129294 .944180-1.149263$

C $-3.1410525 .290826-2.592385$

C $-1.9061725 .104130-3.444298$

C $-1.1036306 .141774-3.735880$

C -1.657582 $3.736205-4.035996$

C -1.006587 2.791277 -3.063980

N -3.339038 2.3829521 .206693

H $2.130830-1.2587142 .641750$

H $-0.799182-1.8732303 .289238$

H $1.179611-2.8180421 .078744$

H -1.498312 -3.969915 2.021048

H $0.056155-4.6115462 .477974$

H -1.325517 -5.934347 -1.195446

H $0.901651-6.223917-0.752912$

H $1.631625-4.733074-0.156490$

H $1.336216-6.0894170 .952492$

H -3.558593 -5.722825 -0.192190

H $-3.073480-4.3888880 .820045$

H - $2.534918-3.121512-1.414956$

H -3.433381 -4.414718 -2.216132

H -5.405716 -3.893893 -0.706158

H $-5.271018-2.849027-2.993122$

H -4.262501 -1.570141 -2.356306

H $-7.1721740 .185444-2.498937$

H $-5.3136510 .083583-0.590637$

H $-7.739273-2.654861-2.801833$
H $-8.484003-1.624613-1.602693$

H -7.601087 -3.076826 -1.091623

H $-7.842985-0.6641160 .415546$

H $-5.958156-0.4039782 .475644$

H $-4.732220-1.6244812 .705645$

H $-6.386295-2.8659111 .989239$

H -1.983565 - $0.736573-1.135719$

H $0.3344461 .261316-1.480480$

H -2.920829 $0.252945-3.317060$

H $-3.5427691 .901219-3.313550$

H $-3.4472481 .030505-1.803737$

H -3.3415980 .1417062 .714694$

H -3.284326 -0.1807400 .980986$

H -2.5905204 .2933461 .400245$

H -4.3542074 .1746621 .564439$

H $-4.5144763 .598579-0.950277$

H -2.099259 $5.458059-0.642136$

H $-3.4641716 .339479-2.634142$

H $-3.9702054 .712173-3.017914$

H $-0.2333256 .021823-4.372594$

H $-1.2961997 .133060-3.339022$

H - $0.9848863 .820137-4.900494$

H $-2.5894203 .345077-4.454178$

H $0.0231383 .060288-2.823116$

H -2.4228231 .9743871 .046453$

C 1.0921711 .1037514 .177005

O 2.1342921 .5291943 .274104

C 1.5544892 .4210042 .302000

C 0.2186152 .8602262 .886331

C $-0.229236 \quad 1.584430 \quad 3.571033$

H 1.3596361 .8266611 .401632

C 2.5128503 .5674601 .971382

H $\quad 0.3400453 .6646073 .621246$

H -0.4755313 .2043672 .115230$

C -1.3348141 .7943924 .590868$

H -0.5845530 .8895112 .800730$

C 3.8920193 .1209321 .446651

O 2.7321044 .3537153 .149428

H 2.0414514 .2405001 .245659

H 4.4757552 .6603832 .252814

O 4.6073684 .2999131 .054873

C 3.8379662 .1613100 .243532

C $5.2294831 .881234-0.354856$

H $3.387126 \quad 1.2166840 .563465$

O $2.9958932 .707605-0.771471$

H $5.6441362 .811041-0.762622$

H $5.897716 \quad 1.5534790 .448576$

H -1.6198790 .8452095 .055558$

H -1.023251 2.4799565 .385962

H -2.2243722 .2178884 .113911$

C $1.190978-0.4009894 .449577$

H 3.0881313 .7621073 .834691

H 4.6360294 .8911361 .826741

H $3.3735123 .563617-1.037176$

H $\quad 0.381623-0.7110695 .120895$

H $2.130821-0.6054954 .977723$

H 1.2777891 .6318695 .121590

C $5.301302-1.169031-0.147017$

O $4.555114-0.383394-1.081145$

C $5.1917020 .832127-1.489836$

C $6.5610290 .528927-2.115447$

C $7.436578-0.319404-1.193459$

C $6.671638-1.555905-0.717832$

H $5.440386-0.5875020 .772825$

C $4.452398-2.3800620 .254250$

H $4.5629461 .252014-2.285089$

O $7.2297811 .741564-2.445478$

H $6.404594-0.016558-3.055318$

H $7.7974430 .271115-0.343847$

O $8.607101-0.731529-1.903238$

H $6.556635-2.242477-1.564748$

O $7.438211-2.2399340 .269925$

C $4.162258-3.307017-0.903723$

H $4.937604-2.9398011 .062286$

H $3.481930-2.0520100 .641595$

H $8.098191 \quad 1.504286-2.813602$

H $8.324662-1.263930-2.666477$

H $7.558862-1.6385371 .024463$ 
O $4.820034-4.488757-0.730650$

O $3.447185-3.012653-1.852769$

C $4.641138-5.450712-1.774734$

H $5.199280-6.351560-1.506337$

H $3.583123-5.710851-1.875753$

H $5.035376-5.061267-2.718113$

SCF Energy (B3LYP/6-31G*//MMFF) $=-3245.91935622$

04_00015

MM̄FF Geometry

C $-0.077653-3.8834880 .786216$

C $0.653875-2.7594800 .781763$

C $1.338266-2.2154872 .006305$

O $1.017393-0.8103872 .123134$

C $2.863449-2.3285151 .854302$

C $3.599627-2.1109193 .160990$

C $4.227276-0.9713653 .510107$

C $3.644503-3.3070564 .077394$

C $4.288671 \quad 0.3241452 .749980$

C 5.6915990 .6139242 .198298

C $6.063483-0.2616560 .990929$

C $7.5008280 .016146 \quad 0.546220$

C $7.863740-0.755497-0.731340$

O $5.156206 \quad 0.056694-0.068237$

O $9.086089-0.206336-1.246627$

C $6.772472-0.503651-1.792443$

C $8.138907-2.236746-0.446396$

O $6.990521-1.309853-2.948407$

C $5.333981-0.713970-1.264050$

C $4.312590-0.192555-2.302215$

O $5.114594-2.106199-1.037500$

C $-0.091238-0.4668772 .824716$

O $-0.894504-1.2307313 .342402$

C -0.2119871 .0037632 .956934$

C $0.480447 \quad 1.881494 \quad 2.213318$

C $0.416788 \quad 3.3421892 .321795$

C $-0.4087393 .922567 \quad 3.441447$

C $2.851420-0.263896-1.837332$

C $1.971518 \quad 0.625767-2.691083$

C $1.3945433 .004538-3.089145$

O $1.277627 \quad 0.176104-3.599400$

C $1.5338594 .300298-2.359872$

C $0.5060784 .971563-1.819606$

C $0.6610996 .276672-1.094375$

C $0.227861 \quad 6.201181 \quad 0.352781$

C $-0.935011 \quad 6.743868 \quad 0.751216$

C 1.1857435 .5913861 .353580

C 1.0908334 .0909841 .422902

N $2.0656551 .962857-2.336915$

H $-0.180831-4.4454351 .711424$

H $0.783517-2.208607-0.146066$

H $1.009262-2.7487502 .906869$

H $3.211638-1.6159411 .099735$

H $3.137643-3.3212641 .472530$

H $4.747459-0.9470554 .467097$

H $4.223587-3.1135704 .986738$

H $2.635109-3.5948544 .386540$

H $4.108297-4.158023 \quad 3.567711$

H $3.5442750 .372781 \quad 1.950310$

H 4.0194491 .1248223 .450653

H $5.7121751 .667947 \quad 1.891711$

H 6.4370570 .4935662 .994184

H $5.949704-1.3167151 .262198$

H $8.209224-0.2179001 .350871$

H 7.6199361 .0903930 .346102

H $9.767602-0.293499-0.558087$

H $6.8707430 .540707-2.120589$

H $8.969848-2.3422880 .261523$

H $8.451640-2.762339-1.355401$

H $7.278461-2.759676-0.023737$

H $7.898458-1.139890-3.252896$

H $4.5586180 .849944-2.541780$

H $4.398074-0.763206-3.235669$

H $4.161788-2.285880-1.061612$

H -0.9250201 .3286633 .707868$

H $1.156917 \quad 1.5051651 .447790$

H -1.456388 $3.616063 \quad 3.349860$

H -0.4078945 .0140193 .464690$
H -0.0280843 .5856844 .412101$

H $2.468967-1.288159-1.904543$

H $2.7411750 .049766-0.793186$

H $0.3504152 .712596-3.240583$

H $1.8695303 .071177-4.073509$

H $2.5382314 .709289-2.275886$

H $-0.5009184 .571793-1.911065$

H $0.0651157 .026692-1.630711$

H $1.6957186 .639268-1.140812$

H $-1.2398476 .727374 \quad 1.792070$

H $-1.612405 \quad 7.2114390 .044293$

H 1.0427296 .0421332 .341868

H 2.2147655 .8625121 .084229

H 1.6596963 .5791710 .646666

H $2.7798172 .234713-1.666435$

C $-2.196840-4.845281-0.158848$

O $-3.013365-3.668816 \quad 0.013595$

C $-4.305017-3.916302-0.578288$

C $-4.308166-5.376696-1.021256$

C $-2.841079-5.614678-1.310252$

H $-4.368739-3.284795-1.471305$

C $-5.432923-3.567183 \quad 0.398854$

H $-4.653666-6.043093-0.222108$

H $-4.950441-5.542078-1.891924$

C $-2.469736-7.086432-1.365934$

H $-2.588673-5.139696-2.267939$

C $-5.463222-2.0945530 .854813$

O $-5.288859-4.3850671 .567532$

H $-6.396230-3.840023-0.049239$

H $-4.605325-1.8865311 .504900$

O $-6.626136-1.9298581 .678575$

C $-5.503210-1.064460-0.290608$

$\begin{array}{llll}C & -5.794501 & 0.363756 & 0.211577\end{array}$

H $-4.544766-1.092833-0.819177$

O $-6.522441-1.447923-1.214200$

H -6.8217090 .4091810 .593349$

H $-5.1263690 .586067 \quad 1.050409$

H - $1.403815-7.214733-1.577185$

H $-2.688772-7.591584-0.419199$

H $-3.032305-7.594834-2.156053$

C $-0.744230-4.453067-0.433933$

H $-6.010566-4.1506882 .175937$

H -7.406832 -2.0543411 .111862$

H $-6.382919-0.945231-2.034215$

H $-0.703892-3.731394-1.258078$

H $-0.173203-5.337562-0.737864$

H -2.268182 -5.4240780 .771951$

C $-3.2662741 .798180-0.694490$

O $-4.3594341 .378990-1.522002$

C $-5.642917 \quad 1.435340-0.892400$

C $-5.9399122 .870680-0.437539$

C $-4.815843 \quad 3.444528 \quad 0.419919$

$\begin{array}{llll}C & -3.462725 & 3.256826 & -0.266647\end{array}$

H -3.2019091 .1548870 .191512$

C $-1.9838721 .603870-1.508097$

H $-6.3771441 .195558-1.671108$

\begin{tabular}{lllll}
\hline & -7.171899 & 2.915497 & 0.274386
\end{tabular}

H $-6.0608103 .503768-1.326464$

H -4.8208132 .9999581 .422239$

O

H $-3.4024543 .929790-1.131640$

O $-2.453242 \quad 3.6443590 .657927$

C $-1.7179310 .131032-1.710654$

H $-2.0642692 .096233-2.484420$

H $-1.1036842 .016454-1.004914$

H $-7.308724 \quad 3.834676 \quad 0.561336$

H $-4.313073 \quad 5.199738 \quad 1.113683$

H $-1.618288 \quad 3.7269220 .168581$

O $-1.929235-0.211472-3.012468$

O $-1.387315-0.627824-0.808713$

C $-1.737734-1.595019-3.322348$

H $-2.456956-2.207736-2.771460$

H $-1.908987-1.730569-4.393407$

H $-0.712861-1.899556-3.090084$

SCF Energy (B3LYP/6-31G**//MMFF) = -3245.91263993

0400016

MM̄FF Geometry 
C $0.120120-2.5311071 .564436$

C $0.925400-1.7803442 .333021$

C $0.611807-0.4246982 .926183$

O -0.6773750 .0525192 .484783$

C 1.6738970 .5860132 .462226

C 1.5241071 .9521553 .098567

C 1.0351053 .0376302 .467609

C 2.0170852 .0589824 .518323

C $0.4847193 .107873 \quad 1.070127$

C -0.8289183 .9021351 .030474$

C $-1.4277223 .924769-0.382296$

C $-2.6084404 .893280-0.458008$

C $-3.2709384 .872427-1.842968$

O $-1.8692452 .598949-0.693320$

O $-4.5188755 .570965-1.720196$

C $-3.5777913 .409159-2.223654$

C $-2.4439925 .646662-2.875832$

O $-4.0760473 .325937-3.557180$

C -2.383865 2.444794-2.021488

C $-2.8682360 .986799-2.185710$

O $-1.3874262 .718744-3.007585$

C $-1.746269-0.1896373 .289694$

O $-1.741597-0.7361894 .380744$

C $-2.952020 \quad 0.2827652 .574621$

C $-4.149524-0.2014662 .935252$

C -5.4296470 .0843252 .285843$

C -5.4613421 .1735331 .248035$

C $-1.840542-0.075178-1.779614$

C $-2.472905-1.451290-1.833185$

C $-4.050187-2.926936-0.577139$

O $-2.323943-2.191602-2.802439$

C $-5.492227-2.523576-0.612180$

C $-6.399588-2.9217250 .292882$

C $-7.843823-2.5048960 .262049$

C $-8.343072-1.9928201 .597621$

C $-9.215446-2.7082062 .328048$

C -7.890576 -0.6195802 .054045$

C $-6.504551-0.6508452 .639377$

N $-3.238519-1.731277-0.712523$

H $-0.885040-2.1947631 .328877$

H $1.921675-2.1603882 .555192$

H $0.639368-0.5097274 .019235$

H 1.6577230 .6659001 .369061

H 2.6743910 .2075112 .707248

H 1.0220343 .9814913 .011236

H 1.9616193 .0833094 .901913

H 1.4178471 .4269425 .180842

H $3.062101 \quad 1.7397844 .584812$

H 1.2256093 .6033160 .431433

H $\quad 0.3116682 .1121590 .653102$

H -1.5453143 .4469661 .726881$

H -0.6416434 .9285401 .369988$

H $-0.6432804 .222389-1.088105$

H -2.296369 $5.912541-0.198075$

H -3.360332 4.6097440 .292144

H $-4.9823705 .498471-2.572403$

H -4.401704 $3.081128-1.574698$

H -2.313360 $6.687359-2.555808$

H -2.959605 $5.695211-3.841412$

H -1.451591 $5.220634-3.036722$

H -3.319252 $3.413886-4.161406$

H -3.768105 $0.840142-1.578382$

H $-3.1596460 .805841-3.228543$

H $-0.8286401 .933071-3.116944$

H $-2.815780 \quad 0.961667 \quad 1.740603$

H $-4.194152-0.914743 \quad 3.757599$

H -4.998055 2.0897311 .631652

H $-6.471797 \quad 1.4542350 .943649$

H $-4.9234050 .858807 \quad 0.350624$

H $-0.979583-0.068274-2.453900$

H $-1.457867 \quad 0.097216-0.767164$

H -3.776279 -3.398832 0.371659

H -3.835435 -3.622075 -1.394279

H -5.805711 -1.872316 -1.425135

H $-6.093944-3.5954361 .090245$

H -8.432620 $-3.377326-0.049961$

H -8.018954 -1.735643 -0.500465

H $-9.596581-2.3386893 .274314$
H $-9.564540-3.6813601 .999246$

H -8.568642 -0.2368092 .827776$

H $-7.984540 \quad 0.070044 \quad 1.210899$

H $-6.382264-1.3961673 .427080$

H -3.290634 -1.043231 0.032266

C $0.378107-3.829530-0.564316$

O $1.113804-2.728646-1.130358$

C $1.918215-3.209850-2.219021$

C $1.328559-4.561520-2.597171$

C $0.917463-5.093228-1.236846$

H $2.926736-3.360893-1.817142$

C $1.974148-2.170143-3.343493$

H $0.447438-4.457939-3.240517$

H $2.051456-5.203003-3.109981$

C $-0.088547-6.229041-1.302587$

H $1.817363-5.444468-0.714281$

C $2.286728-0.735018-2.870998$

O $0.712386-2.125223-4.021091$

H $2.703272-2.486543-4.098135$

H $1.426887-0.320929-2.331278$

O $2.458257 \quad 0.081472-4.036200$

C $3.542165-0.602351-1.991646$

C $3.8469310 .863852-1.629962$

H $3.401353-1.174766-1.069451$

O $4.663859-1.164930-2.670453$

H $4.096174 \quad 1.424593-2.539037$

H $2.9429161 .304401-1.191441$

H $-0.363230-6.563906-0.297319$

H -1.005189 $-5.923766-1.818376$

H $\quad 0.331635-7.084337-1.841571$

C $0.548853-3.8390420 .957217$

H $0.024678-1.967965-3.351845$

H $1.652241-0.007990-4.573304$

H $4.774261-0.680752-3.506715$

H $1.598706-4.0369871 .207166$

H $-0.049452-4.6463831 .394011$

H - $0.675766-3.672017-0.823721$

C 5.6126890 .3893281 .632106

$\begin{array}{lllll}\text { O } & 4.621556 & 0.399417 & 0.595532\end{array}$

C $5.0208320 .998557-0.641261$

C $5.4110462 .467385-0.417235$

C 6.4903422 .5955160 .661176

C 6.1270721 .8084401 .924820

H 5.0784880 .0410552 .525449

C $6.727507-0.6195661 .338276$

H $5.8798530 .454345-1.047390$

O $4.2682443 .234623-0.042353$

H $5.7820632 .883971-1.360992$

H 6.6199683 .6557940 .910131

O 7.7230402 .1178180 .130648

H 6.9834821 .7522382 .606771

O 5.0866822 .5037452 .618185

C $6.182584-2.0273091 .395126$

H $7.208372-0.4667780 .367913$

H $7.522723-0.5533182 .090310$

H 3.8789782 .8224140 .748027

H 8.4018762 .2316310 .817376

H 5.4254983 .3856162 .848555

O $5.890133-2.4674340 .138838$

O $6.008556-2.6468672 .436768$

C $5.329377-3.7814960 .061493$

H $5.982045-4.5087600 .553820$

H $4.332284-3.7887840 .511834$

H $5.241283-4.051371-0.994202$

SCF Energy (B3LYP/6-31G**//MMFF) $=-3245.93815315$

0400017

MM̄FF Geometry

C -3.4905230 .6451962 .860100$

C -3.6261201 .9799412 .896162$

C -4.0161612 .8828031 .745775$

O -4.1374712 .1427350 .511556$

C -2.9162863 .9323851 .509254$

C -3.4186335 .1724810 .798596$

C $-3.3244425 .398786-0.526014$

C $-4.0133506 .222540 \quad 1.702225$

C -2.819876 4.473086-1.597369

C $-1.4449474 .878201-2.145220$ 
C $-0.2834664 .389434-1.266246$

C $1.0561434 .884328-1.814715$

C $2.2407434 .311623-1.022233$

O $-0.3140532 .957377-1.273003$

O $3.4441864 .590961-1.753425$

C $2.0829922 .779575-0.919059$

C 2.4132025 .0203120 .325571

O $3.0789732 .224698-0.063882$

C $0.6792052 .329643-0.454285$

C $0.5352190 .797807-0.630086$

O $0.5209392 .664254 \quad 0.925529$

C $-5.329540 \quad 1.580446 \quad 0.190787$

O $-6.370894 \quad 1.6286260 .822243$

C $-5.1065590 .832561-1.065552$

C $-5.892513-0.209688-1.368637$

C $-5.737072-1.088329-2.530051$

C $-4.644742-0.757655-3.514643$

C $-0.8876120 .263290-0.404975$

C $-0.948105-1.252919-0.498589$

C $-2.536283-3.154365-0.207516$

O $0.028778-1.949899-0.760107$

C $-3.063440-3.589447-1.539026$

C $-4.172456-4.331013-1.687125$

C $-4.681778-4.819385-3.014300$

C $-6.159947-4.560455-3.212438$

C $-7.064056-5.534678-3.014258$

C $-6.580684-3.200162-3.723167$

C $-6.556064-2.154518-2.643337$

$\mathrm{N}-2.224545-1.737009-0.244487$

H $-3.678231 \quad 0.1039301 .937970$

H -3.4560312 .4867963 .844778$

H -4.9688543 .3691501 .989192$

H -2.0990303 .4809990 .937284$

H -2.469446 4.2514492 .460346

H -3.689699 $6.352321-0.906832$

H $-4.358098 \quad 7.102613 \quad 1.149174$

H -4.8732715 .8177262 .244988$

H -3.2698106 .5612022 .431252$

H -2.812922 3.430601-1.269665

H -3.545227 $4.515250-2.420322$

$\mathrm{H}-1.3430724 .426806-3.141054$

H -1.393068 $5.966106-2.275459$

H $-0.4308104 .753858-0.244126$

H $1.0939525 .980740-1.823694$

H $\quad 1.1550344 .573547-2.864313$

H $3.371734 \quad 4.175107-2.629426$

H $2.2625732 .362780-1.919317$

H 2.5955016 .0910500 .172350

H 3.2903604 .6420980 .862326

H 1.5427734 .9234290 .977821

H $3.9429142 .528607-0.390696$

H $\quad 0.829820 \quad 0.522248-1.651410$

H $\quad 1.2223770 .2839570 .051975$

H -0.1196362 .0607491 .331954$

H -4.260865 $1.127167-1.676875$

H $-6.704005-0.464006-0.687919$

H -4.822639 $0.224078-3.967536$

H -4.560409-1.473793 -4.334038

H -3.667514 - $0.742984-3.019074$

H -1.2560000 .5485980 .584946$

H -1.568836 $0.676452-1.157602$

H -3.265547 -3.3046010 .594962$

H -1.629735 -3.7191440 .032869$

H -2.490308 -3.309133 -2.419707

H -4.724252 -4.639974 -0.801918

H -4.485379 $-5.898413-3.066553$

H -4.121052 -4.375413 -3.846228

H $-8.122809-5.366300-3.181340$

H $-6.765943-6.521099-2.674979$

H -7.607931 -3.242854 -4.109722

H $-5.969745-2.938487-4.591133$

H -7.311545 -2.311387-1.871884

H $-2.985159-1.080179-0.097913$

C - $-1.975187-1.1646323 .699927$

O $-0.750404-0.4442013 .459809$

C $0.352571-1.2099443 .997811$

C $-0.236532-2.5390144 .451413$

C $-1.650656-2.1457844 .823060$
H $\quad 0.720387-0.6381254 .858708$

C $1.443787-1.3296092 .925896$

H $-0.248145-3.2632713 .626898$

H $0.319261-2.9815545 .283463$

C $-2.600984-3.3273714 .912997$

H -1.629520 -1.621778 5.788065

C $2.585679-2.2889383 .299629$

O $1.994898-0.0285852 .701777$

H $0.982831-1.6437951 .983415$

H $2.187684-3.3005613 .440602$

O $3.118184-1.8847044 .566777$

C $3.747262-2.3413742 .286384$

C $3.289728-2.6522880 .851096$

H $4.306344-1.3994062 .312523$

O $4.658211-3.3564722 .722700$

H $2.629018-3.5281640 .870334$

H $2.694118-1.8122780 .481090$

H -3.610356 -2.998985 5.179126

H -2.662159 -3.866644 3.961617

H -2.264132 -4.033913 5.678735

C $-3.101604-0.1906914 .048018$

H 1.2560300 .5692142 .495703

H $3.843725-2.4947624 .784422$

H $4.196107-4.2109422 .674827$

H $-2.7912820 .453530 \quad 4.879541$

H $-3.988574-0.7477294 .369473$

H -2.212244 -1.709948 2.777105

C $5.116144-0.679992-0.620306$

O $5.474789-1.946932-0.063794$

C $4.449929-2.941764-0.127134$

C $4.004525-3.159631-1.580481$

C $3.607276-1.852025-2.264630$

C $4.710419-0.806849-2.094837$

H $4.270718-0.275411-0.050237$

C $6.2782420 .297491-0.388665$

H $4.914376-3.8782600 .206531$

O $2.921572-4.083319-1.625995$

H $4.834454-3.610962-2.139859$

H $2.646076-1.485453-1.887433$

O $3.419143-2.120809-3.655766$

H $5.568734-1.091368-2.714337$

O $4.2485230 .457905-2.560478$

C $7.562864-0.077343-1.099991$

H $5.977471 \quad 1.299082-0.716308$

H $\quad 6.5147450 .3396810 .681194$

H $2.654928-4.173240-2.556997$

H $3.063255-1.314175-4.064759$

H $4.0913580 .381202-3.516514$

O $8.3263351 .044158-1.260773$

O $7.876476-1.209440-1.441261$

C $9.5873180 .842815-1.906862$

H $9.4373910 .445907-2.915455$

H $10.089187 \quad 1.811081-1.982194$

H $10.2126010 .168321-1.314297$

SCF Energy $\left(B 3 L Y P / 6-31 G^{* *} / / M M F F\right)=-3245.91493408$

0400018

MM̄FF Geometry

C 1.1201830 .8826942 .929188

C 2.1159400 .0639303 .299928

C $2.229642-1.4219993 .053563$

O $3.452852-1.5962492 .295260$

C $1.080390-2.0697602 .256935$

C $1.076773-3.5843232 .331466$

C $1.516946-4.4085261 .360958$

C $0.477296-4.1592393 .589982$

C $2.164639-4.0454540 .054060$

C $1.270460-4.363562-1.153007$

C $0.129458-3.352089-1.340767$

C $-0.780196-3.767274-2.498540$

C $-1.861005-2.712609-2.783647$

O $0.725476-2.083212-1.625451$

O $-2.450884-3.017850-4.056481$

C $-1.188196-1.330162-2.917600$

C $-2.996978-2.766580-1.755967$

O $-2.162573-0.295783-3.034028$

C $-0.205975-1.004620-1.768704$

C $0.6031440 .268722-2.112756$ 
O $-0.945609-0.784724-0.567609$

C $4.349160-2.5290152 .701182$

O $4.243309-3.2764473 .663785$

C $5.548527-2.5438191 .829746$

C $5.661221-1.826697 \quad 0.700317$

C $6.827671-1.782517-0.185926$

C $8.043187-2.5769710 .214454$

C $1.7671850 .546712-1.151849$

C $2.5732281 .766049-1.564110$

C $4.6587522 .996022-0.962623$

O $2.2628672 .495010-2.502079$

C $5.6948182 .501680-1.922209$

C $6.9802372 .296886-1.597586$

C $8.0064991 .779132-2.564552$

C $8.621407 \quad 0.469253-2.120264$

C $9.8684100 .429240-1.621352$

C $7.830481-0.803221-2.338077$

C $6.766431-1.016321-1.295499$

N $3.6835441 .942885-0.749163$

H $\quad 0.2668300 .492382 \quad 2.384117$

H 2.9513330 .4959073 .849554

H $2.318508-1.8933434 .039924$

H $1.128232-1.7412941 .213248$

H $0.108710-1.7291042 .636800$

H $1.432152-5.4822001 .526734$

H $0.461664-5.2543963 .581705$

H $1.050818-3.8464404 .467765$

H $-0.555951-3.8170643 .708823$

H $2.491046-3.0018120 .028200$

H $3.081668-4.642757-0.028349$

H $1.907742-4.348398-2.047013$

H $0.864574-5.378568-1.061208$

H $-0.447320-3.284457-0.411857$

H $-1.239167-4.744163-2.301911$

H -0.176876 -3.897692 -3.407945

H -2.816451 -3.917889-4.009137

H $-0.625655-1.332579-3.861942$

H -3.471918 -3.754981-1.761134

H $-3.789593-2.053567-2.007080$

H -2.661365 -2.568577 - 0.736393

H -2.752482 $-0.534065-3.769545$

H $1.0250770 .164775-3.121107$

H $-0.0626241 .139843-2.127380$

H $-0.465102-0.161995-0.000648$

H $6.347348-3.1919822 .175645$

H $4.825670-1.2031190 .386832$

H $8.394964-2.2698291 .205764$

H $8.888832-2.451455-0.464954$

H $7.811128-3.6474290 .239081$

H $1.3961130 .719933-0.137925$

H $2.448615-0.311553-1.125604$

H 5.0835493 .2518290 .013103

H $4.1616793 .881311-1.371683$

H $5.3646702 .296224-2.938025$

H $7.3161872 .508866-0.585233$

H $8.7832762 .547822-2.667894$

H $7.5824541 .653479-3.568666$

H $10.331505-0.507485-1.330011$

H $10.4537811 .333216-1.489468$

H $8.499801-1.668783-2.398003$

H $7.350126-0.766002-3.324504$

H $5.840702-0.479008-1.500933$

H $3.894414 \quad 1.242173-0.044329$

C $1.0450703 .181536 \quad 1.936863$

O $-0.2173762 .965522 \quad 1.275649$

C $-0.7624354 .236602 \quad 0.878896$

C $0.391596 \quad 5.225671 \quad 0.968123$

C 1.1432634 .6888642 .171630

H -1.5126834 .5050341 .632581$

C -1.430697 $4.127268-0.495329$

H 1.0297015 .1892270 .077535

H 0.0483016 .2561701 .098731

C 2.5688445 .2031952 .275780

H $0.594278 \quad 4.9645713 .082050$

C -2.458298 $2.979845-0.599130$

O $-0.415726 \quad 3.890266-1.477748$

H $-1.8933305 .086160-0.756387$

H -1.926208 $2.024348-0.549186$
O $\begin{array}{lll}-3.048029 & 3.026909 & -1.903759\end{array}$

C -3.5713013 .0137380 .463659$

$\begin{array}{llll}C & -4.681176 & 1.966516 & 0.273859\end{array}$

H -3.1363472 .8951601 .458583$

$\begin{array}{llll}\text { O } & -4.187903 & 4.306512 & 0.468149\end{array}$

H $-5.408907 \quad 2.103918 \quad 1.083412$

H $-5.2556512 .210648-0.631038$

H 3.0761564 .7736753 .145306

H $3.1533364 .949027 \quad 1.385145$

H 2.5774316 .2927592 .383401

C 1.0948502 .3584363 .224941

H $-0.8587143 .801970-2.339094$

H -3.465289 $3.897992-2.012816$

H $-4.6483614 .422619-0.379956$

H 0.2035792 .5598303 .831790

H 1.9700412 .6451673 .818930

H $1.8283912 .852324 \quad 1.244747$

C $-6.430694-0.4685350 .617442$

O $-5.334143-0.320188-0.291825$

$\begin{array}{llll}\text { C } & -4.247988 & 0.487897 & 0.190678\end{array}$

C $-3.682232-0.150601 \quad 1.466430$

C $-4.763834-0.3442272 .526076$

C $-5.957044-1.0969431 .934764$

H -6.8883920 .5089010 .810379$

C -7.478421 $-1.357490-0.069089$

H $-3.4791740 .418698-0.584547$

$\begin{array}{llll}\text { O } & -2.605626 & 0.610644 & 1.991902\end{array}$

H $-3.272184-1.1363121 .211127$

H -5.0651900 .6159012 .960557$

O $-4.212026-1.1142973 .596575$

H -5.669021 -2.142697 1.769571

O $-7.021457-1.1060892 .883968$

C $-8.138177-0.665893-1.244392$

H $-6.997855-2.267752-0.447256$

H $-8.272134-1.6282840 .635447$

H -2.3004640 .1660072 .801179$

H $-4.924997-1.2667824 .240443$

H $-7.293759-0.1849713 .036116$

O $-8.966275-1.554410-1.870582$

O $-7.962328 \quad 0.500013-1.569904$

C $-9.668607-1.044167-3.008312$

H $-8.960163-0.722811-3.777942$

H $-10.287223-1.848695-3.414948$

H -10.319786 -0.216436 -2.711492

SCF Energy (B3LYP/6-31G**//MMFF)= -3245.91994686

04_00019

MM̄FF Geometry

C $0.592965-0.8858922 .629197$

C $1.445267-0.3015373 .484768$

C $2.141737 \quad 1.0259593 .311370$

$\begin{array}{lllll}\text { O } & 1.586364 & 1.794013 & 2.223031\end{array}$

C 3.6212600 .7862642 .965958

C $4.521508 \quad 1.945552 \quad 3.339207$

C 4.9273072 .9176982 .499601

C 5.0145501 .9277044 .764111

C 4.5168443 .1568361 .073332

C $5.6014862 .764644 \quad 0.059677$

C $5.4819051 .301008-0.389595$

C $6.6469620 .905867-1.296689$

C $6.487408-0.525987-1.828393$

O $4.245186 \quad 1.175024-1.102636$

O $7.455868-0.693757-2.874487$

C $5.082366-0.670076-2.449887$

C $6.825987-1.568415-0.756430$

O $4.840483-2.018774-2.846275$

C $3.938262-0.150762-1.544539$

C $2.625296-0.091883-2.362059$

O $3.773842-1.047655-0.446219$

C $0.495207 \quad 2.5650792 .463115$

O $-0.156208 \quad 2.628526 \quad 3.491865$

C $0.2447393 .338381 \quad 1.226916$

C -0.8802014 .0539821 .093661$

C -1.243106 $4.869914-0.067205$

C $-0.228875 \quad 5.003898-1.174018$

C $1.4396180 .571571-1.645186$

C $0.2054820 .624352-2.534517$

C $-2.2108011 .241670-2.479508$ 
O $\quad 0.2055790 .247262-3.703816$

C -2.469633 $2.655499-2.892200$

C $-3.5654623 .341429-2.531053$

C $-3.8710974 .735136-3.001557$

C $-4.2003745 .683626-1.870496$

C $-5.4746075 .990278-1.573691$

C $-3.0504586 .344961-1.145327$

C -2.457384 $5.456826-0.085239$

$\mathrm{N}-0.897573 \quad 1.139820-1.868104$

H $0.332411-0.3969761 .695137$

H $1.690434-0.8267304 .406715$

H $2.047672 \quad 1.5954434 .244477$

H 3.7121230 .5710841 .896263

H $4.001272-0.1079733 .478650$

H 5.6120263 .6710972 .888463

H 5.6637812 .7797324 .992170

H 4.1709571 .9617455 .460775

H 5.5879711 .0148614 .956286

H 3.5660692 .6731850 .836472

H 4.3278654 .2340650 .977156

H $5.479627 \quad 3.409117-0.821145$

H 6.5994962 .9605100 .469998

H 5.4645890 .6572290 .495132

H $7.6049751 .016677-0.773451$

H $6.6925701 .592881-2.153528$

H $7.311418-1.569805-3.271997$

H $5.085605-0.080903-3.377253$

H $7.854426-1.429337-0.401856$

H $6.787218-2.584350-1.164732$

H $6.165379-1.5215120 .111821$

H $4.623045-2.527338-2.046497$

H $2.8090840 .472661-3.285940$

H $2.332006-1.106875-2.656208$

H $2.891381-0.918954-0.064899$

H $1.003064 \quad 3.2980270 .453092$

H -1.605664 $4.039071 \quad 1.905830$

H $0.6748105 .501253-0.804786$

H $-0.5864895 .583277-2.027517$

H $0.0509844 .021301-1.568258$

H $1.1779830 .016652-0.738676$

H $1.6943291 .598891-1.362982$

H $-2.9400990 .889615-1.745012$

H -2.262375 $0.590679-3.357474$

H -1.741380 $3.122411-3.551047$

H -4.308113 2.859899-1.898670

H $-4.7232784 .671028-3.691123$

H -3.044643 5.148179-3.593164

H $-5.7174426 .692255-0.782816$

H $-6.3034615 .540939-2.110645$

H -3.398818 $7.258233-0.644048$

H -2.314699 6.697145 -1.873441

H -3.1194025 .3014720 .767279$

H -0.792157 1.466919-0.912311

C $0.366784-3.3174701 .907791$

O $-0.075692-3.0085750 .573050$

C $0.867214-3.567448-0.361631$

C $1.891278-4.3421790 .469490$

C $1.861967-3.5951061 .786812$

H $1.370399-2.723017-0.845223$

C $0.172704-4.440435-1.418076$

H $1.584755-5.3854760 .612825$

H $2.880606-4.3482080 .001149$

C $2.446922-4.3784662 .947799$

H $2.425007-2.6621931 .662668$

C $-0.658226-3.678112-2.465865$

O $-0.612554-5.440863-0.778206$

H $0.969560-4.965564-1.962303$

H -1.064012 -4.408913 -3.177660

O $0.256072-2.863101-3.210481$

C -1.817797-2.785001-1.983115

C $-2.903044-3.521013-1.179966$

H -1.424653 -1.938548 -1.411377

O $-2.423770-2.216539-3.153594$

H -3.186932 -4.432465 - 1.720808

H $-2.488775-3.836147-0.218521$

H $2.395533-3.7984603 .874558$

H $1.910540-5.3197223 .108403$

H $3.498148-4.6189552 .758337$
C $-0.042012-2.2221952 .894542$

H $-0.984695-6.011825-1.471661$

H $-0.259878-2.377561-3.876813$

H $-2.801379-2.944445-3.676394$

H $0.183084-2.5577273 .914208$

H - $1.126888-2.0820592 .847514$

H $-0.160701-4.2377322 .194946$

C -3.466938-1.366382 0.938865

O $-3.891152-1.381792-0.425525$

C $-4.181377-2.679813-0.950702$

C $-5.252683-3.377351-0.098849$

C $-4.882302-3.4027261 .381922$

C $-4.513507-1.9999561 .862559$

H -2.535428 -1.9361141 .015319$

$\begin{array}{llll}\text { C }-3.106083 & 0.075008 & 1.329637\end{array}$

H $-4.627987-2.512513-1.938942$

O $-5.473209-4.710463-0.547293$

H -6.204590 -2.840804-0.205507

H $-4.071399-4.1125711 .582994$

O $-6.007219-3.853267 \quad 2.140238$

H $-5.420530-1.3859001 .906121$

O $-3.989449-2.0777093 .185952$

C $-4.255895 \quad 1.056174 \quad 1.227306$

$\begin{array}{llll}\mathrm{H} & -2.723893 & 0.088528 & 2.356752\end{array}$

H -2.317990 0.4461220 .665990

H $-5.732922-4.664075-1.483323$

H $-6.255706-4.7282361 .795523$

H -4.659965 -2.514962 3.738373

O $-3.967694 \quad 2.1596441 .978956$

$\begin{array}{llll}\text { O } & -5.264099 & 0.896406 & 0.553169\end{array}$

C $-4.967796 \quad 3.1829861 .972549$

H $-4.608875 \quad 4.0130042 .586824$

H $-5.134293 \quad 3.5459010 .953910$

H -5.8986592 .8034662 .404421$

SCF Energy (B3LYP/6-31G**//MMFF) = -3245.92736528

0400020

MM̄FF Geometry

C $0.758294-1.8017100 .735355$

C $-0.092336-1.924154-0.295633$

C $0.187041-1.558956-1.736757$

O $1.568178-1.183932-1.928994$

C $-0.652674-0.323708-2.107652$

C $-0.785988-0.107633-3.600111$

C $-0.033166 \quad 0.736454-4.332410$

C $-1.903006-0.879784-4.252949$

C $1.151578 \quad 1.553205-3.896390$

C $0.839013 \quad 3.051010-3.763852$

C $0.2742143 .418541-2.383556$

C $-0.1116284 .897450-2.323291$

C $-0.5765575 .307481-0.917393$

O $1.296893 \quad 3.144664-1.417988$

O $\begin{array}{lllll} & -0.624655 & 6.741889 & -0.894299\end{array}$

$\begin{array}{llll}\text { C } & 0.481520 & 4.849072 & 0.109835\end{array}$

C $-2.0042514 .824683-0.633625$

$\begin{array}{lllll} & 0 & 0.037155 & 5.098644 & 1.441542\end{array}$

C $0.912518 \quad 3.373334-0.058572$

C 2.1305973 .0698910 .845880

$\begin{array}{llll}\text { O } & -0.171801 & 2.530410 & 0.331375\end{array}$

C $2.488749-2.156559-2.148075$

O $2.295152-3.356582-2.239861$

C $3.803362-1.479503-2.199115$

C $4.925760-2.182269-1.992362$

C $6.282941-1.633348-1.940619$

C $6.447911-0.155306-2.185219$

C 2.8129631 .7272320 .544321

C 3.8738511 .3794201 .575208

C $5.395813-0.4822832 .243575$

$\begin{array}{lllll}\text { O } & 4.221861 & 2.150189 & 2.465732\end{array}$

C $6.740742-0.3526851 .599541$

C $7.610627-1.3696861 .497017$

C $8.979451-1.2422470 .889489$

C $9.280354-2.327708-0.121558$

C $10.043256-3.3842350 .206362$

C $8.768863-2.145610-1.533571$

C $7.303616-2.468407-1.655124$

N $4.387150 \quad 0.103992 \quad 1.379512$

H $1.767520-1.4381820 .571799$ 
H $-1.101150-2.281043-0.094066$ H - $0.062554-2.411337-2.380108$ H $-0.2111430 .564351-1.642805$ H -1.664915 -0.402906 -1.687584 H $-0.2626310 .831307-5.393547$ H $-1.950934-0.712368-5.334114$ H -1.772701-1.954550 -4.092235 H -2.865137 $-0.577693-3.828824$ H $1.5999591 .166184-2.977794$ H $1.920671 \quad 1.429389-4.670051$ H $1.7800233 .597493-3.911096$ H $0.1510393 .369587-4.556424$ H $-0.6016332 .793912-2.179750$ H -0.884198 $5.129909-3.066895$ H $0.7583205 .512330-2.594128$ H -0.8451047 .0147230 .013062$ H $1.3637455 .487048-0.037094$ H $-2.7007705 .244437-1.369436$ H -2.3561885 .1761340 .342573$ H -2.104613 $3.737753-0.664846$ H -0.6266094 .4238731 .663521$ H 2.8866293 .8557650 .719456 H 1.8161363 .0864951 .897443 H 0.1748761 .6794790 .641922 H $3.805360-0.406324-2.355391$ H $4.842789-3.254028-1.816843$ H $\quad 6.121515 \quad 0.103214-3.198615$ H $7.4770030 .194247-2.084440$ H $5.8562030 .424695-1.468254$ H 2.0770420 .9194740 .531670 H $3.296471 \quad 1.762620-0.438737$ H $5.114741-1.5277962 .404978$ H 5.3970740 .0337213 .208918 H 7.0228490 .6280951 .223563 H $7.339289-2.3412391 .904166$ H $9.709646-1.2853221 .708368$ H $9.118444-0.2608500 .418970$ H $10.287153-4.152759-0.519511$ H $10.436508-3.5071241 .210092$ H $9.306761-2.814704-2.218743$ H $9.018862-1.138554-1.877699$ H $7.078113-3.522970-1.488503$ H $4.075685-0.4391480 .579867$ C $0.229707-0.7857942 .948403$ O $-0.914372-0.0675622 .439279$ C $-1.806161 \quad 0.214833 \quad 3.529198$ C $-0.936416 \quad 0.1602384 .775307$ C $-0.034659-1.0132774 .438987$ H -2.530363 -0.6072203 .569617$ C -2.5499961 .5315453 .291216$ H $-0.340610 \quad 1.0724104 .895273$ H -1.520235 0.0065945 .687539 C $1.229220-1.0735195 .279428$ H $-0.600734-1.9434394 .583348$ C $-3.2476801 .635326 \quad 1.918877$ O -1.6270082 .6241133 .381569$ H -3.279260 1.6886534 .094288 H -2.5053891 .7996691 .131385$ O -4.0763672 .8038161 .934800$ C -4.1119710 .4236331 .533649$ $\begin{array}{llll}\text { C }-4.859446 & 0.634577 & 0.202916\end{array}$ H -3.471665 -0.459975 1.447660 O $-5.059990 \quad 0.1571792 .566131$ H -5.6468411 .3862160 .335499$ H $-4.145507 \quad 1.007226-0.542102$ H $1.852986-1.9225494 .982418$ H $1.827060-0.1621655 .171240$ H $0.981122-1.1889256 .339482$ C $0.391334-2.0900692 .162903$ H -0.9136872 .4577262 .741392$ H $-3.506173 \quad 3.5597662 .156992$ H -5.604291 0.9553372 .678514 H $-0.540026-2.6676482 .198731$ H $1.178126-2.7104242 .606386$ H $1.101379-0.1351212 .803146$ C $-4.831672-2.859253-1.109504$ O $-4.434106-1.556527-0.664155$ C $-5.500464-0.668998-0.309475$
C $-6.410421-0.428022-1.522725$

C $-6.924523-1.749385-2.103002$

C $-5.794192-2.766492-2.305485$

H $-3.903208-3.327076-1.462322$

C $-5.374370-3.7094980 .044503$

H $-6.087378-1.1106890 .502870$

O $\quad-5.7105650 .295144-2.533167$

H $-7.2583580 .193387-1.212066$

H -7.416220 -1.547991-3.062505

O $-7.904061-2.283209-1.216826$

H $-6.201461-3.760947-2.521922$

O $\quad-5.021446-2.375624-3.444087$

C $-4.313842-3.9138881 .101924$

H $-6.257915-3.2643300 .510479$

H $-5.676236-4.702378-0.308654$

H $-4.915184-0.213847-2.764405$

H -8.242737 -3.103727 -1.613583

H $-5.615954-2.367404-4.213538$

O $-4.694257-3.3143342 .265948$

O $-3.270009-4.525340 \quad 0.912993$

C $-3.772187-3.4354333 .352894$

H $-3.708925-4.4789553 .674884$

H -2.786555 -3.060758 3.063636

H $-4.144893-2.8312274 .184357$

SCF Energy $\left(B 3 L Y P / 6-31 G^{* *} / / M M F F\right)=-3245.93880719$

0400021

MM̄FF Geometry

C -2.080656 3.856721-0.020712

C -2.8675304 .3028830 .970888$

C -3.6588703 .4605651 .947181$

O -3.4051242 .0486691 .770147$

C -5.1623543 .7118301 .726688$

C -6.0559342 .9602052 .694653$

C -6.7959791 .8782372 .378647$

C -6.1113813 .5297384 .089002$

C -6.8653481 .1645601 .056577$

C $-6.458871-0.3076491 .203044$

C $-6.275701-0.992751-0.159898$

C $-5.951370-2.4760080 .028120$

C $-5.611006-3.172133-1.297520$

O $-5.199476-0.332328-0.834691$

O $-5.001931-4.435580-0.988185$

C $-4.552442-2.334746-2.047088$

C $-6.870040-3.486684-2.113972$

O $-4.317099-2.863941-3.349302$

C $-4.911880-0.838493-2.144984$

C $-3.7685070 .024337-2.736418$

O $-6.041896-0.686110-3.013096$

C -2.3439881 .5165082 .438676$

O $-1.5433912 .112443 \quad 3.141363$

C -2.3268970 .0613762 .167408$

C $-1.340369-0.7024182 .660634$

C -1.215504-2.154940 2.510391

C $-2.261837-2.8657061 .693426$

C $-2.4770680 .070692-1.913582$

C $-1.439249-0.966126-2.306504$

C $0.761859-1.871870-1.561996$

O $-1.516792-1.648461-3.323934$

C $0.557929-3.129090-0.778967$

C $1.397708-3.5348120 .185147$

C $1.257222-4.8280980 .932726$

C $1.349437-4.6520282 .431505$

C $2.490415-4.9194573 .089317$

C $0.094268-4.2613933 .175931$

C $-0.186338-2.7840103 .116237$

N $-0.389116-1.005956-1.398539$

H -1.962602 2.790082 -0.185954

H -2.965021 5.3787921 .106539

H $-3.384913 \quad 3.7592542 .966671$

H -5.4224643 .4685420 .691228$

H -5.3775664 .7847931 .826767$

H $-7.422027 \quad 1.441433 \quad 3.155860$

H -6.8173372 .9916834 .730722$

H -5.1280983 .4759784 .566012$

H -6.4282164 .5773734 .059286$

H -7.8946831 .2206610 .682605$

H $\quad-6.237198 \quad 1.6459310 .304567$ 
H $-5.517503-0.3634201 .763316$ H -7.218539 -0.8396351 .789341$ H $-7.196937-0.870339-0.741459$ H $-6.776999-2.9925080 .533503$ H $-5.087524-2.5743100 .697907$ H $-5.629409-4.946768-0.448826$ H -3.612874 -2.438653 -1.491061 H $-6.619827-4.019607-3.037707$ H -7.447005 -2.596495 -2.372663 H -7.532765 -4.156456 -1.552571 H -4.073106 -3.799239 -3.242338 H $-3.575115-0.264725-3.776511$ H $-4.1390721 .058432-2.788091$ H $-6.0596840 .228321-3.340385$ H -3.139282 -0.3470881 .578572$ H $-0.556730-0.2248453 .247496$ H -2.363759 -2.4033050 .705763$ H $-3.230173-2.8275812 .203227$ H -2.032059 -3.916789 1.507222 H -2.000925 $1.045910-2.079126$ H $-2.689518-0.010256-0.843977$ H $1.637768-1.314822-1.218479$ H $0.897212-2.112165-2.621178$ H -0.294121 -3.750175 -1.044046 H $2.259949-2.9183920 .426259$ H $2.057134-5.4967400 .588251$ H $\quad 0.318011-5.3364350 .681959$ H $2.556550-4.8257684 .168139$ H $3.386664-5.2298922 .562239$ H $0.192814-4.5270224 .237473$ H $-0.742892-4.8697022 .821640$ H $0.528403-2.1843003 .681530$ H $-0.432673-0.431086-0.561948$ C $0.1666234 .709694-0.745478$ O $0.6761243 .413778-1.118214$ C $1.9119893 .586483-1.846996$ C $2.2926685 .050279-1.682550$ C $0.9340785 .714372-1.603067$ H $1.6715403 .367643-2.895023$ C $2.9523332 .592107-1.316194$ H $2.8495535 .206621-0.750078$ H $2.9034155 .421740-2.510970$ C $0.9800897 .118128-1.024787$ H $0.5029165 .754073-2.612525$ C $4.3556172 .785548-1.921343$ O $2.472008 \quad 1.281971-1.629730$ H $2.9851402 .667492-0.223583$ H $4.7336343 .781903-1.666080$ O $4.2495072 .758864-3.350661$ C $5.4070641 .735800-1.507607$ C 5.5960731 .6240640 .014539 H $5.1493470 .762789-1.935151$ O $6.6567932 .106498-2.102312$ H 5.8632742 .6132120 .406174 H $4.648353 \quad 1.338548 \quad 0.479114$ H $-0.0219557 .555391-0.977859$ H $1.3969327 .121374-0.011913$ H $1.6031357 .769527-1.646494$ C -1.345059 $4.762332-0.969332$ H $3.0197320 .640882-1.147021$ H $3.8833431 .893032-3.600858$ H $6.9180692 .967861-1.734148$ H -1.585908 4.440019-1.989878 H -1.703951 $5.792426-0.861911$ H $\quad 0.402025 \quad 4.8518310 .317634$ C $5.357786-1.3965320 .486796$ O $6.455818-0.692543-0.105715$ C $6.697628 \quad 0.6172150 .418111$ C 6.9573070 .5419381 .929213 C $5.825262-0.1746372 .659741$ C $5.543291-1.5251972 .004432$ H $4.420487-0.8696600 .270359$ C $5.301567-2.787623-0.167945$ H $7.6305020 .963074-0.044466$ O 7.1359611 .8520642 .455934 H $7.893082-0.0059952 .100910$ H 4.9174750 .4386562 .700588 O $6.210954-0.4161984 .014653$
H $\quad 6.365858-2.217525 \quad 2.225817$

O $4.356265-2.0762252 .572594$

C $4.700393-2.755643-1.557432$

H $6.311494-3.205345-0.254132$

H $4.682469-3.4634660 .431935$

H $7.425136 \quad 1.762528 \quad 3.379382$

H 6.2771990 .4457504 .458996

H $4.494886-2.1368393 .533355$

O $4.556275-4.039207-2.003850$

O $4.395725-1.746030-2.176182$

C $3.983599-4.175998-3.307999$

H $4.620860-3.691959-4.054095$

H $3.918441-5.242178-3.540595$

H $2.975523-3.751129-3.325979$

SCF Energy (B3LYP/6-31G**//MMFF)= -3245.90829372

04 00022

MM̄FF Geometry

C -3.938725 -2.945167 1.892139

C $-2.652208-2.6914811 .606218$

C $-1.627106-2.3536182 .655188$

O $-0.964280-1.1158822 .311050$

C $-0.544867-3.4457622 .717519$

C $0.459656-3.2004243 .827352$

C $1.702809-2.7119123 .647093$

C $-0.009076-3.5741405 .210530$

C $2.360211-2.2851922 .362041$

C $3.519235-3.2263502 .020006$

C $4.268667-2.8262800 .738402$

C $5.339790-3.8792240 .432155$

C $6.223061-3.488332-0.756858$

O $4.870733-1.5436170 .946256$

O $7.353069-4.373594-0.749493$

C $6.740864-2.054452-0.529304$

C $5.503856-3.720886-2.090442$

O $7.485074-1.607190-1.661373$

C $5.626652-1.043379-0.168527$

C 6.2717970 .2992350 .265440

O $4.810660-0.847988-1.320929$

C -1.6075010 .0465822 .595671$

O -2.7680850 .1839942 .945414$

C -0.6146801 .1346582 .459915$

C -1.0103512 .4145212 .513506$

C -0.1341393 .5880242 .460293$

C 1.3507543 .3477812 .520338

C 5.2870041 .4097820 .664484

C $4.8581162 .298491-0.493756$

C $3.4711454 .283582-1.015524$

O $5.1847992 .092165-1.659595$

C $2.9184175 .466370-0.289204$

C $1.6471275 .881865-0.401840$

C $1.1185027 .118848 \quad 0.265867$

C -0.1484666 .8755661 .053226$

C -1.3280117 .3596980 .626961$

C -0.0272086 .1608582 .380798$

C -0.6984034 .8111932 .387733$

N $4.0602083 .353276-0.071351$

H -4.273740 -2.910788 2.925864

H $-2.314923-2.7242150 .573704$

H -2.097711 -2.241901 3.640356

H $-0.034215-3.5191431 .750305$

H -1.009192 -4.427974 2.877850

H $2.334119-2.5893494 .526793$

H $0.770527-3.4331625 .966710$

H $-0.867007-2.9627975 .506312$

H $-0.305852-4.6277845 .238537$

H $1.652879-2.2534961 .529510$

H $2.730085-1.2613372 .493295$

H $4.225842-3.2326442 .861059$

H $3.133264-4.2483141 .911469$

H $3.548964-2.755571-0.085553$

H $4.881377-4.8618170 .262872$

H $5.986348-4.0030731 .312919$

H $7.957629-4.084997-1.454872$

H $7.456227-2.0978640 .303338$

H $5.243995-4.780617-2.201858$

H $6.153959-3.483727-2.939572$

H $4.582204-3.143241-2.188246$ 
H $\quad 6.849411-1.357023-2.353407$ H $6.895751 \quad 0.098917 \quad 1.147062$ H $6.9369030 .670742-0.523046$ H $4.3807870 .017870-1.265222$ H $\quad 0.4250520 .8484982 .344980$ H -2.074320 2.6264882 .611564 H 1.6184662 .8326873 .449504 H 1.9439604 .2632282 .485433 H 1.6749682 .7319921 .675579 H $4.3961540 .997076 \quad 1.149339$ H 5.7877402 .0630911 .390704 H $2.7050133 .746423-1.582777$ H $4.2466614 .603344-1.719803$ H 3.6101206 .0318340 .330840 H $0.9626725 .334977-1.046285$ H $0.9363457 .863875-0.519714$ H 1.8696367 .5658790 .929378 H -2.2357387 .2246831 .205963$ H -1.412878 $7.901610-0.309177$ H $-0.504378 \quad 6.775222 \quad 3.156689$ H 1.0215046 .1062172 .686046 H $-1.787470 \quad 4.8713142 .355547$ H 3.7404783 .3665920 .892621 C $-6.055682-2.2146480 .756599$ O $-5.466857-0.991174 \quad 0.270155$ C $-6.177959-0.569469-0.904959$ C -7.544329-1.227757 -0.798628 C $-7.161623-2.584531-0.237740$ H $-5.646840-0.994339-1.765278$ C $-6.1796290 .957788-1.021525$ H -8.197300 -0.699931 -0.094181 H -8.056183 -1.289648 -1.763404 C $-8.322979-3.3354810 .390259$ H $-6.746818-3.194191-1.052095$ C $-4.7930981 .614205-0.857575$ O $-7.0334391 .516006-0.015101$ H -6.622021 $1.248465-1.981334$ H $-4.498967 \quad 1.6059440 .199641$ O $-4.9105392 .992691-1.229110$ C $-3.6718440 .974614-1.693364$ C $-2.337151 \quad 1.730598-1.561265$ H -3.533355 -0.060778 -1.367698 O $-4.0489290 .933058-3.068701$ $\mathrm{H}-2.3835622 .674061-2.118270$ H -2.175002 $1.961500-0.501584$ H $-7.990488-4.2961780 .796041$ H -8.776359 -2.7631701 .206574$ H $-9.101024-3.533012-0.354345$ C $-4.973658-3.2923570 .860162$ H $-6.705123 \quad 1.2136440 .849264$ H $-5.6122563 .381712-0.679200$ H -4.228724 $1.846032-3.351723$ H -4.490862 $-3.428858-0.114369$ H $-5.425251-4.2522661 .135579$ H $-6.475810-1.9927231 .745693$ C $0.003936-1.170816-1.556971$ O $-1.034240-0.242128-1.227370$ C $-1.1509960 .899292-2.085033$ C $\quad 0.1576201 .706351-2.094307$ C $1.3688570 .823347-2.409446$ C $1.366957-0.464614-1.577114$ H $\quad 0.009145-1.889883-0.727465$ C $-0.300835-1.942449-2.844885$ H -1.379756 $0.564183-3.102408$ O $0.3572332 .340162-0.833274$ H $\quad 0.0751202 .504248-2.841587$ H $2.2848331 .389158-2.204888$ O $1.3579630 .511113-3.798443$ H $2.130184-1.162574-1.938604$ O $1.692508-0.140307-0.222746$ C $-1.559763-2.764320-2.700181$ H $-0.411323-1.286958-3.713266$ H $\quad 0.513370-2.636195-3.085464$ H $0.3891541 .643894-0.155796$ H $2.147471-0.025332-3.984619$ H $2.5584160 .299722-0.222705$ O $-2.556272-2.224350-3.458341$ O $-1.648097-3.759516-1.992932$
C $-3.805895-2.919172-3.424608$

H $-3.688596-3.926837-3.834105$

H $-4.190176-2.960223-2.402534$

H $-4.518478-2.367873-4.043785$

SCF Energy (B3LYP/6-31G**//MMFF) $=-3245.93481008$

0400023

MM̄FF Geometry

C - 0.0454183 .2498582 .085356

C 0.5635222 .1148741 .711787

C 0.8578980 .9857032 .665078

$\begin{array}{llll}\text { O } & 2.276503 & 0.720128 & 2.577583\end{array}$

C $0.094893-0.2782682 .233144$

C $0.049501-1.3505223 .301893$

C $0.819935-2.4560583 .317578$

C $-0.986586-1.1376324 .375463$

C $1.919117-2.8350772 .364029$

C $1.563494-4.0501881 .495238$

C $0.527095-3.7379640 .404723$

C $0.184571-4.995689-0.396916$

C $-0.766451-4.687857-1.564726$

O $1.093061-2.752922-0.464589$

O $-0.779802-5.827260-2.437791$

C $-0.198560-3.503338-2.375925$

C $-2.209010-4.490837-1.084514$

O $-1.120999-3.084102-3.379615$

C $0.213154-2.297361-1.500657$

C $0.988682-1.262695-2.352096$

O $-0.961201-1.688732-0.965396$

C 2.9660030 .5282153 .729790

O 2.5241430 .6025254 .868508

C 4.3885000 .2036073 .470639

C $4.890396-0.0708102 .255880$

C $6.281716-0.4270071 .958287$

C $7.267755-0.4013333 .097636$

C $1.550770-0.089194-1.540004$

C $2.5184630 .753483-2.345418$

C $4.7884580 .736829-3.342298$

O $2.2201141 .874774-2.749645$

C $6.0881760 .070605-3.029693$

C $7.1036570 .681194-2.400939$

C $8.4099580 .012551-2.087415$

C $8.668498-0.094739-0.601145$

C 9.5712660 .6964260 .002229

C $7.953508-1.1850530 .166038$

C $6.612030-0.7501410 .689620$

$\begin{array}{llll}\mathrm{N} & 3.741227 & 0.131835 & -2.541797\end{array}$

H $-0.3149393 .362836 \quad 3.134203$

H $\quad 0.854117 \quad 1.9687890 .676998$

H $0.577292 \quad 1.280328 \quad 3.683189$

H $0.542318-0.6790291 .318913$

H -0.940576 -0.024808 1.967654

H $0.671998-3.170128 \quad 4.127041$

H -1.009542 -1.957587 5.100991

H $-0.784686-0.2152314 .928353$

H -1.983595 -1.064895 3.930294

H $2.230756-1.9957861 .736164$

H $2.798583-3.0898872 .969287$

H $2.490249-4.3920641 .016040$

H $1.205314-4.8704612 .129484$

H $-0.374552-3.3327300 .877118$

H $-0.243568-5.7679160 .254230$

H $1.107180-5.428586-0.808514$

H $-1.065733-6.598934-1.919226$

H $\quad 0.693071-3.866486-2.906149$

H $-2.577480-5.399328-0.593183$

H $-2.886788-4.309690-1.926025$

H $-2.311956-3.668692-0.373984$

H - $-1.345016-3.866531-3.911946$

H $1.818130-1.773150-2.857150$

H $0.338118-0.854778-3.135957$

H $-0.739411-0.798399-0.652892$

H 5.0074880 .1714494 .361900

H $4.225568-0.0521691 .393950$

H 7.2700620 .5812953 .583076

H $8.298057-0.5886652 .788393$

H $7.012834-1.1623063 .843328$

H $0.7404810 .565805-1.205162$ 
H $2.077204-0.435846-0.643512$

H $4.8121101 .814564-3.147236$

H $4.532150 \quad 0.591727-4.396839$

H $6.197815-0.966259-3.338802$

H $6.995787 \quad 1.719751-2.096574$

H $9.2056550 .592727-2.572396$

H 8.464108-0.987765 -2.535191

H 9.7869910 .6019111 .061153

H $10.1118531 .459334-0.547993$

H $8.595955-1.5642810 .968129$

H $7.803423-2.054137-0.487500$

H $5.827027-0.736637-0.066337$

H $3.836586-0.846112-2.282589$

C $-0.2674084 .275944-0.299391$

O $-1.1157493 .225169-0.806908$

C $-1.8312793 .710385-1.957632$

C $-1.1225114 .991243-2.380376$

C $-0.6856985 .548604-1.042417$

H -2.842534 $3.958333-1.612560$

C -1.910790 $2.642161-3.054153$

H $-0.2454944 .787452-3.005472$

H -1.781300 $5.666913-2.934218$

C $0.4145156 .590956-1.148740$

H $-1.5588456 .001057-0.553149$

C $-2.441817 \quad 1.274497-2.587714$

O $-0.5953702 .428950-3.581126$

H -2.513902 $3.023565-3.886942$

H $-1.7074080 .809053-1.923096$

O $-2.5121650 .422166-3.738898$

C $-3.8108731 .298992-1.883391$

C $-4.350305-0.120582-1.622248$

H -3.712559 $1.834223-0.935031$

O $-4.7520232 .024384-2.672840$

H $-4.544965-0.608008-2.584857$

H -3.574925 -0.702473 -1.114409

H $\quad 0.7020306 .958050-0.158645$

H $1.3095186 .181784-1.629377$

H $0.0765667 .447425-1.741241$

C -0.4436874 .4136481 .217656$

H $-0.6636831 .745290-4.269384$

H $-3.123858 \quad 0.829421-4.375257$

H $-4.9034281 .526103-3.493557$

H -1.504056 4.6059571 .425362

H 0.1141995 .2895111 .569704

H $0.7665024 .006845-0.548442$

C $-4.686798-0.157251 \quad 1.416691$

$\begin{array}{llll}\text { O } & -5.516842 & 0.519268 & 0.463809\end{array}$

C $-5.657793-0.137860-0.800343$

C $-6.254065-1.537730-0.590299$

C $-5.430790-2.3585410 .399760$

C $-5.207110-1.5749901 .691999$

H -3.655591 -0.1985981 .045379$

C -4.7054250 .6734562 .709968$

H $-6.3963620 .443037-1.366842$

O $-6.384048-2.232223-1.826303$

H -7.269949 -1.427832 -0.190063

H -4.475038 -2.660524 -0.039622

O $-6.119631-3.5741090 .700601$

H $-6.147452-1.5267682 .255859$

O $-4.260728-2.2705332 .501441$

C -4.0053362 .0057022 .556484$

H -5.7460150 .8737492 .993631$

H $-4.206742 \quad 0.1397543 .525751$

H -5.504853 -2.552069 -2.090665

H -6.985792 -3.343785 1.077800

H $-4.599829-3.1720512 .635726$

O -4.3683742 .8175943 .593302$

O -3.2243492 .2919291 .660446$

C -3.789626 4.1262263 .590522

H -3.9475854 .6189752 .626178$

H -4.2818884 .7165494 .367879$

H -2.7232634 .0602563 .822518$

SCF Energy (B3LYP/6-31G**//MMFF) $=-3245.90592410$

0400024

MM̄FF Geometry

C $0.114378-2.8079332 .122567$

C $0.890750-1.9761102 .833724$
C $0.505915-0.6479613 .441616$

O $-0.896385-0.3799623 .221817$

C 1.3423260 .4676172 .788723

C 1.3920691 .7537603 .588598

C 0.7481622 .8862443 .246146

C 2.2907581 .7298894 .797826

C -0.1931353 .0741052 .090866$

C 0.5327193 .6180480 .854757

C $-0.3719883 .798096-0.375406$

C $-1.3789944 .936550-0.199147$

C $-2.3171055 .059740-1.409135$

O $-1.0494292 .562583-0.615752$

O $-3.4054645 .919661-1.038281$

C $-2.9199943 .672137-1.708754$

C $-1.6278945 .724294-2.606400$

O $-3.6976723 .703597-2.903978$

C $-1.8670332 .542070-1.792196$

C $-2.5821731 .171246-1.840319$

O $-1.0874762 .712235-2.976234$

C -1.5950530 .2106354 .224339$

O

C $-3.0143150 .431148 \quad 3.860329$

C -3.5662450 .0764152 .689301$

C -4.9712720 .2640672 .308354$

C $-5.8841810 .920652 \quad 3.311921$

C $-1.633313-0.031894-1.796729$

C -2.394692 -1.319195 -1.561452

C $-4.020991-2.864415-2.638290$

O $-2.354017-1.903709-0.481455$

C $-5.392050-2.371073-2.298548$

C $-6.058804-2.719731-1.187917$

C -7.426979 -2.203156 -0.847698

C $-7.453675-1.4248520 .449024$

C $-8.139028-1.8752261 .513442$

C $-6.753976-0.0833470 .487354$

C $-5.379721-0.171371 \quad 1.097432$

$\mathrm{N}-3.122150-1.726617-2.667612$

H $-0.925870-2.5607011 .933565$

H $1.922655-2.2719163 .014611$

H $0.715702-0.7249834 .515305$

H $\quad 0.9681490 .656520 \quad 1.775176$

H 2.3788750 .1318672 .657864

H 0.8880493 .7708623 .865489

H 2.3493832 .7062375 .290765

H 1.9249011 .0108575 .536729

H 3.3084481 .4460014 .510559

H -0.7070542 .1363691 .856077$

H -0.9762643 .7698192 .411848$

H 1.0279074 .5682061 .091056

H 1.3254282 .9070150 .585449

H $0.2844084 .011334-1.227712$

H -0.862976 $5.886487-0.012724$

H $-2.0009524 .754523 \quad 0.686720$

H $-3.0321886 .777486-0.771923$

H -3.616954 $3.435175-0.892459$

H - $-1.286036 \quad 6.731649-2.340128$

H -2.325836 $5.855874-3.440608$

H $-0.7607465 .167142-2.966793$

H $-4.3517574 .416562-2.804964$

H $-3.2634411 .094548-0.983162$

H $-3.1949811 .103705-2.747564$

H $-0.6542501 .871766-3.192183$

H -3.5993240 .9103254 .639506$

H $-2.942714-0.4020561 .936524$

H -6.9225390 .9893972 .981930$

H $\quad-5.5498491 .9422933 .523609$

H -5.8981070 .3525294 .248959$

H -1.075164 -0.124985 -2.733720

H $-0.9005500 .069134-0.988526$

H $-3.655549-3.603221-1.917427$

H $-4.019899-3.316428-3.634652$

H -5.855561 -1.685370 -3.004282

H $-5.600421-3.410004-0.483598$

H $-8.104553-3.064957-0.793668$

H -7.820309 -1.565086 -1.649224

H $-8.185091-1.3060702 .435910$

H -8.666788 -2.8227571 .487692$

H $\quad-7.3870820 .6479080 .999792$ 
H $-6.6555540 .313121-0.531504$

H $-4.644571-0.6413720 .445838$

H $-3.132553-1.139450-3.496453$

C $0.410128-4.2120810 .047617$

O $1.130728-3.166332-0.631589$

C $1.724078-3.705730-1.828863$

C $1.174181-5.120902-1.981421$

C $0.940722-5.520012-0.538809$

H $2.802882-3.763406-1.641921$

C $1.453445-2.789091-3.028477$

H $0.228354-5.136402-2.534942$

H $1.874195-5.781473-2.502323$

C $-0.002906-6.699541-0.380600$

H $1.909416-5.770114-0.085660$

C $1.849031-1.316347-2.806627$

O $0.048768-2.818600-3.312968$

H $1.954652-3.192035-3.916463$

H $1.209082-0.879031-2.032739$

O $1.551854-0.595273-4.009519$

C $3.321944-1.085566-2.426423$

C $3.6539820 .412599-2.297793$

H $3.524657-1.580259-1.474470$

O $4.175667-1.687542-3.396828$

H $3.5058650 .899904-3.269053$

H $2.9439260 .866829-1.597910$

H $-0.142504-6.9495850 .675668$

H -0.987702 -6.485848 -0.809523

H $0.399161-7.583895-0.885858$

C $0.620180-4.1090661 .559678$

H $-0.106699-2.231448-4.072594$

H $2.059566-0.999757-4.733120$

H $4.083568-1.190630-4.227234$

H $1.687741-4.2229421 .785938$

H $0.092828-4.9268582 .063774$

H -0.651424 -4.081953 -0.198270

C 4.7780040 .4981360 .545642

O $5.4357440 .002003-0.626232$

C $5.1058940 .680195-1.842027$

C $5.4557802 .170465-1.721877$

C $4.7869362 .807099-0.505706$

C 5.0820281 .9888930 .750454

H 3.6961020 .3442820 .454281

C $5.290010-0.3233001 .740985$

H $5.7617120 .258011-2.614005$

O $5.0769062 .854925-2.910890$

H $6.5444122 .272349-1.622465$

H $3.7069272 .924901-0.652602$

O $5.3124824 .121347-0.307079$

H 6.1347482 .1225451 .031278

O 4.2885472 .4850291 .826425

C $4.786802-1.7515211 .732645$

H $6.385887-0.3581261 .716620$

H 4.9628470 .1251762 .685186

H $5.4236943 .761034-2.853501$

H $5.0230734 .669036-1.056120$

H 4.4804193 .4341421 .916564

O $5.424674-2.4501362 .718737$

O $3.935100-2.1990540 .978279$

C $5.046670-3.8250802 .841118$

H $5.217459-4.354098 \quad 1.898534$

H $5.668819-4.2808793 .615886$

H $3.998062-3.9014053 .142769$

SCF Energy (B3LYP/6-31G**//MMFF) $=-3245.91292326$

04 00025

MM̄FF Geometry

C $\quad 1.3874471 .8706932 .311596$

C 0.5256680 .9907742 .846814

C $0.790330-0.4709363 .125542$

O $2.078374-0.8450752 .590390$

C $-0.265028-1.3661472 .452923$

C -0.479771 -2.665222 3.204083

C $0.105671-3.8412992 .906125$

C -1.465475 -2.580924 4.342083

C $1.117833-4.129374 \quad 1.832602$

C $0.573895-5.0700120 .748088$

C $-0.470134-4.400592-0.157082$

C $-1.027648-5.393385-1.177114$
C $-2.008686-4.716601-2.146993$

O $0.175756-3.323108-0.841321$

O $-2.234138-5.614927-3.244258$

C $-1.347739-3.448268-2.728464$

C $-3.375879-4.475311-1.497820$

O $-2.276878-2.698772-3.508485$

C $-0.696599-2.536307-1.661075$

C $0.160558-1.449453-2.354359$

O $-1.719581-1.910473-0.887107$

C $3.023526-1.3640983 .410955$

O $2.919022-1.5830494 .608936$

C $4.281456-1.6135112 .666576$

C $4.347152-1.5913281 .326569$

C $5.550085-1.8028530 .531810$

C $5.330414-2.702142-0.654295$

C $1.037158-0.628691-1.396484$

C $1.9821540 .297548-2.144255$

C $3.9484461 .784912-1.764165$

O $1.9139980 .490610-3.355269$

C $5.1925820 .992503-2.023047$

C $6.3868351 .291068-1.488398$

C $7.651108 \quad 0.533893-1.785403$

C $8.3854090 .098747-0.535793$

C $9.3948850 .832678-0.037693$

C $8.006941-1.2282970 .084354$

C $6.698796-1.1618240 .824336$

N $2.9017310 .896111-1.293680$

H 2.3827341 .5505192 .016739

H -0.4505661 .3534493 .163463$

H $\quad 0.768817-0.596198 \quad 4.214853$

H $0.022450-1.5708671 .416356$

H -1.233621 -0.8523192 .392662$

H $-0.150068-4.7074343 .515744$

H -1.594370 -3.540149 4.854447

H -1.130493 -1.852207 5.086857

H -2.447672 -2.271062 3.969901

H $1.508335-3.2164941 .376125$

H $1.977024-4.6135962 .313628$

H $1.425093-5.3926050 .134443$

H $\quad 0.148840-5.968503 \quad 1.212256$

H -1.280151 -4.0049790 .465400$

H -1.508228 -6.241563 -0.673857

H $-0.200887-5.820245-1.761972$

H -2.585587 -6.446706 -2.882900

H $-0.561634-3.781977-3.420502$

H $-4.091423-4.076253-2.224753$

H -3.330369 -3.795863 -0.644667

H $-3.805255-5.420913-1.145677$

H -2.649581 -3.302161 -4.173894

H $\quad 0.827849-1.934307-3.079247$

H $-0.486672-0.764408-2.916191$

H -1.382936 -1.075532 -0.526477

H $5.155919-1.8260563 .273934$

H $3.451303-1.4227100 .736299$

H $\quad 6.201636-2.785526-1.306849$

H $4.507336-2.329039-1.274948$

H $5.074802-3.713590-0.319341$

H $0.418519-0.013825-0.734479$

H $1.643252-1.298498-0.778295$

H $4.0995442 .548455-0.995252$

H $3.6242162 .275996-2.687128$

H $5.1120800 .150582-2.706833$

H $6.4710482 .152012-0.828859$

H $8.297597 \quad 1.190057-2.383169$

H $7.455295-0.341460-2.417103$

H 9.9460610 .5146630 .841111

H $9.6891681 .771422-0.495276$

H $8.772875-1.5411740 .806501$

H $8.020392-2.000206-0.690203$

H $6.718730-0.5038911 .693895$

H $2.9051860 .642545-0.310353$

C 1.3284193 .8289740 .720932

O $0.3507623 .261511-0.175481$

C $-0.2567004 .326068-0.928851$

C $0.7042825 .501310-0.824503$

C 1.1888635 .3498520 .602259

H -1.187255 $4.585417-0.410508$

C $-0.5810293 .886090-2.359596$ 
H $1.5457145 .403477-1.520102$ H $0.2134686 .460586-1.013518$ C 2.4720726 .1097420 .892454 H 0.4018425 .7095821 .279101 C -1.387393 $2.578952-2.473941$ O $0.6375193 .696513-3.090237$ H -1.111707 $4.696085-2.873563$ H $-0.7386251 .720513-2.269333$ O $-1.8076602 .444119-3.837381$ C -2.625935 2.491512 -1.567148 C $-3.473957 \quad 1.238499-1.855307$ H $-2.2986002 .481224-0.522584$ O $-3.4425893 .649090-1.738663$ H $-4.0281741 .372165-2.792295$ H -2.799794 $0.382567-1.973132$ H 2.7898445 .9573481 .928662 H 3.2879235 .7836970 .238417 H 2.3262487 .1838190 .737860 C $1.084076 \quad 3.333845 \quad 2.147997$ H $1.1648843 .032907-2.613508$ H -1.007626 2.468563 -4.390096 H -3.688014 $3.699054-2.678565$ H 0.0458623 .5348622 .436335 H $1.729103 \quad 3.8847252 .842666$ H 2.3129873 .5042780 .364765 C $-4.457554 \quad 0.3053831 .618890$ $\begin{array}{lllll}\text { O } & -3.707265 & 0.583244 & 0.431252\end{array}$ C $-4.4757170 .943267-0.722340$ C $-5.449913-0.185368-1.095006$ C $-6.315735-0.5984670 .099194$ C $-5.479662-0.8139281 .367392$ H $-3.716214-0.0711682 .335375$ C -5.0765951 .5759712 .211557$ H $-5.0408391 .857292-0.510493$ O $-4.716875-1.314470-1.561736$ H $-6.0845970 .158313-1.920857$ H -6.849941 -1.523673 -0.148975 O -7.2922940 .4140420 .322969$ H -6.128357 -0.9165502 .245069$ O $-4.748018-2.0368861 .242150$ C -4.0037512 .5729362 .583699$ H $-5.7793662 .056677 \quad 1.525425$ H -5.6402571 .3478343 .123519$ H -5.360522 $-1.989031-1.837161$ H -7.8488370 .1258901 .066468$ H $-5.392870-2.753817 \quad 1.117477$ $\begin{array}{llll}\text { O } & -4.110979 & 3.684418 & 1.800891\end{array}$ O -3.1671492 .3826803 .456949$ C -3.145784 4.7101592 .049339 H -2.134742 4.3221551 .902853 $\mathrm{H}-3.3167685 .519091 \quad 1.334161$ H -3.265766 5.1040193 .062905 SCF Energy (B3LYP/6-31G**//MMFF)= -3245.90384091

\section{4_00026}

\section{MMFF Geometry}

C 1.4160742 .2245192 .174708

C 0.7229361 .2633632 .806679

C $1.136670-0.1855862 .946129$

O $2.338341-0.4008792 .172482$

C $0.038828-1.1265322 .408851$

C $-0.166182-2.3586893 .266871$

C $0.323366-3.5849282 .997232$

C -1.034952 -2.143738 4.480336

C $1.223831-3.9997871 .867069$

C $0.553609-4.9741950 .888208$

C $-0.531263-4.3166190 .023103$

C $-1.146644-5.329160-0.944235$

C $-2.171103-4.670449-1.883071$

O $0.083691-3.261489-0.720635$

O $-2.446847-5.591197-2.949975$

C $-1.537198-3.413888-2.520929$

C $-3.504973-4.422493-1.171932$

O $-2.498961-2.684914-3.281154$

C $-0.825929-2.485673-1.508331$

C $-0.002568-1.412974-2.261788$

O $-1.808761-1.841822-0.697091$

C $3.299217-1.2308822 .645596$
O $3.279864-1.8889703 .671276$

C $4.398571-1.1950391 .658668$

C $5.349209-2.1383001 .660460$

C $6.444032-2.1703580 .697817$

C $6.630119-3.5071290 .035755$

C $0.922328-0.586537-1.356621$

C $1.8100800 .357770-2.146108$

C $3.7744311 .865206-1.837914$

$\begin{array}{llll}\text { O } & 1.681528 & 0.554086 & -3.351173\end{array}$

C $4.9951121 .080608-2.211472$

C $6.2349051 .399169-1.807824$

C $7.470800 \quad 0.671220-2.258896$

C $8.4401260 .386832-1.132933$

C $9.3892461 .279087-0.800976$

C $8.378125-0.967230-0.458944$

C $7.221250-1.0871650 .495147$

N $2.7591420 .962194-1.331862$

H 2.3727741 .9912421 .715352

H -0.2153361 .5313753 .286980$

H $1.338275-0.3621674 .009992$

H $0.268277-1.4200211 .380979$

H $-0.928679-0.6092292 .349943$

H $0.078697-4.3937993 .685050$

H $-1.163105-3.0581675 .069098$

H $-0.595058-1.3877635 .138305$

H $-2.031003-1.8039254 .177954$

H $1.626874-3.1413221 .323705$

H $2.092657-4.5021512 .311475$

H $1.340462-5.3698320 .232678$

H $\quad 0.131165-5.825491 \quad 1.436154$

H $-1.302784-3.9000430 .680201$

H $-1.605503-6.161962-0.397154$

H $-0.352567-5.775815-1.559201$

H $-2.777186-6.416883-2.555939$

H $-0.791110-3.770398-3.244389$

H $-3.922238-5.365117-0.797926$

H -4.253647 -4.019791-1.862901

H -3.411671 -3.744516 -0.322271

H $-3.051492-2.183766-2.657670$

H $0.625984-1.908362-3.013530$

H $-0.676126-0.732791-2.797793$

H $-1.448446-1.005827-0.362729$

H $4.399107-0.3927860 .928178$

H $5.315115-2.9588242 .372756$

H $6.683046-4.3062320 .784280$

H $7.548153-3.572954-0.553019$

H $5.788878-3.718943-0.633087$

H $0.3384620 .018604-0.655399$

H $1.575280-1.251459-0.779968$

H $3.9785082 .600470-1.053267$

H $3.3870732 .387393-2.718744$

H $4.8576130 .237358-2.884449$

H $6.3748882 .268577-1.169198$

H $7.9684341 .297845-3.011348$

H $7.218342-0.263540-2.774548$

H $10.111131 \quad 1.073114-0.017736$

H $9.4610242 .238369-1.303039$

H $9.298279-1.1419810 .113337$

H $8.353600-1.733737-1.240119$

H $7.026542-0.1946161 .090843$

H $2.8091420 .697425-0.352761$

C $0.928334 \quad 4.101663 \quad 0.595011$

$\begin{array}{llllll}\text { O } & -0.139764 & 3.421445 & -0.093541\end{array}$

C $-0.7982324 .357834-0.968799$

C $0.0676615 .612749-0.980093$

C $0.6433795 .592406 \quad 0.421756$

H $-1.7578594 .604836-0.500013$

C -1.033753 $3.730692-2.347679$

H $0.8718045 .547117-1.722185$

H $-0.5133946 .514971-1.194252$

C 1.8633206 .4818930 .587933

H $-0.135507 \quad 5.911194 \quad 1.127584$

C -1.822998 $2.406671-2.308744$

O $0.2432443 .464981-2.940631$

H $-1.5357084 .456270-2.998916$

H -1.220888 $1.623350-1.834774$

O $-2.051598 \quad 1.984770-3.658272$

C $-3.1849822 .498153-1.596274$ 
C $-3.9823261 .184900-1.682956$ H -3.021893 2.748646-0.545979 O $-3.9589773 .554130-2.163375$ H $-4.2664691 .001549-2.726218$ H -3.331285 $0.361290-1.375307$ H 2.2491596 .4298261 .610737 H $2.6701076 .187461-0.091676$ H 1.6094257 .5257570 .376016 C $0.971671 \quad 3.655726 \quad 2.057396$ H $0.0937493 .247468-3.876007$ H $-1.196721 \quad 1.703062-4.025429$ H $-4.0758353 .357700-3.108738$ H -0.0152373 .7862492 .515145$ H 1.6810154 .2750402 .617610 H 1.8609893 .8309320 .082698 C $-4.2891490 .499571 \quad 1.272040$ $\begin{array}{lllll}\text { O } & -5.008743 & 1.506984 & 0.551771\end{array}$ C $-5.268058 \quad 1.203185-0.823292$ C $-6.100619-0.082432-0.918310$ C $-5.416010-1.243320-0.201477$ C $-5.031682-0.8439371 .222880$ H -3.2821650 .3944030 .851047$ C -4.1562190 .9753372 .728021$ H -5.892344 2.021015-1.204435 O $-6.329315-0.410579-2.284357$ H $-7.0816970 .092851-0.457716$ H -4.543301 -1.592998 -0.761278 O $-6.315152-2.351285-0.122201$ H $-5.944285-0.7887951 .829841$ O $-4.217578-1.8667931 .796003$ C -3.2539602 .1811172 .873601$ H -5.1459451 .2547703 .109531$ H -3.736066 $0.181993 \quad 3.355697$ H $-6.956977-1.152214-2.309020$ H -6.458804 -2.678022 -1.026191 H -3.390904 -1.911917 1.285397 O -3.3790482 .6744094 .141589$ O -2.5193132 .6235722 .002516$ C -2.581314 $3.823571 \quad 4.443404$ H -2.705389 4.5974113 .679539 H -2.915592 4.2257725 .403393 H - $1.531403 \quad 3.5312664 .531546$ SCF Energy (B3LYP/6-31G*//MMFF) $=-3245.90985133$

04_00027

MM̄FF Geometry

C $0.0350645 .035356-0.107769$

C $-1.097907 \quad 4.320197-0.048831$

C $-1.721421 \quad 3.879921 \quad 1.251263$

O -2.0321192 .4668651 .168069$

C -3.0289894 .6520241 .495955$

C -3.627753 4.3978772 .867197

C -4.7262753 .6546933 .108096$

C $-2.925203 \quad 5.0859014 .011014$

C -5.571385 2.9052572 .117318

C -5.5490201 .3979772 .400965$

C -6.2913110 .6025601 .317558$

C $-6.305067-0.8884821 .657658$

C $-6.921431-1.7342790 .533372$

$\begin{array}{lllll} & \text { O } & -5.609790 & 0.817589 & 0.077895\end{array}$

O $-6.589622-3.1090320 .784200$

C $-6.258901-1.349287-0.807410$

C $-8.452278-1.6511910 .532959$

O $-6.908794-1.996261-1.898268$

C $-6.2096520 .172412-1.053214$

C $-5.3768440 .563043-2.299628$

O $-7.541364 \quad 0.654200-1.272105$

C $-0.9929121 .592132 \quad 1.280579$

$\begin{array}{lllll}\text { O } & 0.189487 & 1.868583 & 1.408441\end{array}$

C $-1.548130 \quad 0.219951 \quad 1.236771$

C $-0.720388-0.835717 \quad 1.273812$

C -1.116683 -2.246409 1.312151

C $-2.588113-2.5617921 .264610$

C $-3.8946550 .175234-2.252762$

C $-3.579950-1.158454-2.908199$

C $-1.707011-2.774201-3.209074$

O $-4.391348-1.789929-3.579107$

C $-1.797783-3.841326-2.164698$
C $-0.742439-4.560602-1.755176$

C $-0.819283-5.665393-0.743775$

C $0.146765-5.4740660 .402862$

C $1.346968-6.0786970 .402063$

C $-0.322811-4.6732621 .594426$

C $-0.154483-3.1874451 .415022$

N $-2.264761-1.543340-2.682570$

H $0.513538 \quad 5.3489880 .817319$

H $-1.585024 \quad 4.005071-0.968296$

H -1.0309124 .0394372 .088879$

H -3.7466224 .4125250 .704746$

H -2.846953 5.7317111 .403750

H -5.0681243 .5672254 .138977$

H -3.4426824 .9405794 .965247$

H $-1.907894 \quad 4.7007734 .128540$

H -2.871221 6.1643593 .829848

H $-6.601602 \quad 3.2739542 .192720$

H -5.2539753 .0933451 .089641$

H $-4.505678 \quad 1.0616482 .447530$

H $-5.999912 \quad 1.200717 \quad 3.381594$

H -7.3144230 .9890251 .240229$

H $-6.829583-1.0661282 .604734$

H $-5.274994-1.2311591 .820351$

H $-6.935848-3.3456601 .661856$

H $-5.233957-1.738031-0.777423$

H -8.855621 -2.0169111 .485080$

H $-8.883300-2.295816-0.241004$

H $-8.826869-0.6360900 .386338$

H -6.898382 - $2.951611-1.716732$

H $-5.8637660 .194329-3.210510$

H $-5.4094941 .659436-2.378343$

H $-7.4904401 .498685-1.749666$

H -2.626210 $0.115574 \quad 1.204587$

H $0.353157-0.6535501 .310219$

H -2.802277 $-3.627807 \quad 1.161436$

H $-3.060267-2.0770700 .403587$

H -3.080682 -2.2151092 .179056$

H -3.323671 $0.928486-2.810915$

H $-3.5178150 .176008-1.225181$

H $-0.673004-2.564291-3.499728$

H $-2.264429-3.080612-4.100009$

H $-2.779579-4.048021-1.745421$

H $0.230444-4.372584-2.200612$

H $-0.596897-6.607127-1.262703$

H $-1.837227-5.781121-0.351594$

H $2.031174-5.9776361 .237921$

H $1.674121-6.683215-0.437433$

H $0.260316-4.9551222 .482052$

H $-1.348823-4.957721 \quad 1.846291$

H $0.886297-2.8625691 .435611$

H $-1.680740-0.983650-2.067816$

C $2.1459344 .840840-1.408561$

O $2.0862593 .402340-1.448813$

C $3.1908172 .911811-2.241784$

C $4.0111084 .135356-2.630910$

C $2.9633105 .232211-2.634798$

H $2.7370452 .451760-3.127896$

C $3.9456421 .864108-1.415189$

H $4.7809214 .345566-1.877739$

H $4.5098994 .016052-3.597248$

C $3.5510546 .630876-2.572894$

H $2.3526895 .133578-3.542122$

C $5.2502821 .352178-2.051568$

O $3.068057 \quad 0.751521-1.209442$

H $4.1563382 .282592-0.424384$

H $5.9760412 .168869-2.141908$

O $4.982968 \quad 0.890296-3.381815$

C $5.9043870 .173088-1.301816$

C $6.1559830 .438960 \quad 0.192710$

H $5.288287-0.722355-1.432085$

O $7.156595-0.117368-1.934233$

H 6.8078121 .3151570 .296672

H $\begin{array}{llll}5.210611 & 0.683363 & 0.684708\end{array}$

H $2.7603227 .387480-2.570794$

H $4.1556226 .772811-1.670898$

H $4.1928716 .816800-3.440251$

C $0.7290325 .413289-1.386396$

H $2.2601791 .092461-0.788443$ 
H $4.7776321 .664965-3.931117$ H $7.731404 \quad 0.659537-1.826647$ H $\quad 0.1625685 .041311-2.248789$ H $0.7588126 .506204-1.453868$ H $2.6818285 .114768-0.489364$ C $4.824096-2.0707591 .264964$ O $6.125432-1.9866620 .671841$ C $6.815662-0.7572430 .917692$ C $6.999116-0.5597082 .429138$ C $5.677010-0.6803843 .188642$ C $4.928798-1.9541252 .788782$ H $4.185937-1.2686270 .875542$ C $4.212619-3.4105260 .843253$ H $7.816604-0.8758710 .483975$ O $7.598657 \quad 0.7049652 .688953$ H $7.691469-1.3257762 .801607$ H 5.0513300 .2047943 .026784 O $5.942105-0.7129884 .593273$ H $5.444770-2.8305423 .200770$ O $3.618089-1.9242313 .348931$ C $3.938395-3.430617-0.642112$ H $4.899514-4.2368891 .062457$ H $3.274401-3.6197221 .367038$ H 7.6724960 .7995843 .654019 H $6.495838-1.4909854 .776746$ H $3.715454-1.8139694 .310242$ O $2.880274-2.621655-0.934282$ O $4.599012-4.074879-1.446737$ C $2.573466-2.487519-2.325143$ H $3.376047-1.944223-2.832616$ H $2.423995-3.468386-2.785767$ H $1.648676-1.911637-2.414536$ SCF Energy (B3LYP/6-31G**//MMFF) $=-3245.90525060$

\section{4_00028}

\section{MM̄FF Geometry}

C 2.1553473 .9209460 .999656

C $2.6575044 .564433-0.066209$

C $3.5633493 .967094-1.119193$

O $3.4265832 .527548-1.179860$

C $5.0297684 .314000-0.811933$

C $5.9813603 .939810-1.932532$

C $6.8168192 .882023-1.918372$

C $5.9792414 .877897-3.113232$

C $6.9709791 .839362-0.846737$

C $6.6417680 .442475-1.388477$

C $6.677279-0.627022-0.286565$

C $6.380327-2.008798-0.872319$

C $6.266627-3.0903420 .212406$

O $5.688730-0.2822890 .688542$

O $5.661634-4.249267-0.382434$

C $5.304891-2.597921 \quad 1.315507$

C $7.644011-3.5330200 .719902$

O $5.289946-3.5026262 .416764$

C $5.620423-1.1722281 .810650$

C $4.547116-0.6140922 .776946$

O $6.860753-1.1905662 .528098$

C $2.3379672 .048401-1.846952$

O $1.4572832 .706964-2.378468$

C $2.3935530 .569591-1.820013$

C $1.380625-0.132006-2.350701$

C $1.279525-1.590316-2.442669$

C $2.413824-2.407651-1.886656$

C $3.144296-0.4492312 .183183$

C $2.208715-1.6170442 .444454$

C $-0.088254-2.4140221 .888760$

O $2.482354-2.547243 \quad 3.197187$

C $-0.024909-3.4095780 .774858$

C -1.034338 -3.617013-0.084005

C -1.020794 -4.663103-1.159371

C $-1.284930-4.094694-2.534240$

C $-2.513846-4.135992-3.076574$

C $-0.104066-3.571297-3.318485$

C $0.188906-2.124401-3.031730$

N $1.007463-1.4724891 .762034$

H $2.4424692 .889873 \quad 1.183039$

H $2.3837595 .605313-0.226871$

H $3.2810504 .388282-2.092319$
H $5.328567 \quad 3.836473 \quad 0.127062$

H $5.1287125 .392933-0.629573$

H $7.4703532 .735819-2.778002$

H $6.7272244 .603251-3.864746$

H $5.0028484 .875183-3.606895$

H $6.2025445 .899115-2.787233$

H $8.0085241 .857389-0.492185$

H 6.3440042 .0551480 .021030

H $5.6416820 .466052-1.839165$

H $7.3507190 .178199-2.183219$

H $7.668228-0.6121800 .182364$

H $7.138675-2.286768-1.614942$

H $5.429899-1.971067-1.419831$

H $6.217639-4.527020-1.130541$

H $4.295552-2.6097670 .887249$

H $7.553695-4.3352061 .460632$

H $8.221088-2.7176131 .160741$

H $8.238558-3.950493-0.101803$

H $5.066682-4.3818882 .066325$

H $4.529971-1.2001013 .703678$

H 4.8769210 .3911003 .077942

H $6.892166-0.4149933 .112150$

H $3.2645290 .101666-1.378045$

H $0.5407510 .415466-2.777195$

H $2.623278-2.125901-0.849779$

H $3.318380-2.254419-2.483871$

H $2.211542-3.480837-1.870679$

H 2.6774830 .4213572 .659767

H $3.187499-0.2483861 .108646$

H -1.017972 -1.837052 1.873816

H $-0.019244-2.9320592 .850656$

H $0.876564-4.0123450 .695862$

H -1.944367 -3.030534 0.017629

H $-1.791618-5.404290-0.909778$

H - $0.074395-5.217988-1.167156$

H $-2.702870-3.758766-4.076172$

H -3.359185 -4.541182 -2.530468

H $-0.312252-3.650683-4.394458$

H $\quad 0.760787-4.222482-3.162328$

H $-0.582949-1.447639-3.401503$

H $0.890233-0.6940581 .119410$

C -0.1406103 .7919092 .087479$

O $-0.716714 \quad 3.5770690 .781257$

C - 1.0759992 .1828580 .668100

C -1.2096721 .6934512 .100297$

C -0.0580382 .4212352 .762862$

H $-0.2291191 .692530 \quad 0.171010$

C $-2.3411572 .045414-0.183403$

H -2.166576 2.0108892 .532959

H -1.1343440 .6074132 .189550$

C $-0.1580152 .472275 \quad 4.277307$

H 0.8718901 .9104972 .492328

C $-2.8767210 .604261-0.254658$

O $-2.0288582 .479681-1.511319$

H -3.1137012 .7234550 .195197$

H $-3.162830 \quad 0.258657 \quad 0.744423$

O $-1.814079-0.261946-0.671752$

C $-4.0543470 .400541-1.227160$

C $-5.2658101 .325473-1.007030$

H $\quad-3.7021690 .538319-2.256384$

O $-4.475236-0.963563-1.156660$

H -4.965174 2.351144 -1.248684

H $-6.0306521 .046689-1.740714$

H $0.684573 \quad 3.0256664 .703868$

H -1.0827082 .9604534 .603017$

H -0.1462581 .4611554 .697193$

C 1.1878234 .5496701 .966212

H -1.668828 $3.380968-1.444998$

H -1.528965 $0.031060-1.553922$

H $-4.712197-1.152387-0.232986$

H 1.6640184 .6265512 .950136

H 0.9658025 .5737011 .639417

H -0.8499084 .4296092 .630784$

C -7.511103 -0.4744870 .098908$

$\begin{array}{lllll}\text { O } & -6.341316 & -0.014346 & 0.786918\end{array}$

C -5.8825831 .2875420 .411440$

C -6.9971892 .3183220 .638767$

C $-8.2875901 .928315-0.075528$ 
$\begin{array}{llll}C & -8.682271 & 0.498120 & 0.290338\end{array}$ H $-7.288550-0.587093-0.969053$ C $-7.861245-1.8571480 .663264$ H -5.0813051 .5410511 .114932$ O H $\quad-7.1986312 .3894301 .715888$ H -8.191873 2.055736-1.160182 O -9.3158662 .8257930 .349166$ H $-9.0290880 .469606 \quad 1.331525$ O $-9.7672090 .081630-0.536397$ C $-6.886509-2.9035780 .179459$ H -7.861415 -1.850638 1.760045 H -8.858018 -2.1861470 .347956$ H -7.310286 $4.221426 \quad 0.354027$ H -10.106888 $2.639392-0.184053$ H - $-10.5361350 .632715-0.313581$ O $\quad-5.904813-3.094216 \quad 1.106167$ O $-6.976536-3.466579-0.904091$ C $-4.909872-4.0621090 .757851$ H $-4.143189-4.057411 \quad 1.537120$ H $-5.357949-5.0588720 .709815$ H - $4.441292-3.803996-0.196083$

SCF Energy (B3LYP/6-31G**//MMFF) $=-3245.90937253$

04_00029

MM̄FF Geometry

C $-0.025757-0.7837401 .236846$

C $0.047880-1.6197080 .188971$

C $-0.265147-1.265769-1.247725$

O $-0.464317 \quad 0.160534-1.363650$

C $-1.549277-1.970647-1.709403$

C -1.713407 -1.963907 -3.215471

C $-2.526596-1.134329-3.897727$

C $-0.917642-3.012105-3.950312$

C $-3.380401-0.020234-3.358634$

C $-4.880254-0.330092-3.464787$

C $-5.358824-1.366012-2.435271$

C $-6.829250-1.719808-2.667390$

C $-7.364451-2.668010-1.583275$

O $-5.196695-0.789832-1.135136$

O $-8.793308-2.727088-1.707924$

C -7.023760 -2.086903 -0.194077

C $-6.880064-4.105329-1.801760$

O $-7.371085-3.0035810 .841421$

C $-5.544615-1.657411-0.048710$

C $-5.358341-0.8650601 .267906$

O $-4.719361-2.822859-0.016860$

C $0.2493900 .854054-2.282521$

O $1.030148 \quad 0.395111-3.104420$

C $-0.0006042 .311268-2.168414$

C $-0.8613402 .855631-1.293249$

C -1.115823 4.285917-1.101870

C $-0.2822915 .256791-1.896202$

C $-3.985747-0.192370 \quad 1.412574$

C -3.8992160 .6522072 .673248$

C -2.4290122 .3437643 .771044$

O -4.7612380 .6460123 .547953$

C -2.8928363 .7024053 .349288$

C -2.071785 4.7495383 .179754

C -2.5323216 .1094832 .741509$

C $-1.893695 \quad 6.549791 \quad 1.442139$

C -0.8718607 .4223521 .437555$

C $-2.497812 \quad 6.0541560 .146431$

C $-2.0576144 .660220-0.210003$

N -2.731831 1.4019842 .709506

H $-0.376247 \quad 0.2306291 .066811$

H $0.386523-2.6410040 .342679$

H $0.595201-1.585135-1.847641$

H -2.415566 -1.502513 -1.229740

H - $1.555560-3.015988-1.372526$

H $-2.576444-1.244668-4.980579$

H - $1.100424-2.993089-5.029984$ H $\quad 0.155074-2.859792-3.796773$ H - $1.182625-4.011435-3.589853$ H $-3.1153280 .248992-2.332159$ H $-3.1673050 .871931-3.961243$ H $-5.4235430 .609940-3.301814$ H $-5.118270-0.670465-4.480229$
H $-4.738014-2.264877-2.514760$

H -6.974171 -2.155694 -3.663699

H -7.429352 -0.799213 -2.653895

H -9.145613 -1.827229-1.600668

H -7.654514 -1.199416 -0.045419

H -7.222482 -4.478963 -2.774559

H -7.306117 -4.784334 -1.054774

H $-5.792843-4.202590-1.774598$

H $-8.305637-3.2418710 .716577$

H $-6.119644-0.0751581 .320203$

H -5.518356 -1.528822 2.127034

H -3.897592 -2.6193220.456194

H $0.5882462 .922600-2.844892$

H -1.435721 $2.200169-0.640941$

H $0.7853035 .081952-1.719824$

H $-0.4669126 .301825-1.638324$

H $-0.4845505 .149660-2.967424$

H $-3.191330-0.9437571 .462240$

H -3.7912940 .4595610 .553150$

H -1.348034 2.3077643 .939388

H -2.937905 2.0438714 .692230

H -3.958421 3.8274653 .171072

H -1.0087194 .6287453 .371765$

H -2.286255 6.8185033 .542838

H $-3.624026 \quad 6.1497312 .640565$

H $-0.428546 \quad 7.768940 \quad 0.510117$

H -0.452729 7.8060722 .361728

H $-2.2867406 .755374-0.668481$

H -3.5923626 .0627230 .230400$

H -2.604982 3.8810420 .320440

H -2.1257451 .4060921 .894841$

C $1.160046-2.3308902 .943434$

O $2.385168-2.3063692 .186888$

C $3.470336-2.7359353 .025593$

C $2.829336-3.3162144 .281421$

C $1.582650-2.4627554 .407678$

H $4.029784-1.8325313 .296941$

C $4.386199-3.6982712 .262417$

H $2.552715-4.3687154 .152111$

H $3.488224-3.2473455 .152407$

C $0.517956-3.0669995 .306437$

H $1.872653-1.4812654 .806497$

C $4.846247-3.1867220 .881957$

O $3.678380-4.9263232 .043102$

H $5.250170-3.9503152 .888787$

H $3.996786-3.1897750 .188302$

O $5.773097-4.1462420 .354642$

C $5.495118-1.7890080 .875441$

C $6.068947-1.425759-0.507612$

H $4.746472-1.0453541 .162996$

O $6.533682-1.7327801 .851869$

H $6.875861-2.126677-0.752838$

H $5.282751-1.561233-1.257948$

H $-0.363358-2.4199295 .356608$

H $0.195298-4.0483314 .942950$

H $0.901389-3.1950336 .324086$

C $0.359860-1.0557872 .666995$

H $4.277431-5.5249311 .564613$

H $6.526253-4.2016650 .966581$

H $7.249255-2.3243461 .564140$

H $0.960510-0.1953882 .990317$

H -0.554182 -1.051586 3.272110

H $0.597506-3.2173762 .624785$

C $4.6326671 .179828-1.252488$

O $5.6616841 .006868-0.270443$

C $6.6400890 .008533-0.576263$

C $7.3242870 .342243-1.908284$

C $6.3101390 .505100-3.036442$

C $5.2276091 .504067-2.631777$

H $4.0205760 .271561-1.308585$

C $3.7409982 .341164-0.781997$

H 7.4078740 .0834240 .204098

O $8.270500-0.668005-2.241880$

H $7.8836341 .280252-1.795791$

H $5.880825-0.461260-3.325393$

O $7.0030250 .999375-4.185075$

H $5.6524322 .516439-2.628070$

O $4.1799501 .483750-3.599861$ 
C 2.9044601 .9928070 .429498

H $4.3700633 .198285-0.512165$

H $3.0479902 .637923-1.576163$

H $8.659902-0.429198-3.100535$

H $\quad 6.3751151 .004525-4.927024$

H $4.5474561 .806146-4.439739$

$\begin{array}{lllll}\text { O } & 2.243307 & 3.115962 & 0.839254\end{array}$

O 2.8302300 .8868630 .944663

C 1.4132392 .9713991 .995662

H 0.4792232 .4766661 .718055

H 1.9261552 .4142042 .785563

H 1.1840183 .9718532 .371135

SCF Energy (B3LYP/6-31G**//MMFF) $=-3245.90724338$

$04 \quad 00030$

MM̄FF Geometry

C - $-0.0017563 .238425-2.063125$

C $-0.8838973 .665209-2.980871$

C $-2.2382173 .069926-3.289879$

O $-2.6494942 .108563-2.293279$

C $-2.1799532 .362333-4.656113$

C $-3.5166351 .811096-5.113157$

C $-3.8558360 .506327-5.100316$

C -4.483392 $2.835942-5.648620$

C $-3.037979-0.651201-4.597372$

C $-3.799713-1.437771-3.522595$

C $-2.912556-2.492910-2.844926$

C $-3.729517-3.310371-1.842008$

C $-2.857934-4.286224-1.040102$

O $-1.851748-1.803379-2.175066$

O $-3.654957-4.7505320 .060474$

C -1.651511 -3.515743 -0.460916

C $-2.502488-5.528188-1.865123$

O $-0.748285-4.4105820 .184336$

C $-0.912725-2.652453-1.503436$

C $0.175744-1.745974-0.877335$

O $-0.249824-3.522016-2.430753$

C $-3.2769192 .599064-1.187646$

O $-3.4577553 .773202-0.906053$

C $-3.7302471 .447417-0.376103$

C $-4.354511 \quad 1.6598390 .791912$

C $-4.9003900 .632241 \quad 1.682509$

C $-4.709996-0.808311 \quad 1.287549$

C $-0.324727-0.6506600 .068549$

C $-0.370660-1.0480811 .532986$

C $-0.967467-0.1125003 .765710$

O $\quad 0.041575-2.1233541 .957675$

C $-2.288312-0.6834294 .172319$

C $-3.141329-0.0586644 .998104$

C $-4.440981-0.6514375 .459758$

C -5.6259720 .2450285 .182672$

C -6.1499121 .0178986 .149227$

C $-6.261281 \quad 0.1712273 .813379$

C $-5.553818 \quad 1.0250812 .796240$

N -0.899016 -0.0325352 .319466$

H $-0.2256862 .370035-1.450629$

H -0.617798 4.528143 -3.589344

H $-2.9721083 .884544-3.333120$

H -1.425758 $1.568863-4.623581$

H -1.819939 $3.062758-5.422374$

H $-4.8359800 .228697-5.486362$

H -5.407448 2.381585 -6.021640

H -4.760973 $3.548603-4.866100$

H $-4.0308213 .388015-6.478802$

H $-2.814527-1.314287-5.441588$

H $-2.077326-0.322296-4.195859$

H -4.170804 -0.736790 -2.764462

H -4.674379 -1.921609 -3.975042

H -2.488140 -3.143888 -3.618213

H -4.542487 -3.846295 -2.347688

H -4.220361 -2.628020 - 1.135557

H -3.087578 -5.3085880 .619934$

H -2.050732 -2.8638520 .324639$

H -3.414420 -6.055906 -2.169499

H -1.926713 -6.247766 -1.272829

H - $-1.937747-5.294808-2.770024$

H $-0.227001-4.854606-0.506075$

H $\quad 0.947946-2.360735-0.402747$
H $\quad 0.683497-1.230231-1.705174$

H $0.467691-3.027817-2.860580$

H $-3.5575310 .454278-0.772545$

H $-4.4996472 .687273 \quad 1.123408$

H $-5.284152-1.0324050 .382358$

H $-5.023911-1.5172502 .056527$

H -3.653238 -1.022194 1.095821

H $\quad 0.3733600 .1949150 .007558$

H $-1.307800-0.284677-0.237418$

H -0.8138950 .8991174 .154829$

H $-0.158635-0.7521574 .134022$

H -2.539772 -1.671731 3.794584

H $-2.875850 \quad 0.918228 \quad 5.396240$

H $-4.356656-0.8352276 .538978$

H -4.615702 -1.635910 5.007946

H -7.0198801 .6398065 .965897$

H -5.716552 1.0501967 .143401

H -7.2989430 .5287653 .866139$

H $-6.349343-0.8753853 .508730$

H -5.6372612 .0942002 .996997$

H -1.2626690 .8046611 .873473$

C $1.5036934 .401834-0.415739$

O 1.8132073 .2922700 .456999

C 2.9811393 .6273761 .229252

C 3.1010295 .1420921 .154295

C $2.6656885 .389773-0.274773$

H 3.8395343 .1858090 .710432

C 2.8950013 .0624792 .648861

H 2.4168165 .6394791 .851670

H 4.1166095 .4917571 .362065

C $2.2825846 .833789-0.551035$

H $3.4861295 .103513-0.946975$

C 2.6791191 .5384992 .719168

O 1.7980203 .6888513 .325242

H $3.798748 \quad 3.342788 \quad 3.203711$

H 1.6543541 .3019512 .413612

O $2.768780 \quad 1.148768 \quad 4.095339$

C 3.6583670 .6972191 .879021

C $3.539326-0.8107152 .165959$

H 3.4577560 .8850870 .819075

O 5.0016341 .1110112 .127203

H $3.944393-1.0317683 .160960$

H $2.478714-1.0796732 .159860$

H $1.9574526 .960046-1.588474$

H 1.4662407 .1639240 .100162

H $3.1368747 .497270-0.381367$

C $1.3346693 .896292-1.850292$

H 1.7594373 .3160174 .222715

H 3.6609021 .3700204 .411487

H 5.2114720 .9105963 .054880

H $2.1113013 .157708-2.081914$

H $1.4542444 .728467-2.554054$

H $0.5719634 .848338-0.045280$

C $4.192746-2.191412-1.240992$

O $3.619609-1.497556-0.125967$

C $4.290402-1.6653261 .127233$

C $4.318004-3.1499411 .522572$

C $4.921508-4.0144480 .410795$

C $4.306155-3.698399-0.956801$

H $3.466171-2.058562-2.053288$

C $5.512798-1.553103-1.685929$

H $5.316650-1.2923661 .042507$

O $3.002551-3.6137111 .820621$

H $4.908489-3.2594592 .439506$

H $4.757289-5.073590 \quad 0.643646$

O $6.327738-3.7972330 .343454$

H $4.881237-4.177102-1.758049$

O $2.983868-4.242593-1.007770$

C $5.278133-0.147100-2.186231$

H $6.262091-1.522938-0.889657$

H $5.962232-2.117992-2.511024$

H $2.446237-3.4565971 .038955$

H $6.703557-4.0352621 .208017$

H $3.057128-5.203609-0.878761$

O $5.7159850 .756538-1.264108$

O $4.7490170 .114516-3.258846$

C $5.5357072 .131361-1.616216$

H $4.4713882 .354569-1.731821$ 
H $\quad 5.9373312 .745528-0.805918$

H $6.0815912 .362999-2.535677$

SCF Energy (B3LYP/6-31G**//MMFF) $=-3245.92182171$

04 00031

MM̄FF Geometry

C $0.549760-2.452229-0.204037$

C $1.353940-2.7403160 .830593$

C $1.646240-1.8473292 .014811$

O $1.085246-0.5340621 .793612$

C $3.167964-1.6827902 .175447$

C $3.580689-1.0964823 .509695$

C 3.9932200 .1723923 .696764

C $3.563558-2.0620824 .666706$

C 4.0540871 .2862672 .688751

C 5.4903621 .6445222 .281805

C 6.1198540 .6318081 .311685

C 7.5662041 .0162310 .992621

C $8.1835400 .094476-0.070582$

O $5.3401750 .641783 \quad 0.112141$

O $9.3955800 .707335-0.536290$

C $7.2260710 .012087-1.278414$

C $8.582101-1.2654950 .514770$

O $7.687222-0.943892-2.230587$

C $5.762611-0.294535-0.887174$

C $4.837891-0.115907-2.116083$

O $5.675837-1.643003-0.424108$

C 0.3789930 .0575012 .787371

O $0.092038-0.4293823 .872184$

C $-0.023311 \quad 1.4367252 .417287$

C 0.3446022 .0405721 .275979

C 0.0033423 .4041930 .863301

C $-0.8533374 .225921 \quad 1.788932$

C $3.339071-0.195309-1.789698$

C $2.4744880 .149798-2.990520$

C $0.1379380 .810068-3.567235$

O $2.911290 \quad 0.214998-4.136462$

C $0.1848602 .304637-3.636307$

C $-0.8137493 .108561-3.240863$

C $-0.7324314 .608149-3.279935$

C $-0.9062655 .244707-1.916745$

C $-2.0330705 .908541-1.608257$

C $0.2568105 .204774-0.948157$

C $0.4635843 .853006-0.323474$

N $1.1500720 .360343-2.628314$

H $0.025664-1.501144-0.239314$

H $1.844228-3.7122810 .843637$

H $1.206405-2.3224242 .899393$

H $3.548423-1.0638251 .356739$

H $3.671753-2.6529472 .068326$

H 4.2993140 .4677454 .699801

H $3.922396-1.6054835 .595379$

H $2.547734-2.4247354 .850715$

H $4.207278-2.9218564 .453543$

H 3.4429371 .0780311 .805840

H 3.5978862 .1681853 .156776

H 5.4554302 .6265941 .791991

H 6.1177341 .7506563 .175421

H $6.083957-0.3662301 .761722$

H 8.1782351 .0171541 .903141

H 7.5940512 .0473210 .612727

H $9.9812170 .833586 \quad 0.229896$

H $7.2515960 .988463-1.782458$

H $9.318501-1.1355881 .317011$

H $9.069441-1.892814-0.239611$

H $7.737061-1.8182140 .930550$

H $8.602798-0.707474-2.458133$

H $5.0290120 .867590-2.565805$

H $5.073341-0.872900-2.874762$

H $4.775248-1.973060-0.565973$

H $-0.632891 \quad 1.942812 \quad 3.159017$

H 0.9657331 .4941400 .568714

H -1.799359 $3.715078 \quad 1.993366$

H -1.114116 5.2061571 .383921

H -0.3353624 .3985662 .738677$

H $3.069395-1.206253-1.470162$

H $3.0912980 .500352-0.979968$

H $-0.8283680 .439656-3.210589$
H $\quad 0.337246 \quad 0.383397-4.555132$

H $1.0999972 .748456-4.022127$

H $-1.7341442 .670471-2.863921$

H -1.509868 $4.964153-3.968265$

H $0.2192624 .944830-3.710043$

H $-2.1577496 .398097-0.648268$

H -2.859177 $5.975564-2.308147$

H $0.1479815 .974952-0.175876$

H $1.1749335 .484608-1.481194$

H $1.0844793 .186470-0.922072$

H $0.9046010 .360296-1.642723$

C -1.046382 -4.046739-1.291643

O $-2.081927-3.051512-1.360404$

C $-3.203623-3.594099-2.083202$

C $-2.850941-5.042610-2.411277$

C $-1.335170-4.996360-2.451139$

H -3.275137-3.028037-3.018977

C $-4.486041-3.411404-1.261319$

H $-3.190142-5.734043-1.631624$

H $-3.294871-5.369770-3.356699$

C $-0.683945-6.361632-2.316669$

H -1.023534 -4.537422 -3.398927

C $-4.719519-1.965535-0.771297$

O $-4.422492-4.250550-0.100220$

H -5.348371 -3.763829-1.838347

H $-3.956865-1.716692-0.029682$

O $-5.973847-1.936950-0.079786$

C $-4.734976-0.906872-1.884082$

C $-5.0623260 .525320-1.416964$

H -3.772106 -0.897004 -2.405854

O $-5.726105-1.295699-2.841886$

H $-5.3231941 .105147-2.311411$

H $-5.9736900 .517104-0.809666$

H $\quad 0.406907-6.279746-2.349060$

H $-0.957553-6.845647-1.373211$

H $-0.996629-7.018663-3.134804$

C $0.323872-3.377020-1.368404$

H $-3.642278-3.9816860 .414870$

H $-6.671733-2.122823-0.731362$

H $-5.664963-0.682387-3.593998$

H $0.403727-2.780291-2.285315$

H $1.112707-4.136782-1.409851$

H -1.160908 -4.565989 -0.330525

C $-4.5837890 .899887 \quad 1.616886$

$\begin{array}{lllll}\text { O } & -3.603799 & 0.669182 & 0.597842\end{array}$

C $-3.8987671 .230084-0.683729$

C $-4.0811492 .748700-0.551894$

C $-5.153103 \quad 3.0970370 .473702$

C -4.8605582 .3987451 .798993$

H $\quad-5.5095990 .3749241 .356410$

C -4.0378530 .3072992 .922476$

H -3.003214 $1.071356-1.297794$

O $-4.4082533 .319030-1.814575$

H $-3.1296463 .194462-0.236701$

H -6.1532232 .8511790 .098245$

O $\quad-5.1357194 .5117630 .677953$

H -3.998791 2.8839102 .272118

O -5.9680292 .5863522 .679292$

C $-4.064510-1.2014012 .908515$

H -2.9984420 .6137303 .088600$

H $-4.6083350 .644726 \quad 3.795727$

H -4.568673 $4.267543-1.672620$

H $\quad-5.791278 \quad 4.712477 \quad 1.367954$

H -6.7420702 .1514112 .282501$

O $-5.291545-1.6449723 .305134$

O $-3.112883-1.8982862 .580759$

C $-5.442733-3.0658723 .374427$

H $-4.688300-3.4996404 .037879$

H $-5.373408-3.5006012 .374438$

H $-6.433377-3.2824983 .782819$

SCF Energy (B3LYP/6-31G**//MMFF) $=-3245.92309389$

04 00032

MM̄FF Geometry

C $3.075992-3.765520-1.401591$

C $3.867864-4.262294-0.438165$

C $4.629481-3.4530070 .586201$

O $4.001793-2.1699630 .822084$ 
C $6.071923-3.2199400 .107075$

C $6.962810-2.6028081 .167993$

C $7.350472-1.3120841 .197201$

C $7.455864-3.5587752 .224774$

C $6.971585-0.2111840 .246057$

C 6.2791070 .9399520 .986883

C 5.7981892 .0376310 .026254

C 5.1522473 .1839920 .805008

C $4.5419344 .245313-0.121913$

O $4.8451811 .454405-0.866709$

O 3.6860205 .0809760 .673997

C $3.6456723 .548878-1.170110$

C $5.6160565 .166023-0.713929$

O $3.1986174 .505362-2.128330$

C $4.3316582 .350989-1.862528$

C $3.3802501 .526917-2.764859$

O $5.3836802 .840836-2.703406$

C $2.894718-2.1713521 .618707$

O $2.353715-3.1462612 .117635$

C $2.449202-0.770607 \quad 1.793127$

C $1.340150-0.5187582 .505169$

C 0.7885350 .7998692 .826368

C 1.5117272 .0093792 .298636

C $2.1924030 .871295-2.053165$

C $0.9131581 .689347-2.080964$

C $-1.414277 \quad 1.671025-1.185559$

O $0.7877122 .725438-2.727325$

C -1.4741332 .4865540 .066579$

C -2.361009 2.266624 1.048226

C $-2.475048 \quad 3.1127012 .281574$

C -2.3764222 .3064473 .555088$

C -3.4810651 .8796464 .190440$

C -1.0030412 .0870084 .143880$

C -0.3196330 .8659653 .594017$

N $-0.0869791 .101194-1.317885$

H $2.990588-2.689036-1.516853$

H $3.967722-5.341817-0.345325$

H $4.639630-4.0145291 .529119$

H $6.062049-2.602050-0.797125$

H $6.522345-4.173284-0.202143$

H $8.017381-0.9975321 .999397$

H $8.141615-3.0802892 .932219$

H $6.617987-3.9613902 .802143$

H $7.992793-4.3930451 .761388$

H $7.8843600 .161579-0.233735$

H $6.323618-0.573069-0.555242$

H 5.4206780 .5376811 .539066

H 6.9674581 .3709691 .724612

H $6.6590832 .398897-0.548394$

H 5.8744783 .6424551 .492061

H 4.3525362 .7827121 .440745

H 4.2243885 .4728511 .382901

H $2.7554173 .198185-0.634189$

H 6.1590195 .6841370 .085759

H $5.1672175 .954147-1.328359$

H $6.3525474 .632434-1.318172$

H $2.5119224 .084583-2.671839$

H $3.0573792 .123594-3.626277$

H $3.9754020 .706983-3.192976$

H $5.5858692 .165384-3.371760$

H 3.0549400 .0124881 .353868

H $\quad 0.787400-1.3624482 .917338$

H 0.9740722 .9453382 .464744

H 1.6552121 .9337491 .215816

H 2.4910292 .1041192 .778821

H $2.4405570 .617061-1.018457$

H $1.961676-0.065272-2.574414$

H -2.127176 $0.840596-1.175942$

H -1.633467 $2.305313-2.050431$

H -0.7747013 .3143110 .155092$

H $-3.079232 \quad 1.4581430 .940298$

H -3.4441213 .6270502 .245684$

H -1.723028 3.9113172 .290934

H -3.419128 1.3290985 .123215

H -4.471046 2.0641193 .786006

H -1.0867851 .9483155 .230771$

H -0.4035602 .9955284 .035106$

H $-0.777672-0.0676893 .924153$
H $\quad 0.127348 \quad 0.271833-0.771179$

C $0.762687-4.334106-2.287342$

O $0.290163-4.359299-0.923605$

C $-0.464718-3.152436-0.681174$

C $-0.896086-2.674739-2.057953$

C $0.339831-2.986749-2.876538$

H $0.238628-2.439083-0.232423$

C $-1.605788-3.4517720 .297515$

H $-1.754226-3.254700-2.420013$

H -1.161345 -1.614312 -2.078861

C $0.087145-3.021171-4.373876$

H $1.093592-2.220037-2.669648$

C $-2.564461-2.2600030 .487881$

O $-1.017119-3.8015631 .552339$

H -2.170846 -4.328009-0.041942

H $-2.978378-2.002872-0.491493$

O $-1.824338-1.1253350 .934382$

C $-3.734293-2.5225091 .456324$

C $-4.700497-1.3335741 .595078$

H $-4.281301-3.4117141 .123675$

O $-3.224207-2.8124942 .759415$

H -5.484654 -1.592700 2.320469

H -4.182165 -0.494347 2.078310

H $1.004037-3.271171-4.916977$

H $-0.674115-3.763605-4.636054$

H $-0.260398-2.044917-4.727358$

C $2.267766-4.627627-2.333883$

H $-0.405035-4.5403771 .391571$

H -1.481173 -1.336560 1.819468

H -3.980372 -3.033795 3.329329

H $2.641628-4.500365-3.356042$

H $2.416335-5.682542-2.069673$

H $0.238065-5.146511-2.806473$

C $-6.9259580 .884993-0.410543$

$\begin{array}{lllll}\text { O } & -6.231757 & 0.235816 & 0.664441\end{array}$

C $-5.350103-0.8344380 .292704$

C $-6.116488-1.929114-0.466303$

C $-6.892845-1.354235-1.653149$

C -7.719639-0.130510-1.250334

H -7.6556041 .5401560 .082957$

C $-5.9886871 .766873-1.252350$

H $-4.563653-0.421234-0.345557$

O $-7.017297-2.601850 \quad 0.411527$

H $-5.408048-2.679445-0.834048$

H -7.554681 -2.131138 -2.055125

O $-5.964342-0.999813-2.673812$

H $-8.1282120 .368764-2.136589$

O $-8.835014-0.558966-0.463582$

C $-5.5773273 .028375-0.521171$

H $-5.072417 \quad 1.245127-1.539098$

H $-6.5151932 .085051-2.160020$

H -7.608563 -1.934211 0.799868

H $-6.474308-0.660891-3.429341$

H -9.363120 -1.165792 -1.009868

O $-4.8918963 .833879-1.386745$

O $-5.819543 \quad 3.2791550 .651078$

C $-4.4645995 .091958-0.855319$

H $-5.3301165 .682999-0.541305$

H -3.777804 $4.939852-0.018371$

H $-3.9383045 .633277-1.645935$

SCF Energy (B3LYP/6-31G**//MMFF) $=-3245.91309406$

04_00033

MM̄FF Geometry

C -3.1016901 .6585242 .987610$

C -2.4090880 .6443732 .445791$

C -1.320355 -0.104137 3.168166

O -0.0506630 .1872052 .541457$

C -1.559079-1.6171893.040258

C $-0.687994-2.4312633 .973366$

C $0.419110-3.1024683 .601595$

C $-1.175059-2.5101855 .398147$

C $1.061257-3.1508312 .240883$

C $2.591513-3.0825422 .356023$

C $3.287452-3.3423561 .011947$

C $4.790961-3.5343851 .224840$

C $5.531596-3.746692-0.101058$

O $3.056537-2.2244090 .147037$ 
O $6.933955-3.620066 \quad 0.177740$

C $5.133249-2.617604-1.071034$

C $5.326830-5.165862-0.642820$

O $5.698016-2.839830-2.361875$

C $3.605230-2.382806-1.170733$

C $3.349897-1.086936-1.973096$

O $3.007368-3.482501-1.859096$

C 0.6113311 .2979002 .962705

O $\quad 0.224262 \quad 2.121616 \quad 3.776202$

C $1.912943 \quad 1.3371452 .259800$

C 2.6648582 .4451942 .336852

C 3.9702862 .6532561 .706976

C 4.6410851 .4576911 .086431

C $1.888851-0.635146-2.012059$

C $1.7993910 .733258-2.656026$

C $1.9301503 .166420-2.121561$

$\begin{array}{llll}\text { O } & 1.718107 & 0.865148 & -3.875323\end{array}$

C $3.3498383 .629874-2.016654$

C $3.7228824 .744407-1.369082$

C $5.148820 \quad 5.207239-1.266154$

C $5.567537 \quad 5.5270800 .153309$

C $5.8048626 .793654 \quad 0.534447$

C 5.7871454 .3734141 .110732

C 4.5000623 .8942031 .723301

N $1.8702741 .767950-1.736880$

H -2.8678741 .9908613 .995694$

H -2.638192 0.3279291 .431100

H $-1.286197 \quad 0.1814344 .227000$

H $-1.395089-1.9298132 .002458$

H -2.608487 -1.8571003 .259094$

H $0.923690-3.6986544 .361324$

H $-0.539368-3.1482626 .021219$

H $-1.190770-1.5165885 .856177$

H -2.188178 -2.924407 5.430326

H $\quad 0.776010-4.0973351 .766924$

H $0.714062-2.340037 \quad 1.594161$

H $2.875706-2.0937482 .739019$

H $2.932342-3.8291883 .084776$

H $2.850416-4.2426260 .564057$

H $4.986499-4.3705691 .908102$

H $5.204852-2.6427761 .717191$

H $7.408773-3.675103-0.669526$

H $5.600563-1.697293-0.693904$

H $5.679201-5.9059750 .085752$

H $5.920272-5.334544-1.548320$

H $4.282751-5.392500-0.868649$

H $5.163496-3.520866-2.804859$

H $3.948280-0.275673-1.542972$

H $3.694536-1.216776-3.007383$

H $2.136065-3.209126-2.187385$

H $2.2111670 .461064 \quad 1.695161$

H 2.2811953 .2948262 .900711

H 4.7141160 .6370321 .809205

H 5.6588521 .6537050 .744230

H 4.0715951 .1100270 .221854

H $1.273787-1.322541-2.601593$

H $1.456575-0.594792-1.006370$

H $1.2639633 .717354-1.450295$

H $1.5753543 .285503-3.149663$

H $4.1083113 .021699-2.504885$

H $2.9631175 .368697-0.904205$

H $5.2536716 .097315-1.899792$

H $5.8393934 .461563-1.679584$

H 6.1293837 .0284911 .542794

H $5.6752277 .624911-0.150703$

H 6.4448144 .6883171 .931653

H 6.3346853 .5859510 .586565

H 3.9413524 .6847162 .227372

H $1.9745221 .548498-0.751077$

C -3.712249 3.5138831 .391880

$\begin{array}{lllll}\text { O } & -2.994089 & 2.939848 & 0.279425\end{array}$

C -3.729558 $3.212001-0.922581$

C -4.395194 $4.547871-0.647829$

C $-4.860107 \quad 4.334720 \quad 0.783754$

H $-4.5034202 .439876-1.014064$

C $-2.8064543 .142961-2.139777$

H -3.677625 5.375078 -0.692980

H $-5.2167404 .760006-1.338089$
C $-5.141097 \quad 5.627525 \quad 1.530934$

H $-5.781783 \quad 3.7372980 .766393$

C -1.953992 $1.857612-2.204559$

O $-1.903292 \quad 4.254852-2.121355$

H -3.397296 $3.251682-3.056337$

H $-1.1634331 .898056-1.445237$

O $-1.295471 \quad 1.831287-3.475777$

C -2.745494 $0.547889-2.040306$

C $-1.839685-0.695011-2.115761$

H $-3.2551880 .548642-1.072520$

O $-3.7525600 .466445-3.046759$

H $-1.404566-0.779137-3.118601$

H $-1.025922-0.562216-1.393701$

H $-5.452100 \quad 5.419660 \quad 2.559844$

H $-4.2547626 .269546 \quad 1.569200$

H $-5.942648 \quad 6.189498 \quad 1.040518$

C $-4.219076 \quad 2.3746192 .281517$

H $-1.4085344 .218096-1.284574$

H $-0.7986452 .662775-3.562657$

H -3.306867 $0.496419-3.910709$

H -4.7809951 .6487661 .680126$

H -4.9098612 .7634313 .038825$

H -3.006426 $4.151421 \quad 1.937473$

C $-3.819674-3.0311200 .025001$

O $-3.037232-1.923207-0.438006$

C $-2.594301-1.998660-1.797059$

C $-1.708792-3.238941-1.992259$

C $-2.435422-4.513164-1.550578$

C $-3.067234-4.360818-0.162164$

H -3.935445 -2.8571921 .102430$

C $-5.220372-3.033981-0.596272$

H -3.463276 - $2.064314-2.460081$

O $-0.494208-3.105911-1.256059$

H $-1.435705-3.317216-3.051113$

H $-1.719536-5.344420-1.538420$

O $-3.442265-4.817141-2.511341$

H $-3.740287-5.2016930 .041756$

O $-2.013487-4.4279670 .802798$

C $-5.992532-1.808833-0.165459$

H $-5.202883-3.074761-1.689072$

H $-5.793106-3.908320-0.265213$

H $-0.726744-2.967595-0.322058$

H -3.869828 -5.645967 -2.236022

H $-2.419333-4.3969081 .685909$

O $-6.073207-0.920603-1.196274$

$\begin{array}{lllll}0 & -6.450928 & -1.653710 & 0.959139\end{array}$

C $-6.7761420 .292556-0.912117$

H $-6.7103930 .937906-1.792053$

H $-7.8296860 .077587-0.710455$

H $-6.3190280 .810225-0.064163$

SCF Energy (B3LYP/6-31G**//MMFF) $=-3245.93592748$

04_00034

MM̄FF Geometry

C $-2.717140 \quad 3.6985310 .386561$

C $-3.937378 \quad 3.947246 \quad 0.888291$

C $-5.004636 \quad 2.9263941 .216452$

$\begin{array}{llll}\text { O } & -4.480751 & 1.579598 & 1.199519\end{array}$

C $-6.138923 \quad 3.0228310 .179593$

C $-7.325230 \quad 2.1292360 .489036$

C $-7.6332080 .995425-0.172429$

C $-8.2151722 .611071 \quad 1.606621$

C $-6.8791260 .351700-1.302956$

C $-6.362618-1.033960-0.895885$

C $-5.385231-1.615741-1.928433$

C $-4.934832-3.013630-1.501329$

C $-3.846984-3.580849-2.423699$

O $-4.259014-0.737570-1.997556$

O $-3.255916-4.712841-1.765633$

C $-2.728270-2.528279-2.591685$

C $-4.442489-4.106413-3.734899$

O $-1.778483-2.947011-3.567709$

C $-3.250604-1.118147-2.942493$

C $-2.152237-0.030782-2.901818$

O $-3.756920-1.071555-4.282469$

C $-3.872808 \quad 1.142965 \quad 2.338131$

O $-3.7005161 .776907 \quad 3.367460$

C $-3.440496-0.2549342 .115909$ 
C $-2.731419-0.8858763 .063789$ C $-2.246131-2.2668383 .010991$ C $-2.556101-3.0712811 .776885$ C -1.534239 $0.240216-1.527109$ C $-0.278611-0.558210-1.228231$ C $1.330716-0.9988760 .622430$ O $0.330764-1.202788-2.076867$ C $1.053690-2.3638651 .166473$ C $1.410820-2.7510342 .400458$ C $1.215034-4.1420692 .930166$ C $0.507282-4.1666374 .265951$ C $1.197550-4.2947715 .411774$ C $-1.003860-4.1337254 .264835$ C -1.552661 -2.748020 4.064202 N $0.109742-0.419676 \quad 0.098787$ H -2.4195962 .6787050 .164528$ H -4.2166034 .9812481 .083031$ H -5.4008173 .1499052 .214916$ H -5.736960 $2.804914-0.815046$ H -6.5013754 .0584140 .119941$ H $-8.5269580 .451861 \quad 0.132137$ H -9.0956411 .9737701 .741916$ H -7.6704622 .6229442 .555643$ H -8.5734853 .6242121 .396946$ H $-7.5548010 .250056-2.160793$ H $-6.0487740 .974261-1.641370$ H -5.853852 -0.9513590 .072713$ H -7.211086 -1.716026 -0.759812 H $-5.880140-1.647697-2.906144$ H -5.790615 -3.698203 -1.448011 H -4.529956 -2.966154 -0.482669 H -3.963712 -5.351530 -1.573144 H -2.195053 -2.478600 -1.634741 H -5.137372 -4.931553 -3.536223 H -3.665064 -4.520993 -4.385771 H -4.992672 $-3.343902-4.290380$ H -1.449679 -3.821064 -3.295919 H -1.383149 $-0.239689-3.655364$ H -2.622374 $0.909537-3.225160$ H -3.493537 -1.885719 -4.741177 H $-3.719839-0.7275351 .182248$ H -2.486131 -0.3429823 .975870$ H -2.053602 -4.040272 1.748638 H -2.233259 -2.539951 0.875262 H -3.632492 -3.260226 1.709882 H -1.228128 $1.293795-1.495592$ H -2.264873 $0.089176-0.727258$ H $\quad \begin{array}{lll}1.713520 & -0.315829 & 1.387177\end{array}$ H $2.067540-1.068884-0.183055$ H $\quad 0.579334-3.0800450 .499757$ H $1.916506-2.0426353 .052795$ H $2.207772-4.6013613 .026842$ H $0.668378-4.7703702 .216153$ H $\quad 0.695110-4.343021 \quad 6.372217$ H $2.281308-4.3486385 .414952$ H -1.387406 -4.496604 5.228232 H -1.382168 -4.852690 3.532611 H -1.371711 -2.086044 4.912204 H $-0.481790 \quad 0.1002810 .740494$ C $-0.374687 \quad 4.5345650 .774191$ O $0.349247 \quad 3.4883650 .087370$ C $1.6665233 .977367-0.245058$ C 1.9056635 .1454020 .695663 C $\quad 0.5282615 .770578 \quad 0.740334$ H $1.6019204 .326804-1.283858$ C $2.6895412 .839293-0.132787$ H 2.1999444 .7904071 .691156 H 2.6734255 .8346350 .332186 C 0.3260636 .7104151 .916810 H $0.3667906 .328676-0.191952$ C $4.1472703 .305023-0.296950$ O $2.3988781 .880837-1.154442$ H 2.5625832 .3254770 .826606 H 4.4146063 .9761860 .527771 O $4.2368384 .084191-1.497820$ C $5.1896452 .174915-0.397284$ C 5.1633191 .1949820 .787695 H 5.054588 1.636564-1.341192
O $\quad 6.4851502 .782498-0.470939$

H 5.2503421 .7625411 .722538

H $4.1952370 .685590 \quad 0.812426$

H -0.6886007 .1210311 .918209$

H 0.4831696 .1975342 .871657

H 1.0298397 .5475981 .865539

C -1.716442 4.7772920 .076249

H 1.466988 1.622811-1.052608

H $5.1620124 .369612-1.590233$

H $\quad 6.642240 \quad 3.249848 \quad 0.367311$

H $-1.5714134 .796209-1.010856$

H -2.1184135 .7532440 .372946$

H $-0.526274 \quad 4.1924261 .805815$

C $5.311016-1.388521-0.822091$

O $6.392475-0.495622-0.532607$

C $6.3065440 .154088 \quad 0.738712$

C $6.267614-0.8910691 .863078$

C $5.173620-1.9332591 .635479$

C $5.261941-2.5082130 .221022$

H $4.365583-0.833713-0.821271$

C $5.532524-1.939342-2.233836$

H 7.2436720 .7124180 .858479

O $6.082370-0.2444903 .117431$

H $7.238278-1.4025601 .902716$

H $4.180679-1.5112621 .823182$

O $5.338012-3.0092812 .561018$

H $6.153188-3.1432540 .137902$

O $4.124844-3.331791-0.023776$

C $5.205154-0.882758-3.262461$

H $6.566614-2.272549-2.381973$

H $4.885134-2.798188-2.443709$

H $6.183682-0.9152943 .813242$

H $5.155655-2.6618453 .450298$

H $4.096125-4.0043780 .678187$

O $6.313633-0.139058-3.540029$

O $4.087705-0.718529-3.735211$

C $6.1170900 .934518-4.465230$

H $7.0709381 .453856-4.590794$

H $5.7989330 .543960-5.436464$

H $5.3814061 .643248-4.072848$

SCF Energy (B3LYP/6-31G**//MMFF) $=-3245.90717431$

04 00035

MMFF Geometry

C $-4.8253772 .770360-0.955085$

C $-3.5563772 .990448-0.580896$

C $-2.4797683 .427076-1.539650$

O $-1.4080562 .454569-1.509345$

C -1.899521 $4.783350-1.109209$

C $-0.9453135 .358767-2.137286$

C $0.3983485 .334769-2.039888$

C $-1.6028216 .032459-3.315116$

C $1.2380364 .726177-0.950182$

C $2.3202503 .810073-1.531139$

C $3.2093513 .148463-0.464736$

C 4.0401954 .1743130 .304951

C 4.9982863 .5052421 .294384

O $4.0823352 .254578-1.161503$

O 5.9130994 .5243361 .730069

C 5.8135862 .4150100 .563832

C 4.2523623 .0317852 .549148

O 6.5569231 .6554801 .512690

C $4.9682191 .475506-0.333840$

C $5.8260460 .682502-1.353162$

O $4.2392090 .513214 \quad 0.426431$

C $-1.5936961 .306549-2.216002$

O $-2.5895300 .982002-2.843362$

C $-0.3572290 .502083-2.101118$

C $-0.350517-0.753384-2.571761$

C $0.781804-1.681190-2.566585$

C $2.112877-1.162699-2.087779$

C $6.970547-0.178464-0.819726$

C $6.533096-1.3352090 .055029$

C $5.575050-3.608691-0.015305$

O $6.732027-1.3401841 .268116$

C $4.771757-4.450649-0.950653$

C $3.541294-4.903464-0.668751$

C $2.742283-5.778113-1.591449$ 
C $1.449530-5.121365-2.022767$

C $0.281640-5.510704-1.482278$

C $1.565712-4.068670-3.108732$

C $0.572881-2.943332-2.997061$

N $5.912897-2.351792-0.655108$

H -5.110724 2.936190 -1.991296

H -3.2631222 .8230240 .451586$

H -2.870265 $3.498285-2.562748$

H -1.403254 $4.685349-0.136927$

H $-2.7106555 .506974-0.950676$

H $\quad 0.9730845 .817453-2.830052$

H -0.873909 $6.476392-4.001464$

H $-2.1971885 .314564-3.888378$

H $-2.2627136 .836218-2.972598$

H $1.6988305 .551693-0.397719$

H $0.6390684 .153648-0.236913$

H $1.8501483 .022293-2.134835$

H $2.9534534 .369106-2.233761$

H 2.5718042 .5669980 .209562

H 3.4039674 .8898210 .837907

H $4.6313774 .769993-0.405070$

H 6.4923634 .1405162 .409858

H $6.5453682 .925843-0.077702$

H 3.8063703 .8869193 .071668

H 4.9351152 .5666493 .268604

H 3.4490772 .3261482 .329149

H 7.1830002 .2564621 .949781

H $5.1526940 .042274-1.939920$

H $6.2503651 .392901-2.075954$

H $\quad 4.3218190 .725897 \quad 1.368598$

H $\quad 0.5085180 .963519-1.641428$

H -1.269421 -1.148191 -3.004414

H $2.477544-0.374862-2.755949$

H $2.887685-1.930272-2.039690$

H $2.032902-0.748924-1.078318$

H $7.519915-0.600747-1.671733$

H $7.7059250 .422417-0.276188$

H $5.047770-3.3951160 .920784$

H $6.511773-4.1179430 .235920$

H $5.233643-4.718580-1.897920$

H $3.087156-4.6494430 .285836$

H $2.539260-6.721114-1.067543$

H $3.319393-6.054318-2.483045$

H - $0.667037-5.084403-1.787789$

H $0.245135-6.278536-0.715993$

H $1.426523-4.559762-4.080648$

H $2.585614-3.673889-3.140380$

H $-0.429560-3.215749-3.330499$

H $5.956457-2.323505-1.670181$

C $-6.3953070 .891728-0.479586$

O $-5.353519-0.084705-0.275196$

C -5.945321 -1.2981010 .227277$

C $-7.454234-1.1161170 .109551$

C -7.5931130 .3795640 .317204$

H $-5.685573-1.3591201 .290406$

C $-5.384582-2.511978-0.522498$

H -7.821778 -1.398851 -0.883851

H -8.001692 -1.7065690 .850597$

C $-8.9295870 .934127-0.143711$

H $-7.4580240 .601721 \quad 1.384387$

C $-3.848148-2.640524-0.469849$

O $-5.761145-2.425842-1.903385$

H -5.848815 -3.428610 -0.141067

H -3.379548 -1.830049-1.041455

O $-3.486699-3.860158-1.130083$

C $-3.263351-2.6684190 .953441$

C -1.745372 -2.916792 0.964050

H $-3.472822-1.7153401 .443125$

O $-3.908330-3.684028 \quad 1.721004$

H -1.541588 -3.925473 0.586599

H -1.270462 -2.2094220 .276695$

H -8.980356 2.0156120 .016051

H $-9.0979190 .742403-1.208778$

H -9.7504250 .4720560 .414627$

C $-5.9029242 .269556-0.035197$

H -5.410698 $-1.587472-2.250749$

H -3.855516 -3.822390 -2.029248

H $-3.762761-4.5319201 .267307$
H -5.5283152 .2162790 .993417$

H -6.726239 $2.992107-0.049083$

H $-6.6242390 .893548-1.553862$

C $-0.757294-0.4062532 .387280$

O $-1.405125-1.5314082 .991880$

C - $-1.128036-2.7946112 .376930$

C $0.380816-3.0734372 .423360$

C $1.174776-1.9427641 .777499$

C $0.766241-0.5973742 .376692$

H -1.130465 -0.271055 1.364815

C -1.1292480 .8409473 .206032$

H -1.615692 -3.551923 3.003443

O $0.691110-4.2999371 .772162$

H $\quad 0.702638-3.1723083 .468157$

H $1.051380-1.9552090 .690048$

O $2.565117-2.1601802 .025361$

H $1.159314-0.5345893 .399247$

O $1.379804 \quad 0.453205 \quad 1.634578$

C -2.5849501 .2302313 .063292$

H -0.9419330 .6487414 .269385$

H $-0.534598 \quad 1.7021512 .881568$

H $\quad 0.179435-5.0014492 .209697$

H $3.051167-1.4272311 .609571$

H 1.0254190 .4263290 .729776

O -2.8526932 .2539413 .927962$

O $-\begin{array}{llll}-3.382298 & 0.721333 & 2.289004\end{array}$

C $-4.1958552 .747722 \quad 3.907248$

H $-4.3170603 .436716 \quad 4.747427$

H -4.3749743 .2935112 .976778$

H -4.913454 1.9294154 .020904

SCF Energy $(B 3 L Y P / 6-31 G * * / / M M F F)=-3245.92082565$

04_00036

MMFF Geometry

C $1.047172-1.9069632 .733144$

C $-0.023579-2.4436082 .132001$

C $0.000434-2.9174930 .700702$

O $-0.792793-1.993062-0.078857$

C $-0.616216-4.3220980 .594165$

C $-0.553572-4.901928-0.807225$

C $-1.613649-5.057393-1.626242$

C $0.815318-5.345653-1.258460$

C $-3.045525-4.692273-1.352150$

C $-3.475119-3.471026-2.174862$

C $-4.758038-2.834846-1.620926$

C $-5.229642-1.694709-2.524326$

C $-6.430132-0.943541-1.930494$

O $-4.451920-2.331358-0.315887$

O $-6.577990 \quad 0.269390-2.684837$

C $-6.108130-0.558321-0.469560$

C $-7.732454-1.729088-2.121946$

$\begin{array}{lllll}\text { O } & -7.246176 & 0.022881 & 0.161736\end{array}$

C $-5.555999-1.7286010 .369914$

C $-5.045900-1.2888781 .764738$

O $-6.606161-2.6790030 .586271$

C $-0.147025-0.889149-0.551029$

O $1.039999-0.626151-0.435359$

C $-1.144516-0.024748-1.218089$

C $-0.8017631 .225678-1.562048$

C $-1.6596692 .211160-2.223107$

C $-3.0762691 .808097-2.534574$

C $-3.889443-0.2824371 .755843$

C $-4.325586 \quad 1.168158 \quad 1.874711$

C -3.4040733 .4824381 .772394$

O -5.4659651 .5064122 .179035$

C -3.6667054 .0839640 .428404$

C $-2.9283115 .076489-0.091562$

C $-3.2219745 .738101-1.407516$

C $-2.0260415 .764031-2.332295$

C $-1.2379996 .849488-2.413357$

C $-1.8015514 .569005-3.229816$

C $-1.1421463 .422861-2.515290$

N -3.272269 2.0434831 .644288

H $1.974319-1.8221862 .174614$

H $-0.965898-2.5062272 .670426$

H $1.027196-2.9441040 .315396$

H -1.647298 -4.2888090.958604

H $-0.091545-5.0099631 .271117$ 
H $-1.444140-5.490351-2.611325$ H $\quad 0.790720-5.841009-2.235036$ H $1.492249-4.490198-1.342221$ H $1.239294-6.056891-0.541933$ H -3.680098 -5.547324-1.616409 H $-3.219808-4.520084-0.288193$ H $-2.671958-2.723934-2.160915$ H -3.622347 -3.771261 -3.219655 H -5.529796 -3.609320 -1.540587 H -5.466722 -2.066513 -3.529016 H -4.407955 $-0.979311-2.660048$ H $-7.2859730 .790665-2.268456$ H $-5.3521940 .233285-0.519338$ H -7.920648 -1.898806 -3.188965 H $-8.595659-1.163411-1.754514$ H $-7.721811-2.703078-1.628291$ H $-7.853452-0.6980940 .400426$ H $-5.883481-0.9398302 .380494$ H -4.671451 -2.190887 2.270216 H $-6.378641-3.2187031 .361490$ H $-2.131653-0.433446-1.395945$ H $0.2092541 .565663-1.341074$ H $-3.0892741 .010849-3.285221$ H -3.689724 2.625406 -2.919184 H -3.582579 $1.451644-1.631580$ H $-3.254964-0.4790592 .629629$ H -3.265391 -0.4069540 .866698$ H -2.477294 3.8556722 .218791 H -4.2323333 .7164222 .448861$ H $-4.5253063 .711280-0.125086$ H -2.089005 5.4675120 .479571 H -3.548787 $6.764970-1.196429$ H $-4.0681065 .260166-1.916914$ H $-0.3964536 .887645-3.096988$ H -1.414123 $7.722183-1.793013$ H $-1.1452514 .846667-4.065935$ H $-2.7456864 .289776-3.705843$ H $-0.1055293 .623874-2.240882$ H -2.3709891 .6741841 .355559$ C 1.1114810 .1617994 .187610 O $2.198220 \quad 0.670765 \quad 3.383179$ C 1.6931361 .7507132 .576125 C $0.4022212 .197444 \quad 3.249774$ C -0.1596330 .8661943 .704764$ H $1.445396 \quad 1.328676 \quad 1.595449$ C 2.7355242 .8588362 .422376 H 0.5978632 .8442254 .113066 H -0.2589482 .7330472 .562950$ C -1.235983 0.9882394 .769343 H $-0.583180 \quad 0.3558162 .831338$ C 4.0498952 .4325221 .737268 O $3.0667773 .375796 \quad 3.717621$ H 2.2940043 .6978411 .871320 H 4.6398891 .7878582 .399724 O 4.8272273 .6227661 .543035 C 3.8747011 .7344350 .372978 C $5.1888731 .641224-0.428867$ H 3.4688440 .7303340 .535165 O $2.9196312 .452321-0.412394$ H $5.4900702 .653861-0.721270$ H 5.9743301 .2401900 .218823 H -1.603595 0.0005905 .064878 H -0.8589481 .4908005 .666294$ H $-2.086074 \quad 1.5671064 .394927$ C $1.069978-1.3689824 .133485$ H 3.3997602 .6342324 .251985 H 4.9401764 .0402592 .414292 H $3.2562493 .357138-0.531305$ H $0.216297-1.7411014 .711578$ H $1.973254-1.7663064 .613145$ H 1.3330830 .4620405 .220040 C $5.979769-1.297754-0.895736$ O $4.833941-0.602031-1.405335$ C $5.0387880 .783743-1.707729$ C $6.1833740 .941920-2.718389$ C $7.4599970 .261996-2.236369$ C $7.170683-1.189541-1.859970$ H $6.256924-0.8846150 .080961$
C $5.579078-2.770219-0.705680$

H $4.1275391 .121180-2.217033$

O $6.4279772 .319852-2.978272$

H $5.8774970 .488169-3.670195$

H $7.9024910 .808762-1.395987$

O $8.435613 \quad 0.294204-3.280553$

H $6.966698-1.763461-2.772578$

O $8.335696-1.758538-1.264604$

C $4.725175-2.9868290 .524401$

H $5.008192-3.118050-1.574574$

H $6.470857-3.394625-0.581560$

H $7.1763732 .371362-3.597305$

H $8.075372-0.187715-4.044452$

H $8.530640-1.259584-0.452940$

O $4.420113-4.3155990 .615822$

O $4.382022-2.1138461 .308399$

C $3.654264-4.7102321 .758657$

H $3.734400-5.7957501 .861295$

H $4.036685-4.2462312 .672960$

H $2.605043-4.4502731 .602968$

SCF Energy $\left(B 3 L Y P / 6-31 G^{* * / / M M F F}\right)=-3245.92645133$

04 00037

MM̄FF Geometry

C $-4.0999450 .282740 \quad 3.042897$

C $-3.132132-0.4798222 .510855$

C $-1.740559-0.5715473 .077723$

O $-0.834311-0.0150732 .094110$

C -1.340186-2.039109 3.304461

C $-0.204828-2.1714084 .300005$

C $1.103767-2.1991053 .982275$

C -0.635004 -2.287565 5.740043

C $1.725859-2.0619182 .622060$

C $2.589482-3.2809512 .273822$

C $3.289964-3.1044270 .919766$

C $4.075114-4.3602310 .539446$

C $4.859477-4.169007-0.767857$

O $4.179622-1.9896651 .027340$

O $5.785292-5.261552-0.864278$

C $5.673824-2.859232-0.676466$

C $3.941913-4.274625-1.991632$

O $6.314384-2.572288-1.917535$

C $4.844486-1.646807-0.193970$

C $5.771293-0.4408720 .088251$

O $3.916626-1.285173-1.220363$

C -0.6294251 .3293982 .111162$

O -1.0775652 .1327472 .913145$

C 0.2099251 .6649570 .937772

C 0.3688802 .9502640 .587997

C $1.0928773 .452620-0.583343$

C $1.7048022 .439778-1.514544$

C $5.0834140 .691573 \quad 0.863840$

C 5.9478991 .9352540 .965072

C 5.8265924 .3054031 .733320

O 7.0979561 .9947210 .539536

C 5.4422545 .2162010 .602508

C $4.6228354 .943770-0.426076$

C $4.3162125 .916332-1.528441$

C $2.9035596 .451344-1.467527$

C $2.6637907 .723268-1.105881$

C $1.7777965 .545509-1.917190$

C $1.1558724 .787393-0.775465$

N $5.2705462 .973458 \quad 1.592128$

H -3.9000400 .8551383 .945099$

H -3.330063 -1.027979 1.592584

H -1.661894 -0.0096074 .017276$

H -1.066087 -2.512861 2.354218

H -2.194253 -2.615015 3.685751

H $1.823683-2.2978634 .794050$

H $0.216038-2.3630386 .425240$

H -1.219684 -1.410778 6.036289

H -1.252248 -3.180987 5.880094

H $\quad 0.977365-1.9187081 .838265$

H $2.342800-1.1552462 .631098$

H $3.344238-3.4205973 .058963$

H $1.963001-4.1813752 .254186$

H $2.530102-2.8854330 .162207$

H $3.409395-5.2292170 .465083$ 
H $4.789433-4.598503 \quad 1.340205$ H $6.339977-5.109340-1.648815$ H $6.482479-3.0371850 .045568$ H $3.445008-5.252191-2.012099$ H $4.514004-4.212544-2.923822$ H $3.165469-3.507617-2.012228$ H $5.641671-2.214602-2.521915$ H $6.632423-0.7694110 .684643$ H $6.162139-0.048696-0.859232$ H $3.711899-0.339816-1.150372$ H $\quad 0.634650 \quad 0.8446660 .370509$ H -0.0996513 .7157481 .205672$ H $2.3854021 .776910-0.971513$ H $2.2935442 .885350-2.318830$ H $\quad 0.9252601 .831522-1.985037$ H $4.144760 \quad 0.9762970 .379610$ H $4.852400 \quad 0.360314 \quad 1.883150$ H 6.9178514 .2353201 .788515 H 5.4562794 .7153542 .678053 H 5.8942336 .2055720 .648621 H $4.1592163 .965614-0.507174$ H $4.4776085 .420993-2.493956$ H $5.035766 \quad 6.745699-1.507565$ H $1.6557248 .124531-1.090622$ H $3.4682868 .389326-0.811980$ H $2.1283784 .897968-2.725193$ H $0.9840656 .151729-2.375284$ H $0.6707385 .435200-0.043394$ H 4.2960672 .8422721 .847935 C -5.5580541 .7944361 .692266$ O $-4.600647 \quad 1.8087530 .613256$ C $-5.2956612 .040880-0.621024$ C $-6.5571742 .784836-0.217566$ C $-6.9292142 .033577 \quad 1.050344$ H $-5.5780571 .059152-1.019215$ C $-4.3739822 .741416-1.622728$ H -6.3521303 .8366970 .012611$ H $-7.3344272 .742249-0.985982$ C $-7.8983152 .786647 \quad 1.944870$ H $-7.3845301 .074606 \quad 0.767920$ C $-2.9936172 .066055-1.779507$ O $-4.1443004 .090809-1.200303$ H -4.873435 2.812435 -2.595497 H $-2.3833172 .259857-0.889526$ O $-2.3134702 .689228-2.874413$ C $-3.0468500 .548776-2.031598$ C -1.650752 -0.066532 -2.233656 H $-3.5267370 .060165-1.178355$ O $\quad-3.850861 \quad 0.276875-3.178132$ H -1.225722 $0.273730-3.185436$ H $-1.0032390 .280625-1.422264$ H -8.1137882 .2134082 .852128$ H -7.492873 3.7579632 .247567 H -8.8442302 .9650031 .423312$ C -5.4581110 .4504042 .419930$ H -3.757642 $4.057832-0.308256$ H -2.271753 $3.642220-2.684118$ H $-3.452958 \quad 0.743517-3.933157$ H -5.642848 $-0.370261 \quad 1.715807$ H $-6.2190580 .381553 \quad 3.205142$ H -5.2889482 .6182842 .365263$ C $-2.275826-3.438229-0.750627$ O $-2.114961-2.025301-0.928196$ C -1.687354 -1.606705 -2.229178 C $-0.314015-2.215582-2.546450$ C $-0.342352-3.741611-2.414323$ C $-0.981486-4.193008-1.095471$ H $-2.460018-3.5633600 .324352$ C -3.504542 -3.966258 -1.498343 H -2.415872 -1.936660 -2.977120 O $0.679382-1.678770-1.674894$ H $-0.029370-1.938410-3.568119$ H $\quad 0.683777-4.123007-2.476851$ O $-1.073176-4.277527-3.513623$ H -1.182318 -5.270550-1.110435 O $-0.056152-3.958001-0.031113$ C $-4.767718-3.352460-0.940886$ H $-3.460794-3.774016-2.574126$
H $-3.596481-5.052552-1.382645$

H $0.395434-1.847960-0.760181$

H -1.054073 -5.246404 -3.434378

H $0.734415-4.495939-0.207710$

O $-5.300671-2.479311-1.841933$

O $-5.217316-3.6084020 .168849$

C $-6.505669-1.827597-1.429787$

H -7.313683 -2.559141-1.336016

H -6.352299 -1.299558 -0.484360

H -6.777620 -1.098513 -2.197618

SCF Energy $(B 3 L Y P / 6-31 G * * / / M M F F)=-3245.93147345$

04_00038

MMFF Geometry

C $-1.157918-0.967633-1.756809$

C $0.025214-1.525799-1.464252$

C $0.307182-2.260297-0.179164$

O $1.480244-1.6630360 .423124$

C $0.606065-3.738954-0.477615$

C $0.883722-4.5643650 .765372$

C $2.091013-5.0569091 .110102$

C $-0.318001-4.8464061 .631269$

C $3.392190-4.8931670 .375434$

C $4.338147-3.947257 \quad 1.125742$

C $5.453680-3.4146800 .214691$

C $6.451114-2.5782451 .017540$

C $7.501020-1.9072210 .120561$

O $4.830403-2.605996-0.789533$

O $8.165788-0.9195220 .923450$

C $6.780599-1.175165-1.033209$

C $8.581761-2.902474-0.316762$

O $7.723753-0.649151-1.963551$

C $5.729482-2.043960-1.753367$

C $4.876092-1.245669-2.770307$

O $6.404187-3.073578-2.486758$

C $1.293019-0.5194481 .138993$

$\begin{array}{llll}\text { O } & 0.232179 & 0.040938 & 1.364147\end{array}$

C $2.619394-0.0392781 .585516$

C 2.7191721 .1518442 .194309

C 3.9520151 .7802042 .674220

C 5.2481681 .0565762 .423458

C $4.036846-0.107960-2.177539$

C $4.705601 \quad 1.255267-2.229012$

C $4.3990013 .595131-1.422740$

O $5.7182151 .485239-2.884160$

C $5.2699993 .817328-0.226661$

C 5.0448744 .7808340 .679949

C 5.9427865 .0542691 .852934

C 5.1950115 .0971703 .166953

C 4.8086136 .2668433 .704196

C 4.9755753 .7927213 .898937

C 3.8668452 .9732373 .299390

N $4.0068282 .199720-1.487848$

H -1.982187 -1.064156-1.056958

H $\quad 0.850301-1.425809-2.165601$

H $-0.539831-2.1863470 .513548$

H $1.441874-3.798467-1.181287$

H $-0.246808-4.189849-1.003183$

H $2.166438-5.6403382 .026888$

H $-0.083184-5.5219452 .460841$

H $-0.709273-3.9204052 .063359$

H -1.109908 -5.317836 1.040132

H $3.866048-5.8783140 .281665$

H $3.235508-4.549212-0.648857$

H $3.765500-3.0987881 .519905$

H $4.770712-4.4750391 .984790$

H $5.956765-4.264074-0.262309$

H $6.937158-3.187417 \quad 1.789887$

H $5.908083-1.7929291 .559352$

H $8.779205-0.434140 \quad 0.345121$

H $6.281633-0.308788-0.584898$

H $9.091394-3.3200570 .559964$

H $9.361920-2.408613-0.906316$

H $8.185417-3.737512-0.898214$

H $8.035913-1.384983-2.517329$

H $5.503679-0.897693-3.599511$

H $4.166443-1.953993-3.221987$

H $5.808670-3.396894-3.183058$ 
H $3.478413-0.668251 \quad 1.385473$ H 1.8101821 .7297812 .357152 H 5.2797240 .1225902 .994350 H 6.1333091 .6335762 .698058 H $5.3591490 .824438 \quad 1.358912$ H $3.117088-0.013722-2.769239$ H $3.740497-0.333264-1.149569$ H $3.4802864 .188621-1.381977$ H $4.947003 \quad 3.862174-2.332119$ H $6.1437223 .178751-0.119506$ H 4.1876865 .4382330 .552031 H 6.4351496 .0195211 .675532 H $6.7535284 .318001 \quad 1.918011$ H 4.2979056 .3089124 .660447 H 4.9900087 .2082763 .196235 H 4.7025193 .9891914 .944662 H 5.9233113 .2504643 .959880 H 2.8811543 .4232753 .425656 H $3.2041601 .907455-0.938162$ C $-1.8616201 .266434-2.727376$ O $-3.185908 \quad 1.285059-2.161356$ C $-3.2895072 .424503-1.282375$ C $-1.9692943 .188007-1.398432$ C $-0.9985902 .083695-1.768691$ H $-3.3815102 .030025-0.264513$ C $-4.5262283 .256898-1.638476$ H -2.003719 $3.946405-2.189192$ H $-1.7055603 .696180-0.465622$ C $0.3022402 .581134-2.371606$ H $-0.7765481 .515159-0.857650$ C $-5.8577322 .480325-1.614754$ O $-4.3657353 .787102-2.962240$ H -4.589685 4.126263 -0.973936 H $-5.8744311 .726745-2.411502$ O $-6.9012273 .415709-1.924083$ C $-6.1946901 .810652-0.267595$ C -7.603797 $1.184169-0.270370$ H $-5.4556931 .032246-0.052336$ O $-6.1075302 .775761 \quad 0.779515$ H -8.342849 $1.957603-0.511480$ H $-7.6472250 .438689-1.071928$ H $0.9582711 .743040-2.626856$ H $0.1264723 .162247-3.282890$ H $\quad 0.8343283 .222212-1.661179$ C -1.416634 -0.172146-3.003351 H $-4.2576393 .034157-3.568487$ H -6.681235 $3.822412-2.779852$ H $-6.746733 \quad 3.4817470 .581655$ H -0.521195 -0.154044 -3.636291 H -2.202044 -0.689020 -3.568295 H $-1.940113 \quad 1.783470-3.693520$ C $-6.983959-1.6147720 .809447$ O $-7.059082-0.3967621 .558544$ C -8.0114590 .5555941 .081013$ C $-9.415797-0.0643771 .083254$ C $-9.453973-1.3718230 .295695$ C -8.348259 -2.315456 0.767232 H -6.634781 -1.398289-0.207715 C $-5.945266-2.5110001 .494661$ H -8.0224831 .3703451 .816095$ $\begin{array}{llllll}\text { O } & -10.362322 & 0.855638 & 0.550858\end{array}$ H -9.712002 -0.265142 2.120992 H $-9.376869-1.185525-0.781396$ O $-10.722812-2.002278 \quad 0.488539$ H -8.600278 -2.704645 1.762091 O $-8.274895-3.429730-0.119931$ C $-4.544897-1.9914671 .281287$ H $-6.127002-2.5856242 .573925$ H -5.970565 -3.532173 1.096892 H -11.2277160 .4118080 .543162$ H $-10.829745-2.1790391 .438740$ H -9.157203 -3.838057 -0.147341 O $-4.259603-1.0254422 .199679$ O $-3.800063-2.392694 \quad 0.396744$ C -2.955112 -0.4482572 .102268$ H $-2.873423 \quad 0.3387972 .856418$ H -2.194166 -1.207920 2.302943 H $-2.807691-0.0025001 .114580$
SCF Energy (B3LYP/6-31G**//MMFF) $=-3245.93130665$

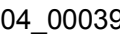

MMFF Geometry

C -0.3117792 .4647230 .303357$

C -1.3385412 .7651861 .113239$

C -1.8084401 .9449842 .292686$

O -1.2580410 .6107332 .206304$

C -3.3423901 .8189722 .263152$

C -3.9343831 .2669163 .543423$

C -4.3865760 .0072613 .698368$

C $-4.0502262 .255546 \quad 4.674634$

C $-4.332363-1.1240942 .709628$

C $-5.711642-1.4907962 .145065$

C $-6.246993-0.4750611 .122524$

C $-7.639419-0.8858690 .636999$

C $-8.1520920 .034956-0.481208$

O $-5.336095-0.4477380 .019350$

O $-9.310027-0.577817-1.068514$

C $-7.0651640 .157598-1.571633$

C $-8.643470 \quad 1.3754350 .075491$

O $-7.4402851 .115816-2.558552$

C $-5.665190 \quad 0.495497-1.009556$

C $-4.6003850 .353963-2.123936$

O $-5.6683091 .837703-0.522642$

C -0.7002370 .0569983 .309683$

O $-0.547434 \quad 0.591763 \quad 4.399243$

C $-0.290458-1.3477213 .064308$

C $-0.486247-1.9981851 .905653$

C $-0.148718-3.3987421 .629413$

C $0.550430-4.1760172 .714253$

C $-3.1576340 .558000-1.641170$

C $-2.1589160 .102644-2.685700$

C -1.204529 -1.940949-3.723327

O $-1.5526680 .900864-3.395996$

C $-1.029265-3.370838-3.327051$

C $0.150657-3.908096-2.984386$

C $0.327630-5.337524-2.564485$

C $0.754294-5.473256-1.119041$

C $2.017840-5.797698-0.797404$

C $-0.307975-5.337032-0.050304$

C $-0.485798-3.9217450 .430790$

N -2.031248 -1.276829 -2.733714

H 0.2791521 .5716250 .483233

H -1.887676 3.6858610 .923877

H -1.4777682 .4569593 .203834$

H -3.634722 1.1946801 .413660

H -3.8026492 .7989022 .077731$

H $-4.823517-0.2650344 .658549$

H -4.5279071 .8215845 .559485$

H -3.0608372 .6114524 .977479$

H -4.6511543 .1172034 .366027$

H -3.624342 -0.9318691 .898496$

H $-3.934129-1.9978273 .241762$

H $-5.612860-2.4676671 .653567$

H $-6.430507-1.6127112 .964820$

H -6.2844810 .5159761 .587648$

H -8.352005 -0.911356 1.470916

H -7.597854 -1.913408 0.249393

H $-9.050538-1.447961-1.416194$

H $-7.004100-0.810524-2.087960$

H $-9.470743 \quad 1.2157620 .777697$

H -9.046443 $2.011160-0.720766$

H -7.8669081 .9338850 .602320$

H $-8.3216470 .866196-2.885120$

H $-4.686594-0.646551-2.566706$

H -4.795551 $1.076676-2.926223$

H $-4.7600122 .176593-0.513713$

H $\quad 0.164786-1.8353983 .920599$

H $-0.959124-1.4709731 .078659$

H $1.440074-3.6416153 .064753$

H $0.900614-5.1581692 .389215$

H -0.120933 -4.332106 3.565611

H $-2.9634611 .614385-1.428163$

H $-2.9587030 .002572-0.717631$

H $-0.252877-1.405899-3.807615$

H - $1.715736-1.874805-4.689297$

H -1.921673 -3.992179 -3.312874 
H $\quad 1.042976-3.289038-3.008498$ H $1.076545-5.789577-3.227764$ H $-0.590448-5.916604-2.725316$ H $2.319802-5.9313340 .236021$ H $2.779294-5.929541-1.558716$ H -0.094124 -6.0221110 .776715$ H -1.269868 -5.688387 -0.445876 H $-0.992441-3.275677-0.285849$ H -2.687074 -1.840234 -2.199170 C $1.2740504 .197587-0.597239$ O $2.4403303 .411719-0.287253$ C $3.5978234 .054696-0.855822$ C $3.1166165 .379192-1.442117$ C $1.6772075 .060888-1.790980$ H $3.9407223 .413327-1.675617$ C 4.7074604 .1971560 .191833 H $3.1590366 .191467-0.707525$ H $3.7109215 .685905-2.308522$ C $0.8155796 .295950-1.988394$ H $1.6645644 .460141-2.710451$ C 5.1170252 .8794770 .878327 O $4.2534715 .091123 \quad 1.217192$ H $5.5805034 .675402-0.268176$ H 4.3085912 .5397041 .536749 O 6.2213563 .1708001 .745802 C $5.5038441 .735591-0.078249$ C $6.086330 \quad 0.521372 \quad 0.669557$ H $4.6140601 .426774-0.636375$ O $6.4545362 .200704-1.035126$ H $7.0279100 .810306 \quad 1.151940$ H 5.3911060 .2345681 .465716 H $-0.2133076 .020113-2.239432$ H $0.7875506 .914211-1.084829$ H $1.2073356 .911386-2.804832$ C $0.0786443 .291826-0.888438$ H 4.9702435 .1724171 .869556 H 6.9497333 .5102941 .198997 H $7.2786152 .408298-0.563340$ H $0.3165482 .600392-1.705525$ H $-0.7751563 .893375-1.221991$ H 1.0688104 .8251370 .280440 C $4.168396-1.661821-0.233584$ O $5.220546-1.077257-1.008660$ C $6.372055-0.680305-0.258850$ C $6.967557-1.8990180 .457794$ C $5.929302-2.6142651 .319113$ C $4.670141-2.9102610 .504172$ H $3.783272-0.9255380 .482486$ C $3.032839-2.023595-1.195099$ H $7.114942-0.344019-0.993083$ O $8.078365-1.5044891 .255973$ H $7.347053-2.602325-0.295018$ H $5.697603-2.0334332 .219570$ O $6.501174-3.8447661 .768893$ H $4.880972-3.712502-0.214942$ O $3.643154-3.3731901 .376880$ C $2.337396-0.789301-1.714673$ H $3.399604-2.606138-2.048971$ H $2.268268-2.630653-0.699179$ H $8.409969-2.3002421 .706270$ H $5.875709-4.2479062 .394393$ H $3.923520-4.2323841 .734331$ O $2.962124-0.338851-2.839330$ O $1.363075-0.281620-1.175559$ C $2.4024710 .839688-3.425254$ H $1.3668430 .659471-3.726846$ H $2.4603711 .674521-2.722154$ H $2.9882951 .089399-4.313691$

SCF Energy (B3LYP/6-31G**//MMFF) $=-3245.90752403$

0400040

MM̄FF Geometry

C 0.2167022 .1869423 .297681

C $\quad 0.864061 \quad 1.2573792 .578841$

C $1.340027-0.0473613 .164300$

O $2.706524-0.2334762 .726946$

C $0.519642-1.2308012 .630625$

C $0.746866-2.4981863 .433500$
C $1.538790-3.5210873 .057007$

C $-0.034655-2.5896184 .719472$

C $2.394344-3.6288491 .825737$

C $1.848989-4.6605880 .827039$

C $0.573271-4.1909360 .112009$

C $0.030926-5.280132-0.813926$

C $-1.183356-4.786666-1.616658$

O $0.913016-3.032674-0.652873$

O $-1.437216-5.735889-2.662486$

C $-0.823219-3.447822-2.295941$

C $-2.450379-4.736481-0.756381$

O $-1.959590-2.875595-2.938356$

C $-0.185494-2.417197-1.336104$

C $0.385319-1.226687-2.142179$

O $-1.179530-1.939187-0.428353$

C $3.710572-0.0629483 .620569$

O $3.596443 \quad 0.2332374 .802386$

C $5.035982-0.2862782 .994623$

C $5.210356-0.5415481 .688201$

C $6.489400-0.7587131 .008109$

C $7.744742-0.7112291 .839253$

C $1.240500-0.259906-1.309534$

C $1.9781610 .735346-2.187526$

C $3.9286692 .283785-2.143522$

O $1.6911120 .932691-3.364875$

C $5.054517 \quad 1.471231-2.704528$

C $6.3370161 .607224-2.334579$

C $7.4522980 .767947-2.890966$

C $8.2411230 .052525-1.814945$

C $9.4594570 .491837-1.454654$

C $7.666801-1.216568-1.222879$

C $6.484921-0.972274-0.324784$

N $2.9900621 .386853-1.493444$

H 0.0023621 .9635194 .342053

H 1.0880321 .4344751 .531256

H $1.285751-0.0131644 .258622$

H $\quad 0.761816-1.4008671 .576889$

H $-0.553423-0.9983012 .657787$

H $1.599426-4.3893933 .712775$

H $\quad 0.144378-3.5311755 .249866$

H $\quad 0.236542-1.7749695 .397437$

H -1.107963 -2.528388 4.513007

H $2.541209-2.6614581 .337100$

H $3.393463-3.9500642 .147153$

H $2.631215-4.8399870 .078117$

H $1.664462-5.6132191 .338325$

H $-0.179663-3.9240730 .860941$

H $-0.224130-6.183705-0.245974$

H $\quad 0.815075-5.582965-1.522350$

H -1.591851 -6.602543 -2.248790

H $-0.099212-3.673374-3.091667$

H -2.691033 -5.733315 -0.368436

H $-3.317684-4.427080-1.348531$

H $-2.357455-4.0665700 .099821$

H -2.320151 -3.547535 -3.541725

H $1.017445-1.617989-2.950068$

H -0.431229 -0.663882 -2.611826

H $-0.952466-1.038127-0.150785$

H $5.875835-0.2157833 .679196$

H $4.336903-0.5925351 .040658$

H 7.8508190 .2673962 .320502

H $8.655977-0.8720261 .259375$

H $7.723481-1.4852512 .614068$

H $0.6157060 .305846-0.611959$

H $1.985148-0.814424-0.728045$

H $4.2681923 .004263-1.392957$

H $3.4228402 .824725-2.949408$

H $4.7975660 .727182-3.455457$

H $6.6030582 .360106-1.596455$

H $8.1123351 .431289-3.464981$

H $7.0778220 .029029-3.610878$

H $10.051806-0.028812-0.709634$

H $9.8918361 .383008-1.896982$

H $8.437790-1.787047-0.691426$

H $7.351455-1.879813-2.039265$

H $5.527388-0.986534-0.846432$

H $3.1968121 .102579-0.540614$

C 0.2315344 .0532981 .485847 
O $-0.496957 \quad 3.3942350 .428331$

C $-0.972308 \quad 4.388696-0.499722$

C $-0.1713965 .648583-0.204078$

C $-0.0230525 .552593 \quad 1.298972$

H -2.023549 $4.574579-0.248544$

C $-0.8679273 .895514-1.946514$

H $0.8144025 .628112-0.682844$

H $-0.6873996 .556873-0.529019$

C 1.0769756 .4409161 .855208

H $-0.978125 \quad 5.8328601 .763954$

C -1.549618 2.539359-2.207967

O $\quad 0.5181103 .755855-2.280786$

H $-1.2703804 .661434-2.620257$

H $-0.9853211 .747446-1.705163$

O $-1.4477782 .258216-3.608911$

C $-3.0233442 .455120-1.773650$

C $-3.6655531 .110380-2.161238$

H $-3.0762102 .579980-0.687984$

O $-3.767863 \quad 3.524556-2.351867$

H -3.719201 $1.030238-3.253689$

H -3.015829 $0.301519-1.811056$

H 1.1567086 .3329042 .941342

H 2.0509626 .1939421 .419781

H $\quad 0.8687667 .4930351 .635476$

C $-0.248834 \quad 3.5480362 .851883$

H $0.566493 \quad 3.413019-3.189672$

H $-1.8993812 .971175-4.091122$

H -3.813183 $3.374713-3.311280$

H $-1.345704 \quad 3.5408192 .873139$

H $0.0840654 .252842 \quad 3.623219$

H 1.2956373 .8297561 .336048

C -4.4859860 .2060340 .621608$

O $-5.1503641 .183534-0.183551$

C $-5.092522 \quad 0.937160-1.592300$

C $-5.729415-0.423680-1.910844$

C $-5.086565-1.551918-1.102830$

C $-5.064920-1.1950550 .384916$

H -3.4192610 .2084390 .366105$

C $-4.554950 \quad 0.6537162 .087835$

H -5.716209 $1.707744-2.063638$

O $-5.624627-0.690884-3.305415$

H $-6.799753-0.379611-1.669023$

H $-4.079528-1.783202-1.465333$

O $-5.856329-2.745575-1.260863$

H -6.091083 -1.2591230 .765099$

O $-4.296896-2.161243 \quad 1.097127$

C $-5.934394 \quad 0.5443362 .696943$

H -3.875803 0.0453932 .697126

H -4.2279881 .6952212 .192219$

H -6.153553 -1.484136 -3.494553

H $-5.776107-3.026041-2.188056$

H -3.372143 -2.078382 0.807780

O -6.7743851 .4608102 .138386$

O $-6.226729-0.2639653 .569342$

C $-8.123603 \quad 1.4307102 .614271$

H $-8.151848 \quad 1.6359443 .688171$

H -8.685165 2.2101132 .092272

H -8.580758 0.4613782 .392648

SCF Energy (B3LYP/6-31G**/MMFF) $=-3245.90940638$

0400041

MMFF Geometry

C $-0.427012-1.6437720 .540979$

C $-0.523018-2.219344-0.667851$

C $-0.940848-1.513164-1.938674$

O $-0.737710-0.090723-1.780074$

C $-2.430684-1.767696-2.218455$

C $-2.845095-1.394182-3.626239$

C $-3.481936-0.256221-3.964509$

C $-2.533305-2.426410-4.679017$

C $-3.852726 \quad 0.900601-3.077607$

C $-5.370857 \quad 1.038518-2.899955$

C $-5.9608110 .005700-1.926976$

C $-7.4884730 .094024-1.910705$

C $-8.102905-0.846780-0.863345$

O $-5.4418360 .293524-0.623622$

O $-9.490290-0.507738-0.717873$

C $-7.402937-0.613116 \quad 0.492323$
C $-8.090794-2.304134-1.337192$

O $-7.838836-1.5640981 .461247$

C $-5.858598-0.6180030 .400964$

C $-5.258492-0.1345821 .744221$

O $-5.410218-1.9485810 .136955$

C $-0.1295980 .606843-2.769698$

$\begin{array}{lllll}\text { O } & 0.256401 & 0.169387 & -3.844578\end{array}$

C $0.0457802 .030966-2.393802$

C $-0.3759562 .549373-1.229457$

C $-0.1982203 .930772-0.774530$

C $0.5344414 .880745-1.684416$

C -3.7466100 .1248931 .704525$

C -3.2155460 .6210373 .040203$

C -1.109172 1.4644524 .087027

O

C -1.0682522 .9558093 .983578$

C 0.0462603 .6541093 .718251

C 0.0831075 .1508793 .605013

C 0.4692495 .6223232 .220165

C 1.6934256 .1183011 .973871

C -0.5913495 .5991631 .140405$

C -0.6719924 .2692140 .443238$

N -1.867479 0.9419802 .966570

H $-0.696569-0.5952280 .632506$

H $-0.291289-3.274991-0.780146$

H $-0.309129-1.899844-2.747205$

H -3.038982 -1.221302 -1.488482

H $-2.671776-2.828185-2.064442$

H $-3.749702-0.113344-5.010987$

H -2.876631 -2.123634 -5.673963

H $-1.454199-2.597462-4.742136$

H -3.022687 -3.375255 -4.436560

H $-3.3545190 .858006-2.104790$

H -3.477208 $1.810996-3.562037$

H -5.567795 $2.045557-2.509478$

H $-5.8673120 .965878-3.875601$

H $-5.643672-0.997638-2.231342$

H $-7.902944-0.116210-2.904618$

H -7.788635 $1.124504-1.673989$

H $-9.5488440 .422464-0.440990$

H $-7.721413 \quad 0.3719180 .860918$

H -8.670277 -2.408600 -2.262533

H $-8.574317-2.961881-0.606347$

H -7.086420 -2.685948 -1.531908

H $-8.810053-1.5238671 .490825$

H -5.7453170 .8046662 .038562$

H -5.470250 -0.871070 2.529733

H -4.499986 -2.048118 0.456154

H $\quad 0.5625642 .633661-3.133634$

H $-0.8910961 .902318-0.521490$

H $1.5406554 .507856-1.901883$

H $0.6624675 .879551-1.261972$

H $-0.0075535 .002143-2.628675$

H -3.207610 -0.7942291 .456471$

H $-3.5116750 .879100 \quad 0.944649$

H -0.1112801 .0189974 .038056$

H -1.5773631 .1662345 .030315$

H -2.0064943 .4851084 .132579$

H 0.9857933 .1258053 .573930

H $\quad 0.799813 \quad 5.5235254 .348259$

H $-0.8812775 .595870 \quad 3.880718$

H 1.9665506 .4840460 .989675

H $2.451346 \quad 6.1640762 .748769$

H -0.4264406 .4164670 .430585$

H -1.5706905 .8212331 .583464$

$\mathrm{H}-1.1901913 .5054221 .022682$

H -1.407447 0.9256822 .061617

C $0.905197-3.517091 \quad 1.717266$

O $2.114519-3.174751 \quad 1.012613$

C $3.238373-3.7613611 .692409$

C $2.655064-4.7476822 .697298$

C $1.358258-4.0583473 .074095$

H $3.731717-2.9507392 .241388$

C $4.216402-4.3655030 .680952$

H $2.446125-5.7217802 .240468$

H $3.319588-4.9115963 .551132$

C $0.350286-4.9780163 .740917$

H $1.589473-3.2272183 .753982$ 
C $4.649158-3.396505-0.438455$

O $3.586091-5.4899010 .053312$

H $5.092251-4.7592161 .210284$

H $3.798704-3.199094-1.101998$

O $5.617442-4.077263-1.248346$

C $5.236265-2.0551370 .044119$

C $5.757177-1.198669-1.125737$

H $4.461428-1.4960980 .578414$

O $6.292209-2.295370 \quad 0.971883$

H $6.542252-1.750429-1.656745$

H $4.935704-1.047072-1.834482$

H $-0.571158-4.4382983 .980869$

H $\quad 0.088070-5.8222393 .094393$

H $\quad 0.757269-5.3826834 .673341$

C $0.016957-2.2770381 .832410$

H $4.220708-5.859547-0.584319$

H $\quad 6.370463-4.311747-0.680055$

H $7.030947-2.7006390 .487314$

H $0.574757-1.5051712 .377723$

H $-0.875349-2.5019422 .427929$

H $0.404565-4.3061091 .142801$

C $4.2977061 .430174-0.565138$

O 5.4469320 .9209960 .120478

C $6.3488690 .158294-0.684138$

C $6.8630461 .020739-1.843733$

C $5.7158421 .605259-2.663585$

C $4.7124802 .311578-1.751828$

H $3.6765890 .594336-0.910417$

C 3.4848842 .2457790 .448705

H $7.211766-0.067232-0.044909$

O $7.7230120 .254986-2.680992$

H $7.4628361 .844689-1.435392$

H $5.2318280 .832128-3.271576$

O $\quad 6.2675512 .552325-3.581584$

H $5.1503523 .249808-1.386919$

O $3.5485822 .644877-2.504739$

C 2.8098921 .3502371 .458692

H 4.1163922 .9625440 .987812

H $2.6962602 .826125-0.041940$

H $8.0055810 .831053-3.411917$

H $5.5500032 .845309-4.167993$

H $3.806728 \quad 3.300577-3.173927$

O 3.6764331 .0495762 .467303

O $1.655698 \quad 0.9572121 .354719$

C 3.1668360 .1836043 .484942

H 2.3548580 .6768754 .026144

H $2.825674-0.7587883 .048624$

H $3.977358-0.0304184 .186452$

SCF Energy (B3LYP/6-31G**//MMFF) $=-3245.90770133$

0400042

MM̄FF Geometry

C 1.2744362 .3524113 .184852

C 1.3941831 .2391532 .446156

C $1.566901-0.1411673 .023148$

O $2.845218-0.6032482 .532829$

C $0.469759-1.0784282 .496438$

C $0.367891-2.3780823 .266321$

C $0.898577-3.5517492 .870890$

C $-0.432335-2.3018804 .541148$

C $1.751040-3.8235941 .662370$

C $1.068241-4.7484170 .645313$

C $-0.078260-4.073352-0.123028$

C $-0.727914-5.059463-1.096034$

C $-1.812477-4.387572-1.950282$

O $0.472472-2.973372-0.851966$

O $-2.131748-5.296959-3.014116$

C -1.232862 -3.101906-2.579544

C $-3.105017-4.190282-1.153034$

O $-2.246788-2.370933-3.266334$

C $-0.488356-2.192841-1.573023$

C $0.279887-1.084850-2.333386$

O $-1.444728-1.589206-0.700823$

C $3.694373-1.2313443 .381147$

O $3.489074-1.5071824 .554502$

C $4.985383-1.5300292 .713729$

C $5.187905-1.3633361 .396899$

C $6.442150-1.5758270 .671918$
C $7.655470-1.9753731 .468628$

C $1.243289-0.276245-1.452955$

C $2.0692130 .709537-2.260938$

C $4.0142152 .254825-2.029473$

O $1.8563050 .953241-3.445532$

C $5.1520011 .507738-2.652622$

C $6.4211031 .572210-2.221749$

C $7.5525860 .815265-2.859000$

C $8.252921-0.120398-1.896575$

C $9.4471380 .200765-1.370998$

C $7.615044-1.463506-1.610792$

C $6.454388-1.361920-0.660668$

N $3.061521 \quad 1.301431-1.490204$

H 1.2799232 .2523534 .268930

H 1.4024951 .3049821 .362986

H $1.560771-0.0964724 .118820$

H $\quad 0.634822-1.2837441 .433394$

H $-0.504297-0.5768002 .551155$

H $0.735477-4.4244863 .502348$

H $-0.496263-3.2687515 .051575$

H $0.023161-1.5916385 .238135$

H -1.455033 -1.973521 4.328841

H $2.084911-2.9044081 .173340$

H $2.665307-4.3171472 .016271$

H $1.836012-5.068258-0.071508$

H $0.701159-5.6510221 .149481$

H -0.818334 -3.701249 0.594181

H -1.140391 $-5.923080-0.559494$

H $\quad 0.040170-5.465530-1.769546$

H -2.764497 -4.850897 -3.603346

H $-0.518929-3.422391-3.350589$

H $-3.480643-5.154379-0.789479$

H $-3.900818-3.781670-1.784684$

H $-2.975486-3.536978-0.288034$

H $-2.782448-1.908712-2.598973$

H $0.872036-1.545828-3.134924$

H $-0.430375-0.395210-2.807360$

H $-1.082144-0.759274-0.353760$

H $5.768829-1.8867573 .374334$

H $4.360984-1.0198180 .777302$

H $7.499919-2.9521461 .939624$

H $7.863884-1.2368592 .250752$

H $8.563943-2.0532420 .867366$

H $\quad 0.6855310 .294731-0.704366$

H $1.935625-0.947071-0.931744$

H $4.3392222 .896039-1.204157$

H $3.5197822 .876539-2.782716$

H $4.9164050 .882460-3.510883$

H $6.6651942 .206176-1.372536$

H $8.2639321 .552833-3.253440$

H $7.2103570 .243053-3.730505$

H $9.971730-0.475167-0.704133$

H $9.9253291 .149231-1.592289$

H $8.360707-2.177590-1.243872$

H $7.259886-1.902062-2.552204$

H $5.521171-1.075941-1.146095$

H $3.2081090 .976835-0.538676$

C 1.1549243 .9832011 .169326

O $-0.115817 \quad 3.5586040 .631817$

C $-0.5939414 .566783-0.278917$

C $0.6024785 .460234-0.575100$

C 1.3090965 .4509260 .765211

H -1.3411785 .1550260 .265897$

C -1.227539 $3.922058-1.515440$

H $1.2485985 .029971-1.349370$

H $0.3043506 .461972-0.898666$

C 2.7513475 .9227810 .690795

H $0.7552076 .094291 \quad 1.462026$

C $-2.4087482 .978500-1.208249$

O $-0.2310783 .158868-2.205435$

H $-1.5453224 .700014-2.218701$

H -2.044457 $2.090941-0.677772$

O $-2.9308772 .512205-2.459312$

C $-3.5595693 .625841-0.414518$

C $-4.8153552 .752713-0.294440$

H -3.2199043 .8986480 .589976$

O $-3.9786334 .834106-1.057695$

H $\quad-5.5897003 .3192330 .242741$ 
H $-5.2622612 .608453-1.288606$ H 3.2271985 .8835651 .675549 H 3.3416135 .3038060 .006589 H 2.7991396 .9567660 .333869 C 1.1651763 .7720052 .687376 H $\quad 0.1260772 .511849-1.573788$ H -2.195273 2.101037 -2.945082 H $-3.2747175 .494113-0.944978$ H 0.2452254 .1923713 .113391 H 2.0066864 .3242663 .122578 H 1.9334803 .3841220 .681327 C $-6.008518-0.550790 \quad 0.899028$ $\begin{array}{llll}\text { O } & -5.934391 & 0.782408 & 0.381619\end{array}$ C -4.6300221 .3797240 .367553$ C $-4.0583571 .444611 \quad 1.792163$ C $-4.088530 \quad 0.073752 \quad 2.476777$ C $-5.451203-0.6134322 .329801$ H $-7.082691-0.7725940 .945073$ C $-5.362779-1.564410-0.048937$ H $-3.9763840 .774733-0.268931$ O -4.7946502 .3803772 .577731$ H -3.0238601 .8039091 .750727$ H -3.853055 0.2026693 .540216 O $-3.072515-0.7416181 .902735$ H $-5.394577-1.6596042 .652247$ O -6.3943740 .0387503 .184696$ C $-6.054800-1.566183-1.391027$ H $-4.298667-1.372162-0.208168$ H -5.435444 -2.579839 0.357607 H -5.7283422 .1082632 .566645$ H -3.085527 -1.5978292 .363490$ H $-6.067995-0.0425834 .097174$ O $-5.258774-0.969336-2.323801$ O $-7.172684-2.026622-1.582096$ C $-5.803115-0.896153-3.644443$ H $-5.070195-0.400507-4.286510$ H $-5.988044-1.901827-4.033923$ H $-6.724354-0.305925-3.642151$

SCF Energy (B3LYP/6-31G**//MMFF) $=-3245.92088270$

\section{3}

MM̄FF Geometry

C $-0.9868390 .597553 \quad 4.432232$

C -1.2251301 .4453223 .420373$

C -0.2551052 .4155142 .786967$

O 0.0377531 .8888851 .468765

C 1.0621462 .6370693 .560571

C 1.7902953 .9146603 .185106

C 2.9477033 .9796952 .498525

C 1.1596355 .1861163 .699754

C 3.7534092 .8520911 .918910

C 4.0877603 .1125300 .443266

C $5.0469302 .056188-0.121924$

C $5.3683872 .346391-1.589040$

C $6.2592641 .256676-2.204483$

O $4.4248310 .774389-0.005928$

O $6.2408911 .424155-3.630263$

C $5.640900-0.124471-1.903486$

C $7.7222101 .401734-1.769224$

O $6.509569-1.173892-2.325258$

C $5.254267-0.320676-0.418341$

C $4.431944-1.617986-0.260171$

O $6.438150-0.4187810 .373571$

C 0.1230052 .7647870 .436739

O $0.0310343 .983304 \quad 0.505220$

C $0.3134192 .081147-0.863161$

C $0.5265930 .764573-1.013790$

C $0.6921820 .065282-2.294096$

C $0.5016670 .867482-3.555296$

C $3.893747-1.8580851 .157735$

C $2.702034-2.7923921 .122978$

C $2.044141-5.0935240 .441364$

O $1.566712-2.3938521 .372298$

C $1.804379-5.053994-1.035883$

C $0.636103-4.707134-1.596635$

C $0.419560-4.631677-3.081026$

C $0.104822-3.230574-3.559076$

C $-1.077192-2.939641-4.128286$
C $1.191674-2.182080-3.448557$

C $0.977078-1.254051-2.281634$

N $3.039112-4.0866790 .760155$

H 0.0046800 .5338984 .870676

H -2.229815 1.4655693 .001486

H -0.8023863 .3620732 .697982$

H 1.7118031 .7648153 .433510

H 0.8578932 .7040474 .637631

H 3.3843804 .9651202 .337547

H 1.7700006 .0701233 .486049

H 0.1799355 .3483163 .240718

H 1.0312855 .1338724 .785950

H 4.6835722 .7699752 .493875

H 3.2359711 .8933192 .006448

H $3.1547913 .110744-0.133584$

H $4.5391354 .106247 \quad 0.329889$

H 5.9628072 .0693390 .480825

H $5.836373 \quad 3.332607-1.698562$

H $4.4327282 .393119-2.164208$

H $6.5593272 .321029-3.830681$

H $4.729255-0.214019-2.511404$

H $8.1172952 .378306-2.074128$

H $8.3566960 .656002-2.260802$

H $7.8590371 .313758-0.689408$

H $\quad 6.714596-1.020138-3.263487$

H $3.581808-1.579948-0.951167$

H $5.042386-2.482881-0.548554$

H $6.219389-0.8532551 .213129$

H $0.2679842 .747318-1.719381$

H $0.5851010 .134771-0.128046$

H $-0.4764501 .362393-3.552904$

H $\quad 0.5326720 .266602-4.466224$

H $1.2831771 .629902-3.646381$

H $4.665757-2.2885581 .805230$

H $3.562768-0.9242611 .625225$

H $1.130179-4.9050551 .013275$

H $2.447725-6.0678230 .732018$

H $2.646188-5.309200-1.675664$

H $-0.207276-4.457072-0.958422$

H $-0.397171-5.319304-3.334809$

H $1.298694-4.997739-3.626581$

H -1.294497 -1.942034-4.495709

H -1.854586 -3.688741 -4.234580

H $1.275471-1.646343-4.398715$

H $2.167082-2.669650-3.323736$

H $1.083317-1.744025-1.314457$

H $4.005059-4.2949030 .524699$

C $-2.044592-1.7254514 .528441$

O $-2.579615-1.765993 \quad 3.190844$

C $-1.858956-2.7628302 .442326$

C $-1.014959-3.5330773 .456356$

C $-0.701371-2.4510824 .470555$

H $-1.190261-2.2167001 .767030$

C $-2.817459-3.6345531 .626272$

H - $-1.582265-4.3418573 .931195$

H $-0.121410-3.9736113 .005532$

C $-0.228182-2.9821875 .811352$

H $0.078496-1.8079394 .044944$

C $-3.789666-2.8603630 .713300$

O $-3.613148-4.4122482 .531099$

H $-2.233793-4.3539221 .038692$

H $-4.566690-2.3798731 .319844$

O $-4.473812-3.835368-0.087751$

C $-3.142029-1.807457-0.206325$

C $-4.122493-1.293284-1.277375$

H $-2.773322-0.9771700 .406063$

O $-2.023289-2.404314-0.855027$

H -4.341450 -2.108730 -1.977585

H $-5.064074-1.024619-0.787201$

H $-0.033181-2.160426 \quad 6.507651$

H $-0.973323-3.6422186 .268065$

H $\quad 0.698560-3.5531475 .693201$

C -2.036157 -0.2867565 .047084$

H $-4.203235-4.9681641 .993605$

H -3.817202 $-4.238520-0.681777$

H -1.505335 -1.689751 -1.259472

H -1.872754 -0.2917426 .131632$

H $-3.0268090 .156864 \quad 4.883511$ 
H $-2.750436-2.2986415 .144833$

C $-4.3578471 .692564-0.670171$

O $-3.2430231 .021053-1.264910$

C -3.583602 -0.095125 -2.093281

C $-4.5228130 .357822-3.221118$

C $-5.768658 \quad 1.048377-2.672574$

C $-5.3721032 .173065-1.717911$

H -4.8545080 .9971570 .018004$

C -3.8233102 .8297170 .214129$

H -2.650987 -0.420092 -2.568740

O $-4.917294-0.744894-4.030639$

H $-3.9937201 .063610-3.874638$

H $-6.4451040 .336701-2.185138$

O $-6.5049251 .621681-3.755621$

H $-4.965748 \quad 3.003315-2.306417$

O $-6.5344082 .661452-1.051364$

C $-3.1459203 .950228-0.548586$

H $-4.653730 \quad 3.2530590 .791239$

H -3.08006324342840 .914458$

H -4.107256 -1.160270 -4.373058

H $-6.7123870 .902029-4.376271$

H -7.170184 2.930371-1.736508

O $\quad-3.195247 \quad 5.092070 \quad 0.199376$

O $-2.6134493 .836067-1.644099$

C $-2.594808 \quad 6.246070-0.396877$

H $-3.1232816 .512240-1.317161$

H -2.679575 7.0762220 .309344

H $-1.5351086 .065047-0.599078$

SCF Energy (B3LYP/6-31G**//MMFF) $=-3245.91522656$

\section{00044}

MM̄̄F Geometry

C $1.7519584 .523954-0.796583$

C $3.0223024 .150983-0.580385$

C 3.6638473 .8025990 .744382

O 3.7520942 .3576350 .821324

C 2.9028554 .2998951 .990038

C 3.6687904 .1123003 .289013

C 3.3380693 .2421844 .264843

C 4.8607695 .0181743 .472169

C 2.1847202 .2793804 .289365

C 2.6699920 .8364734 .108757

C $1.516132-0.1218683 .780666$

C $2.028910-1.5605213 .688248$

C $0.950477-2.5354663 .194368$

O $0.967424 \quad 0.2916952 .525190$

O $1.600923-3.7603462 .821190$

C $0.305632-1.9614421 .915373$

C $-0.046041-2.8937384 .302965$

O $-0.789842-2.7683441 .496466$

C $-0.139400-0.4922052 .062044$

C $-0.613426 \quad 0.1336540 .727565$

O $-1.237191-0.4186042 .977811$

C 4.7790591 .7521480 .164780

O $5.6844072 .296742-0.446669$

C $4.590305 \quad 0.2892190 .286876$

C $5.317461-0.535383-0.481648$

C $5.246878-1.998316-0.495480$

C $4.271921-2.6604220 .440844$

C $0.4488710 .196029-0.375384$

C $0.383417-0.946754-1.373488$

C $1.644106-1.908218-3.298272$

O $-0.559413-1.729824-1.443321$

C $2.391043-3.111382-2.814852$

C $3.476602-3.603356-3.431687$

C $4.190145-4.852636-2.999526$

C $5.680805-4.655121-2.839314$

C $6.531736-4.987686-3.824902$

C $6.189559-4.163800-1.503723$

C $6.049305-2.675376-1.343330$

N $1.490675-0.958524-2.212532$

H 1.0523794 .5982930 .030606

H $3.6816164 .069624-1.443291$

H 4.6718574 .2348760 .749197

H 1.9338963 .7955652 .040158

H 2.6804125 .3704741 .886870

H 3.9648633 .2060755 .155134

H 5.2996944 .9308434 .471979
H 5.6453724 .7780122 .748597

H $4.567503 \quad 6.0643783 .336240$

H 1.6738252 .3715835 .255860

H 1.4339262 .5320963 .538053

H 3.4020260 .8043923 .291956

H 3.1849440 .5080335 .020119

H $0.748583-0.0338204 .558477$

H $2.434168-1.8903484 .653042$

H $2.871762-1.5976132 .985831$

H $2.080470-4.0942163 .598696$

H $1.058803-2.0256531 .121757$

H $-0.789961-3.6157133 .948149$

H $-0.571201-2.0251604 .705489$

H $0.470180-3.3819625 .138371$

H -0.458299 -3.676799 1.393778

H $-1.533016-0.3545970 .384608$

H $-0.902307 \quad 1.172687 \quad 0.942736$

H -1.7404010 .3928252 .797358$

H $3.847227-0.0671190 .990318$

H $\quad 6.036487-0.099128-1.174089$

H $3.263148-2.263740 \quad 0.288633$

H $4.567080-2.4886351 .481227$

H $4.193617-3.7404060 .301090$

H $\quad 0.2847991 .109935-0.957307$

H 1.4567810 .2587270 .045877

H $2.168818-1.392757-4.109040$

H $\quad 0.655167-2.209488-3.658581$

H $1.997737-3.624011-1.940107$

H $3.847760-3.107845-4.326125$

H $3.999742-5.622013-3.759545$

H $3.771649-5.251545-2.067131$

H $7.604753-4.878941-3.706431$

H $6.173241-5.371023-4.774626$

H $7.257487-4.399777-1.399962$

H $5.707271-4.728493-0.700792$

H $6.716975-2.106133-1.991591$

H $2.248937-0.303316-2.045817$

C $0.362083 \quad 3.693122-2.713495$

O $-0.8025923 .492491-1.889019$

C $-1.9535723 .307685-2.738665$

C $-1.4060593 .087282-4.140157$

C $-0.1660553 .957170-4.122753$

H $-2.5121904 .250500-2.686956$

C $-2.8036012 .150423-2.199179$

H -1.133839 2.034989 -4.291358

H $-2.1180403 .371719-4.920551$

C $0.8230143 .624462-5.226431$

H $-0.4728705 .007476-4.218693$

C $-4.0242921 .824441-3.078612$

O $-3.2654632 .509531-0.893017$

H $-2.175176 \quad 1.261556-2.077122$

H -3.692816 $1.466874-4.060073$

O $-4.7520473 .035699-3.324463$

C $-5.0082270 .805796-2.470722$

C $-4.375362-0.562858-2.165857$

H $\quad-5.4639431 .228550-1.568777$

O $-6.0800830 .613999-3.402448$

H $-3.993237-0.993320-3.099598$

H -3.519175 -0.433541-1.498542

H $1.7055844 .268911-5.167566$

H $1.1603432 .584346-5.162791$

H $0.3643153 .769254-6.210125$

C $1.2034074 .843958-2.159926$

H -2.481772 $2.715350-0.355042$

H $-5.0369713 .386055-2.463057$

H $-5.7025810 .242282-4.217969$

H $0.5927585 .750591-2.067545$

H $2.0210885 .076679-2.852106$

H $0.9355602 .757344-2.688853$

C $-5.140402-0.8829480 .765402$

O $-6.008701-1.010508-0.367162$

C $-5.385884-1.551892-1.538084$

C $-4.813735-2.942934-1.234211$

C $-3.904223-2.937091-0.007638$

C $-4.600139-2.2597521 .174266$

H -4.307481 -0.2167820 .511751$

C $-5.950402-0.2156381 .883122$

H $-6.188442-1.693577-2.272859$ 
O $-4.106553-3.439302-2.365899$

H -5.646952 -3.633597 -1.049499

H -2.947741 -2.456031 -0.237310

O $-3.606691-4.293258 \quad 0.329639$

H $-5.419840-2.8978491 .528425$

O $-3.678229-2.1097352 .247682$

C -5.0637230 .4181012 .928819$

H -6.5878910 .5799391 .478061$

H $-6.635382-0.9380792 .343159$

H -3.738895 -4.306048 -2.121912

H $-2.925346-4.2795151 .022862$

H $-3.414553-2.9981452 .540251$

O $-5.357360-0.090558 \quad 4.159337$

O -4.2097891 .2604992 .682722$

C -4.5610270 .4114595 .236977$

H -3.5053220 .1771645 .069390$

H -4.7055991 .4907615 .344125$

H -4.886841-0.079387 6.157847

SCF Energy (B3LYP/6-31G**//MMFF) $=-3245.92002024$

\section{5}

MM̄MF Geometry

C - $-0.8335522 .295595-3.085596$

C $-0.9792681 .223948-2.291389$

C $-0.794551-0.201513-2.741141$

O $-2.072484-0.842056-2.531972$

C $0.255130-0.898126-1.858728$

C $0.748655-2.212621-2.424257$

C $0.339337-3.429110-2.014586$

C $1.811076-2.092693-3.486297$

C $-0.715520-3.759593-0.995483$

C $-0.137894-4.4212690 .263755$

C $0.610536-3.4418981 .181600$

C $1.207676-4.1782352 .383380$

C $1.853943-3.2134703 .390171$

O $-0.330823-2.4626961 .632532$

O $2.079520-3.9352084 .610599$

C $0.855868-2.0806973 .712170$

C $3.227967-2.7268402 .915151$

O $1.466581-1.0802034 .524452$

C $0.233565-1.4339372 .454399$

C $-0.902941-0.4663422 .861000$

O $1.242200-0.7014241 .761281$

C $-2.546739-1.694303-3.472456$

O $-1.990187-2.025999-4.509504$

C $-3.900900-2.181865-3.113280$

C $-4.473268-1.953771-1.920296$

C $-5.820568-2.352999-1.508147$

C $-6.688820-3.047974-2.523026$

C -1.7097930 .0850891 .677303$

C -2.8690930 .9563022 .128031$

C -4.9146412 .0711891 .235122$

O $\begin{array}{llll}-3.020865 & 1.326786 & 3.289037\end{array}$

C $-6.034416 \quad 1.1618371 .635364$

C -7.1296340 .9435910 .891708$

C $-8.244953 \quad 0.025810 \quad 1.307344$

C -8.480259 $-1.099810 \quad 0.322557$

C $-9.522570-1.065697-0.524923$

C -7.564002 $-2.303797 \quad 0.378091$

C $-6.226009-2.042753-0.258726$

N $-3.7052981 .286001 \quad 1.068947$

H - $0.5648352 .131717-4.127493$

H -1.260835 $1.356251-1.251529$

H $-0.510749-0.229578-3.799802$

H - $0.152471-1.050852-0.853741$

H $\quad 1.126042-0.244482-1.724980$

H $\quad 0.791727-4.303382-2.481491$

H $2.131099-3.068346-3.867282$

H $1.441118-1.513881-4.338119$

H $2.696103-1.591286-3.081716$

H - $-1.321725-2.890065-0.726416$

H -1.409740 -4.466398 -1.467936

H $-0.979461-4.8544960 .820248$

H $\quad 0.522976-5.249842-0.019695$

H $\quad 1.402533-2.9476870 .608349$

H $1.932916-4.9332502 .054675$

H $\quad 0.412515-4.7332602 .901189$

H $2.651495-4.6952154 .407658$
H $\quad 0.045786-2.5169584 .313572$

H $3.904650-3.5763972 .763874$

H $3.706665-2.0936763 .670328$

H $3.182871-2.1703431 .977604$

H $1.833771-1.5266125 .306530$

H $-1.601799-0.9924963 .524599$

H $-0.488050 \quad 0.3776623 .426517$

H $\quad 0.812657-0.106050 \quad 1.128685$

H -4.407318 -2.737554 -3.895707

H -3.912591 -1.406461-1.164267

H -6.804648 -2.429159 -3.419926

H -7.697480 -3.258623 -2.160903

H -6.247163 -4.007802 -2.812490

H -1.0729670 .7003751 .034876$

H $-2.114148-0.7389301 .078566$

H -5.1095892 .5784120 .285043$

H -4.7543442 .8275552 .010236$

H -5.9379250 .6584702 .594853$

H -7.237381 $1.453906-0.062559$

H -9.1532600 .6336631 .410578$

H $-8.062629-0.4000752 .301944$

H -9.718403 -1.880680 -1.213577

H -10.201332 -0.219668 -0.552164

H -8.047026 -3.180337 -0.067916

H -7.403059 -2.587337 1.426151

H $-5.520993-1.5389830 .402701$

H -3.5363270 .8692800 .157988$

C $-1.2413004 .041159-1.213375$

O $-0.0723123 .660699-0.465803$

C $0.2226724 .678128 \quad 0.508571$

C -0.9974175 .5892030 .542601$

C -1.457657 $5.522649-0.903256$

H 1.0735675 .2477010 .116019

C 0.6130574 .0254521 .840431

H -1.779696 5.2017331 .205266

H $-0.749636 \quad 6.6034550 .869636$

C $-2.8871875 .993448-1.107080$

H $-0.7846196 .137992-1.515332$

C 1.6662562 .9067681 .684932

O -0.5575753 .4342832 .417043$

H $\quad 0.9535214 .7920242 .545886$

H 1.2132442 .0858351 .123240

O 1.9565012 .3729132 .981502

C 2.9782383 .3477121 .009066

C 4.0052062 .2148440 .828445

H 2.7647643 .7958690 .038662

O $3.5825514 .381973 \quad 1.791928$

H 4.9704672 .6616110 .559153

H 4.1935041 .7443281 .803053

H -3.177919 $5.914160-2.159277$

H $-3.5933625 .399873-0.516599$

H -2.993746 $7.040484-0.805141$

C -1.003622 $3.745626-2.698173$

H -0.2893873 .0095623 .249893$

H 2.2549493 .1055383 .546279

H 3.8990633 .9837892 .620446

H -0.090998 4.264949-3.018463

H -1.832639 4.151155 -3.289724

H $-2.0835483 .451896-0.829378$

C $5.712362-0.086459-0.119334$

O $4.300779-0.1073980 .108466$

C $3.6255331 .123257-0.198008$

C $3.8485531 .467919-1.680207$

C $5.3344281 .517664-2.039045$

C $6.0305380 .233321-1.584972$

H $\quad 6.1597650 .6807150 .525307$

C $6.301919-1.4198770 .356427$

H $2.5618370 .905343-0.074416$

O $3.2168262 .694438-2.015895$

H $3.3732430 .690496-2.291747$

H $5.8227562 .402850-1.615987$

O $5.4766731 .617437-3.457947$

H $5.702306-0.583376-2.238701$

O $7.4389250 .365009-1.760671$

C $5.923871-2.600758-0.507824$

H $7.396944-1.3649850 .377383$

H $5.981411-1.6408021 .382037$

H $3.2503902 .791345-2.982369$ 
H $5.1388512 .488636-3.726031$

H $7.7437651 .095367-1.195682$

O $4.641532-2.984281-0.253025$

O $6.684084-3.119626-1.315597$

C $4.175510-4.109518-1.003546$

H $3.165394-4.350770-0.664080$

H $4.140839-3.862878-2.068715$

H $4.818043-4.977731-0.828525$

SCF Energy (B3LYP/6-31G**//MMFF) $=-3245.89607687$

\section{6}

MM̄FF Geometry

C $1.381939 \quad 1.153628 \quad 2.973751$

C 0.5127441 .8539432 .230013

C $0.8055332 .671697 \quad 0.994229$

O $0.1200462 .012425-0.099626$

C $2.296217 \quad 2.827126 \quad 0.628681$

C $2.5728343 .966119-0.334527$

C $2.9561943 .821327-1.617784$

C $2.460115 \quad 5.3503810 .256318$

C $3.1568532 .553750-2.401620$

C $4.6465552 .289771-2.672108$

C $5.3658351 .688319-1.455352$

C $6.8835481 .726066-1.633512$

C $7.6025651 .045445-0.457869$

$\begin{array}{lllll}\text { O } & 4.927211 & 0.331118-1.332495\end{array}$

O $8.9846740 .887682-0.811791$

C $6.993902-0.356326-0.242593$

C $7.603678 \quad 1.9276810 .795335$

O $7.522659-0.9703230 .930456$

C $5.446958-0.364233-0.193647$

C $4.937713-1.824916-0.246189$

O $5.007406 \quad 0.233657 \quad 1.027683$

C $-0.5187842 .788077-1.011012$

O $-0.5728294 .010652-1.019756$

C $-1.212071 \quad 1.980553-2.039293$

C $-1.0699790 .654946-2.191839$

C $-1.758786-0.168146-3.192210$

C $-2.8286070 .495591-4.021725$

C $3.444516-1.960315-0.570988$

C $2.996990-3.412410-0.566551$

C $0.997084-4.821985-1.016686$

O $3.717691-4.337974-0.203362$

C $0.089571-4.869641-2.202009$

C $-1.248488-4.850183-2.115406$

C $-2.162151-4.885547-3.304763$

C $-2.814292-3.548107-3.572734$

C $-4.108092-3.331848-3.281918$

C $-1.981850-2.489181-4.262292$

C $-1.425843-1.471583-3.303711$

N $1.690266-3.547301-1.014062$

H 2.4303801 .1093862 .695258

H $-0.529387 \quad 1.8628922 .544893$

H $0.356846 \quad 3.6552391 .180013$

H 2.6699361 .8796820 .226006

H 2.8895683 .0303621 .529678

H $3.1501924 .725625-2.194406$

H $2.7675826 .130187-0.448854$

H 1.4292295 .5659610 .551242

H 3.1012055 .4390041 .139593

H $2.6844901 .688129-1.927669$

H $2.6442672 .682957-3.363310$

H $4.7173541 .587546-3.512829$

H $5.1362713 .220995-2.983314$

H $5.0838682 .245643-0.556446$

H $7.2389172 .757069-1.753403$

H $7.1540021 .204967-2.562432$

H $9.0322320 .353741-1.622850$

H $7.312443-0.981662-1.088210$

H 8.1013452 .8829240 .588429

H 8.1759431 .4621631 .605633

H 6.6013432 .1499881 .167315

H $8.492112-0.9519350 .855761$

H $5.485930-2.373626-1.023490$

H $5.148413-2.3221710 .709472$

H $4.137234-0.1277331 .259791$

H - $-1.8595312 .558972-2.691358$

H $-0.3894320 .116496-1.534589$
H -3.561793 $0.995954-3.378677$

H $-3.399759-0.201212-4.638391$

H $-2.3864201 .237775-4.695012$

H $2.836112-1.414908 \quad 0.156331$

H $3.238669-1.547238-1.565627$

H $0.450601-4.897553-0.071278$

H $1.717382-5.645140-1.058353$

H $\quad 0.560313-4.917297-3.181075$

H $-1.718263-4.806542-1.136133$

H $-2.922500-5.655715-3.121907$

H $-1.628424-5.214816-4.205321$

H $-4.583510-2.381322-3.500826$

H $-4.717715-4.096626-2.812361$

H -2.567764 -2.030779-5.064965

H $-1.137929-2.961101-4.781730$

H $-0.644317-1.860085-2.651065$

H $1.133518-2.706153-1.138032$

C $0.775128-1.0840294 .040947$

O $-0.461497-1.3091193 .333409$

C $-0.264270-2.3877582 .400623$

C $1.054878-3.0480852 .796175$

C $1.852424-1.8535303 .277714$

H $-0.149824-1.9248691 .412674$

C $-1.468209-3.3336002 .387205$

H $\quad 0.925560-3.765275 \quad 3.614924$

H $1.525447-3.5752521 .963372$

C $3.066644-2.2158804 .112505$

H $2.184354-1.2938362 .396790$

C $-2.840940-2.6550582 .213475$

O $-1.506759-4.044833 \quad 3.632491$

H $-1.316919-4.0921461 .610010$

H -3.119216 -2.1428863 .142567$

O $-3.807820-3.7044162 .054654$

C $-2.963602-1.6608041 .042012$

C $-4.429009-1.2638620 .773374$

H $-2.371788-0.767807 \quad 1.269075$

O $-2.412963-2.234920-0.140159$

H $-4.978152-2.1475170 .426465$

H $-4.885196-0.9508681 .718105$

H $3.592024-1.3148814 .444659$

H $2.786655-2.7914485 .001083$

H $3.767991-2.8199883 .527534$

C $1.003754 \quad 0.416757 \quad 4.229504$

H -2.263903 -4.6541913 .595527$

H $-3.592094-4.1872401 .238743$

H $-2.987243-2.970716-0.410720$

H $1.8036390 .574746 \quad 4.962972$

H 0.0965110 .8645214 .655371

H $0.631353-1.5300875 .034126$

C -4.5014591 .7659671 .158429$

O -3.9059331 .0504040 .072181$

C $-4.579006-0.157758-0.295349$

C $-6.0354320 .149454-0.673262$

$\begin{array}{llll}\text { C } & -6.766917 & 0.868665 & 0.458516\end{array}$

$\begin{array}{llll}\text { C } & -5.976298 & 2.101786 & 0.894794\end{array}$

H $-4.431277 \quad 1.146642 \quad 2.061280$

C $-3.6444563 .007776 \quad 1.449808$

H $-4.085120-0.524093-1.202472$

O $-6.710870-1.056163-1.014350$

H $-6.043407 \quad 0.788443-1.566019$

H -6.9566030 .2020201 .307785$

O

H -6.0718742 .8695290 .119052$

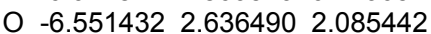

C $-3.636313 \quad 4.042310 \quad 0.342510$

H -4.0075543 .4851452 .367378$

H -2.6028262 .7061531 .602370$

H $-7.581068-0.815209-1.373914$

H $-8.5840250 .515760-0.172588$

H -7.4891202 .8162061 .899462$

$\begin{array}{llll}\text { O } & -3.248756 & 5.244946 & 0.862096\end{array}$

O $-3.903082 \quad 3.824323-0.831174$

C $-3.1938206 .329817-0.069461$

H $-4.185406 \quad 6.514256-0.493482$

H -2.8709597 .2243690 .469634$

H -2.469225 6.116683 -0.860621

SCF Energy (B3LYP/6-31G**//MMFF) $=-3245.90379052$ 


\section{7}

MMFF Geometry

C -0.5080913 .2917621 .766326$

C -1.596370 3.6691082 .456910

C -2.7954812 .8301752 .829774$

O $-2.709366 \quad 1.5070942 .259802$

C -4.0806583 .4841232 .292580$

C -5.3398982 .8712782 .873875$

C -6.1573042 .0274302 .214546$

C $-5.685328 \quad 3.3158504 .272407$

C -5.9782901 .4784720 .826558$

C $-6.353981-0.0092920 .762565$

C $-6.229202-0.560017-0.664365$

C $-6.790662-1.979908-0.750569$

C $-6.614859-2.573943-2.155333$

O $-4.843134-0.565434-1.020502$

O $-6.891095-3.978785-2.052932$

C $-5.142521-2.406262-2.583597$

C $-7.640912-2.004328-3.141662$

O $-4.957148-2.824903-3.934204$

C $-4.584484-0.977798-2.368720$

C $-3.055550-0.987964-2.586499$

O $-5.183506-0.091657-3.315125$

C - $-2.399310 \quad 0.4783623 .091614$

O -2.1536240 .5376234 .285456$

C $-2.381955-0.7569662 .279259$

C -1.669835 -1.807091 2.712693

C -1.496474 -3.079725 2.012598

C -2.413851 -3.3707620 .855948$

C $-2.3430220 .311443-2.193372$

C $-0.8444910 .090058-2.223700$

C $1.007180-0.909768-0.880876$

O $-0.1757640 .349939-3.221058$

C $1.037951-2.406063-0.864609$

C $1.660123-3.1250790 .082004$

C $1.713259-4.6264210 .089385$

C $1.354532-5.2264451 .432435$

C $2.293529-5.7962072 .206679$

C $-0.103451-5.2339141 .844453$

C $-0.526035-3.915606 \quad 2.436417$

N $-0.364504-0.471277-1.050286$

H -0.4065712 .2709661 .410473$

H -1.641926 4.6992132 .808789

H -2.835677 2.7839423 .924561

H $-4.0941283 .428747 \quad 1.197662$

H -4.0895314 .5569392 .529030$

H -7.062414 1.6964252 .722487

H -6.6364242 .8977214 .619201$

H -4.912155 3.0008294 .979682

H -5.7724824 .4065894 .313866$

H $-6.6293302 .043770 \quad 0.149300$

H -4.9508331 .6024690 .472812$

H $-5.696906-0.5733731 .437273$

H -7.384205 -0.1409441 .116336$

H $-6.7758300 .107000-1.341401$

H -7.846969 -2.002334 -0.454667

H -6.264304 -2.623657-0.031392

H -6.708195 -4.379028 -2.920511

H $-4.554891-3.103280-1.970230$

H -8.659771 -2.206175 -2.789930

H -7.562846 -2.490352 -4.120450

H -7.545179 -0.925932 -3.284356

H -5.293316 -2.118324 -4.511525

H -2.620187 -1.807795 -2.003977

H -2.829914 -1.198253 -3.640184

H $-4.6090710 .682305-3.426880$

H -2.913717 $-0.752231 \quad 1.334891$

H -1.124312 -1.713365 3.650996

H -2.168371 -2.729976 0.006094

H -3.459258 -3.195897 1.135203

H $-2.364090-4.4052490 .510422$

H -2.583974 $1.126109-2.884233$

H -2.632056 $0.645206-1.190457$

H $1.372659-0.4773680 .054644$

H $1.619127-0.541374-1.707402$

H $0.539412-2.922472-1.681711$

H $2.182235-2.6070890 .882141$

H $2.732520-4.921175-0.192769$
H $\quad 1.054437-5.051432-0.678187$

H $2.040617-6.2498563 .159401$

H $3.336485-5.8170091 .908656$

H $-0.275586-6.0070032 .604918$

H $-0.707109-5.5349200 .984301$

H $0.069019-3.6175023 .301402$

H -1.011606 -0.654341 -0.289775

C $0.9732904 .368845-0.038386$

O $1.9602073 .387001-0.405811$

C $1.8350753 .130050-1.819001$

C $0.6605703 .973336-2.323571$

C $-0.1465694 .207365-1.061460$

H $1.5818172 .070357-1.917725$

C $3.1544773 .422721-2.548523$

H $0.9936714 .927457-2.747732$

H $0.0919863 .455020-3.102447$

C -1.085373 5.397176-1.148789

H $-0.7284723 .297422-0.868485$

C $4.3933532 .676472-2.016274$

O $3.4423114 .826012-2.463952$

H $3.0287603 .210857-3.616990$

H $4.7001253 .087093-1.046495$

O $5.4734662 .943498-2.922044$

C $4.2316501 .152418-1.890029$

C $5.5670270 .431978-1.620925$

H $3.5254760 .939466-1.082199$

O $3.6704150 .625229-3.093233$

H $6.1733690 .456463-2.534315$

H $6.1190780 .977488-0.849465$

H $-1.6358605 .532121-0.212708$

H $-0.539616 \quad 6.324165-1.354464$

H -1.815626 $5.252243-1.951753$

C 0.6128514 .2449451 .446850

H $3.5175975 .053667-1.521373$

H $5.5709673 .909598-2.979391$

H $4.2643380 .864136-3.825442$

H 0.3495125 .2458721 .812258

H 1.4978353 .9245392 .010368

H $1.4657395 .341869-0.174476$

C $5.386881-0.7309361 .197041$

O $4.655229-1.1538260 .039495$

C $5.362191-1.041830-1.201590$

C $6.654327-1.869533-1.143626$

C $7.517718-1.4888830 .054280$

C $6.689574-1.5294011 .337998$

H 5.6100280 .3397341 .124205

C $4.486311-0.9460322 .422103$

H $4.720699-1.505276-1.961559$

O $7.413079-1.716574-2.338541$

H $6.398304-2.933939-1.060698$

H $7.985397-0.508393-0.093330$

O $8.586135-2.4326590 .160313$

H $6.465766-2.5751501 .583736$

O $7.469970-1.0091102 .412659$

C 3.3247170 .0234092 .461659

H $4.075641-1.9624322 .402770$

H $5.054104-0.8018473 .347695$

H $\quad 6.844773-1.983029-3.081364$

H $9.101478-2.1984790 .951219$

H $7.671372-0.0790062 .212408$

O $2.481268-0.3445793 .471451$

$\begin{array}{lllll}\text { O } & 3.178563 & 0.985739 & 1.721027\end{array}$

C 1.3315590 .4880863 .640550

H 1.6334331 .5177163 .855042

H $\quad 0.707290 \quad 0.446338 \quad 2.744217$

H $\quad 0.7580150 .1073534 .489385$

SCF Energy (B3LYP/6-31G**//MMFF)= -3245.92712077

\section{8}

MM̄FF Geometry

C $3.148973-1.226520-4.078610$

C $3.120694-0.463763-2.976151$

C $3.8896570 .819415-2.806801$

O $4.6325370 .666105-1.577021$

C $2.9097881 .993546-2.649088$

C $3.5685523 .353647-2.742123$

C 3.835414 4.151569-1.689701

C $3.8798373 .819943-4.141058$ 
C $3.6297763 .860106-0.229025$

C $2.510372 \quad 4.7057770 .394858$

C $1.0991524 .254657-0.015312$

C 0.0397715 .1722190 .600680

C $-1.386955 \quad 4.680076 \quad 0.307158$

O $\quad 0.9160182 .917544 \quad 0.459550$

O $-2.2917815 .416691 \quad 1.143772$

C $-1.489699 \quad 3.1842990 .678901$

C -1.807896 $5.010702-1.128827$

$\begin{array}{llll}\text { O } & -2.755851 & 2.650825 & 0.297890\end{array}$

C $-0.3389932 .323552 \quad 0.107164$

C $-0.382950 \quad 0.909553 \quad 0.732430$

O $-0.5014412 .220408-1.308417$

C $5.9082781 .117114-1.511535$

O $6.5342451 .679391-2.398669$

C $6.5118760 .811322-0.191333$

C $5.807911 \quad 0.3309450 .846349$

C $6.334087-0.0387852 .161987$

C $7.819460 \quad 0.0728672 .381153$

$\begin{array}{lllll}\text { C } & 0.813007 & 0.022763 & 0.363461\end{array}$

C $0.793021-1.311708 \quad 1.087985$

C $2.222272-3.3163131 .491505$

O $-0.159268-1.7015181 .757642$

C $2.799152-3.1018122 .856022$

C $4.027815-3.4968863 .223080$

C $4.593512-3.2787754 .598371$

C $5.873808-2.4711134 .587680$

C $7.066574-3.0702484 .742200$

C $5.769003-0.9641224 .488248$

C $5.469072-0.4935173 .092787$

N $1.965801-2.0258830 .878552$

H $3.768315-0.902595-4.913068$

H $2.517309-0.767002-2.127429$

H $4.566598 \quad 0.969947-3.655898$

H $2.3777681 .897391-1.696978$

H $2.1331791 .943535-3.424665$

H $4.2846285 .124317-1.887131$

H $4.3132294 .825600-4.158079$

H $4.5958813 .146173-4.621254$

H $2.9666413 .845556-4.744566$

H $3.4608992 .796578-0.037224$

H 4.5700554 .1023090 .282990

H 2.6058094 .6197951 .485317

H $2.655355 \quad 5.7629900 .140087$

H $1.0226004 .266764-1.107967$

H $0.168756 \quad 6.2051790 .254293$

H $0.179255 \quad 5.2038341 .690435$

H -2.054673 5.248295 2.071560

H -1.455167 3.1215491 .774963

H -1.764051 $6.093350-1.298885$

H -2.849276 $4.723731-1.312496$

H -1.179220 $4.532216-1.882527$

H -2.736432 $2.495738-0.661766$

H $-0.407763 \quad 1.000841 \quad 1.826246$

H -1.3034700 .4019720 .424719$

H $-0.035804 \quad 1.432265-1.627095$

H $7.5779901 .001346-0.122529$

H $4.7364290 .176766 \quad 0.728631$

H 8.1421301 .1163832 .297020

H $8.364458-0.5245411 .641654$

H $8.142103-0.2821333 .362366$

H $0.804993-0.191937-0.708623$

H 1.7526200 .5299620 .610185

H $2.904241-3.8623490 .832193$

H $1.284559-3.8764041 .567652$

H $2.165793-2.5987553 .583265$

H $4.659577-4.0129192 .503885$

H $4.774158-4.2663645 .042690$

H $3.865442-2.7897305 .257612$

H $7.987877-2.4980704 .766758$

H $7.149084-4.1473834 .843190$

H $6.674049-0.4855024 .878319$

H $4.969246-0.6133905 .153320$

H $4.410305-0.5369502 .836544$

H $2.728282-1.5860090 .371216$

C $1.457780-2.993022-3.197873$

O $0.351610-2.076619-3.069297$

C $-0.879752-2.826211-3.014943$
C $-0.472622-4.262238-2.730956$

C $0.834466-4.359368-3.490966$

H $-1.323806-2.740641-4.014613$

C $-1.805042-2.203145-1.963413$

H $-0.304541-4.414943-1.657412$

H $-1.218835-4.987593-3.068391$

C $1.697107-5.534687-3.063830$

H $0.611180-4.449473-4.562548$

C $-3.120438-2.976539-1.756320$

O $-2.119877-0.871547-2.382666$

H $-1.265336-2.114998-1.015090$

H $-2.906646-3.973584-1.354834$

O $-3.737469-3.188238-3.033352$

C $-4.152246-2.272828-0.851073$

C $-3.629961-1.9719140 .563344$

H $-4.500464-1.354000-1.335448$

O $-5.299833-3.123505-0.740130$

H $-3.385024-2.9147461 .067807$

H $-2.715394-1.3798360 .485058$

H $2.635192-5.555858-3.627284$

H $1.945007-5.484098-1.998153$

H $1.174401-6.480034-3.242441$

C $2.399462-2.514869-4.310909$

H $-1.278016-0.401598-2.510608$

H $-3.915940-2.313995-3.420893$

H $-5.016569-3.952285-0.317931$

H $1.817145-2.378607-5.231390$

H $3.145628-3.292824-4.511746$

H $1.976627-3.015193-2.231357$

C $-5.7760690 .952458 \quad 1.454716$

\begin{tabular}{llll}
\hline & -4.820675 & 0.091033 & 0.822415
\end{tabular}

C $-4.647066-1.1970651 .423964$

C $-4.173501-1.0469072 .878143$

C $-5.129300-0.1569743 .679648$

C $-5.441100 \quad 1.149892 \quad 2.943504$

H -5.6522821 .9212350 .953404$

C $-7.2134150 .480705 \quad 1.210307$

H $-5.599435-1.7368331 .408811$

O $-2.866312-0.4776742 .898636$

H -4.107990 -2.042366 3.333207

H -4.6826800 .0802764 .652897$

O $-6.347884-0.8550343 .917721$

H $-6.268488 \quad 1.6713193 .437792$

$\begin{array}{lllll}\text { O } & -4.304318 & 2.013732 & 3.039779\end{array}$

C $-7.5612320 .593689-0.254842$

H $-7.394027-0.5482451 .534066$

H -7.9274801 .1058051 .759554$

H $-2.579972-0.4321973 .826703$

H $-6.131536-1.6634684 .412691$

H -3.5826831 .6139742 .524057$

O $-7.371197-0.606233-0.872997$

O $-7.928374 \quad 1.632235-0.789200$

C $-7.621609-0.621497-2.281529$

H $-7.432434-1.633591-2.649201$

H $-8.665195-0.362811-2.484176$

H $-6.9446290 .069316-2.793142$

SCF Energy (B3LYP/6-31G**//MMFF) = -3245.91483272

0400049

MM̄FF Geometry

C $0.5911790 .644366 \quad 2.712006$

C $1.588792-0.1272323 .168026$

C $1.823788-1.5881722 .869660$

O $3.030963-1.6214112 .072157$

C $0.711077-2.2987922 .076495$

C $0.799852-3.8103202 .138446$

C $1.294454-4.595927 \quad 1.162710$

C $0.225680-4.431746 \quad 3.386491$

C $1.938237-4.179396-0.129401$

C $1.065655-4.488005-1.353852$

C $-0.048575-3.453558-1.581237$

C $-0.915158-3.852698-2.778217$

C $-1.949417-2.772984-3.127913$

O $0.585476-2.196703-1.834892$

O $-2.481096-3.105031-4.419317$

C $-1.228387-1.413032-3.244771$

C $-3.134713-2.794727-2.156751$

O $-2.165090-0.359034-3.455912$ 
C $-0.308399-1.097631-2.043818$

C $0.556090 \quad 0.148445-2.352573$

O $-1.120253-0.844604-0.896001$

C $4.058645-2.4230732 .442241$

O $4.092598-3.1787323 .402495$

C $5.214504-2.2490471 .530043$

C $5.133678-1.5729520 .373963$

C $6.235403-1.348153-0.553384$

C $5.861808-1.579081-1.992244$

C $1.6404520 .427366-1.302824$

C $2.5214081 .606540-1.676470$

C $4.5192272 .860141-0.859328$

O $2.3541732 .277997-2.690704$

C $5.7287272 .303592-1.544580$

C $6.9658712 .351860-1.026528$

C $8.1901911 .845328-1.736299$

C $9.0075910 .889693-0.894816$

C $10.069044 \quad 1.322527-0.193361$

C $8.648960-0.579230-0.938497$

C $7.429043-0.898130-0.118955$

N $3.5188391 .817447-0.732940$

H -0.1601790 .2270072 .049324$

H $2.3282780 .327213 \quad 3.825720$

H $1.979598-2.0878313 .833410$

H $\quad 0.729653-1.9616331 .034544$

H $-0.276379-2.0200552 .467162$

H $1.269916-5.6747141 .314571$

H $\quad 0.281523-5.5254143 .371194$

H $0.768654-4.0881924 .272291$

H $-0.828798-4.1578793 .496480$

H $2.238135-3.127957-0.121638$

H $2.872191-4.749170-0.219017$

H $1.725601-4.490039-2.231639$

H $\quad 0.637023-5.494242-1.269167$

H - $0.660875-3.376569-0.676095$

H -1.409448 -4.815330 -2.596932

H -0.271771 -4.009454 -3.655680

H -3.083885 -2.388323 -4.682965

H $-0.613323-1.455167-4.154080$

H -3.632963 -3.771306 -2.184902

H -3.896574 -2.064160 -2.447690

H -2.846466 -2.599448 -1.121742

H $-2.591769-0.169163-2.603343$

H $1.0579080 .004519-3.318641$

H $-0.0816671 .034494-2.448386$

H $-0.649525-0.249196-0.292974$

H $6.142778-2.7113011 .851899$

H $4.186551-1.1538690 .047289$

H $5.628653-2.636842-2.156919$

H $\quad 6.649083-1.304318-2.697035$

H $4.979001-0.988069-2.262447$

H $1.1889130 .644739-0.330805$

H $2.287294-0.449210-1.193202$

H 4.7428763 .2209080 .149621

H $4.1108023 .689791-1.445379$

H $5.5823551 .866021-2.529456$

H $7.1156132 .816178-0.054378$

H $8.8019092 .716967-2.004385$

H $7.9318321 .364080-2.687760$

H $\quad 10.678640 \quad 0.639917 \quad 0.389258$

H $10.3482642 .370921-0.181927$

H $9.474204-1.181528-0.535692$

H $8.556990-0.894483-1.981731$

H $7.562882-0.7335220 .950811$

H $3.602787 \quad 1.1764220 .050442$

C 0.4901502 .9881611 .826017

$\begin{array}{llll}\text { O } & -0.641328 & 2.723138 & 0.971255\end{array}$

C $-1.217507 \quad 3.9745920 .557537$

C $-0.1858365 .043396 \quad 0.898412$

C 0.4227754 .4775062 .164656

H -2.1068364 .1317311 .179575$

C -1.617669 $3.928178-0.920239$

H 0.5804265 .1347530 .119996

H -0.6402516 .0284181 .041628$

C 1.7676555 .0899552 .517469

H -0.2766424 .6441832 .995027$

C -2.557457 $2.765246-1.294807$

O $-0.4243853 .794019-1.702631$
H $-2.0677154 .887393-1.203674$

H -2.019227 $1.812562-1.245981$

O $-2.9426832 .943680-2.663998$

C $-3.8366012 .657099-0.443019$

C $-4.8379651 .635786-1.018371$

H $-3.5647162 .372170 \quad 0.577419$

O $-4.4743193 .932279-0.366570$

H $-5.2263152 .023151-1.967469$

H $-4.3080580 .703575-1.237214$

H 2.1734174 .6425773 .430115

H $2.4989114 .940973 \quad 1.715752$

H 1.6674096 .1672592 .685321

C 0.4245692 .0953513 .065971

H $-0.6585183 .968875-2.629455$

H $-2.1626772 .759243-3.214045$

H $-4.6794004 .213981-1.274610$

H -0.5511542 .2080073 .553173$

H 1.1888132 .4023833 .789075

H 1.3912142 .7660081 .240714

C $-5.125221-0.5374221 .118289$

O $\begin{array}{llll}-5.618457 & 0.808544 & 1.177081\end{array}$

C $-6.0320241 .368223-0.074800$

C $-7.1443790 .502167-0.681870$

C $-6.712887-0.955314-0.818051$

C $-6.175629-1.4831650 .512116$

H $-4.202950-0.5681880 .526022$

C $-4.785828-0.9568512 .558117$

H -6.4797972 .3423510 .158014$

O $-7.5699961 .018143-1.938554$

H $-8.0191190 .544843-0.020421$

H $-5.968804-1.071693-1.613197$

O $-7.834853-1.745233-1.220336$

H -7.015825 -1.617971 1.205544

O $-5.604953-2.768245 \quad 0.271697$

C $-3.611993-0.1940493 .133643$

H $-5.651314-0.7691183 .205326$

H -4.522964 -2.018506 2.602176

H $-6.9141640 .766609-2.610417$

H -8.524685 -1.656495 -0.540905

H $-5.482374-3.2070481 .129740$

O $-3.496767-0.506454 \quad 4.459057$

O

C -2.4221940 .1289945 .157914$

H -2.4550201 .2134925 .016282$

H -2.536285 -0.0863016 .223717$

$\mathrm{H}-1.466926-0.2797604 .818106$

SCF Energy $(B 3 L Y P / 6-31 G * * / / M M F F)=-3245.91250036$

0400050

MM̄FF Geometry

C $-1.3974141 .232965-2.223927$

C $-0.5044790 .352976-2.701886$

C $-0.577448-1.155058-2.580134$

O $-1.795829-1.577997-1.928669$

C $0.573611-1.668535-1.698046$

C $0.953504-3.104419-1.993039$

C $0.516998-4.175909-1.303465$

C $1.933711-3.282751-3.124120$

C $-0.489785-4.217496-0.188671$

C $0.139749-4.5140231 .178412$

C $0.781034-3.2783451 .829029$

C $1.446939-3.6565973 .153911$

C $1.990532-2.4275873 .896123$

O $-0.261352-2.3237732 .060778$

O $2.307726-2.8501815 .230515$

C $0.874669-1.3646963 .982754$

C $3.302487-1.9346953 .277369$

O $1.370562-0.1559874 .554233$

C $0.175539-1.0855172 .631404$

C $-1.071584-0.1980282 .860365$

O $1.089067-0.396776 \quad 1.774499$

C $-2.922623-1.730219-2.668437$

O $-3.050244-1.584258-3.871818$

C $-4.002997-2.047378-1.709611$

C $-5.271230-1.738742-2.012810$

C $-6.426111-1.902714-1.127138$

C $-6.188533-2.5360990 .219721$

C $-1.986717-0.0611531 .633857$ 
C $-3.1200890 .923228 \quad 1.876111$ C -5.1035511 .9209110 .736144$ O -3.2352251 .5720032 .912719$ C -6.3616701 .1843291 .075693$ C $-7.495937 \quad 1.297517 \quad 0.366706$ C -8.7797680 .6013170 .723489$ C $-9.424226-0.092850-0.457485$ C $-10.4467290 .476138-1.118400$ C $-8.940549-1.480399-0.817526$ C $-7.626885-1.453490-1.547794$ N $-3.975938 \quad 1.0076860 .785755$ H -2.275541 $0.877202-1.694177$ H $0.3655640 .735993-3.232128$ H $-0.524822-1.590727-3.585426$ H $0.302942-1.563381-0.641834$ H $1.470598-1.053520-1.828705$ H $0.895016-5.156362-1.592014$ H $2.178109-4.335361-3.302294$ H $1.522417-2.878514-4.054367$ H $2.869437-2.760617-2.902610$ H $-1.094572-3.308268-0.144312$ H - $-1.194177-5.025303-0.426255$ H $-0.661694-4.8782131 .834657$ H $\quad 0.876249-5.3217601 .088415$ H $1.519114-2.8521171 .141099$ H $2.244522-4.3926832 .992337$ H $\quad 0.710460-4.1527873 .801874$ H $2.580940-2.0626705 .732034$ H $0.125738-1.7467124 .689769$ H $4.056008-2.7312503 .292801$ H $3.730458-1.1081063 .855237$ H $3.184954-1.6076512 .243304$ H $1.8979370 .296423 \quad 3.874396$ H -1.677201 -0.6243423 .671110$ H -0.7533950 .7997043 .186334$ H $0.5913390 .146162 \quad 1.143782$ H -3.712632 -2.464477 - 0.751789 H -5.479082 -1.290669-2.983688 H $-5.815092-3.5592720 .100482$ H -7.084104 -2.597243 0.840677 H $-5.454963-1.9600040 .794653$ H -1.4209550 .2947740 .767667$ H -2.424869 -1.0337781 .381948$ H $-5.1322462 .343022-0.273601$ H -4.9470532 .7343741 .451640$ H -6.348946 0.5525391 .960633 H $-7.515727 \quad 1.958267-0.497197$ H -9.4653491 .3585551 .126198$ H -8.630056 -0.1240311 .532862$ H -10.933016 - $0.025821-1.948332$ H $-10.8168081 .459393-0.847733$ H $-9.667280-1.975502-1.475552$ H $-8.919626-2.0969280 .084993$ H -7.692682 -1.002492 -2.539032 H $-3.8351230 .386229-0.004814$ C $-1.113026 \quad 3.375077-0.986358$ O $\quad 0.251430 \quad 3.207290-0.552736$ C 0.6629434 .3966230 .152620 C $-0.579235 \quad 5.272500 \quad 0.276954$ C -1.355405 $4.881009-0.966017$ H $1.3882384 .910185-0.487773$ C $1.306925 \quad 4.007878 \quad 1.488265$ H -1.1548855 .0338801 .179051$ H $-0.331683 \quad 6.3379590 .311980$ C -2.822268 $5.271681-0.908116$ H $-0.8862155 .350462-1.840956$ C $2.538761 \quad 3.090468 \quad 1.337559$ $\begin{array}{lllll}\text { O } & 0.336409 & 3.310201 & 2.280179\end{array}$ H 1.5726154 .9064042 .056067 H 2.2448142 .1561930 .848140 $\begin{array}{llll}\text { O } & 2.992608 & 2.734815 & 2.649764\end{array}$ C $3.716465 \quad 3.7432120 .586913$ C 4.9573942 .8437140 .473980 H $3.4054634 .052837-0.413351$ $\begin{array}{lllll}\text { O } & 4.102425 & 4.938280 & 1.272923\end{array}$ H 5.8250493 .4511310 .182942 H 5.2365312 .4659941 .466902 H $-3.3472104 .960340-1.816528$
H -3.327193 $4.809351-0.053139$

H $-2.9272046 .357433-0.813581$

C -1.289623 2.726714 -2.358870

H $\quad 0.0349272 .543474 \quad 1.763275$

H 2.2290552 .3745963 .132248

H 4.3592974 .6870502 .176937

H $-0.4514472 .992759-3.014180$

H -2.209381 $3.082133-2.836198$

H $-1.7495862 .883632-0.238777$

C $5.924894-0.409641-1.041425$

O $5.9559000 .827878-0.312845$

C $4.8006891 .659417-0.494420$

C $4.6998582 .082657-1.969492$

C $4.7093470 .875201-2.912351$

C $5.796087-0.144762-2.552237$

H $6.909040-0.858241-0.855678$

C $4.862687-1.348029-0.450745$

H $3.9127241 .088339-0.206913$

O $5.7837432 .946708-2.308939$

H $3.7759232 .650213-2.124114$

H $4.8674911 .229643-3.938610$

$\begin{array}{llll}\text { O } & 3.428212 & 0.259272 & -2.864789\end{array}$

H $5.622800-1.083828-3.089123$

O $7.043794 \quad 0.380010-3.019946$

C $5.101439-2.808289-0.759402$

H $4.878157-1.2400720 .640117$

H $3.857890-1.087240-0.793147$

H $6.6114482 .466785-2.134215$

H $3.424953-0.461030-3.517874$

H $7.723346-0.298632-2.866944$

O $4.151365-3.551139-0.116014$

O $5.994405-3.246614-1.470544$

C $4.237494-4.965808-0.309978$

H $4.101581-5.211476-1.367442$

H $5.198492-5.340920 \quad 0.054478$

H $3.436542-5.4369050 .265705$

SCF Energy (B3LYP/6-31G**//MMFF) $=-3245.93013298$

0400051

MM̄FF Geometry

C $-2.859642 \quad 3.708786 \quad 0.700107$

C $-4.186726 \quad 3.805745 \quad 0.878304$

C -5.2112292 .7910810 .421348$

$\begin{array}{llll}\text { O } & -4.658121 & 1.453970 & 0.425213\end{array}$

C $-5.686874 \quad 3.126067-1.003456$

C $-6.8143432 .235529-1.491575$

C $-6.6828691 .245462-2.397778$

C -8.171631 $2.549349-0.914995$

C $-5.4278600 .777206-3.081887$

C $-5.139712-0.694168-2.754273$

C $-3.761622-1.146567-3.263378$

C $-3.528345-2.620091-2.924404$

C $-2.107009-3.080818-3.278809$

O $-2.780492-0.334490-2.617768$

O $-1.877021-4.342521-2.631482$

C $-1.088957-2.082564-2.684019$

C $-1.958949-3.334247-4.783347$

O $0.233682-2.377784-3.124258$

C $-1.422170-0.605423-2.985253$

C $-0.5394360 .384701-2.193520$

O $-1.187269-0.288529-4.362060$

C $-4.6183750 .813569 \quad 1.627969$

$\begin{array}{lllll}\text { O } & -4.974845 & 1.259149 & 2.707653\end{array}$

C $-4.057406-0.538407 \quad 1.409847$

C -3.812551 -1.330830 2.464489

C $-3.270454-2.6914412 .423497$

C $-2.896059-3.2542171 .078604$

C $-0.7377250 .360154-0.675009$

C $0.296215-0.4434110 .088869$

C $0.886308-1.0937802 .425923$

O $1.262815-0.979651-0.442806$

C $0.530576-2.5406512 .554528$

C $0.244790-3.1298093 .725358$

C $-0.069416-4.5910543 .869720$

C $-1.371950-4.8411494 .596085$

C $-1.383671-5.1897295 .893834$

C $-2.652628-4.775766 \quad 3.795002$

C $-3.134359-3.3670983 .584068$ 
N $0.019009-0.462361 \quad 1.450407$ H -2.458992 2.8582440 .156751 H $-4.586448 \quad 4.679687 \quad 1.388181$ H -6.0640122 .8246431 .111059$ H -4.835806 $3.082272-1.690894$ H $-6.0329384 .168168-1.043788$ H $-7.5774790 .693484-2.684522$ H -8.962286 $1.927248-1.348051$ H -8.17902223826780 .166513$ H $-8.4342303 .594491-1.108713$ H $-5.5577310 .889179-4.164997$ H $-4.5679891 .392441-2.809610$ H $-5.181273-0.826332-1.665653$ H $-5.922535-1.328611-3.188460$ H -3.711344 -0.982197 -4.346017 H -4.273894 -3.254936 -3.419133 H -3.678655 -2.771170 -1.847590 H -2.563822 -4.962923 -2.930406 H -1.096008 -2.230801-1.597608 H -2.632564 -4.138245 -5.104314 H $-0.947825-3.675002-5.030725$ H -2.189212 -2.455282 -5.389400 H $\quad 0.419119-3.302317-2.886333$ H $\quad 0.5147150 .265801-2.472085$ H $-0.8113031 .395687-2.531153$ H $-0.723715-1.033560-4.777391$ H $-3.864141-0.8464060 .389500$ H $-4.036438-0.9516913 .460943$ H -2.371287 -4.210125 1.134276 H -2.222621 -2.5734640 .547902$ H $-3.792983-3.409413 \quad 0.470124$ H $-0.6518241 .387445-0.308772$ H $-1.7354620 .004389-0.405970$ H $\quad 0.765611-0.5506303 .368447$ H $1.925202-0.9958782 .100038$ H $\quad 0.535290-3.1380121 .645677$ H $0.268463-2.5376964 .637462$ H $0.759916-5.0554894 .419167$ H $-0.095260-5.0938892 .894980$ H -2.312186 -5.399733 6.414395 H $-0.465347 \quad-5.2672896 .466262$ H -3.451639 -5.312585 4.324463 H -2.522626 -5.3290692 .860937$ H -3.431882 -2.871000 4.509198 H $-0.839322-0.0362921 .787730$ C $-0.6746164 .078897 \quad 1.886007$ O $\quad 0.1868883 .4861330 .890518$ C 1.5473553 .8989421 .135670 C 1.5585474 .4131522 .564838 C 0.1980135 .0763032 .649267 H 1.7413034 .7185220 .431668 C 2.4924332 .7239280 .853477 H 1.6264963 .5820413 .277765 H 2.3833225 .1044832 .760305 C -0.2638935 .3341564 .073176$ H $0.238066 \quad 6.0312472 .107807$ C 3.9566182 .9938281 .239171 O $2.4363792 .439797-0.548728$ H 2.1278751 .8297001 .370330 H 4.0377833 .1063892 .326652 O 4.3446094 .2525440 .671698 C 4.9629361 .9338670 .748926 C $4.647160 \quad 0.501711 \quad 1.214558$ H $5.0301101 .974589-0.343153$ O 6.2587292 .3029881 .236941 H 4.6085500 .4840752 .310536 H 3.6578630 .2111100 .851939 H -1.256811 5.7945094 .084051 H -0.3163304 .4058534 .652128$ H 0.4281506 .0105844 .585143 C $-1.873354 \quad 4.7338391 .191561$ H $1.5024312 .306205-0.782975$ H $5.272644 \quad 4.4080710 .918089$ H 6.2345402 .2568802 .208046 H -1.527137 5.3087490 .323919 H -2.3695735 .4283041 .879253$ H -1.003372 3.2672812 .547932 C $4.805673-0.956619-1.459950$
O $5.901825-0.465610-0.679298$

C $5.701522-0.5247150 .736482$

C $5.439681-1.9737561 .173535$

C $4.328390-2.6347560 .359727$

C $4.556273-2.432671-1.138732$

H $3.904683-0.369208-1.246181$

C $5.161028-0.750309-2.935401$

H $6.655594-0.2285421 .190701$

O $5.125198-2.0107162 .561575$

H $6.363419-2.5514651 .037282$

H $3.343017-2.2633040 .656205$

O $4.320930-4.0404300 .618974$

H $5.408740-3.041262-1.466485$

O $3.407214-2.884276-1.851910$

C $5.0153010 .706517-3.306930$

H $6.185149-1.073914-3.155737$

H $4.496003-1.314465-3.598912$

H $5.082934-2.9435492 .830707$

H $4.051366-4.1667191 .544269$

H $3.253224-3.810469-1.598131$

O $6.1872331 .366785-3.085104$

O $3.9723271 .202464-3.713921$

C $6.1600992 .772400-3.351000$

H $7.1464673 .181594-3.117071$

H $5.9460492 .953312-4.408596$

H $5.4170193 .264665-2.716005$

SCF Energy $(B 3 L Y P / 6-31 G * * / / M M F F)=-3245.90979084$

0400052

MM̄FF Geometry

C 2.1051010 .2364794 .320200

C 2.5555340 .0759183 .068001

C $3.949391-0.3548762 .699626$

O $4.4130890 .630187 \quad 1.749998$

C $3.894095-1.7251342 .005132$

C $5.251691-2.3583021 .789700$

C $5.900014-2.3985690 .609216$

C $5.849506-3.0206863 .003788$

C $5.488781-1.773919-0.695320$

C $5.086127-2.810579-1.753642$

C $3.708960-3.446600-1.501830$

C $3.399670-4.496979-2.572022$

C $1.978743-5.065862-2.427329$

O $2.732392-2.402210-1.551495$

O $1.675861-5.811471-3.616384$

C $0.976939-3.894358-2.319579$

C $1.898520-6.083065-1.284038$

O $-0.340380-4.372055-2.055092$

C $1.388791-2.822759-1.284355$

C $0.472175-1.582917-1.416125$

O $1.252744-3.3741920 .026380$

C 5.6908531 .0735321 .815960

O $6.555207 \quad 0.7072312 .599045$

C 5.9341212 .1337520 .806353

C $5.0433802 .461347-0.143722$

C $5.1787403 .524913-1.141204$

C $6.4130964 .385180-1.087939$

C $0.905815-0.394894-0.547782$

C $0.0517000 .836872-0.781296$

C $-0.0366263 .270642-0.247903$

O $-0.9837420 .819174-1.439566$

C $0.4803613 .954029-1.475386$

C $1.1990475 .086984-1.457243$

C $1.7127705 .765239-2.696221$

C $3.2184185 .924002-2.699987$

C $3.7859627 .109489-2.420627$

C $4.0632754 .737394-3.112384$

C $4.1759903 .701817-2.027143$

N $0.5726121 .956745-0.148207$

H $2.7901780 .045947 \quad 5.144070$

H 1.8919150 .2704302 .233367

H $4.590482-0.3756013 .588696$

H $3.371088-1.6260721 .048337$

H $3.287384-2.4232052 .598209$

H $6.859954-2.9124050 .570515$

H $6.810401-3.4990502 .786277$

H $6.020524-2.2869843 .797467$

H $5.175466-3.7950293 .384406$ 
H $4.699439-1.027492-0.568107$ H $6.356456-1.217054-1.072131$ H $5.063773-2.294210-2.722501$ H $5.853473-3.591700-1.821894$ H $3.702096-3.904359-0.506576$ H $4.141367-5.305250-2.551261$ H $3.489608-4.036218-3.565818$ H $1.732615-5.208549-4.376935$ H $\quad 0.930477-3.418122-3.308277$ H $2.593205-6.912820-1.463227$ H $0.902981-6.537107-1.226536$ H $2.140588-5.655148-0.309084$ H $-0.384973-4.612979-1.114170$ H $0.466272-1.249108-2.462137$ H $-0.557700-1.852074-1.153019$ H $1.173851-2.6536380 .669751$ H 6.8902302 .6389920 .895968 H $4.1061661 .909672-0.198388$ H $7.3081403 .783639-1.280719$ H $6.5118574 .857595-0.104146$ H $6.4098315 .195534-1.820206$ H $0.812686-0.6541580 .509519$ H $1.949463-0.132337-0.753746$ H $\quad 0.2102493 .8164280 .668002$ H $-1.1246353 .165329-0.310768$ H $0.2441293 .491923-2.431286$ H $1.4248075 .560055-0.504295$ H $1.2300676 .749174-2.759743$ H $1.4055475 .228744-3.602783$ H $4.8623067 .242303-2.449812$ H $3.1868897 .973392-2.151880$ H $5.0536995 .064075-3.448389$ H $3.6176364 .267862-3.998889$ H $3.3226313 .024917-1.983510$ H $1.476647 \quad 1.890347 \quad 0.310669$ C -0.3260030 .8414463 .615760$ O $-0.614900-0.4327043 .000767$ C -2.038382 -0.6526623 .026162$ C $-2.650902 \quad 0.725847 \quad 3.196929$ C $-1.662068 \quad 1.373297 \quad 4.143886$ H $-2.227544-1.2713723 .912808$ C $-2.476673-1.3955901 .757653$ H $-2.678284 \quad 1.262362 \quad 2.240013$ H -3.6648750 .6898563 .603660$ C -1.752758 $2.889702 \quad 4.167590$ H -1.845729 0.9907075 .156988 C $-4.000807-1.598127 \quad 1.674121$ O $-1.843965-2.6788841 .752505$ H -2.112732 -0.8645390 .871901$ H -4.502977 -0.6276521 .618823$ O $-4.455028-2.1967282 .896771$ C $-4.478691-2.4921590 .515769$ C $-4.047295-2.061513-0.897697$ H $-4.131221-3.5188480 .681556$ O $-5.908411-2.5736250 .562442$ H -2.972067-2.235399 -1.016897 H -4.563534 -2.727168 -1.601350 H -1.0090123 .3136444 .849532$ H -1.581698 3.3173443 .173727 H -2.743954 3.2104384 .504758 C $0.714896 \quad 0.6550274 .730471$ H $-0.885491-2.5292171 .820946$ H $-4.002459-3.0528162 .989694$ H $-6.261767-1.675105 \quad 0.446842$ H $0.349789-0.1104265 .427410$ H $\quad 0.809489 \quad 1.589806 \quad 5.295761$ H $\quad 0.0587401 .5021522 .829927$ C $-6.2188490 .962844-1.363182$ O $-5.767379-0.376199-1.119265$ C $-4.364463-0.600209-1.279538$ C $-3.943533-0.284264-2.724788$ C $-4.352138 \quad 1.140330-3.119128$ C $-5.8169521 .427684-2.774050$ H $-7.314470 \quad 0.899746-1.334946$ C $-5.774343 \quad 1.928528-0.252843$ H $-3.8138340 .053210-0.596551$ O $-4.557046-1.215418-3.615227$ H $-2.859588-0.411448-2.817358$
H $-4.205940 \quad 1.279340-4.197317$

$\begin{array}{llll}\text { O } & -3.529040 & 2.085867 & -2.443247\end{array}$

H $-6.0251902 .498474-2.879133$

O $-6.6537790 .758286-3.722410$

C -6.3956411 .5874661 .085919$

H $-4.689328 \quad 1.920179-0.120399$

H $-6.0898142 .947414-0.505399$

H -4.243994 -1.012344 -4.513181

H $-2.6046691 .890062-2.673365$

H $-6.538156-0.199332-3.596937$

O -5.8529512 .3970802 .043553$

$\begin{array}{llll}\text { O } & -7.249636 & 0.731720 & 1.272611\end{array}$

C $-6.3459252 .194786 \quad 3.371748$

H $-5.7690992 .830555 \quad 4.048620$

H -7.3994712 .4842013 .427566$

H -6.2154461 .1516373 .675570$

SCF Energy (B3LYP/6-31G**//MMFF)= -3245.92541676

0400053

MM̄FF Geometry

C $-0.293171-2.5409010 .958267$

C $0.583644-2.126261 \quad 1.886767$

C $0.421387-0.943785 \quad 2.819527$

O $-0.822401-0.2534062 .566906$

C $1.585927 \quad 0.0347132 .583894$

C 1.5566861 .2645503 .467957

C 1.3680522 .5243613 .027357

C 1.8085211 .0115984 .932385

C 1.1017182 .9807431 .619987

C $-0.203384 \quad 3.782752 \quad 1.542315$

C -0.6127104 .0632040 .089089$

C $-1.814856 \quad 5.0085360 .050626$

C $-2.3415105 .211798-1.375510$

O $-0.9466502 .805827-0.505738$

O $-3.6132935 .868056-1.262191$

C $-2.574483 \quad 3.826448-2.012802$

C $-1.4415246 .154994-2.181283$

O $-2.9578623 .959338-3.380273$

C $-1.3684282 .868057-1.871752$

C $-1.7328541 .430617-2.317636$

O $-0.3124003 .332877-2.718896$

C $-1.901253-0.6371823 .304360$

O $-1.913514-1.4281694 .234430$

$\begin{array}{llll}C & -3.098234 & 0.016831 & 2.728958\end{array}$

C $-4.300175-0.5312872 .964757$

C $-5.585813-0.0942932 .416186$

C -5.6159681 .1886631 .630160$

C $-2.6063710 .672439-1.316731$

C $-3.176556-0.618622-1.874275$

C $-4.612357-2.532375-1.141451$

O $-3.107024-0.934632-3.057843$

C $-6.050253-2.148443-0.975444$

C $-6.878413-2.713108-0.083557$

C $-8.309570-2.2934850 .099506$

C -8.646382 -1.949664 1.535492

C $-9.484116-2.7199742 .250258$

C $-8.075042-0.6724832 .121725$

C -6.671972 -0.8639322 .640117$

N $-3.809181-1.353097-0.880579$

H $-1.245780-2.0338510 .838275$

H $1.526157-2.6636451 .980629$

H $\quad 0.457679-1.3094603 .852724$

H 1.6124190 .3109721 .525370

H $2.535701-0.4839532 .767310$

H 1.4026183 .3312163 .758806

H 1.9058881 .9404715 .504379

H $\quad 0.9843800 .440608 \quad 5.370855$

H 2.7357800 .4458895 .068517

H 1.9347973 .6155521 .295808

H 1.0588242 .1451390 .919060

H -1.0027253 .2156512 .038230$

H -0.0812224 .7271652 .087301$

H $0.2396814 .500108-0.444220$

H $-1.5662825 .973874 \quad 0.509321$

H $-2.626624 \quad 4.5892040 .662257$

H -3.992026 $5.932585-2.155912$

H -3.442338 $3.392702-1.502345$

H $-1.3813077 .134113-1.690926$ 
H $-1.8570646 .346236-3.176820$ H $-0.4227595 .779876-2.299050$ H $-2.1556534 .153615-3.894543$ H -2.191733 $1.455439-3.313740$ H $-0.8127900 .847594-2.443648$ H $0.2949952 .594231-2.889460$ H -2.953092 0.8763622 .085275 H $-4.347040-1.4208863 .592516$ H -5.1276751 .9967052 .186610$ H $-6.628015 \quad 1.5412061 .418474$ H $\quad-5.104319 \quad 1.0640280 .671929$ H $-2.0168650 .433554-0.423574$ H -3.458105 $1.285499-1.008695$ H -4.303282 -3.301876 -0.427272 H -4.433202 -2.898354 -2.156734 H $-6.423785-1.353550-1.617261$ H $-6.512349-3.5195720 .547701$ H -8.943649 $-3.117448-0.252281$ H -8.555623 -1.433315 -0.535815 H -9.750112 -2.467672 3.271548 H $-9.918963-3.6221741 .833185$ H -8.696230 -0.3386322 .962923$ H $-8.146628 \quad 0.1143541 .365456$ H -6.550915 -1.755437 3.257772 H -3.813149 -0.9901320 .067617$ C $-0.145196-3.203218-1.447546$ O $0.781099-2.127644-1.691639$ C $1.507468-2.397893-2.901375$ C $0.705712-3.467365-3.630781$ C $0.190927-4.292912-2.466058$ H $2.469851-2.824736-2.595959$ C $1.757292-1.102553-3.680681$ H $-0.135763-3.039089-4.187104$ H $1.318690-4.043970-4.329794$ C $-0.987139-5.183219-2.821400$ H $1.012000-4.919184-2.091751$ C $2.3048360 .056426-2.820465$ O $0.521013-0.662817-4.255651$ H $2.427565-1.310042-4.523163$ H $\quad 1.5123970 .428389-2.160995$ O $2.617098 \quad 1.142127-3.702237$ C $3.542843-0.286976-1.972791$ C $4.0802890 .945508-1.220329$ H $3.279299-1.070984-1.255225$ O $4.555388-0.802855-2.834572$ H $4.4865031 .668060-1.938424$ H $3.2400181 .417068-0.695731$ H -1.330438 -5.742603-1.945395 H -1.832548 $-4.598409-3.199794$ H $-0.704438-5.904893-3.594752$ C $-0.020240-3.6714590 .004662$ H $0.7028690 .159913-4.741244$ H $3.363596 \quad 0.860924-4.259018$ H $5.227970-1.230329-2.278254$ H $0.986918-4.0704330 .178100$ H $-0.733367-4.479790 \quad 0.200599$ H -1.149001 -2.801567 -1.630202 C $5.507044-0.7289411 .802010$ O $4.607889-0.2534560 .791741$ C $5.1843600 .597933-0.203393$ C 5.7750621 .8551350 .452441 C 6.7771531 .4869681 .551905 C 6.2094740 .4400562 .514504 H $4.861742-1.2294362 .535070$ C $6.474716-1.7801941 .248734$ H $5.9818170 .060131-0.725241$ O 4.7382442 .6639441 .004603 H $6.2728132 .455768-0.317714$ H 7.0439602 .3875142 .118684 O $7.967438 \quad 0.971250 \quad 0.963059$ H 7.0040710 .0574063 .165709 O 5.2567121 .0952443 .357216 C $5.718743-3.0266760 .853263$ H $7.051402-1.4305630 .387832$ H $7.206250-2.0762852 .009984$ H 4.2439632 .1203681 .641912 H $8.3330951 .663468 \quad 0.386097$ H 4.9544440 .4479624 .016781
O $5.473399-3.020140-0.487560$

O $5.362460-3.8866341 .648681$

C $4.729405-4.137948-0.981980$

H $3.716555-4.126384-0.568128$

H $4.666455-4.049439-2.069671$

H $5.237447-5.075526-0.736796$

SCF Energy $(B 3 L Y P / 6-31 G * * / / M M F F)=-3245.93374418$

0400054

MMFF Geometry

C $0.394833-1.755220-4.056447$

C $1.039956-1.211148-3.014294$

C $2.509085-1.431089-2.763640$

O $2.663278-2.156822-1.523073$

C $3.229535-0.082384-2.610684$

C $4.730825-0.191191-2.789392$

C $5.631109-0.152835-1.788179$

C $5.201807-0.283290-4.219098$

C $5.373797-0.066056-0.310015$

C $5.711116 \quad 1.319815 \quad 0.260232$

C $4.6528632 .383038-0.073554$

C $5.086394 \quad 3.761968 \quad 0.426421$

C $3.990870 \quad 4.817325 \quad 0.207784$

O $3.438887 \quad 1.991814 \quad 0.573278$

$\begin{array}{lllll}\text { O } & 4.342172 & 5.982668 & 0.969229\end{array}$

C 2.6626524 .2839070 .784110

C $3.9087505 .263627-1.256825$

O $1.5828025 .164596 \quad 0.480204$

C 2.3195522 .8490300 .322493

C 1.1295992 .3066211 .146316

O $1.9658612 .874133-1.060578$

C $2.779315-3.507182-1.601445$

O $2.722517-4.186639-2.618073$

C $3.029571-4.120112-0.277221$

C $2.937122-3.4688280 .892729$

C $3.180838-4.0504702 .216375$

C $3.701756-5.4634312 .275842$

C $0.8136700 .822782 \quad 0.911921$

C $-0.2247810 .309763 \quad 1.894979$

C -1.229542 $-1.812842 \quad 2.722689$

$\begin{array}{llll}\text { O } & -0.845282 & 1.041563 & 2.661900\end{array}$

C $-0.454617-2.1076573 .967796$

C $-0.154627-3.3449254 .389051$

C $0.653445-3.6215825 .624195$

C $1.902837-4.4254085 .335639$

C $1.968624-5.7268415 .664291$

C $3.103255-3.7067554 .755512$

C $2.928099-3.3072063 .314392$

N $-0.378855-1.0673011 .812659$

H $0.939541-2.407899-4.735130$

H $0.498633-0.568789-2.324586$

H $2.957150-1.995476-3.590486$

H $2.9914020 .345713-1.632268$

H $2.8568860 .638370-3.351190$

H $6.687738-0.205200-2.048985$

H $6.294234-0.293473-4.297723$

H $4.831453-1.198049-4.691210$

H $4.8402570 .575979-4.793522$

H $4.351456-0.356719-0.050422$

H $6.020741-0.8080430 .175419$

H 5.7795421 .2187131 .351424

H $6.6965511 .641960-0.098305$

H $4.4969702 .409264-1.157083$

H $6.0219694 .073846-0.054266$

H 5.3054863 .7085951 .502140

H 5.2135946 .2884450 .663827

H 2.7605584 .2811621 .878837

H $4.8649285 .692031-1.580439$

H $3.1658946 .058455-1.386254$

H $3.6647714 .448407-1.941189$

H 1.8328906 .0493990 .796961

$\begin{array}{llll}\text { H } & 1.349780 & 2.437958 & 2.214069\end{array}$

H 0.2309462 .8907450 .924118

H $1.3744162 .129924-1.252052$

H $3.286911-5.174117-0.319764$

H $2.649346-2.4192650 .892545$

H $2.964652-6.162597 \quad 1.866004$

H $3.928736-5.8003783 .289347$ 
H $4.632232-5.5556751 .704455$ H $0.4238560 .665540-0.098648$ H $1.7191490 .217479 \quad 1.031398$ H -1.546825 -2.723387 2.205049 H -2.115062 -1.217781 2.967000 H -0.115513 -1.253739 4.549968 H -0.500534 -4.203299 3.817914 H $\quad 0.008259-4.1612186 .329669$ H $\quad 0.936335-2.6925586 .134817$ H $2.868561-6.3089205 .497354$ H $1.122446-6.2414336 .107712$ H $4.015255-4.3044444 .872874$ H $3.294412-2.8010825 .345935$ H $2.552439-2.2924353 .181521$ H $0.236301-1.5973491 .202179$ C -1.955315 -2.590551-3.796470 O $-2.428907-2.142022-2.505051$ C $-3.853953-2.343918-2.445721$ C $-4.177225-3.323771-3.564453$ C $-3.208058-2.860155-4.632062$ H $-4.322828-1.382659-2.686122$ C -4.285552 -2.808432 -1.053735 H -3.960216 -4.357902 -3.271924 H $-5.223769-3.268404-3.878677$ C $-2.999073-3.873044-5.744600$ H $-3.581004-1.922228-5.065546$ C $-4.004625-1.7892170 .069744$ O $-3.587259-4.020168-0.743674$ H -5.352166 -3.063085 -1.069134 H -2.924068 -1.688315 0.217722 O $-4.515324-2.3414231 .289945$ C $-4.618757-0.393330-0.159196$ C -4.5721040 .5063641 .091396$ H $-4.0838450 .096831-0.978271$ O $-5.978601-0.529757-0.577316$ H -5.2134850 .0702251 .866037$ H -3.5504740 .5209411 .482693$ H -2.272778 -3.505060 -6.476048 H $-2.629806-4.827492-5.354558$ H -3.940026 -4.066560 -6.269924 C $-1.048435-1.514414-4.393334$ H -3.863452 -4.293905 0.147806 H -5.473052 -2.469015 1.182913 H $-6.482786-0.9104430 .161503$ H -1.353666 -0.519910 -4.046451 H -1.121670 -1.506672 -5.487258 H -1.407304 -3.526095 -3.623765 C -2.9450772 .9970890 .308064$ O $-4.2394202 .606029-0.167736$ C $-5.0667091 .941704 \quad 0.794720$ C -5.2571372 .8254862 .034498$ C -3.928037 3.2983662 .612670 C $-3.075583 \quad 3.935311 \quad 1.516127$ H -2.3721932 .1074900 .590695$ C $-2.2154283 .690034-0.854146$ H $-6.055766 \quad 1.846626 \quad 0.329108$ O -6.0048912 .1297523 .026335$ H -5.8485363 .7059561 .750171$ H -3.400039 2.4755903 .108786 O $-4.206554 \quad 4.2714573 .621556$ H -3.5248024 .8877291 .206564$ O -1.7795444 .2190722 .035840$ C $-1.7010032 .705582-1.884851$ H -2.894990 4.382277 -1.364684 H -1.345132 $4.242505-0.483656$ H -6.0828782 .7175093 .797315$ H -3.367104 4.4843524 .062899 H -1.877754 4.9061212 .716215 O $-1.0241893 .396285-2.850626$ O $-1.8626501 .493732-1.852933$ C $-0.4641582 .601128-3.900181$ H $\quad 0.0540993 .269237-4.593093$ H $\quad 0.260015 \quad 1.890147-3.492432$ H -1.257321 $2.078557-4.442995$ SCF Energy $\left(B 3 L Y P / 6-31 G^{* *} / / M M F F\right)=-3245.91221142$

0400055

MM̄MF Geometry
C $-3.3470603 .484296-0.150450$ $\begin{array}{llll}\text { C } & -4.578431 & 3.632178 & 0.363778\end{array}$ C -5.4838092 .5316790 .871774$ O -4.7773851 .2787281 .012067$ C $-6.6452442 .330238-0.118920$ C $-7.685018 \quad 1.332557 \quad 0.354881$ C $-7.8437900 .087775-0.138566$ C $-8.608306 \quad 1.8349701 .435612$ C -7.031142 -0.593670-1.204563 C $-6.320757-1.831309-0.642568$ C $-5.293489-2.408521-1.628192$ C $-4.647607-3.665791-1.043830$ C $-3.511669-4.199848-1.927201$ O $-4.299361-1.405289-1.850819$ O $-2.757896-5.146889-1.153405$ C $-2.551110-3.039297-2.270843$ C $-4.056623-4.967890-3.136840$ O $-1.573531-3.452798-3.221472$ C $-3.267947-1.769506-2.777502$ C $-2.327216-0.550406-2.917334$ O $-3.803200-1.965986-4.092402$ C -4.0880251 .0888452 .172101$ O -3.9799831 .8764783 .098816$ C $-3.473734-0.2565752 .121148$ C $-2.663467-0.6521413 .114299$ C $-1.996083-1.9512073 .225526$ C $-2.217008-2.9485752 .119914$ C $-1.721996-0.022521-1.613405$ C $-0.361553-0.599347-1.266732$ C $1.336577-0.5695240 .557554$ O $0.309296-1.266560-2.048747$ C $1.258261-1.8713591 .289205$ C $1.680925-2.0330672 .552283$ C $1.686579-3.3513953 .270321$ C $1.009623-3.2865614 .620531$ C $1.728730-3.1660985 .749324$ C $-0.491942-3.4525974 .665965$ C $-1.224543-2.1904464 .306688$ N $0.036515-0.2320890 .012975$ H -2.913212 $2.493706-0.243193$ H -4.9962904 .6354000 .426937$ H -5.8829242 .8356001 .847575$ H -6.240942 2.037004-1.093159 H -7.151947 $3.288697-0.297721$ H $-8.644434-0.5270190 .271323$ H -9.3855541 .1066961 .690924$ H -8.048854 2.0514422 .350723 H $-9.1120202 .750770 \quad 1.109439$ H -7.703425 -0.897474 -2.016037 H $-6.3009530 .083721-1.651281$ H $-5.809105-1.5540990 .287602$ H -7.064372 -2.596917 -0.388148 H $-5.798697-2.634310-2.574584$ H -5.400539-4.444941-0.871010 H -4.232080 -3.431594 -0.055732 H $-3.367585-5.842811-0.853535$ H -2.009681 -2.794017 -1.349309 H $-4.628288-5.844281-2.808043$ H $-3.243397-5.357817-3.758547$ H $-4.717035-4.364924-3.763888$ H $-1.123517-4.232549-2.853734$ H -1.553484 -0.752098 -3.668087 H $-2.9282540 .269039-3.338361$ H -3.444606 -2.793248 -4.452264 H -3.707855 -0.8864291 .271563$ H -2.4736430 .0384143 .935209$ H -1.586414 -3.836699 2.199087 H -1.988382 -2.505323 1.144865 H $-3.258310-3.2869482 .119226$ H $-1.5638871 .057693-1.726830$ H -2.406547 $-0.165412-0.772481$ H 1.6406730 .2559401 .208757 H $2.058446-0.651886-0.260478$ H $\quad 0.879059-2.7301760 .740301$ H $2.091402-1.1795473 .087170$ H $2.734600-3.6553563 .394032$ H $1.220736-4.1396362 .665924$ H $1.252508-3.1488316 .723963$ 
H $2.809773-3.0760905 .723101$ H $-0.807603-3.7303635 .680808$ H $-0.781736-4.3064664 .047008$ H -1.119410 -1.402110 5.053372 H -0.6057330 .2858370 .605872$ C $-1.142822 \quad 4.687414 \quad 0.026030$ O $-0.2878263 .662054-0.528907$ C $0.9437114 .273611-0.970199$ C $1.0252895 .590839-0.218528$ C $-0.4264976 .017916-0.219127$ H $0.8196554 .451396-2.046381$ C $2.1181673 .317472-0.724174$ H 1.3798345 .4331190 .807654 H $1.6821576 .318216-0.704393$ C -0.7455907 .0863340 .812683$ H $-0.6780906 .401606-1.217327$ C $3.4936643 .950362-1.000370$ O $1.9551492 .185600-1.583605$ H 2.0747292 .9411450 .304062 H $3.6685514 .770751-0.294192$ O $3.4625694 .541875-2.306429$ C $4.6873652 .975933-0.958066$ C 4.7985612 .1889920 .357973 H $4.6330092 .289191-1.810116$ O $5.8819173 .744679-1.145124$ H 4.7810252 .8945741 .198029 H 3.9206821 .5445040 .463980 H -1.8083427 .3469420 .788787$ H -0.5038916 .7496821 .826551$ H -0.1694807 .9961590 .614890$ C $-2.5169814 .635003-0.648704$ H $1.0708321 .818493-1.413968$ H $4.3364344 .937660-2.466633$ H $5.9680644 .349100-0.388150$ H -2.394648 $4.514667-1.732098$ H -3.049863 $5.578172-0.479703$ H -1.226670 4.4843561 .101318 C $5.390368-0.566114-0.835388$ O $\quad 6.2982320 .531119-0.701570$ C 6.0897221 .3432730 .458162 C 6.1860440 .4885681 .731119 C $5.258512-0.7231821 .675670$ C $5.472283-1.4999220 .377066$ H $4.370970-0.171122-0.922330$ C $5.675860-1.269705-2.166902$ H 6.9277062 .0511070 .486816 O 5.8772541 .2596472 .887409 H 7.2164080 .1286751 .849411 H $4.210772-0.4265371 .787965$ O $5.549624-1.5927782 .771486$ H $6.448422-1.998000 \quad 0.419756$ O $4.479823-2.514600 \quad 0.257207$ C $7.037684-1.924550-2.206280$ H $4.903148-2.017862-2.379668$ H $5.650095-0.549524-2.993995$ H 6.4958402 .0096702 .911318 H $5.438818-1.0754973 .587750$ H $4.521759-3.0594061 .061805$

O $6.903434-3.281387-2.180840$ O $8.093224-1.305278-2.237927$

C $8.128466-4.020769-2.190062$ H $7.882693-5.085906-2.170498$ H $8.693312-3.807433-3.102472$ H $8.721630-3.780954-1.302414$ SCF Energy (B3LYP/6-31G**//MMFF) $=-3245.91149846$

0400056

MM̄MF Geometry

C $0.007657-3.001513 \quad 1.423144$

C $0.020062-2.8597902 .758085$

C $-0.474868-1.6489433 .515600$

O $-1.536936-0.9970312 .780385$

C $0.667980-0.6463343 .731796$

C $\quad 0.307357 \quad 0.440475 \quad 4.724843$

C $-0.084619 \quad 1.686660 \quad 4.395713$

C $0.4532550 .054518 \quad 6.174881$

C -0.2770882 .2561073 .018908$

C -1.6724192 .8728972 .851799$
C -1.8596803 .4714751 .449462$

C -3.1895234 .2195481 .350746$

C $-3.4346664 .757549-0.067021$

O -1.8347892 .3996730 .501631$

O $-4.8092845 .162322-0.153809$

C $-3.2366453 .608264-1.076136$

C $-2.5942706 .005833-0.360661$

O $-3.3099344 .086944-2.417393$

C $-1.9244982 .815243-0.867584$

C $-1.9371501 .547574-1.751916$

O $-0.8166053 .631570-1.248104$

C $-2.781129-1.5366722 .910407$

O $-3.115636-2.4403573 .660924$

C $-3.665675-0.8875441 .919510$

C $-4.763131-1.5486321 .521460$

C $-5.712129-1.1205530 .494442$

C $-5.6183750 .294947-0.003635$

C $-0.8255000 .536549-1.441358$

C -1.079621 -0.749944-2.202224

C $-2.582920-2.743898-2.218029$

O $-0.497697-1.003347-3.254063$

C $-3.935702-2.426777-2.778938$

C $-5.024370-3.184370-2.573134$

C $-6.380748-2.862204-3.136660$

C -7.490311 -2.953737 -2.109032

C $-8.388063-3.952896-2.151314$

C -7.606558-1.848583-1.077830

C $-6.612338-2.0171850 .040552$

N -2.047120 -1.543100 -1.602914

H $-0.344615-2.1856610 .798373$

H $\quad 0.394308-3.6777193 .370015$

H -0.861551 -1.984480 4.486033

H $0.970608-0.2097022 .773273$

H $1.557827-1.1686244 .106696$

H -0.2851942 .3889495 .204150$

H $\quad 0.215702 \quad 0.881764 \quad 6.852152$

H -0.216105 -0.7748966 .422475$

H $1.482929-0.2549806 .382792$

H $0.480403 \quad 3.0346292 .869895$

H -0.1204311 .5039772 .241753$

H -2.4284972 .0965103 .025857$

H -1.820092 3.6541043 .607945

H $-1.0242964 .152458 \quad 1.249339$

H -3.233962 5.0352952 .083011

H -4.0119253 .5377541 .610114$

H -4.9659735 .8387480 .527254$

H -4.081656 $2.917680-0.948892$

H $-2.828646 \quad 6.8021250 .355941$

H -2.828115 $6.415309-1.349703$

H -1.519183 $5.821519-0.308874$

H $-4.1519574 .565065-2.508765$

H -2.899699 $1.041081-1.629872$

H $-1.864274 \quad 1.828702-2.810708$

H -0.097092 $3.060709-1.560686$

H -3.3623040 .0659441 .504339$

H $-4.964391-2.5279291 .955205$

H -6.499813 $0.613487-0.565023$

H $-4.7498960 .407129-0.655364$

H -5.5265830 .9998790 .830416$

H $\quad 0.1573140 .921743-1.731684$

H $-0.7760540 .303157-0.371742$

H -2.628588 -3.514334 -1.441967

H -1.916524 -3.081996 -3.017329

H $-4.022231-1.531758-3.391294$

H $-4.933216-4.095871-1.986662$

H $-6.571931-3.565258-3.957835$

H -6.396338 -1.861947 -3.587558

H -9.200279-4.013355-1.434464

H -8.328868 -4.735821 -2.900147

H $-8.609727-1.851454-0.632197$

H -7.512354 -0.888863 -1.593605

H -6.624109 -3.0105600 .491975$

H -2.489056 -1.218652 -0.748006

C $1.757209-4.180677-0.014015$

O $1.734706-3.179438-1.049415$

C $3.055022-2.583357-1.133924$

C $3.949236-3.433547-0.238741$

C $2.977831-3.8602340 .842990$ 
H $2.943737-1.576090-0.717023$

C $3.476648-2.507309-2.607715$

H $4.318406-4.314880-0.778012$

H $4.812328-2.8884580 .150216$

C $3.472842-5.0210201 .686171$

H $2.786034-2.9975311 .493057$

C $4.907731-2.007363-2.877971$

O $2.561383-1.661870-3.307200$

H $3.364779-3.505722$-3.049985

H $5.024063-1.848571-3.957877$

O $5.813157-3.061680-2.532238$

C $5.353626-0.723919-2.144564$

C $4.5129830 .521252-2.470381$

H $5.375539-0.898799-1.066273$

O $6.713148-0.463790-2.523815$

H $4.5315180 .675989-3.556053$

H $3.4731430 .347229-2.184780$

H $2.731206-5.2979102 .441828$

H $3.677040-5.9051721 .073128$

H $4.398331-4.7510392 .205406$

C $0.415913-4.2602140 .713354$

H $1.664155-1.994150-3.131383$

H $\quad 6.713862-2.743336-2.714295$

H $6.725839-0.285318-3.479714$

H $0.434001-5.1029451 .415100$

H $-0.371189-4.482844-0.018365$

H $1.910192-5.139677-0.528307$

C 3.7308861 .6775110 .259566

O $5.0289881 .687728-0.347089$

C $5.0347891 .802404-1.775281$

C $4.3140903 .088194-2.204795$

C $2.9053593 .160063-1.625984$

C $2.9469392 .941596-0.115770$

H $3.1804380 .784629-0.058904$

C 3.9218491 .6023881 .784722

H $6.085376 \quad 1.922436-2.066912$

O $4.2722373 .170709-3.624984$

H $4.8891953 .952855-1.848118$

H $2.2330352 .441855-2.109297$

O $2.3560604 .456884-1.867993$

H 3.3962063 .8190410 .367586

O 1.6137392 .8222390 .370140

C 4.3912640 .2409602 .248764

H 4.6673302 .3356052 .113155

H 2.9696541 .8046692 .288114

H $3.9247564 .047437-3.859921$

H $2.2186364 .542986-2.826471$

H 1.1528963 .6518080 .157093

O $4.213738 \quad 0.1444853 .599840$

O $4.874132-0.6214461 .529659$

C $4.620559-1.0965594 .185438$

H $5.702856-1.2215694 .086229$

H $4.364602-1.0706045 .247934$

H $4.090507-1.9322643 .718388$

SCF Energy (B3LYP/6-31G**//MMFF)= -3245.92176132

04 00057

MM̄FF Geometry

C $0.051005-3.1759091 .046672$

C $0.185401-3.1411452 .381372$

C $-0.360168-2.0651153 .295097$

O $-1.455731-1.3525902 .670898$

C $0.742165-1.0531963 .651818$

C $0.324188-0.0754374 .736130$

C 0.0105631 .2207664 .536932

C $0.305609-0.6413836 .134103$

C $-0.013967 \quad 1.977721 \quad 3.239735$

C -1.4321812 .4350862 .881905$

C $-1.5008583 .020517 \quad 1.463183$

C -2.9157683 .5130211 .155721$

C $-3.0743013 .962014-0.304611$

O $-1.129174 \quad 1.9815410 .553005$

O $-4.4790784 .077749-0.580071$

C $-2.5238712 .857609-1.232648$

C $-2.4701345 .351342-0.540091$

O $-2.512013 \quad 3.293311-2.589668$

C -1.121742 2.363228 -0.828208

C $-0.6544171 .127134-1.635382$
O $-0.1733913 .409217-1.068490$

C -2.675229 -1.961686 2.697094

O $-2.943244-3.042773 \quad 3.198329$

C $-3.640759-1.0905981 .989813$

C $-4.882221-1.5414411 .752732$

C $-5.964262-0.8089751 .090453$

C -5.6719720 .5851650 .605622$

C $-1.512293-0.131537-1.467002$

C $-2.575351-0.315541-2.535738$

C $-4.495381-1.798849-3.114370$

O $-2.6323620 .363705-3.556706$

C $-5.766431-1.097610-2.753244$

C $-6.911885-1.740687-2.479722$

C $-8.210912-1.046720-2.187419$

C $-8.852363-1.515802-0.901324$

C $-9.826658-2.441383-0.912166$

C $-8.433399-0.8406870 .383904$

C $-7.164436-1.4114960 .955390$

$\mathrm{N}-3.431506-1.365878-2.228781$

H $-0.452152-2.3590780 .537095$

H $\quad 0.700558-3.9612342 .877165$

H -0.727914 -2.549045 4.208444

H $1.052335-0.5232292 .745563$

H $1.639729-1.5828263 .999371$

H -0.2612951 .8206895 .404926$

H $\quad 0.0765150 .119198 \quad 6.888380$

H -0.449268 -1.428309 6.223333

H $1.283480-1.0655386 .384875$

H 0.6291932 .8594313 .343685

H $0.406525 \quad 1.3879852 .422907$

H -2.1104671 .5760422 .952211$

H -1.773939 3.1806403 .610738

H -0.7804743 .8432981 .386034$

H -3.202791 4.3208361 .840485

H -3.628005 $2.699708 \quad 1.343625$

H -4.8568364 .7147460 .050392$

H -3.226169 2.017663 -1.180659

H -2.970856 6.0978420 .088213

H -2.623935 $5.679392-1.573942$

H -1.402250 $5.398196-0.316725$

H $-3.4155713 .576792-2.810975$

H -0.521216 $1.391104-2.691266$

H $0.3509320 .868718-1.271990$

H $0.6972173 .010508-1.230672$

H -3.306791 -0.1060151 .686579$

H $-5.141077-2.5460372 .085583$

H -6.4732891 .0148840 .000712$

H $-4.7801780 .594682-0.029185$

H -5.5091151 .2550051 .456139$

H $-0.855719-1.006880-1.546461$

H $-1.978301-0.164381-0.477653$

H -4.578676 -2.885679 -3.014307

H $-4.226362-1.564024-4.149296$

H $-5.752443-0.010200-2.753638$

H -6.930205 -2.827889-2.510796

H -8.886962 -1.241009 -3.030646

H -8.086012 $0.042831-2.157308$

H $-10.314359-2.7594440 .003253$

H -10.153846 -2.905755 -1.836575

H $-9.214979-0.9714711 .144936$

H $\quad-8.3844790 .2410430 .229417$

H -7.279295 -2.428740 1.332330

H -3.341154 -1.836199-1.332739

C $1.691644-4.017339-0.721168$

O $1.449935-2.848011-1.527846$

C $2.688949-2.099290-1.629045$

C $3.770648-3.036581-1.114997$

C $3.024343-3.773642-0.019068$

H $2.568149-1.256011-0.940272$

C $2.837024-1.598800-3.070459$

H $4.087504-3.737966-1.896286$

H $4.652573-2.511334-0.744216$

C $3.724089-5.0365760 .449629$

H $2.896780-3.0952110 .834140$

C $4.189663-0.985511-3.472371$

O $1.819147-0.623690-3.316553$

H $2.619323-2.435064-3.747699$

H $4.076657-0.495490-4.448441$ 
O $5.135647-2.044750-3.668163$

C $4.8422520 .022306-2.507031$

C $3.9471861 .185154-2.051876$

H $5.227986-0.512254-1.635694$

O $5.997700 \quad 0.562429-3.164846$

H $3.5166131 .677136-2.933141$

H $3.1111050 .796836-1.465774$

H $3.141646-5.5387131 .228301$

H $3.869635-5.744690-0.372949$

H $4.708618-4.7981360 .865411$

C $0.496406-4.3205940 .181849$

H $0.963365-1.043519-3.123843$

H $4.782571-2.630460-4.359425$

H $5.689876 \quad 1.072702-3.933366$

H $\quad 0.709499-5.2070650 .790827$

H $-0.360660-4.581723-0.452686$

H $1.797329-4.853765-1.425543$

C $4.887023 \quad 1.0620700 .868112$

O $5.5620491 .672515-0.236567$

C $4.704648 \quad 2.250770-1.224327$

C $3.8037613 .317999-0.585976$

C $3.0623342 .784793 \quad 0.641074$

C 4.0394122 .1048821 .600699

H 4.2481730 .2456350 .508734

C $5.957536 \quad 0.466933 \quad 1.790346$

H $5.3661492 .772787-1.927789$

O $2.8812323 .810466-1.550744$

H $4.4297654 .165294-0.276080$

H 2.2550442 .0984310 .360280

$\begin{array}{lllll}\text { O } & 2.446373 & 3.873514 & 1.331543\end{array}$

H 4.6864212 .8608332 .064232

O 3.3097791 .4650002 .643341

C $6.505115-0.8199161 .220362$

H $6.790543 \quad 1.162277 \quad 1.948864$

H $\begin{array}{llll}5.550455 & 0.224442 & 2.778627\end{array}$

H $2.4251404 .574562-1.159692$

H $1.740971 \quad 4.221106 \quad 0.760083$

H 2.8335332 .1598123 .128526

O $7.459147-0.551076 \quad 0.284633$

O $6.114992-1.9308661 .556141$

C $8.030929-1.694260-0.358541$

H $8.770641-1.341937-1.082419$

H $8.533156-2.3308030 .376057$

H $7.258216-2.255166-0.892522$

SCF Energy (B3LYP/6-31G**/MMFF)= -3245.91136245

04_00058

MM̄FF Geometry

C $0.920803 \quad 3.813643 \quad 0.523460$

C 0.5841153 .9354881 .816708

C 0.9765952 .9872002 .929497

O 1.8862591 .9662092 .460617

C -0.2999662 .2913123 .442904$

C $-0.049271 \quad 1.2390584 .503915$

C $-0.101329-0.0915134 .289763$

C $0.239141 \quad 1.775428 \quad 5.881509$

C $-0.346181-0.8048782 .986578$

C $0.798343-1.7771672 .671286$

C $0.712707-2.3369991 .242647$

C $1.827985-3.3589261 .009073$

C $1.884808-3.842188-0.446973$

O $0.849712-1.238602 \quad 0.334460$

O $3.139946-4.513422-0.639405$

C $1.882920-2.614812-1.382228$

C $0.792403-4.873317-0.749750$

O $1.781598-3.018324-2.745561$

C $0.774620-1.593788-1.053136$

C $0.893093-0.287166-1.877170$

O $-0.499425-2.169024-1.366137$

C 3.2190132 .2383582 .511288

O 3.7459323 .2720562 .889707

C 3.9354841 .0416472 .014570

C 5.2729441 .0510551 .914919

C $6.113484-0.065717 \quad 1.474132$

C $5.422937-1.3290801 .031754$

C $2.1649110 .532762-1.639892$

C $3.304104 \quad 0.236685-2.599120$

C $5.6967350 .830349-2.988860$
O $3.185556-0.473630-3.592933$

C $6.597632-0.194139-2.375974$

C $7.8416620 .075894-1.952465$

C $8.777458-0.956291-1.393733$

C $9.328660-0.576562-0.038516$

C $10.545362-0.018694 \quad 0.081837$

C $8.512322-0.9359201 .181270$

C $7.4543450 .085791 \quad 1.497730$

N $4.4652330 .902355-2.226913$

H $1.5733583 .005494 \quad 0.207187$

H -0.0852584 .7439792 .104626$

H 1.4395023 .5564723 .744737

H $-0.843703 \quad 1.8552462 .597342$

H -0.9874813 .0428343 .855358$

H $0.063894-0.752973 \quad 5.139448$

H $\quad 0.3700500 .9773286 .619995$

H 1.1567352 .3720185 .875812

H -0.5863972 .4083366 .223474$

H $-1.285906-1.364398 \quad 3.063694$

H $-0.466808-0.1036092 .158245$

H $1.754052-1.2527272 .794624$

H $\quad 0.784789-2.6016833 .395204$

H $-0.270902-2.8008941 .104122$

H $1.722835-4.2103601 .693069$

H $2.794948-2.9002961 .254522$

H $3.188103-5.247238-0.002894$

H $2.859527-2.128762-1.269330$

H $0.898309-5.747586-0.096322$

H $0.879062-5.252961-1.773810$

H $-0.215280-4.479263-0.611394$

H $2.509461-3.639627-2.918636$

H $0.740609-0.495465-2.943055$

H $0.0495110 .354522-1.584381$

H $-1.143549-1.451410-1.484227$

H 3.3398550 .1755811 .752470

H 5.8088841 .9551842 .200709

H $6.099394-2.0661410 .594020$

H $4.675954-1.113096 \quad 0.260217$

H $4.923705-1.8068531 .881309$

H $1.9225081 .591415-1.792353$

H $2.5171340 .423309-0.610262$

H $6.141473 \quad 1.830496-2.980898$

H $5.4758770 .556473-4.025480$

H $6.219214-1.211342-2.307027$

H $8.2266451 .088863-2.048431$

H $9.601738-1.077966-2.108982$

H $8.297307-1.940696-1.330247$

H $10.961170 \quad 0.2261881 .053619$

H $11.149766 \quad 0.210930-0.789481$

H 9.168511-1.000528 2.060249

H $8.111720-1.9471721 .067927$

H $7.8615561 .042174 \quad 1.829078$

H $4.4776881 .431926-1.359815$

C $-0.5684883 .837941-1.444770$

O $-1.7420103 .497819-0.682880$

C $-2.8621753 .375608-1.588289$

C $-2.3114943 .648895-2.983558$

C $-1.1097474 .530726-2.687476$

H $-3.5685444 .161139-1.294156$

C $-3.4778191 .986484-1.382555$

H $-1.9920302 .717240-3.467323$

H $-3.0452314 .131120-3.636187$

C $-0.1210854 .611205-3.836692$

H $-1.4627865 .539351-2.434762$

C $-4.5732301 .587954-2.386509$

O $-4.017971 \quad 1.947256-0.055835$

H -2.671678 $1.244379-1.402875$

H $-4.1530541 .499755-3.395244$

O $-5.5649432 .621191-2.441191$

C $-5.3183110 .285673-2.024568$

C $-4.397931-0.920183-1.763304$

H $-5.9597210 .470322-1.156259$

O $-6.203672-0.035879-3.103560$

H $-3.803602-1.120096-2.663546$

H $-3.693925-0.678225-0.962457$

H $0.730148 \quad 5.247821-3.576240$

H $0.267198 \quad 3.622116-4.102438$

H $-0.5990605 .035030-4.725915$ 
C $0.3694804 .677442-0.578365$ H $-4.7628142 .571729-0.024233$ H $-5.1654733 .395610-2.870738$ H -5.660778 -0.219369-3.889154 H $-0.1694725 .535292-0.158658$ H $1.2056055 .064134-1.170301$ H $-0.0834482 .894054-1.725021$ C $-5.538815-1.697408 \quad 0.942219$ O $-6.121038-1.975092-0.336493$ C $-5.175882-2.203109-1.386606$ C $-4.285752-3.403422-1.031774$ C $-3.635168-3.2481540 .343949$ C $-4.678213-2.8785681 .399660$ H $-4.925574-0.7905280 .882247$ C $-6.686562-1.4313621 .921777$ H $-5.762237-2.486270-2.269825$ O $-3.291635-3.577129-2.035438$ H -4.903546 -4.310881 -1.028191 H -2.823967 -2.5114070 .324613$ O $-3.044417-4.489462 \quad 0.735188$ H $-5.312233-3.7497951 .608196$ O $-4.013216-2.5322762 .612883$ C $-7.405818-0.1494631 .574769$ H -7.424398 -2.2421001 .886109$ H $-6.338320-1.3557622 .958015$ H $-2.821320-4.406554-1.848200$ H -2.336071 -4.6911430 .102100$ H $-3.451874-3.2865162 .861814$ O $-6.655021 \quad 0.9246261 .952376$ O $-8.498313-0.1094341 .023845$ C $-7.2180662 .204471 \quad 1.648788$ H -8.1701602 .3337042 .172113$ H -6.5208802 .9727991 .993303$ H -7.352269 2.3123170 .568116 SCF Energy (B3LYP/6-31G $\left.{ }^{* *} / / \mathrm{MMFF}\right)=-3245.90032163$

0400059

MM̄FF Geometry

C -0.989290 -3.432892 -0.053468

C $-0.822080-3.807758 \quad 1.224217$

C $-1.374334-3.0953182 .439377$

O $-2.339552-2.0841112 .069214$

C $-0.213781-2.4108143 .185764$

C $-0.641925-1.6897364 .448696$

C $-0.746977-0.3511994 .573055$

C $-0.925935-2.5815475 .629822$

C -0.5178990 .6929183 .514916$

C -1.7840801 .5252883 .279189$

C -1.655479 2.4519452 .059454

C -2.9233703 .2951231 .903760$

C $-2.920715 \quad 4.1277770 .614224$

O -1.4600991 .6292720 .904491$

$\begin{array}{lllll}\text { O } & -4.258491 & 4.601658 & 0.392783\end{array}$

C $-2.5679083 .208472-0.574388$

C $-2.035711 \quad 5.372827 \quad 0.743485$

O $-2.4104763 .966212-1.771269$

C -1.313183 2.346636 -0.329039

C $-1.0681831 .299557-1.445050$

O $-0.1639043 .201906-0.294966$

C $-3.625338-2.4939701 .881664$

O $-4.056155-3.6315671 .985916$

C $-4.428764-1.3072871 .510752$

C $-5.722699-1.4543691 .188695$

C $-6.653867-0.3823920 .826899$

C -6.1156831 .0224730 .769955$

C $-2.1590010 .235960-1.606936$

C $-3.2150990 .565416-2.647255$

C $-5.375295-0.300047-3.545897$

O $\quad-3.1191851 .501373-3.435633$

C $-6.5053970 .466770-2.935505$

C $-7.747496-0.024940-2.809661$

C $-8.9054030 .758545-2.262299$

C $-9.6197780 .043669-1.137950$

C $-10.744111-0.655882-1.367232$

$\begin{array}{llll}\text { C } & -9.085903 & 0.222698 & 0.264208\end{array}$

C $-7.940498-0.7017560 .573904$

N -4.262860 -0.346392 -2.616292

H -1.579029-2.551582 -0.287308
H $-0.211178-4.6837091 .433752$

H -1.850277 -3.8338413 .096242$

H $\quad 0.301527-1.7241562 .505587$

H $\quad 0.544535-3.1583153 .457389$

H -1.0398780 .0513745 .542155$

H -1.174889 -2.011871 6.531462

H -1.770294 -3.244439 5.416881

H $-0.049653-3.1959205 .861083$

H $\quad 0.2936911 .3518953 .845316$

H -0.1922670 .2476532 .573185$

H $-2.6306530 .844703 \quad 3.125796$

H -2.0037152 .1191424 .175383$

H -0.7782413 .0950402 .196404$

H -3.0689843 .9410802 .778623$

H -3.7965682 .6305481 .879437$

H -4.5257865 .1198841 .171208$

H -3.428244 $2.547205-0.732753$

H -2.4037996 .0221851 .546975$

H $-2.0689205 .978484-0.168764$

H -0.9929375 .1369070 .965315$

H $-3.2276304 .477742-1.899910$

H $-0.8491781 .802308-2.394802$

H $-0.1458680 .760104-1.184505$

H $0.6189032 .685466-0.547033$

H -3.935228 -0.3430791 .517713$

H $-6.150806-2.4559031 .203840$

H $-6.824971 \quad 1.7460260 .362447$

H $-5.232489 \quad 1.0731720 .124477$

H -5.8405451 .3634691 .773362$

H -1.682387 -0.689984 -1.952990

H $-2.6424630 .016825-0.650338$

H -5.651498 -1.334592 -3.773002

H $-5.0577590 .187101-4.473381$

H $-6.3000481 .485461-2.615285$

H -7.956397 -1.033835 -3.158661

H $-9.6038980 .938817-3.090206$

H -8.591656 $1.753147-1.921772$

H -11.280300 -1.144529-0.560417

H $-11.149928-0.760430-2.368144$

H -9.8785130 .0078390 .994102$

H $-8.842876 \quad 1.2760920 .430636$

H -8.235598 -1.7503760 .633845$

H -4.281712 -1.058108-1.891615

C $0.744071-3.280360-1.831204$

O $1.858624-3.237851-0.918030$

C $3.083629-3.334556-1.665697$

C $2.691038-3.200754-3.132690$

C $1.311076-3.829369-3.137054$

H $3.485775-4.340167-1.489364$

C $4.100553-2.287673-1.189525$

H $2.633703-2.149122-3.438163$

H $3.396809-3.706575-3.798666$

C $0.488541-3.479344-4.364555$

H $1.419515-4.920370-3.072090$

C $4.598442-2.502550 \quad 0.255794$

O $3.530410-0.983940-1.308323$

H $4.959216-2.325842-1.868599$

H $5.097148-3.476387 \quad 0.331185$

O $3.488900-2.5417381 .161971$

C $5.538135-1.3987610 .781523$

C $6.728850-1.082884-0.138437$

H $4.953761-0.4925020 .968371$

O $6.038598-1.8117162 .058653$

H $7.312456-1.997843-0.299316$

H $6.357312-0.763488-1.116142$

H $-0.501537-3.943462-4.316913$

H $0.348734-2.397224-4.459071$

H $0.984559-3.834755-5.273771$

C $-0.367598-4.137542-1.226744$

H $2.754674-0.953106-0.722129$

H $2.933450-3.3015280 .918716$

H $\quad 6.571237-2.6141501 .923923$

H $0.035103-5.108972-0.914740$

H $-1.153146-4.328996-1.965918$

H $0.403854-2.245221-1.968255$

C $6.3503591 .929676-0.178586$

O $\quad 6.951420 \quad 1.165476 \quad 0.873041$

C 7.6664540 .0060190 .434370 
C $8.798474 \quad 0.425226-0.514607$

C $8.2887701 .291700-1.665880$

C $7.4270792 .441187-1.141253$

H $5.6291521 .309160-0.723282$

C 5.5837553 .0880680 .470376

H $8.135626-0.4203671 .329870$

O $9.464908-0.714207-1.047707$

H 9.5453351 .0026120 .045740

H $7.7375870 .695840-2.402780$

O $9.4050851 .852370-2.361039$

H $8.0670053 .177182-0.637951$

O $6.8002383 .098286-2.240303$

C 4.4648622 .5883361 .355237

H 6.2566293 .6810941 .102015

H $5.1516333 .765016-0.274696$

H $9.797280-1.235494-0.297139$

H $9.9623551 .112016-2.656797$

H $7.5048183 .387283-2.845442$

O 3.5053221 .9743270 .605601

O 4.4463242 .7330322 .571117

C 2.4137981 .4183651 .344972

H 1.8346272 .2187631 .814357

H 1.7687900 .8779860 .647402

H $2.778590 \quad 0.7123772 .097447$

SCF Energy (B3LYP/6-31G**//MMFF) $=-3245.91246616$

0400060

MM̄FF Geometry

C -0.7103793 .6983420 .190238$

C -1.6618314 .5724690 .551527$

C $-3.079427 \quad 4.2295490 .956638$

O $-3.2802062 .799091 \quad 1.038535$

C $-4.0570224 .798210-0.087574$

C $-5.514568 \quad 4.537878 \quad 0.239929$

C $-6.2882303 .620208-0.374272$

C -6.0972775 .4190651 .314502$

C $-5.8847392 .651096-1.451180$

C $-6.1251991 .201287-1.010375$

C $-5.5104470 .195803-1.995141$

C $-5.854713-1.237044-1.585880$

C $-5.155574-2.276776-2.474049$

O $-4.090090 \quad 0.382212-1.981788$

O $-5.261786-3.553434-1.824792$

C $-3.651398-1.937729-2.556186$

C $-5.849721-2.423495-3.833329$

O $-2.992858-2.783888-3.495238$

C -3.371061 $-0.458984-2.894731$

C -1.870553 -0.082606 -2.793518

O $-3.772533-0.205903-4.247286$

C -2.9322802 .1870522 .205286$

O $-2.4510102 .713711 \quad 3.195924$

C $-3.232954 \quad 0.7441882 .065222$

C $-2.910396-0.1038643 .053345$

C $-3.183191-1.5434383 .083356$

C -3.843004 -2.156082 1.876524

C $-1.232274-0.249870-1.410340$

C $-0.567104-1.597574-1.187821$

C $0.483743-2.9625140 .614595$

O $-0.370901-2.410174-2.086813$

C $-0.502032-3.822151 \quad 1.338265$

C $-0.361745-4.1849822 .622230$

C -1.302004 -5.111670 3.335856

C -1.865021 -4.5155934 .605038$

C -1.318204 -4.791953 5.801262

C -3.123899-3.687724 4.496536

C $-2.851446-2.2409074 .190109$

N $-0.183873-1.7678530 .135733$

H -0.9328822 .6355010 .160090$

H -1.413422 5.6319900 .569495

H -3.284309 4.6840001 .934056

H $-3.8061774 .399678-1.076145$

H -3.913475 $5.883871-0.177545$

H $-7.3313653 .542950-0.069817$

H -7.164250 5.2301621 .474396

H -5.5876415 .2506652 .268129$

H -5.9878606 .4737591 .041637$

H -6.479334 $2.859963-2.348600$

H $-4.8389242 .777082-1.737898$
H $-5.684317 \quad 1.051433-0.017330$

H $-7.2038251 .022362-0.918726$

H $-5.8947590 .411802-2.999004$

H -6.940719-1.393196-1.593442

H $-5.538179-1.402736-0.547845$

H $-6.206328-3.747352-1.697566$

H -3.222292 -2.164606 -1.572750

H $-6.890016-2.743619-3.698948$

H $-5.373247-3.202508-4.438680$

H $-5.863587-1.496601-4.410323$

H $-3.174228-3.702973-3.234192$

H - $1.296985-0.607791-3.567075$

H $-1.7885300 .984483-3.046801$

H $-3.3067840 .585251-4.565036$

H $-3.7248740 .419506 \quad 1.156540$

H $-2.4187740 .295114 \quad 3.939865$

H -3.895534 -3.246250 1.914692

H $-3.285068-1.9149260 .965352$

H -4.866937 -1.782266 1.772376

H $-0.4359770 .498418-1.305834$

H $-1.961724-0.064599-0.616354$

H $1.305676-2.6420211 .261209$

H $\quad 0.904672-3.519720-0.227791$

H $-1.349653-4.1930970 .767162$

H $0.502290-3.8331023 .181828$

H $-0.747215-6.0295093 .571665$

H -2.124811 -5.428998 2.683062

H -1.738816 -4.395833 6.719631

H $-0.434333-5.4154785 .887456$

H -3.666592 -3.715881 5.451551

H $-3.810412-4.153293 \quad 3.783554$

H -2.370437 -1.708977 5.012279

H $-0.413807-1.0496060 .816398$

C 1.6974883 .7232210 .886371

O 1.6664682 .3062651 .143884

C 3.0195731 .8119931 .236038

C 3.9020993 .0455991 .343682

C 3.1393444 .0421930 .494661

H $3.200994 \quad 1.293930 \quad 0.289017$

C 3.1123100 .8345372 .414523

H 3.9575093 .3975902 .381199

H 4.9201492 .8713790 .985482

C 3.5448845 .4849630 .738572

H $3.3026733 .798263-0.563471$

C 4.4979150 .2053922 .654138

O $2.163783-0.2166132 .224803$

H 2.7980681 .3605223 .325313

H $4.405280-0.5825683 .412888$

O $5.368717 \quad 1.197250 \quad 3.209350$

C $5.213191-0.3946101 .424377$

C $4.411906-1.4769800 .680881$

H $5.494477 \quad 0.409693 \quad 0.737264$

O $6.447119-0.970417 \quad 1.874388$

H $4.117286-2.2554181 .395627$

H $3.491716-1.0405210 .284694$

H $2.959776 \quad 6.1661350 .112805$

H 3.3928825 .7731671 .784180

H 4.6033555 .6319680 .499716

C $0.6869284 .082861-0.203948$

H 1.2964740 .1975222 .075097

H 4.9644261 .5139974 .035051

H $6.230813-1.6986562 .481543$

H $0.9249103 .545956-1.130741$

H $0.7434385 .154195-0.428641$

H $1.4317574 .221647 \quad 1.827713$

C $4.850933-0.456120-2.158318$

O $5.785049-1.187203-1.356627$

C $5.198765-2.144527-0.470954$

C $4.389544-3.172865-1.274347$

C $3.368025-2.499732-2.190366$

C $4.034449-1.412456-3.033721$

H $4.1799320 .119815-1.510976$

C $5.6522370 .534927-3.008292$

H $6.034341-2.685765-0.009496$

O $3.742520-4.077578-0.386456$

H $5.080463-3.762931-1.890479$

H $2.529587-2.084929-1.620593$

O $2.812905-3.470696-3.079329$ 
H $4.678133-1.877572-3.791102$

O $3.026795-0.672927-3.720997$

C $6.1652851 .669275-2.153105$

H $6.5035020 .049786-3.500529$

H $5.0384810 .983052-3.797903$

H $3.328271-4.773604-0.923430$

H $2.270868-4.078181-2.548165$

H $2.502484-1.308418-4.238099$

O $7.377766 \quad 1.344332-1.621232$

O $5.5459182 .707370-1.959060$

C $7.9523322 .325826-0.753219$

H $8.0897303 .272084-1.285187$

H 7.3198822 .4654680 .128328

H $8.9304281 .961034-0.428321$

SCF Energy (B3LYP/6-31G**//MMFF) $=-3245.90844834$

04_00061

MM̄FF Geometry

C $1.172320-1.5338072 .077640$

C $0.014241-2.0676221 .663346$

C $-0.201905-2.5887230 .265386$

O $-1.227770-1.783300-0.360350$

C $-0.683322-4.0490500 .322658$

C $-0.866353-4.682403-1.044041$

C $-2.056667-4.975975-1.605905$

C $0.410402-5.016608-1.773195$

C $-3.426282-4.735587-1.035223$

C $-4.160561-3.629766-1.803686$

C $-5.368476-3.095828-1.019959$

C $-6.144456-2.077884-1.855581$

C $-7.271085-1.410133-1.055699$

O $-4.862846-2.473148 \quad 0.165343$

O $-7.711733-0.277954-1.821831$

C $-6.701507-0.8860240 .281315$

C -8.486261 -2.335071 -0.918417

O $-7.764969-0.393201 \quad 1.092626$

C $-5.863018-1.9361311 .041321$

C $-5.117353-1.3612812 .272216$

O $-6.733038-2.9685661 .520067$

C $-0.826681-0.602128-0.910145$

O $0.309510-0.155798-0.944628$

C $-2.0226880 .076350-1.456181$

C $-1.9150881 .328553-1.925258$

C -2.995828 2.138914 - 2.491528

C $-4.3768631 .540440-2.519844$

C $-4.101898-0.2538321 .968590$

C $-4.657994 \quad 1.154516 \quad 2.089734$

C -4.026646 $3.528741 \quad 1.660790$

$\begin{array}{lllll}\text { O } & -5.747182 & 1.407487 & 2.596851\end{array}$

C $-4.644016 \quad 3.982747 \quad 0.375976$

C $-4.1681125 .007285-0.348271$

C $-4.8102165 .511959-1.608950$

C -3.840028 $5.602884-2.765592$

C $-3.2518486 .768337-3.084282$

C $-3.6241214 .365170-3.606058$

C $-2.7041053 .374390-2.949693$

N $-3.769406 \quad 2.102348 \quad 1.599312$

H $2.007137-1.4757911 .384991$

H $-0.830561-2.1091902 .346894$

H $0.720974-2.530247-0.324770$

H $-1.609899-4.0971510 .902671$

H $\quad 0.039766-4.655978 \quad 0.884534$

H -2.059702 $-5.438303-2.592331$

H $0.225772-5.550351-2.711657$

H $0.966114-4.106344-2.017890$

H $1.045723-5.656965-1.152441$

H $-4.001379-5.667163-1.104789$

H $-3.383377-4.4980260 .029349$

H -3.466517 -2.802294 -1.996614

H -4.483722 -4.017754 -2.777678

H $-6.010667-3.939692-0.741829$

H -6.541963 -2.543520 -2.766086

H $-5.456029-1.295364-2.200392$

H $-8.3791650 .192819-1.293392$

H $-6.069992-0.0252520 .032489$

H -8.886823 -2.586207 - 1.908089

H $-9.303842-1.840532-0.382384$

H $-8.256247-3.272234-0.407043$
H $-7.3720680 .067634 \quad 1.852406$

H $-5.833086-1.0562473 .044728$

H $-4.549047-2.1898502 .719499$

H $-6.294085-3.4262132 .256196$

H -2.959451 -0.467683 -1.449744

H $-0.9393581 .812151-1.895649$

H $-4.4080410 .687449-3.205745$

H $-5.1518202 .240768-2.837664$

H $-4.6702901 .201632-1.520777$

H $-3.288625-0.3212692 .702596$

H $-3.657711-0.3889600 .978881$

H -3.0696304 .0223651 .857826$

H -4.7064873 .7416872 .491956$

H $-5.541562 \quad 3.466498 \quad 0.043479$

H -3.2879985 .5411490 .003743$

H $-5.2255546 .505666-1.394787$

H $-5.6671914 .891451-1.899107$

H -2.577966 $6.848936-3.930811$

H $-3.4271547 .666853-2.501720$

H -3.169559 $4.639147-4.567833$

H $-4.5938783 .936758-3.873971$

H $-1.6731213 .723992-2.878053$

H -2.907608 1.7958601 .157016

C $1.616240 \quad 0.539774 \quad 3.464850$

$\begin{array}{llllll}\text { O } & 2.810066 & 0.885649 & 2.733943\end{array}$

C 2.5481032 .0787601 .971029

C 1.2335332 .6451292 .501725

C 0.4854641 .3808862 .870227

H $2.402221 \quad 1.761204 \quad 0.931813$

C 3.7253703 .0535312 .062884

H 1.3868093 .2669363 .391398

H $\quad 0.712043 \quad 3.2510601 .754325$

C $-0.674188 \quad 1.6110363 .823000$

H $\quad 0.1022190 .933937 \quad 1.945320$

C 5.0884542 .4918721 .613619

$\begin{array}{lllll}\text { O } & 3.872529 & 3.486633 & 3.422817\end{array}$

H 3.4926313 .9575401 .487634

H 5.4661111 .7677862 .345770

O $6.016508 \quad 3.586733 \quad 1.622665$

C 5.1058101 .8489550 .212919

C $6.5376321 .600218-0.303217$

H 4.5519990 .9049860 .244903

O $4.4313862 .700039-0.715517$

H $7.0139692 .569766-0.491348$

H $7.1155801 .099767 \quad 0.479877$

H - -1.1842010 .6693924 .049108$

H $-0.336606 \quad 2.0477714 .768919$

H -1.404787 2.295642 3.381872

C $1.398678-0.9758243 .453110$

H $4.0087802 .693543 \quad 3.969250$

H 6.0070193 .9670032 .517902

H $4.8929073 .556154-0.719522$

H $0.561291-1.2285784 .114164$

H $2.289330-1.4692443 .861647$

H $1.8028260 .846724 \quad 4.502535$

C $6.960323-1.415543-0.665085$

O $6.095193-0.560110-1.423936$

C $6.5676120 .779275-1.613097$

C $7.9337320 .758616-2.312750$

C $8.940539-0.099022-1.552923$

C $8.360125-1.488235-1.294523$

H $7.030338-1.0476410 .365265$

C $6.321239-2.813253-0.634081$

H $5.8654691 .256188-2.308208$

O $8.4320842 .083188-2.464196$

H $7.8070610 .347879-3.323014$

H $9.2410520 .385001-0.616756$

O $10.135382-0.222771-2.327847$

H $8.314505-2.038219-2.242945$

O $9.243279-2.212592-0.439476$

C $5.106792-2.8826400 .265963$

H $6.002119-3.101100-1.642635$

H $7.040125-3.548509-0.255428$

H $9.3081152 .019066-2.881819$

H $9.905701-0.652377-3.169537$

H $9.295683-1.7372520 .407319$

$\begin{array}{llllll}\text { O } & 4.651117 & -4.170929 & 0.282353\end{array}$

O $4.620122-1.9393760 .872721$ 
C $3.499291-4.4160621 .094648$

H $3.364014-5.4978441 .175480$

H $3.633197-4.0049202 .099896$

H $2.616935-3.9876380 .614230$

SCF Energy (B3LYP/6-31G**//MMFF) $=-3245.92975463$

04 00062

MM̄FF Geometry

C $\quad 1.3695013 .3410602 .262102$

C 1.3499054 .2819521 .304547

C $1.9908414 .175628-0.060309$

O $2.1744452 .794985-0.453743$

C $3.3583354 .878817-0.057897$

C $3.9645305 .012054-1.442117$

C $4.9772204 .261154-1.919308$

C $3.3775126 .108463-2.294972$

C $5.6929813 .131727-1.232947$

C $5.538574 \quad 1.823893-2.019640$

C $6.1644450 .631950-1.280767$

C $6.032738-0.644768-2.112825$

C $6.528276-1.885796-1.355673$

O $5.477710 \quad 0.479624-0.034592$

O $6.060548-3.047156-2.059543$

C $5.876325-1.9157350 .043833$

C $8.058999-1.972366-1.345898$

O $6.428086-2.9629200 .837658$

C $5.980558-0.5745120 .797624$

C $5.165839-0.5511652 .114381$

O $7.349999-0.3412931 .149786$

C $1.0682792 .143319-0.913827$

O $-0.0631062 .595566-1.001853$

C $1.4658660 .770156-1.297291$

C $0.522684-0.092878-1.703741$

C $0.731157-1.469583-2.157916$

C $2.139599-1.998475-2.167219$

C $3.652341-0.7366091 .961116$

C $3.174890-2.1673512 .141901$

C $1.105774-3.5504531 .990809$

O $3.902352-3.0795842 .523519$

C $1.025590-4.217800 \quad 0.654644$

C $-0.130951-4.5812780 .080260$

C $-0.226990-5.304080-1.231051$

C $-1.069847-4.566336-2.245472$

C $-2.372658-4.855223-2.404514$

C $-0.377383-3.567692-3.143259$

C $-0.341462-2.183959-2.558969$

N $1.821273-2.2954161 .858907$

H $\quad 1.9298012 .4250472 .099680$

H $\quad 0.8037975 .2050631 .488421$

H $1.3282004 .661749-0.787551$

H 4.0401064 .3491740 .615671

H $3.2600295 .887113 \quad 0.367327$

H $5.340524 \quad 4.472825-2.924511$

H $3.8898466 .206490-3.257972$

H $2.3216885 .911654-2.504198$

H $3.4575157 .072467-1.781826$

H $6.7565263 .389198-1.159925$

H $5.3385192 .989861-0.209867$

H $4.4703151 .632139-2.179895$

H $6.0022031 .930882-3.008300$

H $7.2196950 .857729-1.086955$

H $6.560294-0.541352-3.069297$

H $4.977264-0.799155-2.371829$

H $6.401882-3.004001-2.969151$

H $4.817254-2.158971-0.103595$

H $\quad 8.400607-2.884804-0.845235$

H $8.534085-1.114711-0.865081$

H $8.445317-2.031406-2.370711$

H $6.321474-3.7912610 .339420$

H $5.590141-1.2609352 .834608$

H 5.3132700 .4405562 .566149

H $7.3764630 .289553 \quad 1.888125$

H $2.5167520 .512744-1.249685$

H $-0.5127640 .244891-1.722069$

H $2.607872-1.871074-1.185855$

H $2.738008-1.468639-2.915394$

H $2.203213-3.064830-2.394206$

H $3.158728-0.1529172 .747640$
H $3.299742-0.3524860 .999368$

H $\quad 0.117112-3.3205752 .399705$

H $1.629300-4.2018622 .697885$

H $1.964028-4.4384070 .151549$

H -1.066087 -4.385009 0.599753

H $-0.663621-6.292319-1.034679$

H $0.765953-5.504184-1.652278$

H -2.975331 -4.351843 -3.153259

H $-2.866473-5.595340-1.783548$

H $-0.915056-3.498927-4.099052$

H $\quad 0.613391-3.940633-3.418358$

H -1.324493 -1.712741 -2.510768

H $1.309341-1.4943641 .500442$

C -0.3829722 .3406593 .807508$

O -1.2301072 .1643092 .651481$

C $-1.233251 \quad 0.7628002 .302946$

C -0.8366150 .0399953 .578837$

C 0.2296810 .9728034 .117003

H -0.4558850 .6405511 .537491$

C -2.6010570 .3824691 .728270$

H $-1.684023-0.0192264 .272956$

H $-0.464496-0.9719603 .399604$

C 0.5439800 .7567865 .587141

H 1.1472360 .8195253 .538454

C $-2.778885-1.1295141 .502869$

$\begin{array}{lllll}\text { O } & -2.747521 & 1.051961 & 0.470911\end{array}$

H $-3.393920 \quad 0.7625372 .382255$

H -2.787583 -1.648625 2.468096

O $-1.635774-1.6264240 .797886$

C $-4.027772-1.5311950 .694405$

C $-5.343189-0.9892891 .276644$

H -3.907036 -1.207389-0.345264

O $-4.092452-2.961390 \quad 0.654980$

H -5.458024 -1.352914 2.305341

H $\quad-5.295016 \quad 0.102150 \quad 1.327823$

H $1.301157 \quad 1.4683535 .931387$

H -0.3470250 .8828606 .211383$

H $\quad 0.929623-0.2545795 .752814$

C 0.6233693 .4725203 .562846

H -2.613122 2.0020510 .629711

H $-1.602287-1.170563-0.059820$

H -4.202516 -3.2795581 .567239$

H $1.342743 \quad 3.5189574 .388015$

H 0.0730094 .4222673 .568092

H -1.046441 2.6429224 .628111

C $-6.4016490 .218844-1.300849$

O $-6.429043-1.167139-0.939006$

C $-6.586127-1.4156070 .461578$

C $-7.903182-0.7994920 .952257$

C -8.0132080 .6805030 .588115$

C $-7.7156710 .900941-0.896604$

H $-5.5647270 .708239-0.788846$

C $-6.1427400 .282259-2.811105$

H -6.677040 -2.5036480 .570979$

O $-8.026232-0.9678292 .360503$

H $-8.740109-1.3389450 .489735$

H $-7.357526 \quad 1.2902501 .220871$

O

H $-8.5544510 .509868-1.486533$

O $\quad-7.6417502 .305227-1.127133$

C $-5.2626661 .451747-3.177087$

H $-5.652361-0.631273-3.171568$

H $-7.0756430 .355028-3.382306$

H $-8.860384-0.5475012 .631552$

H -9.4123852 .0435380 .597865$

H -7.588658 2.446135 -2.087297

O $\quad-3.9553721 .139103-2.944699$

O $-5.6884202 .519066-3.598679$

C $-3.0126672 .176091-3.233058$

H $-3.1276262 .993461-2.515208$

H $-2.0076281 .758818-3.134200$

H $-3.1385462 .539623-4.257612$

SCF Energy (B3LYP/6-31G**//MMFF) $=-3245.91795134$

0400063

MM̄FF Geometry

C 2.5643683 .9351150 .624927

C $3.3164614 .330773-0.414231$ 
C $4.1738543 .433503-1.277376$

O $3.6919712 .068614-1.266327$

C $5.6264093 .449227-0.772122$

C $6.5923192 .737134-1.699819$

C $7.1175801 .515161-1.480147$

C $6.9930883 .516944-2.926498$

C $6.8467370 .584277-0.330707$

C $6.302858-0.760488-0.830587$

C $5.922209-1.6982760 .325348$

C $5.414369-3.034814-0.218477$

C $4.886964-3.9535840 .891519$

O $4.898619-1.0569211 .090929$

O $4.156846-5.0093190 .247520$

C $3.896820-3.1635381 .775916$

C $6.034538-4.6277831 .652793$

O $3.512091-3.9629562 .891824$

C $4.448883-1.7971482 .234806$

C $3.399905-0.9172862 .957586$

O $5.520923-2.0160793 .159854$

C $2.6091881 .796766-2.049498$

O $1.9776532 .591899-2.727920$

C $2.321680 \quad 0.348440-1.949045$

C $1.255859-0.158602-2.587292$

C $0.861023-1.568544-2.639105$

C $1.702569-2.558994-1.880465$

C $2.187773-0.5076222 .115372$

C $0.975275-1.4089182 .269188$

C $-1.330771-1.7112221 .364323$

O $0.904211-2.3104893 .099174$

C $-1.318453-2.8069320 .346733$

C $-2.185060-2.875087-0.675015$

C $-2.227953-3.999911-1.666746$

C $-2.124594-3.524622-3.097742$

C $-3.218388-3.406748-3.869440$

C $-0.744440-3.290278-3.665610$

C $-0.214750-1.912355-3.378157$

N $-0.037001-1.0559641 .385989$

H 2.5879772 .8932490 .930113

H $3.3090855 .381170-0.698085$

H $4.1365413 .809391-2.307718$

H $5.668593 \quad 3.0185300 .233951$

H $5.9701414 .486247-0.654649$

H $7.8255021 .125333-2.211125$

H $7.7355822 .987150-3.532883$

H $6.1242603 .706166-3.564156$

H $7.4306454 .478538-2.638944$

H 7.7860020 .4186280 .210470

H $\quad 6.146158 \quad 1.0191610 .385357$

H $5.418195-0.572676-1.452009$

H $7.051092-1.247306-1.468725$

H $6.804813-1.8491060 .957909$

H $6.196040-3.541052-0.798805$

H $4.596736-2.847595-0.926242$

H $3.761662-5.5610640 .944475$

H $2.994365-3.0091021 .172562$

H $6.629357-5.2487210 .971914$

H $5.655421-5.3087512 .422788$

H $6.712255-3.9146482 .126717$

H $2.770919-3.5180323 .335785$

H $3.099527-1.3786553 .905744$

H 3.9070940 .0160883 .243192

H $5.642710-1.2104523 .689137$

H $3.005454-0.263399-1.373535$

H $\quad 0.617617 \quad 0.521522-3.150593$

H 2.691967 -2.645349 -2.340902

H $1.270147-3.561118-1.842505$

H $1.823713-2.246812-0.838344$

H 1.8670570 .4854902 .451144

H $2.448688-0.4250741 .056462$

H -2.078261 -0.9450411 .138356$

H -1.548875 -2.126976 2.353125

H -0.583385 -3.597599 0.476130

H -2.934967 -2.097129 -0.786678

H -3.175715 -4.534453 -1.518980

H - $-1.440941-4.738393-1.469552$

H -3.147002 -3.099014 -4.907432

H -4.209383 -3.612446 -3.479881

H $-0.772343-3.399545-4.758607$
H $-0.071694-4.085618-3.332405$

H $-0.765234-1.121977-3.890767$

H $\quad 0.135280-0.3294350 .696834$

C 0.1912724 .4315551 .393591

O -0.2659534 .2348300 .039050$

C $-0.9114582 .945653-0.043241$

C -1.3064232 .6123491 .383651$

C -0.1184703 .1430642 .156820$

H $-0.1458472 .245195-0.401213$

C -2.072875 $3.014099-1.041037$

H -2.219792 3.1482351 .666138

H -1.4659291 .5431051 .539140$

C -0.3999183 .3529123 .634694$

H 0.7024562 .4262062 .058388

C $-2.9175751 .720010-1.081297$

O $-1.4837803 .274211-2.318169$

H $-2.7115193 .873247-0.803738$

H $-3.3081941 .540241-0.076435$

O $-2.0667810 .619564-1.400330$

C $-4.1094971 .754238-2.059322$

C $-4.9027370 .436903-2.169944$

H -4.783795 $2.569037-1.770707$

O $-3.6270902 .055795-3.372484$

H $-5.5832950 .529094-3.027418$

H -4.208846 $-0.372853-2.429402$

H 0.4821343 .7518824 .145319

H -1.226925 4.0535423 .790969

H $-0.6687992 .405188 \quad 4.112759$

C 1.6643324 .8611631 .398618

H -2.206610 $3.416910-2.952001$

H $-1.7287980 .766922-2.300332$

H -4.399259 $2.179496-3.950256$

H 2.0271904 .9325652 .430129

H 1.7269595 .8689410 .968285

H -0.4080215 .2566461 .799546$

C $-5.450376-0.7869011 .341039$

$\begin{array}{lllll}\text { O } & -4.842797 & -0.373334 & 0.108664\end{array}$

C $-5.7316030 .058188-0.924154$

C $-6.740369-1.054552-1.268051$

C $-7.461314-1.582028-0.025297$

C $-6.478408-1.9060481 .100745$

H -4.628967-1.217742 1.926950

C -6.0292390 .4001412 .128870$

H $-6.2730650 .948446-0.587577$

O $-6.081544-2.137934-1.922698$

H $-7.474178-0.655984-1.978363$

H -8.019506 -2.490198 -0.284678

O $-8.394879-0.6116260 .437900$

H -7.025003 -2.117800 2.027173

O $-5.776833-3.1013150 .746138$

C -4.9523491 .2671332 .746230$

H -6.644992 1.0426471 .494643

H -6.6429120 .0301482 .958559$

H -5.382750 -2.463606 -1.329925

H $-9.031293-0.450491-0.279439$

H $-5.193257-3.3321441 .488784$

O $-5.5282092 .407416 \quad 3.230912$

O $-3.767346 \quad 0.9757342 .821093$

C -4.6443043 .3259573 .881987$

H -4.0336023 .8409533 .135724$

H -5.2525874 .0660794 .408786$

H -4.011692 2.8124434 .612614

SCF Energy (B3LYP/6-31G**//MMFF)= -3245.92271919

0400064

MM̄FF Geometry

C -2.2237105 .1631280 .119629$

C -3.1067804 .1538430 .085653$

C -3.6532053 .4942051 .325672$

O -3.2959922 .0883881 .287224$

C -5.1858953 .6106731 .355599$

C -5.8004173 .0802622 .637247$

C $-6.461371 \quad 1.9110792 .752373$

C -5.6648513 .9858893 .835381$

C -6.7094250 .8807751 .686704$

C $-6.062823-0.4610322 .053606$

C $-6.199216-1.4892720 .921133$

C $-5.602125-2.8343491 .338907$ 
C $-5.605559-3.850898 \quad 0.186757$

O $-5.504068-0.977517-0.220575$

O $-4.772185-4.9570800 .566537$

C $-4.992475-3.195751-1.071607$

C $-6.999396-4.451132-0.026538$

O $-5.125468-4.050695-2.203865$

C $-5.583803-1.806714-1.388175$

C $-4.835600-1.071154-2.527848$

O $-6.943228-1.963302-1.813875$

C $-2.006572 \quad 1.768058 \quad 1.595458$

$\begin{array}{llll}\text { O } & -1.110400 & 2.541962 & 1.895068\end{array}$

C $-1.8632120 .296376 \quad 1.512035$

C $-0.657354-0.261779 \quad 1.702847$

C $-0.352482-1.6953431 .717730$

C $-1.480614-2.6529561 .442736$

C $-3.356547-0.769242-2.262482$

C $-2.398766-1.810115-2.817566$

C $0.029100-2.381881-2.841668$

O $-2.749958-2.732777-3.547029$

C $0.344399-3.368932-1.763458$

C $1.547900-3.467111-1.179248$

C $1.894433-4.497673-0.145731$

C $2.429123-3.8922951 .131393$

C $3.751485-3.8134281 .357680$

C $1.430365-3.4850662 .189441$

C $0.908223-2.0864942 .000794$

N -1.090895 -1.561103 -2.424770

H -1.912443 5.5712641 .078148

H -3.392892 $3.724563-0.871808$

H -3.228633 3.9438182 .232002

H $-5.608827 \quad 3.1033740 .482340$

H $-5.480747 \quad 4.663427 \quad 1.247458$

H -6.8769531 .6525283 .725745$

H -6.184332 3.5925364 .715652

H -4.612532 4.1138334 .106137

H -6.0910444 .9706143 .616979$

H -7.792728 0.7456541 .582066

H -6.345471 1.2103910 .711442

H $-4.999804-0.2951022 .268281$

H -6.523330 -0.8539012 .968601$

H -7.262658 -1.5990790 .678424$

H $-6.128046-3.2408262 .211726$

H -4.565081-2.680155 1.663698

H $-3.876687-4.6191930 .737661$

H -3.917644 -3.087867 -0.885179

H $-7.330341-4.9761100 .877930$

H $-6.989115-5.204985-0.821484$

H -7.757506 -3.703864 -0.269653

H -4.727236 -4.906544 -1.970006

H -4.976699 -1.602451-3.476945

H $-5.332587-0.099598-2.664384$

H -7.200250 -1.177032 -2.323319

H $-2.755043-0.2876701 .320074$

H $\quad 0.1919390 .392925 \quad 1.894828$

H -2.211432 -2.623289 2.257494

H -1.155038 -3.6896741 .332651$

H -1.986251 -2.396842 0.505964

H -3.102452 $0.173896-2.763792$

H -3.166176 $-0.631490-1.193811$

H $0.866745-1.708450-3.046889$

H $-0.223203-2.911804-3.765614$

H $-0.446826-4.056409-1.473640$

H $2.346667-2.799231-1.491460$

H $2.647782-5.165846-0.583720$

H $1.035154-5.1395350 .084619$

H $4.142269-3.4181432 .289370$

H $4.472494-4.1375280 .614491$

H $1.909009-3.5168943 .178111$

H $0.634531-4.2329552 .252119$

H $1.659949-1.3179482 .183646$

H $-0.901977-0.798220-1.781052$

C $-0.1191485 .125527-1.172008$

O $-0.2088323 .709326-1.422056$

C $0.9035693 .308104-2.249522$

C $1.7708124 .547533-2.421012$

C $0.7472195 .664225-2.303398$

H $0.467496 \quad 3.008469-3.210494$

C $1.5784802 .114101-1.568993$
H $2.5117344 .621007-1.614971$

H $2.3069324 .553862-3.374736$

C $1.3620797 .023556-2.023237$

H $0.1658805 .711394-3.233916$

C $2.8936401 .659193-2.220334$

O $0.649668 \quad 1.023652-1.592917$

H $1.7481742 .349915-0.512200$

H $3.6523712 .442977-2.116464$

O $2.6724751 .503845-3.627819$

C $3.4347150 .320373-1.679728$

C $3.5234460 .205606-0.146475$

H $2.794144-0.489591-2.044544$

O $4.7159440 .071841-2.262463$

H 2.5124140 .2677850 .268900

H $3.883472-0.8022970 .085852$

H $0.5873737 .791450-1.933230$

H $1.9411557 .018482-1.093664$

H $2.0342817 .316298-2.836412$

C -1.531402 $5.705101-1.100736$

H $-0.1664111 .330258-1.161013$

H $3.5111981 .209274-4.022436$

H $5.309920 \quad 0.794345-1.996855$

H -2.095183 $5.440334-2.003642$

H $-1.5052696 .797920-1.035101$

H $0.3937225 .250136-0.208038$

C $6.492320-0.0207730 .560235$

O 5.7986341 .1539090 .118332

C 4.4348301 .2493550 .539354

C $4.366388 \quad 1.232616 \quad 2.073036$

C $5.063256 \quad 0.007578 \quad 2.656039$

C $6.479236-0.1225442 .093166$

H $6.014865-0.9063470 .126489$

C $7.9142890 .074797-0.007238$

H $4.0876662 .239293 \quad 0.222338$

$\begin{array}{llll}\text { O } & 3.017311 & 1.264928 & 2.526449\end{array}$

H 4.8587702 .1307822 .468033

H $4.481495-0.9033482 .477461$

O $5.1560250 .151057 \quad 4.075191$

H $7.1061210 .666277 \quad 2.527221$

O $7.031086-1.3682742 .513315$

C $8.667126-1.2317420 .026849$

H $7.8925410 .419632-1.049140$

H $8.4947310 .831547 \quad 0.534414$

H 2.5994512 .0568332 .146930

H $4.251807 \quad 0.269417 \quad 4.413321$

H $6.488448-2.0797052 .132565$

O $8.081695-2.133188-0.812078$

O $9.665294-1.4229170 .709329$

C $8.701892-3.421693-0.860017$

H $8.682880-3.8884440 .129566$

H $8.133218-4.046957-1.553371$

H $9.728849-3.334236-1.227122$

SCF Energy (B3LYP/6-31G**//MMFF)= -3245.91236555

04_00065

MM̄FF Geometry

C $-2.179383 \quad 4.095372 \quad 0.883871$

C $-2.690096 \quad 2.856271 \quad 0.838238$

C -3.016133 2.0456212 .063133

O -2.0954870 .9274652 .063946$

C $-4.443996 \quad 1.4876531 .987008$

C -4.9107390 .9110843 .308546$

C $-4.940591-0.4019853 .608390$

C -5.4095391 .9232874 .308146$

C $-4.486003-1.5574352 .760748$

C $-5.654501-2.4438442 .305823$

C $-6.550806-1.7771221 .250275$

C $-7.737779-2.6772910 .901431$

C $-8.593893-2.081742-0.225850$

O $-5.754139-1.5512790 .083931$

O $-9.476970-3.120847-0.674288$

C $-7.670766-1.699000-1.402436$

C $-9.489567-0.9481520 .287118$

O $-8.404303-1.025880-2.423372$

C $-6.432703-0.873295-0.980267$

C $-5.450526-0.758298-2.169936$

O $-\begin{array}{llll}-6.857987 & 0.433807 & -0.591751\end{array}$

$\begin{array}{llll}\text { C } & -0.913147 & 1.084186 & 2.712683\end{array}$ 
O $-\begin{array}{llll}-0.598693 & 2.014456 & 3.442860\end{array}$

C $0.025741-0.0229562 .417769$

C $-0.147606-0.8935591 .410773$

C $0.781650-1.9561691 .017404$

C $2.049775-2.1124921 .815882$

C $-4.095269-0.131475-1.811553$

C -3.121284 -0.191340 -2.975392

C $-0.6967500 .044600-3.492175$

O $-3.460933-0.480244-4.119898$

C $-0.155126-1.350007-3.453096$

C $1.109679-1.654280-3.126748$

C $1.637285-3.059493-3.080790$

C $2.216637-3.423285-1.731505$

C $3.546077-3.443797-1.541545$

C $1.270090-3.856856-0.633536$

C $0.474055-2.720781-0.051575$

N -1.827356 $0.131026-2.585896$

H -2.031860 4.5820421 .844578

H -2.794801 2.361751-0.124306

H -2.892686 2.6438192 .973996

H -4.5041930 .7293831 .199475$

H -5.146695 2.2781281 .691362

H -5.320269 -0.693989 4.586955

H -5.7730201 .4551675 .229128$

H $-4.6107642 .617446 \quad 4.586308$

H -6.2384632 .4989053 .883206$

H -3.892983 -1.235770 1.899785

H $-3.808330-2.1652223 .373822$

H $-5.225648-3.3612151 .881678$

H -6.255601 -2.739164 3.174911

H $-6.908256-0.8169601 .637857$

H -8.352922 -2.873677 1.788493

H -7.366240 -3.6582890 .572704$

H $-9.971047-2.778181-1.439016$

H -7.324092 -2.639431 -1.852246

H -10.152867 -1.316295 1.079113

H $-10.149740-0.574730-0.503494$

H -8.925335 -0.1052250 .691568$

H -8.550101 $-0.110978-2.128304$

H -5.256875 -1.762830 -2.568984

H $-5.907612-0.169981-2.975692$

H $-6.116879 \begin{array}{lll}1.051581 & -0.689857\end{array}$

H $0.912195-0.0422753 .043804$

H -1.039142 -0.8114890 .791165$

H $1.820412-2.3432172 .861959$

H $2.641843-1.1915961 .779732$

H $2.697949-2.9122371 .451544$

H -4.218587 $0.921182-1.538759$

H -3.645586 -0.659597 - 0.963102

H $0.0401620 .785566-3.170875$

H $-1.0225470 .288688-4.508259$

H - $0.842856-2.153197-3.708908$

H $1.807896-0.855454-2.890236$

H $2.406775-3.150429-3.858749$

H $\quad 0.861979-3.788491-3.347830$

H $3.976661-3.740134-0.591551$

H $4.233700-3.165792-2.332627$

H $1.806662-4.3935760 .157877$

H $0.570515-4.600181-1.037822$

H -0.461636 -2.528324 -0.577119

H $-1.6304790 .282583-1.600640$

C $-0.156688 \quad 4.714967-0.401013$

O $0.2053993 .342771-0.655486$

C $1.3852933 .316200-1.487521$

C $1.8174784 .766829-1.653374$

C $0.4951995 .503594-1.530067$

H $1.0629522 .901997-2.450134$

C $2.4102822 .393323-0.819732$

H $2.4988295 .066663-0.847329$

H $2.3224684 .946072-2.607267$

C $0.6521716 .986327-1.244071$

H $-0.0726815 .369559-2.460320$

C $3.7937232 .384152-1.487732$

O $1.8846461 .062298-0.846170$

H 2.5073212 .6703390 .236441

H $4.2541023 .374093-1.392513$

O $3.6342742 .158260-2.893803$

C $4.7691551 .315894-0.948015$
C 4.9143841 .3288620 .583560

H $4.4562840 .322299-1.289067$

O $6.0496921 .555073-1.541728$

H 5.2149592 .3319510 .910812

H 3.9381861 .1057631 .027099

H $-0.3243797 .471763-1.151160$

H $1.2044047 .159704-0.314317$

H $1.1976047 .478683-2.055794$

C $-1.6798344 .826180-0.332390$

H $1.0032981 .090176-0.436042$

H $3.1879091 .301109-3.004238$

H $6.3649422 .421106-1.231306$

H -2.130058 $4.402093-1.238402$

H $-1.994533 \quad 5.873027-0.266661$

H $0.2899224 .991430 \quad 0.564227$

C $6.362396-2.0786911 .113582$

O $5.503332-0.996326 \quad 0.731870$

C $5.9427340 .311044 \quad 1.114030$

C 6.0953690 .4045462 .641981

C $7.004815-0.7041023 .179563$

C $6.608849-2.0789042 .631980$

H $5.795429-2.9844670 .866418$

C $7.655376-2.1015920 .290688$

H 6.9088740 .5189780 .642478

$\begin{array}{lllll}\text { O } & 4.812285 & 0.309377 & 3.258279\end{array}$

H 6.5098641 .3880212 .894412

H $6.946210-0.7171304 .274819$

O $8.349792-0.4026712 .820665$

H $7.375853-2.8203922 .884077$

O $5.405062-2.4840753 .290274$

C $7.360371-2.413922-1.157112$

H $8.215589-1.1637130 .338505$

H $8.333848-2.8828450 .653800$

H 4.9397070 .3992064 .217898

H $8.915922-1.1008803 .191560$

H $5.183257-3.3772802 .976348$

O $7.335452-1.263232-1.886661$

O $7.154516-3.543697-1.581257$

C $7.048838-1.417714-3.279922$

H $7.149025-0.439418-3.757632$

H $7.759658-2.108087-3.744261$

H $6.022404-1.769857-3.414721$

SCF Energy (B3LYP/6-31G**//MMFF) = -3245.91227060

04_00066

MM̄FF Geometry

C -0.8058172 .7906782 .527776$

C $0.199794 \quad 3.677703 \quad 2.590787$

C 1.3087973 .8831701 .584544

O $1.311902 \quad 2.8101150 .616928$

C 2.6721023 .9071292 .307456

C 3.7739774 .5922051 .521433

C 4.8290563 .9714850 .958165

C 3.6696946 .0961011 .449970

C 5.1348912 .5005180 .929420

C $5.5210252 .041646-0.484229$

C $5.9510730 .568475-0.511537$

C $6.3540910 .148685-1.926330$

C $6.697548-1.346550-2.005203$

O $4.852994-0.228958-0.061051$

O $6.731726-1.719440-3.391211$

C $5.562628-2.159225-1.346954$

C $8.093258-1.640152-1.442354$

O $5.909713-3.539751-1.260694$

C $5.148226-1.6280880 .045445$

C $3.866880-2.348098 \quad 0.521642$

O $6.199142-1.8800150 .979182$

C $1.615483 \quad 3.112985-0.670534$

O $1.8655234 .222411-1.122303$

C $1.6651451 .902851-1.521997$

C $1.1642180 .710053-1.166002$

C $1.186365-0.503208-1.986278$

C $2.070372-0.507947-3.206506$

C $3.293521-1.8089621 .839641$

C $1.867707-2.2793682 .039105$

C $0.503307-4.337196 \quad 2.334791$

O $0.918112-1.5030171 .963324$

C $0.154962-4.768517 \quad 0.944780$ 
C $-0.866894-4.2559440 .243043$

C -1.188944 -4.653302 -1.168975

C -1.051852 -3.505733 -2.146202

C -2.093949 -3.091772 -2.886426

C $0.319813-2.885089-2.319631$

C $0.451921-1.567150-1.598931$

N $1.774527-3.6399372 .286979$

H -0.8948112 .1204421 .677789$

H $\quad 0.2224214 .3568533 .442150$

H 1.1048904 .8417291 .092713

H 2.9589552 .8844412 .575678

H 2.5851984 .4430443 .262874

H 5.5756994 .5924680 .463846

H 4.5350196 .5509580 .956006

H 2.7793786 .3975990 .890458

H 3.6088206 .5209832 .457340

H 5.9719102 .3131911 .612517

H 4.2892311 .9038021 .281378

H $4.6611622 .187916-1.149384$

H $6.3392972 .663593-0.868520$

H $\quad \begin{array}{llll}6.794203 & 0.445362 & 0.178599\end{array}$

H $7.1934920 .755201-2.288206$

H $5.5227110 .349042-2.616276$

H $7.393713-1.161969-3.835222$

H $4.689543-2.101260-2.011908$

H $8.856020-1.086129-2.002518$

H $8.350280-2.699119-1.554827$

H $8.196613-1.369021-0.389610$

H $6.149004-3.834647-2.156087$

H $3.096009-2.250434-0.252006$

H $4.067877-3.4198870 .641132$

H $5.838540-1.8358451 .878471$

H $2.1234262 .058257-2.493888$

H $0.6698350 .610438-0.201380$

H $\quad 1.6612590 .143075-3.985011$

H $2.199861-1.502684-3.640533$

H $3.077950-0.159791-2.953694$

H $3.893018-2.1340442 .696861$

H $3.281276-0.7132691 .848901$

H $-0.258729-3.6795402 .764582$

H $0.620462-5.2096722 .984158$

H $0.788472-5.5266220 .490729$

H -1.494455 -3.4959760 .700790$

H -2.211225 -5.051908 -1.180023

H $-0.540936-5.473816-1.502664$

H - $-1.989495-2.284840-3.604182$

H -3.073484 -3.547584 -2.789888

H $\quad 0.504157-2.769668-3.393833$

H 1.087677 -3.574148 -1.947699

H $-0.120343-1.486661-0.674855$

H $2.618492-4.2046432 .265121$

C -2.164541 1.2726824 .077493

O $-2.9202290 .564186 \quad 3.081244$

C $-2.598518-0.8392903 .171481$

C -1.647559 -0.9909204 .359780$

C -0.9784790 .3715814 .405256$

H -2.057145 -1.089607 2.251270

C $-3.886732-1.6726593 .252259$

H -2.185357 -1.178033 5.296017

H $-0.938825-1.8119914 .220138$

C -0.3100930 .6851995 .731157$

H $-0.227690 \quad 0.4079683 .607080$

C $-4.955575-1.2859752 .208609$

O $-4.476995-1.4804144 .545037$

H -3.638687 -2.738222 3.182085

H $-5.352505-0.2930562 .452145$

O $-6.058456-2.1906072 .348764$

C $-4.471901-1.3023240 .749471$

C $-5.582862-0.862891-0.221628$

H $-3.611726-0.6339640 .649425$

O $-4.042179-2.6209380 .425083$

H -6.400798 -1.593614 -0.200828

H -5.9939240 .0894070 .131523$

H 0.1418961 .6820565 .712460

H -1.026034 $0.654766 \quad 6.559255$

H $0.481197-0.0410165 .943945$

C -1.8625232 .6935613 .595845$

H $\quad-5.286147-2.0192914 .577142$
H $-5.746788-3.0735482 .085283$

H -3.585524 -2.578907 -0.431596

H -1.5748443 .3002834 .463208$

H -2.782285 3.1292983 .185944

H -2.8258691 .3518924 .951411$

C $-3.9925531 .411470-1.486095$

O $-3.8789090 .016705-1.784743$

C $-5.102857-0.717381-1.683473$

C $-6.153134-0.122185-2.633187$

C $-6.3371871 .379636-2.415759$

C $-4.9857832 .095312-2.432282$

H $-4.3426741 .523465-0.452200$

C $-2.5880092 .028946-1.511948$

H $-4.882873-1.729978-2.042341$

O $-7.409284-0.773276-2.475689$

H $-5.834456-0.283203-3.671302$

H $-6.8798171 .588983-1.486576$

O $-7.137191 \quad 1.912377-3.474029$

H -4.602996 $2.103227-3.459979$

O $-5.1600853 .450647-2.028516$

C $-1.9983772 .153335-2.897474$

H -2.612508 $3.033756-1.073441$

H $-1.8935601 .435124-0.905970$

H -7.268765 -1.722160 -2.635785

H $-7.9779551 .423235-3.474345$

H $-5.816083 \quad 3.849157-2.625850$

O $-1.7428150 .920607-3.418507$

O $-1.788964 \quad 3.226216-3.449487$

C $-1.2144000 .911076-4.747798$

H $-0.958005-0.120197-5.004363$

$\mathrm{H}-1.9703201 .271886-5.451373$

H $-0.3089461 .522518-4.807508$

SCF Energy $\left(B 3 L Y P / 6-31 G^{* *} / / M M F F\right)=-3245.91333875$

04_00067

MM̄MF Geometry

C -0.5628232 .5273892 .444701$

C -1.2258161 .5090893 .015029$

C $-0.662547 \quad 0.1833203 .469976$

O $0.757036 \quad 0.132438 \quad 3.211614$

C $-1.352960-0.9633182 .709109$

C -1.287549 -2.296249 3.427693

C $-0.471912-3.3116693 .084084$

C $-2.268734-2.4658334 .558873$

C $0.576409-3.3101272 .008992$

C $0.092376-4.0420710 .751866$

C $1.103790-4.031771-0.406745$

C $2.345595-4.876600-0.111405$

C $3.376124-4.791408-1.248155$

O $1.471037-2.674141-0.660311$

O $4.604924-5.369151-0.781173$

C $3.635224-3.305754-1.575988$

C $2.963058-5.642067-2.453468$

O $4.483332-3.176346-2.714791$

C $2.341589-2.482536-1.781559$

C $2.691471-0.978189-1.847355$

O $1.719446-2.877615-3.003983$

C $1.560925-0.4440004 .139531$

O $1.210854-0.9523835 .196264$

C $2.986644-0.3952153 .736840$

C 3.4297320 .1585872 .596744

C 4.8293310 .2591442 .169527

C $5.880970-0.3123613 .084557$

C $1.471714-0.050378-1.892755$

C $1.8825781 .384204-1.634151$

C $3.1408843 .276709-2.651593$

O $1.6535891 .936248-0.560255$

C $4.5928703 .112839-2.330345$

C $5.1697293 .573218-1.210119$

C $6.6234783 .376785-0.888513$

C 6.8408662 .5653050 .369583

C 7.4326503 .1034051 .449167

C 6.4420651 .1050920 .351491

C 5.1031070 .8721680 .998195

N $2.5390621 .958018-2.710380$

H 0.5049342 .4530412 .260948

H -2.290566 $1.635148 \quad 3.202194$

H -0.8555690 .1172274 .547633$ 
H $-0.930988-1.0462741 .700243$ H -2.415110 -0.7318482 .561166$ H $-0.539399-4.2421663 .645815$ H -2.211864 -3.460857 5.012917 H -2.077116 -1.733491 5.348955 H -3.293052 -2.327691 4.197631 H $0.884083-2.2898491 .762480$ H $1.467019-3.8007032 .417289$ H $-0.179110-5.0770310 .994765$ H -0.823911 -3.5463290 .402866$ H $0.585728-4.427359-1.288592$ H $2.070660-5.9207870 .082228$ H $2.829026-4.5198090 .806898$ H $4.897990-4.8707340 .000654$ H $4.186536-2.872622-0.729282$ H $2.875161-6.696140-2.163720$ H $3.726134-5.611193-3.239305$ H $2.009008-5.337883-2.888540$ H $5.282235-3.703884-2.542732$ H $3.281518-0.713302-0.961056$ H $3.319616-0.779118-2.724403$ H $1.121862-2.171291-3.296022$ H $3.668069-0.8404314 .454999$ H 2.7078230 .5929861 .907400 H $5.828510 \quad 0.157768 \quad 4.072992$ H $6.901572-0.1613222 .728283$ H $5.741229-1.3927633 .200930$ H $0.968054-0.104330-2.863887$ H $\quad 0.733744-0.322942-1.130227$ H $2.6184703 .889558-1.909767$ H $3.0231933 .740694-3.635312$ H $5.1982702 .577674-3.058629$ H $4.5691464 .113271-0.481902$ H $7.0809874 .369525-0.791355$ H $7.1487142 .885738-1.717673$ H 7.6143112 .5157392 .343085 H 7.7458224 .1420021 .464912 H 7.2334480 .5036620 .809188 H $6.3926610 .746165-0.684586$ H 4.2674501 .2571100 .414291 H $2.7208271 .394922-3.535806$ C -0.9744214 .1986490 .595876$ O $-1.5108363 .193506-0.284611$ C -2.157053 $3.839654-1.398265$ C $-1.8035735 .319957-1.299861$ C -1.6629635 .5043870 .196878$ H -3.235783 $3.721621-1.241036$ C $-1.752543 \quad 3.176223-2.720445$ H $-0.8549075 .550269-1.798064$ H -2.574776 $5.958753-1.741332$ C $-0.893657 \quad 6.755590 \quad 0.583811$ H -2.668096 5.5495890 .637501 C -1.916367 $1.644454-2.737608$ O $\quad-0.3706293 .462592-2.969032$ H -2.312696 $3.633658-3.544638$ H -1.209166 $1.195002-2.032242$ O $-1.5237741 .174331-4.033670$ C $-3.3340571 .137991-2.419414$ C $-3.432592-0.395201-2.516190$ H $-3.6035251 .448463-1.408293$ O $-4.2755631 .740986-3.304214$ H $-3.191357-0.705430-3.539848$ H -2.674713 $-0.833831-1.857569$ H -0.8127716 .8440191 .671428$ H 0.1203826 .7469930 .169888 H -1.4020907 .6502290 .209748$ C -1.231821 3.8180362 .055220 H $-0.1289943 .027988-3.804904$ H -2.094526 $1.604916-4.692317$ H -4.119692 $1.386733-4.195729$ H $-2.313116 \quad 3.7424652 .224688$ H -0.8540704 .6084552 .713989$ H $\quad 0.1042814 .2530840 .399961$ C $-4.614270-1.0435010 .240479$ O $-5.299993-0.495001-0.891201$ C $-4.832968-0.944927-2.166937$ C $-4.947461-2.473575-2.254014$ C $-4.227774-3.164647-1.098073$
C $-4.687552-2.578080 \quad 0.235841$

H $-3.567518-0.7161170 .230400$

C $-5.292645-0.4837141 .503156$

H -5.527419 -0.529560 -2.908216

O $-4.460357-2.953367-3.503249$

H $-6.009540-2.747729-2.211903$

H -3.139352 -3.097978 -1.210083

O $-4.554103-4.555559-1.140173$

H $-5.715090-2.9051030 .441723$

O $-3.859936-3.0831591 .281100$

C -4.9955560 .9822661 .738616$

H $-6.380534-0.5890891 .414140$

H -4.948239-1.024471 2.391585

H -3.488846 -2.932861 -3.480234

H -4.016042 -5.004007 -0.466396

H $-4.010565-4.0412701 .342157$

O $\quad-5.660607 \quad 1.3898662 .860986$

O -4.2727001 .6817901 .044413$

C -5.4752172 .7617013 .224346$

H -5.7823593 .4193132 .405515$

H -6.1023602 .9697214 .095269$

H -4.430927 2.9418703 .494454

SCF Energy (B3LYP/6-31G**//MMFF) $=-3245.90479703$

04_00068

MM̄FF Geometry

C $0.240100-1.2415742 .191489$

C $0.902562-2.1111091 .412501$

C $0.407232-2.6806500 .101227$

O $-0.708077-1.917495-0.415967$

C $-0.042875-4.1370290 .306444$

C $-0.437461-4.833160-0.982233$

C $-1.703633-5.105941-1.356816$

C $0.713952-5.258617-1.857844$

C $-2.975110-4.764211-0.630400$

C $-3.841013-3.806408-1.458640$

C $-5.037395-3.270325-0.657893$

C $-5.905664-2.366910-1.535873$

C $-7.034775-1.692847-0.743476$

O $-4.519414-2.5289930 .451237$

O $-7.561514-0.647111-1.574787$

C $-6.437703-1.0381590 .521826$

C $-8.194099-2.662564-0.488344$

O $-7.470165-0.5147361 .353581$

C $-5.514993-1.9785261 .323106$

C $-4.774519-1.2607192 .477825$

O $-6.310621-3.0134721 .914165$

C $-0.404593-0.784223-1.109966$

O $0.710319-0.352186-1.356274$

C $-1.673188-0.137965-1.512749$

C $-1.6405241 .072213-2.091163$

C $-2.7926581 .835672-2.576903$

C $-4.160274 \quad 1.237149-2.385269$

C -3.810562 -0.1485902 .052046$

C -4.4050821 .2487732 .083365$

C -3.8293693 .6019401 .486138$

O -5.4886931 .5109092 .597006$

C -4.4621283 .9657060 .181180$

C $-3.9725364 .908257-0.638609$

C $-4.6358595 .329700-1.917784$

C $-3.7090215 .262975-3.109777$

C $-3.0928376 .365269-3.569486$

C $-3.5759823 .939459-3.826736$

C $-2.5727393 .023373-3.179142$

N -3.549387 2.1795621 .508268

H $-0.757569-0.9229961 .906434$

H $1.892872-2.4408641 .720277$

H $1.230490-2.650515-0.622409$

H $-0.863879-4.1640311 .030192$

H $0.767289-4.7150810 .772118$

H - $-1.856837-5.629916-2.299678$

H $0.381722-5.813834-2.741484$

H $\quad 1.273910-4.386753-2.209689$

H $1.395515-5.908834-1.299530$

H -3.531764 -5.691272 -0.447520

H $-2.775625-4.3298760 .351113$

H -3.221219 -2.963399-1.788184

H $-4.196604-4.320444-2.360277$ 
H $-5.619351-4.121173-0.284425$ H $-6.313136-2.926740-2.386993$ H $-5.277099-1.580783-1.973887$ H -8.226120 -0.163726 -1.054046 H $-5.857385-0.1740640 .179267$ H -8.607486 -3.019423 - 1.439404 H $-9.022770-2.1649530 .027221$ H $-7.900119-3.5382950 .094008$ H -7.873415 -1.260047 1.830307 H $-5.492637-0.910903 \quad 3.228988$ H -4.166301 -2.019586 2.991392 H -5.818298 -3.393095 2.660701 H $-2.595519-0.675313-1.329264$ H -0.672654 $1.549935-2.240285$ H $-4.2737840 .343823-3.007901$ H $-4.9750451 .918354-2.639809$ H $-4.3184870 .960292-1.337727$ H -2.976724 -0.1316972 .765135$ H -3.389002 -0.346274 1.062185 H -2.8786854 .1210081 .644685$ H -4.5054093 .8563822 .308693$ H $-5.3845753 .454498-0.084181$ H $-3.0653755 .438031-0.356284$ H -4.992873 $6.359211-1.781867$ H $-5.5338984 .732705-2.119654$ H -2.451952 $6.330976-4.444310$ H -3.211137 $7.324932-3.077490$ H $-3.2371054 .108728-4.858071$ H $-4.5635683 .482987-3.937497$ H -1.544565 $3.377298-3.266650$ H $-2.699607 \quad 1.8592251 .053178$ C 1.0688090 .8332353 .402737 $\begin{array}{llll}\text { O } & 2.049059 & 1.105460 & 2.382347\end{array}$ C 1.6600142 .3028261 .680089 C 0.5849362 .9614692 .537117 C $-0.121918 \quad 1.744493 \quad 3.104419$ H 1.2028951 .9802810 .736820 C 2.8896433 .1713301 .391663 H 1.0182763 .5603533 .346432 H $-0.0750413 .608371 \quad 1.951266$ C -0.9877512 .0460614 .314385$ H -0.7502431 .3199282 .313803$ C 4.0257072 .4240180 .660560 O 3.4209263 .6598692 .630090 H 2.5905974 .0584680 .822158 H 4.4533301 .6759161 .336699 O $5.076632 \quad 3.361746 \quad 0.398711$ C $3.6049061 .760251-0.662408$ C $4.7576841 .089700-1.423678$ H $2.8240821 .019926-0.471501$ O $3.0301302 .758045-1.510142$ H $4.3843310 .718093-2.388207$ H $5.5003071 .844969-1.716357$ H -1.466896 1.1348924 .686031 H -0.3997032 .4760895 .132016$ H -1.7752662 .7613034 .056283$ C $0.787124-0.6709973 .470089$ H 3.6450442 .8884053 .178506 H $4.7329204 .018051-0.231584$ H $2.7103452 .311632-2.312889$ H $0.076410-0.8797624 .277876$ H $1.720908-1.1871033 .727360$ H 1.5320191 .1321504 .352543 C $7.322151-1.580103-1.033911$ O $6.480954-0.544066-1.559214$ C $5.467774-0.049778-0.672766$ C $4.527335-1.190169-0.252977$ C $5.301823-2.3745300 .333164$ C $6.485177-2.777419-0.552141$ H $7.919529-1.917100-1.891207$ C $8.292874-1.0467490 .031734$ H $\quad \begin{array}{llll}5.956770 & 0.374342 & 0.209188\end{array}$ O $3.761484-1.633996-1.371506$ H $3.818555-0.8194830 .494998$ H $4.625906-3.2314670 .443635$ O $5.788482-2.0354461 .628193$ H $7.134403-3.488396-0.027631$ O $5.987060-3.444351-1.715995$
C $9.283812-0.052599-0.538295$

H $7.765479-0.5465950 .848036$

H $8.871598-1.8812200 .444518$

H $4.382675-1.902868-2.069919$

H $5.020640-1.8125362 .181805$

H $5.503833-4.233467-1.417082$

O $10.101213 \quad 0.379998 \quad 0.467842$

O $9.3407830 .300799-1.707841$

C $11.097114 \quad 1.333410 \quad 0.084129$

H $10.6238492 .238727-0.307910$

H $\quad 11.6757201 .5970770 .973511$

H $11.7729530 .897351-0.657739$

SCF Energy (B3LYP/6-31G**//MMFF) $=-3245.91811563$

04_00069

MM̄FF Geometry

C -0.7003362 .2684703 .002559$

C 0.3440041 .6793463 .606373

C 0.5281020 .2070553 .906469

O $1.500321-0.3240532 .972645$

C $-0.746971-0.6509893 .787430$

C $-0.582140-2.0772624 .286710$

C $-0.628322-3.1804753 .511907$

C $-0.396380-2.2225395 .776359$

C $-0.833971-3.2553822 .025635$

C $0.460954-3.6523551 .305828$

C $0.407582-3.303204-0.187572$

C $1.655040-3.812584-0.909586$

C $1.702227-3.353947-2.374232$

O $0.332179-1.877980-0.279880$

O $3.029643-3.620118-2.852396$

C $1.475187-1.826912-2.426328$

C $0.755098-4.181084-3.250916$

O $1.385416-1.374277-3.774862$

C $0.252169-1.359462-1.612092$

C $0.1590690 .180016-1.487684$

O $-0.939964-1.805824-2.268645$

C $2.818971-0.1578193 .262374$

O $3.295190 \quad 0.392995 \quad 4.241884$

C $3.595770-0.7517352 .151407$

C $4.911193-0.5091342 .054671$

C $5.807886-1.0093541 .009708$

C $5.207039-1.857734-0.079911$

C $1.3381140 .848469-0.771579$

C $2.3987361 .408570-1.703309$

C $4.6577302 .465422-1.648190$

O $2.2571311 .484635-2.920340$

C $5.6952071 .423906-1.927115$

C $6.9736491 .531364-1.533755$

C $8.0375820 .522513-1.860209$

C $8.7974130 .053331-0.639603$

C $9.9850430 .592355-0.315897$

C $8.225257-1.1023580 .149038$

C $7.115536-0.6815011 .070662$

N 3.513971 1.849421-1.002040

H -1.5336941 .6734042 .642391$

H 1.1618162 .3162203 .940283

H $0.9154090 .126184 \quad 4.929525$

H -1.066175 -0.6474772 .743026$

H - $1.563177-0.1912434 .360183$

H $-0.503595-4.1506813 .991423$

H $-0.426908-3.2689726 .098767$

H $0.568500-1.8122746 .088650$

H -1.191806-1.694184 6.312030

H -1.608314 -4.004267 1.816753

H $-1.225251-2.3178981 .627213$

H $1.307032-3.1245841 .763975$

H $0.635326-4.7271821 .438225$

H $-0.495975-3.742964-0.622569$

H $1.722738-4.905919-0.845531$

H $2.549131-3.430083-0.399354$

H $3.094646-3.263009-3.754941$

H $2.379561-1.366597-2.012283$

H $1.028102-5.242451-3.209445$

H $\quad 0.836971-3.894119-4.304988$

H $-0.291347-4.096587-2.950454$

H $0.500081-1.602061-4.106017$

H $-0.0333870 .629106-2.469199$ 
H $-0.7348480 .399139-0.886371$ H -1.671644 -1.219170 -2.015849 H $3.059761-1.3610751 .433362$ H $5.380213 \quad 0.1189542 .811043$ H $4.370566-1.336803-0.557831$ H $4.843829-2.8053550 .331593$ H $5.906486-2.100509-0.882457$ H $0.9565381 .700762-0.196854$ H $1.8067300 .163583-0.059796$ H $5.0263773 .248038-0.977548$ H $4.3406892 .930922-2.586894$ H $5.3876190 .557406-2.508012$ H $7.2850782 .414005-0.979635$ H $8.731400 \quad 0.991346-2.570586$ H $7.620007-0.345006-2.386202$ H $\quad \begin{array}{llll}10.551276 & 0.240733 & 0.540239\end{array}$ H $10.4148411 .401604-0.896943$ H $\quad 9.008007-1.553890 \quad 0.773637$ H $7.931103-1.899919-0.538952$ H $7.454427-0.053451 \quad 1.895882$ H 3.5637321 .6953330 .000771 C $-0.571506 \quad 4.1699041 .351677$ O -1.6071093 .6402810 .502442$ C $-1.9694444 .637805-0.474083$ C $-0.9533775 .765699-0.330984$ C $-0.6093995 .683281 \quad 1.142660$ H -2.957684 $5.013466-0.184780$ C $-2.0363224 .013401-1.873984$ $\mathrm{H}-0.0585135 .589885-0.939050$ H -1.368669 $6.735284-0.622411$ C 0.6849906 .3927531 .501646 H -1.434004 $6.122141 \quad 1.720320$ C $-2.9440242 .770331-1.965142$ O $-0.7190203 .611744-2.271864$ H -2.351925 4.767135 -2.604092 H $-2.5304631 .969617-1.344050$ O $-2.9100262 .292922-3.315850$ C $-4.4127923 .027281-1.584468$ C $-5.3295281 .807479-1.768983$ H $-4.4779693 .369905-0.548184$ O $-4.9314634 .089825-2.389646$ H -6.371586 $2.110983-1.597628$ H $-5.3302311 .496825-2.823059$ H 0.8959056 .3050092 .571939 H 1.5369235 .9741080 .955177 H $\quad 0.619788 \quad 7.4580241 .257654$ C -0.8143253 .7560942 .803539$ H $-0.3766953 .015342-1.585195$ H -1.977132 2.142041 -3.546263 H -4.849235 $3.819524-3.320410$ H -1.8236364 .0532913 .114688$ H -0.1102684 .2774223 .462625$ H $\quad 0.3806503 .7575610 .993769$ C $-5.686978-1.709282-0.610093$ O $-5.909816-0.444411-1.252611$ C $-4.9883510 .593689-0.888637$ C $-5.093013 \quad 0.882810 \quad 0.617190$ C $-4.908855-0.388127 \quad 1.452368$ C $-5.740832-1.5593350 .921729$ H $-6.539617-2.325188-0.922365$ C $-4.409828-2.371193-1.149802$ H -3.976856 $0.256434-1.136636$ O H -4.3277801 .6136710 .900706$ H -5.197982 -0.185765 2.491158 O $-3.535370-0.7602351 .450657$ H -5.425251 -2.489646 1.407002 O $-7.101518-1.3294371 .301973$ C $-4.348476-3.859171-0.886153$ H $-4.362408-2.226268-2.236152$ H $-3.515588-1.911818-0.721153$ H -7.0493370 .8449950 .630412$ H $-3.029385-0.0086371 .802048$ H -7.616174 -2.110551 1.036356 O $-3.127552-4.325592-1.283677$ O $-5.249613-4.531484-0.404824$ C -2.907016 -5.725728 -1.086843 H $-3.636192-6.308572-1.657502$
H $-1.904088-5.965865-1.449551$

H $-2.962702-5.970356-0.021897$

SCF Energy (B3LYP/6-31G**//MMFF) $=-3245.92591655$

04_00070

MM̄FF Geometry

C -4.689598 $2.900620-0.769665$

C $-3.4651703 .004368-0.233233$

C $-2.2834633 .594651-0.956954$

O $-1.2324322 .602207-1.021757$

C $-1.7475684 .803311-0.167884$

C $-0.5995795 .524435-0.848613$

C $0.6356505 .658794-0.325352$

C $-0.9188446 .154043-2.180611$

C 1.1307825 .1659611 .005652

C 1.6540743 .7266421 .001355

C 3.1162533 .5439930 .559027

C $3.3529833 .842260-0.919524$

C $4.7947823 .529885-1.342540$

O $3.4344602 .170113 \quad 0.817837$

O $4.8241573 .564106-2.777732$

C $5.1642092 .101021-0.892296$

C $5.7624614 .621737-0.866670$

O $6.5439711 .859971-1.155092$

C 4.8038901 .7996160 .580380

C 4.8665680 .2908170 .909271

O 5.6872232 .5055431 .450523

C $-1.3635531 .607418-1.941280$

O $-2.2944971 .433105-2.711230$

C $-0.1599460 .751377-1.848389$

C $-0.147479-0.440579-2.463781$

C $0.964571-1.394075-2.484157$

C $2.250359-0.979832-1.820019$

C $6.246163-0.3632670 .884375$

C $6.135367-1.8310141 .246861$

C $5.495223-4.0392910 .320481$

O $6.438774-2.2493702 .361482$

C $4.775881-4.565058-0.879372$

C $3.553576-5.114646-0.829661$

C $2.835379-5.681371-2.020762$

C $1.587635-4.895950-2.363597$

C $0.389510-5.303806-1.910505$

C $1.789922-3.696384-3.269579$

C $0.787131-2.584609-3.095201$

N $5.639071-2.6016670 .205789$

H $-4.8654033 .271925-1.776257$

H -3.287943 2.6281290 .769761

H -2.557069 $3.897768-1.974800$

H -1.4462084 .4609900 .828498$

H -2.555600 $5.530056-0.009173$

H $1.3708506 .219731-0.899514$

H $-0.1091676 .798070-2.540242$

H $-1.0882395 .385768-2.941092$

H -1.817354 $6.774963-2.102106$

H $1.9121395 .847181 \quad 1.364885$

H $0.326176 \quad 5.247744 \quad 1.745932$

H 1.5750953 .3427682 .028314

H 1.0018423 .0728910 .410712

H $3.7478734 .177477 \quad 1.192594$

H $3.1295054 .885346-1.159426$

H $2.6687663 .235319-1.528843$

H $5.7170323 .301304-3.060661$

H $4.6065541 .406179-1.534029$

H $5.4579365 .599114-1.260052$

H $6.7747564 .451579-1.249614$

H $5.814293 \quad 4.7013790 .221288$

H $7.0591722 .289320-0.450831$

H 4.4458220 .1416911 .914515

H $4.182889-0.2483300 .243270$

H 5.6716372 .0688432 .318648

H $\quad 0.6812891 .127987-1.278204$

H -1.039083 -0.754621 -3.005272

H $2.701495-0.136990-2.354599$

H $2.997159-1.775138-1.775029$

H $2.071063-0.678816-0.784169$

H $6.711211-0.298631-0.103196$

H $6.925447 \quad 0.1168331 .596947$

H $4.973063-4.2636871 .257143$ 
H $\quad 6.497009-4.477186 \quad 0.383074$

H $5.295658-4.505457-1.832639$

H $3.042873-5.1824340 .127272$

H $2.579144-6.724671-1.794858$

H $3.490635-5.727354-2.899734$

H $-0.530012-4.787492-2.160114$

H $\quad 0.298277-6.179361-1.275375$

H $1.742631-4.041385-4.310686$

H $2.807234-3.311685-3.148853$

H $-0.181639-2.793572-3.551142$

H $5.550229-2.176469-0.713336$

C $-6.2785660 .976121-0.767729$

O $-5.253285-0.023189-0.590887$

C $-5.880501-1.290797-0.320547$

C -7.368483 -1.094196-0.587103

C $-7.5536600 .358939-0.196766$

H -5.741621 -1.4902000 .748380$

C $-5.222809-2.397038-1.152549$

H $-7.614790-1.238262-1.645513$

H -7.988475 -1.779965 -0.001592

C $-8.835010 \quad 0.972410-0.733651$

H -7.5493550 .4326860 .899047$

C $-3.700896-2.536124-0.944883$

O $-5.442835-2.131687-2.544399$

H -5.715613 -3.354398 -0.948047

H $-3.179381-1.664271-1.358240$

O $-3.259658-3.667142-1.706761$

C $-3.273418-2.7402940 .520022$

C -1.768289 -3.026505 0.661299

H -3.524651 -1.844795 1.092618

O $-4.008433-3.8224941 .090543$

H -1.541105 -3.9899760 .190845$

H -1.212746 -2.257509 0.115053

H $-8.9199082 .022447-0.437498$

H -8.874502 $0.926591-1.827179$

H $-9.7087320 .440146-0.343357$

C $-5.8588502 .268973-0.068468$

H $-5.068153-1.255038-2.738395$

H $-3.526590-3.516043-2.629833$

H $-3.818658-4.6170180 .562469$

H -5.6041902 .0589710 .977275$

H $-6.6847582 .988690-0.066459$

H $-6.3822311 .138635-1.848879$

C $-0.838735-0.7358102 .452631$

O $-1.592576-1.8854262 .856036$

C $-1.302230-3.0877812 .134860$

C $0.179544-3.4446192 .304980$

C $1.082435-2.2966871 .865350$

C $0.670251-1.0039372 .569283$

H -1.101085 -0.4670321 .422145$

C -1.2408450 .4311443 .369550$

H -1.882108 -3.883032 2.619571

O $0.487839-4.622971 \quad 1.569976$

H $\quad 0.374137-3.665383 \quad 3.362705$

H $1.071623-2.1869090 .776323$

O $2.428398-2.6182982 .221709$

H $\quad 0.956268-1.0757273 .626320$

O 1.4047360 .0868692 .019598

C -2.674999 0.8742453 .173451

H $-1.133925 \quad 0.126727 \quad 4.417819$

H $-0.603722 \quad 1.3014553 .176132$

H $1.427815-4.8195191 .722232$

H $2.984853-1.8659181 .957797$

H $1.1144790 .210413 \quad 1.100106$

O $-2.954941 \quad 1.8645474 .072633$

O $-\begin{array}{llll}-3.450190 & 0.422479 & 2.343094\end{array}$

C -4.2849472 .3909704 .021802$

H $-4.421343 \quad 3.0518564 .881867$

H -4.418831 2.9737883 .106421

H -5.0238931 .5858104 .080020$

SCF Energy (B3LYP/6-31G**//MMFF $)=-3245.92912790$

$04 \quad 00071$

MMFF Geometry

C -1.530518 -0.3454343 .453347$

C -2.7253080 .2558543 .558641$

C -3.1281451 .6224213 .066531$

O $-4.0462121 .383046 \quad 1.972394$
C -1.9950302 .5292422 .552032$

C -2.3945483 .9925972 .522499$

C -2.8086934 .6625861 .430039$

C -2.2653844 .7143163 .840493$

C -3.0186114 .1335980 .039388$

C $-2.0568634 .768305-0.973962$

C $-0.6092674 .276297-0.823594$

C $0.3118995 .014825-1.796382$

C $1.7437164 .462306-1.758150$

O $-0.5992802 .874173-1.107437$

O $2.4462344 .976321-2.899981$

C $1.6880032 .929127-1.914137$

C $2.5129374 .944363-0.522213$

O $2.9819132 .359712-1.747057$

C $0.6794102 .243462-0.963761$

C $0.5158740 .754825-1.354787$

$\begin{array}{llll}\text { O } & 1.164134 & 2.328124 & 0.376997\end{array}$

C $-5.2581601 .989449 \quad 1.977454$

O -5.6888242 .7610752 .822798$

C $-6.0667291 .579302 \quad 0.803497$

C $-5.5794410 .852240-0.214723$

C $-6.3251430 .386131-1.386680$

C $-7.7983520 .692140-1.447904$

C $-0.6191400 .031996-0.615595$

C $-0.814718-1.385595-1.123261$

C $-2.430852-3.281917-0.998356$

O $-0.017897-1.948210-1.868772$

C $-3.242077-3.185250-2.251945$

C $-4.550201-3.474315-2.323647$

C $-5.354853-3.367525-3.587663$

C $-6.506607-2.392269-3.470932$

C -7.769705 -2.831264 -3.338025

C $-6.208010-0.913948-3.600135$

C -5.664292-0.316556-2.330855

N -1.980839 -1.952853 -0.628365

H -0.7038170 .1496882 .953834$

H -3.524859 -0.289546 4.059344

H -3.644262 2.1093953 .903256

H - 1.6727642 .2008431 .557950

H -1.111659 2.447231 3.198294

H -3.0512315 .7189851 .541506$

H -2.545644 5.7704953 .766982

H -2.9105264 .2558254 .596184$

H -1.230267 4.6728754 .195232

H -2.953494 $3.043124-0.006985$

H $-4.0457964 .384267-0.254081$

H -2.418890 $4.510155-1.977795$

H $-2.0935005 .861470-0.887384$

H $-0.277427 \quad 4.4400680 .207407$

H $0.3102926 .092811-1.591948$

H $-0.0739114 .904089-2.819675$

H $2.4245115 .947535-2.852847$

H $1.3865672 .716111-2.949406$

H $2.5747766 .039181-0.513221$

H $3.5485074 .586365-0.536753$

H $2.0534904 .633078 \quad 0.418252$

H $3.5784952 .805160-2.372694$

H $0.3059040 .688957-2.430611$

H $1.4529240 .215167-1.173681$

H $\quad 0.780979 \quad 1.608130 \quad 0.901329$

H $-7.098322 \quad 1.9158570 .824070$

H -4.531708 $0.556616-0.196103$

H $-8.3115920 .295270-0.564809$

H $-8.2978480 .255978-2.315631$

H -7.963846 $1.774062-1.494565$

H $-0.403324-0.0306520 .455178$

H -1.562175 $0.574578-0.747073$

H $-3.008940-3.678323-0.157757$

H -1.565165 -3.930472 -1.164706

H -2.720922 -2.860473 -3.149725

H $-5.072534-3.809780-1.430736$

H -5.730627 -4.370841 -3.827526

H -4.725555 -3.079229-4.439095

H $-8.605359-2.141548-3.285434$

H $-7.996341-3.890443-3.274768$

H $-7.095287-0.369730-3.942050$

H $-5.473108-0.761460-4.401142$

H $-4.596028-0.484648-2.195065$ 
H $-2.609970-1.381594-0.071486$ C -1.079161-2.765318 2.923002 $\begin{array}{lllll}\text { O } & 0.194378 & -2.579981 & 2.267227\end{array}$ C $0.941062-3.8044252 .369540$ C $-0.105500-4.8918002 .558653$ C -1.074683 -4.1907603 .487599$ H $1.542140-3.7303683 .284454$ C $1.868858-4.0068591 .168761$ H $-0.604160-5.1454881 .615979$ H $\quad 0.313402-5.8078032 .985357$ C $-2.450349-4.8346703 .530067$ H $-0.652035-4.1927584 .501643$ C $2.800673-2.8170010 .859109$ O $1.071241-4.2457850 .003833$ H $2.456868-4.9193761 .325371$ H $2.215694-2.0149990 .395030$

O $3.732257-3.251155-0.137167$

C $3.572845-2.2621152 .066677$

C $4.654621-1.2097531 .751330$

H $2.860124-1.8112422 .766334$

O $4.203512-3.3351912 .772134$

H $5.071740-0.8979032 .717161$

H $5.486771-1.6948851 .231405$

H -3.118937 -4.2807624 .196479$

H -2.911406 -4.861611 2.536900

H $-2.382135-5.8640093 .896966$

C $-1.256256-1.7120954 .019241$

H $1.680024-4.382254-0.742037$

H $4.243073-3.9923570 .229006$

H $4.887416-3.7132832 .194116$

H $-0.347265-1.6450914 .629516$

H -2.072866 -2.008752 4.688653

H $-1.854068-2.6570562 .155244$

C $5.315681-0.312147-1.120706$

O $4.058822-0.222050-0.436832$

$\begin{array}{llll}\text { C } & 4.131286 & 0.017256 & 0.974592\end{array}$

C 4.9089721 .3135711 .238152

C 6.2948361 .2705100 .607063

C $6.1801050 .933379-0.877420$

H $5.849556-1.208606-0.786196$

C $5.020480-0.463808-2.619297$

H $3.1025410 .196635 \quad 1.312249$

O $5.009517 \quad 1.5522252 .638203$

H $4.350732 \quad 2.1574210 .812441$

H 6.9475250 .5650551 .134513

$\begin{array}{lllll} & 0 & 6.894273 & 2.560174 & 0.747659\end{array}$

H $5.7548741 .796602-1.403932$

O $7.4874620 .731390-1.412534$

C $4.503672-1.844009-2.945707$

H $4.2862540 .281040-2.947243$

H $5.919037-0.316659-3.229345$

H $\begin{array}{llll}5.527091 & 2.367021 & 2.757264\end{array}$

H 7.7554032 .5296490 .296363

H $7.879457-0.035691-0.961438$

O $3.198645-1.777862-3.333742$

O $5.182658-2.860066-2.866637$

C $2.606159-3.024364-3.711076$

H $2.546625-3.689972-2.845660$

H $1.594278-2.824830-4.073225$

H $3.178783-3.491258-4.518297$

SCF Energy (B3LYP/6-31G**//MMFF) $=-3245.91350535$

04_00072

MM̄FF Geometry

C $1.122236 \quad 3.575237-1.376571$

C $2.0059802 .623499-1.043902$

C $3.1354352 .193552-1.945031$

O $2.9656010 .782444-2.213903$

C $4.4871362 .357790-1.233157$

C $5.6528662 .440414-2.197405$

C $6.4243661 .401096-2.571333$

C $5.9516463 .825490-2.711750$

C $6.267053-0.050268-2.213832$

C $7.371299-0.560448-1.278229$

C $7.169481-0.1327250 .183462$

C $8.323810-0.6317661 .055940$

C $8.088976-0.3305842 .544426$

O $5.936590-0.7045350 .634088$
O $9.053635-1.0710373 .307387$

C $6.679350-0.8207152 .942177$

C 8.3616091 .1420392 .869875

O $6.360694-0.4304614 .275779$

C $5.569252-0.3525831 .972889$

C $4.239167-1.0715822 .303946$

O $5.386224 \quad 1.0572772 .121143$

C $2.0199180 .413942-3.116603$

O $1.350143 \quad 1.141507-3.830379$

C $1.890338-1.054689-3.015482$

C $0.733858-1.630950-3.371312$

C $0.400555-3.048588-3.227263$

C $1.473175-3.967872-2.703290$

C $3.144413-0.8936981 .239878$

C $1.828495-1.5198751 .669454$

C $-0.535857-1.777897 \quad 0.930635$

O $1.683840-2.112588 \quad 2.735746$

C $-0.715712-3.1491530 .356862$

C $-1.799926-3.510707-0.346695$

C $-2.034842-4.893131-0.886627$

C $-2.502624-4.888161-2.326541$

C $-3.806491-5.007601-2.628972$

C $-1.452397-4.817316-3.413359$

C $-0.853150-3.444562-3.530641$

N $0.828751-1.3293120 .724193$

H $1.2348554 .098074-2.323469$

H $1.8892962 .092137-0.102719$

H $3.1263632 .748821-2.891425$

H $4.6423921 .529950-0.533129$

H $4.4918763 .269498-0.620762$

H $7.2543611 .600161-3.248614$

H $6.799148 \quad 3.839542-3.405138$

H $5.0848404 .231185-3.243188$

H $6.1941394 .494960-1.879865$

H $5.281310-0.268742-1.795491$

H $6.319996-0.618563-3.151480$

H $7.360741-1.657342-1.328588$

H $8.353346-0.229242-1.637501$

H $7.1027150 .959756 \quad 0.229804$

H $9.277869-0.2043800 .723162$

H $8.425277-1.7192270 .932843$

H $8.934240-2.0163163 .113583$

H $6.698801-1.9194332 .937515$

H 9.4039201 .3957602 .641437

H $8.233347 \quad 1.3415463 .939494$

H 7.7247151 .8316092 .312106

H $7.079549-0.7457834 .850036$

H $4.423366-2.1492732 .405219$

H $3.861521-0.7142543 .270490$

H 4.4657381 .2858931 .919258

H $2.717341-1.605536-2.582250$

H $-0.061629-0.999397-3.765457$

H $2.336879-3.970666-3.377505$

H $1.150714-5.005599-2.600749$

H $1.805554-3.647802-1.709380$

H $2.960593 \quad 0.1680381 .050191$

H $3.452247-1.3632350 .298492$

H $-1.194718-1.0422170 .458821$

H $-0.745458-1.7997342 .005063$

H $0.057940-3.8865960 .556513$

H $-2.587633-2.779787-0.510530$

H $-2.793917-5.371655-0.254011$

H $-1.136382-5.516177-0.797226$

H $-4.148924-5.028249-3.658205$

H $-4.561448-5.080669-1.852993$

H $-1.894875-5.064745-4.387431$

H $-0.706575-5.597445-3.239480$

H $-1.559402-2.696779-3.894685$

H $1.052533-0.870882-0.154106$

C -1.366251 $3.640565-1.272956$

O $-1.5701992 .213002-1.325080$

C $-2.9813861 .934916-1.148702$

C $-3.6747043 .289153-1.183734$

C $-2.6135524 .196905-0.593908$

H $-3.0669321 .488971-0.151299$

C -3.419071 $0.929672-2.223155$

H $-3.8920673 .592279-2.215159$

H - $4.6105603 .300499-0.619592$ 
C $-2.8610455 .671182-0.863384$ H -2.5626584 .0266880 .489725$ C $-4.924736 \quad 0.620837-2.305194$ O $-2.715097-0.297675-2.016626$ H -3.087973 $1.305785-3.200149$ H $-5.068126-0.221538-2.994720$ O $-5.5712951 .744478-2.913720$ C $-5.6568810 .299026-0.985396$ C $-5.090204-0.905794-0.218307$ H $-5.6718061 .184693-0.343362$ O $-7.0278510 .030020-1.310772$ H -5.035431-1.764514-0.898915 H $-4.069226-0.6808130 .100499$ H -2.071564 $6.287305-0.422244$ H -2.892973 $5.883073-1.937416$ H $-3.8155745 .987934-0.431559$ C $-0.0637993 .954809-0.534993$ H -1.766054 -0.087212 -1.982439 H $-6.519318 \quad 1.536337-2.976014$ H -7.049939 -0.769852 -1.863644 H -0.0382963 .4207730 .422673$ H $0.0052755 .026594-0.319058$ H -1.312462 $3.992341-2.312322$ C -5.0426290 .2664322 .602167$ O $-6.189794-0.2104411 .889968$ C $-5.943822-1.3092241 .007477$ C $-5.386221-2.4980911 .800080$ C $-4.149859-2.1047442 .603614$ C $-4.420682-0.8568873 .442835$ H $-4.3062890 .662379 \quad 1.892547$ C -5.5045181 .4189783 .501952$ H $-6.924296-1.6178490 .623720$ O $-5.081253-3.574156 \quad 0.920465$ H $-6.159505-2.8574942 .491317$ H -3.291614 -1.946605 1.945046 O $-3.781987-3.1834903 .465930$ H -5.086139 -1.114262 4.276892 O $-3.192126-0.3967454 .002779$ C -5.8558712 .6457322 .691483$ H $-6.397910 \quad 1.145548 \quad 4.076076$ H $-4.727476 \quad 1.6880844 .226825$ H -4.686576 -4.285058 1.454131 H -4.526339 -3.354812 4.067719 H -2.804353 -1.136467 4.501172 O $-4.949417 \quad 3.6364412 .927486$ O -6.8227072 .7162951 .943876$ C -5.2005944 .8803852 .266002$ H -6.0338805 .3936052 .754508$ H -4.303786 5.4994412 .354384 H -5.4155334 .7272021 .204731$

SCF Energy (B3LYP/6-31G**//MMFF) $=-3245.91300958$

04_00073

MM̄FF Geometry

C $0.5147942 .262462 \quad 2.877673$

C 0.6980491 .0933892 .247465

C $0.674846-0.2389502 .953653$

O $1.834474-0.9873012 .521870$

C $-0.558799-1.0522112 .528643$

C $-0.939604-2.1141453 .540135$

C $-0.515138-3.3929983 .520369$

C $-1.900817-1.652194 \quad 4.605322$

C $0.467417-4.0328682 .578952$

C $-0.169281-5.0909591 .668046$

C $-1.040354-4.4850420 .558294$

C $-1.662238-5.583548-0.305547$

C $-2.470846-4.998293-1.472728$

O $-0.203186-3.654674-0.253566$

O $-2.742198-6.078786-2.378183$

C $-1.601721-3.959974-2.216317$

C $-3.833927-4.482605-1.000720$

O $-2.360809-3.285415-3.217574$

C $-0.899548-2.944077-1.283636$

C $0.137928-2.121914-2.087480$

O $-1.881564-2.059093-0.742022$

C $3.053547-0.6400463 .005232$

O $3.306203 \quad 0.1819043 .869390$

C $4.041370-1.3985752 .209231$
C $5.272414-0.8961682 .042744$

C $6.314061-1.4542081 .178702$

C $6.008520-2.7432920 .460403$

C $1.091935-1.282760-1.222932$

C $2.083590-0.497619-2.066789$

C $4.0852230 .976547-1.857440$

O $2.015465-0.432294-3.291758$

C $5.3241090 .163837-2.077365$

C $6.5600530 .632751-1.841982$

C $7.818913-0.145262-2.111401$

C $8.805800-0.090858-0.963880$

C $9.8365030 .771365-0.980852$

C $8.643332-1.0881070 .162563$

C $7.464545-0.7640741 .036673$

N $3.0398650 .143054-1.288467$

H $\quad 0.305402 \quad 2.238657 \quad 3.945986$

H 0.8978331 .0716941 .181136

H $0.707785-0.1171874 .043679$

H $-0.381423-1.5208041 .555223$

H -1.426163 -0.393375 2.386881

H $-0.886144-4.0650014 .293670$

H -2.140961 -2.443944 5.322753

H -1.473182 -0.815822 5.167443

H $-2.840426-1.3214684 .150352$

H $1.002499-3.2962651 .975624$

H $1.232805-4.5226963 .194384$

H $0.648278-5.6601911 .206574$

H $-0.758189-5.7967362 .266591$

H -1.824065 -3.873592 1.019256

H -2.288562 -6.250081 0.300506

H $-0.864843-6.216823-0.719508$

H -3.190983 -5.705284 -3.156276

H $-0.829015-4.524869-2.755521$

H -4.410292 -5.294292 -0.540591

H -4.439217 -4.129949-1.842706

H $-3.752628-3.677484-0.268378$

H -2.918276 -2.624693 -2.771897

H $\quad 0.756354-2.805915-2.683547$

H $-0.383279-1.459301-2.790496$

H -1.463592 -1.209625 -0.531285

H $3.700337-2.3006721 .713845$

H 5.5256810 .0366512 .545236

H $5.803472-3.5430161 .180789$

H $6.822473-3.096311-0.175215$

H $5.135316-2.624741-0.190932$

H $0.529233-0.562859-0.621313$

H $1.658617-1.932356-0.546416$

H $4.2535191 .805234-1.162383$

H $3.7408991 .380903-2.814678$

H $5.205410-0.838957-2.481063$

H $6.677028 \quad 1.650713-1.476177$

H $8.2808580 .276044-3.014015$

H $7.598236-1.192756-2.351582$

H $10.563826 \quad 0.800114-0.176241$

H $9.9731151 .471550-1.798351$

H $9.534865-1.0886500 .803650$

H $8.601660-2.095285-0.260194$

H 7.5843310 .1647961 .596255

H $3.047088-0.009029-0.284041$

C $1.158423 \quad 3.8009010 .877472$

O $0.2892613 .201143-0.103589$

C $0.0348214 .151866-1.151070$

C $1.1287385 .202790-1.027014$

C $1.2964945 .274058 \quad 0.476277$

H $-0.9308574 .615512-0.916955$

C $-0.0457003 .464715-2.520081$

H $2.065678 \quad 4.876507-1.492501$

H $0.8411686 .158789-1.474701$

C 2.6060845 .9106680 .910538

H $0.463127 \quad 5.856628 \quad 0.892250$

C $-0.9543092 .222286-2.580341$

O $1.2645053 .039256-2.917735$

H $-0.3590814 .195744-3.274285$

H $-0.4601091 .382156-2.077648$

O $\quad-1.0864951 .839887-3.954932$

C $-2.3591952 .392560-1.981129$

C $-3.2260531 .135948-2.191282$

H $-2.2736422 .609490-0.911043$ 
O $-3.042353 \quad 3.487320-2.591032$ H -3.647806 $1.142785-3.203837$ H -2.596617 $0.245313-2.084716$ H 2.6879715 .9306082 .001688 H 3.4684035 .3614540 .517812 H $2.671016 \quad 6.941761 \quad 0.547995$ C $0.567137 \quad 3.645154 \quad 2.284924$ H $1.6013822 .446853-2.223816$ H $-0.1894751 .698822-4.303646$ H -2.559129 4.302607 -2.379739 H -0.4558864 .0393312 .296760$ H 1.1553474 .2602842 .977204 H $2.126033 \quad 3.2882930 .806532$ C $-4.6563020 .722799 \quad 1.220829$ $\begin{array}{llll}\text { O } & -3.764907 & 0.772091 & 0.101181\end{array}$ C $-4.3693431 .065380-1.164457$ C $-5.404344-0.011864-1.516061$ C $-6.434652-0.185475-0.395091$ C $-5.774859-0.3060620 .984173$ H $-4.038976 \quad 0.362627 \quad 2.053842$ C $-5.170502 \quad 2.120571 \quad 1.593213$ H $-4.8596932 .043869-1.119546$ O $-4.757185-1.257118-1.763927$ H $-5.9125050 .274709-2.444125$ H -7.029074 -1.085305 -0.596033 O $-7.3171730 .932673-0.414796$ H $-6.529841-0.2235261 .774643$ O $-5.209222-1.6172151 .080431$ C $-4.027389 \quad 3.056255 \quad 1.926218$ H -5.7437382 .5612350 .773140$ H -5.8232612 .0691972 .471375$ H -4.228648 $-1.476230-0.977546$ H $-7.987947 \quad 0.789180 \quad 0.274430$ H $-4.843217-1.715961 \quad 1.976001$ O $-4.361548 \quad 4.3295051 .565492$ O -2.9784882 .7125182 .454512$ C $-3.358210 \quad 5.321451 \quad 1.803883$ H -3.775126 6.2968581 .539592 H -3.0754945 .3360502 .860827$ H -2.486073 5.129818 1.172004

SCF Energy (B3LYP/6-31G**//MMFF) $=-3245.92727115$

0400074

MMFF Geometry

C $-1.7586150 .241106-3.543471$

C $-0.590015-0.388745-3.357453$

C $-0.432249-1.566523-2.430145$

O $0.428655-1.166048-1.339159$

C $0.228771-2.740373-3.167789$

C $0.372341-3.973507-2.297892$

C $1.522945-4.361337-1.713090$

C $-0.874339-4.805691-2.147890$

C $2.845735-3.647307-1.751844$

C $3.512218-3.613503-0.369053$

C $4.837429-2.840272-0.412035$

C $5.622112-3.0255080 .886648$

C $6.920200-2.2055050 .882809$

O $4.532272-1.453236-0.598528$

O $7.423477-2.2128552 .226961$

C $6.583914-0.7479610 .506265$

C $8.000638-2.8605270 .014833$

$\begin{array}{lllll}\text { O } & 7.772806 & 0.025909 & 0.360871\end{array}$

C $5.682918-0.612219-0.746536$

C $5.2052590 .852266-0.874256$

O $6.438638-0.956389-1.908758$

C $-0.164775-0.496424-0.313603$

O $-1.346090-0.204309-0.216055$

C $0.880848-0.1491560 .671868$

C $0.570315 \quad 0.688383 \quad 1.672326$

C 1.4819921 .1889592 .701796

C 2.8620170 .5923542 .767480

C $4.0877811 .079376-1.901074$

C $3.6288632 .525083-1.854902$

C $2.4244244 .140257-0.373672$

O $3.9497443 .332971-2.722843$

C $2.8754824 .424742 \quad 1.025335$

C 2.0585084 .8486242 .001846

C 2.5135895 .1097923 .410606
C 1.6639784 .4048934 .447861

C 0.8528435 .1002985 .263054

C 1.8062642 .9016724 .590898

C 1.0397622 .1599563 .528082

N $2.8703172 .806317-0.730124$

H $-2.642703-0.118837-3.021803$

H $0.301437-0.023531-3.861590$

H -1.402802 -1.890477-2.032345

H $1.206136-2.434122-3.558458$

H $-0.363882-3.005922-4.053638$

H $1.520637-5.291324-1.146101$

H $-0.709441-5.694561-1.529504$

H $-1.672054-4.223006-1.677820$

H $-1.223870-5.145349-3.128220$

H $3.500665-4.177830-2.453218$

H $2.741118-2.622792-2.119195$

H $2.831511-3.137818 \quad 0.348337$

H $3.691208-4.641000-0.029020$

H $5.423355-3.202032-1.265214$

H $5.840118-4.0858631 .065444$

H $5.004472-2.6988981 .735358$

H $8.203882-1.6326442 .252718$

H $6.049617-0.3180141 .364771$

H $8.213562-3.8749410 .373133$

H $8.948934-2.3161340 .083457$

H $7.720202-2.931144-1.038282$

H $8.165686-0.195072-0.500740$

H 4.8431951 .1913890 .102935

H $6.0532621 .499018-1.134999$

H $6.034922-0.531807-2.682334$

H $1.873885-0.5590410 .530236$

H -0.4459301 .0780161 .725967$

H $2.810121-0.5021992 .797750$

H 3.4211020 .8903623 .657029

H 3.4498210 .8900251 .895397

H $4.4311200 .860754-2.917395$

H $3.2238890 .434179-1.705650$

H $1.3349054 .152972-0.464833$

H $2.8464534 .877351-1.063180$

H 3.9294594 .2703411 .245792

H 1.0090875 .0261651 .777602

H 2.4835816 .1951613 .571906

H 3.5609134 .8140933 .551113

H 0.2581874 .6059946 .024154

H 0.7609546 .1784815 .185045

H 1.4186642 .5780165 .565556

H 2.8723412 .6583994 .597231

H 0.0067172 .4951573 .421333

H $2.6022532 .042927-0.116764$

C $-2.1978432 .738837-3.645311$

O $-3.2758652 .557990-2.703286$

C $-2.8568973 .088033-1.431059$

C $-1.7198654 .047437-1.748953$

C $-1.006483 \quad 3.276233-2.845389$

H $-2.4455762 .251667-0.854411$

C $-4.0454433 .692146-0.680726$

H -2.091492 $5.003962-2.134449$

H $-1.0880764 .247622-0.879177$

C $-0.0469794 .121025-3.665038$

H $-0.4453352 .454963-2.382273$

C $-5.2180972 .710449-0.468382$

O $-4.5447294 .816699-1.414118$

H $-3.710227 \quad 4.0872280 .285181$

H $-5.7086472 .495165-1.425452$

$\begin{array}{lllll}\text { O } & -6.196450 & 3.363156 & 0.349454\end{array}$

$\begin{array}{llll}\text { C } & -4.819207 & 1.388038 & 0.212133\end{array}$

C $-6.0217920 .461550 \quad 0.472180$

H $-4.1053040 .859249-0.426616$

$\begin{array}{llll}\text { O } & -4.157548 & 1.663325 & 1.446016\end{array}$

H $-6.7258990 .941777 \quad 1.162214$

H $-6.5368040 .299985-0.482383$

H $0.427233 \quad 3.520879-4.447903$

H $-0.5608654 .959715-4.146458$

H $0.7437154 .533043-3.030235$

C $-1.9466851 .443231-4.424283$

H $-4.7884524 .503008-2.302114$

H $-6.4364134 .194664-0.094571$

H -4.7789912 .1582372 .007280$ 
H $-1.0933531 .574292-5.099623$ H -2.819434 $1.237972-5.057106$ H -2.540876 $3.502617-4.355335$ C $-4.121822-2.7462940 .630902$ O $-4.705414-1.524904 \quad 0.157027$ C $-5.601605-0.8914131 .075805$ C $-6.804676-1.8094681 .337483$ C $-6.354553-3.1862051 .838638$ C $-5.213779-3.7706040 .992954$ H $-3.568745-3.128091-0.236257$ C $-3.112349-2.465448 \quad 1.755247$ H $-5.077041-0.7021082 .019780$ O $-7.574585-1.968980 \quad 0.147113$ H -7.457010 -1.338776 2.082173 H -7.212759 -3.869449 1.816107 O $\quad-5.941404-3.056900 \quad 3.195867$ H $-4.768139-4.6318801 .503449$ O $-5.790257-4.257567-0.223959$ C -1.943921 -3.426167 1.729559 H -2.717103 -1.450436 1.632911 H $-3.566963-2.5166882 .747565$ H $-6.989045-2.333520-0.538460$ H $-5.699455-3.9432673 .514262$ H -5.084635 -4.699246 -0.726726 O $-0.861096-2.8323382 .312180$ O $-1.982517-4.5643401 .282317$ C $0.328547-3.6262422 .356243$ H $1.142530-2.9995722 .730437$ H $0.189253-4.4694613 .039028$ H $\quad 0.591708-3.9797351 .355259$ SCF Energy (B3LYP/6-31G**//MMFF) $=-3245.93787768$

\section{00075}

MM̄MF Geometry

C $2.797715-2.306576-2.513979$

C $1.925259-2.014844-1.536903$

C $0.511265-1.567210-1.784001$

O $0.429109-0.213285-1.278635$

C $-0.521351-2.434253-1.043939$

C $-0.740888-3.787680-1.682450$

C $-1.636449-4.019653-2.662065$

C $0.080927-4.918955-1.123099$

C $-2.559766-3.023993-3.310579$

C $-4.037667-3.394783-3.128043$

C $-4.504986-3.366290-1.664086$

C $-5.932258-3.907623-1.553259$

C $-6.449807-3.848218-0.110744$

O $-4.455071-2.012775-1.192760$

O $-7.863203-4.093165-0.162578$

C $-6.231123-2.4215610 .430025$

C $-5.851174-4.9647770 .752404$

O $-6.579622-2.3474671 .811315$

C $-4.803331-1.8667900 .191145$

C $-4.783485-0.3646210 .557394$

O $-3.888726-2.5644101 .040521$

C $-0.4124150 .642402-1.917926$

O $-1.179638 \quad 0.380067-2.829892$

C $-0.2058391 .985709-1.335087$

C $-0.8022073 .048582-1.894724$

C $-0.6430284 .444419-1.481838$

C $0.2296904 .721809-0.285232$

C $-3.509053 \quad 0.391759 \quad 0.162494$

C $-3.479121 \quad 1.7269930 .881899$

C -4.6789573 .9065440 .977057$

O $-2.8118481 .895251 \quad 1.900291$

C -3.6426304 .9567990 .736814$

C -3.8904296 .1009340 .081496$

C $-2.8978587 .217672-0.071480$

C $-2.5203597 .473908-1.511847$

C $-3.2760918 .258885-2.298110$

C $-1.2159546 .893495-2.006281$

C -1.275350 $5.401995-2.192597$

N -4.320492 2.6689340 .311076

H $2.488250-2.233567-3.553332$

H $2.249741-2.074175-0.500322$

H $0.304691-1.565145-2.862290$

H -1.473702 -1.895098 -0.979028

H $-0.231800-2.5602390 .007257$
H -1.723435 -5.033558 -3.050096

H $-0.176872-5.882275-1.576370$

H $1.147129-4.750789-1.296987$

H $-0.085432-5.010407-0.044579$

H -2.391262 -2.005270 -2.953694

H $-2.334292-3.009756-4.384063$

H $-4.634691-2.682339-3.711936$

H -4.213516 -4.390626 -3.554262

H -3.828566 -3.982121-1.062046

H $-5.993084-4.933330-1.938348$

H $-6.599661-3.308875-2.189118$

H $-8.215080-3.9804860 .737217$

H $-6.946331-1.772831-0.094369$

H $-6.090532-5.9459600 .325181$

H $-6.285345-4.9648941 .758304$

H $-4.765144-4.8992100 .846867$

H -5.852390 -2.743762 2.320870

H $-5.625840 \quad 0.137577 \quad 0.063371$

H $-4.937839-0.2564661 .639199$

H -3.117472 -2.000823 1.209151

H $0.4613162 .068558-0.486394$

H -1.449878 $2.888086-2.755607$

H $-0.1295154 .167303 \quad 0.588619$

H $0.246767 \quad 5.772907 \quad 0.009234$

H $1.2653614 .426840-0.487019$

H $-2.602550-0.1544130 .436817$

H -3.472185 $0.555024-0.920144$

H -4.7682363 .7192422 .052524$

H -5.6630224 .2043800 .600313$

H $-2.6610804 .793540 \quad 1.174145$

H $-4.8823846 .270323-0.331857$

H -1.995829 7.0327490 .524623

H $-3.343355 \quad 8.1251370 .357568$

H -2.994272 $8.472345-3.323791$

H $-4.1963408 .702424-1.932245$

H $-0.4084617 .204083-1.337035$

H $-0.9514617 .330057-2.978876$

H -1.893219 $5.098053-3.038717$

H $-4.8268762 .429147-0.536110$

C $5.198096-1.591038-2.601798$

O $4.931388-0.457798-1.750238$

C $6.125940-0.130990-1.024159$

C $7.260378-0.665993-1.882599$

C $6.660543-1.978264-2.356192$

H $6.093134-0.697681-0.085907$

C $6.1658021 .365979-0.704201$

H $7.464240-0.014187-2.739679$

H $8.188409-0.796315-1.318338$

C $7.350329-2.560297-3.577743$

H $6.724606-2.706580-1.536343$

C $4.8648771 .916612-0.080790$

O $6.4071172 .107467-1.906352$

H $7.0177831 .576867-0.047795$

H $4.0849381 .990189-0.849131$

O 5.1186713 .2553530 .360949

C 4.3238051 .1026811 .107506

C 3.0676681 .7385151 .731554

H 4.0795290 .0917920 .767030

O 5.3325320 .9760722 .108177

H 3.3370092 .6657172 .251442

H 2.3715901 .9871820 .922864

H $6.867058-3.491856-3.889217$

H $7.321405-1.865771-4.424064$

H $8.400478-2.779528-3.358815$

C $4.220340-2.717218-2.254901$

H $5.7000661 .884204-2.536247$

H $5.4475693 .755564-0.405579$

H $5.587337 \quad 1.8734692 .383675$

H $4.334549-2.996486-1.200417$

H $4.441815-3.610337-2.850119$

H $5.046592-1.253850-3.634851$

C $1.293818-1.3761592 .781581$

O $1.917517-0.3484612 .001812$

C 2.3748640 .7947122 .732788

C 1.1935021 .4737583 .442148

C 0.4247210 .4826604 .321591

C $0.102047-0.817633 \quad 3.574618$

H $0.899564-2.0847642 .043060$ 
C $2.308480-2.1290203 .647825$

H 3.1136540 .4784173 .476691

O 0.3036582 .0478712 .487435

H 1.5759242 .2957294 .058404

H -0.5059730 .9550664 .658980$

O $1.210228 \quad 0.196370 \quad 5.475065$

H -0.264717 -1.582732 4.268552

O $-0.948067-0.5671072 .636398$

C $3.316861-2.8444102 .779028$

H $2.840903-1.4742244 .343332$

H $1.811832-2.8909444 .259941$

H -0.0084121 .3353931 .903703$

H $0.696429-0.4082286 .037234$

H -1.716314 $-0.238813 \quad 3.134169$

O $4.534186-2.2395272 .881755$

O $3.049061-3.8142052 .081045$

C 5.580401-2.822207 2.099292

H $5.800372-3.8315532 .459083$

H $5.301904-2.8418291 .042136$

H $6.475035-2.2044152 .213526$

SCF Energy (B3LYP/6-31G**//MMFF) $=-3245.93771240$

04_00076

MM̄FF Geometry

C $0.571923-0.9309722 .629416$

C $1.421384-0.3486363 .489335$

C $2.117274 \quad 0.9800743 .323960$

O $1.564941 \quad 1.7528502 .237552$

C 3.5982530 .7435872 .982152

C 4.4965261 .8992363 .371115

C 4.9037402 .8814852 .544047

C 4.9854221 .8653264 .797156

C $4.497321 \quad 3.1363171 .119338$

C 5.5873942 .7608350 .105333

C $5.4759611 .301934-0.361106$

C $6.6475990 .922072-1.266078$

C $6.494215-0.503006-1.819108$

O $4.243621 \quad 1.179252-1.082062$

O $7.451592-0.671536-2.875369$

C $5.093771-0.642917-2.450367$

C $6.818486-1.567411-0.764022$

O $4.847553-1.989471-2.851351$

C $3.944566-0.143340-1.540561$

C $2.632742-0.080031-2.360336$

O $3.779294-1.052029-0.452110$

C 0.4773832 .5281722 .479683

O -0.1768052 .5883793 .506867$

C 0.2361193 .3124811 .248598

C $-0.8817934 .039533 \quad 1.118209$

C $-1.2343744 .867099-0.037591$

C $-0.2137105 .005155-1.137835$

C $1.4445810 .576430-1.640925$

C $0.2131300 .637808-2.533592$

C $-2.2013291 .262945-2.482440$

O $0.2157780 .268089-3.705240$

C $-2.4544032 .680724-2.885030$

C $-3.5493903 .367200-2.522370$

C $-3.8496974 .765460-2.983013$

C $-4.1803235 .705955-1.845688$

C $-5.4546546 .014166-1.550925$

C $-3.0311206 .357771-1.110836$

C $-2.4457205 .459996-0.056990$

N $-0.8905881 .151953-1.867256$

H $0.312891-0.4392661 .696398$

H $1.665158-0.8776384 .409353$

H 2.0196521 .5448884 .259537

H 3.6940070 .5390191 .910700

H $3.976344-0.1555633 .487620$

H 5.5865963 .6310812 .943668

H 5.6327282 .7154265 .037090

H 4.1397401 .8898495 .491684

H 5.5595280 .9510194 .980104

H 3.5495852 .6514770 .872927

H 4.3043984 .2138831 .035572

H $5.4661023 .414816-0.768280$

H 6.5829922 .9561380 .521738

H 5.4564900 .6479510 .516060

H $7.6012721 .026883-0.733959$
H $6.698126 \quad 1.620316-2.113382$

H $8.337184-0.515749-2.504682$

H $5.091318-0.041266-3.370286$

H $7.845615-1.444841-0.399996$

H $\quad 6.771233-2.574668-1.192436$

H $6.154768-1.5300820 .102320$

H $5.580054-2.257057-3.432548$

H $2.8174070 .492211-3.279412$

H $2.341163-1.093008-2.663011$

H $2.890656-0.939795-0.080306$

H $0.995876 \quad 3.2711190 .476092$

H -1.609198 4.0250631 .928648

H $0.6921395 .491213-0.758698$

H $-0.5622635 .597127-1.986297$

H $0.0605024 .025015-1.541764$

H $1.1802260 .013147-0.740260$

H $1.6980491 .601087-1.348498$

H $-2.9342180 .907764-1.753189$

H -2.252091 $0.619179-3.365641$

H -1.722515 $3.150437-3.537830$

H $-4.2955982 .882916-1.896136$

H -4.699676 4.708861 -3.675946

H -3.020014 $5.180722-3.568515$

H $-5.6981406 .710489-0.755265$

H $-6.2828595 .571418-2.094318$

H $-3.3780107 .268939-0.604731$

H -2.290678 $6.712139-1.833271$

H -3.1126885 .3010710 .790871$

H $-0.7870821 .472536-0.909155$

C $0.359549-3.3620091 .901318$

O $-0.096358-3.0604430 .569208$

C $0.861679-3.580770-0.372008$

C $1.891576-4.3574800 .448332$

C $1.857251-3.6229921 .772822$

H $1.352642-2.717025-0.833785$

C $0.183608-4.438830-1.452256$

H $1.591009-5.4038200 .581752$

H $2.880392-4.351988-0.020862$

C $2.452921-4.4103482 .925761$

H $2.410355-2.6832501 .655729$

C $-0.653357-3.670040-2.489436$

O $-0.584886-5.490392-0.876328$

H $0.991629-4.929893-2.011222$

H $-1.060287-4.396362-3.205152$

O $0.261635-2.849330-3.229233$

C $-1.809562-2.773644-2.003754$

C $-2.910127-3.506072-1.219153$

H $-1.413853-1.936980-1.419513$

O $-2.401210-2.186658-3.173030$

H -3.192666 -4.413317 -1.767520

H $-2.512970-3.826022-0.253564$

H $2.395657-3.8396623 .857946$

H $1.928024-5.3593223 .077991$

H $3.506755-4.6363332 .732963$

C $-0.058953-2.2706002 .888266$

H $-0.922416-5.186697-0.017685$

H -0.253766 -2.365058 -3.896910

H $-2.780245-2.905518-3.707192$

H $\quad 0.164267-2.6079283 .907753$

H -1.144386 -2.135506 2.837192

H $-0.155295-4.2878072 .192825$

C $-3.477257-1.3650130 .908368$

O $-3.889435-1.365388-0.459688$

C $-4.184967-2.656839-0.998207$

C $-5.271916-3.352115-0.163962$

C $-4.914980-3.394811 \quad 1.321047$

C $-4.536906-1.9988301 .816245$

H $-2.550298-1.9417230 .989121$

C $-3.1095290 .070302 \quad 1.315158$

H -4.618966 -2.479190 -1.990251

O $-5.490648-4.669066-0.658365$

H -6.213828 -2.801009 -0.284248

H $-4.111496-4.1128191 .523462$

O $-6.049415-3.8351702 .070739$

H $-5.438891-1.3772541 .857728$

O $-4.024468-2.0926253 .143358$

C $-4.252798 \quad 1.0594831 .215233$

H $\quad-2.733847 \quad 0.0711882 .344759$ 
H $-2.3148450 .443200 \quad 0.660430$ H $-6.268859-5.030568-0.201974$ H $-6.217117-4.7621941 .831799$ H $-4.705198-2.5247603 .687249$ O -3.9675092 .1493861 .987506$ $\begin{array}{lllll}\text { O } & -5.253923 & 0.915817 & 0.527245\end{array}$ C -4.9624643 .1776141 .986605$ H -5.8970702 .7980792 .410300$ H -4.602459 3.9996682 .611048 H -5.1229693 .5519000 .971015$

SCF Energy $\left(\mathrm{B} 3 L Y P / 6-31 G^{* *} / / \mathrm{MMFF}\right)=-3245.91945737$

04_00077

MMFF Geometry

C $0.130170-1.0273592 .014229$

C $-0.615633-1.9503141 .384848$

C $-0.271379-2.6511600 .087970$

O $1.044975-2.277684-0.371836$

C -1.297495 -2.242144-0.989074

C $-1.022310-2.794235-2.376082$

C $-0.785056-2.026274-3.460226$

C -1.042519-4.294342 -2.500576

C $-0.753413-0.521987-3.502017$

C $0.6084020 .011893-3.059493$

C $0.5650691 .468772-2.589263$

C $0.4436872 .459957-3.745943$

C $0.5410193 .917287-3.262202$

O $1.790607 \quad 1.695305-1.884023$

O $\quad 0.752607 \quad 4.731227-4.425680$

C $1.7752094 .069819-2.342367$

C $-0.7809294 .387122-2.646042$

O $1.8165855 .369962-1.759054$

C $1.8760952 .977320-1.253577$

C $3.2384013 .058066-0.526498$

O $0.8310163 .174117-0.298750$

C $2.099681-3.0295740 .043377$

$\begin{array}{lllll}\text { O } & 2.065554 & -4.003431 & 0.777932\end{array}$

C $3.322980-2.444265-0.552859$

C $4.518028-2.990338-0.283140$

C $5.814337-2.546702-0.803480$

C $5.833484-1.322676-1.680823$

C 3.5286381 .8469460 .373553

C 4.8793921 .9502001 .058703

C 6.4721110 .5927592 .414668

O 5.5535992 .9765521 .064318

C 7.5448560 .1474211 .471056

C $8.286821-0.9551771 .655238$

C $9.405869-1.3651120 .741735$

C $9.271551-2.7859770 .244005$

C $10.021916-3.7738470 .760853$

C $8.337335-3.043767-0.916958$

C $6.913660-3.258310-0.476686$

N $5.228294 \quad 0.761403 \quad 1.687118$

H $1.093060-0.7306731 .607923$

H -1.578063 -2.221034 1.816037

H $-0.321521-3.7353420 .247482$

H -1.350776 -1.148096 -1.016958

H -2.294851 -2.580669-0.683764

H $-0.588471-2.514453-4.413161$

H $-0.960441-4.624898-3.541574$

H $-0.208937-4.738021-1.947600$

H -1.979039 -4.697047 -2.102491

H $-0.967836-0.190995-4.525354$

H -1.561652 -0.111685 -2.886598

H $1.001172-0.591519-2.234609$

H $1.342051-0.106626-3.867908$

H $-0.2685281 .581998-1.886291$

H $-0.4866462 .302563-4.304726$

H $1.2570472 .280947-4.463175$

H $\quad 0.8859935 .646647-4.124758$

H $2.6621704 .000395-2.986714$

H -1.593978 4.306517 -3.377469

H $-0.7371355 .445009-2.365227$

H -1.066071 $3.808072-1.767198$

H $1.1674795 .390434-1.035311$

H $4.0441463 .116479-1.270115$

H 3.2836693 .9735490 .076750

H 1.0846612 .7610480 .541105
H $3.205898-1.584627-1.202927$

H $4.554164-3.8575460 .375220$

H $5.280467-1.506814-2.608393$

H $6.838172-1.002869-1.963903$

H $5.377922-0.470819-1.165450$

H $2.770814 \quad 1.753696 \quad 1.158429$

H $3.5171280 .927967-0.222472$

H $6.289041-0.1366703 .209872$

H 6.7556091 .5463572 .871689

H 7.7435810 .7826460 .610898

H $8.109393-1.5761002 .530489$

H $10.345268-1.2509841 .298806$

H $9.489510-0.688149-0.117669$

H $9.953001-4.790016 \quad 0.386854$

H $10.717149-3.589751 \quad 1.573248$

H $8.650629-3.952928-1.448178$

H $8.446306-2.242695-1.652673$

H $6.788953-4.1338700 .162671$

H $4.639203-0.0569481 .562505$

C $-0.379358 \quad 1.1919873 .058456$

O

C -2.3125582 .4142592 .556130$

C -1.6093433 .1052853 .713930$

C -0.8363151 .9443064 .312233$

H $-3.143506 \quad 1.8173202 .950188$

C -2.8360883 .3302941 .445769$

H -0.9151373 .8788093 .366144$

H -2.3110843 .5631294 .417177$

C 0.3052372 .3722035 .218056

H -1.5332001 .3233644 .891445$

C -3.2657282 .5899750 .162072$

O -1.8180064 .2677381 .075182$

H $-3.666833 \quad 3.9326841 .830742$

H $-2.3803442 .226079-0.373321$

O $-3.898153 \quad 3.539927-0.704268$

C $-4.233573 \quad 1.4162750 .387553$

C $-4.6667830 .756226-0.934433$

H $-3.7486790 .663615 \quad 1.016911$

O -5.3881291 .8728921 .089979$

H $-5.2669001 .460169-1.523377$

H $-3.7632250 .512252-1.506490$

H $\quad 0.840567 \quad 1.4999705 .606641$

H 1.0265643 .0005994 .684888

H -0.0739162 .9449206 .070597$

C $-0.302751-0.3230093 .270248$

H -1.0426243 .7589420 .781830$

H -3.280497 $4.281543-0.822883$

H -5.8047932 .5667180 .550404$

H -1.281797 -0.7009953 .589632$

H $0.413857-0.5538834 .066456$

H $\quad 0.583431 \quad 1.5774542 .700265$

C $-5.269438-2.722260 \quad 0.290584$

O $-4.656037-1.471902-0.047233$

C $-5.495378-0.522149-0.711274$

C $-6.013732-1.106014-2.034039$

C $-6.717494-2.447824-1.810290$

C $-5.876177-3.397968-0.951774$

H -4.440869 -3.349790 0.643609

C -6.261309-2.570893 1.448299

H $-6.344560-0.275105-0.065608$

O $-4.939576-1.278422-2.957198$

H -6.713476 -0.393266 -2.485949

H $-6.916017-2.912108-2.784167$

O $-7.970243-2.203386-1.177778$

H $-6.474456-4.265569-0.650139$

O $-4.809076-3.890900-1.767206$

C $-5.530103-2.2012492 .717280$

H -7.037933 -1.824558 1.258511

H $-6.782527-3.5159571 .642509$

H -4.276301 -1.853872 -2.538785

H -8.418622 -3.059885 - 1.074180

H -4.318094 -4.546796 -1.243470

O $-5.627331-0.8601682 .940482$

O $-4.921055-3.0065573 .410056$

C $-4.948300-0.3795834 .104465$

H $-3.870733-0.5397544 .004311$

H -5.1376810 .6937094 .189704$

H $-5.331494-0.8750225 .001548$ 
H $\quad 6.7474382 .471147-1.653345$

04_00078

MM̄FF Geometry

C 0.9992012 .2000892 .804984

C 0.5245261 .4220733 .791404

C $0.905952-0.0185694 .068925$

O $1.753587-0.5500713 .024648$

C $-0.352979-0.9015184 .154959$

C $-0.063795-2.3135484 .638859$

C $-0.115257-3.424513 \quad 3.875905$

C $0.271694-2.4330386 .104615$

C $-0.451001-3.5202502 .415866$

C $0.767465-3.9261321 .579344$

C $0.528332-3.7003580 .077941$

C $1.736548-4.170968-0.733677$

C $1.608716-3.826093-2.224372$

O $0.321228-2.297396-0.114772$

O $2.905728-4.018173-2.810000$

C $1.241468-2.332292-2.363927$

C $0.664936-4.796473-2.943481$

O $0.987225-1.999695-3.726358$

C $0.062193-1.903632-1.467513$

C $-0.166182-0.372773-1.467224$

O $-1.138920-2.509515-1.959161$

C $3.099039-0.3982543 .171214$

$\begin{array}{lllll}\text { O } & 3.684727 & 0.105864 & 4.116170\end{array}$

C $3.744958-0.9141821 .943611$

C $5.027500-0.6095661 .696401$

C $5.809703-1.0246430 .529690$

C $5.129168-1.887947-0.498808$

C $0.9798820 .458290-0.881912$

C $1.936211 \quad 1.027347-1.914647$

C $4.0718302 .306607-2.070065$

O $1.7245640 .993215-3.123484$

C $5.1581091 .344533-2.435202$

C $6.4558491 .558892-2.168957$

C $7.5609700 .637863-2.601065$

C $8.4811770 .246318-1.466887$

C $9.6442780 .889180-1.266906$

C $8.099070-0.951402-0.628145$

C $7.091993-0.6150450 .434231$

N $3.0414691 .619011-1.315441$

H $1.722987 \quad 1.8011062 .100777$

H -0.1688341 .8644384 .504881$

H $1.432600-0.0437865 .030682$

H -0.847528 -0.9099953 .179496$

H - $-1.080063-0.4528794 .845448$

H $\quad 0.106639-4.3820894 .345358$

H $\quad 0.364814-3.4759046 .426580$

H $1.220708-1.9361946 .326774$

H $-0.514363-1.9735476 .712717$

H -1.246529 -4.264086 2.286201

H -0.859126 -2.580945 2.046826

H $1.633081-3.3321901 .897357$

H $1.007706-4.979547 \quad 1.768527$

H $-0.375379-4.243947-0.221541$

H $1.899736-5.247629-0.599447$

H $2.640794-3.684951-0.344997$

H $2.855725-3.729265-3.737563$

H $2.133569-1.764775-2.075692$

H $1.040113-5.823724-2.860002$

H $0.618492-4.583942-4.017164$

H $-0.351107-4.781636-2.543607$

H $0.097671-2.322630-3.949460$

H $-0.452580-0.030140-2.468668$

H $-1.044317-0.176944-0.836960$

H -1.897561 -2.003610 -1.623995

H $3.143296-1.5106371 .268654$

H 5.5613770 .0085892 .417421

H $4.892081-2.868671-0.073887$

H $5.730540-2.059428-1.393901$

H $4.202521-1.418868-0.845355$

H $0.5432161 .322836-0.368980$

H $1.544343-0.108985-0.136124$

H $4.4376303 .128396-1.446364$

H $3.6327282 .728213-2.980023$

H $4.8675030 .449184-2.979971$

H $8.135717 \quad 1.154619-3.381098$

H $7.165200-0.266595-3.079324$

H $10.3249770 .593494-0.475424$

H $9.9386591 .729503-1.886925$

H $8.986708-1.347865-0.116444$

H $7.776071-1.766543-1.282068$

H 7.4947310 .0172231 .226760

H $3.1578861 .550837-0.308438$

C 0.4532854 .0365541 .171681

O -0.8022883 .5202130 .685285$

C -1.416878 $4.518615-0.153402$

C $-0.3710425 .608063-0.361801$

C 0.3909215 .5465780 .945386

H -2.2475624 .9425680 .422691$

C $-1.9417513 .898227-1.452349$

H $0.2947465 .377950-1.201829$

H $-0.8236956 .586189-0.551186$

C 1.7497296 .2230710 .882403

H -0.2140346 .0203821 .730116$

C $-2.9592192 .757596-1.252501$

O $-0.8294683 .365300-2.183314$

H $-2.3739804 .685525-2.081707$

H $-2.4416711 .878884-0.854259$

O $-3.4356102 .376043-2.550014$

C $-4.1609523 .108608-0.356743$

C $-5.2872092 .064781-0.363043$

H -3.8163253 .2652250 .670956$

O $-4.7180344 .348807-0.802560$

H -6.0857602 .3972190 .315059$

H $-5.7809632 .061442-1.345324$

H $2.2684506 .151704 \quad 1.843303$

H 2.3899865 .7671180 .119606

H 1.6394167 .2845720 .637757

C 0.6478523 .6538232 .640566

H $-1.1805662 .969312-2.999326$

H $-3.9438873 .124414-2.907424$

H $-5.4361434 .584541-0.190591$

H $-0.259013 \quad 3.8908503 .210113$

H $1.4737594 .236543 \quad 3.064017$

H $1.243523 \quad 3.5962430 .549384$

C $-5.916729-1.5612680 .152335$

O $-6.078050-0.152506-0.059471$

C $-4.8753640 .628507-0.012388$

C $-4.2067870 .489406 \quad 1.362453$

C $-3.967306-0.9833171 .712158$

C $-5.218894-1.8378711 .495547$

H $-6.941029-1.9508620 .215354$

C $-5.235125-2.242928-1.039656$

H $-4.1973210 .268352-0.792297$

O -5.0118381 .0980322 .369330$

H -3.248227 1.0195321 .357014

H -3.652580 -1.064385 2.759805

O $-2.920168-1.4969740 .895889$

H $-4.965541-2.9018211 .572463$

O $-6.136370-1.5468692 .553523$

C -6.106537 -2.155455 -2.269610

H $-4.255519-1.821726-1.281041$

H -5.069212 -3.307311 -0.835025

H -5.8851600 .6703352 .351014$

H $-2.131381-0.9514351 .055182$

H -6.898462 -2.142363 2.453670

O $-5.768059-1.056339-3.002450$

O $-6.997696-2.953069-2.530978$

C $-6.545059-0.840182-4.184043$

H $-7.597437-0.692830-3.923156$

H -6.174884 $0.064722-4.673130$

$\mathrm{H}-6.431076-1.683388-4.872000$

SCF Energy $\left(B 3 L Y P / 6-31 G^{* *} / / M M F F\right)=-3245.92459559$

0400079

MM̄FF Geometry

C 0.3169252 .5655503 .201008

C 0.7520821 .4879622 .531084

C 1.0717870 .1751603 .200037

O $2.408759-0.1686212 .768974$

C $0.104543-0.9259832 .733482$

C $0.000821-2.0838743 .704954$ 
C $0.632571-3.266493 \quad 3.570571$

C $-0.934973-1.8561374 .864947$

C $1.619299-3.6790032 .513681$

C $1.077498-4.7747481 .583909$

C $0.028472-4.2602050 .586870$

C $-0.489412-5.394572-0.297744$

C $-1.467787-4.880217-1.366016$

O $0.658543-3.272855-0.232306$

O $-1.649181-5.922771-2.336577$

C $-0.832518-3.680858-2.104466$

C $-2.851374-4.596035-0.774313$

O $-1.769986-3.061083-2.982415$

C $-0.216212-2.622131-1.161816$

C $0.633364-1.608558-1.972824$

O $-1.265877-1.917875-0.494210$

C $3.290345-0.658206 \quad 3.673287$

O $3.079963-0.8762104 .858271$

C $4.615405-0.9033263 .052162$

C $4.864528-0.7404871 .742658$

C $6.148612-0.9466421 .066278$

C $7.337295-1.300847 \quad 1.920978$

C $1.601218-0.798488-1.097766$

C $2.2999320 .362301-1.781045$

C $3.5693011 .028693-3.815192$

O $2.4753911 .423751-1.182387$

C $4.9956870 .578788-3.783186$

C $5.9728521 .229105-3.133474$

C $7.4039700 .774696-3.118759$

C $7.8772820 .368816-1.741195$

C $8.7657011 .115094-1.063668$

C $7.389356-0.951650-1.186152$

C $6.202487-0.788836-0.273686$

N $2.7734070 .086482-3.050426$

H $\quad 0.1710572 .4723494 .276264$

H $0.902576 \quad 1.535678 \quad 1.456824$

H $1.039496 \quad 0.2976094 .289401$

H $0.407283-1.2931721 .749081$

H -0.905647 -0.5160582 .597833$

H $0.450188-4.030763 \quad 4.325315$

H $-0.995835-2.7253985 .528216$

H $-0.600448-1.0059645 .467451$

H $-1.946542-1.6477734 .500864$

H $1.979910-2.8277431 .929492$

H $2.502031-4.0737553 .032779$

H $1.930922-5.1790371 .023712$

H $0.660506-5.5978682 .177206$

H $-0.797302-3.8031131 .143200$

H $-0.958744-6.1790060 .308722$

H $0.356839-5.875942-0.807806$

H -1.985274 -6.708767 -1.872679

H -0.031594 -4.083063 -2.740682

H -3.260633 -5.497427 - 0.302581

H -3.564179 -4.322101-1.559242

H -2.837856 -3.806314 - 0.020823

H -2.124594 -3.755221 -3.564116

H $1.231475-2.162284-2.706284$

H $-0.020113-0.926896-2.530548$

H -0.968695-1.015566 -0.295734

H $5.384888-1.2310853 .743787$

H $4.054396-0.4223491 .088970$

H $7.485254-0.5501352 .705540$

H $8.276375-1.3485851 .366110$

H $7.192257-2.2788832 .392941$

H $1.053472-0.370022-0.252849$

H $2.383165-1.448343-0.688276$

H $3.4456032 .040089-3.415012$

H $3.198604 \quad 1.017793-4.844734$

H $5.234250-0.329906-4.331125$

H $5.7376342 .139650-2.587364$

H $8.0180111 .596706-3.508886$

H $7.562106-0.062363-3.810736$

H $9.1304380 .809577-0.088552$

H $9.1444462 .048583-1.466087$

H $8.217466-1.473890-0.696613$

H $7.101543-1.615732-2.011310$

H $5.282900-0.527820-0.797452$

H $2.679832-0.850363-3.428013$

C 0.3224714 .2134921 .183256 $\begin{array}{lllll}\text { O } & -0.526043 & 3.439177 & 0.313066\end{array}$

C $-0.950725 \quad 4.265847-0.787395$

C $-0.1438745 .555884-0.693081$

C 0.0707575 .6739950 .800865

H -2.007494 $4.497729-0.610974$

C $-0.8030843 .526851-2.122607$

H $0.8205425 .475431-1.207304$

H $-0.6787276 .409816-1.119958$

C $1.1994506 .619988 \quad 1.174665$

H $-0.8621426 .027464 \quad 1.260714$

C $-1.4762962 .141536-2.168007$

O $\quad 0.591046 \quad 3.338809-2.391374$

H -1.184416 $4.162913-2.930544$

H $-0.9390251 .446830-1.511640$

O $-1.3097221 .623094-3.494591$

C $-2.9716352 .135717-1.797780$

C $-3.6343290 .770052-2.057980$

H $-3.0726422 .395039-0.739150$

O $-3.6614093 .141198-2.539095$

H $-3.6066640 .554516-3.132834$

H -3.045983 -0.005582 - 1.558026

H 1.3196296 .6778632 .260805

H 2.1536496 .2945170 .747096

H 0.9925307 .6297210 .805003

C $-0.009234 \quad 3.929415 \quad 2.652442$

H $0.6640882 .857810-3.233432$

H $-1.7404172 .237883-4.112260$

H -3.640894 2.889719-3.477776

H -1.0805294 .0933062 .818459$

H $0.524743 \quad 4.6539573 .279242$

H 1.3599463 .9325670 .960875

$\begin{array}{llll}C & -4.718726 & 0.187124 & 0.727862\end{array}$

O $\begin{array}{llll}-5.264168 & 1.104005 & -0.228258\end{array}$

C $-5.1080360 .713898-1.596281$

C $-5.797053-0.638727-1.828073$

C $-5.302898-1.700821-0.847598$

C $-5.377582-1.1898680 .591246$

H -3.6345010 .1035210 .582619$

C $-4.967217 \quad 0.775525 \quad 2.120033$

H $-5.6535591 .457766-2.190530$

O $-5.621800-1.080682-3.170043$

H $-6.877142-0.509022-1.683602$

H $-4.281911-2.009390-1.093608$

O $-6.107584-2.875066-0.971626$

H $-6.427023-1.1388290 .908038$

O $-4.715201-2.1132721 .452505$

$\begin{array}{llll}C & -4.109160 & 1.997853 & 2.335518\end{array}$

H -6.0206881 .0498952 .252966$

H -4.7188950 .0641472 .915680$

H -4.711892 -1.408629 -3.266486

H $-7.027764-2.631530-0.772824$

H -5.124365 -2.984739 1.314365

$\begin{array}{lllll}\text { O } & -4.837240 & 3.127720 & 2.108928\end{array}$

O $-2.924877 \quad 1.948615 \quad 2.640444$

C $-4.125388 \quad 4.3598642 .254288$

H -3.3227544 .4203011 .514254$

H $-4.8260395 .180472 \quad 2.078854$

H -3.724897 4.4514143 .268437

SCF Energy (B3LYP/6-31G**//MMFF) $=-3245.90473240$

0400080

MMFF Geometry

C $-1.136297 \quad 3.600418 \quad 0.398001$

C $-0.728645 \quad 2.355414 \quad 0.684002$

C $-0.299857 \quad 1.895932 \quad 2.052597$

O $1.0156731 .308762 \quad 1.929155$

C $-1.282661 \quad 0.825408 \quad 2.558847$

C $-0.877273 \quad 0.1890103 .873382$

C $-0.382382-1.0590074 .000892$

C -1.0877021 .0477615 .093016$

C $-0.100224-2.0467502 .903048$

C $1.401663-2.3332112 .783856$

C $1.756933-2.991347 \quad 1.441324$

C $3.239774-3.3663991 .407994$

C $3.674531-3.8953150 .032581$

O $1.464375-2.0432290 .408244$

O $5.109731-3.9438890 .009958$

C $3.205169-2.905720-1.057819$ 
C $3.220737-5.343879-0.176408$

O $3.472965-3.421684-2.358963$

C $1.721364-2.503169-0.925245$

C $1.311861-1.364210-1.892730$

O $0.905337-3.636903-1.245435$

C 2.0778252 .1600131 .986950

O 2.0426683 .3696052 .148209

C 3.3087411 .3601191 .799552

C 4.4988231 .9747561 .726893

C 5.7965951 .3248891 .528420

C $5.804984-0.1651631 .311374$

C $2.042272-0.032253-1.696525$

C $3.2656960 .147650-2.578869$

C $5.1925601 .693849-2.923829$

O $3.538127-0.598208-3.515204$

C $6.4016461 .170599-2.214438$

C $7.4218091 .950151-1.824232$

C $8.6657381 .432698-1.160012$

C 8.9846412 .1547240 .130079

C 9.8749243 .1609450 .156812

C 8.3408211 .6496631 .401160

C 6.9095962 .0879661 .537999

N $3.997996 \quad 1.271230-2.217446$

H -1.160765 $4.351043 \quad 1.183004$

H $-0.7079291 .609980-0.108786$

H -0.2712752 .7289692 .766250$

H -1.420393 0.0627901 .786372

H -2.2758451 .2765592 .686558$

H $-0.138417-1.4169275 .000367$

H $-0.8334310 .523196 \quad 6.020185$

H $-0.465028 \quad 1.9463345 .042467$

H -2.1365601 .3534445 .165551$

H $-0.630693-2.9802493 .126994$

H $-0.490979-1.7033991 .944264$

H $1.956790-1.3917512 .877008$

H $1.713902-2.9782343 .614566$

H $1.129712-3.8802971 .306287$

H $3.474868-4.0965412 .192374$

H $3.839246-2.4773221 .643240$

H $5.447981-3.0445550 .158145$

H $3.825886-2.008765-0.953133$

H $3.649464-5.9925520 .597289$

H $3.585465-5.740153-1.130494$

H $2.135910-5.464279-0.142973$

H $2.798795-4.094077-2.556306$

H $1.369976-1.709842-2.931813$

H $\quad 0.247192-1.161031-1.713509$

H $0.039843-3.319074-1.550933$

H $3.202278 \quad 0.285657 \quad 1.712841$

H $4.530614 \quad 3.059317 \quad 1.823287$

H $5.480709-0.6847952 .219158$

H $6.785421-0.5634591 .042397$

H $5.134610-0.4384750 .489436$

H $1.3547820 .780963-1.963773$

H $2.3227110 .108922-0.649694$

H $5.1762862 .787651-2.962316$

H $5.1699061 .308331-3.948149$

H $6.4533600 .098276-2.041559$

H $7.3796673 .018206-2.026199$

H $9.4956061 .555653-1.868393$

H $8.5996990 .354537-0.968657$

H 10.1320263 .6643911 .082798

H $10.3622703 .507315-0.748706$

H 8.8820972 .0364122 .275441

H $8.463476 \quad 0.564633 \quad 1.461383$

H $6.8002393 .162317 \quad 1.693216$

H $3.7199131 .803988-1.398367$

C $-3.0828064 .195581-1.123708$

O $\quad-3.7608842 .973650-0.762859$

C -4.9075113 .3118820 .040632$

C $-5.1443624 .801199-0.180780$

C -3.718183 $5.306980-0.287968$

H -4.6159323 .1625991 .087058$

C $-6.0975882 .411999-0.304054$

H $-5.6875844 .991490-1.113825$

H $-5.703680 \quad 5.2607280 .639617$

C -3.604022 $6.687702-0.909835$

H $-3.285646 \quad 5.3348700 .719556$
C $-5.8202560 .903449-0.123681$

O $-6.4483952 .639373-1.674302$

H -6.9670872 .7122340 .293274$

H $-5.1139860 .566540-0.888214$

O $\quad \begin{array}{llll}-7.047610 & 0.202246 & -0.352899\end{array}$

C -5.2994020 .5145621 .271530$

C $-5.247010-0.9932441 .581355$

H -4.2961620 .9290831 .410793$

O $-6.132171 \quad 1.1177172 .268618$

H -4.971555-1.100161 2.639578

H $-6.258001-1.4118791 .494254$

H $-2.5564386 .996544-0.981359$

H -4.032716 $6.711622-1.917362$

H $-4.1344077 .428099-0.301946$

C $-1.5693174 .021398-0.978764$

H $-7.3068632 .211945-1.832184$

H $-7.2429190 .257575-1.303568$

H $-7.035324 \quad 0.7804812 .138512$

H -1.055013 $4.952440-1.242675$

H -1.237651 3.267799-1.703976

H $-3.3046964 .361215-2.186324$

C $-4.012842-2.663664-1.537248$

O $-4.811238-1.940120-0.590081$

C $-4.258631-1.7991890 .721278$

C $-3.995425-3.1843671 .339144$

C $-3.149505-4.0696690 .421946$

C $-3.678530-4.070622-1.014537$

H $-4.661842-2.787647-2.413692$

C $-2.772528-1.866286-1.971398$

H $-3.318943-1.2405600 .663933$

O $-5.229807-3.8443241 .619348$

H -3.480191 -3.050758 2.297786

H $-3.150615-5.0927370 .817572$

O $-1.807761-3.5975220 .439426$

H -2.961333 -4.550033 -1.690885

O $-4.881872-4.842561-1.066445$

C -3.128395 -0.664953 -2.822913

H $-2.205391-1.503206-1.110608$

H -2.120102 -2.501892 -2.581135

H $-5.733204-3.8981200 .788897$

H $-1.269546-4.230873-0.065113$

H $-4.662743-5.746422-0.782237$

O $-2.0065430 .092101-3.010249$

O $-4.235231-0.428954-3.287066$

C $-2.176497 \quad 1.247939-3.836115$

H $-1.2276551 .790099-3.861222$

H $-2.4356280 .946323-4.855289$

$\mathrm{H}-2.9465451 .904977-3.421189$

SCF Energy (B3LYP/6-31G**//MMFF $)=-3245.91064069$

04_00081

MMFF Geometry

C $-1.383363-0.585452-2.751584$

C $-0.295255-1.277364-2.384130$

C $-0.239298-2.130837-1.143120$

O $0.819265-1.624736-0.295097$

C $0.081369-3.586681-1.520637$

C $0.127775-4.525538-0.328953$

C $1.251485-5.0721200 .178508$

C $-1.210967-4.8611160 .277936$

C $2.664331-4.869591-0.292827$

C $3.474621-4.0490730 .718319$

C $4.753459-3.4742940 .091758$

C $5.602287-2.7698811 .151027$

C $6.818053-2.0574290 .541290$

O $4.349368-2.540052-0.915764$

O $7.344031-1.1875981 .555748$

C $6.344814-1.179387-0.638325$

C $7.937017-3.0493520 .204415$

O $7.458452-0.602701-1.315618$

C $5.426956-1.922707-1.630530$

C $4.799920-0.988082-2.695398$

O $6.202290-2.900771-2.334412$

C $0.539038-0.5102600 .437461$

$\begin{array}{llll}\text { O } & -0.513374 & 0.108416 & 0.460025\end{array}$

C $1.749850-0.1517091 .207791$

C 1.7500150 .9625701 .954428

C 2.8653801 .4679952 .757895 
C 4.1633340 .7071102 .706915

C $3.8909010 .118421-2.148760$

C $4.5923361 .445907-1.916289$

C $4.2032023 .692395-0.905644$

O $5.7155961 .698388-2.342943$

C 4.8130703 .7373440 .459880

C $4.4322994 .607148 \quad 1.408115$

C 5.0712734 .7019232 .764447

C 4.0651564 .6404553 .892236

C 3.6109045 .7629774 .474814

C 3.6554353 .2770544 .401263

C 2.6799732 .5892443 .485615

N $3.7919062 .334454-1.210004$ H -2.288861 $-0.660110-2.153958$ H $0.615923-1.194670-2.971983$ H -1.188369 -2.095624 -0.595491 H $1.023941-3.612764-2.075553$ H $-0.676256-3.962308-2.221943$ H $1.158112-5.7385661 .034948$ H -1.129897 -5.6021521 .080418$ H -1.678468 -3.9682490 .703199$ H -1.879747 -5.275373 -0.483595 H $3.134105-5.852952-0.419086$ H $2.697435-4.402777-1.279022$ H $2.857629-3.222378 \quad 1.091261$ H $3.726340-4.6815141 .578716$ H $5.316600-4.291160-0.374584$ H $5.919580-3.4761491 .928432$ H $4.986409-2.0211311 .666035$ H $8.067691-0.6741681 .156942$ H $5.791229-0.342825-0.197071$ H $8.262546-3.5768811 .109243$ H $8.825878-2.532569-0.174004$ H $7.636650-3.801878-0.527670$ H $7.851714-1.290775-1.879054$ H $5.580909-0.584357-3.350831$

H $4.172035-1.615001-3.345069$ H $5.740559-3.125007-3.159354$ H $2.610888-0.8043271 .130165$ H $\quad 0.845628 \quad 1.5692621 .982730$ H $4.042282-0.283493 \quad 3.158043$ H 4.9834361 .2027703 .230157 H 4.4974890 .5855641 .671044 H $3.105900 \quad 0.318441-2.889509$ H $3.395125-0.201111-1.228567$ H $3.3136774 .325606-0.982704$ H $4.9354334 .027150-1.647362$ H 5.6253213 .0446250 .667353 H 3.6404345 .3193121 .186083 H 5.6180725 .6531022 .807710 H 5.8279973 .9204522 .906931 H 2.9116875 .7273495 .303569 H 3.9246746 .7433434 .131806 H 3.1673843 .3718065 .380708 H 4.5516502 .6821374 .597209 H 1.7037243 .0752063 .449869 H $2.8897342 .023675-0.861356$ C -1.631302 $1.806338-3.549069$ O $-2.8899061 .968230-2.864813$ C -2.680082 $2.800342-1.708579$ C $-1.3480613 .504025-1.942350$ C $-0.5583962 .413690-2.641122$ H -2.575582 2.125478 -0.849937 C $-3.8759803 .731023-1.486875$ H -1.455557 4.373040-2.601663 H $-0.8851223 .839470-1.009378$ C $0.6620302 .922167-3.388221$ H $-0.2291861 .694420-1.882658$ C $-5.2395933 .011983-1.439672$ O $-3.9251784 .674334-2.565145$ H $-3.7174844 .316029-0.573234$ H -5.487043 $2.632925-2.438369$ O $-6.2406633 .994823-1.143100$ C $-5.3380201 .859065-0.421791$ C $-6.7559831 .257896-0.364582$ H $-4.629154 \quad 1.073222-0.702054$ O $-4.9591132 .320370 \quad 0.873349$ H $-7.4712002 .021374-0.034800$
H $-7.0317180 .950966-1.380925$

H $1.1948622 .095876-3.869121$

H $0.3865203 .644129-4.164179$

H $1.3542803 .418150-2.700992$

C -1.428256 $0.337483-3.936609$

H $-4.6890845 .255490-2.407501$

H $-6.0214284 .395009-0.284862$

H $-5.6368302 .946466 \quad 1.178787$

H $-0.516870 \quad 0.241721-4.538521$

H $-2.2654980 .014956-4.567633$

H $-1.7152172 .393883-4.472602$

C $-5.927553-2.1591920 .915081$

O $-5.987840-0.9681440 .118581$

C $-6.868644 \quad 0.0546130 .591774$

C $-8.310638-0.4739020 .670215$

C $-8.394175-1.7627991 .494846$

C $-7.327458-2.7791981 .075603$

H $-5.314283-2.8562090 .329222$

C $-5.214640-1.9273902 .256983$

H $-6.547040 \quad 0.376387 \quad 1.587575$

O $-8.823581-0.719343-0.638592$

H -8.9455220 .2978201 .120795$

H $-9.386276-2.2133171 .367644$

O $-8.225870-1.4605432 .876595$

H -7.290940 -3.603853 1.797049

O $-7.723179-3.338489-0.180269$

C $-3.744932-1.6115132 .078743$

H $-5.651037-1.1044492 .828034$

H $-5.283423-2.8444502 .854495$

H $-8.231765-1.355534-1.075365$

H $-8.933663-0.8440963 .130727$

H -7.079018 -4.029542 -0.410201

O $-3.108436-1.7776143 .276151$

O $-3.224823-1.2432151 .035151$

C $-1.704461-1.5013373 .275401$

H $-1.532781-0.4387853 .081264$

H $-1.309728-1.7447864 .265242$

H $-1.192418-2.1211362 .533446$

SCF Energy $\left(B 3 L Y P / 6-31 G^{* *} / / M M F F\right)=-3245.91747531$

0400082

MMFF Geometry

C $2.1912644 .132524-0.359705$

C 2.3369014 .6152020 .884422

C 2.9136693 .8905512 .081587

O 3.2690072 .5265801 .763242

C 1.8589233 .8551743 .205052

C 2.3517963 .2152604 .489255

C 1.9690232 .0057874 .946543

C 3.3100424 .0541805 .295813

C 1.0356861 .0243104 .292630

C $1.741103-0.3121934 .027054$

C $0.908188-1.2359233 .125111$

C $1.615966-2.5787802 .940081$

C $0.903547-3.4782981 .919895$

O $0.759538-0.579126 \quad 1.863823$

O $1.801623-4.5455721 .575667$

C $0.645865-2.6705410 .628924$

C $-0.344806-4.1382672 .517956$

O $-0.134431-3.449219-0.274558$

C $-0.010127-1.2974130 .890152$

C $-0.084036-0.395779-0.365992$

O $-1.354000-1.5001911 .343174$

C 4.5314732 .2959911 .308910

O 5.4019553 .1256191 .100111

C $4.6704350 .837217 \quad 1.098544$

C 5.7733470 .3480440 .512559

C $6.064893-1.0657650 .260484$

C $5.024269-2.0771640 .662861$

C $1.262520-0.001194-0.978407$

C $1.728643-0.899606-2.109604$

C $3.683879-1.237395-3.622539$

O $1.035053-1.786166-2.599197$

C $4.555264-2.322384-3.073993$

C $5.872259-2.405471-3.316436$

C $6.743692-3.524899-2.824817$

C $7.955935-3.036040-2.065595$

C $9.153587-2.934934-2.666599$ 
C $7.800074-2.760915-0.587847$

C $7.246109-1.390631-0.305743$

N $3.009330-0.563424-2.529959$

H $2.5274623 .128106-0.599518$

H 1.9984155 .6311141 .082037

H $3.797857 \quad 4.4395662 .427974$

H 0.9598303 .3493412 .838511

H 1.5329814 .8778353 .440184

H 2.3748841 .6674785 .899461

H 3.5743813 .5839036 .249042

H 4.2394564 .2172734 .741860

H 2.8637215 .0277495 .523818

H $0.1812180 .856527 \quad 4.959162$

H $0.6252391 .417144 \quad 3.360534$

H $2.706370-0.1145983 .544061$

H $1.950710-0.8112194 .981343$

H $-0.079873-1.3774523 .578519$

H $1.723213-3.0963843 .901555$

H $2.639367-2.4019002 .584061$

H $2.028296-5.0221782 .392504$

H $1.623556-2.5224310 .154420$

H $-0.073159-4.7611183 .378779$

H $-0.818513-4.8145661 .797844$

H $-1.090353-3.4165882 .857934$

H $-0.152991-2.985903-1.128487$

H $-0.756519-0.830015-1.113726$

H $-0.5659480 .541358-0.054357$

H $-1.870367-0.6978851 .159664$

H 3.8611140 .2023691 .438724

H $6.5464391 .047190 \quad 0.196174$

H $5.251519-3.0930900 .333139$

H $4.052413-1.8296310 .223010$

H $4.921513-2.1029591 .752825$

H $2.0429230 .055450-0.214818$

H $1.1584191 .000020-1.413452$

H $4.257610-0.479840-4.165965$

H $2.942443-1.667380-4.303533$

H $4.073035-3.092954-2.477055$

H $6.346135-1.648592-3.937631$

H $7.063273-4.104909-3.700762$

H $6.179515-4.227169-2.198599$

H $10.035094-2.616081-2.120047$

H $9.277739-3.162990-3.720070$

H $8.779724-2.819674-0.093908$

H $7.215884-3.562415-0.127109$

H $7.933664-0.585048-0.567631$

H $3.5262790 .150502-2.024657$

C $0.3834904 .115182-2.087609$

O $-0.6161113 .861498-1.084320$

C -1.921198 $3.898352-1.702824$

C $-1.6829614 .113641-3.191999$

C $-0.3564994 .850063-3.200790$

H $-2.4342154 .761564-1.262380$

C $-2.6532352 .596827-1.356948$

H -1.589982 3.153914 -3.715987

H -2.491589 $4.678082-3.666130$

C $0.347801 \quad 4.815217-4.545402$

H $-0.533380 \quad 5.894173-2.909945$

C $-4.0377692 .453390-2.010646$

O $-2.8164792 .544470 \quad 0.064753$

H -2.017328 $1.749457-1.632941$

H -3.939219 $2.416395-3.101378$

O $-4.8091383 .626584-1.721631$

C $-4.8463281 .230566-1.530169$

C $-4.138554-0.110143-1.786621$

H $-5.0972101 .337694-0.468869$

O $-6.087127 \quad 1.209225-2.245323$

H $-4.027438-0.256263-2.868051$

H -3.141519 -0.083969-1.337339

H $1.3004705 .352262-4.501404$

H $0.5544693 .787893-4.863768$

H $-0.270503 \quad 5.287929-5.315499$

C $1.5428234 .904458-1.478085$

H -1.9335092 .6400850 .461694$

H $-4.8803283 .699472-0.754181$

H -6.528131 $2.061180-2.084594$

H $1.1766805 .869052-1.105385$

H $2.2990215 .111533-2.243346$
H $0.7380293 .139345-2.444581$

C $-5.531065-2.1930570 .978875$

$\begin{array}{lllll}\text { O } & -4.838983 & -1.185858 & 0.231078\end{array}$

C $-4.907079-1.308423-1.194544$

C $-4.311131-2.650930-1.647193$

C $-4.974273-3.826031-0.922501$

C $-5.041316-3.597910 \quad 0.591644$

H -5.240903 -2.013485 2.022295

C $-7.051479-2.0287200 .880969$

H $-5.952504-1.251511-1.514939$

O $-2.909873-2.661206-1.387263$

H $-4.446916-2.749589-2.730872$

H $-4.407134-4.742075-1.128431$

O $-6.286809-3.999312-1.448261$

H $-5.687210-4.3496021 .058912$

O $-3.735556-3.788987 \quad 1.144517$

C -7.483768 -0.7319401 .523225$

H -7.424213 - $2.050467-0.147134$

H $-7.566001-2.8389331 .411190$

H $-2.556613-3.508436-1.707850$

H $-6.684244-4.764529-0.998761$

H -3.157274 -3.0887590 .795790$

$\begin{array}{lllll}\text { O } & -7.633217 & 0.232658 & 0.571488\end{array}$

O $-7.639924-0.5881802 .728884$

C $-7.997611 \quad 1.527948 \quad 1.057344$

H $-8.0809582 .201077 \quad 0.199874$

H -8.9655121 .4840581 .565573$

H $-7.223725 \quad 1.9111201 .729158$

SCF Energy (B3LYP/6-31G**//MMFF) $=-3245.93271226$

0400083

MMFF Geometry

C 0.9588572 .7436342 .518449

C 0.9493043 .7972601 .686369

C $1.647131 \quad 3.8922640 .349081$

O $2.0192812 .587438-0.150769$

C $2.916567 \quad 4.747350 \quad 0.479323$

C $3.5309765 .098965-0.861019$

C $4.6358294 .519888-1.369858$

C $2.852370 \quad 6.218594-1.607931$

C $5.4347583 .398317-0.766781$

C $5.8767262 .387629-1.834782$

C $6.7186061 .260801-1.221691$

C $7.3454850 .392974-2.313920$

C $8.133780-0.782864-1.717341$

$\begin{array}{lllll}\text { O } & 5.860936 & 0.462401 & -0.398537\end{array}$

O $8.453352-1.691799-2.781470$

C $7.238258-1.522029-0.701360$

C $9.484844-0.330880-1.152635$

O $7.973906-2.527974-0.008889$

C $6.537973-0.5826350 .312109$

C $5.487323-1.3864151 .111840$

O $7.508808-0.0578771 .219463$

C $1.0367051 .869065-0.762161$

$\begin{array}{llll}\text { O } & -0.121516 & 2.214287 & -0.938692\end{array}$

C $1.6039370 .561754-1.160160$

C $0.759430-0.423088-1.500324$

C $1.115107-1.783985-1.905132$

C $2.571162-2.091528-2.128844$

C $4.516706-0.5323361 .939849$

C $3.564585-1.4245842 .714161$

C $1.715525-3.0613442 .385417$

O $3.631648-1.5383133 .935646$

C $1.514529-4.1111371 .339589$

C $0.323013-4.4203150 .805869$

C $0.132239-5.495295-0.226231$

C $-0.572935-5.004805-1.471386$

C $-1.830624-5.387698-1.750092$

C $0.206234-4.132886-2.435593$

C $0.117353-2.677196-2.071088$

N $2.675037-2.0947521 .890704$

H $1.5476601 .865596 \quad 2.270377$

H $\quad 0.3671704 .674303 \quad 1.963404$

H $0.9571314 .363181-0.362400$

H $3.6467694 .230947 \quad 1.113364$

H 2.6883315 .6847251 .005040

H $5.0156704 .893894-2.319813$

H $3.3723156 .473556-2.537509$ 
H $1.8273895 .941023-1.871417$ H $2.8210007 .122560-0.990949$ H $6.3229813 .829601-0.289950$ H 4.8699482 .8719580 .008032 H $4.9864811 .965049-2.319028$ H $6.4609482 .905647-2.605619$ H $7.5007951 .712730-0.600376$ H $7.9901670 .991646-2.969363$ H $6.550626-0.007023-2.958903$ H $7.621819-1.986569-3.190164$ H $6.461039-2.047286-1.273931$ H $10.0945750 .124988-1.942180$ H $10.064570-1.184222-0.783173$ H $9.3920670 .397125-0.344015$ H $8.389494-3.097189-0.679137$ H $4.894515-1.9860710 .410910$ H $5.993093-2.0931821 .782707$ H 7.0701420 .1747042 .052873 H $2.6808180 .441578-1.143099$ H $-0.310830-0.221409-1.458480$ H $3.132520-2.016915-1.194220$ H $3.004974-1.389980-2.850546$ H $2.747666-3.091111-2.530971$ H $5.052566 \quad 0.0958302 .658771$ H $3.9276500 .135258 \quad 1.300844$ H $0.795106-2.5210632 .614578$ H $2.089621-3.5136173 .309349$ H $2.398518-4.6556571 .014980$ H -0.565656 -3.893909 1.145553 H -0.445252 -6.302999 0.241811 H $1.090817-5.944588-0.514901$ H -2.333187 -5.058853 -2.653825 H -2.388276 -6.032051 -1.078644 H $-0.200599-4.246316-3.448897$ H $1.234264-4.500827-2.489069$ H $-0.907533-2.331237-1.928190$ H $2.572673-1.7764260 .932046$ C -0.8971751 .5997563 .823581$ O C -1.8590320 .3507762 .093920$ C $-1.522326-0.5858403 .241148$ C -0.3734160 .1632663 .887920$ H -1.0971180 .2789611 .306561$ C $-3.254720 \quad 0.1768401 .488631$ H -2.366847 -0.6711183 .936390$ H $-1.251065-1.5892912 .905883$ C -0.044739 -0.3148645 .290771$ H $\quad 0.5172700 .044933 \quad 3.259090$ C -3.576789-1.268955 1.070567 O $-3.335504 \quad 1.0201150 .334453$ H -4.0099600 .5390442 .195481$ H -3.648634 -1.901450 1.963249 O $-2.474309-1.7736110 .306043$ C $-4.845532-1.4325110 .212626$ C $-6.109472-0.8173680 .835244$ H $-4.662619-1.010968-0.781070$ O $-5.063015-2.8337140 .009189$ H $-6.291128-1.2807381 .812954$ H $-5.946557 \quad 0.2495231 .013010$ H 0.7730760 .2723325 .720084 H $-0.909079-0.2292945 .957734$ H $\quad 0.265050-1.3650195 .275637$ C 0.1687672 .7006593 .798188 H -3.1141101 .9237430 .617989$ H $-2.690805-2.6868960 .050728$ H $-5.245762-3.2351180 .875927$ H $0.8577002 .576396 \quad 4.641093$ H -0.3358013 .6646663 .943263$ H -1.559982 1.7877324 .678541 C $-6.9604710 .786226-1.597324$ O $-7.142612-0.620830-1.402573$ C -7.367609-1.009215-0.043894 C -8.629120 -0.3175470 .492997$ C $-8.581681 \quad 1.198056 \quad 0.292825$ C -8.214469 $1.543896-1.151947$ H $-6.0940151 .132511-1.021621$ C $-6.6615351 .010478-3.083150$ H -7.575146 -2.086511-0.064577
O $-8.800481-0.630308 \quad 1.871100$

H $-9.502563-0.715558-0.040190$ H -7.887074 1.6740850 .995128 O -9.8710511 .7530370 .562998$ H $-9.0573741 .303372-1.812375$ O $-7.976576 \quad 2.946045-1.251969$ C $-5.3279580 .407999-3.460397$ H $-7.4262500 .534528-3.708972$ H $-6.6413612 .073795-3.346375$ H $-9.665961-0.2850602 .147393$ H $-10.051763 \quad 1.628792 \quad 1.509833$ H $-8.7786313 .399277-0.940134$ O $-4.312276 \quad 1.172344-2.966315$ O $-5.206128-0.625391-4.105511$ C $-2.9923450 .679698-3.217574$ H $-2.8103630 .610606-4.294218$ H $-2.2785831 .386047-2.785990$ H $-2.855181-0.294415-2.738219$ SCF Energy (B3LYP/6-31G*//MMFF) $=-3245.91382513$

\section{4}

MM̄FF Geometry

C $0.673172-1.5638682 .195334$

C $1.217816-2.4937391 .393530$

C $0.639325-3.010902 \quad 0.095827$

O $-0.395536-2.129493-0.395756$

C $0.035310-4.408670 \quad 0.314407$

C $-0.418025-5.074493-0.971189$

C $-1.701761-5.220484-1.356356$

C $0.690833-5.626294-1.831254$

C $-2.939041-4.743884-0.647482$

C $-3.683989-3.699547-1.488287$

C $-4.835968-3.045072-0.711175$

C $-5.578063-2.045171-1.600216$

C $-6.646990-1.257853-0.829266$

O $-4.270458-2.3742720 .419306$

O $-7.033260-0.153267-1.661601$

C $-6.016109-0.6837270 .458548$

C $-7.912287-2.096369-0.615497$

O $-7.006989-0.057406 \quad 1.269326$

C $-5.223458-1.7270291 .271739$

C $-4.440964-1.1061972 .454222$

O $-6.143184-2.6744161 .828561$

C $0.000373-1.129434-1.230839$

O $1.129801-0.925520-1.646970$

C $-1.181882-0.299040-1.548252$

C $-1.0052690 .883567-2.156421$

C $-2.0549611 .814960-2.574545$

C $-3.4851841 .413694-2.332731$

C $-3.346914-0.1050372 .069033$

C -3.7820751 .3498962 .103179$

C $-2.9285203 .632463 \quad 1.570392$

$\begin{array}{lllll}\text { O } & -4.850058 & 1.724601 & 2.578327\end{array}$

C $-3.484104 \quad 4.0841450 .257559$

C $-2.8651514 .967916-0.540109$

C $-3.4511805 .494087-1.818121$

C $-2.5255525 .332605-3.001829$

C $-1.7490146 .348106-3.416657$

C $-2.5782354 .030108-3.765137$

C -1.691795 $2.970413-3.169683$

N $-2.806406 \quad 2.187724 \quad 1.577832$

H $-0.286828-1.1273731 .938922$

H $2.169732-2.9362951 .681337$

H $1.453744-3.075423-0.636272$

H $-0.790460-4.3387801 .029814$

H $0.775013-5.0645240 .793821$

H $-1.898812-5.735933-2.295767$

H $0.310681-6.158333-2.709947$

H $1.338240-4.820707-2.190958$

H $1.299550-6.333481-1.258406$

H $-3.594591-5.605522-0.473223$

H $-2.707767-4.3329410 .337234$

H $-2.973410-2.924040-1.799856$

H $-4.071110-4.170636-2.400581$

H $-5.515755-3.831384-0.362641$

H $-6.021995-2.550401-2.467242$

H $-4.856200-1.329315-2.014231$

H $-7.6543550 .395751-1.152523$ 
H -5.3352630 .1144620 .141440$ H -8.336987 -2.396658 -1.581119 H $-8.694737-1.515549-0.114956$ H -7.731819 -3.004725 -0.036891 H -7.502513 -0.7581461 .726472$ H -5.136824 -0.6853013 .189856$ H -3.935896 -1.932724 2.975103 H $-5.715942-3.1131662 .582733$ H -2.158330 -0.681804-1.278195 H $0.0114321 .200590-2.385787$ H $-3.7465990 .551950-2.955168$ H -4.204035 $2.205306-2.554244$ H -3.642187 $1.151515-1.281655$ H -2.537681 -0.1912262 .804941$ H -2.921570 -0.3378741 .088367$ H $-1.9326794 .044071 \quad 1.759960$ H -3.5935543 .9487132 .380517$ H -4.459321 $3.697685-0.028865$ H -1.903562 $5.375225-0.235196$ H -3.674621 $6.558470-1.665781$ H -4.416393 $5.022465-2.040749$ H -1.107684 $6.250166-4.286329$ H -1.733296 $7.297668-2.891750$ H -2.238532 4.192185-4.797462 H $-3.6177773 .707042-3.871806$ H $-0.6280663 .176982-3.295674$ H -1.9757951 .7818891 .156358$ C 1.5967850 .4062793 .525363 O $2.333755 \quad 0.8242452 .359252$ C 1.8050202 .0929191 .919101 C 0.9709832 .6189323 .079407 C 0.3787401 .3252773 .608299 H 1.1274811 .8819091 .083333 C 2.9411663 .0098951 .454382 H 1.5944723 .0951793 .844981 H 0.2157323 .3406152 .756800 C $-0.217853 \quad 1.449477 \quad 4.998177$ H $-0.401250 \quad 0.9926322 .914698$ C 3.8092882 .3908550 .337373 O 3.7945563 .2962832 .568855 H 2.5338603 .9734251 .128394 H $4.3287831 .518850 \quad 0.741192$ O $4.8270523 .331756-0.018873$ C $3.0226472 .001513-0.924606$ C $3.8649851 .382326-2.054709$ H $2.2351121 .289838-0.661830$ O $2.3756543 .171645-1.432211$ H $3.2029461 .256425-2.921828$ H $4.6426422 .090472-2.367161$ H $-0.616000 \quad 0.4884445 .338509$ H $0.527564 \quad 1.7839345 .727229$ H -1.0387572 .1742944 .999601$ C $1.317460-1.0982083 .472547$ H 4.1468692 .4501042 .894137 H $4.3862484 .106585-0.408074$ H $1.8327172 .902684-2.192636$ H $0.680982-1.3875224 .316331$ H $2.270688-1.6251453 .604990$ H $2.252130 \quad 0.596720 \quad 4.385770$ C $6.408798-0.900316-0.520651$ O $5.709266 \quad 0.264006-0.984744$ C $4.479300 \quad 0.022246-1.676274$ C $4.732294-0.846560-2.917306$ C $5.445865-2.148777-2.544467$ C $6.675744-1.887647-1.671222$ H $7.383874-0.524732-0.184007$ C $5.706672-1.545160 \quad 0.686390$ H $3.785225-0.500267-1.010071$ O $5.506293-0.128688-3.876132$ H $3.770557-1.077102-3.390598$ H $5.754740-2.670030-3.458722$ O $4.551453-3.001590-1.834786$ H $7.065317-2.827560-1.262969$ O $7.711114-1.325524-2.483671$ C $5.839934-0.7058521 .941439$ H $4.639566-1.6967610 .505468$ H $6.171297-2.5137570 .904561$ H $6.3272800 .158670-3.440759$
H $3.784688-3.167432-2.409355$

H $7.919270-1.970752-3.180745$

O $5.163051-1.3192102 .957778$

$\begin{array}{lllll}\text { O } & 6.463916 & 0.342428 & 2.030727\end{array}$

C $5.224031-0.6539574 .223388$

H 4.8512850 .3709384 .136859

H $4.588490-1.1994764 .925786$

H $6.250982-0.6601084 .600439$

SCF Energy (B3LYP/6-31G**//MMFF) $=-3245.91799625$

\section{5}

MM̄FF Geometry

C $1.9935732 .927699-2.467654$

C $2.9296413 .844729-2.176793$

C $3.9143693 .769653-1.032280$

O $4.0651932 .412791-0.557562$

C $3.4042214 .638144 \quad 0.130010$

C 4.3128324 .6157201 .342594

C $4.049576 \quad 3.9208302 .467404$

C $5.5433775 .477294 \quad 1.245749$

C 2.8769613 .0068822 .698692

C 3.2544611 .7976003 .566739

C 2.0798270 .8159783 .688069

C $2.350511-0.2319704 .767916$

C $1.222532-1.2728874 .840680$

$\begin{array}{llllll}\text { O } & 1.917075 & 0.173267 & 2.418343\end{array}$

O $1.668529-2.3561945 .670254$

C $0.957850-1.8244053 .424128$

C $-0.021163-0.7138065 .539895$

O $-0.178903-2.684322 \quad 3.414154$

C $0.801252-0.723998 \quad 2.347188$

C $0.797493-1.3748680 .944438$

O $-0.436634-0.0384142 .544883$

C $5.0127201 .647630-1.164603$

O $5.7403311 .975539-2.088670$

C $5.0040120 .317109-0.515588$

C $5.703129-0.682772-1.073107$

C $5.775862-2.067157-0.600933$

C $5.115784-2.3981300 .711159$

C $0.951796-0.380589-0.212279$

C $0.875031-1.101452-1.543941$

C $2.168561-2.678445-2.984733$

O $-0.118434-1.032771-2.262819$

C $2.920106-3.909431-2.586277$

C $4.062160-4.313014-3.162867$

C $4.821274-5.534970-2.727808$

C $6.271190-5.244933-2.400654$

C $7.261856-5.733585-3.166073$

C $6.578459-4.449666-1.145893$

C $6.430178-2.967474-1.364471$

N $2.011384-1.847603-1.806241$

H $1.9087832 .031393-1.860688$

H $2.9811604 .748247-2.781095$

H $4.8847024 .150554-1.374650$

H $2.392694 \quad 4.3206830 .411862$

H $3.2909185 .678582-0.204013$

H 4.7410604 .0142863 .303228

H 6.1367735 .4589972 .166080

H 6.1886915 .1336630 .431513

H 5.2643706 .5185181 .053880

H $2.088173 \quad 3.5762563 .204597$

H 2.4661122 .6454481 .751147

H 4.1178171 .2864973 .120650

H 3.5521792 .1496954 .562240

H 1.1730391 .3838763 .927485

H 2.4970780 .2419265 .746470

H $3.293147-0.7498724 .541562$

H $2.475852-2.7285355 .276605$

H $1.819865-2.4492483 .151545$

H $-0.794066-1.4831765 .645953$

H -0.4579360 .1430755 .023163$

H $0.225823-0.3967056 .560393$

H $-0.037920-3.3606904 .098557$

H $1.624239-2.0930270 .881760$

H $-0.129058-1.9447470 .800191$

H -0.7572570 .2942951 .691775$

H $4.4069490 .190850 \quad 0.380067$

H $6.259059-0.477069-1.987478$ 
H $4.029258-2.3003140 .634645$ H $5.471208-1.7269441 .501348$ H $5.327596-3.4115571 .059014$ H $\quad 0.1719760 .384106-0.190812$ H $1.9101880 .145601-0.153842$ H $2.702765-2.086238-3.734591$ H $1.185754-2.945670-3.385222$ H $2.502950-4.496115-1.770613$ H $4.473324-3.740139-3.990765$ H $4.754440-6.272345-3.538214$ H $4.350664-6.004833-1.854984$ H $8.303907-5.550027-2.925946$ H $7.054049-6.323767-4.052485$ H $7.612948-4.635858-0.829491$ H $5.944521-4.825942-0.338118$ H $6.911582-2.612334-2.277313$ H $2.827180-1.706830-1.218626$ C $-0.4343313 .306496-3.120420$ O $-0.9065382 .196505-2.331385$ C -1.652124 2.716109-1.207550 C $-1.9245024 .178159-1.530658$ C $-0.6614474 .560337-2.275688$ H $-0.9804032 .634567-0.343461$ C $-2.9076351 .863646-0.989144$ H -2.800704 4.277325 -2.183562 H $-2.0929294 .784075-0.635485$ C $-0.7910725 .836456-3.088062$ H $0.1404114 .688785-1.538616$ C -3.8453372 .4058790 .104680$ O $-2.4922720 .545999-0.617414$ H $-3.4509631 .766594-1.935914$ H $-4.2714113 .363997-0.213858$ O -3.0746082 .6884651 .280834$ C -4.9829221 .4508320 .516999$ C $-5.8580550 .975788-0.655275$ H -4.5582710 .5931591 .048857$ O -5.8130162 .1294351 .466546$ H $-6.3194291 .847501-1.135546$ H $-5.2308880 .490085-1.407935$ H $\quad 0.142126 \quad 6.058205-3.615432$ H -1.590144 $5.759243-3.833035$ H -1.020954 $6.685580-2.436181$ C $1.0040253 .064310-3.590928$ H -1.941260 $0.201998-1.341561$ H -2.660926 1.8561351 .567607 H -6.2100112 .8966441 .019845$ H $1.3044273 .869186-4.272283$ H $1.0389302 .131246-4.167141$ H -1.078858 $3.336352-4.008967$ C -5.653161 -2.008883 -0.129907 O $-6.469275-1.0727110 .583551$ C $-6.9759390 .004642-0.210222$ C $-7.828320-0.553498-1.357394$ C -7.081092 -1.609616 -2.171571 C $-6.462494-2.666199-1.252870$ H -4.780106 -1.495957 -0.551915 C $-5.146229-3.0401630 .882957$ H -7.6485990 .5755770 .442180$ O $\quad-8.257966 \quad 0.505511-2.206576$ H -8.731790 -1.011794 -0.933944 H $-6.322892-1.144513-2.812760$ O $-8.020883-2.235876-3.047963$ H $-7.254973-3.296507-0.829557$ O $-5.599787-3.507188-2.014793$ C $-4.072266-2.4300731 .752963$ H -5.956408 -3.410986 1.522200 H -4.700617 -3.912286 0.391419 H $-8.7788660 .108350-2.925383$ H -7.524337 -2.831778 -3.633733 H $-6.150494-4.001250-2.644889$ O $-4.626498-1.8980352 .879173$ O $-2.887109-2.3996971 .447365$ C $-3.712152-1.2416213 .762509$ H $-4.277630-0.8648944 .618895$ H $-2.959697-1.9490074 .122809$ $\mathrm{H}-3.238494-0.3952953 .256116$

SCF Energy (B3LYP/6-31G**//MMFF) $=-3245.91775187$
04_00086

MMFF Geometry

C $-1.563828-3.1010660 .316055$

C $-0.255276-3.3094250 .522353$

C $0.432133-3.0955441 .846390$

O $1.311606-1.9560541 .705416$

C $1.273014-4.3348842 .201482$

C $2.059997-4.1886303 .489637$

C $3.393324-3.9977663 .558929$

C $1.247156-4.2967194 .753897$

C $4.361150-3.8598502 .416121$

C $4.961265-2.4490002 .365917$

C $5.618496-2.1558221 .008939$

C $6.317559-0.7958691 .033413$

C $6.869404-0.401665-0.343758$

O $4.580629-2.1571700 .021285$

O $7.2072320 .992411-0.274429$

C $5.752266-0.567830-1.397556$

C $8.173650-1.143590-0.657243$

O $6.260453-0.339239-2.709523$

C $5.028854-1.928227-1.320952$

C $3.788488-2.010963-2.246440$

O $5.933804-2.956924-1.741217$

C $0.779631-0.734114 \quad 1.990182$

O $-0.355728-0.4986132 .373680$

C 1.8180590 .2887821 .733185

C 1.5052481 .5871771 .854015

C 2.3901982 .7262221 .605890

C 3.8060232 .4355851 .186731

C $2.669139-1.007463-1.946727$

C $2.7466990 .277287-2.754368$

C $1.6832582 .526819-2.922399$

O $3.491550 \quad 0.422623-3.719544$

C $2.4456173 .558083-2.152710$

C $1.8842624 .667624-1.648233$

C $2.6524445 .749400-0.944628$

C 2.0904676 .0796340 .419434

C 1.2320787 .1000300 .586045

C 2.5981685 .2866701 .601455

C 1.8914203 .9706881 .757987

N $1.8489661 .230982-2.292215$

H $-2.180282-2.7418101 .136242$

H $0.366840-3.630833-0.310230$

H $-0.297146-2.9156662 .646167$

H $1.940189-4.5725111 .367143$

H $\quad 0.615567-5.2102432 .294502$

H $3.851024-3.9190444 .544265$

H $1.869356-4.2494315 .653948$

H $\quad 0.519627-3.4815404 .814536$

H $\quad 0.707369-5.2490094 .777979$

H $5.167498-4.5911852 .550810$

H $3.895874-4.1050921 .459812$

H $4.168925-1.7122112 .545662$

H $5.696464-2.3399273 .172895$

H $6.334924-2.9546460 .784252$

H $7.115524-0.7818261 .786489$

H $5.599511-0.0281351 .349540$

H $7.4856671 .272332-1.163506$

H $5.0285400 .233061-1.207793$

H $8.933576-0.9131980 .099330$

H $8.597746-0.816170-1.612898$

H $8.053319-2.228561-0.685909$

H $6.746958-1.135728-2.982077$

H $4.098386-1.968645-3.297594$

H $3.353314-3.012047-2.113047$

H $5.414845-3.732874-2.010641$

H $2.801473-0.0500811 .432312$

H 0.4951581 .8484622 .164658

H 4.3486121 .9434632 .000634

H 4.3729643 .3277290 .913047

H 3.8233641 .7849270 .306180

H $1.709352-1.471093-2.209446$

H $2.633571-0.768635-0.881142$

H $\quad 0.6112032 .741557-2.950087$

H $2.0523532 .488254-3.952277$

H $3.5161853 .403121-2.040791$

H $\quad 0.8185104 .833494-1.788773$

H $2.6241516 .643452-1.581569$ 
H $3.7145865 .490973-0.851359$ H 0.8487347 .3616631 .566770 H $\quad 0.8890907 .691972-0.256089$ H 2.4352215 .8527162 .528796 H 3.6847235 .1832191 .533221 H 0.8482954 .0770872 .057951 H $1.2943371 .032737-1.464362$ C $-3.606925-3.950190-0.887312$ O $-4.514338-3.000110-0.285427$ C $-5.559214-2.710559-1.227116$ C $-5.672782-3.972905-2.065611$ C $-4.206339-4.315007-2.252733$ H $-5.198926-1.893327-1.864948$ C $-6.839138-2.268676-0.512412$ H -6.181421 -4.777533 -1.522403 H -6.198299 -3.804923 -3.010191 C $-3.963410-5.759427-2.656353$ H -3.792194 -3.661105 -3.032359 C $-6.637360-1.1218780 .501681$ O $-7.375530-3.391031 \quad 0.197579$ H -7.590058 -1.988934 -1.261300 H -6.162722 -1.5054621 .413509$ O $-7.930872-0.6428160 .889294$ C $-5.8199290 .071354-0.025927$ C $-5.7844891 .253878 \quad 0.961525$ H $-4.795954-0.258427-0.229702$ O $\quad-6.3690530 .524279-1.263987$ H -6.7666391 .7411850 .987322$ H -5.5597290 .8691591 .963854$ H -2.891599 -5.958688 -2.756867 H $-4.368157-6.456656-1.915059$ H -4.440795 -5.976132 -3.617575 C -2.226857 -3.298392 -1.018736 H $-8.270873-3.1536270 .491510$ H -8.365652 $-1.347770 \quad 1.397626$ H $-7.2954490 .771963-1.101526$ H -2.335631 -2.311122 -1.482784 H -1.590035 -3.908589-1.670050 H $-3.566911-4.822270-0.223349$ C $-2.3033382 .502267 \quad 0.451376$ O C -4.7215532 .2962590 .561638$ C -4.8597423 .5993531 .361636$ C -3.7093754 .5630241 .053386$ C $-2.3450883 .871702 \quad 1.150087$ H -1.4459101 .9541700 .857266$ C -2.122926 2.591668-1.070361 H $-4.8286642 .514388-0.506057$ O -4.8919993 .3239112 .760511$ H -5.8149084 .0725941 .105560$ H $-3.7497795 .401679 \quad 1.758919$ O $-3.8934765 .087028-0.258487$ H $-1.5545724 .512640 \quad 0.742734$ O -2.0358653 .6542182 .530351$ C $-1.7874451 .242916-1.674564$ H -3.028571 2.961187 -1.558346 H -1.295364 $3.267749-1.310193$ H -4.0833312 .8326952 .985707$ H -3.171303 $5.715965-0.427202$ H -2.0009854 .5242962 .963143$ O $-1.984143 \quad 1.297302-3.025032$ O $-1.378448 \quad 0.273647-1.050302$ C $-1.6937870 .091386-3.739030$ H -1.786296 $0.299326-4.808277$ H $-0.671733-0.242113-3.535413$ $\mathrm{H}-2.415340-0.684872-3.468103$

SCF Energy (B3LYP/6-31G**//MMFF) $=-3245.92016770$

0400087

MMFF Geometry

C $0.9334482 .644103 \quad 3.001917$

C 1.1428501 .4950862 .341563

C $1.3544340 .171278 \quad 3.029467$

O $2.640258-0.3028212 .569417$

C $0.276033-0.8414712 .610413$

C $0.137415-1.9903113 .588411$

C $0.693405-3.2079783 .435508$

C $-0.732041-1.7030234 .785936$
C $1.602176-3.6814842 .335582$

C $0.972916-4.7938631 .485725$

C $-0.165290-4.2988540 .580539$

C $-0.774575-5.461639-0.205337$

C $-1.845881-4.983008-1.196244$

O $0.385352-3.338949-0.325783$

O $-2.120843-6.081953-2.078096$

C $-1.264667-3.827469-2.039734$

C $-3.164864-4.664485-0.484150$

O $-2.263149-3.264509-2.887009$

C $-0.572568-2.729398-1.198893$

C $0.184731-1.750481-2.127857$

O $-1.566993-2.005227-0.470572$

C $3.515700-0.8202833 .465034$

O $3.327490-0.9906124 .661197$

C $4.814005-1.1319412 .818468$

C $4.999220-1.1035091 .489009$

C $6.259311-1.3287350 .777527$

C $7.496342-1.5739801 .599803$

C $1.161715-0.824611-1.389752$

C $1.8109250 .186186-2.317565$

C $3.5806191 .945076-2.320964$

O $1.5258090 .290666-3.507223$

C $4.7862851 .251184-2.874310$

C $6.0401711 .462007-2.445962$

C $7.2360210 .736250-2.995715$

C $7.980806-0.054633-1.940629$

C $9.1522420 .385346-1.451053$

C $7.416186-1.394221-1.515158$

C $6.254052-1.259509-0.570286$

N $2.7481290 .963823-1.649337$

H 0.8980692 .6090464 .089777

H $1.1887891 .487416 \quad 1.256762$

H $1.3623300 .314676 \quad 4.116723$

H $\quad 0.491967-1.2247961 .608123$

H $-0.702580-0.3481172 .532576$

H $\quad 0.500657-3.957048 \quad 4.202953$

H $-0.816313-2.5648715 .456442$

H $-0.321716-0.8723005 .368384$

H -1.744018-1.435653 4.463972

H $1.941790-2.8631961 .694637$

H $2.507444-4.0804902 .810525$

H $1.768685-5.2164790 .858404$

H $\quad 0.611073-5.6001112 .135849$

H $-0.930818-3.8180311 .199503$

H $-1.188694-6.2174390 .473721$

H $\quad 0.017079-5.971813-0.772318$

H $-2.740350-5.765831-2.758256$

H $-0.517262-4.271545-2.711377$

H $-3.530675-5.5475280 .053593$

H $-3.951485-4.409120-1.202271$

H -3.075688 $-3.849907 \quad 0.237333$

H $-2.840123-2.710018-2.335263$

H $\quad 0.770177-2.321196-2.860757$

H $-0.536328-1.145258-2.690857$

H - $-1.265885-1.092635-0.335720$

H $5.618567-1.3746923 .505061$

H $4.151553-0.8731740 .845834$

H $7.393845-2.4974062 .180422$

H $7.671591-0.7416242 .290635$

H $8.403709-1.6751971 .000352$

H $0.650228-0.269004-0.599329$

H $1.959358-1.417571-0.926635$

H $3.8424372 .712071-1.585368$

H $3.0139992 .414219-3.131516$

H $4.6129000 .528413-3.668707$

H $6.2202442 .189495-1.658070$

H $7.8998031 .485054-3.447456$

H $6.9531310 .062479-3.814161$

H $9.709203-0.187211-0.717048$

H $9.579057 \quad 1.330452-1.770159$

H $8.200169-2.026701-1.084252$

H $7.081858-1.944071-2.404324$

H $5.304070-1.079811-1.073617$

H $2.9690060 .744956-0.682195$

C 0.8354794 .1718190 .899754

O $-0.2627243 .480550 \quad 0.276651$

C $-0.8886114 .348395-0.681208$ 
C $0.1068925 .471641-0.931326$ C $0.7234725 .629595 \quad 0.444257$ H -1.781937 $4.755620-0.191101$ C -1.325955 $3.577607-1.935397$ H $0.8750655 .180000-1.656935$ H $-0.3759166 .383596-1.294741$

$\begin{array}{lllll}C & 2.047031 & 6.375087 & 0.434441\end{array}$ H $0.0139756 .170657 \quad 1.085012$ C -2.212753 2.356155-1.609991 O $-0.175647 \quad 3.157918-2.667799$ H - $1.8815474 .268812-2.579932$ H -2.964274 $2.655284-0.871613$ O $-1.4201011 .321881-1.031791$ C $-2.9520141 .784966-2.833128$ C $-3.7224040 .471445-2.594865$ H -3.659129 2.542380 -3.191769 O $-2.0403121 .548263-3.907750$ H $-4.2702750 .251431-3.520983$ H $-3.001642-0.346863-2.474442$ H 2.4589886 .4525561 .445381 H $2.7879775 .868606-0.193379$ H $1.913967 \quad 7.3897370 .045100$ C $0.744153 \quad 4.021862 \quad 2.422440$ H $0.3287052 .555421-2.094752$ H $-1.0600421 .660442-0.194589$ H -1.336055 $0.967032-3.572873$ H -0.2399914 .3719232 .757592$ H $1.493106 \quad 4.6730182 .889384$ H 1.7609653 .7211860 .519656 C $-4.666944 \quad 0.201927 \quad 1.016815$ O $-3.940452 \quad 0.226977-0.218238$ C $-4.698148 \quad 0.495524-1.404159$ C $-5.806088-0.557148-1.568557$ C $-6.683095-0.646877-0.317829$ C $-5.841522-0.7886650 .953600$ H -3.952181 -0.1877451 .753615$ C $-5.082760 \quad 1.6127361 .460586$ H $-5.1495631 .491086-1.336750$ O $-5.236369-1.835052-1.845529$ H -6.424943 -0.287319-2.432318 H $-7.354027-1.509041-0.415259$ O $-7.486371 \quad 0.526910-0.239263$ H -6.466442 -0.6726361 .846599$ O $-5.289951-2.1078761 .002102$ C -3.9031672 .4329161 .945072$ H $-5.558902 \quad 2.1726450 .651747$ H $-5.781818 \quad 1.5367052 .301794$ H -4.633206 - $2.053092-1.114628$ H -8.0566810 .4400420 .543404$ H -6.031840 -2.736490 1.004748 $\begin{array}{llll} & -4.363575 & 3.637807 & 2.396880\end{array}$ O -2.7341792 .0733791 .931939$ C -3.366751 4.5275642 .908608 H $-3.8729005 .406593 \quad 3.316292$ H -2.799015 4.047228 3.711381 H -2.701751 $4.848102 \quad 2.102309$ SCF Energy (B3LYP/6-31G**//MMFF) $=-3245.92856973$

0400088

MM̄FF Geometry

C $-0.8929610 .361956-2.400888$

C $-0.042577-0.675959-2.357464$

C $-0.368856-2.098057-1.960063$

O $-1.794205-2.240731-1.770191$

C $0.338981-2.445906-0.643059$

C $0.366061-3.926445-0.326237$

C $-0.401620-4.5246610 .605728$

C $1.379645-4.730174-1.098383$

C $-1.491839-3.9110361 .439635$

C $-1.078771-3.6785332 .899930$

C $-0.138068-2.4772593 .086197$

C $0.245078-2.3088604 .558362$

C $1.080619-1.0410304 .798717$

O $-0.828884-1.3119022 .624625$

O $1.111145-0.832738 \quad 6.219000$

C $0.3573110 .161557 \quad 4.150798$

C $2.536097-1.2307174 .353926$

$\begin{array}{llll}\text { O } & 1.157694 & 1.339686 & 4.211898\end{array}$
C $-0.065333-0.1017292 .689760$

C $-0.941828 \quad 1.029807 \quad 2.106175$

O $1.111145-0.1866161 .877072$

C $-2.406820-3.349298-2.255022$

O $-1.877728-4.275778-2.853692$

C $-3.866025-3.323005-1.995139$

C $-4.477533-2.393638-1.243535$

C $-5.916395-2.305126-0.976532$

C $-6.813867-3.323631-1.629318$

C $-2.300096 \quad 1.2121302 .782159$

C -3.1016392 .3135952 .116680$

$\begin{array}{llll}C & -4.459651 & 2.809229 & 0.085744\end{array}$

$\begin{array}{llllll}\text { O } & -3.281686 & 3.399117 & 2.664545\end{array}$

C $-5.876232 \quad 2.425123 \quad 0.375704$

C $-6.7305881 .970860-0.553458$

C $-8.1352581 .538091-0.245698$

C $-8.402190 \quad 0.098712-0.633047$

C $-9.226742-0.197437-1.651972$

C $-7.784632-0.997546 \quad 0.209895$

C $-6.365977-1.305836-0.188333$

N $-3.584953 \quad 1.9545760 .868277$

H $-1.9306230 .230575-2.106815$

H $0.988805-0.511599-2.663818$

H $-0.024873-2.742655-2.777670$

H $-0.149081-1.8982270 .166779$

H $1.377411-2.088315-0.659614$

H $-0.264597-5.5924110 .773497$

H $1.400205-5.780999-0.790476$

H $1.155635-4.706602-2.169148$

H $2.383434-4.323643-0.944965$

H $-1.878588-2.9893330 .994235$

H -2.336928 -4.6116521 .428366$

H - $1.997927-3.4977043 .472693$

H $-0.616454-4.5854593 .308778$

H $0.760681-2.6320342 .479405$

H $0.777662-3.1952204 .925496$

H $-0.669384-2.2384895 .164570$

H $1.684215-0.0688126 .400088$

H $-0.537010 \quad 0.358176 \quad 4.754719$

H $3.010932-2.0289584 .936914$

H $3.129025-0.3287244 .541499$

H $2.632916-1.4909463 .297918$

H 1.3259401 .5346695 .148849

H -0.3917331 .9770182 .123693$

H $-1.1115270 .818247 \quad 1.041372$

H $\quad 0.884790 \quad 0.0610160 .965660$

H $-4.419719-4.129490-2.465581$

H $-3.876930-1.612682-0.779995$

H $-6.567868-4.332225-1.279229$

H $-6.703664-3.292210-2.719009$

H $-7.874478-3.167054-1.421829$

H -2.8876590 .2874902 .750120$

H -2.182861 1.4839123 .836085

H $-4.1992582 .666232-0.967564$

H -4.2923413 .8576690 .351250$

H -6.2095002 .5111541 .407403$

H $-6.4034961 .893092-1.587682$

H -8.815739 $2.211390-0.782673$

H -8.3676711 .6670970 .818816$

H $-9.449023-1.225088-1.919019$

H $-9.6961710 .580684-2.244571$

H -8.407233 -1.8986340 .182547$

H $-7.795694-0.6947421 .264946$

H $-5.634754-0.620460 \quad 0.239730$

H $-3.441553 \quad 1.0041840 .539274$

C $-0.6998752 .831223-1.869341$

O $0.354702 \quad 2.755953-0.885366$

C $1.1163423 .974344-0.942553$

C $0.1362155 .018405-1.453044$

C $-0.6025654 .223465-2.509447$

H $1.9023593 .818981-1.692876$

C $1.7675704 .304054 \quad 0.405172$

H $-0.5614675 .339469-0.671140$

H $0.6370595 .904415-1.853991$

C $-1.9527134 .813375-2.882547$

H $0.0216294 .180939-3.412372$

C $2.533767 \quad 3.1421851 .064650$

$\begin{array}{lllll}\text { O } & 0.765771 & 4.731643 & 1.336791\end{array}$ 
H 2.4350875 .1645540 .278466

H 1.8261922 .4279081 .496413

O 3.2781483 .6772462 .166056

C 3.5003192 .3867880 .139290

C 4.3191321 .3223940 .893087

H $2.9258181 .904489-0.657472$

O $4.3952753 .306180-0.484834$

H 5.0589801 .8116691 .538227

H 3.6392040 .7435111 .528374

H -2.454292 $4.192002-3.631334$

H -2.611933 $4.889107-2.011126$

H -1.831689 $5.817942-3.300621$

C $-0.514831 \quad 1.724294-2.912325$

H $\quad 0.087760 \quad 4.0361001 .380247$

H $2.644748 \quad 4.1253362 .752547$

H 4.8724883 .7764110 .220220

H $\quad 0.524881 \quad 1.720077-3.262794$

H -1.150136 $1.928793-3.782692$

H -1.646418 2.708971-1.331743

C $4.551835-1.303684-1.754532$

O $4.059504-0.381015-0.773970$

C $5.0550120 .368897-0.067006$

C $6.003309-0.5783550 .683769$

C $6.612228-1.619395-0.259645$

C $5.542894-2.296689-1.124449$

H $3.667054-1.870242-2.073559$

C $5.113489-0.582241-2.984545$

H $5.6298980 .970406-0.778848$

O $5.312103-1.2416201 .740888$

H $6.8024350 .013377 \quad 1.145422$

H $7.137928-2.3749570 .336791$

O $7.572643-0.974702-1.090843$

H $6.007144-2.898639-1.914248$

O $4.787760-3.193314-0.305114$

C $4.0111320 .122548-3.740324$

H $5.8671630 .172213-2.743096$

H $5.599477-1.295346-3.661499$

H $4.545999-1.6996421 .354305$

H $7.968540-1.653792-1.663092$

H $5.405084-3.8525120 .055075$

O $3.604869-0.642131-4.793709$

O $3.5559331 .213675-3.422289$

C $2.578785-0.071365-5.611894$

H $2.9121390 .883751-6.028910$

H $2.377720-0.761951-6.435283$

H $\quad 1.6609710 .060226-5.032768$

SCF Energy (B3LYP/6-31G**//MMFF) $=-3245.92165318$

0400089

MM̄̄FF Geometry

C $0.806471-1.8767850 .580650$

C $-0.434211-1.5714420 .174840$

C $-0.759594-1.180733-1.241138$

O $-0.996507 \quad 0.244778-1.209968$

C $-2.039790-1.877703-1.724250$

C $-2.233405-1.776398-3.223307$

C $-3.051776-0.899376-3.836754$

C $-1.461284-2.783599-4.036529$

C $-3.8799280 .189290-3.212909$

C $-5.386920-0.084750-3.316429$

C $-5.871437-1.187573-2.362083$

C $-7.361737-1.465035-2.569996$

C $-7.902411-2.480903-1.552204$

O $-5.644067-0.733461-1.024445$

O $-9.336493-2.441154-1.607552$

C -7.492422 -2.032622 -0.133606

C -7.494401 -3.916143 -1.904787

O $-7.846288-3.0178710 .834661$

C $-5.992255-1.678756-0.005294$

C $-5.725274-1.0131511 .366452$

O $-5.219651-2.876045-0.106266$

C $-0.3092851 .038035-2.067942$

O $0.4657190 .674994-2.941892$

C $-0.5747142 .472841-1.806999$

C -1.440759 $2.915002-0.881528$

C $-1.7027954 .314862-0.538508$

C $-0.8918595 .372250-1.240398$

C $-4.316569-0.4202031 .514877$
C $-4.1372590 .298043 \quad 2.841389$

C $-2.543707 \quad 1.8008184 .032526$

O $-4.967483 \quad 0.257744 \quad 3.745590$

C -3.0790193 .1839353 .831922$

C -2.3092734 .2769063 .720000$

C -2.8560165 .6603083 .510537$

C -2.3627816 .2967512 .228998$

C -1.3912987 .2249292 .251465$

C -3.0546525 .9268840 .934588$

C -2.6270784 .5839620 .407381$

N -2.928256 0.9796622 .898887

H $1.616730-1.850602-0.144080$

H -1.240866 -1.546761 0.902139

H $0.084232-1.428145-1.896498$

H -2.906982 -1.459121-1.202492

H $-2.018349-2.942215-1.454772$

H $-3.124081-0.942173-4.923009$

H -1.656461 -2.689888 -5.110217

H $-0.384788-2.655827-3.886387$

H -1.735914 -3.801183 -3.740039

H $-3.5970500 .384568-2.174629$

H $-3.6588471 .116631-3.756865$

H $-5.9080040 .850155-3.071870$

H $-5.648860-0.340577-4.350628$

H -5.289346 -2.098105 -2.540976

H $-7.554508-1.807716-3.594329$

H -7.926337-0.528911-2.456338

H $-9.607108-2.650638-2.518062$

H -8.080582 -1.1360720 .107822$

H -7.879128 -4.191188 -2.894275

H -7.932296 -4.635006 -1.203485

H -6.412487 -4.063424 -1.920101

H -8.795532 -3.200635 0.728405

H $-6.444802-0.1968061 .514447$

H -5.888537 -1.741205 2.171168

H $-4.377254-2.7537660 .357996$

H $0.0091223 .156315-2.415352$

H $-2.0079582 .189583-0.300923$

H $0.1795485 .202192-1.084951$

H -1.097082 $6.384654-0.886301$

H $-1.0976225 .362803-2.316360$

H -3.561050 -1.209804 1.464298

H $-4.121076 \quad 0.2982750 .710510$

H -1.451514 1.7821124 .096067

H $-2.955646 \quad 1.3726864 .951970$

H -4.1602673 .2898873 .777282$

H -1.228608 4.1783643 .788367

H $-2.5587386 .269399 \quad 4.374134$

H -3.953206 5.6622453 .514820

H -1.0519447 .7109431 .342939$

H -0.9104267 .5168873 .179230$

H -2.908571 6.7078390 .180050

H -4.1400855 .9094941 .097987$

H -3.1611193 .7487610 .860766$

H -2.3415571 .0160152 .070079$

C $2.156716-3.3328742 .130002$

O $3.383668-2.9932001 .451970$

C $4.489873-3.2041832 .343622$

C $3.962619-4.1296243 .431822$

C $2.540873-3.6255783 .582921$

H $4.727136-2.2291082 .786976$

C $5.706974-3.7278611 .576358$

H $3.956657-5.1773493 .110181$

H $4.544738-4.0604314 .355621$

C $1.614341-4.6097074 .276022$

H $2.562772-2.6923804 .161838$

C $6.084944-2.897806 \quad 0.332515$

O $5.426290-5.060778 \quad 1.128131$

H $6.559953-3.8083292 .260495$

H $5.325248-3.038346-0.445856$

O $7.291354-3.456473-0.206238$

C $6.282128-1.3879580 .575909$

C $6.804388-0.666593-0.681324$

H $5.327937-0.9451910 .875336$

O $7.192141-1.1864591 .655541$

H $7.789297-1.073572-0.939887$

H $6.131696-0.893239-1.515338$

H $0.599458-4.2056604 .346086$ 
H $1.562072-5.5607623 .735444$ H $1.966053-4.8194365 .291570$ C $1.170365-2.1692292 .009099$ H $6.211212-5.3767970 .648366$ H $7.982139-3.3875330 .474090$ H $8.069586-1.4919711 .369994$ H $1.631668-1.2627462 .419534$ H $0.271472-2.3796742 .599979$ H $1.757639-4.2288121 .637966$ C $4.7295491 .531618-1.195171$ O $5.7193211 .504218-0.159823$ C $6.9507480 .862205-0.505030$ C $7.5773411 .565753-1.716013$ C $6.6165521 .601996-2.900507$ C $5.2737902 .191218-2.472647$ H $4.3928380 .509351-1.406795$ C $3.5292442 .338169-0.669197$ H 7.6276591 .0216830 .343491 O $8.789796 \quad 0.918540-2.087863$ H $7.8361612 .595342-1.436115$ H $6.498306 \quad 0.607215-3.345325$

O $7.1945172 .430304-3.912390$ H $5.3887643 .271572-2.315231$

O $4.3228922 .011779-3.521148$ C $2.796076 \quad 1.650890 \quad 0.460920$ H $3.8763613 .307063-0.290089$ H $2.8026832 .502274-1.472638$ H $9.1369791 .379591-2.870708$ H $6.6286002 .369633-4.700324$ H $4.6221182 .534567-4.283871$ $\begin{array}{lllll} & \text { O } & 1.771329 & 2.457072 & 0.869259\end{array}$ $\begin{array}{lllll}\text { O } & 3.079545 & 0.557359 & 0.927144\end{array}$ C 0.9751901 .9596401 .948768 H $\quad 0.3481841 .1361391 .598164$ H 1.6049101 .6393942 .784333 H 0.3281342 .7707602 .291081 SCF Energy (B3LYP/6-31G*//MMFF) $=-3245.91072976$

0400090

MM̄FF Geometry

C $-0.902580 \quad 0.7907412 .648924$

C $-1.8993490 .120106 \quad 3.248544$

C $-2.496940-1.2104142 .856616$

O $-1.950238-1.7006971 .615026$

C $-4.010757-1.0520852 .624311$

C $-4.790779-2.3332792 .837937$

C $-5.217870-3.1548961 .859563$

C $-5.140979-2.6291054 .274569$

C $-4.944311-3.073291 \quad 0.383922$

C $-6.149831-2.573192-0.426913$

C $-6.227815-1.040528-0.486419$

C $-7.478238-0.575504-1.232414$

C $-7.5098990 .954704-1.386781$

O $-5.059609-0.585459-1.176945$

O $-8.5275291 .277804-2.346760$

C $-6.162277 \quad 1.425803-1.976240$

C $-7.9100801 .650400-0.080309$

O $-6.0893142 .849508-2.014960$

C $-4.925860 \quad 0.836025-1.258667$

C -3.644267 $1.150854-2.066849$

$\begin{array}{llll}\text { O } & -4.811929 & 1.432226 & 0.035123\end{array}$

C $-0.928956-2.5924181 .655488$

O $-0.321815-2.9855062 .636475$

C $-0.684718-3.013275 \quad 0.259354$

C $0.479021-3.583964-0.080289$

C $0.852945-4.022233-1.426714$

C $-0.219052-4.010518-2.486687$

C $-2.4170790 .328450-1.644000$

C $-1.1661790 .748055-2.398303$

C $1.2675630 .224397-2.564750$

O -1.164265 1.625507 -3.257693

C $1.613649-0.931457-3.449701$

C $2.788313-1.577490-3.388325$

C $3.169943-2.721780-4.283122$

C $3.724089-3.903968-3.516802$

C $5.049321-4.105617-3.422477$

C $2.741866-4.894666-2.933273$

C $2.127191-4.406201-1.648542$
N $-0.0530880 .022110-1.998278$ H -0.4050420 .3772061 .776911$ H $-2.344496 \quad 0.566373 \quad 4.137317$ H -2.306325 -1.924498 3.667134 H -4.181642 -0.6740641 .611911$ H $-4.434317-0.2940013 .296909$ H $-5.810124-4.0233482 .147121$ H -5.719827 -3.5527974 .381385$ H $-4.233235-2.7394894 .876022$ H $-5.739738-1.8143744 .694958$ H $-4.051584-2.4797680 .168261$ H $-4.703485-4.0913530 .050521$ H $-6.039375-2.956287-1.449968$ H $-7.081224-2.988332-0.023281$ H $-6.232491-0.6443420 .534170$ H $-8.386651-0.930358-0.729927$ H $-7.495083-1.025243-2.235146$ H $-9.3721650 .924086-2.019004$ H $-6.1441001 .095994-3.024008$ H -8.8909851 .2948930 .257612$ H -8.019259 2.730801 -0.225756 H -7.1997721 .4800300 .731418$ H $-5.9021743 .160751-1.112924$ H $-3.8232730 .940637-3.129567$ H -3.410816 $2.220036-1.982737$ H -3.8835081 .4041840 .315366$ H $-1.470435-2.817067-0.461808$ H $1.235258-3.7233380 .690675$ H $-1.044921-4.671767-2.201262$ H $\quad 0.128778-4.343750-3.466147$ H $-0.615680-2.999673-2.629318$ H $-2.2247850 .438388-0.572761$ H -2.589686 $-0.734269-1.850661$ H $1.9609560 .316511-1.725277$ H $1.2872461 .153015-3.142968$ H $0.875279-1.237670-4.186915$ H $3.537072-1.245936-2.674090$ H $3.924445-2.352510-4.990207$ H $2.322543-3.050312-4.897443$

H $5.454659-4.964820-2.898362$

H $5.758678-3.412191-3.862019$

H $3.250772-5.841623-2.707467$

H $1.999983-5.157285-3.692328$

H $2.830642-4.373528-0.815253$

H $-0.155786-0.688455-1.280374$

C $-0.291760 \quad 3.2193402 .081570$

$\begin{array}{lllll}\text { O } & 0.918912 & 3.036980 & 1.328677\end{array}$

C $0.727916 \quad 3.598238 \quad 0.013642$

C $-0.6993554 .149710-0.028996$

C $-1.398040 \quad 3.327174 \quad 1.038239$

H $0.8032402 .762322-0.690567$

C $1.8256984 .630639-0.291706$

H $-0.738255 \quad 5.2147420 .225036$

H $-1.1518864 .034886-1.019258$

C $-2.6819703 .950358 \quad 1.553668$

H -1.6220032 .3459300 .607441$

C $3.2608774 .139450-0.009686$

O $1.604285 \quad 5.783886 \quad 0.531134$

H $1.7281014 .966557-1.331016$

H 3.4231034 .0635041 .072896

O $4.1769395 .131192-0.488576$

C $3.6186822 .798842-0.670601$

C $5.0725192 .375156-0.390513$

H $2.9435032 .027624-0.290259$

O $3.415362 \quad 2.883758-2.079374$

H $5.7619123 .076452-0.876199$

H 5.2544782 .4390720 .687877

H -3.141472 3.3175402 .319532

H -2.5009794 .9361931 .994572$

H -3.4036124 .0734370 .739353$

C $-0.421812 \quad 2.128005 \quad 3.148751$

H 2.1549926 .5041540 .181097

H 4.0832695 .9154420 .077786

H $3.9852723 .596303-2.416173$

H -1.0995232 .4947123 .929969$

H 0.5571201 .9724053 .618739

H -0.1743394 .1794992 .602998$

C $4.428560-0.2827980 .936925$ 
O $4.420293-0.009632-0.467940$

C $5.3879250 .950634-0.901422$

C $6.7976250 .452443-0.557445$

C 6.9312760 .0910050 .921370

C $5.795669-0.837553 \quad 1.359357$

H $4.2127260 .640048 \quad 1.487966$

C $3.285642-1.2703131 .199506$

H $5.3129610 .986379-1.995675$

O $7.7638651 .436442-0.910261$

H $7.013352-0.440338-1.158933$

H 6.9723280 .9934021 .542553

$\begin{array}{llll}\text { O } & 8.180567 & -0.578624 & 1.107448\end{array}$

H $5.956033-1.8266820 .912664$

O $5.853361-1.0061152 .773176$

C $2.872220-1.3022002 .650522$

H $2.396272-0.9945170 .619346$

H $3.565054-2.2733290 .855384$

H $8.637153 \quad 1.088824-0.660298$

H $8.232690-0.8388422 .043140$

H $5.698194-0.1385903 .184369$

O $2.916397-2.5814423 .119169$

O $2.549537-0.3106093 .292030$

C $2.569903-2.7434854 .498098$

H $1.574033-2.3354114 .696114$

H $2.560593-3.8133264 .722683$

H $3.318474-2.2571615 .130528$

SCF Energy (B3LYP/6-31G*//MMFF) $=-3245.92245210$

0400091

MM̄FF Geometry

C $0.181029-3.250276 \quad 0.836217$

C $-0.495718-3.442628-0.306812$

C $-0.143682-2.909101-1.676171$

O $1.045682-2.091060-1.643446$

C $-1.307182-2.034109-2.185733$

C $-1.102342-1.473979-3.581119$

C $-1.039904-0.158366-3.869990$

C $-0.992026-2.491599-4.686070$

C $-1.181466 \quad 0.990899-2.910842$

C $0.158607 \quad 1.357468-2.278358$

C $0.0421852 .408401-1.168507$

C $-0.2764143 .802939-1.709638$

C $-0.2422514 .870460-0.603682$

O $1.3096942 .423310-0.502953$

O $-0.2264926 .149214-1.255976$

C $1.0718954 .728600 \quad 0.198006$

C $-1.522152 \quad 4.8353850 .239355$

O 1.0824915 .6135841 .315732

C $1.369787 \quad 3.2794810 .643213$

C $2.794794 \quad 3.172787 \quad 1.235036$

O 0.4235902 .9031551 .645062

C $2.241505-2.701090-1.863189$

O $2.438579-3.894131-2.028830$

C $3.283544-1.647422-1.879384$

C $4.567885-1.990374-2.059590$

C $5.709822-1.075980-2.156087$

C $5.435913 \quad 0.399917-2.041053$

C $3.256928 \quad 1.724921 \quad 1.462791$

C $4.6791861 .650656 \quad 1.988715$

C $6.531736 \quad 0.0168412 .336189$

O 5.2712112 .6167912 .462080

C $7.509034 \quad 0.288473 \quad 1.235908$

C $8.329303-0.6400150 .720906$

C $9.370875-0.340103-0.318027$

C $9.273161-1.233020-1.532798$

C $10.137834-2.244966-1.718917$

C $8.241292-0.888180-2.582499$

C $6.935338-1.601011-2.366833$

N $5.194501 \quad 0.364049 \quad 1.895485$

H $1.101855-2.6747460 .840964$

H $-1.409022-4.034865-0.268243$

H $0.000158-3.760611-2.352183$

H $-1.469828-1.223935-1.466204$

H -2.232854 -2.624059-2.191532

H $-0.8787300 .137098-4.905676$

H - $0.988396-2.027664-5.678366$

H - $0.067312-3.068451-4.590151$

H $-1.841456-3.181620-4.652215$
H -1.587767 $1.847013-3.461880$

H $-1.9198860 .759115-2.137070$

H $0.6181550 .461346-1.845675$

H $0.8643901 .699575-3.046900$

H $-0.7233452 .079579-0.457044$

H $-1.246393 \quad 3.818315-2.220518$

H $0.4672434 .073468-2.472402$

H $-0.1361846 .830096-0.567076$

H $1.8848315 .064373-0.460091$

H -2.400184 $5.016202-0.392575$

H -1.5269235 .6324090 .990775$

H -1.6710113 .8807570 .746332$

H 0.5156655 .2266992 .004778

H $3.5092183 .643705 \quad 0.547127$

H $2.846223 \quad 3.7196042 .184874$

H 0.8000052 .1968902 .192442

H $2.963031-0.618166-1.766499$

H $4.810394-3.047002-2.167925$

H $4.801420 \quad 0.738292-2.867516$

H $6.3378681 .014985-2.055102$

H $4.9295170 .625080-1.097697$

H $2.614676 \quad 1.220828 \quad 2.192618$

H 3.2064741 .1644640 .522981

H $6.516874-1.0381592 .627573$

H 6.7891360 .6184123 .214063

H $7.574771 \quad 1.3125300 .875546$

H $8.286867-1.6582561 .100719$

H $10.353470-0.461566 \quad 0.156923$

H $9.3277530 .708905-0.636731$

H $10.094488-2.867437-2.606663$

H $10.903734-2.476638-0.986296$

H $8.614920-1.186848-3.571721$

H $8.1335890 .197658-2.647499$

H $7.023514-2.686067-2.443078$

H $4.653819-0.354006 \quad 1.421864$

C $-0.382985-2.679823 \quad 3.218265$

O $-1.227638-1.6089242 .755446$

C $-2.081949-1.1859713 .831529$

C $-1.556787-1.8701545 .089680$

C $-1.006273-3.164546 \quad 4.526912$

H $-3.080695-1.576323 \quad 3.605413$

C $-2.136993 \quad 0.343268 \quad 3.913078$

H $-0.753859-1.2955045 .564930$

H -2.343068 -2.0247295 .834906$

C $-0.030627-3.8673995 .454577$

H $-1.849095-3.8369264 .317058$

C -2.450242 1.0659012 .589255

$\begin{array}{llll}\text { O } & -0.870095 & 0.842623 & 4.365988\end{array}$

H $-2.8626030 .637883 \quad 4.680258$

H $-1.573641 \quad 1.0431701 .930950$

O -2.6806422 .4459952 .904192$

C $-3.663790 \quad 0.5341801 .809204$

C -4.0312031 .4440710 .620499$

H -3.440840 -0.4744531 .446219$

$\begin{array}{lllll}\text { O } & -4.792544 & 0.430104 & 2.676044\end{array}$

H -4.4640192 .3792240 .996465$

H $-3.115217 \quad 1.6846560 .068068$

H $0.342851-4.7906365 .000620$

H $0.831295-3.232748 \quad 5.685991$

H $-0.518704-4.1290416 .399219$

C $-0.290396-3.7823632 .160926$

H $-0.1913150 .525750 \quad 3.745509$

H -1.8826602 .7779493 .350702$

H $-4.965131 \quad 1.3145713 .042210$

H $-1.271490-4.2580262 .040978$

H $0.406713-4.5586752 .496152$

H $0.608975-2.2440113 .396277$

C $-5.135068-1.033572-1.941327$

O $-4.351103-0.269144-1.017224$

C $-5.0409330 .784732-0.336603$

C $-5.6087521 .792935-1.347970$

C $-6.4822101 .100182-2.398045$

C $-5.788960-0.127470-2.997460$

H $-4.410632-1.674737-2.457690$

C $-6.125635-1.949916-1.216623$

H -5.8606350 .3611580 .253243$

O $-4.5516142 .499065-1.994852$

H -6.204391 2.537321 -0.806696 
H $-6.7146451 .816226-3.195820$

O $\quad-7.7087840 .714169-1.785094$

H $-6.498476-0.707859-3.598617$

O $\quad-4.7700990 .339492-3.887679$

C - $-5.379152-2.980375-0.401737$

H $-6.809842-1.406662-0.558827$

H $-6.751720-2.495160-1.932584$

H -4.004006 $1.847714-2.464795$

H $-8.2644440 .307189-2.471513$

H $-4.367223-0.438637-4.309305$

O $-5.421660-2.651495 \quad 0.920513$

O $-4.802053-3.944567-0.887993$

C $-4.714868-3.5339101 .796938$

H -3.641693 -3.479642 1.593683

H -4.895419 -3.2089692 .824888$

H -5.078966 -4.559854 1.685747

SCF Energy $\left(B 3 L Y P / 6-31 G^{* *} / / M M F F\right)=-3245.93232349$

04_00092

MM̄FF Geometry

C $-0.784129-4.3745150 .870128$

C $-1.963473-3.9815500 .367929$

C $-2.185112-3.692263-1.095063$

O $-2.378480-2.265893-1.242027$

C $-3.448593-4.419607-1.587811$

C $-3.745750-4.194324-3.058677$

C $-4.746711-3.426417-3.535244$

C $-2.842989-4.929917-4.015851$

C $-5.744849-2.623589-2.748416$

C $-5.531503-1.119103-2.957832$

C $-6.270331-0.283765-1.901738$

C $-6.1226491 .209296-2.198938$

C $-6.7205932 .085637-1.089147$

O $-5.687759-0.592749-0.630950$

O $-6.2477623 .422869-1.312866$

C -6.1725661 .6103490 .274735$

C $-8.2488532 .148782-1.188700$

O

C -6.2793640 .0856240 .483828$

C $-5.552580-0.404518 \quad 1.760792$

O $-7.661043-0.2664150 .625058$

C -1.252302 -1.504425 -1.345705

O $-0.096088-1.897770-1.340986$

C -1.664910 -0.088159-1.456490

C $-0.7319840 .875784-1.437669$

C $-0.9726592 .316438-1.545574$

C -2.401740 2.781014-1.639009

C $-4.038523-0.1679061 .789369$

C -3.6176101 .1136032 .488541$

C -1.602023 2.5230122 .903281

$\begin{array}{lllll}\text { O } & -4.382495 & 1.794842 & 3.165561\end{array}$

C -1.585591 3.6644791 .936121

C -0.4696234 .3287911 .598482$

C -0.4460385 .5138230 .675742$

C $0.5484865 .361603-0.453597$

C $1.7680495 .921265-0.381172$

C $0.0941924 .645573-1.704575$

C $0.0883263 .150505-1.553476$

N -2.268141 1.3840062 .299621

H $0.061470-4.5089750 .199813$

H $-2.802116-3.822647 \quad 1.041787$

H -1.325014 -4.014257 -1.695579

H -4.300812 -4.120554-0.969748

H -3.340158 -5.500188 -1.421296

H -4.867155 -3.351730 -4.615387

H $-3.147783-4.798538-5.059653$

H -1.812978 -4.571347 -3.927656

H $-2.858351-6.003863-3.803227$

H $-6.753388-2.896032-3.082475$

H -5.710072 -2.866645 -1.684891

H $-4.458725-0.896629-2.902493$

H -5.870264 -0.840610 -3.963471

H -7.327295 -0.574761 -1.897195

H $-6.5690361 .458995-3.169627$

H $-5.0564541 .453254-2.293161$

H $-6.5575813 .972337-0.572301$

H -5.116632 1.9019890 .301676

H $-8.5497782 .565793-2.157357$
H $-8.6694502 .820904-0.432744$

H -8.725596 $1.171691-1.086357$

H -7.686162 1.9255841 .471514

H $-6.043905-0.0010412 .654412$

H -5.698711-1.493001 1.817269

H $-7.716201-1.1245391 .077371$

H $-2.7231290 .123645-1.548233$

H $\quad 0.3136530 .585840-1.340877$

H -2.845781 $2.456420-2.585841$

H -2.514350 $3.865701-1.582124$

H $-2.9944372 .374900-0.812688$

H -3.574584 -0.986295 2.353840

H -3.617762 -0.190344 0.780636

H -0.5930542 .2013253 .179344$

H -2.1350252 .8181513 .812769$

H -2.5397163 .9795521 .520150$

H 0.4778154 .0314812 .042148

H $-0.1911806 .396531 \quad 1.277156$

H -1.440747 5.7185090 .260669

H $2.4763125 .842889-1.199236$

H 2.0883976 .4635850 .502404

H $\quad 0.7705434 .879988-2.537823$

H $-0.8740005 .045705-2.018707$

H $1.0875632 .720472-1.473226$

H $-1.7222010 .787768 \quad 1.684394$

C $0.232832-3.5027423 .028229$

O $1.451175-3.1965992 .318588$

C $1.565870-1.7658072 .212462$

C $0.623739-1.1994823 .265005$

C $-0.526544-2.1818953 .176411$

H $1.196586-1.4896431 .216316$

C $3.019104-1.3023922 .355220$

H $1.074030-1.2256404 .264547$

H $0.329642-0.1689933 .048432$

C -1.460972 -2.136645 4.372377

H $-1.100388-1.9562302 .269597$

C $3.922348-1.7870061 .202300$

O $3.537963-1.7320233 .611165$

H $3.024407-0.2067442 .364494$

H $3.435170-1.5438620 .249617$

O $4.086857-3.2026451 .248071$

C $5.312522-1.1219191 .210434$

C $6.151157-1.524600-0.016313$

H $5.172332-0.0364871 .221190$

O $6.030024-1.4618882 .393870$

H $6.400897-2.5910260 .045125$

H $5.544279-1.388614-0.917916$

H -2.269300 -2.866216 4.261643

H $-0.931283-2.3575265 .305053$

H -1.912447 -1.144019 4.470711

C $-0.530086-4.6317222 .328178$

H $3.545041-2.7046783 .605042$

H $3.204215-3.6048271 .180535$

H $6.083344-2.4319952 .436375$

H -1.468923 -4.830779 2.858323

H $0.064125-5.5522922 .389418$

H $0.541885-3.8637024 .018077$

C $6.5499411 .257081-1.164471$

O $7.2515420 .692743-0.050576$

C $7.466094-0.720356-0.133536$

C $8.292540-1.043429-1.386680$

C $7.665935-0.457731-2.651964$

C $7.3384351 .024251-2.458642$

H $5.5564230 .800626-1.244743$

C $6.3624072 .752951-0.887557$

H $8.077361-0.987556 \quad 0.737683$

O $8.444505-2.449514-1.549366$

H $9.299742-0.621378-1.274505$

H $6.774593-1.020665-2.953140$

O $8.595172-0.574283-3.732098$

H $8.274570 \quad 1.596357-2.438292$

O $6.5892591 .492736-3.577898$

C $5.4917322 .987330 \quad 0.325175$

H $7.3311883 .227859-0.689228$

H $5.9090873 .275897-1.736891$

H $8.860384-2.793787-0.740371$

H $8.821839-1.516328-3.817644$

H $5.7550450 .994070-3.609230$ 
O 4.2117002 .5898990 .073146

O 5.9033413 .4701391 .372375

C 3.3014722 .7251631 .168894

H 2.3432262 .2907720 .872351

H 3.6735022 .1847582 .044583

H 3.1545003 .7822581 .406535

SCF Energy (B3LYP/6-31G**//MMFF) $=-3245.92015571$

04 00093

MM̄FF Geometry

C $0.773965-2.2172630 .240434$

C $-0.052938-1.971835-0.788304$

C $0.304278-1.266508-2.076654$

O $1.735574-1.088769-2.150906$

C $-0.3513620 .125619-2.094849$

C $-0.4258240 .744736-3.474577$

C $0.394637 \quad 1.709121-3.934450$

C $-1.5604580 .250470-4.334259$

C $1.5894742 .319897-3.256344$

C $1.3332303 .762163-2.793947$

C $0.5754843 .835100-1.459512$

C $0.2361215 .282036-1.098581$

C $-0.420975 \quad 5.3862710 .288071$

O $1.4296293 .275831-0.456379$

O $-0.415158 \quad 6.769403 \quad 0.673715$

C 0.4507294 .6258151 .311154

C -1.8915074 .9518330 .255891$

O -0.1824994 .5810892 .588176$

C 0.8505813 .2051760 .850653

C 1.9117712 .6130561 .808790

O -0.3087502 .3728020 .867677$

C $2.384246-1.389728-3.301968$

O $1.887637-1.811096-4.336656$

C $3.843572-1.171226-3.159033$

C $4.388380-0.522715-2.118210$

C $5.814362-0.281653-1.929294$

C $6.1303861 .126481-1.504435$

C $2.562447 \quad 1.327271 \quad 1.282051$

C 3.5828330 .7512322 .248041

C $5.143003-1.1878602 .468933$

O $3.903994 \quad 1.3020373 .297074$

C $6.455180-0.8849431 .815144$

C $7.244907-1.8195041 .264124$

C $8.580626-1.5248100 .642903$

C $8.707007-2.063514-0.765071$

C $9.321303-3.234976-1.003088$

C $8.205804-1.201844-1.902735$

C $6.714670-1.274899-2.068840$

N $4.099055-0.4509401 .783633$

H $1.814449-1.9115200 .188872$

H -1.094476 -2.273989-0.698412

H - $0.052964-1.891835-2.903091$

H $\quad 0.1924330 .788695-1.413133$

H -1.375052 $0.072901-1.698792$

H $0.2119442 .098409-4.935712$

H - $-1.5785930 .730753-5.318253$

H -1.478555 -0.828271 -4.498223

H $-2.5190410 .459081-3.848718$

H $1.9534741 .704241-2.428907$

H $2.4031532 .332214-3.992890$

H $2.3105944 .246868-2.669749$

H $\quad 0.7917204 .316829-3.570029$

H $-0.3412083 .240344-1.534504$

H - $0.4080315 .733084-1.863742$

H $1.1570935 .882030-1.090843$

H $-0.9014097 .271388-0.002689$

H 1.3666555 .2163221 .450082

H -2.455668 $5.564143-0.457936$

H -2.3716145 .1106721 .227799$

H -2.025052 $3.906344-0.029737$

H -0.8748103 .8995942 .550201$

H 2.7117833 .3487591 .964053

H 1.4592032 .4108912 .787758

H -0.0461051 .4550681 .039398$

H $4.457410-1.558787-3.966743$

H $3.755941-0.100191-1.342898$

H $5.8624761 .830811-2.299861$

H $7.1846671 .285357-1.269323$
H $5.5651851 .395173-0.604796$

H $1.804126 \quad 0.5629901 .091403$

H $3.082817 \quad 1.5333870 .340090$

H $4.884913-2.2498032 .407153$

H $5.175715-0.8964743 .523322$

H 6.7790580 .1532091 .814545

H $6.930525-2.8606161 .287741$

H $9.350395-1.9757591 .282986$

H $8.793482-0.4485580 .641341$

H $9.443834-3.614441-2.012156$

H $9.710410-3.843283-0.193284$

H $8.653284-1.533637-2.849507$

H $8.570380-0.179843-1.766502$

H $6.353561-2.265200-2.349075$

H $3.800398-0.7985250 .877640$

C $0.105468-1.8171622 .612046$

O $-1.053774-1.0308992 .267018$

C $-1.896374-0.9029763 .426617$

C -1.030304 -1.311882 4.610526

C $-0.189364-2.4119143 .988617$

H $-2.700861-1.6398413 .316426$

C -2.4946290 .5069723 .496186$

H $-0.388589-0.4903014 .949113$

H -1.624485 -1.653840 5.463080

C $1.055791-2.752748 \quad 4.788726$

H $-0.808211-3.313413 \quad 3.884649$

C -3.209361 0.9402242 .198556

O -1.4298451 .4334963 .741401$

H -3.1742190 .5764374 .353756$

H -2.4822091 .0400201 .385554$

O -3.7718512 .2408052 .405240$

C $-4.336713-0.0044321 .745880$

$\begin{array}{llll}C & -4.995806 & 0.464323 & 0.437122\end{array}$

H -3.927718 -1.002685 1.587868

O $-5.320176-0.1098172 .772123$

H $-5.442047 \quad 1.4544380 .589735$

H $-4.2134570 .576013-0.321979$

H $1.636775-3.5356234 .291239$

H $1.705172-1.8793314 .912616$

H $\quad 0.784978-3.1144345 .785937$

C $0.340977-2.867237 \quad 1.525178$

H -1.832499 2.2791464 .001265

H -3.0345792 .8702762 .475807$

H $-5.670996 \quad 0.782727 \quad 2.934717$

H $-0.573804-3.4472851 .358693$

H $1.119940-3.5736831 .832926$

H $0.954168-1.1243692 .670135$

C $-4.711939-2.111549-1.213775$

O $-5.663579-1.848442-0.174506$

C $-6.101429-0.489838-0.069146$

C $-6.745317-0.047368-1.389060$

C $-5.804035-0.265313-2.571264$

C $-5.276131-1.699913-2.584008$

H -3.781341 -1.568799-1.007378

C $-4.412544-3.621195-1.185918$

H $-6.892001-0.4842010 .691940$

O $\quad-7.1297901 .321134-1.315243$

H -7.661300 -0.630418 -1.550223

H $-4.9788970 .455731-2.555805$

O $-6.512595-0.012404-3.787709$

H $-6.090249-2.373843-2.880988$

O $-4.261805-1.784040-3.582635$

C $-3.689128-4.0681610 .066593$

H $-5.355221-4.179620-1.235853$

H $-3.778230-3.903197-2.032375$

H $-7.5004811 .566669-2.180258$

H -7.255700 -0.637492 -3.838897

H $-4.098674-2.723896-3.766148$

O $-3.573470-5.429806 \quad 0.046238$

O $-3.267229-3.3303530 .944477$

C $-2.900797-6.0067721 .170051$

H -3.406617 -5.7323842 .100830$

H $-2.931339-7.0942451 .062551$

H $-1.855476-5.6849111 .188129$

SCF Energy $\left(B 3 L Y P / 6-31 G^{* *} / / M M F F\right)=-3245.90952283$

0400094

MM̄FF Geometry 
C $0.103406-1.8289910 .884956$

C $0.581537-0.921796 \quad 0.019770$

C $0.113905-0.636359-1.385510$

O $-0.3461730 .737249-1.347823$

C $-1.036714-1.518154-1.904048$

C $-1.257005-1.401293-3.399195$

C $-2.238249-0.684817-3.981236$

C $-0.305697-2.204070-4.249736$

C $-3.2613450 .197349-3.321488$

C $-4.683155-0.370511-3.429200$

C $-4.936861-1.551506-2.479450$

C $-6.333038-2.135441-2.705094$

C $-6.659359-3.244598-1.694475$

O $-4.826528-1.058413-1.140627$

O $-8.067730-3.502843-1.796753$

C $-6.372560-2.720262-0.271376$

C $-5.954634-4.557773-2.053675$

O $-6.542499-3.7559860 .693959$

C -4.985355 -2.052293 -0.121860

C $-4.890260-1.3435731 .250408$

O $-3.976394-3.060605-0.202405$

C $0.0330651 .591717-2.328407$

O $0.7092881 .325471-3.311931$

C $-0.4487352 .966989-2.054198$

C $-1.3015253 .268309-1.061963$

C $-1.7921124 .603293-0.710726$

C -1.237091 $5.776409-1.474587$

C -3.648288 -0.4562681 .415760$

C $-3.633607 \quad 0.2626802 .753766$

C -2.3453031 .9907584 .007782$

$\begin{array}{llll}\text { O } & -4.478717 & 0.080283 & 3.625835\end{array}$

C -3.041748 3.2933183 .767961

C -2.4089824 .4711553 .657518$

C $-3.1139675 .774725 \quad 3.414405$

C $-2.689165 \quad 6.438575 \quad 2.122537$

C -1.8340847 .4750722 .128719$

C -3.3258355 .9648840 .833993$

C -2.687797 $4.715790 \quad 0.292929$

$\mathrm{N}-2.554113 \quad 1.1300722 .857750$

H $-0.714133-2.4852940 .604680$

H $1.404649-0.2907450 .352035$

H $0.992444-0.728669-2.035938$

H - $1.958062-1.272643-1.365249$

H $-0.834408-2.575309-1.689055$

H -2.307252 -0.703243 -5.068593

H $-0.527163-2.116371-5.318719$

H $0.724261-1.866136-4.099801$

H -0.365634 -3.265453 -3.987669

H $-3.0122050 .416206-2.279169$

H -3.235435 $1.164735-3.839386$

H $-5.3807230 .440096-3.180804$

H $-4.888274-0.668817-4.464848$

H -4.173994 -2.319404-2.648231

H $-6.439268-2.507464-3.731711$

H -7.082367-1.338295 -2.597743

H -8.299328 $-4.149980-1.108479$

H -7.144720 -1.971508 -0.047640

H $-6.258593-4.891693-3.053103$

H -6.244209 $-5.363824-1.370471$

H -4.865737 -4.475706 -2.048070

H $-5.758428-4.3297020 .653402$

H $-5.772953-0.704347 \quad 1.385634$

H $-4.901733-2.0895182 .055219$

H $-3.184569-2.757840 \quad 0.267995$

H $-0.0560043 .726544-2.722403$

H $-1.6812242 .465276-0.432145$

H $-0.1433935 .796904-1.408229$

H $-1.5842106 .742336-1.101524$

H -1.524666 $5.717728-2.529935$

H -2.736997 -1.056971 1.354178

H -3.615932 $0.302144 \quad 0.625202$

H -1.2655172 .1135914 .134393$

H -2.749387 1.5124364 .905598

H $-4.125743 \quad 3.2629583 .683561$

H -1.326338 4.5064643 .753914

H -2.898843 6.4328464 .266452

H $-4.203605 \quad 5.6461363 .412700$

H -1.5487997 .9781101 .210919$
H -1.396522 7.8410793 .051769

H -3.3228016 .7664420 .087256$

H -4.390909 5.7641361 .007956

H $-3.026736 \quad 3.8045090 .785885$

H -1.951914 1.2670212 .050867

C $1.397856-3.2794572 .479402$

O $2.582678-3.243441 \quad 1.657038$

C $3.734719-3.3349302 .508394$

C $3.246141-4.0872693 .735579$

C $1.877273-3.4609013 .925482$

H $4.000319-2.3099242 .797055$

C $4.909800-3.9722781 .762526$

H $3.143910-5.1609673 .540806$

H $3.903432-3.9537124 .599598$

C $0.936037-4.2948164 .777728$

H $2.006739-2.4813744 .405554$

C $5.209948-3.3488920 .383676$

O $4.644755-5.364471 \quad 1.548290$

H $5.804497-3.9345762 .394785$

H $4.453048-3.665425-0.344855$

O $6.455794-3.888912-0.075229$

C $5.306306-1.8129770 .369443$

C $5.747239-1.266809-1.001279$

H $4.331689-1.3881400 .630216$

O $6.233956-1.3774821 .363063$

H $6.797923-1.526873-1.179100$

H $5.136202-1.741845-1.778558$

H $-0.040647-3.8082964 .865794$

H $0.780114-5.2897954 .347162$

H $1.342710-4.4248745 .785948$

C $0.620584-1.9754012 .289416$

H $3.813369-5.4314821 .047644$

H $6.369007-4.857733-0.074714$

H $7.092561-1.7886971 .163937$

H $1.264933-1.1218212 .537168$

H $-0.234096-1.9419122 .975501$

H $\quad 0.803374-4.1386502 .145712$

C $3.8424631 .945363-1.163317$

$\begin{array}{lllll}\text { O } & 4.193101 & 0.556457 & -1.099588\end{array}$

C $5.595617 \quad 0.263947-1.095421$

C $6.2647370 .823562-2.360309$

C $5.9804482 .318620-2.525626$

C $4.4879252 .630454-2.378845$

H $2.7576051 .947957-1.321272$

C 4.1238022 .6613010 .161563

H $6.0576090 .716552-0.211952$

$\begin{array}{llll}\text { O } & 5.812775 & 0.120778 & -3.516232\end{array}$

H $7.3466190 .660506-2.289342$

H $6.3300662 .640297-3.514191$

O $6.7238193 .034938-1.544051$

H $4.3219153 .712197-2.317963$

O $3.8002562 .168875-3.545201$

C 3.1457012 .2088181 .219667

H 5.1297042 .4795650 .549710

H 4.0255843 .7481520 .052670

H $4.843153 \quad 0.190517-3.546577$

H $6.553307 \quad 3.982477-1.680637$

H $4.1735982 .637982-4.310783$

$\begin{array}{lllll}\text { O } & 2.002588 & 2.948748 & 1.138630\end{array}$

$\begin{array}{lllll}\text { O } & 3.363277 & 1.295674 & 2.005319\end{array}$

C 0.9856532 .6213082 .089170

H $\quad 0.6595321 .5861591 .958027$

H 1.3513392 .7848463 .107190

H $\quad 0.1329203 .2812901 .912977$

SCF Energy (B3LYP/6-31G**//MMFF)= -3245.92291514

0400095

MM̄FF Geometry

C $1.022018 \quad 0.7302752 .925911$

C $2.027769-0.077788 \quad 3.293497$

C $2.191878-1.5466542 .980901$

O $3.439334-1.6498592 .249776$

C $1.083222-2.1872992 .123320$

C $1.123143-3.703273 \quad 2.123204$

C $1.607479-4.464781 \quad 1.122968$

C $0.514869-4.3573493 .338092$

C $2.270756-4.018166-0.149788$

C $1.410264-4.298107-1.390455$ 
C $0.246384-3.308591-1.552172$

C $-0.628762-3.688035-2.748434$

C $-1.731655-2.648366-3.002947$

O $0.813902-2.012196-1.761009$

O $-2.287465-2.903619-4.301963$

C -1.094143 -1.243774 -3.052692

C $-2.886272-2.783770-2.003794$

O $-2.093836-0.230526-3.135446$

C $-0.142768-0.952037-1.869437$

C $0.6388260 .357408-2.131944$

O $-0.910884-0.812334-0.674110$

C $4.350324-2.5776352 .634215$

O $4.239966-3.3746453 .555792$

C $5.572111-2.5170541 .796557$

C $5.693236-1.7441170 .705471$

C $6.880710-1.625024-0.145239$

C $8.108071-2.4033050 .250743$

C $1.7806140 .610525-1.138031$

C $2.5549041 .876340-1.461505$

C $4.5970253 .123111-0.753460$

O $2.2357142 .653669-2.356424$

C $5.6705862 .706176-1.708420$

C $6.9522992 .518892-1.359480$

C $8.0169942 .076634-2.322203$

C $8.6576600 .764162-1.924392$

C $9.8923720 .734470-1.395116$

C $7.909036-0.517553-2.223169$

C $6.826139-0.808767-1.218881$

N $3.6482302 .033640-0.619685$

H $\quad 0.200614 \quad 0.3428492 .332077$

H 2.8310890 .3498543 .892140

H $2.268402-2.0618273 .946152$

H $1.147294-1.8060101 .098744$

H $0.092565-1.8941372 .493673$

H $1.551157-5.5473091 .233364$

H $0.531004-5.4506073 .274926$

H $1.060819-4.0733634 .242837$

H $-0.530224-4.0503843 .451213$

H $2.568857-2.966329-0.115509$

H $3.205682-4.585635-0.242625$

H $2.064888-4.221022-2.268469$

H $1.030148-5.326756-1.358477$

H $-0.350516-3.302633-0.633589$

H -1.065111 -4.685167 -2.610752

H $-0.004119-3.756366-3.650195$

H $-2.631355-3.813484-4.307376$

H - $0.514076-1.182735-3.984259$

H $-3.334410-3.782501-2.068918$

H -3.692565 -2.079560 -2.235441

H -2.576510 -2.629198 -0.968753

H -2.662699 $-0.445224-3.894429$

H $\quad 1.0790520 .318589-3.137189$

H -0.048887 1.211185 -2.111228

H -0.463712 $-0.198456-0.071354$

H $6.380339-3.1584022 .133827$

H $4.848060-1.1302690 .398656$

H $8.424503-2.1342131 .264764$

H $8.967236-2.221730-0.398357$

H $7.906797-3.4796720 .218456$

H $1.3909670 .709116-0.121184$

H $2.487357-0.227406-1.154192$

H 4.9893143 .3401200 .244903

H $4.0854144 .014349-1.130419$

H $5.3724032 .543601-2.741844$

H $7.2557462 .688187-0.329093$

H $8.7738152 .870536-2.368090$

H $7.6221641 .987806-3.342147$

H $10.374624-0.202098-1.136149$

H $10.4481861 .646870-1.205274$

H $8.604152-1.360641-2.305585$

H $7.453297-0.446249-3.219132$

H $5.890947-0.288077-1.424256$

H $3.869384 \quad 1.2965620 .043687$

C 0.9182583 .0700372 .037782

O $-0.3095692 .849404 \quad 1.314907$

C $-0.893614 \quad 4.1226100 .985859$

C $0.228867 \quad 5.137844 \quad 1.146548$

C 0.9627374 .5671952 .346091
H -1.6538314 .3242561 .750593$

C -1.564593 $4.066799-0.391816$

H 0.8958415 .1552160 .277788

H $-0.145634 \quad 6.1522001 .312226$

C 2.3682465 .1178462 .516366

H 0.3805244 .7829653 .252035

C $-2.5564192 .893307-0.548903$

O $-0.5687393 .896385-1.406354$

H -2.068985 $5.017150-0.604352$

H -1.994155 $1.953809-0.551641$

O $-3.1605372 .986807-1.844748$

C -3.6627252 .8345580 .520355$

C $-4.739744 \quad 1.7630230 .281006$

H -3.2175512 .6780781 .505360$

O $-4.321044 \quad 4.1044760 .595818$

H -5.4674841 .8353401 .098937$

H $-5.3264142 .034483-0.608144$

H 2.8640424 .6617723 .378985

H 2.9850224 .9242671 .632297

H 2.3400996 .2006402 .675588

C 0.9423752 .1890443 .287819

H $-0.1331024 .754466-1.541674$

H -3.592539 $3.854782-1.912442$

H $-4.7844154 .252522-0.245701$

H $\quad 0.0228492 .3372903 .867391$

H 1.7854752 .4715763 .928408

H $1.7380092 .795707 \quad 1.364336$

C $-6.410603-0.7425000 .490366$

O $-5.319077-0.510181-0.407136$

$\begin{array}{llll}\text { C } & -4.259767 & 0.305044 & 0.121437\end{array}$

C $-3.676685-0.3810651 .364493$

C $-4.753405-0.6668162 .408151$

C $-5.919926-1.4255321 .773127$

H -6.8965890 .2102040 .731749$

C -7.427260 -1.622997 -0.244062

H $-3.4876430 .300694-0.653715$

O

H $-3.232584-1.3373541 .059514$

H $-5.087223 \quad 0.2580342 .892561$

O $-4.178305-1.4747203 .437863$

H $-5.597094-2.4510201 .553035$

O $-6.985857-1.5206472 .715733$

C $-8.076701-0.867006-1.378436$

H $-6.939890-2.506262-0.674760$

H $-8.218777-1.9844860 .422138$

H $-2.311997-0.0881852 .721742$

H -4.886872 -1.684996 4.070210

H -7.288755 -0.618529 2.916212

O $-9.039453-0.032731-0.891565$

O $-7.757014-0.994596-2.553250$

C $-9.7203030 .757236-1.871149$

H -10.456659 $1.378836-1.354722$

H $-9.012016 \quad 1.410198-2.390035$

H -10.243287 $0.110792-2.582395$

SCF Energy (B3LYP/6-31G**//MMFF) $=-3245.90944655$

04_00096

MM̄FF Geometry

C -4.429627-1.500726 2.668130

C $-3.174988-1.5362042 .193737$

C $-1.969501-1.0662742 .964649$

$\begin{array}{llll}\mathrm{O} & -1.413800 & 0.026240 & 2.197117\end{array}$

C $-0.955046-2.2190883 .089779$

C $0.101388-2.0049234 .155770$

C $1.413450-1.8177903 .914062$

C $-0.391010-2.0826445 .579630$

C $2.107722-1.7427102 .584154$

C $2.890490-3.0331262 .314335$

C $3.654196-2.9912830 .983460$

C $4.356235-4.3288660 .732674$

C $5.225072-4.298203-0.532277$

O $4.616906-1.9359471 .057622$

O $6.059892-5.465541-0.495604$

C $6.139808-3.056592-0.473665$

C $4.374098-4.419503-1.801373$

O $6.881405-2.914399-1.683331$

C $5.387757-1.748110-0.135450$

C $6.406668-0.6080800 .128986$ 
O $4.565440-1.401874-1.252698$

C $-0.811937 \quad 1.038395 \quad 2.875685$

O $-0.546542 \quad 1.0749804 .066522$

C $-0.5794552 .144977 \quad 1.920323$

C $0.213512 \quad 3.1661392 .277420$

C $0.465633 \quad 4.3834601 .502125$

C $-0.417042 \quad 4.642987 \quad 0.309705$

C 5.8552990 .6128340 .881648

C $4.7317491 .344842 \quad 0.179229$

C $4.2030192 .617680-1.893197$

O $3.6114501 .435257 \quad 0.676665$

C $4.2897064 .070884-1.551722$

C $3.2534474 .783749-1.085232$

C $3.3248926 .247575-0.763815$

C 3.2204706 .5303450 .717527

C 4.3109576 .8415851 .438661

C 1.8424636 .5532061 .340366

C 1.4398285 .2209081 .916028

N $5.1123791 .876596-1.041892$

H -4.619395-1.112601 3.665367

H -2.997512 -1.916129 1.190010

H -2.288639 -0.7040913 .949172$

H $-0.493630-2.4046662 .114013$

H $-1.481789-3.1493323 .345569$

H $2.080568-1.7040364 .767891$

H $0.427485-2.0342986 .305861$

H $-1.073411-1.2573295 .802304$

H -0.919753 -3.0267405 .747372$

H $1.404823-1.552177 \quad 1.769389$

H $2.787182-0.8822352 .605315$

H $3.602823-3.1912613 .135212$

H $2.199278-3.8854802 .312131$

H $2.942168-2.7771990 .179044$

H $3.627204-5.147160 \quad 0.678114$

H $5.003516-4.5643381 .589563$

H $6.667353-5.417477-1.253905$

H $6.881970-3.2463120 .313813$

H $3.809725-5.359798-1.792395$

H $5.001515-4.458298-2.698570$

H $3.657140-3.604473-1.916640$

H $6.280155-2.555993-2.358317$

H $7.221395-0.9996930 .752078$

H $6.855896-0.286950-0.818779$

H $4.625820-0.449361-1.421288$

H -1.0987742 .1072340 .970022$

H 0.7203973 .1172363 .240585

H $-0.2988593 .853993-0.437842$

H -0.199174 $5.583854-0.199540$

H -1.4695294 .6816780 .612282$

H $5.5024180 .317954 \quad 1.876944$

H 6.6676201 .3316721 .047399

H $3.1900592 .223636-1.767046$

H $4.5123972 .456079-2.930128$

H $5.2485574 .559702-1.706969$

H $2.2951694 .291160-0.947790$

H $2.5204116 .769513-1.296544$

H $4.2554706 .674474-1.161364$

H 4.2393267 .0791772 .494902

H $5.298937 \quad 6.859173 \quad 0.990542$

H 1.1183226 .9452380 .621024

H 1.8257897 .2799722 .164451

H $2.018763 \quad 4.9413682 .797736$

H $6.0901431 .841563-1.314650$

C $-6.505781-0.8268931 .418827$

$\begin{array}{lllll}\text { O } & -5.758527 & 0.020631 & 0.521617\end{array}$

C $-6.4792720 .139636-0.715273$

C $-7.923228-0.189858-0.368183$

C $-7.729351-1.3117680 .633567$

H $-6.087787-0.636281-1.384021$

C $-6.2510321 .514693-1.350278$

H -8.4311600 .6558490 .109312$

H -8.505865 $-0.490723-1.243758$

C $-8.948262-1.574523 \quad 1.500904$

H -7.484251 -2.230294 0.083167

C $-4.7732381 .943766-1.455246$

O $-6.9255952 .517097-0.578682$

H -6.719472 $1.538281-2.341066$

H $-4.3968592 .235652-0.466818$
O $-4.718703 \quad 3.123954-2.266922$

C $-3.8273640 .891255-2.058750$

C $-2.4007521 .437153-2.260070$

H $-3.7937910 .020772-1.395968$

O $-4.3338860 .442710-3.314822$

H -2.390074 2.151321 -3.092296

H $-2.1023261 .966265-1.347099$

H -8.750002 -2.3787352 .216659$

H $-9.237812-0.6828342 .067045$

H $-9.802236-1.8729180 .884062$

C $-5.618902-1.9830661 .886991$

H $-6.5809342 .469508 \quad 0.329770$

H $-5.3074403 .782529-1.859722$

H $-4.418404 \quad 1.220556-3.892544$

H $-5.277297-2.561647 \quad 1.020594$

H $-6.194564-2.6677632 .520424$

H $-6.804798-0.2017602 .269501$

C $-0.410571-1.613986-1.454189$

O $-1.294463-0.489647-1.376206$

C -1.382578 $0.318466-2.554988$

C $-0.001566 \quad 0.884536-2.924562$

C $1.055451-0.219524-3.029896$

C $1.010884-1.161968-1.823027$

H $-0.381047-2.015347-0.432923$

C $-0.959463-2.711048-2.371567$

H $-1.759997-0.290134-3.383748$

$\begin{array}{lllll}\text { O } & 0.420211 & 1.840947 & -1.955025\end{array}$

H $-0.0824681 .415662-3.879966$

H $2.0518120 .234827-3.093299$

O $0.848785-0.975452-4.218382$

H $1.639687-2.042449-1.996628$

O $1.540402-0.475997-0.686009$

C $-2.274172-3.239682-1.848406$

H $-1.105366-2.367689-3.399565$

H $-0.266296-3.558711-2.425048$

H $0.439643 \quad 1.399456-1.088619$

H $0.909805-0.362805-4.971046$

H $2.463498-0.245650-0.884565$

O $-3.293297-2.832070-2.657506$

O $-2.382495-3.919185-0.836002$

C $-4.600535-3.263666-2.270156$

H $-4.659640-4.355922-2.287997$

H $-4.846138-2.879396-1.277176$

H -5.318993 -2.860501-2.988892

SCF Energy (B3LYP/6-31G**//MMFF)= -3245.92825336

04_00097

MM̄FF Geometry

C $1.671326-1.2007032 .492267$

C $0.499865-1.7835442 .200867$

C $0.215467-2.4494790 .880153$

O $-0.762327-1.6476480 .178383$

C $-0.372229-3.850497 \quad 1.106792$

C $-0.631537-4.596639-0.186917$

C $-1.848379-4.747735-0.746169$

C $0.578851-5.228476-0.823300$

C $-3.149939-4.176743-0.254921$

C $-3.991151-3.614507-1.409916$

C $-5.300252-3.000388-0.895106$

C $-6.244962-2.676422-2.052602$

C $-7.533204-2.003202-1.557971$

O $-4.976513-1.799486-0.184817$

O $-8.203922-1.482494-2.715284$

C $-7.153618-0.815197-0.650471$

C $-8.492871-3.016097-0.923254$

O $-8.315462-0.236113-0.060339$

C $-6.101060-1.1600640 .431642$

C $-5.615306 \quad 0.1444491 .103564$

O $-6.702092-1.9953531 .422433$

C $-0.283776-0.615825-0.570320$

O $0.885397-0.298111-0.723367$

C $-1.4413640 .088440-1.163891$

C $-1.2461001 .283520-1.740730$

C $-2.2717602 .138344-2.341466$

C $-3.6586001 .571767-2.487390$

C $-4.376801-0.012178 \quad 1.995556$

C $-3.942337 \quad 1.343842 \quad 2.519627$

C -2.9442763 .5030491 .750731$ 
O $-4.158787 \quad 1.6881243 .678971$ C -3.5202574 .3236560 .639578$ C $-2.796400 \quad 5.151984-0.128752$ C $-3.3771685 .959866-1.255003$ C -2.629802 $5.777969-2.559404$ C -1.893569 $6.775469-3.077818$ C $-2.7942784 .466235-3.302011$ C $-1.9196923 .381452-2.731532$ N -3.3372802 .1213861 .545633$ H $2.468998-1.2109291 .754491$ H $-0.307715-1.7502702 .928291$ H $1.126245-2.5353950 .276566$ H -1.294531 -3.777992 1.694989 H $\quad 0.313938-4.4483701 .721776$ H - $-1.923527-5.345906-1.653307$ H $\quad 0.325628-5.798532-1.723510$ H $1.304263-4.462617-1.114007$ H $1.060164-5.918362-0.122755$ H $-3.710391-4.978300 \quad 0.240771$ H -2.990537-3.386795 0.484210 H $-3.408613-2.851937-1.942910$ H $-4.213594-4.420753-2.120081$ H -5.771311 -3.713484 -0.208308 H -6.482273 -3.578932 -2.629669 H -5.741937-1.995702 -2.753907 H $-8.982122-0.986864-2.406910$ H $-6.733430-0.040719-1.307135$ H -8.749517 -3.801152 -1.644762 H $-9.442434-2.544193-0.647221$ H -8.079342 -3.500806 -0.036398 H $-8.595165-0.8139610 .670017$ H $-5.381447 \quad 0.877250 \quad 0.322982$ H -6.4252470 .5764921 .705826$ H $-6.205007-1.9068722 .251022$ H -2.416992 -0.376940 -1.085191 H $-0.2372481 .695299-1.750877$ H $-3.6288690 .600193-2.993727$ H $-4.3244652 .200340-3.082538$ H $-4.123826 \quad 1.439978-1.507386$ H $-4.583163-0.6607112 .853243$ H -3.539570 -0.4580141 .447074$ H -1.8511803 .5317611 .758414$ H -3.314299 3.8622092 .715575 H $-4.588576 \quad 4.2295820 .458096$ H $-1.7322615 .264806 \quad 0.066111$ H -3.356137 $7.014376-0.950847$ H -4.434647 $5.715003-1.415776$ H $-1.3741116 .661459-4.023540$ H - $1.7891937 .726656-2.566373$ H -2.517551 $4.594905-4.356436$ H -3.855602 $4.203756-3.303139$ H $-0.8764183 .680273-2.615607$ H -3.1247301 .7012520 .646109$ C $2.051131 \quad 1.0295563 .635910$ O $3.056337 \quad 1.3850282 .665201$ C 2.5175052 .4079441 .806099 C 1.3156772 .9798602 .547841 C $0.759984 \quad 1.730806 \quad 3.204458$ H 2.1612561 .9046310 .899052 C $3.596593 \quad 3.4303391 .439713$ H 1.6157853 .7073653 .310801 H 0.6058683 .4664791 .872895 C $-0.194757 \quad 2.010776 \quad 4.351862$ H $0.231946 \quad 1.1493342 .438929$ C 4.8697032 .8226080 .817619 $\begin{array}{llllll}\text { O } & 3.994234 & 4.140821 & 2.619552\end{array}$ H 3.1743224 .1836440 .764691 H $\begin{array}{llll}5.431725 & 2.264282 & 1.576297\end{array}$ $\begin{array}{llllll}\text { O } & 5.713858 & 3.906869 & 0.409327\end{array}$ C $4.6198841 .914480-0.400653$ C $5.9310871 .433556-1.050493$ H $4.0359191 .045117-0.083000$ O $3.8419312 .612046-1.371929$ H $6.4810242 .297836-1.442359$ H $6.5576440 .976214-0.276940$ H $-0.559168 \quad 1.0763264 .790469$ H 0.2894292 .5900695 .145101 H -1.0615342 .5806654 .004355$
C $1.964175-0.4926973 .783806$

H 4.3091153 .4845463 .265065

H 5.8647664 .4662391 .190657

H $4.3402003 .405755-1.632503$

H $1.218379-0.7478524 .545893$

H $2.928361-0.8714574 .145378$

H 2.3967961 .4445734 .591766

C $5.388071-1.561638-0.908651$

O $4.835892-0.635829-1.853888$

C $5.705370 \quad 0.442662-2.215316$

C $6.995787-0.117548-2.833482$

C $7.668099-1.149430-1.927697$

C $6.658894-2.208291-1.478782$

H $5.621244-1.0253210 .018166$

C $4.294315-2.597302-0.607487$

H $5.1857601 .000868-3.004428$

O $7.9049350 .929685-3.155909$

H $6.742596-0.608308-3.782162$

H $8.148223-0.667345-1.068204$

O $8.713978-1.784113-2.666759$

H $6.406954-2.839765-2.339952$

O $7.270098-3.054963-0.509729$

C $4.425946-3.2480470 .752285$

H $3.313819-2.107352-0.633382$

H $4.289620-3.375236-1.379931$

H $8.3009511 .254448-2.329900$

H $9.100450-2.467480-2.092652$

H $7.475171-2.5144970 .272318$

O $3.549320-4.292745 \quad 0.828542$

O $5.173081-2.8758461 .646418$

C $3.527066-4.9876092 .079199$

H $2.799762-5.8002722 .003737$

H $4.510922-5.4157762 .293104$

H $3.215658-4.3117092 .881221$

SCF Energy (B3LYP/6-31G**//MMFF)= -3245.93130360

0400098

MM̄FF Geometry

C $1.7183153 .113803-2.446436$

C $2.7391313 .970923-2.287322$

C $3.9526003 .770966-1.406888$

O $4.0973812 .391336-0.998398$

C $3.8228724 .644035-0.145791$

C $5.071774 \quad 4.651327 \quad 0.715831$

C 5.2050124 .0076841 .893066

C $6.2085995 .489240 \quad 0.187349$

C $4.200133 \quad 3.130352 \quad 2.586188$

C 4.7358411 .7014182 .745069

C $3.662367 \quad 0.7369613 .271745$

C $4.245368-0.6662963 .450297$

C $3.174809-1.7021493 .825151$

$\begin{array}{lllll}\text { O } & 2.599786 & 0.712175 & 2.314931\end{array}$

O $3.739996-3.0087713 .634727$

C $1.988932-1.5785972 .843991$

C $2.788402-1.6086945 .305810$

O $0.907432-2.415020 \quad 3.246420$

C $1.494087-0.1296512 .664570$

C $0.437090 \quad 0.015813 \quad 1.543561$

$\begin{array}{lllll}\text { O } & 0.878372 & 0.308249 & 3.881967\end{array}$

C $4.7028041 .550512-1.883976$

O $5.115693 \quad 1.832852-2.998039$

C $4.7757170 .208076-1.264669$

C $5.225390-0.828719-1.987863$

C $5.388130-2.208619-1.523835$

C $4.994471-2.521186-0.105258$

C $0.920850-0.3225040 .129429$

C $0.619162-1.741910-0.317573$

C $1.034228-3.295530-2.223883$

O $-0.092622-2.5148050 .316669$

C $2.127303-4.237912-1.830413$

C $2.924607-4.855095-2.715597$

C $3.978594-5.854625-2.335395$

C $5.339573-5.518068-2.900550$

C $5.790135-6.111838-4.018807$

C $6.214697-4.568506-2.115749$

C $5.888777-3.123476-2.380461$

N $1.213973-2.029340-1.539758$

H $1.7090152 .172707-1.904343$ 
H $2.7151864 .912469-2.833187$ H $4.8419114 .073287-1.973966$ H 2.9525954 .3172090 .432662 H $3.6029445 .681928-0.431363$ H $6.148074 \quad 4.1118972 .428792$ H $7.057637 \quad 5.522773 \quad 0.878501$ H $6.5751085 .087799-0.762162$ H $5.877375 \quad 6.5203570 .025754$ H 3.9930733 .5529483 .576900 H $3.246343 \quad 3.1121702 .055170$ H 5.0910881 .3472181 .769267 H $5.598071 \quad 1.7045463 .423433$ H 3.2815091 .1211554 .225388 H $5.052003-0.6594174 .194179$ H $4.713622-0.9835282 .509878$ H $4.535145-3.0754784 .190645$ H $2.334051-1.9588871 .875411$ H $3.662345-1.7948105 .941856$ H $2.055407-2.3779185 .572930$ H $2.383604-0.6330845 .582521$ H $1.255243-3.3195623 .327592$ H $-0.472018-0.5365121 .806272$ H $0.137072 \quad \begin{array}{llll}1.072701 & 1.525147\end{array}$ H 0.2794321 .0457103 .679383 H $4.4663180 .110846-0.231265$ H $5.517577-0.651221-3.022335$ H $5.008889-3.5879090 .127889$ H $3.972136-2.1850700 .095850$ H $5.674443-2.0241270 .593984$ H $0.3954730 .331149-0.577766$ H $1.991036-0.1259530 .018762$ H $1.033252-3.086951-3.298325$ H $0.063574-3.722380-1.951051$ H $2.244232-4.449127-0.770151$ H $2.785730-4.664999-3.777616$ H $3.654171-6.835448-2.707629$ H $4.052829-5.964503-1.246338$ H $6.778663-5.899485-4.412436$ H $5.177997-6.819091-4.568432$ H $7.267088-4.716845-2.393940$ H $6.175399-4.828922-1.054232$ H $6.140163-2.802589-3.392448$ H $1.834391-1.345975-1.964196$ C $-0.7811073 .570025-2.641568$ O $-1.1647802 .373925-1.937071$ C $-1.8008452 .757044-0.695700$ C $-2.0262694 .261367-0.786056$ C $-0.8350924 .698797-1.614971$ H $-1.0720442 .530093 \quad 0.090506$ C -3.076596 $1.927549-0.505098$ H $-2.9625204 .485388-1.312456$ H -2.063331 4.7424350 .195797 C - $0.9853836 .082089-2.222048$ H $0.0518624 .685346-0.969401$ C -3.9379902 .3459610 .701197$ O $-2.697720 \quad 0.554760-0.361843$ H -3.667654 $1.986092-1.425610$ H -4.2895593 .3771510 .577270$ O C -5.1487521 .4309910 .975543$ C $-6.051194 \quad 1.208726-0.249872$ H $-4.797845 \quad 0.4704341 .367815$ O $-5.922242 \quad 2.0260672 .023913$ H $-6.336612 \quad 2.183329-0.665508$ H $-5.4803120 .686733-1.023894$ H $-0.1059346 .342410-2.819502$ H -1.865184 $6.142588-2.871472$ H $-1.095416 \quad 6.836855-1.436537$ C $0.5538593 .370202-3.362632$ H -2.2431580 .4635690 .492692$ H -2.4960483 .0547821 .829833$ H -6.2684622 .8729161 .694164$ H $0.752773 \quad 4.239715-4.000701$ H $0.4718952 .502143-4.028832$ H $-1.5539293 .727359-3.406077$ C $-6.385321-1.7948310 .107643$ O $-7.083229-0.768497 \quad 0.821315$ C -7.3408130 .4171980 .065694$
C $-8.189196 \quad 0.075897-1.168054$

C $-7.560711-1.042322-2.000548$

C $-7.196369-2.235317-1.115284$

H $-5.401624-1.424884-0.206474$

C $-6.167781-2.9594961 .078541$

H $-7.954404 \quad 1.0610880 .708500$

O $-8.373956 \quad 1.241714-1.963721$

H $-9.183115-0.249243-0.833697$

H $-6.684542-0.687224-2.555819$

O $-8.501555-1.492278-2.978210$

$\mathrm{H}-8.113765-2.746885-0.796493$

O $-6.429311-3.166243-1.875673$

C $-5.235452-2.5582332 .196643$

H $-7.114593-3.2686191 .538083$

H $-5.750125-3.8413220 .579819$

H $-9.0130001 .024059-2.662890$

H $-8.644952-0.763312-3.604972$

H -6.958346 -3.410278 -2.654466

O $-3.940558-2.6420491 .778537$

O $-5.616577-2.192792 \quad 3.301227$

C $-2.955551-2.2666342 .746457$

H $-1.975717-2.5618492 .364498$

H -2.969444 -1.181871 2.886081

H $-3.124561-2.7818343 .697097$

SCF Energy (B3LYP/6-31G**//MMFF)= -3245.90231892

0400099

MM̄FF Geometry

C $0.232622-3.6651521 .600763$

C $-0.917724-3.5866150 .916648$

C $-0.981184-3.461783-0.584635$

O $-1.511471-2.151292-0.893677$

C $-1.926362-4.528842-1.163698$

C $-2.023436-4.496539-2.678146$

C $-3.097811-4.072936-3.374567$

C $-0.811163-5.012770-3.410989$

C $-4.392324-3.537193-2.830578$

C $-4.524471-2.032341-3.096673$

C $-5.600290-1.388723-2.209861$

C $-5.787370 \quad 0.085303-2.572233$

C $-6.7493930 .801335-1.612971$

O $-5.157286-1.508371-0.853797$

$\begin{array}{lllll}0 & -6.600926 & 2.210203 & -1.847796\end{array}$

C $-6.3145060 .513504-0.158862$

C $-8.2104290 .465039-1.931482$

\begin{tabular}{lllll}
\hline & -7.257098 & 1.050093 & 0.765685
\end{tabular}

C $-6.061680-0.9813770 .124163$

C $-5.432547-1.2376081 .516040$

O $-7.313926-1.6767410 .092897$

C $-0.619257-1.121134-0.915976$

O $0.587808-1.190323-0.744828$

C $-1.369816 \quad 0.129302-1.162564$

C $-0.745537 \quad 1.308050-1.021445$

C $-1.3339882 .633026-1.227918$

C $-2.7974982 .700990-1.573965$

$\begin{array}{llll}C & -4.054170 & -0.605466 & 1.740217\end{array}$

C $-4.092852 \quad 0.7487452 .427664$

C $-2.5949882 .634244 \quad 3.074458$

$\begin{array}{lllll}\text { O } & -5.098560 & 1.203716 & 2.965295\end{array}$

C -2.743070 3.7550952 .095385

C -1.8019014 .6912201 .900373$

C $-1.958608 \quad 5.853651 \quad 0.962343$

C $-0.8162845 .969747-0.021713$

C $0.199714 \quad 6.821993 \quad 0.195598$

C $-0.8989635 .163886-1.297914$

C $-0.5430063 .718201-1.093146$

N $-2.851007 \quad 1.3706822 .409335$

H $1.174536-3.6640561 .057807$

H - $1.860058-3.5652891 .459258$

H $\quad 0.014090-3.575234-1.032303$

H $-2.914607-4.416465-0.707609$

H $-1.582464-5.528353-0.864309$

H $-3.050337-4.103288-4.462508$

H $-0.966620-5.054175-4.494410$

H $0.054252-4.368517-3.228591$

H $-0.568541-6.026809-3.076826$

H $-5.221001-4.064783-3.318792$

H $-4.499546-3.749726-1.765147$ 
H $-3.561913-1.544480-2.899488$ H $-4.760761-1.870397-4.155612$ H $-6.539107-1.940577-2.336165$ H -6.128293 $0.190857-3.609768$ H $-4.8150110 .593378-2.527716$ H -7.147601 2.677176 - 1.192491 H -5.3839511 .0695640 .000582$ H $-8.4551280 .766331-2.957199$ H -8.896930 $1.024104-1.286112$ H -8.435698 $-0.599210-1.836132$ H $-8.022707 \quad 0.4510830 .790018$ H $-6.140067-0.9625432 .307422$ H $-5.297310-2.3245541 .614016$ H -7.216671 -2.5091790 .584418$ H -2.412649 $0.047642-1.443523$ H $0.303306 \quad 1.306694-0.728077$ H -2.976719 2.263468 -2.561740 H $-3.1969603 .717036-1.592000$ H -3.393093 2.157371-0.833175 H -3.477299 -1.2634532 .402787$ H -3.500381 -0.5246490 .801020$ H -1.585328 2.5808603 .493846 H -3.306399 2.7657503 .896022 H -3.6791913 .8174811 .545438$ H -0.8788604 .6424552 .474084$ H $-2.018703 \quad 6.7654871 .571070$ H -2.907898 5.8023920 .414759 H $1.0080106 .932633-0.519693$ H 0.2482147 .4242441 .096921 H $-0.1977405 .566196-2.041792$ H $-1.8858915 .302082-1.748042$ H $0.5043563 .566534-0.830048$ H -2.0828110 .9357691 .906564$ C $0.818529-2.4276653 .739072$ O $2.088496-2.0434853 .175334$ C $2.022785-0.6528202 .809423$ C $0.873443-0.0724043 .623971$ C $-0.115015-1.2221533 .590761$ H $1.750226-0.6137231 .749195$ C $3.377267 \quad 0.0275113 .020731$ H 1.1720160 .1393274 .657044 H $\quad 0.476560 \quad 0.8485843 .186570$ C $-1.179182-1.1453814 .672534$ H $-0.607079-1.2223232 .610871$ C $4.577229-0.6573552 .333333$ O $3.663210 \quad 0.064761 \quad 4.426971$ H 3.3156451 .0753772 .705808 H $4.766169-1.6219972 .821016$ O $5.741180 \quad 0.1471932 .569974$ C $4.485267-0.879518 \quad 0.812422$ C 4.1306930 .3909830 .016654 H $5.463288-1.2395880 .472924$ O $3.541458-1.8999940 .506888$ H $3.107816 \quad 0.699557 \quad 0.261031$ H $4.795368 \quad 1.2053730 .323252$ H -1.867252 -1.993609 4.600302 H -0.735396 -1.155011 5.673855 H -1.763747 -0.2256384 .577808$ C $\quad 0.321619-3.7295583 .099442$ H $3.656083-0.8521124 .751981$ H 5.8176720 .2699683 .531858 H $3.795413-2.6960121 .004111$ H $-0.644054-4.0103203 .536181$ H $1.024490-4.5361543 .343250$ H $1.009012-2.6221924 .802472$ C $6.594600 \quad 0.469105-1.765697$ O $5.461204-0.394312-1.914927$ C $4.2135160 .182864-1.513971$ C $3.9374061 .440103-2.352435$ C $5.1013332 .431028-2.313846$ C $6.4209861 .724386-2.627763$ H $6.7058590 .753161-0.712594$ C $7.837646-0.325864-2.178237$ H $3.440465-0.551970-1.772229$ O $2.7345242 .078861-1.941864$ H $3.7837441 .135314-3.395612$ H $5.1546962 .948303-1.348728$ O $4.8876893 .443466-3.299715$
H $\quad 6.440279 \quad 1.458070-3.691874$

O $7.5032762 .625550-2.404093$

C $8.112095-1.443032-1.199038$

H $7.696811-0.780911-3.166198$

H $8.7327950 .303543-2.234044$

H $2.8957182 .512160-1.086961$

H $4.0236753 .850205-3.114813$

H $7.5027152 .866076-1.461910$

O $8.595514-0.931855-0.030609$

O $7.910896-2.625921-1.441348$

C $8.856794-1.8906210 .998663$

H $9.227401-1.3544931 .876332$

H $7.935252-2.4140001 .271295$

H $\quad 9.622667-2.5997430 .670578$

SCF Energy $\left(B 3 L Y P / 6-31 G^{* *} / / M M F F\right)=-3245.91782634$

0400100

MM̄FF Geometry

C -2.219237-1.477138 3.929782

C $-2.307266-0.6022322 .916397$

C -2.8471310 .7975493 .048672$

$\begin{array}{llll}\text { O } & -4.017331 & 0.843798 & 2.202199\end{array}$

C -1.8172431 .8106042 .520948$

C -2.118431 3.2416622 .913580

C -2.6712904 .1637342 .101247$

C -1.7042843 .6200114 .312611$

C $-3.179793 \quad 3.980670 \quad 0.698241$

C $-2.3425394 .736715-0.343322$

C $-0.9848094 .077688-0.633693$

C $-0.1874194 .907008-1.643670$

C $1.1197574 .212814-2.057733$

O $-1.2409892 .776285-1.170265$

O $1.6245614 .881357-3.223937$

C $0.8061322 .759320-2.471349$

C $2.2036424 .343884-0.980727$

O $2.0068962 .032111-2.721968$

C $-0.0665402 .003238-1.444351$

C $-0.5177100 .644087-2.028941$

O $\quad 0.7013151 .773175-0.263879$

C -5.1268661 .4834952 .644936$

O -5.2565862 .0790013 .705024$

C -6.2417201 .3480331 .675952$

C -6.0914570 .8288180 .447073$

C $-7.1480940 .611944-0.543504$

C $-8.5607090 .953646-0.150325$

C $-1.509819-0.121871-1.144009$

C $-1.962580-1.422797-1.783577$

C $-3.672774-3.219675-1.519012$

O $-1.430641-1.902534-2.781135$

C $-4.714553-2.884516-2.540469$

C $-6.030190-3.082938-2.367586$

C -7.063565 -2.736266 -3.402460

C $-8.086039-1.737663-2.902927$

C $-9.322395-2.133883-2.556956$

C $-7.707852-0.271532-2.887538$

C $-6.8077380 .081835-1.737054$

N -3.021146 -1.995474 -1.090379

H -2.559384 -1.160138 4.914090

H $-1.990892-0.8948701 .920549$

H -3.1113251 .0020224 .093040$

H -1.7493991 .7277851 .431295$

H -0.8153431 .5630682 .897571$

H $-2.8115565 .170628 \quad 2.493317$

H -1.910441 4.6717324 .538099

H -2.2414343 .0149275 .049534$

H -0.6295793 .4593554 .446790$

H -3.271864 2.9260430 .423535

H -4.2021324 .3795470 .672414$

H -2.926932 $4.765498-1.272440$

H -2.195113 $5.775448-0.022156$

H -0.4258083 .9818420 .303798$

H $0.0183605 .909181-1.247247$

H $-0.7948975 .062119-2.546649$

H $1.7573605 .818063-2.998336$

H $0.2619922 .798466-3.425498$

H $2.4319745 .400166-0.793954$

H $3.1440063 .888737-1.310389$

H $1.9184053 .895968-0.026653$ 
H $2.5195722 .535503-3.377343$ H -1.000566 $0.814125-3.000527$ H $\quad 0.355306 \quad 0.006006-2.209408$ H 0.2030021 .1751920 .313235 H -7.2038661 .6959132 .037774$ H $-5.099826 \quad 0.5122850 .127761$ H $-8.8553440 .400727 \quad 0.748703$ H $-9.297177 \quad 0.708853-0.918683$ H -8.654092 2.0266860 .050184 H -1.047684 -0.379208 -0.186727 H $-2.3947080 .494229-0.948635$ H $-4.099153-3.693225-0.629258$ H -2.928928 -3.895127 -1.953555 H $-4.362935-2.456723-3.476773$ H -6.386519 -3.521864 -1.438592 H -7.560002 -3.668967 -3.700796 H -6.598702 -2.346273-4.316721 H -10.072435 -1.422977 -2.226714 H -9.611611 -3.179036 -2.592088 H $-8.6028230 .360730-2.894658$ H $-7.192372-0.021838-3.823956$ H $-5.755433-0.130243-1.926776$ H -3.440012 -1.485195 -0.318227 C -1.059753 -3.341840 2.550604 O $0.157757-2.6028232 .336158$ C $1.181686-3.4983841 .868372$ C $0.467413-4.7968741 .514254$ C $-0.650425-4.8142522 .539348$ H $1.854826-3.6771952 .716556$ C $1.978190-2.8736690 .713119$ H $0.053168-4.7657130 .499492$ H $1.128093-5.6664041 .582760$ C $-1.780032-5.7668212 .187268$ H $-0.230439-5.0965603 .514118$ C $2.728901-1.5880441 .131762$ O $1.075234-2.601217-0.357887$ H $2.704790-3.6117630 .352232$ H $3.357418-1.8234791 .999234$ O $1.802919-0.5899671 .556887$ C $3.635934-1.0018730 .030679$

C $4.394810 \quad 0.251247 \quad 0.509638$ H $4.350487-1.774281-0.271624$ O $2.871837-0.667302-1.123935$ H $3.6745431 .028547 \quad 0.793238$ H $4.959918-0.0055411 .412198$ H $-2.568343-5.7351922 .945738$ H -2.231460 -5.514736 1.221686 H -1.410402 -6.7957812 .126934$ C -1.698627 $-2.892613 \quad 3.869080$ H $1.599959-2.294767-1.116528$ H $1.097600-0.5513540 .889628$ H $2.2834320 .069114-0.884713$ H $-0.954828-2.9892324 .670699$ H -2.532346 -3.559350 4.118576 H -1.724529 -3.133941 1.702714 C $7.200759-0.649870-0.250578$ O $6.193968-0.149842-1.137528$ C $5.3328930 .839853-0.568609$ C $6.1591922 .046835-0.101506$ C 7.3012931 .6322390 .826365 C 8.1126450 .4915480 .209010 H $6.727822-1.1238660 .618368$ C $7.984236-1.726921-1.007643$ H $4.6900291 .190366-1.386015$ O 5.3131652 .9904960 .546201 H $6.5840872 .544665-0.982990$ H $6.930391 \quad 1.3529801 .819611$ O 8.1811032 .7418301 .021112 H $8.6972600 .873340-0.637858$ O $9.033844-0.0041591 .178104$ C $7.149452-2.977635-1.151734$ H $8.284179-1.383249-2.004867$ H $8.900098-2.012878-0.478299$ H 5.8360353 .7930550 .710219 H 7.6912323 .4218301 .513463 H 9.5814040 .7457751 .467798 O $6.417208-2.922998-2.300513$ O $7.126765-3.878503-0.322833$
C $5.539730-4.030966-2.523932$

H $5.009787-3.860407-3.464759$

H $\quad 6.114144-4.958447-2.606887$

H $4.805068-4.101282-1.715824$

SCF Energy (B3LYP/6-31G**//MMFF) $=-3245.92029294$

0400101

MM̄FF Geometry

C $3.102727 \quad 1.2896792 .907008$

C 2.5620110 .2209103 .512919

C $2.839902-1.2329673 .208379$

O $3.908697-1.3424452 .241940$

C $1.587443-1.9114112 .623535$

C $1.580901-3.4131692 .829278$

C $1.898377-4.3269551 .891790$

C $1.135687-3.8654274 .197347$

C $2.376445-4.0926780 .486025$

C $1.369950-4.586182-0.563787$

C $0.127730-3.689788-0.677673$

C $-0.877763-4.274978-1.671371$

C $-2.075630-3.339557-1.898412$

O $0.564872-2.405212-1.130556$

O $-2.771316-3.834786-3.052413$

C $-1.550231-1.924100-2.222432$

C $-3.069045-3.397841-0.731642$

O $-2.618801-0.983276-2.289421$

C $-0.475452-1.426167-1.226724$

C $0.155811-0.111991-1.743781$

O $-1.089503-1.1868410 .039426$

C $4.888630-2.2550082 .457599$

O $4.982017-3.0197893 .407784$

C $5.910340-2.2175091 .384325$

C $5.758007-1.5465480 .231657$

C $6.737831-1.458865-0.855001$

C $8.073077-2.125494-0.650882$

C $1.3968260 .340417-0.961053$

C $1.9931781 .614860-1.534405$

C $4.0441653 .028497-1.385724$

O $1.4233832 .306458-2.374054$

C $4.9313892 .594751-2.509164$

C $6.2676842 .518916-2.420178$

C $7.1472202 .046623-3.542122$

C $7.9475040 .814175-3.177686$

C $9.2642780 .899961-2.924161$

C $7.245040-0.527226-3.194788$

C $6.409534-0.766417-1.966170$

N $3.2325231 .896844-0.977181$

H 3.8105191 .1575792 .093871

H $1.8525770 .393276 \quad 4.320815$

H $3.135198-1.6965414 .157290$

H $1.499718-1.6706601 .558423$

H $0.677394-1.5099093 .089936$

H $1.832910-5.3800452 .164118$

H $1.116338-4.9565104 .290230$

H $1.810857-3.4829354 .968928$

H $\quad 0.124985-3.4999974 .407027$

H $2.636884-3.0464040 .303149$

H $3.309583-4.6566840 .361781$

H $1.886772-4.603430-1.532281$

H $1.070746-5.617158-0.337527$

H $-0.333799-3.5904230 .310881$

H -1.220279 -5.264021 -1.342244

H $-0.379221-4.438123-2.637559$

H -3.564379 -3.288495 -3.185059

H -1.102772 -1.961079 -3.225534

H $-3.472724-4.411846-0.625022$

H -3.932081-2.749600 -0.915972

H -2.626551 -3.1143350 .225587$

H $-3.216689-1.262872-3.002553$

H $\quad 0.459780-0.247219-2.790375$

H $-0.5901600 .691725-1.725864$

H $-0.560235-0.5429840 .535438$

H $\quad 6.805454-2.7940741 .595121$

H $4.830424-1.0040340 .057532$

H $7.949179-3.208928-0.546281$

H $8.563444-1.7384100 .249387$

H $8.770819-1.963832-1.475191$

H 1.1487780 .5356480 .087252 
H $2.165109-0.440884-0.992135$ H $4.6119653 .356762-0.509496$ H $3.3987103 .849768-1.712576$ H $4.4440692 .316708-3.440991$ H $6.7583272 .802019-1.491858$ H $7.8195522 .872586-3.808016$ H $6.5635811 .839270-4.447781$ H $9.8545840 .019610-2.693265$ H $9.7819481 .853395-2.938524$ H $7.965916-1.339314-3.341854$ H $6.593753-0.582276-4.076808$ H $5.416360-0.321467-2.021591$ H $3.651891 \quad 1.223525-0.342656$ C 1.8389633 .3617722 .292721 O 0.5377012 .7584212 .418051 C -0.4701803 .7813092 .325318$ C 0.2568565 .0461751 .884739 C 1.6183654 .8538982 .525292 H -0.8684613 .9252683 .337600$ C $-1.604105 \quad 3.3385301 .389247$ H 0.3526625 .0967520 .793504 H -0.2520865 .9550292 .219756$ C 2.6996535 .7376501 .928171 H 1.5346845 .0595423 .600918 C -2.367416 2.0921791 .896370 O -1.0639163 .0980350 .090962$ H -2.317054 4.1652621 .288793 H -2.7663002 .3085522 .895330$ $\begin{array}{lllll}\text { O } & -1.483937 & 0.978728 & 2.027378\end{array}$ C $-3.539416 \quad 1.6741450 .984455$ C -4.3354180 .4891901 .569948$ H -4.2099902 .5315590 .857300$ O $-3.0606231 .324155-0.310164$ H -3.659587 -0.3444931 .796212$ H -4.7810590 .8208862 .515417$ H 3.6647955 .5541802 .410406 H 2.8198585 .5552730 .854872 H 2.4501456 .7951292 .063414 C 2.7903952 .7105893 .294299 H -0.4440842 .3531970 .169688$ H $-0.807011 \quad 1.2118642 .684800$ H -2.470599 $0.558302-0.206685$ H 2.3482432 .7351024 .298275 H 3.7348563 .2638643 .343685 H 2.1773693 .1833041 .265664 C $-7.2964650 .757062-0.691723$ $\begin{array}{lllll}\text { O } & -6.288027 & 1.053299 & 0.283069\end{array}$ C $-5.426165-0.0364330 .618167$ C $-6.240410-1.1826731 .235585$ C $-7.380535-1.6127780 .303440$ C $-8.178976-0.412779-0.223043$ H -7.923135 $1.657652-0.728957$ C $-6.6936390 .550139-2.089689$ H -4.930420 -0.399590 -0.288133 O $-6.782168-0.7618012 .485949$ H -5.570111 -2.027754 1.432689 H -8.058730 -2.285927 0.841856 O $-6.849240-2.335427-0.803352$ H -8.840014 -0.719227 -1.042318 O $\quad-9.014490 \quad 0.081980 \quad 0.828231$ C $-5.880091 \quad 1.749360-2.528101$ H -6.043428 -0.328860 -2.113053 H -7.485294 $0.396049-2.831080$ H -7.266517 -1.514881 2.864696 H $-6.384342-3.112979-0.450294$ H $-9.615808-0.6366851 .087663$ O $-4.8122691 .328908-3.266969$ O $-6.1593692 .912132-2.268001$ C $-3.9328312 .360926-3.724138$ H -3.093896 $1.887437-4.241150$ H -4.455608 $3.016382-4.426775$ H -3.542982 $2.933583-2.877041$

SCF Energy (B3LYP/6-31G**//MMFF) $=-3245.91417796$

0400102

MM̄MF Geometry

C $5.3138712 .775786 \quad 0.294420$

C 4.0375913 .1353820 .098965
C 3.1444773 .6186811 .209728

$\begin{array}{llll}\text { O } & 2.008621 & 2.725290 & 1.281847\end{array}$

C 2.6220155 .0348730 .922904

C 1.7595475 .5751912 .048924

C 0.4158485 .6783732 .014306

C 2.5159946 .0460813 .265648

C -0.5181105 .2927370 .900969$

C -1.5598244 .2679021 .362599$

C -2.465833 3.7720750 .222568

C $-3.3445934 .890213-0.340929$

C $-4.3130724 .372950-1.410062$

O -3.2943872 .7317430 .760152$

O $-5.2687965 .417498-1.648274$

C $-5.0726553 .148746-0.856211$

C $-3.5926714 .137367-2.744538$

O $-5.8757492 .583165-1.889407$

C $-4.1537772 .090142-0.202282$

C -4.9426661 .0159560 .588116$

O $-3.3867661 .438643-1.215128$

C 2.1930801 .5365501 .918822

O 3.2198051 .1263092 .435695

C 0.9034450 .8158651 .886374

C $0.785834-0.3635872 .513116$

C $-0.437214-1.1583772 .616957$

C $-1.664510-0.6476211 .910913$

C $-5.9244800 .179199-0.228956$

C $-6.352020-1.0698060 .517348$

C $-5.816587-3.4977390 .735228$

O $-7.329955-1.0726611 .261545$

C $-4.894506-3.8443771 .859655$

C $-3.896624-4.7346041 .748199$

C $-3.010152-5.1611962 .882787$

C $-1.661422-4.4756812 .850013$

C $-0.637583-5.0250042 .174489$

C -1.579632 -3.195846 3.656397

C $-0.416639-2.2947913 .344110$

N -5.531248 -2.1565450 .262507$

H $5.7545682 .888248 \quad 1.282243$

H $3.5896613 .008356-0.883052$

H 3.6718273 .6089052 .172065

H $2.0719935 .042198-0.023558$

H $3.4665105 .720722 \quad 0.772227$

H -0.0845186 .1030152 .884225$

H $1.8576916 .493794 \quad 4.017893$

H 3.0388835 .2119283 .742965

H 3.2534016 .8047422 .983745

H -1.016827 6.2085030 .565150

H $0.0147604 .890846 \quad 0.036678$

H - 1.0551533 .4025531 .810913

H -2.177888 4.6893732 .166727

H $-1.8276113 .343833-0.559197$

H -2.741359 $5.706018-0.755139$

H $-3.931358 \quad 5.336018 \quad 0.474537$

H $-5.9310555 .074119-2.272493$

H $-5.7719063 .516098-0.092940$

H $-3.1200845 .064446-3.090859$

H -4.297793 $3.852514-3.533208$

H $-2.8163003 .371749-2.683226$

H -5.291173 $2.066364-2.469708$

H $-4.220746 \quad 0.342827 \quad 1.067029$

H -5.4764781 .4917521 .421738$

H -3.088016 $0.582037-0.867862$

H 0.0778831 .2894551 .368140

H $1.656807-0.7757863 .020909$

H -2.0504230 .2449282 .415006$

H $-2.472649-1.3816111 .864401$

H -1.437338 -0.3889980 .871638$

H $-6.8325290 .750473-0.451287$

H $-5.491418-0.117285-1.191012$

H $-5.691589-4.171975-0.118474$

H $-6.856002-3.5682351 .071404$

H $-5.082966-3.3685842 .818877$

H $-3.716186-5.2135730 .788902$

H $-2.884620-6.2505092 .827800$

H -3.495409-4.979509 3.850382

H $0.348484-4.5758522 .146657$

H $-0.764367-5.953267 \quad 1.626053$

H -1.521015 -3.464903 4.719409 
H $-2.518887-2.6414693 .557857$ H $0.518406-2.599703 \quad 3.814951$ H -4.729930 -2.038154 -0.349958 C $6.5419890 .717820-0.341133$ O $5.367363-0.122413-0.364653$ C $5.762032-1.448524-0.767686$ C $7.281742-1.421057-0.918276$ C $7.5359310 .030785-1.271242$ H $5.318765-1.625871-1.753457$ C $5.264962-2.4873750 .244598$ H $7.784434-1.6823010 .020885$ H $7.632071-2.117765-1.686117$ C $8.9794640 .457467-1.070489$ H $7.2490180 .196098-2.318331$ C $3.730691-2.6265050 .347519$ O $5.763147-2.146928 \quad 1.545357$ H $5.700348-3.4651810 .006594$ H $3.287199-1.7275490 .791193$ O $3.463109-3.6982221 .262729$ C $3.027753-2.940028-0.988534$ C $1.582927-3.458572-0.822623$ H $3.027637-2.037811-1.609394$ O $3.776081-3.933160-1.696669$ H $1.618941-4.442524-0.341440$ H $1.032729-2.785825-0.158146$ H $9.1165351 .511204-1.331911$ H $9.296640 \quad 0.324417-0.030700$ H $9.646892-0.135888-1.703952$ C $6.1750732 .141631-0.759754$ H $5.426398-1.2608951 .764184$ H $3.899505-3.4761792 .103206$ H $3.804962-4.731310-1.141450$ H $5.6506672 .125685-1.722934$ H $7.0759952 .752440-0.881066$ H $\begin{array}{llll}6.920413 & 0.709563 & 0.690521\end{array}$ C $-0.448268-1.566674-2.180501$ O $0.614058-2.320146-2.780345$ C $0.867666-3.599198-2.187004$ C $-0.405650-4.457308-2.178461$ C -1.595439-3.726093-1.572305 C $-1.760658-2.358319-2.229183$ H $-0.198093-1.336135-1.138637$ C $-0.567526-0.245880-2.955272$ H $1.566497-4.108830-2.862103$ O $-0.175940-5.680481-1.487289$ H $-0.652499-4.722602-3.215152$ H -1.492604 -3.640917 -0.484671 O $-2.769952-4.503710-1.811679$ H -2.084824 -2.492277 -3.268702 O $-2.796206-1.646167-1.557752$ C $0.4437240 .780138-2.487973$ H $-0.408700-0.405992-4.027614$ H - $-1.5598210 .190646-2.803078$ H -1.012132 -6.177007 -1.487761 H -3.526918 -3.996274 -1.472169 H $-2.558849-1.597023-0.616977$ O $\quad 0.0415792 .016778-2.907881$ O $1.4597250 .533460-1.852871$ C $0.8856313 .103518-2.519051$ H $0.4123854 .034860-2.841116$ H $0.9959603 .125904-1.431764$ H $1.8609223 .015185-3.006291$

SCF Energy (B3LYP/6-31G**//MMFF) $=-3245.93583313$

04 00103

MM̄FF Geometry

C $0.434218-2.392210-0.138532$

C $1.263971-2.6695520 .878695$

C $1.584518-1.7654542 .047455$

O $1.043240-0.4465241 .811939$

C $3.111660-1.6268452 .185272$

C $3.557913-1.0262373 .502140$

C 4.0059740 .2347243 .658461

C $3.532701-1.9688384 .677817$

C 4.0810061 .3268422 .627849

C 5.5196371 .6378092 .191760

C 6.1068350 .5893921 .233114

C 7.5579160 .9286510 .885141
C $8.134541-0.030347-0.167788$

$\begin{array}{lllll}\text { O } & 5.309457 & 0.596071 & 0.045239\end{array}$

O $9.3552490 .540387-0.663425$

C $7.157245-0.111226-1.359608$

C $8.505846-1.3883410 .439659$

O $7.578680-1.097924-2.298761$

C $5.692004-0.371152-0.940763$

C $4.753965-0.193232-2.159767$

O $5.576894-1.707233-0.448866$

C 0.4119440 .1965382 .824116

O $0.173589-0.248006 \quad 3.938471$

C 0.0277641 .5749732 .432840

C 0.3639062 .1389671 .261847

C 0.0377463 .4986760 .824769

C -0.7732944 .3616051 .754176$

C $3.258459-0.226553-1.810546$

C $2.3850850 .111747-3.007063$

C $0.0524700 .798382-3.568720$

O $2.8088840 .149216-4.159113$

C $0.1201052 .290509-3.667167$

C $-0.8636173 .115907-3.279402$

C $-0.7623064 .613348-3.347331$

C $-0.9081705 .276160-1.993405$

C $-2.0251185 .952539-1.676862$

C $0.2717465 .245424-1.045224$

C $0.4692333 .906435-0.387354$

N $1.0691210 .351646-2.632886$

H $-0.097553-1.445160-0.166771$

H $1.759965-3.6387980 .884725$

H $1.145853-2.2193562 .943669$

H $3.491594-1.0303201 .349965$

H $3.595351-2.6083362 .088212$

H 4.3342080 .5411864 .651111

H $3.915438-1.5037795 .592527$

H $2.510757-2.3023184 .882549$

H $4.151698-2.848403 \quad 4.472840$

H 3.4501281 .1188561 .758865

H 3.6563602 .2297163 .085495

H 5.5032422 .6104141 .682473

H 6.1635391 .7448423 .073431

H $6.051563-0.398126 \quad 1.703917$

H $8.183470 \quad 0.931547 \quad 1.786420$

H 7.6071551 .9507280 .483673

H 9.9554130 .6663730 .091480

H $7.2009090 .853765-1.883923$

H $9.257300-1.2620931 .228410$

H $8.965231-2.043592-0.308640$

H $7.652844-1.9098470 .878843$

H $8.496856-0.890934-2.543829$

H $4.9635680 .775538-2.632392$

H $4.958596-0.971344-2.905914$

H $4.665314-2.015353-0.568872$

H -0.5384972 .1159953 .184329$

H $0.944141 \quad 1.5587760 .546628$

H -1.721525 3.8751502 .003912

H -1.0296215 .3348671 .330220$

H -0.2212424 .5504272 .681497$

H $2.968380-1.222682-1.463219$

H $3.0399990 .494147-1.014391$

H $-0.9148770 .448876-3.194242$

H $\quad 0.2341280 .350127-4.550554$

H $1.0381622 .713930-4.068659$

H -1.786962 2.698725 -2.886855

H -1.544648 $4.967729-4.030925$

H $0.1874914 .928771-3.797288$

H $-2.1294256 .459816-0.723667$

H -2.863637 $6.011512-2.362889$

H $0.1870976 .037343-0.292140$

H $1.1839445 .498246-1.601425$

H $1.0579963 .211518-0.985961$

H $0.8351280 .371716-1.644608$

C $-1.148633-4.033571-1.188352$

O $-2.216158-3.070273-1.205495$

C $-3.309181-3.593556-1.984287$

C $-2.951075-5.040506-2.306341$

C $-1.436270-4.975772-2.355990$

H -3.320813 -3.022721 -2.920409

C $-4.633525-3.380326-1.239490$ 
H -3.267016 -5.732015 -1.518292 H -3.398969 -5.377966 -3.245908 C $-0.766251-6.333669-2.239197$ H -1.136381 -4.505230 -3.301992 C $-4.831569-1.934387-0.733083$ O $-4.672326-4.222442-0.081430$ H -5.477218 -3.679577 -1.872865 H $-4.100346-1.7309560 .052441$ O $-6.117955-1.865058-0.104790$ C $-4.746680-0.857105-1.825792$ C $-5.0388670 .578483-1.346596$ H -3.761253 -0.879807 -2.302930 O $-5.707276-1.189223-2.835394$ H $-5.3093851 .170081-2.230530$ H $-5.9342620 .584886-0.716317$ H $\quad 0.323236-6.236396-2.274779$ H -1.029431 -6.831667-1.299944 H -1.073443 -6.985966 -3.063179 C $0.197843-3.321234-1.297305$ H $-4.930019-5.113255-0.372353$ H $-6.788714-2.006448-0.794889$ H -5.579232 $-0.573246-3.576837$ H $\quad 0.233978-2.719590-2.214010$ H $1.007436-4.056935-1.366154$ H -1.217453 -4.570511 -0.232725 C $-4.4339190 .856624 \quad 1.676345$ $\begin{array}{llll}\text { O } & -3.495796 & 0.663942 & 0.611452\end{array}$ C $-3.8474181 .262230-0.638072$ C $-4.0299702 .775334-0.452769$ C $-5.064474 \quad 3.0848260 .622821$ C -4.7189172 .3468541 .913158$ H $-5.362797 \quad 0.3251521 .441224$ C $-\begin{array}{llll}3.828866 & 0.241331 & 2.944967\end{array}$ H $-2.9782001 .125816-1.293754$ O $-4.4082893 .382732-1.683451$ H $-3.0694203 .216837-0.160553$ H -6.0770582 .8456470 .277681$ $\begin{array}{lllll}\text { O } & -5.046017 & 4.492556 & 0.871238\end{array}$ H -3.8446662 .8263812 .369283$ O $\quad-5.7945282 .4961312 .838804$ C $-3.710534-1.2597592 .852229$ H -2.821041 0.6337103 .123189 H -4.4203700 .4799483 .836750$ H -4.563985 4.326108 -1.506360 H -5.6762174 .6673441 .591268$ H -6.5785592 .0655002 .457218$ O $-4.908287-1.8348063 .153633$ O $-2.675741-1.8403982 .551427$ C $-4.969079-3.2663153 .152339$ H $-4.154449-3.7257222 .585907$ H $-5.919642-3.5647832 .702169$ H -4.943055 -3.618260 4.187010 SCF Energy (B3LYP/6-31G**//MMFF) $=-3245.91162364$

$04 \quad 00104$

MM̄FF Geometry

C $1.113786-0.2683402 .590399$

C $2.296234-0.3516903 .219980$

C 3.4394370 .6324483 .100260

O 3.0669681 .6998912 .200180

C $4.681545-0.0685212 .524714$

C $5.959530 \quad 0.7218692 .716964$

C $6.575151 \quad 1.4363331 .754935$

C $6.5693950 .627244 \quad 4.092124$

C 6.1229761 .6719160 .340527

C $7.0102470 .956371-0.687862$

C $6.780808-0.562451-0.735743$

C $7.754695-1.226411-1.711872$

C $7.470225-2.728421-1.868363$

O $5.434657-0.782791-1.168280$

O $8.224700-3.210986-2.990474$

C $5.969478-2.928155-2.172871$

C $7.981740-3.527129-0.664629$

O $5.630299-4.312900-2.180570$

C $5.036581-2.158897-1.208830$

C $3.580799-2.213555-1.732295$

O $5.094562-2.7659650 .082959$

C 3.3239872 .9827842 .551764
O 3.8840473 .3659463 .569235

C 2.8043803 .9314221 .536448

C 2.2815153 .5504230 .359659

C $1.7237734 .431245-0.670419$

C $1.6609555 .906103-0.372283$

C $2.608451-1.290535-0.983740$

C $1.217752-1.312434-1.595585$

C $-0.960384-0.101408-1.487545$

O $0.875644-2.112689-2.461922$

C $-0.9385400 .952060-2.549499$

C $-1.5343462 .147358-2.424476$

C -1.503922 $3.207794-3.486479$

C $-0.8558104 .490073-3.010672$

C -1.602472 $5.557481-2.681025$

C $0.6561124 .567032-3.002644$

C $1.2711703 .877733-1.815410$

N $0.399260-0.337904-1.041343$

H $\quad 0.9190470 .5613161 .916916$

H $2.467730-1.1902343 .892164$

H $3.634324 \quad 1.0235234 .105831$

H $4.519846-0.2764381 .461762$

H $4.820919-1.0501472 .997438$

H 7.5072261 .9397002 .010054

H 7.5129751 .1781874 .168288

H 5.8888331 .0373094 .844570

H $6.777994-0.4178004 .343906$

H 5.0718051 .4092570 .190698

H $\quad \begin{array}{llll}6.183614 & 2.752507 & 0.157246\end{array}$

H $6.7788191 .381364-1.673360$

H $8.0662511 .171142-0.482052$

H $6.912736-0.9769340 .269592$

H $8.793625-1.062920-1.399585$

H $7.660484-0.748986-2.697260$

H $7.950128-2.712440-3.778905$

H $5.788802-2.559940-3.192359$

H $9.065948-3.401206-0.556894$

H $7.818624-4.601560-0.804456$

H $7.519359-3.228644 \quad 0.278427$

H $6.234625-4.756259-2.800388$

H $3.568961-1.917466-2.789657$

H $3.204338-3.243026-1.677769$

H $4.266381-2.5914330 .556217$

H 2.8759314 .9772711 .817823

H 2.2480802 .4895990 .117170

H 1.0987896 .0893510 .550304

H $1.1660036 .487216-1.153316$

H $2.6697176 .317624-0.257526$

H $2.513500-1.5977870 .062449$

H $2.973713-0.257369-1.007974$

H $-1.5438650 .203228-0.613838$

H - $-1.385335-1.026855-1.887023$

H $-0.4050520 .716508-3.467529$

H -2.079211 2.378877 -1.512835

H -2.540059 $3.398025-3.795700$

H $-0.9885302 .856345-4.388966$

H $-1.1472986 .491283-2.368584$

H -2.686388 $5.519978-2.711773$

H $0.9896445 .608608-3.069232$

H $1.0463834 .100742-3.916661$

H $1.3613732 .798287-1.936896$

H $0.8023740 .332028-0.393166$

C -1.268756 -0.618785 3.276471

O $-1.773990 \quad 0.3352162 .320446$

C -3.2063300 .1801752 .209144$

C $-3.612119-0.6974113 .381475$

C $-2.408196-1.6091183 .508363$

H $-3.369577-0.3480341 .263034$

C $-3.850737 \quad 1.5711052 .168218$

H $-3.730835-0.1012204 .294641$

H $-4.544127-1.2383553 .201154$

C $-2.335771-2.3363464 .839429$

H -2.437739 -2.346716 2.695001

C $-5.388673 \quad 1.6027112 .108996$

O -3.3370082 .2859531 .041920$

H -3.5225832 .1294513 .054632$

H -5.7068422 .6389481 .932859$

O -5.8830441 .2459463 .403740$

C -6.0687300 .6995391 .057014$ 
C $-5.6042480 .960759-0.385181$ H -5.928223 -0.3557381 .314138$ $\begin{array}{llll}\text { O } & -7.478451 & 0.949936 & 1.113478\end{array}$ H -5.668012 $2.037620-0.586112$ H $-4.5531670 .675817-0.482964$ H $-1.448188-2.9747454 .887939$ H $-2.292022-1.6340435 .678666$ H -3.216414 -2.971498 4.979564 C $0.003581-1.2722632 .734260$ H -2.366399 $2.264173 \quad 1.101836$ H -6.8246891 .4853053 .435215$ H $-7.8123650 .557817 \quad 1.937931$ H $-0.189353-1.6975771 .741862$ H $0.312666-2.0941553 .389971$ H -1.048704-0.060919 4.196236 C $-5.417465-1.960559-1.290254$

O $-6.602549-1.167474-1.160142$ C $-6.4392430 .222589-1.456722$ C $-5.925036 \quad 0.395772-2.893696$ C $-4.663393-0.424562-3.150835$ C $-4.867174-1.874854-2.719274$ H $-4.662928-1.617738-0.571536$ C $-5.792768-3.404649-0.941845$ H $-7.4451470 .659991-1.426661$ O $-5.690716 \quad 1.767787-3.194436$ H $-6.7034220 .055228-3.588427$ H $-3.7957200 .009264-2.645310$ O $-4.364703-0.407346-4.547653$ H $-5.551999-2.370868-3.419067$ O $-3.619276-2.562026-2.782684$ C $-6.073798-3.5568040 .534360$ H -6.695864 -3.721777 -1.477603 H $-4.998347-4.107754-1.217878$ H $-4.8831592 .047897-2.731228$ H $-4.2696670 .524002-4.811669$ H -3.279072 -2.463902 -3.688652 O $-4.976637-4.058758 \quad 1.169291$ O $-7.139023-3.2590531 .059290$ C $-5.134888-4.3120432 .568779$ H $-4.155729-4.5707272 .980115$ H $-5.509644-3.424723 \quad 3.086854$ H $-5.813557-5.1570152 .717264$

SCF Energy (B3LYP/6-31G**//MMFF) $=-3245.91477533$

04_00105

MM̄FF Geometry

C $-0.361381-1.812761-0.836649$

C $0.442243-0.952122-0.192243$

C $0.253808-0.407781 \quad 1.204649$

O $-1.033779-0.8187351 .711246$

C $0.290277 \quad 1.129662 \quad 1.178400$

C 0.4858531 .7564492 .543392

$\begin{array}{llll}\text { C } & -0.484916 & 2.353729 & 3.261968\end{array}$

C 1.9000261 .7319673 .064129

C -1.946658 2.4712082 .930275

C -2.357522 3.9069872 .571930

C $-1.863222 \quad 4.354121 \quad 1.187084$

C -2.243097 5.8118370 .917682

C $-1.8618726 .246496-0.506866$

O $-2.479773 \quad 3.506623 \quad 0.213313$

O $-2.4962857 .506335-0.773836$

C -2.404759 5.208939-1.514569

C $-0.3584106 .517666-0.629055$

O $-1.946758 \quad 5.491152-2.834769$

C -2.065674 $3.748454-1.137068$

C $-2.8430452 .771324-2.052062$

O $-0.663873 \quad 3.535714-1.307852$

C -1.127724 -1.280966 2.981286

O $-0.218609-1.3896693 .791799$

C -2.517663 -1.690867 3.297878

C $-3.561848-1.4536402 .487624$

C $-4.951949-1.8546712 .717250$

C $-5.255014-2.6605123 .952769$

C $-2.7706251 .304315-1.604745$

C $-3.6261280 .396800-2.472212$

C $-4.516024-1.935376-2.535853$

O $-4.1552270 .766417-3.516688$

C $-5.876762-1.904489-1.914039$
C $-6.386143-2.905085-1.179718$

C $-7.747538-2.861255-0.545770$

C $-7.699694-3.0274120 .958123$

C $-8.034682-4.1954521 .531940$

C $-7.351967-1.8195971 .802020$

C $-5.880104-1.5107431 .799682$

$\mathrm{N}-3.711228-0.883668-1.942554$

H -1.258372 -2.187099-0.351993

H $1.344792-0.622251-0.698837$

H $1.065577-0.8124121 .820779$

H -0.6299201 .5024010 .718187$

H 1.1044301 .4822850 .532059

H -0.2127372 .7972624 .219155$

H 1.9972372 .2392294 .029892

H $2.2424870 .702002 \quad 3.200179$

H 2.5721602 .2347782 .361275

H $-2.250592 \quad 1.7756532 .142453$

H -2.506944 2.1636853 .822595

H $-3.454465 \quad 3.9475102 .587377$

H $-1.9965384 .600577 \quad 3.341367$

H -0.7755694 .2330441 .139626$

H -1.7846346 .4786451 .658349$

H -3.328750 5.9309521 .040031

H $-3.4571717 .388494-0.682322$

H -3.498388 $5.312897-1.537583$

H $-0.059206 \quad 7.3194950 .056888$

H $-0.1004316 .872306-1.633164$

H $0.2569705 .644217-0.404023$

H -2.182351 $6.413809-3.032636$

H -3.902334 $3.059909-2.067207$

H $-2.4695342 .847198-3.081149$

H $-0.5007122 .592051-1.459517$

H -2.634522 $-2.203657 \quad 4.247114$

H $-3.392903-0.9176871 .555049$

H $-4.653410-3.5760553 .973073$

H $-6.298321-2.9760064 .020998$

H $-5.038418-2.0767954 .854268$

H - $-1.7416690 .936833-1.657885$

H $-3.1179401 .207801-0.569616$

H -3.998273 - $2.882833-2.355832$

H $-4.594636-1.778046-3.616130$

H $-6.471691-1.008537-2.076582$

H -5.797636 -3.806652 -1.025736

H $-8.349120-3.661805-0.995638$

H -8.272049 -1.928313 - 0.787791

H $-8.034141-4.3170332 .610005$

H $-8.313340-5.0581850 .936080$

H -7.723737 -1.9413522 .825612$

H $-7.894081-0.9449431 .419871$

H $-5.561680-0.9251320 .937137$

H $-3.301775-1.066260-1.031151$

C $0.655876-3.644594-2.293104$

O $1.997026-3.454276-1.794608$

C $2.237139-4.431097-0.769504$

C $1.285650-5.576635-1.087829$

C $0.044718-4.806515-1.502111$

H $1.931227-3.973343 \quad 0.179563$

C $3.719364-4.803010-0.692727$

H $1.647618-6.185776-1.924108$

H $1.116992-6.232919-0.228945$

C $-0.953494-5.627376-2.299928$

H $-0.451713-4.448904-0.592416$

C $4.687473-3.603344-0.606107$

O $4.070939-5.553502-1.861518$

H $3.871321-5.4780360 .158155$

H $4.794873-3.146035-1.597330$

O $5.985142-4.121332-0.284797$

C $4.302105-2.5059880 .403453$

C $5.433437-1.488914 \quad 0.650327$

H $3.416068-1.9828320 .030978$

O $3.940562-3.0953901 .653683$

H $6.202136-1.9646491 .270177$

H $5.897711-1.227494-0.305041$

H $-1.814228-5.015498-2.588975$

H $-0.503656-6.031128-3.213095$

H $-1.320665-6.470524-1.705711$

C $-0.101949-2.314659-2.232115$

H $5.011516-5.788811-1.783480$ 
H $5.930569-4.5439150 .588833$ H $4.723884-3.5451032 .012507$ H - $1.064771-2.413185-2.746820$ H $0.472001-1.560053-2.783387$ H $0.769696-3.924755-3.347913$ C $4.7902021 .280166-0.536597$ O 4.0924820 .5786970 .502390 C $4.912110-0.2213931 .366517$ C $6.000283 \quad 0.6431952 .015055$ C 6.8185551 .3943660 .971916 C $5.893902 \quad 2.176021 \quad 0.041945$ H $5.2306260 .561306-1.236629$ C $3.7588192 .130891-1.297440$ H $4.258167-0.5592372 .180057$ O $6.852029-0.1593122 .825175$ H 5.5201721 .3714032 .681831 H 7.4602930 .7097110 .405669 O $7.7021112 .305384 \quad 1.629826$ H 5.4477943 .0170420 .588000 O $6.6640812 .721422-1.027892$ C $2.9037931 .315101-2.243488$ H $3.0921992 .627471-0.582513$ H $4.2597752 .888656-1.910133$ H $7.540633 \quad 0.422640 \quad 3.189747$ H 7.1628522 .9273802 .147400 H $7.3708613 .259812-0.632229$ O $1.9100562 .115650-2.732854$ O $3.0900950 .142180-2.535091$ C $1.0237291 .508516-3.678296$ H $1.5826730 .995707-4.466752$ H $0.3552890 .814911-3.162878$ H $0.4222912 .299238-4.134735$

SCF Energy (B3LYP/6-31G**//MMFF) $=-3245.91375903$

04_00106

MMFF Geometry

C $0.527328-1.6758881 .215440$

C $-0.359977-1.9193730 .237150$

C $-0.047207-2.067814-1.234373$

O $1.362917-2.336646-1.396075$

C $-0.387326-0.758628-1.963618$

C $-0.399318-0.877641-3.472885$

C $0.596182-0.469261-4.284366$

C $-1.661703-1.455176-4.056728$

C $1.937760 \quad 0.087573-3.895499$

C $2.0880331 .581874-4.213328$

C $1.277858 \quad 2.492202-3.277184$

C $1.4736283 .964828-3.647328$

C $0.7734404 .907858-2.654725$

O $1.7348862 .260710-1.940605$

O $1.2733846 .235250-2.880018$

C $1.1793044 .511258-1.218034$

C $-0.7396604 .969512-2.895009$

O $0.4460805 .259806-0.251836$

C $1.0289573 .001786-0.936915$

C 1.6008002 .5821750 .436329

O $-0.3617612 .663837-0.931664$

C $1.755297-3.288519-2.277908$

O $1.032898-3.964287-2.997248$

C $3.228281-3.461417-2.260383$

C $4.062781-2.643550-1.599113$

C $5.518291-2.779143-1.499542$

C $6.159784-3.967955-2.164859$

C $3.091818 \quad 2.8623200 .621781$

C $3.6187142 .244058 \quad 1.901184$

C $4.372303 \quad 0.0744182 .873194$

O 3.8569692 .9260512 .895448

C $5.822377-0.1308642 .565534$

C $6.396395-1.3374372 .443691$

C $7.841135-1.5346852 .081719$

C $8.026554-2.3969460 .850157$

C $8.556859-3.6279520 .947459$

C $7.676935-1.808231-0.501177$

C $6.200994-1.857955-0.787488$

$\begin{array}{lllll}\text { N } & 3.788445 & 0.871397 & 1.808747\end{array}$

H $1.581349-1.5572410 .979973$

H $-1.412226-2.0087240 .500593$

H $-0.643751-2.905845-1.614044$
H $\quad 0.3258000 .012660-1.657285$

H $-1.372554-0.390680-1.645340$

H $0.456433-0.576466-5.359434$

H $-1.636570-1.492169-5.150914$

H -1.816452 -2.477034 -3.697074

H $-2.525300-0.847679-3.767735$

H $2.174994-0.104346-2.845125$

H $2.694183-0.463888-4.468788$

H $3.152983 \quad 1.829805-4.113244$

H $1.8084061 .773146-5.256703$

H $0.2183802 .222704-3.350914$

H $1.1273994 .157838-4.670422$

H $2.5473364 .200178-3.645794$

H $1.0936336 .469985-3.806487$

H $2.2323724 .794633-1.098138$

H $-0.9515655 .341683-3.904739$

H $-1.2202195 .675787-2.209022$

H $-1.2315073 .999946-2.793642$

H $0.5688456 .201681-0.461002$

H $1.029723 \quad 3.055768 \quad 1.245493$

H 1.4299551 .5042070 .559051

H $-0.475843 \quad 1.838315-0.432354$

H $3.591142-4.313097-2.826626$

H $3.647889-1.793213-1.060136$

H $5.996838-3.935588-3.247790$

H $5.740383-4.901637-1.773834$

H $7.239260-4.023461-2.009576$

H $3.6783232 .471712-0.217786$

H 3.2833743 .9395110 .673245

H $3.818321-0.8685772 .915545$

H 4.2623170 .5920223 .831047

H $\quad 6.427416 \quad 0.7613612 .420784$

H $5.797983-2.2316952 .601367$

H $8.340166-1.9918072 .945857$

H $8.345605-0.5739131 .919624$

H $8.721528-4.2437710 .069710$

H $8.835701-4.0497961 .907417$

H $8.249505-2.299075-1.296170$

H $8.006086-0.761757-0.536915$

H $5.643364-1.031103-0.347890$

H $3.6101970 .410697 \quad 0.920690$

C $0.194213-0.084778 \quad 3.130393$

$\begin{array}{lllll}\text { O } & -0.861187 & 0.652538 & 2.477783\end{array}$

C $-1.733496 \quad 1.1911423 .484450$

C -0.8502581 .3431094 .711510$

C $-0.0465400 .057195 \quad 4.638083$

H $-2.490873 \quad 0.424340 \quad 3.689444$

C -2.439976 2.4550182 .983922

H $-0.178227 \quad 2.204423 \quad 4.627413$

H -1.4262861 .4385905 .636310$

C $1.2337850 .090563 \quad 5.455537$

H $-0.673282-0.7692324 .999600$

C -3.0783102 .3074341 .587809$

O -1.5023913 .5332042 .882839$

H -3.199167 2.770288 3.710011

H -2.2956262 .3166720 .823079$

$\begin{array}{lllll}\text { O } & -3.884531 & 3.466849 & 1.345568\end{array}$

C -3.9496181 .0554011 .394288$

C $-4.592558 \quad 1.001667-0.005038$

H -3.3342390 .1616821 .535172$

$\begin{array}{llll}\text { O } & -4.973464 & 1.021051 & 2.386992\end{array}$

H $-5.347737 \quad 1.791344-0.098021$

H -3.809303 $1.182012-0.751775$

H $1.790402-0.8453815 .343495$

H 1.8879810 .9113525 .142813

H 1.0084590 .2269476 .518182

C $0.151778-1.5421852 .664611$

H $-1.310708 \quad 3.842342 \quad 3.784213$

H $-3.307733 \quad 4.2443701 .440988$

H -5.5010051 .8326862 .292652$

H $-0.851746-1.9547072 .824351$

H $\quad 0.847939-2.149198 \quad 3.254992$

H $\quad \begin{array}{llll}1.137919 & 0.388500 & 2.839408\end{array}$

C $-4.668042-2.699512-0.487970$

O $-4.229250-1.347667-0.308200$

C $-5.262965-0.357469-0.281652$

C $-6.031576-0.362651-1.611043$

C $-6.580267-1.758929-1.922958$ 
C $-5.507065-2.844004-1.769008$ H -3.743829 -3.276032 -0.625225 C $-5.371618-3.2358330 .763633$ H $-5.954448-0.5745450 .539573$ $\begin{array}{llll}\text { O } & -5.166696 & 0.059703 & -2.663084\end{array}$ H -6.853756 $0.360305-1.546964$ H $-6.969233-1.769465-2.948416$ O $-7.670860-2.024295-1.045487$ H $-5.959615-3.842081-1.795360$ O $-4.608064-2.765756-2.878449$ C -4.433832 -3.2313291 .949158$ H $-6.265808-2.6595031 .017929$ H $-5.701201-4.2706200 .614487$ H $-5.6871070 .072588-3.484168$ H -8.023624 -2.901409-1.272635 H $-5.127245-2.905886-3.688379$ O $-4.888397-2.3656612 .899485$ O $-3.417170-3.9099172 .018640$ C $-4.088945-2.2695904 .081846$ H -3.058193 -2.007701 3.828763 H $-4.505385-1.4781344 .710718$ H -4.122504 -3.213629 4.633464

SCF Energy (B3LYP/6-31G**//MMFF) $=-3245.91057338$

04_00107

MM̄FF Geometry

C -2.639907 2.557200 2.732238

C $-2.2006501 .363897 \quad 2.301669$

C $-1.315407 \quad 0.4537053 .113423$

O $-0.014855 \quad 0.3596752 .484949$

C $-1.927062-0.958153 \quad 3.145935$

C $-1.228359-1.8963684 .109579$

C $-0.378160-2.8792723 .752330$

C $-1.588096-1.7045845 .561222$

C $0.108907-3.231324 \quad 2.373075$

C $1.641989-3.2778002 .333377$

C $2.187093-3.676290 \quad 0.952417$

C $3.717848-3.7103470 .985927$

C $4.332056-3.964203-0.396740$

O $1.728523-2.717909-0.005023$

O $5.729010-3.646370-0.298772$

C $3.694430-2.991062-1.411505$

C $4.256120-5.447218-0.777111$

O $4.145515-3.277592-2.733155$

C $2.153546-2.967576-1.352279$

C $1.539683-1.869667-2.253337$

O $1.654513-4.224858-1.826152$

$\begin{array}{lllll}\text { C } & 0.871173 & 1.360947 & 2.744138\end{array}$

$\begin{array}{lllll}\text { O } & 0.655890 & 2.387213 & 3.370047\end{array}$

C 2.1798250 .9746852 .167783

C 3.2235941 .8099542 .285413

C 4.6028691 .5513291 .862343

C $4.9049460 .216596 \quad 1.236516$

C $1.843934-0.428253-1.836544$

C $2.9963870 .218186-2.583531$

C $4.2690572 .362802-2.669892$

O $3.594756-0.322433-3.508575$

C $5.5538542 .170459-1.929270$

C $6.1977693 .161631-1.294571$

C $7.5249922 .991889-0.615036$

C $7.504696 \quad 3.4240920 .832481$

C 7.9897714 .6207821 .204547

C 7.0199002 .4266811 .858768

C 5.5357162 .5003412 .089066

N $3.2525521 .497369-2.105511$

H -2.3378682 .9183553 .711987$

H -2.495224 $1.014507 \quad 1.315182$

H $-1.198762 \quad 0.8313704 .137059$ H -1.925143 -1.3800182 .135139$ H -2.985453 -0.9019913 .435408$ H $0.024020-3.5132654 .542109$ H -1.104949 -2.442018 6.211177 H $-1.281544-0.7128365 .906935$ H -2.669685 -1.803342 5.700641 H $-0.293767-4.2149242 .104270$ H $-0.249097-2.5211351 .624207$ H $2.026510-2.2864732 .605260$ H $2.011528-3.9859583 .086013$
H $1.786652-4.6629550 .691205$

H $4.073868-4.4559701 .707982$

H $4.090429-2.7453871 .353027$

H $6.112864-3.730219-1.188696$

H $4.076552-1.991946-1.170090$

H $4.804910-6.055125-0.047708$

H $4.737998-5.635744-1.742705$

H $3.233259-5.827165-0.820257$

H $3.658702-4.057253-3.050186$

H $1.792980-2.053863-3.304262$

H $0.449529-1.984619-2.201990$

H $\quad 0.731575-4.105890-2.105160$

H $2.258766-0.0010271 .703788$

H 3.0633742 .7723312 .770581

H $4.781926-0.5817901 .975308$

H 5.9189630 .1398660 .838313

H $4.236387 \quad 0.0217230 .392651$

H $\quad 0.9622490 .182556-2.061354$

H $2.023149-0.352272-0.760204$

H $3.8980883 .390186-2.597556$

H $4.4119972 .118611-3.727496$

H $5.9926921 .175608-1.947952$

H $5.7706264 .161940-1.299244$

H $8.2601723 .587758-1.172059$

H $7.8796381 .955673-0.680845$

H 8.0100954 .9224742 .246665

H $8.3717125 .327444 \quad 0.475032$

H 7.5064282 .6285022 .823206

H $7.3629661 .424097 \quad 1.588415$

H 5.2186863 .4358322 .552582

H $2.7459521 .833313-1.291289$

C -2.799859 $4.297026 \quad 0.910562$

O $-2.2929953 .422955-0.118950$

C $-2.9607793 .735680-1.350353$

C $-3.2413465 .223209-1.243842$

C -3.7099715 .3106810 .200309$

H $-3.9152333 .195118-1.350665$

C $-2.1229903 .270788-2.543200$

H -2.331746 $5.818664-1.382684$

H -3.994805 $5.563350-1.960084$

C $-3.626535 \quad 6.7112830 .783083$

H $-4.7551094 .976057 \quad 0.249448$

C -1.649206 $1.805502-2.419990$

O $-0.9615594 .102347-2.636901$

H -2.689704 $3.419907-3.469975$

H $-0.8698671 .733956-1.651276$

O $-1.039483 \quad 1.423515-3.657997$

C $-2.7679920 .795404-2.101447$

C $-2.243989-0.648443-1.991814$

H -3.242810 $1.063739-1.153552$

O $-3.7749930 .861275-3.109527$

H $-1.875377-0.985826-2.967719$

H $-1.406979-0.651320-1.283338$

H $-3.954687 \quad 6.7158391 .827431$

H -2.603121 7.0997640 .748881

H -4.2665337 .4004310 .222669$

C $-3.555337 \quad 3.446863 \quad 1.937126$

H $-0.5350793 .916661-3.490148$

H $-0.216283 \quad 1.932057-3.749190$

H $-3.3525930 .651596-3.960152$

H -4.3034072 .8220081 .433127$

H -4.0987694 .0927992 .636798$

H $-1.934664 \quad 4.7884191 .371781$

C $-4.734348-2.0247180 .429894$

O $-3.690781-1.252582-0.176718$

C $-3.324953-1.633916-1.507111$

C $-2.828938-3.087961-1.522879$

C $-3.874785-4.031340-0.920420$

C $-4.388474-3.5232320 .432083$

H $-4.759650-1.6856281 .473705$

C $-6.099552-1.716312-0.193596$

H $-4.195940-1.540265-2.164268$

O $-1.614078-3.186367-0.783441$

H $-2.611491-3.376767-2.558282$

H -3.429867 -5.025814 -0.794638

O $-4.957156-4.155314-1.838191$

H $-5.265736-4.0970260 .752746$

O $-3.374805-3.7276931 .420228$ 
C $-6.485562-0.2778100 .060426$ H $-6.128427-1.904478-1.270601$ H $-6.882626-2.3428510 .249503$ H -1.320843 -4.112622 -0.818614 H $-5.600894-4.773029-1.451420$ H $-3.185531-4.6806451 .455324$ O $-6.3556590 .450371-1.084647$ O $-6.8423890 .149257 \quad 1.151042$ C $-6.6891411 .837259-0.975342$ H $-6.4773662 .314584-1.935758$ H $-7.7540761 .950665-0.752474$ H $-6.0805712 .318070-0.204353$

SCF Energy (B3LYP/6-31G**//MMFF) $=-3245.90734212$

04_00108

MM̄FF Geometry

C $-0.550213-1.7648473 .844315$

C $-0.261124-0.6856293 .101793$

C $-0.718996 \quad 0.7027713 .464197$

O

C 0.4608371 .6892533 .406152

C 0.2820762 .8734824 .333820

C -0.2712694 .0525493 .991065$

C 0.8188992 .6691395 .727599

C -0.9134854 .4389502 .688621$

C -0.0895275 .4612951 .896649$

C 1.0211844 .8111341 .057154

C 1.8739785 .8860720 .380513

C $2.9183435 .278058-0.564680$

O $\quad 0.386975 \quad 3.991017 \quad 0.068018$

O $3.4450456 .355133-1.354082$

C $2.2077334 .292508-1.515445$

C $4.104798 \quad 4.6923880 .209137$

O $3.1533283 .618972-2.342294$

C $1.2851693 .281278-0.792630$

C $0.4377442 .513242-1.835844$

O $2.0942222 .353908-0.067152$

C -2.9781180 .7804382 .631514$

$\begin{array}{lllll}\text { O } & -3.450358 & 0.054247 & 3.489894\end{array}$

C -3.7333301 .4329751 .540322$

C $-4.959150 \quad 0.9975371 .217992$

C $-5.801376 \quad 1.5290620 .143990$

C $-5.2967072 .734116-0.607747$

C $-0.6776991 .637908-1.244410$

C $-1.4187020 .866955-2.326772$

C $-3.292433-0.750417-2.631370$

O $-1.1113680 .916677-3.515169$

C $-4.535844-0.014709-3.021082$

C $-5.768569-0.531068-2.897529$

C $-7.0207700 .181796-3.324760$

C $-8.0870340 .196701-2.250730$

C $-9.126398-0.653792-2.297456$

C $-7.9925931 .252551-1.171459$

C $-6.9690640 .908189-0.123652$

N -2.455770 $0.104062-1.807290$

H -1.155666 -1.648301 4.740017

H $0.328434-0.8009812 .195277$

H $-1.180680 \quad 0.7183354 .460074$

H 0.6062752 .0412362 .378023

H $\quad 1.398671 \quad 1.1882093 .682060$

$\mathrm{H} \quad-0.310506 \quad 4.8350954 .748526$

H $\quad 0.6575693 .541628 \quad 6.369627$

H $\quad 0.327091 \quad 1.814500 \quad 6.203314$

H 1.8963302 .4770645 .695119

H -1.144316 3.5707072 .068044

H -1.8825914 .8920092 .934621$

H -0.7779765 .9841291 .219518$

H 0.3335946 .2138642 .573256

H 1.6434894 .1847711 .705296

H 2.3585616 .5269161 .127886

H $1.2234376 .551272-0.204949$

H $4.0489615 .969881-2.012266$

H $1.5923444 .896780-2.195940$

H 4.5809445 .4689830 .819822

H $4.8838684 .331824-0.471424$

H 3.8213523 .8742640 .874566

H $3.6119622 .961747-1.791945$

H $-0.0394713 .234997-2.512305$
H $\quad 1.092481 \quad 1.883312-2.448269$

H 1.6188781 .5131680 .020691

H -3.244986 2.2448551 .013610

H -5.3778900 .1618311 .777107$

H -5.1814083 .5866400 .070830$

H $-5.9582473 .057835-1.413317$

H $-4.3273132 .524580-1.072796$

H $-0.2693430 .908603-0.537186$

H $-1.4030902 .265044-0.714872$

H -3.511335 -1.649840 -2.047938

H $-2.741093-1.040634-3.531219$

H $-4.4158570 .977261-3.450331$

H -5.884198 -1.536586 -2.498670

H -7.402116 -0.327224 -4.219789

H $-6.8080301 .211633-3.637499$

H $-9.909678-0.631489-1.546990$

H -9.214381-1.395523 -3.084562

H $-8.959147 \quad 1.356820-0.659988$

H -7.820165 2.226535 -1.636980

H -7.2430800 .0397440 .477181$

H $-2.6634560 .157101-0.814773$

C -1.030769 -3.8747892 .566079$

O $-0.738864-3.4359541 .219768$

C $-0.456847-4.592808 \quad 0.413900$

C $-1.145259-5.7459581 .124470$

C $-0.845119-5.3956922 .569011$

H $\quad 0.626066-4.756540 \quad 0.455219$

C $-0.883512-4.365126-1.037025$

H $-2.227121-5.7433030 .948395$

H $-0.747569-6.7205430 .826372$

C $-1.732279-6.1179023 .568371$

H $0.203431-5.6459812 .781237$

C $-0.252262-3.122393-1.699063$

O $-2.306551-4.212597-1.095897$

H $-0.653158-5.257977-1.629822$

H $-0.701295-2.208757-1.293039$

O $-0.586626-3.153624-3.091644$

C $1.278265-3.033133-1.565895$

C $1.888210-1.882746-2.388943$

H $1.535936-2.895561-0.510677$

O $1.870175-4.263020-1.986343$

H $1.797699-2.105490-3.458861$

H $1.327133-0.964799-2.176063$

H -1.493484 -5.8126264 .592057$

H -2.792048 -5.905003 3.391601

H $-1.590669-7.2011873 .495947$

C $-0.086160-3.1587823 .533343$

H $-2.546324-3.471472-0.513161$

H -1.554416 -3.223998 -3.156349

H $1.610986-4.412322-2.912015$

H $0.928400-3.1199303 .117931$

H $-0.023586-3.7039104 .482560$

H $-2.077180-3.6123262 .767137$

C $4.753871-0.930407-0.196201$

O $3.438402-1.151237-0.719357$

C $3.377372-1.668051-2.053449$

C $4.058247-0.700079-3.031972$

C $5.487476-0.368777-2.591222$

C $5.5580030 .012735-1.108130$

H $4.588775-0.4176540 .760007$

C $5.476270-2.2492120 .101070$

H $3.888425-2.635726-2.091596$

O $3.3058670 .504719-3.139616$

H $4.075429-1.157107-4.028207$

H $5.8635450 .462228-3.200839$

O $6.313213-1.501982-2.842060$

H $6.6019970 .050947-0.776388$

O $5.028273 \quad 1.334612-0.973702$

C $4.738598-3.0357851 .159924$

H $5.590943-2.875485-0.788083$

H $6.487507-2.0640150 .481826$

H $3.2143900 .874365-2.244987$

H $7.222247-1.263405-2.592461$

H $5.129587 \quad 1.599870-0.043578$

O $4.215315-4.1721780 .618034$

O $4.631520-2.6763742 .325582$

C $3.489492-5.0090661 .523251$

H $4.162943-5.4086922 .287044$ 
H $2.669825-4.4516151 .984917$

H $3.068825-5.8419010 .953592$

SCF Energy (B3LYP/6-31G**//MMFF) $=-3245.93425305$

04_00109

MM̄FF Geometry

C $0.6508894 .265506-1.756235$

C $1.9486153 .933507-1.839392$

C $3.0255614 .180677-0.804214$

O $3.5287932 .885820-0.387365$

C 2.5625514 .9331050 .460214

C 3.6474125 .1091261 .507459

C 3.6804204 .4704432 .695342

C 4.7261146 .1010061 .155855

C 2.7019013 .4567793 .222222

C 3.3508962 .0732103 .359789

C 2.3012050 .9518163 .382890

C $2.958162-0.3937633 .688208$

C $1.962909-1.5582613 .583951$

O 1.6914590 .9161742 .087793

O $2.719083-2.7796873 .572199$

C $1.224960-1.4699522 .230254$

C $1.039827-1.6313554 .806451$

O $\quad 0.198965-2.4584332 .179523$

C $0.656466-0.0648451 .930445$

C 0.1133090 .0840270 .485416

$\begin{array}{lllll}\text { O } & -0.433774 & 0.198676 & 2.821183\end{array}$

C $4.5540352 .343836-1.100295$

O $5.1108332 .825199-2.073764$

C $4.8742931 .032675-0.493583$

C $5.7208650 .195418-1.110509$

C $6.111955-1.135800-0.639015$

C $5.464794-1.6484270 .621119$

C $1.147909-0.118951-0.626771$

C $1.219122-1.530513-1.181438$

C $2.546718-2.913400-2.778665$

O $0.426601-2.417227-0.879074$

C $3.623755-3.654351-2.050931$

C $4.759445-4.066684-2.634555$

C $5.824591-4.857004-1.929884$

C $7.198049-4.237985-2.062106$

C $8.062492-4.668188-2.996698$

C $7.600753-3.187758-1.052261$

C $7.017178-1.834629-1.355694$

N 2.265406-1.671305-2.084248

H $0.272868 \quad 4.783066-0.879644$

H $2.2841853 .417165-2.737439$

H $3.8283184 .757120-1.281039$

H 1.7036154 .4120330 .890748

H 2.1919095 .9286970 .181493

H 4.5057314 .6873423 .372550

H 5.4339116 .2541711 .977455

H $5.2987505 .756340 \quad 0.289377$

H 4.2844637 .0742150 .918376

H 2.3526163 .7893074 .207643

H 1.8065053 .3947142 .601456

H 4.0331941 .9044572 .517660

H 3.9527332 .0485874 .276629

H 1.5419991 .1920294 .136400

H $3.431036-0.3797464 .678179$

H $3.772226-0.5710912 .972739$

H $3.242351-2.8163164 .391104$

H $1.960108-1.7374311 .461500$

H $1.627850-1.7651295 .722466$

H $\quad 0.375215-2.5004374 .748778$

H $0.428315-0.7361584 .936525$

H $-0.146284-2.4849131 .271612$

H $-0.770438-0.547176 \quad 0.338545$

H $-0.253175 \quad 1.1161040 .386194$

H $-0.999744 \quad 0.8820422 .425581$

H 4.3892940 .7824960 .442219

H $6.1715950 .510447-2.050698$

H $5.775673-1.0465831 .481828$

H $5.709679-2.6880830 .847340$

H $4.373023-1.6082820 .538881$

H $2.1422130 .183531-0.290965$

H $0.8812090 .524316-1.473631$

H $2.840644-2.653758-3.800703$
H $1.640633-3.526043-2.821433$

H $3.447662-3.886825-1.003265$

H $4.919184-3.857139-3.689962$

H $5.829750-5.866122-2.362617$

H $5.586171-4.994129-0.867820$

H $9.060618-4.251023-3.078713$

H $7.788630-5.443594-3.704491$

H $8.693069-3.071433-1.048063$

H $7.360052-3.542583-0.046218$

H $7.422448-1.381239-2.261494$

H $2.899871-0.892126-2.234545$

C $-1.3560362 .889570-2.340666$

O $-2.1067623 .376982-1.212513$

C $-3.4713472 .920353-1.324378$

C $-3.4996921 .953072-2.499262$

C $-2.3992432 .502129-3.384691$

H $-4.0657503 .814857-1.547391$

C -3.9017382 .3084210 .014365$

H -3.256282 $0.933909-2.172822$

H $-4.4745261 .927203-2.994670$

C $-1.9041111 .505326-4.418123$

H -2.772799 $3.399968-3.895186$

C $-5.307217 \quad 1.685177-0.017659$

O -3.8856643 .3434421 .002845$

H -3.1607441 .5654090 .329676$

H $-5.3426580 .867514-0.743912$

O $-6.2390702 .664381-0.496970$

C -5.8174921 .1809091 .344583$

C -4.9002030 .1791912 .070565$

H -5.9957402 .0317992 .013302$

$\begin{array}{lllll}\text { O } & -7.096377 & 0.566746 & 1.152918\end{array}$

H -3.997871 0.6976272 .415693

H $-5.442398-0.1601772 .962886$

H -1.107808 $1.939643-5.030301$

H -1.509598 $0.599579-3.944960$

H $-2.7190901 .207693-5.086142$

C $-0.3695523 .956452-2.817982$

H $-2.993378 \quad 3.7307021 .002150$

H -6.1997863 .4261920 .106719$

H -7.6626591 .2105120 .693743$

H $-0.9023514 .887119-3.048100$

H $0.1239923 .626919-3.739296$

H $-0.8138862 .000203-1.999616$

C $-5.470893-2.9175640 .036192$

O $-5.670515-1.7696270 .871074$

C $-4.488850-1.0591231 .246430$

C -3.543521-1.981794 2.033545

C $-3.236035-3.2607881 .248273$

C $-4.509451-3.9166170 .701742$

H $-6.457463-3.395135-0.025218$

C $-5.051462-2.522696-1.388881$

H $-3.973228-0.7136340 .345659$

O $-4.136704-2.3215313 .284905$

H $-2.616454-1.4372682 .244725$

H $-2.709798-3.9657131 .903349$

O $-2.361615-2.9340210 .173579$

H -4.259977 -4.710422 -0.011905

O $-5.215032-4.533761 \quad 1.782439$

C $-6.108823-1.693797-2.087898$

H -4.127335 -1.938505-1.384915

H $-4.891100-3.423193-1.992343$

H -3.499389-2.871101 3.772106

H -2.141871 -3.760710 -0.288870

H $-4.624231-5.1973792 .177569$

O $-5.560300-1.124381-3.202320$

O $-7.268351-1.570248-1.718507$

C $-6.442053-0.296100-3.966479$

H $-6.8263370 .521803-3.349169$

H $-5.8728260 .130402-4.796638$

$\mathrm{H}-7.263320-0.893847-4.372748$

SCF Energy (B3LYP/6-31G**//MMFF) $=-3245.90657646$

04_00110

MM̄FF Geometry

C $-0.5395415 .246407-0.250598$

C $-1.5696774 .390895-0.331482$

C $-2.311713 \quad 3.8808750 .874955$

O -2.2620212 .4346890 .861719$ 
C -3.7960794 .2813620 .812772$

C -4.4968904 .1064392 .147454$

C -5.1247562 .9834112 .549234$

C -4.4665345 .3183123 .042546$

C -5.2173781 .6725621 .818750$

C -6.6693291 .2855791 .520752$

C $-6.814792-0.0102180 .704027$

C $-6.352749-1.252721 \quad 1.468167$

C $-6.477719-2.5243760 .613256$

O $-6.062644 \quad 0.132510-0.505202$

O $-5.748355-3.5714371 .272751$

C $-5.785518-2.280484-0.744939$

C $-7.928668-3.0076320 .508408$

O $-6.006519-3.373894-1.632122$

C $-6.204878-0.959288-1.425513$

C $-5.347362-0.618629-2.669458$

O $-7.562911-1.071110-1.870024$

C $-1.157976 \quad 1.815201 \quad 1.351744$

O -0.1330912 .3403661 .752680$

C -1.4374800 .3605691 .330058$

C $-0.462433-0.5170421 .606266$

C $-0.607171-1.9733121 .675878$

C $-1.987701-2.5487301 .493737$

C -3.832686 -0.550428 -2.430023

C $-3.096160-1.818752-2.835178$

C $-0.822826-2.833890-2.842295$

O $-3.631830-2.747297-3.434831$

C $-0.752356-3.841494-1.738491$

C $0.403956-4.320683-1.255572$

C $0.503425-5.363879-0.181911$

C $1.317718-4.9022141 .004987$

C $2.628861-5.1821101 .097887$

C $0.588774-4.2014452 .127519$

C $\quad 0.491592-2.7175821 .920139$

N -1.752524 -1.777204 -2.485953

H $-0.2315455 .619550 \quad 0.723342$

H -1.872748 $4.005698-1.302045$

H $-1.860250 \quad 4.2509581 .804677$

H -4.3162243 .7005110 .040536$

H $-3.8939295 .331800 \quad 0.507375$

H -5.5980242 .9833753 .530574$

H -4.968795 5.1426313 .999685

H -3.432834 5.6055273 .260121

H -4.967176 6.1617272 .555910

H -4.652252 1.6929050 .884809

H $-4.748826 \quad 0.9112202 .452293$

H -7.2367131 .1957342 .455434$

H -7.1273842 .1014570 .945226$

H -7.877256 -0.1038480 .449202$

H -6.910432 -1.362604 2.406306

H $-5.298240-1.1472211 .754220$

H -6.125618 -3.685136 2.161962

H $-4.707466-2.253353-0.546497$

H -8.333726 -3.2208591 .505009$

H -7.991654 -3.949531-0.047649

H $-8.590566-2.2813780 .032517$

H -5.708085 -4.180450 -1.178072

H -5.596950 -1.297965 -3.493448

H $-5.6599910 .377809-3.014007$

H -7.709579 -0.421386 -2.577382

H $-2.451506 \quad 0.050964 \quad 1.102897$

H $\quad 0.535937-0.1354091 .814913$

H -2.009719 -3.6405871 .500462$

H -2.410052 -2.241944 0.531060

H -2.655035 -2.206568 2.292442

H $-3.4201090 .251853-3.055381$

H -3.601484 -0.303753 -1.388208

H $\quad 0.145386-2.362160-3.036585$

H -1.165114 -3.320276 -3.761573

H -1.691586 -4.214590 -1.337137

H $1.340804-3.975004-1.685486$

H $\quad 0.967400-6.255057-0.625029$

H $-0.488741-5.689076 \quad 0.154307$

H $3.211140-4.8781931 .961490$

H $3.150074-5.7121350 .307685$

H $1.126574-4.3612553 .072407$

H -0.384404 -4.673744 2.289557

H $1.448656-2.2056492 .027741$
H $-1.402241-0.998990-1.934461$

C $1.6807095 .114765-1.361164$

O $1.6054813 .688866-1.546037$

C $2.7371713 .251161-2.318863$

C $3.6365124 .472486-2.477528$

C $2.6263865 .605200-2.452503$

H $2.3529232 .954334-3.302669$

C $3.4130232 .044184-1.650775$

H $4.3381654 .566012-1.639992$

H $4.2215524 .438344-3.401679$

C $3.2447986 .963806-2.175034$

H $2.1045615 .633991-3.418377$

C $2.5200580 .786945-1.582487$

O $3.8283232 .403068-0.332259$

H $4.3194441 .807206-2.218352$

H $2.2635430 .461977-2.598255$

O $1.2823541 .090731-0.929911$

C $3.131388-0.391757-0.802003$

C $4.508508-0.842381-1.315289$

H $3.184594-0.1361470 .261724$

O $2.224252-1.494154-0.896947$

H $4.431924-1.077317-2.384199$

H $5.219799-0.016941-1.218695$

H $2.4784557 .744976-2.154472$

H $3.7668236 .978354-1.212360$

H $3.9695087 .222582-2.954043$

C $0.2717405 .703064-1.431828$

H 3.0270102 .6203980 .174890

H $0.8140851 .749083-1.470176$

H $2.164901-1.747004-1.833813$

H $-0.2158425 .396481-2.365465$

H $0.3098046 .797770-1.423411$

H $2.1194665 .287623-0.368926$

C $5.933277-0.9747051 .368444$

O $5.006422-1.9336880 .846715$

C $5.047488-2.089366-0.574998$

C $6.450688-2.532440-1.013718$

C $7.538630-1.602991-0.476014$

C $7.368580-1.3873081 .028725$

H 5.7213210 .0140020 .944103

C $5.714729-0.9014722 .883310$

H $4.365136-2.915675-0.811423$

O $6.513355-2.599230-2.434069$

H $6.634256-3.546096-0.633708$

H $7.547701-0.644425-1.008349$

O $8.820486-2.197146-0.692830$

H $7.634840-2.3079401 .563765$

O $8.265319-0.365631 \quad 1.459105$

C $4.378976-0.2736743 .205094$

H $5.722797-1.9050693 .325687$

H $6.492299-0.3169843 .387609$

H $7.365077-3.001404-2.673682$

H $8.970836-2.228825-1.652609$

H $9.163218-0.6441371 .209780$

O 4.4243901 .0680972 .964655

O $3.411179-0.9025143 .612954$

C 3.2012961 .7747983 .190363

H 3.3732712 .8319892 .971601

H 2.4235821 .4005352 .519087

H 2.8919031 .6772184 .235260

SCF Energy $\left(\mathrm{B} 3 \mathrm{LYP} / 6-31 \mathrm{G}^{* *} / / \mathrm{MMFF}\right)=-3245.90919169$

04_00111

MM̄FF Geometry

C $4.754319-2.998376-1.614953$

C $3.572854-3.180088-1.006507$

C $2.328558-3.590347-1.753759$

O $1.338390-2.544216-1.625764$

C $1.733350-4.865965-1.136592$

C $0.644932-5.475554-1.997459$

C $-0.678155-5.349199-1.776180$

C $1.138141-6.316337-3.148326$

C $-1.373085-4.566025-0.694404$

C $-2.495012-3.703107-1.282992$

C $-3.292957-2.892394-0.246387$

C $-4.009862-3.7919270 .765347$

C $-4.947501-3.0055291 .691145$

O $-4.252246-2.132204-0.995645$ 
O $\quad-5.779657-3.966528 \quad 2.358403$

C $-5.855294-2.1038940 .832999$

C $-4.168321-2.2782862 .790773$

O $-6.659774-1.2654891 .660521$

C - $-5.089595-1.266489-0.217905$

C $-6.107656-0.604339-1.181995$

O $-4.335000-0.259604 \quad 0.452247$

C $1.525248-1.403768-2.341988$

O $2.435358-1.144614-3.111855$

C $0.426908-0.487538-1.984181$

C $0.6821170 .824755-1.885716$

C $-0.2771061 .846699-1.465278$

C $-1.721591 \quad 1.432801-1.367375$

C $-5.4935760 .150434-2.370619$

C $-5.3132741 .643757-2.142951$

C $-4.7848723 .730727-3.425871$

O $-5.4356562 .180979-1.046507$

C $-3.3198204 .013799-3.528887$

C $-2.7088345 .010136-2.869566$

C $-1.2373745 .295846-2.981309$

C $-0.5620445 .441491-1.634123$

C $0.0473006 .589075-1.291268$

C $-0.5967224 .268846-0.678997$

C $0.1793183 .085011-1.184438$

N $-4.9854182 .296368-3.323741$

H $4.831821-3.173142-2.685821$

H $3.479802-2.9848070 .058956$

H $2.540298-3.748246-2.818795$

H $1.348456-4.651161-0.132368$

H $2.519239-5.620298-0.994946$

H -1.354343 -5.877913 -2.447830

H $\quad 0.317864-6.767330-3.716847$

H $\quad 1.724979-5.710209-3.845106$

H $1.769319-7.131419-2.779460$

H -1.786686 -5.2899980 .015092$

H $-0.682785-3.923708-0.141428$

H -2.072427 -3.006502 -2.019935

H -3.192235 -4.332228 -1.853857

H $-2.610428-2.2004880 .260423$

H -3.293586 -4.363263 1.366977

H -4.606734 -4.538906 0.222920

H $-6.428799-3.4751372 .890840$

H $-6.555721-2.7668380 .306645$

H -3.599561 -2.995344 3.394819

H -4.842087 -1.769532 3.488548

H -3.464747 -1.5463362 .393573$

H -6.099944 -0.534705 1.974054

H $-6.726857-1.404141-1.610490$

H $-6.7780810 .061223-0.625533$

H $-4.1981030 .486523-0.151960$

H $-0.534753-0.926939-1.747313$

H $\quad 1.693374 \quad 1.176398-2.086963$

H $-1.850550 \quad 0.655772-0.607424$

H $-2.0722341 .040218-2.328472$

H -2.394767 2.248478-1.101491

H $-4.529643-0.276314-2.666837$

H $-6.1772520 .042180-3.223082$

H $-5.2450724 .232586-2.568840$

H -5.288763 $4.057335-4.340821$

H -2.732683 $3.377735-4.187675$

H $-3.2970695 .667025-2.232725$

H -1.124273 $6.218749-3.564562$

H $-0.7261694 .512062-3.553812$

H $\quad 0.532727 \quad 6.706444-0.328038$

H $\quad 0.0762557 .438617-1.965556$

H -1.636756 $4.028678-0.449481$

H -0.1553174 .5522960 .286042$

H $1.2478373 .278081-1.288532$

H $-4.864531 \quad 1.747760-4.170425$

C $6.239945-1.038165-1.256904$

O $5.380552-0.228704-0.424387$

C 6.1380180 .8940710 .066480

C $7.4190400 .926445-0.756232$

C $7.660694-0.555846-0.970725$

H 6.4070020 .6660661 .104312

C 5.2972982 .1728120 .009924

H $7.2707571 .433693-1.716859$

H $8.2387631 .425354-0.230894$
C $8.651652-0.852958-2.082560$

H $8.026141-0.996217-0.033290$

C 3.9969922 .0997090 .839631

O $4.9381602 .418980-1.354802$

H 5.9051223 .0274620 .329639

H 3.3177521 .3696580 .385760

$\begin{array}{lllll}\text { O } & 3.333697 & 3.365397 & 0.734792\end{array}$

C 4.2134941 .7633522 .327394

C 2.9552781 .8609573 .199648

H 4.6256770 .7543072 .418674

O 5.1976122 .6427162 .880558

H 3.2340251 .6704344 .245813

H 2.5964592 .8996753 .223226

H $8.779194-1.931951-2.214611$

H $8.320286-0.433595-3.038486$

H $9.631290-0.423366-1.848962$

C $6.002295-2.513879-0.933246$

H $4.3861253 .219593-1.369897$

H $3.9382784 .049517 \quad 1.068327$

H 4.8260723 .5409592 .890305

H $5.923438-2.6578740 .151299$

H $6.836147-3.129998-1.287404$

H $5.971274-0.817570-2.298948$

C -0.4551810 .4407503 .602050$

O $0.768424 \quad 1.163882 \quad 3.785480$

C 1.7892610 .9428642 .801183

C $2.164622-0.5467902 .738403$

C $0.932362-1.4350432 .547345$

C $-0.189465-1.0713973 .523835$

H -1.0332440 .6281824 .516433$

C -1.2626150 .9816242 .413629$

H 1.4135161 .2720991 .827575

O $2.846451-0.9352653 .929662$

H $2.856209-0.7110311 .904299$

H $1.222946-2.4827772 .691514$

O $0.471445-1.2912261 .208763$

H -1.115729 -1.591683 3.256928

O $0.171736-1.5122274 .835689$

C -1.8542232 .3467272 .694681$

H -0.6599561 .0743111 .509165$

H -2.0962320 .3016302 .212080$

H $2.267662-0.729748 \quad 4.683796$

H $-0.296115-1.8771991 .097115$

H $\quad 0.294760-2.4761494 .797273$

O $-2.8637392 .583697 \quad 1.805172$

O -1.4705053 .1166113 .564312$

C -3.521605 3.8473861 .938494

H $-4.2458863 .940478 \quad 1.125094$

H -4.0545053 .8933392 .892707$

H -2.7998394 .6660631 .859033$

SCF Energy (B3LYP/6-31G**//MMFF) $=-3245.91526423$

0400112

MM̄FF Geometry

C $-0.514710-2.933883-2.173521$

C $-1.571188-3.445792-2.825169$

C $-2.976407-2.891881-2.878763$

O $-3.073960-1.589084-2.263433$

C $-3.925439-3.850959-2.135774$

C $-5.387854-3.460305-2.235883$

C $-6.115965-2.929586-1.232835$

C $-6.037599-3.746463-3.565733$

C $-5.652104-2.5695340 .151148$

C $-5.889994-1.0819180 .442862$

C $-5.201160-0.6351011 .740998$

C -5.5198470 .8303392 .039959$

C -4.7333501 .3605673 .246053$

O $-3.792790-0.805408 \quad 1.558663$

O -4.8669882 .7904953 .235111$

C -3.2372021 .0224433 .061859$

C -5.3439740 .8824924 .568659$

O -2.5184361 .3856204 .238215$

C $-2.987322-0.4555602 .692323$

C $-1.518000-0.7593472 .306545$

O $-3.304529-1.277748 \quad 3.821678$

C $-2.799780-0.512331-3.051998$

O $-2.399505-0.529131-4.205189$

C $-3.0884770 .708393-2.266765$ 
C $-2.824126 \quad 1.915414-2.788851$

C $-3.0910153 .210165-2.156795$

C $-3.6784913 .203877-0.770445$

C $-0.997173-0.0190951 .070879$

C $-0.278551 \quad 1.283190 \quad 1.373123$

C 0.7736453 .2203760 .208760

O $\quad-0.0059721 .6568412 .510156$

C -0.2005814 .3500000 .106505$

C $-0.1523585 .281578-0.857719$

C $-1.0858706 .454980-0.932461$

C $-1.7940656 .557727-2.263827$

C -1.354453 $7.395366-3.218379$

C $-3.0663395 .763538-2.449049$

C -2.811413 4.335497-2.847548

N $0.054523 \quad 1.9620530 .208470$

H $-0.611073-2.013400-1.606112$

H -1.430643 -4.372430 -3.380096

H -3.278825 -2.823362 -3.931234

H $-3.614058-3.929015-1.088887$

H -3.818602 -4.867262 -2.539872

H $-7.168351-2.718922-1.420227$

H -7.108729 -3.517643 -3.563774

H -5.576247 -3.147624 -4.356820

H $-5.929559-4.805640-3.821655$

H $-6.210679-3.1742290 .875627$

H $-4.597048-2.8088020 .297491$

H -5.501238 -0.488245 -0.394089

H $-6.968175-0.8891560 .506082$

H $-5.540870-1.2799132 .559954$

H $-6.597621 \quad 0.9713492 .190249$

H -5.2611161 .4415991 .165428$

H -4.3175603 .1426683 .956664$

H -2.8711601 .6686292 .254885$

H -6.3742951 .2457044 .664918$

H -4.801662 1.2920625 .428013

H $-5.366702 \quad-0.2052534 .662244$

H -1.569462 1.3160854 .041201

H $-0.856817-0.6241093 .170629$

H -1.464178 -1.832190 2.070132

H -2.847601 -2.129373 3.722862

H $-3.529577 \quad 0.581727-1.285588$

H -2.385285 $1.965310-3.784594$

H -4.696264 $2.800222-0.791512$

H -3.729964 4.194084 -0.312948

H -3.071045 2.592801-0.094384

H $-0.264084-0.6610550 .567233$

H - $1.803753 \quad 0.1714130 .358442$

H $1.4709313 .195781-0.634519$

H 1.3477323 .3156791 .135493

H $-0.960304 \quad 4.4226610 .881229$

H $0.6258435 .221775-1.615435$

H $-0.4927387 .362006-0.755482$

H -1.828355 $6.429017-0.125200$

H - $1.8764777 .498498-4.163937$

H $-0.4593137 .992097-3.077446$

H $-3.6731426 .215327-3.245804$

H -3.685823 $5.853196-1.552298$

H -2.382492 $4.236920-3.845733$

H $-0.2575761 .596302-0.686672$

C $1.350305-4.024038-0.801561$

O $1.859314-2.895840-0.068513$

C $1.732714-3.1804831 .340560$

C $1.097734-4.5681001 .457817$

C $0.356536-4.6966860 .140478$

H $1.034434-2.4408061 .745099$

C $3.089736-3.0491302 .044781$

H $1.851832-5.3579561 .550979$

H $\quad 0.437957-4.6431902 .328118$

C $0.015752-6.127637-0.234043$

H $-0.568309-4.1115780 .218953$

C $3.808889-1.7039261 .816211$

O $3.958253-4.0818741 .557754$

H $2.960950-3.2372843 .117415$

H 4.167119-1.668086 0.783782

O $4.978357-1.6984432 .644344$

C $2.970625-0.4525362 .116239$

C 3.7409850 .8825682 .092276

H $2.147072-0.3956191 .397441$
O $2.388544-0.597048 \quad 3.414942$

H 3.0361021 .6729482 .381800

H 4.5105000 .8688072 .874965

H $-0.506833-6.165855-1.194967$

H $\quad 0.915607-6.746438-0.315217$

H $-0.635040-6.5782510 .522267$

C $0.837889-3.592419-2.178570$

H $4.812134-3.9808112 .012314$

H $4.681700-1.6734963 .570366$

H 1.7911700 .1576373 .554748

H $0.817961-4.474517-2.830761$

H $1.547954-2.884440-2.623027$

H $2.212203-4.685648-0.964492$

C $6.2744240 .642010-0.658582$

O $5.5638160 .465795 \quad 0.575369$

C $4.364513 \quad 1.231794 \quad 0.729721$

C 4.6891172 .7312620 .630594

C $5.4172343 .058201-0.674725$

C $6.6069952 .123081-0.907058$

H $7.2274140 .118453-0.508836$

C $5.545069-0.026474-1.835083$

H $3.6500970 .960654-0.054991$

O $5.484141 \quad 3.142127 \quad 1.741334$

H 3.7564743 .3027760 .679124

H $5.7648704 .097686-0.637690$

O $4.4921822 .944322-1.752128$

H $7.0025662 .243086-1.922222$

O $7.6599652 .480819-0.006322$

C $5.592468-1.539041-1.748232$

H $4.4941200 .270714-1.877909$

H $6.0296800 .250340-2.778345$

H 6.2796612 .5830841 .760705

H $4.9632203 .180351-2.569425$

H $7.9061933 .402492-0.194611$

O $4.751106-2.065330-2.687330$

O $6.291416-2.181282-0.976412$

C $4.714008-3.494901-2.742085$

H $4.465535-3.910812-1.761210$

H $3.938883-3.788913-3.454550$

H $5.676746-3.880018-3.090877$

SCF Energy $(B 3 L Y P / 6-31 G * * / / M M F F)=-3245.92935560$

04_00113

MM̄FF Geometry

C $4.339155-3.011758-1.865850$

C $3.072447-3.135119-1.443024$

C $1.893861-3.225011-2.376883$

O $0.953008-2.184629-2.024484$

C $1.189327-4.582969-2.207417$

C $0.071696-4.807003-3.208264$

C $-1.240627-4.861388-2.906805$

C $0.515546-5.011832-4.635681$

C $-1.904007-4.728094-1.563832$

C $-2.639769-3.392772-1.449353$

C $-3.373100-3.201307-0.112216$

C $-4.573143-4.1442180 .024747$

C $-5.412101-3.8473011 .276292$

O $-3.809224-1.835756-0.088540$

O $-6.647933-4.5638821 .131237$

C $-5.738359-2.3398241 .307801$

C $-4.744385-4.3975622 .541658$

O $-6.421569-1.9938012 .510227$

C $-4.499947-1.4421721 .104320$

$\begin{array}{llll}\text { C } & -4.878270 & 0.049387 & 0.957279\end{array}$

O $-3.650948-1.5531632 .252022$

C $1.208262-0.928488-2.483434$

O $2.151617-0.570771-3.170090$

C $0.127218-0.049411-1.987142$

C $0.197828 \quad 1.276489-2.177111$

C $-0.7900972 .259307-1.724470$

C $-2.0602931 .728291-1.115739$

C $-5.7330420 .375268-0.270123$

C $-5.764773 \quad 1.866784-0.560237$

C $-6.1817553 .457740-2.438401$

$\begin{array}{lllll}\text { O } & -5.468615 & 2.719765 & 0.272047\end{array}$

C $-4.8487653 .705486-3.074924$

C $-4.1415064 .833726-2.909734$

C $-2.8049715 .073477-3.554796$ 
C -1.775569 $5.620856-2.589084$ C $-1.2655736 .853193-2.754360$ C -1.339482 $4.752261-1.429410$ C $-0.5135513 .572629-1.868236$ N $-6.1815222 .127220-1.858328$ H $4.547434-2.992516-2.932740$ H $2.862618-3.152687-0.376001$ H $2.209529-3.092561-3.418891$ H $0.814464-4.664087-1.182105$ H $\quad 1.916480-5.397928-2.323924$ H -1.942171 -5.031545 -3.723495 H $-0.312803-5.301730-5.291195$ H $0.948826-4.093742-5.043108$ H $1.265918-5.807460-4.689930$ H -2.607663 -5.562010 -1.463791 H -1.195399 -4.829502 -0.737986 H -1.921279 -2.573024 -1.574386 H -3.353491 -3.276265 -2.276365 H -2.659310 -3.3555360 .705323$ H $-4.256459-5.1935610 .029846$ H -5.220316 -4.033555 -0.857071 H -7.216427 -4.3255191 .883791$ H $-6.450717-2.1581020 .493460$ H -4.626335 -5.485305 2.466621 H $-5.367342-4.2268603 .426477$ H $-3.756518-3.9703442 .726232$ H $-5.763353-1.9583513 .225108$ H $-5.3729630 .409278 \quad 1.868468$ H -3.9498390 .6298040 .888281$ H -3.062332 -0.7804922 .274754$ H $-0.696057-0.518653-1.461577$ H $1.0655171 .685284-2.693000$ H $-2.5899211 .086817-1.829183$ H $-2.7626272 .507608-0.818479$ H -1.839064 $1.143446-0.217073$ H -5.335145 $-0.137761-1.152877$ H $-6.7656300 .043828-0.116525$ H $-6.4127034 .193961-1.661722$ H $-6.9686553 .479867-3.198383$ H -4.448330 $2.919032-3.710889$ H $-4.5521445 .634286-2.298707$ H -2.960153 5.779272 -4.381195 H -2.415889 4.155178 -4.011450 H $-0.5284377 .257309-2.068397$ H -1.572347 $7.487161-3.579708$ H $-2.2158494 .468800-0.841698$ H $-0.7182705 .335459-0.735941$ H $\quad 0.4388813 .853669-2.319848$ H $-6.3440881 .346305-2.487452$ C $6.234142-1.549233-1.137119$ O $5.373748-0.463873-0.731482$ C 6.1499430 .4871700 .019166 C $7.6096960 .087702-0.166746$ C $7.495617-1.417982-0.284715$ H 5.8831350 .3458391 .072341 C $5.8299581 .922430-0.409486$ H $8.0339480 .509565-1.085276$ H 8.2368020 .4078010 .670969

C $8.724847-2.074201-0.888968$ H $7.319155-1.8359930 .715700$ C $4.3435352 .319789-0.304440$ O $6.2261602 .089808-1.777045$ H 6.4472672 .6159830 .174554 H $3.7690111 .825500-1.097126$ O $4.2555103 .723000-0.588609$ C 3.6786872 .0269991 .054125 C 2.3160492 .7273541 .218724 H 3.5520780 .9438541 .155275 O 4.5350282 .4458412 .118418 H 2.4761603 .8054001 .342700 H 1.7233922 .5664080 .311303 H $8.593120-3.158159-0.962418$ H $\quad 8.931275-1.690800-1.893774$ H $9.606633-1.883144-0.268680$ C $5.517722-2.886414-0.944182$ H $6.0195473 .006268-2.029151$ H 4.7650174 .1980260 .089427 H 4.6373153 .4106692 .059870
H $\quad 5.192411-2.9865780 .098043$

H $6.202879-3.715649-1.152718$

H $6.468539-1.389287-2.197920$

C $0.305214 \quad 0.220801 \quad 3.113985$

O 1.0313270 .9083972 .087184

C 1.5422732 .1982432 .441944

C 0.3936653 .1343172 .845479

C -0.4570202 .5218293 .961482$

C -0.8574011 .0764443 .644718$

H $-0.127269-0.6531812 .609290$

C $1.238638-0.2907164 .216261$

H 2.2344022 .0956093 .284362

O -0.4315723 .4246971 .720502$

H $0.8169784 .086426 \quad 3.186313$

H -1.3573593 .1333854 .097788$

O $0.2858972 .564918 \quad 5.176032$

H -1.2932790 .5919954 .526055$

O $-1.868844 \quad 1.0926512 .634107$

C $2.219336-1.2921383 .652164$

H 1.7956440 .5141104 .704298

H $0.672849-0.7994095 .005418$

H -0.7585342 .5800231 .367513$

H -0.2787192 .2001825 .878558$

H -2.6236181 .5980692 .981138$

O $3.473693-0.758186 \quad 3.633612$

O $1.903005-2.4061313 .254534$

C $4.491340-1.6001223 .084448$

H $5.449281-1.0817523 .177872$

H $4.548120-2.5430863 .636398$

H $4.291562-1.7825802 .025051$

SCF Energy $\left(B 3 L Y P / 6-31 G^{* *} / / M M F F\right)=-3245.93003121$

04 00114

MMFF Geometry

C $3.756600-3.074311-1.516001$

C $2.639599-2.916247-0.788797$

C $1.269825-3.308491-1.282077$

O $0.426448-2.130623-1.307655$

C $0.625446-4.321817-0.319226$

C $-0.281191-5.301509-1.034503$

C $-1.623819-5.222901-1.093037$

C $0.430963-6.464517-1.680980$

C $-2.530733-4.163151-0.530762$

C $-3.450104-3.608852-1.628330$

C $-4.616512-2.784256-1.063952$

C $-5.533759-2.331674-2.200064$

C $-6.681673-1.456999-1.686896$

O $-4.089869-1.645001-0.383457$

O $-7.300487-0.862296-2.837698$

C $-6.099764-0.322411-0.816602$

C $-7.765226-2.300536-1.002922$

O $-7.1669150 .411285-0.220364$

C $-5.080057-0.8046570 .242983$

C -4.2725650 .3575410 .861886$

O $-5.763940-1.5020951 .284511$

C $0.619571-1.251008-2.328230$

O $1.403600-1.361622-3.257694$

C $-0.272317-0.093028-2.102266$

C $0.0201441 .072700-2.698900$

C $-0.7257522 .326567-2.572712$

C $-2.0423612 .279254-1.850556$

C -5.0584491 .4151891 .633767$

C -4.1201092 .4208862 .274071$

C $-2.6063304 .313717 \quad 1.746297$

O $-3.8743352 .402816 \quad 3.478008$

C -2.3277015 .2265310 .595835$

C -1.106402 5.4165650 .071791

C $-0.825096 \quad 6.390511-1.037037$

C $-0.0412485 .785843-2.180018$

C $1.2346126 .145631-2.403949$

C $-0.7690304 .842999-3.113860$

C $-0.1976053 .449401-3.101961$

N $-3.589438 \quad 3.3198591 .360376$

H $3.692231-3.510758-2.509558$

H $2.703308-2.4661120 .198458$

H $1.325647-3.723936-2.296372$

H $\quad 0.077684-3.7995210 .472925$

H $1.395948-4.9103670 .197568$ 
H -2.150955 -6.025362 -1.609275 H $-0.259836-7.152256-2.180257$ H $1.142269-6.110666-2.433817$ H $0.980064-7.038723-0.927455$ H $-3.134768-4.6235090 .260138$ H -1.974075 -3.340332 -0.074007 H $-2.846977-2.986834-2.302981$ H $-3.859367-4.434314-2.225040$ H -5.166710 -3.408096 -0.349823 H -5.926932 -3.193640 -2.753684 H -4.951924 -1.749154 -2.928638 H -7.983421 $-0.246712-2.519896$ H $-5.5892370 .376289-1.493272$ H -8.150072 -3.059712 -1.694353 H -8.630107 -1.687972 -0.724886 H -7.405877 -2.814495 -0.108832 H -7.489026 -0.1006110 .541189$ H -3.529643 -0.0747201 .546093$ H -3.6836010 .8347440 .071127$ H -5.206347 -1.486322 2.080150 H -1.115231 -0.222622 -1.433119 H $\quad 0.9092621 .124664-3.326765$ H -2.699365 $1.519081-2.287890$ H -2.595794 3.219231-1.883582 H -1.878615 2.041231 -0.795808 H $-5.738001 \quad 1.968714 \quad 0.978381$ H -5.6629880 .9585422 .424772$ H - 1.7090313 .7886222 .086191 H -3.0040984 .8812672 .594183$ H -3.1709085 .7849530 .195784$ H $-0.257414 \quad 4.8802120 .489687$ H $-0.2685477 .230965-0.601980$ H $-1.7533186 .821850-1.432811$ H $1.7945505 .748323-3.244222$ H $1.7484666 .845162-1.752922$ H $-0.6917635 .231858-4.138428$ H -1.841707 $4.853062-2.900690$ H $\quad 0.7678473 .374330-3.604720$ H -3.7842193 .1848040 .372307$

C $5.599661-1.396832-1.771378$

O $4.753584-0.292969-1.387192$

C $5.5689760 .724086-0.784360$

C $6.9474110 .519617-1.389152$

C $7.029367-0.997069-1.386810$

H 5.6173730 .5009630 .288414

C $4.9386172 .105819-0.979390$

H $7.0011800 .899172-2.415925$

H $7.7390700 .993543-0.801727$

C $8.089709-1.552666-2.321877$

H $7.252006-1.333439-0.365052$

C $3.4461772 .178088-0.593156$

O $5.0417152 .492010-2.355097$

H $5.5108562 .851641-0.415912$

H $2.8404851 .681048-1.360515$

O $3.0501953 .554127-0.600344$

C 3.1068691 .5754080 .780894

C 1.6204291 .7368951 .141684

H 3.3585120 .5107570 .775416

O $3.9029692 .188543 \quad 1.793412$

H 1.4043872 .7842201 .384970

H 1.0227691 .4562890 .267509

H $8.091900-2.647127-2.299130$

H $7.917652-1.236967-3.356273$

H $9.084616-1.205038-2.025395$

C $5.123793-2.660949-1.049716$

H $4.5986521 .807797-2.886210$

H $3.2507413 .908053-1.483895$

H $3.7221783 .143921 \quad 1.770668$

H $5.105946-2.4881840 .032941$

H $5.818132-3.488653-1.234150$

H $5.500512-1.509949-2.858117$

C $1.088815-1.4951902 .937137$

O $1.379740-0.511871 \quad 1.936742$

C $1.215437 \quad 0.8530192 .337976$

C -0.2383381 .1035992 .759668$

C $-0.701546 \quad 0.1104653 .828676$

C $-0.348121-1.3350293 .461515$

H $1.151372-2.4553072 .409984$
C $2.144056-1.5067214 .048117$

H $1.886077 \quad 1.0703953 .175839$

O -1.0944721 .0077401 .624686$

H -0.3234652 .1259143 .144898$

H $-1.7880050 .201227 \quad 3.951142$

O $-0.092496 \quad 0.461903 \quad 5.067297$

H $-0.506724-1.9940974 .322856$

O $-1.253711-1.7578852 .437515$

C $3.506088-1.8483673 .489661$

H $2.208816-0.5506294 .575254$

H $1.905235-2.2617224 .806280$

H -0.9792560 .1231341 .237195$

H $-0.426499-0.1524385 .743011$

H -1.087920 -2.701373 2.271044

O $4.331305-0.7666593 .580933$

O $3.797176-2.9409813 .020222$

C $5.660845-0.9592413 .089731$

H $6.187668-1.6894543 .710910$

H $5.640686-1.2842082 .046352$

H $6.186469-0.0024243 .147233$

SCF Energy $(B 3 L Y P / 6-31 G * * / / M M F F)=-3245.92947841$

04 00115

MMFF Geometry

C $-3.911138-3.3894211 .421860$

C $-2.637792-3.0114911 .231634$

C -1.696929 -2.695826 2.364537

O $-1.115153-1.3875202 .161148$

C $-0.541790-3.7112142 .396288$

C $0.378922-3.5024883 .582960$

C $1.582100-2.8982903 .525221$

C $-0.125750-4.0553214 .891255$

C $2.257619-2.2845682 .328792$

C $3.513401-3.0783071 .957073$

C $4.274277-2.4810520 .761846$

C $5.469073-3.3799930 .424439$

C $6.365478-2.783771-0.665657$

O $4.718987-1.167311 \quad 1.118939$

O $7.587489-3.537371-0.662238$

C $6.702258-1.329284-0.282706$

C $5.753656-2.965874-2.058769$

O $7.446156-0.700067-1.325040$

C $5.461678-0.4854540 .096123$

C 5.9246560 .8768680 .673860

O $4.680034-0.279496-1.077071$

C $-1.872946-0.3013812 .465102$

O $-3.051274-0.2876832 .781715$

C -0.9916150 .8839242 .378361$

C -1.5248512 .1128202 .430155$

C -0.7842203 .3763952 .408216$

C 0.7165293 .3032552 .504317

C 4.8034371 .8595391 .046252

C $4.4091792 .789017-0.093326$

C $3.2072884 .895472-0.570921$

O $4.7534122 .610370-1.259003$

C 2.3264705 .8743790 .134725

C $1.0723566 .160541-0.246327$

C 0.2161757 .2006050 .416562

C -1.0594016 .6295180 .991445$

C $-2.2349296 .806246 \quad 0.363501$

C -0.9666805 .9434382 .337545$

C -1.481440 4.5284862 .325084

N $3.607234 \quad 3.837718 \quad 0.337807$

H -4.293271 -3.485855 2.435326

H $-2.250493-2.9160530 .220359$

H $-2.224204-2.702723 \quad 3.326940$

H $\quad 0.026612-3.6587401 .460669$

H $-0.939463-4.7341112 .438706$

H $2.156574-2.8169604 .447497$

H $\quad 0.595695-3.9237195 .704814$

H - $1.051973-3.5552245 .190068$

H $-0.323087-5.1282944 .798552$

H $1.590916-2.2270151 .465097$

H $2.523901-1.2518682 .583620$

H $4.180413-3.1063862 .829305$

H $3.232520-4.1145301 .728394$

H $3.590874-2.408721-0.092579$

H $5.130474-4.3835430 .136785$ 
H $\quad 6.079216-3.5223521 .328087$ H $8.193543-3.118496-1.297520$ H $7.374747-1.3703750 .585045$ H $5.615911-4.031804-2.276874$ H $\quad 6.422015-2.587375-2.839894$ H $4.783523-2.476932-2.167419$ H $6.820558-0.453380-2.027556$ H $\quad 6.489840 \quad 0.673811 \quad 1.593543$ H $6.6120701 .368739-0.024594$ H $4.0516010 .439557-0.909264$ H $\quad 0.075024 \quad 0.7107632 .290128$ H -2.608203 2.2064712 .496274 H 1.0179142 .7867443 .422555 H 1.2003324 .2810482 .523176 H 1.1322962 .7623971 .648176 H $3.911837 \quad 1.3348001 .404421$ H 5.1685902 .4925181 .865096 H $2.7217584 .439448-1.440521$ H $4.1150505 .397799-0.922709$ H 2.7570756 .4012020 .983200 H $0.6476845 .652609-1.108938$ H $-0.0161137 .966536-0.334955$ H $0.7650467 .726976 \quad 1.207739$ H -3.164303 6.4386090 .785536 H -2.295055 $7.326430-0.586886$ H $-1.560061 \quad 6.5167873 .062564$ H 0.0563405 .9979252 .720769 H -2.5690814 .4656302 .265205$ H 3.4836443 .9773981 .336949 C $-6.066674-2.7447810 .312274$ O $-5.612678-1.415272-0.018058$ C $-6.307353-0.973441-1.194990$ C $-7.609359-1.758356-1.195406$ C $-7.116411-3.119312-0.741435$ H -5.703412 -1.290131-2.055042 C $-6.4498680 .551191-1.214346$ H -8.326951 -1.356764 -0.470836 H -8.086506 -1.780318 -2.179624 C $-8.217457-4.022014-0.211533$ H -6.634065 -3.617855 -1.593197 C $-5.1369051 .319610-0.954073$ O $\quad-7.384500 \quad 0.944643-0.202558$ H $\quad-6.8900790 .856315-2.171239$ H $-4.895492 \quad 1.2757510 .115134$ O $\quad-5.3731772 .704174-1.236034$ C $-3.9205890 .827807-1.757046$ C $-2.6874301 .734333-1.590950$ H -3.671895 $-0.186268-1.427818$ $\begin{array}{lllll}O & -4.246374 & 0.741770 & -3.144517\end{array}$ H -2.808182 $2.639863-2.197372$ H $-2.6037502 .029532-0.538359$ H -7.806977 -4.9806380 .121177$ H $-8.737576-3.5650060 .637043$ H -8.959046 -4.223085 -0.991548 C $-4.870826-3.701780 \quad 0.309054$ H $-7.4603881 .913616-0.238366$ H -5.622513 2.780725 -2.172387 H $-4.4317361 .639828-3.466575$ H $-4.353248-3.644505-0.656927$ H $-5.216930-4.7341030 .435373$ H $-6.515668-2.6875951 .311655$ C $-0.013443-0.833356-1.276448$ O $-1.1367890 .013834-1.016538$ C -1.399252 $1.005414-2.017510$ C $-0.2031621 .958787-2.132825$ C $1.0854841 .186343-2.432911$ C $1.268370-0.011169-1.493929$ H $0.112112-1.408398-0.351550$ C $-0.342522-1.823398-2.396658$ H -1.569774 $0.511711-2.981498$ O $-0.0623592 .683946-0.914826$ H -0.401441 $2.684592-2.930586$ H $1.9416181 .864405-2.330648$ O $1.044636 \quad 0.745900-3.786784$ H $2.069629-0.660520-1.861831$ O $1.6934040 .504533-0.228787$ C $0.449136-3.098087-2.243644$ H $-1.404145-2.100470-2.366417$
H $-0.185973-1.404401-3.394823$

H $0.7212713 .251896-0.998336$

H $1.8812940 .285794-3.970813$

H $1.913703-0.2528660 .338335$

O $1.511891-3.096948-3.096581$

O $0.167431-3.980095-1.442483$

C $2.347346-4.256758-3.032983$

H $3.158871-4.131369-3.754491$

H $2.778687-4.359137-2.032395$

H $1.775604-5.150436-3.300385$

SCF Energy $(B 3 L Y P / 6-31 G * * / / M M F F)=-3245.91621714$

04_00116

MMFF Geometry

C -0.7358522 .2561312 .609131$

C -1.4381491 .1657592 .955017$

C $-0.916079-0.2355313 .172392$

O $0.512424-0.2727832 .966821$

C -1.602321-1.1839472.172111

C $-1.463381-2.6524232 .513388$

C $-0.724501-3.5425971 .823420$

C $-2.281893-3.1160833 .693022$

C $0.142069-3.3052200 .618671$

C $1.588057-3.7298910 .900581$

C $2.481974-3.610019-0.340512$

C $3.902976-4.077485-0.017002$

C $4.848969-3.904250-1.212350$

O $2.501810-2.241573-0.760562$

O $6.185648-4.070465-0.716040$

C $4.713858-2.463585-1.746126$

C $4.639140-5.004182-2.259204$

O $5.479440-2.293439-2.937216$

C $3.248457-2.011565-1.963047$

C $3.220551-0.499106-2.271279$

O $2.701213-2.725157-3.072711$

C $1.266405-0.9978713 .831704$

O $\quad 0.857853-1.6898524 .754707$

C $2.716585-0.846176 \quad 3.563243$

C $3.223486-0.1298602 .547907$

C 4.6464700 .0889722 .266797

C $5.652111-0.545643 \quad 3.189933$

C $1.8205340 .122373-2.354188$

C $1.9629921 .616068-2.567076$

C $2.6010133 .683753-1.329474$

O $1.9886502 .108675-3.692413$

C $4.0945083 .639613-1.230820$

C $4.7800773 .940205-0.117478$

C 6.2677873 .7749600 .005464

C 6.6532622 .7288571 .032168

C 7.3130993 .0693272 .151970

C 6.3427801 .2764890 .729057

C $4.9769260 .869407 \quad 1.217135$

N $2.1275472 .311087-1.378577$

H 0.3390212 .1986762 .465109

H -2.512685 1.2750353 .096463

H -1.163356 -0.5038614 .206291$

H -1.230572 -0.9759601 .163256$

H -2.676939 -0.9640332 .135498$

H $-0.740787-4.5800532 .157037$

H -2.239939 -4.202161 3.827694

H -1.922676 -2.657232 4.618785

H -3.333990 -2.845198 3.556937

H $-0.259360-3.898875-0.211365$

H $0.125285-2.2615830 .295694$

H $1.988754-3.0992961 .702732$

H $1.607744-4.7662181 .261338$

H $2.046784-4.225702-1.136525$

H $3.903540-5.1201340 .324976$

H $4.296056-3.4877200 .823703$

H $6.797333-3.887544-1.450017$

H $5.172987-1.803317-0.997389$

H $4.811129-5.991386-1.813560$

H $5.362829-4.917605-3.077200$

H $3.634173-5.003198-2.686410$

H $4.983412-2.700320-3.667945$

H $3.7811750 .030468-1.491674$

H $3.737482-0.305200-3.220262$

H $1.934214-2.238980-3.413088$ 
H $3.358499-1.3586704 .273048$ H 2.5383920 .3615491 .859117 H $5.492403-0.2128804 .221698$ H $6.686933-0.2988202 .944032$ H $5.568044-1.6374513 .154887$ H $1.241419-0.284136-3.189368$ H $1.245573-0.063664-1.440203$ H $2.1412294 .160520-0.458769$ H $2.2973514 .222627-2.231947$ H $4.6330993 .292550-2.110020$ H 4.2441894 .2785960 .766248 H 6.6931554 .7516230 .269054 H $6.7173783 .503675-0.958153$ H 7.6135352 .3213182 .878374 H 7.5643124 .1020792 .369509 H $7.1281260 .632844 \quad 1.138107$ H $6.3909781 .109799-0.354741$ H $4.165188 \quad 1.2742630 .616191$ H $2.1456501 .786975-0.508501$ C -1.1582374 .1084240 .956884$ O $-1.5929703 .125832-0.000622$ C $-2.3706533 .770219-1.024251$ C $-2.1346405 .266476-0.851278$ C -1.958102 5.3753570 .650365 H $-3.4207503 .543447-0.802259$ C $-2.0077713 .208850-2.405397$ H -1.223056 $5.600873-1.358971$ H $-2.9688555 .863970-1.231224$ C - -1.2744436 .6567581 .094293$ H $-2.9496285 .318831 \quad 1.119346$ C $-1.9457701 .669591-2.479733$ O $-0.7182263 .697700-2.794005$ H -2.707904 $3.596896-3.153883$ H $-1.0793521 .300388-1.918393$ O $-1.7213081 .305491-3.847514$ C $-3.2100640 .949959-1.983689$ C $-3.131471-0.576943-2.183310$ H $-3.3546551 .166797-0.920363$ O $-4.3515711 .456297-2.674485$ H -3.112781 -0.811029 -3.254369 H -2.197018 $-0.936782-1.735044$ H -1.162710 6.6817472 .182788 H $-0.277834 \quad 6.7540240 .650702$ H -1.862449 7.5304200 .794678 C -1.3859413 .5937322 .380374$ H $-0.0903883 .445856-2.095745$ H - $0.9097161 .756750-4.137382$ H $-4.2139161 .298739-3.624315$ H -2.463720 3.5168952 .571570 H -0.9832924 .3110253 .104653$ H $-0.087152 \quad 4.2683530 .780685$ C -5.330138-1.654150 0.627632 O $-4.237443-1.123999-0.131792$ C $-4.327502-1.306292-1.547263$ C $-4.338719-2.805812-1.873788$ C $-5.479139-3.518881-1.139053$ C $-5.520352-3.1555110 .351439$ H -5.020681 -1.5485611 .674979$ C $-6.603227-0.8197180 .435158$ H -5.250513 -0.847631 -1.917888 O $-3.092882-3.400670-1.515107$ H -4.458729 -2.934572 -2.955765 H $-5.350560-4.602238-1.252086$ O $-6.710982-3.161851-1.759197$ H $-6.457160-3.4929210 .809676$ O $-4.457042-3.8455521 .015930$ C $-6.360581 \quad 0.6536540 .677147$ H -6.995471 $-0.913212-0.581818$ H -7.396625 -1.1540681 .113027$ H -2.939296 -3.223571 -0.571077 H -7.420222 -3.646939-1.303971 H -4.604071 -4.7989520 .894358$ O $-5.856910 \quad 0.859414 \quad 1.926846$ O $-6.608971 \quad 1.522877-0.149224$ C -5.5506592 .2197122 .248656$ H -4.8155982 .6206291 .543758$ H -6.4626982 .8238512 .242670$ H -5.1199832 .2429723 .253286$
SCF Energy (B3LYP/6-31G**//MMFF) $=-3245.92942082$

04_00117

MMFF Geometry

C $-0.732282-0.0305184 .392039$

C $-0.4859360 .813249 \quad 3.379558$

C -1.168522 2.1489683 .237869

O -1.9026172 .1725881 .995543$

C -0.1056153 .2557263 .145109$

C -0.6363394 .6387753 .458653$

C -1.0183695 .5486262 .541499$

C $-0.6602234 .989816 \quad 4.924414$

C -1.1297685 .3836641 .051435$

C 0.0140726 .0511900 .274589

C 1.2361115 .1348540 .108836

C $2.3711805 .860312-0.614257$

C $3.5611324 .926623-0.879988$

O $0.8173374 .000235-0.658279$

O $4.4289195 .606680-1.799142$

C $3.0440033 .646024-1.569663$

C 4.3837524 .6862070 .391189

O $4.0963172 .695788-1.723720$

C $1.8239943 .004438-0.865829$

C $1.2204411 .904295-1.771802$

O $2.2561862 .419350 \quad 0.363134$

C -3.1609221 .6640291 .978253$

O $-3.749781 \quad 1.1079262 .889808$

C $-3.7000671 .917506 \quad 0.624331$

C $-4.8313291 .320540 \quad 0.224315$

C $-5.4582681 .471401-1.090914$

C $-4.8266862 .439320-2.058795$

C $-0.1212581 .329777-1.289991$

C $-0.6642320 .293620-2.262727$

C $-2.472033-1.408030-2.496786$

O $-0.1442620 .061419-3.351270$

C $-3.590565-0.845759-3.315963$

C $-4.827352-1.367311-3.326475$

C $-5.952230-0.842738-4.173755$

C $-7.221298-0.604346-3.383869$

C $-8.225246-1.497208-3.404246$

C $-7.3583680 .705420-2.639973$

C $-6.5513820 .731591-1.371866$

N $-1.799829-0.340087-1.776996$

H -1.483600 0.2319415 .133181

H $\quad 0.2624870 .5520592 .635046$

H -1.8501342 .3417234 .075783$

H $\quad 0.3356293 .2460892 .143977$

H 0.7237353 .0520633 .836211

H $-1.337525 \quad 6.5275772 .898511$

H -1.042157 6.0000365 .105595

H -1.3008704 .2929125 .474001$

H $0.349904 \quad 4.9386555 .343997$

H -1.236569 4.3342240 .765987

H -2.0722555 .8614620 .752685$

H $-0.3697856 .299867-0.723838$

H $\quad 0.304801 \quad 6.9937790 .754146$

H 1.5729724 .8078001 .097517

H $2.6936876 .743115-0.048325$

H $2.0058186 .240487-1.578735$

H $5.1474444 .994542-2.033992$

H $2.7471423 .932969-2.587729$

H 4.7548465 .6380580 .789688

H 5.2749234 .0850250 .179689

H $3.8157684 .192887 \quad 1.182840$

H $4.2476812 .280705-0.857489$

H $1.0608182 .320273-2.775448$

H $1.9332991 .077678-1.871672$

H 1.6488891 .7032510 .605838

H $-3.1361712 .585161-0.017682$

H -5.3380490 .6482690 .915433$

H $-4.8593013 .457916-1.656084$

H -5.316186 2.467100 -3.034094

H $-3.7814932 .172242-2.249483$

H $-0.0067190 .852456-0.311292$

H $-0.8669522 .127284-1.202781$

H $-2.830649-2.124661-1.751730$

H $-1.754683-1.911986-3.152382$

H $-3.3679630 .009431-3.949632$ 
H $-5.037060-2.243566-2.716782$ H -6.140866 -1.578805 -4.966223 H $-5.667100 \quad 0.082203-4.690294$ H -9.149227 -1.321838 -2.863201 H -8.143491 -2.426493 -3.958295 H $-8.407036 \quad 0.873122-2.359517$ H -7.119402 $1.529576-3.317443$ H $-6.9295480 .053844-0.605100$ H -2.189103 -0.049885 -0.885208 C $-0.830471-2.5364414 .231185$ O $-0.724248-2.7521242 .804805$ C $-0.390206-4.1332762 .574597$ C $-0.742920-4.8611593 .863294$ C $-0.337997-3.8265024 .891601$ H $0.695718-4.1799322 .436213$ C $-1.098089-4.674570 \quad 1.331261$ H -1.817543 -5.0671143 .931426$ H $-0.204744-5.807843 \quad 3.969174$ C $-0.927097-4.0764316 .269339$ H $\quad 0.757983-3.8154704 .968068$ C $-0.753325-3.9455020 .017557$ O $-2.516027-4.5853221 .519808$ H $-0.877958-5.743255 \quad 1.223797$ H -1.203339 -2.945993 0.006426 O $-1.370444-4.676755-1.050252$ C $0.753587-3.824397-0.280128$ C $1.046307-3.370982-1.722997$ H $\quad 1.198407-3.1148200 .425787$ O $1.391742-5.084918-0.066584$ H $0.784034-4.178283-2.417740$ H $0.422874-2.500344-1.950832$ H - $0.624326-3.2934816 .971500$ H -2.021702 -4.097524 6.240052 H $-0.583351-5.037416 \quad 6.665763$ C $0.006098-1.3177884 .620585$ H $-2.730847-3.6513891 .687023$ H $-2.322493-4.723947-0.856550$ H $\quad 0.971727-5.731588-0.659501$ H $0.949294-1.3133504 .061216$ H $\quad 0.264469-1.3544255 .685477$ H -1.893855 -2.374683 4.450260 C $4.098063-1.267755-1.307079$ O $2.752440-1.760067-1.267529$ C $2.527535-3.009370-1.929450$ C $2.889268-2.895382-3.417787$

C $4.322743-2.390124-3.605817$

C $4.608125-1.148611-2.754622$ H $4.034854-0.256216-0.886531$

C $5.024390-2.088496-0.403108$ H $3.148725-3.783243-1.466560$ O $1.982120-2.022355-4.086335$ H $2.784160-3.882432-3.883145$ H $4.479755-2.152785-4.665263$

O $5.222520-3.437336-3.255180$ H $5.682210-0.929035-2.753628$ O $3.956626-0.039398-3.379885$ C $4.579048-1.9907571 .037461$ H $5.066918-3.143093-0.689807$ H $6.053060-1.712427-0.450895$ H $2.008801-1.161008-3.635209$ H $6.125518-3.113714-3.414634$ H $4.204890 \quad 0.762542-2.888954$ O $4.086394-3.190941 \quad 1.456637$ O $4.643568-0.9648431 .702848$ C $3.608519-3.2230862 .804574$ H $4.415403-2.9748073 .500526$ H $2.769489-2.5317632 .922224$ H $3.260550-4.237123 \quad 3.018424$

SCF Energy (B3LYP/6-31G**//MMFF) $=-3245.93882759$

\section{8}

MM̄FF Geometry

C $4.824064-2.594732-2.072381$

C $3.610733-2.806025-1.540587$

C $2.372301-2.993346-2.380744$

O $1.398668-1.976643-2.050621$

C $1.733322-4.360601-2.071282$

C $0.577895-4.701479-2.991963$
C $-0.716986-4.772820-2.625237$

C $0.963133-5.013006-4.417525$

C $-1.340804-4.553835-1.273230$

C $-2.181110-3.274945-1.261232$

C $-3.103836-3.130198-0.038817$

C $-4.264734-4.131534-0.088730$

C $-5.310845-3.8817401 .006197$

O $-3.602570-1.785107-0.077644$

O $-6.467741-4.6612580 .665550$

C $-5.714684-2.3937840 .970418$

C $-4.831406-4.3998472 .366777$

O $-6.611118-2.0858152 .035264$

C $-4.506014-1.4362520 .979786$

$\begin{array}{llll}C & -4.922186 & 0.038413 & 0.766778\end{array}$

O $-3.866287-1.5180532 .258445$

C $1.580312-0.724909-2.552561$

O $2.528436-0.314300-3.200923$

C $0.3941590 .069793-2.168567$

C $0.4376841 .409630-2.156238$

C $-0.6691762 .287570-1.764406$

C $-2.0216721 .652013-1.570577$

C $-5.5942620 .332328-0.576828$

C $-5.6901021 .824817-0.851072$

C $-6.0372903 .421949-2.738171$

O -5.5160472 .6805740 .012276$

C $-4.6639243 .772404-3.222065$

C $-4.0180584 .900092-2.887997$

C $-2.6279065 .228741-3.357659$

C -1.718160 $5.670103-2.230286$

C $-1.2284716 .921021-2.191887$

C $-1.3846474 .674711-1.141007$

C $-0.4301713 .605973-1.602826$

N $-6.0054562 .082316-2.178647$

H $4.928793-2.549554-3.154084$

H $3.497927-2.843452-0.460205$

H $2.611360-2.921091-3.448733$

H $1.409737-4.375366-1.025080$

H $2.485978-5.155347-2.162770$

H $-1.443138-5.037430-3.394570$

H $\quad 0.116647-5.382361-5.006540$

H $1.345990-4.120089-4.920472$

H $1.737150-5.787186-4.441360$

H -1.963642 -5.429676-1.062951

H $-0.599899-4.508482-0.470678$

H -1.505485 -2.412332 -1.294166

H $-2.786106-3.198493-2.175287$

H -2.511020 -3.258487 0.874051

H $-3.902848-5.164266-0.027739$

H -4.765936 -4.051860 -1.064102

H $-7.163220-4.454548 \quad 1.313712$

H $-6.284047-2.2471140 .044403$

H -4.658163 -5.4819262 .321350$

H $-5.593924-4.2533323 .139577$

H -3.903584 -3.9317212 .702139$

H $-6.088640-2.0185182 .852487$

H $-5.5641800 .375182 \quad 1.590887$

H -4.0187140 .6580890 .833604$

H -3.434816 -0.669792 2.450114

H $-0.491558-0.480736-1.877973$

H $1.3689011 .906064-2.424738$

H $-2.3564481 .176308-2.499354$

H $-2.7980142 .361387-1.284663$

H $-1.9912600 .892258-0.784429$

H $-5.024110-0.128776-1.391399$

H $-6.610893-0.074422-0.593003$

H $-6.4005134 .123283-1.980023$

H -6.738676 $3.409353-3.577656$

H -4.173534 $3.056344-3.878040$

H $-4.5144205 .628875-2.251308$

H -2.712684 $6.024076-4.109458$

H $-2.1716784 .374253-3.872339$

H $-0.5783977 .249745-1.387657$

H -1.464712 $7.645842-2.963789$

H -2.307335 $4.268108-0.719403$

H $-0.9058615 .188762-0.296225$

H $0.5817243 .974224-1.777539$

H $-6.0598641 .304213-2.829530$

C $6.516672-0.940449-1.289452$ 
O $5.708785-0.206097-0.345591$

C $6.556636 \quad 0.706795 \quad 0.378466$

C $7.8890580 .719187-0.359323$

C $7.962597-0.713548-0.851811$

H $6.7187950 .269981 \quad 1.370491$

C 5.8743472 .0711420 .508846

H 7.877507 1.415081-1.206489

H 8.7231330 .9941180 .293368

C $8.984459-0.922224-1.955760$

H $8.206643-1.368248-0.004290$

C $4.5055312 .010557 \quad 1.220393$

O $5.6654822 .598129-0.807157$

H $6.5426302 .773095 \quad 1.021227$

H 3.8027381 .4393740 .604168

$\begin{array}{lllll}\text { O } & 3.984303 & 3.344614 & 1.277397\end{array}$

C 4.5577061 .4313822 .647134

C 3.2255851 .5091183 .405841

H 4.8977890 .3922412 .616654

O $\begin{array}{lllll}5.532431 & 2.163961 & 3.396134\end{array}$

H 3.3820451 .1894784 .445625

H 2.9194632 .5590863 .512790

H 8.990722 -1.963966 -2.291414

H $8.771991-0.289924-2.824390$

H $9.990279-0.676647-1.599636$

C $6.085369-2.406425-1.277360$

H $5.2156093 .454713-0.708013$

H 4.5685553 .8625501 .857516

H $5.600744 \quad 1.7499214 .273326$

H $5.937968-2.749542-0.245880$

H $6.857540-3.041124-1.726455$

H $6.345002-0.489252-2.276079$

C $-0.297370 \quad 0.3458213 .210230$

$\begin{array}{lllll}\text { O } & 0.930666 & 0.954274 & 3.629826\end{array}$

C 2.0683380 .7071602 .791032

C $2.344397-0.8025622 .689631$

C $1.093788-1.5896852 .280831$

C $-0.134668-1.1781923 .096069$

H -1.0044310 .5486574 .025146$

C $-0.845492 \quad 0.993773 \quad 1.936233$

H 1.8618971 .1138011 .796948

O $2.821596-1.3014703 .938625$

H $3.136741-0.9753031 .952807$

H $1.276726-2.6620832 .420348$

O $0.814495-1.373030 \quad 0.902638$

H -1.043640 -1.610966 2.663340

O $-0.012318-1.6973514 .422764$

C -1.286617 2.4129692 .198290

H -0.1206621 .0339131 .119931$

H $-1.699896 \quad 0.426661 \quad 1.554015$

H $2.152175-1.0988714 .614480$

H $1.591202-1.662050 \quad 0.394915$

H $0.037996-2.6660384 .354387$

O -2.6273132 .4471122 .440914$

O $-0.525913 \quad 3.372121 \quad 2.203095$

C -3.175194 3.7440482 .694121

H -4.2513373 .6331592 .851302$

H -2.730000 4.1727523 .596933

H -3.012849 4.3996781 .833523

SCF Energy (B3LYP/6-31G**/MMFF) $=-3245.92334725$

04_00119

MM̄FF Geometry

C $4.134553-2.897975-1.767331$

C $2.862797-2.884113-1.338757$

C $1.687926-2.933900-2.278025$

O $0.908946-1.727687-2.095793$

C $0.763751-4.122839-1.966824$

C $-0.221266-4.360511-3.096184$

C $-1.481388-3.884868-3.139560$

C $0.310778-5.186222-4.241044$

C $-2.199184-3.067386-2.098009$

C $-3.696774-3.404195-2.095303$

C $-4.479459-2.714747-0.967348$

C $-5.961839-3.077175-1.081427$

C $-6.819326-2.351588-0.040256$

O $-4.314161-1.298285-1.075896$

O $-8.186809-2.525675-0.441759$

C $-6.493079-0.842030-0.079184$
C $-6.683849-2.9999551 .343814$

O $-7.162792-0.178656 \quad 0.990148$

C $-4.975339-0.540609-0.042808$

C $-4.645108 \quad 0.944662-0.335308$

O $-4.454675-0.8693841 .246377$

C $1.314115-0.610215-2.755798$

O $2.299430-0.486380-3.464816$

C $0.328905 \quad 0.451787-2.460535$

C $0.5683721 .710986-2.854685$

C $-0.3059302 .862033-2.621712$

C $-1.6563822 .591485-2.014496$

C $-5.2387351 .955755 \quad 0.642814$

C -4.5268523 .2940510 .592164$

C -2.7606904 .6051691 .772908$

O $-4.892528 \quad 4.192786-0.161666$

C $-1.469415 \quad 4.6525201 .021977$

C $-1.040855 \quad 5.742154 \quad 0.365621$

C $0.2843175 .826406-0.337036$

C $0.1635306 .301767-1.769919$

C $0.6882967 .478455-2.151435$

C $-0.555118 \quad 5.414380-2.764501$

C $0.141544 \quad 4.091751-2.950194$

N -3.4657443 .3707691 .480043$

H $4.338750-2.986082-2.831746$

H $2.652384-2.773610-0.277954$

H $2.027473-2.989353-3.320310$

H $\quad 0.234612-3.950358-1.022759$

H $1.351496-5.038349-1.819240$

H $-2.065874-4.095034-4.035032$

H $-0.435991-5.339648-5.027339$

H $1.176298-4.699256-4.700643$

H $\quad 0.617662-6.174454-3.882932$

H - $1.790291-3.244923-1.099824$

H $-2.066541-2.005609-2.333329$

H $-4.117739-3.099851-3.063335$

H $-3.831449-4.490589-2.011664$

H $-4.069679-3.052380-0.008318$

H $-6.104846-4.162661-1.006884$

H $-6.326622-2.798234-2.080439$

H $-8.755440-2.1315450 .240817$

H $-6.901764-0.431405-1.012846$

H -7.013606 -4.0452991 .308211$

H -7.330497 -2.507998 2.078772

H -5.660357 -2.9876531 .724598$

H $-8.118871-0.2750560 .845858$

H $-3.5537201 .052330-0.340985$

H $-4.9579171 .199020-1.356717$

H $-3.613763-0.3966881 .361435$

H $-0.5632350 .169332-1.912602$

H $1.4979651 .926102-3.379983$

H $-2.2115671 .863542-2.616878$

H $-2.2937543 .473750-1.939360$

H $-1.5412472 .194730-1.001390$

H -6.2945762 .1398950 .414146$

H -5.1991361 .5829681 .673025$

H -2.5580844 .6059212 .848695$

H $-3.406023 \quad 5.4586201 .539646$

H -0.8409493 .7661801 .049957$

H -1.6560406 .6391700 .366536$

H $0.8043004 .861385-0.315969$

H $\quad 0.917042 \quad 6.515707 \quad 0.237341$

H $0.6108307 .827264-3.175808$

H $1.2072418 .121465-1.448258$

H $-0.5980615 .899020-3.748932$

H $-1.5976685 .310549-2.454880$

H $1.1351304 .181195-3.391612$

H -3.2056722 .5455802 .012660$

C $5.969359-1.356088-1.090085$

O $5.040822-0.334483-0.670784$

$\begin{array}{llll}\text { C } 5.692847 & 0.526120 & 0.276261\end{array}$

$\begin{array}{llll}\text { C } 7.179072 & 0.355132 & 0.006246\end{array}$

C $7.242555-1.137966-0.267989$

H 5.4743990 .1248911 .272950

C 5.1385881 .9508930 .178613

H $7.4989800 .916520-0.879156$

H 7.7977700 .6632810 .853981

C $8.511991-1.575047-0.977879$

H $7.169419-1.6740340 .687807$ 
C 3.5952532 .0239470 .222993

O $5.5686022 .523957-1.060923$

H 5.5766072 .5656930 .974032

H $3.1779831 .692446-0.735976$

O 3.2144553 .3950720 .379475

C 2.9525931 .2106531 .360155

C 1.4236861 .3771051 .429418

H $3.1866180 .151478 \quad 1.216432$

O 3.5185861 .5978782 .612254

H $1.1737292 .378467 \quad 1.798808$

H 1.0135201 .2672970 .419337

H $8.496976-2.652124-1.172371$

H $8.633056-1.060363-1.936996$

H $9.391296-1.353552-0.364405$

C $5.328160-2.729372-0.869956$

H $5.351273 \quad 3.470990-1.038851$

H $3.4486693 .860942-0.440826$

H 3.3585292 .5506252 .724525

H $5.023373-2.8373160 .177682$

H $6.046578-3.527472-1.087331$

H $6.162816-1.187506-2.157165$

C $0.530035-2.0800272 .519154$

O $0.995851-0.956294 \quad 1.762273$

C $\quad 0.784036 \quad 0.3285812 .359650$

C -0.7175940 .5701122 .564772$

C $-1.352576-0.5613123 .381330$

C $-0.968678-1.9433582 .840631$

H $0.654755-2.9379571 .846007$

C $1.397911-2.3294943 .757715$

H $1.2941940 .368733 \quad 3.327942$

O $\begin{array}{llll}1.1 .378990 & 0.675394 & 1.306845\end{array}$

H -0.8569251 .5265643 .081495$

H $-2.443846-0.4584983 .360526$

O $-0.935304-0.4649454 .740036$

H -1.271675 -2.722411 3.550111

O $-1.706693-2.1625101 .635199$

C 2.819451-2.656099 3.360488

H $1.408724-1.4724864 .436966$

H $1.020085-3.1811754 .335054$

H -1.195139 $-0.136210 \quad 0.803407$

H $-1.218557 \quad 0.4032745 .073699$

H -1.526844 -3.072028 1.341953

O $3.670074-1.7125423 .855737$

O $3.134991-3.6311252 .690477$

C $5.055541-1.9133603 .561171$

H $5.605736-1.0295963 .894511$

H $5.428351-2.7872524 .103135$

H $5.207971-2.0339972 .485393$

SCF Energy (B3LYP/6-31G**//MMFF) $=-3245.91636673$

0400120

MMFF Geometry

C -1.8493693 .2899121 .335655$

C - 1.2266812 .1023421 .303692

C -0.6549581 .4161752 .516145$

O $0.731960 \quad 1.1176352 .234867$

C -1.396620 0.0941882 .764925

C $-0.902875-0.6394583 .995172$

C $-0.079745-1.7061103 .968036$

C $-1.434870-0.1247555 .307386$

C $0.545726-2.3428742 .758272$

C $2.048206-2.5824612 .959411$

C $2.689320-3.2013161 .708664$

C $4.130854-3.6244181 .991309$

C $4.816033-4.1813140 .734084$

O $2.669003-2.2189780 .667198$

O $6.224736-4.2600021 .000326$

C $4.626389-3.175314-0.419871$

C $4.354128-5.6083560 .416920$

O $5.129491-3.703806-1.645282$

C $3.167752-2.684114-0.593342$

C $3.140060-1.504607-1.591933$

O $2.374166-3.751647-1.113295$

C 1.6376032 .1010312 .487260

O 1.4197073 .1908162 .992475

C 2.9502271 .6390271 .985659

C 3.92255725428481 .793915

C 5.2451892 .2854561 .223008
C 5.6478610 .8524991 .003208

C $1.797820-0.768380-1.679552$

C $1.9440230 .474129-2.535735$

C $2.9764052 .749090-2.508506$

O $1.5725310 .497383-3.706699$

C $4.4728412 .760627-2.584325$

C $5.2296943 .803165-2.208133$

C $6.7321633 .805176-2.266407$

C $7.3757324 .276150-0.977944$

C $8.0014625 .463777-0.914137$

C 7.3585253 .3424030 .217002

C 6.0233513 .3425510 .910562

N $2.5628911 .516361-1.862580$

H -1.9665133 .8099442 .282515$

H -1.1132531 .5879690 .351352$

H -0.7288102 .0532483 .406264$

H -1.320998 -0.5491521 .881209$

H -2.4702030 .2889122 .887828$

H $0.175052-2.1800354 .915047$

H -1.084463 -0.716905 6.159463

H -1.1160540 .9089505 .472619$

H -2.529386 -0.1569255 .312232$

H $0.047592-3.3039522 .582988$

H $0.401147-1.7353631 .861356$

H $2.536484-1.6266983 .189731$

H $2.197679-3.2471633 .819456$

H $2.089939-4.0671581 .403094$

H $4.169976-4.3588042 .805553$

H $4.704126-2.7551392 .343692$

H $6.352474-4.8384711 .771693$

H $5.257883-2.306871-0.187140$

H $4.550324-6.2721861 .267560$

H $4.917454-6.028145-0.423782$

H $3.288654-5.6727380 .186522$

H $4.479248-4.344416-1.980200$

H $3.909162-0.779636-1.304944$

H $3.404317-1.860113-2.596506$

H $1.588974-3.377526-1.543849$

H $3.0603660 .590667 \quad 1.734229$

H 3.7163093 .5853922 .033860

H $5.4869790 .259601 \quad 1.910844$

H $\quad 6.7038520 .732400 \quad 0.751492$

H 5.0634500 .4139430 .190996

H $1.030898-1.403598-2.132263$

H $1.431780-0.470803-0.690491$

H $2.5878333 .577043-1.907493$

H $2.5501122 .807746-3.514323$

H $4.9578691 .865543-2.967577$

H $4.7450174 .709157-1.851257$

H $7.0294344 .459323-3.096266$

H $7.1213422 .809518-2.513495$

H $8.4851175 .799483-0.002762$

H $8.040396 \quad 6.129827-1.769757$

H $8.112923 \quad 3.654144 \quad 0.950928$

H $7.6645512 .348953-0.122335$

H 5.6526554 .3420501 .144064

H $2.8345421 .382514-0.893286$

C $-3.9093313 .854769-0.045613$

O $-4.3009572 .467311-0.080858$

$\begin{array}{llll}\text { C } & -5.478884 & 2.306778 & 0.732627\end{array}$

C -6.0361503 .7104270 .935585$

C -4.7534984 .5148701 .045126$

H $-5.142867 \quad 1.9281551 .705390$

C -6.4428591 .3057040 .089362$

H $-6.6226194 .043756 \quad 0.071397$

H -6.6676813 .7835921 .826140$

C -4.9494406 .0083910 .853183$

H -4.3188804 .3381712 .036627$

C $-5.809316-0.076086-0.184060$

O $-6.8834101 .848240-1.160974$

H $-7.336434 \quad 1.2008490 .716308$

H $-5.0605390 .017621-0.975586$

O $-6.833084-0.944115-0.681672$

C $-5.180004-0.7381361 .054186$

C $-4.695311-2.1879320 .870226$

H $-4.332003-0.1380141 .397728$

O $-6.135832-0.7413852 .120087$

H -4.410234 -2.565352 1.861684 
H $-5.535983-2.812613 \quad 0.542611$ H $-3.993720 \quad 6.5376880 .920961$ H -5.391875 6.233694 -0.122898 H -5.6147486 .4109321 .623983$ C -2.3910843 .9647570 .106289$ H $-7.623767 \quad 1.301496-1.473275$ H -7.062152 -0.642959-1.577111 H $-6.907802-1.2506871 .818726$ H -2.088895 5.0181830 .120125 H -1.920615 $3.519602-0.779343$ H -4.182650 4.270519-1.024415 C $-2.993319-2.312915-2.466410$ O $-3.981661-2.211355-1.431269$ C $-3.503206-2.341939-0.089814$ C $-2.815077-3.7046940 .100055$ C $-1.720177-3.933434-0.944013$ C $-2.216176-3.636046-2.360612$ H $-3.569121-2.348224-3.400300$ C $-2.080778-1.076147-2.516441$ H $-2.786615-1.5415110 .120382$ O $-3.772343-4.759840 \quad 0.022164$ H -2.379402 -3.7430631 .105335$ H -1.376943 -4.973999-0.892965 O $-0.608827-3.091511-0.660414$ H -1.377964 -3.624944 -3.067273 O $-3.097799-4.683957-2.774769$ C $-2.7954030 .149207-3.047889$ H $-1.681700-0.821261-1.531454$ H -1.245928 -1.269738 -3.199962 H $-4.226745-4.686387-0.834618$ H $-0.251093-3.3621670 .201769$ H $-2.592627-5.514819-2.759765$ O $-1.9293461 .206298-3.028600$ O $-3.9513690 .179385-3.446395$ C $-2.4478612 .432960-3.551698$ H $-1.6904053 .208963-3.413260$ H $-2.6513822 .327210-4.621386$ $\mathrm{H}-3.3543622 .726846-3.014369$

SCF Energy $\left(B 3 L Y P / 6-31 G^{* *} / / M M F F\right)=-3245.92088216$

\section{1}

MM̄FF Geometry

C $2.230562-3.825983-0.504580$

C $2.842737-4.052835-1.677418$

C $3.885625-3.171157-2.331507$

O $4.121256-1.978008-1.550448$

C $3.390793-2.733194-3.720531$

C $4.425336-1.942952-4.497185$

C $4.397485-0.605873-4.663737$

C $5.510082-2.764405-5.144478$

C $3.4034420 .363920-4.087115$

C $4.1004761 .601467-3.503956$

C $3.0861152 .578025-2.892451$

C $3.7622493 .893150-2.503697$

C $2.7799324 .854262-1.817910$

O $2.5234601 .960051-1.730123$

O $3.5435915 .910473-1.216089$

C $2.0662464 .103805-0.675577$

C $1.8325675 .520894-2.822302$

O $1.0367644 .906565-0.101553$

C $1.4935492 .727868-1.094218$

C 1.0327721 .9608110 .165403

O $0.3732232 .923796-1.958068$

C $5.116174-2.017832-0.624228$

O $5.893359-2.934616-0.412990$

C $5.048840-0.7569400 .146834$

C $5.589918-0.7202681 .373499$

C 5.5434170 .4058462 .307688

C 5.0135701 .7179521 .795767

C $0.6613730 .493061-0.077681$

C $0.355188-0.176611 \quad 1.246448$

C $1.488558-1.1037823 .271497$

O $-0.797713-0.3166571 .647584$

C $2.117171-0.1066924 .195328$

C $3.098938-0.4061755 .059700$

C 3.7296250 .5993475 .982235

C 5.2435070 .5751605 .942359

C 5.9533160 .1052226 .982390
C $5.938413 \quad 1.165304 \quad 4.731249$

C 5.9656450 .2055713 .573584

N $1.501801-0.5524181 .929404$

H $2.483488-2.9463910 .080379$

H $2.577029-4.950754-2.232763$

H $4.812279-3.748491-2.436228$

H $2.464652-2.155638-3.617741$

H $3.113935-3.613873-4.316078$

H $5.170519-0.152431-5.283093$

H $6.198678-2.151972-5.736460$

H $6.103153-3.284396-4.385802$

H $5.072505-3.509844-5.816366$

H $2.7248120 .677200-4.889381$

H $2.791720-0.099143-3.308223$

H $4.8153161 .281579-2.734787$

H $4.6681582 .105757-4.295964$

H $2.2928852 .760131-3.627105$

H $4.2179674 .371825-3.379255$

H $4.5891333 .687076-1.809408$

H $4.0549976 .347982-1.918229$

H $2.809348 \quad 3.940587 \quad 0.118047$

H $1.1844856 .252876-2.327802$

H $1.2019984 .807114-3.356293$

H $2.4030816 .083749-3.570940$

H 1.4403055 .7523600 .158710

H 1.8358701 .9877850 .910295

H 0.1719952 .4683120 .618562

H $-0.2090122 .149687-1.902881$

H $4.5075730 .076379-0.285868$

H $6.078284-1.6179351 .751548$

H $3.941117 \quad 1.6438401 .600506$

H 5.5200282 .0055580 .867210

H 5.1635212 .5482492 .488853

H $-0.2194970 .398295-0.720516$

H $1.475751-0.051685-0.568860$

H $2.044140-2.0463963 .240578$

H $0.460564-1.3089233 .584865$

H 1.7407640 .9130564 .153601

H $3.456972-1.4314305 .122723$

H 3.3769450 .3810126 .998656

H $3.385071 \quad 1.616246 \quad 5.756785$

H 7.0382700 .1067576 .972502

H $5.466304-0.2964327 .864841$

H 6.9783591 .4147954 .979105

H 5.4565452 .1159814 .488138

H $6.362771-0.7769443 .834143$

H $2.407114-0.3936051 .497398$

C $-0.096772-3.924560 \quad 0.384714$

O $-0.703790-3.484367-0.845831$

C $-2.137523-3.615435-0.730799$

C $-2.413064-3.9381730 .730636$

C $-1.174266-4.7250201 .109978$

H $-2.409400-4.466264-1.367996$

C $-2.795972-2.330124-1.246295$

H -2.480550 -3.019415 1.326971

H $-3.339400-4.5034710 .869114$

C $-0.962172-4.8322422 .610069$

H -1.249396 -5.7329050 .680365$

C $-4.316013-2.274059-1.015392$

O $-2.543418-2.239807-2.652647$

H $-2.310165-1.461942-0.788110$

H $-4.531677-2.2732420 .058568$

O $-4.908663-3.476163-1.526202$

C $-5.029413-1.082356-1.682915$

C $-4.4541130 .310740-1.368781$

H $-5.016258-1.216999-2.771138$

O $-6.411563-1.117527-1.320922$

H $-3.4696230 .390333-1.843588$

H $-5.096576 \quad 1.050785-1.860155$

H $-0.051688-5.3954402 .837089$

H $-0.871413-3.8443413 .074285$

H - $1.804693-5.3487803 .081401$

C $1.172972-4.720140 \quad 0.082194$

H $-1.579292-2.275598-2.776441$

H $-4.699707-3.525820-2.474894$

H $-6.461724-1.094623-0.350487$

H $0.943371-5.541088-0.607790$

H $1.570919-5.1592951 .003671$ 
H $\quad 0.149523-3.0239210 .958651$

C $-6.520888 \quad 1.6215630 .524059$

$\begin{array}{lllll}\text { O } & -5.574744 & 0.590400 & 0.832749\end{array}$

C -4.3301930 .6662160 .131552$

$\begin{array}{llll}\text { C } & -3.656608 & 2.021340 & 0.396970\end{array}$

C $-4.588062 \quad 3.189746 \quad 0.087297$

C -5.9280553 .0086240 .796763$

H $-6.8207061 .541552-0.527928$

C $\quad-7.759894 \quad 1.385943 \quad 1.397097$

H -3.683747 -0.0932740 .585490$

O $-2.4630242 .137661-0.368659$

H -3.3651222 .0690331 .454290$

H $-4.7291603 .304707-0.993384$

$\begin{array}{lllll}O & -3.981905 & 4.406520 & 0.530748\end{array}$

H $-5.795138 \quad 3.1558121 .876298$

$\begin{array}{llllll}0 & -6.837872 & 4.006366 & 0.337956\end{array}$

C $-8.544047 \quad 0.187792 \quad 0.919152$

H -7.483252 1.2326022 .447509

H $-8.4444772 .241591 \quad 1.371656$

H -2.093443 $3.021620-0.201400$

H -3.835617 4.3380561 .489641

H $-6.423618 \quad 4.872096 \quad 0.495613$

O $-8.166584-0.925146 \quad 1.610544$

$\begin{array}{lllll}0 & -9.375037 & 0.233529 & 0.021360\end{array}$

C $-8.834585-2.1358681 .241798$

H $-8.420469-2.9489571 .843862$

H $-9.905350-2.0536731 .450463$

H -8.663105 -2.360404 0.184806

SCF Energy (B3LYP/6-31G**/MMFF) $=-3245.92521243$

0400122

MMFF Geometry

C $1.886636-3.103369-1.498491$

C $1.763122-4.040641-0.544879$

C $2.233429-3.8916260 .884052$

O $2.243361-2.4991581 .277425$

C $3.656782-4.4603391 .036224$

C $4.230979-4.3205412 .435117$

C $5.329140-3.5963512 .734551$

C $3.502572-5.0689553 .520584$

C $6.197987-2.8328121 .772765$

C $5.659198-1.4253401 .521351$

C $6.310620-0.7345550 .317664$

C $7.768037-0.349185 \quad 0.575523$

C $8.359402 \quad 0.459615-0.591738$

$\begin{array}{lllll}\text { O } & 5.534170 & 0.441406 & 0.057963\end{array}$

O $9.6004941 .033052-0.153452$

C $7.3968351 .612559-0.955670$

C $8.729602-0.448514-1.769887$

O $7.8329812 .289535-2.132027$

C $5.9278311 .159022-1.118401$

C $5.0003722 .389286-1.256860$

O $5.8136010 .356098-2.295330$

C $1.051034-1.9460001 .632467$

O $-0.038132-2.4979631 .644296$

C $1.299302-0.5349592 .009123$

C 0.2702190 .2170792 .426870

C 0.3259951 .6102092 .876651

C 1.6575822 .3093992 .824390

C $3.5041342 .053890-1.166989$

C $2.6321623 .288891-1.310468$

C $0.2459213 .998840-1.184453$

O $3.0748384 .393133-1.615291$

C $0.016187 \quad 4.619203 \quad 0.156886$

C -1.175805 4.6332730 .771955

C -1.4305695 .3200482 .081167$

C -1.9858774 .3903663 .133940$

C $-3.298063 \quad 4.376700 \quad 3.423851$

C -1.0050313 .5505413 .920180$

C $-0.810030 \quad 2.1812403 .329786$

N $1.2934933 .001484-1.081423$

H $2.408687-2.179179-1.266973$

H $1.265283-4.977619-0.783839$

H $1.544402-4.4407281 .537945$

H $4.306506-3.9638920 .307170$

H $3.660733-5.5244040 .765381$

H $5.651940-3.5477993 .773266$

H $4.035881-5.0356604 .476699$
H $2.508207-4.6426483 .684215$

H $3.391248-6.1227973 .245608$

H $7.209248-2.7760552 .193336$

H $6.302877-3.384850 \quad 0.832238$

H $4.579376-1.4631501 .339986$

H $5.775966-0.8067452 .421089$

H $6.231502-1.404609-0.546547$

H $8.384749-1.2338820 .773185$

H 7.8223510 .2628301 .486604

H 9.4184971 .6022530 .613653

H $7.4403372 .348535-0.140791$

H $9.1899740 .124443-2.582363$

H $7.876800-0.997094-2.174899$

H $9.481020-1.185471-1.461284$

H $8.7551232 .561578-1.985067$

H $5.2259193 .105346-0.455617$

H $5.1967182 .894848-2.210874$

H $4.9024690 .395999-2.624234$

H $2.315381-0.1627291 .950518$

H $-0.718290-0.2385682 .463944$

H 2.0725582 .2719051 .812304

H $2.367456 \quad 1.835308 \quad 3.511004$

H 1.6058683 .3668543 .091524

H $3.2141951 .355725-1.958739$

H $3.281377 \quad 1.587202-0.201224$

H - $0.6471513 .496987-1.569235$

H $0.5453554 .770667-1.900993$

H 0.8636315 .1124200 .627240

H -2.029736 $4.164918 \quad 0.288340$

H $-2.1385886 .138066 \quad 1.893077$

H -0.5229245 .8033882 .463805$

H -3.696976 3.7341754 .201972

H -4.002642 5.0012972 .884711

H -1.3853693 .4107204 .941529$

H -0.0696354 .1010794 .049838$

H -1.7220481 .5823053 .328962$

H $1.0327952 .073799-0.759093$

C $0.285417-2.170933-3.232295$

O $-0.772073-2.173366-2.248864$

C $-0.995081-0.812923-1.819552$

C $-0.4372450 .049987-2.938966$

C $0.805206-0.732771-3.313998$

H $-0.402657-0.685034-0.904694$

C $-2.482363-0.607140-1.518169$

H $-1.1357880 .092147-3.783885$

H $-0.2179491 .071956-2.622125$

C $1.376932-0.359553-4.670235$

H $1.570024-0.546794-2.551174$

C $-2.8441840 .849345-1.177655$

O $-2.824874-1.433184-0.400602$

H $-3.079080-0.959405-2.366802$

H $-2.6651301 .489392-2.049020$

O $-1.956614 \quad 1.317058-0.154167$

C $-4.2851221 .065503-0.675390$

C $-5.3625720 .539370-1.638577$

H -4.3997010 .6078850 .312670$

O $-4.482093 \quad 2.470978-0.482932$

H $-5.2365161 .023023-2.615326$

H $-5.219370-0.534069-1.792261$

H $2.257476-0.967416-4.901091$

H $0.644056-0.507839-5.470328$

H $1.6793840 .692876-4.682890$

C $1.328638-3.241410-2.888095$

H $-2.569912-2.344898-0.624023$

H -2.092582 0.7574030 .629450

H $-4.3889182 .905852-1.347665$

H $2.153023-3.204475-3.609068$

H $0.856708-4.226770-2.992277$

H $-0.186516-2.448159-4.183804$

C -6.975964 -1.050089 0.387340

$\begin{array}{lllll}\text { O } & -6.996487 & 0.370748 & 0.209258\end{array}$

C $-6.8018770 .805419-1.139743$

C $-7.9020510 .221978-2.036350$

C $-8.013047-1.296487-1.900110$

C $-8.097197-1.703795-0.427205$

H $-6.005225-1.4495660 .069797$

C $-7.135095-1.3264101 .886095$

H $-6.9371281 .894431-1.132845$ 
O $-7.6636210 .575083-3.394906$ H $-8.8628780 .670797-1.752174$ H $-7.178816-1.796770-2.405944$ O $-9.202188-1.713380-2.575103$ H $-9.076105-1.417609-0.021813$ O $-7.981296-3.120670-0.320596$ C $-5.920504-0.8531002 .649813$ H -8.005613 -0.7949542 .289484$ H $-7.277004-2.3916152 .099495$ H $-8.3718600 .174751-3.927900$ H $-9.215969-2.685279-2.572643$ H $-8.763359-3.513131-0.743611$ O $-4.877620-1.707552 \quad 2.443589$ O $-5.898847 \quad 0.161433 \quad 3.334411$ C $-3.647835-1.338307 \quad 3.074385$ H $-2.891587-2.0808932 .806949$ H $-3.319131-0.3586242 .715506$ H -3.766759-1.332305 4.161885 SCF Energy (B3LYP/6-31G**//MMFF) $=-3245.91053181$

0400123

MM̄FF Geometry

C $0.712259-0.5147931 .747429$

C $1.401868-1.3938851 .002796$

C $0.914046-2.083219-0.252600$

O $-0.373571-1.568323-0.663339$

C $0.776426-3.5924040 .014470$

C $0.384791-4.391158-1.213817$

C $-0.834971-4.923132-1.432075$

C $1.489040-4.601344-2.218614$

C $-2.054584-4.823735-0.557614$

C $-3.170391-4.038621-1.258189$

C $-4.321089-3.687911-0.302297$

C $-5.437258-2.962965-1.057368$

C $-6.544885-2.458256-0.121974$

$\begin{array}{llllll} & 0 & -3.789277 & -2.842452 & 0.723022\end{array}$

O $-7.342732-1.535438-0.879564$

C $-5.899329-1.6797931 .045652$

C $-7.484564-3.5951890 .294996$

O $-6.883167-1.3060642 .006983$

C $-4.736591-2.4362941 .719148$

C $-3.978254-1.5758492 .759539$

O $-5.265989-3.5697102 .418144$

C $-0.372703-0.488687-1.493985$

O $0.6015130 .067070-1.975548$

C $-1.776778-0.069486-1.700507$

C -2.031083 $1.099557-2.307817$

C $-3.3528201 .656905-2.603996$

C $-4.5590200 .858456-2.188200$

C -3.263305 $-0.341176 \quad 2.201457$

$\begin{array}{llll}\text { C } & -4.061399 & 0.947884 & 2.286383\end{array}$

C -3.9349253 .3421451 .596062$

O -5.1060551 .0556482 .921865$

C $-4.830486 \quad 3.545162 \quad 0.415564$

C $-4.6590484 .529886-0.479770$

C $-5.5942044 .790266-1.625409$

C $-4.8853164 .868158-2.958319$

C -4.554985 $6.053948-3.497375$

C $-4.6499113 .578247-3.709008$

C -3.424731 2.846345 -3.237408

$\mathrm{N}-3.434073 \quad 1.9812521 .601513$

H -0.302496 -0.2499011 .470519$

H $2.418148-1.6423141 .302917$

H $1.653597-1.917314-1.045802$

H $\quad 0.060508-3.7507050 .827213$

H $1.728421-3.9917510 .391046$

H $-0.989229-5.492264-2.348210$

H $1.175423-5.235009-3.055152$

H $1.817991-3.645181-2.636986$

H $2.347357-5.087411-1.743161$

H $-2.408235-5.837906-0.335450$

H $-1.824524-4.3670180 .406676$

H -2.749731 -3.112159-1.667681

H $-3.552644-4.623220-2.104342$

H $-4.697499-4.6128630 .150088$

H -5.857681 -3.605596 -1.841024

H $-5.011348-2.098328-1.582469$

H $-8.001909-1.151573-0.275687$
H $-5.518736-0.743666 \quad 0.621869$

H $-7.958749-4.039797-0.588431$

H $-8.306058-3.2243490 .917688$

H $-6.975224-4.3958550 .835136$

H -7.092417 -2.092966 2.538548

H $-4.641309-1.3186623 .594250$

H $-3.197430-2.2145253 .197429$

H -4.622998 -3.8446443 .092667$

H $-2.559467-0.727684-1.344049$

H $-1.1884251 .710516-2.629792$

H $-4.621715-0.064966-2.772963$

H $-5.5029161 .391694-2.319275$

H $-4.5055030 .601631-1.125193$

H -2.363184 -0.1697712 .805715$

H -2.938732 -0.5038261 .169453$

H $-3.0659104 .007381 \quad 1.575517$

H -4.4937073 .5276472 .519067$

H -5.6812642 .8753370 .314733$

H $-3.825148 \quad 5.217597-0.358293$

H $-6.1084245 .739283-1.423494$

H $-6.3866654 .033279-1.676444$

H $-4.0752246 .119529-4.468329$

H $-4.7515436 .985211-2.976472$

H $-4.507420 \quad 3.789852-4.777783$

H $-5.5543102 .964095-3.673267$

H $-2.4939573 .355288-3.492199$

H $-2.6018181 .782261 \quad 1.054153$

C 1.6254111 .6234392 .760807

O $2.575476 \quad 1.730034 \quad 1.681681$

C 2.1468622 .7870360 .802356

C 1.2107773 .6481041 .637383

C 0.4746042 .5730012 .420267

H 1.5571582 .3208920 .003651

C 3.3642763 .4920050 .197795

H 1.7634914 .3060422 .318030

H 0.5468214 .2632321 .022879

C $-0.270553 \quad 3.097178 \quad 3.634477$

H -0.2445602 .0896851 .748151$

C $4.3400092 .524074-0.511886$

$\begin{array}{lllll}\text { O } & 4.085829 & 4.160161 & 1.238871\end{array}$

H $3.0354434 .275632-0.493838$

H $4.811161 \quad 1.8861430 .239959$

O $5.394802 \quad 3.295567-1.094711$

C $3.6848181 .658859-1.601079$

C $4.631830 \quad 0.699516-2.343192$

H $2.8833101 .060693-1.158403$

O $3.0811602 .528330-2.562448$

H $4.0700300 .269799-3.183292$

H $5.4589111 .266051-2.788843$

H $-0.7693982 .280253 \quad 4.165661$

H 0.4057673 .5947894 .337328

H $-1.034287 \quad 3.821788 \quad 3.334083$

C 1.2636610 .1520772 .977085

H 4.3592773 .4870991 .885677

H $5.0027463 .846525-1.793773$

H $2.5857641 .974255-3.189616$

$\begin{array}{llll}\text { H } & 0.534201 & 0.058312 & 3.789547\end{array}$

H $2.165056-0.3833623 .301243$

H $2.143370 \quad 1.9826963 .659228$

C $6.898899-0.8651220 .195164$

O $6.2893580 .055918-0.721320$

C $5.159735-0.436878-1.448748$

C $5.558725-1.666045-2.279145$

C $6.191072-2.746933-1.398536$

C $7.297871-2.173777-0.509636$

H $7.826972-0.3716200 .511522$

C $6.029631-1.0853161 .444081$

H $4.369141-0.723080-0.746798$

O $6.466978-1.293437-3.313277$

H $4.663731-2.068146-2.768647$

H $6.609703-3.536562-2.034134$

O $5.193129-3.335531-0.568906$

H $7.610980-2.9105610 .239207$

O $8.443211-1.889735-1.319306$

$\begin{array}{llll}\text { C } & 5.994938 & 0.135741 & 2.341051\end{array}$

H $4.997399-1.3293161 .181905$

H $6.446901-1.9038772 .041992$

H $7.228499-0.854641-2.896750$ 
H $\quad 4.510964-3.711384-1.151097$

H $8.730784-2.726047-1.723590$

O $5.136950-0.101387 \quad 3.377717$

O 6.6443221 .1584552 .172010

C 5.0219370 .9570484 .334030

H 4.7482281 .8948373 .841172

H 4.2328390 .6908795 .042242

H 5.9629181 .0695784 .880389

SCF Energy $\left(B 3 L Y P / 6-31 G^{* *} / / M M F F\right)=-3245.91928224$

\section{00124}

MM̄FF Geometry

C $0.030800-3.266769-0.246348$

C $-0.044991-3.8334820 .968090$

C $-0.532813-3.1478192 .222726$

O $-1.599426-2.218526 \quad 1.915175$

C $0.622479-2.3851172 .894200$

C $0.261200-1.8252824 .257047$

C $0.011312-0.5268274 .519621$

C $0.225537-2.8362795 .375114$

C 0.0041850 .6248293 .554197

C $-1.399370 \quad 1.2284423 .424993$

C -1.482870 2.2715322 .299495

C -2.8892412 .8704492 .233764$

C -3.0752713 .8026361 .027488$

O $-1.1686691 .610171 \quad 1.070241$

O $-4.483310 \quad 4.030833 \quad 0.859233$

C $-2.5820783 .082864-0.246457$

C -2.439655 5.1766651 .269846

O $-2.5943873 .965830-1.365250$

C $-1.1840302 .453959-0.088635$

C $-0.7652861 .580396-1.297586$

O -0.2169093 .5039920 .024206$

C -2.834731 $-2.760617 \quad 1.713547$

O $-3.131026-3.9444591 .766356$

C $-3.779343-1.6637911 .404714$

C $-5.042751-1.9633291 .065538$

C $-6.112876-1.0128300 .754523$

C -5.7814450 .4539320 .799041$

C $-1.6498300 .358058-1.566534$

C $-2.7557640 .594411-2.580639$

C $-4.755239-0.530301-3.558728$

O $-2.812127 \quad 1.588639-3.298680$

C $-5.9826930 .051155-2.931607$

C -7.156273 -0.596365 -2.869515

C $-8.4136650 .003003-2.308422$

C $-9.063985-0.869671-1.259107$

C $-10.083919-1.686038-1.573873$

C $-8.596350-0.7227630 .170284$

C $-7.337186-1.4936170 .452318$

N $-3.650163-0.467809-2.620855$

H - $0.249059-2.224599-0.371279$

H $0.261066-4.8708391 .085331$

H -0.917959 -3.912685 2.908648

H $\quad 0.969816-1.5896862 .227106$

H $1.486383-3.0525103 .019169$

H $-0.221536-0.2474015 .546614$

H $0.027731-2.3742336 .348289$

H -0.558604 -3.578979 5.199636

H $1.187045-3.3548325 .448675$

H $\quad 0.696110 \quad 1.390491 \quad 3.925051$

H $\quad 0.374751 \quad 0.3279722 .571269$

H -2.113083 0.4208393 .220541

H -1.691882 1.6862384 .378139

H $-0.739356 \quad 3.0543272 .489427$

H $-3.131306 \quad 3.3947293 .166707$

H -3.621587 2.0573292 .154058

H $-4.8261634 .412278 \quad 1.685612$

H -3.305681 $2.289982-0.466717$

H -2.904566 5.6660502 .134328

H -2.6097915 .8468940 .420160$

H -1.365829 5.1247921 .461344

H -3.495674 4.322834 -1.442627

H $-0.6506992 .202999-2.193000$

H $0.2422971 .194561-1.083327$

H $0.6506883 .149636-0.228326$

H -3.414240 -0.646228 1.469169

H $-5.332074-3.0127401 .020372$
H $-6.580198 \quad 1.094860 \quad 0.420003$

H -4.9053900 .6702990 .179652$

H $-5.5743190 .763738 \quad 1.828249$

H - $-1.019312-0.432505-1.993878$

H $-2.077617-0.032461-0.638338$

H -4.888475 -1.581375 -3.833759

H $-4.4994310 .035874-4.460291$

H $-5.9124021 .065286-2.545274$

H -7.231446 -1.598686-3.285461

H $-9.1086900 .156816-3.144547$

H $-8.2310071 .001331-1.891762$

H -10.576819 -2.291516 -0.820434

H -10.444759-1.771294 -2.593661

H $-9.366289-1.1027360 .855817$

H -8.5119880 .3389980 .419169$

H $-7.480641-2.5749410 .437408$

H -3.554693 -1.225259-1.950474

C $1.876227-3.662065-1.962517$

O $1.976435-2.279155-2.350514$

C $3.323223-1.820517-2.065023$

C $4.096780-3.052212-1.606922$

C $3.008508-3.888957-0.966261$

H $3.217714-1.105749-1.240892$

C $3.880731-1.119817-3.312775$

H $4.521766-3.590418-2.462768$

H $4.910328-2.809241-0.918942$

C $3.390893-5.345303-0.775071$

H $2.766501-3.4487360 .008056$

C $5.356314-0.678776-3.244436$

O $3.0729540 .024193-3.598497$

H $3.756742-1.795666-4.168813$

H $5.589295-0.067333-4.125924$

O $6.192573-1.838139-3.332449$

C $5.7948560 .108300-1.991946$

C $5.0770681 .455957-1.816843$

H $5.682523-0.506452-1.093153$

O $7.1971560 .382235-2.121866$

H $5.2109402 .045394-2.732175$

H $4.0050021 .284943-1.694432$

H $2.570066-5.907479-0.319097$

H $3.639431-5.824234-1.728128$

H $4.263434-5.430100-0.118942$

C $0.465531-4.002608-1.481117$

H $2.148382-0.274999-3.640867$

H $5.989310-2.283000-4.173008$

H $7.649069-0.472122-2.230486$

H $0.389656-5.085277-1.322177$

H $-0.251908-3.753598-2.273167$

H $2.064018-4.244845-2.875031$

C $4.217951 \quad 1.3285191 .106447$

O 5.5431201 .5479840 .608923

C $5.6123692 .278047-0.620003$

C $4.9542013 .654950-0.452368$

C 3.5302783 .5362950 .083679

C 3.5056112 .6651261 .339009

H 3.6509380 .7195300 .392499

C 4.3383010 .5344092 .411144

H $6.6777262 .461090-0.807454$

O $4.9603904 .349605-1.694623$

H 5.5499694 .2487490 .253215

H $2.8488033 .143836-0.679580$

O $3.0426864 .831902 \quad 0.437005$

H 3.9785573 .2058802 .168981

O 2.1524152 .4158951 .706169

C $4.770921-0.8843882 .129988$

H 5.0587810 .9974843 .095982

H 3.3823140 .4771062 .944079

H $4.6534625 .256440-1.527764$

H $2.9470055 .343350-0.384000$

H 1.7248773 .2786121 .844285

O $6.124469-0.9933872 .248599$

O $3.998186-1.7798681 .815070$

C $6.665503-2.2908121 .981842$

H $7.732143-2.2694102 .220394$

H $6.182276-3.0468882 .608207$

H $6.547731-2.5333090 .921990$

SCF Energy (B3LYP/6-31G**//MMFF)= -3245.91443255 
0400125

MM̄FF Geometry

C $-0.834460-4.3674530 .447752$

C -1.947043 -3.764209 0.005640

C $-2.250548-3.493389-1.444491$

O $-2.413598-2.059599-1.574772$

C $-3.568135-4.177233-1.845542$

C $-3.975093-3.916320-3.284512$

C $-5.011933-3.140926-3.663008$

C $-3.139742-4.614757-4.326638$

C $-5.959174-2.382421-2.776039$

C $-5.663580-0.877283-2.801443$

C $-6.266208-0.158012-1.586148$

C $-6.0697501 .354298-1.700522$

C $-6.5306222 .095961-0.436621$

O $-5.592286-0.653339-0.423408$

O $-6.012706 \quad 3.434148-0.497364$

C $-5.896906 \quad 1.4239580 .800738$

C $-8.0571632 .224450-0.376957$

O $-6.418510 \quad 1.9809092 .004571$

C $-6.058922-0.1095100 .817902$

C $-5.252294-0.7923511 .950780$

O $-7.438521-0.4323791 .030922$

C -1.276594 -1.313714-1.661696

O $-0.132311-1.727203-1.761666$

C $-1.6635250 .110888-1.553121$

C $-0.7110591 .044192-1.407844$

C $-0.9232082 .483500-1.237480$

C $-2.3433972 .979196-1.188754$

C $-3.732865-0.5959381 .891974$

C -3.2157790 .5499672 .744549$

C -1.1132551 .7828473 .261724$

O $-3.909917 \quad 1.1525113 .558706$

C -1.146134 3.0907822 .537331

C -0.0432873 .7944922 .239539$

C -0.0654965 .1429101 .579404$

C 0.8091115 .2071170 .348318

C 2.0556615 .7047450 .411654

C $0.2038534 .777359-0.968575$

C $0.1571013 .283172-1.120009$

N -1.866583 0.7915052 .517646

H $-0.109157-4.752786-0.263998$

H $-2.657227 \quad-3.3703400 .729239$

H -1.437243 -3.835811-2.096007

H -4.356659-3.861506-1.155744

H -3.478342 $-5.262905-1.704279$

H -5.208309 -3.026030 -4.728180

H -3.529703 -4.466882 -5.339429

H -2.111961 -4.239590 -4.312828

H -3.120845 -5.693003 -4.137918

H $-6.982795-2.554904-3.131423$

H -5.943509 -2.764951-1.753351

H $-4.578326-0.718611-2.805649$

H $-6.058432-0.447523-3.730317$

H -7.332405 -0.405458 -1.522223

H $-6.5847201 .746757-2.586316$

H -5.004797 $1.569924-1.857187$

H $-6.3419003 .846158-1.314573$

H $-4.8281541 .667000 \quad 0.781893$

H -8.428568 2.780949-1.245933

H -8.3718912 .7981470 .501768$

H -8.568576 $1.259726-0.361893$

H -6.2752592 .9419851 .964975$

H -5.660072 -0.5092902 .928596$

H -5.430406 -1.874174 1.865487

H $-7.493910-1.3378161 .378669$

H -2.720230 $0.349399-1.558247$

H $\quad 0.330506 \quad 0.725617-1.405029$

H -2.429731 $4.045123-0.969811$

H -2.901713 $2.462477-0.401435$

H $-2.8420842 .806001-2.148326$

H -3.254452 -1.500695 2.288536

H -3.390205 -0.4696730 .861148$

H -0.0949901 .4003263 .377622$

H -1.554897 1.9024354 .256439

H -2.122246 3.4958072 .281210

H $\quad 0.9301453 .4013172 .524413$

H 0.2784975 .8792862 .317661
H -1.0872005 .4452311 .317962$

H $2.6798485 .782685-0.472025$

H 2.4819646 .0403191 .351386

H $0.8029255 .168438-1.801638$

H $-0.7737855 .252322-1.085702$

H $1.1448512 .822868-1.157990$

H -1.3824550 .2760601 .788131$

C $0.833451-3.7405722 .200348$

O $0.780522-2.3818911 .729281$

C $1.601499-1.5575252 .583578$

C $2.155037-2.4780283 .668653$

C $1.153262-3.6166803 .685832$

H $0.927222-0.8097383 .014424$

C $2.689482-0.8892621 .729575$

H $3.150480-2.8511923 .396386$

H $2.244229-1.9715234 .634681$

C $1.689213-4.8892144 .316738$

H $\quad 0.262097-3.2860804 .235898$

C 3.6344710 .0216612 .532193

O $2.055453-0.1158560 .706046$

H $3.250873-1.6747051 .213968$

H $4.082583-0.5333253 .364433$

O $2.8450721 .064963 \quad 3.116144$

C 4.7563760 .6673881 .691322

C $5.728188-0.372111 \quad 1.104147$

H $4.3265651 .290543 \quad 0.901110$

O 5.4921721 .5512002 .545619

H $6.125803-0.9735781 .931049$

H 5.180111-1.052608 0.447667

H $0.932389-5.6796494 .304776$

H $2.571884-5.2598423 .784771$

H $1.974466-4.7113835 .358761$

C $-0.487442-4.4566441 .908348$

H $1.561751 \quad 0.6002341 .141723$

H 3.4517151 .6533063 .597385

H 5.8943961 .0167973 .251722

H -1.288271 -4.007704 2.509523

H -0.417184 -5.511175 2.196322

H $1.654363-4.2355461 .663666$

C $5.9350560 .301046-1.876167$

O $6.4879001 .041055-0.780612$

C $6.914910 \quad 0.253508 \quad 0.335655$

C $7.972427-0.764839-0.114560$

C $7.460060-1.634208-1.258734$

C $6.925819-0.755068-2.387196$

H $4.999649-0.178080-1.562794$

C $5.614508 \quad 1.304485-2.996244$

H 7.4172730 .9443871 .023775

O $8.365891-1.5756690 .987169$

H $8.865892-0.223076-0.451929$

H $6.697552-2.344858-0.918979$

O $8.536528-2.415291-1.782205$

H $7.767871-0.265208-2.893526$

O $6.272849-1.579914-3.351200$

C $4.4412492 .202453-2.670229$

H $6.483737 \quad 1.949520-3.172659$

H $5.3618770 .773748-3.921054$

H $9.122483-2.1147380 .701577$

H $8.796592-3.052940-1.096235$

H $6.916703-2.246505-3.646257$

O $4.2661653 .079925-3.703443$

O $3.7625582 .143534-1.655257$

C $3.1808973 .999950-3.557766$

H $3.2939134 .581127-2.638180$

H $3.1994404 .685942-4.408741$

H $2.2298493 .460099-3.563123$

SCF Energy (B3LYP/6-31G**//MMFF) $=-3245.91168746$

\section{6}

MM̄MF Geometry

C $1.375562-0.3065241 .976770$

C $0.151932-0.8327441 .824136$

C $-0.207654-1.7818840 .711135$

O $-1.396867-1.2757430 .059330$

C $-0.522593-3.1718361 .282094$

C $-0.776514-4.2028840 .200376$

C -1.996345 -4.649173 -0.158222

C $0.459024-4.764181-0.455630$ 
C $-3.326983-4.2015490 .380154$

C $-4.325278-3.918365-0.751648$

C -5.679807-3.457191-0.195953

C $-6.729578-3.379638-1.304506$

C $-8.073306-2.858923-0.774110$

O $-5.501680-2.1630760 .390454$

O $-8.880903-2.544714-1.918415$

C $-7.825646-1.5529950 .009315$

C -8.831022 $-3.940905 \quad 0.003684$

O $-9.025051-1.1011660 .634418$

C $-6.671111-1.6449981 .036830$

C $-6.347807-0.2319751 .572359$

O $-7.078213-2.4714372 .128693$

C -1.223056 $-0.296252-0.869051$

O $-0.1643340 .176598-1.250346$

C $-2.5622990 .141863-1.318411$

C $-2.6714221 .297510-1.990865$

C $-3.9131771 .921674-2.449764$

C $-5.1837731 .124778-2.331180$

C $-5.055211-0.1362112 .391899$

C -4.7666411 .3158012 .720318$

C -3.9715333 .4337141 .658836$

O -5.0638721 .8019163 .808692$

C $-4.906160 \quad 4.054884 \quad 0.666704$

C $-4.517023 \quad 4.924377-0.278415$

C $-5.452620 \quad 5.531904-1.286274$

C $-4.9453975 .418344-2.709318$

C -4.525255 6.504211-3.380266

C $-4.9922094 .059765-3.381512$

C $-3.8543723 .176532-2.942465$

N $-4.194279 \begin{array}{llll}1.999313 & 1.658566\end{array}$

H $2.168209-0.5935821 .292468$

H $-0.643030-0.5482142 .509509$

H $0.605820-1.858210-0.020429$

H -1.377911-3.111093 1.964705

H $0.315261-3.521437 \quad 1.900546$

H -2.052297 $-5.425218-0.920585$

H $0.223289-5.543962-1.187786$

H $\quad 1.008575-3.976777-0.980700$

H $\quad 1.120078-5.2079870 .295935$

H $-3.722707-5.0004101 .018883$

H $-3.233695-3.3082941 .003258$

H -3.911436 -3.143478-1.409850

H $-4.462010-4.826664-1.351767$

H $-5.995351-4.1669230 .577872$

H $-6.861221-4.355222-1.788792$

H -6.377683 -2.698549-2.092382

H $-9.705702-2.142404-1.595626$

H $-7.567507-0.785574-0.733363$

H -9.008176 $-4.815012-0.634348$

H $-9.823187-3.589560 \quad 0.308194$

H -8.298456 -4.2808280 .894352$

H -9.170008 $-1.647542 \quad 1.425634$

H $-6.2651120 .458647 \quad 0.726130$

H -7.1787520 .1310682 .191322$

H $-6.545789-2.2458242 .907988$

H -3.416918 $-0.466225-1.045505$

H $-1.7651111 .867773-2.192433$

H -5.464062 $1.011352-1.281460$

H $-5.0605380 .128495-2.770761$

H $-6.0309181 .578059-2.850375$

H -5.135396 -0.6919753 .331848$

H -4.199503 -0.5489591 .845439$

H -2.922791 3.5993161 .394693

H -4.1566923 .8439272 .656080$

H $-5.955054 \quad 3.7737390 .728905$

H -3.473173 $5.226573-0.324739$

H -5.587445 6.587653-1.017646

H $-6.4493375 .076933-1.227931$

H -4.182322 $6.436241-4.407504$

H -4.509845 $7.485063-2.916907$

H $-4.9208964 .175164-4.470696$

H -5.974740 $3.618951-3.192199$

H -2.876121 $3.654029-3.019650$

H -3.9938991 .4953220 .800150$

C 2.2961002 .0015022 .468931

O $3.593317 \quad 1.774597 \quad 1.884425$

$\begin{array}{llll}\text { C } 3.759345 & 2.682488 & 0.775574\end{array}$
C 2.5310913 .5927610 .770375

C $1.4746602 .695053 \quad 1.384574$

H $3.7514812 .073050-0.134965$

C 5.0921003 .4285810 .898256

H 2.6806134 .4844511 .390203

H $2.273977 \quad 3.930459-0.238477$

C $\quad 0.259730 \quad 3.439745 \quad 1.906579$

H 1.1542031 .9807480 .616573

C 6.3341952 .5200180 .992383

$\begin{array}{lllll}\text { O } & 5.064698 & 4.238393 & 2.082604\end{array}$

H 5.2032204 .1270040 .060824

H 6.3195151 .9491461 .928674

O 7.4837953 .3762421 .061758

C $6.5232061 .554883-0.195285$

C $7.8545840 .782077-0.106025$

H $5.6947470 .839750-0.219961$

O $6.4823492 .290053-1.417186$

H $8.6840991 .496448-0.041925$

H $7.855826 \quad 0.2066220 .826160$

$\begin{array}{llll}\text { H } & -0.463515 & 2.744748 & 2.344808\end{array}$

H 0.5351384 .1698552 .674910

H $-0.239066 \quad 3.978442 \quad 1.094423$

C $1.7294760 .696797 \quad 3.035446$

H 4.9141383 .6461222 .839240

H 7.3550683 .9730841 .818960

H $7.2048632 .940819-1.392247$

H 0.8536620 .9243303 .654712

H 2.4792050 .2353303 .689775

H 2.4613282 .6886203 .310056

C $6.876150-2.079414-0.600211$

O $7.052975-1.046124-1.575306$

C $8.126799-0.139646-1.315999$

C $9.451664-0.910207-1.227646$

C $9.382791-2.032354-0.196089$

C $8.158031-2.910522-0.447610$

H $6.594045-1.6303090 .360065$

C $5.720069-2.967859-1.075359$

H $8.1957820 .510444-2.197301$

O $10.518325-0.021385-0.913671$

H $9.675526-1.345781-2.210268$

H $9.389357-1.6314970 .824061$

O $10.562835-2.829613-0.319967$

H $8.330055-3.504521-1.354175$

O $8.016857-3.8337650 .630626$

C $4.391918-2.264709-0.940926$

H $5.847284-3.269559-2.122251$

H $5.653856-3.888748-0.484398$

H $11.329900-0.553256-0.847252$

H $10.482475-3.5652820 .311284$

H $7.851868-3.3230661 .441506$

O $4.187518-1.442856-2.009017$

O $3.632596-2.425328 \quad 0.005545$

C $2.957057-0.714480-2.001864$

H $2.878079-0.104928-1.097336$

H $2.948389-0.051415-2.870850$

H $2.113500-1.406463-2.079130$

SCF Energy (B3LYP/6-31G**//MMFF) $=-3245.93636234$

\section{4_00127}

MM̄FF Geometry

C $0.973093 \quad 4.906260 \quad 0.201145$

C $2.109166 \quad 4.1949870 .251285$

C $2.907808 \quad 3.824556-0.971826$

O $2.9942712 .379046-1.023049$

C $4.3319574 .390294-0.869747$

C $5.1057914 .255315-2.165897$

C $6.0456623 .320706-2.406935$

C $4.7954995 .298055-3.209867$

C $6.4948302 .210167-1.498882$

C $6.5253500 .865116-2.238928$

C $7.082802-0.253515-1.348301$

C $7.276488-1.542498-2.148347$

C $7.770002-2.692005-1.256554$

O $6.156857-0.481131-0.280455$

O $7.660515-3.913797-2.002139$

C $6.854573-2.796419-0.018590$

C $9.259954-2.552128-0.926349$

O $7.363262-3.7468740 .914258$ 
C $6.603876-1.4410480 .686694$

C $5.496217-1.6135821 .750308$

O $7.803200-1.0141801 .335222$

C $1.9020341 .714190-1.491541$

O $0.881966 \quad 2.206403-1.948178$

C $2.1371580 .265470-1.304867$

C $1.083391-0.564620-1.331012$

C $1.107367-2.013203-1.120472$

C $2.452460-2.684789-1.063746$

C $4.949982-0.3010552 .324952$

C $3.809144-0.6001383 .277154$

C $1.415124-1.3103363 .294279$

O $3.981340-0.6488924 .492751$

C $1.075553-2.6738372 .776240$

C $-0.146941-3.0238472 .348582$

C $-0.492990-4.3919481 .834232$

C $-1.172327-4.3610260 .481889$

C -2.487234 -4.6120620 .363285$

C $-0.312662-4.124517-0.742593$

C $-0.069306-2.662100-0.994797$

N $2.615272-0.8517972 .619001$

H $0.6164685 .279155-0.755928$

H 2.4555063 .8055221 .205874

H $2.4235304 .191546-1.885413$

H $4.8699413 .904397-0.047607$

H $4.2956625 .455736-0.604757$

H $6.563173 \quad 3.356225-3.365136$

H $5.4131855 .185799-4.107388$

H $3.7489605 .232203-3.522424$

H $4.9768336 .301097-2.809805$

H $7.5036382 .451608-1.143150$

H $5.8559572 .118428-0.616549$

H $5.5065590 .612363-2.560060$

H $7.1443880 .956153-3.140355$

H $8.0400340 .083411-0.933234$

H $7.967192-1.384664-2.985989$

H $6.318311-1.834609-2.600660$

H $6.729874-4.032289-2.257548$

H $5.886301-3.187787-0.361082$

H $9.855930-2.551910-1.847100$

H $9.617051-3.406229-0.340216$

H $9.495919-1.637239-0.379043$

H $7.498068-4.5817380 .433862$

H $4.658645-2.1632261 .307290$

H $5.869766-2.2285712 .579678$

H $7.572260-0.4137222 .061582$

H $3.150292-0.067828-1.112834$

H $0.093362-0.139390-1.494375$

H $3.048081-2.434091-1.948959$

H $2.397597-3.774449-1.032070$

H $2.998563-2.363742-0.173658$

H $5.720564 \quad 0.2481142 .875791$

H $4.5844460 .362914 \quad 1.533281$

H $0.627016-0.5800873 .088756$

H $1.582800-1.3533564 .374598$

H $1.874375-3.4120082 .759045$

H -0.952544 -2.296083 2.396322

H - $-1.150836-4.8662372 .574282$

H $\quad 0.396383-5.031607 \quad 1.773408$

H $-2.976176-4.617305-0.605400$

H $-3.106413-4.8083181 .232380$

H $-0.812090-4.530970-1.632083$

H $0.608676-4.703200-0.639186$

H $-0.988404-2.079747-1.077497$

H $2.577329-0.7514501 .608882$

C $-1.209274 \quad 4.3859431 .270611$

O $-0.927723 \quad 2.972811 \quad 1.311933$

C -1.952948 2.3110012 .082859

C $-3.021290 \quad 3.361072 \quad 2.349241$

C $-2.195767 \quad 4.632543 \quad 2.408117$

H -1.470056 2.0069713 .019880

C -2.4281001 .0817611 .300515$

H -3.738152 3.4077871 .519786

H -3.579099 3.1677953 .270288

C -3.019025 5.8986912 .252078

H -1.6635074 .6626223 .368241$

C $-3.622656 \quad 0.3523931 .936883$

$\begin{array}{llll}\text { O } & -1.328857 & 0.170528 & 1.208278\end{array}$
H -2.669320 1.3776020 .273456

H -4.5003111 .0084811 .943830$

O -3.3102920 .0919003 .311889$

C $-3.975628-0.9938971 .274441$

C $-4.168731-0.961271-0.252652$

H $-3.187903-1.7201941 .502222$

O $-5.152049-1.515396 \quad 1.898577$

H -3.204717 -0.733783 -0.720910

H $-4.433495-1.975418-0.572316$

H -2.3801396 .7866682 .287464$

H -3.5603745 .9109241 .300103$

H -3.7552245 .9802003 .058275$

C 0.0993165 .1673831 .396235

H -0.5752180 .6605610 .837002$

H $-4.071022-0.3748913 .698595$

H -5.867851 -0.8699551 .770510$

H $0.621438 \quad 4.8748392 .315710$

H $-0.0976556 .243094 \quad 1.457321$

H $-1.683895 \quad 4.5885120 .300867$

C $-7.188128-1.394472-0.701736$

O $-6.533915-0.219794-0.212235$

C $-5.2389670 .030456-0.765369$

C $-5.3256490 .120312-2.297390$

C $-5.993047-1.110662-2.904740$

C $-7.337658-1.371705-2.227721$

H $-6.594748-2.272105-0.416623$

C -8.525971-1.547656 0.038226

H $-4.9480701 .026097-0.412018$

O $-4.0343310 .284736-2.874410$

H $-5.9153361 .003816-2.575010$

H $-5.344080-1.992268-2.845685$

O $-6.231428-0.879220-4.294990$

H -8.049719 -0.599462 -2.541194

O $-7.853050-2.626512-2.666414$

C $-9.515615-0.430027-0.221857$

H $-8.987819-2.499631-0.248297$

H $-8.348657-1.5608801 .120213$

H -3.637996 $1.081580-2.482098$

H $-5.373637-0.672033-4.704150$

H $-7.903920-2.595760-3.637296$

O $-10.779414-0.887808 \quad 0.022026$

O $-9.221090 \quad 0.708160-0.560227$

C $-11.827307 \quad 0.068633-0.165561$

H $-11.8378780 .421424-1.201379$

H $-12.779647-0.4244240 .046642$

$\mathrm{H}-11.7034130 .9061170 .527494$

SCF Energy (B3LYP/6-31G*//MMFF) $=-3245.92046670$

\section{8}

MM̄FF Geometry

C $-4.233757-3.0259141 .425568$

C $-2.934235-2.9271861 .105895$

C $-1.833750-2.9590712 .132611$

O $-0.908397-1.875307 \quad 1.879347$

C $-1.047753-4.2780402 .019034$

C $-0.085319-4.4813133 .173232$

C $1.224581-4.1657733 .148316$

C $-0.691022-5.0980044 .407803$

C $1.991978-3.5245552 .026822$

C $3.213649-4.3622741 .623985$

C $3.927091-3.7674300 .401190$

C $5.185017-4.5600010 .049152$

C $5.945018-3.924490-1.125962$

O $4.287271-2.4194210 .707383$

O $7.240281-4.542339-1.168284$

C $6.145401-2.413814-0.853912$

C $5.270651-4.250699-2.464837$

O $6.712781-1.782013-1.998011$

C $4.858979-1.693126-0.393419$

$\begin{array}{llll}C & 5.128207 & -0.275626 & 0.157941\end{array}$

O $3.938988-1.605721-1.481152$

C -1.220091 -0.6435532 .364630$

$\begin{array}{llll}\text { O } & -2.271885 & -0.298232 & 2.877827\end{array}$

$\begin{array}{llll}\text { C }-0.013499 & 0.204045 & 2.215180\end{array}$

$\begin{array}{llll}\text { C } & 0.011973 & 1.435162 & 2.748719\end{array}$

C 1.1625862 .3449792 .769030

C $2.458424 \quad 1.8353892 .197674$

C $5.534500 \quad 0.787538-0.862763$ 
C $5.4875002 .175842-0.242965$

C $4.9426744 .549049-0.795741$

O 5.8004102 .3878420 .926368

C $3.5533564 .787485-0.290025$

C 3.2683305 .6040940 .736464

C 1.8700275 .8667281 .223480

C 1.7617565 .8987852 .733464

C 1.4123547 .0287663 .371951

C 2.0640894 .6353903 .508896

C 1.0168533 .5692553 .318703

N $5.0943423 .148494-1.149015$

H $-4.519537-3.1344362 .469220$

H -2.641786 -2.831475 0.063151

H -2.239808 -2.8542443 .147151$

H $-0.510888-4.315674 \quad 1.063810$

H -1.740485 -5.1303941 .995847$

H $1.814360-4.3569814 .044081$

H $\quad 0.042630-5.2314055 .209939$

H -1.494224 -4.465544 4.798409

H -1.106760 -6.083584 4.174138

H $1.367431-3.350374 \quad 1.146534$

H $2.321115-2.5371462 .372745$

H $3.907670-4.3993552 .474022$

H $2.909241-5.3929221 .403237$

H $3.220971-3.773026-0.437664$

H $4.939787-5.606586-0.170278$

H $5.854741-4.5857880 .920385$

H $7.756030-4.098509-1.863523$

H $6.894978-2.326970-0.055881$

H $5.210932-5.336585-2.606386$

H $5.858829-3.874275-3.309047$

H $4.258515-3.848328-2.543208$

H $\quad 6.001881-1.655007-2.649204$

H 4.2086940 .0666680 .651717

H $\quad 5.872877-0.3291190 .963464$

H $3.263152-0.947765-1.248863$

H $0.842540-0.2310331 .711152$

H $-0.884651 \quad 1.8064143 .243504$

H 2.7744350 .9206382 .711410

H 3.2863472 .5401522 .282891

H 2.3452091 .6202171 .131650

H $6.5600400 .624693-1.208709$

H $4.8729820 .751848-1.734249$

H $5.1106875 .128443-1.708620$

H $5.7017914 .818875-0.054295$

H $2.7400454 .284219-0.806357$

H 4.0774896 .1396991 .227871

H $\quad 1.1690905 .1213720 .831444$

H $1.5528556 .830574 \quad 0.804473$

H 1.3326547 .0664524 .453363

H 1.1968737 .9428942 .828861

H 2.1016844 .8547454 .584718

H 3.0733444 .2973143 .263038

H 0.0384293 .8454373 .714271

H $4.7985882 .869497-2.079569$

C $-6.317061-1.8604030 .658440$

O $-5.635581-0.609496 \quad 0.437017$

C $-6.4284730 .202640-0.444792$

C $-7.828157-0.392996-0.403901$

C $-7.508223-1.871706-0.302779$

H $-6.0181450 .065490-1.452004$

C $-6.3305491 .679901-0.054437$

H -8.385046 -0.0661210 .481674$

H -8.416209 -0.138978 -1.290876

C $-8.676226-2.7172970 .174191$

H -7.190537 -2.229139-1.291727

C -4.8906872 .2070520 .112621$

O

H $-6.8687162 .287009-0.791628$

H -4.4580271 .8333401 .049100$

O -4.9624623 .6322030 .246244$

C -3.939492 $1.870398-1.048392$

C $-2.5680072 .554541-0.903350$

H -3.799072 $0.785565-1.093166$

O $-4.5263342 .269299-2.287041$

H -2.675031 3.633416-1.069312

H -2.204506 2.3947660 .119268

H $\quad-8.390415-3.7713470 .246445$
H $-9.028968-2.395387 \quad 1.159681$

H -9.516104 -2.641912 -0.524341

C $-5.348554-3.0197510 .418844$

H -6.5939461 .3083311 .852022$

H -5.5535513 .8229930 .994945$

H $-4.7007703 .224828-2.235259$

H $-4.934444-2.954925-0.594272$

H $-5.881879-3.9743550 .493086$

H $-6.660128-1.8537441 .701026$

C $-0.323002-0.055029-2.345368$

O $-1.2413530 .656814-1.505995$

C $-1.5391441 .999409-1.905938$

C $-0.2560562 .841420-1.930832$

C $0.8179042 .197138-2.812657$

C $1.0029950 .710566-2.488126$

H $-0.115477-0.986066-1.801779$

C $-0.960096-0.429302-3.687630$

H $-1.9883561 .991262-2.904631$

O $0.2505683 .002833-0.608857$

H $-0.4946753 .842712-2.307251$

H $1.7709182 .719704-2.666227$

O $0.4589652 .329437-4.184312$

H $1.6265190 .224775-3.247507$

O $1.6933300 .592801-1.241342$

C -2.153212 -1.332865 -3.476966

H $-1.2784380 .447866-4.258080$

H -0.249109 -0.971624 -4.321695

H $0.3851702 .116329-0.232876$

H $\quad 0.3720583 .278872-4.375576$

H $2.5112491 .114397-1.307011$

O $-3.295431-0.692152-3.856739$

O $-2.079372-2.465691-3.018530$

C $-4.503844-1.440298-3.696202$

H $-4.487553-2.326162-4.337931$

H $-4.637415-1.724250-2.649008$

$\mathrm{H}-5.340737-0.803798-3.995477$

SCF Energy $(B 3 L Y P / 6-31 G * * / / M M F F)=-3245.94090392$

0400129

MM̄FF Geometry

C -1.608799-3.065102 -2.410097

C $-2.637294-3.924895-2.341366$

C $-3.943299-3.722328-1.604829$

O $-4.105370-2.357824-1.154199$

C $-3.996253-4.650069-0.377663$

C $-5.354407-4.6784020 .299339$

C $-5.646743-4.0927711 .478105$

C $-6.413740-5.471338-0.424564$

C $-4.741458-3.2709982 .351921$

C $-5.284731-1.8460762 .523527$

C $-4.284382-0.9340213 .249092$

C $-4.8721840 .465122 \quad 3.441107$

C -3.8500261 .4516244 .026371$

O $-3.105578-0.8664452 .442930$

O -4.3712682 .7790353 .856201$

C $-2.5456711 .372248 \quad 3.203812$

C $-3.663681 \quad 1.2489475 .534547$

O -1.5172212 .1571003 .802196$

C $-2.046378-0.0700972 .987497$

C $-0.860413-0.1571051 .997051$

O $-1.593749-0.6020814 .237938$

C $-4.590498-1.464368-2.062331$

O $-4.872099-1.685502-3.229862$

C $-4.718636-0.151700-1.390192$

C $-5.0287890 .934049-2.115024$

C $-5.2228232 .294871-1.607174$

C $-5.0272012 .525939-0.132940$

C -1.1706700 .3004220 .567620$

C -0.7353841 .7209550 .254817$

C $-0.7880533 .386198-1.601046$

O -0.1084102 .4270901 .038902$

C $-1.9174094 .334196-1.348910$

C $-2.5716904 .982504-2.324223$

C $-3.6618995 .983973-2.074407$

C $-4.9350745 .666882-2.824814$

C $-5.2316286 .290075-3.977963$

C $-5.9091414 .702873-2.187467$

C $-5.5772993 .263888-2.477256$ 
N -1.110174 2.093183 -1.029931 H -1.657823 -2.113473-1.888515 H $-2.554620-4.871156-2.873568$ H -4.761777 -3.974372 -2.290697 H $-3.212872-4.3589470 .329704$ H $-3.747267-5.678106-0.674795$ H $-6.656886-4.208527 \quad 1.869820$ H $-7.349756-5.5296740 .141256$ H -6.644165 -5.015244 -1.392003 H $-6.070379-6.497040-0.595044$ H $-4.673277-3.7539053 .334216$ H $-3.724352-3.2348951 .956675$ H $-5.500862-1.4282021 .532086$ H -6.231014 -1.876488 3.077831 H -4.035624 -1.384698 4.217133 H $-5.771727 \quad 0.4253704 .067900$ H $-5.205230 \quad 0.8530472 .469634$ H -5.2323462 .8229174 .306279$ H -2.755620 1.8261132 .228097 H $-4.612993 \quad 1.4037436 .061421$ H -2.9678821 .9864095 .949699$ H -3.3046150 .2503845 .791818$ H -1.8634173 .0603343 .902557$ H $\quad 0.0189140 .3513202 .409821$ H $-0.575619-1.2169171 .930925$ H $-0.982329-1.3355234 .059484$ H $-4.564660-0.116240-0.318578$ H $-5.1689980 .816749-3.189078$ H -5.0590093 .5793080 .152799$ H -4.0480552 .1576200 .189522$ H -5.8048162 .0087150 .438551$ H $-0.624921-0.346860-0.128841$ H -2.2344730 .1939620 .337986$ H $-0.6048403 .233143-2.669295$ H $\quad 0.1282463 .771581-1.142284$ H $-2.1870164 .519707-0.311821$ H $-2.2820884 .816423-3.359527$ H -3.284075 $6.968095-2.381941$ H -3.883284 $6.076863-1.003796$ H -6.158865 $6.091665-4.505100$ H -4.550672 $7.007911-4.423197$ H $-6.9194124 .884976-2.579043$ H $-5.9892984 .918889-1.118414$ H -5.692025 $2.997685-3.529146$ H -1.681792 $1.461299-1.583128$ C $0.921613-3.398639-2.387494$ O $1.198904-2.128687-1.769733$ C $1.792122-2.366575-0.478786$ C $2.082353-3.863702-0.413420$ C $0.956840-4.426960-1.260326$ H $1.028718-2.1206020 .268160$ C $3.022630-1.476159-0.252111$ H $3.054796-4.105132-0.859064$ H $2.079302-4.2419170 .613437$ C $1.194173-5.851271-1.727684$ H $0.033071-4.391630-0.669351$ C $2.7126310 .035071-0.234303$ O $4.001927-1.746818-1.254705$ H $3.460910-1.7634010 .709474$ H 1.9806380 .2505480 .552166 O $2.1158570 .430057-1.475116$ C $3.9452620 .941000-0.045016$ C 4.7758990 .6198091 .208784 H $4.5721140 .890405-0.941654$ O 3.4887442 .2966530 .042000 H 4.1301440 .6900332 .092920 H $5.133861-0.4119841 .153941$ H $0.360474-6.202310-2.344196$ H $2.110653-5.932095-2.321633$ H $1.288527-6.526351-0.870974$ C $-0.364118-3.327852-3.212811$ H $3.613356-1.503117-2.112587$ H $1.280087-0.056424-1.571140$ H 2.9185622 .3690990 .826598 H -0.469771 -4.253018 -3.792169 H $-0.272609-2.509089-3.937753$ H $1.753527-3.581633-3.081449$ C $7.5033170 .492650-0.123248$
O $\quad \begin{array}{llll}6.776411 & 1.679297 & 0.215387\end{array}$

C 5.9788821 .5730761 .398540

C 6.8721651 .2353222 .601307

C 7.7377640 .0016732 .343061

C 8.4710090 .1258081 .006188

H $6.803961-0.333749-0.297254$

C $8.2486120 .767938-1.433359$

H 5.5638662 .5728941 .578577

O $\quad 6.068358 \quad 1.042417 \quad 3.760079$

H 7.5298602 .0912282 .802470

H $7.144610-0.9197772 .377565$

O $8.720914-0.1131173 .374285$

H 9.2602070 .8840101 .089980

O $9.100274-1.1165310 .699904$

C $7.2796430 .936106-2.580107$

H $8.8344491 .692242-1.359699$

H $8.944264-0.037883-1.692319$

H 6.6645900 .9593424 .522969

H $8.253687-0.3021184 .205406$

H $9.689325-1.3339891 .442656$

O $6.758366-0.273812-2.933081$

O $7.0118162 .015185-3.092478$

C $5.793634-0.242890-3.989265$

H $5.455199-1.266572-4.170488$

H $4.9315380 .364169-3.696411$

H $6.2472460 .145044-4.906215$

SCF Energy $(B 3 L Y P / 6-31 G * * / / M M F F)=-3245.91205358$

\section{0}

MM̄FF Geometry

C $-1.8327490 .801345-2.523011$

C - $-1.089142-0.218657-2.979242$

C $-1.311191-1.688149-2.697739$

O $-2.646434-1.893791-2.186167$

C $-0.297492-2.160573-1.646332$

C $-0.193572-3.663795-1.503124$

C $-0.726511-4.374729-0.490190$

C $0.618342-4.357454-2.565892$

C -1.604036 -3.879993 0.625649

C $-0.891498-3.8330171 .984551$

C $0.084698-2.6539482 .130696$

C $0.736765-2.6640753 .516630$

C $1.612856-1.4237183 .756588$

O $-0.664484-1.4484331 .950799$

O $1.905735-1.3542535 .160789$

C $0.797045-0.1610063 .403214$

C $2.962182-1.5309343 .039060$

O 1.6081541 .0093813 .469139

C $0.108856-0.2446522 .024862$

C $-0.856094 \quad 0.933076 \quad 1.761877$

O $1.111192-0.2047381 .002365$

C -3.349221 -2.966747 -2.628004

O $-2.972827-3.807133-3.433932$

C $-4.702753-3.017915-2.026624$

C $-5.115714-2.207696-1.039179$

C $-6.449233-2.196094-0.430529$

C $-7.477359-3.152812-0.975127$

C $-2.033730 \quad 1.034123 \quad 2.730723$

C -2.969189 2.1630792 .346375

C -4.7734912 .7634020 .735423$

O $-\begin{array}{llll}-3.010957 & 3.210897 & 2.987483\end{array}$

C -6.0814102 .3356851 .322298$

C -7.1378421 .9506750 .590627$

C -8.4280521 .4702291 .190570$

C -8.7907930 .0666950 .752484$

C $-9.846338-0.151110-0.049959$

C -7.986074 -1.090119 1.308207

C $-6.698143-1.3130430 .559260$

N -3.7378841 .8742671 .229946$

H $-2.6822210 .606472-1.873571$

H $-0.2377840 .006113-3.619335$

H -1.180174 -2.220469 -3.647477

H $-0.552227-1.709657-0.683022$

H $0.699634-1.778163-1.895398$

H $-0.551956-5.449899-0.471978$

H $0.674501-5.439518-2.406372$

H $0.177477-4.195114-3.554213$

H $1.642690-3.973428-2.574318$ 
H $-2.058300-2.9123030 .393148$ H -2.445826 -4.580597 0.704053 H -1.670039 -3.740359 2.753532 H - $0.367935-4.7792412 .168812$ H $\quad 0.850489-2.7255471 .350315$ H $1.320132-3.5815973 .663882$ H $-0.047694-2.6829304 .286519$ H $2.351064-2.1805935 .415336$ H $0.031361-0.0534354 .181112$ H $3.590900-0.6569853 .242205$ H $2.860422-1.6408711 .958697$ H $3.525474-2.3971733 .406595$ H 2.0186381 .0283114 .350669 H -0.3019571 .8777111 .754023$ H -1.2540370 .8263860 .743780$ H $\quad 0.7130350 .1380870 .185322$ H -5.352631 -3.777718 -2.449316 H $-4.421440-1.471940-0.636793$ H -7.153374 -4.188924 -0.827330 H -7.633809 -2.981778-2.046028 H -8.455973 -3.057819 -0.500220 H -2.607706 0.1008322 .757846 H - 1.6886831 .2302123 .751163 H -4.765511 $2.697852-0.357083$ H $-4.5518013 .793761 \quad 1.029510$ H -6.1558482 .3274032 .407516$ H -7.071211 $1.967385-0.494701$ H -9.2145622 .1779000 .898459$ H -8.3929101 .5000372 .286902$ H -10.134133 -1.154160 -0.346365 H $-10.4458630 .668760-0.431545$ H -8.591119 -2.0033001 .332321$ H $-7.749989-0.8950562 .362322$ H $-5.885007-0.665510 \quad 0.886637$ H -3.6747120 .9497410 .813392$ C $-1.1943143 .086273-1.668333$ O $0.1876562 .837103-1.322129$ C $0.8649384 .102157-1.195765$ C $-0.2344565 .141337-1.025066$ C $-1.2992004 .588268-1.947298$ H $1.3713574 .284249-2.151231$ C $1.8928734 .085867-0.060205$ H -0.6062005 .1743930 .005752$ H $0.0954626 .147143-1.301770$ C $-2.6801475 .168265-1.693216$ H -1.009422 $4.798530-2.985922$ C $2.9740752 .991649-0.175545$ O 1.2023983 .8933231 .179471 H 2.3664705 .0730390 .009258 H 2.5215182 .0101850 .002841 O 3.8894943 .2230950 .903845 C $3.7399392 .997059-1.511939$ C $4.9893272 .106372-1.552227$ H $3.0600602 .706069-2.319746$ O $4.1625614 .336721-1.790522$ H $5.4752372 .220160-2.531831$ H $5.7457772 .500938-0.859002$ H -3.424298 $4.713370-2.354292$ H $-3.0001195 .001750-0.659650$ H -2.682122 6.248113-1.874131 C $-1.5882692 .240834-2.882055$ H 1.8708523 .8823871 .885870 H 4.4293722 .4230291 .014469 H $4.5795544 .336811-2.668974$ H $-0.8091872 .312380-3.651314$ H $-2.5158272 .624822-3.322581$ H -1.794798 $2.817243-0.791454$ C $6.149986-1.388382-1.076902$ O $6.0751630 .013771-1.359989$ C $4.7750320 .613452-1.259350$ C $3.787022-0.072245-2.214996$ C $3.778227-1.591596-2.017172$ C $5.193115-2.176844-1.984509$ H $7.175022-1.675754-1.344601$ C $5.954097-1.6812970 .418337$ H $4.4237700 .509852-0.228256$ O $4.1426430 .230175-3.562631$ H $2.7814940 .328429-2.040450$
H $3.209322-2.052739-2.833264$

O $3.107125-1.888170-0.797445$

H $5.170255-3.225009-1.664039$

O $5.738497-2.156053-3.306369$

C $7.057278-1.0942531 .273777$

H $5.011839-1.2697940 .786142$

H $5.955637-2.7647450 .583060$

H $3.479294-0.182900-4.141168$

H $3.073688-2.855591-0.706096$

H $5.155640-2.689736-3.872785$

O $6.758680-1.3176352 .588723$

O $8.051963-0.5206290 .853014$

C $7.710227-0.8120053 .530349$

H 7.7958810 .2746533 .436051

H $7.352328-1.0495034 .535738$

H $8.682262-1.2914953 .381042$

SCF Energy (B3LYP/6-31G**//MMFF) $=-3245.89851702$

0400131

MM̄FF Geometry

C -2.4393954 .8135400 .050549$

C -3.3371953 .8212420 .139150$

C -3.8523513 .2767721 .446865$

O -3.5233341 .8656061 .484374$

C -5.3801613 .4296061 .524582$

C -5.9641262 .9629162 .845874$

C -6.6876141 .8380873 .017651$

C -5.7106163 .8759094 .018878$

C -7.0594720 .8171011 .979954$

C $-6.301574-0.4985502 .197195$

C $-6.421066-1.4278510 .981196$

C $-5.744814-2.7705071 .254587$

C $-5.737103-3.674750 \quad 0.012612$

O $-5.777673-0.776585-0.118388$

O $-4.816872-4.7497330 .260410$

C $-5.188922-2.878662-1.193208$

C $-7.106330-4.323005-0.225952$

O $-5.325586-3.653493-2.382269$

C $-5.843526-1.489375-1.360830$

C $-5.149735-0.598561-2.421548$

O $-7.202474-1.661808-1.780543$

C -2.2438931 .5423191 .826177$

O -1.3634792 .3104642 .181701$

C -2.0792590 .0811661 .654382$

C $-0.853756-0.4558491 .752610$

C $-0.503787-1.8693271 .594662$

C -1.612984 -2.832991 1.269454

C $-3.671816-0.286479-2.157002$

C $-2.705752-1.221101-2.865150$

C $-0.271304-1.719575-3.045590$

O $-3.059437-2.055060-3.694100$

C $0.091190-2.850294-2.135925$

C $1.324673-3.032381-1.640691$

C $1.723529-4.206021-0.793883$

C $2.323229-3.8013920 .533017$

C $3.654351-3.7040590 .691051$

C $1.383939-3.6110771 .701452$

C $0.786621-2.2332381 .747292$

N $-1.390893-0.987492-2.484645$

H -2.0903455 .3045410 .955582$

H $-3.6659443 .316751-0.766788$

H -3.384206 3.7843002 .299183

H -5.8374892 .8973560 .685039$

H $-5.6550384 .483792 \quad 1.383740$

H -7.0582581 .6126154 .016831$

H -6.2276253 .5422924 .925014$

H -4.6421303 .9216314 .249750$

H -6.0636014 .8879493 .795307$

H -8.1381450 .6289982 .048580$

H $-6.8916691 .192106 \quad 0.968400$

H $-5.242180-0.2785762 .378037$

H -6.688789 -0.9988493 .093395$

H -7.483527 -1.569262 0.750860

H -6.222144 -3.281797 2.099920

H $-4.704963-2.5967211 .561006$

H $-5.112717-5.2199431 .058495$

H $-4.113586-2.755600-1.019530$

H $-7.400675-4.9262460 .641476$ 
H -7.073584 -5.016260 -1.073823 H -7.900079-3.594665 -0.404691 H -4.841960 -3.199093 -3.092077 H -5.304920 -1.010105 -3.425751 H $-5.6765440 .366969-2.423850$ H -7.501649 $-0.840118-2.204013$ H -2.961939 -0.5070061 .434229$ H $-0.015517 \quad 0.2049501 .971039$ H -2.314934 -2.903154 2.106932 H -1.263316 -3.844596 1.053004 H $-2.158308-2.505357 \quad 0.378217$ H -3.453855 -0.279867 -1.084852 H $-3.457507 \quad 0.719491-2.540748$ H $0.551479-1.010305-3.176048$ H $-0.544917-2.113343-4.029800$ H $-0.687771-3.573606-1.906371$ H $2.108385-2.324677-1.900287$ H $2.454373-4.793600-1.365426$ H $\quad 0.877044-4.882617-0.623324$ H $4.091735-3.4453981 .649704$ H $4.335268-3.870195-0.137186$ H $1.930361-3.7569342 .643529$ H $0.630244-4.4036171 .699630$ H $1.518715-1.4576961 .973848$ H -1.198705 -0.315065 -1.747650 C $-0.3165464 .859573-1.240426$ O $-0.182733 \quad 3.429862-1.123637$ C $0.9406332 .994285-1.918812$ C $1.5991054 .260782-2.449495$ C $0.4258725 .220959-2.522301$ H $0.5106242 .410269-2.741472$ C $1.8401502 .114255-1.042785$ H $2.3538934 .633108-1.745566$ H $2.0868964 .104468-3.416284$ C $0.8385436 .679175-2.611383$ H $-0.1852264 .962478-3.397396$ C $3.1493351 .658331-1.717784$ O $1.0693010 .974922-0.651398$ H $2.0766692 .651765-0.116679$ H $3.7220102 .539035-2.031277$ O $2.8532210 .902595-2.892186$ C $4.0507920 .811835-0.793254$ C $5.3763600 .425395-1.475939$ H 4.2631931 .3848790 .116659 O $3.394626-0.391862-0.405165$ H $5.170490-0.197725-2.354626$ H 5.867640 1.346011-1.813048 H $-0.0392247 .331580-2.651449$ H $1.4423386 .979046-1.748393$ H $1.4319296 .856236-3.514470$ C -1.799483 $5.235474-1.243244$ H $\quad 0.9320090 .429312-1.443894$ H $2.4490781 .504616-3.539206$ H $2.611183-0.1478090 .115461$ H -2.304218 $4.750252-2.087892$ H -1.922632 $6.317033-1.363771$ H $0.1845935 .300146-0.367811$ C $7.510612-0.0630281 .554003$ $\begin{array}{lllll}\text { O } & 6.689337 & 0.518174 & 0.532347\end{array}$ C $6.316761-0.356704-0.537819$ C $7.566394-0.884296-1.260614$ C $8.526780-1.551746-0.271692$ C $8.790596-0.6681800 .953473$ H 7.8066640 .7839082 .186977 C $6.723789-1.0463042 .429221$ H $5.761992-1.208018-0.130556$ O $8.257494 \quad 0.174405-1.918868$ H $7.264066-1.611214-2.024406$ H $9.473433-1.764088-0.783501$ O $7.965534-2.7955700 .138486$ H $9.324381-1.2315291 .727801$ O $\quad 9.638701 \quad 0.4187730 .571922$ C $5.708284-0.3119433 .271936$ H $6.204632-1.8229741 .861657$ H $7.395055-1.5681563 .121913$ H $7.7234350 .446758-2.683848$ H $8.598065-3.2171010 .744772$ H 10.4636220 .0393410 .224209
O $4.542017-0.1680522 .581367$

O $5.935894 \quad 0.104146 \quad 4.400749$

C 3.5114950 .5643203 .251753

H 3.8879341 .5297863 .603720

H 2.7046550 .7479792 .537355

H $3.118959-0.0254744 .085094$

SCF Energy (B3LYP/6-31G**//MMFF) $=-3245.90399160$

0400132

MM̄FF Geometry

C 2.2529920 .2892753 .209836

C $3.130209-0.6279133 .646593$

C $4.360670-1.1270212 .925497$

O $4.654980-0.3355401 .754388$

C $4.130526-2.5668602 .434701$

C $5.413470-3.3620212 .306670$

C $6.118842-3.5215061 .170380$

C $5.861477-4.0452363 .573819$

C $5.881015-2.892289-0.173582$

C $5.346574-3.885875-1.214292$

C $3.821907-4.059272-1.141940$

C $3.349198-5.130209-2.126478$

C $1.817095-5.238926-2.158999$

O $3.233544-2.796491-1.475084$

O $1.454295-6.021991-3.306353$

C $1.222788-3.830526-2.366680$

C $1.269162-5.981972-0.934927$

O $-0.199369-3.855668-2.262354$

C $1.804045-2.764702-1.408554$

C $1.339558-1.356277-1.850537$

O $1.329488-3.019412-0.084830$

C $5.3804310 .801954 \quad 1.904841$

O 5.8131361 .2754692 .941779

C 5.5274221 .3891350 .555487

C 5.8423402 .6848160 .421682

C $5.9676593 .409669-0.844784$

C $5.8217912 .618987-2.119498$

C $2.060646-0.197203-1.145045$

C $1.4549661 .148573-1.512705$

C $1.5682903 .565337-0.906533$

O $\quad 0.5867301 .280265-2.371652$

C $2.458604 \quad 4.276286-1.877102$

C $3.0194765 .470276-1.629489$

C $3.875708 \quad 6.212573-2.616997$

C $5.1862026 .685718-2.025623$

C $5.3507967 .964016-1.645280$

C $6.3306135 .698860-1.954917$

C $6.1861334 .740520-0.803384$

N $1.9995402 .185971-0.767789$

H 2.3889920 .7579572 .239775

H $2.957972-1.0814224 .621460$

H $5.210361-1.0845273 .618366$

H $3.605066-2.5483221 .473624$

H $3.468525-3.1126483 .120684$

H $7.006933-4.1520461 .207503$

H $6.791921-4.6069793 .438975$

H $6.033893-3.3084424 .364739$

H $5.096627-4.7502113 .915929$

H $5.233892-2.014178-0.107514$

H $6.850976-2.515357-0.522929$

H $5.606208-3.493938-2.206662$

H $5.847314-4.855827-1.107413$

H $3.541021-4.336252-0.120359$

H $3.798363-6.102600-1.889128$

H $3.699353-4.877491-3.137302$

H $1.883261-6.890969-3.223790$

H $\quad 1.452010-3.529335-3.398659$

H $0.182129-6.102216-1.000043$

H $1.505906-5.4874570 .009347$

H $1.680482-6.997544-0.888243$

H $-0.523913-4.525877-2.887851$

H $1.512084-1.238101-2.928541$

H $\quad 0.259732-1.261970-1.683704$

H $1.287507-2.1845210 .406610$

H $5.3331640 .743109-0.293280$

H $5.9971573 .277614 \quad 1.322347$

H $4.8446502 .125442-2.161062$

H $6.6029381 .853482-2.185615$ 
H $5.8964623 .226066-3.023517$ H $2.004659-0.310019-0.058117$ H $3.117695-0.180544-1.433397$ H $1.598004 \quad 4.0163220 .090292$ H $0.5367663 .588405-1.271715$ H $2.6264543 .800943-2.840818$ H $2.8239125 .960523-0.678321$ H $3.2967947 .074642-2.973788$ H $4.0793775 .603866-3.506729$ H $6.2955868 .318061-1.246221$ H $4.5422598 .683912-1.719161$ H $7.2806636 .231983-1.814679$ H $6.4335935 .199799-2.922088$ H $6.273123 \quad 5.2204970 .172605$ H $2.7545101 .988430-0.118147$ C $-0.2885780 .370401 \quad 3.365099$ O -0.4723361 .0919242 .133110$ C $-1.1896620 .242771 \quad 1.208755$ C -1.626170 -0.9820402 .002702$ C $-0.512150-1.1007723 .023885$ H $-0.460814-0.0429630 .443859$ C $-2.336897 \quad 1.0413650 .580784$ H -2.586776 -0.8027002 .501623$ H $-1.729270-1.8725861 .375349$ C $-0.865353-1.967558 \quad 4.218727$ H $\quad 0.365729-1.5242282 .521347$ C $-3.2363410 .205760-0.346608$ O $-1.769630 \quad 2.111776-0.180919$ H -2.9319271 .5064231 .374095$ H -3.716704 -0.6025850 .215097$ O $-2.416814-0.442369-1.328925$ C $-4.3103941 .014621-1.097239$ C $-5.2424791 .862886-0.213437$ H -3.830983 $1.678963-1.826624$ O $-5.1064580 .110973-1.866487$ H $-4.6616852 .693700 \quad 0.203268$ H -5.999745 $2.311191-0.867230$ H $-0.033268-2.0093844 .928526$ H $-1.742783-1.5827074 .749057$ H -1.088368 -2.9907573 .898798$ C 1.0546870 .7289054 .005139 H -1.194716 2.6197900 .416866 H -1.930819 $0.250221-1.809200$ H -4.501852 - $-0.403613-2.428273$ H 1.0927130 .3085225 .017356 H 1.1191131 .8191104 .111766 H -1.0834530 .7203374 .037885$ C -7.901857 $0.296538-0.239200$ $\begin{array}{llll}\text { O } & -6.709501 & -0.018510 & 0.489558\end{array}$ C $-5.949410 \quad 1.106414 \quad 0.937084$ C -6.8185912 .0006351 .834191$ C -8.119907 2.402083 1.144098 C $-8.841387 \quad 1.1685860 .602484$ H -7.640229 $0.816570-1.168409$ C $-8.578214-1.029438-0.607085$ H -5.1614910 .6994141 .580925$ $\begin{array}{lllll}\text { O } & -6.092892 & 3.160349 & 2.226885\end{array}$ H -7.060382 1.4489122 .751962 H $-7.933993 \quad 3.1296920 .345964$ $\begin{array}{llll}\text { O } & -8.967318 & 3.066536 & 2.084696\end{array}$ H -9.248664 0.5853021 .438195 O $-9.9413921 .585949-0.203290$ C $-7.782137-1.772437-1.653180$ H -8.694169-1.674183 0.272840 H -9.581281 -0.873402 -1.020274 H -6.6893293 .7093442 .764304$ H -9.1535012 .4480152 .811692$ H -10.502289 2.160484 0.345577 O $-6.939350-2.662072-1.055359$ O $-7.882410-1.567573-2.856046$ C $-6.113105-3.421436-1.943142$ H $-5.471530-4.068918-1.339624$ H $-6.732939-4.046942-2.592452$ H -5.479324-2.756834-2.537485 SCF Energy (B3LYP/6-31G**//MMFF) $=-3245.91896108$

0400133

MM̄FF Geometry
C $-0.863320-3.105770-0.344979$

C $-1.179559-3.4167330 .922462$

C $-0.342579-3.0969552 .141384$

O $0.573119-2.0078231 .882961$

C $0.465186-4.341768 \quad 2.547890$

C $1.327178-4.1314623 .776993$

C $2.663591-3.9526123 .755465$

C $0.589814-4.1666005 .090464$

C $3.559805-3.8725262 .549934$

C $4.327552-2.5444732 .519685$

C $5.070250-2.3400381 .190350$

C $5.899519-1.0551271 .234049$

C $6.555872-0.739891-0.117152$

O $4.089586-2.259217 \quad 0.149695$

O $7.0223100 .616606-0.048340$

C $5.482389-0.816820-1.225219$

C $7.797072-1.608207-0.352172$

O $6.076729-0.660767-2.511255$

C $4.629546-2.101701-1.169391$

C $3.442092-2.088082-2.164894$

O $5.455837-3.218609-1.522493$

C $0.071040-0.7438391 .979878$

O $-1.083024-0.427517 \quad 2.221601$

C $1.184887 \quad 0.202407 \quad 1.746724$

C 0.9653701 .5239451 .813335

C 1.9687442 .5777721 .639882

C 3.3787772 .1553801 .323774

C $2.394524-0.993817-1.937074$

C $2.6339820 .283178-2.723455$

C $1.7727522 .615217-2.928164$

O $3.4623900 .376166-3.624482$

C $2.543923 \quad 3.557027-2.059538$

C $2.0319434 .698179-1.574768$

C $2.8080055 .680965-0.747750$

C $2.1396105 .994790 \quad 0.571226$

$\begin{array}{llll}\text { C } & 1.371558 & 7.088223 & 0.714634\end{array}$

C 2.4461235 .0987121 .748345

C 1.5886343 .8644631 .785112

N $1.7760921 .298310-2.321629$

H $\quad 0.084818-2.607467-0.533667$

H $-2.107614-3.9408041 .133519$

H $-1.012566-2.8115552 .962329$

H $1.077239-4.6734621 .702412$

H $-0.218811-5.1789732 .743793$

H $3.180717-3.8395804 .707618$

H $1.262158-4.0592745 .948309$

H $-0.141125-3.3537365 .142343$

H $0.061304-5.1187475 .204037$

H $4.274167-4.7035462 .590248$

H $2.998873-3.9907861 .620625$

H $3.620218-1.7196252 .668934$

H $5.040600-2.5168653 .353141$

H $5.715284-3.2086911 .012962$

H $6.656447-1.1071112 .026644$

H $5.247136-0.2145061 .503674$

H $7.3709950 .852771-0.925258$

H $4.8296080 .052367-1.082867$

H $8.537066-1.4355330 .438615$

H $8.297168-1.341787-1.289745$

H $7.575328-2.677203-0.370137$

H $6.497002-1.504932-2.748158$

H $3.814065-2.081016-3.196562$

H $2.917281-3.047983-2.053073$

H $4.881807-3.949942-1.804715$

H $2.162555-0.2151501 .539561$

H $-0.042664 \quad 1.8720052 .035363$

H 3.8171791 .6282202 .177677

H 4.0405022 .9893341 .080660

H 3.3992001 .4931840 .451558

H $1.422270-1.373773-2.276312$

H $2.299973-0.757444-0.873828$

H $0.7275712 .918776-3.040960$

H $2.2297662 .568729-3.921597$

H $3.5783623 .301120-1.843005$

H $1.0067834 .963507-1.820160$

H $2.9137246 .600897-1.337982$

H $3.8319555 .331619-0.565778$

H 0.9152867 .3373601 .666871 
H $1.1754967 .753600-0.119797$ H 2.2642625 .6431872 .685391 H 3.5167984 .8760161 .769797 H 0.5417854 .0729482 .007851 H $1.1456761 .136431-1.541223$ C -3.127445 -3.822464 -1.385258 O $-3.853828-2.813731-0.657140$ C $-5.072940-2.513111-1.354733$ C $-5.304293-3.688416-2.293192$ C -3.880495 -4.013084 -2.705374 H $-4.878685-1.614816-1.954048$ C $-6.198637-2.226835-0.355182$ H $-5.750076-4.544323-1.773656$ H $-5.948590-3.427684-3.138021$ C $-3.720481-5.397290-3.309879$ H -3.550669 -3.265058 -3.439113 C $-5.830323-1.2089220 .745367$ O $-6.573200-3.4432880 .304262$ H -7.092022 -1.892999-0.895212 H $-5.138750-1.666478 \quad 1.463697$ O $-7.023146-0.9020381 .477426$ C $-5.223173 \quad 0.105473 \quad 0.227136$ C -4.9484451 .1161341 .357385$ H $-4.283198-0.111813-0.289742$ O H $-5.895359 \quad 1.458226 \quad 1.791710$ H $-4.369977 \quad 0.6081132 .138576$ H -2.675199 -5.588839-3.572674 H -4.041614 -6.179057 -2.613332 H -4.321107 -5.491165 -4.220507 C $-1.681277-3.358151-1.585018$ H $-5.777773-3.7999040 .736298$ H $-7.385742-1.742981 \quad 1.805277$ H $-6.9523710 .857067-0.286977$ H -1.694997 -2.411429 -2.139237 H - $-1.142025-4.084780-2.203785$ H -3.162016 -4.742383 -0.788816 C $-2.1562462 .887786-0.336526$ $\begin{array}{lllll}\text { O } & -2.876168 & 1.909672 & 0.426885\end{array}$ C -4.1712682 .3413620 .851284$ C -3.9982043 .4405751 .912703$ C -2.8494154 .3964231 .559566$ C $-2.510840 \quad 4.3307560 .071510$ H -1.092909 2.711085 -0.136067 C $-2.3998572 .642160-1.832548$ H $-4.7038532 .761725-0.011360$ O $-3.7075182 .858213 \quad 3.185090$ H -4.948552 3.9752572 .028830 H -1.957068 4.1800362 .159635 O -3.2505625 .7224431 .905471$ H $-3.3574674 .717611-0.509553$ O $-1.4043785 .189992-0.189956$ C $-1.843141 \quad 1.311584-2.298034$ H -3.474020 $2.637939-2.048176$ H -1.914963 $3.428415-2.421260$ H $-3.634413 \quad 3.5847103 .827592$ H -2.529743 6.3201431 .642184 H -1.246872 $5.184062-1.148889$ O $-2.0010201 .220002-3.652088$ O C -1.532956 $0.007508-4.251105$ H -2.198688 -0.817231 -3.982129 H - $-1.553048 \quad 0.135573-5.336492$ H $-0.504812-0.208470-3.945249$ SCF Energy $\left(B 3 L Y P / 6-31 G^{* *} / / M M F F\right)=-3245.91336710$

0400134

MM̄FF Geometry

C $-0.272800-4.221550 \quad 0.992787$

C $0.396682-3.0640620 .890004$

C $1.032084-2.3693292 .064797$

O $\quad 0.523947-1.0160792 .130514$

C $2.555898-2.2957101 .865910$

C $3.291165-1.7865193 .089614$

C $3.764744-0.5335273 .238042$

C $3.518707-2.8110214 .171406$

C 3.6264620 .6243192 .290214

C 4.9691721 .1229601 .742113
C 5.7015950 .1063620 .852259

C 7.0047130 .7111660 .323056

C $7.719642-0.228270-0.657890$

O $4.840774-0.238970-0.236544$

O $8.7327790 .530316-1.336691$

C $6.709197-0.690582-1.729036$

C $8.445621-1.3676840 .067161$

O $7.289575-1.678778-2.578131$

C $5.379209-1.213876-1.137171$

C $4.329291-1.500710-2.242441$

O $5.631831-2.453825-0.464715$

C $-0.698373-0.8215542 .691014$

O $-1.451110-1.6891373 .113646$

C -1.051058 0.6173502 .759968

C -0.2167411 .6007722 .390258$

C -0.4636183 .0412912 .477136$

C -1.8309303 .4994092 .905861$

C $3.723551-0.263951-2.915934$

C $2.5576030 .336426-2.146498$

C $1.0597492 .320968-2.119828$

O $2.010906-0.228360-1.202775$

C $1.5748473 .374038-1.192066$

C $1.0655504 .614378-1.143763$

C $1.5251365 .682372-0.191462$

C $0.469893 \quad 6.0238970 .839815$

C -0.4881096 .9269180 .570403$

C 0.5666825 .3826152 .207431

C 0.5458303 .8803412 .164697

N $2.169477 \quad 1.564407-2.665759$

H $-0.359378-4.7052601 .962598$

H $0.482993-2.578742-0.079138$

H $\quad 0.801626-2.8872653 .004201$

H $2.785964-1.6704880 .997397$

H $2.951699-3.2904561 .619450$

H $4.295569-0.2925694 .158225$

H $4.089451-2.4071115 .014360$

H $2.564547-3.1743714 .565168$

H $4.078951-3.6638713 .774167$

H $2.9474240 .404912 \quad 1.462315$

H 3.1589841 .4469252 .845959

H 4.7624832 .0281731 .155861

H $5.619711 \quad 1.4214302 .573847$

H $5.915048-0.7921601 .442068$

H $7.6718280 .982194 \quad 1.150915$

H $6.7826251 .651388-0.201462$

H $9.3362290 .892115-0.665200$

H $6.4993070 .176868-2.366740$

H $9.201320-0.9647040 .752199$

H $8.991358-2.001970-0.640082$

H $7.775777-2.0000270 .653418$

H $8.116356-1.307955-2.931506$

H $4.786507-2.139009-3.009276$

H $3.517850-2.108779-1.820708$

H $4.787637-2.921068-0.352294$

H $-2.034293 \quad 0.826128 \quad 3.169279$

H $\quad 0.761080 \quad 1.334791 \quad 1.996954$

H -1.998783 3.2655443 .962655

H -2.609227 3.0073922 .312872

H -1.983857 4.5738682 .777089

H $4.4786780 .506257-3.096279$

H $3.322612-0.565597-3.891918$

H $0.402814 \quad 1.644469-1.567761$

H $0.5065122 .745601-2.963100$

H $2.3697603 .096544-0.503917$

H $0.2581814 .881251-1.822336$

H $1.7746886 .576329-0.778120$

H $2.458107 \quad 5.396777 \quad 0.310383$

H -1.234689 $7.198392 \quad 1.309273$

H $-0.5492537 .410743-0.398824$

H $\quad-0.208765 \quad 5.7676472 .878499$

H 1.5140375 .6931252 .667785

H 1.5029873 .4467071 .872770

H $2.7015591 .977597-3.426164$

C $-2.449801-4.9863930 .026774$

O $-3.014444-3.6592350 .008084$

C $-4.194935-3.665999-0.811809$

C $-4.536604-5.132075-1.044237$

C $-3.156597-5.755669-1.088267$ 
H -3.913461 -3.205769-1.767679

C $-5.327141-2.846684-0.177184$

H -5.115886 $-5.549091-0.211998$

H -5.108218 -5.285057 -1.964632

C $-3.163232-7.261826-0.893601$

H -2.696615 -5.520877 -2.057685

C $-4.955911-1.3630330 .036179$

O $-5.713189-3.438631 \quad 1.061726$

H -6.195214 -2.902604 -0.844742

H -4.476340 -0.995732 -0.876646

O $-4.020723-1.229521 \quad 1.103589$

C $-6.178356-0.4733280 .330423$

C -5.8766851 .0187450 .572553$

H -6.871008 $-0.548114-0.516566$

O $-6.886083-0.956076 \quad 1.473528$

H $-6.846433 \quad 1.5246870 .670817$

H $-5.377787 \quad 1.1369291 .543726$

H -2.145593 -7.663591 -0.920187

H -3.610588 -7.540508 0.066437

H -3.739329 -7.749518 -1.686744

C $-0.934957-4.907647-0.167222$

H -4.947968 -3.3848701 .659799$

H $-3.223507-1.7309480 .861559$

H -6.257985 -0.9840002 .215727$

H $-0.706629-4.374591-1.097830$

H $-0.512018-5.914512-0.256127$

H -2.689937 -5.4195301 .006627$

C $-2.7093842 .039104-1.121501$

O $-3.6667011 .506820-0.198004$

C $-5.0469221 .679749-0.540263$

C $-5.3704993 .173863-0.686197$

C $-4.4396813 .842220-1.701197$

C $-2.9695073 .527485-1.412775$

H $-1.7512501 .975548-0.591285$

C $-2.6082841 .176940-2.389168$

H $-5.2586571 .176499-1.489783$

O

H $-6.410048 \quad 3.279077-1.018734$

H -4.599903 $4.926713-1.670325$

O $-4.783747 \quad 3.384643-3.005917$

H $-2.332543 \quad 3.854122-2.242847$

O $-2.5583354 .267554-0.261197$

C $-1.902519-0.139423-2.125613$

H -3.588453 $0.941947-2.811115$

H -2.016253 $1.712103-3.140961$

H -4.3674603 .6855810 .912808$

H -4.203215 $3.840381-3.639042$

H -2.679091 $5.211468-0.460237$

O $-1.618112-0.734310-3.322161$

O $-1.642161-0.595245-1.020779$

C $-0.940866-1.991931-3.238990$

H $-0.695678-2.313867-4.254533$

H $-0.011465-1.891795-2.670447$

H -1.595821 -2.738840 -2.782411

SCF Energy (B3LYP/6-31G**//MMFF) $=-3245.91060722$

0400135

MM̄FF Geometry

C 1.3369971 .9094633 .543155

C 1.5416000 .9427692 .635669

C $1.792687-0.5015832 .980396$

O $3.102452-0.8068892 .452153$

C $0.755710-1.3985682 .283368$

C $0.709142-2.8073742 .837269$

C $1.271151-3.8841042 .254161$

C $-0.070967-2.9643624 .117402$

C $2.125138-3.9310831 .017750$

C $1.454672-4.672527-0.147005$

C $0.340851-3.863093-0.829627$

C $-0.295992-4.673242-1.962579$

C $-1.322876-3.852511-2.758030$

O $0.933264-2.672276-1.357877$

O $-1.603907-4.585838-3.959898$

C $-0.683508-2.505884-3.159430$

C $-2.653774-3.735550-2.007102$

O $-1.641996-1.658589-3.789405$

C $0.008056-1.777016-1.984872$

C $0.805182-0.560286-2.511528$
O $-0.992089-1.327915-1.070977$

C $3.954296-1.5544403 .195550$

O $3.729366-2.0526404 .289482$

C $5.273797-1.6801242 .530188$

C $5.507581-1.2734111 .272318$

C $6.791759-1.3091280 .568995$

C $7.993655-1.8095311 .325219$

C $1.7139430 .091804-1.460848$

C $2.4774891 .279732-2.019391$

C $4.3262722 .845451-1.424787$

O $2.2649191 .753955-3.132005$

C $5.5180232 .290802-2.140890$

C $6.7675172 .312371-1.652568$

C $7.9506091 .736118-2.378678$

C $8.6455890 .641911-1.596045$

C $9.8135330 .876933-0.974595$

C $8.035698-0.744225-1.604260$

C $6.839379-0.854485-0.700723$

N $3.4171901 .756965-1.114817$

H 1.3546871 .6377264 .597042

H 1.5437161 .1853821 .578041

H $\quad 1.770097-0.6361104 .068277$

H $0.954202-1.4237281 .206498$

H $-0.248773-0.9652642 .385368$

H $1.143680-4.8515652 .738928$

H $-0.135535-4.0087434 .440763$

H $0.401072-2.3979034 .925978$

H -1.093873 -2.596807 3.988966

H $2.453398-2.9370110 .701482$

H $3.043583-4.4705501 .283455$

H $2.236410-4.889816-0.886787$

H $1.060775-5.6363250 .198780$

H $-0.414675-3.594116-0.083041$

H $-0.754633-5.591194-1.573888$

H $\quad 0.489974-5.004405-2.656314$

H $-2.193716-4.039065-4.507168$

H $\quad 0.072169-2.726925-3.925599$

H -3.072308 -4.730805-1.815196

H $-3.403926-3.207320-2.605922$

H -2.557866 -3.225166 -1.047741

H -2.195912 -1.268001 -3.092279

H $1.443045-0.879880-3.346281$

H $\quad 0.1134410 .195652-2.902148$

H $-0.679298-0.533174-0.611986$

H $6.052211-2.1218723 .143857$

H $4.685820-0.8539250 .694383$

H $7.857924-2.8586571 .610264$

H $8.153263-1.2161422 .232518$

H $8.921748-1.7542660 .752078$

H $1.1234300 .451678-0.613908$

H $2.442430-0.637671-1.088544$

H $4.5952713 .327610-0.479751$

H $3.8167873 .577196-2.059879$

H $5.339317 \quad 1.844180-3.116545$

H $6.9539302 .766969-0.682501$

H $8.6485842 .558922-2.581136$

H $7.6642081 .345265-3.363084$

H $\quad 10.3355890 .093058-0.436073$

H $10.272478 \quad 1.860014-0.986631$

H $8.787409-1.501392-1.354448$

H $7.726303-0.996237-2.626860$

H $5.912361-0.514594-1.162707$

H $3.571391 \quad 1.247892-0.249183$

C $0.9069803 .792674 \quad 1.811694$

O $-0.289624 \quad 3.1912591 .281692$

C -1.0304384 .1766120 .542508$

C -0.0775095 .3477090 .344846$

C 0.7245625 .3002421 .631122

H -1.8626044 .4899381 .186148$

C $-1.5955283 .581770-0.755885$

H $0.5803615 .192211-0.518485$

H $-0.606177 \quad 6.2949550 .201635$

C 2.0292606 .0750131 .561459

H 0.1083205 .7059832 .444684

C $-2.5468302 .387081-0.516634$

O $-0.5171083 .185983-1.601281$

H -2.144574 4.369685-1.284669

H $\quad-3.2991392 .694467 \quad 0.218731$ 
$\begin{array}{llll}\text { O } & -1.833778 & 1.285092 & 0.042516\end{array}$ C $-3.276323 \quad 1.903529-1.788622$ C $-4.1743150 .677041-1.566701$ H -3.875265 $2.724392-2.198780$ O $-2.341573 \quad 1.528496-2.802344$ H -4.632262 $0.392378-2.524559$ H -3.552691 -0.195097-1.321098 H 2.5791795 .9992032 .504621 H 2.6773025 .6989220 .762535 H 1.8367937 .1351491 .366849 C 1.0799123 .3720793 .275306 H $-0.0284922 .485686-1.136302$ H $-1.460337 \quad 1.5728940 .892400$ H $-1.8189472 .315656-3.030008$ H $\quad 0.168275 \quad 3.636421 \quad 3.826855$ H 1.9049713 .9382283 .722975 H 1.7484603 .4402891 .201937 C -6.975904 -0.5598220 .537332$ O $-5.958604-0.434990-0.464600$ C $-5.2625610 .819236-0.492945$ C $-6.2478241 .967428-0.754412$ C $-7.4064351 .951946 \quad 0.248400$ C -8.0239100 .5568990 .393745$ H $-7.466900-1.5162340 .315650$ C $-6.383075-0.6600401 .947400$ H -4.7717190 .9624620 .474215$ O $-6.7644961 .881776-2.080971$ H $-5.7190002 .923878-0.677678$ H $-8.1750892 .659571-0.086034$ O $-6.9182572 .400743 \quad 1.509478$ H $-8.7121140 .534543 \quad 1.246827$ O $-8.8025820 .300765-0.778845$ C $-5.581864-1.931166 \quad 2.098074$ H -5.7432570 .1855122 .215456$ H -7.180302 -0.6901972 .699909$ H $-7.1910701 .013288-2.178545$ H -7.6706242 .4196322 .125071$ H $-9.246780-0.555567-0.655797$ O $-4.277096-1.702398 \quad 1.773735$ O $-6.064910-3.0029772 .439831$ C $-3.415250-2.8412561 .852907$ H $-2.416954-2.5347761 .529886$ H $-3.358160-3.1991412 .885030$ H $-3.770510-3.6352431 .189258$ SCF Energy (B3LYP/6-31G**//MMFF) $=-3245.93119800$

04_00136

MM̄FF Geometry

C -4.097895 0.2854913 .047295

C $-3.126831-0.4734272 .516218$

C $-1.734632-0.5573803 .082754$

O $-0.831051-0.0029592 .095536$

C -1.329409-2.022429 3.316341

C $-0.200624-2.1485524 .320128$

C $1.110169-2.1747694 .011461$

C $-0.640526-2.2636205 .757312$

C $1.740904-2.0367922 .655645$

C $2.573923-3.2729062 .294199$

C $3.269417-3.1054760 .935970$

C $4.042352-4.3691810 .555996$

C $4.826768-4.188246-0.752973$

O $4.169040-1.9982611 .037099$

O $5.743819-5.288640-0.846834$

C $5.652026-2.884829-0.667015$

C $3.907116-4.290633-1.975203$

O $6.293079-2.606948-1.909775$

C $4.833415-1.664735-0.186942$

C $5.769511-0.4647090 .089757$

O $3.905987-1.299406-1.212417$

C -0.6313851 .3423792 .103961$

O -1.0839412 .1491362 .900022$

C $0.209606 \quad 1.6731820 .930521$

C 0.3749302 .9573610 .579560

C $1.1043053 .455176-0.590381$

C $1.7152122 .438531-1.518261$

C $5.0904310 .674607 \quad 0.863033$

C $5.962263 \quad 1.9134760 .958599$

C 5.8578294 .2853131 .724440
O 7.1111491 .9656250 .529247

C 5.4748095 .1983360 .595242

C $4.6502384 .931215-0.430613$

C $4.3464705 .906143-1.531678$

C $2.9356686 .445618-1.469711$

C $2.6997647 .717458-1.104870$

C $1.8070925 .544897-1.922585$

C $1.1734374 .789426-0.784021$

N 5.2929442 .9569381 .585779

H -3.9003790 .8606813 .948246$

H $-3.322323-1.0244711 .599154$

H -1.6569540 .0098314 .019217$

H -1.046755 -2.497796 2.369417

H -2.183515 -2.601017 3.693463

H $1.825185-2.2730884 .827741$

H $0.205811-2.3372126 .448356$

H -1.228472 -1.387257 6.048304

H -1.257596 -3.157631 5.894335

H $0.999402-1.8623511 .872091$

H $2.380858-1.1463372 .678471$

H $3.330357-3.4332993 .073741$

H $1.928130-4.1594432 .273862$

H $2.507360-2.8820650 .181478$

H $3.368447-5.2320930 .484704$

H $4.755872-4.6127131 .355885$

H $6.297893-5.144159-1.633261$

H $6.460077-3.0669780 .054648$

H $3.402280-5.264087-1.991927$

H $4.478646-4.235998-2.908320$

H $3.136883-3.517426-1.997153$

H $5.622097-2.247474-2.514857$

H $6.629304-0.7974240 .685762$

H $6.161309-0.078278-0.859691$

H $3.714155-0.350820-1.150001$

H $\quad 0.6322310 .8506730 .364904$

H $-0.0911493 .725651 \quad 1.195606$

H $2.3913731 .773810-0.971960$

H $2.3083102 .880625-2.321264$

H $0.9347651 .832730-1.990417$

H 4.1522470 .9633600 .380053

H 4.8596540 .3480151 .884008

H 6.9489474 .2085331 .775357

H 5.4939404 .6976322 .670637

H 5.9333036 .1848830 .639888

H $4.1802403 .955990-0.510298$

H $4.5058405 .411392-2.497944$

H $5.0687146 .733147-1.509963$

H $1.6930648 .121845-1.089018$

H $3.5062938 .379996-0.808962$

H $2.1570394 .895244-2.729167$

H $1.0178256 .154837-2.383685$

H $0.6888335 .440193-0.054227$

H 4.3184392 .8319501 .844964

C -5.5626981 .7870761 .693372$

O -4.6062651 .8020820 .613508$

C $-5.3030672 .030326-0.620255$

C $-6.5673562 .769646-0.216966$

C -6.9350612 .0190711 .052612$

H -5.581877 $1.047134-1.017359$

C $-4.3852622 .733269-1.623371$

H -6.3667803 .8228430 .011028$

H -7.345117 $2.722401-0.984595$

C -7.9064092 .7695691 .947034$

H $-7.3865231 .057766 \quad 0.772330$

C $-3.0035532 .061314-1.783891$

O $-4.1576854 .083045-1.201372$

H -4.887259 2.803412 -2.595006

H -2.389709 2.259263 -0.897389

O $-2.3305322 .684166-2.883504$

C $-3.0530070 .543267-2.032098$

C $-1.656925-0.066430-2.252014$

H $-3.5198740 .054106-1.171848$

O $-3.8704990 .265416-3.167870$

H -1.248692 $0.271459-3.211956$

H $-0.9988670 .287927-1.452302$

H -8.1187732 .1969462 .855414$

H -7.504900 3.7429972 .247819

H -8.8536332 .9430661 .426005$ 
C $-5.457068 \quad 0.4454452 .424497$ H $-3.7710044 .050760-0.309310$ H -2.291002 $3.637727-2.695615$ H -3.485375 $0.733374-3.928558$ H $-5.638471-0.3779251 .722668$ H $-6.217286 \quad 0.375297 \quad 3.210441$ H -5.2963392 .6136542 .363945$ C $-2.249672-3.435898-0.752632$ O $-2.072864-2.024025-0.926633$ C -1.687750 -1.605441 -2.241151 C $-0.324624-2.214991-2.599059$ C $-0.345597-3.740334-2.457949$ C $-0.975561-4.206525-1.140064$ H -2.405986 -3.566797 0.325967 C -3.505001-3.943693 -1.470375 H $-2.437442-1.938556-2.966273$ O $0.707924-1.671422-1.780089$ H $-0.079765-1.948040-3.633890$ H $0.680101-4.121135-2.530389$ O $-1.087456-4.275329-3.552631$ H -1.202298 -5.277556-1.192027 O $-0.012588-4.061927-0.093798$ C $-4.744257-3.296529-0.896658$ H -3.473152 -3.763210 -2.548610 H $-3.618434-5.026456-1.341421$ H $0.362095-1.570150-0.878216$ H -1.051535 -5.244744 -3.485506 H $-0.017216-3.1359930 .197550$ O $-5.337998-2.507887-1.836635$ O $-5.132257-3.466178 \quad 0.252287$ C $-6.530789-1.838618-1.416835$ H -7.332564 -2.567246 -1.265591 H $-6.351764-1.267822-0.501356$ $\mathrm{H}-6.828219-1.145271-2.207968$ SCF Energy (B3LYP/6-31G**//MMFF)= -3245.92617767

0400137

MM̄FF Geometry

C -0.028246 -0.027423 3.835879

C $0.873509-0.2140352 .860773$

C $2.174526-0.9444503 .054864$

O $3.219278-0.0256902 .664485$

C $2.227476-2.1743202 .133211$

C $3.309582-3.1625892 .515417$

C $4.491163-3.2937771 .881906$

C $2.965333-4.0738103 .665818$

C $5.026647-2.477000 \quad 0.738952$

C $5.118610-3.280575-0.565433$

C $3.751963-3.536496-1.220292$

C $3.907007-4.417663-2.462286$

C $2.576772-4.591154-3.211219$

O $3.202967-2.266570-1.587830$

O $2.858086-5.177820-4.490920$

C $1.947367-3.201170-3.444661$

C $1.655945-5.590544-2.502587$

O $0.645306-3.320118-4.012335$

C $1.894288-2.326751-2.169281$

C $1.476803-0.884306-2.542372$

O $0.938138-2.877042-1.263192$

C 4.3446750 .0370923 .418326

O $4.586084-0.6068864 .429996$

C 5.2999561 .0352892 .881156

C $5.159427 \quad 1.6325171 .687055$

C 6.0554702 .6375541 .109286

C 7.2207483 .1009891 .943547

C $1.5688470 .119015-1.384180$

C $1.2554651 .535936-1.833728$

C $1.3780543 .894093-1.030545$

O $0.8013281 .805101-2.942685$

C $2.6380784 .438738-1.626292$

C $3.4407825 .318736-1.008909$

C $4.7063915 .853704-1.615826$

C $5.9340305 .534071-0.790345$

C $6.5201466 .478419-0.035226$

C $6.5356394 .150208-0.909829$

C $5.7786143 .116039-0.122011$

N $1.5294562 .463665-0.837367$

H $\quad 0.175130-0.4029834 .835459$
H $\quad 0.664348 \quad 0.167401 \quad 1.864290$

H $2.283150-1.2323364 .107306$

H $2.358627-1.8493691 .095690$

H $1.266673-2.7068062 .154665$

H $5.177551-4.0627932 .235055$

H $3.760318-4.7972993 .876164$

H $2.798252-3.4950964 .579418$

H $2.055784-4.6407103 .441876$

H $4.457621-1.5561510 .582666$

H $6.037733-2.1536431 .018010$

H $5.743273-2.705855-1.261765$

H $5.631437-4.232672-0.380644$

H $3.090804-4.021402-0.493906$

H $4.328585-5.395311-2.197512$

H $4.633492-3.954707-3.144863$

H $3.462055-4.586007-4.970864$

H $2.563447-2.681499-4.191635$

H $2.135009-6.575559-2.445887$

H $\quad 0.728252-5.741848-3.065659$

H $1.395861-5.291309-1.485208$

H $0.724944-3.872685-4.808628$

H $2.128635-0.521552-3.348330$

H $0.450639-0.882932-2.929613$

H $0.600220-2.175451-0.685856$

H 6.1442341 .2462233 .529676

H 4.3063131 .3690951 .063926

H 6.8730273 .5020622 .902197

H 7.8055103 .8936991 .472242

H 7.9081572 .2704052 .137763

H $0.856682-0.138209-0.593871$

H $2.5780700 .111866-0.956825$

H $1.1547694 .334968-0.054080$

H $0.5369314 .086928-1.703626$

H $2.9010474 .089684-2.622334$

H $3.1760115 .679199-0.017638$

H $4.5914256 .940675-1.719073$

H $4.8517065 .476351-2.635902$

H 7.4187886 .2693450 .535319

H 6.1121937 .4815140 .032588

H $7.5939124 .162067-0.625760$

H $6.5436523 .849783-1.965588$

H $4.9116892 .716828-0.648483$

H 1.9741562 .1495040 .020539

C $-2.555838-0.1996843 .929924$

O -3.7342420 .6119243 .716385$

C -4.5221250 .0050262 .672083$

C $-4.201197-1.4718632 .784088$

C $-2.702497-1.4288493 .022155$

H -4.1369080 .4151681 .732246$

C -5.9940660 .3873162 .858024$

H $-4.712064-1.9147343 .648095$

H -4.470342 -2.044211 1.893282

C $-2.170401-2.7211773 .622325$

H -2.199295 -1.251440 2.062510

C $-6.977285-0.2331911 .849063$

O -6.1138581 .8103862 .788371$

H $-6.3024160 .105828 \quad 3.873223$

H $-7.9574840 .243803 \quad 1.978883$

O $-7.154282-1.6099242 .201060$

C $-6.591125-0.1607250 .354443$

C $-6.3353121 .259849-0.173957$

H $-5.722093-0.7972100 .168601$

O $-7.665317-0.741661-0.397400$

H $-7.216399 \quad 1.8774310 .040297$

H $-5.493361 \quad 1.7018240 .363736$

H -1.088936 -2.673669 3.776984

H -2.638826 -2.936210 4.588773

H -2.374792 -3.565379 2.955398

C $-1.3342240 .676093 \quad 3.628769$

H $-5.4898772 .186033 \quad 3.432955$

H $-7.783735-1.9937191 .566677$

H -8.452810 -0.185951-0.267798

H $-1.362117 \quad 1.559093 \quad 4.280120$

H -1.413111 1.0740092 .608131

H -2.561058 -0.4854224 .988837$

C $-3.6816500 .854658-1.657809$

O $-4.9858300 .452158-2.092411$

C $-6.0565101 .314894-1.694622$ 
C $-5.811188 \quad 2.732870-2.229987$

C $-4.4482343 .268160-1.801881$

C $-3.3481052 .268866-2.151956$ H -3.631269 $0.819162-0.563844$ C $-2.672328-0.158049-2.223956$ H $-6.9553590 .936008-2.196762$ O $-6.824802 \quad 3.629219-1.787515$ H $-5.8492032 .719695-3.327050$ H $-4.428638 \quad 3.516686-0.734303$ O $-4.1812394 .486940-2.500177$ H -3.196331 2.261890 -3.239090 O $-2.1251202 .693813-1.553338$ C $-2.808750-1.529952-1.601436$ H $-2.833201-0.260929-3.304208$ H -1.645577 $0.174171-2.043162$ H -7.679337 $3.274299-2.086778$ H $-4.9098415 .099539-2.300160$ H $-1.9427623 .593407-1.874939$ O -2.207050 -2.452199-2.409076 O $-3.350233-1.765254-0.530952$ C $-2.263215-3.804344-1.942756$ H -1.732111 -4.434901-2.660375 H $-1.773714-3.892313-0.968116$ H $-3.303171-4.139422-1.883700$

SCF Energy (B3LYP/6-31G*//MMFF)= -3245.92140135

0400138

MM̄FF Geometry

C -1.275989 4.1615500 .936160

C $0.023472 \quad 3.8306690 .900500$

C $0.8210653 .755993-0.376065$

O $1.292400 \quad 2.399197-0.548954$

C $2.0445884 .681197-0.287639$

C $2.7756274 .812584-1.608990$

C $3.9312334 .188295-1.908635$

C $2.1498645 .761226-2.599320$

C $4.6924713 .214405-1.052208$

C $5.2191512 .034490-1.882138$

C $6.0454151 .067767-1.022866$

C $6.7591430 .037501-1.899166$

C $7.544016-0.976326-1.055337$

O $5.1575750 .402462-0.117475$

O $7.920612-2.048356-1.932433$

C $6.606025-1.5503820 .025873$

C $8.850469-0.377579-0.521984$

O $7.322424-2.400670 \quad 0.918926$

C $5.813697-0.4739970 .808171$

C $4.738149-1.1654921 .676094$

O $6.710273 \quad 0.239037 \quad 1.662106$

C $0.4295141 .522881-1.130958$

O $-0.708722 \quad 1.756511-1.505702$

C $1.103590 \quad 0.209621-1.230412$

C $0.371030-0.861457-1.569476$

C $0.841981-2.240417-1.698902$

C $2.327306-2.478640-1.669226$

C $3.702850-0.2176352 .294868$

C $2.671647-1.0154013 .070419$

C $0.771878-2.6008612 .733227$

O $2.681155-1.0621874 .297991$

C $0.825842-3.8529871 .915620$

C $-0.227719-4.366875 \quad 1.262661$

C $-0.166835-5.6321490 .454836$

C $-0.706119-5.466269-0.949456$

C $-1.864432-6.039890-1.316975$

C $0.134830-4.700964-1.951230$

C $-0.078861-3.215248-1.850246$

N $1.789010-1.6906582 .243188$

H -1.7956694 .3872940 .009209$

H $0.5408313 .591892 \quad 1.826656$

H $0.206593 \quad 4.043823-1.238286$

H 2.7268374 .3247530 .493257

H 1.7348145 .6854680 .032365

H $4.3894894 .402837-2.873397$

H $2.7450445 .860934-3.513258$

H $1.1552105 .412202-2.892782$

H $2.0532176 .759444-2.159973$

H $5.5398823 .745954-0.603139$

H $4.078752 \quad 2.827902-0.233718$
H $4.3689441 .501862-2.327805$

H $5.8392872 .416709-2.702752$

H $6.7768141 .651443-0.451298$

H $7.4231920 .529418-2.621055$

H $6.016095-0.507667-2.498441$

H $8.351037-2.733604-1.392518$

H $5.887479-2.198979-0.494102$

H $9.480048-0.036702-1.352757$

H $9.443241-1.129404 \quad 0.010874$

H 8.6917090 .4716190 .145941

H $7.820011-1.8332521 .532119$

H $4.203133-1.8966671 .059186$

H $5.220087-1.7304332 .484774$

H 6.2063540 .6231302 .396834

H $2.1625210 .156340-1.006773$

H $-0.693964-0.722215-1.749253$

H $2.837952-1.826760-2.387369$

H $2.609025-3.499601-1.934780$

H $2.728959-2.279118-0.673022$

H 4.1725650 .4928612 .982727

H 3.1863950 .3690341 .526838

H $-0.189797-2.0908962 .643685$

H $0.948120-2.8323063 .787943$

H $1.782171-4.3690551 .868708$

H $-1.190299-3.865657 \quad 1.327644$

H $-0.744226-6.3952230 .992891$

H $0.858608-6.0184830 .397572$

H -2.245381 -5.944678 -2.328535

H $-2.461845-6.610517-0.613775$

H $-0.133779-5.003326-2.971921$

H $1.180170-4.994175-1.823255$

H -1.129799 -2.925766 -1.895093

H $1.791672-1.4791951 .250054$

C -3.0433553 .0540462317230$

O -2.2764891 .8759572 .651967$

C $-2.812503 \quad 1.3135043 .855696$

C -4.2459741 .8107303 .936336$

C -4.0737643 .2329043 .437286$

H $-2.249833 \quad 1.764392 \quad 4.685758$

C -2.602730 -0.1992063 .919069$

H -4.9075601 .2525013 .265242$

H $-4.656572 \quad 1.7602644 .948996$

C $-5.369682 \quad 3.8772912 .975408$

H -3.646752 3.8396634 .247488

C -3.423510 -1.082529 2.958874

O $-2.892766-0.6232235 .259176$

H $-1.538451-0.4108153 .764256$

H $-4.479739-1.0748113 .253951$

O $-2.978232-2.434848 \quad 3.153632$

C -3.321974 $-0.754527 \quad 1.454972$

C $-4.010729-1.8346600 .598462$

H $-3.7854180 .216112 \quad 1.251924$

O $-1.956041-0.6806601 .053592$

H -3.471918 -2.7836890 .709985$

H $-5.031176-1.9721050 .977178$

H -5.1891304 .8915742 .605274$

H $-5.838230 \quad 3.302702 \quad 2.169324$

H -6.0841163 .9402613 .802575$

C $-2.093109 \quad 4.2464182 .192988$

H -3.842011 -0.4848145 .417748$

H $-3.068375-2.6373774 .100631$

H $-1.569770 \quad 0.111424 \quad 1.463692$

H -1.4318104 .2913843 .067350$

H $-2.658793 \quad 5.1848152 .163888$

H -3.5412282 .8482971 .362419$

C $-5.1397490 .069176-2.423043$

O $-4.945943-0.382051-1.074476$

C $-4.062145-1.495287-0.903936$

C $-4.539306-2.707575-1.723201$

C $-4.768146-2.344407-3.190710$

C $-5.617760-1.079031-3.330029$

H $-5.9550980 .801132-2.356779$

C $-3.9016950 .791502-2.977318$

H $-3.059575-1.209380-1.234858$

O $-5.743166-3.240890-1.173346$

H $-3.782659-3.497341-1.654268$

H $-5.270729-3.180985-3.691448$

O $-3.501969-2.155125-3.814887$ 
H $-5.632489-0.749003-4.375265$

O $-6.965020-1.408554-2.980655$

C $-3.7730642 .191090-2.414196$

H -2.965889 $0.270182-2.764257$

H $-4.0052770 .884695-4.065332$

H $-6.406744-2.530142-1.165423$

H -3.664358 -1.952528 -4.751878

H -7.510109-0.617562 -3.131554

O $-3.1897652 .997250-3.349175$

O $-4.1134162 .524490-1.287598$

C $-2.9978144 .366681-2.979420$

H -3.850787 4.755864 -2.415092

H $-2.0768054 .458683-2.399046$

H -2.893787 $4.952890-3.896514$

SCF Energy (B3LYP/6-31G**//MMFF) $=-3245.92841720$

04 00139

MM̄FF Geometry

C $5.097921-1.423263$-2.114171

C $3.969619-1.930704-1.594685$

C $2.742319-2.202978-2.421197$

O $1.659033-1.401527-1.893687$

C $2.316858-3.676011-2.327605$

C $1.286787-4.034491-3.382712$

C $-0.037149-4.161225-3.167425$

C $1.848881-4.298132-4.758076$

C $-0.800310-3.977237-1.884791$

C $-1.986380-3.020196-2.066237$

C $-2.930037-2.974544-0.850970$

C $-3.705659-4.284772-0.689787$

C $-4.729138-4.2129470 .447372$

O $-3.852299-1.896821-1.071437$

O $-5.582790-5.3599450 .314075$

C $-5.597457-2.9502350 .268143$

C $-4.047913-4.3428491 .815997$

O $-6.460546-2.8087941 .393935$

C $-4.779373-1.6635120 .006928$

C $-5.661820-0.470842-0.443488$

O $-4.091216-1.2893721 .200811$

C $1.547086-0.127260-2.355838$

O $2.294387 \quad 0.445664-3.132317$

C $0.3162350 .446584-1.772578$

C $-0.2156651 .545468-2.326908$

C -1.461436 2.195575-1.923350

C $-2.1269851 .717132-0.659714$

C $-6.742978-0.0428720 .548144$

C -7.281324 1.3399290 .240764

C -6.9084043 .7496640 .772390$

O $-8.2725571 .505650-0.466129$

C $-6.2323524 .384142-0.398988$

C $-5.1952325 .228249-0.290556$

C -4.520102 $5.876061-1.465648$

C $-3.1772055 .242539-1.766990$

C $-2.0415845 .860001-1.398603$

C $-3.250483 \quad 3.924177-2.512862$

C $-1.9504793 .189987-2.693326$

N -6.5415722 .3475750 .838048$

H $5.154772-1.233600-3.183494$

H $3.898748-2.103919-0.524527$

H $2.917468-1.934344-3.470590$

H $1.933979-3.893714-1.323890$

H $3.186959-4.332434-2.462056$

H $-0.659323-4.456462-4.012312$

H $1.077423-4.605800-5.472132$

H $2.327262-3.400063-5.160441$

H $2.593549-5.099816-4.715693$

H -1.150950 -4.968315 -1.580609

H -0.166964 -3.602455-1.076399

H -1.607908 -2.008623 -2.263376

H -2.566553 -3.288474 -2.959617

H $-2.336459-2.7526950 .042901$

H -3.034572 -5.135012 -0.525917

H $-4.241367-4.505053-1.624107$

H $-6.280477 \quad-5.2876920 .988132$

H $-6.251161-3.123517-0.597251$

H $-3.496228-5.2887451 .878403$

H $-4.783963-4.3763582 .626692$

H $-3.344223-3.5341432 .021393$
H $-5.933679-2.4495002 .128153$

H $-5.0055120 .385653-0.649349$

H $-6.128768-0.703967-1.409860$

H $-3.873758-0.3438821 .146390$

H $-0.141503-0.075118-0.940026$

H $\quad 0.286402 \quad 1.991450-3.184709$

H $-2.4526600 .677337-0.764178$

H $-3.0038932 .306330-0.379879$

H -1.4324491 .7863510 .184389$

H -7.591522 -0.7353360 .519764$

H $-6.363593-0.0449221 .576598$

H -6.6082894 .2093441 .719602$

H $-7.994541 \quad 3.8449290 .673297$

H -6.623245 4.142116-1.384415

H -4.8013995 .4676410 .694453$

H $-4.4073876 .945584-1.246238$

H -5.152046 $5.828199-2.361797$

H -1.060503 $5.448838-1.606607$

H $-2.0662606 .808728-0.871502$

H $-3.6611784 .118700-3.512628$

H $-3.979543 \quad 3.264553-2.030337$

H -1.392647 $3.492975-3.579766$

H -5.6912522 .1078701 .339598$

C $6.4085740 .508408-1.269692$

O $5.2767101 .054953-0.564342$

C 5.7223252 .1394670 .270255

C $7.1619742 .422358-0.140895$

C $7.6371021 .033736-0.531711$

H $5.725127 \quad 1.7643991 .300289$

C 4.7501053 .3190240 .148808

H $7.2142053 .098824-1.001894$

H 7.7463982 .8645170 .671643

C $8.9064401 .037411-1.364779$

H 7.8057800 .4480970 .382103

C 3.2740472 .9429350 .404934

O $4.8289213 .860416-1.176184$

H 5.0574804 .1260020 .823407

H $2.9056932 .297904-0.401913$

O $2.491840 \quad 4.141553 \quad 0.350632$

C 3.0245862 .2608081 .760859

C $1.5447681 .907717 \quad 1.984434$

H 3.6103241 .3421301 .811539

O 3.4792263 .1064522 .815620

H 0.9480302 .8276581 .996493

H 1.1983311 .3075401 .136746

H $9.1994570 .017832-1.634095$

H $8.7770331 .606826-2.291323$

H $9.7320521 .489220-0.805278$

C $6.293560-1.016160-1.299479$

H $4.6173283 .143806-1.799111$

H $2.6589724 .556927-0.512781$

H 2.9807303 .9397552 .758582

H $6.203839-1.405559-0.279138$

H $7.189254-1.462963-1.744207$

H $6.3738860 .914343-2.289739$

C $1.832288-1.0865732 .560494$

O $2.131964-0.0069633 .452749$

C 1.3017831 .1514423 .310998

C -0.1634460 .7715253 .553604$

C $-0.608064-0.3736782 .650196$

C $0.374337-1.5399632 .728234$

H $2.020079-0.7732571 .526203$

C $2.782261-2.2487852 .897828$

H 1.5936421 .8341514 .119090

O $-1.0042951 .903353 \quad 3.355752$

H -0.2812180 .4630324 .600669$

H -0.744982 -0.033626 1.618487

O $-1.889910-0.8164873 .099930$

H $0.247380-2.0415923 .695983$

O $0.042417-2.4959771 .722296$

C $4.230556-1.9548482 .571140$

H $2.726847-2.4636253 .971953$

H $2.501347-3.1447902 .333186$

H -1.9213541 .6127663 .498973$

H -2.154558 -1.558936 2.530291

H $0.164095-2.0714530 .855849$

O $5.001861-2.9894153 .021282$

O $4.639378-0.9610781 .989063$ 
C $6.407160-2.8602472 .782985$

H $\quad 6.610706-2.9159881 .709942$

H $\quad 6.783977-1.9213693 .200394$

H $6.913782-3.6916233 .280211$

SCF Energy (B3LYP/6-31G**//MMFF) $=-3245.93642977$

04_00140

MM̄FF Geometry

C -0.539362 -1.505505 0.194220

C $-1.341485-0.914466-0.705993$

C $-0.9189900 .112936-1.733180$

O $0.5217150 .207537-1.803100$

C $-1.4719401 .491207-1.332719$

C -1.154006 $2.574793-2.342811$

C $-0.1715983 .486251-2.199932$

C $-2.0576142 .617564-3.546595$

C $0.8197613 .589648-1.073774$

C $2.2481483 .792112-1.599294$

C $3.2590973 .881355-0.447668$

C $4.6393334 .289920-0.962695$

C 5.6782024 .3206040 .167736

O 3.3379232 .5944260 .175179

O $6.9687484 .417669-0.453104$

C 5.6164202 .9836390 .935160

C 5.5255105 .5709091 .041353

O 6.4706043 .0141852 .076487

C 4.1830922 .5515751 .331986

C 4.2131661 .0994821 .859986

O 3.7028973 .4112322 .366747

C $1.161477-0.650678-2.642822$

O $0.650116-1.444131-3.416489$

C $2.613761-0.504520-2.401711$

C $3.436098-1.489177-2.792558$

C $4.878667-1.559299-2.554252$

C $5.553104-0.344248-1.977010$

C 2.8335800 .4593272 .055590

C $2.994759-1.0109172 .389613$

C $3.585294-3.1975021 .334570$

O $2.935251-1.4200033 .546470$

C $5.023532-3.3549270 .947150$

C $5.457482-4.2365520 .033153$

C $6.900330-4.380398-0.363974$

C $7.106082-4.377393-1.864935$

C $7.470929-5.496897-2.512750$

C $6.962098-3.062811-2.606967$

C $5.520532-2.708727-2.849943$

N $3.249553-1.7871071 .269596$

H $\quad 0.517728-1.2615240 .224389$

H -2.400834 -1.162456 -0.701022

H -1.322516 -0.178170 -2.710735

H $-1.0909811 .770645-0.343115$

H -2.562092 $1.438834-1.212590$

H $-0.0654314 .247921-2.971399$

H -1.776072 $3.407676-4.250810$

H -2.023667 $1.667884-4.089226$

H -3.089458 2.806375 -3.235269

H $0.5400784 .447185-0.450124$

H $0.7986222 .704654-0.432179$

H $2.5106422 .954579-2.258356$

H $2.2881124 .710810-2.197830$

H $2.891164 \quad 4.6150330 .279267$

H $4.5966675 .261767-1.469964$

H $4.9722193 .569139-1.723038$

H $7.638914 \quad 4.3609750 .249611$

H 6.0359782 .2185010 .267406

H $5.6281326 .476440 \quad 0.431244$

H 6.3194705 .6284581 .794263

H $4.5620495 .621308 \quad 1.552887$

H 6.0217393 .5301432 .767768

H 4.7805650 .4786371 .157882

H 4.7508091 .0614612 .816479

H 3.0000932 .9528472 .854216

H $2.954670 \quad 0.368483-1.857803$

H $3.006243-2.353937-3.297179$

H $5.3052050 .550574-2.559484$

H $6.643112-0.410175-1.978025$

H $5.234738-0.187698-0.943507$

H $2.282196 \quad 0.9381672 .871501$
H $2.2164900 .548446 \quad 1.154460$

H $2.912975-3.7231850 .649315$

H $3.426450-3.5762582 .348655$

H $5.744838-2.7104831 .445080$

H $4.741396-4.900772-0.445515$

H $7.268298-5.3217390 .064303$

H $7.515259-3.5872740 .079494$

H $7.643663-5.498980-3.583910$

H $7.600257-6.437748-1.988060$

H $7.457665-3.125714-3.584301$

H $7.502290-2.293934-2.047911$

H $4.943872-3.519279-3.298966$

H $3.278909-1.3406850 .357846$

C -1.005771-1.858276 2.622084

O $-1.979147-0.7941422 .653139$

C $-2.981589-1.1206573 .626649$

C $-2.253303-1.9855924 .641569$

C $-1.410778-2.8500923 .719367$

H -3.739055 -1.729570 3.116555

C -3.6360430 .1539514 .165263$

H $-1.606012-1.3882345 .293959$

H -2.937075 -2.565837 5.267804

C $-0.225838-3.5054554 .408344$

H -2.049655 -3.638058 3.298144

C $-4.117497 \quad 1.130796 \quad 3.069643$

O -2.7020800 .8636554 .987685$

H -4.469742 -0.1141074 .824283$

H -3.2580631 .6424072 .618805$

O -4.9023072 .1486283 .702215$

C $-4.9579930 .483778 \quad 1.955368$

C -5.5003041 .5111300 .944252$

H -4.343256 -0.2477051 .421755$

O $-6.053803-0.2275952 .530363$

H -6.2630422 .1357851 .424654$

H $-4.6729262 .156530 \quad 0.624729$

H $0.362582-4.090633 \quad 3.694261$

H $\quad 0.437271-2.760576 \quad 4.861250$

H $-0.565934-4.1789645 .201633$

C -1.015085 -2.487833 1.226657

H -1.9169711 .0520524 .445053$

H -4.3507362 .5505464 .395167$

H $-6.5809090 .409327 \quad 3.042653$

H -2.028711 -2.826083 0.978989

H -0.360668 -3.366484 1.200060

H $-0.031673-1.4038522 .838245$

C $-5.452571-0.553321-2.170172$

O $-5.0632040 .189700-1.007992$

C $-6.1242190 .826233-0.286893$

C $-6.8537381 .825498-1.195292$

C $-7.352878 \quad 1.145243-2.473537$

C $-6.2507190 .322311-3.150156$

H -4.505693 -0.820120 -2.657864

C $-6.167404-1.854687-1.791888$

H -6.8342210 .0683760 .060031$

O $-5.9924872 .910285-1.534769$

H $-7.7035552 .248845-0.647148$

H -7.718777 $1.913577-3.165456$

O $-8.4500240 .299836-2.139453$

H $-6.669113-0.307722-3.943666$

O $\quad-5.3241431 .215330-3.774440$

C $-5.207189-2.799633-1.108310$

H -7.012828 -1.706329 -1.114400

H $-6.569546-2.354828-2.681209$

H $-5.1927812 .537723-1.943943$

H -8.774405 -0.099906 -2.964404

H $-5.8161681 .737994-4.430261$

O $-4.592546-3.587745-2.036234$

O $-5.007811-2.8137660 .099361$

C $-3.638969-4.522248-1.521578$

H $-2.758696-3.989470-1.152022$

H $-4.083035-5.133160-0.729554$

H -3.332976 -5.180197 -2.339366

SCF Energy (B3LYP/6-31G**//MMFF) $=-3245.93235314$

0400141

MM̄FF Geometry

C -1.420782 3.5734002 .259947

C -2.5932084 .2259022 .230097$ 
C $-3.944473 \quad 3.6559951 .866217$

O

C -4.4354034 .2875340 .552835$

C -5.8989094 .0058290 .278552$

C $-6.3526183 .098129-0.607397$

C -6.8726954 .8704611 .038362$

C $-5.5434312 .151610-1.451516$

C $-6.1918630 .760008-1.495888$

C -5.455869 -0.182261-2.458682

C $-6.256222-1.469987-2.664601$

C $-5.513587-2.458419-3.574730$

O $-4.172725-0.490545-1.902177$

O $-6.183897-3.725524-3.498634$

C $-4.075701-2.638376-3.047310$

C $-5.596830-2.045218-5.047917$

O $-3.299959-3.430049-3.944344$

C $-3.347976-1.303972-2.749651$

C $-2.027398-1.604674-2.005812$

O $-3.047429-0.643064-3.979656$

C $-4.077762 \quad 1.4362882 .760902$

O $-4.2659851 .799092 \quad 3.910824$

C -3.9873440 .0301812 .310002$

C $-3.824907-0.9400393 .221504$

C -3.679744 -2.370646 2.945508

C $-3.922377-2.8347641 .534676$

C $-1.300288-0.373668-1.455675$

C $-0.138444-0.834436-0.599146$

C $0.437877-1.4940141 .735453$

O $0.945518-1.131014-1.096401$

C $0.047476-2.9176611 .987690$

C $-0.129414-3.4344273 .213507$

C $-0.515344-4.8651373 .464774$

C $-1.677284-5.0068684 .425457$

C -1.491390 -5.486224 5.667121

C $-3.065925-4.6682093 .922949$

C $-3.332817-3.1880553 .961429$

N $-0.462570-0.9327920 .744827$

H -1.3736572 .5188392 .003996$

H -2.599797 5.2828112 .492434

H $-4.643663 \quad 3.8925182 .677758$

H -3.815152 $3.937845-0.281285$

H -4.3000295 .3774330 .579620$

H -7.430230 $3.028464-0.752751$

H -7.9131274 .6654420 .764625$

H $-6.778370 \quad 4.7027022 .115504$

H -6.6818125 .9287520 .832472$

H -5.495483 $2.560627-2.467744$

H -4.516376 $2.056332-1.087539$

H $-6.1913150 .333826-0.484189$

H -7.237685 $0.857142-1.813861$

H $-5.3210680 .337456-3.414619$

H -7.252315 -1.252822 -3.070170

H $-6.427318-1.948744-1.690158$

H $-6.178910-4.017635-2.571160$

H -4.140206 -3.208277 -2.109721

H -5.115451 -2.787201 -5.694842

H -5.146072 -1.071137 -5.248492

H $-6.643529-2.000356-5.372538$

H -3.786903 -4.257990 -4.096511

H -2.235258 -2.278592 -1.166857

H -1.339571 -2.139769 -2.673937

H -2.307049 -0.032722 -3.836098

H $-4.025902-0.163138 \quad 1.243873$

H -3.761425 -0.6597624 .272420$

H -3.154499 -2.434921 0.868309

H $-4.903367-2.498567 \quad 1.179930$

H -3.913411 -3.920248 1.421544

H $-0.9011750 .254160-2.259146$

H $-1.969737 \quad 0.255254-0.858670$

H $\quad 0.357475-0.8767572 .635587$

H $1.466859-1.4529001 .367098$

H - $0.085871-3.5578221 .118368$

H $\quad 0.033209-2.8034724 .084449$

H $0.368036-5.3797733 .864993$

H $-0.763784-5.3801532 .528353$

H $-2.322215-5.614875 \quad 6.352981$

H $-0.504376-5.7560936 .027650$

H $-3.822253-5.1555614 .552283$
H -3.191959 -5.105018 2.929096

H -3.204016 -2.750340 4.952767

H -1.409768 -0.7198491 .041990$

C $0.9322814 .218011 \quad 1.530404$

O $1.3808062 .872778 \quad 1.283332$

C $1.6369592 .727623-0.131662$

C $1.5408094 .126355-0.728034$

C $0.508446 \quad 4.774758 \quad 0.173107$

H $0.8285362 .092306-0.512919$

C $2.9958662 .045707-0.325395$

H $2.5011694 .651474-0.650404$

H $1.2442654 .114008-1.781108$

C 0.4972756 .2910000 .103066

H $-0.4791894 .395543-0.117034$

C $3.4270511 .919322-1.797655$

O 2.9208650 .7363200 .244500

H 3.7590692 .5892460 .243202

H $3.6159432 .915210-2.214481$

O $2.3402491 .374939-2.558297$

C $4.6563461 .020750-2.038350$

C $5.8956821 .427217-1.223320$

H $4.388495-0.020953-1.833077$

O $4.9830411 .075114-3.431731$

H $6.1764062 .454589-1.486209$

H $5.6501751 .425129-0.157841$

H $-0.254406 \quad 6.7060820 .781751$

H 1.4699446 .7132450 .377488

H $0.2594626 .627322-0.911398$

C -0.1231724 .2334882 .638642$

H 2.6403830 .8378371 .170229

H $2.1317660 .500046-2.187048$

H $5.2284711 .991845-3.644157$

H -0.2985055 .2703722 .949897$

H 0.2699713 .6997163 .512927

H 1.8124424 .7628911 .897998

C $6.484725-1.2809680 .022543$

O $6.774574-0.880861-1.321740$

C $7.1088370 .501812-1.475603$

C $8.3461290 .832392-0.630858$

C 8.1782770 .4090290 .828218

C $7.704029-1.0432240 .920320$

H $5.625607-0.7144220 .402189$

C $6.091486-2.761191-0.013914$

H $7.3930070 .633063-2.527310$

O $8.6317132 .225106-0.706425$

H $9.2116450 .304228-1.052200$

H $7.496513 \quad 1.0862871 .356262$

O $9.4485650 .533870 \quad 1.471842$

H $8.522019-1.7165110 .633819$

O $7.350761-1.3399002 .269321$

C $4.714545-2.921286-0.614681$

H $6.806582-3.353875-0.596919$

H $6.054798-3.1996880 .989546$

H $9.4099242 .392834-0.147671$

H 9.3159620 .3621032 .419208

H $8.162068-1.2972882 .802759$

O $4.811473-3.134050-1.957783$

O $3.677581-2.8317780 .030183$

C $3.567534-3.250042-2.655377$

H $3.783364-3.525144-3.691148$

H $2.945175-4.031293-2.208377$

H $3.046458-2.288472-2.648954$

SCF Energy (B3LYP/6-31G**//MMFF) $=-3245.91602588$

04 00142

MM̄MF Geometry

C 0.3139881 .3653452 .552685

C 1.4363850 .8864333 .109418

C $1.951960-0.5329563 .071580$

O $3.156399-0.4586262 .269456$

C $1.003823-1.5794602 .450710$

C $1.278296-3.0035602 .893511$

C $1.883919-3.9461912 .145977$

C $0.752723-3.3572434 .262435$

C $2.506677-3.798367 \quad 0.787624$

C $1.704143-4.497546-0.318689$

C $0.472462-3.700324-0.778034$

C $-0.270793-4.448718-1.887460$ 
C $-1.414569-3.615171-2.485118$ O $0.943215-2.444404-1.270796$ O $-1.817764-4.267969-3.698712$ C $-0.875477-2.215380-2.854822$ C $-2.644543-3.613171-1.570943$ O $-1.930302-1.367468-3.302845$ C $-0.077364-1.544237-1.714992$ C $0.633396-0.271110-2.232480$ O $-0.979341-1.194640-0.664290$ C $4.242879-1.1823672 .637670$ O $4.337671-1.9621523 .570347$ C $5.319188-0.8459071 .681163$ C $6.410804-1.6181661 .595584$ C $7.543547-1.3626510 .709442$ C $8.870892-1.7082441 .329157$ C $1.5926100 .355490-1.210056$ C $2.4066931 .492514-1.798307$ C $4.3958112 .906830-1.279999$ O $2.1656862 .000181-2.889837$ C $5.5309942 .285723-2.033520$ C $6.8119262 .340508-1.637405$ C $7.9528351 .776755-2.436353$ C $8.8350620 .843229-1.637881$ C $9.9805071 .282685-1.089337$ C $8.448933-0.616637-1.563465$ C $7.379964-0.889491-0.543327$ N $3.4292551 .880579-0.941612$ H -0.3487510 .7050912 .002290$ H 2.0784541 .5856443 .643670 H $2.204068-0.8035904 .104241$ H $1.043461-1.5057021 .359619$ H $-0.036498-1.3701262 .730350$ H $1.983736-4.9455932 .568423$ H $\quad 0.936027-4.4054634 .521468$ H $1.231613-2.7418955 .030083$ H $-0.328875-3.192104 \quad 4.308480$ H $2.693445-2.7513150 .532286$ H $3.498967-4.2653270 .839526$ H $2.378727-4.623405-1.176091$ H $1.404501-5.5018070 .005476$ H $-0.188757-3.5312220 .079271$ H $-0.645527-5.413099-1.522231$ H $0.434200-4.691256-2.695581$ H -2.490263 -3.708905 -4.124778 H $-0.208434-2.347297-3.717709$ H $-3.005278-4.636994-1.414714$ H -3.476853 -3.072522 -2.033527 H $-2.450797-3.176691-0.588899$ H -2.425355 -1.073720 -2.519635 H $\quad 1.216869-0.527017-3.126848$ H $-0.1060920 .480116-2.531803$ H - $0.617349-0.447140-0.164349$ H 5.1912150 .0384651 .066101 H $6.509445-2.4919832 .236423$ H $8.986050-1.1995542 .293329$ H $9.725992-1.4135700 .717067$ H 8.943967 -2.788088 1.499265 H $\quad \begin{array}{llll}1.036337 & 0.759144 & -0.359161\end{array}$ H $2.292374-0.400332-0.835810$ H $4.7226663 .370669-0.344078$ H $3.9134233 .669358-1.900031$ H $5.2880721 .795278-2.973490$ H $7.0609332 .855756-0.712650$ H $8.5478682 .623600-2.803548$ H $7.5957461 .259903-3.335786$ H $10.6408110 .615010-0.546198$ H $\quad 10.2818372 .322040-1.167867$ H $9.332032-1.239299-1.380043$ H $8.086372-0.947793-2.545102$ H $6.370355-0.690914-0.901776$ H $3.5771051 .359883-0.082157$ C -0.1785573 .4312581 .226254$ O -1.2332632 .8096020 .464535$ C -1.927538 $3.823302-0.286077$ C $-1.1116415 .102676-0.133954$ C -0.5146604 .9212911 .247152$ H -2.897596 3.9679410 .203090 C $-2.1387493 .370942-1.734873$
H $-0.3158155 .171571-0.884667$

H -1.730896 $6.000741-0.220523$

C 0.6782835 .8228961 .513710

H -1.293330 5.1209031 .995544

C $-2.8575312 .014573-1.878967$

O $-0.8532963 .252777-2.357305$

H -2.680100 $4.150557-2.284519$

H $-2.2087301 .203872-1.529766$

O $-3.0810141 .785337-3.275747$

C $-4.2124601 .919283-1.152388$

C $-4.9631860 .613087-1.480595$

H $-4.0440071 .979494-0.072606$

O $-5.0315523 .030506-1.513774$

H $-5.2105360 .599263-2.548640$

H -4.295362 -0.233778 -1.290665

H 1.0797365 .6543882 .517777

H $1.484803 \quad 5.644708 \quad 0.794365$

H $0.3882426 .876167 \quad 1.439397$

C -0.1039832 .8082712 .621102$

H $-0.9964663 .166605-3.314731$

H $-2.2181511 .602264-3.684319$

H $-5.1559823 .002868-2.477985$

H -1.0871572 .8487763 .103291$

H $0.590513 \quad 3.3777573 .248906$

H 0.7573353 .2557260 .679136

C $-5.346306-0.526696 \quad 1.320691$

$\begin{array}{llll}\text { O } & -6.069109 & 0.560415 & 0.728761\end{array}$

C $-6.2764640 .453583-0.682391$

C -7.063156 -0.828103 -0.988496

C $-6.394548-2.065391-0.393932$

C $-6.079359-1.8548761 .086828$

H $-4.336697-0.5744390 .894290$

C $-5.216259-0.2133272 .814957$

H $-6.919573 \quad 1.297478-0.961856$

O $-7.252400-0.992914-2.390006$

H $-8.065485-0.736418-0.550712$

H $-5.490122-2.336309-0.948981$

O $-7.283479-3.178953-0.510289$

H -7.016356 -1.886251 1.657982

O $-5.270085-2.9470251 .519481$

C -4.2808290 .9520243 .029006$

H -6.1920120 .0243673 .255840$

H $-4.805588-1.0553343 .381877$

H -6.408985 -1.277718 -2.780291

H -7.503737 -3.276420 -1.452696

H $-5.259594-2.9442162 .490920$

O -4.9931122 .0960803 .232196$

O -3.0611550 .8560542 .992121$

C -4.2140193 .2803793 .424720$

H -3.614150 3.4865422 .534238

H -4.8996984 .1162913 .586528$

H -3.5741773 .1751794 .306088$

SCF Energy (B3LYP/6-31G**//MMFF)= -3245.91369272

04_00143

MM̄FF Geometry

C -1.914585 2.6415990 .807552

C -3.0526362 .7923991 .502387$

C -3.6703701 .7763232 .437242$

O -2.8705850 .5730082 .447864$

C -5.0774361 .4045801 .937058$

C -5.9125130 .6660282 .962191$

C $-6.154848-0.6591962 .945731$

C -6.5363921 .5338454 .024745$

C $-5.607924-1.6910341 .998790$

C $-6.671457-2.2349201 .034624$

C -7.016552 -1.260714 -0.103005

C $-8.133175-1.829527-0.981139$

C $-8.418046-0.934994-2.197357$

O $-5.832970-1.073572-0.885044$

O $-9.228930-1.683608-3.115815$

C $-7.086820-0.629673-2.915746$

C $-9.2302780 .307869-1.814499$

O $\quad-7.279198 \quad 0.313888-3.967487$

C $-5.968881-0.142098-1.965627$

C $-4.617822-0.096606-2.720685$

O $-6.2912501 .169026-1.498797$

C $-2.587678-0.0229043 .631222$ 
O $\quad \begin{array}{llll}-2.922872 & 0.360187 & 4.743144\end{array}$ C -1.781466 -1.252742 3.434778 C $-1.492223-1.7689442 .229268$ C $-0.710060-2.9795811 .965847$ C $-0.131757-3.7127503 .147271$ C $-3.4029940 .154725-1.815648$ C $-2.0927290 .089690-2.582564$ C $0.370050-0.039898-2.190222$ O $-2.030806 \quad 0.052637-3.808341$ C $0.707698-1.495900-2.254002$ C $1.609291-2.094567-1.461147$ C $1.910276-3.564975-1.518683$ C $1.642626-4.274124-0.207969$ C $2.658851-4.7127240 .553371$ C $0.208167-4.5727270 .172498$ C $-0.534730-3.3684400 .685113$ N $-0.9986900 .095466-1.727592$ H $-1.342179 \quad 1.7230100 .899068$ H -3.604131 3.7246101 .394372 H -3.7094762 .2300583 .434362$ H $-4.9874720 .808608 \quad 1.022865$ H -5.6303532 .3075411 .644403$ H -6.800460 -1.070146 3.721317 H $-7.166370 \quad 0.960923 \quad 4.713477$ H -5.7633442 .0277504 .621358$ H -7.1663642 .3027473 .565452$ H -4.736719 -1.326542 1.446932 H -5.237921 -2.524215 2.610224 H $-6.274310-3.1606630 .597728$ H -7.578715 -2.5023431 .590301$ H $-7.324952 \quad-0.3015680 .326912$ H -9.047889 -1.984780 -0.395442 H $-7.839360-2.823944-1.345897$ H -10.042159-1.946891 -2.651820 H -6.752424 -1.561300 -3.393291 H $-10.1924890 .016187-1.376525$ H $-9.4737200 .908194-2.698063$ H $-8.7204910 .947887-1.091492$ H -7.978333 -0.036037-4.545864 H -4.458446 -1.056975 -3.228735 H $-4.649286 \quad 0.680717-3.494678$ H -5.474125 $1.636330-1.266290$ H -1.446594 -1.724360 4.353217 H -1.864835 -1.266294 1.338330 H $\quad 0.524400-3.0532143 .726088$ H $0.469810-4.5803022 .867713$ H - $0.930925-4.0776573 .801713$ H -3.466131 $1.145615-1.356314$ H -3.366517 -0.594254-1.016325 H $\quad 1.0078170 .503787-1.487485$ H $0.4729960 .413115-3.181236$ H $0.169893-2.090793-2.988970$ H $2.154435-1.503620-0.729149$ H $2.964691-3.675781-1.803843$ H $1.336405-4.058891-2.312903$ H $2.483844-5.2546921 .476410$ H $3.690667-4.543408 \quad 0.267844$ H $0.160634-5.392480 \quad 0.898873$ H - $0.322006-4.961200-0.706928$ H $-0.994500-2.772809-0.103515$ H $-1.1588930 .037981-0.726356$ C $0.011474 \quad 4.158667 \quad 0.261252$ O $0.957894 \quad 3.0733490 .200284$

C $2.1751943 .543086-0.403463$

C $2.0535865 .061133-0.473070$

C $0.5620895 .237991-0.669988$

H $2.1958693 .135677-1.422333$

C 3.4106793 .0496930 .363084

H 2.3720275 .5364220 .462115

H $2.6489095 .487655-1.286189$

C $0.0701246 .639875-0.354641$

H $0.3156814 .996486-1.712792$

C 3.5308091 .5097580 .392838

O 3.3608353 .5718771 .690389

H $4.3003493 .469512-0.122353$

H $3.4197531 .134196-0.631550$

O $2.469390 \quad 0.943426 \quad 1.159176$

C 4.8861581 .0117540 .936493
C $4.982226-0.5256870 .922878$

H $5.678123 \quad 1.4511160 .320154$

O 5.0686661 .4776012 .271307

H $4.285766-0.9401001 .662063$

H $4.663504-0.889065-0.059780$

H $-1.0114886 .717116-0.502495$

H $\quad 0.289284 \quad 6.9185420 .681485$

H $\quad 0.553057 \quad 7.372939-1.009010$

C -1.374520 3.663836-0.153005

H 4.1828223 .3141382 .140385

H 2.5446601 .2948072 .062955

H 6.0002071 .3369572 .510140

H -1.318994 $3.189062-1.140383$

H -2.064478 $4.511202-0.237529$

H $-0.002974 \quad 4.5125281 .300463$

C $7.347555-0.808913-0.962738$

O $7.400864-0.4357580 .419589$

C $6.402732-1.0456401 .243929$

C $6.549453-2.5715861 .188093$

C $6.531083-3.093255-0.247236$

C $7.532480-2.323648-1.111016$

H $6.384223-0.504594-1.390279$

C $8.450875-0.035086-1.690737$

H $6.627332-0.7422912 .273737$

O $5.520825-3.1885831 .953153$

H $7.504797-2.8518391 .651105$

H $5.520704-3.045900-0.669264$

O $6.893272-4.475459-0.227965$

H $8.550385-2.610612-0.819590$

O $7.365144-2.707599-2.474017$

C $8.0666301 .419402-1.826494$

H $9.408709-0.102252-1.161251$

H $8.616741-0.417946-2.704099$

H $5.645111-4.1507661 .884541$

H $\quad 6.916023-4.781417-1.151013$

H $6.468595-2.450476-2.749415$

O $8.5206492 .126776-0.753146$

O $7.4103041 .864067-2.759588$

C $8.1734953 .514867-0.740393$

H $7.0855983 .633331-0.727817$

H $8.6081224 .021893-1.607040$

H 8.5846013 .9598020 .169613

SCF Energy (B3LYP/6-31G**//MMFF) $=-3245.91831637$

04 00144

MM̄FF Geometry

C $1.776349-3.696104-0.637861$

C $1.159366-2.583397-0.211353$

C $-0.112994-2.057736-0.820707$

O $0.118198-0.711084-1.303974$

C $-1.253352-1.9673910 .209725$

C -1.691779 $-3.298830 \quad 0.776734$

C $-2.490997-4.1688680 .129667$

C -1.194411-3.609860 2.164006

C $-3.095665-4.006478-1.237930$

C $-4.609619-4.260911-1.244626$

C $-5.418410-3.242222-0.425598$

C $-6.899569-3.629266-0.421362$

C -7.759920 $-2.592326 \quad 0.311087$

O $-5.249357-1.950873-1.020245$

O $-9.129320-2.888344-0.001604$

C -7.433034 -1.194549-0.252330

C $-7.628140-2.7242621 .832703$

O $-8.120488-0.1844640 .484096$

C $-5.916655-0.886817-0.326390$

C $-5.7025530 .421040-1.129080$

O $-5.412941-0.7253740 .998201$

C $0.788089-0.572606-2.478261$

O $1.251335-1.471771-3.166635$

C $0.9546890 .851156-2.843605$

C $0.150078 \quad 1.824899-2.391294$

C $0.2555643 .250840-2.715803$

C $1.4475183 .696322-3.522235$

C $-4.2411120 .846451-1.317365$

C $-3.6853841 .638274-0.148052$

C -3.9000573 .8401491 .017565$

O -2.7999991 .1824010 .571654$

C -3.3584465 .0588320 .341037$ 
C -2.1048025 .5090010 .498551$ C -1.559571 $6.706138-0.224351$ C $-0.4404546 .350468-1.178736$ C $0.8164336 .771001-0.958175$ C $-0.7934245 .580641-2.433957$ C $-0.6914364 .090844-2.247246$ N $-4.2751712 .883124-0.007998$ H $1.354661-4.260298-1.465901$ H $1.588145-2.0135800 .609162$ H $-0.438742-2.678323-1.665258$ H -2.112572 -1.463456 -0.249599 H $-0.965123-1.2894861 .025170$ H $-2.736691-5.1066850 .626144$ H $-1.559366-4.5755232 .529746$ H $-0.101312-3.6417912 .186660$ H -1.533676 -2.842343 2.867455 H $-2.890040-3.022477-1.669298$ H -2.621024 -4.740063 -1.901073 H $-4.943636-4.228006-2.289966$ H -4.809256 $-5.274721-0.874706$ H $-5.033372-3.2203390 .599881$ H $-7.041894-4.6245840 .018130$ H $-7.257761-3.706981-1.457760$ H $-9.679414-2.1913420 .395794$ H -7.844629-1.156655 -1.270315 H $-7.933130-3.7270852 .155314$ H -8.297845 -2.029284 2.351200 H $-6.610807-2.5549252 .191623$ H $-7.642045-0.0513041 .320228$ H $-6.1202200 .273636-2.133894$ H $-6.264233 \quad 1.235887-0.658402$ H $-4.491603-0.4275730 .938666$ H $1.7583381 .049444-3.545226$ H $-0.6689071 .560804-1.724046$ H $2.3791943 .369688-3.046309$ H $1.5241024 .780639-3.625372$ H $1.4040143 .278603-4.533927$ H -3.592002 -0.014024 -1.508690 H $-4.1651551 .490489-2.203148$ H -3.180490 3.3930261 .710239 H -4.8061804 .0962081 .574922$ H $-4.0323605 .589350-0.328066$ H $-1.427598 \quad 4.986818 \quad 1.169101$ H $-1.210853 \quad 7.4222890 .530651$ H -2.348406 $7.225573-0.783112$ H $1.6174496 .542856-1.653396$ H $1.0745297 .346044-0.075158$ H $-0.1774895 .927059-3.270274$ H - $-1.8200675 .823892-2.736905$ H -1.509866 $3.664760-1.667907$ H $-4.9995383 .160681-0.663976$ C $4.247749-3.991329-0.946224$ O $4.480831-2.572018-1.063557$ C $5.824114-2.287738-0.642656$ C $6.581443-3.590788-0.841454$ C $5.539960-4.594916-0.383697$ H $5.780447-2.0636750 .430023$ C $6.380801-1.071849-1.388091$ H $6.830449-3.760140-1.895271$

H $7.505083-3.632771-0.256864$

C $5.808737-6.012721-0.857664$

H $5.510536-4.5908090 .714501$

C $5.4603140 .166554-1.373328$

O $6.609166-1.413634-2.760870$

H $7.364013-0.813448-0.978262$

H $4.6184590 .016426-2.060940$

O $6.2072991 .273768-1.892264$

C $4.9122260 .550958 \quad 0.011774$

C $4.1092991 .864765-0.016833$

H $4.270308-0.2569470 .376987$

$\begin{array}{lllll}\text { O } & 5.986709 & 0.684881 & 0.941379\end{array}$

H $4.7902182 .714582-0.146869$

H $3.4254551 .833986-0.873793$

H $5.019606-6.691422-0.518862$

H $5.855704-6.068522-1.950433$

H $6.762325-6.376569-0.461317$

C $3.043248-4.228532-0.032642$

H $5.763178-1.717995-3.132366$
H $6.5312561 .016890-2.772725$

H 6.5902381 .3652710 .596744

H $3.213161-3.7515360 .939715$

H $2.913190-5.3006920 .153833$

H $4.046188-4.364815-1.958019$

C 1.4444841 .0411912 .458427

O $2.296626 \quad 1.0469151 .307830$

C 3.2954662 .0726631 .275098

C 2.6427343 .4631201 .326399

C 1.6712533 .5954762 .503440

$\begin{array}{llll}\text { C } & 0.726021 & 2.392058 & 2.604689\end{array}$

$\begin{array}{llll}\text { H } & 0.688671 & 0.276162 & 2.240377\end{array}$

C 2.1921280 .6089953 .724320

H 3.9647821 .9534812 .133872

$\begin{array}{lllll}\text { O } & 1.944156 & 3.724968 & 0.112764\end{array}$

H 3.4313894 .2199571 .409317

H 1.0820024 .5121512 .375625

$\begin{array}{lllll} & & 2.426263 & 3.728246 & 3.703366\end{array}$

H $0.1742042 .421660 \quad 3.551292$

O $-0.2348622 .505967 \quad 1.551775$

C $2.736639-0.7930963 .578124$

H 3.0183961 .2812343 .971543

H 1.5227690 .6091314 .592429

H $1.2547603 .046124 \quad 0.017489$

H 1.7961953 .8434334 .435014

H -0.8698121 .7758491 .649359$

O $4.099984-0.7625753 .572066$

O $2.035861-1.791557 \quad 3.474915$

C $4.747047-2.0316643 .445939$

H $5.827057-1.8629423 .431886$

H $4.500645-2.6685284 .300647$

H $4.456702-2.5133562 .509217$

SCF Energy (B3LYP/6-31G**//MMFF) $=-3245.94068982$

04_00145

MM̄FF Geometry

C -1.413603 $4.827616-0.929500$

C $-2.5805814 .183249-1.078856$

C -3.5746093 .9926530 .036455$

O -3.4936862 .6095520 .459108$

C $-4.9993324 .262896-0.469630$

C $-6.040447 \quad 4.160070 \quad 0.626149$

C $-6.853172 \quad 3.100453 \quad 0.806277$

C $-6.164116 \quad 5.371045 \quad 1.513724$

C -6.8620651 .8151940 .024261$

C -7.0283280 .5993620 .947062$

C $-7.080191-0.7097530 .146640$

C $-7.521686-1.8714461 .037880$

C $-7.525604-3.2019920 .271865$

O $-5.772366-0.969942-0.378404$

$\begin{array}{llll}0 & -7.645782 & -4.263133 & 1.231285\end{array}$

C $-6.162446-3.374336-0.426053$

C $-8.733179-3.324199-0.664737$

O $-6.164058-4.521840-1.272807$

C $-5.702459-2.127734-1.222454$

C $-4.235803-2.327126-1.670588$

O $-6.522489-1.978811-2.383113$

C -2.5516652 .3113401 .395632$

$\begin{array}{lllll}\text { O } & -1.803652 & 3.095154 & 1.959701\end{array}$

C -2.551585 0.8458701 .599626

$\begin{array}{llll}C & -1.488062 & 0.288324 & 2.197470\end{array}$

C $-1.279564-1.1339112 .474034$

C $-2.429738-2.0724572 .231444$

C $-3.544985-1.065733-2.203693$

C $-2.133908-1.398031-2.652637$

C $0.107050-2.039023-1.768168$

O $-1.834745-1.468449-3.842485$

C $0.476535-2.988586-0.673758$

C $1.458974-2.7700650 .213497$

C $1.838847-3.7552901 .282110$

C $1.777078-3.1738192 .676572$

C $2.896887-2.9711413 .391613$

C $0.411561-2.9013323 .276299$

C $-0.076865-1.5180922 .949701$

$\mathrm{N}-1.277668-1.645294-1.591433$

H $-1.166126 \quad 5.2737010 .030474$

H -2.804092 $3.706299-2.030628$

H -3.3534804 .6580290 .880389$ 
H $-5.242603 \quad 3.574942-1.288207$ H $-5.0556905 .267441-0.910307$ H $-7.599023 \quad 3.159511 \quad 1.598118$ H -6.9611535 .2624912 .257115$ H -5.230546 5.5467962 .056910 H $-6.391451 \quad 6.2591610 .915080$ H $-7.7025181 .851089-0.679218$ H $-5.9490661 .691051-0.564791$ H $-6.1910440 .568703 \quad 1.656415$ H $-7.9525090 .711668 \quad 1.527686$ H $-7.785126-0.580431-0.682949$ H -8.510822 -1.6780261 .471271$ H $-6.832400-1.9607471 .889437$ H -8.470280 -4.125510 1.728490 H -5.420294 -3.5721610 .360558$ H $-9.667799-3.246794-0.096384$ H -8.758534 -4.306482 -1.149541 H -8.754186 -2.556374 -1.440784 H -6.441821 -5.280804 -0.731903 H -3.651244 -2.695896-0.819863 H -4.188703 -3.105319-2.443765 H -6.025713 -1.492497 -3.060012 H -3.3975650 .2745501 .236768$ H -0.6673730 .9390512 .499335$ H -3.323240 -1.732643 2.767537 H $-2.237900-3.0918362 .573128$ H -2.665063 -2.126667 1.166172 H $-4.083538-0.646382-3.059668$ H $-3.495718-0.283897-1.437623$ H $\quad 0.715572-1.131434-1.754223$ H $0.234379-2.520481-2.742916$ H $-0.087136-3.917558-0.622828$ H $2.039723-1.8522990 .154104$ H $2.855418-4.1057501 .061415$ H $1.202618-4.6484761 .243396$ H $2.857260-2.5773464 .401851$ H $3.877452-3.1882572 .982089$ H $0.465881-2.9870044 .369402$ H $-0.277714-3.6853412 .950765$ H $0.667211-0.743623 \quad 3.137011$ H -1.577645 -1.382676 -0.657470 C $0.7765913 .916439-1.831297$ O $1.4648534 .144039-0.585685$ C 1.6859342 .8717120 .058623 C $1.4728831 .818371-1.018995$ C $0.3702812 .440922-1.846802$ H $\quad 0.9169552 .7925650 .836825$ C 3.0868562 .8407300 .681337 H $2.3787571 .685628-1.623296$ H $1.1939870 .848331-0.598824$ C $0.2454001 .862242-3.245475$ H $-0.5733112 .279573-1.314559$ C 3.4157071 .4934321 .348544 O 3.1723153 .8727991 .667891 H $3.8268463 .084456-0.088387$ H 3.3051450 .6729480 .631421 O 2.4398551 .2558322 .371655 C 4.8099581 .4242531 .994799 C 5.9912041 .7032521 .044028 H 4.8561312 .1225542 .839190 O 4.9534010 .1179302 .562116 H 6.0059322 .7750940 .814222 H 6.9191531 .4888641 .587003 H $-0.5611492 .353887-3.798569$ H $\quad 1.171714 \quad 1.986845-3.816141$ H $0.0213570 .792873-3.203300$ C $-0.3702664 .919868-2.007437$ H 2.9247154 .7082601 .235637 H $2.676267 \quad 0.4178762 .805117$ H $5.7845980 .108403 \quad 3.066485$ H $-0.8270254 .784527-2.995043$ H $0.0344815 .939185-1.983305$ H $1.5169504 .106668-2.619374$ C $7.008853-1.1615570 .438133$ O $5.845814-0.506065-0.073725$ C $5.9815770 .901135-0.279982$

C $7.1680131 .196714-1.212134$ C $8.4544390 .521732-0.743403$
C $8.210418-0.965584-0.490329$

H $7.251755-0.7425101 .422190$

C $6.658604-2.6332710 .686683$

H $5.0832741 .209279-0.827279$

O $7.3905312 .597873-1.329925$

H $6.9345030 .824649-2.218437$

H 8.8647201 .0104390 .148061

O $9.4480950 .644089-1.763489$

H $8.049501-1.470559-1.450463$

O $9.367255-1.5406390 .110819$

C $6.310327-3.389241-0.574401$

H $7.488603-3.1565461 .175272$

H $5.801797-2.7112481 .367119$

H $6.5656382 .998450-1.654025$

H $9.5629081 .593273-1.942410$

H $10.117525-1.372488-0.484971$

O $5.014775-3.146755-0.924292$

O $7.100091-4.102566-1.179670$

C $4.567308-3.793953-2.119202$

H $4.578670-4.879574-1.985350$

H $3.540540-3.474526-2.315628$

H $5.192548-3.501626-2.968385$

SCF Energy (B3LYP/6-31G**//MMFF) $=-3245.90899081$

0400146

MM̄FF Geometry

C 2.6650353 .8329151 .341873

C 2.9763444 .5118200 .226430

C $3.6222703 .936438-1.013431$

O $3.3961642 .510660-1.116916$

C $5.1376474 .198452-0.986391$

C $5.8306563 .847282-2.289352$

C $6.5936172 .754223-2.490562$

C $5.6514404 .851322-3.400004$

C $6.8941911 .644935-1.521517$

C $6.4039780 .295832-2.062879$

C $6.589593-0.837872-1.043292$

C $6.120308-2.168209-1.633052$

C $6.156406-3.307302-0.603714$

O $5.815843-0.5145350 .114887$

O $5.396765-4.403670-1.137594$

C $5.440813-2.8482190 .686323$

C $7.581371-3.832053-0.389144$

O $5.599472-3.8376061 .700643$

C $5.910362-1.4644721 .185490$

C $5.063753-0.9081312 .356639$

O $7.258473-1.5733891 .658405$

C $2.1718152 .120255-1.573539$

O $1.2364072 .846710-1.872431$

C $2.1581610 .642329-1.652576$

C $1.0279190 .014555-2.011577$

C $0.845943-1.429192-2.180999$

C $2.030424-2.319352-1.918237$

C $3.584542-0.6606472 .044056$

C $2.661303-1.8072532 .416571$

C $0.268099-2.4920472 .257935$

O $3.030130-2.7928713 .048457$

C $0.089424-3.4134341 .093743$

C $-1.071595-3.5558190 .436741$

C $-1.282413-4.523629-0.690246$

C $-1.785368-3.857571-1.950059$

C $-3.094819-3.848162-2.251802$

C $-0.760478-3.297575-2.908769$

C $-0.360164-1.885614-2.579678$

N $1.360669-1.5770841 .986808$

H 2.9288112 .7821131 .416927

H 2.7299985 .5703410 .171426

H $3.1786834 .426267-1.889551$

H $5.5872123 .654654-0.148883$

H $5.3300175 .259479-0.775777$

H $7.0595712 .629491-3.467464$

H $6.2215264 .586672-4.296902$

H $4.5988784 .922350-3.690043$

H $5.9914645 .840466-3.075960$

H $7.9791301 .603029-1.367626$

H $6.4490851 .831891-0.541920$

H $5.3403920 .384240-2.317471$

H $6.9407080 .052191-2.988303$ 
H $7.649371-0.890170-0.767593$ H $6.712279-2.430729-2.518896$ H $5.087694-2.060419-1.988858$ H $5.795015-4.659273-1.987194$ H $4.370653-2.8064910 .450635$ H $7.994371-4.213802$-1.330658 H $7.590847-4.6790280 .305754$ H $8.267464-3.069208-0.015528$ H $5.004732-3.6104232 .434590$ H $5.192249-1.5287333 .251309$ H $5.490914 \quad 0.0695742 .624271$ H $7.436405-0.8324742 .261225$ H $3.0774190 .114544-1.430062$ H $0.1455340 .618236-2.221800$ H $2.805289-2.150436-2.672982$ H $1.787819-3.384079-1.931701$ H $2.452747-2.120758-0.927888$ H $3.438256-0.4014210 .991431$ H 3.2538620 .1977062 .641059 H -0.624235 -1.887595 2.447906 H $\quad 0.490967-3.073738 \quad 3.158120$ H $0.946149-4.0149770 .799451$ H -1.934771 -2.9746950 .752779$ H $-2.009032-5.273940-0.351304$ H $-0.366745-5.085366-0.913028$ H -3.460191 -3.401324 -3.170531 H -3.830618 -4.281021-1.582294 H - $-1.176561-3.283475-3.925754$ H $\quad 0.092042-3.979547-2.976021$ H -1.160101-1.161586 -2.741435 H $1.160933-0.7567281 .421493$ C 0.6271103 .7423282 .861267 O -0.1994283 .6114831 .684962$ C -0.6257112 .2356521 .581060$ C $-0.510474 \quad 1.6820962 .991119$ C 0.7728362 .3396543 .455832 H $\quad 0.096747 \quad 1.743990 \quad 0.916615$ C -2.0273712 .1786600 .966075$ H -1.356688 2.0085323 .608363 H -0.4622890 .5908293 .019531$ C 0.9549222 .3252124 .963508 H 1.6156091 .8106452 .999008 C $-2.623103 \quad 0.7576380 .944184$ O $-1.9306582 .675549-0.371542$ H $-2.7033602 .853647 \quad 1.504353$ H -2.6975150 .3952581 .975756$ O $-1.726478-0.1197830 .263881$ C $-4.023627 \quad 0.6574070 .305115$ C -4.586503 $-0.773590 \quad 0.408623$ H $-4.698621 \quad 1.3584250 .809489$ O $-3.9804211 .010503-1.075596$ H -3.959404 -1.458574 -0.174350 H -4.547232 -1.0839061 .459875$ H 1.8841452 .8290585 .247436 H $\quad 0.1274082 .8297795 .473289$ H 1.0004741 .2961525 .334971 C 1.9429814 .4491242 .510400 H -1.532943 $3.562005-0.325021$ H -1.692252 $0.168009-0.664430$ H $-3.6860001 .935104-1.133963$ H 2.6080734 .4530443 .381271 H 1.7141965 .4976382 .280656 H $\quad 0.068370 \quad 4.3830473 .555698$ C $-8.239044 \quad 0.007270 \quad 0.367806$ O $-6.855987-0.0711450 .735319$ C $-6.033432-0.880980-0.109946$ C $-6.539530-2.331414-0.114054$ C -8.019462 -2.398081 -0.504807 C $-8.865967-1.3954520 .289170$ H -8.7202640 .5412031 .197569$ C $-8.4446810 .841785-0.906185$ H $-6.062815-0.486876-1.131143$ O $-6.358444-2.904618 \quad 1.178941$ H -5.937194 -2.913357 -0.821293 H -8.391036 -3.414710 -0.327696 O $-8.132828-2.134414-1.900197$ H -9.873884 -1.319144 -0.134760 O $-9.012991-1.8724861 .630069$
C $-8.0047992 .280587-0.727636$

H $-7.8835960 .433470-1.750645$

H $-9.5095640 .855208-1.165566$

H -6.654339 -3.829611 1.134141

H $-9.073445-2.203506-2.136487$

H $-9.453497-2.7381941 .586802$

O $-8.1282622 .930714-1.923331$

O -7.6103212 .7768200 .318363$

C $-7.7414194 .308520-1.916234$

H $-8.3726224 .875553-1.225342$

H $-6.6860144 .404350-1.643818$

H -7.880652 4.705987-2.925164

SCF Energy (B3LYP/6-31G**//MMFF) $=-3245.91534015$

0400147

MM̄FF Geometry

C $0.652450-3.6137781 .784482$

C $0.254866-2.3487911 .583107$

C $0.141232-1.3182862 .677641$

O $-1.084883-0.5805792 .473654$

C $1.309440-0.3296052 .535624$

C 1.4835030 .6036853 .716514

C 1.1040731 .8968533 .723137

C 2.2016020 .0149904 .901928

C 0.3428162 .6106882 .642613

C 1.2495593 .1696661 .538192

C 0.4769213 .7532900 .342361

C -0.2123415 .0733510 .689498$

C -1.039408 $5.609694-0.487942$

O $-0.4806922 .779310-0.079992$

O $-1.876195 \quad 6.6657360 .008416$

C -1.971502 $4.484942-0.982424$

C $-0.1543166 .231394-1.574026$

O $-2.6876874 .894820-2.146276$

C $-1.2536303 .136075-1.229856$

C $-2.3117382 .025381-1.439616$

O $-0.4369843 .248090-2.394827$

C $-2.247834-1.1531582 .880797$

O $-2.380319-2.2045833 .485241$

C $-3.348350-0.2838192 .405524$

C $-4.609593-0.7384282 .435132$

C $-5.796588-0.0326431 .945818$

C $-5.5991891 .336193 \quad 1.350710$

C $-1.7284770 .621955-1.644873$

C $-2.752774-0.484121-1.454371$

C $-4.919765-1.350864-2.347069$

O $-2.556805-1.405553-0.664133$

C $-6.218322-0.712478-1.962984$

C $-7.171749-1.348556-1.264115$

C $-8.503861-0.738498-0.928977$

C $-8.939507-1.0020620 .496199$

C $-9.887945-1.9139400 .770569$

C $-8.341452-0.1493641 .592963$

C $-6.992698-0.6508022 .034200$

N -3.857674 -0.362420 -2.278560

H $0.886214-3.9212422 .802366$

H $0.025149-1.9991540 .581136$

H $0.138391-1.7792083 .672777$

H $1.182051 \quad 0.246463 \quad 1.611497$

H $2.251503-0.8789982 .410603$

H 1.3221962 .4995994 .603006

H $2.337698 \quad 0.7429645 .708635$

H $1.638036-0.8287455 .311803$

H $3.194494-0.3400554 .607115$

H -0.4112961 .9373832 .223463$

H -0.2203683 .4254643 .112080$

H 1.9326343 .9228131 .949735

H 1.8766372 .3555051 .155223

H $1.1998363 .911095-0.467109$

H 0.5213505 .8203071 .017190

H -0.8922994 .9295641 .539290$

H -1.300362 7.3506930 .389468

H -2.730076 $4.334962-0.201480$

H $\quad 0.4507427 .044574-1.155224$

H $-0.7606006 .688272-2.363976$

H $0.5292755 .514184-2.033129$

H -2.076716 $4.847800-2.901174$

H $-2.9681181 .995212-0.561546$ 
H $-2.9353772 .274213-2.306615$ H $-0.2227812 .357396-2.714309$ H -3.084198 0.6880352 .003740 H -4.792258 -1.7339492 .838347$ H -6.5236421 .8064391 .010991$ H -4.9420391 .2793800 .477419$ H -5.1528292 .0158392 .085001$ H -1.311092 $0.520231-2.651754$ H $-0.9174330 .435729-0.931764$ H -4.679693 -2.210855 -1.714051 H -4.968880 -1.683752 -3.388665 H $-6.4014940 .304126-2.303334$ H -7.005896 -2.379807 -0.960265 H -9.242194 -1.158722 -1.624592 H $-8.5033750 .343425-1.110830$ H $-10.226353-2.0887911 .786615$ H -10.343108 -2.510177 -0.013227 H $-8.995348-0.1619842 .475427$ H -8.332084 0.8944521 .269248 H -7.027755 -1.644655 2.483359 H $-3.8851760 .392840-2.956692$ C $0.558399-4.319159-0.716652$ O $1.383936-3.223502-1.147442$ C $1.723926-3.399968-2.533275$ C $1.021911-4.675235-2.994690$ C $0.870704-5.447483-1.697982$ H $2.808646-3.555058-2.567976$ C $1.355136-2.141541-3.331328$ H $\quad 0.037751-4.469527-3.430202$ H $\quad 1.605380-5.214342-3.747734$ C $-0.190706-6.532074-1.759830$ H $1.839087-5.903018-1.450583$ C $1.862183-0.814569-2.730712$ O $-0.073355-2.035740-3.417600$ H $\quad 1.708147-2.247083-4.363646$ H $\quad 1.300063-0.574517-1.820017$ O $1.5658850 .228191-3.670054$ C $3.368054-0.771078-2.420036$ C $3.8204700 .622405-1.942649$ H $3.598434-1.510812-1.649651$ O $4.109942-1.133457-3.583792$ H $3.6880861 .338429-2.762197$ H $3.1695330 .942720-1.121796$ H $-0.261930-7.064944-0.806579$ H -1.177456 -6.114791 -1.987126 H $0.052230-7.263949-2.537099$ C $0.871014-4.679348 \quad 0.740043$ H $-0.421646-2.027837-2.509321$ H $\quad 0.621717 \quad 0.160917-3.891852$ H $3.880958-0.501899-4.287245$ H $1.925220-4.9749120 .811733$ H $\quad 0.273233-5.551463 \quad 1.029781$ H $-0.487844-4.000387-0.813423$ C 5.0159350 .0121640 .811671

O $5.586458-0.289027-0.468114$

C $5.3006850 .660506-1.502070$

C $5.8047892 .050724-1.086040$

C 5.2480262 .4786510 .269918

C $5.490249 \quad 1.385617 \quad 1.308787$

H $3.922408-0.0049380 .738588$

C $5.457816-1.0899171 .788275$

H $5.896314 \quad 0.353112-2.370864$

O $5.5008463 .027121-2.076734$

H $6.8993362 .017869-1.012597$

H 4.1841992 .7330180 .205661

O $5.918703 \quad 3.6649900 .700172$

H 6.5618741 .3499261 .542054

O 4.8162881 .7285462 .518011

C $4.790944-2.4213391 .517011$

H $6.541285-1.2385321 .706067$

H $5.205932-0.8132142 .817892$

H $4.5541663 .239072-2.016260$

H 5.7975224 .3346840 .005351

H 3.8627931 .7535502 .331429

O $5.352182-3.3672092 .327808$

O $3.885718-2.6106720 .717649$

C $4.812337-4.6866652 .203862$

H $4.833984-5.0178501 .161106$
H $\quad 5.431230-5.3640512 .798174$

H $3.791652-4.7079072 .596202$

SCF Energy (B3LYP/6-31G**//MMFF)= -3245.91730982

04 00148

MM̄MF Geometry

C 1.4141641 .6670943 .722625

C 1.6004310 .7905962 .724018

C $1.728716-0.6957152 .932253$

O $2.978826-1.0767912 .315082$

C $0.581396-1.4330032 .222345$

C $0.401171-2.8584172 .701324$

C $0.804049-3.9548702 .030185$

C $-0.326994-3.0020984 .013456$

C $1.573770-4.0234950 .740716$

C $0.749118-4.606119-0.415452$

C $-0.317962-3.640736-0.953875$

C $-1.125542-4.298913-2.075056$

C $-2.123061-3.321546-2.716511$

O $0.356299-2.484728-1.459195$

O $-2.587212-3.904514-3.943448$

C - $-1.383524-2.017949-3.085447$

C $-3.362119-3.115371-1.839267$

O $-2.299611-1.022718-3.537809$

C $-0.513967-1.452591-1.938437$

C $0.367540-0.296152-2.467041$

O $-1.367723-0.959522-0.905675$

C $3.774291-1.9768722 .943149$

O $3.547936-2.5417564 .003997$

C $5.034398-2.1918162 .191733$

C $5.238197-1.7336210 .946410$

C $6.467554-1.8636710 .161031$

C $7.651540-2.5265250 .813668$

C $1.4304870 .190486-1.472279$

C $2.3042611 .284842-2.059688$

C $4.3388112 .629436-1.535566$

O $2.0997351 .792728-3.158984$

C $5.4203351 .976581-2.338184$

C $6.6919661 .853189-1.928444$

C $7.7640001 .186255-2.743706$

C $8.382500-0.003984-2.041791$

C $9.6011690 .084211-1.482448$

C $7.631123-1.318431-2.063331$

C $6.488360-1.350270-1.086822$

N $3.3360621 .635519-1.198610$

H $1.349479 \quad 1.2803544 .738368$

H $1.672725 \quad 1.1397381 .698746$

H $1.744610-0.9196694 .005521$

H $0.745886-1.4139301 .140140$

H $-0.368189-0.9049292 .384668$

H $0.590625-4.9288652 .469270$

H -0.504194 -4.049823 4.278662

H $\quad 0.250548-2.5504954 .825720$

H -1.301695 -2.506192 3.964424

H $1.999434-3.0563110 .458678$

H $2.434788-4.6818220 .914493$

H $1.449211-4.847725-1.226069$

H $\quad 0.279949-5.547133-0.102398$

H $-0.979620-3.345332-0.132518$

H -1.645393 -5.192153 -1.706645

H $-0.439802-4.653342-2.857604$

H -2.992309 -4.763358 -3.732669

H $-0.726774-2.244768-3.937065$

H $-3.873703-4.069434-1.664401$

H -4.095191 -2.467941-2.332785

H $-3.121424-2.686779-0.866174$

H -2.812211 -1.411373 -4.267154

H $\quad 0.891543-0.629945-3.372605$

H $-0.2652900 .553104-2.752490$

H $-0.876098-0.323646-0.363911$

H $5.793320-2.7526342 .727624$

H $4.434323-1.1960750 .446094$

H $7.922405-2.0071041 .739741$

H $8.547087-2.5301740 .188460$

H $7.423711-3.5716351 .050226$

H $0.9583430 .594955-0.572343$

H $2.081601-0.640507-1.177803$

H $4.7126203 .049889-0.596728$ 
H $3.8748223 .431353-2.118859$

H $5.1386071 .584696-3.312939$

H $6.9822422 .253069-0.959686$

H $8.5325101 .939574-2.960657$

H $7.3822080 .866869-3.721497$

H $10.066986-0.770471-1.003570$

H $10.1591341 .014737-1.487125$

H $8.313328-2.158789-1.893176$

H $7.234956-1.491084-3.072384$

H $5.577462-0.891191-1.471308$

H $3.4679461 .103706-0.343138$

C 1.3403983 .7824262 .216991

O $\quad 0.190244 \quad 3.370917 \quad 1.452604$

C -0.3625254 .5154810 .778388$

C 0.6894355 .6123310 .883497

C 1.2972295 .3119972 .239127

H $-1.2468004 .818701 \quad 1.351857$

C $-0.7765054 .150383-0.650612$

H 1.4520835 .5246750 .101116

H $\quad 0.253253 \quad 6.613757 \quad 0.818963$

C 2.6516495 .9654242 .454174

H 0.6044925 .6579463 .018035

C -1.683712 $2.907472-0.748605$

O $\quad 0.4066703 .879495-1.411759$

H -1.254723 5.015717-1.124531

$\mathrm{H}-1.1073982 .014286-0.484893$

O $-2.0473092 .741019-2.125200$

C -2.9596542 .9599460 .112818$

C $-3.8534011 .721197-0.100993$

H -2.676449 $2.998051 \quad 1.170289$

O $-3.6726874 .158362-0.168056$

H -4.139271 $1.647942-1.155996$

H -3.2440240 .8377610 .124244$

H 3.0558095 .7123823 .439231

H 3.3771355 .6429391 .699473

H 2.5666267 .0553632 .394094

C 1.2764443 .1644413 .618048

H $0.1260313 .635531-2.310466$

H $-2.5281793 .538103-2.405198$

H -4.065648 4.072558-1.053040

H 0.3140483 .4251944 .077360

H 2.0627013 .6055984 .241966

H 2.2315453 .4358961 .678214

C $-5.654602-0.6302430 .441758$

O

C $-5.128348 \quad 1.7052800 .776262$

C -6.4298572 .1344970 .077098$

C $-6.9185021 .087274-0.924249$

C $-6.967845-0.295887-0.277489$

H $-4.846711-0.751068-0.290296$

C $-5.787239-1.9385591 .235686$

H -4.9630082 .3885341 .618162$

O $-6.2580563 .384209-0.577294$

H $-7.2050332 .276040 \quad 0.841880$

H $-6.2979331 .074848-1.827915$

O $-8.2418481 .420588-1.350847$

H -7.804231 -0.3284430 .432065$

O $-7.237797-1.271979-1.282682$

C -4.445592 -2.482783 1.680111

H $-6.394722-1.7893442 .135395$

H $-6.259848-2.7025780 .607713$

H -7.132663 $3.686471-0.874104$

H -8.185366 $2.240232-1.870061$

H -6.498176 -1.263168 -1.914215

O $-4.560677-3.828577 \quad 1.885286$

O $-3.434200-1.8152531 .847301$

C -3.368291 -4.499337 2.302828

H $-3.557719-5.5757302 .278731$

H $-2.540487-4.2769701 .623509$

H $-3.120637-4.2091443 .327406$

SCF Energy (B3LYP/6-31G**//MMFF) $=-3245.90603304$

04_00149

MM̄FF Geometry

C $0.708822-2.8458690 .664871$

C $0.893214-3.170451-0.625517$

C $-0.044341-2.836954-1.765094$

O $-0.948468-1.786074-1.358089$
C $-0.874154-4.071207-2.151717$

C $-1.515925-3.937765-3.518344$

C $-2.785288-3.546152-3.742025$

C $-0.629246-4.317377-4.677160$

C $-3.808604-3.109736-2.730948$

C $-4.315995-1.698383-3.054068$

C $-5.346065-1.201546-2.030486$

C $-5.8679330 .180408-2.431992$

C $-6.8257890 .759369-1.381204$

O $-4.709712-1.131226-0.750357$

O $-7.0348422 .147010-1.684017$

C -6.1633630 .6631380 .008707$

C $-8.2123540 .112928-1.465993$

O $\quad-7.0735601 .0525081 .034786$

C $-5.576507-0.735100 \quad 0.319935$

C $-4.739503-0.662311 \quad 1.617243$

O $-6.645627-1.6638220 .504898$

C $-0.716959-0.526707-1.813967$

O $0.080763-0.179592-2.669131$

C $-1.5782760 .381239-1.027302$

C $-1.5841971 .696733-1.281806$

C $-2.3150292 .686769-0.495927$

C $-3.0201353 .720686-1.326315$

C $-3.918641-1.9215451 .928272$

C $-3.143674-1.7084643 .213917$

C $-1.257867-0.357774 \quad 4.126564$

O $-3.551199-2.1331604 .292463$

C $-1.794926 \quad 1.024428 \quad 4.337489$

C -1.1160102 .1440454 .046137$

C -1.7048523 .5218144 .148218$

C -1.8527964 .1880272 .795180$

C -1.138288 5.2801952 .475951

C -2.8819793 .6252041 .832781$

C -2.2699632 .6618630 .851489$

N -1.999672 $-0.948073 \quad 3.027117$

H $-0.204679-2.3261940 .945517$

H $1.787775-3.712684-0.919900$

H $\quad 0.568156-2.509532-2.613595$

H $-1.636241-4.265798-1.388021$

H $-0.239818-4.968092-2.163476$

H $-3.137223-3.522966-4.773069$

H -1.137559-4.216320 -5.641975

H $\quad 0.259377-3.679049-4.705730$

H $-0.306432-5.359303-4.582360$

H -4.647375 -3.815349 -2.760699

H $-3.411449-3.134351-1.713062$

H -3.461338 -1.010816 -3.075071

H $-4.763779-1.689533-4.056025$

H -6.167459 -1.926300 -1.986694

H $-6.3534260 .144957-3.415297$

H $-5.0175930 .867660-2.544356$

H $-6.1727692 .596419-1.663448$

H $-5.342146 \quad 1.3925010 .032253$

H -8.654969 $0.289122-2.453929$

H $-8.9038180 .562741-0.744811$

H $-8.195011-0.966061-1.299478$

H -7.410662 1.9351630 .804061

H -4.0444810 .1832661 .544079$

H -5.398193 -0.4577992 .471460$

H -6.334776 -2.401645 1.051988

H $-2.156808-0.046112-0.216164$

H -1.009106 2.097395 -2.112916

H -3.663139 $3.237487-2.071110$

H $-2.2934834 .347361-1.855082$

H $-3.6638824 .380634-0.740874$

H -4.557169 -2.800589 2.060540

H -3.216024 -2.150626 1.119045

H $-0.201319-0.3557203 .844318$

H -1.382474 -0.9561035 .034050$

H $-2.816096 \quad 1.102444 \quad 4.703844$

H -0.0980322 .0702183 .673351$

H -1.057605 4.116607 4.804997

H $-2.686247 \quad 3.497808 \quad 4.638478$

H -1.259135 5.7702361 .515267

H -0.4147945 .7047323 .163944$

H $-3.3879874 .455076 \quad 1.330029$

H -3.666420 3.1041302 .395599

H -1.7109771 .8527001 .319621$ 
H $-1.790392-0.6044872 .094489$

C $3.061869-3.5560531 .480248$

O $3.730221-2.5148810 .741697$

C $5.092465-2.4175861 .196021$

C $5.318964-3.604606 \quad 2.126240$

C $3.933867-3.8001962 .711663$

H $5.162882-1.4953191 .783968$

C $6.048687-2.3424060 .001830$

H $5.629014-4.5008801 .576698$

H $6.080835-3.3967832 .883772$

C $3.733974-5.1608153 .355794$

H $3.756160-3.0169283 .460778$

C $5.720448-1.221460-1.006429$

O $5.990813-3.587878-0.706147$

H $7.078348-2.2421740 .365236$

H $4.790779-1.462264-1.535826$

O $6.745960-1.228490-2.008742$

C $5.6064340 .190503-0.399002$

C $5.4385741 .280608-1.475474$

H 4.7456140 .2179610 .276723

$\begin{array}{lllll} & \text { O } & 6.762711 & 0.472689 & 0.387860\end{array}$

H $6.3183401 .281685-2.130273$

H $4.5740341 .025622-2.098108$

H $2.719814-5.2589723 .755513$

H $3.893030-5.9723202 .637726$

H $4.437512-5.3015744 .182986$

C $1.637640-3.1112461 .821948$

H $6.602442-3.521928-1.459610$

H $7.595736-1.058885-1.568182$

H $7.5243220 .542401-0.212196$

H $1.693034-2.1776652 .395904$

H $1.160271-3.8558612 .469158$

H $3.047696-4.4516920 .846277$

C $2.9042662 .663361-0.469554$

$\begin{array}{llllll}\text { O } & 4.224233 & 2.781113 & 0.073337\end{array}$

C $5.2823682 .700174-0.884852$

C $5.1199533 .806169-1.935792$

C $3.7359763 .781904-2.580341$

C $2.6471523 .763813-1.507182$

H $2.7810971 .674382-0.928225$

C $1.9183772 .773120 \quad 0.698315$

H $6.2076732 .915784-0.335863$

O $6.127660 \quad 3.686612-2.934368$

H $5.2650724 .779797-1.449384$

H $3.6370392 .933449-3.267516$

O $3.5981794 .963035-3.373696$

H $2.6030264 .743366-1.013973$

O $1.3824513 .533994-2.122507$

C $1.970254 \quad 1.5339161 .558179$

H $2.1332553 .647427 \quad 1.324722$

H 0.8857332 .8792710 .350514

H $5.9749324 .391120-3.587280$

H $2.7610434 .892229-3.862348$

H $1.1821724 .302572-2.682655$

O $2.876593 \quad 1.7032552 .562401$

$\begin{array}{lllll}\text { O } & 1.301267 & 0.531030 & 1.346973\end{array}$

C 3.0484520 .5865133 .439064

H 3.8775450 .8096094 .115600

H 2.1424720 .4330614 .031480

H $3.295655-0.3135182 .870499$

SCF Energy (B3LYP/6-31G**//MMFF) $=-3245.90223008$

0400150

MM̄FF Geometry

C $-3.6320443 .233406-1.023803$

C $-4.965796 \quad 3.161215-0.888891$

C $-5.775108 \quad 1.885447-0.811463$

O $-5.001338 \quad 0.811219-0.227406$

C $-6.226913 \quad 1.461202-2.220154$

C -7.170072 $0.272398-2.222301$

C $-6.837057-0.981170-2.591246$

C $-8.5865240 .579873-1.806869$

C $-5.492526-1.490939-3.032481$

C $-4.975209-2.573067-2.075365$

C $-3.520170-2.969070-2.373224$

C $-3.056247-4.053997-1.399571$

C $-1.563244-4.377209-1.555247$

O $-2.718730-1.797180-2.218837$
O $-1.152150-5.133362-0.405006$

C $-0.755707-3.060096-1.534486$

C $-1.308225-5.272164-2.773488$

O $0.616254-3.296306-1.838708$

C $-1.319849-1.973569-2.474780$

C $-0.651472-0.595498-2.274049$

O $-1.083555-2.299882-3.849251$

$\begin{array}{llll}C & -4.911406 & 0.794447 & 1.132721\end{array}$

O $\quad-5.3954331 .6034391 .909138$

C $-4.110045-0.3860351 .526017$

C $-3.784080-0.5629722 .815436$

C $-3.009813-1.6746313 .373325$

C $-2.482083-2.7088542 .415615$

C $-0.9045120 .058444-0.912610$

C $0.212359-0.1210620 .097564$

$\begin{array}{lllll}\text { C } & 0.780067 & 0.454974 & 2.460274\end{array}$

O $1.288563-0.644537-0.171793$

C $0.681920-0.8178193 .239109$

C $0.462941-0.8579144 .562009$

C $0.406804-2.1278665 .361305$

C $-0.853855-2.2435246 .188597$

C $-0.846138-1.9634557 .502763$

C -2.098954-2.769982 5.511089

C $-2.808862-1.716168 \quad 4.707437$

N $-0.1322240 .412146 \quad 1.334000$

H $-3.0587772 .316700-1.122590$

H $-5.538607 \quad 4.084183-0.829354$

H $-6.6552132 .071429-0.183233$

H $-5.346256 \quad 1.256642-2.837733$

H $-6.7350872 .299829-2.715740$

H $-7.615462-1.743336-2.570829$

H $-9.244320-0.291583-1.892501$

H -8.619162 $0.913718-0.765353$

H $-9.005183 \quad 1.369289-2.439776$

H $-5.592660-1.914099-4.039179$

H $-4.762095-0.683173-3.110017$

H $-5.040685-2.193417-1.047812$

H $-5.620797-3.458167-2.135886$

H $-3.452249-3.319543-3.409559$

H -3.661182 -4.962253 -1.513466

H $-3.226812-3.712204-0.370585$

H $-1.710898-5.927716-0.352538$

H $-0.786035-2.684846-0.504535$

H $-1.819925-6.235025-2.655028$

H $-0.243887-5.509812-2.875425$

H $-1.658400-4.830248-3.708896$

H $\quad 0.943431-3.959685-1.207329$

H $0.418195-0.650766-2.510116$

H $-1.0765330 .078888-3.032023$

H $-0.484508-3.062663-3.888817$

H $-3.812731-1.0771940 .746770$

H $-4.121790 \quad 0.175601 \quad 3.541709$

H $-1.798491-3.4246682 .876656$

H -1.917636 -2.2347201 .606180$

H -3.309507 -3.280154 1.982517

H $-0.9941131 .137567-1.071282$

H - $-1.845376-0.282057-0.472494$

H 0.5146661 .3309163 .060638

H 1.8005800 .5840792 .091358

H $0.825899-1.7484082 .695115$

H $\quad 0.3487470 .0750805 .109539$

H $1.287012-2.1457196 .017445$

H $0.501078-3.0113054 .717661$

H $-1.739241-2.0722458 .109192$

H $0.052220-1.6133848 .000385$

H $-2.809809-3.1326476 .265981$

H -1.843645 -3.6577344 .925891$

H $-3.228979-0.9164155 .319316$

H $-1.0696150 .778458 \quad 1.472441$

C -1.621858 $4.480984-0.176236$

O $-0.604525 \quad 3.682420-0.818470$

C $0.642358 \quad 4.408673-0.809085$

$\begin{array}{lllll}\text { C } & 0.467698 & 5.489713 & 0.243341\end{array}$

C $-0.990448 \quad 5.855814 \quad 0.049088$

H $0.7342534 .856551-1.807036$

C $1.7965883 .429598-0.560058$

H $\quad 0.632116 \quad 5.084559 \quad 1.249544$

H 1.1436316 .3371590 .097236 
C -1.5844026 .6210021 .219159$ H -1.082281 $6.464958-0.860304$ C $3.1605304 .109077-0.350956$ O $1.8898622 .560362-1.693727$ H 1.5583522 .7974880 .302126 H 3.1405104 .6961380 .574933 O $3.3656745 .044712-1.417861$ C $4.3696543 .151656-0.331932$ C 4.2522922 .0172920 .700152 H $4.5263832 .739850-1.335132$ O $5.5363003 .929507-0.037798$ H 4.0763732 .4544911 .690767 H 3.3810091 .4023790 .459664 H -2.641367 6.8443441 .043448 H -1.511245 6.0497182 .150805 H -1.057873 7.5697461 .364894 C -2.866254 4.528195-1.069737 H $1.0132782 .162545-1.829796$ H $4.2315755 .463680-1.273547$ H 5.4362374 .2869570 .861075 H -2.566594 4.704385 -2.109887 H -3.515887 $5.355588-0.761430$ H -1.8484313 .9980250 .783233$ C $5.027853-0.353457-1.069292$ O $5.8995080 .639405-0.520865$ C 5.5151801 .1267890 .768963 C $5.425339-0.0329211 .773179$ C $4.540180-1.1705971 .265953$ C $4.944712-1.576990-0.151021$ H $4.0271320 .079187-1.190892$ C $5.507769-0.686555-2.486619$ H 6.3359631 .7722121 .106322 O $4.943351 \quad 0.447903 \quad 3.023774$ H $6.436088-0.4253661 .946313$ H $3.480658-0.9023051 .306094$ O $4.696934-2.3102952 .114137$ H $5.908913-2.098002-0.108216$ O $3.986075-2.492441-0.674896$ C $6.869415-1.342351-2.508648$ H $4.782428-1.330954-2.997014$ H $\quad 5.590475 \quad 0.227502-3.087144$ H $5.012315-0.2779863 .666211$ H $4.319403-2.0846582 .981049$ H $3.923938-3.236424-0.051534$ O $6.748796-2.656290-2.853560$ O $7.912195-0.761854-2.235620$ C $7.972123-3.397569-2.887721$ H $7.738209-4.426760-3.172947$ H $8.652004-2.972875-3.632335$ H $8.438162-3.405089-1.897758$ SCF Energy $\left(\mathrm{B} 3 \mathrm{LYP} / 6-31 \mathrm{G}^{* *} / / \mathrm{MMFF}\right)=-3245.90801782$

04 00151

MM̄FF Geometry

C $-0.583985-0.0327012 .710035$

C $-1.787459-0.1920563 .281363$

C -2.9232780 .8026943 .332364$

O -4.0341890 .1605172 .657739$

C -2.6654972.156582 2.643675

C -3.6972713 .2148022 .981116$

C -4.6917873 .6114882 .163811$

C -3.5177303 .8786104 .322975$

C $-5.056686 \quad 3.0684070 .810584$

C $-4.7363024 .052877-0.323270$

C $-3.2434774 .090363-0.686768$

C $-2.9708185 .159685-1.747113$

C -1.509874 $5.132507-2.224170$

O $-2.8969582 .799752-1.197518$

O $-1.4073265 .956963-3.394817$

C $-1.1352453 .686824-2.616863$

C $-0.5698585 .771239-1.196240$

O $0.2524903 .584511-2.927255$

C -1.516654 2.640016-1.545390

C -1.343115 $1.212662-2.117493$

O $-0.6611622 .799617-0.412069$

C $-5.2515280 .162768 \quad 3.255079$

O

C $-6.261599-0.5487072 .435529$
C $-6.028488-1.0227381 .201216$

C $-6.983430-1.7481630 .357835$

C $-8.349287-2.0289290 .927787$

C $-1.9343500 .110045-1.228511$

C $-1.734610-1.274310-1.819959$

C $-2.346269-3.656697-1.399686$

O $-1.127547-1.480790-2.866901$

C $-3.580092-3.910502-2.206705$

C $-4.604689-4.668667-1.788684$

C $-5.853537-4.889445-2.592697$

C $-7.097691-4.391965-1.888292$

C $-7.967643-5.255491-1.337561$

C $-7.381833-2.904823-1.901317$

C $-6.588569-2.147676-0.869644$

N -2.320408 -2.253976 -1.029548

H -0.3331380 .8973832 .209922$

H -1.995587 -1.141464 3.773180

H -3.1539300 .9557314 .393556$

H -2.614458 2.0090351 .559761

H -1.687641 2.5569052 .940770

H -5.3506724 .4068202 .511007$

H -4.2567154 .6672814 .500065$

H -3.618549 3.1480255 .131278

H -2.525806 4.3375294 .390105

H -4.6012032 .0924530 .618531$

H -6.1396052 .8896300 .817690$

H -5.306106 $3.733595-1.205872$

H $-5.0864385 .057926-0.057396$

H $-2.657911 \quad 4.294370 \quad 0.216103$

H -3.234021 $6.156878-1.373002$

H $-3.6237244 .984254-2.613564$

H -2.008833 $5.601700-4.071089$

H $-1.677828 \quad 3.445807-3.541458$

H $-0.8458506 .819254-1.027532$

H $0.4629155 .789852-1.562041$

H $-0.5828555 .266381-0.228038$

H $0.4485804 .254586-3.604380$

H $-1.8429201 .150496-3.093277$

H $-0.2793301 .007631-2.284757$

H -0.5402551 .9420290 .023653$

H $-7.229259-0.6598782 .914681$

H $-5.045820-0.8747260 .756798$

H $-8.268120-2.5995091 .859672$

H -8.987106 -2.614670 0.262537

H -8.879514 -1.092409 1.133040

H $-1.4706410 .121182-0.238484$

H -3.011964 $0.266818-1.101850$

H -2.327301 -4.240166 -0.473871

H -1.455874 -3.902355 -1.987051

H -3.635222 -3.433654 -3.182752

H $-4.550012-5.151579-0.815820$

H $-5.934111-5.965748-2.793306$

H -5.784702 -4.411445 -3.577900

H $-8.878745-4.912663-0.859086$

H -7.786793 -6.325241 -1.349874

H $-8.455203-2.716410-1.787582$

H -7.141706 -2.499315 -2.892815

H $-5.579337-1.894940-1.194385$

H $-2.863103-1.972383-0.217978$

C $0.757995-1.5966621 .289252$

O $1.338965-0.5435560 .493579$

C $2.479979-1.068607-0.219653$

C $2.337610-2.579255-0.146600$

C $1.749888-2.7572791 .238689$

H $3.355770-0.739540 \quad 0.350666$

C $2.504727-0.473255-1.632217$

H $1.633334-2.942330-0.905704$

H $3.287738-3.102359-0.278486$

C $1.113909-4.1194941 .456090$

H $2.544531-2.6070341 .981664$

C $3.632250-0.972269-2.553527$

O $2.5930950 .950080-1.533330$

H $1.541904-0.684843-2.113023$

H $3.631728-0.363133-3.467068$

O $3.306495-2.305017-2.964110$

C $5.063685-0.980061-1.974315$

C $5.5415230 .393589-1.476270$

H $5.134369-1.730816-1.180203$ 
O $5.928538-1.419297-3.029245$

H $5.3883021 .132033-2.273321$

H $4.9215350 .701866-0.630429$

H $0.692783-4.1973012 .463219$

H $\quad 0.307600-4.3050290 .738367$

H $1.856812-4.9149261 .337899$

C $0.487257-1.0893682 .706089$

H $1.866867 \quad 1.247879-0.958827$

H $4.010020-2.603264-3.565818$

H $6.772064-1.685185-2.625105$

H $1.396890-0.6436583 .126923$

H $0.201642-1.9251113 .355068$

H $-0.183017-1.880156 \quad 0.804128$

C $6.820904-0.547147 \quad 1.133925$

O $7.385999-0.628565-0.179339$

C $7.0288370 .439725-1.059489$

C $7.468347 \quad 1.783073-0.459482$

C $6.9250551 .976987 \quad 0.953823$

C $7.237256 \quad 0.758992 \quad 1.821513$

H $5.728092-0.6099851 .069702$

C $7.323927-1.7594011 .925298$

H $7.6179140 .293092-1.973335$

O $7.0445642 .870286-1.275140$

H $8.5646721 .820341-0.416508$

H 5.8493442 .1883600 .947395

$\begin{array}{lllll}\text { O } & 7.551636 & 3.118016 & 1.545243\end{array}$

H 8.3110430 .7395562 .048558

O 6.5408990 .8775423 .060610

C $6.695730-3.0388631 .423831$

H $8.410646-1.8746981 .831926$

H $7.105945-1.6590932 .994810$

H $7.4135822 .729884-2.163885$

H 7.3759883 .8775560 .963360

H $6.806379 \quad 1.722444 \quad 3.462861$

O $5.721309-3.4497342 .284382$

$\begin{array}{lllll}\text { O } & 7.028674 & -3.601601 & 0.388754\end{array}$

C $5.085723-4.6877221 .951378$

H $4.255264-4.8407952 .645478$

H $4.690498-4.6601090 .932067$

H $5.795833-5.5119982 .065093$

SCF Energy (B3LYP/6-31G**//MMFF) $=-3245.91806964$

0400152

MM̄FF Geometry

C $0.180877-2.160453-0.089383$

C $1.132530-2.5837730 .757332$

C $1.651190-1.8405931 .966504$

O $1.210695-0.4649141 .927852$

C $3.189332-1.8419501 .979825$

C $3.778840-1.4720053 .326515$

C $4.360649-0.2916503 .614508$

C $3.736230-2.5620804 .367226$

C $4.4980970 .916196 \quad 2.730469$

C 5.9439601 .1414752 .264196

C 6.4018350 .1370701 .194524

C 7.8692140 .3659310 .825832

C $8.315908-0.547191-0.327276$

$\begin{array}{lllllll}\text { O } & 5.577459 & 0.330192 & 0.042008\end{array}$

O $9.591380-0.083258-0.795128$

C $7.302901-0.428465-1.487104$

C $8.559546-1.9819880 .152884$

O $7.596191-1.367263-2.519080$

C $5.830819-0.577465-1.037820$

C $4.883505-0.193287-2.199611$

O $5.589444-1.933724-0.661676$

$\begin{array}{llll}\text { C } & 0.658411 & 0.075458 & 3.041567\end{array}$

O $0.446996-0.492324 \quad 4.104294$

C $0.306810 \quad 1.501002 \quad 2.835166$

C $0.674842 \quad 2.2126121 .757791$

C $0.376575 \quad 3.625828 \quad 1.509702$

C $-0.494144 \quad 4.3454902 .506133$

C $3.401117-0.113775-1.802948$

C $2.5548900 .491890-2.909351$

C $0.338732 \quad 1.565244-3.286360$

$\begin{array}{llll}\text { O } & 2.956652 & 0.630700 & -4.061757\end{array}$

C $0.579712 \quad 3.034951-3.128260$

C $-0.3406503 .899289-2.675855$

C $-0.0913635 .368542-2.493225$
C $-0.3565345 .830401-1.076093$

C $-1.4977916 .467336-0.763180$

C $0.7330395 .636998-0.044448$

C $0.880352 \quad 4.207518 \quad 0.400736$

N $1.2908250 .848430-2.457618$

H $-0.289926-1.1918540 .053535$

H $1.549045-3.577546 \quad 0.600377$

H $1.255867-2.3573692 .848727$

H $3.555255-1.1638831 .202908$

H $3.575075-2.8344561 .710759$

H $4.777351-0.1572654 .612270$

H $4.235992-2.2698215 .296895$

H $2.702314-2.8174214 .617974$

H $4.237115-3.4618873 .995088$

H $3.8167100 .883691 \quad 1.875196$

H $4.184551 \quad 1.7873003 .320039$

H $5.9993702 .155174 \quad 1.846190$

H $\quad 6.622727 \quad 1.108636 \quad 3.125489$

H $6.263117-0.8804651 .575106$

H 8.5181530 .2269461 .699439

H $8.005344 \quad 1.4114830 .515846$

H $9.4909500 .838174-1.089132$

H $7.4271510 .568799-1.931633$

H $9.344665-1.9992480 .918561$

H $8.924397-2.615741-0.663081$

H $7.670560-2.4499340 .580896$

H $8.525422-1.236779-2.774540$

H $5.1796010 .791364-2.585402$

H $4.988200-0.911274-3.022776$

H $4.647335-2.133741-0.775413$

H -0.2587351 .9470523 .647133$

H $1.263314 \quad 1.7265310 .981458$

H -1.442134 3.8142762 .647213

H $-0.756957 \quad 5.3601992 .198865$

H 0.0131324 .4216223 .474118

H $3.004333-1.111337-1.588933$

H $3.2808130 .505052-0.906646$

H $-0.6634101 .269977-2.962437$

H $\quad 0.4710521 .276148-4.333664$

H $1.5667193 .405163-3.396311$

H $-1.3323403 .536455-2.422709$

H $-0.7438395 .906748-3.193064$

H $0.9330525 .640304-2.776683$

H $-1.686184 \quad 6.8299850 .241700$

H -2.272422 $6.634935-1.504035$

H $0.576648 \quad 6.298578 \quad 0.815244$

H $1.6899435 .968091-0.468828$

H $1.4992603 .604734-0.264201$

H $1.0758290 .755960-1.468941$

C $-1.449033-3.923256-0.810816$

O $-2.560635-3.168622-0.298542$

C $-3.781469-3.866618-0.616586$

C $-3.374475-5.148672-1.339374$

C $-2.036885-4.759658-1.943073$

H $-4.328042-3.230397-1.320508$

C $-4.617643-4.069327 \quad 0.655096$

H $-3.250723-5.988478-0.646259$

H $-4.112138-5.448413-2.090479$

C $-1.186006-5.950125-2.348074$

H -2.219585 -4.126722 -2.821990

C $-4.834121-2.7768821 .470073$

O $-3.934307-5.0001391 .505050$

H $-5.577301-4.5315460 .396128$

H $-3.872539-2.4423131 .877449$

O $-5.647348-3.0918372 .606651$

C $-5.485296-1.6263660 .683334$

C $-5.595131-0.3405571 .522241$

H $-4.878854-1.412381-0.199716$

O $-6.772199-2.0212970 .216159$

H $-6.192645-0.5332612 .421825$

H $-4.589711-0.0662421 .861505$

H $-0.233434-5.622572-2.775892$

H $-0.966222-6.596234-1.491383$

H $-1.703095-6.553999-3.100910$

C $-0.324188-2.982650-1.243558$

H $-4.473651-5.1107362 .306815$

H $-6.479921-3.4735652 .280946$

H -7.362349 -2.087505 0.985907 
H $-0.679497-2.291051-2.016706$

H $\quad 0.501259-3.554301-1.683702$ H - $1.101266-4.5743210 .003011$ C $-4.3703331 .430250-0.635081$ O C $-6.242617 \quad 0.8383420 .761213$ C -6.1678222 .1806791 .501376$ C -4.7599552 .7776531 .466598$ C -4.2154982 .7987020 .036797$ H $-3.7150020 .699543-0.144943$ C $-3.9943861 .492507-2.118415$ H -7.3050710 .5875160 .648146$ O -6.5976162 .0195672 .849056$ H -6.8609812 .8861141 .024871$ H -4.0873202 .2364552 .142276$ O $-4.8355614 .116277 \quad 1.962996$ H $-4.7394413 .568096-0.544601$ O -2.8325953 .1419910 .062132$ C -3.883682 $0.103061-2.700735$ H $-4.7387012 .064058-2.686124$ H $-3.0278451 .978795-2.275346$ H -6.5160662 .8849453 .285429$ H -3.9275584 .4566012 .030037$ H $-2.7604604 .053436 \quad 0.390873$ O $-4.939235-0.144886-3.526629$ O $-2.975753-0.674446-2.436048$ C $-4.955470-1.438572-4.137848$ H $-4.012260-1.633384-4.657362$ H $-5.146190-2.205046-3.381762$ H -5.767360 -1.458048 -4.869805

SCF Energy (B3LYP/6-31G**//MMFF) $=-3245.91359682$

04_00153

MM̄MF Geometry

C $-0.8717813 .549370-0.688532$

C $-1.9930524 .287287-0.631763$

C $-3.267356 \quad 3.9553040 .111667$

O -3.2290262 .6022620 .612877$

C $-4.4706184 .065481-0.841670$

C $-5.8008493 .826071-0.153744$

C $-6.5224742 .692694-0.262464$

C -6.3246404 .9833990 .656058$

C $-6.1450561 .445585-1.013057$

C $-6.4583530 .178887-0.202739$

C $-5.985388-1.082304-0.939105$

C $-6.475202-2.347167-0.234466$

C $-5.943869-3.616524-0.916674$

O $-4.554216-1.066083-0.958864$

O $-6.204712-4.710704-0.024988$

C $-4.414777-3.489657-1.082678$

C $-6.707201-3.923715-2.210137$

O $-3.892313-4.586584-1.829175$

C $-3.959535-2.142379-1.694098$

C $-2.424112-2.013618-1.573828$

O $-4.324174-2.103322-3.074103$

C -3.0212272 .4178841 .942975$

O $-2.864910 \quad 3.2745042 .797331$

C $-2.969840 \quad 0.9585002 .172393$

C $-2.3350800 .488503 \quad 3.255501$

C $-2.093063-0.9221553 .560476$

C $-2.812028-1.9540032 .733429$

C -1.869215 -0.633102 -1.949269

C $-0.430512-0.506159-1.488536$

C $0.928336-0.2471890 .591961$

O $0.512297-0.630206-2.265838$

C $1.035343-1.5010181 .404653$

C $1.401932-1.5186942 .695595$

C $1.485284-2.7710773 .523121$

C $0.821145-2.6328354 .878986$

C $1.553057-2.6191646 .006011$

C $-0.693605-2.5766684 .936990$

C $-1.216398-1.2221904 .541041$

N $-0.331083-0.274564-0.125441$

H $-0.8030152 .609845-0.150212$

H -2.007445 5.234325 -1.169398

H $-3.382334 \quad 4.6771170 .929161$

H -4.341913 $3.370701-1.679875$

H $-4.4957995 .063597-1.299984$
H $\quad-7.4885902 .650963 \quad 0.238767$

H $-7.3117384 .778614 \quad 1.084033$

H -5.6478035 .2094781 .485679$

H -6.4172235 .8755610 .028139$

H $-6.7140391 .421370-1.950182$

H $-5.0843451 .443556-1.277998$

H $\quad \begin{array}{llll}-5.962030 & 0.243775 & 0.774194\end{array}$

H $-7.5390880 .121280-0.022957$

H -6.364089-1.046695-1.967516

H -7.571066 -2.368512 -0.184720

H $-6.128108-2.3406710 .808579$

H -5.804999-5.508475 -0.412453

H -3.980932 -3.577886 -0.077125

H -7.776582 -4.046915 -2.000905

H $-6.378989-4.872549-2.648942$

H $-6.603208-3.141395-2.964871$

H -4.092432 -4.427733 -2.767408

H $-2.132773-2.237285-0.541417$

H - $1.933446-2.763941-2.207410$

H -3.760371 -1.457461 -3.528709

H $-3.388127 \quad 0.3121391 .409443$

H -1.896156 1.2020143 .951793

H -2.746479 -2.962368 3.148185

H $-2.402032-1.9853031 .720723$

H -3.882480 -1.726490 2.671746

H -1.900290 $-0.476640-3.032790$

H -2.448303 $0.176918-1.491533$

H 0.9257040 .6503861 .217936

H $1.754484-0.181713-0.118981$

H $0.799139-2.4396540 .908186$

H $1.671175-0.5833053 .181479$

H $2.548259-3.0130853 .649699$

H $1.040566-3.6243392 .996055$

H $1.085962-2.5416186 .982356$

H $2.635983-2.6803165 .978518$

H $-1.043149-2.7822745 .956927$

H -1.088610 -3.387074 4.318333

H $-0.769804-0.4005945 .103598$

H $-1.181892-0.2099130 .423704$

C $1.4708154 .477987-0.635300$

O $2.2030463 .381678-0.042741$

C $3.6137533 .654272-0.163878$

C $3.7299825 .115931-0.576777$

C $2.4980545 .276641-1.439437$

H $3.9864493 .032790-0.984648$

C 4.3643173 .3229041 .129313

H 3.6800935 .7868320 .289084

H $4.6614585 .323734-1.112187$

C $2.1119046 .727475-1.673632$

H $2.6786394 .790211-2.407671$

C 4.3115561 .8457861 .562388

O 3.8248534 .1097392 .199262

H 5.4115103 .6328161 .029956

H 3.3064031 .5859831 .914896

O 5.1823011 .6998602 .692754

C 4.7491440 .8403470 .480233

C $4.939656-0.5836981 .034949$

H $3.9978460 .830856-0.315213$

O $5.978327 \quad 1.262842-0.111680$

H $5.840564-0.6174951 .659955$

H $4.077825-0.8300191 .663566$

H $1.2114096 .799582-2.291208$

H $1.9151027 .247599-0.730127$

H $2.9174977 .260678-2.189132$

C $0.3280303 .948477-1.504000$

H 2.8763503 .9036902 .264263

H 4.8846762 .3305993 .370647

H 6.6404351 .3254670 .597997

H $0.6577243 .070422-2.073167$

H $0.0288374 .708612-2.235580$

H 1.0821525 .0849730 .193019

C $3.738398-2.641023-1.842385$

O $3.815011-1.722153-0.746219$

C $5.078452-1.637018-0.079714$

C $5.479528-3.0093460 .483034$

C $5.453327-4.088675-0.604022$

C $4.149438-4.057754-1.405628$

H $2.673323-2.667849-2.106245$ 
C $4.508324-2.130173-3.064755$

H $5.840800-1.298108-0.789086$

O $4.607738-3.3862301 .547385$

H $6.487109-2.9355400 .908461$

H $5.564646-5.076375-0.139840$

O $6.546806-3.898058-1.496707$

H $4.231205-4.710466-2.282643$

O $3.113131-4.595526-0.578032$

C $3.802143-0.939291-3.668426$

H $5.542106-1.848649-2.845668$

H $4.555721-2.899807-3.844466$

H $3.699246-3.3913101 .200157$

H $7.365339-3.955024-0.974863$

H $2.301089-4.634592-1.111533$

O $4.3057330 .216527-3.150497$

O $2.891368-1.031285-4.481557$

C $3.6968811 .422918-3.621632$

H $3.6354021 .430852-4.714275$

H $2.7034371 .531889-3.177118$

H $4.3200992 .263404-3.305323$

SCF Energy $\left(B 3 L Y P / 6-31 G^{* *} / / M M F F\right)=-3245.92949813$

04_00154

MMFF Geometry

C -0.2144224 .0348243 .122075$

C -0.0271792 .7705102 .715871$

C $0.496106 \quad 1.686743 \quad 3.624544$

O $1.434406 \quad 0.8837962 .870244$

C -0.6498690 .7747854 .085886$

C $-0.201589-0.2493545 .110707$

C $0.034824-1.5482064 .840308$

C $-0.070753 \quad 0.265693 \quad 6.520905$

C $-0.050479-2.2256103 .502005$

C $1.145569-3.1528023 .248692$

C $1.013047-3.8683611 .896365$

C $2.103448-4.9267391 .729673$

C $2.020122-5.6126270 .358629$

O $1.125231-2.889480 \quad 0.856665$

O $3.236941-6.3543320 .187421$

C $1.963630-4.527245-0.736212$

C $0.880541-6.6369140 .307763$

O $1.752892-5.112996-2.019274$

C $0.924677-3.411620-0.463427$

C $1.128547-2.265611-1.479971$

O $-0.390227-3.945556-0.623726$

C 2.7067851 .3504892 .759312

O 3.1684552 .3596473 .267330

C 3.4389400 .4294861 .862469

C 4.6049580 .8272021 .332337

C 5.4206810 .0787450 .374520

C $5.023124-1.3420210 .077709$

C $0.321642-0.994721-1.190963$

C $0.7493120 .101654-2.145688$

C $2.5954281 .735614-2.541966$

O $0.1426520 .315876-3.192481$

C $3.8601231 .109682-3.044719$

C $5.0626691 .701007-2.972742$

C $6.3323581 .068688-3.471013$

C $7.4588111 .122338-2.459656$

C $8.5175811 .928182-2.648572$

C $7.3962550 .188872-1.266768$

C $6.4705220 .704056-0.197695$

N $1.8937740 .761548-1.725151$

H -0.0122234 .2704734 .165868$

H -0.2423382 .4850321 .690500$

H 1.0035192 .1184234 .496594

H -1.103447 0.2803923 .220261

H -1.454946 1.3760104 .529825

H $0.310013-2.2015095 .667244$

H $0.205551-0.5236537 .228049$

H $0.698667 \quad 1.0415606 .578326$

H -1.021010 $0.691715 \quad 6.859024$

H - $0.975084-2.8145693 .478666$

H $-0.104183-1.4983572 .688375$

H $2.068903-2.5596443 .267179$

H $1.210427-3.8950954 .053803$

H $\quad 0.021277-4.3336751 .849613$

H $2.053290-5.6713922 .533789$
H $3.090012-4.4508021 .822610$

H $3.229299-6.730577-0.709641$

H $2.961360-4.068662-0.776174$

H $1.018690-7.3986761 .084541$

H $0.877212-7.180031-0.643737$

H $-0.105054-6.1893070 .451687$

H $0.811486-5.347062-2.086575$

H $2.190401-1.996395-1.503347$

H $0.871560-2.613872-2.488959$

H -1.001322 -3.216903 -0.814337

H $2.978052-0.5227291 .625984$

H 4.9827441 .8154561 .592417

H $4.914318-1.9160261 .005014$

H $5.754115-1.882734-0.526879$

H $4.072907-1.365458-0.461098$

H $-0.751348-1.161970-1.325913$

H $0.468563-0.647812-0.161961$

H $2.7889302 .609164-1.911745$

H $1.9687122 .037445-3.386465$

H $3.7815680 .122481-3.494800$

H $5.1384162 .700107-2.549477$

H $6.6257291 .595398-4.388442$

H $6.1679010 .024042-3.763820$

H $9.3401241 .955427-1.941531$

H $8.5838652 .582559-3.511362$

H $8.3926480 .082765-0.818318$

H $7.126455-0.807989-1.625023$

H $\quad 6.691016 \quad 1.7272110 .111290$

H $2.3298000 .483130-0.851306$

C $-0.4243295 .134576 \quad 0.785461$

$\begin{array}{lllll}\text { O } & -1.350723 & 4.225072 & 0.158027\end{array}$

C -1.848939 $4.825293-1.051798$

C $-0.9740576 .044376-1.314791$

C $-0.640610 \quad 6.483572 \quad 0.094571$

H -2.868441 $5.164670-0.834280$

C $-1.8694823 .811993-2.201301$

H $-0.0577315 .781000-1.855544$

H $-1.4961306 .812400-1.893645$

C 0.5553327 .4183440 .166020

H -1.517945 6.9880440 .521763

C $-2.6509192 .513428-1.920786$

O $-0.5239543 .436827-2.523811$

H $-2.2664154 .294729-3.102136$

H $-2.0860681 .880400-1.226930$

O $-2.729848 \quad 1.786141-3.154031$

C $-4.0800392 .720148-1.389587$

C $-4.9528881 .456850-1.372855$

H $-4.034743 \quad 3.135589-0.377470$

O $-4.7563883 .691667-2.193973$

H $-5.9351661 .711206-0.950156$

H $\quad-5.196250 \quad 1.163227-2.404098$

H $\quad 0.765067 \quad 7.7096691 .199768$

H $1.4569976 .947834-0.240557$

H $0.3644608 .330730-0.408561$

C -0.6789455 .2042702 .295732$

H $-0.1215053 .067521-1.718954$

H -1.818722 $1.639428-3.462023$

H $-4.7609303 .363245-3.109541$

H -1.748926 5.3466202 .491399

H -0.1594456 .0848522 .693353$

H 0.5865424 .7585490 .580742

C $-5.010378-2.068333-0.196258$

O $-5.363965-0.793879-0.748869$

C $-4.371540 \quad 0.237601-0.642914$

C -4.0245050 .4969610 .830615$

C $-3.620256-0.7986061 .541760$

C $-4.619927-1.9309291 .285778$

H $-5.931835-2.662286-0.247672$

C $-3.944098-2.784034-1.039672$

H -3.476662 -0.086791-1.182780

O -5.1334101 .0881341 .504015$

H $-3.196503 \quad 1.2121750 .889265$

H -3.550366 -0.608683 2.619728

O $-2.328896-1.179174 \quad 1.077721$

H $-4.217153-2.8813301 .654785$

O $-5.799867-1.6544462 .045420$

C $-4.444518-3.148069-2.421648$

H $-3.055495-2.163999-1.175340$ 
H $-3.652648-3.717143-0.543669$ H $-5.892676 \quad 0.487557 \quad 1.411390$ H $-2.103813-2.0278961 .494373$ H -6.406683 -2.405524 1.930444 O $-3.421667-3.726686-3.119785$ O $-5.578218-2.957186-2.838800$ C $-3.737747-4.125492-4.457142$ H $-4.534503-4.875432-4.450599$ H -4.029924 -3.255444 -5.052713 $\mathrm{H}-2.842081-4.569156-4.900142$

SCF Energy (B3LYP/6-31G**//MMFF) = -3245.93318566

04_00155

MM̄MF Geometry

C $-2.1574693 .876476 \quad 1.469852$

C -2.5766752 .6284061 .212540$

C $-2.916243 \quad 1.6126482 .268023$

O -2.0368590 .4902262 .022320$

C -4.3698861 .1417492 .108670$

C $-4.880974 \quad 0.3671593 .305545$

C $-4.993214-0.9742083 .363471$

C -5.3255291 .2123124 .471753$

C $-4.585001-1.9845032 .327528$

C $-5.787605-2.6891081 .684727$

C $-6.560428-1.7975460 .700036$

C $-7.789699-2.5314680 .159601$

C $-8.522470-1.709662-0.910796$

O $-5.675625-1.472731-0.376709$

O $-9.452229-2.594327-1.553896$

C -7.498065 -1.244121-1.967619

C $-9.359360-0.588756-0.283680$

O $-8.110206-0.372965-2.916221$

C $-6.231150-0.594199-1.362567$

C $-5.168433-0.390055-2.468783$

O $-6.5831810 .673908-0.807057$

C -1.426896 -0.1108823 .072258$

$\begin{array}{lllll}\text { O } & -1.553008 & 0.175400 & 4.254677\end{array}$

C $-0.518028-1.1920752 .619316$

C $-0.441995-1.6186901 .348253$

C $0.459868-2.6517590 .831436$

C $1.455502-3.2542481 .787027$

C $-3.7944550 .058478-1.950064$

C $-2.7661420 .142800-3.065428$

C $-0.3164140 .427283-3.429371$

O $-3.052977 \quad 0.026803-4.254121$

C $0.222574-0.959618-3.592795$

C $1.443762-1.342679-3.190817$

C $1.972358-2.739374-3.351546$

C $2.313954-3.394753-2.030532$

C $3.591294-3.507002-1.628536$

C $1.189034-4.007757-1.224076$

C $0.377112-2.987237-0.473285$

N $-1.4883210 .377043-2.573592$

H -2.072075 4.2184432 .497698

H -2.6375542 .2969640 .178674$

H -2.7403732 .0356553 .264129$

H -4.4620640 .5376941 .199899$

H -5.0334602 .0039591 .957124$

H $-5.401940-1.4138204 .272697$

H -5.7199780 .6095545 .296711$

H -4.4883841 .7975894 .864350$

H -6.116971 1.9019354 .160554

H -3.937722 -1.553549 1.558445

H $-3.972797-2.7401012 .836236$

H -5.404416 -3.564805 1.144628

H $-6.462784-3.0603382 .465720$

H -6.864397 -0.8775611 .211046$

H -8.474191 -2.7985420 .974456$

H -7.475847 -3.483977 -0.290603

H $-9.867748-2.104579-2.284533$

H -7.195393 -2.137116 -2.531420

H -10.100990 -1.008581 0.406756

H -9.933940 -0.048834-1.044392

H $-8.759555 \quad 0.1342490 .273062$

H $-8.204790 \quad 0.499829-2.498076$

H $-5.026737-1.336945-3.006505$

H $-5.5274850 .347945-3.197291$

H $\quad-5.7962881 .239748-0.779696$
H $\quad 0.097407-1.6214673 .403506$

H -1.093487 -1.167660 0.601662

H $2.060428-2.4704652 .255696$

H $2.160504-3.9375381 .308501$

H $\quad 0.941037-3.8182992 .572428$

H $-3.8630191 .049135-1.490768$

H $-3.423814-0.647640-1.198240$

H $0.404757 \quad 1.102566-2.959541$

H $-0.5931180 .835846-4.406439$

H $-0.430656-1.687678-4.068744$

H $2.105373-0.614771-2.729168$

H $2.864594-2.685131-3.988835$

H $1.262771-3.375290-3.895755$

H $3.851042-4.002277-0.698890$

H $4.406682-3.098696-2.216644$

H $1.575111-4.777947-0.546927$

H $0.518988-4.555112-1.899802$

H $-0.376325-2.492327-1.085885$

H $-1.3388540 .384706-1.568574$

C -0.2244595 .0624940 .414468$

O 0.4501063 .8004950 .249055

C $1.4616593 .927260-0.759432$

C $1.6790585 .425163-0.929309$

C $0.2724685 .955590-0.722773$

H $1.0334353 .513945-1.681907$

C $2.7228173 .126280-0.397638$

H $2.3503625 .825633-0.160642$

H $2.0947265 .677602-1.909273$

C $0.2180147 .439958-0.407292$

H $-0.3091065 .763757-1.634494$

C $2.4512361 .631622-0.134226$

O $3.3496253 .694258 \quad 0.752262$

H $3.4266323 .234492-1.229084$

H $1.909657 \quad 1.204607-0.985512$

O $1.587121 \quad 1.5079491 .001711$

C 3.7106550 .7909980 .154633

C $4.7456870 .824143-0.983440$

H 4.1693331 .1175791 .094706

O $3.290803-0.5610520 .368488$

H $4.2481700 .595675-1.933950$

H $5.1552251 .837903-1.051920$

H $-0.8146367 .766466-0.249313$

H $\quad 0.7893427 .6797750 .495657$

H $0.6352408 .024463-1.233780$

C -1.7375614 .8344630 .388716$

H 2.7226403 .6164751 .491977

H 1.4522100 .5584121 .160930

H $2.866971-0.873131-0.448557$

H $-2.0334604 .437855-0.590903$

H -2.2690595 .7826790 .526393$

H 0.0846985 .4606661 .389571

C $7.628773-0.7250070 .818126$

O $6.572797 \quad 0.1630840 .428267$

C $5.896003-0.179886-0.783493$

C $6.878957-0.180069-1.963008$

C $8.064686-1.111291-1.685034$

C $8.676134-0.860069-0.301551$

H $8.113958-0.2282801 .668563$

C $7.083175-2.0742011 .313083$

H $5.462648-1.179669-0.686161$

O $7.3524011 .145167-2.191525$

H $6.347169-0.501779-2.866344$

H $8.835267-0.962354-2.451046$

O $7.637297-2.468475-1.760729$

H $9.383129-1.657168-0.042932$

O $9.4192290 .362301-0.338740$

C $6.213684-1.9219692 .544480$

H $6.481771-2.5690350 .546063$

H $7.916093-2.7335821 .582269$

H $7.9544741 .114802-2.954256$

H $7.276770-2.613925-2.652035$

H $10.117955 \quad 0.260395-1.006999$

O $5.567990-3.1028292 .779903$

O $6.124711-0.9100693 .226100$

C $4.716185-3.1201793 .929887$

H $3.998337-2.2950813 .892460$

H $4.163912-4.0636193 .929715$

H $5.319888-3.0642504 .840399$ 
C $1.690576-2.981306-2.456438$

C $2.246957-4.339846-2.136104$

C $3.140225-4.335516-0.913585$

C $4.476180-4.254137-1.033591$

C $2.488682-4.5091520 .440744$

C $1.849371-3.2395050 .932094$ N -1.335179 -1.090294 -2.202341 H -1.984879 4.2287772 .110643 H -3.019894 2.379023 -0.103229 H -2.8231982 .1514422 .999685$ H $-5.045170 \quad 0.9704291 .258074$ H -5.2158172 .6026961 .858715$ H -5.954279 -0.3886914 .646329$ H -5.8827201 .7841055 .366108$ H -4.5743872 .7312734 .652530$ H -6.2390352 .9173984 .058208$ H -4.581485 -1.183079 2.017277 H $-4.868918-2.1363413 .446803$ H -6.427244 -2.992161 1.965567 H $-7.417091-1.9134072 .954122$ H -7.145242 -0.1418661 .135265$ H -9.175487 -1.626464 1.165896 H -8.296204 -2.8549150 .279970$ H -10.085362 -1.586987 -2.327691 H -7.506474 -2.312486 -2.163296 H -10.219485 $0.259166-0.072904$ H -9.698734 $0.693942-1.683605$ H -8.617309 $0.968582-0.304159$ H $-7.7835970 .380983-2.964593$ H $-5.190099-2.209455-2.528629$ H -5.192064 -0.572956 -3.196017 H $-5.3979761 .005136-1.198648$ H -1.967159 -1.781600 1.401125 H $\quad 0.972057-0.957013 \quad 1.788933$ H $-1.153075-4.1563441 .666589$ H -0.208598 -4.9001990 .385603$ H -1.237342 -3.538569-0.000270 H -3.539591 $0.113580-1.413454$ H -3.551971 -1.551588 -0.794262 H $0.583974-0.573283-2.749413$ H $-0.381416-1.318270-4.046564$ H $-0.332256-3.534747-2.556388$

H $2.818077-4.679283-3.010294$

H $1.447336-5.078687-2.001463$

H $5.124463-4.277850-0.164008$

H $4.950905-4.154705-2.004145$

H $3.239417-4.8034451 .186138$

H $1.794734-5.3530650 .401356$

H $2.572300-2.4638831 .187421$

H $-1.218360-0.764172-1.247540$

C $-0.2754034 .982370-0.067272$

O $0.3298753 .733325-0.451967$

C $1.4216453 .994968-1.351335$

C $1.6132675 .507677-1.354155$

C $0.1999345 .996577-1.104500$

H $1.0956273 .668731-2.346890$

C $2.6732253 .204717-0.942157$

H $2.2780195 .830636-0.544011$

H $2.0338015 .870099-2.297063$

C $0.1306887 .440382-0.639231$

H $-0.3791135 .886982-2.031417$

C $2.5034571 .674092-1.020460$

O 3.0544553 .5704720 .384520

H $3.4904953 .508303-1.605170$

H $2.2595151 .379418-2.048492$

O $1.4032481 .254188-0.204815$

C $3.7250180 .870423-0.534059$

C $5.0296511 .213576-1.270219$

H 3.8521250 .9955390 .547636

O $3.448527-0.516183-0.753297$

H $4.8781851 .092132-2.349903$

H $5.2804802 .261763-1.075100$

H $-0.9055577 .744669-0.461649$

H $\quad 0.6897237 .5898990 .290601$

H $0.5533668 .108932-1.396469$

C $-1.7935404 .816035-0.018381$

H $2.328853 \quad 3.308990 \quad 0.977368$

H $0.5955251 .657209-0.565288$

H $2.588602-0.706833-0.341533$

H $-2.1553884 .426188-0.978033$

H $-2.277451 \quad 5.7852410 .145359$

H 0.1165115 .2363200 .926798

C $7.486368-0.2156091 .156586$

O 6.4539250 .5731840 .551430

C $6.2103370 .322306-0.836189$

C $7.4762860 .597383-1.660672$

C $8.663056-0.212159-1.125308$

C $8.814402-0.0660530 .391676$

H 7.6235810 .2207842 .154423

C $7.051724-1.6783361 .344792$

H $5.921426-0.725027-0.971136$

O $7.7829251 .989153-1.617618$

H $7.2800250 .335430-2.707282$

H $9.5870090 .125707-1.610435$

O $8.485345-1.590362-1.439997$

H $9.544440-0.792756 \quad 0.767230$

O $9.347948 \quad 1.235026 \quad 0.655158$

C $5.942488-1.8174402 .368164$

H $6.693526-2.1289200 .416349$

H $7.906999-2.2582301 .711886$

H $8.5796552 .131430-2.156276$

H $8.418668-1.664536-2.407269$

H $9.502517 \quad 1.301348 \quad 1.612799$

O $5.777429-3.1413192 .663837$

O $5.298991-0.8928502 .844628$

C $4.782205-3.4351803 .649487$

H $3.825439-2.9745673 .387546$

H $4.649229-4.5196713 .685311$

H $5.119337-3.0882234 .630598$

SCF Energy (B3LYP/6-31G**//MMFF) $=-3245.91975857$

0400157

MMFF Geometry

C $0.971048-3.4804632 .002170$

C $-0.259576-3.5765121 .479920$

C $-0.527843-3.539377-0.001630$

O $-1.045746-2.233545-0.339734$

C $-1.582731-4.593470-0.373742$

C $-1.896658-4.616550-1.856089$ 
C $-2.998452-4.064885-2.402485$

C $-0.903754-5.352941-2.715917$

C $-4.064553-3.285047-1.681997$

C $-4.566435-2.099796-2.518921$

C $-5.591287-1.264454-1.738126$

C $-6.320287-0.292453-2.665864$

C $-7.3067180 .592963-1.892368$

O $-4.888471-0.533664-0.725039$

O $-7.7152401 .641267-2.783578$

C $-6.5635021 .236940-0.705033$

C $-8.580004-0.172440-1.515040$

$\begin{array}{lllll}\text { O } & -7.466665 & 1.969574 & 0.120552\end{array}$

C $-5.733573 \quad 0.2368800 .139411$

C -4.8409461 .0286741 .122937$

O $-6.621270-0.597548 \quad 0.884524$

C $-0.143590-1.308946-0.769117$

O $1.067831-1.442312-0.843371$

C $-0.888224-0.087570-1.146848$

C $-0.2005441 .021615-1.455209$

C $-0.7613442 .322685-1.822821$

C $-2.2445192 .404013-2.066760$

C -3.7842760 .1956411 .860633$

C -2.8974531 .1055902 .691942$

C -1.153280 2.8830092 .446694

O $-3.005117 \quad 1.172913 \quad 3.913862$

C -1.2784534 .0929461 .574458$

C -0.2309424 .7327691 .032944$

C -0.3602615 .9285120 .132399$

C $0.3591525 .750089-1.188100$

C $1.4712566 .450128-1.469109$

C $-0.2497244 .812513-2.212791$

C $0.0792213 .373484-1.926221$

N $-2.0229201 .849171 \quad 1.916306$

H $1.818149-3.3529681 .335643$

H -1.112942 -3.678118 2.146000

H $\quad 0.389481-3.741985-0.568090$

H $-2.500150-4.4239720 .202768$

H - $-1.235535-5.591288-0.072883$

H $-3.153685-4.182830-3.473892$

H $-1.202027-5.374358-3.769500$

H $0.079563-4.875293-2.662325$

H $-0.805733-6.390353-2.379800$

H -4.905107 -3.959116-1.477423

H -3.704720 -2.909822 -0.719643

H -3.714493 -1.469095 -2.804151

H -5.021941 -2.482194 -3.440948

H -6.307511 -1.945571-1.263579

H $-6.839403-0.831138-3.468476$

H $-5.5857190 .355107-3.165399$

H -8.280143 2.252789-2.280130

H $-5.8770711 .981425-1.131809$

H -9.070977 -0.562021 -2.414997

H $-9.313306 \quad 0.487745-1.038613$

H -8.391479 $-1.015367-0.846747$

H $-7.954877 \quad 1.3290330 .665276$

H -4.3248191 .8201840 .567060$

H -5.4679001 .5299431 .871964$

H $-6.147249-0.9506401 .654138$

H -1.970874 -0.127798 -1.144650

H $\quad 0.8867390 .983982-1.409359$

H $-2.5638291 .629283-2.773350$

H -2.560570 $3.354759-2.501360$

H -2.796746 2.272200-1.132779

H -4.250467 -0.533429 2.531238

H -3.152935 $-0.364617 \quad 1.162948$

H -0.1351382 .4832802 .447885$

H -1.440723 3.1252713 .474186 H -2.2837494 .4562651 .373284$ H $0.775744 \quad 4.3849941 .251209$ H $\quad 0.0450826 .7948370 .671030$ H -1.413054 $6.165169-0.066264$ H $1.9798586 .344002-2.421625$ H $1.9016927 .140657-0.751541$ H $0.1365785 .048022-3.212917$ H -1.325434 5.003374 -2.256869 H $1.1441343 .197625-1.766433$ H -1.940794 1.6290010 .928882 C $1.437498-2.1797214 .138632$
O $2.353955-1.3361503 .407539$

C $1.791220-0.0103603 .348110$

C 0.7007430 .0234364 .413348

C $0.143514-1.378864 \quad 4.294871$

H 1.3213900 .0904762 .362289

C 2.8687841 .0598453 .530300

H 1.1169910 .1842185 .414947

H -0.0450470 .7999794 .229832$

C -0.709971 -1.798426 5.478410

H $-0.463593-1.4306713 .383131$

C 3.9939471 .0532592 .476353

O 3.4801950 .8971024 .816952

H 2.3955722 .0490093 .549727

H 4.6543650 .1897412 .621044

O 4.8001422 .2157812 .717399

C 3.5161581 .0851481 .010799

C 4.6432451 .4422140 .019166

H 3.0882600 .1102660 .754802

O 2.4726312 .0511900 .866875

H 4.9195292 .4910760 .175161

H 5.5241690 .8330120 .242552

H -1.077870 -2.821272 5.350482

H $-0.145699-1.7570036 .416026$

H -1.577676-1.138182 5.579368

C $1.288236-3.5463263 .467078$

H $3.847236-0.0030234 .854303$

H 5.1051412 .1702783 .640029

H 2.8329812 .9160921 .127723

H $0.539438-4.1458743 .997848$

H $2.237398-4.0899323 .559425$

H $1.892783-2.3361035 .125700$

C $5.158144-0.931684-1.868463$

O $3.979911-0.119909-1.771355$

C $4.2095641 .258792-1.452976$

C $5.1464921 .892118-2.492380$

C $6.4431641 .105875-2.649107$

C $6.131601-0.365924-2.911932$

H $5.653809-0.981272-0.891984$

C $4.712203-2.350097-2.259807$

H $3.2425651 .762468-1.565483$

O $5.4176773 .254130-2.177937$

H $4.6321531 .900453-3.462200$

H $7.0898911 .229370-1.772541$

O $7.1633471 .637869-3.762565$

H $5.707079-0.467537-3.918671$

O $7.350878-1.107020-2.898407$

C $4.164458-3.130729-1.084146$

H $3.928682-2.304088-3.024930$

H $5.564396-2.921566-2.645042$

H $6.0968993 .278209-1.483195$

H $7.9609031 .093253-3.878509$

H $7.733976-1.035980-2.007371$

O $3.908453-4.409294-1.491867$

O $3.989231-2.6837500 .040245$

C $3.429813-5.317047-0.493543$

H $3.665137-6.333450-0.821093$

H $3.911163-5.1463170 .474262$

H $2.345186-5.220206-0.406684$

SCF Energy $\left(B 3 L Y P / 6-31 G^{* *} / / M M F F\right)=-3245.92810658$

0400158

MMFF Geometry

C $-0.832302 \quad 2.570378 \quad 0.200196$

C -1.8890472 .8322020 .984862$

C -2.4471361 .9589822 .084748$

O -1.7454670 .6972402 .132162$

C -3.9331721 .6651011 .810735$

C -4.6855341 .1420923 .017233$

C $-5.067208-0.1383223 .190679$

C -5.0601822 .1838824 .040252$

C $-4.778599-1.3207062 .308476$

C -6.019849-1.813536 1.550731

C $-6.413792-0.9085350 .372402$

C -7.686127 -1.422963 -0.304158

C $-8.035638-0.610566-1.560859$

O $-5.330342-0.921650-0.561258$

O $-9.028779-1.341046-2.296788$

C $-6.784798-0.516829-2.460479$ 
C $-8.6644520 .742839-1.208869$

$\begin{array}{lllll}\text { O } & -7.017770 & 0.348236 & -3.569921\end{array}$

C $-5.510873-0.075760-1.703785$

C $-4.268562-0.247476-2.611414$

O $-5.6385151 .298095-1.334639$

C - $-1.3799850 .205526 \quad 3.342200$

O

C $-0.771938-1.1409033 .223761$

C $-0.787315-1.8707692 .097219$

C $-0.218710-3.2101191 .925047$

C $0.485838-3.8306393 .102843$

C $-2.930695-0.030515-1.888261$

C $-1.744540-0.395605-2.763934$

C $0.682376-0.947223-2.603217$

O $-1.831801-0.561060-3.977705$

C $0.720758-2.442904-2.550560$

C $1.609294-3.144163-1.831705$

C $1.624614-4.643199-1.754520$

C $1.518011-5.153976-0.333274$

C $2.594684-5.6495590 .299736$

C $0.153765-5.1780540 .321890$

C $-0.336893-3.813586 \quad 0.723457$

N $-0.571856-0.498199-2.026637$

H -0.2792081 .6448010 .317470$

H -2.405463 3.7807500 .844471

H -2.3225912 .5119483 .023029$

H $-4.009197 \quad 0.957280 \quad 0.979650$

H $-4.4473282 .574093 \quad 1.470032$

H $-5.635645-0.3851734 .086756$

H -5.6549191 .7672044 .860221$

H -4.1644262 .6322374 .480561$

H -5.654012 2.9772033 .574619

H $-3.954328-1.1281281 .615894$

H $-4.427435-2.1310412 .960386$

H -5.790395 -2.815592 1.164935

H -6.864048 -1.918905 2.243289

H $-6.566817 \quad 0.1122030 .739251$

H $-8.527783-1.4240080 .399577$

H -7.543112 -2.471734 -0.600582

H -9.796894 -1.471991 -1.714720

H $-6.608259-1.517356-2.879765$

H $-9.5863070 .598172-0.632672$

H -8.958914 $1.288517-2.112002$

H $-8.0046451 .382021-0.618475$

H -7.817197 $0.029192-4.022778$

H -4.265221 $-1.266557-3.020421$

H $-4.325870 \quad 0.444997-3.460687$

H $-4.755511 \quad 1.688532-1.244187$

H $-0.326170-1.5152634 .139861$

H -1.265252 -1.4588731 .210230$

H $1.305742-3.1891023 .444527$

H $\quad 0.928359-4.8039802 .880435$

H $-0.213217-3.9803053 .933061$

H -2.816316 $1.018787-1.599892$

H -2.886739 -0.644388 -0.981616

H $1.491375-0.488421-2.026196$

H $0.753154-0.607745-3.641223$

H $-0.030219-2.975372-3.130061$

H $2.365356-2.615977-1.259634$

H $2.560219-4.989579-2.212349$

H $\quad 0.819317-5.083804-2.355529$

H $2.526553-6.0482451 .306314$

H $3.570973-5.665129-0.173384$

H $\quad 0.144381-5.8602541 .179893$

H -0.570699 -5.614461 -0.378170

H $-0.863194-3.285705-0.071947$

H -0.618227 -0.419095-1.014984

C $0.921566 \quad 4.233787-0.460147$

O $2.0505673 .340112-0.543398$

C $3.1975654 .090261-0.995931$

C $2.7594115 .549511-1.071824$

C $1.2793615 .410529-1.365543$

H $3.4137113 .749629-2.014337$

C $4.4019143 .830482-0.082365$

H $2.9099776 .075326-0.123092$

H $3.3008096 .102283-1.845945$

C $0.4899646 .678177-1.086460$

H $1.1522775 .127148-2.419121$
C $4.8818242 .362022-0.087553$

O $4.049164 \quad 4.1511301 .269020$

H $5.2340524 .491218-0.353888$

H $4.086710 \quad 1.7186860 .294754$

O 5.9634762 .2611890 .849124

C $5.3625181 .855400-1.460878$

C $6.0269090 .463038-1.448899$

H $4.5318621 .857117-2.174190$

O $6.3324702 .791432-1.949572$

H $6.7302920 .417460-2.290137$

H $6.6470500 .352150-0.553512$

H $-0.5720466 .536961-1.309362$

H $0.5761476 .981788-0.037720$

H $0.8564857 .503035-1.706200$

C $-0.3570473 .507601-0.876636$

H 4.0309085 .1186841 .352272

H 6.6953632 .8052020 .510691

H $6.5435662 .543890-2.866043$

H $-0.1845612 .919474-1.786211$

H -1.146090 4.232729-1.106456

H 0.8522324 .5674780 .584250

C $4.637857-1.2183960 .756094$

O $4.103283-0.795730-0.502330$

C $5.033440-0.710533-1.580898$

C $5.711273-2.071702-1.794254$

C $6.391386-2.557186-0.519404$

C $5.395178-2.5499420 .636007$

H $5.296491-0.4358161 .148939$

C $3.459851-1.3927031 .723721$

H $4.437359-0.509517-2.480590$

O $6.656515-1.993563-2.855745$

H $4.953104-2.806220-2.095631$

H $7.282646-1.961411-0.292016$

O $6.846196-3.895336-0.733815$

H $4.683548-3.3701520 .483856$

O $6.084420-2.8291921 .853327$

C $2.683468-0.1152271 .915123$

H $2.750056-2.1348291 .338194$

H $3.790723-1.7576382 .702894$

H $7.076290-2.867246-2.935858$

H $7.231612-4.2072580 .103027$

H $6.717430-2.1076772 .009753$

O 3.1599080 .5705062 .990763

O 1.7653920 .2312991 .183192

C 2.4809991 .7924353 .300179

H 1.5615021 .5678553 .847547

H 2.2550182 .3670162 .397059

H 3.1353512 .3918393 .938634

SCF Energy (B3LYP/6-31G**//MMFF) $=-3245.91462553$

0400159

MM̄FF Geometry

C $-1.326388-3.6206851 .272713$

C $-0.526245-2.5704691 .033458$

C $0.304815-1.9215472 .110581$

O $0.143443-0.4841762 .051665$

C $1.795176-2.2388521 .886183$

C $2.659737-1.8750653 .075896$

C $3.483310-0.8112123 .139804$

C $2.584842-2.8328714 .239027$

C 3.7237580 .2572102 .108505

C 5.2084140 .3826961 .745639

C $5.721478-0.7506690 .842907$

C $7.251950-0.7368260 .828834$

C $7.825942-1.769403-0.145119$

O $5.208967-0.527607-0.476913$

O $9.223485-1.488100-0.314500$

C $7.154781-1.569480-1.515443$

C $7.732344-3.1966300 .406562$

O $7.565043-2.590111-2.425096$

C $5.606875-1.499888-1.455030$

C $5.082229-1.036263-2.839794$

O $5.093237-2.793278-1.144832$

C -0.9809050 .0553572 .592695$

O $-1.941107-0.5579393 .038876$

C -0.9355381 .5382472 .585895$

C 0.2011052 .2290872 .409227

C 0.3608953 .6848412 .406468 
C $-0.868550 \quad 4.5342172 .577764$

C $3.577536-1.148301-3.093868$

C $2.682061-0.383574-2.144831$

C $2.0314741 .890341-1.436307$

O $1.795837-0.956255-1.513124$

C $2.7948263 .013158-0.816524$

C $2.4354334 .298398-0.957802$

C $3.0863265 .444558-0.235747$

C 2.1222326 .1341330 .710662

C 1.4101307 .2005270 .308777

C 2.0231415 .6293562 .135869

C 1.5986294 .1897052 .222063

N $2.9308350 .979898-2.115132$

H -1.376823 -4.027345 2.280380

H $-0.452004-2.1644350 .028418$

H $-0.008434-2.269712 \quad 3.102693$

H $2.151236-1.7300550 .985525$

H $1.928292-3.3113871 .689588$

H $4.051137-0.6684304 .059080$

H $3.282972-2.5698835 .040983$

H $1.579646-2.8419614 .670690$

H $2.832522-3.8477423 .910824$

H $3.1219430 .125501 \quad 1.206269$

H 3.4090961 .2076342 .554729

H 5.3470321 .3409561 .228253

H $5.800391 \quad 0.4278492 .668694$

H $5.354282-1.7136931 .213925$

H $7.651720-0.9008191 .837585$

H 7.6051860 .2594010 .526450

H $9.647677-1.5397490 .559196$

H $7.525079-0.620343-1.928333$

H $8.283998-3.2787981 .350848$

H $8.199183-3.915169-0.276154$

H $6.705912-3.5175950 .595991$

H $8.537340-2.586735-2.444677$

H $5.3933120 .003289-3.004307$

H $5.559757-1.637208-3.624089$

H $4.127598-2.769977-1.237972$

H -1.882956 2.0306062 .778221

H $\quad 1.122081 \quad 1.6747742 .248316$

H -1.626642 4.2758681 .832373

H -0.6739135 .6031482 .460650$

H -1.296966 4.3893593 .575449

H $3.358941-0.786104-4.106664$

H $3.270765-2.200737-3.081916$

H $1.4760981 .353222-0.660366$

H $1.3181432 .247332-2.185738$

H $3.6225552 .756564-0.160659$

H $1.5919554 .544684-1.599388$

H $3.450876 \quad 6.156359-0.987523$

H $3.975617 \quad 5.1193410 .318352$

H $0.731268 \quad 7.7141710 .981329$

H $1.4877957 .580869-0.704403$

H $1.363144 \quad 6.2644892 .737358$

H 3.0094455 .7240852 .608747

H 2.4301903 .4931512 .113177

H $3.569017 \quad 1.372426-2.800910$

C $-3.636870-4.3298210 .625174$

O $-4.180030-2.998050 \quad 0.530319$

C $-5.501328-3.075204-0.038999$

C $-5.863732-4.555178-0.078045$

C $-4.503898-5.189744-0.292824$

H -5.420707 -2.713032 -1.070603

C $-6.476656-2.1954540 .749931$

H $-6.296708-4.8941630 .870256$

H $-6.577611-4.786238-0.874451$

C -4.470709 -6.673372 0.029972

H -4.206215 $-5.040557-1.339538$

C $-6.041854-0.7196940 .865301$

O $-6.588880-2.7204302 .079007$

H -7.476277 -2.2665210 .304937$

H -5.151853 -0.645777 1.501670

O $-7.079608-0.0152851 .559831$

C $-5.773407-0.024909-0.482248$

C $-5.4418041 .467874-0.309472$

H $-4.942814-0.528191-0.982188$

O $-6.928856-0.167813-1.306863$

H -6.3148801 .9857720 .105321$
H $-4.630913 \quad 1.560596 \quad 0.421415$

H -3.470024 -7.086552 -0.130100

H -4.751149 -6.863948 1.071364

H $-5.168269-7.221779-0.611484$

C $-2.159744-4.3137080 .232164$

H -7.204660 -2.146576 2.566102

H -7.866203 -0.0149480 .987631$

H $-6.6727240 .059481-2.216329$

H -2.038594 -3.822452 -0.739602

H -1.786129 -5.3389490 .132907$

H $-3.754874-4.6493251 .669222$

C $-2.7077961 .570548-1.687337$

O $-3.9915611 .490126-2.317613$

C $-5.0497302 .166764-1.631485$

C $-4.7077213 .654087-1.476388$

C $-3.3556543 .855862-0.799369$

C $-2.2795413 .034948-1.507853$

H $-2.7403261 .066652-0.713571$

C -1.705906 $0.830733-2.589060$

H $-5.9249582 .112337-2.290426$

O $-5.7287324 .317802-0.738671$

H -4.677991 4.117414 -2.471289

H -3.411035 3.6120410 .267627

O $-3.0225885 .243610-0.881169$

H -2.062120 $3.485421-2.485078$

O $-1.0786043 .071718-0.741866$

C -1.853469 -0.674292 -2.544424

H $-1.841776 \quad 1.147312-3.630035$

H $-0.6818861 .048550-2.274031$

H $-5.4614645 .248097-0.644463$

H $-2.2117675 .382933-0.363537$

H $-0.7617193 .990319-0.729560$

O $-0.963994-1.224952-3.424210$

O $-2.626908-1.291216-1.827151$

C $-0.951380-2.654490-3.485338$

H $-0.496472-3.058121-2.576558$

H -1.962986 -3.049072 -3.621178

$\mathrm{H}-0.343006-2.951834-4.343565$

SCF Energy (B3LYP/6-31G**//MMFF) $=-3245.91246787$

0400160

MM̄FF Geometry

C $-0.596605 \quad 3.083842 \quad 0.127284$

C 0.3653803 .2986531 .038443

C 0.6300242 .4981692 .294649

O 1.7558721 .6264042 .028858

C -0.5447801 .6182582 .763326$

C -0.2858880 .8854604 .068448$

C $-0.154467-0.451874 \quad 4.185912$

C $-0.214478 \quad 1.7559895 .296507$

C $-0.223732-1.4772523 .089596$

C $1.163756-2.0391332 .759053$

C $1.211357-2.6325381 .343542$

C $2.545043-3.3399581 .101929$

C $2.688507-3.823178-0.348477$

O $1.056930-1.5446870 .423641$

O $4.065130-4.172436-0.562012$

C $2.369347-2.650192-1.299600$

C $1.872287-5.094183-0.610047$

O $2.344890-3.089224-2.655272$

C $1.052345-1.922122-0.959374$

C $0.839826-0.631772-1.791252$

O $-0.049408-2.789859-1.247365$

C 3.0080962 .1377552 .172387

O 3.3086543 .2626572 .537617

C 3.9640591 .0815781 .770740

C 5.2611911 .3799221 .608097

C $6.3130890 .451088 \quad 1.187379$

C $5.909713-0.9659580 .873999$

C $1.9079760 .452374-1.611699$

C $3.0376730 .397091-2.626396$

C $5.2615151 .421078-3.105981$

O $2.997994-0.290080-3.643114$

C $6.3590570 .535469-2.605668$

C $7.5923910 .981072-2.320554$

C $8.7221870 .096597-1.875391$

C $9.3927270 .591245-0.612990$

C $10.511431 \quad 1.333555-0.670230$ 
C 8.8182630 .1483450 .713195

C 7.5782400 .9102561 .088840

N $4.0814961 .245824-2.280040$

H $-1.2799112 .247775 \quad 0.240545$

H 1.0507524 .1282470 .872536

H 0.8840403 .2033283 .095671

H -0.7810770 .9042301 .971486$

H -1.4436582 .2358942 .892118$ H $\quad 0.025475-0.8676575 .176308$

H $-0.152626 \quad 1.168574 \quad 6.218896$ H 0.6663732 .4039805 .260967 H -1.1085912 .3837225 .369441$ H $-0.876355-2.2959463 .418062$ H $-0.700113-1.0758652 .193679$ H $1.911108-1.2395652 .833651$ H $1.427794-2.8027933 .500873$ H $\quad 0.375690-3.332127 \quad 1.223839$ H $2.673263-4.1764761 .800244$ H $3.367685-2.6452371 .316934$ H $4.303286-4.8605360 .082777$ H $3.200596-1.940081-1.214162$ H $2.207956-5.9061220 .046332$ H $2.021376-5.456126-1.633330$ H $0.801791-4.958945-0.443382$ H $3.197876-3.519902-2.836068$ H $0.694196-0.883484-2.848695$ H $-0.112740-0.189993-1.464129$ H $-0.847844-2.248480-1.364019$ H 3.5685560 .0880261 .595753 H 5.5867352 .4025571 .794098 H $5.552404-1.4697451 .778549$ H $6.720150-1.5727950 .465649$ H $5.112858-0.9830160 .122432$ H $1.4323121 .431442-1.748806$ H $2.3215950 .428764-0.600259$ H $5.5328122 .480624-3.062279$ H $5.0246801 .164264-4.143441$ H $6.138576-0.524705-2.505289$ H $7.8171302 .037118-2.453096$ H $9.4550080 .058484-2.692303$ H $8.388669-0.938458-1.731042$ H $11.0158361 .667083 \quad 0.230636$ H $10.9447091 .628630-1.620191$ H 9.5520420 .3113811 .514387 H $8.667025-0.9345270 .697167$ H 7.7698881 .9574761 .327340 H $4.0447251 .745065-1.396039$ C $-2.2194644 .390002-1.333622$ O $-3.067812 \quad 3.257876-1.608669$ C $-4.3411853 .474171-0.969748$ C $-4.3483814 .932406-0.519093$ C $-2.8838815 .149736-0.186660$ H $-4.3564982 .835643-0.078357$ C $-5.486953 \quad 3.082705-1.909177$ H -4.658616 $5.607032-1.325362$ H -5.0129115 .0991220 .334194$ C -2.483214 $6.611507-0.101108$ H $-2.677323 \quad 4.6660910 .776174$ C $-5.3925031 .644384-2.461177$ O $-5.482290 \quad 3.976532-3.029624$ H -6.444886 $3.234687-1.397730$ H $-4.5513631 .573079-3.160970$ O $-\begin{array}{llll}-6.567504 & 1.398363 & -3.244429\end{array}$ C $-5.254903 \quad 0.549298-1.387475$ C $-5.293919-0.873945-1.979607$ H $-4.3051640 .688053-0.861269$ O $-6.2924340 .691137-0.417926$ H $-6.264743-1.053338-2.456944$ H $-4.515623-0.945560-2.748800$ H $-1.416527 \quad 6.7092510 .124221$ H -2.679568 $7.137902-1.041107$ H -3.044141 7.1182300 .690976 C $-0.778438 \quad 3.926170-1.105614$ H $-6.2127043 .711905-3.614789$ H -7.340778 $1.505205-2.665278$ H $-7.1368280 .477438-0.849339$ H $-0.118864 \quad 4.801309-1.074499$ H $-0.4688563 .315186-1.962764$
H -2.235788 $5.002689-2.245121$

C $-3.426049-2.6398590 .726752$

O $-3.766852-1.775143-0.363677$

C $-5.075752-1.958419-0.909392$

C $-5.207485-3.377411-1.480041$

C $-4.868876-4.433341-0.420082$

C $-3.558511-4.117930 \quad 0.311620$

H -2.366862 -2.434490 0.924582

C $-4.209594-2.2975772 .004324$

H $-5.823385-1.816713-0.121998$

O $-4.347653-3.541194-2.606183$

H $-6.233534-3.523425-1.836578$

H $-4.782365-5.415886-0.900447$

O $-5.922742-4.5075590 .535608$

H $-3.453560-4.7634121 .191638$

O $-2.483338-4.440549-0.574442$

C $-3.969781-0.878096 \quad 2.475687$

H -5.288043 -2.407867 1.864923

H $-3.890379-2.9733422 .806531$

H $-3.437888-3.357878-2.315195$

H $-6.735321-4.747606 \quad 0.058476$

H $-1.650889-4.295715-0.093179$

O $-4.555427-0.715117 \quad 3.699302$

O $-3.369379-0.0153761 .850811$

C $-4.445750 \quad 0.594842 \quad 4.265012$

H $-4.863942 \quad 1.3427553 .584239$

H -5.0174880 .6114245 .196638$

H -3.400077 0.8197314 .491018

SCF Energy (B3LYP/6-31G**//MMFF)= -3245.91536095

0400161

MMFF Geometry

C $-4.365758-1.3145542 .720347$

C $-3.124862-1.4341012 .224320$

C -1.895673 -0.9559682 .950899$

O $-1.120616-0.1113752 .070155$

C -1.019970 -2.164619 3.326989

C $0.125550-1.7992304 .248672$

C $1.403647-1.6339463 .856465$

C $-0.241355-1.6626525 .704344$

C $1.961162-1.7076712 .462746$

C $2.904108-2.9073602 .301561$

C $3.580046-2.9242300 .922165$

C $4.464423-4.1618530 .763329$

C $5.256671-4.136422-0.552460$

$\begin{array}{llllll}\text { O } & 4.380332 & -1.744933 & 0.822242\end{array}$

O $6.258652-5.160246-0.457309$

C $5.978273-2.775478-0.683743$

C $4.369784-4.511873-1.746199$

O $6.608110-2.685256-1.959479$

C $5.046944-1.567528-0.433916$

C $5.851321-0.245007-0.350032$

O $4.121090-1.469716-1.517881$

C $-1.436879 \quad 1.2102182 .013213$

O -2.3759701 .7686082 .555858$

C $-0.3995001 .871901 \quad 1.188787$

C $-0.437740 \quad 3.197143 \quad 0.980212$

$\begin{array}{lllll}\text { C } & 0.544351 & 3.976081 & 0.217890\end{array}$

C $1.7619703 .246980-0.285348$

$\begin{array}{llll}\text { C } 5.042151 & 0.900687 & 0.272812\end{array}$

$\begin{array}{llll}\text { C } 5.755415 & 2.241355 & 0.242463\end{array}$

C $5.4761004 .564214 \quad 1.133149$

O $6.790422 \quad 2.448710-0.381893$

C 4.2777485 .3743641 .515935

C 3.8271806 .4353470 .829499

C $2.583363 \quad 7.187743 \quad 1.214898$

C 1.6482297 .4389870 .049206

C $1.2664238 .691058-0.256876$

C $1.1588256 .264588-0.773839$

C $0.3219405 .292556 \quad 0.015386$

N $5.051562 \quad 3.1853090 .977747$

H $-4.512426-0.8336553 .684425$

H -2.975436 -1.924156 1.265063

H $-2.165243-0.3940313 .853898$

H $-0.636682-2.6429482 .418656$

H -1.628017 -2.931654 3.825847

H $2.144208-1.3904504 .617719$

H $0.626953-1.4393086 .333328$ 
H $-0.965154-0.8542105 .845093$ H $-0.683300-2.5940386 .073140$ H $1.174225-1.7576611 .705568$ H $2.502333-0.7722792 .275275$ H $3.675743-2.8592323 .081501$ H $2.343252-3.8376232 .452566$ H $2.801663-2.9033500 .150725$ H $3.868262-5.0797860 .836824$ H $5.181105-4.2053321 .595757$ H $\quad 6.806057-5.111597-1.260005$ H $6.782854-2.7654910 .064823$ H $3.973348-5.526816-1.620907$ H $4.944754-4.528239-2.678659$ H $3.519402-3.839630-1.878121$ H $7.178075-1.898159-1.955836$ H $6.743790-0.3923210 .271503$ H $6.1934170 .049451-1.349759$ H $3.837799-0.546896-1.616023$ H $\quad 0.387887 \quad 1.243736 \quad 0.788721$ H $-1.2615203 .768631 \quad 1.405566$ H $1.4906562 .533735-1.067159$ H 2.2433082 .6999680 .531634 H $2.5270273 .901946-0.704598$ H $4.0907661 .028235-0.255125$ H $4.818517 \quad 0.663158 \quad 1.320074$ H 5.9426324 .9066820 .203821 H 6.2259374 .5937461 .929756 H 3.7362805 .0552072 .404265 H $4.3716296 .776488-0.047667$ H 2.9000878 .1401171 .659437 H $2.0304726 .654447 \quad 1.998324$ H $0.6007888 .893201-1.089519$ H $\quad 1.6091079 .5447640 .318242$ H $2.0150465 .792599-1.261800$ H $\quad 0.5304016 .622517-1.600551$ H $-0.589421 \quad 5.7322280 .422761$ H 4.3123152 .8519251 .590311 C $-6.569637-0.7453381 .645306$ $\begin{array}{lllll}\text { O } & -5.985508 & 0.038471 & 0.583580\end{array}$ C $-6.816317-0.084190-0.581117$ C $-8.208329-0.349545-0.031307$ C -7.886573 -1.3117541 .095947$ H -6.470863 -0.973132 -1.124614 C -6.685885 $1.141552-1.487958$ H -8.6671900 .5606390 .371468$ H $-8.882327-0.775339-0.780190$ C -8.985344 -1.417886 2.139984 H $-7.718003-2.3082340 .665465$ C $-5.2334061 .548819-1.808612$ O $-7.3131752 .261319-0.851359$ H $-7.2481840 .962964-2.412445$ H $-4.7941752 .046411-0.935177$ O $-5.2773232 .548627-2.834535$ C $-4.3040580 .401004-2.243230$ C $-2.933100 \quad 0.907372-2.725501$ H $-4.163319-0.279802-1.397356$ O $-4.911908-0.357748-3.288218$ H $-3.0379391 .359831-3.719000$ H -2.578895 $1.677331-2.028688$ H -8.695339 -2.107380 2.939274 H $-9.201627-0.4454922 .595104$ H -9.910395 -1.791170 1.688602 C $-5.593180-1.8548682 .043737$ H -7.222531 $3.023325-1.448923$ H -5.688685 2.152445 -3.621050 H $-5.0041930 .219129-4.064933$ H -5.304960 -2.430462 1.154722 H $-6.081637-2.5497482 .737021$ H -6.750026 -0.062898 2.484905 C $-0.627995-1.653757-1.301808$ O $-1.568718-0.582896-1.448434$ C -1.892390 -0.226908 -2.798885 C $-0.6284010 .216533-3.551970$ C $0.480465-0.835771-3.468110$ C $0.686476-1.338690-2.035963$ H $-0.410894-1.680666-0.227352$ C -1.272412 -2.992016-1.674602 H $-2.336125-1.092031-3.304295$
O $-\begin{array}{llll}0.148517 & 1.450417 & -3.022680\end{array}$

H $-0.8865070 .397968-4.601788$

H $1.415332-0.397293-3.839066$

O $0.141103-1.922366-4.323633$

H $1.341926-2.215975-2.036367$

O $1.368023-0.310162-1.311326$

C $-0.450991-4.164240-1.200025$

H -2.275459-3.070368 -1.236122

H -1.428650 -3.096828 -2.752100

H $-0.0382301 .332052-2.063934$

H $0.867003-2.568069-4.280114$

H $1.589212-0.665908-0.433894$

O $-0.590740-4.3188490 .146947$

O $0.240708-4.852933-1.938889$

C $0.160638-5.390680 \quad 0.724452$

H $-0.227415-6.3509960 .372564$

H $1.222486-5.2909460 .480350$

H $\quad 0.045343-5.3399971 .810373$

SCF Energy (B3LYP/6-31G**//MMFF) $=-3245.91757139$

0400162

MMFF Geometry

C -1.258955 -0.267754 3.256975

C $-2.398919 \quad 0.421643 \quad 3.417937$

C $-2.698141 \quad 1.8335602 .972668$

O -3.8469121 .7288682 .094110$

C -1.5736772 .5513592 .202687$

C -1.8041784 .0413892 .043825$

C -2.2274204 .6406650 .914041$

C -1.4687794 .8732373 .255723$

C $-2.6381383 .997929-0.381031$

C $-1.6284994 .248458-1.509923$

C $-0.3707503 .372173-1.402037$

C $0.6307443 .725474-2.503718$

C $1.8438222 .782635-2.498933$

O $-0.7856942 .009959-1.541264$

O $2.5559192 .972682-3.731135$

C $1.3399291 .324691-2.488699$

C $2.8275023 .122453-1.373873$

O $2.4197850 .409586-2.337541$

C $0.2662521 .046636-1.414602$

C $-0.348164-0.356364-1.638386$

O $0.8733361 .089620-0.121572$

C -4.8990952 .5613202 .291446$

O -4.9980503 .4248853 .152358$

C -5.9890612 .3004811 .320858$

C -5.8899351 .4403120 .294769$

C $-6.9403831 .129737-0.679530$

C $-8.2766891 .803781-0.508674$

C $-1.617999-0.614204-0.817292$

C $-2.194628-1.996289-1.069254$

C $-4.202039-3.374872-0.523972$

O $-1.654578-2.833123-1.787099$

C $-5.167806-3.164167-1.646877$

C $-6.496458-3.075407-1.484316$

C $-7.454761-2.828111-2.613743$

C $-8.253585-1.554797-2.433617$

C $-9.547378-1.600548-2.073706$

C $-7.583271-0.236380-2.757585$

C $-6.6702390 .242834-1.660680$

N $-3.390446-2.179903-0.387497$

H -0.4084890 .1846302 .756825$

H -3.222818 -0.0720963 .931945$

H -2.956337 2.3964983 .877757

H -1.4482312 .0812281 .221959$

H -0.6145752 .4287922 .721711$

H $-2.322767 \quad 5.7260820 .916973$

H -1.611738 5.9444773 .078323

H -2.1031384 .5939374 .102395$

H -0.4219924 .7244493 .540545$

H $-2.8360342 .927993-0.268383$

H -3.598271 $4.443846-0.670909$

H $-2.1344804 .023625-2.458098$

H -1.349395 $5.308956-1.535108$

H $0.0854723 .515597-0.416436$

H 0.954948 4.769771 -2.414478

H $0.1378193 .648212-3.483266$

H $2.8185073 .907666-3.784071$ 
H $\quad 0.9002301 .125585-3.476364$ H $3.1778774 .156999-1.471256$ H $3.7248092 .498776-1.435484$ H $2.3962143 .013571-0.376796$ H $3.0495030 .584235-3.057485$ H $-0.613953-0.471217-2.697522$ H $0.394815-1.129038-1.403816$ H 0.4083450 .4797170 .471988 H $-6.8951272 .870348 \quad 1.500827$ H -4.9551450 .9023710 .146993$ H -8.698539 1.5783950 .477238 H -9.021084 $1.487507-1.242371$ H -8.174246 $2.890021-0.608303$ H $-1.409305-0.5259410 .251962$ H -2.389075 $0.120936-1.076337$ H $-4.706648-3.5389470 .433305$ H -3.562046 -4.237149 -0.735671 H $-4.746959-3.067577-2.645201$ H -6.920814 -3.178136 -0.488311 H -8.127248 -3.693660 -2.674990 H $-6.934511-2.791034-3.579161$ H -10.138861 $-0.696926-1.972764$ H -10.044032 -2.543100 -1.868386 H -8.329939 $0.526177-3.006122$ H -6.995194 -0.346919-3.677971 H $-5.671500-0.191449-1.703111$ H $-3.787668-1.3994350 .127814$ C $-0.791181-2.6381882 .581468$ O $0.513161-2.3684802 .022166$ C $1.265873-3.5928242 .006747$ C $0.225527-4.7021832 .051072$ C $-0.780340-4.1066423 .015752$ H $1.842548-3.6189922 .939458$ C $2.223252-3.6497120 .813241$ H $-0.239402-4.8627901 .071205$ H $0.641379-5.6535532 .395745$ C -2.144833 -4.7721572 .959027$ H -0.384316 -4.194116 4.036534 C $3.178907-2.4436130 .686810$ O $1.452987-3.718333-0.393188$ H $2.796518-4.5836160 .862032$ H $2.620811-1.5833130 .299990$ O $4.143440-2.766410-0.320213$ C $3.906879-2.0461411 .982356$ C $5.027894-0.9960421 .840113$ H $3.169972-1.6536722 .692392$ O $4.477021-3.2064432 .595403$ H $5.364391-0.7644652 .858332$ H $5.892448-1.4658021 .361194$ H -2.842754 -4.290264 3.650847 H $-2.575907-4.7184371 .953774$ H -2.068837 -5.8288713 .235409$ C $-1.065534-1.6787623 .741914$ H $2.079076-3.748886-1.136848$ H $4.642969-3.544893-0.021841$ H $5.166559-3.5505852 .003046$ H $-0.216855-1.6789864 .436847$ H -1.947619-2.012603 4.300601 H $-1.519592-2.4844141 .778613$ C $6.1209810 .194528-0.779462$ O $4.7643020 .180643-0.312380$ C $4.5958550 .292163 \quad 1.107622$ C 5.2680151 .5768361 .611951 C 6.7321661 .6504841 .197355 C $6.8543131 .458394-0.312582$ H $6.646584-0.693139-0.411099$ C $6.0900930 .126996-2.312965$ H 3.5205240 .4214491 .284354 O $5.147592 \quad 1.6730453 .027283$ H 4.7388492 .4415301 .190440 H 7.3359030 .9208131 .749583 O $7.2348462 .941726 \quad 1.546300$ H $6.4454862 .341661-0.819088$ O $8.2349851 .381577-0.662933$ C $5.706019-1.253982-2.793200$ H $5.3782070 .861547-2.707056$ H $7.0688830 .348385-2.752620$ H 5.6045152 .4861693 .302965
H $8.1533772 .989753 \quad 1.229854$

H $8.6105920 .597162-0.227768$

O $4.625773-1.178016-3.620559$

O $6.301242-2.277034-2.479394$

C $4.145095-2.427431-4.125632$

H $3.277066-2.226002-4.758908$

H $4.916631-2.913528-4.730195$

H $3.833785-3.075596-3.300865$

SCF Energy (B3LYP/6-31G*//MMFF) $=-3245.91417178$

\section{3}

MMFF Geometry

C $-2.547715-2.2736282 .591011$

C $-2.603850-1.560188 \quad 3.726981$

C $-3.164754-0.164443 \quad 3.894208$

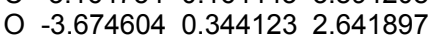

C $-2.0425590 .782617 \quad 4.355942$

C -2.5597592 .1095034 .875977$

C $-2.560026 \quad 3.255165 \quad 4.167360$

C -3.040476 2.0995506 .303581

C -2.1523013 .4279352 .732015$

C $-0.7319623 .991372 \quad 2.604797$

C -0.2150594 .0518331 .156780$

C $-1.0221175 .023557 \quad 0.290634$

C $-0.5647165 .008931-1.174352$

$\begin{array}{llll}\text { O } & -0.257501 & 2.725566 & 0.620012\end{array}$

O $-1.5596115 .691187-1.954068$

C $-0.5388943 .545176-1.659260$

C $0.7460325 .776037-1.379401$

O $-0.0182253 .459478-2.983176$

C $0.2410342 .599545-0.717013$

C $0.0989431 .117706-1.139031$

O $1.6334742 .928087-0.778611$

C $-4.9715630 .086733 \quad 2.327139$

O $-5.808669-0.4619463 .024361$

C -5.1684750 .5243410 .927306$

C $-6.131196-0.0567940 .196553$

C $-6.3889280 .163286-1.228398$

C $-5.605748 \quad 1.245117-1.923886$

C $-1.2921550 .516670-0.920233$

C $-1.416516-0.881730-1.501778$

C $-3.134189-2.660495-1.871365$

O $-0.500042-1.455146-2.083178$

C $-4.032791-2.357123-3.030238$

C $-5.274827-2.847978-3.161024$

C $-6.178070-2.525110-4.318269$

C $-7.549997-2.051186-3.885223$

C $-8.637002-2.817674-4.077528$

C $-7.675361-0.658702-3.298592$

C $-7.296203-0.625168-1.842306$

N $-2.687678-1.402259-1.301731$

H $-2.943510-1.8640571 .666940$

H -2.202656 -2.007309 4.635182

H $-3.965140-0.1968994 .643406$

H -1.335362 0.9471973 .532964

H -1.4531650 .3155815 .156963$

H -2.915404 4.1643034 .649934

H -3.3694213 .0892266 .638274$

H -3.8868241 .4156006 .419338$

H $-2.2369001 .775436 \quad 6.972925$

H -2.2395192 .4859612 .183345$

H -2.8739564 .1098132 .267825$

H -0.6831494 .9902983 .055788$

H $-0.052198 \quad 3.346848 \quad 3.178376$

H 0.8319694 .3748551 .200613

H -0.9735826 .0399140 .700865$

H -2.083778 4.7461120 .304270

H $-1.6457676 .594886-1.604988$

H $-1.5822913 .212261-1.718094$

H $0.6209236 .828493-1.097562$

H $1.0392605 .781132-2.435020$

H $1.5754895 .376915-0.792450$

H $-0.5354544 .067353-3.538625$

H $0.413742 \quad 0.999502-2.183644$

H $0.8105630 .515862-0.559473$

H $2.1485602 .156910-0.489152$

H $-4.469947 \quad 1.245674 \quad 0.518636$

H -6.761906 -0.8059290 .674287$ 
H $-5.9445451 .440541-2.943332$ H $-4.5453600 .979747-1.979435$ H $-5.7011172 .195622-1.386819$ H -1.5130380 .4616980 .152279$ H -2.058493 $1.134518-1.397773$ H $-3.651457-3.210547-1.079160$ H -2.274079 -3.247776 -2.206901 H -3.639801-1.697641-3.800920 H -5.661036 -3.526617 -2.403947 H $-6.267822-3.431853-4.930403$ H $-5.731090-1.765202-4.971412$ H -9.626596 -2.476074 -3.792503 H -8.561809-3.805612 -4.519494 H -8.713136 -0.309896 -3.377980 H $-7.086626 \quad 0.027977-3.912375$ H -7.829427 -1.361481 -1.238427 H -3.378153 -0.837357 -0.817419 C $-0.800232-3.6717801 .470306$ O $0.238644-2.7566481 .866013$ C $1.514002-3.4020091 .733226$ C $1.261253-4.6506980 .896883$ C $-0.131734-5.0404431 .350022$ H $1.820022-3.6964542 .745343$ C $2.571289-2.4518261 .150285$ H $1.260207-4.425298-0.176097$ H $2.006986-5.4306951 .077884$ C $-0.840120-5.9883030 .397757$ H $-0.058452-5.5133052 .338493$ C $2.784290-1.1603661 .968681$ O $2.226524-2.102664-0.190123$ H $3.510070-3.0121821 .099088$ H $3.022674-1.4133253 .009053$ O $1.575074-0.3925441 .998250$ C $3.878366-0.2281891 .405607$ C $5.263025-0.8912081 .313036$ H 3.5697790 .1547210 .428483 O 3.9743190 .9133382 .266536 H $5.540020-1.2575802 .309256$ H $5.206669-1.7610290 .653744$ H -1.844832 -6.2267870 .760839$ H $-0.938139-5.553262-0.602650$ H $-0.283191-6.9261270 .303398$ C -1.932498 -3.642369 2.498451 H $1.350602-1.680732-0.163951$ H $0.895921-0.9266412 .443532$ H 4.2443450 .6000653 .146597 H -1.547602 -3.9461303 .479885$ H -2.713640 -4.357111 2.216072 H -1.163457 -3.3333470 .492409$ C $6.105447-0.295173-1.564849$ O $6.0670350 .627533-0.469248$ C 6.3703020 .0650170 .811688 C $7.772417-0.5603490 .790562$ C $7.914024-1.582629-0.334066$ C $7.480475-0.969446-1.664331$ H $5.321826-1.051849-1.438714$ C $5.8108880 .498267-2.848316$ H $6.4095250 .906706 \quad 1.514290$ O $8.052424-1.1651532 .048288$ H $8.513650 \quad 0.236010 \quad 0.642275$ H $7.350852-2.499079-0.122337$ O $9.284606-1.969990-0.455068$ H $8.233552-0.239623-1.988982$ O $7.427175-1.994653-2.655080$ C $4.3786410 .981015-2.929597$ H $6.4602461 .381336-2.886960$ H $5.989138-0.122239-3.733493$ H $8.986592-1.4333192 .045591$ H $9.522722-2.4603980 .349658$ H $8.304504-2.413518-2.686548$ O $4.2617291 .821946-4.000466$ O $3.477150 \quad 0.658612-2.169415$ C $2.9606962 .376672-4.214764$ H $2.7467743 .117616-3.439957$ H $2.9598392 .875716-5.187522$ H $2.1975581 .592443-4.224713$ SCF Energy (B3LYP/6-31G**//MMFF) $=-3245.92515991$
0400164

MM̄FF Geometry

C $-1.526435-3.658004-0.788167$

C $-2.352209-4.133078-1.733915$

C $-3.584817-3.443954-2.276175$

O $-3.596673-2.035611-1.949420$

C $-4.842701-4.098929-1.677695$

C $-6.139889-3.562608-2.252718$

C $-6.985447-2.732359-1.609787$

C $-6.478697-4.055632-3.636551$

C $-6.826028-2.130715-0.240837$

C $-6.768764-0.599802-0.319464$

C -6.3816310 .0358501 .024406$

C -6.3877491 .5608290 .911028$

C -5.8598392 .2437712 .180693$

O $-5.070952-0.427896 \quad 1.360290$

O $\quad-5.5897183 .6176101 .858209$

C -4.5129681 .6003502 .578631$

C -6.9130092 .2599243 .295231$

O $-4.0837602 .123776 \quad 3.833506$

C -4.5593830 .0573082 .609211$

C $-3.171731-0.6005582 .814818$

O $-5.387152-0.3613603 .701064$

C $-2.874751-1.212756-2.761688$

O $-2.193749-1.541987-3.720423$

C $-3.0531940 .175673-2.281247$

C $-2.3777201 .171857-2.873677$

C $-2.4620112 .596411-2.542809$

C $-3.3718422 .999820-1.413474$

C $-2.146525-0.3418711 .706741$

C $-1.2442960 .854728 \quad 1.945365$

C 0.5163282 .2000690 .803082

O $-1.172388 \quad 1.4418603 .021053$

C $-0.078913 \quad 3.4724310 .291995$

C $0.4504384 .177672-0.719233$

C $-0.0977295 .489650-1.201031$

C $-0.3721475 .499707-2.687581$

C $0.5070056 .037987-3.549639$

C $-1.7063514 .971739-3.162108$

C $-1.7321323 .471546-3.266068$

N $-0.490275 \quad 1.1575160 .818948$

H -1.732744 -2.698644 -0.323613

H $-2.140069-5.108338-2.168057$

H $-3.594378-3.560114-3.367268$

H $-4.825692-3.986591-0.588706$

H $-4.816097-5.183444-1.852280$

H -7.900161 -2.436074 -2.122369

H -7.455120 -3.696603 -3.979156

H $-5.732057-3.715298-4.360412$

H $-6.507810-5.150013-3.653553$

H $-7.682300-2.4312620 .374794$

H $-5.936401-2.5093320 .265921$

H $-6.031381-0.311946-1.079092$

H $-7.741520-0.212934-0.647984$

H $-7.091573-0.2983381 .790027$

H -7.3922941 .9276490 .665267$

H -5.7498091 .8619020 .070258$

H -6.4158394 .0187121 .538213$

H -3.7797051 .9279111 .831932$

H -7.8069262 .8054092 .969152$

H -6.5454442 .7916774 .179683$

H -7.231655 1.2605623 .598524

H -3.164092 1.8435173 .974455

H $-2.771679-0.350846 \quad 3.804614$

H -3.332441 -1.688408 2.837935

H $-5.159274-1.2777763 .929242$

H -3.740874 $0.341781-1.461118$

H -1.703612 $0.925952-3.693373$

H -4.416890 $2.821392-1.687450$

H $-3.2831814 .051869-1.134946$

H $-3.1415062 .429726-0.507403$

H -2.634188 -0.2464400 .732331$

H -1.477862 -1.210439 1.654146

H 1.3352301 .8445560 .170748

H $\quad 0.8970892 .354618 \quad 1.817511$

H -0.9638873 .8489630 .799423$

H $1.3532493 .813569-1.204691$

H $0.6371726 .266941-0.953358$ 
H $-1.0108455 .766313-0.659453$ H $\quad 0.3029906 .076942-4.614574$ H $1.4530626 .446516-3.209752$ H -1.929490 5.367371-4.162411 H -2.502421 $5.371340-2.527637$ H -1.096373 $3.082241-4.062644$ H $-0.6482170 .639060-0.040302$ C $0.951338-3.537914-0.390836$ O $0.940882-2.551690 \quad 0.665231$ C $2.203416-2.6016961 .364055$ C $3.153071-3.3230590 .422908$ C $2.232182-4.351204-0.197299$ H $2.024946-3.2052782 .263623$ C $2.632945-1.1820601 .754798$ H $3.538732-2.638906-0.343282$ H $4.001628-3.7747230 .944150$ C $2.778964-4.956038-1.479162$ H $2.061044-5.1547000 .531802$ C $4.069234-1.1136302 .307547$ O $1.711521-0.7277402 .750813$ H $2.512109-0.5230490 .888571$ H $4.777513-1.4735131 .554860$ O $4.185069-2.0258693 .408544$ C 4.5301920 .2702102 .798490 C 4.3752151 .4248351 .793293 H 3.9959770 .5374023 .718258 O 5.9113580 .1819663 .167765 H 3.3130631 .6659781 .687578 H 4.8659092 .3027572 .233586 H $2.073695-5.678237-1.901933$ H $2.968983-4.187634-2.236215$ H $3.722401-5.477341-1.286304$ C $-0.312038-4.398807-0.300451$ H $1.859870 \quad 0.2233232 .883020$ H $3.529153-1.7625584 .076816$ H $5.996515-0.5492723 .803214$ H $-0.491486-4.6869490 .742603$ H $-0.178182-5.319265-0.880720$ H $0.982271-2.988034-1.340373$ C $7.0867550 .648716-0.675956$ O $6.382516 \quad 0.9639920 .532645$ C 4.9762101 .1759990 .394402 C $4.7055932 .351625-0.559436$ C $5.3982782 .138795-1.908993$ C $6.868977 \quad 1.739613-1.738659$ H $8.1477660 .665673-0.395043$ C $6.757740-0.766001-1.179254$ H $4.5163110 .275840-0.022144$ O $\quad 5.1682103 .5641320 .031087$ H $3.6231932 .446728-0.704419$ H $5.3334893 .063748-2.494856$ O $4.6968361 .125379-2.623650$ H $7.2920731 .406053-2.693322$ O $7.6171912 .888581-1.330066$ C $7.186620-1.839073-0.200176$ H $5.685598-0.891498-1.351390$ H $7.287475-0.954379-2.120056$ H $4.9509774 .289485-0.578705$ H $5.1338771 .018089-3.485651$ H $7.5202883 .563685-2.023180$ O $6.688381-3.041315-0.616942$ O $7.875953-1.6562220 .793607$ C $7.005880-4.1605710 .216086$ H $6.649398-3.9904821 .236617$ H $\quad 6.498653-5.040079-0.189219$ H $8.084911-4.3407550 .208218$ SCF Energy (B3LYP/6-31G**//MMFF) $=-3245.89537049$

\section{5}

MM̄FF Geometry

C $0.198663-1.0803974 .148858$

C $1.106784-0.9477753 .170689$

C $2.456435-0.3108243 .365010$

O $2.591593 \quad 0.7836792 .430988$

C $3.554649-1.3262393 .012874$

C $4.922225-0.9458913 .539435$

C $5.881582-0.3239932 .826627$

C $5.193978-1.3714914 .959848$
C $5.799790 \quad 0.2319211 .431749$

C $6.529115-0.6304840 .391949$

C $5.631918-1.728359-0.199077$

C $6.425395-2.636293-1.139231$

C $5.519245-3.674903-1.817984$

O $4.578857-1.077533-0.921657$

O $6.270271-4.303730-2.867363$

C $4.313760-2.949505-2.452560$

C $5.138070-4.807444-0.858658$

O $3.370994-3.885208-2.971335$

C $3.606499-1.958801-1.494817$

C $2.598422-1.101536-2.298820$

O $2.905196-2.695009-0.492570$

C 1.9907321 .9659512 .717998

O 1.2936812 .2293383 .683236

C 2.3441412 .8946351 .621567

C 1.7330464 .0830001 .519163

C 1.9935545 .0933410 .490923

C $3.0749994 .801220-0.517738$

C $1.9634570 .064676-1.524384$

C $1.0405320 .883212-2.416864$

C $-0.5588582 .793512-2.347849$

O $0.8930540 .652929-3.615055$

C $0.0781914 .123162-2.599479$

C $-0.4925125 .287329-2.252716$

C $0.1002086 .634534-2.553758$

C $0.1634947 .534482-1.339405$

C $-0.7747418 .472138-1.124196$

C $1.3611447 .406664-0.425878$

C 1.2567546 .2236990 .496215

N $0.3984361 .898648-1.721378$

H $0.422889-0.7116325 .146289$

H $0.872660-1.3117542 .172575$

H 2.5844270 .0629354 .388509

H $3.596312-1.4493121 .925812$

H $3.303812-2.3179043 .413626$

H $6.841093-0.1469763 .312082$

H $6.195502-1.0845675 .297291$

H $4.472478-0.9102035 .641611$

H $5.113674-2.4596165 .052362$

H $4.7674130 .420575 \quad 1.127087$

H 6.2798851 .2190331 .458622

H $6.8471560 .033684-0.422504$

H $7.435789-1.0686600 .826592$

H $5.207310-2.3194970 .618087$

H $7.244717-3.133183-0.604965$

H $6.902735-2.024761-1.917676$

H $\quad 6.556677-3.615335-3.491424$

H $4.691002-2.381658-3.314430$

H $6.037888-5.321023-0.498748$

H $4.541076-5.572656-1.367622$

H $4.581589-4.4631350 .015553$

H $3.852882-4.480800-3.570505$

H $3.114284-0.675429-3.169786$

H $1.794415-1.743625-2.680084$

H $2.185875-2.147276-0.141468$

H 3.1036322 .5682720 .919570

H 0.9704614 .3413212 .252709

H $4.0495014 .726771-0.022618$

H $3.1670925 .563210-1.294216$

H $2.8751873 .859064-1.040000$

H $1.376049-0.307733-0.678766$

H $2.7402910 .734957-1.140207$

H $-1.4196112 .871724-1.678303$

H $-0.8959452 .369234-3.297870$

H $1.0313554 .130928-3.122584$

H -1.462902 $5.274936-1.761068$

H $-0.5200647 .102930-3.329446$

H $1.1016056 .543108-2.992477$

H $-0.7206879 .141188-0.271743$

H - $-1.6210538 .587850-1.793220$

H $1.451030 \quad 8.301828 \quad 0.204443$

H $2.2753767 .402722-1.025982$

H $\quad 0.490203 \quad 6.3396591 .263830$

H $0.6028572 .034510-0.735679$

C $-2.273777-0.692326 \quad 3.971312$

O -2.1154230 .2079492 .855411$

C $-3.295881 \quad 0.1424812 .031744$ 
C $-4.393580-0.3201082 .972051$

C $-3.651630-1.3411453 .811481$

H $-3.086183-0.6223911 .276456$

C $-3.515740 \quad 1.5060701 .369631$

H -4.7438280 .5082993 .599950$

H $-5.252614-0.7487592 .449380$

C $-4.334697-1.6588045 .130409$

H -3.562176 -2.269490 3.232777

C -4.7517361 .6118860 .456761$

O $-2.355522 \quad 1.8405740 .605966$

H -3.5872632 .2673762 .156908$

H -4.719066 2.569406 -0.079199

O

C $-4.9510770 .486881-0.582967$

C $-3.773707 \quad 0.292449-1.554354$

H $-5.177302-0.451700-0.067179$

O $\quad-6.1228820 .802244-1.347395$

H $-3.552597 \quad 1.250693-2.040448$

H -2.882099 $0.003363-0.992504$

H $-3.750102-2.3841185 .705331$

H $-4.457366-0.760906 \quad 5.745385$

H -5.327898 -2.085468 4.956295

C -1.143366 -1.723797 3.945639

H $-1.586570 \quad 1.7702821 .197669$

H -5.8464612 .4108891 .871344$

H $-5.9498941 .629786-1.828025$

H -1.153981 -2.259631 2.987550

H -1.293245 -2.470453 4.733977

H $-2.223606-0.0833534 .882379$

C $-3.545848-2.743385-1.376663$

O $-4.518298-1.999823-2.119105$

C $-4.050931-0.759642-2.654554$

C $-2.867047-1.016133-3.597183$

C $-1.759671-1.814439-2.910736$

C $-2.332818-3.071051-2.254321$

H -3.228736 -2.165192 -0.501499

C -4.230333-4.020432 -0.877477

H $-4.869022-0.364867-3.269962$

O $-2.363163 \quad 0.221282-4.084290$

H -3.223737 -1.584023 -4.466469

H $-1.217440-1.206063-2.177878$

O $-0.798695-2.227042-3.884702$

H $-2.613233-3.793946-3.031184$

O $-1.323117-3.677439-1.449346$

C $-5.205375-3.7069510 .232491$

H $-4.770160-4.531927-1.683523$

H -3.504904 -4.736031 -0.473614

H $-1.6786580 .023927-4.745420$

H - $0.343257-1.429506-4.203585$

H $-0.557129-3.848023-2.024240$

O $-6.408907-3.336988-0.290218$

O $-4.921511-3.7632401 .421892$

C $-7.410946-2.9761310 .665234$

H -7.090006 -2.099846 1.235971

H $-8.324656-2.7239520 .120602$

$\mathrm{H}-7.620819-3.8174031 .332683$

SCF Energy (B3LYP/6-31G**//MMFF) $=-3245.91993496$

0400166

MM̄FF Geometry

C $3.521036-3.6803560 .243604$

C $2.601853-2.7735370 .606741$

C $1.119120-2.9704040 .459242$

O $0.652064-2.011672-0.517730$

C $0.407519-2.6586451 .786830$

C $-0.975821-3.2720881 .865519$

C $-2.123158-2.6565251 .518450$

C $-1.011511-4.6767552 .412968$

C $-2.301089-1.2823210 .933196$

C $-2.944431-1.311519-0.461828$

C $-4.419671-1.744682-0.477681$

C $-4.917855-1.863325-1.919323$

C $-6.400726-2.252872-1.969349$

O $-5.191409-0.7592820 .214330$

O $-6.868737-2.040521-3.310769$

C $-7.200787-1.296710-1.059717$

C -6.604767 -3.743631-1.674369

O $-8.554625-1.727101-0.935774$
C $-6.583932-1.0791580 .347688$

C -7.2922950 .1154451 .034104$

O $-6.782934-2.2112231 .196222$

C $0.652653-2.374908-1.825523$

O $0.980521-3.459804-2.284857$

C $0.139153-1.280161-2.680976$

C $0.1426490 .006973-2.299778$

C $-0.3785721 .139893-3.069144$

C $-1.1510460 .830590-4.324273$

C -6.8056680 .4470442 .452525$

C -5.3850150 .9704222 .536104$

C $-3.8608342 .716513 \quad 1.644857$

O -4.5538140 .4494613 .277136$

C $-3.779773 \quad 3.466857 \quad 0.354455$

C $-2.9701763 .124881-0.659332$

C $-2.9069263 .875448-1.960547$

C $-1.5371734 .455600-2.232484$

C $-1.1742785 .638808-1.709429$

C $-0.6224233 .699753-3.172280$

C $-0.1710472 .386960-2.597014$

N -5.1556932 .0700531 .726916$

H $3.204859-4.629371-0.181035$

H $2.930350-1.8151050 .998868$

H $0.885204-3.9911470 .131806$

H $0.348198-1.5746961 .941253$

H $0.994207-3.0418442 .633186$

H -3.051048 -3.205392 1.670925

H -2.025665 -5.089002 2.441047

H $-0.402696-5.3431601 .793776$

H $-0.618475-4.695756 \quad 3.434715$

H $-2.913287-0.6881881 .616794$

H -1.344179 -0.7592900 .851201$

H -2.870801 $-0.299094-0.879059$

H -2.363029 -1.975930-1.111753

H $-4.519919-2.7071100 .033372$

H $-4.313899-2.584683-2.484078$

H $-4.791868-0.899183-2.431746$

H $-6.317267-2.573523-3.908882$

H -7.231814 -0.322366 -1.568019

H $-6.087025-4.355971-2.422669$

H -7.662506 -4.020028 -1.742897

H $-6.230367-4.042438-0.693043$

H $-8.912780-1.819430-1.835303$

H -7.195739 1.0047810 .400186

H -8.364382 -0.1058511 .115358$

H $-7.262402-2.8940760 .701724$

H -0.224750 -1.591995 -3.654291

H $0.5642330 .264896-1.329384$

H $-0.4953460 .378915-5.076568$

H $-1.6080191 .708498-4.784755$

H $-1.9714100 .135839-4.109842$

H -7.458779 1.2131722 .888303

H $-6.885940-0.4363303 .096113$

H -3.0734481 .9612531 .738928$

H -3.7730753 .4049222 .491345$

H -4.4189824 .3409790 .254551$

H -2.337672 2.246844 -0.556580

H -3.651803 4.682251-1.977005

H $-3.1953363 .199090-2.774653$

H $-0.2042936 .077017-1.918937$

H -1.839850 $6.193034-1.055921$

H $\quad 0.2792684 .290192-3.381892$

H -1.126345 $3.592104-4.136304$

H $0.4033982 .500072-1.678668$

H -5.9574242 .5597361 .340013$

C $5.566118-2.830270-0.923875$

O $5.038861-1.495848-1.075496$

C $6.121716-0.589651-1.342331$

C $7.282460-1.465389-1.792380$

C $7.088194-2.679889-0.903358$

H $6.386655-0.120932-0.386664$

C $5.6774920 .485029-2.337705$

H $7.192955-1.750128-2.847059$

H $8.251847-0.978480-1.650800$

C $7.833958-3.912625-1.383299$

H $7.427781-2.4299650 .111000$

C $4.3896121 .228573-1.922811$

O $5.426373-0.141178-3.602167$ 
H $6.497018 \quad 1.194556-2.502150$

H $3.5339510 .545741-1.986972$

O $4.1474292 .248707-2.900942$

C $4.4329341 .868047-0.520547$

C $3.1540292 .668203-0.217647$

H $4.554698 \quad 1.081150 \quad 0.227904$

O $5.5700432 .724848-0.446414$

H $3.0510533 .472355-0.955921$

H $2.2992421 .996845-0.345428$

H $7.645503-4.763898-0.721672$

H $7.528047-4.198118-2.395413$

H $8.912929-3.727725-1.398192$

C $5.001005-3.4421470 .358315$

H $5.137617 \quad 0.554538-4.217589$

H $4.8617582 .904236-2.822443$

H 5.7224632 .9320840 .490822

H $5.199131-2.7757811 .206392$

H $5.489906-4.3975250 .579688$

H $5.242504-3.402991-1.802630$

C 2.2558991 .4140172 .430107

O 3.3197102 .3558112 .239711

C 3.1194913 .3057661 .189624

C 1.8517604 .1217591 .468400

C 0.6366883 .2212151 .654372

C 0.9219852 .1368832 .691718

H 2.1759340 .7669361 .548018

C 2.6310090 .5385033 .639454

H 3.9628474 .0050841 .243432

O 1.6199375 .0458410 .410990

H 2.0006124 .7108572 .382835

H 0.3127532 .7874780 .702551

O $-0.449580 \quad 4.0314432 .111253$

H 0.9253552 .5988253 .687702

O -0.1619251 .2100132 .652701$

C $3.860409-0.3167363 .415296$

H $2.838109 \quad 1.1749434 .508122$

H $1.806730-0.1419733 .878159$

H 0.7971335 .5215680 .616984

H -1.1959513 .4360142 .293933$

H -0.1109700 .6538653 .447632$

O $3.996792-1.1702064 .474259$

O $4.612173-0.2443392 .454240$

C $5.126283-2.0476764 .425869$

H $6.045909-1.4902974 .223114$

H $5.221229-2.5320455 .401408$

H $4.963657-2.8170303 .666439$

SCF Energy (B3LYP/6-31G**//MMFF) $=-3245.91172275$

0400167

MM̄FF Geometry

C $2.3186554 .583714-0.211991$

C $3.5664904 .092352-0.175088$

C 4.3082693 .5564321 .029285

O 4.3154552 .1098340 .931758

C 3.7027133 .9364162 .395661

C 4.5669123 .5339493 .579160

C 4.2638022 .5641814 .465951

C 5.8326554 .3349843 .754949

C $3.052427 \quad 1.674976 \quad 4.483065$

C 3.4220820 .2334474 .113381

C $2.182209-0.6100073 .782677$

C $2.580902-2.0595583 .501220$

C $1.397048-2.9046533 .008594$

O $1.574902-0.0309952 .623873$

O $1.923299-4.1243212 .462310$

C $0.695232-2.1604131 .852492$

C $0.469077-3.3098694 .159709$

O $-0.496571-2.837347 \quad 1.464096$

C $0.382308-0.6855552 .174223$

C $-0.133287 \quad 0.1053650 .947096$

O $-0.642045-0.6309843 .173826$

C 5.2485441 .5354340 .124460

O $6.1260542 .103281-0.506122$

C 4.9959550 .0770930 .112740

C $5.620054-0.694141-0.790063$

C $5.474971-2.143801-0.941964$

C $4.536977-2.855312-0.003802$

C $0.8547160 .220453-0.219129$
C $0.650049-0.806432-1.319022$

C $1.704161-1.617357-3.428592$

O $-0.336020-1.534260-1.389287$

C $2.420882-2.897148-3.133025$

C $3.431705-3.366627-3.880368$

C $4.110828-4.684186-3.636575$

C $5.616734-4.566403-3.562925$

C $6.383916-4.831124-4.634102$

C $6.235391-4.235695-2.224184$

C $6.180724-2.765152-1.910085$

N $1.687176-0.781379-2.243085$

H 1.7163654 .6238010 .690441

H $4.1242674 .053759-1.109602$

H 5.3366953 .9351670 .981220

H 2.7068763 .4926652 .480132

H 3.5527955 .0230592 .446224

H 4.9636682 .3753185 .279295

H 6.3547964 .0882674 .685756

H 6.5273514 .1479022 .930793

H 5.6042645 .4054083 .783866

H 2.6226341 .6910205 .492238

H 2.2669152 .0519663 .825132

H 4.0869800 .2466153 .240567

H $3.979353-0.2237144 .940341$

H $1.481715-0.5572444 .624470$

H $3.037274-2.515750 \quad 4.388610$

H $3.359259-2.0751742 .727216$

H $2.435362-4.5686953 .159644$

H $1.371173-2.2000440 .990409$

H $1.015579-3.9071674 .899669$

H $-0.347008-3.9484083 .804189$

H $\quad 0.036708-2.4546524 .683279$

H $-0.253257-3.754736 \quad 1.252079$

H -1.103796 -0.2882490 .624669$

H $-0.336161 \quad 1.1316621 .286059$

H $-1.079947 \quad 0.234694 \quad 3.122284$

H $4.292714-0.3190540 .835426$

H $6.305895-0.220096-1.491273$

H $4.388034-3.909038-0.248184$

H $3.542990-2.397266-0.031555$

H $4.918944-2.8104321 .021435$

H 1.8901900 .1796380 .131534

H $\quad 0.712027 \quad 1.198676-0.693042$

H $2.190960-1.043589-4.223556$

H $\quad 0.675436-1.833969-3.734192$

H $2.066787-3.482842-2.287901$

H $3.761943-2.794424-4.744564$

H $3.832620-5.356822-4.458758$

H $3.736849-5.165346-2.724416$

H $7.466286-4.779486-4.581023$

H $5.945649-5.099659-5.589631$

H $7.294601-4.527190-2.219082$

H $5.778530-4.857358-1.448947$

H $6.828934-2.162033-2.547542$

H $2.490988-0.183709-2.073583$

C $0.6828834 .064796-2.040936$

O $-0.4045293 .853253-1.120006$

C $-1.6407863 .779048-1.861917$

C $-1.2458833 .698354-3.328850$

C $0.0321994 .511966-3.348193$

H $-2.1663934 .719500-1.655287$

C $-2.4576412 .584349-1.355204$

H $-1.0431872 .660258-3.621725$

H -2.017886 $4.095503-3.994523$

C $0.8855794 .269056-4.580869$

H $-0.2286505 .577446-3.293674$

C $-3.7581902 .342182-2.140834$

O -2.7920022 .8251080 .015302$

H $-1.8320471 .685477-1.373779$

H -3.525222 $2.075277-3.177996$

O $-4.4962483 .570083-2.205425$

C $-4.6910831 .272197-1.540460$

C $-4.027862-0.105979-1.390375$

H $-5.0892501 .614289-0.578466$

O $-5.8122231 .123573-2.419996$

H $-3.720827-0.467819-2.379419$

H $-3.133453-0.006066-0.767671$

H $1.8009384 .867963-4.547102$ 
H $\quad 1.174598 \quad 3.216251-4.667660$ H $0.3369784 .542510-5.488099$ C $1.6635755 .088662-1.468620$ H -1.958953 2.9761180 .494227 H -4.683114 $3.847652-1.291983$ H -6.224798 $1.999411-2.512952$ H $1.1387586 .019109-1.219129$ H $2.4240645 .339361-2.217259$ H $1.182073 \quad 3.096217-2.175282$ C $-6.094153-1.5529471 .364339$ O $-5.204506-0.728140 \quad 0.601450$ C $-4.964873-1.148138-0.746756$ C $-4.353928-2.557877-0.769624$ C $-5.221666-3.5512680 .008444$ C $-5.604894-3.0106931 .390613$ H $-6.028801-1.1604362 .387593$ C -7.546599-1.405072 0.898413 H $-5.911177-1.158169-1.297205$ O $-3.049782-2.518127-0.196943$ H -4.249441 -2.881077 -1.812292 H $-4.672813-4.493792 \quad 0.125056$ O $-6.394881-3.821428-0.752615$ H $-6.374117-3.6451421 .844848$ O $-4.461728-3.0863422 .248390$ C $-8.049041-0.0086001 .177373$ H -7.686562 $-1.620557-0.164778$ H -8.201874 -2.096974 1.440815 H $-2.668240-3.409545-0.268826$ H $-6.922367-4.471400-0.257785$ H $-3.786197-2.4798621 .898849$ O $\begin{array}{llll}\text { O } & -8.488055 & 0.352730 & 2.261520\end{array}$ C -8.3062252 .1348850 .214825$ H $-8.1435012 .641804-0.739974$ H -9.3690342 .1937340 .467432$ H -7.6979852 .6259940 .980272$ SCF Energy (B3LYP/6-31G**//MMFF) $=-3245.91811547$

04_00168

MM̄FF Geometry

C $1.4969123 .310809-1.926466$

C $2.3289142 .317152-1.581163$

C $2.7115771 .176948-2.487718$

O $2.322968-0.049966-1.826957$

C $4.2381231 .125856-2.653769$

C $4.6823600 .293558-3.838524$

C $5.046603-1.002396-3.780944$

C $4.7483301 .039458-5.146551$

C $4.997945-1.933568-2.601510$

C $6.385271-2.286605-2.049729$

C $6.959321-1.202010-1.124927$

C $8.363957-1.585742-0.653244$

C $8.917397-0.5843240 .371980$

O $6.081423-1.0846330 .001448$

O $10.091434-1.1575350 .967211$

C $7.867479-0.3699461 .483856$

C $9.3959820 .705565-0.302848$

O $8.277250 \quad 0.6604172 .380151$

C $6.449736-0.0701950 .943424$

C $5.426254-0.1012522 .105182$

$\begin{array}{lllll}\text { O } & 6.443425 & 1.231147 & 0.352351\end{array}$

C $1.018273-0.421661-1.863480$

O $0.1124490 .107821-2.485021$

C $0.881851-1.562989-0.932146$

C $-0.326237-1.856676-0.433080$

C $-0.620347-2.8712770 .579361$

C $0.514781-3.7449011 .043470$

C $3.955689-0.0855551 .658116$

C 3.0062680 .0346632 .839669

C $0.589974 \quad 0.333522 \quad 3.375169$

O $3.387344 \quad 0.0412684 .007762$

C $0.020811-0.9911503 .776551$

C -1.297509-1.234709 3.841464

C -1.888706 -2.541013 4.291423

C -2.922346 -3.081222 3.326985

C $-4.236233-2.9367063 .569088$

C -2.429301-3.852635 2.122034

C $-1.878806-2.9436681 .059823$
N 1.6812790 .1402052 .436449

H $1.0792383 .341575-2.929222$

H $2.7298542 .292444-0.570680$

H $2.2126351 .247968-3.462101$

H $4.6956020 .736011-1.737999$

H $4.6441742 .138610-2.779997$

H $5.383847-1.477789-4.701658$

H $5.0731210 .401320-5.975125$

H $3.7638421 .441254-5.406766$

H $5.4557581 .872074-5.073609$

H $4.354549-1.555515-1.803592$

H $4.519887-2.859103-2.947935$

H $6.281110-3.216308-1.474728$

H $7.080316-2.491207-2.873149$

H $6.987469-0.250112-1.666220$

H $9.049168-1.681911-1.504763$

H $8.328756-2.579582-0.185200$

H $\quad 9.840002-1.9966971 .389059$

H $7.820744-1.2944562 .075851$

H $10.1982730 .486129-1.017794$

H $\quad 9.828610 \quad 1.3977530 .428088$

H $8.6048461 .227535-0.844970$

H $9.167306 \quad 0.4311112 .698221$

H $5.576122-1.0132192 .698024$

H 5.6069210 .7522632 .771213

H $5.556427 \quad 1.6156190 .429341$

H $1.785750-2.078035-0.627240$

H $-1.180622-1.271991-0.770208$

H $0.941486-4.2986280 .199618$

H $\quad 0.220562-4.4876161 .787233$

H $1.305254-3.1395441 .500912$

H 3.7655380 .7588520 .989700

H $3.714589-1.0100941 .121370$

H $-0.152514 \quad 0.9684702 .883284$

H 0.9667040 .8549944 .261019

H $0.720059-1.7749554 .057871$

H -1.995601 -0.4389723 .592216$

H -2.348317 -2.375203 5.274606

H -1.111202 $-3.298357 \quad 4.451254$

H $-4.983808-3.3421692 .895647$

H $-4.593926-2.4033084 .443598$

H -3.254528 -4.4200801 .671668$

H -1.714078 -4.609093 2.455016

H -2.623005 -2.254073 0.658693

H 1.4535330 .0737831 .448945

C $-0.4420114 .310251-0.692069$

O $-0.7789023 .019699-0.148257$

C -1.7204973 .1895090 .930731$

C -2.1588634 .6460940 .882002$

C $-0.916977 \quad 5.328120 \quad 0.343574$

H -1.1583602 .9857021 .849610$

C -2.8639712 .1817740 .757258$

H $-2.998867 \quad 4.7781230 .188598$

H -2.4655735 .0244451 .861825$

C $-1.180096 \quad 6.711718-0.224112$

H -0.1836125 .4072221 .157319$

C -3.9263682 .2606581 .865720$

O -2.3065680 .8644510 .759766$

H $-3.3209452 .326525-0.227357$

H $-4.367733 \quad 3.263671 \quad 1.894314$

O $-3.2669762 .070783 \quad 3.123750$

C -5.0496201 .2093671 .743791$

C -5.9030521 .3898420 .474785$

H -4.6296560 .1987251 .789978$

O

H -6.3143332 .4067120 .478276$

H -5.266735 $1.303172-0.409780$

H $-0.2573837 .157496-0.608861$

H -1.905485 $6.677937-1.043815$

H -1.5785847 .3755750 .550038$

C $1.0621774 .382648-0.965121$

H -1.601294 0.8500450 .090335

H -3.9495902 .0945083 .816016$

H -6.2948082 .2314272 .865277$

H $1.6114284 .278762-0.020761$

H $1.3238585 .359979-1.385721$

H -1.003970 $4.416598-1.629351$

C $-5.957143-1.379265-0.847918$ 
O $-6.616147-0.972638 \quad 0.360099$

C -7.0728510 .3826910 .383524$

C -8.038652 $0.635611-0.782050$

C $-7.4179190 .254366-2.121928$

C $-6.869387-1.171148-2.066892$

H $-5.032574-0.802425-0.965662$

C $-5.580887-2.854949-0.649245$

H -7.6611100 .4903851 .303058$

O $-8.4408612 .000865-0.791645$

H -8.944142 $0.034117-0.627466$

H -6.637358 $0.963401-2.422073$

O $-8.4204320 .300386-3.139926$

H -7.705715 -1.881306 -2.035955

O $-6.139989-1.423309-3.265647$

C $-4.717639-3.453531-1.733541$

H $-5.056190-2.9855410 .303979$

H $-6.494578-3.457232-0.566000$

H -9.147419 2.096122 -1.452169

H -8.680338 $1.229951-3.253708$

H -6.745457-1.272198 -4.011784

O $-3.628724-2.671305-1.973756$

O $-4.968079-4.521965-2.277699$

C -2.740436 -3.145008 -2.991048

H $-2.227706-4.047683-2.646544$

H -3.285679 -3.339532 -3.919801

H $-1.996508-2.367186-3.182491$

SCF Energy (B3LYP/6-31G**/MMFF)= -3245.91948884

0400169

MM̄FF Geometry

C $0.040799-2.9627161 .550536$

C $0.026331-2.7697662 .879019$

C $-0.521518-1.5505813 .584982$

O $-1.577661-0.9470602 .801498$

C $0.587561-0.511632 \quad 3.806814$

$\begin{array}{llll}\text { C } & 0.176628 & 0.572892 & 4.782979\end{array}$

C -0.2494531 .8031504 .436761$

C $0.3093960 .204608 \quad 6.239070$

C $-0.433557 \quad 2.359413 \quad 3.053764$

C -1.853927 2.9023092 .846859

C $-2.0329873 .487557 \quad 1.437950$

C $-3.3921324 .176203 \quad 1.304302$

C -3.627660 $4.693995-0.123129$

O $-1.939988 \quad 2.414164 \quad 0.496023$

O $-5.0083625 .069363-0.238976$

C $-3.3509623 .552454-1.124331$

C $-2.835140 \quad 5.976703-0.396662$

O $-3.4103794 .025210-2.468161$

C $-2.0093982 .821184-0.876934$

C $-1.938172 \quad 1.554603-1.759522$

O $-0.9316243 .688326-1.228581$

C $-2.810949-1.5155332 .910918$

O $-3.144247-2.4071903 .676213$

C $-3.682739-0.9168621 .877700$

C $-4.745913-1.6209651 .461141$

C $-5.673706-1.245726 \quad 0.394834$

C $-5.612436 \quad 0.162390-0.128011$

C $-0.7946260 .592363-1.414204$

C $-0.977942-0.710765-2.167074$

C $-2.391180-2.769448-2.191354$

O $-0.363423-0.949099-3.203669$

C $-3.737250-2.515583-2.798683$

C $-4.800453-3.314085-2.614932$

C $-6.149752-3.056512-3.226863$

C $-7.288247-3.182682-2.235179$

C $-8.140515-4.220193-2.292438$

C $-7.486494-2.070110-1.224515$

C $-6.526143-2.181781-0.071528$

$\mathrm{N}-1.922873-1.539246-1.579666$

H $-0.326697-2.1840490 .888213$

H $0.415708-3.5521653 .527079$

H $-0.929057-1.8699014 .552460$

H $0.890986-0.0759722 .848101$

H $1.487242-1.0013354 .201971$

H -0.4878652 .5038435 .236261$

H $0.033705 \quad 1.0296326 .904497$

H $-0.337387-0.6436336 .483305$

H $1.344599-0.0697136 .467382$
H $\quad 0.284107 \quad 3.178348 \quad 2.927441$

H $-0.212083 \quad 1.6185972 .281526$

H -2.573356 2.0882573 .003282

H $-2.061803 \quad 3.6762783 .596263$

H - $1.2222124 .202473 \quad 1.254557$

H -3.490620 4.9936692 .029187

H -4.1867203 .4585421 .552521$

H $-5.5535314 .287500-0.047525$

H $-4.1644572 .821971-1.018958$

H $-3.132793 \quad 6.7652230 .305044$

H $-3.0512726 .369789-1.396326$

H $-1.7551295 .843349-0.305791$

H -4.266795 4.471901-2.581190

H $-2.881026 \quad 1.006814-1.667620$

H $-1.8444041 .839031-2.815681$

H $-0.1445763 .150506-1.410720$

H $-3.3952170 .035801 \quad 1.449561$

H -4.930588 -2.5962401 .910785$

H $-5.565120 \quad 0.8858220 .693685$

H $-6.4889810 .441528-0.717525$

H $-4.7315060 .289929-0.759902$

H $0.1770451 .014706-1.688419$

H $-0.7586450 .371036-0.341489$

H $-2.430033-3.529531-1.404922$

H $-1.686928-3.091692-2.964311$

H $-3.840180-1.634396-3.428146$

H $-4.691240-4.212299-2.011476$

H -6.283782 -3.776754 -4.044318

H $-6.192098-2.063412-3.691414$

H $-8.972949-4.306312-1.601885$

H -8.022605 $-5.009495-3.027357$

H $-8.503875-2.109093-0.814196$

H -7.414117 -1.113910 - 1.750048

H $-6.518903-3.1666710 .397980$

H -2.398587 -1.224737 -0.739416

C $1.849225-4.1486700 .192228$

O $1.814605-3.200412-0.891451$

C $3.122530-2.580064-0.993830$

C $4.022703-3.352367-0.035893$

C $3.043656-3.7464491 .051304$

H $2.982334-1.552033-0.641304$

C $3.563711-2.585638-2.463948$

H $4.427405-4.251245-0.517416$

H $4.862264-2.7611950 .336629$

C $3.555023-4.8440561 .966111$

H $2.815444-2.8546931 .648412$

C $4.987367-2.072276-2.746842$

O $2.641028-1.805110-3.226305$

H $3.478871-3.611767-2.845061$

H $5.114312-1.975436-3.832969$

O $5.909921-3.084433-2.328442$

C $5.397274-0.738621-2.086041$

C $4.532110 \quad 0.466051-2.489310$

H $5.413154-0.850418-0.999392$

O $6.754127-0.469773-2.468755$

H $4.5568200 .558831-3.581900$

H $3.493786 \quad 0.285194-2.202061$

H $2.807613-5.1001592 .723408$

H $3.794914-5.7545361 .406894$

H $4.462869-4.5193382 .484936$

C $0.498003-4.2363160 .899375$

H $1.748088-2.142030-3.038456$

H $6.806124-2.758614-2.519786$

H $6.772543-0.351183-3.434023$

H $0.527631-5.0488231 .635531$

H $-0.269232-4.5118000 .164553$

H $2.041699-5.126200-0.271421$

C 3.6960741 .7668710 .163736

O $4.9998591 .767131-0.433100$

C $5.0193171 .795916-1.865479$

C $4.2756873 .036958-2.377169$

C $2.8621923 .114730-1.814109$

C $2.8920452 .992944-0.292768$

H $3.1652620 .847293-0.109193$

C $3.8814441 .773122 \quad 1.691418$

H $6.0704401 .921593-2.152767$

O $4.2146593 .049137-3.799308$

H $4.8207153 .939562-2.071530$ 
H $2.2091782 .355681-2.259793$

O $2.2925434 .382042-2.151374$

H 3.3189963 .9133370 .127249

O 1.5469862 .8898350 .163882

C 4.3856860 .4491992 .224293

H 4.6034912 .5420671 .988316

H 2.9228731 .9705332 .183203

H $5.1281583 .014080-4.131040$

H $2.3103364 .457618-3.120970$

H 1.5328703 .1618601 .095788

O $4.1290790 .379483 \quad 3.564162$

O $4.955709-0.4056761 .562267$

C $4.557190-0.8239964 .209900$

H $4.249767-0.7747875 .257830$

H $4.083105-1.6949693 .746908$

H $5.647185-0.9077584 .165752$

SCF Energy (B3LYP/6-31G**//MMFF) $=-3245.91757062$

04_00170

MM̄FF Geometry

C $0.167269-1.4802222 .689120$

C $0.427588-2.6334272 .053415$

C $-0.092934-3.0582270 .698999$

O $-0.941365-2.047943 \quad 0.109739$

C $-0.924475-4.3432970 .847196$

C $-1.409951-4.891933-0.480044$

C $-2.670224-4.761101-0.939861$

C $-0.387007-5.662451-1.272841$

C $-3.798285-4.013761-0.283602$

C $-4.622790-3.219344-1.306760$

C $-5.725113-2.404202-0.616360$

C $-6.706526-1.833435-1.640216$

C $-7.773210-0.955372-0.968575$

O $-5.098562-1.3344360 .101537$

O $-8.480605-0.247447-1.997559$

C $-7.0732450 .078874-0.062355$

C $-8.834789-1.798934-0.254641$

$\begin{array}{lllll}\text { O } & -8.025224 & 0.817810 & 0.699627\end{array}$

C $-6.002382-0.5336390 .874408$

C $-5.195996 \quad 0.6061051 .539064$

O $-6.644200-1.3023541 .892551$

C $-0.339272-1.089996-0.644681$

O $0.856131-0.965588-0.855450$

C -1.397594 -0.187471-1.149865

C $-1.0426510 .943001-1.777179$

C $-1.9421591 .985724-2.271424$

C -3.420533 $1.705035-2.252789$

C -3.9333860 .1549722 .285789$

C -3.1491041 .3670582 .753613$

C -1.7901113 .2680301 .859495$

O $-3.155238 \quad 1.728096 \quad 3.927743$

C -2.3449584 .2282210 .853322$

C -1.5865384 .9408990 .006376$

C -2.146677 $5.880278-1.024556$

C $-1.6065225 .619894-2.415835$

C $-0.7613686 .485434-3.000944$

C $-2.0995544 .392775-3.158246$

C $-1.3981363 .142831-2.703353$

N -2.491494 2.0060221 .714920

H $-0.485418-0.7397582 .236229$

H $1.091100-3.3478742 .538205$

H $\quad 0.764963-3.2532140 .043209$

H -1.772757 -4.1596501 .517273$

H $-0.329697-5.1206961 .345785$

H -2.922558 -5.238873 -1.885482

H $-0.803464-6.077225-2.196956$

H $0.450138-5.015055-1.551728$

H $\quad 0.000687-6.498717-0.682129$

H -4.449965 $-4.742003 \quad 0.213679$

H -3.431752 -3.327025 0.484519

H $-3.957879-2.545933-1.863157$

H -5.070382 -3.915790 -2.026657

H $-6.249854-3.0580670 .090026$

H -7.181024 -2.635487 -2.218986

H -6.155199 -1.222949-2.369165

H $-7.838391 \quad 0.284041-2.498202$

H $-6.5757880 .804324-0.721187$

H $-9.333930-2.466409-0.967597$
H $-9.624975-1.1670590 .165951$

H -8.426046 -2.416780 0.547507

H $-8.670840 \quad 1.1902970 .074820$

H -4.8977621 .3227920 .765350$

H -5.8362641 .1540052 .242802$

H $-6.052900-1.3637632 .659285$

H -2.430184-0.452683 -0.954817

H $\quad 0.017321 \quad 1.148630-1.910230$

H -3.791976 $1.672587-1.225352$

H $-3.6390280 .744716-2.733915$

H $-4.0097122 .450612-2.791198$

H $-4.185074-0.4507373 .162474$

H -3.283242 -0.4541571 .648125$

H -0.7282083 .0675241 .691805$

H -1.9235913 .6614602 .871430$

H -3.4266904 .3366550 .815842$

H -0.5041834 .8520970 .059706$

H -1.901121 $6.902638-0.709236$

H -3.242324 $5.830348-1.054771$

H $-0.3870496 .318016-4.005474$

H $-0.4200157 .378062-2.487213$

H -1.915286 $4.504151-4.234520$

H $-3.1857254 .337877-3.046344$

H $-0.3101993 .229538-2.708601$

H $-2.497024 \quad 1.576396 \quad 0.795236$

C $1.964534-0.2397774 .000171$

O $3.073981-0.9575923 .426666$

C $3.860319-0.0397302 .630502$

C 3.3214371 .3486352 .949214

C 1.8555781 .0628273 .212542

H $3.651111-0.3037221 .587336$

C $5.344287-0.2544182 .955807$

H 3.7926601 .7530463 .853664

H 3.4820232 .0595162 .135324

C 1.1424822 .1740383 .961762

H 1.3601900 .9020922 .247169

C 6.3376300 .6574542 .213376

O $5.686263-1.6164052 .689459$

H $5.482492-0.1144264 .035940$

H 7.3554350 .3277842 .460348

O 6.2112331 .9813502 .741662

C 6.2139350 .7319330 .675138

C $6.327345-0.629400-0.031078$

H 5.2760661 .2258890 .400084

O 7.2618351 .5893310 .203686

H $7.193330-1.1659720 .376634$

H $5.440494-1.2250040 .204227$

H $0.093366 \quad 1.9145734 .134862$

H 1.6069682 .3655854 .934802

H 1.1726893 .1061073 .388246

C $0.723672-1.1338784 .041811$

H $5.050287-2.1748513 .168651$

H 6.8524052 .5438122 .274237

H 8.1106241 .1626050 .412046

H -0.067703 -0.642512 4.620255

H $0.973212-2.0590994 .577199$

H $2.254479-0.0231525 .037228$

C $4.191390-0.228823-2.187402$

O $5.5220940 .298793-2.181190$

C $6.502128-0.538132-1.564063$

C $6.554427-1.901358-2.269661$

C $5.176645-2.557444-2.334607$

C $4.151147-1.581711-2.910271$

H $3.834213-0.337286-1.156183$

C $3.3001970 .790950-2.904829$

H $7.470754-0.054140-1.741779$

O $7.479212-2.755547-1.605430$

H $\quad 6.925758-1.755868-3.292671$

H $4.856955-2.927724-1.353504$

O $5.232776-3.695811-3.197250$

H $4.346841-1.441697-3.981273$

O $2.847881-2.145687-2.787002$

C $3.2298602 .101696-2.159248$

H $3.6980561 .010556-3.903602$

H $2.2784430 .420932-3.042693$

H $7.581570-3.557117-2.145248$

H $5.797392-4.360828-2.768595$

H $2.861371-3.009570-3.233628$ 
O $2.440532 \quad 1.975550-1.055618$

O $3.801888 \quad 3.121016-2.524174$

C $2.241144 \quad 3.178457-0.307943$

H $1.7864593 .947778-0.939354$

H $1.5594872 .956906 \quad 0.516831$

H 3.1920543 .5298220 .103334

SCF Energy (B3LYP/6-31G*//MMFF) $=-3245.90750603$

04_00171

MMFF Geometry

C $0.0948261 .991101-3.277755$

C $-0.437697 \quad 1.093507-2.434444$

C $-0.616465-0.368927-2.749836$

O $-2.034289-0.635733-2.639802$

C $0.118298-1.230992-1.708411$

C $0.230728-2.691394-2.095465$

C $-0.514244-3.684659-1.572340$

C $1.299352-3.010586-3.108828$

C $-1.645855-3.577915-0.589249$

C $-1.300128-4.1642610 .786576$

C $-0.362326-3.2746121 .618649$

C $-0.054447-3.933816 \quad 2.965614$

C $0.771444-3.0194943 .882792$

O $-1.025518-2.025977 \quad 1.831087$

O $0.726334-3.5935015 .197651$

C $0.098623-1.6307293 .944551$

C $2.247199-3.0032873 .472180$

O $0.902128-0.7102404 .679777$

C $-0.261259-1.0547082 .556171$

C $-1.146756 \quad 0.204422 \quad 2.716990$

O $0.945037-0.7055351 .874704$

C -2.623006 -1.415975 -3.579103

O $-2.086665-1.908831-4.562026$

C $-4.062071-1.623979-3.289909$

C $-4.663420-1.239827-2.152167$

C $-6.075988-1.438097-1.810775$

C $-6.965312-2.060532-2.855822$

C $-1.6897390 .744401 \quad 1.389069$

C $-2.683038 \quad 1.868323 \quad 1.596334$

C $-5.0158402 .323442 \quad 2.308902$

O $-2.382773 \quad 3.043076 \quad 1.396968$

$\begin{array}{llll}\text { C } & -6.303686 & 1.568147 & 2.256778\end{array}$

$\begin{array}{lllll}C & -7.234932 & 1.750881 & 1.308757\end{array}$

C $-8.527926 \quad 0.990762 \quad 1.255966$

C $-8.648787 \quad 0.129367 \quad 0.018611$

C $-9.4706290 .474462-0.986747$

C $-7.892687-1.180727-0.010270$

C $-6.509614-1.047567-0.592975$

$\mathrm{N}-3.926152 \quad 1.4156342 .007856$

H $0.4201051 .645250-4.257362$

H $-0.7883361 .413472-1.460126$

H $-0.254828-0.577862-3.763354$

H $-0.383897-1.137219-0.740343$

H $1.132348-0.846076-1.546257$

H $-0.317875-4.702095-1.909225$

H $1.343550-4.079151-3.345255$

H $1.116145-2.476088-4.045739$

H $2.282570-2.719523-2.728663$

H -2.014462 -2.553136 -0.489656

H -2.486234 -4.149343 -1.004458

H $-2.244715-4.2876071 .332602$

H $-0.862776-5.1635310 .669049$

H $0.561974-3.1028991 .057019$

H $0.454225-4.8952292 .820681$

H $-0.998222-4.1707823 .477079$

H $1.186202-2.9844825 .800940$

H $-0.827488-1.7510244 .522954$

H $2.665318-4.0164303 .507523$

H $2.848443-2.4078744 .167959$

H $2.400117-2.6156702 .464430$

H $1.632207-0.4276474 .102818$

H -1.996513 -0.0382493 .367444$

H $-0.580251 \quad 1.0036303 .211143$

H $0.750183-0.0392481 .198072$

H $-4.604942-2.143517-4.073287$

H $-4.072158-0.742499-1.385133$

H -6.651429 $-3.089220-3.063618$

H -6.925769-1.485088 -3.787647
H -8.017815 -2.098747 -2.567684

H $-0.876231 \quad 1.1314770 .770355$

H -2.185200 -0.0422300 .808221$

H $-4.992327 \quad 3.1611421 .603334$

H -4.852006 2.7254553 .313955

H -6.4762540 .8341333 .040424$

H -7.0641302 .4881390 .527636$

H -9.3456881 .7220551 .293672$

H $-8.6575380 .361742 \quad 2.145686$

H $-9.587947-0.155970-1.861723$

H $-10.0413351 .396839-0.959520$

H $-8.479452-1.940950-0.536797$

H $-7.802143-1.5741581 .010601$

H $-5.787964-0.6031210 .092437$

H $-4.0214670 .441672 \quad 2.282417$

C $0.0771703 .978057-1.605289$

O $1.1356193 .482649-0.761040$

C 1.6020434 .5525740 .085762

C $0.5637745 .659384-0.034094$

C $0.1348705 .501804-1.481166$

H $2.5428924 .910040-0.347850$

C $1.844046 \quad 4.030427 \quad 1.507824$

H -0.2880395 .4923080 .635514$

H $\quad 0.9805686 .6465350 .186972$

C $-1.1758606 .198596-1.802923$

H $0.9252445 .902995-2.129716$

C $2.8024932 .821227 \quad 1.558147$

O 0.5942273 .6208462 .073475

H 2.2150564 .8375932 .148711

H 2.3803241 .9987420 .971362

$\begin{array}{llll}\text { O } & 2.874941 & 2.352975 & 2.910332\end{array}$

C $4.2318903 .145052 \quad 1.083047$

C $5.1754361 .932904 \quad 1.101198$

H 4.2108583 .5723130 .077781

$\begin{array}{lllll}\text { O } & 4.775095 & 4.152031 & 1.942180\end{array}$

H 6.2137372 .2716120 .982327

H $5.173361 \quad 1.4723562 .098491$

H -1.453296 $6.044891-2.850460$

H $-1.9938825 .822840-1.179136$

H -1.090382 $7.276541-1.631065$

C $0.2911533 .468854-3.035460$

H $\quad 0.1961792 .976348 \quad 1.464566$

H 1.9652372 .1718023 .203179

H 5.6542024 .3873451 .599886

H $1.319733 \quad 3.703861-3.339069$

H $-0.3785224 .001632-3.720542$

H $-0.8709003 .611804-1.193254$

C $5.534357-1.389508-0.526785$

$\begin{array}{llllll}\text { O } & 5.762632 & -0.227231 & 0.285595\end{array}$

C 4.8589690 .8606120 .044622

C $5.0242921 .356519-1.401499$

C $4.856056 \quad 0.223168-2.416815$

C $5.634322-1.037995-2.021838$

H $6.368266-2.059621-0.280840$

C $4.231497-2.083772-0.105606$

H 3.8366000 .5040860 .203877

O $6.3102661 .950516-1.573206$

H $4.2800722 .132548-1.609773$

H $5.1988300 .575332-3.397578$

O $3.469341-0.074055-2.531006$

H $5.313515-1.893692-2.625979$

O $7.021115-0.820950-2.306758$

C $4.174840-3.546355-0.485192$

H $4.146112-2.0262060 .985948$

H $3.355802-1.585987-0.529890$

H $6.9787501 .283036-1.341378$

H $3.368939-0.724875-3.246215$

H $7.102958-0.665470-3.263152$

O $3.004872-4.074177-0.016549$

O $5.033729-4.151357-1.110910$

C $2.793234-5.460377-0.298865$

H $2.749595-5.623567-1.379744$

H $3.585635-6.0648040 .152603$

H $1.835700-5.7535710 .139602$

SCF Energy (B3LYP/6-31G*//MMFF)= -3245.92151866

0400172

MM̄FF Geometry 
C -1.8195963 .6572611 .503293$ C -1.1422342 .5259741 .256871$ C $-0.590653 \quad 1.638326 \quad 2.342776$ O $0.787101 \quad 1.3333032 .021618$ C $-1.3670790 .314614 \quad 2.394817$ C -1.021694 -0.5101723 .619647$ C $-0.210247-1.5858253 .623372$ C $-1.703573-0.079014 \quad 4.893698$ C $0.538101-2.1789532 .463596$ C $2.018642-2.4055442 .797280$ C $2.754052-3.0737111 .626865$ C $4.178550-3.4587352 .023148$ C $4.947509-4.0660550 .841715$ $\begin{array}{llllll}\text { O } & 2.791213 & -2.147437 & 0.537123\end{array}$ O $6.330112-4.1168851 .225198$ C $4.823636-3.125131-0.375413$

C $4.521316-5.5144620 .573202$ O $5.411108-3.741357-1.519362$ C $3.370063-2.672052-0.665761$ C $3.370224-1.545096-1.723663$ O $2.622490-3.779017-1.170966$ C $1.722768 \quad 2.2652182 .347093$ O $1.539703 \quad 3.320846 \quad 2.932110$ C 3.0209561 .7941131 .817466 C 4.0144662 .6803061 .654108 C 5.3317292 .4065751 .077901 C 5.7032250 .9689720 .834779 C $2.030877-0.813667-1.882521$ C $2.2164280 .399699-2.772563$ C $3.1930522 .699464-2.755590$ O $1.9340300 .371846-3.967943$ C $4.6907202 .732324-2.770269$ C $5.4155793 .796209-2.390846$ C $6.9185723 .825296-2.399559$ C $7.5105324 .342981-1.104759$ C $8.1085635 .545336-1.052133$ C $7.477157 \quad 3.438302 \quad 0.111134$ C 6.1332213 .4521910 .786509 N $2.7674681 .478024-2.096089$ H -2.003867 3.9551832 .532512 H $-0.9600642 .224800 \quad 0.227908$ H -0.6358902 .1395843 .317734$ H $-1.188886-0.2578281 .478052$ H -2.446927 0.5117852 .409579 H $-0.074034-2.1169404 .564845$ H $-1.467071-0.7376915 .736120$ H -1.396592 0.9333295 .172945 H $-2.790873-0.0901344 .764549$ H $0.074785-3.1436152 .224227$ H $0.464128-1.5557821 .568581$ H $2.485140-1.4392003 .027639$ H $2.100960-3.0359913 .691366$ H $2.187705-3.963587 \quad 1.327394$ H $4.175429-4.1507402 .874598$ H $4.716228-2.5633322 .366102$ H $6.836529-4.4381130 .459238$ H $5.432433-2.237850-0.151051$ H $4.702233-6.134754 \quad 1.459226$ H $5.117212-5.961066-0.230547$ H $3.465684-5.6094630 .310369$ H $5.499061-3.064848-2.211316$ H $4.133964-0.807252-1.455390$ H $3.655766-1.949388-2.703326$ H $1.880527-3.443033-1.698860$ H $3.1055660 .752518 \quad 1.529786$ H 3.8337373 .7189321 .929071 H 5.5375360 .3669901 .735668 H $\begin{array}{llll}6.753073 & 0.828921 & 0.569354\end{array}$ H $\begin{array}{llll}5.101536 & 0.554742 & 0.022412\end{array}$ H $1.274197-1.462279-2.336133$ H $1.632353-0.480149-0.917667$ H $2.768166 \quad 3.537026-2.193741$ H $2.8087632 .726174-3.779632$ H $5.2044001 .837352-3.114775$ H $4.9028934 .701892-2.074304$ H $7.2313804 .462787-3.236770$ H $7.3339992 .831198-2.607010$ H $8.5564345 .913922-0.135192$
H $8.1595716 .190582-1.922802$ H $8.220216 \quad 3.7695450 .848079$ H $7.7929812 .437865-0.196578$ H $5.780464 \quad 4.4554291 .031926$ H $2.9754281 .384082-1.106457$ C $-3.805933 \quad 4.434538 \quad 0.101309$ O $-4.0411343 .174768-0.553200$ C $-5.3606272 .713146-0.196917$ C $-6.007335 \quad 3.8347950 .613972$ C $-4.7965194 .484163 \quad 1.260569$ H $-5.221931 \quad 1.8458160 .458445$ C $-6.1279662 .300497-1.459845$ H $-6.5286874 .554670-0.027610$ H $-6.7303293 .453211 \quad 1.341560$ C $-5.059256 \quad 5.881778 \quad 1.790713$ H -4.4671843 .8409152 .086038$ C $-5.380218 \quad 1.282514-2.346092$ O $-6.373592 \quad 3.463927-2.261377$ H $-7.1144451 .910072-1.185207$ H $-4.4807801 .743859-2.771443$ O $-6.2265870 .951253-3.453979$ C $-4.999610-0.021270-1.624264$ C $-4.249262-1.002344-2.543750$ H $-4.3605040 .210906-0.767122$ O $-6.177996-0.646050-1.118904$ H $-4.881718-1.279679-3.395711$ H -3.364184 $-0.485196-2.933834$ H -4.1522306 .3067552 .232129$ H $-5.396064 \quad 6.5562600 .996296$ H $-5.833878 \quad 5.860322 \quad 2.564073$ C -2.3206914 .5841380 .433010$ H $-5.5116853 .861345-2.474263$ H $-6.4613991 .784242-3.897762$ H $-6.759847-0.830753-1.876188$ H -2.118208 5.6125510 .755346 H $-1.7375434 .424978-0.483501$ H $-4.0607615 .208003-0.636226$ C -2.552886 -3.0146240 .114684$ O $-2.996853-1.930810-0.711332$ C $-3.823135-2.294759-1.821411$ C $-3.068976-3.253328-2.756860$ C $-2.529223-4.468804-1.994782$ C $-1.790072-4.059684-0.717255$ H $-1.842550-2.5549140 .809434$ C $-3.690918-3.6138640 .948697$ H $-4.726998-2.789610-1.451032$ O $-1.985956-2.578486-3.395150$ H $-3.750479-3.583303-3.549529$ H $-1.842430-5.029161-2.641267$ O $-3.606270-5.333444-1.646118$ H $-1.578696-4.944087-0.104862$ O $-0.529200-3.502354-1.098495$ C $-4.131847-2.6532122 .027603$ H $-4.568619-3.8960610 .360880$ H $-3.354477-4.5258441 .456918$ H $-1.406596-2.221428-2.700311$ H $-4.039525-5.610348-2.471334$ H $-0.033275-3.312070-0.284232$ O $-5.064360-1.7910901 .533797$ O $-3.689440-2.662674 \quad 3.168814$ C $-5.568985-0.8257362 .461346$ H $-4.764610-0.1646482 .795212$ H $-6.325486-0.225142 \quad 1.949409$ H $-6.036782-1.3262823 .314385$ SCF Energy (B3LYP/6-31G**//MMFF) $=-3245.92350504$

04_00173

MM̄FF Geometry

C $-0.752461-4.0778310 .295028$

C $-2.051866-3.7648810 .188475$

C $-2.751439-3.572167-1.133122$

O $-3.086557-2.169653-1.253378$

C $-4.049173-4.398130-1.168723$

C $-4.803443-4.287633-2.481312$

C $-5.960885-3.617230-2.653386$

C $-4.184010-5.024061-3.641844$

C $-6.731811-2.835425-1.627002$

C $-6.673262-1.330605-1.918444$ 
C -7.102172 $-0.496395-0.702632$ C $-7.1350700 .991491-1.054584$ C -7.4133251 .8727210 .172575$ O $-6.144268-0.7350860 .334016$ O $-7.0987443 .229267-0.178842$ C $-6.451014 \quad 1.4683971 .310434$ C $-8.896726 \quad 1.8576230 .559473$ O $-\begin{array}{llll}6.773528 & 2.154180 & 2.517447\end{array}$ C -6.406962 -0.0514771 .565746$ C $-5.298268-0.4672522 .564720$ O $-7.657426-0.4687752 .126587$ C $-2.108414-1.347587-1.728518$ O $-0.990432-1.672900-2.097113$ C -2.622604 $0.039258-1.704049$ C $-1.7865821 .054797-1.967431$ C $-2.1267072 .479091-1.980401$ C $-3.5377752 .864128-1.624225$ C $-3.863907-0.1523962 .124414$ C -3.3219361 .1643612 .653929$ C -1.342065 2.665721 2.446383 O $-3.879681 \quad 1.8202653 .529120$ C -1.6756273 .8083451 .540699$ C -0.7494794 .5178510 .877715$ C -1.064450 5.7058040 .014428 C $-0.4756045 .595022-1.374111$ C $0.6845716 .198566-1.683181$ C -1.277492 $4.861894-2.425032$ C $-1.1641183 .368778-2.301804$ N -2.1112031 .4979812 .060511$ H - $0.164244-4.223562-0.607857$ H -2.634594 -3.5951081 .091081$ H -2.106267 -3.872909-1.968081 H $-4.685282-4.103245-0.328674$ H -3.817197 -5.457941 -0.995781 H -6.412681 -3.617122 -3.644685 H $-4.809505-4.986083-4.540173$ H -3.212939-4.590945 -3.900118 H -4.039582 -6.079418 -3.388528 H -7.777185 -3.167326 -1.648065 H $-6.379416-3.042586-0.614722$ H $-5.647644-1.056303-2.195020$ H -7.314725 -1.101891-2.778457 H -8.089930 $-0.837322-0.370673$ H -7.871104 $1.187594-1.844296$ H -6.164274 $1.284566-1.475092$ H $-7.6449313 .474917-0.945188$ H -5.4511001 .8079871 .015946$ H $-9.5113592 .234648-0.266862$ H -9.0915312 .5256191 .405614$ H -9.2634640 .8610620 .813236$ H -6.7706003 .1063162 .319498$ H -5.515618 -0.0639213 .561021$ H $-5.357312-1.5597252 .675879$ H -7.521521 -1.3088282 .595259$ H -3.667445 $0.188178-1.460664$ H $-0.7472690 .824635-2.199515$ H -3.688001 $3.941728-1.532265$ H $-3.8207602 .437823-0.656129$ H $-4.2352302 .502514-2.386994$ H -3.203341 -0.9329122 .522828$ H $-3.772019-0.1751231 .035219$ H -0.2841782 .3901132 .393028$ H -1.579593 2.9341133 .480856 H -2.723436 4.0860011 .453184 H $\quad 0.300260 \quad 4.2565150 .991999$ H -0.6639996 .5961760 .516902$ H -2.146386 $5.872224-0.060175$ H $1.0986306 .148988-2.684733$ H $1.2488986 .751193-0.939297$ H $-0.9173215 .131433-3.427303$ H -2.312240 $5.215060-2.402009$ H $-0.1676412 .992026-2.537119$ H $-1.7439970 .914474 \quad 1.314230$ C $0.910213-3.0473321 .913389$ O $1.848743-2.8482910 .838202$ C $1.929298-1.4403880 .551777$ C $1.334300-0.7395071 .766977$ C $0.225581-1.6994322 .149506$
H $1.290214-1.256276-0.321253$

C $3.365088-1.0099800 .223227$

H $2.064378-0.6561492 .580691$

H $0.9713920 .265072 \quad 1.530429$

C $-0.279887-1.5136283 .569944$

H $-0.608746-1.5534221 .453166$

C $3.938773-1.651060-1.057210$

O $4.219208-1.3074891 .327509$

H 3.3607810 .0789650 .106419

H $3.303640-1.397904-1.914816$

O $3.921251-3.078660-0.941365$

C $5.398831-1.260563-1.365008$

C $5.6391740 .256540-1.450505$

H $6.058864-1.709956-0.615431$

O $5.762398-1.853727-2.617447$

H $4.9765180 .680623-2.215142$

H $5.3699010 .722401-0.498670$

H -1.078586 -2.227631 3.794018

H $0.519964-1.6607324 .303470$

H $-0.678449-0.5044423 .710468$

C $-0.023664-4.2216911 .601039$

H $4.214794-2.2731431 .445113$

H $2.993381-3.357337-0.861521$

H $5.193211-1.468334-3.305241$

H $-0.729765-4.3626672 .427864$

H $0.571100-5.1414831 .535435$

H $1.511599-3.3163982 .791639$

C 8.0428580 .4059610 .401002

O $8.043460-0.042686-0.959008$

C $7.0999490 .619851-1.806368$

C $7.3897742 .127753-1.831008$

C $7.4576992 .720530-0.423229$

C $8.3907561 .896450 \quad 0.465747$

H 7.0563990 .2341850 .847883

C $9.064763-0.4418651 .165553$

H $7.2813340 .241981-2.820351$

O $6.3985592 .796942-2.602855$

H $8.3540392 .292793-2.329564$

H 6.4613702 .8015190 .027174

O $7.9780314 .050057-0.492487$

H 9.4306592 .0566410 .153232

O $8.2733872 .347631 \quad 1.813282$

C $8.611465-1.8800061 .257670$

H $10.034824-0.4350920 .653786$

H $9.231624-0.0725262 .183544$

H $6.6779253 .722212-2.704936$

H $7.3267064 .596027-0.964162$

H 8.4762163 .2989311 .819199

O $7.590624-1.9921522 .155031$

O $9.100794-2.7911960 .602723$

C $7.046973-3.3071082 .306058$

H $6.241064-3.2588403 .043125$

H $6.631945-3.6564361 .355682$

H $7.815457-3.9957122 .669805$

SCF Energy (B3LYP/6-31G**//MMFF $)=-3245.90998020$

0400174

MM̄MF Geometry

C $-0.483161-4.3877380 .463221$

C $-1.678601-3.9795850 .013687$

C $-1.931566-3.546244-1.408067$

O $-2.153744-2.118337-1.413737$

C $-3.192296-4.247865-1.946195$

C $-3.552357-3.855970-3.366673$

C $-4.587382-3.061746-3.708612$

C $-2.673891-4.450103-4.437493$

C $-5.564072-2.376797-2.792513$

C $-5.424087-0.851568-2.879366$

C $-6.115968-0.140573-1.706071$

C $-6.0337051 .377190-1.879917$

C $-6.5690402 .136066-0.657728$

O $-5.439573-0.538900-0.508984$

O $-6.1494113 .502214-0.797896$

C $-5.906901 \quad 1.564800 \quad 0.615342$

C $-8.1015912 .157903-0.640763$

O -6.4696452 .1549991 .784422$

C $-5.963226 \quad 0.026230 \quad 0.699044$

C $-5.133297-0.5455131 .875364$ 
O $-7.322492-0.3756580 .907638$ C -1.047931-1.328583 -1.521009 O $0.114255-1.695937-1.593232$ C $-1.494593 \quad 0.081429-1.530767$ C $-0.5828371 .064838-1.543317$ C -0.861262 $2.502417-1.538835$ C $-2.2996832 .938111-1.458576$ C $-3.623319-0.296132 \quad 1.797739$ C $-3.142780 \quad 0.929598 \quad 2.555786$ C $-1.084750 \quad 2.2934832 .923681$ O $\begin{array}{lllll} & -3.850741 & 1.562011 & 3.334147\end{array}$ C -1.164262 3.5211032 .071367 C -0.088337 4.225818 1.687618 C $-0.159847 \quad 5.494362 \quad 0.885253$ C $0.720328 \quad 5.466129-0.344934$ C $1.9392496 .032012-0.336291$ C $0.1509604 .861622-1.608594$ C $0.1791513 .359523-1.590914$ N $-1.808005 \quad 1.2079242 .287128$ H $0.365998-4.401828-0.215713$ H $-2.520704-3.9488260 .701600$ H - $1.079465-3.795744-2.053187$ H $-4.030790-4.055505-1.269383$ H -3.047969 -5.336707 -1.915300 H $-4.755355-2.864895-4.766831$ H $-3.017666-4.196341-5.446010$ H - $1.646899-4.085217-4.339323$ H -2.665535 -5.542177 - 4.359336 H $-6.580851-2.664145-3.086724$ H -5.448012 -2.706724 -1.758669 H $-4.359002-0.588474-2.877053$ H -5.842975 -0.502063 -3.831210 H -7.160926 -0.469028 -1.658393 H $-6.5584891 .693207-2.790182$ H $-4.9850791 .664663-2.029817$ H -6.416456 3.9763300 .008479 H -4.8586661 .8834350 .589403$ H -8.484618 $2.649546-1.543286$ H -8.4816792 .7459290 .201650$ H -8.543965 $1.160596-0.594422$ H -7.3314821 .7338281 .943840$ H $-5.552323-0.2130112 .832647$ H $-5.268273-1.6369451 .864500$ H $-7.325476-1.2707201 .285608$ H $-2.560417 \quad 0.273772-1.519352$ H $0.4726620 .795437-1.555031$ H -2.828810 $2.666598-2.377875$ H - $2.4243524 .013676-1.318417$ H -2.801358 $2.464182-0.608436$ H -3.112836 -1.152634 2.254185 H $-3.288141-0.2363710 .759644$ H -0.0534281 .9562323 .067094$ H -1.525556 2.4970323 .904928 H -2.1552323 .8656801 .785564$ H 0.8995533 .8971622 .002498 H $0.148566 \quad 6.3186021 .541846$ H $-1.191200 \quad 5.7241140 .590034$ H $2.5656186 .039479-1.222076$ H 2.3418596 .4916750 .560280 H $0.7386865 .185186-2.478414$ H $-0.8483555 .268479-1.786300$ H $1.1860322 .946061-1.648690$ H -1.3141920 .6544601 .592806$ C $0.473660-3.8500682 .764717$ O $1.705580-3.3998432 .169382$ C $1.881366-2.0048832 .493648$ C $0.817244-1.6697753 .534226$ C $-0.312589-2.5854863 .105140$ H $1.655665-1.4347291 .586329$ C $3.319356-1.7424712 .948597$ H $1.148104-1.9191834 .549322$ H $\quad 0.543422-0.6107903 .520439$ C $-1.375018-2.7804534 .171845$ H $-0.776056-2.1619032 .205715$ C $4.409681-2.0987511 .915064$ O $3.576977-2.5173874 .129189$ H $3.427060-0.6964273 .256056$ H $4.416887-3.1843951 .757099$
O $5.683149-1.7625002 .482642$

C $4.325234-1.3899320 .548578$

$\begin{array}{llll}\text { C } & 4.191926 & 0.140262 & 0.663732\end{array}$

H $5.244699-1.614873-0.004468$

O $3.243596-1.903976-0.220022$

H 3.2620770 .3844321 .189602

H 5.0169390 .5239431 .273686

H -2.168574 -3.4440663 .814330$

H $-0.954059-3.2175265 .083439$

H $-1.831683-1.8220244 .439977$

C $-0.231377-4.865721 \quad 1.864485$

H $3.430145-3.4522313 .903699$

H $5.748975-2.2206653 .338039$

H $3.364839-2.865819-0.294360$

H $-1.166944-5.1901882 .335386$

H $0.398709-5.7608911 .783198$

H $0.755726-4.3658873 .692775$

C $6.5211180 .881184-1.164705$

O $5.2144870 .454301-1.566446$

C $4.1544700 .865303-0.699234$

C $4.1226572 .398751-0.599763$

C $5.4853212 .985892-0.228986$

C $6.5864382 .412438-1.123357$

H $6.7572260 .467755-0.176182$

C $7.5221920 .306107-2.171304$

H $3.2228480 .556639-1.190471$

$\begin{array}{lllll}\text { O } & 3.132775 & 2.829126 & 0.328433\end{array}$

H $3.8281882 .802701-1.576809$

H 5.7134582 .8077840 .828107

$\begin{array}{lllll}\text { O } & 5.444181 & 4.406713 & -0.380791\end{array}$

H $6.4786072 .821706-2.135425$

O $7.8569672 .837071-0.634853$

C $7.667947-1.184213-1.973790$

H $7.2106220 .498696-3.204921$

H $8.5193750 .744259-2.050282$

H 3.4629832 .6632361 .227231

H $5.2392804 .604267-1.310708$

H 7.9709272 .4725560 .259409

O $6.776195-1.850604-2.760911$

O $8.457550-1.691219-1.187417$

C $6.780833-3.274988-2.625646$

H $7.759100-3.679049-2.902741$

H $6.520603-3.558476-1.601240$

H $6.027174-3.683396-3.304092$

SCF Energy (B3LYP/6-31G*//MMFF) $=-3245.90912943$

0400175

MM̄FF Geometry

C $1.1848213 .182697 \quad 1.464717$

C 1.4131564 .1986690 .618495

C $2.2688094 .115255-0.629073$

O $2.5340432 .735385-0.984558$

C $3.6071084 .836792-0.402522$

C $4.4507814 .938631-1.659641$

C $5.5493444 .200428-1.915746$

C $3.9987315 .979507-2.652459$

C $6.1604333 .129214-1.057506$

C $6.0494331 .753286-1.726121$

C $6.5000880 .626954-0.785638$

C $6.447200-0.725877-1.494041$

C $6.789727-1.884853-0.545532$

$\begin{array}{lllll}\text { O } & 5.616216 & 0.617069 & 0.339594\end{array}$

O $6.401692-3.107857-1.191905$

C $5.938647-1.761800 \quad 0.738757$

C $8.299834-1.987057-0.297187$

O $6.366875-2.7329561 .690949$

C $5.950999-0.3436281 .351341$

C $4.934334-0.1524652 .504675$

O $7.251691-0.0829641 .893852$

C $1.5266862 .059615-1.608962$

O $0.4554282 .517577-1.975697$

C $1.927816 \quad 0.639314-1.726104$

C $1.011309-0.270903-2.090085$

C $1.230692-1.708223-2.270365$

C $2.604456-2.249106-1.978917$

C $3.464632-0.397468 \quad 2.143221$

C $2.976491-1.8013302 .459086$

C $0.961036-3.2491192 .232331$ 
O $3.658316-2.6378263 .044646$

C $1.042584-4.0688400 .983443$

C $-0.034903-4.493146 \quad 0.306236$

C $0.028683-5.372982-0.907084$

C $-0.646301-4.761251-2.112739$

C -1.932643 -5.028494 -2.396009

C $0.200843-3.930608-3.048098$

C $0.200474-2.468075-2.698110$

N $1.670867-2.0003532 .032143$

H 1.6858522 .2306221 .309510

H $0.907145 \quad 5.1470100 .784912$

H $1.7245394 .594978-1.452168$

H 4.1578354 .3334360 .398404

H $3.4252355 .854421-0.030668$

H $6.0781484 .375676-2.852050$

H $4.6786156 .062614-3.507171$

H $3.0073635 .734407-3.045103$

H $3.9514696 .963928-2.175272$

H $7.2183953 .372866-0.900859$

H $5.7087403 .099844-0.063958$

H $5.0071291 .583528-2.021985$

H $6.6546331 .739839-2.641097$

H $7.5193810 .845687-0.446415$

H $7.111282-0.734579-2.367406$

H $5.435346-0.889442-1.887742$

H $6.881494-3.166361-2.035779$

H $4.912959-2.0272140 .456989$

H $8.833810-2.138763-1.243139$

H $8.540724-2.8570550 .323632$

H $8.717349-1.0966890 .177334$

H $5.730264-2.7327132 .424933$

H $5.238403-0.7374203 .380975$

H 5.0059010 .8976352 .823968

H 7.1750750 .6271312 .552418

H $2.9558320 .381392-1.503024$

H $-0.0047610 .068622-2.288959$

H $3.328217-1.863685-2.704469$

H $2.658890-3.339372-2.011182$

H $2.926103-1.961335-0.972559$

H $3.271055-0.1723731 .089651$

H $2.845522 \quad 0.2815312 .743727$

H $-0.070208-2.9994502 .500085$

H $1.407938-3.8011663 .065332$

H $2.034687-4.3530050 .640460$

H -1.026023 -4.2301100 .668383$

H $-0.459048-6.323861-0.654117$

H $1.063832-5.636815-1.156615$

H - $2.412866-4.620474-3.279258$

H -2.535583 -5.653871 -1.745771

H $-0.193736-4.015923-4.070267$

H $1.207576-4.353404-3.114051$

H -0.760962 -1.983462 -2.874380

H $1.197919-1.2643691 .514925$

C -1.057760 2.4549632 .220899

O $-0.7318831 .090678 \quad 1.898233$

C $-1.862362 \quad 0.254227 \quad 2.224345$

C -2.923096 1.1778892 .815232

C -2.0983562 .3444303 .327779$

H -1.499959 -0.453653 2.979250

C $-2.298937-0.4845740 .952234$

H -3.6223331 .5177082 .040857$

H -3.5063930 .6883073 .600668$

C -2.9123423 .6062453 .551107$

H -1.616604 2.0481164 .269021

C -3.565112 -1.337639 1.140507

O $-1.208070-1.3203360 .560742$

H -2.433650 $0.247038 \quad 0.148183$

H $-4.405217-0.702371 \quad 1.437040$

O $-3.360388-2.2367382 .238717$

C -3.977607 -2.178665-0.081585

C $-4.112701-1.410571-1.409276$

H -3.263919 -2.997913 -0.227718

O $-5.231181-2.808987 \quad 0.204044$

H -3.116714 -1.118685 -1.761453

H -4.525377 -2.112564 -2.145678

H -2.2787774 .4204573 .916299$

H -3.393702 3.9422812 .626534

H -3.6974483 .4305374 .293913$
C 0.2095573 .2195412 .606298

H -1.394884 -1.661202 -0.329390

H -2.584681 -2.784466 2.028428

H $-5.130316-3.2909491 .042671$

H 0.6899942 .7554183 .475828

H -0.0353864 .2523812 .877159$

H -1.5025652 .9004931 .319849$

C $-7.2916760 .494044-0.846196$

O $-6.339342-0.565599-1.008322$

C $-5.015513-0.160268-1.361387$

C $-5.0292670 .584415-2.706210$

C $-6.0082091 .763804-2.672884$

C $-7.3778401 .353671-2.119444$

H $-8.257181-0.013148-0.720811$

C $-7.024977 \quad 1.3137350 .426582$

H $-4.6289370 .514058-0.592667$

O $\quad-5.400498-0.316171-3.747654$

H $-4.0148040 .939878-2.923105$

H -6.135449 2.163405 -3.686235

O $-5.4755542 .807002-1.861843$

H $-7.9924672 .239125-1.918698$

O $\quad-8.0660310 .582105-3.108518$

C $-7.1341270 .474875 \quad 1.682931$

H $-6.025014 \quad 1.7553370 .414401$

H -7.7613882 .1210660 .508390$

H $-5.3752650 .176638-4.585315$

H -4.621968 $3.071036-2.245559$

H $-8.170813 \quad 1.142151-3.896387$

O $-6.623143 \quad 1.1835302 .733527$

O $-7.613853-0.6486791 .743820$

C $-6.6412160 .510113 \quad 3.996042$

H -6.062889 -0.4171903 .940021$

H -6.1785491 .1666814 .737624$

H -7.671963 0.3061134 .300642

SCF Energy $\left(B 3 L Y P / 6-31 G^{* *} / / M M F F\right)=-3245.90027143$

0400176

MM̄FF Geometry

C -3.426368 -0.989089 3.157413

C $-3.7588190 .288944 \quad 3.396309$

C -4.6365031 .1778562 .546768$

O $-5.151907 \quad 0.4416231 .415373$

C -3.8250762 .3743612 .017303$

C -4.6914763 .5488291 .608995$

C $-4.981708 \quad 3.8908640 .338882$

C -5.1995084 .3953732 .748745$

C $-4.5884203 .184617-0.928221$

C $-3.5747293 .990785-1.752828$

C $-2.1566443 .963668-1.161435$

C $-1.2189204 .870821-1.960971$

C $0.2313794 .774607-1.464619$

O $-1.6941802 .611206-1.221364$

O $1.0552875 .411672-2.452684$

C $0.6337153 .286654-1.396354$

C $0.4349685 .559188-0.163791$

O $1.9327203 .137207-0.827132$

C $-0.3939272 .396538-0.660854$

C $-0.0359170 .905919-0.865439$

$\begin{array}{lllll}\text { O } & -0.358558 & 2.706747 & 0.733006\end{array}$

C -6.4577260 .6007651 .084311$

O -7.2807071 .2961661 .664240$

C $-6.822994-0.205119-0.104328$

C $-5.933770-0.838763-0.885234$

C $-6.245879-1.646869-2.068276$

C -7.697714 -1.841500 -2.420609

C $-1.111030-0.070026-0.368172$

C $-0.724775-1.517314-0.619008$

C $-1.677214-3.820150-0.489292$

O $0.375507-1.857942-1.044843$

C $-2.189278-4.143747-1.856624$

C $-3.314717-4.835951-2.088130$

C $-3.840339-5.126134-3.464253$

C $-5.210679-4.529213-3.702665$

C $-6.305020-5.308509-3.728038$

C $-5.303531-3.048222-4.003063$

C $-5.228089-2.191095-2.768192$

N $-1.758910-2.384440-0.295946$

H $-3.790231-1.4934392 .266749$ 
H $-3.3764600 .756609 \quad 4.302527$ H -5.457006 1.5111383 .193305 H -3.199914 2.0481171 .179068 H -3.124045 2.7334832 .782998 H -5.5956814 .7764040 .176703$ H -5.774988 5.2600932 .401235 H -5.8515273 .8101653 .404242$ H $-4.3612254 .776243 \quad 3.341404$ H $-4.2176342 .172362-0.743137$ H -5.500182 $3.059543-1.525994$ H -3.547892 $3.554129-2.759693$ H -3.919677 $5.026934-1.857873$ H -2.194991 4.288974 -0.116157 H -1.566146 $5.911282-1.934074$ H -1.242681 4.575741-3.019765 H $1.9828855 .286974-2.187444$ H $0.7162862 .935493-2.433616$ H $0.1860426 .616478-0.314472$ H 1.4847115 .5426870 .149779 H $-0.176873 \quad 5.1882200 .661132$ H 1.8475573 .2409830 .135957 H $\quad 0.1080070 .715342-1.937255$ H $0.9140050 .679324-0.366826$ H $-0.621473 \quad 1.9253511 .243890$ H -7.887464 $-0.232655-0.315685$ H $-4.874301-0.762595-0.646509$ H -8.164553 -0.882077 -2.668980 H -8.240852 -2.291339-1.581905 H -7.853725 -2.501001-3.276870 H -1.2768830 .0480060 .706926$ H -2.059481 $0.120873-0.883585$ H -2.271774 -4.2907160 .300009$ H $-0.638450-4.150457-0.389202$ H $-1.607210-3.777244-2.699217$ H -3.895636 -5.208386 -1.247605 H -3.873215 -6.216771-3.585069 H -3.152732 -4.765279-4.239384 H -7.288098 $-4.897051-3.930649$ H $-6.243292-6.375820-3.543523$ H -6.209944 -2.827316 -4.577861 H $-4.483621-2.763736-4.675358$ H -4.209364 -1.991282 -2.436621 H -2.655664 -1.998787 -0.014235 C -1.212952 -2.133673 3.431759 O $-0.459930-0.9225203 .229017$ C $0.919903-1.1586993 .582750$ C $1.049235-2.6636353 .765508$ C $-0.324705-3.0297544 .290476$ H $1.073601-0.6409484 .537591$ C $1.818146-0.5589712 .495392$ H $1.235400-3.1585762 .803914$ H $1.856504-2.9359844 .451948$ C $-0.643579-4.5100874 .173486$ H $-0.390514-2.7278685 .344315$ C $3.319423-0.8581092 .682648$ $\begin{array}{llll}\text { O } & 1.585614 & 0.852359 & 2.506410\end{array}$ H $1.486599-0.9280061 .518097$ H $3.468212-1.9411702 .764590$ O $3.786353-0.2722723 .897655$ C $4.197321-0.3592921 .515493$ C $5.685690-0.7005521 .725034$ H $3.838102-0.8202560 .588535$ $\begin{array}{lllll}\text { O } & 4.070101 & 1.052469 & 1.357134\end{array}$ H $6.075363-0.1351162 .580130$ H $5.767757-1.7702701 .953413$ H $-1.647762-4.7244464 .552140$ H $-0.596264-4.8499353 .133320$ H $0.070031-5.1041644 .753692$ C -2.555434-1.799267 4.080244 H $2.059215 \quad 1.234673 \quad 1.748570$ H $3.341355-0.7225394 .634969$ H 4.3414091 .4668642 .194397 H -2.386720 -1.250085 5.015124 H $-3.093142-2.7188194 .337286$ H -1.368261 -2.585021 2.445270 C $6.787475-1.064571-1.824621$ O $6.138547-1.255762-0.561246$

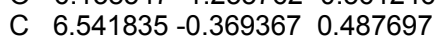

C $8.043650-0.5249700 .767707$

C $8.864241-0.319202-0.510601$

C $8.318189-1.145828-1.678640$

H $6.464410-1.916142-2.437142$

C $6.3062870 .218218-2.521022$

H 6.3364610 .6642320 .190911

O $8.297720-1.8230321 .300585$

H $8.3387110 .207061 \quad 1.529023$

H $9.907224-0.605754-0.327615$

$\begin{array}{llll}\text { O } & 8.850027 & 1.058326 & -0.873897\end{array}$

H $8.798433-0.836058-2.614323$

O $8.681999-2.511493-1.454867$

C $4.836866 \quad 0.152732-2.885644$

H $6.4460291 .099577-1.889890$

H $6.8662660 .358417-3.452796$

H $9.251386-1.8855761 .479405$

H $9.2278381 .561591-0.132715$

H $8.393486-3.023482-2.229461$

O $4.470487 \quad 1.339499-3.455205$

O $4.104707-0.810747-2.706440$

C $3.1026011 .429034-3.865504$

H $2.8928252 .469645-4.126670$

H $2.9394620 .802754-4.747296$

H $2.4331411 .130892-3.053375$

SCF Energy (B3LYP/6-31G*//MMFF)= -3245.91532614

04_00177

MM̄FF Geometry

C $0.7028320 .367472-3.242259$

C $1.878625-0.206410-3.541260$

C $2.462182-1.466490-2.949186$

O $1.629722-1.968729-1.880442$

C $3.873976-1.215485-2.387002$

C $4.716387-2.476773-2.358973$

C $5.011596-3.190366-1.255689$

C $5.282399-2.897985-3.692875$

C $4.579640-2.9434190 .162988$

C $5.774847-2.5820531 .058037$

C $6.287727-1.1545710 .814810$

C $7.617099-0.9221891 .532076$

C $8.106673 \quad 0.5209701 .344012$

O $5.303845-0.254784 \quad 1.329287$

$\begin{array}{llll}\text { O } & 9.175650 & 0.750288 & 2.275804\end{array}$

C $6.966766 \quad 1.4905861 .723596$

C $8.7003640 .740818-0.052596$

O $7.307242 \quad 2.8356391 .396553$

C $5.5879261 .132771 \quad 1.112712$

C 4.4818281 .9356821 .830271

O $5.506437 \quad 1.475813-0.271075$

C $1.363564-3.299757-1.855877$

O $1.715989-4.132253-2.681058$

C $0.549195-3.679581-0.679517$

C $0.303949-2.8703720 .362725$

C $-0.481490-3.2166861 .551625$

C $-1.142888-4.5708071 .580983$

C $3.062767 \quad 1.5878851 .361893$

C $2.0119592 .316262 \quad 2.182405$

C -0.4527922 .4071002 .532869$

$\begin{array}{lllll}\text { O } & 2.286425 & 3.177962 & 3.013587\end{array}$

C -0.9175531 .4091543 .543478$

C $-2.0477210 .697216 \quad 3.415835$

C -2.541878 -0.2749794 .445857$

C $-2.590062-1.696886 \quad 3.935178$

C $-3.760301-2.2871223 .639502$

C $-1.290263-2.4670313 .868472$

C $-0.583201-2.3128072 .549066$

N $0.7288901 .895066 \quad 1.867784$

H $0.067655-0.054212-2.469714$

H $2.4691580 .231747-4.344917$

H $2.496995-2.187389-3.774791$

H $3.801328-0.771123-1.388091$

H $4.409503-0.477315-2.999210$

H $5.641130-4.071943-1.374745$

H $5.938844-3.770856-3.610072$

H $4.481282-3.156792-4.391336$

H $5.873695-2.085023-4.127026$

H $3.797625-2.1827330 .238954$

H $4.132224-3.8731760 .536110$ 
H $5.451784-2.6751132 .102984$ H $6.583437-3.3069790 .901475$ H $6.405562-0.987463-0.260726$ H $8.378037-1.6321931 .184948$ H $7.493738-1.1131022 .607313$ H 9.8695540 .0905492 .104949 H 6.8708071 .4546372 .818210 H $9.5674680 .087087-0.206662$ H $9.0739841 .764065-0.167815$ H $7.9918340 .536873-0.857976$ H $8.1581273 .031476 \quad 1.825031$ H 4.5405701 .7445422 .910112 H 4.6433153 .0108211 .679521 H $6.3750261 .784891-0.570998$ H $0.177471-4.699074-0.707176$ H $0.720065-1.8643390 .359211$ H -1.772496 -4.712685 0.695285 H -1.797743 -4.718833 2.441959 H $-0.389142-5.3651951 .607734$ H 2.9304621 .8666090 .310528 H 2.8828020 .5110551 .459282 H -1.205040 2.5870651 .763585 H -0.2317673 .3603103 .023023$ H -0.3029001 .2836054 .431666$ H $-2.663840 \quad 0.8283272 .529836$ H $-3.5426340 .051964 \quad 4.757273$ H -1.927953 -0.239227 5.354640 H -3.804205 -3.3182893 .305825$ H -4.700046 -1.750399 3.717182 H -1.465490 -3.520097 4.112584 H $-0.617278-2.1188974 .662865$ H $-0.072698-1.3568422 .432374$ H $\quad 0.603458 \quad 1.153305 \quad 1.184467$ C $-0.3154702 .717894-3.131971$ O $-1.5609552 .396139-2.483236$ C -1.641822 $3.174665-1.270129$ C $-0.3681804 .021136-1.204072$ C $0.6071173 .189742-2.014284$ H -1.626499 2.457181-0.445467 C -2.931785 4.004854-1.240795 H $-0.5126725 .004744-1.667094$ H $-0.0378724 .189242-0.174446$ C $1.8245303 .960438-2.489498$ H $0.9319922 .345035-1.393982$ C $-4.2314603 .172967-1.224294$ O $-2.9535134 .839911-2.406123$ H -2.911148 $4.683903-0.380237$ H $-4.3327562 .626390-2.168978$ O $\quad-5.3304904 .093458-1.182347$ C $-4.3582512 .192187-0.042353$ C $-5.742847 \quad 1.5183560 .028844$ H -3.581891 $1.424125-0.116475$ O -4.1693132 .8923181 .189204$ H -6.4940772 .2726670 .291486$ H $-6.0035641 .125148-0.958640$ H $2.4865353 .318032-3.078561$ H $1.5410424 .815188-3.112621$ H $2.3955004 .340738-1.636040$ C $0.1680951 .554606-3.996109$ H $-3.7783805 .354468-2.378278$ H $-5.2960024 .552368-0.325287$ H -3.244850 $3.185252 \quad 1.229474$ H $0.9297031 .930763-4.690276$ H -0.672374 $1.199135-4.605469$ H $-0.5397013 .555318-3.807753$ C $-5.184722-1.476440-0.318174$ O $-4.835914-0.6423960 .792894$ C $-5.777391 \quad 0.3939451 .091385$ C -7.164846 -0.2057201 .360104$ C $-7.618288-1.1318690 .235106$ C $-6.532024-2.161032-0.074640$ H -5.243963 -0.869906 -1.230203 C $-4.054628-2.496818-0.493051$ H $-5.445374 \quad 0.8410702 .036970$ O H $-7.121124-0.7831702 .293208$ H -7.891788 $-0.568007-0.664777$ O $-8.790608-1.8392750 .644459$
H $-6.451302-2.8760040 .754229$

O $-6.904019-2.892364-1.241305$

C $-2.977947-1.917174-1.379833$

H $-3.592363-2.7669410 .462358$

H $-4.408928-3.427811-0.950150$

H -8.9441060 .4269831 .856280$

H -9.510395 -1.1912090.727497

H $-7.772680-3.293809-1.066904$

O $-3.075960-2.454698-2.629507$

O $-2.178207-1.064479-1.017538$

C $-2.152755-1.951220-3.598433$

H $-1.133760-2.239718-3.328532$

H $-2.398098-2.397347-4.565721$

H $-2.242767-0.864287-3.682639$

SCF Energy (B3LYP/6-31G**//MMFF) $=-3245.91083568$

0400178

MM̄FF Geometry

C -0.769046 $2.378037 \quad 0.284944$

C $-1.9332962 .796060 \quad 0.808617$

C -2.6794472 .1794001 .969878$

O -2.1048550 .8950992 .293425$

C $-4.1577441 .960914 \quad 1.599377$

C $-5.058571 \quad 1.7511172 .800019$

C -5.5754030 .5660673 .179529$

C -5.4163793 .0039203 .558713$

C $-5.335831-0.7888382 .574428$

C $-6.572924-1.3395721 .849877$

C $-6.865988-0.6322240 .517157$

C $-8.132487-1.196362-0.130804$

C $-8.378911-0.594122-1.523819$

O $-5.744694-0.851474-0.342802$

O $-9.398515-1.370154-2.171220$

C $-7.084123-0.704151-2.359548$

C $-8.9504900 .824667-1.429518$

O $-7.221322-0.025176-3.605544$

C $-5.827347-0.198081-1.615603$

C $-4.552667-0.567102-2.412478$

O $-5.9029331 .221959-1.481070$

C -1.9016290 .5776113 .595558$

O -2.1299801 .2841704 .567466$

C -1.320212 -0.7790763 .731822$

C $-1.261823-1.6624372 .722579$

C $-0.691663-3.0102142 .778931$

C $-0.049984-3.4486984 .068665$

C $-3.246164-0.307290-1.648532$

C $-2.031751-0.831736-2.393168$

C $0.374148-1.373958-2.018769$

O $-2.060791-1.175511-3.571501$

C $0.417413-2.842067-1.733991$

C $1.287670-3.412194-0.887413$

C $1.321090-4.885787-0.596535$

C $1.175873-5.1953520 .878244$

C $2.241004-5.5573261 .613273$

C $-0.211861-5.1714481 .482323$

C $-0.742852-3.7770421 .669650$

N $-0.905397-0.849674-1.581760$

H $-0.261534 \quad 1.5085740 .692470$

H -2.3867933 .6930010 .389382$

H -2.585199 2.8742802 .812702

H -4.2355081 .1131170 .912174$

H -4.545846 2.8254891 .044068

H -6.2365870 .5510804 .045461$

H -6.1153892 .8085394 .379013$

H -4.5222673 .4615813 .992492$

H -5.8901633 .7301662 .890206$

H -4.465357 -0.7994161 .912189$

H -5.084947 -1.471329 3.396614

H $-6.389373-2.4041771 .653587$

H -7.448783 -1.278146 2.507530

H -6.9807240 .4417550 .699656$

H -9.005017 -1.039975 0.515449

H $-8.030183-2.285551-0.235690$

H $-9.088778-2.289828-2.231470$

H $-6.936616-1.766137-2.600397$

H $-9.9088320 .814541-0.896181$

H $-9.1664351 .231358-2.423766$

H $-8.2903441 .521215-0.908469$ 
H -8.018003 -0.374609-4.040231 H -4.584578 -1.635646 -2.663075 H -4.526774 -0.010536 -3.357799 H $-5.006828 \quad 1.585191-1.409085$ H $-0.953988-1.0185944 .724883$ H -1.668586 -1.380309 1.752820 H $\quad 0.751503-2.7584904 .354657$ H $0.402322-4.4411834 .013204$ H -0.791246 -3.480894 4.874652 H $-3.0973790 .766001-1.492716$ H $-3.278775-0.796361-0.668246$ H $\quad 1.149496-0.824437-1.477234$ H $0.497762-1.198491-3.091609$ H - $0.305342-3.466845-2.253752$ H $2.018151-2.788778-0.377112$ H $2.274898-5.277754-0.972779$ H $\quad 0.542285-5.421262-1.153830$ H $2.143745-5.8108772 .663532$ H $3.235354-5.6063591 .182397$ H $-0.237452-5.7279502 .426181$ H $-0.898627-5.7206520 .825138$ H -1.228665 -3.3718510 .781764$ H -1.001904 -0.610954 -0.599034 C $1.0349313 .984259-0.370592$ O $2.1857803 .196162-0.003605$ C $3.3758623 .947094-0.327108$ C $2.9058045 .304698-0.841830$ C $1.5486734 .962217-1.423160$ H $3.8687533 .420323-1.151276$ C 4.3044524 .0302050 .890445 H $2.8032836 .032763-0.028366$ H $3.5951925 .727188-1.579513$ C $0.6632456 .177942-1.635497$ H $1.6929504 .443395-2.380495$ C 4.8201232 .6622671 .384084 O $3.5818714 .647770 \quad 1.963216$ H 5.1489454 .6921540 .664688 H 3.9789052 .0493661 .727797

O 5.6362732 .8896812 .540292

C $5.636621 \quad 1.882186 \quad 0.335520$

C 6.2321750 .5741790 .888163

H $4.9895071 .642253-0.509956$

O $6.6854022 .708290-0.169535$

H $6.897008 \quad 0.8048951 .729043$

H $5.416604-0.0432901 .280203$

H $-0.3057175 .890287-2.054592$

H $\quad 0.4790136 .709184-0.695654$

H $1.135067 \quad 6.879614-2.331245$

C $-0.0922043 .077215-0.863780$

H 4.1771474 .6826972 .731576

H 6.3587463 .4873482 .283766

H 7.3296652 .8450040 .545321

H $\quad 0.2985862 .313397-1.546873$

H $-0.8328403 .660534-1.422720$

H 0.7205124 .5319420 .528600

C $5.244804-1.415897-1.234878$

O $6.298762-0.454327-1.363764$

C $7.046515-0.207273-0.168706$

C $7.666756-1.5120580 .347339$

C $6.623513-2.6131590 .514363$

C $5.805542-2.766397-0.767488$

H $4.496470-1.053064-0.519812$

C $4.577084-1.552074-2.613552$

H $7.8806380 .442150-0.463282$

O $8.339068-1.2825041 .581123$

H $8.424404-1.851139-0.371408$

H $5.980811-2.4192531 .381211$

O $7.311742-3.8362080 .785799$

H $6.434570-3.205066-1.552879$

O $4.724597-3.663186-0.530932$

C $3.777317-0.325309-3.000510$

H $5.349843-1.701332-3.377447$

H $3.885259-2.400828-2.629041$

H $8.697581-2.1357421 .880130$

H $6.642641-4.5087530 .996929$

H $5.101570-4.543189-0.363374$

O $3.454659-0.405022-4.325666$

O $3.4506990 .575695-2.241068$
C $2.6839970 .687796-4.836009$

H $1.7330960 .766856-4.300462$

H $3.251276 \quad 1.619928-4.754773$

H $2.4766110 .493848-5.891690$

SCF Energy $(B 3 L Y P / 6-31 G * * / / M M F F)=-3245.91399359$

0400179

MM̄FF Geometry

C -4.325697 -2.278941 2.240502

C $-3.049695-2.0608911 .888514$

C $-2.031575-1.4016642 .782043$

O $-1.628456-0.1984312 .087925$

C $-0.832982-2.3463532 .989030$

C $0.136858-1.9036374 .066135$

C $1.412034-1.5277943 .844859$

C $-0.384049-1.9818525 .479788$

C $2.139357-1.4445802 .531852$

C $3.086465-2.6394722 .375684$

C $3.853071-2.6233651 .045570$

C $4.729856-3.8740860 .934049$

C $5.606361-3.856978-0.324274$

O $4.666993-1.4475931 .011858$

O $6.584460-4.896000-0.168021$

C $6.350924-2.506870-0.387451$

C $4.796898-4.213536-1.576117$

O $7.090551-2.396966-1.602031$

C $5.427350-1.280308-0.193111$

C $6.2871990 .000869-0.039462$

O $4.587927-1.165094-1.339596$

C -1.3614840 .9069402 .831894$

O -1.2566450 .9723884 .046221$

C -1.280693 2.0636591 .911054

C $-0.838683 \quad 3.2410912 .377568$

C -0.7927694 .5077181 .642875$

C $-1.5361154 .577431 \quad 0.335547$

C 5.5076681 .2759850 .317680

C $4.9209582 .010370-0.876065$

C $3.4109773 .896027-1.436562$

O $5.1065811 .663996-2.039277$

C $3.2375015 .281500-0.905422$

C $2.0452955 .818485-0.606015$

C $1.8625477 .226300-0.119125$

C $1.4437007 .302788 \quad 1.331064$

C 2.2922867 .7528222 .271121

C 0.0128596 .9534771 .677250

C -0.1297475 .5466952 .192982$

N $4.1711343 .111455-0.483458$

H -4.670638 -1.980775 3.227145

H $-2.714661-2.3549580 .896032$

H -2.501566 -1.143858 3.738806

H $-0.313574-2.4846372 .036292$

H -1.197051 -3.344418 3.270315

H $2.023453-1.2599834 .705936$

H $0.387408-1.745406 \quad 6.220483$

H -1.209240 -1.281460 5.634110

H $-0.743053-2.9937735 .694627$

H $1.449907-1.3972971 .685825$

H $2.705012-0.5053082 .513613$

H $3.804082-2.6282653 .207178$

H $2.513348-3.5723872 .452707$

H $3.128105-2.5851620 .224486$

H $4.118518-4.7845730 .964170$

H $5.389752-3.9323211 .811697$

H $7.191812-4.844953-0.926343$

H $7.096315-2.5139030 .419560$

H $4.354857-5.211773-1.471840$

H $5.437940-4.263157-2.463091$

H $3.985080-3.511534-1.776003$

H $6.462054-2.182847-2.312595$

H $6.997200-0.1673180 .781302$

H $6.8745190 .172109-0.949431$

H $4.242297-0.261547-1.387853$

H -1.6260181 .9255010 .893073$

H -0.4859513 .2918773 .407242$

H $-1.0956973 .892100-0.393003$

H - $1.5250455 .568363-0.122426$

H $-2.588977 \quad 4.3078120 .476206$

H 4.7095061 .0616571 .036486 
H $\quad 6.203115 \quad 1.9765220 .797833$

H $2.4555233 .384935-1.589486$

H $3.9370533 .922765-2.396424$

H $4.1382595 .878182-0.781881$

H $1.1476595 .224036-0.749170$

H $1.1082467 .721885-0.742690$

H $2.7862427 .799095-0.276408$

H 1.9871587 .8427523 .308546

H 3.3103908 .0362672 .025904

H $-0.635843 \quad 7.1659170 .823352$

H -0.3436027 .6293802 .466840$

H 0.3627995 .3893183 .154006

H 3.9457743 .2140980 .502417

C $-6.439446-1.9416910 .925024$

O $-5.867771-0.8828390 .127346$

C $-6.519805-0.867253-1.152216$

C $-7.883747-1.491886-0.902559$

C $-7.514307-2.6071580 .056641$

H -5.940237 -1.526973 -1.810363

C $-6.5406970 .546055-1.743104$

H $-8.570143-0.789828-0.415762$

H -8.354153 -1.853401-1.821603

C $-8.692314-3.145940 \quad 0.850162$

H $-7.074783-3.431669-0.521028$

C $-5.1765961 .266645-1.757653$

O $-7.443603 \quad 1.367900-0.992128$

H $-6.9522610 .505707-2.758417$

H $-4.926140 \quad 1.617192-0.748467$

O $\quad-5.3124322 .442376-2.565725$

C $-4.0044070 .432570-2.300052$

C $-2.7092221 .253932-2.448126$

H -3.827187 -0.410262 -1.624172

O $-4.343796-0.117552-3.573044$

H $-2.7739811 .891041-3.338311$

H -2.596492 $1.897084-1.566867$

H -8.368868 -3.932467 1.539457

H $-9.173583-2.3579451 .439151$

H $-9.445830-3.5714930 .179398$

C $-5.336168-2.9225561 .333245$

H -7.145672 $1.360939-0.066100$

H $-6.0454512 .964309-2.196382$

H -4.567918 $0.622540-4.162946$

H -4.835882 -3.3084420 .435731$

H -5.771331 -3.7815421 .856898$

H $-6.883407-1.4681931 .809516$

C $-0.210333-1.205787-1.208403$

O $-1.282938-0.261044-1.285115$

C - $-1.4746840 .343177-2.569830$

C $-0.2155201 .121471-2.979782$

C $1.0342010 .235521-2.933410$

C $1.121361-0.562747-1.627553$

H -0.137982 -1.445796 -0.141866

C $-0.577330-2.493610-1.950759$

H -1.678881 -0.437854 -3.311267

O $-0.0256992 .243691-2.122667$

H $-0.3565631 .513927-3.993449$

H $1.9287520 .862820-3.031813$

O $1.020816-0.672256-4.030209$

H $1.894419-1.334353-1.699347$

O $1.5064640 .325186-0.572006$

C $0.273882-3.658181-1.512154$

H -1.629499 -2.752630 -1.775903

H $-0.488756-2.392648-3.036282$

H $0.0518391 .912049-1.211650$

H $1.005551-0.146008-4.847643$

H $2.3606120 .719963-0.814859$

O $-0.132373-4.097419-0.287159$

O $1.192465-4.118210-2.177552$

C $0.608303-5.1956690 .252819$

H $0.402380-6.102049-0.323783$

H $1.679566-4.9757810 .255159$

H $\quad 0.282036-5.3507901 .284601$

SCF Energy (B3LYP/6-31G**//MMFF) $=-3245.93300607$

04 00180

MM̄FF Geometry

C -2.559257 -0.287632 3.549054

C $-3.858115-0.0830593 .281071$
C -4.4994871 .1387772 .666768$

O $-5.140561 \quad 0.675041 \quad 1.452262$

C -3.5498612 .2928252 .293652$

C -4.2695333 .6009142 .025963$

C -4.5080714 .1182730 .805260$

C $-4.6815534 .366613 \quad 3.258283$

C $-4.2027223 .516152-0.537581$

C $-3.0796444 .261383-1.273585$

C -1.682952 $3.947077-0.716199$

C $-0.6114004 .777888-1.425454$

C $0.8042974 .398396-0.964350$

O $-1.4405062 .554592-0.935858$

O $1.7225515 .000551-1.888792$

C $0.9658512 .866735-1.062788$

C $1.1267224 .993600 \quad 0.411101$

O $2.2170932 .452809-0.519549$

C $-0.1948692 .076118-0.418103$

C $-0.0787520 .579782-0.791140$

O -0.1188902 .2230641 .000585$

C -6.4282091 .0288931 .216128$

O $\quad-7.1406541 .7201201 .931204$

C $-6.9269890 .448708-0.053563$

C $-6.152504-0.209984-0.930416$

C $-6.594056-0.822748-2.186416$

C $-8.060872-0.754890-2.522252$

C $-1.289480-0.264990-0.371489$

C $-1.155596-1.714313-0.804363$

C $-2.454939-3.843069-0.860348$

O $-0.149782-2.166916-1.344018$

C $-3.000491-3.925496-2.250993$

C $-4.217262-4.403397-2.552160$

C $-4.761987-4.459644-3.950664$

C $-6.024684-3.642451-4.120304$

C $-7.223953-4.241512-4.213457$

C $-5.891275-2.142167-4.273752$

C $-5.676250-1.437282-2.962263$

N $-2.297727-2.444851-0.504726$

H -1.8229330 .4746353 .314351$

H -4.560183 -0.8749373 .539989$

H -5.2497681 .4858613 .387588$

H -2.9496212 .0043241 .424706$

H -2.836391 2.4817623 .105779

H -5.0090725 .0843790 .749730$

H -5.1550735 .3238093 .015344$

H -5.3971153 .7884833 .850743$

H -3.8072624 .5820933 .881171$

H -3.979337 $2.447264-0.471151$

H $-5.1177803 .587965-1.139261$

H -3.117662 $3.955018-2.327293$

H -3.264344 $5.342292-1.243072$

H $-1.670697 \quad 4.1542700 .359358$

H $-0.7890155 .850559-1.278970$

H $-0.6788764 .609218-2.509640$

H $2.6181444 .707830-1.646385$

H $1.0045412 .619482-2.132298$

H $1.048658 \quad 6.0871780 .380076$

H 2.1597924 .7776440 .705156

H $0.461724 \quad 4.6355791 .199810$

H 2.1356822 .4574450 .449326

H $0.0261900 .488679-1.880411$

H $0.8271330 .156078-0.342738$

H $-0.483370 \quad 1.432527 \quad 1.427131$

H $-7.9870050 .600496-0.230816$

H $-5.091145-0.321855-0.715310$

H $-8.3781250 .286219-2.647007$

H $-8.660007-1.212925-1.727256$

H -8.321245 -1.278299 -3.444593

H -1.408067 $-0.254700 \quad 0.715513$

H -2.204836 $0.137253-0.820910$

H $-3.125013-4.295528-0.122789$

H $-1.484798-4.347331-0.808424$

H -2.358529 -3.565903 -3.052171

H $-4.859213-4.771554-1.755282$

H -4.955925 -5.513522 -4.189646

H $-4.017350-4.124773-4.683770$

H $-8.132191-3.668630-4.367410$

H $-7.325344-5.318873-4.134484$

H $-6.756973-1.729216-4.803880$ 
H $-5.041656-1.920240-4.932419$

H -4.636816 -1.431782 -2.634497

H -3.107016 -1.961687 -0.125168

C -1.052369 -2.282039 3.281051

O $0.157119-1.5107253 .137129$

C $1.291875-2.3690943 .345395$

C $0.755681-3.7896203 .229115$

C $-0.622099-3.6381293 .840669$

H $1.637636-2.1922104 .371878$

C $2.432623-2.0482352 .368291$

H $0.673680-4.1045202 .182011$

H $1.382474-4.5161323 .754610$

C -1.568298 -4.7746863 .492502$

H $-0.518030-3.5793514 .932446$

C $3.010539-0.6250732 .509470$

O $1.985621-2.246761 \quad 1.028368$

H $3.231831-2.7756452 .547294$

H $3.403291-0.4855963 .524212$

O $1.976743 \quad 0.3502992 .336361$

C $4.107915-0.2706071 .484755$

C $5.273715-1.2722181 .453522$

H $3.670041-0.1664400 .484781$

O 4.6349691 .0137521 .837467

H $5.690869-1.3738172 .463012$

H $4.886727-2.2488621 .143894$

H $-2.553773-4.6137323 .940680$

H -1.702145 -4.8678432 .409410$

H -1.178583 -5.7272953 .865953$

C -2.032285 -1.541840 4.193408

H $1.215619-1.6692950 .892670$

H $1.324088 \quad 0.219077 \quad 3.044741$

H 3.8921451 .6415701 .834600

H $-1.527893-1.2494875 .122788$

H $-2.861070-2.2053464 .466008$

H -1.477902 -2.399025 2.277502

C $6.763804-0.381961-1.859170$

O $5.860469-0.800696-0.827736$

C $6.407859-0.8574380 .493537$

C $7.587698-1.8423760 .545567$

C $8.642649-1.500529-0.511140$

C $8.014516-1.276777-1.891417$

H $6.212754-0.546265-2.793972$

C $7.0913421 .112368-1.760230$

H $6.759836 \quad 0.1384320 .781913$

O $7.112050-3.1696300 .328636$

H $8.031349-1.8092311 .547977$

H $9.370754-2.318645-0.570468$

O $9.338004-0.329153-0.094878$

H $8.752630-0.846753-2.577585$

O $7.645429-2.545859-2.440022$

C $5.8537781 .955216-1.953201$

H $7.5210391 .390058-0.793572$

H $7.8252421 .406620-2.520058$

H $7.872672-3.7702710 .407661$

H $\quad 10.018820-0.139136-0.762524$

H $6.935565-2.913994-1.886380$

O $5.5204342 .001763-3.274506$

O $5.2536772 .500181-1.036104$

C $4.3497532 .762369-3.588290$

H $3.4653152 .276380-3.167190$

H $4.4447603 .786524-3.214988$

H $4.2472882 .795133-4.676184$

SCF Energy (B3LYP/6-31G**//MMFF)= -3245.92889438

0400181

MM̄FF Geometry

C $1.3144451 .805903-2.721722$

C $0.8280620 .583262-2.989978$

C $1.595998-0.721263-3.021410$

O $1.007951-1.610064-2.039217$

C $3.108774-0.630872-2.731792$

C $3.895057-1.842780-3.196710$

C $4.419312-2.784198-2.388274$

C $4.138633-1.916282-4.683802$

C $4.292220-2.910546-0.896500$

C $5.595336-2.557820-0.162597$

C $5.826073-1.043379-0.045316$

C $7.168597-0.7393690 .622369$
C 7.3557090 .7680520 .865345

O $4.762344-0.5165360 .751527$

O $8.4850590 .942957 \quad 1.734043$

C 6.1046841 .3258581 .580921

C $7.7225501 .504819-0.427388$

O 6.1670812 .7455501 .692038

C 4.7767910 .9056480 .912163

C 3.5775331 .2851081 .813404

O $4.6470891 .583062-0.339254$

C $0.722530-2.879259-2.431319$

O $0.789387-3.324385-3.569980$

C $0.314411-3.740377-1.298404$

C $0.390518-3.387418-0.005994$

C $0.011153-4.2262871 .135883$

C $-0.461264-5.6284630 .852036$

C $2.2622840 .604576 \quad 1.405239$

C 1.1034291 .0160242 .294551

C -1.2898450 .4587702 .713792$

O $1.2113501 .820563 \quad 3.215726$

C -1.278608 -0.622235 3.745699

C $-2.111731-1.6730983 .733837$

C $-2.045580-2.7873664 .737198$

C $-1.706366-4.1194494 .102865$

C $-2.638430-5.0803693 .987021$

C $-0.271763-4.3697953 .684809$

C $0.085962-3.7069422 .379308$

N $-0.0679880 .361850 \quad 1.937878$

H $2.3520961 .922656-2.426611$

H $-0.2111270 .504655-3.306278$

H $1.442908-1.116947-4.032937$

H $3.258817-0.465859-1.660563$

H $3.5479620 .236968-3.239331$

H $4.997372-3.585751-2.847646$

H $4.775828-2.763223-4.960127$

H $3.195084-2.027141-5.226060$

H $4.636334-1.005470-5.032715$

H $3.452378-2.329493-0.504504$

H $4.047482-3.958968-0.682350$

H $5.528312-2.9834310 .847228$

H $6.449439-3.034039-0.659501$

H $5.791311-0.596853-1.044969$

H $7.999870-1.1395460 .028675$

H $7.216476-1.2573401 .590581$

H 8.3075250 .4677102 .563376

H $\quad 6.1116700 .9343322 .607576$

H $8.6614921 .112293-0.836388$

H $7.9010402 .569362-0.239111$

H $6.961114 \quad 1.415866-1.204915$

H 7.0128372 .9661812 .118389

H 3.7914490 .9864022 .848218

H $3.4393412 .373871 \quad 1.811575$

H $3.7061421 .714995-0.532983$

H $-0.038463-4.722989-1.595863$

H $\quad 0.762222-2.3969990 .248268$

H -1.379055 -5.611196 0.255972

H $-0.684187-6.2046721 .752211$

H $0.304766-6.1904830 .306445$

H 2.0017580 .8433470 .370100

H $2.365542-0.4842671 .484544$

H $-2.127073 \quad 0.3565512 .018317$

H $-1.346073 \quad 1.437378 \quad 3.199141$

H -0.527213 -0.547087 4.528407

H -2.864416 -1.751389 2.952818

H -3.018070 -2.838944 5.243489

H -1.313772 -2.572071 5.525838

H $-2.401817-6.051023 \quad 3.564626$

H -3.661361 -4.917817 4.310155

H $-0.060806-5.4446413 .644336$

H $0.401970-3.9931074 .465421$

H $0.448703-2.6859022 .491959$

H $-0.036579-0.3362331 .200623$

C $0.2620443 .936843-1.699142$

O $-0.9142823 .453967-1.031920$

C -0.8692973 .9064840 .337868$

C 0.4934404 .5768930 .540690

C $1.3180894 .018220-0.605735$

H -0.9204153 .0082030 .960213$

C $-2.075054 \quad 4.8116240 .639162$ 
H $0.4304835 .668658 \quad 0.474185$

H $0.919645 \quad 4.341151 \quad 1.521292$

C $2.5261574 .864725-0.961408$

H $1.6510663 .016494-0.317353$

C -3.434992 $4.224230 \quad 0.212471$

O $-1.9117376 .046437-0.073029$

H -2.079726 $5.071871 \quad 1.703909$

H -3.492338 $4.216878-0.882905$

O $-4.458872 \quad 5.127648 \quad 0.648850$

$\begin{array}{llll}\text { C } & -3.745521 & 2.815472 & 0.746931\end{array}$

$\begin{array}{llll}C & -5.114132 & 2.314636 & 0.253932\end{array}$

H $-2.9682942 .119016 \quad 0.419414$

O -3.7613542 .8219612 .172428$

H -5.9082282 .9287520 .696292$

H $-5.1665232 .453411-0.831277$

H $3.0790634 .424963-1.797502$

H $2.2339635 .880531-1.247773$

H $3.2082554 .938237-0.107966$

C $0.5587103 .089223-2.937780$

H -2.679460 $6.605657 \quad 0.136784$

H $-4.475052 \quad 5.103793 \quad 1.621123$

H -2.872790 3.0654982 .480261

H $1.1672583 .691504-3.624869$

H $-0.383762 \quad 2.881545-3.460391$

H $0.0122374 .948150-2.050616$

C $-4.163116-0.188043-1.192413$

$\begin{array}{llll}\text { O } & -4.316455 & -0.031433 & 0.221297\end{array}$

C -5.3864150 .8351160 .614510$

$\begin{array}{llll}C & -6.718317 & 0.299352 & 0.067788\end{array}$

C $-6.6654480 .091774-1.446739$

C $-5.443182-0.745555-1.827033$

H $-3.9337460 .789639-1.634177$

C -2.922939-1.054575-1.446472

H -5.4363730 .7809801 .709335$

O

H $-6.937987-0.6621950 .550029$

H $-6.6706921 .045210-1.987449$

O $-7.832344-0.617163-1.870095$

H $-5.617671-1.779627-1.506781$

O $-5.294663-0.755788-3.244436$

C $-3.115753-2.489732-1.014185$

H $-2.645640-1.030362-2.506950$

H -2.060341 $-0.667539-0.889913$

H -8.6118850 .7648720 .163730$

H -8.597048 $-0.034150-1.728295$

H -6.127417 -1.088361 -3.621089

O $-3.325051-3.275563-2.108748$

O $-3.109955-2.8581250 .152942$

C -3.605930 -4.656754-1.859099

H $-4.141064-5.053961-2.725796$

H -2.666475 -5.202907 -1.747386

H $-4.236534-4.788330-0.974375$

SCF Energy (B3LYP/6-31G*//MMFF) $=-3245.90893710$

05_00001

MM̄FF Geometry

C -3.215706 -3.105265 -2.099118

C $-2.274703-3.156652-1.145098$

C $-0.801366-3.288882-1.433445$

O $-0.111926-2.152706-0.861347$

C $-0.255391-4.562772-0.763698$

C $1.207829-4.829539-1.059150$

C $2.187890-4.835784-0.134236$

C $1.539674-5.133462-2.498028$

C $2.071384-4.5968911 .344975$

C $2.310476-3.1432741 .781629$

C $3.721021-2.6163621 .469785$

C $3.963679-1.2764902 .167905$

C $5.333445-0.6794291 .807438$

O $3.837477-2.4409710 .055165$

$\begin{array}{lllll}O & 5.340042 & 0.692917 & 2.230529\end{array}$

C $5.497109-0.678478 \quad 0.271936$

C $6.473511-1.3718222 .563645$

O $6.817609-0.292308-0.100016$

C $5.137297-2.026018-0.387309$

C $5.095646-1.954390-1.933991$

O $6.131175-2.997949-0.039394$

C $-0.185620-0.973290-1.535922$
O $-0.785486-0.753887-2.576177$

C $0.591850 \quad 0.026992-0.774117$

C $0.3893341 .331848-1.006675$

C $1.0728932 .440192-0.339824$

C $2.3015392 .116593 \quad 0.467790$

C $4.079046-0.968593-2.521726$

C $4.6613130 .389983-2.877889$

C $3.9652362 .637682-3.707856$

O $5.8639390 .636097-2.858746$

C $3.8869743 .580047-2.548487$

C $3.1141834 .677034-2.538066$

C $3.0673535 .657680-1.400944$

C $1.6618885 .916135-0.905441$

C $0.9777647 .002693-1.301881$

C $1.0862224 .963756 \quad 0.117406$

C $0.5868783 .688231-0.501040$

N $3.6729331 .288691-3.259440$

H $-2.921771-3.177238-3.143352$

H $-2.566769-3.079415-0.101652$

H $-0.612916-3.319958-2.513487$

H $-0.832971-5.435212-1.097632$

H $-0.420779-4.4868390 .316479$

H $3.202918-5.047495-0.469088$

H $2.575397-5.467161-2.623814$

H $0.892552-5.931045-2.877370$

H $1.404776-4.245046-3.122187$

H $1.093844-4.9223651 .715796$

H $2.796633-5.247693 \quad 1.850361$

H $1.561099-2.5016881 .303660$

H $2.138064-3.0905072 .863811$

H $4.451054-3.3606541 .808837$

H $3.865666-1.3795913 .255743$

H $3.184715-0.564577 \quad 1.864592$

H $5.166800 \quad 0.7124243 .187376$

H $4.8263220 .095298-0.116929$

H $6.328567-1.2752553 .646427$

H $7.436841-0.8966362 .347931$

H $6.554669-2.4366422 .336010$

H $6.9948100 .570143 \quad 0.313176$

H $6.103284-1.783119-2.331312$

H $4.808518-2.952893-2.294696$

H $\quad 6.105937-3.713528-0.695993$

H $1.298471-0.335689-0.037150$

H $-0.3658591 .620347-1.736911$

H $2.9993841 .518524-0.126381$

H $2.0394331 .552751 \quad 1.367668$

H 2.8590202 .9996120 .786753

H $3.695146-1.388656-3.460519$

H $3.225341-0.835800-1.850940$

H $3.2407872 .883286-4.490800$

H $4.9700982 .669075-4.141026$

H $4.5185803 .365330-1.689634$

H $2.5052844 .904938-3.410196$

H $3.5154686 .595872-1.753922$

H $3.6943425 .329744-0.562510$

H $-0.0193927 .210417-0.928331$

H $1.3978527 .705615-2.013677$

H $\quad 0.2325025 .430575 \quad 0.627684$

H $1.8195784 .795443 \quad 0.911431$

H $-0.3023943 .823046-1.118536$

H $2.6974431 .012942-3.192837$

C $-5.187396-1.591833-2.358859$

O $-4.617692-0.514201-1.587527$

C $-5.634168 \quad 0.478916-1.353092$

C $-6.818498 \quad 0.090823-2.231071$

C $-6.700718-1.420547-2.243078$

H $-5.9335810 .379086-0.303416$

C $-5.080823 \quad 1.884944-1.609527$

H $-7.7701790 .445347-1.823508$

H $-6.7195400 .488137-3.248038$

C $-7.488287-2.081452-3.360839$

H $-7.050281-1.808524-1.276764$

C $-3.8244342 .230238-0.784706$

O $-4.729945 \quad 1.986526-2.996015$

H $-5.8686442 .627721-1.437156$

H $-2.9845101 .614536-1.127217$

O $-3.4651573 .584678-1.088815$

$\begin{array}{llll}\text { C } & -3.979687 & 2.081592 & 0.740927\end{array}$ 
C $-2.7120112 .523472 \quad 1.492203$

H -4.1987651 .0406420 .989453$ O H -1.851443 2.0080401 .055889 H -2.560403 3.5990911 .341170 H -8.556018 -1.860521 -3.261106 H -7.162375 -1.727963 -4.344856 H $-7.365350-3.168743-3.336044$ C $-4.682423-2.931311-1.821826$ H -4.373199 2.879798 -3.140054 H -4.165959 4.159545 -0.736174 H -5.8908852 .4830660 .866902$ H $-5.218600-3.760062-2.297246$ H -4.873105 -2.993065 -0.744004 H -4.863539 -1.446690 -3.398239 C -1.954029 -0.0222612 .998602$ $\begin{array}{lllll}\text { O } & -3.029195 & 0.871381 & 3.313104\end{array}$ C -2.7837862 .2502913 .012851$ C -1.5557452 .7396313 .791657$ C -0.3360821 .8677553 .514233$ C $-0.669467 \quad 0.393266 \quad 3.731911$ H - $1.788347-0.0330121 .914543$ C $-2.378286-1.4345163 .434212$ H -3.6492462 .8054813 .395722$ O -1.2725334 .0952283 .460969$ H -1.7778882 .7097014 .866227$ H 0.0445972 .0482652 .505262 O 0.7085842 .2501914 .410829 H $-0.777187 \quad 0.2125784 .808859$ O $0.427102-0.4037303 .287798$ C $-3.504140-2.0015322 .596399$ H -1.533499 -2.127145 3.348318 H $-2.724628-1.4087724 .474469$ H -0.4720414 .3526953 .949715$ H 1.4654651 .6636104 .239381 H $0.525948-0.2675452 .330272$ O $-3.853238-3.222842 \quad 3.100512$ O $-4.008764-1.4586331 .624351$ C -4.909240 -3.899718 2.411287 H -4.555131 -4.240300 1.434489 H $-5.196813-4.7721533 .004131$ H -5.782475 -3.249182 2.301855

SCF Energy (B3LYP/6-31G**//MMFF) $=-3245.91613420$

05_00002

MM̄FF Geometry

C $2.1602933 .828485-0.512676$

C $1.2567144 .649302-1.069179$

C $-0.2199524 .348062-1.226807$

O $-0.5074752 .958625-0.946487$

C $-1.0202455 .211270-0.234451$

C $-2.5183114 .990365-0.295588$

C $-3.2559194 .480676 \quad 0.710750$

C -3.186399 $5.402256-1.581842$

C -2.7895504 .0388762 .070540$

C -2.4377392 .5465132 .176097$

C -3.6317551 .6035751 .954510$

C -3.2549990 .1623412 .304383$

C -4.403930 -0.817932 2.016947

O $-4.019718 \quad 1.671098 \quad 0.579801$

O $-3.868809-2.1497902 .057051$

C $-4.921973-0.5845740 .581182$

C $-5.489031-0.7552253 .098585$

O $-6.096426-1.3525610 .332082$

C -5.1848600 .8997630 .254453$

C $-5.4995361 .147546-1.241766$

O -6.3224451 .3503531 .000539$

C $-0.2608472 .055882-1.934968$

O $\quad 0.1241202 .289687-3.069213$

C $-0.4883380 .708719-1.369322$

C $0.077586-0.355352-1.957568$

C $-0.023156-1.743591-1.504409$

C $-1.007618-2.045911-0.407341$

C $-4.400915 \quad 0.728747-2.225343$

C $-4.594938-0.655860-2.822701$

C $-3.448162-2.307898-4.298068$

O $-5.621347-1.314691-2.681572$

C $-2.802211-3.357103-3.449227$
C $-1.760848-4.098374-3.857608$

C $-1.134942-5.189200-3.036057$

C $0.360679-5.023706-2.884130$

C $1.215341-5.704435-3.666409$

C $0.863638-4.138158-1.767634$

C $0.753964-2.675134-2.094649$

N $-3.493827-1.052922-3.570696$

H $1.8391872 .868707-0.118281$

H $1.5858625 .615652-1.444861$

H $-0.5200634 .581281-2.255754$

H $-0.8213056 .274882-0.422059$

H $-0.647655 \quad 5.0132970 .775782$

H -4.3283324 .3711690 .552106$

H -4.278162 $5.335287-1.522939$

H -2.935389 $6.439901-1.824931$

H $-2.8633724 .760659-2.407373$

H -1.9272184 .6303162 .395850$

H -3.5814404 .2743612 .793163$

H -1.642533 $2.314857 \quad 1.457866$

H $-2.0268262 .372620 \quad 3.178473$

H -4.4604541 .9447432 .585967$

H -2.939032 0.0846883 .352230

H $-2.383903-0.1389171 .707324$

H -3.468793 -2.284769 2.933136

H -4.151465 -0.965065 -0.098438

H $-5.065261-0.9987784 .080233$

H -6.271108 -1.500848 2.917640

H $-5.959498 \quad 0.226937 \quad 3.177734$

H $-5.881261-2.2815790 .523343$

H $-6.4675590 .703036-1.502289$

H $-5.6361242 .231550-1.366744$

H -6.6864972 .1374010 .562550$

H $-1.0857410 .635043-0.468164$

H $\quad 0.696871-0.194935-2.839229$

H -2.003872 -1.684136 -0.680337

H $-0.703622-1.5605160 .523822$

H -1.119548 -3.111269-0.196881

H -4.411416 $1.427411-3.072056$

H -3.412456 $0.792929-1.761888$

H -2.893928 -2.125709 -5.224351

H $-4.465982-2.617877-4.556028$

H -3.229892 -3.535213 -2.465346

H -1.354162 -3.937548 -4.853734

H $-1.354721-6.144717-3.530534$

H -1.596536 -5.258809 -2.043225

H $2.289531-5.612956-3.544051$

H $0.859438-6.364246-4.450761$

H $1.924336-4.345854-1.571462$

H $\quad 0.356290-4.406685-0.836917$

H $1.430122-2.358142-2.889878$

H $-2.666362-0.464028-3.589543$

C $4.4747923 .172224-1.188264$

O $4.2521461 .813713-0.760714$

C $5.5056331 .108062-0.771131$

C $6.5223552 .046409-1.414678$

C $5.9752083 .406453-1.030820$

H $5.7842760 .949576 \quad 0.277046$

C $5.352806-0.240400-1.483280$

H $7.5373411 .870948-1.044911$

H $6.5428071 .940141-2.505423$

C $6.5197914 .541816-1.879838$

H 6.2176383 .5959310 .023499

C $4.246584-1.156600-0.923201$

O $5.049000-0.011124-2.866676$

H $6.313219-0.768959-1.473003$

H $3.257174-0.754928-1.171398$

O $4.351154-2.413748-1.606162$

C $4.318722-1.4174160 .592735$

C $3.358031-2.5349701 .045486$

H $4.085513-0.491647 \quad 1.127579$

O $5.650876-1.7857730 .952905$

H $2.363420-2.3353860 .636982$

H $3.700563-3.4865940 .622192$

H $7.6081084 .609009-1.779462$

H $6.2883294 .396661-2.940413$

H $6.0930795 .500964-1.570544$

C $3.6216554 .132661-0.358735$

H $4.2314990 .514862-2.903704$ 
H $4.271398-2.233194-2.558624$

H $5.880438-2.5869830 .451397$

H $3.8336015 .168934-0.644697$

H 3.8684604 .0294810 .705047

H $4.1831973 .224436-2.245756$

C $1.356675-1.2902623 .012751$

O $2.760115-1.5051513 .211937$

C $3.292935-2.6774082 .583125$

C $2.543930-3.9253023 .070955$

C $1.035837-3.7933142 .886115$

C $0.544838-2.491783 \quad 3.517141$

H $1.157647-1.1245581 .948200$

C $0.966664-0.0141723 .775545$

H $4.326222-2.7719582 .939505$

O $3.021358-5.0836232 .394523$

H $2.756735-4.0694184 .138452$

H 0.760663 -3.858443 1.826912

O $0.411439-4.9009343 .538299$

H $0.619097-2.5635644 .609871$

O $-0.829276-2.2988053 .192361$

C $1.459300 \quad 1.2436523 .092776$

H $-0.123848 \quad 0.0692123 .843428$

H $1.395141-0.0346464 .784328$

H $2.506953-5.8425302 .718875$

H $-0.534806-4.875898 \quad 3.317442$

H $-1.335110-3.0103093 .619542$

O 1.0230352 .3260913 .803443

O $2.132261 \quad 1.2790192 .072547$

C 1.3898363 .6028713 .272765

H $\quad 0.8764663 .7695532 .322564$

H 1.0710684 .3705823 .982606

H 2.4745063 .6712883 .145948

SCF Energy (B3LYP/6-31G**//MMFF) $=-3245.91812588$

05_00003

MM̄FF Geometry

C $2.2043623 .773290-0.585048$

C $1.3085524 .597653-1.148822$

C $-0.1721424 .312993-1.297284$

O $-0.4764012 .931994-0.994838$

C $-0.9580905 .200918-0.315326$

C $-2.4592265 .001339-0.371849$

C $-3.203150 \quad 4.5174070 .642524$

C -3.122839 5.404116-1.663296

C -2.7416384 .0891622 .008295$

C -2.4127832 .5933862 .135824$

C -3.621284 1.6657841 .927016

C -3.2681820 .2251082 .301262$

C -4.428864 -0.7444352 .027647$

O

O $-3.886109-2.0711172 .113839$

C $-4.940926-0.5276960 .585853$

C $-5.510376-0.6455803 .110037$

O $-6.131988-1.2730200 .346388$

C -5.1793180 .9556180 .235303$

C $-5.4877031 .184693-1.265305$

O $-6.311551 \quad 1.434508 \quad 0.972424$

C $-0.2459242 .011706-1.970961$

O $0.1346182 .223802-3.110952$

C $-0.4857670 .676340-1.383142$

C $0.067320-0.403022-1.955524$

C $-0.047607-1.782331-1.479072$

C $-1.032983-2.055260-0.375276$

C $-4.3940300 .733109-2.240006$

C $-4.606672-0.659189-2.812637$

C $-3.481080-2.353605-4.255909$

O $-5.642168-1.301192-2.660735$

C -2.847345 -3.394682 -3.388065

C -1.816004 -4.156736 -3.783759

C -1.201987 -5.239217 -2.942436

C $0.296132-5.089870-2.797551$

C $1.139638-5.795125-3.569977$

C $0.813467-4.190618-1.698580$

C $0.718006-2.732321-2.054768$

N -3.510481 -1.084875 -3.551878

H $1.8741422 .822548-0.176546$

H $1.6481605 .555132-1.537959$

H $-0.4724614 .534627-2.328622$
H $-0.743924 \quad 6.259017-0.517374$

H -0.5867575 .0106880 .696855$

H -4.2771434 .4214730 .486494$

H -4.215446 $5.356124-1.601587$

H -2.855331 $6.433450-1.923098$

H $-2.8121274 .744370-2.479192$

H -1.8697874 .6718312 .323568$

H -3.5287414 .3477742 .728235$

H -1.620826 2.3393401 .421584

H -2.0051142 .4278513 .140937$

H -4.4460302 .0308662 .550220$

H $-2.954910 \quad 0.159842 \quad 3.350761$

H -2.400015 -0.096715 1.710703

H -4.612613 -2.703639 1.983875

H $-4.176400-0.932484-0.086282$

H $-5.090358-0.8964924 .091668$

H $-6.314285-1.3690932 .935115$

H $-5.9540200 .349566 \quad 3.183025$

H $-5.917529-2.2148870 .452639$

H $-6.4621750 .751255-1.520814$

H $-5.6070152 .268603-1.407590$

H -6.6621062 .2208100 .522253$

H -1.081075 $0.623414-0.479143$

H $0.686264-0.263563-2.841104$

H -1.162656 -3.116164 -0.152840

H $-2.024443-1.679649-0.647841$

H $-0.715880-1.564600 \quad 0.548847$

H $-4.393851 \quad 1.416680-3.098886$

H -3.405309 $0.791657-1.776364$

H -2.925643 -2.195419 -5.185756

H $-4.502908-2.655761-4.507218$

H -3.275391 -3.548408 -2.400349

H -1.409083 -4.020128 -4.783290

H -1.435182 $-6.201240-3.417553$

H -1.661784 -5.283359-1.947314

H $2.215252-5.714769-3.452410$

H $0.773003-6.464183-4.341446$

H $1.872328-4.407358-1.502266$

H $0.306486-4.436821-0.761395$

H $1.396433-2.436294-2.856258$

H -2.674995 -0.507666 -3.580951

C $4.5105313 .083925-1.258367$

O $4.279697 \quad 1.734310-0.807295$

C $5.5293511 .022036-0.802273$

C $6.5498021 .939589-1.469922$

C $6.0135593 .310789-1.111610$

H $5.8081010 .892970 \quad 0.249591$

C $5.373179-0.345165-1.476983$

H $7.5651681 .764923-1.100821$

H $6.564678 \quad 1.811310-2.558388$

C $6.5622934 .425107-1.985473$

H $6.2615533 .519903-0.062308$

C $4.260183-1.235712-0.889349$

O $5.062950-0.133495-2.861760$

H $6.334449-0.872511-1.457052$

H $3.281315-0.811569-1.141058$

O $4.324617-2.495942-1.573065$

C $4.329497-1.4829870 .630203$

C $3.304406-2.5392751 .088769$

H $4.157606-0.5448471 .167727$

O $5.627310-1.9619250 .985649$

H $2.323121-2.2857720 .677945$

H $3.593643-3.5113350 .672457$

H $7.6513564 .486782-1.890905$

H $6.3254484 .260055-3.041941$

H $6.1434375 .393189-1.693774$

C $3.6693964 .064221-0.439676$

H $4.967714-1.008983-3.274792$

H $5.157084-2.927333-1.314134$

H $6.258970-1.2332750 .868632$

H $3.8909355 .094291-0.740764$

H 3.9185813 .9734460 .624784

H $4.2141383 .121104-2.315160$

C $1.356229-1.1908973 .044799$

O $2.749128-1.4653393 .244824$

C $3.229415-2.6662912 .627723$

C $2.425311-3.8753533 .125220$

C $0.924945-3.6779282 .939111$ 
C $0.493155-2.3510073 .560230$

H $1.162667-1.0251791 .978935$

C $1.0223640 .107122 \quad 3.797162$

H $4.256359-2.8039842 .988049$

O $2.850636-5.0595122 .458831$

H $2.631602-4.0201844 .193897$

H $0.647606-3.7387831 .880322$

O $0.251629-4.7515323 .599244$

H $0.566003-2.4170634 .653416$

O $-0.870782-2.0994373 .235286$

C 1.5603201 .3382983 .099738

H -0.0638730 .2333573 .868941$

H $1.4540210 .080825 \quad 4.804344$

H $2.302492-5.7919342 .789054$

H $-0.693499-4.6816663 .383291$

H -1.410482 -2.779597 3.671751

O 1.1190052 .4458533 .767483

O $2.271586 \quad 1.3356632 .105209$

C $1.5270593 .703393 \quad 3.220946$

H 2.6139813 .7401533 .100435

H 1.0241583 .8700542 .265161

H $1.225568 \quad 4.4904063 .917221$

SCF Energy (B3LYP/6-31G**//MMFF)= -3245.91092016

05_00004

MM̄FF Geometry

C $3.590085-0.382311-2.138209$

C $2.571872-0.939193-2.810476$

C $1.860217-2.179939-2.344951$

O $0.460903-1.998193-2.679131$

C $2.372112-3.462453-3.022127$

C $3.726964-3.920261-2.523024$

C $3.916593-4.624363-1.388800$

C $4.894339-3.601478-3.419993$

C $2.865706-5.083235-0.415769$

C $3.251755-4.8697721 .054060$

C $3.276124-3.4026391 .520870$

C $3.773011-3.3484432 .972317$

C $3.696721-1.9406123 .574469$

O $1.948716-2.8680621 .425235$

O $3.880058-2.0802244 .991757$

C $2.278534-1.3850283 .332058$

C $4.845517-1.0538703 .080460$

O $2.181440-0.0366883 .785332$

C $1.821756-1.5062001 .862625$

C $0.355260-1.0595521 .664148$

O $2.628263-0.6237861 .072629$

C $-0.457708-2.621948-1.895224$

O $-0.238137-3.339885-0.933075$

C -1.802291-2.279905 -2.406462

C $-2.876061-2.685949-1.713141$

C $-4.277993-2.456751-2.061355$

C $-4.574093-1.717263-3.339329$

C $-0.708483-1.9221682 .347582$

C -2.086020 -1.293220 2.206333

C $-4.307686-1.2350833 .329683$

O $-2.336474-0.4144531 .386101$

C $-5.346871-1.9424992 .519635$

C $-6.223019-1.2978591 .733141$

C $-7.353157-1.9645821 .000763$

C $-7.311440-1.726744-0.491729$

C $-7.854209-0.623406-1.033557$

C $-6.718383-2.810556-1.361125$

C $-5.227256-2.910945-1.217152$

N -2.999792 -1.822003 3.105260

H $3.969942-0.879488-1.249338$

H $2.210816-0.466106-3.720089$

H $1.951514-2.256547-1.255852$

H $2.396819-3.326420-4.112093$

H $1.644749-4.272535-2.879601$

H $4.930316-4.939439-1.145425$

H $\quad 5.843770-3.963191-3.011084$

H $4.756454-4.072699-4.398973$

H $4.992101-2.522748-3.567881$

H $1.896167-4.622353-0.608097$

H $2.731056-6.161093-0.571904$

H $2.518228-5.417217 \quad 1.661106$

H $4.229053-5.3328251 .241082$
H $3.941844-2.8271390 .867881$

H $4.794990-3.7415533 .046671$

H $3.154809-4.0170433 .588695$

H $3.757976-1.2019565 .392103$

H $1.603322-1.9666643 .970542$

H $5.810120-1.4809073 .380711$

H $4.805619-0.0594453 .538333$

H $4.862695-0.9346451 .995124$

H $2.6219970 .527882 \quad 3.128248$

H $0.247859-0.0146501 .983225$

H $0.135015-1.0464450 .588135$

H $2.121054-0.350307 \quad 0.290850$

H $-1.871197-1.712355-3.326966$

H $-2.716629-3.244347-0.790949$

H $-5.637726-1.522276-3.492839$

H $-4.222603-2.291571-4.203473$

H $-4.081693-0.739468-3.346152$

H $-0.740010-2.9223161 .902662$

H $-0.490864-2.0237513 .414999$

H $-4.267003-0.1639903 .103841$

H $-4.532443-1.3523324 .394730$

H $-5.418967-3.0216412 .629369$

H $-6.163232-0.2145631 .654382$

H $-8.294273-1.5691061 .405660$

H -7.380156 -3.0419311 .206234$

H $-7.852097-0.460097-2.106093$

H $-8.306757 \quad 0.143953-0.414040$

H $-7.010519-2.674893-2.407679$

H -7.158335 -3.776254 -1.079995

H $-4.911818-3.431248-0.312181$

H $-2.712375-2.580706 \quad 3.716496$

C $4.4780991 .800430-1.311117$

O $3.2181032 .184740-0.725766$

C $3.3521323 .513077-0.181654$

C $4.7988333 .940822-0.423660$

C $5.1770273 .116108-1.637062$

H $2.6926494 .160250-0.769627$

C 2.9466513 .5318751 .298599

H $4.885098 \quad 5.018557-0.593888$

H 5.4440483 .6897560 .426620

C $6.6767662 .995587-1.842241$

H $4.7234273 .572642-2.527055$

C $1.492594 \quad 3.1088791 .586190$

$\begin{array}{lllll}\text { O } & 3.803892 & 2.641225 & 2.027762\end{array}$

H 3.1281314 .5286431 .717137

H 1.3644962 .0410801 .376535

$\begin{array}{lllll}\text { O } & 1.264691 & 3.272793 & 2.992240\end{array}$

C 0.4155753 .9038250 .825221

C $-1.012133 \quad 3.5784331 .311656$

H $0.4973323 .689369-0.245251$

$\begin{array}{llll}\text { O } & 0.616875 & 5.306568 & 1.005229\end{array}$

H $-1.1325282 .492306 \quad 1.374763$

H -1.1485243 .9817682 .322442$

H $7.1262963 .984182-1.981554$

H $7.1627452 .523252-0.981900$

H $6.9038102 .395409-2.728779$

C $4.2554560 .904305-2.530639$

H 3.7271141 .7618251 .618745

H 1.9434372 .7556643 .458964

H 1.4254415 .5579190 .529343

H $5.2182240 .665406-2.995531$

H $3.6448791 .431641-3.272776$

H $5.0359901 .252747-0.538859$

C $-2.3135852 .352850-1.155154$

O -1.961805 3.728283 -0.958947

C $-2.090722 \quad 4.1925290 .389138$

C -3.538195 4.0131070 .869446

C -4.0456112 .5893050 .659697$

C $-3.7790452 .125315-0.772287$

H $-1.6677001 .709869-0.546185$

C $-2.0531172 .023461-2.629621$

H $-1.9014415 .273026 \quad 0.357736$

O $-3.669103 \quad 4.3579012 .244339$

H -4.1895644 .6939020 .305868$

H $-3.603057 \quad 1.9003491 .386985$

O -5.4571642 .5551050 .884178$

H $-4.4400322 .664897-1.462383$

O $-4.0863770 .739094-0.880521$ 
C $-0.5686192 .024252-2.926532$

H $-2.4347921 .034984-2.901788$

H $-2.5441182 .771104-3.264208$

H -3.353794 5.2720592 .347549

H -5.6131042 .8771941 .788551$

H $-5.0078590 .620765-0.593412$

O $-0.3351012 .659430-4.110695$

O $0.2792621 .510982-2.207980$

C $1.0382572 .747645-4.502589$

H $1.6386473 .200715-3.707348$

H $1.4173751 .753785-4.756076$

H $1.0984483 .383254-5.389981$

SCF Energy (B3LYP/6-31G**//MMFF) $=-3245.93536213$

05 00005

MM̄FF Geometry

C $2.6972461 .741892-2.725353$

C $2.7482113 .082279-2.778293$

C $1.8561014 .049786-2.038724$

O $0.7396063 .358577-1.439980$

C $2.6740464 .712430-0.910850$

C $1.8877895 .698079-0.069454$

C 1.5996885 .5264151 .236781

C $1.4391446 .947650-0.781800$

C 2.0004194 .3817412 .128614

C 0.9059363 .3179852 .192570

C 1.1971742 .1736473 .173490

C $2.384976 \quad 1.3118142 .733027$

C 2.5748720 .0821013 .636332

O $0.0043331 .376352 \quad 3.222441$

O $3.475174-0.8189262 .973667$

C $1.223445-0.6496893 .771830$

C $3.2368740 .449224 \quad 4.969397$

O $1.310608-1.7243644 .702938$

C $0.056548 \quad 0.2847124 .152243$

C $-1.318258-0.4240304 .114542$

$\begin{array}{lllll}\text { O } & 0.242917 & 0.759961 & 5.490377\end{array}$

C $-0.5185763 .727004-1.802449$

O $-0.8397234 .522177-2.670044$

C $-1.4555253 .007652-0.911286$

C $-2.7692283 .276412-0.943518$

C $-3.7841972 .672149-0.075268$

C $-3.2959991 .740076 \quad 1.001642$

C -1.651917 -1.112683 2.789539

C $-3.130820-1.4419152 .654637$

C $-4.762157-2.2984310 .959605$

O $-3.948977-1.2566913 .550778$

C $-5.208580-1.260015-0.021624$

C $-6.374910-0.6010930 .053574$

C $-6.8049150 .424980-0.958199$

C $-7.2606001 .726645-0.332317$

C -8.512983 $2.175610-0.522853$

C -6.2802552 .5119380 .513641$

C $-5.0826192 .986756-0.267839$

N -3.419809 -1.969149 1.404393

H $1.940298 \quad 1.244361-2.125917$

H $3.5325813 .546262-3.374270$

H $1.5145974 .806242-2.754522$

H $3.5390375 .237476-1.338168$

H $3.0920753 .921293-0.278638$

H 1.0154876 .2967101 .738924

H $0.9661067 .665538-0.102997$

H $2.2965967 .449477-1.241965$

H $0.7132546 .709941-1.564588$

H 2.9515723 .9488391 .808035

H $2.1832294 .783073 \quad 3.133284$

H $-0.048513 \quad 3.7854162 .471276$

H $\quad 0.7418762 .893314 \quad 1.196367$

H 1.3751152 .6050314 .165408

H 3.3114351 .8965172 .701203

H 2.2171410 .9617891 .704439

H $4.307683-0.3439782 .808429$

H $1.011668-1.0995992 .794005$

H 4.2305650 .8800834 .797091

H $3.399803-0.4403705 .587619$

H 2.6621161 .1737395 .549706

H $2.053789-2.2878064 .426603$

H $-1.390943-1.1522014 .932997$
H $-2.089695 \quad 0.3301334 .324644$

H -0.6175591 .0491775 .836985$

H -1.030092 2.281122 -0.228644

H -3.136415 4.011681-1.657993

H -4.0874961 .3467831 .640766$

H -2.5884892 .2509681 .664362$

H -2.7960310 .8768960 .551462$

H -1.369050 -0.471571 1.947729

H -1.092571-2.051352 2.708356

H $-5.434998-2.3740991 .819413$

H $-4.708510-3.2733950 .465804$

H $-4.538614-1.047133-0.851504$

H -7.064455 -0.8248230 .864075$

H -7.618678 $-0.017512-1.547398$

H $-5.9989950 .636115-1.672022$

H $-8.8546703 .105703-0.080808$

H $-9.2270071 .624162-1.125597$

H -6.0122051 .9129661 .386979$

H -6.7669013 .4063870 .925225$

H $-5.3331703 .681520-1.070567$

H $-2.668897-2.0557410 .725654$

C $4.5711940 .037396-2.474175$

O $3.812839-1.033743-1.884510$

C $4.326111-1.282497-0.559129$

C $5.546703-0.379104-0.384925$

C $5.2099760 .780941-1.301978$

H $3.545173-0.9561200 .137012$

C $4.606853-2.778312-0.359262$

H $5.688132-0.0753460 .656920$

H $6.470690-0.867137-0.715181$

C $6.4084481 .629319-1.686212$

H $4.4747251 .409985-0.785143$

C $3.432540-3.702387-0.736172$

O $5.717832-3.148644-1.187218$

H $4.921845-2.9553620 .675989$

H $3.291856-3.683653-1.823650$

O $3.812043-5.046899-0.414941$

C $2.100802-3.374789-0.040483$

C $0.992154-4.362858-0.448182$

H $1.805354-2.354264-0.300975$

O $2.294879-3.4264521 .370899$

H $0.998169-4.462088-1.539363$

H $1.212319-5.354287-0.033446$

H $6.8662252 .073323-0.796208$

H $7.1738121 .035496-2.196933$

H $6.1112082 .443396-2.354849$

C $3.6936050 .859204-3.425027$

H $5.883212-4.096256-1.043580$

H $3.889376-5.1053400 .552917$

H $1.478331-3.1145901 .794703$

H $3.1343600 .181083-4.081052$

H $4.3438421 .463539-4.069356$

H $5.350836-0.448312-3.076990$

C $-0.854572-2.285054-1.668321$

O $-0.703384-2.570373-0.272278$

C $-0.414532-3.9388260 .030006$

C $-1.549238-4.834695-0.486720$

C $-1.840237-4.593161-1.967284$

C $-2.014260-3.100898-2.253521$

H $0.076885-2.533978-2.189725$

C $-1.081596-0.771916-1.788680$

H -0.413963 -4.017201 1.124002

O $-1.242094-6.210936-0.290392$

H -2.463807 $-4.626240 \quad 0.082646$

H -1.059771 -5.024061-2.605306

O $-3.055847-5.258587-2.319584$

H -2.967133 -2.762322 -1.829855

O $-2.079889-2.898817-3.662289$

C $-0.747840-0.226062-3.158089$

H $-2.120710-0.532773-1.534307$

H $-0.451442-0.235294-1.068560$

H -1.079056 -6.341407 0.659425

H -2.942541 -6.200270 -2.103401

H $-2.806690-3.448962-4.001482$

O $\quad-1.578840 \quad 0.816792-3.446078$

O $0.156076-0.640366-3.871514$

C -1.342184 $1.465027-4.699462$

H $-1.4894480 .760677 \quad-5.523634$ 
H $-2.0628052 .280809-4.800905$ H $-0.3319471 .884661-4.724015$

SCF Energy (B3LYP/6-31G**//MMFF)= -3245.92591184

05_00006

MM̄MF Geometry

C $0.606892-2.956135-1.620880$

C $-0.399185-3.774524-1.280990$

C -1.290622 -3.531432 -0.093908

O $-2.609364-3.249648-0.621924$

C $-1.379759-4.7578440 .833388$

C $-0.189873-4.928277 \quad 1.756681$

C $-0.152279-4.4746553 .025931$

C $0.963285-5.7256751 .206755$

C -1.214812 -3.6756943 .727570$

C $-0.660542-2.5923654 .664022$

C $0.235252-1.5419223 .987244$

C $0.667993-0.4887965 .014514$

C $1.488341 \quad 0.6426204 .380538$

O $-0.492606-0.9245292 .918617$

O $1.553226 \quad 1.7045095 .345047$

C $0.726416 \quad 1.1687973 .145755$

C 2.9372180 .2123854 .121754

O 1.5028462 .1413322 .452960

C 0.2664260 .0496122 .186391

C $-0.605158 \quad 0.5809571 .026501$

O $1.427902-0.5421401 .589243$

C $-3.402185-2.4217740 .110705$

O $-3.131461-1.9056071 .183039$

C $-4.676559-2.240382-0.617452$

C $-5.599031-1.412016-0.105388$

C $-6.911752-1.108801-0.674361$

C $-7.292570-1.778704-1.967588$

C -1.9058821 .2786031 .430512$

C -2.7029791 .7224580 .212396$

C $-4.6093723 .194582-0.409035$

O $-2.3683651 .459854-0.939797$

C $-6.0666643 .068954-0.097527$

C -6.993456 $2.825004-1.037232$

C $-8.4683552 .725474-0.764514$

C $-9.0717341 .447130-1.305583$

C $-9.6406011 .417015-2.522662$

C $-9.0824050 .225441-0.414623$

C -7.706676 $-0.234592-0.023265$

N -3.830644 2.4523110 .567138

H $\quad 0.776687-2.056142-1.038220$

H - $0.585779-4.667092-1.872845$

H $-0.931639-2.6616100 .469768$

H -1.520161 -5.6730200 .241509$

H -2.301395 -4.699988 1.426471

H $0.725789-4.7030123 .628235$

H $\quad 0.615623-6.6981710 .843177$

H $\quad 1.443410-5.1952130 .380474$

H $1.733023-5.9166421 .962359$

H -1.896155 -3.204137 3.016018

H -1.815592 -4.3680344 .329998$

H -1.523637 -2.088433 5.118934

H $-0.104896-3.0697695 .481376$

H $1.116282-2.0472263 .575334$

H $1.226882-0.9536275 .836488$

H $-0.226561-0.0467025 .476279$

H 2.0058072 .4562804 .925105

H -0.1537591 .6993363 .528600$

H $3.426102-0.0608275 .064791$

H 3.5292451 .0363573 .708337

H $3.019231-0.6431653 .448291$

H 2.1816321 .6674541 .943574

H -0.0078831 .2544690 .400612$

H $-0.861192-0.2618040 .370571$

H $1.158268-0.9911690 .772449$

H -4.819596 -2.785570 -1.542925

H -5.373554 -0.898305 0.828916

H $-8.275437-1.476640-2.337350$

H -7.319080 -2.866859-1.843745

H -6.572611 -1.531613 -2.755727

H -2.532916 0.6105592 .029790

H -1.686339 2.173448 2.022372

H $-4.3711202 .851725-1.421099$
H $-4.3040284 .242640-0.323218$

H -6.3775413 .2074090 .935038$

H $-6.6786142 .716725-2.073038$

H -8.953736 $3.591321-1.233661$

H $-8.6864962 .809248 \quad 0.307309$

H $-10.098176 \quad 0.513566-2.911780$

H $-9.6575002 .295575-3.159284$

H $-9.644820-0.597551-0.871444$

H -9.6367980 .4650920 .502339$

H -7.3432830 .2126740 .902644$

H -3.9600522 .6917741 .546205$

C $2.980956-3.349880-2.281038$

O $3.444702-2.107069-1.714068$

C $4.805579-1.885746-2.126775$

C $5.265061-3.178864-2.791178$

C $3.970332-3.685307-3.394947$

H $4.780449-1.093439-2.883778$

C $5.660502-1.447236-0.933270$

H $6.048835-3.006287-3.535092$

H $5.650104-3.897996-2.058741$

C $4.010252-5.157060-3.767508$

H $3.743933-3.091737-4.290872$

C $5.158947-0.183189-0.205306$

O $5.701350-2.5078700 .031111$

H $6.696622-1.300067-1.259537$

H $4.230880-0.3973200 .338780$

$\begin{array}{lllll}\text { O } & 6.135800 & 0.156513 & 0.788031\end{array}$

C $4.9445991 .040278-1.115513$

C $4.6639492 .330144-0.321395$

H $4.1092210 .838824-1.792400$

O $6.1016941 .244418-1.927257$

H 3.8737332 .1313140 .408728

H $5.5642682 .607646 \quad 0.239613$

H $4.788501-5.343185-4.514831$

H $4.223670-5.785983-2.896537$

H $3.053570-5.479965-4.189390$

C $1.541501-3.191442-2.772244$

H $4.784363-2.7040210 .289952$

H $\quad 6.247497-0.621187 \quad 1.361487$

H $6.8586881 .379738-1.331611$

H $1.235502-4.078733-3.337609$

H $1.468976-2.329474-3.445435$

H $3.031720-4.102561-1.482955$

C $1.8763553 .196705-1.245235$

O $3.0936493 .243923-1.999856$

C $4.2734213 .514734-1.234351$

C $4.1273894 .856111-0.501870$

C 2.8593034 .9042140 .343687

C $1.6451064 .516161-0.497068$

H $1.9197492 .367363-0.530638$

C $0.7305612 .929933-2.234355$

H $5.0860743 .637398-1.961259$

O 5.2538685 .1121490 .329900

H $4.0817525 .668544-1.238928$

H 2.9444144 .2683251 .232168

O $2.662612 \quad 6.2368850 .821988$

H $1.4238525 .320204-1.210866$

O $0.509756 \quad 4.378852 \quad 0.355926$

C $0.674540 \quad 1.487630-2.692317$

H $-0.2349043 .149181-1.765857$

H $0.8528043 .557691-3.124898$

H $6.0450385 .090191-0.235187$

H 3.4600116 .4849741 .320739

H $0.4057425 .217247 \quad 0.837823$

O $-0.3652841 .349232-3.568534$

O $1.426940 \quad 0.595715-2.326776$

C $-0.5794390 .026831-4.069968$

H $-0.847980-0.646332-3.250739$

H $-1.4103260 .062693-4.779648$

H $\quad 0.312314-0.331471-4.592684$

SCF Energy (B3LYP/6-31G**//MMFF) $=-3245.93701431$

05_00007

MM̄MF Geometry

C -4.139123 -3.084135 -1.802973

C $-3.134946-3.189803-0.920205$

C $-1.708447-3.485996-1.307800$

O $-0.894821-2.338549-0.967134$ 
C $-1.189420-4.693212-0.506125$

C $0.235197-5.086179-0.847330$

C $1.268296-5.0428640 .017034$

C $0.459804-5.577433-2.254410$

C $1.250430-4.6258181 .460904$

C $1.557983-3.1406951 .702776$

C $2.980878-2.7178501 .302179$

C $3.275289-1.2998901 .795129$

C $4.655865-0.8030851 .338693$

O $3.085199-2.756030-0.123099$

O 4.7060020 .6163451 .553056

C $4.800439-1.035169-0.180902$

C $5.784890-1.4090252 .180621$

O $6.127062-0.750053-0.616437$

C $4.392623-2.453000-0.630228$

C $4.336922-2.610219-2.170121$

O $5.359864-3.394045-0.147885$

C $-0.903776-1.289672-1.834527$

O $-1.525025-1.203046-2.881740$

C $-0.033551-0.234731-1.273120$

C $-0.1998321 .032964-1.677905$

C $0.5531552 .195081-1.204892$

C $1.7883461 .940249-0.384047$

C $3.352928-1.682823-2.892890$

C $3.985682-0.419161-3.454144$

C $3.3736391 .705366-4.609702$

O $5.198062-0.227143-3.485808$

C $3.3700372 .815883-3.607028$

C $2.6558083 .942327-3.754328$

C $2.6850875 .086032-2.780836$

C $1.3055555 .499104-2.318325$

C $0.6743006 .546299-2.875730$

C $0.6989674 .755475-1.150679$

C $0.1219193 .426516-1.547928$

N $3.030598 \quad 0.456041-3.955577$

H $-3.940244-3.233003-2.861411$

H -3.335190 -3.019192 0.134378

H -1.626601 -3.687576 -2.382908

H -1.836001 -5.562744 -0.684705

H -1.277608 -4.4654390 .561697$

H $2.248576-5.353593-0.342842$

H $1.465898-5.987255-2.395624$

H $-0.252564-6.372473-2.497823$

H $\quad 0.335241-4.761974-2.973288$

H $0.286194-4.868128 \quad 1.920488$

H $1.982886-5.2391652 .001109$

H $0.825696-2.5319821 .159952$

H $1.413734-2.9429042 .772113$

H $3.689735-3.4296031 .741273$

H $3.191610-1.2441242 .887611$

H $2.512584-0.6150611 .401006$

H $4.543976 \quad 0.7832652 .497427$

H $4.148421-0.307267-0.675366$

H $5.656338-1.1476453 .237932$

H $6.758683-1.0008481 .888664$

H $5.832046-2.4979622 .113869$

H $6.3343750 .158666-0.338583$

H $5.346060-2.539815-2.593200$

H $4.007968-3.639017-2.376771$

H $5.305554-4.197703-0.691020$

H $0.692685-0.522847-0.522281$

H $-0.9827211 .248474-2.404300$

H $2.441766 \quad 1.224872-0.892742$

H $1.526217 \quad 1.535176 \quad 0.596248$

H $2.3941432 .833202-0.217040$

H $2.940462-2.222824-3.754990$

H $2.513922-1.415263-2.244138$

H $2.6415331 .865356-5.407558$

H $4.3674461 .619884-5.060952$

H $4.0085902 .700434-2.734344$

H $2.0408254 .067663-4.642931$

H $3.1786455 .931578-3.277883$

H $3.3077114 .853927-1.907729$

H $-0.3025856 .867325-2.529520$

H $1.1182577 .102266-3.694760$

H $-0.1185795 .344512-0.713238$

H $1.4368834 .675297-0.347824$

H $-0.7767513 .510107-2.160860$
H $\quad 2.0452480 .238737-3.836750$

C $-5.832196-1.256754-1.786836$

O $-5.076112-0.398118-0.908923$

C $-5.9020240 .713470-0.513423$

C $-7.1390320 .655467-1.401349$

C -7.290333 $-0.841480-1.602792$

H -6.2099950 .5238740 .521401$

C $-5.0978972 .016331-0.586960$

H $-8.0112631 .116637-0.928264$

H $-6.9738551 .153966-2.363570$

C $-8.191222-1.209035-2.768616$

H $-7.693546-1.282722-0.681387$

C $-3.774471 \quad 1.969752 \quad 0.207739$

O $-4.7695682 .267750-1.959620$

H $-5.7197002 .856784-0.257665$

H $-3.105478 \quad 1.246094-0.267699$

$\begin{array}{llll}\text { O } & -3.133425 & 3.243905 & 0.068566\end{array}$

C -3.9381381 .6498431 .706461$

C -2.6203441 .6770432 .501525$

H -4.4260850 .6817321 .830502$

$\begin{array}{llll}\text { O } & -4.817114 & 2.627224 & 2.273815\end{array}$

H -2.146832 2.6593682 .370805

H -2.8554991 .6390683 .572658$

H $-9.203413-0.823565-2.608346$

H -7.818541 -0.793184 -3.710762

H $-8.258079-2.295364-2.883212$

C -5.547497-2.714946-1.425882

H $-4.2435143 .085362-1.986612$

H -3.6885113 .9016630 .521754$

H -4.9694992 .3812713 .202172$

H $-6.231936-3.386645-1.955490$

H -5.701316 -2.871718 -0.350889

H $-5.499050-1.040107-2.810721$

C $\quad 0.0287301 .1598643 .824576$

O -0.2700521 .0019152 .434057$

C -1.6070300 .5680082 .149704$

C -1.836254 -0.8025962 .803603$

C $-1.514835-0.7862184 .297223$

C $-0.134747-0.1796624 .550936$

H $-0.634277 \quad 1.9138984 .266708$

C 1.4724661 .6714293 .931250

H -1.637411 0.4191361 .068326

O $-3.168545-1.2597632 .620478$

H -1.180088 -1.540393 2.323971

H $-2.286220-0.2563084 .868025$

O $-1.506261-2.1289744 .789279$

H $0.638821-0.8846624 .220672$

O $0.0401130 .020643 \quad 5.951623$

C 1.6432973 .0541303 .337394

H 1.7884821 .7224584 .978520

H 2.1382080 .9953863 .384042

H -3.330587 -1.304792 1.662834

H $-2.375169-2.5162504 .586398$

H $-0.093442-0.8392006 .386339$

O $2.9781323 .333745 \quad 3.251635$

O $0.7321373 .797836 \quad 3.001742$

C 3.3059494 .6148892 .705382

H 2.8771565 .4115643 .320784

H 4.3943674 .7169992 .707972

H 2.9496244 .6884631 .673976

SCF Energy (B3LYP/6-31G**//MMFF) $=-3245.91609669$

05_00008

MM̄MF Geometry

C $-4.072725-3.392917-1.338443$

C $-2.925466-3.324442-0.646927$

C $-1.571190-3.553268-1.267108$

O $-0.776494-2.351938-1.115031$

C $-0.844771-4.700304-0.541378$

C $0.485044-5.073292-1.172018$

C $1.696588-4.865053-0.617232$

C $0.388933-5.763658-2.510051$

C $2.022135-4.2317450 .706948$

C $2.637711-2.8381340 .561902$

C $4.039556-2.776134-0.067726$

C $5.098103-3.4160480 .836670$

C $6.526996-3.1933610 .319306$

O $4.324015-1.382345-0.258827$ 
O $7.437207-3.526429 \quad 1.379277$

C $6.719253-1.6930650 .017403$

C $6.861896-4.129140-0.848110$

O $7.986921-1.453288-0.589110$

C $5.596218-1.100453-0.857486$

C $5.717136 \quad 0.434717-1.004213$

O $5.680923-1.658008-2.173653$

C $-1.076155-1.298078-1.924794$

O $-1.991315-1.222678-2.729250$

C $-0.066265-0.245079-1.684852$

C $-0.2061230 .958259-2.260615$

C $0.7571292 .059792-2.192034$

C $2.0727651 .781575-1.515775$

C 5.5505701 .2200350 .300020

C 5.3239172 .7017610 .046193

C 4.3383154 .7170391 .142438

O $5.5940653 .250389-1.018707$

C 2.9024984 .7385770 .718676

C $2.3893475 .605313-0.167061$

C $0.9462775 .590298-0.590904$

C $0.777612 \quad 5.634461-2.094227$

C $0.1930056 .687249-2.690607$

C $1.2944784 .469613-2.909051$

C $0.4399443 .238063-2.768889$

N $4.793973 \quad 3.337983 \quad 1.160410$

H -4.045013 -3.656290 -2.393101

H -2.947832 -3.0474540 .402711$

H $-1.662442-3.783813-2.335720$

H $-1.479863-5.596590-0.535619$

H $-0.712940-4.4272390 .510385$

H $2.571885-5.199060-1.173384$

H $1.361721-6.120124-2.865434$

H $-0.271379-6.634484-2.441664$

H $-0.007235-5.083162-3.269532$

H $1.133390-4.1449291 .337915$

H $2.698029-4.8969361 .255208$

H $1.959321-2.203529-0.020470$

H $2.681232-2.3704671 .554372$

H $4.019455-3.260643-1.049607$

H $4.911367-4.4883560 .965971$

H $5.026612-2.9781521 .842343$

H $7.274817-4.4507801 .634913$

H $6.732349-1.1699270 .982225$

H $6.797911-5.176558-0.529404$

H $7.892496-3.982873-1.189242$

H $6.192970-4.006452-1.702353$

H $8.664088-1.839096-0.007261$

H $6.6694450 .697533-1.482732$

H $4.9445880 .771092-1.708220$

H $5.223436-1.064160-2.791604$

H $0.786874-0.499119-1.068351$

H - $-1.0957911 .151155-2.858824$

H $2.5742350 .932715-1.993128$

H $1.9235911 .548795-0.456254$

H $2.7698052 .618496-1.558181$

H 4.6938650 .8348730 .864559

H 6.4455851 .1211270 .923420

H $4.975605 \quad 5.3033470 .472757$

H 4.4340695 .1025322 .161674

H 2.2469293 .9932611 .159770

H $3.0311686 .369467-0.599001$

H $\quad 0.4562126 .456248-0.127555$

H $0.4280864 .705522-0.201520$

H $0.0693006 .730403-3.767769$

H -0.176814 7.530116 - 2.116121

H $2.3448044 .289502-2.666552$

H $1.3039584 .732920-3.975691$

H $-0.540457 \quad 3.337332-3.237351$

H 4.5462762 .7789531 .971821

C $-5.968546-1.778046-1.372255$

O $-5.165959-0.662179-0.934647$

C $-6.033140 \quad 0.448511-0.638056$

C $-7.4146340 .055862-1.149330$

C $-7.394900-1.446574-0.940560$

H -6.0793150 .5282340 .454366$

C $-5.4652791 .742290-1.232318$

H -8.217639 $0.556608-0.600048$

H $-7.5369790 .290803-2.213147$
C $-8.467666-2.182357-1.723910$

H $-7.522010-1.6553860 .130292$

C -4.021978 $2.060637-0.787076$

O $\quad-5.461598 \quad 1.622330-2.660584$

H $-6.1329502 .578807-0.994524$

H $-3.3337781 .326281-1.221283$

O $-3.6576343 .323358-1.359288$

C -3.8188632 .1181100 .738186$

C -2.3914172 .5483681 .118809$

H $-4.030413 \quad 1.132917 \quad 1.159542$

$\begin{array}{lllll}\text { O } & -4.758317 & 3.038989 & 1.289829\end{array}$

H $-1.6827451 .930527 \quad 0.557490$

H -2.232481 3.5885660 .809457

H $-9.464467-1.847743-1.418220$

H $-8.369976-2.006937-2.800547$

H $-8.406724-3.261402-1.551690$

C $-5.422496-3.070008-0.762547$

H $-5.0899442 .446317-3.019567$

H $-4.216573 \quad 4.003863-0.946195$

H -4.7738092 .9048582 .252304$

H $-6.095404-3.908844-0.970993$

H $-5.353782-2.966290 \quad 0.326369$

H $-5.898397-1.810837-2.467922$

C $-1.495910 \quad 0.1048152 .708581$

O $-2.373998 \quad 1.1411893 .163179$

C -2.1023682 .4440152 .635056$

C $-0.6833632 .870463 \quad 3.029874$

C 0.3527941 .8545912 .560497

C $-0.031786 \quad 0.4501593 .021936$

H -1.633075 -0.0470341 .630936$

C $-1.890572-1.1931193 .433610$

H -2.7928103 .1300803 .140383$

$\begin{array}{llll}\text { O } & -0.393368 & 4.156419 & 2.494808\end{array}$

H $-0.627392 \quad 2.956845 \quad 4.122938$

H $0.4850931 .901618 \quad 1.474234$

$\begin{array}{lllll} & \text { O } & 1.616511 & 2.199382 & 3.133118\end{array}$

H $\quad 0.1447740 .377557 \quad 4.102705$

O $0.834404-0.4964412 .400189$

C $-3.255608-1.7126553 .037202$

H -1.166319 -1.984457 3.209467

H $-1.912738-1.0155894 .515720$

H $0.506494 \quad 4.3922962 .777955$

H $2.257751 \quad 1.5281082 .842693$

H $0.684919-0.4520011 .440376$

O $-3.521901-2.839177 \quad 3.763773$

O $-3.991760-1.2102692 .201010$

C $-4.783511-3.463276 \quad 3.504593$

H $-4.951269-4.221448 \quad 4.274227$

H -5.598117 -2.733893 3.550738

H -4.757964 -3.954136 2.527901

SCF Energy (B3LYP/6-31G**//MMFF) $=-3245.92225438$

05_00009

MMFF Geometry

C $4.683166-2.5231031 .519582$

C $3.385830-2.7765491 .294425$

C $2.370468-2.9431462 .395212$

O $1.324725-1.9621662 .205021$

C $1.731276-4.3409082 .320373$

C $0.715381-4.5960543 .417985$

C $-0.604907-4.7852373 .223346$

C $1.277384-4.6632234 .816274$

C $-1.370831-4.8137061 .929528$

C $-2.277394-3.5892011 .788328$

C $-3.262336-3.6902510 .612592$

C $-2.559191-3.725366-0.746710$

C $-3.554667-3.690214-1.917905$

O $-4.118674-2.5417820 .694078$

O $-2.818206-3.381609-3.112024$

C $-4.557099-2.538096-1.697441$

C $-4.204340-5.059359-2.151747$

O $-5.593314-2.564213-2.676025$

C $-5.170909-2.519800-0.281687$

C $-6.031174-1.261072-0.018290$

O $-6.035226-3.652842-0.130209$

C $1.579156-0.6928882 .625811$

O $2.603370-0.277106 \quad 3.143239$

$\begin{array}{llll}\text { C } & 0.382260 & 0.120723 & 2.325877\end{array}$ 
C $0.473842 \quad 1.458676 \quad 2.332544$

C -0.6097802 .3867352 .012849$

C -2.0070831 .8285341 .929151$

C $-5.2935110 .071769-0.163911$

C -6.1168241 .2488680 .332462$

C -6.0112643 .7184690 .580802$

O

C -4.9241474 .7366550 .697032$

C -4.4359935 .1715201 .868794$

C -3.3122756 .1620821 .991479$

C -2.0220975 .4998502 .432889$

C -1.6014875 .6093433 .704462$

C -1.2677434 .7620431 .344800$

C -0.3172063 .6886801 .808304$

N $-5.4271842 .446400 \quad 0.197388$

H $5.042551-2.4552602 .543632$

H $3.021918-2.841416 \quad 0.272527$

H $2.831891-2.7968263 .379635$

H $2.511861-5.1103802 .390095$

H $1.276133-4.4675941 .333202$

H -1.226791-4.960713 4.101166

H $\quad 0.533430-4.9962085 .548090$

H $2.111863-5.3710614 .856714$

H $1.635383-3.6805115 .137812$

H $-0.707598-4.8925111 .065977$

H - $-1.981756-5.7249601 .925670$

H -2.856374 -3.444783 2.710980

H -1.669723 -2.680807 1.683722

H -3.869291 -4.5922350 .754448$

H -1.912969-4.606045 -0.837471

H -1.891922 -2.857757 -0.830090

H -2.127243 -4.057318 -3.221286

H -4.005149 -1.605677 -1.859571

H -3.440444 -5.810553 -2.386099

H -4.875509 -5.036145 -3.017260

H $-4.765637-5.420588-1.287626$

H -5.166617 -2.567627 -3.550021

H $-6.916099-1.267443-0.668099$

H -6.423556 -1.327466 1.006448

H $-6.661888-3.4689770 .589163$

H $-0.533167-0.4034432 .076713$

H 1.4369571 .9180782 .550484

H -2.7703922 .5998461 .810715$

H -2.2600791 .2865562 .847438$

H -2.103771 1.1435051 .082445

H -4.3615680 .0518800 .411406$

H $-5.051950 \quad 0.256135-1.216317$

H -6.5637943 .5895981 .517937$

H -6.727929 4.011913 -0.193065

H -4.507103 5.120206-0.231086

H $-4.845964 \quad 4.7779292 .796093$

H -3.617672 6.9367622 .706245

H -3.140952 6.6883891 .043768

H -0.6732485 .1656594 .046524$

H $-2.180078 \quad 6.1578044 .441166$

H $-0.700218 \quad 5.4967650 .758538$

H -1.972041 4.3197880 .630637

H $\quad 0.7161314 .0203611 .909808$

H $-4.5700112 .452506-0.349326$

C $6.282048-0.8892740 .514004$

$\begin{array}{llll}\text { O } & 5.277424 & 0.077378 & 0.142538\end{array}$

C $5.8980711 .111402-0.645995$

C $7.4001370 .856001-0.581237$

C $7.445338-0.653143-0.446724$

H $5.5663280 .959756-1.679411$

C $5.4693452 .497287-0.150721$

H $7.9211751 .224822-1.470169$

H $7.856081 \quad 1.3309200 .295370$

C $8.781076-1.1764630 .051358$

H $7.222562-1.101229-1.424513$

C $3.9458342 .736309-0.172475$

O 5.9156932 .6497081 .203282

H $5.9845363 .271032-0.732090$

H 3.4634472 .0908080 .570980

O 3.7127724 .0809790 .267866

C $3.2719512 .533691-1.543202$

C $1.7777672 .901186-1.513516$

H $3.3806641 .492170-1.856007$
O $3.9051353 .352087-2.526304$

H $1.3079042 .388543-0.668839$

H $1.6743173 .979184-1.341032$

H $9.582817-0.906750-0.643977$

H $9.033869-0.761737 \quad 1.033082$

H $8.765636-2.2671220 .140436$

C 5.698154-2.3004620.434069

H 5.6271373 .5277201 .506557

H $4.0800734 .677812-0.406690$

H $4.7944732 .993823-2.684182$

H $6.490265-3.0478400 .553760$

H $5.241834-2.458076-0.550209$

H $6.584615-0.6558241 .543778$

C $0.4595040 .264041-2.291334$

O $1.1665621 .156407-3.161036$

C $1.0491642 .545215-2.830581$

C $-0.4273502 .963921-2.864119$

C $-1.2788702 .080432-1.960274$

C -1.035856 $0.603982-2.263344$

H $\quad 0.8766740 .324272-1.278974$

C $0.675416-1.164751-2.816832$

H $1.5562153 .093807-3.634058$

O $-0.5842014 .322314-2.468137$

H $-0.8078862 .880563-3.890369$

H -1.089726 2.297259 -0.905722

O $-2.6619922 .363682-2.182438$

H -1.500881 $0.348230-3.224109$

O $-1.667785-0.185587-1.259417$

C $2.090829-1.659030-2.605902$

H $\quad 0.012737-1.868605-2.301926$

H $0.469906-1.193116-3.893629$

H $-0.0372804 .864608-3.061733$

H -2.792931 $3.312343-2.011450$

H -2.596097 $0.097695-1.212637$

O $2.241331-2.852239-3.254478$

O $2.949315-1.090362-1.946836$

C $3.532649-3.459123-3.142573$

H $3.565019-4.315836-3.820865$

H $4.319102-2.755090-3.431610$

H $3.687931-3.814636-2.120096$

SCF Energy $(B 3 L Y P / 6-31 G * * / / M M F F)=-3245.92532119$

05_00010

MMFF Geometry

C $-3.066636-2.760037-2.866809$

C $-2.177295-2.923652-1.876565$

C $-0.686262-2.949147-2.095938$

O $-0.078205-1.905176-1.299753$

C $-0.114874-4.298984-1.625936$

C $1.361700-4.473696-1.924736$

C $2.324354-4.600623-0.990566$

C $1.730483-4.536928-3.385373$

C $2.174836-4.6035620 .504473$

C $2.363383-3.2322961 .170338$

C $3.753126-2.6103640 .953651$

C $3.953492-1.4026661 .870207$

C $5.300901-0.7080561 .619534$

O $3.851690-2.187528-0.408530$

O 5.2638320 .5672532 .279861

C $5.456226-0.4310870 .107520$

C $6.466371-1.4855662 .243301$

O $6.767700 \quad 0.060156-0.159570$

C $5.131828-1.654803-0.778228$

C $5.059977-1.323660-2.290122$

O $6.162037-2.636711-0.612579$

C $-0.182232-0.628724-1.759485$

O $-0.749142-0.253105-2.773207$

C $0.5141380 .252336-0.798146$

C $0.2588221 .568446-0.810151$

C $0.8652012 .569808 \quad 0.067148$

C 2.0736822 .1578680 .865279

C $4.010458-0.278932-2.686531$

C $4.549901 \quad 1.138347-2.790064$

C $3.7832503 .479425-3.183298$

O $5.7452971 .411460-2.727369$

C $3.6530444 .186254-1.871028$

C $2.8476695 .242200-1.677862$

C $2.7453585 .992169-0.380293$ 
C 1.3213116 .1170310 .114133

C $0.6093827 .235687-0.103960$

C 0.7561194 .9818830 .936708

C $0.333333 \quad 3.8093260 .095121$

N $3.5334842 .061746-2.998089$

H -2.713901 -2.654357 -3.889859

H $-2.530409-3.023612-0.853814$

H $-0.443520-2.782959-3.152549$

H -0.655990 -5.122062 -2.111734

H $-0.305178-4.403934-0.552311$

H $3.351474-4.727392-1.331741$

H $2.776760-4.823755-3.537543$

H $\quad 1.112067-5.277980-3.902296$

H $1.585952-3.563726-3.863957$

H $1.201292-5.0101120 .798026$

H $2.911016-5.3062750 .915786$

H $1.591235-2.5505010 .796253$

H $2.188844-3.3608062 .245916$

H $4.511421-3.3754541 .157366$

H $3.862177-1.6922422 .924454$

H $3.152085-0.6744781 .689501$

H 5.1048680 .4103353 .226465

H $4.7619990 .382826-0.129907$

H $6.319603-1.5929993 .324813$

H $7.411683-0.9447182 .123187$

H $6.585757-2.4874651 .825939$

H $6.7904610 .364566-1.081971$

H $6.054737-1.064809-2.671621$

H $4.787114-2.253813-2.809874$

H $\quad 6.150101-3.229427-1.382353$

H $1.207155-0.205431-0.102181$

H -0.479606 $1.949784-1.514339$

H 2.8159931 .6821100 .216100

H $1.7968791 .452007 \quad 1.653378$

H 2.5870072 .9956121 .341879

H $3.631354-0.532840-3.684908$

H $3.159500-0.296482-1.999696$

H $3.0614943 .841864-3.922118$

H $4.7925233 .624540-3.581642$

H $4.2717703 .830996-1.050414$

H $2.2516795 .613058-2.508867$

H $3.1717866 .991266-0.540191$

H 3.3630685 .5302190 .399878

H -0.4023727 .3446390 .272479$

H $1.0208578 .065475-0.669084$

H $-0.133975 \quad 5.3206251 .484346$

H 1.4710174 .7089991 .718279

H $-0.5340884 .016158-0.533087$

H $2.5669691 .749137-2.984729$

C $-5.090843-1.302561-3.000435$

O $-4.619201-0.346596-2.027399$

C $-5.7171960 .496474-1.634144$

C $-6.8214220 .250868-2.654791$

C $-6.616927-1.219527-2.960513$

H $-6.0597380 .131028-0.658621$

C $-5.2660421 .955330-1.513277$

H -7.814881 $0.473584-2.253904$

H $-6.6793450 .850307-3.561511$

C -7.295095 -1.673825 -4.241316

H -7.004312 -1.810836 -2.119817

C $-4.0702922 .182695-0.564943$

O $-4.8811792 .421921-2.813150$

H -6.117603 2.574175-1.205310

H -3.156512 $1.786168-1.024486$

O $-3.8934653 .602205-0.470437$

C -4.2489691 .5841830 .844341$

C -3.130976 2.0031471 .817050

H -4.2766980 .4928810 .756283$

O

H -2.165862 1.8775291 .316783

H -3.2401673 .0682072 .055158$

H -8.378297 -1.528737 -4.174779

H $-6.933366-1.111053-5.108439$

H -7.107554 -2.735916-4.427067

C -4.552264 -2.691774 -2.655018

H $-4.6084993 .350640-2.717877$

H -3.011514 $3.766374-0.096277$

H $\quad-5.7012301 .4675372 .150467$
H $-5.021779-3.451785-3.289272$

H -4.796305 -2.936250 -1.613614

H $-4.713738-0.973468-3.977724$

C -1.874383 -0.709738 2.425064

O $-3.117607-0.2079132 .925103$

C $-3.147081 \quad 1.2072243 .143546$

C -2.0437261 .6100564 .132377$

C -0.6700901 .1086633 .691846$

C $-0.723721-0.3886163 .386189$

H $-1.674817-0.2469941 .451230$

C $-2.032400-2.2075252 .136466$

H $-4.105131 \quad 1.4220583 .632378$

O -2.0197713 .0250724 .289371$

$\mathrm{H}-2.2766121 .1820945 .116245$

H -0.3047931 .6830152 .834626$

O $\quad 0.251954 \quad 1.342570 \quad 4.757823$

H $-0.840639-0.9369194 .328879$

O $0.502090-0.8040522 .794567$

C -2.164134 -3.055930 3.378745

H -2.917682 -2.388524 1.514787

H $-1.170201-2.5808931 .571203$

H -1.296294 3.2373184 .903610

H 1.1403381 .1225234 .430505

H $1.203112-0.6951783 .458728$

O $-3.417413-2.935713 \quad 3.900865$

O $-1.255310-3.7399563 .832118$

C $-3.659973-3.6826645 .096746$

H -2.987001 -3.351047 5.893246

H -4.691036 -3.498324 5.409472

H $-3.536894-4.7531624 .906589$

SCF Energy $\left(B 3 L Y P / 6-31 G^{* *} / / M M F F\right)=-3245.90557396$

05_00011

MMFF Geometry

C $-3.536937-2.640301-2.543743$

C $-2.533695-2.813887-1.671323$

C -1.090502 -2.942034 -2.072175

O $-0.317547-1.923785-1.396432$

C $-0.467519-4.314366-1.733053$

C $-0.561211-4.755295-0.282270$

C $0.297956-4.3907620 .690798$

C - $1.681868-5.7148980 .029261$

C $1.491134-3.4823570 .573564$

C $2.767313-4.1674761 .074712$

C $4.043366-3.3237310 .915883$

C $4.043796-2.0665551 .787020$

C $5.328137-1.2441701 .590368$

O $4.184063-2.964761-0.462302$

O 5.1208500 .0441602 .190772

C $5.542357-1.0092380 .079661$

C $6.528820-1.8688832 .310004$

O $6.800839-0.387412-0.167392$

C $5.412026-2.289148-0.774031$

C $5.400067-2.001136-2.295973$

O $6.536315-3.139956-0.516253$

C $-0.388402-0.645877-1.850406$

O $-1.070533-0.221579-2.768329$

C $0.5262980 .159167-1.012329$

C $0.4243391 .495381-1.014374$

C $1.2487822 .417262-0.232464$

C 2.4615861 .8519170 .459659

C $4.318596-1.021790-2.773294$

C $4.8301860 .395593-2.987981$

C $4.0368082 .677487-3.602443$

O $6.0215560 .693339-2.966554$

C $4.1143223 .511215-2.362120$

C $3.4257124 .650699-2.192930$

C $3.5363515 .520714-0.972709$

C $2.1924745 .855004-0.363271$

C $1.6146417 .049407-0.575500$

C 1.5615124 .8378070 .560166

C $0.8955463 .718410-0.190404$

N $3.799161 \quad 1.287712-3.253893$

H $-3.322375-2.613479-3.609121$

H $-2.753572-2.820865-0.608042$

H $-0.972912-2.792546-3.153881$

H $0.589489-4.303202-2.028951$

H $-0.933195-5.081855-2.365974$ 
H $0.126278-4.776513 \quad 1.695155$ H $-1.727417-5.9716621 .093065$ H -2.650524 $-5.289360-0.245095$ H $-1.544861-6.645975-0.530517$ H $1.646405-3.152574-0.455910$ H $1.286594-2.5883711 .170776$ H $2.651313-4.4567782 .126812$ H $2.902560-5.0947060 .501428$ H $4.881785-3.9717631 .198475$ H $3.908593-2.3237932 .844724$ H $3.193544-1.4256821 .520415$ H $4.915663-0.0934073 .131373$ H $4.776197-0.293350-0.236860$ H $6.328701-1.9537273 .384846$ H $7.416709-1.2331412 .220320$ H $6.780255-2.8654141 .941581$ H 6.8327080 .4248890 .366375 H $6.399578-1.694569-2.626590$ H $5.210181-2.957672-2.804010$ H $6.646659-3.741008-1.271612$ H $1.248197-0.371731-0.402179$ H $-0.3478461 .958764-1.627042$ H $3.086657 \quad 1.307749-0.254946$ H $2.1702281 .163163 \quad 1.258411$ H 3.1051142 .6125670 .906346 H $3.953291-1.361009-3.751265$ H $3.461140-1.008722-2.092235$ H $3.2217352 .991454-4.262162$ H $4.9805042 .750870-4.152770$ H $4.7924003 .179767-1.579033$ H $2.7722094 .998770-2.989855$ H $4.0501516 .444200-1.270679$ H $4.1754425 .060012-0.209201$ H $0.6644217 .306718-0.119323$ H $2.0760657 .794591-1.215042$ H $0.7894365 .316943 \quad 1.177620$ H 2.3078504 .4871911 .278655 H $0.0032044 .031403-0.734702$ H $2.8361680 .968773-3.199334$ C $-5.438814-1.025227-2.446741$ O $-4.755434-0.091834-1.584677$ C $-5.6932090 .908448-1.144007$ C $-6.9594110 .696690-1.966750$ C $-6.927749-0.802704-2.188270$ H $-5.9194800 .686499-0.094524$ C $-5.0800852 .307868-1.263416$ H -7.856868 $1.035130-1.439945$ H -6.914534 $1.224096-2.926689$ C -7.830644 -1.267989-3.317314 H -7.227095 -1.302476 -1.257087 C $-3.7515412 .485844-0.499931$ O $-4.8238462 .573441-2.648600$ H -5.811469 $3.056342-0.935702$ H -2.970262 $1.880388-0.974169$ O $-3.3337433 .847792-0.659509$ C -3.8193992 .1445791 .000965$ C -2.516839 2.4903371 .743726 H -4.022532 1.0769431 .110559 O $-4.908438 \quad 2.8377531 .608867$ H -1.6796472 .0349241 .206969$ H -2.3665093 .5763291 .720205$ H -8.874256 -1.013786 -3.104523 H $-7.557816-0.799023-4.268680$ H -7.767657-2.352916 -3.446454 C -4.970589-2.447197-2.136237 H $-4.4283993 .460191-2.705590$ H -4.029104 $4.418803-0.291810$ H -4.7054323 .7882941 .593396$ H -5.580566 -3.178846 -2.677492 H $-5.089321-2.650323-1.065175$ H $-5.183381-0.752165-3.479328$ C -1.678620 -0.2109892 .893418$ O -2.7453890 .6242613 .359857$ C -2.522419 $2.031782 \quad 3.220192$ C -1.266431 2.4345824 .002608 C -0.0524211 .6193663 .569558$ C $-0.365428 \quad 0.124526 \quad 3.617156$ H $-1.559818-0.0885561 .810068$
C $-2.074530-1.6716443 .168735$

H -3.3740322 .5262463 .703813$

O $-1.0075913 .824448 \quad 3.833003$

H -1.4432682 .2684645 .073261$

H $\quad 0.2850851 .9281332 .576113$

$\begin{array}{lllll}\text { O } & 1.021049 & 1.908037 & 4.467699\end{array}$

H $-0.421963-0.1989194 .664597$

O $0.696762-0.6010103 .002595$

C $-3.258120-2.1339292 .345937$

H $-1.238376-2.3409262 .937982$

H $-2.348944-1.7828114 .224817$

H -0.1890024 .0290014 .316760$

H $1.820246 \quad 1.480567 \quad 4.116504$

H $1.496844-0.4649223 .537145$

O $-3.569539-3.4148772 .706954$

O $-3.838302-1.4690531 .500010$

C $-4.695127-3.9884092 .034952$

H $-4.848604-4.9964412 .429164$

H $-5.594507-3.3953892 .226565$

H $-4.500441-4.0580640 .961485$

SCF Energy (B3LYP/6-31G**//MMFF) $=-3245.90805196$

05_00012

MMFF Geometry

C $4.478520-2.0067832 .004046$

C $3.236534-2.2626981 .563878$

C $2.048821-2.323192 \quad 2.488334$

O $1.051044-1.3746882 .042699$

C $1.398584-3.7189932 .473433$

C $0.403322-3.906406 \quad 3.605014$

C $-0.927057-4.0602293 .458803$

C $0.999497-3.949800 \quad 4.991771$

C $-1.743687-4.1368652 .200532$

C $-2.483634-2.8289331 .910850$

C $-3.779225-3.0156961 .104524$

C $-3.570649-3.752927-0.219820$

C $-4.869872-3.840642-1.036897$

O $-4.310542-1.7048090 .860881$

O $-4.524231-4.247688-2.370240$

C $-5.488959-2.431736-1.141886$

C $-5.820408-4.912242-0.490499$

O $-6.768701-2.476915-1.767745$

C $-5.592866-1.6983860 .213696$

C $-6.036307-0.2219190 .061660$

O $-6.577616-2.3466851 .027702$

C $1.198404-0.0804032 .437638$

$\begin{array}{lllll}\text { O } & 2.145318 & 0.405345 & 3.034948\end{array}$

$\begin{array}{llll}\text { C } & -0.037039 & 0.639449 & 2.057617\end{array}$

C $-0.2357291 .895866 \quad 2.483399$

C -1.4425352 .6983402 .264467$

C -2.5538692 .0707981 .465932$

C $-5.160903 \quad 0.610634-0.874735$

C $-5.4376512 .101976-0.796860$

C $-4.6268664 .244424-1.809067$

O -6.2152762 .6075560 .006242$

C $-3.3953474 .711788-1.098192$

C $-3.3850295 .689535-0.179901$

C -2.1411126 .1313870 .539368$

C -2.3222506 .1881782 .041420$

C -2.200102 7.3508942 .704172

C -2.6561014 .9084562 .777518$

C -1.505734 3.9343212 .804029

N $-4.6788932 .796037-1.730162$

H $4.644586-1.8623363 .069347$

H $3.056139-2.4001580 .501620$

H $2.354494-2.0650963 .509354$

H $2.168051-4.4963582 .569501$

H $0.918668-3.8800581 .502134$

H $-1.523718-4.1745814 .364282$

H $\quad 0.273875-4.2739975 .745899$

H $1.835167-4.6567855 .024501$

H $1.363218-2.9628935 .291970$

H $-1.136116-4.4214451 .336157$

H -2.465453 -4.9517292 .340376$

H -2.742044 - 2.3137452 .846236

H - $1.814993-2.1387311 .381955$

H $-4.483457-3.5684551 .737807$

H $-3.165679-4.758821-0.058420$ 
H $-2.819543-3.221314-0.817745$ H $-4.065760-5.103424-2.313005$ H $-4.836532-1.857263-1.810684$ H -5.340658 -5.898130 -0.515319 H -6.716669 -5.000275 - 1.114268 H $-6.133129-4.7251660 .538798$ H $-6.660122-2.938423-2.616964$ H -7.080853 $-0.173477-0.272289$ H -6.0256520 .2415431 .058099$ H $-6.885663-1.7153771 .699088$ H $-0.773500 \quad 0.0973291 .476494$ H 0.5429312 .3743003 .076159 H -2.9027501 .1482831 .943223$ H -2.209116 1.8350290 .453174 H -3.4287262 .7123901 .356585$ H $-4.1056950 .463689-0.623557$ H $-5.3197200 .297123-1.912438$ H -5.540263 4.672170-1.383759 H $-4.5681504 .509566-2.868929$ H -2.460022 4.218002 -1.352029 H $-4.311866 \quad 6.2045400 .061758$ H -1.869276 7.1215490 .151258 H -1.295049 5.4729950 .306764 H -2.330491 7.4055423 .779922 H - $-1.965588 \quad 8.2755812 .187277$ H -3.5711954 .4800662 .361753$ H -2.907697 5.1318493 .823222 H -0.6461864 .2952793 .370938$ H $-4.0381412 .275055-2.321140$ C $6.175306-0.4092221 .132346$ $\begin{array}{llll}\text { O } & 5.196542 & 0.422729 & 0.480047\end{array}$ C $5.8723271 .351514-0.387614$ C $7.3434051 .286243-0.001129$ C $7.479520-0.1808610 .369887$ H $5.7705210 .961412-1.407197$ C $5.2061052 .729539-0.300500$ H $8.0030931 .586324-0.820641$ H 7.5661561 .9192450 .865546

C $8.728934-0.4914241 .174905$

H $7.486147-0.779212-0.551207$

C $3.6716822 .682183-0.474656$

O 5.4762623 .2876330 .990857 H $5.6607223 .407114-1.032531$ H 3.2245042 .1919480 .398655 O $3.1787324 .026610-0.462756$ C $3.1998621 .979177-1.759717$

C $1.6683111 .892966-1.842352$ H $3.6074220 .968220-1.779621$ O $3.7184182 .680334-2.886326$ H $1.3044681 .422037-0.922351$ H $1.2455952 .904474-1.875991$ H $9.627643-0.2456380 .599824$ H 8.7590920 .0827072 .106949 H $8.772885-1.5542861 .432437$ C $5.685745-1.8580521 .121154$ H $5.0349594 .153451 \quad 1.030372$ H $3.5149774 .468173-1.261571$ H $3.5815562 .122459-3.670436$ H $6.468387-2.5305481 .489041$ H $5.441791-2.1643810 .098250$ H $6.267353-0.0427982 .163512$ C $1.526298-1.136055-2.264476$ O $1.806341-0.147985-3.262002$ C $1.1527011 .111908-3.074411$ C $-0.3664300 .907812-3.082296$ C $-0.799088-0.113364-2.034698$ C $0.015896-1.398680-2.161601$ H $1.925316-0.804509-1.298240$ C $2.246743-2.434300-2.671772$ H $1.4005021 .717000-3.955493$ O $-1.0265882 .148360-2.857059$ H $-0.6748420 .553184-4.074519$ H $-0.7357390 .306956-1.026002$ O $-2.177814-0.418016-2.253262$ H $-0.329359-1.943461-3.049463$ O $-0.251906-2.235345-1.037309$ C $3.756015-2.337675-2.618509$ H $1.951945-3.249908-2.001627$
H $1.977156-2.695863-3.702140$

H $-1.9822211 .970747-2.855363$

H $-2.435338-1.078003-1.587330$

H $0.071182-1.779462-0.241356$

O $4.285912-3.552511-2.952870$

O $4.395289-1.336877-2.331231$

C $5.715525-3.618601-2.965960$

H $6.006258-4.620216-3.293902$

H $6.124733-2.887079-3.669308$

H $\quad 6.110618-3.450403-1.959973$

SCF Energy $\left(B 3 L Y P / 6-31 G^{* *} / / M M F F\right)=-3245.92811220$

05_00013

MMFF Geometry

C $-2.353895-3.6394840 .743861$

C $-2.629914-3.6861002 .055728$

C $-2.075815-2.7611243 .114129$

O $-1.159399-1.8092222 .529245$

C $-3.244100-1.9656833 .734930$

C $-2.818296-0.9558284 .781795$

C $-2.9217010 .381854 \quad 4.646264$

C $-2.266125-1.5410916 .056483$

C -3.4865991 .1665573 .492026$

C -2.3766411 .7703742 .631251$

C -2.8796982 .7625001 .571756$

C -3.7311832 .0921940 .489699$

C $-4.1153633 .073049-0.631051$

O

O $-4.6225192 .307403-1.734468$

C $-2.8412683 .788826-1.127467$

C $-5.2480134 .013876-0.202958$

O $-3.1589844 .809428-2.069983$

C -1.9865294 .3765520 .014658$

C $-0.6268494 .930082-0.474549$

O -2.6875395 .4702980 .618281$

C $0.114214-1.7587463 .001822$

O $0.641509-2.5132373 .802055$

C $0.746300-0.5600212 .407652$

C $1.864247-0.0542252 .949610$

C 2.5402681 .1761832 .529852

C 1.8516572 .0126701 .486001

C $0.2338593 .928158-1.246709$

C $1.6947514 .341188-1.327388$

C $3.9067873 .439154-2.064773$

O $2.1267385 .393738-0.866647$

C $4.4872132 .518471-1.037369$

C $5.4164862 .869649-0.136140$

C $5.971617 \quad 1.9113800 .881930$

C 5.9012132 .4441592 .297340

C 7.0227172 .6492023 .009044

C 4.5423542 .7483142 .891124

C 3.7094381 .5130013 .114277

N $2.458773 \quad 3.376413-1.970361$

H -1.634755 -2.918019 0.366823

H -3.356875 -4.416368 2.406538

H $-1.582847-3.3731783 .878059$

H -3.961877 -2.658256 4.194954

H -3.792349 -1.469375 2.927251

H -2.5716681 .0048275 .469091$

H -2.061768 -0.7745006 .811638$

H $-2.983442-2.2448796 .491413$

H -1.328974 -2.072095 5.866849

H -4.1578330 .5590962 .880656$

H $-4.1078711 .969673 \quad 3.908069$

H -1.647506 2.2855023 .271506

H -1.8149900 .9703662 .135519$

H -3.448503 3.5486162 .081791

H -4.6343491 .6405360 .915742$

H $-3.163277 \quad 1.263640 \quad 0.044096$

H $-5.3934781 .804224-1.421118$

H $-2.2494533 .039474-1.668313$

H -6.1461123 .4391960 .053772$

H -5.541974 $4.677003-1.024049$

H -4.9935664 .6275260 .663355$

H -3.694291 $4.400018-2.771115$

H $-0.7805005 .828734-1.086088$

H -0.0683205 .2698690 .408848$

H $-2.042446 \quad 6.0348691 .075791$ 
H $\quad 0.243547-0.096722 \quad 1.566433$ H $2.315276-0.5720343 .794812$ H 2.3666422 .9470691 .261404 H 0.8446362 .2885591 .817201 H $1.773417 \quad 1.4590150 .544645$ H $0.1945682 .948674-0.759884$ H $-0.1445413 .819171-2.269418$ H $4.2464004 .471526-1.935154$ H $4.1823373 .098591-3.067233$ H $4.1211771 .495595-1.034858$ H $5.8084313 .883741-0.137673$ H 7.0135241 .7047310 .604638 H 5.4534130 .9453180 .839288 H 6.9874693 .0322024 .023692 H 8.0030412 .4385472 .594801 H 4.0407703 .4960382 .272175 H 4.6588403 .2337953 .869613 H 4.1227810 .8417113 .868464 H $2.0057392 .523043-2.284971$ C -3.815958 -3.660851-1.322513 O $-2.891821-2.855127-2.083074$ C $-3.304350-1.480887-1.989034$ C $-4.801106-1.541027-1.719609$ C $-4.873138-2.706248-0.749825$ H -2.798138 -1.057786 -1.111845 C $-2.883612-0.694804-3.232275$ H -5.189536 -0.609949-1.297758 H $-5.369852-1.773618-2.627038$ C $-6.254765-3.327649-0.637423$ H $-4.582016-2.3381220 .240824$ C $-1.416865-0.907745-3.655624$ O $-3.706445-1.096207-4.334946$ H -3.087551 $0.370150-3.072594$ H -1.311798 $-1.901126-4.108532$ O $-1.1360640 .028163-4.705597$ C $-0.368095-0.749484-2.537580$ C $1.061136-0.891185-3.092742$ H $-0.543757-1.509010-1.769055$ O $-0.5275350 .529373-1.931171$ H $1.112478-1.820332-3.671006$ H $1.262048-0.067158-3.788460$ H -6.978221 -2.592962 -0.268814 H -6.611319 -3.693037 -1.606239 H $-6.243386-4.1720170 .059199$ C $-3.042086-4.491200-0.287882$ H $-3.428770-0.575806-5.108396$ H -1.161135 $0.919575-4.316888$ H $-0.0001180 .535135-1.114726$ H -2.268039 $-5.074754-0.800997$ H $-3.726017-5.2029010 .189187$ H $-4.274247-4.349434-2.043245$ C $2.021314-3.179068-1.301456$ O $1.913544-1.796949-0.947042$ C $2.167590-0.880634-2.015090$ C $3.577314-1.113428-2.581258$ C $3.783480-2.564943-3.010398$ C $3.404805-3.514164-1.874723$ H $1.253940-3.401000-2.054044$ C $1.649672-4.023545-0.072184$ H $2.1576420 .118969-1.566235$ O $3.828605-0.261036-3.693554$ H $4.323447-0.872378-1.813204$ H $3.226019-2.801460-3.923915$ O $5.163715-2.771029-3.321538$ H $4.176499-3.451900-1.098665$ O $3.396350-4.855575-2.357005$ C $2.714299-4.1109680 .999733$ H $0.744663-3.6326490 .404455$ H $1.436565-5.049585-0.395906$ H $3.6906650 .654803-3.398274$ H $5.401344-2.132551-4.015818$ H $4.273046-5.032214-2.739306$ O $3.155658-2.8720521 .352601$ O $3.083532-5.1700831 .492742$ C $4.183162-2.8415472 .348268$ H $5.018361-3.4880122 .061882$ H 4.550959-1.815049 2.426464 H $3.772632-3.1451413 .315580$
SCF Energy (B3LYP/6-31G**//MMFF) $=-3245.91481963$

05 00014

MM̄FF Geometry

C $2.698521 \quad 1.738720-2.734531$

C $2.7506703 .079470-2.778984$

C $1.8642704 .042793-2.027389$

O $0.748003 \quad 3.349922-1.430384$

C $2.6905894 .690541-0.896694$

C $1.9116365 .665852-0.037018$

C 1.6313995 .4765141 .268382

C $1.461116 \quad 6.926012-0.729243$

C 2.0347064 .3190392 .142697

C 0.9358983 .2593792 .202764

C 1.2235162 .1100043 .178693

C 2.4014231 .2394702 .730573

C 2.5923310 .0122523 .638237

O $0.025948 \quad 1.320726 \quad 3.230351$

O $3.485549-0.8931542 .971745$

C $1.237000-0.7127823 .786413$

C 3.2696960 .3854644 .961860

O $1.328743-1.7896244 .715493$

C 0.0727190 .2294614 .159959

C -1.304949 -0.4724164 .122904$

O $0.261878 \quad 0.7036225 .498409$

C $-0.5101193 .730919-1.779444$

O $-0.8321144 .537772-2.636011$

C $-1.4454243 .009287-0.888397$

C $-2.7577383 .286329-0.909273$

C $-3.7702972 .680471-0.039322$

C -3.2804151 .7366251 .026653$

C -1.646926 -1.147692 2.793375

C $-3.128326-1.4657752 .660267$

C $-4.771965-2.2880050 .960001$

O $-3.940715-1.2924083 .563996$

C $-5.217601-1.238264-0.009464$

C $-6.381706-0.5768030 .075509$

C $-6.8109100 .460945-0.924479$

C $-7.2542371 .759724-0.283898$

C $-8.5047042 .218692-0.462833$

C -6.2636192 .5303450 .563584$

C $-5.0678643 .004602-0.220839$

N -3.426712 $-1.969171 \quad 1.402428$

H $1.9424451 .237613-2.137158$

H $3.5352533 .546484-3.372122$

H $1.5226474 .807626-2.734325$

H $3.5532705 .220015-1.323218$

H $3.1120523 .891281-0.277458$

H 1.0518016 .2410921 .784617

H $0.9917207 .634339-0.038140$

H $2.3172517 .433608-1.185756$

H $0.7319116 .700280-1.512495$

H 2.9811193 .8851171 .809381

H 2.2275314 .7076383 .150495

H -0.0164713 .7292112 .484531$

H 0.7694452 .8399941 .204720

H 1.4093212 .5370104 .171062

H 3.3310281 .8185782 .686941

H 2.2214510 .8860251 .705318

H $4.318598-0.4217392 .800402$

H $1.026507-1.1709542 .812267$

H 4.2504790 .8392104 .774962

H $3.465290-0.5042125 .570415$

H 2.6892031 .0930555 .557150

H $1.330158-1.4094505 .610494$

H -1.377710 -1.206691 4.935881

H $-2.0721250 .283503 \quad 4.342103$

H $-0.596106 \quad 1.002648 \quad 5.842861$

H $-1.0198772 .274259-0.215146$

H -3.125212 $4.030027-1.614666$

H -2.5652522 .2380031 .688589$

H -2.7885390 .8740230 .566558$

H -4.0698831 .3435731 .668260$

H -1.362287 $-0.501371 \quad 1.956028$

H -1.094141 -2.089168 2.702978

H $-5.441938-2.3692211 .821541$

H -4.724771 -3.2583900 .456397$

H $-4.548954-1.018457-0.838471$ 
H $-7.069829-0.806597 \quad 0.885387$ H $-7.6309660 .029224-1.512757$ H $-6.0079780 .673411-1.641115$ H -8.837493 $3.147039-0.010452$ H -9.225912 $1.677585-1.066395$ H $-5.9942051 .921172 \quad 1.429414$ H -6.7419733 .4239490 .986564$ H $-5.3190913 .707804-1.016110$ H -2.679262 -2.0492120 .719080$ C $4.5815620 .045460-2.483469$ O $3.832427-1.036222-1.901065$ C $4.325155-1.268747-0.565200$ C $5.541554-0.361504-0.383583$ C $5.2078280 .794112-1.307136$ H $3.532890-0.9346100 .114421$ C $4.603571-2.761378-0.341263$ H $5.673698-0.0539620 .658371$ H $\quad 6.469717-0.846624-0.705997$ C $6.4055551 .646974-1.683689$ H $4.4665271 .421564-0.797266$ C $3.445696-3.696566-0.739392$ O $5.738883-3.137115-1.133887$ H $4.890550-2.9260910 .704224$ H $3.343746-3.700928-1.831937$ O $3.837369-5.022429-0.361581$ C $2.095095-3.355077-0.086990$ C $0.978629-4.331336-0.498017$ H $\quad 1.808267-2.340930-0.379621$ O $2.225465-3.3604061 .332526$ H $0.980008-4.424760-1.589425$ H $1.187505-5.326657-0.087581$ H $6.8535262 .096169-0.791303$ H $7.1781901 .055164-2.185726$ H $6.1101772 .457179-2.357669$ C $3.6984690 .860523-3.435381$ H $5.910067-4.079651-0.965169$ H $3.255349-5.646567-0.826864$ H $2.529319-4.2453821 .597567$ H $3.142481 \quad 0.178722-4.090357$ H $4.3448381 .468170-4.080486$ H $5.368584-0.430257-3.084478$ C $-0.872462-2.248317-1.702420$ O $-0.703519-2.526238-0.307639$ C $-0.417569-3.893967-0.001242$ C $-1.565159-4.784429-0.494466$ C -1.876495 -4.553635 -1.973432 C -2.044120 -3.061124 -2.269511 H $\quad 0.050680-2.503929-2.235045$ C -1.093797 -0.734286 -1.825912 H $-0.396575-3.964497 \quad 1.093487$ O $-1.252141-6.155128-0.269308$ H -2.464270 -4.5622320 .093817$ H - $1.105799-5.002881-2.610848$ O $-3.096557-5.234879-2.276221$ H -2.989761 -2.712607 -1.837781 O $-2.117603-2.863304-3.678294$ C $-0.766664-0.191684-3.198319$ H -2.130294 -0.490104-1.565711 H $-0.456154-0.199226-1.111244$ H $-1.994710-6.682747-0.609845$ H $-3.236614-5.174215-3.236061$ H -2.932116 -3.288634 -3.994615 O $-1.5787220 .869975-3.471234$ O $0.118730-0.621928-3.925237$ C $-1.3443331 .519133-4.724454$ H $-0.3267131 .920080-4.758837$ H -1.514163 $0.821498-5.549863$ $\mathrm{H}-2.0506982 .348775-4.814168$

SCF Energy (B3LYP/6-31G**//MMFF) $=-3245.92311736$

0500015

MM̄FF Geometry

C $2.1604913 .810341-0.545027$

C $1.2606144 .630936-1.107820$

C $-0.2160834 .333067-1.269582$

O $-0.5089342 .947196-0.977870$

C -1.018177 $5.207574-0.288481$

C $-2.5165024 .990576-0.354598$
C -3.2605324 .4944760 .653935$

C $-3.1773885 .389802-1.648543$

C -2.8017034 .0676232 .020935$

C -2.4487252 .5771042 .144238$

C -3.6413181 .6304391 .930314$

C -3.2599550 .1929212 .288204$

C $-4.417130-0.7842732 .027245$

O -4.0269581 .6825310 .555631$

O $-3.887599-2.1189142 .066925$

C $-4.959729-0.5632960 .596664$

C $-5.474039-0.7093673 .135551$

O $\quad-6.152969-1.3111770 .380742$

C $\begin{array}{llll}-5.200136 & 0.920050 & 0.237941\end{array}$

C $-5.4962261 .148119-1.262512$

O -6.3508121 .4418120 .914954$

C $-0.2647462 .035271-1.958498$

O $\quad 0.1203082 .258047-3.094931$

C $-0.4950150 .694040-1.380283$

C $0.065676-0.377379-1.959909$

C $-0.038995-1.760700-1.492813$

C $-1.025264-2.049691-0.393777$

C $-4.3942800 .705392-2.231152$

C $-4.597043-0.686155-2.809165$

C $-3.465191-2.361194-4.269881$

O $-5.623414-1.341158-2.650196$

C $-2.818134-3.400545-3.410007$

C $-1.777659-4.146828-3.811468$

C $-1.150463-5.227622-2.977913$

C $0.344854-5.057875-2.827686$

C $1.200785-5.746124-3.601956$

C $0.845887-4.158461-1.721456$

C $0.736994-2.699962-2.072208$

N -3.502583 -1.096239-3.559705

H $1.8368502 .852417-0.147931$

H $1.5935215 .595144-1.485974$

H $-0.5108754 .558108-2.301776$

H $-0.8151056 .268815-0.484878$

H -0.6506475 .0173340 .724978$

H $-4.332371 \quad 4.3860150 .491433$

H -4.269661 $5.328040-1.593543$

H $-2.9211646 .423482-1.902831$

H -2.853259 $4.737276-2.465025$

H -1.9417284 .6635022 .344378$

H -3.5976374 .3101762 .736745$

H -1.6513512 .3381911 .430804$

H -2.0403202 .4147593 .149572$

H $-4.471857 \quad 1.9751232 .557252$

H -2.9294150 .1244043 .332080$

H $-2.399075-0.1162031 .680987$

H -3.461679 -2.246427 2.931847

H $-4.207977-0.971443-0.088218$

H -5.035081 -0.9855774 .101985$

H $-6.284116-1.4256202 .960458$

H -5.9078860 .2866123 .247283$

H $-5.951523-2.2417680 .579164$

H $-6.4655300 .708012-1.526981$

H $-5.6256032 .231587-1.401648$

H $-6.7906580 .716282 \quad 1.385930$

H -1.090052 $0.630545-0.476786$

H $\quad 0.683598-0.227334-2.844471$

H $-2.018626-1.678054-0.665264$

H $-0.713887-1.5656120 .535630$

H -1.148418 -3.113559 -0.181538

H $-4.3914331 .392155-3.087350$

H $-3.4089310 .765663-1.760837$

H $-2.915787-2.193940-5.201724$

H $-4.485447-2.671258-4.517922$

H $-3.243957-3.566796-2.423360$

H $-1.372519-3.997896-4.809961$

H -1.368735 -6.188739 -3.461643

H $-1.612128-5.286915-1.984445$

H $2.274773-5.651313-3.480363$

H $0.845971-6.415533-4.378490$

H $1.906043-4.364336-1.520495$

H $0.336515-4.414889-0.788202$

H $1.414074-2.392700-2.870466$

H -2.673380 -0.510027 -3.591867

C $4.4778363 .152691-1.211669$ 
O $4.256938 \quad 1.794816-0.780533$

C $5.5130401 .093753-0.779690$

C $6.5293002 .031101-1.426598$

C $5.9776703 .391586-1.051207$

H 5.7875830 .9513360 .271558

C $5.373424-0.264259-1.477991$

H $7.5437011 .860552-1.053239$

H $6.5528491 .919542-2.516651$

C $6.521864 \quad 4.524031-1.904361$

H $6.216877 \quad 3.5871350 .002740$

C $4.268582-1.175236-0.905429$

O $5.064626-0.026164-2.858375$

H $6.340239-0.781743-1.462865$

H $3.280790-0.762527-1.143982$

O $4.354640-2.435979-1.584122$

C $4.347481-1.4404830 .611333$

C $3.341271-2.5192381 .061010$

H $4.160317-0.5120151 .160626$

O $5.653252-1.901961 \quad 0.961491$

H $2.355541-2.2806180 .651916$

H $3.648807-3.4824770 .637117$

H $7.6097354 .593789-1.802014$

H $6.2928384 .373325-2.964690$

H $6.0926555 .483784-1.600444$

C $3.6214654 .113868-0.386193$

H $5.192882-0.862791-3.336073$

H $4.052977-2.298181-2.497794$

H $6.274409-1.1640030 .847448$

H $3.8346805 .149830-0.672724$

H $3.864368 \quad 4.0119760 .678713$

H $4.1883853 .201220-2.270029$

C $1.354350-1.2397333 .026507$

O $2.754880-1.4770133 .224352$

C $3.268764-2.6598832 .598749$

C $2.498126-3.8960463 .085583$

C $0.993883-3.7356922 .901702$

C $0.526507-2.4274863 .533715$

H $1.156061-1.0724871 .962059$

C 0.9846570 .0429093 .787824

H $4.298559-2.7711302 .960071$

O $2.929200-5.0723122 .409621$

H $2.700142-4.0497014 .153742$

H $0.707740-3.7882491 .844709$

O $0.321798-4.813618 \quad 3.556674$

H $0.603184-2.5012744 .626245$

O $-0.847556-2.2207103 .216874$

C $\quad 1.490887 \quad 1.292332 \quad 3.099376$

H -0.1046860 .1393343 .858755$

H $1.415933 \quad 0.0207554 .795268$

H $3.887765-5.1555622 .550968$

H $0.661007-5.6406213 .172822$

H $-1.334846-3.0072843 .516541$

O 1.0300242 .3834373 .780858

O 2.1949891 .3149142 .100013

C 1.4052813 .6544253 .242246

$\begin{array}{lllll}\mathrm{H} & 0.904498 & 3.811288 & 2.283694\end{array}$

H $1.077192 \quad 4.4294993 .939732$

H 2.4915233 .7218843 .129103

SCF Energy (B3LYP/6-31G**/MMFF) $=-3245.90928636$

$05 \_00016$

MMTFF Geometry

C $-1.131368-2.8462760 .604120$

C $-0.485812-2.400116-0.483792$

C $-1.028393-1.348179-1.410585$

O $-0.123281-0.213700-1.409109$

C $-1.096974-1.834709-2.867251$

C $-2.119837-2.916202-3.125397$

C $-3.405302-2.676600-3.447951$

C $-1.602661-4.331452-3.082357$

C $-4.090333-1.342094-3.578182$

C $-5.451410-1.324758-2.866950$

C $-5.344460-1.142671-1.343416$

C $-6.681043-1.496915-0.686835$

C $-6.685547-1.2029140 .816989$

O $-5.0179020 .230748-1.097893$

O $-8.049907-1.304401 \quad 1.251594$

C $-6.2178010 .249758 \quad 1.029170$
C $-5.894399-2.2541561 .603991$

O $-6.074400 \quad 0.5412452 .418395$

$\begin{array}{llll}\text { C }-4.902914 & 0.592086 & 0.285374\end{array}$

C -4.6696892 .1222860 .354180$

$\begin{array}{llllll}\text { O } & -3.823869 & -0.091171 & 0.920541\end{array}$

C $-0.1135110 .586313-0.308849$

$\begin{array}{lllll}\text { O } & -0.758885 & 0.437768 & 0.716000\end{array}$

C $0.8308391 .693630-0.571340$

C 1.0026672 .6502530 .352726

C $1.9068793 .797505 \quad 0.252252$

C $2.7515393 .913789-0.989750$

C $-3.4347502 .627727-0.403543$

C $-3.3794424 .146685-0.450841$

C $-1.9556506 .014804-1.254120$

O $-4.2986854 .863866-0.063730$

C $-0.5018456 .329289-1.102884$

C $-0.0262907 .180926-0.181332$

C $1.4201717 .565095-0.047275$

C 1.9987287 .1965541 .300838

C 1.8752298 .0207162 .355022

C 2.7936645 .9143611 .400327

C $1.9335624 .688318 \quad 1.266008$

N $-2.1817194 .606795-0.985238$

H $-2.116388-2.4561450 .844984$

H $\quad 0.506815-2.779951-0.704534$

H -2.019402 $-0.998901-1.096839$

H $-0.105713-2.178666-3.194203$

H -1.292309-0.973690 -3.520515

H $-4.041758-3.534033-3.664653$

H $-2.370543-5.062534-3.357233$

H $-0.770547-4.452419-3.783925$

H $-1.250943-4.590291-2.080168$

H -3.470987 -0.514955 -3.218894

H $-4.262364-1.169810-4.647823$

H $-6.033838-0.491739-3.282500$

H $-5.997623-2.246785-3.102716$

H $-4.545569-1.780797-0.949833$

H $-6.937566-2.547981-0.870532$

H $-7.482883-0.909440-1.156989$

H -8.070478 -1.1792102 .215279$

H -7.0121540 .9093700 .652424$

H $-6.343174-3.2459131 .471511$

H -5.927591 -2.051064 2.680091

H -4.847659-2.320772 1.300082

H $-6.945750 \quad 0.4318822 .834549$

H $-5.5528282 .626002-0.061006$

H $-4.5766722 .439302 \quad 1.400773$

H -2.9939990 .3698020 .723691$

H $1.3618031 .688204-1.515808$

H 0.4292152 .5894291 .276980

H $3.3366284 .834201-1.037924$

H $2.124013 \quad 3.907143-1.888050$

H $3.4596203 .080658-1.047391$

H -2.5238442 .2792460 .089210$

H -3.437758 2.260671-1.435610

H -2.581287 $6.623269-0.592446$

H -2.273058 $6.206727-2.284277$

H $0.1839195 .861022-1.804328$

H $-0.722788 \quad 7.6603340 .503360$

H $1.4948618 .650812-0.193777$

H $2.0269277 .123591-0.847207$

H 2.3119087 .7782693 .318110

H 1.3302188 .9558062 .277485

H 3.2950005 .8519702 .375536

H 3.6086645 .9439230 .671724

H 1.2769444 .5224532 .121230

H $-1.5520163 .933086-1.412386$

C $-0.031821-3.1453902 .825053$

O $1.050320-2.2593492 .473960$

C $2.128685-2.4369513 .408843$

C $1.538269-3.2130704 .578836$

C $0.541740-4.1079273 .864779$

H $2.874809-3.0684862 .911637$

C $2.753539-1.0818933 .758030$

H $2.298202-3.7716715 .133382$

H $1.017504-2.5555185 .284120$

C $-0.502457-4.7143964 .785645$

H $1.092049-4.9166943 .365242$ 
C $3.136953-0.2345922 .526175$

O $1.804162-0.3246114 .519503$

H $3.621444-1.2336864 .410221$

H 2.2247610 .0960792 .016491

O 3.7741240 .9595512 .997750

C $4.062932-0.9397411 .517440$

C $4.413351-0.0265820 .330644$

H $3.564894-1.8371731 .145148$

O $5.251196-1.3454552 .191060$

H $3.4766580 .316693-0.119334$

H $4.938367 \quad 0.8629420 .699827$

H $-0.026804-5.3448885 .543815$

H -1.077916 -3.9405245 .304712$

H -1.205478 -5.335833 4.221972

C $-0.539146-3.8417991 .561676$

H 2.2144650 .5334054 .723478

H 4.6188350 .6990373 .403633

H $5.727752-1.9578291 .605675$

H -1.303854 -4.585794 1.810741

H $0.286489-4.3756131 .076656$

H $-0.822089-2.5234793 .265606$

C $3.586037-1.913858-1.935020$

O $4.804209-1.959955-1.183460$

C $5.301560-0.691461-0.746054$

C $5.5683490 .206461-1.961729$

C $4.3222660 .358069-2.828762$

C $3.735950-1.009908-3.170232$

H $2.773544-1.555091-1.291845$

C $3.256309-3.348502-2.384017$

H $6.272486-0.889932-0.275605$

O $6.025893 \quad 1.485550-1.535694$

H $6.370209-0.238073-2.565528$

H $3.581227 \quad 1.001961-2.345277$

O $4.6941491 .022946-4.038472$

H $4.386755-1.493554-3.909709$

O $2.466773-0.827640-3.796795$

C $2.900309-4.279181-1.244416$

H $2.400752-3.334244-3.068879$

H $4.122798-3.784721-2.895545$

H $6.1539382 .028868-2.332040$

H $3.8992531 .074410-4.596542$

H $1.869766-0.417476-3.147869$

O $2.498665-5.476326-1.768071$

O $2.968813-4.007173-0.054942$

C $2.132808-6.478564-0.814432$

H $1.956241-7.412641-1.354661$

H $2.940103-6.639282-0.093517$

H $1.209964-6.188597-0.304724$

SCF Energy (B3LYP/6-31G**//MMFF) $=-3245.92704809$

0500017

MM̄FF Geometry

C $1.881422-2.146670-2.055715$

C $1.165933-3.280300-2.086844$

C $0.319797-3.741840-0.929401$

O $-1.076011-3.658507-1.312031$

C $0.577655-5.210649-0.557687$

C $1.918935-5.4732630 .087084$

C $2.136477-5.4187191 .415180$

C $3.026259-5.893860-0.843761$

C $1.152991-5.0348142 .489288$

C $1.803572-4.1626653 .573445$

C $2.066950-2.716333 \quad 3.120880$

C $3.086099-2.0616434 .054737$

C $3.311957-0.5871583 .707344$

O $0.822579-2.0089673 .180180$

O $4.044598 \quad 0.0113194 .787562$

C 1.9437510 .1160943 .643131

C $4.173742-0.4175242 .452613$

O $2.0840621 .458743 \quad 3.181217$

C $0.888012-0.6296682 .788229$

C -0.5014830 .0008873 .058352$

O $1.221243-0.4928931 .407854$

C -1.653450 -2.427121 -1.281376

O $-1.109548-1.368364-1.012422$

C $-3.081186-2.588636-1.629868$

C $-3.865317-1.502450-1.692215$

C $-5.294237-1.479410-2.006708$
C $-5.986393-2.804685-2.189748$

C $-1.676661-0.6782112 .343123$

C $-3.019100-0.1954462 .870026$

C $-5.451470-0.6042382 .662467$

O -3.1283730 .6339083 .769990$

C $-6.406231-0.8931411 .549052$

C -7.2725390 .0138931 .071806$

C $-8.291280-0.2644960 .002519$

C $-8.0964760 .598445-1.224406$

C $-8.6236621 .832592-1.293347$

C $-7.358588-0.002922-2.399407$

C $-5.913911-0.283271-2.091650$

N $-4.085806-0.7965102 .210552$

H $1.842262-1.528246-1.162638$

H $\quad 1.165033-3.890844-2.986059$

H $0.475844-3.117160-0.040004$

H $0.459406-5.850279-1.443639$

H $\quad-0.225407-5.552900 \quad 0.109469$

H $3.128055-5.6872041 .778085$

H $3.943990-6.155194-0.306027$

H $2.724035-6.774129-1.420659$

H $3.275801-5.089421-1.541148$

H $0.273677-4.5240182 .085075$

H $\quad 0.802167-5.9605862 .961113$

H $1.132655-4.1500144 .442407$

H $2.739085-4.6360563 .897825$

H $2.437401-2.7100002 .089936$

H $4.037940-2.6072894 .036395$

H $2.725721-2.1241855 .091403$

H $4.886761-0.4666954 .879264$

H 1.5670450 .1829134 .673601

H $5.129326-0.9424742 .569228$

H 4.4320690 .6350992 .298926

H $3.696254-0.7970141 .546702$

H 2.7479951 .8920033 .744453

H $-0.692890-0.0395124 .139090$

H -0.4942251 .0594322 .769392$

H $\quad 0.423723-0.6356250 .874478$

H -3.446844 -3.590819-1.821034

H $-3.420540-0.529398-1.491780$

H -7.065942 -2.720488 -2.327865

H -5.841351 -3.439473 -1.308145

H $-5.589702-3.326487-3.067518$

H -1.642132 $-0.451697 \quad 1.274512$

H -1.634158 -1.764733 2.474827

H $-5.5645950 .412970 \quad 3.052841$

H -5.623435 -1.300487 3.490059

H $-6.410051-1.9021481 .144482$

H -7.276346 1.0149311 .498012

H $-9.286169-0.0781470 .428045$

H $-8.292801-1.323728-0.282847$

H -8.511808 $2.448082-2.179727$

H $-9.1770612 .255440-0.461246$

H $-7.380740 \quad 0.683615-3.256145$

H -7.900247 -0.891011-2.736199

H $-5.3227130 .620180-1.934296$

H -3.890495 -1.586182 1.601175

C $4.026328-1.032708-2.722813$

O $3.752680 \quad 0.134541-1.920297$

C $4.6147281 .206895-2.336635$

C $5.6963630 .560879-3.193246$

C $4.916399-0.550388-3.868455$

H $4.0113561 .869410-2.968905$

C $5.1281231 .979434-1.118616$

H $6.1419021 .266154-3.901310$

H $6.5030360 .138819-2.582854$

C $5.795396-1.635827-4.465060$

H $4.300034-0.111160-4.664480$

C $4.0189192 .485855-0.174215$

O $5.9780031 .111275-0.356614$

H $5.7589202 .811845-1.452205$

H 3.5405431 .6341050 .321345

O $4.657610 \quad 3.238007 \quad 0.866864$

C $2.9332203 .355228-0.835544$

C 1.9589073 .9350250 .208414

H $2.3840942 .751689-1.563816$

O $3.5703584 .419053-1.540288$

H 1.5760323 .1113030 .820258 
H 2.5123414 .6068880 .875379

H $6.451252-1.218845-5.236284$

H $6.427798-2.104055-3.703289$

H $5.187643-2.420391-4.926608$

C $2.705061-1.635738-3.203397$

H 6.2857531 .6133670 .417430

H $5.0360514 .036563 \quad 0.460134$

H $2.9119464 .815152-2.135261$

H $2.897702-2.439461-3.922908$

H $2.119372-0.861642-3.712709$

H $4.564060-1.743071-2.081414$

C $-0.7776192 .915670-0.750300$

O $0.0224353 .952418-1.332156$

C $0.785212 \quad 4.730279-0.404483$

C $-0.143110 \quad 5.374448 \quad 0.634797$

C -1.0234494 .3396331 .329001$

C $-1.751446 \quad 3.4911750 .288872$

H $-0.1280892 .165599-0.281949$

C $-1.5594852 .244780-1.891435$

H $1.2244755 .552199-0.982623$

$\begin{array}{lllll} & \text { O } & 0.619772 & 6.094006 & 1.597841\end{array}$

H $-0.788408 \quad 6.104357 \quad 0.128627$

H -0.4382573 .7203342 .018851$

O $-1.983302 \quad 5.0380352 .125127$

H -2.515183 4.102119 -0.209744

O $-2.421823 \quad 2.415178 \quad 0.939821$

C $-0.695516 \quad 1.355487-2.758723$

H -2.354752 $1.613109-1.482355$

H -2.001958 $3.009656-2.540492$

H $-0.003922 \quad 6.4611602 .247576$

H -2.4659494 .3769562 .649351$

H $-3.109762 \quad 2.796207 \quad 1.510914$

O $-1.4852790 .778280-3.713459$

O $\quad 0.506165 \quad 1.180081-2.620114$

C $-0.820559-0.114940-4.611907$

H $-1.531867-0.406005-5.389344$

H $\quad 0.0334020 .378190-5.086318$

H $-0.501114-1.012687-4.075691$

SCF Energy (B3LYP/6-31G**/MMFF) $=-3245.93241945$

0500018

MMFF Geometry

C $2.475695-3.139615-2.462087$

C $1.147975-3.123160-2.651044$

C $0.157765-3.139348-1.512888$

O $-0.803418-2.074751-1.705634$

C $-0.609080-4.473904-1.506228$

C $-1.514392-4.649461-0.301955$

C $-2.857002-4.747071-0.357509$

C $-0.817011-4.7507891 .031145$

C $-3.743980-4.712439-1.569646$

C $-4.304533-3.325194-1.919605$

C $-5.155085-2.688846-0.807785$

C $-5.904640-1.463459-1.333981$

C $-6.691952-0.751772-0.224019$

O $-4.283747-2.2885740 .252953$

O

C $-5.759000-0.4991220 .981684$

C $-7.986822-1.5005120 .114551$

O $-6.517937 \quad 0.0130452 .074142$

C $-4.950872-1.7461301 .401833$

C $-3.856471-1.4518962 .457969$

O $-5.841917-2.7081011 .979384$

C $-0.383322-0.801449-1.475021$

O $0.731115-0.445486-1.128313$

C $-1.5253860 .100380-1.738725$

C $-1.3077741 .412890-1.907578$

C $-2.3294972 .427448-2.175003$

C $-3.7717542 .014323-2.047122$

C $-2.806850-0.4142512 .045111$

C -3.1211240 .9994942 .506256$

C -2.330906 $3.348726 \quad 2.228410$

O $-3.9940401 .260763 \quad 3.329356$

C -3.0985194 .0593531 .159485$

C $-2.619741 \quad 5.120853 \quad 0.493242$

C $-3.3924975 .874842-0.550093$

C $-2.6449205 .991391-1.859287$

C $-1.9438437 .097910-2.159130$
C $-2.7837914 .862159-2.854822$

C $-1.9318673 .674309-2.502977$

N -2.2778391 .9325501 .919752$

H $2.864251-3.196792-1.447980$

H $0.748191-3.076233-3.660874$

H $0.665998-2.987278-0.553032$

H $0.100442-5.312194-1.516823$

H $-1.183894-4.551594-2.435333$

H $-3.396847-4.8777610 .580122$

H $-1.500479-5.0416271 .836380$

H $-0.024044-5.5046520 .989906$

H $-0.374238-3.7905041 .310401$

H -3.224728 -5.117074-2.444673

H $-4.580438-5.401185-1.393203$

H -3.470172 -2.660816 -2.172781

H $-4.915861-3.435132-2.823939$

H $-5.862652-3.440726-0.439140$

H $-6.571856-1.738497-2.160434$

H $-5.182599-0.752951-1.758622$

H $-7.5316591 .012396-0.032021$

H -5.0699660 .2968210 .676493$

H -8.629859 -1.568319 -0.771257

H -8.572436 -0.9613940 .867377$

H -7.812804 -2.5159640 .476847$

H -5.8958130 .2861612 .768814$

H $-4.315206-1.2033393 .422269$

H $-3.315408-2.3935902 .631873$

H $-5.322469-3.3191602 .527825$

H $-2.512761-0.342245-1.798561$

H $-0.2832371 .780437-1.865065$

H $-3.9467311 .527382-1.081858$

H $-4.0440861 .318106-2.847485$

H -4.471241 $2.851646-2.090172$

H $-1.852419-0.6800282 .517499$

H -2.649108 -0.4239100 .962788$

H -1.297622 3.7025972 .300847

H -2.8190873 .4989053 .196313$

H -4.1019523 .7008930 .942534$

H $-1.6263175 .492944 \quad 0.734004$

H -3.600887 $6.876081-0.150469$

H $-4.3746245 .419755-0.729455$

H -1.428104 $7.200891-3.108147$

H $-1.8649277 .922913-1.458713$

H $-2.468920 \quad 5.200267-3.851437$

H -3.842020 $4.612165-2.970564$

H $-0.8623073 .875324-2.567565$

H -1.6321651 .6314351 .196379$

C $4.504401-1.944557-3.414067$

O $5.272249-2.135200-2.208365$

C $5.354386-0.871816-1.509152$

C $5.0146380 .181623-2.551338$

C $3.931876-0.528376-3.342559$

H $4.572333-0.911706-0.742473$

C $6.737800-0.758358-0.859888$

H $4.6728861 .122785-2.113331$

H $5.8798510 .390554-3.192558$

C $3.655144 \quad 0.093113-4.699688$

H $3.008547-0.508021-2.751862$

C $7.034250 \quad 0.554411-0.115442$

O $6.905460-1.8433470 .057183$

H $7.498106-0.900374-1.638945$

H 7.9845620 .4384140 .422197

O $7.2536451 .576602-1.093727$

C 5.9695851 .0606500 .882091

C $5.5532350 .041368 \quad 1.953938$

H $5.091213 \quad 1.4069510 .331466$

$\begin{array}{lllll}\text { O } & 6.505449 & 2.222010 & 1.529848\end{array}$

H $5.038631-0.7947061 .473507$

H $6.456472-0.3658802 .425509$

H $3.3052211 .124428-4.585813$

H $4.5540510 .110645-5.324910$

H $2.882649-0.469235-5.233697$

C $3.487416-3.080191-3.572131$

H $6.722077-2.665569-0.429095$

H $7.4548892 .399048-0.615152$

H $7.276693 \quad 1.9412142 .051473$

H $4.025356-4.036350-3.569348$

H $2.990622-2.990157-4.545364$ 
H $\quad 5.223930-2.015595-4.240140$

C 2.5320420 .6111181 .906899

O 3.5521031 .3800112 .553461

C 4.6536020 .6269813 .066410

C $4.153488-0.4173624 .075175$

C $3.063577-1.3039703 .477819$

C $1.960687-0.4501422 .856011$

H $2.9441250 .130577 \quad 1.011344$

C 1.4284071 .5922301 .481264

H 5.2724721 .3398663 .625948

O $5.222642-1.2377574 .533975$

H 3.7400190 .0947174 .953801

H $3.472531-2.0104902 .746217$

O $2.481264-2.0965134 .515523$

H 1.3802120 .0308363 .654054

O $1.068299-1.2932482 .129568$

C 1.8676072 .5101290 .361697

H $\quad 0.541903 \quad 1.0580671 .128695$

H 1.1498922 .2135882 .341480

H $5.893621-0.6517794 .924068$

H $3.200436-2.6053204 .927898$

H $\quad 0.735117-1.9647802 .749077$

O 1.1279793 .6574490 .403448

O $2.7286672 .244994-0.465351$

C $1.4484774 .631296-0.594883$

H $0.7162455 .440040-0.530075$

H $2.4446255 .042438-0.406500$

H $1.3974504 .191674-1.595475$

SCF Energy (B3LYP/6-31G**//MMFF) $=-3245.93076575$

0500019

MM̄FF Geometry

C $4.015398-3.8187630 .139510$

C $2.975044-3.543834-0.660546$

C $1.555498-3.952590-0.361448$

O $0.781263-2.753520-0.123544$

C $0.952984-4.678081-1.578288$

C $-0.480181-5.131396-1.375244$

C -1.526960 -4.702094 -2.107901

C $-0.698393-6.150089-0.286208$

C $-1.514553-3.730082-3.254614$

C -1.711816 -2.261562 -2.850991

C $-3.098429-1.947858-2.267866$

C $-3.278027-0.438905-2.087240$

C $-4.623111-0.091833-1.430173$

O $-3.219595-2.593585-0.998739$

O $-4.5392891 .284892-1.030194$

C $-4.806378-0.953072-0.159792$

C $-5.778545-0.184822-2.433834$

O $-6.117021-0.8025390 .379420$

C $-4.507680-2.450132-0.384350$

C $-4.482592-3.2698160 .929290$

O $-5.534861-3.013883-1.209840$

C $0.866651-2.1944661 .114613$

O $1.513259-2.6025072 .066196$

C $0.048815-0.9632541 .108066$

C $0.269153-0.0254862 .041120$

C -0.4230821 .2595852 .151183$

C -1.640943 1.4710641 .291928

C -3.449404 -2.818091 1.968120

C $-4.011199-1.9019323 .043740$

C -3.289332 -0.569214 5.024096

O $-5.209381-1.6668103 .173423$

C $-3.223670 \quad 0.8716094 .627316$

C -2.4602111 .7769195 .258378$

C -2.4261743 .2361724 .904226$

C -1.024944 3.7454194 .650705

C -0.3445204 .4022305 .605530$

C -0.4503573 .5720873 .263464$

C $0.0536972 .177113 \quad 3.017725$

N $-3.011964-1.4067603 .871783$

H $3.855481-4.3858271 .053102$

H $3.142638-2.957916-1.560647$

H $1.510043-4.6022990 .521163$

H $1.559975-5.560568-1.820822$

H $1.022785-4.014649-2.447256$

H -2.513732 -5.099153 -1.872031

H $-1.718038-6.550619-0.290638$
H $-0.015668-6.996346-0.414827$

H $-0.525814-5.7059700 .698804$

H $-0.582288-3.820811-3.822744$

H -2.304147 -4.022169 -3.958696

H $-0.935231-1.984222-2.129192$

H $-1.557979-1.647258-3.746377$

H -3.856369 -2.343240 -2.954213

H -3.175562 $0.084134-3.046291$

H $-2.470090-0.052476-1.450631$

H $-5.3951001 .540619-0.646821$

H $-4.111953-0.5622110 .591371$

H $-5.6196480 .514624-3.263327$

H $-6.7285550 .108119-1.973393$

H $-5.896923-1.183547-2.859274$

H -6.2309560 .1312760 .623150$

H $-5.490542-3.327243 \quad 1.357393$

H $-4.224581-4.3040150 .658323$

H $-5.545490-3.975964-1.074591$

H $-0.686358-0.8490680 .320240$

H $1.053865-0.1976122 .776914$

H $-1.366487 \quad 1.5218210 .234234$

H -2.191429 2.3835851 .529657

H $-2.3486880 .647914 \quad 1.429822$

H -3.076561 -3.7073452 .492679$

H -2.590909 -2.337887 1.489573

H $-2.552462-0.8212255 .793292$

H $-4.287856-0.7998675 .409241$

H $-3.856896 \quad 1.1909113 .802993$

H -1.850054 1.4605176 .101532

H -2.878133 3.7901275 .737576

H $-3.055733 \quad 3.4521414 .032078$

H 0.6493864 .7964705 .421862

H -0.7645994 .5552386 .594138$

H $0.401213 \quad 4.251765 \quad 3.122015$

H -1.186173 3.8981742 .522909

H 0.9385491 .9257643 .604360

H -2.040156 -1.608806 3.655407

C $5.776472-2.1860500 .811379$

O $5.011378-1.0164500 .449927$

C $5.867448 \quad 0.139743 \quad 0.515122$

C $7.149387-0.3218231 .198108$

C $7.240443-1.7591650 .723528$

H $6.1034670 .417016-0.518836$

C $5.147861 \quad 1.295408 \quad 1.218820$

H 8.0140980 .2830310 .908795

H $7.063049-0.2861642 .290320$

C $8.186205-2.6123891 .550382$

H $7.571719-1.764317-0.323607$

C 3.8145861 .7112850 .562399

O 4.8603800 .9137492 .571085

H 5.8160802 .1613091 .287345

H 3.0788630 .9091820 .686752

O $3.3079472 .842953 \quad 1.281223$

C $3.9240462 .096796-0.924234$

C $2.6566972 .726295-1.529786$

H $4.2096991 .222296-1.513663$

O $4.9873463 .042546-1.091159$

H $2.5004413 .718304-1.082614$

H $2.8649602 .951975-2.583135$

H $9.204844-2.2137881 .502442$

H $7.885361-2.6400682 .603192$

H $8.209101-3.6418181 .179622$

C $5.415243-3.339581-0.125306$

H 4.3136710 .1097522 .538879

H 3.2288522 .5811522 .214683

H $4.7841103 .813696-0.534231$

H $6.098680-4.1836780 .018756$

H $5.510918-3.016117-1.169368$

H $5.510880-2.4329261 .847987$

C $0.0776843 .174439-3.041745$

O $0.2162622 .766761-1.676540$

C $1.3464841 .924077-1.404504$

C $1.2251200 .644741-2.240729$

C $1.0957630 .958879-3.729766$

C $-0.043216 \quad 1.953032-3.960956$

H $0.9408803 .783017-3.337541$

C $-1.179818 \quad 4.052563-3.128335$

H $\quad 1.241924 \quad 1.643062-0.353747$ 
O $2.322641-0.222179-2.005206$

H $\quad 0.3227230 .108429-1.921859$

H $2.0370661 .335077-4.146993$

O $0.786314-0.238273-4.445882$

H $-1.0040301 .449388-3.793247$

O $-0.0203872 .385400-5.319637$

C $-1.010688 \quad 5.371523-2.402619$

H -1.416532 4.283541-4.172508

H $-2.0261943 .527578-2.668978$

H $2.132755-1.064571-2.450760$

H $1.562819-0.820449-4.393920$

H $-0.0779811 .590998-5.877812$

O $-2.2079506 .030218-2.400067$

O $0.0317075 .779618-1.909579$

C $-2.207707 \quad 7.302993-1.745780$

H $-1.9455057 .185997-0.689923$

H $-1.5121607 .984956-2.243972$

H -3.216191 7.719880-1.812645

SCF Energy (B3LYP/6-31G**/MMFF) $=-3245.91230718$

0500020

MM̄FF Geometry

C -3.144924 -0.9822112 .146441$

C -2.027032 -1.463865 2.709598

C $-1.133762-2.4828422 .058011$

O $0.224751-2.0614432 .338696$

C -1.332409 -3.899249 2.626211

C $-2.641241-4.5524342 .244310$

C $-2.861900-5.1700581 .067306$

C $-3.706858-4.5465723 .308906$

C $-1.913250-5.303583-0.095353$

C $-2.597917-5.012737-1.438975$

C $-2.887135-3.519793-1.678827$

C $-3.987453-3.375933-2.733609$

C $-4.284646-1.908331-3.052645$

O $-1.679391-2.901362-2.145557$

O $-5.091170-1.896876-4.242758$

C $-2.956864-1.199810-3.380540$

C $-5.141654-1.256532-1.963663$

O $-3.1520640 .201923-3.547726$

C $-1.788055-1.493116-2.401305$

C $-0.472858-1.032710-3.073926$

O $-1.892749-0.748078-1.190046$

C $1.206521-2.5179201 .517994$

O $1.071837-3.216636 \quad 0.526883$

C $2.497236-2.0352032 .053166$

C $3.633165-2.4224341 .456182$

C $4.990830-2.0370151 .838197$

C $5.160270-1.0843162 .991664$

C $0.805956-1.279890-2.262045$

C $2.029408-0.792516-3.025808$

C $4.483732-0.608542-2.919610$

O $1.952612-0.210636-4.106400$

C $5.635448-1.311662-2.278356$

C $6.646884-0.668239-1.674168$

C $7.862403-1.342692-1.100809$

C $7.983176-1.152716 \quad 0.395047$

C $8.569363-0.0583850 .908941$

C $7.485565-2.2637321 .291801$

C $6.018871-2.5323801 .119916$

N $3.223504-1.081108-2.375676$

H -3.465695 -1.374473 1.186016

H $-1.726520-1.0971183 .688183$

H $-1.287565-2.4686540 .972444$

H $-1.227908-3.8780403 .720025$

H $-0.506911-4.5480502 .305282$

H -3.827646 -5.6527270 .921102$

H $-4.624740-5.0464322 .981652$

H -3.349464 -5.0667614 .203723$

H -3.973466 -3.523344 3.587595

H $-1.029982-4.6707760 .011511$

H -1.560303 -6.342031-0.111006

H $-1.944231-5.384368-2.238819$

H -3.528114 -5.592934-1.490072

H -3.195924 -3.045621 - 0.741832

H $-4.904871-3.886338-2.413706$

H -3.672206 $-3.879250-3.658797$

H $-5.223007-0.968124-4.500399$
H $-2.657877-1.571051-4.371119$

H $-6.109212-1.767880-1.886318$

H $-5.377367-0.216874-2.212938$

H $-4.681043-1.283666-0.975699$

H -3.223834 $0.601024-2.665561$

H $-0.363276-1.549906-4.036726$

H $-0.5346310 .042608-3.288724$

H $-2.826311-0.593060-0.989314$

H $2.479263-1.3846822 .919464$

H $3.565640-3.0854380 .594153$

H $6.199963-0.7979773 .167230$

H $4.790226-1.5353223 .918789$

H $4.611341-0.1541282 .810912$

H $0.763409-0.747004-1.305833$

H $\quad 0.926900-2.350530-2.066788$

H $4.5204790 .475148-2.772846$

H $4.492188-0.797142-3.998923$

H $5.662022-2.395649-2.356155$

H $6.6266460 .417809-1.621765$

H $8.746891-0.922492-1.597515$

H $7.877026-2.412563-1.343276$

H 8.6801350 .0745191 .979956

H 8.9485200 .7307160 .267848

H $7.723992-2.0656532 .342637$

H $8.034765-3.183327 \quad 1.050742$

H $5.798604-3.2117920 .295593$

H $3.189049-1.374659-1.403199$

C -4.5481601 .0632001 .747227$

$\begin{array}{llll}\text { O } & -3.472875 & 1.734422 & 1.065248\end{array}$

C $-3.876614 \quad 3.089016 \quad 0.782419$

C $-5.318948 \quad 3.224961 \quad 1.265987$

C -5.3872052 .1804972 .361692$

H -3.2372683 .7397381 .389705$

C $-3.6862123 .401145-0.707990$

H -5.5384314 .2359801 .623194$

H -6.0396492 .9932130 .473322$

C $-6.804806 \quad 1.771704 \quad 2.721471$

H -4.886962 2.5786173 .254854

C -2.260560 $3.164927-1.244017$

O $-4.5691612 .573817-1.477404$

H $-3.9931584 .433914-0.909506$

H $-2.0287752 .093750-1.252460$

O $-2.2342623 .593075-2.611833$

C -1.158111 $3.915572-0.478934$

C $0.2219643 .788670-1.152478$

H -1.1034413 .5234340 .540262$

O $-1.498907 \quad 5.298336-0.376775$

H $0.4237892 .731054-1.352923$

H $0.2017774 .304801-2.119925$

H -7.3758742 .6361393 .075954$

H -7.3320541 .3509381 .858668$

H -6.8053321 .0194843 .516378$

C -4.0004920 .0770932 .780855$

H $-4.4097131 .653164-1.209163$

H -2.925140 $3.097323-3.083920$

H $-1.5858695 .647042-1.280648$

H $-4.834507-0.414766 \quad 3.293741$

H -3.4168390.620962 3.533680

H $-5.124927 \quad 0.5245530 .983381$

C 1.8599302 .4669351 .052090

$\begin{array}{lllll}\text { O } & 1.411949 & 3.826884 & 1.011832\end{array}$

C $1.3513594 .403546-0.295884$

C $2.7392834 .365238-0.945013$

C $3.3386452 .961545-0.942922$

$\begin{array}{llll}\text { C } 3.267127 & 2.342899 & 0.453722\end{array}$

H $1.163112 \quad 1.8272530 .497307$

$\begin{array}{llll}\text { C } & 1.847840 & 2.033219 & 2.525327\end{array}$

H $1.0974685 .461346-0.151367$

O $2.6728764 .867053-2.275794$

H $3.4074155 .032929-0.385002$

H $2.8502432 .324074-1.689371$

O $4.707534 \quad 3.076399-1.339507$

H 4.0003442 .8301451 .109244

$\begin{array}{llllll} & \text { O } & 3.609934 & 0.961414 & 0.372453\end{array}$

C 0.4386151 .8836443 .058157

H 2.3464691 .0698142 .663028

H 2.3656102 .7896503 .127753

H $3.5663504 .805218-2.655046$ 
H $5.0847472 .181977-1.363181$

H 4.5482450 .9016250 .126704

O 0.4411132 .0965704 .406546

O $-0.536748 \quad 1.5863742 .382316$

C -0.8324341 .9909965 .050957$

H -0.7193482 .3304246 .083956$

H -1.5726792 .6258464 .554034$

H -1.159868 0.9479025 .057861

SCF Energy (B3LYP/6-31G**//MMFF) $=-3245.92196656$

\section{000021}

MM̄FF Geometry

C $-3.138086 \quad 1.7720862 .465597$

C -2.2025492 .2795461 .651109$

C -1.4604883 .5559641 .940287$

O -0.0466493 .2324431 .991900$

C -1.6451344 .6091140 .835022$

C -3.0519645 .1652650 .734606$

C $-3.9354984 .868372-0.239630$

C -3.4330026 .1401691 .819748$

C $-3.7416353 .931518-1.397138$

C $-4.6641572 .708522-1.289395$

C $-4.2282441 .593850-2.249057$

C $-5.196278 \quad 0.411843-2.203421$

C $-4.705689-0.759295-3.070762$

O $-2.9288851 .163359-1.833173$

O $-5.477039-1.919604-2.723615$

C $-3.236239-1.071548-2.713748$

C $-4.951391-0.505480-4.562916$

O $-2.690581-2.044683-3.601698$

C $-2.3265250 .179498-2.678545$

C $-0.952289-0.189467-2.066635$

O $-2.1464730 .664681-4.007324$

C 0.4427472 .7544743 .166984

O $-0.1774902 .611511 \quad 4.212237$

C 1.8846082 .4344703 .070394

C 2.5161492 .2057021 .908591

C 3.9375761 .8836301 .748722

C 4.8082941 .9329612 .977119

C $-0.0293350 .993889-1.740756$

C $0.829144 \quad 1.474273-2.897251$

C $2.6225823 .139128-3.394084$

O $0.8198480 .956006-4.009606$

C $3.9368952 .487802-3.098668$

C $4.949178 \quad 3.107903-2.473435$

C $6.2550172 .445931-2.141957$

C $6.4921152 .343627-0.650445$

C $7.3551113 .163940-0.028098$

C 5.7999651 .2290910 .103351

C $4.397578 \quad 1.587346 \quad 0.515234$

N $1.6185072 .554011-2.525165$

H -3.416602 2.3179883 .363971

H $-1.907448 \quad 1.7216580 .766834$

H -1.7597303 .9886802 .902898$

H -1.290133 4.204001-0.118791

H -0.9559535 .4439841 .026025$

H -4.915603 5.342437 -0.207799

H -4.429873 $6.566801 \quad 1.665105$

H -3.435033 5.6481962 .797220

H -2.7219046 .9723361 .849143$

H $-3.9662684 .472092-2.324774$

H -2.700162 $3.615173-1.483777$

H $-4.6430942 .325196-0.261871$

H -5.696918 $3.009726-1.504078$

H -4.170106 2.008631 -3.262304

H $-6.2039950 .720964-2.507330$

H -5.290990 $0.057510-1.167218$

H -6.414914 -1.712411 -2.875990

H -3.234831-1.528151-1.713871

H $-6.021046-0.359005-4.755048$

H $-4.655534-1.371554-5.165183$

H $-4.4254600 .373824-4.940363$

H -3.283848 -2.815132 -3.587611

H -1.136599 -0.699181-1.111970

H $-0.431111-0.893930-2.723074$

H $-1.3640831 .236860-4.027512$

H 2.3957972 .3579154 .024586

H 1.9440302 .2495710 .983259
H 4.5217291 .1442353 .681329

H $4.7150192 .903213 \quad 3.478026$

H 5.8714881 .8042782 .763755

H $-0.5941911 .842587-1.342536$

H $0.6675880 .668478-0.956608$

H $2.6398044 .215350-3.195089$

H $2.3518992 .981574-4.442711$

H $4.0513991 .449464-3.400990$

H $4.8328974 .146081-2.170879$

H $7.0550773 .029237-2.615881$

H $6.3198361 .445140-2.587001$

H 7.5520633 .0743631 .035110

H $7.8796733 .946135-0.566628$

H 6.4059750 .9173660 .960313

H $5.7597360 .338357-0.533981$

H $3.6817901 .571259-0.305586$

H $1.6002512 .864396-1.557804$

C $-3.212818-0.5619603 .266605$

O $-1.873985-0.9186172 .864839$

C $-1.608822-2.2668313 .304275$

C $-2.869579-2.7408024 .023699$

C $-3.944875-1.8937663 .364500$

H -1.476968 -2.875271 2.404205

C $-0.335737-2.2801954 .160876$

H -3.034700 -3.815620 3.899481

H $-2.820487-2.5356705 .099823$

C -5.239681-1.840477 4.155044

H $-4.144068-2.2896072 .359674$

C $0.929812-1.8391033 .391606$

O $-0.517038-1.3882415 .268467$

H $-0.183104-3.2748394 .593898$

H $\quad 0.799498-0.8277892 .988673$

O $2.018367-1.7723014 .321161$

C $1.328896-2.8085392 .262723$

C $2.587404-2.3578481 .498511$

H $0.509300-2.8921031 .547489$

O $1.537434-4.1093182 .812228$

H $2.357192-1.4205960 .979110$

H $3.390563-2.1367602 .211510$

H -5.664268 -2.843804 4.264985

H $-5.081281-1.4307855 .158301$

H -5.981641 -1.215001 3.649310

C $-3.791348 \quad 0.4326842 .262461$

H $-0.673604-0.4990404 .906053$

H $1.753058-1.1657135 .033631$

H $2.255402-4.0390183 .464704$

H -4.870442 0.5532792 .404015

H -3.6274730 .0782551 .237310$

H -3.140677 -0.1007224 .261962$

C $1.619452-3.041349-1.358845$

O $2.090187-3.939447-0.348105$

C $3.113878-3.4191150 .504578$

C $4.321755-2.963359-0.324145$

C $3.919216-1.998745-1.435697$

C $2.777206-2.588668-2.259851$

H $1.151110-2.169862-0.885879$

C $\quad 0.559505-3.794262-2.181599$

H $3.455482-4.2664831 .112855$

O $5.297378-2.3614770 .519804$

H $4.791780-3.846043-0.777522$

H $3.650251-1.017744-1.029254$

O $5.055028-1.793866-2.278309$

H $3.150322-3.435841-2.849604$

O $2.300286-1.605073-3.173088$

C $-0.741959-3.990588-1.432361$

H $0.315918-3.246844-3.098366$

H $0.944764-4.786406-2.446961$

H $6.039548-2.085483-0.044963$

H $4.831180-1.085223-2.904808$

H $3.013733-1.416944-3.805695$

O $-1.517063-4.883978-2.116752$

O $-1.057888-3.419987-0.398384$

C $-2.795246-5.159927-1.534756$

H $-2.669037-5.631030-0.555375$

H $-3.382318-4.240742-1.448153$

H $-3.325796-5.853228-2.192902$

SCF Energy (B3LYP/6-31G**//MMFF) $=-3245.93051832$ 
05_00022

MM̄FF Geometry

C $2.392925-2.4081980 .541844$

C $1.630509-2.0376651 .580934$

C $1.786853-0.7061722 .265925$

O $0.503932-0.0304212 .307348$

C $2.245682-0.8405513 .726148$

C $3.655824-1.3553173 .898297$

C $4.753909-0.5764453 .856971$

C $3.781896-2.8258924 .201546$

C 4.8245390 .9058503 .600361

C 5.9346901 .2603072 .599444

C 5.5524640 .9881261 .134036

C 6.8157160 .9549410 .269890

C $6.4958580 .821589-1.222504$

O $4.6873252 .049722 \quad 0.710395$

O $7.717591 \quad 1.083757-1.929317$

C $5.4762701 .912908-1.599315$

C $6.074032-0.605337-1.590500$

O $5.0368651 .755884-2.947657$

C $4.2499361 .971883-0.653578$

C $3.4418863 .255675-0.967655$

O $3.4365150 .822703-0.880500$

C $0.008763 \quad 0.465576 \quad 1.141963$

O $0.476823 \quad 0.3281930 .023620$

C -1.2088201 .2372541 .472790$

C $-1.907343 \quad 1.827008 \quad 0.491837$

C -3.1201122 .6289630 .661461$

C -3.5629242 .9365412 .068822$

C $2.2309313 .510109-0.060501$

C $1.6022904 .867904-0.334264$

C $-0.285344 \quad 6.317227 \quad 0.354434$

O $2.1056025 .700262-1.084971$

C - -1.7431116 .0986710 .601377$

C $-2.692916 \quad 6.381443-0.303392$

C $-4.1679996 .241462-0.055522$

C $-4.8300205 .264349-1.001053$

C $-5.2837745 .662864-2.201508$

C $-5.0453253 .849550-0.514928$

C $-3.7674283 .060519-0.441213$

N $0.413253 \quad 5.0453470 .363252$

H $3.181436-1.7426370 .201286$

H $0.840844-2.6942891 .934944$

H $2.487579-0.0571891 .726488$

H $1.544043-1.4788754 .281563$

H $\quad 2.148874 \quad 0.1353924 .221430$

H 5.717154-1.048904 4.046581

H $4.822490-3.1308934 .355890$

H $3.228840-3.0727895 .113794$

H $3.383619-3.4292793 .381155$

H 3.8702091 .3202883 .262232

H 5.0607021 .3891314 .556434

H 6.1603382 .3286842 .714586

H $6.846903 \quad 0.7104172 .863177$

H 5.0180420 .0348391 .061104

H 7.4837440 .1450050 .589652

H 7.3827951 .8846700 .421304

H $7.5531770 .949061-2.877794$

H $5.9979162 .879282-1.551906$

H $6.883825-1.310871-1.369169$

H $5.881357-0.697037-2.665222$

H $5.185081-0.941708-1.053851$

H $5.8145191 .843721-3.523793$

H $4.1159264 .118275-0.880276$

H $3.0854923 .226114-2.005362$

H $2.5331801 .007582-0.580620$

H -1.493592 1.3046552 .516645

H -1.567311 $1.705788-0.536013$

H -4.398362 3.6377122 .120278

H $-2.746877 \quad 3.3967772 .637562$

H -3.8758982 .0233372 .584607$

H $1.4650842 .749356-0.230638$

H 2.5258503 .4821760 .994081

H $-0.1001226 .831143-0.595031$

H $\quad 0.1404496 .932225 \quad 1.154261$

H -2.0301415 .7283861 .582118$

H -2.397190 $6.772353-1.274721$

H $-4.6237157 .233504-0.174765$
H -4.3734225 .9532370 .982600$

H $-5.7867654 .975707-2.873686$

H $-5.1573586 .686124-2.539738$

H $-5.7176873 .311543-1.196969$

H -5.5835103 .8742590 .437103$

H -3.361415 2.797962 -1.419220

H $\quad 0.1677904 .368703 \quad 1.080865$

C $1.854774-3.377626-1.682636$

O $0.526320-2.819215-1.737759$

C $-0.150829-3.345081-2.895083$

C $0.897837-4.116110-3.689715$

C $1.812979-4.607623-2.584449$

H $-0.896822-4.058437-2.526230$

C $-0.841195-2.214816-3.666487$

H $0.457733-4.927909-4.277083$

H $1.447404-3.463772-4.378413$

C $3.174729-5.060091-3.081496$

H $1.321883-5.440419-2.063146$

C $-1.837184-1.390816-2.822664$

O $0.165728-1.316927-4.151074$

H $-1.339720-2.626626-4.552037$

H -1.292398 -0.815013 -2.065633

O $-2.442485-0.422331-3.689350$

C $-2.941648-2.222346-2.144198$

C $-3.972242-1.340213-1.416550$

H $-2.480456-2.911101-1.432835$

O $-3.600380-3.002872-3.140639$

H $-3.435735-0.653652-0.753692$

H -4.508422 $-0.729968-2.153194$

H $3.070121-5.894130-3.783096$

H $3.702882-4.251107-3.597308$

H $3.802743-5.395694-2.250424$

C $2.221663-3.684217-0.230390$

H $-0.288904-0.602387-4.629293$

H -2.980315 -0.906878 -4.339041

H $-4.119575-3.686659-2.685159$

H $3.163129-4.242139-0.182779$

H $1.444112-4.3050120 .228745$

H $2.535720-2.623016-2.099790$

C $-3.750763-2.4518071 .430201$

O $-4.402176-3.0633390 .309726$

C -5.012706 -2.151701-0.610799

C $-6.057960-1.2915310 .113138$

C $-5.467683-0.5907011 .331794$

C $-4.744166-1.5940012 .227480$

H $-2.915465-1.8337271 .079478$

C -3.187490 -3.577512 2.312873

H $-5.561327-2.768855-1.332827$

O $-6.610123-0.332098-0.782142$

H $-6.883227-1.9367390 .441474$

H $-4.8053590 .224506 \quad 1.026570$

O

H $-5.489108-2.2350942 .715239$

O $-4.062938-0.8888373 .263201$

C -1.995270 -4.273309 1.691528

H $-2.853741-3.1790583 .277221$

H -3.963910 -4.333662 2.480685

H $-7.2417930 .207934-0.276860$

H -6.1428960 .4250922 .856647$

H -3.373195 -0.3438882 .848155$

O $-1.674160-5.3612022 .453564$

O $-1.405202-3.9060090 .685958$

C $-0.544628-6.1180922 .006787$

H $-0.490220-7.0316852 .604679$

H $-0.654511-6.3957830 .953922$

H $\quad 0.371763-5.5415882 .161867$

SCF Energy (B3LYP/6-31G**//MMFF)= -3245.92133637

0500023

MM̄FF Geometry

C $-3.609101-2.919428-2.041146$

C $-2.534313-2.264326-2.509177$

C $-1.090400-2.717253-2.516060$

O $-0.327999-1.758547-1.735803$

C $-0.829695-4.111643-1.913048$

C $0.620838-4.552622-1.993188$

C $1.397309-4.822870-0.925188$

C $1.182878-4.714869-3.382339$ 
C $1.020336-4.7681650 .528430$

C $1.286891-3.417868 \quad 1.209622$

C $2.774942-3.046698 \quad 1.315581$

C $2.958649-1.7961382 .178587$

C $4.428010-1.3493942 .239982$

O $3.281023-2.7948940 .002698$

O $4.460058-0.0170452 .775230$

C $4.996571-1.2739380 .805923$

C $5.247398-2.2213813 .198757$

O $6.401122-1.0336520 .826137$

C $4.688706-2.522073-0.045894$

C $5.073031-2.352909-1.536478$

O $5.443842-3.631496 \quad 0.457111$

C $0.044374-0.598515-2.341523$

O $-0.162078-0.270412-3.499036$

C $0.7252810 .237108-1.329563$

C $0.7802281 .565037-1.510309$

C $1.3956352 .533411-0.602137$

C 2.2848461 .9966890 .486049

C $4.376883-1.201376-2.270944$

C $5.2000240 .074864-2.345572$

C $5.0484112 .434556-3.137662$

O $6.3745540 .143306-1.994450$

C $4.806343 \quad 3.320041-1.956419$

C $4.228674 \quad 4.527846-2.045483$

C $4.0252955 .447359-0.875254$

C $2.5908585 .904895-0.732400$

C $2.198307 \quad 7.104062-1.194386$

C 1.6355835 .0103440 .024015

C $1.157440 \quad 3.846602-0.797904$

N $4.473246 \quad 1.125180-2.891422$

H -3.482827 -3.912024-1.616694

H -2.680201 -1.278566 -2.943667

H $-0.741660-2.702108-3.555935$

H $-1.435457-4.863294-2.435561$

H $-1.167713-4.107121-0.871640$

H $2.425111-5.135542-1.106485$

H $2.169775-5.189947-3.378144$

H $\quad 0.522352-5.342964-3.988975$

H $1.290622-3.742212-3.872055$

H $-0.034789-5.0312690 .661124$

H $1.575735-5.5551901 .054595$

H $0.744523-2.6313160 .671584$

H $\quad 0.863192-3.4708012 .219568$

H $3.309219-3.8963251 .756914$

H $2.568011-1.9600273 .190275$

H $2.363336-0.9744041 .758065$

H $4.034739-0.0352033 .649827$

H $4.538937-0.4001730 .329700$

H $4.828979-2.1743254 .211299$

H $6.276675-1.8557993 .284073$

H $5.277208-3.2721612 .903058$

H $6.549657-0.2267821 .348301$

H $6.163377-2.300731-1.641139$

H $4.777483-3.278372-2.051966$

H $5.520153-4.296860-0.246651$

H $1.135351-0.257715-0.456982$

H $0.3040021 .990506-2.392905$

H 3.0399791 .3269510 .063040

H 1.6993681 .4410611 .222290

H 2.8359532 .7707481 .023971

H $4.202630-1.510424-3.309727$

H $3.401218-0.982182-1.827740$

H $4.5866652 .825420-4.050013$

H $6.1251582 .333317-3.307823$

H $5.1491252 .963535-0.987883$

H $3.9130364 .894041-3.019948$

H $4.6805986 .316614-1.018701$

H $4.354426 \quad 4.9823290 .062322$

H $1.1781117 .451827-1.069955$

H $2.8875657 .762593-1.712543$

H $\quad 0.7451225 .5793950 .323951$

H 2.0957514 .7051990 .968108

H $0.5099954 .138335-1.626017$

H $3.4886750 .992172-3.103514$

C $-5.247670-0.942373-2.378683$

O $-4.539391-0.098265-1.452721$

C $-5.286776 \quad 1.116282-1.259584$
C $-6.5035271 .025239-2.176713$

C $-6.698367-0.476321-2.291590$

H $-5.6313371 .111462-0.219693$

C $-4.3765632 .326963-1.501687$

H -7.373959 $1.539476-1.757642$

H $-6.3051321 .461517-3.162479$

C $-7.558440-0.887011-3.473343$

H $-7.156743-0.843182-1.363503$

C $-3.0721492 .317987-0.675075$

$\begin{array}{llll}\text { O } & -4.000710 & 2.365343 & -2.885437\end{array}$

H $-4.9342403 .252082-1.316304$

H $-2.4102051 .517229-1.025687$

O $-2.3855963 .549438-0.930764$

C -3.2790132 .1785340 .843468$

C $-1.9526792 .267476 \quad 1.624738$

H -3.7566311 .2155311 .047868$

O $-4.167076 \quad 3.205624 \quad 1.280233$

H -1.2677101 .5058091 .236126$

H $-1.4888243 .244593 \quad 1.443543$

H $-8.559722-0.451740-3.389687$

H $-7.124318-0.552342-4.421498$

H -7.667137 -1.975069 -3.518359

C $-5.032593-2.411362-2.003977$

H $-3.545193 \quad 1.530303-3.089279$

H -2.251022 $3.611555-1.892004$

H -4.4688402 .9744512 .174709$

H -5.633111 -3.051566 -2.660279

H $-5.396382-2.568783-0.980775$

H $-4.850395-0.741540-3.382288$

C $-2.286485-0.306037 \quad 3.259907$

$\begin{array}{llll}\text { O } & -2.924852 & 0.957923 & 3.478557\end{array}$

C -2.124624 2.0986813 .150686

C -0.8138012 .0786593 .953768$

C $-0.081160 \quad 0.741704 \quad 3.846580$

C -1.033857 -0.4167264 .133704$

H $-2.009931-0.4038942 .202756$

C -3.306434 -1.398962 3.598574

H -2.688361 $2.973983 \quad 3.497259$

O $0.045316 \quad 3.145614 \quad 3.565158$

H -1.054218 $2.248113 \quad 5.011223$

H 0.3861690 .6157532 .865024

$\begin{array}{lllll}O & 0.977946 & 0.704552 & 4.804307\end{array}$

H $-1.307063-0.4174545 .196551$

O $-0.371213-1.6467023 .857285$

C $-4.357084-1.4957202 .515980$

H $-2.842990-2.3883533 .676083$

H $-3.790818-1.182136 \quad 4.558318$

H 0.4033532 .9432082 .684572

H $1.553516 \quad 1.468695 \quad 4.628381$

H $\quad 0.427063-1.6767794 .412085$

O $-5.597901-1.298523 \quad 3.043909$

O $-4.103048-1.7220601 .339803$

C $-6.679943-1.3663372 .109606$

H -7.614016 -1.229682 2.660695

H $-6.589144-0.5665291 .369320$

H $-6.700943-2.3449611 .620376$

SCF Energy (B3LYP/6-31G**//MMFF)= -3245.91295595

0500024

MM̄FF Geometry

C $-1.582078-3.462626-2.290491$

C $-0.816881-3.095802-1.252258$

C $0.540191-2.471613-1.401740$

O $0.575820-1.218435-0.680187$

C $1.709818-3.339051-0.892077$

C $1.547488-4.0459910 .440621$

C $1.853452-3.5199481 .643214$

C $1.090984-5.4815360 .351761$

C $2.304056-2.1254181 .967626$

C $3.763204-2.0329252 .430509$

C $4.788903-2.1685511 .292263$

C $6.206163-1.9425881 .829730$

C $7.259807-1.9094950 .711888$

O $4.475831-1.186810 \quad 0.299034$

O $8.469238-1.3628731 .260922$

C $6.787246-0.936448-0.390679$

C $7.608490-3.3181090 .217294$

O $7.664268-0.971794-1.513650$ 
C $5.336732-1.195622-0.846506$

C $4.812437-0.123531-1.830227$

O $5.285534-2.453302-1.531423$

C $0.034917-0.121530-1.272499$

O $-0.593053-0.080719-2.317789$

C $0.3737441 .044304-0.425903$

C $-0.1862252 .235272-0.681326$

C $0.068287 \quad 3.480866 \quad 0.045438$

C 1.2069543 .4949681 .031582

C $4.7000511 .290424-1.254776$

C $4.0865332 .264081-2.248969$

C $3.4876454 .665068-2.480942$

O $3.7912311 .955486-3.400188$

C $2.8571005 .681944-1.583949$

C $1.6741356 .262251-1.837986$

C $1.0515817 .302360-0.950740$

C $-0.3915117 .002593-0.609876$

C -1.391478 $7.729117-1.137667$

C $-0.672388 \quad 5.917214 \quad 0.405962$

C $-0.714664 \quad 4.547904-0.217292$

N $3.9148793 .524610-1.691116$

H -1.208263 -3.332297 -3.303052

H - $1.196962-3.216700-0.242688$

H $0.749641-2.251483-2.457484$

H $2.605829-2.712246-0.845059$

H $1.932741-4.099099-1.653506$

H $1.734937-4.1550282 .520752$

H $0.993870-5.9503291 .336781$

H $0.116221-5.551457-0.139064$

H $1.811340-6.071871-0.224290$

H $2.133131-1.4341991 .142074$

H $1.665811-1.7678782 .785206$

H $3.886475-1.0453972 .895113$

H $3.964725-2.7796893 .208276$

H $4.700244-3.1680960 .851756$

H $6.461916-2.7025692 .578641$

H $6.242951-0.9799902 .359366$

H $8.737086-1.9211992 .010909$

H 6.8559920 .0761550 .026162

H $8.032874-3.9158171 .033145$

H $8.378783-3.284775-0.560805$

H $6.745796-3.862900-0.171560$

H $8.564032-0.805559-1.183892$

H $5.429615-0.115671-2.738079$

H $3.810022-0.426392-2.166382$

H $4.530083-2.448431-2.141751$

H $1.081460 \quad 0.892125 \quad 0.381233$

H $-0.9090852 .306778-1.493208$

H $2.125102 \quad 3.1175120 .569224$

H $0.9755862 .868357 \quad 1.898243$

H 1.4464054 .4922521 .406195

H $4.0744701 .285328-0.354988$

H $5.6905991 .673234-0.988171$

H $2.8105614 .328132-3.273088$

H $4.3827295 .085537-2.950639$

H $3.4078005 .969835-0.691234$

H $1.1365405 .998246-2.745942$

H $1.1222068 .266066-1.471958$

H $1.6197087 .423806-0.019971$

H -2.427027 $7.537301-0.876764$

H - $1.1981468 .527678-1.846139$

H -1.651430 6.0904790 .873270

H 0.0444115 .9960611 .227588

H $-1.514724 \quad 4.431829-0.950345$

H $4.3459453 .708832-0.789164$

C $-4.007186-3.069994-2.722027$

O $-4.062117-1.879623-1.910193$

C $-5.436910-1.478846-1.762648$

C $-6.240338-2.350077-2.721902$

C $-5.427843-3.630550-2.718350$

H $-5.729341-1.731368-0.736690$

C $-5.5790440 .029734-1.990523$

H -7.271694 -2.493839-2.385555

H $-6.272809-1.923113-3.730903$

C $-5.736949-4.548481-3.887836$

H -5.618834 -4.165990 -1.778505

C $-4.6736680 .894749-1.088746$

O $-5.2355140 .317960-3.352079$
H -6.629453 $0.320225-1.869820$

H -3.624964 $0.760776-1.379957$

O $-4.9814862 .268555-1.361253$

C $-4.826736 \quad 0.630532 \quad 0.421086$

C $-4.004144 \quad 1.618503 \quad 1.266758$

H $-4.509690-0.3926570 .636354$

$\begin{array}{lllll}\text { O } & -6.206808 & 0.741629 & 0.765369\end{array}$

H -2.974071 1.6168610 .895312

H $-4.4006212 .630753 \quad 1.122165$

H $-6.787725-4.855541-3.868130$

H $-5.549154-4.053949-4.846812$

H $-5.120978-5.452237-3.848905$

C $-2.966068-4.030946-2.149100$

H $-5.322891 \quad 1.278698-3.475769$

H $-5.8905202 .430678-1.055444$

H -6.3239520 .3493841 .646741$

H -2.998658 -4.990252 -2.677313

H -3.186757 -4.229722 -1.093592

H $-3.722537-2.753420-3.734568$

C $-2.207235-0.2879172 .845262$

O $-3.596827-0.0279183 .077158$

C -4.0253501 .3053332 .780993$

C $-3.2344502 .305048 \quad 3.633622$

C $-1.731474 \quad 2.137008 \quad 3.441461$

C -1.3246870 .6794843 .649796$

H $-1.989906-0.2015151 .773687$

C -1.926144 -1.736574 3.280776

H $-5.070612 \quad 1.370747 \quad 3.106415$

O $-3.622411 \quad 3.637121 \quad 3.313821$

H -3.478186 2.1441294 .691961

H -1.4232642 .5035572 .457742$

O -1.0669792 .9573864 .404660$

H $-1.3837100 .437667 \quad 4.719004$

$\begin{array}{lllll}\text { O } & 0.032081 & 0.505849 & 3.246673\end{array}$

C -2.565803 -2.7708172 .380080$

H $-0.847503-1.9278533 .272669$

H -2.316215 -1.9009384 .292389$

H -3.076177 4.2361023 .851223

H $-0.1155852 .929902 \quad 4.207977$

H $0.587991 \quad 1.034216 \quad 3.843325$

O $-2.197655-4.0139262 .813482$

O $-3.293351-2.531795 \quad 1.427421$

C $-2.733321-5.1150152 .072730$

H $-2.492748-6.0352672 .611569$

H -3.821376 -5.0341231 .988556$

H $-2.270701-5.1575331 .083265$

SCF Energy (B3LYP/6-31G**//MMFF) $=-3245.91131593$

\section{5}

MM̄FF Geometry

C $-5.435846 \quad 2.259403 \quad 1.124335$

C $-4.1767732 .642047 \quad 0.864193$

C -3.185221 3.0160721 .936053

O $-2.0303642 .153436 \quad 1.821692$

C -2.7123494 .4669701 .735766$

C $-1.738136 \quad 4.9298202 .802380$

C $-0.432818 \quad 5.1984352 .598999$

C $-2.331995 \quad 5.121244 \quad 4.175902$

C 0.3601655 .1188541 .323161

C 1.4649284 .0632311 .417044

$\begin{array}{llll}\text { C } 2.477527 & 4.129414 & 0.262781\end{array}$

C $1.8482523 .829119-1.099892$

C $2.8981503 .785960-2.222966$

$\begin{array}{lllll}\text { O } & 3.497019 & 3.162019 & 0.554103\end{array}$

O $2.2889793 .196097-3.381131$

C $4.0473482 .850876-1.794418$

C $3.3434255 .191608-2.643187$

O $5.1143612 .881796-2.738578$

C $4.5866503 .152927-0.380700$

C 5.6118092 .1018390 .104066

O $5.2621774 .416430-0.392388$

C $-2.094756 \quad 0.937927 \quad 2.430778$

$\begin{array}{lllll}\text { O } & -3.044020 & 0.462081 & 3.032313\end{array}$

$\begin{array}{lll}\text { C }-0.789842 & 0.272827 & 2.225870\end{array}$

C $-0.658550-1.0372882 .480643$

C $0.552949-1.8311572 .267717$

C $1.819540-1.0935551 .917378$

$\begin{array}{llll}\text { C } & 5.101930 & 0.659159 & 0.118474\end{array}$ 
C $6.037495-0.276938 \quad 0.865218$

C $6.266723-2.6616781 .524161$

$\begin{array}{llll}\text { O } & 7.075265 & 0.093023 & 1.407118\end{array}$

C $5.313429-3.7878771 .752751$

C $4.738236-4.0486682 .935836$

C $3.740753-5.1510803 .151755$

C $2.348292-4.6071633 .399422$

C $1.854997-4.5596974 .649042$

C $1.585749-4.1717452 .161785$

C $0.484116-3.1728172 .398757$

N $5.557581-1.578791 \quad 0.869339$

H -5.7768412 .2178022 .156153$

H -3.832690 $2.683149-0.166303$

H -3.6279282 .9062572 .933730$

H -3.577177 5.1439351 .743188

H -2.271476 4.5655770 .738852

H $0.152323 \quad 5.5366813 .454415$

H -1.623194 5.5762134 .876263

H -3.2055515 .7794254 .123736$

H -2.641692 4.161879 4.601063

H $-0.274143 \quad 4.9116100 .459279$

H $\quad 0.808673 \quad 6.104659 \quad 1.148694$

H $2.014706 \quad 4.1788782 .361360$

H 1.0222813 .0603031 .462290

H $2.929393 \quad 5.1283620 .266557$

H $1.0684184 .557459-1.349650$

H $1.3456392 .853498-1.054895$

H $1.4986373 .717649-3.603152$

H $3.645617 \quad 1.830249-1.812928$

H $2.4883435 .767711-3.016940$

H $4.0593355 .148212-3.471395$

H $3.7919695 .762845-1.828137$

H $4.7345242 .683431-3.611579$

H $6.5275642 .162319-0.498527$

H 5.9166182 .3740961 .124462

H 5.8733594 .4464290 .362306

H $\quad 0.021760 \quad 0.873423 \quad 1.832181$

H $-1.525246-1.5893542 .841478$

H $2.029954-0.3144302 .658935$

H $1.734896-0.6240950 .931748$

H $2.701153-1.7372721 .897042$

H 4.1214330 .6083360 .604665

H $5.0015370 .284860-0.906299$

H $6.706129-2.2923332 .457235$

H $7.084836-2.9791540 .869739$

H $5.071110-4.4080900 .894838$

H $4.975082-3.4232263 .793508$

H $4.080327-5.7547504 .003093$

H $3.712302-5.8383102 .296529$

H $0.856344-4.1973224 .864523$

H $2.444593-4.8927185 .497443$

H $\quad 1.147680-5.0638931 .695359$

H $2.279478-3.7777171 .411195$

H $-0.470354-3.6248172 .669749$

H $4.781509-1.8112540 .255380$

C $-6.8148960 .397166 \quad 0.185563$

O $-5.707850-0.413824-0.256624$

C $-6.215764-1.505841-1.048556$

C $-7.728513-1.492209-0.867554$

C $-7.994099-0.008511-0.696998$

H $-5.989034-1.268821-2.094429$

C $-5.524579-2.813428-0.644598$

H $-8.252503-1.928150-1.723512$

H $-8.036310-2.039848 \quad 0.030660$

C $-9.3543780 .297696-0.094810$

H $-7.9150400 .479327-1.677827$

C $-3.984552-2.750816-0.719112$

O $-5.877587-3.1301930 .708589$

H $-5.900401-3.641121-1.256350$

H $-3.616748-2.0378950 .026494$

O $-3.465299-4.032242-0.344441$

C $-3.432713-2.400328-2.112807$

C $-1.898862-2.419067-2.219916$

H $-3.800287-1.423182-2.429943$

O $-3.944629-3.334997-3.069791$

H $-1.537999-3.444350-2.056844$

H -1.631656 -2.216694 -3.264557

H - $-10.154049-0.081149-0.739643$
H $-9.470284-0.1650500 .891038$

H $-9.497070 \quad 1.3765840 .020737$

C -6.4377301 .8745110 .071007$

H $-5.607469-2.380077 \quad 1.265995$

H $-3.823810-4.2442240 .534580$

H $-3.667833-4.224086-2.788288$

H -7.3234362 .5056750 .204108$

H $-6.0338732 .080103-0.928013$

H -7.0114020 .1273141 .231903$

C $0.998497-1.584451-2.426230$

O $0.258226-1.836406-1.226707$

C $-1.129114-1.470154-1.278359$

C $-1.2350760 .034497-1.562464$

C $-0.500667 \quad 0.412688-2.845423$

C $0.940753-0.096204-2.793389$

H $0.592942-2.188864-3.246840$

C $2.445309-2.035193-2.171853$

H -1.505492 -1.644353-0.267209

$\begin{array}{llll}\mathrm{O} & -2.581856 & 0.474322 & -1.638158\end{array}$

H $-0.7699440 .589996-0.738497$

H $-1.0201380 .038002-3.734792$

O $-0.472672 \quad 1.835782-2.968720$

H $1.5074190 .491186-2.060102$

$\begin{array}{llll}\text { O } & 1.554820 & 0.098671 & -4.064777\end{array}$

C $2.561481-3.540620-2.050147$

H $3.090170-1.723197-3.000667$

H $2.814865-1.599078-1.236850$

H $-3.0066420 .263166-0.789087$

H $-1.3942902 .145806-2.947334$

H $1.503071 \quad 1.048435-4.268285$

O $3.877508-3.871240-1.890337$

O $1.626305-4.328274-2.089000$

C $4.147146-5.272700-1.782919$

H $3.846213-5.786881-2.700467$

H $5.224008-5.402019-1.645261$

H $3.629148-5.694009-0.915804$

SCF Energy (B3LYP/6-31G**//MMFF)= -3245.93364119

0500026

MM̄FF Geometry

C $0.282712 \quad 5.356123 \quad 1.019717$

C $1.165661 \quad 4.783413 \quad 0.187836$

$\begin{array}{llll}\text { C } & 2.316800 & 3.928036 & 0.653314\end{array}$

$\begin{array}{lllll}\text { O } & 2.196853 & 2.619726 & 0.046414\end{array}$

C $3.643547 \quad 4.5524490 .183708$

C 4.8761583 .8148160 .668919

C $5.771500 \quad 3.208478-0.135116$

C 5.0925693 .8116242 .160700

C $5.7653723 .123678-1.635810$

C $5.0881631 .866909-2.204462$

C $5.7764080 .549496-1.811598$

C $5.191720-0.624070-2.599741$

C $5.817459-1.962572-2.176384$

$\begin{array}{lllll}\text { O } & 5.563956 & 0.325477 & -0.416963\end{array}$

O $4.999922-3.019139-2.705244$

C $5.765672-2.087020-0.635869$

C $7.210085-2.149098-2.790482$

O $6.506651-3.218801-0.189208$

C $6.243050-0.8218710 .111575$

C $5.953185-0.8552181 .629588$

$\begin{array}{llllllll}\text { O } & 7.664816 & -0.665131 & 0.020128\end{array}$

C 1.3306621 .7388840 .618463

$\begin{array}{lllll}\text { O } & 0.628932 & 1.926930 & 1.599319\end{array}$

C $1.3704990 .491213-0.177160$

C $0.406319-0.427706-0.017263$

C $0.290834-1.693893-0.745040$

C $1.456093-2.115016-1.599531$

C $4.477803-0.9980182 .019887$

C $4.054754-2.4288572 .309938$

C $2.038823-3.7813802 .866530$

O $4.843918-3.3676492 .370183$

C $1.518848-4.4240721 .620178$

C $0.250209-4.8365351 .478573$

$\begin{array}{llll}\text { C }-0.274020 & -5.525419 & 0.252227\end{array}$

C $-1.477118-4.826842-0.338687$

C $-2.723510-5.254657-0.075987$

C $-1.228337-3.691606-1.305113$

C $-0.843099-2.413397-0.613174$ 
N $2.687043-2.5284192 .528898$ H 0.3876535 .2144182 .092522 H $1.0486554 .914203-0.885513$ H 2.3139793 .8259701 .745250 H 3.7106385 .5903380 .536931 H $3.6349934 .605759-0.910361$ H 6.6239792 .7160740 .332071 H 6.0715453 .4055352 .437463 H 5.0405024 .8316412 .554962 H 4.3332483 .2026502 .660337 H $5.2932794 .010007-2.072358$ H $6.8066253 .158578-1.981208$ H $4.0413881 .849585-1.878800$ H $5.0851411 .957416-3.297918$ H $6.8503510 .647958-2.008639$ H $5.313816-0.469325-3.679090$ H $4.108603-0.675381-2.425759$ H $4.950051-2.905944-3.669946$ H $4.718213-2.275725-0.373431$ H $7.147338-2.158524-3.885445$ H $7.642195-3.115468-2.508471$ H $7.910672-1.359488-2.510913$ H $6.153273-3.997739-0.652167$ H $6.574265-1.6178062 .115292$ H $\quad 6.3007250 .1031442 .042817$ H $8.037201-1.450109-0.412094$ H $2.1800340 .373028-0.888010$ H $-0.394215-0.2225610 .692410$ H $2.384271-2.088859-1.018787$ H $1.561205-1.448968-2.462546$ H $1.371387-3.134460-1.981375$ H $4.310178-0.4365672 .948122$ H $3.822204-0.5704921 .255383$ H $1.241757-3.5532103 .580447$ H $2.759470-4.4480443 .351333$ H $2.224310-4.5819810 .807747$ H $-0.446878-4.7029142 .302773$ H $-0.540528-6.5521330 .535840$ H $\quad 0.503327-5.625287-0.515488$ H -3.590830 -4.778914 -0.521201 H $-2.904658-6.0878840 .594941$ H -2.141253 -3.483256 -1.879271 H - $0.497211-4.011624-2.052341$ H -1.627849 -2.0229780 .033935$ H $2.100586-1.7084362 .403406$ C $-2.187895 \quad 5.3890260 .694785$ O $-2.1896284 .321783-0.272701$ C $-3.5116974 .182924-0.822630$ C -4.4092015 .0923190 .009353$ C $-3.446808 \quad 6.198506 \quad 0.398897$ H $-3.4696994 .559024-1.852394$ C -3.940151 $2.708768-0.834033$ H $-5.2729505 .452969-0.557342$ H $-4.7839414 .578440 \quad 0.902678$ C -3.931395 7.0418831 .564843 H $-3.2820756 .845874-0.472907$ C $-3.1001151 .815603-1.772236$ O H -4.988452 $2.664695-1.148714$ H -3.227867 2.146536 -2.810086 O $-1.7054871 .951020-1.472440$ C -3.404698 $0.309535-1.669340$ C $-4.881209-0.057141-1.875015$ H -3.048079 -0.071898 -0.707548 O $-2.644781-0.361168-2.682767$ H $-5.5019000 .492659-1.162898$ H $-5.1854280 .254205-2.881734$ H -4.8748397 .5379871 .314209$ H -4.099524 6.4321672 .458958 H -3.200146 7.8159861 .817765 C $-0.887962 \quad 6.1802740 .559270$ H -2.9516912 .2221630 .779199$ H -1.452637 2.873919-1.643048 H -1.711142 $-0.123568-2.548567$ H $-0.9247507 .092227 \quad 1.165166$ H $-0.7431606 .486739-0.484262$ H $-2.255470 \quad 4.9238291 .687513$ C $-5.461190-1.6440000 .677056$
O $-4.691065-2.071962-0.452970$

C $-5.139357-1.574868-1.719683$

C $-6.593116-1.998956-1.966283$

C $-7.506026-1.598490-0.809980$

C $-6.926468-2.0712600 .523029$

H $-5.401133-0.5537260 .775993$

C $-4.837226-2.2821701 .928612$

H $-4.529226-2.080883-2.478324$

O $-7.069591-1.436528-3.183833$

H -6.626162 -3.090069-2.082666

H $-7.676148-0.515919-0.800211$

O $-8.793463-2.191097-0.998791$

H $-7.009353-3.1634570 .593477$

O $-7.693197-1.5141191 .588485$

C $-3.517846-1.6437442 .308678$

H $-5.498057-2.1764762 .795558$

H $-4.659001-3.3478541 .741305$

H -7.997932 -1.707480 -3.286417

H $-8.681063-3.156872-1.013167$

H $-8.619368-1.7757231 .448054$

O $-2.827990-2.4955823 .123634$

O $-3.150382-0.5280541 .967826$

C $-1.571390-2.0053273 .601513$

H $-0.880924-1.8597592 .766163$

H $-1.710908-1.0717274 .154866$

$\mathrm{H}-1.151382-2.7532164 .279100$

SCF Energy (B3LYP/6-31G**//MMFF) $=-3245.92521537$

0500027

MM̄FF Geometry

C $-3.713377-1.824987-2.782294$

C $-2.598030-2.134508-2.103402$

C $-1.218060-2.025775-2.699398$

O $-0.456529-1.083214-1.903244$

C $-0.498822-3.385597-2.657045$

C $0.541136-3.505531-3.753844$

C $1.859811-3.278102-3.604972$

C $0.000155-3.930007-5.096952$

C $2.594832-2.849147-2.366485$

C $3.750260-3.815128-2.060012$

C $4.634042-3.394027-0.874210$

C $3.906508-3.5193420 .465555$

C $4.768414-3.0208771 .633966$

O $5.065139-2.050805-1.100884$

O $3.921548-2.8987802 .786105$

C $5.275792-1.6048351 .290103$

C $5.866053-4.0210332 .012692$

O $6.173728-1.1287152 .289639$

C $5.939650-1.513185-0.101902$

C $6.255257-0.053117-0.505298$

O $7.181573-2.225708-0.072756$

C $-0.635430 \quad 0.239144-2.178508$

O $-1.3168320 .714246-3.073934$

C $0.1108411 .027008-1.169508$

C $-0.1045922 .349962-1.097736$

C $0.4823203 .291710-0.141860$

C 1.5342882 .7827740 .804587

C $5.0225110 .830208-0.720192$

C $5.3907972 .205504-1.249039$

C $4.3449604 .231076-2.265552$

O $6.5278162 .665278-1.185591$

C $3.8851955 .156052-1.183400$

C $2.8804346 .032566-1.331053$

C $2.4376126 .974419-0.249053$

C 0.9526426 .9007520 .025317

C $0.1359607 .912302-0.315466$

C 0.4260535 .6957610 .773561

C $0.0392154 .567021-0.145220$

N $4.2878662 .869318-1.768914$

H -3.634623 -1.497717 -3.816387

H $-2.669274-2.447847-1.065403$

H -1.273208 -1.662836 -3.733786

H -1.216703 -4.206135 -2.792268

H $-0.048336-3.549139-1.671493$

H $2.495502-3.390159-4.483041$

H $0.784135-4.005338-5.857981$

H $-0.477643-4.912166-5.018876$

H $-0.741342-3.211406-5.459589$ 
H $2.987629-1.841901-2.548253$ H $1.928545-2.775122-1.504737$ H $3.361473-4.826897-1.889672$ H $4.393199-3.868806-2.949455$ H $5.513969-4.048425-0.879025$ H $3.582724-4.5530840 .637981$ H $2.990431-2.9169860 .448726$ H $3.526676-3.7699272 .962542$ H $4.403272-0.940072 \quad 1.315533$ H $5.423111-4.9750452 .323198$ H $6.443839-3.6650372 .872791$ H $6.558328-4.2326461 .195512$ H $5.718950-1.2013893 .146173$ H $6.930250 \quad 0.4031530 .230180$ H $\quad 6.823125-0.067207-1.446579$ H $7.747910-1.890884-0.787440$ H $0.7761540 .497603-0.498059$ H $-0.8132962 .793686-1.796828$ H 2.2522302 .1353790 .292886 H 1.0691292 .2141791 .613758 H $2.127823 \quad 3.5811221 .256397$ H $4.3377050 .356160-1.432453$ H 4.4888250 .9798390 .223820 H $3.7064634 .281313-3.152969$ H $5.3708364 .478081-2.555860$ H $4.4167345 .111741-0.235343$ H $2.3645036 .096036-2.286271$ H $2.7145477 .989761-0.561036$ H $2.981598 \quad 6.791194 \quad 0.686134$ H $-0.9261527 .878214-0.095429$ H $0.5088968 .793883-0.826094$ H -0.4761835 .9785981 .333129$ H 1.1469775 .3992651 .540439 H $-0.7368754 .840963-0.861557$ H $3.3766552 .421969-1.731174$ C $-5.632873-0.441274-1.998121$ O $-4.866801 \quad 0.215008-0.968528$ C $-5.7637950 .932640-0.100691$ C $-7.1075750 .956398-0.817085$ C $-7.084522-0.386278-1.525765$ H $-5.870026 \quad 0.3299140 .809116$ C -5.1714372 .3010350 .255253$ H -7.946864 $1.063110-0.123354$ H -7.165952 $1.770530-1.548520$ C -8.104185 -0.500755 -2.645015 H $-7.269671-1.178013-0.787133$ C $-3.7261302 .227756 \quad 0.792013$ O $-5.150423 \quad 3.109736-0.927925$ H -5.8265052 .8149170 .968012$ H -3.059384 $1.920613-0.022092$ O -3.3180743 .5529121 .151885$ C -3.5263261 .2917141 .998201$ C -2.0535301 .2188952 .429233$ H $-3.859540 \quad 0.2851691 .741919$ O $-4.306206 \quad 1.7406293 .103396$ H $-1.454147 \quad 0.9424491 .556165$ H -1.718702 2.211980 2.752718 H $-9.120038-0.387645-2.252517$ H $-7.950961 \quad 0.269973-3.408024$ H -8.038309 -1.477311 -3.134840 C $-5.097256-1.859653-2.196644$ H -4.757276 $3.966095-0.686616$ H $-3.8511953 .825047 \quad 1.918611$ H -5.243139 1.6349392 .868064 H -5.745191 -2.423531-2.876763 H $-5.085567-2.393173-1.239796$ H $-5.5058360 .149653-2.915023$ C -1.674573 -1.816912 2.277927 O $-2.328494-1.0853673 .322576$ C $-1.801425 \quad 0.219827 \quad 3.583076$ C $-0.327907 \quad 0.103498 \quad 3.986999$ C $0.482101-0.6337912 .925907$ C $-0.172095-1.9676892 .570510$ H -1.826621 -1.304814 1.319894 C -2.345158 -3.200579 2.201499 H -2.3481050 .6005064 .455024$ O $\quad 0.221527 \quad 1.3966904 .215315$ H $-0.258194-0.4473944 .934122$
H $\quad 0.624861-0.0160732 .034224$

O $1.788268-0.8744953 .452921$

H $-0.007809-2.6707683 .397332$

O $0.499708-2.4858711 .423496$

C $-3.791482-3.1478521 .757020$

H -1.818659 -3.842336 1.487529

H -2.326894 -3.668595 3.193375

H $1.162021 \quad 1.2788164 .432876$

H $2.282405-1.3781342 .783353$

H $\quad 0.254308-3.4209421 .328424$

O $-4.312126-4.4107281 .804093$

O $-4.390861-2.1427381 .405548$

C $-5.682841-4.5264111 .409371$

H -6.315817 -3.890087 2.035410

H $-5.793987-4.2634600 .353483$

H $-5.988974-5.5670511 .545963$

SCF Energy (B3LYP/6-31G**//MMFF) $=-3245.92579132$

0500028

MM̄FF Geometry

C $0.147980-2.667393-1.794070$

C $-0.935611-3.435352-1.610914$

C $-1.783331-3.393425-0.370017$

O $-3.099080-2.965900-0.797149$

C $-1.898693-4.7741540 .301055$

C $-0.633627-5.2507540 .980711$

C $-0.322598-4.9709292 .261615$

C $0.253325-6.1545470 .165351$

C -1.103807-4.100592 3.209324

C $-0.205929-3.3413044 .196307$

C $0.686593-2.2735713 .541953$

C $1.714057-1.7722754 .561478$

C $2.609272-0.6744113 .978645$

O $-0.144118-1.1869063 .111662$

O $3.297792-0.0666945 .084940$

C 1.7133360 .4045223 .338540

C $3.704246-1.2600803 .080376$

O $2.496113 \quad 1.3702662 .641960$

C $0.567484-0.1358492 .443312$

C $-0.430184 \quad 1.0145682 .173959$

O $1.038126-0.5522071 .162224$

C $-3.899630-2.3917990 .139454$

O $-3.652612-2.2389191 .324775$

C $-5.139515-1.950477-0.533594$

C $-5.985725-1.1566100 .138106$

C $-7.230402-0.585594-0.371051$

C $-7.688918-0.993163-1.745452$

C $-1.6489020 .631017 \quad 1.324616$

C -2.4249611 .8691540 .899257$

C $-4.0490882 .660297-0.811866$

O -2.2492172 .9737891 .408990$

C $-5.3957542 .872738-0.194717$

C $-6.5139683 .018155-0.923249$

C $-7.8774563 .286707-0.347054$

C $-8.9049202 .270084-0.797599$

C $-9.6238252 .469354-1.915425$

C $-9.146246 \quad 1.0542190 .070101$

C -7.8903690 .3007440 .402498$

$\mathrm{N}-3.3333181 .605121-0.116205$

H $0.434094-1.958539-1.022809$

H - $1.230186-4.136289-2.388454$

H -1.380840 -2.6544070 .335133$

H $-2.231147-5.521445-0.432996$

H $-2.717158-4.7602441 .032551$

H $0.579657-5.4168462 .677828$

H $1.110074-6.5232220 .739582$

H $-0.309413-7.027985-0.179958$

H $0.648864-5.628848-0.707858$

H - $-1.732242-3.3842372 .675039$

H -1.770705 -4.750354 3.788812

H $-0.858289-2.8588284 .935772$

H $\quad 0.415126-4.0654844 .738857$

H $1.194424-2.7050252 .672554$

H $2.326974-2.6006784 .939086$

H $1.188046-1.3660435 .437245$

H 3.8162070 .6788394 .735713

H $1.2517280 .949081 \quad 4.174239$

H $4.369232-1.9062113 .666836$ 
H $4.345069-0.4724962 .669863$ H $3.316089-1.8602982 .255882$ H $2.7645110 .977912 \quad 1.794710$ H $-0.797436 \quad 1.4143983 .128352$ H 0.0982431 .8285351 .662675 H $1.939697-0.8921161 .244203$ H -5.305884 -2.261068 -1.558311 H $-5.730612-0.8708461 .158254$ H -8.649297 -0.553698 -2.026476 H -7.812795 -2.080298 -1.803329 H $-6.961120-0.680891-2.502364$ H $-1.333803 \quad 0.0924420 .424433$ H -2.320735 -0.0124561 .900876$ H -4.120146 2.361815 -1.862896 H -3.468369 $3.586856-0.752732$ H -5.4509242 .9451780 .888462$ H $-6.4440532 .974781-2.008334$ H $-8.1857814 .289764-0.670186$ H -7.851578 $3.326870 \quad 0.749005$ H - $-10.377546 \quad 1.758852-2.238070$ H $-9.471575 \quad 3.347447-2.534293$ H $-9.8805650 .375264-0.379950$ H $-9.6043101 .387517 \quad 1.010566$ H $-7.479490 \quad 0.5504541 .381280$ H -3.441159 $0.653721-0.454508$ C $2.456369-3.061478-2.667592$ O $3.070065-1.941454-1.993752$ C $4.374234-1.718796-2.559438$ C $4.696479-2.965092-3.373713$ C $3.324267-3.337537-3.895567$ H $4.281032-0.867969-3.244478$ C $5.393027-1.402015-1.461686$ H $5.421065-2.766155-4.169120$ H $5.095605-3.769381-2.744673$ C $3.236330-4.765603-4.406019$ H $3.057305-2.648356-4.707984$ C $5.054949-0.173122-0.594830$ O $5.504140-2.529417-0.582496$ H $6.385062-1.274161-1.910454$ H $4.203013-0.3896920 .060452$

O $6.175512 \quad 0.0608750 .268684$

C $4.7673171 .118081-1.383871$ C $4.7159932 .366000-0.482434$ H $3.8167591 .005444-1.914402$ O $5.7786991 .315816-2.373123$ H 4.0883592 .1521900 .386479 H $5.7257412 .577650-0.111050$ H $3.927369-4.918172-5.241479$ H $3.490685-5.488061-3.623191$ H $2.225483-4.993342-4.758203$

C $1.009699-2.716800-3.022933$ H $4.618097-2.709205-0.223409$ H $\quad 6.331279-0.7582260 .769639$ H $6.6343631 .368125-1.913505$ H $0.611724-3.449839-3.733908$ H $0.967591-1.732414-3.504102$ H 2.493052 -3.910492 -1.972298 C $1.8464163 .413878-0.678752$ O $2.8530573 .442975-1.698783$ C $4.1955393 .614556-1.229054$ C $4.3095624 .919280-0.427521$ C 3.2911654 .9753750 .705269 C 1.8887684 .6933900 .168666 H $1.9945302 .536209-0.039583$ C $0.4851323 .279472-1.379177$ H $4.8186313 .735530-2.123900$ O $5.6191095 .076754 \quad 0.108664$ H $4.1319615 .772135-1.095713$ H 3.5610684 .2848411 .512986 O $3.3165606 .287257 \quad 1.272535$ H $1.5593215 .551269-0.431162$ O $\quad 0.9844654 .5852201 .266590$ C $0.233217 \quad 1.885085-1.912486$ H $-0.3264853 .501620-0.678653$ H $0.4332663 .973888-2.226177$ H $6.2438155 .057584-0.636276$ H 2.6325456 .3167731 .963334 H 1.2828753 .8491811 .827797
O $\quad-0.979718 \quad 1.873510-2.542404$

O $\quad 0.9859160 .929494-1.787857$

C $-1.3885450 .612206-3.079609$

H $-2.3425270 .755447-3.593891$

H $-0.6522970 .247923-3.801852$

$\mathrm{H}-1.532910-0.109684-2.271180$

SCF Energy (B3LYP/6-31G**//MMFF) $=-3245.92791697$

0500029

MM̄FF Geometry

C $-3.295041 \quad 1.7582052 .351851$

C -2.1444631 .4492111 .734503$

C -0.8572971 .1668832 .462532$

O $-0.409598-0.1475342 .050893$

C 0.2259062 .2042432 .102599

C -0.1087873 .6182692 .528407$

C -0.3937354 .6309911 .686413$

C $-0.069073 \quad 3.8671984 .014784$

C -0.4654954 .5993310 .185161$

C $0.6031345 .486844-0.470550$

C $2.0438835 .023435-0.200762$

C $3.0486475 .975189-0.848371$

C $4.4910615 .493341-0.644296$

O $2.2019243 .715411-0.753027$

O $5.3197916 .276297-1.516891$

C $4.5992094 .014628-1.080509$

C 4.9796475 .7774600 .781896

O $5.8943843 .512479-0.761817$

C $3.4839333 .108837-0.506695$

C $3.4147141 .735716-1.212830$

O $3.6947042 .907783 \quad 0.890771$

C $0.467420-0.7820032 .877532$

O $0.908386-0.3650153 .937479$

C $0.822856-2.0804752 .261964$

C $1.873232-2.7622332 .743428$

C $2.375108-4.0527422 .263737$

C $1.628085-4.7164261 .138613$

C $4.5810260 .776192-0.969908$

C $4.344121-0.570606-1.634230$

C $5.262274-2.865159-1.767072$

O $3.482047-0.753479-2.490149$

C $5.916884-3.820027-0.822748$

C $5.266329-4.832669-0.230105$

C $5.923476-5.8491100 .657255$

C $5.605228-5.6627922 .122458$

C $6.580099-5.3962553 .008871$

C $4.176104-5.8716812 .577182$

C $3.471193-4.5713962 .856658$

N $5.228542-1.540098-1.179032$

H $-3.327568 \quad 1.789477 \quad 3.438214$

H $-2.111303 \quad 1.4258110 .648961$

H -1.036281 1.1526463 .545144

H 0.4477692 .1365331 .031632

H 1.1750361 .9226152 .576944

H -0.6081995 .6082532 .117914$

H -0.2222564 .9226634 .264205$

H -0.8492873 .2935594 .523958$

H $0.903576 \quad 3.5729104 .422673$

H $-0.4057463 .584726-0.216905$

H -1.454033 $4.975086-0.106780$

H $0.4180255 .479117-1.552603$

H $0.4784646 .522207-0.129160$

H 2.2081614 .9836940 .881317

H $2.9263116 .995404-0.463607$

H $2.8516816 .036388-1.928047$

H $6.2264235 .929702-1.451854$

H $4.5275893 .996844-2.176427$

H 4.9020496 .8483821 .005181

H 6.0403025 .5274860 .896272

H $4.4146355 .235587 \quad 1.543354$

H 5.9072943 .3097280 .189225

H $2.4959781 .236446-0.876801$

H $3.2719601 .889542-2.290996$

H $3.2076852 .110907 \quad 1.159103$

H $\quad 0.221545-2.4325991 .432393$

H $2.431293-2.3303453 .573781$

H $2.036404-5.6886420 .855983$

H $1.657645-4.089621 \quad 0.241491$ 
H $\quad 0.581547-4.8852541 .415766$

H $5.5073341 .176510-1.392182$

H 4.7250160 .6169840 .104355

H $5.837712-2.802608-2.696579$

H $4.243689-3.172002-2.027025$

H $6.981960-3.691609-0.646320$

H $4.204998-4.964852-0.423639$

H $7.009606-5.8348730 .496190$

H $5.597677-6.8493600 .344779$

H $\quad 6.365449-5.2926574 .067574$

H $7.612092-5.2737132 .697454$

H $4.178270-6.4543283 .508665$

H $3.646921-6.5017721 .857586$

H $3.924957-4.0059013 .672419$

H $6.022673-1.242870-0.618422$

C -5.6501771 .0613351 .817162$

O $-5.273015-0.1351231 .106918$

C $-6.384651-0.5735480 .304471$

C -7.6039150 .1445300 .864771$

C $-7.001826 \quad 1.4854601 .238229$

H $-6.201329-0.208305-0.713459$

C $-6.466696-2.1032950 .291536$

H $-8.413751 \quad 0.224306 \quad 0.133581$

H -7.997709 -0.3541341 .757786$

C $-7.8580002 .290822 \quad 2.200215$

H -6.8507532 .0695700 .320245$

C $-5.135720-2.795627-0.065187$

O $-6.846563-2.5518131 .598778$

H $-7.265056-2.422662-0.388538$

H $-4.421439-2.6504350 .754248$

O $-5.378766-4.206796-0.125139$

C $-4.500275-2.333648-1.389836$

C $-3.171398-3.057377-1.659411$

H $-4.318159-1.258294-1.342343$

O $-5.418510-2.575833-2.451489$

H $-2.520356-2.898105-0.792615$

H -3.353047 -4.136927 -1.730001

H $-8.8312182 .516316 \quad 1.752102$

H $-8.035743 \quad 1.744718 \quad 3.132642$

H -7.373993 3.2395532 .452363

C -4.5635262 .1200961 .631441$

H $-6.889830-3.5230371 .570448$

H $-5.962029-4.370605-0.886264$

H -5.087395 -2.112774 -3.239349

H $-4.906153 \quad 3.0862662 .019177$

H -4.3590842 .2551460 .563386$

H -5.749686 0.7873892 .875358

C - $-1.461542-0.564338-2.131509$

O $-2.333601-1.191395-3.077669$

C $-2.450280-2.612317-2.952373$

C $-1.071394-3.260768-3.133693$

C $-0.059650-2.697845-2.140395$

C $-0.051472-1.172124-2.191542$

H -1.882503 -0.668098 -1.124039

C $-1.3867970 .933757-2.477296$

H -3.075180 -2.942548 -3.791466

O $-1.169888-4.673005-2.982672$

H $-0.715564-3.068746-4.154211$

H $-0.253518-3.065327-1.126850$

O $1.236838-3.183607-2.496639$

H $0.452924-0.856758-3.113841$

O $0.730144-0.679415-1.105492$

C $-2.6838241 .677885-2.244765$

H $-0.6210681 .418889-1.860709$

H -1.130586 $1.056703-3.536433$

H $-0.273489-5.038468-3.076784$

H $1.877629-2.758740-1.900804$

H $\quad 0.245897-0.860459-0.282735$

O $-2.470023 \quad 3.008847-2.471139$

O $-3.7462931 .170444-1.917361$

C $-3.6095643 .857393-2.301166$

H $-4.4367593 .521883-2.933981$

H $-3.9088763 .876073-1.249592$

H $-3.3265314 .869371-2.602629$

SCF Energy $\left(B 3 L Y P / 6-31 G^{* *} / / M M F F\right)=-3245.93466703$

0500030

MM̄FF Geometry
C $3.955514 \quad 3.8464772 .198031$

C 3.3111622 .9742902 .989150

C 3.7305571 .5674543 .332779

O 2.7352530 .6961312 .737702

C $5.102931 \quad 1.1225092 .786394$

C $5.463725-0.3052973 .154526$

C $5.601993-1.3152662 .271901$

C $5.690548-0.5604404 .623067$

C $5.480450-1.2715360 .772756$

C $4.118837-1.7831140 .300143$

C $4.038199-2.011627-1.217027$

C $4.175632-0.715096-2.019382$

C $3.965889-0.941458-3.526002$

O $2.761055-2.614225-1.476449$

O $3.7735920 .342459-4.141144$

C $2.664447-1.743016-3.741532$

C $5.205579-1.555953-4.186739$

O $2.506400-2.110182-5.109525$

C $2.559081-2.996039-2.846260$

C $1.180870-3.694280-2.941070$

O $3.537739-3.958505-3.257815$

C 1.8562620 .0505463 .548046

O $1.7430230 .157954 \quad 4.757518$

C $1.070120-0.8603312 .686084$

C $0.308816-1.8204823 .231130$

C $-0.449676-2.8330482 .490682$

C $-0.305781-2.839570 \quad 0.992490$

C $-0.021753-2.779667-2.705850$

C $-1.306545-3.542582-2.423367$

C $-3.687368-3.105305-1.783844$

O $-1.371350-4.767174-2.373993$

C $-3.786388-2.892281-0.305998$

C $-4.160337-3.8368870 .569922$

C $-4.225084-3.6037152 .053820$

C $-3.510098-4.6743292 .851308$

C $-4.184987-5.4485223 .718036$

C $-2.022071-4.8612462 .643863$

C $-1.209910-3.7122323 .177006$

N -2.370313 -2.675137 -2.216583

H 4.9109853 .5863921 .753350

H 2.3466853 .2735253 .397849

H 3.7263871 .4796324 .425548

H $5.8901681 .785266 \quad 3.168147$

H 5.1013431 .2397361 .698292

H $5.850147-2.3020392 .661959$

H $6.079749-1.5661924 .814553$

H 6.4195170 .1516735 .023449

H $4.756863-0.4584115 .183816$

H $5.669477-0.2671210 .385372$

H $\quad 6.275767-1.9033520 .357345$

H $3.876964-2.7277580 .806391$

H $3.332559-1.0814610 .601979$

H $4.825865-2.721911-1.494826$

H $5.149809-0.242807-1.847403$

H $3.4279140 .009225-1.669009$

H $4.5544450 .888323-3.945562$

H $1.835970-1.063819-3.504540$

H $6.071300-0.893433-4.066968$

H $5.063050-1.668588-5.267072$

H $5.476528-2.528139-3.769982$

H $2.571416-1.295899-5.637415$

H $1.074471-4.188418-3.915807$

H $1.163051-4.504223-2.198605$

H $3.259434-4.834014-2.941220$

H $1.172455-0.7390321 .613663$

H $0.250986-1.8885094 .316464$

H $0.744674-2.9619310 .705905$

H $-0.680306-1.9016880 .567700$

H $-0.852493-3.6469350 .505063$

H $0.167305-2.120589-1.851967$

H $-0.189715-2.156934-3.591722$

H -3.848976 -4.150288 -2.065990

H -4.420604 -2.478372 -2.300171

H $-3.529363-1.9025110 .063185$

H $-4.440170-4.822340 \quad 0.205213$

H $-5.285070-3.5632772 .335815$

H -3.805270 -2.626226 2.317007

H $-3.686324-6.2210004 .294090$ 
H $-5.251952-5.3279383 .873128$ H - $-1.827411-5.0689511 .588894$ H -1.679762 -5.7641063 .167876$ H -1.245022 -3.626590 4.264072 H $-2.192970-1.675110-2.235180$ C 2.5333665 .1206540 .526690 $\begin{array}{lllll}\text { O } & 1.392423 & 4.265621 & 0.755391\end{array}$ C $1.3885293 .234379-0.251520$ C $2.1195433 .848753-1.431686$ C $3.2416934 .588699-0.725429$ H 1.9630092 .3964430 .164678 C $-0.0541282 .800813-0.518069$ H $2.4855523 .101470-2.141772$ H $1.4710484 .552320-1.968208$ C $3.8813125 .677794-1.569481$ H $4.0165363 .859463-0.460994$ C $-0.1913781 .777033-1.657038$ O -0.5606552 .2120010 .684515$ H -0.670468 $3.683314-0.721632$ H $0.0984092 .238296-2.607962$ O $0.7407190 .710045-1.434834$ C $-1.5889301 .144507-1.795901$ C $-2.7231692 .157779-2.016902$ H -1.797584 $0.515604-0.924013$ O $-1.558524 \quad 0.260983-2.923962$ H $-2.6869032 .934153-1.249692$ H -2.568300 $2.649765-2.984630$ H $4.3341205 .250631-2.470204$ H $3.1459206 .425235-1.885696$ H $4.6661846 .192914-1.006458$ C 3.3823815 .1819351 .804837 H -0.4560542 .8673061 .395696$ H $0.5197590 .299231-0.581166$ H -1.349075 $0.789399-3.712929$ H 2.7611475 .5603702 .626773 H 4.2048765 .8947641 .676028 H 2.1252556 .1211440 .336709 C $-4.652178 \quad 1.7867040 .333914$ O $-4.4049860 .871458-0.741727$ C $-4.1133691 .479087-2.007356$ C $-5.2684302 .396522-2.429885$ C $-5.5972823 .423225-1.352565$ C $-5.8134762 .730663-0.009160$ H -3.7469602 .3712060 .537555$ C $-4.9788740 .956263 \quad 1.586545$ H $-4.078071 \quad 0.660225-2.736634$ O $-4.9666403 .073451-3.645455$ H $-6.1646821 .789920-2.614684$ H -4.820504 4.193282 -1.278386 O $-6.8051114 .102628-1.703325$ H $-6.7584872 .172679-0.032660$ O C $-3.729657 \quad 0.4499472 .276839$ H -5.5212581 .5753612 .310556$ H -5.5949450 .0854491 .336151$ H $-4.7786132 .396267-4.317634$ H -6.666905 $4.503657-2.578665$ H -6.6565714 .3151520 .757538$ O $-3.979888 \quad 0.320575 \quad 3.613273$ O -2.6788690 .1764521 .714228$ C $-2.885885-0.1359824 .414461$ H -1.9715640 .4201894 .185545$ H -3.1406810 .0327995 .464174$ H -2.739542 -1.207227 4.257701

SCF Energy (B3LYP/6-31G**//MMFF) $=-3245.92671524$

05_00031

MM̄MF Geometry

C $4.769116-2.4044481 .877527$

C $3.506608-2.6918381 .526282$

C $2.367800-2.7183542 .509407$

O $1.342878-1.8017722 .066371$

C $1.743858-4.1259212 .563271$

C $0.671700-4.260423 \quad 3.628582$

C $-0.639729-4.457874 \quad 3.389427$

C $1.163053-4.1882365 .053412$

C -1.345361-4.613892 2.070999

C $-2.148731-3.3614601 .716909$
C $-3.165697-3.5802810 .587459$

C $-2.511315-3.955649-0.743279$

C $-3.538990-4.061715-1.882342$

O $-3.890228-2.3503140 .441943$

O $-2.789318-4.100733-3.106736$

C $-4.411971-2.787761-1.893207$

C $-4.326490-5.375291-1.811183$

O $-5.485607-2.899956-2.823445$

C $-4.970522-2.414556-0.501950$

C $-5.682587-1.040280-0.483949$

O $-5.940708-3.389851-0.101381$

C $1.343160-0.5548112 .610723$

O $2.206105-0.0549403 .313819$

C 0.0490700 .0728862 .263955

C -0.3993991 .1054792 .993509$

C -1.7046461 .7573122 .854130$

C $-2.659131 \quad 1.1801791 .843139$

C $-4.8638760 .106712-1.075923$

C $-5.3734701 .482885-0.683333$

C $-4.8238823 .895517-1.061050$

O $-6.271893 \quad 1.6684460 .131337$

C $-3.7300654 .311593-0.127854$

C -3.9287875 .0046031 .002984$

C -2.8152075 .3989731 .932675$

C -3.1040755 .0454893 .376003$

C -3.2125516 .0060704 .309590$

C -3.2789923 .5870863 .741310$

C -1.9938132 .8064653 .652642$

N -4.669215 $2.480549-1.343267$

H $4.992992-2.1570382 .912469$

H $3.280109-2.9390590 .491970$

H $2.720665-2.4366873 .508967$

H $2.522386-4.8731442 .767218$

H $1.342015-4.3733811 .575297$

H -1.305453 -4.533408 4.249198

H $0.380622-4.4428775 .776504$

H $1.987557-4.8920465 .207202$

H $1.513652-3.1798735 .292867$

H $-0.650892-4.8573041 .263386$

H -2.020229 -5.474586 2.158212

H -2.691347 -2.997699 2.600430

H -1.466228 -2.548777 1.441937

H -3.861414 -4.364153 0.909354

H -1.950391 -4.893912 -0.661544

H -1.772369 -3.187416 -1.010818

H $-3.414946-4.221710-3.840724$

H -3.769113 -1.974339-2.251963

H $-3.647724-6.231457-1.906079$

H $-5.033084-5.459662-2.644217$

H $-4.880686-5.493319-0.877812$

H $-5.100092-3.033837-3.705293$

H -6.644502 -1.105617 -1.009149

H -5.933336 -0.8043350 .559410$

H -6.515316 -2.9918520.573618

H $-0.526007-0.3715341 .460504$

H $\quad 0.232602 \quad 1.491799 \quad 3.792199$

H -2.883158 0.1333972 .077315

H $-2.227125 \quad 1.229408 \quad 0.838928$

H -3.615956 1.7012031 .796115

H $-3.8214790 .039213-0.744819$

H $-4.8841810 .039678-2.169683$

H $-5.8188104 .084619-0.645971$

H -4.725596 4.428766 -2.011219

H $-2.7159574 .028530-0.398899$

H -4.9366795 .3143931 .269140$

H $-2.666566 \quad 6.4815191 .829119$

H -1.8673254 .9343431 .633900$

H -3.4219835 .7663485 .346740$

H $-3.0936007 .055110 \quad 4.059410$

H -4.0837453 .1589483 .139124$

H -3.6324713 .4993334 .777676$

H -1.234316 3.1411654 .360908

H -3.918978 2.211178 -1.972251

C $6.465707-1.0292000 .642882$

O $5.516221-0.332093-0.192029$

C $6.1831590 .082529-1.395103$

C $7.6542760 .168253-1.020015$

C $7.788330-1.053699-0.132817$ 
H $6.053076-0.725534-2.125979$

C $5.569503 \quad 1.371333-1.945562$

H $8.3123890 .139940-1.893344$

H $7.8769451 .076179-0.447910$

C $9.018960-1.0295230 .757481$

H $7.833868-1.946329-0.771612$

C $4.0369981 .316607-2.128885$

O $5.8656062 .439399-1.038188$

H $6.0594071 .627545-2.892831$

H $3.5471741 .367206-1.151418$

O $3.6536462 .490375-2.854296$

C $3.5258330 .091474-2.904556$

C $2.0278330 .098975-3.266399$

H $3.737791-0.822050-2.338861$

O $4.256087-0.012185-4.133279$

H $1.7938271 .012463-3.823590$

H $1.873222-0.715598-3.986240$

H $9.931188-1.0043220 .152311$

H $9.024662-0.1504071 .410616$

H $9.057291-1.9208901 .391630$

C $5.934488-2.4342590 .930731$

H $5.6287673 .273653-1.476734$

H $3.7780173 .254111-2.266060$

H $4.0991040 .805495-4.636365$

H $6.719537-3.0503971 .384300$

H $5.640827-2.921261-0.007938$

H $6.564233-0.4483411 .568368$

C $0.4436572 .212316-1.689998$

O $1.029862 \quad 1.012306-1.172758$

C $1.065244-0.097420-2.073321$

C $-0.371201-0.440830-2.498477$

C $-1.0435740 .759683-3.155746$

C $-0.9734621 .968104-2.226510$

H $1.0800402 .615270-2.484912$

C $0.400933 \quad 3.241229-0.552366$

H $1.445446-0.950530-1.496992$

O $-0.394235-1.543328-3.397864$

H $-0.958075-0.731829-1.617283$

H $-0.6043640 .986434-4.133955$

O $-2.419270 \quad 0.460540-3.396609$

H $-1.6656831 .813597-1.390942$

O $-1.409667 \quad 3.130872-2.928362$

C $1.7831653 .578473-0.048391$

H $-0.067492 \quad 4.178717-0.873160$

H $-0.194723 \quad 2.865667 \quad 0.287493$

H $0.023661-2.296706-2.946926$

H $-2.448762-0.344813-3.941294$

H $-2.2852512 .935331-3.303434$

O $2.0273882 .912531 \quad 1.115970$

O $2.557830 \quad 4.325860-0.630832$

$\begin{array}{llll}\text { C } 3.311860 & 3.133553 & 1.706039\end{array}$

H 3.2999212 .7088142 .713283

H 4.0803142 .6238081 .119278

H $3.528313 \quad 4.2034161 .782289$

SCF Energy (B3LYP/6-31G**/MMFF) $=-3245.92253997$

05_00032

MM̄FF Geometry

C -3.287981 -0.822783 -2.650254

C $-2.761552-1.968654-3.108230$

C $-1.539065-2.660935-2.549289$

O $-0.670450-1.735019-1.858579$

C $-1.992761-3.736629-1.544364$

C $-0.861962-4.567784-0.972865$

C $-0.518671-4.599423 \quad 0.330099$

C $-0.117312-5.426088-1.962895$

C $-1.143404-3.8512641 .475254$

C $-0.465585-2.5132641 .810021$

C $0.978776-2.6457612 .318886$

C $1.512237-1.2932752 .794795$

C $2.982443-1.3773333 .231162$

O $1.796963-3.1233641 .250674$

O $3.457817-0.0274393 .351482$

C $3.807267-2.0766972 .126042$

C $3.118103-2.0059134 .623321$

O $5.133824-2.3100282 .592791$

C $3.160413-3.3816391 .613324$

C $3.848817-3.969515 \quad 0.357615$
O $3.240700-4.3771432 .640671$

C $0.195760-1.012619-2.621141$

O $0.308387-1.045069-3.836301$

C $0.985736-0.151033-1.713881$

C $1.726673 \quad 0.841137-2.229857$

C $2.537347 \quad 1.796643-1.472429$

C $2.7752241 .506517-0.014943$

C $3.881567-3.046783-0.866143$

C $5.181447-2.275685-1.020050$

C $6.251764-0.551468-2.464722$

O $6.167185-2.464861-0.312751$

C $6.198646 \quad 0.756448-1.741290$

C $6.2594471 .943554-2.362930$

C $6.2559933 .263194-1.647500$

C $5.1649754 .187354-2.137642$

C $5.4298785 .161079-3.025045$

C $3.7891994 .037927-1.530487$

C $3.0184492 .883962-2.110941$

N $5.120867-1.369499-2.070330$

H -2.813465 -0.308401 -1.818631

H $-3.265836-2.488281-3.920282$

H $-0.990591-3.124998-3.377886$

H -2.704844 -4.419780 -2.027007

H $-2.549352-3.244125-0.739525$

H $\quad 0.306765-5.247777 \quad 0.622540$

H $0.588498-6.106780-1.474641$

H $-0.818728-6.040617-2.536824$

H $0.455472-4.804952-2.658309$

H $-2.207159-3.671898 \quad 1.287427$

H $-1.117798-4.5005382 .359728$

H $-0.490061-1.8701150 .922163$

H - $-1.066469-2.0208142 .583806$

H $0.987152-3.371636 \quad 3.140415$

H $0.893846-0.8936773 .608013$

H $1.434648-0.5652651 .975842$

H $4.407050-0.0674083 .560990$

H $3.887878-1.3618841 .299807$

H $2.571758-1.4102975 .364603$

H $4.161708-2.0122194 .956770$

H $2.735656-3.0277384 .671193$

H $5.664031-2.6235611 .841274$

H $4.846524-4.3468340 .611197$

H $3.273547-4.8595380 .063162$

H $3.151547-5.2528982 .229439$

H $0.918863-0.341009-0.648856$

H $1.7145040 .993953-3.308316$

H 3.1364660 .4813650 .114353

H 1.8514261 .6280550 .559109

H 3.5321512 .1489900 .439760

H $3.786457-3.666111-1.767437$

H $3.039971-2.346747-0.860302$

H $6.200564-0.427621-3.551088$

H $7.182181-1.072371-2.216146$

H $6.1359970 .725529-0.656037$

H $6.3493881 .971115-3.446655$

H $7.2380653 .728581-1.803497$

H $6.1618833 .131639-0.562389$

H $4.6584125 .846857-3.359372$

H $6.4234755 .290464-3.441277$

H $3.1981434 .944873-1.717637$

H $3.8798103 .988177-0.441884$

H $2.7907683 .004340-3.171107$

H $4.242460-1.234749-2.562773$

C $-5.737583-0.295654-2.233489$

O $-5.4139420 .321242-0.968645$

C $-5.668474-0.636795 \quad 0.072543$

C $-6.712160-1.582649-0.506250$

C $-6.208785-1.723492-1.928967$

H $-4.735621-1.1938340 .224599$

C $-6.0719240 .045141 \quad 1.381511$

H $-6.757293-2.5354420 .029663$

H -7.713203 -1.136174 -0.503145

C $-7.254645-2.240923-2.901647$

H $-5.365842-2.424412-1.923414$

C $-5.102701 \quad 1.137617 \quad 1.877287$

$\begin{array}{llll}\text { O } & -7.356366 & 0.653800 & 1.200001\end{array}$

H -6.202338 -0.7225552 .154338$

H -5.2505582 .0635121 .307458$ 
$\begin{array}{llll}\text { O } & -5.456530 & 1.440380 & 3.234201\end{array}$ C -3.607666 $0.766311 \quad 1.840202$ C $-2.731497 \quad 1.7649102 .620907$ H -3.2785500 .7163390 .797133$ O $-3.414182-0.531605 \quad 2.404386$ H -3.093881 2.780442 2.436544 H -2.8461831 .5688003 .693614$ H -7.591222 -3.240766 -2.608670 H $-8.132707-1.587113-2.933320$ H $-6.844043-2.304605-3.914425$ C $-4.544663-0.197649-3.192268$ H -7.6868230 .9136732 .076163$ H $-6.328755 \quad 1.868733 \quad 3.222703$ H $-3.769008-0.5161613 .309928$ H $-4.3259800 .860363-3.378443$ H $-4.813621-0.640056-4.158720$ H $-6.5639780 .292817-2.651630$ C -1.142737 3.4842030 .670623 $\begin{array}{llll}\text { O } & -0.978571 & 2.078599 & 0.902315\end{array}$ C -1.2357251 .6492272 .246248$ C $-0.290277 \quad 2.3801413 .208201$ C -0.4202903 .8929373 .071652$ C $-0.250683 \quad 4.307523 \quad 1.612144$ H -2.194924 3.7607710 .802570 C $-0.7662423 .775300-0.789161$ H -0.9749150 .5852892 .284318$ O -0.5432442 .0066004 .558217$ H 0.7472572 .0979352 .986731 H $-1.372328 \quad 4.2552863 .476868$ $\begin{array}{lllll}\text { O } & 0.608941 & 4.524882 & 3.836374\end{array}$ H 0.8030594 .1923201 .325858 O -0.5777655 .6893451 .476174$ C $-1.6586203 .074626-1.788591$ H $-0.8256824 .849301-0.996260$ H $0.2578443 .435668-0.966420$ H -0.4452371 .0408504 .617185$ H $0.517692 \quad 4.215464 \quad 4.754129$ H -0.0119036 .1835282 .094016$ O $-1.071794 \quad 3.148680-3.020449$ O $-2.7327732 .550343-1.531544$ C $-1.790082 \quad 2.538518-4.096331$ H $-2.8294792 .879315-4.114985$ H $-1.738055 \quad 1.450812-4.003494$ $\mathrm{H}-1.3108462 .832950-5.033853$ SCF Energy (B3LYP/6-31G**/MMFF) $=-3245.92294676$

05_00033

MMFF Geometry

C -3.968612 -3.650288 -1.338453

C $-2.821422-3.475470-0.665789$

C $-1.462035-3.636855-1.297121$

O $-0.730339-2.394077-1.163012$

C $-0.664650-4.737568-0.574163$

C $0.662052-5.061825-1.238826$

C $1.879344-4.806225-0.717682$

C $0.558540-5.761102-2.572010$

C $2.216355-4.1625170 .598134$

C $2.747990-2.7363290 .442399$

C $4.117970-2.587999-0.240455$

C $5.244498-3.1786070 .614161$

C $6.638427-2.8640460 .051132$

O $4.314175-1.177561-0.420052$

O $7.604314-3.1602271 .072061$

C $6.731770-1.350239-0.229356$

C $6.984380-3.759345-1.144339$

O $7.961600-1.026565-0.873630$

C $5.546105-0.810900-1.055419$

C $5.5715250 .731299-1.177096$

O $5.618174-1.340796-2.383601$

C -1.097964-1.362466 -1.972749

O $-2.026277-1.341570-2.765309$

C $-0.146938-0.252993-1.747186$

C $-0.3738680 .943301-2.309982$

C $0.5174802 .103733-2.249522$

C $1.8698291 .898972-1.621628$

$\begin{array}{llll}\text { C } & 5.388354 & 1.483633 & 0.144474\end{array}$

C $5.0705112 .953874-0.077535$

C 3.9901744 .8900221 .072555
O $5.2941093 .537266-1.134837$

C $2.544861 \quad 4.8423610 .684346$

C $1.9683485 .685380-0.185312$

C $0.5174955 .600780-0.573238$

C $0.3065665 .667914-2.070666$

C $-0.358134 \quad 6.695143-2.626338$

C $0.8731414 .557159-2.927098$

C $0.105473 \quad 3.269287-2.791208$

N $4.519436 \quad 3.537308 \quad 1.055170$

H -3.934612 $-3.957186-2.381197$

H $-2.853964-3.1571250 .372099$

H $-1.553470-3.878399-2.363285$

H $-1.257236-5.661614-0.535909$

H $-0.515094-4.438950 \quad 0.468242$

H $2.751740-5.105763-1.297521$

H $1.533581-6.092965-2.944920$

H $-0.075870-6.649414-2.486975$

H $\quad 0.129413-5.095467-3.326739$

H $1.347067-4.1306151 .260752$

H $2.947683-4.7918341 .117017$

H $2.012061-2.134823-0.104730$

H $2.807384-2.2774341 .437915$

H $4.086927-3.059924-1.228052$

H $5.124861-4.262003 \quad 0.730751$

H $5.184475-2.7615181 .629382$

H $7.505117-4.0963751 .316474$

H $6.747816-0.8426600 .743697$

H $6.994674-4.813735-0.842467$

H $7.991085-3.546082-1.520086$

H $6.277865-3.663483-1.971316$

H $8.679829-1.381189-0.322136$

H $6.4946301 .058875-1.672593$

H $4.7640441 .032597-1.857227$

H $5.104018-0.766199-2.974613$

H $0.731256-0.458292-1.147410$

H $-1.2868571 .084648-2.886895$

H $2.404703 \quad 1.088102-2.127673$

H $1.7722471 .644900-0.561095$

H $2.5132492 .777211-1.676631$

H 4.5682091 .0396800 .720030

H 6.3010901 .4267180 .747082

H $4.578275 \quad 5.5211340 .398772$

H 4.0907115 .2633642 .095953

H 1.9364854 .0668021 .140655

H $2.5613646 .480961-0.630083$

H $-0.0062336 .428769-0.078397$

H $0.0589024 .680608-0.191208$

H $-0.5129436 .755113-3.698663$

H $-0.7645727 .499770-2.022509$

H $1.9400734 .442377-2.719946$

H $0.8320534 .842611-3.987273$

H $-0.8940593 .312895-3.226313$

H 4.3173962 .9503801 .859377

C $-5.991982-2.201664-1.448201$

O $-5.271983-0.994875-1.118559$

C $-6.2191270 .036090-0.784903$

C $-7.571480-0.470833-1.268643$

C $-7.434435-1.958932-1.004855$

H $-6.248797 \quad 0.090048 \quad 0.310067$

C $-5.766212 \quad 1.387793-1.349777$

H $-8.405546-0.012178-0.729175$

H $-7.718859-0.293934-2.339469$

C $-8.458477-2.804382-1.741742$

H $-7.531778-2.1363680 .074637$

C $-4.3388641 .799411-0.929579$

O $-5.7659691 .337764-2.781603$

H $-6.4769092 .172479-1.063163$

H -3.599631 $1.164278-1.432461$

O $-4.110058 \quad 3.132964-1.402478$

C -4.0860021 .7711990 .587940$

$\begin{array}{llll}\text { C } & -2.706607 & 2.335639 & 0.975991\end{array}$

H -4.1632800 .7373710 .938257$

$\begin{array}{llll}\text { O } & -5.098135 & 2.520032 & 1.260265\end{array}$

H $-1.942406 \quad 1.858408 \quad 0.354384$

H -2.675271 3.4103960 .759963

H - $-9.473462-2.538455-1.428514$

H $-8.392844-2.660648-2.825484$

H $-8.308671-3.868394-1.533493$ 
C $-5.330198-3.395248-0.756349$ H $-6.6886431 .403295-3.079881$ H $-4.249705 \quad 3.125593-2.365074$ H $-5.060386 \quad 3.4320780 .924271$ H $-5.936195-4.298419-0.887378$ H $-5.250280-3.2041510 .321020$ H $-5.940642-2.312453-2.539591$ C $-1.528180-0.1200532 .339041$ O $-2.522882 \quad 0.7596652 .872892$ C -2.388814 2.129177 2.475828 C -1.019924 2.6656992 .913369 C $0.121808 \quad 1.792086 \quad 2.400093$ $\begin{array}{llll}C & -0.121062 & 0.325461 & 2.754673\end{array}$ H $-1.601871-0.116006 \quad 1.244727$ C -1.878151-1.550699 2.765760 H -3.148319 2.6859993 .039005 O -0.8542434 .0061622 .465128$ H $-0.9818862 .687175 \quad 4.010400$ H $\quad 0.2638801 .927203 \quad 1.322281$ $\begin{array}{lllll}\text { O } & 1.333870 & 2.223164 & 3.023888\end{array}$ H 0.0323030 .2042093 .833806 O $0.860999-0.4600162 .087639$ C $-1.691639-1.8054274 .242694$ H -2.921330 -1.782367 2.516856 H -1.260989 -2.276565 2.223651 H 0.0265124 .3020932 .751935 H 2.0458401 .6448812 .700108 H $\quad 0.794947-1.367538 \quad 2.429434$ O $-2.733093-1.279327 \quad 4.946837$ O $-0.727282-2.3962224 .712136$ C $-2.659310-1.4402846 .366639$ H -3.543923 -0.9737696 .808124$ H $-2.655247-2.5028526 .627804$ H $-1.767338-0.9419876 .758250$

SCF Energy (B3LYP/6-31G*//MMFF) $=-3245.91850320$

0500034

MM̄FF Geometry

C $3.821146-1.5118821 .788447$

C $3.408029-2.2933672 .797259$

C $2.057422-2.2732783 .470417$

O $1.180052-1.2846352 .888343$

C $1.361041-3.6409113 .319727$

C $0.111124-3.7656744 .171700$

C -1.143441 -3.904658 3.700280

C $0.341565-3.7583665 .663489$

C $-1.620354-4.0042732 .278330$

C - $2.321486-2.720722 \quad 1.828075$

C $-3.279928-2.921803 \quad 0.643845$

C $-2.581941-3.465906-0.603484$

C $-3.530041-3.546213-1.809921$

O $-3.865112-1.641596 \quad 0.365332$

O $-2.709972-3.748243-2.971409$

C $-4.255512-2.193181-1.970223$

C $-4.450499-4.768428-1.718134$

O $-5.241232-2.260428-2.997508$

C $-4.871761-1.661968-0.657851$

C $-5.431838-0.224787-0.794508$

O $-5.969298-2.501446-0.279654$

C $0.951652-0.1435293 .592513$

$\begin{array}{lllll}\text { O } & 1.518545 & 0.226226 & 4.607676\end{array}$

C $-0.175088 \quad 0.5560972 .935041$

C $-0.793361 \quad 1.5657873 .566035$

C -1.983193 2.2787193 .091807

C -2.611086 1.7975461 .811123

C $-4.4348750 .791963-1.348923$

C $-4.8504442 .235483-1.129427$

C $-3.9421814 .547505-1.422883$

O $-5.8993142 .564524-0.584554$

C -3.106567 $4.897511-0.231138$

C -3.5492125 .6176830 .810514$

C -2.7030005 .9418422 .010121$

C -3.3782055 .5827183 .316999$

C $-3.694161 \quad 6.534813 \quad 4.211289$

C -3.6990414 .1283783 .587641$

C -2.469314 $3.297242 \quad 3.832797$

N -3.885432 $3.109939-1.613977$

H $3.148488-0.774267 \quad 1.360327$
H $4.109035-3.0223063 .201188$

H $2.230941-2.0599354 .531562$

H $2.046981-4.4488533 .607407$

H $1.128634-3.8025432 .261746$

H -1.950266 -3.978746 4.429868

H $-0.560261-4.0256746 .224877$

H $1.116137-4.4842315 .932161$

H $0.656258-2.7673026 .003483$

H $-0.808149-4.2485081 .588419$

H $-2.321041-4.8473652 .230368$

H $-2.895524-2.2846132 .657323$

H $-1.570262-1.9641021 .569163$

H $-4.072384-3.6075110 .966877$

H $-2.138668-4.449915-0.411990$

H $-1.743948-2.807287-0.863096$

H $-3.291484-3.723026-3.750863$

H $-3.497740-1.484272-2.325160$

H $-3.856592-5.689660-1.681320$

H $-5.082463-4.857974-2.608483$

H $-5.094976-4.754177-0.836708$

H $-6.012698-2.730048-2.637149$

H $-6.334897-0.228025-1.418719$

H -5.7628320 .1063950 .199536$

H $-6.544158-2.0011490 .323206$

H $-0.500796 \quad 0.1830561 .971336$

H -0.4151421 .8826674 .537175$

H $-2.8675590 .734527 \quad 1.880188$

H -1.9227341 .9408410 .971551$

H -3.5340512 .3183541 .554901$

H $-3.4609730 .657521-0.869052$

H $-4.3171010 .641406-2.427618$

H $-4.9834694 .864601-1.308427$

H $-3.524898 \quad 5.013249-2.320664$

H $-2.0807754 .534929-0.225783$

H $-4.5671316 .000303 \quad 0.800527$

H -2.482658 7.0166221 .978130

H -1.7321045 .4340691 .958007$

H $-4.180510 \quad 6.290600 \quad 5.149931$

H -3.4734487 .5808674 .026721$

H -4.3241003 .7397112 .780031$

H -4.3260774 .0423574 .485610$

H $-1.944600 \quad 3.564715 \quad 4.751173$

H $-3.0267792 .724812-1.997455$

C $5.951755-0.2870231 .238387$

$\begin{array}{lllll}\text { O } & 5.330710 & 0.656090 & 0.339846\end{array}$

C $6.3064661 .081194-0.626232$

C 7.6458540 .8921510 .066216

C $7.408584-0.4266740 .777550$

H $6.2422880 .384038-1.471431$

C $6.0037712 .499819-1.117214$

H $8.4817460 .856333-0.638341$

H $7.841705 \quad 1.6847750 .797384$

C $8.383776-0.692247 \quad 1.911764$

H $7.494166-1.2395950 .043550$

C $4.5464372 .716815-1.574055$

O $6.2633643 .437552-0.065460$

H $6.6948902 .761064-1.926868$

H $3.8846942 .758926-0.699886$

O $4.4656294 .002507-2.200113$

C $4.0150791 .658801-2.556213$

C $2.5797761 .961002-3.020830$

H $4.0339650 .680644-2.067081$

O $4.8698311 .575476-3.694603$

H $1.9721722 .208683-2.143817$

H $2.5816922 .843180-3.672542$

H $9.409789-0.7429501 .533194$

H 8.3435130 .0981172 .668842

H $8.156692-1.6428862 .404579$

C $5.192334-1.6155281 .176542$

H $5.713908 \quad 3.184407 \quad 0.696351$

H $4.7984274 .656793-1.561912$

H $4.8997182 .456900-4.104899$

H $5.774628-2.3971731 .678594$

H $5.064041-1.9213890 .130675$

H 5.8965520 .1460822 .244867

C $1.226585-0.517237-1.893620$

O $2.031392-0.454495-3.073859$

$\begin{array}{llll}\text { C } & 1.943180 & 0.781532 & -3.792747\end{array}$ 
C $0.4891491 .028609-4.218155$

C $-0.467120 \quad 0.986776-3.026984$

C $-0.256473-0.288160-2.208359$

H $1.5683100 .257595-1.195662$

C $1.501870-1.848623-1.179476$

H $2.5349510 .645904-4.706792$

O $\quad 0.3575652 .284493-4.875916$

H $0.1832160 .255289-4.934776$

H $-0.3660211 .879768-2.399291$

O $-1.811690 \quad 0.991790-3.511167$

H - $0.673565-1.132349-2.768270$

O $-0.992193-0.197263-0.991129$

C $1.100007-3.084421-1.956917$

H $2.577592-1.942060-0.992152$

H $0.976088-1.855729-0.217397$

H $0.9612812 .279246-5.638318$

H $-1.9173051 .784118-4.065255$

H $-0.6424180 .556549-0.486746$

O $0.974649-4.130641-1.086842$

O $0.951780-3.140587-3.169601$

C $0.625434-5.386532-1.676980$

H $0.475201-6.109920-0.871234$

H $-0.304988-5.298914-2.245728$

H $1.438723-5.737280-2.318971$

SCF Energy (B3LYP/6-31G**/MMFF) $=-3245.93312431$

0500035

MM̄FF Geometry

C $-3.744643-2.495626 \quad 0.839479$

C $-4.312890-2.2751192 .035518$

C $-3.732243-1.4488043 .160906$

O $-2.404360-0.9867682 .831251$

C -4.617946 $-0.209513 \quad 3.391419$

C $-4.091663 \quad 0.7191304 .468365$

C $-3.632547 \quad 1.9680174 .251251$

C $-4.119907 \quad 0.163655 \quad 5.869617$

C -3.5531412 .7199312 .950660$

C -2.106464 2.858861 2.472411

C $-1.966143 \quad 3.709171 \quad 1.200359$

C $-2.5515543 .019886-0.036499$

C $-2.3040343 .831937-1.318497$

O $-0.562373 \quad 3.940491 \quad 1.008352$

O -2.589233 $2.984032-2.441291$

C $-0.8072304 .198721-1.397277$

C $-3.257965 \quad 5.027304-1.429982$

O $-0.5504395 .074137-2.492220$

C $-0.255740 \quad 4.809141-0.092464$

C $1.2773325 .005155-0.129681$

O $-0.834783 \quad 6.1061230 .099614$

C -1.346279 $-1.685745 \quad 3.322397$

O $-1.378138-2.6802644 .028313$

C $-0.120197-1.0257452 .823240$

C $1.077910-1.5953733 .018366$

C $2.355161-1.0753722 .529602$

C $2.3629390 .303525 \quad 1.922621$

C $2.100143 \quad 3.730596-0.340836$

C $3.5879053 .982976-0.152696$

C $5.7738472 .804704-0.112689$

$\begin{array}{llllll}\text { O } & 4.059203 & 5.098116 & 0.055407\end{array}$

C 6.2132261 .4783580 .416543

C 6.4770301 .2511531 .712594

C $6.907129-0.0790242 .263187$

C $5.774348-0.7898392 .975917$

C $5.678512-0.7473994 .315624$

C $4.824191-1.5476052 .072368$

C $3.459242-1.8442742 .636771$

N $4.327847 \quad 2.811545-0.238335$

H $-2.764999-2.081625 \quad 0.618235$

H $-5.296484-2.699478 \quad 2.228637$

H -3.712998 -2.063878 4.068324

H -5.632783 -0.524095 3.670035

H -4.7278820 .3222092 .441094$

H -3.275779 $2.536323 \quad 5.109723$

H -3.8352370 .9101936 .618835$

H $-5.128180-0.1820656 .120394$

H -3.427458 -0.6780885 .965831$

H -4.1677392 .2571302 .176837$

H -3.9810003 .7171093 .113878$
H -1.498340 3.3133403 .266625

H $-1.666646 \quad 1.8687882 .300638$

H -2.459390 $4.671967 \quad 1.378134$

H -3.624495 2.8298930 .080536

H -2.084315 $2.032182-0.156366$

H -3.511388 $2.685149-2.364724$

H $-0.2639053 .271256-1.609280$

H $-4.2996834 .684715-1.450674$

H $-3.1032285 .570071-2.369024$

H $-3.1615795 .733329-0.602779$

H $-0.9070584 .650401-3.291733$

H $1.5409445 .747571-0.894632$

H 1.5839745 .4539500 .826182

H -0.2626496 .6142980 .698303$

H $-0.240023-0.0974362 .276683$

H $1.127259-2.5453503 .548489$

H 3.3625200 .6487251 .652349

H $1.966777 \quad 1.0373892 .633758$

H $1.7521350 .334514 \quad 1.015783$

H 1.7923642 .9590560 .373237

H $1.9548353 .345051-1.355580$

H 6.0916923 .6288450 .535129

H $6.2001292 .977453-1.106072$

H $6.3143180 .668223-0.301416$

H 6.3640852 .0624762 .428351

H 7.7498940 .0904122 .945817

H $7.301230-0.7273371 .470167$

H $4.892627-1.2628194 .855993$

H $6.397138-0.1930184 .911064$

H $5.296345-2.4996251 .796483$

H $4.703153-1.0118411 .124977$

H $3.377291-2.8214013 .112573$

H $3.8681571 .967526-0.568763$

C $-4.600958-2.453034-1.546768$

O $-3.325855-2.124697-2.136910$

C $-3.355970-0.746441-2.549146$

C $-4.827999-0.344751-2.561327$

C $-5.358988-1.133065-1.381566$

H $-2.842325-0.176528-1.765348$

C $-2.636153-0.549150-3.885781$

H $-4.9659790 .735172-2.453794$

H $-5.330656-0.661156-3.482435$

C $-6.869244-1.288932-1.373358$

H $-5.054161-0.612048-0.465939$

C $-1.193191-1.090006-3.946738$

O $-3.383779-1.215137-4.912503$

H $-2.6518640 .516904-4.143174$

H $-1.209596-2.183795-4.023475$

O $-0.627228-0.626650-5.181909$

C $-0.274561-0.682986-2.779272$

C $1.209100-0.978266-3.075032$

H $-0.601073-1.204450-1.873221$

O $-0.4183430 .718194-2.551721$

H $1.291106-1.966794-3.537415$

H $1.571741-0.244919-3.805405$

H $-7.356415-0.309817-1.318384$

H $-7.226515-1.791385-2.278423$

H $-7.193855-1.877526-0.509452$

C $-4.399354-3.273761-0.268209$

H -2.920831-1.061934 -5.754141

H $-0.5449280 .340686-5.121628$

H $-0.0380630 .916260-1.679847$

H $-3.755576-4.132627-0.492778$

H $-5.366585-3.6711460 .061401$

H $-5.111516-3.089078-2.281875$

C $2.098563-3.251123-1.237253$

O $1.764659-1.910797-0.853901$

C $2.099660-0.901378-1.814582$

C $3.606328-0.934908-2.101981$

C $4.063729-2.319630-2.543214$

C $3.598208-3.375592-1.543539$

H $1.508689-3.543384-2.113773$

C $1.714627-4.186647-0.079907$

H $1.8962140 .062477-1.334612$

$\begin{array}{llll}\text { O } & 3.961716 & 0.017955 & -3.098026\end{array}$

H $4.154640-0.662855-1.192106$

H $3.716937-2.555686-3.556075$

O $5.492393-2.349204-2.588877$ 
H $\quad 4.179787-3.279995-0.617723$

O $3.861392-4.671449-2.078693$

C $0.219029-4.3074780 .115948$

H $2.094489-5.196346-0.272804$

H $2.139533-3.8159720 .857934$

H $3.6679940 .890939-2.786276$

H $5.776297-1.646017-3.198034$

H $4.812151-4.714924-2.279477$

O $-0.014000-5.200637 \quad 1.123970$

O $-0.632066-3.706575-0.523629$

C $-1.388531-5.4578321 .424899$

H -1.937823 -5.7366690 .520817$

H $-1.431315-6.2924122 .129691$

H -1.834942 -4.579066 1.895650

SCF Energy (B3LYP/6-31G**//MMFF) $=-3245.91311615$

0500036

MM̄MF Geometry

C $2.6534503 .178602-1.493410$

C $2.4059394 .348586-0.884445$

C 1.3565724 .6146800 .164065

O $\quad 0.2758993 .6600180 .089138$

C 1.9735084 .5194951 .572422

C 1.0086464 .9246002 .671255

C 0.5070094 .0934013 .606009

C 0.6246526 .3831742 .690797

C 0.7882352 .6308813 .812065

C -0.3339811 .7566663 .250420$

C $-0.299220 \quad 0.305686 \quad 3.753463$

C $0.978320-0.4329323 .347926$

C $0.941257-1.9124963 .762516$

O $-1.446288-0.3510263 .194212$

O $2.013501-2.5845883 .085474$

C $-0.378071-2.5356903 .260358$

C $1.199934-2.0947655 .263011$

O $-0.524863-3.873557 \quad 3.728533$

C -1.626451-1.706943 3.631720

C $-2.919033-2.2449942 .971623$

O $-1.828674-1.7648495 .048591$

C $-0.8445474 .038589-0.584769$

O $-0.9954355 .041459-1.263636$

C -1.892600 $3.034883-0.298076$

C $-3.1708873 .295576-0.610984$

C $-4.3196472 .430685-0.327098$

C $-4.0470331 .144080 \quad 0.405609$

C $-2.872829-2.3184851 .445089$

C $-4.243794-2.5208570 .819210$

C $-5.332482-2.545626-1.439041$

O $-5.262612-2.7229791 .472798$

C $-5.546505-1.209623-2.077949$

C $-6.720040-0.559713-2.085394$

C $-6.9110320 .786953-2.725178$

C $-7.5640121 .792373-1.800054$

C $-8.7393782 .358094-2.123385$

C $-6.8694492 .142445-0.500538$

C $-5.5481292 .834083-0.714825$

N $-4.178018-2.452170-0.564927$

H $2.0724642 .297022-1.238685$

H $3.0248905 .206456-1.141790$

H $0.9724545 .628312-0.002537$

H 2.8606175 .1633481 .639761

H 2.3313293 .4966301 .730395

H -0.1888644 .5094834 .334525$

H 0.0504576 .6498873 .584702

H 1.5208677 .0120772 .681625

H 0.0109916 .6348851 .820672

H 1.7494542 .3413183 .379033

H 0.8841962 .4628644 .892171

H -1.312992 2.1851123 .506021

H $-0.292268 \quad 1.7619892 .154727$

H $-0.402830 \quad 0.3242304 .844900$

H $1.8681570 .048403 \quad 3.770278$

H $1.097591-0.3801382 .256492$

H $2.847464-2.1633423 .356034$

H - $0.302597-2.5960072 .167922$

H $2.188870-1.7035085 .531140$

H $1.214658-3.1560565 .534643$

H $0.466092-1.5871235 .891965$
H $\quad 0.277042-4.360128 \quad 3.471337$

H -3.172469 -3.2309273 .382296$

H $-3.748121-1.5850113 .263641$

H $-2.757979-1.5523415 .236313$

H $-1.5881712 .120800 \quad 0.197215$

H $-3.4054204 .238865-1.102468$

H -4.9413050 .5538590 .609966$

H -3.5775221 .3421611 .375713$

H $-3.3791120 .508960-0.184860$

H -2.454266 -1.391337 1.043092

H $-2.235981-3.1511831 .126003$

H $-6.207357-2.884536-0.875812$

H $-5.096280-3.288581-2.206972$

H $-4.687314-0.753218-2.565010$

H -7.588885 -1.020384 -1.621358

H $-7.5255180 .640723-3.622813$

H -5.955832 $1.195210-3.077468$

H -9.220191 $3.078424-1.469970$

H $-9.2492002 .114691-3.049818$

H $-6.786740 \quad 1.240660 \quad 0.110608$

H -7.4952472 .8250790 .089928$

H -5.638749 $3.790770-1.231371$

H -3.285588 -2.242423-1.000121

C $4.9680852 .256041-2.053088$

O $4.6581620 .874508-1.798361$

C $5.4718530 .420895-0.696891$

C $6.4452671 .554409-0.377855$

C $5.6385342 .773169-0.781555$

H 4.7939090 .2936810 .155600

C $6.140440-0.918317-1.030703$

H 6.7378091 .5620310 .676590

H $7.3607811 .490457-0.976987$

C $6.4758634 .022725-0.986043$

H 4.8982982 .9610830 .005660

C $5.171973-2.009224-1.527678$

O $7.106055-0.702389-2.068505$

H $6.702034-1.270909-0.157306$

H $4.825388-1.757727-2.537320$

O $5.920791-3.224035-1.664488$

C $3.956991-2.261038-0.618576$

C $3.093990-3.429681-1.128954$

H $3.356503-1.347101-0.570791$

O $4.419382-2.5482390 .699340$

H $2.903227-3.280908-2.197410$

H $3.651520-4.368860-1.027499$

H $6.9910294 .296062-0.059384$

H $7.2350023 .875172-1.761542$

H $5.8480804 .867916-1.285160$

C $3.7272853 .013075-2.533649$

H $7.521670-1.560571-2.260684$

H $6.190168-3.503789-0.772682$

H $3.648452-2.5369801 .290893$

H $3.2813412 .474591-3.379365$

H $4.0353543 .994494-2.914857$

H $5.6937802 .250775-2.878001$

C $0.523064-1.857023-1.529265$

O $1.042473-2.340295-0.284214$

C $1.756495-3.576834-0.370669$

C $0.835545-4.672380-0.923577$

C $0.200279-4.262933-2.249847$

C $-0.445349-2.882133-2.135063$

H $1.350686-1.668280-2.223491$

C $-0.152163-0.514784-1.226053$

H $2.006155-3.8613760 .658097$

O $1.544990-5.893273-1.102953$

H $\quad 0.031913-4.868901-0.201662$

H $0.926749-4.288453-3.070361$

O $-0.820523-5.204444-2.590630$

H -1.342094 -2.961967-1.511589

O $-0.857703-2.453540-3.429134$

C $-0.4085850 .305794-2.465233$

H $-1.084722-0.671726-0.673833$

H $0.4757740 .092115-0.561317$

H $1.925598-6.139229-0.242601$

H $-0.403225-6.082516-2.625126$

H $-1.452978-3.135090-3.785629$

O $-1.749018 \quad 0.496590-2.626378$

O $\quad 0.4741540 .721773-3.204256$ 
C $-2.1300091 .265790-3.770715$

H $-1.8391010 .745299-4.687818$

H $-3.2176241 .376495-3.758797$

H -1.6762092 $2.260595-3.729451$

SCF Energy (B3LYP/6-31G**//MMFF) $=-3245.92428205$

05 00037

MM̄FF Geometry

C -3.533759 -2.628702 -2.531665

C $-2.525076-2.800087-1.665103$

C $-1.084378-2.930659-2.074804$

O $-0.304861-1.916680-1.400182$

C $-0.463601-4.306147-1.744071$

C $-0.551680-4.752573-0.294723$

C $0.313309-4.3943400 .675522$

C $-1.674248-5.7094510 .018789$

C $1.508192-3.4884650 .556099$

C $2.783485-4.1751991 .056985$

C $4.059163-3.3295700 .906338$

C $4.056173-2.0776431 .784997$

C $5.340290-1.2530401 .597149$

O $4.203619-2.961961-0.469177$

O $5.130178 \quad 0.0316792 .204271$

C $5.559058-1.0091500 .088310$

C $6.539335-1.8809622 .316723$

O $6.817753-0.385034-0.151194$

C $5.432452-2.284134-0.773033$

C $5.425613-1.987328-2.293112$

O $6.556179-3.136047-0.516460$

C $-0.377370-0.636850-1.848447$

O $-1.063877-0.208616-2.761265$

C $0.5405520 .164980-1.010812$

C $0.4369191 .501058-1.005735$

C $1.2632422 .420247-0.222642$

C 2.4774551 .8527920 .464833

C $4.345944-1.005257-2.768587$

C $4.8588270 .412955-2.975275$

C $4.0683432 .697227-3.584199$

O $\quad 6.0501590 .710318-2.947411$

C $4.1393093 .526373-2.340538$

C $3.4471244 .663445-2.169713$

C $3.5519785 .530158-0.946758$

C $2.2056885 .858843-0.340100$

C $1.6249277 .052160-0.551077$

C 1.5754184 .8378430 .579605

C $0.9094043 .721116-0.174273$

N $3.8289981 .306261-3.241838$

H -3.325982 -2.605460 -3.598471

H -2.737988 -2.804854 -0.600394

H $-0.972680-2.777883-3.156574$

H $0.591838-4.296857-2.045214$

H - $0.934761-5.069727-2.377662$

H $\quad 0.144990-4.7832031 .679248$

H - $1.712910-5.9732231 .081047$

H -2.642973 -5.277684 -0.245405

H - $1.545531-6.637492-0.547954$

H $1.663157-3.159545-0.473803$

H $\quad 1.305493-2.593898 \quad 1.152887$

H $2.664997-4.4704432 .107157$

H $2.920860-5.0992080 .479025$

H $4.897279-3.9787421 .187207$

H $3.918137-2.3413182 .840748$

H $3.206314-1.4357111 .519686$

H $4.921099-0.1116633 .143254$

H $4.793470-0.291912-0.226186$

H $6.335935-1.9725243 .390499$

H $7.426899-1.2440262 .233775$

H $\quad 6.792547-2.8752231 .943125$

H $\quad 6.8476450 .4240420 .387563$

H $6.426352-1.678720-2.618403$

H $5.237564-2.941006-2.807384$

H $\quad 6.669109-3.732893-1.274895$

H $1.265953-0.368074-0.406547$

H $-0.3387071 .966546-1.612611$

H $3.1013891 .311323-0.252962$

H 2.1878961 .1609791 .261729

H 3.1215122 .6120510 .912875

H $3.982885-1.339778-3.749010$
H $3.486562-0.994831-2.089752$

H $3.2566993 .013324-4.247121$

H $5.0149012 .772791-4.129492$

H $4.8158543 .194057-1.556632$

H $2.7951415 .012400-2.967496$

H $4.0640906 .455791-1.240579$

H $4.1902615 .069191-0.182733$

H $0.6727527 .305647-0.096839$

H $2.0858197 .800121-1.187752$

H $\quad 0.8035295 .314501 \quad 1.199207$

H $2.3219994 .484617 \quad 1.296562$

H $0.0157294 .036129-0.715208$

H $2.8657730 .987583-3.191929$

C $-5.444622-1.021963-2.445092$

O $-4.754735-0.067673-1.610871$

C $-5.6971560 .924446-1.161421$

C $-6.9689140 .697879-1.970800$

C $-6.931537-0.804856-2.170453$

H $-5.9158200 .693721-0.112323$

C $-5.0912142 .329458-1.269241$

H $-7.8633571 .040060-1.441502$

H -6.936846 $1.205161-2.940903$

C -7.845795 -1.292647 -3.280697

H -7.215784 -1.292608 -1.228347

C $-3.7745742 .508861-0.484944$

O $-4.7882472 .613106-2.641452$

H -5.820998 $3.081910-0.947119$

H $-2.9851551 .909954-0.954221$

O $-3.3652663 .875853-0.631887$

C -3.8488812 .1682351 .016716$

C -2.5316342 .4890061 .745273$

H -4.0812921 .1079811 .143443$

O $-4.8898842 .921126 \quad 1.638301$

H -1.7071872 .0232611 .197562$

H -2.3660943 .5729641 .725682$

H $-8.888134-1.041534-3.058222$

H -7.588195 -0.836562 -4.242514

H -7.777643 -2.378999 -3.394303

C $-4.964812-2.435583-2.115492$

H $-5.6205312 .840108-3.088419$

H $-4.0218734 .428747-0.174372$

H -5.7394592 .5836821 .308824$

H $-5.575488-3.179352-2.639154$

H -5.073671 -2.621306 -1.040332

H $-5.205116-0.767118-3.486118$

C $-1.699085-0.2223992 .875155$

O $-2.7573830 .616267 \quad 3.354279$

C -2.5275572 .0232393 .220312$

C -1.2626592 .4149283 .994165$

C -0.0572231 .5957063 .546044$

C -0.3779570 .1024673 .589008$

H -1.588315 -0.095479 1.791268

C $-2.101018-1.6820683 .146386$

H -3.3719262 .5198913 .714283$

O $-0.9973793 .804278 \quad 3.830382$

H $-1.4311312 .243570 \quad 5.065341$

H $\quad 0.2721401 .9072332 .550950$

O $1.026156 \quad 1.874123 \quad 4.435161$

H $-0.427492-0.2259824 .635245$

O $0.675172-0.6255782 .961947$

C $-3.292075-2.1342662 .329143$

H -1.269816 -2.354535 2.906700

H -2.369285 -1.797157 4.203626

H -0.1733084 .0014114 .307667$

H 1.8203921 .4470494 .072420

H $1.480793-0.4950753 .489525$

O $-3.600302-3.4192942 .678200$

O $-3.879882-1.4594331 .496323$

C $-4.730374-3.9867792 .008593$

H $-4.532708-4.0676590 .936662$

H $-4.895052-4.9898552 .410845$

H -5.624762 -3.383806 2.192350

SCF Energy (B3LYP/6-31G**//MMFF) $=-3245.90173955$

0500038

MM̄FF Geometry

C -3.066221 -2.753643 -2.872487

C $-2.178020-2.921711-1.881830$ 
C $-0.686680-2.945699-2.099968$

O $-0.079938-1.903027-1.300719$

C $-0.114513-4.296247-1.632638$

C $1.361163-4.471488-1.936037$

C $2.326591-4.601323-1.004990$

C $1.725980-4.531989-3.397785$

C $2.181860-4.6073700 .490410$

C $2.366905-3.2363631 .158020$

C $3.752915-2.6073900 .937788$

C $3.944743-1.3941941 .848225$

C $5.296829-0.7073891 .604385$

O $3.845896-2.184157-0.423193$

O 5.2579550 .5768022 .248725

C $5.472154-0.4438870 .090312$

C $6.445398-1.4824392 .261898$

O $6.7949200 .017056-0.171856$

C $5.126136-1.658402-0.802769$

C $5.032750-1.317641-2.308777$

O $6.139735-2.668519-0.724944$

C $-0.186233-0.625256-1.756546$

O $-0.754145-0.247470-2.768900$

C $0.5096950 .253919-0.793163$

C $0.2578991 .570605-0.805202$

C 0.8660332 .5702690 .072986

C 2.0763012 .1568430 .867632

C $3.981893-0.267950-2.686546$

C $4.5256101 .147882-2.788987$

C $3.7655283 .490852-3.183066$

O $5.7214151 .418344-2.720862$

C $3.6400224 .195717-1.869396$

C $2.8354155 .251261-1.671582$

C $2.7372845 .998587-0.372283$

C 1.3153216 .1183930 .129340

C $0.5996667 .236022-0.081711$

C 0.7566514 .9790130 .950518

C 0.3342623 .8097450 .104444

N $3.5119952 .073731-2.999771$

H -2.712428 -2.643511 -3.894701

H $-2.532149-3.025948-0.859965$

H $-0.443295-2.776529-3.155958$

H $-0.657434-5.118776-2.117577$

H $-0.301471-4.401802-0.558485$

H $3.352598-4.727975-1.349539$

H $2.771146-4.820642-3.553377$

H $\quad 1.104756-5.270608-3.914823$

H $1.582208-3.557260-3.873484$

H $1.210615-5.0181750 .786020$

H $2.921918-5.3077270 .898504$

H $1.590234-2.557500 \quad 0.787691$

H $2.196890-3.3673042 .234030$

H $4.516865-3.3668631 .140312$

H $3.842882-1.6759772 .903617$

H $3.147349-0.6652081 .654090$

H 5.0724450 .4339393 .192617

H $4.7970150 .384346-0.153481$

H $6.299987-1.5339563 .347794$

H $7.403900-0.9732842 .113787$

H $6.534988-2.5076441 .896370$

H $6.8278820 .318869-1.095012$

H $\quad 6.022478-1.057679-2.703250$

H $4.754405-2.246252-2.829014$

H $6.859982-2.346336-0.160383$

H $1.200314-0.205514-0.096070$

H $-0.4785591 .954280-1.510338$

H 2.8153801 .6768110 .217575

H 1.7997681 .4543151 .658799

H 2.5936912 .9946261 .339976

H $3.588918-0.515780-3.681079$

H $3.140188-0.284838-1.988543$

H $3.0433443 .856566-3.920001$

H $4.7743623 .633625-3.583363$

H $4.2614923 .838820-1.051438$

H $2.2363365 .623422-2.499778$

H $3.1603246 .999090-0.532398$

H 3.3600285 .5369770 .404077

H -0.4105127 .3414010 .299660$

H $1.0066918 .068678-0.646031$

H -0.1317145 .3137891 .503181$
H $1.4758364 .705458 \quad 1.728050$

H $-0.5344304 .017694-0.521811$

H $2.544771 \quad 1.763205-2.989489$

C $-5.088828-1.293792-3.002492$

O $-4.616946-0.342033-2.025310$

C $-5.7143620 .500705-1.629734$

C $-6.8180590 .260219-2.652320$

C $-6.614732-1.209144-2.963730$

H $-6.0580950 .131905-0.656006$

C $-5.2620381 .958703-1.503030$

H $-7.8116160 .482465-2.251416$

H $-6.6743090 .862939-3.556598$

C $-7.292421-1.657464-4.247008$

H -7.003540 -1.803427 -2.125792

C $-4.0666012 .181705-0.553104$

O $-4.8762112 .429992-2.800921$

H $-6.1132152 .577068-1.193044$

H $-3.1528071 .786385-1.013659$

O $-3.8891643 .600670-0.453212$

C $-4.246186 \quad 1.577987 \quad 0.853841$

C -3.1288321 .9938191 .828784$

H $-4.2739880 .487090 \quad 0.762108$

O

H -2.1634481 .8714551 .328231$

H -3.239149 3.0576882 .071195

H $-8.375659-1.511728-4.180819$

H -6.929421 -1.091501-5.111519

H -7.105691 -2.719031-4.437106

C -4.552077 -2.684862 -2.661759

H $-4.6032583 .358338-2.701845$

H $-3.0073343 .763176-0.078301$

H -5.6987961 .4575602 .159412$

H -5.021767 -3.441819 -3.299698

H $-4.797308-2.933491-1.621618$

H $-4.710490-0.961367-3.978175$

C $-1.867169-0.7188672 .426012$

O $-3.111719-0.2219652 .927382$

C $-3.144473 \quad 1.192163 \quad 3.151803$

C -2.0426101 .5932504 .143005$

C $-0.6676871 .096816 \quad 3.701218$

C $-0.717749-0.3994193 .389402$

H -1.668010 -0.251433 1.454337

C $-2.021739-2.2155612 .130495$

H -4.1032821 .4027983 .640749$

O -2.0218973 .0076374 .306010$

H -2.2750391 .1604545 .124864$

H -0.3029621 .6755562 .846582$

O $\quad 0.253274 \quad 1.328068 \quad 4.768976$

H $-0.834281-0.9517604 .329662$

O $0.509497-0.8097242 .796936$

C $-2.151923-3.0702453 .368748$

H $-2.906370-2.3956631 .507804$

H $-1.158555-2.5845961 .563698$

H -1.2994963 .2188674 .922022$

H 1.1422921 .1109344 .441435

H $1.209774-0.7021423 .462093$

O $-3.406328-2.9570883 .889477$

O $-1.241105-3.7528793 .820214$

C -3.647924-3.711172 5.081069

H $-2.975991-3.3825405 .879818$

H $-4.679568-3.5305785 .394409$

H -3.522468 -4.780201 4.884873

SCF Energy (B3LYP/6-31G**//MMFF) $=-3245.90772208$

05_00039

MM̄MF Geometry

C $-0.1442405 .307749 \quad 0.144117$

C $0.8832724 .721850-0.488148$

C 1.9885723 .9922210 .232766

O $2.1741742 .697028-0.386801$

C 3.3044984 .7789110 .094159

C $4.444558 \quad 4.2170590 .921417$

C 5.5900813 .7226230 .412960

C 4.2561764 .2675042 .416552

C $5.9963763 .613076-1.029409$

C $5.6418122 .275946-1.698369$

C $6.2818851 .046855-1.031684$

C $6.102327-0.196758-1.905359$ 
C $6.640725-1.464640-1.223020$

O 5.6438730 .8364250 .231019

O $6.141010-2.600225-1.946486$

C $6.065218-1.5531060 .207628$

C $8.170311-1.544072-1.296319$

O $6.667565-2.6214330 .933217$

C $6.194382-0.2386461 .003827$

C $5.441835-0.2643152 .357380$

O $7.576338-0.0022881 .300174$

C $1.2591931 .726445-0.114930$

O $0.286522 \quad 1.8121490 .617151$

C $1.6424770 .527945-0.893901$

C $0.763994-0.471551-1.064678$

C $1.005049-1.703934-1.820396$

C $2.415743-1.975230-2.273807$

C $3.931999-0.5132972 .268621$

C $3.536226-1.9707162 .439169$

C $1.571928-3.4886852 .228512$

O $4.309940-2.8375632 .836568$

C $1.663000-4.1826050 .906318$

C $0.614912-4.7646880 .303950$

C $0.707548-5.492750-1.006527$

C $-0.332204-5.037053-2.006134$

C -1.453342 -5.751088 -2.202948

C $-0.028998-3.817098-2.846684$

C $-0.030841-2.536203-2.057503$

N $2.198755-2.1833322 .130937$

H $-0.181850 \quad 5.2874201 .230777$

H $0.9273014 .742577-1.574458$

H 1.7369003 .8498651 .290559

H 3.1500535 .8211870 .404820

H $3.5792154 .815638-0.965597$

H 6.3399863 .3532581 .112157

H 5.1726954 .0119192 .959295

H 3.9638825 .2751842 .729525

H 3.4818423 .5624422 .733330

H $5.5664244 .431056-1.617213$

H $7.0822113 .763945-1.085505$

H $4.5512472 .164374-1.708030$

H $5.9701322 .331491-2.743716$

H $7.3473581 .252364-0.875457$

H $6.578855-0.057259-2.883669$

H $5.033777-0.341540-2.112879$

H $6.433555-2.519391-2.870412$

H $5.002781-1.8036780 .106060$

H $8.503387-1.552630-2.341182$

H $8.539298-2.477861-0.857884$

H $8.667499-0.708445-0.799552$

H $6.542304-3.4328450 .411805$

H $5.928181-0.9659973 .045756$

H 5.5699030 .7269782 .816180

H 7.6300890 .6065682 .055302

H $2.6379670 .509205-1.322256$

H $-0.230570-0.372909-0.631450$

H $2.715962-1.260253-3.047061$

H $2.560040-2.977523-2.681990$

H $3.109386-1.895322-1.430044$

H 3.4421240 .0306573 .086462

H $3.524868-0.1272551 .329678$

H $0.536101-3.3299482 .543373$

H $2.084458-4.0819882 .992641$

H $2.640961-4.2290580 .432706$

H $-0.356651-4.7448730 .792586$

H $0.580982-6.563693-0.799966$

H $\quad 1.705470-5.393431-1.451650$

H $-2.187903-5.458006-2.945015$

H -1.662780 -6.645386 -1.625187

H $-0.784502-3.705900-3.636337$

H $\quad 0.909679-3.983173-3.382453$

H -1.013852 -2.259852 -1.675880

H $\quad 1.645447-1.4179551 .756909$

C $-2.6096195 .302098-0.213583$

O $-2.6174653 .965042-0.749011$

C $-3.9579543 .643858-1.182547$

C $-4.8288424 .828295-0.784430$

C $-3.8371505 .975094-0.818694$

H -3.904039 $3.554882-2.274502$

C $-4.3599762 .305642-0.551712$
H $-5.6756234 .971367-1.462489$

H $\quad-5.2260054 .698378 \quad 0.230188$

C $-4.3090847 .205976-0.065406$

H -3.642893 $6.241940-1.866224$

C $-5.8280461 .910317-0.782620$

O $-3.5167211 .287896-1.102576$

H -4.1474042 .3395860 .522940$

H $-6.4882132 .629278-0.283472$

O $-6.1073902 .010765-2.184433$

C $-6.1857600 .479682-0.331284$

C $-5.828871 \quad 0.184073 \quad 1.135029$

H -5.703343 -0.242065 -0.997667

O

H -4.7582720 .3438311 .288650$

H $\quad-6.3566270 .896457 \quad 1.781088$

H $-5.2371917 .590592-0.500966$

H -4.5003656 .9823700 .989523$

H -3.560558 8.003101 -0.110267

C -1.286995 $5.985734-0.558989$

H $-2.5954261 .553102-0.937583$

H $-7.0345661 .747772-2.315317$

H -8.0525450 .9203890 .081278$

H -1.308587 $7.035718-0.247591$

H -1.129030 $5.964711-1.644352$

H -2.7202135 .2130380 .875802$

C $-4.246676-2.3835250 .721446$

O $-5.671906-2.2424530 .687370$

C $-6.200735-1.2504761 .572854$

C $-5.812472-1.5852203 .020357$

C $-4.305433-1.7784163 .175781$

C $-3.776909-2.7607752 .131111$

H -3.772403 -1.445510 0.409188

C $-3.873482-3.474049-0.290799$

H -7.292785 -1.330594 1.504762

O $-6.247057-0.565653 \quad 3.913967$

H $-6.312189-2.514306 \quad 3.324077$

H $-3.769461-0.8234933 .121491$

O $-4.032031-2.3162294 .471948$

H $-4.110823-3.7757022 .382410$

O $-2.351197-2.7586672 .164313$

C $-4.103449-3.012055-1.713492$

H -2.818855 -3.755096 -0.210271

H -4.483436 -4.366802 -0.106033

H -7.209782 -0.476652 3.809102

H -4.405539-1.699456 5.124810

H $-2.081719-2.978528 \quad 3.072712$

O $-4.654022-4.024953-2.442841$

O $-3.806483-1.903250-2.138103$

C $-4.899759-3.726050-3.820531$

H -3.960052 -3.493225 -4.330450

H $-5.341751-4.610656-4.286579$

H -5.604623 -2.893707 -3.907969

SCF Energy (B3LYP/6-31G**//MMFF) $=-3245.91358465$

05_00040

MM̄FF Geometry

C $\quad 0.712738-2.6368332 .089137$

C $1.259085-3.1612340 .981344$

C $0.659767-3.162736-0.404518$

O $-0.660702-2.581498-0.390518$

C $1.545832-2.300738-1.325426$

C $1.048828-2.208799-2.753203$

C $0.611735-1.076178-3.338826$

C $1.086970-3.495014-3.537193$

C $0.5364290 .306997-2.749943$

C $-0.8491570 .587094-2.168294$

C - $-1.0986212 .061626-1.820477$

C $-0.1837112 .572705-0.704506$

C $-0.5357584 .009490-0.284705$

O $-2.4707102 .151473-1.407869$

O 0.1477654 .2663240 .950980

C $-2.0514754 .094247-0.007425$

C $-0.0003315 .033843-1.291278$

O -2.4474615 .4363880 .262850$

C $-2.9196823 .484885-1.127663$

C $-4.4169043 .404482-0.746377$

O $-2.8288754 .312056-2.293681$

C $-1.723034-3.373470-0.693713$ 
O $-1.716003-4.570964-0.925290$

C $-2.917127-2.499193-0.721573$

C $-4.110846-2.985027-1.093533$

C -5.350206 -2.209193 -1.202200

C $-5.252432-0.725297-0.965713$

C -4.7084912 .6538410 .555022$

C -6.1771182 .2917740 .708831$

C -7.6856110 .9786142 .210518$

O $-7.0439822 .627756-0.092679$

C -7.691512 -0.4732311 .844845$

C $-8.653629-1.0618411 .117635$

C $-8.651145-2.5269270 .778618$

C $-8.873811-2.798322-0.694841$

C $-9.941703-3.502482-1.107273$

C $-7.871498-2.260556-1.694632$

C $-6.497193-2.848543-1.514200$

N -6.3950831 .5422341 .856777$

H $-0.276741-2.1895312 .052618$

H $2.250857-3.6031291 .054118$

H $0.635042-4.197808-0.764309$

H $2.563196-2.711454-1.344120$

H $1.635392-1.300622-0.886899$

H $\quad 0.267273-1.134967-4.370766$

H $\quad 0.828102-3.346789-4.591090$

H $2.092168-3.928179-3.506059$

H $0.380638-4.221380-3.124754$

H $\quad 1.3135370 .461377-1.995697$

H $\quad 0.7547751 .023599-3.551600$

H -1.624810 $0.264202-2.876590$

H -1.003937 -0.023344 -1.270797

H $-0.9648882 .654504-2.732883$

H $0.8709672 .517564-0.998592$

H -0.2840691 .9194730 .173820$

H $-0.1216695 .148576 \quad 1.259811$

H $-2.221243 \quad 3.5366570 .921801$

H $1.0906204 .952914-1.371699$

H -0.198276 $6.058841-0.959139$

H $-0.4170344 .908761-2.292865$

H $-2.4860725 .910110-0.585477$

H -4.847587 4.412905 -0.691789

H $-4.9474882 .903761-1.568435$

H -3.602291 4.136708 -2.855354

H -2.774897 -1.459398 -0.448989

H -4.190353 -4.040507 -1.349159

H -4.535602 -0.269296-1.657966

H $-4.924740-0.5289530 .060383$

H -6.193730 -0.192157 -1.104019

H $-4.125831 \quad 1.7268910 .595155$

H $-4.4282253 .273211 \quad 1.414574$

H -8.485044 1.5355051 .711810

H $-7.8005861 .088183 \quad 3.293130$

H -6.864165 -1.076428 2.212509

H -9.497311 -0.4704820 .770192$

H -9.441364 -3.001919 1.374338

H -7.713582 -3.002616 1.091832

H - $10.114871-3.704897-2.158961$

H $-10.668728-3.893446-0.403136$

H -7.879594 -1.169221-1.647075

H -8.190993 -2.499865 -2.717822

H -6.461139 -3.926775 -1.675993

H $-5.598422 \quad 1.2833372 .431898$

C $1.559060-1.1231733 .887907$

O $2.308434-0.3498392 .930672$

C $3.138304 \quad 0.5917663 .636777$

C 2.7702900 .4685985 .113019

C $2.319541-0.9766795 .202886$

H 4.1739090 .2580013 .503447

C 2.9617671 .9981263 .049356

H 3.6163500 .6961905 .768835

H 1.9486681 .1402615 .386919

C $1.490516-1.2789246 .438566$

H $3.208690-1.6213815 .196961$

C 3.1884712 .0937381 .526808

O 1.6246232 .4499603 .306760

H 3.6209372 .7007903 .571824

H 2.3853671 .5701920 .993991

O 3.0852013 .4747731 .156671

C 4.5494101 .5651821 .041570
C $4.7343661 .774327-0.473113$

H 4.6210670 .5009811 .275676

O $5.5858462 .236727 \quad 1.754125$

H $3.8723941 .337173-0.989522$

H $4.7313092 .848989-0.691843$

H $2.068382-1.0801777 .347188$

H $\quad 0.585047-0.6640536 .474662$

H $1.185019-2.3297676 .456794$

C $1.430214-2.5710783 .409562$

H 1.0153191 .8184222 .886941

H 2.2130223 .7886511 .451508

H 6.4136981 .7519391 .598460

H $0.870463-3.1626514 .142255$

H $2.427599-3.0183413 .318084$

H $0.565584-0.6605603 .963387$

C $5.325012-1.107933-1.325245$

O $6.222086-0.197031-0.679268$

C $6.0467581 .178654-1.030911$

C $6.2182141 .358776-2.545431$

C $5.2975380 .432563-3.335802$

C $5.453914-1.008728-2.852456$

H $4.295607-0.885644-1.020996$

C $5.683373-2.525203-0.847046$

H $6.8697921 .723751-0.552483$

O $5.9779282 .714259-2.908935$

H $7.2591861 .134942-2.813126$

H $4.2547570 .766297-3.276302$

O $5.6689620 .512061-4.713811$

H $6.429342-1.396852-3.173151$

O $4.449451-1.820324-3.456360$

C $5.297424-2.7765930 .595294$

H $5.163886-3.278485-1.449391$

H $6.766300-2.675937-0.932479$

H $6.0768502 .778395-3.874263$

H $5.0137130 .007037-5.224089$

H $4.617776-1.836407-4.413336$

O $5.813278-3.9794490 .987827$

O $4.616725-2.0335761 .287273$

C $5.516735-4.3652722 .333760$

H $6.031331-5.3078472 .538282$

H $5.878345-3.6088563 .037014$

H $4.440305-4.5201762 .451745$

SCF Energy (B3LYP/6-31G**//MMFF) $=-3245.93028019$

05_00041

MM̄FF Geometry

C $4.682467-2.6692071 .382301$

C $3.383381-2.9247931 .171284$

C $2.387579-3.1070432 .287757$

O $1.349337-2.1116042 .134411$

C $1.730952-4.4959032 .204728$

C $0.733913-4.752678 \quad 3.319804$

C $-0.592757-4.9210473 .149592$

C $1.321464-4.8413334 .706454$

C $-1.383614-4.9215731 .871016$

C $-2.247708-3.6648301 .746714$

C $-3.258851-3.7320890 .591370$

C $-2.585668-3.776745-0.782518$

C $-3.606931-3.718391-1.930753$

O $-4.084107-2.5626110 .693842$

O $-2.892049-3.420473-3.140477$

C $-4.582985-2.548625-1.685554$

C $-4.288477-5.074048-2.152129$

O $-5.643676-2.555784-2.638034$

C $-5.159929-2.518292-0.255376$

C $-5.987711-1.2428000 .026689$

O $-6.044138-3.632897-0.081658$

C $1.619916-0.8563862 .585863$

O $2.649184-0.4660273 .112975$

C $0.434235-0.0205702 .302924$

C 0.5366601 .3153592 .360831

C -0.5338232 .2645732 .059023$

C $-1.929106 \quad 1.717506 \quad 1.902989$

C $-5.2325860 .077230-0.146635$

C $-6.035201 \quad 1.266986 \quad 0.354357$

C -5.9006293 .7357250 .590972$

O $\quad-7.1614491 .1689410 .834130$

C -4.7947494 .7232160 .773538$ 
C $-4.340298 \quad 5.1128681 .974401$ C -3.2014476 .0739192 .168066$ C -1.9360505 .3659502 .609881$ C $-1.5538085 .396913 \quad 3.897902$ C -1.1619434 .6770411 .504168$ C -0.2307653 .5742561 .932820$ N -5.3322102 .4556320 .211875$ H $5.056255-2.6238702 .402644$ H $3.002267-2.9648050 .154285$ H $2.869628-2.9827203 .265296$ H $2.503135-5.2754272 .248300$ H $1.254006-4.6025301 .225624$ H $-1.199944-5.0974064 .037343$ H $\quad 0.586172-5.1679305 .449960$ H $2.144628-5.5630424 .724982$ H $1.701536-3.8674235 .029436$ H $-0.739893-5.0260540 .995257$ H $-2.026633-5.8106851 .879630$ H -2.802004 -3.499091 2.680752 H -1.609736 -2.7794891 .627931$ H -3.885656 $-4.619010 \quad 0.741632$ H -1.960085 -4.670383 -0.889727 H -1.902208 -2.922678 -0.877677 H $-2.222109-4.113177-3.271234$ H -4.020385 -1.625645 -1.861773 H -3.544902 -5.839688 -2.404404 H -4.978174 -5.036335 -3.002303 H -4.837608 -5.425685 -1.276064 H $-5.239137-2.566456-3.522226$ H $-6.891417-1.236419-0.597088$ H $-6.351189-1.2976781 .062813$ H $-6.649790-3.4343580 .651541$ H $-0.482068-0.5269002 .022107$ H 1.4998591 .7577312 .611655 H -2.684403 $2.496251 \quad 1.782762$ H $-2.218671 \quad 1.1437882 .790680$ H -1.995509 1.0634201 .028825 H -4.2892150 .0495770 .409412$ H $-5.0091350 .250269-1.204949$ H -6.4989773 .6091281 .499978$ H $-6.5728484 .060053-0.209977$ H -4.335563 $5.122064-0.127733$ H -4.792822 4.7021412 .874308 H -3.508956 6.8219572 .909945 H $-2.997323 \quad 6.638816 \quad 1.249517$ H -0.6441364 .9188484 .243190$ H -2.147222 5.9126114 .646240 H $-0.575400 \quad 5.4348880 .968425$ H -1.853689 $4.278016 \quad 0.753513$ H 0.8001423 .8948052 .083083 H $-4.4808842 .450310-0.343908$ C $6.244284-0.9813450 .424217$ O $5.224168-0.0143160 .098809$ C $5.8365931 .085116-0.603912$

C $7.341090 \quad 0.833723-0.567064$

C $7.397055-0.680983-0.529677$ H $5.5014171 .020516-1.645295$ C 5.4034972 .4208540 .010916 H $7.8531331 .262356-1.434126$ H 7.8011961 .2556940 .334232 C $8.740544-1.224835-0.076459$ H $7.167255-1.068130-1.531462$ C $3.8796132 .664921-0.006201$ O 5.8364112 .4494201 .377290 H $5.9241973 .242709-0.494789$ H $3.382321 \quad 1.9512560 .660746$ O 3.6433303 .9607690 .560635 C $3.2364012 .601943-1.404796$ C $1.7552823 .024821-1.387674$ H $3.3296531 .584036-1.791861$ O $3.9619853 .466776-2.278821$ H $1.2469982 .477775-0.588433$ H $1.6885114 .092851-1.148579$ H $9.534548-0.905756-0.759826$ H $8.998947-0.8708770 .927237$ H $8.733617-2.318922-0.055982$ C $5.678520-2.3959040 .290563$ H 5.5427093 .2953131 .756968
H $4.0310234 .618804-0.041718$

H $3.7045793 .249944-3.190479$

H $6.480571-3.1377690 .369621$

H $5.208944-2.518715-0.692437$

H $6.550236-0.7871641 .461333$

C $0.3881390 .478957-2.379581$

O $1.1384361 .417975-3.161299$

C $1.0367482 .780519-2.734638$

C $-0.4302273 .233085-2.765112$

C $-1.3301282 .290815-1.971730$

C $-1.1033640 .841057-2.397832$

H $\quad 0.7559240 .475717-1.346501$

C $0.635730-0.912668-2.984039$

H $1.5636763 .377341-3.489039$

O $-0.5383154 .560744-2.262558$

H $-0.7726163 .253366-3.807956$

H $-1.1734872 .408249-0.896100$

O $-2.6994552 .618442-2.217120$

H -1.527307 $0.692853-3.399567$

O $-1.8208580 .004661-1.494355$

C $2.045201-1.407705-2.733750$

H $-0.036729-1.660945-2.553235$

H $0.479082-0.868871-4.068610$

H -1.452981 $4.857361-2.403958$

H -2.866488 $3.491695-1.824293$

H $-1.876321-0.878889-1.893478$

O $2.321450-2.423462-3.604313$

O $2.796276-0.986057-1.865798$

C $3.618210-3.013882-3.470660$

H $3.753725-3.725374-4.289635$

H $4.399262-2.250100-3.537928$

H $3.685378-3.553578-2.521537$

SCF Energy (B3LYP/6-31G**//MMFF) $=-3245.91644361$

05 00042

MM̄FF Geometry

C -1.604763 -3.068638 3.023809

C $-0.276846-3.056363 \quad 3.213123$

C $0.714614-2.9758902 .079989$

O $1.248231-1.6309242 .053404$

C $1.880698-3.9518832 .319010$

C $2.836723-4.0525251 .146125$

C $4.126501-3.6639701 .167228$

C $2.266395-4.663187-0.108495$

C $4.900516-3.0595752 .304116$

C $4.891343-1.5232192 .351035$

C $5.437406-0.8418281 .084588$

C 5.6221110 .6601541 .317395

C $6.060848 \quad 1.3952750 .040404$

O $4.500555-1.0476900 .023872$

O 5.8864042 .8037610 .260419

C $5.1195121 .000419-1.118805$

C $7.5511671 .185049-0.252970$

O $5.585701 \quad 1.531083-2.356419$

C $4.910894-0.522229-1.245609$

C $3.820925-0.906632-2.276319$

O $6.131853-1.130779-1.683607$

C $0.491066-0.6830641 .436270$

O $-0.564373-0.8512540 .845844$

C $1.153085 \quad 0.624748 \quad 1.618437$

C $0.4221521 .737387 \quad 1.460386$

C 0.9012233 .1159941 .551239

C 2.3893053 .3284831 .629273

C $2.420640-0.356782-1.986152$

C $2.0864470 .923518-2.732759$

C $0.3109042 .664546-2.902537$

O $2.7959431 .393953-3.617657$

C $0.7811103 .866369-2.144093$

C $-0.0427104 .832868-1.710315$

C $0.419336 \quad 6.067682-0.988265$

C $-0.3102206 .287660 \quad 0.319188$

C - -1.3591057 .1246090 .392834$

C 0.2241785 .5952961 .554394

C -0.0096544 .1109361 .519565$

N $0.8767041 .464080-2.314732$

H -1.999085 -3.049715 2.010494

H $0.116562-3.0405534 .226740$

H $\quad 0.227904-3.2007751 .123281$ 
H $1.489428-4.9579212 .521633$

H $2.416168-3.6463213 .224683$

H $4.711546-3.7935190 .256832$

H $3.034528-4.853668-0.865852$

H $\quad 1.786207-5.6209640 .116604$

H $1.525714-3.994905-0.556060$

H $4.545077-3.4476363 .264717$

H $5.938972-3.4062622 .224356$

H $3.865846-1.1841882 .537129$

H $5.493196-1.2146363 .214979$

H $6.394713-1.3084660 .824419$

H 6.3375730 .8426412 .128973

H 4.6719541 .0940871 .656434

H 6.4150853 .0534571 .037532

H $4.1518771 .476391-0.918545$

H $8.161281 \quad 1.5602800 .577521$

H $7.8646671 .754684-1.134771$

H $7.8147350 .136615-0.406472$

H $5.6814762 .492049-2.241843$

H $4.154600-0.656649-3.290460$

H $3.736498-2.003194-2.262064$

H $5.922623-1.994252-2.076607$

H 2.2064490 .6355001 .868098

H $-0.638647 \quad 1.6319921 .238840$

H 2.8886522 .8396800 .785694

H 2.7886912 .9151732 .561662

H 2.6857274 .3783631 .593818

H $1.684194-1.100596-2.311456$

H $2.274949-0.201850-0.914450$

H $-0.7779662 .557881-2.876191$

H $0.6309672 .739988-3.946786$

H $1.8502713 .961165-1.968643$

H -1.108367 $4.750390-1.913047$

H $\quad 0.2533826 .924259-1.654688$

H $1.5003586 .043223-0.802140$

H $-1.872603 \quad 7.3074561 .330861$

H -1.730596 $7.641965-0.485552$

H -0.2746895 .9826412 .452559$

H 1.2774225 .8611811 .674441

H -1.0659913 .8423901 .465063$

H $\quad 0.376427 \quad 1.030998-1.543879$

C -3.189389-1.665167 4.425318

O $-3.743622-1.075013 \quad 3.230955$

C $-3.374627 \quad 0.318424 \quad 3.199883$

C $-2.879250 \quad 0.654887 \quad 4.601844$

C $-2.200172-0.642974 \quad 4.989452$

H -2.5316580 .4058532 .505549$

C -4.5381191 .1864952 .715889$

H -2.201752 1.5142204 .607750

H $-3.708270 \quad 0.870140 \quad 5.285983$

C $-1.950366-0.7811016 .480779$

H -1.239761 -0.6925194 .463117$

C $-5.073616 \quad 0.816154 \quad 1.318417$

O $\quad-5.619003 \quad 1.0549423 .647948$

H -4.2386452 .2412632 .738899$

H $-5.577125-0.156787 \quad 1.360104$

O $-6.093473 \quad 1.7694320 .988353$

C $-4.013700 \quad 0.799816 \quad 0.198424$

C $-4.6492600 .608831-1.191691$

H -3.299205 -0.0057390 .396325$

$\begin{array}{llll}\text { O } & -3.301881 & 2.036179 & 0.226074\end{array}$

H $-5.306311-0.266859-1.154924$

H -5.278312 $1.477316-1.421903$

H -1.294180 $0.020124 \quad 6.836423$

H -2.883695 -0.7285307 .051390$

H -1.468931 -1.737405 6.708010

C -2.616190 -3.056849 4.133349

H -6.3482431 .6125093 .326274$

H -5.662456 2.6356320 .886093

H -2.513203 $1.936948-0.333149$

H $-3.433630-3.7222593 .828604$

H -2.191077 -3.484199 5.049059

H -4.030601-1.785635 5.120942

C $-3.204528-1.917103-2.143430$

O $-2.672579-0.590835-2.071096$

C $-3.6162110 .453040-2.331181$

C $-4.2430560 .266105-3.720603$

C $-4.867068-1.117812-3.880552$
C $-3.855439-2.200227-3.505472$

H -3.956036 -2.033902 -1.352443

C $-2.084136-2.911689-1.800974$

H $-3.0399281 .385293-2.367698$

O $-5.2209881 .273844-3.955803$

H $-3.4622410 .392711-4.481973$

H $-5.789247-1.205883-3.294851$

O $-5.244941-1.285146-5.249012$

H -3.099466 -2.251574-4.296805

O $-4.507673-3.468200-3.481101$

C $-0.933780-2.911351-2.787595$

H - $1.658105-2.668155-0.821866$

H -2.504720 -3.922684 -1.751648

H $-5.6053281 .106509-4.833431$

H $-5.595607-2.187451-5.344008$

H $-5.189789-3.440194-2.788504$

O $-0.295516-4.117329-2.731932$

O $-0.614191-1.966645-3.496359$

C $0.827602-4.260009-3.607480$

H $0.513152-4.146425-4.649245$

H $1.600648-3.528369-3.354620$

H $1.238424-5.263987-3.472058$

SCF Energy (B3LYP/6-31G**//MMFF $)=-3245.91487161$

05 00043

MM̄FF Geometry

C $4.174662-2.5219712 .202956$

C $2.871877-2.7569341 .987020$

C $1.849210-2.7398073 .092140$

O $0.956127-1.6280112 .847533$

C $1.007140-4.0278713 .096931$

C $0.061343-4.1014184 .282510$

C $-1.283242-4.0805754 .197987$

C $\quad 0.717277-4.2261555 .635309$

C $-2.140620-4.0397512 .965047$

C $-2.479706-2.6120422 .533729$

C $-3.716901-2.5202621 .626373$

C $-3.589863-3.3615680 .354293$

C $-4.793071-3.172121-0.583298$

O $-3.881669-1.1349081 .287434$

O $-4.450171-3.729532-1.861503$

C $-5.022824-1.662395-0.796362$

C $-6.026839-3.940471-0.095914$

O $-6.208500-1.423102-1.549524$

C $-5.061236-0.8525370 .518305$

C -5.1126890 .6756790 .277033$

O $-6.242543-1.196073 \quad 1.252558$

C $1.338022-0.4047393 .305882$

O $2.360055-0.130043 \quad 3.913837$

C 0.2787670 .5575122 .931601

C 0.3971101 .8485713 .276254

C -0.5737172 .9075542 .988398$

C -1.8135922 .5146492 .230596$

C $-3.9943471 .221441-0.610874$

C $-3.9257342 .738985-0.615285$

C $-2.5219904 .589105-1.549932$

O $-4.708223 \quad 3.4545490 .002534$

C -1.347163 $4.880972-0.670608$

C -1.2719205 .9386210 .151346$

C $-0.080108 \quad 6.2229381 .021837$

C -0.4517916 .4876812 .466059$

C -0.1634017 .6668843 .042348$

C $-1.1570175 .398003 \quad 3.246618$

C -0.3116094 .1621023 .411916$

N -2.875355 $3.189477-1.402173$

H $4.523345-2.3522583 .219287$

H $2.510994-2.8971210 .971732$

H $2.338146-2.6124584 .065411$

H $1.663944-4.9070253 .126270$

H $0.449564-4.0915372 .155860$

H - $1.852838-4.1204885 .126111$

H -0.005253 -4.450367 6.427510

H $1.451563-5.0384335 .628024$

H $1.225033-3.2967075 .909436$

H -1.667713 -4.585524 2.141934

H -3.063651 -4.588442 3.192379

H -2.653411 -1.976348 3.412729

H -1.617989 -2.166893 2.020763 
H $-4.584782-2.8450392 .212748$ H -3.468838 -4.4254960 .589419$ H $-2.677647-3.067157-0.181643$ H -4.220991 -4.665285-1.728843 H -4.186528 -1.306429-1.410257 H $-5.813543-5.015229-0.047228$ H $-6.861572-3.832652-0.797295$ H $-6.364862-3.6281610 .894151$ H -6.141166 -1.943252 -2.368585 H $-6.0861670 .956898-0.145887$ H $-5.061735 \quad 1.1776721 .253582$ H $-6.439794-0.4743811 .872600$ H -0.5755350 .1741082 .385303$ H 1.2837242 .1630293 .825241 H -2.498252 3.3434022 .046107 H -2.382332 1.7579662 .782632 H -1.541969 2.1057931 .251668 H $-3.0243740 .837760-0.275465$ H -4.154893 $0.903401-1.646191$ H -3.385734 5.218127-1.312390 H -2.247515 4.743054 -2.597796 H -0.508956 4.190069 -0.720604 H $-2.095914 \quad 6.6473400 .185848$ H 0.4363567 .0937570 .597450 H 0.6418635 .3980050 .983992 H -0.4238807 .8711114 .075732$ H 0.3404528 .4567642 .495131 H -2.1227765 .1972492 .776976$ H -1.402064 5.7539874 .256316 H 0.6142514 .3394193 .960919 H $-2.2600492 .506669-1.835572$ C $5.543494-0.9129650 .908390$ O $4.450092-0.2634280 .224708$ C $4.9912220 .698985-0.700950$ C $6.4892940 .760299-0.423949$ C $6.766899-0.6600510 .033167$ H $4.849908 \quad 0.286823-1.706074$ C $4.2539752 .036258-0.568121$ H $7.0629741 .047144-1.310450$ H $6.724140 \quad 1.4739750 .374691$ C $8.093100-0.8140160 .756371$ H $6.749227-1.325026-0.840640$ C $2.7384441 .951555-0.858916$ O $4.4216462 .512873 \quad 0.772854$ H $4.7236702 .780902-1.221730$ H $2.2436881 .351139-0.086665$ O $2.1982513 .273054-0.736295$ C $2.3962161 .392756-2.253105$ C $0.8884311 .441282-2.569561$ H $2.746057 \quad 0.358312-2.310353$ O $3.1105222 .147700-3.231813$ H $0.3437990 .938733-1.762616$ H $0.5506852 .484416-2.587754$ H $8.923880-0.5407480 .097633$ H $8.143292-0.1740161 .643695$ H $8.245218-1.8487521 .078696$ C $5.205468-2.3894801 .117879$ H 3.9316223 .3498960 .846426 H $2.5816933 .813993-1.447849$ H $3.0989731 .641735-4.061545$ H $6.093471-2.9540461 .421847$ H $4.837178-2.8327510 .185629$ H $5.661525-0.4040901 .875300$ C $0.537712-1.513624-3.253067$ O $1.118044-0.500426-4.084154$ C $0.541610 \quad 0.799559-3.934565$ C $-0.955511 \quad 0.748423-4.265437$ C $-1.682787-0.334471-3.470086$ C $-0.951222-1.673524-3.581186$ H $0.656346-1.241601-2.197179$ C $1.325316-2.804566-3.508775$ H $1.0084971 .429200-4.702300$ O $-1.5441302 .021861-4.030693$ H -1.070633 $0.533032-5.336034$ H -1.806141 -0.045225 -2.420268 O $-2.998365-0.507826-4.002294$ H -1.086694 -2.072151 -4.594935 O $-1.561644-2.583001-2.669826$
C $2.723189-2.692168-2.942765$

H $0.863502-3.676279-3.034903$

H $1.385578-3.000228-4.586196$

H -2.455546 $1.994359-4.366788$

H $-3.4908670 .315072-3.846453$

H $-1.271773-3.480263-2.903724$

O $3.646909-2.947619-3.911777$

O $2.965126-2.412452-1.775764$

C $5.012921-2.856863-3.494170$

H $5.212335-3.565494-2.684498$

H $5.646957-3.111996-4.347394$

H $5.243426-1.835047-3.178584$

SCF Energy (B3LYP/6-31G**//MMFF) $=-3245.91637150$

05 00044

MM̄̄FF Geometry

C 2.3464790 .5951573 .462492

C 1.5410410 .3679574 .511466

C $0.203711-0.3341834 .396723$

O $-0.371985-0.0860873 .090567$

C $0.393741-1.8514944 .568651$

C $-0.879395-2.6519344 .381142$

C $-1.078690-3.5390183 .385785$

C $-1.957678-24150445.405901$

C $-0.119217-3.9304732 .295743$

C $-0.192878-3.0637141 .029562$

C - $1.517460-3.1866720 .259471$

C $-1.431855-2.457799-1.083260$

C $-2.762933-2.504270-1.848555$

O $-2.563151-2.6084381 .044649$

O $-2.690224-1.546545-2.916331$

C -3.899916 -2.048918 -0.908580

C $-2.993311-3.869424-2.507328$

O $-5.172991-2.236573-1.520700$

C $-3.872693-2.7384540 .472191$

C $-4.877809-2.1258451 .478939$

O $-4.227477-4.1180050 .314889$

C $-0.912494 \quad 1.1446842 .874383$

O -1.0998802 .0225313 .701507$

C -1.1639551 .2687241 .422103$

C -1.3133682 .4888250 .886089$

C -1.520292 $2.792856-0.530965$

C $-1.6853411 .633119-1.476480$

C $-4.687094-0.6338411 .774979$

C -5.5565830 .2807150 .926928$

C -5.9048952 .6925760 .402850$

O $-6.493294-0.1236320 .243509$

C $-5.2506143 .007793-0.905495$

C $-4.9067414 .251273-1.274553$

C $-4.2970244 .594169-2.604055$

C $-3.0223815 .398121-2.472880$

C $-3.0353486 .737257-2.582760$

C $-1.7244944 .642672-2.300477$

C - $-1.5594024 .086577-0.912943$

N $-5.189924 \quad 1.613673 \quad 1.059397$

H 2.0582840 .2040012 .488889

H 1.8238250 .7278215 .496866

H -0.4753830 .0535395 .165654$

H $0.796556-2.0637525 .567797$

H $1.159645-2.1895823 .862260$

H $-2.036370-4.0566703 .344823$

H -2.802636 -3.101756 5.286011

H -1.559828 -2.559297 6.415709

H -2.349408 -1.396409 5.325756

H $0.909755-3.9241582 .672220$

H $-0.314684-4.9771542 .029327$

H -0.014165 -2.016676 1.298508

H $0.629331-3.3693190 .372451$

H - $-1.723918-4.2518760 .102660$

H -0.626135 -2.867688 -1.701186

H $-1.166011-1.406806-0.910330$

H -1.926721 -1.776093 -3.473396

H $-3.778050-0.969153-0.767040$

H -2.181010 -4.097976 -3.207971

H -3.911857 -3.869807 -3.104497

H $-3.048169-4.689278-1.788248$

H $-5.157071-1.767349-2.372479$

H $-5.905560-2.3484731 .167625$ 
H $-4.742835-2.6562182 .432788$

H -4.536772 $-4.456081 \quad 1.171655$ H -1.1776810 .3601130 .832743$ H -1.257402 $3.355038 \quad 1.544537$ H -2.495349 $0.979020-1.138557$ H $-0.7611711 .048696-1.534655$ H -1.943421 $1.930045-2.494830$ H -4.980106 -0.447365 2.816284 H $-3.637167-0.3445201 .675444$ H $-5.8985573 .548281 \quad 1.085564$ H -6.9429942 .3899220 .231568$ H $-5.0804682 .181800-1.591954$ H $-5.1073405 .078837-0.597484$ H $-5.0405795 .168488-3.172287$ H -4.101407 $3.694188-3.200206$ H -2.122254 7.319557 -2.517991 H $-3.9615597 .281810-2.734538$ H $-0.8715865 .310472-2.482340$ H -1.647855 $3.879527-3.079745$ H -1.433997 $4.856500-0.150202$ H $-4.369621 \quad 1.8533331 .608502$ C 3.5259692 .5807182 .557651 O 3.2952262 .1308091 .207525 C 4.0290182 .9707980 .300360 C 4.6115224 .1027211 .142036 C 4.7952733 .4240382 .487041 H $4.8553282 .363663-0.086417$ C $3.1218623 .420943-0.851586$ H 5.5465264 .4885730 .724188 H 3.9148294 .9440451 .232745 C 4.9603064 .3964523 .641341 H 5.6774412 .7719582 .433802 C $2.4342982 .264312-1.609631$ O $2.0914674 .256864-0.307638$ H $3.6950524 .047766-1.545183$ H $1.6807601 .791413-0.968135$ O $1.7249192 .829908-2.719627$ C $3.3842031 .178010-2.151995$ C $2.6480070 .143107-3.028964$ H $3.8727090 .662486-1.319212$ O $4.4031381 .778836-2.952865$ H $1.778332-0.235816-2.482257$ H $2.2772170 .645843-3.929878$ H 5.8466485 .0221143 .493124 H 4.0932415 .0587313 .735305 H 5.0797373 .8615224 .588715 C $3.627975 \quad 1.3745103 .493747$ H $1.6497094 .700582-1.051109$ H $0.9777863 .337682-2.362177$ H $4.9831412 .291690-2.366184$ H 3.8617491 .7013904 .512659 H $4.4337720 .709498 \quad 3.162590$ H 2.6648473 .2031812 .836810

C $3.065892-2.620139-1.712766$

O $4.041689-1.776235-2.338866$

C $3.568801-1.021782-3.461405$

C $2.969570-1.955001-4.524967$

C $1.926241-2.899917-3.939824$

C $2.501536-3.624200-2.725471$

H $2.253175-2.008451-1.302790$

C $3.759007-3.347520-0.550029$

H $4.459194-0.569834-3.915908$

O $2.385295-1.215241-5.591612$

H $3.773278-2.562135-4.962054$

H $1.000951-2.370726-3.683139$

O $1.574930-3.878653-4.919975$

H $3.288503-4.316972-3.050240$

O $1.477542-4.401645-2.111876$

C $4.004955-2.4421220 .638485$

H $3.134809-4.174354-0.193265$

H $4.729737-3.738100-0.876807$

H $3.075759-0.636339-5.957785$

H $1.253707-3.400120-5.703412$

H $1.117526-4.996883-2.791605$

O $4.560825-3.1825811 .643769$

O $3.745564-1.248240 \quad 0.687046$

C 4.844551-2.470969 2.852078

H $3.912272-2.1390963 .317563$
H $5.354354-3.1540103 .536813$

H $5.503739-1.6212192 .650954$

SCF Energy (B3LYP/6-31G**//MMFF) $=-3245.90799341$

05_00045

MM̄FF Geometry

C $-3.484936-3.014191-0.862218$

C $-2.850804-3.938982-1.598602$

C -1.425428 -3.858858 -2.099654

O $-0.830431-2.569021-1.832837$

C $-0.567292-4.927376-1.395427$

C $0.857917-5.006032-1.913827$

C $1.960698-4.659727-1.218501$

C $1.001622-5.555575-3.311297$

C $2.050283-4.1228850 .183566$

C $2.469531-2.6508400 .227002$

C $3.934153-2.360329-0.143353$

C $4.900345-2.8601020 .936544$

C $6.351193-2.4328310 .670357$

O $4.043340-0.934704-0.280891$

O $7.086335-2.6722241 .880462$

C $6.379930-0.9153170 .390344$

C $7.003034-3.308830-0.405662$

O $7.691201-0.4932450 .023250$

C $5.345426-0.469089-0.663923$

C $5.2833521 .069486-0.810640$

O $5.727247-0.994364-1.941001$

C -1.066572 -1.570632 -2.728509

O $-1.783157-1.619433-3.715046$

C $-0.268510-0.399330-2.303814$

C $-0.2540040 .714416-3.051644$

C $0.5658481 .902914-2.800635$

C $1.5274911 .843779-1.645098$

C $4.819608 \quad 1.8226740 .439127$

C 4.4918703 .2755150 .131438

C 3.1123065 .1724150 .983257

O $4.9077903 .859308-0.866018$

C 1.8184265 .0574590 .238363

C $1.5159325 .761726-0.862726$

C $0.2332155 .583955-1.628595$

C $0.4587455 .434586-3.119045$

C $-0.0141786 .351339-3.980424$

C $1.2486334 .243085-3.613987$

C $0.4475982 .967355-3.622020$

N 3.6899653 .8450081 .111518

H $-2.959986-2.113132-0.557970$

H -3.394155 -4.838421 -1.882522

H -1.431182 -4.040945 -3.181320

H -1.027974 -5.916716 -1.520650

H $-0.580190-4.734068-0.318311$

H $2.926440-4.784495-1.706936$

H $2.049224-5.704084-3.594805$

H $0.501896-6.526620-3.391101$

H $0.558682-4.872873-4.042582$

H $1.095348-4.2166050 .705520$

H $2.751439-4.7432050 .751745$

H $1.811713-2.066565-0.427711$

H $2.281974-2.2666241 .238122$

H $4.159973-2.817619-1.112216$

H $4.845690-3.9493811 .045716$

H $4.596135-2.4488271 .909678$

H $7.990304-2.3386971 .746526$

H $6.158033-0.4174061 .342434$

H $7.016253-4.357606-0.084935$

H $8.051630-3.032922-0.561999$

H $6.486449-3.264398-1.366605$

H $7.839376-0.756681-0.900983$

H $6.2557221 .456379-1.142264$

H $4.5902691 .306394-1.629439$

H $5.289762-0.471189-2.633028$

H $0.319359-0.499694-1.399209$

H $-0.872250 \quad 0.751590-3.947362$

H $2.1735540 .962934-1.720866$

H $0.9795331 .800440-0.698866$

H $2.1907202 .706088-1.589198$

H $3.9254301 .345580 \quad 0.853149$

H $5.6012491 .813867 \quad 1.206071$

H 3.8250655 .8303260 .475601 
H $2.932194 \quad 5.551707 \quad 1.993471$ H 1.0952314 .3408000 .617666 H $2.2248276 .496676-1.236855$ H -0.397592 $6.458969-1.425715$ H $-0.3271254 .713915-1.264647$ H $\quad 0.146315 \quad 6.258582-5.049472$ H $-0.5742887 .215054-3.637642$ H $2.1819024 .153408-3.051607$ H $1.5787854 .417722-4.647468$ H $-0.2834522 .919516-4.430179$ H 3.3369303 .2549361 .859338 C $-5.792228-2.037068-0.983505$ O $-5.325320-0.749428-0.532888$ C $-6.421315-0.0380180 .064195$ C $-7.675145-0.688770-0.500417$ C -7.251746 -2.145246 -0.532133 H $-6.374308-0.2347661 .142689$ C $-6.284761 \quad 1.467755-0.183458$ H -8.556373 -0.5097860 .122670$ H -7.894613 -0.338728 -1.515562 C $-8.110426-3.011373-1.437591$ H -7.300879 -2.5452280 .489778$ C $-4.898512 \quad 2.0519180 .161276$ O $-6.5287761 .750066-1.567435$ H -7.066072 2.0004090 .370860 H $-4.1664491 .753730-0.599655$ O $-4.993123 \quad 3.4796830 .087426$ C -4.3626661 .6673951 .550905$ C -3.0280102 .3610501 .879861$ H -4.2234990 .5828591 .589229$ O $\quad-5.3221642 .0033172 .551901$ H -2.3532272 .2536491 .023943$ H -3.200042 3.4345922 .024373 H -9.150445 -3.012805 -1.095298 H -8.097137 -2.648114 -2.470814 H $-7.753112-4.046009-1.439435$ C $-4.907163-3.138044-0.392495$ H $-5.8928281 .231132-2.089563$ H $-5.3208393 .702294-0.800938$ H -5.4903502 .9591872 .488832$ H $-5.315551-4.122375-0.648909$ H $-4.897456-3.0621160 .702032$ H -5.720048 -2.034019-2.078524 C -1.216643 -0.0784332 .160133$ O $-2.203495 \quad 0.369774 \quad 3.094321$ C $-2.366961 \quad 1.792333 \quad 3.157198$ C - 1.0326202 .4587313 .523851 C 0.0939672 .0316312 .585869 C 0.1608550 .5089442 .486356 H $-1.514780 \quad 0.238061 \quad 1.152545$ C $-1.236387-1.6132532 .122818$ H -3.062736 1.9828003 .984051 O -1.1458183 .8777053 .498906$ H -0.7550492 .1755594 .547537$ H $-0.0201352 .481170 \quad 1.593500$ O $1.3411162 .501672 \quad 3.101627$ H $\quad 0.545307 \quad 0.1082503 .431499$ O $1.080727 \quad 0.143373 \quad 1.463626$ C $-0.764875-2.2707193 .402273$ H -2.257401 -1.970653 1.946714 H $-0.606040-1.9572501 .294180$ H -1.857392 4.124483 4.113986 H 1.2659993 .4661463 .203082 H 1.9393190 .5383241 .691675 O $-0.146140-3.4494213 .100976$ O $-0.944392-1.8232484 .526879$ C $0.355488-4.1876764 .218994$ H $0.847374-5.0869483 .838648$ H $1.090583-3.5918024 .768398$ H $-0.468027-4.4872934 .874056$

SCF Energy (B3LYP/6-31G**//MMFF) $=-3245.93647549$

0500046

MM̄FF Geometry

C $-2.857378-2.405104-2.125245$

C $-1.970242-3.236438-2.692251$

C $-0.489797-3.319071-2.394245$

O $-0.033433-2.220472-1.573450$
C $-0.228036-4.627982-1.623035$

C $1.234610-4.922478-1.361777$

C $1.787526-5.022380-0.137008$

C $2.084125-5.150772-2.585647$

C $1.122174-4.8570091 .201863$

C $1.220987-3.4393381 .788940$

C $2.655183-2.9993592 .127974$

C $2.655186-1.6563972 .861643$

C $4.080479-1.1450633 .129228$

O $3.387585-2.8705050 .908655$

O $3.9633840 .232844 \quad 3.515842$

C $4.897411-1.2034961 .817645$

C $4.718983-1.8711634 .319099$

O $6.265162-0.8831772 .057568$

C $4.773600-2.5501741 .076342$

C $5.410271-2.536315-0.333505$

O $5.450476-3.5617931 .832430$

C $0.316917-1.066197-2.205002$

O $0.250559-0.832421-3.401064$

C $0.796623-0.117864-1.173687$

C $0.9625191 .177295-1.483535$

C $1.4186762 .231634-0.572634$

C 1.9610151 .8046490 .764743

C $4.815776-1.516636-1.311571$

C $5.610671-0.225934-1.411034$

C $5.5443522 .008823-2.507874$

O $6.705786-0.062943-0.880326$

C $5.1108813 .006511-1.481744$

C $4.5514804 .185032-1.793585$

C $4.1584665 .217798-0.778185$

C $2.7075105 .622835-0.894328$

C $2.3525206 .753770-1.527602$

C $1.6778284 .763153-0.198102$

C $1.3348893 .517767-0.973971$

N $4.9645280 .715382-2.201372$

H $-2.526386-1.674713-1.392427$

H -2.335040 -3.965125 -3.414196

H $0.057784-3.322508-3.344221$

H $-0.643814-5.478230-2.181022$

H $-0.785084-4.587520-0.680602$

H $2.849371-5.259300-0.077155$

H $3.090526-5.502117-2.333427$

H $1.626366-5.908054-3.230632$

H $\quad 2.195363-4.223953-3.156529$

H $\quad 0.067507-5.146576 \quad 1.150531$

H $1.578920-5.5703531 .899580$

H $\quad 0.773387-2.7312161 .081303$

H $\quad 0.613939-3.4105372 .702335$

H $3.114284-3.7755412 .751562$

H $2.089233-1.7235763 .799093$

H $2.128529-0.9116442 .249892$

H 4.8634420 .5887923 .613501

H $4.509521-0.4050971 .175601$

H $4.114098-1.7263005 .222360$

H $5.706164-1.4581714 .553689$

H $4.823675-2.9462334 .158703$

H $6.688661-1.6659822 .449264$

H $6.499846-2.440565-0.254831$

H $5.239671-3.529615-0.773745$

H $5.658823-4.3022481 .238861$

H $0.988834-0.510560-0.181878$

H $\quad 0.7190991 .506247-2.493154$

H $\quad 1.1678341 .3737721 .384077$

H 2.4127812 .6178341 .335907

H 2.7490191 .0563270 .631886

H $4.825253-1.957271-2.316905$

H $3.773885-1.286841-1.065678$

H $5.2174862 .281139-3.516413$

H $6.6361161 .927408-2.504546$

H $5.2919662 .761995-0.437677$

H $4.3961294 .438977-2.839754$

H $4.8045856 .093000-0.926289$

H 4.3600004 .8733710 .243980

H $1.3150587 .065387-1.589178$

H $3.0914107 .390014-2.003481$

H $0.7471625 .331803-0.073831$

H $2.015904 \quad 4.558188 \quad 0.820782$

H $0.9279183 .722479-1.965198$ 
H $4.0308660 .518239-2.549888$

C $-5.183096-2.758354-1.172250$

O $-5.058529-1.688724-0.211717$

C $-4.747956-2.2643931 .068119$

C $-5.217428-3.7120420 .985711$

C $-4.835159-4.059127-0.439545$

H $-3.654608-2.2507741 .160157$

C -5.362962 -1.452295 2.209145

H $-4.732012-4.3514221 .728948$

H $-6.302437-3.7975321 .114794$

C - $-5.551051-5.280335-0.989912$

H -3.755878 -4.250874 -0.461405

C -5.0650850 .0599162 .172733$

O $-6.788008-1.6054382 .169470$

H -5.038885 -1.881531 3.165137

H $-5.6815750 .538414 \quad 1.402194$

O

C $-3.591796 \quad 0.4539391 .948954$

C -3.3539541 .9520792 .222107$

H $-3.305237 \quad 0.1977190 .923430$

O $-2.772955-0.3096912 .835268$

H -4.1866972 .5257511 .803335$

H -3.3597312 .1124553 .307002$

H $-5.309123-6.168063-0.396433$

H -6.638432 -5.150873 -0.972410

H $-5.250217-5.471945-2.024876$

C $-4.334772-2.449948-2.411294$

H -7.155344 -1.095307 2.911747

H $-4.9478630 .246903 \quad 4.121646$

H -1.848051 -0.189465 2.562688

H $-4.625368-1.469920-2.805830$

H $-4.554858-3.188681-3.191133$

H $-6.238578-2.765144-1.472945$

C $-2.8428143 .335602-0.454634$

O

C -2.0152122 .4721141 .651634$

C -1.644066 3.8839352 .124606

C -2.5517004 .9369391 .500140$

C $-2.5764574 .784062-0.017981$

H -3.887089 $3.066205-0.257131$

C $-2.5919653 .195640-1.964756$

H -1.226705 1.7999042 .008242

O -1.7159773 .9855273 .542755$

H -0.6073124 .1036841 .840916$

H -3.5647814 .8961201 .917123$

O -2.0436536 .2375311 .807366$

H -1.621173 $5.129248-0.431802$

O $-3.5944515 .627508-0.555476$

C $-3.0538801 .869888-2.525879$

H -3.128174 $3.978876-2.512094$

H - $1.5197023 .282105-2.170278$

H -1.121282 3.3115483 .914295

H -1.993803 6.3064252 .776341

H -3.410439 $6.533368-0.252651$

O $-2.7192261 .821802-3.850204$

O $-3.6371540 .998820-1.897349$

C $-3.109010 \quad 0.638493-4.552571$

H $-2.903790 \quad 0.791665-5.615355$

H $-4.1796390 .450418-4.428327$

H -2.520106 -0.212426 -4.201993

SCF Energy $\left(B 3 L Y P / 6-31 G^{* *} / / M M F F\right)=-3245.91856556$

$05 \quad 00047$

MM̄FF Geometry

C $0.751933-3.804067-0.054672$

C $1.317904-3.534527-1.241372$

C $0.690184-2.775433-2.386252$

O $-0.561810-2.181314-1.980458$

C $1.618170-1.624646-2.824766$

C $1.110112-0.877158-4.042888$

C $0.7359420 .417336-4.049114$

C $1.055512-1.674917-5.321192$

C $0.7730831 .404114-2.915889$

C $-0.5623741 .476730-2.176306$

C $-0.7327612 .745633-1.327689$

C $0.3445622 .894816-0.251434$

C $0.090772 \quad 4.1122190 .650640$

O $-2.0287262 .659441-0.718491$
O $0.966716 \quad 3.986486 \quad 1.779922$

C -1.363180 4.0521641 .164937

C $0.4828135 .419872-0.046590$

O -1.6878645 .2207161 .913443$

C -2.4020563 .8135160 .048108$

C -3.8257913 .5684120 .599981$

O $-2.4712404 .979103-0.782276$

C $-1.719344-2.690528-2.477604$

O $-1.852789-3.653725-3.213389$

C $-2.815064-1.837998-1.964201$

C $-4.091791-2.095533-2.284295$

C $-5.252130-1.309066-1.854041$

C $-4.976547-0.050341-1.074102$

C -3.9434632 .3996051 .581039$

C $-5.3874131 .995747 \quad 1.833682$

C -6.7167300 .1944862 .944930$

O -6.3497422 .6166451 .391351$

C $-6.907313-0.9637332 .015514$

C $-8.025134-1.1807381 .305338$

C $-8.209337-2.3648280 .397145$

C $-8.690752-1.985031-0.987885$

C $-9.874657-2.426382-1.446049$

C $-7.812371-1.099276-1.847071$

C $-6.489860-1.739754-2.176877$

N -5.4651490 .8541412 .619502$

H $-0.268017-3.4893800 .147663$

H $2.331306-3.889318-1.420176$

H $\quad 0.546581-3.478809-3.214417$

H $2.617719-2.014642-3.057082$

H $1.747351-0.940199-1.979341$

H $0.3717250 .838640-4.985638$

H $0.828100-1.049626-6.191362$

H $2.021184-2.155085-5.510199$

H $\quad 0.284386-2.448803-5.263734$

H $1.5818861 .172723-2.216353$

H $1.0192212 .385186-3.341309$

H -1.397088 $1.427640-2.888882$

H $-0.6759550 .593706-1.535388$

H $-0.7188513 .607316-2.005643$

H $1.3428402 .966931-0.696319$

H $\quad 0.358591 \quad 1.991973 \quad 0.375047$

H 0.7598094 .7096652 .396645

H $-1.4011103 .217718 \quad 1.875887$

H $1.5466485 .403927-0.313407$

H $\quad 0.356880 \quad 6.279130 \quad 0.621195$

H $-0.0845995 .607163-0.960370$

H -1.844470 5.9431641 .281542

H -4.2120884 .4831121 .068471$

H $-4.4902483 .375213-0.254204$

H $-3.3230884 .973334-1.249740$

H $-2.535353-1.003336-1.330936$

H -4.310516 -2.955878 -2.914905

H $-4.3308470 .625693-1.646173$

H $-4.481417-0.293464-0.128299$

H $-5.8718630 .520310-0.824754$

H -3.4084921 .5254741 .193690$

H -3.502871 2.6728692 .546318

H -7.5408710 .9126622 .886772$

H $-6.634345-0.1664223 .974606$

H -6.085260 -1.671952 1.936893

H $-8.860390-0.4910591 .400883$

H -8.932594 -3.036323 0.877934

H -7.280438 -2.941499 0.307664

H -10.232622 -2.163412 -2.435965

H $-10.514730-3.061369-0.842457$

H $-7.709344-0.128620-1.356611$

H -8.310085 -0.882591 -2.801614

H $-6.582950-2.660231-2.754908$

H -4.6052580 .3882872 .894864$

C $1.368588-3.7472082 .372266$

O $1.968157-2.4469922 .225494$

C $2.636821-2.0996373 .452735$

C $2.325421-3.2153434 .446935$

C $2.118200-4.4039813 .528256$

H $3.711553-2.1097713 .237093$

C $2.207167-0.6992413 .907522$

H $3.137095-3.3644755 .165722$

H $1.411809-3.0121855 .017246$ 
C $1.372121-5.5537984 .181576$

H $3.100628-4.7571443 .187120$

C 2.3690520 .4015472 .839464

O $0.820602-0.7280584 .275296$

H $2.752379-0.4238294 .817526$

H $1.647748 \quad 0.2473732 .027318$

O 2.0253291 .6509953 .451436

C 3.7847670 .5210622 .247868

C 3.8923331 .6875961 .247484

H $4.040200-0.4145321 .745554$

O $4.713953 \quad 0.708503 \quad 3.313211$

H 3.1063331 .5710360 .493917

H 3.7028012 .6296571 .775397

H $1.925679-5.9275775 .049141$

H $0.378889-5.2446944 .524223$

H $1.243375-6.3844793 .480788$

C $1.465171-4.5230671 .057780$

H $0.317444-1.0273563 .498508$

H 1.1214821 .5643843 .799740

H 5.6062290 .5627582 .956573

H $1.013702-5.5142071 .176763$

H $2.519570-4.6691540 .793863$

H $0.315324-3.5851692 .638465$

C $4.9496730 .176662-1.210167$

O $5.6795090 .557877-0.037592$

C 5.2707371 .7936060 .558528

C $5.3871542 .936285-0.462193$

C $4.6557252 .625752-1.766386$

C $5.0722471 .254332-2.296298$

H $3.8946370 .021703-0.954828$

C $5.537156-1.155711-1.704923$

H 6.0006672 .0057621 .349338

O 4.9204534 .1642850 .088113

H $6.4495703 .087530-0.693631$

H $3.5703162 .689630-1.634971$

O $5.006218 \quad 3.628899-2.721990$

H $6.1052651 .302933-2.664046$

O $4.2400360 .899653-3.397839$

C $5.176333-2.323166-0.811365$

H $5.164897-1.398053-2.706110$

H $6.631060-1.081600-1.734085$

H 3.9490484 .1362530 .115671

H $4.4529503 .491089-3.509068$

H $4.4145851 .530797-4.115881$

O $5.951002-3.396538-1.148988$

O $4.310474-2.3119780 .051577$

C $5.708768-4.590479-0.397691$

H $\quad 6.449852-5.336415-0.696792$

H $5.817483-4.3993150 .674386$

H $4.710683-4.977159-0.622383$

SCF Energy $\left(B 3 L Y P / 6-31 G^{* *} / / M M F F\right)=-3245.91721142$

05_00048

MM̄MF Geometry

C $-0.4722392 .868967-1.648858$

C $0.5244443 .752100-1.487449$

C $1.3856493 .789369-0.255723$

O $2.7277363 .445769-0.673514$

C 1.4061085 .1740880 .421070

C $0.060125 \quad 5.8116870 .693091$

C -0.8233435 .3843451 .615589$

C $-0.2449637 .034068-0.133438$

C $-0.699861 \quad 4.1895082 .518985$

C - -1.9011103 .2525902 .331629$

C -1.725799 1.8466562 .924592

C -1.2626921 .8475944 .383555$

C -1.052210 0.4223054 .920070

O $-0.790720 \quad 1.1333392 .104476$

$\begin{array}{lllll}\text { O } & -0.325817 & 0.542376 & 6.153199\end{array}$

C $-0.167179-0.3583313 .921504$

C $-2.384380-0.2484685 .274297$

O $-0.043907-1.7234834 .312566$

C $-0.650003-0.2499522 .458937$

C $0.320995-0.9113381 .458184$

O $-1.897455-0.9443212 .332189$

C 3.5587502 .9365480 .275303

O $3.3298142 .810608 \quad 1.467710$

C $4.8003572 .507082-0.401353$
C $5.5512681 .557744 \quad 0.176088$

C $6.7856740 .984860-0.359320$

C $7.3603801 .596928-1.609328$

C $1.725356-0.3142121 .409906$

C $2.519447-0.9229680 .270341$

C $3.645460-3.044065-0.415989$

O $2.717307-0.313094-0.777687$

C $5.093176-3.122598-0.045230$

C $6.089535-2.962443-0.929715$

C $7.546152-3.111699-0.589276$

C $8.378981-1.934817-1.047104$

C $8.965656-1.937917-2.256183$

C $8.603872-0.796622-0.078307$

C $7.341524-0.0636540 .282753$

N $2.943186-2.2128520 .543456$

H $-0.6497982 .121816-0.878579$

H $0.7191864 .492828-2.258790$

H 1.0377153 .0262470 .449052

H $2.0167265 .855605-0.188504$

H 1.9614475 .1095611 .366194

H -1.7473315 .9481901 .739431$

H $\quad 0.502425 \quad 7.8125470 .052054$

H $-0.2326586 .786553-1.199615$

H -1.228679 7.4579630 .094772

H 0.2366393 .6513152 .357744

H -0.6767434 .5496993 .554485$

H -2.785415 3.7209882 .783042

H $-2.1101093 .143871 \quad 1.258688$

H -2.701595 1.3528162 .841441

H -1.969143 2.3995305 .015774

H -0.3044522 .3766214 .470216$

H $-0.121036-0.3567246 .463078$

H $\quad 0.8394850 .070502 \quad 3.998447$

H $-2.908448 \quad 0.331180 \quad 6.043928$

H -2.225978 -1.242163 5.707425

H $-3.057172-0.3475274 .420151$

H $-0.870876-2.1757874 .073193$

H $\quad 0.373117-1.989676 \quad 1.652811$

H $-0.110997-0.8213570 .452098$

H -2.051564 -1.117412 1.389149

H $5.0366252 .949872-1.361607$

H $5.219303 \quad 1.1399581 .126193$

H $8.2997831 .136959-1.924430$

H $7.5666852 .662115-1.456445$

H $6.6614161 .490407-2.446382$

H $1.677674 \quad 0.766057 \quad 1.250551$

H $2.268802-0.4838502 .345157$

H $3.496626-2.651493-1.427197$

H $3.198014-4.041453-0.359798$

H $5.332659-3.3611710 .988293$

H $5.844969-2.757506-1.969875$

H $7.909037-4.027857-1.074057$

H $7.693475-3.2707140 .486195$

H $9.590051-1.113904-2.585032$

H $8.831139-2.764570-2.946104$

H $9.355571-0.093600-0.456488$

H $9.044248-1.1998970 .843008$

H $6.848079-0.453648 \quad 1.173477$

H $2.746714-2.6138141 .455969$

C $-2.8505652 .963311-2.387254$

O $-3.2565001 .736178-1.743438$

C $-4.5683841 .377369-2.213781$

C $-5.1012692 .600830-2.948783$

C $-3.8275273 .165585-3.544408$

H $-4.4344810 .566583-2.939281$

C $-5.4443170 .905337-1.049819$

H -5.850519 2.339159-3.702205

H $-5.5529113 .323809-2.259281$

C $-3.9591114 .609087-3.998663$

H -3.531474 $2.543742-4.400063$

C $-4.892089-0.308976-0.275555$

O $\quad-5.5987591 .979933-0.112937$

H $-6.4526240 .681140-1.417142$

H $-4.003304-0.0232640 .299738$

$\begin{array}{lllll}\text { O } & -5.884816 & -0.693598 & 0.684831\end{array}$

C $-4.561678-1.532887-1.151128$

C -4.244942 -2.792641-0.323533

H $-3.708286-1.291865-1.791292$ 
O $-5.663718-1.819532-2.013656$ H $-3.509215-2.5389800 .444507$ H $-5.155495-3.1182310 .193377$ H $-4.7220744 .698261-4.778882$ H -4.246834 $5.265630-3.170636$ H -3.013652 $4.978296-4.407668$ C -1.393047 2.847414 -2.835050 H -4.7089642 .2435190 .179495$ H -6.0723500 .0862351 .235138$ H -6.439981 - $1.988346-1.452524$ H - $1.1430633 .662538-3.523423$ H $-1.2398261 .901837-3.367408$ H $-2.9644723 .764939-1.645175$ C $-1.362726-3.489900-1.098745$ O $-2.537060-3.629406-1.908221$ C $-3.733268-3.963539-1.194027$ C $-3.536511-5.278151-0.425564$ C $-2.310187-5.2258300 .479600$ C $-1.084941-4.779865-0.314566$ H -1.493469 -2.653418 -0.404002 C $-0.188115-3.165547-2.035306$ H $-4.499915-4.152144-1.955951$ O $-4.682786-5.591717 \quad 0.358252$ H $-3.402712-6.100013-1.141123$ H -2.475246 -4.5752681 .346559$ O $-2.053281-6.5321580 .999880$ H - $0.783772-5.581128-1.001908$ O $-0.000455-4.5601930 .585258$ C $-0.218208-1.742115-2.550509$ H $0.762474-3.288483-1.506087$ H $-0.209208-3.833332-2.904497$ H -5.445413 -5.633419-0.243759 H $-2.856685-6.8201591 .466301$ H $0.137021-5.3857141 .080803$ O $0.870295-1.547057-3.353617$ O $-1.071401-0.907597-2.284734$ C $0.999169-0.236673-3.912995$ H $1.1137890 .504267-3.116723$ H $1.896351-0.220471-4.537405$ H $0.132098-0.004181-4.538447$

SCF Energy (B3LYP/6-31G**//MMFF) $=-3245.93345552$

05_00049

MM̄FF Geometry

C -2.949785 -3.756294 -2.129408

C $-2.113678-3.648835-1.085728$

C $-0.611669-3.673898-1.217648$

O $-0.082973-2.401559-0.780823$

C $-0.033415-4.772549-0.305825$

C $1.462802-4.972470-0.452249$

C $2.360856-4.7852820 .534911$

C $1.932004-5.449237-1.803435$

C $2.106300-4.3529651 .951496$

C $2.177967-2.8356152 .179111$

C $3.561329-2.2196221 .917755$

C $3.593193-0.7543202 .358902$

C $4.937130-0.0839462 .029285$

O $3.836163-2.2974170 .517694$

O 4.7661651 .3353342 .166396

C $5.291080-0.3548830 .549878$

C $6.032095-0.4865033 .024430$

$\begin{array}{lllll}\text { O } & 6.607507 & 0.099996 & 0.248663\end{array}$

C $5.140308-1.8346300 .142819$

C $5.285608-2.069396-1.380462$

O $6.163436-2.6058380 .784418$

C $-0.109384-1.379980-1.680165$

O $-0.548226-1.412080-2.818910$

C $0.488476-0.192806-1.032296$

C $0.311094 \quad 1.015745-1.586397$

C $0.8314982 .284530-1.074750$

$\begin{array}{llll}\text { C } & 1.834094 & 2.219893 & 0.046076\end{array}$

C $4.280435-1.317548-2.260486$

C $4.815581-0.013682-2.829118$

C $4.0937161 .958129-4.169436$

O $5.991298 \quad 0.329611-2.742065$

C $3.8592953 .101286-3.233830$

C $3.083898 \quad 4.152782-3.538798$

C $2.8756135 .330765-2.631508$
C $1.4135995 .609684-2.367588$

C $0.7553486 .560087-3.052402$

C $0.7406574 .846356-1.250086$

C $0.3980073 .433032-1.635506$

N $3.8264270 .707421-3.484919$

H $-2.544314-3.853044-3.133518$

H $-2.522633-3.537554-0.084106$

H $-0.315368-3.857948-2.257610$

H $-0.522554-5.731348-0.524756$

H $-0.287799-4.5318140 .732180$

H $3.410897-4.9705180 .310568$

H $2.989120-5.736257-1.798286$

H $1.358306-6.327334-2.117476$

H $1.810737-4.662889-2.554583$

H $1.133835-4.7191932 .297719$

H $2.842670-4.8497702 .596221$

H $1.430348-2.3478081 .542900$

H $1.896019-2.6424103 .220979$

H $4.307069-2.8021022 .471346$

H $3.372186-0.6641953 .429827$

H $2.795571-0.2028251 .843019$

H 4.4693631 .5151003 .074990

H $4.6067880 .250192-0.055438$

H $5.745435-0.202698 \quad 4.044249$

H 6.9680020 .0445812 .818597

H $6.236423-1.5591133 .026356$

H $6.652937 \quad 1.039670 \quad 0.495045$

H $6.320021-1.883063-1.693543$

H $5.118967-3.141801-1.558615$

H $6.284602-3.4319090 .287494$

H $1.045453-0.344096-0.115194$

H $-0.2893951 .090300-2.492172$

H $2.6701751 .569622-0.230480$

H $1.370776 \quad 1.8266450 .956483$

H $2.2741903 .186595 \quad 0.297856$

H $4.038909-1.948893-3.125365$

H $3.345817-1.128037-1.723564$

H $3.4369801 .996481-5.044294$

H $5.1334891 .970042-4.512271$

H $4.3722223 .077349-2.275191$

H $2.5948044 .186954-4.509843$

H $3.3420906 .203516-3.106996$

H $3.4002435 .200069-1.676882$

H $-0.2862866 .785203-2.848573$

H $1.2425017 .130472-3.836346$

H $-0.2002935 .341111-0.973836$

H $1.3605324 .908513-0.351767$

H $-0.329223 \quad 3.369669-2.446444$

H $2.8664730 .375682-3.458584$

C $-5.068056-2.429230-2.405175$

O $-4.756178-1.447320-1.391530$

C $-5.984705-1.028413-0.777488$

C $-7.024961-1.182266-1.874337$

C $-6.599989-2.507300-2.478466$

H $-6.202881-1.7446760 .025065$

C $-5.8571640 .375260-0.180175$

H -8.047572 -1.196044 -1.486143

H $-6.947200-0.385074-2.622583$

C $-7.129033-2.733940-3.884639$

H $-6.967169-3.317817-1.834306$

C -4.6447930 .5749170 .756149$

O $-5.746133 \quad 1.337004-1.236411$

$\begin{array}{llll}\mathrm{H} & -6.784842 & 0.623578 & 0.348620\end{array}$

H -3.7391470 .6851100 .149879$

O -4.8282941 .8240201 .431941$

C $-4.437151-0.5295921 .802871$

C $-3.322835-0.2648112 .834575$

H $-4.216545-1.475138 \quad 1.294667$

O $-5.662762-0.7122362 .518407$

H -3.6251630 .5628543 .483804$

H $-3.297450-1.1412583 .494487$

H -8.223855 -2.746871 -3.886708

H $-6.800673-1.944555-4.569120$

H $-6.777535-3.691926-4.280973$

C $-4.446268-3.770072-2.003900$

H $-4.9626301 .104972-1.764076$

H -5.5954761 .7301562 .022341$

H -5.540062 -1.461834 3.125539 
H -4.830561 -4.572989-2.644005

H -4.728060 -4.017379 -0.972319

H -4.641595 -2.064292 -3.347355

C -1.6579832 .3151642 .753171$

O $\begin{array}{llll}-1.744853 & 1.306297 & 1.738497\end{array}$

C -1.926995 -0.0334422 .213576$

C $-0.759244-0.4081613 .136206$

C -0.6264470 .5772964 .292170$

C -0.5363242 .0034493 .753938$

H -2.617913 2.3959923 .275454

C - -1.3884213 .6586402 .064450$

H -1.855443 -0.6874001 .334973$

O $-0.933352-1.7341813 .622592$

H $0.170700-0.3975512 .553831$

H -1.451106 0.4773975 .007748

O $\quad 0.577084 \quad 0.304667 \quad 5.013142$

H 0.4421202 .1485683 .278212

O -0.6154692 .9202714 .844189$

C $-2.5803534 .136473 \quad 1.271761$

H -1.1586324 .4441952 .793966$

H -0.5249303 .5960681 .394498$

H $-0.143171-1.9679074 .137178$

H $0.463058-0.5497135 .462381$

H 0.0953822 .6919915 .467512

O $-2.5025963 .679925-0.009721$

O -3.4740704 .8294271 .740537$

C $-3.5485964 .104296-0.888915$

H $-3.4228795 .164899-1.124493$

H -3.469698 $3.525894-1.813205$

H $-4.5325273 .923726-0.446567$

SCF Energy (B3LYP/6-31G**//MMFF) $=-3245.90490786$

05_00050

MM̄FF Geometry

C $1.858227-4.298162-1.160391$

C $0.562342-4.165863-1.479759$

C $-0.493055-3.756990-0.482647$

O $-1.185784-2.590324-0.984021$

C $-1.523796-4.888340-0.319073$

C $-2.548751-4.625797 \quad 0.767487$

C $-3.870745-4.473674 \quad 0.555478$

C $-2.010306-4.5694852 .174849$

C $-4.616527-4.527409-0.747874$

C $-4.816129-3.166444-1.433210$

C $-5.612617-2.149470-0.599008$

C $-5.994333-0.934152-1.447373$

C $-6.7115270 .144179-0.619231$

O $-4.796512-1.7249850 .496229$

O $-6.7337391 .352687-1.394915$

C -5.8848790 .4396370 .651410$

C $-8.175396-0.224630-0.350191$

O

C -5.446701 -0.830236 1.409093

C $-4.450461-0.5432442 .559711$

O $-6.598259-1.4468191 .998597$

C $-0.534367-1.399026-0.904411$

O $0.584834-1.198722-0.459319$

C -1.421346 -0.362742 -1.474542

C $-0.8857730 .802498-1.864132$

C $-1.6016741 .948671-2.424356$

C $-3.1054521 .892245-2.438156$

C -3.1384410 .1320102 .144848$

C -3.1487241 .6450122 .289115$

C - -1.7486413 .6479921 .799829$

O -4.0440832 .2631302 .858326$

C -2.2036374 .2966550 .531116$

C $-1.4122175 .070737-0.227091$

C $-1.8778305 .786842-1.462810$

C -1.021435 $5.488069-2.672298$

C $-0.0314116 .318117-3.041628$

C $-1.3704104 .272098-3.500048$

C $-0.8800682 .996198-2.874147$

N -2.0129772 .2231561 .738472$

H $2.171795-4.123522-0.133933$

H $0.240365-4.341140-2.503394$

H $-0.040934-3.5149860 .486998$

H -1.010292 -5.828542 -0.076552

H $-2.018244-5.053242-1.282520$
H -4.507905 -4.294956 1.421234

H -2.808876 -4.540424 2.924224

H $-1.400132-5.4540842 .384561$

H -1.395125 -3.6762532 .318875$

H -4.125139 -5.209960 -1.449234

H $-5.598727-4.978077-0.555187$

H $-3.833907-2.749603-1.684482$

H $-5.341711-3.346557-2.379229$

H $-6.512522-2.645221-0.216263$

H -6.613184 -1.234504 -2.302031

H $-5.085439-0.493041-1.877928$

H -7.187495 $1.162119-2.233807$

H -4.9909730 .9852660 .327227$

H -8.718488 $-0.348992-1.294826$

H -8.6928010 .5772850 .187921$

H $-8.287043-1.1504700 .217788$

H -6.8298222 .1045521 .022793$

H $-4.9523520 .007743 \quad 3.364106$

H -4.178511-1.513650 2.999819

H -6.302978 -2.019995 2.725295

H $-2.478041-0.585569-1.558603$

H $\quad 0.191050 \quad 0.928351-1.772711$

H -3.489449 $1.677136-1.435510$

H $-3.4537251 .112141-3.123434$

H $-3.5755492 .828090-2.747104$

H $-2.340022-0.2297332 .805616$

H $-2.862548-0.1378071 .121383$

H -0.6750273 .7728051 .968976$

H -2.284864 4.0846732 .648570

H $-3.242650 \quad 4.1533140 .243783$

H -0.3813665 .2358830 .078850$

H -1.855931 $6.863819-1.250102$

H $-2.9256805 .554385-1.690455$

H $0.5696646 .124277-3.923874$

H $0.2043867 .206766-2.465497$

H $-0.903474 \quad 4.346335-4.491372$

H -2.445615 $4.268584-3.698656$

H $0.2071712 .938022-2.814995$

H -1.3393561 .6356271 .255460$

C $4.042973-3.637961-2.275805$

O $4.703935-3.437216-1.010710$

C $4.968095-2.022298-0.845643$

C $4.764279-1.411344-2.223221$

C $3.618165-2.250927-2.755306$

H $4.195615-1.661129-0.156973$

C $6.360466-1.853528-0.228335$

H $4.529552-0.345877-2.181842$

H $5.655076-1.546930-2.848925$

C $3.427453-2.146680-4.257464$

H $2.695898-1.929242-2.256612$

C $6.887362-0.413186-0.091302$

O $6.338643-2.4753131 .060113$

H $7.068517-2.434346-0.833892$

H $7.819096-0.4429870 .488861$

O $7.2527750 .051321-1.395652$

C 5.9537270 .6357220 .549651

C 5.3742080 .2344321 .914806

H $5.1438840 .876600-0.145780$

O $\quad 6.709179 \quad 1.845063 \quad 0.698920$

H $4.657732-0.5788591 .770020$

H $6.181225-0.1624082 .543890$

H $3.194748-1.116084-4.544737$

H $4.329029-2.450758-4.799425$

H $2.602173-2.784400-4.589691$

C $2.932389-4.683085-2.137282$

H $7.243766-2.4496261 .414316$

H $7.6030900 .953261-1.296011$

H $7.418047 \quad 1.674675 \quad 1.342527$

H $3.374075-5.619815-1.774930$

H $2.503156-4.891874-3.124216$

H $4.806575-4.045878-2.951497$

C 2.6512311 .5629781 .423200

O $3.8539942 .196628 \quad 1.868692$

C 4.6986831 .3889712 .689982

C 3.9371420 .9368983 .944108

C 2.6156610 .2533843 .591060

C 1.8040151 .1135372 .621301

H 2.9004210 .7019390 .791324 
C $\quad 1.8880942 .5868950 .576529$

H 5.5087542 .0479873 .028255

$\begin{array}{lllll}\text { O } & 4.747981 & 0.062252 & 4.721210\end{array}$

H 3.7263661 .8170164 .565325

H $2.789715-0.7459713 .176608$

$\begin{array}{lllll}\text { O } & 1.858823 & 0.047247 & 4.786482\end{array}$

H 1.4199141 .9899323 .158188

$\begin{array}{lllll}\text { O } & 0.670512 & 0.375189 & 2.172103\end{array}$

C $2.6576322 .991249-0.657651$

H $\begin{array}{llll}0.908871 & 2.212438 & 0.262847\end{array}$

H $1.712593 \quad 3.5019151 .155998$

H $4.218892-0.2225685 .486120$

H 1.6754640 .9170975 .180566

H $0.993217-0.3979681 .677901$

O $2.6805941 .968284-1.557179$

O $3.1685164 .094201-0.806442$

C $3.3443252 .252718-2.792738$

H $4.4202332 .352793-2.622376$

H $3.1697621 .414636-3.472615$

H $2.9385363 .160303-3.250271$

SCF Energy (B3LYP/6-31G**//MMFF) $=-3245.90795533$

05_00051

MMFF Geometry

C $3.599145-1.027287-3.427086$

C $4.914906-1.186949-3.216101$

C $5.728657-0.498498-2.142035$

O $4.9071770 .413584-1.380172$

C $6.291539-1.542282-1.157982$

C $7.153060-0.929122-0.070276$

C $6.840957-0.9282941 .240890$

C $8.441618-0.299875-0.534625$

C $5.642812-1.546628 \quad 1.907544$

C $4.420387-0.6287341 .881410$

C $3.324924-1.0190052 .884570$

C $2.788402-2.4351962 .663260$

C $1.619379-2.7590273 .607617$

$\begin{array}{llllll}\text { O } & 2.267567 & -0.062302 & 2.723508\end{array}$

O $0.986198-3.9567453 .131239$

C $0.576580-1.6273683 .512390$

C $2.098308-3.0610025 .032124$

O $-0.466477-1.8054374 .466976$

C $1.182112-0.2144603 .649716$

C 0.1529550 .9008003 .348806

O $1.629646-0.0211654 .996503$

C $4.865827 \quad 1.719971-1.752361$

O $5.4865922 .254504-2.655610$

C $3.8786032 .381426-0.869663$

C $3.4930043 .644312-1.105289$

C $2.4848904 .384918-0.340276$

C $1.864886 \quad 3.683410 \quad 0.839733$

C $-0.495783 \quad 0.820047 \quad 1.964732$

C - $-1.2974862 .065011 \quad 1.623380$

C -2.585999 $3.064437-0.267491$

O $-1.444672 \quad 3.0082212 .395192$

C $-1.6632913 .893527-1.105123$

C $-1.5980155 .232644-1.043980$

C $-0.702386 \quad 6.057104-1.926038$

C $0.1335717 .061180-1.159470$

C $0.0005198 .378846-1.388358$

C $1.1273316 .553678-0.133688$

C $2.1572685 .634160-0.732040$

N $-1.829175 \quad 1.9892020 .344364$

H $3.029460-0.348982-2.797926$

H $5.467419-1.865672-3.862819$

H $6.5499000 .042509-2.626766$

H $6.892924-2.284207-1.699726$

H $5.455456-2.096177-0.715466$

H $7.529105-0.4409571 .930634$

H 9.0819590 .0001590 .301942

H $9.012289-1.008945-1.143198$

H $8.2429630 .594556-1.132791$

H $5.409312-2.5150631 .452251$

H $5.919281-1.7654852 .946858$

H $4.719701 \quad 0.4082502 .086880$

H $3.990436-0.6121160 .872531$

H $3.740994-0.9191423 .893919$

H $3.579712-3.1844472 .782948$
H $2.436125-2.5321611 .626564$

H $1.655655-4.6618423 .110676$

H $0.111236-1.7162252 .524958$

H $2.776995-3.9224735 .033078$

H $1.259707-3.3403515 .679465$

H $2.627552-2.2251875 .494013$

H $-0.830168-2.6978284 .335382$

H -0.6256880 .9118214 .122460$

H $\quad 0.664784 \quad 1.869480 \quad 3.436625$

H $1.6758970 .933428 \quad 5.171482$

H $3.4659141 .790502-0.059332$

H $3.9346894 .177584-1.945668$

H 2.6347543 .3870391 .561166

H 1.3332962 .7875010 .505674

H 1.1473884 .2919011 .391999

H $\quad 0.2713180 .688053 \quad 1.193426$

H $-1.179224-0.0342971 .917318$

H -3.0842343 .6529240 .509564$

H $-3.3462642 .602345-0.903438$

H $-1.0349893 .364737-1.818666$

H $-2.2462055 .767266-0.353557$

H $-1.3447076 .575291-2.649966$

H $-0.0386495 .417971-2.521329$

H $0.5933719 .110224-0.848863$

H $-0.7046628 .758571-2.120310$

H 0.5651386 .0919370 .681352

H $1.668248 \quad 7.3945090 .319720$

H $2.6819036 .061191-1.588009$

H $-1.6063211 .183780-0.233642$

C $1.660951-2.571647-3.963867$

O $0.759166-1.752958-3.190430$

C $0.391416-2.478970-1.995318$

C $0.778801-3.925258-2.262946$

C $2.052727-3.744705-3.065997$

H $1.011126-2.062811-1.189827$

C $-1.091708-2.228991-1.702405$

H $0.931667-4.500061-1.344758$

H $0.012766-4.430149-2.864530$

C $2.478152-4.988050-3.826486$

H $2.856867-3.457490-2.376843$

C $-1.687714-3.141167-0.617401$

O $-1.225737-0.865347-1.285623$

H $-1.669566-2.330146-2.628537$

H $-1.713936-4.174665-0.983385$

O $-0.806602-3.137638 \quad 0.511047$

C $-3.089618-2.735282-0.119099$

C $-4.101862-2.529064-1.255549$

H $-3.004844-1.8318050 .491949$

O $-3.542945-3.780308 \quad 0.745706$

H -3.784922 -1.667927 -1.852141

H $-4.070978-3.403382-1.918211$

H $2.680241-5.811760-3.134049$

H $1.700754-5.318484-4.523571$

H $3.389679-4.798883-4.402232$

C $2.817424-1.725099-4.504295$

H $-0.847373-0.309256-1.988296$

H -1.193334 $-3.723093 \quad 1.184713$

H $-4.297365-3.434578 \quad 1.252350$

H $2.405310-0.944034-5.155407$

H $3.472440-2.347398-5.124711$

H $1.077812-2.936654-4.819625$

C $-5.435898-0.007475-0.134470$

$\begin{array}{llll}\text { O } & -5.699911 & -1.361751 & 0.248374\end{array}$

C $-5.564050-2.328565-0.796477$

C $-6.527841-1.994961-1.943933$

C $-6.322615-0.570172-2.450727$

C $-6.3550780 .421152-1.289583$

H $-4.3827610 .093587-0.423712$

C $-5.6903310 .885810 \quad 1.092651$

H $-5.893356-3.284781-0.370712$

O $-6.365999-2.903301-3.028075$

H -7.563065 -2.093024-1.591996

H $-5.393220-0.473194-3.023855$

O $-7.381460-0.235407-3.351659$

H $-7.3876540 .517496-0.929181$

O $-5.949387 \quad 1.705353-1.759540$

C -4.7390180 .6275702 .241655$

H $-5.586747 \begin{array}{lll}1.940291 & 0.812765\end{array}$ 
H $-6.704017 \quad 0.710212 \quad 1.472594$

H $-6.516162-3.799836-2.682488$

H $-7.373196-0.896511-4.065115$

H -6.537412 $1.943673-2.496816$

O $-4.980051 \quad 1.5453643 .225511$

O $-3.908715-0.2677062 .290258$

C -4.1857841 .4043764 .407589$

H -4.3883650 .4411664 .885336$

$\mathrm{H}-3.1236851 .4994314 .167218$

H -4.461203 2.2045095 .099701

SCF Energy $\left(\mathrm{B} 3 L \mathrm{LP} / 6-31 \mathrm{G}^{* *} / / \mathrm{MMFF}\right)=-3245.93507147$

05_00052

MM̄FF Geometry

C $-1.090819-4.221069-1.561473$

C $-0.469502-3.036389-1.476780$

C $0.070224-2.292722-2.669460$

O $-0.746524-1.106591-2.818257$

C $1.524568-1.837780-2.461241$

C $2.505731-2.988846-2.381375$

C $3.019838-3.485973-1.239072$

C $2.900171-3.586121-3.707546$

C $2.754749-3.0190020 .164521$

C $4.028099-2.8779201 .008950$

C $5.089515-1.9413600 .412158$

C $6.287048-1.8332671 .359888$

C $7.348356-0.8589520 .832532$

O $4.509846-0.6490350 .197828$

O $8.254572-0.568177 \quad 1.907587$

C 6.6591750 .4660800 .451440

C $8.190328-1.480828-0.287899$

O $7.5800591 .350599-0.184565$

C $5.3996910 .284285-0.431478$

C $4.6777631 .645035-0.573179$

O $5.805453-0.164698-1.727004$

C $-1.871755-1.221850-3.572632$

O $-2.176026-2.166285-4.289659$

C $-2.771327-0.058090-3.407921$

C $-2.5868280 .899565-2.486791$

C $-3.4827512 .027204-2.225134$

C $-4.7436372 .136514-3.040823$

C $3.3032071 .591562-1.255678$

C $2.7929032 .990092-1.571240$

C $1.0330264 .179813-2.866823$

O $3.3662134 .011811-1.199963$

C $0.0553184 .757949-1.892934$

C -1.119117 $5.288498-2.267348$

C $-2.1017145 .909527-1.314544$

C $-3.5082935 .386264-1.508983$

C $-4.4010166 .070003-2.244745$

C $-3.9044274 .114548-0.795161$

C $-3.1375312 .910440-1.266823$

N $1.6273262 .969858-2.325530$

H -1.202936 -4.700332 -2.530763

H $-0.389358-2.546514-0.509065$

H $0.011731-2.898209-3.582353$

H $1.586883-1.182646-1.585310$

H $1.815029-1.179360-3.291588$

H $3.697483-4.335912-1.311135$

H $3.637263-4.389117-3.599959$

H $2.027317-4.006973-4.216190$

H $3.342780-2.818989-4.351464$

H $2.216812-2.0700470 .184225$

H $2.102250-3.7548990 .650206$

H $3.725526-2.5040961 .995464$

H $4.469148-3.8704131 .167568$

H $5.414361-2.352927-0.550061$

H $6.730364-2.8203591 .542064$

H $5.945211-1.4741352 .340466$

H $8.641692-1.4081212 .208557$

H $\quad 6.355994 \quad 0.9576351 .386340$

H $8.704333-2.3795630 .073798$

H $8.980577-0.796034-0.614858$

H $7.599681-1.768406-1.160069$

H 8.3433151 .4506360 .409896

H 4.5287032 .0829160 .422856

H $5.3236392 .331706-1.135754$

H $5.134590 \quad 0.088229-2.379466$
H -3.638556 -0.062592-4.060431

H - $-1.7112610 .849657-1.841519$

H $-5.3364003 .023401-2.804985$

H $-5.3876981 .266692-2.870722$

H -4.507463 2.197444 -4.109031

H $3.3531331 .034988-2.196658$

H $2.5773531 .096493-0.602676$

H $0.5587343 .911390-3.816225$

H $1.8277744 .907118-3.063411$

H $0.3330664 .774070-0.842452$

H $-1.3777475 .306188-3.324013$

H -2.083773 $6.995019-1.478159$

H $-1.7985875 .760994-0.270456$

H -5.419495 $5.717738-2.370580$

H $-4.1318576 .996280-2.741523$

H $-4.9831073 .935005-0.871767$

H -3.7251714 .2423790 .280130$

H $-2.1974762 .756691-0.737369$

H $1.1830422 .081014-2.537246$

C $-3.215298-4.696489-0.347547$

O $-3.484371-3.3387780 .069911$

C $-4.526851-3.3572961 .061828$

C $-5.155352-4.7422430 .977888$

C -3.942133 -5.591955 0.655334

H -4.036480 -3.260776 2.037693

C $-5.503462-2.1964610 .850894$

H $-5.649374-5.0325101 .909952$

H -5.891320 -4.805365 0.167802

C $-4.290920-6.9681420 .115694$

H $-3.337750-5.7053601 .565468$

C $-4.856310-0.7952710 .896771$

O $-6.123275-2.350456-0.431918$

H $-6.309578-2.2632591 .591480$

H -4.242034 -0.6402950 .002175$

$\begin{array}{llll}\text { O } & -5.914622 & 0.168480 & 0.813464\end{array}$

C $-4.010036-0.5149842 .153728$

C -3.5955610 .9647422 .273006$

H -3.122207 -1.155786 2.125173

O $-4.771951-0.874523 \quad 3.306725$

H -3.2216601 .3053081 .303268$

H -4.4790351 .5687592 .512565$

H -4.864428 -7.536681 0.855057

H $-4.892728-6.900769-0.796858$

H -3.385676 -7.536502 -0.119278

C $-1.703004-4.924457-0.383858$

H $-6.736245-1.604506-0.549292$

H -6.4456850 .0913151 .624736$

H $-4.163932-0.9114774 .063918$

H -1.472433 $-5.992183-0.469628$

H -1.246078 -4.5657870 .547078$

H $-3.650567-4.812685-1.348977$

C $-0.562940 \quad 0.7591832 .046314$

O $-1.368711 \quad 0.384443 \quad 3.167694$

C -2.5278911 .2026073 .366533$

C -2.1144032 .6716203 .549285$

C -1.2121343 .1642872 .420974$

C -0.0522332 .1958622 .197965$

H -1.1637580 .6811851 .131916$

C $0.555038-0.2742451 .883785$

H $-2.9690550 .880316 \quad 4.317456$

O -3.2564003 .5174873 .634572$

H -1.566841 2.7755094 .495206

H $-1.7766243 .317917 \quad 1.497128$

O -0.6703024 .4395562 .771696$

H 0.6424922 .2753943 .042822

O 0.6569532 .5679341 .021118

C $1.545904-0.2611013 .023266$

H $0.137568-1.2876741 .840541$

H $1.085799-0.1158090 .938750$

H -3.8026673 .1972194 .372663$

H -1.420565 5.0359062 .937885

H 0.9629383 .4829371 .143972

O $2.701418 \quad 0.345717 \quad 2.628342$

O $1.312858-0.715578 \quad 4.135723$

C 3.7154340 .4494853 .631717

H 3.3520391 .0402894 .477984

H $4.026446-0.5466153 .960185$

H 4.5767000 .9596053 .193300 
H $-11.2839120 .756570 \quad 0.494934$

H $-10.512222-0.251224-0.707768$

H -10.669103 $3.895158-1.341052$

H $-11.310573 \quad 3.1664270 .236440$

H $-9.5002672 .579240-2.923868$

H $-9.9861180 .911442-2.909771$

H $-7.9184130 .140322-2.135364$

H -6.416552 -2.277165 0.847793

C 1.7167673 .9169221 .620324

O $2.8087723 .392400 \quad 0.836144$

C 4.0281414 .0397201 .242853

C 3.6024545 .2651782 .040518

C 2.3612294 .7450522 .735387

H 4.5453463 .3448591 .914984

C $4.9089304 .348340 \quad 0.028455$

H 4.3783985 .5987842 .736141

H 3.3463696 .1076041 .387530

C 1.4671235 .8434113 .285069

H 2.6706414 .0899133 .560941

C $5.2612503 .126201-0.841914$

O $4.2306505 .290911-0.813907$

H 5.8270194 .8492920 .356140

H $4.3652152 .770366-1.363390$

O $6.1665293 .569341-1.862221$

C $5.9125221 .947220-0.092611$

C $6.3326940 .820629-1.054982$

H $5.212173 \quad 1.546750 \quad 0.645844$

O 7.0766802 .3890490 .604214

H $5.4724300 .549951-1.675327$

H $7.1124631 .197499-1.727635$

H 2.0031526 .4372734 .032456

H $1.132506 \quad 6.5224272 .493456$

H 0.5784315 .4206383 .763819

C 0.8720382 .7603402 .161845

H $3.3773144 .897631-1.065875$

H $5.7348324 .301907-2.334587$

H 6.7904602 .9770021 .322686

H 0.1438013 .1424222 .886950

H 1.5347662 .0758642 .705622

H 1.1246484 .5654730 .963416

C $4.803335-1.5938190 .045080$

O $5.961072-0.9733380 .616161$

C $6.887024-0.427079-0.329495$

C $7.376416-1.531168-1.277941$

C $6.211977-2.243845-1.958844$

C $5.199778-2.719454-0.919584$

H $4.209082-0.841038-0.481869$

C $3.958772-2.1555301 .198948$

H $7.756268-0.0962300 .252383$

O $8.247372-1.004271-2.273157$

H $7.950964-2.272733-0.707730$

H $5.733022-1.607444-2.712004$

O $6.705071-3.389567-2.657367$

H $5.623234-3.563385-0.359556$

O $4.032006-3.194939-1.587615$

C $3.272506-1.0769372 .008174$

H $3.170504-2.8074760 .810523$

H $4.601737-2.7265391 .879446$

H $8.982978-0.564070-1.813959$

H $7.377885-3.076315-3.286282$

H $4.314461-3.889842-2.206554$

O $2.712440-1.6494473 .115493$

O $3.213950 \quad 0.106110 \quad 1.705725$

C $2.011121-0.7576813 .987327$

H $1.115954-0.3750333 .489332$

H $1.705688-1.3198654 .873750$

H 2.6614580 .0646354 .301283

SCF Energy (B3LYP/6-31G**//MMFF) $=-3245.92478384$

\section{4}

MM̄FF Geometry

C $-2.808101-0.100752-3.249982$

C $-2.6844320 .941311-2.413456$

C $-1.595897 \quad 1.984646-2.480769$

O $-0.3939821 .378085-3.012502$

C $-1.978648 \quad 3.197463-3.344050$

C $-3.1124804 .025275-2.781731$ 
C $-2.9589894 .972938-1.836574$

C $-4.4680533 .772455-3.388399$

C $-1.6879305 .381406-1.141888$

C -1.846602 5.4324580 .384136

C -1.8891124 .0501391 .060257$

C -2.3320174 .2117762 .516327$

C -2.2867242 .8874913 .282056$

O $-0.572733 \quad 3.4891520 .999960$

O $-2.464650 \quad 3.2027694 .671675$

C -0.8861042 .2680293 .109348$

C $-3.452151 \quad 1.9703272 .896654$

O -0.8653310 .9720513 .707483$

C -0.4173232 .2091041 .633319$

C 1.0797971 .8213141 .526680

O

C $0.7987501 .880243-2.596334$

O $0.9793572 .850319-1.878374$

C $1.8655971 .018884-3.151782$

C $3.1349831 .222178-2.769489$

C $4.3068960 .464735-3.211436$

C $4.085559-0.677602-4.167687$

C 2.0477212 .9231981 .965223

C 3.3076512 .3482052 .575669

C 5.4271961 .1993812 .050039

O 3.4770372 .3301963 .793615

C 6.3947661 .1504290 .913266

C 6.8542810 .0077740 .380462

C $7.888691-0.056714-0.706802$

C $7.368832-0.694936-1.974975$

C $7.437845-2.023958-2.159899$

C $6.8559890 .217961-3.064763$

C $5.5185650 .821203-2.736389$

N $4.205174 \quad 1.8627201 .637127$

H -2.095208 -0.193568 -4.067427

H -3.398881 $1.071650-1.605202$

H -1.393028 2.302057 -1.453458

H -2.224930 $2.865962-4.362399$

H -1.099161 $3.837710-3.490788$

H $-3.8401965 .537121-1.532958$

H $-5.2398794 .426384-2.968727$

H $-4.4383783 .950792-4.468472$

H -4.784995 2.740025 -3.215726

H $-0.8421514 .742605-1.405918$

H -1.437664 6.390704-1.491932

H $-0.993611 \quad 5.995126 \quad 0.786173$

H $-2.751868 \quad 6.0009570 .633190$

H $-2.5841573 .397503 \quad 0.520924$

H -3.3368174 .6498142 .571149$

H -1.667904 4.9275593 .021805

H -2.3628772 .3772375 .176133$

H -0.1958682 .8883963 .693889$

H -4.409714 2.4648053 .099913

H -3.4530901 .0604403 .504779$

H -3.4445651 .6822701 .843464$

H $0.0627460 .702355 \quad 3.806223$

H 1.2621080 .8886452 .071265

H 1.3161041 .5773590 .482635

H $-0.650057 \quad 0.8454930 .225972$

H $\quad \begin{array}{llll}1.577486 & 0.239328 & -3.847572\end{array}$

H $3.3416072 .023407-2.060569$

H $4.991950-1.248592-4.381102$

H $3.703371-0.306997-5.125019$

H $3.366144-1.393614-3.756842$

H 2.3170673 .5554431 .110419

H 1.6065313 .5911512 .711775

H 5.1568650 .2029602 .411539

H 5.8658951 .7488072 .890024

H 6.7639392 .1015230 .537297

H $6.497141-0.9419390 .772918$

H $8.740704-0.635074-0.325203$

H $8.2937450 .937960-0.931308$

H $7.095038-2.485689-3.079843$

H $7.834564-2.679603-1.391805$

H $6.832162-0.302527-4.028198$

H $7.5749101 .033456-3.217361$

H $5.5775221 .661798-2.044365$

H 3.8905871 .7478610 .677380

C $-4.702649-1.307205-1.945909$
O $-3.878206-1.577282-0.793076$

C $-4.495003-2.613031-0.007442$

C $-5.908850-2.773611-0.554013$

C $-5.701199-2.464596-2.021626$

H $-3.933350-3.532703-0.206254$

C $-4.432528-2.2781741 .486516$

H $-6.309765-3.776392-0.377406$

H -6.603316 -2.050719 -0.110641

C $-6.985724-2.127629-2.759062$

H -5.232651 -3.336348 -2.498296

C $-3.025278-1.9757172 .036831$

O $-5.240920-1.1202461 .742458$

H -4.889762 -3.091369 2.062107

H -2.680255 -1.005696 1.661017

O $-3.144279-1.8347053 .459919$

C $-1.949237-3.0392071 .747614$

C $-0.614629-2.7006092 .442718$

H $-1.785574-3.1158460 .667528$

O $-2.380666-4.3154152 .217635$

H $-0.315983-1.6868942 .154077$

H $-0.764443-2.6966323 .529130$

H -7.683722 -2.970288 -2.719954

H -7.484407 -1.257397 -2.319205

H $-6.785251-1.904441-3.811644$

C -3.832337 -1.202564 -3.202268

H $-4.898927-0.3962121 .190050$

H -3.854419-1.193066 3.631291

H $-3.136880-4.5950931 .675843$

H $-4.472729-1.097848-4.085901$

H -3.279543 -2.142845 -3.319307

H $-5.227806-0.362658-1.756463$

C $1.304498-2.7047860 .076234$

O $0.734759-3.8513410 .716995$

C $0.511283-3.7093262 .121759$

C $1.841287-3.4171502 .829159$

C $2.552068-2.2094902 .225747$

C $2.653927-2.342176 \quad 0.707233$

H $0.610488-1.8579790 .149279$

C $1.479186-3.050477-1.405583$

H $0.169675-4.6897122 .476716$

O $1.643378-3.1934904 .221080$

H $2.501412-4.2892772 .734218$

H $2.058138-1.2734482 .506999$

O $3.877619-2.1373922 .755478$

H $3.402849-3.1053460 .458972$

O $3.105614-1.1066710 .156018$

C $0.139462-3.150371-2.090364$

H $2.053936-2.283107-1.934365$

H $2.015673-3.998129-1.536376$

H $1.197958-3.9787284 .582685$

H $3.799079-2.0796703 .723383$

H $3.959198-0.8992180 .572120$

O $-0.302500-4.439461-2.070001$

O $-0.452932-2.188615-2.560780$

C -1.586193 -4.661883 -2.660513

H $-2.358933-4.156454-2.075092$

H -1.597097 -4.315094 -3.698248

$\mathrm{H}-1.784644-5.736854-2.648656$

SCF Energy (B3LYP/6-31G**//MMFF) $=-3245.91592414$

0500055

MMFF Geometry

C $4.198670-2.5162741 .869000$

C $2.905846-2.6216781 .529131$

C $1.786281-2.8124512 .515131$

O $0.736933-1.8776202 .177711$

C $1.220811-4.2395422 .366694$

C $0.046384-4.5387083 .278282$

C $-1.201653-4.8189822 .851316$

C $0.350859-4.5348774 .754403$

C $-1.693572-4.9233841 .433028$

C $-2.399900-3.6404410 .997908$

C $-2.977347-3.699362-0.423764$

C $-1.885331-3.747793-1.496875$

C $-2.463144-3.654573-2.918724$

O $-3.767174-2.512614-0.585524$

O $-1.379076-3.374839-3.818290$

C $-3.428035-2.452641-2.990781$ 
C $-3.070817-4.985980-3.374990$

O $-4.102137-2.407731-4.245564$

C $-4.456782-2.424020-1.841068$

C $-5.293994-1.123283-1.815053$

O $-5.376961-3.510234-1.997934$

C $0.371861-0.957336 \quad 3.110418$

O $0.926307-0.7141514 .169593$

C $-0.888545-0.3380682 .640998$

C $-1.646331 \quad 0.3669193 .495078$

C $-2.9674350 .933184 \quad 3.205649$

C -3.5864440 .5870101 .878126$

C $-4.4679480 .163559-1.778160$

C $-5.260624 \quad 1.370032-1.301297$

C $-4.916368 \quad 3.790282-0.759637$

O $-6.4406101 .318478-0.968508$

C $-4.258116 \quad 4.0148510 .566327$

C -4.9128504 .3694061 .682468$

C -4.2267904 .5953183 .001331$

C $-4.877778 \quad 3.846483 \quad 4.145388$

C $-5.399443 \quad 4.5113705 .190437$

C -4.9347622 .3346394 .085566$

C -3.5711351 .6956314 .141838$

N -4.471204 $2.511027-1.281797$

H $4.479726-2.5447162 .918755$

H $2.632857-2.6201880 .477899$

H $2.147745-2.6559973 .538127$

H $2.011243-4.9734772 .574155$

H $0.940907-4.3928051 .319226$

H $-1.969278-5.0084973 .600880$

H $-0.498743-4.8782445 .354366$

H $1.192247-5.2016574 .969526$

H $0.606947-3.5277505 .095743$

H $-0.880751-5.1755950 .748316$

H $-2.395393-5.765014 \quad 1.378667$

H -3.213833 -3.4126481 .699881$

H -1.711031 -2.790972 1.067609

H -3.634094 -4.574132 -0.495282

H - $1.273457-4.652231-1.402970$

H $-1.195920-2.904978-1.348703$

H $-0.717584-4.081010-3.719789$

H $-2.807168-1.549016-2.938707$

H $-2.306730-5.772805-3.381769$

H -3.440629 -4.919662-4.404155

H $-3.887110-5.326835-2.734889$

H $-3.422149-2.423704-4.940630$

H -5.978237 -1.093957 -2.673025

H $-5.939790-1.159550-0.926676$

H $-6.189813-3.298200-1.509753$

H $-1.188297-0.5299111 .617084$

H $-1.284067 \quad 0.520158 \quad 4.510774$

H -3.656423 -0.4995951 .754957$

H $-2.9814340 .994917 \quad 1.063549$

H -4.596622 0.9762021 .745096

H -3.613903 $0.043111-1.103369$

H $-4.0825340 .385823-2.780013$

H $-6.0084003 .808957-0.690136$

H $-4.5934134 .558871-1.468330$

H $-3.178616 \quad 3.8867460 .608692$

H $-5.9884194 .524797 \quad 1.644454$

H -4.2413695 .6752573 .198016$

H $-3.167778 \quad 4.3140952 .948267$

H -5.870167 3.9886306 .016504

H -5.3680905 .5945115 .245634$

H -5.5122992 .0367493 .207346$

H -5.5043951 .9439174 .939579$

H -3.0441291 .8771285 .079594$

H -3.496035 $2.431282-1.555182$

C $6.203363-1.213768 \quad 1.074931$

O $5.502456-0.023758 \quad 0.658502$

C $6.4060960 .805250-0.097480$

$\begin{array}{llll}\text { C } 7.796880 & 0.215350 & 0.104814\end{array}$

C $7.479801-1.2590490 .233625$

H $6.1289930 .692048-1.152123$

$\begin{array}{llll}\text { C } & 6.283769 & 2.272422 & 0.322522\end{array}$

H $8.4681170 .440693-0.729545$

H $8.266951 \quad 0.5845301 .023842$

C $8.605674-2.0674760 .855579$

H $7.258555-1.659490-0.764964$
C 4.8679332 .8646540 .185646

$\begin{array}{lllll}\text { O } & 6.669482 & 2.384319 & 1.699030\end{array}$

H $7.0027932 .873774-0.247189$

H 4.2044022 .4185930 .936025

$\begin{array}{lllllll}\text { O } & 4.957570 & 4.256148 & 0.524243\end{array}$

C $4.2318392 .721816-1.211319$

C $2.9250873 .527179-1.329013$

H $4.0422101 .662880-1.410145$

O $5.1620203 .190221-2.186865$

H $2.2917843 .288253-0.468151$

H $3.1616134 .596661-1.272139$

H $9.510483-2.0004720 .242686$

H $8.852257-1.7057051 .859462$

H $8.331358-3.123726 \quad 0.936686$

C $5.315897-2.4401150 .865898$

H 6.5884463 .3211811 .947371

H $5.4951574 .690000-0.160675$

H $4.8522342 .891974-3.058345$

H $5.912293-3.3534580 .973376$

H $4.909991-2.432535-0.153431$

H $6.447764-1.0864912 .137585$

C $0.8405381 .379581-1.919930$

O $1.8142821 .898869-2.833912$

C $2.1532043 .276649-2.644806$

C $0.8958334 .142177-2.796581$

C $-0.2012563 .703660-1.833150$

C $-0.4558522 .203105-1.966145$

H $1.2557951 .377359-0.904998$

C $0.549598-0.075764-2.323377$

H $2.8242923 .544723-3.469614$

O $1.2144795 .513416-2.584908$

H $0.5230364 .054009-3.825480$

H $0.042726 \quad 3.983529-0.801850$

O $-1.396118 \quad 4.412871-2.167294$

H $-0.9833692 .019226-2.910593$

O $-1.3286001 .785021-0.919723$

C $1.741317-0.990020-2.138113$

H $-0.270147-0.485190-1.722770$

H $0.271547-0.107190-3.383985$

H $0.3879556 .019699-2.665440$

H $-2.0851254 .124141-1.545921$

H $-0.8754521 .936718-0.072728$

O $1.480909-2.179304-2.757807$

O $2.758448-0.709393-1.520503$

C $2.519230-3.162688-2.698952$

H $2.305198-3.925251-3.452605$

H $3.496183-2.721405-2.919279$

H $2.522022-3.635372-1.713673$

SCF Energy (B3LYP/6-31G**//MMFF) = -3245.92210487

0500056

MMFF Geometry

C $-2.334271-3.6578720 .733236$

C $-2.603878-3.7099362 .046333$

C $-2.047729-2.7863493 .104872$

O $-1.136471-1.8301762 .519144$

C $-3.215660-1.9959773 .732772$

C $-2.788379-0.985904 \quad 4.778680$

C $-2.896358 \quad 0.351515 \quad 4.645138$

C $-2.228656-1.570946 \quad 6.050171$

C $-3.468313 \quad 1.136507 \quad 3.494578$

C $-2.363906 \quad 1.751692 \quad 2.634537$

C $-2.8760202 .744718 \quad 1.580382$

C $-3.7154442 .071451 \quad 0.491748$

C $-4.124890 \quad 3.065596-0.607453$

$\begin{array}{llll}0 & -1.715038 & 3.343766 & 0.989340\end{array}$

O $-4.6214812 .312234-1.724565$

C $-2.8687103 .818643-1.099342$

C $-5.2796083 .965262-0.151123$

O $-3.215723 \quad 4.863066-2.004228$

$\begin{array}{lllll}\text { C } & -1.996485 & 4.386178 & 0.043084\end{array}$

C $-0.6391014 .937111-0.447210$

O -2.6289855 .5016130 .682352$

C $0.138178-1.7760392 .988802$

O $0.670584-2.5311453 .784908$

C $0.763351-0.5718272 .398426$

C $1.879376-0.0626632 .940943$

C $2.547751 \quad 1.1735392 .525993$ 
C 1.8508182 .0127191 .489855

C $0.2193713 .938186-1.225717$

C $1.6796024 .352885-1.309771$

C $3.8897933 .458238-2.060878$

O $2.1120655 .403915-0.846019$

C $4.4772652 .537022-1.037966$

C $5.4087562 .889429-0.139363$

C 5.9714571 .9309490 .874196

C 5.9018792 .4594812 .291345

C 7.0240562 .6690803 .000466

C 4.5427982 .7538182 .889557

C 3.7173351 .5129713 .108076

N $2.4423393 .391882-1.959877$

H $-1.619342-2.9326280 .355444$ H $-3.326820-4.4437442 .398059$ H -1.549918 -3.399151 3.865052 H -3.928746 -2.691839 4.195358 H -3.769965 -1.500601 2.928526 H -2.5447250 .9745995 .467200$ H -2.021637 $-0.804427 \quad 6.804673$ H -2.942560 -2.276138 6.488430 H $-1.291525-2.1000125 .855656$ H -4.1358220 .5266602 .881507$ H $-4.094763 \quad 1.933482 \quad 3.914387$ H -1.6374972 .2693853 .275993$ H -1.7972480 .9578282 .134644$ H -3.4519603 .5233242 .093704$ H -4.606992 1.5926460 .912325 H -3.1306291 .2643110 .029041$ H -5.374062 $1.779051-1.416507$ H -2.275302 $3.094742-1.672352$ H -6.1694783 .3633560 .070310$ H $-5.5782364 .659441-0.944253$ H $-5.045643 \quad 4.5417200 .746329$ H -3.750463 $4.466521-2.713281$ H $-0.7923375 .837841-1.056151$ H $-0.080448 \quad 5.2762910 .436715$ H $-3.406610 \quad 5.757676 \quad 0.160826$ H $0.256920-0.107643 \quad 1.559905$ H $2.334601-0.5818453 .783177$ H 2.3597222 .9513161 .269427 H $0.8434292 .280837 \quad 1.826281$ H 1.7721391 .4646250 .545171 H $0.1823102 .956640-0.743114$ H $-0.1620533 .833056-2.247705$ H $4.2275304 .491100-1.931109$ H $4.1612783 .120127-3.065275$ H $4.1151571 .512746-1.036725$ H $5.7968143 .905015-0.139753$ H 7.0136241 .7306520 .593632 H 5.4582080 .9623240 .829822 H 6.9893993 .0490654 .016161 H 8.0044452 .4651832 .582748 H 4.0361323 .5016892 .275120 H 4.6589773 .2356053 .870020 H 4.1368720 .8394763 .856858 H $1.9895892 .538849-2.275487$ C $-3.807577-3.675551-1.325049$ O $-2.890760-2.860535-2.084566$ C $-3.308203-1.488619-1.977642$ C $-4.803628-1.557269-1.702914$ C $-4.866912-2.730259-0.741924$ H -2.800365 - $1.070855-1.098961$ C $-2.895161-0.691252-3.216145$ H $-5.194241-0.631000-1.272325$ H $-5.374920-1.785100-2.609951$ C $-6.245305-3.358871-0.629911$ H $-4.574402-2.368540 \quad 0.250675$ C $-1.428902-0.894646-3.645830$ O $-3.720023-1.087967-4.318867$ H $-3.1029740 .371505-3.047743$ H $-1.320978-1.884625-4.105466$ O $-1.1558590 .049052-4.690866$ C $-0.376920-0.738696-2.530205$ C $1.050888-0.872193-3.091032$ H $-0.547140-1.502855-1.765342$ O $-0.539208 \quad 0.536455-1.916322$ H $1.103369-1.797593-3.675171$
H $1.246479-0.043195-3.782506$

H $-6.970889-2.630418-0.253491$

H $-6.603283-3.718572-1.600441$

H $-6.228020-4.2084680 .060201$

C $-3.024645-4.507610-0.298535$

H -3.445953 -0.562144 -5.090058

H $-1.1826240 .937769-4.296144$

H $-0.0082590 .540196-1.102039$

H $-2.251080-5.085622-0.818602$

H $-3.702928-5.2244800 .178857$

H $-4.265385-4.362481-2.047661$

C $2.026179-3.168679-1.318414$

O $1.914467-1.789338-0.954527$

C $2.161194-0.864877-2.017258$

C $3.569570-1.088951-2.590526$

C $3.779182-2.536895-3.029940$

C $3.408649-3.494768-1.898989$

H $1.256949-3.388359-2.069768$

C $1.662360-4.022627-0.093477$

H $2.1494060 .131517-1.561608$

O $3.813400-0.228502-3.698141$

H $4.317747-0.850026-1.823799$

H $3.218666-2.769550-3.942578$

O $5.158852-2.735953-3.348084$

H $4.183037-3.434344-1.125473$

O $3.403127-4.833136-2.389402$

C $2.731645-4.1144270 .973283$

H $\quad 0.758288-3.6376910 .389743$

H $1.450656-5.047092-0.423390$

H $3.6729150 .684866-3.396475$

H $5.391017-2.092879-4.039965$

H $4.279201-5.004237-2.775879$

O $3.170056-2.8764901 .333573$

O $3.106799-5.1758821 .456441$

C $4.201567-2.8499242 .325202$

H $5.038567-3.4901042 .029715$

H $4.565266-1.8225362 .410547$

H $3.796561-3.1634203 .291684$

SCF Energy $\left(B 3 L Y P / 6-31 G^{* *} / / M M F F\right)=-3245.91394946$

\section{7}

MM̄FF Geometry

C $2.1572953 .812775-0.540979$

C $1.2549634 .633142-1.100403$

C $-0.2224064 .335033-1.255451$

O $-0.5134872 .948092-0.967075$

C -1.019310 $5.205580-0.266629$

C $-2.5184304 .993024-0.329034$

C -3.2605364 .4950610 .679865$

C $-3.1823855 .399316-1.619204$

C -2.7982664 .0616902 .043650$

C -2.4420532 .5713192 .157865$

C -3.6329941 .6231651 .940648$

C -3.2471150 .1845512 .289172$

C $-4.399986-0.7958522 .022440$

O -4.0222251 .6827000 .567384$

O $-3.864385-2.1286192 .048600$

C $-4.947971-0.5656700 .595368$

C $-5.453913-0.7355923 .134472$

O $-6.139375-1.3159120 .377509$

C -5.1943390 .9190250 .248185$

C $-5.4964361 .156835-1.249556$

O $-6.344207 \quad 1.432526 \quad 0.932842$

C $-0.2695972 .039219-1.950606$

O $0.1135932 .265647-3.086947$

C $-0.4979510 .696025-1.375942$

C $0.063556-0.373130-1.958949$

C $-0.040738-1.758103-1.496689$

C -1.025834-2.050930 -0.397616

C $-4.3967160 .723500-2.225106$

C $-4.597957-0.664920-2.811379$

C $-3.465187-2.330352-4.282332$

O $-5.623888-1.321616-2.656604$

C $-2.822589-3.376434-3.427291$

C $-1.784646-4.124684-3.831631$

C $-1.162171-5.212022-3.002880$

C $0.333935-5.049362-2.851674$

C $1.187031-5.738032-3.628694$ 
C $0.838888-4.156846-1.741646$

C $0.733490-2.695607-2.081264$ N $-3.503074-1.069639-3.564210$ H $1.8351202 .855382-0.141690$ H $1.5860755 .597049-1.480609$ H $-0.5225414 .563515-2.285347$ H $-0.814336 \quad 6.267532-0.457122$ H -0.6488145 .0080490 .744568$ H $-4.333154 \quad 4.389780 \quad 0.520436$ H -4.274606 5.342149-1.560319 H $-2.9225896 .432684-1.871121$ H $-2.8644104 .748013-2.439076$ H -1.9389384 .6577762 .368425$ H -3.593255 4.2985902 .762398 H -1.645556 2.3380591 .441585 H -2.031403 2.4041423 .161492 H -4.4628931 .9615722 .571840$ H -2.917174 0.1118703 .332851 H -2.384512 -0.1177321 .680777$ H -3.428627 -2.2612632 .907830$ H -4.197074 -0.965951 -0.095071 H -5.010657 -1.018370 4.097036 H -6.261335 -1.453901 2.955577 H $-5.891474 \quad 0.2575803 .256259$ H $-5.933913 \quad-2.247526 \quad 0.567317$ H $-6.4653640 .715921-1.513647$ H $-5.6289682 .240925-1.380643$ H $-6.779000 \quad 0.702917 \quad 1.402206$ H $-1.0929770 .629247-0.472676$ H $0.681204-0.220040-2.843059$ H - $-2.019785-1.680851-0.668265$ H $-0.715376-1.5677200 .532551$ H -1.146660 -3.115249 -0.186816 H -4.399232 $1.415591-3.077133$ H $-3.4098800 .783946-1.758176$ H -2.912525 -2.157789 -5.211275 H -4.485185 -2.637102 -4.535507 H -3.249516 -3.545840 -2.441547 H -1.378373 -3.972605 -4.829190 H -1.384449 -6.169958 -3.491301 H -1.624356 -5.273985 -2.009817 H $2.261423-5.648499-3.506660$ H $\quad 0.829447-6.402436-4.408389$ H $1.899252-4.365412-1.544035$ H $\quad 0.330970-4.417316-0.808835$ H $1.409585-2.385782-2.879365$ H -2.674344 -0.482752 -3.592877 C $4.4711993 .152840-1.216872$

O $4.2505661 .795869-0.783091$

C $5.5057151 .092913-0.787967$

C $6.5208432 .029038-1.438414$

C $5.9717763 .390523-1.062486$ H 5.7842430 .9490320 .262032

C $5.361361-0.264170-1.487332$

H $7.5361791 .857483-1.067778$

H $6.541497 \quad 1.916407-2.528517$

C $6.5138064 .521527-1.918923$

H $6.2149043 .586962-0.009603$

C $4.258776-1.175154-0.910446$

O $5.045442-0.023793-2.865707$

H $6.327885-0.782329-1.477751$

H $3.270163-0.761440-1.143803$

O $4.340817-2.434997-1.591303$

C $4.344812-1.4425280 .605452$

C $3.341970-2.5232451 .058618$

H $4.159340-0.5149321 .156786$

O $5.652697-1.9029530 .949048$

H $2.354881-2.2882840 .650686$

H $3.651827-3.4860420 .635409$

H $7.6021914 .590551-1.820539$

H $6.2810254 .369659-2.978268$

H $6.0866165 .481913-1.614524$

C $3.6190184 .115932-0.389456$

H $5.170201-0.859980-3.345301$

H $4.035709-2.295742-2.503501$

H $6.272442-1.1641220 .833826$

H $3.8317115 .151242-0.678716$

H 3.8668064 .0158100 .674493
H $4.1776923 .200651-2.274043$

C $1.345753-1.2545953 .022251$

O $2.748747-1.4814883 .219903$

C $3.272690-2.6607652 .597244$

C $2.515991-3.9018343 .091202$

C $1.009309-3.7567322 .905513$

C $0.527637-2.4500123 .533479$

H $1.145481-1.0931211 .957489$

C 0.9786050 .0338873 .775296

H $4.304409-2.7614762 .956469$

O $2.990170-5.0631172 .418624$

H $2.728092-4.0409774 .159600$

H $\quad 0.724450-3.8143641 .848334$

O $0.345257-4.836503 \quad 3.566052$

H $\quad 0.602860-2.5316374 .625754$

O $-0.850271-2.2902903 .205418$

C 1.5064751 .2772503 .091264

H -0.1086280 .1483403 .831812$

H $1.3970070 .008764 \quad 4.788194$

H $2.586898-5.8374612 .845652$

H $0.556812-5.651372 \quad 3.080237$

H -1.229765 -1.644047 3.823615

O 1.0753312 .3728193 .785122

O 2.1983541 .2925332 .083384

C 1.4698023 .6399863 .251258

H 2.5543313 .6820833 .112340

H 0.9498643 .8199092 .307062

H 1.1784534 .4150983 .964830

SCF Energy $\left(B 3 L Y P / 6-31 G^{* *} / / M M F F\right)=-3245.89746396$

\section{8}

MM̄FF Geometry

C $-3.516335-2.085397 \quad 0.829600$

C $-3.826782-1.9434002 .126832$

C $-2.966768-1.287403 \quad 3.182614$

O $-1.704920-0.8609572 .622080$

C -3.698648 $-0.032307 \quad 3.703353$

C $-2.9185830 .757574 \quad 4.735162$

C -2.4315512 .0003174 .543218$

C $-2.7190450 .068536 \quad 6.060704$

C $-2.5616192 .879038 \quad 3.327461$

C -1.2736832 .8852832 .503988$

C $-1.274417 \quad 3.9029651 .353502$

$\begin{array}{llll}C & -2.283061 & 3.553166 & 0.255287\end{array}$

C $-2.182576 \quad 4.508152-0.945468$

$\begin{array}{llllll} & 0 & 0.054170 & 3.902225 & 0.810680\end{array}$

O $-2.9330543 .915817-2.016119$

C $-0.7073554 .595787-1.391968$

C $-2.8527475 .854673-0.649090$

O $-0.542407 \quad 5.564283-2.424441$

C $0.2711994 .866301-0.229702$

C $1.7543834 .764296-0.657089$

$\begin{array}{lllll}\text { O } & 0.067967 & 6.198891 & 0.255318\end{array}$

C $-0.554689-1.344227 \quad 3.163645$

O $-0.440524-2.2037734 .021441$

C $0.561495-0.586768 \quad 2.553507$

C $1.788637-0.6367763 .093427$

C 2.9455500 .1545032 .661749

C $2.712025 \quad 1.174258 \quad 1.579532$

C $2.1388853 .441553-1.322519$

C $3.6393423 .199069-1.337765$

C $5.2881411 .418214-1.939857$

O $4.458088 \quad 3.992057-0.882155$

C $5.3877140 .383349-0.864144$

$\begin{array}{llll}\text { C } & 6.354153 & 0.333415 & 0.064467\end{array}$

C $6.410646-0.7309181 .126033$

C $6.535642-0.1672802 .525418$

C $7.615195-0.437323 \quad 3.278883$

C $5.4240320 .713198 \quad 3.054917$

C $4.134427-0.0344203 .273018$

N $3.9469201 .974601-1.915217$

H -2.549185 -1.7515790 .465182$

H $-4.803542-2.2843052 .465615$

H $-2.818208-2.0068233 .996124$

H $-4.661730-0.3209844 .145643$

$\mathrm{H}-3.9484110 .6021982 .846459$

H -1.877519 2.4620025 .360261

H -2.2324690 .7169136 .797273$ 
H -3.684686 -0.2337496 .479258$ H -2.095209 -0.8224545 .946062$ H -3.4125192 .5863182 .708266$ H -2.782159 3.8968303 .672873 H -0.4163703 .0980843 .157511$ H $-1.088177 \quad 1.8850042 .096795$ H -1.483488 4.8941251 .772746 H -3.3088843 .5462310 .641386$ H -2.090531 $2.530586-0.098667$ H -2.812893 $4.472968-2.804462$ H $-0.4680773 .626551-1.846651$ H $-3.9140215 .707402-0.414151$ H -2.831190 $6.510929-1.526132$ H -2.398655 6.3849930 .190308 H $-0.5620586 .444090-2.011015$ H $2.0156935 .597515-1.322558$ H 2.3712474 .9031520 .241798 H $\quad 0.869334 \quad 6.479074 \quad 0.728293$ H $\quad 0.327147 \quad 0.038578 \quad 1.699519$ H $1.956458-1.2856053 .951784$ H 1.9403611 .8898901 .884431 H 2.3908440 .6827960 .655468 H 3.5959001 .7622101 .330871 H $1.6746492 .604676-0.790608$ H $1.7839643 .428959-2.359383$ H $6.0278572 .215703-1.818368$ H $5.4268250 .950078-2.919086$ H $4.613283-0.377699-0.848652$ H 7.1484221 .0757140 .052894 H $7.268332-1.3769530 .898089$ H $5.527406-1.3803831 .082106$ H $7.719311-0.0386514 .282598$ H $8.420802-1.0639132 .910632$ H 5.3110961 .5796972 .399019 H $5.712016 \quad 1.1388274 .025797$ H $4.198975-0.7920174 .055147$ H $3.1872641 .375703-2.226515$ C $-4.731708-1.647857-1.349164$ O $-3.511484-1.414463-2.082428$ C $-3.2875980 .004814-2.153821$ C $-4.6676490 .628003-1.996353$ C $-5.269449-0.272779-0.932985$ H $-2.6745540 .271745-1.283516$ C $-2.5285110 .374850-3.430498$ H $-4.6232281 .677217-1.691884$ H $-5.2532860 .560506-2.920187$ C $-6.785333-0.209320-0.859295$ H -4.8643050 .0344980 .038483$ C -1.287154 -0.497038 -3.708387 O H $-2.2539841 .435749-3.395553$ H -1.611774 -1.492033-4.038711 O $-0.610530 \quad 0.099575-4.822072$ C $-0.311165-0.635401-2.524172$ C $0.933682-1.467843-2.882045$ H $-0.830011-1.114810-1.688958$ O $\quad 0.0957840 .655762-2.077696$ H $0.605558-2.402038-3.350335$ H $1.535308-0.926455-3.621962$ H $-7.1161270 .803364-0.607040$ H $-7.246028-0.484938-1.813894$ H -7.164128 -0.891449 -0.091452 C $-4.462045-2.633821-0.203881$ H $-2.9137030 .483843-5.346210$ H $0.000109-0.563126-5.186397$ H $0.5099821 .110515-2.830953$ H -4.007641-3.542922 -0.615688 H $-5.414103-2.9236280 .255978$ H -5.418743 -2.125614 -2.058950 C $0.626062-3.663036-0.783752$ O $1.114991-2.333146-0.565696$ C $1.836583-1.769930-1.664458$ C $3.049898-2.651174-1.989986$ C $2.652537-4.106486-2.231530$ C $1.788240-4.622411-1.079266$ H $-0.074493-3.648643-1.626634$ C $-0.143635-4.0646850 .484075$ H $2.218066-0.803735-1.314326$
O $3.739327-2.135807-3.123882$

H $3.749245-2.617947-1.144424$

H $2.146179-4.222295-3.196851$

O $3.850768-4.882432-2.308373$

H $2.414678-4.741490-0.185766$

O $1.268615-5.905493-1.413559$

C $-1.264557-5.0513240 .242543$

H $0.542605-4.4803791 .231020$

H -0.601291 -3.1721990 .923194$

H $4.490699-2.727569-3.300543$

H $3.599549-5.786404-2.562356$

H $2.017932-6.521131-1.476085$

O $-1.813617-5.392478 \quad 1.446822$

O $-1.631899-5.456834-0.851012$

C $-2.915781-6.3029141 .385047$

H $-3.738847 \quad-5.8632120 .813839$

H -2.599504 -7.251332 0.940852

H -3.259217 -6.490543 2.405905

SCF Energy (B3LYP/6-31G**//MMFF) $=-3245.92729800$

\section{9}

MM̄FF Geometry

C $-1.648915-3.219035-2.398647$

C $-0.993706-2.188137-1.845023$

C $-0.358544-1.080959-2.642742$

O $-0.921250 \quad 0.185246-2.217824$

C $1.158206-0.991452-2.392245$

C $1.932989-2.192059-2.890271$

C $2.371104-3.202191-2.113280$

C $2.204352-2.206845-4.372259$

C $2.192232-3.363119-0.629259$

C $3.457172-3.8649150 .080271$

C $4.672904-2.931634-0.039191$

C $5.856125-3.524306 \quad 0.731665$

C $7.069844-2.5870340 .737010$

$\begin{array}{llllll}\text { O } & 4.321780 & -1.652777 & 0.497329\end{array}$

O $7.990987-3.0623321 .730544$

C $6.608856-1.1854091 .182248$

C $7.820227-2.615065-0.599718$

O $7.676356-0.245641 \quad 1.070586$

C $5.365014-0.6685490 .420423$

C $4.846823 \quad 0.6219651 .103798$

O $5.726819-0.391613-0.930723$

C -2.157456 $0.517101-2.673306$

O $-2.890867-0.178650-3.362857$

C $-2.5523251 .881058-2.249366$

C $-1.7293172 .723476-1.606808$

C $-2.0219714 .106969-1.225556$

C $-3.3607784 .677347-1.608951$

C $3.626097 \quad 1.2692630 .436553$

C $3.9751672 .283056-0.642531$

C $2.8918213 .835304-2.268387$

O $5.1258152 .635366-0.887220$

C $2.4617995 .107831-1.606600$

C $1.5176665 .921933-2.103676$

C $1.0292197 .157418-1.399967$

C $-0.4755417 .169069-1.211999$

C $-1.2474698 .017285-1.912325$

C $-1.082236 \quad 6.252665-0.168512$

C -1.061542 $4.810632-0.591124$

N $2.8424832 .765927-1.284892$

H $-1.732151-3.283171-3.480657$

H $-0.924217-2.122043-0.763065$

H $-0.543474-1.198052-3.718194$

H $1.344492-0.798973-1.330101$

H $1.548613-0.089311-2.884144$

H $2.896540-4.025038-2.596634$

H $2.792754-3.077993-4.679415$

H $1.266236-2.228299-4.935261$

H $2.765357-1.312622-4.663227$

H $1.859407-2.440221-0.149045$

H $1.398095-4.102238-0.465925$

H $3.203847-3.9914561 .141129$

H $3.720382-4.858539-0.304355$

H $4.931631-2.818022-1.097931$

H $6.131277-4.5063170 .326680$

H $5.557201-3.7051841 .774070$

H $8.233891-3.9759391 .501492$ 
H $6.358472-1.2445842 .251016$ H $8.181816-3.627990-0.814168$ H $8.712511-1.980417-0.565501$ H $7.204404-2.300602-1.444775$ H $8.429357-0.6015881 .572630$ H 4.5498680 .3628002 .128832 H 5.6539341 .3597771 .180349 H $5.0024190 .097860-1.350681$ H $-3.5579142 .172306-2.535824$ H $-0.7253502 .385888-1.356847$ H -3.483809 5.724101-1.322091 H -4.168385 4.118735-1.123087 H -3.503745 $4.631257-2.694305$ H 2.9501720 .5121540 .026868 H 3.0791391 .8261601 .207482 H $2.226273 \quad 3.551499-3.089624$ H $3.9096583 .940635-2.655927$ H $2.9336945 .360045-0.659492$ H $1.0558205 .680622-3.058432$ H $1.3446118 .024824-1.993802$ H $1.5087527 .273833-0.420107$ H -2.322447 8.052558 -1.770250 H -0.823128 $8.694152-2.646499$ H -2.099911 6.5677720 .088474 H $-0.518516 \quad 6.352790 \quad 0.768045$ H $-0.1274814 .304671-0.348715$ H $1.9271152 .447800-0.979352$ C $-3.831825-4.158207-1.635510$ O $-4.205062-2.968417-0.909724$ C $-5.277348-3.289249-0.006887$ C $-5.835732-4.621428-0.487727$ C $-4.569749-5.312873-0.956960$ H $-4.823813-3.4377150 .980763$ C -6.285787 -2.1386970 .057620$ H $-6.355615-5.1663420 .305763$ H $-6.530134-4.493549-1.326111$ C $-4.825899-6.500680-1.867976$ H -4.007829 -5.652572 -0.076431 C $-5.658029-0.7669120 .384934$ O $-6.932535-2.024879-1.216057$ H $-7.071907-2.3840810 .781425$ H -5.066432 $-0.419678-0.470748$ $\begin{array}{lllll}\text { O } & -6.724427 & 0.180216 & 0.524650\end{array}$ C $-4.779653-0.7431101 .650131$ C -4.2928540 .6735632 .006570$ H $-3.910699-1.3885941 .487595$ O $-5.502630-1.2872262 .753308$ H -3.814105 1.1112841 .123903 H -5.155316 1.3047692 .252515 H $-5.406176-7.268065-1.345286$ H $-5.385603-6.207169-2.762429$ H -3.883662 -6.952564 -2.193431 C -2.310577 -4.307842 -1.602275 H -7.565285 -1.288601-1.155497 H $-7.300327-0.122221 \quad 1.246907$ H -6.230979 -0.6791602 .964371$ H - $2.009720-5.273640-2.023318$ H $-1.955745-4.279910-0.564199$ H -4.186006 -4.025936 -2.666259 C -1.2757250 .1862592 .041349$ $\begin{array}{lllll} & -1.275725 & 0.186259 & 2.041349\end{array}$ C -3.323946 0.6883603 .212052 C -2.8080322 .0855843 .586592$ C -1.785448 2.6008492 .577730 C $-0.681792 \quad 1.5659592 .355467$ H -1.7821040 .2262091 .068585$ C - $-0.225927-0.9275001 .909532$ H -3.8888370 .3125374 .074446$ O -3.8824323 .0151723 .681585$ H -2.329563 2.0437234 .573861 H -2.272336 2.8768421 .636332 O -1.2030153 .8000133 .094000$ H $-0.051973 \quad 1.5365803 .251949$ O $\quad 0.1342052 .0104111 .276662$ C $0.663971-1.0950493 .123001$ H $-0.722684-1.8920541 .752237$ H $0.403835-0.722774 \quad 1.036956$ H -4.5041612 .6754324 .347611$
H -0.5356864 .0966682 .451646$

H $0.8825941 .396776 \quad 1.200384$

O $1.887692-1.5513592 .724066$

$\begin{array}{lllll}\text { O } & 0.319700 & -0.886307 & 4.278467\end{array}$

C $2.832918-1.7786313 .774004$

H $3.056997-0.8396464 .288931$

H $2.448770-2.5241394 .476800$

H $3.754002-2.1617583 .327142$

SCF Energy (B3LYP/6-31G**//MMFF) $=-3245.90076385$

\section{0}

MM̄FF Geometry

C $-2.410652-3.097029-2.284684$

C $-1.552384-2.254270-1.691882$

C $-0.531102-1.422629-2.418294$

O $-0.877017-0.032622-2.210476$

C $0.859171-1.656684-1.799892$

C $2.006318-1.219590-2.690441$

C $2.955603-2.041471-3.181371$

C $2.074256 \quad 0.256180-2.995004$

C $3.082507-3.529369-3.015352$

C $4.441924-3.989071-2.469075$

C $4.818106-3.563347-1.038772$

C $3.764087-3.9259370 .010185$

C $4.186418-3.4824501 .421545$

O $5.085196-2.156188-1.013843$

O $3.033150-3.554626 \quad 2.273432$

C $4.614007-1.9988911 .367356$

C $5.227465-4.4265082 .033719$

O $5.143790-1.5723632 .619566$

C $5.621145-1.6930010 .237810$

C $5.933376-0.1861750 .095523$

O $6.868125-2.3380020 .530975$

C $-1.8465420 .505177-2.994495$

O $-2.448686-0.055262-3.899920$

C $-2.1617351 .893369-2.585919$

C -1.360607 $2.634630-1.805093$

C $-1.6278213 .996327-1.334475$

C $-2.9485994 .618316-1.705438$

C $4.7178750 .722699-0.092267$

C $5.1128952 .127317-0.517114$

C $4.1301084 .312715-1.168988$

O $6.2777252 .464165-0.714389$

C $3.2251795 .229484-0.410263$

C $2.3898396 .096718-1.003230$

C $1.5419517 .085135-0.255218$

C $0.0766527 .001537-0.617776$

C $-0.4786787 .882871-1.466533$

C -0.7666315 .9603150 .078956$

C $-0.6907414 .612953-0.584092$

N $4.0097552 .962568-0.649536$

H $-2.389833-3.222620-3.363700$

H $-1.611083-2.116194-0.616296$

H $-0.512885-1.650345-3.491858$

H $0.957019-2.713190-1.537461$

H $0.945739-1.106770-0.854404$

H $3.731665-1.601587-3.807560$

H $3.0344460 .545511-3.435713$

H $1.2882040 .538514-3.701733$

H $1.9490220 .843212-2.079658$

H $2.282644-3.964118-2.412838$

H $2.961627-3.970814-4.013519$

H $4.460335-5.085917-2.513517$

H $5.225443-3.635306-3.152764$

H $5.753523-4.089125-0.811300$

H $3.549043-5.001286-0.008510$

H $2.817347-3.424244-0.221894$

H $2.691130-4.4644842 .239812$

H $3.701236-1.4131481 .198595$

H $4.819691-5.4411172 .118924$

H $5.485562-4.121403 \quad 3.053834$

H $6.147606-4.4918711 .449872$

H $4.479253-1.776343 \quad 3.299668$

$\begin{array}{llll}\text { H } & 6.514480 & 0.159429 & 0.960924\end{array}$

H $6.599891-0.063443-0.769887$

H $7.569912-1.8963630 .024368$

H -3.098421 $2.278008-2.976494$

H $-0.4189392 .207780-1.464023$ 
H -3.062487 $5.645060-1.351476$ H -3.777551 $4.039038-1.283993$ H -3.063599 4.656984 -2.794501 H $4.0558210 .312169-0.859232$ H 4.1624280 .8005500 .847949 H $3.8807934 .271418-2.234428$ H $5.1647524 .656985-1.072251$ H 3.2941525 .2128430 .674820 H $2.3475016 .134515-2.089548$ H $1.9252718 .088304-0.484437$ H $\quad 1.655664 \quad 6.9697470 .829855$ H -1.536765 $7.848184-1.704177$ H $0.1132648 .655065-1.946686$ H -1.801189 6.3074860 .169441 H -0.4293725 .8534401 .118291$ H $\quad 0.238714 \quad 4.080972-0.380557$ H $3.0796372 .558348-0.583555$ C $-4.787077-3.111002-1.497653$ O $-4.617988-1.876994-0.769830$ C $-5.676086-1.7481380 .193612$ C $-6.748937-2.736416-0.243324$ C $-5.898112-3.874609-0.775127$ H $-5.267678-2.074784 \quad 1.157819$ C $-6.125844-0.2879270 .292438$ H -7.400957 -3.0355590 .582642$ H $-7.377469-2.329091-1.043617$ C $-6.659080-4.841084-1.665632$ H $-5.481649-4.4277640 .077552$ C $-4.9756990 .709787 \quad 0.546274$ O $-6.7493040 .081232-0.944587$ H $-6.897121-0.193516 \quad 1.065944$ H $-4.3388150 .770042-0.344142$ O $\quad \begin{array}{lllll}-5.561798 & 2.010498 & 0.693137\end{array}$ C $-4.1044110 .402470 \quad 1.780600$ C -3.0472691 .4962232 .017051$ H $-3.607015-0.5605431 .636634$ O H -2.451151 1.5933821 .103451 H -3.5531712 .4563162 .175278$ H -7.474574 -5.315282 -1.109834 H -7.094689 -4.331066 -2.531421 H $-5.998560-5.630900-2.036891$ C $-3.455288-3.862628-1.520873$ H $-7.025048 \quad 1.010531-0.863884$ H -6.0847072 .0044501 .513423$ H -4.435309 -0.1168003 .634795$ H -3.574571 -4.848757 -1.983109 H -3.107969 -4.029232 -0.493838 H -5.095940 -2.840732 -2.515633 C $-0.508749-0.2098912 .172505$ O $-1.489988-0.0379013 .201201$ C $-2.125191 \quad 1.2440003 .232916$ C -1.0698882 .3391393 .441571$ C 0.0087332 .2764762 .367183 C 0.5849750 .8677342 .266004 H $-0.999166-0.178097 \quad 1.191858$ C $0.125704-1.5993852 .352862$ H $-2.765431 \quad 1.2467454 .123576$ O $-1.6864693 .622506 \quad 3.439495$ H $-0.602622 \quad 2.2065774 .425967$ H $-0.3849212 .611176 \quad 1.404485$ O 1.0520043 .1891992 .712758 H 1.2188310 .6836563 .142719 $\begin{array}{lllll}\text { O } & 1.434415 & 0.799347 & 1.122154\end{array}$ C $-0.825815-2.7435252 .077301$ H $0.970844-1.7041101 .663261$ H $0.483646-1.7210123 .381918$ H -0.9805624 .2846603 .535401$ H 1.7398543 .1152062 .028852 H $\quad 0.890400 \quad 0.9750660 .335559$ O $-0.099460-3.8933631 .944623$ O $-2.043041-2.6534212 .008380$ C $-0.851443-5.0847381 .695523$ H $-1.676614-5.1838942 .407389$ H $-1.224474-5.0765100 .668183$ H $-0.181319-5.9397921 .818487$

SCF Energy (B3LYP/6-31G**//MMFF) $=-3245.91935030$
0500061

MMFF Geometry

C -1.496515 -3.554462 -2.185835

C $-0.724952-3.196129-1.148963$

C $0.645343-2.601707-1.307890$

O $0.703428-1.338494-0.605550$

C $1.801472-3.479885-0.786264$

C $1.623849-4.1755730 .549945$

C $1.917289-3.6414181 .752083$

C $1.163169-5.6100830 .466079$

C $2.369868-2.2461512 .071462$

C $3.819045-2.1620402 .566487$

C $4.867118-2.3260981 .452488$

C $6.275834-2.1160022 .016761$

C $7.354685-2.1114250 .923157$

O $4.590985-1.3503130 .442999$

O $8.548722-1.5890891 .526573$

C $6.921749-1.143272-0.201451$

C $7.688671-3.5335970 .458101$

O $7.814047-1.209672-1.310899$

C $5.475956-1.385683-0.683147$

C $4.988388-0.317116-1.689642$

O $5.419431-2.650754-1.354313$

C $0.209350-0.234888-1.225452$

O $-0.392614-0.190062-2.285654$

C $0.5602990 .931052-0.383956$

C $0.0377332 .133669-0.662962$

C $0.301913 \quad 3.3766860 .064964$

C 1.4191493 .3702291 .075849

C 4.893499 1.105719-1.133520

C $4.3121862 .078493-2.147578$

C $3.7681364 .489366-2.416643$

O $4.0218231 .760426-3.297474$

C $3.1433515 .526410-1.538916$

C $1.9741226 .124419-1.814572$

C $1.3568807 .182079-0.944530$

C $-0.0935896 .905136-0.615887$

C $-1.0776467 .644431-1.155462$

C -0.4000005 .8272810 .400879$

C $-0.4561124 .456988-0.216028$

N $4.1629143 .349882-1.608495$

H -1.114860 -3.444038 -3.198053

H -1.109488 -3.297064 -0.137811

H $0.858224-2.402033-2.367010$

H $2.706008-2.864736-0.739229$

H $2.017733-4.247642-1.541712$

H $1.786916-4.2697182 .632939$

H $1.064941-6.0752851 .452801$

H $\quad 0.188139-5.677751-0.024755$

H $1.881961-6.205010-0.107387$

H $2.227127-1.564376 \quad 1.232936$

H $1.713855-1.8722022 .867901$

H $3.943075-1.1692393 .019261$

H $3.994827-2.8996963 .359048$

H $4.772161-3.3287411 .020667$

H $6.504233-2.8705432 .780167$

H $6.314798-1.1480862 .536877$

H $9.264026-1.6347920 .869893$

H $6.999507-0.1267460 .203836$

H $8.099547-4.1188651 .289734$

H $8.464526-3.526132-0.315277$

H $6.821998-4.073900 \quad 0.071559$

H $8.697306-0.955681-0.995086$

H $5.620694-0.332089-2.586932$

H $3.986181-0.605847-2.038923$

H $4.675610-2.641920-1.978718$

H 1.2427260 .7683550 .442802

H $-0.6629702 .216651-1.492975$

H 2.3419172 .9840230 .630216

H 1.1624322 .7403941 .933621

H 1.6625634 .3618061 .462567

H $4.2554551 .123406-0.242687$

H $5.887171 \quad 1.473706-0.857429$

H $3.0968444 .156627-3.215190$

H $4.6776434 .888531-2.877132$

H $3.6863045 .813327-0.641278$

H $1.4442035 .860702-2.727231$

H $1.4463938 .139239-1.474620$ 
H $1.9167817 .304788-0.008955$ H -2.118461 $7.468966-0.903751$ H -0.865916 $8.437452-1.864897$ H -1.3813186 .0158090 .857141$ H $0.309793 \quad 5.8993831 .229179$ H -1.243912 4.352491-0.963805 H $4.5870543 .536383-0.703845$ C $-3.900295-3.108121-2.671300$ O $-3.927214-1.882890-1.912350$ C $-5.279166-1.385786-1.880462$ C $-6.096096-2.300726-2.787972$ C $-5.341316-3.610725-2.663474$ H $-5.636115-1.511529-0.852194$ C $-5.307500 \quad 0.097519-2.268567$ H -7.140117 -2.374088 -2.467708 H $-6.090551-1.953996-3.827877$ C -5.662581-4.601927 -3.767940 H $-5.576912-4.061958-1.690260$ C $-4.3996950 .993791-1.399672$ $\begin{array}{lllll}\text { O } & -4.864867 & 0.218177 & -3.626668\end{array}$ H -6.340888 $0.463198-2.246616$ H -3.348309 $0.744210-1.585116$ O $-4.5662912 .345479-1.847326$ C $-4.690784 \quad 0.923218 \quad 0.110614$ C $-3.812616 \quad 1.897817 \quad 0.917388$ H $-4.515032-0.0991650 .456744$ O -6.0680041 .2248110 .325909$ H -2.764010 1.6969830 .674248 H -4.0237092 .9279220 .605470$ H -6.726946 -4.858437 -3.756268 H $-5.426138-4.192615-4.755879$ H -5.091943 -5.527184-3.641516 C $-2.897857-4.080609-2.049013$ H $-4.878704 \quad 1.163988-3.852946$ H -5.479029 $2.611366-1.641516$ H $-6.2865250 .971373 \quad 1.238382$ H -2.948867 -5.053542 -2.549931 H $-3.140422-4.243273-0.992331$ H $-3.592941-2.845399-3.692786$ C $-2.714570-0.1677812 .866632$ $\begin{array}{llll}\text { O } & -4.001195 & 0.459514 & 2.922765\end{array}$ C $-4.031004 \quad 1.8075962 .445449$ C $-3.0739492 .675928 \quad 3.273864$ C -1.667695 2.0842783 .324855 C $-1.7084600 .608963 \quad 3.722854$ H $-2.368029-0.2094441 .826535$ C -2.883867-1.604320 3.371631 H -5.0452792 .1781852 .639762$ O $-3.029255 \quad 3.9968062 .743983$ H -3.461248 2.7528894 .298189 H -1.159541 2.2193952 .366414 $\begin{array}{lllll}\text { O } & -0.906011 & 2.811953 & 4.289855\end{array}$ H -1.974096 0.5322614 .784543 O -0.4050180 .0496083 .576733$ C -3.708042 -2.411121 2.395297 H $-1.922240-2.1166853 .484133$ H -3.376976 -1.609365 4.351207 H -2.386838 4.5009473 .272091 H -0.0250552 .4019484 .331856$ H -0.1624210 .0970172 .636172$ $\begin{array}{llll}\text { O } & -4.873481 & -2.811338 & 2.977365\end{array}$ O $-3.362939-2.6441071 .244299$ C $-5.751956-3.5634162 .133991$ H $-5.258601-4.4770181 .788731$ H -6.632047 -3.8401342 .720466$ H -6.073733 -2.953404 1.284638 SCF Energy (B3LYP/6-31G*//MMFF) $=-3245.91618936$

\section{2}

MM̄FF Geometry

C $-4.410186-2.500032-2.155526$

C $-3.156493-2.725738-1.734572$

C -1.980241-2.809396 -2.674678

O $-0.987531-1.842087-2.256738$

C $-1.338019-4.205829-2.617419$

C $-0.248018-4.398284-3.656256$

C $1.066714-4.532964-3.391859$

C $-0.727503-4.477041-5.085199$
C $1.765401-4.539625-2.061031$

C $2.702241-3.338102-1.912633$

C $3.657637-3.451735-0.713763$

C $2.921553-3.5164060 .625975$

C $3.890281-3.5161001 .819134$

O $4.506694-2.294810-0.749659$

O $3.126724-3.257610 \quad 3.007519$

C $4.879154-2.3448161 .654626$

C $4.552653-4.8841842 .019917$

O $5.888145-2.3783582 .660329$

C $5.530721-2.2827820 .256836$

C $6.377427-1.0047090 .051389$

O $6.416480-3.3976220 .098123$

C $-1.188017-0.547806-2.629026$

O $-2.124304-0.104011-3.274599$

C $-0.061763 \quad 0.251758-2.099050$

C $-0.1282721 .590903-2.139321$

C $0.8864072 .518091-1.638496$

C $2.2247021 .947769-1.249349$

C $5.6246550 .309674 \quad 0.268043$

C $6.4222531 .519976-0.187902$

C $6.2871234 .002084-0.268810$

O $7.521677 \quad 1.442656-0.728542$

C $5.1677564 .989391-0.336458$

C $4.6480135 .442055-1.487364$

C $3.4945076 .401376-1.565034$

C $2.2343385 .725238-2.065675$

C $1.8541515 .866961-3.347281$

C $1.4569464 .938693-1.027410$

C $0.5880883 .832605-1.567653$

N $5.7387902 .701237 \quad 0.065713$

H $-4.603527-2.404136-3.221600$

H -2.953563 -2.811869 -0.669958

H $-2.291093-2.585022-3.702402$

H $-2.103297-4.976118-2.782545$

H $-0.952688-4.381874-1.608341$

H $1.740195-4.676657-4.236899$

H $0.072619-4.760230-5.777665$

H $-1.518737-5.228205-5.179782$

H $-1.120169-3.511576-5.417324$

H $1.057536-4.561272-1.230207$

H $2.345911-5.468283-1.995983$

H $3.307362-3.217280-2.821683$

H $2.115690-2.413739-1.832281$

H $4.274872-4.346427-0.857429$

H $2.266991-4.3936290 .682932$

H $2.262892-2.641847 \quad 0.710443$

H $2.461977-3.9622463 .095521$

H $4.303976-1.4260551 .821355$

H $3.793743-5.6522552 .211942$

H $5.204906-4.8816112 .900201$

H $5.137265-5.2099521 .157287$

H $5.437972-2.4133243 .521766$

H $7.263419-1.0319170 .699372$

H $6.767652-1.018211-0.976017$

H $7.059949-3.183138-0.597683$

H $\quad 0.780009-0.280545-1.671369$

H -1.021652 $2.057252-2.552801$

H $2.9764312 .715637-1.057329$

H $2.6298551 .324889-2.055008$

H $2.1396261 .337968-0.345059$

H $4.6819150 .302282-0.289906$

H $5.3983230 .441943 \quad 1.331715$

H $6.8371923 .929839-1.213324$

H 6.9985034 .2837070 .514131

H 4.7542125 .3323080 .608852

H $5.0574935 .089806-2.431471$

H $3.7825097 .226806-2.228695$

H $3.2937846 .865107-0.590893$

H $0.9473485 .416935-3.735170$

H $2.4456946 .449905-4.046253$

H $0.8180785 .638176-0.472472$

H $2.1414674 .530609-0.275879$

H $-0.3997484 .164020-1.889420$

H 4.9154242 .6648640 .660708

C $-6.109510-0.882477-1.333629$

O $\begin{array}{lllll}-5.138627 & 0.010820 & -0.754567\end{array}$

C $-5.8316201 .061159-0.053021$ 
C $-7.312205 \quad 0.880744-0.368325$ C $-7.398220-0.623403-0.558821$ H -5.6849430 .8732221 .015610$ C $-5.2349782 .422701-0.429235$ H $-7.9546591 .249703 \quad 0.437177$ H -7.598608 1.399732 -1.290431 C $-8.657937-1.071995-1.277505$ H $-7.349189-1.1079080 .425726$ C $-3.7067232 .501707-0.223980$ O $-5.5004012 .664017-1.816712$ H -5.7425793 .2183360 .128207$ H $-3.210843 \quad 1.810827-0.915517$ O $-3.2748303 .814241-0.603356$ C $-3.245976 \quad 2.216791 \quad 1.217060$ C -1.7133412 .2530001 .348646$ H -3.603298 1.2289861 .517983 $\begin{array}{llll}\text { O } & -3.840256 & 3.176784 & 2.086795\end{array}$ H -1.2987261 .4970510 .673621$ H -1.3354613 .2260781 .012701$ H -9.547899 $-0.786971-0.706947$ H $-8.736313-0.618616-2.271370$ H -8.671134 -2.159264 -1.401531 C $-5.592540-2.318451-1.244383$ H $-5.0999093 .520861-2.043372$ H -3.658435 4.4429190 .032063 H -3.700367 2.8740042 .999779 H -6.371348 -3.027047 - 1.546463 H $-5.313510-2.554532-0.210806$ H $-6.232244-0.584600-2.384032$ C -1.575692 -0.3612042 .929134$ $\begin{array}{llll}\text { O } & -1.875989 & 0.941475 & 3.441314\end{array}$ C -1.195939 2.0141532 .784422 C 0.3256601 .8197562 .880935 C 0.7576350 .4300012 .409485 C $-0.082143-0.6520963 .088507$ H $-1.856564-0.4196181 .869999$ C $-2.427724-1.3688323 .706843$ H -1.4391762 .9201853 .354026$ $\begin{array}{lllll}\text { O } & 0.990351 & 2.828273 & 2.127465\end{array}$ H $0.630267 \quad 1.945362 \quad 3.928123$ H 0.6963900 .3363261 .319516 $\begin{array}{lllll}\text { O } & 2.126096 & 0.213466 & 2.758622\end{array}$ H $0.182957-0.7106684 .152000$ O $0.214537-1.9151012 .500533$ C -3.886386 -1.2211923 .341704$ H $-2.145813-2.4040733 .485164$ H -2.310757 -1.227393 4.788076 H 1.9443842 .7400862 .290064 H 2.6654150 .8184452 .222040 H $1.162076-2.0801592 .643673$ O $-4.566110-0.6217614 .360022$ O $-4.357899-1.574725 \quad 2.268577$ C $-5.962208-0.4072934 .130514$ H $-6.410991-0.0625235 .065946$ H $-6.0990860 .365953 \quad 3.369674$ H $-6.453701-1.3377563 .829874$

SCF Energy (B3LYP/6-31G**//MMFF) $=-3245.92069792$

0500063

MM̄FF Geometry

C $-4.105855-1.4952122 .647906$

C $-5.315186-1.7537032 .125405$

C $-5.877250-1.1655900 .851379$

O $-4.992649-0.153951 \quad 0.324077$

C $-6.002176-2.276830-0.210222$

C $-6.515066-1.783458-1.549704$

C $-5.795730-1.793627-2.690276$

C $-7.930412-1.267128-1.556084$

C $-4.395417-2.308000-2.889194$

C $-3.351498-1.226812-2.612868$

C $-1.913499-1.629899-2.969856$

C $-1.413170-2.820322-2.146788$

C $0.074151-3.116711-2.397205$

O $-1.103142-0.474728-2.708295$

O $0.528000-4.003850-1.362815$

C $0.877045-1.808891-2.249535$

C $0.294757-3.850574-3.724819$

O $2.240152-1.998691-2.616726$
C $0.282220-0.627927-3.044998$

C $0.9871680 .713882-2.732488$

O $0.443882-0.870398-4.447032$

C $-5.293079 \quad 1.153207 \quad 0.540577$

$\begin{array}{lllll}\text { O } & -6.248768 & 1.604971 & 1.148647\end{array}$

C $-4.2225791 .948388-0.102308$

C $-4.2391363 .288425-0.046510$

C $-3.2293444 .178968-0.627782$

C $-2.0904413 .532157-1.372349$

C $0.9853591 .102379-1.253255$

C $1.4205642 .538736-1.019086$

C 1.6414584 .1999310 .839985

O $1.8332493 .273284-1.911496$

C $0.3813184 .901993 \quad 1.237566$

C $0.074733 \quad 6.1565090 .872657$

C $-1.178262 \quad 6.859952 \quad 1.314678$

C $-1.947588 \quad 7.490205 \quad 0.172077$

C -2.1842848 .8131990 .155732$

C $-2.4497906 .604637-0.950420$

C -3.363620 $5.511848-0.464017$

$\begin{array}{llll}\text { N } 1.301282 & 2.888780 & 0.318743\end{array}$

H -3.440793 -0.7884652 .159132$

H -5.957686 -2.471559 2.631640

H $-6.864405-0.7413481 .069132$

H -6.676236 -3.0646920 .151661$

H $-5.022983-2.754028-0.330471$

H $-6.258282-1.398280-3.593660$

H -8.283562 -1.036992 -2.567026

H $-8.609248-2.016438-1.135937$

H $-8.010822-0.350724-0.963807$

H $-4.220846-3.192172-2.268857$

H $-4.304741-2.651707-3.927111$

H -3.605027 -0.313428 -3.168580

H $-3.382418-0.939108-1.555756$

H $-1.874050-1.855873-4.041888$

H $-2.008197-3.719127-2.345603$

H $-1.548815-2.602596-1.078660$

H $-0.023317-4.804594-1.391242$

H $0.873522-1.560949-1.180825$

H $-0.238225-4.809052-3.725371$

H $1.352517-4.096679-3.869444$

H $-0.050394-3.282620-4.591158$

H $2.576325-2.749693-2.098137$

H $2.0183200 .695524-3.108722$

H $0.4814511 .506108-3.302268$

H $0.400220-0.019292-4.913844$

H $-3.4333941 .399555-0.604197$

H $-5.057427 \quad 3.7799050 .477465$

H $-1.361694 \quad 4.237905-1.773484$

H -2.465568 $2.953364-2.223851$

H $-1.5414742 .860095-0.705999$

H $-0.0160260 .974621-0.827783$

H $1.6780970 .458992-0.702004$

H 2.2158694 .7634420 .098071

H 2.2691974 .0404521 .722278

H $-0.3058374 .355841 \quad 1.880435$

$\begin{array}{lllll}H & 0.770948 & 6.718451 & 0.254640\end{array}$

H -0.8822057 .6285512 .040467$

H $-1.8425816 .176403 \quad 1.857679$

H $-2.7343889 .280398-0.654414$

H -1.8333199 .4614530 .951857$

H $-1.5852316 .225244-1.499709$

H -3.017492 7.199495-1.678062

H $-4.234710 \quad 5.8808750 .079401$

H 0.9466252 .2007590 .977155

C $-2.314408-2.9988113 .628057$

O $-1.223433-2.1589153 .201461$

C $-0.473140-2.858600 \quad 2.190253$

C $-0.982810-4.2964952 .202510$

C -2.439075 -4.096949 2.572403

H $-0.730382-2.3920171 .231442$

C $1.037991-2.7310722 .425887$

H $-0.848382-4.7925291 .236511$

H $-0.474165-4.8983302 .964853$

C $-3.125303-5.3564633 .069129$

H $-2.966380-3.7309261 .682804$

C $1.557721-1.2811682 .312622$

O $1.363951-3.2767903 .702709$ 
H $1.552023-3.3433651 .675141$ H $1.215737-0.8647081 .357677$ O $1.016891-0.4634573 .348430$ C $3.097587-1.1877292 .355697$ C 3.5869750 .2525792 .122834 H $3.500675-1.8521601 .586985$ O $3.574959-1.6468333 .617767$ H $3.083240 \quad 0.652657 \quad 1.238566$ H 3.2893360 .8753642 .975196 H -3.117852 -6.129645 2.293925 H $-2.625926-5.763672 \quad 3.954470$ H $-4.167864-5.1527493 .333167$ C -3.569012 -2.157975 3.885465 H $0.934368-2.7197334 .374491$ H $0.048367-0.4861943 .268291$ H $4.532883-1.7893243 .538518$ H -3.326285 -1.363523 4.601788 H $-4.337221-2.7869574 .350580$ H -1.998762 -3.435580 4.585065 C $5.242898-0.145414-0.416433$ O $5.620552-0.4994880 .919685$ C 5.1182880 .3653131 .944751 C 5.6128691 .7987481 .704336 C 5.2552962 .2922080 .305950 C $5.6991621 .283331-0.751019$ H $4.155466-0.232638-0.526873$ C $5.908657-1.150985-1.371881$ H 5.5740040 .0245062 .882260 O 5.0688822 .6956552 .667063 H $6.704651 \quad 1.8307121 .814591$ H 4.1857542 .5054340 .219635 O 5.9280423 .5288490 .057752 H $6.7929601 .316443-0.833464$ O $5.1774481 .675226-2.020211$ C $5.297476-2.534136-1.301588$ H $5.804558-0.809068-2.407861$ H $6.972596-1.249643-1.125685$ H 5.3214082 .3678663 .547143 H 5.6569904 .1499680 .755526 H $4.2068871 .655889-1.963644$

O $5.909463-3.333810-2.225634$

O $4.400220-2.877285-0.545650$

C $5.422165-4.677707-2.292925$

H $5.579912-5.186071-1.336914$

H $4.361966-4.683232-2.563921$

H $5.984817-5.205007-3.067962$

SCF Energy (B3LYP/6-31G**//MMFF) $=-3245.92224589$

0500064

MM̄FF Geometry

C $0.090763 \quad 1.5223531 .143625$

C $-0.2502951 .891229-0.100361$

C $-1.0417410 .999291-1.023302$

O $-2.3054531 .639743-1.333970$

C $-0.344597 \quad 0.769079-2.374873$

C $0.9734340 .035119-2.303444$

C $1.101794-1.304280-2.342402$

C $2.2023070 .904612-2.267535$

C $0.013062-2.338361-2.426395$

C $0.233317-3.480918-1.423724$

C $-0.233419-3.1425610 .001718$

C $0.303874-4.1877410 .982982$

C $-0.251172-3.9929192 .398412$

O $-1.665812-3.157522-0.007325$

O $0.058888-5.1695953 .160338$

C -1.786252 -3.902575 2.311316

C $0.416635-2.8179823 .121410$

O $-2.348355-3.599186 \quad 3.586927$

C $-2.289644-2.8896191 .255660$

C -3.817515 -3.064515 1.069293

O $-2.007991-1.5693031 .714291$

C $-3.2855791 .599597-0.392416$

O $-3.203673 \quad 1.1474210 .737945$

C $-4.5017052 .191378-0.992705$

C $-5.6574982 .165542-0.312391$

C $-6.9429022 .696137-0.772974$

C $-6.9950613 .286072-2.158616$

C $-4.442196-2.172271-0.012043$
C $-5.890768-2.539684-0.291256$

C $-7.825227-1.864685-1.695536$

O $-6.443491-3.5173550 .205867$

C $-8.496350-0.548469-1.924653$

C $-9.613622-0.172865-1.283520$

C $-10.3430871 .116235-1.534530$

C $-10.4120901 .993906-0.304903$

C -11.3832161 .8288430 .609136$

C $-9.4060513 .115682-0.182253$

C -8.0075532 .6181950 .053267$

N $-6.490743-1.645203-1.169752$

H -0.2293040 .5410921 .488708$

H $0.0294642 .868234-0.482098$

H $-1.2506440 .024921-0.564401$

H $-0.2045441 .731130-2.888004$

H $-1.0339260 .229637-3.038763$

H $2.110169-1.714063-2.332935$

H $3.1240740 .327027-2.388656$

H $2.1763881 .639185-3.079173$

H $2.2691101 .437290-1.316497$

H $-0.984759-1.909009-2.296606$

H $\quad 0.048072-2.761683-3.437929$

H $-0.333900-4.350284-1.782210$

H $1.292147-3.769269-1.419303$

H $\quad 0.116940-2.1420130 .278288$

H $1.400826-4.1786250 .998504$

H $0.020341-5.1917810 .636294$

H $1.024845-5.2821923 .158109$

H -2.158578 -4.900262 2.038859

H $1.495303-2.9909293 .215630$

H $\quad 0.039653-2.7180394 .145137$

H $0.279695-1.8639282 .608522$

H -2.016926 -4.263713 4.215028

H $-4.016967-4.1113010 .804186$

H -4.335892 -2.863964 2.015523

H $-2.601874-0.9439421 .271397$

H -4.413809 $2.623075-1.983253$

H -5.6682591 .7119570 .678032$

H -7.997139 $3.586852-2.470002$

H $\quad-6.6535692 .555774-2.900941$

H $-6.3584234 .175396-2.221178$

H -4.422634 -1.126925 0.306575

H -3.888306 -2.261499 -0.952981

H $-8.393013-2.505403-1.012482$

H $-7.719087-2.398610-2.645517$

H $-8.0607680 .109433-2.672463$

H -10.053120 $-0.846293-0.550514$

H $-11.3602750 .868958-1.866204$

H $-9.8953691 .673610-2.366438$

H $-11.4575292 .472218 \quad 1.479512$

H -12.1239841 .0424820 .507452$

H -9.6644003 .7674990 .663119$

H $-9.4845943 .759092-1.063057$

H -7.8707972 .1626901 .035038$

H -5.905396 -0.954844 -1.632182

C $1.6589913 .523077 \quad 1.686434$

O 2.7605043 .0923130 .857860

C 3.9580043 .7666241 .286182

C 3.4963614 .9136792 .176410

C 2.2877654 .2984702 .848120

H 4.5206893 .0521411 .898550

C 4.8065944 .2100580 .091006

H 4.2696115 .2276302 .884078

H 3.1981685 .7903641 .589573

C 1.3608855 .3209633 .483476

H 2.6353103 .6011173 .622609

C $5.2383933 .078973-0.862991$

O $4.0500715 .156631-0.675826$

H 5.6888024 .7491800 .457702

H $4.3724422 .722663-1.430229$

O $6.1193993 .663036-1.833962$

C $5.9421981 .881423-0.193903$

C $6.5479880 .913984-1.231245$

H $\quad 5.219715 \quad 1.353797 \quad 0.438007$

O $6.9813082 .377626 \quad 0.648927$

H $5.7697350 .645267-1.953447$

H $7.3386411 .433570-1.786105$

H 1.8859625 .8839964 .261996 
H $\quad 0.987584 \quad 6.0379112 .744495$

H $\quad 0.497077 \quad 4.8324113 .944847$

C 0.8637922 .3064222 .167722

H $4.6067015 .434873-1.423285$

H $6.9281643 .937251-1.368081$

H 7.2579031 .6527661 .234468

H 0.1537502 .6119802 .945262

H 1.5630121 .6026682 .634299

H 1.0357014 .1908581 .079894

C $5.162193-1.696484-0.473968$

O $6.241526-1.0639780 .224116$

C $7.165747-0.359954-0.610447$

C $7.785625-1.323937-1.632782$

C $6.721369-2.073647-2.429771$

C $5.700813-2.713043-1.488035$

H $4.561980-0.935134-0.983861$

C $4.270429-2.3776950 .567359$

H $7.981248-0.0305690 .045034$

O $8.649655-0.615732-2.515128$

H $8.404726-2.055646-1.097338$

H $6.237780-1.412121-3.158056$

O $7.366564-3.100023-3.186666$

H $6.176195-3.551753-0.964180$

O $4.630303-3.257055-2.256407$

C $3.592290-1.3432091 .432026$

H $3.482310-2.9783230 .100437$

H $4.853707-3.0521991 .205782$

H $9.002992-1.258248-3.154094$

H $6.671819-3.595312-3.653746$

H $4.226922-2.530713-2.761267$

O $4.223599-1.2581192 .637120$

O $2.638191-0.6705351 .064749$

C $3.707757-0.2742593 .537896$

H $4.257154-0.3541794 .479630$

H $3.862370 \quad 0.7271853 .125801$

H $2.646888-0.4545483 .735655$

SCF Energy (B3LYP/6-31G**//MMFF) $=-3245.92165945$

0500065

MM̄FF Geometry

C $0.798273 \quad 4.7919590 .067539$

C 1.7274764 .8022121 .035201

C 2.8136693 .7684321 .242013

$\begin{array}{lllll}\text { O } & 2.572710 & 2.568447 & 0.470705\end{array}$

C 4.1664474 .3581610 .802473

C 5.3580183 .4815781 .133701

C $6.1758522 .921743 \quad 0.220745$

C 5.6304303 .2761162 .602284

C $6.1048463 .020792-1.277206$

C $5.3202071 .888039-1.958074$

C $5.9144200 .487364-1.735496$

C $5.220718-0.544976-2.627223$

C $5.726843-1.971906-2.358469$

O $5.726799 \quad 0.130047-0.364044$

O $4.811016-2.888180-2.978448$

C $5.682170-2.248847-0.839251$

C $7.093788-2.221759-3.006813$

O $6.300627-3.494922-0.530324$

C $6.311911-1.1247250 .008363$

C $6.084763-1.3010701 .529724$

O $7.729135-1.114021-0.203416$

C 1.6828851 .6682730 .974451

O 1.0573361 .7572522 .019020

C $1.578424 \quad 0.5500350 .010128$

C $0.537387-0.2931440 .083960$

C $0.290016-1.441600-0.792472$

C $1.383618-1.833348-1.749810$

C $4.619114-1.3486581 .976257$

C $4.068116-2.7565302 .129490$

C $1.942314-3.9700932 .587559$

O $4.768093-3.7642592 .082009$

C $1.375282-4.4473001 .288265$

C $0.084403-4.7740121 .126258$

C $-0.493905-5.292843-0.157905$

C $-1.672354-4.475614-0.635427$

C $-2.932417-4.861793-0.373442$

C -1.388624 -3.261790 -1.490979

C $-0.887248-2.092929-0.689029$
N $2.699588-2.7528582 .365885$

H $0.8029224 .000616-0.677369$

H 1.7114965 .6108961 .763330

H 2.8415393 .5101112 .307492

H 4.3239895 .3325751 .284324

H $4.1231084 .557781-0.273610$

H 7.0077552 .3192340 .584568

H 6.5849492 .7690452 .780871

H 5.6722324 .2399323 .120086

H 4.8459172 .6655383 .059457

H $5.6791673 .982014-1.583595$

H $7.1319633 .029313-1.664379$

H $4.2841251 .911146-1.600479$

H $5.2953392 .100673-3.034084$

H $6.9865660 .527588-1.961276$

H $5.342833-0.288885-3.687003$

H $4.139390-0.519247-2.437197$

H $4.771622-2.676840-3.926888$

H $4.625045-2.348672-0.566497$

H $7.034326-2.078265-4.092458$

H $7.417552-3.257995-2.860139$

H $7.874983-1.558561-2.629786$

H $5.859305-4.177584-1.064144$

H $6.649513-2.1661361 .897486$

H $6.535944-0.4294182 .025928$

H $8.149898-0.6629280 .547107$

H $2.3539350 .460088-0.741180$

H $-0.219173-0.1184960 .848099$

H $2.327556-1.974449-1.212861$

H $1.523577-1.059143-2.511846$

H $1.192385-2.772282-2.272919$

H $4.544388-0.8842472 .967954$

H $3.979832-0.7754461 .298009$

H $1.163406-3.7394323 .320914$

H $2.600592-4.7380263 .006527$

H $2.060839-4.5527550 .450681$

H $-0.592994-4.6954831 .973512$

H $-0.805339-6.3318530 .012600$

H $0.263793-5.339409-0.950099$

H $-3.784437-4.299536-0.739599$

H $-3.139797 \quad-5.7481120 .217029$

H -2.309344 -2.938974 -1.994197

H $-0.713050-3.547099-2.301575$

H -1.613004 -1.729886 0.037943

H $2.189560-1.8749112 .332211$

C -1.6562305 .1879160 .209763$

O $-1.9481654 .213884-0.807461$

C $-3.3693014 .180850-1.031069$

C $-3.9792415 .198357-0.068827$

C $-2.827418 \quad 6.161493 \quad 0.154089$

H $-3.5336374 .514033-2.063142$

C $-3.9117402 .751539-0.862922$

H $-4.8661175 .681978-0.490126$

H -4.2774354 .7303650 .877003$

C $-2.9876887 .018451 \quad 1.397138$

H $-2.735676 \quad 6.810359-0.727160$

C $-3.3424971 .751835-1.893473$

O $-3.6157862 .307498 \quad 0.461516$

H $-5.0019332 .799284-0.960589$

H -3.575946 $2.096810-2.908314$

O $-1.9126691 .702062-1.799154$

C $-3.8212290 .297287-1.730998$

C $-5.347340 \quad 0.124436-1.740775$

H $-3.393030-0.135881-0.821755$

O $-3.293136-0.457665-2.829480$

H $\quad-5.7958890 .737660-0.955022$

H $-5.7366180 .486189-2.700238$

H $-3.893514 \quad 7.629627 \quad 1.328225$

H -3.0642336 .4044752 .300818$

H -2.1351037 .6933751 .519900$

C $-0.2923355 .820211-0.063190$

H -4.0944181 .4763290 .614791$

H -1.570350 $2.586897-2.010043$

H $-2.326490-0.349259-2.815991$

H -0.1177096 .6550720 .624453$

H $-0.2560126 .220335-1.083540$

H -1.6425004 .6583981 .172345$

C $-5.820294-1.4169020 .867325$ 
O $-5.239494-1.911760-0.345283$

C $-5.772487-1.350932-1.550873$

C $-7.286126-1.592141-1.624383$

C $-8.005128-1.106197-0.369651$

C $-7.333544-1.6716810 .881311$

H $-5.627223-0.3418850 .958304$

C $-5.131877-2.1395832 .036164$

H -5.323558 -1.920134 -2.374608

O $-7.829010-0.956924-2.776951$

H -7.466210 -2.669298 -1.737992

H -8.051316 - $0.011213-0.342284$

O $-9.358050-1.564207-0.421864$

H $-7.535539-2.7485260 .940048$

O $-7.921806-1.0740702 .035185$

C $-3.733747-1.6186742 .297324$

H $-5.693480-2.0036722 .966654$

H $-5.059992-3.2105431 .811159$

H $-8.787600-1.121397-2.775123$

H $-9.789409-1.2874390 .404861$

H -7.740701-0.119136 2.002639

O $-3.062139-2.5174353 .076683$

O $-3.296116-0.5458061 .905653$

C -1.738589-2.131652 3.461023

H -1.104985 -2.013769 2.577872

H $-1.767267-1.2045444 .041473$

H -1.322351 -2.924767 4.087761

SCF Energy (B3LYP/6-31G**/MMFF)= -3245.91776659

0500066

MMFFF Geometry

C $-2.159475-2.6468880 .354861$

C $-1.543912-2.483552-0.825713$

C $-1.938107-1.425910-1.822577$

O $-0.791052-0.584766-2.113707$

C $-2.395448-2.033258-3.159403$

C $-3.724762-2.753916-3.089667$

C $-4.919744-2.157792-3.268948$

C $-3.652421-4.243950-2.873285$

C $-5.191471-0.706579-3.556107$

C $-6.307989-0.126475-2.674583$

C $-5.855290 \quad 0.246717-1.252449$

C $-7.061993 \quad 0.706055-0.431796$

C $-6.659343 \quad 1.2464290 .944808$

O $-4.912661 \quad 1.314046-1.369622$

O -7.8181731 .9016091 .482743$

$\begin{array}{llll}\text { C } & -5.547160 & 2.304061 & 0.764768\end{array}$

C $-6.310250 \quad 0.108226 \quad 1.912485$

O -5.0566562 .7158972 .037502$

C $-4.383796 \quad 1.819062-0.129694$

C $-3.4373602 .967111-0.558756$

O $\begin{array}{llll}-3.642612 & 0.819874 & 0.563979\end{array}$

C $-0.354427 \quad 0.247199-1.128653$

$\begin{array}{lllll}\text { O } & -0.789180 & 0.333210 & 0.008756\end{array}$

C $0.7644921 .048709-1.666151$

C $1.5188091 .757601-0.813020$

C $2.6768292 .579483-1.162466$

C $2.8767362 .913752-2.617333$

C -2.5260853 .5640550 .514981$

C $-1.700636 \quad 4.720769-0.029838$

C $-0.007940 \quad 6.3967190 .702307$

O $-1.8661195 .197223-1.149915$

C $1.2621716 .089370-0.026370$

C 2.4780296 .3559140 .474699

C $3.7654016 .163527-0.275546$

C $4.672163 \quad 5.140213 \quad 0.368144$

C 5.4241715 .4527181 .437079

C $4.767325 \quad 3.780797-0.283889$

C $3.4924702 .993269-0.170029$

$\begin{array}{llll}\mathrm{N} & -0.784137 & 5.187589 & 0.902327\end{array}$

H -2.998199 -2.004634 0.611877

H $-0.705903-3.122220-1.088439$

H -2.736048 $-0.782590-1.435859$

H -1.623155 -2.711125 -3.549096

H -2.441299-1.238915 -3.916054

H $-5.810059-2.785061-3.239226$

H $-4.639446-4.717503-2.911095$

H -3.037755 -4.709686 -3.650653

H $-3.216260-4.479743-1.899214$
H -4.292432 -0.088992 -3.476875

H $-5.521092-0.644795-4.601009$

H $-6.6733470 .781848-3.172524$

H $-7.150947-0.827526-2.629676$

H -5.371267 $-0.617151-0.783628$

H $-7.792265-0.106361-0.327060$

H $-7.5859791 .506506-0.974065$

H -7.609632 2.1913602 .386812

H $-5.998746 \quad 3.189803 \quad 0.297143$

H $-7.177658-0.5469542 .057721$

H -6.0577390 .4946852 .906078$

H $-5.481152-0.5117221 .564402$

H -5.7906583 .1361852 .515833$

H $-2.7903102 .591106-1.365222$

H $-4.0349573 .759566-1.029728$

H $-2.7398660 .805017 \quad 0.208031$

H $0.9501621 .016687-2.733346$

H 1.2912631 .7076400 .251282

H $3.6542623 .661171-2.788452$

H $1.9576913 .331300-3.044725$

H $3.1514752 .019632-3.186624$

H $-3.1141803 .963776 \quad 1.345446$

H -1.843996 2.8020300 .905979

H $\quad 0.176310 \quad 6.830546 \quad 1.690453$

H -0.5998907 .1105770 .119672$

H $1.1752565 .682310-1.030736$

H $2.5553726 .787044 \quad 1.470636$

H $4.2821227 .131583-0.316654$

H $3.5775235 .894310-1.322166$

H $6.098308 \quad 4.7310381 .886218$

H 5.3793956 .4389931 .887299

H $5.5584703 .185268 \quad 0.191186$

H $5.0953963 .906832-1.319584$

H 3.2545382 .7001510 .853675

H $-0.692606 \quad 4.707648 \quad 1.793018$

C $-1.239981-2.9917852 .650311$

O $0.022688-2.3505182 .376516$

C $0.914232-2.5852083 .483102$

C $0.063200-3.1924374 .592522$

C $-0.963282-3.968924 \quad 3.791486$

H $1.636961-3.3386123 .148889$

C $1.643723-1.2934653 .867548$

H $0.650290-3.8233195 .266915$

H $-0.429503-2.4217535 .196446$

C $-2.195065-4.3517534 .593111$

H $-0.489527-4.8795803 .400661$

C $2.415099-0.6346962 .706184$

O $0.672426-0.3473294 .333893$

H $2.314251-1.4868314 .713051$

H $1.701013-0.2525041 .967343$

$\begin{array}{llll}\text { O } & 3.087830 & 0.517712 & 3.230752\end{array}$

C $3.443459-1.5411432 .003135$

C $4.218397-0.7864390 .909820$

H $2.934516-2.3972591 .554381$

O $4.385555-2.0409242 .950689$

H $3.497621-0.295807 \quad 0.249727$

H $4.8247350 .000168 \quad 1.374960$

H $-1.920215-4.9928035 .437101$

H $-2.703673-3.4685474 .993748$

H $-2.910732-4.8999473 .972615$

C $-1.757288-3.6679931 .380333$

H 1.1495240 .4684114 .563885

H 3.7727790 .2021413 .845086

H $3.919023-2.6607653 .535793$

H $-2.633333-4.2857661 .606211$

H $-0.984927-4.3299780 .972081$

H $-1.932922-2.2054702 .978311$

C $3.557648-2.439262-1.570824$

O $4.463313-2.797441-0.519787$

C $5.155749-1.7014320 .088660$

C $5.977203-0.956545-0.972166$

C $5.103449-0.504841-2.137036$

C $4.286285-1.672420-2.684034$

H $2.740132-1.832137-1.163346$

C $2.958900-3.740332-2.131600$

H $5.868030-2.1450460 .795408$

$\begin{array}{llll}\text { O } & 6.629027 & 0.178535 & -0.411875\end{array}$

H $6.760619-1.619834-1.361076$ 
H $4.4521380 .324368-1.847300$

O $5.935646-0.008997-3.187845$

H $4.950583-2.351169-3.234645$

O $3.323766-1.173871-3.611961$

C $2.019302-4.429794-1.165636$

H $2.385078-3.530047-3.041273$

H $3.764825-4.446615-2.364367$

H $7.182208-0.1361040 .323481$

H $6.4693580 .714120-2.815824$

H $3.807224-0.670789-4.289694$

O $1.549197-5.571007-1.752580$

O $1.726571-4.026593-0.049502$

C $0.633505-6.343643-0.969535$

H $-0.334759-5.837298-0.929479$

H $\quad 0.502665-7.312496-1.458708$

H $1.024596-6.5127130 .038520$

SCF Energy (B3LYP/6-31G**//MMFF) $=-3245.92612856$

05_00067

MM̄FF Geometry

C - $0.1295503 .010435-0.654582$

C $0.9492323 .522490-0.044411$

C 1.7830452 .7706710 .956509

O 3.1440542 .8194580 .457575

C 1.7468623 .4238562 .351206

C 0.5262713 .0686983 .177389

C 0.4972582 .0753464 .088461

C -0.6771023 .9558992 .997103$

C 1.6043511 .1102334 .406280

C $1.118513-0.3207574 .674882$

C $0.363564-0.983306 \quad 3.510314$

C $-0.034042-2.4120653 .902375$

C $-0.703306-3.1707992 .749041$

O $1.207890-0.9978092 .351694$

O $-0.730661-4.5578753 .119285$

C $0.185526-3.0314231 .495855$

C $-2.165716-2.7480132 .569919$

O $-0.433137-3.6320330 .363088$

C $0.590293-1.5733731 .190059$

C $1.573030-1.4733280 .000410$

O $-0.584094-0.842116 \quad 0.813795$

C 3.9770751 .8031740 .805339

O $3.706141 \quad 0.836514 \quad 1.498565$

C 5.3012842 .0690500 .201925

C $6.292034 \quad 1.1861090 .397332$

C $7.6621521 .289619-0.104510$

C $8.0224322 .496375-0.929599$

C $2.913072-2.1909760 .180159$

C $3.802881-2.033033-1.044123$

C $5.949397-2.860963-1.984449$

O $3.449233-1.435528-2.057355$

C $7.360199-2.752559-1.500912$

C $8.296745-2.034960-2.140574$

C $9.728480-1.927647-1.696022$

C $10.180781-0.491105-1.547189$

C $10.8073620 .139898-2.554809$

C $9.9754600 .191327-0.213557$

C 8.5295410 .2971550 .184042

N $5.032381-2.658610-0.876048$

H $-0.4438281 .996494-0.427248$

H $1.2511914 .542884-0.270079$

H 1.4564801 .7249821 .012003

H 1.8235834 .5163772 .261243

H 2.6554613 .1549192 .904748

H -0.4110101 .9413994 .674334$

H -0.3978725 .0083163 .111280$

H -1.116556 3.8151392 .007280

H -1.460775 3.7465603 .733265

H 2.3523261 .0810233 .611892

H 2.1153731 .4687965 .308211

H $2.005516-0.9220494 .914668$

H $0.481705-0.3239265 .568857$

H $-0.533033-0.3923243 .291565$

H $-0.684879-2.4059314 .785924$

H $0.866496-2.9674504 .201509$

H - $1.082852-5.0573482 .362303$

H $1.088990-3.6206481 .693586$

H $-2.737271-2.9626713 .481081$
H $-2.649685-3.3233711 .774378$

H $-2.284874-1.6853652 .349446$

H -1.136965 -3.035154 0.056621

H $1.077833-1.832577-0.911962$

H $1.781613-0.411949-0.191082$

H $-0.313527-0.0773440 .280896$

H $5.4262112 .978378-0.374202$

H 6.0840350 .2946030 .988433

H $9.0554892 .483681-1.285444$

H $7.8985383 .413827-0.343893$

H $7.3861402 .558229-1.819467$

H $3.444977-1.7995191 .053329$

H $2.754272-3.2646600 .324348$

H $5.729988-2.148582-2.786433$

H $5.766575-3.870938-2.365962$

H $7.630192-3.301605-0.602215$

H $8.027092-1.510071-3.054643$

H $10.349825-2.436695-2.444477$

H $9.899872-2.465294-0.755177$

H $11.1587371 .161174-2.452150$

H $10.979310-0.350541-3.507242$

H $10.4478311 .180669-0.194066$

H $10.503292-0.3831620 .558932$

H $8.178773-0.5464840 .779561$

H $\quad 5.170245-3.227400-0.045305$

C $-2.2864904 .218336-1.020767$

O $-3.1086973 .075254-0.711653$

C $-4.4871453 .424234-0.945268$

C $-4.5043734 .906540-1.307411$

C $-3.1399065 .085283-1.942251$

H $-4.8154972 .850617-1.818920$

C -5.3422243 .0688810 .275847$

H $-5.3287895 .156680-1.982303$

H -4.599105 $5.540466-0.417885$

C -2.697853 $6.536049-2.023610$

H $-3.1607324 .653957-2.952096$

C -5.3312011 .5759090 .664623$

O -4.8753263 .8138861 .408814$

H -6.3747083 .3970780 .107449$

H -4.3516651 .2895191 .066171$

O -6.2700521 .4053171 .734782$

C $-5.7159350 .617223-0.477525$

C $-5.940549-0.831744-0.001205$

H $-4.9250970 .633392-1.234426$

O $-6.9107831 .080673-1.108982$

H $-5.070430-1.1552180 .579555$

H $-6.808415-0.8607950 .668329$

H -3.401543 $7.118605-2.627146$

H -2.645310 $6.995919-1.031027$

H -1.709465 $6.619517-2.485796$

C $-0.9662293 .769258-1.644765$

H -3.9397033 .5848261 .544758$

H -6.0075682 .0110452 .449163$

H -7.609680 $1.105639-0.432916$

H $-0.4114444 .637273-2.018903$

H $-1.1523803 .111140-2.501179$

H -2.098661 $4.743782-0.074781$

C $-3.920697-2.405521-1.673255$

O $-5.132573-1.790205-2.127696$

C $-6.203869-1.799313-1.177805$

C $-6.535053-3.240744-0.764323$

C $-5.298104-4.006821-0.304814$

C $-4.178709-3.877740-1.336840$

H -3.542915 -1.877209-0.789311

C $-2.880155-2.257701-2.786806$

H $-7.083809-1.416662-1.710319$

O $-7.512611-3.2676050 .269952$

H $-6.965159-3.770662-1.624439$

H $-4.962607-3.6714560 .683319$

O $-5.625659-5.391629-0.170975$

H $-4.450579-4.432068-2.244327$

O $-2.991142-4.465321-0.817289$

C $-2.475151-0.809700-2.938429$

H -1.967776 -2.826236 -2.574415

H $-3.272110-2.623503-3.743525$

H $-8.296087-2.792853-0.056439$

H -6.364394 -5.4539130 .458736$

H $-3.200124-5.385725-0.582483$ 
O - -3.095502 -0.263016 -4.022603

O $-1.721276-0.228851-2.168077$

C $-2.817137 \quad 1.119877-4.259595$

H $-1.7439991 .272688-4.408182$

H $-3.3435211 .422264-5.168752$

H -3.182781 $1.724855-3.425562$

SCF Energy (B3LYP/6-31G**//MMFF) $=-3245.93271132$

05_00068

MMFF Geometry

C $4.619737-0.334509-1.621098$

C $4.167817-1.507778-2.088893$

C $3.649743-2.605386-1.199524$

O $2.453751-3.107068-1.846803$

C $4.650615-3.750568-0.993557$

C $5.808202-3.375308-0.092125$

C $5.737495-3.3297441 .253649$

C $7.109549-3.087434-0.793740$

C $4.545734-3.6330542 .121425$

C $4.291386-2.5711493 .200973$

C $3.696953-1.2456412 .687713$

C $3.680609-0.2274443 .834821$

C 2.9759661 .0794063 .453505

O $2.364081-1.4956102 .220302$

$\begin{array}{lllll}\text { O } & 2.742441 & 1.824341 & 4.658877\end{array}$

C 1.5947580 .7330802 .864695

C 3.8528291 .9646242 .559911

O 0.9468131 .9065782 .377454

C $1.657454-0.3357111 .751573$

C $0.245089-0.7731531 .292453$

$\begin{array}{lllll}\text { O } & 2.300691 & 0.246509 & 0.613203\end{array}$

C $1.485182-3.646976-1.060975$

O $1.544231-3.8657450 .137898$

C $0.301509-3.892379-1.913632$

C $-0.840588-4.304585-1.343732$

C $-2.104189-4.572737-2.031444$

C $-2.181289-4.257145-3.502395$

C $-0.545692-1.6009782 .308229$

C $-1.978646-1.8276791 .854410$

C $-4.206421-2.4178122 .792441$

O $-2.381373-1.5418710 .730521$

C $-4.671205-3.555321 \quad 1.940049$

C -5.449942 -3.3862900 .860004$

C $-6.023390-4.5096440 .044935$

C $-5.568611-4.484906-1.396418$

C $-6.155093-3.678345-2.296989$

C $-4.496538-5.464876-1.809968$

C $-3.132890-5.072471-1.315258$

N $-2.757945-2.3804092 .862629$

H $4.701305-0.183829-0.547991$

H $4.094591-1.661125-3.162932$

H $3.359280-2.177032-0.235007$

H $5.022306-4.104645-1.964731$

H $4.137147-4.626116-0.575572$

H $6.643353-3.077621 \quad 1.803585$

H $7.910158-2.824518-0.094195$

H $7.438002-3.968418-1.354998$

H $6.998665-2.253975-1.493034$

H $3.637361-3.7930481 .539660$

H $4.755388-4.5828572 .629782$

H $3.591783-3.0079863 .926224$

H $5.227692-2.3748163 .739035$

H $4.305382-0.8726651 .857083$

H $4.700594-0.0226024 .184361$

H $3.153727-0.6616034 .696681$

H 3.6020021 .9792515 .086788

H $0.9775300 .358702 \quad 3.690298$

H 4.7716012 .2485853 .087143

H 3.3459662 .9043162 .314577

H 4.1486041 .4776761 .628554

H 0.9304342 .5516213 .105191

H $-0.3301640 .110670 \quad 0.985526$

H $0.336941-1.3737890 .378502$

H $1.996058-0.208349-0.188880$

H $0.396770-3.712315-2.978217$

H $-0.856155-4.464987-0.266116$

H - $1.529617-4.927511-4.073022$

H - $-1.873857-3.222749-3.692886$
H -3.188173 -4.351086 -3.915287

H $-0.074198-2.5795992 .451587$

H $-0.580465-1.0891573 .274946$

H $-4.560735-1.4508732 .419383$

H $-4.580058-2.5480473 .813128$

H $-4.397788-4.5595272 .253708$

H $-5.736155-2.3778120 .568429$

H $-7.118021-4.4290590 .081446$

H $-5.791342-5.4830650 .494883$

H $-5.851985-3.678847-3.338687$

H $-6.948103-2.995944-2.009056$

H $-4.504653-5.611192-2.895118$

H $-4.739278-6.455303-1.403402$

H $-2.983173-5.257960-0.251130$

H -2.333816 - 2.5407113 .771621

C $4.0567982 .031000-2.123354$

O $2.6721901 .662120-2.273989$

C $1.9447812 .772882-2.834732$

C $2.9116383 .948948-2.842578$

C $4.2488323 .253764-3.015553$

H $1.6933292 .472956-3.859430$

C $0.6705752 .996058-2.009893$

H $2.6936034 .665131-3.640448$

H $2.880588 \quad 4.485714-1.886144$

C $5.4380084 .117510-2.635110$

H $4.3483632 .942941-4.064136$

C $-0.1678864 .195485-2.482645$

O $-0.1308291 .813545-2.099532$

H $0.9383893 .112418-0.953611$

H $0.402388 \quad 5.122709-2.352580$

O $-0.3951324 .054107-3.891347$

C $-1.541615 \quad 4.330476-1.796673$

C -1.458337 $4.470755-0.266846$

H $-2.176135 \quad 3.481251-2.072542$

O $-2.184805 \quad 5.494723-2.330431$

H $-0.9566883 .595193 \quad 0.154700$

H $-0.8400255 .344096-0.025239$

H $5.4805145 .012120-3.264755$

H $5.3796724 .443549-1.591195$

H $6.3760673 .568508-2.764689$

C $4.9504190 .843619-2.491941$

H $0.4143481 .069563-1.790635$

H $-0.9349734 .811586-4.175509$

H $-1.6484676 .268669-2.086916$

H $6.0055701 .104888-2.357488$

H $4.8046720 .582284-3.547238$

H $4.2000322 .298125-1.067498$

C -3.434336 2.3173120 .603656

\begin{tabular}{lllll}
\hline & -3.747738 & 3.595219 & 0.036769
\end{tabular}

C -2.846098 $4.648340 \quad 0.391776$

C $-2.812248 \quad 4.821179 \quad 1.916840$

C -2.512492 $3.506508 \quad 2.633227$

C -3.4395362 .3986292 .133721$

H -2.4502191 .9867850 .250699$

C $-4.485924 \quad 1.3242310 .096584$

H -3.276568 $5.569848-0.019767$

$\begin{array}{lllll}O & -1.850416 & 5.809112 & 2.270302\end{array}$

H -3.7912315 .1891052 .251244$

H -1.4624083 .2179872 .516858$

O $-2.734163 \quad 3.667074 \quad 4.035972$

H -4.4583282 .5802072 .499532$

O $-3.006294 \quad 1.1527592 .673184$

C $-4.297958 \quad 1.035339-1.374275$

$\begin{array}{llll}\text { H } & -4.450649 & 0.370248 & 0.632753\end{array}$

H $-5.496972 \quad 1.7293860 .226276$

H $-1.944193 \quad 5.9861223 .221334$

H -2.065297 $4.288254 \quad 4.369653$

H $-2.996757 \quad 1.2423353 .641503$

O $-3.2152580 .227353-1.555823$

O $-5.026077 \quad 1.476382-2.253902$

C $-2.921949-0.122245-2.911476$

H $-3.725080-0.742180-3.320740$

H $-2.7794670 .778138-3.517051$

H $-1.993088-0.698651-2.919072$

SCF Energy (B3LYP/6-31G**//MMFF)= -3245.91094121

0500069

MM̄FF Geometry 
C $-1.2665682 .950385-1.347270$ C $-1.3977001 .922423-2.200108$ C $-0.502806 \quad 0.706289-2.301394$ O $\quad 0.6031390 .776639-1.375768$ C - $-1.335512-0.540216-1.946042$ C $-0.587720-1.851269-2.073579$ C $-0.355096-2.698215-1.051657$ C $-0.124586-2.207962-3.462146$ C $-0.759717-2.5444260 .388185$ C $0.297013-1.8687151 .276154$ C $1.598119-2.6746461 .427949$ C $2.513491-2.0367112 .475596$ C $3.859196-2.7721492 .588418$ O $2.269712-2.698990 \quad 0.166380$ O $4.759146-1.9327973 .328856$ C $4.463091-2.9453401 .177134$ C $3.729598-4.0762473 .384564$ O $5.624044-3.770691 \quad 1.217226$ C $3.460207-3.4979020 .143366$ C $3.999571-3.473190-1.307739$ O $3.167467-4.8652790 .456529$ C $1.7318211 .414068-1.788623$ O $1.935307 \quad 1.925917-2.877426$ C $2.6758741 .419240-0.649475$ C $3.6729802 .315731-0.624301$ C 4.6614372 .4752890 .444462 C 4.7083751 .4150791 .512725 C $4.385787-2.089857-1.843473$ C $5.866727-1.771409-1.718966$ C $7.4891910 .082273-2.093190$ O $6.709473-2.597734-1.380286$ C $7.7898120 .730416-0.779054$ C $8.2623391 .981177-0.667364$ C 8.6126522 .6306380 .640299 C 7.9197263 .9605170 .833738 C 8.5533875 .1154680 .568487 C 6.5251323 .9516721 .417063 C 5.4782953 .5491740 .413916 N $6.142515-0.456826-2.071528$ H $-0.4299202 .983609-0.655355$ H $-2.245614 \quad 1.925947-2.882649$ H $-0.1299280 .629277-3.329772$ H -2.216298 $-0.593814-2.599118$ H $-1.721978-0.416196-0.928612$ H $\quad 0.183023-3.620821-1.266173$ H $0.279731-3.224431-3.516939$ H - $0.960089-2.150770-4.167462$ H $0.662835-1.524275-3.793857$ H - $1.700016-1.9888720 .468437$ H $-0.985505-3.5414740 .787461$ H $0.521723-0.8757980 .868805$ H - $0.147198-1.7178412 .267891$ H $1.335214-3.6973911 .722852$ H $2.017308-1.9946293 .453174$ H $2.711830-0.9928712 .196344$ H $4.356815-1.7534844 .195993$ H $4.796971-1.9533500 .851688$ H $3.363684-3.8711784 .397877$ H $4.704089-4.5605793 .511562$ H $3.044738-4.7924582 .926028$ H $6.238638-3.3754911 .859132$ H $4.817971-4.194694-1.418124$ H $3.194731-3.847786-1.956947$ H $2.826879-5.298790-0.343397$ H 2.5027980 .7094510 .151066 H $3.7589543 .021404-1.449786$ H 5.5697181 .5032562 .178140 H 4.7803160 .4202881 .060794 H 3.8078561 .4548262 .134600 H $4.158371-2.059754-2.916935$ H $3.799179-1.301039-1.363148$ H $7.5420200 .790178-2.926393$ H $8.203154-0.727136-2.276775$ H $7.638414 \quad 0.1355290 .118634$ H $8.4394412 .563413-1.569119$ H 9.7014072 .7713700 .660578 H $8.385017 \quad 1.9736621 .488812$ H $8.073328 \quad 6.0745800 .731756$
H $9.5650725 .127004 \quad 0.176586$ H $6.2606094 .957307 \quad 1.771223$ H 6.5175253 .3308532 .317211 H $5.3693964 .259097-0.407322$ H $5.3750910 .173217-2.286373$ C -2.7440834 .2508250 .193607$ O $-3.378614 \quad 3.0434690 .653850$ C -4.4632673 .3935431 .534605$ C -4.4385004 .9145191 .671222$ C -3.7993815 .3393710 .362783$ H -5.3869743 .1024421 .021534$ C -4.3361212 .6292182 .859001$ H -5.4408195 .3304761 .813588$ H -3.8239975 .2396142 .518521$ C $-3.240848 \quad 6.750946 \quad 0.389814$ H $-4.5506095 .261749-0.434648$ C -4.2009241 .1009592 .699457$ O $\quad-3.1605353 .0920503 .538394$ H -5.1832222 .8767743 .509223$ H -3.2318130 .8680562 .241928$ O $-4.157426 \quad 0.5308024 .014627$ C -5.3232610 .4203341 .894606$ C $-5.131575-1.1057311 .823493$ H -5.3479290 .8241840 .880004$ O H $-4.124445-1.3158191 .447974$ H -5.195957 -1.524481 2.835053 H -4.0360897 .4750770 .594832$ H -2.4748246 .8647191 .164093$ H -2.788570 $7.010920-0.572251$ C $-2.2605074 .075219-1.247014$ H -3.094724 2.597386 4.373216 H -5.0308490 .6656824 .420934$ H -6.7781631 .6326612 .386359$ H -1.782001 $4.996796-1.596393$ H $-3.1179543 .878301-1.901811$ H -1.8891164 .4366410 .857841$ C $-5.116712-1.550786-1.209290$ O $-6.249878-1.262507-0.380845$ C $-6.191096-1.8066690 .942321$ C $-6.044394-3.3333670 .877480$ C $-4.860227-3.752090 \quad 0.009415$ C $-4.926795-3.066485-1.354737$ H -4.216994 -1.101812 -0.772730 C $-5.371384-0.902240-2.579963$ H $-7.167321-1.6002511 .399118$ O $-5.905521-3.8717122 .187703$ H $-6.963245-3.7578570 .452667$ H -3.909758 -3.5370610 .510125$ O $-4.887998-5.169514-0.175463$ H $-5.750792-3.494037-1.939984$ O $-3.719402-3.323778-2.067316$ C $-5.2592120 .607330-2.542313$ H $-4.642775-1.255857-3.317368$ H $-6.383103-1.153906-2.920315$ H $-5.786286-4.8326082 .097047$ H -5.722961 -5.399030 -0.617867 H $-3.603181-4.288531-2.106046$ O $\quad-5.7084991 .114270-3.728990$ O $-4.8157661 .261693-1.609621$ C $-5.6486452 .539133-3.850656$ H -4.606673 $2.871834-3.849020$ H $-6.1049002 .818214-4.803972$ $\mathrm{H}-6.2087923 .017150-3.041060$ SCF Energy $(B 3 L Y P / 6-31 G * * / / M M F F)=-3245.91160537$

$05 \_00070$

MM̄FF Geometry

C $3.0847633 .223760-1.142241$

C $2.8603102 .559279-2.285322$

C $3.1321381 .091174-2.469704$

O $1.8533040 .509585-2.831859$

C $4.1478460 .812701-3.589699$

C $5.5869241 .039823-3.176325$

C $6.3802560 .084240-2.652310$

C $6.1404802 .413362-3.451287$

C $6.006422-1.338752-2.339807$

C $6.562104-1.830036-0.996129$ 
C $5.946618-1.1535800 .241280$

C $6.693968-1.6197081 .496472$

C $6.067875-1.0749172 .785623$

O $4.560876-1.5155560 .321798$

O $6.624151-1.8052623 .889949$

C $4.557137-1.3787272 .759786$

C 6.4225810 .3980603 .018653

O $3.900598-0.7792053 .874944$

C $3.865878-0.9510681 .444869$

C $2.386706-1.4092131 .395043$

O $3.858727 \quad 0.4804751 .380664$

C $1.638308-0.796999-2.527869$

O $2.434488-1.580203-2.037608$

C $0.238430-1.116248-2.882641$

C $-0.237841-2.344620-2.630405$

C $-1.589774-2.824632-2.919709$

C $-2.582273-1.832007-3.465307$

C $2.165527-2.9214431 .301165$

C $0.699057-3.2914151 .469863$

C $-0.738589-5.2707881 .970630$

O $-0.207116-2.4630991 .485217$

C -1.412964 -5.8097890 .749476$

C $-2.698392-5.5612280 .454764$

C $-3.429771-6.154194-0.715485$

C $-3.957236-5.107146-1.670270$

C $-5.130059-4.491338-1.444888$

C -3.169942 -4.844449 -2.932439

C $-1.879674-4.119283-2.673849$

N $0.529204-4.6613801 .614401$

H $3.5343992 .706806-0.299766$

H $2.3999613 .082739-3.120543$

H $3.4675610 .645485-1.525340$

H $3.9142561 .419524-4.475455$

H $4.027414-0.216660-3.950527$

H $7.4166230 .340951-2.436905$

H $7.2070402 .486352-3.212491$

H $6.0254752 .664149-4.510856$

H $5.6212363 .169309-2.856479$

H $4.926562-1.492425-2.358506$

H $6.428231-1.971051-3.130947$

H $6.372359-2.910161-0.940005$

H $7.651260-1.695189-0.988289$

H $6.027585-0.0660460 .135105$

H $7.754455-1.3433161 .441309$

H $6.675058-2.7179831 .542454$

H $7.589527-1.6884823 .870903$

H $4.450430-2.4627872 .885244$

H 7.5085190 .5191993 .113706

H 5.9975620 .7626093 .960239

H 6.0898231 .0542092 .211816

H $4.362808-1.0762504 .677488$

H $1.851189-0.9988712 .261580$

H $1.900057-0.9486900 .525035$

H $3.112100 \quad 0.762587 \quad 0.827846$

H -0.359482 -0.335405 -3.338539

H $0.427148-3.077204-2.174203$

H -2.289773 -1.508789 -4.470123

H -2.641630 -0.950825 -2.816805

H $-3.598718-2.226213-3.532833$

H $2.504017-3.2956310 .328669$

H $2.726688-3.4413152 .083397$

H -1.359613 -4.538947 2.497454

H - $0.515562-6.0928572 .658416$

H $-0.833807 \quad-6.4657490 .104433$

H -3.280154 -4.928638 1.121779

H $-4.267955-6.746100-0.324439$

H -2.798807 -6.869859-1.257090

H -5.528776 -3.761099-2.141153

H $-5.713597-4.699719-0.554101$

H -3.783163 -4.315755 -3.669940

H $-2.936795-5.803877-3.412798$

H -1.094813 -4.758820 -2.268664

H $\quad 1.338759-5.2704501 .538861$

C 1.5003104 .7221490 .038749

O $0.347614 \quad 4.141583-0.601222$

C $-0.8357304 .845357-0.162891$

C -0.3659425 .8639070 .867195$

C 1.0607746 .1307400 .420963
H $-1.2292415 .350633-1.053293$

C $-1.8346553 .804140 \quad 0.352821$

H $-0.988547 \quad 6.7635090 .878797$

H -0.3761095 .4330561 .876319$

C 1.9183386 .7823251 .490846

H $1.0380426 .773188-0.469423$

C -3.0936454 .3875221 .014331$

O $-2.2344812 .995768-0.760290$

H -1.3210053 .1369141 .054383$

H -2.8182734 .9213521 .931429$

$\begin{array}{lllll}\text { O } & -3.656200 & 5.364221 & 0.129898\end{array}$

C $-4.188728 \quad 3.344971 \quad 1.325105$

C -3.6952572 .1628762 .176871$

H -4.6239862 .9890720 .385355$

O $\quad-5.2492834 .005607 \quad 2.024274$

H $-2.8424841 .688847 \quad 1.684201$

H -3.3374282 .5416883 .142318$

H 1.5029037 .7539671 .777435

H 1.9761526 .1623512 .391941

H $2.938026 \quad 6.9456481 .128530$

C $2.6907454 .658057-0.917605$

H -1.426591 $2.623672-1.153995$

H -4.457752 5.7137850 .555885

H -4.8940234 .3241002 .871687$

H $3.5509295 .195443-0.504372$

H $2.4316105 .132866-1.871971$

H 1.7025934 .1271710 .940228

C $-4.592113-0.0674630 .334665$

$\begin{array}{llll}\text { O } & -5.428916 & 0.672821 & 1.230253\end{array}$

C -4.7865051 .0983222 .435723$

C $-4.291373-0.1302023 .212666$

C -3.393540-1.020445 2.352719

C $-4.059719-1.3339591 .011862$

H $-3.753047 \quad 0.5592330 .010483$

C $-5.434843-0.409330-0.898574$

H $-5.5618531 .579393 \quad 3.045030$

O

H $-5.159882-0.7156883 .541397$

H -2.406529 -0.5678662 .200814$

O $-3.167570-2.259783 \quad 3.028540$

H $-4.873654-2.0534531 .167187$

O $-3.104851-1.9466290 .147459$

C $-5.7281800 .831803-1.707241$

H -4.937728 -1.131252 -1.554541

H $-6.396364-0.848190-0.605573$

H $-3.396990-0.5058864 .906892$

H -2.624001-2.071709 3.811962

H $-2.693188-2.6789670 .636534$

O $-4.6193801 .203342-2.409004$

O $-6.8079451 .408442-1.703163$

C $-4.7541852 .396430-3.186811$

H $-5.5307382 .267783-3.946724$

H $-4.9839663 .247165-2.538009$

$\mathrm{H}-3.8006942 .585256-3.687097$

SCF Energy (B3LYP/6-31G**//MMFF) $=-3245.91707571$

05_00071

MM̄FF Geometry

C -4.174956 -2.480607 2.037470

C $-3.173811-1.5880682 .058324$

C $-1.784223-1.9003962 .543730$

O $-0.921242-1.8606731 .380048$

C $-1.291966-0.8252043 .527137$

C $-0.384162-1.3966424 .596277$

C $0.950551-1.5346124 .485370$

C $-1.085251-1.8151435 .863038$

C $1.817524-1.2192783 .298934$

C $2.529368-2.4904462 .815576$

C $3.411371-2.2442971 .584496$

C $4.113150-3.5388751 .165161$

C $5.103117-3.3171040 .011870$

O $4.380153-1.2493201 .921002$

O $5.947369-4.475462-0.074962$

C $6.014557-2.1191130 .354525$

C $4.386775-3.203842-1.337706$

O $6.846215-1.776974-0.752293$

C $5.238637-0.8714730 .837880$

C 6.2301830 .1987831 .365943 
O $4.502892-0.343514-0.267293$

C $-0.774823-3.001300 \quad 0.659645$

O $-1.179872-4.114410 \quad 0.967230$

C $-0.100541-2.751418-0.635976$

C $0.378796-1.558980-1.024783$

C $0.972244-1.253450-2.331249$

C $1.017062-2.362027-3.351397$

C 5.6087281 .2997292 .240060

C 4.6999342 .2388151 .478173

C $4.742427 \quad 3.973826-0.307332$

O 3.4776362 .1899641 .595636

C $4.8338343 .352489-1.665015$

C $3.7797972 .895681-2.357996$

C $3.8920712 .298445-3.730696$

C $3.460156 \quad 0.851172-3.775094$

C $4.365502-0.139496-3.841846$

C $1.9784830 .561817-3.845433$

C $1.424669-0.002708-2.564200$

$\begin{array}{lllll}N & 5.396788 & 3.093682 & 0.640261\end{array}$

H -4.008817 -3.4869692 .412661$

H $-3.340325-0.5973381 .643236$

H -1.745489 -2.893292 3.009972

H -2.140095 -0.344665 4.033559

H $-0.776526-0.0189322 .992032$

H $1.493163-1.9395385 .339216$

H $-0.398318-2.2493176 .597109$

H $-1.569080-0.9519866 .331968$

H $-1.850631-2.5664515 .642731$

H $1.249148-0.7799742 .474891$

H $2.554713-0.4684823 .607074$

H $3.152554-2.8729073 .635238$

H $1.788266-3.2655712 .582693$

H $2.777942-1.8692170 .773738$

H $3.378738-4.3096690 .899487$

H $4.671312-3.9420912 .022092$

H $5.377778-5.251290-0.214990$

H $6.688258-2.4415811 .160852$

H $3.817678-4.117209-1.548468$

H $5.103934-3.102163-2.159572$

H $3.690030-2.365757-1.379960$

H $7.319474-2.583679-1.019134$

H $6.978006-0.2984681 .997344$

H 6.7658340 .6525040 .523218

H $4.4208840 .616994-0.179409$

H $-0.052680-3.620868-1.284109$

H $0.312527-0.714815-0.340646$

H $1.520484-2.084085-4.279364$

H $0.002807-2.679301-3.617787$

H $\quad 1.564745-3.225937-2.958638$

H $5.048867 \quad 0.874242 \quad 3.080212$

H $6.412216 \quad 1.8991042 .686897$

H $5.274748 \quad 4.929758-0.297227$

H $3.7074834 .149691-0.000861$

H $5.8270403 .286787-2.103801$

H $2.7853782 .967220-1.926398$

H $4.9210482 .393026-4.102587$

H $3.2799402 .885505-4.426601$

H $4.060288-1.177992-3.913681$

H $5.4307120 .065315-3.822141$

H $1.775919-0.081619-4.707215$

H $1.4289381 .486230-4.065941$

H $1.3612390 .729299-1.759471$

H $6.409223 \quad 3.0235800 .594280$

C -5.573195 -2.660490 -0.016465

O $-4.683334-1.838962-0.801364$

C $-5.375191-1.387946-1.977448$

C $-6.575663-2.312180-2.120744$

C $-6.951178-2.520519-0.664128$

H $-5.740266-0.376245-1.762109$

C $-4.415330-1.344067-3.169894$

H $-7.379201-1.864537-2.713009$

H -6.300717 -3.267735 -2.581996

C $-7.862849-3.712934-0.433588$

H $-7.449164-1.613490-0.295630$

C $-3.124908-0.537940-2.902154$

O $-4.028198-2.684860-3.494864$

H -4.939864 -0.952434 -4.049304

H $-2.496339-1.082821-2.189171$
O $-2.378526-0.490866-4.124524$

C $-3.355908 \quad 0.895917-2.389707$

C $-2.0388641 .677761-2.224611$

H $-3.8738040 .849257-1.426280$

O $-4.205873 \quad 1.575589-3.311627$

H $-1.3671941 .092140-1.588956$

H $-1.552077 \quad 1.785597-3.201310$

H $-8.811005-3.580429-0.964858$

H $-7.405766-4.642546-0.789061$

H $-8.085418-3.8327990 .631421$

C $-5.523620-2.190388 \quad 1.438894$

H $-3.410467-2.634448-4.244408$

H $-2.8896860 .040597-4.758880$

H $-4.5304822 .381240-2.875348$

H $-6.288201-2.6973122 .037879$

H $-5.728121-1.1134801 .493250$

H $-5.209679-3.692968-0.101495$

C -2.3696452 .4960030 .708393$

O $-3.023655 \quad 3.062460-0.430534$

C $-2.235347 \quad 3.088347-1.624305$

C $-0.932128 \quad 3.865195-1.384675$

C $-0.1664753 .353599-0.166572$

C $-1.084268 \quad 3.257582 \quad 1.050550$

H -2.1183081 .4505900 .488289$

C $-3.3745952 .443261 \quad 1.868687$

H $-2.8137193 .663390-2.357940$

O $-0.083124 \quad 3.803444-2.526233$

H $-1.1707494 .924074-1.219029$

H $\quad 0.3167072 .391670-0.368831$

$\begin{array}{lllll}O & 0.883412 & 4.272439 & 0.145242\end{array}$

H -1.3105494 .2695381 .405762$

$\begin{array}{llll}\text { O } & -0.402157 & 2.581382 & 2.103621\end{array}$

C -3.8558383 .7988012 .344549$

H $-4.267453 \quad 1.8886001 .556137$

H -2.918351 1.9171032 .714853

H $-0.5844614 .152634-3.282731$

H $1.4054144 .401271-0.664871$

H $0.432377 \quad 3.0553882 .261785$

O $-4.294128 \quad 3.688258 \quad 3.633806$

O $-3.8804324 .816787 \quad 1.666638$

C -4.8005344 .8928914 .217049$

H $-5.1024334 .672475 \quad 5.244356$

H -4.0215015 .6611294 .237335$

H -5.675489 5.2434053 .661290

SCF Energy (B3LYP/6-31G**//MMFF) = -3245.90816107

05_00072

MM̄FF Geometry

C 1.2861725 .2607640 .974178

C $2.189964 \quad 4.6863700 .166683$

C 3.2466463 .7298520 .657655

$\begin{array}{lllll}\text { O } & 3.009130 & 2.445891 & 0.030149\end{array}$

$\begin{array}{llll}\text { C } 4.644135 & 4.216852 & 0.237502\end{array}$

C $5.771428 \quad 3.353487 \quad 0.771449$

C 6.6142732 .6323950 .006162

C 5.9420933 .3526892 .269458

C $6.6469732 .524674-1.492015$

C $5.8319531 .360174-2.075552$

C $6.290148-0.030622-1.606228$

C $5.622871-1.127363-2.439124$

C $5.983718-2.534894-1.939646$

O $5.923428-0.189524-0.232798$

O $5.072099-3.442254-2.578196$

C $5.745298-2.606799-0.414543$

C $7.391989-2.945840-2.385633$

O $6.233625-3.8329190 .123380$

C $6.360580-1.4211990 .358043$

C $5.949866-1.3876691 .851432$

$\begin{array}{llllll}\text { O } & 7.789422 & -1.527984 & 0.321208\end{array}$

C 1.9920541 .6874430 .524659

$\begin{array}{lllll}\text { O } & 1.282051 & 1.940001 & 1.485109\end{array}$

C $1.8647770 .492050-0.336552$

C $0.734337-0.228398-0.296441$

C $0.447643-1.430150-1.081682$

C $1.583964-2.036490-1.861784$

C $4.443851-1.2895952 .119342$

C $3.766944-2.6299792 .356359$

C $1.506865-3.6332382 .674264$ 
O $4.384676-3.6790692 .516214$ C $1.027898-4.2116571 .380071$ C $-0.266029-4.4500041 .116687$ C $-0.764965-5.070643-0.156605$ C $-1.812898-4.226126-0.846956$ C $-3.122679-4.484212-0.693119$ C $-1.337373-3.138568-1.783243$ C $-0.802005-1.938898-1.050456$ N $2.384374-2.5082762 .408636$ H 1.3319505 .0788802 .045179 H $2.1223254 .842279-0.907456$ H 3.1976993 .6147761 .747202 H 4.8037545 .2430690 .594496 H $4.6838194 .266642-0.855946$ H 7.3874662 .0509440 .507693 H 6.8609272 .8449842 .582318 H $5.993434 \quad 4.3791502 .647014$ H 5.1051302 .8415302 .754497 H $6.313075 \quad 3.459579-1.954776$ H $7.6953402 .416112-1.798749$ H $4.7753151 .505656-1.823428$ H $5.9134051 .416136-3.168293$ H $7.380570-0.087145-1.704506$ H $5.879475-1.021728-3.500653$ H $4.532093-1.008654-2.384040$ H $5.308369-4.346192-2.310136$ H $4.659599-2.605599-0.263181$ H $7.456474-2.957056-3.480278$ H $7.629145-3.965168-2.061493$ H $8.171542-2.277477-2.014079$ H $5.738112-4.557001-0.294431$ H $6.401516-2.2322082 .385770$ H $6.406518-0.4890802 .291144$ H $8.156103-1.0087321 .056007$ H $2.6953670 .248135-0.988164$ H -0.0681650 .1007990 .361997$ H $2.447158-2.207400-1.209581$ H $1.886289-1.373073-2.679195$ H $1.345219-3.006267-2.302378$ H $4.293048-0.7076183 .037649$ H $3.935318-0.7594481 .309490$ H $0.682686-3.2623753 .291967$ H $2.051470-4.396195 \quad 3.239464$ H $1.779532-4.4703890 .637939$ H -1.011218 -4.2171371 .873880$ H - $-1.185322-6.0532670 .094660$ H $0.057862-5.272490-0.853628$ H $-3.878204-3.900827-1.208517$ H $-3.468475-5.280614-0.042432$ H -2.169161 -2.791553 -2.410040 H $-0.619555-3.566346-2.488260$ H $-1.554476-1.426244-0.449412$ H $1.955684-1.6070622 .218661$ C $-1.151504 \quad 5.2323530 .541731$ O $-1.0703744 .174148-0.435069$ C $-2.3816193 .956103-1.001744$ C $-3.3333124 .831568-0.198097$ C $-2.4317495 .986190 \quad 0.199842$ H $-2.3227154 .312366-2.037926$ C -2.670924 2.451261-0.971396 H $-4.2044665 .147545-0.779735$ H -3.691693 4.3029620 .694331 C -2.985091 6.8217501 .340273 H $-2.2693546 .627698-0.676594$ C -4.123371 2.081091-1.329670 O $-1.7564771 .846652-1.891020$ H $-2.422740 \quad 2.056251 \quad 0.021027$ H $-4.7924632 .560585-0.606510$ O $-4.4545872 .610367-2.612271$ C $-4.401710 \quad 0.564184-1.300928$ C $-5.8985160 .256257-1.490787$ H $-4.0529090 .172218-0.339594$ O $-3.655667-0.079687-2.330098$ H $-6.4709130 .848177-0.768062$ H $-6.2126480 .579466-2.490920$ H $-3.942748 \quad 7.2710941 .057804$ H -3.149631 6.2167802 .238272 H -2.296646 7.6314631 .601272
C $\quad 0.1233916 .0744050 .477448$

H $-1.8552070 .882716-1.815725$

H -3.868162 2.184567 -3.261374

H $-3.699619-1.037169-2.171026$

H $0.0328906 .970073 \quad 1.101188$

H $0.3055256 .405257-0.552384$

H -1.2444024 .7526051 .525892$

C $-6.245156-1.3448051 .072346$

O $-5.670927-1.812350-0.152981$

C $-6.242104-1.243535-1.334854$

C $-7.743864-1.559614-1.393974$

C $-8.467061-1.143875-0.112572$

C $-7.738449-1.6846211 .118972$

H $-6.108448-0.2600441 .155517$

C -5.483973 -2.0132782 .222792$

H $-5.774619-1.763161-2.180474$

O $-8.327500-0.918146-2.522544$

H $-7.870703-2.641138-1.534760$

H $-8.581581-0.055241-0.050502$

O $-9.788660-1.688704-0.115120$

H $-7.877520-2.7717301 .178787$

O $-8.313514-1.1132642 .291899$

C $-4.048348-1.5458312 .282746$

H $-5.944255-1.8106903 .196157$

H -5.467567 -3.101893 2.088416

H $-9.242480-1.235488-2.603173$

H -10.282747 -1.257542 -0.832590

H $-9.262770-1.3248522 .282131$

O $-3.993492-0.2374702 .662992$

O $-3.089221-2.2624942 .028489$

C $-2.680044 \quad 0.3259192 .737114$

H -2.1591150 .2088251 .782368$

H -2.7789471 .3932972 .951611$

H -2.114791-0.146531 3.545753

SCF Energy (B3LYP/6-31G**//MMFF)= -3245.90423141

0500073

MM̄FF Geometry

C 3.0592111 .8600062 .976167

C 1.7301962 .0462412 .976132

C 0.6538551 .0278223 .283360

$\begin{array}{llll}\text { O } & -0.196210 & 0.925215 & 2.111924\end{array}$

C $1.163897-0.3923393 .601766$

C $0.064514-1.3742033 .962467$

C $-0.238925-2.4793663 .253169$

C $-0.696253-1.0690735 .227649$

C $0.411306-2.9823701 .995038$

C $-0.271746-2.5223230 .699298$

C -1.699849 -3.0569790 .511707$

C $-2.206554-2.748857-0.899446$

C $-3.666494-3.187064-1.095950$

O $-2.551948-2.4290971 .471925$

O $-4.152788-2.571459-2.298954$

C $-4.521497-2.6393540 .067997$

C $-3.781634-4.701418-1.307587$

$\begin{array}{llll}0 & -5.845824 & -3.163215 & 0.016649\end{array}$

C -3.906565 -2.900316 1.458453

C $-4.655622-2.1775662 .605060$

O $-3.964703-4.3038181 .742314$

C -1.2027991 .8293581 .972460$

\begin{tabular}{llll}
\hline & -1.505614 & 2.724573 & 2.744486
\end{tabular}

C $-1.856563 \quad 1.5658220 .672805$

$\begin{array}{llll}C & -2.526970 & 2.554356 & 0.062965\end{array}$

C -3.173955 $2.474083-1.248083$

C $-3.2916251 .114275-1.883385$

C $-4.719980-0.6506752 .490972$

C $-5.994581-0.1327001 .845302$

C $-7.031577 \quad 1.977014 \quad 1.018977$

O $-6.969839-0.8415241 .612542$

C $-6.8547712 .062080-0.464201$

C $-6.9077613 .216800-1.145356$

C $-6.7739993 .317058-2.637622$

C $-5.701106 \quad 4.294982-3.060723$

C $-6.0131265 .554037-3.411752$

C $-4.280478 \quad 3.786938-3.158437$

C -3.632072 $3.611001-1.812615$

N $-5.927891 \quad 1.231476 \quad 1.594734$

H $3.4789800 .891223 \quad 3.230005$ 
H 1.3599723 .0377102 .719106 H 0.0701871 .4032344 .132694 H $1.865966-0.3569144 .444850$ H $1.731908-0.7582492 .740083$ H - $1.046495-3.1131773 .618331$ H - $1.365374-1.8865975 .517567$ H $-0.002421-0.9063556 .058869$ H $-1.311018-0.1727005 .102611$ H $1.467050-2.6933731 .961450$ H $0.419426-4.0792682 .030475$ H $-0.277235-1.4268380 .669669$ H $0.343229-2.864971-0.139536$ H $-1.687916-4.1396710 .684128$ H -1.562515 -3.216048 -1.654955 H -2.140759 -1.667621-1.080117 H -3.577229 -2.849591-3.031980 H $-4.607509-1.557217-0.084431$ H -3.209623 -5.008850 -2.191352 H $-4.818129-4.995501-1.505864$ H -3.413455 -5.282144 - 0.459257 H $-6.206135-2.955116-0.862343$ H -5.651566 -2.616182 2.740128 H -4.113044-2.400467 3.535256 H -3.901902 -4.4270362 .704020$ H - 1.7273780 .5842610 .233046 H -2.576383 3.5270070 .550902 H -3.746356 $0.404436-1.184392$ H -2.304872 $0.739074-2.174941$ H -3.922455 $1.100824-2.774396$ H -4.699665 -0.2269573 .503393$ H $-3.850734-0.2582861 .955191$ H -7.038911 2.963670 1.492902 H -7.9746961 .4715041 .249491$ H $-6.7021401 .130768-1.004588$ H $-7.0887304 .143056-0.604423$ H -7.745739 $3.634053-3.038396$ H -6.574201 2.337618 -3.089978 H -5.253371 $6.253759-3.743669$ H $-7.0357625 .914465-3.369907$ H -3.663938 4.501139-3.720823 H -4.268793 $2.875254-3.761941$ H -3.500930 4.547963 -1.269306 H -5.0603321 .7292201 .772623$ C 4.7950902 .5549881 .329868 O 5.7195881 .4792231 .605653 C $6.955875 \quad 1.760414 \quad 0.935880$ C $6.6662062 .886119-0.045875$ C 5.6395813 .6829530 .738031 H 7.6339542 .1609981 .702966 C $7.611995 \quad 0.506175 \quad 0.355377$ H $7.5582783 .466827-0.298939$ H $6.2266572 .504786-0.974518$ C $4.8635844 .671772-0.113259$ H $6.1515354 .227821 \quad 1.542845$ C $6.838438-0.286009-0.721583$ O $8.8667820 .916557-0.205770$ H $7.853591-0.1721431 .183509$ H $6.7611480 .307843-1.639028$ O $7.665955-1.410396-1.067526$ C $5.439535-0.806516-0.330046$ C $4.890225-1.842208-1.333879$ H $4.7379160 .029621-0.262471$ O $5.500649-1.4362700 .950245$ H $4.919247-1.412229-2.340290$ H $5.549313-2.718137-1.331179$ H $5.5373715 .423635-0.536955$ H $4.3554644 .174127-0.944917$ H 4.1078375 .1935740 .481771 C 4.0481642 .9343312 .608020 H $9.3027660 .120096-0.554364$ H $7.711068-1.991281-0.288502$ H $5.616640-0.7374911 .616148$ H 3.5341923 .8928802 .474622 H 4.7520173 .0488343 .440961 H 4.0891322 .1815370 .579577 C $2.170480-0.772306-2.327701$ O $2.513090-1.245010-1.018796$ C $3.461196-2.317302-0.982243$
C $2.962790-3.508356-1.814460$

C $2.573295-3.094378-3.229356$

C $1.611212-1.909306-3.191624$

H $3.058608-0.348262-2.810701$

$\begin{array}{llll}\text { C } & 1.123726 & 0.339926 & -2.153835\end{array}$

H $3.487707-2.6606660 .059403$

O $3.950919-4.532382-1.857958$

H $2.084613-3.941033-1.319480$

H $3.460545-2.861111-3.829014$

O $1.937054-4.194372-3.884532$

H $0.637403-2.234914-2.804656$

O $1.406707-1.433549-4.520488$

C $1.738792 \quad 1.635017-1.670826$

H $\quad 0.6261300 .565954-3.103041$

H $0.3729540 .023465-1.420303$

H $3.603287-5.249880-2.415004$

H $1.142125-4.429013-3.375821$

H $1.085497-2.182620-5.051213$

O $\quad 0.748566 \quad 2.451079-1.205296$

O $2.928337 \quad 1.912781-1.728405$

C $1.1720673 .734010-0.735120$

H $1.6635504 .290293-1.538755$

H $0.2863364 .289396-0.415569$

H 1.8412173 .6193990 .121727

SCF Energy (B3LYP/6-31G**//MMFF)= -3245.91821126

0500074

MM̄FF Geometry

C $-2.883330-1.9416493 .076120$

C $-1.892896-1.3619423 .770044$

C $-0.496730-1.177197 \quad 3.243927$

O $-0.235785 \quad 0.245774 \quad 3.330718$

C $0.552899-1.9244744 .085030$

C $0.478268-3.4328564 .011826$

C $1.071689-4.1691913 .052482$

C $-0.243923-4.1088895 .147634$

C $1.829338-3.6710211 .853439$

C $3.327727-3.5344392 .138674$

C $4.168664-3.454307 \quad 0.855347$

C $5.636456-3.1838551 .199654$

C $6.507610-3.027773-0.055377$

O $3.657332-2.415475 \quad 0.010699$

O $7.758834-2.447156 \quad 0.344615$

C $5.829083-2.021835-1.009683$

C $6.844098-4.382603-0.689101$

O $6.522293-1.952257-2.253334$

C $4.333119-2.320423-1.253573$

C $3.642770-1.229989-2.103651$

O $4.218063-3.544037-1.993384$

C $0.7593290 .762182 \quad 2.562989$

$\begin{array}{lllll}\text { O } & 1.487217 & 0.157077 & 1.793455\end{array}$

C $0.8059142 .217067 \quad 2.830458$

C 1.7045732 .9853702 .197895

C $1.855297 \quad 4.434212 \quad 2.349221$

C $0.9377795 .139606 \quad 3.313649$

C $3.5846790 .165884-1.480636$

C $2.9220691 .169600-2.412060$

C $2.3123983 .569141-2.572887$

$\begin{array}{llll}\text { O } & 2.457547 & 0.867902 & -3.508381\end{array}$

C $2.8676654 .845946-2.031973$

C $2.1136255 .815132-1.491857$

C $2.6764597 .087983-0.926461$

C $2.3105117 .290707 \quad 0.528384$

C 1.3570768 .1696560 .880055

C 3.1019546 .5396371 .578006

$\begin{array}{llll}\text { C } 2.787740 & 5.068832 & 1.608851\end{array}$

N $2.9255352 .451332-1.878647$

H -2.682574 -2.3323202 .081705$

H -2.098525 -0.9737594 .765202$

H $-0.445994-1.4801402 .190703$

H $0.487430-1.5997135 .133116$

H $1.557400-1.6013403 .785444$

H $1.000375-5.2541133 .117411$

H $-0.218974-5.2008305 .064309$

H $0.219234-3.8423206 .103380$

H - $-1.294773-3.8073785 .175327$

H $1.664298-4.3843391 .036121$

H $1.427436-2.7150971 .503587$ 
H $3.482548-2.6268912 .736080$ H $3.682027-4.3799672 .742049$ H $4.070804-4.4149090 .335330$ H $6.036019-3.9734881 .848420$ H $5.705642-2.2548331 .783257$ H $8.162493-3.0326691 .008080$ H $5.923657-1.032435-0.545407$ H $7.403063-5.0072580 .018242$ H $7.496988-4.260440-1.560267$ H $5.960399-4.946031-0.995127$ H $7.454307-1.757735-2.055174$ H $4.118906-1.180554-3.092031$ H $2.611520-1.550520-2.302111$ H $3.342684-3.569789-2.413932$ H 0.0902742 .6190673 .539103 H 2.3897122 .5134441 .495037 H 1.0803434 .7550244 .329686 H $-0.109524 \quad 4.9955713 .027128$ H 1.1022616 .2182503 .360072 H $3.0165570 .138963-0.544935$ H $4.5925520 .535120-1.266661$ H $1.2294213 .493190-2.433878$ H $2.5247493 .486925-3.643948$ H $3.9459914 .976983-2.083923$ H $1.0338135 .690735-1.451576$ H $2.2938637 .919120-1.532907$ H $3.7681157 .124541-1.030381$ H 1.1057158 .3464451 .920418 H $0.8033428 .731174 \quad 0.134725$ H 2.9602576 .9832112 .570497 H 4.1734646 .6647151 .374572 H 3.4099474 .4762020 .938218 H $3.1676632 .566276-0.898223$ C $-5.334307-1.3777532 .713605$ O $-5.416953-2.0354751 .433794$ C $-5.411262-1.0290220 .397926$ C $-5.797777 \quad 0.2663891 .095470$ C $-5.093403 \quad 0.1061312 .428412$ H $-4.374358-0.9794820 .047932$ C $-6.341477-1.469770-0.737954$ H $-5.476755 \quad 1.1581260 .550442$ H -6.8834190 .3220841 .242740$ C -5.6126661 .0318853 .514037$ H -4.0272950 .3115092 .273544$ C $-6.431960-0.503958-1.932751$ O $-5.917814-2.747477-1.218743$ H -7.347535 -1.619520 -0.325463 H -7.000991 $-0.993658-2.733726$ O $-7.2075870 .628777-1.527510$ C $-5.0971510 .005592-2.522000$ C $-4.138090-1.106866-2.976811$ H $-4.6027790 .663485-1.801313$ O $-5.4124230 .831920-3.650652$ H -3.839950 -1.703588 -2.111346 H -4.675932 -1.773845 -3.661847 H -5.4655262 .0787943 .229190$ H -6.6821490 .8801983 .694154$ H $-5.0833020 .859374 \quad 4.456460$ C $-4.289631-2.0741013 .592636$ H -5.864361 -3.343249 -0.451707 H $-7.2673091 .228510-2.290861$ H $-5.8302740 .267969-4.323935$ H -4.519852 -3.145607 3.640411 H -4.362155 -1.682513 4.614365 H -6.317960 -1.515159 3.181036 C -1.486792 $-0.139543-1.758267$ O $-2.158341 \quad 0.376657-2.915010$ C $-2.873788-0.584435-3.695429$ C -1.922099-1.691288 -4.167588 C $-1.181788-2.332746-2.998918$ C $-0.511146-1.262688-2.140642$ H -2.224240 -0.513652 -1.039094 C $-0.729921 \quad 1.039955-1.127888$ H -3.214732 -0.055474 -4.594087 O $-2.642814-2.681295-4.893797$ H - $1.186743-1.258095-4.858226$ H -1.851426 -2.964836 -2.404188 O $-0.176683-3.202150-3.527475$
H $\quad 0.342837-0.855417-2.695057$

O $-0.005497-1.900525-0.968788$

C $-1.6459062 .041930-0.461049$

H $-0.0293940 .700042-0.361833$

H $-0.1744761 .564861-1.913350$

H $-2.008490-3.372319-5.150838$

H $0.314503-3.568370-2.771986$

H $\quad 0.623916-1.294133-0.544366$

O $-0.9677893 .215981-0.291315$

O $-2.7939761 .819868-0.103811$

C -1.7014664 .2643010 .348498$

H -1.0529165 .1419940 .414398$

H $-2.5847274 .524286-0.242714$

H -1.9885273 .9622591 .360141$

SCF Energy $\left(B 3 L Y P / 6-31 G^{* *} / / M M F F\right)=-3245.92955020$

0500075

MM̄FF Geometry

C -4.181523 -2.690021-2.079266

C $-3.019797-2.810368-1.418709$

C $-1.700526-3.044809-2.108792$

O $-0.845041-1.903461-1.858864$

C $-0.999529-4.290781-1.538988$

C $0.344597-4.574017-2.188589$

C $1.535822-4.528032-1.557827$

C $0.290644-4.954432-3.647131$

C $1.811292-4.232397-0.110684$

C $2.370881-2.8284240 .131727$

C $3.741605-2.517544-0.491717$

C $4.840454-3.4385280 .046910$

C $6.235290-3.029779-0.446940$

O $4.030236-1.155157-0.145569$

O $7.207902-3.7278700 .346929$

C $6.438678-1.523994-0.179593$

C $6.468537-3.467998-1.897548$

O $7.658299-1.063081-0.754914$

C $5.265350-0.644439-0.665475$

C $5.3959600 .820210-0.196617$

O $5.226773-0.567903-2.095509$

C $-1.063913-0.783530-2.601333$

O $-1.942437-0.606219-3.429811$

C $-0.0241970 .201860-2.235735$

C $-0.1213861 .467038-2.671977$

C $0.8500662 .535790-2.427066$

C $2.1195192 .152069-1.717020$

C 5.3529251 .0282711 .318458

C 5.1556612 .4940721 .658494

C $3.4094334 .262247 \quad 1.782538$

O 6.0815823 .1982272 .053523

C 2.1332294 .5472821 .057500

C 1.9308365 .6153740 .271473

C $0.6354065 .867990-0.449056$

C $0.8189276 .107184-1.932836$

C $0.4452027 .271730-2.489820$

C $1.4382895 .011647-2.774851$

C $0.5753583 .782920-2.864289$

N 3.8526222 .9200331 .449726

H -4.196888 -2.805860 -3.160268

H $-2.998177-2.661025-0.342032$

H -1.839532 -3.160212 -3.190914

H $-1.640948-5.171830-1.673738$

H $-0.888114-4.164510-0.457621$

H $2.429149-4.751628-2.140235$

H $1.261998-5.291467-4.024993$

H $-0.419281-5.774024-3.799775$

H $-0.020789-4.102238-4.258548$

H $\quad 0.910805-4.3551280 .497249$

H $2.505537-4.9899310 .270317$

H $1.645337-2.080829-0.214788$

H $2.444299-2.6684911 .214621$

H $3.665498-2.591155-1.581988$

H $4.647311-4.484483-0.217869$

H $4.838334-3.4018331 .145395$

H $7.040629-4.6817400 .256920$

H $6.542348-1.4054400 .906650$

H $6.425337-4.561018-1.978987$

H $7.467916-3.181621-2.242384$

H $5.729972-3.060955-2.591621$ 
H $8.371895-1.629345-0.413930$

H $6.3132901 .267183-0.602529$

H $4.5834601 .394009-0.656850$

H $6.043020-0.956635-2.449178$

H $0.800875-0.146362-1.624954$

H $-0.9885721 .749489-3.267431$

H $2.6520221 .375992-2.278396$

H $1.8971281 .769550-0.714852$

H $2.8203632 .977988-1.589342$

H $4.5405740 .455981 \quad 1.781065$

H 6.2863540 .6945051 .784283

H 4.2030394 .9753841 .536266

H $3.242794 \quad 4.2938072 .864020$

H $1.323248 \quad 3.833573 \quad 1.186157$

H $2.724134 \quad 6.348770 \quad 0.148874$

H $0.1613496 .738857 \quad 0.021673$

H $-0.0623185 .034061-0.307437$

H $0.5719607 .458486-3.551083$

H $0.0026768 .066334-1.898213$

H $2.4442454 .802038-2.403219$

H $1.5912925 .367245-3.802879$

H $-0.3733313 .954563-3.374770$

H 3.1408092 .2111191 .295346

C $-5.760022-0.818900-1.676638$

O $-4.781841-0.030012-0.970220$

C $-5.428026 \quad 1.125212-0.404225$

C $-6.8315601 .153441-0.997155$

C -7.112122 $-0.330675-1.165008$

H $-5.5158080 .941840 \quad 0.672754$

C $-4.5686652 .371227-0.649080$

H $-7.5491461 .655635-0.341300$

H $-6.8525471 .657783-1.970106$

C -8.272328 $-0.628195-2.097762$

H $-7.319802-0.765336-0.177880$

C -3.106377 2.211410 -0.176970

O $-4.5419762 .632951-2.057927$

H -5.036202 3.245071-0.181316

H -2.610291 $1.460812-0.801791$

O $-2.421786 \quad 3.444026-0.426059$

C $-2.9407491 .832917 \quad 1.305747$

C -1.4670941 .6170661 .692995$

H -3.4867230 .9119431 .514465$

$\begin{array}{llll}\text { O } & -3.487116 & 2.857820 & 2.133284\end{array}$

H -1.037735 0.8604251 .026883

H -0.9081702 .5466041 .532826$

H -9.198512 - $-0.190881-1.710509$

H -8.099116 $-0.217057-3.097866$

H -8.424932 -1.707172 -2.198911

C $-5.480051-2.301863-1.427672$

H $-3.9730573 .409422-2.197819$

H -2.801920 4.1153220 .166234

H -4.4498822 .8636802 .001215$

H -6.279249 -2.924524 -1.843651

H $-5.434724-2.503458-0.351224$

H -5.649270 -0.582968 -2.743874

C $-1.725056-1.1784122 .956197$

O $-2.116468 \quad 0.0760553 .525926$

C $-1.291962 \quad 1.191731 \quad 3.170444$

C 0.1607020 .9235703 .584352

C $0.680914-0.3968573 .022437$

C $-0.287428-1.5290663 .363202$

H $-1.805850-1.1305551 .863123$

C $-2.701544-2.2483513 .475730$

H -1.645252 2.0335543 .779562

$\begin{array}{lllll}\text { O } & 0.997438 & 1.995745 & 3.169142\end{array}$

$\begin{array}{lllll}\mathrm{H} & 0.213050 & 0.883924 & 4.680387\end{array}$

H $0.857063-0.3264561 .942572$

O $1.950707-0.659715 \quad 3.624102$

H $-0.241843-1.7347974 .440690$

O $\quad 0.107267-2.7163872 .682676$

C $-4.087703-2.1244342 .881352$

H -2.341170 -3.252664 3.227964

H $-2.797217-2.1556194 .564507$

H $1.906832 \quad 1.7763473 .435259$

H $2.315329-1.4575383 .206326$

H $0.951310-3.0082703 .066062$

O $-4.931424-2.9719943 .542073$

O $-4.395261-1.3993481 .946908$
C $-6.284789-2.9761403 .076232$

H $-6.326065-3.3269142 .040709$

H -6.858024 -3.6642793 .703091$

H $-6.720688-1.9763143 .163483$

SCF Energy (B3LYP/6-31G**//MMFF) = -3245.92116730

0500076

MM̄FF Geometry

C $2.693076-2.3864031 .716139$

C $1.832933-2.5229582 .735182$

C $0.736290-1.5294493 .012932$

O $-0.506929-2.267148 \quad 2.947316$

C $0.876974-0.8704164 .396218$

C 2.0955400 .0182634 .524023

C 2.1701401 .2797474 .056498

C $3.268037-0.5874915 .251595$

C $1.1052282 .073774 \quad 3.349670$

C 1.4810782 .3381811 .884547

C 0.6479843 .4360501 .197352

C 1.0443824 .8339701 .683800

C $0.187646 \quad 5.931172 \quad 1.039119$

O $-\begin{array}{llll}0.741355 & 3.193160 & 1.433844\end{array}$

O $0.4269157 .138347 \quad 1.778519$

C $-1.298528 \quad 5.555599 \quad 1.209188$

C $0.626946 \quad 6.216748-0.401558$

\begin{tabular}{llll}
\hline & -2.132164 & 6.493483 & 0.530505
\end{tabular}

C $-1.630044 \quad 4.1077810 .772609$

C $-3.0806703 .762907 \quad 1.199990$

O $-1.5122714 .024985-0.646306$

C -1.626227 -1.564401 2.625657

O $-1.716598-0.3560662 .481962$

C -2.729287 -2.532928 2.436940

C $-3.926612-2.0905112 .024588$

C $-5.102222-2.9113751 .728429$

C $-4.988259-4.3994251 .929565$

$\begin{array}{llll}\text { C } & -3.519217 & 2.322885 & 0.904226\end{array}$

C $-4.0764862 .112661-0.494331$

C $-4.671257 \quad 0.276211-2.080742$

O $-4.484540 \quad 3.031701-1.198853$

C $-6.023431-0.285111-1.771298$

C $-6.356005-1.571333-1.956720$

C $-7.697150-2.147473-1.601755$

C $-7.593954-3.280182-0.601740$

C $-7.640230-4.560366-1.007679$

C -7.520178 -2.934412 0.870400

C $-6.213250-2.302722 \quad 1.262760$

N $-4.0941980 .770141-0.844304$

H $2.591877-1.5255121 .059254$

H $1.910203-3.3841843 .393884$

H $0.727188-0.7628422 .228025$

H $0.891562-1.6441465 .176416$

H $-0.025673-0.2914094 .627752$

H 3.0998931 .8266894 .210256

H $4.1124380 .105540 \quad 5.330067$

H $2.976673-0.8667666 .269611$

H $3.626801-1.4828534 .735830$

H $\quad 0.131323 \quad 1.577558 \quad 3.393216$

H $0.991793 \quad 3.0157093 .896403$

H 2.5459422 .5874571 .793916

H $1.3343461 .402043 \quad 1.329822$

H $0.8403803 .354120 \quad 0.120875$

H $2.1096685 .021731 \quad 1.500061$

H 0.9146144 .9033642 .771389

H -0.1699607 .8216461 .427545$

H $-1.535530 \quad 5.6601292 .276814$

H $1.6741196 .542303-0.421855$

H $0.0511927 .041089-0.836401$

H $0.5373415 .349155-1.058610$

H $-2.103393 \quad 6.282217-0.418231$

H $-3.150810 \quad 3.894682 \quad 2.287997$

H -3.7860804 .4648380 .740507$

H $-1.9307703 .204351-0.947583$

H -2.517173 -3.5820552 .607760$

H $-4.058368-1.0206921 .868810$

H -5.897782 -4.9436741 .666009$

H $-4.773600-4.6294232 .979023$

H $-4.186244-4.8127721 .307718$

H -2.6994911 .6247091 .090214$ 
H -4.3322912 .0596891 .593126$ H -3.988116 $-0.480885-2.478304$ H $-4.7579251 .088234-2.809014$ H -6.759282 $0.400141-1.357041$ H -5.624483 -2.253850 -2.382218 H -8.163391 -2.504876 -2.529261 H -8.374055 -1.378405 -1.209377 H -7.596251 -5.380845 -0.299391 H -7.719360 -4.816677 -2.058996 H -7.724833 -3.8136361 .492280$ H $-8.330860-2.2347651 .112625$ H $-6.200632-1.2190221 .143643$ H $-3.7675690 .080369-0.174251$ C 5.150553-2.675091 1.283002 O $5.099994-1.6650210 .255810$ C $6.081185-1.968169-0.747055$ C $7.139721-2.776321-0.013773$ C $6.268753-3.6436590 .878866$ H $5.589321-2.610211-1.489065$ C $6.558428-0.684030-1.432581$ H $7.770682-3.356613-0.693306$ H $7.786271-2.1388350 .600069$ C $7.010909-4.2428262 .061181$ H $5.857012-4.4622770 .273029$ C $5.4137260 .261221-1.857320$ O $7.3944810 .042615-0.523465$ H $7.190466-0.941650-2.290505$ H $4.9799730 .724313-0.962562$ O $5.9896721 .336118-2.609896$ C $4.292517-0.395943-2.683905$ C $3.1963100 .618750-3.058099$ H $3.850431-1.211551-2.103905$ O $4.859780-0.958646-3.863942$ H $2.8772701 .132502-2.144169$ H $3.6156591 .381144-3.726165$ H $7.820941-4.8941361 .717335$ H $7.450719-3.4652022 .694687$ H $6.334736-4.8404682 .680844$ C $3.784172-3.3622261 .379567$ H $7.6846790 .851199-0.979668$ H $6.3283520 .961804-3.441359$ H $4.179599-1.511346-4.284252$ H $3.825796-4.1719992 .116814$ H $3.528178-3.8092210 .410686$ H $5.387893-2.1612282 .222695$ C $0.864270-0.911640-1.816222$ O $1.484539-1.174448-3.077722$ C $1.971341-0.013542-3.759065$ C $0.8174440 .971684-4.003836$ C $0.077611 \quad 1.316774-2.712073$ C $-0.3219400 .045135-1.965641$ H $1.607202-0.464748-1.143773$ C $0.477143-2.251676-1.178514$ H $2.314819-0.359098-4.741930$ O $1.2913472 .172997-4.603156$ H $\quad 0.0998590 .523222-4.703206$ H $\quad 0.6708751 .978670-2.071037$ O $-1.1163642 .035389-3.032431$ H -1.146558 $-0.435900-2.505420$ O $-0.8003440 .386603-0.668494$ C $-0.611596-2.977565-1.934662$ H $1.340842-2.926660-1.147652$ H $\quad 0.155501-2.098676-0.142427$ H $1.7413741 .928959-5.430004$ H $-0.8511132 .828846-3.528631$ H $-1.5281001 .018468-0.790684$ O $-1.750571-3.019043-1.185140$ O $-0.471339-3.440072-3.059420$ C $-2.865435-3.671781-1.799188$ H -2.633978 -4.725992 -1.978417 H -3.714972 -3.611253 -1.114792 H -3.131967 -3.169687 -2.734141

SCF Energy (B3LYP/6-31G**//MMFF) $=-3245.92885008$

0500077

MM̄MF Geometry

C -2.488960 -2.662020 -2.140327

C $-1.629002-3.327188-2.926744$
C $-0.120090-3.296918-2.800337$

O $0.319889-2.185576-1.985126$

C $0.367241-4.599275-2.139939$

C $1.875499-4.698111-2.016806$

C $2.542613-4.772079-0.848023$

C $2.637782-4.737214-3.316208$

C $1.971916-4.7863520 .542665$

C $1.826778-3.4014801 .191489$

C $3.161961-2.6909421 .466092$

C $2.936087-1.4127952 .275978$

C $4.247378-0.6477182 .512626$

$\begin{array}{llll}\text { O } & 3.766510 & -2.352013 & 0.217674\end{array}$

O 3.9108470 .6722282 .969350

C $4.993236-0.4797501 .168441$

C $5.075554-1.2906263 .631604$

O 6.3012830 .0470531 .370872

C 5.074931-1.774569 0.328950

C $5.588332-1.543484-1.110674$

O $6.002678-2.7082920 .896220$

C $0.386346-0.963100-2.580792$

O $0.148951-0.698814-3.748551$

C $0.7617990 .020658-1.541763$

C $0.5414471 .325299-1.762469$

C $0.8266822 .418576-0.831354$

C 1.6378322 .0954110 .393725

C $4.749810-0.588577-1.967531$

C $5.2759150 .837359-1.997991$

C $4.7206073 .088141-2.913779$

O $6.3480561 .173370-1.502665$

C $4.1469933 .917601-1.809070$

C $3.3460424 .973286-2.019866$

C $2.8045795 .845353-0.923773$

C $1.2990175 .976274-0.971597$

C $0.7150857 .050990-1.528201$

C $0.4755354 .904436-0.295529$

C $0.3626963 .652038-1.120077$

N $4.4168881 .688641-2.680952$

H -2.108024 -2.068802 -1.312558

H -2.022255 -3.935852 -3.738167

H $\quad 0.310351-3.200319-3.804618$

H $\quad 0.014373-5.464233-2.717326$

H $-0.103113-4.682836-1.154198$

H $3.629227-4.842945-0.883471$

H $3.701198-4.953852-3.167498$

H $2.234630-5.517499-3.969988$

H $2.569328-3.775443-3.833706$

H $0.997793-5.2872520 .551873$

H $2.617965-5.413987 \quad 1.169723$

H $1.194798-2.7717680 .554184$

H $1.295257-3.5357662 .141333$

H $3.813622-3.3824832 .012884$

H $2.449408-1.6383413 .232548$

H $2.242484-0.7560361 .734106$

H $3.3634170 .582653 \quad 3.768254$

H $4.4399930 .273190 \quad 0.596302$

H $4.520314-1.2720644 .577412$

H $5.997512-0.7286443 .816184$

H $5.336588-2.331148 \quad 3.427014$

H $\quad 6.205493 \quad 0.878228 \quad 1.866778$

H $6.644183-1.247205-1.091495$

H $5.574195-2.520493-1.615899$

H $6.451433-2.2854991 .645451$

H $1.181110-0.357227-0.616559$

H $0.0812461 .623296-2.703854$

H $2.562506 \quad 1.5811440 .113658$

H 1.0695561 .4510891 .069418

H 1.9441032 .9757370 .962278

H $4.775536-0.944310-3.005672$

H $3.703136-0.585093-1.649258$

H $4.3034343 .352793-3.890547$

H $5.8064903 .223575-2.950111$

H $4.4269663 .656081-0.791289$

H $3.0904955 .249675-3.040354$

H $3.2681406 .835279-1.027518$

H 3.1074505 .4811140 .065786

H $-0.3637507 .165874-1.540138$

H $1.3033857 .837446-1.989302$

H $-0.5464655 .269165-0.124676$ 
H $\quad 0.872380 \quad 4.7226120 .707175$ H $-0.2070643 .788532-2.040298$ H $3.5222001 .335914-3.008629$ C $-4.501618-1.275125-2.660642$ O $-4.129134-0.336125-1.635963$ C $-5.1919250 .618678-1.461576$ C $-6.2323890 .305464-2.533311$ C $-6.022578-1.182846-2.746716$ H $-5.6312060 .416970-0.478486$ C -4.625515 2.044960-1.490148 H $-7.2472440 .552840-2.206502$ H $-6.0415600 .852116-3.463651$ C $-6.604392-1.697846-4.051132$ H -6.479776 -1.723913 -1.907253 C $-3.4100042 .253445-0.562331$ O $-4.1950492 .330233-2.827935$ H $-5.4217512 .762466-1.260261$ H $-2.5511521 .694568-0.953765$ O $-3.0482773 .638973-0.604861$ C -3.6378811 .8654190 .910353$ C -2.3512842 .0218691 .744129$ H $-3.9639840 .823586 \quad 0.975181$ O $\quad-4.6625572 .6767191 .478958$ H -1.585194 $1.363173 \quad 1.319861$ H -1.9744593 .0481601 .653913$ H $-7.686306-1.532429-4.081227$ H -6.162294 -1.191025 -4.915381 H $-6.424551-2.771657-4.162031$ C -3.982722 -2.670316 -2.306504 H -4.046309 $3.288765-2.890196$ H $-2.7029273 .825179-1.493936$ H -5.4963742 .4601101 .029264$ H -4.285042 -3.389582 -3.076086 H -4.419519 -3.006169 -1.359072 H -4.038814 -0.928676 -3.594595 C $-2.565841-0.6910553 .150359$ O C -2.5452601 .7179793 .246684$ C -1.2435561 .7132134 .063567$ C $-0.425856 \quad 0.442184 \quad 3.839224$ C $-1.298717-0.8005264 .002736$ H -2.302062 -0.6904712 .085133$ C -3.513936-1.865965 3.409419 H -3.170665 2.5264893 .646292 O $-0.4383612 .845108 \quad 3.750572$ H $-1.489047 \quad 1.7784135 .131681$ H 0.0589380 .4443762 .857726 $\begin{array}{lllll}\text { O } & 0.627299 & 0.382802 & 4.803563\end{array}$ H -1.561543 -0.928791 5.060553 O $-0.559394-1.9501143 .600363$ C $-4.640701-1.8657102 .403281$ H -3.005475 -2.832390 3.322186 H -3.933719 -1.8107534 .421202$ H -0.9741853 .6394913 .916131$ H 1.1494551 .1982104 .710779 H $0.238952-1.9890124 .154336$ O $-5.808862-1.5041373 .005306$ O $-4.493053-2.1329961 .217879$ C $-6.951893-1.4457702 .146501$ H -7.828683 -1.228089 2.762083 H $-6.829330-0.6429041 .414146$ H -7.105596 -2.406416 1.645474 SCF Energy (B3LYP/6-31G**//MMFF) $=-3245.90674913$

05_00078

MM̄MF Geometry

C -4.328423 -1.963660 0.819652

C $-4.345806-2.0791942 .155553$

C $-3.203206-1.7832403 .095422$

O $-1.957736-1.5970852 .386779$

C $-3.480622-0.4902163 .884945$

C $-2.456670-0.2350584 .975459$

C -1.5439820 .7568274 .974535$

C -2.525553 -1.177272 6.152521

C -1.3098631 .8355053 .952499$

C 0.0697601 .7020083 .300167

C 0.5538042 .9868392 .609759

C -0.3585923 .4354841 .466987$
C 0.1989384 .6705920 .740009

O 1.8697252 .7169272 .102506

O $-\begin{array}{lllll}0.552455 & 4.816832 & -0.474828\end{array}$

C $1.6709694 .403548 \quad 0.359049$

C -0.0389625 .9527101 .545838$

O $2.2734235 .566187-0.203293$

C 2.5234993 .8587001 .526155

C 3.9390283 .4137781 .083590

O 2.7002574 .8920512 .502811

C -1.090870 -2.645217 2.352198

O $-1.298164-3.7904652 .719032$

C $0.202232-2.1265521 .855006$

C $1.330152-2.7962372 .136449$

C $2.694683-2.3551571 .834466$

C $2.855320-1.0475581 .107485$

C $3.9617042 .464957-0.115060$

C $5.2639901 .694740-0.259669$

C $6.3322780 .042079-1.803681$

$\begin{array}{lllll}\text { O } & 6.117134 & 1.633637 & 0.620348\end{array}$

C $5.784489-1.302155-1.436434$

C $6.429173-2.193403-0.669225$

C $5.832592-3.511266-0.258810$

C $5.938758-3.7542131 .231490$

C $6.685382-4.7625151 .712818$

C $5.203262-2.8190292 .165695$

C $3.729076-3.1165252 .250218$

N $5.326121 \quad 1.037548-1.481896$

H -3.428913 -1.615071 0.320671

H -5.262042 -2.420980 2.633596

H -3.120427 -2.629177 3.788134

H $-4.473194-0.5393124 .352550$

H $-3.523190 \quad 0.3525013 .187062$

H $-0.875760 \quad 0.8251315 .833480$

H -1.861648 -0.8732826 .969051$

H -3.543070 -1.207408 6.555975

H $-2.236322-2.1898575 .856120$

H $-2.081275 \quad 1.8452573 .179086$

H -1.3804432 .7987064 .472761$

H $\quad 0.8206541 .4174854 .050064$

H $\quad 0.058780 \quad 0.8777492 .577277$

H $\quad 0.6245603 .769292 \quad 3.374374$

H -1.375311 3.6401161 .822039

H -0.4526402 .6160520 .741763$

H $-0.1576445 .548792-0.979313$

H $1.6429283 .658493-0.445771$

H $-1.1122486 .101761 \quad 1.714928$

H $0.300324 \quad 6.836876 \quad 0.994951$

H 0.4523105 .9446532 .520944

H 2.4801316 .1747860 .526393

H 4.5580114 .2920150 .857883

H 4.4217802 .9279651 .942449

H 3.4780204 .6729543 .042697

H $0.196114-1.1757411 .335285$

H $1.253872-3.7349602 .683765$

H $2.435230-0.2240211 .694660$

H $2.348152-1.0847480 .137844$

H $3.894562-0.7900450 .901530$

H $3.162426 \quad 1.722231-0.019169$

H $3.7921653 .034714-1.035966$

H $7.2612210 .272411-1.272699$

H $6.5114820 .093033-2.881599$

H $4.791416-1.547020-1.805896$

H $7.429924-1.963658-0.310935$

H $6.356792-4.300612-0.812374$

H $4.778750-3.579288-0.556162$

H $6.773620-4.9414802 .779414$

H $7.224686-5.4335311 .052429$

H $5.412013-1.7803221 .896256$

H $5.607336-2.9175473 .182848$

H $3.507952-4.0507812 .768438$

H $4.5441821 .124679-2.123464$

C $-6.077862-1.100351-0.812699$

O $-5.065635-0.504414-1.649822$

C $-5.0392180 .908164-1.383540$

C $-6.4083151 .234154-0.801971$

C -6.6395010 .0170120 .072610$

H $-4.2830161 .067927-0.604910$

C $-4.657946 \quad 1.699687-2.634925$ 
H $-6.4121642 .173534-0.241465$

H -7.178040 $1.292464-1.580288$ C $-8.091301-0.1874840 .469111$ H $-6.042177 \quad 0.1333130 .985105$ C $-3.3492181 .246103-3.313972$ O $\quad-5.7106681 .574014-3.599164$ H -4.603122 $2.765512-2.381111$ H $-3.521797 \quad 0.312972-3.864726$ O $-3.045308 \quad 2.244819-4.297111$ C $-2.1508981 .070284-2.361026$ C $-0.8088320 .891852-3.093674$ H $-2.3332090 .203060-1.717483$ O $-2.0478362 .210769-1.507211$ H $-0.9398670 .177501-3.911958$ H $-0.5217591 .852590-3.536895$ H -8.4543610 .6650321 .052198$ H -8.736914 -0.293004 -0.409122 H -8.202912 -1.088544 1.080667 C $-5.498193-2.303758-0.064088$ H -5.455233 $2.099689-4.376701$ H $-2.3929701 .867123-4.910934$ H -1.927522 $2.994044-2.070912$ H -5.142790 -3.038049 -0.797515 H $-6.293077-2.7907520 .513050$ H $-6.855687-1.464113-1.496360$ C $0.286833-1.942678-2.649668$ O $0.107315-0.910789-1.670653$ C $0.3155690 .426679-2.140773$

C $1.7266790 .558522-2.720922$

C $1.988557-0.467783-3.815571$

C $1.679022-1.869578-3.295102$ H $-0.486957-1.857111-3.421407$ C $0.108520-3.293699-1.937732$ H $0.2778721 .072198-1.254482$ O $1.9372331 .876391-3.214126$ H $2.4492690 .397500-1.913287$ H $1.416824-0.235587-4.721706$ O $3.369861-0.389554-4.173628$ H $2.444837-2.166226-2.566767$ O $1.735081-2.796685-4.377667$ C -1.329388-3.574139-1.559654 H $0.428929-4.113491-2.590294$ H $\quad 0.705730-3.308812-1.019098$ H $2.8305831 .905514-3.597061$ H $3.510001-0.977852-4.934581$ H $2.655288-2.831800-4.688433$ O $-1.375737-4.756172-0.875969$ O $-2.285333-2.864742-1.837921$ C -2.674907 -5.204274-0.477067 H -2.600060 -6.260860 -0.206272 H -3.003482 -4.6410790 .399395$ H -3.396421 -5.106175 -1.293944 SCF Energy (B3LYP/6-31G**//MMFF) $=-3245.92969954$

05_00079

MM̄MF Geometry

C -0.002896 -3.218479 1.475291

C $-0.963019-3.0295912 .393393$

C $-1.409271-1.6867142 .928848$

O $-0.447906-0.6790542 .533952$

C $-2.788926-1.2819212 .378616$

C $-3.932773-2.1520412 .852196$

C $-4.555480-3.0812192 .100425$

C $-4.392884-1.8897524 .262851$

C $-4.237522-3.4924710 .689437$

C $-5.373227-3.178411-0.294314$

C $-5.631617-1.675438-0.481770$

C $-6.782290-1.444922-1.460496$

C $-7.0067730 .049956-1.724960$

O $-4.440114-1.077546-0.997552$

O $\quad-7.869148 \quad 0.170056-2.866880$

C $-5.6608710 .708793-2.102776$

C $-7.7343270 .731171-0.558606$

O $\quad-5.8252902 .119655-2.205222$

C $-4.5007910 .355350-1.138607$

C $-3.1156190 .762471-1.705582$

$\begin{array}{llll}\text { O } & -4.708948 & 0.982323 & 0.124010\end{array}$

C $-0.299386 \quad 0.421915 \quad 3.314613$
O $\quad \begin{array}{llll}0.926566 & 0.706113 & 4.321121\end{array}$

C $0.787124 \quad 1.2276662 .712094$

C 1.0868242 .4392183 .202881

C 2.1204113 .3405212 .684140

C 2.9842752 .8277291 .560290

C $-2.7523512 .250290-1.690339$

C $-2.2437292 .745610-0.346782$

C $-1.6717154 .850316 \quad 0.857414$

O -1.9271921 .9927670 .570555$

C -0.2175765 .1486540 .674126$

C $0.337276 \quad 6.3326230 .973190$

C 1.8030146 .6347950 .833129

C 2.4738516 .8560422 .172468

C 2.5029078 .0754422 .736161

C 3.1753875 .6796922 .819044

C 2.2263204 .5787573 .211706

N -2.160450 $4.130851-0.304049$

H $0.504486-2.3586731 .045581$

H -1.471515 -3.899891 2.802438

H -1.420376-1.751056 4.023803

H $-2.741796-1.2340401 .284577$

H -3.005858 -0.2444962 .667395$

H -5.380375 -3.633732 2.548834

H $-5.254061-2.5065134 .541529$

H -3.590789 -2.104393 4.975639

H $-4.688891-0.8417824 .376625$

H -3.302699 -3.055094 0.330550

H -4.072864 -4.577344 0.691681

H -5.099195 -3.619322 -1.261727

H $-6.294371-3.6740580 .036861$

H $-5.869915-1.2331890 .491642$

H -7.704871 -1.913965 -1.096529

H $-6.553370-1.935410-2.417187$

H -8.697721 $-0.298483-2.667155$

H $-5.3911540 .352835-3.106832$

H $-8.7024390 .248790-0.377448$

H -7.961672 $1.777462-0.790832$

H -7.1698990 .7000020 .375692$

H $-6.5433902 .283570-2.840218$

H $-2.3424930 .206520-1.157352$

H $-3.0445170 .393193-2.737493$

H $-3.8741460 .942148 \quad 0.619097$

H 1.3078550 .7987591 .862711

H 0.5128102 .8164844 .048054

H 3.7142423 .5531271 .197436

H 2.3681492 .5541980 .697392

H 3.5480691 .9450041 .881991

H -1.931404 2.408913 -2.401401

H -3.583648 $2.875603-2.021276$

H -1.8126894 .2357141 .752388$

H -2.2715015 .7606110 .954007$

H $\quad 0.415727 \quad 4.3442950 .309640$

H $-0.2941837 .134176 \quad 1.350126$

H $1.905813 \quad 7.5337170 .211233$

H 2.3249085 .8416560 .283016

H 3.0031628 .2503763 .682814

H 2.0231308 .9259292 .262950

H 3.6951475 .9995263 .731940

H 3.9683215 .3369922 .149046

H 1.5483804 .8581584 .019415

$\mathrm{H}-2.3741134 .668072-1.139390$

C $1.897036-4.8377811 .262812$

O $2.728741-3.8171430 .678340$

C $3.900743-4.4288910 .112129$

C $3.887790-5.8825470 .574256$

C $2.400138-6.1573150 .679923$

H $3.770280-4.402737-0.976268$

C $5.155059-3.6385450 .501782$

H $4.398664-6.544901-0.131284$

H $4.364676-6.0054211 .553275$

C $2.064727-7.3756191 .522171$

H $1.998670-6.298483-0.332577$

C $5.076217-2.1263640 .206273$

O $5.366630-3.7850001 .912608$

H $6.030406-4.0820780 .012315$

H $4.357143-1.6560660 .887817$

O $6.348641-1.5533860 .537276$

C $4.710970-1.759002-1.244406$ 
C $4.796658-0.242107-1.500826$

H $3.694412-2.102705-1.453648$

O $5.575199-2.444332-2.149497$

H $4.1974110 .275289-0.743933$

H $5.8354570 .084100-1.371222$

H $2.512152-8.2767341 .090189$

H $2.440073-7.2716122 .545880$

H $0.982299-7.5283651 .574419$

C $0.426544-4.557897 \quad 0.947044$

H $6.166811-3.2809512 .139757$

H $7.023063-1.983462-0.014969$

H $6.472898-2.089782-2.034543$

H $-0.203938-5.3564371 .353990$

H $0.276708-4.545604-0.139101$

H $2.064797-4.802543 \quad 2.347449$

C $1.980377 \quad 0.470700-2.480244$

O $2.993249-0.243539-3.198383$

C $4.3381870 .159056-2.921219$

C $4.513577 \quad 1.651556-3.236563$

C $3.4932432 .508527-2.494230$

C $2.0805891 .981432-2.741325$

H $2.0743830 .266727-1.406583$

C $0.618518-0.057565-2.960016$

H $4.972095-0.391957-3.627034$

O $5.826512 \quad 2.091993-2.905342$

H $4.3831381 .812801-4.314798$

H $3.7259792 .556771-1.424825$

O $3.5887383 .846415-2.988604$

H $1.7868972 .202731-3.775703$

O $1.1650872 .658630-1.882660$

C $0.301448-1.444653-2.444722$

H $-0.1822860 .600943-2.609288$

H $0.599450-0.100781-4.055298$

H $6.4536561 .543831-3.407303$

H $3.0251564 .406767-2.428992$

H $1.1578623 .596229-2.138483$

O $-0.950190-1.791491-2.870775$

O $1.042841-2.135295-1.761256$

C $-1.418096-3.073014-2.440885$

H -2.411264 -3.233667 -2.868803

H $-0.749229-3.862568-2.795932$

H $-1.499643-3.095145-1.351156$

SCF Energy (B3LYP/6-31G**//MMFF) $=-3245.91696060$

05_00080

MM̄FF Geometry

C 3.7448784 .2293501 .841752

C 3.1580743 .3851542 .704594

C 3.6511022 .0294873 .142368

O $2.693974 \quad 1.0656952 .634054$

C 5.0370461 .6087922 .611529

C 5.4852290 .2512813 .122927

C $5.668404-0.8409262 .354224$

C 5.7510970 .1704884 .605410

C $5.525887-0.9747040 .862879$

C $4.189710-1.6098950 .474107$

C $4.135903-2.091707-0.984115$

C $4.292942-0.951571-1.992549$

C $4.109126-1.426920-3.443122$

O $2.862868-2.731019-1.161907$

O $3.952251-0.244911-4.243695$

C $2.807391-2.252055-3.543716$

C $5.362059-2.141899-3.963004$

O $2.671205-2.852081-4.829147$

C $2.685742-3.336821-2.451761$

C $1.309338-4.045424-2.454805$

O $3.671514-4.352110-2.678708$

C $1.843308 \quad 0.463458 \quad 3.505842$

$\begin{array}{lllll}\text { O } & 1.726509 & 0.681056 & 4.700278\end{array}$

C $1.094138-0.5566252 .738462$

C $0.357708-1.477758 \quad 3.377421$

C $-0.368915-2.581748 \quad 2.743547$

C $-0.204268-2.7470301 .256941$

C $0.101596-3.108986-2.400981$

C $-1.190111-3.824728-2.038449$

C $-3.575404-3.311556-1.486602$

O $-1.261667-5.034283-1.841952$

C $-3.677386-2.963504-0.034716$
C $-4.052930-3.8231290 .924120$

C $-4.125878-3.4529112 .379499$

C $-3.399800-4.4343653 .275584$

C $-4.068452-5.1376104 .205325$

C -1.906972 -4.6127413 .096297$

C $-1.120267-3.3978203 .512784$

N -2.253144 -2.935756 -1.952738

H 4.7009663 .9811621 .391632

H $2.1908413 .670368 \quad 3.117054$

H 3.6649602 .0268054 .238477

H 5.7917172 .3502222 .903897

H 5.0093371 .6094241 .517280

H $5.978775-1.7624932 .846431$

H $6.212525-0.7803344 .893375$

H 6.4346200 .9689394 .912487

H $4.8209290 .267944 \quad 5.172971$

H $5.651596-0.0118090 .360548$

H $6.351534-1.6048520 .508608$

H $3.973557-2.4661321 .127603$

H $3.375787-0.8965740 .650837$

H $4.927507-2.837739-1.121565$

H $5.265323-0.456730-1.885395$

H $3.541839-0.178919-1.781184$

H $3.891582-0.516769-5.174929$

H $1.977045-1.542213-3.438094$

H $6.219870-1.458611-3.957601$

H $5.236183-2.456041-5.004921$

H $5.633009-3.018076-3.370244$

H $2.649144-2.137966-5.487893$

H $1.223036-4.697137-3.334126$

H $1.275499-4.718596-1.586764$

H $3.387780-5.162647-2.224037$

H $1.199109-0.5423031 .659451$

H $0.293501-1.4342084 .463554$

H $0.853058-2.8699660 .997119$

H $-0.598079-1.8718990 .728153$

H $-0.721273-3.6184710 .855200$

H $0.268424-2.320876-1.658719$

H $-0.043702-2.638090-3.379716$

H -3.745241 -4.376666 -1.672096

H $-4.301668-2.728982-2.061235$

H -3.422546 $-1.943608 \quad 0.241363$

H $-4.329898-4.8389050 .651828$

H -5.187327 -3.4002592 .653870$

H -3.720556 -2.449329 2.551353

H $-3.561518-5.8454734 .852760$

H $-5.138670-5.021874 \quad 4.342007$

H $-1.702433-4.9229992 .068729$

H -1.551135 -5.4501423 .711798$

H $-1.170878-3.1963844 .583832$

H $-2.072257-1.944372-2.078759$

C $2.250775 \quad 5.356792 \quad 0.124297$

$\begin{array}{llllll}\text { O } & 1.168335 & 4.440365 & 0.396158\end{array}$

C $1.2365183 .364143-0.560710$

C $1.9064383 .980129-1.775781$

C $2.9805684 .826275-1.115614$

H $1.8785602 .595809-0.110588$

C $-0.1690812 .805839-0.789321$

H $2.317773 \quad 3.235138-2.462784$

H $1.2020564 .613439-2.329110$

C $3.5300065 .923315-2.011658$

H $3.8095424 .166808-0.832777$

C $-0.247777 \quad 1.717743-1.873858$

O $-0.620363 \quad 2.246240 \quad 0.448922$

H $-0.8503323 .629552-1.028683$

H $-0.0006112 .136580-2.856720$

O $0.7295640 .703432-1.600151$

C $-1.6032360 .990333-1.955988$

C $-2.7975801 .915028-2.233218$

H $-1.7679890 .398229-1.048890$

O $-1.5323660 .059083-3.044813$

H $-2.7999962 .749490-1.529007$

H -2.681651 2.337320 -3.238693

H $4.0026575 .493378-2.900793$

H $2.7388976 .602231-2.347338$

H $4.2816536 .516177-1.480705$

C $3.1075905 .516293 \quad 1.388567$

H -0.5589822 .9442001 .123629$ 
H $1.6052761 .077325-1.792376$ H $-0.700544-0.434670-2.948587$ H 2.4757305 .9055462 .197381 H 3.8946956 .2598911 .218382 H $1.7790676 .322535-0.095843$ C -4.7031801 .6583640 .154177$ O $-4.3999220 .661010-0.830253$ C $-4.1455631 .159976-2.150254$ C $-5.3530471 .963318-2.651567$ C $-5.7394713 .068920-1.676741$ C $-5.9162022 .497374-0.272245$ H -3.8338482 .3108150 .299439$ C $-4.985406 \quad 0.933151 \quad 1.480659$ H $-4.0634190 .278089-2.797677$ O $\quad-5.0937372 .535157-3.929108$ H $-6.2125581 .290580-2.770096$ H $-5.0073703 .885072-1.680990$ O $-6.9837753 .642295-2.084686$ H $-6.827728 \quad 1.886761-0.237349$ O C -3.7109450 .5732902 .214654$ H -5.5689591 .5837952 .142310$ H -5.5458630 .0060251 .316030$ H $-4.8623111 .808500-4.532732$ H $-6.8683333 .964039-2.995345$ H -6.8482104 .0982130 .342783$ O $-3.9506740 .586168 \quad 3.558985$ O $-2.649555 \quad 0.288731 \quad 1.677805$ C $-2.834404 \quad 0.2766874 .398838$ H $-3.0884850 .567845 \quad 5.421567$ H $-2.648752 \quad-0.799703 \quad 4.377033$ H $-1.943087 \quad 0.8322894 .091314$ SCF Energy $\left(B 3 L Y P / 6-31 G^{* *} / / M M F F\right)=-3245.92107807$

05_00081

MM̄FF Geometry

C -2.9379792 .1874962 .182077$

C -2.0900522 .6075151 .232580$

C - -1.4522823 .9700381 .232586$

O $-0.020696 \quad 3.7457881 .219752$

C $-1.8270954 .797089-0.009877$

C $-3.2869695 .196576-0.073905$

C $-4.2069154 .635471-0.883821$

C $-3.678234 \quad 6.331826 \quad 0.837013$

C $-4.0087083 .496847-1.843746$

C $-4.8950112 .298129-1.475908$

C $-4.4870691 .039438-2.252667$

C $-5.442788-0.116970-1.965839$

C $-4.986395-1.412938-2.653146$

O $-3.1692810 .683798-1.826555$

O $-5.740638-2.497339-2.089099$

C $-3.500721-1.670884-2.317522$

C $-5.299789-1.401400-4.154543$

O $-3.026910-2.782622-3.075442$

C $-2.593081-0.430453-2.517874$

C $-1.196368-0.682147-1.894950$

O $-2.458143-0.171826-3.913615$

C 0.7674354 .6845391 .801562

O $\quad 0.393183 \quad 5.7218342 .332720$

C 2.2006444 .3129201 .750033

C 2.6900373 .2637481 .070101

C 4.1023182 .8754200 .991582

C 5.0930593 .6731241 .800131

C $-0.2665800 .539853-1.842644$

C $0.6087040 .722204-3.069656$

C $2.5092492 .121441-3.879647$

O $0.5464430 .000033-4.059915$

C $3.7779411 .460679-3.440982$

C $4.8776752 .131911-3.068358$

C $6.1379071 .463559-2.600679$

C $6.5454711 .894064-1.208114$

C $7.5731802 .740908-1.027182$

C $5.8304431 .275824-0.025698$

C 4.4518541 .8393760 .199958

N $1.4727991 .798708-2.917199$

H -3.204622 2.8612072 .993180

H -1.820107 1.9311330 .427358

H -1.7240944 .5086602 .149140$
H -1.504786 $4.266720-0.912985$

H -1.219993 $5.712737-0.029296$

H $-5.2237555 .024990-0.854037$

H -4.7282216 .6191880 .716963$

H -3.532262 6.0520351 .884926

H -3.069304 7.2166990 .624346

H -4.267519 $3.841404-2.852234$

H -2.962564 $3.186932-1.888462$

H $-4.8099562 .100408-0.400129$

H $-5.9440382 .544321-1.681678$

H $-4.4749561 .277310-3.322884$

H $-6.4664200 .138661-2.266602$

H -5.484683 -0.296552 -0.882115

H $-6.684257-2.315821-2.238738$

H -3.460359-1.970345 -1.260775

H $-6.375808-1.268891-4.320001$

H $-5.042537-2.359548-4.619659$

H -4.782433 -0.605353 -4.694095

H -2.163000 -3.043946 -2.717649

H -1.347339 $-0.996803-0.853891$

H $-0.690888-1.500121-2.417977$

H -1.677287 $0.385935-4.053894$

H 2.8506924 .9808912 .306806

H 2.0045862 .6307760 .509446

H 4.8389813 .6357322 .865310

H 5.1010224 .7195341 .475291

H 6.1194373 .3114081 .712181

H $-0.8257841 .465233-1.675261$

H $\quad 0.4147750 .406667-0.991598$

H $2.5981893 .211773-3.910917$

H $2.2235541 .763082-4.873462$

H $3.7862900 .373589-3.421269$

H $4.8748343 .219122-3.097321$

H $6.9301001 .707998-3.320446$

H $6.0450170 .371293-2.625931$

H $7.8998873 .031764-0.034590$

H $8.1108833 .165240-1.868635$

H $\quad 6.434863 \quad 1.3614880 .884792$

H $5.7429790 .194789-0.187019$

H $3.6683581 .318772-0.350684$

H $1.4872172 .294945-2.030518$

C -2.9949260 .0254093 .404324$

O $-1.662329-0.4288283 .091145$

C -1.444331-1.695554 3.747870

C $-2.711921-1.9921034 .545037$

C $-3.763726-1.2462043 .744377$

H -1.351816 -2.449615 2.959985

C $-0.162574-1.6306884 .587771$

H -2.910121 -3.065847 4.619221

H -2.649739 -1.595027 5.565329

C $-5.052168-1.0127734 .513121$

H -3.980946 -1.811867 2.828351

C $1.109377-1.3720953 .749555$

O $-0.304902-0.5621025 .532040$

H $-0.052667-2.5513535 .172535$

H $1.010510-0.4264463 .204203$

O $2.210486-1.1941574 .649691$

C $1.446916-2.5115352 .767579$

C $2.710895-2.2323341 .933495$

H $0.612456-2.6508812 .078553$

O $1.595347-3.7327693 .490489$

H $2.508092-1.3761821 .279637$

H $3.531186-1.9356822 .598063$

H $-5.504085-1.9667574 .803795$

H $-4.876144-0.4322945 .425120$

H $-5.779632-0.4684793 .903177$

C $-3.5528320 .815173 \quad 2.222124$

H $0.520590-0.5154356 .044038$

H $2.264164-1.9826855 .215659$

H $2.410412-3.6707994 .016612$

H -4.6371690 .9384842 .314157$

$\begin{array}{llll}H & -3.358887 & 0.280565 & 1.284047\end{array}$

H -2.9176860 .6662894 .293767$

C $1.648803-3.333221-0.754051$

O $2.132234-4.0725820 .371993$

C $3.188007-3.4490831 .107271$

C $4.384455-3.1591190 .190229$

C $3.970633-2.368205-1.048203$ 
C $2.789257-3.045816-1.738580$

H $1.199532-2.392330-0.413360$

C $0.564596-4.190841-1.429305$

H $3.527163-4.1980921 .834378$

O $5.390902-2.4599120 .913881$

H $4.821901-4.113689-0.130772$

H $3.735152-1.325969-0.804708$

O $5.057676-2.333366-1.975577$

H $3.127846-3.979588-2.205883$

O $2.308445-2.198941-2.778754$

C $-0.715153-4.272279-0.624065$

H $\quad 0.297577-3.780074-2.408700$

H $0.941082-5.212991-1.559218$

H $6.179993-2.4081140 .349038$

H $5.778332-1.826607-1.565636$

H $3.052026-2.027980-3.381922$

O $-1.509605-5.249460-1.154428$

O $-1.002123-3.5589050 .326073$

C -2.771663 -5.435299 -0.505578

H $-2.620078-5.7503930 .531144$

H $-3.361154-4.514758-0.547072$

H $-3.314025-6.222356-1.036322$

SCF Energy (B3LYP/6-31G**//MMFF)= -3245.91219429

05 00082

MM̄FF Geometry

C 1.7780825 .5741320 .475115

C 2.5947264 .5241490 .302739

C 3.2686553 .7917081 .429755

O 2.7573862 .4393301 .433400

C 4.7886563 .7156931 .193690

C 5.5243112 .9794742 .297908

C 6.1612351 .8012382 .144743

C 5.5263793 .6598423 .643477

C 6.3287360 .9874010 .890857

C $5.263687-0.1037260 .773092$

C $5.570539-1.157285-0.302546$

C $5.643512-0.564399-1.711982$

C $5.833494-1.649229-2.785239$

O $4.518477-2.132187-0.235173$

O $5.566194-1.054402-4.064962$

C $4.777073-2.753260-2.572350$

C $7.279880-2.155121-2.837604$

O $4.998342-3.853338-3.451022$

C $4.696573-3.253489-1.114411$

C $3.517158-4.227169-0.879097$

O $5.895161-3.969182-0.791789$

C 1.7419792 .1405582 .287063

O 1.1587852 .9015813 .041308

C 1.4817930 .6895972 .157357

C 0.6868670 .0605063 .035817

C $0.391995-1.3757183 .043127$

C $1.032073-2.2127431 .967850$

C $2.144237-3.693650-1.293776$

C $1.000461-4.533894-0.747333$

C $-1.498524-4.547277-0.647858$

O $1.166880-5.541518-0.066158$

C $-2.003546-3.6831720 .464298$

C -2.455461 -4.1450221 .639807$

C -2.973101 -3.241892 2.723972

C $-2.334672-3.4980494 .072109$

C -3.080634 -3.887648 5.119751

C $-0.840043-3.3016774 .216773$

C $-0.418872-1.8721574 .001111$

N $-0.231857-4.010336-1.112391$

H 1.5869625 .9511211 .476466

H $2.7572184 .138625-0.701607$

H 3.0654644 .2828312 .389058

H 5.2054834 .7281281 .112358

H 4.9717263 .2351310 .226505

H 6.6392061 .3621023 .019919

H 6.1858693 .1594774 .360840

H $5.8765654 .692743 \quad 3.545963$

H 4.5209723 .6699384 .074882

H 6.3260361 .6264330 .003146

H 7.3254460 .5296150 .923541

H $5.143477-0.6192541 .735860$

H 4.2882640 .3511710 .564018
H $\quad 6.518089-1.642120-0.039531$

H $6.4456630 .178437-1.790612$

H $4.711083-0.023432-1.925834$

H $6.176538-0.305935-4.180822$

H $3.809861-2.318552-2.854866$

H $7.964760-1.332087-3.074891$

H $7.411482-2.893119-3.636590$

H $7.614124-2.599217-1.897876$

H $5.037148-3.498454-4.355733$

H $3.707597-5.179968-1.390478$

H $3.490983-4.4708020 .192444$

H $5.710748-4.553818-0.037869$

H 1.9787560 .1684251 .347132

H $0.222903 \quad 0.641723 \quad 3.831195$

H $2.124486-2.1481932 .024816$

H $0.709373-1.8669410 .980161$

H $\quad 0.782039-3.2725622 .026646$

H $2.012357-2.667636-0.933492$

H $2.059736-3.688426-2.386300$

H -1.372219-5.589545 -0.338579

H $-2.193741-4.508670-1.491800$

H -2.008718 -2.6100270 .288881$

H -2.479201 -5.216401 1.822485

H $-4.059454-3.3870672 .783023$

H -2.824531 -2.189059 2.457279

H $-2.637807-4.0727386 .092712$

H -4.152546 -4.029696 5.030025

H $-0.325495-4.0046253 .557485$

H $-0.518108-3.5801255 .229239$

H $-0.825118-1.1831194 .742607$

H $-0.258735-3.142859-1.641189$

C $-0.4127345 .808816-0.696111$

O $-0.4740964 .396494-0.980110$

C -1.441292 4.161387-2.014756

C $-2.2759815 .433384-2.084379$

C $-1.2253526 .489956-1.796381$

H $-0.8812734 .052761-2.952937$

C $-2.2302702 .869221-1.755095$

H -2.759738 $5.561464-3.057327$

H $-3.0522255 .454642-1.311789$

C $-1.8055827 .833521-1.391025$

H $-0.6068986 .623633-2.694105$

C $-1.3527501 .599579-1.805295$

O $-2.8650362 .927614-0.478496$

H $-3.0157342 .789101-2.514823$

H $-0.9021981 .509565-2.800891$

O $-0.2577941 .722800-0.888807$

C $-2.0825120 .288125-1.453940$

C $-3.3199270 .013696-2.322106$

H $-2.3476710 .278467-0.391882$

O $-1.159457-0.789865-1.653138$

H $-4.0160420 .852940-2.247779$

H $-3.003903-0.056368-3.370156$

H -2.423535 $8.244182-2.196194$

H -2.432455 $7.747002-0.497153$

H $-1.0089998 .552694-1.175632$

C $1.0521876 .246507-0.657317$

H -3.597891 $3.562519-0.537136$

H $-0.6313121 .909642-0.010221$

H $-0.384055-0.613153-1.092901$

H $1.1279257 .331721-0.527260$

H $1.5398345 .999077-1.608772$

H -0.8848455 .9564910 .283923$

C $-5.506381-0.397397-0.221307$

O $-4.444312-1.300075-0.554408$

C $-4.047731-1.293550-1.931111$

C $-5.245327-1.641721-2.824936$

C $-6.440603-0.733526-2.553556$

C $-6.756344-0.702722-1.058748$

H $-5.1825800 .635330-0.396578$

C $-5.804982-0.565996 \quad 1.277157$

H -3.322192 -2.107926 -2.045722

O $-4.872820-1.564526-4.196766$

H $-5.540844-2.681302-2.631167$

H $-6.267526 \quad 0.273835-2.949967$

O $-7.569385-1.253912-3.259413$

H $-7.179415-1.671459-0.764427$

O $\quad-7.7613370 .280123-0.816035$ 
C $-4.740155 \quad 0.050878 \quad 2.159777$

H $-6.750649-0.0794191 .539564$

H $-5.865375-1.6334561 .520903$

H -5.662354 -1.774579-4.724463

H -8.332264 -0.691951-3.039505

H -7.396317 $1.147522-1.061259$

O $-4.976507-0.3141843 .455333$

O $\begin{array}{llll}-3.832676 & 0.774662 & 1.775572\end{array}$

C $-4.058999 \quad 0.208692 \quad 4.421459$

H -4.250728 -0.2881895 .376151$

H -3.025691 0.0079704 .124902

H -4.2223101 .2834094 .542749$

SCF Energy (B3LYP/6-31G**//MMFF) $=-3245.92148019$

05_00083

MMFF Geometry

C $4.018387-2.1662882 .599134$

C $2.710026-2.1638062 .306451$

C $1.642490-1.5636993 .182276$

O $1.134615-0.4224472 .453907$

C $0.510867-2.5878333 .399321$

C $-0.542039-2.1930684 .418914$

C $-1.870973-2.3345834 .239021$

C $-0.034929-1.6862445 .745690$

C -2.602152 -2.923971 3.064281

C $-3.395747-1.8533222 .318455$

C $-4.323534-2.4092111 .225563$

C $-3.555144-3.1212750 .110191$

C $-4.445203-3.486453-1.085913$

O $-5.030737-1.276793 \quad 0.708594$

O $-3.585952-3.813107-2.190168$

C $-5.259315-2.244089-1.503397$

C $-5.284770-4.738285-0.806394$

O $-6.198182-2.572904-2.524825$

C $-5.980893-1.562232-0.320579$

C $-6.666962-0.232666-0.733968$

O $-7.012372-2.434096 \quad 0.157174$

C 1.3514320 .8111922 .984513

O $1.746801 \quad 1.073272 \quad 4.109499$

C 1.0890491 .8130471 .926113

C 1.0398993 .1144712 .249909

C $0.882714 \quad 4.235432 \quad 1.319716$

C $1.0662693 .945777-0.145799$

C $-5.7276110 .919122-1.108773$

C -5.0857491 .6009950 .090783$

C -3.1263112 .9950520 .708847$

O $-5.5188561 .493994 \quad 1.235307$

C $-2.548413 \quad 4.2493110 .139105$

C $-2.607490 \quad 5.4374840 .759085$

C -2.001182 $6.697551 \quad 0.212161$

C -0.9682327 .2964231 .142242$

C $-1.277814 \quad 8.3275831 .946398$

C $0.444638 \quad 6.758268 \quad 1.072456$

C $0.612791 \quad 5.460218 \quad 1.818715$

N $-3.9957252 .378456-0.275584$

H $4.363605-1.7226463 .529263$

H $2.380112-2.597348 \quad 1.367448$

H $2.081146-1.2678834 .141772$

H $0.945869-3.537502 \quad 3.739327$

H $0.044554-2.7821392 .427252$

$\mathrm{H}-2.529416-2.0273605 .051662$

H $-0.824670-1.6422586 .503644$

H $0.745353-2.3498606 .133012$

H $0.376411-0.6784775 .645332$

H -1.923103 -3.4423162 .383758$

H $-3.289342-3.6887603 .447030$

H -4.008525 -1.281282 3.029296

H -2.708711 -1.117694 1.878528

H -5.044416 -3.0913811 .691702$

H $-3.050396-4.0184450 .487271$

H $-2.757693-2.463291-0.250577$

H -3.003889 -4.541091-1.912331

H $-4.551925-1.534589-1.949940$

H -4.634344 -5.601793 -0.621269

H -5.897308 $-5.006311-1.674069$

H $-5.939135-4.630560 \quad 0.061044$

H -5.703871 -2.996458 -3.247466

H $-7.346600-0.428026-1.573273$
H -7.3166350 .1023720 .085884$

H -7.648274 -1.9085320 .670038$

H 0.9822601 .4543190 .908674

H $1.1293013 .393898 \quad 3.299165$

H $0.9497574 .821240-0.787003$

H $0.3289413 .214512-0.486110$

H $2.0686353 .545406-0.332524$

H $-4.9552520 .584736-1.806594$

H $-6.3145201 .691607-1.621697$

H $-2.324967 \quad 2.2809270 .924902$

H $-3.680833 \quad 3.1738581 .635853$

H $-2.0316494 .171857-0.814517$

H -3.122686 5.5138491 .714098

H $-2.815836 \quad 7.413862 \quad 0.042981$

H $-1.5485106 .529967-0.773156$

H -0.5357058 .7818662 .594401$

H $-2.282356 \quad 8.7366101 .978565$

H $1.1423287 .478784 \quad 1.520548$

H $\quad 0.751950 \quad 6.693748 \quad 0.025221$

H 0.5052855 .5651952 .899419

H $-3.6449342 .297321-1.225860$

C $5.950635-1.6564591 .084026$

O $5.172204-0.8364350 .187286$

C $5.939256-0.590140-1.006268$

C $7.357729-1.059014-0.702134$

C $7.096444-2.2157260 .241041$

H $5.517957-1.234301-1.786932$

C $5.8324450 .879708-1.427350$

H $7.899088-1.354205-1.606051$

H $7.944431-0.283286-0.196701$

C $8.315194-2.6287761 .047757$

H $6.746421-3.074955-0.347101$

C $4.3899921 .387027-1.622744$

$\begin{array}{llll}\text { O } & 6.437470 & 1.686820 & -0.407819\end{array}$

H $6.4219961 .043357-2.337274$

H $3.8858611 .431942-0.649818$

$\begin{array}{llll}\text { O } & 4.469893 & 2.743248 & -2.082632\end{array}$

C $3.5274890 .573565-2.607146$

C $2.139826 \quad 1.208189-2.806019$

H $3.407837-0.446198-2.233886$

$\begin{array}{lllll}\text { O } & 4.167620 & 0.501373-3.879684\end{array}$

H $1.7014141 .397518-1.821306$

H $2.2552862 .179068-3.303043$

H $9.123191-2.9540720 .384170$

H $8.693297-1.8004531 .656348$

H $8.076201-3.4587141 .719915$

C $5.064459-2.7465361 .688664$

H $6.3597432 .614873-0.688571$

H $4.8579142 .727484-2.974449$

H $4.963577-0.047395-3.782180$

H $5.669396-3.4508232 .270737$

H $4.585817-3.3184140 .884408$

H $6.336135-0.9933071 .869521$

C $0.316884-1.024103-1.863916$

O $0.998096-0.974256-3.122295$

C $1.1850520 .339372-3.658195$

C $-0.1863390 .982263-3.899470$

C $-1.0133861 .022037-2.619308$

C $-1.063185-0.350962-1.953340$

H $0.931070-0.542422-1.093520$

C $0.152848-2.504572-1.479639$

H $1.6600110 .206813-4.638119$

O $-0.0487342 .305784-4.405146$

H $-0.7360240 .403239-4.652666$

H $-0.638013 \quad 1.778399-1.924188$

O $-2.3518181 .411601-2.938274$

H -1.757607 -0.994889-2.508787

O $-1.586839-0.200005-0.634613$

C $1.466647-3.225541-1.267934$

H $-0.413431-2.586212-0.545762$

H $-0.380668-3.033908-2.278335$

H $0.4771692 .253212-5.221491$

H -2.302195 $2.271571-3.390086$

H $-2.4685480 .202158-0.715919$

O $1.202895-4.528799-0.950691$

O $2.577286-2.723373-1.360499$

C $2.349142-5.353515-0.718295$

H $2.006884-6.385265-0.602375$ 
H $\quad 3.037479-5.307179-1.567858$

H $2.849868-5.0440730 .203259$

SCF Energy (B3LYP/6-31G**//MMFF) $=-3245.92047088$

$05 \quad 00084$

MM̄FF Geometry

C $-1.2114172 .687468-1.687981$

C $-0.1918533 .556898-1.629691$

C $0.7980243 .602036-0.496671$

O $2.1098563 .287371-1.027984$

C 0.9109745 .0054920 .122445

C -0.2776245 .4256210 .957052$

C -0.3605215 .2530142 .290271$

C -1.376711 6.1513200 .224235

C $0.6396304 .587876 \quad 3.197917$

C -0.0404663 .6569474 .212268$

C -0.4609932 .3043723 .613242$

C -1.4396371 .6052834 .559956$

C -1.7898790 .1916114 .085326$

O 0.7267731 .5188353 .454942

O $-2.458213-0.4829935 .162115$

C $-0.479781-0.5703383 .817746$

C $-2.767467 \quad 0.2044572 .906492$

O $-0.747239-1.8533783 .256593$

C $0.5218300 .201642 \quad 2.924502$

C $1.884738-0.5334752 .953929$

O $0.0227250 .243692 \quad 1.588826$

C $2.4034771 .976577-1.242508$

O $1.6744761 .017412-1.049621$

C $3.7862161 .911636-1.765033$

C $4.3445190 .719604-2.023837$

C $5.6905950 .495031-2.556360$

C $6.5469381 .707552-2.816782$

C 3.0131370 .1532052 .173769

C $4.366216-0.4783962 .461495$

C $6.779005-0.2244071 .934483$

O $4.519931-1.4021513 .256643$

C $7.517013-0.1413030 .637461$

C $8.085145-1.2035940 .046764$

C $8.879452-1.141721-1.226520$

C $8.262586-1.963687-2.335980$

C $8.533430-3.274193-2.458518$

C $7.403165-1.245098-3.350850$

C $6.095090-0.773683-2.778280$

N $5.383620 \quad 0.113003 \quad 1.722679$

H -1.338701 $1.965389-0.885098$

H $-0.0455064 .263536-2.443132$

H 0.5529092 .8787330 .291528

H $1.0916595 .751945-0.664080$

H 1.8264935 .0526180 .726770

H -1.240016 5.6478282 .798050

H -2.142119 6.5403990 .904433

H $-0.9679097 .005047-0.326270$

H -1.879661 $5.487880-0.484113$

H 1.4054174 .0358112 .644880

H 1.1532885 .3814393 .754139

H 0.6709083 .4769795 .029202

H -0.9083534 .1666344 .649372$

H -0.9248182 .4601482 .632855$

H -2.3515572 .2026474 .685829$

H -0.9902881 .5331815 .560740$

H -3.254090 0.0285385 .388085

H $-0.004545-0.7529664 .791932$

H -3.698669 0.7124123 .185052

H -3.055452 -0.8143712 .626675$

H $-2.370086 \quad 0.7074062 .022414$

H -1.370245 -2.305587 3.851002

H $2.203003-0.6261524 .001011$

H $1.770776-1.5504772 .558244$

H $\quad 0.7603580 .4025030 .979415$

H $4.3051932 .849385-1.928674$

H $3.760714-0.180570-1.835026$

H $7.562011 \quad 1.466789-3.138108$

H $6.6518162 .307916-1.905979$

H $6.1010492 .330991-3.599486$

H 2.8310540 .0680861 .099205

H 3.0745151 .2147802 .437229

H $6.857995-1.2165732 .391222$
H 7.1881310 .5032022 .643162

H 7.6129540 .8409300 .181849

H $7.999282-2.1795090 .520010$

H $9.890123-1.513239-1.011758$

H $9.017877-0.106526-1.561780$

H $8.118690-3.865248-3.268107$

H $9.174263-3.785291-1.747495$

H $7.165612-1.913858-4.189189$

H $7.985614-0.435620-3.799936$

H $5.405374-1.586844-2.547319$

H $5.1727830 .955831 \quad 1.195177$

C $-3.6337032 .597847-2.353701$

O $-3.896106 \quad 1.362755-1.655379$

C $-5.160740 \quad 0.834710-2.094886$

C $-5.8354331 .953939-2.878714$

C $-4.6404192 .644583-3.503356$

H $-4.936114 \quad 0.010317-2.781758$

C $-5.971190 \quad 0.309834-0.906376$

H -6.547091 $1.572369-3.617253$

H $-6.3719712 .647499-2.221040$

C $-4.9429234 .046381-4.004170$

H $-4.2815672 .033028-4.342361$

C $-5.231441-0.722399-0.032598$

O $\quad-6.3116041 .419330-0.063721$

H -6.920286 -0.104151-1.267134

H $-4.421091-0.2256760 .511519$

O $-6.155694-1.1707590 .968517$

C $-4.665554-1.942442-0.785712$

C $-4.107579-3.0034750 .183675$

H -3.877342 -1.605608 -1.467420

O $-5.708002-2.517235-1.571362$

H -3.398862 -2.514976 0.860355

H $-4.926266-3.3945510 .799939$

H $-5.7178474 .019449-4.777199$

H $-5.2978044 .693614-3.195089$

H $-4.0502774 .508025-4.437504$

C -2.181666 $2.620782-2.833790$

H $-6.825668 \quad 1.0683160 .683593$

H $-6.857676-1.6696610 .516125$

H -5.298006 -3.138019-2.196758

H $-2.026597 \quad 3.468412-3.511058$

H -1.967353 $1.706007-3.397541$

H -3.817458 $3.413632-1.642285$

C $-1.250158-3.223716-0.855976$

O $-2.423401-3.793633-1.447735$

C $-3.429939-4.201575-0.518561$

C $-2.852744-5.249500 \quad 0.442850$

C $-1.585609-4.7513381 .134396$

C $-0.587752-4.2178680 .105883$

H -1.516549 -2.301807 -0.323822

C $-0.296323-2.855596-1.997169$

H -4.206331 -4.704991-1.107609

O $-3.828782-5.6147121 .412993$

H $-2.610706-6.156900-0.126051$

H -1.825785 -3.997507 1.891994

O $-1.001485-5.8530481 .833122$

H $-0.160861-5.058094-0.456709$

O $0.484925-3.561910 \quad 0.778186$

C $-0.844259-1.695719-2.790531$

H $0.689234-2.553506-1.624832$

H $-0.136111-3.704325-2.673158$

H -3.415553 -6.259374 2.012663

H $-0.258929-5.5078962 .356824$

H $0.955779-4.2285141 .305572$

O - $-1.535965-2.151655-3.872858$

O $-0.698057-0.525042-2.465974$

C -2.136102 -1.142810 -4.690414

H $-2.611263-1.634221-5.543539$

H $-2.902243-0.607058-4.123193$

H $-1.375467-0.450357-5.063534$

SCF Energy (B3LYP/6-31G**//MMFF) $=-3245.91860612$

05 00085

MM̄MF Geometry

C $2.145447-4.8265250 .719261$

C $0.902940-4.3227210 .699984$

C $0.023684-4.1808081 .912042$

O $-0.356855-2.7896812 .019045$ 
C $-1.266719-5.002044 \quad 1.729657$ C $-2.232715-4.8643072 .891587$ C $-3.453559-4.2987692 .807438$ C $-1.754170-5.4219754 .207510$ C $-4.132600-3.7377821 .587842$ C $-3.849245-2.2459431 .410768$ C $-4.737878-1.5627680 .360139$ C $-4.551283-2.145013-1.043476$ C $-5.340004-1.363794-2.106338$ O $-4.380488-0.1726010 .369199$ O $-4.851567-1.760681-3.397522$ C $-5.0315970 .139549-1.951921$ C $-6.832436-1.713296-2.082184$ O $-5.8439330 .922901-2.822196$ C $-5.1717440 .648910-0.501912$ C $-4.6912812 .110189-0.332870$ O $-6.5520600 .618395-0.120004$ C $0.408644-1.9660892 .782676$ O $1.429234-2.2521493 .385993$ C $-0.234076-0.632227 \quad 2.754626$ $\begin{array}{llll}C & 0.273300 & 0.386186 & 3.465091\end{array}$ C $-0.269678 \quad 1.7481353 .516576$ C - $\begin{array}{lll}1.507566 & 2.026600 & 2.704397\end{array}$ C $-3.249833 \quad 2.365552-0.774642$ C $-2.7306103 .725516-0.340394$ C $-0.6548515 .114653-0.460903$ $\begin{array}{lllll}\text { O } & -3.390114 & 4.522169 & 0.320597\end{array}$ C 0.3571074 .7562680 .580836 C 0.5688605 .4626811 .701564 C 1.6205905 .1029302 .713668 C $1.099715 \quad 5.0733174 .135044$ C 1.6222225 .8839295 .071116 C -0.0205374 .1150274 .484243$ C $0.355265 \quad 2.6714924 .277555$ N $-1.4306053 .930851-0.780637$ H $2.569381-5.1830641 .654181$ H $0.494078-3.968595-0.243227$ H $0.548765-4.5022422 .819633$ H -1.018273 -6.064355 1.606555 H $-1.749620-4.6947010 .795363$ H $-4.053641-4.2379203 .714747$ H -2.540449 -5.419232 4.970032 H - $-1.425381-6.4589964 .082968$ H $-0.917866-4.8336034 .596699$ H -3.845691 -4.2972660 .692334$ H -5.212010 -3.897632 1.703556 H -3.980656 -1.722141 2.367587 H - $2.796836-2.0962201 .141416$ H $-5.781522-1.6609140 .681491$ H $-4.834393-3.203537-1.075574$ H $-3.486710-2.111622-1.310262$ H $-4.968117-2.722801-3.479327$ H -3.994924 $0.280731-2.282411$ H $-6.978768-2.781015-2.285979$ H -7.375622 -1.181440 -2.871119 H $-7.309516-1.494165-1.124895$ H $-5.7245290 .574161-3.722237$ H $-5.3662142 .793521-0.864553$ H $-4.783542 \quad 2.3767590 .729519$ H $-6.680397 \quad 1.234150 \quad 0.620524$ H $-1.119901-0.5234252 .138848$ H 1.1675650 .2120534 .061472 H -2.333487 1.3813363 .024189 H $-1.309026 \quad 1.8442121 .644343$ H -1.8692223 .0528482 .780977$ H $-2.5879901 .605778-0.347026$ H -3.176374 2.315271-1.866620 H $-1.3179705 .922345-0.135579$ H $-0.1463745 .423262-1.379437$ H $0.968584 \quad 3.8789240 .387194$ H $-0.016706 \quad 6.360097 \quad 1.885895$ H 2.4280645 .8409772 .621813 H 2.0750484 .1326702 .481893 H $1.260273 \quad 5.875226 \quad 6.094033$ H 2.4245596 .5760134 .837664 H $-0.9119224 .409672 \quad 3.925910$ H -0.2945534 .2239165 .542016$ H 1.2383752 .3651354 .839983
H $-0.9628373 .189275-1.294369$

C $4.272767-4.046661-0.351421$

O $3.903791-2.672800-0.128542$

C $4.766224-1.819630-0.898229$

C $5.880834-2.716098-1.434181$

C $5.154286-4.037147-1.599523$

H $4.188048-1.461598-1.755050$

C $5.225088-0.626917-0.058024$

H $6.302161-2.336854-2.370327$

H $6.703208-2.829298-0.719027$

C $6.082252-5.234006-1.711562$

H $4.530250-3.980734-2.501554$

C 4.1513890 .1100700 .771036

O $6.199118-1.1103920 .884439$

H $5.7568730 .082082-0.703993$

H $3.773311-0.5414801 .568086$

$\begin{array}{lllll}\text { O } & 4.854495 & 1.167281 & 1.463244\end{array}$

C $2.9708640 .779198 \quad 0.039490$

C $1.922022-0.177276-0.550746$

H $3.3486671 .457211-0.733468$

O $2.286654 \quad 1.5941051 .003049$

H $2.345731-0.709480-1.401000$

H $1.660243-0.9306640 .200835$

H $6.733411-5.134920-2.586193$

H $6.720338-5.331452-0.826671$

H $5.511516-6.161594-1.819042$

C $3.014436-4.902077-0.505838$

H $6.512457-0.3452201 .396763$

H $5.1925101 .784974 \quad 0.792021$

H 2.9398472 .2055251 .383669

H $3.288301-5.949042-0.677365$

H $2.448626-4.566298-1.384256$

H $4.846155-4.3686380 .527749$

C $1.3979391 .372847-3.122322$

O $0.8824241 .672741-1.820976$

C $0.6204490 .529530-1.003720$

C $-0.396059-0.388971-1.694698$

C $0.024945-0.747632-3.117699$

C $0.3969680 .512604-3.901476$

H $2.3534290 .841974-3.028295$

C $1.6633872 .706727-3.825577$

H $0.1354010 .909064-0.096892$

O $-0.588978-1.568320-0.924906$

H $-1.3643690 .123466-1.740486$

H $0.845036-1.475164-3.113017$

O $-1.078018-1.390993-3.760372$

H $-0.514548 \quad 1.086731-4.109717$

$\begin{array}{lllll}\text { O } & 0.949105 & 0.133732 & -5.160248\end{array}$

C $2.8786473 .374900-3.226995$

H $1.8648012 .573691-4.894357$

H $\quad 0.804643 \quad 3.383497-3.741469$

H $-1.181140-2.153165-1.427374$

H $-0.812943-1.571087-4.678766$

H $1.765473-0.366682-4.990295$

O $2.5008994 .255459-2.257619$

$\begin{array}{lllll} & 0 & 4.028401 & 3.113598 & -3.557059\end{array}$

C $3.5709884 .913855-1.572857$

H $4.2097475 .447551-2.283089$

H $3.1371755 .639592-0.880051$

H $4.1524724 .185377-0.999925$

SCF Energy (B3LYP/6-31G**//MMFF)= -3245.91995053

05_00086

MM̄FF Geometry

C -3.093779 -2.739892 -2.706650

C $-2.183353-2.683059-1.723844$

C $-0.699150-2.746775-1.952737$

O $-0.110888-1.530847-1.431868$

C $-0.006118-3.965615-1.307107$

C $-0.161741-4.141422 \quad 0.192625$

C $0.711653-3.6780231 .108453$

C $-1.321683-4.996536 \quad 0.637914$

C $1.956304-2.8712810 .865517$

C $3.214599-3.7040461 .139044$

C $4.533043-2.978190 \quad 0.824317$

C $4.779485-1.7761491 .737742$

C $6.071632-1.0323901 .367069$

O $4.499159-2.568989-0.545362$ 
$\begin{array}{llll}\text { O } & 6.061168 & 0.233164 & 2.046201\end{array}$

C $6.054053-0.731458-0.146708$

C $7.321789-1.7742951 .853346$

O $7.296917-0.173958-0.567205$

C $5.707482-1.957149-1.018011$

C $5.480485-1.592484-2.506367$

O $6.798360-2.885082-0.971872$

C $-0.152358-0.428272-2.226983$

O $-0.581117-0.371455-3.372128$

C $0.3763290 .772044-1.543483$

C $0.830810 \quad 0.781456-0.282518$

C $1.348621 \quad 1.960717 \quad 0.401694$

C 2.6278181 .7070991 .148514

C $4.329833-0.614678-2.776645$

C $4.7656340 .835435-2.915099$

C $3.8114743 .112675-3.274658$

O $5.939781 \quad 1.193732-2.913475$

C $3.6978133 .817409-1.959840$

C $2.8113754 .796344-1.722477$

C $2.7325805 .562299-0.432978$

C $1.351664 \quad 5.5349290 .181480$

C $0.4776616 .532706-0.033202$

C 1.0201364 .3963661 .117256

C $0.678447 \quad 3.129980 \quad 0.385684$

N $3.6772061 .683000-3.071984$

H -2.767861 -2.870860 -3.735336

H -2.519292 -2.512040 -0.705262

H $-0.481833-2.804449-3.028083$

H $1.063382-3.916537-1.548948$

H $-0.363827-4.878975-1.801818$

H $0.523256-3.9006672 .158086$

H - $1.350652-5.1243971 .725440$

H -2.276370 -4.5595170 .338922$

H $-1.246127-5.9945630 .193530$

H $1.984189-2.472403-0.151504$

H $1.926822-2.0056241 .535275$

H $3.222889-4.0405732 .183433$

H $3.169377-4.6057100 .513218$

H $5.338079-3.7120830 .951079$

H $4.800241-2.0852222 .789993$

H $3.949838-1.0641721 .650995$

H 5.9962730 .0602043 .001096

H $5.2949690 .042960-0.306410$

H $7.297284-1.8912662 .943515$

H $8.230326-1.2024431 .634516$

H $7.427291-2.7700521 .418185$

H $7.4665980 .606950-0.012995$

H $6.418837-1.250537-2.959246$

H $5.229445-2.525227-3.032019$

H $6.753518-3.454192-1.758071$

H $0.3705831 .680359-2.140013$

H $\quad 0.849400-0.1289910 .309205$

H 2.4476591 .0483792 .004979

H 3.1009402 .6184441 .520383

H 3.3599951 .2256920 .491531

H $3.867528-0.885876-3.734825$

H $3.553605-0.688321-2.007120$

H $3.0276693 .415722-3.976213$

H $4.7848773 .329265-3.726259$

H $4.4014853 .534120-1.180577$

H $2.1281665 .095923-2.514175$

H $3.023016 \quad 6.599926-0.644639$

H $3.465968 \quad 5.196520 \quad 0.296388$

H $-0.504300 \quad 6.533350 \quad 0.428292$

H $\quad 0.7230527 .367868-0.681068$

H 0.1498324 .6586511 .733909

H 1.8365914 .2662241 .833553

H $-0.255136 \quad 3.186374-0.174864$

H $2.7387321 .298004-3.028639$

C $-5.028185-1.174484-2.861940$

O $-4.492329-0.225696-1.915639$

C $-5.5132660 .734756-1.587513$

C $-6.6390640 .511794-2.590231$

C $-6.543039-0.985210-2.816164$

H $-5.8827450 .473094-0.589178$

C $-4.927136 \quad 2.150470-1.575378$

H -7.611226 $0.827053-2.199354$

H $-6.4612021 .050559-3.528182$
C $-7.264894-1.458285-4.065673$

H $-6.960381-1.501470-1.941073$

C $-3.7263842 .325597-0.621968$

O $-4.4795932 .465324-2.900545$

H $-5.7151212 .874430-1.337436$

H $-2.8741421 .747639-0.998746$

O $-3.3222913 .700070-0.681971$

C $-3.9964721 .944614 \quad 0.846774$

C -2.776144 2.2109301 .745026

H -4.2605540 .8865140 .911886$

O -5.0959262 .7006931 .351766$

H -1.9094701 .7040011 .310590$

H -2.560412 3.2860921 .749312

H $-8.334170-1.231994-4.000506$

H $-6.872542-0.971301-4.964767$

H -7.156047 -2.539791-4.193841

C $-4.571026-2.582037-2.478325$

H $-4.0999243 .360338-2.873111$

H $-4.036647 \quad 4.232445-0.291564$

H -5.9010492 .3896970 .905164$

H $-5.093391-3.336486-3.076707$

H $-4.810448-2.778380-1.426555$

H $-4.636197-0.896693-3.849584$

C -2.264232 $-0.544412 \quad 2.973752$

$\begin{array}{lllllll}\text { O } & -3.322078 & 0.363461 & 3.304448\end{array}$

$\begin{array}{llll}\text { C } & -2.986887 & 1.751939 & 3.206774\end{array}$

C $-1.809750 \quad 2.069274 \quad 4.137633$

C $-0.612674 \quad 1.165688 \quad 3.861098$

C $-1.037738-0.3014693 .866195$

H $-1.993669-0.4292361 .916899$

C - $-2.796922-1.9723223 .182014$

H $-3.8544482 .303076 \quad 3.590743$

O $-1.432792 \quad 3.4356864 .004433$

H -2.1299211 .9247055 .177813$

H -0.1268561 .4378992 .919557$

O $0.354575 \quad 1.3831194 .890408$

H $-1.255798-0.612254 \quad 4.896151$

O $0.041104-1.105146 \quad 3.395365$

C -3.857485 -2.364320 2.175468

H $-1.981830-2.6983753 .089311$

H -3.245799 -2.054732 4.179135

H -0.6669943 .5843764 .585123$

H 1.1587470 .8953304 .645146

H $0.769732-1.0266624 .033595$

O $-4.298932-3.6234772 .471298$

O $-4.248901-1.6698241 .248791$

C $-5.310481-4.1427141 .602270$

H $-4.900706-4.2944580 .599654$

H $-5.635149-5.1085621 .998268$

H -6.172710 -3.469624 1.570578

SCF Energy (B3LYP/6-31G**//MMFF) $=-3245.90558444$

05_00087

MM̄FF Geometry

C $2.168478-0.631614-2.653832$

C $2.165409-1.905681-3.071230$

C $1.484594-2.998714-2.292260$

O $0.094060-2.998050-2.688908$

C $2.080260-4.393502-2.555302$

C $3.428973-4.630690-1.906573$

C $3.590661-5.165478-0.679694$

C $4.633937-4.303791-2.748913$

C $2.507807-5.551750 \quad 0.289034$

C $2.843402-5.2014181 .746303$

C $3.035124-3.7002862 .020129$

C $3.391421-3.4831273 .495251$

C $3.500646-1.9954233 .854683$

O $1.819954-3.0143811 .695723$

O $3.523036-1.9115925 .287959$

C $2.229053-1.2771103 .356142$

C $4.824867-1.3932383 .371051$

O $2.330895 \quad 0.1299053 .561050$

C $1.881393-1.5930181 .884045$

C $0.525482-0.9841831 .455774$

O $2.886144-1.0028401 .048956$

C $-0.811424-3.345085-1.733786$

O $-0.572012-3.733931-0.601605$

C $-2.166341-3.147477-2.291561$ 
C $-3.225417-3.340784-1.491513$

C $-4.633034-3.183838-1.855253$

C $-4.951874-2.700694-3.245016$

C -0.706095 -1.531766 2.183004

C $-1.971548-0.7878281 .783199$

C $-4.303714-0.4037232 .583815$

$\begin{array}{lllll}\text { O } & -2.007995 & 0.023758 & 0.862473\end{array}$

C $-5.426317-1.3094902 .189771$

C $-6.353757-0.9679201 .281354$

C -7.545630 -1.813235 0.929372

C -7.644079 -2.097190 -0.553071

C $-8.293165-1.255865-1.375663$

C $-7.063289-3.393449-1.067768$

C $-5.569346-3.460636-0.924246$

N $-3.048953-1.1336552 .588240$

H $1.598333-0.373276-1.764777$

H $2.698477-2.172730-3.979409$

H $1.560779-2.766144-1.222189$

H $2.150364-4.576193-3.636538$

H $1.371211-5.160189-2.216765$

H $4.605470-5.349683-0.329879$

H $5.569550-4.619253-2.274558$

H $4.572646-4.812280-3.716637$

H $4.704492-3.226591-2.923277$

H $1.549931-5.0962690 .027597$

H $2.370525-6.6379920 .224205$

H $2.019536-5.5763152 .368010$

H $3.746243-5.7466142 .049860$

H $3.841339-3.3237551 .380754$

H $4.319810-4.0083603 .752721$

H $2.611052-3.9332924 .125375$

H $3.514768-0.9688985 .527958$

H $1.411627-1.6176714 .002105$

H $5.670663-1.9127923 .837360$

H $4.918117-0.3440343 .672407$

H $4.956317-1.4542162 .288809$

H $2.921630 \quad 0.4850382 .875280$

H $\quad 0.567044 \quad 0.107710 \quad 1.558483$

H $\quad 0.383670-1.1621170 .381199$

H $2.524544-0.9072260 .153859$

H $-2.254679-2.842717-3.327767$

H -3.048326 -3.645594-0.460409

H $-6.018486-2.532121-3.410779$

H -4.620139 -3.430890 -3.991118

H $-4.455307-1.744215-3.443924$

H $-0.844881-2.5942141 .956758$

H -0.591055 -1.421077 3.265596

H -4.2290840 .4692121 .929979$

H -4.464518 -0.0494023 .607243$

H -5.508619 -2.2632282 .705149$

H $-6.282477 \quad 0.0020460 .793899$

H -8.445290 -1.2767461 .259274$

H -7.547447 -2.756883 1.488776

H -8.392758 -1.464439 -2.435554

H -8.735719 -0.336534-1.006314

H $-7.363706-3.577711-2.105349$

H -7.502152 -4.226728 -0.503473

H -5.242078 -3.792058 0.061890

H -2.907584 -1.814020 3.329575

C $3.8301991 .173684-2.284466$

O $3.0165531 .837984-1.296221$

C $3.6375183 .086767-0.942136$

C $5.0010663 .093580-1.624562$

C $4.7238712 .265198-2.865674$

H $3.0246763 .880053-1.383749$

C 3.6722723 .2400170 .582186

H $5.3447574 .107225-1.851879$

H $5.7671042 .610680-1.006785$

C $5.9808581 .745434-3.540483$

H $4.1555312 .876674-3.579208$

C 2.2941203 .1202571 .266611

O 4.5138432 .2193881 .137557

H 4.1425214 .1932040 .848790

H 1.9343022 .0872461 .198527

O 2.4767473 .3910842 .662399

C 1.2076234 .0688170 .725127

C -0.1127433 .9500631 .512911$

H $1.0128223 .842889-0.328247$
O $1.6548385 .421434 \quad 0.807325$

H -0.4347772 .9029921 .505349$

H 0.0627304 .2279692 .559099

H $6.6145002 .577279-3.865378$

H $6.5702101 .118476-2.862950$

H $5.7322061 .146888-4.422314$

C $2.9279250 .485542-3.310495$

H $4.152336 \quad 1.358780 \quad 0.862976$

H 3.1642242 .7856812 .988923

H 2.3842215 .5344060 .175468

H $3.5253920 .104774-4.146060$

H $2.2034861 .200449-3.714920$

H $4.4358500 .428675-1.750341$

C $-2.0702973 .419939-0.785445$

O $-1.4445224 .663017-0.445165$

C -1.2282514 .8645180 .955074$

C -2.5608984 .7825951 .715452$

C -3.3302683 .5044801 .391419$

C $-3.4465783 .321510-0.121020$

H -1.436532 $2.586455-0.459259$

C -2.184485 $3.367325-2.311876$

H -0.8690075 .8960521 .059704$

O -2.3551024 .8600213 .121868$

H -3.1875105 .6395221 .435812$

H -2.8653162 .6290391 .859009$

O $-4.649247 \begin{array}{llll}3.597857 & 1.933808\end{array}$

H -4.121503 $4.083399-0.531784$

O $-4.0188792 .047292-0.402414$

C $-0.8273983 .130194-2.929843$

H -2.832655 2.551300 -2.650308

H $-2.6054634 .300591-2.704717$

H -1.879341 5.6889373 .302146

H -4.5575883 .7491282 .890292$

H -4.8779522 .0088970 .051761$

O $-0.3955734 .255167-3.566171$

O $-0.2177682 .072182-2.839741$

C $0.8900944 .155326-4.185843$

H $1.6570603 .949764-3.434303$

H $\quad 0.882172 \quad 3.375192-4.953047$

H $1.1140175 .113617-4.661892$

SCF Energy $($ B3LYP/6-31G**//MMFF $)=-3245.92497803$

05_00088

MMFF Geometry

C $-2.269565-2.9483040 .984142$

C $-1.042731-3.4730731 .118478$

C $0.055899-3.2590120 .106864$

O $1.080301-2.4406590 .717105$

C $0.685897-4.608260-0.284292$

C $1.664834-4.509427-1.438674$

C $2.985725-4.760099-1.351335$

C $1.067744-4.133194-2.771565$

C $3.777493-5.178427-0.145221$

C $4.397884-4.0222500 .654664$

C $5.353920-3.129490-0.154299$

C $6.119417-2.1808260 .771825$

C $7.008524-1.200825-0.010525$

O $4.579954-2.364964-1.083326$

O $7.415967-0.1594040 .890642$

C $6.169152-0.532191-1.121457$

C $8.295568-1.871829-0.505393$

O $6.9924520 .258648-1.974321$

C $5.353775-1.536412-1.961588$

C $4.367435-0.856376-2.943309$

O $6.250644-2.325102-2.753655$

C $0.878131-1.0944660 .711808$

O $-0.060899-0.4917980 .215972$

C $2.000181-0.4647441 .440513$

C 1.8593640 .7909651 .888666

C 2.8627031 .5751052 .608946

C 4.2680271 .0373162 .643219

C $3.3131440 .049979-2.298678$

C $3.6933201 .521206-2.271282$

C $2.9188133 .710163-1.366478$

O $4.6581931 .980343-2.876424$

C $3.7201494 .019556-0.140939$

C 3.3264894 .9000810 .791871

C 4.1342055 .2502932 .009555 
C $3.349016 \quad 5.092628 \quad 3.293217$

C $2.756506 \quad 6.1493353 .874681$

C $3.317226 \quad 3.7256303 .940886$

C 2.4822412 .7420783 .169572

N $2.7998142 .271857-1.517964$

H -2.483366 -2.331985 0.114029

H $-0.804123-4.0602402 .001827$

H $-0.333729-2.756702-0.786794$

H $-0.101236-5.317930-0.572861$

H $1.173259-5.0377600 .597699$

H $3.583539-4.660064-2.257026$

H $1.784987-4.239600-3.592626$

H $0.213304-4.778017-3.001653$

H $0.730876-3.092262-2.765652$

H $3.166651-5.7895100 .527766$

H $4.579415-5.846277-0.485578$

H $3.590044-3.4115571 .073526$

H $4.942156-4.4599401 .500727$

H $6.051685-3.774777-0.700743$

H $6.718704-2.7448981 .497446$

H $5.402873-1.5991991 .366702$

H $7.881559-0.5741151 .637093$

H $5.477800 \quad 0.158821-0.624178$

H $8.877844-2.2542940 .341746$

H $8.946462-1.152061-1.014147$

H $8.110405-2.709107-1.181355$

H $7.4688700 .892337-1.411099$

H $4.921294-0.335184-3.733329$

H $3.819070-1.659797-3.456505$

H $5.753925-2.702368-3.498472$

H $2.899622-1.0482411 .593565$

H $0.911476 \quad 1.2982531 .720302$

H 4.6205130 .8296661 .627189

H $4.3125640 .111798 \quad 3.227457$

H $4.9919061 .727903 \quad 3.079638$

H $2.391891-0.013906-2.891920$

H $3.073969-0.285923-1.286122$

H $1.9038084 .116227-1.311478$

H $3.4168524 .127822-2.247446$

H $4.6775613 .516213-0.027435$

H 2.3816995 .4230880 .660931

H $4.465474 \quad 6.2914191 .901384$

H 5.0524604 .6528052 .068247

H 2.2129146 .0508004 .808387

H 2.7980157 .1365213 .426388

H $2.886613 \quad 3.7942614 .948698$

H 4.3453013 .3873274 .093408

H 1.4352743 .0382503 .085431

H $2.0562161 .801041-1.010771$

C $-4.708341-3.4444801 .327409$

O $-5.111943-2.349351 \quad 0.479534$

C $-6.488626-2.0301850 .742752$

C $-7.048447-3.2191221 .513320$

C $-5.843716-3.6332902 .335299$

H $-6.486582-1.1521901 .399754$

C $-7.221048-1.699023-0.561204$

H $-7.914390-2.9478112 .124718$

H $-7.347193-4.0351290 .845413$

C $-5.942738-5.0418352 .894991$

H $-5.729415-2.9258033 .167825$

C $-6.531982-0.622083-1.424160$

O $-7.301518-2.892421-1.352568$

H $-8.254995-1.410971-0.337435$

H -5.601854 -1.029254-1.838412

O $-7.382249-0.372119-2.552528$

C $-6.2311680 .713374-0.715002$

C $-5.6245351 .746278-1.684269$

H -5.5372740 .5476600 .113992$

O $-7.4343051 .259055-0.176596$

H -4.748885 $1.300011-2.167762$

H $-6.3549211 .966327-2.472219$

H $-6.806222-5.1306383 .562147$

H $-6.056802-5.7834632 .097219$

H $-5.045685-5.2979123 .467399$

C $-3.371880-3.1084001 .992942$

H -7.769230 -2.664366 -2.174265

H -8.190688 $0.054864-2.220475$

H -7.7242160 .6828270 .549984$
H $-3.113054-3.8820352 .724717$

H -3.465318 -2.1591862 .533032$

H $-4.605872-4.3264890 .682377$

C -2.983570 $2.526923-0.324700$

$\begin{array}{llllll}\text { O } & -4.309118 & 2.900522 & 0.070963\end{array}$

C $-5.2416563 .078853-1.000374$

C $-4.7274334 .152766-1.969458$

C $-3.326286 \quad 3.828726-2.477714$

C $-2.3904643 .551683-1.303054$

H -3.005052 $1.532138-0.785887$

$\begin{array}{llll}\text { C }-2.124716 & 2.458175 & 0.948580\end{array}$

H $-6.1571973 .475748-0.544186$

O $-5.6246474 .298021-3.065151$

H $-4.7002925 .118413-1.447756$

H $-3.3461582 .991724-3.185306$

O $-2.8396754 .958237-3.206136$

H $-2.1892104 .495974-0.781633$

O $-1.1389883 .082726-1.803555$

C $-2.449933 \quad 1.261168 \quad 1.815113$

H -1.0643562 .3829760 .684642$

H -2.285946 3.3580831 .553959

H $-5.2493864 .968736-3.661198$

H $-1.9301124 .753412-3.483422$

H $-1.2966022 .236500-2.256123$

O $-1.651974 \quad 1.2943452 .923811$

$\begin{array}{llll}0 & -3.281397 & 0.403552 & 1.555451\end{array}$

C $-1.806707 \quad 0.201197 \quad 3.833592$

H $-1.437010-0.7194913 .373572$

H -1.2077620 .4129204 .723392$

H -2.853243 $0.092424 \quad 4.133933$

SCF Energy (B3LYP/6-31G**//MMFF)= -3245.92028983

05_00089

MMFF Geometry

C $4.031901-2.428227-2.762650$

C $2.920865-1.736752-3.055009$

C $1.539826-2.341087-3.080869$

O $0.786309-1.782954-1.978952$

C $0.823960-1.963545-4.390706$

C $-0.582514-2.521092-4.504142$

C $-1.695232-1.767615-4.610144$

C $-0.694068-4.023875-4.519902$

C $-1.792124-0.268079-4.657197$

C $-1.9322830 .406917-3.285380$

C $-3.2571630 .109605-2.566262$

C $-3.3903980 .971628-1.308282$

C $-4.667138 \quad 0.643017-0.518338$

O $-3.281684-1.271774-2.200472$

$\begin{array}{llll}O & -4.545922 & 1.241416 & 0.781622\end{array}$

C $-4.754122-0.884523-0.306573$

C $-5.911414 \quad 1.262299-1.165855$

O $-6.005853-1.249442 \quad 0.268678$

C $-4.508107-1.700379-1.591981$

C $-4.384468-3.222096-1.333245$

O $-5.614581-1.516081-2.483743$

C $0.975203-2.342091-0.752351$

O $1.693370-3.289541-0.474993$

C $0.172386-1.5714120 .220135$

C $0.491942-1.6288951 .521234$

C $-0.171902-0.9031162 .605196$

C $-1.459893-0.1939182 .282635$

C $-3.256345-3.639767-0.382510$

C $-3.706571-3.8672341 .051986$

C $-2.792906-4.434481 \quad 3.302094$

O $-4.884589-3.8727901 .398015$

C $-2.744611-3.1845124 .122619$

C $-1.916765-3.0176775 .165468$

C $-1.897430-1.7904206 .031187$

C $-0.518908-1.1805236 .153895$

C $0.252522-1.424838 \quad 7.226619$

C $-0.074113-0.2175265 .076989$

C $0.392006-0.9182093 .830807$

N $-2.630848-4.1055311 .897938$

H $3.960976-3.492366-2.551507$

H $2.997268-0.669137-3.245136$

H $1.586127-3.432656-2.983277$

H $1.406533-2.326001-5.248061$

H $0.807924-0.871303-4.474912$ 
H $-2.654411-2.278551-4.685726$ H - $1.708356-4.362379-4.757677$ H $-0.024103-4.446788-5.275611$ H -0.430355 -4.441583 -3.543446 H $-0.9228630 .153126-5.174218$ H $-2.6517880 .000548-5.284351$ $\mathrm{H}-1.0922130 .100225-2.652479$ H -1.847672 $1.489938-3.436631$ H $-4.0789740 .318943-3.261013$ H $-3.3634072 .038444-1.562416$ H $-2.5237210 .798774-0.655493$ H -4.4119672 .1971860 .660361$ H $-3.986450-1.1461000 .429443$ H -5.810627 2.352884 -1.222571 H $-6.8059451 .076378-0.561218$ H $-6.0955900 .895428-2.177650$ H $-6.110046-0.7341941 .086859$ H -5.352469 -3.630905-1.019589 H -4.171093 -3.696883 -2.302063 H -5.634088 -2.259542 -3.108801 H $-0.632611-0.955680-0.163328$ H $1.341183-2.2416751 .821736$ H $-1.973717 \quad 0.2017813 .161073$ H -2.163455 -0.884274 1.807573 H -1.281882 0.6450371 .603605 $\mathrm{H}-2.852136-4.599964-0.728633$ H -2.436276 -2.916000 -0.398791 H $-1.993692-5.1342563 .565729$ H -3.755860 -4.934096 3.449377 H -3.445196 -2.393096 3.866696 H -1.237749 -3.8227105 .437408$ H -2.265662 -2.079781 7.024414 H -2.600578 -1.031228 5.666716 H $1.230540-0.9677117 .335969$ H -0.074494 -2.092293 8.017055 H $\quad 0.767709 \quad 0.3881545 .439190$ H -0.8708990 .5072914 .887380$ H $1.323581-1.4696633 .966257$ H -1.685110 -4.026757 1.535409 C $5.842453-1.723475-1.195569$

O $5.056071-0.726339-0.508633$

C $5.9246340 .051090 \quad 0.338018$

C $7.255242-0.6909830 .370881$

C $7.296657-1.288161-1.022582$

H $6.0767511 .012632-0.166135$

C 5.2787850 .2774121 .709674

H $8.097379-0.0240240 .578835$

H $7.259211-1.4856941 .125912$

C $8.303234-2.416011-1.169249$

H $7.539238-0.492709-1.740143$

C 3.8888390 .9456381 .647105

O $5.120725-0.9950412 .350613$

H $5.956610 \quad 0.8610882 .343511$

H $3.1830190 .247873 \quad 1.185249$

O 3.4251651 .1325172 .990076

C 3.8549072 .2966800 .907730

C 2.5158643 .0501830 .990151

H $4.1172262 .148729-0.142128$

$\begin{array}{lllll}\text { O } & 4.870643 & 3.156431 & 1.437437\end{array}$

H 2.3517793 .3801672 .025732

H 2.6260723 .9911260 .436682

H $9.316697-2.055539-0.965437$

H $8.091925-3.235523-0.474114$

H $8.288288-2.823759-2.184744$

C $5.396897-1.807331-2.656880$

H $4.698588-0.8355533 .212190$

H 4.0721961 .6885113 .455913

H 4.6354753 .3690682 .356382

H $6.094938-2.419630-3.238403$

H $5.390533-0.804869-3.102837$

H $5.665115-2.678072-0.682189$

C -0.1905124 .2235790 .281800$

O $0.070994 \quad 2.9796180 .943361$

C 1.2587052 .2949210 .515006

C $1.1458901 .994914-0.984011$

C $0.9030973 .264285-1.793939$

C $-0.3009634 .024203-1.237444$

H $0.6165384 .928914 \quad 0.511643$
C $-1.492893 \quad 4.7676290 .887694$

H $1.2399381 .339613 \quad 1.045745$

O $2.292979 \quad 1.329152-1.485962$

H $0.2941941 .323840-1.147733$

H $1.7943423 .901698-1.822631$

O $0.6190522 .910913-3.149743$

H -1.216859 $3.470141-1.478271$

O $-0.3904865 .291253-1.883129$

C -1.703298 6.2419330 .632789

H -2.3447464 .1932840 .502285$

H -1.494733 4.6262221 .975541

H $2.3777850 .488892-1.004153$

H $1.3655442 .376688-3.471757$

H $-0.4362125 .127115-2.840790$

O

$\begin{array}{lllll}\text { O } & -0.813741 & 7.082425 & 0.646579\end{array}$

C -3.3743967 .8510210 .178872$

H $-2.8641478 .216414-0.717458$

H -4.4539117 .9054660 .014595$

H -3.1199708 .4662591 .047092$

SCF Energy (B3LYP/6-31G**//MMFF) $=-3245.91134155$

05_00090

MMFF Geometry

C $-0.812188 \quad 4.868571-0.414770$

C $-1.3344834 .539336-1.606989$

C $-2.4240883 .517803-1.841022$

O $-2.4029072 .484303-0.829054$

C $-3.801578 \quad 4.202984-1.810124$

C $-4.944072 \quad 3.296177-2.223972$

C $-5.9457012 .903211-1.412922$

C $-4.9353742 .851877-3.664799$

C $-6.164542 \quad 3.249032 \quad 0.032977$

C $-5.5859582 .234868 \quad 1.032671$

C -6.1688450 .8176330 .904161$

C $-5.739261-0.0553602 .085589$

C -6.229151 -1.505311 1.938763

O $-5.6858710 .238868-0.311139$

O $-5.518860-2.3090732 .893814$

C $-5.848785-2.029968 \quad 0.536567$

C $-7.717674-1.6402202 .281869$

O $-6.431936-3.3077340 .295401$

C $-6.219598-1.059647-0.603289$

C $-5.654857-1.487978-1.979931$

O $-7.645757-1.010594-0.734266$

C $-1.443564 \quad 1.524762-0.949601$

O $-0.5904001 .444192-1.819629$

C -1.5960610 .5839390 .181868$

C $-0.596076-0.2649710 .460549$

C $-0.570160-1.2627161 .531727$

C $-1.841435-1.4869072 .304461$

C $-4.129043-1.614917-2.056543$

C $-3.616758-3.033488-1.868616$

C $-1.490205-4.321200-1.703856$

O $-4.352099-4.014941-1.807286$

C $-1.189943-4.598153-0.265121$

C $0.045159-4.8536590 .192187$

C $0.357049-5.1881091 .622351$

C $1.379744-4.2547952 .228695$

C $2.675577-4.6013582 .307690$

C $0.883019-2.9531302 .816129$

C $0.582298-1.9259451 .761614$

N $-2.229492-3.078149-1.815520$

H $-1.2148564 .411827 \quad 0.484353$

H $-0.9477695 .022028-2.501968$

H $-2.254398 \quad 3.054815-2.820725$

H -3.802074 $5.072625-2.480936$

H $-3.9711144 .599300-0.803212$

H $-6.7152622 .255565-1.832439$

H $-5.8564602 .328548-3.943815$

H $-4.8389923 .716917-4.329045$

H $-4.1018492 .168947-3.854263$

H -5.762382 4.2415370 .261510

H -7.2461293 .3365910 .197994$

H -4.4974962 .1990510 .909451$

H -5.7839372 .6151922 .042625$

H $-7.261666 \quad 0.895730 \quad 0.865228$

H $-6.0889960 .372336 \quad 3.033539$ 
H -4.642966 -0.0646932 .149578$ H -5.689654 -1.943888 3.778925 H -4.762504 -2.177839 0.537296 H -7.903285 -1.317861 3.313559 H -8.041563 -2.685609 2.233105 H -8.363343 -1.047258 1.630905 H -6.154251 -3.894309 1.019812 H -6.155899 -2.400142 -2.325538 H -5.941931 - $0.709715-2.701897$ H -7.862800 -0.689250 -1.625019 H -2.5153620 .6198800 .753632$ H $0.306925-0.214231-0.145490$ H -2.108626 -0.5907552 .874919$ H -1.783282 -2.314346 3.013922 H $-2.663343-1.7280841 .622313$ H -3.807013 -1.305722 -3.059359 H -3.640153 -0.951536 -1.337300 H $-0.581590-4.214398-2.304276$ H -2.086339 -5.140005 -2.119513 H $-2.029688-4.6203030 .425368$ H $0.879396-4.856152-0.505918$ H $\quad 0.730770-6.2202071 .649011$ H $-0.548883-5.1879982 .241152$ H $3.409826-3.9451332 .763182$ H $3.032363-5.5456321 .909580$ H $1.649823-2.5238003 .475045$ H $\quad 0.032180-3.1613453 .469695$ H $1.447664-1.6831251 .142826$ H -1.701548 -2.210221 -1.820889 C 1.5073355 .2799280 .547041 O 1.9185434 .0069040 .005880 C 2.0416923 .0690031 .097159 C 2.2068953 .9250532 .341897 C 1.2527175 .0635862 .040842 H 1.0892212 .5245011 .132699 C 3.1918662 .0986200 .808898 H 1.9538313 .3917673 .262746 H 3.2360644 .2959482 .426137 C 1.4930986 .3002952 .889982 H 0.2288504 .7127942 .216778 C 3.5482921 .1854811 .995605 O $2.8053351 .282402-0.302410$ H 4.0771342 .6604500 .490078 H 3.9868331 .7863252 .801197 O 2.3363360 .6356812 .522932 C 4.4889790 .0137491 .651102 C $5.833876 \quad 0.467393 \quad 1.060550$ H $3.982998-0.6809610 .971939$ O $4.736779-0.7211532 .855736$ H 5.6548361 .0402560 .146245 H $6.321417 \quad 1.1432221 .773970$ H 1.3520286 .0711343 .951400 H 2.5107636 .6840932 .762306 H 0.7947837 .0988082 .620418 C $0.3233985 .842289-0.249412$ H $2.567075 \quad 1.878891-1.032874$ H 2.5783880 .0532683 .263308 H $5.194917-0.1291953 .476583$ H $0.6913606 .137420-1.240486$ H $-0.051416 \quad 6.7511390 .234617$ H 2.3611385 .9566860 .413009 C $5.991446-1.270230-1.447580$ O $6.193487-1.689914-0.092604$ C $6.789991-0.7097390 .762834$ C $8.155055-0.2846390 .203613$ C $8.0534530 .182884-1.245131$ C $7.320306-0.855839-2.091415$ H $5.284541-0.432095-1.471385$ C $5.368375-2.449835-2.201815$ H $6.981330-1.2148621 .717867$ $\begin{array}{llll}\text { O } & 8.729127 & 0.753753 & 0.990445\end{array}$ H $8.845167-1.1375920 .243626$ H $7.5683891 .163339-1.319387$ O $9.3692010 .345755-1.779685$ H $7.963384-1.734785-2.228710$ O $7.063324-0.308010-3.383104$ C $3.914935-2.627159-1.832808$ H $5.424266-2.322019-3.289102$
H $\quad 5.885718-3.387638-1.965482$

H 8.8001130 .4235691 .902312

H $9.8368400 .978902-1.208039$

H $7.918334-0.034043-3.757331$

O $3.144555-1.788585-2.582587$

O $3.519365-3.414184-0.983218$

C $1.730026-1.866588-2.377825$

H $1.479515-2.075105-1.334763$

H $1.292993-0.903516-2.654558$

H $1.317224-2.642038-3.028317$

SCF Energy $(B 3 L Y P / 6-31 G * * / / M M F F)=-3245.90927340$

05_00091

MMFF Geometry

C $3.0012563 .574840-0.502273$

C $4.3056853 .661962-0.197455$

C $5.2553932 .492960-0.068124$

O $4.579867 \quad 1.322448 \quad 0.445589$

C $5.8541012 .140757-1.441674$

C $6.8929881 .036921-1.379611$

C $6.682551-0.243878-1.746029$

C $8.2551051 .469035-0.900168$

C $5.403358-0.869331-2.236140$

C $5.024271-2.090844-1.384471$

C $3.623198-2.659653-1.671854$

C $3.506325-3.254088-3.076260$

C $2.083891-3.759270-3.364218$

O $2.669933-1.609707-1.493533$

O $1.981665-4.009331-4.774476$

C $1.082702-2.634147-3.031478$

C $1.793749-5.092178-2.664963$

O $-0.261377-3.094995-3.155581$

C $1.297942-2.004879-1.635419$

C $0.438360-0.723125-1.506202$

O $0.911518-2.944199-0.633506$

C 4.3972331 .2538621 .793444

O 4.6745332 .1086232 .619491

C $3.799876-0.0646092 .095694$

C $3.338012-0.3293423 .325953$

C $2.725484-1.5862873 .759767$

C $2.670631-2.7231862 .773923$

C $0.6870400 .087708-0.225576$

C $-0.336771-0.1484070 .868104$

C $-1.256748 \quad 0.9233512 .921160$

O $-1.096845-1.1114810 .875780$

C -0.7462090 .1825604 .114271$

C -1.243188 -0.996737 4.517294

C $-0.778801-1.7423825 .736378$

C $0.081590-2.9366845 .389360$

C $-0.463996-4.0634014 .900573$

C $1.566480-2.8494285 .664590$

C $2.229367-1.6666965 .011323$

N $-0.2915120 .840979 \quad 1.841813$

H $2.5699302 .598864-0.705395$

H $4.7439064 .644561-0.036929$

H 6.0597782 .7704590 .625032

H $6.3210713 .031503-1.883752$

H $5.0507801 .867139-2.134759$

H $7.522112-0.935607-1.685539$

H $8.9826640 .650660-0.919743$

H $8.6450742 .270311-1.536491$

H $8.2017351 .836176 \quad 0.129323$

H $4.579383-0.152421-2.233636$

H $5.555634-1.177150-3.276629$

H $5.773532-2.881973-1.514051$

H $5.058239-1.797070-0.328293$

H $3.432763-3.435747-0.920746$

H $4.237909-4.058640-3.220035$

H $3.748136-2.490367-3.827021$

H $2.658533-4.666087-5.012115$

H $1.211056-1.848248-3.789191$

H $2.501337-5.860913-2.997976$

H $0.798566-5.467346-2.928017$

H $1.862500-5.030647-1.576868$

H $-0.359256-3.465936-4.049214$

H $0.693586-0.063474-2.346691$

H -0.626128 -0.967551-1.598706

H $0.588645-2.4640850 .144922$ 
H $3.746561-0.785921 \quad 1.289888$ H $3.406174 \quad 0.4472074 .086522$ H $3.679749-3.0055882 .453787$ H $2.089742-2.4358341 .892294$ H $2.202408-3.6241913 .177322$ H $1.680680-0.0967440 .186771$ H $0.6381221 .147684-0.489356$ H -2.2185840 .5303362 .576516$ H -1.392749 1.9811553 .167099 H 0.0513550 .6523524 .683954 H -2.043923 -1.457260 3.942603 H -1.663473 -2.076598 6.294086 H $-0.244570-1.0756406 .424622$ H $0.142811-4.9320084 .667980$ H -1.531561 -4.140282 4.721544 H $2.082435-3.7753145 .383421$ H $1.712545-2.7645596 .749516$ H $2.297003-0.7906815 .657075$ H $\quad 0.4173081 .5663001 .782346$ C 0.7482734 .5265390 .083010 O $-0.0258363 .583069-0.685845$ C -1.368460 $4.084910-0.844491$ C -1.5119555 .1893860 .188124$ C $-0.1165445 .783173 \quad 0.183986$ H -1.413122 4.498229-1.860099 C $-2.3571342 .922160-0.697708$ H $-2.2872305 .915225-0.074027$ H -1.7491234 .7720971 .174880$ C $\quad 0.1819316 .638829 \quad 1.402804$ H $0.002396 \quad 6.394519-0.720896$ C $-3.8344423 .345088-0.738668$ O $-2.1206452 .002107-1.768179$ H -2.1469672 .3838810 .231345$ H -4.0669523 .9778050 .126155$ O $-4.0300364 .160110-1.902584$ C $-4.8355942 .174321-0.812026$ C -4.7392041 .2085330 .383360$ H $-4.7096101 .644255-1.763011$ O $-6.1459002 .753920-0.831166$ H -3.7199650 .8208800 .460582$ H $-4.943603 \quad 1.768207 \quad 1.304324$ H -0.5041337 .4907151 .451912$ H $0.074236 \quad 6.0667932 .330510$ H 1.2037237 .0295361 .364881 C $2.0884244 .766327-0.619011$ H -1.171699 $1.792609-1.770908$ H -4.967357 4.419511-1.919478 H $-6.7598642 .081362-1.171301$ H $2.5756605 .654343-0.200128$ H $1.9149794 .949316-1.686541$ H $\quad 0.9032334 .0817761 .074534$ C $-4.389029-1.475740-1.038114$ O $-5.587202-0.695113-0.938489$ $\begin{array}{llll}\text { C } & -5.733525 & 0.027716 & 0.288651\end{array}$ C $-5.702655-0.9392381 .481948$ C $-4.486774-1.8599981 .450534$ C -4.345659-2.521700 0.080602 H -3.512778 $-0.819607-0.971591$ C $-4.384466-2.138360-2.419173$ H -6.7410610 .4607050 .269616$ O $\quad-5.718144-0.2299672 .716296$ H -6.604972 -1.564510 1.460448 H -3.574350 -1.320236 1.721482 O $-4.648034-2.8875612 .430990$ H -5.149530 -3.256420 -0.056157 O $-3.107912-3.2262090 .022166$ C $-4.097368-1.121713-3.497969$ H $-3.633090-2.932523-2.496347$ H -5.356675 -2.593592 -2.643018 H -6.5134380 .3297082 .721457$ H $-4.760791-2.4493243 .292024$ H -3.091701 -3.848192 0.769713 O $-2.752687-0.916963-3.595884$ O $-4.964047-0.567434-4.161341$ C $-2.341720 \quad 0.033537-4.582937$ H $-2.8322080 .997557-4.416870$ H -1.260808 $0.170599-4.492674$ H $-2.566775-0.346459-5.583831$
SCF Energy (B3LYP/6-31G**//MMFF) $=-3245.91539296$

0500092

MMFF Geometry

C $-3.269570-2.218750-2.532298$

C $-2.163519-1.957410-1.819386$

C $-0.860884-1.515572-2.426621$

O $-0.656948-0.166148-1.935577$

C $0.326065-2.388800-1.987487$

C $0.167343-3.876497-2.210705$

C $0.147293-4.797806-1.226753$

C $0.085436-4.307353-3.651953$

C $0.236326-4.5618530 .255242$

C $1.533327-5.091476 \quad 0.884014$

C $2.828525-4.5262570 .274497$

C $4.045992-5.0057641 .067240$

C $5.353421-4.3914720 .546334$

O $2.770957-3.0988020 .303049$

O $6.353205-4.6503521 .543942$

C $5.182120-2.8599550 .420451$

C $5.826442-5.086033-0.736779$

O $6.326131-2.281387-0.198987$

C $3.891231-2.447245-0.326755$

C $3.607888-0.930221-0.208258$

O $3.986912-2.800306-1.706488$

C $0.227010 \quad 0.639437-2.579074$

O $\quad 0.931475 \quad 0.354417-3.532841$

C $0.1651501 .959521-1.911889$

C $0.9736822 .958783-2.294033$

C $0.9609244 .321338-1.752987$

C $-0.0090624 .617054-0.637399$

C $4.474546-0.008243-1.069240$

C $5.1234451 .073474-0.232517$

C 4.6099943 .0982011 .101393

O $6.324038 \quad 1.053367 \quad 0.028954$

C 3.7243914 .2829690 .891470

C 4.1641955 .4443540 .384673

C 3.3133436 .6681220 .208194

C $3.1722627 .072599-1.241645$

C $4.1174587 .809342-1.849824$

C $1.8915856 .703930-1.953097$

C $1.7988925 .239090-2.280028$

N 4.2227152 .0413540 .188256

H -3.241096 -2.144223 -3.616366

H -2.206948 -2.005352 -0.734112

H $-0.934659-1.485905-3.521136$

H $0.559964-2.153685-0.945176$

H $1.220005-2.062711-2.532928$

H $\quad 0.051560-5.846311-1.506123$

H $\quad 0.059277-5.396964-3.759312$

H $-0.818021-3.909092-4.123577$

H $0.957169-3.943788-4.205963$

H $\quad 0.107188-3.5086760 .516270$

H $-0.610596-5.0774650 .725978$

H $1.497490-4.8406031 .952369$

H $1.551674-6.1866470 .816559$

H $2.899158-4.865411-0.765100$

H $4.107173-6.1013491 .063279$

H $3.927669-4.7177982 .121535$

H $7.206556-4.3284721 .207834$

H $5.135255-2.4477581 .438035$

H $6.012099-6.150174-0.547342$

H $6.780181-4.673314-1.083427$

H $5.104929-5.015292-1.553405$

H $7.091851-2.4501220 .375117$

H $2.564458-0.756023-0.505362$

H $3.647521-0.6460220 .850962$

H $3.400993-2.216714-2.215369$

H $-0.5767142 .082299-1.131663$

H $1.6926822 .773094-3.090575$

H $0.0937245 .621151-0.221427$

H 0.1481123 .9269840 .198664

H -1.042238 $4.515847-0.987291$

H $5.262176-0.540195-1.610392$

H $3.8569180 .476091-1.836353$

H 5.6683923 .3395050 .954878

H 4.4931612 .7169952 .120903

H 2.6845674 .1870261 .193348 
H 5.2105565 .5334200 .099793 H 3.7767557 .4873860 .773773 H 2.3224856 .5325670 .658520 H $4.0094188 .131012-2.880186$ H $5.0230898 .103706-1.329402$ H $1.8174147 .250013-2.903473$ H $1.0399707 .061493-1.366993$ H $2.4749314 .928342-3.077546$ H 3.2304031 .8753570 .043226 C -5.515034 -1.324857 -1.940281 O $-4.945501-0.293446-1.111334$ C $-5.9911910 .334744-0.350295$ C -7.301733 -0.169141-0.942108 C $-6.913167-1.571541-1.378295$ H $-5.904593-0.0425130 .674439$ C $-5.792112 \quad 1.853552-0.349190$ H $-8.118074-0.156000-0.213626$ H -7.613113 $0.424542-1.809171$ C -7.879288 -2.188910 -2.373636 H -6.854132 -2.213080 -0.489169 C -4.3628262 .2904680 .039533$ O $-6.0470622 .347539-1.670190$ H -6.5352082 .3233770 .305750$ H $-3.6633061 .995908-0.752084$ O $-4.338694 \quad 3.723723 \quad 0.065862$ C $-3.864747 \quad 1.7502151 .394281$ C -2.4329472 .2260591 .700694$ H $-3.880148 \quad 0.656267 \quad 1.376550$ O -4.7601192 .1830542 .414722$ H $-1.778023 \quad 1.849757 \quad 0.908193$ H -2.3921463 .3211621 .655350$ H -8.878104 -2.278972 -1.934497 H -7.964616 -1.580852 -3.280524 H -7.548490 -3.189595 -2.668817 C $-4.598570-2.548442-1.908933$ H $-5.9087193 .310060-1.651100$ H -4.9043394 .0118370 .803002$ H -4.5562971 .6769533 .219390$ H -5.046883 -3.383611 -2.457940 H -4.452989 -2.879552 -0.873596 H - $-5.576222-0.918235-2.958700$ C - $-1.325183-0.5025242 .582500$ O $-2.115906 \quad 0.407937 \quad 3.354055$ C -1.894649 1.7931343 .081449 C -0.4194192 .1492633 .314520$ C 0.5161071 .2369402 .524465 C $0.169752-0.2273992 .787732$ H -1.586563 -0.4101641 .520795$ C -1.672579-1.922278 3.051698 H -2.477118 2.3462623 .829009 O -0.1821853 .5104332 .972464$ H -0.1944922 .0438354 .383949$ H $0.4890581 .471243 \quad 1.454042$ O 1.8541001 .4914022 .957306 H $0.462112-0.4926073 .812220$ O $0.914596-1.0544441 .899005$ C -2.990529-2.401772 2.493414 H $-0.915609-2.6445992 .725074$ H -1.721686 -1.9857104 .145561$ H 0.7654553 .6835893 .106428 H 2.4484930 .9666682 .395048 H $1.842615-1.0284852 .185897$ O $-4.025543-1.7904583 .134855$ O -3.084158 -3.238308 1.604259 C $-5.336412-2.1911122 .723581$ H $-5.412806-2.2388211 .633596$ H -5.577074 -3.161706 3.166042 H -6.049368 -1.447877 3.090163

SCF Energy (B3LYP/6-31G**//MMFF $)=-3245.90739393$

0500093

MM̄MF Geometry

C $3.562800-0.7248872 .818648$

C $2.306521-0.6565752 .353119$

C $1.171766-0.0110653 .101900$

O $0.753417 \quad 1.113803 \quad 2.290131$

C -0.024383 -0.967005 3.289860

C $0.308880-2.2766103 .976533$
C $0.184907-3.495478 \quad 3.413113$

C $0.795833-2.1533655 .398098$

C $-0.331413-3.8241372 .038953$

C -1.262001-5.048579 2.061349

C $-2.152688-5.1848450 .813576$

C $-1.356409-5.214061-0.493261$

C $-2.266477-5.224622-1.731325$

O $-3.071757-4.0866460 .827044$

O $-1.457158-4.907731-2.875179$

C -3.308337 -4.092892 -1.591669

C $-2.859969-6.613099-1.992964$

O $-4.258047-4.138785-2.653337$

C $-4.038462-4.099845-0.231906$

C $-4.955376-2.869127-0.036663$

O $-4.873889-5.260542-0.152349$

C 0.0735472 .1204802 .899888

O $-0.251741 \quad 2.1853804 .074138$

C $-0.2157763 .143697 \quad 1.869887$

C -1.0808134 .1332232 .138536$

C $-1.4871815 .195577 \quad 1.214754$

C $-0.7166985 .330480-0.072879$

C $-4.232085-1.520900-0.062107$

C $-5.105345-0.3787010 .431352$

C -4.9374232 .0197591 .082330$

O $-6.281349-0.519840 \quad 0.754371$

C -4.4626683 .2209220 .329044$

C -5.2272584 .3026050 .115682$

C $-4.7689755 .532564-0.617590$

C $-4.586444 \quad 6.719974 \quad 0.304773$

C -5.6336927 .4769430 .673286$

C -3.1776227 .0703080 .738378$

C -2.5355455 .9777331 .550127$

N $-4.4027460 .819606 \quad 0.464912$

H $3.796845-0.3040963 .793299$

H $2.078721-1.0675021 .373573$

H 1.5207360 .3622094 .073184

H -0.496000 -1.120263 2.314369

H $-0.800687-0.4605983 .878696$

H $0.486760-4.3613704 .001623$

H $0.920793-3.1287545 .880698$

H $1.762594-1.6426655 .435444$

H $0.076797-1.5835035 .995818$

H $-0.865350-2.9783801 .601191$

H $\quad 0.529066-4.0372721 .394465$

H -0.658329 -5.957112 2.179650

H -1.919095 -4.980973 2.938925

H -2.724139 -6.1141220 .925021$

H $-0.668606-6.068520-0.508595$

H $-0.723841-4.322131-0.563104$

H $-0.742389-5.565196-2.928052$

H $-2.765154-3.145192-1.700128$

H -2.061297 -7.341559-2.177944

H $-3.480676-6.613953-2.895650$

H -3.458334 -6.988536-1.160477

H -3.759899 -4.132378 -3.488540

H $-5.763356-2.875003-0.779536$

H -5.456199 -2.971883 0.936630

H $-5.565114-5.0999240 .511144$

H $\quad 0.2759843 .0411970 .908739$

H -1.563106 4.1531443 .114934

H $-0.9306074 .487041-0.735806$

H 0.3615405 .3621210 .121078

H $-0.9469476 .241688-0.627908$

H -3.349010 -1.561059 0.584952

H -3.909194 -1.282476 -1.081214

H -4.5470192 .0465812 .105017$

H -6.029678 1.9630761 .128535

H -3.439112 $3.217011-0.035670$

H $-6.247416 \quad 4.3122860 .493454$

H -5.517486 $5.771403-1.384591$

H $-3.8414035 .342424-1.171742$

H $-5.508854 \quad 8.344053 \quad 1.313241$

H $-6.640583 \quad 7.2433720 .343049$

H -3.1790967 .9791941 .354080$

H -2.602343 $7.328181-0.154485$

H -3.0266535 .8047942 .508869$

H $-3.398796 \quad 0.7859920 .310574$

C $5.677379-0.2789361 .559407$ 
$\begin{array}{llll}\text { O } & 5.024055 & 0.531619 & 0.562077\end{array}$

C $5.9159350 .715475-0.552522$

C $7.2894720 .277060-0.062205$

C $6.916564-0.8560830 .875898$

H $5.5891030 .019676-1.334556$

C $5.8251932 .153617-1.075299$

H $7.942566-0.038654-0.881259$

H $7.799177 \quad 1.0740520 .491167$

C $8.028253-1.2422501 .835674$

H $6.640399-1.7331790 .274952$

C $4.3829562 .624719-1.359950$

O $6.377300 \quad 3.033731-0.088206$

H $6.4511562 .260037-1.969010$

H $3.8422652 .730182-0.411645$

O $4.4524853 .942563-1.918468$

C $3.5843911 .713828-2.309695$

C $2.1555352 .234829-2.537199$

H $3.5344630 .712384-1.879950$

O $4.2774961 .622384-3.551300$

H $1.6861952 .377685-1.557358$

H $2.1988613 .218512-3.020604$

H $8.909245-1.587426 \quad 1.284755$

H $8.332528-0.3948982 .459271$

H $7.706987-2.0508412 .499674$

C $4.703027-1.3456982 .060566$

H $6.3069653 .939388-0.435753$

H $4.8748493 .867809-2.791489$

H $3.8753420 .899626-4.061676$

H $5.215275-2.0494102 .726110$

H $4.316450-1.9236931 .213267$

H 5.9663230 .3937372 .377580

C $0.685543-0.313748-1.721171$

O $1.300576-0.057062-2.988751$

C $1.2804401 .308167-3.413774$

C $-0.172766 \quad 1.775002-3.547536$

C $-0.9388841 .586035-2.243371$

C $-0.7758220 .166731-1.703342$

H $1.2591500 .180032-0.927504$

C $0.741111-1.834226-1.490363$

H $1.7163871 .321607-4.420451$

O $-0.2130653 .142200-3.942949$

H $-0.6653511 .194594-4.338517$

H $-0.6341902 .327702-1.498519$

O $-2.3238801 .831031-2.503216$

H -1.410460 -0.508971 -2.291226

O $-1.2714410 .164659-0.364537$

C $2.143315-2.379786-1.327365$

H $0.194629-2.096227-0.579927$

H $0.290071-2.350665-2.346414$

H -1.148426 $3.405482-3.984568$

H -2.812600 $1.632229-1.686755$

H -1.296642 $-0.754892-0.053991$

O $2.068073-3.734188-1.158994$

O $3.170511-1.718110-1.336986$

C $3.318977-4.404252-0.976912$

H $3.786398-4.080002-0.042687$

H $3.123048-5.478067-0.916742$

H $3.981326-4.215866-1.827252$

SCF Energy (B3LYP/6-31G**//MMFF) $=-3245.92220635$

05_00094

MMFF Geometry

C $2.870948-3.329163-1.507113$

C $2.318224-2.139219-1.230448$

C $1.257448-1.483924-2.074174$

O $0.167475-1.091178-1.208607$

C $1.828470-0.211046-2.724917$

C $0.8581520 .479088-3.664857$

C $0.383101 \quad 1.728046-3.491110$

C $0.444783-0.312170-4.879472$

C $0.703246 \quad 2.691360-2.383799$

C $-0.2251852 .602591-1.163503$

C $-1.6898252 .960462-1.460497$

C $-2.490203 \quad 3.076414-0.162180$

C $-3.9777723 .355624-0.429093$

O $-2.2559431 .930355-2.272990$

O $-4.692168 \quad 3.127707 \quad 0.796268$

C $-4.5120642 .336916-1.461395$
C $-4.221192 \quad 4.822782-0.805059$

O $-5.839506 \quad 2.690680-1.842668$

C $-3.6024842 .182841-2.700299$

C $-4.0055281 .009658-3.627816$

O $-3.6755943 .379563-3.485156$

C $-0.713297-2.056441-0.831996$

O $-0.715562-3.228188-1.173140$

C $-1.667208-1.4379890 .113586$

C $-2.396771-2.2221110 .920537$

C $-3.347758-1.7661571 .935787$

C $-3.679870-0.2979041 .968464$

C $-4.021623-0.374638-2.969833$

C $-5.388945-0.799888-2.460833$

C $-6.497957-2.606818-1.151571$

O $-6.423270-0.189882-2.717831$

C $-6.701257-2.1335550 .253173$

C $-6.882795-2.9658051 .289784$

C $-7.131843-2.5091222 .698726$

C $-6.174333-3.1311993 .690466$

C $-6.527580-4.2109954 .408140$

C $-4.842346-2.4501603 .909878$

C $-3.880969-2.6780892 .775558$

N $-5.322465-1.968138-1.713367$

H $2.555974-3.871496-2.395061$

H $2.644422-1.595755-0.348857$

H $0.886788-2.172333-2.843093$

H $2.734174-0.458344-3.294834$

H $2.1470420 .473525-1.930919$

H $-0.3100172 .120063-4.234696$

H $-0.1144190 .294931-5.599627$

H $1.326950-0.701180-5.398374$

H $-0.194814-1.152871-4.594529$

H $1.7401812 .562987-2.056883$

H $\quad 0.6612183 .708127-2.795070$

H $-0.1672721 .591874-0.742823$

H $0.1605513 .291735-0.403133$

H $-1.7078423 .911034-2.006556$

H -2.066508 3.8505690 .489752

H -2.4087102 .1363980 .400146$

H -4.3209063 .7178531 .474242$

H $-4.580217 \quad 1.375522-0.939369$

H $-3.8751045 .485814-0.002960$

H $-5.2909085 .029013-0.921310$

H -3.709585 $5.120847-1.722480$

H $-6.2003701 .965055-2.378626$

H $-4.954428 \quad 1.230823-4.130795$

H $-3.2579620 .965476-4.433541$

H $-3.392862 \quad 3.172119-4.391267$

H $-1.715775-0.3558130 .144301$

H -2.266170 $-3.301560 \quad 0.853261$

H -3.9981650 .0435650 .977952$

$\begin{array}{llll}H & -2.807460 & 0.286793 & 2.279446\end{array}$

H $-4.497322-0.0486062 .647913$

H -3.733270 -1.119264 -3.723047

H -3.291994 -0.432376 -2.156996

H $-6.336640-3.688232-1.205312$

H -7.374753 -2.353451 -1.756237

H $-6.727371-1.0593870 .420911$

H $-6.885096-4.0392441 .113008$

H -8.163820 -2.779601 2.958473

H -7.083960 -1.416272 2.781161

H $-5.857757-4.6469295 .141834$

H $-7.493644-4.6864784 .273689$

H $-4.365086-2.8404204 .818945$

H -5.013994 -1.390999 4.119686

H -3.578062 -3.720737 2.667712

H $-4.415720-2.376769-1.506275$

C $5.332097-3.415006-0.805651$

O $5.402067-2.102762-0.210492$

C $6.088524-1.226531-1.119950$

C $6.842799-2.139195-2.080905$

C $5.854223-3.278445-2.237748$

H $5.313871-0.689459-1.681086$

C $6.962629-0.221962-0.365489$

H $7.081327-1.642913-3.026348$

H $7.777337-2.508993-1.643501$

C $6.466119-4.553524-2.790556$

H $5.061126-2.945985-2.918510$ 
C $6.2381090 .580924 \quad 0.735239$

O $8.040410-0.9293560 .261562$

H $7.4270290 .458899-1.089333$

H $6.074162-0.0569151 .612058$

O $7.1432451 .606411 \quad 1.169541$

C 4.8987881 .2216740 .323636

C 4.3983432 .2478691 .361134

H 4.1564240 .4296800 .177612

O $5.079843 \quad 1.887778-0.927361$

H $4.555808 \quad 1.8467942 .367035$

H 5.0056783 .1561311 .270048

H $6.874808-4.381512-3.791651$

H $7.279463-4.918013-2.154095$

H $5.713332-5.344986-2.863128$

C $3.918800-3.984305-0.649903$

H $8.590103-0.2717150 .721444$

H $7.2587182 .227416 \quad 0.429704$

H $4.2006962 .098081-1.283288$

H $3.612587-3.9059860 .400891$

H $3.930287-5.053969-0.891644$

H $6.015229-4.040060-0.215903$

C 1.9159841 .1544642 .820388

O $2.0449401 .506574 \quad 1.435558$

C 2.9107292 .6163301 .162367

C 2.4479513 .8588371 .936023

C $2.304846 \quad 3.579782 \quad 3.427611$

C $1.4259682 .350423 \quad 3.649421$

H 2.8836340 .8137913 .205864

C $0.916092-0.0096962 .912837$

H 2.7786852 .8516100 .101126

O 3.3493244 .9393861 .721526

H $1.4739574 .176243 \quad 1.542200$

H 3.2862973 .4554703 .899327

O $1.705754 \quad 4.7108504 .063751$

H $\quad 0.390196 \quad 2.593662 \quad 3.380949$

O 1.4294322 .0195355 .037010

C $1.539125-1.3442492 .562129$

H $0.534303-0.106383 \quad 3.935308$

H 0.0785110 .1628972 .227516

H 3.0348545 .6912132 .252206

H 0.8253964 .8452993 .673297

H $2.342214 \quad 1.798796 \quad 5.289712$

O $0.578144-2.310908 \quad 2.653317$

O $2.711288-1.5235452 .263588$

C $1.005368-3.6474652 .373525$

H $1.899609-3.9007562 .950977$

H $\quad 0.199680-4.3278472 .662058$

H $1.191217-3.7595491 .302493$

SCF Energy (B3LYP/6-31G**/MMFF) $=-3245.91070281$

0500095

MMFF Geometry

C $4.095902-3.317399-1.794654$

C $3.399728-2.246899-2.205414$

C $1.907275-2.237101-2.401414$

O $1.385598-1.473707-1.288879$

C $1.550871-1.537913-3.726098$

C $0.106888-1.709004-4.155637$

C $-0.803851-0.718519-4.235177$

C $-0.281798-3.107338-4.569483$

C $-0.6325310 .743899-3.934027$

C $-1.1939101 .188363-2.575582$

C $-2.7240791 .095324-2.444373$

C $-3.1856791 .735762-1.131209$

C $-4.6959171 .574744-0.898640$

O $-3.112923-0.280985-2.469253$

O $-4.963934 \quad 1.875952 \quad 0.480117$

C $-5.090470 \quad 0.098314-1.122164$

C $-5.505728 \quad 2.580784-1.725139$

O $-6.505655-0.061393-1.079747$

C $-4.532536-0.492218-2.433002$

C $-4.786953-2.013125-2.582825$

O $-5.175750 \quad 0.141514-3.546367$

C $0.251886-1.909752-0.680981$

O $-0.473020-2.825999-1.031923$

C $0.050177-1.0722790 .522617$

C $-1.157296-1.0198431 .101519$

C $-1.512277-0.2486922 .294251$
C -0.3971750 .4221653 .052230$

C $-4.216168-2.894272-1.468351$

C $-5.201606-3.183815-0.347328$

C $-5.278973-3.9769462 .016670$

O $-6.417392-3.063750-0.472536$

C $-5.478986-2.7351412 .826566$

C $-4.875388-2.5033724 .001970$

C $-5.074845-1.2509294 .806897$

C $-3.802707-0.4451654 .955840$

C $-3.071984-0.5092356 .081540$

$\begin{array}{llll}\text { C }-3.424806 & 0.502414 & 3.838227\end{array}$

C $-2.809279-0.2029732 .662717$

N $-4.564513-3.6389690 .798870$

H $3.582376-4.257811-1.612255$

H $3.920939-1.303973-2.349634$

H $1.528144-3.265342-2.376193$

H $2.180747-1.934734-4.534275$

H $1.806769-0.476891-3.643040$

H $-1.800751-0.979631-4.588761$

H $-1.296484-3.153288-4.979607$

H $0.399089-3.476576-5.343486$

H $-0.244501-3.791892-3.717452$

H $0.421657 \quad 1.033338-3.983078$

H $-1.123399 \quad 1.315139-4.732024$

H $-0.717140 \quad 0.598543-1.786448$

H $-0.8930712 .231340-2.422775$

H $-3.1724181 .616175-3.298657$

H -2.909344 $2.796526-1.100391$

H $-2.6549681 .266498-0.292970$

H $-4.6379752 .774548 \quad 0.659635$

H -4.684347 - $0.469527-0.276238$

H $-5.2351483 .607486-1.450114$

H $-6.5779702 .489862-1.519834$

H $-5.3483602 .477042-2.800749$

H $-6.8125550 .299318-0.230361$

H $-5.856657-2.200862-2.735982$

H $-4.300282-2.331210-3.516149$

H $-5.077035-0.430177-4.325745$

H $0.900241-0.5157910 .897298$

H $-1.970522-1.5876330 .654525$

H $\quad 0.053145 \quad 1.2172382 .448464$

H $0.382026-0.3023743 .314691$

H -0.7218100 .8748443 .991696$

H -3.954222 $-3.871021-1.895048$

H -3.297666 -2.461327 -1.063384

H $-4.684836-4.7273242 .547578$

H $-6.249181-4.4162841 .763163$

H $-6.157017-1.9876722 .420788$

H $-4.200947-3.2513544 .412225$

H $-5.453934-1.5455295 .794260$

H $-5.857323-0.6162114 .372492$

$\begin{array}{llll}H & -2.174569 & 0.087469 & 6.207298\end{array}$

H $-3.351745-1.1642806 .899999$

H -2.7686251 .2920074 .216991$

H -4.3215131 .0345843 .495538$

H $-3.537122-0.7150592 .034381$

H -3.549402 $-3.643378 \quad 0.833174$

C $5.781908-3.1297070 .025455$

O $5.290955-1.8426940 .449952$

C $6.184015-1.2983091 .438871$

C $7.164177-2.414508 \quad 1.782071$

C $7.243726-3.162728 \quad 0.462295$

H $6.737882-0.4889060 .949449$

C $5.373755-0.7377472 .614106$

H $8.131697-2.0276172 .116306$

H $6.775430-3.0714602 .568523$

C $7.821098-4.5611270 .590191$

H $7.858481-2.579814-0.236668$

C $4.265146 \quad 0.2493912 .183496$

$\begin{array}{lllll} & \text { O } & 4.738181 & -1.832476 & 3.287026\end{array}$

H $6.047475-0.2738363 .343711$

H $3.513279-0.2942811 .602939$

O $3.590647 \quad 0.697706 \quad 3.366284$

C 4.7716021 .4753111 .398101

C 3.6576892 .4601490 .997368

H $5.316348 \quad 1.1514570 .510164$

$\begin{array}{lllll}\text { O } & 5.709042 & 2.172466 & 2.224677\end{array}$

H 3.0763282 .7238381 .890974 
H 4.1160673 .4082650 .689809 H $8.845634-4.5204660 .973935$ H $7.229970-5.178573 \quad 1.274874$ H $7.844625-5.064029-0.381646$ C $5.567502-3.271535-1.482218$ H $4.218614-1.4594214 .019713$ H $4.225071 \quad 1.2239813 .882701$ H 6.0765242 .9037571 .699391 H $6.033577-4.188867-1.857955$ H $6.033895-2.428934-2.007907$ H $5.202087-3.8886180 .567990$ C $1.3507303 .951288-0.224640$ $\begin{array}{llllll}\text { O } & 1.404875 & 2.544312 & 0.039071\end{array}$ C $2.7059821 .957085-0.109541$ C $3.1834562 .155942-1.556686$ C $3.1395553 .622997-1.979864$

C $1.7758184 .237050-1.669353$ H $2.007384 \quad 4.4868210 .472237$ C $-0.092544 \quad 4.409802 \quad 0.023182$ H 2.5600050 .8838350 .036248 O $4.501063 \quad 1.661313-1.750698$ H $2.5268501 .588857-2.229492$ H $3.9449944 .200930-1.511981$ O $3.354862 \quad 3.710629-3.391047$ H $1.0302213 .839647-2.369512$ O $1.839623 \quad 5.647315-1.870032$ C $-0.487207 \quad 4.297175 \quad 1.479869$ H $-0.2320525 .455144-0.271474$ H $-0.7733903 .780085-0.559184$ H $4.4913200 .714194-1.532844$ H $4.2069143 .281247-3.580209$ H $2.1340055 .797089-2.784937$ O $-1.849236 \quad 4.248943 \quad 1.569096$ $\begin{array}{lllll}\text { O } & 0.295330 & 4.285455 & 2.420191\end{array}$ C $-2.383732 \quad 4.1654562 .893683$ H -2.3307035 .1475313 .371886$ H $-3.431846 \quad 3.8638522 .818996$ H -1.8482773 .4205143 .489290$

SCF Energy (B3LYP/6-31G**/MMFF) $=-3245.91518737$

0500096

MMFF Geometry

C $2.650794-1.1595152 .854316$

C $1.415452-1.3527362 .369954$

C $0.171378-1.312596 \quad 3.216831$

O $-0.740921-0.4275352 .519898$

C $-0.490031-2.699307 \quad 3.356455$

C $0.394467-3.7859293 .934084$

C $0.804181-4.8788103 .258461$

C $0.794209-3.5947275 .374151$

C $0.470654-5.2478311 .840578$

C $1.645731-4.9793570 .891591$

C $1.166305-4.784724-0.553677$

C $2.353644-4.694410-1.511521$

C $1.897219-4.401801-2.947800$

O $0.427394-3.560036-0.590247$

O $3.053848-4.025896-3.711910$

C $0.953318-3.180618-2.935364$

C $1.321321-5.651466-3.623646$

O $0.374051-2.968455-4.220355$

C $-0.158157-3.242305-1.859086$

C $-0.815691-1.849802-1.712514$

O $-1.187357-4.165362-2.207827$

C -1.800916 $0.077493 \quad 3.201991$

O $-2.064155-0.0763484 .382968$

C -2.6226320 .8250312 .224806$

C -3.9179581 .0539342 .484051$

C $-4.869866 \quad 1.721113 \quad 1.592277$

C $-4.365452 \quad 2.173340 \quad 0.246580$

C $-1.925708-1.764807-0.658167$

C $-3.311428-2.120841-1.167846$

C $-5.670463-2.299963-0.371364$

O $-3.548820-2.423607-2.333389$

C $-6.373352-1.007730-0.649015$

C $-7.447449-0.586300 \quad 0.036119$

C $-8.1934250 .680383-0.274592$

C $-8.390083 \quad 1.5616380 .939071$

C -9.5418601 .5369401 .631072$
C $-7.289328 \quad 2.536138 \quad 1.293241$

C -6.1432451 .8773512 .010580$

N $-4.259399-2.042244-0.154164$

H $2.781497-0.9835613 .919396$

H $1.284414-1.5415341 .306912$

H $0.391566-0.8851404 .203171$

H $-0.903956-2.9809312 .384294$

H - $1.377726-2.6127613 .997026$

H $1.444217-5.5951313 .771664$

H $1.365174-4.4445325 .763115$

H $1.415599-2.7017355 .489373$

H $-0.095733-3.4828986 .002087$

H $0.222576-6.3164871 .809940$

H $-0.434869-4.7404661 .499428$

H $2.184392-4.0784301 .210660$

H $2.350835-5.8176390 .949054$

H $0.507277-5.617578-0.825140$

H $2.953187-5.612596-1.478189$

H $3.024398-3.885664-1.188630$

H $3.696934-4.753740-3.660572$

H $1.574790-2.299665-2.727807$

H $2.083122-6.438035-3.686396$

H $1.022912-5.441514-4.656493$

H $\quad 0.463766-6.069396-3.092029$

H $1.104021-2.901920-4.859626$

H $-0.036530-1.142094-1.402121$

H $-1.204378-1.510404-2.679781$

H $-0.981850-4.553575-3.072672$

H $-2.146594 \quad 1.1291591 .299513$

H -4.3274350 .7040203 .430984$

H $-3.9323811 .331464-0.305012$

H -3.599746 2.9472150 .366054

H -5.143059 $2.588792-0.397095$

H $-1.693766-2.386550 \quad 0.211840$

H $-1.983228-0.724430-0.317958$

H $-6.052562-2.7943170 .527271$

H $-5.792387-2.977375-1.222607$

H $-5.998689-0.405581-1.473586$

H $-7.839027-1.2050030 .840595$

$\begin{array}{llll}H & -9.170531 & 0.396247 & -0.687333\end{array}$

H $-7.6958911 .255006-1.065728$

$\begin{array}{llll}H & -9.703078 & 2.187358 & 2.484413\end{array}$

H $-10.347116 \quad 0.861129 \quad 1.362618$

H -7.679979 3.3175541 .959022

H $-6.979932 \quad 3.0723790 .392117$

H $-6.411603 \quad 1.5070123 .001202$

H -3.976087 -1.7110180 .763484$

C 4.6975990 .1103342 .198889

O $3.984197 \quad 1.2170021 .615100$

C 4.9421252 .1476751 .070892

C 6.3238591 .6010021 .419972

C $6.055348 \quad 0.1100891 .503495$

H $4.8263562 .118150-0.018274$

C 4.6587983 .5606001 .596750

H $7.072288 \quad 1.8606860 .664757$

H $6.680463 \quad 1.9809932 .384386$

C $7.137240-0.6577892 .242014$

H $5.956144-0.2885910 .484822$

C $3.2283724 .061717 \quad 1.302826$

O 4.8356103 .5558113 .019296

H 5.4009034 .2602271 .194387

H $2.5025263 .447007 \quad 1.847943$

O 3.1080245 .3844041 .841947

C $2.8668254 .094637-0.192741$

C $1.4412984 .623798-0.440348$

H $2.9487453 .084143-0.600934$

$\begin{array}{lllll}\text { O } & 3.816446 & 4.914850 & -0.871249\end{array}$

H 0.7363503 .9826330 .101105

H $1.3441565 .633012-0.022256$

H $8.102124-0.543707 \quad 1.737030$

H $7.251979-0.3003333 .270860$

H $6.902439-1.7258922 .280977$

C $3.897806-1.1787642 .013895$

H 4.6424474 .4541013 .337916

H 3.7046495 .9620861 .335520

H $3.7225674 .748270-1.824358$

H $4.497832-2.0424442 .321083$

H $3.641080-1.3110350 .955592$ 
H $4.819412 \quad 0.333461 \quad 3.267775$

C $0.5942842 .354264-2.326250$

O $1.4071883 .492506-2.636444$

C $1.0626264 .690747-1.936570$

C $-0.3994465 .069590-2.212196$

C $-1.354143 \quad 3.904447-1.955719$

C $-0.8715132 .640279-2.668255$

H $0.6759512 .120286-1.258019$

C $1.1478631 .177440-3.143521$

H $1.6798545 .485100-2.375111$

O $-0.7731826 .192070-1.420724$

H $-0.4899475 .374014-3.262948$

H -1.470219 $3.724617-0.880924$

O $-2.6567374 .246448-2.434747$

H $-0.9940292 .754025-3.752745$

O $-1.6747601 .537496-2.255769$

C $2.364506 \quad 0.561534-2.482785$

H $\quad 0.4123330 .375881-3.255147$

H $1.4302641 .527606-4.143933$

H -1.709471 6.380105-1.604995

H $-2.5924684 .407670-3.391532$

H $-2.5995841 .758541-2.460196$

O $3.2170030 .091385-3.439283$

O $2.526610 \quad 0.463429-1.273641$

C $4.396861-0.550111-2.943691$

H $5.0019300 .161131-2.373147$

H $4.130067-1.412767-2.325341$

H $4.978971-0.899849-3.800254$

SCF Energy (B3LYP/6-31G**//MMFF) $=-3245.91912744$

0500097

MM̄FF Geometry

C -2.230697 -0.4536343 .708914$

C $-0.916844-0.6084893 .928212$

C $-0.000324-1.3355012 .976789$

O $0.964605-0.3930412 .455082$

C $0.763460-2.4383153 .732303$

C $1.605925-3.3251572 .836408$

C $2.948261-3.4228802 .895841$

C $0.844135-4.161418 \quad 1.840569$

C $3.893912-2.7032673 .815301$

C $4.450264-1.3826033 .259151$

C $5.241370-1.5313531 .948629$

C $5.961158-0.2266871 .598644$

C $6.680587-0.310544 \quad 0.242437$

O $4.323243-1.8609320 .902873$

O $7.0348461 .024453-0.151571$

C $5.697207-0.848095-0.820771$

C $7.994463-1.0945010 .343192$

O $6.367318-1.099813-2.052929$

C $4.935462-2.110946-0.369203$

C $3.808961-2.530559-1.345621$

O $5.850807-3.210634-0.291549$

C $\quad 0.545637 \quad 0.440813 \quad 1.463839$

$\begin{array}{lllll}\text { O } & -0.559714 & 0.476469 & 0.946846\end{array}$

C 1.6724081 .3345761 .121433

C 1.4305892 .4566720 .427717

C 2.4272973 .4410820 .002503

C 3.8786163 .0790950 .175049

C $2.715247-1.482761-1.578997$

C $2.938989-0.619646-2.809508$

C $2.0179471 .383549-3.970309$

O $3.792586-0.863087-3.658090$

C $2.8717342 .565572-3.633584$

C $2.4549703 .834289-3.764320$

C $3.3112605 .033495-3.471788$

C $2.6504436 .001944-2.516075$

C $1.9952347 .083253-2.971345$

C $2.8204535 .759639-1.032923$

C $1.9979134 .599970-0.539490$

N $2.0411210 .438056-2.869410$

H -2.674841-0.875755 2.810589

H $-0.468677-0.1729954 .818239$

H $-0.566224-1.7731052 .146311$

H $0.052906-3.0820974 .267953$

H $1.385941-1.9683224 .501410$

H $3.440251-4.1030032 .200986$

H $1.479924-4.9051681 .348117$
H $\quad 0.032960-4.7038522 .336903$

H $\quad 0.417337-3.5275671 .058521$

H $3.422403-2.5113784 .785003$

H $4.730402-3.3792384 .035058$

H $3.618229-0.6844823 .110090$

H $5.102663-0.9477324 .026543$

H $5.965285-2.3453062 .072327$

H 6.6678330 .0525702 .390006

H 5.2283650 .5900681 .551873

H 7.5953251 .4042870 .546669

H $4.973657-0.047769-1.015823$

H $8.672268-0.6148361 .059609$

H $8.526850-1.098813-0.614348$

H $7.853695-2.1282640 .665316$

H $6.811864-0.275397-2.314603$

H $4.240561-2.883389-2.289954$

H $3.313311-3.409475-0.907879$

H $5.343961-4.038106-0.340580$

H 2.6630961 .0508001 .456067

H $0.4016902 .687073 \quad 0.153323$

H $4.0871612 .107532-0.285664$

H 4.1385813 .0279681 .237822

H $4.5656393 .786653-0.293107$

H $1.766201-2.006826-1.745900$

H $2.585889-0.845236-0.699664$

H $0.9724251 .661534-4.137570$

H $2.4004660 .898687-4.874286$

H $3.8860762 .370628-3.293024$

H $1.4497664 .025762-4.133685$

H $3.5176045 .535782-4.426112$

H $4.2910534 .740285-3.074722$

H $1.5414377 .797700-2.292640$

H $1.8938237 .275150-4.034516$

H $2.5015336 .645122-0.466819$

H $3.8858075 .655239-0.810607$

H $0.9227684 .763733-0.626246$

H $1.3982550 .591214-2.097841$

C -3.6340301 .6301564 .077716$

O -4.3002411 .4498552 .812523$

C -3.9234322 .5350011 .940671$

C -3.2458113 .5743162 .827222$

C -2.552262 2.6845043 .841230

H -3.1740602 .1363841 .246669$

C -5.1387483 .0449561 .160445$

H -2.5534334 .2099582 .267004$

H -3.9757794 .2230573 .325155$

C -2.1135113 .4145645 .097867$

H $-1.6714212 .240853 \quad 3.361145$

C -5.8592971 .9555230 .338611$

O -6.0827033 .5846992 .093781$

H -4.8360323 .8755680 .511955$

H -6.3386061 .2390791 .015991$

O $-6.9254822 .589035-0.380301$

C $-4.9552591 .203490-0.655910$

C $-5.7414580 .186434-1.504808$

H $-4.1715660 .681452-0.097832$

O $-4.3228212 .156789-1.507885$

H -6.262545 $-0.499165-0.827167$

H $-6.5101720 .708722-2.087272$

H -1.3962674 .2048014 .852691$

H -2.9626423 .8789775 .610255$

H -1.631065 2.726575 5.799341

C $-3.156160 \quad 0.285578 \quad 4.632217$

H -6.8487773 .8974951 .582665$

H $-6.5230513 .188168-1.032400$

H $-3.599914 \quad 1.703020-1.972947$

H -4.027592 -0.360767 4.796262

H $-2.6881550 .436818 \quad 5.611999$

H $-4.3929662 .027903 \quad 4.764793$

C $-3.903156-2.196310-0.950710$

O $-3.676206-1.121896-1.867163$

C $-4.856210-0.608358-2.491595$

C $-5.591325-1.727043-3.244884$

C $-5.858019-2.942303-2.357694$

C $-4.573466-3.382222-1.653154$

H $-4.551344-1.840868-0.139662$

C $-2.568526-2.554483-0.289968$

H $-4.5122660 .108709-3.247627$ 
O $-6.815183-1.234104-3.779772$

H -4.973622 -2.043073 -4.095738

H $-6.660118-2.736350-1.639449$

O $-6.326179-4.008737-3.186213$

H -3.901141 -3.823641 -2.398138

O $-4.877416-4.404674-0.708106$

C - $1.538631-3.059273-1.273954$

H $-2.133825-1.6681570 .185908$

H -2.715260 -3.2947760 .504935$

H -7.253923 -1.974850 -4.232269

H $-6.447309-4.786843-2.615228$

H -5.475966 -4.027211 -0.041010

O $-1.311259-4.388184-1.071079$

O $-1.007125-2.358889-2.125936$

C $-0.342655-4.985024-1.938705$

H $-0.669729-4.910221-2.980089$

H $0.632349-4.506952-1.803402$

H $-0.254279-6.041762-1.673446$

SCF Energy (B3LYP/6-31G**//MMFF) $=-3245.91326478$

\section{00098}

MM̄MF Geometry

C $3.407214-0.6541143 .441428$

C $2.576447-1.3764082 .673388$

C $1.125980-1.6029283 .012031$

O $0.325226-0.959301 \quad 1.989491$

C $0.789701-3.1049562 .995481$

C $-0.356386-3.4371953 .929742$

C -1.661378 -3.389551 3.601432

C $0.058641-3.8327175 .323862$

C $-2.266116-2.9793232 .287805$

C - $-3.323252-3.9889201 .815484$

C $-4.178989-3.4954810 .636246$

C $-3.365192-3.299606-0.644483$

C $-4.225752-2.738742-1.786753$

O $-4.803370-2.272675 \quad 1.034912$

O $-3.347197-2.304833-2.836085$

C $-4.959425-1.484009-1.273943$

C $-5.137853-3.806063-2.400626$

O $-5.872527-0.992874-2.253414$

C $-5.692702-1.7000430 .070501$

C $-6.215584-0.3658570 .656561$

O $-6.823574-2.549040-0.153324$

C $0.074120 \quad 0.3701142 .133830$

O 0.3993521 .0823513 .070601

C -0.6308230 .8310650 .915659$

C -0.6408462 .1433510 .636440$

C $-1.2158482 .786655-0.547024$

C -1.934330 $1.916577-1.543053$

C $-5.1177950 .555730 \quad 1.197732$

C -5.6239971 .9544471 .495968$

C -4.7657824 .2378262 .012390$

O -6.8099742 .2677901 .448269$

C $-4.545578 \quad 4.9187340 .697861$

C $-3.606570 \quad 5.853327 \quad 0.488374$

C $-3.3853016 .520118-0.838628$

C -1.969282 $6.360540-1.346254$

C $-1.1436367 .417146-1.435480$

C -1.532843 $4.990169-1.826006$

C $-1.0417624 .116600-0.701881$

N $-4.5806762 .811473 \quad 1.824142$

H $3.037664-0.2139334 .364292$

H $2.940476-1.7951841 .738216$

H $0.883843-1.1739633 .993338$

H $1.659693-3.6988863 .307028$

H $0.555159-3.4383631 .977786$

H -2.388254 -3.631711 4.376182

H $-0.799234-4.0319255 .974716$

H $0.669141-4.7412375 .293770$

H $\quad 0.646193-3.0336075 .787319$

H $-2.725688-1.9950152 .433976$

H -1.505292 -2.8654831 .512365$

H -2.839680 $-4.937871 \quad 1.552278$

H -4.003561 -4.198462 2.652260

H -4.960153 $-4.246778 \quad 0.469310$

H $-2.889495-4.240567-0.946238$

H -2.546116 -2.590710 -0.466309

H -2.811611 -3.068052 -3.113194
H $-4.198552-0.705123-1.148934$

H $-4.540398-4.631871-2.805398$

H $-5.703200-3.402203-3.247750$

H $-5.844722-4.231249-1.685224$

H $-5.372413-0.864575-3.077657$

H $-6.8296580 .150084-0.092441$

H -6.901226 -0.5806691 .488157$

H -7.461990 -2.410604 0.565483

H -1.0718800 .0830000 .267206$

H -0.1486552 .8214721 .333422$

H $-2.8437831 .500199-1.101165$

H $-1.2945221 .090633-1.871573$

H $-2.2294802 .448468-2.450301$

H -4.7036840 .1408792 .124168$

H -4.3017890 .6491710 .477495$

H -4.0511374 .5634062 .774617$

H -5.7806024 .4364682 .371016$

H $-5.1947454 .625398-0.124104$

H -2.9663436 .1613751 .311836$

H $-3.6313047 .583323-0.721138$

H $-4.0784356 .135787-1.597524$

H $-0.1351257 .313834-1.822357$

H - $1.4546748 .407263-1.119360$

H $-0.7118265 .097002-2.546896$

H -2.364606 $4.538325-2.374899$

H -0.4560164 .6472760 .050451$

H -3.626244 2.4677721 .767703

C 4.9839121 .0492532 .520518

O $4.2683941 .138397 \quad 1.272893$

C 5.0623381 .8727440 .323019

C 6.1992642 .4969231 .121919

C 6.4227401 .4426832 .192005

H $5.4880291 .133858-0.365494$

C $4.1746772 .849694-0.457721$

H 7.0856582 .6816860 .507734

H 5.9024083 .4461751 .582128

C 7.2144071 .9459813 .385945

H $\quad 6.9516400 .5917841 .741762$

C $2.9073612 .194248-1.045742$

O $3.744248 \quad 3.885350 \quad 0.435387$

H $4.7614363 .342128-1.241303$

H $2.2504011 .897322-0.221037$

O $2.1896023 .192699-1.780181$

C $3.1601700 .982068-1.959987$

C $1.8435720 .300795-2.375317$

H $3.7769740 .246196-1.438415$

O $3.8667321 .388712-3.128519$

H $1.328384-0.030171-1.466571$

H $1.1874621 .030258-2.866044$

H 8.2116332 .2742213 .075130

H 6.7168932 .7935793 .868958

H 7.3367321 .1562784 .133833

C $4.838759-0.3649193 .084276$

H $3.1668334 .482933-0.070214$

H $2.7268293 .428258-2.555971$

H $4.7503231 .684653-2.851987$

H $5.452531-0.4859653 .983704$

H $5.192725-1.1000262 .353368$

H 4.5297871 .7813513 .201581

C $2.870494-2.508621-1.756352$

O $3.104731-1.754763-2.951233$

C $2.035563-0.891110-3.340589$

C $0.766233-1.717795-3.584452$

C $0.426492-2.612229-2.393088$

C $1.646322-3.412619-1.935333$

H $2.714645-1.827893-0.910604$

C $4.139996-3.322707-1.485108$

H $2.331835-0.460579-4.305665$

O $-0.329630-0.858078-3.878746$

H $0.922638-2.352362-4.466636$

H $0.000502-2.026275-1.569983$

O $-0.592523-3.528164-2.802443$

H $1.850772-4.200268-2.672051$

O $1.311963-4.048179-0.703713$

C $5.307541-2.408900-1.196305$

H $4.027271-3.984328-0.619779$

H $4.392713-3.958181-2.342591$

H $-1.117413-1.417268-3.991238$ 
H $-0.755221-4.130420-2.056275$

H $1.998633-4.708303-0.512071$

O $6.046914-2.220154-2.326155$

O $5.517125-1.898032-0.104275$

C $7.161655-1.333429-2.191025$

H $7.840362-1.687760-1.409308$

H $7.700647-1.318077-3.141992$

H $6.811185-0.321130-1.970076$

SCF Energy $\left(B 3 L Y P / 6-31 G^{* *} / / M M F F\right)=-3245.92450164$

\section{5_00099}

MM̄FF Geometry

C $3.822360-3.1181951 .428357$

C $2.495814-3.0170581 .260845$

C $1.502890-2.8716382 .380966$

O $0.543600-1.8676581 .977601$

C $0.751240-4.2056222 .565050$

C $-0.333006-4.1673753 .624349$

C -1.648409-4.333298 3.378108

C $0.144880-3.9529155 .037602$

C $-2.314697-4.6202982 .059275$

C $-2.870428-3.3458551 .425203$

C $-3.641238-3.5867350 .118869$

C $-2.730208-4.050452-1.021251$

C $-3.477163-4.152969-2.360853$

O $-4.252087-2.335513-0.228308$

O $-2.473450-4.279342-3.380295$

C $-4.250056-2.838868-2.607102$

C $-4.332841-5.423176-2.432187$

O $-5.087433-2.936426-3.756098$

C $-5.089600-2.389811-1.392534$

C $-5.715097-0.987505-1.578467$

O $-6.173873-3.306530-1.200459$

C $0.344531-0.7930342 .786513$

O $0.983795-0.478672 \quad 3.776557$

C $-0.854863-0.0889102 .279318$

C $-1.445410 \quad 0.8684883 .010437$

C -2.6859501 .5680432 .658624$

C -3.4190081 .0903331 .432877$

C $-4.7203880 .120554-1.932286$

C $-5.2802131 .512813-1.686451$

C $-4.5680013 .908506-1.735552$

O $-6.4141991 .721053-1.264889$

C -3.786872 4.295261-0.519832

C -4.3025434 .9035510 .558789$

C -3.4789785 .2700661 .763083$

C -4.1176464 .8529173 .071441$

C $-4.449775 \quad 5.7724573 .993797$

C -4.3833423 .3828343 .317324$

C -3.1203332 .5713543 .450399$

N $-4.3473702 .495308-1.988197$

H $4.241101-3.0871902 .430768$

H $2.087222-3.0859790 .256246$

H $2.012780-2.5875943 .308860$

H $1.464205-4.9973002 .831866$

H $0.328954-4.4999011 .598334$

H -2.338835 -4.273988 4.218825

H $-0.664777-4.0494885 .768909$

H $0.907535-4.6950335 .295757$

H $0.575654-2.9547695 .156342$

H - $-1.633480-5.1334911 .376216$

H -3.134105 -5.326747 2.241889

H -3.534769 -2.838305 2.138075

H -2.055965 -2.6389261 .232222$

H $-4.429070-4.3235840 .313877$

H $-2.253734-5.009469-0.787383$

H -1.908425 -3.330138 -1.138900

H -2.922864 -4.402280 -4.233461

H -3.500088 -2.068982 -2.829206

H -3.698434 -6.314253 -2.353061

H $-4.843179-5.503824-3.398422$

H -5.083331 -5.479969-1.641122

H -4.518555 -3.121626-4.521787

H $-6.505998-1.023003-2.339125$

H $-6.223559-0.722998-0.640638$

H $-6.867488-2.859517-0.687541$

H -1.251495 -0.4147581 .324674$

H -0.9971821 .1525213 .961403$
H $-3.6685370 .027219 \quad 1.522779$

H $-2.8011901 .232476 \quad 0.540183$

H -4.3588421 .6121391 .248925$

H -3.808151 $0.016726-1.335020$

H $-4.4456830 .049214-2.990900$

H $-5.6380944 .108773-1.622756$

H $-4.1952504 .456744-2.606049$

H $-2.7257604 .065481-0.534055$

H -5.3579555 .1645980 .572232$

H $-3.3336696 .358011 \quad 1.742447$

H -2.4758054 .8307331 .703077$

H -4.9099605 .4901604 .934948$

H -4.2688096 .8296663 .830798$

H -5.054092 3.0079202 .540876

H -4.944052 3.2529954 .252978

H -2.5212182 .8385354 .321903$

H -3.421589 $2.213889-2.298673$

C $5.979582-2.4116320 .326906$

O $5.563590-1.0513790 .098267$

C $6.530705-0.407285-0.753466$

C $7.696940-1.379860-0.898880$

C $7.000552-2.717349-0.769084$

H $6.052121-0.280721-1.731521$

C $6.9310810 .956168-0.181534$

H $8.219828-1.257528-1.852585$

H $8.434431-1.256610-0.097411$

C $7.944668-3.861076-0.440149$

H $6.484623-2.936028-1.713796$

C 5.7641631 .9318670 .059752

O 7.5895810 .7627841 .078478

H $7.6749011 .423429-0.837486$

H $5.147706 \quad 1.5884790 .899678$

O 6.3351563 .1826460 .470187

C $4.8592972 .181784-1.162817$

C $3.8795523 .343643-0.914876$

H $4.3087451 .263749-1.391886$

O $5.6798472 .491192-2.288431$

H 3.3879093 .1810620 .050172

H $4.4499884 .277158-0.836630$

H $8.697867-3.975809-1.226631$

H $8.469685-3.6886390 .505412$

H $7.400333-4.806710-0.355373$

C $4.772446-3.3479170 .285826$

H $\quad 6.9721980 .2885601 .661822$

H 6.8918103 .0056991 .247861

H $5.1250712 .433883-3.084132$

H $5.113198-4.3881930 .341938$

H $4.246333-3.224355-0.669278$

H $6.454811-2.4399011 .316331$

C $1.1426302 .018946-1.149283$

O $2.0274882 .348606-2.226771$

C $2.8224653 .521926-2.027607$

C $1.9073424 .735771-1.817546$

C $0.9575674 .515979-0.646009$

C $0.2121883 .193965-0.810924$

H $1.7297951 .730860-0.269070$

C $0.3044630 .808357-1.594266$

H $3.3640403 .690826-2.966063$

O $2.6866065 .907551-1.602997$

H $1.3183984 .903197-2.728863$

H 1.4909024 .5645520 .310124

O $0.0096115 .586191-0.627225$

H $-0.5395953 .312270-1.601565$

O -0.4954752 .9132260 .394253$

C $1.113360-0.465184-1.713248$

H $-0.5010790 .611436-0.879179$

H $-0.1324111 .015745-2.579253$

H $2.0693416 .642856-1.447240$

H -0.6061725 .4140790 .105674$

H 0.1568562 .8050151 .107352

O $0.355605-1.401066-2.358565$

O $2.246245-0.625428-1.282384$

C $0.958888-2.680157-2.584147$

H $\quad 0.748129-3.331015-1.731987$

H $\quad 0.502569-3.115119-3.477636$

H $2.037711-2.602396-2.750235$

SCF Energy (B3LYP/6-31G**//MMFF)= -3245.91828382 
05_00100

MM̄FF Geometry

C $2.9232393 .202292-0.883909$

C $2.7055914 .233260-0.053471$

C 1.6684744 .3031801 .038065

O $0.515062 \quad 3.488671 \quad 0.732021$

C 2.2481373 .8117242 .377606

C 1.3236394 .0840743 .549755

C 0.6835183 .1419364 .269554

C 1.1540055 .5380823 .916452

C 0.7446261 .6460484 .136909

C -0.4584431 .0903053 .372069$

C $-0.758812-0.3842883 .680716$

C $0.403855-1.3165513 .333876$

C $0.039152-2.7935243 .557629$

O $-1.921304-0.7413412 .917404$

O $1.042021-3.5992262 .921541$

C - $-1.296889-3.0900832 .844958$

C $0.065120-3.1718345 .043299$

O $-1.750580-4.4102653 .131153$

C $-2.407956-2.0687703 .173329$

C $-3.684877-2.2757242 .322884$

O $-2.797390-2.2141294 .544506$

C -0.5168254 .0928560 .080982$

O $-0.5258945 .210433-0.409735$

C $-1.669306 \quad 3.1658940 .119061$

C $-2.8786533 .581597-0.286268$

C $-4.1161752 .796512-0.249691$

C $-4.038916 \quad 1.4120130 .337360$

C $-3.449108-2.2961170 .813321$

C $-4.727287-2.1603610 .004258$

C $-5.470827-1.882693-2.367777$

O $-5.845125-2.105146 \quad 0.507656$

C $-5.447022-0.436479-2.751326$

C $-6.5371320 .344321-2.791745$

C $-6.4996881 .794161-3.185408$

C $-7.1862702 .694719-2.180371$

C $-8.2648893 .416068-2.529707$

C $-6.6326092 .762705-0.772126$

C $-5.2516303 .354565-0.720667$

N -4.455512 -2.108162 -1.356384

H $2.3329912 .295959-0.788367$

H $3.3301275 .119311-0.153061$

H 1.3601805 .3517041 .130390

H 3.2093724 .3032882 .578144

H 2.4654762 .7410522 .298063

H 0.0362363 .4775925 .079911

H 0.6085135 .6685624 .857480

H 2.1317726 .0149364 .040499

H 0.5989816 .0730503 .140183

H 1.6755901 .3188203 .664613

H 0.7646001 .2323535 .153149

H -1.361203 1.6752413 .595218

H -0.2935891 .2109102 .294559$

H -1.002745 -0.4579154 .747200$

H $1.302695-1.0638003 .908399$

H $0.674762-1.1791342 .278420$

H $1.905333-3.3584913 .299283$

H -1.089008 -3.0588651 .767778$

H $1.067343-3.0155735 .460291$

H $-0.154502-4.2360135 .183397$

H -0.635133 -2.592089 5.647741

H -1.027584 -5.020389 2.905373

H -4.195007 -3.199507 2.625828

H -4.385591 -1.462801 2.559899

H -3.684986 -1.833874 4.653933

H -1.498900 2.1708510 .511433

H -2.979588 $4.597047-0.667095$

H -4.9996980 .8976410 .381485$

H $-3.6614701 .447941 \quad 1.365582$

H $-3.3712510 .783102-0.259454$

H -2.787804 -1.472319 0.529713

H -2.973555 -3.239640 0.523196

H $-6.448542-2.196744-1.988838$

H -5.209905 -2.500557 -3.232196

H -4.482004 -0.012210 -3.019737

H -7.507848 -0.081411 -2.548602

H $-6.9838231 .881416-4.166706$
H $-5.4679112 .138149-3.328645$

H $-8.7665714 .064833-1.819324$

H $-8.6720533 .375237-3.534691$

H $-6.6891801 .767893-0.324368$

H $-7.274723 \quad 3.394221-0.143875$

H -5.203342 $4.372545-1.110108$

H -3.487212 -2.101637-1.662646

C $5.2014552 .387390-1.706561$

O $4.8669190 .988829-1.648173$

C $5.7112690 .361509-0.661228$

C $6.7521401 .402864-0.256765$

C $5.9681432 .690268-0.420484$

H 5.0723020 .1578650 .205897

C $6.295225-0.950350-1.198272$

H $7.117550 \quad 1.247572 \quad 0.763074$

H $7.6187921 .397871-0.927991$

C $6.8379233 .932279-0.494762$

H 5.2844122 .7811800 .432543

C $5.245418-1.939856-1.741131$

O $7.191954-0.644538-2.274307$

H $6.903244-1.424756-0.418950$

H $4.836552-1.554326-2.682909$

O $5.927864-3.153677-2.081045$

C $4.088773-2.262345-0.779281$

C $3.126383-3.312133-1.364716$

H $3.532232-1.345892-0.558500$

O $4.598883-2.772413 \quad 0.452073$

H $2.852175-3.015530-2.382597$

H $3.639319-4.278994-1.434198$

H $7.4206374 .049188 \quad 0.424794$

H $7.5405753 .881118-1.333288$

H $6.2250064 .830014-0.622570$

C $3.9564283 .234486-1.976521$

H $7.550401-1.487446-2.601374$

H $6.243651-3.553905-1.252648$

H $5.078390-2.0551610 .898437$

H $3.4707112 .872529-2.890991$

H $4.2634524 .268641-2.175688$

H $5.8719182 .497529-2.569852$

C $0.569841-1.665540-1.407800$

O $1.192234-2.247891-0.255977$

C $1.859925-3.490601-0.496615$

C $0.859610-4.524162-1.028352$

C $0.108070-4.007361-2.253279$

C $-0.483100-2.623485-1.979829$

H $1.330531-1.450013-2.167254$

C $-0.025468-0.329554-0.949966$

H $2.201928-3.8505620 .481022$

O $1.536632-5.737272-1.336930$

H $0.132599-4.750737-0.237187$

H $0.750444-3.988612-3.141590$

O $-0.972877-4.893209-2.554723$

H -1.316872 -2.722901 -1.276223

O $-1.006988-2.099984-3.196664$

C $-0.4410220 .564766-2.090169$

H $-0.888104-0.490702-0.294715$

H $0.6946250 .222112-0.331666$

H $\quad 0.863767-6.406764-1.545322$

H $-0.588441-5.740372-2.835992$

H $-1.660601-2.738211-3.530734$

O $0.6594461 .079843-2.707332$

O

C $0.3908631 .960293-3.802452$

H $1.3463782 .261905-4.239362$

H $-0.1975801 .447316-4.569317$

$\mathrm{H}-0.1310822 .853744-3.446654$

SCF Energy (B3LYP/6-31G**//MMFF) $=-3245.91641388$

0500101

MM̄FF Geometry

C $-2.730107-2.415512-2.644530$

C $-1.699640-1.894347-1.962763$

C $-0.564100-1.140774-2.599390$

O $-0.5530440 .175752-1.997813$

C $0.792183-1.818732-2.327346$

C $0.939068-3.178486-2.975791$

C $0.889217-4.354905-2.320370$

C $1.189424-3.153926-4.461749$ 
C $0.649992-4.589466-0.854674$

C $1.840357-5.267147-0.160743$

C $3.122637-4.420455-0.157590$

C $4.245457-5.1461210 .582527$

C $5.524950-4.3006720 .629326$

O $2.842568-3.1884450 .506412$

O $6.388255-4.907810 \quad 1.606167$

C $5.184719-2.8756421 .129470$

C $6.286011-4.375973-0.700092$

O $6.323092-2.028768 \quad 1.011851$

C $3.923103-2.2383900 .489899$

C $3.388884-1.0455321 .325316$

O $4.158786-1.757322-0.829928$

C $-0.0146491 .189606-2.728384$

O $0.463316 \quad 1.115489-3.849063$

C $-0.0973502 .421683-1.911848$

C $0.4416363 .560619-2.372799$

C $0.4803024 .846648-1.671140$

C $-0.1427484 .906750-0.301274$

C 4.1698210 .2688951 .255689

C 3.8589301 .1073580 .026202

C $4.6049563 .173133-1.149213$

O $2.9194240 .874268-0.729593$

C $3.8676224 .354350-0.600963$

C $4.2064165 .624352-0.868667$

C $3.4602736 .821966-0.350688$

C $2.7663377 .590374-1.455451$

C $3.4058288 .563460-2.126036$

C $1.3088137 .281032-1.727379$

C $1.1005295 .888907-2.264711$

N $4.7386132 .174104-0.105159$

H -2.776178 -2.296003 -3.723958

H -1.664386 -2.018632 -0.884172

H - $0.739194-1.040825-3.678009$

H $\quad 0.970215-1.860184-1.246900$

H $1.602368-1.172796-2.691833$

H $1.019831-5.268336-2.899675$

H $1.351184-4.155992-4.873306$

H $0.336648-2.715917-4.989192$

H $2.081298-2.559627-4.686235$

H $\quad 0.383379-3.672620-0.322323$

H $-0.223257-5.247558-0.762920$

H $1.540284-5.4730320 .874773$

H $2.040453-6.235965-0.635297$

H $3.416908-4.218712-1.193151$

H $4.447437-6.1238520 .127451$

H $3.928076-5.3548301 .613900$

H $7.177647-4.3455751 .691531$

H $4.996339-2.9696912 .207908$

H $6.601158-5.408141-0.897423$

H $7.209032-3.787234-0.666913$

H $5.694423-4.048417-1.556763$

H $\quad 6.401018-1.7558160 .082328$

H $2.351198-0.8514801 .023758$

H $3.318991-1.3591292 .375361$

H $4.854328-2.285442-1.245836$

H $-0.5842572 .352252-0.945959$

H $0.9195553 .550413-3.351606$

H -0.0718245 .8865110 .173863$

H 0.3534624 .1999380 .372427

H -1.208785 $4.658492-0.351194$

H 3.8797970 .8815722 .119343

H 5.2469890 .1050691 .327207

H $4.0500162 .748939-1.991976$

H $5.6139563 .434689-1.482380$

H 2.9999684 .1580030 .024214

H $5.0684405 .820826-1.502575$

H 4.1799207 .4738180 .161740

H 2.7302996 .5374290 .417299

H $2.9098059 .137552-2.901527$

H $4.4425158 .804576-1.915396$

H $\quad 0.9027397 .979380-2.471030$

H $0.7368867 .474820-0.816219$

H $1.5284415 .740215-3.257214$

H 5.4396882 .3307260 .613184

C $-5.165900-2.418751-2.050008$

O $-5.079734-1.263491-1.189995$

C $-6.255015-1.209035-0.361980$
C $-7.264627-2.144063-1.014383$

C $-6.353624-3.236310-1.538810$

H $-5.972804-1.6256030 .612709$

C $-6.7282890 .237846-0.187368$

H $-8.012100-2.510530-0.304424$

H -7.791816 -1.660849-1.844920

C $-7.000619-4.112125-2.597773$

H $-6.041277-3.867130-0.695609$

C $-5.6374001 .204796 \quad 0.318723$

O $\quad-7.1817440 .719731-1.458564$

H $\quad \begin{array}{llll}-7.597919 & 0.257233 & 0.480554\end{array}$

H $-4.8951181 .380826-0.470010$

O -6.2580882 .4700350 .579447$

C $-4.914526 \quad 0.745588 \quad 1.600081$

C -3.9411801 .8175272 .126968$

H $-4.365989-0.1773931 .385558$

O

H -3.2936582 .1316411 .300901$

H -4.5096792 .6996192 .445859$

H -7.877379 -4.625069-2.189133

H $-7.328013-3.522838-3.460790$

H $-6.300223-4.873178-2.955786$

C $-3.845803-3.188241-1.998448$

H $-7.6703161 .545207-1.302117$

H $-6.5226402 .848906-0.275459$

H $-5.439296-0.018716 \quad 3.321479$

H -3.942692 -4.145742 -2.522662

H $-3.589390-3.413761-0.955529$

H -5.359221 -2.046916 -3.064557

C $-1.373690 \quad 0.165323 \quad 2.100594$

O -2.4272420 .0991793 .065245$

C $-3.083717 \quad 1.3433843 .323610$

C -2.0637182 .3809153 .812535$

C -0.8986682 .5368752 .837196$

C -0.2896551 .1734452 .506704$

H -1.8001230 .4691181 .135809$

C $-0.834352-1.2559761 .879384$

H -3.7748321 .1589074 .155186$

O -2.7036893 .6369434 .013339$

H -1.669816 2.0609914 .786011

H -1.2168913 .0643311 .931422$

O 0.0915363 .3601493 .457369

H $\quad 0.2739420 .8163293 .375959$

O $0.632426 \quad 1.3134501 .429965$

C $-0.178250-1.8809973 .093511$

H $-1.661451-1.9194251 .600002$

H -0.107176 -1.239789 1.060193

H $-2.0217204 .267715 \quad 4.301428$

H 0.7842823 .5345632 .798229

H 1.3621381 .8780351 .734867

O $0.623213-2.9087022 .684321$

O $-0.365467-1.5398584 .253163$

C $1.317618-3.6020963 .725704$

H $1.956435-2.9101334 .282817$

H $\quad 0.603140-4.0927324 .393262$

H $1.949177-4.3655673 .264300$

SCF Energy (B3LYP/6-31G**//MMFF)= -3245.89531266

\section{2}

MMFF Geometry

C $-4.542007-0.449542 \quad 1.802735$

C $-4.418705 \quad 0.364392 \quad 2.861821$

C -3.1816691 .1555013 .216503$

$\begin{array}{llll}\text { O } & -1.998714 & 0.601216 & 2.598685\end{array}$

C -3.3537692 .6015492 .712818$

C -2.1728803 .5022503 .016948$

C $-1.265570 \quad 3.921174 \quad 2.110955$

C $-2.075248 \quad 3.962538 \quad 4.449030$

C $-1.181524 \quad 3.571702 \quad 0.648272$

C 0.1170692 .8388440 .296271

$\begin{array}{llll}\text { C } & 1.389719 & 3.701040 & 0.279816\end{array}$

C $1.4103004 .670598-0.906556$

C $2.7493335 .413682-1.031646$

$\begin{array}{lllll}\text { O } & 2.489411 & 2.783911 & 0.183117\end{array}$

O $2.7658156 .030393-2.328480$

C $3.8987044 .383637-0.981573$

C $2.8518436 .555800-0.014224$

O $5.1659655 .036365-0.974770$ 
$\begin{array}{llll}\text { C } 3.784362 & 3.398508 & 0.200271\end{array}$

C 4.8411182 .2725140 .139706

$\begin{array}{lllll}\text { O } & 4.002950 & 4.110970 & 1.423657\end{array}$

C -1.345840 -0.3856623 .272724$

O $-1.701771-0.9369954 .301547$

C $-0.074822-0.6394122 .559044$

C $0.897695-1.3481633 .151696$

C $2.245324-1.5707422 .618963$

C $2.600060-0.8838091 .327370$

C $4.754078 \quad 1.367624-1.092434$

C $5.5980940 .114102-0.928970$

C $5.791532-2.210068-1.803840$

O $6.464198-0.005297-0.066368$

C $4.939263-2.994800-0.856082$

C $5.409183-3.6928690 .188152$

C $4.519193-4.4128601 .164087$

C $4.814495-4.0374862 .600675$

C $5.310588-4.9428473 .461070$

C $4.559685-2.6114003 .036467$

C $3.105934-2.3365473 .322421$

N $5.269162-0.855896-1.866879$

H $-3.695453-0.5923741 .136668$

H -5.2822840 .5238143 .504129$

H $-3.059074 \quad 1.153928 \quad 4.306731$

H -4.252066 3.0455983 .163646

H -3.556865 $2.589608 \quad 1.636477$

H $-0.484340 \quad 4.5968222 .456065$

H -1.243288 4.6570114 .607880

H -2.994353 4.4781624 .745989

H $-1.922533 \quad 3.1093145 .116996$

H -2.0107482 .9272390 .344364$

H $-1.282736 \quad 4.4889910 .059454$

H 0.2638052 .0073990 .995116

H $0.0005582 .366609-0.688007$

H 1.4738524 .2419921 .227793

H $0.5864095 .390969-0.845694$

H $1.2435984 .106856-1.835462$

H $3.6385116 .443603-2.446799$

H $3.853613 \quad 3.821327-1.922415$

H $2.046508 \quad 7.282522-0.176337$

H $3.7857147 .115212-0.136960$

H $2.785605 \quad 6.215524 \quad 1.021290$

H $5.3162655 .381930-0.078357$

H $5.8503442 .696248 \quad 0.222507$

H 4.7181501 .6449851 .034003

H 4.2540413 .4724812 .111620

H $0.046662-0.189027 \quad 1.581547$

H $0.707555-1.7725984 .136611$

H $2.466772 \quad 0.199397 \quad 1.416394$

H $1.968567-1.2512550 .512697$

H $3.632553-1.0457061 .018834$

H $3.7152251 .063070-1.263453$

H $5.1108321 .896305-1.982764$

H $6.839903-2.179884-1.490192$

H $5.729026-2.635233-2.809686$

H $3.866861-2.960098-1.022544$

H $\quad 6.481952-3.7422800 .358288$

H $4.663250-5.4903491 .013186$

H $3.460032-4.217247 \quad 0.955418$

H $5.531514-4.6818394 .490880$

H $5.505826-5.9657403 .156799$

H $4.988120-1.9146042 .311055$

H $5.111960-2.4023933 .963230$

H $2.753265-2.7946764 .247518$

H $4.483388-0.688618-2.488838$

C $-6.437510-0.6307470 .130642$

O $-5.495765-0.743813-0.958680$

C $-5.345567 \quad 0.555273-1.555523$

C $-6.6448551 .284912-1.242330$

C -6.8877740 .8347780 .184562$

H $-4.5229241 .054150-1.027841$

C $-5.0057530 .454397-3.042662$

H $-6.5496632 .370482-1.339962$

H $-7.4655100 .950541-1.887261$

C $-8.323203 \quad 1.0142230 .648580$

H $-6.237903 \quad 1.4238740 .842048$

C -3.798914 -0.442662 -3.380936

O $-6.141534-0.074816-3.739596$
H $-4.8490331 .464516-3.441067$

H $-4.089840-1.497644-3.299309$

O $-3.513740-0.209797-4.767799$

C $-2.530040-0.192311-2.540348$

C $-1.287965-0.904725-3.108552$

H $-2.711514-0.528399-1.514123$

O $-2.256287 \quad 1.208505-2.479764$

H -1.562532 -1.925264 -3.392076

H $-0.972848-0.384104-4.020675$

H $-8.607072 \quad 2.0713430 .621709$

H -9.0233320 .4624350 .012503$

H $-8.4458080 .656222 \quad 1.675795$

C $-5.811163-1.1629691 .425933$

H $-5.912261-0.107349-4.684226$

H -2.965393 -0.946909-5.085236

H $-2.1522221 .528867-3.392172$

H $-5.560791-2.2219951 .291439$

H $-6.545484-1.1089212 .238168$

H $-7.283175-1.277296-0.134790$

C $-0.442044-3.139625-1.200453$

O $-0.376108-1.727599-0.962817$

C $-0.103733-0.921683-2.115366$

C $1.229557-1.349536-2.740342$

C $1.227028-2.832679-3.093449$

C $0.838243-3.660743-1.870295$

H $-1.312553-3.366953-1.826703$

C $-0.634463-3.829647 \quad 0.160083$

H $\quad 0.0288770 .103824-1.750589$

O $1.504233-0.567361-3.897540$

H $2.038358-1.154576-2.024870$

H $\quad 0.566369-3.035164-3.944567$

O $2.545385-3.191118-3.512031$

H $1.662019-3.653904-1.146803$

O $0.631198-5.016085-2.264823$

C -2.007516 $-3.597820 \quad 0.750539$

H $-0.511597-4.9139020 .060815$

H $\quad 0.105309-3.4470600 .872376$

H $2.342224-0.888120-4.272820$

H $2.516097-4.112656-3.819439$

H $1.485893-5.370398-2.562393$

O $-2.028523-4.1110652 .016709$

O $-2.942949-3.0603790 .175324$

C $-3.275423-4.0170772 .711913$

H -3.216947 -4.652302 3.599818

H -3.442915 -2.985511 3.030309

H $-4.101223-4.3694812 .086502$

SCF Energy (B3LYP/6-31G**//MMFF) $=-3245.92573751$

0500103

MM̄FF Geometry

C $-0.202059 \quad 5.2598750 .248975$

C $0.865316 \quad 4.728166-0.364877$

C $1.9600303 .994092 \quad 0.367584$

O $2.1750192 .720162-0.284986$

C 3.2723994 .7943870 .289829

C $4.3967504 .200879 \quad 1.117398$

C $5.5517393 .724008 \quad 0.613332$

C $4.178013 \quad 4.1929462 .609226$

C $5.9887963 .673668-0.823001$

C $5.6517372 .365430-1.554731$

C $6.2882951 .111089-0.933485$

C $6.130898-0.090871-1.867147$

C $6.689372-1.377934-1.240723$

$\begin{array}{lllll}\text { O } & 5.627150 & 0.833706 & 0.302864\end{array}$

O $6.213245-2.489531-2.016457$

C $6.109059-1.5455690 .182499$

C $8.220002-1.422451-1.321380$

O $6.742034-2.621822 \quad 0.868434$

C $6.180021-0.2643481 .042246$

C $5.385728-0.3658522 .365178$

$\begin{array}{lllll}\text { O } & 7.524891 & 0.016990 & 1.450669\end{array}$

C $1.2727981 .728978-0.048609$

$\begin{array}{lllll}\text { O } & 0.298360 & 1.776037 & 0.684625\end{array}$

C $1.6699700 .565957-0.872175$

C $0.794885-0.426790-1.089500$

C $1.041033-1.626414-1.893029$

C $2.454622-1.884967-2.343197$

C $3.884171-0.6348262 .218435$ 
C $3.505178-2.1034912 .318889$

C $1.546881-3.6245822 .062165$

O $4.290730-2.9813822 .665233$

C $1.614137-4.2631420 .710857$

C $0.549687-4.7981850 .093306$

C $0.615062-5.477101-1.245128$

C $-0.386990-4.922677-2.233136$

C $-1.547189-5.558372-2.468991$

C -0.003839 -3.686755 -3.015142

C $0.002111-2.439662-2.175184$

N $2.167513-2.3136712 .008926$

H -0.2800415 .1956951 .331775$

H $0.9485414 .792031-1.447104$

H $1.6807013 .819913 \quad 1.413714$

H 3.1038965 .8216110 .640089

H $3.5700284 .876889-0.761233$

H $6.2865933 .325407 \quad 1.312452$

H 5.0822053 .9116583 .159876

H 3.8834015 .1888252 .955976

H 3.3944483 .4797862 .882145

H $5.5694654 .514605-1.385734$

H $7.0752573 .828719-0.849966$

H $4.5620962 .249264-1.587316$

H $5.9963792 .468906-2.591245$

H $7.3487571 .315322-0.745433$

H $6.6096160 .101656-2.835336$

H $5.065826-0.243600-2.086009$

H $6.497551-2.356886-2.937069$

H $5.057054-1.8282880 .059094$

H $8.548728-1.410558-2.367586$

H $8.612937-2.352207-0.895870$

H $8.699862-0.579553-0.819444$

H $6.644205-3.4157750 .315069$

H $5.861011-1.0923283 .035646$

H 5.4870200 .6039232 .874156

H $8.089508-0.7303651 .196625$

H $2.6683700 .568080-1.293856$

H $-0.203033-0.345979-0.659802$

H $3.139855-1.846865-1.489438$

H $2.766999-1.137388-3.080089$

H $2.597259-2.868262-2.795519$

H $3.360281-0.1291203 .039731$

H $3.500748-0.2210931 .281656$

H $0.516877-3.4828282 .403470$

H $2.074771-4.2480702 .790988$

H $2.587867-4.310517 \quad 0.228922$

H - $0.418268-4.7762640 .588962$

H $0.426963-6.546792-1.083933$

H $1.621751-5.416110-1.677222$

H -2.258230 -5.186362 -3.199085

H - $1.815168-6.462403-1.932203$

H - $0.720558-3.519903-3.830726$

H $0.951102-3.868047-3.515668$

H $-0.980824-2.169690-1.789958$

H $1.603926-1.5348051 .681230$

C $-2.6579975 .215750-0.176434$

O $-2.6300163 .886741-0.731665$

C $-3.9580943 .544437-1.188606$

C $-4.8546514 .715499-0.805109$

C $-3.8794555 .876731-0.806078$

H $-3.8847783 .459075-2.279618$

C $-4.3553022 .200104-0.564850$

H -5.686036 $4.851079-1.503615$

H -5.275996 4.5737790 .198112

C $-4.3879477 .094448-0.054783$

H -3.663532 $6.154835-1.846414$

C $-5.8169251 .787873-0.810607$

O $-3.483187 \quad 1.194070-1.092590$

H -4.1541502 .2429970 .511170$

H $-6.4920172 .509733-0.336451$

O $-6.070646 \quad 1.858745-2.219083$

C $-6.1698970 .362986-0.338187$

C $-5.8249050 .096719 \quad 1.136572$

H -5.674236 $-0.366490-0.986519$

O $\quad-7.5754840 .168639-0.529510$

H $-4.7521290 .244600 \quad 1.289029$

H $-6.342284 \quad 0.833257 \quad 1.764011$

H $-5.3120057 .467492-0.508393$
H $-4.5992096 .859757 \quad 0.993907$

H -3.651557 $7.903600-0.077045$

C -1.338727 $5.928643-0.472791$

H $-3.6693891 .114392-2.043868$

H $-6.9951131 .591476-2.360766$

H -8.0439550 .7897210 .054056$

H - $1.3887186 .972111-0.142948$

H -1.151602 $5.930491-1.553508$

H -2.7976045 .1082770 .908048$

C $-4.325519-2.5073710 .714440$

O $-5.747832-2.3429270 .732314$

C $-6.219896-1.3195881 .613859$

C $-5.790918-1.6383403 .053244$

C $-4.283829-1.8683803 .163136$

C $-3.812712-2.8748502 .112068$

H $-3.850299-1.5797160 .374272$

C $-4.000304-3.609236-0.298592$

H $-7.315627-1.3696411 .583276$

O $-6.190031-0.5868723 .926197$

H $-6.311323-2.5464903 .384647$

H $-3.735553-0.9222823 .086034$

O $-4.002879-2.3864244 .465506$

H $-4.167207-3.8743232 .393555$

O $-2.387538-2.9233122 .114459$

C $-4.396093-3.213912-1.702227$

H $-2.933551-3.855864-0.302938$

H $-4.548789-4.526706-0.052417$

H $-5.883891-0.8193074 .819728$

H -3.048017 -2.567614 4.504941

H -2.057469-2.048955 1.845556

O $-3.627902-2.174308-2.133253$

O $-5.275860-3.774291-2.343238$

C $-3.900586-1.711479-3.459060$

H $-4.922503-1.325635-3.521226$

H -3.204341 -0.899419-3.684640

H $-3.744984-2.517623-4.182231$

SCF Energy $(B 3 L Y P / 6-31 G * * / / M M F F)=-3245.91359950$

0500104

MM̄FF Geometry

C 1.7697853 .0213252 .124004

C 0.5282762 .8582681 .644618

C 0.0639403 .4084260 .322177

O $-0.1779232 .289678-0.561300$

C -1.2574154 .1736180 .512171$

C $-1.7705804 .801196-0.768987$

C $-2.7366474 .266648-1.541994$

C $-1.1482066 .123130-1.133094$

C $-3.4277472 .947883-1.341630$

C $-4.9050533 .121187-0.975231$

C $-5.5461071 .770182-0.626524$

C $-7.0609281 .907311-0.474439$

C $-7.7085570 .584978-0.037401$

$\begin{array}{llll}\text { O } & -4.972085 & 1.321390 & 0.607402\end{array}$

$\begin{array}{llll}\text { O } & -9.052966 & 0.867540 & 0.379336\end{array}$

C $-6.9557140 .046747 \quad 1.196883$

C -7.813851 -0.411298 -1.197629

O $-7.424531-1.2520561 .554168$

C -5.4149860 .0273451 .032573$

C $-4.759349-0.2938542 .401899$

O $-5.067761-0.9849020 .088128$

C $0.8447961 .883878-1.357591$

O $1.9679492 .367617-1.401936$

C $0.4661200 .725835-2.201663$

C $-0.7673760 .199723-2.241465$

C $-1.198087-0.964471-3.019894$

C $-0.144130-1.713184-3.792123$

C $-3.255328-0.0073902 .518600$

C $-2.354486-0.9550801 .755938$

C $-1.721487-3.3530601 .549463$

O $-1.614757-0.5517290 .861341$

C $-2.559867-3.9245510 .450266$

C $-2.223187-3.855203-0.846652$

C $-3.050479-4.430188-1.959029$

C $-3.880334-3.390320-2.679153$

C $-5.212390-3.337972-2.509786$

C $-3.185619-2.469803-3.660696$

C $-2.501525-1.314892-2.980339$ 
N -2.449201 -2.269484 2.181754 H 2.5036273 .5693061 .538174 H -0.1930692 .2873062 .225299$ H $0.8139104 .081462-0.112144$ H -1.1242814 .9672181 .260099$ H -2.0190043 .5076450 .936024$ H -3.048775 $4.811014-2.432029$ H -1.574251 $6.544843-2.049668$ H -1.306059 $6.851155-0.330680$ H -0.071472 $6.008993-1.292927$ H $-3.3528312 .380376-2.277670$ H -2.924004 2.341611 -0.584591 H $-4.9875883 .798201-0.114995$ H -5.435269 $3.584392-1.816362$ H -5.304482 $1.054133-1.421145$ H $-7.5157412 .267261-1.405798$ H $-7.284610 \quad 2.671128 \quad 0.283421$ H $-9.518827 \quad 1.277362-0.369514$ H -7.204175 0.7073602 .039556 H $-8.4227300 .007462-2.007974$ H $-8.322001-1.330277-0.885568$ H -6.843634 -0.678939-1.620026 H $-8.389476-1.1925001 .660401$ H $-5.237996 \quad 0.3323023 .166667$ H -4.962893 -1.337453 2.668183 H $-4.136015-1.2226300 .205238$ H $1.277704 \quad 0.318367-2.795768$ H -1.548093 $0.652631-1.636081$ H $-0.525225-2.589883-4.318736$ H $0.641101-2.073408-3.118335$ H $0.311425-1.064950-4.548828$ H -3.029104 1.0173212 .202014 H -2.962669 -0.0700993 .574812$ H -1.525320 -4.1144912.310106 H -0.763978 -2.977089 1.177976 H $-3.485396-4.4178020 .737182$ H -1.292726 -3.367439-1.125753 H -3.698057 -5.224813 -1.564724 H $-2.387297-4.930954-2.675305$ H -5.821432 -2.616287 -3.043667 H $-5.723297-4.010399-1.828581$ H -3.920220 -2.045958 -4.358535 H -2.512836 -3.064058 -4.284117 H -3.182982 $-0.697251-2.392725$ H -3.109719 -2.510946 2.914181 C 2.9537461 .1457453 .285878 O 1.9816250 .1042893 .036610 C $2.244956-0.9888793 .926873$

C $3.669387-0.7935264 .422826$

C 3.7223800 .7179614 .538077 H $1.570662-0.8532954 .784559$ C $1.926019-2.3473033 .296366$ H $3.860816-1.3038245 .371397$ H 4.405068-1.140265 3.689036 C 5.1351151 .2721614 .608867 H 3.1729901 .0228355 .439024 C $2.820641-2.8192982 .130491$ O $2.017868-3.3216504 .344765$ H $0.880482-2.3423352 .966667$ H $3.832643-3.0201832 .498753$ O $2.313641-4.0917721 .697718$ C $2.887238-1.875614 \quad 0.917227$ C $3.714617-2.470115-0.238127$ H $3.337648-0.9293831 .225990$ O $1.560396-1.6099430 .471174$ H $4.720117-2.6898790 .137972$ H $3.274686-3.423597-0.554487$ H 5.6545640 .8890945 .493309 H 5.7208750 .9915593 .726920 H 5.1226662 .3649404 .670741 C 2.2294622 .4818253 .449163 H $1.789115-4.1845543 .958732$ H $1.441864-3.9377821 .294926$ H $1.596252-0.830842-0.108409$ H 2.8931233 .2279953 .900827 H 1.3705202 .3643364 .121830 H 3.6232581 .1710362 .418003 C $5.473511-0.004713-0.716432$
O $4.124374-0.205074-1.144733$

C $3.794443-1.554460-1.480333$

C $4.726713-2.070868-2.585615$

C $6.198966-1.902058-2.215170$

C $6.480537-0.464496-1.777372$

H $5.640741-0.5822280 .202205$

C $5.6359161 .468718-0.313278$

H $2.785227-1.523342-1.906890$

O $4.470568-3.442589-2.867916$

H $4.537308-1.510705-3.510670$

H $6.504216-2.614825-1.440289$

O $7.007345-2.186263-3.359632$

H $6.4516090 .186647-2.657756$

O $7.797751-0.384823-1.236931$

C $5.3428022 .469501-1.413492$

H $4.928087 \begin{array}{lll}1.687767 & 0.494726\end{array}$

H 6.6529431 .6448010 .053536

H $3.533686-3.519133-3.117606$

H $6.788058-3.087973-3.651140$

H $8.410570-0.711913-1.917820$

O $5.5859333 .726944-0.934994$

O $4.9359472 .200148-2.534613$

C $5.3228224 .794061-1.851242$

H $5.5545895 .737509-1.349968$

H $5.9604464 .700336-2.735507$

H $4.2658384 .797588-2.134551$

SCF Energy $\left(B 3 L Y P / 6-31 G^{* *} / / M M F F\right)=-3245.90837456$

0500105

MM̄FF Geometry

C $-1.994751-1.4514582 .187010$

C $-1.568868-0.1807812 .129071$

C $-1.0264970 .587945 \quad 3.302489$

O $0.204124 \quad 1.2057142 .847820$

C $-1.978138 \quad 1.711888 \quad 3.756154$

C -3.2589321 .2357774 .409299$

C $-4.4798191 .271742 \quad 3.838524$

C $-3.1036690 .730545 \quad 5.821103$

C -4.8303561 .7179192 .446309$

C -5.4737770 .5709311 .654501$

C -5.4870010 .8426950 .143706$

C $-6.162255-0.308779-0.604597$

C $-6.066428-0.142567-2.128769$

$\begin{array}{llll}0 & -4.126826 & 0.965107 & -0.282050\end{array}$

O $-6.413402-1.398396-2.732350$

C $-4.5998810 .151141-2.511660$

C $-7.076183 \quad 0.885026-2.653318$

O $-4.4874830 .454596-3.900252$

C $-3.956418 \quad 1.277524-1.669436$

C $-2.4336921 .340734-1.948635$

O $-4.560732 \quad 2.522949-2.010527$

C 1.1511601 .4872643 .777598

$\begin{array}{lllll}\text { O } & 1.105636 & 1.213583 & 4.969535\end{array}$

C $2.3108642 .193705 \quad 3.185254$

C 2.3296052 .7046781 .943615

C 3.4490693 .4235831 .325975

C 4.6971153 .6128742 .149505

C $-1.6523572 .329357-1.070147$

C $-1.5692403 .740024-1.624888$

C $-0.6316085 .979301-1.047821$

O $-2.0090314 .062339-2.724417$

C $0.7391396 .049150-1.642205$

C $1.7763166 .661678-1.052383$

C $3.1527586 .720252-1.647929$

C $4.200746 \quad 6.090257-0.757204$

C $5.0389836 .854851-0.037098$

C $4.3438724 .584383-0.777729$

C 3.3152193 .8761050 .060926

N $-0.927654 \quad 4.593879-0.737315$

H $-1.978023-1.9766393 .138574$

H -1.6159700 .3451831 .179874$

H $-0.802657-0.0975064 .129206$

H -2.1722402 .3860612 .914114$

H -1.4544852 .3581644 .474274$

H $-5.3305800 .926603 \quad 4.424934$

H $-4.0624250 .452987 \quad 6.272137$

H $-2.461796-0.155438 \quad 5.843997$

H $-2.654607 \quad 1.5029836 .453944$ 
H -5.5361452 .5547662 .510197$ H -3.954425 2.0969551 .916323 H $-4.914881-0.3522941 .845565$ H -6.4971130 .4076252 .015006$ H $-6.0124031 .787330-0.038699$ H -7.208863 $-0.413941-0.292858$ H $-5.674440-1.255379-0.332557$ H $-7.310513-1.633927-2.439662$ H -4.026825 -0.772884 -2.348984 H $-8.0998700 .571094-2.416122$ H $-7.0323420 .960972-3.745302$ H $-6.932447 \quad 1.881680-2.230844$ H $-4.893890-0.280097-4.391180$ H -2.018195 $0.347101-1.740152$ H -2.249682 $1.557581-3.007093$ H $-4.001593 \quad 3.242268-1.678763$ H 3.1585852 .2964433 .855421 H 1.4490702 .5932811 .313126 H 5.1274142 .6440562 .426223 H 4.4725224 .1729583 .064067 H 5.4811484 .1693081 .632107 H -2.064434 2.371321 -0.056727 H $-0.6177331 .972243-0.990722$ H $-0.7095206 .547326-0.115391$ H -1.368143 6.368581-1.757686 H $0.882725 \quad 5.568272-2.607208$ H $1.6313387 .151766-0.092460$ H $3.3928427 .776386-1.827109$ H $3.1832826 .241579-2.634847$ H 5.8186936 .4172120 .576985 H $4.9627147 .937150-0.043180$ H $5.3573874 .288592-0.483602$ H $4.2524404 .226698-1.811695$ H $2.3738403 .694647-0.457706$ H $-0.528413 \quad 4.2088350 .114041$ C $-2.099550-3.6458190 .946315$ O $-0.686568-3.7368680 .680760$ C $-0.449421-4.897362-0.143239$ C -1.797233 -5.592759-0.319149 C $-2.765793-4.437552-0.177066$ H $-0.121418-4.525850-1.120007$ C $0.632258-5.7926650 .473222$ H -1.874323 -6.101520-1.285145 H -1.975681 -6.3401440 .462801$ C -4.189231 -4.8754340 .120993$ H $-2.754417-3.853221-1.107071$ C $1.993228-5.1022790 .687256$ O $0.168198-6.2448981 .752700$ H $\quad 0.756746-6.691583-0.142391$ H $1.900308-4.3439841 .473670$ O $2.897771-6.0895601 .201351$ C $2.610084-4.462984-0.571288$ C $4.027629-3.919261-0.309208$ H $1.966814-3.648474-0.918715$ O $2.698769-5.428269-1.618790$ H $4.000305-3.2720180 .573851$ H $4.701246-4.753677-0.078599$ H $-4.570144-5.515496-0.681660$ H -4.247552 -5.4398291 .057753$ H $-4.856164-4.0118840 .205799$ C $-2.549987-2.1857800 .997556$ H $0.864801-6.8085562 .131090$ H $3.049566-6.7429290 .496928$ H $1.796350-5.629153-1.917609$ H -3.641881-2.151534 1.066431 H $-2.257879-1.6757030 .071799$ H -2.277092 -4.133076 1.914697 C $3.586046-1.016528-1.086013$ O $3.727632-2.124443-1.982332$ C $4.606538-3.155233-1.522628$ C $6.010037-2.577409-1.299532$ C $5.988274-1.354914-0.383950$ C $4.943426-0.342985-0.858494$ H $3.174282-1.362206-0.129788$ C $2.573349-0.050278-1.707788$ H $4.684342-3.879112-2.343610$ O $6.871595-3.575772-0.763223$ H $6.428000-2.281531-2.270803$
H $5.815402-1.6495490 .657874$

O $7.283286-0.751084-0.422885$

H $5.2878130 .136454-1.783720$

$\begin{array}{lllll}\text { O } & 4.795617 & 0.673130 & 0.127847\end{array}$

C $1.189441-0.655707-1.670686$

H $2.5239730 .899169-1.163916$

H $2.8338290 .187365-2.746155$

H $7.741162-3.163736-0.621008$

H $7.293807-0.036217 \quad 0.235627$

H $5.627616 \quad 1.174222 \quad 0.163553$

O $0.895946-1.220836-2.874879$

O $0.477024-0.650398-0.675515$

C $-0.365438-1.889527-2.998369$

H -1.067086 -1.226846 -3.511393

H $-0.215338-2.783905-3.608994$

$\mathrm{H}-0.777391-2.195267-2.032904$

SCF Energy (B3LYP/6-31G**//MMFF) $=-3245.90698581$

0500106

MM̄MF Geometry

C $-2.760861-3.8000862 .809392$

C $-2.419275-2.6922713 .485630$

C $-3.280147-1.4805323 .744599$

O $-2.740173-0.4199292 .918070$

C $-4.771040-1.6297613 .375643$

C $-5.588094-0.3692963 .600830$

C $-6.208900 \quad 0.3175262 .619669$

C -5.6961170 .0963815 .029366$

C $-6.259282-0.0255561 .155162$

C $-5.122706 \quad 0.640886 \quad 0.380134$

C $-5.1093690 .303947-1.118293$

C $-4.768905-1.166958-1.380533$

C $-4.612454-1.468744-2.879267$

O $-4.1122371 .148234-1.712427$

O $-3.986940-2.755424-3.006329$

C $-3.647977-0.436360-3.497362$

C $-5.968837-1.575357-3.585411$

O $-3.575604-0.583137-4.913034$

C $-3.9984411 .023090-3.137826$

C $-2.9200562 .028926-3.607672$

O $-5.2228811 .390903-3.783316$

C $-1.9482070 .526106 \quad 3.485972$

O $-1.5763230 .586966 \quad 4.645475$

C -1.6330541 .5067182 .422645$

C -1.0195602 .6635042 .713893$

C $-0.7127733 .728301 \quad 1.752857$

C $-1.1393623 .505323 \quad 0.325537$

C $-1.5109541 .734595-3.092267$

C $-0.5524282 .899894-3.276680$

C $1.8047393 .541356-2.708478$

O $-0.857807 \quad 3.946571-3.839259$

C $2.1579743 .817842-1.281104$

C $2.3111105 .046951-0.765092$

C 2.6626435 .2941410 .675684

C 1.7715806 .3209851 .342772

C 2.2972107 .4367691 .876947

C 0.2789276 .0715791 .393619

C -0.0868154 .8428742 .186289$

N $0.6893912 .612668-2.729223$

H $-3.761164-3.9138962 .403495$

H -1.397155 -2.612999 3.853224

H -3.194938 -1.227654 4.808132

H -5.219524 -2.442759 3.960981

H $-4.840547-1.9292212 .325412$

H $-6.756227 \quad 1.2201532 .887692$

H -6.4061890 .9226175 .143526$

H -6.042289 -0.7214445 .669783$

H $-4.726580 \quad 0.443898 \quad 5.398531$

H $-6.257407-1.1096951 .015492$

H -7.2226220 .3169980 .756788$

H $-5.189757 \quad \begin{array}{lll}1.731173 & 0.498120\end{array}$

H -4.1545390 .3637150 .811860$

H $-6.0879480 .560248-1.540605$

H $-5.520177-1.835474-0.945520$

H $-3.822703-1.414164-0.878266$

H -4.544931 -3.406760 -2.547681

H $-2.649118-0.671292-3.108294$

H $-6.565186-2.385117-3.147911$ 
H $-5.844568-1.833351-4.642822$ H -6.560212 $-0.659832-3.519564$ H $-3.348233-1.510609-5.097026$ H -2.907157 $2.086309-4.703895$ H -3.217841 $3.030065-3.265521$ H $-5.2580162 .359739-3.847570$ H -1.9468441 .2565661 .415123$ H -0.7328682 .8591303 .745999$ H -2.225913 3.3778880 .261000 H $-0.6549732 .607963-0.073110$ H $-0.8819814 .323186-0.348616$ H $-1.5451601 .492500-2.024605$ H -1.090256 $0.874846-3.626178$ H $1.5522564 .451667-3.260619$ H $2.6439813 .046748-3.207005$ H $2.3000372 .953663-0.636044$ H $2.1966755 .915216-1.409314$ H 3.7097125 .6221940 .706857 H 2.6198714 .3644691 .254560 H 1.6733658 .1831452 .357310 H 3.3643297 .6307091 .847455 H $-0.110886 \quad 6.0535000 .373191$ H -0.2293546 .9202371 .871022$ H 0.1776824 .9107353 .242358 H $0.8242291 .710423-2.282885$ C $-0.990113-4.6752101 .214364$ O $-0.069274-3.5853131 .419555$ C $-0.067047-2.749748 \quad 0.241984$ C $-0.740181-3.571071-0.847501$ C $-1.781197-4.334025-0.049763$ H $-0.676493-1.8729680 .496067$ C $1.376029-2.332438-0.055620$ H $-1.175626-2.948906-1.635126$ H $-0.027317-4.264547-1.310690$ C $-2.339110-5.548421-0.769904$ H $-2.605579-3.6457260 .172476$ C $1.545582-1.529203-1.355963$ O $1.834006-1.5276081 .035898$ H $2.010881-3.225112-0.076516$ H $1.284180-2.152171-2.218725$ O $0.610005-0.442840-1.353300$ C $2.951408-0.929966-1.566889$ C $4.078582-1.974810-1.529733$ H $3.132774-0.136269-0.833601$ O $2.983260-0.309788-2.857456$ H $4.036118-2.533517-0.591513$ H $3.918026-2.693383-2.342926$ H -2.837165 -5.250828 -1.698207 H -1.548681-6.261307-1.027315 H -3.071676 -6.067662 -0.143897 C $-1.798320-4.9114132 .495739$ H $1.718261-2.0479061 .849555$ H $\quad 0.7846300 .092767-0.560100$ H $2.2488780 .325411-2.894959$ H -1.108287 -5.0372933 .339846$ H $-2.365719-5.8455402 .410070$ H $-0.366912-5.5617621 .038208$ C $5.844777-0.745406 \quad 0.618714$ O $5.716513-0.303828-0.738084$ C $5.483383-1.348774-1.689516$ C $6.642991-2.354898-1.644643$ C $6.902941-2.877467-0.231640$ C $7.023494-1.7162410 .757259$ H $4.917665-1.2365700 .937014$ C $6.0464860 .498198 \quad 1.491249$ H $5.509331-0.874266-2.678353$ O $6.414400-3.437033-2.541585$ H $7.553188-1.851541-1.995525$ H $6.122944-3.5832510 .077497$ O $8.128627-3.612304-0.249514$ H $7.970357-1.1873110 .588709$ O $7.045555-2.2291932 .087516$ C 4.7912821 .3364041 .538975 H 6.3212650 .2431582 .520923 H 6.8486311 .1292251 .089306 H $5.741904-4.019830-2.150586$ H $8.236939-4.0254430 .623624$ H $7.861967-2.7463332 .190607$
O $3.869103 \quad 0.751638 \quad 2.355867$

O 4.6485752 .3811690 .917660

C 2.6199821 .4403962 .471440

H 2.7727112 .4278912 .916576

H 2.1407941 .5254961 .491658

H $\quad 1.9697480 .8566393 .128500$

SCF Energy (B3LYP/6-31G**//MMFF) $=-3245.91553416$

0500107

MM̄FF Geometry

C $1.405725-2.384430-2.223842$

C $0.595452-3.446832-2.116928$

C $-0.326707-3.652786-0.944992$

O $-1.670423-3.424186-1.431364$

C $-0.237499-5.079477-0.372378$

C $0.981022-5.3274560 .493735$

C $0.980974-5.2299441 .838404$

C $2.222380-5.783317-0.227487$

C $-0.164656-4.8031772 .713569$

C $0.265413-3.937533 \quad 3.906787$

C $0.929311-2.6029963 .529344$

C $1.316499-1.8387764 .800862$

C $1.894480-0.4509214 .493978$

O $0.007809-1.8331672 .747057$

O $1.9179090 .275970 \quad 5.732193$

C $0.9309930 .277978 \quad 3.533358$

C $3.351036-0.5376674 .022558$

O 1.4746651 .5305613 .127577

C $0.529511-0.5701652 .306403$

C $-0.5394180 .120203 \quad 1.427163$

O $1.686194-0.7513711 .480124$

C $-2.574615-2.948506-0.532357$

O $-2.391194-2.7476120 .657645$

C $-3.842279-2.690442-1.248175$

C $-4.856911-2.126281-0.576564$

C $-6.177061-1.793235-1.110050$

C $-6.437422-2.068208-2.567250$

C -1.8739300 .4161992 .117562$

C -2.8560301 .0823251 .165504$

C -4.7445582 .7135751 .164606$

O $-2.7932600 .954963-0.054260$

C -6.1232792 .1344481 .193910$

C -6.9345702 .1285810 .124419$

C $-8.354926 \quad 1.6374730 .144719$

C $-8.6373660 .617392-0.935706$

C $-9.0695121 .000027-2.149229$

C $-8.500794-0.845969-0.586224$

C $-7.085695-1.245479-0.276732$

N -3.8149351 .8250911 .838029$

H $1.365946-1.615470-1.458000$

H $0.607523-4.210266-2.890616$

H $-0.097401-2.920266-0.160277$

H $-0.267212-5.817753-1.185795$

H $-1.148366-5.3006170 .198328$

H $1.894699-5.4888002 .371749$

H $3.020823-6.0728810 .464280$

H $2.002105-6.654965-0.852399$

H $2.617109-4.987274-0.864175$

H $-0.926678-4.2646362 .145753$

H $-0.642261-5.7086463 .107535$

H $-0.635723-3.7335544 .500040$

H $\quad 0.944690-4.5152344 .546764$

H $1.823085-2.8102142 .930174$

H $2.021934-2.4222685 .405993$

H $0.423915-1.7082505 .429302$

H $2.210828 \quad 1.1826855 .535719$

H $\quad 0.033877 \quad 0.5125294 .118203$

H $3.974142-0.9978134 .798932$

H 3.7749370 .4583403 .854041

H $3.475061-1.1242703 .110001$

H 2.1887941 .3479422 .494126

H -0.1276361 .0467331 .004978$

H $-0.737660-0.5217650 .558474$

H $1.389883-0.9662990 .581629$

H $-3.903610-2.961933-2.295419$

H $-4.710863-1.8810340 .475087$

H -7.420857 -1.730662 -2.902624

H $-6.378171-3.142633-2.772192$ 
H $-5.703403-1.549528-3.194294$ H $-2.335140-0.5065572 .485054$ H -1.713605 1.0849132 .969188 H -4.398801 2.9091370 .144091 H -4.734279 3.6577261 .718736 H $-6.487871 \quad 1.7482432 .142553$ H $-6.5748532 .549049-0.812476$ H -9.0105082 .5076370 .006626$ H $-8.622240 \quad 1.222261 \quad 1.124286$ H -9.302122 $0.275111-2.922144$ H $-9.1960382 .048719-2.397580$ H $-8.918930-1.482404-1.374467$ H $-9.120374-1.0588350 .294794$ H -6.802193 -1.0693520 .761542$ H $-3.809643 \quad 1.8367172 .853849$ C $3.804885-2.022734-2.762289$ O $3.879079-0.823813-1.966322$ C $5.156639-0.196399-2.179601$ C $5.979063-1.177413-3.007631$ C $4.898137-1.872156-3.816437$ H $4.9699820 .698501-2.784191$ C $5.7685520 .201068-0.831049$ H $6.723506-0.671332-3.629692$ H $6.506529-1.901630-2.376184$ C $5.350513-3.183853-4.433159$ H $4.562289-1.194095-4.612354$ C 4.8398121 .0612670 .052415 O $6.081621-0.985837-0.089119$ H $6.7231940 .714401-0.990695$ H 3.9867050 .4600550 .388340 O 5.5643691 .4214021 .235128 C $4.3205372 .349281-0.613610$ C 3.4058653 .1537280 .328643 H $3.7552492 .098022-1.516642$ O $5.4154103 .177290-0.999785$ H 2.5770072 .5085250 .635922 H 3.9600153 .4263621 .234679 H $6.184372-3.016922-5.122809$ H $5.683617-3.893292-3.668174$ H $4.536830-3.652537-4.995268$ C $2.393596-2.164539-3.334231$ H $5.257344-1.4913510 .018388$ H $5.8706880 .596601 \quad 1.649643$ H $5.8933882 .725037-1.714771$ H $2.356148-2.990352-4.053086$ H $2.107089-1.246705-3.861721$ H $4.031165-2.864756-2.094084$ C $1.0176053 .502613-1.554386$ O $2.261198 \quad 4.215651-1.587870$ C $2.8608434 .449656-0.310932$ C 1.8989665 .2412960 .586593 C 0.5252614 .5814080 .674254 C $-0.0149984 .274019-0.722370$ H $1.1793842 .508254-1.122008$ C $0.5884903 .327465-3.016797$ H $3.7280665 .095633-0.497225$ O $2.4291325 .390703 \quad 1.899315$ H 1.7695756 .2509980 .175322 H $\quad 0.552573 \quad 3.6754291 .290785$ O -0.3851645 .4757521 .318484$ H $-0.2808735 .212573-1.225076$ O $-1.2058983 .504145-0.595990$ C $-0.5664162 .379855-3.227517$ H $0.3230454 .301319-3.447162$ H $1.4298192 .962660-3.619780$ H 3.2944355 .8268221 .816820 H -0.0109215 .6893802 .190522$ H -1.823793 $4.010240-0.041166$ O $-0.263726 \quad 1.144280-2.740639$ O $-1.6022552 .699402-3.797231$ C $-1.271256 \quad 0.137057-2.887546$ H $-2.2824070 .552028-2.834016$ H -1.123478 $-0.381475-3.838531$ $\mathrm{H}-1.152614-0.577995-2.070791$ SCF Energy (B3LYP/6-31G $\left.{ }^{* *} / / \mathrm{MMFF}\right)=-3245.92436393$

0500108

MM̄FF Geometry
C $-1.8330394 .225044-0.090584$

C $-0.8616744 .183202-1.014734$

C $0.337723 \quad 3.276941-0.903408$

O $0.2285472 .225504-1.896349$

C $1.6558494 .022184-1.176255$

C 2.1673444 .8015360 .017096

C 3.0530044 .3100380 .906102

C 1.6749326 .2186650 .142818

C 3.6499352 .9299640 .919660

C 5.1005032 .9654410 .427490

C 5.7409081 .5706550 .380466

C $7.2114631 .684384-0.029271$

C $7.8712250 .310000-0.205582$

O $5.0219160 .784955-0.575000$

O $9.1195720 .505797-0.887565$

C $6.980856-0.549522-1.126160$

C $8.210864-0.334174 \quad 1.143635$

O $7.490796-1.878412-1.223557$

C $5.488538-0.564582-0.715231$

C $4.651268-1.234120-1.834695$

O $5.355197-1.2987090 .499869$

C $-0.5871001 .181386-1.582515$

O $-1.2863871 .073766-0.587153$

C $-0.4757580 .159784-2.645201$

C $-1.324965-0.878150-2.615285$

C $-1.331186-2.039655-3.504636$

C $-0.221726-2.152842-4.514641$

C $3.142342-1.316932-1.559776$

C $2.716764-2.588021-0.840353$

C $0.672503-3.752521-0.011216$

O $3.501171-3.477221-0.520959$

C $0.192943-4.653235-1.106545$

C $-1.074833-5.076320-1.224458$

C $-1.549668-5.974547-2.332443$

C $-2.736490-5.408252-3.082951$

C $-3.961648-5.941187-2.939783$

C $-2.491236-4.269402-4.054006$

C $-2.309512-2.955459-3.344636$

N $1.349217-2.614671-0.605629$

H -1.7629123 .5797350 .781949$

H $-0.9376654 .812378-1.897871$

H $0.3964492 .809470 \quad 0.088483$

H $1.5504974 .691935-2.040822$

H $2.4158353 .300806-1.504006$

H $3.3867554 .955529 \quad 1.717195$

H $2.1667506 .757087 \quad 0.960068$

H $1.8740746 .773999-0.779545$

H $\quad 0.599376 \quad 6.240651 \quad 0.338317$

H 3.6179362 .5526491 .948806

H 3.0553212 .2304310 .323827

H $5.1195783 .404188-0.579297$

H 5.6917083 .6197841 .080635

H $5.6518081 .108731 \quad 1.370367$

H 7.7713572 .2887630 .695513

H $7.2818532 .224446-0.984173$

H $9.6636941 .107239-0.350950$

H $7.057900-0.123551-2.135989$

H $8.886590 \quad 0.312231 \quad 1.716806$

H $8.748640-1.2787421 .006857$

H $7.331269-0.5215641 .763040$

H $7.245880-2.349503-0.408909$

H $4.774938-0.636349-2.747853$

H $5.039757-2.236317-2.049942$

H $4.419047-1.5132260 .632294$

H $\quad 0.3089750 .267349-3.383936$

H $-2.085690-0.897985-1.836156$

H $\quad 0.748577-2.226787-4.012140$

H $-0.210127-1.277625-5.173745$

H $-0.312858-3.024273-5.166214$

H $2.793177-0.445353-0.996924$

H $2.620848-1.318396-2.525121$

H $-0.149805-3.3625910 .595908$

H $1.366749-4.2938090 .638969$

H $0.929348-4.978305-1.838217$

H - $1.809340-4.771848-0.482240$

H -1.811618 -6.942051-1.884935

H $-0.744599-6.184857-3.047775$

H $-4.813333-5.556441-3.490793$ 
H $-4.142250-6.771160-2.264689$

H -3.346371 -4.164285 -4.734182

H -1.638154 -4.535363 -4.684117

H -3.082363 -2.755685 -2.600213

H $0.774676-1.850518-0.947100$

C $-4.3054074 .420724-0.669189$

O $-4.546144 \quad 3.1885180 .042527$

C $-4.9537472 .188617-0.913204$

C $-5.3346632 .949324-2.177160$

C $-4.3096734 .065990-2.157027$

H $-4.0674981 .583295-1.135873$

C $-6.063217 \quad 1.299966-0.344846$

H $-5.2739822 .324807-3.073459$

H $-6.3492933 .359624-2.113497$

C $-4.6561875 .228119-3.070934$

H $-3.3412293 .650341-2.461231$

C -5.6740370 .5388290 .940061$

O $-7.1975602 .122096-0.046287$

H $-6.3902510 .592326-1.116237$

H $-5.581698 \quad 1.242734 \quad 1.775575$

O $-6.764356-0.326143 \quad 1.281825$

C $-4.379375-0.287646 \quad 0.824672$

C $-4.131000-1.2067732 .034201$

H -3.5375610 .4028210 .720832$

O $-4.418137-1.083501-0.360238$

H $-4.238200-0.6218862 .953322$

H -4.897240 -1.9908272 .055350$

H $-4.7156004 .893569-4.111881$

H $-5.6206485 .674147-2.806134$

H -3.894257 $6.011473-3.011510$

C $-3.0398135 .115416-0.157936$

H $-7.8892291 .541620 \quad 0.314818$

H $-6.897360-0.9458180 .544742$

H -5.119230 -1.748128 -0.253796

H -3.2238115 .4729660 .863459$

H $-2.8311396 .006562-0.761415$

H $-5.1613805 .071582-0.446170$

C -1.453229 -0.1805953 .110607$

O $-1.678731-0.9184271 .905166$

C -2.738811-1.880606 1.976409

C $-2.471565-2.8831883 .107754$

C $-2.213479-2.1865874 .441413$

C - $-1.129307-1.1207854 .278682$

H $-2.356326 \quad 0.3941693 .349880$

C $-0.361427 \quad 0.8644252 .852527$

H -2.699901 -2.445665 1.036355

O -3.563608 -3.788073 3.230139

H - $-1.588703-3.4816692 .847447$

H $-3.138081-1.7632374 .850729$

O $-1.768537-3.1668815 .381598$

H $-0.170861-1.6268884 .116026$

O $-1.010208-0.3786575 .489455$

C $0.9442840 .259678 \quad 2.395807$

H -0.6810831 .5488642 .058023$

H -0.1862241 .4880093 .736644$

H $-3.361181-4.3900243 .966772$

H -1.561700 -2.700326 6.209662

H -1.8564690 .0737195 .647426$

O $1.7770890 .079115 \quad 3.459447$

O $1.185956-0.0355121 .232884$

C $3.028961-0.5440993 .157724$

H $2.864257-1.5460992 .749851$

H 3.5976360 .0698082 .454604

H $3.598242-0.6323754 .086699$

SCF Energy (B3LYP/6-31G**//MMFF) $=-3245.92138922$

05 00109

MM̄MF Geometry

C -3.647432 -0.307951 -3.630708

C $-2.709394-1.006890-2.973649$

C $-1.257836-1.041231-3.366993$

O $-0.456488-0.557153-2.261835$

C $-0.751628-2.445510-3.762348$

C $-0.992927-3.554356-2.755006$

C $-0.176021-3.844966-1.724041$

C $-2.216443-4.402241-3.000516$

C $1.107580-3.162993-1.337652$

C $2.254651-4.178063-1.253877$
C $3.585678-3.600152-0.747167$

C $3.519535-3.1971390 .728252$

C $4.848160-2.6042921 .217027$

O $3.945112-2.479280-1.560404$

O $4.611504-1.9812292 .489213$

C $5.273587-1.4885890 .241331$

C $5.910765-3.6833451 .453651$

O $6.573238-0.9990760 .567192$

C $5.224918-1.912281-1.247635$

C $5.461638-0.705911-2.188348$

O $6.277543-2.853806-1.489946$

C $-0.4049130 .788591-2.068072$

O $-0.981075 \quad 1.646319-2.718785$

C $0.4826451 .048482-0.911456$

C $0.5276522 .287074-0.398147$

C 1.3570832 .7494110 .716117

C 2.2981021 .7628901 .352811

C $4.3036920 .296458-2.248217$

C $4.7630041 .695815-2.622814$

C $3.9767404 .062005-2.542455$

O $5.8929521 .948872-3.031069$

C $4.2421704 .591554-1.166436$

C $3.5444405 .588780-0.601747$

C 3.7995036 .0966010 .789464

C 2.5552106 .0995461 .652851

C 1.9706007 .2534472 .016914

C 2.0295954 .7689682 .156206

C 1.2420444 .0367241 .104719

N $3.7521122 .631180-2.444059$

H $-3.3761010 .265188-4.513209$

H $-2.991509-1.544804-2.071587$

H -1.076014 -0.386281-4.229944

H $\quad 0.323313-2.385104-3.974807$

H $-1.214868-2.732881-4.716040$

H -0.459185 -4.667421 -1.067662

H $-2.334742-5.190002-2.248772$

H $-3.126983-3.797692-2.983039$

H -2.145476 -4.889840 -3.978500

H $1.380114-2.372426-2.041327$

H $\quad 0.946882-2.681766-0.368408$

H $1.968211-5.025812-0.618440$

H $2.420109-4.580221-2.262745$

H $4.339508-4.385294-0.881487$

H $3.226420-4.0503791 .352154$

H $2.743182-2.4355850 .874467$

H $4.292883-2.6645283 .103492$

H $4.584862-0.6507310 .400411$

H $5.563230-4.4041612 .203581$

H $6.830575-3.2488601 .860482$

H $6.163825-4.2438460 .551649$

H $6.557281-0.7344331 .502959$

H $6.403892-0.217892-1.909345$

H $\quad 5.625875-1.071209-3.211659$

H $6.470154-2.864007-2.442004$

H $1.0708120 .224968-0.523782$

H $-0.1054583 .052828-0.846115$

H 3.1008041 .4981530 .658562

H $1.7684360 .849628 \quad 1.642250$

H 2.7663372 .1371692 .265812

H $3.565417-0.033521-2.988741$

H $3.7935870 .362923-1.284293$

H $3.0816164 .503519-2.991132$

H $4.8351814 .260521-3.191426$

H $5.0489324 .125636-0.604670$

H $2.7505636 .070347-1.168074$

H 4.2026817 .1139460 .702169

H 4.5777235 .5083571 .291650

H 1.0926527 .2653072 .654495

H 2.3529468 .2119741 .682183

H 1.3651324 .9285063 .015264

H 2.8759464 .1894562 .534863

H 0.4998884 .6602720 .603392

H $2.8626862 .324576-2.062552$

C $-5.3409511 .019998-2.359863$

O $-4.5593810 .973932-1.150585$

C $-5.2964111 .613948-0.091922$

C $-6.6023082 .109477-0.710505$

C $-6.7824731 .162215-1.882144$ 
H $-5.526286 \quad 0.8364690 .645425$ $\begin{array}{llll}C & -4.436584 & 2.710494 & 0.547837\end{array}$ H -7.432932 2.0718360 .001238 H -6.520613 $3.143774-1.064045$ C $-7.7412201 .680775-2.938946$ H $-7.1487230 .199789-1.500228$ C -3.063085 2.220270 1.049706 O $-4.195170 \quad 3.724062-0.439033$ H $-4.994336 \quad 3.196007 \quad 1.356791$ H -2.459151 1.8982290 .194711 O -2.3826983 .3541791 .606460$ C -3.103661 1.0953902 .102754 C -1.6883590 .7039672 .574671$ H -3.5928290 .2080461 .687853$ O -3.8618501 .5250793 .231847$ H $-1.1400800 .297167 \quad 1.718174$ H -1.150478 1.6022702 .900656 H -8.736874 $1.837092-2.511109$ H -7.400981 2.634465 -3.356291 H -7.837349 $0.967674-3.763541$ C $-5.081129-0.239048-3.185203$ H -3.638446 $4.405434-0.024381$ H -2.863840 3.6187952 .409225 H $-4.793977 \quad 1.5746172 .961155$ H $-5.722815-0.258623-4.073129$ H $-5.325859-1.130112-2.597532$ H $-5.0254531 .913032-2.916330$ C $-1.991225-2.3558842 .525336$ O $-2.497867-1.4425923 .504317$ C $-1.677003-0.2992013 .749313$ C $-0.274308-0.735830 \quad 4.194314$ C $0.352304-1.739530 \quad 3.228924$ C $-0.617240-2.8876132 .951474$ H $-1.920577-1.8535401 .552225$ C $-3.002120-3.5007402 .405259$ H -2.1294720 .2195644 .604228$ O 0.5726560 .3998314 .338199 H $-0.350726-1.2038985 .184605$ H $\quad 0.669335-1.251657 \quad 2.300350$ O $1.535856-2.2553293 .841923$ H $-0.715497-3.5089423 .850930$ O $-0.095037-3.7127691 .914078$ C $-4.279101-3.0252761 .756229$ H $-2.603036-4.3363201 .818767$ H -3.268712 -3.902924 3.390108 H 1.4532670 .0759394 .594262 H $1.968531-2.8410633 .199169$ H $\quad 0.713960-4.1343142 .249069$ O $-4.269595-3.332057 \quad 0.428032$ O $-5.170328-2.4371652 .355392$ C $-5.445413-2.953100-0.291809$ H $-5.579898-1.868128-0.251997$ H -5.318070 -3.257488 -1.333817 H $-6.321827-3.4650610 .116931$

SCF Energy (B3LYP/6-31G**//MMFF) $=-3245.91021810$

\section{5_00110}

MM̄FF Geometry

C $1.620334-4.3172620 .040401$

C $0.812893-3.246217 \quad 0.063264$

C $-0.054798-2.811593-1.089542$

O $-1.351675-2.528997-0.518590$

C $0.545502-1.529094-1.689774$

C $-0.027447-1.113040-3.029123$

C $-0.7589070 .002001-3.225295$

C $0.346094-1.988547-4.196554$

C $-1.2040540 .978474-2.173986$

C $-0.115158 \quad 2.007544-1.845563$

C $-0.5624983 .017951-0.778957$

C $0.6125013 .895416-0.340934$

$\begin{array}{llll}\text { C } & 0.235815 & 4.837916 & 0.811797\end{array}$

O $\begin{array}{lllll} & -1.060975 & 2.289994 & 0.344270\end{array}$

O 1.4518065 .3448891 .384151

C $-0.476752 \quad 4.028872 \quad 1.920422$

C $-0.542353 \quad 6.057193 \quad 0.303044$

O -0.9940274 .8933352 .928693$

C -1.600832 3.1095251 .388635

$\begin{array}{llll}\text { C }-2.112772 & 2.161498 & 2.498897\end{array}$
O $-2.7189923 .876146 \quad 0.939135$

C $-2.459260-2.788455-1.259363$

O $-2.503320-3.176298-2.415023$

C $-3.631005-2.546434-0.385534$

C $-4.870324-2.718009-0.868164$

C $-6.119918-2.582188-0.114474$

C $-6.020057-2.2625201 .354329$

C -3.1360011 .1203642 .013705$

C $-4.4768851 .741748 \quad 1.668269$

C $-6.1767341 .877735-0.146288$

$\begin{array}{llll}\text { O } & -5.051081 & 2.515488 & 2.432329\end{array}$

C $-7.344673 \quad 1.048422 \quad 0.284498$

C $-8.2251490 .507246-0.571102$

C $-9.433580-0.272310-0.138909$

C $-9.522038-1.632955-0.791640$

C $-10.379734-1.862206-1.800535$

C $-8.684664-2.750183-0.211861$

C $-7.286680-2.770848-0.766213$

N $-4.956379 \quad 1.3443190 .429513$

H $1.633213-4.933072-0.857064$

H $0.782912-2.6050470 .939897$

H $-0.127530-3.616677-1.830098$

H $1.625681-1.662402-1.834846$

H $0.444316-0.719980-0.957739$

H $-1.0850440 .240129-4.236225$

H $-0.012779-1.584719-5.149347$

H $1.434805-2.080539-4.269147$

H $-0.080928-2.989154-4.083260$

H $-2.1016701 .494621-2.536318$

H $-1.5164330 .434299-1.275493$

H $0.772757 \quad 1.475895-1.482046$

H $0.1853852 .543734-2.754421$

H $-1.3699323 .627649-1.201087$

H $1.0140494 .462119-1.190190$

H 1.4333013 .2534600 .004848

H 1.9600315 .7764250 .676030

H 0.2809843 .3992882 .407742

H $0.0740046 .642594-0.390051$

H $-0.796397 \quad 6.736765 \quad 1.123742$

H $-1.460195 \quad 5.788635-0.224331$

H -0.2549295 .4376293 .250294$

H -1.2611961 .6065592 .913125$

H -2.555447 2.7471213 .313792

H -2.633976 4.7795461 .284796

H -3.439111 -2.262075 0.643068

H $-4.984834-3.003834-1.913249$

H $-5.443227-1.3462241 .515685$

H -5.528626 -3.081842 1.890563

H $-6.987658-2.0985141 .832740$

H $-2.7332970 .551593 \quad 1.169103$

H -3.3241590 .3978962 .817202$

H $-6.0491851 .881680-1.233424$

H -6.3130682 .9098190 .192862$

H $-7.4927430 .915711 \quad 1.353767$

H -8.094425 $0.664641-1.639385$

H $-10.3196690 .323753-0.394172$

H $-9.463458-0.3923850 .951291$

H - $10.470025-2.844335-2.252747$

H -11.006091-1.071764-2.200662

H $-9.142198-3.719580-0.452845$

H -8.714065 -2.6978500 .879790$

H -7.250769 $-3.003541-1.831607$

H $-4.4508990 .643695-0.104396$

C $2.672445-3.8740992 .371009$

O $3.106979-2.5509052 .010982$

C $3.923516-2.0173013 .067844$

C $3.963131-3.0793644 .164841$

C $3.707551-4.356007 \quad 3.385309$

H $4.930313-1.9014232 .650796$

C $3.383819-0.6513153 .513633$

H $4.921145-3.0862194 .694285$

H $3.177186-2.9268794 .913196$

C $3.241391-5.5121624 .252397$

H $4.634518-4.6407322 .869629$

C 3.1470120 .3513002 .365594

O $2.120201-0.8505934 .163850$

H $4.052456-0.2232834 .269812$

H 2.2900340 .0201751 .766317 
O $2.746120 \quad 1.5953132 .957028$

C 4.3492980 .5994071 .435616

C 4.0401861 .6870810 .390290

H $4.621836-0.3289680 .925907$

O $5.477811 \quad 1.0276642 .196415$

H $3.0825501 .453513-0.085429$

H 3.9257292 .6503970 .901829

H $3.994765-5.7527745 .009734$

H $2.307043-5.2731704 .771377$

H $3.071732-6.4097363 .649663$

C $2.579271-4.7548451 .120235$

H 1.7958640 .0234834 .441233

H 3.5121531 .9492043 .441068

H 5.7671350 .2816382 .747543

H $2.306414-5.7752321 .413517$

H $3.571413-4.8083260 .654551$

H $1.686472-3.7801662 .844969$

C $4.3017520 .222038-2.273409$

O $5.3685010 .612711-1.400290$

C $5.1473141 .829904-0.678882$

C $4.9228762 .985698-1.664698$

C $3.7918892 .679025-2.642193$

C $4.0099681 .320344-3.304818$

H $3.4017010 .012648-1.684477$

C $4.730557-1.074620-2.978950$

H $6.0811922 .042802-0.143726$

O $4.6470404 .186077-0.950806$

H $5.8476523 .152076-2.232389$

H $2.8154422 .720731-2.150171$

O $3.7665793 .671525-3.670119$

H $4.838798 \quad 1.394284-4.020722$

O $2.8401170 .968008-4.041942$

C $4.762797-2.267598-2.047632$

H $4.033102-1.320538-3.787159$

H $5.738294-0.949732-3.393220$

H $4.6379734 .915908-1.592564$

H $3.5069104 .513107-3.258672$

H $2.6606121 .691918-4.666032$

O $5.296379-3.332693-2.716894$

O $4.360141-2.276442-0.893275$

C $5.383534-4.551449-1.972046$

H $5.958852-5.269627-2.562043$

H $5.898835-4.389230-1.020279$

H $4.381175-4.956078-1.806174$

SCF Energy (B3LYP/6-31G**//MMFF)= -3245.91368995

05 00111

MM̄FF Geometry

C -2.544395 -1.918069 -2.667313

C $-1.544539-1.470584-1.893798$

C $-0.342032-0.742829-2.422430$

O $-0.2621970 .559876-1.793457$

C $0.967804-1.455882-2.051433$

C $1.310240-2.634464-2.931425$

C $1.155712-3.926106-2.585353$

C $1.910564-2.280131-4.268407$

C $0.553993-4.488235-1.328830$

C $1.497847-5.437875-0.577722$

C $2.490867-4.7993110 .410311$

C $1.782849-4.1352281 .594617$

C $2.779424-3.5284912 .592543$

O $3.339581-3.859593-0.260160$

O $2.050331-2.6660043 .479791$

C $3.769141-2.6395081 .812249$

C $3.434382-4.5957243 .476406$

O $4.800961-2.1555992 .668870$

C $4.388212-3.3378830 .577398$

C $5.269649-2.374776-0.254718$

O $5.253738-4.389141 \quad 1.029790$

C -1.019327 $1.568036-2.295000$

O $-1.9141421 .471662-3.122360$

C $-0.6243462 .866702-1.701953$

C $0.6023743 .068730-1.195349$

C $1.1238554 .310841-0.624233$

C $0.2262665 .518848-0.633034$

C $4.530537-1.210885-0.913626$

C $5.475220-0.221202-1.574903$

C $5.4618132 .002267-2.711131$
O $6.689803-0.389732-1.639121$

C $5.6316583 .087203-1.693303$

C $5.2036384 .347057-1.869558$

C 5.376125 5.436874-0.848991

C $4.0750306 .127332-0.497529$

C $3.7894147 .346483-0.985271$

C 3.1438895 .4480290 .483083

C 2.382444 4.309209-0.137025

N $4.7918190 .874037-2.089106$

H -2.498359-1.771740 -3.743435

H $-1.602508-1.605381-0.817958$

H $-0.398122-0.598769-3.508584$

H $\quad 0.964910-1.730455-0.989148$

H $1.787516-0.728338-2.123785$

H $1.457370-4.683818-3.308222$

H $2.188128-3.166482-4.848754$

H $1.200074-1.704065-4.868928$

H $2.816732-1.680795-4.131724$

H $\quad 0.158232-3.716922-0.666298$

H -0.319796 -5.073509-1.645390

H $0.883051-6.156243-0.019531$

H $2.067866-6.025851-1.309286$

H $3.114086-5.623448 \quad 0.778831$

H $1.116995-4.8447152 .101032$

H $1.141405-3.3240811 .231866$

H $1.357597-3.195703 \quad 3.910703$

H $3.204309-1.7601471 .480255$

H $2.673056-5.1303114 .057413$

H $4.106753-4.1405764 .211998$

H $3.996432-5.3406582 .909854$

H $4.370117-1.7180333 .423098$

H $6.090532-1.9996820 .370453$

H $5.759997-2.948861-1.053884$

H $5.872925-4.6083580 .313994$

H -1.367598 $3.654201-1.759792$

H $1.3141832 .244251-1.207033$

H $0.7051146 .417977-0.238837$

H $-0.6660705 .337870-0.023492$

H $-0.0885575 .757815-1.655071$

H $3.863800-1.600988-1.686603$

H $3.936302-0.656065-0.181876$

H $4.8444192 .321122-3.556759$

H $6.4407231 .689555-3.087795$

H $6.1400712 .823841-0.768506$

H $4.7156304 .616966-2.803298$

H $6.0881196 .164887-1.259678$

H 5.8397655 .0552070 .069277

H $2.8736817 .862779-0.717167$

H $4.4630447 .854462-1.667478$

H 2.4613706 .1740820 .938686

H 3.7290945 .0593561 .326667

H $2.9487643 .378032-0.171125$

H $3.7899890 .947209-1.936669$

C $-4.984547-1.661260-2.210448$

O $-4.771035-0.523915-1.350756$

C $-5.978765-0.254825-0.617093$

C -7.068932 -1.080565 -1.288464

C $-6.285165-2.303175-1.728629$

H $-5.822874-0.6421950 .397004$

C $-6.2362551 .254554-0.558633$

H $-7.890036-1.318099-0.605515$

H -7.491163 -0.567839-2.160398

C $-6.993386-3.132979-2.784890$

H $-6.091333-2.931083-0.848491$

C $-5.0312992 .077214-0.054636$

O $-6.5477541 .715278-1.879746$

H -7.123449 1.4522580 .054290

H $-4.2306742 .049472-0.803476$

O -5.4436223 .4478630 .024320$

C -4.4741731 .6358151 .311631$

C -3.3044842 .5247181 .768090$

H -4.1327340 .6011221 .238877$

O

H -2.5440152 .5181610 .979708$

H -3.6545213 .5591611 .869932$

H -7.946807 -3.510894 -2.401517

H -7.202507 -2.544130 -3.684327

H $-6.383229-3.992647-3.079186$ 
C $-3.773866-2.590855-2.124663$ H $-6.7023972 .673745-1.823333$ H -6.1130543 .5115910 .727270$ H -5.2269091 .2036353 .062129$ H -3.950619 -3.508050 -2.697054 H -3.607710 -2.885324-1.081805 H -5.099595 -1.272457 -3.230840 C $-1.234398 \quad 0.331822 \quad 2.316975$ $\begin{array}{lllll}\text { O } & -2.328466 & 0.712091 & 3.158677\end{array}$ C -2.6770752 .0998443 .116158$ C -1.471844 2.9546073 .532487 C -0.2478102 .6481292 .675721$ C 0.0253221 .1462922 .655877 H $-1.513294 \quad 0.474217 \quad 1.265724$ C $-0.958147-1.1619622 .550123$ H -3.4486372 .2441003 .882650$ O $-1.796654 \quad 4.3378613 .442891$ H -1.231814 2.7474214 .583393 H -0.3717143 .0478901 .665267$ O $\quad 0.879157 \quad 3.3233643 .237898$ H 0.4103490 .8482283 .639452 O $1.057827 \quad 0.8698321 .710752$ C -2.040854 -2.076969 2.021853 H -0.030031 -1.441092 2.041149 H $-0.859800-1.3612773 .623759$ H $-0.999551 \quad 4.8398403 .684552$ H 1.6543253 .0900732 .698606 H $0.706126 \quad 1.0546520 .823660$ O $-1.616453-3.3700162 .149643$ O $-3.114388-1.7184841 .561094$ C $-2.518492-4.3738751 .674779$ H -3.516856 -4.234290 2.100546 H -2.555594 -4.350061 0.582369 H -2.137307 -5.348457 1.990966 SCF Energy (B3LYP/6-31G**//MMFF)= -3245.91737862

05 00112

MM̄FF Geometry

C -1.705553 $5.472790 \quad 0.270919$

C $-0.5420934 .987988-0.187496$

C 0.5778864 .5164120 .701038

O $0.920406 \quad 3.163471 \quad 0.320405$

C 1.8227025 .3976940 .490196

C 2.9991685 .0063991 .366060

C 4.1255414 .4033910 .933087

C 2.8614245 .3760712 .821751

C $4.4704743 .964184-0.464140$

C $4.5911242 .443021-0.594523$

C 5.8095631 .7958730 .084991

C $7.1268492 .210543-0.578206$

C $8.3308011 .426243-0.033393$

O $5.618955 \quad 0.378707-0.037929$

O $9.4356451 .637542-0.926333$

C $8.004020-0.081051-0.072275$

C 8.7769951 .9482851 .337404

O $9.036768-0.8440050 .546675$

C $6.642271-0.4244660 .564283$

C $6.253518-1.9080340 .361538$

O $\quad 6.710741-0.1968481 .976418$

C 0.1812442 .1479760 .846368

$\begin{array}{lllll}\text { O } & -0.801571 & 2.240988 & 1.563013\end{array}$

C 0.8240220 .8837920 .424949

C $0.485355-0.2806710 .998495$

C $1.139202-1.5701810 .752997$

C $2.319404-1.576227-0.182162$

C $6.067938-2.333268-1.098622$

C $5.373928-3.681473-1.206792$

C $3.939788-5.002565-2.760178$

O $5.344051-4.494780-0.286546$

C $2.552059-4.671408-2.303838$

C $1.858406-5.408645-1.423373$

C $0.499974-5.022771-0.907355$

C $0.429548-5.0486000 .605386$

C $-0.344370-5.943591 \quad 1.242652$

C $1.270397-4.0590281 .381651$

C $0.687976-2.6686321 .394683$

N $4.801951-3.872439-2.458402$

H -1.8671855 .5589511 .342376$
H $-0.3889154 .897560-1.260755$

H 0.2816524 .5357351 .757106

H 1.5725556 .4465990 .700825

H $2.1055635 .377321-0.567133$

H 4.9049384 .2024661 .667078

H 3.7669695 .1530033 .395939

H 2.6653926 .4483722 .924453

H 2.0367944 .8247003 .283540

H $3.7180084 .296918-1.183827$

H $5.4015004 .454627-0.766930$

H $3.6814061 .973923-0.201240$

H $4.6048442 .181326-1.661308$

H 5.8099222 .0535631 .149341

H $7.3037563 .286656-0.469117$

H $7.0600702 .027409-1.659984$

H $9.6049362 .594164-0.972864$

H $7.988855-0.378199-1.128674$

H 9.0846862 .9984641 .265280

H 9.6551221 .4037741 .701436

H 7.9933701 .8887912 .095453

H $9.874386-0.5999320 .117048$

H $6.985241-2.5620290 .853287$

H $5.307392-2.0871890 .891831$

H $6.009458-0.7130292 .407199$

H $1.6074690 .954827-0.320272$

H $-0.309310-0.2874221 .742918$

H $3.093334-0.8860340 .169224$

H $2.012720-1.279354-1.191305$

H $2.794380-2.552167-0.273230$

H $5.467035-1.588880-1.632814$

H $7.037457-2.417890-1.600734$

H $4.334749-5.897973-2.269204$

H $3.956003-5.153376-3.843410$

H $2.108398-3.759614-2.697948$

H $2.294245-6.327837-1.038658$

H $-0.231159-5.720315-1.335588$

H $0.211808-4.026148-1.261413$

H $-0.398281-5.9747602 .325763$

H $-0.945302-6.6630310 .696289$

H $2.302330-4.0814291 .020908$

H $1.349503-4.3798632 .429797$

H -0.177944 -2.566154 2.050065

H $4.815120-3.106727-3.125604$

C $-4.0444604 .962224-0.444163$

O $-3.6810773 .620600-0.822366$

C $-4.7611113 .032853-1.576421$

C $-5.9366953 .989144-1.441641$

C $-5.2342265 .328094-1.329333$

H $-4.4111202 .993670-2.615354$

C $-5.0187701 .621357-1.039473$

H $-6.6224743 .933804-2.292346$

H $-6.5083943 .779050-0.528904$

C $-6.1122226 .429887-0.762749$

H $-4.8841125 .623945-2.327299$

C $-6.2012420 .902998-1.712077$

O $-3.835604 \quad 0.844128-1.251465$

H -5.1704801 .6720540 .044185$

H -7.135249 $1.436892-1.503309$

O $-6.0248220 .953978-3.134068$

C $-6.361169-0.578043-1.315251$

C $-6.568473-0.7857650 .193472$

H -5.502531 -1.156947 -1.673259

O $-7.513828-1.098039-1.987480$

H $-5.755074-0.3111560 .747657$

H -7.498356 -0.2880350 .495121$

H $-6.9808356 .599185-1.407462$

H -6.4801656 .1757710 .237004$

H -5.558394 7.371014 -0.688696

C $-2.8398225 .886006-0.625631$

H $-3.100879 \quad 1.309012-0.815114$

H $-5.1811370 .515557-3.340264$

H -7.382607 -0.953678 -2.940313

H -3.113209 $6.920200-0.388899$

H -2.513846 $5.866394-1.673210$

H -4.3434784 .9261950 .611827$

C $-4.287693-2.7023460 .786578$

O $-5.518201-3.0208260 .126783$

C $-6.653685-2.2790800 .585004$ 
C $-6.864738-2.5276792 .084248$

C $-5.602697-2.2422282 .896428$

C $-4.398903-2.9672322 .292844$

H -4.034008 -1.650224 0.609903

C $-3.193090-3.567524 \quad 0.156282$

H $-7.524187-2.6964530 .063800$

O $-7.945140-1.7342322 .564228$

H -7.148264 -3.578167 2.231526

H $-5.422513-1.1636482 .975756$

O $-5.819630-2.713764 \quad 4.228368$

H $-4.487362-4.0449962 .480791$

O $-3.206157-2.5136952 .930020$

C -2.845314 -3.079765 -1.229154

H -2.276161 -3.5623440 .752981$

H -3.514542 -4.6123450 .069398$

H -8.033545 -1.9092633 .516856$

H -5.056404 -2.440701 4.764570

H -3.242086 -2.797087 3.858991

O $-1.990506-2.020240-1.147550$

O $-3.286345-3.575955-2.257604$

C $-1.571928-1.468137-2.399299$

H $-0.976163-2.200977-2.951625$

H $-2.438694-1.151789-2.987630$

H $-0.950083-0.592765-2.194068$

SCF Energy (B3LYP/6-31G**//MMFF) $=-3245.91961256$

0500113

MM̄FF Geometry

C $3.096725-3.3167911 .519357$

C $2.048080-4.0749551 .878772$

C $0.599551-3.6706681 .711166$

O $\quad 0.512715-2.738591 \quad 0.609467$

C $-0.292374-4.8875531 .387411$

C -1.771635 -4.649545 1.641631

C $-2.737029-4.6321010 .700986$

C $-2.156237-4.4734283 .091482$

C $-2.614479-4.847257-0.780261$

C $-2.671545-3.566511-1.625543$

C -4.009509-2.806902 -1.574564

C $-4.021276-1.693054-2.626484$

C $-5.296429-0.839416-2.556561$

O $-4.177588-2.235050-0.273494$

O $-5.087957 \quad 0.334681-3.357540$

C $-5.535797-0.393432-1.095662$

C $-6.485194-1.559115-3.204069$

O $-6.798010 \quad 0.261371-0.996583$

C $-5.429302-1.554269-0.081094$

C $-5.486344-1.0931921 .397745$

O $-6.533789-2.447352-0.274569$

C $-0.281320-1.6481060 .742625$

O $-1.009151-1.3736501 .681575$

C $-0.099044-0.830285-0.478323$

C $-0.9248420 .200233-0.702117$

C $-0.8889461 .118731-1.838877$

C $0.225150 \quad 0.961215-2.835967$

C $-4.400515-0.103081 \quad 1.827060$

C -4.8058261 .3595801 .731245$

C -3.8165073 .6541891 .828812$

O -5.9700131 .7293541 .607040$

C $-3.7685794 .134026 \quad 0.412875$

C $-2.7216574 .778978-0.124920$

C $-2.6772135 .251065-1.551057$

C $-1.6408004 .520131-2.377967$

C $-0.4583665 .088104-2.668687$

C $-2.0075053 .159745-2.933474$

C $-1.8414642 .069827-1.913054$

N -3.711553 $2.206171 \quad 1.848647$

H $2.924186-2.3320411 .092155$

H $2.238987-5.0354872 .351855$

H $0.295631-3.1969222 .651715$

H $\quad 0.003203-5.7467472 .004408$

H $-0.116012-5.1875800 .349505$

H -3.761797 -4.471904 1.035102

H -3.242160 $-4.460853 \quad 3.235471$

H - 1.759588 -5.298270 3.692408

H - $1.765341-3.5323183 .488587$

H -1.689353 -5.379603 -1.022047

H -3.423154 -5.520165 -1.092907
H $-1.860300-2.903851-1.310993$

H -2.466710 -3.851100 -2.665417

H $-4.817846-3.522139-1.766091$

H -3.894202 -2.106920 -3.634658

H -3.155106 -1.038112 -2.465341

H $-4.3254750 .817862-2.996796$

H $-4.7701010 .357967-0.866973$

H $-6.276241-1.763766-4.261257$

H -7.382709-0.930674-3.194665

H $-6.725266-2.510938-2.725902$

H $-6.8623380 .656274-0.111191$

H $-6.488908-0.7222121 .641778$

H -5.353891 -1.991383 2.018623

H $-6.650818-2.9729990 .534258$

H $\quad 0.698857-1.103405-1.159364$

H -1.7075250 .3995800 .025817$

H $\quad 0.1035350 .029329-3.398728$

H $1.1944660 .936447-2.330443$

H $\quad 0.279826 \quad 1.776502-3.560536$

H -4.172332 -0.280424 2.885827

H $-3.480071-0.2720931 .264122$

H -2.9888934 .0491202 .426685$

H -4.7585043 .9606382 .294516$

H -4.644262 $3.936640-0.201380$

H -1.844832 4.9768950 .487355

H $-2.4639206 .327885-1.539689$

H -3.657970 $5.154200-2.033322$

H $0.2780154 .577354-3.280326$

H $-0.1972286 .072372-2.294479$

H -1.432861 2.951711 -3.841820

H $-3.0528373 .172018-3.267266$

H -2.616720 $2.070734-1.148410$

H -2.7778461 .8065751 .872440$

C $5.356335-3.566694 \quad 0.395974$

O $5.413143-2.1767790 .011234$

C $5.189218-2.086113-1.408356$

C $5.412626-3.492782-1.953048$

C $4.847838-4.335810-0.825258$

H $4.132909-1.823904-1.544911$

C $6.075130-1.002965-2.032034$

H $4.903831-3.654829-2.907995$

H $6.477702-3.711596-2.092289$

C $5.288474-5.788365-0.863514$

H $3.753197-4.297570-0.886156$

C $5.9357410 .388423-1.379053$

O $7.442344-1.412043-1.903254$

H $5.869030-0.941716-3.107553$

H $6.384000 \quad 0.383787-0.377718$

O $6.698077 \quad 1.317125-2.160712$

C $4.4900620 .914321-1.285563$

C $4.4235362 .369882-0.783353$

H $3.9178790 .270470-0.610128$

O $3.8706550 .839147-2.568923$

H 5.0386842 .4576920 .118139

H $4.8605093 .034081-1.539054$

H $4.950481-6.268503-1.787653$

H $6.378844-5.879100-0.817641$

H $4.867294-6.344152-0.019667$

C $4.534793-3.7230431 .680375$

H $7.976197-0.837457-2.477393$

H $7.6379051 .103232-2.035963$

H $4.3984801 .378148-3.182787$

H $4.968610-3.0785832 .455038$

H $4.607934-4.7572842 .035945$

H $6.391926-3.8568620 .616745$

C 2.7850582 .0484051 .767600

$\begin{array}{llll}\text { O } & 2.293748 & 1.991239 & 0.424908\end{array}$

C $2.9787602 .846234-0.498176$

C $2.8815434 .304585-0.026537$

C 3.3992314 .4736241 .400158

C 2.7205593 .4728622 .334977

H 3.8278841 .7073161 .772039

C 2.0220911 .0190792 .614389

H $2.4311032 .779605-1.445545$

O $3.6070415 .170157-0.893018$

H $1.8322094 .626318-0.054274$

H 4.4902504 .3796191 .447880

O 3.0908975 .7920891 .859094 
H $1.682208 \quad 3.7869782 .490395$

O 3.3646693 .4988693 .606616

C 0.5611151 .3515332 .838556

H 2.0522880 .0376402 .127480

H 2.5132140 .9324713 .590806

H $3.2441125 .057564-1.788206$

H 3.4907386 .4173941 .230391

H 3.3367704 .4167093 .927151

$\begin{array}{lllll}\text { O } & 0.161076 & 0.817392 & 4.030223\end{array}$

O $-0.148147 \quad 1.9701932 .057102$

C -1.206212 1.0438604 .385382

H -1.4544142 .1076214 .319811$

H -1.347453 0.7162235 .418828

H -1.858749 0.4528853 .737790

SCF Energy (B3LYP/6-31G**//MMFF) $=-3245.91806467$

05_00114

MMFF Geometry

C $0.665082-3.8227850 .634222$

C $1.300142-3.669016-0.538421$

C $0.698904-3.131708-1.816519$

O $-0.577525-2.509473-1.553331$

C $1.616854-2.049639-2.419292$

C $1.129707-1.520660-3.754675$

C $0.740957-0.250580-3.981667$

C $1.117622-2.517835-4.885452$

C $0.7367390 .907866-3.023598$

C $-0.6222361 .081721-2.346579$

C $-0.8188582 .458037-1.694064$

C $0.2089312 .752766-0.599258$

C -0.0760344 .0840360 .113026$

O $-2.1424262 .461337-1.140160$

O 0.7431934 .1135181 .290586

C $-1.553277 \quad 4.1006630 .560094$

C $0.361504 \quad 5.281416-0.738402$

O $\begin{array}{llll}0.1 .901593 & 5.362735 & 1.123161\end{array}$

C -2.540260 $3.712649-0.561716$

C $-3.9911903 .552100-0.051139$

O $-2.5579394 .750848-1.549009$

C -1.702791-3.082712 -2.054567

O $-1.792038-4.128281-2.675858$

C $-2.826593-2.179519-1.719008$

C $-4.080250-2.475307-2.092733$

C $-5.260678-1.642094-1.842969$

C $-5.027158-0.291219-1.219316$

C -4.1673322 .5362161 .079959$

C $-5.6247552 .168571 \quad 1.309473$

C -7.0208060 .5356932 .586677$

$\begin{array}{lllll}\text { O } & -6.558869 & 2.718609 & 0.732947\end{array}$

C -7.158269 -0.7453361 .823803$

C $-8.228993-1.0671241 .081532$

C $-8.359705-2.3699710 .342399$

C -8.749881-2.194824-1.110772

C $-9.904364-2.702262-1.575542$

C $-7.814336-1.437090-2.029798$

C $-6.478176-2.112944-2.186025$

N $-5.750633 \quad 1.1493582 .243379$

H $-0.388229-3.5705010 .720078$

H $2.347034-3.961273-0.598866$

H $\quad 0.599082-3.970025-2.515586$

H $2.626460-2.454710-2.563863$

H $1.716297-1.234296-1.694566$

H $0.3955360 .006266-4.982638$

H $0.900617-2.048268-5.850904$

H $2.095072-3.003561-4.973130$

H $0.358173-3.287009-4.717450$

H $1.5251120 .802447-2.272908$

H $0.988193 \quad 1.810263-3.595016$

H - $-1.4323020 .928177-3.072775$

H $-0.7589650 .299337-1.589882$

H $-0.7660213 .215835-2.484661$

H $1.2274502 .759885-1.002605$

H $\quad 0.185987 \quad 1.9454530 .146074$

H $\quad 0.5173804 .9186651 .787469$

H -1.6332023 .3730941 .377068$

H $1.4368355 .226078-0.947135$

H $0.2108616 .225439-0.203174$

H $-0.1589775 .341102-1.696412$
H -2.021991 5.9907450 .390584

H $-4.391277 \quad 4.525250 \quad 0.262379$

H $-4.6148783 .240502-0.900939$

H $-3.386053 \quad 4.681957-2.052677$

H -2.585293 -1.274785 -1.172256

H $-4.261795-3.405455-2.628784$

H $-4.3382740 .302993-1.830516$

H $-4.599004-0.406697-0.218130$

H $-5.9319690 .307508-1.109617$

H $-3.616528 \quad 1.6170760 .851626$

H -3.7776852 .9467582 .018143$

H $-7.838605 \quad 1.2326142 .377735$

H -7.0002160 .3269753 .660588$

H $-6.336497-1.4542311 .900637$

H -9.065152 $-0.374277 \quad 1.022728$

H $-9.115231-2.9692110 .867049$

H -7.429382 -2.948803 0.395886

H -10.197265 -2.585263 -2.613687

H -10.584629-3.246955-0.929355

H $-7.734930-0.406634-1.675971$

H $-8.250964-1.357664-3.034333$

H -6.540661 -3.101756 -2.642543

H -4.9089720 .7294002 .627699$

C $1.089709-3.4001173 .065489$

O $1.661857-2.1072812 .795129$

C $2.235493-1.5871404 .009212$

C $1.850362-2.5599315 .120218$

C $1.746717-3.8690424 .361560$

H $3.323560-1.6202293 .878414$

C $1.783301-0.1385224 .232541$

H $2.594642-2.5857675 .922147$

H $\quad 0.884489-2.3059755 .571431$

C $0.966556-4.9404765 .102654$

H $2.761395-4.2365884 .157594$

C 2.0084020 .7883593 .020427

O $0.377679-0.1431364 .514853$

H $2.278761 \quad 0.264413 \quad 5.124120$

H 1.3196320 .5228932 .208522

O 1.6725562 .1220273 .421525

C 3.4503950 .7992362 .482499

C 3.6308361 .7862871 .313870

H $3.714482-0.2056912 .139493$

$\begin{array}{lllll}\text { O } & 4.352600 & 1.137324 & 3.534191\end{array}$

H $2.903798 \quad 1.5359840 .534120$

H 3.4040232 .8039441 .653343

H $1.448401-5.1757476 .057263$

H $-0.058247-4.6170325 .313899$

H $0.914274-5.8625454 .515299$

C $1.333537-4.3359921 .880539$

H $0.143296 \quad 0.738617 \quad 4.849764$

H 0.7064122 .1638313 .520510

H 4.1037932 .0186263 .861840

H $0.932675-5.3310512 .103040$

H $2.412149-4.4423891 .710529$

H $\quad 0.012060-3.2479543 .213824$

C $4.890353-0.148368-0.697663$

$\begin{array}{llllll}\text { O } & 5.522634 & 0.463569 & 0.429696\end{array}$

C 5.0630221 .7837560 .733799

C $5.2517882 .706201-0.480280$

C $4.6191542 .128173-1.744762$

C $5.0823870 .688696-1.967548$

H $3.816906-0.244213-0.490750$

C $5.414612-1.586229-0.825686$

H 5.7222062 .1584901 .527108

O $4.7001893 .995393-0.232867$

H $6.3250432 .845108-0.665247$

H $3.5262232 .187104-1.709090$

O $5.0235642 .907903-2.872567$

H $6.1339910 .697737-2.276550$

$\begin{array}{lllll}\text { O } & 4.333271 & 0.107690 & -3.031448\end{array}$

C $6.897832-1.689024-1.117788$

H $5.243342-2.124140 \quad 0.114447$

H $4.866041-2.099894-1.622768$

H 5.1302614 .3486430 .564756

H $4.7563303 .826828-2.698477$

H $4.4338590 .684874-3.807655$

O $7.154114-2.898461-1.699238$

O $7.732446-0.838089-0.842486$ 
C $8.526346-3.148554-2.019812$

H $8.893089-2.399176-2.727770$

H $9.133078-3.150541-1.109358$

H $8.591815-4.134499-2.487480$

SCF Energy $\left(B 3 L Y P / 6-31 G^{* *} / / M M F F\right)=-3245.92063290$

05 00115

MM̄MF Geometry

C $\quad 0.0678285 .567510 \quad 0.151763$

C $0.6356324 .729026-0.727951$

C $2.0094554 .133505-0.551520$

O $1.8790512 .694083-0.488273$

C $2.8817504 .482586-1.771324$

C $4.3123623 .991890-1.665855$

C $4.8748453 .089365-2.493718$

C $5.1358924 .606528-0.563007$

C $4.2479252 .386355-3.665323$

C $3.6091651 .028661-3.332583$

C $4.603961-0.027037-2.822585$

C $3.936452-1.401268-2.729540$

C $4.878879-2.460225-2.134268$

O $5.057827 \quad 0.367525-1.525854$

O $4.087426-3.605299-1.780604$

C $5.492345-1.914787-0.825736$

C $5.908643-2.946671-3.161045$

O $6.499113-2.793227-0.330371$

C $6.060841-0.488049-0.962172$

$\begin{array}{llll}\text { C } & 6.485867 & 0.135417 & 0.389817\end{array}$

O $7.229520-0.524405-1.790637$

C $1.5145192 .152503 \quad 0.706274$

O 1.2978942 .7480021 .749734

C $1.3967210 .689490 \quad 0.518587$

C $0.745354-0.0416201 .435697$

C $0.516173-1.4871041 .385498$

C $1.233198-2.2676820 .316337$

C $5.3741600 .255851 \quad 1.438040$

C $5.334693-0.8966742 .427797$

C $3.958247-1.8050234 .294580$

O $6.219163-1.7441372 .512985$

C $3.109021-2.9177323 .766651$

C $1.993581-3.3430484 .378632$

C $1.151241-4.4848323 .887109$

C $-0.303632-4.1060253 .727772$

C -1.200156 -4.386074 4.688822

C $-0.732076-3.4807552 .420062$

C $-0.312001-2.0410702 .295811$

N $4.207757-0.8434173 .237357$

H 0.6108425 .8533111 .049114

H $0.0791994 .432417-1.614357$

H 2.4827234 .5013960 .366980

H $2.9074025 .572017-1.909033$

H $2.4034794 .076131-2.669073$

H $5.9167662 .820608-2.322262$

H $6.1941294 .332363-0.632781$

H $5.0789515 .698962-0.609385$

H 4.7772104 .2772960 .417017

H $3.4976353 .025094-4.143383$

H $5.0238422 .241556-4.428168$

H $2.8182881 .181433-2.588911$

H $3.1270190 .655294-4.244471$

H $5.453917-0.061940-3.514285$

H $3.570193-1.723808-3.712113$

H $3.047201-1.329485-2.088789$

H $3.633206-3.915093-2.582890$

H $4.689680-1.907015-0.079049$

H $5.404308-3.386918-4.029711$

H $6.536122-3.742723-2.745221$

H $6.558793-2.149510-3.527219$

H $6.095015-3.672608-0.234725$

H $7.363408-0.3885790 .787161$

H $\quad \begin{array}{llll}\text { H } & 830961 & 1.158128 & 0.178789\end{array}$

H $7.7619010 .267550-1.607919$

H $\quad \begin{array}{llll}1.825760 & 0.259639 & -0.378701\end{array}$

H $\quad 0.312567 \quad 0.4687532 .295351$

H $2.308196-2.0615100 .349751$

H $0.853784-1.999516-0.675537$

H $1.131818-3.3495830 .421320$

H 5.5564291 .1599412 .033308
H $4.3940980 .368808 \quad 0.964530$

H $3.473652-1.2666385 .115225$

H $4.910971-2.2072584 .654205$

H $3.439227-3.4121652 .856070$

H $1.682248-2.8647585 .304710$

H $1.245546-5.3043274 .611622$

H $1.530436-4.8850632 .938608$

H -2.250933 -4.142835 4.570809

H -0.901852 -4.860118 5.617964

H -1.826433 -3.502314 2.338558

H $-0.379084-4.1040031 .594202$

H $-0.782933-1.3875153 .031739$

H $3.498459-0.1382003 .059540$

C -2.3098075 .4362410 .912106$

O -2.4886224 .0752690 .469378$

C $-3.8930103 .772907 \quad 0.430275$

C $-4.5717784 .872075 \quad 1.235571$

C $-3.7023716 .066557 \quad 0.893345$

H $-4.2033943 .849380-0.619848$

C $-4.1649322 .348706 \quad 0.932973$

H -5.6203885 .0077260 .954378$

H -4.5309704 .6670172 .311749$

C $-3.864674 \quad 7.232459 \quad 1.852917$

H $-3.9502486 .401773-0.122859$

C $-3.491316 \quad 1.259603 \quad 0.069327$

O $-3.7355772 .234122 \quad 2.288491$

H -5.2486122 .1836950 .925042$

H $-3.758598 \quad 1.431733-0.980806$

$\begin{array}{llll}\text { O } & -2.070995 & 1.347872 & 0.165395\end{array}$

C $-3.922200-0.1744330 .440959$

C $-3.274763-1.220260-0.486019$

H $-5.013530-0.2428840 .372746$

O $-3.552990-0.4907171 .781209$

H -3.436280 -0.919557-1.526564

H -2.191155 -1.230826 -0.319362

H -4.8950257 .6025241 .837574$

H -3.627481 6.9415092 .881882

H -3.204052 8.0600691 .576201

C -1.314133 $6.135866-0.013667$

H -2.7715682 .3648842 .299518$

H -1.805642 2.229376 -0.146570

H -4.0109190 .1330412 .369436$

H -1.2726907 .2085950 .205633$

H -1.637839 $6.029912-1.056977$

H -1.9196245 .3895191 .937162$

C $-5.778961-2.496018-1.644976$

O $-5.246088-2.705560-0.332326$

C $-3.818356-2.647834-0.249440$

C $-3.201392-3.719413-1.158325$

C $-3.727282-3.623011-2.590374$

C $-5.256388-3.567782-2.607542$

H -5.493872 -1.500879-2.005939

C $-7.306890-2.540701-1.534074$

H $-3.564715-2.9159570 .782757$

O $-1.782122-3.614083-1.149624$

H $-3.451971-4.709069-0.754919$

H -3.300747 -2.754970 -3.105921

O $-3.290212-4.765212-3.330907$

H -5.663483 -4.550001-2.335870

O $-5.700392-3.269749-3.928938$

C $-7.829228-1.386321-0.710854$

H -7.794275 -2.505512 -2.514552

H -7.629019 -3.466270 -1.041382

H -1.435241 -4.293519-1.752779

H -3.650461 -5.558892 -2.899799

H $-5.327326-3.945993-4.520259$

O $-7.666867-0.214290-1.389036$

O $-8.322547-1.5133740 .402432$

C $-8.0981580 .961594-0.697110$

H $-7.8540901 .829035-1.316032$

H $-9.1809030 .931379-0.543537$

H -7.5744651 .0558240 .259111$

SCF Energy (B3LYP/6-31G**//MMFF) $=-3245.92084541$

05 00116

MM̄FF Geometry

C $2.199779-3.0804941 .870602$

C $1.205842-2.2467911 .529453$ 
C $0.257810-1.6364032 .527800$ O $0.200594-0.1986942 .348298$ C - $1.174219-2.1638212 .319623$ C -1.371891-3.592310 2.780316 C -1.406801-4.669055 1.970897 C $-1.570750-3.7608684 .265004$ C -1.216554 -4.715624 0.480310 C $-2.454072-5.242047-0.259933$ C $-3.658931-4.292231-0.188145$ C $-4.858807-4.882969-0.927344$ C $-6.049111-3.914712-0.933612$ O $-3.282598-3.057237-0.800451$ O $-6.997369-4.425228-1.882889$ C $-5.575541-2.528827-1.428760$ C $-6.764529-3.9003360 .423382$ O $-6.635158-1.584222-1.300653$ C $-4.284556-2.027510-0.740556$ C $-3.654380-0.815702-1.464365$ O $-4.567009-1.6672590 .612291$ C $1.259725 \quad 0.5340262 .781857$ O $2.334424 \quad 0.104463 \quad 3.178346$ C 0.9769621 .9851872 .714209 C -0.2610202 .4842012 .860825$ C -0.6215943 .9049122 .900845$ C 0.4475574 .9072822 .554573 C $-4.4638420 .481243-1.440984$ C $-3.6486581 .651413-1.963994$ C $-3.5214514 .129131-1.874380$ O $-2.6920061 .513227-2.721589$ C $-3.8344975 .144503-0.824825$ C -2.9822985 .4944880 .150723$ C -3.2946676 .5313061 .189008$ C -3.6082045 .9298822 .539120$ C -4.8804385 .7623202 .938718$ C -2.4547585 .6201623 .465058$ C -1.8798834 .2430433 .254606$ N $-4.1301812 .867254-1.502388$ H $2.351243-3.3289262 .918144$ H $1.044890-2.0034970 .483677$ H $0.577144-1.8324703 .559241$ H -1.472365 -2.032709 1.272299 H -1.877244 -1.520545 2.867521 H -1.568222 -5.6453582 .426970$ H -1.747389 -4.804599 4.546262 H -0.687799 -3.418101 4.813091 H $-2.436157-3.1796314 .600057$ H $-0.922289-3.7469940 .068227$ H $-0.378055-5.3936430 .276603$ H -2.171055 -5.383894 -1.311097 H -2.731711 -6.2270150 .135796$ H $-3.910626-4.1160040 .863384$ H -5.149660 -5.847913 -0.493598 H -4.578381 -5.095294 -1.968747 H -7.723541 -3.781359-1.949584 H -5.378407 -2.620968 -2.505389 H $-7.101231-4.9102000 .687117$ H -7.670777 -3.2856220 .389007$ H $-6.132476-3.536191 \quad 1.236149$ H $-6.683941-1.318820-0.366445$ H -2.679085 $-0.616698-0.996885$ H -3.418350 -1.090554-2.501297 H -3.862950 -1.073144 0.921683 H 1.8506532 .6227632 .629531 H -1.089052 1.7924453 .009066 H 0.0672545 .9242912 .436411 H 0.9195634 .6530801 .599672 H 1.2187554 .9313133 .331838 H $-5.3481760 .398784-2.080409$ H -4.791561 $0.704887-0.419856$ H $-3.9397804 .436679-2.838114$ H $-2.4435603 .992767-2.008263$ H -4.811103 $5.619532-0.877827$ H $-2.008095 \quad 5.0164360 .204359$ H -4.1350737 .1550800 .855352$ H -2.4459687 .2209661 .274259$ H -5.1164405 .3633053 .919695$ H -5.7135886 .0150042 .291233$ H -2.8003185 .6660804 .507200$
H -1.7051496 .4130363 .393094$ H -2.584355 3.4422893 .484376 H $-5.0337902 .887656-1.037475$ C $4.565084-3.2768531 .062623$ O $4.682116-1.9016820 .644459$ C $5.851030-1.764190-0.184160$ C $6.660556-3.0383360 .018456$ C $5.556769-4.0605250 .201550$ H $5.498942-1.736211-1.222323$ C $6.594383-0.4667730 .146013$ H $7.313015-3.256081-0.832518$ H $7.283235-2.9877870 .919096$ C $6.029820-5.3594190 .830718$ H $5.113127-4.278021-0.779561$

C 5.7147450 .7981010 .082009 O $7.112478-0.5648681 .479008$ H $7.463110-0.364948-0.515076$ H 4.9951630 .7879980 .909434 O $6.566824 \quad 1.926655 \quad 0.320095$ C $4.9652290 .999390-1.248839$ C $4.1945102 .330234-1.273275$ H $4.2687250 .170092-1.394241$

O $5.9108110 .969874-2.315566$

H $3.5542142 .370589-0.385169$

H $4.9077493 .159618-1.196177$

H $6.782963-5.8409760 .198512$

H $6.477510-5.1886831 .815546$

H $5.197289-6.0586270 .956066$

C $3.119854-3.7399390 .883080$

H 7.5771020 .2677091 .671075

H $7.1775691 .996028-0.433777$

H $5.4156020 .874011-3.146447$

H $3.047636-4.823671 \quad 1.029043$

H $2.789429-3.526357-0.139758$

H $4.857518-3.3155502 .120049$

C $1.3754131 .317621-1.886708$

O $2.4529921 .463066-2.818684$

C $3.3494652 .546424-2.550077$

C $2.5769243 .872139-2.556549$

C $1.4204643 .845013-1.563130$

C $0.5503112 .612244-1.796663$

H $1.7767601 .045896-0.902587$

C $0.4773120 .168643-2.375287$

H $4.0492602 .581649-3.393888$

O $3.4534854 .953388-2.257446$

H $2.1782974 .049855-3.563861$

H $1.7863693 .891182-0.531789$

O $0.6311365 .019959-1.760080$

H $-0.0181422 .758103-2.723838$

O $-0.4027792 .509577-0.741342$

C $1.141943-1.189609-2.334335$

H $-0.4158250 .106514-1.744487$

H $0.1778860 .349795-3.414652$

H $2.9190865 .766059-2.246100$

H $-0.1207064 .968226-1.146047$

H 0.0861192 .3503830 .082826

O $0.223664-2.133245-2.702878$

O $2.305984-1.403773-2.029515$

C $0.699320-3.482108-2.737848$

H $1.546856-3.570716-3.424058$

H $0.979318-3.808779-1.733091$

H -0.112549-4.119091 -3.098691

SCF Energy $\left(B 3 L Y P / 6-31 G^{* *} / / M M F F\right)=-3245.92316453$

0500117

MM̄FF Geometry

C $1.715574-3.871399-1.975646$

C $0.872939-3.415883-2.914844$

C $-0.442995-2.758974-2.579638$

O $-0.312980-1.343023-2.857488$

C $-1.582461-3.321195-3.453125$

C $-2.911751-3.365105-2.721425$

C $-3.920807-2.486309-2.878710$

C $-3.072690-4.525274-1.769536$

C $-3.975431-1.283891-3.777996$

C $-3.5561470 .006217-3.072544$

C $-4.394270 \quad 0.403940-1.849475$

C $-5.7801520 .921910-2.237298$ 
C $-6.553216 \quad 1.473183-1.027375$

O $-3.6589701 .438463-1.184850$

O $-7.6630972 .217026-1.553369$

C $-5.6458992 .451432-0.245212$

C $-7.1494580 .339820-0.183444$

$\begin{array}{lllll}\text { O } & -6.261320 & 2.861666 & 0.973751\end{array}$

C $-4.2414891 .876740 \quad 0.047712$

C -3.3189462 .9636860 .645693$

$\begin{array}{lllll}\text { O } & -4.368191 & 0.811677 & 0.991972\end{array}$

C $0.287125-0.580002-1.902268$

O $0.663383-0.939007-0.798109$

C $0.4665670 .780961-2.445716$

C $1.5466231 .477095-2.061407$

C $1.9190022 .818708-2.509625$

C $0.8523543 .642437-3.180192$

C -1.8394262 .5518310 .701798$

C -0.9721073 .6086111 .360419$

C 1.4090604 .0675521 .937021

O $-1.4197784 .648741 \quad 1.834987$

C 1.9697764 .9556040 .872511

C 3.2713614 .9965590 .551549

C $3.8424875 .913899-0.490662$

C $4.6062065 .172600-1.563760$

C $5.9496215 .137156-1.556909$

C $3.8151264 .544945-2.691515$

C $3.1850553 .235745-2.297139$

N $0.366406 \quad 3.239418 \quad 1.363698$

H $1.438380-3.784119-0.927902$

H $1.154327-3.475962-3.963175$

H $-0.682820-2.894946-1.519601$

H - $-1.355867-4.348701-3.769047$

H -1.665035 -2.739571-4.377559

H -4.825571 -2.639116 -2.291104

H $-4.042630-4.516656-1.261003$

H -2.994418 -5.472602 -2.313106

H $-2.298183-4.505544-0.997147$

H -3.342298 -1.427558 -4.659646

H -4.995477 -1.186605 -4.167760

H $-2.512543-0.084344-2.756720$

H -3.559086 $0.829432-3.799121$

H $-4.472986-0.451027-1.170678$

H $-6.3665760 .143433-2.739468$

H $-5.6700951 .732442-2.971428$

H -8.200705 $2.527097-0.805292$

H $-5.5300893 .356266-0.858127$

H $-7.862035-0.243602-0.778930$

H -7.7201880 .7341610 .664554$

H $-6.396169-0.3509730 .200469$

H -7.0905713 .3151340 .747689$

H $-3.384367 \quad 3.8742300 .035764$

H -3.6593943 .2225491 .656323$

H -3.521278 0.6831961 .446449

H $-0.255421 \quad 1.146009-3.165690$

H $2.2500151 .007288-1.375677$

H $-0.0476983 .686201-2.555801$

H $0.5847653 .209597-4.150216$

H $1.1497734 .676965-3.361537$

H -1.713662 1.6231811 .268206

H - $1.4582782 .388793-0.312776$

H 2.1634403 .3967952 .359759

H 0.9906824 .6761422 .744937

H 1.2736845 .6130310 .356643

H 3.9711094 .3555421 .083095

H $4.505463 \quad 6.624710 \quad 0.019824$

H $3.0609786 .525122-0.959167$

H $6.5059074 .630712-2.338757$

H $6.5232135 .609004-0.765975$

H $4.4800444 .341722-3.541461$

H $3.0905235 .277573-3.056310$

H $3.8798722 .541095-1.821449$

H 0.6480402 .3903370 .881402

C $4.235439-3.543754-1.986356$

O $4.149560-2.965223-0.665777$

C $4.441381-1.557707-0.771073$

C $5.138378-1378761-2.113462$

C $4.373883-2.373899-2.963875$

H $3.478300-1.039006-0.818301$

C $5.241170-1.0595960 .435905$
H $5.069360-0.352006-2.485326$

H $6.198015-1.655231-2.060296$

C $5.074895-2.732673-4.262223$

H $3.392715-1.943445-3.198840$

C $4.529638-1.2458031 .793357$

O $6.481630-1.7736800 .476900$

H $5.495118-0.0023970 .288632$

H $4.494148-2.3080232 .064278$

O $5.319994-0.5948762 .795628$

C $3.106912-0.6569621 .846978$

C $2.492712-0.6684383 .260130$

H $2.465591-1.2344471 .175116$

O $3.1241100 .687693 \quad 1.365384$

H $2.583363-1.6762163 .678690$

H 3.0620010 .0073353 .909400

H $5.204478-1.843165-4.887354$

H $6.065821-3.161318-4.078991$

H $4.491137-3.463029-4.831201$

C $3.056614-4.487906-2.250839$

H $7.061002-1.3200641 .111842$

H $6.140428-1.1054622 .899445$

H $3.7249421 .196821 \quad 1.936121$

H $3.146510-5.358802-1.589216$

H $3.106967-4.870569-3.276845$

H $5.156751-4.141190-1.995153$

C $-0.039952-2.3315432 .802657$

O $0.213189-0.9934032 .364902$

C $1.014726-0.2114553 .257012$

C $0.358772-0.1477284 .643777$

C $0.026922-1.5350335 .188185$

C $-0.760723-2.3394404 .154035$

H $0.915156-2.8624622 .900239$

C $-0.807182-3.0696481 .701724$

H 1.0042880 .8092062 .857266

O $1.1941520 .532841 \quad 5.574001$

H -0.5754590 .4250564 .573385$

H $0.931024-2.0723515 .497995$

O $-0.782082-1.3997406 .358823$

H -1.768116 -1.9157884 .059858$

O $-0.899637-3.6837664 .605826$

C $-2.133311-2.4289211 .365022$

H $-0.220463-3.0729500 .777421$

H $-0.961927-4.1216601 .968142$

H $1.369445 \quad 1.419228 \quad 5.214353$

H $-0.285638-0.8518796 .990757$

H -1.328503 -3.653525 5.478242

O $-3.154884-3.1882321 .852747$

O $-2.245462-1.3759750 .750698$

C $-4.471500-2.6862991 .604777$

H -4.642270 -1.783963 2.198569

H $-5.189927-3.4505391 .912814$

H -4.615245 -2.482176 0.539542

SCF Energy $\left(B 3 L Y P / 6-31 G^{* *} / / M M F F\right)=-3245.90682869$

05_00118

MM̄FF Geometry

C $3.401939-2.7705872 .125498$

C $2.590437-2.4533881 .105068$

C $1.090317-2.4475791 .199484$

O $0.631324-1.1735120 .694251$

C $0.413288-3.6132440 .442535$

C $0.547239-3.612785-1.070045$

C $-0.372151-3.114912-1.922163$

C $1.761159-4.317706-1.622912$

C $-1.688854-2.462023-1.597577$

C $-2.847279-3.243593-2.228128$

C $-4.249606-2.756481-1.831076$

C $-4.530971-1.310738-2.242307$

C $-5.943364-0.869834-1.821455$

O $-4.405159-2.903178-0.414218$

O $-6.0175850 .558144-1.952519$

C $-6.143019-1.197269-0.324809$

C $-7.024659-1.441639-2.745704$

O $-7.487416-0.9477720 .076406$

C $-5.739469-2.6400180 .053215$

C $-5.760492-2.9037891 .580259$

O $-6.672646-3.557447-0.533671$

C $-0.535579-0.6870061 .193868$ 
O $-1.242872-1.2005632 .046272$

C $-0.831814 \quad 0.5814330 .491647$

C $-2.089381 \quad 1.0465300 .506605$

C $-2.5736782 .279734-0.113434$

C $-1.5631063 .189386-0.755845$

C $-4.940140-1.9286302 .431877$

C $-5.768629-0.7888393 .006910$

C -5.5453421 .5011293 .967501$

O $-6.987346-0.8522683 .149771$

C -6.0221342 .3982922 .868739$

C -5.5079593 .6109712 .613413$

C -5.9846854 .4964361 .496545$

C -4.9011104 .7903260 .480824$

C -4.2552775 .9685250 .483421$

C $-4.6234683 .754078-0.587494$

C $-3.8931862 .552320-0.053532$

$\begin{array}{llll}\text { N } & -4.986040 & 0.291629 & 3.388821\end{array}$

H $2.973949-3.0482133 .085351$

H $3.028505-2.1602630 .155901$

H $0.811403-2.5271752 .259207$

H $-0.650295-3.6230410 .707770$

H $\quad 0.798997-4.5659550 .830470$

H $-0.168499-3.188527-2.990232$

H $1.751480-4.366117-2.717399$

H $2.683231-3.812409-1.331812$

H $1.800270-5.347771-1.253506$

H -1.850473 -2.381643 -0.521916

H -1.665617 -1.441579-1.995412

H $-2.754523-3.219402-3.321534$

H -2.759356 -4.295487 -1.923286

H $-4.957783-3.428264-2.331020$

H -4.391021 -1.177694 -3.321976

H -3.811865 - $0.637391-1.758349$

H $-5.8092810 .786050-2.874810$

H $-5.514423-0.4945670 .234807$

H -6.856660 -1.113957 -3.778841

H -8.016923 -1.066964 -2.471166

H -7.056776 -2.532863 -2.748140

H $-7.692167-0.025381-0.154293$

H $-6.797325-2.9685801 .932040$

H $-5.336573-3.9053111 .741959$

H -6.629718 -4.394941-0.042991

H $-0.0200601 .081627-0.023183$

H -2.848853 0.4627581 .024404

H -1.148820 $2.714373-1.650274$

H $-0.7451323 .417214-0.063493$

H -1.978763 4.151439-1.063746

H -4.537923 -2.470377 3.297336

H -4.087016 -1.538603 1.868555

H -4.7624561 .9667014 .574168$

H -6.3835041 .2368474 .620362$

H -6.834890 2.0266382 .248568

H -4.7010253 .9907053 .235760$

H -6.3482555 .4306561 .943998$

H -6.8510874 .0611840 .982764$

H -3.497182 $6.199570-0.257470$

H -4.467147 6.7271191 .229659

H -4.082997 4.197962 -1.431080

H -5.575897 $3.420469-1.019128$

H -4.5472841 .8250620 .425870$

H $-3.9915620 .283675 \quad 3.182432$

C $5.529423-1.6279392 .800569$

O $5.215237-0.3869582 .136561$

C 6.4108530 .4086992 .040098

C $7.412468-0.2283582 .993957$

C $7.056174-1.6957382 .854967$

H $\quad 6.783416 \quad 0.295273 \quad 1.015089$

C 6.0913281 .8814492 .317325

H $8.448000-0.0080972 .717889$

H 7.2587430 .1023954 .027488

C $7.596191-2.5617123 .979654$

H $7.450699-2.0637291 .898079$

C 4.9517182 .4554001 .450800

O 5.6983492 .0275133 .688880

H 6.9970642 .4870732 .200539

H 3.9989661 .9956541 .741911

O 4.8304793 .8516491 .750553

C $5.1458032 .298586-0.069257$
C $3.9657192 .891691-0.857729$

H $5.2365031 .240193-0.321455$

O $6.3437262 .949941-0.484910$

H $3.0409892 .432965-0.490599$

H $3.8978053 .966905-0.652662$

H $8.690121-2.5205594 .005121$

H $7.224935-2.2301614 .955208$

H $7.300589-3.6067893 .844147$

C $4.902006-2.7871982 .026839$

H $4.932478 \quad 1.4465823 .838212$

H 4.7013903 .9315862 .711330

H $7.0940782 .468234-0.098736$

H $5.256735-3.7458512 .421717$

H $5.207295-2.7389030 .975220$

H $5.108704-1.5642883 .812443$

C $3.1972590 .458414-2.546333$

O $4.3047501 .338878-2.767398$

C $4.0887412 .701527-2.387923$

C $2.9055143 .275341-3.178360$

C $1.6494712 .431137-2.981751$

C $1.9406360 .960443-3.273173$

H $3.0064430 .369586-1.469612$

C $3.592543-0.926683-3.086925$

H $4.9844353 .251173-2.703658$

O $2.6645384 .620742-2.781716$

H $3.1622853 .291815-4.245581$

H $1.2373722 .554475-1.974455$

O $0.6362052 .866620-3.890897$

H $2.0556840 .825204-4.356621$

O $\quad 0.823078 \quad 0.172556-2.864624$

C $4.699909-1.588053-2.295273$

H $2.729328-1.600726-3.061510$

H $3.946810-0.829306-4.120463$

H $1.9981554 .991518-3.384089$

H $\quad 0.3689713 .762459-3.624734$

H $0.0376020 .523933-3.317958$

O $4.986372-2.795592-2.867924$

O $5.242965-1.120761-1.304947$

C $6.011911-3.557143-2.223112$

H $5.673387-3.876019-1.233213$

H $6.207623-4.445168-2.829904$

H $6.934786-2.974106-2.147984$

SCF Energy (B3LYP/6-31G**//MMFF) $=-3245.91064904$

05 00119

MM̄MF Geometry

C $0.825280-0.6331872 .292500$

C $-0.066467-0.4937931 .300040$

C $-0.737054-1.6632030 .627482$

O $-2.113041-1.6292241 .081967$

C $-0.732782-1.564442-0.911747$

C $0.615996-1.334541-1.558526$

C $0.951133-0.230246-2.256142$

C $1.602779-2.462142-1.407489$

C $0.0970470 .979843-2.519092$

C $0.5948352 .186691-1.716611$

C $-0.5012553 .240097-1.507042$

C $0.0767794 .482818-0.827702$

C $-1.0108195 .507977-0.479159$

O $-1.5062102 .652753-0.671400$

O -0.4400996 .4663930 .424854$

C -2.1458354 .7914930 .280904$

C $-1.4707526 .293917-1.712511$

O

C $-2.6355353 .495537-0.409861$

C -3.5915532 .7312780 .543602$

O $-3.3234123 .838621-1.610704$

C $-2.858697-2.7581990 .963139$

O $-2.496344-3.8459780 .547080$

C $-4.229230-2.4144521 .400082$

C $-5.260343-3.1816301 .019402$

C $-6.672549-2.9355371 .321138$

C -7.008235 -1.723039 2.150542

C -4.0447991 .3459640 .058439$

C $-5.267751 \quad 1.359142-0.843616$

C $-6.705117-0.179297-2.183282$

O $-5.8903202 .380832-1.119389$

C $-7.897585-0.574219-1.369177$ 
C $-8.620484-1.680403-1.603709$

C $-9.847965-2.058722-0.823597$

C $-9.806959-3.482760-0.313493$

C $-10.455033-4.466808-0.959595$

C $-9.089240-3.7485880 .991180$

C $-7.593651-3.7884750 .825944$

N $-5.5829540 .086187-1.301689$

H $1.073704-1.6406332 .622055$

H -0.3721790 .4973860 .979001$

H $-0.277227-2.6065480 .949358$

H -1.464005 $-0.806307-1.207350$

H -1.149588 -2.491765 -1.327473

H $1.950910-0.175129-2.684821$

H $2.530722-2.277599-1.959149$

H $1.866632-2.605226-0.355158$

H $1.174940-3.394923-1.788883$

H $\quad 0.1442921 .214175-3.589832$

H $-0.9575080 .786218-2.314122$

H $0.948117 \quad 1.850612-0.735275$

H $1.4501372 .630781-2.239846$

H $-0.9379563 .495398-2.479548$

H $0.8520274 .943067-1.452834$

H $\quad 0.579718 \quad 4.1870980 .103643$

H $0.3285556 .870545-0.013076$

H -1.757219 $4.527125 \quad 1.274769$

H $-0.6275206 .836662-2.156514$

H -2.208470 $7.057361-1.442242$

H -1.899432 $5.658894-2.490473$

H -2.8963926 .4650210 .945783$

H -3.058666 2.5664921 .489836

H -4.4708883 .3467060 .767456$

H -3.836326 $3.070341-1.905304$

H $-4.354512-1.5143661 .990834$

H -5.056021 -4.0611580 .410093$

H -6.622876 -0.8122851 .678755$

H $-6.570836-1.8111303 .151173$

H -8.080212 -1.5659542 .283891$

H $-3.2298770 .818785-0.447442$

H -4.3230920 .7567590 .940811$

H -6.395334 -0.970128 -2.873609

H $-6.9354370 .720885-2.762068$

H -8.197672 $0.096521-0.567485$

H $-8.337723-2.333660-2.426273$

H -10.714107 -1.926961 -1.485444

H -10.013482 -1.375845 0.018965

H $-10.454857-5.485064-0.585212$

H -10.995804 -4.276868 -1.880852

H $-9.395245-4.721901 \quad 1.398005$

H -9.420317 -3.0212741 .737308$

H -7.247945 -4.634335 0.229699

H -5.045462 $-0.708578-0.967769$

C 1.8040351 .7673202 .304432

O 2.7535461 .5049471 .250406

C 3.8586712 .4173081 .375214

C 3.3460333 .5616722 .239071

C 2.4504702 .8213733 .210480

H 4.6453661 .8810881 .920180

C $4.3866332 .839850-0.000244$

H 4.1567184 .1074552 .731194

H 2.7554124 .2800431 .659325

C 1.4458303 .7178843 .914860

H 3.0827602 .3349723 .965535

C $4.6757471 .670439-0.961110$

O $3.4098353 .679397-0.627207$

H 5.2795203 .4627530 .128668

H $3.731917 \quad 1.188488-1.240617$

O $5.1991652 .231451-2.173170$

C $5.6575380 .605494-0.435712$

C $5.927803-0.482421-1.491501$

H 5.2522320 .1384180 .465154

O $6.899103 \quad 1.216718-0.090381$

H $4.968710-0.913495-1.798448$

H $6.371892-0.016689-2.379549$

H 1.9604194 .4822334 .506278

H 0.7936594 .2297493 .198921

H 0.8119603 .1360234 .591466

C 1.5077660 .4674863 .059350

H $3.7585013 .930609-1.499607$
H $\quad 6.0711612 .610124-1.967318$

H $\quad 6.749215 \quad 1.771745 \quad 0.693120$

H $0.9065770 .676863 \quad 3.952124$

H 2.4617110 .0574473 .413280

H 0.8999322 .1709111 .834868

C $5.275987-3.037328 \quad 0.066527$

O $6.446842-2.223270 \quad 0.199217$

C $6.888891-1.592702-1.006670$

C $7.193916-2.657135-2.070774$

C $5.992413-3.567925-2.310912$

C $5.486160-4.135183-0.986079$

H $4.423214-2.406386-0.208184$

C $4.990573-3.6686731 .439397$

H $7.840407-1.103463-0.763092$

O $7.591060-2.030186-3.285267$

H $8.040349-3.267654-1.729890$

H $5.189992-3.048494-2.847536$

O $6.385521-4.666106-3.137139$

H $6.202830-4.879046-0.614009$

O $4.249900-4.811448-1.209245$

C $4.531568-2.6592762 .468325$

H $4.200213-4.4228321 .356243$

H $5.904062-4.1387731 .823187$

H $7.897392-2.726017-3.890556$

H $6.597292-4.310235-4.016399$

H $4.407189-5.485842-1.892182$

O $4.411722-3.2858583 .676550$

O $4.297313-1.4802532 .246864$

C $3.978970-2.4606894 .762900$

H $4.072457-3.0384715 .686217$

H $4.605413-1.5671214 .845471$

H $2.929290-2.1865834 .625060$

SCF Energy (B3LYP/6-31G**//MMFF) $=-3245.91649637$

05 00120

MM̄̄FF Geometry

C $-2.2149433 .402967-0.293641$

C $-1.7754162 .155526-0.072310$

C $-0.8559031 .422334-1.016073$

O $0.3252571 .007708-0.293489$

C $-1.5615080 .157785-1.539546$

C $-0.794495-0.569355-2.626384$

C $-0.312608-1.822984-2.517044$

C $-0.6119870 .187380-3.917469$

C $-0.418272-2.760988-1.348203$

C $0.760429-2.699201-0.365121$

C $2.121761-3.058708-0.981480$

C $3.179461-3.2259560 .112294$

C $4.575807-3.492847-0.471699$

O $2.513136-2.002709-1.862725$

O $5.535856-3.3087530 .580483$

C $4.882641-2.433106-1.552141$

C $4.726824-4.942959-0.946439$

O $6.094133-2.740320-2.236635$

C $3.738379-2.242343-2.568619$

C $3.961135-1.042578-3.522414$

O $3.637174-3.413463-3.388207$

C $1.2895021 .944666-0.084096$

O $1.2727633 .113465-0.435164$

C $2.3807861 .298198 \quad 0.675213$

C 3.2573462 .0571791 .348713

C 4.3777161 .5691112 .155394

C 4.6991950 .0997322 .082509

C $4.1096140 .322333-2.841135$

C $5.5511260 .735231-2.592827$

C $6.8975982 .492679-1.448733$

O $\quad 6.5144640 .145642-3.074852$

C $7.3584121 .975410-0.122462$

C 7.7385282 .7748220 .885942

C 8.2443802 .2741842 .208507

C 7.4981792 .8705533 .381061

C 7.9914823 .9273214 .048663

C 6.2252632 .1890803 .829862

C 5.0684202 .4548092 .903469

N $5.6338471 .870997-1.798044$

H -1.874024 $3.930032-1.181201$

H $-2.100528 \quad 1.6244050 .817048$

H $-0.5629042 .065974-1.854303$ 
H $-2.5482410 .420404-1.944085$ H -1.746242 -0.509776 -0.692358 H $0.218236-2.241037-3.371961$ H $-0.206079-0.444276-4.715060$ H $-1.5728880 .577020-4.269116$ H $\quad 0.078249 \quad 1.025273-3.781215$ H -1.349829 -2.591709 -0.798139 H $-0.503579-3.782135-1.741531$ H $0.806388-1.6932350 .067977$ H $0.544113-3.3960380 .454046$ H $2.009905-3.989397-1.549969$ H $2.896848-4.0256600 .808318$ H $3.224948-2.3090930 .715249$ H $5.309477-3.9191421 .302933$ H $5.051818-1.486135-1.026310$ H $4.566971-5.637174-0.112614$ H $5.743234-5.137358-1.305940$ H $4.022948-5.210975-1.736990$ H $6.792475-2.826910-1.565240$ H $4.797993-1.250132-4.199999$ H $3.074316-0.976049-4.169474$ H $3.175989-3.176081-4.209769$ H $2.4208800 .215340 \quad 0.674032$ H 3.1334473 .1391611 .325489 H $3.898857-0.4922032 .539166$ H $5.630309-0.1703202 .584632$ H $4.818706-0.2142101 .040098$ H $3.6854501 .087916-3.503473$ H $3.5494750 .356391-1.902145$ H $6.7344183 .574889-1.437563$ H $7.6439952 .259425-2.214796$ H 7.4067800 .8965050 .005139 H 7.7162783 .8531810 .743910 H 9.3096412 .5315242 .276331 H 8.2011581 .1794022 .267074 H 7.4770304 .3444214 .907951 H 8.9190274 .4025993 .746648 H 5.9323272 .5549524 .823244 H 6.4233881 .1235263 .974315 H 4.7602283 .5012802 .883094 H $4.7855082 .264911-1.401553$ C $-4.577115 \quad 4.291196 \quad 0.064245$ O -5.2182082 .9990600 .014240$ C $-5.8085272 .834880-1.286245$ C $-5.9717234 .246342-1.838732$ C $-4.7029054 .905224-1.334218$ H $-5.0708412 .296893-1.895078$ C $-7.1003902 .014931-1.220923$ H $-6.0595384 .260044-2.929317$ H -6.848265 $4.751785-1.417522$ C $-4.7561356 .423288-1.331367$ H -3.881516 4.587798 -1.986062 C $-7.0079980 .698332-0.424717$ O $-8.1229662 .808107-0.603212$ H $-7.4466101 .819730-2.243282$ $\begin{array}{llll}\text { H } & -7.029970 & 0.919427 & 0.649547\end{array}$ O $-8.209197-0.040437-0.689277$ C $-5.786806-0.189587-0.723756$ C $-5.913465-1.578203-0.065373$ H $-4.883990 \quad 0.322629-0.375068$ O $-5.670945-0.357982-2.136344$ H -6.391459 -1.467592 0.913225 H -6.570812 -2.205178 -0.680001 H -4.902356 $6.805180-2.347036$ H -5.578632 $6.795635-0.711432$ H -3.822955 $6.842788-0.942336$ C $-3.153564 \quad 4.1456940 .616722$ H $-8.9376192 .276841-0.596322$ H -8.184734 -0.314164 -1.622451 H -4.838442 $-0.827497-2.312097$ H -3.2032083 .6326821 .585670$ H -2.730248 5.1393190 .804838 H $-5.160073 \quad 4.8799760 .784276$ C -3.990200 -1.476960 2.307075 O $-3.652245-1.5296420 .918068$ C $-4.550955-2.2876700 .099498$ C $-4.648659-3.727096 \quad 0.623940$ C $-5.041397-3.7701752 .098734$
C $-4.110749-2.8786812 .921518$

H $-4.949977-0.9569132 .415639$

C $-2.965976-0.5857923 .026746$

H $-4.092352-2.338681-0.895515$

O $-5.582939-4.468286-0.153672$

H $-3.672440-4.2150240 .503416$

H $-6.092783-3.4917862 .234835$

O $-4.921497-5.1188402 .556897$

H $-3.132413-3.3665362 .983803$

O $-4.604966-2.7865314 .256280$

C $-1.527123-1.0452372 .904075$

H -3.0132100 .4186882 .591353$

H $-3.216432-0.5159694 .091046$

H $-5.629219-5.3636140 .223418$

H $-5.129864-5.1211673 .507014$

H $-5.480072-2.3638984 .222624$

O $-0.732359-0.2000563 .626115$

O $-1.141162-2.0101432 .260260$

C $0.665652-0.5078093 .619419$

H $\quad 0.854389-1.3692314 .266174$

H $1.020223-0.7072732 .604198$

H 1.2066900 .3563554 .014137

SCF Energy (B3LYP/6-31G**//MMFF) $=-3245.91018417$

$05 \_00121$

MM̄MF Geometry

C -3.343089-2.854936-1.102496

C $-2.419892-3.811905-1.284569$

C $-0.926351-3.638159-1.096740$

O $-0.640885-2.234414-0.906158$

C $-0.479644-4.4267110 .150448$

C $1.016199-4.6450210 .262001$

C $1.804336-4.1463091 .235120$

C $1.616669-5.548373-0.787321$

C $1.422776-3.2853882 .406673$

C $1.775534-1.7989062 .249777$

C $3.282480-1.4937562 .211806$

C 3.5195800 .0187882 .254159

C 5.0057130 .3830012 .116411

O $3.836307-2.0299061 .007467$

O $5.0585581 .791174 \quad 1.839056$

C $5.600373-0.3687830 .904070$

C $5.759106 \quad 0.1712503 .434727$

O $7.013348-0.1987100 .838142$

C $5.258139-1.8733110 .891781$

C $5.706400-2.596378-0.403641$

O $5.936753-2.5180621 .977593$

C $0.469727-1.700501-1.472033$

O $1.367918-2.287364-2.051288$

C $0.384896-0.236349-1.274875$

C $1.4953640 .511207-1.335057$

C $1.5596921 .963349-1.165694$

C $0.2621212 .726286-1.206550$

C $5.109762-2.053592-1.705171$

C $5.957614-0.980484-2.367798$

C $5.7922730 .897511-3.999619$

O $7.160065-0.848629-2.155190$

C $5.7171722 .132066-3.157865$

C $4.9608923 .197871-3.460723$

C $4.8900354 .436051-2.613260$

C $3.4978324 .700360-2.083089$

C $2.6897995 .591280-2.682150$

C $3.0761464 .001633-0.809543$

C $2.7677822 .543992-1.015247$

N $5.221695-0.213578-3.260447$

H $-3.027710-1.860877-0.797044$

H -2.749331 -4.810486 -1.563943

H - $0.440455-4.001838-2.009351$

H $-0.949043-5.4202020 .151192$

H $-0.865303-3.9157641 .038585$

H $2.860824-4.4113521 .210717$

H $2.671286-5.770774-0.591252$

H $1.082518-6.503844-0.815272$

H $1.560248-5.088867-1.778094$

H $0.352804-3.3649682 .620871$

H $1.923488-3.6835903 .298429$

H $1.296819-1.4166971 .342158$

H $1.331992-1.2637303 .098720$ 
H $3.751643-1.9772293 .076683$ H $3.103247 \quad 0.452737 \quad 3.171994$ H $2.971127 \quad 0.4914661 .428836$ H 5.9929432 .0542451 .789032 H 5.1813720 .0994280 .005322 H 5.3418410 .8146954 .218844 H $\quad \begin{array}{llll}6.812114 & 0.458782 & 3.340711\end{array}$ H $5.714524-0.8590553 .793515$ H 7.1915840 .7490330 .718104 H $6.801390-2.638586-0.450397$ H $5.378730-3.642791-0.320022$ H $5.991074-3.4689961 .785811$ H $-0.5936190 .183518-1.069585$ H $24491900.015286-1.501805$ H $0.3922743 .809614-1.160262$ H $-0.3787672 .442149-0.367080$ H $-0.2748522 .518170-2.139054$ H $5.046678-2.874782-2.430548$ H $4.092520-1.687847-1.541603$ H $5.2272160 .995072-4.931886$ H $6.8362950 .677710-4.245166$ H $6.3209212 .148612-2.253382$ H $4.3634443 .186885-4.369463$ H $5.2262895 .281019-3.228358$ H $5.5950614 .387988-1.773798$ H $1.7015525 .810781-2.292027$ H $2.9971896 .118048-3.579630$ H $2.2298374 .518798-0.343390$ H $3.8833404 .084872-0.070170$ H $3.6500151 .904291-1.004681$ H $4.217555-0.352720-3.323609$ C - $-5.493715-2.023995-2.115997$ O $-5.230545-0.697464-1.615001$ C $-6.4650580 .032057-1.532941$ C $-7.456865-0.740100-2.394371$ C $-7.016826-2.169563-2.148718$ H $-6.786378-0.017700-0.485550$ C $-6.2585861 .495439-1.938072$ H -8.494714 -0.552834 -2.102696 H -7.354244 -0.494919-3.457551 C $-7.518507-3.151393-3.193411$ H -7.386793 -2.481223-1.162576 C $-5.0832922 .202419-1.233572$ O $\quad-5.9950691 .545531-3.347575$ H -7.191738 2.050219-1.783933 H -4.137856 $1.798481-1.615611$ O $-5.1091733 .577460-1.643577$ C -5.0734912 .1363260 .306579$ C -3.917752 2.9688330 .894170 H -4.9782891 .0971190 .633607$ $\begin{array}{lllll}\text { O } & -6.297934 & 2.650310 & 0.827051\end{array}$ H -2.9892502 .6798600 .390778$ H -4.0936094 .0275940 .668672$ H -8.613119 -3.172130 -3.207585 H -7.174579 -2.880050 -4.197179 H -7.163962 -4.164353 -2.978101 C -4.826378 -3.064345 -1.212064 H $-5.8767392 .480669-3.587296$ H $-5.9056673 .982323-1.258838$ H -7.0101422 .0403410 .573062$ H -5.052111 -4.073766 - 1.574461 H -5.237782 -2.981049 -0.198547 H -5.070484 -2.070416 -3.127327 C -2.2629270 .9293852 .439810$ O -3.5261951 .4743682 .837736$ C -3.7658272 .8237982 .426529$ C -2.6892873 .7345343 .029990$ C -1.2885823 .2817022 .629458$ C -1.0955431 .7977012 .940567$ H $-2.232116 \quad 0.834292 \quad 1.347574$ C $-2.151465-0.4810853 .044581$ H -4.724261 3.1162232 .873206 O -2.9027775 .0821732 .623527$ H -2.772624 3.7116444 .124383 H -1.092479 3.5056321 .576108 O -0.3437074 .0473183 .381560$ H $-0.978120 \quad 1.673290 \quad 4.025057$ O 0.1086291 .3385622 .328261
C $-3.181328-1.4525512 .511478$

H -1.164095 -0.902312 2.828493

H -2.293795 -0.426997 4.130761

H -2.1866445 .6178483 .005832$

H 0.5430493 .8284753 .049062

H $\quad 0.8494641 .8222552 .730257$

O $-2.962725-2.6743843 .083258$

O $-4.066436-1.1808331 .713651$

C $-3.879980-3.7123982 .723392$

H $-4.914260-3.3560602 .755019$

H $-3.632343-4.0927431 .729627$

H -3.771280 -4.525203 3.446466

SCF Energy (B3LYP/6-31G**//MMFF) $=-3245.90751750$

05 00122

MM̄FF Geometry

C $-2.1548690 .275447 \quad 3.167610$

C $-0.876521-0.0733193 .373038$

C $-0.104086-0.9573202426492$

O $1.159764-0.3245612 .115085$

C $0.192395-2.307573 \quad 3.102605$

C $0.846649-3.3216472 .184083$

C $2.072646-3.8475592 .374801$

C $0.019153-3.7693271 .005696$

C $3.038169-3.5685063 .492478$

C $4.035048-2.4330663 .212376$

C $4.979775-2.7000472 .028851$

C $6.073378-1.6313511 .958391$

C $6.977129-1.8095730 .727509$

O $4.212094-2.6743590 .823486$

O $7.740087-0.6040040 .562683$

C $6.094649-1.959700-0.531252$

C $7.993077-2.9405440 .926920$

O $6.883638-2.292941-1.670143$

C $4.955346-2.985425-0.362273$

C $3.953450-2.987165-1.542318$

O $5.515201-4.302418-0.287580$

C $1.1369670 .737902 \quad 1.265447$

$\begin{array}{lllll}\text { O } & 0.157990 & 1.232346 & 0.729957\end{array}$

C 2.5276041 .2160671 .107561

C 2.7506532 .4154080 .550202

C 4.0580693 .0367260 .329957

C 5.2801462 .1944840 .582318

C $3.228663-1.660223-1.792861$

C $3.881640-0.788726-2.852432$

C $3.7187901 .479303-3.870323$

O $4.773506-1.186210-3.596958$

C $4.8146782 .323822-3.300471$

C $4.7964073 .665288-3.324176$

C $5.9100404 .528099-2.802946$

C $5.4288275 .570674-1.818656$

C $5.1893356 .833403-2.211029$

C $5.3085355 .169019-0.366057$

C $4.1003794 .315232-0.100222$

N $3.3168840 .479323-2.898749$

H $-2.678269-0.1150512 .299001$

H $-0.348914 \quad 0.3060994 .244633$

H $-0.661720-1.1133871 .495481$

H $-0.741040-2.7490263 .477173$

H $0.817561-2.1261263 .983596$

H $2.433940-4.5751211 .648613$

H $0.464728-4.6250290 .486909$

H - $0.980137-4.0729401 .334398$

H $-0.082091-2.9612700 .275197$

H $2.500215-3.3493454 .420938$

H $3.594158-4.4921833 .698445$

H $3.475968-1.5062473 .037025$

H $4.632009-2.2827124 .120484$

H $5.424628-3.6935252 .159162$

H $6.673698-1.6273672 .876665$

H $5.606440-0.6387521 .903816$

H $8.245256-0.4548361 .380304$

H $5.657901-0.974523-0.731532$

H $8.633700-2.7312811 .792114$

H $8.668533-3.0216170 .068126$

H $7.525385-3.9133011 .092009$

H $7.572582-1.611808-1.754824$

H $4.440746-3.361534-2.450570$ 
H $3.176486-3.728434-1.304816$ H $4.824095-4.945256-0.518100$ H $3.325704 \quad 0.576732 \quad 1.466297$ H 1.8936043 .0114160 .238350 H $5.213413 \quad 1.2503910 .031628$ H 5.3807151 .9731931 .650204 H 6.2109722 .6644710 .258406 H $2.222889-1.886216-2.168909$ H $3.110954-1.090538-0.865868$ H $2.8291592 .066840-4.118768$ H $4.0734300 .980112-4.777862$ H $5.6749731 .810471-2.877363$ H $3.9485704 .175588-3.775924$ H $6.3804505 .019813-3.664651$ H $6.7011963 .926064-2.338991$ H $4.8710647 .594275-1.506199$ H $5.3013897 .132891-3.247945$ H $5.215896 \quad 6.0630850 .265249$ H $6.2431924 .700029-0.046701$ H $3.1568854 .839357-0.260261$ H $2.6182380 .740443-2.208993$ C -3.478962 2.4296073 .350937 O -4.4178852 .0420552 .329250$ C -4.2631202 .9283341 .201703$ C -3.3420084 .0532361 .666839$ C $-2.453826 \quad 3.3259312 .658682$ H -3.7502592 .3570350 .419383$ C $-5.637805 \quad 3.3882780 .703537$ H -2.7851914 .5009860 .838137$ H -3.8972274 .8536092 .169621$ C -1.700574 4.247562 3.600610 H -1.7311282 .7277662 .091812$ C -6.5995082 .2347720 .353362$ O $-6.263815 \quad 4.173027 \quad 1.728126$ H $-5.5197834 .055259-0.157849$ H $-6.831014 \quad 1.6513871 .252705$ O $-7.8375232 .812614-0.082925$ C $-6.092931 \quad 1.294747-0.757542$ C $-7.0898230 .156968-1.043418$ H $-5.1395340 .853096-0.457952$ O $-5.8552162 .044207-1.946598$ H -7.180275 -0.447585 -0.133841 H $-8.0819280 .581080-1.240465$ H -1.037593 4.9132853 .038405 H -2.384846 4.8707014 .186028 H $-1.086572 \quad 3.6712324 .299892$ C -2.933428 1.1911194 .067717 H -6.3286903 .6177372 .524231$ H -8.145673 3.4047510 .624442 H $-6.6974132 .455132-2.206975$ H $-3.776035 \quad 0.6132204 .467035$ H -2.323138 1.5047134 .922749 H -4.0532743 .0162584 .080656$ C - $-5.048653-2.131476-1.199950$ O $-5.358995-1.180725-2.223434$ C $-6.715332-0.732912-2.250102$ C -7.657266 -1.929338 -2.449349 C $-7.430946-3.000730-1.384096$ C $-5.950407-3.369883-1.307264$ H $-5.160380-1.660975-0.215513$ C $-3.578003-2.538467-1.384946$ H -6.807178 -0.100332 -3.142242 O $-9.010610-1.489070-2.429151$ H -7.472907 -2.366516 -3.439325 H -7.807899 -2.685767 -0.404228 O $-8.159231-4.180164-1.734210$ H -5.680761 -3.954371 -2.196673 O $-5.731548-4.203984-0.170920$ C $-2.627409-1.371814-1.229521$ H -3.279457 -3.303609 -0.660452 H -3.449408 -2.948484 -2.394825 H -9.570172 -2.242361 -2.681813 H $-9.106075-3.969559-1.671637$ H -6.323932 -4.970713 -0.255527 O $-1.580996-1.527921-2.092162$ O $-2.771330-0.457234-0.430422$ C $-0.583947-0.501647-2.071766$ H $-0.019791-0.558260-3.006671$
H $-1.0327830 .493660-1.998265$

H $0.099499-0.681257-1.238712$

SCF Energy (B3LYP/6-31G**//MMFF) $=-3245.91918805$

05_00123

MM̄MF Geometry

C $3.9349380 .875871-2.549932$

C $4.4955422 .083512-2.386597$

C $3.8647853 .270043-1.697397$

O $2.4966672 .987195-1.329025$

C $4.6457613 .570162-0.401992$

C 4.0772674 .7226200 .402694

C 3.5070524 .6031661 .618740

C $4.1962546 .080383-0.240946$

C 3.3233893 .3581032 .444581

C 1.8816052 .8551392 .372389

C 1.5933041 .6559403 .287698

C 2.3322420 .3882032 .848473

C $1.929214-0.8361253 .687357$

O $\quad 0.175877 \quad 1.436437 \quad 3.232098$

O $2.406636-2.0103923 .013904$

C $0.389806-0.9337523 .722440$

C $2.598699-0.8285605 .066534$

O $-0.040749-1.9698544 .601090$

C $-0.297248 \quad 0.395404 \quad 4.099890$

C $-1.8362880 .339293 \quad 3.958922$

$\begin{array}{llll}\text { O } & -0.016675 & 0.704539 & 5.470208\end{array}$

C $1.5022453 .642528-1.985525$

O $1.6107544 .388314-2.944661$

C $0.2401353 .333589-1.276562$

C $-0.8408004 .106323-1.461178$

C $-2.1129003 .987213-0.741522$

C -2.2008782 .9323630 .329451$

C $-2.337788-0.1180472 .588151$

C -3.7918470 .2476552 .337794$

C -5.5124720 .1151950 .523429$

$\begin{array}{llll}\text { O } & -4.486868 & 0.857329 & 3.144803\end{array}$

C $-5.3249871 .097851-0.589965$

C $-6.0091262 .245781-0.706428$

C $-5.7820263 .226894-1.823101$

C $-5.6085724 .649243-1.333263$

C $-6.4590375 .615165-1.720612$

C $-4.4665394 .958472-0.388788$

C $-3.1154214 .839233-1.044033$

N $-4.208119-0.1838281 .087514$

H $2.9240220 .696099-2.197121$

H $5.5127312 .236129-2.742476$

H $3.9199524 .129656-2.375391$

H $5.6924633 .799445-0.643049$

H $4.6772442 .658280 \quad 0.203224$

H 3.1257795 .5063682 .094454

H 3.8680816 .8861490 .424430

H $5.2382716 .282702-0.509410$

H $3.5852146 .134186-1.146921$

H 4.0219652 .5729422 .147971

H 3.5755333 .6030343 .483946

H 1.1925353 .6696192 .635383

H 1.6312392 .5857021 .340159

H 1.8681981 .9316254 .312435

H 3.4189840 .5246332 .888413

H 2.0950310 .1790091 .795966

H $3.370519-1.9275172 .913840$

H $0.070841-1.2289062 .714318$

H $3.690304-0.8492604 .961938$

H $2.336268-1.7255795 .638028$

H 2.3436460 .0491905 .663636

H $0.412542-2.7864994 .329854$

H $-2.265309-0.3055254 .736942$

H $-2.227014 \quad 1.3455094 .164944$

H $-0.695477 \quad 1.3211625 .791763$

H $0.2533392 .507777-0.574636$

H $-0.7802584 .925926-2.175827$

H -3.160948 2.9086620 .846279

H -1.437916 3.0971661 .098546

H -2.053924 $1.937702-0.104483$

H $-1.7391460 .338905 \quad 1.792574$

H -2.243728 -1.206880 2.504310

H $-6.1832780 .491672 \quad 1.301747$ 
H $-5.919193-0.8210600 .129931$ H $-4.581167 \quad 0.851142-1.344257$ H -6.7727052 .4897990 .028311$ H $-6.6433103 .160448-2.500066$ H $-4.9050542 .948118-2.420271$ H $-6.3497216 .637867-1.375135$ H $-7.2845395 .406962-2.393237$ H -4.5644624 .3422490 .508248$ H -4.551074 $5.990894-0.022892$ H -2.948647 5.574480-1.832626 H $-3.542372-0.6712630 .495202$ C $4.786849-1.483055-2.201728$ O $3.489255-1.998038-1.841009$ C $3.488956-2.298731-0.433572$ C $4.952287-2.287496-0.003009$ C $5.514554-1.193383-0.887829$ H $2.962316-1.4737690 .062347$ C $2.760948-3.616065-0.134188$ H $5.070030-2.0799501 .064753$ H $5.444761-3.242872-0.219387$ C $7.028032-1.213362-1.004494$ H $5.204765-0.228158-0.469090$ C $1.261330-3.575629-0.509505$ O $3.428504-4.668819-0.829155$ H $2.854863-3.8211390 .938760$ H $0.833198-2.660157-0.088291$ O $1.116254-3.500014-1.925284$ C $0.462800-4.7797340 .024643$ C $-1.029790-4.809327-0.359040$ H $0.555279-4.8062531 .117215$ O $1.049905-5.986791-0.471135$ H -1.119514 -4.860357-1.449265 H -1.427448 -5.7670320 .000066$ H $7.491287-1.068573-0.023047$ H $7.387667-2.165786-1.408244$ H $7.377414-0.413208-1.664671$ C $4.642704-0.299408-3.164259$ H $3.004135-5.505394-0.575289$ H $1.532307-4.293658-2.303412$ H $\quad 0.595928-6.733462-0.044579$ H $4.059416-0.615389-4.037073$ H $5.634123-0.006791-3.529714$ H $5.292752-2.296950-2.737884$ C -2.179372 -2.329939-1.776835 O $-1.665522-2.409280-0.441166$ C $-1.869479-3.6556770 .230356$ C $-3.374787-3.954948 \quad 0.294287$ C $-3.993141-3.976482-1.098681$ C $-3.671610-2.681067-1.837581$ H -1.612185 -3.006183-2.426443 C $-1.955255-0.891353-2.260900$ H -1.528603 -3.509701 1.263313 O $-3.626130-5.198891 \quad 0.939321$ H -3.878226 -3.1815120 .887930$ H -3.665430 -4.851010 -1.673051 O $-5.414144-4.079734-0.982237$ H -4.267087 -1.865842 -1.412989 O $-4.061112-2.811811-3.204467$ C $-0.515949-0.666292-2.650141$ H $-2.581576-0.645295-3.126504$ H $-2.199303-0.164373-1.477998$ H -3.230326 -5.1529721 .826444$ H -5.604086 -4.885587 -0.471680 H - $-5.004574-3.048225-3.216065$ O $-0.345455-0.969270-3.968782$ O $0.350165-0.286425-1.873459$ C $0.978902-0.795190-4.478225$ H $0.961511-1.006346-5.550698$ H $1.3119550 .236316-4.330960$ H $1.661389-1.498610-3.994426$ SCF Energy (B3LYP/6-31G $\left.{ }^{* *} / / M M F F\right)=-3245.92251228$

\section{$05 \_00124$}

\section{MMFF Geometry}

C 0.9716555 .3471911 .002028

C $1.858998 \quad 4.7237690 .213201$

C $2.8984643 .762546 \quad 0.732163$

O 2.7284992 .4937760 .058378
C 4.3051984 .2943680 .403034

C 5.4284993 .4570250 .983139

C 6.3481072 .7965670 .252657

C 5.5040383 .4149582 .488357

C $6.4776992 .734739-1.243268$

C $5.7572621 .548197-1.902460$

C $6.2708220 .170270-1.452911$

C $5.687729-0.938405-2.332095$

C $6.107028-2.336800-1.850143$

O $5.870306-0.040984-0.096391$

O $5.268472-3.299301-2.508235$

C $5.826787-2.455998-0.335862$

C $7.548076-2.669735-2.254929$

O $6.355477-3.6722680 .185482$

C $6.355067-1.2601700 .482355$

C $5.897089-1.2796061 .961662$

O $7.787825-1.2907120 .489299$

C 1.7365551 .6748200 .503908

O $\quad 0.969791 \quad 1.881175 \quad 1.430921$

C $1.7292980 .474659-0.361241$

C $0.674426-0.353678-0.339851$

C $0.511408-1.558051-1.156783$

C $1.708574-2.037720-1.934051$

C $4.380400-1.2657942 .182881$

C $3.773328-2.6453822 .378578$

C $1.566602-3.7831042 .590742$

O $4.443663-3.6608262 .543693$

C $1.204121-4.3725321 .264282$

C $-0.052662-4.6909640 .919336$

C $-0.426586-5.314287-0.394952$

C $-1.514877-4.548372-1.112895$

C $-2.795657-4.948802-1.051950$

C $-1.108047-3.374361-1.972550$

C $-0.688387-2.175732-1.167318$

N $2.385214-2.6024302 .386219$

H 0.9958285 .1725022 .075032

H $1.8298004 .890914-0.860981$

H 2.7929903 .6174761 .814097

H $4.415148 \quad 5.3172290 .787935$

H $4.4042164 .368163-0.685383$

H 7.1131452 .2328040 .785886

H 6.4222462 .9354892 .844903

H 5.4869464 .4298782 .898784

H 4.6608792 .8542152 .902731

H $6.1251693 .664724-1.701868$

H $7.5466122 .685885-1.487737$

H $4.6833451 .627511-1.696827$

H $5.8864581 .643313-2.987766$

H $7.3652560 .171757-1.516823$

H $5.973545-0.794096-3.381539$

H $4.591365-0.878499-2.311074$

H $5.378594-3.186337-3.467854$

H $4.738182-2.512860-0.219339$

H $7.656460-2.636723-3.345861$

H $7.816255-3.690009-1.959193$

H $8.282674-1.982267-1.830664$

H $5.979739-4.399972-0.338945$

H $6.376826-2.1100202 .493656$

H $6.291716-0.3677602 .433218$

H $8.103548-0.7717591 .247467$

H $2.5832460 .318183-1.010017$

H $-0.163107-0.1149550 .314627$

H $2.577720-2.133758-1.274640$

H $1.952331-1.335803-2.738637$

H $1.570649-3.020740-2.388667$

H $4.170179-0.7087203 .105065$

H $3.867457-0.7502191 .365491$

H $\quad 0.685556-3.4728343 .161199$

H $2.125541-4.5170693 .180208$

H $2.013849-4.5676550 .565051$

H $-0.857671-4.5208441 .631058$

H $-0.762907-6.339695-0.192937$

H $0.444244-5.413442-1.055006$

H $-3.580318-4.422579-1.585448$

H $-3.088476-5.810356-0.461196$

H -1.952246 -3.057090 -2.599742

H $-0.343207-3.703403-2.681109$

H - $1.492781-1.756460-0.561328$ 
H $\quad 1.912507-1.7254462 .187982$

C -1.4785945 .6584100 .661487$

O $-1.6295334 .571590-0.274144$

C $-2.9671734 .592561-0.805322$

C $-3.7279095 .633761 \quad 0.008434$

C -2.6264136 .6161590 .353759$

H -2.892068 $4.932399-1.845859$

C $-3.5961543 .192729-0.768088$

H $-4.5462436 .086524-0.559553$

H -4.1527295 .1973310 .920589$

C -2.9829257 .5482191 .498446$

H -2.391699 $7.211630-0.538635$

C $-2.9342552 .175059-1.720337$

O -3.5574362 .6909740 .568297$

H -4.651730 $3.295876-1.042389$

H -3.037212 $2.518991-2.756821$

O $-1.5284742 .091887-1.458360$

C $-3.4755030 .735548-1.601317$

C $-5.0023630 .626744-1.722231$

H $-3.1331630 .289698-0.660446$

O $-2.898800-0.040389-2.658113$

H $-5.4731411 .248015-0.956046$

H $-5.3121401 .025690-2.696145$

H $-3.859420 \quad 8.152693 \quad 1.243151$

H -3.213846 6.9910672 .412742

H -2.1561278 .2302311 .719751$

C $-0.0925416 .280451 \quad 0.496238$

H -2.6212432 .6077960 .819613$

H -1.141349 2.966841-1.629744

H -1.933255 $0.058881-2.596309$

H -0.0204127 .2155921 .062301$

H $0.0859686 .520659-0.559150$

H $-1.594247 \quad 5.2336151 .667795$

C $-5.534873-0.9771670 .811757$

O $-5.025071-1.485200-0.425311$

C $-5.524484-0.824730-1.594006$

C $-7.055843-0.938182-1.641825$

C $-7.705205-0.420805-0.358485$

C -7.064650 -1.072837 0.867108

H -5.2350280 .0740730 .904580$

C $-4.826490-1.7027801 .965703$

H $-5.129492-1.382480-2.452217$

O $-7.585103-0.222374-2.752871$

H -7.338125 -1.990963 -1.771880

H $-7.6560360 .672296-0.292390$

O $-9.094889-0.757010-0.366918$

H -7.389488 -2.118904 0.915256

O $-7.530353-0.4271752 .049240$

C -5.322703 -3.104396 2.240275

H -3.747455 -1.766289 1.780824

H -4.966669 -1.127957 2.889347

H -7.168007 -0.579479 -3.555531

H -9.480434 - $0.369890-1.171678$

H $-8.501790-0.4763802 .043321$

O $\quad-5.236555-3.877453 \quad 1.122482$

O $-5.717277-3.4741653 .339571$

C -5.707623 -5.2212661 .265998$

H $-5.717350-5.6859730 .276645$

H $-5.031469-5.7853161 .914890$

H -6.726811 -5.232858 1.664536

SCF Energy $\left(B 3 L Y P / 6-31 G^{* *} / / M M F F\right)=-3245.91693993$

05 00125

MM̄FF Geometry

C $-1.3902965 .392929-1.330343$

C $-2.0331724 .720846-0.363715$

C $-3.1977973 .795938-0.608965$

O $-2.7990432 .460297-0.222790$

C $-4.384807 \quad 4.2192760 .275608$

C $-5.623867 \quad 3.3643200 .096621$

C -6.1771372 .6104541 .067123$

C $-6.2627553 .413554-1.267472$

C -5.7182412 .4497262 .490040$

C -4.7384391 .2882902 .720508$

C $-5.338310-0.1018072 .454479$

C $-4.371125-1.2035612 .894284$

C $-4.905131-2.6058802 .559255$

O $\quad-5.593250-0.226782 \quad 1.053765$
O $-3.818715-3.5351992 .695078$

C $-5.344804-2.6403631 .078642$

C $-5.985212-3.0549213 .550616$

O $-6.001643-3.8669890 .771109$

C $-6.239199-1.4498860 .675831$

C $-6.510532-1.371431-0.846285$

O $-7.512956-1.5785491 .319855$

C $-2.0876301 .737335-1.130581$

O $-1.7772182 .075665-2.261637$

C $-1.7121310 .457688-0.490169$

C $-0.719879-0.272520-1.021069$

C $-0.194813-1.533635-0.494304$

C $-0.938012-2.1735520 .648059$

C $-5.265366-1.251863-1.731966$

C $-4.770761-2.581461-2.276407$

C $-2.868436-3.577027-3.537203$

O $-5.413648-3.624670-2.196437$

C $-1.922593-4.207831-2.564996$

C $-0.643368-4.481888-2.860903$

C $0.313271-5.148449-1.914837$

C $1.599620-4.370382-1.752466$

C $2.677493-4.663178-2.499094$

C $1.652457-3.309599-0.676320$

C $0.912404-2.057844-1.061081$

N $-3.537623-2.457524-2.902238$

H $-1.7100205 .289013-2.363838$

H -1.689259 4.8151650 .664182

H -3.494787 $3.805512-1.664746$

H -4.6556685 .2610870 .057096$

H -4.0610014 .2056291 .321966$

H -7.073665 2.0413860 .822935

H -7.229548 $2.899163-1.292266$

H $-6.4382164 .451649-1.568102$

H $-5.6192932 .936193-2.012841$

H -5.2635383 .3771552 .854461$

H -6.6056202 .3038843 .119337$

H $-3.854767 \quad 1.4373512 .089020$

H $-4.401546 \quad 1.3378203 .763426$

H -6.282012 -0.1798203 .007138$

H -4.150862 -1.123832 3.966085

H $-3.409205-1.0680012 .381415$

H -3.480914 -3.470531 3.604836

H $-4.428824-2.6161980 .476570$

H $-5.583171-3.0763544 .570740$

H $-6.315931-4.0776923 .338709$

H $-6.860705-2.4024413 .555979$

H $-5.397228-4.5886951 .015597$

H -7.151528 -2.204877 -1.158122

H -7.109135 -0.465546 -1.021231

H -8.162490 -1.0502920.827046

H -2.2356140 .1715490 .414299$

H $-0.2204550 .101849-1.914183$

H -1.998462 -2.2874160 .398737$

H $-0.852861-1.5620211 .552748$

H $-0.579045-3.1742780 .896928$

H $-5.520713-0.642032-2.608236$

H -4.452588 -0.742410 -1.205333

H -2.355271 -3.188670 -4.422635

H $-3.612646-4.313048-3.858158$

H $-2.312043-4.473316-1.584944$

H $-0.267128-4.241655-3.853111$

H $0.532548-6.148669-2.311172$

H $-0.142758-5.314719-0.931068$

H $3.611348-4.126035-2.381626$

H $2.646053-5.441956-3.254050$

H $2.693561-3.019669-0.482858$

H $1.306023-3.738706 \quad 0.267922$

H $1.375894-1.518376-1.888386$

H $-3.050034-1.566768-2.872843$

C $1.1033795 .597028-1.450565$

O $1.3202094 .469788-0.576337$

C $2.6382054 .555578-0.013776$

C $3.4032845 .499161-0.930555$

C $2.3235006 .504220-1.281396$

H 2.5302635 .0166360 .976648

C 3.2740323 .1675710 .149358

H $4.2682895 .951673-0.436759$

H $3.7531634 .986383-1.834290$ 
C $2.6427307 .339213-2.509252$

H $2.1781407 .174892-0.423752$

C 2.4898232 .2246101 .086169

O $3.4161832 .547497-1.128777$

H 4.2836793 .3176700 .544621

H 2.3622092 .6930372 .069774

O 1.1757182 .0019050 .564215

C $3.1283640 .831971 \quad 1.264549$

C 4.5444240 .8868521 .860155

H $3.1308860 .291646 \quad 0.310998$

O $2.304126 \quad 0.0784122 .161836$

H 5.1969291 .4582821 .194730

H 4.5046581 .4262122 .814691

H $3.5520947 .927433-2.348441$

H $2.8009326 .710719-3.391982$

H $1.8257078 .032783-2.731436$

C $-0.2088186 .284462-1.067308$

H $2.5222162 .425597-1.492778$

H 0.7144162 .8572860 .546671

H 1.4116670 .0475931 .775952

H $-0.3390417 .209630-1.640110$

H -0.185182 $6.562597-0.005899$

H $1.0488325 .200873-2.472511$

C $6.026375-0.929603-0.099510$

O $5.127642-1.3302370 .938945$

C $5.157461-0.5097692 .110996$

C $6.577191-0.4745012 .696950$

C $7.613684-0.0542681 .657033$

C $7.476931-0.9059900 .395823$

H $5.7431050 .073607-0.441818$

C $5.813733-1.851389-1.307090$

H 4.519425-1.0100972.850056

O 6.6481150 .4101173 .810079

H $6.842455-1.4748413 .063474$

H 7.5394811 .0135051 .420370

O $8.924154-0.2559042 .191604$

H $7.829492-1.9210340 .615724$

O $8.313054-0.375247-0.629069$

C $6.283237-3.270764-1.083087$

H $4.752654-1.890924-1.581100$

H $6.349180-1.460131-2.180317$

H 5.9925870 .1106264 .463030

H $8.9818820 .258167 \quad 3.015461$

H $9.219629-0.346665-0.277991$

O $5.458676-3.909865-0.206389$

O $7.265967-3.753792-1.630883$

C $5.798333-5.2691570 .082209$

H $5.057022-5.6631490 .782589$

H $5.770772-5.869497-0.832179$

H $\quad 6.786183-5.3208190 .549925$

SCF Energy $\left(\mathrm{B} 3 \mathrm{LYP} / 6-31 \mathrm{G}^{* *} / / \mathrm{MMFF}\right)=-3245.91819350$

05 00126

MM̄FF Geometry

C $1.316774 \quad 3.361862-2.088653$

C $2.5581183 .868784-2.158914$

C $3.7516363 .425040-1.345262$

O $3.3479402 .553433-0.266816$

C $4.7500032 .652340-2.227190$

C $6.0230592 .275847-1.491047$

C $6.4071061 .012142-1.221991$

C $6.8959703 .428288-1.063540$

C $5.719016-0.266643-1.611161$

C $4.684601-0.704311-0.574315$

C $4.205756-2.154128-0.743575$

C $3.566084-2.412213-2.110499$

C $2.982369-3.829140-2.216310$

O $3.251126-2.389127 \quad 0.302425$

O $2.155808-3.853541-3.390442$

C $2.078368-4.081985-0.993326$

C $4.078497-4.876319-2.441668$

O $1.595268-5.422448-0.982672$

C $2.742456-3.7294040 .354407$

C $1.752348-3.8114861 .541005$

O $3.793167-4.6660030 .618961$

C 3.0645403 .1182240 .937466

O $3.112575 \quad 4.3016601 .229994$

C 2.6645362 .0218171 .847244
C 2.2939642 .2911053 .108004

C 1.8555751 .3051214 .100378

C $1.842409-0.1387843 .673298$

C $0.503037-2.9413851 .391226$

C $-0.309850-2.8510252 .671942$

C $-2.302189-1.6481173 .594769$

O $-0.051923-3.4948893 .684332$

C $-1.968792-0.2958984 .142093$

C -1.847119-0.027783 5.451293

C $-1.549751 \quad 1.3442635 .988295$

C -0.3895611 .3662586 .961997$

C -0.5668981 .7812838 .227952$

C 0.9757370 .9162086 .482566

C $1.4822691 .734798 \quad 5.324077$

N -1.368635 -1.965494 2.530639

H $1.1072302 .526187-1.426178$

H $2.7440954 .697629-2.839227$

H $4.2321944 .323012-0.938492$

H $5.0238703 .255042-3.103176$

H $4.2533701 .756838-2.617329$

H $7.3346910 .864979-0.669975$

H $7.8652573 .093720-0.677966$

H $7.0954774 .089131-1.913416$

H $6.4118294 .009574-0.273116$

H $5.260332-0.169703-2.600393$

H $6.489456-1.041180-1.715090$

H $5.093234-0.592950 \quad 0.439361$

H $3.816638-0.035125-0.611196$

H $5.066468-2.815965-0.591126$

H $4.281664-2.245621-2.924038$

H $2.754902-1.688031-2.271757$

H $1.715492-4.720382-3.422822$

H $1.199993-3.442390-1.134514$

H $4.625332-4.660989-3.367603$

H $3.651086-5.876199-2.574629$

H $4.806550-4.917475-1.628827$

H $2.318953-5.995266-0.676532$

H $1.456913-4.8547681 .713240$

H $2.284107-3.5036182 .452306$

H $3.972966-4.6635841 .573878$

H 2.6754061 .0145651 .445958

H 2.3004743 .3267013 .444781

H $2.847371-0.4650323 .382798$

H $1.170664-0.2694712 .819639$

H $1.504642-0.8289324 .447683$

H $\quad 0.783662-1.9261791 .089653$

H -0.154399 -3.359377 0.621897

H $-2.272000-2.4268494 .363129$

H $-3.301736-1.6293123 .149746$

H -1.8419230 .5106503 .423280$

H $-2.000240-0.8239926 .175893$

H -2.4621371 .7082046 .478461$

H -1.3470122 .0507225 .173910$

H $\quad 0.2541521 .8039298 .936887$

H -1.537453 2.1080408 .586390

H $0.926820-0.152566 \quad 6.262456$

H 1.7127561 .0127327 .290706

H 1.5436262 .8027945 .537814

H $-1.456601-1.4463421 .662317$

C $-0.9466384 .397406-1.933222$

O $-1.6393023 .272184-1.350790$

C -3.044525 $3.583692-1.277525$

C $-3.1761925 .055689-1.650863$

C $-2.0285675 .221243-2.627181$

H $-3.5430972 .987985-2.049975$

C -3.6016093 .2302730 .105367$

H -4.151649 5.286155 -2.089772

H -3.036335 $5.708000-0.780666$

C -1.648284 $6.671576-2.870036$

H $-2.3041334 .753470-3.581913$

C -3.4845201 .7389190 .485152$

O $-2.9020803 .989417 \quad 1.100137$

H $-4.6496913 .544760 \quad 0.170754$

H $-2.433742 \quad 1.4633830 .636531$

O $-4.132576 \quad 1.5639941 .751893$

C $-4.1264860 .774164-0.530933$

C $-4.173819-0.678494-0.018555$

H $-3.5562170 .819120-1.464400$ 
O $-5.454090 \quad 1.218102-0.809960$ H $-3.167004-0.9702740 .293831$ H -4.819051 -0.7283930 .866599$ H -2.492971 $7.224153-3.294262$ H -1.353366 $7.170298-1.940529$ H $-0.811746 \quad 6.745632-3.571458$ C $0.151972 \quad 3.899802-2.874004$ H $-1.956968 \quad 3.7726561 .023173$ H -3.7136362 .1783382 .378963$ H $-5.7552770 .763426-1.614414$ H $0.4847954 .712050-3.529777$ H $-0.229923 \quad 3.094742-3.511823$ H $-0.5113174 .969213-1.102286$ C $-2.684175-2.051534-2.308955$ O $-4.032217-1.566398-2.316416$ C $-4.715288-1.679562-1.063728$ C $-4.740647-3.146135-0.604835$ C $-3.354868-3.790419-0.619480$ C $-2.666683-3.543662-1.962826$ H $-2.091336-1.492302-1.574645$ C $-2.093496-1.788848-3.698102$ H $-5.757290-1.396580-1.258100$ O $-5.331776-3.2686120 .684982$ H $-5.384528-3.710683-1.291920$ H $-2.743380-3.4257230 .213632$ O $-3.515666-5.195947-0.419933$ H $-3.171514-4.121240-2.747717$ O $-1.317729-3.992658-1.895687$ C $-1.854026-0.309575-3.897202$ H $-1.128629-2.288254-3.838623$ H $-2.770155-2.156462-4.478963$ H $-4.691043-2.953147 \quad 1.344319$ H $-2.629352-5.581482-0.317012$ H $-1.331481-4.957867-1.782677$ O $-2.6550520 .165774-4.892413$ O $-1.0573790 .345896-3.237387$ C $-2.527843 \quad 1.563735-5.169893$ H $-1.5098011 .793773-5.497780$ H $-3.221467 \quad 1.814907-5.976667$ H -2.791418 2.150785-4.285787

SCF Energy (B3LYP/6-31G**//MMFF) $=-3245.92559545$

0500127

MM̄FF Geometry

C $-1.183547-1.8852722 .786543$

C $-1.420959-0.867746 \quad 3.629184$

C -0.7212360 .4720203 .647878$

$\begin{array}{llll}\text { O } & 0.297129 & 0.505692 & 2.615526\end{array}$

C -1.674134 1.6568823 .376642

C -2.8549191 .8497804 .306357$

C $-4.0801852 .210788 \quad 3.871920$

C -2.594197 1.6978455 .781610

C -4.4542412 .5680382 .460301$

C $-5.189300 \quad 1.433974 \quad 1.738343$

C $-5.176403 \quad 1.657138 \quad 0.218595$

C $-6.1474950 .714209-0.488872$

C $-6.0619230 .851860-2.016799$

O $-3.8399021 .407032-0.234162$

O $-6.749490-0.265943-2.599038$

C $-4.5850680 .750532-2.452803$

C $-6.7791872 .111851-2.516682$

O $-4.4447771 .033790-3.844148$

C $-3.6265311 .645721-1.631350$

C $-2.1582351 .263165-1.956204$

O $-3.8610533 .010676-1.970596$

C 1.3295041 .3754222 .774260

O 1.5693362 .0617633 .754460

C 2.0974261 .3849111 .509110

C $3.0939862 .268372 \quad 1.350418$

C 3.9235362 .4356390 .155288

C $3.6045691 .580575-1.042191$

C $-1.0858731 .990470-1.129688$

C $-0.646616 \quad 3.322320-1.715367$

C $0.8543485 .254586-1.235783$

O $-1.059427 \quad 3.754920-2.787947$

C $2.1591765 .034754-1.935218$

C $3.2961485 .659415-1.592013$

C $4.6036685 .466555-2.306859$
C $5.7436495 .138981-1.367386$

C $6.6400796 .076450-1.016155$

C $5.8909883 .706849-0.901810$

C 4.9126023 .3533150 .183013

N $0.2681883 .970676-0.896065$

H $-0.438295-1.7825102 .003184$

H -2.167582 -1.020394 4.405432

H $-0.2391050 .579447 \quad 4.627713$

H $-2.007008 \quad 1.5591752 .336364$

H $-1.0911172 .587406 \quad 3.398497$

H -4.8809052 .3267344 .600094$

H $-3.4534902 .006406 \quad 6.387024$

H -2.369852 $0.659726 \quad 6.040157$

H $-1.7458332 .322740 \quad 6.079987$

H -5.1044263 .4520142 .492099$

H -3.5743442 .8899401 .893763$

H $-4.706127 \quad 0.476474 \quad 1.968401$

H $-6.220067 \quad 1.3834332 .109444$

H $-5.4419712 .701150 \quad 0.013547$

H $-7.1753740 .885431-0.145687$

H $-5.913161-0.323252-0.218595$

H -7.667139 $-0.254032-2.276965$

H $-4.275830-0.294524-2.323443$

H $-7.8360482 .092111-2.224439$

H $-6.7731522 .162231-3.611006$

H $-6.3459373 .034953-2.125988$

H $-5.0483330 .440151-4.322760$

H $-2.0385630 .193058-1.744457$

H $-1.9589891 .406331-3.024698$

H -3.113337 $3.541876-1.656156$

H $1.8085810 .680306 \quad 0.737365$

H $3.3238092 .943996 \quad 2.173394$

H $2.5473671 .669171-1.315710$

H $3.8256880 .531228-0.824813$

H $4.1695381 .850437-1.936381$

H $-1.4160792 .141378-0.096945$

H $-0.1903951 .356081-1.100779$

H $0.9740485 .814284-0.302745$

H $0.1718845 .804695-1.891352$

H $2.1650514 .345475-2.776487$

H $3.2828096 .370992-0.769474$

H $4.8234186 .394194-2.851451$

H $4.5301624 .683657-3.071904$

H $7.4758145 .843906-0.364635$

H $6.5552227 .099285-1.368055$

H $6.8978273 .546096-0.493525$

H $5.8323923 .042293-1.767846$

H 5.0625953 .9239401 .100949

H $0.6024323 .503725-0.058280$

C $-2.685098-3.5825741 .623688$

$\begin{array}{llll}0 & -1.807333 & -3.708767 & 0.485967\end{array}$

C $-2.382950-2.974441-0.609811$

C $-3.860711-2.837179-0.268335$

C $-3.783672-2.593811 \quad 1.225917$

H -1.921003 -1.979592 -0.592824

C $-2.081682-3.646054-1.950992$

H $-4.342430-2.024171-0.813737$

H -4.414937 -3.760955 -0.470586

C $-5.097206-2.8090811 .956135$

H -3.457193 -1.559299 1.385633

C $-0.597892-4.000474-2.171596$

O $-2.828421-4.866496-2.034442$

H $-2.449703-3.007154-2.762989$

H $-0.335357-4.867508-1.553293$

O $-0.460232-4.438341-3.530153$

C $0.404597-2.863470-1.897608$

C $1.838763-3.268234-2.287299$

H $\quad 0.362487-2.600553-0.835578$

O $0.016090-1.714613-2.647909$

H $2.036177-4.272067-1.896109$

H $1.912697-3.325429-3.380209$

H $-5.861611-2.1177551 .587486$

H $-5.472224-3.8285091 .816953$

H $-4.976065-2.6370223 .030319$

C -1.881534 -3.216132 2.873473

H -2.631049 $-5.268668-2.897835$

H $-0.622271-3.669057-4.103115$

H $0.548139-0.963262-2.336173$ 
H -1.104501 -3.975402 3.028440 H -2.540675 -3.2506193 .749061$ H $-3.116954-4.5801341 .777116$ C $3.195992-3.1808150 .443313$ O $2.828093-2.061153-0.366744$ C $2.916894-2.285079-1.777830$

C $4.351198-2.684775-2.150568$

C $4.831395-3.895801-1.352062$

C $4.622644-3.6650470 .145758$

H $2.494694-4.0010450 .243539$

C $2.970607-2.8070151 .916927$

H $2.716849-1.319048-2.256911$

O $4.434849-2.955152-3.545955$

H $5.019902-1.838245-1.946594$

H $4.336840-4.813598-1.690762$

O $6.226113-4.071058-1.612997$

H 5.366331 -2.941549 0.496391

O $4.850804-4.881861 \quad 0.853620$

C $3.827933-1.6667342 .428290$

H $1.928489-2.4932232 .046313$

H $\quad 3.154617-3.6840512 .547755$

H $5.350220-3.223189-3.735717$

H $6.504861-4.895324-1.179789$

H $5.785232-5.1216430 .735558$

O $3.657923-1.5638363 .780667$

O $4.538531-0.9456921 .742619$

C $4.410743-0.5280164 .419941$

H $4.148009-0.5226965 .481086$

H $5.481886-0.7283984 .323323$

H $4.1612740 .447508 \quad 3.992978$

SCF Energy $\left(\mathrm{B} 3 \mathrm{LYP} / 6-31 \mathrm{G}^{* *} / / \mathrm{MMFF}\right)=-3245.91293453$

05 00128

MM̄FF Geometry

C 0.8311513 .1092573 .073039

C 0.7519731 .9105442 .473992

C $1.168220 \quad 0.624376 \quad 3.140672$

O $0.102159-0.3288422 .919994$

C $2.478561 \quad 0.0813742 .539593$

C 3.6783510 .9659542 .810233

C 4.3275221 .6930391 .879958

C 4.1646560 .9708514 .237448

C 4.0306231 .7995190 .409915

C $5.1697341 .230155-0.448532$

C $5.377529-0.280909-0.260747$

C $6.618736-0.756466-1.016856$

C $6.808597-2.274883-0.887476$

O $4.216894-0.957051-0.755961$

O $7.790894-2.680557-1.852602$

C $5.486772-2.972931-1.264354$

C $7.360297-2.6716850 .486889$

O $5.562707-4.375801-1.018343$

C $4.239444-2.377906-0.563560$

C $2.965779-2.975022-1.204440$

O $4.267246-2.719448 \quad 0.823128$

C $0.010214-1.3695663 .788147$

O $0.786021-1.6297414 .698426$

C - $-1.219907-2.1633533 .568398$

C -2.014701-2.042012 2.494026

C $-3.295659-2.7261812 .296437$

C $-3.793486-3.6225893 .400277$

C $1.652067-2.310902-0.775352$

C $0.518221-2.897255-1.591693$

C $-0.926248-4.920301-1.749429$

O $0.169871-2.398271-2.659797$

C $-2.308633-4.354246-1.706711$

C $-3.363874-4.994604-1.183371$

C $-4.767276-4.460348-1.227387$

C $-5.387102-4.3372980 .144356$

C $-6.016446-5.3821550 .708746$

C $-5.368210-2.9851230 .816868$

C -3.989989-2.493925 1.163165

N $-0.003539-4.055970-1.037641$

H 1.1910293 .1437304 .100172

H $\quad 0.4068921 .8313731 .448064$

H 1.2713510 .7836424 .221544

H $2.336444-0.1065991 .469943$

H $2.687970-0.9151892 .949417$
H 5.1832172 .2859852 .201563

H 5.0907181 .5428824 .359401

H $3.416207 \quad 1.4142634 .900808$

H $4.366467-0.0519744 .572627$

H $3.085573 \quad 1.324200 \quad 0.137755$

H 3.9107932 .8631600 .169672

H $4.9300961 .435944-1.499698$

H $6.099621 \quad 1.764692-0.216437$

H $5.493221-0.4960200 .806665$

H $7.514929-0.228228-0.668387$

H $6.516967-0.506673-2.082242$

H $8.611509-2.192218-1.668031$

H $5.358592-2.856730-2.349954$

H $8.323219-2.1794920 .669315$

H $7.560612-3.7478060 .535819$

H $6.693338-2.4079871 .310221$

H $6.346112-4.710062-1.487707$

H $3.039414-2.879453-2.296395$

H $2.907387-4.048238-0.981409$

H $3.363281-2.6874391 .173447$

H -1.454969-2.850737 4.375105

H -1.730808 -1.3512061 .701628$

H -4.760335 -4.081772 3.182895

H -3.914362 -3.0576354 .331167$

H -3.089926 -4.444361 3.574209

H $1.452885-2.4569390 .291431$

H $1.671053-1.231429-0.955700$

H $-0.870146-5.911047-1.286977$

H $-0.595981-5.004849-2.790321$

H -2.459751 -3.389186 -2.179449

H $-3.222175-5.977105-0.738730$

H $-5.367593-5.145423-1.840669$

H $-4.814040-3.493593-1.743940$

H $-6.498183-5.2984251 .677100$

H $-6.062142-6.3466040 .213577$

H $-6.003544-2.9781421 .711257$

H -5.835116 -2.2523190 .145272$

H -3.556322 -1.833346 0.414130

H $0.376584-4.398387-0.160083$

C 0.0568784 .5003571 .023803

O 1.1107514 .0300340 .156550

C $1.2274054 .922882-0.960144$

C $-0.0286825 .782008-0.955087$

C -0.2818105 .9119630 .535702$

H $2.0805915 .577870-0.734127$

C $1.5420494 .178225-2.262552$

H $0.1189656 .745501-1.451793$

H $-0.8720595 .274731-1.435553$

C -1.690685 6.3676810 .873557

H $0.435006 \quad 6.6331850 .951028$

C $0.4167853 .327082-2.890406$

O $1.9244865 .171658-3.223827$

H $2.4227893 .544140-2.101055$

H $-0.3677313 .985800-3.279794$

O $0.9705802 .673124-4.042559$

C $-0.2098162 .264774-1.972879$

C -1.248301 $1.396204-2.706025$

H $-0.6913152 .765121-1.131137$

O $0.8280651 .432957-1.461779$

H - $1.9539662 .055613-3.223494$

H $-0.744251 \quad 0.795153-3.472603$

H -1.879259 7.3654110 .463679

H -2.442792 5.6893650 .458644

H -1.838132 6.4145041 .956866

C 0.5194724 .4607432 .482874

H $2.1553484 .705542-4.045766$

H $1.6286382 .030552-3.725699$

H $0.4536630 .901081-0.739884$

H $-0.2443344 .926526 \quad 3.116531$

H 1.4320275 .0629582 .580477

H $-0.802951 \quad 3.8351130 .885457$

C $-3.6484721 .973865-0.893340$

O $-2.5441231 .108438-0.616509$

C $-2.0144450 .436793-1.765841$

C $-3.111770-0.380572-2.466176$

C $-4.3662690 .442586-2.749136$

C $-4.8160811 .185681-1.491321$

H $-3.3310662 .738979-1.613465$ 
C $-4.003971 \quad 2.7448380 .380341$

H -1.279894 -0.280853 -1.381482

O $-2.637260-0.937642-3.687536$

H -3.398586 -1.219617 -1.823028

H $-4.2108221 .139059-3.581515$

O $-5.421150-0.434862-3.150468$

H $-5.2096320 .460643-0.769303$

O $-5.8713412 .082928-1.824277$

C $-4.401048 \quad 1.8703051 .546677$

H -3.141636 $3.345703 \quad 0.692129$

H -4.8178003 .4573880 .203167$

H $-1.838009-1.453117-3.483983$

H $-5.104383-0.932392-3.923945$

H $-6.5809901 .557494-2.232037$

O $-3.354057 \quad 1.7473162 .412386$

O -5.5110281 .3725331 .681217$

C -3.6280831 .0305643 .620014$

H $-2.677040 \quad 0.832005 \quad 4.120972$

H $-4.249911 \quad 1.644838 \quad 4.277582$

H -4.1219300 .0777353 .410311$

SCF Energy (B3LYP/6-31G**//MMFF) $=-3245.92052597$

\section{5_00129}

MM̄FF Geometry

C -4.025632 -1.829522 -2.521801

C $-3.128207-2.197517-1.594925$

C $-1.759306-2.735211-1.924083$

O $-0.765877-1.860215-1.336917$

C $-1.584418-4.128626-1.291121$

C $-0.640934-5.012258-2.078917$

C $0.662555-5.203512-1.802163$

C $-1.276136-5.736566-3.240112$

C $1.497118-4.577253-0.718964$

C $2.612349-3.737170-1.342886$

C $3.733612-3.337912-0.373404$

C $3.243500-2.4698760 .785705$

C $4.401550-1.9290761 .639237$

O $4.682151-2.618444-1.169183$

O $3.881349-0.8891452 .481018$

C $5.444552-1.2743870 .710015$

C $4.966632-3.0017972 .577341$

O $6.603079-0.8796321 .440790$

C $5.851915-2.171733-0.474604$

C $6.731120-1.429418-1.509511$

O $6.612351-3.2814080 .015713$

C $-0.503336-0.684206-1.970974$

O $-0.962963-0.297304-3.033018$

C $0.4139890 .086308-1.103813$

C $0.5038141 .417438-1.240092$

C $1.2777702 .305599-0.371090$

C $2.395171 \quad 1.7018540 .436334$

C $6.047710-0.247687-2.209457$

C $6.4492631 .116922-1.675440$

C $6.0424523 .536978-2.121942$

O $7.320727 \quad 1.286049-0.827965$

C $5.2545594 .097216-0.981598$

C $4.3904905 .114878-1.115419$

C 3.7088155 .7906670 .037243

C $2.2035315 .777495-0.070611$

C $1.5424446 .788285-0.660646$

C 1.4613574 .6608550 .621757

C $0.9416703 .610709-0.321616$

N $5.7371072 .132363-2.301878$

H -3.771731 -1.910818 -3.575704

H -3.380586 -2.105437 - 0.542369

H $-1.602818-2.779207-3.009276$

H -2.549275 -4.651739-1.236494

H $-1.246882-4.035241-0.252476$

H $1.217053-5.892493-2.439637$

H $-0.560336-6.361205-3.785017$

H -2.080427 -6.389562 -2.885517

H - $-1.698231-5.022015-3.953989$

H $0.899705-3.957408-0.045998$

H $1.929236-5.383123-0.114033$

H $3.073275-4.285151-2.177250$

H $2.183335-2.835054-1.800830$

H $4.209452-4.2511680 .002937$

H $2.532824-3.0160301 .416822$
H $2.691329-1.6127920 .383820$

H $3.168941-1.2721163 .020804$

H $4.994611-0.3528630 .321302$

H $4.189344-3.3559133 .265379$

H $5.762905-2.5941793 .209731$

H $5.356893-3.8730842 .047434$

H $6.305708-0.3052672 .167035$

H $7.690423-1.146829-1.060341$

H $6.985405-2.154525-2.296352$

H $7.154853-3.630353-0.710611$

H $0.931248-0.451931-0.320158$

H $-0.1003651 .909233-2.001173$

H 3.0789222 .4503620 .844830

H $3.010071 \quad 1.045933-0.190119$

H $2.0015391 .119207 \quad 1.273259$

H $6.346767-0.258362-3.265996$

H $4.956267-0.336281-2.181075$

H $5.8175194 .041276-3.067287$

H $7.1124643 .651599-1.919011$

H 5.4568373 .6936460 .007561

H $4.2163015 .536581-2.102907$

H 4.0639956 .8297140 .067742

H 4.0184905 .3559130 .995741

H $0.4581706 .805915-0.699138$

H $2.0714257 .616374-1.120723$

H 0.5921165 .0845901 .144776

H 2.0715654 .2362811 .424800

H $0.1280333 .959645-0.958690$

H $5.0044961 .890344-2.962603$

C $-5.4865430 .206167-2.456601$

\begin{tabular}{lllll}
\hline & -4.648539 & 0.905568 & -1.513984
\end{tabular}

C $-5.3959641 .991265-0.936877$

C -6.597052 2.194795-1.849597

C $-6.8966650 .764777-2.257353$

H -5.7550381 .6419430 .038721$

C $-4.497188 \quad 3.217010-0.742728$

H $-7.4325082 .680767-1.336860$

H $-6.3432442 .793591-2.731901$

C $-7.7824220 .654474-3.486059$

H $-7.3871200 .255890-1.416407$

C $-3.180352 \quad 2.9147320 .000879$

$\begin{array}{llll}\text { O } & -4.150224 & 3.737357 & -2.032714\end{array}$

H $-5.0588114 .008736-0.233409$

H $-2.5351262 .310567-0.647782$

O $-2.491909 \quad 4.1569490 .191754$

C $-3.3389792 .212944 \quad 1.362357$

C -1.9768451 .8876091 .995210$

H -3.8900941 .2799321 .234842$

$\begin{array}{lllll}\text { O } & -4.078322 & 3.039974 & 2.256634\end{array}$

H -1.412082 1.2698281 .289691

H -1.4105832 .8160212 .137931$

H $-8.7553701 .121302-3.301586$

H $-7.3309321 .149978-4.352141$

H $-7.954812-0.393736-3.749627$

C $-5.392763-1.297418-2.195416$

H $-3.5735854 .507439-1.889165$

H -3.0120234 .6861640 .820689$

H -4.9840513 .1137501 .911959$

H $-6.124630-1.837733-2.806502$

H $-5.631691-1.506609-1.146327$

H $-5.1107690 .449227-3.458819$

C -2.451326 -1.0859712 .578315$

O $-2.936644 \quad 0.0267653 .337672$

C $-2.079254 \quad 1.172594 \quad 3.361714$

C -0.7177340 .7880793 .955678$

C $-0.091838-0.3846813 .206912$

C -1.077994 -1.543131 3.093130

H $-2.390074-0.8090121 .518829$

C -3.468359 $-2.231766 \quad 2.725372$

H $-2.545051 \quad 1.884608 \quad 4.054474$

$\begin{array}{lllll} & 0.183290 & 1.890297 & 3.928052\end{array}$

H -0.8455050 .5010325 .007450$

H $0.268204-0.0855572 .217889$

O $1.054621-0.8425663 .927438$

H -1.185658 -2.022127 4.074923

O $-0.547302-2.5207632 .199461$

C $-4.807452-1.9424052 .081642$

H -3.075425 -3.142082 2.258313 
H $-3.656649-2.4216043 .789082$ H -0.2339252 .6207434 .416044$ H $1.634515-0.0732954 .063376$ H $\quad 0.324909-2.7826862 .540634$ O $-5.612770-3.0360882 .234816$ O $-5.117541-0.9015401 .521310$ C $-6.928166-2.9158241 .683813$ H $-6.873012-2.8378750 .594189$ H -7.488603 -3.818058 1.943022 $\mathrm{H}-7.444128-2.0493542 .108415$

SCF Energy (B3LYP/6-31G**//MMFF) $=-3245.91562035$

05_00130

MM̄FF Geometry

C 4.0636553 .8952702 .276712

C 3.4560383 .0125193 .084662

C 3.9330731 .6309993 .454798

O $2.992231 \quad 0.7029822 .857570$

C $5.334371 \quad 1.2428262 .940324$

C $5.754362-0.1600483 .340930$

C $5.963481-1.1744102 .477651$

C $5.954635-0.3831404 .818421$

C $5.876990-1.1535020 .975885$

C $4.536041-1.6951210 .478630$

C $4.488168-1.921681-1.040118$

C $4.599869-0.618965-1.836620$

C $4.441777-0.853083-3.347537$

O $3.232995-2.558164-1.323122$

O $4.2524820 .436142-3.951234$

C $3.165533-1.685669-3.593913$

C $5.720846-1.431228-3.963584$

O $3.043259-2.039516-4.969063$

C $3.061178-2.938497-2.696929$

C $1.692828-3.651371-2.818609$

O $4.060292-3.884933-3.095250$

C 2.0464820 .1279223 .645562

$\begin{array}{lllll}\text { O } & 1.840110 & 0.335171 & 4.829367\end{array}$

C $1.317907-0.8437042 .798978$

C $0.477405-1.7310883 .351664$

C $-0.238909-2.7902352 .633934$

C $0.025739-2.919121 \quad 1.157084$

C $0.482173-2.752399-2.566222$

C $-0.803294-3.527556-2.330457$

C $-3.205077-3.107533-1.759898$

O $-0.861198-4.752865-2.298217$

C $-3.373969-2.868399-0.292722$

C $-3.835131-3.7825330 .574060$

C -3.996359 -3.513191 2.044526

C $-3.353256-4.5726302 .913951$

C $-4.096992-5.317226 \quad 3.749714$

C -1.856802 -4.7801942 .816802$

C -1.069757 -3.600575 3.323529

N $-1.875848-2.667503-2.140917$

H 5.0267463 .6649681 .832046

H 2.4822033 .2816003 .491866

H 3.9139801 .5550074 .548395

H 6.0826351 .9486573 .323533

H $5.346589 \quad 1.340237 \quad 1.850337$

H $6.247832-2.1431652 .887656$

H $6.393102-1.3634125 .034557$

H $\quad \begin{array}{lll}6.632191 & 0.373142 & 5.228007\end{array}$

H $5.001566-0.3258725 .352478$

H $6.059465-0.1505570 .581609$

H $6.692742-1.7762570 .587282$

H $4.308093-2.6461390 .979155$

H $3.727682-1.0133070 .767698$

H $5.299606-2.609172-1.306197$

H $5.549125-0.108444-1.637881$

H $3.8129190 .074534-1.507635$

H $4.0788380 .295329-4.897996$

H $2.323107-1.017856-3.378931$

H $6.563052-0.747133-3.803352$

H $5.626227-1.536215-5.049929$

H $5.997624-2.401433-3.545780$

H $3.658271-2.772639-5.141841$

H $1.598092-4.122896-3.805450$

H $1.677986-4.478789-2.095602$

H $3.790851-4.764883-2.783025$
H $\quad 1.524049-0.824777 \quad 1.734622$

H $0.316770-1.6986734 .428163$

H $-0.282948-2.0066570 .635106$

H $-0.506850-3.7458010 .685998$

H $1.091472-3.0905860 .967849$

H $0.656341-2.129948-1.682218$

H $\quad 0.322642-2.092954-3.426977$

H -3.342644 -4.159311-2.029249

H -3.920712 -2.497856 -2.319750

H -3.104593 -1.881260 0.075880

H $-4.129338-4.7637580 .209557$

H -5.072486 -3.4534232 .252145$

H -3.583764 -2.533609 2.314187

H -3.650079 -6.081052 4.377460

H -5.170606 -5.180696 3.826762

H $-1.599507-5.0569241 .791622$

H -1.556769 -5.6476863 .420243$

H -1.189975 -3.427992 4.393961

H -1.704294 -1.666028 -2.149613

C 2.5826125 .0937750 .602218

O 1.4650674 .2159220 .856655

C $1.4256583 .212883-0.179003$

C $2.1557913 .837439-1.355651$

C $3.2889044 .557095-0.647439$

H $1.9849572 .351703 \quad 0.209506$

C $-0.0316682 .823189-0.437992$

H $2.5091163 .096056-2.078389$

H $1.5109684 .554312-1.878610$

C $3.9468965 .638516-1.486681$

H $4.0497073 .813303-0.382365$

C $-0.2181461 .859640-1.621376$

O -0.5338322 .1945860 .747107$

H $-0.6284003 .729555-0.589549$

H $0.0663332 .359535-2.554795$

O $0.6908350 .764125-1.454204$

C $-1.6376831 .273582-1.753154$

C $-2.7364372 .338476-1.905672$

H $-1.8453040 .620894-0.898034$

O $-1.6601840 .429484-2.910698$

H -2.679172 $3.049841-1.078090$

H $-2.5556192 .902755-2.828756$

H $4.3932025 .207238-2.388688$

H $3.2241686 .399201-1.800504$

H $4.7399706 .138484-0.921303$

C 3.4407525 .2033541 .869735

H -0.3938292 .8124921 .485170$

H $\quad 0.5820300 .182735-2.225773$

H -1.480032 $0.984760-3.688497$

H 2.8157065 .5734512 .692579

H 4.2373675 .9415031 .721497

H $2.147518 \quad 6.079867 \quad 0.396348$

C $-4.6020621 .632788 \quad 0.406514$

O $-4.4342180 .911321-0.817853$

C $-4.1554181 .725271-1.963072$

C $-5.2783292 .753293-2.167635$

C $-5.5330693 .578382-0.906790$

C $\begin{array}{llll}-5.736711 & 2.657587 & 0.296623\end{array}$

H -3.6650502 .1533280 .639871$

C $-4.8022990 .626683 \quad 1.547182$

H $-4.1766161 .051848-2.829040$

O $-4.9586003 .606036-3.261690$

H $-6.2005882 .219701-2.432815$

H $-4.7233234 .293744-0.720613$

O $-6.7239724 .350172-1.076210$

H -6.7060352 .1541980 .196716$

O -5.7801023 .4377921 .488629$

C $-6.116962-0.1163611 .487409$

H -3.998363 -0.1180791 .550702$

H $-4.756474 \quad 1.1390212 .515656$

H $-5.7393624 .154386-3.446928$

H $-6.5493425 .011735-1.766589$

H $-6.4909164 .092826 \quad 1.380815$

O $-6.112346-1.0054570 .455384$

$\begin{array}{llll}0 & -7.037641 & 0.071176 & 2.272635\end{array}$

C $-7.307169-1.7758200 .297328$

H -7.491413 -2.3778721 .192237$

H -8.156871 -1.118123 0.091181

H $-7.168046-2.447225-0.554238$ 
H $\quad 0.2061516 .704041-0.853430$

05 00131

MM̄FF Geometry

C $2.852160-2.353379-3.318929$

C $4.092954-2.836342-3.149585$

C $5.148635-2.277233-2.222884$

O $4.694582-1.053696-1.603945$

C $5.434557-3.288844-1.094763$

C $6.514546-2.827662-0.134913$

C $6.311221-2.5498371 .168278$

C $7.896572-2.696393-0.722303$

C $5.033587-2.661888 \quad 1.954034$

C $4.187878-1.3915111 .862091$

C $3.129029-1.2636352 .967434$

C $2.125524-2.4196072 .974802$

C $1.015266-2.2062154 .017655$

O $2.448379-0.0223622 .733750$

O $-0.028507-3.1560063 .753707$

C $0.411245-0.8009363 .816209$

C $1.503660-2.4890015 .443425$

O $-0.533045-0.4963654 .839317$

C 1.4712930 .3171913 .727774

C $0.8581561 .681746 \quad 3.333352$

O 2.0856990 .4885365 .010317

C $5.0607500 .130614-2.161157$

O $5.758781 \quad 0.303364-3.146337$

C $4.4303291 .196661-1.349808$

C $4.5734872 .487544-1.685589$

C $3.9755513 .623871-0.977379$

C 3.1498393 .3119850 .243592

C 0.1222851 .6939021 .990737

C $-0.210298 \quad 3.104251 \quad 1.530717$

C $-1.0770704 .328394-0.468327$

O $-0.0708834 .095326 \quad 2.241587$

C $0.0086224 .693673-1.432253$

C $0.5561055 .916152-1.516154$

C $1.6077386 .283553-2.525904$

C $2.8241546 .944831-1.911608$

C $3.1683848 .197465-2.256599$

C $3.6472356 .170749-0.901769$

C $4.1795734 .871896-1.450506$

N -0.6989653 .1153010 .232699$

H $2.533005-1.470461-2.772048$

H $4.385320-3.723347-3.708281$

H $6.058807-2.096407-2.806633$

H $5.742238-4.251720-1.524002$

H $4.501703-3.483761-0.553260$

H $7.162934-2.2115691 .757120$

H $8.654265-2.4925010 .041760$

H $8.184344-3.624671-1.226577$

H $7.933388-1.877533-1.446987$

H $4.454963-3.5342941 .632221$

H $5.304573-2.8543053 .000002$

H $4.832040-0.5023491 .901940$

H $3.693921-1.3465150 .883515$

H $3.651078-1.2024443 .929399$

H $2.624255-3.3792463 .154340$

H $1.660421-2.5029481 .982565$

H $0.352232-4.0479723 .825750$

H $-0.150524-0.8318032 .875170$

H $1.846599-3.5268605 .531589$

H $0.689968-2.3799516 .168927$

H $2.329067-1.8444465 .751390$

H -1.183394 -1.219351 4.856289

H $\quad 0.1889892 .0377684 .127889$

H 1.6721212 .4191333 .286649

H 2.4762031 .3775975 .048675

H $3.8440570 .881964-0.493299$

H $5.1710272 .736843-2.561462$

H 3.7582482 .8058101 .001486

H $2.3125542 .661422-0.027796$

H 2.7205804 .1898230 .727891

H $\quad 0.735331 \quad 1.214967 \quad 1.219145$

H $-0.821790 \quad 1.1441382 .070629$

H -1.277575 5.1281140 .252126

H -1.999387 4.110102 -1.015894

H $\quad 0.3417173 .917000-2.117029$

H $1.1385366 .957796-3.254649$

H $1.9281105 .404856-3.098813$

H $4.0350308 .686431-1.824346$

H $2.5896238 .766825-2.977137$

H $3.0438666 .038960-0.000584$

H $4.5150996 .763924-0.585004$

H $4.8046724 .993648-2.336467$

H $-0.7531232 .239889-0.280905$

C $0.576217-3.460743-3.479023$

O $-0.098026-2.350421-2.852968$

C $-0.498967-2.746967-1.522871$

C $-0.397013-4.265746-1.498035$

C $0.813287-4.497911-2.380257$

H $0.240481-2.302034-0.844493$

C $-1.896088-2.187959-1.227081$

H $-0.271373-4.664394-0.486814$

H $-1.289225-4.723846-1.941964$

C $0.936839-5.920807-2.895070$

H $1.709424-4.255232-1.796103$

C $-2.484935-2.6775740 .106588$

O $-1.804405-0.759725-1.192148$

H -2.570498 -2.438785 -2.053408

H $-2.636850-3.7631150 .067959$

O $-1.513678-2.442027 \quad 1.133279$

C $-3.795965-1.9917700 .535913$

C -4.905296-2.085173 -0.524107

H $-3.592284-0.9502110 .803084$

O $-4.230078-2.6470961 .732756$

H -4.579715 -1.561418-1.428203

H $-5.046125-3.138912-0.795897$

H $1.043208-6.623261-2.061973$

H $0.055952-6.217108-3.474284$

H $1.815504-6.024728-3.539675$

C $1.824937-2.975683-4.223065$

H $-1.421550-0.473964-2.039443$

H -1.893070 -2.758721 1.971313

H -4.886392 -2.071663 2.160665

H $1.530916-2.212036-4.953684$

H $2.259329-3.809727-4.786968$

H $-0.128061-3.857430-4.222002$

C $-5.8467310 .824905-0.476631$

O $-6.146673-0.1973780 .481433$

C $-6.264318-1.516454-0.058896$

C $-7.362531-1.551921-1.131191$

C $-7.146159-0.486196-2.202601$

C $-6.9304040 .882597-1.559149$

H $-4.8709830 .626834-0.936450$

C -5.7666552 .1554590 .286392$

H $-6.608385-2.1542850 .764499$

O $-7.430778-2.832154-1.750508$

H $-8.336460-1.371963-0.657416$

H $-6.311308-0.744289-2.864799$

O $-8.307376-0.411677-3.032784$

H $-7.8758821 .236780-1.128625$

O $-6.5411641 .816641-2.563760$

C -4.5541062 .2109561 .189282$

H $-5.6969203 .008988-0.395834$

H -6.6658122 .2718740 .903945$

H -7.587895 -3.488413-1.050088

H $-8.450139-1.297234-3.409278$

H $-7.2408581 .823438-3.239284$

O -4.8483682 .9412082 .303978$

$\begin{array}{llll}\text { O } & -3.471510 & 1.703539 & 0.930958\end{array}$

C -3.7793873 .0852753 .244800$

H -3.3700352 .1079243 .519329$

H -3.000474 3.7275832 .824448

$\mathrm{H}-4.1807773 .5603124 .143619$

SCF Energy $(B 3 L Y P / 6-31 G * * / / M M F F)=-3245.93214862$

0500132

MM̄FF Geometry

C $4.423912-2.8224380 .685232$

C $3.452237-3.5200890 .080193$

C $1.993458-3.3259390 .404255$

O $1.448851-2.513959-0.660026$

C $1.266043-4.6826200 .454264$

C $-0.079401-4.6430951 .154129$ 
C $-1.272259-4.859625 \quad 0.565487$ C $-0.026888-4.3890442 .641271$ C $-1.570786-5.173868-0.872928$ C $-2.122570-3.991963-1.685192$ C $-3.490329-3.468982-1.214251$ C $-4.054918-2.463845-2.221521$ C $-5.383305-1.856748-1.748267$ O $-3.332875-2.8257430 .053958$ O $-5.646065-0.722328-2.589445$ C $-5.225726-1.346101-0.297888$ C $-6.550460-2.828394-1.962481$ O $-6.496294-0.9349340 .200471$ C $-4.562716-2.3749830 .644223$ C $-4.220139-1.8150142 .048792$ O $-5.465128-3.4702320 .848129$ C $0.559108-1.544203-0.318787$ O $0.090316-1.3277620 .787000$ C $0.245470-0.772605-1.542291$ C $-0.8231260 .037402-1.544448$ C $-1.2842990 .870323-2.655667$ C $-0.3911630 .983028-3.861997$ C -3.314616 -0.5794712 .070822$ C -4.076014 0.7358312 .063924 C -3.777452 3.1585451 .568040 $\begin{array}{llll}\text { O } & -5.261925 & 0.825661 & 2.371351\end{array}$ C -4.2483143 .3507780 .160827$ C -3.659203 $4.175321-0.718205$ C $-4.1226104 .356184-2.134590$ C $-3.1130303 .854155-3.143792$ C $-2.2534754 .696684-3.740928$ C $-3.1619142 .391694-3.528484$ C -2.468992 $1.504664-2.532112$ N -3.267736 1.8063311 .709140 H $4.154454-2.0907651 .441170$ H $3.709954-4.235460-0.696452$ H $1.910128-2.8020761 .362538$ H $1.884728-5.4161390 .989559$ H $1.164467-5.065258-0.566343$ H $-2.157510-4.8311041 .200109$ H $-0.999552-4.5363553 .123293$ H $0.679336-5.0748653 .120545$ H $0.286489-3.3623172 .849525$ H $-0.684005-5.559712-1.384377$ H $-2.296941-5.996332-0.896150$ H -1.390257 -3.179338 -1.664853 H -2.208382 -4.320985 -2.728479 H $-4.166248-4.325781-1.109832$ H $-4.176086-2.926483-3.209119$ H -3.332873 -1.648903 -2.362608 H $-6.449079-0.287358-2.254321$ H $-4.600069-0.446916-0.354547$ H -6.649754 -3.074833 -3.026511 H -7.504320 -2.374635 - 1.671632 H -6.434027 -3.765842 -1.414668 H $-6.358585-0.5150241 .065532$ H -5.139324 -1.648376 2.623128 H -3.683156 -2.608112 2.589351 H -5.201967 -3.939115 1.657389 H $0.888639-0.903041-2.404686$ H $-1.4331970 .091767-0.644983$ H $-0.3318850 .023047-4.386234$ H $0.6198661 .284855-3.567310$ H $-0.7301521 .728262-4.585068$ H -2.734219 -0.5865353 .002152$ H -2.601802 -0.607364 1.242579 H -2.9682233 .8449671 .833315$ H $-4.607856 \quad 3.3164902 .263477$ H $-5.116602 \quad 2.774507-0.150400$ H -2.795833 $4.758351-0.407632$ H -4.312872 $5.426120-2.290857$ H $-5.0875223 .862761-2.305838$ H - $-1.5476294 .347923-4.487357$ H -2.232961 $5.752340-3.491577$ H $-2.7602522 .249841-4.537340$ H $-4.2092502 .072461-3.608125$ H -3.040748 $1.348445-1.617825$ H -2.3141651 .6287711 .406763$ C $6.410822-1.934825-0.627996$
O $5.882419-0.614702-0.369795$

C $5.413214-0.068073-1.617225$

C $6.100214-0.882862-2.705755$

C $6.075232-2.263709-2.084674$

H $4.335905-0.265994-1.667675$

C $5.6800811 .434984-1.705253$

H $5.571487-0.831360-3.662233$

H $7.133663-0.553558-2.865860$

C $7.038564-3.241639-2.735061$

H $5.057227-2.662257-2.169167$

C $4.9348572 .292202-0.661346$

O $7.0867131 .655156-1.542776$

H $5.4312451 .786438-2.714524$

H 5.3599862 .1251520 .335815

O $5.2183823 .657140-1.000349$

C $3.4062452 .076016-0.627271$

$\begin{array}{llll}\text { C } & 2.636980 & 3.181594 & 0.123729\end{array}$

H $3.1958141 .104368-0.167120$

$\begin{array}{llll}\text { O } & 2.903564 & 2.021582 & -1.965648\end{array}$

H $3.1189883 .361576 \quad 1.088848$

H $2.7048624 .108342-0.457620$

H $\quad 6.785870-3.385209-3.790684$

H $8.071885-2.882417-2.683381$

H $6.995557-4.217724-2.241766$

C $5.891171-2.9475580 .398188$

H $7.2391022 .613410-1.609698$

H $5.0096284 .203206-0.223682$

H $3.1208652 .865861-2.396838$

H $6.411674-2.7790781 .349953$

H $6.144590-3.9685050 .090162$

H $7.499628-1.856080-0.511010$

C $1.1919742 .109876 \quad 2.622232$

O $0.958601 \quad 1.761451 \quad 1.250921$

C $1.1441302 .824016 \quad 0.308574$

C $0.236948 \quad 4.007273 \quad 0.664961$

C $0.4437784 .468364 \quad 2.101145$

C $0.305383 \quad 3.2847913 .055031$

H 2.2459522 .3771062 .761412

C 0.8959950 .8636853 .473071

H $0.7843272 .444266-0.654291$

O $0.444241 \quad 5.102513-0.219417$

H $-0.808490 \quad 3.698764 \quad 0.550519$

H 1.4109564 .9684202 .229145

$\begin{array}{lllll}\text { O } & -0.557902 & 5.431071 & 2.436048\end{array}$

H $-0.7444782 .969228 \quad 3.100416$

O $0.683223 \quad 3.6991704 .366900$

C $2.021291-0.1467663 .412388$

H 0.7794001 .1464824 .525415

H -0.0186980 .3696983 .126530$

H $0.2742164 .784126-1.122563$

H $-0.490776 \quad 6.1545521 .789352$

H 0.1202314 .4556864 .605372

O $1.810642-1.1008654 .367139$

$\begin{array}{llll}\text { O } & 2.961700 & -0.107468 & 2.631637\end{array}$

C $2.825697-2.1033834 .482207$

H $2.790291-2.7718663 .618696$

H $2.624900-2.6867365 .384806$

H $3.815261-1.6455094 .576151$

SCF Energy (B3LYP/6-31G**//MMFF) = -3245.92934969

05_00133

MMFF Geometry

C -3.546253 -3.190199 -0.611867

C $-3.077933-4.234247-1.312437$

C $-1.646376-4.405243-1.778199$

O $-0.904260-3.167518-1.694185$

C $-0.954545-5.437768-0.867356$

C $0.502766-5.681758-1.203279$

C $1.548172-5.271682-0.455954$

C $0.743203-6.471805-2.464339$

C $1.531903-4.4829290 .827811$

C $2.026185-3.0435520 .643508$

C $3.519011-2.8999850 .306104$

C $4.407274-3.1960721 .518934$

C $5.893094-2.9287871 .231441$

O $3.724332-1.546890-0.132994$

O $6.587618-2.8991802 .488424$

C $6.037135-1.5252770 .605252$ 
C $6.527557-4.0611360 .415199$

O $7.378850-1.2881300 .186873$

C $5.065745-1.277246-0.568553$

C $5.125710 \quad 0.173927-1.095947$

O $5.433378-2.123760-1.666103$

C -1.111902 -2.234469-2.663995

O $-1.847882-2.319946-3.632858$

C $-0.313528-1.047851-2.295125$

C $-0.726817 \quad 0.178076-2.646555$

C $-0.077576 \quad 1.429343-2.252555$

C $1.2558331 .336171-1.557008$

C $4.7399411 .262247-0.092438$

C $4.7666212 .643287-0.727518$

C $4.3405305 .037170-0.192843$

O $5.0994942 .840801-1.893344$

C $2.9678175 .384169-0.676127$

C $2.6620095 .559454-1.971010$

C $1.3004415 .948247-2.472584$

C $0.5507834 .771504-3.061136$

C $0.6065964 .521976-4.380589$

C $-0.258803 \quad 3.969515-2.064199$

C $-0.6945132 .600849-2.512504$

N 4.3851613 .6330020 .167745

H -2.870395 -2.384836 -0.337446

H -3.755876 -5.048570 -1.558565

H -1.644037 -4.754120 -2.818030

H -1.487632 -6.396679 -0.924183

H -1.056834 -5.1172530 .174572$

H $2.547144-5.535083-0.801279$

H $1.805309-6.684615-2.626586$

H $\quad 0.220069-7.432446-2.416307$

H $\quad 0.381887-5.920962-3.338132$

H $\quad 0.528940-4.4415041 .260498$

H $2.145792-5.0087171 .567275$

H $1.434462-2.559756-0.140324$

H $1.809076-2.4726491 .555823$

H $3.763243-3.568551-0.525341$

H $4.272510-4.226511 \quad 1.867774$

H $4.099829-2.5546012 .356928$

H $6.440526-3.7518922 .932493$

H $5.826036-0.7999271 .401099$

H $6.469988-5.0079720 .965797$

H $7.594448-3.8777220 .247205$

H $6.045925-4.215976-0.552315$

H $7.954597-1.4509990 .953519$

H $6.1256350 .377796-1.501694$

H $4.4467480 .254550-1.956744$

H $5.051348-1.756976-2.480557$

H $0.566241-1.211748-1.686424$

H -1.644889 $0.277347-3.223798$

H $1.7319182 .307339-1.404378$

H $1.9591650 .745260-2.154072$

H $\quad \begin{array}{llll}1.147051 & 0.865112 & -0.574848\end{array}$

H $3.731497 \quad 1.0811350 .292774$

H 5.4392691 .2742880 .749680

H $5.1031575 .242745-0.951816$

H $4.5821015 .621444 \quad 0.700332$

H 2.1962125 .5157330 .078118

H $3.4364635 .423357-2.722680$

H $\quad 1.4262736 .738211-3.224294$

H $0.700614 \quad 6.404085-1.674369$

H $0.0662193 .701958-4.839523$

H $1.2002045 .144670-5.042549$

H -1.157405 $4.546762-1.809869$

H $0.2942153 .884877-1.121860$

H -1.648180 $2.588454-3.040204$

H 4.0697713 .3676931 .096179

C $-5.633993-1.801599-0.672682$

O $-4.897023-0.624501-0.288241$

C -5.8248440 .4245020 .041302$

C $-7.212040-0.096133-0.323803$

C -7.045530 -1.589826 -0.128359

H $-5.7724350 .558213 \quad 1.128054$

C $-5.430651 \quad 1.728921-0.658789$

H $-7.993844 \quad 0.3353910 .308874$

H -7.470729 $0.122294-1.366197$

C -8.111683 -2.416262 -0.825613

H $-7.069326-1.8050320 .948490$
C $-3.9723602 .173073-0.427996$

O $-5.6010681 .556163-2.072818$

H $-6.1253302 .523646-0.361530$

H -3.294260 $1.499053-0.964011$

O $-3.8227093 .455670-1.053686$

C -3.5313322 .2724861 .045091$

C -2.1310442 .9049921 .179723$

H $-3.534822 \quad 1.2707791 .487847$

O $-4.4824793 .067337 \quad 1.750887$

H -1.435967 2.3442050 .547018

H $-2.1637593 .931850 \quad 0.796286$

H $-9.105820-2.163044-0.442732$

H $-8.112740-2.240116-1.906555$

H -7.948747 -3.485628 -0.658764

C $-4.956781-3.054919-0.115872$

H $-5.3508302 .393632-2.499406$

H $-4.3666354 .089655-0.555335$

H -4.3312092 .9335442 .701677$

H $-5.544473-3.943673-0.372252$

H $-4.912574-2.9992530 .978222$

H $-5.639556-1.830344-1.770292$

C -0.6443870 .7441712 .746704$

O $-1.6067121 .673600 \quad 3.258147$

C -1.601570 2.9574252 .629695

C -0.2176313 .6088382 .773199$

C 0.8975432 .6959712 .268130

C $\quad 0.774911 \quad 1.3058512 .890964$

H $\quad-0.862814 \quad 0.5274391 .693639$

C $-0.794151-0.5571013 .543164$

H -2.301114 3.5805413 .200076

O -0.1925104 .8509652 .077921$

H -0.040382 3.8287623 .834245

H 0.9041062 .6388241 .174542

O 2.1643203 .2367822 .650585

H 1.0511141 .3580603 .952063

O 1.6962590 .4231212 .254492

C -2.072464 -1.270668 3.177351

H $0.028541-1.2525303 .340699$

H $-0.794127-0.3698424 .623860$

H 0.6389345 .3005542 .303244

H 2.2652634 .0928682 .201678

H 2.5852140 .7996272 .370960

O $-3.100737-0.813361 \quad 3.946839$

O $-2.147671-2.1191602 .298664$

C $-4.378475-1.3984023 .680090$

H $-4.334647-2.4854913 .796672$

H -5.094118 -0.996739 4.402164

$\mathrm{H}-4.708851-1.1304712 .673257$

SCF Energy (B3LYP/6-31G**//MMFF) $=-3245.91228958$

0500134

MM̄FF Geometry

C $-4.170851-2.669041-1.932337$

C $-3.138195-2.738427-1.078385$

C $-1.729342-3.066986-1.505177$

O $-0.852997-1.983509-1.113576$

C $-1.254310-4.342178-0.783330$

C $0.054097-4.893581-1.318897$

C $1.239245-4.856768-0.676830$

C $-0.040620-5.566997-2.666088$

C $1.560009-4.2651160 .668412$

C $2.342120-2.9525300 .562494$

C $3.755286-3.060701-0.031139$

C $4.721621-3.7821800 .913034$

C $6.167310-3.7643480 .391168$

O $4.206706-1.716395-0.264201$

O $7.031823-4.1460881 .472764$

C $6.542750-2.3176890 .004865$

C $6.384057-4.799092-0.719663$

O $7.823560-2.271587-0.618966$

C $5.492450-1.634003-0.895419$

C $5.808229-0.144009-1.156482$

O $5.481937-2.279997-2.175405$

C $-0.886019-0.841717-1.855007$

O $-1.596820-0.604262-2.818080$

C $0.1012720 .091446-1.272816$

C $0.048007 \quad 1.401274-1.554347$

C $0.9435872 .414274-0.996442$ 
C $2.2446891 .945345-0.397367$

C $5.842495 \quad 0.755857 \quad 0.081425$

C $6.085653 \quad 2.209877-0.292927$

C 6.3009554 .4727410 .722309

O $6.2931392 .580600-1.445738$

C 4.9966295 .1911210 .576438

C $4.5675325 .718013-0.580695$

C $3.2869406 .487631-0.736825$

C $2.2020595 .666665-1.401088$

C $2.0509365 .695789-2.736348$

C $1.3198374 .880306-0.453489$

C $0.5839753 .713057-1.056805$

N 6.0615953 .0446940 .816198

H $-4.004589-2.866156-2.988615$

H -3.300377-2.531828 -0.023801

H -1.668484 -3.199066 -2.592192

H $-2.012714-5.131234-0.881191$

H $-1.179032-4.1395130 .290217$

H $2.095618-5.314014-1.171803$

H $0.903289-6.035101-2.965417$

H $-0.801858-6.353813-2.644616$

H $-0.307539-4.844397-3.442902$

H $0.651186-4.0779041 .246408$

H $2.124691-5.002817 \quad 1.249162$

H $1.764420-2.244680-0.040481$

H $2.407579-2.4934661 .557773$

H $3.699307-3.574691-0.996160$

H $4.401943-4.8149301 .094386$

H $4.705035-3.2874831 .894370$

H $6.756376-5.0266841 .780385$

H $6.631716-1.7525660 .941117$

H $6.189512-5.810687-0.343102$

H $7.425442-4.801961-1.059707$

H $5.737275-4.642532-1.585220$

H $8.453668-2.707046-0.019721$

H $6.754762-0.058586-1.706776$

H $5.0436200 .254045-1.838997$

H $5.082933-1.675810-2.823295$

H $0.832370-0.316426-0.586405$

H $-0.7430011 .770600-2.204862$

H $2.7415131 .232008-1.064684$

H 2.0798191 .4620840 .570049

H $2.9597952 .757151-0.244498$

H 4.8941230 .6916090 .625618

H $6.651413 \quad 0.452127 \quad 0.753724$

H $6.9735014 .675428-0.118357$

H $6.801756 \quad 4.7899841 .642237$

H 4.3905565 .3016551 .472240

H $5.1771465 .602751-1.474346$

H $3.4997037 .388272-1.327368$

H $2.9279846 .858790 \quad 0.231740$

H $1.2710005 .139653-3.243762$

H $2.710734 \quad 6.289917-3.360839$

H $0.5785685 .566494-0.023059$

H 1.9122454 .5356610 .401782

H $-0.3620853 .976267-1.529871$

H $5.8184782 .659795 \quad 1.724271$

C $-5.958162-0.926368-2.055264$

O $-5.160794 \quad 0.074806-1.392542$

C $-6.0076101 .176135-1.014387$

C $-7.3183980 .969614-1.761653$

C -7.411314 $-0.545266-1.776576$

H -6.2004601 .0713780 .059823$

C $-5.2919632 .505303-1.282460$

H -8.163805 $1.448411-1.258566$

H -7.269671 $1.356297-2.786015$

C -8.398230 -1.084269 -2.797024

H $-7.704892-0.889628-0.775612$

C $-3.8747152 .586051-0.677954$

O $-5.1610792 .693525-2.697611$

H $-5.9060593 .338319-0.922557$

H -3.209450 $1.887634-1.199861$

O $-3.3559303 .897881-0.925840$

C -3.811994 2.317200 0.835676

C -2.3734612 .3669371 .372100$

H -4.2228291 .3288241 .041124$

O $-4.620217 \quad 3.2621951 .531450$

H -1.7686191 .6699350 .785129$
H $-1.961856 \quad 3.370884 \quad 1.214037$

H $-9.409542-0.724682-2.580800$

H $-8.138826-0.767153-3.812706$

H $-8.418163-2.178463-2.780542$

C $-5.573529-2.309467-1.528466$

H $-4.671276 \quad 1.931031-3.051214$

H -3.396259 $4.048020-1.885935$

H -4.2777394 .1490061 .325490$

H $-6.251719-3.072326-1.926791$

H $-5.665408-2.331644-0.436549$

H $-5.739078-0.848804-3.128386$

C -2.321168 $-0.391357 \quad 2.709238$

O $\begin{array}{lllll} & -2.918274 & 0.805516 & 3.224877\end{array}$

C -2.2564352 .0259042 .874222$

C $-0.811967 \quad 1.991505 \quad 3.391851$

C -0.0631610 .7630012 .881683$

C $-0.856171-0.5114083 .160663$

H -2.382294 -0.3909111 .613943$

C -3.143152 $-1.578696 \quad 3.243320$

H -2.7785612 .8229153 .418487$

O $-0.109958 \begin{array}{llll}H & 3.179126 & 3.038179\end{array}$

H $-0.829461 \quad 1.9561774 .488443$

H $0.1654880 .847063 \quad 1.815152$

$\begin{array}{lllll}O & 1.195362 & 0.667648 & 3.552660\end{array}$

H $-0.799736-0.732903 \quad 4.234404$

O $-0.217133-1.5801802 .464571$

C $-4.552364-1.6349982 .693556$

H -2.665020 -2.527161 2.978007

H $-3.221569-1.5040204 .334761$

H $\quad 0.1006323 .1387012 .090127$

H $1.674398 \quad 1.497846 \quad 3.387591$

H $-0.555078-2.4169072 .824423$

O $-5.225599-2.651383 \quad 3.311280$

O $-5.008069-0.9010101 .829476$

C $-6.580648-2.8367892 .889616$

H $-7.160404-1.9237433 .055460$

H $-6.610645-3.1278501 .835472$

H -7.015707 -3.641498 3.488159

SCF Energy (B3LYP/6-31G**//MMFF)= -3245.91631154

0500135

MMFF Geometry

C $0.381836 \quad 0.398828 \quad 3.362281$

C $1.370298-0.4379123 .712949$

C $1.758581-1.6407722 .900070$

O $3.118164-1.4078672 .459295$

C $1.736228-2.9500103 .707007$

C $0.372648-3.3978894 .182930$

C $-0.526528-4.0297963 .403403$

C $0.081055-3.1577345 .640266$

C $-0.397750-4.3148031 .932075$

C $-1.698515-3.9727751 .193972$

C $-1.549514-3.750959-0.318911$

C $-0.736412-4.838234-1.026050$

C $-0.564825-4.543977-2.525240$

O $-0.949222-2.462185-0.520207$

O $0.472916-5.401220-3.027283$

C $-0.067983-3.090244-2.694613$

C $-1.828080-4.886207-3.323652$

O $-0.028506-2.724491-4.071930$

C $-0.899572-2.060905-1.897585$

C $-0.316477-0.631188-1.979085$

O $-2.215337-1.993673-2.464813$

C $3.503085-1.9702611 .283065$

O $2.843887-2.695182 \quad 0.555855$

C $4.877906-1.5046731 .005598$

C $5.291584-1.433746-0.267606$

C $6.579600-0.913278-0.721598$

C $7.650503-0.6793810 .310677$

C $1.057492-0.431609-1.337172$

C $1.5909600 .972988-1.574371$

C $3.5251942 .439078-1.002655$

O $1.0449741 .783151-2.318690$

C $4.569718 \quad 2.265783-2.061779$

C $5.8734292 .518333-1.868771$

C $6.9260442 .353384-2.930851$

C $8.0654551 .460247-2.486834$

C $9.1884141 .988845-1.971455$ 
C $7.940635-0.033846-2.701589$

C $6.739089-0.640989-2.033274$

N $2.767392 \quad 1.207231-0.877223$

H -0.1874610 .2027012 .457173$

H $1.946641-0.2383564 .613076$

H $1.105070-1.7139302 .023015$

H $2.420164-2.8653784 .563342$

H $2.183315-3.7534933 .105799$

H $-1.457795-4.3641363 .858483$

H $-0.911618-3.5179055 .930679$

H $0.816602-3.6752146 .264758$

H $0.121295-2.0883045 .868722$

H $0.433983-3.7613891 .491889$

H $-0.179123-5.3814121 .803083$

H $-2.418820-4.7828941 .366639$

H $-2.130833-3.0628131 .626849$

H $-2.566925-3.720201-0.727176$

H - $1.192849-5.824020-0.874123$

H $0.268365-4.900906-0.588011$

H $0.212178-6.321556-2.851919$

H $0.968504-3.064648-2.336215$

H -2.063033 -5.953293 -3.228281

H - $1.679466-4.707727-4.394352$

H $-2.707988-4.327664-2.998692$

H $\quad 0.508710-3.391104-4.533424$

H $-0.300570-0.305988-3.027597$

H - $-1.0102310 .058560-1.478549$

H -2.637675 -1.173244 -2.161899

H $5.481552-1.1753461 .843450$

H $4.603204-1.734475-1.056861$

H $8.618365-0.421685-0.126414$

H $7.813939-1.5831460 .908585$

H 7.3675080 .1394430 .980842

H $0.991230-0.601378-0.257342$

H $1.789598-1.127671-1.757639$

H $3.9598532 .655619-0.021781$

H $2.8519823 .256604-1.279001$

H $4.2345071 .926844-3.039453$

H $6.2049232 .879464-0.897687$

H $7.3104673 .352055-3.176777$

H $6.4997321 .963609-3.863687$

H $10.0229681 .363600-1.672130$

H $9.2967393 .059765-1.834203$

H $8.846437-0.564621-2.383456$

H $7.865717-0.222328-3.780592$

H $5.907944-0.830622-2.713134$

H $3.1723030 .457532-0.326212$

C $0.1655872 .931507 \quad 3.367405$

O $-0.809547 \quad 3.0187152 .308965$

C $-0.1965193 .673001 \quad 1.180236$

$\begin{array}{llll}\text { C } & 1.147808 & 4.207577 & 1.670722\end{array}$

C $1.524124 \quad 3.1702622 .709083$

H -0.0110452 .8925190 .433320$

C -1.119337 4.7472590 .594633

H 1.8790064 .2953850 .862267

H 1.0490565 .1934282 .139765

C $2.607468 \quad 3.6257503 .669453$

H 1.8695032 .2736712 .179700

C -2.521168 $4.253813 \quad 0.184486$

O $-1.297567 \quad 5.779195 \quad 1.574129$

H -0.620143 $5.220153-0.260110$

H -3.1175334 .0444901 .080502$

O $-3.1852945 .343726-0.470137$

C $-2.5404373 .016556-0.731420$

C -3.929060 2.745460 -1.342845

H $-2.2120442 .147346-0.152817$

O $-1.6024423 .183476-1.795268$

H $-4.6918042 .856453-0.566342$

H $-4.1320053 .501453-2.110556$

H 3.5311193 .8525963 .127036

H 2.3082264 .5268974 .215010

H 2.8299922 .8449424 .403599

C $0.005221 \quad 1.623140 \quad 4.146644$

H -1.876022 6.4552081 .181258

H -2.671098 $5.575631-1.261721$

H - $-1.8896743 .938088-2.336058$

H $-1.043886 \quad 1.511422 \quad 4.446414$

H 0.5979751 .6805395 .067447
H -0.0665313 .7585654 .052093$

C $-4.9985690 .146195-0.130924$

$\begin{array}{lllll}\text { O } & -3.879216 & 0.300768 & -1.012848\end{array}$

C $-4.009697 \quad 1.343551-1.987636$

C $-5.2560121 .103701-2.850821$

C $-6.5092230 .928056-1.999980$

C $-6.280279-0.133863-0.924985$

H -5.1273951 .0543680 .469674$

C $-4.680052-1.0126810 .826518$

H $-3.143343 \quad 1.248765-2.654355$

O $-5.4563112 .171538-3.770270$

H $-5.1097970 .192549-3.445640$

H $-6.8160061 .879860-1.550973$

O $-7.5975690 .521917-2.833576$

H $-6.225456-1.125901-1.390969$

O $-7.394057-0.139344-0.034031$

C $-3.748335-0.5818381 .939794$

H $-5.603338-1.3719561 .295218$

H $-4.203128-1.8432340 .295099$

H $-4.6496882 .249247-4.307928$

H $-7.353504-0.317174-3.259869$

H $-8.192971-0.287511-0.568572$

O $-3.933968-1.3994213 .018370$

O $\begin{array}{llll}-2.945107 & 0.337666 & 1.866910\end{array}$

C $-3.120245-1.1125604 .159251$

H $-2.066165-1.2610453 .913883$

H -3.296969-0.0902314.506623

H -3.395095 -1.807343 4.957124

SCF Energy (B3LYP/6-31G**//MMFF) $=-3245.91060083$

\section{5_00136}

MMFF Geometry

C $0.618268-2.0197142 .681982$

C $1.275396-2.6583921 .701418$

C $0.732579-3.003888 \quad 0.334266$

O $-0.627527-2.5418550 .194756$

C $1.583062-2.286613-0.732555$

C $1.137104-2.541713-2.157356$

C $0.645487-1.597618-2.984298$

C $1.294049-3.959457-2.643277$

C $0.453487-0.130081-2.710259$

C $-0.9653720 .163051-2.222888$

C $-1.3249131 .655461-2.198664$

C $-0.4794672 .454626-1.203263$

C $-0.9411263 .917548-1.100130$

O $-2.711323 \quad 1.729269-1.833866$

O $-0.309906 \quad 4.477501 \quad 0.061319$

C $-2.4663003 .946199-0.866042$

C $-0.450545 \quad 4.744729-2.293430$

O $-2.9614565 .282541-0.888239$

C $-3.258385 \quad 3.055306-1.845764$

C $-4.7568412 .946169-1.477078$

O -3.191175 3.626145 -3.158002

C $-1.615289-3.460176 \quad 0.027743$

O $-1.511768-4.675308 \quad 0.040439$

C $-2.867203-2.705219-0.204723$

C $-4.010941-3.346956-0.486486$

C $-5.298005-2.706387-0.774375$

C $-5.312321-1.202605-0.854813$

C $-5.0337212 .456151-0.053932$

$\begin{array}{llll}C & -6.478377 & 2.028209 & 0.149656\end{array}$

C $-7.9375290 .941658 \quad 1.865985$

$\begin{array}{llll}\text { O } & -7.342898 & 2.132890 & -0.715588\end{array}$

C -7.833636 $-0.550876 \quad 1.812549$

C $-8.731433-1.346308 \quad 1.210707$

C $-8.619425-2.8455471 .187301$

C $-8.783250-3.434667-0.198709$

C $-9.788443-4.285953-0.466109$

C $-7.794124-3.046074-1.277752$

C $-6.387509-3.480897-0.960214$

N $-6.678193 \quad 1.5120331 .422737$

H $-0.420499-1.7314232 .547390$

H $2.315521-2.9324761 .870246$

H $0.800487-4.0900780 .204665$

H $2.627468-2.608058-0.640784$

H $1.579906-1.212437-0.516973$

H $0.345669-1.901861-3.986573$

H $1.068442-4.060078-3.710356$ 
H $2.323934-4.299917-2.494178$ H $0.622264-4.631571-2.101241$ H $\quad 1.1948850 .240839-1.996561$ H $\quad 0.644450 \quad 0.412076-3.644726$ H -1.694648 $-0.357099-2.859427$ H $-1.106038-0.255378-1.219283$ H -1.205465 $2.051846-3.213792$ H $0.5844692 .416589-1.464926$ H $-0.5597711 .994078-0.208432$ H $-0.652891 \quad 5.3803730 .176773$ H -2.6233483 .5829910 .156995$ H $0.6451334 .730188-2.340855$ H $-0.7292555 .799015-2.187874$ H $-0.8287554 .381821-3.251407$ H -3.011375 5.564274 -1.817592 H $-5.2565593 .910681-1.636399$ H $-5.2284492 .251490-2.186478$ H $-3.9338213 .281027-3.681229$ H -2.808487 -1.623843 -0.153056 H -4.007393 -4.435451 -0.515982 H $-4.608951-0.848922-1.617162$ H $-5.030327-0.7715900 .111372$ H -6.284184 - $0.782474-1.116309$ H -4.3910901 .6010020 .182890$ H $-4.820413 \quad 3.2555460 .664723$ H -8.7574881 .3229251 .249354$ H -8.092931 1.2649812 .899709 H -6.978596 -1.002736 2.310801 H $-9.602955-0.9030940 .734612$ H -9.391009 -3.241839 1.860186 H $-7.660248-3.1757151 .604625$ H -9.918779 -4.716206 -1.453591 H $-10.505218-4.5735530 .295957$ H -7.880385 -1.972856 -1.462416 H -8.067534 -3.518437 -2.230728 H $-6.271025-4.563307-0.890342$ H -5.8827851 .4332712 .050098$ C $1.239525-0.0871514 .129664$ O $1.9920520 .518773 \quad 3.061072$ C 2.7069901 .6564503 .577794

C 2.2395091 .8395705 .019260

C 1.8908430 .4168945 .414636 H 3.7678561 .3801593 .587809 C 2.4942682 .8727642 .667245 H 3.0151362 .2831495 .651202 H 1.3521452 .4795975 .083806 C $0.999051 \quad 0.327540 \quad 6.640303$ H $2.822730-0.1323185 .604362$ C 2.8093252 .6118771 .179349 O $1.117578 \quad 3.2628292 .755471$ H 3.0825933 .7182903 .043399 H 2.0555391 .9433590 .745414 O 2.7016063 .8584710 .480653 C $4.2123512 .038501 \quad 0.910642$ C $4.4978901 .881353-0.595292$ H 4.3007341 .0602941 .392901 O 5.1979882 .8883601 .494488 H $3.7106781 .258382-1.034339$ H $4.4418312 .861427-1.084233$ H $1.494167 \quad 0.7733267 .509177$ H $\quad 0.0513220 .8544086 .486408$ H $0.769484-0.7150576 .881142$ C $1.261971-1.6092143 .978268$ H 1.0290734 .1304972 .326625 H 1.7604554 .1000870 .455337 H 5.1028463 .7682721 .091186 H $\quad 0.719475-2.0818984 .804335$ H $2.297537-1.9693054 .014957$ H $\quad 0.210256 \quad 0.2874094 .047248$

C $5.374464-1.049894-0.571509$

O $6.1369390 .083413-0.148254$

C $5.8913851 .281276-0.890655$

C $6.1687051 .047545-2.383738$

C $5.399381-0.155426-2.927318$

C 5.635809-1.381609-2.045905

H $4.309729-0.821993-0.439709$

C $5.666143-2.217548 \quad 0.382368$

H $6.6286532 .013107-0.537204$
O $5.8354282 .199265-3.151455$

H $7.2413620 .863821-2.528019$

H $4.3283380 .058910-3.022293$

O $5.866017-0.455984-4.244659$

H $6.663981-1.731946-2.192743$

O $4.767217-2.434112-2.455589$

C $7.102057-2.700494 \quad 0.361323$

H $5.455229-1.9097601 .413539$

H $5.010667-3.0595780 .131822$

H $6.3520772 .944075-2.799195$

H $5.7471820 .345632-4.782650$

H $4.924510-2.589175-3.402777$

O $7.145775-3.9859810 .821882$

O $8.067478-2.0308690 .020335$

C $8.448475-4.5758410 .877443$

H $8.892749-4.608419-0.121864$

H $9.087656-4.0167561 .567476$

H $8.342713-5.5989831 .247685$

SCF Energy (B3LYP/6-31G**//MMFF) $=-3245.93437223$

0500137

MMFF Geometry

C $2.262788-2.588967-2.611995$

C $0.986508-2.911929-2.869206$

C $-0.070746-2.974702-1.795597$

O $-0.914977-1.807531-1.936858$

C $-0.945027-4.229852-1.962412$

C $-1.861648-4.484223-0.780337$

C $-3.205527-4.398111-0.811690$

C $-1.178200-4.8925200 .500741$

C $-4.093295-4.042155-1.968870$

C $-4.450441-2.551813-2.080523$

C $-5.086585-1.947350-0.817071$

C $-5.669520-0.564037-1.118057$

C $-6.2195600 .120247 \quad 0.143400$

O $-4.072759-1.8289820 .187019$

O $-6.4301671 .507445-0.163657$

C $-5.150470 \quad 0.062916 \quad 1.256751$

C $-7.586755-0.4450810 .546507$

O -5.6780620 .5398692 .491574$

C $-4.543349-1.3420861 .451868$

C $-3.345530-1.3631912 .435531$

O $-5.541179-2.2144431 .997467$

C $-0.452324-0.650363-1.388841$

O $0.569292-0.501889-0.736846$

C $-1.3950960 .434724-1.728393$

C $-0.9648811 .702490-1.652907$

C $-1.7579722 .907059-1.895655$

C $-3.2491212 .740684-2.015739$

C $-2.156743-0.4756502 .052526$

C -2.1804150 .9041962 .689125$

C -1.017731 3.1067492 .599734

O $-2.950048 \quad 1.2157703 .593873$

C -1.8025554 .0362341 .727485$

C $-1.2820285 .146826 \quad 1.182874$

C $-2.0609286 .113670 \quad 0.335799$

C -1.393429 $6.391030-0.993710$

C $-0.6034987 .465394-1.158877$

C -1.702092 $5.464018-2.149460$

C -1.116855 $4.092952-1.954860$

N $-1.210621 \quad 1.7402472 .150499$

H $2.564526-2.392658-1.586119$

H $0.672124-3.075871-3.896860$

H $0.390534-2.976011-0.802498$

H $-0.308061-5.115745-2.088713$

H $-1.524923-4.138431-2.887217$

H -3.749807 -4.610475 0.108506

H -1.890606 -5.2168421 .267028$

H $-0.496647-5.7295340 .318366$

H $-0.608746-4.0576830 .919272$

H $-3.653783-4.375134-2.915135$

H -5.020638 -4.620798 -1.867024

H -3.543379 -1.993797 -2.336956

H $-5.143154-2.441624-2.924108$

H $-5.874131-2.624849-0.466558$

H $-6.448668-0.629623-1.887781$

H $-4.8845420 .074740-1.543941$

H $-7.0439231 .558151-0.916311$ 
H -4.3528530 .7577340 .965781$

H -8.314491 $-0.298279-0.260846$ H -7.9935390 .0847351 .414806$ H -7.559727 -1.5127180 .773811$ H $-6.026594 \quad 1.4337312 .331923$ H -3.692051 -1.155115 3.455031 H -2.969777 -2.396353 2.462474 H $-5.095008-2.9691272 .416264$ H $-2.4025870 .166063-2.018862$ H $0.0750421 .882005-1.383292$ H $-3.6474242 .233897-1.129814$ H $-3.5011252 .149527-2.902829$ H -3.791616 $3.684107-2.098233$ H -1.234601 -0.9568702403280 H $-2.082619-0.3761230 .967500$ H $0.056146 \quad 3.3136792 .561514$ H -1.356458 3.2003083 .636497 H -2.8503383 .7999501 .556619$ H -0.2412765 .3944531 .380467$ H $-2.160197 \quad 7.0485200 .903078$ H $-3.085810 \quad 5.7620480 .163731$ H - $0.1403627 .686278-2.114766$ H $-0.3986838 .146096-0.339038$ H -1.284275 $5.866582-3.081531$ H $-2.7841645 .449865-2.302270$ H $-0.0298654 .097425-1.856952$ H -0.6410021 .4103641 .376701$

C $3.633914-0.954034-3.982692$

O $3.957447-0.218126-2.783150$

C $3.2574721 .041088-2.821550$

C $2.8437361 .241309-4.274166$

C $2.507249-0.181585-4.674291$

H $2.3500900 .917054-2.221120$

C $4.1102912 .169711-2.236522$

H $1.9982471 .928454-4.375427$

H $3.670951 \quad 1.622709-4.884004$

C $2.443273-0.396176-6.176190$

H $1.533070-0.435877-4.239982$

C $4.5835381 .925035-0.788946$

O $5.2705602 .327861-3.061641$

H $3.5535523 .112948-2.297784$

H $5.3353791 .126552-0.765115$

O $5.2402653 .116154-0.336956$

C 3.4554491 .5918020 .207653

C 3.9543381 .5208901 .663196

H $3.013270 \quad 0.629496-0.066722$

$\begin{array}{lllll}\text { O } & 2.423546 & 2.573497 & 0.117848\end{array}$

H $4.8127600 .842142 \quad 1.706527$

H 4.3100392 .5107381 .974076

H $\quad 1.6650910 .232317-6.621427$

H $3.393847-0.145696-6.658801$

H $2.210094-1.439563-6.410500$

C $3.327261-2.421157-3.657001$

H $5.6937243 .167411-2.815314$

H $6.0727553 .197708-0.832024$

H 2.8194583 .4364670 .328771

H $4.235940-2.896876-3.267051$

H $3.060853-2.958817-4.574390$

H $4.537171-0.926412-4.606189$

C $3.080502-1.3130282 .365131$

O $2.235061-0.1585792 .272701$

C 2.8577531 .0719462 .657558

C 3.3315970 .9805584 .114834

C $4.231094-0.2337564 .344976$

C $3.568960-1.5045473 .808438$

H $3.934812-1.1758731 .693057$

C $2.262574-2.5173801 .872284$

H 2.0713171 .8352432 .622782

O 4.0146072 .1729934 .485482

H 2.4519280 .8989284 .766511

H $5.216367-0.0826333 .890051$

O $4.471756-0.3814585 .746616$

H $2.723766-1.7626154 .458573$

O $4.493005-2.5859883 .882884$

C $3.096701-3.5856131 .197520$

H $1.709565-2.9730752 .701585$

H $1.526022-2.1753881 .137291$

H 4.3204052 .0607195 .401906
H $3.616060-0.5259356 .185333$

H $5.240727-2.3809893 .295820$

O $2.270713-4.5935950 .787374$

O $4.307992-3.5449501 .034408$

C $2.907947-5.6696070 .092162$

H $3.409160-5.297784-0.806740$

H $3.619784-6.1755230 .751247$

H $2.137049-6.384211-0.207716$

SCF Energy (B3LYP/6-31G**//MMFF) $=-3245.90997316$

\section{8}

MM̄FF Geometry

C -4.9680922 .6082241 .440865$

C -3.6968492 .9264431 .155388$

C -2.6273833 .0730192 .203088$

O -1.5584882 .1509801 .897618$

C -2.0406354 .4964542 .173895$

C -0.9918764 .7275873 .246036$

C 0.3225964 .9193633 .017520

C -1.5131044 .7601194 .661465$

C 1.0522154 .9728441 .703667

C 1.9308433 .7367811 .500274

C 2.9091793 .8656210 .323309

C $2.2031383 .992712-1.028873$

C $3.2016834 .023447-2.197314$

O 3.7294312 .6869840 .333231

O $2.4415143 .829232-3.400028$

C $4.1763402 .835324-2.053093$

C $3.8699645 .396556-2.330559$

O $5.2023302 .892206-3.040379$

C $4.7858432 .701719-0.639624$

C $5.6121401 .405925-0.457362$

O $5.6796593 .797405-0.407124$

C -1.5721220 .9449782 .526519$

O $-2.454306 \quad 0.488402 \quad 3.234992$

C -0.2730640 .2964322 .247063$

C $0.137173-0.7279883 .008825$

C $1.428864-1.4094292 .905078$

C $2.413163-0.8818351 .894863$

C $4.8884070 .124774-0.869244$

C $5.582457-1.139655-0.392293$

C $5.355875-3.626614-0.561715$

O $6.562052-1.1390800 .346904$

C $4.521721-4.1145830 .582478$

C $5.036259-4.7057821 .672697$

C $4.279262-5.2377162 .862509$

C $2.918550-4.6164773 .107813$

C $1.802346-5.2751292 .750494$

C $2.942189-3.2708963 .809213$

C $1.677326-2.4611293 .713541$

N $4.954488-2.272424-0.891880$

H -5.2579082 .4360612 .474590$

H -3.4068213 .0954870 .120998$

H -3.0340012 .8656953 .200519$

H -2.842633 5.2338722 .311942

H -1.6271664 .6903611 .179112$

H 0.9698215 .0732023 .880963

H -0.7449545 .0638935 .380735$

H -2.338039 5.4750664 .745952

H $-1.873095 \quad 3.7725204 .964961$

H $0.365845 \quad 5.0884140 .862238$

H 1.6789535 .8734101 .709064

H 2.5120743 .5347682 .410495

H 1.3011972 .8512201 .356944

H $3.5446304 .739416 \quad 0.509794$

H $1.5638294 .882188-1.065368$

H $1.5307383 .134180-1.166866$

H $3.0703743 .770804-4.139803$

H $3.5887841 .936819-2.278165$

H $3.1137256 .172890-2.498367$

H $4.5316435 .433357-3.203007$

H $4.4466135 .680774-1.447982$

H $5.8456803 .565317-2.760398$

H $6.5565401 .480914-1.012420$

H $5.898528 \quad 1.3312050 .600825$

H $6.290786 \quad 3.547690 \quad 0.306147$

H $\quad 0.3318170 .711704 \quad 1.449792$

H $-0.522223-1.0898453 .796477$ 
H 2.7215080 .1369532 .154348 H $1.968792-0.8676360 .894731$ H $3.320496-1.4827611 .815166$ H $3.8693990 .117637-0.468499$ H $4.8320520 .077234-1.962360$ H $6.426448-3.645560-0.333937$ H $5.169918-4.243727-1.446070$ H $3.446318-3.9877450 .489981$ H $6.114013-4.8526071 .716736$ H $4.891728-5.0813143 .760177$ H $4.196465-6.3267392 .752419$ H $\quad 0.809947-4.8786422 .931550$ H $1.855888-6.2433372 .262625$ H $3.792844-2.6846143 .447235$ H $3.151486-3.4440074 .873192$ H $\quad 0.900922-2.7774194 .411084$ H $4.124761-2.158164-1.466110$ C $-6.529540 \quad 1.0453490 .255389$ O $-5.4945690 .268405-0.385856$ C $-6.056270-0.385549-1.535804$ C $-7.557246-0.421186-1.286231$ C $-7.7695330 .931113-0.637043$ H $-5.8602780 .265516-2.396682$ C -5.407226 -1.752588-1.766658 H -8.131514 -0.551131 -2.208395 H -7.836523 $-1.221512-0.591465$ C $-9.082047 \quad 1.0452620 .119225$ H $-7.7433231 .702563-1.418631$ C $-3.865310-1.728848-1.861303$ O $-5.767251-2.613642-0.679908$ H $-5.835876-2.203231-2.670312$ H -3.431869 -1.586810 -0.865384 O $-3.450626-3.020813-2.319624$ C $-3.298405-0.680147-2.831622$ C -1.788799 -0.773252 -3.127795 H $-3.5132660 .324980-2.453156$ O $-3.977968-0.795725-4.088278$ H -1.564411 -1.755604 -3.557370 H -1.587291 $-0.061795-3.939477$ H $-9.9301830 .906801-0.559390$ H -9.1572890 .2905070 .909115$ H -9.1787012 .0311470 .584883$ C -6.0531092 .4903930 .409527$ H -5.515813 -3.519813 -0.924914 H -3.633636 -3.658739 -1.609728 H $-3.822545-1.695661-4.423388$ H $-6.885017 \quad 3.1324020 .721627$ H $-5.6946692 .868212-0.556424$ H $-6.710757 \quad 0.5861561 .235485$ C $-0.225385-2.712114-1.321936$ O $-0.842850-1.478484-0.934883$ C $-0.863452-0.453514-1.932837$ C $0.580918-0.132429-2.349221$ C $1.282810-1.379590-2.876352$ C $1.204321-2.496398-1.839701$ H $-0.834842-3.199900-2.090109$ C $-0.194219-3.628976-0.092735$ H $-1.267810 \quad 0.443767-1.447380$ O $\quad 0.6176650 .884077-3.344642$ H $1.1402360 .246101-1.483494$ H $0.868383-1.702983-3.838174$

O $2.659703-1.085695-3.113845$ H $1.869251-2.254259-1.002770$ O $1.681531-3.711388-2.414507$ C -1.578365 -3.932169 0.425035 H $\quad 0.289451-4.586778-0.316599$ H $0.385186-3.1702910 .715844$ H $\quad 0.179413 \quad 1.670019-2.975878$ H $2.692877-0.332970-3.729028$ H $2.579762-3.542729-2.746910$ O $-1.865291-3.1203971 .482440$ O $-2.319535-4.776139-0.061086$ C $-3.153964-3.3036402 .074591$ H -3.243007 -2.610708 2.915426 H $-3.938844-3.0764651 .348641$ H $-3.257766-4.3262272 .449261$

SCF Energy (B3LYP/6-31G**//MMFF) $=-3245.93262491$
0500139

MMFF Geometry

C $3.309595-2.7699761 .372049$

C $2.405022-3.3507212 .175447$

C $1.172104-2.6871112 .749565$

O $1.228429-1.2655762 .494692$

C $-0.085719-3.2742862 .081778$

C $-1.382894-2.8962482 .768854$

C -2.276211-2.014283 2.278531

C $-1.670518-3.6274424 .054456$

C $-2.156578-1.2002751 .020600$

C $-2.765511-1.888633-0.207868$

C $-4.269927-2.174785-0.090238$

C $-4.773438-2.916245-1.330367$

C $-6.289405-3.150941-1.275732$

O $-4.955973-0.9272030 .047321$

O $-6.693021-3.576171-2.586598$

C $-6.990316-1.809011-0.972608$

C $-6.653301-4.295967-0.323423$

O $-8.386068-2.025736-0.779621$

C $-6.374842-1.0486600 .225815$

C -6.9521330 .3781780 .406781$

O $-6.668236-1.765518 \quad 1.432744$

C $0.775860-0.4170003 .453332$

O $0.288201-0.7068924 .533402$

C 0.9688130 .9579532 .943999

C 0.2418901 .9580973 .462689

C $0.297973 \quad 3.3641603 .058787$

C 1.3971483 .7757342 .114508

C $-6.9495451 .288730-0.823015$

C $-5.5724381 .551198-1.395810$

C $-3.4356982 .722172-0.934216$

O $-5.2055221 .036443-2.450008$

C $-3.1238284 .103273-0.454920$

C $-2.129754 \quad 4.3827770 .401263$

C -1.7842955 .7713720 .858499$

C -1.9767395 .9670042 .346303$

C $-3.127114 \quad 6.4629172 .832344$

C -0.8032165 .6776783 .256914$

C -0.6408564 .2083543 .535891$

N $-4.8156612 .410669-0.616466$

H $3.174900-1.7346781 .070140$

H $2.545810-4.3944422 .449715$

H $1.177776-2.8772493 .829439$

H $-0.025516-4.3711232 .066079$

H $-0.111212-2.9691921 .028887$

H -3.192789-1.841353 2.840775

H -2.643886 -3.356953 4.477626

H -1.676212 -4.708775 3.882726

H $-0.911040-3.3993824 .808126$

H $-2.640576-0.2301181 .187125$

H -1.106617 -0.9672960 .815395$

H -2.589622 -1.234349-1.071662

H $-2.231470-2.827477-0.397352$

H $-4.444909-2.7843960 .802878$

H $-4.242768-3.867875-1.459351$

H $-4.545268-2.322684-2.227075$

H -7.662240 -3.658341 -2.583889

H $-6.890462-1.204488-1.882065$

H $-6.181722-5.229419-0.653622$

H $-7.731889-4.488228-0.328341$

H -6.342142 -4.114211 0.707406

H -8.821148 $-1.157579-0.747748$

H $-7.9826800 .306640 \quad 0.778293$

H -6.3888350 .8753661 .208430$

H $-6.576242-1.1539482 .182223$

H $1.683898 \quad 1.1020112 .142018$

H $-0.482336 \quad 1.7235374 .242105$

H 1.3984984 .8424051 .882089

H 1.3010653 .2501791 .159264

H 2.3784963 .5454682 .544035

H -7.588026 $0.885503-1.616106$

H -7.391873 2.256359 -0.552854

H $-3.2800842 .655664-2.015857$

H $-2.8139581 .961422-0.451900$

H $-3.7334414 .913426-0.848214$

H -1.512362 3.5674550 .766306

$\mathrm{H} \quad-2.382686 \quad 6.5112780 .310111$ 
H $-0.743318 \quad 5.9863370 .587672$ H -3.264111 6.6365873 .894455 H $-3.959217 \quad 6.7008932 .177977$ H -0.9444316 .1783324 .224440$ H 0.1002946 .1348962 .843632 H -1.403539 3.8099264 .206762 H -5.1587282 .6598240 .307055$ C $5.811936-2.7390331 .148474$ O $5.791515-1.4240910 .555997$ C $6.953355-1.267229-0.273750$ C $7.960745-2.2711340 .265212$ C $7.051817-3.4432760 .586213$ H $6.659548-1.567312-1.287286$ C $7.4099490 .195809-0.288270$ H $8.738313-2.516788-0.463938$ H $8.446322-1.9110471 .179296$ C $7.668316-4.4505131 .541600$ H $6.800167-3.956141-0.352053$ C $6.2826971 .211886-0.571485$ O $7.973841 \quad 0.5315830 .986284$ H $8.2228750 .317360-1.012946$ H 5.6331041 .2824100 .309562 O $6.8819972 .505322-0.715627$ C $5.4159130 .914162-1.809330$ C $4.3297941 .986189-2.016725$ H $4.937106-0.063247-1.695717$ O $6.2271930 .870504-2.981371$ H $3.8066602 .146643-1.067785$ H $4.8040672 .936619-2.290873$ H $8.572846-4.8888441 .107437$ H $7.944359-3.9839752 .493233$ H $6.966879-5.2631541 .755490$ C $4.511599-3.4714240 .803657$ H 7.2911580 .3740051 .661013 H $7.4161522 .491441-1.528419$ H $6.829240 \quad 0.112258-2.901286$ H $4.561414-4.5066421 .160415$ H $4.383836-3.503029-0.285721$ H $5.896510-2.5970462 .233055$

C $1.851614 \quad 0.218248-1.806897$ O $2.749514 \quad 0.308366-2.916716$ C $3.3187291 .606467-3.124045$ C $2.2031562 .639530-3.338612$ C $1.1792572 .621573-2.204687$ C $0.6800231 .197361-1.955565$ H $2.4035370 .454254-0.888338$ C $1.413427-1.244790-1.650850$ H $3.8869221 .544138-4.060826$ O $2.7619123 .942286-3.471053$ H $1.6886072 .413355-4.281729$ H $1.5942043 .068060-1.294418$ O $0.0748623 .444782-2.585804$ H $\quad 0.0316430 .909521-2.790484$ O $-0.1288741 .176765-0.782947$ C $0.580460-1.777704-2.797967$ H $2.297516-1.889283-1.582501$ H $0.838120-1.345775-0.724175$ H $2.0236034 .564594-3.587605$ H $-0.5918123 .376714-1.881471$ H $0.432308 \quad 1.418873-0.027270$ O $-0.181124-2.818531-2.348742$ O $0.614621-1.360692-3.947488$ C - $-1.035771-3.423976-3.323967$ H -1.748617 -2.689250 -3.710363 H $-0.440616-3.851708-4.136169$ H - $1.592756-4.227727-2.835033$

SCF Energy (B3LYP/6-31G**//MMFF) $=-3245.90789869$

\section{0}

MM̄̄FF Geometry

C $-0.121764-2.0261672 .215311$

C -1.058150-1.511628 3.027012

C $-1.437911-0.0570113 .017835$

O $-2.879907-0.0114732 .881948$

C $-1.059601 \quad 0.685113 \quad 4.310254$

C $0.422090 \quad 0.7714904 .601952$

C 1.2811101 .5698103 .938027

C $0.896667-0.0638605 .760526$
C 0.9585792 .4763322 .782083

C 1.9258492 .2550001 .612361

C $1.4502442 .806100 \quad 0.256521$

C $0.7959164 .189200 \quad 0.339175$

C $0.2665944 .665006-1.023214$

O $0.5410871 .851116-0.312027$

O $-0.6210355 .770578-0.790979$

C $-0.5802753 .535198-1.651884$

C $1.3922815 .198918-1.916785$

O $-0.9792653 .879156-2.976586$

C $0.1355352 .167833-1.652666$

C $-0.7580921 .025415-2.184380$

O $1.2631282 .240918-2.536074$

C -3.4202231 .0577772 .239294$

O $-2.8308582 .015001 \quad 1.764681$

C -4.8802180 .8374692 .177888$

C -5.5746151 .3717351 .163073$

C $-7.001308 \quad 1.1804650 .909621$

C -7.8398840 .5873062 .010568$

C $-1.9728380 .667424-1.329027$

C $-2.775472-0.462892-1.955540$

C $-4.753843-1.916083-1.515859$

O $-2.545502-0.909409-3.076678$

C $-5.962311-1.242280-2.088920$

C $-7.211718-1.456268-1.649204$

C $-8.428242-0.779492-2.218732$

C $-9.253094-0.080101-1.158744$

C $-10.311900-0.692527-0.601788$

C $-8.9040131 .347839-0.793027$

C $-7.4943581 .520226-0.299430$

N $-3.792985-0.901980-1.121824$

H $0.427444-1.3632671 .550298$

H -1.604770 -2.167059 3.700363

H -0.9871220 .4224332 .142151$

H $-1.5811030 .221541 \quad 5.159593$

H $-1.473386 \quad 1.7023814 .280739$

H $2.322236 \quad 1.5860654 .257294$

H 1.9780150 .0133485 .914639

H $0.405794 \quad 0.258916 \quad 6.684584$

H $0.659373-1.1191055 .594269$

H -0.0715662 .3465022 .446431$

H 1.0461793 .5128683 .129775

H 2.8822912 .7295631 .867219

H 2.1197621 .1803961 .498302

H $2.3374942 .850747-0.386555$

H 1.4935684 .9223300 .762249

H $-0.0613574 .159606 \quad 1.023961$

H $-0.1243106 .459283-0.316742$

H $-1.5010863 .453517-1.060325$

H $1.8751096 .064592-1.447023$

H $1.0003525 .558706-2.874305$

H $2.1713664 .460580-2.116566$

H -1.428731 $4.740368-2.929202$

H $-1.0697101 .252534-3.212444$

H $-0.1462430 .117708-2.269890$

H $1.5211361 .337498-2.783601$

H $-5.327820 \quad 0.1952302 .927424$

H -5.0400551 .9601010 .418036$

H $-8.9113500 .619149 \quad 1.797548$

H $-7.696474 \quad 1.1402142 .945718$

H -7.571131 -0.4614792 .177072$

H -1.647876 $0.348960-0.332922$

H -2.641212 $1.527767-1.224069$

H $-4.990622-2.504221-0.623816$

H $-4.305474-2.576343-2.264827$

H $-5.801014-0.548741-2.911028$

H -7.374845 -2.166509-0.841581

H $-9.034920-1.547730-2.715860$

H $-8.159820-0.061568-3.003692$

H -10.928035 -0.1955520 .140103$

H -10.584285 - $1.707053-0.873393$

H -9.604462 $1.758115-0.055007$

H $-9.0341141 .973483-1.685914$

H $-6.8153791 .936035-1.044308$

H $-3.934830-0.438446-0.230630$

C $0.149247-4.0506250 .709982$

O $1.222638-3.502374-0.083247$

C $0.704954-3.172308-1.385142$ 
C $-0.638372-3.887553-1.495920$

C -1.141947-3.790314 -0.069418

H $\quad 0.526715-2.090598-1.382044$

C $1.709401-3.526036-2.486081$

H -1.304442 -3.412556-2.221804

H $-0.518462-4.938639-1.782759$

C -2.264104 -4.759202 0.255752

H -1.501693 -2.767025 0.091809

C $3.118104-2.924550-2.302526$

O $1.855073-4.951465-2.532350$

H $1.293902-3.230114-3.457040$

H $3.639784-3.447014-1.491582$

O $3.869102-3.216309-3.489158$

C $3.158956-1.410091-2.028460$

C $4.579243-0.819504-2.131034$

H $2.748294-1.221698-1.031018$

O $2.318986-0.725631-2.958747$

H $5.287998-1.500004-1.650197$

H $4.858330-0.752089-3.189277$

H $-3.133213-4.569663-0.382458$

H $-1.955231-5.7984870 .101712$

H -2.579785 -4.651238 1.298373

C $0.207488-3.4902822 .135516$

H $2.486421-5.155726-3.243425$

H $3.418886-2.793908-4.240065$

H $2.677344-0.867499-3.850959$

H $1.222712-3.6249052 .525916$

H $-0.468511-4.0685802 .776367$

H $0.343509-5.1300230 .762588$

C $5.548786-0.0394820 .667165$

O $4.4701280 .543224-0.076488$

C $4.6623190 .587785-1.496347$

C $5.9408001 .368647-1.828658$

C $7.1524470 .815220-1.088043$

C 6.8582540 .7149890 .407095

H $5.668944-1.0920770 .385246$

C 5.1706430 .0094082 .156119

H $3.8258071 .170506-1.899529$

O $\quad 6.2003171 .357416-3.228155$

H $5.8085382 .418771-1.536510$

H $7.464074-0.154509-1.494033$

O $8.2587561 .702373-1.266761$

H $\quad 6.807214 \quad 1.7221410 .840666$

O 7.9322940 .0268961 .045965

C $4.192698-1.0868402 .523499$

H $6.065998-0.1364582 .771163$

H 4.7114570 .9689012 .416018

H $5.4194431 .729187-3.672878$

H $8.4082791 .788039-2.224075$

H 8.7496860 .5136240 .843684

O $4.279898-1.329383 \quad 3.864861$

O $3.441223-1.6505651 .740097$

C $3.448779-2.3850804 .354982$

H $2.397619-2.1624754 .157886$

H $3.734760-3.335273 \quad 3.894109$

H $3.594602-2.4599265 .435723$

SCF Energy (B3LYP/6-31G**//MMFF) $=-3245.92087720$

0500141

MM̄MF Geometry

C $2.294822-3.581395-2.002175$

C $0.972436-3.516331-2.216372$

C $-0.034031-3.361678-1.103277$

O $-0.911822-2.253275-1.410598$

C $-0.894713-4.633184-0.998296$

C -1.849291 -4.6281920 .180320$

C -3.192684 -4.655497 0.078802

C -1.206212 -4.623138 1.544096

C $-4.030157-4.696224-1.168229$

C $-4.462455-3.319981-1.698163$

C $-5.333674-2.512222-0.721933$

C $-5.911788-1.274490-1.410919$

C $-6.726180-0.407155-0.438007$

O $-4.517975-2.0977320 .375214$

O $-6.943755 \quad 0.869311-1.060193$

C $-5.889605-0.1459640 .835949$

C -8.115800 -1.003749 -0.184337

O $-6.6726620 .492346 \quad 1.840374$
C $-5.224181-1.4123741 .418382$

C $-4.198079-1.1127112 .534991$

O $-6.189204-2.2724322 .037559$

C $-0.408799-0.998343-1.261407$

O $0.717755-0.694703-0.903218$

C -1.473693 -0.041344-1.632342

C $-1.1451051 .231900-1.893790$

C $-2.0622132 .307815-2.270594$

C $-3.5380392 .018309-2.209360$

C $-3.020807-0.2198402 .129093$

C -3.2242371 .2521422 .449388$

C -2.1558073 .4764382 .095103$

O $-4.141151 \quad 1.6701993 .150634$

C -2.813295 4.1793920 .950253

C -2.2163745 .1513810 .243605$

C $-2.8832755 .908516-0.868911$

C $-2.0773805 .898568-2.148081$

C $-1.2786916 .930051-2.470471$

C $-2.2658014 .732800-3.091860$

C -1.540661 $3.498160-2.634204$

N -2.2289342 .0421921 .890806$

H $2.667708-3.546614-0.981300$

H $0.589442-3.557894-3.232922$

H $0.468294-3.163475-0.148399$

H $-0.246799-5.514736-0.901670$

H - $-1.443465-4.761738-1.937576$

H -3.773161 -4.655517 1.001023

H -1.933168 -4.789823 2.346512

H $-0.456711-5.4181941 .614389$

H $-0.719292-3.6628971 .739342$

H $-3.510908-5.237763-1.966225$

H $-4.924768-5.295464-0.954858$

H $-3.566635-2.743523-1.957576$

H $-5.021515-3.483181-2.628037$

H $-6.138696-3.160666-0.357085$

H $-6.525146-1.559191-2.274844$

H $-5.091089-0.666843-1.814703$

H $-7.3939370 .717026-1.908778$

H -5.1042200 .5646980 .553101$

H -8.687439 -1.053410 -1.119192

H $-8.702241-0.3708180 .490485$

H -8.079065 $-2.013814 \quad 0.229307$

H $-7.043728 \quad 1.3013021 .448135$

H -4.710664 -0.725156 3.423826

H -3.773036 -2.078128 2.846934

H -7.045143 -1.815455 2.057819

H -2.490491 -0.410950 -1.689525

H $-0.0957811 .515030-1.843787$

H $-3.8098691 .622587-1.224883$

H $-3.8175421 .284693-2.973002$

H $-4.1635542 .900312-2.361176$

H -2.136981 -0.533581 2.699306

H $-2.783110-0.3371291 .067688$

H -1.097305 3.7380342 .188011

H -2.662339 3.7398483 .029131

H -3.8371213 .8995910 .713756$

H -1.202274 5.4484830 .502595

H -3.025759 $6.942487-0.528022$

H -3.890861 $5.523300-1.068932$

H $-0.7189446 .943215-3.399728$

H $-1.1625377 .782032-1.808596$

H -1.873277 $4.987046-4.085724$

H $-3.3350764 .568555-3.250603$

H $-0.4552523 .608412-2.637908$

H $-1.5480871 .616147 \quad 1.269704$

C $4.373428-2.588067-3.072369$

O $5.118291-2.633378-1.838477$

C $5.250235-1.285740-1.329730$

C $4.952604-0.381983-2.514945$

C $3.848462-1.158323-3.207153$

H $4.467429-1.183264-0.569658$

C $6.637532-1.129425-0.698132$

H $4.6451980 .621769-2.214926$

H $5.827002-0.296100-3.171803$

C $3.599628-0.726624-4.641149$

H $2.924007-1.023597-2.633286$

C $6.9855590 .267238-0.154249$

O $6.765289-2.0736170 .368609$ 
H $7.390948-1.412779-1.444764$

H $7.934627 \quad 0.195227 \quad 0.392990$

O $7.2363261 .127778-1.270779$

C 5.9482760 .9541920 .761303

C 5.4941370 .1094331 .961963

H 5.0823891 .2604440 .166004

O $\quad 6.5379702 .168772 \quad 1.242944$

H $4.918338-0.7455571 .597912$

H $6.379165-0.2959712 .468798$

H $3.2868380 .321872-4.678636$

H $4.501072-0.829916-5.254316$

H $2.809584-1.331772-5.096975$

C $3.320946-3.701450-3.093672$

H $6.551845-2.9510320 .007310$

H $7.4735952 .002064-0.916833$

H 7.2943011 .9286961 .805330

H $3.826310-4.665521-2.955750$

H $2.839026-3.727145-4.077921$

H $5.101490-2.794634-3.867755$

C $2.561407 \quad 0.9780701 .826912$

O 3.6434581 .6965492 .426553

C $4.661211 \quad 0.8823173 .010471$

C $4.052857-0.0094034 .101364$

C $2.872439-0.8268673 .576975$

C $1.869008 \quad 0.0770982 .859527$

H 2.9364850 .3768470 .989894

C 1.5787252 .0166901 .275960

H 5.3530411 .5734333 .508704

O $5.048472-0.8757714 .635825$

H $3.703708 \quad 0.626576 \quad 4.925251$

H $3.213527-1.6413512 .927583$

O $2.233876-1.4463264 .696276$

H $1.3402900 .691113 \quad 3.600060$

O $0.895670-0.7256062 .195524$

C 2.2046322 .8869940 .213511

H $\quad 0.685270 \quad 1.5487980 .851334$

H 1.2415692 .6846112 .078354

H $4.618434-1.4337825 .306379$

H $1.549949-2.0441324 .350643$

H $\quad 0.392500-1.2037362 .875677$

O $2.3447812 .187721-0.947195$

$\begin{array}{lllll}\text { O } & 2.522137 & 4.055613 & 0.394949\end{array}$

C $2.8729172 .931117-2.049747$

H $2.2851913 .838356-2.220654$

H $3.9222133 .178781-1.864071$

H $2.8096892 .304370-2.943088$

SCF Energy (B3LYP/6-31G**//MMFF) $=-3245.90601444$

0500142

MM̄FF Geometry

C $-5.1975442 .766384-0.226540$

C -3.896326 $3.089541-0.261429$

C -3.1535753 .5847960 .950707$

O $-2.2175042 .552581 \quad 1.344416$

C -2.3467064 .8567790 .638634$

C -1.7482915 .4791551 .887060$

C -0.4314505 .5428092 .163259$

C -2.7378516 .0781592 .856104$

C 0.7267235 .0919791 .319996

C 1.1603003 .6569131 .627765

C 2.6400293 .3855281 .313907

C $3.0051713 .666121-0.145043$

C $4.4821593 .353297-0.429949$

O 2.8905932 .0052791 .616631

O $4.6330463 .328851-1.860103$

C 4.8071241 .9377340 .096640

C 5.3969384 .4797850 .062758

O $6.1984501 .650930-0.000558$

C $4.273157 \quad 1.6283181 .516876$

C 4.3615280 .1220641 .867293

O 5.0493462 .2644842 .538228

C -2.6945901 .5432712 .123402$

O -3.8329391 .4111612 .543899$

C - -1.5765820 .6096082 .377543$

C -1.790373 -0.4966293 .105845$

C $-0.785297-1.4998333 .463437$

C $0.621703-1.2630212 .985218$

C $3.688816-0.8107980 .861305$
C $3.572324-2.2448801 .345837$

C $2.568451-4.4244630 .632980$

O $4.020755-2.6373852 .418395$

C $1.159022-4.5044041 .128999$

C $0.781303-5.2331542 .190071$

C $-0.637211-5.3040842 .681185$

C $-0.759219-5.0439504 .168078$

C $-1.247011-5.9873934 .991510$

C $-0.315309-3.7004254 .707794$

C -1.165132 -2.5651974.200109

N $2.903790-3.0301300 .415969$

H -5.7591352 .9143210 .693146$

H -3.323416 2.914877 -1.167933

H -3.8516503 .7791301 .773784$

H $-2.9898805 .604510 \quad 0.156791$

H $-1.5612174 .613369-0.085614$

H -0.1315485 .9994203 .106364$

H -2.2468146 .6598143 .643937$

H -3.420055 6.7556062 .331996

H -3.326826 5.2971893 .345585

H 0.5047325 .1993320 .253020

H 1.5550795 .7838831 .520097

H 0.9881243 .4181772 .685992

H 0.5312392 .9545831 .066640

H 3.2405234 .0089511 .987006

H $2.7874094 .703651-0.423909$

H $2.3797723 .042964-0.798017$

H $5.5482323 .062547-2.055172$

H $4.3168941 .258782-0.609786$

H $5.1923655 .403351-0.492943$

H $6.4510284 .248770-0.124293$

H 5.2723934 .7073851 .122769

H 6.6517842 .1027690 .730558

H $5.412364-0.1730771 .989354$

H $3.900062-0.0284482 .853516$

H 5.4701423 .0568872 .177243

H -0.6068380 .8612541 .964150$

H -2.793726 -0.6857213 .485163$

H $1.006366-0.3104203 .366482$

H $\quad 0.650409-1.2409471 .890517$

H $1.326963-2.0305733 .304950$

H $2.683355-0.4463190 .624441$

H $4.278550-0.840490-0.059180$

H $3.281956-4.8708461 .332682$

H $2.651217-4.930888-0.333435$

H $0.409429-3.9365810 .582592$

H $1.523732-5.8220632 .723588$

H -1.020586 -6.303474 2.437899

H -1.277967 -4.596476 2.141302

H -1.338899 -5.815002 6.058779

H $-1.566660-6.9546324 .618306$

H $\quad 0.748592-3.5678814 .497789$

H $-0.388587-3.6905665 .803702$

H $-2.210849-2.6374444 .503022$

H $2.520934-2.584810-0.413352$

C $-6.1295350 .619893-1.032515$

O $-4.864698-0.066413-1.169050$

C $-5.085574-1.306079-1.865173$

C $-6.592346-1.528791-1.851913$

C $-7.094877-0.101925-1.970307$

H $-4.777969-1.143624-2.904607$

C -4.254425 -2.433289-1.241979

H $-6.925522-2.172558-2.671432$

H $-6.927618-1.975695-0.908534$

C $-8.5580310 .059250-1.597013$

H $-6.9455870 .242995-3.002405$

C -2.730614 -2.170553-1.238085

O $-4.679762-2.6086120 .114320$

H $-4.473945-3.374496-1.760756$

H -2.484296-1.401127 -0.498494

O $-2.077821-3.370513-0.807126$

C $-2.158755-1.784686-2.613581$

C $-0.628212-1.762900-2.724330$

H -2.548565 -0.809436 -2.916932

O $-2.631012-2.716137-3.597172$

H $-0.244533-2.790588-2.649846$

H $-0.392818-1.481983-3.758083$

H $-9.192240-0.533828-2.263980$ 
H $-8.749240-0.270738-0.570363$

H -8.868722 $1.105527-1.677560$

C $-5.9342392 .103029-1.354655$

H -4.284098 -3.4333200 .442796$

H $-2.286376-3.496071 \quad 0.133691$

H -2.338954 -3.603340 -3.325303

H $-6.8989162 .606915-1.477856$

H -5.376912 2.216962 -2.292406

H -6.4486880 .4885040 .010356$

C $2.138825-0.688966-3.135562$

O $1.577863-1.134572-1.892764$

C $0.167758-0.882634-1.739596$

C $-0.0583760 .633096-1.797507$

C $0.4803491 .230175-3.094877$

C $1.9386030 .822136-3.305912$

H $1.674836-1.227223-3.969822$

C $3.632470-1.045828-3.142500$

H $-0.084546-1.220175-0.727636$

O $-1.427440 \quad 0.975313-1.655641$

H $0.4735441 .106271-0.960925$

H $-0.1373300 .946746-3.954864$

O $\quad 0.4256382 .656809-3.013495$

H $2.5721451 .363749-2.593303$

O $2.3508101 .206086-4.615711$

C $3.867679-2.538425-3.049091$

H $4.111408-0.694047-4.062581$

H $4.122256-0.572658-2.286057$

H $-1.7087080 .695073-0.768324$

H $-0.4973582 .900193-2.826445$

H $2.1995872 .163422-4.698076$

O $5.165246-2.761776-2.685989$

O $3.029597-3.399314-3.279734$

C $5.538780-4.136535-2.551885$

H $5.421231-4.656038-3.507580$

H $6.591061-4.175661-2.258181$

H $4.939431-4.617745-1.773390$

SCF Energy (B3LYP/6-31G**//MMFF) $=-3245.92122940$

0500143

MM̄FF Geometry

C -0.5327495 .0572530 .586891$

C $-1.0062154 .838345-0.650235$

C $-1.8650563 .676785-1.100059$

O $-1.9339612 .637800-0.097878$

C $-3.2969244 .170078-1.377163$

C $-4.1770613 .138992-2.056862$

C $-5.2735122 .581376-1.507491$

C $-3.7794012 .765513-3.462616$

C $-5.8653252 .832338-0.149780$

C -5.4003411 .8590440 .944582$

C -5.7272870 .3838910 .659008$

C $-5.485368-0.4744321 .901760$

C $-5.719080-1.9665281 .622998$

O $-4.880604-0.071587-0.399831$

O $-5.173124-2.6892662 .737562$

C $-4.933779-2.3799880 .357478$

C -7.215219-2.302240 1.585481

O $-5.276858-3.716550-0.000223$

C $-5.138049-1.415752-0.831636$

C $-4.196382-1.691819-2.030545$

O $-6.479832-1.550532-1.316641$

C $-0.9031521 .749835-0.049286$

O $0.0820791 .725054-0.769404$

C - $-1.165121 \quad 0.817361 \quad 1.068835$

C -0.1612530 .0620451 .537048$

C $-0.237869-0.8912872 .644407$

C -1.607811-1.298634 3.115947

C $-2.696102-1.630817-1.722961$

C $-2.076746-2.979634-1.398365$

C $0.054735-4.004946-0.620146$

O $-2.675363-4.043958-1.528195$

C $-0.012993-4.2245200 .857900$

C $1.077322-4.3571821 .627596$

C $1.039185-4.6145503 .105976$

C $1.820486-3.5854843 .891302$

C $3.090720-3.8169324 .263774$

C $1.100779-2.3226364 .308054$

C $0.913041-1.3651253 .165458$
N $-0.759891-2.857764-0.975311$

H -0.7905184 .3773351 .393258$

H $-0.7409765 .542516-1.437038$

H -1.424228 $3.266486-2.016615$

H -3.269931 $5.060670-2.019501$

H $-3.7422384 .494515-0.430528$

H $-5.8284721 .856741-2.102964$

H $-4.5330392 .142281-3.956085$

H -3.650588 $3.664826-4.073720$

H -2.841173 $2.203844-3.462788$

H $-5.666723 \quad 3.8578400 .179076$

H $-6.9573842 .770821-0.242347$

H -4.3200111 .9774541 .086298$

H -5.8837292 .1605941 .882149$

H $-6.775870 \quad 0.3168920 .346187$

H $-6.112825-0.1386942 .736881$

H $-4.448066-0.3410982 .237156$

H -5.253524 -3.638489 2.540451

H -3.874519 -2.390789 0.640222

H -7.684515 -2.055593 2.545521

H $-7.378312-3.376127 \quad 1.441511$

H -7.756025 -1.764552 0.803890

H -4.682462 -3.996782 -0.715883

H -4.470542 -2.633891-2.520084

H $-4.394028-0.911405-2.780138$

H $-6.513549-1.213819-2.227406$

H $-2.162710 \quad 0.795178 \quad 1.490572$

H 0.8206150 .1643521 .079652

H -2.219999 -1.626573 2.269103

H -2.110567 -0.460584 3.610287

H $-1.596834-2.1334753 .819627$

H -2.175801 -1.265765 -2.616948

H -2.488996 -0.927191 -0.911802

H $1.074327-3.799660-0.959124$

H $-0.314415-4.893038-1.142803$

H $-0.998912-4.3008461 .310202$

H $2.059695-4.3069211 .164775$

H $1.457695-5.6141323 .282241$

H $0.008724-4.6566823 .480146$

H $3.649578-3.093746 \quad 4.848315$

H $3.601921-4.7329473 .986227$

H $1.680044-1.7969275 .078992$

H $\quad 0.161800-2.5933544 .798295$

H $1.854126-1.0033522 .750711$

H $\quad-0.358289-1.932565-0.852921$

C 1.8114955 .8113921 .227816

O 2.3585195 .1050010 .095178

C 2.9510493 .8722040 .566717

C 3.2592104 .1334802 .031314

C 2.0278414 .9160962 .449596

H 2.1659363 .1141770 .463530

C $4.1413093 .523180-0.330225$

H 3.3943913 .2236382 .620224

H 4.1598944 .7512242 .135184

C 2.2041625 .6846393 .747674

H 1.1974964 .2103212 .571824

C 4.9319542 .2458240 .014521

O $3.6567883 .371342-1.671001$

H $4.8328524 .375291-0.343681$

H $5.6669682 .104850-0.789173$

O 5.6616772 .4542191 .219567

C 4.0780410 .9620210 .104872

C $4.930033-0.3192970 .066507$

H $3.3721230 .942954-0.729504$

O $3.3143300 .961941 \quad 1.306636$

H $5.570642-0.288124-0.821249$

H $5.589312-0.3430130 .941974$

H 2.4074694 .9983334 .576333

H $3.0377276 .392174 \quad 3.685977$

H 1.2984926 .2497553 .990108

C 0.3618176 .2165490 .937487

H $3.1621054 .178470-1.894124$

H $\quad 6.279808 \quad 1.712241 \quad 1.326290$

H 3.9376140 .9857172 .052635

H $\quad 0.365519 \quad 6.933098 \quad 0.105928$

H -0.0609176 .7412541 .801892$

H $2.4077506 .727491 \quad 1.327667$

C $3.641474-1.765067-2.305668$ 
O $3.104051-1.630685-0.984401$

C $4.076078-1.6098670 .067093$

C $4.901759-2.9033130 .043630$

C $5.535483-3.143023-1.323372$

C $4.478484-3.048488-2.422736$

H $4.257731-0.889520-2.542686$

C $2.460665-1.808602-3.289697$

H $3.507815-1.6123981 .004848$

O $5.921757-2.876814 \quad 1.036087$

H $4.251617-3.7562030 .274979$

H $6.363753-2.449626-1.509983$

O $6.098471-4.456722-1.354903$

H $3.825178-3.928127-2.361789$

O $5.120160-3.097647-3.696042$

C $1.737399-0.484117-3.407666$

H $2.805001-2.071891-4.295795$

H $1.734400-2.556663-2.952473$

H $5.487354-2.7499861 .896993$

H $6.739217-4.514029-0.625443$

H $5.703117-2.322596-3.766883$

O $0.540346-0.689747-4.034065$

O $2.1725830 .595368-3.033071$

C $-0.2492320 .482414-4.257849$

H $0.314219 \quad 1.220266-4.836772$

H $-1.1348730 .191627-4.829153$

H $-0.5723530 .904323-3.302739$

SCF Energy (B3LYP/6-31G*//MMFF) $=-3245.92081230$

05_00144

MM̄FF Geometry

C $-0.475970 \quad 5.237994 \quad 0.216272$

C $0.657555 \quad 4.5209790 .226089$

C 1.2767213 .9752551 .484836

O $1.5409302 .566913 \quad 1.284120$

C $2.622725 \quad 4.6648981 .772461$

C $3.246336 \quad 4.221258 \quad 3.083421$

C 4.4124403 .5550043 .194477

C 2.4813374 .5919724 .329341

C $5.362466 \quad 3.1498252 .102832$

C 5.1111701 .7189931 .625634

C $6.276886 \quad 1.125042 \quad 0.820120$

C $6.5914001 .918409-0.450344$

C $7.6877481 .244949-1.292179$

O $5.901599-0.217822 \quad 0.479585$

O $7.6892141 .862506-2.588825$

C $7.316645-0.237922-1.499519$

C $9.0846021 .473841-0.703279$

O $8.367519-0.944976-2.152921$

C $6.927135-0.963713-0.194009$

C $6.382536-2.390719-0.442027$

O $8.087052-1.1005850 .635916$

C $\quad 0.548777 \quad 1.6808851 .568729$

$\begin{array}{lllll}\text { O } & -0.585498 & 1.933268 & 1.940049\end{array}$

C $1.1093190 .325721 \quad 1.365738$

C $0.408692-0.7629791 .717892$

C $0.877268-2.149347 \quad 1.615370$

C $2.245014-2.3707601 .023942$

C $5.204577-2.469715-1.414732$

C $4.519642-3.826573-1.400615$

C $2.592343-5.014775-2.463959$

O $4.857847-4.748396-0.663990$

C $1.299403-4.713535-1.773905$

C $0.700154-5.540051-0.903004$

C $-0.608199-5.216501-0.239126$

C $-0.593061-5.4321161 .258557$

C $-1.421557-6.3227361 .830157$

C $0.369364-4.6215312 .099971$

C $0.079628-3.1438332 .059214$

N $3.474566-3.872318-2.312903$

H -0.9663895 .4732361 .157860$

H $1.1500204 .283522-0.713759$

H $\quad 0.597670 \quad 4.1059042 .336054$

H $2.4860435 .753624 \quad 1.809577$

H 3.3036994 .4703420 .936804

H 4.7474853 .2761924 .193204

H 3.0567654 .4011645 .241807

H 2.2337895 .6585814 .319881

H 1.5535834 .0167164 .401374
H 5.3180323 .8489141 .262122

H 6.3803013 .2336642 .504690

H 4.9174681 .0608022 .483775

H 4.1954231 .6840291 .022465

H 7.1545071 .0899261 .476687

H $6.8855692 .947887-0.215198$

H $5.6817191 .997797-1.062062$

H $7.8478692 .814332-2.467141$

H $6.459726-0.250703-2.184628$

H $9.3108122 .546104-0.660477$

H $9.8587831 .030145-1.338982$

H $9.196114 \quad 1.0744320 .306811$

H $8.579438-0.460393-2.969025$

H $7.190313-3.046700-0.792126$

H $6.067616-2.8054700 .525872$

H $7.932995-1.8270311 .262610$

H $2.109510 \quad 0.262541 \quad 0.951816$

H $-0.587101-0.6311872 .138543$

H $3.013611-1.8763501 .628405$

H $2.285167-1.9695890 .005438$

H $2.531474-3.4208700 .954398$

H $4.454380-1.711950-1.163502$

H $5.552366-2.285590-2.437433$

H $3.074332-5.914374-2.068563$

H $2.417772-5.148347-3.535853$

H $\quad 0.822147-3.766077-2.015796$

H $1.154885-6.501310-0.676721$

H - $1.379966-5.842036-0.705825$

H $-0.896346-4.179469-0.440525$

H $-1.424476-6.4863972 .902637$

H $-2.118142-6.9084101 .239410$

H $1.391659-4.8659631 .802107$

H $0.303502-4.930668 \quad 3.151807$

H $-0.893100-2.8798532 .476559$

H $3.252381-3.036466-2.846237$

C $-2.5465385 .099104-1.157803$

O $-2.4258693 .672035-1.306882$

C $-3.4590013 .205691-2.201636$

C $-4.324056 \quad 4.417378-2.521661$

C $-3.3332995 .557936-2.381217$

H $-2.9370222 .855438-3.100552$

C $-4.1958952 .047937-1.519168$

H $-4.7740404 .358496-3.517202$

H $-5.1346874 .523463-1.789751$

C $-3.9915446 .917133-2.224590$

H $-2.6821385 .569099-3.265473$

C $-5.413151 \quad 1.521980-2.297940$

O $-3.2626160 .976245-1.346125$

H $-4.5004832 .361576-0.514362$

H $-6.1859102 .297809-2.350908$

O $-5.003746 \quad 1.265106-3.647611$

C $-6.0196960 .220343-1.735599$

C $-6.4366320 .327638-0.258355$

H $-5.313378-0.602437-1.892081$

O $-7.177987-0.076445-2.523210$

H -5.5815210 .6530090 .339797$

H $-7.2092341 .100482-0.161261$

H -4.603454 $7.151402-3.101795$

H $-4.6411326 .949580-1.343428$

H $-3.2398797 .705364-2.117797$

C $-1.1541825 .718367-1.036877$

H $-2.505473 \quad 1.327857-0.846682$

H $-5.7818570 .933485-4.127738$

H $-7.427001-0.998764-2.344033$

H $-1.2244006 .810817-0.997823$

H $-0.5521645 .456061-1.915614$

H $-3.1244835 .278700-0.240815$

C $-4.868884-2.0559640 .774699$

O $-6.114911-2.1009390 .069194$

C $-6.985395-0.994407 \quad 0.325888$

C $-7.318373-0.932252 \quad 1.824278$

C $-6.061207-0.9238332 .693631$

C $-5.114299-2.054412 \quad 2.287635$

H $-4.313971-1.155190 \quad 0.487380$

C $-4.050334-3.2758240 .343563$

H $-7.922473-1.211435-0.201137$

$\begin{array}{lllll}\text { O } & -8.100765 & 0.218540 & 2.125045\end{array}$

H $-7.918615-1.8098972 .097435$ 
H -5.5536850 .0471542 .652421$

O $-6.434974-1.1210214 .059343$

H $-5.546228-3.0128412 .601451$

O $-3.877642-1.9070502 .982828$

C $-3.622181-3.155830-1.099694$

H $-3.150255-3.404860 \quad 0.953851$

H $-4.641422-4.194727 \quad 0.437182$

H -8.9022390 .1784021 .575695$

H $-7.063026-0.4155594 .291764$

H -3.476333 -1.065839 2.705214

O $-2.626565-2.230871-1.215553$

O $-4.115424-3.804826-2.012895$

C $-2.129983-2.023203-2.541141$

H $-1.741458-2.959219-2.953690$

H $-2.920188-1.616855-3.179509$

H $-1.313175-1.298437-2.487817$

SCF Energy (B3LYP/6-31G**//MMFF) $=-3245.91752379$

0500145

MMFF Geometry

C 3.2040092 .5554402 .342976

C 3.5024101 .3296632 .802911

C 2.5310420 .4152623 .515844

$\begin{array}{lllll}\text { O } & 1.190310 & 0.776491 & 3.117322\end{array}$

C $2.758707-1.0480393 .090786$

C $1.945108-2.0435723 .895366$

C $0.874028-2.7227453 .437734$

C $2.448929-2.2956935 .294497$

C $0.221171-2.6383882 .084114$

C -1.301492 -2.514269 2.202588

C $-2.037639-2.6482550 .859328$

C $-1.701674-1.516667-0.114087$

C $-2.551775-1.571442-1.392460$

O $-3.440986-2.6385031 .161292$

O $-2.416803-0.311029-2.067172$

C $-4.038465-1.716316-1.004468$

C $-2.041293-2.638807-2.366942$

O $-4.845665-1.923880-2.160310$

C $-4.293968-2.8374430 .023463$

C -5.756771 $-2.876520 \quad 0.533610$

O $-4.038406-4.107010-0.591200$

C $0.235386 \quad 0.968273 \quad 4.057882$

O 0.3533820 .8369905 .266965

C $-1.053107 \quad 1.3190943 .414051$

C -1.1236041 .8123272 .166894$

C -2.3425542 .1066901 .414677$

C -3.6618131 .7711882 .057970$

C $-6.263833-1.5918931 .197361$

C -7.036909 -0.6742890 .262101$

C $-7.956721 \quad 1.6451050 .148695$

O $-7.436630-1.018238-0.846578$

C $-6.9827772 .372618-0.722156$

C -6.454196 $3.567617-0.418507$

C $-5.4394774 .269098-1.276981$

C $-4.0515844 .211284-0.670801$

C $-3.5204205 .298493-0.085843$

C $-3.3383592 .880389-0.809350$

C -2.228064 2.6216290 .171789

$\begin{array}{llll}\text { N } & -7.261639 & 0.570390 & 0.834747\end{array}$

H 2.2172742 .9732282 .526852

H 4.5074580 .9454392 .644403

H 2.6794070 .5374794 .594856

H $3.819183-1.3116313 .203093$

H $2.545195-1.1533842 .021676$

H $0.410002-3.4432354 .110963$

H $1.876011-3.0738795 .809915$

H $3.493930-2.6217705 .268587$

H $2.382664-1.3864645 .899267$

H $0.609257-1.7985241 .503290$

H $\quad 0.461192-3.5567341 .536367$

H - $1.686231-3.2895292 .879736$

H -1.568498 -1.562089 2.677656

H - $1.777936-3.6202770 .423392$

H $-0.637334-1.517369-0.373932$

H $-1.887812-0.5562630 .378961$

H - $1.471316-0.162435-2.241278$

H $-4.349600-0.757040-0.571720$

H $-1.014645-2.412431-2.674237$
H -2.632617 -2.649713 -3.288961

H $-2.041340-3.644747-1.942289$

H $-4.670632-1.187982-2.771676$

H $-6.425928-3.206003-0.270393$

H $-5.810254-3.6673641 .295729$

H $-4.505620-4.793569-0.087059$

H -1.9355791 .1440514 .019824$

H $-0.199623 \quad 1.997044 \quad 1.620747$

H -4.5211002 .0119941 .429124$

H -3.7900322 .3336942 .989242$

H -3.7191480 .7004542 .281893$

H $-6.966741-1.8684511 .993841$

H -5.442392 -1.0397401 .665280$

H $-8.3929942 .295464 \quad 0.913449$

H -8.766868 $1.230940-0.459809$

H $-6.6856561 .878124-1.644147$

H -6.7406194 .0596620 .508051$

H -5.766452 $5.308128-1.410358$

H $-5.407800 \quad 3.839176-2.286273$

H $-2.522373 \quad 5.2981270 .337962$

H $-4.0756406 .228990-0.022601$

H -2.926097 2.814871-1.824905

H $-4.0558202 .054539-0.744407$

H $-1.2346262 .841373-0.218950$

H $-6.8303550 .787068 \quad 1.728670$

$\begin{array}{llll}\text { C } 3.439334 & 3.884874 & 0.254341\end{array}$

O $3.0508712 .752872-0.545552$

C $3.1734183 .101928-1.937118$

C $3.5761644 .574033-1.979767$

C $4.3138584 .733964-0.662628$

H $3.9998462 .503598-2.337683$

C $1.8744932 .761446-2.678752$

H $4.1976344 .805826-2.850260$

H $2.7029395 .235657-2.010562$

C $4.4620006 .178959-0.221068$

H $5.3089814 .279734-0.759834$

C $1.4042031 .303252-2.489501$

O $0.8323653 .615203-2.187986$

H $1.9884382 .994806-3.743953$

H $1.0766641 .153655-1.453600$

O $0.2353911 .115390-3.297900$

C $2.4522820 .237192-2.858320$

C $1.892749-1.190713-2.730940$

H $3.3204530 .350047-2.205126$

$\begin{array}{llll}\text { O } & 2.883830 & 0.466892 & -4.198328\end{array}$

H $1.447671-1.301106-1.736474$

H $1.090555-1.328019-3.465748$

H $5.0303866 .750848-0.961801$

H $3.4873156 .663038-0.098109$

H $4.9927196 .243117 \quad 0.733884$

C 4.1378413 .4073801 .529117

H $0.0163383 .378209-2.661150$

H $0.509324 \quad 1.184416-4.228674$

H $3.688336-0.058589-4.344027$

H 4.4472244 .2673832 .132920

H 5.0378742 .8380791 .269327

H $2.517742 \quad 4.4256410 .510466$

C $3.899776-2.385445-0.740702$

O $4.107014-2.126673-2.134619$

C $2.950992-2.293281-2.962296$

C $2.407481-3.722404-2.826550$

C $2.147350-4.096188-1.369246$

C $3.391942-3.817947-0.526698$

H $3.176387-1.667355-0.336513$

C $5.245769-2.180377-0.026019$

H $3.298488-2.185284-3.997077$

O $1.217250-3.870599-3.592636$

H $3.144306-4.421640-3.243134$

H $1.268165-3.570997-0.977990$

O $1.842000-5.491128-1.317686$

H $4.177659-4.538218-0.789004$

O $3.086731-4.000790 \quad 0.852758$

C $5.657871-0.7257690 .044481$

H $5.196776-2.5447931 .005825$

H $6.030249-2.724264-0.565953$

H $0.892428-4.776841-3.454194$

H $1.568289-5.698021-0.408150$

H $2.872253-4.9392120 .986746$ 
O $6.958536-0.653764 \quad 0.456294$

O $4.933425 \quad 0.227478-0.202013$

C $7.490742 \quad 0.668073 \quad 0.589841$

H $7.3644161 .231334-0.339939$

H 7.0041021 .1826871 .423228

H 8.5593520 .5838520 .804975

SCF Energy (B3LYP/6-31G**//MMFF)= -3245.91667622

05_00146

MMFF Geometry

C $3.014178 \quad 0.7538803 .993237$

C $2.451890-0.214543 \quad 3.253493$

C $1.005058-0.6225883 .374088$

O $0.313581-0.2648572 .154732$

C $0.912849-2.1502373 .547302$

C $-0.493368-2.6569643 .803930$

C $-1.164332-3.4981782 .992806$

C $-1.135253-2.1897265 .085542$

C $-0.700229-4.1091251 .701079$

C $-1.066599-3.3061980 .443582$

C $-2.577038-3.1808500 .191204$

C $-2.848190-2.543455-1.173468$

C $-4.351489-2.332307-1.419109$

O $-3.144900-2.3619331 .214968$

O $-4.493941-1.449852-2.543103$

C $-4.970553-1.613108-0.199746$

C $-5.053870-3.640061-1.803498$

O $-6.390300-1.554162-0.308171$

C $-4.572184-2.2396891 .151826$

C $-5.011284-1.3938532 .371812$

O $-5.203874-3.5195101 .280334$

C -0.1286151 .0188102 .050393$

O 0.0168201 .9150802 .867019

C $-0.828554 \quad 1.1589310 .754373$

$\begin{array}{llll}\text { C } & -1.089548 & 2.387970 & 0.283729\end{array}$

C $-1.7707262 .708378-0.971677$

C $-2.5089791 .597519-1.667951$

C $-4.444647 \quad 0.0297382 .423834$

C $-5.388768 \quad 1.0893361 .879789$

C $-5.487892 \quad 3.5312391 .394142$

$\begin{array}{lllll}\text { O } & -6.554880 & 0.861354 & 1.570041\end{array}$

C $-5.3057913 .725901-0.077614$

C $-4.8725804 .873902-0.619873$

C $-4.7305375 .100509-2.097362$

C -3.346892 $5.571512-2.484045$

C -3.093344 $6.874453-2.693460$

C $-2.282798 \quad 4.522971-2.713381$

C $-1.7196103 .976765-1.429346$

N -4.7815652 .3368001 .817312$

H 2.4090571 .2955664 .715865

H $3.056934-0.7393962 .517745$

H $0.531692-0.1226134 .227727$

H $1.542687-2.4669664 .389602$

H $1.335473-2.6271142 .656058$

H -2.168497 -3.799765 3.289698

H $-2.071750-2.7170975 .297094$

H $-0.464706-2.3653395 .933088$

H - $-1.366257-1.1214615 .035528$

H $\quad 0.382792-4.2703861 .718534$

H -1.134368 -5.1143901 .625638$

H $-0.616208-2.3089900 .512997$

H $-0.607770-3.810803-0.414233$

H $-3.016300-4.1843200 .237998$

H -2.408856 -3.144499 -1.979433

H -2.348350 -1.566664 -1.223680

H $-4.043615-1.857121-3.302899$

H $-4.614088-0.577507-0.228096$

H $-4.609029-4.060348-2.713446$

H -6.109617 -3.467097 -2.039764

H -4.994470 -4.403549 -1.025023

H $-6.598740-1.130446-1.158446$

H -6.104292 -1.400464 2.461482

H -4.645060 -1.910843 3.270791

H -5.239879 -3.7524662 .222906$

H $-1.0985820 .254900 \quad 0.221251$

H $-0.751352 \quad 3.2487940 .859401$

H $-3.1452601 .058952-0.958555$

H $-1.8076910 .889344-2.117624$
H -3.176702 $1.948201-2.457931$

H -4.2675950 .2922363 .474878$

H -3.4831750 .0943581 .904869$

H $-5.0884014 .368091 \quad 1.975908$

H $-6.553116 \quad 3.4242491 .622657$

H $-5.5689502 .895118-0.728154$

H $-4.6366445 .712606 \quad 0.031615$

H $-5.4758255 .851208-2.391644$

H $-4.981025 \quad 4.197791-2.668120$

H $-2.1106177 .217525-2.999331$

H $-3.8622707 .627871-2.558039$

H $-1.4432094 .957614-3.272736$

H -2.679300 $3.745167-3.372324$

H $-1.1673314 .715830-0.847251$

H -3.7938592 .4223522 .039056$

C 4.6838962 .1930432 .786371

$\begin{array}{lllll}\text { O } & 4.565910 & 1.535912 & 1.506547\end{array}$

$\begin{array}{llll}\text { C } 5.773508 & 1.755815 & 0.759148\end{array}$

C 6.3686843 .0254861 .344972

C 6.0863472 .8092562 .820830

H 6.4462130 .9179450 .980666

C $5.4688711 .786694-0.741022$

H 7.4343303 .1314361 .121835

H $5.8485803 .921466 \quad 0.986993$

C $6.1753384 .078098 \quad 3.650998$

H 6.8078302 .0795743 .212912

C $4.6876850 .550077-1.244745$

O $4.6869502 .948678-1.038418$

H $6.4021831 .901006-1.303729$

H $3.6686490 .586881-0.846526$

O $4.5596870 .650630-2.666659$

C $5.338624-0.797739-0.890163$

C $4.643997-2.050656-1.457671$

H $5.383963-0.9064080 .198689$

O $6.694160-0.801687-1.349649$

H $4.619643-1.992646-2.550562$

H $5.301246-2.900461-1.233748$

H 7.1855334 .4978553 .604995

H 5.4773204 .8420193 .292308

H 5.9408073 .8730604 .700437

C 4.4589601 .1567843 .889379

H $3.8815362 .906974-0.494444$

H $5.4523330 .716707-3.045092$

H $6.679980-0.811053-2.321700$

H $4.768714 \quad 1.5585604 .861121$

H 5.0706180 .2651583 .700691

H 3.9126122 .9729152 .817808

C $1.875820-1.702228-2.772971$

O $2.239889-1.443256-1.411189$

C $3.242229-2.304900-0.862618$

C $2.774655-3.763775-0.954060$

C $2.417978-4.147926-2.385282$

C $1.416272-3.152995-2.966237$

H $2.729166-1.491355-3.427774$

C $0.739516-0.736495-3.129597$

H $3.302721-2.0634750 .206288$

O $3.772904-4.639182-0.441558$

H $1.889173-3.889937-0.319776$

H $3.315475-4.213820-3.010880$

O $1.838105-5.454615-2.393495$

H $0.436189-3.300878-2.496857$

O $1.258330-3.410237-4.360741$

C $1.2710850 .634899-3.466035$

H $0.176902-1.071426-4.008892$

H $0.019473-0.653200-2.307250$

H $3.446107-5.548911-0.549016$

H $1.041341-5.434205-1.836264$

H $\quad 0.992137-4.340801-4.456349$

O $1.204537 \quad 1.448994-2.376018$

O $1.7033520 .940245-4.570370$

C $1.6440002 .793979-2.588998$

H $0.9474813 .311442-3.255115$

H $1.6545483 .305912-1.623244$

H $2.6550082 .811010-3.006098$

SCF Energy (B3LYP/6-31G*//MMFF)= -3245.90301306

0500147

MM̄FF Geometry 
C $-2.884304-3.294267-0.811708$ C $-2.209048-4.246465-1.473758$ C $-0.809952-4.123476-2.036943$ O $-0.326550-2.762719-1.994254$ C $0.162724-4.993982-1.218859$ C $1.590175-4.968796-1.737326$ C $2.654513-4.508200-1.049026$ C $1.787452-5.535425-3.120720$ C $2.688772-3.9592220 .349494$ C $2.793009-2.4328750 .398920$ C $4.090921-1.825467-0.161506$ C $5.322759-2.2572660 .641605$ C $6.598342-1.5264330 .194493$ O $3.931746-0.398947-0.095270$ O $7.618793-1.7686071 .175667$ C $6.320029-0.0093860 .153859$

C $7.150437-2.109308-1.111000$ O $7.4318520 .699796-0.386609$ C $5.0358290 .351809-0.621461$ C $4.6856921 .853864-0.510652$ O $5.2301170 .070592-2.012790$ C $-0.722132-1.929964-2.995703$ O $-1.490719-2.186516-3.908433$ C -0.047309-0.631814 -2.783371 C $-0.4461950 .446062-3.475638$ C $0.1482861 .782907-3.400395$ C $1.3964871 .929027-2.573253$ C 4.4318042 .3620720 .909431 C 3.7880563 .7366320 .895488 C 1.6093974 .8802390 .534546 O 4.4168734 .7499221 .191356 C $0.3915234 .626362-0.295243$ C $-0.0306875 .433564-1.280020$ C -1.255050 5.152087-2.106167 C $-0.9855585 .198700-3.595870$ C $-1.6199706 .087527-4.379180$ C $0.0175204 .227905-4.183582$ C $-0.4303172 .793851-4.082232$ N 2.4568913 .7012090 .508602 H -2.412670 -2.335352 -0.614874 H $-2.695398-5.204783-1.645479$ H - $0.828745-4.467631-3.078496$ H $-0.182366-6.036552-1.217434$ H $0.123145-4.664115-0.176107$ H $3.629651-4.544761-1.533565$ H $2.846629-5.630835-3.383704$ H $1.344475-6.534521-3.188557$ H $1.319820-4.892480-3.872528$ H $1.804584-4.2648750 .914487$ H $3.528755-4.4186960 .882289$ H $1.938931-1.992637-0.132649$ H $2.675548-2.1106091 .442285$ H $4.191995-2.112940-1.213772$ H $5.478145-3.3405530 .581941$ H $5.150927-2.038607 \quad 1.704837$ H $7.300760-1.4396222 .033566$ H $\quad 6.212598 \quad 0.3261111 .192969$ H $7.423257-3.162516-0.972105$ H $8.071512-1.599658-1.414310$ H $6.440313-2.061113-1.939038$ H 8.2170430 .4435100 .126760 H $5.4701712 .460126-0.982270$ H $3.7901392 .037062-1.115334$ H $4.5936420 .594918-2.526398$ H $\quad 0.765224-0.602517-2.066140$ H -1.283663 $0.343953-4.164534$ H $1.8198442 .934245-2.585552$ H $2.1801461 .257771-2.942430$ H $1.1918381 .685187-1.525339$ H 3.7789491 .6825751 .468362 H 5.3711872 .4359501 .468199 H 2.1851605 .7442360 .186335 H $1.3238755 .056117 \quad 1.576722$ H $-0.1804253 .728304-0.073827$ H $0.5195346 .346054-1.496945$ H $-2.0144235 .896203-1.833057$ H -1.687057 4.176120 -1.852224 H -1.436143 $6.133422-5.447509$
H $-2.3390786 .790273-3.971433$

H $0.9937814 .403844-3.725615$

H $0.1681024 .440294-5.250577$

H -1.330215 $2.579132-4.660470$

H 2.0027292 .7930240 .468612

C $-5.229960-2.410843-0.882548$

O $-4.870123-1.106497-0.379950$

C $-6.037527-0.4893610 .189141$

C $-7.219370-1.213569-0.437611$

C $-6.690946-2.634679-0.481159$

H $-6.019124-0.7125751 .263341$

C $-6.0094641 .029053-0.013251$

H -8.133969 -1.112222 0.153875

H -7.424385 -0.856129-1.453308

C $-7.455946-3.540716-1.430652$

H $-6.741576-3.0584920 .531028$

C -4.6971041 .7064630 .434291$

O $-6.1842601 .312435-1.406828$

H -6.8682601 .4774700 .500483$

H $-3.9051121 .478260-0.289492$

$\begin{array}{lllll}\text { O } & -4.891351 & 3.124454 & 0.363175\end{array}$

C -4.2169911 .3272671 .846461$

C -2.9705282 .1272922 .269324$

H -3.9993180 .2549901 .864715$

O

H -2.2777842 .1764261 .422216$

H -3.264058 3.1565272 .509088

H -8.503061 -3.624789-1.121964

H -7.437674 -3.154867 -2.455469

H -7.024445 -4.546509-1.441518

C $-4.285600-3.463141-0.293961$

H -6.169042 2.279513 -1.510147

H -5.5474873 .3641491 .040206$

H -5.0068951 .1944643 .630450$

H -4.658704 -4.468437 -0.521622

H $-4.249631-3.3624460 .797816$

H -5.128494 -2.369224-1.974555

C $-0.994855-0.1750602 .331143$

$\begin{array}{llll}\text { O } & -1.980872 & 0.121854 & 3.324377\end{array}$

C -2.2527651 .5183543 .497367$

C -0.9628562 .2434143 .907843$

C 0.1762751 .9912052 .920844

C 0.3473210 .4959492 .653320

H $-1.353808 \quad 0.187778 \quad 1.359881$

C $-0.899342-1.6998092 .178948$

H -2.9484361 .5934544 .341980$

O -1.1871863 .6420144 .054028$

H -0.6512961 .8748094 .893524$

H 0.0152632 .5349221 .985630

O 1.3960382 .4917253 .471673

H $0.813206 \quad 0.038414 \quad 3.532817$

$\begin{array}{llll}\text { O } & 1.252308 & 0.315337 & 1.566976\end{array}$

C $-0.383446-2.4233543 .404972$

H -1.895059 -2.110011 1.974165

H $-0.245774-1.9345991 .331175$

H -1.2791394 .0304773 .167786$

H 1.2601143 .4343653 .668921

H $0.848246 \quad 0.7010580 .771161$

O $0.140245-3.6253813 .023211$

O $-0.459444-2.0090454 .553358$

C $0.669737-4.4317874 .079738$

H $1.479051-3.9024804 .591714$

H $-0.123296-4.7012534 .783530$

H $1.073879-5.3468193 .638500$

SCF Energy (B3LYP/6-31G**//MMFF) $=-3245.90680464$

0500148

MM̄FF Geometry

C $0.8884974 .194876-1.637669$

C $0.6201633 .301136-2.602271$

C $-0.5646782 .359975-2.614266$

O $-1.0388482 .098259-1.272016$

C $-1.7202642 .971108-3.426459$

C $-2.8517091 .998438-3.697042$

C $-4.1027632 .116459-3.211089$

C $-2.5139910 .843437-4.605048$

C $-4.6601723 .189477-2.319723$

C $-4.5746852 .892237-0.814163$ 
C $-5.2768651 .594818-0.377906$

C -5.3577041 .5129341 .148263$

C $-5.952116 \quad 0.179553 \quad 1.625181$

$\begin{array}{lllll}\text { O } & -4.520968 & 0.486991 & -0.872974\end{array}$

$\begin{array}{lllll}\text { O } & -5.691570 & 0.086874 & 3.034506\end{array}$

C $-5.215085-0.9862890 .927701$

C -7.4776590 .1585951 .469592$

O $-5.859747-2.217318 \quad 1.245100$

C $-5.091830-0.800403-0.599982$

C $-4.180843-1.849538-1.283778$

O $-6.390166-0.924097-1.192985$

C $-0.2796801 .265650-0.507924$

O $0.7381230 .684003-0.847607$

C $-0.879727 \quad 1.200696 \quad 0.840460$

C -0.1400490 .7185881 .849648$

C $-0.567870 \quad 0.544695 \quad 3.237532$

C -2.028392 0.7419023 .542997

C $-2.730728-1.880707-0.790605$

C $-2.457510-2.9233930 .279542$

C $-0.647892-3.7155241 .800023$

O $-3.272985-3.7798050 .610144$

C $-0.960714-3.1979513 .169319$

C $-0.047554-3.1037694 .148213$

C $-0.356833-2.6287575 .540340$

C $0.552590-1.5063085 .990373$

C $1.630247-1.7542146 .753914$

C $0.168642-0.0907015 .617922$

C 0.3574130 .1844114 .151128

N -1.175199 -2.8047980 .800589$

H $\quad 0.1821334 .315637-0.821664$

H $1.3076553 .204011-3.439535$

H $-0.2437731 .412633-3.063081$

H $-1.3489003 .331245-4.395300$

H -2.091595 $3.855339-2.896960$

H -4.828609 $1.350786-3.484140$

H $-3.398127 \quad 0.260237-4.884482$

H -2.057090 $1.206606-5.531179$

H -1.815713 $0.161181-4.112869$

H $-4.1761904 .151342-2.520912$

H $-5.7129243 .338426-2.593020$

H -3.518507 2.853389-0.523999

H $-5.0225243 .741318-0.283004$

H $-6.2815891 .578935-0.816256$

H -5.9320112 .3552191 .553943$

H -4.3484111 .6123291 .569820$

H $-6.001536-0.7851553 .334219$

H -4.213349 -1.0309401 .371865$

H -7.9318140 .9593892 .065783$

H $-7.903528-0.7756841 .851818$

H $\quad-7.8052600 .2887240 .436051$

H $-5.299604-2.9407360 .918124$

H -4.645729 -2.841776 -1.245859

H -4.141824 -1.592848 -2.352800

H $-6.280177-1.134099-2.135221$

H $-1.898924 \quad 1.5445800 .963834$

H $\quad 0.8857490 .417483 \quad 1.641773$

H -2.641328 0.0953382 .906201

H -2.3202561 .7836503 .371307$

H $-2.298737 \quad 0.5011184 .572710$

H -2.085574 -2.139787 -1.638136

H $-2.417225-0.897553-0.430973$

H $0.429295-3.8021691 .625294$

H -1.106168 -4.700688 1.665781

H -1.990343 -2.9144313 .374587$

H $0.975519-3.4156423 .949803$

H $-0.249815-3.4875946 .216024$

H -1.402350 -2.309013 5.632567

H $2.273763-0.9531257 .101983$

H $1.895662-2.7655037 .043868$

H $0.7920320 .628276 \quad 6.165900$

H -0.8518220 .0961895 .961660$

H $\quad \begin{array}{llll}1.391327 & 0.070987 & 3.821497\end{array}$

H $-0.582815-2.036790 \quad 0.498098$

C $2.9796714 .881626-0.359421$

O $3.4956143 .536670-0.282020$

C 3.5322333 .1436031 .104891

C 3.2285724 .4004111 .918957

C 2.3044595 .1534030 .984757
H $2.7137732 .429210 \quad 1.248983$

C 4.8721822 .4908241 .462081

H 2.7677884 .1653692 .883397

H 4.1327144 .9886502 .114856

C 2.1627616 .6275081 .320127

H 1.3179394 .6791121 .037880

C $5.230753 \quad 1.237528 \quad 0.639530$

O $5.909123 \quad 3.4599421 .258737$

H 4.8790472 .2502642 .532460

H $5.4825251 .513537-0.391789$

O $6.4215800 .677095 \quad 1.210428$

C 4.1469340 .1418190 .620494

C $4.661776-1.1949340 .051605$

H 3.2977880 .4938350 .027306

O $3.670086-0.0879181 .947313$

H $5.200407-0.999812-0.880756$

H $5.379613-1.6258660 .759489$

H 1.7484096 .7550092 .325543

H $3.128957 \quad 7.141947 \quad 1.287534$

H 1.4915047 .1247640 .612962

C $2.1137615 .064101-1.611563$

H 6.7125003 .1257351 .691726

H 7.1554901 .2818511 .009354

H $4.430509-0.3544872 .492147$

H $2.7313674 .851900-2.493380$

H $1.7964016 .110521-1.687214$

H $3.8581855 .532614-0.465957$

C $3.095148-1.787974-2.519213$

O $2.598201-1.779100-1.176875$

C $3.526378-2.219741-0.179117$

C $4.017423-3.637324-0.507303$

C $4.614342-3.716271-1.908729$

C $3.622789-3.168885-2.932748$

H $3.907830-1.055265-2.595857$

C $1.990810-1.272436-3.456530$

H $2.958489-2.2882250 .757302$

O $4.986125-4.075848 \quad 0.439243$

H $3.173253-4.336938-0.449828$

H $5.575462-3.192429-1.969658$

O $4.879760-5.083237-2.231880$

H $2.799798-3.884246-3.044995$

O $4.264220-3.062881-4.202148$

C $0.755500-2.149263-3.496787$

H $1.663402-0.278547-3.135038$

H $2.394011-1.186206-4.472231$

H $4.576327-4.0269461 .319939$

H $5.474907-5.431351-1.545759$

H $4.614528-3.942548-4.424458$

O $0.117757-1.970568-4.691030$

O $0.380823-2.878856-2.589032$

C $-1.068905-2.748557-4.878609$

H $-0.835080-3.815995-4.824233$

H $-1.466878-2.526422-5.872159$

$\mathrm{H}-1.821530-2.479158-4.131885$

SCF Energy (B3LYP/6-31G**//MMFF)= -3245.92062074

0500149

MM̄MF Geometry

C $3.124892-3.2821910 .635844$

C $2.572662-4.1682581 .479147$

C $1.334903-3.9296022 .318074$

O $0.874352-2.5738162 .121525$

C $0.220557-4.9041601 .900308$

C -1.035561-4.800099 2.743402

C $-2.244869-4.4226862 .282125$

C $-0.887789-5.2341504 .180424$

C $-2.618718-4.0162170 .882539$

C $-3.569231-2.8137910 .869430$

C $-4.208051-2.567564-0.507451$

C $-3.181802-2.174204-1.572586$

C $-3.847868-1.824700-2.912948$

O $-5.170530-1.517874-0.338001$

O $-2.870434-1.174614-3.739534$

C $-4.972266-0.800571-2.656264$

C $-4.288547-3.078779-3.677011$

O $-5.714872-0.545923-3.845993$

C $-5.928534-1.210715-1.515654$

C $-6.952286-0.101009-1.165407$ 
O - $-6.692010-2.350324-1.935399$

C $0.819015-1.7515813 .202149$

O $0.955398-2.0596544 .375018$

C $0.614827-0.3739022 .699083$

C $0.4299520 .627196 \quad 3.572569$

C $0.271514 \quad 2.0459693 .243621$

C 0.5478862 .4610491 .822684

C $-6.3956121 .266873-0.766284$

C $-5.664391 \quad 1.2349870 .559844$

C -3.3824991 .3381511 .552022$

O $-6.265565 \quad 1.1022331 .623867$

C -3.0301902 .7508341 .891916$

C -3.2008253 .2945743 .105909$

C $-2.799895 \quad 4.7017523 .444299$

C $-1.742493 \quad 4.764356 \quad 4.524927$

C -2.0373495 .2144965 .756171$

C $-0.324103 \quad 4.3822664 .153025$

C -0.0924112 .8966204 .226566$

$\begin{array}{llll}\mathrm{N} & -4.290996 & 1.342796 & 0.422999\end{array}$

H $2.668357-2.3046020 .508719$

H $3.039473-5.1447911 .590644$

H $1.617861-4.1019593 .363111$

H $0.589050-5.9366681 .972978$

H $-0.007809-4.752663 \quad 0.840465$

H $-3.079359-4.4405282 .982664$

H $-1.847432-5.2614324 .707911$

H $-0.460358-6.2410444 .230351$

H $-0.233723-4.5507874 .728819$

H $-1.736421-3.7726730 .286236$

H $-3.112262-4.8725160 .407462$

H $-4.377458-2.9637441 .598117$

H $-3.040540-1.9117011 .204247$

H -4.736977 -3.481453 -0.802640

H $-2.440707-2.967216-1.723936$

H $-2.618929-1.297668-1.223615$

H $-2.110971-1.774934-3.834285$

H $-4.4798410 .142212-2.391060$

H -3.423247 -3.715602 -3.897650

H $-4.722661-2.817282-4.648353$

H -5.010603 -3.686087 -3.127811

H $-5.078726-0.290129-4.535804$

H $-7.6335680 .037003-2.015355$

H $-7.582445-0.464018-0.341171$

H $-7.490935-2.404613-1.385145$

H $0.649112-0.2189161 .626464$

H $\quad 0.3882790 .3891654 .634830$

H $\quad 0.4338083 .532383 \quad 1.647753$

H $-0.133893 \quad 1.9560581 .131717$

H 1.5762912 .2041121 .544939

H -5.767568 $1.691090-1.555230$

H -7.228929 $1.970859-0.644989$

H $-2.484557 \quad 0.7931581 .245141$

H $-3.839824 \quad 0.8094742 .394472$

H -2.577660 3.3491351 .104711

H -3.646729 2.7008443 .900323

H -3.703535 $5.238767 \quad 3.760519$

H -2.433982 5.2354932 .558006

H $-1.2777575 .286676 \quad 6.527556$

H -3.045112 5.5156316 .022139

H 0.3829154 .8572364 .846417

H -0.0865084 .8065173 .174032$

H -0.2493202 .4886635 .226509$

H -3.890456 $1.492416-0.498099$

C $5.469525-2.5572800 .145736$

O $5.052108-1.208907-0.149742$

C $6.150128-0.499348-0.749636$

C $7.370059-1.405418-0.614504$

C $6.738130-2.781429-0.675300$

H $5.908673-0.391393-1.813553$

C $6.3140720 .882296-0.108381$

H $8.102813-1.232878-1.408730$

H $7.879092-1.2642950 .345915$

C $7.635382-3.885500-0.143871$

H $6.476495-3.000549-1.719233$

C $5.0499391 .766409-0.137535$

O 6.6811910 .7048791 .266742

H $7.1537911 .403529-0.583943$

H 4.3015721 .3690810 .558446 $\begin{array}{llll}\text { O } & 5.422506 & 3.049460 & 0.386378\end{array}$

C $4.4071131 .951412-1.526115$

C $3.3209733 .045815-1.520008$

H $3.9840660 .996087-1.852511$

O $5.4293572 .316802-2.453543$

H $2.6523772 .874428-0.670704$

H $3.8037624 .018120-1.365577$

H $8.558635-3.945813-0.729527$

H $7.911202-3.7103290 .901453$

H $7.135579-4.857375-0.201893$

C $4.347955-3.539316-0.196685$

H $6.786916 \quad 1.590451 \quad 1.654983$

H $6.0476433 .451713-0.241107$

H $5.0765552 .187070-3.349590$

H $4.695359-4.569901-0.063402$

H $4.060018-3.422757-1.248681$

H $5.692141-2.5937261 .220416$

C $0.6986881 .617485-2.239322$

O $1.812108 \quad 1.888005-3.100405$

C $2.5076953 .111082-2.832592$

C $1.5395764 .301358-2.915192$

C $0.3228984 .107455-2.017307$

C $-0.3277572 .757028-2.302014$

H $1.0508331 .484949-1.209719$

C $0.0586490 .301160-2.704756$

H $3.2263873 .238147-3.650645$

O $2.1891295 .518759-2.565380$

H $1.1899444 .411521-3.950192$

H $0.5826814 .205323-0.956944$

O $-0.635034 \quad 5.131600-2.293697$

H $-0.8017922 .782519-3.291865$

O $-1.3568402 .520294-1.344564$

C $0.880203-0.916891-2.344249$

H $-0.9185740 .173629-2.227705$

H $-0.0643020 .308412-3.794115$

H $2.9485865 .626868-3.163120$

H $-0.1935465 .986165-2.148949$

H - $-1.9761763 .268616-1.393419$

O $0.203541-2.032291-2.751199$

O $1.963732-0.900068-1.778615$

C $0.829740-3.284421-2.457301$

H $1.862335-3.297844-2.819021$

H $0.791161-3.471215-1.381073$

H $0.269977-4.071530-2.969365$

SCF Energy (B3LYP/6-31G**//MMFF) = -3245.92194419

0500150

MM̄FF Geometry

C $1.292434-2.277042-2.205292$

C $0.295433-3.164723-2.327572$

C $-0.789026-3.307035-1.294073$

O $-2.056390-2.916142-1.879411$

C $-0.969439-4.759047-0.817608$

C $0.178147-5.326352-0.011898$

C $0.245091-5.2908151 .333269$

C $1.241534-6.050338-0.797203$

C $-0.724354-4.6351712 .280919$

C $-0.017266-4.033717 \quad 3.507309$

C $0.817575-2.7868153 .178391$

C $1.734703-2.4307134 .350128$

C $2.518920-1.1375374 .085847$

O $-0.090505-1.7081112 .929715$

O $3.107104-0.7173935 .326312$

C $1.528075-0.0365473 .658917$

C $3.679186-1.3582333 .108835$

$\begin{array}{lllll} & & 2.217287 & 1.144500 & 3.255160\end{array}$

C $0.542168-0.4794132 .552017$

$\begin{array}{llll}\text { C } & -0.550627 & 0.603196 & 2.365721\end{array}$

O $1.252536-0.6201151 .318012$

C $-2.325158-1.585768-1.970427$

O $-1.565188-0.660279-1.737892$

C $-3.750228-1.448360-2.338755$

C $-4.376759-0.290033-2.083625$

C $-5.806914-0.028670-2.255799$

C $-6.628073-1.086629-2.945480$

C $-1.769156 \quad 0.131982 \quad 1.559888$

C -2.916134 -0.3788682 .419565$

C $-5.274228-1.1459502 .236107$ 
O $-2.845315-0.514363 \quad 3.637608$ C $-6.398546-0.9195121 .276338$ C -7.2893220 .0769641 .396830$ C $-8.434724 \quad 0.309717 \quad 0.450471$ C $-8.3065431 .613935-0.308868$ C -8.7369022 .7695190 .225188$ C $-7.7498571 .563477-1.716981$ C $-6.3146611 .114919-1.749217$ N $-4.041355-0.6527731 .652640$ H $1.299826-1.617501-1.341656$ H $\quad 0.254059-3.814384-3.197588$ H $-0.598450-2.671633-0.419117$ H -1.172007 -5.411960 -1.678498 H -1.897124 -4.820921 -0.232002 H $1.084390-5.7935151 .812273$ H $1.973281-6.543299-0.148190$ H $0.789876-6.825581-1.424920$ H $1.792510-5.358703-1.439972$ H -1.319913 -3.861894 1.784996 H -1.419166 -5.4066082 .634290$ H $-0.788676-3.7667864 .241366$ H $0.616602-4.8011203 .969466$ H $1.413660-2.9751392 .278759$ H $2.423103-3.2558434 .571250$ H $1.130705-2.2922065 .258006$ H $3.683443-1.4343575 .642447$ H $\quad 0.944007 \quad 0.2356554 .549429$ H $4.362399-2.1254533 .492406$ H $4.281603-0.4496493 .003810$ H $3.352490-1.6742102 .116024$ H $2.819871 \quad 1.390743 \quad 3.977664$ H -0.8849360 .9728833 .342390$ H -0.1041661 .4564331 .840507$ H $\quad 0.650022-0.4254220 .582393$ H -4.258710 -2.321616 -2.730814 H $-3.8021750 .527573-1.649091$ H -7.668078 $-0.798667-3.108642$ H -6.642903 -2.008644 -2.353865 H $-6.212409-1.310470-3.934588$ H -2.155736 0.9822260 .985090 H - $-1.494860-0.6545310 .852056$ H -5.441620 -0.6454203 .196205$ H -5.152590 -2.215793 2.434164 H $-6.486070-1.6188760 .448944$ H $-7.198422 \quad 0.767472 \quad 2.232570$ H $-9.362703 \quad 0.314551 \quad 1.037357$ H -8.544271 $-0.522624-0.255813$ H $-8.6734273 .704674-0.320931$ H -9.1603412 .8046151 .223642$ H -7.793056 $2.556383-2.183280$ H $-8.4056030 .933359-2.323279$ H -5.632992 $1.814694-1.263274$ H $-3.941464-0.6799460 .642430$

C $3.763403-2.027745-2.487536$

O $3.806531-0.844564-1.662453$

C $5.054147-0.164808-1.879086$

C $5.950899-1.163396-2.599995$

C $4.940970-1.904882-3.454404$

H $4.843750 \quad 0.671891-2.556153$

C $5.6096070 .367458-0.555410$

H $6.732965-0.671934-3.186419$

H $\quad 6.435215-1.855267-1.901291$

C $5.448690-3.236298-3.980050$

H $4.664789-1.266059-4.304176$

C $4.628674 \quad 1.2554190 .238406$

O $5.948696-0.749151 \quad 0.277819$

H $6.5465920 .905100-0.743912$

H 3.8142380 .6401500 .636774

O $\quad 5.3375581 .7521991 .382296$

C $4.0332992 .441639-0.544595$

C 3.2120613 .3755140 .365758

H $3.4035772 .057835-1.352260$

O $5.104478 \quad 3.175812-1.136114$

H 2.4888432 .7711630 .923002

H $3.8858483 .836607 \quad 1.097535$

H $6.327558-3.088658-4.616132$

H $5.733895-3.907728-3.163164$

H $4.680647-3.738756-4.576352$
C $2.408573-2.103241-3.194498$

H $6.289637-0.392277 \quad 1.115883$

H 6.0294942 .3544371 .058754

H $4.7241873 .758851-1.814373$

H $2.407848-2.922161-3.922460$

H $2.228966-1.169605-3.739734$

H $3.896575-2.887992-1.818488$

C $0.3996393 .367117-0.878039$

O $1.5802144 .003863-1.387398$

C $2.4838714 .505577-0.397149$

C 1.7639935 .5118510 .510866

C 0.4994224 .9177621 .120049

C -0.3881394 .3293880 .025198$

H $0.6793732 .466354-0.319246$

C $-0.4459802 .940651-2.089221$

H $3.2504285 .070570-0.940734$

O $2.6392555 .963307 \quad 1.538845$

H $1.4893636 .392726-0.084131$

H $0.740944 \quad 4.1728111 .887387$

O -0.2113935 .9645691 .785615$

H $-0.8128075 .152184-0.564757$

O -1.4697753 .6525030 .660313$

C $0.1991921 .836282-2.898421$

H -1.422552 $2.564071-1.768501$

H $-0.5874953 .800329-2.755314$

H 2.1347426 .5742892 .102865

H -1.031548 5.5775952 .137661

H $-2.1547063 .488204-0.008926$

O $-0.5289981 .646936-4.039246$

O $1.1939871 .206464-2.569826$

C $-0.0575160 .616415-4.912700$

H $-0.6456980 .656311-5.833317$

H $\quad 0.9956640 .774811-5.164125$

H $-0.202431-0.361965-4.446315$

SCF Energy $\left(B 3 L Y P / 6-31 G^{* *} / / M M F F\right)=-3245.92139507$

0500151

MM̄FF Geometry

C - $0.6692713 .968011-2.428833$

C $0.6575373 .917594-2.616769$

C $1.6562153 .890388-1.492261$

O $2.6655162 .921762-1.882454$

C $2.3451765 .246338-1.263834$

C $1.5156416 .231305-0.464613$

C 1.5110376 .2910150 .882557

C $0.7303277 .233551-1.268490$

C 2.2735575 .4024811 .825256

C 1.4913405 .0112433 .085870

C 0.2296134 .1637772 .844282

C -0.4498693 .8787494 .190524$

C -1.6567072 .9419144 .052552$

O $\quad 0.6017592 .9358632 .203451$

O $-2.035727 \quad 2.5029375 .366444$

C -1.2317121 .7039393 .235833$

C -2.8836543 .6803563 .507348$

O $-2.353808 \quad 0.8690812 .962818$

C -0.5044732 .0621861 .920746$

C 0.0292460 .8088161 .185973

O -1.4433992 .6866651 .035180$

C $3.2854032 .216120-0.900683$

O 3.0855102 .2941010 .300097

C $4.2764601 .321718-1.538006$

C $5.0349600 .535193-0.759925$

C $6.074477-0.390823-1.208690$

C $6.315750-0.517406-2.689431$

C 1.1026380 .0058211 .924940

C $1.523894-1.2236991 .133680$

C $2.944125-3.2539791 .337639$

O $0.998573-1.5490850 .072018$

C $4.429236-3.3948821 .431025$

C $5.169249-3.9351220 .450198$

C $6.656909-4.1347110 .523952$

C $7.382777-3.502406-0.643298$

C $7.646131-4.210176-1.754830$

C $7.868200-2.078381-0.496827$

C $6.755301-1.092183-0.278683$

N $2.541333-1.9266361 .767467$

H -1.059555 $4.066145-1.419933$ 
H $1.0479373 .843931-3.629209$

H $1.1660793 .544025-0.574117$

H $2.6331375 .692627-2.225742$

H $3.3064785 .089473-0.756769$

H $\quad 0.921954 \quad 7.071791 \quad 1.361174$

H $\quad 0.1967377 .948648-0.633194$

H $1.4010667 .807679-1.916187$

H $-0.0140606 .736467-1.895218$

H 2.6244624 .4960451 .331079

H 3.1687555 .9510092 .143980

H 2.1797904 .4454643 .727550

H 1.2192435 .9211453 .636113

H -0.4492404 .7188542 .187147$

H $-0.746608 \quad 4.8137104 .682542$

H $\quad 0.2780703 .4070624 .865858$

H -1.273653 2.0529565 .769336

H -0.5547231 .1179623 .868959$

H -3.1863204 .4766084 .198409$

H -3.7467023 .0101583 .427831$

H -2.7128284 .1407022 .532193$

H -2.7842870 .6733823 .812610$

H -0.8136580 .1555230 .922073$

H $\quad 0.454278 \quad 1.1238760 .222704$

H -1.1745252 .5068720 .119953$

H $4.362668 \quad 1.342891-2.618164$

H 4.8881290 .5756430 .318979

H $6.6552920 .436913-3.106691$

H $5.397201-0.819948-3.204712$

H $7.072451-1.264082-2.941731$

H $1.986706 \quad 0.6254822 .107284$

H $0.724314-0.3461332 .889560$

H $2.575837-3.4469810 .325627$

H $2.465891-3.9653692 .018511$

H $4.912961-3.0686432 .348284$

H $4.673371-4.285657-0.452803$

H 6.849188 -5.215662 0.537287

H $7.068529-3.7512451 .465723$

H $8.187876-3.775442-2.588214$

H $7.321423-5.240497-1.857037$

H $\quad 8.482915-1.776346-1.352978$

H $8.548138-2.0261680 .363604$

H $6.504858-0.9458440 .772636$

H $2.827319-1.6278622 .695586$

C -2.679827 $2.749010-3.248772$

O $-1.9913471 .489532-3.143851$

C $-2.8487990 .444516-3.651927$

C $-4.1583911 .118867-4.045925$

C $-3.7137762 .537003-4.349056$

H -2.338503 $0.045381-4.536575$

C -2.992777 -0.635995 -2.571902

H $-4.6396930 .628031-4.897348$

H -4.868628 $1.113394-3.209503$

C $-4.8497373 .544050-4.340243$

H -3.225153 $2.548246-5.332501$

C $-3.965940-1.769840-2.938836$

O $-1.696315-1.187892-2.319338$

H -3.305184 -0.154932-1.638691

H -4.976964 -1.367573 -3.070922

O $-3.570593-2.300686-4.209930$

C -3.999861 -2.933889-1.928091

C $-4.362614-2.495094-0.500100$

H -3.040416 -3.458996-1.939227

O $-4.973810-3.882261-2.380466$

H -3.649576 -1.740841 -0.157568

H -5.351364 -2.020347 -0.520080

H $-5.5972193 .287215-5.098089$

H -5.353064 $3.573139-3.367989$

H -4.480934 4.551108 -4.558757

C $-1.6782693 .868378-3.537798$

H -1.413233 -1.654520 -3.124368

H $-4.183612-3.024453-4.425151$

H -5.843157 -3.446650 -2.365085

H $-2.2002674 .827347-3.626099$

H -1.170828 $3.675825-4.491012$

H $-3.1747312 .925579-2.283757$

C $-2.061914-3.6479451 .145711$

O $-3.148653-4.3730140 .557089$

C $-4.394072-3.6689710 .504803$
C $-4.824739-3.267540 \quad 1.921217$

C $-3.740437-2.4649792 .634134$

C $-2.405617-3.2068192 .576470$

H -1.825042 -2.771189 0.530943

C $-0.840301-4.5814911 .153357$

H $-5.137514-4.3868070 .136519$

O $-6.035058-2.5193131 .871297$

H -5.032761 -4.176226 2.501217

H -3.659624 -1.456653 2.213056

O $-4.133604-2.3035693 .998678$

H -2.446372 -4.080914 3.239223

O $-1.369143-2.3526043 .056140$

C $-0.247135-4.788112-0.224308$

H $-0.046356-4.1656701 .782285$

H -1.131764 -5.565265 1.540360

H $-6.257347-2.2651942 .783417$

H -3.496761 -1.701179 4.418375

H -1.547039 -2.169124 3.993631

O $0.752150-5.716688-0.137182$

O $-0.594144-4.205968-1.241876$

C $1.436213-6.000698-1.361700$

H $\quad 0.742079-6.427559-2.091783$

H $1.902662-5.092644-1.755852$

H $2.219800-6.733335-1.151174$

SCF Energy (B3LYP/6-31G $\left.{ }^{* *} / / M M F F\right)=-3245.91049366$

05 00152

MM̄FF Geometry

C $4.240384-2.4487092 .149654$

C $2.934525-2.6874011 .958406$

C $1.930828-2.6614463 .080614$

O $1.022805-1.5621352 .833412$

C $1.101386-3.9569443 .120781$

C $0.174267-4.0179774 .321471$

C $-1.171564-4.0097344 .256980$

C $0.851520-4.1133505 .666241$

C $-2.047565-3.9973743 .036335$

C $-2.412226-2.5804012 .591053$

C $-3.662567-2.5185111 .699321$

C $-3.535589-3.3646480 .431770$

C $-4.767523-3.222787-0.474940$

O $-3.853311-1.1421381 .344557$

O $-4.413001-3.795313-1.744048$

C $-5.059252-1.722085-0.693936$

C $-5.947746-4.0458360 .054558$

O $-6.289469-1.525469-1.384460$

C $-5.057757-0.883807 \quad 0.606344$

C $-5.118240 \quad 0.637245 \quad 0.336423$

O $-6.215041-1.1530101 .407593$

C $1.404459-0.3259873 .256020$

O $2.438632-0.0292813 .832065$

C 0.3265520 .6180552 .888686

C 0.4366841 .9160263 .209253

C -0.5537742 .9579622 .926103$

C -1.8032242 .5376962 .199343$

C $-4.0185641 .173850-0.580758$

C $-3.9643922 .692024-0.613814$

C $-2.5922134 .539666-1.598116$

O $-4.7484713 .411304-0.002157$

C -1.410539 $4.863867-0.739713$

C -1.3397675 .9365800 .062888$

C $-0.139812 \quad 6.2545620 .910418$

C -0.4931796 .5335192 .356351$

C -0.2151997 .7253192 .911696$

C -1.1677665 .4435073 .163084$

C -0.3005124 .2217643 .326881$

N $-2.9263213 .138810-1.419110$

H $4.607278-2.2713163 .158016$

H $2.555956-2.8354320 .950376$

H $2.434616-2.5128744 .043358$

H $1.766967-4.8292723 .155576$

H $0.530526-4.0425432 .189464$

H -1.726972 -4.038104 5.194084

H $\quad 0.143022-4.3303076 .473001$

H $1.592945-4.9191375 .661700$

H $1.354573-3.1747655 .916465$

H -1.579301 -4.547988 2.213925

H $-2.959586-4.5552413 .284786$ 
H $-2.583663-1.9357293 .463939$ H -1.563697 -2.129712 2.061219 H -4.517571 -2.850186 2.300016 H -3.372346 -4.4223570 .669417$ H -2.647097 -3.042288 -0.127828 H $-5.198775-3.772530-2.315903$ H $-4.265522-1.351090-1.352397$ H $\quad-5.697963-5.1137100 .052614$ H -6.826026 -3.942336 -0.591997 H $-6.235388-3.7774281 .073332$ H $-6.221375-1.973063-2.244354$ H $-6.0998930 .906875-0.075524$ H $-5.056610 \quad 1.1563441 .303366$ H $-6.800821-1.7523470 .918576$ H -0.5350120 .2153772 .368063$ H 1.3310542 .2502493 .733423 H -2.352103 1.7842002 .775322 H -1.5456182 .1150811 .222470$ H -2.501183 3.3548862 .013706 H -3.039983 $0.805098-0.253713$ H -4.191553 $0.835434-1.608110$ H -3.460745 $5.162744-1.362372$ H $-2.3325344 .676549-2.652206$ H - $0.5625224 .185063-0.790014$ H $-2.173957 \quad 6.6332730 .097304$ H $0.354395 \quad 7.1277910 .465249$ H 0.5959735 .4420330 .873150 H -0.4626827 .9397543 .946101$ H 0.2668948 .5155242 .345668 H -2.138973 5.2233432 .713816 H -1.399347 5.8083774 .172679 H 0.6336434 .4190313 .854820 H $-2.3078662 .454989-1.846148$ C $5.589083-0.8433130 .830849$ O $4.473670-0.1817940 .195952$ C $4.983198 \quad 0.770712-0.757170$ C $6.4898040 .830601-0.532787$ C $6.779368-0.592645-0.089999$ H $4.8149410 .341709-1.751145$ C $4.2346362 .103304-0.624416$ H $7.0323081 .117975-1.438535$ H $\quad 6.759747 \quad 1.5367820 .259754$ C $8.131367-0.7544810 .581898$ H $6.726173-1.255469-0.964000$ C $2.7191951 .996352-0.908260$ O 4.3681422 .5934090 .715747 H $4.6801022 .860158-1.280789$ H $2.2353491 .382460-0.138560$ O $2.1559383 .308669-0.790917$ C $2.3673351 .443361-2.302368$ C $0.8516831 .435711-2.582271$ H $2.744024 \quad 0.421424-2.392817$ O $2.9986802 .228303-3.314239$ H $0.3412130 .914225-1.765476$ H $\quad 0.476467 \quad 2.465907-2.597148$ H $8.937683-0.484120-0.107715$ H $8.218785-0.1162631 .467617$ H $8.290281-1.7905820 .896468$ C $5.249202-2.3189321 .043647$ H 5.2774372 .9170390 .825586 H 2.3636373 .6330760 .102261 H $3.9539342 .055809-3.272497$ H $6.141208-2.8886571 .325851$ H $4.857716-2.7566090 .118468$ H $5.748292-0.3426511 .795915$ C $0.502685-1.530058-3.237161$ O $1.093712-0.526251-4.070209$ C $0.5151250 .776608-3.942089$ C $-0.978650 \quad 0.718861-4.282231$ C $-1.714904-0.354533-3.483232$ C $-0.982679-1.694369-3.576330$ H $0.611359-1.250409-2.182297$ C $1.278683-2.833401-3.475666$ H $0.9901921 .395193-4.714068$ O $-1.5791631 .990981-4.069032$ H -1.085163 $0.491532-5.351249$ H -1.847237 -0.045641 -2.440231 O $-3.025098-0.489290-4.038585$
H $-1.098406-2.108576-4.585947$

O $-1.563635-2.616950-2.659212$

C $2.667593-2.762757-2.877982$

H $0.778556-3.694006-3.019793$

H $1.367996-3.010984-4.554303$

H $-2.5245841 .906243-4.281047$

H $-3.509766-1.137158-3.501015$

H $-2.471183-2.799667-2.955117$

O $3.550855-3.422284-3.681669$

O $2.937168-2.217545-1.816021$

C $4.906918-3.430313-3.223482$

H $5.517769-3.933277-3.977931$

H $5.274508-2.406911-3.100044$

H $4.982769-3.984260-2.283149$

SCF Energy (B3LYP/6-31G**//MMFF)= -3245.91654676

05_00153

MM̄FF Geometry

C $-2.6119882 .215603-2.956063$

C $-2.1758611 .140357-2.281423$

C $-0.9847210 .315370-2.701135$

$\begin{array}{llll}\text { O } & 0.002346 & 0.376687 & -1.645257\end{array}$

C $-1.405844-1.155366-2.873326$

C $-0.284636-2.058080-3.350901$

C $0.229446-3.082193-2.641530$

C $0.243588-1.770218-4.733062$

C $-0.175162-3.559040-1.275031$

C $0.614050-2.933593-0.115047$

C $2.114104-3.267528-0.123939$

C $2.774951-2.8118571 .178341$

C $4.289458-3.0719361 .169722$

O $2.725180-2.588787-1.222092$

O $4.871086-2.3300082 .253862$

C $4.897377-2.507046-0.135148$

C $4.606047-4.5476251 .440704$

O $6.261781-2.893480-0.272407$

C $4.114592-2.892193-1.409894$

C $4.576560-2.128620-2.672173$

O $4.299005-4.275163-1.738560$

C $0.8111031 .470225-1.608924$

O $0.8165842 .410569-2.386962$

C $1.681247 \quad 1.341425-0.420057$

C 2.2943802 .4302550 .066839

C $3.1460692 .480841 \quad 1.256529$

C 3.5444821 .1710951 .882678

C $4.458455-0.602331-2.603317$

C $5.739130 \quad 0.097068-2.177745$

C $6.6140042 .356429-1.595119$

O $6.819208-0.477421-2.072237$

C $6.6685082 .484030-0.105568$

C 6.6814793 .6650190 .531029

C 6.7795973 .8126712 .022328

C 5.6723174 .6676702 .596487

C 5.8724885 .9691332 .864672

C $4.363306 \quad 3.9926452 .938063$

C 3.5339183 .6869631 .721569

N $5.5390821 .454710-1.964958$

H -2.079486 $2.502199-3.861485$

H $-2.6676340 .829115-1.365009$

H $-0.5513690 .694627-3.634591$

H $-2.230106-1.223975-3.595772$

H -1.807955 -1.516961 -1.921066

H $1.033086-3.663040-3.093202$

H $0.936552-2.543511-5.082258$

H $-0.580119-1.722651-5.452819$

H $0.781270-0.817409-4.751146$

H -1.244158 -3.391247 -1.111306

H $-0.053689-4.649621-1.246385$

H $0.474160-1.846615-0.133630$

H $\quad 0.175930-3.298650 \quad 0.821253$

H $2.226609-4.350271-0.253030$

H $2.308180-3.2978832 .044223$

H $2.606755-1.7353531 .314180$

H $4.438253-2.6129893 .077590$

H $4.889990-1.415398-0.035623$

H $4.237515-4.8423262 .430808$

H $5.686890-4.7245681 .459943$

H $4.155395-5.2233460 .710789$ 
H $6.728088-2.6005220 .529269$ H $5.589574-2.441989-2.952872$ H $3.933582-2.460767-3.500447$ H $4.971774-4.648377-1.146984$ H 1.7625750 .3649430 .042138 H $2.1334873 .384682-0.433135$ H $3.9777900 .504097 \quad 1.129948$ H 2.6745530 .6790832 .331062 H 4.3007991 .2715232 .663655 H $4.227772-0.227703-3.608884$ H $3.638796-0.299379-1.945458$ H $6.4172363 .314618-2.086346$ H $7.5653281 .961958-1.966257$ H 6.7265661 .5655380 .473793 H $6.6519004 .582089-0.053280$ H 7.7542664 .2648522 .248507 H 6.7842702 .8366892 .523392 H 5.0927196 .5830543 .302929 H 6.8200666 .4508612 .646781 H 3.7600064 .6466413 .582113 H 4.5662533 .1105713 .551481 H 3.1940304 .5790381 .193403 H $4.5991861 .835412-2.024208$ C $-4.6272252 .700342-1.407190$ O $-5.4476941 .574760-1.789536$ C $-6.7796461 .801013-1.310332$ C $-6.6820572 .929352-0.294466$ C $-5.5985493 .781108-0.929308$ $\mathrm{H}-7.3528772 .181010-2.168184$ C $-7.4670590 .519822-0.834624$ H $-7.6268713 .464939-0.163734$ H -6.3544692 .5596280 .683903$ C -4.9868974 .7917210 .025311$ H $-6.0227244 .312637-1.791755$ C $-6.820294-0.2624340 .329264$ O H -7.574852 -0.155831-1.692622 H $-6.872494 \quad 0.3300661 .250184$ O $-7.672403-1.4010890 .541236$ C $-5.373534-0.753076 \quad 0.101351$ C $-4.917811-1.8012621 .137919$ H $-4.6882430 .098676 \quad 0.137315$ O $-5.262498-1.351664-1.190924$ H -5.062886 -1.393684 2.143613 H -5.555436 -2.687961 1.044998 H -5.7449965 .5039570 .367091$ H -4.563905 4.3044310 .909616 H $-4.1874965 .357936-0.462611$ C -3.759371 $3.125856-2.593115$ H $-9.2553080 .070924-0.175231$ H $-7.516670-1.7208181 .446053$ H -5.295609 -0.637662 -1.849774 H $-3.3319364 .116543-2.398357$ H -4.394144 $3.214291-3.483918$ H -4.006294 2.379005 -0.566008 C $-2.332510-0.6968242 .407178$ O $-2.527290-1.1694111 .068765$ C $-3.452801-2.252300 \quad 0.933650$ C $-3.023645-3.4381441 .809291$ C $-2.790946-3.0284613 .259156$ C -1.848071 -1.8285213 .323059$ H -3.273959 -0.290553 2.795111 C $-1.293231 \quad 0.4342732 .345473$ H -3.371004 -2.592676 -0.105573 O $-3.993313-4.4789231 .743714$ H $-2.090754-3.8529321 .408496$ H -3.740820 -2.822886 3.766886 O $-2.195536-4.1348013 .940623$ H - $0.834536-2.1392023 .038156$ O $-1.791972-1.3461284 .663921$ C -1.8827291 .7249831 .819798$ H -0.8918420 .6584623 .339500$ H $-0.470907 \quad 0.137093 \quad 1.683989$ H $-3.686879-5.1989712 .321277$ H $-2.151490-3.9085314 .884893$ H $-1.370501-2.0333315 .206966$ O $-0.8688152 .550810 \quad 1.428723$ O $\quad-3.0759001 .9918801 .790858$
C -1.2670853 .8297050 .925733$

H $-1.8058803 .708948-0.017657$

H -1.8836794 .3567361 .659970$

H $-0.364754 \quad 4.4183350 .740851$

SCF Energy (B3LYP/6-31G**//MMFF) $=-3245.91272249$

0500154

MM̄FF Geometry

C $3.851283-0.766229-3.181255$

C $5.162802-0.645550-2.919082$

C $5.7785590 .236224-1.856321$

O $4.7441970 .958344-1.155688$

C $6.508939-0.651338-0.826938$

C $7.097854 \quad 0.119619 \quad 0.338121$

C 6.6751720 .0079201 .613890

C $8.2343831 .047576-0.003110$

C $5.596346-0.8950142 .150525$

C $4.209691-0.2652792 .026408$

C $3.097181-1.0272002 .759698$

C $2.893684-2.4434942 .215030$

C $1.686573-3.1404672 .861751$

O $1.901454-0.2543152 .577330$

O $1.382658-4.3114442 .088837$

C $0.461953-2.2079302 .764948$

C $2.002936-3.6315844 .279616$

O $-0.645432-2.7506053 .478372$

C $0.745550-0.7706923 .251038$

C -0.4287860 .1961712 .967209$

O $0.945180-0.7835974 .669400$

C $4.6951062 .311845-1.244506$

O $5.4294503 .045430-1.883688$

C $3.5575712 .743067-0.401091$

C $3.2140894 .035932-0.310178$

C 2.0952844 .5589280 .479886

C $1.3627673 .582788 \quad 1.363979$

C -0.8127350 .3310391 .490973$

C -1.7338441 .5169311 .247521$

C $-2.5362082 .996564-0.602993$

O -2.3885972 .0498302 .139317$

C -1.614632 $4.105469-1.002546$

C $-1.7614695 .380415-0.609200$

C $-0.8753146 .498209-1.084043$

C $-0.260814 \quad 7.3019340 .043225$

C -0.5019358 .6194770 .154024$

C $0.641006 \quad 6.5989671 .038470$

C 1.7769095 .8659490 .373994

N -1.756199 $1.885603-0.089605$

H $3.127650-0.168109-2.634364$

H $5.863818-1.258154-3.482915$

H $6.4846370 .921556-2.339011$

H $7.316863-1.207158-1.321273$

H $5.807789-1.410512-0.461290$

H 7.1597480 .6225632 .371501

H 8.6815961 .5010630 .887975

H $9.0267950 .500160-0.523901$

H $7.8907701 .861553-0.648081$

H $5.631882-1.8686461 .651379$

H $5.817543-1.0943383 .206550$

H 4.2321280 .7660682 .404511

H $3.933305-0.1821850 .969608$

H $3.343380-1.0526823 .827589$

H $3.792881-3.0553632 .351831$

H $2.725992-2.3943921 .130009$

H $2.175744-4.8740082 .071704$

H $\quad 0.168263-2.1777591 .707800$

H $2.829987-4.3515334 .259355$

H $1.150720-4.1677334 .711601$

H $2.285896-2.8263454 .960622$

H $-0.789156-3.6540813 .148476$

H $-1.307688-0.0943243 .556828$

H $-0.146338 \quad 1.1912513 .339954$

H 0.7747820 .1090695 .013368

H $3.013787 \quad 1.965266 \quad 0.123757$

H $3.7844534 .771670-0.874854$

H 0.5725014 .0322931 .966878

H 2.0546193 .1088582 .069789

H $\quad 0.8954412 .8028430 .756497$

$\mathrm{H} \quad 0.0865010 .4514310 .876790$ 
H -1.341098 $-0.566877 \quad 1.152300$ H $-3.273558 \quad 3.3160870 .139928$ H -3.064609 2.632421 -1.489011 H -0.806495 $3.851952-1.685312$ H -2.5853235 .6450670 .049495$ H -1.486983 7.149041-1.722527 H $-0.0751426 .118583-1.731359$ H $-0.066668 \quad 9.2096900 .953659$ $\mathrm{H}-1.1413139 .139760-0.551474$ H $0.0163875 .946722 \quad 1.653274$ H 1.0837737 .3256061 .731938 H $2.3858166 .496099-0.275779$ H $-1.1734131 .379966-0.750318$ C $2.329655-2.760645-3.537047$ O $1.120126-2.111058-3.098059$ C $0.691909-2.716964-1.863216$ C $1.559421-3.959824-1.677605$ C $2.859153-3.525735-2.324206$ H $\quad 0.916068-1.993852-1.068492$ C $-0.817251-3.006494-1.870705$ H $1.673703-4.232505-0.625249$ H $1.145624-4.827146-2.205033$ C $3.786687-4.674799-2.674991$ H $3.372660-2.854475-1.624986$ C -1.694495 -1.750959-2.078706 O $-1.104291-3.973363-2.880917$ H -1.077659 -3.462927 -0.908214 H $-1.369508-0.970838-1.381002$ O $-1.524568-1.236587-3.398778$ C $-3.196925-2.015676-1.835097$ C $-4.056064-0.756689-2.066765$ H -3.314425 -2.370279 -0.805363 O $-3.650765-3.051150-2.703847$ H $-3.6349940 .067090-1.482504$ H -3.996438 $-0.468293-3.123185$ H $4.072037-5.227253-1.773783$ H $3.310303-5.379705-3.364492$ H $4.701630-4.304880-3.148706$ C $3.283698-1.742637-4.172963$ H $-0.900851-3.564786-3.739883$ H $-0.584515-1.016636-3.511500$ H -4.496663 -3.380189-2.356311 H $2.741553-1.166127-4.932361$ H $4.090392-2.278975-4.686675$ H $2.020294-3.466796-4.319294$ C $-5.394896-0.5438020 .666689$ O $-5.715741-1.468305-0.379452$ C $-5.545747-0.958716-1.704931$ C $-6.4290640 .280876-1.907225$ C $-6.2058371 .331249-0.822709$ C $-6.283000 \quad 0.7017330 .567559$ H $-4.338322-0.2584170 .594739$ C $-5.603029-1.2697411 .999854$ H -5.933104 -1.731355 -2.380500 O $-6.1978940 .870997-3.182074$ H -7.484464 $-0.020755-1.879751$ H $-5.2535701 .851106-0.964866$ O $-7.2233122 .330569-0.920560$ H $-7.3261590 .439797 \quad 0.784480$ O -5.8840511 .6653851 .540612$ C $-4.440327-2.1879122 .286500$ H $-5.663235-0.5670772 .838814$ H $-6.527925-1.8583022 .005949$ H $-6.3668890 .189053-3.854639$ H $-7.1825802 .697223-1.820588$ H -4.9518641 .8889241 .376860$ O $-4.601873-3.3731361 .633322$ O $-3.488112-1.8733012 .988273$ C -3.530293 -4.309159 1.785156 H $-2.607354-3.8963651 .366756$ H $-3.396189-4.5667072 .840021$ H -3.790217 -5.215847 1.232488 SCF Energy (B3LYP/6-31G**//MMFF) $=-3245.91137384$

\section{$05 \_00155$}

MM̄FF Geometry

C -3.206993 -2.483175 -2.479544

C $-1.955991-2.867671-2.773217$
C $-0.772097-1.943347-2.660713$

O $0.126550-2.555472-1.708088$

C $-0.068923-1.786882-4.025572$

C $0.716349-0.495071-4.177723$

C $2.048627-0.405074-4.362221$

C $-0.1107110 .768876-4.173167$

C $3.061206-1.507716-4.479125$

C $3.890336-1.764977-3.212586$

C $4.777396-0.591208-2.759999$

C $5.723150-1.049070-1.644030$

C $6.5605250 .105455-1.074060$

O $3.942431 \quad 0.467265-2.278689$

O $7.141365-0.3391330 .162264$

C $5.6247691 .284096-0.732094$

C $7.7308660 .467713-1.995725$

O $6.3746062 .432803-0.346293$

C $4.6547321 .647491-1.875032$

C $3.6101472 .719060-1.468902$

O $5.4009622 .195196-2.969470$

C $0.855036-1.723973-0.919883$

O $0.881513-0.505443-0.968840$

C $1.623947-2.5548530 .032658$

C $2.573512-1.9716970 .778492$

C $3.419105-2.6202691 .779815$

C $3.115732-4.0516372 .133055$

C $2.6956452 .340994-0.301085$

C 3.2013042 .7842961 .060843

C 2.9000722 .2984113 .490491

O 4.0260353 .6807801 .213757

C 4.0705521 .4451173 .866494

C 4.0070070 .4315884 .743352

C $5.190035-0.4095695 .132334$

C $5.007671-1.8718464 .788437$

C $4.591816-2.7515715 .715085$

C $5.390962-2.3299153 .398427$

C $4.402903-1.8958222 .351994$

N 2.5855272 .0841632 .090383

H $-3.383590-1.460058-2.155299$

H -1.769123 $-3.886419-3.103397$

H -1.116807 -0.977476 -2.274081

H $-0.813795-1.797077-4.833163$

H $\quad 0.572138-2.658289-4.193729$

H $2.4745720 .591529-4.477267$

H $0.4754931 .650465-4.454741$

H $-0.9348660 .686346-4.889242$

H $-0.5283040 .960706-3.181137$

H $2.583147-2.446162-4.777700$

H $3.738936-1.256655-5.305052$

H $3.211867-2.046136-2.401879$

H $4.528841-2.635386-3.410592$

H $5.351161-0.238024-3.624828$

H $6.376535-1.857049-1.996316$

H $5.133321-1.481817-0.825631$

H $7.669260-1.134595-0.023524$

H 5.0416810 .9796930 .145834

H $8.398920-0.392839-2.121841$

H $8.3464941 .262141-1.559828$

H $7.4114620 .783225-2.991053$

H 6.9534642 .1676200 .388797

H $4.1063023 .681631-1.295590$

H $2.9577612 .879893-2.339457$

H $4.7955882 .710761-3.527791$

H $1.390968-3.6114100 .092632$

H $2.759778-0.9080560 .640567$

H $3.306684-4.7069451 .276311$

H $2.067178-4.1612212 .432261$

H $3.712597-4.4278032 .967075$

H $1.7268702 .837635-0.438523$

H $2.5131401 .265097-0.292542$

H 2.0037442 .0512114 .068105

H 3.1415923 .3533853 .654341

H 5.0230601 .6836383 .398599

H $3.057996 \quad 0.1989175 .220739$

H $5.339567-0.2941856 .213967$

H $6.113691-0.0395974 .669919$

H $4.486435-3.8068285 .486314$

H $4.342680-2.4344996 .722370$

H $5.539272-3.4151303 .372914$ 
H $\quad 6.374513-1.9151553 .141978$

H $4.542327-0.8653412 .024907$

H $\quad \begin{array}{llll}1.957570 & 1.318677 & 1.862891\end{array}$

C $-5.161091-3.563471-1.267057$

O $-5.547155-2.291276-0.710961$

C $-5.392506-2.3525240 .722621$

C $-5.178180-3.8240261 .059458$

C $-4.402315-4.291694-0.159588$

H $-4.470998-1.8126660 .964736$

C $-6.588712-1.6866801 .411314$

H $-4.630533-3.960048 \quad 1.996813$

H $-6.128410-4.3649541 .139047$

C $-4.368364-5.800798-0.320132$

H -3.374061-3.915995 -0.081798

C $-6.799755-0.2176740 .984292$

O $-7.768012-2.423418 \quad 1.065791$

H $-6.481876-1.7593682 .499770$

H -7.000995 $-0.175289-0.092342$

$\begin{array}{lllll}\text { O } & -7.985913 & 0.266444 & 1.627170\end{array}$

C $-5.6261590 .716701 \quad 1.338158$

C -5.8397342 .1401320 .792845$

H -4.7040930 .3170710 .911149$

$\begin{array}{llll}\text { O } & -5.472284 & 0.740263 & 2.754831\end{array}$

H $-5.8501142 .080821-0.301801$

H $-6.8281772 .507921 \quad 1.094707$

H $-3.879846-6.2678690 .541263$

H -5.377071 -6.219252 -0.401690

H $-3.811664-6.084344-1.218807$

C $-4.416442-3.366279-2.589828$

H -8.521404 -1.979757 1.491838

H $-7.813276 \quad 0.2825062 .584394$

H -4.6224231 .1696502 .951631$

H -5.096119 -2.883175 -3.303226

H -4.152207 -4.341917 -3.013790

H $-6.096951-4.095923-1.484185$

C $-2.8986472 .465256-0.054415$

O $-3.4562332 .674808 \quad 1.246938$

C $-4.793698 \quad 3.176079 \quad 1.262525$

C $-4.862317 \quad 4.5162910 .516279$

C $-4.2804984 .411423-0.892501$

C $-2.8931903 .771256-0.856940$

H $-3.4778351 .700446-0.586202$

C $-1.468619 \quad 1.943780 \quad 0.144614$

H -5.0226803 .3839772 .315649$

O

H -4.2891865 .2706161 .070968$

H $-4.9484403 .860474-1.564783$

O $-4.1516845 .723495-1.445596$

H -2.177429 $4.480099-0.421103$

O $-2.4652603 .497651-2.189522$

C $-1.463631 \quad 0.6053480 .849051$

H $-0.9438421 .816912-0.807689$

H -0.8983552 .6612390 .748037$

H -6.5433475 .0607751 .341935$

H $-5.0343016 .131943-1.428245$

H $-2.4938714 .336457-2.681183$

O -0.6057790 .6436551 .908695$

O $-2.131974-0.358200 \quad 0.498588$

C $-0.524563-0.5507742 .693145$

H $0.367306-0.4838853 .322127$

H $-1.406599-0.6250393 .335545$

H - $0.438416-1.4382712 .059987$

SCF Energy (B3LYP/6-31G**//MMFF)= -3245.92266303

\section{00156}

MM̄FF Geometry

C -1.621785 -3.235240 2.906537

C $-2.703653-2.8862423 .617903$

C $-3.243427-1.4845583 .784195$

O $-2.476057-0.5364493 .007568$

C $-4.694511-1.4346643 .263454$

C $-5.336547-0.065394 \quad 3.377317$

C -5.7089970 .6995872 .331502$

C -5.5701230 .4246334 .783792$

C $-5.609647 \quad 0.387956 \quad 0.862680$

C -4.4363391 .1253460 .216579$

C $-4.3889290 .997685-1.313372$

C $-4.108780-0.434419-1.776905$
C $-3.933195-0.523904-3.301824$

O $-3.340606 \quad 1.869339-1.761104$

O $-3.360234-1.805988-3.603850$

C $-2.9141510 .542805-3.754416$

C $-5.278147-0.467302-4.035302$

O $-2.826161 \quad 0.600932-5.175709$

C $-3.2036051 .949947-3.188078$

C $-2.0703112 .962154-3.487853$

O $-4.3966312 .467122-3.788424$

C $-1.7018200 .367118 \quad 3.665938$

O -1.4745380 .4164574 .863361$

C -1.2200421 .3392392 .658002$

C -0.6843042 .5061113 .047299$

C -0.2758233 .6014302 .161341$

C $-0.556403 \quad 3.4387080 .690786$

C $-0.6880862 .520272-3.007107$

C $0.3228123 .650907-2.930314$

C $2.6891824 .042205-2.207680$

O $0.0571614 .818980-3.195026$

C $2.8451244 .113787-0.721250$

C $3.0114475 .250740-0.029520$

C 3.1721415 .2849811 .465076

C 2.2044156 .2315312 .142450

C 2.6542877 .2875412 .841398

C 0.7196115 .9670022 .009870

C 0.2876544 .7024142 .702727

N $1.558473 \quad 3.181892-2.504756$

H -1.025600 -2.467454 2.421650

H -3.277200 -3.671980 4.106036

H -3.219985 -1.233752 4.851060

H $-5.314589-2.1524543 .816920$

H -4.701094 -1.777484 2.223523

H -6.1525741 .6720682 .544068$

H $-6.142361 \quad 1.3582184 .809181$

H $-6.134723-0.3191845 .355620$

H -4.6194250 .6092285 .292862$

H -5.534234 -0.6870320 .684265$

H -6.5480610 .7030940 .389250$

H -4.479579 2.1921710 .475725

H -3.4913430 .7625120 .637278$

H $-5.342771 \quad 1.357000-1.717044$

H -4.900295 -1.120120 -1.453590

H -3.187137 -0.796167 -1.300152

H -3.955912 -2.490740 -3.254230

H $-1.9329170 .207506-3.397339$

H $-5.919738-1.300362-3.723303$

H $-5.143925-0.582600-5.116519$

H $-5.828520 \quad 0.457667-3.851548$

H -2.639030 -0.299694 -5.491629

H $-2.0326293 .184524-4.562133$

H $-2.3292003 .912538-3.000689$

H -4.385603 $3.435651-3.710770$

H -1.3715761 .0893071 .614565$

H -0.5642072 .6950264 .113144$

H -0.2821754 .3065640 .089745$

H -1.6247393 .2678510 .516881$

H 0.0047362 .5894870 .289536

H $-0.7660412 .094171-2.002955$

H $-0.2870011 .752785-3.678904$

H $2.5418595 .028179-2.658948$

H $3.5740893 .575085-2.650413$

H $2.8231233 .171068-0.180742$

H $3.0549626 .198783-0.560331$

H 4.2073405 .5818851 .677576

H 3.0533274 .2847601 .898635

H 1.9721447 .9749753 .330623

H 3.7154257 .4897972 .941819

H 0.4478105 .9903520 .952081

H 0.1490846 .7896512 .461789

H $0.4362134 .728696 \quad 3.783144$

H $1.6580802 .193343-2.291897$

C -1.151059-5.066959 1.217155

O $-0.281283-4.1813600 .481907$

C $-1.034927-3.609372-0.601932$

C $-2.114262-4.635872-0.904807$

C $-2.509663-5.0448990 .504500$

H -1.516041 -2.701595 -0.215957

C $-0.111977-3.230847-1.760692$ 
H $-2.945195-4.215118-1.478508$ H -1.713379 $-5.497323-1.451279$ C $-3.253560-6.3674460 .571898$ H -3.156955 -4.263104 0.920432 C $1.097774-2.368149-1.333646$ O $0.397036-4.429070-2.357176$ H $-0.693504-2.723245-2.539319$ H $\quad 1.809717-3.004517-0.799577$ O $1.763136-1.940685-2.527460$ C $0.741187-1.137970-0.480942$ C $1.907510-0.184914-0.155280$ H $0.287654-1.4619920 .461693$ O $-0.253449-0.390251-1.185174$ H $2.3836050 .143854-1.084706$ H $1.4712260 .723896 \quad 0.275528$ H -4.192054 -6.309967 0.010891 H -2.659626 -7.1848020 .149504$ H -3.493169 -6.624232 1.608728 C -1.169871 -4.6551392 .695858$ H $0.983984-4.163094-3.085800$ H $\quad 1.166070-1.330008-2.993004$ H $-0.5945460 .287065-0.576841$ H $-0.151513-4.7308813 .096904$ H -1.794075 -5.3554453 .262952$ H $-0.699890-6.0643161 .142500$ C $4.664482-1.460081-0.697685$ O $3.696755-1.8424790 .283658$ C $2.932878-0.7702450 .839655$ C $3.8752210 .271874 \quad 1.462831$ C 4.9180770 .7618310 .461992 C $5.656778-0.426831-0.150850$ H $4.142341-1.023690-1.557351$ C $5.339480-2.732060-1.232809$ H $2.352744-1.2036091 .664498$ O $3.147871 \quad 1.3845341 .971488$ H $4.401573-0.1801232 .313734$ H $4.4685681 .384807-0.319149$ O $5.870857 \quad 1.584880 \quad 1.137258$ H $6.310521-0.8711900 .608493$ O $6.4899830 .029962-1.213603$

C $6.132300-3.503133-0.196944$ H $4.575301-3.419286-1.614991$ H $6.008894-2.463801-2.058385$ H 2.5005021 .0435892 .612134 H 5.3790022 .3153491 .550578 H $7.0825790 .708264-0.846340$

O $7.051867-4.287618-0.833719$

O $5.950085-3.454526 \quad 1.011834$

C $7.869276-5.0943370 .020151$

H $8.444240-4.4617820 .703366$

H $7.249500-5.8030190 .577755$

H $8.566109-5.656036-0.607721$

SCF Energy (B3LYP/6-31G**//MMFF) $=-3245.92245465$

05_00157

MM̄FF Geometry

C $1.383985-4.826262-2.861332$

C $1.763071-3.646075-3.376068$

C $3.077508-2.936794-3.155449$

O $2.762368-1.659830-2.544842$

C $4.073093-3.655881-2.219681$

C $5.371353-2.899297-2.007055$

C $5.778957-2.391618-0.826249$

C $6.244309-2.749764-3.226951$

C $5.094462-2.4763890 .511400$

C $4.337883-1.1878930 .833393$

C $3.773357-1.1394292 .260853$

C $2.662979-2.1681712 .492441$

C $2.019944-2.0204673 .881844$

O $3.254894 \quad 0.1855842 .448061$

O $0.802726-2.7828943 .888515$

C $1.628762-0.5435374 .100332$

C $2.905087-2.6117084 .985180$

O $1.163336-0.3251315 .429462$

C $2.7635610 .447403 \quad 3.771094$

C 2.3019381 .9235143 .810048

O $3.8086990 .311567 \quad 4.741296$

C $2.975023-0.524252-3.262366$
O $3.309890-0.436702-4.432028$

C $2.7808650 .614871-2.337008$

C $3.1927811 .844351-2.681774$

C $3.1574743 .031427-1.821581$

C $2.6939992 .836562-0.402620$

C 1.0976422 .2471622 .922030

C 0.9427323 .7380562 .664988

C -0.4373865 .3234931 .310121$

O 1.6841404 .5882643 .149201

C $0.0255345 .406553-0.110660$

C $0.8210546 .371421-0.596251$

C $1.2666436 .419494-2.032028$

C $2.7628796 .596702-2.183621$

C $3.2648307 .676262-2.807050$

C $3.6816925 .538375-1.611505$

C $3.5679254 .215740-2.323011$

N -0.1251893 .9989741 .817961$

H $2.060049-5.389610-2.225469$

H $1.054835-3.112799-4.008647$

H $3.541059-2.796638-4.139100$

H $4.319802-4.645040-2.626677$

H $3.579766-3.832582-1.258777$

H $\quad 6.727852-1.856661-0.800811$

H $7.220321-2.313500-2.988256$

H $\quad 6.428080-3.727747-3.683897$

H $5.768574-2.101364-3.968336$

H $4.428458-3.3409800 .565385$

H $5.866330-2.6500331 .271752$

H $4.999819-0.3225890 .690390$

H $3.518752-1.0484440 .118847$

H $4.601309-1.2969532 .961807$

H $3.034074-3.1911692 .361299$

H $1.880347-2.0341671 .732473$

H $1.024415-3.7037303 .667457$

H $0.779144-0.3412213 .437361$

H $3.073611-3.6809184 .808709$

H $2.415914-2.5437065 .963038$

H $3.883756-2.1323685 .053632$

H $0.448277-0.9633505 .594746$

H 2.0846642 .2250734 .843121

H 3.1514722 .5463583 .496394

H 4.3325301 .1299674 .746118

H $2.3450300 .398309-1.368287$

H $3.6247381 .992769-3.670472$

H 3.3185882 .0960100 .109483

H $1.6545502 .492617-0.386142$

H 2.7342053 .7430470 .202242

H 1.1965651 .7483841 .951918

H $\quad 0.177098 \quad 1.890174 \quad 3.397818$

H 0.0256496 .0872191 .942602

H -1.5245025 .4399601 .353577$

H $-0.3211904 .626815-0.783819$

H $1.154688 \quad 7.171140 \quad 0.060364$

H $0.7351387 .250929-2.513050$

H $0.9596705 .514432-2.570785$

H $4.3344367 .818073-2.921317$

H $2.6184778 .444176-3.219070$

H $3.5121575 .462898-0.534781$

H $4.7283955 .858580-1.704150$

H $3.8950764 .251535-3.363085$

H $-0.6573083 .218007 \quad 1.444055$

C $-0.953027-5.192382-1.939189$

O $-1.254909-3.784660-1.865714$

C $-1.202949-3.372629-0.483871$

C $-1.293288-4.6550460 .328393$

C $-0.499319-5.612837-0.539498$

H $-0.221622-2.899200-0.350426$

C $-2.324645-2.362674-0.226259$

H $-0.879445-4.5472241 .335450$

H $-2.334577-4.9890490 .416638$

C $-0.752405-7.077404-0.228319$

H $0.565421-5.400112-0.387281$

C $-2.438394-1.9148651 .241540$

O $-2.071400-1.211555-1.037771$

H $-3.275882-2.784674-0.568900$

H $-2.736497-2.7639681 .868015$

O $-1.135175-1.5152141 .681942$

C $-3.396010-0.7294451 .471859$ 
C $-4.837519-1.0091121 .014720$

H -3.0006530 .1681690 .983230$

O $-3.437973-0.4474042 .875182$

H $-4.840098-1.347938-0.024013$

H -5.251011 -1.8244431 .621257$

H $-0.463467-7.3071820 .802468$

H -1.809921 $-7.336353-0.346004$

H $-0.170506-7.721694-0.894978$

C $0.031219-5.444025-3.086522$

H -1.998295 -1.515246 -1.958951

H -1.157487 -1.4702822 .652747$

H -2.571283 -0.0854283 .126172$

H $-0.397979-5.047022-4.015330$

H $0.162992-6.522014-3.235622$

H -1.900381 -5.687728 -2.189262

C $-5.2074371 .277088-0.951531$

O

C $\begin{array}{llll}-5.753706 & 0.229240 & 1.152204\end{array}$

C -7.204288 -0.0013750 .704899$

C $-7.329966-0.079937-0.815293$

C $-6.6354311 .109736-1.478723$

H $-4.5923640 .424787-1.263911$

C $-4.5525512 .550890-1.493225$

H -5.7826690 .4877452 .218124$

O -7.732588 -1.191900 1.279271

H $-7.8277390 .830248 \quad 1.058588$

H -6.935927 -1.027466 -1.201602

O $-8.713359-0.045960-1.174616$

H -7.216346 $2.023005-1.296506$

O $-6.5943410 .900695-2.888517$

C -3.068376 2.534402 -1.214016

H -4.698058 $2.664302-2.573486$

H $-4.9686993 .447052-1.017571$

H -7.656681 -1.1086282 .245322$

H $-9.148844-0.785136-0.716230$

H $-7.5103360 .771706-3.189285$

O $-2.4109291 .898620-2.225155$

O $-2.562723 \quad 3.005441-0.204004$

C $-0.9921881 .777005-2.074415$

H $-0.7245221 .468807-1.058886$

H $-0.6419631 .011309-2.771965$

H -0.514997 $2.727241-2.327810$

SCF Energy (B3LYP/6-31G**//MMFF)= -3245.92541035

05_00158

MM̄FF Geometry

C 1.5818183 .5762230 .885444

C 1.8678292 .9809412 .054036

C 0.9809872 .0276212 .825641

O $-0.212396 \quad 1.7078652 .075458$

C 1.7529330 .7160393 .063075

C $1.019845-0.2726073 .946126$

C $0.430489-1.4049973 .514097$

C 1.0124230 .0643885 .415458

C $0.327676-1.9211162 .104426$

C $-1.131672-1.9326811 .653737$

C -1.353304 -2.455396 0.227921

C $-0.702693-1.574124-0.841814$

C - $-1.081065-2.018413-2.263935$

O $-2.773877-2.4724840 .044246$

O $-0.715998-0.965024-3.168393$

C -2.612976 -2.173037 -2.355237

C $-0.292156-3.260004-2.697529$

O $-2.995281-2.723619-3.613879$

C -3.216992 -3.009945-1.207604

C $-4.760023-2.902105-1.225586$

O $-2.834566-4.376273-1.364620$

C -1.3324582 .4438052 .312469$

O -1.4737053 .3276273 .141803$

C -2.3577862 .0106951 .335342$

C -3.4851932 .7213661 .183735$

C -4.5515392 .4462590 .215767$

C $-4.4879851 .146367-0.541448$

C -5.439606 -3.649199-0.070291

C $-6.780636-3.0402190 .281252$

C $-7.800927-1.1175101 .497904$

O $-7.838569-3.564427-0.058515$

C $-8.198230-0.0917830 .484419$
C $-8.372168 \quad 1.207228 \quad 0.772537$

C $-8.8613052 .215204-0.226628$

C $-8.0128263 .463630-0.276844$

C -8.4862564 .6390220 .171699$

C $-6.6579413 .372487-0.941540$

C $-5.517728 \quad 3.3726120 .044016$

N $-6.658863-1.8658261 .009657$

H 0.6130543 .4258060 .418254

H 2.8419383 .1721142 .501061

H 0.7231912 .4882923 .786587

H 2.7215820 .9322693 .533889

H 1.9980890 .2654062 .096359

H -0.047011 -2.045243 4.255249

H $0.535462-0.7151816 .019167$

H 2.0370870 .1818945 .783263

H 0.4681710 .9965695 .594630

H $0.931491-1.3330641 .412163$

H $\quad 0.729381-2.9409622 .079114$

H -1.722314 -2.552284 2.343444

H -1.561283 -0.927594 1.741862

H $-0.973388-3.4820610 .170662$

H $\quad 0.388035-1.558510-0.736625$

H -1.030945 -0.534752 -0.706833

H $0.243460-0.825761-3.094808$

H -3.047267 -1.164643 -2.314316

H $\quad 0.785392-3.058049-2.672317$

H $-0.521571-3.532200-3.733684$

H $-0.476970-4.128426-2.061800$

H -2.603169 -2.163869 -4.305890

H -5.040716 -1.842567-1.188966

H $-5.156433-3.304205-2.166343$

H -3.461934 -4.938562 -0.883849

H $-2.137300 \quad 1.1399780 .730078$

H -3.626978 3.6091491 .798630

H $-5.3895930 .935275-1.120245$

H -4.3681310 .3040060 .149089$

H $-3.6434791 .149228-1.238931$

H $-5.598142-4.700810-0.336067$

H -4.824954 $-3.646700 \quad 0.837372$

H -7.511802 -0.666996 2.452563

H $-8.635153-1.8045531 .672904$

H -8.397410 -0.443077 -0.525537

H -8.2093321 .5541261 .790388$

H -9.8934522 .4724610 .045175$

H -8.918182 $1.782849-1.233555$

H -7.8957925 .5468430 .103462$

H -9.471516 4.7198980 .618609

H -6.524662 4.246556-1.593922

H $-6.6335862 .516552-1.621470$

H -5.4635014 .2845100 .641040$

H $-5.733782-1.4864521 .188960$

C $2.6218284 .023776-1.343474$

O $3.0127292 .644314-1.461160$

C $3.7850822 .481607-2.666166$

C $3.8446363 .854171-3.335204$

C $3.6565654 .792906-2.158345$

H $4.7965732 .198689-2.352672$

C $3.1770351 .373046-3.535331$

H $4.7912374 .012686-3.861482$

H $3.0379823 .989696-4.064695$

C $3.2216106 .191020-2.560378$

H $4.6029454 .852131-1.604161$

C $2.9703060 .031782-2.802554$

O $1.8895961 .808915-3.993614$

H $3.7920611 .231900-4.431944$

H $2.1819950 .143278-2.048599$

O $2.466108-0.908382-3.760649$

C $4.232034-0.550012-2.142077$

C $3.965286-1.932070-1.516303$

H $4.5839350 .141999-1.373271$

O $5.254658-0.658126-3.130596$

H $3.095591-1.853157-0.854847$

H $3.705636-2.642714-2.310366$

H $3.9705786 .654372-3.210883$

H $2.2695636 .175159-3.101505$

H $3.0983076 .830056-1.680526$

C $2.560324 \quad 4.4330690 .129428$

H $1.5204911 .095683-4.542437$ 
H $3.171932-1.071710-4.409860$

H $6.096867-0.808038-2.669505$

H 2.2474155 .4796990 .211734

H 3.5576494 .3465990 .577577

H $1.6266564 .115528-1.799936$

C $4.816662-1.3968981 .380881$

O $5.668940-1.585126 \quad 0.244485$

C $5.174114-2.499750-0.738993$

C $4.925171-3.875388-0.105060$

C $4.031674-3.7840741 .129252$

C $4.572454-2.7317232 .096784$

H $3.860907-0.9680691 .057937$

C $5.512550-0.3958492 .317656$

H $5.983729-2.631945-1.467168$

O $4.349990-4.759745-1.061243$

H $5.890434-4.3075440 .190459$

H $2.992599-3.5769550 .848040$

O $4.031512-5.0613501 .770038$

H $5.504994-3.0939512 .548321$

O $3.634558-2.5297023 .149885$

C 5.4856411 .0193111 .779979

H $5.022172-0.3704363 .296608$

H $\quad 6.561950-0.6876642 .446397$

H $4.192248-5.608916-0.614056$

H $3.377113-5.0291232 .488162$

H $3.578962-3.3553973 .659329$

O 6.3670121 .7824612 .492030

O $4.766768 \quad 1.4178380 .874911$

C 6.4368883 .1594062 .107638

H $6.6901403 .248967 \quad 1.046717$

H 5.4866203 .6569892 .321648

H 7.2240063 .6371262 .696789

SCF Energy (B3LYP/6-31G**//MMFF)= -3245.91674801

05_00159

MM̄FF Geometry

C $0.239487 \quad 5.4743290 .473578$

C $1.279071 \quad 4.875377-0.125647$

C 22578543.9890950 .601913

O $2.3701722 .739038-0.119598$

C 3.6459154 .6534770 .622028

C 4.6768463 .8917301 .432769

C 5.7995443 .3456650 .925964

C 4.4025893 .7900862 .911826

C $6.2803983 .363662-0.497398$

C $5.8463982 .153712-1.339122$

C $6.3543360 .802576-0.809365$

C $6.113630-0.306007-1.836609$

C $6.524356-1.687110-1.301032$

O $5.647383 \quad 0.494826 \quad 0.395319$

O $5.969708-2.681680-2.176170$

C $5.884274-1.9018670 .087866$

C $8.044974-1.881142-1.333909$

O $6.372595-3.0954530 .694101$

C $6.078482-0.7085531 .045675$

C $5.266293-0.8382192 .358164$

O $7.459936-0.6238921 .417035$

C $1.357609 \quad 1.8384210 .010837$

O $\quad 0.356416 \quad 1.946313 \quad 0.700115$

C $1.6727900 .690500-0.868054$

C $0.712197-0.186719-1.195731$

C $0.878437-1.351325-2.069322$

C $2.282057-1.722762-2.471101$

C $3.747528-0.9543012 .185944$

C $3.236992-2.3856402 .159149$

C $1.172176-3.7041131 .704472$

O $3.926921-3.3536022 .467420$

C $1.233357-4.2230270 .302367$

C $0.151266-4.630575-0.377981$

C $0.197521-5.184769-1.773020$

C $-0.757379-4.483819-2.713977$

C -1.953549-5.021309-3.007372

C $-0.287697-3.215922-3.390720$

C $-0.217568-2.036404-2.458423$

N $1.900776-2.4516331 .786407$

H 0.0981485 .3528181 .544783

H $1.4199114 .993491-1.197500$

H 1.9171693 .7849881 .624071
H 3.5683585 .6649881 .042854

H $3.9869404 .780348-0.411174$

H 6.4680152 .8257821 .611759

H 5.2540423 .3785283 .464590

H 4.1923634 .7803913 .328847

H 3.5441073 .1391583 .102497

H $5.9622044 .281750-1.002916$

H $7.3767143 .414537-0.482306$

H $4.7517912 .139137-1.400084$

H $6.2246832 .306659-2.357628$

H $7.4250650 .895836-0.593224$

H $6.638626-0.088275-2.775012$

H $5.046125-0.336572-2.092623$

H $6.306632-2.513419-3.072790$

H $4.811234-2.052236-0.079009$

H $8.420757-1.786042-2.359820$

H $8.322172-2.889387-1.006887$

H $8.583327-1.157565-0.718216$

H $6.208313-3.8247460 .071932$

H $5.667426-1.6547452 .970462$

H 5.4471170 .0767452 .941006

H $7.526408-0.1185212 .244309$

H $2.6876810 .611607-1.240652$

H $-0.294203-0.027598-0.810768$

H $2.926162-1.795626-1.588331$

H $2.699106-0.972499-3.151115$

H $2.352765-2.691826-2.969259$

H $3.263460-0.4765573 .047434$

H $3.412501-0.4260281 .288436$

H $\quad 0.145835-3.5048192 .028258$

H $1.620850-4.4325152 .387473$

H $2.212577-4.287211-0.166318$

H $-0.821101-4.5940130 .106961$

H $-0.054428-6.251948-1.714081$

H $1.212261-5.143561-2.188029$

H $-2.633540-4.543982-3.705068$

H -2.282878 -5.948807 -2.550501

H $-0.979586-2.942061-4.198935$

H $\quad 0.661193-3.414725-3.896946$

H -1.193133 -1.697283 -2.108168

H $1.421489-1.6040221 .497118$

C $-2.1687435 .632912-0.133799$

O $-2.1559964 .351434-0.790717$

C $-3.4533884 .115524-1.381849$

C $-4.3220955 .303811-0.987012$

C $-3.2947716 .406154-0.810835$

H -3.288587 $4.102403-2.466117$

C $-3.9631602 .753100-0.894930$

H $-5.0780285 .537324-1.742863$

H $-4.8405105 .111903-0.039127$

C $-3.8076077 .590017-0.010585$

H -2.973085 $6.749287-1.803217$

C $-5.4060142 .433686-1.325375$

O $-3.0701661 .765353-1.422022$

H -3.8669482 .7183910 .196087$

H $-6.0928753 .178705-0.906994$

O $\quad-5.4843012 .566451-2.750364$

C $-5.9021071 .022344-0.950281$

C -5.8213230 .7371120 .559795$

H $-5.349290 \quad 0.273594-1.528680$

O $-7.2695860 .937679-1.366763$

H -4.786196 $0.847756 \quad 0.894151$

H -6.4130431 .4906391 .094707$

H -4.666668 $8.048906-0.510826$

H -4.1246427 .2889020 .993547$

H -3.0320378 .3548580 .096376$

C $-0.7928466 .286281-0.258429$

H $-3.2393160 .930956-0.954689$

H $-6.4006672 .368605-3.009366$

H -7.522351 -0.000941-1.357773

H $-0.808481 \quad 7.2949410 .168492$

H -0.519355 $6.379061-1.316628$

H -2.4093385 .4508420 .922660$

C $-4.365710-1.9371230 .414475$

O $-5.753674-1.6995360 .162608$

C $-6.336326-0.6640020 .960251$

C $-6.174745-0.9900682 .453035$

C $-4.721511-1.2821912 .825568$ 
C $-4.127907-2.3256451 .878477$

H -3.802394 -1.024207 0.186896

C $-3.866269-2.986443-0.581782$

H -7.412284 -0.6797070 .746485$

O $-6.675861 \quad 0.0822893 .244145$

H $-6.782313-1.8737072 .688470$

H -4.125046-0.362656 2.836548

O $-4.694041-1.7927414 .160290$

H $-4.585749-3.2961452 .102942$

O $-2.730995-2.4558312 .130377$

C $-4.514970-4.337813-0.392557$

H -4.085853 -2.673596-1.609935

H -2.778221 -3.089695 -0.512969

H $-6.540432-0.1569694 .177079$

H -3.770005 -2.016771 4.365505

H - $2.309621-1.6021781 .931688$

O $-3.627493-5.2054140 .171812$

O $-5.674732-4.587218-0.694905$

C $-4.123111-6.5258950 .410601$

H $-4.427136-6.992928-0.531060$

H -3.315654 -7.117138 0.850522

H $-4.959719-6.4941801 .115125$

SCF Energy (B3LYP/6-31G**//MMFF) $=-3245.89758216$

05_00160

MM̄FF Geometry

C $3.101832-3.6472900 .146912$

C $2.331758-4.6194500 .659081$

C $0.832899-4.5600390 .862757$

O $0.312493-3.224967 \quad 0.673173$

C $0.147835-5.483613-0.162496$

C $-1.361337-5.544593-0.027766$

C $-2.230184-5.142576-0.976618$

C $-1.879595-6.1253191 .262783$

C $-1.921785-4.574969-2.334605$

C $-1.858479-3.040757-2.386672$

C $-3.208201-2.347623-2.137617$

C $-3.094968-0.843506-2.388529$

C $-4.412528-0.114833-2.088170$

O $-3.591676-2.564946-0.780186$

O $-4.109566 \quad 1.289023-2.039475$

C $-4.931784-0.533856-0.691686$

C $-5.413999-0.292871-3.236035$

O $-6.245333-0.019697-0.489561$

C $-4.890269-2.058877-0.437625$

C $-5.139796-2.454306 \quad 1.036497$

O $-5.917783-2.738592-1.169926$

C $0.382758-2.3666391 .727050$

O $0.863341-2.5855502 .827274$

C -0.214949-1.085019 1.294220

C 0.0692200 .0406321 .965768

C -0.4188301 .3809001 .635057$

C -1.5472041 .4858780 .644411$

C $-4.149526-1.8787392 .055106$

C $-4.635071-0.6147102 .746328$

C $-3.8922101 .087944 \quad 4.411607$

O $-5.760587-0.1490092 .591164$

C -3.483023 2.3167183 .663010

C $-2.586515 \quad 3.2017204 .125041$

C -2.2116804 .4650633 .404718$

C -0.7214504 .5969143 .186380$

C 0.0288525 .3764383 .983198

C -0.1203523 .9030971 .986708$

C $0.156164 \quad 2.4465312 .230165$

N -3.664597 -0.0838803 .586399$

H $2.648795-2.713947-0.175413$

H $2.804589-5.5527500 .959076$

H $0.606968-4.8980571 .881526$

H $0.540162-6.504746-0.064366$

H $0.428831-5.149366-1.167096$

H $-3.294802-5.241520-0.767835$

H $-2.964957-6.2723131 .245974$

H -1.421030 -7.101539 1.451250

H -1.652411 -5.4626782 .103489$

H - $0.979816-4.981705-2.717363$

H -2.688142 $-4.930661-3.035096$

H -1.118732 -2.687941 -1.658093

H $-1.492502-2.756859-3.381202$
H $-3.953803-2.794876-2.805223$

H $-2.772679-0.641180-3.417520$

H -2.310085 -0.427532 -1.743309

H $-4.9245751 .757481-1.789180$

H $-4.285875-0.0317460 .035826$

H $-5.0154720 .145025-4.159406$

H $-6.3504410 .238516-3.034526$

H -5.646431 -1.339850 -3.442701

H $-6.484993-0.1717180 .439978$

H -6.178142 -2.241488 1.317705

H $-5.050249-3.5494661 .091680$

H -6.436929 - $2.084468-1.663197$

H $-0.849261-1.0985550 .415412$

H $0.750429-0.0148642 .813843$

H -1.9804072 .4861410 .579050$

H -2.367053 0.8220410 .933489

H $-1.2115051 .207555-0.358160$

H -4.001788 -2.622342 2.848941

H -3.173538 -1.691222 1.597568

H -3.317069 0.9530975 .333116

H $-4.954251 \quad 1.1504454 .669965$

H -3.970858 2.5022652 .708942

H -2.119396 3.0287755 .092098

H -2.5769115 .3090574 .004621$

H -2.728458 4.5451842 .440162

H 1.0930205 .5009253 .812098

H -0.4035015 .9030804 .827558$

H $0.837304 \quad 4.372927 \quad 1.723467$

H $-0.7553604 .073578 \quad 1.113080$

H 0.9619532 .2706122 .943875

H -2.738505 -0.5014523 .602005$

C $5.352447-2.7538210 .806108$

O $4.997912-1.4169430 .406361$

C $6.192063-0.6210090 .300468$

C $7.292442-1.4277840 .977760$

$\begin{array}{llll}\text { C } 6.870125 & -2.846147 & 0.642504\end{array}$

H $6.419851-0.543562-0.769721$

C $5.947864 \quad 0.779997 \quad 0.873134$

H $8.287111-1.1734740 .599299$

H $7.296800-1.2836272 .064128$

C $7.523268-3.8989521 .520829$

H $7.123481-3.047512-0.407136$

C 4.6582001 .4478790 .350256

$\begin{array}{lllll}\text { O } & 5.825183 & 0.677421 & 2.297988\end{array}$

H $6.821877 \quad 1.4139840 .684462$

H 3.7997430 .9112480 .766463

$\begin{array}{lllll}\text { O } & 4.593534 & 2.770049 & 0.899049\end{array}$

C $4.5459361 .531853-1.183450$

C $3.2933622 .275359-1.682383$

H $4.5777200 .529824-1.615580$

O $5.6973092 .225295-1.676649$

H $3.3251773 .310257-1.314503$

H $3.3632392 .384415-2.772012$

H $8.611908-3.8731251 .406800$

H $7.292355-3.7381982 .579271$

H $7.178876-4.9018361 .249632$

C $4.588354-3.764927-0.050656$

H 5.6643831 .5736152 .640222

H 5.3232943 .2832690 .511490

H $5.6490742 .220031-2.647737$

H $4.928360-4.7813980 .178250$

H $4.792540-3.583564-1.113220$

H $5.068481-2.8565161 .861561$

C $0.5470083 .124241-2.619078$

O $0.9045362 .639611-1.321748$

C $1.9374351 .644621-1.303553$

C $1.4855330 .428842-2.125583$

C $1.0614850 .822606-3.540308$

C $0.0518781 .971562-3.501667$

H $1.4124163 .612811-3.083360$

C $-0.5548764 .175277-2.421759$

H $1.999747 \quad 1.324755-0.260788$

O $2.502081-0.561120-2.177737$

H $0.625427-0.030932-1.622262$

H $1.932872 \quad 1.084768-4.150648$

O $0.465202-0.306642-4.183405$

H $-0.9052281 .593155-3.121687$

O $-0.1845812 .437399-4.828098$ 
C $-0.0579445 .382874-1.654635$ H $-0.9369884 .530532-3.384468$ H -1.377760 $3.730006-1.849041$ H $2.163887-1.300669-2.711074$ H $-0.313370-0.570836-3.664749$ H $0.6556402 .778254-5.179394$ O $-1.1289846 .054384-1.137305$ O $1.1157785 .707439-1.537413$ C $-0.8118697 .217406-0.366215$ H $-0.3300387 .968837-0.998692$ H -1.7454547 .6317020 .023480$ H $-0.168013 \quad 6.9510730 .477591$

SCF Energy (B3LYP/6-31G**//MMFF) $=-3245.92272995$

05 00161

MM̄FF Geometry

C $1.698745-3.2954611 .813395$

C $0.634517-3.6578022 .546444$

C $-0.768943-3.0997562 .455029$

O $-0.886781-2.1290131 .391713$

C $-1.741552-4.2537182 .138049$

C $-3.204783-3.8589182 .165247$

C $-4.036073-3.9269541 .106907$

C $-3.733758-3.3977293 .499431$

C $-3.725628-4.380760-0.292126$

C $-3.340064-3.248577-1.257302$

C $-4.462115-2.227524-1.508861$

C $-4.074873-1.259169-2.628067$

C $-5.136561-0.168287-2.837673$

O $-4.683142-1.490996-0.304761$

O $-4.5389440 .841657-3.665471$

C $-5.4859740 .477466-1.476580$

C $-6.342352-0.704414-3.619452$

O $-6.5850391 .370596-1.639137$

C $-5.770509-0.557738-0.367046$

C $-5.908936 \quad 0.059713 \quad 1.045716$

O $-7.005985-1.224329-0.654200$

C $-0.706097-0.818037 \quad 1.704364$

O $-0.457516-0.3495572 .803172$

C $-0.834508-0.0422720 .450059$

C $-0.396511 \quad 1.2244380 .409825$

C $-0.4167752 .112179-0.754637$

C $-1.2232981 .671787-1.946400$

C $-4.6917420 .847800 \quad 1.542231$

C $-4.8048482 .348578 \quad 1.334519$

C -3.5137954 .4533161 .664425$

O

C -2.9420734 .8607330 .343832$

C -1.861548 5.6461570 .221650

C -1.298294 $6.096118-1.095233$

C $0.1518265 .706179-1.268534$

C $1.1391566 .592572-1.055731$

C $0.4508654 .310223-1.768376$

C $0.2839103 .264262-0.698372$

N -3.643230 3.0092921 .711023

H $1.615178-2.4942341 .084778$

H $0.763844-4.4584643 .273323$

H - $-1.023628-2.6473753 .420504$

H -1.604394 -5.067744 2.862640

H -1.473126 -4.671381 1.161624

H $-5.073508-3.6286611 .255155$

H -4.822631 -3.278374 3.496109

H -3.488621 -4.126788 4.278653

H -3.300229 -2.4312203 .773631$

H -2.928757 -5.131769 -0.289297

H $-4.607538-4.904194-0.683249$

H -2.453633 -2.735969 -0.865147

H -3.052784 -3.706703 -2.211995

H -5.372759 -2.776417 -1.775914

H -3.890099 -1.798954 -3.565147

H $-3.123307-0.774112-2.372559$

H -5.178318 $1.570060-3.748957$

H -4.622308 $1.090121-1.191894$

H -6.026151 -1.065857 -4.605417

H $-7.0746280 .087200-3.812993$

H $-6.850941-1.527042-3.112201$

H -6.699999 $1.857601-0.806300$

H -6.8315820 .6480871 .115386$
H $-6.049406-0.775044 \quad 1.748175$

H -7.344600 -1.614587 0.168627

H $-1.257539-0.543157-0.412697$

H $\quad 0.043543 \quad 1.6487751 .309723$

H $-2.255177 \quad 1.460514-1.647411$

H $-0.7926640 .768290-2.391539$

H - $-1.2830512 .420020-2.738558$

H -4.6006790 .6953602 .625575$

H -3.7679210 .4847931 .081736$

H -2.8752734 .7495382 .502562$

H -4.4990874 .9118451 .796856$

H -3.453879 $4.514814-0.551305$

H -1.3652076 .0100641 .118603$

H -1.402470 $7.187843-1.146891$

H -1.883323 $5.704629-1.936854$

H $2.1803906 .325774-1.203634$

H $0.9279947 .603319-0.722524$

H $1.4901154 .254474-2.117998$

H $-0.1608004 .121391-2.654175$

H $\quad 0.8510823 .476676 \quad 0.209325$

H -2.8310962 .4683151 .993934$

C $3.526954-4.5333030 .552962$

O $3.928778-3.439794-0.295978$

C $3.323335-3.614884-1.588505$

C $2.968674-5.095409-1.671998$

C $2.523128-5.370832-0.248192$

H $2.397652-3.025001-1.578796$

C $4.236476-3.092977-2.703660$

H $2.189598-5.296490-2.413243$

H $3.840663-5.712970-1.914960$

C $2.522503-6.8428200 .125571$

H $1.502884-4.984868-0.138611$

C $4.856618-1.707148-2.435239$

O $5.320073-4.015636-2.876078$

H $3.681190-3.091499-3.649507$

H $5.669121-1.800488-1.703370$

O $5.461682-1.252454-3.652644$

C $3.875136-0.623420-1.952638$

C $4.5641500 .747313-1.813031$

H $3.458658-0.920576-0.985756$

O $2.782989-0.509793-2.862462$

H $5.5123140 .610232-1.282730$

H $4.8048741 .137329-2.809700$

H $1.829769-7.401553-0.512194$

H $3.516828-7.2871590 .011003$

H $2.209544-6.9777771 .165970$

C $3.032928-3.9877691 .899975$

H $5.768725-3.791738-3.708530$

H $6.220051-1.831044-3.838399$

H $3.149627-0.293806-3.737035$

H $3.759543-3.2605082 .282195$

H $2.986650-4.8135022 .620083$

H $4.441407-5.1122580 .735476$

C $4.273417 \quad 1.196128 \quad 1.183474$

O 3.2430181 .3273880 .199774

C $3.6910131 .789958-1.079383$

C $4.3605503 .163115-0.924720$

C 5.4992353 .1243890 .093154

C 5.0182712 .5185871 .412409

H 4.9911160 .4410430 .838448

C 3.6539950 .6049322 .459503

H $2.7917711 .928030-1.690900$

O $4.8637263 .627687-2.172540$

H $3.6181033 .895731-0.583320$

H $6.3695882 .582433-0.294409$

O $5.9440224 .458510 \quad 0.351806$

H 4.3799953 .2498081 .919306

O 6.1388292 .2811272 .261785

C 2.6225091 .4823003 .139722

H $3.146112-0.3332952 .205031$

H $4.4492260 .390128 \quad 3.182472$

H $4.1165933 .659517-2.794259$

H $6.2051794 .845943-0.501215$

H $6.608708 \quad 3.1263982 .365136$

O 2.2987520 .9242894 .344917

O 2.1453552 .5098682 .680179

C 1.3274791 .6412295 .113841

H 1.7315702 .6120565 .415572 
H $\quad 1.1064481 .0576136 .011285$

H 0.4030401 .7691174 .542778

SCF Energy (B3LYP/6-31G**//MMFF) $=-3245.92270806$

05 00162

MM̄FF Geometry

C $\quad 0.111151 \quad 1.5127331 .147432$

C $-0.2296571 .879701-0.097186$

C -1.025202 $0.987583-1.016424$

O $-2.2872581 .631439-1.326784$

C $-0.332394 \quad 0.750517-2.368812$

C $0.9874070 .019246-2.300431$

C $1.120169-1.319790-2.340561$

C $2.2141740 .891318-2.267820$

C $0.034310-2.357102-2.419976$

C $0.256376-3.494415-1.411758$

C $-0.214130-3.1519150 .011670$

C $0.317451-4.1969780 .996474$

C $-0.240791-3.9978412 .409938$

O $-1.646623-3.163606-0.001350$

O $0.064722-5.173510 \quad 3.175248$

C - $-1.775427-3.9046542 .318162$

C $0.427606-2.822743 \quad 3.132342$

O $-2.340647-3.5979363 .591485$

C $-2.273581-2.8926821 .259441$

C $-3.801185-3.0651811 .068707$

O $-1.990836-1.5723961 .717175$

C $-3.2663431 .597195-0.383914$

O $-3.1852021 .147291 \quad 0.747300$

C $-4.4804322 .192806-0.984538$

C $-5.6372682 .168441-0.305746$

C $-6.9205652 .702770-0.767567$

C $-6.9681133 .298535-2.150878$

C $-4.421225-2.172595-0.015015$

C $-5.869493-2.537583-0.298405$

C $-7.799930-1.856353-1.705563$

O $-6.424405-3.5159940 .194810$

C $-8.468016-0.538466-1.934599$

C $-9.588050-0.162977-1.298235$

C $-10.3148251 .127677-1.549665$

C $-10.3923332 .000703-0.317118$

C -11.3703891 .8326190 .588946$

C $-9.3866523 .121290-0.182483$

C -7.987535 2.6227570 .055314

N -6.466439 -1.640206 -1.176169

H -0.2121270 .5331041 .494466$

H $0.0543012 .854268-0.482041$

H - $1.2364120 .015262-0.554482$

H $-0.1961501 .710166-2.887650$

H -1.023893 $0.206978-3.027079$

H $2.130444-1.726299-2.336425$

H $3.1348320 .317974-2.414025$

H $2.1755201 .640260-3.065565$

H $2.2936321 .406380-1.308091$

H $-0.964724-1.929578-2.292661$

H $0.070528-2.784819-3.429508$

H -0.307502 -4.367007-1.767661

H $\quad 1.316015-3.779317-1.403829$

H $\quad 0.138119-2.151750 \quad 0.287223$

H $1.414180-4.1915151 .015072$

H $\quad 0.031723-5.2007170 .651034$

H $1.030585-5.2877813 .176438$

H -2.148902 -4.902093 2.046366

H $1.505982-2.9969773 .228671$

H $\quad 0.048829-2.7207754 .155313$

H $0.292909-1.8693322 .617878$

H -2.011640 -4.261360 4.221866

H -4.001601 -4.111846 0.803849

H -4.322001 -2.863180 2.013287

H $-2.583417-0.946696 \quad 1.273017$

H -4.3905242 .625665 -1.974398

H $-5.650614 \quad 1.7130080 .683797$

H -7.968403 $3.604655-2.462813$

H $-6.6279792 .569947-2.895649$

H $-6.3281434 .185774-2.208883$

H $-4.400715-1.1271570 .302898$

H -3.864727 -2.263329 -0.954269

H -8.370465 -2.496998-1.024895
H -7.692020 -2.389158 -2.655973

H -8.027689 $0.120552-2.678637$

H -10.032299-0.837744 -0.569335

H -11.329698 $0.882391-1.889799$

H $-9.8605541 .687996-2.376157$

H $-11.4508592 .472589 \quad 1.461283$

H -12.110858 1.0472930 .478518

H $-9.6485103 .767660 \quad 0.665986$

H -9.461170 $3.770598-1.059175$

H -7.8543212 .1628651 .035526$

H $-5.879003-0.949793-1.635861$

C 1.6641943 .5254221 .692062

O 2.7510953 .1207020 .831672

C 3.9545133 .7874091 .255625

C 3.5022504 .9198942 .168688

C 2.3108494 .2855132 .854268

H 4.5187253 .0636661 .856136

C 4.7998634 .2289870 .055681

H 4.2861135 .2304182 .865960

H 3.1803215 .8014671 .604388

C 1.3897935 .2906873 .524757

H 2.6780363 .5779863 .610245

C $5.2113213 .093336-0.903001$

O $4.0584625 .171559-0.730236$

H $5.6968894 .752920 \quad 0.408481$

H $4.3369672 .748718-1.464544$

O $6.0935403 .665825-1.880481$

C $5.9057331 .884736-0.242874$

C $6.4778530 .906582-1.289540$

H 5.1850821 .3678770 .399934

O 6.9651102 .3646990 .583633

H $5.676414 \quad 0.633131-1.983921$

H $7.2503821 .419857-1.874772$

H 1.9266545 .8452164 .301482

H 0.9960336 .0164852 .805290

H 0.5392314 .7881393 .995609

C 0.8899382 .2938212 .169149

H $4.0994726 .031248-0.278962$

H $6.9065653 .934449-1.419198$

H $7.2390051 .635576 \quad 1.164967$

H 0.1875802 .5825182 .960135

H 1.6031481 .5927422 .618008

H 1.0209244 .1972951 .111079

C $5.119080-1.692923-0.437973$

O $6.228385-1.0529550 .202958$

C $7.115894-0.363655-0.681240$

C $7.686152-1.341914-1.720264$

C $6.581404-2.096346-2.457986$

C $5.608128-2.723561-1.460625$

H $4.497331-0.938149-0.931579$

C $4.276238-2.3595270 .652540$

H $7.961435-0.029985-0.067307$

O $8.510746-0.641313-2.644918$

H $8.326261-2.069621-1.204214$

H $\quad 6.052212-1.447382-3.165725$

O $7.159726-3.150990-3.230104$

H $6.099847-3.560289-0.947747$

O $4.489193-3.254153-2.167080$

C $3.618045-1.3126721 .516861$

H $3.477722-2.9795600 .231359$

H $4.892605-3.0116681 .283060$

H $8.952259-1.300784-3.205947$

H $7.686821-2.742063-3.937023$

H $4.832547-3.884098-2.823760$

O $4.271564-1.2134482 .708849$

O $2.657324-0.6440961 .159495$

C $3.769610-0.2215203 .608623$

H $4.337193-0.2893574 .540467$

H 3.9123300 .7760063 .182959

H $2.713312-0.4037053 .828434$

SCF Energy (B3LYP/6-31G**//MMFF) $=-3245.91003923$

05_00163

MMFF Geometry

C -1.9695462 .6705401 .110330$

C -1.4256102 .0238222 .153110$

C -1.904145 0.6704242 .606816

O $-0.734927-0.1780802 .730021$ 
C $-2.6330350 .713597 \quad 3.963054$ C -3.8249191 .6419154 .054270$ C -5.0069391 .4417103 .439043$ C -3.631995 2.8395114 .947796 C -5.3795120 .3260612 .501102$ C -5.9034820 .8986801 .175984$ C $-5.924604-0.086296-0.002697$ C $-6.614345-1.4137890 .316680$ C $-6.570879-2.381570-0.877743$ O $-4.571118-0.312281-0.419407$ O $-6.928363-3.688147-0.399929$ C $-5.116817-2.472865-1.391937$ C -7.604721 -2.017712 -1.949595 O $-5.044025-3.249095-2.585224$ C $-4.455984-1.095185-1.618657$ C $-2.955918-1.205886-1.980689$ O $-5.096650-0.443684-2.723200$ C $-0.937967-1.5218402 .660310$ O $-2.005957-2.1038712 .549806$ C $0.380204-2.1865782 .705895$ C $0.530555-3.3431132 .043396$

C $1.752524-4.1412951 .960641$ C $2.863450-3.8191462 .922870$ C $-2.087869-1.879763-0.919872$ C $-0.611503-1.738278-1.232879$ C $1.142773-2.533981-2.820453$ O $0.119722-1.004134-0.572541$ C $1.759027-3.860825-2.508790$ C $2.942710-4.002397-1.892935$ C $3.590103-5.332322-1.627812$ C $3.991888-5.513286-0.181031$ C $5.252044-5.2677510 .215854$ C $2.966823-6.0646200 .784695$ C $1.822794-5.1240021 .039001$ N $-0.215646-2.509336-2.312640$ H -2.7951352 .2075200 .574709$ H -0.6125992 .4830332 .709231$ H $-2.550470 \quad 0.2459051 .831528$ H $-1.9060680 .967846 \quad 4.747628$ H -2.960587 -0.2980474 .236713$ H $-5.8029932 .164773 \quad 3.614898$ H -4.517518 3.4830874 .981300 H -3.4177982 .5172905 .972159$ H -2.795068 3.4489584 .592134 H -4.543682 -0.3534282 .323642$ H $-6.168469-0.2700852 .975347$ H -6.9204521 .2789561 .338916$ H $-5.285876 \quad 1.7584630 .884162$ H $-6.452880 \quad 0.421682-0.818695$ H -7.648563 -1.2461140 .641437$ H $-6.109358-1.9048011 .158868$ H -7.812387 -3.628691 0.001459 H -4.546850 -3.019384 -0.630017 H -8.617922 -2.054176 -1.531451 H -7.593845 -2.742811 -2.770846 H $-7.458743-1.019800-2.367793$ H -5.464482 -4.106166 -2.399222 H -2.843809 -1.716667 -2.945594 H $-2.562609-0.192382-2.145725$ H $-4.4981170 .240121-3.066684$ H $1.181899-1.6967223 .244813$ H - $0.318272-3.7348921 .483141$ H $3.707828-4.5090662 .858406$ H $2.501907-3.8604933 .956377$ H $3.257067-2.8180412 .729783$ H $-2.266117-1.4235630 .056574$ H -2.318928 -2.946510 -0.829738 H $1.716314-1.704745-2.396921$ H $1.084088-2.399902-3.904921$ H $1.218951-4.747591-2.832578$ H $3.494389-3.114099-1.594216$ H $4.477656-5.402340-2.270596$ H $2.941034-6.162984-1.931685$ H $5.563127-5.423778 \quad 1.243419$ H $6.000564-4.899452-0.477904$ H $3.432337-6.3588751 .732978$ H $2.563446-7.0005200 .376328$ H $\quad 0.970229-5.2823730 .377758$
H $-0.900512-3.101223-2.773814$ C $-1.2670574 .014555-0.880056$ O $-0.1107633 .206619-1.171563$ C $0.6260153 .823516-2.246182$ C $-0.2150315 .002000-2.726455$ C $-0.9479575 .391412-1.457462$ H $1.5500444 .216141-1.807849$ C $0.9595892 .790347-3.328910$ H $0.4008045 .810227-3.132669$ H $-0.9288534 .704091-3.503220$ C $-2.1669496 .262197-1.706336$ H $-0.2502455 .923550-0.796774$ C $1.7154251 .551484-2.806486$ O $-0.2676122 .333049-3.912552$ H $1.5247363 .272873-4.135056$ H $1.0513540 .964350-2.163766$ O $2.0121890 .717212-3.933803$ C $3.0199791 .866425-2.053792$ C $3.7499590 .590775-1.591619$ H $2.7858872 .483330-1.181674$ O $3.8672382 .628470-2.911231$ H $3.0643800 .007628-0.966385$ H $3.996202-0.029026-2.462355$ H $-1.8774157 .197258-2.196884$ H -2.896326 $5.757526-2.348644$ H $-2.6655226 .516312-0.765940$ C -1.5366454 .0250090 .625340$ H $-0.038271 \quad 1.672260-4.588357$ H $2.6517351 .194235-4.490199$ H $4.5765923 .006512-2.364533$ H $-2.339174 \quad 4.7352970 .853850$ H -0.6412054 .3531641 .165042$ H -2.114252 $3.564859-1.415738$ C $4.114517 \quad 1.4684341 .312301$ O $4.894427 \quad 1.877204 \quad 0.183022$ C $5.0603300 .874331-0.822914$ C $5.732621-0.371326-0.225768$ C $5.011882-0.8693861 .027110$ C 4.7831080 .2807902 .008698 H 3.1052361 .1880450 .986167 C 4.0026912 .6755902 .250412 H $5.7641431 .291800-1.554114$ O $5.796711-1.402227-1.205011$ H $6.766519-0.1170620 .042578$ H $4.066005-1.3637680 .776121$ O $5.821428-1.8481341 .682102$ H $5.742706 \quad 0.5880132 .444027$ O $3.951406-0.1664333 .076018$ C 3.0904813 .7237031 .656481 H 3.5825502 .4042223 .224959 H 4.9925483 .1135302 .426882 H $\quad 6.320747-2.131703-0.834265$ H $5.874185-2.6228941 .098342$ H $4.409208-0.9082193 .507212$ O $3.7689124 .891073 \quad 1.472284$ O 1.9137943 .5248561 .383920 C 3.0064235 .9602310 .903209 H 3.6440106 .8472070 .860270 H $2.6962315 .701197-0.113197$ H 2.1360496 .1842321 .527696 SCF Energy (B3LYP/6-31G**//MMFF) $=-3245.91577996$

$05 \_00164$

MM̄MF Geometry

C $0.447978-2.831972-1.787106$

C $-0.481230-3.763901-1.527941$

C $-1.281460-3.788132-0.256149$

O $-2.655525-3.550214-0.650512$

C $-1.217929-5.1347530 .493025$

C $0.136943-5.4881661 .075688$

C $0.478814-5.3527702 .373087$

C $1.126237-6.1107150 .121323$

C $-0.343693-4.8411073 .522300$

C $-0.812132-3.3872273 .447748$

C $0.251653-2.3488253 .067255$

C $1.349850-2.2128274 .121810$

C $2.337834-1.0876813 .770999$

O $-0.437952-1.0991522 .921906$ 
O $3.124168-0.8112664 .940344$

C $1.546693 \quad 0.1977753 .445033$

C $3.324648-1.5170712 .678269$

O $2.412357 \quad 1.2223982 .959568$

C $0.384931-0.0254412 .450366$

C $-0.491616 \quad 1.2469822 .362774$

O $\quad 0.930567-0.3023601 .158331$

C $-3.362100-2.6250850 .050557$

O -3.001272 -2.022011 1.047806

C $-4.660379-2.431377-0.631339$

C $-5.501409-1.497562-0.162857$

C $-6.801130-1.133372-0.726142$

C $-7.237770-1.820357-1.992604$

C -1.824902 1.0425841 .627809

C -2.5466532 .3553451 .374815$

C $-4.3822633 .323898-0.002510$

O -2.2039013 .4163031 .890189$

C -5.8430923 .0837100 .208469$

C $-6.7409003 .048073-0.788478$

C $-8.2205692 .875702-0.584320$

C $-8.7690591 .650797-1.282844$

C $-9.2211951 .721138-2.546128$

C $-8.8683080 .366276-0.491453$

C $-7.525064-0.181800-0.101240$

N -3.6262352 .1980590 .514952$

H $0.629189-2.045570-1.059036$

H -0.674987 -4.547295 -2.256322

H $-0.936945-2.9860280 .405380$

H -1.549094 $-5.944761-0.171819$

H - $-1.977333-5.1284611 .285208$

H $1.479621-5.6698172 .666288$

H $1.971719-6.5714390 .644248$

H $0.647181-6.900314-0.466762$

H $1.537287-5.364100-0.561529$

H -1.213711 -5.4956513 .657468$

H $0.250900-4.965048 \quad 4.436654$

H -1.644256 -3.316356 2.741078

H -1.257145 -3.1105994 .413337$

H $\quad 0.683116-2.6197402 .098445$

H $1.888256-3.1578004 .259718$

H $0.893854-1.9826035 .094768$

H $3.576982-1.6325945 .197892$

H $\quad 1.1253370 .5697174 .389564$

H $3.891486-2.3994132 .999077$

H $4.069948-0.7360502 .492702$

H $2.838685-1.7650051 .732313$

H 3.1217051 .3372133 .614705

H -0.7277031 .6006003 .374892$

H $0.0749712 .042272 \quad 1.865292$

H $\quad 0.304499-0.0158300 .475642$

H $-4.876045-3.037484-1.503421$

H -5.207382 -0.9298640 .719590$

H -8.179895 -1.436698 -2.391054

H -7.374048 -2.893519-1.819766

H $-6.490846-1.683720-2.782814$

H $-1.662767 \quad 0.5512260 .664129$

H -2.488626 0.4101682 .227874

H -4.128235 $3.418650-1.063517$

H -4.0829194 .2478750 .501841$

H $-6.1816082 .968787 \quad 1.235531$

H $-6.4009843 .185709-1.812598$

H -8.718781 $3.774864-0.970474$

H -8.4760212 .8378170 .482056$

H -9.641230 $0.854755-3.046303$

H -9.177029 2.647339-3.109534

H $-9.455022-0.386387-1.030213$

H -9.4424130 .5578740 .424560$

H $-7.121254 \quad 0.2666360 .807073$

H $-3.787794 \quad 1.288510 \quad 0.091536$

C $2.767246-2.910427-2.696172$

O $3.211896-1.697930-2.052443$

C $4.509021-1.349135-2.568988$

C $4.977316-2.545968-3.388982$

C $3.661894-3.076017-3.922410$

H $4.360179-0.501549-3.248030$

C $5.454448-0.954091-1.431551$

H $5.678150-2.258652-4.178776$

H $5.467122-3.301042-2.762846$
C $3.748928-4.501514-4.439558$

H $3.317436-2.417800-4.731472$

C $4.9712590 .236067-0.577346$

O $5.612258-2.080987-0.559372$

H $6.448673-0.743433-1.843729$

H $4.112020-0.0656110 .032143$

$\begin{array}{lllll}\text { O } & 6.020301 & 0.542047 & 0.351841\end{array}$

C $4.6076681 .504548-1.373043$

C $4.4249702 .735745-0.464158$

H $3.6941641 .313795-1.945886$

O $5.6594191 .771690-2.302518$

H 3.8085262 .4547710 .395610

H $5.4071823 .036196-0.080357$

H $4.461376-4.566642-5.268534$

H $4.079683-5.192848-3.657177$

H $2.776099-4.846164-4.803607$

C $1.281167-2.797770-3.035757$

H $6.206735-1.8089330 .160856$

H $6.7791210 .870218-0.160644$

H $5.3221812 .402899-2.959821$

H $0.984729-3.607862-3.711356$

H $1.082565-1.848611-3.547797$

H $2.939419-3.729875-1.985605$

C $1.4705553 .562686-0.629546$

O $2.4677693 .653258-1.657728$

C $3.7959493 .937847-1.204644$

C $3.8137375 .252515-0.415564$

C 2.8012845 .2406510 .721451

C 1.4185324 .8580890 .197142

H $1.6932152 .713566 \quad 0.027014$

C $0.1293683 .293953-1.332934$

H $4.3948434 .112373-2.106165$

O 5.1199345 .5062070 .090807

H $3.5700146 .078253-1.096842$

H 3.1239124 .5745741 .530638

O 2.7413306 .5547661 .281661

H $1.0232695 .686452-0.404960$

O 0.5667634 .6989381 .329300

C $-0.0193661 .851324-1.771369$

H $-0.7037393 .502985-0.656191$

H $0.0328583 .922466-2.225612$

H 5.0774216 .3309550 .604554

H 2.0653156 .5393301 .980883

H -0.3531784 .6928521 .017387$

O $-1.3170521 .638537-2.144763$

O $0.8767281 .019369-1.790125$

C -1.633694 $0.304527-2.554188$

H -1.006465 $0.004066-3.398666$

H -1.509851 -0.383978 -1.713732

H -2.680335 $0.285232-2.869585$

SCF Energy (B3LYP/6-31G**//MMFF) $=-3245.92395797$

05_00165

MMFF Geometry

C -3.0883693 .0589740 .460707$

C -2.6243244 .0910001 .182011$

C -1.398853 4.1091292 .063194

O -0.6440252 .8834481 .943894$

C -0.4665145 .2648701 .646426$

C 0.7261205 .4411972 .568351

C 2.0140285 .2809902 .205143

C 0.3992755 .8524113 .982334

C 2.5705304 .9357030 .851779

C 2.9957423 .4688990 .781701

C $3.8273073 .126069-0.463025$

C $3.0425843 .296236-1.766329$

C $3.8517682 .837263-2.989696$

O $4.2393731 .759596-0.315063$

O $2.9325012 .752484-4.088977$

C $4.4048761 .421762-2.718132$

C $4.9025773 .878421-3.392041$

O $5.2624320 .996834-3.773907$

C $5.1125051 .288958-1.352337$

C $5.494963-0.170435-1.012393$

O $6.3302802 .043047-1.379122$

C -0.6140552 .0430923 .013028$

O $-1.271610 \quad 2.1239284 .037592$

C 0.4261301 .0254952 .739196 
C $\quad 0.859765 \quad 0.229725 \quad 3.728438$

C $1.952809-0.7428413 .634521$

C $2.724088-0.7892662 .342545$

C $4.334225-1.165763-1.063423$

C $4.640274-2.469000-0.342644$

C $3.529451-4.6232260 .274111$

O $5.690742-2.681795 \quad 0.255679$

C $2.650676-4.4533491 .474321$

C $3.002508-4.8078532 .719269$

C $2.109923-4.6175133 .913829$

C $2.810146-3.9327735 .068916$

C $2.970234-4.5634826 .244776$

C $3.334177-2.5269904 .867038$

C $2.232098-1.5137684 .706855$

N $3.579495-3.358549-0.436913$

H -2.5690532 .1049870 .467770$

H $-3.187379 \quad 5.0231101 .159722$

H -1.7406954 .2635883 .093214$

H -1.024484 6.2106351 .630936

H $-0.138661 \quad 5.0913190 .616281$

H 2.7795135 .4294452 .966639

H 1.2942476 .1180314 .555372

H -0.2568176 .7290893 .980792$

H -0.1020845 .0401794 .516894$

H 1.8610555 .1675370 .053587

H 3.4400175 .5814610 .675554

H 3.5825333 .2047561 .672355

H 2.1100352 .8230950 .816514

H $4.7184863 .764561-0.462154$

H $2.7172824 .333917-1.904355$

H $2.1218632 .697884-1.714287$

H $3.4176072 .392315-4.851401$

H $3.5406010 .747167-2.740075$

H $4.4186684 .831389-3.638322$

H $5.4367183 .573610-4.298750$

H $5.6369124 .073178-2.607779$

H $6.1165491 .447805-3.661342$

H $6.300348-0.515338-1.673877$

H $5.924677-0.176085-0.001011$

H $6.9174911 .702766-0.683714$

H $0.8455190 .995501 \quad 1.740170$

$\begin{array}{lllll}H & 0.394070 & 0.315961 & 4.709279\end{array}$

H $3.149297 \quad 0.193723 \quad 2.110324$

H $2.068852-1.0921181 .519820$

H $3.558942-1.4907522 .353784$

H $3.444233-0.731250-0.595667$

H $4.092782-1.403111-2.105923$

H $4.541853-4.9413560 .542411$

H $3.094792-5.362344-0.404987$

H $1.668662-4.0153221 .308418$

H $3.972772-5.2688182 .888843$

H $1.753581-5.6094914 .220334$

H $1.213146-4.0437293 .649308$

H $3.471840-4.0857917 .079897$

H $2.602821-5.5723196 .401235$

H $4.043338-2.5243744 .035990$

H $3.927980-2.2179655 .737890$

H $1.619073-1.3962915 .601663$

H $2.736719-3.064111-0.922608$

C -5.5058832 .4115760 .233394$

$\begin{array}{lllll}\text { O } & -5.270396 & 0.990027 & 0.189169\end{array}$

C $-6.4443010 .340933-0.332566$

C $-7.567216 \quad 1.361745-0.217744$

C $-6.8196272 .646106-0.515862$

H $-6.2520160 .151205-1.395684$

C $-6.694478-0.9866870 .389620$

H -8.383304 $1.164996-0.919261$

H $-7.986873 \quad 1.394470 \quad 0.794310$

C -7.563460 $3.897003-0.080392$

H -6.631454 2.700240 -1.596770

C $-5.479107-1.9349490 .423227$

O $-7.068669-0.7270681 .748637$

H -7.553204 -1.494188 -0.065008

H $-4.721859-1.5519361 .118572$

O $-5.916647-3.1901020 .957810$

C $-4.826682-2.187148-0.946511$

C $-3.687018-3.218591-0.874416$

H $-4.430995-1.242017-1.330195$
O $-5.809683-2.640337-1.876073$

H $-3.012475-2.941105-0.057436$

H $-4.100632-4.205167-0.632420$

H -8.519954 $3.978301-0.607001$

H -7.7718143 .8862750 .994753$

H $-6.9789834 .795713-0.300928$

C $-4.3232363 .151032-0.394310$

H $-6.352767-0.2100452 .156695$

H $-6.319951-3.0108711 .824624$

H -6.199358 -3.456017-1.517227

H $-4.5861854 .202350-0.560687$

H $-4.0833362 .719979-1.374243$

H -5.6132162 .6831391 .291647$

C $-1.389882-1.480748-1.874537$

O $-2.433628-2.057802-2.664795$

C $-2.903925-3.329578-2.202465$

C $-1.744208-4.334702-2.162210$

C $-0.565914-3.813286-1.341841$

C $-0.172244-2.410329-1.804406$

H $-1.770000-1.301926-0.861156$

C $-1.061239-0.093528-2.440361$

H $-3.614594-3.684461-2.959336$

O $-2.168814-5.583495-1.626349$

H $-1.394780-4.525643-3.185355$

H $-0.784098-3.829116-0.267691$

O $0.554301-4.679077-1.536396$

H $\quad 0.309039-2.495976-2.785459$

O $0.795602-1.867119-0.911595$

C $-0.532467-0.147772-3.854779$

H $-1.9652840 .527774-2.467840$

H $-0.3433190 .433891-1.801211$

H $-2.905788-5.901249-2.175583$

H $0.279819-5.572340-1.266425$

H $0.387046-1.802780-0.031536$

O $0.827391-0.045526-3.851452$

O $-1.242577-0.291578-4.841804$

C $1.452609-0.147533-5.134596$

H $1.164258-1.080357-5.628968$

H $1.1853480 .715807-5.750901$

H $2.535665-0.154122-4.986899$

SCF Energy (B3LYP/6-31G**//MMFF) = -3245.93641456

05_00166

MMFF Geometry

C -3.864674 -3.575835 -1.728916

C $-2.729370-3.369616-1.044876$

C $-1.365424-3.385684-1.686475$

O $-0.714545-2.112285-1.460143$

C $-0.488856-4.481656-1.054327$

C $0.883196-4.604525-1.694908$

C $2.052711-4.277145-1.107203$

C $0.888433-5.188152-3.086248$

$\begin{array}{llll}\text { C } 2.282485 & -3.714579 & 0.268019\end{array}$

C $2.966975-2.343804 \quad 0.258715$

C $4.418513-2.294756-0.247629$

C $5.361303-3.138787 \quad 0.614893$

C $6.836839-2.9386180 .238036$

O $4.801559-0.913070-0.186555$

O $7.640511-3.4939691 .290689$

C $7.141990-1.4279070 .189069$

C $7.205068-3.704022-1.038632$

O $8.465648-1.193298-0.287307$

C $6.127052-0.625041-0.653352$

C $6.3588370 .894778-0.459228$

O $6.289821-0.961862-2.031068$

C $-1.165449-1.040478-2.168187$

O $-2.137937-0.995130-2.904278$

C $-0.232013 \quad 0.078313-1.922014$

C $-0.5524851 .319460-2.317583$

C $0.3163832 .494353-2.221862$

C $1.7599432 .243027-1.880552$

C $5.3905281 .765695-1.268481$

C $5.1864703 .142303-0.666673$

C 3.9311694 .3091021 .153162

O $5.6520424 .151525-1.190053$

C 2.4656794 .4401600 .876629

C 1.8862975 .5090250 .309176

$\begin{array}{llll}\text { C } & 0.409023 & 5.591490 & 0.036448\end{array}$ 
C $0.0874985 .970386-1.393782$

C $-0.5690317 .109731-1.670174$

C $0.5244625 .040109-2.505059$

C $-0.1962263 .715696-2.480653$

N $4.413516 \quad 3.1133110 .483412$

H -3.814247 -3.800639 -2.791731

H $-2.779571-3.1287650 .013094$

H -1.444341 -3.549693 -2.768474

H $-0.993170-5.453740-1.140476$

H $-0.400394-4.2951630 .020636$

H $2.966362-4.450199-1.674345$

H $\quad 1.902725-5.365967-3.459740$

H $\quad 0.364190-6.149441-3.097149$

H $\quad 0.394541-4.512323-3.790791$

H $1.343240-3.605300 \quad 0.816023$

H $2.875543-4.4327040 .844441$

H $2.363965-1.639799-0.329995$

H $2.950553-1.9440061 .281532$

H $4.448714-2.618060-1.293481$

H $5.104533-4.2029060 .556812$

H $5.235157-2.8579191 .670090$

H $7.405393-4.4330871 .384112$

H $7.111975-1.0506731 .220968$

H $7.047504-4.780376-0.898611$

H $\quad 8.267584-3.586387-1.278094$

H $6.617848-3.397503-1.906781$

H $9.070671-1.7091030 .272910$

H $6.263701 \quad 1.1329910 .607567$

H $7.3794481 .163959-0.758432$

H $5.888504-0.268322-2.577559$

H $0.713271-0.158411-1.447648$

H -1.530098 $1.493397-2.764484$

H $2.1994281 .521316-2.578707$

H $1.8506141 .846463-0.863490$

H $2.384743 \quad 3.135008-1.932991$

H $5.7605641 .894327-2.292503$

H $4.4069751 .291869-1.350323$

H 4.4966305 .1841900 .818220

H 4.0952624 .1713852 .226186

H 1.8415903 .5965681 .160238

H $2.495727 \quad 6.3674320 .037630$

H $-0.013914 \quad 6.3290280 .730630$

H $-0.088034 \quad 4.6418920 .267299$

H -0.805745 $7.393906-2.690192$

H $-0.8862037 .785831-0.882943$

H $1.6117064 .934754-2.476871$

H $0.3183955 .496261-3.482895$

H - $1.2562713 .790848-2.726626$

H 4.0572302 .2196500 .810662

C $-5.956381-2.227587-1.688731$

O $-5.298373-1.036650-1.209367$

C $-6.295590-0.088780-0.787677$

C $-7.619078-0.603742-1.337882$

C -7.411995 -2.104094-1.240563

H $-6.333381-0.1372330 .307200$

C $-5.902517 \quad 1.324976-1.230691$

H -8.476866 -0.248790 -0.758863

H -7.768009 -0.307941 -2.382754

C -8.388346 -2.908307 -2.080695

H -7.508441 -2.405973 -0.188873

C $-4.4878241 .757130-0.789219$

O $-5.9455221 .401485-2.661302$

H $-6.6452892 .045072-0.868795$

H -3.731468 $1.193814-1.348410$

O $-4.3119903 .132551-1.148081$

C -4.2207711 .6074950 .718008$

C -2.8324932 .1279831 .132172$

H $-4.298503 \quad 0.5498150 .986533$

O $\quad-5.2221422 .3040491 .457969$

H -2.079634 1.6747020 .479403

H -2.782806 3.2129090 .979082

H -9.417207 -2.727581-1.752685

H -8.321658 -2.640352 -3.140514

H -8.188869 -3.980757 -1.991315

C $-5.238985-3.458736-1.131531$

H $-5.340140 \quad 0.724204-3.009638$

H $-4.4731473 .204803-2.104613$

H -5.1866833 .2388631 .191493$
H $-5.798168-4.370273-1.368836$

H $-5.170176-3.388145-0.038869$

H $-5.890849-2.206765-2.784676$

C -1.768228 -0.4519242 .356204$

O $-2.708490 \quad 0.4505942 .946592$

C -2.5081651 .8287172 .614741$

C -1.106454 2.2717423 .056509

C -0.0189561 .3672312 .480973$

C $-0.335264-0.1005142 .771022$

H $-1.853880-0.3911701 .264040$

C $-2.186244-1.8814642 .721729$

H $-3.2316132 .394253 \quad 3.215457$

O -0.8547493 .6182212 .672543$

H -1.0432252 .2329284 .151899$

H 0.1170991 .5446861 .407720

O $1.223897 \quad 1.7026543 .103865$

H $-0.174295-0.2806833 .840778$

O $0.592326-0.9052992 .052116$

C -2.027496 -2.200787 4.189427

H -3.235768 -2.055723 2.453028

H -1.597163 -2.613712 2.157802

H -1.552245 4.1703743 .065212

H 1.8988011 .1025072 .742652

H $0.499624-1.8192422 .369454$

O $-3.067962-1.6807604 .899900$

O $-1.083002-2.8309984 .647866$

C $-3.018876-1.9003146 .312902$

H -3.041392 -2.972366 6.530780

H -3.899839 -1.432666 6.760446

H -2.122443 -1.437664 6.736994

SCF Energy (B3LYP/6-31G**//MMFF) $=-3245.91567245$

05_00167

MMFF Geometry

C $-4.186357-2.833041-2.100520$

C $-3.210976-2.927790-1.184691$

C -1.786216 -3.292307-1.509536

O $-0.937598-2.182331-1.138646$

C $-1.366008-4.514536-0.673643$

C $0.054361-4.975535-0.933219$

C $1.044394-4.954515-0.019207$

C $0.325178-5.510775-2.315907$

C $0.979868-4.4952241 .410968$

C $1.389089-3.0309181 .634794$

C $2.844136-2.7210191 .246186$

C $3.256592-1.3376811 .752989$

C $4.676540-0.9594241 .303417$

O $2.950258-2.755604-0.179752$

O 4.8511640 .4469951 .531788

C $4.804010-1.186926-0.218300$

C $5.746620-1.6691522 .141406$

O $6.151175-1.010389-0.648622$

C $4.280350-2.561684-0.680793$

C $4.217740-2.703129-2.221950$

O $5.162489-3.584966-0.202776$

C $-0.896393-1.117800-1.985318$

O $-1.485162-0.992952-3.046986$

C $-0.016796-0.101151-1.373064$

C $-0.0975111 .173912-1.779151$

C $0.6858772 .289570-1.248132$

C $1.8428201 .953947-0.346489$

C $3.314145-1.695311-2.941182$

C $4.044588-0.472545-3.472506$

C $3.6028981 .723384-4.569473$

O $5.267993-0.371783-3.491857$

C $3.6261902 .805050-3.536142$

C $2.9913873 .978209-3.681573$

C $3.0448845 .090439-2.673711$

C $1.6721265 .573531-2.261628$

C $1.1298976 .670399-2.817205$

C $0.9695224 .837814-1.143727$

C $0.3466403 .546818-1.600337$

N $3.1616680 .481801-3.961377$

H $-3.968388-3.036084-3.145594$

H $-3.435562-2.702875-0.144049$

H - $-1.663915-3.505753-2.578495$

H $-2.041573-5.355361-0.880737$

H $-1.501549-4.2750850 .386637$ 
H $\quad 2.025423-5.318569-0.322977$ H $1.321986-5.956936-2.400854$ H $-0.402455-6.287695-2.572330$ H $0.260934-4.710729-3.059596$ H $-0.021283-4.6506101 .826129$ H $1.635667-5.1457802 .003969$ H $0.708756-2.3817091 .071670$ H $1.244229-2.8052592 .698722$ H $3.489919-3.4930171 .680765$ H $3.176928-1.2866812 .845602$ H $2.556771-0.5852081 .365873$ H 4.6938140 .6184722 .476133 H $4.215767-0.401268-0.705629$ H $5.640086-1.4054113 .200680$ H $6.753189-1.3462741 .853470$ H $5.697889-2.7574062 .066754$ H $6.433776-0.125477-0.360832$ H $\quad 5.230950-2.710305-2.641599$ H $3.808155-3.700843-2.437277$ H $5.040701-4.377820-0.751007$ H $0.652879-0.425129-0.584904$ H $-0.8227971 .432609-2.549471$ H $2.4991371 .219264-0.824063$ H 1.4828781 .5375720 .598747 H $2.4749822 .811565-0.109810$ H $2.874824-2.189698-3.817392$ H $2.488717-1.377867-2.297694$ H $2.911027 \quad 1.947246-5.387744$ H $4.6059381 .590521-4.987356$ H $4.2149642 .625158-2.639682$ H $2.4269024 .165938-4.592420$ H $3.6108695 .917607-3.122106$ H $3.6110654 .796373-1.781068$ H $0.1585977 .040542-2.506435$ H $1.6434827 .219385-3.599554$ H $\quad 0.1601905 .458671-0.735756$ H $1.6608884 .705628-0.306376$ H $-0.5033593 .686685-2.269666$ H $2.1619520 .335251-3.855818$ C $-5.902972-1.007400-2.236017$ O $-5.048792-0.072457-1.539490$ C $-5.8740360 .789660-0.744453$ C $-7.1973350 .844572-1.492116$ C -7.348721 $-0.604108-1.913872$ H $\quad-6.025077 \quad 0.2859800 .218646$ C $-5.2025652 .145251-0.508950$ H $-8.020617 \quad 1.196750-0.863615$ H -7.137694 $1.488102-2.377288$ C $-8.308263-0.805430-3.074615$ H -7.718952 -1.178976-1.054257 C -3.7547912 .0918660 .021749$ O $-5.1712202 .886551-1.735729$ H -5.8252622 .7351460 .174280$ H -3.061013 $1.863515-0.796480$ O $-3.418048 \quad 3.412770 \quad 0.469485$ C $-3.499271 \quad 1.1022521 .175029$ C -2.1268701 .3078971 .839032$ H -3.5807520 .0840060 .781731$ O -4.5100631 .2653542 .171463$ H -1.4390071 .7789621 .124968$ H -2.2307372 .0214302 .663510$ H -9.314871 -0.469475 -2.805024 H -7.991618 $-0.242621-3.959174$ H -8.365943 -1.863239 -3.350444 C -5.591555 -2.429671 -1.759913 H $-4.6886982 .351272-2.389321$ H -3.544239 4.014465 -0.284296 H -4.495200 2.1943862 .458917 H -6.281592 -3.144070 -2.223742 H $-5.740458-2.496650-0.674323$ H -5.685898 $-0.900316-3.305775$ C $-0.031411 \quad 1.0785184 .018439$ O $-0.087354 \quad 0.352616 \quad 2.781730$ C - $-1.415958 \quad 0.0256242 .332857$ C -2.103664 -0.8380553 .398312$ C $-2.122753-0.1411654 .755419$ C $-0.706266 \quad 0.2847725 .143484$ H -0.5318862 .0468393 .906913$
C 1.4484641 .3420094 .338275 H -1.283899 -0.6069361 .446258$ O $-3.414661-1.2046052 .998766$ H -1.536391 -1.773429 3.499303 H $-2.807516 \quad 0.714956 \quad 4.760870$ O $-2.595411-1.0494475 .752245$ H $-0.110114-0.6040215 .387602$ O $-0.767596 \quad 1.094048 \quad 6.316605$ C 2.0356982 .4448883 .481691 H $1.570517 \quad 1.6574465 .380151$ H $2.030910 \quad 0.430276 \quad 4.165540$ H -3.749960 -1.854582 3.638687 H -3.536181 -1.216466 5.573265 H -1.219545 0.5747157 .003759 O $3.3907182 .465758 \quad 3.654859$ O 1.3936153 .2099172 .775519 C 4.0867223 .4907652 .938028 H 3.7434114 .4783603 .260623 H 5.1524563 .3984823 .163374 H 3.9444453 .3645211 .860894 SCF Energy $(B 3 L Y P / 6-31 G * * / / M M F F)=-3245.91082080$

$05 \_00168$

MMFF Geometry

C $-0.8963585 .083781 \quad 1.716690$

C $-0.229067 \quad 4.6651280 .630591$

C 1.2180374 .2429070 .643757

O 1.2761222 .8193890 .393587

C $1.9764664 .962728-0.486701$

C $3.4588214 .648011-0.532909$

C $4.0782404 .036525-1.561841$

C 4.2611565 .0949820 .662133

C $3.4742623 .545109-2.847894$

C $2.9904612 .087083-2.812740$

C $4.1101071 .055574-2.599748$

C $3.576212-0.368550-2.772576$

C $4.651861-1.426586-2.476344$

O $4.6237221 .210833-1.275310$

O $3.994704-2.696129-2.338224$

C $5.312895-1.110960-1.116149$

C $5.639200-1.575047-3.640015$

O $6.429076-1.965028-0.880921$

C $5.739000 \quad 0.363939-0.968034$

$\begin{array}{llll}C & 6.205638 & 0.726964 & 0.462727\end{array}$

O $6.8405750 .625897-1.846165$

C $1.0678021 .996698 \quad 1.457974$

O 0.8609422 .3271242 .615069

C 1.1088280 .6019160 .968032

C $0.590793-0.3718001 .731317$

C $0.541007-1.7982041 .405002$

C $1.294566-2.2591060 .186131$

C 5.1664920 .5090261 .568377

C $5.313047-0.8158552 .298241$

C $4.193838-2.2070854 .035028$

O $6.273902-1.5659432 .150378$

C $3.440845-3.3020223 .348127$

C $2.425542-3.965873 \quad 3.920914$

C $1.691890-5.1008253 .266819$

C $0.195699-4.8861623 .237274$

C $-0.601544-5.4438754 .164182$

C $-0.381627-4.0993472 .083043$

C -0.156721 -2.619442 2.217506

N 4.256978-1.043542 3.170802

H -0.3874065 .1339482 .675641$

H $-0.7565854 .591986-0.318156$

H 1.6844954 .4701751 .610171

H $1.8637836 .049447-0.374411$

H $1.5029204 .707115-1.440921$

H $5.1522693 .870111-1.484407$

H $5.3392504 .980274 \quad 0.505051$

H 4.0734576 .1530250 .872183

H 3.9955924 .5087411 .547046

H $2.6423174 .189084-3.152958$

H $4.2248543 .657298-3.640832$

H $2.2377211 .982867-2.022830$

H $2.4899011 .878496-3.766333$

H $4.9053371 .256291-3.327357$

H $3.164542-0.511544-3.779407$ 
H $2.736961-0.527676-2.082213$

H $3.505401-2.871380-3.160307$

H $4.572796-1.345155-0.342301$

H $5.111069-1.870303-4.554582$

H $6.364611-2.372275-3.443685$

H $6.186285-0.655592-3.858063$

H $6.114152-2.881876-0.959427$

H 7.1529550 .2232900 .689518

H $6.442812 \quad 1.8008850 .461604$

H $7.3124471 .411516-1.523448$

H $1.5472230 .418968-0.005559$

H $0.142487-0.0988922 .686185$

H $0.843163-1.850255-0.723749$

H $1.323563-3.3445890 .073460$

H $2.338270-1.9322750 .237796$

H 5.3014121 .2899812 .328028

H 4.1478970 .6044951 .179993

H $3.712418-1.8938644 .966842$

H $5.209601-2.5459434 .263033$

H $3.763572-3.5813592 .347890$

H $2.124300-3.7032844 .932757$

H $1.925630-6.0153463 .827730$

H $2.055287-5.2798072 .247243$

H $-1.678888-5.3190734 .132890$

H $-0.193555-6.0337834 .978294$

H $-1.466807-4.2548262 .033052$

H $0.004607-4.5086291 .146047$

H $-0.664506-2.1842663 .079766$

H $3.474662-0.3956553 .187462$

C -3.2504224 .3018522 .092253$

O -3.2066423 .3036601 .048909$

C -4.5422013 .0953380 .551857$

C $-5.4462363 .490786 \quad 1.704217$

C -4.7198704 .7133942 .231763$

H $-4.6616173 .789166-0.290725$

C -4.6816381 .6483240 .069984$

H -6.4704863 .7083101 .388000$

H -5.4757722 .7013652 .465224$

C -5.1144395 .0926683 .648741$

H -4.9371995 .5614671 .568256$

C $-6.1064261 .275154-0.377760$

O $-3.7909561 .473871-1.038048$

H -4.3413010 .9638680 .855140$

H $-6.778168 \quad 1.2692200 .488612$

O $-6.5849122 .305210-1.254180$

C $-6.212038-0.061467-1.137549$

C $-5.710420-1.280586-0.345355$

H $-5.6826540 .024915-2.092751$

O $-7.589561-0.275400-1.471596$

H -4.702652 -1.0922720 .033001$

H -6.358128 -1.431220 0.526983

H -6.179852 5.3400793 .698621

H -4.9265724 .2731114 .350522$

H -4.547239 5.9648793 .989182

C -2.3475835 .4735561 .695963$

H $-2.897751 \quad 1.708658-0.732432$

H -7.483052 2.055877-1.531900

H -8.089604 $-0.347351-0.640611$

H -2.487188 6.3152212 .384109

H -2.616456 5.8298110 .693100

H -2.886035 3.8212543 .008700

C -3.544474 -2.297791-2.221282

O $-4.960576-2.412274-2.410746$

C $-5.713753-2.569865-1.201516$

C $-5.250646-3.831462-0.460016$

C $-3.739314-3.852004-0.244212$

C $-2.999075-3.563661-1.550637$

H $-3.331393-1.426740-1.591833$

C $-2.922108-2.046880-3.600168$

H -6.752339 -2.743597 -1.509743

O $-5.903529-3.9531610 .799066$

H -5.524257 -4.716067-1.050114

H -3.436600 -3.1476710 .539747$

$\begin{array}{lllll}\text { O } & -3.351460 & -5.151347 & 0.209226\end{array}$

H $-3.102166-4.423498-2.224846$

O $-1.612438-3.400084-1.272935$

C -1.567683 -1.384794 -3.520348

H $-2.827865-2.982189-4.164639$
H $-3.570395-1.404308-4.209717$

H $-6.861434-3.9616130 .631430$

H $-3.846582-5.3313791 .026898$

H $-1.302572-4.220181-0.852239$

O $-1.701602-0.099827-3.082832$

O $-0.518183-1.940639-3.817423$

C $-0.4823710 .635399-2.944091$

H $-0.0120700 .771521-3.922307$

H $-0.7214271 .617666-2.527789$

H $\quad 0.1960650 .121373-2.257619$

SCF Energy $(B 3 L Y P / 6-31 G * * / / M M F F)=-3245.90888365$

05_00169

MMFF Geometry

C $0.4352165 .215115-1.704599$

C $-0.0659934 .774433-0.540778$

C -1.496379 $4.337851-0.355196$

O $-1.4976412 .897774-0.210793$

C -2.071663 4.9523420 .933561

C -3.5190234 .5824151 .193896$

C -3.9457663 .8617892 .250032$

C -4.5172505 .0992650 .189874$

C -3.1271923 .2864723 .372154$

C -2.6199781 .8555013 .133160$

C -3.7343420 .8053452 .993240$

C $-3.146540-0.6080742 .973487$

C $-4.225190-1.6785382 .740635$

O $-4.434377 \quad 1.0452491 .770067$

O $-3.561426-2.9080652 .408443$

C $-5.077432-1.2809931 .515054$

C $-5.035927-1.9546964 .012546$

O $-6.192187-2.155801 \quad 1.364537$

C $-5.559896 \quad 0.183614 \quad 1.552271$

C $-6.2382240 .639610 \quad 0.237292$

O

C $-1.4625142 .161789-1.355565$

O $-1.5001882 .579802-2.502164$

C $-1.3284110 .739240-0.974488$

C $-0.909472-0.143089-1.893766$

C $-0.711319-1.579900-1.692559$

C $-1.159813-2.164175-0.379660$

C $-5.3683960 .541834-1.020762$

C $-5.588641-0.728591-1.825691$

C $-4.705718-1.941090-3.815255$

O $-6.498440-1.521861-1.600464$

C $-3.820055-3.052118-3.347030$

C $-2.886664-3.622865-4.123509$

C $-2.023155-4.772395-3.690224$

C $-0.547478-4.485809-3.853601$

C $0.128538-4.924933-4.928637$

C $0.159326-3.766149-2.727252$

C $-0.154296-2.296753-2.691327$

N $-4.668267-0.851172-2.858345$

H $-0.2120595 .295845-2.573882$

H 0.6003864 .6605290 .311694

H $-2.1117284 .628454-1.215351$

H -2.001544 6.0472420 .885401

H $-1.4433834 .646778 \quad 1.777240$

H -5.0136333 .6617772 .332998$

H -5.5516314 .9279540 .506718$

H -4.3933136 .1782060 .051622$

H -4.385283 $4.603772-0.776741$

H -2.2726263 .9341683 .596000$

H -3.7406423 .3031954 .282168$

H $-1.989526 \quad 1.8469972 .236558$

H -1.9779981 .5842223 .980578$

H -4.4231340 .9201063 .838515$

H -2.596664 -0.814316 3.900164

H $-2.406055-0.6820832 .165461$

H -2.960439 -3.131416 3.139931

H $-4.446006-1.4240560 .630099$

H -4.377855 -2.303332 4.817620

H $-5.762388-2.7588823 .851671$

H $-5.568783-1.0756824 .380846$

H -5.844814 -3.063102 1.321010

H $-7.197097 \quad 0.123210 \quad 0.109128$

H $-6.496298 \quad 1.701698 \quad 0.359467$

H $\quad-7.063431 \quad 1.1243292 .410189$ 
H $-1.557818 \quad 0.4642140 .047792$ H $-0.6733730 .221880-2.892798$ H $-0.585077-1.7352320 .448210$ H -1.045308 -3.247739 -0.316954 H $-2.222360-1.956876-0.214538$ H $-5.6314341 .372670-1.688196$ H $-4.3070350 .642908-0.776433$ H $-4.387379-1.535906-4.780986$ H -5.733631 -2.305450 -3.911291 H -3.973258 $-3.423324-2.336499$ H $-2.755801-3.267418-5.143341$ H $-2.302610-5.645091-4.294948$ H -2.226487 -5.057564 -2.650425 H $1.193401-4.748174-5.038395$ H $-0.369661-5.466884-5.725702$ H $1.246836-3.862629-2.843000$ H $-0.075161-4.275791-1.789905$ H $\quad 0.140889-1.771577-3.601147$ H -3.915912 -0.173340 -2.939328 C $2.6640354 .309276-2.377148$ O $2.7585023 .369102-1.284172$

C $4.1396622 .998709-1.103756$

C $4.8118523 .330274-2.423928$

C $4.1029944 .620215-2.791988$

H $4.5223333 .648266-0.305481$

C $4.2084641 .531552-0.667810$

H $5.8949923 .450804-2.331951$

H $4.6101402 .552397-3.170820$

C $4.2508145 .000011-4.255086$

H $4.5057705 .432003-2.171299$

C $5.6337850 .956373-0.618236$

$\begin{array}{llll}\text { O } & 3.629691 & 1.442039 & 0.639722\end{array}$

H $3.5824360 .920281-1.328182$

H $6.0344980 .879195-1.635971$

O 6.4734531 .8890110 .076492

C $5.761178-0.3997560 .102221$

C $4.828966-1.499266-0.435404$

H $5.596628-0.2510561 .174321$

O $7.118318-0.840814-0.027548$

H $3.789294-1.165528-0.371372$

H $5.047961-1.668696-1.496782$

H $5.3041295 .163273-4.505442$

H $3.866804 \quad 4.214398-4.914489$

H $3.7034605 .922047-4.475096$

C $1.8882985 .542960-1.906290$

H 2.7282411 .8033480 .583302

H 7.3697011 .5119190 .098660

H $7.291756-0.994009-0.972115$

H $1.9602796 .350335-2.643529$

H $2.3140475 .920983-0.968141$

H $2.1281913 .799483-3.188275$

C $3.582383-2.3325452 .215873$

O $4.893355-2.664384 \quad 1.743338$

C $4.994111-2.8356370 .325998$

C $4.051681-3.957316-0.131465$

C $2.622111-3.7513640 .365082$

C $2.599755-3.4525821 .863929$

H $3.251110-1.3892751 .764553$

C $3.678785-2.1229843 .730205$

H $6.017413-3.1821740 .134083$

O $4.040548-4.068498-1.550673$

H $4.415927-4.9150940 .262985$

H $2.113858-2.960653-0.198736$

O $1.873659-4.949551 \quad 0.144219$

H $2.847514-4.3633862 .423891$

O $1.282388-3.0605742 .243113$

C $4.346880-0.8030144 .034567$

H $2.690054-2.0950964 .202043$

H $4.243526-2.9277514 .215725$

H $4.956340-4.223480-1.838540$

H $1.966198-5.180133-0.796007$

H $\quad 0.678060-3.7728561 .971840$

O $5.697412-0.9693204 .119794$

$\begin{array}{lllll}\text { O } & 3.740376 & 0.254364 & 4.149727\end{array}$

C $6.4500210 .224993 \quad 4.353609$

H $\quad 6.172490 \quad 0.664457 \quad 5.316367$

H $7.509956-0.0412074 .381187$

H 6.2892430 .9398593 .540830
SCF Energy (B3LYP/6-31G**//MMFF)= -3245.91093520

05_00170

MM̄FF Geometry

C $3.317787 \quad 0.369426 \quad 2.396628$

C $2.949649-0.2423643 .533035$

C $1.617924-0.9093313 .783978$

O $0.801437-0.8420452 .591844$

C $1.719765-2.379608 \quad 4.254545$

C $2.826178-3.2192103 .642968$

C $2.748776-3.8712102 .467970$

C $4.074206-3.3576884 .481808$

C $1.599998-3.9399851 .505743$

C $1.932173-3.210584 \quad 0.199061$

C $1.071305-3.693942-0.974220$

C $1.347336-2.859544-2.225635$

C $0.463708-3.292057-3.404963$

O $-0.310277-3.595497-0.619040$

O $0.535048-2.266849-4.407936$

C $-1.004429-3.363888-2.934000$

C $0.979105-4.578202-4.061626$

O $-1.839602-3.916040-3.949286$

C $-1.196908-4.139242-1.608282$

C $-2.648563-3.971387-1.107046$

O $-0.948847-5.528387-1.837409$

C $-0.533949-0.6462582 .750020$

O $-1.160095-0.6384803 .797521$

C $-1.102812-0.3736671 .411513$

C $-2.226596 \quad 0.353851 \quad 1.331681$

C -2.9075800 .7893950 .111682$

C $-2.3757650 .300848-1.207260$

C $-2.893046-4.4819860 .317812$

C $-4.328515-4.2098320 .720052$

C $-5.846411-2.3560191 .417824$

O $-5.193418-5.079636 \quad 0.647032$

C $-6.234499-1.4273130 .309581$

C $-6.667300-0.1735170 .510996$

C $-7.0346650 .769685-0.599102$

C $-6.3207722 .101004-0.496903$

C $-6.9861073 .216354-0.151764$

C $-4.8509572 .154816-0.864747$

C -3.967172 1.6169860 .229059

N $-4.533456-2.8986341 .120094$

H $2.6121590 .448701 \quad 1.574129$

H $3.660688-0.2784374 .354960$

H $1.126775-0.3309034 .577990$

H $\quad 0.757977-2.8853894 .103620$

H $1.867980-2.3751005 .343395$

H $3.614979-4.4536082 .153609$

H $4.827837-3.9951004 .006786$

H $4.539882-2.3854884 .661208$

H $3.829153-3.8077755 .449597$

H $1.406811-5.0010031 .303837$

H $0.677372-3.5369801 .933743$

H $1.782124-2.1353020 .355398$

H $2.987793-3.352218-0.064060$

H $1.320907-4.746403-1.155259$

H $2.408062-2.905653-2.501975$

H $1.139995-1.802752-2.008049$

H $1.468519-2.157618-4.658624$

H $-1.346012-2.331043-2.781503$

H $2.001635-4.435893-4.431669$

H $0.378248-4.842118-4.938927$

H $0.990779-5.432152-3.381113$

H $-1.700349-3.391157-4.756168$

H $-2.917329-2.910610-1.132822$

H $-3.338994-4.488200-1.786292$

H $-1.409189-6.048965-1.160808$

H $-0.557446-0.7258880 .544301$

H $-2.688400 \quad 0.6899322 .259589$

H $-2.582686-0.764097-1.325635$

H $-1.2946730 .454858-1.279596$

H -2.807591 $0.818121-2.066954$

H $-2.718037-5.5597450 .396399$

H -2.225145 -3.9941791 .036957$

H $-5.774184-1.8418432 .381326$

H $-6.578491-3.1648151 .501275$

H $-6.153017-1.804321-0.707433$ 
H $-6.770770 \quad 0.195807 \quad 1.528937$

H $-8.1214770 .919212-0.561326$

H $-6.8268940 .328774-1.582095$

H -6.487215 $4.178385-0.098582$

H -8.0433563 .1923360 .090424$

H -4.549485 $3.193036-1.054905$

H -4.719852 $1.621288-1.810673$

H -4.2446251 .9587881 .227403$

H -3.747132 -2.255431 1.126082

C 5.2806480 .4980590 .798405

O $4.5335251 .111382-0.273262$

C $4.1612960 .090838-1.216398$

C $5.154221-1.042291-0.989519$

C $5.291967-1.0094720 .521661$

H $3.158252-0.249342-0.929992$

C $4.1097220 .652382-2.640624$

H $4.788749-2.001018-1.368557$

H $6.123635-0.835393-1.457115$

C $6.527578-1.7188061 .046394$

H $4.409343-1.4953070 .951335$

C $3.3369201 .980743-2.775381$

O $5.4495530 .895266-3.088710$

H $3.695230-0.107649-3.313737$

H $3.9253862 .786695-2.320277$

O $3.2600852 .303466-4.169864$

C $1.9212781 .990541-2.168434$

C $1.2046783 .334086-2.406479$

H $1.9950991 .808390-1.091929$

O $1.145827 \quad 0.928500-2.717667$

H $1.8715584 .143630-2.090548$

H $\quad 1.0250373 .466986-3.480377$

H $6.505073-2.7798440 .777142$

H $7.445139-1.2864440 .633611$

H $6.580581-1.6472212 .137564$

C 4.6901920 .9316282 .147934

H $\quad 5.393910 \quad 1.244264-3.994831$

H $2.7862571 .582355-4.617595$

H $0.9827751 .127517-3.654999$

H 4.6068352 .0251302 .164505

H $5.375930 \quad 0.6377022 .951341$

H 6.2964210 .9059620 .721662

C $0.670877 \quad 4.0891590 .497144$

O $-0.0494313 .128387-0.280106$

C $-0.1517653 .444147-1.672900$

C $-0.8505394 .799988-1.848580$

C $-0.1520935 .907066-1.059945$

C 0.0419015 .4859940 .398123

H 1.7014494 .1379940 .122921

C $0.7920953 .562511 \quad 1.934401$

H $-0.8070672 .685045-2.115368$

O $-0.9072595 .144391-3.228831$

H -1.886597 4.713306 -1.495487

H $0.7988896 .184530-1.528840$

O $-0.9735867 .076143-1.103300$

H -0.9331635 .5168500 .894338$

O $0.866831 \quad 6.441981 \quad 1.060735$

C -0.5180533 .3832642 .673729$

H 1.2658202 .5747681 .903629

H 1.4245224 .2390622 .520142

H -1.338707 $6.013765-3.293538$

H $-0.5396357 .755125-0.558672$

H $1.737101 \quad 6.437943 \quad 0.626719$

O -0.2441393 .0044363 .958630$

O -1.6337883 .5387812 .199163$

C -1.3843032 .8036984 .799753$

H -1.0336412 .4175865 .760389$

H -1.896330 3.7557874 .967650

H -2.066805 2.0724154 .357490

SCF Energy (B3LYP/6-31G**//MMFF) $=-3245.91099150$

0500171

MM̄FF Geometry

C $5.2997142 .953549-0.790411$

C $4.1036293 .144861-0.215730$

C $2.9111873 .743789-0.917101$

O $1.9069262 .713470-1.062386$

C $2.3194814 .870384-0.048879$

C $1.0603345 .491759-0.621515$
C $-0.1788145 .341423-0.109936$

C $1.2698846 .355336-1.839250$

C $-0.6029774 .543576 \quad 1.094747$

C -1.3616373 .2672340 .719001$

C -2.7621883 .4788630 .124117$

C -3.7606293 .9633551 .181115$

C -5.1970414 .0326810 .640339$

O $-3.1815602 .209527-0.403574$

O $-6.085592 \quad 4.1566801 .762100$

C $-5.5347122 .696619-0.054198$

C $-5.4191885 .278488-0.225647$

O $-6.8053612 .763080-0.696693$

C $-4.4562912 .243295-1.061057$

C $-4.7407970 .841127-1.643894$

O $-4.4417083 .152034-2.170004$

C $2.0571721 .827675-2.085492$

O $2.947471 \quad 1.797255-2.919065$

C $0.9508770 .855465-1.983608$

C $1.119751-0.397642-2.429344$

C $0.150553-1.481757-2.268971$

C $-1.238927-1.120294-1.810999$

C $-4.739126-0.313691-0.638578$

C $-4.982047-1.646870-1.329275$

C $-5.099208-4.089452-0.875698$

O $-5.253539-1.742448-2.523994$

C $-3.797048-4.698825-1.288931$

C $-3.433711-4.882723-2.567759$

C $-2.148379-5.534942-2.992423$

C $-1.093008-4.523058-3.387385$

C $-0.930559-4.177918-4.676010$

C $-0.256118-3.997305-2.239950$

C $0.540647-2.750736-2.509511$

N $-4.881343-2.718714-0.452857$

H $5.4535763 .249256-1.824828$

H 3.9590332 .8256680 .813767

H $3.1812114 .136111-1.905322$

H 3.0679665 .6633540 .084877

H 2.1294044 .4828770 .957033

H $-0.9979635 .857009-0.609893$

H $0.3479346 .850428-2.162627$

H $2.0047127 .138832-1.627197$

H $1.6316895 .755147-2.679704$

H $\quad 0.2556014 .2585541 .708142$

H $-1.2165195 .183087 \quad 1.738558$

H $-0.7584602 .684793 \quad 0.014131$

H -1.4507662 .6314291 .608998$

H -2.696421 $4.190984-0.704958$

H -3.4667394 .9378861 .587483$

H -3.7492243 .2692772 .033386$

H -5.8344094 .9554162 .256755$

H $-5.624547 \quad 1.9406190 .735891$

H $-5.254766 \quad 6.1885250 .364072$

H $-6.4544435 .334895-0.579465$

H -4.754204 $5.325200-1.090310$

H $-7.4532103 .046690-0.029150$

H $-5.691006 \quad 0.858225-2.194415$

H -3.976848 $0.622779-2.403465$

H $-4.0193082 .711157-2.925790$

H $0.0497781 .175940-1.476322$

H $2.071030-0.671241-2.883123$

H - $1.662812-0.342215-2.455571$

H -1.228079 -0.753479 -0.779854

H -1.936169-1.960323 -1.852972

H $-3.777836-0.361581-0.115577$

H $-5.533279-0.1806140 .103028$

H $-5.831897-4.107688-1.689783$

H -5.519064 -4.640025 -0.028300

H -3.128964 -5.020903 -0.494674

H $-4.099303-4.554035-3.362770$

H $-2.368470-6.209343-3.830223$

H $-1.755768-6.183420-2.198661$

H $-0.171751-3.473505-4.996713$

H -1.560254 -4.601897 -5.451780

H $\quad 0.443351-4.786598-1.934396$

H $-0.891840-3.831103-1.362267$

H $1.556466-2.935124-2.858477$

H $-4.588127-2.5545760 .505752$

C $6.6809650 .877863-0.567521$ 
O $5.5962630 .041996-0.109075$

C $6.138575-1.194480 \quad 0.392125$

C $7.593117-1.228847-0.060410$

C $7.9547420 .242270-0.011534$

H $6.113316-1.1298611 .486228$

C $5.295007-2.382963-0.081967$

H $8.216368-1.8477110 .592321$

H $7.692136-1.610464-1.083258$

C $9.210236 \quad 0.585135-0.794434$

H $8.0943920 .535163 \quad 1.037844$

C $3.801627-2.2997640 .295537$

O $5.370086-2.469628-1.511676$

H $5.725002-3.316804 \quad 0.297849$

H $3.329860-1.486839-0.266454$

O $3.172587-3.512242-0.139019$

C $3.524627-2.1309871 .800785$

C $2.043425-2.2502792 .205406$

H $3.917446-1.1715512 .144726$

O $4.246635-3.1311062 .529154$

H $1.711097-3.2859122 .045777$

H $1.978911-2.1260493 .293740$

H $10.074306 \quad 0.048290-0.389481$

H $9.1111440 .313811-1.850855$

H $9.4236691 .657282-0.740776$

C $6.4614892 .312387-0.083940$

H $5.052660-1.624304-1.873895$

H $3.351303-3.606483-1.090402$

H $3.951520-3.9999032 .205983$

H $7.3518982 .922450-0.272496$

H 6.2889392 .3163390 .999701

H $6.6752550 .833862-1.664624$

C $-0.822705-1.6589002 .989910$

O $-0.294798-1.7750861 .664388$

C $1.051636-1.3040361 .500837$

C $1.112875 \quad 0.1806591 .880124$

C 0.5898950 .4209093 .293219

C $-0.798453-0.1976193 .456100$

H $-0.237593-2.2811743 .678262$

C -2.261259-2.1981112.954396

H $\quad 1.245515-1.3776150 .429217$

$\begin{array}{llll}\text { O } & 2.425831 & 0.705994 & 1.767887\end{array}$

H $\quad 0.4836740 .7521891 .186519$

H 1.2837470 .0379574 .050419

O $\quad 0.481924 \quad 1.827333 \quad 3.524516$

H -1.529019 0.3906432 .886060

O $-1.180113-0.1367684 .828833$

C -2.310346 -3.702430 2.782532

H $-2.776810-1.9636133 .892243$

H -2.806958 -1.7510442 .115089$

H 2.7070010 .5932800 .843874

H 1.3580662 .2157573 .358239

H -1.1328260 .7955455 .101796$

O $-3.616377-4.1043952 .799903$

O $-1.337708-4.4333582 .657257$

C -3.830323 -5.515655 2.694112

H -3.475314 -6.013809 3.600991

H -4.904935 -5.6897022 .594100$

H -3.327127 -5.920416 1.811298

SCF Energy (B3LYP/6-31G**//MMFF) $=-3245.92498664$

0500172

MMFF Geometry

C $2.6056843 .706568-1.363928$

C $3.1452073 .172703-2.472119$

C $4.1014642 .002535-2.537229$

O $4.2045401 .397064-1.230908$

C $3.5828670 .946245-3.529576$

C $4.656292-0.009694-4.015204$

C $4.763807-1.295547-3.627943$

C $5.5998520 .548152-5.049395$

C $3.924047-2.000579-2.603184$

C $2.718647-2.701112-3.239439$

C $1.886394-3.465402-2.200329$

C $0.643271-4.080304-2.846662$

C $-0.264679-4.762774-1.811919$

O $1.500332-2.543220-1.178703$

O $-1.534300-5.013088-2.434877$

C $-0.521461-3.784432-0.645631$
C $0.285555-6.128484-1.384389$

O $-1.222956-4.430536 \quad 0.413313$

C $0.762020-3.126719-0.099064$

C $0.466081-1.9989890 .914372$

$\begin{array}{llll}\text { O } & 1.539994 & -4.117665 & 0.582726\end{array}$

C $5.431287 \quad 1.050226-0.764431$

O $6.4979341 .118857-1.351364$

C 5.2404850 .5961940 .629845

C $6.2984690 .302281 \quad 1.398756$

C $6.197089-0.0630212 .808367$

C 7.1603590 .6876413 .686223

C $-0.274361-0.787862 \quad 0.336636$

C -0.3066920 .3418901 .345517$

C 1.1990451 .9480532 .513445

O $\begin{array}{llll}1.317291 & 0.605653 & 1.991921\end{array}$

C 1.5778661 .1944063 .751029

C 2.8396791 .0409204 .179854

C 3.2109560 .2422725 .397155

C $3.614276-1.1786775 .063476$

C $2.740976-2.1906345 .202607$

C $5.045162-1.4384134 .640613$

C $5.311739-0.988198 \quad 3.229765$

N 0.9161150 .9851491 .465740

H $2.8493343 .301549-0.385958$

H $2.9055303 .630296-3.430695$

H $5.0711192 .402568-2.856689$

H $3.1569451 .432749-4.417735$

H $2.7527120 .394429-3.072309$

H $5.545772-1.908939-4.072654$

H $6.308339-0.203473-5.413796$

H $5.0400900 .917906-5.914702$

H $6.1842181 .374496-4.633792$

H $4.554123-2.738341-2.090993$

H $3.599244-1.299207-1.827551$

H $2.088158-1.943646-3.723293$

H $3.053649-3.392699-4.022592$

H $2.519011-4.245396-1.760693$

H $0.924760-4.786379-3.637770$

H $0.063007-3.290034-3.343881$

H $-1.379453-5.569059-3.217736$

H $-1.185134-3.000370-1.026871$

H $0.373723-6.794004-2.251603$

H $-0.397876-6.630709-0.690984$

H $1.270060-6.066766-0.915982$

H -2.024959 -4.8265380 .031568$

H $-0.085390-2.397847 \quad 1.775870$

H $1.424578-1.6573341 .329530$

H $2.142079-3.6695241 .199395$

H 4.2262810 .5516751 .014185

H 7.3087320 .3901031 .005931

H 7.0066961 .7678553 .582592

H 8.1937250 .4592213 .402043

H 7.0595710 .4537334 .747591

H $0.204537-0.420715-0.578044$

H $-1.306912-1.043047 \quad 0.080174$

H 0.3136112 .5632502 .701780

H 2.0080142 .5943582 .159084

H 0.7671860 .7319264 .309601

H 3.6500251 .5000393 .620168

H $4.0278980 .748116 \quad 5.926227$

H 2.3718090 .2373576 .106035

H $3.024012-3.2159704 .989102$

H $1.720441-2.0148945 .527078$

H $5.264424-2.5133324 .686196$

H $5.714329-0.9783855 .372363$

H $4.689198-1.4851842 .484293$

H 1.7062310 .6361730 .931154

C $0.4173524 .667117-0.551441$

O $-0.3429663 .562751-1.078884$

C $-1.717468 \quad 3.969512-1.234051$

C $-1.870305 \quad 5.231510-0.399831$

C $-0.5151515 .880489-0.578305$

H $-1.8421974 .190508-2.301296$

C -2.647790 $2.827471-0.805799$

H $-2.6952375 .864752-0.739434$

H -2.0398214 .9832830 .655496$

C $-0.196838 \quad 6.9248150 .477975$

H $-0.4865436 .356091-1.567985$ 
C $-4.1403993 .178583-0.945492$

O $-2.3676481 .685680-1.620060$

H -2.415607 2.5431970 .225534

H $-4.3862144 .038176-0.311026$

O $-4.370323 \quad 3.593849-2.299077$

C -5.107359 $2.019674-0.634685$

C $-5.009346 \quad 1.5330730 .821435$

H $-4.9428031 .201291-1.344579$

O $-6.4333382 .511010-0.864273$

H -3.983401 1.2206241 .032292

H -5.2328682 .3738511 .489835$

H $-0.928140 \quad 7.7390740 .446564$

H $-0.215498 \quad 6.494897 \quad 1.485201$

H $\quad 0.795818 \quad 7.355668 \quad 0.313875$

C $1.6788714 .890128-1.390670$

H - $1.4183871 .492851-1.532476$

H $-5.3152103 .810251-2.378509$

H -7.021579 $1.741330-0.945162$

H $2.2246665 .759313-1.005598$

H $1.3930215 .113040-2.426472$

H $0.6850814 .403500 \quad 0.479527$

C $-4.610609-1.4425160 .354528$

O $-5.840224-0.7169990 .234946$

C $-5.980364 \quad 0.375730 \quad 1.147905$

C $-5.895889-0.1358302 .592900$

C $-4.632159-0.9555122 .835528$

C $-4.471092-2.0314871 .762627$

H -3.767716 -0.7743410 .143560$

C $-4.624563-2.544638-0.709645$

H $-6.9975330 .762291 \quad 1.011201$

O $-5.9372330 .943543 \quad 3.520198$

H $-6.764977-0.7732052 .802241$

H $-3.744008-0.3160032 .886078$

O $-4.728796-1.6035504 .106368$

H $-5.219143-2.8192661 .918612$

O $-3.184190-2.6293841 .895283$

C $-4.456500-1.960689-2.092310$

H -3.828254 -3.280562 -0.556179

H $-5.574900-3.091818-0.695300$

H -6.7579351 .4386743 .355731$

H -4.864014 -0.9095194 .774337$

H $-3.112587-2.9684212 .803954$

O $-3.145297-1.659549-2.312871$

O $-5.380455-1.785566-2.875774$

C $-2.846007-1.096296-3.593189$

H $-1.778615-0.860588-3.618478$

H $-3.065231-1.820830-4.383016$

H -3.413287 -0.173233 -3.746230

SCF Energy (B3LYP/6-31G**//MMFF) $=-3245.91240664$

0500173

MM̄FF Geometry

C $3.984609-3.458617-0.717064$

C $2.883843-3.295107-1.466019$

C $1.499762-3.237007-0.876295$

O $0.799803-2.191259-1.591240$

C $0.752774-4.570699-1.066313$

C $-0.381831-4.781342-0.078095$

C $-1.687113-4.892022-0.394769$

C $0.036848-4.9296811 .365620$

C $-2.336516-4.849525-1.747681$

C $-3.044503-3.528393-2.081959$

C $-4.221281-3.168215-1.157680$

C $-5.009655-1.992601-1.742657$

C $-6.146914-1.539901-0.815418$

O $-3.709171-2.8011780 .127039$

O $-6.598333-0.252054-1.265254$

C $-5.589748-1.3432630 .612141$

C -7.356124 -2.477439 -0.913078

O $-6.642427-1.0966891 .539802$

C $-4.718900-2.5183811 .109316$

C $-4.001738-2.2295092 .450180$

O $-5.515052-3.6807501 .375439$

C $-0.014421-1.367977-0.882876$

O $-0.239499-1.4075280 .315428$

C $-0.614695-0.408339-1.838393$

C $-1.645643 \quad 0.347547-1.435999$

C $-2.3920481 .316418-2.239376$
C $-1.9190861 .585878-3.642380$

C $-3.056168-1.0254182 .455044$

$\begin{array}{llll}\text { C } & -3.709310 & 0.278261 & 2.886028\end{array}$

C -3.3607162 .7485372 .792579$

$\begin{array}{lllll}\text { O } & -4.765056 & 0.327707 & 3.511279\end{array}$

C $-4.231538 \quad 3.2240021 .672527$

C $-3.871360 \quad 4.169937 \quad 0.791672$

C $-4.7480174 .635631-0.336339$

C $-4.167197 \quad 4.322823-1.699055$

C $-3.5419795 .269618-2.418737$

C $-4.3898452 .937602-2.267012$

C $-3.4593241 .917147-1.673823$

N $-2.948684 \quad 1.3824452 .524486$

H $3.877628-3.6122020 .354106$

H $2.980386-3.159414-2.540389$

H $1.586991-2.9702400 .182745$

H $1.446914-5.412105-0.935445$

H $0.393294-4.632457-2.098354$

H $-2.389152-5.066194 \quad 0.420382$

H $-0.787638-5.2631772 .005420$

H $0.836160-5.6727011 .455318$

H $\quad 0.396288-3.978668 \quad 1.768803$

H - $1.611077-5.063335-2.538577$

H $-3.065478-5.668575-1.797658$

H $-2.304743-2.722575-2.066222$

H -3.412282 -3.606588 -3.112828

H $-4.865615-4.049435-1.056893$

H $-5.403593-2.243233-2.735554$

H $-4.332248-1.143321-1.897995$

H $-6.867245-0.336308-2.196134$

H $-4.974232-0.4357590 .584469$

H -7.763115 $-2.472225-1.931558$

H $-8.172111-2.141577-0.264030$

H $-7.115882-3.513058-0.663598$

H -7.143743 -0.3303891 .212353$

H $-4.736114-2.1625063 .262210$

H $-3.392061-3.1144272 .684218$

H -6.451235 -3.4243831 .358407$

H $-0.218532-0.375452-2.846657$

H $-1.993816 \quad 0.238227-0.411346$

H $-2.0630190 .699048-4.269051$

H $-0.856892 \quad 1.851165-3.655570$

H $-2.4437802 .413911-4.124454$

H -2.253990 -1.213238 3.179972

H -2.587608 -0.9020351 .476427$

H -2.4527653 .3501772 .896062$

H $-3.913747 \quad 2.787428 \quad 3.736397$

H -5.2112352 .7601081 .582593$

H -2.8949604 .6389410 .882481$

H $-4.887821 \quad 5.718802-0.224177$

H $-5.7539584 .203489-0.264347$

H -3.148394 $5.059472-3.407644$

H -3.402689 $6.273457-2.031269$

H $-4.3205362 .956223-3.359558$

H -5.422937 $2.626375-2.065010$

H $-3.7279651 .626143-0.658963$

H -2.1217041 .2467091 .950616$

C $6.236363-2.432594-0.440509$

O $5.703398-1.100308-0.557551$

C $6.792771-0.160073-0.485927$

C $8.067842-0.981716-0.301033$

C $7.681222-2.315244-0.912936$

H $6.8375220 .341924-1.458951$

$\begin{array}{llll}\text { C } & 6.529624 & 0.865294 & 0.623992\end{array}$

H $8.928913-0.518874-0.793459$

H $8.323869-1.1059370 .757586$

C $8.576123-3.462767-0.479480$

H $7.713145-2.221793-2.006687$

C $5.180872 \quad 1.607616 \quad 0.496594$

$\begin{array}{lllll}\text { O } & 6.526561 & 0.180255 & 1.884008\end{array}$

H $7.359647 \quad 1.5804000 .666155$

H 4.3642790 .9012520 .669708

O $5.1209092 .561227 \quad 1.565216$

C $4.9741372 .339656-0.838631$

C $3.7161783 .228722-0.914716$

H $4.9520821 .615854-1.660534$

$\begin{array}{llll}\text { O } & 6.113447 & 3.182084 & -1.053253\end{array}$

H $3.6760173 .887974-0.041207$ 
H $3.8496113 .903175-1.770178$ H $9.613523-3.274159-0.774865$ H $8.555455-3.5996310 .606938$ H 8.259805-4.402191-0.943309 C $5.386973-3.409961-1.254479$ H $6.338147 \quad 0.8407232 .572795$ H 5.8108413 .2274491 .403082 H $6.0370983 .557889-1.946951$ H $5.816401-4.416224-1.200368$ H $5.375934-3.105328-2.308171$ H $6.202942-2.7003320 .624648$ C 1.5840842 .4349581 .186753 O 2.0164351 .6813570 .048051 C $2.3938512 .450897-1.095991$ C $1.2156333 .334040-1.533907$ C $0.7327784 .225984-0.395890$ C $0.4242093 .375360 \quad 0.833014$ H 2.4278533 .0105091 .583706 C 1.1342351 .4364472 .261602 H $2.5661511 .729503-1.905219$ O $1.5785964 .117333-2.664740$ H $0.3896802 .686710-1.845922$ H $1.4581045 .013299-0.159901$ O $-0.4725294 .886207-0.785702$ H -0.4896442 .7949820 .650208$ O $\quad 0.166677 \quad 4.232821 \quad 1.943573$ C 2.2905460 .6137562 .776401 H $0.690497 \begin{array}{lll}1.942185 & 3.126785\end{array}$ H $\quad 0.3672990 .762444 \quad 1.862351$ H $\quad 0.7728304 .548308-2.995786$ H $-0.2497305 .507635-1.499124$ H -0.5510004 .8358681 .684671$ O $2.193684-0.6658942 .316293$ O 3.1782221 .0599863 .491646 C $3.224509-1.5622792 .741830$ H $3.400347-1.4790913 .818804$ H $2.897611-2.5822282 .523405$ H $4.141792-1.3584052 .183745$

SCF Energy (B3LYP/6-31G**//MMFF) $=-3245.90980946$

0500174

MMFF Geometry

C -2.862803 -2.639387 -2.688466

C -1.939329 -2.770900 -1.724348

C $-0.464642-2.953317-1.965703$

O $0.236144-1.919206-1.234423$

C -0.015214 -4.314544 -1.405980

C $1.466483-4.586619-1.576592$

C $2.337625-4.736580-0.559150$

C $1.948545-4.712664-2.998942$

C $2.056798-4.6836470 .916942$

C $2.239994-3.2990151 .557066$

C $3.682902-2.7708281 .508849$

C $3.829471-1.5190772 .376868$

C $5.241603-0.9191282 .285689$

O $4.004718-2.447626 \quad 0.153641$

$\begin{array}{lllll}\text { O } & 5.197095 & 0.399551 & 2.852687\end{array}$

C $5.627274-0.7576740 .799168$

C $6.253938-1.7146773 .118328$

O $6.990705-0.3645010 .666250$

C $5.358574-2.018439-0.048007$

C $5.544482-1.782816-1.567449$

O $6.283156-3.045251 \quad 0.332231$

C $0.294227-0.680674-1.793717$

O $-0.143667-0.340361-2.880717$

C $0.9552410 .212643-0.817309$

C $0.8367051 .541129-0.953510$

C $1.3731022 .552457-0.042221$

C 2.3638812 .1009030 .996760

C $4.644769-0.704604-2.182318$

C $5.2980980 .664469-2.282883$

C $4.7720433 .024693-2.890463$

O $6.4926960 .859381-2.076282$

C $4.5260073 .813315-1.643123$

C $3.8099734 .947940-1.618291$

C $3.5914195 .774858-0.383485$

C $2.1287316 .054795-0.119218$

C $1.5695147 .218542-0.491735$
C 1.3394565 .0223920 .653068

C $0.9667143 .830957-0.186779$

N $4.3910831 .641440-2.672619$

H -2.570968 -2.688288 -3.733480

H $-2.254584-2.714019-0.684683$

H $-0.222964-2.880943-3.032991$

H $-0.571920-5.121268-1.901498$

H $-0.292524-4.364807-0.347352$

H $3.380926-4.931846-0.805364$

H $2.990344-5.046549-3.056356$

H $1.341519-5.443842-3.542526$

H $1.884811-3.750159-3.515465$

H $1.044576-5.0424881 .131148$

H $2.721615-5.4015651 .414272$

H $1.568287-2.5868131 .064322$

H $1.920360-3.3728292 .603972$

H $4.351815-3.5626361 .865879$

H $3.572260-1.7355453 .421192$

H $3.108769-0.7606252 .043130$

H 4.8812600 .3212203 .769342

H 5.0251040 .0685510 .404806

H $5.953977-1.7303004 .172990$

H $7.242092-1.2422573 .096176$

H $6.358931-2.7508892 .790474$

H 7.1113900 .4451401 .191294

H $6.602602-1.605560-1.794854$

H $5.294274-2.726766-2.073111$

H $6.349016-3.685690-0.395441$

H $1.481459-0.2459690 .011913$

H $0.2644161 .929153-1.794585$

H $3.157551 \quad 1.5049260 .534715$

H 1.8707031 .4947221 .762511

H 2.8684162 .9252021 .505130

H $4.403996-1.001034-3.211338$

H $3.700092-0.621770-1.637029$

H $4.1838173 .393515-3.736696$

H $5.8331543 .074661-3.154877$

H $4.9800023 .449281-0.724297$

H $3.3814165 .324421-2.544525$

H $4.1329056 .720682-0.516947$

H 4.0378065 .3024420 .500365

H $0.5283317 .439082-0.280724$

H $2.1397177 .974703-1.021181$

H 0.4019795 .4621281 .020070

H 1.8892514 .7520831 .559069

H $0.2503424 .061580-0.976655$

H $3.409748 \quad 1.398530-2.772764$

C $-4.804188-1.043042-2.946172$

O $-3.8973620 .012164-2.570704$

C $-4.5313190 .823394-1.569798$

C $-6.0009180 .780601-1.947247$

C $-6.178808-0.666486-2.371671$

H $-4.3501420 .317362-0.616708$

C -3.902731 $2.222252-1.568849$

H $-6.6615991 .056480-1.120463$

H $-6.2031501 .451880-2.790726$

C -7.318387 -0.870709 -3.357355

H $-6.396286-1.264440-1.478047$

C $-4.3850003 .148954-0.437643$

O $-2.4832852 .104858-1.489510$

H $-4.1115642 .700126-2.534525$

H -3.786925 $4.069346-0.456329$

O $-5.7303513 .536664-0.737249$

C -4.3504002 .5718900 .997389$

C -2.9452082 .1635191 .476612$

H -5.0571151 .7406101 .077150$

O -4.8403623 .6065561 .861627$

H $-2.519908 \quad 1.4319230 .787539$

H -2.3027453 .0520431 .449464$

H $-8.271021-0.561780-2.914796$

H $-7.166867-0.287956-4.272179$

H -7.400809 -1.925570 -3.638533

C $-4.310164-2.381563-2.376888$

H $-2.2012751 .502182-2.199153$

H $-6.0240904 .134538-0.028441$

H -5.1213703 .1876162 .692475$

H -4.918368 -3.199716-2.779566

H $-4.450274-2.389949-1.287896$ 
H $-4.811629-1.081051-4.041788$

C $-3.199855-0.7675102 .340388$

O $-3.7160590 .386625 \quad 3.017255$

C $-2.917306 \quad 1.5709712 .905357$

C -1.5038371 .3068193 .448218$

C -0.8570230 .0986692 .781906$

C -1.789790 -1.108355 2.843984

H -3.180067 -0.5854861 .260095$

C -4.158913 -1.939257 2.608804

H -3.3717182 .3117393 .573673$

O -0.6666892 .4448863 .269202$

H $-1.560643 \quad 1.1170184 .528094$

H $-0.569457 \quad 0.329628 \quad 1.751318$

O $0.351972-0.2125903 .477857$

H -1.838006 -1.466694 3.880003

O $-1.231889-2.1663502 .066437$

C $-5.481780-1.7978841 .885939$

H $-3.702991-2.8756972 .267586$

H $-4.378412-2.0120613 .680231$

H -1.0943743 .1956773 .715034$

H $0.731525-1.0034383 .057798$

H -1.122401 -1.841913 1.156426

O $-6.150564-2.9876621 .952851$

O $-5.890721-0.7786531 .348871$

C -7.432895 -3.011017 1.317701

H $-8.084626-2.241173 \quad 1.742059$

H -7.319973 -2.8711510 .238774$

H -7.884021 -3.990113 1.499201

SCF Energy (B3LYP/6-31G**//MMFF)= -3245.91709201

\section{5}

MM̄FF Geometry

C $1.8646005 .268444-1.144104$

C $0.9768044 .593503-1.890048$

C $-0.4629864 .279948-1.553419$

O $-0.5435192 .884947-1.165763$

C $-1.0625335 .106887-0.397821$

C $-2.5467584 .867859-0.182432$

C $-3.087684 \quad 4.3648710 .945059$

C $-3.4480955 .264817-1.324130$

C -2.3876193 .9560732 .210088$

C -2.0294232 .4650192 .294569$

C -3.2425051 .5211992 .296070$

C -2.8125380 .0969262 .656519$

C -3.984714 -0.8941752 .580469$

O $-3.825653 \quad 1.5262490 .990384$

O $-3.448275-2.2244212 .647468$

C $-4.703012-0.7296661 .222184$

C $-4.904265-0.7696993 .800220$

O $-5.895758-1.508228 \quad 1.179508$

C -5.0193060 .7389030 .869956$

C $-5.545410 \quad 0.918148-0.575788$

O $-6.0424551 .222040 \quad 1.749456$

C $-0.5197151 .940878-2.143168$

O $-0.4392872 .121775-3.347099$

C $-0.5881660 .621930-1.479935$

C $-0.134221-0.465101-2.120071$

C $-0.127127-1.828960-1.588794$

C $-0.944553-2.093687-0.353294$

C $-4.5903920 .466449-1.686643$

C $-4.850120-0.941605-2.197756$

C $-3.914786-2.631153-3.776715$

O $-5.824972-1.611466-1.868822$

C $-3.112761-3.630029-3.003851$

C $-2.116948-4.351842-3.540293$

C $-1.336912-5.391640-2.787918$

C $0.157122-5.167061-2.857057$

C $0.920347-5.852223-3.725296$

C $0.775221-4.215539-1.858765$

C $0.583334-2.773992-2.238378$

N $-3.875153-1.348653-3.099462$

H $1.5804235 .666248-0.174702$

H $1.3193304 .197279-2.844941$

H -1.058442 4.440981 -2.460321

H $-0.9192536 .177819-0.592089$

H -0.5091704 .8783520 .518536$

H $-4.169928 \quad 4.243457 \quad 0.980198$

H $-4.5072335 .238298-1.045484$
H $-3.2236436 .286080-1.648859$

H $-3.3171484 .588621-2.174357$

H -1.478395 4.5479482 .359220

H -3.0366154 .2182943 .055529$

H -1.363523 2.2119661 .461121

H -1.4624032 .3157573 .221370$

H -3.9711131 .8958893 .024501$

H -2.3517950 .0672343 .651739$

H $-2.031523-0.2293611 .956965$

H -2.826488 -2.340159 1.909252

H $-4.036149-1.1399300 .456104$

H $-4.345438-0.9769934 .721052$

H $-5.709315-1.5122173 .766715$

$\begin{array}{llll}H & -5.352888 & 0.220533 & 3.902308\end{array}$

H $-5.651349-2.4257811 .389086$

H $-6.5342230 .455161-0.677879$

H $-5.7133281 .994537-0.725890$

H -6.4707891 .9883161 .333094$

H $-0.9909350 .586026-0.474287$

H $0.297638-0.344313-3.112786$

H $-1.974099-1.751562-0.499422$

H $-0.524273-1.5681300 .508620$

H -1.012317 -3.150944 -0.090224

H $-4.7258401 .131961-2.549146$

H $-3.5475410 .552044-1.368687$

H $-3.522208-2.477003-4.786799$

H -4.952232 -2.972135 -3.854323

H -3.382702 -3.786231-1.962031

H -1.867489 -4.213171 -4.589983

H -1.586674 -6.370045 -3.219132

H $-1.648679-5.444881-1.737396$

H $1.996423-5.717902-3.760545$

H $\quad 0.484890-6.558566-4.424354$

H $1.857918-4.390125-1.793884$

H $\quad 0.399900-4.448347-0.858426$

H $1.133789-2.483534-3.134433$

H -3.075001-0.746954-3.271994

C $4.2266634 .307918-1.219254$

O $3.8995573 .174280-2.045286$

C $3.8150011 .987895-1.220303$

C 4.5714792 .3549930 .050503

C 4.1962623 .8144530 .224219

H $2.7482071 .841113-1.010460$

C $4.3628600 .791943-2.011557$

H 4.2859451 .7659990 .923335

H $5.6534212 .252638-0.098207$

C 5.1209554 .5804621 .153743

H $3.1797703 .853547 \quad 0.632177$

C $3.993392-0.606202-1.476348$

O $3.792150 \quad 0.871919-3.332414$

H $5.4457320 .892503-2.149938$

H $2.920105-0.764030-1.628159$

O $4.642303-1.576674-2.310293$

C $4.324932-0.906664-0.010202$

C $3.999120-2.3597870 .384422$

H $3.736499-0.2376500 .618053$

O $5.700401-0.642080 \quad 0.249733$

H $2.992906-2.6046880 .029254$

H $4.695164-3.042186-0.118177$

H 4.8138725 .6285441 .228527

H 5.0997014 .1484972 .159606

H $6.157177 \quad 4.5558950 .800041$

C $3.2970005 .480809-1.555639$

H $4.1544490 .131997-3.849522$

H $5.600935-1.426358-2.251913$

H $6.230375-1.281282-0.255379$

H $3.3320235 .669289-2.636469$

H $3.6613176 .392025-1.066709$

H $5.2487534 .593341-1.499595$

C $1.898232-1.8026682 .516544$

O $3.316495-1.6511072 .655590$

C $4.095994-2.5936251 .909486$

C $3.746750-4.0221072 .351334$

C $2.244878-4.2913682 .279676$

C $1.464025-3.1913222 .998766$

H $1.616566-1.6707731 .466642$

C $1.229542-0.6920593 .339629$

H $5.141651-2.4133292 .189453$ 
O $4.431337-4.984777 \quad 1.557003$

H $4.073610-4.1699563 .388969$

H $1.905696-4.3972571 .242874$

O $1.959123-5.5352732 .923061$

H $1.617604-3.2811334 .081750$

O $0.071321-3.3640672 .747454$

C 1.4019310 .6689552 .696627

H $\quad 0.152851-0.8605673 .442055$

H $1.679177-0.6639744 .339634$

H $\quad 5.383617-4.803498 \quad 1.634824$

H $2.488911-6.2188592 .477886$

H $-0.166768-4.2617773 .036547$

O $1.447331 \quad 1.6255523 .668735$

O 1.4593620 .8688081 .490826

C 1.6183152 .9751413 .221204

H 1.0346413 .1812712 .318924

H 1.2729353 .6418164 .015775

H 2.6801503 .1615963 .041231

SCF Energy (B3LYP/6-31G**//MMFF) $=-3245.90213782$

\section{6}

MM̄FF Geometry

C $-3.2071654 .076017-0.381605$

C $-2.2504933 .880202-1.301419$

C $-0.7892693 .806687-0.946951$

O $-0.2134172 .670973-1.633391$

C $-0.0384985 .067609-1.411890$

C $1.2410155 .297626-0.627754$

C $2.4894765 .077238-1.082337$

C 1.0473735 .8514610 .763710

C $2.9271074 .575082-2.427000$

C $3.3454653 .096786-2.467965$

C $4.4191552 .695804-1.442669$

C $5.0334171 .342632-1.811282$

C $6.0404210 .857103-0.757662$

O $3.7996342 .601064-0.153318$

O $6.307412-0.531464-1.009762$

C 5.3888340 .9435180 .639222

C $7.3850021 .584091-0.877213$

O $6.3403870 .658893 \quad 1.660925$

C 4.7082522 .3006160 .917173

C 3.9005902 .3226692 .240519

O 5.7126543 .3170981 .031853

C $-0.2448751 .473759-0.990863$

O $-0.749557 \quad 1.2287610 .092836$

C $0.4985430 .503811-1.821781$

C $1.178461-0.463561-1.192367$

C $2.035154-1.478863-1.804758$

C $2.028699-1.599332-3.303310$

C 2.8126801 .2514322 .377213

C $3.274336-0.0064233 .100180$

C $2.600428-2.3803513 .491737$

O $4.306942-0.064240 \quad 3.762272$

C $3.431139-3.1645962 .524479$

C $2.961493-4.1733061 .773855$

C $3.793312-4.9142910 .765329$

C $3.349197-4.662209-0.661647$

C $2.694693-5.604479-1.360557$

C $3.744051-3.349803-1.309905$

C $2.784256-2.240148-0.981507$

N 2.373946-1.051810 2.947581

H -2.919288 4.2172410 .657862

H $-2.522543 \quad 3.757697-2.346567$

H -0.6706853 .6756220 .135514$

H $-0.6675915 .958471-1.281162$

H $0.1660605 .000794-2.485741$

H $3.3175205 .300405-0.409552$

H $\quad 1.998374 \quad 6.068340 \quad 1.262171$

H $\quad 0.479492 \quad 6.786824 \quad 0.723307$

H $\quad 0.5041775 .1418891 .394631$

H $2.1532884 .738044-3.183544$

H $3.7778675 .189988-2.748347$

H $2.4542732 .480808-2.318609$

H $3.7127352 .891109-3.481207$

H $5.1928613 .472153-1.424856$

H $5.5072331 .386570-2.800036$

H $4.2354820 .594333-1.897405$

H $6.643452-0.611993-1.918799$
H 4.6325430 .1498540 .683590

H $7.8243141 .415271-1.867796$

H $8.1120711 .191754-0.157642$

H $7.3038862 .663444-0.733470$

H $6.725454-0.2122271 .463890$

H 4.5858102 .3077583 .096803

H 3.3934943 .2971182 .291931

H 5.3344934 .0695591 .516620

H $0.5228270 .652110-2.894305$

H $1.125628-0.498238-0.104902$

H $2.480257-0.710876-3.758370$

H $1.007234-1.701562-3.683765$

H $2.580128-2.467582-3.670097$

H 1.9935661 .6615812 .981645

H $2.396851 \quad 0.9913131 .398992$

H $1.620606-2.8372743 .661685$

H $3.120261-2.3012464 .451771$

H $4.472689-2.8676162 .421994$

H $1.919978-4.4706641 .867815$

H $3.730771-5.9842201 .002578$

H $4.855671-4.6559620 .859917$

H $2.400903-5.438491-2.391751$

H $2.431164-6.558221-0.915655$

H $3.852820-3.489161-2.389528$

H $4.743629-3.062820-0.960145$

H $2.727942-2.0163660 .081229$

H $1.575007-0.9258792 .333679$

C $-5.464414 \quad 3.2131260 .258901$

O $-5.1549251 .831691-0.013247$

C $-6.358608 \quad 1.052706 \quad 0.130009$

C -7.4239121 .9986360 .676278$

C -6.9784753 .3268780 .098266$

H $-6.6548770 .745030-0.879336$

C -6.103034 -0.181006 1.002648

H $-8.432178 \quad 1.709720 \quad 0.363808$

H $-7.4123802 .036203 \quad 1.771874$

C $-7.592404 \quad 4.527078 \quad 0.797813$

H -7.242912 $3.354009-0.967379$

C $-4.982966-1.1110340 .487517$

O

H -7.038234 -0.7416591 .120201$

H $-4.014729-0.6132160 .602759$

O $-4.954126-2.252576 \quad 1.353110$

C $-5.167134-1.589382-0.961070$

C $-4.186557-2.680695-1.432438$

H $-5.099368-0.733612-1.641970$

O $-6.492416-2.120061-1.085552$

H $-4.257445-3.548077-0.767872$

H -4.557039 -3.039751 -2.400998

H $-8.6839584 .503613 \quad 0.714243$

H $-7.337270 \quad 4.544831 \quad 1.862733$

H -7.240876 5.4625520 .351687

C $-4.6776344 .123702-0.685501$

H $-5.564902-0.5348272 .851063$

H $-5.786997-2.738818 \quad 1.227236$

H $-6.641421-2.322429-2.024917$

H -5.012912 $5.160170-0.569557$

H $-4.8583063 .826607-1.725829$

H -5.1780083 .4047991 .302048$

C $-1.747224-3.1971140 .388352$

O $-2.052065-2.006422-0.347435$

C -2.727531-2.201987-1.593045

C $-1.879665-3.108004-2.498168$

C $-1.577878-4.440360-1.820845$

C $-0.940951-4.192911-0.456602$

H $-2.677378-3.6602270 .736055$

C $-0.927432-2.7887331 .618680$

H $-2.768931-1.218375-2.078121$

O $-2.538744-3.316379-3.741887$

H $-0.934271-2.600230-2.719424$

H $-2.474832-5.064231-1.730672$

O $-0.646068-5.177866-2.614119$

H $0.079481-3.818164-0.600878$

O $-0.839543-5.4290380 .248103$

C $-1.716691-1.9142892 .561333$

H $-0.594913-3.6612852 .192337$

H $-0.023812-2.2504931 .311198$

H $-1.917697-3.776301-4.331261$ 
H -1.098470 -5.433264 -3.435753

H $-0.328571-6.039683-0.310440$

O $-1.279361-0.6261532 .474789$

O $-2.617072-2.325548 \quad 3.281302$

C $-1.9507010 .314733 \quad 3.317282$

H $-1.859146 \quad 0.0201434 .366992$

H -1.474562 1.2898453 .184037

H -3.001893 0.3951173 .027735

SCF Energy (B3LYP/6-31G**//MMFF) $=-3245.90343562$

\section{7}

MM̄FF Geometry

C -3.455922 -2.779263 -0.710359

C $-3.238814-3.616903-1.735859$

C $-2.083113-3.567941-2.711892$

O $-1.250940-2.402261-2.515100$

C $-1.197601-4.815473-2.538973$

C $-0.110553-4.930788-3.590789$

C $1.213450-4.864658-3.349125$

C $-0.595862-5.170805-4.999101$

C $1.925504-4.687070-2.037008$

C $2.584174-3.309614-1.945108$

C $3.583678-3.183514-0.784970$

C $2.924125-3.3327630 .587813$

C $3.907688-3.0601461 .736341$

O $4.185684-1.887413-0.905975$

O $3.122515-2.8989372 .927574$

C $4.648061-1.7304701 .474288$

C $4.822612-4.2653161 .986156$

O $5.664851-1.5421042 .455609$

C $5.227763-1.6265310 .046369$

C $5.809176-0.230799-0.279946$

O $6.300560-2.565784-0.094433$

C $-1.676162-1.231693-3.065722$

O $-2.699293-1.043639-3.704047$

C $-0.684289-0.191704-2.721049$

C $-1.072048 \quad 1.091217-2.667731$

C $-0.2210342 .224971-2.309393$

C $1.2691002 .003002-2.290011$

C $4.8316410 .933028-0.106989$

C $5.3739692 .236785-0.669841$

C $4.7626994 .631101-0.939655$

O $6.4755522 .337629-1.202607$

C $3.4900675 .412544-0.985347$

C $2.8706595 .759553-2.123919$

C $1.5615256 .496312-2.172529$

C $0.4226625 .593800-2.605340$

C $-0.0713075 .672156-3.852706$

C $-0.1058524 .656647-1.537458$

C $-0.7965503 .414101-2.031937$

N $4.4663873 .275585-0.514000$

H -2.744667 -1.987384 -0.492352

H -3.971705 -4.398625 -1.927773

H -2.500999 -3.545525 -3.725824

H -1.814035 -5.723093 -2.588962

H $-0.767281-4.801852-1.532599$

H $1.888656-4.966167-4.198896$

H $\quad 0.223613-5.403999-5.687405$

H -1.289835 $-6.017483-5.022850$

H -1.108516 -4.286231 -5.388675

H $1.259959-4.842344-1.185301$

H $2.692890-5.468301-1.968844$

H $3.115708-3.084287-2.880020$

H $1.814705-2.531366-1.856493$

H $4.359924-3.945709-0.922130$

H $2.479348-4.3276200 .708336$

H $2.088441-2.6257190 .666734$

H $3.729041-2.6635973 .650818$

H $3.912827-0.9325671 .631310$

H $4.227528-5.1430732 .265743$

H $5.499076-4.0833592 .828550$

H $5.422288-4.5359141 .114589$

H $5.999974-0.6343312 .367058$

H $6.713046-0.0474890 .315338$

H $6.150910-0.242957-1.324815$

H $6.862543-2.283184-0.834919$

H $0.323637-0.513809-2.486623$

H $-2.1157851 .331512-2.866888$
H $1.8373572 .926630-2.166044$

H $1.6050741 .563207-3.236012$

H $1.5557401 .334637-1.474597$

H $3.8877510 .714253-0.617978$

H $4.624707 \quad 1.0913140 .956669$

H $5.2682754 .596751-1.910909$

H $5.4556385 .071721-0.215587$

H $3.0458025 .685885-0.031109$

H $3.3094205 .475375-3.077652$

H $1.6727347 .342888-2.862056$

H $1.3180986 .939861-1.198613$

H $-0.8986835 .055953-4.186222$

H $0.3409536 .367788-4.576595$

H $-0.8066485 .214619-0.902686$

H $0.7048324 .358783-0.862711$

H $-1.8809333 .503985-2.096247$

H 3.6549053 .1222270 .079179

C $-5.621896-1.683800-0.120002$

$\begin{array}{llll}\text { O } & -4.983998 & -0.433076 & 0.202648\end{array}$

C -5.9192500 .4018590 .909186$

C $-7.283719-0.2494560 .725207$

C $-6.906678-1.7193650 .707379$

H $-5.6479380 .344324 \quad 1.970547$

C $-5.806674 \quad 1.854458 \quad 0.431707$

H -7.9791820 .0072851 .529862$

H $-7.7465760 .033646-0.227071$

C -7.988342 -2.617374 0.132629

H $-6.683424-2.0379571 .734579$

C -4.363360 2.4010770 .419981

O $-6.3058661 .938408-0.908908$

H $-6.4572002 .491121 \quad 1.042910$

H -3.803846 $1.930748-0.397763$

O $-4.422877 \begin{array}{llll}3.795937 & 0.098787\end{array}$

C -3.5942062 .2135731 .739041$

C -2.1827942 .8230001 .674920$

H -3.5231251 .1436281 .955841$

O -4.3332922 .8235132 .794801$

H -1.7050832 .4945080 .746462$

H -2.254999 3.9166411 .633598

H -8.902706 -2.548889 0.731188

H $-8.238354-2.336719-0.896054$

H -7.665782 -3.6632510 .127444$

C $-4.667473-2.8419400 .178422$

H $-6.2237372 .865537-1.191109$

H -4.8599284 .2483730 .840699$

H -3.9273992 .5527793 .635246$

H -5.186466 -3.798999 0.052324

H $-4.322098-2.7920481 .218228$

H -5.857456 -1.651725 -1.192162

C $-0.652161 \quad 0.2341792 .142017$

O $-1.314457 \begin{array}{llll}1.023901 & 3.133713\end{array}$

C -1.2981402 .4341282 .882048$

C 0.1503602 .9343312 .785064

C $0.9671012 .134911 \quad 1.769637$

C 0.8233270 .6316442 .012935

H -1.1505380 .3898381 .176556$

C $-0.863276-1.2515722 .467186$

H -1.743652 2.9076823 .765606

O $\quad 0.1689074 .3165652 .443630$

H 0.6221442 .8402283 .771618

H 0.6749042 .3931410 .747495

O $2.3427382 .504957 \quad 1.881185$

H 1.3808620 .3682352 .918292

O $1.426316-0.0783610 .936265$

C $-0.264158-1.7017783 .783617$

H - $1.938329-1.4598962 .527304$

H -0.436029 -1.8608891 .662161$

H 1.1001854 .5831262 .356355

H 2.6442962 .2766802 .777017

H 0.8681360 .0386330 .149409

O $-0.143653-3.062807 \quad 3.772352$

O $0.025619-0.9668434 .717359$

C $0.377915-3.6460314 .970746$

H $\quad 0.511219-4.7170864 .797087$

H $1.349021-3.2086485 .221588$

$\mathrm{H}-0.331000-3.5076625 .792409$

SCF Energy (B3LYP/6-31G**//MMFF) $=-3245.92011621$ 
05_00178

MM̄FF Geometry

C 3.0534894 .8738872 .110526

C 3.1489943 .6381452 .624054

C 4.0391902 .5103682 .152763

O 3.1455431 .4671121 .683865

C 4.9812542 .8610310 .983440

C 5.7513861 .6757190 .431682

C $5.6503361 .237213-0.839519$

C $6.682927 \quad 0.988447 \quad 1.394814$

C $4.8165771 .824558-1.946134$

C $3.3766391 .294080-2.017415$

C $3.273943-0.161721-2.497966$

C $1.810817-0.561716-2.700662$

C $1.672273-2.033806-3.118869$

O $3.870296-1.016908-1.520256$

O $0.294449-2.406031-2.967713$

C $2.486835-2.915113-2.146939$

C $2.015607-2.240826-4.598826$

O $2.525924-4.266966-2.596563$

C $3.920298-2.399890-1.900975$

C $4.658363-3.160949-0.772441$

O $4.693087-2.574525-3.095519$

C 2.6138840 .6195892 .603754

O $2.895131 \quad 0.540218 \quad 3.788134$

C $1.553551-0.1484921 .918668$

C $0.561747-0.6839312 .644875$

C $-0.600009-1.3959192 .113279$

C $-0.603529-1.7250180 .645013$

C $3.974595-3.1322710 .599208$

C $3.121846-4.3573960 .888705$

C $1.556986-5.2717232 .599197$

O $3.112748-5.3577640 .176721$

C $0.150553-5.0771252 .128247$

C $-0.904080-5.0446612 .957013$

C $-2.326323-4.8948932 .499524$

C $-3.046736-3.7642673 .198906$

C $-3.845649-4.0006854 .253124$

C $-2.913506-2.3762432 .618694$

C -1.602199-1.723213 2.955522

N $2.398965-4.2169002 .065954$

H 3.7133935 .1919011 .310140

H 2.4553203 .3699433 .419956

H 4.6292202 .1473303 .003080

H 5.7012903 .6245091 .304619

H 4.3860963 .3137870 .184099

H $6.2426160 .371823-1.133903$

H 7.3019190 .2302270 .903252

H 7.3602701 .7169341 .852329

H $6.118970 \quad 0.4874102 .187535$

H $4.7906342 .916632-1.858393$

H $5.3250831 .632279-2.899411$

H $2.9019781 .396842-1.034444$

H $2.8188061 .934527-2.712036$

H $3.825545-0.247292-3.441748$

H $1.3265480 .091221-3.437253$

H $1.259437-0.414210-1.761735$

H $-0.242547-1.798244-3.504539$

H $1.944616-2.919647-1.194931$

H $1.354599-1.637586-5.232799$

H $1.851223-3.281020-4.901009$

H $3.044583-1.968709-4.842558$

H $1.605874-4.560367-2.712208$

H $4.881120-4.185913-1.092719$

H $5.638551-2.678415-0.646139$

H $5.634628-2.576168-2.855717$

H $1.584830-0.1965490 .836186$

H $\quad 0.578607-0.5552903 .726320$

H $\quad 0.338014-2.2012450 .353716$

H $-0.740735-0.8198880 .044596$

H - $-1.386828-2.4316520 .368392$

H $4.752997-3.1154421 .372857$

H $\quad 3.372291-2.2273140 .722165$

H $1.638355-5.2308283 .690103$

H $1.928711-6.2433672 .258145$

H $-0.010337-4.9864321 .056544$

H $-0.741348-5.1652234 .025896$

H $\quad-2.841359-5.8438302 .699173$
H $-2.386498-4.7503681 .413651$

H -4.390099 -3.199090 4.741226

H -3.968618 -5.0012134 .654702$

H $-3.706201-1.7264783 .013288$

H -3.101428 -2.415090 1.543078

H -1.506122 -1.4546514 .008369$

H $2.424666-3.3319362 .564181$

C 0.7950895 .8526461 .530882

O $\quad 0.208513 \quad 4.532943 \quad 1.454678$

C 0.1185124 .1501080 .065575

C $0.1231535 .462782-0.701154$

C 1.1293906 .2700920 .095463

H $1.0269813 .572234-0.149717$

C $-1.1257013 .278677-0.148192$

H $0.4123955 .341560-1.749238$

H $-0.8652035 .937685-0.665810$

C $1.0362837 .767821-0.140763$

H $2.1337095 .936689-0.190233$

C -1.396137 $2.946547-1.625768$

O -0.9448532 .0547760 .569325$

H -1.9962593 .7766130 .293607$

H -1.578859 $3.875230-2.179565$

O $-0.2091982 .371242-2.185591$

C $-2.5632301 .969157-1.881313$

C $-3.8673812 .386904-1.184088$

H $-2.2772310 .959897-1.568317$

O $-2.7694751 .904143-3.296082$

H $-3.7569292 .200275-0.110472$

H -3.997839 $3.470157-1.303540$

H $1.2353108 .004726-1.191034$

H $\quad 0.041442 \quad 8.152917 \quad 0.107141$

H 1.7683518 .3032140 .472262

C 1.9757515 .8434622 .514793

H -0.7526842 .2853121 .494565$

H $-0.3983852 .162335-3.116461$

H $-3.0535022 .784700-3.595914$

H 1.6005785 .5761143 .510924

H 2.4092386 .8464492 .597757

H 0.0133546 .5049421 .940110

C $-4.967459-0.427051-0.574063$

O $\quad \begin{array}{llll}-5.020445 & 0.277699 & -1.818017\end{array}$

C $-5.1436251 .697044-1.716380$

C $-6.4134712 .062311-0.934578$

C -6.4367041 .3888810 .435150$

C $-6.197994-0.1124830 .290379$

H $-4.045891-0.161705-0.040799$

C $-4.934098-1.930646-0.900576$

H $-5.2884742 .060975-2.741495$

O $-6.5060833 .475665-0.793428$

H -7.291102 $1.735372-1.507517$

H $-5.709770 \quad 1.837412 \quad 1.122043$

O $\quad-7.7219291 .5732281 .033455$

H $-7.090923-0.577443-0.147654$

O $-6.016442-0.6831321 .585430$

C $-3.636906-2.378762-1.539147$

H $-5.064614-2.5116320 .019433$

H -5.739941 -2.182672 -1.600156

H $-7.3757333 .677758-0.409429$

H -7.8215572 .5202601 .228361$

H -6.801436 -0.459038 2.114123

O $-3.579991-3.743991-1.502716$

O $-2.793345-1.639550-2.025435$

C $-2.415011-4.335308-2.086849$

H -1.533010 -4.096197-1.486497

H $-2.551297-5.419970-2.091401$

$\mathrm{H}-2.286718-3.995203-3.118907$

SCF Energy (B3LYP/6-31G**//MMFF) $=-3245.90409598$

\section{9}

MM̄FF Geometry

C $2.7977083 .522715-0.371905$

C $2.3574624 .437568-1.249816$

C $1.0493804 .380749-2.009408$

O $0.3552043 .135553-1.766303$

C $0.1283655 .533865-1.581038$

C $-1.1396745 .606444-2.410555$

C $-2.3598395 .217563-1.990074$

C $-0.9730206 .198716-3.787109$ 
C $-2.7340214 .623930-0.660488$

C -3.513678 $3.314386-0.821068$

C -4.0863942 .7977640 .507584$

C $-2.9997692 .356997 \quad 1.492861$

C -3.6003841 .7660172 .778786$

$\begin{array}{lllll}\text { O } & -4.943577 & 1.687653 & 0.199387\end{array}$

O $-2.549207 \quad 1.0962833 .490523$

C $-4.640603 \quad 0.6928492 .394041$

C $-4.1256242 .856586 \quad 3.720276$

$\begin{array}{lllll}\text { O } & -5.328406 & 0.210688 & 3.545283\end{array}$

C $-5.655583 \quad 1.173182 \quad 1.332590$

C $-6.616544 \quad 0.053027 \quad 0.861935$

O $-6.4869512 .188787 \quad 1.914985$

C $0.7113552 .070036-2.535224$

O $1.5193372 .060250-3.450332$

C $-0.024790 \quad 0.889330-2.032877$

C $0.358113-0.331839-2.433059$

C $-0.239200-1.607501-2.032597$

C $-1.446839-1.555900-1.137128$

C $-5.993293-1.2090040 .264453$

C $-5.582322-1.101571-1.194881$

C $-4.700352-2.525882-3.037180$

O $-5.747586-0.098881-1.881794$

C $-3.870662-3.765054-3.138157$

C $-2.718920-3.851373-3.820747$

C $-1.894996-5.107607-3.865845$

C $-0.434773-4.888116-3.536540$

C $0.527202-5.367757-4.343981$

C $-0.078930-4.175274-2.247719$

C $0.322580-2.744635-2.492967$

N $-5.023714-2.294077-1.640461$

H $2.1766072 .667361-0.120735$

H $3.0011855 .281987-1.488052$

H $1.274196 \quad 4.471977-3.079345$

H $0.6570086 .492247-1.674543$

H $-0.1084775 .439346-0.515836$

H -3.198947 $5.350789-2.672259$

H -1.926489 $6.294330-4.317616$

H $-0.5346767 .199741-3.719223$

H $-0.3186645 .572259-4.400644$

H $-1.8552884 .443053-0.038810$

H -3.356262 $5.355446-0.130704$

H -4.346169 $3.459553-1.523069$

H -2.879056 2.545054 -1.278966

H -4.6948353 .6000480 .941442$

H -2.327586 3.1844021 .746532

H -2.369178 1.5915351 .019106

H -1.844582 1.7439323 .663743

H -4.074845 -0.1554141 .990977$

H -3.311134 3.5269684 .020553

H -4.5135852 .4234624 .648839$

H -4.9078823 .4729013 .273022$

H -4.655386 -0.0840254 .182573$

H -7.238290 -0.2465091 .716705$

H -7.318222 0.4765410 .130207

H -7.293680 2.2701261 .379655

H $-0.8383511 .054646-1.336629$

H $1.201389-0.410357-3.118745$

H -1.858796 $-2.539076-0.902544$

H -2.250275 $-0.980247-1.609142$

H $-1.194764-1.080700-0.183364$

H $-5.142884-1.5628850 .853231$

H $-6.754821-1.9997610 .305669$

H -4.194914 -1.641315 -3.438278

H -5.644523 -2.658360 -3.575303

H $-4.236714-4.645630-2.614179$

H $-2.355840-2.988043-4.372671$

H $-1.999846-5.536911-4.870440$

H -2.291161 -5.855223 -3.166669

H $1.578099-5.237489-4.106694$

H $0.287387-5.900233-5.258293$

H $0.774581-4.683041-1.778492$

H $-0.894918-4.277858-1.527340$

H $1.208749-2.653892-3.124061$

H $-5.097233-3.104640-1.031316$

C $5.0722942 .514939-0.335960$

O $4.5587951 .189316-0.100938$

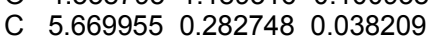

C $6.936722 \quad 1.111407-0.165694$

C $6.4796852 .496798 \quad 0.250718$

H $5.654297-0.078624 \quad 1.072271$

C $5.517930-0.896497-0.930082$

H 7.7728030 .7382200 .433998

H $7.2583681 .112906-1.213747$

C $7.3827723 .608396-0.253886$

H 6.4305372 .5368151 .347088

C $4.196964-1.680457-0.779951$

O $5.570909-0.393100-2.271561$

H $6.375870-1.570611-0.820346$

H $3.358038-1.062918-1.121276$

O $4.254414-2.792693-1.683430$

C $3.910234-2.1959540 .643140$

C $2.685227-3.1295130 .690016$

H $3.751053-1.3373951 .303869$

O $5.058787-2.901573 \quad 1.111694$

H $1.842231-2.6210530 .212190$

H $2.895012-4.0329980 .105005$

H $8.398523 \quad 3.4848110 .135709$

H $7.4401123 .613660-1.347571$

H 7.0160524 .5877790 .068662

C 4.1472063 .5608520 .287571

H $5.468926-1.152560-2.870373$

H $4.957370-3.387892-1.370802$

H $4.969221-3.0054142 .073871$

H 4.5886294 .5596830 .197902

H 4.0066883 .3597291 .356167

H $5.1149462 .653317-1.425379$

C $0.971064-1.6454202 .731713$

O $2.130385-2.4446552 .997043$

C $2.301128-3.5636442 .122214$

C $1.075642-4.4863982 .199703$

C $-0.227792-3.7278951 .967757$

C $-0.299332-2.492767 \quad 2.864317$

H $1.035213-1.2221931 .721676$

C $0.976370-0.488213 \quad 3.734570$

H $3.149371-4.1347662 .518794$

O $1.171933-5.546724 \quad 1.254677$

H $1.032688-4.9463883 .195560$

H $-0.345661-3.453722 \quad 0.914626$

O $-1.331614-4.577825 \quad 2.285936$

H $-0.440630-2.8040743 .907106$

O $-1.431052-1.7097892 .493708$

C $2.0400420 .520713 \quad 3.371280$

$\begin{array}{llll}\mathrm{H} & 0.023104 & 0.049640 & 3.746715\end{array}$

H $1.153839-0.8496444 .754572$

H $2.000050-6.0241031 .434347$

H $-1.253507-5.3721111 .729919$

H -2.214511 -2.282733 2.555976

$\begin{array}{lllll} & \text { O } & 3.117627 & 0.385085 & 4.194799\end{array}$

O $1.931131 \quad 1.3197312 .449942$

C 4.1989301 .2880323 .945049

H 4.5854701 .1459632 .932493

H 3.8705362 .3214194 .091076

H 4.9974111 .0688294 .658816

SCF Energy (B3LYP/6-31G**//MMFF) $=-3245.90879381$

\section{0}

MM̄FF Geometry

C $-1.962697 \quad 4.078117-1.474301$

C $-0.7042294 .416419-1.792364$

C $0.415272 \quad 4.477792-0.789284$

O $1.5519943 .846790-1.428144$

C $0.7899715 .908199-0.376517$

C $-0.252654 \quad 6.5770630 .492928$

C $-0.397513 \quad 6.3514751 .814000$

C -1.128667 $7.584501-0.204646$

C 0.4066525 .4244462 .686391

C $-0.471523 \quad 4.5103803 .554793$

C $-1.096818 \quad 3.3222962 .800838$

C $-2.1410852 .645217 \quad 3.692999$

C $-2.737916 \quad 1.3981783 .032683$

O $-\begin{array}{lll}-0.050067 & 2.399683 & 2.482359\end{array}$

O $-3.477171 \quad 0.678002 \quad 4.032017$

C -1.5853220 .4792452 .582808$

C $-3.743764 \quad 1.7626931 .934876$

O $-2.083277-0.6088941 .806159$ 
C $-0.460674 \quad 1.203827 \quad 1.802660$

C $0.7847590 .294481 \quad 1.660714$

O

C $2.4764033 .244279-0.635184$

O 2.5047263 .2250530 .584236

C $3.4571502 .584978-1.525174$

C $4.4792331 .899690-0.992251$

C $5.5233361 .194317-1.736649$

C $5.3979831 .133048-3.236674$

C 1.6318230 .1764542 .929959

C $2.410986-1.1214312 .972462$

C $4.242291-2.3822821 .896141$

O $2.109077-2.0198853 .756540$

C $5.604861-2.0507431 .382014$

C $6.101274-2.5487370 .239210$

C $7.502016-2.306562-0.246773$

C $7.543279-1.611996-1.588490$

C $7.501776-2.315189-2.732630$

C $7.720986-0.111941-1.601427$

C $6.5330260 .622537-1.048612$

N $3.463443-1.1706862 .070422$

H $-2.2207263 .906736-0.433518$

H $-0.4487334 .599370-2.833472$

H $\quad 0.145292 \quad 3.887647 \quad 0.091565$

H $0.987816 \quad 6.516428-1.269734$

H $1.751756 \quad 5.9032220 .152736$

H -1.163046 6.9164312 .344843

H -1.8603248 .0390680 .471749$

H $-0.5172058 .392454-0.619797$

H -1.684618 $7.116584-1.022051$

H 1.1195054 .8259602 .115896

H 1.0020516 .0558933 .357839

H $\quad 0.1618174 .1174364 .361477$

H -1.258536 5.1103804 .029026

H -1.5586343 .6794301 .875626$

H -2.9372203 .3504513 .963371$

H -1.672753 2.3453394 .641259

H -4.1551681 .2750384 .392321$

H -1.157585 $0.035823 \quad 3.490578$

H -4.585985 2.323582 2.357745

H -4.1794320 .8660291 .480665$

H -3.308756 2.3756721 .144113

H -2.778118 -1.041931 2.330789

H $0.476068-0.6954131 .302012$

H 1.4283510 .6902640 .863865

H $-1.791363 \quad 1.2058680 .342678$

H $3.3096422 .679024-2.595066$

H 4.5673581 .8543480 .092532

H $5.4879362 .134516-3.671269$

H $4.429451 \quad 0.709221-3.525313$

H $6.1571310 .506331-3.710232$

H $2.333871 \quad 1.0156633 .001861$

H 1.0135430 .2091263 .833234

H $3.690279-3.0319711 .210560$

H $4.325758-2.9025262 .856154$

H $6.227756-1.4109262 .002208$

H $5.481284-3.207770-0.364969$

H $8.004938-3.279936-0.320511$

H $8.085806-1.734806 \quad 0.485437$

H $7.560694-1.827205-3.699795$

H $7.403755-3.395833-2.726152$

H $7.9700820 .247176-2.606406$

H $8.599121 \quad 0.146871-0.995210$

H $\quad \begin{array}{lll}6.535958 & 0.705342 & 0.038635\end{array}$

H $3.524180-0.4530731 .353338$

C $-3.6602082 .455849-2.307234$

O $-2.6269991 .455491-2.369874$

C $-3.1099650 .321177-3.115513$

C $-4.596756 \quad 0.562940-3.335513$

C $-4.6617192 .077185-3.394881$

H $-2.5685900 .343626-4.069242$

C -2.781837 -0.958252 -2.336205

H $-4.969706 \quad 0.081065-4.244227$

H -5.182796 $0.188037-2.486943$

C $-6.0556902 .637088-3.174453$

H $-4.2931062 .403380-4.376597$

C -3.262853 -2.243250 -3.032183

O $-1.361329-1.030189-2.174419$
H $-3.206795-0.886247-1.328949$

H -4.356251 -2.241084 -3.110475

O $-2.759087-2.236390-4.374552$

C $-2.790939-3.546771-2.358585$

C $-3.299005-3.713557-0.916219$

H -1.697799 -3.606326 -2.395995

O $-3.274008-4.648141-3.138498$

H -3.023866 -2.838555 -0.322182

H $-4.394755-3.761280-0.931865$

H $-6.7417682 .276473-3.947655$

H $-6.4589642 .338256-2.201153$

H $-6.0469493 .730834-3.214117$

C $-3.048348 \quad 3.847897-2.486707$

H $-1.077369-0.211420-1.732465$

H -3.054869 -3.059509-4.799693

H $-4.245994-4.620757-3.122706$

H -3.820246 $4.616025-2.368158$

H $-2.6382243 .943817-3.499735$

H $-4.1200292 .372981-1.313604$

C $-0.715360-4.0603160 .656175$

O $-1.319951-5.016257-0.223024$

C $-2.751307-4.994538-0.245678$

C $-3.298462-5.2607011 .161944$

C $-2.712286-4.3011192 .193433$

C -1.186419-4.278762 2.099802

H $-0.966324-3.0445760 .328149$

C $0.802361-4.2348510 .544508$

H $-3.060956-5.840967-0.871220$

O $-4.719256-5.169793 \quad 1.160388$

H -3.043650 -6.288606 1.451922

H $-3.138738-3.2987442 .078182$

O $-3.100052-4.7565963 .491648$

H $-0.782141-5.2224262 .488394$

O $-0.680194-3.2245042 .915039$

C $1.319676-3.671393-0.758110$

H $1.332991-3.7373971 .363309$

H $1.084208-5.2940970 .580723$

H $-5.021538-5.3286902 .071063$

H -2.807128 -4.0906324 .136219$

H $-0.874667-3.4468543 .840855$

O $1.559759-2.336107-0.623085$

O $1.482045-4.336432-1.772770$

C $2.081913-1.676000-1.780152$

H $3.101682-2.020420-1.975808$

H $1.440685-1.852343-2.649061$

H $2.105894-0.602459-1.575694$

SCF Energy $\left(B 3 L Y P / 6-31 G^{* *} / / M M F F\right)=-3245.91499385$

0500181

MM̄FF Geometry

C -1.680668 -3.047942 -1.494175

C $-1.399428-4.003534-0.593250$

C $-1.889393-3.9995480 .836839$

O $\quad-2.079642-2.639747 \quad 1.290975$

C $-3.238762-4.7372720 .935052$

C $-3.867714-4.6682452 .313579$

C $-5.009783-4.0074592 .593883$

C $-3.133247-5.4057213 .402168$

C $-5.891336-3.253317 \quad 1.633428$

C $-5.376691-1.8383981 .371627$

C $-6.315286-0.9566930 .536248$

C $-6.590368-1.540047-0.852603$

C $-7.391936-0.575790-1.740594$

$\begin{array}{llll}\text { O } & -5.654269 & 0.312423 & 0.418251\end{array}$

O $-7.319529-1.057578-3.091740$

C $-6.7063930 .805259-1.718283$

C $-8.878698-0.556152-1.367440$

O $-7.4810931 .774522-2.419399$

C $-6.3944451 .311501-0.294320$

C $-5.5481132 .606464-0.295971$

O $-\begin{array}{llll}-7.622007 & 1.614190 & 0.378912\end{array}$

C $-0.999455-1.9542621 .750622$

O $0.141058-2.367926 \quad 1.876240$

C $-1.458990-0.5781522 .046134$

C -0.5909420 .3522092 .470091$

C -0.9125911 .7531352 .756840$

C -2.3529312 .1763312 .621639$

C $-4.1985492 .500405-1.011727$ 
C $-3.3390903 .738040-0.806499$

C $-1.1091014 .666203-1.441849$

O $-3.7025844 .711044-0.151803$

C $-0.0354684 .301284-0.466828$

C $0.402087 \quad 5.115420 \quad 0.506291$

C $1.523103 \quad 4.7547131 .439737$

C 1.1489234 .8600812 .902671

C 1.8557935 .6467923 .731982

C $-0.031650 \quad 4.0614943 .421212$

C $0.0789042 .588477 \quad 3.133551$

N $-2.1174833 .622776-1.454224$

H $-2.345543-2.236178-1.211513$

H $-0.758583-4.834061-0.878814$

H $-1.144141-4.4917341 .473489$

H -3.106504 -5.7917310 .658956$

H -3.924931 -4.3212730 .188505$

H $-5.366086-4.0078823 .623051$

H -3.683846 -5.403779 4.349014

H $-2.980050-6.4512013 .114892$

H -2.157037 -4.9477703 .588681$

H $-5.993152-3.8160290 .699845$

H $-6.899489-3.2039442 .063334$

H -5.178557-1.330622 2.325592

H $-4.408612-1.882483 \quad 0.861818$

H -7.250179 -0.8210641 .092336$

H $-7.114596-2.500134-0.782935$

H $-5.634254-1.755704-1.350397$

H $-7.678813-1.961249-3.107399$

H $-5.7664170 .696598-2.273698$

H $-9.317985-1.553120-1.493270$

H -9.444236 $0.106015-2.032301$

H $-9.058678-0.246864-0.335843$

H -7.653988 $1.419785-3.308342$

H $-6.1299593 .433701-0.723194$

H -5.3610912 .8922940 .749019$

H -7.4323642 .2347901 .101894$

H $-2.508647-0.3602831 .882803$

H 0.4524570 .0708792 .603788

H -2.998693 1.5512643 .249109

H -2.680717 2.0850291 .582426

H -2.540384 3.2065732 .931004

H $-3.639146 \quad 1.632077-0.645867$

H -4.351498 2.384042 -2.090238

H - $-1.5729215 .629451-1.206027$

H $-0.6879574 .718871-2.450598$

H $0.4291173 .326309-0.589047$

H $-0.039906 \quad 6.1025490 .617826$

H 2.3651515 .4214251 .212549

H $1.8866503 .740708 \quad 1.241472$

H 1.6028635 .7335224 .783518

H 2.7047096 .2233683 .379667

H $-0.935476 \quad 4.4969352 .985451$

H -0.1203254 .1799904 .508485$

H 1.0830842 .1797213 .252574

H $-1.8939232 .756603-1.935911$

C $-0.163025-1.842263-3.126429$

O $0.953286-1.948943-2.218129$

C $1.158824-0.663835-1.595526$

C $0.491522 \quad 0.339747-2.521116$

C $-0.740281-0.434090-2.949258$

H $0.631149-0.708373-0.634577$

C $2.656967-0.448484-1.365644$

H $0.248593 \quad 1.281362-2.021902$

H $1.1300330 .558896-3.385678$

C -1.408524 $0.118133-4.195990$

H -1.462536 -0.401082 -2.125429

C $3.0051210 .948534-0.825029$

O $3.099371-1.425574-0.417459$

H $3.201884-0.641901-2.296350$

H $2.7687631 .709724-1.577465$

$\begin{array}{llll}\text { O } & 2.163113 & 1.229500 & 0.300711\end{array}$

C $4.4624191 .126868-0.359523$

C $5.5052820 .762106-1.427333$

H 4.6319290 .5488390 .555262

O $4.6508452 .503023-0.010808$

H $5.355708-0.273096-1.746624$

H $5.3529081 .396438-2.309395$

H $-1.7509001 .144406-4.026324$
H $-0.7213160 .130058-5.048543$

H $-2.277894-0.487677-4.470472$

C $-1.131228-3.008360-2.892265$

H $2.860280-2.300514-0.768830$

H 2.3227280 .5388630 .966810

H 3.9980622 .7185430 .677074

H $-0.602939-3.947206-3.101465$

H -1.966689-2.947200 -3.598850

H $0.258666-1.946660-4.134508$

C $7.157159-1.1281050 .281901$

$\begin{array}{llll}\text { O } & 7.187396 & 0.303386 & 0.326335\end{array}$

C $6.9596260 .943990-0.933175$

C $8.0310560 .503063-1.940837$

C $8.133566-1.018396-2.041074$

C $8.253457-1.648305-0.652584$

H $6.176348-1.468037-0.071015$

C $7.347071-1.6372991 .714376$

H $7.1026612 .017967-0.759440$

O $7.7676561 .039288-3.232903$

H $9.0071920 .892546-1.623276$

H $7.283590-1.441760-2.589360$

O $9.302377-1.361636-2.789287$

H $9.244380-1.429528-0.234333$

O $8.138989-3.064543-0.768785$

C $6.156598-1.2803802 .574090$

H $7.481450-2.7240051 .754015$

H $8.233085-1.1830512 .174146$

H $7.7252812 .006707-3.144210$

H $9.228847-0.925552-3.655642$

H $8.828108-3.361253-1.387678$

O $5.096317-2.078767 \quad 2.260074$

O $6.166029-0.3901753 .414249$

C $3.888815-1.8109752 .980186$

H $4.043381-1.9708514 .051449$

H $3.550369-0.7886142 .785959$

H $3.122175-2.5060762 .628100$

SCF Energy (B3LYP/6-31G**//MMFF) $=-3245.92022330$

0500182

MM̄FF Geometry

C $-0.5064014 .937429-0.281289$

C $0.721151 \quad 4.524648-0.630308$

$\begin{array}{lllll}\text { C } & 1.671496 & 3.848289 & 0.324647\end{array}$

O $2.2210212 .676452-0.326162$

C 2.8397624 .7889060 .667696

C 3.7662874 .2379421 .735209

C 5.0633763 .9286721 .541823

C 3.1590074 .0681673 .105045

C $5.8723684 .059107 \quad 0.282925$

C $5.9074452 .800853-0.597861$

C 6.4812841 .5556190 .098730

C $6.7562260 .447736-0.920946$

C $7.239952-0.847302-0.250107$

O $5.525232 \quad 1.0933881 .057632$

O $7.155743-1.903578-1.219571$

C $6.274053-1.2119110 .898085$

C $8.713065-0.7575920 .165707$

O $6.774019-2.312351 \quad 1.652864$

C $5.960431-0.0294771 .837282$

C $4.840650-0.3440582 .860888$

$\begin{array}{lllll}\text { O } & 7.135159 & 0.295446 & 2.591082\end{array}$

C $1.4042761 .600448-0.488566$

O $0.249628 \quad 1.484790-0.110370$

C $2.162498 \quad 0.580812-1.245846$

C $1.501724-0.403383-1.872329$

C $2.104875-1.488636-2.648985$

C $3.603481-1.628257-2.602922$

C $3.482748-0.7230182 .259250$

C $3.263561-2.2200852 .111751$

C $1.670012-3.8613291 .120305$

O $3.998719-3.0623192 .619613$

C $2.197456-4.321685-0.202452$

C $1.426757-4.886238-1.144504$

C $1.941255-5.388024-2.463408$

C $1.192085-4.804813-3.641320$

C $0.176747-5.473273-4.213840$

C $1.675332-3.487181-4.204944$

C $1.293723-2.317586-3.338539$ 
N $2.120697-2.5054291 .375519$ H -0.8295724 .8375820 .752061$ H $1.0481894 .629008-1.662310$ H 1.1491173 .5350611 .236535 H 2.4514225 .7524741 .024055 H $3.3959065 .006366-0.250627$ H 5.6270323 .5411822 .390238 H 3.9105123 .8299813 .865722 H 2.6619354 .9922843 .418019 H 2.4251663 .2566673 .106065 H $5.5214434 .904793-0.318198$ H $6.898874 \quad 4.322466 \quad 0.569441$ H $4.8923212 .590017-0.953538$ H $6.5162583 .033844-1.480294$ H 7.4077641 .8398920 .611271 H $7.4815200 .782115-1.673267$ H $5.8334520 .227948-1.474123$ H $7.702355-1.653070-1.984040$ H $5.341912-1.5505040 .430815$ H $9.346839-0.569169-0.709267$ H $9.062934-1.7055350 .588927$ H $8.907066 \quad 0.0367730 .889303$ H $6.934351-3.0420191 .030106$ H $5.187694-1.0956403 .580371$ H 4.6782000 .5691693 .451621 H $\quad \begin{array}{llll}6.865458 & 0.781345 & 3.388058\end{array}$ H $3.2415360 .674712-1.274711$ H $0.413702-0.411605-1.822357$ H $4.082974-0.782753-3.107572$ H $\quad 3.973117-2.540312-3.075733$ H $3.953448-1.664340-1.565770$ H $2.693517-0.3670292 .934032$ H $3.332535-0.2324511 .293804$ H $\quad 0.575731-3.8501511 .148450$ H $2.034544-4.5225621 .912930$ H $3.263442-4.207457-0.384292$ H $0.366829-5.027416-0.945220$ H $1.842108-6.481436-2.465186$ H $3.014096-5.189602-2.577646$ H $-0.348443-5.075249-5.075802$ H $-0.155739-6.431661-3.828910$ H $\quad 1.231145-3.314305-5.194537$ H $2.750939-3.550366-4.391543$ H $0.218944-2.135235-3.306814$ H $1.597293-1.7445210 .952534$ C $-2.8390104 .749894-1.159222$ O $-2.6468383 .341874-1.386922$ C $-3.8122412 .812296-2.055142$ C $-4.7866723 .975690-2.206564$ C $-3.8630005 .177934-2.205589$ H $-3.4614172 .467817-3.035116$ C -4.362152 $1.639291-1.231247$ H $-5.3888833 .897608-3.116915$ H -5.474507 $4.023597-1.352951$ C $-4.5679396 .485197-1.890374$ H $-3.3853435 .251458-3.191844$ C $-5.6429891 .021946-1.818367$ O $-3.3577230 .621795-1.166762$ H $-4.5350681 .975844-0.203272$ H -6.436972 $1.775255-1.870095$ O $-5.384557 \quad 0.626551-3.171912$ C $-6.174323-0.210696-1.059596$ C -6.5200610 .0889530 .408777$ H -5.459276 -1.038148 -1.127339 O $-7.371838-0.646148-1.713460$ H $-5.619677 \quad 0.4198610 .933915$ H -7.2354370 .9201690 .439515$ H -5.343481 $6.693071-2.634771$ H -5.046315 $6.455972-0.905607$ H -3.861940 7.321397 -1.897163 C $-1.5036985 .490396-1.260541$ H $-2.5518101 .032011-0.807930$ H -4.656744 -0.018817 -3.153944 H -7.145366 -0.824953 -2.642375 H -1.650619 $6.555890-1.052031$ H -1.111033 5.403579 -2.281279 H -3.251846 4.857021 -0.146901 C $-5.096372-2.2291051 .789264$
O $-6.323287-2.288842 \quad 1.052070$

C -7.140509-1.118794 1.147883

C $-7.497918-0.8466002 .616296$

C $-6.257989-0.7860823 .504618$

C $-5.375849-2.0135393 .280411$

H -4.474399-1.411711 1.404002

C $-4.356002-3.5483821 .548270$

H $-8.078978-1.3592160 .632790$

$\begin{array}{llll}\text { O } & -8.226562 & 0.369223 & 2.748544\end{array}$

H -8.146540 -1.651829 2.985457

H -5.6916520 .1385223 .341946$

O $\quad-6.662472-0.7717954 .875728$

H $-5.864197-2.8992953 .706393$

O $-4.139591-1.8326963 .967752$

C $-3.805282-3.5934010 .143539$

H $-3.521913-3.6903952 .244779$

H -5.026103 -4.407901 1.669610

H -9.0181950 .2973932 .188264$

H -7.253914 -0.008830 4.994189

H -4.349325 -1.672657 4.903992

O $-2.616976-2.9266930 .092930$

O $-4.374283-4.132044-0.796822$

C -1.999029 -2.864763-1.194839

H -2.593907 -2.238153 -1.865732

H -1.012793 -2.412348 -1.070104

$\mathrm{H}-1.874137-3.868010-1.613111$

SCF Energy $(B 3 L Y P / 6-31 G * * / / M M F F)=-3245.91657053$

0500183

MM̄FF Geometry

C $-0.7309795 .358241-1.346203$

C $-0.9727784 .207220-1.992668$

C $-2.2664473 .426376-2.029219$

O $-2.1417142 .313894-1.107630$

C $-3.5219714 .222951-1.620319$

C $-4.8200703 .460510-1.815898$

C $-5.6675933 .124350-0.823428$

C $-5.1638463 .102055-3.239446$

C $-5.543093 \quad 3.4220810 .644313$

C -4.8635752 .3186231 .469266$

C $-5.6228870 .981611 \quad 1.476854$

C -5.0374220 .0337772 .526269$

C $-5.719505-1.3432622 .501998$

$\begin{array}{llll}\text { O } & -5.507474 & 0.384846 & 0.182020\end{array}$

O $-4.909449-2.2493303 .267192$

C $-5.739484-1.8720291 .051288$

C $-7.093456-1.3108143 .182086$

O $-6.511779-3.0659010 .955680$

C $-6.243580-0.8366190 .025382$

C $-6.066369-1.296423-1.443041$

O $-7.645582-0.6187580 .227841$

C $-1.4529671 .214054-1.512533$

O $-0.933917 \quad 1.025346-2.600476$

C $-1.4102850 .276763-0.368599$

C $-0.483363-0.691664-0.341963$

C $-0.292016-1.6683810 .731679$

C $-1.377117-1.7884871 .767466$

C $-4.626360-1.603592-1.869428$

C $-4.250467-3.073130-1.768570$

C $-2.289407-4.594684-2.002373$

O $-5.064329-3.964271-1.542219$

C -1.696966 -4.872334 -0.656810

C $-0.432397-5.285452-0.482577$

C $0.172249-5.6042890 .854528$

C $1.431442-4.8120261 .126196$

C $2.646074-5.3398450 .898438$

C $1.280615-3.4366511 .735651$

C $0.834706-2.4098370 .732814$

N $-2.896403-3.276405-2.000254$

H $-1.5134265 .839536-0.767518$

H $-0.1488313 .750996-2.539577$

H -2.396562 $3.045984-3.049681$

H $-3.5842305 .147932-2.208281$

H $-3.4189804 .524697-0.573289$

H $-6.5729342 .579042-1.088839$

H $-6.1842902 .715536-3.335355$

H -5.089592 $3.985113-3.882656$

H $-4.4860442 .332030-3.619553$ 
H $-5.009748 \quad 4.3651300 .804514$ H -6.5515493 .5978221 .040364$ H -3.8466142 .1657581 .089403$ H -4.772304 2.685306 2.499237 H -6.6780401 .1851311 .694397$ H $-5.1022330 .474486 \quad 3.528977$ H $-3.965636-0.1058242 .330644$ H -4.827466 -1.890581 4.167485 H $-4.708059-2.1463750 .801189$ H $-6.995628-0.9935414 .227323$ H $-7.542426-2.3096733 .214408$ H $-7.796691-0.6312242 .696429$ H $-6.146730-3.6995591 .596752$ H $-6.739611-2.134699-1.659349$ H $-6.414904-0.472647-2.082773$ H -8.022037 $-0.253304-0.589919$ H -2.1219090 .4286270 .434614$ H $\quad 0.224621-0.758497-1.167241$ H -2.352850 -1.913256 1.286196 H -1.407439 -0.8936302 .398334$ H -1.256554 -2.650452 2.426970 H -4.514050 -1.333808 -2.927411 H -3.911209-1.002302 -1.300932 H -1.535777 -4.602879 -2.796176 H -3.048894 -5.348305 -2.233893 H $-2.342501-4.7503560 .209828$ H $\quad 0.200803-5.432484-1.354661$ H $0.397063-6.6787970 .871124$ H $-0.544936-5.4410601 .668550$ H $3.553382-4.7847901 .113260$ H $2.759396-6.3361160 .483917$ H $2.244587-3.0975802 .138208$ H $0.621662-3.5002142 .605734$ H $1.560135-2.242641-0.062405$ H $-2.287846-2.473956-2.134383$ C $1.5782095 .488695-0.269280$ O $1.9534434 .136352-0.602708$ C 1.8043453 .3136840 .572667 C 1.8309584 .2838401 .743012 C 1.0572435 .4569621 .170072 H 0.8164312 .8439430 .483926 C 2.9036972 .2473580 .585760 H 1.3728763 .8732812 .647325 H 2.8602564 .5804281 .980097 C 1.2672006 .7581621 .924354 H -0.0097225 .2048631 .198039$ C 2.9119731 .3521221 .839036 O $2.7312661 .429847-0.576623$ H 3.8746422 .7404580 .468289 H 3.1483251 .9435782 .731700 O 1.6014910 .8091462 .041562 C 3.8734010 .1496701 .755895 C 5.3421820 .5525031 .551305 H $3.549600-0.5416660 .970611$ O $3.786797-0.5689612 .992764$ H 5.4359321 .1609380 .648163 H 5.6525451 .1766622 .398235 H 0.9261316 .6616512 .960290 H 2.3238807 .0453791 .944307 H 0.7045397 .5722751 .456614 C $0.6149226 .032231-1.331618$ H $1.891810 \quad 0.949931-0.476754$ H 1.0217651 .5298982 .338950 H $2.854231-0.8161293 .117539$ H $1.0755855 .924165-2.321991$ H $0.4596587 .106134-1.174394$ H $2.505496 \quad 6.073724-0.320306$ C $6.192576-1.031400-0.933987$ O $5.933909-1.543147 \quad 0.379835$ C $6.287550-0.667598 \quad 1.456925$ C $7.777637-0.3067671 .377670$ C 8.1523370 .2594270 .012371 C $7.675690-0.674009-1.098001$ H $5.575887-0.142686-1.112801$ C $5.785769-2.121439-1.939547$ H $6.153554-1.2490792 .377432$ $\begin{array}{lllll}\text { O } & 8.132000 & 0.632921 & 2.386655\end{array}$ H $8.378504-1.2085581 .554063$
H $7.7559241 .271968-0.127241$

O $9.5748510 .368729-0.076934$

H $8.286448-1.585984-1.096360$

O $7.866292-0.033864-2.358586$

C $4.284431-2.247952-2.085378$

H $\quad 6.178755-1.899627-2.937677$

H $6.176533-3.089983-1.604589$

H 7.8960840 .2421423 .245370

H 9.8675970 .9328450 .659420

H $8.8075530 .201990-2.424546$

O $3.997597-3.423077-2.720444$

O $3.467823-1.412056-1.725283$

C $2.609909-3.664732-2.972809$

H $2.057919-3.717117-2.031122$

H $2.199688-2.882048-3.617928$

H $2.519199-4.625918-3.485624$

SCF Energy (B3LYP/6-31G**//MMFF) $=-3245.90986515$

0500184

MM̄FF Geometry

C -0.4045152 .0921872 .049292$

C -1.4201502 .9540401 .893587$

C $-2.064843 \quad 3.2659700 .571284$

O -3.4415132 .8377440 .703667$

C $-2.0455304 .769017 \quad 0.244414$

C $-0.6732525 .343322-0.031623$

C $-0.1154335 .394389-1.256849$

C $0.0326495 .964410 \quad 1.145704$

C $-0.6925734 .846859-2.534403$

C $0.3920404 .398061-3.525880$

C $1.1687633 .148457-3.079675$

C $2.3725342 .930516-4.000217$

C $3.1249851 .637850-3.663724$

O $0.2832462 .025792-3.155963$

O $4.029221 \quad 1.358411-4.743310$

C $2.1124440 .478479-3.610466$

C $3.9847751 .786376-2.404590$

O $2.738744-0.721934-3.162671$

C $0.8624640 .776973-2.747241$

C $-0.192069-0.334012-2.987498$

O $1.2368320 .797257-1.371202$

C $-4.1161952 .493231-0.423641$

O $-3.7332662 .586860-1.577774$

C $-5.403603 \quad 1.9108770 .008500$

C $-5.9883600 .986830-0.766657$

C $-7.1780930 .214815-0.415041$

C -8.0225770 .7007430 .732806$

C $-1.549160-0.115274-2.302112$

C $-1.697330-0.895526-1.006450$

C $-3.410231-2.0281010 .405926$

O $-0.748857-1.137841-0.265883$

C $-4.623525-2.8356640 .065966$

C -5.745067-2.851005 0.802582

C $-6.975432-3.6342780 .431081$

C $-8.229147-2.7822490 .408692$

C $-9.103991-2.8279681 .427953$

C $-8.511015-1.931052-0.814704$

C $-7.436039-0.918333-1.100446$

N $-3.011224-1.269480-0.766429$

H -0.0085401 .5689041 .184100$

H -1.8187503 .4670632 .766199$

H -1.583332 $2.691095-0.230072$

H -2.526176 5.3335961 .055740

H $-2.7000634 .959817-0.616276$

H $0.8487955 .890814-1.357758$

H $\quad 0.976921 \quad 6.4400070 .859629$

H -0.5965246 .7359641 .601633$

H $\quad 0.2649055 .2125501 .904409$

H - 1.3781434 .015545 -2.344181

H $-1.2728795 .647337-3.009218$

H $-0.1014404 .188811-4.484042$

H $1.0874785 .229687-3.696966$

H $1.5021973 .275830-2.043954$

H $3.0534143 .790106-3.960525$

H $2.0284382 .869857-5.042511$

H $4.6301482 .117745-4.835190$

H $1.7821290 .288033-4.641418$

H $4.7259672 .583486-2.537109$ 
H $4.5586360 .873538-2.212564$ H $3.4009642 .024887-1.513353$ H $3.510304-0.877731-3.733792$ H $-0.392439-0.373453-4.066849$ H $\quad 0.215941-1.309767-2.698117$ H $0.4591140 .581552-0.833306$ H -5.7830122 .1879700 .985395$ H $-5.5130040 .716825-1.708724$ H -8.9769710 .1740810 .812857$ H $-8.269644 \quad 1.7611780 .608576$ H $-7.493816 \quad 0.5718951 .683212$ H $-1.7308770 .940806-2.087396$ H -2.332998 -0.449485 -2.993624 H -3.594914 -1.312707 1.213819 H -2.590939 -2.687896 0.705275 H -4.578911 -3.434995 -0.840863 H $-5.785364-2.2695281 .720924$ H -7.085838 -4.4450891 .162901$ H -6.859228 -4.125808 -0.542840 H -10.017844 -2.243382 1.416199 H -8.924676 -3.4489682 .299406$ H -9.481966 -1.425985 -0.741551 H -8.600016 -2.595466 -1.684070 H -6.769247 -1.202431-1.915092 H -3.743675 -0.872089-1.347505 C 1.7081732 .1886793 .391216 O $2.462061 \quad 1.2867452 .555686$ C 3.6912090 .9484973 .226160 C 3.8036931 .8995454 .411411 C 2.3461222 .0948344 .776766 H $3.573064-0.0722783 .607598$ C 4.8668631 .0120282 .246256 H 4.3994111 .4775885 .226619 H 4.2555342 .8561974 .124512 C 2.1003103 .3085385 .656459 H 1.9888131 .1948185 .295210 C 4.7113820 .1134101 .003960 $\begin{array}{lllll}\text { O } & 5.017480 & 2.361159 & 1.782937\end{array}$ H $5.798468 \quad 0.7679572 .769473$ H 3.8981320 .4837380 .369548 $\begin{array}{lllll}\text { O } & 5.914540 & 0.227623 & 0.233058\end{array}$ C $4.475348-1.3749661 .313813$ C $4.433600-2.2386440 .039604$ H $3.530205-1.4852941 .850347$ O $5.505859-1.8545572 .176415$ H $3.688583-1.817027-0.643194$ H $5.405226-2.184379-0.465679$ H 2.6419793 .2128616 .603163 H 2.4344254 .2310495 .169834 H 1.0358543 .4161885 .886795 C 0.2320021 .7918373 .377655 H 4.1796922 .6214761 .362365 H $6.041997 \quad 1.1711810 .034558$ H $6.353991-1.7330671 .715918$ H -0.3041462 .3133924 .178743$ H 0.1318450 .7163143 .562626 H 1.8406683 .1964332 .975381 C $1.707111-3.6115570 .314579$ O $2.900190-3.8970941 .053843$ C $4.122207-3.7245510 .329307$ C $4.124251-4.621010-0.915610$ C $2.894042-4.385140-1.787001$ C $1.621375-4.482925-0.946854$ H $1.690850-2.5510190 .036140$ C $0.515155-3.8966451 .244273$ H $4.920266-4.0917220 .986844$ O $5.309976-4.406119-1.673954$ H $4.134580-5.671296-0.595961$ H $2.959345-3.423869-2.308780$ O $2.870641-5.393464-2.800018$ H $1.447582-5.530437-0.668535$ O $0.507050-4.055013-1.727924$ C $0.306736-2.8174912 .286387$ H $-0.411545-3.9572590 .663177$ H $\quad 0.672574-4.8432131 .774465$ H $5.258370-4.974835-2.461260$ H $2.149124-5.176022-3.413795$ H $0.397640-4.687647-2.457443$
O $-0.794132-3.1360413 .031391$

O $1.000746-1.8211302 .427374$

C $-1.154557-2.1855374 .038280$

H -1.974980 -2.607011 4.625094

H $-0.310059-1.9911984 .706522$

$\mathrm{H}-1.498857-1.2596823 .568548$

SCF Energy (B3LYP/6-31G**//MMFF) $=-3245.93078396$

0500185

MM̄FF Geometry

C $-4.107150-1.3789150 .997423$

C $-4.395588-1.0365362 .262762$

C $-3.436969-0.4383313 .267273$

O $-2.131205-0.2429792 .680284$

C $-3.9620220 .938895 \quad 3.715277$

C -3.0411471 .6499604 .687666$

C -2.3154972 .7493924 .399807$

C $-2.988177 \quad 1.0558086 .072128$

C -2.2385433 .5017813 .098996$

C -0.8201383 .4774242 .524571$

C -0.6743194 .2854341 .225608$

C -1.4298833 .6550990 .050853$

C -1.175542 $4.405521-1.266636$

O $\quad 0.7290724 .3384920 .929354$

O $-1.6438913 .579394-2.342590$

C $0.3451714 .573675-1.459581$

C $-1.9762585 .710543-1.345169$

O $0.6325605 .383781-2.596263$

C 1.061025 5.139535-0.215049

C $2.6008175 .136850-0.358906$

O $0.6646916 .502489-0.019618$

C $-1.156649-1.1395382 .988840$

O $-1.256636-2.1208473 .706947$

C $0.073569-0.7136802 .285265$

C $1.188299-1.4516542 .389115$

C $2.465493-1.1711541 .734356$

C 2.6567350 .1975791 .134991

C $3.2159913 .767824-0.653872$

C $4.7252313 .756140-0.479721$

C $6.6676942 .204774-0.599007$

O $5.3775334 .743767-0.154370$

C $6.8321790 .746948-0.315068$

C $7.041148 \quad 0.253077 \quad 0.914584$

C $7.160690-1.2127891 .219459$

C $5.932227-1.7387011 .934537$

C $5.944568-1.8999413 .268974$

C $4.756256-2.0800561 .039241$

C $3.412187-2.1331291 .716058$

N $5.2508542 .493601-0.715741$

H $-3.093841-1.2689610 .621418$

H $-5.418693-1.1633562 .612363$

H -3.381686 -1.108384 4.133336

H -4.9455520 .8240004 .191081$

H -4.1446001 .5565402 .830342$

H -1.700726 3.1747295 .192645

H -2.3783281 .6530816 .758444$

H $-3.9950680 .994718 \quad 6.497857$

H -2.559460 0.0493826 .043826

H -2.9421623 .1121082 .362757$

H -2.5360134 .5399643 .290461$

H -0.1162693 .8785523 .266883$

H -0.4975192 .4435752 .350611$

H -1.032486 5.3040741 .415247

H $-2.5071143 .606366 \quad 0.245729$

H -1.101397 2.613548 -0.074560

H $-2.5905713 .410023-2.199494$

H $\quad 0.7452633 .575562-1.679132$

H $-3.0517705 .503480-1.287137$

H $-1.8201316 .212426-2.306344$

H $-1.7343376 .412673-0.544711$

H $0.1676044 .992949-3.355752$

H $2.9097895 .853696-1.130950$

H 3.0279355 .5175200 .579658

H 1.3404046 .9457270 .519788

H $\quad 0.025072 \quad 0.1856541 .682382$

H $1.160436-2.3603982 .988193$

H $3.6888770 .392848 \quad 0.835903$

H $2.4018370 .976740 \quad 1.862720$ 
$\begin{array}{llll}\text { H } & 2.025688 & 0.328082 & 0.250424\end{array}$

H 2.7973613 .0133190 .020942

H $2.9966283 .471251-1.685503$

H 7.1053082 .8318050 .185358

H $7.1482612 .466468-1.547015$

H $6.7533640 .066993-1.159601$

H 7.1070610 .9362391 .758536

H $8.059960-1.3618841 .830859$

H $7.327295-1.7984570 .306484$

H $5.093387-2.2923223 .813770$

H $6.821272-1.6445063 .855876$

H $4.947135-3.0593720 .580863$

H $4.712403-1.3810430 .197137$

H $3.197031-3.0891042 .194150$

H $4.6497801 .789559-1.135631$

C $-5.246451-0.990643-1.234508$

O $-4.019890-1.047031-1.991592$

C -3.599871 $0.298195-2.280592$

C $-4.860721 \quad 1.145516-2.165022$

C $-5.5392970 .491755-0.975893$

H $-2.9031470 .587159-1.483083$

C $-2.8778180 .372567-3.628579$

H $-4.6411192 .204808-2.004229$

H $-5.495176 \quad 1.056690-3.054164$

C $-7.0197120 .810071-0.859719$

H $-5.0365410 .843195-0.066906$

C - $-1.773855-0.684469-3.828216$

O $-3.836522 \quad 0.187313-4.678097$

H $-2.472518 \quad 1.381197-3.765464$

H $-2.238415-1.655834-4.038078$

O $-1.055675-0.334805-5.019144$

C $-0.780101-0.847043-2.662781$

C $0.354340-1.825157-3.023894$

H $-1.322797-1.204500-1.782079$

O $-0.2237490 .426479-2.342821$

H $-0.076136-2.679090-3.557496$

H $1.052134-1.332112-3.712094$

H -7.172545 $1.886456-0.729930$

H $-7.5691830 .498528-1.754295$

H -7.4591790 .2985090 .002570$

C $-5.129030-1.8775830 .012381$

H $-3.3581810 .251686-5.522603$

H $-0.5764670 .492376-4.838943$

H $0.2863860 .328146-1.521430$

H -4.825052 -2.886413 - 0.292661

H $-6.113594-1.9632020 .487140$

H -6.017359-1.419389-1.887489

C $-0.328836-4.116656-1.144976$

O $0.298205-2.881914-0.785626$

C $1.147974-2.327349-1.796060$

C $2.260872-3.327964-2.140450$

C $1.694149-4.685296-2.553914$

C $0.701514-5.194172-1.507594$

H $-0.976448-3.940144-2.013151$

C -1.283976 -4.526535 -0.013367

H $1.627491-1.449413-1.349995$

O $3.088711-2.828446-3.185206$

H $2.903328-3.475386-1.263065$

H $1.231875-4.644406-3.546923$

O $2.762581-5.630618-2.651039$

H $1.261491-5.519915-0.624790$

O $0.019977-6.338256-2.017550$

C $-0.617292-4.839798 \quad 1.310385$

H $-1.982086-3.702550 \quad 0.176382$

H $-1.856177-5.408165-0.324060$

H $3.451864-1.976860-2.887420$

H $3.405633-5.274667-3.288164$

H $0.696221-6.990315-2.269470$

O $-1.551991-5.364747 \quad 2.158430$

O $\quad 0.558467-4.6425301 .580911$

C $-1.071246-5.725852 \quad 3.457097$

H $-0.352384-6.5464353 .375143$

H $-0.616089-4.8633283 .952800$

H $-1.923392-6.0625504 .053392$

SCF Energy (B3LYP/6-31G*//MMFF) $=-3245.91414650$

0500186

MM̄FF Geometry
C $-3.182875-0.498258 \quad 3.086272$

C $-2.385103 \quad 0.3309723 .775490$

C $-0.9187010 .499522 \quad 3.475665$

$\begin{array}{lllll}\text { O } & -0.710777 & 1.908444 & 3.218577\end{array}$

C $-0.0252220 .046895 \quad 4.645988$

C $-0.122084-1.4310084 .955167$

C $0.450061-2.4024714 .217152$

C $-0.909994-1.7820306 .190288$

C $1.285120-2.2507802 .973206$

C $2.624533-2.9802123 .132964$

C $3.454005-3.0031221 .839083$

C $4.702977-3.8638772 .048959$

C $5.630452-3.8494780 .829819$

O $3.821083-1.6620351 .502402$

O $6.873114-4.4316291 .251188$

C $5.893831-2.3835440 .434017$

C $5.092511-4.734280-0.301001$

O $6.640981-2.314012-0.779645$

C $4.604088-1.536466 \quad 0.302468$

C $4.991879-0.0419740 .160476$

O $3.880979-1.969607-0.848732$

C $0.384003 \quad 2.247728 \quad 2.486311$

$\begin{array}{lllll}\text { O } & 1.261093 & 1.497287 & 2.090168\end{array}$

C 0.3120963 .6986572 .204458

C 1.2494154 .2552451 .423139

C 1.2879955 .6427240 .959823

C $0.184796 \quad 6.563912 \quad 1.407698$

C $3.8177180 .910242-0.102808$

C $3.6319861 .261374-1.572472$

C $2.2878352 .782773-3.031789$

$\begin{array}{llll}\text { O } & 4.321468 & 0.792316 & -2.473360\end{array}$

C $2.7825194 .194990-3.010754$

C $1.9791055 .267981-3.073304$

C $2.4752226 .685852-3.023140$

C $1.8711027 .474829-1.880088$

C $0.8428718 .313405-2.093000$

C $2.5063877 .357923-0.510582$

$\begin{array}{llll}\text { C } 2.282361 & 6.014168 & 0.126183\end{array}$

N $2.6033632 .174805-1.751866$

H -2.761768 -1.0865512 .274741$

H $-2.7967120 .910564 \quad 4.598081$

H $-0.662782-0.0603572 .567914$

H $-0.2655290 .636878 \quad 5.541395$

H 1.0221560 .3032924 .442158

H $0.296261-3.4345614 .530782$

H $-0.948321-2.862156 \quad 6.367267$

H $-0.454373-1.3196547 .072252$

H - $1.941179-1.4262196 .105958$

H $0.727464-2.6851692 .135363$

H $1.467866-1.2013982 .727708$

H $3.198276-2.4845043 .927404$

H $2.441403-4.0126223 .458170$

H $2.837138-3.4177541 .034980$

H $4.427125-4.8943062 .306231$

H $5.263861-3.4823162 .914245$

H $7.457697-4.4900900 .476831$

H $6.523944-1.9365001 .215764$

H $5.021033-5.7764940 .032812$

H $5.774605-4.740130-1.158353$

H $4.103670-4.430360-0.651327$

H $7.495098-2.751625-0.626125$

H $5.4573150 .271944 \quad 1.104683$

H $5.7493050 .081380-0.621604$

H $3.234302-1.288943-1.090867$

H -0.5304374 .2532082 .600985$

H 2.0609993 .6265091 .060497

H $-0.790752 \quad 6.180323 \quad 1.088411$

H 0.2710007 .5737831 .000453

H 0.1848556 .6607062 .499013

H 2.8806490 .5017800 .286930

H 4.0162401 .8539040 .421423

H $1.2031762 .727662-3.163369$

H $2.7658402 .229271-3.845681$

H $3.8574634 .338449-2.929691$

H $0.9049515 .123636-3.164028$

H $2.2251577 .160083-3.981126$

H $3.5692546 .725992-2.951635$

H $0.4195588 .905789-1.288837$ 
H $\quad 0.3982718 .423415-3.076702$ H $2.163864 \quad 8.160270 \quad 0.152844$ H $3.5875547 .523573-0.604405$ H $3.0340685 .273590-0.148107$ H $2.0836022 .496711-0.941024$ C -5.580370 -0.2787002 .261451$ O $-5.233746-0.9136421 .015069$ C $-5.480798 \quad 0.022315-0.056688$ C $-6.227226 \quad 1.1967150 .568192$ C $-5.628465 \quad 1.217645 \quad 1.963393$ H $-4.5041350 .385690-0.392360$ C $-6.210390-0.673294-1.211639$ H $-6.0686692 .128866 \quad 0.017531$ H $-7.306792 \quad 1.0114850 .616359$ C -6.4375252 .0257772 .961727$ H -4.6144151 .6329911 .900804$ C $-5.431700-1.870998-1.799476$ O $-7.466002-1.156131-0.719045$ H $-6.4418610 .054786-1.997754$ H -5.294317 -2.634319-1.024801 O $-6.240537-2.481616-2.812033$ C $-4.066769-1.494522-2.407426$ C $-3.290664-2.717514-2.929563$ H $-3.458995-1.007347-1.643092$ O $-4.250238-0.546092-3.456240$ H -3.112692 -3.394974 -2.086598 H -3.906146 $-3.266088-3.653053$ H -6.5025003 .0722922 .646116$ H -7.4579131 .6401323 .058321$ H -5.9719732 .0006523 .951959$ C $-4.637407-0.7225763 .381772$ H $-7.910846-1.610480-1.454870$ H -6.458085 -1.800694 -3.470965 H $-4.672688-1.004320-4.202257$ H $-4.763608-1.8018463 .535907$ H $-4.924776-0.2362364 .321328$ H $-6.588753-0.6347702 .513481$ C $-0.635571-1.877913-1.657351$ O $-1.191687-1.394298-2.884911$ C $-1.955320-2.353439-3.620212$ C $-1.075808-3.561161-3.972041$ C $-0.393778-4.151211-2.738195$ C $0.304969-3.056736-1.929390$ H -1.442860 -2.189354-0.983038 C $0.111403-0.708253-1.004777$ H $-2.222134-1.864252-4.565850$ O $-1.852900-4.558600-4.625949$ H $-0.301851-3.240074-4.681462$ H $-1.110466-4.707710-2.123117$ O $0.579773-5.102243-3.175469$ H $1.190446-2.716501-2.480569$ O $0.766593-3.608660-0.699387$ C $-0.8538910 .394222-0.632082$ H $0.635319-1.011348-0.092149$ H $0.858352-0.309583-1.701199$ H -1.263876 $-5.309536-4.813289$ H $1.038090-5.432234-2.383511$ H $-0.009727-3.899274-0.190834$ O $-0.512268 \quad 1.553959-1.258604$ O C $-1.3904452 .663377-1.041372$ H -1.639902 2.7723280 .018118 H $-0.8799533 .570968-1.374511$ H -2.299383 2.530259-1.634913 SCF Energy (B3LYP/6-31G**/MMFF) $=-3245.92092725$

05_00187

MM̄FF Geometry

C $-4.543817-2.723940-1.545605$

C $-3.379549-2.925063-0.910268$

C $-2.120346-3.349925-1.621924$

O $-1.072964-2.392033-1.340938$

C $-1.653148-4.718126-1.097919$

C $-0.631195-5.371968-2.006295$

C $0.697226-5.405734-1.785305$

C $-1.207154-6.065640-3.216075$

C $1.477065-4.794631-0.653084$

C $2.608652-3.910191-1.186572$
C $3.649899-3.525903-0.124625$

C $3.073355-2.659400 \quad 0.996834$

C $4.162382-2.1872261 .974850$

O $4.684201-2.810726-0.815146$

O $3.604308-1.1468462 .791618$

C $5.318263-1.5561551 .169431$

C $4.590885-3.3054102 .933922$

O $6.413926-1.2355102 .022939$

C $5.803063-2.4422920 .004208$

C $6.832349-1.731520-0.907248$

O $6.452120-3.604050 \quad 0.536554$

C $-1.112207-1.206112-2.006733$

O $-1.947305-0.840896-2.818429$

C $0.049288-0.409676-1.558287$

C $0.0734590 .911635-1.784777$

C $1.1375371 .826723-1.373010$

C $2.4516701 .224674-0.945406$

C $6.332033-0.464714-1.610139$

C $6.7975310 .832415-0.966075$

C $6.8172483 .293026-1.414970$

$\begin{array}{lllllllll}\text { O } & 7.442247 & 0.880810 & 0.076888\end{array}$

C $5.6092924 .098367-1.060290$

C $4.9653724 .885886-1.935128$

C $3.7734015 .731856-1.592429$

C $2.4718995 .073088-1.995537$

C $1.9692565 .262166-3.227800$

C $1.7976774 .256940-0.911909$

C $0.8939013 .154198-1.393291$

N $6.4105601 .933800-1.717276$

H $-4.596653-2.885333-2.619966$

H $-3.316680-2.7679950 .163182$

H $-2.279538-3.393586-2.706737$

H $-2.508021-5.402293-1.008320$

H $-1.254113-4.613679-0.082175$

H $1.312583-5.947898-2.503284$

H $-0.437935-6.558144-3.820398$

H $-1.923370-6.834222-2.907299$

H -1.722848 -5.349847 -3.863357

H $\quad 0.839571-4.2062580 .011467$

H $1.899172-5.612322-0.056829$

H $3.135086-4.426400-2.001689$

H $2.192313-3.003901-1.645576$

H $4.074870-4.4507770 .282928$

H $2.283116-3.187307 \quad 1.543177$

H $2.595568-1.7737880 .557999$

H $2.881332-1.5340483 .314614$

H $4.946972-0.6056360 .765225$

H $3.735698-3.647568 \quad 3.529182$

H $5.331917-2.9443293 .655609$

H $5.002391-4.1778802 .422777$

H $6.071852-0.6637072 .731305$

H $7.763781-1.551371-0.357037$

H $7.102612-2.443334-1.701010$

H $7.037744-3.971515-0.145960$

H $0.840150-0.926683-1.027787$

H $-0.7786581 .373896-2.281334$

H $3.2586891 .958227-0.882509$

H $2.7879770 .473535-1.669067$

H $2.3581780 .748151 \quad 0.035580$

H $6.733544-0.464445-2.632201$

H $5.240070-0.450108-1.688941$

H $7.317443 \quad 3.684963-2.306964$

H $7.5372843 .309341-0.590827$

H $5.2615094 .041513-0.031999$

H $5.3149644 .932754-2.964155$

H $3.8828536 .700437-2.097440$

H $3.7545515 .970355-0.521220$

H $1.0272514 .827354-3.542185$

H $2.4936245 .870679-3.958059$

H $1.2034954 .939111-0.289659$

H $2.5541193 .847899-0.233717$

H $-0.0857243 .499788-1.725091$

H $5.8812011 .784079-2.571572$

C -6.264911 $-0.916570-1.406864$

$\begin{array}{llll}\text { O } & -5.358867 & 0.109852 & -0.952927\end{array}$

C $-6.1239821 .267909-0.566768$

C $-7.5495371 .013182-1.041940$

C $-7.644550-0.493751-0.905248$ 
H $-6.124900 \quad 1.2921480 .529064$

C $-5.4821142 .545471-1.119047$ H $-8.2894081 .547985-0.438560$ H -7.689638 1.310259 -2.087880 C -8.801165 -1.100446-1.680435 H $-7.751270-0.7453730 .158604$ C $-4.0100332 .742101-0.703107$ O $-5.5252052 .490435-2.551042$ H -6.082334 $3.415044-0.827102$ H -3.390447 $1.976867-1.186099$ O $-3.5696413 .996664-1.239412$ C -3.7530542 .7269720 .815529$ C -2.2754122 .9895441 .150876$ H $-4.0456831 .758957 \quad 1.228102$ O H -1.6581402 .3090360 .556236$ H -2.011896 4.0121900 .854694 H $-9.755468-0.701077-1.321804$ H -8.726036 -0.880890 -2.750871 H -8.824774 -2.187921 -1.560709 C $-5.811450-2.276598-0.874638$ H $-5.1050083 .301486-2.885078$ H -4.062422 4.699609 -0.782047 H $-5.4713503 .461607 \quad 1.395486$ H -6.579261 -3.034342 -1.066025 H -5.666869 -2.222037 0.210110 H $-6.237633-0.903279-2.504645$ C $-1.579527 \quad 0.4349982 .655022$ O -2.3416521 .5370743 .160780$ C -1.9600042 .8218822 .655993$ C -0.4957193 .1007523 .019132$ C 0.4214861 .9964602 .506823 C $-0.083811 \quad 0.6229832 .941622$ H -1.7477840 .3336791 .575849$ C -2.092096 $-0.840372 \quad 3.345057$ H -2.5728943 .5557073 .194042$ O $-0.065110 \quad 4.3484872 .485867$ H -0.3929553 .1620414 .110191$ H 0.5284112 .0431681 .420472 O 1.7334992 .1816403 .042226 H $0.1163160 .487677 \quad 4.012205$ O $0.644245-0.3844212 .241846$ C -3.487357 -1.231368 2.908310 H -1.436683 -1.688189 3.116587 H -2.118054 -0.6826284 .430144$ H -0.6621025 .0347272 .829724$ H 2.0322603 .0675022 .773283 H $1.589324-0.1755442 .334388$ O $-3.908986-2.2815893 .674200$ O $-4.122357-0.7030762 .007231$ C $-5.216337-2.781802 \quad 3.375947$ H $-5.199865-3.3103832 .418830$ H $-5.497069-3.4876934 .162211$ H $-5.949996-1.9700663 .358029$ SCF Energy (B3LYP/6-31G**//MMFF)= -3245.91701961

0500188

MM̄MF Geometry

C $0.7923964 .649749-0.299274$

C 1.7169024 .7764040 .665201

C 2.8129373 .7867490 .999094

$\begin{array}{lllll}\text { O } & 2.689056 & 2.564515 & 0.237341\end{array}$

C 4.1871534 .4039380 .679393

C 5.3565833 .5761721 .177918

C 6.2766072 .9891430 .387532

C 5.4783353 .4532092 .675956

C $6.3680383 .012925-1.111794$

C $5.6944861 .825832-1.817464$

C $6.2755040 .451997-1.442301$

C $5.739643-0.633640-2.378937$

C $6.223302-2.035609-1.974915$

O $5.8927420 .149994-0.097558$

O $5.426721-2.997838-2.683607$

C $5.953863-2.249277-0.469491$

C $7.676472-2.280267-2.398890$

O $6.537374-3.467914-0.016023$

C $6.431604-1.0776510 .412643$

C $5.978310-1.2020921 .888388$
O $7.864146-1.0459430 .417087$

C 1.8074011 .6308630 .687928

O 1.1047611 .6987361 .683819

C $1.8176420 .508022-0.274653$

C $0.774558-0.333278-0.308270$

C $0.622984-1.490997-1.190125$

C $1.829313-1.926365-1.977876$

C $4.462468-1.2508992 .110456$

C $3.896086-2.6558942 .236564$

C $1.721036-3.8547722 .462157$

O $4.595883-3.6609232 .325669$

C $1.311232-4.3963481 .129089$

C $0.041593-4.6991880 .818071$

C $-0.384419-5.290621-0.495217$

C $-1.441359-4.463566-1.192288$

C $-2.743174-4.786080-1.108253$

C $-0.976525-3.309544-2.051888$

C $-0.573006-2.114072-1.231662$

N $2.507727-2.6493472 .278276$

H $0.8034163 .783154-0.954373$

H $1.6789855 .656677 \quad 1.304522$

H 2.7441013 .5555212 .068826

H 4.2659245 .4014731 .132385

H $4.2557494 .557291-0.402947$

H 7.0750612 .4248920 .868716

H 6.4216592 .9862112 .979198

H 5.4408404 .4428083 .143195

H 4.6665282 .8422223 .081812

H $5.9548213 .944751-1.512040$

H $7.4313463 .034472-1.383965$

H $4.6212171 .846225-1.595358$

H $5.8032251 .979399-2.898307$

H $7.3683340 .507878-1.509181$

H $6.013914-0.420025-3.419606$

H $4.641793-0.624950-2.352624$

H $5.527299-2.827201-3.635723$

H $4.869551-2.360696-0.354218$

H $7.779195-2.183608-3.486481$

H $7.991768-3.301632-2.159051$

H $8.380518-1.583842-1.938936$

H $6.192713-4.181561-0.579821$

H $6.486032-2.0469442 .369215$

H $6.343848-0.3079062 .413892$

H $8.159376-0.5565191 .202654$

H $2.6709790 .414321-0.935392$

H $-0.059700-0.1499380 .365793$

H $2.089446-1.173616-2.729795$

H $1.692422-2.873392-2.503018$

H $2.687059-2.070723-1.312536$

H $4.236291-0.7467333 .058932$

H $3.932103-0.7128541 .320186$

H $0.859911-3.5877843 .082906$

H $2.317446-4.6019232 .995680$

H $2.096581-4.5743110 .398339$

H $-0.737176-4.5449381 .561734$

H $-0.770416-6.298946-0.296594$

H $0.471012-5.430823-1.167845$

H $-3.503290-4.210123-1.625671$

H -3.078376 $-5.628386-0.512111$

H -1.785465 -2.990148 -2.721133

H $-0.185485-3.661988-2.718900$

H -1.383859-1.712610 -0.622408

H $2.009504-1.7734672 .149201$

C -1.661155 $5.075313-0.109554$

O $-2.0082703 .951132-0.939638$

C $-3.4275373 .961943-1.178097$

C $-3.9934585 .094322-0.325428$

C $-2.8214016 .054039-0.261497$

H $-3.5669944 .205604-2.238667$

C $-4.0556412 .589269-0.884178$

H $-4.8890655 .538608-0.770636$

H -4.2586884 .7469050 .680299$

C -2.9315667 .0700230 .861796$

H $-2.7454426 .581277-1.221972$

C $-3.5543771 .472799-1.824229$

O

H $-5.1416812 .693887-0.984829$

H -3.776776 1.750171 -2.861598 
O $-2.1270581 .376045-1.741221$

C $-4.1195700 .065711-1.545288$

C $-5.6568210 .003952-1.539953$

H -3.706431 -0.312760 -0.603676

O $-3.628722-0.789111-2.584161$

H $-6.0450020 .674817-0.768450$

H $-6.0304140 .371618-2.503736$

H $-2.063618 \quad 7.736540 \quad 0.873328$

H -3.827876 7.6863010 .735885

H -2.994343 6.5816071 .840046

C $-0.309195 \quad 5.644731-0.541195$

H $-4.322427 \quad 1.4721540 .703186$

H -1.897417 $1.233635-0.806911$

H -3.751824 -1.707431 -2.290691

H $-0.1045046 .578143-0.004688$

H $-0.3196005 .879231-1.612480$

H -1.6046694 .7053790 .923345$

C $-6.011282-1.4895881 .092452$

O $-5.624778-2.051018-0.167837$

C $-6.214629-1.420325-1.309042$

C $-7.745509-1.499657-1.222054$

C $-8.274146-0.9727830 .111376$

C -7.526006 -1.608938 1.284095

H $-5.716543-0.4343161 .132731$

C $-5.244404-2.2414742 .184967$

H $-5.919079-2.020754-2.178388$

O $-8.335748-0.781954-2.300288$

H -8.048351 -2.549160 - 1.331576

H $-8.207106 \quad 0.1203300 .154101$

O $-9.666422-1.2788790 .220530$

H -7.813921 -2.663499 1.379350

O $-7.901534-0.9490482 .490684$

C -3.782208-1.864561 2.159975

H $-5.630918-2.0276613 .187687$

H $-5.310188-3.3261252 .036794$

H -9.300811 - $0.838437-2.194366$

H $-9.765354-2.2457340 .189458$

H -8.869011 -1.012536 2.567554

O $-3.607172-0.6455162 .746075$

O $-2.907589-2.5595611 .660515$

C $-2.267405-0.1433322 .741168$

H $-1.985896 \quad 0.1367391 .722855$

H -2.236373 0.7491623 .371531

H - $1.570876-0.8833413 .147033$

SCF Energy (B3LYP/6-31G**//MMFF) $=-3245.89923541$

05_00189

MM̄FF Geometry

C $-0.311642-2.9265571 .619204$

C $0.575808-2.073797 \quad 1.087245$

C $1.053628-2.158409-0.337907$

O $0.726048-0.895538-0.967883$

C $2.577301-2.361278-0.398163$

C $3.068454-3.6947420 .118350$

C $3.852078-3.8493711 .203357$

C $2.683557-4.891612-0.712194$

C $4.329460-2.7909942 .159056$

C $5.797539-2.4002521 .934470$

C $6.015482-1.5049770 .703037$

C $7.504627-1.214190 \quad 0.505310$

C $7.747246-0.228276-0.647388$

O $5.306454-0.2815820 .923561$

$\begin{array}{lllll}\text { O } & 9.106368 & 0.226203 & -0.560964\end{array}$

C $6.844347 \quad 1.006525-0.445937$

C $7.601037-0.902029-2.016834$

O $6.9300431 .888287-1.563356$

C $5.3649840 .652978-0.162368$

C $4.596921 \quad 1.9241840 .273292$

O $4.773674 \quad 0.128718-1.351774$

C $0.704115-0.863071-2.326518$

O $0.914958-1.803997-3.080429$

C $0.424508 \quad 0.495429-2.844377$

C $-0.0568361 .494419-2.089783$

C $-0.3880652 .843097-2.554350$

C $0.136960 \quad 3.269046-3.899395$

C 3.1580361 .6699630 .751068

C $2.546996 \quad 2.915111 \quad 1.373115$

C 0.5913003 .6603852 .719573
O $3.0811924 .020301 \quad 1.321674$

C $-0.444084 \quad 4.2601591 .824318$

C $-0.2940505 .436267 \quad 1.196503$

C $-1.346791 \quad 6.070474 \quad 0.333068$

C $-0.9809906 .063582-1.134823$

C $-0.1655136 .998119-1.651446$

C -1.634554 $5.024629-2.017929$

C $-1.129723 \quad 3.634488-1.752032$

N $1.3338872 .647262 \quad 1.993911$

H $-0.723564-3.7204971 .001157$

H $0.983656-1.2756221 .703416$

H $0.526806-2.960106-0.868993$

H $3.057562-1.525965 \quad 0.119524$

H $2.924289-2.257336-1.434378$

H $4.180514-4.8563501 .458139$

H $3.172281-5.809829-0.369429$

H $1.603122-5.058936-0.668170$

H $2.971262-4.738585-1.757476$

H $3.684154-1.9072452 .151348$

H $4.234741-3.2063973 .170912$

H $6.130906-1.8564992 .828228$

H $6.415462-3.3034061 .856288$

H $5.610362-2.006497-0.182175$

H $8.063956-2.1435480 .340109$

H $7.916435-0.7769001 .425871$

H $9.686749-0.552442-0.614075$

H $7.238611 \quad 1.561540 \quad 0.417055$

H $8.319748-1.724274-2.117424$

H $7.831567-0.202401-2.827831$

H $6.604300-1.313640-2.188749$

H $7.8710302 .093728-1.698589$

H 5.1446782 .3983301 .098768

H $4.5656812 .644858-0.553715$

H $3.8187740 .296683-1.335299$

H $0.5986020 .619922-3.908568$

H $-0.256512 \quad 1.308678-1.037135$

H $\quad 0.036815 \quad 4.341697-4.081514$

H $-0.3933132 .745663-4.702241$

H $1.2074063 .049090-3.984246$

H $2.5215111 .366510-0.085999$

H 3.1419450 .8741121 .504009

H 1.2825804 .4185213 .103609

H 0.1108903 .1758503 .575355

H -1.3737193 .7099021 .711060$

H 0.6355095 .9876781 .319693

H $-1.489068 \quad 7.1046440 .673120$

H -2.3202145 .5850420 .478297$

H $0.0772857 .015153-2.708691$

H $\quad 0.2802707 .764237-1.025520$

H $-1.5317695 .296070-3.073449$

H $-2.7174215 .036434-1.836036$

H $-1.4654813 .226478-0.799474$

H 0.9322541 .7164641 .926366

C $-2.134187-2.1585813 .141229$

O $-1.909481-0.7303053 .113997$

C $-2.731014-0.1191244 .118652$

C $-3.741567-1.1743184 .543641$

C $-2.898461-2.4319274 .435875$

H $-2.0719790 .070995 \quad 4.977714$

C $-3.305287 \quad 1.229157 \quad 3.673173$

H $-4.131828-1.0044675 .551452$

H $-4.587912-1.2219353 .849070$

C -3.718704 -3.7100514 .410219$

H $-2.201366-2.4671365 .283991$

C $-4.250868 \quad 1.2399902 .450998$

O $-4.016387 \quad \begin{array}{llll}1.771081 & 4.793827\end{array}$

H -2.469422 $1.912333 \quad 3.477650$

H -5.1865670 .7254122 .695085$

O -4.6156722 .6105822 .221869$

C -3.663066 $0.661463 \quad 1.152001$

C $-4.5631190 .890482-0.077438$

H -3.517417 -0.4135041 .280723$

$\begin{array}{lllll}\text { O } & -2.387252 & 1.253636 & 0.926543\end{array}$

H -5.5170840 .3796950 .095268$

H $-4.786823 \quad 1.958987-0.181290$

H $-4.292114-3.8171875 .336787$

H $-4.426559-3.7160543 .574404$

H -3.072176 -4.5873324 .309765$ 
C $-0.789313-2.875933 \quad 3.043907$ H -4.3605302 .6404584 .525697$ H -3.8138803 .0843381 .941103$ H $-1.900770 \quad 0.668610 \quad 0.321675$ H $-0.872424-3.9086263 .400859$ H $-0.043757-2.373634 \quad 3.672771$ H -2.766435 -2.401560 2.277800 C $-4.288953-1.953527-1.209999$ O $-3.346151-0.882848-1.297562$ C $-3.925590 \quad 0.418610-1.404131$ C $-4.8561190 .496015-2.622470$ C $-5.899150-0.619376-2.617760$ C $-5.228684-1.979268-2.421025$ H $-4.889855-1.821728-0.300641$ C -3.512381-3.262775 -1.007831 H $-3.0931421 .103098-1.603983$ O $-5.5177261 .755292-2.678461$ H $-4.2592820 .404948-3.539464$ H $-6.668545-0.447951-1.855986$ O $-6.577678-0.634144-3.875875$ H -4.686444 -2.246469 -3.335003 O $-6.229831-2.973552-2.216062$ C - $-2.505591-3.583448-2.094798$ H -2.943214 -3.186083 -0.074822 H -4.211368 -4.101978 -0.922532 H $-4.8318602 .444468-2.702466$ H $-6.9642590 .248949-4.005672$ H -6.829804 -2.942860 -2.981069 O $-1.961044-4.810778-1.837718$ O $-2.213999-2.856958-3.034174$ C $-0.975741-5.256012-2.775265$ H -1.422860 -5.368812 -3.767442 H $-0.135873-4.556016-2.807292$ $\mathrm{H}-0.606481-6.229853-2.443379$ SCF Energy (B3LYP/6-31G $\left.{ }^{* *} / / \mathrm{MMFF}\right)=-3245.91200948$

05_00190

MM̄MF Geometry

C $2.572839-4.007518-1.112573$

C $1.293310-3.988728-1.513821$

C $0.194722-3.355922-0.700989$

O $-0.248699-2.156592-1.378759$

C $-1.009335-4.306013-0.581624$

C -2.012495 -3.8180090 .444470$

C $-3.066941-3.0235120 .173886$

C $-1.753294-4.2789031 .855868$

C $-3.462084-2.443711-1.156983$

C $-4.940655-2.661417-1.518436$

C $-5.931605-1.958087-0.577148$

C $-7.364563-2.067261-1.103591$

C $-8.357437-1.287140-0.225494$

O $-5.556261-0.581849-0.483114$

O $-9.586701-1.158443-0.956354$

C $-7.816500 \quad 0.1418160 .002950$

C -8.697293 -2.047055 1.062392

$\begin{array}{lllll}\text { O } & -8.624297 & 0.851292 & 0.939135\end{array}$

C $-6.3367430 .177727 \quad 0.446679$

C $-5.806672 \quad 1.6314870 .439267$

O $-6.233703-0.3426031 .774769$

C $0.471931-1.020679-1.176073$

O $1.489125-0.902442-0.512395$

C $-0.1940760 .074298-1.913847$

C $0.4592891 .231700-2.093713$

C $-0.0413432 .405243-2.812076$

C -1.497598 2.419021-3.195309

C -4.2774891 .7297240 .550944$

C $-3.7996213 .161487 \quad 0.714129$

C $-1.701750 \quad 4.496080 \quad 0.886916$

O $-4.561763 \quad 4.112213 \quad 0.867389$

C $-1.5455895 .195900-0.426165$

C $-0.3783855 .687723-0.868473$

C $-0.2333596 .451099-2.153279$

C $0.8658865 .911879-3.039073$

C $2.0377716 .558456-3.159159$

C $0.5724884 .673619-3.855635$

C $0.822774 \quad 3.404727-3.087314$

N -2.412963 $3.246410 \quad 0.694127$

H $2.835702-3.557496-0.157975$
H $\quad 1.026627-4.416488-2.476874$

H $\quad 0.550961-3.0955490 .302919$

H $-0.673174-5.310016-0.289297$

H -1.493521 -4.431979-1.557108

H -3.689823 -2.716949 1.012259

H -2.478763 -3.8719282 .567970$

H $-1.810018-5.3708461 .912864$

H $-0.757833-3.9658152 .185300$

H -3.241550 -1.369862 -1.139492

H -2.860964 -2.860397 -1.970130

H $-5.080911-2.270508-2.534970$

H -5.163230 -3.734874 -1.555032

H $-5.863352-2.4227180 .412368$

H -7.668827 -3.117764 -1.190171

H -7.411027 -1.656654 -2.122161

H -9.902472 -2.052976 -1.171403

H $-7.9027770 .680072-0.951554$

H -9.144191 -3.020236 0.825849

H $-9.447864-1.5094211 .652250$

H -7.826534 -2.230252 1.695214

H -9.5410400 .8158860 .615912$

H -6.096683 $2.125396-0.497276$

H -6.2704192 .1933321 .259971$

H -5.4659540 .0526042 .216226$

H -1.189321 -0.106858 -2.302809

H $1.4708751 .324280-1.699843$

H -2.127789 2.197408 -2.326548

H $-1.697327 \quad 1.672857-3.971859$

H -1.838236 $3.384821-3.574622$

H -3.9161041 .1657681 .416682$

H $-3.810413 \quad 1.312420-0.348819$

H -0.7379364 .2558741 .346820$

H -2.2717815 .1290811 .574956$

H -2.441910 $5.339099-1.025129$

H $0.5130075 .575160-0.255414$

H $-0.0257247 .497479-1.893742$

H -1.174329 $6.467634-2.717326$

H $2.8196686 .192513-3.816552$

H $2.2438707 .464390-2.598968$

H $1.2259714 .650952-4.738453$

H $-0.4402164 .739905-4.261988$

H $1.8595203 .291910-2.765811$

H -1.865713 2.4154210 .488136

C $4.570581-3.559429-2.615851$

O $4.997112-2.526853-1.702083$

C $4.832005-1.251582-2.352838$

C $4.700577-1.559054-3.838779$

C $3.901229-2.846256-3.792885$

H $3.880131-0.835736-2.003233$

C $5.978654-0.302583-1.995573$

H $4.196116-0.758084-4.387434$

H $5.677736-1.732183-4.304911$

C $3.934475-3.632346-5.091770$

H $2.859697-2.596410-3.556752$

C $6.122776-0.011148-0.486704$

O $7.213957-0.863564-2.455649$

H $5.8545630 .638899-2.543376$

H $6.478518-0.9076880 .034388$

O $7.1603020 .964741-0.327824$

C 4.8353070 .4927630 .195451

C $5.0665180 .977121 \quad 1.639432$

H $4.104713-0.3220140 .204487$

O $4.266197 \quad 1.556012-0.568898$

H 5.5765330 .1862942 .199540

H $5.732571 \quad 1.8481801 .626692$

H $3.504472-3.043217-5.908501$

H $4.958285-3.899775-5.373795$

H $3.356473-4.557312-5.001975$

C $3.706553-4.601027-1.897649$

H $7.319892-1.730183-2.026821$

H $6.8976691 .763392-0.815838$

H $4.8752632 .312519-0.529922$

H $4.334330-5.147857-1.182541$

H $3.337967-5.341884-2.616627$

H $5.486950-4.056855-2.960001$

C $3.084915-0.7770923 .189756$

$\begin{array}{lllll}\text { O } & 2.784651 & 0.327047 & 2.331241\end{array}$

C 3.7516061 .3830452 .345489 
C 3.9355451 .9187793 .772802

C 4.2538650 .8056984 .768059

C $3.228201-0.3216994 .646272$

H $4.025378-1.2366452 .861170$

C $2.011685-1.8534512 .996712$

H 3.3171342 .1997551 .755146

$\begin{array}{lllll}\text { O } & 4.960636 & 2.906430 & 3.798385\end{array}$

H 3.0067182 .4140924 .085133

H $5.277162 \quad 0.436758 \quad 4.632408$

$\begin{array}{lllll}\text { O } & 4.194633 & 1.351964 & 6.087414\end{array}$

H 2.2693510 .0374095 .037531

O $3.629751-1.4160325 .466264$

C $0.614263-1.3760803 .318671$

H $2.016529-2.2006081 .957052$

H $2.218492-2.7372383 .610836$

H 5.0548013 .2038574 .719591

H 4.3494450 .6212806 .710379

H $4.482197-1.7435255 .131837$

O $0.048544-0.8131122 .213774$

O $0.094058-1.481864 \quad 4.421564$

C -1.282682 $-0.315978 \quad 2.375537$

H -1.934969 -1.081492 2.806564

H $-1.2709330 .579613 \quad 3.003024$

H $-1.664791-0.049913 \quad 1.386674$

SCF Energy (B3LYP/6-31G*//MMFF) $=-3245.90875977$

0500191

MM̄FF Geometry

C 0.2012785 .3822161 .051710

C 1.2763864 .6007680 .871243

C $2.050527 \quad 3.9674691 .994744$

O 2.0511332 .5407361 .766342

C 3.5153484 .4449081 .974288

C $4.354713 \quad 3.8436943 .086682$

C 5.3941283 .0063892 .897179

C 3.9705264 .2522444 .486198

C 5.9751752 .5234641 .596608

C 5.3689951 .1888151 .162811

C 6.1359750 .4958310 .026267

C $6.1669891 .324883-1.260701$

C $6.824526 \quad 0.561304-2.422479$

O $5.471597-0.754739-0.210792$

O $6.5557691 .279777-3.636424$

C $6.179077-0.836565-2.536435$

C $8.3515710 .546738-2.294112$

O $6.851938-1.636140-3.505229$

C $6.108398-1.588769-1.190645$

C $5.298373-2.903817-1.283625$

O $7.432444-1.944519-0.775432$

C 1.2822511 .7555542 .566176

$\begin{array}{lllll}\text { O } & 0.513484 & 2.113837 & 3.442564\end{array}$

C 1.5732790 .3526582 .195253

C $1.060951-0.6640732 .904571$

C $1.312972-2.0868462 .655099$

C $2.286446-2.4270481 .557671$

C $3.872297-2.747613-1.816860$

C $3.018410-3.981024-1.568269$

C $0.645906-4.747237-1.741891$

O $3.458922-5.022296-1.089782$

C $-0.116863-4.375221-0.509049$

C $-0.404957-5.2269660 .487058$

C $-1.211344-4.8354951 .693997$

C $-0.533626-5.1744873 .004368$

C -1.096227 -6.042973 3.861852

C $0.786865-4.5099503 .333161$

C $0.678268-3.0073823 .410867$

$\mathrm{N} \quad 1.700630-3.771976-1.951123$

H $-0.147658 \quad 5.5907492 .060056$

H $1.6195404 .394777-0.140144$

H 1.5922124 .2104192 .960558

H 3.5528915 .5385532 .064484

H 3.9495134 .2063410 .997085

H 5.9100952 .6250653 .777859

H $4.694603 \quad 3.9081065 .232645$

H 3.9193855 .3432994 .562300

H 2.9963483 .8356514 .758710

H $5.856524 \quad 3.276090 \quad 0.811552$

H 7.0574542 .4099481 .737513
H 5.3221320 .4998632 .017382

H $4.327776 \quad 1.3386370 .853765$

H 7.1539430 .2931150 .379143

H $6.6811752 .280878-1.107927$

H $5.1373591 .580825-1.546947$

H $5.5917291 .343055-3.745919$

H $5.159746-0.684451-2.912540$

H $8.7451201 .570410-2.314233$

H $8.8152700 .032139-3.142941$

H $8.702710 \quad 0.077458-1.372969$

H $6.880118-1.126883-4.333250$

H $5.839705-3.637999-1.894626$

H $5.249889-3.341182-0.276351$

H $7.370269-2.683825-0.148083$

H $2.2284290 .193233 \quad 1.346094$

H $0.406266-0.4393923 .745172$

H $3.265338-1.9754381 .754688$

H $1.913994-2.0552480 .597769$

H $2.461048-3.4965891 .435176$

H $3.382102-1.891845-1.339902$

H $3.893049-2.572916-2.898446$

H $\quad 1.075592-5.751709-1.676054$

H $-0.014464-4.700563-2.613226$

H $-0.468865-3.348429-0.440731$

H $-0.078307-6.2616340 .418025$

H $-2.178282-5.350621 \quad 1.622865$

H $-1.443739-3.7638971 .679781$

H $-0.623569-6.293706 \quad 4.805754$

H $-2.042979-6.5245813 .640630$

H $1.534760-4.8488582 .612652$

H $1.153898-4.8561164 .308496$

H $-0.000578-2.6584464 .190133$

H $1.428020-2.854499-2.292266$

C $-2.0016715 .483365-0.145315$

O $-1.988614 \quad 4.086722-0.505102$

C $-2.9797453 .855069-1.528245$

C $-3.8502195 .101786-1.550610$

C $-2.8425626 .181255-1.214326$

H -2.420415 $3.758103-2.467179$

C $-3.7316182 .555779-1.214948$

H $-4.3383415 .257952-2.517142$

H $-4.627435 \quad 5.049106-0.777947$

C -3.476911 $7.481178-0.751123$

H $-2.2308946 .378462-2.104963$

C $-4.8975202 .267574-2.176505$

O $-2.7987301 .472538-1.287159$

H $-4.0933302 .589700-0.181230$

H $-5.6602383 .049781-2.081928$

O $-4.3994342 .347950-3.518926$

C $-5.5552140 .884765-2.001651$

C $-6.0873280 .640947-0.579056$

H $-4.8463200 .104270-2.299736$

$\begin{array}{lllll} & \text { O } & -6.647926 & 0.825375 & -2.925117\end{array}$

H -5.2588720 .7069110 .132075$

H $-6.794898 \quad 1.439327-0.322576$

H $-4.1147487 .899138-1.536794$

H -4.0962507 .3303720 .139584$

H - $2.7112608 .224225-0.507014$

C $-0.567238 \quad 6.010312-0.077089$

H -2.067018 $1.679115-0.680311$

H $-5.1456942 .166429-4.115663$

H $-6.904841-0.107785-3.016666$

H -0.5704857 .0954240 .076267$

H $-0.0578695 .817542-1.029690$

H -2.4863845 .5547850 .837089$

C $-4.847114-2.102185-0.146010$

O $-6.020644-1.805683-0.912150$

C $-6.802439-0.718470-0.409350$

C $-7.260102-1.0196771 .025398$

C $-6.091933-1.3951191 .935503$

C $-5.231594-2.4813931 .288759$

H $-4.184012-1.228987-0.134462$

C $-4.117917-3.249592-0.851148$

H -7.707528 $-0.680046-1.028015$

$\begin{array}{llll}\text { O } & -7.948586 & 0.093570 & 1.585389\end{array}$

H $-7.966527-1.859973 \quad 1.009561$

H $-5.487849-0.5171132 .192956$

O $-6.604065-1.9036913 .169418$ 
H $-5.785932-3.4281301 .293952$

O $-4.058654-2.6810782 .074589$

C $-3.514526-2.787951-2.156797$

H -3.312703 -3.666258 -0.238179

H $-4.810402-4.069063-1.078487$

H -8.6985290 .2972501 .000704$

H -7.176018 -1.215349 3.550469

H $-3.549418-1.8526962 .069648$

O $-2.429081-1.998436-1.915647$

O $-3.957635-3.090464-3.256940$

C $-1.766073-1.488539-3.076268$

H -1.396787 -2.311914 -3.694925

H $-2.444265-0.847457-3.647532$

H $-0.913369-0.890211-2.744298$

SCF Energy (B3LYP/6-31G**//MMFF) $=-3245.91660510$

05_00192

MM̄FF Geometry

C -1.429630 $2.424155-0.001764$

C $-1.6112501 .383768-0.831053$

C $-0.6678940 .215969-1.024998$

O $\quad 0.6247770 .472542-0.433112$

C $-1.268297-1.027375-0.339778$

C $-0.445118-2.289038-0.506890$

C $0.148658-2.9551510 .502936$

C $-0.332741-2.817247-1.913593$

C $0.142210-2.6228431 .968931$

C $1.345318-1.7904362 .440095$

C $2.701629-2.4983982 .284058$

C $3.811312-1.7033222 .975854$

C $5.194959-2.3384502 .760572$

O $2.998380-2.6095210 .890121$

O $6.186842-1.3744573 .147298$

C $5.398120-2.6138811 .254265$

C $5.405604-3.5615873 .661061$

O 6.593407 -3.356395 1.028712

C $4.202546-3.3300120 .594768$

C $4.316527-3.418330-0.946903$

O $4.130503-4.6743051 .085864$

C $1.5204291 .183667-1.171150$

O $1.3660901 .616710-2.301866$

C $2.7255351 .382683-0.336442$

C $3.5936052 .354458-0.654129$

C 4.8100002 .7002460 .084500

C 5.2514321 .7622951 .176525

C $4.406594-2.074559-1.678780$

C $5.827063-1.637751-1.996515$

C $7.0977420 .286858-2.940612$

O $6.806673-2.362532-1.845544$

C $7.6667891 .090118-1.814257$

C $8.0173542 .379513-1.934488$

C $8.6327093 .187165-0.828352$

C $7.8804824 .470250-0.556340$

C $8.2947555 .638020-1.076425$

C 6.7019994 .4112160 .388610

C $5.4732033 .826064-0.253710$

N $5.866489-0.347720-2.509112$

H -0.5285202 .4900500 .601497$

H -2.534638 $1.332481-1.404364$

H $-0.5514450 .034284-2.100155$

H $-2.272136-1.223794-0.738115$

H -1.409478 -0.8013940 .722720$

H $\quad 0.705269-3.8592340 .257891$

H $\quad 0.208000-3.768798-1.958895$

H -1.327917 -2.989048 -2.333722

H $0.202989-2.109371-2.552921$

H - $0.780232-2.1020652 .246267$

H $\quad 0.114636-3.5658172 .529972$

H $1.357156-0.8420131 .890343$

H $1.187928-1.5481503 .498469$

H $2.619498-3.4997822 .722646$

H $3.602780-1.5944154 .047433$

H $3.831908-0.6826492 .570803$

H $6.025697-1.1349144 .076061$

H $5.539666-1.6405520 .770498$

H $5.318430-3.2788354 .717093$

H $6.415562-3.9697393 .543863$

H $4.685631-4.3610393 .474033$
H $7.323749-2.8611821 .437516$

H $5.137732-4.089082-1.226627$

H $3.403515-3.912586-1.309782$

H $3.622348-5.2073220 .452238$

H 2.8487190 .7453540 .531463

H $3.3822992 .976892-1.522999$

H 5.3019900 .7351900 .800619

H 4.5513611 .7953132 .018245

H 6.2456731 .9855061 .568835

H $3.896364-2.173195-2.645673$

H $3.896445-1.284437-1.119558$

H $6.8562900 .905639-3.810647$

H $7.816417-0.480277-3.246191$

H $7.8230300 .581323-0.865904$

H $7.8858452 .877453-2.892761$

H $9.6664533 .416084-1.119169$

H 8.7095702 .6056750 .098790

H $7.7777496 .567738-0.862993$

H $9.1575225 .689020-1.732366$

H 6.4391595 .4237220 .723618

H 7.0002603 .8871741 .300839

H $5.0753304 .437144-1.065226$

H $5.0114810 .199527-2.548299$

C -3.0986683 .3810581 .580554$

O -3.8480642 .1518831 .628385$

C -5.1039362 .3892472 .289786$

C -4.9908943 .7729992 .917085$

C -4.1045274 .4825691 .907561$

H -5.8695582 .4296971 .506166$

C $-5.413431 \quad 1.233438 \quad 3.249720$

H -5.9662554 .2518343 .045313$

H -4.498692 3.7393963 .895769

C -3.4749395 .7572962 .440122$

H -4.7039914 .7204521 .018440$

C $-5.272774-0.1600572 .596774$

O $-4.487928 \quad 1.2964404 .341818$

H -6.4125621 .3664403 .680144$

H $-4.215847-0.3541242 .373731$

O $-5.673886-1.1518953 .548459$

C $-6.106297-0.356971 \quad 1.316303$

C $-5.828965-1.7167790 .654319$

H $\quad-5.8640530 .423190 \quad 0.594057$

O $-7.496882-0.2583921 .611566$

H $-4.755834-1.7833840 .442302$

H $-6.069138-2.5216731 .359608$

H -4.2482576 .4807202 .718406$

H -2.862151 5.5625203 .326574

H $-2.834848 \quad 6.221672 \quad 1.683439$

C -2.4451743 .5153690 .204747$

H $-4.8115480 .696825 \quad 5.035010$

H $-5.011799-1.1603324 .259907$

H -7.676005 0.6534551 .895559

H -1.9410614 .4834410 .110801$

H -3.210193 $3.468601-0.579111$

H -2.3313923 .3169602 .363779$

C $-5.290342-0.688467-2.197918$

O $-6.561962-0.859084-1.561692$

C $-6.643965-1.950998-0.638858$

C $-6.306175-3.268583-1.351338$

C $-4.953275-3.197201-2.054518$

C $-4.885745-1.963401-2.951343$

H -4.534621 -0.437069-1.444553

C $-5.4135740 .488431-3.182225$

H -7.697240 -2.010192 -0.336542

O $-6.298915-4.359079-0.436242$

H -7.076534 -3.483849 -2.103104

H -4.122938 -3.209236 -1.339094

O $-4.788891-4.355412-2.876447$

H $-5.542035-2.110738-3.819075$

O $-3.556616-1.812827-3.445242$

C $-5.5331091 .833844-2.499614$

H $-4.5275660 .537901-3.825124$

H $-6.3070660 .355832-3.804191$

H -7.173149 -4.389015 -0.011300

H $-4.873896-5.133324-2.298717$

H -3.321962 -2.638882 -3.902097

O $-5.6614662 .802960-3.454710$

O $-5.5113752 .023146-1.292562$ 
C $-5.7771024 .141043-2.959956$

H $-4.8729244 .420718-2.411050$

H $-5.8891364 .811583-3.816059$

H -6.661252 4.235088 -2.322219

SCF Energy (B3LYP/6-31G**//MMFF) $=-3245.90683274$

05_00193

MM̄FF Geometry

C -0.534187 $5.341105-0.021117$

C $0.4754654 .641403-0.560229$

C 1.5884834 .0128930 .238407

O $1.8283632 .685553-0.287941$

C 2.8848804 .8237600 .066809

C 4.0579554 .2660050 .851032

C 5.1929083 .7933820 .298696

C 3.9096564 .2822742 .351031

C $5.5574763 .730896-1.157769$

C $5.2154492 .403353-1.850993$

C $5.9824391 .190495-1.299081$

C $5.807213-0.021594-2.216996$

C $6.498459-1.273328-1.653883$

O $5.4711890 .879104 \quad 0.000145$

O $6.020477-2.409423-2.391101$

C $6.062250-1.472726-0.186582$

C $8.016915-1.229403-1.862141$

O $6.806841-2.5212860 .427665$

C $6.159373-0.190108 \quad 0.665719$

C $5.524079-0.3438492 .069947$

$\begin{array}{lllll}\text { O } & 7.540264 & 0.137415 & 0.865545\end{array}$

C 0.9708801 .6927170 .069147

O $0.025019 \quad 1.772355 \quad 0.835890$

C $1.368838 \quad 0.483417-0.684802$

C $0.498787-0.526572-0.827657$

C $0.728793-1.759739-1.582630$

C $2.126030-2.028558-2.074559$

C $4.039157-0.7241332 .083100$

C $3.782137-2.2155952 .232487$

C $1.916093-3.8710952 .205484$

O $4.660151-3.0274572 .510369$

C $1.743116-4.4887500 .854632$

C $0.580887-5.0010570 .421976$

C $0.407133-5.679028-0.906545$

C $-0.695554-5.065146-1.738873$

C -1.918571 -5.619770 -1.788049

C $-0.351230-3.862355-2.586315$

C $-0.315471-2.586667-1.795950$

N $2.440243-2.5261132 .058485$

H -0.5540265 .5138781 .051881$

H $0.4970844 .484294-1.636507$

H 1.3184213 .9415121 .299040

H 2.7205805 .8606000 .389315

H $3.1255954 .873562-1.000551$

H 5.9674533 .4170980 .966153

H 4.8405514 .0156452 .863130

H 3.6246465 .2821772 .693888

H 3.1440683 .5694132 .671932

H $5.0864414 .551098-1.709909$

H $6.6360203 .915441-1.245128$

H $4.1360672 .227447-1.772993$

H $5.4479772 .519334-2.916877$

H $7.0426031 .458673-1.221575$

H $6.1770780 .196832-3.226510$

H $4.736356-0.238054-2.331509$

H $6.223502-2.263816-3.331063$

H $5.017886-1.804593-0.211167$

H $8.254833-1.154354-2.930089$

H $8.490460-2.154591-1.515240$

H $8.494961-0.386709-1.358353$

H $6.699087-3.314911-0.123974$

H $6.122712-1.0296492 .681377$

H 5.6037380 .6332442 .568450

H 7.6111580 .7085621 .648342

H $2.3551490 .473638-1.134415$

H -0.487622 $-0.431609-0.376586$

H $2.845798-1.935376-1.254368$

H $2.398816-1.320155-2.863860$

H $2.260044-3.035072-2.476621$

H $3.567839-0.2474222 .952324$
H $3.529409-0.3512421 .189945$

H $0.969189-3.7931712 .748231$

H $2.607162-4.4753402 .801690$

H $2.622050-4.5456180 .216917$

H $-0.289215-4.9665381 .073761$

H $\quad 0.183110-6.736281-0.712550$

H $1.341283-5.678079-1.481863$

H -2.705973 -5.199309 -2.405021

H $-2.162605-6.501112-1.204174$

H -1.106874 -3.731372 -3.372933

H $\quad 0.578957-4.056325-3.127489$

H -1.286851-2.301871-1.393598

H $1.793834-1.7939281 .780820$

C $-3.0325115 .531250-0.215783$

O $-3.1375924 .109803-0.032200$

C $-4.4950933 .699833-0.280900$

C $-5.2899554 .975699-0.556693$

C $-4.2213565 .907168-1.096553$

H -4.474894 3.095806-1.194968

C -5.0156072 .8540030 .889381$

H $-6.1065404 .804756-1.265295$

H -5.7299325 .3942290 .355343$

C $-4.6033377 .375331-1.032317$

H $-4.0178535 .632454-2.140288$

C $-4.077348 \quad 1.716857 \quad 1.344135$

O $-5.195300 \quad 3.7228562 .018496$

H -6.0093202 .4618140 .642774$

H -3.1666212 .1552261 .772591$

$\begin{array}{llll}\text { O } & -4.718561 & 0.999612 & 2.407912\end{array}$

C -3.6721910 .6745600 .288389$

C $-4.8626050 .010066-0.427181$

H -3.098699 -0.1018350 .805652$

O $-2.806530 \quad 1.241851-0.688499$

H $-5.628940-0.2590800 .306455$

H $-5.315490 \quad 0.728039-1.120926$

H $-5.5054907 .564143-1.623312$

H $-4.8026007 .693414-0.003443$

H $-3.8016238 .005147-1.430456$

C -1.677542 $5.887380-0.832007$

H -5.7486763 .2522282 .664304$

H -4.7453641 .5866943 .182122$

H -2.065493 $1.661012-0.218473$

H -1.576583 $6.976618-0.896400$

H -1.626037 $5.491964-1.854182$

H -3.1242305 .9894040 .778061$

C $-4.538443-2.8506830 .571997$

O $-3.731868-2.185135-0.405590$

C $-4.430619-1.242489-1.225564$

C $-5.564285-1.951045-1.977015$

C $-6.473609-2.744071-1.039289$

C $-5.648592-3.647753-0.119810$

H -4.981755 -2.113842 1.252973

C $-3.611205-3.7538431 .393911$

H -3.709937 -0.900734 -1.979439

O $-6.328646-1.004517-2.716253$

H $-5.122651-2.644487-2.704971$

H -7.121107 -2.072185 -0.463226

O $-7.338940-3.549906-1.842417$

H $-5.217578-4.473155-0.700711$

O $-6.500986-4.213340 \quad 0.873100$

C $-2.900329-2.9482832 .455673$

H $-4.168088-4.5393101 .917284$

H $-2.861876-4.2518820 .769821$

H $-7.041322-1.492249-3.163646$

H $-7.983828-3.970064-1.248808$

H $-7.124703-4.8069640 .422562$

O $-1.824675-2.3033311 .921265$

O $-3.278903-2.8776783 .618145$

C -1.128950-1.432829 2.819014

H $-0.336999-0.9287222 .260810$

H $-1.809216-0.6734353 .217120$

H -0.676982 -2.010216 3.630647

SCF Energy (B3LYP/6-31G**//MMFF)= -3245.90955657

05 00194

MM̄MF Geometry

C 0.4225044 .4972351 .770362

C 0.1962723 .1879921 .962032 
C $-0.2594282 .614443 \quad 3.278780$

O $-1.496944 \quad 1.9110573 .007315$

C 0.7482231 .6100863 .872185

C 2.1069772 .1779964 .228690

C 3.2877401 .6268013 .883598

C 2.0972593 .4076285 .103501

C $3.545697 \quad 0.3474813 .138477$

C 4.4628200 .5568221 .925005

C $4.925822-0.7880641 .345171$

C $5.906882-0.5945600 .189280$

C $6.333156-1.939221-0.422847$

O $3.776246-1.5052100 .885426$

O $6.974405-1.668384-1.678621$

C $5.074338-2.778908-0.729830$

C $7.371310-2.6582230 .447502$

O $5.426786-4.100275-1.135278$

C $4.064584-2.8364510 .439645$

C $2.742008-3.487712-0.030566$

O $4.617571-3.6349641 .490931$

C -2.3994351 .8014164 .015968$

O -2.2749682 .2209295 .158218$

C -3.6444251 .1488063 .550913$

C -3.6984120 .3582612 .467821$

C $-4.903247-0.2586191 .907674$

C -6.2215880 .0524302 .566587$

C $1.572918-3.2913600 .946935$

C $0.426744-4.251447 \quad 0.678834$

C $-1.759364-4.963317 \quad 1.637351$

O $0.451579-5.100586-0.208334$

C $-2.800646-4.5670330 .639748$

C $-4.103520-4.4571470 .940306$

C $-5.169824-4.165096-0.076112$

C $-6.150614-3.1118330 .385100$

C -7.279221 -3.4587931 .027339$

C $-5.881364-1.6751030 .010815$

C $-4.781034-1.0425980 .816198$

N $-0.624242-4.0624491 .567264$

H $\quad 0.2792825 .1685742 .615769$

H $\quad 0.3244292 .4830861 .146561$

H $-0.4617163 .423453 \quad 3.991824$

H 0.8251040 .7618393 .184005

H $\quad 0.3176581 .1808974 .787003$

H 4.1956402 .1220484 .228221

H 3.0939273 .6554685 .484461

H 1.7341334 .2786034 .551173

H 1.4496783 .2487345 .972324

H $4.030612-0.3425353 .840633$

H $2.623604-0.1419532 .816366$

H 3.9167731 .1288481 .164868

H 5.3408751 .1471972 .215013

H $5.405151-1.3534912 .153297$

H $6.786261-0.0249210 .514263$

H $5.4331710 .007440-0.596659$

H $7.730656-1.080996-1.508294$

H $4.577752-2.318747-1.593254$

H $8.261776-2.0314370 .577732$

H $7.721712-3.577996-0.033949$

H $6.996716-2.9101551 .441713$

H $6.045918-4.018910-1.880807$

H $2.434621-3.055598-0.991564$

H $2.912477-4.558762-0.197481$

H $3.898062-4.0609061 .982840$

H -4.5198591 .3545584 .157961$

H -2.780478 0.1605891 .916040

H -7.070065 -0.454064 2.101578

H $-6.428774 \quad 1.1275282 .524198$

H -6.211455 -0.264085 3.615527

H $1.898771-3.4536651 .980181$

H $1.192956-2.2660680 .869089$

H -2.136868 -4.933456 2.664361

H $-1.415360-5.9804281 .421458$

H -2.480573 -4.423620 -0.388901

H -4.429016 -4.649151 1.960459

H -5.708135 -5.102312 -0.270771

H -4.735876 -3.869730 -1.039593

H $-8.010242-2.716681 \quad 1.329752$

H -7.495289-4.494605 1.267856

H $-6.798834-1.0783910 .072771$
H $-5.603842-1.627025-1.050239$

H -3.784072 -1.2201220.415608

H $-0.572489-3.3095482 .247349$

C $0.9727404 .336134-0.758664$

O $2.0800293 .419927-0.630467$

C $2.8607443 .467983-1.832831$

C 1.995364 4.154351-2.878371

C $1.2557965 .161524-2.018178$

H $3.7110574 .129705-1.615272$

C $3.4207092 .093375-2.206088$

H $2.5851174 .618993-3.674068$

H $1.2840743 .460382-3.338976$

C $0.0123575 .727011-2.682818$

H $1.9391725 .987538-1.778595$

C $2.428544 \quad 1.031897-2.725435$

O $4.4046072 .308550-3.226872$

H $3.9548961 .689833-1.338450$

H $2.072428 \quad 1.318242-3.721409$

O $3.173223-0.179373-2.921303$

C $1.2266830 .728203-1.815250$

C $0.367312-0.428708-2.358623$

H $\quad 0.6133531 .627247-1.729480$

O $1.7056180 .392163-0.515785$

H $0.143288-0.232781-3.413026$

H $\quad 0.943439-1.361041-2.318835$

H $0.278165 \quad 6.265510-3.598335$

H $-0.6934424 .935308-2.953785$

H $-0.5037996 .425969-2.017587$

C 0.8576635 .1911200 .505368

H $4.791301 \quad 1.442591-3.443039$

H $3.438155-0.496983-2.041359$

H $\quad 0.945270 \quad 0.3834150 .089191$

H $\quad 0.1409216 .0009140 .322980$

H 1.8284125 .6585540 .714315

H $0.0651923 .737019-0.896767$

C $-2.2664231 .098945-2.585817$

O $-1.6737230 .580784-1.390837$

C $-0.946639-0.640547-1.571433$

C $-1.859715-1.726801-2.162473$

C $-2.600905-1.252351-3.411110$

C $-3.2714930 .098957-3.163721$

H -1.476895 $1.282975-3.325307$

C $-2.8640502 .476371-2.284102$

H $-0.662139-0.975607-0.566344$

O $-1.119270-2.900078-2.483312$

H $-2.605636-2.016798-1.414247$

H -1.933358 -1.204031-4.279522

O $-3.618619-2.201451-3.739433$

H -4.117661 -0.040769 -2.480822

O $-3.7865760 .603893-4.392337$

C $-3.9796882 .445309-1.268638$

H -2.092139 $3.136312-1.870914$

H -3.221046 2.961351 -3.200193

H $-0.666135-3.191056-1.673884$

H -3.183711 -3.064381-3.850572

H $-4.393989-0.066493-4.749527$

O $\quad-5.1880692 .446327-1.899179$

O $-3.7958172 .418576-0.058924$

C $-6.3322232 .429396-1.040384$

H $-6.374377 \quad 1.486037-0.489979$

H $-6.3067703 .278534-0.350754$

$\mathrm{H}-7.2262732 .511627-1.664081$

SCF Energy (B3LYP/6-31G**//MMFF) $=-3245.90662645$

05_00195

MM̄MF Geometry

C -2.340354 -1.275662 2.897007

C $-1.107474-1.7821043 .042845$

C $-0.257831-2.2366391 .883379$

O $0.853744-1.3194001 .760308$

C $0.305338-3.6425992 .156583$

C $1.131180-4.2003751 .012967$

C $2.450596-4.4665801 .078542$

C $0.376553-4.490347-0.259674$

C $3.378386-4.2955162 .248565$

C $4.136545-2.9592792 .281891$

C $5.059116-2.7271851 .073913$

C $5.973581-1.5228351 .314467$ 
C $6.844176-1.2094440 .086435$

O $4.245240-2.483046-0.076635$

O $7.449431 \quad 0.077306 \quad 0.285082$

C $5.944150-1.132199-1.167705$

C $8.016485-2.189194-0.035845$

O $6.729468-1.008078-2.350043$

C $4.976873-2.326679-1.300467$

C $3.939766-2.149826-2.437090$

O $5.728816-3.508538-1.603064$

C $0.620741-0.1475991 .107411$

O $-\begin{array}{llll}0.416072 & 0.204702 & 0.567730\end{array}$

C $1.853654 \quad 0.6633121 .191841$

C 1.7826551 .9878070 .993802

C 2.9037002 .9275511 .052415

C 4.2918372 .3482041 .124314

C $3.018793-0.931774-2.306625$

C $3.494246 \quad 0.290507-3.074587$

C $2.9336102 .692416-3.434549$

$\begin{array}{lllll}\text { O } & 4.432537 & 0.268342 & -3.866175\end{array}$

C $3.801173 \quad 3.542772-2.561312$

C $3.4777504 .793313-2.198403$

C $4.3560435 .677310-1.360039$

C $3.6379466 .234258-0.151240$

C $3.1054247 .467982-0.171587$

C 3.6138985 .3946681 .105760

C 2.6403684 .2507171 .020202

N $2.717078 \quad 1.407408-2.796545$

H -2.771871 -1.202965 1.902418

H $-0.664179-1.8279484 .034725$

H $-0.835250-2.242056 \quad 0.951953$

H $-0.517861-4.3411492 .355423$

H $0.899551-3.6083153 .075929$

H $2.933024-4.8717730 .189303$

H $1.004119-4.979166-1.012892$

H $-0.465643-5.158839-0.055872$

H $-0.005898-3.567524-0.705727$

H $2.840290-4.4212673 .193788$

H $4.104480-5.1184342 .225343$

H $3.410254-2.1415132 .355478$

H $4.736132-2.9427783 .200505$

H $5.655577-3.6332640 .916085$

H $6.603379-1.6798862 .198954$

H $5.356824-0.642481 \quad 1.540457$

H $\quad \begin{array}{lllll}6.741170 & 0.733785 & 0.395473\end{array}$

H $5.357348-0.210128-1.083425$

H $8.656822-2.1295200 .852748$

H $8.660214-1.932600-0.884568$

H $7.700412-3.228502-0.145232$

H $7.311485-0.237859-2.232671$

H $4.440675-2.176503-3.412348$

H $3.290259-3.037088-2.421369$

H $5.131573-4.159032-2.007997$

H 2.7783310 .1528081 .433811

H 0.8094092 .4326080 .789505

H $4.439667 \quad 1.6109640 .327607$

H 4.4558701 .8595492 .090633

H 5.0824183 .0901290 .994799

H $2.039268-1.192864-2.727967$

H $2.862982-0.666597-1.257062$

H $1.9493243 .140659-3.603101$

H $3.4197982 .543059-4.403921$

H $4.7493593 .121316-2.235773$

H $2.5416015 .221393-2.550088$

H $4.7046846 .500410-1.997488$

H $5.2627625 .151558-1.035886$

H $2.613493 \quad 7.8865070 .700231$

H $3.1453488 .085057-1.063131$

H 3.3115836 .0105631 .963653

H 4.6324545 .0739071 .341126

H $1.599074 \quad 4.5703570 .958396$

H $1.989407 \quad 1.344829-2.090407$

C -3.3400650 .7765873 .996318$

O $-3.963913 \quad 1.187302 \quad 2.762598$

C -3.260076 2.3379912 .256490

C -2.4527992 .8798293 .429941$

C -2.0507251 .5918904 .122490$

H -2.5615941 .9693631 .496011$

C -4.2325453 .3361881 .622318$
H $-1.5968973 .479188 \quad 3.105175$

H -3.0668773 .4940984 .098535$

C -1.5819521 .7851645 .553726$

H -1.238319 1.1352213 .544768

C -5.1405022 .7289510 .533906$

O -5.0816403 .8603782 .651346$

H -3.6723474 .1898751 .222955$

H -5.8645142 .0516381 .002738$

O $-5.9139813 .797058-0.028566$

C $-4.4065901 .985770-0.599115$

C $-5.3842511 .464896-1.668914$

H $-3.8478661 .141163-0.183382$

O $-3.4739902 .858220-1.234268$

H $-6.1632180 .874987-1.173597$

H $-5.8816332 .313849-2.153763$

H -0.6958422 .4275575 .584493$

H -2.3567102 .2523536 .170758$

H -1.3199660 .8252726 .009687$

C $-3.174121-0.7466214 .028798$

H -5.6931824 .4886042 .230276$

H $-5.3021274 .378199-0.512359$

H -2.764597 $3.050610-0.598699$

H -4.162554 -1.215995 3.947950

H $-2.755370-1.0502804 .995396$

H -4.0383621 .0597044 .794823$

C $-4.585768-1.473837-1.580662$

O $-3.867672-0.413442-2.224731$

C $-4.6906760 .624861-2.766749$

C $-5.6519040 .036172-3.809019$

C $-6.460054-1.131619-3.245971$

C $-5.537287-2.152100-2.576858$

H $-5.153272-1.059025-0.739778$

C $-3.537061-2.447252-1.022118$

H $-4.0128391 .303365-3.299888$

O $-6.527491 \quad 1.047876-4.295267$

H $-5.067146-0.321481-4.666648$

H -7.233932 -0.777151 -2.555073

O $-7.148830-1.762953-4.327988$

H -4.962476 -2.668630 -3.355586

O $-6.329651-3.139665-1.923895$

C $-4.032155-3.2721180 .145635$

H -3.188580 -3.117691-1.816577

H $-2.665668-1.883031-0.668760$

H $-7.1285480 .629198-4.935104$

H -7.622904 -2.528905 -3.961215

H $-6.826665-2.701097-1.212282$

O $-3.147999-4.286690 \quad 0.377533$

O $-5.042460-3.042410 \quad 0.795817$

C $-3.464766-5.1383281 .482904$

H $-2.692960-5.9096521 .549697$

H $-3.467645-4.5636302 .413908$

H -4.432712 -5.623096 1.323673

SCF Energy (B3LYP/6-31G**//MMFF) $=-3245.91604697$

0500196

MM̄FF Geometry

C -2.008670 -3.527262 -1.380440

C $-1.153840-4.100884-2.241051$

C $0.270521-3.644928-2.482969$

O $0.508693-2.346654-1.890038$

C $1.258473-4.653593-1.871044$

C $2.714706-4.235348-1.961444$

C $3.555857-4.221915-0.908424$

C $3.216500-3.839673-3.325697$

C $3.262946-4.6805500 .491740$

C $2.782125-3.5814971 .448441$

C $3.832036-2.4892891 .703962$

C $3.519804-1.7397683 .001211$

C $4.513097-0.5974773 .255101$

O $3.809679-1.5719740 .603791$

O 3.9631460 .1986654 .314923

C $4.5960120 .274711 \quad 1.986174$

C $5.863852-1.1203883 .758125$

O $5.621071 \quad 1.2591612 .103961$

C $4.800943-0.5395820 .684321$

C $4.6339170 .399439-0.532674$

O $6.123891-1.0809560 .670432$

C $0.100597-1.265604-2.611410$ 
O $-0.397282-1.273697-3.726589$

C $0.328522-0.057273-1.789746$

C $-0.214206 \quad 1.102155-2.190069$

C $-0.0825162 .403368-1.533976$

C $0.7155552 .468662-0.260974$

C $4.496768-0.314658-1.883154$

C $4.5942040 .677074-3.028671$

C $3.4452742 .657853-4.022143$

O $5.531723 \quad 0.652568-3.822963$

C $2.936799 \quad 3.883219-3.328429$

C $1.8994854 .615169-3.763701$

C $1.3944075 .843625-3.058359$

C $-0.0979715 .808954-2.801018$

C $-0.9365236 .597041-3.494759$

C $-0.6113654 .915724-1.689165$

C $-0.6681663 .471660-2.113723$

N $3.5486811 .585509-3.048795$

H -1.672455 -2.694724 -0.768444

H $-1.493917-4.941312-2.841803$

H $\quad 0.436365-3.586682-3.566402$

H $1.146077-5.624889-2.370378$

H $0.974202-4.809156-0.824463$

H $4.580045-3.888344-1.068706$

H $4.302394-3.696658-3.342437$

H $2.979241-4.619419-4.056799$

H $2.758451-2.901141-3.652226$

H $2.526642-5.4923380 .483214$

H $4.175851-5.1372520 .895713$

H $1.860649-3.1331581 .055529$

H $2.528177-4.0712702 .396735$

H $4.820074-2.9596221 .768945$

H $3.505417-2.4265683 .856749$

H $2.507219-1.3173052 .939748$

H 4.6001970 .9012084 .527957

H 3.6460840 .8218791 .905648

H $5.733780-1.6648364 .701210$

H $6.549892-0.2956063 .980439$

H $6.354888-1.7923953 .051192$

H 5.4047591 .8211462 .866704

H $3.7327991 .006503-0.388883$

H $5.4826021 .094286-0.580702$

H $6.405616-1.206758-0.249401$

H $0.929682-0.154098-0.893287$

H $-0.808564 \quad 1.106237-3.103398$

H $0.345108 \quad 1.7343870 .461961$

H $0.6624413 .438167 \quad 0.237854$

H $1.7713892 .264013-0.457539$

H $5.285095-1.061960-2.020582$

H $3.537381-0.839184-1.953928$

H $2.7697532 .316805-4.813172$

H $4.4279342 .855401-4.461535$

H $3.4552774 .187681-2.421781$

H $1.3953664 .328335-4.683736$

H $1.6485626 .710371-3.682138$

H $1.9157365 .997810-2.105316$

H -2.004042 6.595788 -3.300220

H $-0.5759797 .257667-4.276161$

H -1.627707 $5.215343-1.401653$

H $0.0050185 .081363-0.801875$

H -1.249980 3.309859-3.022706

H $2.7499761 .419172-2.444782$

C $-4.376837-2.812466-1.657876$

O $-4.133757-1.607428-0.905644$

C $-5.380028-0.908202-0.731459$

C $-6.438460-1.713074-1.483433$

C $-5.857071-3.113662-1.447012$

H $-5.611510-0.9410860 .339005$

C -5.240952 $0.548009-1.193594$

H -7.421444 -1.644731-1.006986

H -6.550476-1.373113 -2.519442

C $-6.458320-4.046927-2.482749$

H $-6.015782-3.533105-0.444335$

C $-4.100958 \quad 1.332187-0.509358$

O $-4.9816280 .547753-2.603850$

H -6.197538 $1.066034-1.053727$

H $-3.1268490 .958931-0.847798$

O $-4.1862702 .694223-0.949087$

C -4.1391781 .3149941 .031772$
C -3.132326 2.3064461 .650052

H -3.9265500 .2979321 .378264$

\begin{tabular}{llll}
\hline & -5.456687 & 1.656427 & 1.461632
\end{tabular}

H -2.152095 2.1412351 .191554

H -3.439095 3.3296651 .402168

H $-7.536365-4.153999-2.324128$

H $-6.303728-3.670719-3.499623$

H $-6.008630-5.042811-2.420113$

C $-3.442167-3.928513-1.187868$

H $-5.103241 \quad 1.457190-2.924356$

H $-3.9181632 .717847-1.883080$

H $-5.533793 \quad 1.4154302 .399912$

H $-3.660827-4.857189-1.726467$

H $-3.592536-4.124757-0.120014$

H $-4.178054-2.576524-2.712370$

C -1.3737160 .4419213 .310228$

$\begin{array}{lllll}\text { O } & -2.705840 & 0.870755 & 3.617577\end{array}$

C $-3.0274992 .197344 \quad 3.188426$

C $-2.063800 \quad 3.204566 \quad 3.832041$

C -0.6039522 .8381703 .582931$

C -0.3469951 .3792323 .957439$

H $-1.236370 \quad 0.419726 \quad 2.222288$

C $-1.214623-0.987757 \quad 3.837232$

H -4.0234892 .4165593 .591786$

O $-2.328415 \quad 4.516867 \quad 3.347809$

H -2.242432 3.2210424 .915186

H $-0.321637 \quad 3.0443432 .545339$

O $0.216574 \begin{array}{llll}3 & 0.681169 & 4.394536\end{array}$

H $-0.384197 \quad 1.2815825 .049651$

$\begin{array}{llllll} & 0 & 0.972089 & 1.020004 & 3.554737\end{array}$

C $-2.014369-1.9552472 .999165$

H $-0.171177-1.3212043 .801882$

H $-1.540517-1.0685454 .881376$

H -1.6865795 .1161183 .766015$

H $1.1391043 .405054 \quad 4.257976$

H 1.0151061 .0696092 .585058

O $-3.249336-2.1388943 .546379$

O $-1.597132-2.4642841 .967190$

C $-4.117411-3.0196042 .827464$

H $-3.656780-4.0058582 .717111$

H $-5.042950-3.1268873 .399263$

H $-4.356698-2.5930161 .850011$

SCF Energy (B3LYP/6-31G**//MMFF) $=-3245.89828883$

05_00197

MMFF Geometry

C 1.5229874 .7436230 .184816

C $2.3320074 .687931 \quad 1.254342$

C $3.3698723 .614227 \quad 1.505541$

$\begin{array}{lllll}\text { O } & 3.107200 & 2.437626 & 0.704374\end{array}$

C $4.7729014 .135103 \quad 1.151518$

C 5.8817123 .1546581 .484018

C 6.6781392 .5595510 .574305

C 6.0858512 .8725342 .950746

C $6.670666 \quad 2.729827-0.918597$

C $5.8191901 .704163-1.682687$

C $6.278644 \quad 0.247154-1.507913$

C $5.581293-0.663719-2.521954$

C $5.954834-2.140695-2.317783$

O $5.949230-0.178119-0.181869$

O $5.058987-2.940353-3.105066$

C $5.756124-2.513248-0.831495$

C $7.353143-2.448071-2.864976$

O $6.248233-3.823858-0.566033$

C $6.394949-1.5027790 .144334$

C $6.020606-1.7659141 .624417$

O $7.821946-1.5993990 .052651$

C 2.0954501 .6213861 .110771

$\begin{array}{lllll}\text { O } & 1.420605 & 1.729164 & 2.122124\end{array}$

C 1.9109820 .5897130 .068323

C $0.741071-0.060994-0.011906$

C $0.399168-1.098211-0.986732$

C $1.507911-1.620817-1.861723$

C $4.522551-1.718643 \quad 1.945944$

C $3.839728-3.0769031 .931948$

C $1.581488-4.1147622 .132553$

O $4.450458-4.138607 \quad 1.845163$

C $1.002081-4.3780360 .778563$ 
C $-0.314627-4.502396 \quad 0.552199$

C $-0.909771-4.817665-0.789782$

C -1.944341 -3.804034-1.224485

C $-3.255589-4.028675-1.036269$

C -1.461850 -2.575195 -1.960342

C $-0.873528-1.543500-1.037448$

N $2.462030-2.9630712 .067269$

H $1.6395584 .016145-0.614815$

H 2.2127065 .4249242 .045354

H 3.3263513 .3362422 .565739

H 4.9718015 .0717271 .689307

H $4.7908884 .389310 \quad 0.086277$

H 7.4414231 .8716800 .937180

H 6.9870462 .2784563 .137933

H 6.1926273 .8095933 .506915

H 5.2370092 .3162683 .359781

H $6.3422373 .737877-1.192447$

H $7.7087702 .666069-1.269939$

H $4.7741031 .803719-1.367193$

H $5.861970 \quad 1.969332-2.746399$

H $7.3655530 .209090-1.645949$

H $5.807615-0.351673-3.549103$

H $4.493422-0.558800-2.411354$

H $4.150101-2.757440-2.812751$

H $4.675233-2.542544-0.651911$

H $7.396632-2.234835-3.939969$

H $7.593596-3.512107-2.762169$

H $\quad 8.142297-1.868451-2.381735$

H $5.810724-4.429961-1.188111$

H $\quad 6.478526-2.7010941 .968654$

H $6.495071-0.9739892 .222161$

H $8.208487-1.2358200 .866532$

H $2.7323950 .411946-0.615527$

H $-0.0495290 .199940 \quad 0.690857$

H $2.363260-1.931456-1.251984$

H $1.839020-0.848914-2.564571$

H $1.226049-2.496890-2.449155$

H $4.399865-1.3280162 .964318$

H $3.997921-1.0388151 .269422$

H $0.810965-3.8917362 .876749$

H $2.145463-4.9906712 .468504$

H $1.699005-4.500747-0.047075$

H -1.007127 -4.4086091 .385579$

H - $1.369564-5.812573-0.723812$

H $-0.135087-4.903408-1.561884$

H $-4.003517-3.321769-1.378046$

H -3.609331 -4.922962 -0.534149

H $-2.299315-2.098976-2.485846$

H $-0.776472-2.878928-2.756423$

H -1.604354 -1.103172 -0.357854

H $2.038140-2.0400002 .089908$

C -0.9388654 .9583450 .118063$

O $-1.0754694 .071663-1.009341$

C $-2.4792443 .946440-1.333286$

C $-3.2260764 .794610-0.309931$

C -2.1848515 .8331530 .067581$

H -2.593197 4.373729 -2.337202

C $-2.8349712 .454189-1.343680$

H $-4.1413635 .232453-0.719586$

H -3.5008294 .1973050 .568593$

C -2.4958846 .5559141 .365850$

H -2.100298 $6.563937-0.747666$

C $-4.3457782 .166459-1.448069$

O $-2.1354111 .878302-2.451259$

H -2.431465 $1.980163-0.440049$

H $-4.8428132 .609633-0.577948$

O $-4.8848692 .808404-2.602151$

C $-4.6880510 .663417-1.477420$

C $-6.2044290 .421888-1.366419$

H $-4.1657650 .177178-0.648716$

O $-4.2067820 .087970-2.689446$

H -6.595009 $1.012642-0.530740$

H $-6.6954480 .784091-2.277955$

H -1.718502 7.2891061 .601646

H -3.449359 7.0889671 .289912

H -2.566986 5.8581822 .207026

C $\quad 0.3869275 .7148580 .025504$

H $-2.2584040 .915012-2.409683$
H $-4.4523032 .415989-3.379996$

H -4.281059-0.877442 -2.610226

H $0.436716 \quad 6.4946520 .793192$

H $0.4856246 .200650-0.952207$

H -0.9520744 .3329121 .022010$

C $-6.231573-1.2100121 .222757$

O $-5.846814-1.660667-0.081548$

C $-6.566867-1.067649-1.168046$

C $-8.069290-1.339865-1.014352$

C $-8.588430-0.9152200 .357583$

C $-7.717783-1.4991981 .470451$

H $-6.044272-0.1335571 .314826$

C $-5.350109-1.9505942 .242110$

H -6.242655 -1.595760 -2.073007

O $-8.796630-0.674410-2.040963$

H -8.246212 -2.415850 -1.142174

H -8.6421630 .1765440 .436081$

O $-9.930771-1.3806110 .518396$

H -7.884684 -2.581733 1.539498

O $-8.107145-0.9248202 .716229$

C $-3.932479-1.4167292 .287449$

H -5.754785 -1.846878 3.254722

H $-5.301859-3.0140781 .978626$

H -9.741095 -0.850251-1.889892

H $-9.922703-2.3509340 .453867$

H $-9.057730-1.0962182 .830925$

O $-3.151002-2.2923122 .987233$

O $-3.559710-0.355377 \quad 1.807804$

C -1.789006-1.891322 3.167530

H $-1.740353-0.939573 \quad 3.705191$

H $-1.286640-2.6563003 .765098$

H -1.285805-1.813584 2.200033

SCF Energy $\left(B 3 L Y P / 6-31 G^{* *} / / M M F F\right)=-3245.91306873$

05 00198

MM̄̄FF Geometry

C -1.621335 $4.918430-0.917984$

C $-2.1146714 .397554-2.052951$

C $-3.0489943 .212608-2.141722$

O $-2.7956472 .277188-1.068001$

C $-4.5127733 .678219-2.049722$

C $-5.5183982 .577463-2.327442$

C $-6.3882602 .084879-1.423861$

C $-5.5318162 .046100-3.738553$

C -6.5672722 .4874440 .012594$

C -5.7620931 .6537411 .021894$

C $-6.097416 \quad 0.1528601 .014824$

C $-5.456138-0.5499582 .213929$

C $-5.690506-2.0691322 .185940$

O $-5.591632-0.418296-0.195137$

O $-4.797123-2.6707003 .136020$

C $-5.303441-2.6162370 .794305$

C $-7.110094-2.4337562 .636984$

O $-5.663280-3.989483 \quad 0.669313$

C $-5.905772-1.806635-0.372147$

C $-5.358621-2.229537-1.757544$

O $-7.323678-2.011507-0.404720$

C $-1.7341551 .437463-1.221009$

O $-0.9815101 .372004-2.180218$

C -1.6240720 .6178560 .005794$

C $-0.516730-0.1117180 .205279$

C $-0.228396-0.9561481 .365798$

C $-1.347097-1.2052292 .340163$

C $-3.840948-2.106377-1.933545$

C $-3.083859-3.406112-1.712601$

C $-0.760526-4.309042-1.654371$

O $-3.635550-4.489887-1.542872$

C $-0.336433-4.462461-0.228073$

C $0.948134-4.4889710 .158725$

C $1.391738-4.6853401 .580012$

C $2.263177-3.5550472 .079188$

C $3.600351-3.6787512 .126018$

C $1.575835-2.3118492 .600236$

C $1.017206-1.4580351 .495897$

N -1.709014 -3.216298 -1.765663

H -1.9376374 .5046280 .035082$

H -1.818744 $4.832979-3.004979$

H $-2.8733122 .710111-3.100567$ 
H $-4.6934724 .489327-2.767855$

H -4.680657 4.110679-1.057625 H -7.067057 $1.296614-1.748390$ H $-6.3740641 .369413-3.919751$ H $-5.6171402 .870078-4.454505$ H -4.614508 $1.489335-3.951611$ H -6.3249103 .5462140 .153059$ H $-7.6349732 .406710 \quad 0.253883$ H -4.6936231 .7943890 .822927$ H -5.9603312 .0621572 .020547$ H -7.1875580 .0420931 .046065$ H -5.822653 -0.1242003 .156396$ H -4.373686 -0.3654392 .205336$ H -4.978212 -2.2787994 .007554$ H -4.209662 -2.5727550 .728827$ H -7.289515 -2.079782 3.659364 H -7.248088 -3.520159 2.669018 H -7.886211 -2.0050301 .999739$ H -5.244245 -4.466969 1.405769 H -5.716540 -3.234095 -2.013489 H - $5.816688-1.561721-2.501692$ H -7.646162 -1.794432 -1.295163 H -2.4416790 .6521980 .715639$ H $0.271591-0.073087-0.545476$ H -2.208762 -1.640744 1.823766 H $-1.658395-0.2701442 .818525$ H -1.086028 -1.8990583 .141162$ H -3.636890 -1.807950-2.970004 H -3.429645 -1.329615 -1.282829 H $0.081408-4.076269-2.314001$ H - $-1.229425-5.235581-2.000821$ H -1.119926 -4.5836330 .516167$ H $1.730098-4.391534-0.590883$ H $1.942726-5.6336131 .628542$ H $0.534774-4.8078632 .254141$ H $4.229642-2.8803172 .505124$ H $4.096596-4.5782991 .777048$ H $2.292162-1.6942323 .157936$ H $0.822124-2.6143513 .331715$ H $\quad 1.753249-1.1993630 .733836$ H $-1.339285-2.274707-1.859257$ C $0.6057585 .767687-0.023602$ O $1.1760994 .485831-0.361380$ C 1.3787303 .7444950 .861589 C 1.4318964 .7948431 .957767 C 0.3589575 .7573441 .487389 H 0.4875903 .1141410 .980445 C 2.6269862 .8671620 .725366 H $1.228414 \quad 4.3858132 .951592$ H 2.4116625 .2878551 .977943 C 0.4473847 .1299722 .132874 H $-0.6217615 .325014 \quad 1.719839$ C 3.0594942 .1831552 .035231 O $2.3418611 .860273-0.251693$ H 3.4557323 .4626630 .325647 H 3.4488502 .9359392 .731111 O 1.8945211 .6249982 .659002 C 4.0820911 .0430311 .871108

C 5.3574311 .4475551 .114439 H 3.5934950 .1939061 .383286 O $4.4382200 .608501 \quad 3.188294$ H 5.0877741 .8634210 .139605 H $5.8703352 .240676 \quad 1.672576$ H 0.3190577 .0536023 .217543 H 1.4169867 .6022221 .942311 H -0.3327797 .7927681 .745604$ C $-0.6292426 .050041-0.889092$ H $2.0539992 .314335-1.062315$ H $2.1837741 .195553 \quad 3.482324$ H $4.869054-0.2590863 .105537$ H $-0.2862186 .251946-1.911978$ H -1.129866 $6.958291-0.535208$ H $\quad 1.3719936 .515127-0.266833$ C $5.265207-0.778341-0.974593$ $\begin{array}{llll}\text { O } & 5.681972 & -0.891043 & 0.389097\end{array}$ C 6.3388770 .2690480 .911869

C 7.5730220 .6082010 .063565 C $7.2388790 .727103-1.422807$
C $6.456658-0.498984-1.898250$

H $4.5505130 .049884-1.058875$

C $4.472102-2.036864-1.353996$

H $6.705811-0.0099881 .907423$

O $8.167362 \quad 1.815526 \quad 0.528987$

H $8.318675-0.1873010 .193117$

H $6.6943591 .655791-1.629426$

O $8.464750 \quad 0.812134-2.153008$

H $7.137604-1.356512-1.931098$

O $6.004115-0.280104-3.232492$

C $5.231155-3.339930-1.209848$

H $3.600230-2.113906-0.694195$

H $4.119292-1.952720-2.387816$

H $8.9327102 .001218-0.041726$

H $8.2370680 .839488-3.098290$

H $5.3977920 .480169-3.221881$

O $4.472673-4.347088-1.738078$

O $6.325882-3.472945-0.681530$

C $5.036894-5.658430-1.642026$

H $5.178571-5.933455-0.592368$

H $4.336767-6.363615-2.097682$

H $5.985243-5.703791-2.185746$

SCF Energy (B3LYP/6-31G**//MMFF) $=-3245.91233700$

05_00199

MM̄MF Geometry

C $0.4052562 .833943-2.147539$

C $-0.6005402 .512223-1.320360$

C -1.992982 $3.070850-1.411734$

O $-2.8430371 .922088-1.647566$

C $-2.4375063 .755288-0.101408$

C $-1.482784 \quad 4.7976170 .443550$

C -0.7405994 .6519261 .560692$

C -1.415275 $6.076643-0.349784$

C -0.6917703 .4620852 .479664$

C 0.6644412 .7469882 .409386

C 0.5807551 .3153672 .955134

C 1.9691850 .6763493 .017603

C $1.898501-0.8017023 .428386$

O -0.2556800 .5761392 .057692$

O $3.178637-1.3944013 .164268$

C $0.872415-1.5176922 .525758$

C $1.644485-0.9681294 .931312$

O $0.675226-2.8646302 .949090$

C $-0.487999-0.7860602 .431752$

C $-1.354187-1.4359641 .322210$

O $-1.157614-0.8796953 .686361$

C $-4.1053952 .126938-2.101369$

O $-4.6264703 .189982-2.393106$

C $-4.7612050 .801556-2.137554$

C $-6.0990680 .719273-2.132625$

C $-6.882845-0.517927-2.101641$

C $-6.128578-1.822784-2.110727$

C $-2.675734-0.7197131 .002442$

C $-3.844173-1.1242891 .885416$

C $-6.238728-0.5652582 .310403$

O $-3.770215-2.0048022 .738039$

C $-7.112674-1.5103281 .546159$

C $-8.388919-1.2442201 .226963$

C $-9.289027-2.2070750 .505074$

C $-9.986379-1.584391-0.684774$

C $-11.257510-1.157579-0.594633$

C $-9.240509-1.537427-1.999852$

C $-8.227869-0.425332-2.044919$

N $-4.984614-0.3834771 .602237$

H $\quad 0.2356613 .537477-2.957812$

H $-0.4208571 .789570-0.527530$

H $-2.0788103 .761833-2.259816$

H -2.6636462 .9813850 .639214$

H $-3.4109344 .237521-0.263129$

H -0.0902735 .4738251 .857198$

H $-0.717656 \quad 6.800010 \quad 0.085315$

H -1.086742 $5.878639-1.374545$

H -2.401807 $6.550053-0.387790$

H -0.8611633 .8094863 .506536$

H -1.5024942 .7606952 .273297$

H $0.9976682 .699327 \quad 1.366990$

H 1.4095373 .3268002 .967615 
H $\quad 0.118931 \quad 1.3402803 .949101$ H $2.625551 \quad 1.2342113 .696854$ H $2.443346 \quad 0.7368382 .027445$ H $3.837319-0.9418533 .718604$ H $1.306753-1.5688651 .517962$ H $2.449608-0.4991225 .509914$ H $1.647516-2.0257475 .217274$ H $\quad 0.703047-0.5237905 .260377$ H $1.548631-3.2920362 .970014$ H $-0.768857-1.4197880 .394205$ H -1.554541 -2.485533 1.566993 H -2.092104 -0.6550113 .558416$ H -4.120384 -0.072741 -2.122504 H $-6.6761171 .643148-2.125923$ H -5.433766 -1.874607-1.265085 H -5.557664 -1.928076 -3.039989 H $-6.771086-2.701500-2.030641$ H $-2.554647 \quad 0.367815 \quad 1.040220$ H -2.961783 -0.984534 -0.023284 H -6.6955820 .4232892 .419894$ H $-6.039252-0.971577 \quad 3.307029$ H -6.684687 -2.4706791 .268293$ H -8.825184 -0.2962851 .534210$ H -10.035438 -2.565964 1.225957 H -8.740974 -3.099584 0.178433 H $-11.774750-0.736339-1.450346$ H -11.807515 -1.214988 0.338752 H $-9.944270-1.368050-2.826130$ H $-8.808619-2.521097-2.202751$ H -8.678212 $0.568456-2.036130$ H -4.9668680 .2813120 .834390$ C $2.9146533 .274903-1.963278$ O $4.1277512 .538014-1.686001$ C $4.5546402 .877229-0.357437$

C $4.1662924 .336997-0.218699$

C $2.7851954 .332314-0.854678$

H 3.9581092 .2793160 .343702

C $6.0376242 .557120-0.161801$

H 4.1549234 .6729690 .822267

H $4.8405864 .988854-0.786012$

C $2.3702315 .703901-1.365470$

H $2.0565484 .009214-0.104314$

C $6.4217251 .113609-0.548971$

O $6.8144603 .444485-0.974570$

H 6.3201672 .7656210 .876952

H $6.399406 \quad 1.012047-1.640730$

O $7.7881360 .911314-0.167170$

C 5.5466350 .0142670 .082782

C $6.043284-1.395210-0.286636$

H $4.5142210 .141680-0.257858$

O $5.561452 \quad 0.1727401 .499267$

H $6.183394-1.437569-1.372313$

H $7.024501-1.5679600 .172471$

H $2.3147576 .420946-0.539761$

H $3.0848406 .090885-2.099813$

H $1.3879565 .663798-1.844520$

C $1.7772572 .245843-2.016178$

H $7.7506173 .220772-0.834876$

H 7.8259340 .9297460 .804720

H $4.877017-0.4111151 .866240$

H $1.8383451 .596322-1.132259$

H $1.9538421 .577832-2.869256$

H $3.0409803 .739472-2.948321$

C $3.486045-2.385961-1.624273$

O $3.734824-2.295014-0.217696$

C $5.093599-2.5289320 .166907$

C $5.531202-3.927270-0.291345$

C $5.309457-4.130030-1.789307$

C $3.875065-3.765738-2.175944$

H $4.079036-1.616451-2.134668$

C $2.019190-2.003768-1.874855$

H $\quad 5.106217-2.5316401 .263531$

O $6.900754-4.1410220 .033551$

H $4.949032-4.6792050 .257141$

H $6.039756-3.562392-2.377428$

O $5.539449-5.508342-2.093049$

H $3.210407-4.551677-1.799746$

O $3.781626-3.772844-3.598461$
C $1.016527-3.017440-1.363236$

H $1.788222-1.054748-1.376751$

H $1.864959-1.869299-2.951789$

H $7.141961-5.025039-0.292460$

H $5.359079-5.629087-3.041223$

H $2.841292-3.700597-3.833701$

O $-0.098681-2.983498-2.150998$

O $1.175993-3.727007-0.379471$

C $-1.138347-3.899086-1.791260$

H -1.977821 -3.745191 -2.474404

H $-0.784348-4.929107-1.895171$

H -1.479745 -3.709869-0.769690

SCF Energy (B3LYP/6-31G**//MMFF) $=-3245.92009911$

05_00200

MM̄MF Geometry

C $0.689371-3.0477861 .893185$

C $-0.074625-2.1298181 .281132$

C -1.059097-2.491358 0.197403

O $-2.373892-2.2052490 .742811$

C $-0.901421-1.652956-1.086274$

C $0.435789-1.703583-1.794869$

C $1.047602-0.608872-2.291138$

C $1.046588-3.064475-1.993924$

C $0.5077850 .794248-2.283214$

C $1.0941511 .618057-1.134459$

C $0.2396472 .856250-0.831642$

C 0.9839603 .8072990 .104787

C 0.1086994 .9935270 .530061

O $-0.9612202 .392790-0.202325$

O $0.7622075 .652907 \quad 1.625485$

C -1.232917 4.4519091 .063140

C $-0.0278586 .035495-0.585794$

O -2.1369595 .5192621 .342063$

C -1.9058903 .4190210 .126646$

C -3.0899932 .7482430 .871285$

O $-2.3808984 .085294-1.040737$

C $-3.437954-2.8608650 .207091$

O $-3.411795-3.741561-0.637197$

C $-4.660654-2.2774260 .803657$

C $-5.863251-2.6230590 .321113$

C -7.157543 -2.097143 0.762736

C $-7.149332-1.0159111 .811598$

C -3.7765861 .5967620 .121428$

C $-4.9184442 .028400-0.783073$

C $-6.6476241 .073132-2.306532$

O $-5.2654193 .198166-0.919998$

C $-7.9055430 .863278-1.522561$

C $-8.859063-0.014160-1.871271$

C $-10.138569-0.203794-1.106805$

C $-10.402407-1.650559-0.751662$

C -11.283239-2.384305 -1.452769

C $-9.710180-2.2198850 .467420$

C $-8.278155-2.5906970 .195239$

N $-5.5059090 .942682-1.420019$

H $\quad 0.602330-4.0816241 .560598$

H -0.033854 -1.087885 1.583913

H $-0.994090-3.561605-0.037268$

H -1.175712 $-0.623300-0.830208$

H -1.665753 -1.971754-1.807682

H $2.008727-0.725308-2.787551$

H $1.913724-3.033538-2.661863$

H $\quad 1.379537-3.488308-1.042937$

H $\quad 0.315817-3.744810-2.443528$

H $\quad 0.7645541 .270549-3.237936$

H $-0.5865330 .793607-2.254301$

H $1.1477990 .996471-0.231300$

H $2.116611 \quad 1.914687-1.398193$

H $-0.0107683 .358573-1.773363$

H $1.9140014 .161505-0.356938$

H 1.2861263 .2621161 .008831

H 1.6436795 .9324901 .323848

H -1.026839 3.9621572 .025499

H $0.9578356 .423485-0.869735$

H $-0.6045716 .903360-0.247339$

H $-0.4981695 .638984-1.487920$

H -1.681763 6.1353361 .941234

H -2.6971972 .3179741 .802565$ 
H -3.8350943 .5023241 .151067$ H -3.033160 $3.517493-1.479457$ H - $-4.535621-1.5558861 .603044$ H -5.908231 -3.353025 -0.486166 H -6.545898 -0.1629051 .481739$ H -6.734431-1.396617 2.751301 H -8.140014 -0.6178192 .038892$ H -3.056992 $1.015729-0.464194$ H $-4.213906 \quad 0.9238330 .869603$ H -6.527428 $0.332283-3.103166$ H $-6.6522172 .072305-2.753255$ H -8.048236 $1.478609-0.637191$ H -8.728929 $-0.609277-2.772498$ H -10.956347 $0.182967-1.728931$ H -10.151453 $0.401020-0.191566$ H -11.499080 -3.414054-1.187713 H -11.804178 -1.973341 -2.311357 H -10.222956 -3.1334890 .797044$ H $-9.823361-1.5203661 .299593$ H -8.173159-3.374384 -0.556922 H $-5.1904030 .004582-1.191381$ C $1.843435-1.4522463 .601645$ O $2.593260-0.6427112 .671461$ C $3.671947-0.0000413 .377470$ C $3.324759-0.1094134 .856021$ C $2.657454-1.4664654 .900507$ H $4.572851-0.5937193 .181385$ C 3.8789631 .4361952 .888651 H $4.208965-0.0425505 .497101$ H 2.6189650 .6685945 .169063 C $1.822464-1.6898886 .150331$ H $3.435613-2.2402384 .848873$ C 4.1110641 .5694411 .372896 $\begin{array}{lllll}\text { O } & 2.708373 & 2.197626 & 3.215124\end{array}$ H $4.705796 \quad 1.8950833 .443503$ H 3.1879381 .3132780 .845676 O 4.3480932 .9568181 .096817 C 5.2695680 .7355790 .794313 C $5.480807 \quad 1.022858-0.704864$ H $5.067073-0.3302750 .933411$ $\begin{array}{llll}\text { O } & 6.481238 & 1.044497 & 1.481262\end{array}$ H $4.5270360 .888836-1.226019$ H $5.7752952 .071928-0.828390$ H $2.448549-1.6293007 .046519$ H $1.030151-0.9394476 .242750$ H $1.350650-2.6772326 .134388$ C $1.653208-2.8618243 .033604$ H $2.862253 \quad 3.1092412 .912759$ H 5.1995153 .1944301 .502971 H 6.4043510 .7045512 .388411 H $1.312628-3.5260753 .837460$ H $2.625591-3.2410122 .697775$ H $0.878350-0.9564633 .762211$ C $5.199813-1.743322-1.996792$ O $6.294209-1.259603-1.208611$ C $6.5734360 .137298-1.345530$ C $6.872830 \quad 0.473735-2.813813$ C $5.749136 \quad 0.011783-3.737831$ C $5.433297-1.461415-3.486457$ H $4.269264-1.267035-1.666816$ C $5.089160-3.256066-1.748337$ H $7.4983800 .319346-0.784117$ O $7.0895141 .873041-2.957936$ H $7.803407-0.028884-3.108779$ H $4.8509180 .630360-3.625315$ $\begin{array}{llll}\text { O } & 6.166168 & 0.145998 & -5.098229\end{array}$ H $6.259007-2.079767-3.861677$ O $4.264735-1.820422-4.221242$ C $4.550011-3.582536-0.372542$ H $4.411118-3.719580-2.472972$ H $6.081649-3.714417-1.835690$ H $7.4020542 .031838-3.864409$ H $6.2535901 .095876-5.285496$ H $4.433381-1.610303-5.155675$ O $4.672826-4.926690-0.160545$ O $4.064419-2.7752290 .406536$ C $4.187191-5.4020361 .098579$ H $4.445223-6.4609691 .184511$
H $4.658843-4.859518 \quad 1.923632$

H $3.098984-5.3016041 .139568$

SCF Energy (B3LYP/6-31G**//MMFF) $=-3245.91809881$

05_00201

MM̄FF Geometry

C -2.079192 1.7487912 .404916

C -0.8166401 .4927422 .030003$

C $0.201725 \quad 0.8074172 .899417$

O $0.833636-0.1842102 .050626$

C 1.2899681 .7797143 .404007

C 0.7779093 .0279364 .093185

C $0.854874 \quad 4.2728293 .580049$

C 0.1654052 .8044745 .451399

C 1.4494024 .6800512 .261067

C 0.3718925 .1079051 .255473

C $0.9146475 .089800-0.180147$

C $-0.0962985 .684293-1.158832$

C $0.4115345 .601872-2.606427$

O $1.1592643 .722821-0.527598$

O $-0.695088 \quad 5.878435-3.479435$

C $0.8646194 .154931-2.903248$

C $1.4668166 .676192-2.895973$

O $1.510097 \quad 4.070473-4.171539$

C $1.7691523 .531535-1.810596$

C $1.8901322 .004471-2.039113$

O $3.0889794 .071338-1.846448$

C $1.570840-1.1609132 .639475$

O $1.673532-1.3932623 .832428$

C $2.300479-1.8585751 .557485$

C $3.416024-2.5424151 .849716$

C $4.298740-3.2101770 .890266$

C $3.952362-3.109680-0.572528$

C $2.6725631 .248879-0.955446$

C $4.1827041 .299376-1.116084$

$\begin{array}{llll}\text { C } & 6.280490 & 0.693752 & 0.092442\end{array}$

O $4.741302 \quad 1.727540-2.122256$

C $6.743928-0.629843-0.431324$

C $7.532598-1.467726 \quad 0.259461$

C $8.042876-2.772324-0.284366$

C $7.786923-3.9403670 .642636$

C $8.760788-4.419723 \quad 1.434851$

C $6.431077-4.6081780 .584624$

C $5.388942-3.8516801 .360461$

N $4.834741 \quad 0.775444-0.006555$

H -2.4087191 .4653663 .401017$

H -0.4844451 .8064801 .042603$

H $-0.2889540 .299392 \quad 3.739185$

H 1.9588682 .0169892 .570819

H 1.9457291 .2493414 .107686

H $0.4514725 .098127 \quad 4.165104$

H $-0.154375 \quad 3.7402195 .922127$

H $-0.713122 \quad 2.1565115 .377994$

H $\quad 0.8914372 .330864 \quad 6.120262$

H 2.1315825 .5217072 .435175

H 2.0729083 .8888301 .839552

H $-0.487223 \quad 4.429762 \quad 1.321465$

H 0.0166836 .1126591 .515252

H $1.857257 \quad 5.648802-0.205817$

H $-0.3343436 .721831-0.893770$

H -1.043376 $5.130652-1.089799$

H $-1.0454676 .756465-3.250854$

H $-0.0451813 .544509-2.980141$

H $1.0411717 .677619-2.758346$

H $1.7996136 .634189-3.938750$

H $2.3429826 .601974-2.248280$

H $0.9006524 .441905-4.832270$

H $0.8788341 .579499-2.048227$

H $2.3381891 .804148-3.019638$

H $3.1528004 .697002-2.585073$

H $1.931921-1.7409940 .545509$

H $3.731019-2.5977932 .891216$

H $3.819332-2.064342-0.871279$

H $3.027772-3.654218-0.785199$

H $4.722742-3.513631-1.232351$

H 2.3993391 .6030630 .044018

H $2.394430 \quad 0.189417-1.015363$

H $6.5432700 .837671 \quad 1.145136$ 
H $6.7305491 .500718-0.494788$

H $6.439949-0.898238-1.440391$

H $7.862027-1.1851041 .256804$

H $9.122327-2.660744-0.451517$

H $7.612989-2.990995-1.269875$

H $8.594122-5.2753272 .080908$

H $\quad 9.744807-3.9632941 .459444$

H $6.490201-5.6152751 .019322$

H $6.154545-4.776128-0.459812$

H $5.561059-3.8586812 .437776$

H 4.2858910 .3671530 .744385

C -4.4698781 .8535341 .607958$

O $-4.471271 \quad 0.4984931 .120066$

C $-5.709170 \quad 0.2595540 .420567$

C -6.5528911 .5216450 .582986$

C -5.4917032 .5899630 .744187$

H $-5.4522290 .144439-0.638756$

C $-6.390742-1.0130240 .938783$

H $-7.2070401 .692603-0.277707$

H $-7.187351 \quad 1.4774131 .475700$

C -6.0171043 .8770221 .355894$

H -5.062011 $2.807504-0.243104$

C $-5.527427-2.2874750 .858924$

O $-6.735986-0.8133222 .316514$

H -7.336569-1.161192 0.403798

H $-4.703229-2.2213361 .579087$

O $-6.341769-3.3839251 .297271$

C $-4.964348-2.604294-0.538661$

C $-4.261556-3.975187-0.580202$

H -4.264564 -1.812839-0.823187

O $-6.037291-2.594899-1.479119$

H -3.563544 -4.0352260 .261864$

H -5.005557 -4.769223 -0.440995

H -6.8050074 .3069430 .728850$

H $-6.437018 \quad 3.7052972 .352712$

H -5.2194984 .6203191 .449844$

C -3.0678782 .4595061 .523272$

H $-7.169457-1.6265962 .627384$

H -7.050193 -3.5029290 .641288$

H -5.649395 -2.594155 -2.370148

H $-3.1053353 .508477 \quad 1.838662$

H -2.720940 2.4380990 .483326

H -4.8044321 .8177982 .653558$

C -1.489483 -3.039060-1.400536

O $-2.630187-3.175972-2.256837$

C $-3.516048-4.243312-1.907435$

C $-2.760808-5.578915-1.952182$

C - $1.494391-5.546432-1.097830$

C $-0.645053-4.316691-1.424401$

H -1.818272 -2.834248 -0.374524

C $-0.692770-1.826832-1.899135$

H -4.277351 -4.281505 -2.696199

O $-3.611192-6.638058-1.526925$

H -2.480712 -5.789984 -2.992452

H -1.742269 -5.576015 -0.030639

O $-0.726389-6.726475-1.347404$

H $-0.174553-4.441763-2.407678$

O $0.397178-4.204428-0.458967$

C -1.447683 -0.549768 -1.608009

H $0.283003-1.743764-1.409918$

H $-0.524419-1.908679-2.979742$

H -3.086950 -7.457016 -1.542242

H $-0.487512-6.736166-2.289956$

H $0.893257-5.040962-0.467190$

O

O $-1.884997-0.253772-0.503892$

C $-2.2818811 .429192-2.585619$

H $-3.3127911 .227503-2.278606$

H $-1.7816932 .073881-1.856754$

H $-2.2960781 .938682-3.552617$

SCF Energy $\left(\mathrm{B} 3 \mathrm{LYP} / 6-31 \mathrm{G}^{* *} / / \mathrm{MMFF}\right)=-3245.91071449$

05 00202

MM̄FF Geometry

C -1.104104 -2.215589 1.726783

C $-0.384050-2.4294030 .615164$

C $-0.872016-2.079419-0.761027$

O $0.016010-1.040547-1.241835$
C $-0.837699-3.252452-1.753591$

C $-1.559392-4.510378-1.324129$

C $-2.898657-4.643009-1.256806$

C $-0.664613-5.679525-1.004267$

C $-3.937637-3.586938-1.518447$

C $-4.900319-3.474149-0.327144$

C $-5.704577-2.166380-0.256636$

C $-6.387353-1.787054-1.572478$

C $-7.127116-0.442387-1.467645$

O $-4.813257-1.1252830 .164609$

O $-7.440458-0.006388-2.799924$

C $-6.1686100 .609109-0.864418$

C $-8.466251-0.580725-0.734386$

O $-6.8481301 .837490-0.617240$

C $\begin{array}{llll}-5.463108 & 0.129338 & 0.422638\end{array}$

C $-4.394606 \quad 1.1270670 .926506$

O $-6.4332000 .003191 \quad 1.470186$

C $-0.486504-0.140933-2.127255$

O $-1.585373-0.153608-2.657551$

C $0.5312290 .914986-2.300164$

C $0.1302102 .169228-2.552227$

C $1.0002603 .334880-2.707320$

C $2.4836813 .095724-2.810286$

C $-3.2516661 .392185-0.051114$

C -2.1131052 .1300610 .623684$

C -1.4977024 .3723241 .532007$

$\begin{array}{llll}\text { O } & -1.068288 & 1.560649 & 0.930003\end{array}$

C -1.0741685 .4457770 .580463$

C 0.2070905 .7769360 .359398

C $0.6345456 .889688-0.555902$

C $1.6492116 .445177-1.585570$

C $2.9645156 .619731-1.371462$

C $1.1422835 .882568-2.894711$

C $0.4402924 .561892-2.745900$

N -2.401888 3.4647650 .851743

H $-2.106777-1.8028141 .647612$

H $\quad 0.625510-2.8201330 .701843$

H -1.884014 -1.669147 -0.688573

H $\quad 0.209007-3.490699-1.989995$

H -1.247179 -2.921339-2.717641

H $-3.303150-5.613688-0.972270$

H -1.229494 -6.565109-0.694599

H $-0.071864-5.954433-1.882958$

H $0.020384-5.423875-0.189400$

H -3.484568 -2.618463 -1.738391

H $-4.502524-3.876311-2.412827$

H $-5.599570-4.319465-0.365018$

H $-4.332306-3.5710750 .608211$

H $-6.457925-2.3084690 .527741$

H -7.070595 -2.580982 -1.898128

H $-5.638292-1.688974-2.369141$

H -7.977486 -0.698266 -3.223069

H $-5.4091760 .821618-1.627561$

H -9.124374 -1.276755 -1.268398

H $-9.0016470 .374731-0.706207$

H $-8.361302-0.9485490 .288110$

H -7.273115 2.105877 -1.449774

H -4.8726872 .0714661 .216809$

H $-3.9608260 .728677 \quad 1.855075$

H $-5.969951 \quad 0.0257662 .323941$

H $1.5691570 .640691-2.163690$

H $-0.9385932 .368747-2.623507$

H $3.0535983 .999245-3.037761$

H $2.7052542 .381412-3.611050$

H $2.8744952 .701550-1.866750$

H $-2.8536330 .450972-0.436584$

H -3.589125 $1.979627-0.911762$

H $-0.645328 \quad 3.820770 \quad 1.939272$

H -2.0537784 .8159152 .363834$

H $-1.8609265 .996607 \quad 0.070312$

H 0.9925225 .2438530 .890855

H 1.0633017 .6856230 .067380

H $-0.2246127 .345347-1.063693$

H $3.7021146 .335319-2.114181$

H $3.3333547 .048608-0.445586$

H $1.9501495 .798451-3.631615$

H $0.4399336 .599902-3.339144$

H $-0.6427674 .646485-2.653273$ 
H $-3.305321 \quad 3.8297430 .563897$

C $-0.324492-1.117528 \quad 3.820007$

O $0.715202-0.4014263 .122952$

C $1.645295 \quad 0.129779 \quad 4.082127$

C $0.955997 \quad 0.0164095 .435810$

C $0.168332-1.2686065 .258522$

H $2.516063-0.5370834 .076779$

C 2.0782341 .5427293 .677058

H $1.670028-0.0267456 .263802$

H 0.2743650 .8550185 .618372

C $-0.944515-1.446847 \quad 6.276187$

H $0.861146-2.1177915 .331846$

C 2.5858031 .6546812 .222963

O $0.954243 \quad 2.421322 \quad 3.809860$

H $2.8372381 .907044 \quad 4.379398$

H $1.7461321 .528043 \quad 1.529572$

O 3.0509792 .9965672 .028344

C 3.7086220 .6669251 .853160

C 4.2311380 .8962730 .422676

H $3.327524-0.3553141 .944692$

$\begin{array}{lllll}\text { O } & 4.776936 & 0.821128 & 2.785194\end{array}$

H $3.3758340 .882318-0.260747$

H $4.684506 \quad 1.8925570 .354651$

H $-0.534786-1.486644 \quad 7.290753$

H -1.661759 -0.6203186 .235503$

H $-1.492061-2.3773806 .096044$

C $-0.577416-2.4512043 .114188$

H 1.2480883 .3094183 .543607

H 3.8359673 .1181012 .589570

H 5.3727450 .0604122 .679805

H - $-1.307755-3.049307 \quad 3.670065$

H $\quad 0.353446-3.0301583 .067745$

H -1.222913 -0.4871923 .783401$

C $3.796169-1.924051-0.672565$

O $4.849427-1.4875210 .190729$

C $5.284693-0.142174-0.025867$

C $5.7619750 .030225-1.475723$

C $4.703814-0.413322-2.483036$

C $4.191904-1.814047-2.149861$

H $2.912104-1.299935-0.490975$

C $3.388336-3.345897-0.258653$

$\begin{array}{llll}\mathrm{H} & 6.162269 & 0.001567 & 0.616479\end{array}$

O $6.1143921 .384654-1.738592$

H $6.664375-0.574421-1.634595$

H $3.878410 \quad 0.303022-2.536270$

O $5.282556-0.448646-3.789884$

H $4.968827-2.544574-2.403152$

O $3.054942-2.107143-2.959466$

C $4.471710-4.389023-0.439780$

H $3.121585-3.3569480 .804848$

H $2.510876-3.650182-0.840437$

H $6.8000341 .637716-1.097024$

H $5.6344190 .439511-3.973484$

H $3.322989-1.995120-3.887665$

O $3.888988-5.618495-0.564721$

O $5.674327-4.165049-0.436677$

C $4.794003-6.715165-0.726038$

H $4.204422-7.629989-0.828892$

H $5.394796-6.581178-1.630675$

H $5.436077-6.8083210 .154938$

SCF Energy (B3LYP/6-31G**//MMFF) $=-3245.90635214$

05_00203

MMFF Geometry

C -0.3057802 .9531311 .148998$

C $-1.3096382 .066661 \quad 1.204798$

C -2.654008 2.3272251 .822844

$\begin{array}{lllll}\text { O } & -3.607552 & 2.072642 & 0.763431\end{array}$

C -2.941452 1.3676662 .997816

C -1.8785461 .3307764 .077590$

C $-1.0209710 .309194 \quad 4.279424$

C -1.833276 2.5444554 .968755

C $-0.909289-0.9639123 .485921$

C $0.421667-1.0301132 .723679$

C $0.366964-2.0260671 .556006$

C $1.734584-2.1386850 .881410$

C $1.687781-3.018657-0.375933$

O $-0.596393-1.533248 \quad 0.618696$
O $2.903160-2.804954-1.110290$

C $0.527070-2.544045-1.275697$

C $1.648293-4.510774-0.026040$

O $0.353061-3.421460-2.386254$

C $-0.813195-2.371466-0.521672$

C $-1.843823-1.665020-1.442745$

O $-1.304336-3.655544-0.143745$

$\begin{array}{llll}\text { C } & -4.886257 & 2.498850 & 0.916244\end{array}$

O $-5.350884 \quad 3.142427 \quad 1.841783$

C $-5.640583 \quad 1.987102-0.248465$

C $-6.9665881 .814663-0.158393$

C $-7.8306361 .253066-1.199246$

C $-7.191608 \quad 0.884811-2.513660$

C $-3.163842-1.252946-0.770238$

C $-4.221142-2.343293-0.723964$

C $-6.499778-2.7974860 .187924$

O $-4.077552-3.442584-1.251632$

C $-7.536904-2.513366-0.853401$

C $-8.827032-2.278648-0.566615$

C $-9.887626-2.048090-1.606178$

C $-10.728453-0.820518-1.330217$

C -11.932092 -0.926191-0.742099

C $-10.213427 \quad 0.516084-1.815601$

C $-9.1390491 .074734-0.921999$

N $-5.349549-1.937080-0.022648$

H $-0.436767 \quad 3.9451701 .570498$

H -1.1585341 .0772850 .780685$

H -2.741247 3.3736382 .140707

H -3.1510650 .3696022 .598576$

H -3.892946 1.6508973 .467371

H -0.3033190 .3929615 .094613$

H $-1.0729712 .457503 \quad 5.752233$

H -1.602562 3.4410974 .385030

H -2.8000492 .6889345 .461992$

H $-0.971633-1.8137314 .176652$

H $-1.748077-1.0825992 .797489$

H $0.663928-0.0373092 .327616$

H $1.224065-1.3055693 .419157$

H $0.039684-3.0002231 .937820$

H $2.482006-2.5179211 .588102$

H $2.079448-1.1374440 .587120$

H $3.647837-3.043192-0.531969$

H $0.815876-1.568992-1.693481$

H $2.538381-4.7933890 .548769$

H $1.668047-5.129282-0.930140$

H $\quad 0.773462-4.7895710 .565067$

H $1.211222-3.489286-2.838924$

H $-1.384549-0.736633-1.808325$

H $-2.056635-2.290618-2.317634$

H -2.244282 -3.5760010 .080096$

H $-5.0721551 .718177-1.131551$

H -7.4632442 .0833130 .773129$

H $-6.3844690 .159424-2.362337$

H $-6.776401 \quad 1.774730-2.999444$

H $-7.8824380 .427413-3.224540$

H $-2.989538-0.8786520 .243719$

H $-3.597774-0.431433-1.354470$

H $-6.867611-2.6041681 .200582$

H $-6.188833-3.8450330 .122147$

H -7.218844 - $2.533510-1.893155$

H $-9.149296-2.2927680 .472362$

H -10.529740 -2.938651-1.626492

H $-9.454078-1.973645-2.611226$

H -12.554531-0.054972 -0.566677

H -12.319557-1.886439-0.417697

H -11.030201 $1.250364-1.835985$

H $-9.9010850 .423377-2.859226$

H $-9.503888 \quad 1.3658740 .064220$

H $-5.400407-0.9849200 .327570$

C $1.303887 \quad 3.210435-0.805952$

O $2.4461552 .517082-1.355962$

C $3.4242263 .481776-1.772378$

C $2.6541364 .781069-1.951078$

C $1.6571364 .701450-0.808230$

H $4.1314723 .598527-0.941968$

C $4.1654612 .987644-3.017884$

H $3.3021675 .660784-1.894412$

H $2.1241394 .810069-2.910087$ 
C $0.4693475 .630350-1.002958$

H $\quad 2.171553 \quad 4.9706180 .123719$

C $4.7777101 .578354-2.875728$

O $3.2400372 .944417-4.112321$

H $4.9361923 .716055-3.296637$

H $3.9762420 .830917-2.836766$

O $5.5045881 .306360-4.082271$

C $5.7140201 .389882-1.665886$

C $6.377888-0.000228-1.669567$

H $\quad 5.1409101 .522377-0.744314$

O $6.7171352 .403001-1.706881$

H $5.589096-0.758404-1.727143$

H $6.990072-0.099914-2.573904$

H $0.8054546 .668708-1.092889$

H -0.090922 $5.379920-1.910187$

H $-0.2203605 .576918-0.156603$

C 1.0349012 .6120300 .574603

H $3.7282882 .628894-4.892070$

H $6.2613391 .916790-4.111378$

H $7.1481112 .426881-0.835977$

H 1.8123562 .9314201 .278937

H 1.1481651 .5214810 .510283

H $0.4654993 .000799-1.481974$

C $5.622170-1.0812391 .105226$

O $6.610825-0.0908210 .798205$

C $7.287857-0.268684-0.449436$

C $7.979273-1.638372-0.479367$

C $6.998714-2.771763-0.195163$

C $6.229953-2.4923621 .094757$

H $4.803006-1.0165820 .379510$

C $5.065743-0.7589622 .503346$

H $8.0843040 .484955-0.475342$

O $8.612391-1.842356-1.738239$

H $8.769649-1.6541200 .282645$

H $6.321543-2.931597-1.042244$

O $7.750402-3.978407-0.045339$

H $6.903681-2.6164311 .952581$

O $5.180204-3.4477931 .233410$

C 4.1968750 .4796112 .525205

H $4.449375-1.5870402 .869786$

H $5.896572-0.5901193 .199023$

H $9.015612-2.727266-1.721013$

H $7.117018-4.7120140 .028343$

H $5.589411-4.322017 \quad 1.346868$

O $3.801093 \quad 0.7147993 .811922$

O $3.896397 \quad 1.1509741 .549112$

C 2.9739101 .8660314 .007351

H 2.8249011 .9986715 .082195

H 3.4595212 .7617513 .608151

H 1.9999741 .7114623 .535732

SCF Energy (B3LYP/6-31G**//MMFF) $=-3245.92220892$

05_00204

MMFF Geometry

C $2.560703-4.096261-0.042345$

C $1.298393-3.6479920 .028242$

C $0.639834-3.218121 \quad 1.310947$

O $-0.146500-2.041428 \quad 1.012858$

C $-0.321057-4.3090361 .819072$

C $-0.971783-3.9626423 .145600$

C $-2.295893-3.790887 \quad 3.329445$

C $-0.034449-3.8333694 .319804$

C $-3.402798-3.9448062 .323969$

C $-3.774434-2.6121831 .673643$

C $-5.108768-2.6474920 .912630$

C $-5.104326-3.641020-0.251417$

C $-6.402044-3.577315-1.073099$

O $-5.336270-1.3189530 .420821$

O $-6.155300-4.306314-2.285560$

C -6.686574 -2.106217-1.445312

C $-7.556279-4.293331-0.361711$

O $-7.957496-1.966556-2.074954$

C $-6.597547-1.142936-0.241643$

C $-6.7030440 .343552-0.657266$

$\begin{array}{llll}\text { O } & -7.685386 & -1.402726 & 0.653918\end{array}$

C $0.173562-0.8684551 .618618$

O $1.115785-0.6473662 .359928$

C $\begin{array}{llll}0.869925 & 0.109568 & 1.238601\end{array}$
C $-0.837754 \quad 1.363356 \quad 1.713784$

C $-1.8415912 .398698 \quad 1.448984$

C $-3.076197 \quad 1.979156 \quad 0.695679$

C $-5.685108 \quad 0.791454-1.708314$

C $-5.5988812 .303918-1.832475$

C $-4.2371204 .063512-2.970208$

O $-6.3152813 .076512-1.202498$

C $-3.0111884 .364907-2.166055$

C $-2.8965815 .407483-1.329039$

C $-1.6474745 .692506-0.543608$

C $-1.908228 \quad 5.956916 \quad 0.924348$

C $-1.5989607 .145054 \quad 1.470927$

C -2.5221814 .8571361 .764253$

C $-1.623777 \quad 3.6530541 .897435$

N $-4.6150182 .681561-2.736078$

H $3.145991-4.1840100 .869416$

H $0.703225-3.587243-0.880030$

H $1.404999-3.0003392 .065101$

H $\quad 0.220103-5.256548 \quad 1.941429$

H $-1.082607-4.4893421 .052487$

H -2.642897 -3.5271664 .328187$

H $-0.570543-3.7465455 .271102$

H $0.609538-4.7161654 .390347$

H $0.597295-2.9462454 .218270$

H $-3.141695-4.6863141 .563018$

H $-4.273543-4.3552292 .850680$

H -3.833567 -1.8227982 .435415$

H -2.975586 -2.297292 0.991093

H -5.899347 -2.898292 1.629692

H $-4.938928-4.6661920 .099755$

H $-4.259849-3.412237-0.916818$

H $-6.980955-4.329067-2.797965$

H $-5.933570-1.820094-2.190272$

H $-7.317839-5.354787-0.223119$

H $-8.470539-4.267992-0.964890$

H $-7.781585-3.8738490 .621008$

H -7.948230 -2.506463 -2.882920

H $-7.7179210 .565690-1.012322$

H -6.5687540 .9561590 .245464$

H $-7.830045-0.6120001 .199837$

H $-1.660447-0.2413520 .584497$

$\begin{array}{llll}H & -0.009944 & 1.660062 & 2.355968\end{array}$

H -3.5813591 .1534541 .209725$

H -2.810496 $1.654307-0.315924$

H -3.818052 2.7712670 .588905

H $-4.6880280 .416063-1.452867$

H $-5.9617190 .393504-2.691227$

H $-5.0719674 .725014-2.718028$

H $-4.0154124 .163772-4.036908$

H -2.165494 $3.691577-2.289235$

H -3.726981 6.100979 -1.220777

H -1.159813 $6.561360-1.004287$

H $-0.9352774 .864593-0.633672$

H -1.778285 7.3493762 .521214

H -1.1575207 .9419450 .881588$

H $-3.5086304 .614451 \quad 1.362286$

H -2.7180395 .2220612 .781579$

H -0.6985513 .8562112 .438496$

H -4.042982 $1.961066-3.167261$

C $4.618979-3.896602-1.462632$

O $4.524590-2.462169-1.539715$

C $5.538025-1.978675-2.442856$

C $6.354878-3.194596-2.874505$

C $5.345615-4.316744-2.738094$

H $5.011722-1.581445-3.318316$

C $6.362191-0.869433-1.777597$

H $6.743260-3.088432-3.892257$

H $7.210505-3.369612-2.212321$

C $5.978974-5.695334-2.671060$

H $4.658180-4.275053-3.593639$

C $5.5224160 .301941-1.226400$

O $7.083210-1.443124-0.679186$

H $7.116303-0.502290-2.484189$

H $4.927743-0.040188-0.374899$

O $6.4360101 .289260-0.733270$

C $4.6097290 .976643-2.262514$

C $3.9063422 .265427-1.790316$

H $3.8494430 .269135-2.608903$ 
O $5.396909 \quad 1.320082-3.410010$ H $4.6395522 .943044-1.339849$ H $3.5570992 .786649-2.691283$ H $6.552778-5.899133-3.581016$ H $6.659596-5.783294-1.817550$ H $5.214954-6.472867-2.573964$ C $3.225435-4.510992-1.325209$ H $7.743696-0.791599-0.389262$ H $6.8286660 .941737 \quad 0.085282$ H $6.089817 \quad 1.936013-3.115312$ H $3.300972-5.604063-1.332208$ H $2.605627-4.214353-2.180470$ H $5.227030-4.131596-0.578588$ C $3.766030 \quad 2.3621231 .304568$ O 3.0599491 .4839090 .420121 C $2.7034392 .037885-0.848870$

C $1.8424623 .294912-0.641949$ C $2.548772 \quad 4.314026 \quad 0.245146$ C $2.981236 \quad 3.6568751 .551924$ H $4.7483792 .595136 \quad 0.879344$ C $3.978697 \quad 1.6191892 .630628$ H $2.0646641 .291198-1.337676$ O $1.5086113 .904913-1.884042$ H $0.8978443 .013649-0.162341$ H $3.4018554 .774396-0.266678$ $\begin{array}{llll}\text { O } & 1.647929 & 5.377056 & 0.559887\end{array}$ H 2.0939533 .4489562 .163844 O 3.7994714 .5659772 .285845 C $4.910694 \quad 0.4420772 .470669$ H $4.4174462 .270453 \quad 3.395200$ H 3.0209221 .2642763 .029672 H $1.0384423 .241589-2.417431$ H $1.3642405 .772778-0.281945$ H $3.285814 \quad 5.3823982 .410599$ O $4.209155-0.7234292 .557159$ $\begin{array}{lllll}\text { O } & 6.115302 & 0.547995 & 2.279331\end{array}$ C $4.974372-1.9255472 .430784$ H $4.338023-2.7641932 .725065$ H $5.277918-2.0621181 .390015$ H $5.848115-1.9048613 .089296$

SCF Energy (B3LYP/6-31G**//MMFF) $=-3245.92008350$

0500205

MMFF Geometry

C $3.767266-2.581706-2.413626$

C $4.897084-2.946616-1.787644$

C $5.503025-2.266669-0.581854$

O $4.803408-1.040127-0.279272$

C $5.374321-3.1916850 .644524$

C $5.944739-2.5931371 .916036$

C $5.213524-2.2629832 .999534$

C $7.436733-2.3818381 .923340$

C $3.732868-2.4362823 .204591$

C $2.957435-1.1849282 .793711$

C $1.474079-1.2097973 .189688$

C $0.690402-2.314806 \quad 2.478773$

C $-0.810045-2.2541632 .806882$

$\begin{array}{lllll}\text { O } & 0.938720 & 0.068650 & 2.823002\end{array}$

O $-1.500583-3.0887401 .862479$

C $-1.320265-0.8136872 .584243$

C $-1.107059-2.8354914 .193829$

O $-2.657702-0.6589323 .048940$

C $-0.425310 \quad 0.2782393 .215982$

C $-0.813248 \quad 1.704977 \quad 2.766679$

$\begin{array}{lllll}\text { O } & -0.548817 & 0.300706 & 4.643072\end{array}$

C $5.362263 \quad 0.138327-0.661832$

O $6.4071180 .301965-1.269022$

C $4.4579691 .213241-0.194502$

C $4.7783702 .503336-0.374372$

C 3.9709623 .6503790 .053763

C $2.6840913 .351794 \quad 0.777261$

C -0.8287631 .9114561 .252067$

C $-0.911663 \quad 3.3733540 .848286$

C $-0.9340984 .793684-1.212095$

O $-0.9616394 .297221 \quad 1.654354$

C $0.4388405 .069251-1.741069$

C $1.0977156 .223706-1.556461$

C $2.4667536 .489558-2.118486$
C $3.4367077 .042113-1.094676$

C $3.9993568 .250235-1.268469$

C 3.7611356 .2094850 .128226

C $4.4114824 .897107-0.216979$

N $-0.9209543 .511264-0.533217$

H $3.211812-1.713158-2.070412$

H $5.429594-3.825651-2.145595$

H $6.560259-2.066816-0.792589$

H $5.884600-4.1444040 .450190$

H $4.316340-3.4416150 .781529$

H $5.730097-1.822313 \quad 3.851574$

H $7.806293-2.0647302 .904548$

H $7.952910-3.3125271 .666072$

H $7.722532-1.6117531 .200607$

H $3.365437-3.3170422 .670328$

H $3.563218-2.6435654 .268697$

H $3.421001-0.2948253 .241132$

H $3.034093-1.0376881 .710052$

H $1.410244-1.3228314 .278175$

H $1.084323-3.3071892 .725752$

H $\quad 0.813579-2.2039431 .392845$

H -1.133313 -3.986903 1.930010

H -1.349195 -0.666153 1.497695

H $-0.819115-3.8931074 .235379$

H $-2.179927-2.8095704 .413339$

H $-0.574248-2.3207964 .996252$

H -3.191153 -1.359365 2.636162

H -1.7917801 .9796623 .182694$

H -0.0977752 .4085873 .215067$

H -1.238232 -0.329449 4.906296

H 3.5406940 .9080910 .296614

H $5.7136002 .745048-0.876988$

H 2.1258584 .2400351 .076007

H 2.8777842 .7835251 .694076

H 2.0202902 .7658890 .133481

H $\quad 0.074371 \quad 1.487008 \quad 0.800300$

H -1.698103 $1.403616 \quad 0.824068$

H -1.281345 $5.575309-0.529152$

H -1.641329 $4.710984-2.043045$

H $0.9098944 .280161-2.323468$

H $0.6211607 .027510-1.000178$

H $2.3475567 .199306-2.947470$

H $2.8941325 .581188-2.560777$

H $4.6960248 .660041-0.544634$

H $3.7771768 .859020-2.138665$

H $2.851528 \quad 6.0931530 .721875$

H 4.4586546 .7508730 .781226

H $5.3627525 .007422-0.739453$

H $-0.8475202 .677927-1.109946$

C $1.807091-3.941439-3.271871$

O $0.855154-2.910393-2.942199$

C $0.040404-3.370471-1.847616$

C $0.299421-4.868603-1.732638$

C $1.763112-4.946609-2.120436$

H $0.416881-2.871697-0.945886$

C $-1.434277-2.999624-2.050722$

H $0.099753-5.248284-0.725927$

H $-0.314133-5.439523-2.439529$

C $2.224221-6.341965-2.501207$

H $2.359284-4.595597-1.269097$

C $-1.675697-1.473644-2.061940$

O $-1.891426-3.593486-3.264025$

H -2.011495 -3.443154-1.230045

H -1.221359-1.042590 -1.160799

O $-1.046333-0.865313-3.188068$

C $-3.170831-1.097310-2.071744$

C $-3.3842160 .421561-1.942777$

H -3.657550 -1.609569-1.236037

O $-3.797056-1.555480-3.267082$

H $-2.8487810 .772223-1.056037$

H $-2.9391880 .931014-2.806257$

H $2.103651-7.030126-1.658305$

H $1.649067-6.739463-3.344211$

H $3.281222-6.337952-2.785607$

C $3.175625-3.324952-3.578467$

H $-2.845955-3.424082-3.334454$

H $-0.095980-1.063954-3.139599$

H $-3.332173-1.145786-4.016998$ 
H $3.066651-2.605782-4.399643$

H $3.858900-4.108138-3.927219$

H $1.433724-4.411882-4.191166$

C $-5.2408590 .270267 \quad 0.458155$

O

C $-4.8737130 .825236-1.858602$

C $-5.1085682 .318170-1.587033$

C $-4.7992902 .688421-0.136241$

C $-5.495918 \quad 1.7329450 .834043$

H -4.1830250 .0229860 .609905$

C $-6.077941-0.683257 \quad 1.318132$

H $-5.3135680 .600517-2.838671$

O $-4.3179553 .100142-2.475743$

H -6.160349 2.554054-1.795072

H -3.7210662 .7071910 .049108$

O

H -6.5737781 .9395360 .840584$

O -5.0053241 .9681822 .152602$

C $-5.594514-2.1067751 .165429$

H $-6.006798-0.4334072 .382900$

H -7.140354 -0.643966 1.049307

H $-4.5733284 .030418-2.358412$

H $-4.7384814 .625937-0.398385$

H -5.1518262 .9085312 .353664$

O $-6.158318-2.679613 \quad 0.064138$

O $-4.791023-2.6368331 .921806$

C $-5.734894-4.016897-0.217947$

H $-4.653479-4.046104-0.382811$

H $-6.019710-4.6842200 .600963$

H $-6.236965-4.346793-1.131401$

SCF Energy $\left(B 3 L Y P / 6-31 G^{* *} / / M M F F\right)=-3245.92733012$

05 00206

MMFF Geometry

C $3.599650-4.361421-2.196088$

C $3.990267-3.167931-2.669053$

C $4.950683-2.199008-2.027221$

O $4.174379-1.019707-1.696040$

C $5.617222-2.677610-0.721092$

C $6.559594-1.651857-0.117945$

C $6.366229-1.0341461 .064830$

C $7.793025-1.344885-0.928483$

C $5.245935-1.2312332 .049369$

C $4.163363-0.1615091 .898399$

C $3.153307-0.1393963 .055508$

C $2.315363-1.4175473 .136811$

C $1.239308-1.3301894 .232078$

O $2.295774 \quad 0.9902242 .834221$

O $\quad 0.310656-2.4060384 .023757$

C $0.450361-0.0152314 .060006$

C $1.831791-1.5344565 .631343$

$\begin{array}{lllll}\text { O } & -0.453060 & 0.188598 & 5.143541\end{array}$

C 1.3507471 .2268483 .889631

C 0.5514312 .5011423 .524154

O $2.022855 \quad 1.4977085 .125682$

C $4.2738650 .076271-2.492908$

O $4.918400 \quad 0.190398-3.521854$

C $3.4670011 .141879-1.856718$

C $3.5359022 .404748-2.303073$

C $2.8443973 .556782-1.716234$

C $2.0270823 .308960-0.475703$

C -0.3374392 .3642122 .287627$

C -0.8215823 .6997231 .747433$

C $-2.1222334 .616468-0.186838$

O $-0.533886 \quad 4.7807752 .251745$

C $-1.2781274 .730991-1.417845$

C $-0.7218865 .875072-1.844265$

C $0.1301535 .966872-3.079625$

C $1.4444486 .680270-2.840041$

C $1.7357067 .817818-3.493869$

C $2.4195856 .090556-1.843276$

C $2.9877864 .768074-2.294311$

N -1.609382 3.5239950 .618628

H $4.010452-4.749066-1.269113$

H $3.537984-2.814209-3.594740$

H $5.725784-1.960656-2.765124$

H $6.183997-3.599154-0.905559$

H $4.833598-2.935102-0.001522$
H $7.107512-0.3023741 .384917$

H $8.499710-0.708751-0.384580$

H $8.319247-2.270493-1.184149$

H $7.529639-0.824395-1.854104$

H $4.813480-2.2317661 .965685$

H $5.677869-1.1794653 .056861$

H 4.6257590 .8323111 .823206

H $3.627198-0.3055610 .953068$

H 3.7097000 .0177883 .986956

H $2.946233-2.2977923 .305947$

H $1.816999-1.5869252 .172060$

H $0.808185-3.2412564 .052497$

H $-0.165728-0.1371813 .160700$

H $2.311085-2.5181795 .704252$

H $1.048484-1.5265426 .397312$

H $2.580121-0.7840205 .894198$

H -0.990099 -0.617637 5.229158

H $-0.057706 \quad 2.823995 \quad 4.378615$

H 1.2712633 .3139443 .353872

H 2.2981062 .4297095 .128101

H $2.8549560 .859639-1.007465$

H $4.1712232 .622301-3.160370$

H 2.6506252 .8884960 .321418

H $1.2119692 .609463-0.691653$

H $1.5683924 .208708-0.064572$

H $\quad 0.212033 \quad 1.862157 \quad 1.484478$

H -1.221066 1.7633892 .529156

H -2.131761 5.5406200 .399304

H -3.149292 $4.360180-0.463858$

H -1.118845 $3.820528-1.991995$

H $-0.8975386 .793376-1.288818$

H -0.454493 $6.495283-3.843769$

H $0.3350264 .971308-3.492286$

H $2.6723408 .340689-3.330934$

H $1.0450888 .253363-4.208413$

H $1.9409816 .039162-0.862424$

H $3.2703586 .770609-1.700391$

H $3.6074104 .844097-3.188933$

H -1.744376 2.5823330 .264114

C $1.125512-4.899376-2.314201$

O $0.777119-3.531665-2.618890$

C $0.340765-2.888270-1.404512$

C $-0.118812-4.022614-0.504163$

C $0.927480-5.079244-0.805332$

H $1.227234-2.395639-0.983950$

C $-0.729845-1.849808-1.747761$

H -0.146201 -3.7414350 .552731$

H $-1.116211-4.373221-0.797248$

C $0.509639-6.484882-0.409544$

H $1.838760-4.820735-0.253214$

C $-1.364907-1.190098-0.512571$

O $-0.115420-0.827606-2.540898$

H $-1.498845-2.312173-2.375885$

H $-1.871669-1.9418180 .102076$

O $-0.319911-0.6574840 .312973$

C $-2.343619-0.045481-0.831517$

C $-3.488317-0.400356-1.799916$

H $-1.7850900 .802235-1.246719$

$\begin{array}{lllll}\text { O } & -2.886533 & 0.414447 & 0.407161\end{array}$

H $-4.1418220 .476220-1.878651$

H $-3.061083-0.556135-2.797122$

H $0.335419-6.5448990 .669840$

H $-0.413257-6.787458-0.915463$

H $1.289649-7.208009-0.668041$

C $2.532518-5.196902-2.850248$

H $0.299342-1.263683-3.305195$

H $0.180912-0.018211-0.222425$

H $-3.451072 \quad 1.1822130 .215303$

H $2.547127-5.014777-3.932433$

H $2.774941-6.255788-2.704621$

H $0.409233-5.520880-2.866438$

C $-5.981008-0.5720120 .013049$

O $-4.918693-1.525039-0.107055$

C $-4.337402-1.633017-1.408229$

C $-5.418585-2.003130-2.435607$

C $-6.600102-1.038867-2.391141$

C $-7.118419-0.898241-0.961166$

H $\quad-5.5983940 .435868-0.186094$ 
C $-6.474421-0.6161061 .463803$

H -3.653861 -2.488309-1.360849

O $-4.885164-2.030190-3.755108$

H -5.791029-3.013195 -2.220112

H $-6.338725-0.061172-2.812648$

O $-7.659064-1.555310-3.200505$

H -7.619796 -1.827371 -0.660694

O $-8.088808 \quad 0.145781-0.915089$

C -5.432306 -0.0560882 .402462$

H -7.384909 -0.0222751 .602993$

H $-6.713321-1.6416801 .770911$

H $-4.148330-2.664713-3.759326$

H -7.303704 -1.676399-4.097944

H -8.783781 -0.075825 -1.558405

O $-4.680245-1.0677702 .922113$

O $\quad \begin{array}{llll}-5.286673 & 1.139669 & 2.621804\end{array}$

C -3.627839 -0.6644343 .803235$

H -2.9330930 .0033053 .286114$

H -4.043655 -0.177296 4.690280

H -3.084401 -1.560004 4.115831

SCF Energy (B3LYP/6-31G**//MMFF) $=-3245.91438159$

0500207

MMFF Geometry

C $-0.625397-2.769273 \quad 1.225947$

C $-0.971862-2.1820552 .382193$

C $-0.430632-0.8627542 .892018$

O $0.243489-0.1729291 .807595$

C -1.5371040 .0790043 .414271$

C $-2.395944-0.4328594 .552787$

C $-3.742839-0.3632784 .557679$

C $-1.663866-0.9998975 .740206$

C $-4.605600 \quad 0.271321 \quad 3.503481$

C $-5.182606-0.7624222 .530501$

C $-5.610692-0.101414 \quad 1.212215$

C $-6.458204-1.0586380 .375784$

C $-6.784805-0.476513-1.006826$

$\begin{array}{lllll}\text { O } & -4.410874 & 0.239224 & 0.505787\end{array}$

O $-7.288924-1.542003-1.826653$

C $-5.4801790 .019665-1.663146$

C $-7.895410 \quad 0.578116-0.933850$

O

C $-4.6180530 .915152-0.740798$

C $-3.2293111 .142569-1.394832$

O $-5.2772962 .165440-0.555021$

C 1.1556090 .7832692 .120897

O 1.5554821 .0836573 .233672

C 1.5415531 .4502610 .856991

C 2.2562882 .5837810 .902053

C $2.6482743 .408650-0.242804$

C $2.1288033 .016895-1.601228$

C $-2.2114161 .912713-0.540394$

C $-2.2823853 .423676-0.685781$

C -1.221403 5.5061560 .188070

O $-3.0569483 .991646-1.450605$

C $-0.1301745 .921139-0.748956$

C $0.9029006 .697347-0.386707$

C $1.9797917 .149494-1.332210$

C $3.3738896 .871586-0.813819$

C $4.1101857 .847224-0.255430$

C $3.9457045 .486448-1.021149$

C $3.4196804 .493025-0.018717$

N $-1.370170 \quad 4.0631430 .144005$

H $0.101863-2.2843220 .580995$

H -1.659634 -2.712173 3.036869

H $0.296160-1.0803103 .684528$

H -2.145856 0.3651742 .550491

H -1.0776331 .0176093 .750857$

H -4.284565 -0.7638165 .412754$

H -2.336628 -1.213414 6.577883

H -1.159601 -1.934856 5.478246

H -0.913932 -0.2863976 .097644$

H $-5.431176 \quad 0.7953664 .002006$

H -4.0587781 .0565122 .971666$

H -4.430774 -1.533522 2.316077

H $-6.037690-1.2579143 .006199$

H $-6.1741710 .811921 \quad 1.436725$

H $-7.380375-1.3260170 .906779$
H $-5.912507-2.0008750 .238267$

H -8.072066 -1.914313 -1.386172

H $-4.890793-0.867765-1.929447$

H $-8.8131880 .139563-0.523845$

H $-8.1592680 .944003-1.932161$

H $-7.6325561 .434888-0.309900$

H $-6.2815370 .097942-3.440130$

H $-2.7851280 .157404-1.586871$

H $-3.3462781 .640974-2.364144$

H $-4.6389292 .803726-0.200907$

H $1.1912091 .014046-0.071582$

H 2.5707662 .9599601 .874989

H $1.0418882 .881714-1.579721$

H $2.5912192 .082741-1.932119$

H $2.3208433 .764363-2.373805$

H -2.3034001 .6498370 .517769$

H -1.206410 $1.619002-0.868333$

H -1.0025245 .7786531 .225262$

H -2.162702 $5.980012-0.108217$

H $-0.2068975 .586627-1.780882$

H $0.962953 \quad 7.0558560 .638454$

H $1.8496238 .228240-1.490224$

H $1.8633166 .689696-2.321587$

H 5.1211297 .6636170 .093146

H $3.7164028 .850334-0.129214$

H $5.0380095 .510353-0.908104$

H $3.7824355 .180760-2.058174$

H 3.7362174 .7005371 .004632

H -0.7238093 .5045660 .693420$

C $-1.507796-4.168860-0.713238$

O $-0.376722-3.788105-1.524909$

C $-0.786684-2.717012-2.392397$

C $-2.288230-2.899946-2.549649$

C $-2.679585-3.274138-1.130323$

H $-0.600218-1.778932-1.853916$

C $0.025925-2.720415-3.688641$

H $-2.786529-1.995987-2.910620$

H $-2.526877-3.721708-3.234783$

C $-4.026984-3.970058-1.038213$

H -2.711605 -2.360885 -0.523041

C $1.554233-2.771076-3.478635$

O $-0.341075-3.869422-4.461394$

H $-0.251041-1.845938-4.289567$

H $1.843871-3.777994-3.154635$

O $2.176400-2.586617-4.756278$

C $2.115560-1.743110-2.477197$

C $3.655116-1.751685-2.426931$

H $1.722483-1.970991-1.481681$

O $1.658898-0.434671-2.814085$

H $3.997069-2.786234-2.317297$

H $4.051137-1.378491-3.379110$

H -4.818961 -3.335063-1.445585

H -4.034104 -4.909193 -1.601824

H $-4.273186-4.1998270 .003296$

C -1.123706 -4.114213 0.770704

H $\quad 0.183251-3.847962-5.280279$

H $1.913108-1.713731-5.093798$

H $2.066868-0.181805-3.659309$

H $-0.303947-4.8250290 .935755$

H - $1.971710-4.444846 \quad 1.381446$

H $-1.720952-5.213853-0.971349$

C $4.137900-2.4946460 .499049$

O $3.687159-1.235703-0.007418$

C $4.222372-0.875981-1.285440$

C $5.755092-0.841251-1.220236$

C $6.335931-2.154803-0.699294$

C $5.667086-2.5482150 .618983$

H $3.807373-3.286486-0.185238$

C $3.411891-2.7759861 .822962$

H $3.8868310 .149164-1.479054$

O $6.292000-0.544389-2.505185$

H $6.062348-0.028621-0.548960$

H $\quad 6.246267-2.949737-1.448904$

O $7.735407-1.962600-0.480217$

H $6.022631-1.8796651 .410935$

O $6.055129-3.8733070 .974593$

C $3.685871-1.7770682 .928549$

H $2.331459-2.7524831 .647123$ 
H $3.685279-3.774263 \quad 2.182797$

H $7.261126-0.549707-2.423231$

H $8.116360-2.824226-0.241335$

H $7.011103-3.8639991 .149569$

O $3.208658-2.2847804 .104522$

O $4.219419-0.6861092 .785805$

C $3.385554-1.4523755 .254863$

H $3.021970-1.9993176 .128789$

H $4.445440-1.2232085 .401146$

H $2.801981-0.5337105 .148847$

SCF Energy (B3LYP/6-31G**//MMFF) $=-3245.91064237$

05_00208

MMFF Geometry

C $2.778674 \quad 3.980367 \quad 1.024023$

C $1.894906 \quad 2.9747310 .938739$

C $1.074358 \quad 2.4799172 .101626$

$\begin{array}{llll}\text { O } & -0.253279 & 2.176150 & 1.615806\end{array}$

C 1.6900381 .1794002 .648470

C $0.9060190 .573478 \quad 3.796091$

C $0.246297-0.6009113 .744289$

C $0.920207 \quad 1.3678535 .077350$

C $0.140605-1.5577572 .589523$

C $-1.284309-1.6077392 .037289$

C -1.446012 -2.5935170 .870108$

C $-0.734149-2.116856-0.400320$

C $-1.008877-3.040032-1.598084$

O $-2.854522-2.7108160 .624889$

O $-0.591494-2.354797-2.788904$

C $-2.530795-3.262463-1.719868$

C $-0.179054-4.326939-1.527037$

O $-2.830501-4.226231-2.726110$

C $-3.200000-3.665933-0.389535$

C $-4.743341-3.707978-0.483999$

O $-2.768782-4.982344-0.023830$

C $-1.162971 \quad 3.186679 \quad 1.590937$

O $-0.9912494 .343108 \quad 1.940326$

C -2.4217022 .6295921 .046114$

C $-3.490693 \quad 3.4226490 .879757$

C $-4.788237 \quad 3.0119580 .339221$

C -4.9847181 .5514740 .024853$

C $-5.395947-2.399148-0.934089$

C $-6.898418-2.386030-0.706279$

C $-8.879170-0.907637-0.983121$

O $-7.517138-3.322711-0.209474$

C $-9.0879760 .571385-1.029250$

C $-9.276832 \quad 1.3267780 .063272$

$\begin{array}{llll}C & -9.457616 & 2.817830 & 0.031874\end{array}$

C $-8.238975 \quad 3.5450310 .563409$

C $-8.215604 \quad 3.9872251 .832579$

C $-7.1154603 .750988-0.434579$

C -5.7463793 .9459290 .159136$

N $-7.460581-1.182450-1.109007$

H 2.9010114 .4722901 .987513

H 1.7540582 .4447040 .001850

H $1.020828 \quad 3.2337852 .896477$

H 2.7132891 .3714332 .997619

H $1.7922800 .465474 \quad 1.825857$

H $-0.277000-0.9321044 .640782$

H 0.4446750 .8306115 .904951

H 1.9506131 .5839755 .378316

H $\quad 0.3863742 .3145354 .951378$

H $0.845865-1.3125971 .794867$

H $0.426868-2.5538912 .949245$

H -1.978101-1.894596 2.839478

H $-1.606840-0.6078211 .719700$

H -1.061412 -3.5694771 .188290$

H $0.346173-2.026028-0.243617$

H $-1.084992-1.106757-0.654542$

H $\quad 0.350167-2.130628-2.692143$

H $-2.960221-2.312026-2.061261$

H $0.891696-4.091291-1.534644$

H $-0.352141-4.958087-2.405566$

H $-0.378540-4.918049-0.630911$

H $-2.396176-3.933918-3.545784$

H $-5.059762-4.524334-1.146271$

H $-5.134451-3.970370 \quad 0.509079$

H $-3.419902-5.3603690 .590451$
H -2.430685 1.5774740 .784056

H $-3.408217 \quad 4.4740601 .151550$

H $-6.011752 \quad 1.306253-0.251624$

H -4.7522190 .9318920 .898511$

H -4.340156 $1.245089-0.806020$

H $-4.970229-1.554471-0.381256$

H $-5.217603-2.239578-2.003336$

H $-9.251154-1.350752-0.052854$

H $-9.396727-1.393424-1.816495$

H $-9.0679751 .042363-2.008935$

H $-9.2863310 .852903 \quad 1.042385$

H $-10.346132 \quad 3.065647 \quad 0.626729$

H $-9.677973 \quad 3.172097-0.983125$

H -7.3712904 .5309442 .240961$

H -9.0546903 .8185142 .500084$

H $-7.3555324 .631051-1.045512$

H $-7.0889072 .917541-1.145058$

H -5.5289564 .9792890 .430919$

H $-6.893477-0.544490-1.660977$

C $3.4825753 .947827-1.477941$

O $3.7354852 .533933-1.505498$

C $4.2197982 .171170-2.811198$

C $4.3809273 .472075-3.597908$

C $4.4609964 .518235-2.501438$

H $5.2065621 .718708-2.661691$

C $3.2781461 .145776-3.458505$

H $5.2730983 .455304-4.232381$

H $3.5252413 .667358-4.253657$

C $4.1232615 .919638-2.979408$

H $5.4793354 .511056-2.089835$

C $2.916453-0.054970-2.561719$

O $2.045521 \quad 1.799361-3.794196$

H $3.7115500 .804771-4.406233$

H $2.2348410 .273436-1.767387$

O $2.161090-0.972409-3.364806$

C $4.109198-0.796559-1.932494$

C $3.647963-2.047495-1.160768$

H $4.638677-0.115205-1.258700$

O $5.011352-1.176793-2.968774$

H $2.856707-1.749860-0.464342$

H $3.206880-2.764686-1.863658$

H $4.8089476 .230424-3.774563$

H $3.1032495 .973456-3.374556$

H $4.2065406 .643383-2.162860$

C $3.6849424 .500468-0.063700$

H $1.4709441 .136442-4.214368$

H $2.759761-1.328451-4.043899$

H $5.841352-1.458585-2.548683$

H $3.5673455 .590246-0.089156$

H 4.7140524 .2962610 .258045

H $2.4454354 .106977-1.802063$

C $4.809216-1.3735281 .574436$

O $5.525904-1.8779170 .441423$

C $4.782038-2.765753-0.397385$

C $4.312997-3.9804910 .416920$

C $3.535262-3.5597331 .660978$

C $4.325407-2.5263362 .462568$

H $3.956265-0.774637 \quad 1.233036$

C $5.759751-0.4529842 .347110$

H $5.486825-3.138194-1.150878$

O $3.499895-4.842462-0.371711$

H $5.186548-4.5639610 .735725$

H $2.540441-3.1806641 .402177$

O $3.322191-4.7026062 .492446$

H $5.182372-3.0166882 .942267$

O $3.498426-2.0032253 .499289$

C $5.983165 \quad 0.842847 \quad 1.606031$

H $5.356148-0.1894153 .331635$

H $6.731646-0.9311972 .519455$

H $4.021709-5.106789-1.148627$

H $2.849933-5.3641851 .958188$

H $3.176916-2.7565904 .023531$

$\begin{array}{llllll}\text { O } & 7.030324 & 0.716736 & 0.742552\end{array}$

$\begin{array}{lllll}\text { O } & 5.297742 & 1.843752 & 1.768251\end{array}$

C $7.3461581 .887589-0.016751$

H $6.4679652 .241397-0.563269$

H 7.7275212 .6701630 .645637

H $8.1244381 .625601-0.738366$ 
C $0.8318435 .018421-2.411004$

C $-0.0540004 .058238-2.720257$

C $-1.4131253 .807974-2.105353$

O $-1.3918212 .489411-1.497701$

C -1.833159 $4.809774-1.011084$

C $-3.2436594 .596243-0.493391$

C -3.5539414 .2553630 .772923$

C $-4.3487734 .824440-1.493446$

C -2.6231084 .0160041 .928199$

C -2.2360582 .5448202 .144877$

C -3.4209371 .6235822 .481229$

C -2.9235690 .2473772 .927976$

C $-4.081473-0.7288883 .187998$

O $-4.229938 \quad 1.476977 \quad 1.311219$

O $-3.529604-2.0518023 .277495$

C $-5.033253-0.7187591 .972162$

C $-4.767713-0.4538274 .531059$

O $-6.208546-1.4795742 .238698$

C -5.4195670 .6990821 .504747$

C $-6.197605 \quad 0.7077750 .165467$

O -6.2712381 .3011542 .487425$

C $-1.5653621 .411052-2.308864$

O $-1.7543251 .417055-3.514835$

C $-1.4592460 .196529-1.471891$

C -1.125606 -0.968090 -2.047359

C $-0.986929-2.256534-1.366631$

C $-1.545269-2.3688040 .026451$

C $-5.4531210 .109042-1.033301$

C $-5.781597-1.350223-1.304698$

C $-5.104445-3.246714-2.776032$

O $-6.686131-1.956259-0.737221$

C $-4.172375-4.144678-2.025352$

C $-3.286033-4.954710-2.624472$

C $-2.378849-5.896634-1.885690$

C $-0.923780-5.722152-2.259840$

C $-0.339201-6.531529-3.159270$

C $-0.126307-4.668662-1.525585$

C $-0.403816-3.278478-2.026897$

N $-4.963507-1.886458-2.290853$

H $0.6090545 .747253-1.638598$

H $\quad 0.223733 \quad 3.355451-3.504814$

H $-2.1529603 .824118-2.915028$

H -1.775972 $5.834643-1.400365$

H -1.112605 4.749388 -0.188916

H -4.6079674 .1410231 .024722$

H -5.339952 4.814039-1.026995

H $-4.2281145 .798704-1.978272$

H -4.341797 4.046070 -2.262449

$\mathrm{H}-1.7098064 .6106061 .822684$

H -3.106419 4.3981112 .836566

H -1.723930 2.1775331 .247949

H -1.5104912 .5108712 .967353$

H -4.009419 2.0938343 .277738

H $-2.2875990 .332383 \quad 3.817889$

H $-2.284275-0.1770772 .142784$

H -2.868978 -2.053334 3.991303

H $-4.509749-1.2301381 .156557$

H -4.048767 -0.546073 5.354077

H -5.550156 -1.193387 4.733970

H -5.2100010 .5427084 .590589$

H -5.923066 -2.370456 2.504379

H -7.1851790 .2500830 .299502$

H -6.398059 $1.759943-0.083734$

H -6.7740362 .0162432 .063340$

H -1.643589 $0.296654-0.408995$

H $-0.913963-0.979422-3.115943$

H $-0.955480-1.7728730 .727171$

H -1.569786 -3.391807 0.407383

H $-2.580320-2.0129770 .051786$

H $-5.7522210 .659249-1.934831$

H $-4.3712440 .225949-0.923501$

H -4.890476 -3.231689-3.849410

H -6.136319 -3.582213 -2.630968

H $-4.250322-4.149312-0.940559$
H $-2.702175-6.919695-2.118481$

H $-2.491060-5.792660-0.799308$

H $0.711403-6.431774-3.411270$

H $-0.900228-7.308697-3.667683$

H $0.948409-4.850892-1.659902$

H $-0.290114-4.775930-0.449657$

H $-0.045157-3.102540-3.042019$

H $-4.206057-1.324792-2.668814$

C $3.3425344 .689147-2.202021$

O $3.1090763 .349946-1.718585$

C $3.4089693 .315877-0.305458$

C $4.2799644 .535113-0.052449$

C $3.6282135 .551135-0.972082$

H 2.4449413 .4245720 .209207

C 4.0335871 .9593190 .030627

H 4.2800944 .8482090 .995478

H $5.3160664 .345531-0.359338$

C $4.4987816 .762162-1.258396$

H $2.6940935 .889699-0.507343$

C 4.6475141 .8675761 .437904

O $3.0012150 .973286-0.090108$

H $4.7929131 .710067-0.719820$

H 5.5358242 .5079991 .491348

O 3.7068552 .4026042 .378940

C 4.9997230 .4402021 .901673

C $5.850083-0.3652630 .905825$

H $4.073222-0.0943062 .130833$

O $5.708164 \quad 0.5378793 .142431$

H $5.347197-0.398018-0.064401$

H $\quad \begin{array}{llll}6.808697 & 0.145532 & 0.752339\end{array}$

H $4.7188857 .304663-0.333165$

H $5.4524976 .473392-1.712820$

H $3.9935407 .451017-1.942764$

C $2.1736635 .135924-3.084612$

H $2.6349521 .043648-0.988465$

H 4.1108242 .3386483 .261362

H 6.5472120 .9985752 .970072

H $2.1754824 .519255-3.992963$

H $2.3197806 .171454-3.412213$

H $4.2414704 .632509-2.829788$

C $4.004472-2.7709120 .771114$

O $4.939303-2.4801141 .816722$

C $6.128435-1.8102061 .384341$

C $6.866427-2.6828800 .358731$

C $5.955868-3.108334-0.793532$

C $4.646395-3.697625-0.265985$

H $3.685753-1.8404900 .287429$

C $2.771450-3.4145011 .415977$

H $6.772964-1.7307152 .268836$

O $7.999131-2.000877-0.168990$

H $7.237838-3.5887240 .855386$

H $5.759948-2.274694-1.478027$

O $6.616739-4.112267-1.567748$

H $4.840054-4.6838330 .174920$

O $3.742865-3.883120-1.352982$

C $2.086925-2.4672682 .374994$

H $2.035334-3.7325180 .670161$

H $3.064251-4.3024611 .989740$

H $8.570501-1.7608490 .580463$

H $7.462150-3.735793-1.867237$

H $4.185754-4.453659-2.004354$

O $1.578018-1.3967551 .701697$

O $2.010558-2.6619093 .581511$

C $0.933592-0.4079772 .511775$

H 1.6580370 .0546113 .188390

H $\quad 0.528517 \quad 0.3624591 .850060$

H $\quad 0.109808-0.8553563 .075750$

SCF Energy (B3LYP/6-31G**//MMFF) $=-3245.90792601$

0500210

MM̄FF Geometry

C $0.7853794 .992918-0.474387$

C 1.0630054 .7521860 .817156

C 1.9007373 .6097071 .348018

O 2.0113362 .5465240 .374644

C 3.3174654 .1074691 .683285

C 4.1739043 .0706542 .384429 
C 5.2892662 .5190271 .867553

C 3.7228832 .6788863 .768828

C 5.9282312 .7892510 .534921

C $5.4888081 .844393-0.594231$

C $5.8101470 .362467-0.339574$

C $5.595425-0.463060-1.610454$

C $5.825011-1.963427-1.367831$

O $4.943583-0.1210890 .691168$

O $5.292329-2.678058-2.493986$

C $5.014855-2.405381-0.129612$

C $7.317810-2.307991-1.303490$

O $5.331118-3.7471820 .231579$

C $5.195831-1.4764851 .088566$

C $4.232848-1.8031642 .257443$

O $6.528584-1.6212351 .595965$

C 0.9739061 .6686160 .296195

$\begin{array}{lllll}\text { O } & -0.028391 & 1.647818 & 0.992494\end{array}$

C $1.2502970 .747177-0.826666$

C $0.2358030 .069680-1.383516$

C $0.336205-0.865409-2.505228$

C $1.715002-1.302703-2.923710$

C $2.738252-1.7339151 .924019$

C $2.129098-3.0713131 .535428$

C $0.026999-4.0636810 .639904$

O $2.716309-4.1422141 .663244$

C $0.135893-4.226842-0.843179$

C $-0.931000-4.359962-1.645450$

C $-0.847633-4.555337-3.131950$

C -1.642007 -3.521714 -3.898727

C $-2.896166-3.778192-4.307510$

C $-0.951194-2.226789-4.262584$

C $-0.800910-1.299360-3.087926$

N $0.831473-2.9316051 .060649$

H $1.2211964 .359584-1.241425$

H $0.6380685 .407401 \quad 1.574983$

H 1.4142953 .2226042 .251499

H 3.2605514 .9929142 .330595

H 3.7977134 .4413890 .757250

H 5.8223681 .7858082 .472414

H 4.4571982 .0483584 .281990

H 3.5719903 .5704084 .386263

H 2.7842782 .1182983 .726195

H 5.7510913 .8235420 .221700

H 7.0161182 .7141630 .661527

H $4.4122591 .966373-0.759698$

H $5.9945952 .169456-1.512033$

H $6.8526550 .287476-0.008445$

H $6.240932-0.104204-2.421867$

H $4.565353-0.322535-1.964669$

H $5.742477-2.355819-3.293661$

H $3.959066-2.401016-0.425218$

H $7.814962-2.032071-2.241285$

H $7.470115-3.387041-1.191345$

H $7.841173-1.798708-0.491587$

H $5.177935-4.302167-0.552307$

H $4.501210-2.7662542 .708209$

H 4.414608-1.055147 3.042932

H $6.541819-1.3159992 .518385$

H $2.2723930 .670362-1.177627$

H $-0.769827 \quad 0.229225-0.997287$

H $2.281190-1.663632-2.058226$

H $2.261324-0.469954-3.379017$

H $1.712790-2.124246-3.642951$

H $2.197826-1.4034772 .820510$

H $2.546391-0.999878 \quad 1.136679$

H $-1.000847 \quad-3.867716 \quad 0.958198$

H $0.381066-4.9715721 .138422$

H $1.134202-4.257492-1.273155$

H -1.925712 -4.359672 -1.206147

H -1.228748 -5.559924 -3.358139

H $0.192612-4.550536-3.480197$

H -3.464167 -3.053016 -4.880472

H $-3.386058-4.716786-4.069839$

H - $-1.534225-1.690443-5.023576$

H $-0.000053-2.454449-4.751710$

H - $-1.751988-0.924352-2.708729$

H $\quad 0.438616-2.001398 \quad 0.948247$

C $-1.3923765 .596305-1.636932$
O $-2.0907154 .673698-0.774578$

C $-2.3601163 .474062-1.518975$

C $-2.3450223 .903985-2.977872$

C $-1.1822314 .878873-2.974173$

H -1.520931 $2.791163-1.336590$

C -3.655395 2.794613-1.060967

H -2.197709 $3.062595-3.661550$

H $-3.2735614 .417236-3.254423$

C -1.163913 $5.812338-4.172494$

H $-0.2497384 .302417-2.968913$

C -3.6138012 .3387120 .411510$

O $-4.7641123 .670427-1.258156$

H $-3.8185901 .929356-1.710896$

H -2.7171501 .7381140 .598543$

O $-3.518604 \quad 3.481121 \quad 1.271352$

C -4.8559051 .5502300 .862829$

C $\quad \begin{array}{llll}-5.183963 & 0.288810 & 0.042963\end{array}$

H -5.7343962 .2070290 .855188$

O -4.6761141 .1619752 .227319$

H -5.989810 -0.2450070 .560615$

H $-5.5905050 .603438-0.925225$

H -1.064330 $5.242810-5.102294$

H -2.084764 $6.401663-4.237243$

H $-0.3211976 .508179-4.109232$

C $-0.1213346 .099855-0.941171$

H $-4.6192474 .452267-0.697728$

H -2.678727 3.9288731 .072627

H -4.4733351 .9672162 .733889$

H $-0.4217346 .708182-0.078371$

H $0.4376006 .759235-1.614823$

H $-2.0674176 .450443-1.775763$

C -4.111789-1.999254 1.832029

O $-3.358491-1.1003571 .008959$

C $-4.003410-0.683076-0.195199$

C $-4.358224-1.906644-1.049325$

C $-5.175230-2.928920-0.268088$

C $-4.486662-3.2684021 .053399$

H $-5.020349-1.4995212 .187924$

C $-3.236509-2.3309903 .046839$

H -3.248444 -0.126954 -0.760689

O $-5.059319-1.505018-2.220931$

H -3.428445 -2.382814 -1.381632

H $-6.200594-2.575431-0.108251$

O $-5.278096-4.115811-1.058862$

H $-3.595307-3.8730820 .843837$

O $-5.384245-4.0722201 .816650$

C $-3.008226-1.1136803 .910636$

H $-3.674298-3.1137683 .675379$

H -2.250282 -2.691441 2.731366

H $-5.276413-2.310961-2.720208$

H $-5.769783-4.770543-0.533776$

H -4.881830 -4.468881 2.547438

O $-4.064990-0.9366394 .754190$

O $-2.015243-0.4016613 .835278$

C -3.9665870 .1860425 .635882$

$\begin{array}{llll}\text { H } & -4.888400 & 0.240404 & 6.221025\end{array}$

$\begin{array}{llll}H & -3.123049 & 0.053761 & 6.320147\end{array}$

H -3.858611 1.1133925 .065428

SCF Energy (B3LYP/6-31G**//MMFF $)=-3245.91492299$

0500211

MMFF Geometry

C $2.918775-3.270702-1.550709$

C $2.353110-2.092756-1.250358$

C $1.296796-1.425384-2.090205$

O $0.193727-1.061773-1.229012$

C $1.866475-0.132972-2.702739$

C $0.9002460 .578078-3.631068$

C $0.4204271 .820806-3.428194$

C $0.496992-0.182898-4.868102$

C $0.7314502 .755989-2.294349$

C $-0.2038452 .632220-1.082497$

C $-1.6658843 .003978-1.375216$

C $-2.4737223 .072755-0.077693$

C $-3.9548923 .382208-0.345692$

O $-2.2304732 .004935-2.225960$

$\begin{array}{llll}\text { O } & -4.689057 & 3.104278 & 0.857580\end{array}$

C $-4.4903332 .416945-1.428564$ 
C $-4.1711374 .870312-0.646368$

O $-5.8004752 .791422-1.844233$

C $-3.5687562 .283810-2.661708$

C $-3.9799781 .140686-3.617727$

O $-3.6089463 .463790-3.474522$

C $-0.690835-2.039716-0.896179$

O $-0.683545-3.201157-1.270522$

C $-1.663168-1.4506190 .049384$

C -2.402022 -2.258509 0.823607

C $-3.367889-1.8326531 .838025$

C $-3.709393-0.3674811 .901908$

C $-4.004103-0.262213-3.001409$

C $-5.376482-0.697609-2.513544$

C -6.501653 -2.552695 -1.287693

O $-6.406400-0.071058-2.747396$

C $-6.715772-2.1339550 .132668$

C $-6.899982-3.0053571 .136104$

C $-7.160820-2.6027832 .559340$

C -6.203664 -3.252789 3.533196

C $-6.553744-4.3579104 .212865$

C $-4.876474-2.5721913 .780567$

C $-3.904038-2.7673022 .650339$

N -5.319694 -1.896044 -1.814248

H $2.615759-3.794263-2.453833$

H $2.668933-1.566529-0.354644$

H $0.941257-2.097164-2.880670$

H $2.777519-0.361270-3.272266$

H $2.1763170 .531567-1.888525$

H $-0.2692632 .229994-4.165544$

H $-0.0619250 .440258-5.574637$

H $1.383706-0.553636-5.392600$

H $-0.139558-1.034215-4.608608$

H $1.7670862 .622041-1.964563$

H $\quad 0.6890393 .783005-2.678984$

H $-0.1509101 .608936-0.692675$

H $0.1794663 .296804-0.299379$

H -1.679426 $3.972851-1.888029$

H -2.0465153 .8133660 .609939$

H -2.4079892 .1085320 .443840$

H $-4.3073553 .641343 \quad 1.572992$

H $-4.582567 \quad 1.434184-0.951773$

H -3.8675875 .4820050 .211921$

H $-5.2312385 .092056-0.810432$

H -3.604271 $5.219085-1.512096$

H -6.358022 $2.827002-1.048223$

H $-4.9317561 .382225-4.106346$

H -3.237965 $1.119646-4.429363$

H -4.295033 $4.056582-3.128469$

H - $1.715671-0.3698530 .109319$

H -2.266494 -3.335347 0.729567

H $-4.011245-0.0019370 .914737$

H -2.846249 0.2137772 .243994

H -4.540986 -0.1408032 .571891$

H -3.713512 -0.984691 -3.774913

H -3.280223 -0.347305 -2.185973

H $-6.342886-3.631468-1.382416$

H -7.372563 -2.273353 -1.889234

H $-6.748255-1.0671090 .340570$

H -6.895766 -4.0714460 .919395$

H -8.191983 -2.890811 2.802952

H -7.122566 -1.513244 2.682102

H -5.884137 -4.814619 4.934011

H -7.516704 -4.833723 4.058745

H -4.405340 -2.983392 4.683578

H -5.053548 -1.519135 4.015602

H -3.593766 -3.805349 2.521155

H -4.416339 -2.319116-1.622390

C $5.383838-3.360142-0.867321$

O $5.457010-2.057181-0.251538$

C $6.092397-1.154469-1.171830$

C $6.852749-2.040133-2.152734$

C $5.888790-3.201114-2.304330$

H $5.285674-0.644831-1.711388$

C $6.950072-0.122027-0.435131$

H $7.064182-1.530178-3.097349$

H $7.802165-2.392623-1.733454$

C $6.523166-4.459152-2.871214$

H $5.082048-2.883648-2.975830$
C 6.2369520 .6284590 .708211

$\begin{array}{lllll}\text { O } & 8.080627 & -0.792552 & 0.138604\end{array}$

H $7.3576260 .589899-1.163012$

H $6.113312-0.0457311 .564325$

O $7.1294931 .659349 \quad 1.155912$

C 4.8726641 .2557360 .365492

C 4.3493322 .1571541 .503217

H 4.1440930 .4661940 .153750

O $4.9866032 .074938-0.799581$

H 4.4964401 .6514302 .461862

H 4.9479813 .0755431 .519808

H $6.916380-4.272756-3.875925$

H $7.351943-4.808306-2.246166$

H $5.787663-5.267084-2.939394$

C $3.972067-3.933539-0.705817$

H $8.619493-0.1193310 .588650$

H 7.2071262 .3112680 .438193

H $5.1550621 .497701-1.561857$

H $3.673472-3.8653620 .347872$

H $3.982938-5.000698-0.958411$

H $6.073822-3.992813-0.294089$

C 1.8553910 .9990022 .896227

O 1.9999921 .4022301 .526971

C 2.8613252 .5272181 .305920

C 2.3836473 .7359382 .123024

C 2.2309423 .3998003 .600597

C 1.3533752 .1617863 .764273

H 2.8188490 .6465213 .281018

C $0.858578-0.1709772 .933899$

H $2.7390282 .801144 \quad 0.251774$

O 3.2734614 .8379511 .981072

H 1.4076364 .0656281 .744297

H 3.2050513 .2638604 .085081

O 1.5947214 .4919554 .267492

H $\quad 0.3203042 .415253 \quad 3.494447$

O 1.3418451 .7765165 .138232

C $1.491585-1.4897002 .543428$

H $0.463931-0.3061423 .947083$

H $\quad 0.029413 \quad 0.023873 \quad 2.244639$

H 3.3425275 .0371991 .031814

H 2.1434275 .2809994 .117576

H 2.2524561 .5494625 .392784

O $0.532033-2.4615332 .579513$

O $2.669698-1.6548692 .260572$

C $0.969340-3.7845252 .254924$

H $1.198760-3.8481161 .188269$

H $1.839326-4.0654312 .856165$

H $\quad 0.152416-4.4756842 .479064$

SCF Energy (B3LYP/6-31G**//MMFF) $=-3245.90907841$

0500212

MMFF Geometry

C $-3.029365-2.092712-2.784448$

C $-1.906426-1.800926-2.111429$

C $-0.587159-1.527040-2.781688$

O $-0.264063-0.144255-2.501028$

C $0.533624-2.431803-2.234593$

C $0.303911-3.911944-2.451610$

C $0.117171-4.812608-1.467430$

C $0.344598-4.367347-3.887997$

C $0.065659-4.5703260 .015121$

C $1.258107-5.1963620 .751371$

C $2.611220-4.5532540 .404936$

C $3.745185-5.2867771 .118820$

C $5.102139-4.6248340 .853699$

O $2.579552-3.1898950 .830552$

O $6.030453-5.2109061 .778739$

C $4.999149-3.1141501 .161708$

C $5.620867-4.949445-0.553510$

O $6.196441-2.4567600 .754161$

C $3.764587-2.4300870 .523960$

C $3.490234-1.0260741 .113812$

O $3.948770-2.314824-0.887110$

C $0.6280680 .464543-3.327671$

O $1.218407-0.036332-4.271716$

C $0.7699241 .867506-2.881233$

C $1.8229472 .581759-3.304950$

C $2.1189443 .975898-2.968890$ 
C $1.0448264 .765111-2.268071$

C 4.5491970 .0465440 .863071

C 4.1252101 .3599531 .490325

C 2.6229543 .3142571 .149652

O 4.5517581 .7152212 .587259

C 3.2980754 .5150390 .558100

C $4.4216234 .557319-0.175676$

C $4.9941905 .833533-0.727544$

C $5.1441655 .811549-2.233032$

C $6.3587775 .828975-2.807440$

C $3.8845005 .848903-3.073909$

C $3.3251454 .473226-3.314479$

N 3.2092022 .0592300 .722643

H -3.005548 -2.133176 -3.870834

H -1.931755 -1.747971 -1.026316

H $-0.684401-1.649596-3.867981$

H $\quad 0.704882-2.189657-1.180005$

H $1.483511-2.164565-2.715700$

H - $0.019607-5.856173-1.749374$

H $0.289135-5.457321-3.980532$

H $-0.494730-3.947481-4.450595$

H $1.278422-4.046731-4.361496$

H $-0.019919-3.5099730 .266939$

H $-0.853102-5.0368730 .390864$

H $\quad 1.073275-5.087125 \quad 1.828074$

H $1.294057-6.2721350 .537580$

H $2.760527-4.591621-0.679259$

H $3.771460-6.3444050 .827614$

H $3.560348-5.2794722 .202445$

H $6.913828-4.8519861 .590036$

H $4.929163-2.9997602 .252294$

H $5.719681-6.033856-0.682349$

H $\quad 6.623757-4.537585-0.712069$

H $4.969057-4.576402-1.346533$

H $\quad 6.927788-2.8195351 .281344$

H $2.535244-0.6692490 .702025$

H $3.310838-1.1184332 .193825$

H $3.381093-1.596407-1.211813$

H $\quad 0.0152412 .266094-2.213248$

H $2.5544532 .094161-3.948839$

H $1.3204065 .803543-2.074925$

H $0.8009214 .318656-1.298989$

H $0.1335544 .790336-2.876481$

H $5.508619-0.2211211 .314543$

H $4.7211870 .195378-0.208456$

H 2.6539143 .3836652 .241110

H $1.5744773 .293702 \quad 0.836974$

H 2.7855945 .4548890 .758011

H $4.9717433 .645106-0.389494$

H $5.9687685 .993360-0.248608$

H $4.3796536 .698717-0.448101$

H $6.4723025 .839083-3.886437$

H $7.2664925 .828849-2.213068$

H $4.1062036 .288950-4.055474$

H $3.1671686 .532724-2.612725$

H $4.0203223 .814966-3.838480$

H $2.9538381 .704760-0.194523$

C $-5.311015-1.197612-2.281087$

O $-4.894410-0.134276-1.397733$

C $-6.0527150 .389321-0.721355$

C $-7.258747-0.160245-1.471886$

C $-6.748844-1.528070-1.880923$

H $-6.050420-0.0386180 .288078$

C $-5.9846391 .917322-0.641504$

H -8.154348 $-0.203933-0.844778$

H -7.493965 $0.439506-2.358929$

C -7.565591 -2.174580 -2.986229

H $-6.752750-2.184207-0.999793$

C $-4.7162592 .457997 \quad 0.052013$

O $-6.0111492 .442347-1.974811$

H $-6.8836982 .297996-0.142413$

H -3.837922 $2.252349-0.571145$

O -4.8310573 .8861930 .108110$

C -4.4805941 .9135251 .473903$

C -3.2745542 .5794952 .160934$

H $-4.320651 \quad 0.8345291 .418349$

O $-5.657325 \quad 2.1401312 .248313$

H -2.4076142 .4997121 .495810$
H $-3.483537 \quad 3.6475702 .296187$

H $-8.600378-2.325137-2.661645$

H -7.583439 -1.553307 -3.887986

H -7.151750 -3.150551 -3.258128

C $-4.353165-2.379286-2.134112$

H $-5.9624213 .411237-1.905324$

H -5.5704874 .0970220 .703878$

H -5.5959431 .5898043 .047039$

H $-4.771208-3.274944-2.607433$

H -4.207986 -2.611973-1.073086

H $-5.280103-0.795039-3.302157$

C $-1.5592890 .130328 \quad 2.821963$

O $-2.7466520 .558757 \quad 3.499007$

C -2.9421471 .9758843 .545386$

C -1.7546402 .6420554 .251057$

C -0.4246502 .2635013 .606871$

C -0.3096230 .7460043 .468815$

H $-1.616400 \quad 0.410623 \quad 1.763019$

C -1.498671-1.404650 2.913515

H -3.8190392 .1423694 .182812$

O -1.9137224 .0569404 .250002$

H -1.742404 2.3219275 .301281

H -0.2958752 .7663842 .641604$

O $\quad 0.6216892 .7423164 .455709$

H $-0.144090 \quad 0.3033944 .459704$

O 0.8233750 .4315242 .663448

C -2.590556 -2.090548 2.121233

H $-0.541852-1.7740222 .528004$

H -1.611159-1.710609 3.960753

H -1.1329394 .4375704 .687551$

H 1.4690622 .5344504 .028436

H 1.6183690 .7117253 .147247

O $-2.556207-3.4295862 .391135$

O $-3.366455-1.5360701 .356673$

C $-3.536903-4.2205571 .712215$

H $-3.341766-4.2156540 .636075$

H $-3.457371-5.2475742 .078270$

H $-4.544099-3.8495871 .924845$

SCF Energy $\left(B 3 L Y P / 6-31 G^{* *} / / M M F F\right)=-3245.91676574$

0500213

MMFF Geometry

C $-3.193671-1.864816-3.394210$

C $-2.165417-2.157367-2.584318$

C $-0.728639-1.888888-2.942523$

O $-0.095830-1.123347-1.888037$

C $0.107329-3.160326-3.203140$

C $0.078388-4.232226-2.130351$

C $0.939499-4.304809-1.097090$

C $-0.948485-5.321119-2.320932$

C $2.052350-3.356243-0.750401$

C $3.411654-4.061529-0.827037$

C $4.612011-3.177268-0.452590$

C $4.580817-2.7352651 .012887$

C $5.771153-1.8304331 .361491$

O $4.634878-2.040695-1.322738$

O $5.502962-1.2203712 .633946$

C $5.841744-0.6927580 .322102$

C $7.071380-2.6247441 .528684$

O 7.0122690 .0988480 .516652

C $5.773619-1.186783-1.144152$

C $5.657030-0.007638-2.140464$

O $6.990403-1.879448-1.450281$

C $-0.4248380 .194862-1.796891$

O $\quad-1.255011 \quad 0.791259-2.465427$

C $0.4214300 .810269-0.749803$

C $0.1407892 .058896-0.347901$

C 0.8957012 .8684820 .610515

C 2.1492662 .2833641 .203674

C $4.3041920 .710377-2.131361$

C $4.3796422 .102724-2.736906$

C $3.0240124 .191312-2.922859$

O $5.3909842 .555771-3.265515$

C $3.1836304 .981309-1.659914$

C $2.2702135 .850374-1.200466$

C 2.4301136 .6261020 .077145

C 1.2467856 .4784941 .011256

C 0.4076207 .5051201 .229527 
C $1.0782995 .165847 \quad 1.751142$

C 0.4432464 .1092150 .889488

N $3.1766392 .783311-2.603502$

H -2.997161 -1.435019-4.373545

H -2.365661 -2.574950 -1.601571

H $-0.671656-1.288343-3.861163$

H $1.148431-2.862412-3.382244$

H $-0.224665-3.610993-4.148562$

H $0.834103-5.136925-0.401445$

H $-0.881993-6.094961-1.548502$

H -1.963842 -4.918068 -2.288852

H $-0.801978-5.812920-3.288357$

H $2.058344-2.475279-1.396652$

H $1.866311-2.9985950 .266825$

H $3.408993-4.952704-0.186486$

H $3.554925-4.410896-1.858598$

H $5.511841-3.775972-0.639218$

H $4.548565-3.6045231 .681123$

H $3.664635-2.1659121 .214716$

H $5.378233-1.9308833 .286300$

H $4.989755-0.0312800 .519266$

H $6.966262-3.3653492 .330684$

H $7.896828-1.9712061 .831417$

H $7.368715-3.1596750 .624545$

H 7.0124480 .3933651 .443483

H $6.4816320 .694128-1.962637$

H $5.818581-0.379559-3.162029$

H $7.101026-1.898433-2.415175$

H $1.2589870 .238059-0.369237$

H $-0.7255732 .556522-0.783659$

H 2.9371412 .2246260 .447813

H 1.9697051 .2787831 .599281

H 2.5422342 .8636112 .041404

H $3.5686910 .125898-2.696618$

H $3.9298400 .818821-1.110852$

H $2.0329824 .319588-3.369082$

H $3.7835854 .492777-3.650637$

H 4.099768 4.827588-1.093902

H $1.3631036 .022289-1.775142$

H $2.5699747 .681038-0.191693$

H 3.3418426 .3298100 .610820

H -0.4283147 .4153191 .915366$

H 0.5339658 .4567030 .723809

H 0.4323745 .3076922 .627274

H 2.0524294 .8676072 .147640

H $-0.492220 \quad 4.4317750 .429040$

H $2.4105652 .331293-2.114798$

C $-5.372126-0.741115-2.916570$

O $-4.888510-0.018791-1.764282$

C $-6.0144760 .512032-1.042376$

C $-7.2084490 .390649-1.980857$

C -6.878487 -0.897269-2.709105

H $-6.178223-0.153252-0.186411$

C $-5.7234231 .933148-0.549379$

H -8.159276 $0.349089-1.440973$

H -7.256395 $1.225709-2.689244$

C -7.663702 -1.086239-3.995371

H -7.080305 -1.742871 -2.037757

C -4.442956 2.0634890 .300751

O $-5.5734472 .788273-1.690077$

H -6.5904492 .3116510 .004979$

H -3.564089 $1.929023-0.341154$

O $-4.375236 \quad 3.4149960 .774699$

C -4.3480851 .1021631 .500491$

C -3.1047351 .3770882 .366217$

H -4.3178620 .0695481 .138028$

O $\quad-5.497436 \quad 1.2415472 .335206$

H $-2.228124 \quad 1.448806 \quad 1.714972$

H -3.217875 2.3468232 .866220

H -8.737987 -1.125599-3.787598

H $-7.487171-0.264710-4.697787$

H -7.381382 -2.020060 -4.491561

C -4.635193 -2.076835 -3.028196

H $-5.3859703 .683339-1.358928$

H -5.1081183 .5425991 .401394$

H $-6.266807 \quad 0.907574 \quad 1.845157$

H -5.094320 -2.702524 -3.801678

H $-4.706893-2.621734-2.078569$
H $-5.168590-0.112504-3.793636$

C -1.653669 -1.288547 2.109723

O $-2.825690-1.0242752 .886346$

C -2.8849930 .2924843 .447824$

C $-1.6646180 .536200 \quad 4.348319$

C $-0.353250 \quad 0.264222 \quad 3.612707$

C $-0.387012-1.1155392 .954477$

H $-1.625449-0.5900261 .265113$

C -1.788379 -2.678145 1.477478

H $-3.770696 \quad 0.308156 \quad 4.095397$

O -1.6870541 .8708274 .842335$

H -1.727558 -0.1317985 .217342$

H -0.1426541 .0446902 .874839$

$\begin{array}{lllll}\mathrm{O} & 0.730502 & 0.282947 & 4.543314\end{array}$

H $-0.335036-1.8810953 .738041$

O $0.758180-1.2751322 .123310$

C -1.681265 -3.813568 2.466840

H -2.750272 -2.776402 0.959292

H -1.009247 -2.824994 0.721786

H -0.9772491 .9544615 .500800$

H $\quad 0.823787 \quad 1.1949694 .866265$

H $1.543439-1.1302002 .678570$

O $-2.832212-3.919507 \quad 3.189054$

O $-0.687742-4.5197672 .583446$

C -2.845874 -4.955676 4.175497

H $-2.057173-4.7853944 .914570$

H -3.813714 -4.9289614 .683314$

H -2.725655 -5.933475 3.699565

SCF Energy (B3LYP/6-31G**//MMFF $)=-3245.91091556$

\section{4}

MM̄FF Geometry

C $2.137112-4.8050460 .745084$

C $0.892378-4.3070820 .724750$

C $0.011391-4.1654881 .935465$

O $-0.371895-2.7748262 .038636$

C $-1.277106-4.9895801 .752493$

C $-2.247768-4.8474542 .909865$

C $-3.468670-4.2829802 .818155$

C $-1.773925-5.3992084 .229976$

C $-4.142665-3.7276291 .593191$

C $-3.857915-2.2368371 .409650$

C $-4.739344-1.5589200 .349444$

C $-4.544232-2.149187-1.049545$

C $-5.324715-1.372937-2.122229$

O $-4.380787-0.1690290 .353120$

O $-4.827776-1.777821-3.407673$

C $-5.0158600 .130852-1.974188$

C $-6.817593-1.721011-2.106480$

O $-5.8212350 .910106-2.854716$

C $-5.1654000 .648248-0.527925$

C $-4.6849642 .110079-0.363652$

O $-6.5483280 .620872-0.155346$

C $0.389278-1.9484522 .803407$

O $1.406557-2.2320853 .413623$

C $-0.252881-0.6145542 .765819$

C 0.2494510 .4063343 .476503

C $-0.293021 \quad 1.7688273 .517966$

C - 1.5232932 .0450162 .693713

C $-3.2404182 .362232-0.797074$

C $-2.7238283 .724525-0.366653$

C $-0.6462705 .111595-0.477513$

$\begin{array}{lllll}\text { O } & -3.388247 & 4.525568 & 0.283841\end{array}$

C 0.3577324 .7580320 .573530

C 0.5604325 .4695001 .692853

C 1.6041435 .1143942 .714997

C 1.0726035 .0940574 .132690

C 1.5887525 .9102085 .067619

C -0.0510354 .1389964 .479416$

C 0.3261832 .6948524 .280391

N $-1.4201573 .926784-0.797056$

H $2.562380-5.1582891 .680534$

H $0.482201-3.957866-0.219720$

H $0.535655-4.4842812 .844458$

H $-1.026404-6.0521511 .635470$

H - $1.757084-4.6879160 .814848$

H -4.072406 -4.218440 3.722901

H $-2.562762-5.3922994 .989969$ 
H -1.445292 -6.436894 4.111590 H $-0.938525-4.8093074 .619074$ H -3.852642 -4.2919010 .701699$ H -5.222720 -3.886157 1.705604 H -3.995339 -1.7077112 .362689$ H -2.803919 -2.0887921 .146047$ H $-5.785192-1.6544940 .664688$ H $-4.828372-3.207442-1.077786$ H -3.478003 -2.118413 -1.309528 H -4.944023 -2.740384 -3.484551 H $-3.9767770 .269136-2.298275$ H $-6.963624-2.789683-2.304888$ H -7.354972 -1.193224 -2.902096 H -7.301067 -1.495672 -1.153736 H $-5.6960570 .555922-3.751841$ H $-5.3558582 .791046-0.903624$ H -4.7841372 .3825230 .696621$ H $-6.681172 \quad 1.2404490 .581190$ H -1.133653 -0.507566 2.142520 H 1.1389720 .2341544 .080679 H -2.3526531 .4018843 .008866$ H -1.315598 1.8578221 .636357 H -1.884366 3.0720042 .762745 H $-2.5817791 .604491-0.360988$ H -3.159635 2.305999-1.888243 H -1.311256 $5.921199-0.160867$ H $-0.1310565 .415784-1.393644$ H 0.9708693 .8800010 .388439 H $-0.026821 \quad 6.3675401 .868583$ H 2.4130585 .8510722 .624842 H 2.0591024 .1420642 .492598 H 1.2192115 .9084216 .087747 H 2.3933356 .6002464 .835846 H -0.9384084 .4319293 .913837$ H $-0.332145 \quad 4.2534125 .534740$ H 1.2042742 .3901664 .851518 H $-0.9481703 .181771-1.302281$ C $4.274603-4.040876-0.319213$ O $3.926204-2.663966-0.079447$ C $4.782119-1.815544-0.862659$ C $5.892746-2.718588-1.394400$ C $5.153658-4.031539-1.569301$ H $4.197621-1.472846-1.721460$ C $5.231390-0.603176-0.043105$ H $6.325459-2.339524-2.325175$ H $6.706029-2.850163-0.673093$ C $6.069628-5.236650-1.691183$ H $4.528828-3.963086-2.470069$ C $4.1508910 .124943 \quad 0.785513$ O $6.212611-1.0388290 .914187$ H $\quad \begin{array}{llll}5.737986 & 0.116369 & -0.698439\end{array}$ H $3.779030-0.5271871 .585012$ O 4.8401371 .1942551 .474109 C 2.9639310 .7810020 .051704 C $1.923285-0.183661-0.539450$ H $3.3367341 .461916-0.721132$ O $2.270875 \quad 1.590728 \quad 1.013605$ H $2.354154-0.718880-1.384067$ H $1.659492-0.9335330 .215084$ H $6.720763-5.137596-2.565835$ H $6.707540-5.347105-0.807944$ H $5.489420-6.157729-1.804770$ C $3.005471-4.878514-0.480465$ H $7.069640-1.0812490 .457160$ H 5.1845661 .8052320 .800150 H 2.9185082 .2069831 .395924 H $3.266956-5.927043-0.662094$ H $2.444150-4.527291-1.355592$ H $4.845302-4.3803100 .555253$ C $1.4089601 .344879-3.125733$ O $0.886231 \quad 1.654406-1.829420$ C $0.6220520 .517229-1.004144$ C $-0.390348-0.406968-1.693111$ C $0.038918-0.777756-3.110416$ C $0.4139340 .476032-3.903056$ H $2.3652240 .817141-3.022388$ C $1.6749692 .673535-3.838815$ H $0.1318560 .903070-0.102763$
O $-0.588046-1.579508-0.914365$

H -1.358412 $0.105152-1.748945$

H $0.859608-1.504457-3.094716$

O $-1.059933-1.427831-3.753598$

H $-0.4970931 .047008-4.121834$

O $0.9741110 .086866-5.155003$

C $2.8882883 .347663-3.242728$

H $1.8793612 .532099-4.905950$

H $0.8154893 .350587-3.762229$

H $-1.176330-2.169250-1.415468$

H $-0.789552-1.615775-4.668866$

H $1.789953-0.411147-4.975725$

O $2.508258 \quad 4.226877-2.273163$

O $4.0384853 .091361-3.575161$

C $3.5766794 .890929-1.591263$

H $4.1659654 .165245-1.022582$

H $3.1408575 .611875-0.894883$

H $4.2086905 .430761-2.303046$

SCF Energy (B3LYP/6-31G**//MMFF)= -3245.91059177

\section{5}

MM̄FF Geometry

C $0.455360-0.6203881 .832594$

C $1.137015-1.5411021 .132063$

C $0.686032-2.181055-0.160648$

O $-0.723234-1.959413-0.378977$

C $1.461218-1.527492-1.324142$

C $0.997317-1.947863-2.704845$

C $0.432559-1.109626-3.598421$

C $1.215149-3.395930-3.053956$

$\begin{array}{llll}\text { C } & 0.181791 & 0.364898 & -3.423410\end{array}$

C $-1.186106 \quad 0.625206-2.794218$

C -1.457542 $2.095963-2.446430$

C $-0.5439952 .605897-1.325672$

C $-0.9257194 .019024-0.860540$

O $-2.8278242 .149005-2.023654$

O $-0.227605 \quad 4.256542 \quad 0.371311$

C $-2.4371304 .051371-0.557658$

C $-0.4351345 .084933-1.845987$

O $-2.865013 \quad 5.372077-0.234965$

C $-3.3057803 .457125-1.685788$

C $-4.7912843 .315162-1.275678$

O $-3.260507 \quad 4.328925-2.820674$

C $-1.594701-2.9260070 .013373$

O $-1.330836-3.9790660 .569568$

C $-2.950031-2.468174-0.369811$

C $-4.013621-3.262923-0.178046$

C $-5.401066-2.939991-0.527218$

C $-5.660566-1.584861-1.133545$

C $-5.0264272 .444174-0.039346$

$\begin{array}{llll}\text { C } & -6.489922 & 2.090884 & 0.166934\end{array}$

C $-7.933800 \quad 0.715562 \quad 1.678791$

$\begin{array}{lllll}\text { O } & -7.399689 & 2.548699 & -0.517719\end{array}$

C $-7.814534-0.7463001 .973323$

C $-8.683320-1.6718861 .538970$

C $-8.537979-3.1376881 .839821$

C $-8.701042-4.0202380 .619272$

C $-9.606462-5.0136140 .614528$

C $-7.834544-3.765019-0.597603$

C $-6.360143-3.862624-0.299270$

N $-6.644366 \quad 1.198461 \quad 1.218729$

H $-0.530110-0.3025631 .502817$

H $2.134668-1.8169511 .465608$

H $0.901995-3.255603-0.125443$

H $2.528817-1.759995-1.233211$

H $1.391179-0.439354-1.216402$

H $0.119008-1.511080-4.560713$

H $0.922584-3.622547-4.084739$

H $2.273491-3.655155-2.949720$

H $0.629093-4.045082-2.396374$

H $0.9891310 .820783-2.844412$

H $\quad 0.2270050 .843840-4.409411$

H $-1.9715250 .270596-3.476091$

H $-1.3097790 .027471-1.884527$

H $-1.3413672 .699753-3.353711$

H $0.5092432 .588358-1.627963$

H $-0.616227 \quad 1.927064-0.463773$

H -0.5108195 .1237000 .708908$ 
H $-2.575600 \quad 3.4583240 .354292$ H $0.6563055 .037911-1.944673$ H $-0.657338 \quad 6.094277-1.483010$ H $-0.8632834 .974294-2.844442$ H -2.929585 5.873665 -1.065508 H $-5.2387534 .305957-1.123881$ H $-5.3383392 .873947-2.120711$ H $-4.0416574 .156606-3.372143$ H $-3.033806-1.482630-0.814343$ H $-3.857330-4.2442870 .267883$ H -5.142979 -1.488960 -2.094516 H -5.304602 $-0.795438-0.464685$ H $-6.714870-1.376202-1.321124$ H $-4.461626 \quad 1.508942-0.123715$ H -4.6886242 .9703710 .860412$ H -8.7053640 .9307790 .932936$ H $-8.172463 \quad 1.2622132 .596475$ H -6.969428 -1.061643 2.581681 H -9.546656 -1.362767 0.954747 H -9.291450 -3.393498 2.595842 H -7.563533 -3.348089 2.298116 H -9.739729 -5.654863 -0.250495 H - $-10.236251-5.2106201 .475920$ H -8.120701 -2.802652 -1.028323 H -8.053044 -4.506169-1.377985 H $-6.063587-4.8213310 .128768$ H -5.8314340 .9787891 .788077$ C 1.3357431 .5518092 .666941 O 2.3590921 .5735281 .652093 C 3.3613172 .5404492 .011296 C 2.7284373 .4025083 .094536 C 1.8878002 .3736103 .830989 H 4.1945181 .9808692 .453085 C 3.8498173 .2790590 .759197 H 3.4748243 .8851473 .732347 H 2.0850524 .1823202 .671256 C $0.815342 \quad 2.9836374 .715997$ H $2.552036 \quad 1.7532544 .447781$

C $4.2343322 .343197-0.407761$ O $2.814768 \quad 4.1472350 .283354$ H 4.6917943 .9309461 .017417 H $3.3369431 .844679-0.795369$ O $4.7388953 .149062-1.479368$ C $5.2937821 .286096-0.048264$ C $5.5984450 .351337-1.230604$ H $4.925990 \quad 0.685514 \quad 0.784728$ O $\quad 6.480075 \quad 1.947104 \quad 0.382159$ H $4.660483-0.128850-1.531676$ H $5.9421870 .945278-2.086073$ H 1.2676453 .6071415 .493976 H 0.1282603 .6116014 .138897 H $\quad 0.2261732 .2033685 .207924$ C 1.0173160 .0985443 .028529 H $2.0245343 .603037 \quad 0.123592$ H $4.0591363 .812948-1.687054$ H 7.0568921 .2811310 .792703 H $\quad 0.285791 \quad 0.0552003 .842847$ H $1.925797-0.4070413 .376578$ H 0.4520092 .0399302 .235504

C $5.334967-2.2907790 .324686$

O $6.463050-1.4098490 .300545$

C $6.673729-0.720620-0.935483$

C $6.862824-1.732776-2.073098$

C $5.696190-2.714366-2.154108$

C $5.437416-3.346571-0.787198$

H $4.415346-1.7063870 .210134$

C $5.308576-2.9796111 .701342$

H $7.624544-0.184253-0.824449$

O $7.016142-1.052040-3.314186$

H $7.788941-2.295978-1.898468$

H $4.797009-2.228931-2.550672$

O $6.044804-3.737929-3.089277$

H $6.244963-4.054092-0.557710$

O $4.219405-4.087610-0.831116$

C $4.932744-2.0501432 .835684$

H $4.573091-3.7919161 .700341$

H $\quad 6.300575-3.3877451 .929151$

H $7.109677-1.728219-4.006994$
H $5.260234-4.297146-3.218520$

H $4.341517-4.820207-1.457842$

O $4.895536-2.7678323 .998419$

O $4.700125-0.8552942 .729559$

C $4.547023-2.0223415 .169366$

H $4.566118-2.703666 \quad 6.024197$

H $5.274286-1.2230615 .341165$

H $3.537693-1.6121575 .070182$

SCF Energy (B3LYP/6-31G**//MMFF) $=-3245.92834708$

\section{6}

MMFF Geometry

C $0.5578595 .377053 \quad 0.327637$

C $1.4336724 .690759-0.421566$

C 2.5756833 .8954810 .160082

O $2.547207 \quad 2.556403-0.386727$

C $3.9096714 .548514-0.248021$

C $5.125766 \quad 3.9413820 .423592$

C $6.1293063 .318593-0.224682$

C $5.1960894 .102969 \quad 1.921058$

C $6.276706 \quad 3.077607-1.700590$

C $5.7202971 .731117-2.190226$

C $6.3800650 .502354-1.542639$

C $5.948598-0.782772-2.252858$

C $6.507837-2.038731-1.564357$

O $5.9752830 .445561-0.172614$

O $5.798247-3.176463-2.079145$

C $6.204332-1.965530-0.051435$

C $7.986632-2.261411-1.903318$

O $6.848939-3.0256740 .649422$

C $6.579030-0.6115860 .583876$

C $6.091904-0.4630762 .046345$

O $8.005699-0.4796220 .603928$

C 1.6330681 .6866710 .123941

$\begin{array}{lllll}\text { O } & 0.813566 & 1.908479 & 1.000914\end{array}$

C $1.7965330 .399061-0.587420$

C $0.832890-0.531288-0.511701$

C $0.855201-1.848773-1.153125$

C $2.149727-2.292898-1.781370$

C $4.576585-0.5704222 .249943$

C $4.103587-1.9636672 .629047$

C $2.017670-3.2901262 .934889$

O $4.865205-2.8671472 .963178$

C $1.812988-4.1118231 .701627$

C $0.629450-4.6418951 .358283$

C $0.421021-5.4991680 .143071$

C $-0.742557-5.036039-0.704661$

C $-1.945801-5.624012-0.596253$

C $-0.487192-3.960893-1.737525$

C $-0.264663-2.601965-1.129319$

N $2.719405-2.0682442 .589151$

H $\quad 0.6640605 .372290 \quad 1.409828$

H $1.3355824 .699588-1.504597$

H 2.4966123 .8402831 .252535

H 3.8922125 .6179410 .002425

H $4.0052914 .494066-1.337981$

H $6.9570732 .932962 \quad 0.369909$

H 6.1676833 .7998712 .326344

H 5.0408395 .1506472 .198680

H 4.4327643 .4922402 .412607

H $5.8115883 .885819-2.274979$

H $7.3451313 .133446-1.946142$

H $4.6395981 .709066-2.006659$

H $5.8643021 .687073-3.276840$

H $7.4682060 .623063-1.599658$

H $6.243613-0.761707-3.309234$

H $4.852329-0.846141-2.251535$

H $5.918204-3.193042-3.044076$

H $5.125673-2.1251250 .063096$

H $8.117418-2.380180-2.985695$

H $8.360060-3.188222-1.454215$

H $8.628712-1.438313-1.583180$

H $\quad 6.568647-3.859636 \quad 0.235054$

H $6.639370-1.1545432 .698193$

H 6.3828250 .5427482 .382536

H 8.2453280 .1784251 .277396

H $2.7039000 .255998-1.162608$

H $-0.065632-0.3054890 .061417$ 
H $2.973734-2.195825-1.066644$ H $2.375984-1.687157-2.665309$ H $2.148613-3.338925-2.094533$ H $4.292213 \quad 0.0829213 .084941$ H $4.034063-0.2239971 .365232$ H $1.071319-3.0011023 .402667$ H $2.610447-3.8561103 .660743$ H $2.681954-4.3005781 .075413$ H $-0.232016-4.4780102 .001965$ H $\quad 0.243953-6.5264730 .487883$ H $1.325727-5.547006-0.475676$ H $-2.779218-5.325598-1.221543$ H $-2.125305-6.4157250 .123604$ H -1.349653 -3.873502 -2.412067 H $\quad 0.337739-4.277664-2.381812$ H -1.150977 -2.193713 -0.642750 H $2.169724-1.2860722 .245479$ C -1.9293155 .6644330 .247601$ O $-2.1991504 .385890-0.362928$ C $-3.5677644 .366235-0.822978$ C $-4.2377475 .570990-0.180938$ C $-3.0973616 .567167-0.146823$ H -3.517096 4.493295-1.911718 C $-4.1984673 .013805-0.468880$ H $-5.1009145 .927355-0.750835$ H -4.5715925 .3355680 .837488$ C $-3.3336607 .726654 \quad 0.805442$ H $-2.9437696 .960393-1.160857$ C $-5.7099252 .930684-0.750957$ O $-3.5183502 .001255-1.218704$ H -3.9999662 .7969760 .586441$ H -6.246767 $3.633174-0.102235$ O $-5.9311243 .365689-2.098512$ C $-6.3158841 .521226-0.603148$ C -6.0584590 .8843120 .772568$ H $-5.945148 \quad 0.869782-1.402904$ O $\quad-7.7331391 .623272-0.777659$ H -4.9809650 .8097600 .944063$ H -6.4647521 .5409361 .552067$ H -4.2203888 .2949540 .506185$ H -3.4889747 .3777961 .831911$ H -2.479464 8.4108000 .807963 C $-0.5758176 .187423-0.234669$ H -3.709289 2.154639 -2.159961 H $-6.8861443 .510855-2.206437$ H $-7.8993941 .828753-1.713031$ H -0.4369897 .2263070 .085402$ H $-0.5417336 .173741-1.331154$ H -1.917895 5.5031381 .333528 C $-5.005873-1.808826-0.196729$ O $-6.368643-1.376272-0.171130$ C $-6.702133-0.5142530 .921068$ C $-6.402583-1.2111962 .256786$ C $-4.965116-1.7281882 .324261$ C $-4.635485-2.5624141 .085027$ H $-4.355908-0.929518-0.290914$ C $-4.779838-2.618183-1.481640$ H $-7.788707-0.368087 \quad 0.873327$ O $-6.657569-0.3162613 .334149$ H -7.088547 -2.060717 2.372417 H $-4.247238-0.9084742 .445067$ O $-4.815027-2.5675123 .471515$ H -5.167152 -3.518482 1.146844 O $-3.240555-2.8533581 .072833$ C $-5.616326-3.876455-1.597724$ H $-5.038593-1.994173-2.345843$ H $-3.722570-2.894575-1.555619$ H -6.586411 -0.8215974 .161172$ H -4.913855 -2.007502 4.259685 H $-3.030838-3.3096851 .905741$ O $-5.130063-4.633104-2.627018$ O $-6.584377-4.153966-0.904107$ C $-5.823015-5.861554-2.868863$ H $-5.771836-6.505590-1.985884$ H $-6.863155-5.661741-3.143159$ H -5.331772 -6.370406 -3.702563

SCF Energy (B3LYP/6-31G**//MMFF)= -3245.89956406

\section{7}

MMFF Geometry

C $-0.457313 \quad 4.737343 \quad 0.248207$

C $-0.8473794 .569297-1.025337$

C -1.945495 $3.646665-1.503598$

O $-2.1693222 .562682-0.573117$

C $-3.2556684 .440594-1.652796$

C $-4.3826693 .654428-2.292924$

C $-5.5380963 .332606-1.678996$

C $-4.1660803 .253407-3.729979$

C $-5.9667513 .654422-0.274717$

C -5.6409892 .5660060 .760612$

C $-6.327148 \quad 1.2161720 .492929$

C -6.1438720 .2681681 .680288$

C $-6.738121-1.1228651 .403849$

O

O $-6.239128-2.0221672 .406312$

C $-6.220202-1.6347120 .041205$

C -8.265436-1.125896 1.541395

O $-6.878048-2.842333-0.332691$

C $-6.344392-0.596296-1.091759$

C $-5.641143-1.028597-2.401641$

O $-7.729618-0.405422-1.405590$

C $-1.2909981 .521780-0.611543$

O $-0.3274831 .398785-1.351211$

C -1.6935850 .5540420 .432786$

C $-0.839689-0.412510 \quad 0.802281$

C $-1.079061-1.4313881 .827516$

C $-2.469820-1.5339882 .394519$

C $-4.137347-1.300867-2.283567$

C $-3.792127-2.766856-2.080618$

C $-1.851646-4.258968-1.621569$

O $-4.613811-3.674045-2.178498$

C $-1.840918-4.591496-0.163034$

C $-0.750235-5.034840 \quad 0.479571$

C $-0.725183-5.4073541 .933602$

C $0.332458-4.6522832 .706133$

C $1.533466-5.2015862 .953874$

C $-0.034223-3.2940693 .260158$

C $-0.052880-2.2233262 .202573$

N - $2.441472-2.947318-1.813457$

H $-0.9781574 .207343 \quad 1.040177$

H $-0.3362095 .126426-1.808095$

H -1.644739 $3.227600-2.471535$

H -3.081844 $5.336355-2.264434$

H $-3.5547904 .804804-0.664080$

H -6.278362 $2.772160-2.249202$

H $-5.0675372 .826215-4.182509$

H $-3.8824244 .124599-4.329636$

H $-3.3747002 .501670-3.806685$

H -5.5307764 .6025340 .057330$

H -7.050451 $3.828313-0.283686$

H -4.5542542 .4271350 .797699$

H -5.9528592 .9386131 .744354$

H -7.3928341 .4007550 .313109$

H -6.5797710 .6962742 .591521$

H -5.0718630 .1519801 .888998$

H -6.494057 -1.671766 3.276937

H $-5.161727-1.8853130 .178760$

H $-8.559086-0.8229162 .553791$

H $-8.673119-2.1331531 .401513$

H -8.760754 -0.451811 0.839756

H $-6.755170-3.4764170 .394354$

H $-6.172703-1.874806-2.853201$

H $-5.755139-0.200989-3.116924$

H $-7.794704-0.034419-2.301205$

H -2.672996 0.6742950 .880539

H $0.139715-0.4509330 .326570$

H $-3.202478-1.6449541 .588069$

H -2.716865 -0.638165 2.974305

H $-2.613228-2.3946283 .050676$

H -3.657199-1.003672 -3.224885

H -3.688196 -0.703252 -1.484572

H $-0.843880-4.227316-2.047403$

H $-2.438679-5.005777-2.165663$

H $-2.775017-4.4848150 .383334$

H $0.174715-5.167719-0.076924$

H $-0.535446-6.4867901 .998415$ 
H $-1.703425-5.2517702 .405264$

H $2.288213-4.6761283 .529612$

H $1.791049-6.1871662 .580075$

H $0.702454-2.9862894 .014520$

H -0.977152 -3.376685 3.806900

H $\quad 0.915110-2.0735141 .723942$

H -1.843037 -2.134809-1.695301

C 1.8147904 .8935371 .376204

$\begin{array}{lllll}\text { O } & 2.246231 & 3.758588 & 0.597387\end{array}$

C 2.3363232 .6134861 .473289

C 2.4315433 .1890812 .876431

C 1.4805764 .3655552 .772816

H 1.3927272 .0662701 .349415

C $3.513807 \quad 1.7384971 .034192$

H 2.1397942 .4736483 .650663

H 3.4509843 .5344673 .088226

C 1.6593395 .3929923 .877252

H 0.4533053 .9838092 .811750

C 3.8580510 .6063432 .017404

O $3.1742801 .161626-0.232412$

H 4.3953032 .3672430 .864959

H 4.2591881 .0319442 .944791

O $2.643492-0.0644432 .373701$

C $4.829335-0.4538881 .464540$

C 6.1695440 .1192860 .974186

H $4.333199-1.0136710 .665389$

O $5.089184-1.4034862 .505253$

H 5.9878060 .8996260 .230460

H $6.685516 \quad 0.593117 \quad 1.818251$

H 1.4641794 .9428974 .856206

H 2.6776645 .7958533 .890690

H 0.9667426 .2301563 .745357

C 0.6831915 .6316570 .652652

H $2.9502451 .892078-0.834403$

H $2.876073-0.7800452 .989956$

H $5.537931-0.935243 \quad 3.229913$

H $1.1047876 .106109-0.242946$

H $\quad 0.298127 \quad 6.438597 \quad 1.286176$

H 2.6792695 .5678201 .434544

C $6.167764-0.992662-1.857635$

O $6.433389-1.720235-0.652470$

C $7.091312-0.9662730 .371186$

C $8.438403-0.443591-0.148046$

C $8.2879570 .320086-1.463405$

C $7.479949-0.497011-2.473087$

H $5.513446-0.139551-1.640750$

C $5.417119-1.932841-2.807087$

H $7.313689-1.6757741 .178171$

$\begin{array}{lllll}O & 9.048151 & 0.382216 & 0.838016\end{array}$

H $9.107372-1.298501-0.312348$

H $7.8326251 .305078-1.305998$

O $9.5806730 .557807-2.024896$

H $8.079588-1.347101-2.822780$

O $7.189075 \quad 0.319067-3.605984$

C $3.964062-2.034514-2.406082$

H $5.433506-1.565996-3.839740$

H 5.853282 -2.938709 -2.816279

H 9.9470830 .5913420 .533743

H $\quad 10.049257 \quad 1.170883-1.433838$

H $8.0374530 .637018-3.959695$

O $3.809780-2.973226-1.429975$

O $3.077438-1.339761-2.886308$

C $2.479556-3.118731-0.923496$

H $1.826570-3.529801-1.698757$

H $2.511517-3.815489-0.081977$

H $2.097944-2.157431-0.566663$

SCF Energy (B3LYP/6-31G**//MMFF) $=-3245.90299393$

\section{8}

MMFF Geometry

C $4.299707-3.5179831 .702365$

C $3.164690-3.4694710 .988355$

C $1.792047-3.5959781 .597120$

O $1.032658-2.3957121 .331201$

C $1.051708-4.7814940 .947463$

C $-0.333307-5.0289321 .516335$

C -1.492960 -4.819238 0.860009

C $-0.358645-5.5909502 .915787$
C $-1.695734-4.284986-0.532177$

C $-2.368885-2.910250-0.535771$

C $-3.866848-2.895818-0.189200$

C $-4.712896-3.505019-1.312834$

C $-6.219761-3.327288-1.077190$

O $-4.212152-1.5165230 .000044$

O $-6.876628-3.640182-2.315220$

C $-6.500247-1.844612-0.753024$

C $-6.746787-4.333039-0.047389$

O $-7.872024-1.650893-0.416818$

C $-5.581396-1.2770560 .348349$

C -5.7603770 .2471330 .533577$

O $-5.914347-1.8963821 .595942$

C $1.203945-1.3490862 .186084$

O $2.042460-1.2462173 .066676$

C $0.130361-0.3696351 .906447$

C -0.0380170 .6955762 .704654$

C -1.1307791 .6691232 .614584$

C -2.2246901 .3832381 .620673$

C $-5.3359321 .094994-0.669370$

C $-5.2314142 .567395-0.307985$

C $-4.0781664 .659293-1.021100$

O $-5.795022 \quad 3.0597450 .666007$

C -2.824873 $4.714440-0.203896$

C -2.6906795 .4358200 .919385$

C -1.4284925 .4728491 .735490$

C -1.6671815 .1801113 .201203$

C -1.4439156 .1206694 .134782$

C -2.1731983 .8076423 .589739$

C -1.1271712 .7349663 .443116$

N $-4.4455373 .267886-1.212865$

H $4.250109-3.6257582 .782972$

H $3.219622-3.352831-0.091677$

H $1.856819-3.7542992 .681005$

H $1.641297-5.6995271 .076859$

H $1.001580-4.621456-0.134214$

H -2.418393 -5.064542 1.379811

H -1.371188 -5.8522583 .241602$

H $\quad 0.246878-6.5015192 .970665$

H $\quad 0.037740-4.8638563 .630817$

H $-0.747232-4.191274-1.066960$

H -2.284977 -5.009172 -1.104341

H -1.836606 -2.244087 0.153590

H -2.237620 -2.456965 -1.527298

H $-4.029869-3.4250570 .755329$

H $-4.478268-4.566288-1.455362$

H -4.456185 -3.015111 -2.262977

H -7.825733 -3.461638 -2.198330

H $-6.335219-1.283942-1.681487$

H $-6.583050-5.358656-0.399909$

H $-7.829446-4.2345860 .086986$

H -6.266652 -4.239369 0.928799

H -8.001414 -1.966912 0.493543

H $-6.797237 \quad 0.4767630 .811312$

H $\quad-5.1600450 .558758 \quad 1.399264$

H $-5.589673-1.3323002 .317198$

H $-0.532357-0.5812631 .076574$

H 0.6663710 .8550323 .519853

H $-2.6860770 .411326 \quad 1.828201$

H $-1.827711 \quad 1.3707390 .601424$

H -3.0280672 .1196691 .632429$

H $-4.3610880 .760332-1.041469$

H $-6.0644121 .002009-1.481587$

H $-4.9072235 .192174-0.544351$

H $-3.8997165 .090794-2.010553$

H $-1.9828754 .125821-0.558131$

H -3.524561 6.0420251 .265840

H -0.9862116 .4700141 .613934$

H $-0.6853944 .766909 \quad 1.348742$

H -1.6134875 .9239235 .188234$

H -1.0855417 .1094343 .868066$

H -3.090438 3.5899523 .037223

H -2.4828083 .8074284 .643809$

H $-0.2913762 .848257 \quad 4.135138$

H $-3.9636952 .755303-1.945902$

C $6.328625-2.0961821 .253710$

O $5.637400-1.1501570 .409683$

C $6.553070-0.685936-0.594666$ 
C $7.915845-0.771313 \quad 0.071994$

C $7.792937-2.1020860 .790842$

H $6.514542-1.406561-1.421806$

C $6.147666 \quad 0.696371-1.112275$

H $8.739079-0.746783-0.647937$

H $8.064650 \quad 0.0357780 .798559$

C $8.786855-2.2755211 .926898$

H $7.954755-2.9078210 .061933$

C $4.664256 \quad 0.804642-1.528510$

$\begin{array}{lllll}\text { O } & 6.378498 & 1.657032 & -0.074741\end{array}$

H $6.8038920 .976558-1.945110$

H $4.0418080 .852151-0.629128$

O $4.4931332 .044364-2.223490$

C $4.166253-0.319357-2.450167$

C $2.722735-0.165725-2.969295$

H $4.236741-1.282523-1.933848$

O $5.028193-0.405107-3.589859$

H $2.6405120 .757496-3.552516$

H $2.563506-0.973085-3.695616$

H $9.813837-2.2376711 .549167$

H $8.676286-1.4885242 .680327$

H $8.642871-3.2406132 .423338$

C $5.671913-3.4693761 .092534$

H $6.3175722 .541510-0.472224$

H $4.6728282 .761516-1.594628$

H $4.9989000 .454720-4.043928$

H $6.283669-4.2404011 .575615$

H $5.610082-3.7290440 .027680$

H $6.236128-1.7352662 .285183$

C $1.0824872 .109847-1.700964$

$\begin{array}{llll}\text { O } & 1.577688 & 0.931994-1.054387\end{array}$

C $1.643453-0.242866-1.867666$

C $0.239036-0.567502-2.392433$

C $-0.3437720 .603060-3.174987$

C $-0.2993401 .871755-2.328161$

H $1.7954752 .436863-2.465848$

C $0.9840823 .210606-0.638833$

H $1.931735-1.064231-1.199758$

O $0.250883-1.728644-3.214606$

H $-0.423835-0.780248-1.544901$

H $0.1695390 .746897-4.132724$

O $-1.7116010 .326272-3.486511$

H $-1.0605201 .801144-1.542074$

O $-0.6419442 .993230-3.140980$

C $2.333515 \quad 3.637017-0.115400$

H $0.4710494 .098349-1.026601$

$\begin{array}{lllll}\mathrm{H} & 0.404828 & 2.865263 & 0.224488\end{array}$

H $0.609973-2.459217-2.682377$

H -1.731516 $-0.512652-3.978401$

H $-1.5146732 .813166-3.530687$

O $2.7835684 .717959-0.813490$

O $2.912634 \quad 3.079527 \quad 0.807906$

C $4.0163025 .285920-0.358225$

H $4.7815404 .517290-0.218300$

H $4.3657645 .990913-1.117136$

H 3.8458425 .8287680 .575936

SCF Energy (B3LYP/6-31G**/MMFF) $=-3245.91435422$

0500219

MM̄FF Geometry

C $-4.699602 \quad 2.744110-0.014375$

C $-3.398967 \quad 3.024993 \quad 0.151351$

C $-2.780132 \quad 3.4708971 .447704$

O $-1.7356882 .528593 \quad 1.783400$

C $-2.121553 \quad 4.852271 \quad 1.269276$

C -1.414133 5.3474382 .516900

C -0.0843905 .5489912 .612153$

C -2.3022895 .6308343 .701179$

C 0.9567995 .3783361 .539342

C 1.5643253 .9759611 .555592

C $2.794567 \quad 3.8157920 .649465$

C $2.480664 \quad 4.057360-0.829617$

C $3.6878333 .764888-1.734410$

O $3.263525 \quad 2.472345 \quad 0.838910$

O $3.196063 \quad 3.714043-3.082728$

C $4.2508572 .371978-1.382327$

C $4.7091824 .907233-1.693480$

O $5.4482462 .106802-2.108565$
C $4.4717622 .160556 \quad 0.131076$

C $4.8588230 .704330 \quad 0.481061$

O $\begin{array}{lllll}5.551564 & 2.994963 & 0.567120\end{array}$

C -2.044053 1.4875132 .601011

$\begin{array}{llll}\text { O } & -3.134128 & 1.216936 & 3.076609\end{array}$

C -0.7933270 .7255392 .819755$

C $-0.776844-0.3420113 .631635$

C $0.391314-1.1822603 .916564$

C $1.682798-0.8218553 .229747$

C $3.862701-0.3553520 .007901$

C $4.130221-1.7252990 .608619$

C $3.211187-4.0528700 .560702$

O $5.025651-1.9459351 .418579$

C $1.997926-4.2655171 .408641$

C $2.013345-4.8398112 .621010$

C $0.772706-5.0520543 .443738$

C $0.937919-4.6417054 .892509$

C $0.767128-5.5379345 .879529$

C $1.302434-3.2063215 .210500$

C $0.253270-2.2245724 .763181$

N $3.231842-2.6675410 .129931$

H -5.3860892 .8478490 .821493$

H -2.727005 $2.915347-0.696519$

H $-3.528705 \quad 3.5127682 .248102$

H -2.880434 $5.592008 \quad 0.982062$

H -1.4192114 .7997920 .430049$

H $\quad 0.315325 \quad 5.8900523 .566458$

H $-1.7587776 .110493 \quad 4.522278$

H $-3.1161156 .304008 \quad 3.412510$

H -2.735275 4.7048214 .091152

H 0.5409745 .6124410 .554903

H $1.743136 \quad 6.1234381 .713539$

H 1.8525573 .7048892 .580679

H $\quad 0.8070923 .238757 \quad 1.265170$

H 3.5664784 .5119750 .998142

H $2.1295135 .081521-1.000567$

H $1.6528743 .403405-1.134818$

H $3.9388173 .461435-3.657919$

H $3.5128511 .646322-1.743775$

H $4.2463675 .843191-2.029270$

H $5.5399764 .722723-2.383464$

H $5.1197905 .079958-0.696578$

H $6.1687652 .601619-1.682455$

H 5.8567810 .4737690 .085705

H 4.9535920 .6323001 .573696

H 5.9053852 .6316471 .395982

H $\quad 0.092722 \quad 1.0672742 .297077$

H $-1.700486-0.6342074 .129056$

H 2.0014670 .1883453 .509932

H $1.554050-0.8617372 .143880$

H $2.513928-1.4872543 .467506$

H $2.844225-0.0637140 .284413$

H $3.910611-0.454757-1.082228$

H $4.137268-4.2980461 .089621$

H $3.145337-4.669560-0.340880$

H $1.048566-3.9425310 .990197$

H $2.955052-5.1964723 .030868$

H $0.516372-6.1175183 .380284$

H $-0.080070-4.5102273 .016416$

H $0.882696-5.2612956 .922285$

H $0.507256-6.5700855 .668919$

H $2.290157-2.9974964 .793472$

H $1.419025-3.0778936 .295016$

H $-0.724177-2.3966995 .215986$

H $2.519733-2.373898-0.533200$

C $-5.8565260 .859541-1.201186$

O $-4.831306-0.064549-0.789547$

C $-4.945549-1.272306-1.559580$

C $-6.277626-1.177493-2.300472$

C $-6.3927380 .317441-2.525827$

H $-4.146608-1.259279-2.306522$

C $-4.784176-2.496308-0.656725$

H $-6.275356-1.753968-3.230614$

H -7.117386 -1.528905 -1.691019

C $-7.8014660 .777545-2.857847$

H $-5.7205220 .598213-3.347883$

C $-3.613639-2.4955790 .349221$

O $-5.982076-2.6362840 .129335$ 
H $-4.734831-3.398403-1.277742$ H $-3.767639-1.7333591 .123129$ O $-3.692098-3.762210 \quad 1.040247$ C $-2.173320-2.402755-0.193008$ C $-1.753028-1.028663-0.742463$ H -2.014982 -3.180642 -0.947952 O $-1.291378-2.7179260 .895278$ H $-2.264235-0.841025-1.685648$ H $-2.075088-0.248896-0.042225$ H $-8.153500 \quad 0.303531-3.779904$ H $-8.505770 \quad 0.522631-2.058823$ H -7.834018 $1.862016-3.002048$ C $-5.2690792 .266474-1.321767$ H $-6.081891-1.8251310 .657163$ H -4.577999 $-3.819717 \quad 1.437760$ H -1.468692 -2.0849481 .611025$ H $-6.0435402 .971663-1.643444$ H -4.486652 2.271329 -2.091372 H $-6.6346730 .836434-0.427016$ C $-0.025627-2.044534-3.062098$ O $0.329546-1.985326-1.678587$ C $-0.226988-0.880263-0.962445$ C $0.1922400 .442695-1.620020$ C $-0.1430040 .476076-3.109596$ C $0.393094-0.772925-3.810998$ H - $-1.114484-2.158678-3.141523$ C $0.567379-3.331927-3.654704$ H $0.236552-0.8987620 .031195$ O $-0.4277131 .548674-0.978372$ H $\quad 1.2751270 .571981-1.508152$ H $-1.2222980 .580378-3.268472$ O $\quad 0.4475861 .635801-3.702891$ H $1.483419-0.699274-3.884816$ O $-0.117497-0.823281-5.141179$ C $2.078364-3.431210-3.581747$ H $0.173899-4.188498-3.093377$ H $0.264986-3.429506-4.703320$ H $-0.180507 \quad 1.518357-0.039029$ H $1.4076911 .596237-3.553263$ H $\quad 0.1264970 .010952-5.577834$ O $2.475537-4.560215-4.242063$ O $2.818302-2.643691-3.009858$ C $3.885145-4.807877-4.241079$ H $4.247337-4.931105-3.215836$ H $4.067923-5.734524-4.791537$ H $4.413300-3.991329-4.742653$

SCF Energy (B3LYP/6-31G**//MMFF) $=-3245.93307810$

0500220

MMFF Geometry

C $3.926227-0.3768452 .797955$

C $3.490895-0.3382484 .066797$

C $2.039158-0.4082504 .478835$

$\begin{array}{lllll}\text { O } & 1.190125 & 0.255131 & 3.515078\end{array}$

C $1.597011-1.8802294 .586268$

C $0.118565-2.0548234 .873100$

C $-0.793040-2.5022763 .984937$

C $-0.304712-1.708768 \quad 6.277641$

C $-0.579328-2.9075072 .550941$

C $-1.273758-1.9802451 .548151$

C $-2.810040-1.9538721 .594280$

C -3.422223 -3.314708 1.247762

C $-4.953852-3.257197 \quad 1.150367$

O $-3.228118-0.9684560 .635564$

O $-5.378851-4.4787130 .526602$

C $-5.349349-2.088217 \quad 0.224629$

C $-5.603281-3.2374422 .538870$

O $-6.764228-1.918577 \quad 0.195990$

C $-4.646915-0.760456 \quad 0.578437$

C $-4.9236230 .347301-0.463435$

O $-5.147243-0.2859091 .834265$

C 1.0767881 .6082853 .616841

O $1.703868 \quad 2.3465134 .359412$

C 0.0126762 .0355162 .682562

C -0.3039353 .3337472 .567800$

C $-1.372618 \quad 3.8839101 .728867$

C -2.2669912 .8997611 .022055$

C $-4.4910630 .010672-1.891137$
C $-4.439024 \quad 1.248717-2.766599$

C $-3.0441223 .250200-3.245148$

O $-5.271194 \quad 1.457461-3.646017$

C $-2.0789034 .096013-2.478937$

C $-2.2881005 .384595-2.170912$

C $-1.3133246 .211744-1.380186$

C -1.951333 $6.884557-0.182699$

C $-1.9896788 .224606-0.088539$

C -2.5524086 .0145590 .901656$

C -1.5137395 .2242351 .652439$

N $-3.3739192 .079041-2.454530$

H $3.200139-0.4791911 .994849$

H $4.215896-0.2837474 .875267$

H 1.9210360 .0814565 .453670

H $2.170466-2.3791635 .379311$

H $1.870927-2.4079783 .667485$

H -1.822460 -2.603161 4.326540

H -1.365402 -1.915188 6.455940

H $0.269788-2.2948287 .002409$

H -0.138956 -0.645869 6.478299

H $0.482413-2.9328482 .292228$

H $-0.929714-3.9376802 .424955$

H $-0.898013-0.9584881 .678233$

H $-0.959986-2.2651910 .536882$

H -3.129825 -1.624065 2.588367

H $-3.126715-4.0811681 .973317$

H -3.026045 -3.6518880 .279240$

H $-6.340635-4.4216230 .392245$

H $-5.060257-2.387666-0.790449$

H -5.331904 -4.141046 3.098178

H $-6.696335-3.2498532 .466765$

H -5.306104 -2.375435 3.139780

H -7.026088 -1.4728191 .019574$

H $-5.9889530 .613098-0.458236$

H $-4.4059801 .257192-0.135819$

H $-4.9712590 .667675 \quad 1.895205$

H -0.5041491 .2622902 .127867$

H $\quad 0.2612574 .062747 \quad 3.146813$

H -2.7496872 .2313531 .744171$

H -1.6896572 .2930290 .316502$

H -3.0694283 .3676370 .450378$

H -3.502857 -0.461592 -1.908891

H -5.191382 -0.696857 -2.348864

H -3.967505 $3.782156-3.497397$

H -2.584326 $2.902566-4.176032$

H $-1.1549213 .621036-2.157856$

H -3.200112 $5.872937-2.505865$

H $-0.8906976 .963043-2.059644$

H -0.465331 $5.605084-1.039185$

H $-2.445440 \quad 8.7194820 .762697$

H $-1.5658978 .858995-0.860022$

H -3.334026 5.3880810 .465588

H -3.0755626 .6386321 .638911$

H -0.8167795 .8496212 .212277$

H -2.668664 $1.724432-1.815220$

C $5.6191710 .582247 \quad 1.211072$

$\begin{array}{llll}\text { O } & 4.946700 & 0.040154 & 0.056143\end{array}$

C $5.892443-0.098686-1.016826$

C $7.0203550 .862999-0.676961$

C 7.0987570 .6898210 .829089

H $6.275373-1.125736-0.972763$

C $5.1963080 .131144-2.361811$

H $7.9538090 .610625-1.188417$

H $6.7616551 .898997-0.923938$

C 7.8320621 .8166791 .535852

H $7.612411-0.2565971 .045732$

C $3.902321-0.693677-2.544074$

O $4.8419521 .513927-2.482990$

H $5.895109-0.070319-3.181487$

H $3.136880-0.321961-1.857451$

O $3.415987-0.457787-3.870035$

C $4.084231-2.208607-2.358968$

C $2.832968-3.069140-2.625953$

H $4.437166-2.423316-1.345211$

O $5.113361-2.657311-3.248695$

H $2.406055-2.807952-3.600002$

H $3.176190-4.106188-2.735015$

H $8.869797 \quad 1.8759631 .191444$ 
H 7.3587802 .7855491 .344700 H 7.8430671 .6545282 .618319 C $5.377309-0.3449362 .405331$ H $4.3184671 .749896-1.698480$ H $3.2822160 .501318-3.962569$ H $4.823985-2.455661-4.155304$ H $5.997891-0.0326333 .253011$ H $5.665670-1.3686122 .135547$ H 5.1867581 .5734681 .396402 C $0.233033-1.414501-2.484576$ O $1.118806-1.737098-1.407032$ C $1.770172-3.005987-1.505456$ C $0.714455-4.119962-1.586582$ C $-0.251159-3.890784-2.743938$ C $-0.848597-2.489170-2.662626$ H $0.808749-1.300688-3.409981$ C $-0.422237-0.067599-2.160489$ H $2.306443-3.147128-0.558240$ O $1.322687-5.399075-1.729980$ H $\quad 0.137810-4.145648-0.653133$ H $0.232651-4.058305-3.713339$ O - $-1.321195-4.834958-2.658403$ H -1.562283 -2.448385 - 1.829863 O $-1.581613-2.220300-3.856724$ C $0.573621 \quad 1.066011-2.177131$ H $-1.1966500 .183370-2.893129$ H -0.908762 -0.098325 -1.177648 H $1.922656-5.523329-0.974891$ H $-0.924129-5.722763-2.667937$ H $-2.248459-2.921905-3.951688$ O $0.849127 \quad 1.458374-0.902194$

O $1.0439471 .537612-3.203812$ C $1.7556602 .553677-0.722516$ H 2.4936952 .2644620 .030600 H $2.2780292 .834102-1.640981$ H $1.1915883 .413591-0.352650$ SCF Energy $\left(B 3 L Y P / 6-31 G^{* *} / / M M F F\right)=-3245.92846568$

0500221

MM̄MF Geometry

C $-2.924683-3.804893-2.088305$

C $-2.091051-3.677472-1.044763$

C $-0.588605-3.692217-1.174400$

O $-0.069316-2.412184-0.748578$

C $-0.003523-4.778159-0.251772$

C $1.493307-4.973150-0.398579$

C $2.392359-4.7724570 .584876$

C $1.962137-5.462014-1.745564$

C $2.139520-4.3261941 .997544$

C $2.207839-2.8064332 .208845$

C $3.587369-2.1886451 .930824$

C $3.615881-0.7198622 .355101$

C $4.962882-0.0580582 .024870$

O $3.846204-2.2712520 .530030$

O 4.7923851 .3642832 .141953

C $5.332784-0.3455730 .549678$

C $6.039717-0.4458443 .046094$

$\begin{array}{lllll}\text { O } & 6.663240 & 0.093314 & 0.288027\end{array}$

C $5.150462-1.8242890 .134615$

C $5.250213-2.066696-1.388797$

O $6.173092-2.6547130 .698631$

C $-0.105839-1.397553-1.655401$

O $-0.548998-1.440526-2.792114$

C $0.488956-0.202491-1.019322$

C $0.3029101 .001610-1.580227$

C $0.8229282 .275548-1.080854$

C $1.8380242 .221176 \quad 0.029350$

C $4.239139-1.299203-2.247603$

C $4.782356-0.002652-2.824447$

C $4.0743691 .959642-4.186150$

O $5.957260 \quad 0.340624-2.725718$

C $3.8328463 .108944-3.259926$

C $3.0476844 .151200-3.571126$

C $2.8328795 .334783-2.672854$

C $1.3699155 .598134-2.398413$

C $0.6940286 .534490-3.085590$

C $0.7164854 .836481-1.268261$

C $0.3787303 .419030-1.643489$
N $3.8002710 .713869-3.495296$

H -2.516870 -3.910824 -3.090538

H $-2.502288-3.557227-0.045114$

H $-0.289648-3.883033-2.212169$

H $-0.488195-5.741819-0.459507$

H $-0.257243-4.5276130 .784073$

H $3.442706-4.9558570 .360458$

H $3.020066-5.745997-1.739130$

H $1.390503-6.344911-2.050118$

H $1.837722-4.683655-2.504479$

H $1.168728-4.6912362 .349667$

H $2.878620-4.8141232 .645743$

H $1.454896-2.3287471 .571234$

H $1.930522-2.6022993 .250187$

H $4.341772-2.7642902 .479406$

H $3.391932-0.6182233 .424178$

H $2.821349-0.1729301 .829890$

H 4.4840361 .5569153 .043934

H $4.6707020 .281567-0.058236$

H $5.752588-0.1120024 .050731$

H $\quad \begin{array}{llll}6.991300 & 0.051231 & 2.828091\end{array}$

H $6.212380-1.5229683 .097799$

H $6.8128830 .036092-0.670476$

H $6.278797-1.905934-1.733649$

H $5.059770-3.137388-1.554840$

H $6.804262-2.0928651 .175531$

H $1.052916-0.345079-0.105097$

H $-0.3040901 .067712-2.482432$

H $2.6711551 .567630-0.249569$

H 1.3841041 .8372270 .948539

H 2.2810523 .1903550 .266472

H $3.970043-1.926543-3.107113$

H $3.319493-1.094207-1.691078$

H $3.4249061 .992606-5.066639$

H $5.1167241 .968573-4.520493$

H $4.3487783 .097223-2.302673$

H $2.5546554 .173188-4.540532$

H $3.2844986 .208819-3.160404$

H $3.3674375 .219539-1.721752$

H $-0.3484176 .748518-2.874621$

H $1.1672187 .103803-3.878812$

H $-0.2229375 .328072-0.982137$

H $1.3478974 .906264-0.378456$

H $-0.3570183 .348049-2.446069$

H $2.8399050 .383242-3.474402$

C $-5.052908-2.499964-2.388347$

O $-4.751023-1.500347-1.389230$

C $-5.984516-1.078595-0.786972$

C $-7.019795-1.258623-1.884546$

C $-6.583765-2.591250-2.463573$

H $-6.200892-1.7838340 .025903$

C $-5.8702930 .333715-0.208914$

H $-8.043670-1.272520-1.499705$

H $-6.944731-0.474305-2.646548$

C -7.107738 -2.847479-3.866453

H $-6.946268-3.392735-1.805610$

C $-4.653940 \quad 0.553477 \quad 0.717191$

O $\quad-5.7636601 .269286-1.288182$

H $-6.801403 \quad 0.5799170 .316071$

H $\quad-3.7520120 .6521200 .102901$

O $-4.8346561 .817177 \quad 1.366057$

C $-4.443488-0.5303721 .785029$

C $-3.327094-0.2473782 .809024$

H -4.223786 -1.485475 1.294615

O $-5.667408-0.6997922 .506688$

H $-3.633396 \quad 0.584521 \quad 3.450598$

H -3.292953 -1.116938 3.477595

H $-8.202422-2.868768-3.870903$

H $-6.783719-2.068245-4.564499$

H $-6.747926-3.809851-4.244556$

C $-4.421241-3.829324-1.965344$

H $-5.7430182 .159256-0.896916$

H $-5.595107 \quad 1.733672 \quad 1.966634$

H $-5.543326-1.4386593 .126717$

H $-4.797740-4.644923-2.593946$

H $-4.703159-4.062911-0.930605$

H -4.627293 -2.145875 -3.335038

C -1.6556772 .3280712 .753261$ 
O

C -1.936330 -0.012599 2.178330

C $-0.761885-0.4043913 .083884$

C $-0.618196 \quad 0.5597154 .256041$

C -0.5293881 .9965073 .744862$

H $-2.6130812 .406243 \quad 3.280352$

C -1.381009 3.6798552 .083978

H -1.873950 -0.6529991 .289227$

O $-0.926416-1.7373993 .554539$

H $\quad 0.162657-0.3849732 .493381$

H -1.444394 0.4415064 .966267

O $\quad 0.571271 \quad 0.2431374 .982965$

H $0.4455772 .142023 \quad 3.263533$

O -0.5760952 .8931344 .853494$

C -2.5801314 .1821301 .318273$

H -1.1329834 .4508142 .822881$

H -0.5276933 .6191931 .400458$

H $-0.197227-1.9214294 .170962$

H 1.3276830 .3420214 .380113

H -1.4359792 .7797645 .293383$

O -2.5112943 .7709250 .021236$

O -3.4711094 .8562021 .818723$

C $-3.5615314 .222998-0.839058$

H $-4.5406844 .120884-0.362140$

H $-3.3796005 .264575-1.118306$

H $-3.5479493 .607479-1.742527$

SCF Energy (B3LYP/6-31G**//MMFF) $=-3245.91112231$

0500222

MM̄FF Geometry

C $3.967859-4.386976-1.659639$

C $4.316150-3.215220-2.213151$

C $5.190046-2.142046-1.615018$

O $4.341768-0.981170-1.427159$

C $5.815020-2.466864-0.242718$

C $6.696398-1.3518250 .290657$

C $6.434972-0.6287001 .397838$

C $7.951354-1.078916-0.499322$

C $5.284836-0.7697622 .356956$

C 4.1617700 .2213362 .047908

C 3.1364760 .3646533 .182736

C $2.371380-0.9311163 .461550$

C $1.284888-0.7365684 .531586$

O $2.219246 \quad 1.3942092 .784253$

O $0.444870-1.9004624 .488969$

C 0.4225570 .4864384 .153390

C $1.885761-0.6928215 .941350$

O -0.5168810 .7882055 .181920$

C 1.2489331 .7377953 .784947

C 0.3778762 .8847043 .219539

O 1.8868772 .2416604 .964803

C $4.4130460 .034439-2.327045$

O $5.0895930 .081926-3.340679$

C $3.5239781 .109310-1.831865$

C $3.5147152 .312051-2.425510$

C $2.7277693 .471486-1.994037$

C $1.9007283 .314210-0.745196$

C -0.4927262 .4974912 .022963$

C -1.062520 3.7019561 .291733

C $-2.3519204 .251756-0.781229$

O $-0.8976264 .859866 \quad 1.663645$

C -1.465258 4.304350-1.986000

C $-0.9931825 .439629-2.523069$

C $-0.1005445 .469754-3.732533$

C $1.1387116 .317314-3.532775$

C $1.3615897 .396632-4.301844$

C $2.1175765 .927919-2.445119$

C $2.7948994 .610823-2.714895$

N -1.780265 3.3153540 .168873

H $4.346030-4.669284-0.682202$

H $3.900216-2.970300-3.189669$

H $5.988032-1.927101-2.335511$

H $\quad 6.421480-3.379027-0.312655$

H $5.010175-2.6828090 .467492$

H $7.137587 \quad 0.158610 \quad 1.669724$

H $8.616694-0.3729710 .009252$

H $8.514623-2.005800-0.649060$

H $7.709273-0.653701-1.477803$
H $4.901196-1.7937742 .370434$

H $5.673287-0.5861223 .366742$

H $4.582178 \quad 1.2138971 .835769$

H $3.645140-0.0784851 .128390$

H $3.671636 \quad 0.6976884 .079834$

H $3.048903-1.7385893 .762554$

H $1.890311-1.2737642 .534390$

H $-0.288038-1.7576445 .112497$

H -0.1715840 .1804103 .285241$

H $2.424691-1.6240786 .154158$

H $1.102996-0.6216526 .704601$

H 2.5836860 .1345596 .084838

H $-0.037413 \quad 1.2391445 .897690$

H -0.2557873 .3034994 .012298$

H 1.0485663 .7017752 .918191

H 2.1133693 .1749254 .816098

H $2.9128720 .886476-0.964464$

H $4.1521122 .471307-3.294130$

H 2.5340213 .0421230 .106933

H $1.1453362 .533443-0.886284$

H $1.3667134 .219492-0.454538$

H $0.090710 \quad 1.9109601 .304990$

H $-1.337397 \quad 1.8880812 .361832$

H -2.474278 $5.232699-0.311431$

H -3.337153 $3.868294-1.062491$

H -1.201619 $3.353796-2.444769$

H -1.273022 $6.394431-2.084590$

H $-0.6946305 .858255-4.569834$

H $0.203274 \quad 4.456862-4.023968$

H $2.2439408 .014016-4.169489$

H $0.6676277 .688756-5.083064$

H $1.6068405 .955956-1.479759$

H $2.9099906 .684006-2.362074$

H $3.4298494 .624795-3.601859$

H -1.804084 2.330483 -0.079560

C $1.553244-5.139309-1.846518$

O $1.113947-3.817629-2.226206$

C $0.542849-3.173833-1.068420$

C $0.138631-4.312110-0.146403$

C $1.294893-5.274311-0.343430$

H $1.351254-2.582667-0.618299$

C $-0.593700-2.253047-1.521171$

H $\quad 0.023265-3.9950790 .894279$

H $-0.802572-4.767422-0.478754$

C $0.990677-6.6955140 .097809$

H $2.147534-4.9045330 .238446$

C -1.378979-1.625714 -0.353857

O $-0.015469-1.217099-2.320952$

H -1.282750 -2.803556 -2.172679

H -1.817124 -2.436700 0.240165

O $-0.465626-0.9247860 .489581$

C $-2.517543-0.686499-0.805796$

C $-3.372128-0.2015160 .379335$

H -3.152657 -1.223915 -1.518644

O $-2.0002260 .464768-1.468597$

H $-3.677227-1.0680770 .975164$

H $-2.763757 \quad 0.4361221 .029371$

H $\quad 0.765177-6.725868 \quad 1.168703$

H $\quad 0.128901-7.106971-0.438184$

H $1.848006-7.350485-0.086983$

C $3.002692-5.345760-2.303466$

H $0.482909-1.646480-3.037469$

H $-0.924113-0.7302131 .323577$

H -1.505624 $0.160534-2.247956$

H $3.050501-5.225223-3.393480$

H $3.324717-6.370461-2.085511$

H $0.915578-5.838345-2.402412$

C $-6.098717-1.247705-0.459089$

O $-5.418297-0.112254-1.005614$

C $-4.6105210 .607649-0.069285$

C -5.4828341 .1197621 .085287$

C $-6.291071-0.0044341 .730417$

C $-7.040178-0.8107610 .668178$

H $-5.367306-1.967695-0.073699$

C $-6.866920-1.919018-1.602930$

H $-4.2379031 .488728-0.605165$

O -4.6893261 .7579322 .080421$

H $\quad-6.184489 \begin{array}{lll}1.872231 & 0.702357\end{array}$ 
H $-5.654552-0.6570372 .339552$ O H -7.862261 -0.2071840 .262739$ O $-7.614530-1.9661111 .274732$ C $-5.923209-2.485298-2.638675$ H $-7.508528-2.733206-1.248590$ H -7.511134 -1.190667 -2.110553 H -4.1951662 .4740941 .645608$ H -6.766933 1.1021633 .268106 H -8.185126 -1.659121 2.000213 O $-5.236725-3.543885-2.120906$ O $-5.812872-2.039510-3.773580$ C $-4.284365-4.149983-3.000184$ H -3.564815 -3.406560 -3.356644 H -3.744083 -4.917252 -2.439399 $\mathrm{H}-4.799316-4.623805-3.841108$ SCF Energy $\left(B 3 L Y P / 6-31 G^{* *} / / M M F F\right)=-3245.93541766$

0500223

MM̄FF Geometry

C $-3.9303972 .916135-0.926453$

C $-3.7708704 .172282-0.482524$

C $-2.525055 \quad 4.6897170 .205012$

O -1.7591353 .6061950 .782457$

C $-1.6285135 .422858-0.809427$

C $-0.3163545 .908937-0.221739$

C $0.9027805 .449399-0.570736$

C $-0.437773 \quad 6.9979370 .813729$

C $1.2455494 .409989-1.602022$

C $1.6250033 .054238-1.000683$

C $2.8881503 .024845-0.123558$

C $4.1432823 .421001-0.908189$

C $5.4296883 .232871-0.091729$

O 3.0078721 .6761650 .356595

O $6.5273283 .331516-1.012198$

C 5.4347551 .8095710 .502048

C $5.6193394 .365510 \quad 0.923474$

O $6.556726 \quad 1.622546 \quad 1.361430$

C 4.1233121 .4445701 .227851

C $4.088454-0.0397621 .658373$

O 4.0159762 .2310922 .420052

C -2.2062333 .0860581 .958687$

O -3.1921153 .4307982 .590202$

C -1.284208 1.9945122 .338246

C $-1.634867 \quad 1.134726 \quad 3.306731$

C $-0.8230080 .019592 \quad 3.800572$

C $0.559798-0.125573 \quad 3.225859$

C $4.167728-1.0468390 .510480$

C $3.777531-2.4380870 .973810$

C $1.791615-3.8553781 .461218$

O $4.616613-3.3088081 .190037$

C $0.421828-3.6049592 .005001$

C $-0.023411-4.083893 \quad 3.176213$

C -1.402245 -3.806503 3.707612

C -1.393176 -3.283043 5.128744

C -1.996976 -3.967807 6.114945

C $-0.685847-1.9751605 .416032$

C $-1.340805-0.7988984 .740742$

N $2.407732-2.582421 \quad 1.132557$

H $-3.1039252 .217038-0.835597$

H $-4.5856874 .883380-0.595883$

H -2.823655 5.3784421 .005226

H -2.166771 $6.285503-1.224356$ H -1.445730 $4.755846-1.657470$

H $1.7706755 .880060-0.072439$

H $\quad 0.5374847 .391326 \quad 1.119999$

H -1.017516 7.8371670 .415462

H $-0.937064 \quad 6.6228801 .712235$

H $0.4203374 .254588-2.302931$

H $2.0640534 .794938-2.220781$

H $0.7799532 .664380-0.417823$

H $1.7571292 .337146-1.821078$

H 2.7409933 .6866940 .736919

H $4.0796924 .455803-1.263965$

H $4.2139082 .798909-1.811779$

H $7.3435583 .141138-0.518372$

H $5.5820101 .122254-0.340294$

H 5.6878135 .3308670 .407367
H $6.5612674 .254659 \quad 1.471747$

H 4.8041254 .4349861 .646706

H 6.3637332 .0704112 .202583

H $4.887616-0.2431272 .383195$

H $3.158945-0.2113142 .212774$

H $3.407540 \quad 1.784336 \quad 3.031471$

H -0.3426081 .9278691 .805121$

H $-2.605810 \quad 1.255213 \quad 3.785385$

H $1.149150 \quad 0.778238 \quad 3.417085$

H $0.509912-0.2894692 .143737$

H $1.126460-0.9591093 .642940$

H $3.506962-0.760467-0.315448$

H $5.183920-1.097630 \quad 0.104677$

H $2.433139-4.3972812 .163936$

H $1.726518-4.437350 \quad 0.536911$

H $-0.244714-2.9952161 .400431$

H $0.626975-4.7141483 .778057$

H -1.972542 -4.742527 3.649767

H -1.934360 -3.090326 3.069713

H -1.997583 -3.605620 7.137708

H $-2.505706-4.9068435 .923552$

H $0.371951-2.0796075 .163194$

H $-0.695580-1.7696096 .494984$

H $-2.353270-0.6024075 .096534$

H $1.809391-1.8563380 .748031$

C $-5.6743081 .140444-0.784160$

O $-4.7090720 .076944-0.920793$

C $-5.398972-1.150021-1.216263$

C $-6.866768-0.884609-0.907604$

C $-6.9920910 .573166-1.311218$

H $-5.296596-1.315818-2.295412$

C $-4.762567-2.317751-0.452708$

H -7.535656-1.545365 -1.467108

H -7.087090 -1.002031 0.159685

C $-8.2268451 .255886-0.749324$

H $-7.0156460 .634031-2.407577$

C $-3.234866-2.437696-0.651166$

O $-5.005304-2.1262990 .946547$

H $-5.263057-3.252952-0.730991$

H -2.734264 -1.612614 -0.132537

O $-2.794942-3.644346-0.016948$

C $-2.786619-2.487382-2.122603$

C $-1.286003-2.743672-2.337950$

H -3.064687 -1.562663 -2.632542

O $-3.500942-3.531584-2.795349$

H $-1.040174-3.759347-1.997049$

H -1.113074 -2.789916 -3.420449

H $-9.1359670 .766064-1.113163$

H -8.2415661 .2213090 .345231$

H $-8.2644292 .306548-1.053669$

C $-5.1854532 .373704-1.548955$

H $-4.787957-2.9583371 .399386$

H $-2.917179-3.5347110 .940676$

H $-3.305204-4.365308-2.333904$

H $-5.968003 \quad 3.140339-1.570045$

H $-4.9533952 .102307-2.585894$

H $-5.751788 \quad 1.362213 \quad 0.288518$

C $1.617591-2.354025-3.042835$

O $1.038433-2.287450-1.733011$

C $-0.296618-1.757686-1.682684$

C $-0.270000-0.322580-2.221062$

C $0.311975-0.262829-3.630680$

C $1.674858-0.956136-3.677174$

H $1.030245-3.029736-3.675539$

C $3.021336-2.959174-2.891509$

H $-0.545001-1.704760-0.618055$

O

H $\quad 0.3646710 .287413-1.565618$

H $-0.375767-0.694458-4.367132$

O $0.493307 \quad 1.104864-4.006760$

H $2.409076-0.326451-3.157535$

O $2.075044-1.049691-5.042669$

C $2.979656-4.373058-2.350581$

H $3.541379-2.996701-3.853724$

H $3.604465-2.349101-2.193016$

H $-1.8768620 .268887-1.297718$

H $-0.3702981 .544520-3.922890$

H $3.017847-1.283730-5.061512$ 
O $4.212268-4.698484-1.860782$

O $2.000444-5.106136-2.371748$

C $4.325858-6.010535-1.301489$

H $5.348175-6.136164-0.934896$

H $3.635571-6.125301-0.460295$

H $4.131565-6.766674-2.068012$

SCF Energy (B3LYP/6-31G**//MMFF) $=-3245.91587635$

05 00224

MM̄FF Geometry

C $-0.2050573 .174506-0.606421$

C $0.8728913 .738443-0.041779$

C 1.6310263 .0980751 .087013

O 3.0084042 .9846110 .660024

C 1.5864813 .9402972 .375410

C 0.2051274 .1890162 .941166

C -0.4984813 .2803153 .643737$

C $-0.345165 \quad 5.5741712 .725387$

C -0.0979351 .8617783 .940100$

C $-1.2543070 .894423 \quad 3.654772$

C $-0.853264-0.5879213 .579106$

C $0.063013-1.0403424 .719961$

C $0.486819-2.5107384 .572939$

O $-0.221764-0.8136992 .309558$

O $1.591959-2.7426465 .461029$

C $1.010091-2.7372093 .136785$

C - $0.624289-3.4722995 .010243$

O $1.282078-4.1176382 .907677$

C $0.057562-2.1974422 .047348$

C $0.647023-2.3230640 .625295$

O $-1.145395-2.9795772 .057148$

C 3.7537131 .9972921 .226307

O $3.397234 \quad 1.1972382 .076424$

C 5.0920512 .0364320 .598950

C 5.8681350 .9447340 .659239

C 7.1782010 .7857990 .031187

C $7.8467702 .012178-0.530274$

C $1.892610-1.4860260 .330953$

C $2.406981-1.741932-1.078450$

C $4.066266-0.890850-2.734039$

O $2.005435-2.665970-1.781567$

C $5.376818-1.594656-2.563694$

C $6.541769-1.107128-3.018048$

C $7.865999-1.801175-2.852731$

C $8.912581-0.908863-2.218333$

C $9.784377-0.227415-2.981181$

C $8.999208-0.852944-0.707147$

C $7.711750-0.451293-0.041400$

N $3.385873-0.833397-1.452012$

H $-0.5394212 .199187-0.259946$

H $1.2090744 .715400-0.380216$

H 1.2320332 .0926741 .266694

H 2.0940014 .8996922 .200928

H 2.2067823 .4617773 .145298

H -1.464268 3.5789354 .049246 H $-1.340925 \quad 5.6992973 .163872$ H $\quad 0.3139536 .3188873 .183630$ H -0.4277595 .7942331 .656732$ H 0.7842821 .5639683 .369222 H 0.1716151 .8010375 .001112 H $-2.009156 \quad 1.0159144 .442559$ H $-1.733647 \quad 1.1700642 .705584$ H -1.788270 -1.160676 3.600729 H -0.415306 -0.869990 5.692155 H $0.980442-0.4374634 .725068$ H $1.302389-2.5232206 .363330$ H $1.971264-2.2135673 .059241$ H $-0.873581-3.3131816 .066522$ H $-0.297083-4.5150464 .934511$ H -1.545799 -3.355876 4.436404 H $1.887304-4.4139583 .608995$ H $0.837628-3.3812930 .403472$ H - $0.123926-2.014649-0.093732$ H -1.589227 -2.8783061 .198798$ H 5.3777022 .9354560 .065098 H 5.4891950 .0639471 .176778 H $8.8713091 .827486-0.862899$ H 7.9068252 .8003650 .228628
H $7.2887992 .394832-1.391753$

H $1.657447-0.4207910 .431417$

H $2.703771-1.7330391 .022687$

H $4.1920920 .139767-3.080342$

H $3.446280-1.431389-3.456095$

H $5.366370-2.553735-2.050923$

H $6.545971-0.159412-3.552224$

H $8.202330-2.119236-3.848272$

H $7.767920-2.722517-2.265247$

H $10.5545610 .396988-2.540788$

H $9.744953-0.276541-4.064451$

H $9.809133-0.192875-0.372871$

H $\quad 9.275767-1.850627-0.341430$

H $7.155820-1.2879490 .382770$

H $3.689868-0.132353-0.784526$

C $-2.4261904 .145757-1.178541$

O $\quad-3.1538092 .923091-0.942742$

C $-4.513413 \quad 3.100267-1.380873$

C $-4.6689654 .582075-1.705949$

C $-3.2716024 .937116-2.173895$

H $-4.6215162 .528332-2.309776$

C $-5.4898122 .575942-0.323597$

H $-5.4358784 .760756-2.465824$

H $-4.9337265 .167850-0.818018$

C $-2.9948956 .430496-2.175518$

H $-3.1275524 .540789-3.188123$

C $-5.290044 \quad 1.092644 \quad 0.053239$

O

H $-6.5185472 .746020-0.663169$

H $-4.3559770 .972786 \quad 0.614902$

$\begin{array}{llll}\text { O } & -6.340511 & 0.741348 & 0.964461\end{array}$

C $-5.3026690 .115430-1.138220$

C $-5.332659-1.358694-0.689148$

H $-4.420640 \quad 0.300894-1.759152$

O $-6.459673 \quad 0.385719-1.930711$

H $-4.539765-1.5193550 .048924$

H -6.289306 -1.557701 -0.191619

H -3.684124 $6.948201-2.850775$

H -3.115633 $6.862178-1.176251$

H $-1.9743186 .639178-2.511057$

C $-1.0219513 .821486-1.687718$

H $-5.9527213 .005587 \quad 1.529174$

H -7.1800220 .7857130 .474979$

H $-6.322707-0.021214-2.802514$

H $-0.5284244 .733144-2.043116$

H $-1.0773103 .125532-2.532714$

H -2.372217 $4.675500-0.217614$

C $-2.771551-2.575917-1.883226$

O $-3.954570-2.158884-2.577107$

C $-5.181071-2.351713-1.863770$

C $-5.347659-3.828039-1.474682$

C $-4.131726-4.361493-0.726678$

C $-2.857803-4.061947-1.512161$

H $-2.644161-1.975491-0.974495$

C -1.576928 -2.314967 -2.815424

H $-5.984599-2.129519-2.576009$

O $-6.510212-4.021719-0.676186$

H -5.481668 -4.427642 -2.384694

H $-4.072235-3.9556980 .290231$

O $-4.249089-5.779333-0.589369$

H $-2.831251-4.679984-2.418831$

O $-1.728670-4.418199-0.718493$

C $-1.155466-0.859860-2.831520$

H $-0.706674-2.891227-2.484023$

H -1.829156 -2.600004 -3.843320

H $-7.271386-3.692224-1.183739$

H $-5.083245-5.955890-0.121228$

H -1.825716 $-5.355661-0.478291$

O $-0.019464-0.741859-3.582514$

O

C $0.5283950 .576723-3.670876$

H $-0.1944011 .258329-4.128932$

H $0.8217120 .930220-2.678091$

H $1.4186380 .533076-4.303671$

SCF Energy $\left(B 3 L Y P / 6-31 G^{* *} / / M M F F\right)=-3245.92248489$

0500225

MM̄MF Geometry 
C $2.782677-4.0719240 .017983$

C $2.125414-2.903508-0.018861$

C $1.124524-2.527697-1.079957$

O $0.039314-1.802547-0.454764$

C $1.783830-1.584533-2.102760$

C $0.890469-1.231136-3.276126$

C $0.4894010 .018469-3.582162$

C $0.471285-2.383199-4.153047$

C $0.8278291 .299115-2.873207$

C $-0.1583921 .703784-1.767912$

C $-1.5847521 .984079-2.267213$

C $-2.4323482 .608393-1.157902$

C $-3.8875912 .825626-1.601212$

O $-2.1756590 .746947-2.670174$

O $-4.6654703 .104265-0.426016$

C $-4.4370471 .514749-2.207810$

C $-4.0268114 .051060-2.512894$

O $-5.7240381 .749569-2.774497$

C $-3.486420 \quad 0.870250-3.240822$

C $-3.917024-0.550719-3.681867$

O $-3.4576101 .683750-4.420176$

C $-0.886606-2.5182520 .236513$

O $-0.934592-3.7303810 .368150$

C $-1.828897-1.550252 \quad 0.840313$

C $-2.639246-1.9471871 .832175$

C $-3.608645-1.1089032 .540703$

C $-3.874544 \quad 0.266646 \quad 1.988528$

C $-4.034246-1.582233-2.554029$

C $-5.442049-1.737197-2.003337$

C $-6.699724-2.889552-0.186638$

O $-6.429755-1.226799-2.524790$

C $-6.928227-1.9266970 .935419$

C $-7.186359-2.3129422 .194125$

C $-7.462688-1.3618173 .323155$

C $-6.570471-1.6033954 .520085$

C $-7.000378-2.3270175 .567758$

C $-5.214788-0.9345524 .537743$

C $-4.221084-1.6123823 .632755$

N $-5.469726-2.547456-0.876107$

H $2.602790-4.812071-0.757372$

H $2.320804-2.1638730 .750970$

H $0.728288-3.421899-1.575650$

H $2.697576-2.044880-2.501749$

H $2.101470-0.676633-1.579664$

H $-0.1513260 .150156-4.453572$

H -0.021604 -2.047357 -5.071954

H $1.344804-2.972905-4.449721$

H $-0.230913-3.035656-3.625324$

H $1.8359611 .248652-2.449799$

H $\quad 0.8763122 .099268-3.622960$

H -0.181938 $0.915521-1.006505$

H $0.2365012 .607648-1.288267$

H - $1.5228602 .659831-3.128133$

H -1.991783 $3.552709-0.814364$

H -2.432880 $1.943166-0.283982$

H $-4.287683 \quad 3.8905800 .004027$

H $-4.5838270 .824430-1.369151$

H -3.675982 $4.953462-1.997570$

H $-5.076375 \quad 4.240442-2.763672$

H $-3.4601793 .961089-3.441915$

H -6.103658 $0.889872-3.021104$

H -4.831882 -0.501762 -4.284138

H -3.142169 - $0.922871-4.368076$

H $-3.1504601 .138628-5.163484$

H $-1.812525-0.5324250 .468022$

H -2.575008 $-2.979123 \quad 2.175039$

H -4.1125180 .2113930 .921026$

H -2.998546 0.9099612 .121115

H -4.7228950 .7694102 .457330$

H -3.755067 -2.565378 -2.954294

H -3.343282 $-1.353523-1.737269$

H $-6.597758-3.9206880 .166224$

H -7.537618 -2.841874 -0.889509

H $-6.907498-0.8658870 .696866$

H $-7.234973-3.3752302 .422757$

H -8.514563 -1.485601 3.612325

H $-7.366611-0.3174173 .001246$

H $-6.377527-2.4815386 .442508$
H -7.983676 -2.785643 5.571384

H $-4.795120-0.9624165 .552427$

H $-5.3381480 .129636 \quad 4.318588$

H $-3.965616-2.6247163 .949294$

H $-4.595760-2.884049-0.482483$

C $5.227996-4.0501290 .757196$

O $5.355568-2.6138540 .710050$

C $6.055215-2.258367-0.495165$

C $6.812515-3.514446-0.904280$

C $5.791526-4.585530-0.562060$

H $5.289145-2.051695-1.253262$

C $6.908986-1.003358-0.286941$

H $7.091795-3.509061-1.961915$

H $7.721081-3.654249-0.307472$

C $6.378386-5.981905-0.454479$

H $5.024372-4.587632-1.345922$

C 6.1501320 .1892530 .338365

O $7.999211-1.3246630 .585135$

H $7.359332-0.712513-1.243565$

H 6.0105750 .0001251 .407739

$\begin{array}{lllll}\text { O } & 7.002442 & 1.338233 & 0.257028\end{array}$

C $4.8002690 .513121-0.321445$

C 4.1131851 .7936650 .174112

H $4.115517-0.329067-0.193057$

O $5.012170 \quad 0.652900-1.731578$

H $4.6684312 .667233-0.196079$

H $3.1531111 .866180-0.350172$

H $6.814276-6.290685-1.410231$

H $7.166326-6.0297310 .304682$

H $5.604650-6.707644-0.184288$

C $3.780127-4.4296351 .086280$

H $8.528696-0.5165110 .696879$

H $7.0983901 .565663-0.683934$

H $4.1439490 .785508-2.148216$

H $3.489886-3.9343702 .022270$

H $3.713267-5.5082591 .270596$

H $5.861242-4.3749591 .592917$

C 2.0125043 .4788211 .545312

O $3.3674343 .272751 \quad 1.969990$

C 3.9023081 .9654561 .693450

C 3.0412150 .9254392 .420855

C 1.5783831 .0215601 .995671

C 1.0712842 .4518742 .190486

H 1.9496043 .4066270 .453703

C 1.6186134 .9096261 .943409

H $4.895871 \quad 1.9533332 .156418$

$\begin{array}{llll}\text { O } & 3.534935 & -0.388100 & 2.223300\end{array}$

H 3.1025951 .1205253 .499945

H $1.4465050 .691126 \quad 0.959658$

$\begin{array}{lllll}O & 0.790549 & 0.132694 & 2.790739\end{array}$

H 0.9713852 .6594843 .263530

O $-0.226067 \quad 2.573667 \quad 1.613215$

C $2.3477385 .955421 \quad 1.124927$

H $0.5457945 .067763 \quad 1.788640$

H 1.8659385 .0812852 .997769

H $2.957524-0.9952272 .717009$

H $\quad 0.8952430 .389164 \quad 3.722847$

H -0.7853191 .8821042 .006577$

O $2.009754 \quad 7.1944341 .591471$

$\begin{array}{llllllll} & \text { O } & 3.107740 & 5.723672 & 0.194923\end{array}$

C 2.6209608 .2954800 .911429

H 2.2713239 .2200631 .378554

H 3.7096368 .2433431 .008065

H $2.3256248 .300986-0.142146$

SCF Energy (B3LYP/6-31G*//MMFF)= -3245.91109244

0500226

MM̄FF Geometry

C $3.448944-3.8109830 .605422$

C $2.137317-3.5521460 .494696$

C $1.247325-3.2531591 .671578$

O $0.420979-2.122574 \quad 1.311638$

C $0.322185-4.455708 \quad 1.946257$

C $-0.575626-4.2752463 .155660$

C $-1.922802-4.244564 \quad 3.116297$

C $0.132712-4.162468 \quad 4.482387$

C -2.829046 -4.4289891 .930242$

C $-3.338520-3.0935251 .387840$ 
C $-4.504534-3.2367520 .396745$

C $-4.115346-3.995228-0.874348$

C $-5.256031-4.024672-1.904971$

O $-4.925361-1.9069700 .061098$

O $-4.679399-4.473162-3.141392$

C $-5.781938-2.587691-2.112483$

C $-6.330091-5.051895-1.527853$

O $-6.943118-2.573898-2.938718$

C $-6.080717-1.850109-0.789117$

C $-6.429817-0.357703-1.000501$

O $-7.213026-2.455835-0.153596$

C $0.390689-1.0503052 .147166$

O $1.079390-0.8582523 .135456$

C $-0.697333-0.1642641 .677116$

C $-1.0606390 .902232 \quad 2.405277$

C -2.1953351 .7844542 .119717$

C -3.1093931 .3795520 .994872$

C $-5.3869770 .442753-1.783809$

C $-5.5617531 .944699-1.625939$

C $-4.3797694 .081046-2.155504$

O $-6.4874702 .456421-1.002890$

C $-3.3660534 .358904-1.089725$

C $-3.5633935 .188709-0.053912$

C $-2.5267975 .446357 \quad 1.004767$

C -3.0534575 .2545232 .410651$

C -3.1116026 .2868553 .268968$

C -3.5146653 .8743122 .826205$

C -2.3884402 .8756142 .890457$

N $-4.5458212 .642649-2.265002$

H $3.911305-3.8082651 .589514$

H $1.669372-3.578887-0.486614$

H $1.860583-3.0307692 .552426$

H $\quad 0.925588-5.3597342 .104070$

H $-0.276453-4.6441281 .048709$

H -2.456458 -4.0974754 .054925$

H $-0.563556-4.1838245 .327890$

H $0.826855-4.9993394 .612985$

H $0.696739-3.2282664 .547400$

H -2.338211 -5.0023541 .139131$

H -3.678227 -5.0424602 .256755$

H -3.666809 -2.450865 2.216306

H -2.516117 -2.5500370 .906732$

H $-5.326385-3.7464840 .913297$

H -3.795979 -5.018803 -0.645932

H -3.244863 -3.505509-1.333341

H $-5.393448-4.555260-3.795745$

H -5.001497 -2.042853 -2.658698

H -5.899106 -6.059953-1.499213

H -7.125927 -5.087815 -2.279980

H $-6.785898-4.859007-0.554570$

H $-6.698681-2.953810-3.799020$

H -7.407091 -0.263438 -1.491861

H $-6.5558440 .100208-0.009312$

H -7.599929 -1.8127770 .463643$

H -1.211048 -0.4491470 .765838$

H -0.4997131 .1360213 .308951$

H -3.5015200 .3702821 .162054$

H -2.572361 1.3953420 .040279

H -3.977599 2.0287130 .881256

H -4.378206 $0.183980-1.443903$

H $-5.4601210 .207852-2.851737$

H -5.344947 $4.551456-1.943194$

H -4.012987 $4.441495-3.121217$

H -2.412755 $3.843165-1.178562$

H $-4.507110 \quad 5.7220220 .030914$

H -2.1654196 .4739560 .869959$

H -1.652475 4.8002940 .866347

H -3.4867776 .1627164 .279335$

H -2.785096 7.2809672 .981984

H -4.329752 3.5570362 .171252

H -3.9649943 .9121083 .827443$

H -1.672888 3.0790333 .688373

H $-3.7957472 .118997-2.707310$

C $5.558995-3.243345-0.628701$

O $5.151707-1.907090-0.981056$

C $6.109970-1.357529-1.905742$

C $7.270115-2.346038-1.961851$

C $6.561946-3.660342-1.703138$
H $\quad 5.621877-1.337164-2.887070$

C $6.5054280 .062879-1.487877$

H $7.789822-2.319189-2.924605$

H $8.010475-2.152959-1.177120$

C $7.498281-4.778966-1.280037$

H $6.031722-3.959553-2.617369$

C $5.3108871 .024891-1.317373$

O $7.191675-0.012651-0.232037$

H $7.2251900 .468308-2.209209$

H $4.7100300 .718335-0.457093$

O $5.8429532 .320965-1.018789$

C $4.4144531 .152060-2.560007$

C $3.3318112 .247334-2.490766$

H $3.9302920 .193470-2.772798$

O $5.2430521 .447926-3.691735$

H $3.7827553 .189324-2.160966$

H $3.0021082 .437865-3.520619$

H $8.238633-4.976770-2.062199$

H $8.038970-4.524287-0.362291$

H $6.944750-5.705443-1.098500$

C $4.335037-4.156187-0.558744$

H $7.6025070 .853123-0.069445$

H $6.1879762 .293151-0.110297$

H $5.6949812 .290160-3.510787$

H $4.653110-5.198041-0.438875$

H $3.769169-4.088879-1.496236$

H $6.050319-3.1809230 .351476$

C 2.7009312 .9454800 .461823

O $2.3851241 .737934-0.241750$

C $2.0976961 .888189-1.633971$

C $0.9245892 .866004-1.815174$

C $1.2289104 .210174-1.162085$

C 1.5925893 .9958410 .304469

H 3.6497983 .3461280 .088363

C 2.8788732 .5856001 .942460

H $1.7533190 .905937-1.981952$

O $\quad 0.6275503 .069023-3.192145$

H $0.0219492 .447961-1.350324$

H $2.0235974 .748368-1.691268$

O $0.0742225 .046098-1.225105$

H 0.6976703 .6857490 .859363

O 2.0340935 .2305330 .864903

C 4.0970321 .7205322 .162579

H 3.0042563 .4780612 .565988

H 1.9956272 .0568662 .320413

H $\quad 0.4336302 .198652-3.580224$

H $-0.1752475 .125412-2.161814$

H 1.3200265 .8784230 .736486

O $3.7171820 .432321 \quad 2.393771$

O 5.2469692 .1380562 .118027

C $4.775120-0.4985892 .640324$

H $4.327918-1.4412532 .966304$

H $5.336322-0.6760471 .719934$

H $5.436009-0.1340223 .432667$

SCF Energy (B3LYP/6-31G**//MMFF) $=-3245.92086431$

0500227

MM̄FF Geometry

C $2.579786-1.304876-2.651307$

C $1.788733-0.647810-1.788909$

C $0.822199-1.234825-0.784779$

O $-0.521192-0.867319-1.185304$

C $0.865166-2.770230-0.652393$

C $0.014016-3.3124400 .480902$

C $-1.038097-4.1391410 .321587$

C $0.431492-2.9003351 .870127$

C $-1.597997-4.695704-0.957263$

C $-2.734672-3.865012-1.573191$

C $-3.986880-3.755656-0.687449$

C $-5.144355-3.120682-1.461715$

C $-6.384049-2.911762-0.576607$

O $-3.671990-2.9418640 .445335$

O $-7.278615-2.029891-1.272623$

C $-5.963109-2.1920680 .723758$

C $-7.151851-4.220492-0.355197$

O $-7.048573-2.1296481 .645050$

C $-4.731812-2.8238611 .404089$

C $-4.188250-1.9872032 .588448$ 
O $-5.092460-4.1065791 .931399$

C $-0.9744540 .365952-0.833921$

O $-0.3804821 .215016-0.189581$

C $-2.3307270 .522910-1.401759$

C $-2.8292471 .755486-1.577975$

C $-4.1428962 .082465-2.135910$

C $-5.1270420 .954856-2.300492$

C $-3.750088-0.5600452 .241896$

C -4.8190130 .4910522 .491669$

C -5.2764362 .9150952 .142591$

$\begin{array}{llll}\text { O } & -5.865520 & 0.260425 & 3.091351\end{array}$

C $-6.1310593 .076878 \quad 0.925191$

C -6.2180284 .2248250 .236125$

C -7.099495 $4.417684-0.964511$

C $-6.3415054 .942130-2.163452$

C $-6.3388456 .252816-2.459922$

C $-5.6584073 .937092-3.063465$

C $-4.4082373 .367130-2.453166$

N $-4.446461 \quad 1.7340201 .997254$

H $2.550439-2.388716-2.709556$

H $1.8486110 .438764-1.775877$

H $1.052031-0.7850760 .189034$

H $1.896870-3.101112-0.474947$

H $\quad 0.555707-3.210977-1.605579$

H -1.565991 -4.469658 1.215752

H $-0.095690-3.4652562 .646557$

H $1.503020-3.0735322 .013782$

H $0.221011-1.8399122 .038225$

H $-0.806772-4.824639-1.703308$

H -1.963409 $-5.710458-0.753610$

H $-2.355769-2.862377-1.803474$

H -3.009459-4.333218 -2.526555

H $-4.260175-4.762796-0.351186$

H $-5.400523-3.722338-2.342634$

H -4.827223 -2.143580 -1.850253

H -7.494737 -2.436967 -2.129088

H $-5.725378-1.1573220 .450119$

H -7.482549-4.637063 -1.314290

H -8.064430 -4.050050 0.226602

H -6.558422 -4.9861650 .148622$

H $-7.793259-1.703847 \quad 1.186910$

H -4.903882 -2.001234 3.419366

H -3.295748 -2.506596 2.966906

H -4.454255 -4.3459802 .623777$

H -2.869960 -0.375231 -1.678427

H $-2.2095762 .610631-1.310124$

H $-5.2265350 .395449-1.364090$

H $-4.7980350 .268047-3.087581$

H $-6.1345501 .287614-2.557839$

H -2.903867 -0.291405 2.887408

H $-3.404602-0.4941701 .206079$

H -4.605409 3.7664992 .294191

H -5.9119542 .8083193 .027511$

H -6.7230482 .2204210 .611046$

H -5.6460025 .0878140 .570134$

H $-7.8913475 .125309-0.685905$

H -7.616275 $3.489083-1.237028$

H $-5.8209206 .634529-3.333453$

H $-6.8509886 .974393-1.831847$

H -5.365746 $4.415593-4.007737$

H $-6.3820393 .170023-3.352403$

H -3.627654 4.111940 -2.290543

H -3.5832591 .8222991 .469107$

C $4.983006-1.109785-3.330275$

O $5.376430-0.834491-1.973541$

C $6.756559-0.424249-1.957444$

C $7.280915-0.626158-3.375802$

C $6.025868-0.412596-4.201117$

H $6.758308 \quad 0.648847-1.732602$

C $7.520452-1.182289-0.864284$

H $8.0827660 .076170-3.623316$

H $7.666934-1.640145-3.530130$

C $6.124716-0.963138-5.612737$

H $\quad 5.817537 \quad 0.664867-4.247545$

C $6.837749-1.1488390 .518393$

O $7.623700-2.559323-1.252012$

H $8.547168-0.803643-0.801695$

H $5.917280-1.7439120 .478452$
O $7.697163-1.824127 \quad 1.446078$

C 6.5150650 .2550891 .061459

C 5.8054320 .1840782 .424563

H $5.8697250 .785596 \quad 0.358518$

O 7.7148371 .0107051 .207600

H $4.912370-0.4415242 .316763$

H $6.462412-0.3112513 .149669$

H $6.937120-0.474258-6.160079$

H $6.321268-2.040554-5.610528$

H $5.195318-0.791526-6.164732$

C $3.555403-0.614934-3.566938$

H $8.107993-3.023190-0.547433$

H $8.496592-1.2802251 .552960$

H 8.0711891 .1765740 .318838

H $3.257651-0.809172-4.603361$

H $3.5124100 .469640-3.411495$

H $5.033898-2.198742-3.463038$

C 3.3419691 .9050961 .800056

O 4.6733882 .3623112 .064722

C 5.4218991 .5697832 .992064

C 4.6876541 .5067124 .338134

C 3.2578780 .9993164 .174851

C $2.528517 \quad 1.8029513 .099786$

H 3.3807370 .9298451 .299265

C 2.6894922 .9227520 .849055

H 6.3614782 .1099373 .163801

O 5.4006290 .6759425 .248304

H 4.6593042 .5129884 .776048

H $3.241694-0.0746873 .956471$

O 2.5780391 .1631105 .421746

H 2.3202332 .8068243 .490840

O $1.265575 \quad 1.1917862 .838888$

C $3.2216342 .845174-0.565792$

H 1.6100712 .7483890 .793968

H 2.8727093 .9410201 .212500

H 4.8901430 .6463716 .075638

H 1.6576280 .8779425 .288829

H 1.4327560 .3061032 .473951

O $2.5442343 .746276-1.338813$

O $4.1083972 .097997-0.952307$

C $2.9280113 .788238-2.716752$

H $2.6582102 .849268-3.208403$

H $2.3821504 .604961-3.196653$

H $4.0010883 .981110-2.811708$

SCF Energy (B3LYP/6-31G**//MMFF) $=-3245.91415787$

05_00228

MM̄FF Geometry

C -3.786487 -2.699415 -2.196302

C $-2.625710-2.049145-2.370778$

C $-1.231772-2.635461-2.275908$

O $-0.434005-1.753180-1.444741$

C $-1.161858-4.043708-1.648676$

C $0.236293-4.624521-1.565048$

C $0.866071-4.942433-0.416859$

C $0.916143-4.881592-2.885644$

C $0.365848-4.7904590 .993459$

C $0.804060-3.4880251 .683054$

C $2.317190-3.3926111 .940870$

C $2.652401-2.1485832 .766794$

C $4.168571-1.9772752 .961581$

O $2.988896-3.3180160 .682696$

O $4.402785-0.6435873 .439913$

C $4.875521-2.0956401 .592467$

C $4.712985-2.9250454 .037348$

O $6.291788-2.1171621 .749418$

C $4.419359-3.3178620 .771219$

C $4.963394-3.322767-0.677597$

O $4.897115-4.5128641 .401463$

C $0.199536-0.714254-2.055208$

O $0.161554-0.422025-3.239907$

C $0.9418960 .034062-1.016730$

C $1.3975601 .264962-1.293997$

C $2.0993622 .153891-0.366484$

C 2.6146171 .5542310 .914002

C $4.556473-2.120197-1.536860$

C $5.607773-1.025127-1.598998$

C $5.9782831 .252201-2.537128$ 
O $6.733349-1.139284-1.122243$

C $5.7958832 .241722-1.430738$

C $5.483307 \quad 3.529092-1.640644$

C $5.3414074 .544162-0.544332$

C $4.0015465 .242378-0.570601$

C $3.8662926 .460878-1.120840$

C $2.840217 \quad 4.565140 \quad 0.119609$

C $2.2416713 .453414-0.701353$

N $5.1476210 .089592-2.287381$

H -3.778511-3.764010 -1.985470

H -2.667980 - $0.991351-2.618784$

H $-0.811563-2.668824-3.288686$

H $-1.768127-4.744921-2.236624$

H $-1.617741-4.000521-0.654245$

H $1.863776-5.374471-0.488671$

H $1.857919-5.428716-2.768963$

H $\quad 0.269919-5.479815-3.536283$

H $1.146552-3.938651-3.390883$

H $-0.726162-4.8586911 .028455$

H $0.722922-5.6486441 .577003$

H $0.476789-2.6368831 .074151$

H $0.275585-3.4210392 .642145$

H $2.631850-4.2991262 .471054$

H $2.142023-2.1762873 .737554$

H $2.266432-1.2580602 .252733$

H $3.905559-0.5305404 .268009$

H $4.638910-1.1839341 .032284$

H $4.218923-2.7372824 .998284$

H $5.780684-2.7531244 .212227$

H $4.567649-3.9793423 .793256$

H $6.540048-1.3204762 .249028$

H $6.049725-3.471653-0.672264$

H $4.552813-4.214811-1.172814$

H $4.911442-5.2256330 .741488$

H $1.061895-0.430803-0.045293$

H $1.2041021 .682264-2.281422$

H 3.2815732 .2173551 .468693

H 3.1975100 .6518360 .703035

H $1.7852991 .289596 \quad 1.578250$

H $4.416514-2.466266-2.569242$

H $3.604556-1.694409-1.203474$

H $5.6852161 .658294-3.510334$

H $7.0280380 .945615-2.588183$

H $5.9546101 .890387-0.413868$

H $5.3510263 .883977-2.660382$

H $6.1486145 .278661-0.663967$

H $5.498027 \quad 4.0933600 .443532$

H $2.9138366 .980326-1.117638$

H $4.7044956 .963507-1.592014$

H 2.0399855 .2951400 .301240

H 3.1542954 .2441331 .116302

H $1.8292433 .789205-1.654058$

H $4.1798110 .121822-2.594557$

C $-5.944771-2.020896-1.006091$

O $-5.404226-1.012530-0.128546$

C $-5.411836-1.525947 \quad 1.215404$

C $-6.301495-2.7671651 .192338$

C $-6.015539-3.313793-0.190714$

H $-4.381040-1.8330861 .430101$

C -5.848237 -0.4619742 .227297$

H $-6.053283-3.4698231 .993684$

H $-7.364140-2.5143331 .282213$

C $-7.054317-4.300839-0.692322$

H -5.042016 $-3.815222-0.148093$

C $-5.123092 \quad 0.893757 \quad 2.134113$

$\begin{array}{llll}\text { O } & -7.247322 & -0.199018 & 2.047430\end{array}$

H -5.748142 -0.8775453 .237698$

H -5.5235001 .4683591 .289900$

O -5.4839101 .6376813 .308323$

C -3.589188 0.8430162 .014464

$\begin{array}{llll}C & -2.950585 & 2.225037 & 2.259981\end{array}$

H -3.323134 0.4582361 .024200

O $-3.077935-0.0705632 .985930$

H $-3.6080903 .001948 \quad 1.858271$

H -2.878380 2.3887793 .342083

H $-6.802190-4.651885-1.697980$

H -7.106736 $-5.172990-0.032464$

H $-8.051462-3.849607-0.731752$
C $-5.148870-2.072634-2.315787$

H -7.5081380 .4560052 .717408$

H -5.0828981 .1899164 .073199$

H $-2.125576-0.1744772 .822805$

H $-5.055738-1.054966-2.712714$

H $-5.714573-2.651746-3.055624$

H $-6.960668-1.680687-1.247904$

C -2.191303 $3.427478-0.440588$

O $-1.5885552 .296327 \quad 0.202042$

C - 1.5446212 .3565101 .633605

C -0.7771993 .6089062 .080156$

C $-1.360811 \quad 4.8764201 .466725$

C $-1.479537 \quad 4.729451-0.048139$

H $-3.2513103 .487985-0.167864$

C -2.107038 $3.202819-1.958946$

H $-0.959715 \quad 1.4882401 .959984$

$\begin{array}{lllll}\text { O } & -0.776182 & 3.732234 & 3.498403\end{array}$

H $0.270616 \quad 3.5254841 .769416$

H $-2.3285395 .131792 \quad 1.914364$

$\begin{array}{llll}\text { O } & -0.489367 & 5.976136 & 1.740524\end{array}$

H $-0.4805294 .762697-0.498663$

O $-2.2132125 .838581-0.565986$

C $-3.1098312 .180521-2.450690$

H $-2.321628 \quad 4.138920-2.486832$

H $-1.1091392 .850045-2.240002$

H -0.3862952 .9187713 .861751$

H $-0.388634 \quad 6.0312242 .706424$

H $-1.7503716 .647539-0.287578$

O $-3.1339462 .215630-3.816526$

O $-3.777567 \quad 1.444644-1.738052$

C $-4.0641191 .335615-4.455811$

H $-5.0379151 .355937-3.956573$

H $-4.1971491 .674990-5.486661$

H $-3.6578870 .321346-4.470890$

SCF Energy (B3LYP/6-31G**//MMFF)= -3245.90437155

0500229

MM̄FF Geometry

C -4.175365 $2.095416-2.334413$

C $-5.2130951 .292443-2.052310$

C $-5.110059-0.100245-1.460037$

O $-3.729751-0.462801-1.231573$

C $-5.826806-0.118493-0.095765$

C $-5.700010-1.4324910 .650630$

C $-5.094481-1.5692891 .847515$

C $-6.316822-2.630458-0.022911$

C $-4.464138-0.4906852 .685309$

C $-2.979213-0.2332652 .388612$

C $-2.046675-1.3736832 .828058$

C -0.582121 $-0.961800 \quad 2.662977$

C $0.381773-2.1026003 .022262$

O $-2.310798-2.5261062 .024903$

O $1.682129-1.7503672 .526325$

C $-0.051273-3.385608 \quad 2.280233$

C $0.526920-2.2673934 .539553$

O $0.707714-4.5090002 .719367$

C -1.558728 -3.6898112 .399812$

C $-2.019951-4.8515781 .485289$

O $-1.853125-4.0772813 .747869$

C $-3.039817-1.028291-2.257331$

O $-3.475103-1.376324-3.342258$

C $-1.619991-1.062245-1.846351$

C $-0.664924-1.052803-2.787078$

C $0.777360-1.001530-2.543538$

C $1.247491-1.138121-1.119863$

C $-1.772805-4.649244-0.014169$

C $-0.490181-5.288455-0.522905$

C $0.910790-5.455092-2.579006$

O $0.192386-6.058460 \quad 0.147120$

C $2.074014-4.522494-2.453362$

C $2.746554-4.036957-3.508179$

C $3.946445-3.140118-3.397511$

C $3.808457-1.875271-4.215484$

C $4.367752-1.774811-5.433226$

C $3.102320-0.698806-3.583173$

C $1.605964-0.841880-3.596409$

N $-0.219533-4.927976-1.836620$

H $-3.1619611 .739496-2.174006$ 
H $-6.2215231 .662578-2.224991$ H -5.582907 -0.816938 -2.142165 H $-6.893850 \quad 0.100491-0.234647$ H -5.4303720 .7029850 .511336$ H -5.055682 -2.562707 2.292701 H $-6.298090-3.5205530 .615103$ H -7.363719 -2.428425 -0.271898 H $-5.778516-2.875732-0.943541$ H -5.017785 0.4481322 .572809 H $-4.583867-0.7623453 .741894$ H $-2.852710-0.040567 \quad 1.316820$ H -2.6878940 .6839202 .916050$ H $-2.260799-1.6039423 .878403$ H -0.359752 -0.0731063 .264815$ H $-0.401644-0.6695041 .619677$ H $1.939360-0.9040112 .930872$ H $\quad 0.195854-3.2375101 .223399$ H $0.902096-1.3408674 .990953$ H $1.265302-3.0388994 .784765$ H -0.413222 -2.518401 5.034906 H $1.647478-4.2879232 .601383$ H -1.599634 -5.800139 1.840391 H -3.107514 -4.9508711 .614471$ H -2.683986 -4.580942 3.749249 H -1.403011 -1.028912 -0.785017 H $-0.965532-1.044043-3.834145$ H $0.804462-2.024212-0.654570$ H $0.966531-0.256694-0.534426$ H $2.327391-1.265533-1.028756$ H -2.589801-5.130813 -0.566740 H - $1.783821-3.587310-0.275616$ H $\quad 0.588005-5.576701-3.617837$ H $1.182246-6.438045-2.180907$ H $2.395803-4.258462-1.448675$ H $2.440304-4.327360-4.510768$ H $4.819813-3.710292-3.740312$ H $4.157430-2.878054-2.353167$ H $4.299143-0.860463-6.013299$ H $4.902174-2.607033-5.879349$ H $3.3432430 .223398-4.129248$ H $3.503087-0.534239-2.580574$ H $1.170124-0.763680-4.593291$ H $-0.811536-4.242922-2.297367$ C -3.571252 $4.511625-1.918343$ O $-2.1850194 .136521-1.752773$ C $-1.8986174 .070438-0.344827$ C -2.931391 4.9784020 .306514 C $-4.1639254 .640617-0.511015$ H -2.075185 $3.033997-0.026870$ C $-0.4423864 .441685-0.046156$ H -3.0552924 .7738461 .374054$ H -2.6697206 .0360600 .184113$ C $-5.2706815 .675677-0.407098$ H $-4.5552263 .677430-0.161239$ C $0.5777653 .444529-0.640751$ O $-0.1815625 .760996-0.519754$ H -0.3135824 .4618191 .042163$ H $0.2906222 .429128-0.339792$ O $\quad 0.5546423 .487285-2.065922$ C $2.0214753 .693606-0.155849$ C $3.0063452 .639188-0.696781$ H 2.0290593 .6776080 .939408 O $2.4860414 .976563-0.570184$ H $2.5973821 .641166-0.511868$ H $3.1008062 .754580-1.783396$ H -5.6207315 .7621060 .626731$ H $-4.9278216 .664625-0.729079$ H $-6.1245145 .394015-1.031601$ C $-4.2795483 .507099-2.838201$ H $-0.2832145 .747163-1.487230$ H $-0.3438393 .253651-2.354238$ H $1.9054095 .645223-0.169504$ H -3.799735 $3.533703-3.824346$ H -5.325848 $3.802509-2.975990$ H $-3.5620795 .489407-2.416264$ C 4.0082601 .4852761 .939752 O $4.3637792 .749574 \quad 1.366977$ C $4.4127422 .765107-0.064701$
C $5.435458 \quad 1.734275-0.563898$

C 5.1981870 .3487790 .035685

C 5.0466280 .4268101 .555052

H $3.017723 \quad 1.1799171 .582423$

C 3.9297701 .6673683 .459673

H $4.7949993 .754842-0.345043$

O $5.4096191 .664177-1.985336$

H $6.4400352 .072874-0.278697$

H $4.319675-0.124302-0.412432$

O $6.301147-0.501088-0.286523$

H $\quad 6.016170 \quad 0.6624592 .011697$

O $4.634668-0.8436012 .051152$

C 2.8193892 .6149023 .851927

H 3.7609640 .7184723 .980461

H 4.8681712 .0901923 .838900

H $6.0420280 .976593-2.255602$

H $7.103513-0.1124320 .101949$

H $5.289644-1.4983651 .754425$

O 1.6074282 .0940553 .506567

O 3.0095273 .6857894 .414465

C 0.4738062 .9145843 .806513

H -0.4010062 .4806813 .314846$

H 0.3026822 .9282644 .886656

H 0.6166923 .9304483 .425310

SCF Energy $\left(B 3 L Y P / 6-31 G^{* *} / / M M F F\right)=-3245.91813426$

0500230

MM̄FF Geometry

C $3.591347-4.198329-2.256934$

C $4.323815-3.092758-2.052901$

C $5.050578-2.696883-0.789531$

O $4.531968-1.398962-0.407372$

C $4.857543-3.6361330 .420117$

C $5.591495-3.1764491 .667454$

C $4.988813-2.8058632 .815214$

C $7.095753-3.1660571 .573909$

C $3.516681-2.7999593 .126042$

C $2.862210-1.4719932 .747945$

C $1.426427-1.3053383 .267903$

C $0.470757-2.3669492 .716870$

C $-0.987660-2.1006653 .122437$

$\begin{array}{lllll}\text { O } & 1.005066 & 0.002517 & 2.855534\end{array}$

O $-1.827494-2.9429862 .319157$

C -1.340233 -0.640063 2.773667

C $-1.258824-2.4899504 .580075$

O $-2.641629-0.2974893 .242623$

C -0.3066090 .3791413 .296662$

C -0.5738331 .8124442 .779814$

$\begin{array}{lllll}\text { O } & -0.371427 & 0.428625 & 4.726238\end{array}$

C $5.272140-0.292494-0.680784$

O $6.348743-0.241598-1.251751$

C $4.5222430 .867629-0.149588$

C $4.9928912 .114104-0.304976$

C $4.337773 \quad 3.3377380 .168172$

C $3.067443 \quad 3.1722200 .959944$

C -0.5747921 .9520821 .256099$

C -0.5457723 .4040910 .805824$

C $-0.3229594 .769131-1.278888$

O -0.6526874 .3522631 .578185$

C $1.0827724 .976631-1.748013$

C $1.7864656 .100183-1.538374$

C $3.1840936 .305166-2.053281$

C $4.1533826 .777162-0.989036$

C $4.7991447 .947981-1.124097$

C 4.3867255 .9052880 .228266

C $4.8920524 .532012-0.128315$

N $-0.397723 \quad 3.504872-0.570472$

H $3.496072-4.939801-1.470157$

H $4.409915-2.379706-2.871742$

H $6.118278-2.630781-1.030276$

H $5.210888-4.644910 \quad 0.171071$

H $3.785375-3.7247790 .626268$

H $5.617651-2.4774183 .641942$

H $7.570064-2.9651022 .540629$

H $7.461049-4.1386671 .228375$

H $7.435888-2.3957800 .875469$

H $3.015652-3.6379712 .631877$

H $3.399338-2.9767834 .202523$ 
H $3.465988-0.6352243 .125317$ H $2.859264-1.3571581 .657378$ H $1.451124-1.3392974 .363399$ H $0.766756-3.3724523 .037810$ H $0.529461-2.3729591 .619155$ H -1.557732 -3.865515 2.467229 H $-1.378227-0.5775461 .679911$ H -1.070997 -3.559884 4.731516 H $-2.310836-2.3311074 .841568$ H -0.638264 -1.944382 5.293718 H -3.263018-0.949783 2.876102 H -1.5192012 .1921863 .188764$ H 0.2059682 .4715503 .187184 H $-0.004321 \quad 1.2779275 .022762$ H 3.5860290 .6607180 .356983 H $5.9345142 .256188-0.833049$ H 2.6278294 .1106951 .300311 H 3.2474412 .5704651 .858113 H 2.3102272 .6723780 .349059 H 0.2962611 .4439870 .828047 H -1.4766991 .4929990 .837972$ H $-0.6710135 .582658-0.634713$ H $-0.9925004 .694190-2.141333$ H $1.5383004 .165399-2.311860$ H $1.3269366 .925882-1.000328$ H $3.1275197 .040941-2.866188$ H $3.5743005 .386854-2.509188$ H $5.4976028 .299921-0.371941$ H $4.6450058 .583718-1.989846$ H $3.465148 \quad 5.8821220 .814166$ H 5.1366546 .3640710 .886255 H $5.8239484 .543377-0.695327$ H $-0.3121252 .653917-1.119299$ C $1.347050-4.429223-3.440186$ O $0.924720-3.081184-3.152216$ C $-0.194681-3.134342-2.238415$ C $-0.631856-4.593290-2.197107$ C $0.687324-5.314765-2.384376$ H $0.204005-2.827259-1.263092$ C -1.278550 -2.149667-2.704014 H $-1.132251-4.855762-1.260098$ H -1.313724 -4.820491-3.026265 C $0.538447-6.766879-2.801302$ H $1.231348-5.270658-1.433605$ C $-2.566575-2.229609-1.861826$ O $-0.728839-0.833150-2.614164$ H -1.508336 -2.326133 -3.761337 H -2.933270 -3.262026 -1.915101 O $-2.249074-1.967860-0.497111$ C $-3.732328-1.322935-2.303275$ C $-3.4619250 .190223-2.207661$ H -4.586381-1.576426 -1.664192 O $-4.087781-1.665680-3.641268$ H $-2.8830010 .402692-1.303793$ H $-2.8589750 .504965-3.067210$ H $-0.005354-7.332394-2.037575$ H - $0.012021-6.860300-3.743491$ H $1.518656-7.235382-2.934890$ C $2.874159-4.499936-3.544526$ H $0.084243-0.820737-3.147913$ H $-3.062256-2.0798600 .024002$ H -4.920650 -1.212492 -3.853756 H $3.203158-3.796417-4.319979$ H $3.174431-5.500170-3.877727$ H $0.933784-4.665821-4.429991$ C -5.2192530 .8921710 .166946$ O $-5.666490 \quad 0.617133-1.166297$ C $-4.7639591 .028174-2.199445$ C $-4.5432582 .544835-2.112488$ C $-4.0901892 .971013-0.719340$ C -5.0091482 .3978970 .358262$ H -4.2843630 .3536660 .363783$ C $-6.2827050 .353929 \quad 1.128640$ H $-5.275770 \quad 0.831799-3.149307$ O $-3.580212 \quad 2.976507-3.067766$ H $-5.4827823 .063141-2.344003$ H -3.054452 $2.673874-0.538420$ O $-4.1217024 .396289-0.625645$
H -5.9731112 .9217840 .333685$

O -4.4270012 .6286731 .639848$

C $-6.302790-1.1559961 .107925$

H -6.1102550 .6818832 .160107$

H -7.2843480 .6997320 .845617$

H -3.897953 $2.705250-3.945955$

H $-3.5505114 .744913-1.331445$

H $-4.266165 \quad 3.5847801 .718188$

O $-5.374154-1.6469641 .977402$

O $-7.039015-1.817100 \quad 0.387106$

C $-5.291649-3.0738402 .047968$

H -6.274533 -3.5081912 .254779$

H $-4.882587-3.4696621 .113715$

H $-4.617808-3.3350832 .868065$

SCF Energy (B3LYP/6-31G**//MMFF)= -3245.91777326

05_00231

MM̄FF Geometry

C $0.221547 \quad 4.379644 \quad 1.222117$

C 0.1419853 .9700762 .498138

C -0.6349242 .7727493 .005523$

$\begin{array}{llll}\text { O } & -1.365026 & 2.130486 & 1.937604\end{array}$

C $\quad 0.3848801 .755976 \quad 3.559898$

C $-0.2112790 .444076 \quad 4.024993$

C $0.104901-0.7569453 .500633$

C $-1.1943700 .537125 \quad 5.161923$

C $1.074498-1.0475832 .386479$

C $0.450337-1.0100020 .982749$

C $-0.535604-2.1580180 .711497$

C $-0.994845-2.148405-0.748027$

C $-2.033462-3.247184-1.028173$

O $-1.671238-2.004448 \quad 1.564856$

O $-2.643086-2.963149-2.297170$

C $-3.151634-3.1731650 .036005$

C $-1.377908-4.626939-1.161741$

O $-4.042016-4.279680-0.081273$

C $-2.620500-3.0766421 .480638$

C $-3.728121-2.7895532 .522963$

O $-2.019295-4.3258751 .843999$

C $-2.6212902 .577758 \quad 1.670492$

$\begin{array}{llll}\text { O } & -3.245348 & 3.434345 & 2.274819\end{array}$

C $-3.0851151 .873413 \quad 0.454283$

C $-4.0860362 .397276-0.268587$

C $-4.613887 \quad 1.862330-1.525904$

C $-4.1151270 .513885-1.973192$

C $-4.510010-1.4881992 .311766$

C $-5.841252-1.6760821 .602616$

C $-7.777673-0.3645330 .741991$

O $-6.315655-2.7761201 .333638$

C $-7.651415-0.268757-0.745290$

C $-8.2270720 .700943-1.472059$

C $-8.1546970 .778508-2.969706$

C $-7.6356132 .110689-3.460706$

C $-8.4782143 .068685-3.882366$

C $-6.1380502 .295210-3.547809$

C $-5.5118132 .597992-2.214345$

$\mathrm{N}-6.458851-0.460468 \quad 1.338234$

H $-0.358849 \quad 3.8826820 .449967$

H 0.7504394 .4795803 .243573

H -1.3199093 .0917673 .800089$

H 0.9271282 .2024764 .404603

H 1.1398511 .5740572 .786645

H $-0.378827-1.6404823 .915294$

H -1.499516 -0.4489395 .528616$

H $-0.750444 \quad 1.0772146 .004432$

H -2.1000921 .0640494 .846122$

H $1.918873-0.3503562 .421759$

H $1.517875-2.0357992 .563545$

H $-0.052609-0.0463540 .837977$

H $1.265861-1.0596600 .250423$

H $-0.031791-3.1020290 .947240$

H $-0.139807-2.247455-1.427946$

H $-1.449299-1.175206-0.979319$

H $-1.939328-2.934421-2.967878$

H $-3.740655-2.276109-0.184640$

H $-0.645825-4.625306-1.978320$

H $-2.116817-5.391862-1.424886$

H $-0.859069-4.945063-0.255182$ 
H $-4.373087-4.289661-0.995630$ H -4.394762 -3.6555902 .612974$ H $-3.234051-2.7034703 .501760$ H -1.994482 -4.385829 2.813329 H $-2.557931 \quad 0.9722140 .162780$ H -4.541782 3.3263350 .071910 H $-3.0558690 .565974-2.246947$ H $-4.6551540 .111044-2.832456$ H -4.235659 -0.221865-1.171623 H -4.747570 -1.065511 3.296685 H -3.911512 -0.7466891 .774161$ H -8.2665490 .5140791 .174393$ H -8.360709 -1.2529201 .005476$ H -7.087459 -1.049607 -1.249936 H -8.814516 $1.464651-0.967330$ H $-9.165977 \quad 0.605431-3.360527$ H -7.537603 $-0.028422-3.384058$ H $-8.1120934 .016001-4.264167$ H $-9.5536772 .928914-3.849673$ H $-5.9035353 .137924-4.212293$ H $-5.6956961 .425343-4.041383$ H -5.814991 $3.562160-1.803561$ H $\begin{array}{llll}-5.965917 & 0.403929 & 1.542898\end{array}$ C $2.1991444 .770285-0.203358$ O 2.9095843 .7341850 .500058 C $4.2425843 .630705-0.042495$ C $4.3329584 .670359-1.154099$ C $3.2923685 .692459-0.727519$ H 4.9191693 .8853940 .782063 C $4.4559942 .179437-0.490281$ H $5.3377695 .093391-1.247225$ H $4.0625484 .231631-2.122843$ C $2.8472026 .611672-1.850280$ H 3.7049796 .2927920 .094172 C $5.8015651 .920718-1.185061$ O 4.3715251 .3444810 .670285 H $3.6315151 .881594-1.148110$ H $5.8448652 .474106-2.130335$ O $6.8442872 .451245-0.357212$ C $6.1124610 .430974-1.436677$ C $5.032237-0.302763-2.251278$ H $6.279731-0.073092-0.478523$ O $7.3555050 .354556-2.144734$ H $4.070112-0.229573-1.736787$ H $4.9184240 .197469-3.220930$ H $3.6957887 .186000-2.236096$ H $2.4159266 .047649-2.684153$ H $2.0923157 .321269-1.497367$ C 1.1910365 .4259500 .742158 H 3.5120471 .5182411 .091297 H $7.6894902 .269009-0.802603$ H $7.2337630 .787473-3.007009$ H $0.639173 \quad 6.2204150 .229032$ H $1.7144105 .878173 \quad 1.593231$ H $1.672174 \quad 4.288434-1.038908$ C $4.533850-2.702716-0.426196$ O $5.665991-2.480937-1.276398$ C $5.375048-1.790180-2.496340$ C $4.320492-2.562131-3.304703$ C $3.084451-2.897083-2.472261$ C $3.491366-3.555461-1.154785$ H $4.092384-1.740973-0.138255$ C $5.038170-3.3954540 .843282$ H $6.301211-1.808241-3.084340$ O $3.921827-1.829025-4.458071$ H $4.759007-3.504028-3.659554$ H $2.468176-2.008414-2.291800$ O $2.262699-3.815194-3.196421$ H $3.891090-4.558439-1.352223$ O $2.341497-3.707080-0.328767$ C $5.826101-2.4319571 .698585$ H $4.219829-3.7939371 .453452$ H $5.697399-4.2364680 .597023$ H $4.721690-1.652327-4.982425$ H $2.044160-3.397691-4.047349$ H $1.690772-4.227742-0.829990$ O $4.974673-1.6084092 .375052$ O $7.048944-2.4068441 .748023$
C $5.599245-0.6259223 .206636$

H 6.2636390 .0099182 .613340

H $4.814480-0.0017213 .642322$

H $6.151347-1.1138704 .015293$

SCF Energy (B3LYP/6-31G**//MMFF)= -3245.91166337

05 00232

MM̄FF Geometry

C 3.7632042 .6903200 .483642

C 3.7183763 .1980761 .725284

C 2.6958202 .8177172 .769511

O 1.4078192 .5593522 .166376

C 3.1579601 .5466583 .507685

C $2.1300950 .992003 \quad 4.474021$

C $1.447012-0.1576034 .296244$

C 1.9071021 .8168745 .715355

C $1.536027-1.1259663 .147604$

C $0.290800-1.1221762 .255696$

C -1.011011-1.6198812.905862

C $-0.922280-3.0946043 .313141$

C $-2.264033-3.645563 \quad 3.816725$

O $-2.046210-1.4395641 .926090$

O $-2.137972-5.0752583 .863377$

C -3.359487 -3.299068 2.787836

C $-2.550733-3.1943345 .253486$

O $-4.645020-3.6890593 .264660$

C -3.358443-1.813739 2.369264

C $-4.349410-1.5290061 .218103$

O $-3.772301-1.0148843 .484245$

C 0.6310843 .6414341 .883521

O 0.9389524 .8174941 .994020

C -0.6860713 .1408121 .433178$

C -1.6179474 .0047701 .002665$

C -2.977395 3.6614910 .576342

C $-3.3937882 .221593 \quad 0.709513$

C $-4.065105-2.282896-0.081735$

C $-4.871019-1.714274-1.234851$

C $-4.9354990 .196230-2.824038$

O $-5.836484-2.308511-1.708107$

C $-4.4628541 .614231-2.821247$

C $-5.2744362 .680974-2.871018$

C $-4.7705684 .097488-2.860219$

C $-5.4103154 .943021-1.779062$

C $-6.1384166 .026689-2.097808$

C $-5.2174244 .538948-0.332053$

C -3.7832654 .6418900 .116025$

N -4.397946 -0.482023 -1.659282

H $3.020511 \quad 1.9565860 .182409$

H 4.4836773 .9048072 .037188

H 2.5941673 .6413003 .487762

H 4.0829691 .7590504 .060813

H $3.438246 \quad 0.7875712 .771288$

H $0.742581-0.4534785 .072652$

H $1.2156621 .336376 \quad 6.415864$

H 2.8537201 .9714916 .243383

H 1.4871832 .7941325 .457776

H $2.401299-0.9208292 .511923$

H $1.715576-2.1279303 .552242$

H $0.128102-0.1120991 .859033$

H $0.496542-1.7398391 .373260$

H - $1.241596-0.9893213 .771130$

H -0.149634 -3.251976 4.074370

H $-0.609670-3.6897012 .443191$

H -3.005290 -5.439409 4.111357

H -3.163685 -3.920470 1.905266

H -1.764656 -3.553769 5.928661

H -3.484629 -3.627846 5.627687

H -2.605917 -2.109027 5.359833

H $-4.928613-3.0321473 .923145$

H $-5.377484-1.7337251 .544507$

H $-4.331080-0.4519001 .014145$

H -4.089324 -0.1593093 .150338$

H $-0.8601382 .072728 \quad 1.486752$

H -1.3702315 .0648200 .966784$

H -3.3255711 .8976091 .754098$

H -2.7492041 .5786890 .101604$

H -4.4184052 .0272050 .389798$

H $-3.003160-2.233865-0.347939$ 
H $-4.322250-3.3428770 .019355$ H $-6.0272850 .113032-2.813707$ H -4.560613 $-0.324117-3.711453$ H $-3.3885181 .774854-2.778950$ H $-6.3498032 .533303-2.934272$ H $-4.9737354 .526687-3.849895$ H $-3.6807504 .128260-2.738735$ H $-6.6036346 .641025-1.334009$ H $-6.2864756 .325975-3.130046$ H $-5.6424973 .543679-0.182693$ H -5.8042995 .1956820 .324103$ H -3.3870535 .6575070 .070885$ H $-3.492617-0.179798-1.311301$ C $5.8094851 .920848-0.804988$ O $5.1414460 .749375-1.318993$ C $5.686040-0.408588-0.659022$ C $6.9859380 .051705-0.005982$ C 6.6235441 .4625540 .407512 H $4.975287-0.6805180 .130684$ C $5.857559-1.579353-1.630227$ H $7.275043-0.5835130 .836876$ H $7.8178240 .068745-0.719501$ C 7.8238752 .3414310 .711852 H 5.9958651 .3997821 .304390 C $4.582290-2.012078-2.380129$ O $6.832692-1.213859-2.616796$ H $6.280414-2.433909-1.087295$ H $4.340577-1.274768-3.155885$

O $4.925770-3.223815-3.068252$

C $3.346379-2.250747-1.488922$

C $2.210742-2.983690-2.231141$

H $2.996318-1.284227-1.110217$

O $3.736845-3.040953-0.364132$

H $2.116392-2.567687-3.238877$

H $2.486695-4.039852-2.335561$

H 8.4036561 .9262331 .542546

H $8.4900662 .425914-0.153316$

H 7.5056413 .3503790 .992743

C $4.8042803 .045971-0.538942$

H $\quad 6.946066-1.977747-3.208042$

H $4.257327-3.373673-3.757683$

H $3.017498-3.0063570 .288056$

H $4.2758933 .282502-1.470869$

H $5.3419103 .953718-0.240175$

H $6.4861552 .252397-1.603473$

C $-0.060300-0.979151-2.652807$

O $0.377813-1.547557-1.412460$

C $0.855431-2.895534-1.493755$

C $-0.240131-3.804616-2.064862$

C $-0.757115-3.296752-3.406207$

C -1.167555-1.830552 -3.289909

H $\quad 0.786576-0.898275-3.344279$

C $-0.5735790 .438094-2.352767$

H $1.026461-3.226090-0.462989$

O $0.246118-5.135951-2.198076$ H -1.076217 -3.840182 -1.353863 H - $0.014522-3.447503-4.198485$ O $-1.898772-4.079083-3.763643$ H -2.084303 -1.758273 -2.694721 O $-1.457643-1.317333-4.588563$ C $0.5461151 .442235-2.185247$ H -1.190789 $0.793701-3.184931$ H -1.163852 $0.443015-1.430143$ H - $0.471776-5.670966-2.577995$ H -2.163231 -3.819582 -4.662147 H $-2.245596-1.780287-4.919310$ O $0.0059562 .697422-2.160731$ O $1.7338051 .168797-2.088427$ C $0.9407853 .775724-2.060845$ H $1.691103 \quad 3.710541-2.854738$ H $\quad 0.390454 \quad 4.713394-2.175807$ H $1.4171793 .767754-1.078050$

SCF Energy (B3LYP/6-31G**//MMFF) $=-3245.90905186$

05 00233

MM̄MF Geometry

C -3.452801-1.914006 2.627934

C $-4.785067-2.0828682 .600728$
C $-5.631570-1.9995001 .351645$

O $-5.081499-1.0077740 .455980$

C $-5.644905-3.3588660 .625104$

C $-6.384876-3.329682-0.698401$

C $-5.795118-3.487789-1.900973$

C $-7.873086-3.115222-0.607966$

C $-4.338584-3.747016-2.183190$

C $-3.508113-2.464923-2.146600$

C $-2.066914-2.617069-2.651979$

C $-1.260741-3.636723-1.845522$

C $0.218311-3.664109-2.261575$

O $-1.474142-1.316674-2.530284$

O $0.939952-4.399229-1.260680$

C $0.769716-2.223039-2.250717$

C $0.426828-4.414507-3.582461$

O $2.099915-2.208745-2.762145$

C $-0.126999-1.219528-3.010885$

C $0.3111310 .251840-2.822736$

O $-0.064229-1.500926-4.413662$

C $\begin{array}{llll}-5.439116 & 0.290420 & 0.640257\end{array}$

O -6.2306640 .7305621 .457199$

C $-4.6524691 .094623-0.321585$

C $-4.7214322 .433546-0.293087$

C $-3.9533223 .348820-1.140733$

C $-3.0533152 .739112-2.183848$

C $0.3855390 .721092-1.367639$

C $0.6085862 .220054-1.254272$

C 0.8835524 .0013750 .472807

O $0.7457002 .958458-2.225460$

C -0.4306234 .6620420 .743515$

C $-0.794225 \quad 5.8412180 .215719$

C $-2.092747 \quad 6.525608 \quad 0.537524$

C $-2.9009746 .887105-0.691461$

C $-3.2391448 .164876-0.934931$

C $-3.3314605 .783836-1.639655$

C $-4.0842784 .678027-0.947140$

N 0.6541692 .6269340 .071341

H -2.921134 -1.737006 1.695916

H $-5.313826-2.2989603 .525781$

H $-6.653364-1.7139931 .629840$

H $-6.104575-4.1194921 .269895$

H -4.611746 -3.689953 0.468903

H $-6.420187-3.434613-2.791376$

H $-8.365390-3.206493-1.582126$

H $-8.323474-3.8593300 .057060$

H $-8.096933-2.117298-0.218973$

H -3.947253 -4.489626-1.480043

H $-4.262434-4.206248-3.176636$

H -4.001976 -1.681588 -2.737893

H -3.471408 -2.075066 -1.123690

H $-2.099269-2.897122-3.711308$

H -1.689645 -4.641688 -1.934742

H -1.315969 -3.380339 -0.778068

H $0.557161-5.291740-1.209188$

H $\quad 0.835842-1.930026-1.195852$

H $\quad 0.083197-5.452019-3.491702$

H $1.490163-4.473712-3.839664$

H $-0.107781-3.964114-4.421261$

H $2.473688-1.326988-2.598424$

H $1.2717500 .428409-3.321519$

H $-0.4083940 .890822-3.354399$

H $-0.322941-0.701046-4.900761$

H -4.007897 $0.559119-1.009833$

H -5.3784782 .9070710 .435214$

H -2.266853 2.146965 -1.708301

H $-2.5635543 .473345-2.826549$

H -3.626387 $2.086238-2.852098$

H $-0.5421150 .474010-0.839050$

H $1.2190100 .227921-0.855555$

H $1.4569564 .519916-0.302518$

H 1.4796033 .9739721 .390240

H -1.104482 4.1587841 .433252

H $-0.1118826 .360680-0.452867$

H -1.851562 7.4294411 .112242

H -2.7089565 .9075331 .202278$

H $-3.8195248 .440465-1.809251$

H -2.942941 $8.966582-0.266533$

H $-2.4329435 .417359-2.144176$ 
H -3.986206 $6.188810-2.421558$ H $-4.7994105 .023602-0.199325$ H $0.461397 \quad 1.9485510 .802426$ C -1.601701 -0.8326393 .930488$ O $-0.556527-1.0804022 .961831$ C $0.712589-1.0935723 .645484$ C $0.483580-0.2762944 .904028$ C $-0.908734-0.7302005 .292565$ H $0.905771-2.1445793 .897813$ C $1.809757-0.5656652 .713197$ H $1.226503-0.4731255 .682309$ H $\quad 0.490514 \quad 0.797257 \quad 4.678047$ C $-1.605826 \quad 0.207450 \quad 6.263565$ H - $0.836424-1.7249705 .753137$ C $3.173921-0.3975823 .404640$ O $1.950839-1.4925341 .632100$ H 1.4939690 .3854502 .270974 H $3.109250 \quad 0.4013194 .152895$ O $3.463992-1.6032594 .126341$ C $4.362191-0.1318452 .461773$

C 4.1756281 .0756471 .527641 H 4.570622 -1.035507 1.879932 O 5.5218200 .0865393 .275356 H 3.3068180 .9086330 .883957 H $3.962397 \quad 1.9652902 .132834$ H -1.0460980 .2748897 .202161$ H -1.694098 1.2184055 .851691 H -2.613654 -0.1514886 .495073$ C -2.622069-1.975648 3.878009 H $1.073968-1.6003911 .225627$ H $4.325898-1.4816104 .560468$ H $5.3752290 .898643 \quad 3.789710$ H -3.269774 -1.939193 4.761641 H -2.096228 -2.9382783 .882947$ H -2.0696270 .1213143 .656467$ C $5.036629-0.280733-1.065758$

O $5.888854 \quad 0.177651-0.009378$

C 5.4273281 .3553840 .662289

C $5.2834132 .508072-0.342494$

C $4.4479022 .114882-1.560414$

C $4.9331730 .791825-2.153860$

H $4.039263-0.504426-0.668298$

C $5.635592-1.580714-1.612503$

H 6.2279851 .6421161 .355845

O 4.7383603 .6657010 .281336

H $6.2855352 .788877-0.690929$

H $3.3889122 .048716-1.297677$

O $4.5392193 .139308-2.551932$

H $5.9076540 .938446-2.636517$

O $4.016930 \quad 0.362382-3.156211$

C $5.576224-2.683749-0.581821$

H $5.117642-1.930493-2.512105$

H $6.689946-1.436235-1.877899$

H 3.7915193 .5096590 .434764

H $5.473304 \quad 3.228307-2.807134$

H $3.940634 \quad 1.080383-3.807828$

O $4.285522-3.080538-0.391232$

O $6.561706-3.135937-0.013259$

C $4.095491-4.0993960 .595178$

H $3.021515-4.2669310 .709884$

H $4.564782-5.0315640 .267054$

H 4.502007 -3.779106 1.559475

SCF Energy (B3LYP/6-31G**//MMFF) $=-3245.90976874$

05 00234

MM̄MF Geometry

C $3.0230663 .564522-0.487435$

C $4.3293593 .636926-0.186781$

C $5.2704532 .458437-0.081678$

O $4.590924 \quad 1.289144 \quad 0.429809$

C $5.8484092 .113739-1.465979$

C $6.8786031 .000737-1.427032$

C $6.652702-0.274880-1.802215$

C $8.2502251 .417028-0.960870$

C $5.362259-0.885045-2.281997$

C $4.983276-2.111674-1.437485$

C $3.573929-2.665787-1.712870$

C $3.434536-3.246157-3.121100$
C $2.003967-3.735519-3.396329$

O $2.632451-1.609007-1.513537$

O $1.882594-3.972285-4.807262$

C $1.017700-2.603873-3.041855$

C $1.709511-5.071584-2.704947$

O $-0.332335-3.050742-3.154172$

C $1.255333-1.989589-1.643081$

C $0.410551-0.700464-1.492543$

O $0.871023-2.934082-0.645228$

C $4.421707 \quad 1.211240 \quad 1.778908$

O 4.7143452 .0571632 .608752

C $3.817103-0.1047292 .076640$

C $3.360361-0.3736703 .307898$

C $2.741481-1.6289053 .737629$

C $2.672762-2.7591732 .744902$

C $0.6819850 .095535-0.206881$

C $-0.333557-0.1404890 .894088$

C -1.2347640 .9247042 .958129$

O $-1.096213-1.1016220 .904439$

C -0.7202900 .1708694 .141463$

C $-1.221164-1.0091394 .537525$

C $-0.751648-1.7691065 .745627$

C $0.096928-2.9664405 .380026$

C $-0.461406-4.0844654 .885436$

C $1.584627-2.8926605 .643213$

C $2.252454-1.7136224 .991689$

N $-0.277803 \quad 0.844611 \quad 1.871306$

H $2.5841782 .595151-0.705942$

H $4.7754764 .613290-0.010709$

H 6.0860612 .7218730 .603488

H $6.3174293 .004548-1.905754$

H $5.0340501 .853281-2.151209$

H $7.487144-0.974190-1.758607$

H $8.9706520 .592951-0.997536$

H $8.6386782 .221319-1.594309$

H 8.2129441 .7746260 .072681

H $4.544516-0.161303-2.262168$

H $5.498605-1.183993-3.327271$

H $5.724233-2.907804-1.583931$

H $5.032723-1.828301-0.378975$

H $3.385586-3.446772-0.966465$

H $4.156766-4.056083-3.281079$

H $3.674362-2.477949-3.867909$

H $2.550677-4.633082-5.058574$

H $1.144772-1.812300-3.793856$

H $2.405470-5.844160-3.053541$

H $\quad 0.707495-5.434783-2.958699$

H $1.792420-5.020190-1.617313$

H $-0.444033-3.412341-4.050011$

H $0.663510-0.035531-2.329548$

H $-0.657296-0.932909-1.575535$

H $0.562876-2.4580160 .141610$

H $3.754007-0.8206291 .266859$

H 3.4381390 .3978924 .072750

H $3.677992-3.0471792 .417940$

H $2.089622-2.4617791 .868102$

H $2.199472-3.6590053 .144995$

H $1.677244-0.1046460 .193795$

H $\quad 0.643130 \quad 1.158327-0.459961$

H -2.2013770 .5400472 .617122$

H -1.3624621 .9814493 .213336$

H 0.0844190 .6307274 .709200

H -2.028742 -1.459639 3.964278

H -1.633918 -2.101972 6.308124

H $-0.206215-1.1127486 .435067$

H $0.136801-4.9553024 .639304$

H -1.530866 -4.151718 4.714636

H $2.090926-3.8205355 .351250$

H $1.740898-2.8165176 .727466$

H $2.330176-0.8421305 .642407$

H 0.4329421 .5679021 .810114

C 0.7786364 .5196460 .124110

O $-0.0020363 .592037-0.656937$

C -1.344292 $4.099630-0.799587$

C $-1.478821 \quad 5.189187 \quad 0.250091$

C $-0.081051 \quad 5.7779280 .248539$

H -1.394266 $4.528049-1.808560$

C $-2.3345172 .936908-0.663413$ 
H $-2.252707 \quad 5.9216640 .002271$ H -1.7130704 .7581661 .231516$ C 0.2257396 .6137121 .479149 H $0.0360686 .402395-0.647456$ C $-3.8112743 .362130-0.695819$ O $-2.1020402 .029067-1.744998$ H $-2.1227152 .387414 \quad 0.258678$ H $-4.041047 \quad 3.9848770 .176811$ O $-4.0082634 .191403-1.849387$ C $-4.8140062 .193292-0.781518$ C $-4.719616 \quad 1.2144410 .403616$ H $-4.6886251 .673042-1.738103$ O $-6.1232722 .775393-0.795046$ H -3.7004980 .8264720 .479423$ H $-4.926907 \quad 1.7647331 .329456$ H -0.4570647 .4671371 .544353$ H $\quad 0.1200006 .0278332 .398403$ H $1.2487467 .001378 \quad 1.442789$ C $2.1177344 .764066-0.578532$ H -1.152755 $1.820848-1.754597$ H -4.945269 4.452214 -1.861337 H -6.738667 2.106764 -1.140304 H $2.6121405 .641055-0.145325$ H $1.9414124 .967707-1.641946$ H 0.9350304 .0582491 .107776 C $-4.360040-1.458143-1.039819$ O $-5.559493-0.678682-0.937848$ C $-5.713247 \quad 0.0342550 .293347$ C $-5.694032-0.9424961 .477847$ C $-4.477742-1.8640801 .447628$ C $-4.323185-2.5145720 .071892$ H -3.484231 -0.802982 -0.962138 C $-4.354647-2.095351-2.433222$ H $-6.7201090 .468417 \quad 0.271398$ O $-5.736123-0.2181972 .702697$ H $-6.601662-1.5592511 .437290$ H -3.567772 -1.326638 1.731534 O $-4.648515-2.9041882 .413724$ H $-5.124946-3.251479-0.066288$ O $-3.081801-3.2156870 .054308$ C $-4.111904-1.052057-3.498080$ H -3.583082 -2.864818 -2.541075 H -5.317273 -2.573194 -2.651779 H -5.844249 -0.861594 3.423071 H -4.606162 -2.495895 3.294795 H $-3.086995-3.814905-0.710364$ O $-2.776207-0.796212-3.600101$ O $-5.002160-0.521854-4.149620$ C -2.406928 $0.181307-4.577191$ H $-2.9316841 .124299-4.395835$ H $-1.3312990 .357196-4.491222$ H -2.623839 -0.193924 -5.581566 SCF Energy (B3LYP/6-31G**//MMFF) $=-3245.90740428$

\section{$05 \_00235$}

MM̄FF Geometry

C -1.003550 $3.257862-0.570432$

C $-1.1129612 .488523-1.664557$

C $-0.2129631 .335597-2.052553$

O $0.9001841 .196771-1.141698$

C -1.042901 $0.037097-2.020716$

C $-0.265147-1.229707-2.317292$

C $-0.268201-2.313480-1.515712$

C $0.513986-1.247955-3.605372$

C -1.040255 -2.490162 -0.237263

C $-0.331991-1.9466251 .010168$

C $0.857401-2.8014141 .475728$

C $1.438313-2.2310842 .772939$

C $2.683308-3.0013183 .235404$

O $1.855706-2.8020380 .453546$

O $3.333743-2.2201834 .250014$

C $3.666864-3.1121162 .051946$

C $2.318415-4.3403013 .886843$

O $4.772164-3.9487832 .387129$

C $3.003916-3.6073950 .744233$

C $3.970970-3.515595-0.460783$

O $2.647150-4.9855730 .900883$

C $2.0187501 .919423-1.428833$
O $2.1786662 .674797-2.375223$

C $3.0258921 .648828-0.376839$

C $4.1561242 .372104-0.364875$

C 5.2783452 .2411050 .567222

C 5.1715871 .2158121 .663763

C $4.226026-2.091035-0.960624$

C $5.502202-1.972497-1.774601$

C $7.022433-0.225720-2.703413$

O $6.202388-2.933499-2.079515$

C $7.9322400 .224171-1.602071$

C $8.4659351 .452699-1.527838$

C $9.3541651 .904815-0.403762$

C 8.8530213 .1643130 .270788

C 9.5055914 .3302180 .127207

C 7.6387113 .0587161 .173721

C 6.3480953 .0471960 .398037

N $5.769239-0.650893-2.107915$

H $-0.184736 \quad 3.1029760 .126420$

H -1.943519 $2.671579-2.343411$

H $\quad 0.1539291 .505688-3.072507$

H $-1.8575660 .110587-2.753045$

H -1.523002 -0.032840 -1.038174

H $\quad 0.322007-3.178939-1.812931$

H $0.929472-2.237403-3.824567$

H $-0.133988-0.972744-4.443938$

H $1.350488-0.543765-3.562090$

H -2.019391 -2.007566 -0.328344

H -1.260359 -3.556927 -0.105294

H $0.003208-0.9197050 .816869$

H -1.068564 -1.896199 1.822082

H $0.502194-3.8265551 .633862$

H $0.680302-2.2167373 .566074$

H $1.722691-1.1812742 .612600$

H $2.698181-2.0781554 .972399$

H $4.081245-2.1102651 .887951$

H $\quad 1.679217-4.177723 \quad 4.763054$

H $3.211097-4.8546644 .259522$

H $1.786589-5.0150213 .213091$

H $5.163141-3.5986383 .205961$

H $4.908343-4.027887-0.210748$

H $3.555456-4.083757-1.304787$

H $2.535556-5.3781920 .019398$

H 2.8146540 .8671980 .343616

H $4.2851713 .137930-1.129416$

H 5.2289770 .2051381 .249993

H 4.2259651 .3212682 .207320

H 5.9582581 .3061252 .416457

H $3.386552-1.759635-1.583335$

H $4.311996-1.398073-0.120664$

H $6.7935040 .579934-3.407846$

H $7.478174-1.057056-3.249923$

H $8.156938-0.499356-0.821476$

H $8.2572272 .173118-2.315574$

H $10.3568412 .069619-0.819002$

H $9.469841 \quad 1.1190310 .353358$

H 9.1672635 .2320760 .626617

H $\quad 10.389620 \quad 4.410842 \quad-0.496492$

H $7.606500 \quad 3.916720 \quad 1.857474$

H 7.7596422 .1729501 .803953

H $6.3098723 .791109-0.399364$

H $5.1464130 .074922-1.767160$

C -2.5458934 .0980251 .207011$

O -3.2270432 .8309421 .275654$

C -4.3579442 .9569512 .158794$

C -4.3000154 .3687172 .738102$

C $-3.583603 \quad 5.129679 \quad 1.639118$

H -5.2533022 .8737571 .532369$

C $-4.341816 \quad 1.834572 \quad 3.205657$

H -5.2966494 .7653342 .955189$

H -3.7201284 .4063653 .667483$

C -2.9947676 .4511552 .100109$

H -4.2931435 .3145690 .821420$

C -4.2601380 .4141322 .608816$

O -3.1914832 .0198904 .041568$

H -5.2177101 .9288923 .858516$

H -3.2793310 .2630772 .140088$

O $-4.341876-0.5258073 .687789$

C $-5.3647820 .071861 \quad 1.591097$ 
C $-5.253686-1.3800851 .090604$ H $\quad-5.3000190 .7489650 .736174$ $\begin{array}{lllll}\text { O } & -6.651948 & 0.241618 & 2.182587\end{array}$ H $-4.230423-1.5534770 .742728$ H -5.433461 -2.0652161 .928009$ H -3.782828 7.1143612 .471492 H $-2.268506 \quad 6.3072202 .907135$ H -2.485932 6.9610451 .276210 C -1.998209 4.324932 -0.203509 H -3.3020651 .4515934 .822209$ H -3.518592 -0.4564854 .199636$ H -6.7842091 .1909762 .341439$ H -1.500822 $5.299322-0.260880$ H -2.825328 $4.332240-0.923475$ $\mathrm{H}-1.7204484 .0567841 .930641$ C $-4.973571-0.941969-1.923921$ O $-6.167384-0.814439-1.142145$ C $-6.265257-1.711425-0.030697$ C $-6.198616-3.163218-0.525364$ C $-4.953337-3.415303-1.370049$ C $-4.834773-2.367432-2.475113$ H -4.101693 -0.689981-1.309116 C $-5.066617 \quad 0.073237-3.074969$ H -7.266564 -1.562561 0.392472 O $-6.215340-4.0775760 .565516$ H -7.082689-3.378822 -1.139446 H $-4.048130-3.442207-0.753432$ O $-5.054731-4.701884-1.985249$ H -5.604565 -2.553473 -3.235069 O $-3.566043-2.501189-3.112895$ C $-4.9327171 .506208-2.605011$ H -4.270164 -0.098021 -3.807100 H $-6.040993-0.025218-3.569022$ H -7.021567 -3.9029021 .080534$ H -5.168747 -5.354142 -1.272714 H -3.491491 -3.420030 -3.422618 O $-5.2464072 .342074-3.639276$ O $-4.5794621 .854855-1.487690$ C $-5.1558503 .740802-3.349981$ H -4.112624 4.019542 -3.176305 H -5.526592 4.291906 -4.218267 H -5.774026 $3.995809-2.483770$

SCF Energy (B3LYP/6-31G**//MMFF) $=-3245.90751303$

05_00236

MM̄FF Geometry

C $3.707549-3.023024-1.147354$

C $2.908626-3.833978-0.436503$

C $1.441232-3.606308-0.146003$

O $0.950304-2.516666-0.959108$

C $0.617323-4.872741-0.459817$

C $-0.699783-4.9549670 .292198$

C $-1.925230-4.946276-0.268845$

C $-0.579672-5.1165391 .789020$

C $-2.298985-4.861990-1.720535$

C $-2.829595-3.492720-2.171605$

C $-4.129715-3.040272-1.483715$

C $-4.709816-1.815662-2.199515$

C $-5.958884-1.267652-1.491728$

O $-3.844180-2.705187-0.120646$

O $-6.2515690 .026206-2.041864$

C -5.654437 -1.093778 0.012966

C $-7.194834-2.121449-1.795067$

O $-6.833298-0.7419740 .731500$

C $-4.998363-2.3357460 .650842$

C $-4.538668-2.1019542 .113057$

O $\quad-5.952890-3.404853 \quad 0.688045$

C $0.084737-1.634028-0.398743$

O $-0.371156-1.665808 \quad 0.732214$

C $-0.230230-0.612385-1.424356$

C $-1.2328070 .249022-1.201290$

C $-1.7040141 .298413-2.105556$

C $-0.9346511 .529122-3.378182$

C -3.527176 -0.970909 2.319954

C -4.1560180 .3753652 .642951$

C -3.619832 2.813290 2.666201

O $\quad-5.3074010 .5000843 .050996$

C -4.1818133 .3608021 .392105$
C -3.5704124 .2935660 .646610$

C $-4.1231894 .828864-0.643078$

C $-3.2667444 .469475-1.838458$

C $-2.3989885 .352992-2.359408$

C $-3.4820793 .118608-2.486123$

C $-2.7952692 .005066-1.744800$

N -3.261192 1.4213942 .461177

H $3.307701-2.113883-1.588623$

H $3.349523-4.7182740 .020073$

H $1.379288-3.3457230 .915940$

H $1.188796-5.772112-0.192475$

H $0.457924-4.929573-1.541358$

H -2.782593 -5.0415140 .397131$

H $-1.541377-5.3449362 .261332$

H $0.101958-5.9391132 .029389$

H $-0.200258-4.2011742 .251743$

H -1.454474 -5.132840 -2.361234

H -3.064546 -5.623717 -1.915968

H $-2.048429-2.744427-2.009627$

H -3.000689 -3.549093 -3.254118

H $-4.842501-3.872669-1.514119$

H -4.936373 -2.046643 -3.247927

H -3.950045 -1.023779 -2.228390

H $-5.4811120 .600486-1.894972$

H $-4.965655-0.2450660 .102509$

H -7.401747 -2.122274 -2.872275

H $-8.090199-1.705578-1.319977$

H -7.088470 -3.161318 -1.479811

H $-7.204997 \quad 0.0513130 .309255$

H -5.411664 -1.982624 2.766209

H $-4.048526-3.0273142 .448600$

H -5.667859 -4.0490101 .357097$

H $\quad 0.350030-0.614641-2.339852$

H $-1.7778690 .172891-0.262983$

H $-1.041570 \quad 0.670339-4.049555$

H $0.129757 \quad 1.677336-3.169245$

H -1.260448 $2.415266-3.927477$

H -2.894689 -1.220666 3.181272

H -2.871299 -0.8786331 .451249$

H -2.7139713 .3411962 .980653$

H -4.3638862 .8893723 .465416$

H -5.1385372 .9606501 .064005$

H -2.6176284 .6980980 .977480$

H -4.199809 $5.919743-0.546757$

H $-5.1494144 .479747-0.812862$

H $-1.8023795 .110946-3.232608$

H $-2.2591576 .334367-1.918300$

H $-3.178350 \quad 3.148129-3.537534$

H $-4.5576912 .902188-2.520282$

H $-3.287328 \quad 1.738692-0.810189$

H $-2.351573 \quad 1.2279602 .052986$

C $6.033791-2.393046-0.463797$

O $5.777236-1.004168-0.734594$

C $7.005082-0.262576-0.639049$

C $8.053686-1.252454-0.135842$

C $7.533599-2.569075-0.678315$

H $7.2672680 .060053-1.653574$

C $6.8312710 .958717 \quad 0.272251$

H $9.058222-1.006651-0.494195$

H $8.088459-1.2778820 .960112$

C $8.123213-3.7859570 .012404$

H $7.757110-2.618356-1.752400$

C $5.9301012 .102577-0.243389$

O 6.3657430 .5460721 .558516

H 7.8306631 .3849570 .428011

H $5.9367132 .872870 \quad 0.539610$

O $6.5096342 .687127-1.409053$

C $4.4527301 .764097-0.530484$

C $3.5892753 .039771-0.597981$

H $4.058138 \quad 1.109908 \quad 0.250750$

O $4.3159331 .096861-1.783417$

H 3.7347513 .6168140 .321552

H $3.9350663 .662877-1.431862$

H $9.210358-3.810726-0.114439$

H $7.909987-3.7803731 .086632$

H $7.713842-4.710181-0.407304$

C $5.173533-3.272715-1.371539$

H 5.5671520 .0071401 .427476 
H $7.4118252 .968591-1.181014$ H $4.7025290 .211363-1.694427$ H $5.411303-4.329830-1.205982$ H $5.388436-3.055141-2.424947$ H $5.771177-2.5673100 .588414$ C 1.4511872 .3951301 .508108 O $1.578001 \quad 1.8369840 .195035$ C $2.0878002 .731195-0.800801$

C $1.1929523 .973291-0.906323$ C $0.987503 \quad 4.6440490 .447970$ $\begin{array}{lllll}\text { C } & 0.524669 & 3.617257 & 1.480868\end{array}$ H 2.4400442 .6833751 .884418 C 0.8805411 .3013962 .424380 H $2.0055372 .199396-1.756173$ O $1.7432204 .900228-1.835717$ H $0.2141753 .671506-1.298892$ H 1.8977295 .1606920 .773552 $\begin{array}{llllll}\text { O } & -0.015422 & 5.651426 & 0.303735\end{array}$ H $-0.499128 \quad 3.3058601 .240822$ $\begin{array}{lllll}\text { O } & 0.482986 & 4.233821 & 2.765677\end{array}$ $\begin{array}{lllll}\text { C } & 1.885904 & 0.209025 & 2.724858\end{array}$ H 0.5733221 .7219293 .387942 H 0.0135120 .8382081 .940133 H $1.1510165 .671440-1.861636$ H -0.1743296 .0302951 .185190$ H 1.3880994 .5042272 .996780 O $1.233953-0.8512483 .288139$ $\begin{array}{lllll}\text { O } & 3.090108 & 0.281634 & 2.524597\end{array}$ C $2.061478-1.9612103 .650402$ H $1.410653-2.7857673 .953089$ H $2.666924-2.2879002 .799402$ H $2.700850-1.6876274 .494779$ SCF Energy (B3LYP/6-31G**//MMFF) $=-3245.91951559$

\section{5_00237}

\section{MM̄FF Geometry}

C -2.766589 -2.748181-2.280241

C $-3.031415-1.666999-3.028393$

C $-3.570713-0.373667-2.483725$

O $-2.6188110 .641950-2.894427$

C $-4.947478-0.012854-3.068209$

C $-6.112126-0.693392-2.378507$

C $-6.824534-0.133960-1.379661$

C $-6.510274-2.037398-2.928561$

C $-6.5694711 .196805-0.728111$

C -6.8263491 .2060490 .785519$

C $-5.972937 \quad 0.2169221 .596211$

C -6.3185970 .3346653 .085496$

C $-5.435066-0.5670233 .959092$

O $-4.585257 \quad 0.5083021 .384227$

O $-5.618055-0.1792345 .329788$

C $-3.956760-0.3285673 .583500$

C $-5.883527-2.0307613 .893555$

O $-3.103927-1.2440364 .266195$

C -3.693685 -0.3917882 .061966$

C $-2.237217-0.0152751 .700347$

O $-3.889295-1.7401541 .615391$

C $-2.467768 \quad 1.731103-2.096987$

O $-3.0588251 .969142-1.056960$

C -1.445443 $2.604065-2.714333$

C $-1.0836813 .730815-2.083373$

C $-0.1006574 .709453-2.548139$

C $0.5858344 .451670-3.863209$

C -1.8050251 .4077062 .063650$

C $-0.351799 \quad 1.6662401 .693285$

C 1.4249623 .3791831 .963920

$\begin{array}{lllll}\text { O } & 0.383249 & 0.803811 & 1.218803\end{array}$

C 1.5427774 .7848551 .470525

C $2.439426 \quad 5.157414 \quad 0.543854$

C 2.6100216 .5659580 .049476

C $2.4828196 .668991-1.454844$

C $3.5690556 .609656-2.243407$

C $1.1097906 .911050-2.039943$

C $0.151505 \quad 5.783955-1.772470$

N 0.0316182 .9713351 .980781

H -2.983616 -2.732388 -1.216571

H -2.811359-1.694193 -4.093559

H -3.611176 $-0.409764-1.388066$
H $-4.975635-0.235604-4.144030$

H $-5.085606 \quad 1.075367-3.033555$

H -7.683316 -0.679919 -0.991919

H $-7.423529-2.422214-2.462123$

H $-6.698333-1.967949-4.005053$

H -5.722407 - $2.775713-2.758838$

H $-5.5502411 .543006-0.910111$

H -7.240622 $1.931828-1.189075$

H -6.6279592 .2258601 .140809$

H $-7.889314 \quad 1.0053290 .971379$

H $-6.184252-0.7984181 .242365$

H $-7.3792930 .113800 \quad 3.258674$

H -6.1755841 .3763173 .405701$

H -5.3737800 .7578465 .416833$

H -3.693687 0.6718353 .947533

H $-6.908985-2.1316114 .269660$

H -5.265208 -2.663740 4.539758

H -5.862423 -2.444598 2.883554

H $-3.303355-1.1741915 .215461$

H -1.550693 -0.741784 2.155222

H -2.102670 -0.1398280 .616952$

H $-3.409871-1.8589270 .779746$

H -1.023044 $2.299681-3.665001$

H $-1.5527153 .964226-1.127877$

H $-0.1429154 .446356-4.681278$

H $1.1026313 .486192-3.847828$

H $1.3402865 .201779-4.112032$

H -2.434459 2.1426121 .551700

H - $1.892226 \quad 1.5701793 .142708$

H 2.0070532 .6793411 .358185

H 1.7849903 .3200942 .996138

H $0.8843685 .531488 \quad 1.907358$

H 3.1137384 .4106850 .130325

H 3.6036126 .9125520 .362581

H 1.8964637 .2492810 .526480

H $3.4924116 .706276-3.321275$

H $4.5599216 .458087-1.827890$

H $1.1662177 .121680-3.114134$

H $0.6958997 .827894-1.600334$

H $-0.3884195 .885605-0.830483$

H -0.6236653 .5646552 .482017$

C $-0.843000-4.340410-2.099616$

O $0.091422-3.252168-2.227966$

C $1.411718-3.786012-2.456129$

C $1.301210-5.290326-2.246972$

C $-0.127830-5.561867-2.672236$

H $1.646279-3.558330-3.503188$

C $2.403734-3.091400-1.513571$

H $2.037055-5.849838-2.832423$

H $1.440649-5.548904-1.189834$

C $-0.670070-6.889020-2.171755$

H $-0.173704-5.545052-3.769448$

C $3.846173-3.611867-1.640386$

O $2.383070-1.688488-1.795965$

H $2.045522-3.200484-0.484587$

H $3.884199-4.679263-1.393658$

O $4.236820-3.496895-3.014830$

C $4.877604-2.847266-0.787480$

C $4.590727-2.9149500 .722725$

H $4.940205-1.806830-1.124343$

O $6.165958-3.424026-1.035003$

H $3.600769-2.4982630 .926835$

H $4.566939-3.9679541 .029361$

H $-0.073403-7.719950-2.562075$

H $-0.648541-6.944478-1.078172$

H $-1.704980-7.035252-2.496237$

C $-2.143844-4.000760-2.830554$

H $2.719484-1.566034-2.700385$

H $5.153059-3.815880-3.083428$

H $6.141849-4.347631-0.731476$

H $-2.859089-4.824015-2.725601$

H - $-1.941731-3.876524-3.901798$

H -1.034077 -4.469957 -1.026056

C 4.6882310 .0353701 .505408

O $5.797149-0.808938 \quad 1.173202$

C $5.653829-2.1769751 .567424$

C $5.436459-2.2677653 .083532$

C $4.279299-1.3890153 .551158$ 
C 4.4416990 .0350473 .018982

H $3.789607-0.3155720 .984019$

C 5.0248301 .4424840 .998937

H $6.617373-2.6581121 .357812$

O $5.209914-3.6204123 .465932$

H $6.353541-1.9411893 .591382$

H $3.314936-1.8201893 .257584$

O $4.293370-1.3645614 .980180$

H 5.2727220 .5299383 .537624

$\begin{array}{lllll}\text { O } & 3.256053 & 0.776855 & 3.294691\end{array}$

C $4.8432161 .534217-0.497595$

H 4.3974112 .2139991 .457580

H 6.0660731 .7027191 .224415

H $5.059611-3.6295724 .426804$

H $3.495261-0.8937135 .273619$

H 3.1809030 .8662504 .259448

O $3.5159111 .623718-0.797931$

O $5.7678841 .524955-1.299517$

C $3.1981261 .722156-2.189106$

H $2.1132501 .637486-2.294841$

H $3.5162412 .695228-2.574246$

H $3.6678480 .910459-2.753022$

SCF Energy (B3LYP/6-31G*//MMFF) $=-3245.90510153$

05_00238

MM̄FF Geometry

C $0.121393 \quad 3.2507351 .204648$

C $1.083048 \quad 3.298255 \quad 2.139202$

C 1.5671022 .0977132 .923661

$\begin{array}{lllll}\text { O } & 0.479730 & 1.154481 & 3.082469\end{array}$

C $2.703677 \quad 1.3929442 .161870$

C 3.5987250 .5337603 .034785

C 4.9361620 .6725353 .135715

C $2.913652-0.5731393 .796240$

C 5.8258861 .7119232 .514530

C $6.886616 \quad 1.1487381 .557587$

C 6.4102720 .6369690 .185552

C $5.6393701 .688106-0.617814$

C $5.1608131 .147310-1.974268$

O $5.626715-0.5500720 .354236$

O $4.172726 \quad 2.054804-2.485796$

C $4.452967-0.206395-1.747779$

C $6.2906451 .115932-3.009777$

O $4.110112-0.815028-2.990154$

C $5.276370-1.188258-0.886780$

C $4.511792-2.493308-0.571188$

O $6.449266-1.576992-1.615668$

C -0.4039151 .3738884 .089229$

O $-0.302243 \quad 2.209014 \quad 4.977862$

C -1.592075 0.4952623 .978232

C $-1.799153-0.3699032 .972522$

C $-2.989274-1.2047992 .785878$

C $-4.106839-1.0687013 .787061$

C $3.208217-2.3267520 .209561$

C $2.619252-3.6705510 .609378$

C $0.824673-4.6656142 .024437$

$\begin{array}{llll}\text { O } & 3.078691 & -4.743491 & 0.225747\end{array}$

C $-0.490336-4.8488561 .336185$

C $-1.643011-5.0325571 .997612$

C $-2.969393-5.2472771 .326785$

C $-4.052352-4.3450631 .876951$

C $-4.935144-4.8044352 .780125$

C $-4.169293-2.9444711 .321621$

C -3.035386 -2.044949 1.730680

N $1.520123-3.5305301 .445352$

H -0.3222672 .2895340 .955385$

H 1.5564804 .2490942 .370617

H 1.9174082 .4310653 .908775

H 3.2959822 .1479981 .640784

H 2.2818120 .7444621 .381432

H $5.469186-0.0255463 .781213$

H $3.624204-1.3033004 .198313$

H $2.347848-0.1628694 .638413$

H $2.222917-1.1181773 .146962$

H 5.2707382 .5217732 .037743

H 6.3592052 .1942133 .345258

H $7.625696 \quad 1.941516 \quad 1.381893$

H 7.4178930 .3338232 .067196
H $7.3219870 .364647-0.360768$

H $6.2445672 .592916-0.754503$

H $4.7498112 .004670-0.063686$

H $4.5803432 .935153-2.554961$

H $3.5072710 .009610-1.234236$

H $6.6750602 .127810-3.186696$

H $5.9273860 .760390-3.980217$

H $7.1357570 .494354-2.707365$

H $3.593199-0.166226-3.497903$

H $4.320133-3.045937-1.500538$

H $5.176693-3.1422670 .017115$

H $6.787769-2.405479-1.237731$

H -2.3232220 .6332664 .768742$

H -1.042030 -0.4702572 .196801$

H $-4.949457-1.7354013 .591644$

H $-4.502801-0.0470533 .784432$

H -3.751531-1.307358 4.795703

H $3.395621-1.7532361 .120578$

H $2.462113-1.796882-0.390490$

H $0.704792-4.457608 \quad 3.092590$

H $1.429982-5.5704761 .911489$

H $-0.492566-4.8575750 .249322$

H - $-1.630645-5.0584493 .085233$

H $-3.249999-6.2983901 .475197$

H $-2.898125-5.1102580 .240813$

H $-5.735164-4.1777243 .159590$

H $-4.875497-5.8177943 .163513$

H -5.132457 -2.493412 1.587580

H $-4.193304-2.9999200 .226186$

H -2.177957 -2.084388 1.058360

H $1.182029-2.5999051 .671794$

C -1.921031 4.5540560 .605170

O $-2.557511 \quad 3.3755210 .073187$

C $-3.7404043 .761252-0.647996$

C $-3.956106 \quad 5.240661-0.347252$

C $-2.5299025 .724816-0.162756$

H $-3.507473 \quad 3.649773-1.713551$

C $-4.9088142 .842847-0.273544$

H $-4.4837145 .754646-1.156537$

H -4.5291965 .3908820 .574939$

C $-2.428547 \quad 7.0580800 .556949$

H $-2.0588115 .811641-1.151088$

C $-4.6034511 .336935-0.418696$

$\begin{array}{llll}\text { O } & -5.250753 & 3.078553 & 1.099104\end{array}$

H $-5.7925673 .115801-0.862245$

H $-3.877590 \quad 1.0348150 .345465$

O $-5.808406 \quad 0.621892-0.113017$

C $-4.0952710 .904692-1.807685$

C $-3.934744-0.623790-1.905541$

H -3.136794 $1.392054-2.005987$

O $-5.0247351 .352632-2.792313$

H -3.328678 $-0.959091-1.058985$

H $-4.920570-1.094220-1.809859$

H $-2.9438807 .840703-0.009389$

H -2.8803607 .0116261 .553528$

H $-1.383195 \quad 7.3598190 .674867$

C $-0.403484 \quad 4.436240 \quad 0.446304$

H -5.9897342 .4858541 .319476$

H $-6.4541610 .825430-0.811525$

H $-4.5977561 .269500-3.661431$

H $0.083685 \quad 5.358573 \quad 0.781753$

H $-0.151148 \quad 4.294244-0.610551$

H $-2.1880104 .607418 \quad 1.669086$

C $-0.967098-0.891920-2.617156$

O $-2.033337-0.483026-3.483514$

C $-3.297192-1.104949-3.227854$

C $-3.165931-2.631398-3.340255$

C $-2.049903-3.174703-2.453091$

C $-0.752847-2.408708-2.702121$

H $-1.198010-0.601213-1.585207$

C $0.301671-0.147068-3.059812$

H $-3.964935-0.785841-4.037200$

O $-4.401122-3.257466-3.009269$

H $-2.944861-2.891071-4.383846$

H $-2.335865-3.144452-1.396730$

O $-1.850147-4.552936-2.773868$

H - $0.364363-2.680202-3.691676$

O $0.221507-2.820508-1.747152$ 
C $\quad 0.2264041 .337122-2.777902$

H $1.182962-0.528244-2.533866$

H $\quad 0.440231-0.280070-4.139664$

H -4.265252 -4.218700 -3.068589

H $-1.106293-4.871078-2.233857$

H $-0.098855-2.567846-0.864270$

O $1.142998 \quad 1.986786-3.553859$

O $-0.5190741 .859776-1.961608$

C $1.1981343 .406661-3.383672$

H $1.5688253 .648367-2.383280$

H $1.8946933 .810512-4.122981$

H $0.2129353 .852936-3.550143$

SCF Energy (B3LYP/6-31G**//MMFF) $=-3245.91703571$

05 00239

MM̄FF Geometry

C -2.499625 -5.371049-0.181660

C $-2.173171-4.8528241 .012086$

C -3.056501-4.058197 1943964

O $-2.603756-2.6799321 .888006$

C $-4.559907-4.0637771 .605705$

C $-5.382977-3.1711432 .517461$

C $-5.894199-1.9753412 .158225$

C $-5.642503-3.7233753 .894751$

C $-5.740176-1.2718730 .836015$

C -5.2664890 .1757181 .006206$

C $-5.0440160 .890908-0.337322$

C $-3.8127400 .363919-1.081964$

C $-3.5482641 .147122-2.374647$

O $-4.8722852 .286872-0.051676$

O $-2.236173 \quad 0.780924-2.827705$

C -3.533203 $2.656046-2.052990$

C $-4.5142850 .732593-3.491156$

$\begin{array}{lllll}\text { O } & -3.433850 & 3.394327 & -3.268593\end{array}$

C $-4.7479783 .118970-1.218115$

C $-4.6450874 .588973-0.733631$

O $-5.9249163 .035716-2.033479$

C $-1.546369-2.3220812 .664843$

O $-0.894145-3.0449823 .400639$

C -1.325524 -0.8719802 .470051$

C $-0.297201-0.2673293 .081956$

C 0.0196901 .1622993 .031392

C -0.9450692 .0644092 .308086$

C -3.468366 4.9134590 .192310

C $-2.1994655 .341967-0.526911$

C $0.2329425 .704295-0.078453$

O $-2.1479885 .569718-1.731693$

C 1.0503524 .5041290 .280520

C 2.1264664 .5438911 .079861

C 2.8842263 .3193671 .509684

C 2.9607253 .1966973 .016465

C 4.1400823 .2764183 .655231

C 1.6694832 .9936263 .782020

C 1.1386381 .5916443 .651472

N -1.130905 5.4498660 .350602

H -3.510173 $-5.281924-0.567311$

H -1.138556 -4.951422 1.337981

H -2.924665 -4.448125 2.961246

H -4.948904 -5.088646 1.670108

H -4.710221 -3.7641050 .564551$

H $-6.497372-1.4369152 .888194$

H -6.291473 -3.071212 4.488877

H $-6.132667-4.7000163 .826072$

H -4.703931 -3.842074 4.444738

H $-5.046265-1.8015780 .182828$

H $-6.714427-1.2738770 .332447$

H $-6.0114180 .736781 \quad 1.586904$

H $-4.346194 \quad 0.214477 \quad 1.601810$

H -5.949285 $0.766062-0.942760$

H -3.900576 $-0.704232-1.309293$

H $-2.9308800 .458616-0.433337$

H -2.030136 $1.325970-3.606688$

H -2.610482 2.839911-1.488909

H -4.397789 -0.334255 -3.717506

H $-4.2930881 .261240-4.424935$

H $-5.5626350 .904857-3.239099$

H -3.266419 4.323866 -3.041137

H $-4.6796945 .274887-1.588661$
H $-5.5582524 .801788-0.158826$

H $-6.6073233 .612134-1.651289$

H $-2.024808-0.3367861 .839436$

H $0.374457-0.8730473 .689222$

H $-0.975851 \quad 1.820511 \quad 1.240832$

H $-0.690740 \quad 3.1220582 .386199$

H -1.9553231 .9605362 .719905$

H -3.7563245 .7588940 .830483$

H -3.2450584 .0668180 .850681$

H $\quad 0.5735116 .607643 \quad 0.436995$

H $0.2700335 .876938-1.158006$

H $0.7147033 .546800-0.111292$

H 2.4686255 .5006891 .467317

H 3.8899153 .3749751 .074294

H 2.4288112 .4091411 .103652

H 4.2049253 .1908244 .734804

H 5.0683763 .4241093 .113305

H 0.9408493 .7531563 .488048

H 1.8368343 .1747034 .852593

H 1.7605900 .8511854 .154242

H $-1.2891415 .287423 \quad 1.341175$

C $-0.737297-4.991761-1.971908$

O $0.121819-4.187663-1.135133$

C $-0.115483-2.796542-1.432474$

C $-0.711589-2.789231-2.830797$

C $-1.599666-4.018010-2.781750$

H -0.851021 -2.449652 -0.695779

C $1.195309-2.016597-1.284963$

H -1.266848 -1.873537 -3.051017

H $\quad 0.071785-2.910793-3.589057$

C $-1.992850-4.545644-4.150494$

H -2.513544 -3.750070 -2.238290

C $1.062083-0.524565-1.632698$

O $1.632612-2.1286090 .073497$

H $1.965434-2.488989-1.904008$

H $\quad 0.761971-0.409353-2.680912$

O $-0.0027320 .019219-0.840794$

C $2.3228420 .319108-1.361808$

C $3.552823-0.156136-2.151724$

H $2.5242900 .345641-0.286436$

O $2.0109261 .658331-1.763722$

H $3.745091-1.208944-1.930231$

H $3.323730-0.086123-3.222249$

H $-2.563858-3.792635-4.703622$

H -1.113223 -4.806031-4.748757

H $-2.614968-5.441297-4.056023$

C -1.495156-6.007493 -1.103996

H $1.682896-3.0764380 .285269$

H $-0.0952990 .956368-1.084368$

H $2.6696292 .248623-1.361154$

H $-0.774926-6.582093-0.507850$

H -2.019867 -6.725302 -1.745325

H $-0.065358-5.546735-2.638833$

C $5.677157-0.6111240 .008216$

O $5.1839640 .645526-0.473682$

C $4.8277200 .669673-1.860762$

C $6.0367520 .283233-2.724682$

C $6.621328-1.061199-2.304320$

C $6.898462-1.068170-0.802797$

H $4.883971-1.366276-0.051559$

C $6.059775-0.4237551 .486344$

H $4.5985031 .714741-2.100316$

O $5.661961 \quad 0.253861-4.097539$

H $6.809651 \quad 1.055718-2.617447$

H $5.967563-1.894433-2.587491$

O $7.862376-1.272879-2.981205$

H $7.756579-0.418077-0.587902$

O $7.255585-2.391078-0.404059$

C $4.860073-0.4351802 .409642$

H $6.714604-1.2427141 .805968$

H 6.5796680 .5300001 .632968

H $6.4714320 .135976-4.622371$

H $7.665716-1.382521-3.926767$

H $8.020100-2.659772-0.942078$

O $5.294452-0.6533553 .686762$

O $3.700681-0.2582812 .064998$

C $4.285043-0.7226494 .698859$

H 3.9981160 .2894484 .994739 
H $\quad 3.413074-1.2889894 .357332$ H $4.710762-1.2339365 .566415$

SCF Energy (B3LYP/6-31G**//MMFF) $=-3245.92376181$

05 00240

MM̄FF Geometry

C -0.464535 -2.332991 0.091092

C $0.137985-1.945655-1.043294$

C $-0.567418-1.494463-2.304174$

O $-1.989128-1.774225-2.242168$

C $-0.4442150 .021967-2.523816$

C $0.936040 \quad 0.498380-2.914017$

C $1.7617921 .187698-2.104388$

C $1.343466 \quad 0.206727-4.334963$

C $1.548421 \quad 1.551296-0.663183$

C $1.3492513 .057395-0.455065$

C $-0.0252343 .557437-0.924809$

C $-0.1222775 .077142-0.775527$

C -1.520981 $5.598275-1.135388$

O $-1.0207712 .922367-0.115267$

O $-1.619386 \quad 6.954260-0.674023$

C $-2.5712934 .778883-0.358130$

C $-1.7447925 .638348-2.651635$

O $-3.8926285 .129020-0.764975$

C $-2.3712993 .248199-0.467357$

C -3.3083702 .5311160 .533711$

O $-2.6883482 .830546-1.795923$

C $-2.414859-2.992242-2.663418$

O $-1.716246-3.896308-3.102205$

C $-3.880688-3.146753-2.512069$

C $-4.708400-2.174661-2.096409$

C $-6.160628-2.294649-1.925201$

C $-6.778203-3.646940-2.175735$

C -3.0775571 .0180690 .660578$

C $-3.9987940 .398028 \quad 1.698936$

C $-4.729049-1.8154172 .575566$

O -4.7317241 .0616892 .428031$

C $-5.955257-2.2026411 .811048$

C $-7.131488-1.5697781 .934818$

C $-8.366158-1.9139881 .152186$

C $-8.711986-0.8451760 .137775$

C $-9.380047 \quad 0.2624210 .502454$

C $-8.347879-1.095052-1.309764$

C $-6.862884-1.208630-1.535597$

N -3.895782 -0.9865091 .724527$

H -1.548258 -2.3246560 .150824$

H $1.224647-1.963143-1.087483$

H $-0.139156-2.027782-3.161189$

H $-0.8177030 .546443-1.637502$

H -1.145293 $0.325403-3.314446$

H $2.720846 \quad 1.512345-2.505855$

H $2.3153200 .644297-4.587380$

H $1.417153-0.872325-4.500765$

H $\quad 0.6075250 .618015-5.033561$

H $\quad 0.7323290 .987283-0.202918$

H $2.451051 \quad 1.245818-0.124784$

H 1.4521943 .2584690 .619243

H $2.1465353 .611586-0.965788$

H - $0.1725763 .271578-1.971936$

H $0.6419775 .577570-1.383257$

H $\quad 0.093495 \quad 5.3560800 .265567$

H -0.912467 7.468016-1.100956

H -2.4881215 .0629100 .700479$

H -1.005581 $6.291740-3.130361$

H -2.723824 $6.065564-2.895194$

H -1.669871 $4.656235-3.122896$

H -3.979704 $6.092768-0.667211$

H $-3.1675112 .972774 \quad 1.529271$

H -4.3545522 .7000270 .247883$

H -2.951108 1.897508 -1.784357

H -4.250284 -4.133774 -2.772709

H -4.296186 -1.196132-1.858207

H -7.844656 -3.693735 -1.948004

H -6.302088 -4.409281 - 1.548545

H $-6.662395-3.932603-3.227079$

H $-3.2601200 .519843-0.296580$

H -2.045248 0.8126630 .965770

H $-4.974968-1.2703593 .493117$
H $-4.155348-2.7067252 .847064$

H $-5.862150-3.0345281 .117918$

H $-7.212160-0.7373782 .630924$

H -9.197132 -2.037409 1.859033

H $-8.264598-2.8860460 .654212$

H $-9.6545611 .023052-0.220646$

H -9.6651310 .4328011 .535584$

H $-8.706150-0.270299-1.940215$

H -8.896723 -1.973945 -1.658194

H $-6.324850-0.274622-1.367634$

H $-3.342899-1.4507941 .011665$

C $0.072507-1.8712942 .498791$

O $1.122238-0.8779412 .458115$

C $1.791825-0.8692343 .733165$

C $0.798843-1.4759684 .711984$

C $0.198476-2.5718583 .855358$

H $2.649890-1.5473683 .645388$

C 2.2849440 .5339384 .096771

H $1.280917-1.8533915 .618502$

H $\quad 0.025290-0.7566375 .005330$

C $-1.119415-3.1079174 .388915$

H $\quad 0.917877-3.3991723 .786679$

C 3.1848271 .1875823 .028869

O 1.1476041 .3845714 .285970

H 2.8048840 .4963055 .061539

H $2.570767 \quad 1.4787642 .171432$

O 3.6911712 .4115603 .575663

C 4.3612490 .3172232 .550462

C 5.2702821 .0588311 .552827

H $3.961078-0.5860642 .079201$

O $5.125242-0.0837283 .686193$

H 4.6471861 .4795030 .757117

H $5.758056 \quad 1.9015172 .058164$

H -0.979528 -3.558232 5.376961

H -1.866429 -2.312665 4.484966

H $-1.527454-3.8739553 .721952$

C $\quad 0.241760-2.8329171 .318660$

H 1.4816632 .2687964 .515105

H 4.2908612 .1799974 .305660

H $5.734677-0.7847273 .399885$

H $-0.196166-3.8091831 .558324$

H $1.306317-2.9945731 .109433$

H $-0.878103-1.3267882 .437958$

C $5.053541-0.986057-0.715563$

O $5.858198-1.0843240 .462352$

C 6.3721920 .1626150 .940594

C $7.2049370 .848179-0.154071$

C $6.4366150 .979584-1.469346$

C $5.840361-0.369176-1.878179$

H $4.186486-0.352457-0.496015$

C $4.473033-2.372184-1.030875$

H $7.061500-0.0867091 .756879$

O $7.6335902 .138564 \quad 0.268939$

H $8.1092990 .254742-0.342660$

H $5.6637351 .754168-1.398706$

O $7.3530361 .413754-2.477029$

H $6.650020-1.034224-2.200048$

O $4.965895-0.191635-2.988963$

C $5.508817-3.421592-1.377591$

H $3.927265-2.748673-0.157294$

H $3.769293-2.284659-1.866783$

H 8.1422612 .0228921 .089689

H $6.8389861 .600917-3.280342$

H $5.5022430 .106407-3.742552$

O $4.912196-4.412047-2.105289$

O $6.680951-3.398911-1.028233$

C $5.767991-5.491519-2.493107$

H $6.587044-5.120876-3.117020$

H $\quad 6.156727-6.002863-1.607406$

H $5.176291-6.201662-3.076781$

SCF Energy $\left(B 3 L Y P / 6-31 G^{* *} / / M M F F\right)=-3245.89937468$

05_00241

MM̄MF Geometry

C -5.2688862 .3282411 .175563$

C -4.0210162 .7590910 .940612$

C -3.043472 3.1184332 .028554

O $-1.8827962 .266474 \quad 1.890462$ 
C $-2.5890224 .579326 \quad 1.867489$ C -1.583999 5.0126682 .917500 C -0.2875805 .2942082 .677903$ C $-2.1325195 .150576 \quad 4.315525$ C $0.4529405 .257937 \quad 1.369228$ C 1.5271304 .1678801 .365481 C 2.4192254 .1983670 .114853 C $1.6609333 .826013-1.161862$ C $2.5929923 .774238-2.383034$ O 3.4756573 .2548050 .337164 O $1.8891063 .120546-3.450965$ C $3.8150892 .892119-2.045197$ C $2.9322405 .179806-2.893228$ O $4.7886672 .940574-3.084083$ C $4.4778003 .235699-0.691434$ C $5.5530442 .211801-0.266762$ O $5.1759704 .485803-0.751208$ C $-1.948206 \quad 1.0300782 .456791$ O -2.8890920 .5473973 .066596$ C $-0.660758 \quad 0.3511142 .193128$ C $-0.507980-0.9400852 .523002$ C $0.691189-1.7469272 .293782$ C $1.917041-1.0420321 .775085$ C $5.0675630 .764121-0.186937$ C $6.053874-0.1504570 .518822$ C $6.331712-2.5245371 .205643$ O $7.127098 \quad 0.232977 \quad 0.974810$ C $5.393705-3.6326591 .559396$ C $4.954612-3.8611732 .806340$ C $3.986891-4.9518263 .168141$ C $2.616551-4.4001193 .505662$ C $2.244889-4.2464434 .788181$ C $1.731255-4.0850482 .315897$ C $0.647901-3.0699272 .558916$ N $5.574951-1.4518750 .588664$ H -5.6218832 .2422162 .200256$ H $-3.6663792 .831987-0.084639$ H -3.4887502 .9780183 .021238$ H $-3.4594695 .246677 \quad 1.926219$ H -2.183046 4.7181480 .860918 H 0.3290695 .6032583 .521642 H -1.400172 5.5766885 .009785 H -3.0056895 .8113294 .317367$ H -2.430860 4.1754734 .712038 H -0.2217625 .1190870 .523190$ H $\quad 0.9255696 .237360 \quad 1.225144$ H $2.167750 \quad 4.2763542 .251597$ H 1.0633423 .1783461 .463314 H 2.8546545 .2009160 .031482 H $0.8353134 .519909-1.355865$ H $1.1990152 .837120-1.034179$ H $1.0644313 .610572-3.611394$ H $3.4491531 .858394-2.008444$ H $2.0225585 .698096-3.220401$ H $3.5834085 .135433-3.773076$ H $3.4100685 .807023-2.137753$ H $4.3393712 .703503-3.913469$ H $6.4172632 .270125-0.941869$ H $5.9344412 .513303 \quad 0.719074$ H $5.2076614 .779379-1.675906$ H $\quad 0.1213340 .927227 \quad 1.712679$ H -1.345148 -1.465435 2.980491 H $2.195580-0.2153562 .438589$ H $1.736805-0.6398890 .773551$ H $2.793101-1.6899171 .712490$ H 4.1164590 .7110860 .354290 H $4.9181380 .370088-1.197814$ H $6.863857-2.1340572 .079982$ H $7.079082-2.8721500 .485246$ H $5.049448-4.266608 \quad 0.745875$ H $5.293420-3.2186283 .615930$ H $4.402132-5.5069174 .018996$ H $3.892273-5.6862262 .358072$ H $1.264862-3.8757175 .066360$ H $2.919264-4.4973175 .600948$ H $1.259878-5.0179451 .979575$ H $2.346112-3.7632751 .468880$ H $-0.275898-3.4967292 .950311$
H $\quad 4.752832-1.6949670 .042497$ $\begin{array}{llll}\text { C }-6.470717 & 0.410424 & 0.125751\end{array}$ O $-5.261731-0.279226-0.251210$ C $-5.597418-1.375553-1.120090$ C $-7.115164-1.503852-1.066541$ C $-7.533468-0.060983-0.863805$ H -5.296463 - $1.083000-2.133947$ C $-4.843589-2.647577-0.710488$ H -7.526261 -1.947294-1.978480 H -7.437344 -2.118445 -0.217549 C $-8.9590230 .093176-0.363934$ H -7.424792 $0.474356-1.816624$ C $-3.310279-2.543386-0.892612$ O $-5.166560-2.9631610 .642867$ H -5.208168 -3.476856 -1.328476 H $-3.111847-2.242514-1.926492$ O $-2.765609-1.540292-0.039355$ C $-2.582760-3.870889-0.606430$ C $-1.059195-3.856480-0.834449$ H -3.022247 -4.660686 -1.226692 O $-2.792966-4.2607770 .754501$ H - $-0.606556-3.099913-0.188405$ H $-0.678557-4.815041-0.459827$ H $-9.666915-0.327800-1.085374$ H $-9.107337-0.4210780 .591466$ H $-9.2110241 .148624-0.220914$ C -6.2275551 .9191870 .092818$ H $-4.823444-2.2420411 .198382$ H $-3.184149-0.693578-0.268885$ H $-2.474828-3.5348511 .318558$ H -7.1668622 .4626570 .243429$ H -5.834169 2.211824 -0.888630 H $-6.7210070 .082646 \quad 1.143573$ C $0.286838-1.428551-2.181943$ O $-0.709648-2.300155-2.732233$ C $-0.641304-3.666593-2.307359$ C $0.742104-4.245783-2.643191$ C $1.859177-3.406784-2.035758$ C $1.701251-1.952047-2.464849$ H $0.130182-1.328173-1.102358$ C $0.102035-0.048129-2.823742$ H -1.368481 -4.215506 -2.919074 O $0.864167-5.590153-2.191392$ H $0.874334-4.262876-3.732965$ H $1.886013-3.496326-0.944053$ O $3.120391-3.879474-2.512595$ H $1.927577-1.863282-3.535495$ O $2.649389-1.152255-1.764036$ C $-1.1399580 .642303-2.314185$ H $0.9423590 .618258-2.601334$ H $\quad 0.042487-0.138449-3.915294$ H $\quad 0.142590-6.101127-2.596107$ H $3.186521-4.819119-2.270025$ H $3.530769-1.515970-1.955442$ O $-2.048206 \quad 0.772916-3.322321$ O $-1.2818701 .026508-1.160638$ C $-3.2590531 .451522-2.977622$ H $-3.7283870 .983139-2.109600$ H $-3.0511212 .506944-2.779514$ H $-3.9442141 .379613-3.826299$ SCF Energy (B3LYP/6-31G**//MMFF) $=-3245.92344406$

05_00242

MM̄FF Geometry

C -2.576492 -2.816093 -2.202611

C $-1.687138-3.152409-3.149519$

C $-0.283903-2.596877-3.271230$

O $-0.098575-1.461243-2.393683$

C $0.748957-3.667058-2.878055$

C $2.168768-3.227133-3.178172$

C $2.981763-2.599151-2.305062$

C $2.646182-3.529748-4.575150$

C $2.664872-2.192834-0.891340$

C $3.762371-2.5544570 .121814$

C $5.066392-1.760821-0.056321$

C $6.054854-2.0774991 .068577$

C $7.332333-1.2299670 .962829$

O $4.746891-0.365925-0.041118$ 
O $8.028896-1.3656962 .210872$

C $6.9378590 .254363 \quad 0.795880$

C $8.276947-1.772320-0.116037$

O 8.0890581 .0612490 .558010

C $5.864306 \quad 0.493781-0.291372$

C $5.3712441 .959188-0.239602$

O $6.4455710 .249241-1.575041$

C $-0.537058-0.248981-2.827301$

O $-1.0120010 .018745-3.918924$

C $-0.4018950 .692665-1.695229$

C $-1.1824321 .782650-1.659955$

C $-1.2313992 .774261-0.584170$

C $-0.213290 \quad 2.654418 \quad 0.517017$

C $4.1022782 .223592-1.065174$

C $3.7798063 .706084-1.156418$

C $2.1298945 .276155-2.170179$

O $4.4803314 .579298-0.650967$

C $1.2342335 .732625-1.061739$

C $0.0138316 .252918-1.262597$

C $-0.8616906 .770493-0.157481$

C $-2.2603136 .197738-0.194158$

C $-3.2961616 .928769-0.639894$

C $-2.472147 \quad 4.8118390 .370947$

C $-2.1830853 .729449-0.631613$

N $2.6218993 .940171-1.887017$

H $-2.288700-2.118344-1.420932$

H $-1.981930-3.864020-3.917786$

H $-0.119879-2.283294-4.310003$

H $0.550254-4.602773-3.417701$

H $0.644704-3.918585-1.816208$

H $3.967531-2.305784-2.660863$

H $3.671527-3.186441-4.749135$

H $2.624797-4.609256-4.756843$

H $2.004457-3.039541-5.313944$

H $2.486142-1.111637-0.874514$

H $1.739094-2.658584-0.546504$

H $3.359342-2.3458861 .121319$

H $3.971833-3.6304510 .079704$

H $5.507135-2.021645-1.024287$

H $6.301153-3.1466091 .082296$

H $5.581270-1.8676212 .038180$

H $8.801289-0.7749502 .183145$

H 6.5325580 .5859531 .761645

H $8.557997-2.8079650 .110241$

H $9.216226-1.208903-0.141653$

H $7.838859-1.758320-1.116210$

H $8.3504260 .937359-0.370375$

H $\begin{array}{llll}5.145737 & 2.234657 & 0.798978\end{array}$

H $6.1742662 .623156-0.584289$

H $5.9758280 .771294-2.244001$

H $\quad 0.2887280 .433533-0.900907$

H -1.879648 1.948677 -2.480392

H $-0.238864 \quad 3.479712 \quad 1.231192$

H 0.8018692 .6360300 .106969

H -0.3757251 .7313191 .079486$

H $4.216607 \quad 1.847430-2.087268$

H $3.2465251 .713659-0.607431$

H $1.6103965 .235139-3.132661$

H $2.9809165 .959250-2.259335$

H $1.6214335 .665109-0.047643$

H $-0.3611696 .351725-2.278924$

H $-0.902774 \quad 7.863497-0.253707$

H $-0.418930 \quad 6.576973 \quad 0.827477$

H -4.306786 $6.533976-0.640716$

H -3.156038 $7.935554-1.019048$

H -3.519847 4.6938410 .679336

H -1.900423 4.7042481 .296733

H -2.876106 $3.726815-1.474355$

H $2.0661933 .151476-2.205462$

C $-4.995507-2.187100-2.229774$

O $-4.818338-1.274920-1.125656$

C -6.093511-1.031336 -0.509563

C $-7.130646-1.456067-1.539931$

C $-6.448033-2.661894-2.157679$

H $-6.158241-1.7070310 .351829$

C $-6.1953670 .422425-0.031930$

H -8.097110 -1.693915 -1.085827

H $-7.290458-0.683147-2.300372$
C $-7.028936-3.070409-3.500222$

H $-6.533190-3.506991-1.461230$

C $-5.0145550 .904544 \quad 0.838054$

O $\quad-6.263765 \quad 1.290372-1.172009$

H -7.1403150 .5642350 .505097$

H $-4.134692 \quad 1.0501180 .198745$

O $\begin{array}{lllll}-5.369378 & 2.199243 & 1.338253\end{array}$

C $-4.643875-0.0218612 .012121$

C $-3.496790 \quad 0.5430812 .871831$

H $-4.350672-0.9993421 .616807$

$\begin{array}{llll}\text { O } & -5.767826 & -0.219237 & 2.868921\end{array}$

H -2.7306920 .9659562 .214077$

H -3.877729 1.3607823 .495707

H $-8.083110-3.346891-3.394961$

H $-6.966379-2.255494-4.229387$

H $-6.492836-3.931784-3.910784$

C $-3.984222-3.332917-2.121410$

H $-5.463157 \quad 1.140445-1.703881$

H -4.5646632 .6102611 .696149$

H $-6.453829-0.6869832 .364708$

H $-4.171272-4.074923-2.905908$

H $-4.094128-3.838467-1.154183$

H $-4.820126-1.609298-3.146469$

C $-1.296224-1.4370532 .210680$

O $-2.423410-1.6778853 .059925$

C $-2.872654-0.5321693 .794289$

C -1.737295 $-0.019497 \quad 4.689947$

C $-0.4624220 .255496 \quad 3.894944$

C $-0.090856-0.947746 \quad 3.025129$

H -1.566347 -0.6767791 .467870$

C $-1.034061-2.7120831 .401901$

H $-3.674443-0.8860124 .454757$

O $-2.149607 \quad \begin{array}{lll}1.158108 & 5.375427\end{array}$

H - $1.519721-0.7768975 .454402$

H $-0.5666551 .168340 \quad 3.299781$

$\begin{array}{lllll} & 0 & 0.598128 & 0.506556 & 4.820743\end{array}$

H $0.291556-1.7383073 .682202$

$\begin{array}{lllll} & 0 & 0.969862 & -0.561420 & 2.155171\end{array}$

C $-0.459359-3.8356862 .231322$

H $-1.966290-3.0894800 .964553$

H $-0.361885-2.4967800 .564472$

H $-1.398306 \quad 1.4583785 .915182$

H 1.4077760 .6482554 .300611

H $1.276554-1.3585461 .691990$

O $0.834556-4.0650381 .866448$

O $-1.077799-4.424332 \quad 3.108225$

C $1.509282-5.0933632 .596943$

H $1.556650-4.8342213 .658855$

H $1.003843-6.0532102 .454527$

H $2.528428-5.1721912 .209369$

SCF Energy (B3LYP/6-31G**//MMFF) = -3245.91415263

05_00243

MM̄FF Geometry

C $0.684756 \quad 3.155123-0.736990$

$\begin{array}{llll}\text { C } & 1.260612 & 3.269230 & 0.468574\end{array}$

C $1.8140642 .095306 \quad 1.230754$

$\begin{array}{llll}\text { O } & 3.239040 & 2.323528 & 1.354124\end{array}$

C $1.2076591 .972642 \quad 2.642649$

C -0.1605231 .3190242 .689013$

$\begin{array}{lll}\text { C }-0.363675 & 0.013281 & 2.957240\end{array}$

C -1.3398832 .2354232 .499667$

C $0.689671-1.0366153 .172392$

C $0.329258-2.4054212 .576959$

C $0.044767-2.402741 \quad 1.066214$

C $-0.232681-3.8313600 .585021$

C $-0.439873-3.901934-0.934394$

O $1.176094-1.8582410 .375276$

O $-0.356287-5.280577-1.328493$

C $0.724312-3.164411-1.627021$

C $-1.835834-3.420198-1.343628$

O $0.508671-3.077918-3.033357$

C $0.992415-1.759184-1.045884$

C $2.247095-1.096792-1.661503$

O $-0.118024-0.908581-1.361270$

C $4.036056 \quad 1.2233831 .423859$

$\begin{array}{lllll}\text { O } & 3.678203 & 0.056564 & 1.414045\end{array}$

C $5.4408331 .677147 \quad 1.514078$ 
C $6.415606 \quad 0.7559911 .539462$

C 7.8534811 .0097811 .623205

C 8.3152842 .4406761 .695351

C $3.569944-1.833921-1.435721$

C $4.741213-1.090955-2.061824$

C $7.164402-1.357607-2.531243$

O $4.609133-0.056406-2.711036$

C $8.358680-1.636714-1.675662$

C $9.366979-0.763917-1.523802$

C $10.591934-1.024054-0.692284$

C $10.848937 \quad 0.0739250 .317658$

C 11.6868561 .0856460 .034148

C $10.194757-0.0329201 .677176$

C 8.693073-0.046428 1.612411

N $5.951166-1.733891-1.826067$

H $0.6332962 .178845-1.210801$

H 1.3100524 .2447020 .946309

H $1.642508 \quad 1.1695660 .668131$

H 1.1570192 .9608423 .120873

H $\quad 1.9008611 .4184243 .288265$

H -1.389990 -0.343194 3.031565

H -2.292671 1.7350492 .704463

H -1.271832 3.0934603 .176062

H -1.3803372 .6009381 .471452$

H $1.653391-0.7241442 .764608$

H $0.828026-1.1627484 .253094$

H $1.171668-3.0786762 .783542$

H $-0.540366-2.8134183 .108012$

H $-0.829440-1.7691970 .878115$

H -1.096743 -4.257777 1.109819

H $0.620844-4.4734270 .845224$

H - $1.034237-5.774649-0.836328$

H $\quad 1.618216-3.784814-1.486788$

H $-2.604034-4.060063-0.894322$

H -1.980061 -3.497034 -2.426871

H $-2.044860-2.392846-1.038503$

H $\quad 0.348200-3.980407-3.358195$

H $2.087007-0.940232-2.736735$

H $2.349799-0.087347-1.241102$

H $\quad 0.1723840 .014710-1.287406$

H 5.6329932 .7429281 .553970

H $6.136686-0.2962411 .489897$

H 7.9175632 .9282042 .592275

H 7.9802952 .9984230 .813727

H 9.4025522 .5421061 .734754

H $3.764200-1.955603-0.365288$

H $3.536696-2.826662-1.896336$

H $7.106983-0.306678-2.833363$

H $7.207200-1.971201-3.437166$

H $8.409768-2.601589-1.177255$

H $9.3239280 .188729-2.047800$

H $11.447536-1.107542-1.375349$

H $10.530658-1.989890-0.175742$

H 11.8981821 .8657620 .757868

H $12.177667 \quad 1.158151-0.930773$

H $10.538506 \quad 0.7614512 .350320$

H $10.527175-0.9646882 .153528$

H $8.270142-1.049517 \quad 1.546454$

H $5.925163-2.655007-1.397660$

C -1.479844 $4.193677-1.337854$

O $-1.9544932 .973810-1.932751$

C $-3.3602043 .142077-2.232355$

C $-3.6637624 .626526-2.029411$

C $-2.2838995 .267305-2.055187$

H -3.482944 $2.855145-3.282984$

C $-4.1326102 .188340-1.311067$

H -4.329965 5.019316 -2.803891

H -4.146175 4.802358 -1.060219

C -2.239469 $6.633434-1.395083$

H -1.953447 $5.351793-3.098971$

C $-5.6614332 .335736-1.349779$

O $-3.7935940 .847457-1.686403$

H $-3.7738692 .323986-0.284149$

H $-5.9516243 .324876-0.977773$

O $-6.0868162 .276365-2.715850$

C $-6.4209201 .245112-0.564459$

C -5.9551841 .0974480 .894926$

H $-6.3330330 .291328-1.095116$
O $\quad-7.8135131 .575508-0.576445$

H -4.8856240 .8716800 .914285$

H -6.0894882 .0556911 .412208$

H -2.916947 7.327791-1.902723

H -2.537929 $6.580795-0.342650$

H $-1.2307317 .055682-1.438275$

C $0.0362914 .289927-1.481927$

H $-2.8253780 .767906-1.637683$

H -7.056378 $2.352963-2.721470$

H -7.924923 2.412691-0.094294

H $0.3957115 .253355-1.103823$

H $0.3302594 .218004-2.535523$

H $-1.7488654 .170144-0.272755$

C $-5.493481-1.9011330 .845169$

O $-6.757536-1.2393770 .970577$

C $-6.7155800 .003287 \quad 1.677329$

C $-6.188745-0.2288413 .101681$

C $-4.845598-0.9609173 .099908$

C $-4.914114-2.2158402 .228042$

H $-4.795488-1.2646390 .288398$

C $-5.729579-3.1788450 .033167$

H $-7.755391 \quad 0.342178 \quad 1.765618$

O -6.0717931 .0167643 .780756$

H $-6.919764-0.8330033 .654840$

H $-4.030872-0.3044572 .772897$

O $-4.522922-1.3680814 .431447$

H -5.523684 -2.977948 2.730160

O $-3.600735-2.7501322 .072534$

C $-6.072059-2.851107-1.401340$

H $-4.855514-3.8382480 .030988$

H $-6.567323-3.7521950 .448671$

H $-5.8574950 .827714 \quad 4.709694$

H -4.365076 -0.563966 4.954011

H -3.244159 -2.908933 2.963369

O $-4.964019-2.398144-2.053973$

O $-7.191609-2.976769-1.880128$

C $-5.162463-2.017779-3.418624$

H $-5.500983-2.876368-4.006232$

H -5.882874 -1.196582 -3.481218

H $-4.204319-1.674575-3.817967$

SCF Energy $(B 3 L Y P / 6-31 G * * / / M M F F)=-3245.91079519$

05_00244

MM̄FF Geometry

C 0.0047425 .9641220 .164360

C $0.9710735 .171267-0.322953$

C 2.0950944 .6121780 .510018

O 2.0103713 .1677810 .491378

C $3.4519865 .014221-0.096767$

C 4.6432264 .4198500 .631988

C 5.4856143 .5005730 .117621

C 4.8732144 .9468672 .025946

C $5.4648662 .875529-1.250410$

C $5.0153621 .411067-1.246675$

C $5.9070820 .426446-0.470173$

C $7.3294540 .363215-1.035754$

C $8.174997-0.735166-0.374005$

O $5.272908-0.857915-0.577347$

O $9.349760-0.931904-1.176437$

C $7.383247-2.057557-0.404728$

C $8.666400-0.3166261 .016806$

O $8.067226-3.0796720 .316236$

C $5.940566-1.9172620 .122924$

C $5.115122-3.208841-0.079294$

O $5.976269-1.6577951 .531175$

C 1.1061162 .5817851 .322794

O 0.2937183 .1399462 .042406

C 1.2927991 .1181361 .218326

C 0.4139110 .2860421 .796944

C $0.503788-1.1763521 .817672$

C $1.732121-1.7963621 .208404$

C $4.947690-3.649038-1.534794$

C $3.846538-4.683930-1.669166$

C $1.372201-4.913641-1.776620$

O $4.094251-5.878011-1.818388$

C $0.214479-4.185838-1.171471$

C $-0.606181-4.715401-0.251267$

C $-1.763029-3.9685240 .351135$ 
C $-1.760092-3.988176 \quad 1.864404$ C $-2.776578-4.5455162 .544056$ C $-0.586108-3.3723342 .595013$ C $-0.489983-1.8818732 .397164$ N $2.578303-4.129045-1.582853$ H $0.0202106 .248721 \quad 1.213327$ H $0.9350614 .870731-1.367793$ H $2.0344264 .965217 \quad 1.547202$ H $3.5501536 .108099-0.089229$ H $3.4645424 .727412-1.152635$ H $6.308800 \quad 3.1605550 .745299$ H 5.8066564 .5756222 .462461 H 4.9313996 .0402492 .013528 H $4.058218 \quad 4.6471832 .691714$ H $4.8146203 .427258-1.934575$ H $6.4668092 .964111-1.685198$ H $3.9930041 .346398-0.852557$ H $4.9362801 .067853-2.287286$ H 5.9212360 .7168420 .586042 H $7.8384611 .329104-0.938765$ H $7.2803590 .157702-2.114352$ H $9.821881-0.083291-1.230370$ H $7.349920-2.384783-1.451780$ H $\quad 9.2978970 .5773780 .946528$ H $9.296537-1.0934831 .463751$ H $7.853572-0.0888911 .709247$ H $8.967070-3.138599-0.047861$ H $5.548974-4.030547 \quad 0.505288$ H $4.122509-3.0494730 .357713$ H $5.120812-1.9124291 .914799$ H 2.1635490 .7645430 .678722 H -0.4462160 .7111812 .312708$ H $2.634677-1.4325791 .712222$ H $1.800155-1.5470270 .143827$ H $1.755619-2.8847741 .276615$ H $4.704195-2.801321-2.185581$ H $5.875157-4.089514-1.916771$ H $1.516035-5.909894-1.345046$ H $1.223314-5.026435-2.855266$ H $0.039152-3.168268-1.513380$ H $-0.449827-5.7401750 .077107$ H $-2.684490-4.423578-0.034018$ H $-1.776508-2.926500 \quad 0.013638$ H -2.788935 -4.5662963 .628786$ H -3.623219 -4.990747 2.032007 H $0.327103-3.9010462 .312057$ H $-0.683440-3.5439753 .675545$ H - $1.334093-1.3325112 .814032$ H $2.497803-3.117211-1.633621$ C $-2.4239935 .670035-0.329886$ O $-2.2519334 .302699-0.754328$ C -3.414359 $3.882642-1.485953$ C $-4.4901674 .911446-1.163410$ C $-3.6643336 .179940-1.062372$ H -3.158832 $3.950171-2.551462$ C $-3.7950182 .431930-1.152699$ H $-5.263664 \quad 4.961676-1.935572$ H $-4.977710 \quad 4.696643-0.205039$ C $-4.3768107 .316482-0.350476$ H -3.395442 $6.505453-2.076276$ C $-2.7017791 .414278-1.544434$ $\begin{array}{lllll}\text { O } & -4.068425 & 2.336591 & 0.244922\end{array}$ H $-4.7233912 .204783-1.687685$ H -2.489253 $1.507676-2.616181$ O $-1.4787851 .735201-0.871758$ C $-3.017774-0.059110-1.221538$ C $-4.313946-0.565968-1.871407$ H $-3.038968-0.215863-0.138730$ O $-1.937243-0.854231-1.726102$ H $-5.1517950 .062412-1.559732$ H $-4.218470-0.465856-2.959549$ H $-5.2863497 .598545-0.890832$ H $-4.664856 \quad 7.0344050 .667819$ H -3.733808 $8.199859-0.286265$ C $-1.1539716 .457771-0.656742$ H $-4.465058 \begin{array}{lll}1.465718 & 0.413059\end{array}$ H $-1.672272 \quad 1.7782130 .080533$ H $-1.122275-0.537760-1.299473$
H -1.297550 $7.524057-0.449958$

H $-0.9205386 .362045-1.724643$

H -2.5959745 .6473360 .754122$

C $-5.917666-1.688258 \quad 0.484274$

O $-4.751568-2.260995-0.122567$

C $-4.621517-2.043022-1.531791$

C $-5.843054-2.614155-2.261629$

C $-7.144519-2.046591-1.707332$

C $-7.196889-2.211011-0.189107$

H $-5.874646-0.5953860 .409087$

C $-5.888052-2.0708901 .973312$

H $-3.755899-2.633691-1.855498$

O $-5.748657-2.351316-3.657465$

H $-5.851861-3.705319-2.140515$

H $-7.276216-0.999127-2.003251$

O $-8.232142-2.767259-2.292421$

H $-7.351745-3.2720230 .046343$

$\begin{array}{llllll} & \text { O } & -8.335156 & -1.491994 & 0.282949\end{array}$

C $-4.799020-1.3575342 .747359$

H $-6.834863-1.8102772 .457044$

H $-5.714201-3.1488322 .072855$

H $-6.550408-2.709537-4.075585$

H $-9.052293-2.418565-1.902664$

H $-8.524124-1.7916161 .187672$

O $-4.814622-1.8092014 .037279$

O $-4.043471-0.5106782 .293221$

C $-3.860929-1.2124394 .922132$

H $-3.868004-0.1218204 .831508$

H $-4.137493-1.4772635 .946185$

H -2.865143 -1.611971 4.713728

SCF Energy (B3LYP/6-31G**//MMFF) = -3245.91671762

05_00245

MMFF Geometry

C $-1.413116 \quad 3.837926-2.163221$

C $-2.2197734 .787594-1.664284$

C $-3.2662924 .580423-0.596498$

O $-3.6309543 .185898-0.506848$

C -2.6990465 .0101840 .770330$

C $-3.683044 \quad 4.838721 \quad 1.910904$

C -3.5384813 .9576902 .920983$

C $-4.885407 \quad 5.7467591 .872921$

C $-2.403305 \quad 3.000598 \quad 3.166691$

C -2.6949801 .6194012 .579793$

C -1.7285380 .5230803 .050781$

$\begin{array}{llll}C & -0.285321 & 0.768611 & 2.603018\end{array}$

C $0.639786-0.4009202 .979603$

O $-2.209932-0.708892 \quad 2.492443$

\begin{tabular}{llllllll}
\hline & 1.865889 & -0.246248 & 2.250828
\end{tabular}

C $0.000881-1.7208222 .501745$

C $1.015200-0.3783154 .465547$

O $0.744270-2.8478482 .957609$

C $-1.479470-1.8718162 .909375$

C $-2.154201-3.1043802 .260847$

O $-1.565504-2.0471784 .328597$

C $-4.8149472 .792383-1.047048$

O $-5.616800 \quad 3.478742-1.658486$

C $-4.974596 \quad 1.354035-0.736625$

C $-6.1195870 .717765-1.025608$

C $-6.409093-0.690850-0.739205$

C $-5.345488-1.476875-0.019036$

C $-2.053825-3.1627170 .734957$

C $-3.001988-4.1800210 .120636$

C $-3.780698-4.971332-2.120779$

O $-3.731372-4.911672 \quad 0.783541$

C $-4.874328-4.106328-2.665527$

C $-6.176623-4.427866-2.625471$

C $-7.258882-3.556712-3.200202$

C $-8.401104-3.310219-2.236571$

C $-9.648546-3.709941-2.537314$

C $-8.111360-2.605374-0.927657$

C $-7.596310-1.205247-1.123394$

N $-2.929750-4.163841-1.265589$

H $-1.4928782 .815217-1.803978$

H $-2.1034165 .810493-2.016563$

H $-4.1405735 .191128-0.850124$

H $-2.388964 \quad 6.063038 \quad 0.733056$

H -1.7858654 .4372930 .965872$ 
H -4.3224613 .9136913 .676408$ H -5.4980505 .6626962 .777080$ H $-4.568926 \quad 6.791716 \quad 1.789676$ H -5.525822 5.5058651 .019168 H -1.461068 3.3961772 .776965 H -2.2648692 .9200484 .252138$ H -3.7180121 .3110052 .835604$ H -2.671958 1.6697321 .484991 H -1.789184 0.4631564 .143862 H $\quad 0.107584 \quad 1.7032283 .019877$ H -0.2601030 .8933881 .511214$ H 2.2371350 .6264752 .466424 H $\quad 0.072389-1.7223171 .406778$ H 1.5403870 .5519944 .713006 H $1.711158-1.1882704 .709956$ H $0.150288-0.4543215 .127661$ H $1.660561-2.7308572 .653184$ H $-1.744776-4.0283832 .689769$ H -3.216151 -3.095762 2.544058 H -2.413014 -2.475338 4.534541 H $-4.1394670 .855213-0.258303$ H -6.920903 $1.275597-1.507707$ H $-5.115203-1.0198450 .950143$ H - $4.430825-1.507779-0.619889$ H -5.621927 $-2.511806 \quad 0.186212$ H $-2.283886-2.1823850 .303516$ H - 1.035698 -3.438844 0.438028 H -4.169062 -5.827159-1.559634 H -3.157232 $-5.339985-2.941204$ H -4.575638 -3.170910 -3.133826 H $-6.479489-5.374317-2.183701$ H -7.629547 -4.051134 -4.107572 H $-6.854723-2.591485-3.529445$ H -10.477063 -3.540535 -1.857704 H -9.871585 -4.216274 -3.470761 H $-7.438760-3.230006-0.335574$ H $-9.027842-2.528731-0.327467$ H $-8.304236-0.548916-1.631462$ H -2.318817 -3.488879-1.716860 C $1.0731574 .039211-2.635908$ O $1.3488082 .738690-2.078866$ C $2.1267112 .911685-0.871559$ C $2.5828974 .364339-0.873530$ C $1.4069285 .049611-1.539134$ H $1.4318322 .728814-0.041712$ C $3.2617161 .881064-0.854676$ H 2.7861004 .7434770 .132352 H $3.4906654 .488369-1.477385$ C $1.7217116 .442095-2.055691$ H $0.5944945 .116861-0.805526$ C 4.2758592 .0840870 .284575 O $2.6766720 .581299-0.725571$ H $3.7777801 .897087-1.821443$ H 4.8061343 .0332200 .141444 O $3.5543052 .209316 \quad 1.516541$ C 5.3017510 .9453150 .450655 C $6.0861920 .647416-0.838877$ H 4.7933370 .0476900 .820197 O $\quad 6.213836 \quad 1.354057 \quad 1.475319$ H $5.3882450 .304774-1.608915$ H $6.5341251 .579359-1.206174$ H $2.0240707 .098185-1.232933$ H $2.5361236 .424641-2.787737$ H $0.8445786 .886271-2.536830$ C $-0.3517954 .098861-3.194539$ H $2.035094 \quad 0.477947-1.449205$ H 4.2094552 .3407212 .223405 H 6.7039380 .5651641 .763095 H $-0.4631563 .333563-3.972848$ H $-0.5146705 .068350-3.679982$ H $1.7701104 .160562-3.476023$ C $5.855058-2.385863-0.709286$ O $6.770198-1.5650910 .025788$

C $7.213017-0.394925-0.665241$

C $7.912482-0.792659-1.973266$

C $7.042176-1.707853-2.832477$

C $6.506151-2.877261-2.006409$

H $4.946823-1.814666-0.938355$
C $5.462370-3.5571310 .196270$

H $7.9750300 .068156-0.025913$

O $8.269390 \quad 0.358244-2.731341$

H $8.843433-1.324830-1.738131$

H $6.221753-1.154834-3.304886$

O $7.831103-2.239889-3.899517$

H $7.325471-3.571468-1.779434$

O $5.540494-3.590357-2.775949$

C $4.590326-3.0727711 .329864$

H $4.892147-4.319941-0.345711$

H $6.346297-4.0541230 .613910$

H $8.8376660 .912916-2.169952$

H $8.193940-1.485107-4.394289$

H $5.974558-3.867263-3.601210$

O $5.353560-2.8150952 .429835$

O $3.380904-2.9100571 .233722$

C $4.649901-2.3003913 .564353$

H $4.260507-1.3032783 .341012$

H $3.841136-2.9773933 .855625$

H $5.356066-2.2222224 .395200$

SCF Energy $(B 3 L Y P / 6-31 G * * / / M M F F)=-3245.92845804$

05_00246

MMFF Geometry

C $-3.937430-1.8990572 .463109$

C $-5.261120-2.0396872 .282767$

C $-5.934376-2.0940140 .931251$

O $-5.288979-1.1624700 .033944$

C $-5.824529-3.509097 \quad 0.329584$

C $-6.375237-3.612226-1.080159$

C $-5.622775-3.857663-2.172448$

C -7.864344 -3.428835 -1.212698

C $-4.137630-4.102769-2.226348$

C $-3.341030-2.799260-2.204788$

C $-1.831447-2.959810-2.430604$

C $-1.169951-3.856256-1.380331$

C $0.360855-3.873442-1.511367$

O $-1.289126-1.632667-2.362291$

O $0.898919-4.461324-0.316445$

C $0.869824-2.419701-1.565062$

C $0.826858-4.754295-2.676019$

O $2.271443-2.376042-1.814441$

C $0.122334-1.543722-2.592047$

C $0.511236-0.049431-2.493677$

O $0.452957-1.979763-3.915260$

C $\begin{array}{rlll}-5.684250 & 0.137446 & 0.067577\end{array}$

$\begin{array}{lllll}\text { O } & -6.574146 & 0.622001 & 0.746876\end{array}$

C $-4.8026370 .881507-0.860012$

C $-4.8966422 .216273-0.950078$

C $-4.0544153 .080922-1.779942$

C $-3.0392652 .415297-2.672443$

C $0.2756820 .589096-1.122046$

C $0.4825902 .094748-1.139219$

C 0.5024414 .0539370 .407294

O $0.7480082 .729257-2.156175$

C -0.8442694 .7040790 .440517$

C $-1.1563935 .808041-0.255765$

C $-2.4969506 .482475-0.177318$

C $-3.1451026 .687803-1.531077$

C $-3.4814047 .920844-1.946824$

C $-3.4166885 .480286-2.408597$

C $-4.2262794 .417646-1.708876$

N $0.3409942 .640067 \quad 0.128418$

H -3.285667 -1.857573 1.593295

H -5.912846 -2.115322 3.149428

H $-6.989854-1.8183971 .045145$

H -6.355689 -4.225890 0.969646

H $-4.774590-3.8224340 .344596$

H $-6.118473-3.896575-3.141308$

H $-8.216343-3.616543-2.232768$

H $-8.392274-4.123838-0.551588$

H -8.153066 -2.407056 -0.948348

H -3.838592 -4.762638-1.405400

H -3.915998 -4.653751 -3.148805

H -3.734878 -2.108344 -2.962852

H $-3.487916-2.289758-1.246739$

H -1.670694 -3.359043 -3.438892

H -1.556819-4.880577 -1.432809 
H -1.427715 -3.489112 -0.376835 H $\quad 0.526265-5.355356-0.228199$ H $0.718620-2.000615-0.563922$ H $0.501896-5.791025-2.525814$ H $1.920474-4.788611-2.732276$ H $\quad 0.440535-4.426700-3.643325$ H $2.704425-2.925653-1.139007$ H $1.5587020 .086909-2.791188$ H $-0.0764590 .505397-3.238674$ H $0.286887-1.247481-4.531855$ H $-4.0747480 .309227-1.424455$ H $-5.6402252 .729272-0.341755$ H $-3.5265161 .692420-3.336711$ H -2.291499 $1.889048-2.073100$ H -2.504633 $3.109935-3.323681$ H $-0.7459520 .392495-0.777970$ H $0.9739410 .170263-0.389600$ H $1.1615824 .504323-0.342034$ H $0.9790104 .137040 \quad 1.388970$ H -1.592194 4.2584861 .092717 H $-0.403377 \quad 6.271786-0.888494$ H -2.3478337 .4484880 .322209$ H -3.1835135 .9185010 .466089$ H -3.947959 $8.085570-2.912329$ H -3.297847 8.795903 -1.332167 H -2.449145 $5.100508-2.750188$ H -3.971269 $5.780784-3.306488$ H -5.023835 4.803524-1.072771 H 0.0566392 .0383050 .895800 C $-2.242647-0.6705193 .837489$ O $-1.073442-1.0728823 .087450$ C $0.093927-0.8952243 .916696$ C -0.3283090 .0974704 .985395$ C -1.750893 -0.3508505 .250889$ H $0.295236-1.8773684 .364193$ C $1.279352-0.4599493 .046681$ H $\quad 0.3034050 .0574325 .877434$ H -0.3134891 .1221024 .593557$ C -2.5913050 .6887945 .972355$ H -1.723450 -1.267679 5.855351 C $2.524565-0.0602533 .855894$ O $1.623458-1.5560492 .192998$ H $0.974596 \quad 0.3641802 .392589$ H $2.311433 \quad 0.844171 \quad 4.437294$ O $2.806242-1.0874044 .816985$ C 3.8089220 .1614413 .034290 C 3.6468301 .1708381 .886323 H $4.179078-0.7948812 .647163$ O $44.813840 \quad 0.6563573 .927342$ H $2.9316330 .775824 \quad 1.158647$ H 3.2208912 .1000872 .284972 H -2.165243 0.9122326 .956007 H -2.6423901 .6258335 .407722$ H -3.614262 0.3285776 .120516 C -3.276494 -1.801427 3.809006 H $0.813303-1.8256701 .727477$ H $2.954571-1.9144514 .326948$ H 4.9094130 .0062994 .644584 H -4.032123 -1.638986 4.586227 H -2.781896 -2.759004 4.011907 H $-2.636947 \quad 0.2313493 .351581$ C $5.148615-0.395999-0.289222$ O $5.706487 \quad 0.3291940 .810026$ C 4.9828011 .5066351 .182224 C $4.8502262 .459364-0.015670$ C $4.2873891 .758818-1.249628$ C $5.0683030 .479841-1.544131$ H $4.138060-0.726619-0.016835$ C $5.962626-1.682329-0.489709$ H 5.6058682 .0212321 .924843 O 4.0235863 .5725800 .306901 H $5.8409902 .857925-0.270466$ H $3.2224141 .540159-1.133270$ O $4.4000942 .626665-2.378678$ H $6.0684420 .747082-1.904019$ O $4.420443-0.243528-2.586210$ C $7.405017-1.453487-0.893405$ H $5.987602-2.2516340 .447200$
H $\quad 5.476975-2.298479-1.255318$

H 4.4167414 .0138851 .079194

H $3.9130023 .441303-2.165498$

H $4.3373030 .355516-3.347908$

O $7.867396-2.562069-1.544159$

O $8.057160-0.449608-0.641126$

C $9.231708-2.499987-1.972095$

H $9.469464-3.435046-2.486335$

H $9.372694-1.669588-2.670547$

H $9.894703-2.395977-1.107991$

SCF Energy $(B 3 L Y P / 6-31 G * * / / M M F F)=-3245.91769644$

05_00247

MMFF Geometry

C $-1.957820-1.106441-3.403789$

C $-1.270814-2.259530-3.424928$

C $-0.050108-2.619752-2.606830$

O $0.427500-1.511725-1.811361$

C $-0.377878-3.781913-1.648939$

C $0.853823-4.465639-1.082397$

C $1.189222-4.4992410 .221952$

C $1.720949-5.186978-2.084514$

C $0.457414-3.9018811 .388150$

C $0.919875-2.4924121 .782417$

C $2.400279-2.3878002 .179888$

C $2.686581-1.0253932 .816704$

C $4.177029-0.8419883 .141437$

O $3.197159-2.5390081 .002049$

O 4.3994840 .5495713 .418472

C $5.011854-1.1911981 .890292$

C $4.582344-1.6057434 .407573$

O $6.404220-1.1955972 .193480$

C $4.611922-2.5309531 .238445$

C $5.306991-2.780990-0.122857$

O $4.992646-3.6075172 .104375$

C $1.233117-0.603557-2.427274$

O $1.583290-0.602219-3.596878$

C $1.6097820 .417872-1.426151$

C $2.0369701 .618312-1.844484$

C $2.4611852 .729091-0.990715$

C 2.7072162 .4325820 .464751

C $5.043962-1.724266-1.201429$

C $6.107421-0.640833-1.276454$

C $6.5821681 .540825-2.383273$

O $7.181131-0.707209-0.684443$

C $6.2083552 .662544-1.466090$

C $5.9666963 .910900-1.894306$

C $5.6255115 .058869-0.987629$

C $4.3554345 .768243-1.401570$

C $4.4000306 .884790-2.147983$

C $3.0416815 .229040-0.881895$

C $2.6370383 .943828-1.551643$

N $5.7296520 .396716-2.118471$

H $-1.668775-0.308785-2.725856$

H - $1.588108-3.030582-4.125601$

H $0.734756-2.922922-3.309802$

H $-0.966182-4.548709-2.170863$

H -1.017674 -3.402304 -0.844776

H $2.098874-5.0313840 .499872$

H $2.476546-5.816853-1.602382$

H $1.110101-5.840602-2.715912$

H $2.250393-4.474747-2.724243$

H $-0.621307-3.8837491 .200330$

H $0.585311-4.5735662 .246886$

H $0.714159-1.8122630 .948106$

H $0.303152-2.1686752 .629269$

H $2.628784-3.1952952 .885148$

H $2.078024-0.8806383 .718121$

H $2.383042-0.2301812 .122453$

H 3.8220890 .8038714 .158673

H $4.850649-0.3820851 .167728$

H $4.000404-1.2550515 .268579$

H $5.631889-1.4213444 .661683$

H $4.430671-2.6839274 .325349$

H $6.620100-0.3283542 .576940$

H $6.380577-2.9472250 .027242$

H $4.918490-3.732742-0.513467$

H $5.055612-4.4204391 .575983$ 
H $\quad 1.517947 \quad 0.158142-0.378465$ H $2.0657081 .816182-2.915378$ H $3.353604 \quad 1.554843 \quad 0.573725$ H 1.7622902 .2415570 .982553 H 3.2133913 .2416240 .995302 H $5.047092-2.220150-2.180620$ H $4.059621-1.265684-1.070379$ H $6.4504451 .806209-3.437014$ H $7.6285711 .264120-2.220107$ H $6.1573132 .444390-0.401919$ H $6.0468874 .130621-2.956765$ H $6.4682825 .762340-1.009330$ H 5.5388524 .7343500 .056788 H $3.4960407 .415643-2.427278$ H $5.3425327 .290205-2.500906$ H $2.2375825 .954482-1.064708$ H 3.0986945 .1439990 .206701 H $2.4405664 .054526-2.618979$ H $4.8015490 .390591-2.531557$ C -4.428649 -0.488427 -3.616335 O $-4.322441 \quad 0.766738-2.920218$ C $-5.0643860 .670857-1.681149$ C $-5.860886-0.624522-1.774060$ C $-4.929724-1.499887-2.590235$ H $-4.3028880 .591208-0.898612$ C $-5.8946491 .947694-1.494775$ H -6.096103 -1.048658 -0.794220 H $-6.801676-0.467648-2.316013$ C $-5.605641-2.713270-3.203238$ H -4.113959-1.833301-1.937126 C $-6.8002681 .985665-0.246937$ O $-4.9937363 .057641-1.463355$ H $-6.5101182 .084043-2.393292$ H $-7.2159572 .995145-0.132486$ O $-7.9188431 .116441-0.457736$ C -6.1565921 .5818541 .095881$ C -4.9307582 .4198021 .488124$ H -5.9036140 .5173551 .085120$ O -7.1445411 .7514462 .121053$ H $-4.1490312 .294444 \quad 0.735590$ H -5.2121153 .4802091 .489952$ H -6.021992 -3.359306 -2.423995 H -6.424875 -2.422943 -3.869593 H $-4.890539-3.303305-3.785163$ C $-3.115100-0.824369-4.324151$ H -5.527786 $3.868728-1.417523$ H -8.382006 $1.422412-1.256246$ H -7.9126931 .2081131 .874320$ H -2.825591 $0.028240-4.951295$ H -3.281853 -1.672933 -4.998603 H $-5.193652-0.334025-4.389544$ C -3.033923 0.1540272 .229655 O $-4.1076620 .662551 \quad 3.028203$ C -4.3595442 .0634662 .880712$ C -3.1032012 .8599663 .261434$ C -1.881904 2.399798 2.470062 C -1.724556 0.8831122 .556965 H $-3.274908 \quad 0.271707 \quad 1.167591$ C $-2.907227-1.3459932 .521026$ H -5.1336902 .3138503 .616670$ O $-3.2983414 .253973 \quad 3.049255$ H -2.895279 2.7222124 .330525 H -1.9307582 .7294531 .425642$ O -0.7081773 .0013823 .022399$ H -1.3819500 .6078243 .562948$ O $-\begin{array}{llll}0.718890 & 0.470997 & 1.634397\end{array}$ C -4.041902 -2.134075 1.906057 H -1.965497 -1.732911 2.120137 H -2.915088 -1.550110 3.597924 H -4.073254 4.523126 3.571580 H -0.8361513 .9651282 .991405$ H $0.1024420 .929804 \quad 1.879515$ O $-3.544883-3.0548841 .031395$ O $-5.222503-1.9658822 .181861$ C $-4.506081-3.9151410 .412095$ H $-4.028333-4.395631-0.445921$ H -4.816382 -4.686384 1.122476 H $-5.373512-3.3515550 .057278$
SCF Energy (B3LYP/6-31G**//MMFF)= -3245.89991514

0500248

MMFF Geometry

C $3.708089-1.1078502 .305928$

C $3.111115-1.3761373 .476709$

C $1.726029-0.9460823 .904536$

O $1.137846-0.0714852 .914422$

C $0.827216-2.1866514 .070365$

C $-0.501970-1.9059454 .743890$

C $-1.714024-2.1397514 .202759$

C $-0.415747-1.4078736 .166558$

C $-2.057546-2.7214462 .859130$

C $-3.082589-1.8600662 .114181$

C $-3.820865-2.6240241 .003190$

C $-2.893105-3.091448-0.118418$

C $-3.666414-3.748772-1.272666$

O $-4.812746-1.7313860 .481104$

O $-2.776134-3.852062-2.395410$

C $-4.821420-2.816943-1.697347$

C $-4.097402-5.179350-0.929915$

O $-5.662126-3.445408-2.661717$

C $-5.670319-2.309559-0.512049$

C $-6.712055-1.239005-0.925812$

O $-6.409657-3.4072630 .040280$

C $0.6866521 .143483 \quad 3.328559$

O $\quad 0.6758321 .575376 \quad 4.470571$

C 0.2188441 .8895852 .136830

C -0.2611593 .1330622 .295360$

C $-0.7361904 .034636 \quad 1.241896$

C $-0.6645123 .543003-0.178626$

C $-6.201173-0.037806-1.724522$

C $-5.0633100 .709892-1.064685$

C -4.4880262 .1235850 .896752$

O $-3.9291600 .694593-1.538137$

C -4.3103963 .4904590 .316046$

C -4.4799144 .6265391 .008242$

C -4.2754445 .9912950 .417027$

C -3.1472806 .7519481 .076877$

C -3.3960087 .7947401 .886969$

C -1.7273956 .3583990 .724784$

C -1.200843 5.2508441 .600386

N $-5.435401 \quad 1.3778130 .089554$

H $3.179275-0.5359641 .548299$

H $3.657624-1.9668094 .210288$

H $1.847850-0.4277324 .862497$

H $1.346637-2.9413004 .677231$

H $\quad 0.679907-2.6451953 .087417$

H -2.586261 -1.930004 4.822406

H -1.392009-1.394742 6.663408

H $0.238167-2.058226 \quad 6.757051$

H -0.019329 -0.3899266 .202405$

H -1.177317 -2.856296 2.226571

H -2.479044 -3.718617 3.036330

H -3.836132 -1.472030 2.813440

H $-2.592361-0.9698781 .697379$

H $-4.328141-3.4805211 .462728$

H $-2.125747-3.7780920 .257528$

H $-2.352133-2.225882-0.518988$

H -2.000395 -4.368108 -2.116346

H $-4.359627-1.959761-2.201845$

H -3.220251 -5.802458 -0.717046

H $-4.602330-5.653877-1.778438$

H $-4.756512-5.232753-0.061152$

H $-5.092988-3.743167-3.392041$

H -7.512416 -1.718706 -1.504718

H -7.196494 -0.864112 -0.013597

H -7.163179 -3.0496660 .538614$

H $\quad 0.299548 \quad 1.4069341 .170019$

H -0.3066883 .5437013 .303700$

H -1.008032 $4.272747-0.914044$

H -1.289731 $2.653988-0.305227$

H $0.3668263 .288681-0.447775$

H $-5.876925-0.350373-2.723493$

H $-7.0245600 .669444-1.884767$

H -3.5282501 .5960580 .899629$

H -4.876243 2.1512871 .919606

H $-3.9939603 .548826-0.723074$ 
H -4.7850274 .5782552 .050841$ H -5.2193796 .5426760 .517676$ H -4.083636 $5.933469-0.661932$ H $-2.589508 \quad 8.3622452 .339668$ H -4.4104448 .1007142 .120007$ H -1.061793 7.2217190 .859450 H -1.675309 $6.126975-0.342062$ H -1.193856 5.5103102 .660369 H -6.4147361 .4041470 .357614$ C $6.013811-0.4848131 .486416$ O $5.467962 \quad 0.157517 \quad 0.317311$ C $6.4567950 .158325-0.724954$ C $7.789224-0.041854-0.016464$ C $7.395021-1.0102631 .081143$ H $6.249385-0.716415-1.354317$ C $6.3497301 .430008-1.571806$ H $8.561047-0.437458-0.683265$ H 8.1627210 .8895040 .424068 C $8.388549-1.0700802 .228876$ H $7.302974-2.0128880 .641857$ C $4.9199841 .765801-2.039762$ O $6.8101642 .542067-0.792082$ H $7.0308941 .354701-2.427760$ H $4.3276802 .104797-1.181014$ O $5.0125942 .897216-2.916753$ C $4.1689320 .626861-2.756510$ C $2.7892081 .097615-3.249426$ H $4.048647-0.213690-2.068125$ O $4.953145 \quad 0.181786-3.859933$ H $2.2619041 .546232-2.400290$ H $2.928126 \quad 1.888333-3.997006$ H $9.368143-1.4055391 .872662$ H $8.519215-0.0891732 .697908$ H $8.050905-1.7701212 .999648$ C $5.078567-1.6084101 .939256$ H $6.7413803 .335631-1.350357$ H $5.4773362 .606359-3.720343$ H $4.562904-0.647398-4.183594$ H $5.530751-2.1483262 .779431$ H $4.955405-2.3280381 .122100$ H 6.0947280 .2846392 .264249 C $0.980697-0.928941-1.859446$ O $1.748814-1.153187-3.046176$ C $1.918191-0.008930-3.887962$ C $0.5426880 .493022-4.350011$ C $-0.3521830 .820302-3.157411$ C $-0.408535-0.363926-2.194564$ H $1.525652-0.247037-1.194872$ C $0.817527-2.276999-1.135127$ H $2.449861-0.359644-4.780877$ O $0.698606 \quad 1.638013-5.180955$ H $\quad 0.061820-0.285657-4.956302$ H $-0.025831 \quad 1.730615-2.642470$ O $-1.6802851 .076892-3.618843$ H -1.035386 -1.150748 -2.634533 O $-1.0358080 .046732-0.981566$ C $2.119127-2.871724-0.647293$ H $0.171358-2.148734-0.259335$ H $0.366603-3.010223-1.814686$ H $-0.1739451 .856667-5.548969$ H - $-1.6594781 .904819-4.127653$ H -1.915070 $0.392748-1.212050$ O $1.835565-3.9841450 .094314$ O $3.233826-2.436361-0.894657$ C $2.956615-4.7097590 .609992$ H $3.767486-4.771567-0.122088$ H $3.303789-4.2354881 .531150$ H $2.623500-5.7246990 .842878$

SCF Energy (B3LYP/6-31G**//MMFF) $=-3245.92729399$

0500249

MM̄MF Geometry

C $3.201498 \quad 3.153746-0.726894$

C 3.0958264 .1169980 .200793

C 2.0835454 .1679991 .317373

O $0.868346 \quad 3.472551 \quad 0.958454$

C 2.6361023 .5148142 .597372

C 1.7518883 .7681323 .804101
C 1.0269392 .8317254 .446392

C 1.7275925 .1929224 .301160

C 0.9421131 .3539234 .186362

C $-0.2964740 .977242 \quad 3.368503$

C $-0.848717-0.4189273 .696367$

C $0.163520-1.5413913 .458343$

C $-0.453904-2.9232863 .734269$

O $-2.003926-0.6213672 .868963$

O $0.457092-3.9220883 .251719$

C $-1.777786-3.0474012 .946941$

C $-0.579952-3.1970745 .237346$

O $-2.431962-4.267173 \quad 3.286232$

C $-2.721329-1.8369783 .143148$

C $-3.953729-1.8683272 .207090$

O $-3.222059-1.8424294 .485485$

C $-0.1087534 .199566 \quad 0.350961$

O $-0.0422315 .361022-0.018112$

C $-1.303905 \quad 3.3343200 .243330$

C $-2.4669493 .855134-0.175840$

C $-3.7423583 .141634-0.279605$

C $-3.762357 \quad 1.700707 \quad 0.154393$

C $-3.625663-1.9905300 .718708$

C $-4.826624-1.718222-0.172496$

C $-5.385019-1.363936-2.584793$

O $-5.962665-1.5438750 .258515$

C $-5.2114270 .083804-2.922470$

C $-6.2241810 .960160-2.999619$

C $-6.0362272 .413477-3.333611$

C $-6.7038103 .336343-2.335583$

C $-7.6942694 .160070-2.718378$

C $-6.2348003 .305746-0.896175$

C $-4.8237113 .808197-0.736334$

N $-4.466900-1.700285-1.512848$

H $2.5350532 .297153-0.691150$

H 3.7893284 .9547350 .162970

H 1.8561155 .2240271 .506082

H 3.6393793 .9019282 .819146

H 2.7553602 .4394762 .425487

H $0.419907 \quad 3.155370 \quad 5.292147$

H 1.2034225 .2902475 .258298

H 2.7481615 .5596894 .452644

H 1.2229075 .8473263 .584455

H $1.8439880 .974147 \quad 3.696135$

H $\quad 0.9095340 .8618795 .167064$

H $-1.103740 \quad 1.705428 \quad 3.526577$

H -0.0600491 .0374102 .298564$

H -1.166915 -0.406785 4.745507

H $1.064447-1.4072324 .068429$

H $\quad 0.501203-1.5081342 .413037$

H $0.557649-3.8012132 .293635$

H $-1.501180-3.1233451 .888003$

H $0.408011-3.1704625 .713173$

H $-0.973846-4.2024395 .424069$

H -1.214785 -2.477254 5.757382

H -3.171653 -4.391273 2.668763

H $-4.634871-2.6769022 .502382$

H $-4.521758-0.9401892 .363880$

H -4.037871 -1.315213 4.511871

H -1.2004922 .2963290 .536705$

H -2.496765 $4.907859-0.453759$

H $-4.748719 \quad 1.2399110 .095137$

H -3.439396 1.6049831 .197134

H -3.094962 $1.104592-0.477029$

H $-2.835370-1.2814330 .451866$

H -3.273228 -3.0030490 .493509$

H $-6.409866-1.600386-2.281593$

H -5.117559-1.981850 -3.447301

H -4.197806 $0.427510-3.117870$

H -7.241565 $0.616052-2.828170$

H $-6.4486222 .577052-4.337553$

H $-4.9716042 .668867-3.401009$

H -8.181953 $4.825155-2.013360$

H $-8.0410644 .189633-3.746037$

H $-6.3793012 .299523-0.495937$

H $-6.8755763 .947682-0.276653$

H $-4.7044384 .855845-1.016492$

H -3.485581 -1.787010 -1.756386

C $5.3611352 .219625-1.714091$ 
$\begin{array}{llll}\text { O } & 4.895449 & 0.856470 & -1.737747\end{array}$

C $5.6648230 .099973-0.783079$

C $6.8383140 .987851-0.377130$

C $6.2069302 .363159-0.448586$

H $5.018203-0.0388050 .090900$

C $6.074870-1.260375-1.355552$

H 7.2207640 .7401720 .617797

H $7.6706870 .915110-1.086721$

C $7.2129113 .498988-0.501759$

H 5.5724942 .4900650 .437053

C $4.908371-2.129872-1.863727$

O $6.961103-1.040321-2.461100$

H $6.656055-1.807739-0.603643$

H $4.511969-1.705197-2.793807$

O $5.455325-3.406621-2.222658$

C $3.751487-2.337721-0.869659$

C $2.735561-3.384643-1.367107$

H $3.242707-1.384163-0.690797$

O $4.258458-2.808407 \quad 0.381189$

H $2.515349-3.203058-2.423114$

H $3.192050-4.378627-1.291556$

H 7.8369403 .5021890 .398020

H $7.8752403 .409187-1.369227$

H $6.7046974 .466473-0.563713$

C $4.1928423 .196544-1.856593$

H $7.215528-1.913080-2.807084$

H $5.757750-3.836539-1.404211$

H $4.764141-2.0894200 .793721$

H $3.6420292 .963008-2.776567$

H $4.5860634 .213262-1.977561$

H $6.0004362 .330437-2.600375$

C $0.042780-2.047258-2.005741$

O $0.694787-2.152135-0.732628$

C $1.439390-3.359656-0.526064$

C $0.531312-4.583852-0.705062$

C $-0.193011-4.561390-2.044948$

C $-0.911512-3.227221-2.225869$

H $0.791107-2.032960-2.806651$

C $-0.712339-0.708071-2.029984$

H $1.746954-3.3489190 .525737$

O $1.270115-5.794799-0.589841$

H $-0.223128-4.5942710 .092219$

H $0.491006-4.759004-2.878712$

O $-1.172391-5.602165-2.067351$

H $-1.749620-3.171490-1.521976$

O $-1.449768-3.160379-3.544366$

C $0.191155 \quad 0.449582-2.398814$

H $-1.508973-0.740352-2.781722$

H $-1.147719-0.487200-1.050061$

H $1.709200-5.7876880 .277975$

H $-0.706023-6.440595-1.907096$

H $-2.028602-3.933223-3.661040$

O $-0.5827001 .504401-2.793217$

O $1.4127430 .436487-2.341519$

C $\quad 0.1278092 .666782-3.230524$

H $0.8068382 .414444-4.050596$

H $-0.6030203 .395180-3.591950$

H $0.678066 \quad 3.107597-2.396378$

SCF Energy (B3LYP/6-31G**/MMFF) $=-3245.91261015$

05_00250

MM̄FF Geometry

C $-2.790113-3.7351682 .959377$

C $-2.492389-2.6024803 .614209$

C $-3.374734-1.3940753 .804460$

O $-2.799358-0.3350443 .000639$

C $-4.842316-1.5548323 .357275$

C $-5.680794-0.3100163 .589306$

C $-6.253316 \quad 0.4171612 .608824$

C $-5.871977 \quad 0.093173 \quad 5.028938$

$\begin{array}{llll}C & -6.233815 & 0.147851 & 1.129058\end{array}$

C -5.0464580 .8210440 .440863$

C $-5.1104960 .776584-1.093257$

C $-5.070897-0.650565-1.645551$

C $-5.003818-0.677742-3.180746$

O $-3.9764221 .516403-1.568711$

O $-4.660877-2.022374-3.551304$

C $-3.8658850 .254610-3.647222$
C $-6.370632-0.386461-3.811120$

$\begin{array}{lllll}\text { O } & -3.853132 & 0.388813 & -5.066032\end{array}$

C $-3.9153651 .654307-2.995864$

C $-2.6698212 .511009-3.328078$

O $-5.0558602 .366667-3.489695$

C -1.970427 0.5642143 .591560

$\begin{array}{llll}\text { O } & -1.614839 & 0.592209 & 4.757626\end{array}$

C $-1.572091 \quad 1.5283262 .541554$

C -0.7908162 .5779112 .836168$

C $-0.343143 \quad 3.599522 \quad 1.884515$

C $-0.826688 \quad 3.4717010 .463781$

C $-1.3349201 .857129-2.969435$

C $-0.165908 \quad 2.826659-3.017433$

C $2.2803552 .901491-2.491403$

O $-0.268725 \quad 3.994762-3.379011$

C $2.5599363 .147273-1.042355$

C $2.9592664 .326846-0.542784$

C 3.2740064 .5406950 .911457

C $2.5563285 .731673 \quad 1.510122$

C 3.2502906 .7647432 .017322

C 1.0418645 .7347101 .530200

C 0.4669314 .5876092 .319435

N $1.0093802 .212161-2.608341$

H $-3.767919-3.8714662 .508126$

H - $-1.492023-2.5054294 .034237$

H $-3.348843-1.1280614 .867990$

H $-5.308370-2.3892023 .896837$

H -4.858537 -1.8218192 .295550$

H $-6.819707 \quad 1.3038962 .891127$

H -6.6060930 .8984015 .140131$

H $-6.231578-0.7576675 .616785$

H $-4.930810 \quad 0.4456325 .461410$

H -6.240958 -0.9287180 .933552$

H -7.1711150 .5311120 .706130$

H $-4.977427 \quad 1.8715280 .755284$

H -4.1098560 .3572970 .772567$

H $-6.0257721 .289324-1.411096$

H -5.932924 -1.234857-1.303578

H $-4.184121-1.165846-1.249578$

H -4.669964 - $2.080220-4.521539$

H $-2.925700-0.238157-3.369588$

H $-7.098723-1.149464-3.510355$

H -6.321092 -0.434104 -4.904462

H $-6.7752960 .587294-3.527757$

H $-3.722263-0.496071-5.445833$

H $-2.6717202 .785108-4.391107$

H $-2.7538913 .460425-2.780753$

H $-4.9021153 .317885-3.364205$

H $-1.943747 \quad 1.346732 \quad 1.539148$

H -0.447486 2.7033053 .861995

H -1.9211373 .5100940 .422525$

H -0.4918852 .5201520 .038282$

H $-0.4641664 .256362-0.201434$

H $-1.3827541 .433364-1.960240$

H $-1.115817 \quad 1.047513-3.674626$

H $2.2603243 .827478-3.074376$

H $3.0447962 .237134-2.906096$

H $2.4479332 .299648-0.369662$

H $3.0996245 .171092-1.213432$

H $4.360897 \quad 4.6698700 .995998$

H 3.0334763 .6487661 .501223

H 2.7503467 .6244582 .451109

H 4.3352136 .7759362 .010938

H 0.6801735 .7685910 .499972

H $0.670426 \quad 6.6590051 .992537$

H 0.7546834 .5954583 .371671

H $0.966971 \quad 1.253815-2.274239$

C $-1.032705-4.7630561 .442314$

O $-0.201358-3.5844611 .444311$

C $-0.263180-2.971695 \quad 0.137130$

C $-0.883411-4.017233-0.778646$

C $-1.862541-4.696105 \quad 0.160357$

H $-0.935526-2.1104180 .242478$

C $1.143943-2.509334-0.258239$

H $-1.367178-3.575890-1.655139$

H $-0.126251-4.731498-1.125605$

C $-2.346034-6.048736-0.330817$

H $-2.727768-4.0335240 .285083$ 
C $1.267477-2.006814-1.708054$

O $1.527540-1.4624850 .640896$

H $1.845108-3.332638-0.084396$

H $1.080116-2.832888-2.404338$

O $0.232906-1.041377-1.927645$

C $2.619134-1.341490-2.039689$

C $3.826339-2.261226-1.793422$

H $2.724250-0.407186-1.476835$

O $2.622873-0.991041-3.427727$

H $3.811365-2.621056-0.761565$

H $3.740398-3.141792-2.442148$

H $-2.875637-5.945047-1.283484$

H $-1.512438-6.742346-0.484066$

H -3.033345 -6.5010540 .391098$

C -1.806003 -4.854899 2.761526

H $0.937185-0.7058640 .481693$

H $0.155662-0.911656-2.887848$

H $\quad 1.973924-0.278863-3.553700$

H -1.089327 -4.8548163 .592983$

H -2.346561 -5.8071102 .813929$

H $-0.342528-5.615831 \quad 1.394823$

C $5.432716-0.4600180 .051025$

O $5.315656-0.329484-1.370905$

C $5.181290-1.569363-2.075065$

C $6.405837-2.455046-1.801690$

C $6.650412-2.645413-0.305657$

C $6.664096-1.2973630 .416164$

H $4.531427-0.946340 \quad 0.440828$

C 5.5046510 .9576290 .632890

H $5.195064-1.317948-3.142983$

O $6.260125-3.730940-2.416333$

H $7.296842-1.986278-2.239584$

H $5.910075-3.3200480 .140038$

O $7.923379-3.266998-0.113451$

H $7.581924-0.7561470 .152720$

O $6.685636-1.5185751 .823623$

C 4.9129721 .0468602 .018509

H 6.5386731 .3204490 .668309

H $4.9691931 .672615-0.002166$

H $\quad 6.123478-3.582622-3.367693$

H $7.913199-4.104787-0.607567$

H $7.462149-2.0706352 .019095$

O $3.561880 \quad 0.868721 \quad 1.960958$

O 5.5710671 .2611303 .027929

C 2.8751280 .9216143 .214942

H 3.3002490 .1974013 .916568

H 2.9239291 .9340663 .625989

H $1.8280330 .662343 \quad 3.038819$

SCF Energy (B3LYP/6-31G**//MMFF) $=-3245.90962777$

0500251

MM̄FF Geometry

C -1.448619-3.712056 1.269882

C $-0.508269-2.7559981 .292519$

C $-0.074621-2.0588652 .556245$

O $1.320345-1.6930412 .445272$

C $-0.877444-0.7569502 .720142$

C $-0.675588-0.0927274 .066951$

C 0.0822421 .0035374 .265409

C -1.444492 -0.7023245 .209674$

C $0.919623 \quad 1.7206383 .244888$

C 0.1844302 .9330742 .663472

C 1.0515903 .6922471 .649307

C 0.3099904 .9185941 .112439

C $1.0953475 .627317-0.002592$

O 1.3632232 .7976310 .576650

O $0.2077756 .546802-0.657246$

C $1.5635884 .586173-1.044239$

C 2.2210936 .4934240 .573365

O $2.4310255 .181699-2.006132$

C $2.2411113 .348408-0.413309$

C $2.4907322 .267306-1.492616$

O $3.4967693 .737914 \quad 0.148010$

C $2.253205-2.6724992 .567214$

O $2.059621-3.8604302 .765351$

C $3.573778-2.0341222 .379825$

C $4.609909-2.7749011 .961308$

C $5.958243-2.2804971 .682153$
C $6.291026-0.8702672 .092693$

C $2.9014650 .902414-0.921003$

C $3.180421-0.119509-2.010099$

C $3.799213-2.529114-2.271635$

O $3.1672640 .149002-3.207799$

C $5.259269-2.863001-2.184139$

C $6.267144-2.000338-1.977151$

C $7.710633-2.408617-1.871985$

C $8.352799-1.923055-0.589780$

C $9.055703-0.777592-0.568656$

C $8.246683-2.7886640 .648188$

C $6.833363-3.0978081 .060236$

N $3.459893-1.367269-1.471446$

H -1.922137 -4.018200 2.199431

H $-0.057620-2.4291860 .358766$

H $-0.206054-2.7092713 .429805$

H -1.951391 -0.9545302 .599635$

H $-0.614366-0.0623011 .913295$

H $\quad 0.1275291 .4285005 .266938$

H -1.307108 -0.1490436 .144804$

H -2.516204 -0.7086594 .985621$

H -1.119782 -1.732127 5.386715

H 1.8457262 .0485463 .733190

H 1.2323951 .0407112 .445988

H -0.7321502 .5845692 .169924$

H $-0.1152463 .610527 \quad 3.472719$

H 1.9795833 .9954052 .148545

H 0.0783925 .6191491 .924493

H $-0.661647 \quad 4.605596 \quad 0.709921$

H $-0.5430216 .042470-1.014612$

H $0.6762134 .248061-1.596659$

H $1.8075707 .268774 \quad 1.229720$

H $2.7553827 .028336-0.219568$

H 2.9477555 .9224301 .155080

H $1.9650115 .948252-2.381610$

H $1.5750772 .118538-2.079275$

H $3.2672472 .612319-2.187078$

H 4.0900032 .9711860 .170837

H $3.643958-0.9651802 .546938$

H $4.449287-3.8354371 .771476$

H $5.713522-0.1511401 .501351$

H $7.347323-0.6229381 .962088$

H $6.067605-0.7138243 .154007$

H $3.8089980 .992274-0.314741$

H $2.1028030 .506385-0.283073$

H $3.505813-2.382503-3.315415$

H $3.215768-3.360232-1.861928$

H $5.497916-3.918665-2.297285$

H $6.057597-0.937273-1.887783$

H $8.240619-1.989176-2.737184$

H $7.830381-3.496326-1.950588$

H $9.542993-0.4287010 .335684$

H $9.156636-0.160922-1.455771$

H $8.785581-2.3470551 .495655$

H $8.761686-3.7376570 .448720$

H $6.508812-4.1026980 .788914$

H $3.477925-1.473202-0.461748$

C $-3.109130-3.570869-0.592044$

O $-4.219697-3.531276 \quad 0.329710$

C $-5.434402-3.727976-0.408160$

C $-5.072117-3.595731-1.881314$

C -3.664461-4.164694-1.885949

H $-5.724772-4.773231-0.232295$

C $-6.576882-2.8346890 .093917$

H $-5.759001-4.142266-2.534250$

H $-5.056044-2.548068-2.201103$

C -2.869496 -3.801963 -3.127476

H $-3.723984-5.258042-1.801741$

C $-6.450956-1.311663-0.129032$

O $-7.779457-3.250450-0.569329$

H $-6.731229-3.0151701 .165108$

H -6.508799 -1.079845 -1.198878

O $-7.601945-0.6943800 .468824$

C $-5.200648-0.6512140 .474149$

$\begin{array}{llll}\text { C } & -5.193577 & 0.882026 & 0.313971\end{array}$

H $-4.316165-1.051618-0.021737$

O $-5.098230-0.9797251 .858111$

H $-5.329196 \quad 1.123658-0.746114$ 
H $-6.043970 \quad 1.3141870 .855097$ H -3.345716 -4.214800 -4.022789 H $-2.801566-2.717059-3.255145$ H -1.851629 -4.200200 -3.072109 C -1.947144 -4.361294 0.009576 H $-7.926506-4.186569-0.350663$ H $-8.389030-1.1040170 .070275$ H $-5.899092-0.6469832 .298399$ H -1.130441-4.448135 -0.716447 H $-2.266387-5.3803460 .259312$ H $-2.798226-2.532958-0.763123$ C $-2.4644371 .096233-1.036796$ $\begin{array}{llll}\text { O } & -2.719844 & 0.895345 & 0.358876\end{array}$ C -3.8992081 .5349530 .854040$ C -3.8063493 .0501190 .626957$ C $-3.4851273 .397306-0.826098$ C $-2.2831872 .591926-1.322772$ H $-3.3048160 .697717-1.616781$ C $-1.2104530 .279057-1.375537$ H -3.8983671 .3725901 .939463$ O H -3.011732 3.4536221 .267827 H $-4.3608983 .245724-1.467737$ O $-3.1736524 .790958-0.895101$ H $-1.3808142 .960059-0.822669$ O $-2.1069352 .827394-2.715657$ C -1.125659 -0.155996 -2.821111 H $-0.3088080 .842997-1.116813$ H -1.208684 -0.634073 -0.770845 H $-4.922826 \quad 4.6326260 .849169$ H -2.931017 $4.987692-1.816280$ H -2.883935 $2.471218-3.179518$ O $0.016302-0.886415-2.992755$ O $-1.9508790 .094058-3.688272$ C $0.237900-1.380762-4.317246$ H $1.130900-2.010602-4.300387$ H $-0.610249-1.987528-4.648202$ H $0.408082-0.546475-5.003992$ SCF Energy $\left(B 3 L Y P / 6-31 G^{* *} / / M M F F\right)=-3245.91637811$

\section{2}

MM̄FF Geometry

C $1.5417615 .018651-0.909909$

C $0.6481644 .582828-0.011051$

C $-0.7754094 .212302-0.337543$

O $-0.9443662 .799239-0.078007$

C $-1.725866 \quad 4.990178 \quad 0.591345$

C $-3.196194 \quad 4.7245440 .338339$

C -4.0358054 .1671321 .233124$

C $-3.7180345 .158164-1.007397$

C -3.7262013 .6933142 .626450$

C -3.3165422 .2149132 .723889$

C -4.4192071 .2287862 .305220$

C $-4.006019-0.2126382 .610318$

C $-5.044037-1.2315972 .111247$

O $-4.641437 \quad 1.3689470 .900785$

O $-4.410445-2.5193912 .158634$

C $-5.395123-0.9212000 .637143$

C $-6.252442-1.3068743 .052534$

O $-6.483993-1.723380 \quad 0.188580$

C $-5.712750 \quad 0.5674390 .387876$

C $-5.8686570 .922452-1.110364$

O

C $-0.5598861 .933699-1.056116$

O $-0.1170932 .218138-2.157541$

C $-0.7453290 .558617-0.539167$

C $-0.236767-0.477847-1.222931$

C $-0.299302-1.890041-0.835917$

C - $-1.097725-2.2378550 .391754$

C $-4.6494050 .620873-1.988448$

C $-4.742513-0.701527-2.731263$

C $-3.417513-2.179193-4.234420$

O $-5.756651-1.393372-2.755998$

C $-2.894781-3.313171-3.411216$

C -1.833894 -4.051685-3.769271

C $-1.321038-5.218923-2.976910$

C $0.139133-5.075370-2.614946$

C $1.094319-5.695045-3.328682$
C $0.484202-4.280957-1.374990$

C $0.367286-2.793265-1.585996$

N $-3.563421-1.001153-3.400515$

H $1.2471065 .131174-1.950038$

H 0.9569904 .4732351 .025483

H -1.011062 $4.430926-1.386181$

H -1.5520906 .0687550 .478495$

H -1.4698254 .7512631 .629364$

H $-5.0771694 .034693 \quad 0.941147$

H -4.808212 $5.076369-1.076112$

H $-3.4570786 .204382-1.197489$

H -3.293075 $4.539935-1.803938$

H $-2.9427714 .310523 \quad 3.078947$

H -4.6145943 .8628473 .248158$

H -2.4235452 .0531982 .109024$

H -3.0326732 .0138363 .764271$

H $-5.335217 \quad 1.4868942 .849645$

H -3.823152 $-0.347003 \quad 3.683795$

H $-3.047493-0.4244982 .117545$

H -5.062722 -3.187066 1.887115

H $-4.523777-1.2062260 .035256$

H -5.935767 -1.623411 4.053669

H $-6.972510-2.0604342 .714588$

H $-6.775446-0.3538573 .155923$

H $-6.209163-2.6536300 .246067$

H $-6.7822690 .468041-1.512055$

H $-6.0375612 .007758-1.168364$

H $-7.311451 \quad 1.698080 \quad 0.607031$

$\begin{array}{llll}H & -1.272800 & 0.440233 & 0.399954\end{array}$

H $\quad 0.298760-0.274537-2.149693$

H $-2.138790-1.9221920 .267875$

H $-0.680815-1.7429871 .275977$

H -1.121958 -3.305827 0.614387

H $-4.5782831 .398287-2.760144$

H -3.722343 $0.651515-1.407460$

H $-2.742313-1.915493-5.054613$

H $-4.391458-2.448305-4.655843$

H $-3.427218-3.554531-2.494203$

H -1.318925 $-3.824500-4.700249$

H -1.470037 -6.123386 -3.581171

H -1.909312 $-5.374576-2.064039$

H $2.142203-5.618093-3.058049$

H $0.849601-6.288628-4.203324$

H $1.522066-4.485341-1.083216$

H $-0.127157-4.649469-0.547554$

H $0.936245-2.428995-2.442915$

H -2.752276 -0.402162 -3.275982

C $3.9518804 .424186-1.249711$

O $3.8511743 .098543-0.688660$

C $5.1683312 .516268-0.630337$

C $6.1074783 .511630-1.307145$

C $5.4093904 .830168-1.050195$

H 5.4341122 .4316650 .430611

C $5.1928731 .127295-1.286128$

H $7.1190193 .472306-0.891011$

H $6.1843053 .322415-2.384766$

C $5.8771405 .948945-1.964856$

H $5.5798685 .120572-0.004898$

C $4.4370010 .041081-0.485261$

O $4.6823791 .232380-2.614219$

H $6.2378260 .806283-1.375546$

H $4.896575-0.0043300 .506377$

O $3.0746730 .410433-0.294065$

C $4.506387-1.352403-1.140707$

C $3.880579-2.498534-0.322520$

H $5.553961-1.595020-1.355779$

O $3.819558-1.307533-2.396217$

H $2.834426-2.258905-0.116187$

H $3.850383-3.376696-0.979275$

H $6.9480996 .132692-1.830362$

H $5.7080995 .702851-3.018576$

H $5.3462406 .880813-1.747465$

C $2.9623575 .362671-0.559384$

H $3.7754961 .577968-2.553445$

H $2.6441580 .404904-1.165730$

H $3.961497-2.160072-2.841636$

H $3.1424056 .398707-0.867231$

H 3.1055685 .3153630 .527400 
H $3.719106 \quad 4.339255-2.319798$

C $3.088805-1.9761352 .608292$

O $4.398889-1.9223342 .029880$

C $4.646082-2.8503920 .969992$

C $4.407420-4.2828341 .473805$

C $3.006410-4.4448542 .053931$

C $2.759588-3.3824073 .121581$

H $2.345754-1.6691791 .862955$

C $3.062928-0.9662693 .761636$

H $5.715704-2.7744580 .735755$

O $4.622502-5.2147650 .419752$

H $5.143627-4.5093862 .256427$

H $2.236756-4.4038521 .275284$

O $2.891260-5.7269282 .674009$

H $3.361732-3.6109364 .010481$

O $1.387832-3.424182 \quad 3.512932$

C 2.9123720 .4387363 .235560

H $2.237608-1.1533944 .458560$

H $3.988560-1.0048894 .348361$

H $4.590639-6.1070790 .803614$

H $2.926032-6.3965091 .970280$

H $1.199248-4.3299543 .812871$

O $1.5892150 .725272 \quad 3.070104$

O 3.8527011 .1805052 .984075

C 1.3058482 .0294552 .561610

H $\quad 1.7150242 .1326481 .553139$

H 0.2204062 .1490612 .517561

H $\quad 1.7152012 .797153 \quad 3.225194$

SCF Energy (B3LYP/6-31G**//MMFF) $=-3245.90188318$

0500253

MM̄FF Geometry

C $-0.2990155 .463898-0.441738$

C $-0.0825994 .361998 \quad 0.294532$

C 1.2352913 .8313290 .814843

O $1.5583952 .633667 \quad 0.063457$

C 2.4328604 .7938660 .687594

C 3.6918174 .2953981 .374465

C 4.8478094 .0087720 .744160

C 3.6106554 .1575102 .873852

C $5.1587664 .116692-0.721638$

C $4.9278902 .827912-1.525163$

C $5.7912241 .638295-1.073172$

C $5.7031220 .493894-2.085148$

C $6.485228-0.746795-1.623728$

O $5.312323 \quad 1.186521 \quad 0.196822$

O $6.082202-1.850769-2.449768$

C $6.074589-1.091698-0.174783$

C $7.994089-0.576467-1.837371$

O $6.885110-2.1423170 .346086$

C 6.0811520 .1240380 .776812

C $5.472130-0.1838402 .167166$

O $7.436908 \quad 0.5334700 .998004$

C $0.921145 \quad 1.4769630 .389590$

O $\quad 0.117270 \quad 1.302538 \quad 1.290974$

C $1.3515120 .449444-0.582800$

C $0.594509-0.640756-0.772949$

C $0.879268-1.728279-1.711235$

C $2.240641-1.755646-2.353922$

C $4.014630-0.6579732 .159319$

C $3.854289-2.1693932 .176669$

C $2.110854-3.9396051 .975833$

O $4.785144-2.9441522 .379141$

C $2.091646-4.4527340 .570505$

C $1.037714-5.0826580 .029350$

C $1.022028-5.637216-1.366760$

C $-0.160962-5.153312-2.175292$

C $-1.254217-5.920346-2.325361$

C $-0.040024-3.822608-2.883352$

C $-0.078225-2.650957-1.938550$

N $2.533819-2.5512641 .977034$

H $0.5476836 .083016-0.727364$

H -0.9341693 .7455880 .571003$

H 1.0918603 .5709721 .870595

H 2.1784085 .7670761 .127230

H $2.6269924 .972327-0.374974$

H 5.6857413 .6628651 .348863

H 4.5898243 .9669923 .326677
H $3.2204595 .077953 \quad 3.320079$

H 2.9554423 .3264253 .151822

H $4.5866494 .929884-1.180599$

H $6.2093894 .418349-0.822335$

H $3.8668992 .558848-1.465527$

H $5.1456703 .051229-2.576986$

H $6.8280381 .980511-0.974283$

H $6.0518930 .819665-3.073005$

H $4.6507660 .210412-2.220156$

H $\quad 6.268443-1.616557-3.375192$

H $5.056604-1.493865-0.231209$

H $8.211429-0.381333-2.894460$

H $8.535112-1.495939-1.587964$

H $8.4191060 .244876-1.256852$

H $7.744050-1.7595520 .593228$

H $6.119963-0.8769622 .717298$

H $5.494926 \quad 0.7526772 .743341$

H $7.471106 \quad 1.0547141 .817237$

H $2.2669530 .631281-1.133536$

H $-0.332864-0.736758-0.209236$

H $2.354860-0.918874-3.051223$

H $2.443980-2.673385-2.909489$

H $3.023776-1.688591-1.591108$

H $3.525969-0.2908703 .071005$

H $3.471692-0.2409541 .306772$

H $1.123177-3.9791702 .445323$

H $2.806914-4.5344862 .575820$

H $2.995203-4.322773-0.020739$

H $\quad 0.144877 \quad-5.2367870 .631344$

H $\quad 0.997108-6.732148-1.288613$

H $1.949444-5.400731-1.903251$

H -2.095808 $-5.594807-2.927835$

H -1.334988 -6.888892 -1.843055

H $-0.872801-3.693647-3.587862$

H $\quad 0.856175-3.836635-3.509573$

H -1.026560 -2.545312 -1.412499

H $1.836367-1.8427441 .768684$

C $-2.8675605 .152409-0.577079$

O $-2.8254973 .859990-1.209570$

C $-4.1814633 .406856-1.426233$

C $-5.0901604 .534354-0.947428$

C $-4.1880255 .750819-1.050808$

H $-4.2791023 .262812-2.508735$

C $-4.3604952 .075333-0.684411$

H $-5.9982474 .620543-1.551765$

H -5.3958634 .3771450 .094426$

C $-4.6743156 .931700-0.229295$

H $-4.1119086 .045166-2.105981$

C $-5.8037821 .546498-0.661048$

O $-3.5241401 .099593-1.316808$

H $-3.9886882 .187797 \quad 0.340658$

H -6.445619 $2.244710-0.111521$

O $-6.2969021 .517788-2.005691$

C $-5.9596890 .131169-0.066846$

C $-5.326542-0.0258681 .326070$

H $-5.541197-0.603260-0.762595$

O $-7.358866-0.1593570 .019035$

H -4.2502590 .1560331 .252843$

H -5.7358810 .7424621 .994036$

H $-5.6690347 .246690-0.561140$

H $-4.738516 \quad 6.6812680 .834954$

H -3.999256 $7.786468-0.333895$

C -1.627476 $5.962510-0.963097$

H -2.615697 $1.447454-1.308765$

H $-7.2055611 .172388-1.974245$

H -7.7541520 .4653920 .650853$

H -1.745233 $6.993882-0.611186$

H -1.548450 $5.998618-2.057052$

H -2.8966324 .9933950 .509462$

C $-3.876927-2.6402580 .771334$

O $-5.267458-2.4861901 .075396$

C $-5.571001-1.4104971 .966744$

C $-4.841391-1.6206783 .302300$

C $-3.339792-1.8306343 .104147$

C $-3.072829-2.9051802 .048365$

H $-3.502575-1.7348810 .278704$

C $-3.755414-3.810306-0.210116$

H $-6.646941-1.4778212 .171409$ 
O $-5.048817-0.5153934 .175039$ H -5.253087 -2.507409 3.801460 H $-2.844257-0.8907862 .835581$ O $-2.749171-2.2357304 .341872$ H -3.325502 -3.892189 2.456111 O $-1.682409-2.9158491 .731535$ C $-4.376649-3.477273-1.546520$ H -2.713046 -4.095203-0.381462 H $-4.274408-4.6941870 .180792$ H -6.008348 -0.4179654 .299789$ H -3.171804 -3.0663394.619421 H -1.196036 -3.0459782 .563559$ O $-3.639598-2.523102-2.182194$ O $-5.393648-4.007470-1.974621$ C $-4.133733-2.107195-3.458476$ H $-4.145841-2.952201-4.153368$ H $-5.133830-1.675478-3.355040$ H -3.459726 -1.340359-3.849607

SCF Energy (B3LYP/6-31G**//MMFF) $=-3245.90605332$

\section{4}

MM̄FF Geometry

C -3.116702 0.3998862 .794216

C $-2.007854 \quad 0.3913892 .039761$

C $-0.626712 \quad 0.116105 \quad 2.561938$

O $-0.177304-1.1015391 .918877$

C $0.351045 \quad 1.246373 \quad 2.187861$

C 0.4288512 .3389303 .227413

C -0.2329383 .5097603 .173492$

C $1.3533342 .047598 \quad 4.382497$

C $-1.201358 \quad 3.990045 \quad 2.127352$

C -0.9119835 .4294831 .674461$

C 0.1086795 .5972190 .534749

C $-0.4849805 .204357-0.819777$

C $0.525797 \quad 5.385718-1.961622$

O $1.287648 \quad 4.831803 \quad 0.801882$

O $\quad 0.0104804 .704513-3.116585$

C $1.8401734 .680872-1.570782$

C $0.6876626 .855856-2.362936$

O $2.8649204 .934697-2.528058$

C $2.3439155 .040402-0.153450$

C $3.558558 \quad 4.1829130 .282788$

$\begin{array}{lllll}\text { O } & 2.777308 & 6.406406 & -0.137177\end{array}$

C $0.755136-1.8378652 .583550$

O $1.201918-1.6249753 .699532$

C $1.192403-2.9258151 .681941$

C $2.328020-3.5822001 .962328$

C $2.982307-4.5784051 .112679$

C $2.227464-5.093232-0.083604$

C 3.3663752 .6765360 .114343

C 4.4306291 .8443500 .806992

C $5.063238-0.5362651 .167670$

O $5.294602 \quad 2.3204561 .536819$

C $5.119878-1.752217 \quad 0.297689$

C $6.212551-2.521406 \quad 0.171535$

C $6.271488-3.775558-0.658680$

C $6.435486-5.0225730 .188569$

C 7.656143 $-5.471903 \quad 0.525260$

C $5.184818-5.7840630 .589306$

C $4.247972-4.9395971 .412901$

$\begin{array}{llll}\mathrm{N} & 4.283134 & 0.494231 & 0.507874\end{array}$

H -3.046697 0.2087503 .861580 H -2.099162 0.5628730 .970772 H $-0.662693-0.0430053 .647045$ H $\quad 0.108521 \quad 1.653552 \quad 1.199074$ H 1.3601830 .8331942 .055930 H $-0.0928204 .203734 \quad 4.002135$ H 1.3944272 .8718005 .102542 H $1.024128 \quad 1.1547354 .922987$ H 2.3720231 .8781424 .018076 H - 1.2659113 .3200351 .267478 H -2.193790 3.9824022 .596357 H $-1.856004 \quad 5.897524 \quad 1.366715$ H $-0.558206 \quad 6.0092182 .537475$ H $\quad 0.3755526 .6611180 .517269$ H $-1.4004605 .772380-1.026840$ H $-0.785022 \quad 4.150620-0.795101$ H $-0.8607135 .083814-3.324202$
H $1.6328533 .604306-1.614051$

H $-0.2728757 .270509-2.691847$

H $1.3662346 .959183-3.216869$

H $1.0569597 .485385-1.550938$

H $2.5131424 .691105-3.401480$

H $4.4563134 .494999-0.266978$

H 3.7691504 .4013001 .338770

H 3.3861446 .5252900 .610744

H $\quad 0.614687-3.1034600 .782806$

H $2.873564-3.3138402 .866536$

H $2.200830-4.337833-0.874074$

H $\quad 1.198930-5.3582330 .186375$

H $2.662322-6.002603-0.505427$

H 2.3974432 .3799050 .524012

H $3.3882752 .417706-0.950431$

H $4.546856-0.7728532 .103833$

H $6.057688-0.146557 \quad 1.408053$

H $4.211129-2.038716-0.225854$

H $7.119150-2.2454270 .705597$

H $7.116948-3.682179-1.352843$

H $5.383468-3.876130-1.295368$

H $7.785571-6.375403 \quad 1.111727$

H $8.553839-4.9427720 .222599$

H $5.443862-6.6691531 .183748$

H $4.719736-6.159955-0.326078$

H $4.705398-4.5311702 .315248$

H $3.4299430 .205416 \quad 0.036140$

C -5.250973 -0.6901842 .081379$

O $-4.567631-1.5266561 .126618$

C $-5.529333-2.0940720 .220202$

C $-6.890241-1.8631110 .863956$

C $-6.671120-0.5222951 .541323$

H $-5.476075-1.505254-0.703490$

C $-5.172688-3.553904-0.081743$

H -7.700596 -1.8470630 .129026$

H $-7.124364-2.6298491 .611294$

C -7.702768 -0.2020422 .608412$

H -6.6917020 .2646950 .775356$

C $-3.704260-3.762641-0.511510$

O $-5.385824-4.330096 \quad 1.104315$

H $-5.859394-3.951325-0.838291$

H $-3.044898-3.5737430 .344113$

O $-3.535948-5.148792-0.834936$

C $-3.253203-2.905245-1.708341$

C $-1.779584-3.157912-2.070242$

H $-3.381657-1.850933-1.458057$

O $-4.092202-3.196031-2.822732$

H $-1.174123-2.965245-1.177698$

H -1.645158 $-4.215574-2.327638$

H $-8.705650-0.1543112 .171655$

H -7.715336 -0.9629383 .396030$

H $-7.4918790 .764425 \quad 3.076812$

$\begin{array}{llll}\text { C } & -4.491050 & 0.628870 & 2.227603\end{array}$

H $-5.149566-5.2500450 .894502$

H $-4.064325-5.329040-1.631475$

H $-3.937703-2.511206-3.494989$

H $-5.032091 \quad 1.3146182 .888938$

H $-4.410347 \quad 1.120803 \quad 1.250638$

H $-5.259652-1.2358793 .033905$

C $-0.847201-0.233297-2.084764$

O $-1.556884-0.916814-3.124852$

C $-1.262397-2.310514-3.256030$

C $0.232399-2.498534-3.543737$

C $1.092740-1.830847-2.476915$

C $0.672949-0.378308-2.269082$

H $-1.151161-0.633118-1.109939$

C $-1.252188 \quad 1.250021-2.154701$

H $-1.803946-2.653609-4.146333$

O $0.545378-3.884958-3.626162$

H $\quad 0.468177-2.055723-4.520094$

H $1.057125-2.389800-1.537044$

O $2.454949-1.867853-2.909746$

H $1.0157560 .213803-3.127600$

O $1.3569210 .102432-1.113363$

C $-2.7014881 .505390-1.798592$

H $-0.6488531 .837874-1.456353$

H $-1.097308 \quad 1.625433-3.173333$

H $1.503800-3.958452-3.774409$ 
H $2.983845-1.399605-2.241193$

H $1.2612541 .068477-1.092237$

O $-2.9434452 .847618-1.886627$

O $\quad-3.517730 \quad 0.654070-1.479148$

C $-4.2715143 .259320-1.549527$

H $-4.3344714 .342773-1.680880$

H $-4.9981642 .781938-2.213712$

H $-4.4864803 .019975-0.503899$

SCF Energy (B3LYP/6-31G**//MMFF) $=-3245.91576523$

0500255

MM̄FF Geometry

C $1.004714-2.9759551 .605685$

C $0.013635-3.0230532 .508699$

C $-0.738941-1.8329413 .058974$

O $-0.046336-0.6130992 .701443$

C $-2.172621-1.7540002 .498674$

C $-3.089038-2.8572822 .980144$

C $-3.464442-3.9223022 .245098$

C $-3.616612-2.6833764 .381241$

C $-3.042391-4.274788 \quad 0.845870$

C $-4.224799-4.340368-0.129989$

C $-4.892902-2.980663-0.388649$

C $-6.086856-3.148370-1.327661$

C $-6.730213-1.799985-1.668989$

O $-3.926321-2.116297-0.989652$

O $-7.636242-2.033839-2.757868$

C $-5.635460-0.826274-2.159009$

C $-7.582010-1.275946-0.505548$

O $-6.2026870 .467758-2.345906$

C $-4.386553-0.774789-1.246902$

C $-3.180255-0.070389-1.916974$

O $-4.708729-0.110771-0.025821$

C -0.1848620 .4595863 .524596$

O -0.8301240 .5178074 .558469$

C 0.5737231 .5770942 .918781

C 0.3983122 .8223793 .384951

C 0.9981274 .0401112 .833974

C 2.0673633 .8764231 .785124

C $-3.3389431 .383872-2.365128$

C $-3.6316262 .365829-1.248917$

C -2.6295103 .3512930 .815214$

O $-4.6894892 .989348-1.200478$

C -1.639202 4.4535650 .613207

C $-1.845976 \quad 5.7265970 .982337$

C $-0.820090 \quad 6.8148950 .821762$

C $-0.298073 \quad 7.3236182 .150117$

C -0.8275878 .4155202 .726855$

C $0.888676 \quad 6.6093282 .769239$

C $0.5299365 .232333 \quad 3.261108$

N $-2.594808 \quad 2.473072-0.339507$

H $1.312078-2.0185181 .193140$

H $-0.288058-3.9937572 .897152$

H $-0.745017-1.9265804 .151853$

H -2.135171 $-1.704818 \quad 1.403818$

H $-2.621733-0.7907342 .777169$

H -4.138999 -4.645976 2.701473

H -4.311662 -3.480264 4.666439

H -2.796181 -2.688507 5.105368

H -4.152880 -1.732856 4.469187

H $-2.277541-3.5984220 .457168$

H -2.571783 $-5.265306 \quad 0.884190$

H -3.843681 -4.738608 -1.079598

H $-4.969306-5.0546740 .243564$

H $-5.217637-2.5537950 .566459$

H $-6.830435-3.831169-0.897610$

H -5.753242 -3.620526 -2.262665

H -7.995170 -1.172389 -3.032484

H -5.320580 -1.167873 -3.154209

H -8.355792 -2.006623 -0.240740

H $-8.120001-0.363543-0.785593$

H $-6.997277-1.0684350 .393179$

H $-6.2786980 .887717-1.472057$

H -2.327447 $-0.134361-1.227214$

H $-2.879117-0.660583-2.793278$

H $\quad-3.878954 \quad 0.1717450 .393767$

H 1.2149381 .3515142 .073995

H -0.2917902 .9750504 .213952$
H $2.5308054 .817043 \quad 1.481731$

H $1.655363 \quad 3.4156250 .881273$

H 2.8786703 .2426282 .161022

H -2.408055 $1.709597-2.847554$

H $-4.1119101 .477998-3.133709$

H -2.3386322 .7455671 .678661$

H -3.6439873 .7297060 .974432$

H -0.6808004 .1871360 .176462$

H -2.794385 6.0035581 .436486

H $-1.285598 \quad 7.6351750 .259776$

H $\quad 0.023050 \quad 6.481577 \quad 0.203457$

H -0.4440058 .8025583 .664978$

H -1.660333 8.9440852 .274773

H 1.2697957 .1759073 .628706

H 1.7036986 .6043932 .040979

H -0.2419245 .2376224 .032560$

H $-1.7448151 .938496-0.494415$

C $3.222150-4.1294451 .390008$

$\begin{array}{llll}\text { O } & 3.806512 & -2.963729 & 0.777437\end{array}$

C $5.057699-3.3266190 .168833$

C $5.383169-4.7273490 .674921$

C $3.997116-5.3219560 .830365$

H $4.876357-3.374601-0.911706$

C $6.122591-2.2658280 .466091$

H $6.014790-5.284100-0.024062$

H $5.894047-4.7044781 .644300$

C $3.959332-6.5553551 .715833$

H $3.616049-5.583789-0.165985$

C $5.691060-0.8207590 .141314$

O $6.434847-2.3109621 .865055$

H $7.048557-2.520792-0.062958$

H $4.928629-0.4952880 .859310$

$\begin{array}{lllll}\text { O } & 6.826290 & 0.025457 & 0.370826\end{array}$

C $5.172287-0.596470-1.291870$

C $4.8804780 .891150-1.563990$

H $4.262940-1.185183-1.441024$

O $\quad 6.155507-1.069768-2.210037$

H $4.2125431 .261665-0.778547$

H $5.8166831 .457260-1.487899$

H $4.584859-7.3496771 .295626$

H $4.326128-6.3366302 .724338$

H $2.938992-6.9409711 .804813$

C $1.725990-4.1844501 .077280$

H $7.114638-1.6355582 .031967$

H $7.499075-0.201542-0.293987$

H $5.733776-1.142420-3.082650$

H $1.291800-5.1041581 .485462$

H $1.576929-4.208734-0.008365$

H $3.381649-4.0343212 .472088$

C $1.9331230 .824229-2.426065$

O $3.0727600 .416735-3.192188$

C $4.2683941 .166766-2.955627$

C $4.0222642 .656004-3.241111$

C $2.8383873 .194818-2.444460$

C $1.6137662 .308215-2.660282$

H $2.1200260 .640462-1.360737$

C $0.741656-0.040279-2.871573$

H $4.9971870 .820631-3.698461$

O $5.179278 \quad 3.430844-2.943733$

H $3.8114302 .790748-4.310222$

H $3.0867103 .288626-1.380989$

O $2.5504354 .515298-2.910173$

H $1.2365532 .453296-3.681088$

O $0.5797812 .703362-1.762070$

C $0.816675-1.468175-2.377867$

H $-0.1901940 .380059-2.479706$

H $0.692460-0.073188-3.966474$

H $5.9114163 .079836-3.479011$

H $1.8730834 .891947-2.323562$

H $0.2990913 .599551-2.012752$

O $-0.327604-2.116682-2.749997$

O $1.746954-1.956112-1.753347$

C $-0.427819-3.485120-2.346149$

H $0.424203-4.060069-2.721336$

H $-0.488395-3.548375-1.256864$

$\mathrm{H}-1.345859-3.898932-2.771904$

SCF Energy (B3LYP/6-31G**//MMFF)= -3245.92082768 
0500256

MM̄FF Geometry

C $-3.101563-2.728474-2.708850$

C $-2.189661-2.672733-1.727214$

C $-0.705773-2.734276-1.958726$

O $-0.116530-1.520972-1.432598$

C $-0.011151-3.956701-1.321639$

C $-0.163959-4.1424300 .176984$

C $0.712284-3.6861311 .093948$

C $-1.323916-4.9991860 .619067$

C $1.956354-2.8780580 .853084$

C $3.215840-3.7127741 .114934$

C $4.532600-2.9842560 .799306$

C $4.776675-1.7827111 .712847$

C $6.078578-1.0527161 .351132$

O $4.493153-2.567499-0.566479$

O $6.074150 \quad 0.2168102 .024160$

C $6.080302-0.753963-0.165013$

C $7.311346-1.8058871 .863770$

O $7.339091-0.230214-0.579453$

C $5.703114-1.965525-1.047829$

C $5.454328-1.583770-2.525571$

O $6.765891-2.926083-1.094545$

C -0.162505-0.413649-2.221011

O $-0.598675-0.350100-3.363214$

C $0.3712290 .782669-1.534646$

C $0.840050 \quad 0.784423-0.279015$

C 1.3642091 .9598540 .406951

C $2.6516841 .703277 \quad 1.138376$

C $4.305777-0.597196-2.770609$

C $4.7493070 .851178-2.904861$

C $3.8072213 .133048-3.266896$

O $5.9248641 .204625-2.892966$

C $3.6980443 .835575-1.950632$

C $2.8101824 .811764-1.707355$

C $2.7356185 .574575-0.415975$

C 1.3593125 .5371950 .208464

C 0.4794026 .5324530 .006599

C 1.0399034 .3916181 .139883

C 0.6930223 .1286450 .405722

N $3.665531 \quad 1.703718-3.066915$

H -2.776842 -2.856511 -3.738282

H - $2.524307-2.504802-0.707693$

H $-0.490254-2.785766-3.034748$

H $1.057966-3.905613-1.565205$

H $-0.369143-4.867190-1.821412$

H $\quad 0.526367-3.9156362 .142545$

H -1.350203 -5.1349781 .705800$

H -2.278869 -4.5588250 .325884$

H -1.250818 -5.9941840 .167293$

H $1.980387-2.470640-0.160752$

H $1.929746-2.0178961 .530003$

H $3.228943-4.0562472 .157010$

H $3.167995-4.6102150 .483085$

H $5.339554-3.7168820 .919295$

H $4.785484-2.0891242 .766043$

H $3.953613-1.0644781 .615580$

H 5.9868100 .0504332 .978333

H $5.3429390 .040892-0.326739$

H $7.285470-1.8825892 .957579$

H $8.233632-1.2656461 .624122$

H $7.389856-2.8205771 .467912$

H $7.5243470 .549395-0.028235$

H $6.386977-1.240656-2.989928$

H $5.194988-2.511641-3.056211$

H $7.530669-2.569132-0.615612$

H $0.3576831 .695021-2.125040$

H $0.866964-0.1300820 .305908$

H $3.375766 \quad 1.2210200 .472602$

H 2.4800601 .0444291 .996366

H 3.1305962 .6138631 .504951

H $3.826876-0.859077-3.723099$

H $3.541643-0.670206-1.989156$

H $3.0248373 .441675-3.967449$

H $4.7816773 .345219-3.718565$

H $4.4061373 .552758-1.175213$

H $2.1219375 .110613-2.494956$

H $3.0181686 .614394-0.627555$
H $3.4762945 .211906 \quad 0.307557$

H -0.4990936 .5259810 .475585$

H $\quad 0.716168 \quad 7.372797-0.637598$

H $\quad 0.175927 \quad 4.6484701 .767621$

H 1.8641714 .2579091 .846510

H $-0.2466503 .187718-0.144474$

H $2.7251621 .322672-3.030255$

C $-5.037224-1.164379-2.858424$

O $-4.499680-0.217306-1.911515$

C $-5.5200650 .741880-1.578874$

C $-6.6477930 .520864-2.579853$

C $-6.551773-0.975592-2.809390$

H $-5.8874260 .477542-0.580573$

C $-4.9340662 .157407-1.564055$

H $-7.6192680 .834937-2.186322$

H $-6.4718751 .061678-3.516991$

C $-7.275773-1.446003-4.058664$

H $-6.967417-1.493801-1.934644$

C $-3.7300412 .329496-0.614064$

O $-4.4910432 .476634-2.889587$

H $-5.7213812 .880359-1.320889$

H $-2.8789271 .753134-0.995803$

O $-3.326330 \quad 3.704342-0.670383$

C -3.9955031 .9430990 .854001$

C -2.7716992 .2040181 .749274$

H -4.2603410 .8852110 .916085$

O $\quad-5.0923992 .6985261 .365493$

H $-1.907427 \quad 1.6961391 .310678$

H -2.553033 3.2784741 .755708

H -8.345075 -1.220553 -3.991051

H $-6.885423-0.956363-4.957186$

H -7.166431 -2.527004-4.189782

C $-4.578695-2.572433-2.478540$

H $-4.1119243 .371832-2.860619$

H $-4.0397634 .234910-0.275850$

H -5.8992682 .3896410 .920799$

H -5.101217 -3.326045 -3.077839

H -4.816731 -2.770849 -1.426741

H $-4.647406-0.884526-3.846345$

C $-2.265176-0.5565712 .969924$

O $\quad-3.3192020 .354150 \quad 3.304954$

C -2.9793781 .7416933 .210039$

C -1.7986732 .0526834 .138516$

C $-0.605496 \quad 1.1451103 .857670$

C -1.036463 -0.3203293 .861312$

H $-1.996032-0.4395381 .913016$

C $-2.802557-1.9831333 .175351$

H -3.8439892 .2946943 .597964$

O -1.4166603 .4178764 .007228$

H $-2.116797 \quad 1.907440 \quad 5.179230$

H -0.1207401 .4169942 .915603$

O $0.364943 \quad 1.356847 \quad 4.885163$

H $-1.254614-0.6315964 .891102$

O $0.038522-1.1276983 .387983$

C $-3.865289-2.3694452 .168894$

H $-1.989978-2.7118223 .080130$

H -3.250986 -2.066494 4.172704

H -0.6484553 .5622234 .585999$

H 1.1665680 .8662114 .637260

H $\quad 0.767873-1.0539104 .025947$

O $-4.307734-3.6292582 .459940$

O $-4.257544-1.6703631 .246027$

C $-5.321533-4.1433811 .590494$

H $-4.911173-4.2986860 .588773$

H $-5.652398-5.1068091 .987257$

$\mathrm{H}-6.179776-3.4654801 .556690$

SCF Energy $\left(B 3 L Y P / 6-31 G^{* *} / / M M F F\right)=-3245.90613631$

\section{7}

MM̄FF Geometry

C $2.7422583 .656752-1.242606$

C $4.0785163 .774188-1.191005$

C $5.0570102 .638481-1.372992$

O $4.6167591 .468381-0.644593$

C $5.1980602 .266690-2.859319$

C $6.2657671 .219196-3.111790$

C $6.025866-0.086504-3.347391$

C $7.680717 \quad 1.739146-3.123025$ 
C $4.697587-0.794107-3.371887$

C $4.673012-1.955797-2.367314$

C $3.293709-2.614991-2.188947$

C $2.822600-3.329530-3.457050$

C $1.420324-3.930889-3.285462$

O $2.356858-1.605136-1.803159$

O $0.936635-4.300863-4.585836$

C $0.477795-2.834953-2.748499$

C $1.442325-5.215439-2.449263$

O $-0.807193-3.375470-2.448771$

C $1.034696-2.084222-1.515518$

C $0.148978-0.846182-1.221406$

O $1.023946-2.964098-0.393133$

C $4.836707 \quad 1.4593130 .699702$

O 5.3169602 .3575251 .372699

C 4.3948960 .1473621 .219169

C $4.359618-0.0725392 .541140$

C $3.964624-1.3214003 .193947$

C $3.668893-2.5120962 .321239$

C $0.6599080 .068196-0.097400$

C $0.041590-0.2101141 .261860$

C -0.3438210 .8758473 .469515$

O $-0.572053-1.240371 \quad 1.524096$

C $0.5808990 .267074 \quad 4.474160$

C $0.343960-0.9107155 .071514$

C $1.225072-1.5247266 .122579$

C $2.023220-2.6966455 .596635$

C $1.435664-3.8804985 .352491$

C $3.513707-2.5191835 .413048$

C $3.880894-1.3504354 .539632$

N $0.262908 \quad 0.8312032 .153580$

H $2.3020012 .688393-1.467716$

H $4.5164794 .749368-0.990250$

H $6.0325032 .951077-0.979032$

H $5.4458813 .162150-3.445356$

H $4.2327391 .927529-3.250785$

H $6.879408-0.734510-3.543772$

H $8.4103800 .956049-3.355473$

H $7.7903162 .524881-3.877620$

H $7.9457492 .154797-2.146048$

H $3.872308-0.110834-3.160382$

H $4.540813-1.176744-4.386456$

H $5.406227-2.717681-2.660940$

H $4.994160-1.572653-1.391304$

H $3.387428-3.335222-1.367354$

H $3.540218-4.103473-3.756493$

H $2.780473-2.619584-4.293332$

H $1.563164-4.939046-4.968113$

H $0.326670-2.107280-3.558236$

H $2.076904-5.971794-2.926742$

H $\quad 0.443535-5.660918-2.383638$

H $1.819043-5.062418-1.435896$

H -1.129434 -3.820312 -3.251287

H $0.121933-0.232675-2.132339$

H $-0.881058-1.156278-1.010407$

H $0.923281-2.4430960 .418257$

H $4.106006-0.6070240 .498578$

H $4.644703 \quad 0.7367173 .212100$

H $4.541151-2.7698441 .710444$

H $2.826362-2.2970821 .657352$

H $3.402806-3.4082792 .886966$

H $1.7472480 .025800-0.002457$

H $\quad 0.3964751 .097248-0.363668$

H -1.310702 $0.361973 \quad 3.438285$

H -0.5258341 .9264183 .716704$

H $1.468470 \quad 0.835467 \quad 4.739679$

H - $0.550434-1.4685784 .801680$

H $0.589621-1.8583956 .953525$

H $1.894159-0.7739316 .560801$

H $2.004634-4.7324114 .995382$

H $0.370783-4.0228455 .504832$

H $3.983808-3.4342025 .032921$

H $3.966712-2.3596146 .400544$

H $4.099229-0.4385355 .096371$

H 0.8423841 .6158611 .870446

C $0.6934474 .396365-0.017924$

O $-0.2840903 .595160-0.714365$

C $-1.6067093 .999263-0.297281$
C -1.4004734 .8989130 .910653$

C $-0.087527 \quad 5.5724810 .562312$

H $-2.0205094 .574675-1.135241$

C -2.449295 $2.743453-0.047962$

H -2.221207 5.6077331 .054135

H -1.2950284 .3044381 .826922$

C $0.585108 \quad 6.235074 \quad 1.752098$

H $-0.2699906 .324836-0.216747$

C -3.8063343 .0103810 .620927$

O $-2.6725492 .107127-1.311013$

H -1.8754042 .0371790 .559933$

H -3.646784 3.3868531 .638446

O $-4.4668094 .059558-0.098941$

C $-4.7599051 .800308 \quad 0.662412$

C -4.1232630 .5270351 .247206$

H $-5.1467301 .615259-0.345460$

O

H -3.2859620 .2198090 .612973$

H -3.7073150 .7556192236440$

H -0.0541517 .0218672 .165754$

H 0.7894595 .5138462 .550692

H 1.5358026 .6916981 .459885

C $1.7973354 .802121-0.996480$

H $-1.8008141 .930928-1.705185$

H -5.3195504 .2198540 .340699$

H -6.5926651 .5436531 .302360$

H $2.3501155 .663466-0.605228$

H $1.3566155 .092311-1.957774$

H 1.1006493 .7756920 .791121

C $-5.195292-1.343930-0.910104$

O $-5.910569-0.8404980 .224226$

C $-5.111849-0.6520381 .395917$

C $-4.461330-1.9804791 .811733$

C $-3.724184-2.6518640 .653608$

C $-4.610702-2.721567-0.589344$

H $-4.386549-0.652147-1.175737$

C $-6.177382-1.405609-2.084162$

H $-5.808345-0.3757392 .197552$

O $-3.556101-1.7891552 .893804$

H -5.241934 -2.667726 2.163490

H $-2.784554-2.1390350 .425020$

O $-3.371585-3.984577 \quad 1.029891$

H $-5.414988-3.450744-0.428995$

O $-3.834619-3.171723-1.696448$

C $-6.573517-0.015860-2.524490$

H $-5.757574-1.928172-2.950780$

H -7.094156 -1.934903 -1.797205

H $-4.053520-1.3805423 .622619$

H -2.822206 -3.922043 1.830152

H -3.427717 -4.017625 -1.441829

O $-5.5422460 .572468-3.195942$

O $\quad-7.663013 \quad 0.489321-2.287402$

C $-5.7752341 .916963-3.626667$

H -5.980135 $2.561029-2.766036$

H -4.870386 2.275827 -4.124214

H $-6.6046511 .947316-4.339395$

SCF Energy (B3LYP/6-31G**//MMFF) $=-3245.91880923$

0500258

MM̄FF Geometry

C $-2.824897 \quad 2.317867 \quad 1.577457$

C -2.2432491 .6068442 .555358$

C $-2.326530 \quad 0.108214 \quad 2.648021$

O $-0.987020-0.3665672 .931194$

C -3.268461 -0.3581193 .774272$

$\begin{array}{llll}C & -4.713217 & 0.067313 & 3.631962\end{array}$

C -5.576796 -0.4557282 .739843$

C $-5.172962 \quad 1.119204 \quad 4.606896$

C $-5.292606-1.5018051 .696253$

C $-5.687687-0.9922580 .303786$

C $-5.196682-1.851340-0.872858$

C $-5.489325-3.344391-0.706758$

C $-4.952725-4.169100-1.887556$

O $-3.787988-1.632710-1.038069$

O $-4.966926-5.552812-1.501974$

C $-3.476136-3.785302-2.130657$

C $-5.854442-4.057731-3.122300$

O $-2.973648-4.418677-3.305192$ 
C $-3.241756-2.258978-2.210290$

C $-1.740717-1.899495-2.313025$

O $-3.863170-1.759404-3.402551$

C -0.716621 -1.669416 2.650002

O $-1.485620-2.5001442 .192697$

C $0.699743-1.9204122 .989706$

C $1.311940-2.9886192 .458518$

C $2.697556-3.3981882 .686782$

C $3.481917-2.6667813 .744640$

C $-0.909719-2.200056-1.066474$

C $0.566897-1.972044-1.326063$

C $2.506812-2.952005-2.560530$

O $1.158863-0.992301-0.880090$

C $3.279940-3.993613-1.814515$

C $4.440223-3.742921-1.189600$

C $5.262675-4.782278-0.482342$

C $5.514774-4.4388700 .969133$

C $6.585816-3.7119951 .330132$

C $4.574660-5.0078462 .007105$

C $3.198982-4.4063321 .943076$

N $1.127442-2.965874-2.112709$

H $-3.404238 \quad 1.7991170 .817533$

H -1.691156 2.1230693 .336419

H -2.636164 -0.2965581 .677371$

H -2.872027 -0.0141664 .740303$

H -3.234085 -1.452110 3.860216

H $-6.605084-0.0961482 .750756$

H $-6.223313 \quad 1.3927564 .460625$

H -5.0655010 .7556665 .634182$

H $-4.5770132 .030597 \quad 4.496724$

H $-4.245711-1.8134001 .702426$

H $-5.886104-2.3900341 .943034$

H $-6.782523-0.9241700 .255799$

H -5.2974070 .0254250 .169450$

H -5.707114 -1.468068 -1.764784

H $-6.563938-3.517166-0.570579$

H $-5.004166-3.7209690 .203415$

H $-5.880509-5.788581-1.265494$

H $-2.900044-4.191041-1.290143$

H $-6.861770-4.426951-2.894404$

H $-5.484019-4.685929-3.939780$

H $-5.956334-3.035210-3.490916$

H -3.137007 -5.372922 -3.212016

H $-1.309786-2.380067-3.200514$

H $-1.648241-0.821414-2.509313$

H $-3.470835-0.896980-3.617285$

H $1.195748-1.2199263 .651134$

H $\quad 0.747290-3.6235551 .776262$

H $4.515389-3.0091723 .833082$

H $3.013907-2.8000094 .726361$

H $3.531201-1.5950913 .525876$

H $-1.215690-1.540688-0.248484$

H $-1.037658-3.230724-0.722047$

H $2.929450-1.950299-2.435333$

H $2.499787-3.194685-3.627866$

H $2.882494-5.005669-1.813696$

H $4.846119-2.735617-1.218801$

H $6.220990-4.867983-1.011596$

H $4.801101-5.774934-0.553001$

H $6.790327-3.4833902 .370768$

H $7.278316-3.3236300 .590646$

H $4.996047-4.9123063 .014013$

H $4.486015-6.0910401 .851483$

H $2.554856-4.8719091 .196396$

H $0.548027-3.747456-2.405045$

C $-2.616505 \quad 4.2963090 .040893$

O $-1.3480953 .888428-0.507443$

C $-0.8567694 .938529-1.363732$

C $-1.9556835 .995903-1.431188$

C -2.641491 $5.817491-0.092814$

H $0.0134915 .372830-0.858514$

C $-0.449743 \quad 4.380322-2.732355$

H $-1.5506477 .002341-1.575965$

H -2.659124 $5.801175-2.249189$

C $-4.0313876 .427808-0.040272$

H $-2.015621 \quad 6.273700 \quad 0.685918$

C $0.626598 \quad 3.277427-2.688177$

O -1.613168 3.824838 -3.360466
H $-0.1188115 .205201-3.375029$

H $0.2041602 .361963-2.257151$

O $0.9578252 .952294-4.045622$

C $1.9123563 .656180-1.929705$

C $3.0294062 .612371-2.120536$

H $1.6737293 .768232-0.867634$

O $2.3706514 .920679-2.408596$

H $2.6084291 .613144-1.967021$

H $3.3900372 .658021-3.155346$

H -3.985101 $7.505454-0.228272$

H $-4.6934265 .983721-0.791241$

H $-4.4887096 .277704 \quad 0.942488$

C $-2.756897 \quad 3.815865 \quad 1.486447$

H $-1.3372793 .478773-4.226535$

H $1.3900543 .731253-4.436193$

H $3.0426465 .246640-1.787007$

H $-3.679390 \quad 4.2204841 .918056$

H $-1.915072 \quad 4.1881502 .082306$

H $-3.396582 \quad 3.845077-0.587257$

C 3.3948751 .6072700 .723696

$\begin{array}{lllllllllll}\text { O } & 3.844573 & 2.862872 & 0.200376\end{array}$

C $4.2335232 .834363-1.176560$

C $5.3823991 .835594-1.370318$

C $5.0247000 .451099-0.836350$

C 4.4922020 .5452110 .592873

H $2.494171 \quad 1.2839240 .189471$

C 3.0220421 .8312762 .196201

H $4.6382443 .828460-1.401779$

O $5.731264 \quad 1.760138-2.748239$

H $6.2658592 .204719-0.832871$

H $4.305728-0.057591-1.488677$

O $6.199394-0.363341-0.813147$

H $\begin{array}{llll}5.320217 & 0.772450 & 1.276598\end{array}$

$\begin{array}{llll}\text { O } & 3.959340 & -0.720716 & 0.971363\end{array}$

C 1.8067932 .7230632 .333577

H 2.7904830 .8885212 .702560

H 3.8642942 .3089122 .711840

H $6.5331401 .215787-2.820819$

H $6.460295-0.527771-1.734926$

H $4.672758-1.3753300 .882576$

$\begin{array}{lllll}\text { O } & 1.936812 & 3.501540 & 3.446894\end{array}$

$\begin{array}{lllll}\text { O } & 0.850257 & 2.716403 & 1.570562\end{array}$

C $0.861168 \quad 4.4118313 .696135$

H $1.1662375 .085500 \quad 4.501237$

H $0.642673 \quad 5.0095682 .805685$

H -0.0253203 .8588934 .018167$

SCF Energy (B3LYP/6-31G**//MMFF) $=-3245.90892380$

0500259

MMFF Geometry

C $3.685518-4.275739-0.228317$

C $2.973817-3.520000-1.076895$

C $1.469644-3.504995-1.143004$

O $1.069542-2.265785-0.514590$

C $1.016157-3.513465-2.615429$

C $-0.466512-3.753250-2.820728$

C $-1.332920-2.859952-3.339120$

C $-0.953766-5.134065-2.456016$

C $-1.063788-1.462764-3.824862$

C $-1.479992-0.355360-2.845362$

C $-2.998558-0.214957-2.645371$

C $-3.3134361 .021763-1.798328$

C $-4.813793 \quad 1.149057-1.489458$

O $-3.492198-1.384282-1.986228$

O $-4.966807 \quad 2.107688-0.430888$

C $-5.337409-0.201229-0.952195$

C $-5.5946551 .701357-2.687757$

O $-6.754640-0.176148-0.807683$

C $-4.917375-1.407799-1.816000$

C $-5.293083-2.774833-1.190940$

O $-5.587571-1.337445-3.081232$

C $-0.030380-2.2698330 .282534$

$\begin{array}{lllll}\text { O } & -0.807603 & -3.190785 & 0.472078\end{array}$

C $-0.122391-0.931168 \quad 0.907436$

C $-1.284270-0.5254871 .438345$

C $-1.539643 \quad 0.7660972 .078342$

C -0.3601331 .6598692 .353762$

C $-4.708951-3.0522540 .197340$ 
C $-5.632270-2.6634801 .341263$

C $-5.602485-2.200968 \quad 3.792737$

O $-6.840915-2.4951901 .203118$

C $-5.657101-0.7091853 .889268$

C -4.9434920 .0107524 .767946$

C -4.9934901 .5096654 .848350$

C -3.6686512 .1566934 .506533$

C -2.8595262 .6255125 .471305$

C -3.32381023424263 .044799$

C $-2.810371 \quad 1.0815622 .403204$

N -4.952682 -2.571259 2.548224

H $3.174476-4.9446260 .458955$

H $3.502298-2.837940-1.738993$

H $1.069198-4.365135-0.593953$

H $\quad 1.551797-4.302397-3.161357$

H $1.322785-2.570124-3.079231$

H $-2.368251-3.176323-3.460738$

H -2.003352 -5.290595 -2.728023

H $-0.364830-5.894142-2.980116$

H $-0.867534-5.308129-1.379909$

H $-0.005319-1.330823-4.066552$

H -1.593237-1.323992 -4.776026

H $-0.995126-0.536646-1.881013$

H $-1.0864740 .593960-3.229788$

H -3.466327 -0.128203-3.633065

H $-2.9442291 .932120-2.286984$

H $-2.7703810 .956018-0.846754$

H -4.564642 $2.943765-0.722762$

H $-4.921053-0.3237240 .054976$

H -5.224343 $2.697304-2.959655$

H $-6.654177 \quad 1.833688-2.442760$

H -5.520842 $1.070268-3.575746$

H $-6.9744490 .581191-0.238410$

H -6.381859-2.905867-1.203060

H -4.899699 -3.554240 -1.859441

H $-5.588173-2.223673-3.479486$

H $0.764901-0.3099420 .896794$

H $-2.140193-1.1952831 .384047$

H $\quad 0.0423172 .0561801 .416381$

H $0.432738 \quad 1.1102462 .873846$

H -0.6033492 .5130482 .991155$

H -4.547956 -4.133189 0.298165

H -3.735574 -2.567606 0.314021

H -5.026442 -2.648618 4.608784

H -6.616458 -2.612995 3.814196

H -6.317768 -0.1961023 .194030$

H -4.284477 -0.4995975 .466444$

H -5.2964291 .7792705 .868500$

H $-5.774317 \quad 1.9191234 .195111$

H -1.9227643 .1180435 .233226$

H -3.113755 2.522684 6.521076

H -2.615655 3.1687972 .919306

H -4.219565 2.6695482 .501347

H -3.594654 0.3692392 .150515

H -3.940787 -2.661102 2.555195

C $5.630924-3.4343631 .099081$

O $5.244419-2.0503640 .962827$

C $6.331247-1.2139531 .400627$

C $7.325519-2.1413652 .090142$

C $7.146305-3.4194621 .294394$

H $6.799520-0.8092800 .495612$

C $5.809569-0.0677802 .274128$

H $8.347625-1.7522852 .055645$

H $7.064523-2.3096213 .141439$

C $7.692929-4.6521221 .993107$

H $7.649411-3.3027130 .324993$

C 4.7134810 .7910991 .607617

O $5.250862-0.6279393 .470012$

H 6.6494630 .5613062 .592155

H 3.8055920 .1877831 .499689

O 4.3711801 .8459502 .515934

C 5.1004771 .4003650 .246447

C $4.1085522 .440960-0.305962$

H $5.2344830 .601095-0.486197$

O $\quad 6.378112 \quad 2.039246 \quad 0.355174$

H 4.1270343 .3296280 .340698

H $4.5049152 .810300-1.260190$

H $8.769663-4.5511992 .164177$
H $7.211696-4.8095952 .964231$

H $7.530206-5.5489871 .387231$

C $5.185315-4.214495-0.139020$

H 4.9241970 .1111194 .011338

H 5.1726752 .3702012 .681914

H 6.2863142 .7944760 .960141

H $5.571026-5.239609-0.108551$

H $5.593378-3.741716-1.041500$

H $5.129160-3.8184281 .997041$

C $1.9171923 .874815-1.810879$

O $1.7798503 .133202-0.593481$

C $2.6429931 .991012-0.476725$

C $2.3436251 .021892-1.627658$

C $2.4577891 .702523-2.988905$

C $1.6192812 .979885-3.019779$

H $2.9327434 .282108-1.886635$

C $0.9324705 .051759-1.742113$

H $2.341016 \quad 1.504146 \quad 0.454914$

O $3.195863-0.111323-1.599288$

H $1.3163640 .649885-1.523483$

H $3.5021721 .910104-3.248923$

O $1.961098 \quad 0.820637-3.998665$

H $0.5540322 .716119-3.039711$

O $1.9034183 .698739-4.217869$

C $1.3299466 .073785-0.697080$

H $0.8878495 .572019-2.704932$

H $-0.0659554 .681050-1.480017$

H $3.057167-0.558035-0.746664$

H $2.463537-0.009542-3.930129$

H $1.7285673 .099526-4.963869$

O $0.3456517 .017210-0.606744$

O $2.3675646 .057471-0.049356$

C 0.5784418 .0595710 .345800

H 1.4804818 .6196670 .081435

H $-0.276794 \quad 8.7400430 .320980$

H 0.6644127 .6401421 .352757

SCF Energy $(B 3 L Y P / 6-31 G * * / / M M F F)=-3245.91391570$

0500260

MM̄FF Geometry

C $4.4285944 .142245-0.168013$

C 3.1920393 .9398330 .311974

C $1.9242034 .342547-0.394249$

O $1.199258 \quad 3.134837-0.728937$

C 1.0395825 .1805620 .547766

C $-0.3088035 .545386-0.045933$

C -1.4952015 .0406880 .352341$

C $-0.2642746 .575181-1.146387$

C $-1.765666 \quad 4.0276201 .432652$

C $-2.2339092 .677187 \quad 0.881474$

C -3.6371792 .6545850 .251152$

C -4.7341002 .8711251 .298920$

C $-6.1454102 .661237 \quad 0.730197$

O $-3.7807651 .362999-0.360288$

O -7.0540282 .5444801 .836425$

C $-6.1855941 .317647-0.026412$

C $-6.6129393 .870820-0.087745$

O $-7.4365251 .141571-0.686882$

C $-5.0304721 .153592-1.034218$

C $-4.998616-0.257434-1.668109$

O $-5.2012932 .088668-2.105502$

C $1.5713292 .469779-1.857064$

O $2.4739592 .763436-2.624137$

C $0.6760741 .300737-1.995996$

C $0.9097890 .377089-2.939961$

C $0.092406-0.811842-3.192875$

C -1.184402 -0.950328 -2.408076

C $-4.765734-1.402179-0.679294$

C $-4.355817-2.688900-1.377817$

C $-3.175726-4.838086-0.904113$

O $-4.485905-2.871998-2.585000$

C -1.743861 -4.535679-1.226777

C $-1.114666-4.977119-2.326384$

C $0.311714-4.632659-2.657150$

C $0.492216-4.217754-4.102691$

C $1.229184-4.962208-4.944555$

C $-0.190691-2.955368-4.580795$

C $0.508229-1.705673-4.114308$ 
N -3.825421 -3.608963 -0.483647

H $4.5648134 .657855-1.114624$

H 3.0737973 .4000411 .249430

H $2.1372294 .910357-1.308492$

H 1.5640156 .1072430 .817422

H 0.9084924 .6406931 .490786

H -2.392119 $5.401679-0.149672$

H -1.264460 $6.870226-1.481435$

H $0.2443897 .480368-0.798961$

H $\quad 0.2715206 .185538-2.017361$

H -0.8772203 .8484212 .043554$

H -2.5091104 .4426312 .121505$

H -1.507891 2.3175830 .143666

H -2.201050 1.9366821 .691792

H -3.693193 $3.414983-0.534776$

H -4.6587933 .8671321 .750290$

H -4.5898102 .1606702 .123565$

H -6.9828993 .3545702 .369947$

H -6.1165180 .5220630 .726873$

H -6.6552094 .7664590 .543931$

H $-7.6305753 .723591-0.465714$

H -5.958274 $4.097226-0.931782$

H -8.134803 $1.250579-0.018693$

H $-5.918078-0.438370-2.239772$

H -4.192808 $-0.274886-2.413892$

H $-4.696701 \quad 1.774394-2.873897$

H $-0.156803 \quad 1.225330-1.308184$

H $1.7773850 .498378-3.586867$

H -1.812204 -0.062211 -2.536829

H $-0.967229-1.077186-1.342454$

H - $-1.790783-1.803892-2.710751$

H -3.970016 -1.131611 0.022596

H -5.677183 -1.604899-0.106715

H $-3.713113-5.255387-1.761693$

H -3.232063 -5.538970 -0.066006

H -1.197317 $-3.910663-0.524733$

H -1.647360 -5.623245 -3.020381

H $0.924741-5.516382-2.438161$

H $\quad 0.686690-3.833699-2.005640$

H $1.361924-4.677013-5.982952$

H $1.718310-5.873049-4.615391$

H -1.248782 -2.978782 -4.306969

H $-0.198878-2.926511-5.679185$

H $1.454037-1.520636-4.625668$

H -3.729694 -3.343812 0.492045

C $6.2886232 .459763-0.260987$

O $5.3158121 .406972-0.413286$

C 5.8469130 .1893340 .132307

C 7.3571330 .3699700 .082482

C 7.4855341 .8346950 .463866

H 5.5311510 .1431921 .181971

C $5.286800-1.023630-0.617779$

H $7.881693-0.3005880 .769524$

H $7.7544840 .210027-0.926135$

C 8.8198842 .4517850 .082073

H 7.3562791 .9224831 .550995

C $3.754266-1.017889-0.821300$

O $5.881813-1.060249-1.923532$

H $5.607835-1.943324-0.115685$

H $3.517530-0.214346-1.528512$

O $3.380208-2.233130-1.483043$

C $2.860416-0.8459370 .423133$

C $3.063240-1.9227181 .503269$

H $1.817608-0.9055360 .089498$

O 3.0305420 .4431830 .997493

H $2.759908-2.8917881 .092158$

H $4.127989-2.0046001 .750335$

H $\quad 9.641078 \quad 1.9405300 .594757$

H $9.0008812 .381527-0.995780$

H $8.851913 \quad 3.509730 \quad 0.361137$

C 5.6621773 .6217910 .518663

H $5.520016-1.835808-2.385871$

H $3.701564-2.978510-0.948506$

H $2.8929881 .098077 \quad 0.292803$

H 6.3836154 .4397010 .625101

H 5.4037853 .2868891 .531481

H $6.5628702 .784008-1.272669$

C $0.064449-2.1826732 .105742$
O $0.962459-1.1827152 .589168$

C $2.298842-1.6360412 .817304$

C $2.304114-2.8008093 .817828$

C $1.354805-3.9234323 .398955$

C $-0.032762-3.3628613 .080168$

H $\quad 0.432671-2.5500141 .139202$

C -1.283886-1.512955 1.808825

H $2.821435-0.7992263 .298762$

O $3.625792-3.3088953 .967847$

H $1.991111-2.4237654 .800300$

H $1.763598-4.4885032 .553368$

O $1.247693-4.8488534 .483070$

H $-0.508008-3.0587334 .019309$

O $-0.844352-4.3937712 .522601$

C $-1.991640-0.9474003 .022599$

H -1.134682 -0.6741801 .118689$

H -1.943383 -2.243176 1.331310

H $3.582434-4.0498754 .596507$

H $0.606430-5.5308144 .219135$

H $-0.420406-4.6970781 .701726$

O $-3.326676-0.8462892 .750648$

O $-1.446322-0.6045484 .062337$

C $-4.132557-0.3111403 .804723$

H -3.7789260 .6847584 .088665$

H $-5.158987-0.2266143 .437842$

$\mathrm{H}-4.120221-0.9856744 .665936$

SCF Energy $\left(B 3 L Y P / 6-31 G^{* *} / / M M F F\right)=-3245.90988601$

0500261

MM̄FF Geometry

C $-0.3625482 .890375-1.614087$

C $0.6303043 .768411-1.408217$

C $1.4625513 .772815-0.156195$

O $2.8144103 .440837-0.550892$

C 1.4659175 .1378090 .559616

C 0.1143785 .7745020 .806735

C $-0.8010785 .325791 \quad 1.687234$

C -0.1579467 .0220400 .006776$

C -0.7136604 .1052222 .559575$

C $-1.917913 \quad 3.1850892 .314690$

C -1.7696581 .7616012 .872362$

C -1.3378021 .7193624 .339541$

C $-1.1637560 .276368 \quad 4.840001$

O $-0.819906 \quad 1.0646392 .057860$

O $-0.4502720 .322677 \quad 6.086041$

C $-0.269981-0.4957203 .841648$

C $-2.514008-0.3832115 .143596$

O $-0.207306-1.8797594 .175869$

C $-0.703711-0.3323962 .367280$

C $0.293197-0.956515 \quad 1.372707$

O $-1.934733-1.0218362 .113380$

C 3.6194462 .8970510 .400994

O $3.3605592 .732823 \quad 1.582773$

C $4.8746412 .482058-0.259207$

C 5.5964381 .4976620 .295999

C $6.8364010 .929565-0.231333$

C $7.4557281 .589142-1.435129$

C $1.698606-0.3604341 .377249$

C $2.510833-0.9183300 .224114$

C $3.626419-3.014891-0.549454$

O $2.734947-0.258210-0.787555$

C $5.062874-3.145339-0.150968$

C $6.083189-2.961425-1.003239$

C $7.527984-3.160487-0.638336$

C $8.395682-1.979785-1.013838$

C $9.016395-1.935232-2.205084$

C $8.614503-0.8953320 .016070$

C $7.356662-0.1573870 .375466$

N $2.918482-2.2242960 .439751$

H $-0.5561072 .122755-0.867883$

H $0.8401644 .530846-2.153873$

H 1.0981882 .9913090 .519371

H $2.0980945 .833425-0.010798$

H $1.9907095 .043727 \quad 1.519598$

H -1.725668 5.8915051 .797286

H -1.1458697 .4457440 .216407$

H $0.587548 \quad 7.7900640 .237863$

H $-0.1136356 .805163-1.065209$ 
H 0.2218053 .5625142 .408455 H -0.7154984 .4361813 .605039$ H -2.8095703 .6487902 .756383$ H -2.100031 3.1086161 .234196 H -2.7461501 .2771642 .751802$ H -2.049932 2.2652104 .970502 H -0.3729792 .2288724 .461614$ H $-0.955811 \quad 0.881986 \quad 6.700495$ H $0.747590-0.1006033 .955395$ H -3.034169 0.1600295 .941902 H -2.381075 -1.405081 5.515095 H -3.182294 -0.4111264 .280417$ H $0.080896-1.9410415 .102765$ H $0.343576-2.0402951 .533499$ H $-0.118171-0.8350850 .361187$ H -2.117284 -1.614316 2.860568 H $5.1425932 .962286-1.192894$ H 5.2320101 .0434251 .217153 H $8.3982661 .130708-1.743023$ H $7.6711682 .643309-1.228062$ H $6.7806441 .530275-2.296021$ H 1.6567840 .7261861 .266993 H $2.224669-0.5746282 .313413$ H $3.509467-2.563732-1.540092$ H $3.157931-4.004116-0.559554$ H $5.272928-3.4428280 .873638$ H $5.867530-2.697098-2.036163$ H $7.882858-4.057701-1.162640$ H $7.646417-3.378716 \quad 0.430297$ H $9.665365-1.108927-2.475392$ H $8.885739-2.724031-2.938683$ H $9.389622-0.189733-0.306192$ H $9.020685-1.3522410 .928010$ H $6.832043-0.5797241 .233136$ H $2.696854-2.6703951 .325034$ C $-2.7249053 .036772-2.384124$ O $-3.158847 \quad 1.806714-1.765550$ C $-4.4855691 .497183-2.229858$ C $-4.9791312 .739907-2.961443$ C $-3.686728 \quad 3.273177-3.546818$ H $-4.3838580 .684815-2.958807$ C -5.372906 $1.051316-1.064613$ H -5.730702 $2.503272-3.720666$ H -5.415491 $3.471214-2.270883$ C $-3.7762824 .722459-3.992484$ H $-3.4023022 .648029-4.403880$ C $-4.852957-0.184938-0.301202$ O $-5.4704742 .133237-0.129126$ H $-6.3903340 .869092-1.431200$ $\begin{array}{lllll}\text { H } & -3.949117 & 0.077648 & 0.260934\end{array}$ O $-5.839993-0.522087 \quad 0.683477$ C $-4.571247-1.418926-1.181927$ C $-4.293475-2.684159-0.348160$ H -3.719398 -1.201823 -1.833123 O $-5.708135-1.651619-2.014107$ H -3.551004 -2.444996 0.418753 H -5.215026 -2.976333 0.169441 H -4.533067 $4.837572-4.775458$ H -4.049229 $5.381905-3.161765$ H -2.819165 $5.067786-4.395457$ C $-1.2638342 .904352-2.815166$ H -6.0306881 .8307230 .605994$ H $-6.638973-0.8111190 .209942$ H - -5.433938 -2.250207 -2.728883 H $-0.9903103 .727373-3.484777$ H $-1.1177591 .966066-3.362029$ H -2.834656 $3.828577-1.630664$ C $-1.431866-3.465848-1.132189$ O $-2.617293-3.589173-1.928548$ C -3.813254 -3.883459-1.197219 C $-3.640450-5.186968-0.403305$ C $-2.401024-5.1508770 .484126$ C $-1.176589-4.753056-0.336057$ H -1.538739 -2.618837 -0.446175 C $-0.258435-3.178924-2.082668$ H $-4.589321-4.075996-1.947526$ O $-4.783749-5.4544050 .401492$ H -3.538416 -6.026184-1.103798
H -2.536035 -4.479901 1.340791

O $-2.171950-6.4538921 .025292$

H $-0.911901-5.571649-1.017974$

O $-0.070746-4.5559520 .542430$

C $-0.253430-1.757367-2.604160$

H $0.693105-3.325453-1.561010$

H $-0.305114-3.849338-2.948705$

H -5.555395 -5.487870 -0.189439

H $-2.974886-6.7097821 .510848$

H $0.046780-5.3771921 .050075$

O $0.864118-1.578575-3.370669$

O $-1.106659-0.912387-2.374469$

C $1.026339-0.272299-3.930976$

H $1.941268-0.270407-4.529297$

H $\quad 0.180835-0.030154-4.581778$

H $1.1277100 .469996-3.134307$

SCF Energy $\left(B 3 L Y P / 6-31 G^{* *} / / M M F F\right)=-3245.92023772$

0500262

MM̄FF Geometry

C $-3.176221-3.0677650 .535454$

C $-2.086894-3.807173 \quad 0.791828$

C $-0.772367-3.5502700 .103092$

O $0.001874-2.7419351 .017127$

C $-0.014981-4.858528-0.184001$

C $1.108267-4.695009-1.192221$

C $2.425126-4.784283-0.919807$

C $0.665067-4.457675-2.615858$

C $3.100554-5.0562060 .393684$

C $3.702024-3.8190361 .077911$

C $4.821424-3.1172280 .289043$

C $5.511034-2.0671041 .167022$

C $6.573051-1.2699940 .393203$

O $4.252623-2.478984-0.859630$

O $6.945016-0.1334291 .188342$

C $5.958911-0.754188-0.927678$

C $7.863764-2.0780530 .220100$

O $6.954424-0.149782-1.748298$

C $5.213008-1.847214-1.721058$

C $4.452836-1.297152-2.954906$

O $6.162543-2.800226-2.216105$

C $0.545606-1.5941580 .534396$

O $0.545111-1.203976-0.622054$

C $1.138069-0.8664321 .678346$

C 1.9957620 .1333271 .433040

C 2.6459790 .9894832 .423679

C 2.1575070 .9135383 .844858

C $3.394594-0.229903-2.661157$

C $3.8998231 .199549-2.781773$

C $3.2570073 .540907-2.198817$

O $4.9467321 .499310-3.349105$

C $4.0812823 .886568-0.999315$

C 3.6198354 .5733850 .057116

C 4.4580114 .9160841 .256586

C 3.9696864 .2484712 .524547

C 3.2331084 .9239203 .422440

C 4.4073542 .8241782 .792938

C 3.6144791 .8260291 .998647

N $3.0161422 .109333-2.215941$

H $-3.094555-2.261754-0.190783$

H $-2.126062-4.5827281 .551881$

H -0.966517 -3.010582 -0.831208

H $-0.707596-5.612235-0.582362$

H $0.363171-5.2673650 .758570$

H $3.119523-4.669178-1.751809$

H $1.502574-4.477938-3.321756$

H $-0.041344-5.233842-2.928223$

H $\quad 0.179700-3.482573-2.715823$

H $2.413972-5.5390881 .095769$

H $3.896764-5.7908560 .218056$

H $2.896805-3.1076901 .283401$

H $4.099746-4.1420272 .048365$

H $5.542344-3.877272-0.034192$

H $5.955566-2.5321512 .055825$

H $4.756652-1.3651941 .546279$

H $\quad \begin{array}{lll}6.147823 & 0.398883 & 1.349917\end{array}$

H $5.2476160 .037287-0.661976$

H $8.290391-2.3281211 .199204$ 
H $8.631210-1.492895-0.298510$

H $7.717159-3.013547-0.323395$

H $7.3881190 .540529-1.218076$

H $5.166729-0.956981-3.714769$

H $3.924682-2.146393-3.412273$

H $5.759485-3.279370-2.959054$

H $0.852732-1.1678962 .679400$

H 2.2489480 .3546110 .398412

H $2.409950-0.0571904 .285154$

H 1.0705621 .0456263 .888235

H 2.5840951 .6838974 .490978

H $2.588811-0.326349-3.400020$

H $2.951628-0.386936-1.674692$

H $2.282128 \quad 4.037678-2.188616$

H $3.7864293 .834947-3.110869$

H $5.1166573 .553306-1.004434$

H $2.586527 \quad 4.9123280 .063949$

H $4.4418846 .007617 \quad 1.373547$

H 5.5119034 .6572271 .094195

H 2.9034374 .4599264 .346157

H 2.9384585 .9539713 .250975

H 4.3673192 .6063203 .865300

H 5.4679162 .7160002 .531725

H 3.8976641 .7844930 .947518

H $2.202568 \quad 1.763437-1.715435$

C $-5.675754-3.024468 \quad 0.286231$

O $-5.618558-1.701658-0.285976$

C $-6.908688-1.079837-0.177061$

C $-7.885107-2.209778 \quad 0.124423$

C $-7.031670-3.1180200 .988960$

H $-6.861006-0.4098590 .690017$

C $-7.216200-0.268010-1.439238$

H $-8.787215-1.8552970 .631965$

H $-8.192946-2.737900-0.785269$

C $-7.568778-4.5339441 .103512$

H $-6.965474-2.6801561 .994135$

C $-6.1011710 .715676-1.852577$

O $-7.404492-1.179228-2.531034$

H $-8.169814 \quad 0.257974-1.313405$

H $-5.2327360 .149577-2.210493$

O $-6.5805811 .448738-2.988991$

C $-5.6458901 .711343-0.766842$

C -4.587142 2.693348-1.307088

H $\quad-5.229348 \quad 1.1696810 .087261$

O $-6.7641752 .466460-0.303780$

H $-3.7551172 .115262-1.723300$

H $-5.0256443 .271060-2.129450$

H -8.562910 -4.532336 1.562320

H $-7.652533-5.0119420 .121694$

H $-6.911127-5.1510771 .723651$

C $-4.494300-3.2163951 .240780$

H -7.598945 -0.647549-3.321892

H -7.310007 2.015705 -2.684635

H -7.353197 1.8619650 .177516

H $-4.562165-4.1940551 .731038$

H $-4.536040-2.4474482 .021210$

H -5.614148 $-3.735182-0.547793$

C -2.242581 $2.391340 \quad 0.663911$

$\begin{array}{lllll}\text { O } & -3.500101 & 3.037996 & 0.893122\end{array}$

C -4.071998 $3.690583-0.244027$

C $-3.0959344 .741083-0.791446$

C $-1.7296954 .137606-1.102840$

C $-1.201078 \quad 3.3758990 .110460$

H $-2.3797801 .556657-0.034423$

C -1.766951 1.8315192 .014684

H $-4.947956 \quad 4.2370800 .127597$

O $-3.6368095 .356081-1.956108$ H $-2.9698625 .532746-0.041126$ H $-1.7729523 .499553-1.993285$ O $-0.834022 \quad 5.207417-1.414337$ H $-0.908788 \quad 4.091155 \quad 0.890435$ O $-0.0322902 .647568-0.263053$ C -2.521891 0.5919412 .440324 H $-0.710007 \quad 1.5560511 .954642$ H -1.8991122 .5839272 .801137$ H -2.979402 $5.995786-2.279140$ H $0.0062614 .812209-1.701262$ H $\quad 0.664765 \quad 3.293926-0.463904$ $\begin{array}{llll}\text { O } & -1.946131 & 0.099721 & 3.577664\end{array}$

O -3.4888110 .1180621 .861946$

C $-2.541876-1.0842614 .116343$

H $-2.315249-1.9374853 .471737$

H $-2.105827-1.2645205 .102585$

H $-3.623083-0.9599964 .230544$

SCF Energy (B3LYP/6-31G**//MMFF) $=-3245.91045428$

05_00263

MM̄FF Geometry

C $1.055436-2.907355-1.410589$

C $0.097121-3.825823-1.218583$

C $-0.769453-3.8785040 .011255$

O $-2.148987-3.688265-0.391640$

C $-0.696750-5.2457120 .711898$

C $0.613648-5.5149881 .418876$

C $0.853577-5.2025102 .707184$

C $1.658348-6.2464950 .615849$

C $-0.073056-4.516827 \quad 3.673677$

C $0.635860-3.4243104 .487069$

C $0.879588-2.1229413 .704065$

C $1.748088-1.1802054 .537609$

C 1.9079420 .1927763 .879329

O $-0.394559-1.523272 \quad 3.458266$

$\begin{array}{lllll}\text { O } & 2.486563 & 1.056458 & 4.869524\end{array}$

C 0.5109620 .7406513 .516140

C $2.896050 \quad 0.143600 \quad 2.708168$

O $0.646393 \quad 1.9517162 .780001$

C $-0.367322-0.2733772 .742883$

C -1.8525420 .1646602 .671788$

$\begin{array}{llllllll}\text { O } & 0.145647 & -0.442027 & 1.424046\end{array}$

C -2.547351-2.414355 -0.656658

O $-1.867868-1.402936-0.589805$

C $-3.967618-2.457031-1.063116$

C $-4.541252-1.347192-1.552087$

C $-5.930449-1.214268-1.994862$

C $-6.836500-2.402625-1.802441$

C $-2.193473 \quad 1.3057391 .714294$

C $-3.554121 \quad 1.8909092 .035631$

C -5.9794821 .4016572 .005485$

$\begin{array}{llll}\text { O } & -3.662550 & 2.971712 & 2.612701\end{array}$

C -6.9248410 .8319450 .997590$

C $-7.706993 \quad 1.5909580 .214923$

C $-8.7085201 .051701-0.767194$

C $-8.3615651 .400142-2.197743$

C $-8.6996792 .590515-2.721660$

C $-7.690758 \quad 0.337810-3.040019$

C $-6.328559-0.037883-2.523878$

N $-4.611747 \quad 1.0890851 .633145$

H $1.221069-2.144518-0.654140$

H $-0.081628-4.579357-1.981914$

H $-0.502814-3.0962280 .732005$

H $-0.903051-6.050910-0.007212$

H $-1.528440-5.3249121 .424374$

H $1.816283-5.4889363 .129025$

H $2.539325-6.5009931 .215025$

H $1.249145-7.183903 \quad 0.225177$

H $2.002795-5.639778-0.225519$

H $-0.955440-4.0980833 .181063$

H $-0.433419-5.2806724 .373897$

H $0.000602-3.1985875 .354109$

H $1.584846-3.8126524 .877816$

H $1.363740-2.3528652 .748517$

H $2.730513-1.6280424 .734004$

H $1.285113-1.0369625 .524652$

H 2.6678861 .9159624 .453168

H $-0.0013870 .996314 \quad 4.453955$

H $3.884701-0.1797463 .055684$

H $3.042798 \quad 1.1379402 .273179$

H $2.584743-0.5364361 .912215$

H 1.0786392 .6038553 .356095

H $-2.451719-0.7072312 .371197$

H -2.2026530 .3995753 .686263$

H $-0.575421-0.7519350 .852161$

H $-4.496670-3.396930-0.956742$

H $-3.936943-0.444866-1.641897$

H -7.878084 -2.208165 -2.064891

H $-6.841554-2.716527-0.752367$ 
H $-6.501857-3.243908-2.419366$ H $-1.4772612 .124941 \quad 1.782868$ H -2.1864330 .9655110 .673515$ H -6.0926302 .4860922 .110840$ H -6.1642820 .9512352 .986410$ H $-6.990380-0.2510800 .933461$ H -7.6450872 .6739150 .299273$ H $-9.6910491 .471663-0.514464$ H -8.824524 $-0.034208-0.663493$ H $-8.4777892 .839027-3.754034$ H $-9.2048853 .344107-2.126421$ H $-7.5605510 .692902-4.070930$ H -8.363421 $-0.520490-3.119827$ H $-5.5963320 .766008-2.612665$ H $-4.409551 \quad 0.149963 \quad 1.302047$ C $3.406888-2.840139-2.270632$

O $3.744425-1.565140-1.683260$

C $4.946838-1.072778-2.300567$

C $5.562451-2.265005-3.021083$

C $4.320902-2.997619-3.486655$

H $4.637854-0.333359-3.049112$

C $5.859408-0.413952-1.263458$

H $\quad 6.217427-1.961428-3.843215$

H $6.142829-2.897559-2.339400$

C $4.579653-4.441687-3.880836$

H $3.897935-2.461518-4.347118$

C $5.2034880 .734696-0.470322$

O $6.271592-1.412069-0.320398$

H $6.773645-0.061688-1.756238$

H 4.4497110 .3276190 .212006

O $\quad 6.2187661 .3051990 .366908$

C $4.5670861 .844949-1.329355$

C $4.1523463 .067777-0.487208$

H $3.6979691 .435517-1.854111$

O $5.5182332 .256020-2.311821$

H 3.5657502 .7235620 .370446

H $5.0571653 .546655-0.094409$

H $5.287081-4.491532-4.715022$

H $5.000586-5.015552-3.048395$

H $3.653251-4.933352-4.193849$

C $1.922317-2.854364-2.636523$

H $6.847507-0.9754270 .330461$

H $6.876644 \quad 1.723826-0.214717$

H $5.0386452 .743572-3.002244$

H $1.705318-3.707258-3.289745$

H $1.669137-1.941267-3.187910$

H $3.629126-3.607601-1.517786$

C $1.1102453 .324946-0.864080$

O $2.1398143 .588654-1.826488$

C $3.3554504 .121577-1.289845$

C $3.0745235 .421640-0.521392$

C 1.9888215 .2392990 .533316

C $0.7499144 .605707-0.096706$

H $1.4437092 .547473-0.166320$

C $-0.1136122 .795407-1.628566$

H $3.9717294 .400874-2.152565$

O $4.2566475 .914673 \quad 0.100923$

H $2.7411806 .192446-1.228742$

H 2.3560324 .6501241 .382175

O $1.648546 \quad 6.528466 \quad 1.048529$

H $0.2754025 .327809-0.773886$

O $-0.1921124 .294193 \quad 0.924585$

C $0.115228 \quad 1.422323-2.223939$

H $-0.9825182 .716548-0.966895$

H $-0.3478053 .480611-2.452483$

H $4.9212986 .045498-0.596845$

H 1.0427946 .3946971 .796700

H -0.5014265 .1320281 .307669$

O $-0.881413 \quad 1.162870-3.122262$

O $1.0332860 .668860-1.932901$

C $-0.794890-0.093779-3.800470$

H $0.127459-0.143477-4.386863$

H $-0.842344-0.917823-3.084291$

$\mathrm{H}-1.647533-0.172549-4.480089$

SCF Energy (B3LYP/6-31G**//MMFF) $=-3245.91668075$

05 00264

MM̄FF Geometry
C $3.152806-3.3782111 .388868$

C $2.354856-4.2455792 .030673$

C $0.868971-4.0950952 .264633$

O $0.388161-2.8114471 .809900$

C $0.112304-5.1833391 .478982$

C $-1.382343-5.1952521 .742806$

C $-2.330849-4.7924690 .872539$

C $-1.787617-5.7478293 .086562$

C $-2.150477-4.224337-0.508842$

C $-2.632532-2.774614-0.621284$

C $-4.150698-2.551313-0.517582$

C $-4.906905-3.159116-1.702967$

C $-6.397970-2.788259-1.698213$

O $-4.337706-1.128596-0.491095$

O $-6.919413-3.135691-2.990299$

C $-6.531222-1.259650-1.528159$

C $-7.179581-3.628424-0.681701$

O $-7.899372-0.879863-1.401755$

C $-5.698813-0.700880-0.356103$

C $-5.7030930 .844657-0.311043$

O $-6.263859-1.1584900 .877914$

C $0.352833-1.7926292 .711460$

O $0.736001-1.8154353 .869503$

C $-0.282003-0.6384212 .038761$

C $-0.6089180 .455700 \quad 2.741830$

C $-1.343827 \quad 1.6135242 .226489$

C $-1.887606 \quad 1.5171920 .826042$

C $-5.0964201 .527647-1.540629$

C $-4.8157632 .999690-1.285036$

C $-3.3225264 .842983-2.048960$

O $-5.347803 \quad 3.636027-0.378959$

C -2.175002 $4.742022-1.091985$

C $-2.0643875 .471126 \quad 0.028757$

C -0.9602795 .2815591 .032414$

C -1.4829155 .1262252 .444830$

C -1.2104486 .0464263 .385177$

C -2.3392863 .9205512 .762536$

C -1.5314042 .6795723 .032578$

N -3.904744 $3.519401-2.196287$

H $2.741904-2.4567520 .986768$

H $2.794528-5.1618472 .420509$

H $0.675403-4.2103603 .337944$

H $0.510380-6.1742861 .737248$

H $\quad 0.316182-5.0622010 .410074$

H -3.370833 -4.886105 1.182412

H $-2.875016-5.8174623 .197903$

H -1.380268 -6.7551213 .221604$

H -1.416315 -5.109750 3.894055

H -1.101299-4.245973 -0.815181

H -2.679042 -4.863804 -1.223648

H $-2.138056-2.1704780 .148382$

H -2.280574 -2.359407-1.575564

H $-4.512964-2.9640070 .429768$

H -4.793969-4.248958 -1.729519

H -4.464260 -2.791192 -2.639535

H $-7.845693-2.839953-3.023126$

H $-6.178763-0.810431-2.464847$

H -7.098170 -4.694327 -0.927042

H $-8.249960-3.397404-0.713922$

H $-6.827978-3.4980510 .343787$

H $-8.184193-1.094943-0.497330$

H -6.721874 $1.216060-0.140482$

H $-5.1304891 .159430 \quad 0.573107$

H $-5.967424-0.5679101 .590334$

H $-0.505886-0.7385400 .983280$

H $-0.3466860 .497381 \quad 3.797964$

H -2.509232 0.6229720 .713091

H $-1.069926 \quad 1.473400 \quad 0.098154$

H -2.515691 2.3629520 .548961

H $-4.1554271 .038076-1.815636$

H $-5.7810091 .462247-2.392993$

H -4.092752 $5.538852-1.700959$

H -2.968567 $5.162128-3.033580$

H -1.415303 $3.996838-1.316554$

H $-2.816371 \quad 6.2247680 .250565$

H $-0.291223 \quad 6.1485470 .963392$

H $-0.351304 \quad 4.4036590 .787270$

H $-1.5859985 .946818 \quad 4.398331$ 
H -0.6047296 .9191243 .164923$ H $-3.0888893 .768611 \quad 1.980610$ H -2.936276 4.1169283 .664113 H -1.089857 2.6591714 .029806 H $-3.4616782 .888802-2.858324$ C $5.015699-3.622360-0.317880$

O $4.845385-2.311270-0.894306$ C $4.249195-2.459837-2.195650$

C $4.398385-3.933995-2.562388$

C $4.238675-4.594210-1.208085$

H $3.182766-2.233451-2.076364$

C $4.872729-1.491276-3.204142$

H $3.648966-4.258183-3.290927$

H $5.389571-4.154962-2.974627$

C $4.751666-6.022175-1.153386$

H $3.170828-4.593460-0.958179$

C $4.872323-0.008947-2.781517$

O $6.238909-1.872330-3.418946$

H $4.368701-1.608545-4.171413$

H $5.6279790 .153853-2.002846$

O $5.312810 \quad 0.727828-3.931455$

C $3.511910 \quad 0.538737-2.306280$

C $3.4968312 .073878-2.171729$

H $3.2612630 .080823-1.343950$

O $2.4898230 .157739-3.229127$

H $4.4048502 .396048-1.653095$

H $3.5200352 .512666-3.176229$

H $4.202703-6.655410-1.857984$

H $5.814840-6.077620-1.410388$

H $4.624416-6.442390-0.150702$

C $4.622015-3.606607 \quad 1.162420$

H $6.609275-1.261705-4.079185$

H $5.5964371 .606820-3.628612$

H $2.7329620 .512607-4.101536$

H $5.161745-2.7960951 .667127$

H $4.940170-4.5465141 .628964$

H $6.091040-3.835226-0.380603$

C 3.1755342 .7990890 .785830

O $2.2042742 .177105-0.065639$

C $2.2396492 .593214-1.436543$

C $2.0411324 .111481-1.527676$

C $3.0425124 .871117-0.665130$

C 3.0332764 .3274070 .762405

H 4.1849842 .5164980 .464511

C 2.9609342 .2593292 .209386

H $1.3699692 .130469-1.919629$

O $2.1321164 .541826-2.881724$

H $1.0287534 .355646-1.187687$

H $4.0465614 .835171-1.104091$

O $2.6549006 .247063-0.648254$

H 2.1049474 .6263751 .264632

O 4.1177554 .9030241 .489267

C 3.5107760 .8594972 .383573

H 3.4814792 .8981272 .932011

H 1.8945232 .2337972 .455321

H $2.0162415 .507578-2.888073$

H $3.3490376 .742077-0.181436$

H 3.9426045 .8552611 .575002

O $3.588594 \quad 0.5807303 .718867$

O 3.8266720 .1060521 .473620

C $4.150327-0.6911214 .056142$

H $4.257860-0.7372405 .143062$

H $3.481561-1.4929653 .736018$

H $5.138998-0.8075583 .601998$

SCF Energy (B3LYP/6-31G**//MMFF) $=-3245.92513960$

05 00265

MM̄MF Geometry

C -3.158009-0.604773 3.317066

C $-2.112905-0.0244373 .925386$

C $-0.692669-0.1582913 .450175$

O -0.2055891 .1954603 .281200$

C $0.206365-0.8843744 .466875$

C $-0.145433-2.3339674 .709665$

C $0.220126-3.3427013 .895770$

C $-0.894218-2.6185025 .984731$

C $0.956951-3.2435622 .589758$

C $2.412952-3.6981782 .724902$
C $3.070507-3.9249381 .355974$

C $4.508001-4.4222431 .527270$

C $5.209748-4.6211350 .175611$

O $3.057982-2.6981830 .611568$

O $6.616841-4.7632620 .425727$

C $5.028000-3.342419-0.669154$

C $4.763026-5.912707-0.519138$

O $5.530272-3.527310-1.990361$

C $3.566411-2.839062-0.724325$

C $3.436674-1.483870-1.456951$

O $2.782929-3.779473-1.473521$

C $0.878506 \quad 1.3879552 .484945$

O $1.5101220 .538625 \quad 1.878233$

C 1.1660222 .8387392 .484153

C 2.2421333 .3034431 .833787

C 2.6336984 .7085691 .716162

C 1.7535615 .7425612 .368437

C $4.176779-0.299830-0.833947$

C $4.2478040 .828737-1.843824$

C $2.9486912 .733270-2.782002$

O $5.1831890 .915528-2.637970$

C $3.7728803 .927940-2.419799$

C $3.2680375 .154330-2.216672$

C $4.1115366 .352395-1.879981$

C $3.7340956 .982102-0.556925$

C $2.9756148 .090335-0.514656$

C 4.2999656 .3766040 .710102

C 3.7393545 .0128061 .006073

N $3.1518981 .674854-1.807769$

H -2.987709 -1.235871 2.448197

H $-2.2847850 .603743 \quad 4.796432$

H $-0.672538-0.6596812 .474782$

H $\quad 0.203859-0.3349705 .418804$

H $1.253724-0.8244924 .142750$

H -0.052618 -4.358132 4.180140

H -1.100762 -3.685882 6.116196

H $-0.309248-2.2871656 .849180$

H -1.854662 -2.094988 5.994591

H $\quad 0.431657-3.8789081 .866010$

H $0.928156-2.2298922 .181967$

H $2.967451-2.9318193 .282028$

H $2.464321-4.6275073 .306276$

H $2.474202-4.6741150 .821274$

H $4.532976-5.3510672 .110802$

H $5.079771-3.6863862 .110222$

H $6.740134-5.5212751 .022362$

H $5.654876-2.572435-0.204604$

H $4.995061-6.7833700 .106222$

H $5.307879-6.065281-1.457443$

H $3.692704-5.938815-0.733047$

H $6.450472-3.832033-1.910826$

H 3.742652 -1.612481-2.504109

H $2.373994-1.211969-1.507980$

H $1.969140-3.337897-1.766759$

H 0.4750393 .4878693 .010140

H 2.8927632 .5916591 .327427

H 1.7095415 .5812913 .451208

H 0.7367045 .6949491 .963648

H $2.101826 \quad 6.7667812 .217419$

H 3.6768550 .0376000 .079969

H $5.207310-0.544331-0.562782$

H $1.8780562 .958422-2.805270$

H $3.2487982 .366262-3.769273$

H $4.8476883 .781321-2.341285$

H $2.1966295 .313519-2.312505$

H $3.9927177 .081127-2.692383$

H $5.1793826 .100204-1.866865$

H 2.7222238 .5676350 .425965

H $2.5879408 .544137-1.420746$

H 4.1555057 .0437691 .568073

H 5.3896726 .2924770 .608092

H 4.3172274 .1994340 .566726

H $2.4441321 .546210-1.090857$

C -5.4844960 .1754052 .664526$

O $-5.636071-0.7675311 .584795$

C $-5.463437-0.0681830 .332836$

C -5.6488221 .4051270 .665800$

C $-5.006971 \quad 1.4858312 .036515$ 
H $-4.429203-0.2664410 .030524$

C $-6.442445-0.635265-0.702190$ H $-5.1775812 .070288-0.063059$ H -6.7139941 .6600120 .729608$ C -5.3962242 .7224882 .826702$ H -3.9190621 .4862801 .899526$ C $-6.3662430 .000678-2.102019$ O $-6.226900-2.042347-0.828632$ H -7.462919 $-0.518867-0.314852$ H -7.006121 -0.576210 -2.782400 O $\quad-6.943160 \quad 1.308479-2.024446$ C $-4.9624130 .123292-2.737412$ C $-4.220998-1.214717-2.897991$ H $-4.3556880 .830419-2.164476$ O $\quad \begin{array}{llll}-5.127580 & 0.713273 & -4.034277\end{array}$ H -4.048427 -1.654469-1.912906 H -4.867008 -1.904509 -3.455007 H -5.0810253 .6297992 .300907$ H -6.479721 2.7799212 .975697 H -4.9192392 .7192103 .812016$ C $-4.584358-0.416873 \quad 3.754831$ H $-6.273433-2.4248330 .064500$ H $-6.899346 \quad 1.698937-2.914150$ H $-5.6469420 .095779-4.577338$ H -4.971382 -1.402158 4.042262 H $-4.6311690 .222042 \quad 4.644723$ H -6.4893870 .3051913 .086777$ C -1.436561 $-0.606248-1.754719$ O $-1.978904-0.172446-3.009439$ C $-2.873351-1.090024-3.645545$ C $-2.165618-2.429570-3.888046$ C - $-1.573322-2.993927-2.600240$ C $-0.702494-1.948053-1.906212$ H -2.241606 -0.702004-1.017190 C $-0.4691760 .492149-1.284632$ H $-3.093257-0.667488-4.633732$ O $-3.069061-3.366646-4.464020$ H -1.356937 -2.276033-4.614426 H -2.361261 -3.356474 -1.930383 O $-0.774863-4.139039-2.912507$ H $0.219363-1.815770-2.486322$ O $-0.341967-2.463547-0.626238$ C $-1.1801361 .766475-0.883750$ H $0.1068380 .167112-0.414455$ H $\quad 0.2200320 .734426-2.102584$ H $-2.590132-4.206680-4.568525$ H - $0.059909-3.855107-3.507306$ H $\quad 0.381425-1.919083-0.274270$ O $-0.2793472 .793335-0.874142$ O $-2.3638261 .850355-0.589068$ C $-0.8081354 .073249-0.514430$ H $-1.5878404 .373206-1.220929$ H -1.2008004 .0478360 .506463$ H $0.0051984 .802099-0.560648$ SCF Energy (B3LYP/6-31G**//MMFF)= -3245.92527064

0500266

MM̄FF Geometry

C -2.274029-1.067015 3.357623

C $-1.083664-0.6575683 .819573$

C $0.182289-0.7010673 .006680$

O $\quad 0.584904 \quad 0.6667912 .743859$

C $1.337808-1.3723003 .769047$

C $1.298427-2.885413 \quad 3.723854$

C $1.921627-3.6190682 .780632$

C $0.557026-3.5608444 .845970$

C $2.694357-3.0943741 .603605$

C $4.201894-3.3012951 .787140$

C $5.004070-2.6002110 .682191$

C $6.485400-2.9687350 .769903$

C $7.328028-2.191254-0.251950$

O $4.841754-1.1898950 .864624$

O $8.710263-2.352876 \quad 0.100951$

C $7.001928-0.688585-0.125949$

C $7.172667-2.755350-1.669117$

O $7.6534960 .060602-1.149917$

C $5.485485-0.378915-0.125748$

C 5.2593921 .1054210 .259127
O $4.958990-0.620810-1.428950$

C -0.0065591 .2960011 .690362$

O $-\begin{array}{llll}0.828281 & 0.826692 & 0.918923\end{array}$

C 0.5132052 .6808111 .646125

C -0.0305433 .5626040 .794148$

C 0.3698514 .9613170 .620662

C 1.5928735 .4324091 .362501

C $3.791087 \quad 1.5130470 .443917$

C $3.1268921 .945570-0.848026$

C $3.1561433 .762369-2.555635$

O $2.3058121 .230998-1.417826$

C $2.8199775 .205737-2.355129$

C $1.6903735 .771216-2.806925$

C $1.3545897 .223167-2.621209$

C $-0.0140457 .435677-2.012981$

C $-1.0373277 .890672-2.755726$

C $-0.1757007 .199043-0.526656$

C $-0.3675225 .743185-0.195991$

N $3.5530643 .189429-1.282930$

H -2.346127 -1.475622 2.353253

H - $1.019533-0.2282264 .816673$

H $0.034141-1.2159202 .048940$

H $1.361461-1.0315434 .813407$

H $2.293414-1.0135133 .363916$

H $1.864821-4.7045352 .842283$

H $0.638989-4.6520554 .798832$

H $\quad 0.960591-3.2440795 .813235$

H $-0.507130-3.3113424 .813374$

H $2.364773-3.6346110 .709013$

H $2.466943-2.0406171 .414953$

H $4.504402-2.9006752 .763775$

H $4.422250-4.3759361 .787408$

H $4.592731-2.892070-0.291089$

H $6.626373-4.0491010 .641207$

H $\quad 6.860413-2.7358181 .776559$

H $8.911251-3.3044720 .096692$

H $7.424867-0.3419020 .827557$

H $7.485767-3.805870-1.699216$

H $7.822565-2.229414-2.377187$

H $6.146892-2.706785-2.040453$

H $8.601040-0.154119-1.107358$

H 5.7559911 .2852011 .221880

H $5.7355841 .755932-0.483806$

H $4.068207-0.240756-1.477752$

H 1.3249542 .9368702 .317196

H -0.8562493 .2338180 .163974$

H 2.4527754 .7922421 .136460

H 1.4163835 .4124972 .443508

H $1.8958726 .449227 \quad 1.105863$

H 3.1960310 .7145360 .899233

H 3.7414882 .3636351 .135846

H $2.3226803 .195256-2.982355$

H $4.0150123 .672327-3.228231$

H $3.5455855 .818660-1.825532$

H $0.9763105 .164638-3.359633$

H $1.4079247 .704080-3.606512$

H $2.1043267 .730429-2.001010$

H -2.014900 8.070712 -2.320799

H $-0.9224428 .088093-3.816309$

H $-1.0623147 .733422-0.159454$

H $0.6665287 .655990-0.000823$

H -1.241106 $5.301962-0.679031$

H $4.3012853 .651811-0.774179$

C -4.5048180 .1109943 .621648$

$\begin{array}{llll}\text { O } & -4.856346 & -0.143170 & 2.247071\end{array}$

C -4.8354241 .1078291 .531593$

C -4.8252732 .1994852 .595681$

C $-3.979571 \quad 1.5475443 .672497$

H -3.8828661 .1439070 .989096$

C $-5.998581 \quad 1.1832010 .538484$

H -4.4018843 .1377622 .224667$

H -5.8316772 .4068832 .977220$

C -4.1085992 .2039405 .035483$

H -2.9309101 .5946003 .354930$

C $-6.068810-0.006032-0.440890$

O

H $-5.9482432 .130677-0.011322$

H $-6.347902-0.914740 \quad 0.105617$ 
O $-7.145810 \quad 0.240642-1.353892$ C $-4.774349-0.267245-1.233966$ C $-4.936649-1.402703-2.261132$ H -3.977686 -0.533930 -0.532596 O $-4.3622440 .925305-1.898825$ H - $-5.304954-2.292548-1.738975$ H $-5.699396-1.124147-2.998421$ H -3.7822613 .2480594 .991835$ H -5.143985 2.190335 5.391902 H -3.4898131 .6871975 .775910$ C $-3.552843-0.9714684 .139999$ H $-7.952417 \quad 1.2553270 .630884$ H $-6.957017 \quad 1.071803-1.821338$ H $-5.0102171 .118751-2.597172$ H -4.048256 -1.947845 4.068568 H -3.345081 -0.7993395 .202684$ H $-5.441576 \quad 0.0385834 .190035$ C $-2.577016-3.122729-1.363331$ O $-2.519707-1.923964-2.141797$ C $-3.630540-1.725972-3.022242$ C $-3.749822-2.908433-3.994674$ C $-3.812212-4.247374-3.260893$ C $-2.658039-4.361755-2.263811$ H $-3.464938-3.082677-0.720028$ C -1.376966 -3.135722 -0.409497 H $-3.389148-0.838586-3.621042$ O $-4.898598-2.749418-4.820477$ H -2.875183 -2.910401-4.658290 H -4.783165 -4.384217 -2.771037 O $-3.700843-5.294584-4.227546$ H -1.728813 -4.492155 -2.830354 O $-2.830303-5.533116-1.470443$ C $-0.051115-3.296108-1.114335$ H -1.337710 -2.204503 0.168086 H -1.468011 $-3.950006 \quad 0.318726$ H $-4.946365-3.525766-5.404389$ H -3.691967 -6.137116 -3.741536 H -3.654151 -5.432612 -0.963753 O $0.373013-2.095378-1.600550$ O $0.530476-4.367591-1.228690$

C $1.607604-2.125795-2.323079$

H $2.435364-2.319674-1.635672$ H $1.575818-2.882194-3.113371$ H $1.755084-1.147452-2.787092$

SCF Energy (B3LYP/6-31G**//MMFF)= -3245.91235506

0500267

MM̄FF Geometry

C $2.3059513 .405907-0.764682$

C $1.3902224 .321088-1.116500$

C $-0.1044204 .088776-1.190556$

O $-0.4032782 .673588-1.214567$

C -0.7785614 .7169690 .043843$

C $-2.294846 \quad 4.7100130 .013732$

C -3.0726774 .2642991 .019751$

C $-2.9408375 .290983-1.218106$

C -2.6455343 .7096752 .349883$

C -2.5894332 .1756332 .413859$

C -3.9450361 .4875512 .175553$

C $-3.858766-0.0004372 .520138$

C $-5.150773-0.7558882 .172954$

$\begin{array}{llll}\text { O } & -4.282736 & 1.642763 & 0.796042\end{array}$

O $-4.861971-2.1623302 .211292$

C $-5.559171-0.419880 \quad 0.722033$

C $-6.248920-0.5103113 .214333$

O $-6.837622-0.9693610 .413167$

C $-5.5491401 .090636 \quad 0.417693$

C $-5.7575921 .409906-1.083439$

O $-6.619476 \quad 1.717799 \quad 1.134805$

C $-0.4244482 .067627-2.431815$

O $-0.3427772 .619951-3.521392$

C $-0.5001050 .594084-2.321521$

C $-0.593127-0.079174-1.165631$

C $-0.628482-1.534850-1.066148$

C $-1.717188-2.055222-0.173269$

C $-4.6479770 .926139-2.024084$

C $-4.916767-0.416999-2.681586$

C $-3.849112-2.031566-4.260759$
O $-5.956654-1.051162-2.531610$

C $-3.123599-3.097582-3.503092$

C $-2.002940-3.687986-3.945479$

C $-1.306167-4.807426-3.227453$

C $0.139130-4.495407-2.914256$

C $1.128703-4.907239-3.724773$

C $0.441650-3.795696-1.610456$

C $0.280624-2.303441-1.698310$

N -3.852088 -0.808209-3.482946

H $1.9823332 .407140-0.483072$

H $1.7227605 .326537-1.365969$

H $-0.4741144 .566909-2.105328$

H -0.4577515 .7627990 .143953$

H $-0.412322 \quad 4.1910570 .932698$

H -4.1532604 .3222170 .891562$

H $-4.0195895 .435155-1.093085$

H -2.507504 $6.269707-1.449073$

H -2.797328 $4.628956-2.077662$

H -1.671491 4.113576 2.645799

H -3.347709 4.0757053 .109802

H -1.8586261 .8181991 .676751$

H -2.212862 1.8976313 .406097

H -4.7033231 .9792802 .796100$

H $-3.604721-0.1408783 .578243$

H -3.033987 -0.451064 1.955155

H $-4.528983-2.3758403 .099821$

H $-4.839131-0.9182850 .063811$

H $-5.918722-0.8455854 .204944$

H -7.148049 -1.093283 2.986189

H -6.5305080 .5412383 .300450$

H $-6.794722 \quad-1.9236140 .596227$

H $-6.7466821 .068226-1.411291$

H $-5.7852302 .505283-1.178012$

H -6.8257722 .5643280 .705330$

H $-0.4561630 .063258-3.269077$

H $-0.6629160 .446537-0.216661$

H -2.673415 -1.589054 -0.430656

H -1.492863 -1.829316 0.872547

H -1.869138 $-3.133680-0.257990$

H $-4.5552351 .652454-2.842429$

H $-3.6806150 .887920-1.512872$

H -3.368881 -1.805405 -5.218150

H $-4.877767-2.350635-4.456801$

H -3.562255 -3.420858 -2.562075

H -1.580559 -3.377654 -4.898683

H $-1.363281-5.698254-3.866974$

H -1.831551 -5.079147 -2.303457

H $2.170010-4.715566-3.487781$

H $0.918358-5.440325-4.646076$

H $1.481253-3.992481-1.316647$

H $-0.155046-4.239603-0.807715$

H $1.037275-1.815086-2.312740$

H $-3.018025-0.229733-3.513665$

C $4.5747262 .678112-1.541863$

O $4.3606871 .337714-1.059023$

C $5.6058790 .618003-1.096079$

C $6.5933811 .511181-1.839721$

C $6.0857082 .894419-1.480563$

H $5.9386300 .516602-0.056959$

C $5.398695-0.771535-1.709551$

H $7.6266011 .337032-1.524091$

H $6.5456331 .361074-2.924343$

C $6.5943563 .987046-2.404462$

H $6.3912423 .122410-0.450441$

C $4.258090-1.583729-1.063221$

O $5.075256-0.605546-3.096703$

H $6.340839-1.331508-1.676268$

H $3.292300-1.127597-1.312985$

O $4.256513-2.886968-1.660766$

C $4.349220-1.7543680 .466899$

C $3.218070-2.6537391 .000826$

H $4.289719-0.7757450 .954752$

O $5.596149-2.3474340 .823553$

H $2.261473-2.2453660 .660437$

H $3.318506-3.655170 \quad 0.565670$

H $7.6872174 .043129-2.368197$

H $6.3012843 .802586-3.443369$

H $6.1970254 .963603-2.110907$ 
C $3.7836963 .669715-0.687320$

H $5.151561-1.476376-3.521693$

H $3.941714-2.789567-2.575205$

H $6.299765-1.7083450 .622312$

H $4.0098754 .695808-0.998376$

H 4.0810293 .5729060 .363126

H $4.2202362 .704197-2.580738$

C $1.940619-0.8249563 .088320$

O $3.175645-1.5402963 .211012$

C $3.217660-2.8015962 .538298$

C $2.106083-3.715496 \quad 3.074827$

C $0.736268-3.0494902 .981441$

C $0.771564-1.6627793 .620767$

H $1.771018-0.5611632 .036894$

C 2.0811820 .4733893 .890828

H $4.174323-3.2628562 .813369$

O $2.072131-4.9508632 .367976$

H $2.305441-3.9520894 .128129$

H $\quad 0.387963-2.996738 \quad 1.944233$

O $-0.223001-3.8413563 .685465$

H $0.849366-1.7678244 .710656$

O $-0.452418-0.9849513 .344761$

C 2.9999881 .4440453 .191189

H $1.116996 \quad 0.9801304 .013284$

H $2.4712810 .283881 \quad 4.898241$

H $2.951302-5.3584052 .449981$

H $-0.206972-4.7310203 .292297$

H $-1.176317-1.5515553 .662377$

O $4.291399 \quad 1.2227093 .567098$

O $2.613042 \quad 2.2864212 .392060$

C 5.2651702 .0757002 .958639

H 5.0507183 .1239113 .187850

H 6.2454991 .8200353 .369075

H 5.2842461 .9126711 .877933

SCF Energy (B3LYP/6-31G*//MMFF) $=-3245.89714021$

0500268

MM̄FF Geometry

C $-0.9008685 .133289-1.231246$

C $-1.8444284 .673968-0.395900$

C $-2.974568 \quad 3.777456-0.833628$

O $-2.8519652 .522428-0.121681$

C $-4.3260724 .410777-0.459131$

C $-5.524353 \quad 3.624474-0.955650$

C $-6.4374973 .033854-0.159979$

C $-5.6827713 .548848-2.453096$

C -6.4904313 .0176341 .341601$

C -5.7911191 .8183292 .000214$

C $-6.3725590 .450815 \quad 1.604409$

C $-5.803034-0.6563662 .494823$

C $-6.288160-2.0501082 .064570$

O

O $-5.464741-3.0258352 .722301$

C $-6.060514-2.2186090 .546260$

C -7.726704 -2.318126 2.522909

$\begin{array}{lllllll}0 & -6.647900 & -3.428417 & 0.074825\end{array}$

C -6.573129-1.026740 -0.287095

C $-6.166309-1.103866-1.779416$

O $-8.005484-1.004978-0.246363$

C -1.918315 $1.638300-0.569835$

O $-1.1929541 .762081-1.543815$

$\begin{array}{llll}C & -1.907112 & 0.488166 & 0.360432\end{array}$

C $-0.875670-0.369095 \quad 0.336247$

C $-0.709607-1.5455421 .193006$

C -1.868179-1.935782 2.071272

C $-4.658516-1.151632-2.050432$

C $-4.106910-2.555967-2.236395$

C $-1.949812-3.769391-2.532347$

O $-4.817416-3.551141-2.348412$

C $-1.544033-4.361177-1.219545$

C $-0.281845-4.712172-0.929990$

C $0.135067-5.347300 \quad 0.365812$

C $1.255455-4.5953591 .048721$

C $2.531621-4.999705 \quad 0.932806$

C $0.884964-3.4241921 .931195$

C $0.458852-2.2183541 .141650$

N -2.719735 -2.560781-2.303395

H $-0.9553214 .887102-2.288805$
H -1.7727864 .8989960 .665854$

H -2.931085 $3.588832-1.913110$

H $-4.3902575 .424515-0.876574$

H $-4.368310 \quad 4.5277760 .629183$

H -7.258679 2.498826 -0.636496

H $-6.6425123 .110576-2.747575$

H -5.634931 $4.550981-2.891806$

H $-4.8935082 .933672-2.895450$

H $-6.0739883 .942887 \quad 1.753844$

H -7.5463943 .0244321 .641334$

H $-4.723807 \quad 1.8515181 .752432$

H -5.8735361 .9435043 .087130$

H $-7.4633810 .496714 \quad 1.703774$

H $-6.049517-0.4742953 .548362$

H $-4.706330-0.6384432 .438428$

H $-5.539649-2.8836403 .681530$

H -4.978564 -2.317368 0.397395

H -7.798359 -2.254406 3.615409

H -8.042052 -3.3344022 .261992$

H -8.448618 -1.613835 2.104507

H $-6.281483-4.1552350 .607202$

H -6.692904 -1.930561 -2.271376

H -6.543972 -0.191555 -2.263790

H $-8.327825-0.494340-1.007515$

H $-2.7382830 .387947 \quad 1.048243$

H $-0.063182-0.185431-0.365992$

H -2.773315 -2.069914 1.469260

H -2.057446 -1.164334 2.825558

H -1.717834 -2.875939 2.605008

H -4.459234 -0.621185 -2.990453

H $-4.100496-0.639374-1.261657$

H -1.087025 -3.492896 -3.146593

H -2.558929 -4.489307 -3.088481

H $-2.327080-4.533904-0.484783$

H $\quad 0.494770-4.563344-1.677149$

H $0.455124-6.3740880 .145234$

H -0.712024 -5.444193 1.056389

H $3.338121-4.480767 \quad 1.440022$

H $2.799354-5.8545330 .320586$

H $1.749293-3.1228422 .538325$

H $0.132126-3.7528432 .652217$

H $1.230581-1.8555680 .463381$

H -2.210077 -1.695592 -2.149947

C $1.5412335 .030860-0.793712$

O $1.418463 \quad 4.032844 \quad 0.240271$

C 2.7077723 .8381480 .860916

C $3.7014754 .618240 \quad 0.012993$

C $2.8434305 .764545-0.488646$

H 2.6271324 .2814531 .861329

C 2.9682622 .3315330 .961912

H 4.5717854 .9516130 .586122

H $4.0586224 .010771-0.828134$

C $3.4401846 .492058-1.680219$

H 2.6906886 .4775250 .332556

C 4.3897351 .9596671 .419452

O $2.0264021 .786973 \quad 1.893952$

H $2.7503821 .864184-0.004985$

H 5.1213882 .2917830 .674067

O 4.6775912 .6909842 .617822

C 4.5863220 .4594051 .717176

C $4.120306-0.4975150 .605161$

H 4.0522050 .1959982 .638807

O $5.9679510 .213771 \quad 1.981113$

H $4.312374-1.5204470 .948259$

H $3.034574-0.4063340 .507749$

H $4.4063836 .934216-1.416097$

H $3.6007165 .814231-2.525348$

H $2.7808297 .298400-2.016040$

C $0.2984505 .921165-0.782468$

H 1.1368682 .0198551 .576712

H 5.6298252 .6056212 .792618

H 6.1962040 .6668882 .810073

H $0.4249196 .774832-1.457123$

H $\quad 0.131708 \quad 6.3201330 .225714$

H $1.6206054 .493542-1.748537$

C $6.649884-1.802609-0.547462$

O $6.198907-0.458598-0.754544$

C $4.779335-0.287830-0.778087$ 
C $4.170072-1.150407-1.895112$

C $4.576916-2.615143-1.759784$

C $6.095757-2.738335-1.630545$

H $6.335477-2.1472000 .444756$

C $8.182243-1.783485-0.578902$

H $4.6061150 .755785-1.063432$

O $2.749597-1.054846-1.901362$

H $4.520289-0.780320-2.867505$

H $4.071102-3.082738-0.908461$

O $4.141704-3.338812-2.913199$

H $6.557026-2.505039-2.598239$

O $6.432977-4.090431-1.326019$

C $8.735983-1.1080600 .652997$

H $8.602385-2.795451-0.603825$

H $8.554212-1.261920-1.469171$

H $2.519517-0.114481-1.993733$

H $4.568325-2.946339-3.693793$

H $6.026721-4.314590-0.471346$

$\begin{array}{llllll}0 & 9.036334 & 0.193282 & 0.378848\end{array}$

O $8.861562-1.6661731 .735538$

$\begin{array}{lllll}\text { C } & 9.556308 & 0.949474 & 1.476698\end{array}$

H 9.7466781 .9679111 .127941

H 8.8269320 .9884712 .291147

H $10.498870 \quad 0.5147381 .822583$

SCF Energy (B3LYP/6-31G**/MMFF) $=-3245.89271471$

0500269

MMFF Geometry

C $1.875891-3.3913150 .821420$

C $0.840994-3.905763 \quad 0.141264$

C $0.377626-3.504658-1.238665$

O $-0.879020-2.805841-1.070352$

C $1.321601-2.564173-2.018623$

C $0.837805-2.252037-3.422984$

C $0.489302-1.024453-3.857365$

C $0.779902-3.422940-4.370553$

C $0.536790 \quad 0.276467-3.104726$

C $-0.7998360 .597848-2.437011$

C $-0.918412 \quad 2.052370-1.958808$

C $0.1296302 .420152-0.905825$

C $-0.0748893 .843109-0.364275$

O $-2.2362602 .196758-1.409838$

O 0.7497223 .9689240 .803131

C $-1.546408 \quad 4.000458 \quad 0.073856$

C $0.427074 \quad 4.898994-1.355544$

O $\begin{array}{llll} & -1.819410 & 5.341023 & 0.472936\end{array}$

C -2.560941 $3.530565-0.991789$

C $-4.0152633 .515087-0.465786$

O $-2.527563 \quad 4.436927-2.100853$

C $-2.033140-3.437066-1.409993$

O $-2.165902-4.584237-1.801852$

C $-3.124688-2.452582-1.235052$

C $-4.392890-2.774455-1.530258$

C $-5.545278-1.873846-1.425651$

C $-5.265007-0.442599-1.049729$

$\begin{array}{llll}\text { C } & -4.236237 & 2.667768 & 0.789341\end{array}$

C $-5.7083902 .408237 \quad 1.067426$

C -7.1721751 .0606442 .581303$

$\begin{array}{lllll}\text { O } & -6.618002 & 2.904481 & 0.409134\end{array}$

C -7.366329 -0.3243512 .046792$

C $-8.455536-0.7238261 .372246$

C $-8.643749-2.1269420 .865635$

C $-9.043773-2.185927-0.594097$

C $-10.219776-2.723656-0.960431$

C $-8.093731-1.627601-1.633185$

C $-6.779903-2.359450-1.673804$

N $-5.876869 \quad 1.551566 \quad 2.146570$

H $2.475320-2.5979960 .385194$

H $0.251229-4.6874060 .617163$

H $0.238001-4.428524-1.812503$

H $2.318268-3.016711-2.095639$

H $1.442384-1.636302-1.450243$

H $0.141619-0.925723-4.885275$

H $0.579400-3.112501-5.401731$

H $1.735663-3.957096-4.372619$

H $-0.010900-4.120396-4.078964$

H $1.3433120 .274249-2.365717$

H $\quad 0.794288 \quad 1.066038-3.822054$
H -1.628267 $0.394040-3.129331$

H $-0.959717-0.075969-1.586494$

H $-0.8283162 .704214-2.835777$

H $1.1450802 .318820-1.303388$

H $0.0643771 .713614-0.066309$

H 0.5752964 .8417951 .195576

H $-1.662666 \quad 3.3871880 .975839$

H $1.4958964 .754173-1.555686$

H $\quad 0.3365415 .908561-0.939974$

H $-0.0959014 .872039-2.313676$

H $-1.907138 \quad 5.877899-0.333060$

H $-4.3616984 .540555-0.282584$

H -4.659686 $3.127918-1.267697$

H $-3.3615354 .352099-2.592078$

H $-2.847891-1.468934-0.872388$

H $-4.609712-3.782164-1.881276$

H $-4.5710660 .016219-1.763275$

H $-4.822739-0.395852-0.049219$

H $-6.1514070 .192568-1.033429$

H -3.7348201 .6992850 .684651$

H -3.8190643 .1780361 .664642$

H -7.9594661 .7463612 .252162$

H -7.158751 1.0368663 .675063

H $-6.572625-1.0426362 .241324$

H $-9.264411-0.018097 \quad 1.198738$

H $-9.414950-2.5987641 .488369$

H -7.735248 -2.7241021 .012040$

H - $10.520066-2.773160-2.001872$

H -10.910925 -3.127303-0.227896

H -7.980524 $-0.554561-1.462573$

H $-8.536630-1.709004-2.634922$

H $-6.876712-3.410613-1.949027$

H $-5.053585 \quad 1.1605042 .595717$

C $1.774101-2.8688433 .276579$

O $2.190764-1.5239542 .981709$

C $2.691591-0.9080494 .180380$

C $2.260347-1.8141865 .327607$

C $2.321529-3.1802434 .670078$

H $3.785907-0.9308034 .104202$

C 2.2211250 .5497204 .271266

H $2.918395-1.7247626 .197234$

H $1.236348-1.6029585 .655609$

C $1.543734-4.2517925 .413693$

H $3.374364-3.4856704 .601270$

C 2.3865931 .3527472 .964125

$\begin{array}{lllll} & 0 & 0.825811 & 0.558974 & 4.599801\end{array}$

H $2.737592 \quad 1.046128 \quad 5.101419$

H 1.6374631 .0273042 .230902

$\begin{array}{lllll}\text { O } & 2.104868 & 2.727531 & 3.251626\end{array}$

C $3.7845501 .273248 \quad 2.327130$

C 3.8737612 .0935001 .026693

H $4.019686 \quad 0.229352 \quad 2.100921$

$\begin{array}{lllll}\text { O } & 4.765446 & 1.734124 & 3.253289\end{array}$

H 3.0597491 .7820450 .363487

H $3.7203873 .156226 \quad 1.249943$

H $1.943610-4.3857896 .424114$

H $\quad 0.484249-3.9893115 .503460$

H $1.609926-5.2126784 .893840$

C $2.286457-3.8278382 .199458$

H $0.587610 \quad 1.4688284 .844779$

H 1.1515962 .8017963 .425826

H 4.5427522 .6524463 .483596

H $1.933573-4.8450282 .405496$

H $3.383003-3.8624462 .214800$

H $0.676329-2.8633153 .282217$

C $4.837912-0.162017-0.804709$

$\begin{array}{llllll}\text { O } & 5.612384 & 0.570086 & 0.149068\end{array}$

C $5.232586 \quad 1.9408450 .307521$

C $5.3153382 .673433-1.040893$

C $4.5304121 .953183-2.136857$

$\begin{array}{llll}\text { C } 4.920971 & 0.476481 & -2.195448\end{array}$

H $3.790093-0.171872-0.477718$

C $5.290439-1.629223-0.774680$

H $5.9863982 .389708 \quad 0.966744$

$\begin{array}{lllll}\text { O } & 4.834693 & 4.008862 & -0.926427\end{array}$

H $6.3656132 .735120-1.354392$

H $3.4497242 .066870-1.998697$

O $4.8349122 .546626-3.401205$ 
H $5.9335950 .392925-2.606334$

O $4.045290-0.213731-3.082651$

C $6.725733-1.852684-1.205056$

H $5.207134-2.0178960 .247340$

H $4.635135-2.220315-1.424269$

H $5.3606454 .450229-0.237855$

H $4.6179373 .492545-3.335601$

H $4.0813230 .242500-3.940737$

O $6.868095-3.144899-1.625075$

O $7.617790-1.017650-1.148757$

C $8.184633-3.512944-2.048199$

H $8.498328-2.893341-2.893623$

H $8.889320-3.418864-1.216431$

H $8.158656-4.557575-2.369722$

SCF Energy (B3LYP/6-31G**//MMFF) $=-3245.91990159$

0500270

MM̄FF Geometry

C -2.041784 -2.363119 -3.045717

C $-1.183490-3.393145-2.979259$

C $0.147004-3.447245-2.262594$

O $0.504402-2.185478-1.658633$

C $0.078151-4.500132-1.138527$

C $1.423936-4.829092-0.519781$

C $1.747826-4.6252970 .772032$

C $2.431070-5.466081-1.444061$

C $0.895883-4.0480721 .866110$

C $1.078965-2.5395172 .087732$

C $2.491063-2.1289362 .534344$

C $2.516107-0.6572562 .953457$

C $3.933269-0.1867803 .318543$

O $3.387264-2.3237741 .438096$

O 3.9196341 .2481543 .374785

C $4.907070-0.5842492 .187384$

C $4.352002-0.6711364 .711876$

O $6.257092-0.3209072 .560595$

C $4.762667-2.0527831 .739117$

C $5.582586-2.3872270 .468790$

O $5.238421-2.9113432 .783021$

C $1.137027-1.271835-2.443492$

O $1.389085-1.369699-3.633767$

C $1.470088-0.107788-1.594600$

C $1.7215511 .077239-2.169482$

C $2.0642352 .315658-1.468343$

C $2.4760692 .205887-0.024225$

C $5.221743-1.575358-0.780634$

C $6.112762-0.365860-1.011492$

C $6.3511261 .605666-2.518085$

O $7.130927-0.142526-0.362682$

C $5.7951872 .809473-1.825844$

C $5.3535163 .895640-2.477810$

C $4.8351755 .128690-1.795379$

C $3.4596505 .528759-2.279133$

C $3.3046456 .495425-3.199492$

C $2.2636534 .869754-1.631108$

C $2.0144153 .479636-2.149191$

N $5.652780 \quad 0.419149-2.060750$

H $-1.814697-1.424245-2.549607$

H $-1.452454-4.313655-3.496086$

H $0.910363-3.732266-2.996322$

H $-0.343511-5.437199-1.526585$

H $-0.623235-4.145268-0.375617$

H $2.745783-4.917372 \quad 1.097947$

H $3.309163-5.840940-0.906889$

H $1.982914-6.317371-1.967020$

H $2.785904-4.744691-2.186182$

H $-0.163472-4.2537281 .682155$

H $1.131467-4.5802442 .796712$

H $\quad 0.819585-2.0138081 .161445$

H $0.359001-2.2278922 .854082$

H $2.789223-2.7706273 .371856$

H $1.826027-0.4774403 .787306$

H $2.148119-0.0384342 .124056$

H 3.2550021 .5158854 .032458

H 4.6867830 .0692401 .335924

H $3.657081-0.2952375 .472492$

H $5.336990-0.2785434 .987575$

H $4.376813-1.7593694 .797774$
H $\quad \begin{array}{lll}6.311038 & 0.617475 & 2.810167\end{array}$

H $6.655812-2.3393570 .688851$

H $5.384452-3.4413910 .225806$

H $5.465244-3.7725772 .394897$

H $1.486325-0.254590-0.521012$

H $1.6454351 .158302-3.253092$

H 3.2453121 .4359450 .094273

H $1.620377 \quad 1.9444100 .605612$

H $2.910046 \quad 3.124792 \quad 0.375724$

H $5.351684-2.219535-1.659893$

H $4.174081-1.259674-0.759205$

H $\quad 6.2266131 .657442-3.604347$

H $7.4183881 .511015-2.293183$

H $5.7802522 .797914-0.738508$

H $5.3955613 .915474-3.564616$

H $5.5489025 .940400-1.988574$

H $4.8166235 .006561-0.705155$

H $2.3199816 .808477-3.530643$

H $4.1598366 .991603-3.646591$

H $1.3569685 .455992-1.834007$

H $2.3763594 .906511-0.543751$

H $1.7154823 .453890-3.198018$

H $4.7687440 .188419-2.505161$

C $-4.588676-2.194133-2.925960$

O $-4.709687-0.796612-2.604108$

C $-5.256125-0.672465-1.270558$

C $-5.663857-2.081462-0.851471$

C $-4.661604-2.939577-1.596019$

H $-4.426603-0.311032-0.653886$

C $-6.4040740 .348797-1.278860$

H $-5.629322-2.2254790 .231650$

H $-6.680313-2.312612-1.193547$

C $-5.072341-4.395182-1.724587$

H $-3.708077-2.885809-1.058940$

C -7.061072 0.6115410 .094583

$\begin{array}{lllll}\text { O } & -5.913692 & 1.572406 & -1.826638\end{array}$

H -7.167575 $-0.010039-1.981020$

H $-7.7934411 .423261-0.002747$

O $-7.802096-0.5498270 .483925$

C -6.1084020 .9669851 .258211$

C -5.3744452 .3050801 .071887$

H $-5.3982520 .152721 \quad 1.436217$

$\begin{array}{llll}\text { O } & -6.897325 & 1.083612 & 2.450960\end{array}$

H $-4.8091892 .290046 \quad 0.139444$

H -6.1237413 .1016290 .986715$

H $-5.177940-4.853501-0.735851$

H $-6.029401-4.498464-2.246711$

H $-4.320817-4.963380-2.282019$

C $-3.348336-2.440882-3.790034$

H $-6.6615952 .191446-1.879527$

H $-8.463939-0.720454-0.207749$

H $-7.3683010 .240687 \quad 2.568454$

H $-3.315163-1.693683-4.592532$

H $-3.448120-3.421934-4.270646$

H -5.471436 - $2.431683-3.535425$

C -2.4276561 .6023551 .349011$

O -3.3962001 .6548552 .403429$

$\begin{array}{llll}C & -4.417692 & 2.644224 & 2.239426\end{array}$

C -3.7788954 .0370002 .143206$

C -2.7374684 .1006311 .029353$

C $-1.733613 \quad 2.957731 \quad 1.171577$

H $-2.916847 \quad 1.3073150 .413671$

C $-1.4113200 .514158 \quad 1.710713$

H $-5.0116532 .630063 \quad 3.161489$

O $-4.787923 \quad 5.018917 \quad 1.935359$

H -3.2917544 .2706663 .099081$

H $-3.205844 \quad 4.0898250 .037980$

O $-2.016140 \quad 5.331393 \quad 1.120905$

H -1.0709583 .1562502 .023881$

O $-0.922502 \quad 2.911329-0.000232$

C $-2.017504-0.8573991 .544731$

H -0.5283600 .5504931 .063269$

H -1.0539550 .6226632 .741968$

H -4.3691805 .8929182 .007266$

H $-2.636510 \quad 6.0508830 .915333$

H $-0.4991703 .781758-0.095588$

O $-2.565551-1.2736782 .720988$

O $-2.016302-1.4755550 .488295$ 
C $-3.177901-2.5661582 .691781$

H $-2.463793-3.3226932 .353020$

H $-3.494983-2.8149953 .707963$

H -4.057957 -2.548312 2.043836

SCF Energy (B3LYP/6-31G**//MMFF) $=-3245.89745897$

05 00271

MM̄MF Geometry

C -4.989801 $1.254909-0.931431$

C $-5.7858520 .413603-1.609867$

C $-5.584201-1.083536-1.684473$

O $-4.184966-1.412309-1.516696$

C $-6.397218-1.777150-0.577172$

C $-6.141808-3.268548-0.481988$

C $-5.417486-3.8632040 .488204$

C $-6.781259-4.098069-1.564964$

C $-4.705447-3.2087161 .641579$

C $-3.194995-3.4506131 .576089$

C -2.410164 -2.691343 2.658711

C $-2.386609-1.1797192 .406505$

C $-1.494746-0.4356913 .412863$

O $-1.070891-3.2061792 .635954$

O -1.2768740 .8896652 .905434$

C $-0.118401-1.1293043 .469983$

C $-2.180498-0.2714174 .773606$

O $0.699328-0.5701534 .494195$

C $-0.210328-2.6602203 .648606$

C $1.163368-3.361373 \quad 3.518111$

O $-0.707441-2.9579224 .958467$

C $-3.404823-1.381448-2.633189$

O $-3.757801-1.110950-3.770111$

C $-2.025627-1.733269-2.228772$

C $-1.065216-1.844925-3.159132$

C $0.336766-2.189739-2.912957$

C $0.733980-2.524068-1.499280$

C $1.910120-3.0443422 .223261$

C $3.035973-4.0171531 .918552$

C $4.772360-4.4350790 .175189$

O $3.295673-4.9992082 .606063$

C $4.903195-4.055612-1.265231$

C $4.362649-4.762613-2.269014$

C $4.433911-4.356637-3.713871$

C $3.081173-3.922322-4.243909$

C $2.339076-4.770836-4.976342$

C $2.676956-2.497247-3.914863$

C $1.196993-2.212699-3.953401$

N $3.692232-3.6579810 .750491$

H $-4.1554940 .851096-0.362449$

H $-6.6409410 .810573-2.151587$

H -5.925921 -1.440714 -2.664013

H $-7.469489-1.612987-0.750973$

H $-6.194706-1.2916070 .382717$

H $-5.315067-4.9474740 .458542$

H -6.611827 -5.170671-1.422556

H -7.863938 -3.935490 -1.578417

H $-6.375861-3.828627-2.545150$

H -4.909059 -2.139059 1.684269

H -5.099424 -3.632301 2.573420

H -2.995319 -4.525832 1.680758

H -2.804404 -3.180899 0.587321

H $-2.858859-2.9177903 .633015$

H -3.395608 -0.7528532 .422723$

H -1.998993 -0.9884001 .395865$

H -2.144094 1.3155752 .793720

H $0.381142-0.9083232 .518074$

H -3.1099090 .3011784 .667305$

H -1.553612 0.3023465 .465089

H -2.433731 -1.2235005 .244411$

H $\quad 0.7429810 .3886924 .338397$

H $1.799685-3.1141904 .377731$

H $0.996408-4.4457153 .578840$

H -0.443761 -3.864205 5.188968

H -1.836367 -1.889283-1.173605

H -1.324842 -1.665506 -4.201610

H $0.096372-3.318282-1.094711$

H $0.647681-1.641483-0.856071$

H $1.758503-2.891180-1.415901$

H $1.218005-3.0755431 .374789$
H $2.340107-2.0378692 .281045$

H $4.560568-5.5013080 .309288$

H $5.692450-4.2015670 .720016$

H $5.442257-3.137438-1.486158$

H $3.812994-5.673428-2.041850$

H $4.819799-5.208751-4.287890$

H $5.158759-3.546980-3.866643$

H $1.377081-4.490535-5.390191$

H $2.683868-5.778330-5.187018$

H $3.175264-1.825311-4.625747$

H $3.076882-2.216150-2.936038$

H $\quad 0.820597-1.976424-4.949128$

H $3.500250-2.7411740 .357481$

C $-3.8904313 .458618-1.329654$

O $-2.833670 \quad 3.191527-0.386492$

C $-2.0641844 .396146-0.185305$

C $-2.5592535 .388440-1.226606$

C $-4.0121964 .979153-1.376364$

H $-2.3123884 .742244 \quad 0.825754$

C $-0.5768204 .040342-0.282265$

H $-2.4359466 .427762-0.907766$

H -2.025079 $5.257530-2.176126$

C $-4.6644715 .511125-2.640361$

H -4.571061 $5.338746-0.501935$

C $0.3711095 .249795-0.223258$

O $\quad-0.264397 \quad 3.1607780 .803070$

H -0.404071 $3.473095-1.203887$

H $\quad 0.2178915 .885227-1.103385$

O $\quad 0.017091 \quad 6.043234 \quad 0.916561$

C $1.8644234 .884940-0.095970$

C $2.3733303 .953851-1.209036$

H $2.046453 \quad 4.4437230 .889533$

O $2.6234826 .098993-0.129162$

H $1.7635783 .047023-1.236104$

H $2.2485014 .453647-2.177670$

H $-4.6555836 .606075-2.645916$

H $-4.1405005 .166269-3.538132$

H $-5.7057815 .182241-2.711071$

C $-5.1674122 .746656-0.878938$

H $-0.8694292 .401256 \quad 0.746432$

H 0.6307496 .7971810 .947222

H $2.4853136 .512672-0.998265$

H $-6.0133103 .055477-1.503154$

H -5.3998743 .0183050 .157845$

H $-3.5585463 .068659-2.301275$

C 3.5465121 .7599090 .528727

$\begin{array}{lllll}\text { O } & 4.138679 & 3.036873 & 0.261649\end{array}$

C $3.8600453 .566281-1.039064$

C $4.3830502 .600587-2.112219$

C $3.8443711 .184931-1.916293$

C $4.0546700 .720004-0.475166$

H $2.454616 \quad 1.8375570 .468674$

C 3.9116121 .3672011 .964892

H $4.4422694 .492216-1.124429$

O $4.0400453 .053469-3.417548$

H $5.4790342 .562458-2.061901$

H $2.787743 \quad 1.112415-2.200177$

O $4.5517550 .291272-2.779606$

H $5.1216240 .523306-0.308903$

O $3.358429-0.506638-0.274251$

C 3.3577732 .3529922 .966434

H 3.5425410 .3691762 .225799

H 5.0012101 .3547382 .091505

H $4.4106973 .946202-3.523408$

H $4.4298540 .612024-3.689773$

H $3.652959-1.118362-0.970248$

O 2.0005762 .2454703 .037441

O 4.0539953 .1294143 .608567

C 1.3620323 .1647643 .929506

H 0.2817023 .0173553 .857735

H 1.6753712 .9688534 .959201

H 1.5921064 .1957103 .643276

SCF Energy (B3LYP/6-31G**//MMFF) $=-3245.92326830$

0500272

MM̄MF Geometry

C -0.132898 $3.210560-1.693441$

C $-1.1031472 .645826-0.959060$ 
C $-2.5271853 .121699-0.900168$ O $-3.3156132 .008059-1.386911$ C -2.978364 3.4469460 .540711 C $-2.045506 \quad 4.344018 \quad 1.327125$ C $-1.247803 \quad 3.9285392 .332668$ C -2.070605 5.7951110 .924962 C -1.112133 2.5322042 .876418 C 0.2781421 .9421902 .602670 C $0.303634 \quad 0.426102 \quad 2.840235$ C $1.723551-0.1254002 .705575$ C $1.750102-1.6560562 .836798$ O $-0.542833-0.1777721 .853882$ O $3.036484-2.1145112 .397347$ C $0.702012-2.2579471 .876624$ C $1.605803-2.1088874 .294475$ O $0.591156-3.6673952 .061213$ C $-0.693536-1.5963311 .985491$ C $-1.600373-2.0969310 .830013$ O $-1.280398-1.9476793 .237195$ C $-4.6029762 .233715-1.751913$ O $-5.184094 \quad 3.304717-1.798725$ C $-5.2001400 .910109-2.035429$ C $-6.5325710 .765544-2.015413$ C -7.264758 -0.489912 -2.199687 C $-6.459916-1.733134-2.477852$ C $-2.925172-1.3384780 .645968$ C $-4.065141-1.8337861 .519355$ C $-6.444132-1.3258832 .076537$ O $-3.972205-2.8112412 .256704$ C $-7.341395-2.1556651 .211673$ C $-8.627118-1.8531150 .973177$ C $-9.545024-2.7037780 .140993$ C $-10.288675-1.913590-0.913979$ C $-11.570006-1.552430-0.730802$ C $-9.574753-1.617260-2.214505$ C $-8.609345-0.470910-2.087418$ N $-5.206001-1.0558221 .368331$ H $-0.3573114 .070951-2.317472$ H $-0.867017 \quad 1.770582-0.358174$ H -2.674964 $3.986233-1.559402$ H -3.180068 2.5091011 .069129 H -3.965659 3.9272130 .504759 H -0.620412 4.6669412 .830161 H -1.362434 $6.399376 \quad 1.501678$ H $-1.8141795 .906850-0.132882$ H -3.069973 $6.213271 \quad 1.083739$ H -1.280169 2.5664693 .960132 H - 1.8842651 .8694112 .481037 H $\quad 0.559457 \quad 2.138058 \quad 1.562602$ H $1.0166822 .440093 \quad 3.242687$ H -0.0976120 .2227583 .839999$ H $2.3935530 .335603 \quad 3.441920$ H 2.1298720 .1451191 .720373 H $3.712405-1.6905132 .953500$ H $1.075500-2.1088450 .853953$ H $2.421490-1.7018134 .904143$ H $1.684487-3.1984814 .379040$ H $0.665411-1.7942634 .751553$ H $1.484025-4.0423551 .969915$ H -1.046767 -1.974302 -0.108493 H $-1.800844-3.1685580 .948158$ H -2.236746 -1.795979 3.187107 H -4.522394 $0.080219-2.201055$ H -7.146897 $1.643138-1.817356$ H $-5.741288-1.917816-1.671883$ H $-5.910407-1.630135-3.420184$ H -7.064656 -2.637958 -2.561263 H -2.791887 -0.2627430 .801064$ H -3.252495 $-1.479973-0.392302$ H $-6.891405-0.359303 \quad 2.328727$ H $-6.224062-1.8642653 .003825$ H $-6.921066-3.0623220 .782522$ H -9.055492 -0.963324 1.429331 H $-10.262085-3.1803130 .822418$ H -9.001552 -3.526670 -0.339981 H -12.119135-1.010158-1.493302 H -12.096793 -1.786098 0.188502 H -10.302810 -1.342820 -2.989669
H -9.109258 -2.534814 -2.583960

H $-9.0971940 .482812-1.880254$

H $-5.206827-0.3043560 .684998$

C $2.3587773 .736266-1.427293$

O $3.6175093 .025522-1.339435$

C $4.1481433 .204968-0.017845$

C $3.612246 \quad 4.5548390 .426812$

C $2.1944424 .506927-0.109766$

H 3.7175232 .4204570 .616945

C $5.6742293 .099879-0.017125$

H 3.6535854 .6849961 .512456

H $4.1639715 .380229-0.037930$

C $1.5907555 .893261-0.277080$

H 1.5754603 .9355930 .590321

C $6.2329451 .770538-0.563151$

$\begin{array}{lllll}\text { O } & 6.205178 & 4.158157 & -0.825780\end{array}$

H 6.0463953 .2767320 .999332

H $6.0766831 .717846-1.647079$

O $7.655792 \quad 1.809618-0.378590$

$\begin{array}{llll}\text { C } & 5.668560 & 0.494803 & 0.096257\end{array}$

C $6.470360-0.761337-0.301518$

H $4.6174430 .382073-0.187249$

O 5.7227910 .6534541 .514078

H $6.567031-0.785845-1.391967$

H $7.480172-0.6740720 .115997$

H 1.5242236 .4022750 .690247

H $2.1972346 .516304-0.943174$

H $0.5841015 .838830-0.698858$

C $1.2731292 .690156-1.706351$

H $7.1744104 .078040-0.807980$

H 7.8271801 .8029880 .578971

H $5.138865-0.0160721 .908229$

H $1.3841241 .866577-0.988868$

H $1.4700342 .242181-2.688722$

H $2.4420084 .421342-2.279511$

C $4.435618-2.606507-1.682687$

O $4.527634-2.291019-0.287879$

C $5.853856-2.0825090 .209825$

C $6.712477-3.330219-0.048678$

C $6.699383-3.733384-1.520295$

C $5.262457-3.854582-2.022877$

H $4.783482-1.752905-2.276912$

C $2.951301-2.851550-2.002376$

H $5.762588-1.9916451 .298635$

O $8.048108-3.140080 \quad 0.405826$

H $6.303295-4.1604460 .541254$

H $7.267277-3.022803-2.131497$

O $7.358280-4.992455-1.674502$

H $4.794876-4.745481-1.584002$

O $5.278490-4.034656-3.437875$

C $2.143070-1.573535-2.043645$

H $2.851620-3.322847-2.986723$

H $2.503394-3.500522-1.240597$

H $8.517524-2.592472-0.245755$

H $6.887656-5.647341-1.131180$

H $5.827718-4.815133-3.625376$

O $0.880663-1.867799-2.476463$

O $2.555875-0.464993-1.735445$

C $-0.011063-0.754421-2.582570$

H $-0.994342-1.131474-2.875960$

H $-0.104025-0.251757-1.616590$

H $0.343659-0.060285-3.349869$

SCF Energy (B3LYP/6-31G**//MMFF) $=-3245.91297461$

05_00273

MM̄FF Geometry

C -2.313582 -2.880783 1.015987

C $-1.093643-3.422292 \quad 1.146622$

C $0.003087-3.2259180 .129372$

O $1.047415-2.4309250 .736710$

C $0.603564-4.585503-0.272279$

C $1.571516-4.500471-1.436977$

C $2.889087-4.772965-1.364579$

C $0.966390-4.111815-2.762660$

C $3.687009-5.207203-0.168435$

C $4.334839-4.063604 \quad 0.627579$

C $5.291952-3.180737-0.190910$

C $6.086240-2.2496010 .728735$ 
C $6.977916-1.278200-0.061379$

O $4.515565-2.398542-1.103214$

O $7.414668-0.2486280 .839835$

C $6.131945-0.590029-1.155387$

C $8.246767-1.965341-0.580196$

O $6.954096 \quad 0.193599-2.015980$

C $5.288471-1.576230-1.988284$

C $4.297874-0.875025-2.950796$

O $6.161015-2.373666-2.798731$

C $0.868689-1.081527 \quad 0.743674$

$\begin{array}{llll}\text { O } & -0.064219 & -0.459125 & 0.260828\end{array}$

C $2.008108-0.4761841 .465647$

C $1.890746 \quad 0.776197 \quad 1.929881$

C $2.915008 \quad 1.5387662 .643639$

C $4.3125100 .979753 \quad 2.654675$

C $3.2684370 .044571-2.285094$

C $3.6741091 .508878-2.253037$

C $2.9552003 .703163-1.316101$

O $4.6359121 .956394-2.871424$

C $3.7774693 .986443-0.098137$

C $3.412902 \quad 4.866438 \quad 0.846774$

C $4.242984 \quad 5.1911162 .056591$

C 3.4715965 .0389833 .349139

C 2.9062286 .1023313 .945336

C 3.4232803 .6684723 .988542

$\begin{array}{llll}\text { C } & 2.559913 & 2.706850 & 3.218651\end{array}$

N $2.8068892 .268758-1.478369$

H $-2.522657-2.2647570 .144270$

H $-0.859015-4.0103732 .030305$

H $-0.381649-2.711393-0.759444$

H $-0.199563-5.279516-0.554794$

H $1.092104-5.0270590 .603083$

H $3.478311-4.680322-2.276662$

H $1.672838-4.228307-3.591804$

H $0.098945-4.742023-2.984437$

H $0.646810-3.065596-2.751220$

H $3.073789-5.8102580 .509613$

H $4.474164-5.887015-0.519415$

H $3.541762-3.4436591 .060562$

H $4.884639-4.5131501 .463925$

H $5.971188-3.833028-0.752184$

H $6.687954-2.8271691 .441465$

H $5.387992-1.6608641 .338376$

H $7.885522-0.6748901 .576282$

H $5.4588720 .108169-0.643290$

H $8.836186-2.3618530 .255467$

H $8.900526-1.252366-1.094380$

H $8.038728-2.795422-1.258216$

H $7.449706 \quad 0.815596-1.456123$

H $4.847656-0.358210-3.746656$

H $3.728575-1.666580-3.459618$

H $5.647074-2.739271-3.537787$

H $2.901541-1.0736971 .599290$

H $0.947518 \quad 1.2982491 .780955$

H $4.649136 \quad 0.777447 \quad 1.632209$

H 4.3502750 .0478473 .228963

H 5.0522001 .6547873 .088910

H $2.3377470 .000568-2.865459$

H $3.038227-0.294494-1.271730$

H $1.9489344 .128006-1.244776$

H $3.4499434 .119224-2.199554$

H $4.7261353 .463549-0.000722$

H 2.4770525 .4091530 .732002

H $4.593166 \quad 6.226398 \quad 1.951958$

H 5.1500144 .5751772 .098588

H 2.3732556 .0075624 .885646

H 2.9600927 .0917513 .503083

H 3.0058843 .7379115 .001845

H $4.446873 \quad 3.3109754 .126751$

H 1.5165163 .0196903 .150775

H $2.0647681 .806279-0.961240$

C $-4.751273-3.3735791 .375821$

O $-5.151731-2.3090110 .488289$

C $-6.521998-1.9636540 .753011$

C $-7.089483-3.1272871 .555352$

C -5.886799 -3.5230692 .390206$

H $-6.500556-1.0741281 .394473$

C $-7.254621-1.633344-0.552692$
H $-7.955174-2.8353532 .157174$

H $-7.385882-3.9646930 .914756$

C $-5.993974-4.9124252 .994575$

H $-5.767416-2.7905113 .200026$

C $-6.541721-0.583186-1.430422$

O $-7.361611-2.818177-1.353367$

H $-8.279812-1.308982-0.337504$

H $-5.622315-1.016962-1.841860$

O $-7.387463-0.324064-2.560632$

C $-6.2065540 .754342-0.739726$

C $-5.575924 \quad 1.757997-1.724754$

H $-5.5151060 .583614 \quad 0.090029$

O $-7.394856 \quad 1.336967-0.207115$

H $-4.7087311 .284512-2.197481$

H $-6.2994771 .978868-2.518755$

H $-6.857322-4.9745243 .665082$

H $-6.113372-5.6783032 .221000$

H -5.097967 -5.1556923 .574165$

C $-3.413780-3.0198422 .029908$

H $-8.089441-3.352618-0.993888$

H $-8.1879070 .119563-2.231336$

H $-7.6985380 .776973 \quad 0.526500$

H -3.152708 -3.7752732 .779683$

H $-3.507617-2.0588522 .548260$

H $-4.651130-4.2784940 .762774$

C $-2.9155952 .512487-0.381883$

$\begin{array}{lllll}\text { O } & -4.234442 & 2.920467 & 0.005757\end{array}$

C $-5.1670303 .095067-1.064681$

C $-4.6343904 .141590-2.051717$

C $-3.2455303 .773605-2.561079$

C $-2.3030503 .497686-1.390837$

H $-2.9550101 .505907-0.815717$

C -2.070039 2.4604850 .901894

H $-6.0738513 .516799-0.613630$

O $-5.534234 \quad 4.290582-3.144749$

H $-4.5808355 .114457-1.545707$

H $-3.2900832 .923102-3.251393$

O $-2.738674 \quad 4.876342-3.317365$

H $-2.0600624 .449924-0.901587$

O $-1.0916652 .967485-1.928335$

C $-2.446934 \quad 1.3104251 .810571$

H $-1.0107632 .335167 \quad 0.658411$

H -2.203932 3.3881161 .471196

H $-5.1450994 .940137-3.755162$

H $-1.840568 \quad 4.639476-3.606235$

H $-0.4118923 .009878-1.235710$

$\begin{array}{llll}\text { O } & -1.653076 & 1.353369 & 2.921997\end{array}$

$\begin{array}{llll}0 & -3.310298 & 0.477202 & 1.577945\end{array}$

C -1.8535120 .3010243 .870310$

H -1.504890 -0.6468243 .450776$

H -1.2611420 .5290194 .760367$

H -2.906978 $0.234053 \quad 4.158785$

SCF Energy (B3LYP/6-31G**//MMFF) $=-3245.90674090$

05_00274

MM̄FF Geometry

C 0.2044625 .3979120 .455409

C $1.0730854 .826869-0.391754$

C 2.2986854 .0730830 .059659

O $2.2272022 .731237-0.477136$

C $3.5627604 .744845-0.506833$

C $4.853868 \quad 4.1842110 .056989$

C $5.770763 \quad 3.497684-0.652568$

C 5.1056644 .4698531 .516226

C $5.739828 \quad 3.125661-2.108058$

C $5.1145491 .755461-2.416564$

C $5.776536 \quad 0.572723-1.690323$

C $5.257719-0.762015-2.233276$

C $5.828433-1.961627-1.458253$

O $5.4676930 .668009-0.296096$

O $5.046902-3.118153-1.797225$

C $5.640264-1.7212010 .056076$

C $7.269548-2.276218-1.876491$

O $6.296185-2.7299920 .818511$

C $6.104992-0.3252200 .518759$

C $5.754249-0.0076081 .994452$

O $7.531601-0.241507 \quad 0.408627$

C 1.3768591 .8562300 .125180 
$\begin{array}{lllll}\text { O } & 0.700392 & 2.048313 & 1.122736\end{array}$

C $1.3741980 .618421-0.680642$

C $0.337138-0.224938-0.590920$

C $0.207312-1.438519-1.385569$

C $-1.084245-1.523972-2.148121$

C $4.270514-0.1198112 .360774$

C $3.888926-1.5012552 .865332$

C $1.983839-3.0969882 .942579$

O $4.687515-2.2517003 .421465$

C $2.171808-3.9667371 .738995$

C $1.162726-4.553398 \quad 1.079203$

C $1.350625-5.381173-0.159703$

C $0.572210-4.840921-1.340419$

C $-0.577538-5.420447-1.725512$

C $1.162478-3.689097-2.125772$

C $1.161812-2.389400-1.370055$

N $2.547764-1.7897892 .656182$

H 0.3912165 .3491641 .525441

H $0.882283 \quad 4.867057-1.461631$

H $2.348427 \quad 4.022037 \quad 1.153740$

H $3.5435755 .821278-0.289612$

H $3.5507674 .656932-1.598834$

H $6.6637823 .154242-0.130604$

H $6.113195 \quad 4.175127 \quad 1.829496$

H 5.0032965 .5412791 .717702

H 4.3961513 .9251462 .146258

H $5.2232793 .893173-2.694203$

H $6.7737053 .134370-2.476797$

H $4.0499091 .793001-2.163272$

H $5.1804681 .596727-3.500116$

H $6.8610680 .645872-1.832845$

H $5.477379-0.858311-3.303839$

H $4.162845-0.788402-2.150716$

H $5.089482-3.236943-2.761503$

H $4.566588-1.8229680 .255659$

H $7.313849-2.515586-2.945833$

H $7.647483-3.163087-1.356156$

H $7.959018-1.448990-1.696019$

H $5.957576-3.5893780 .514281$

H $6.379316-0.6106462 .664069$

H 6.0491221 .0350922 .181393

H 7.8430390 .4776310 .983124

H $2.1986290 .443723-1.361805$

H $-0.504127-0.000887 \quad 0.061127$

H -1.111694 -0.760226 -2.933295

H $-1.936916-1.357431-1.482594$

H -1.239414 -2.493294 -2.626680

H 4.0550710 .5715043 .184969

H 3.6383850 .1735601 .517198

H $0.928119-2.9517493 .191723$

H $2.494179-3.5375363 .804913$

H $3.189946-4.1060831 .382223$

H $0.146383-4.4282521 .442936$

H $1.028197-6.4048220 .070791$

H $2.410335-5.459584-0.433443$

H - $1.131306-5.063234-2.587215$

H $-0.994915-6.262786-1.183568$

H $0.650118-3.567572-3.087900$

H $2.197888-3.934637-2.394772$

H $2.049913-2.224414-0.760187$

H $1.978523-1.1403932 .121112$

C -2.289058 5.3331420 .520086

O $-2.3887584 .064438-0.159497$

C $-3.774020 \quad 3.814997-0.485053$

C $-4.573475 \quad 4.885774 \quad 0.242875$

C $-3.605176 \quad 6.051430 \quad 0.234054$

H -3.852943 $3.950017-1.571049$

C $-4.1214602 .371475-0.099056$

H -5.522011 5.112487 -0.253044

H -4.791209 4.5779501 .273362

C -3.9528967 .1354791 .239230$

H -3.585954 $6.486086-0.774394$

C $-5.6183682 .035256-0.211258$

O $-3.3855581 .493870-0.958305$

H -3.770532 2.1768550 .921055

H -6.1859962 .6354070 .509955$

O $-6.0736352 .430322-1.511864$

C $-5.9684820 .545770-0.024797$
C $-5.401359-0.056893 \quad 1.271825$

H $-5.626349-0.017018-0.900077$

O

H $-4.308066-0.0372891 .222080$

H -5.692496 0.5772372 .118823

H -4.9392717 .5583691 .022356$

H -3.9730176 .7433482 .261625$

H -3.222239 7.9494001 .205092

C $-1.057054 \quad 6.089408 \quad 0.020879$

H $-2.4445581 .724712-0.871290$

H $-7.0213752 .217611-1.563719$

H -7.631283 $-0.482162-0.129755$

H -1.044538 7.1079720 .423982

H $-1.0870626 .168231-1.072874$

H $-2.1972605 .114051 \quad 1.592492$

C $-4.477289-2.652442-0.020762$

O $-5.806401-2.3404590 .410724$

C $-5.877442-1.4982691 .563607$

C $-5.168045-2.1704402 .747964$

C $-3.743000-2.5961152 .396620$

C $-3.723323-3.3909691 .089907$

H $-3.949690-1.727323-0.280050$

C $-4.589878-3.507023-1.287027$

H $-6.940669-1.4288441 .826315$

O $-5.154679-1.2949353 .870566$

H $-5.738644-3.0612323 .041717$

H -3.075507 -1.727859 2.347038

O $-3.246091-3.420784 \quad 3.452982$

H $-4.174714-4.3757751 .263200$

O $-2.371374-3.6147630 .700140$

C $-5.033576-2.663435-2.458949$

H -3.627198 -3.953222 -1.560763

H $-5.301236-4.331558-1.158017$

H $-4.683259-1.7505894 .588963$

H -2.356211 $-3.716708 \quad 3.194743$

H $-1.953171-2.7492860 .553770$

O $-6.393627-2.568885-2.480249$

O $-4.260648-2.129164-3.243681$

C $-6.936607-1.746331-3.517441$

H $-6.573264-0.719133-3.415766$

H $-6.676726-2.153837-4.499111$

H -8.024947 -1.744175 -3.414494

SCF Energy (B3LYP/6-31G**//MMFF)= -3245.90557996

05_00275

MM̄FF Geometry

C -2.168034 -1.616004 -2.149442

C $-1.332009-2.304478-2.941926$

C $0.142840-2.532888-2.696351$

O $0.678156-1.587229-1.743733$

C $0.346961-3.950680-2.131661$

C $1.801569-4.335883-1.947291$

C $2.378295-4.602355-0.758845$

C $2.614149-4.443497-3.212236$

C $1.746137-4.5834760 .604837$

C $1.881932-3.2486931 .353871$

C $3.330893-2.8609011 .691563$

C $3.363051-1.6518002 .629038$

C $4.799278-1.1849232 .916921$

$\begin{array}{lllll}\text { O } & 4.009344 & -2.535393 & 0.476154\end{array}$

$\begin{array}{lllll}0 & 4.726738 & 0.117785 & 3.516981\end{array}$

C $5.557164-1.0240861 .580799$

C $5.499430-2.0924583 .935490$

O $6.938816-0.7582361 .807228$

C $5.402249-2.2335860 .636337$

C $5.981860-1.982283-0.777435$

O $6.109649-3.3520231 .185780$

C $1.029700-0.360222-2.216740$

$\begin{array}{lllll}\text { O } & 0.945100 & 0.038407 & -3.367306\end{array}$

C $1.5133680 .439286-1.070700$

C $1.5525321 .775795-1.173122$

C $1.9598832 .709306-0.121913$

C 2.6213002 .1357041 .102479

C $5.356297-0.813783-1.547739$

C $6.1300160 .489351-1.431126$

C $5.9846752 .893998-2.071130$

O $7.244763 \quad 0.570155-0.922892$

C $5.5214603 .683494-0.887853$ 
C $4.9292394 .883117-0.989529$

C 4.5015525 .7092200 .189470

C 3.0489726 .1231520 .115850

C $2.6989767 .340505-0.332572$

C 2.0119485 .1585900 .644184

C $1.7328214 .026760-0.305321$

N $5.4398541 .551027-2.001708$

H -1.796295 -1.164914 -1.235831

H -1.730692 -2.767463 -3.842412

H $0.673144-2.426788-3.650432$

H $-0.116842-4.689103-2.799569$

H $-0.192891-4.025229-1.181334$

H $3.432681-4.877091-0.754553$

H $3.610319-4.862089-3.031829$

H $2.111605-5.097307-3.932506$

H $2.751358-3.458404-3.668350$

H $0.685663-4.8499120 .544406$

H $2.204923-5.3813681 .202841$

H $1.417575-2.4571790 .754444$

H $\quad 1.306637-3.3292842 .284554$

H $3.817076-3.7228542 .163533$

H $2.836736-1.8708233 .566402$

H $2.814890-0.8199522 .166640$

H 4.1869110 .0469514 .322789

H $5.145699-0.1355521 .088882$

H $4.944980-2.1048994 .881514$

H $6.498509-1.7151304 .180039$

H $5.593690-3.125843 \quad 3.595674$

H $\quad 6.9929790 .0236372 .383155$

H $7.074056-1.897596-0.727773$

H $5.788818-2.888200-1.370403$

H $6.300602-3.9789910 .468525$

H $1.789038-0.089265-0.165808$

H $1.2208372 .236146-2.103093$

H $3.439348 \quad 1.4665960 .816685$

H $1.899828 \quad 1.5725081 .702661$

H 3.0682652 .8920181 .751074

H $5.350835-1.067995-2.615584$

H $4.317306-0.652098-1.245400$

H $5.6509253 .331041-3.017572$

H $7.0783552 .844734-2.075927$

H $5.706743 \quad 3.2623770 .097534$

H $4.7710785 .314262-1.975637$

H $5.141510 \quad 6.6009120 .220192$

H $4.6835495 .183917 \quad 1.135284$

H $1.6613097 .656143-0.361639$

H $3.4427038 .047052-0.685965$

H 1.0600105 .6821890 .806548

H 2.3113684 .8174881 .639260

H $1.2528334 .346242-1.231509$

H $4.4979531 .402417-2.352122$

C -4.559534 -2.147731-1.462075

O $-4.445172-1.604067-0.133669$

C $-4.767999-2.6433720 .811304$

C $-5.110693-3.887752-0.013244$

C $-4.344961-3.648053-1.297849$

H -3.857838 -2.838746 1.390742

C $-5.914113-2.2261151 .747208$

H -4.815976 -4.810792 0.495959

H -6.187056 -3.952431 -0.214363

C $-4.836553-4.480962-2.467600$

H -3.288170 -3.875244 -1.109634

C $-5.561896-1.1700512 .811726$

O $-7.038898-1.8114570 .977900$

H $-6.222008-3.1326742 .285978$

H $-6.448962-0.9911683 .433770$

O $-4.567177-1.7283823 .682701$

C $-5.032188 \quad 0.200452 \quad 2.353180$

C -5.9302720 .9443051 .353314$

H -4.0233220 .0901451 .945232$

O

H -5.8930940 .4331300 .387478$

H $-6.9715710 .893116 \quad 1.696536$

H -4.736971 -5.549061 -2.247891

H -5.890192 -4.280243 -2.689008

H $-4.255712-4.267041-3.370136$

C -3.629177 -1.427653 -2.439933

H $-7.762454-1.6129991 .596325$
H $-4.936747-2.5409164 .068407$

H -4.3062350 .5535884 .137246$

H $-3.833991-0.352063-2.398520$

H -3.859171 - $1.752939-3.461717$

H $-5.592231-1.950944-1.782063$

C $-3.5570172 .192529-0.134361$

O -4.1623932 .6632601 .073106$

C $-5.5706232 .437608 \quad 1.165744$

C $-6.2937273 .121045-0.004046$

C $-5.6891582 .737984-1.356213$

C $-4.1689582 .910219-1.341165$

H $-3.7100331 .111617-0.228037$

C $-2.0516582 .456255-0.027739$

H -5.8963542 .9474442 .081786$

O

H -6.2138834 .2085210 .122305$

H $-5.9611131 .712456-1.631073$

O $-6.247171 \quad 3.572230-2.374255$

H -3.919786 $3.978571-1.312447$

O $-3.6197392 .374894-2.541576$

C -1.4381851 .6963351 .125997$

H -1.522259 2.176901 -0.944767

H $-1.8607803 .522107 \quad 0.148143$

H -8.092631 $3.228789-0.748212$

H $-6.0201414 .494363-2.165235$

H $-4.0579972 .821102-3.286411$

$\begin{array}{lllll}0 & -1.434550 & 0.360601 & 0.859316\end{array}$

O -1.0141542 .2298402 .143091$

C $-0.915368-0.4800111 .894074$

H $-1.485864-0.3435782 .817759$

H $-1.018800-1.5188881 .569845$

H $0.144262-0.2661452 .058958$

SCF Energy $\left(B 3 L Y P / 6-31 G^{* *} / / M M F F\right)=-3245.90677528$

05 00276

MM̄FF Geometry

C $-0.1665403 .796770 \quad 0.465615$

C 0.3312223 .5907041 .695389

C -0.1280022 .5471232 .686939$

O -1.3019141 .8677602 .193764$

C 0.9902211 .5007152 .861648

C 0.6446060 .3696693 .810249

C $0.528674-0.9219133 .439620$

C $0.448693 \quad 0.762620 \quad 5.250820$

C $0.746449-1.4994582 .067125$

C $-0.545043-1.5198021 .252467$

C $-0.377789-2.022903-0.189027$

C $0.446956-1.059064-1.050138$

C $0.468994-1.469051-2.531076$

O $-1.706498-2.138216-0.720777$

O $0.972230-0.343791-3.266938$

C $-0.976093-1.732537-3.001825$

C $1.451142-2.616888-2.782153$

O $-0.996273-2.238609-4.334102$

C $-1.773498-2.654606-2.056679$

C $-3.269148-2.755788-2.441201$

O $-1.231873-3.978706-2.122343$

C -2.4666801 .9827082 .884605$

O -2.6799182 .6564843 .878455$

C -3.4486551 .0999772 .215207$

C -4.6685280 .9081512 .739103$

C -5.7016670 .0253052 .187630$

C $-5.328084-0.7933310 .979901$

C $-3.989944-1.410946-2.559422$

C $-5.504842-1.543596-2.555525$

C $-7.556134-0.105547-2.597322$

O $-6.089900-2.619040-2.469542$

C $-7.8962220 .582112-1.311486$

C $-8.8617730 .176722-0.472080$

C $-9.195798 \quad 0.896816 \quad 0.805381$

C $-9.306380-0.0246472 .002837$

C -10.452922 -0.1039262 .699803$

C $-8.104747-0.8563682 .400747$

C $-6.913013-0.0177392 .781328$

N $-6.118198-0.301899-2.650822$

H $-0.995847 \quad 3.196356 \quad 0.102271$

H 1.1651244 .2071592 .026839

H -0.3318403 .0481783 .640149$ 
H 1.9039841 .9891883 .225318 H 1.2370371 .1023431 .871888 H $\quad 0.262247-1.6556934 .198899$ H $0.310621-0.1066525 .902728$ H 1.3242301 .3093845 .616209 H -0.4328811 .4002635 .363239$ H $1.540782-0.9590311 .546789$ H $1.117466-2.5245192 .185980$ H -1.288185 -2.146952 1.764116 H -0.988039-0.519132 1.220798 H $0.082716-3.017338-0.162804$ H $1.472784-0.966153-0.675917$ H $0.013081-0.051345-0.978332$ H $0.928702-0.566437-4.212915$ H -1.464634 -0.751467 -3.044260 H $2.467339-2.311129-2.508563$ H $1.497717-2.877069-3.845289$ H $1.207788-3.517736-2.215916$ H $-0.748272-3.178150-4.297772$ H $-3.381807-3.314477-3.379466$ H -3.772895 -3.362988 -1.676264 H -1.909620 -4.602857 -1.813331 H -3.1274320 .6087341 .303504$ H $-4.934604 \quad 1.4339073 .654792$ H $-4.468220-1.4366001 .198611$ H $-5.070062-0.131800 \quad 0.147258$ H $-6.122581-1.4503530 .623613$ H $-3.709754-0.757834-1.725720$ H $-3.701144-0.916408-3.494014$ H -8.072833-1.064224-2.705355 H $-7.8239780 .537305-3.441286$ H -7.324198 $1.473998-1.064360$ H $-9.458227-0.696377-0.725812$ H $-10.1456861 .423037 \quad 0.644913$ H -8.453955 1.6751581 .023287 H - $10.549349-0.7590183 .559327$ H -11.3231310 .4841972 .428041$ H -7.889757 -1.566341 1.598703 H -8.347277 -1.480583 3.271262 H $-7.082700 \quad 0.609138 \quad 3.657728$ H $-5.5354940 .528388-2.712000$ C $0.8931094 .187491-1.780645$ O $1.9990743 .306096-1.490879$ C $3.1223983 .689453-2.301265$ C $2.5302454 .470349-3.465887$ C $1.4207625 .232450-2.769693$ H $3.731148 \quad 4.367227-1.689796$ C $3.9637972 .471349-2.699210$ H $3.2642795 .122848-3.947836$ H $2.107143 \quad 3.807154-4.229020$ C $0.3615715 .769661-3.716731$ H $1.8670416 .075465-2.225043$ C $4.3552761 .534236-1.537170$ O $3.2432651 .680813-3.652972$ H $4.8646472 .812859-3.222596$ H $3.4808560 .950160-1.228200$ O $5.3140130 .599409-2.045787$ C $4.9661372 .237663-0.315717$ C $5.5366301 .317464 \quad 0.783516$ H 4.2070292 .8793730 .145284 O $6.0236473 .102073-0.742264$ H 6.4453510 .8336430 .411640 H 5.8817781 .9766851 .590106 H $0.8040836 .472953-4.429732$ H $-0.1110774 .964051-4.288622$ $\mathrm{H}-0.4232486 .296251-3.164346$ C $0.380476 \quad 4.824856-0.484712$ H $2.4060051 .410145-3.237686$ H $4.9002610 .141003-2.797331$ H $6.6848832 .553064-1.197786$ H $-0.4160775 .542678-0.711540$ H $1.1943875 .381272-0.003030$ H $0.1112463 .570617-2.241561$ C $5.440574-1.732627 \quad 0.375346$ O $4.336949-0.824520 \quad 0.457999$ C $4.5268740 .290187 \quad 1.338252$ C $4.845245-0.2193872 .752179$ C $6.047291-1.1586522 .756413$
C $5.832647-2.2877821 .749851$

H $6.301757-1.202970-0.048565$

C $5.102032-2.827021-0.648928$

H 3.5607700 .8036751 .397678

$\begin{array}{lllll}\text { O } & 5.088653 & 0.861619 & 3.645784\end{array}$

H $3.978201-0.7673273 .143819$

H $6.980796-0.6206432 .553959$

O $6.187643-1.7396154 .054641$

H $5.060841-2.9640922 .135019$

O $7.033824-3.0454661 .623386$

C $3.950574-3.725181-0.248608$

H $4.823681-2.365457-1.603347$

H $5.989343-3.449720-0.812627$

H $4.302018 \quad 1.433126 \quad 3.633720$

H $6.274583-1.0082934 .690058$

H $7.276699-3.3544092 .513059$

O $4.064536-4.918633-0.902898$

O $3.045973-3.4127710 .512533$

C $3.036973-5.874598-0.622070$

H $2.057523-5.476039-0.902152$

H $3.234093-6.770113-1.217286$

H $3.054292-6.1462310 .437736$

SCF Energy (B3LYP/6-31G**//MMFF)= -3245.93640322

$05 \quad 00277$

MM̄FF Geometry

C $1.4376325 .634662-0.020427$

C $1.7812574 .662157-0.878046$

C $2.9827543 .770044-0.694473$

O $2.5254022 .401702-0.586310$

C $3.8945263 .874581-1.930766$

C $5.1769603 .073381-1.819849$

C $5.5082362 .044158-2.624201$

C $6.1332593 .513671-0.741010$

C $4.7224201 .470376-3.770141$

C $3.7965630 .303172-3.392672$

C $4.532148-0.935428-2.855085$

C $3.572116-2.117899-2.714445$

C $4.259572-3.345753-2.095519$

O $5.076669-0.617232-1.573121$

O $3.232852-4.272029-1.706060$

C $5.000357-2.922243-0.806696$

C $5.137883-4.078826-3.117294$

O $5.778389-4.012480-0.318449$

C $5.870173-1.658513-0.986711$

C $6.435072-1.0939320 .339935$

O $6.993450-1.977879-1.817047$

$\begin{array}{llll}\text { C } & 2.057679 & 1.994290 & 0.625574\end{array}$

O 1.9914322 .6554341 .649709

C $1.610804 \quad 0.590574 \quad 0.486307$

$\begin{array}{llll}\text { C } & 0.823734 & 0.053475 & 1.430589\end{array}$

C $0.278359-1.305688 \quad 1.432010$

C $0.793647-2.261978 \quad 0.389937$

C $5.386780-0.7033821 .387804$

C $5.109023-1.7885982 .414022$

C $3.602738-2.3092324 .328475$

O $5.779049-2.8131672 .510507$

C $2.513454-3.2147403 .847609$

C $1.339374-3.3517544 .481947$

C $0.251818-4.2875954 .039310$

C $-1.079885-3.5920853 .870872$

C $-2.004597-3.623537 \quad 4.845407$

C $-1.370532-2.9352542 .540823$

C $-0.642137-1.630800 \quad 2.363895$

N $4.041323-1.4588013 .238148$

H 2.0468765 .8124690 .862179

H $1.1573414 .476949-1.749549$

H 3.5412824 .0435880 .208959

H $4.1662244 .924691-2.103136$

H $3.3236283 .561914-2.811798$

H $6.4637391 .549720-2.451650$

H $7.1007533 .004741-0.810632$

H $6.3249544 .588889-0.818236$

H 5.7224883 .3001210 .250422

H $4.1304862 .249493-4.261908$

H $5.4348421 .131968-4.533464$

H $3.0697580 .653151-2.650135$

H $3.2316780 .022770-4.290274$ 
H $\quad 5.344304-1.182430-3.548818$ H $3.127056-2.376222-3.683372$ H $2.732238-1.828774-2.068307$ H $2.714563-4.495684-2.498086$ H $4.226310-2.726786-0.055203$ H $4.536512-4.406357-3.973931$ H $5.566535-4.991225-2.688197$ H $5.952149-3.462245-3.503357$ H $6.117569-3.7685070 .558729$ H $7.189184-1.7743380 .752797$ H $6.985393-0.1750150 .089896$ H $7.688867-1.317063-1.663026$ H $1.9223040 .046916-0.397770$ H $\quad 0.5263090 .6750222 .274524$ H $1.887997-2.2982960 .413206$ H $0.472924-1.950853-0.610073$ H $\quad 0.456230-3.2901770 .534042$ H 5.7662990 .1582961 .952092 H $4.449152-0.3968360 .914351$ H $3.269394-1.6528665 .138410$ H $4.447012-2.9067654 .687282$ H $2.708497-3.8033912 .954242$ H $1.158735-2.7824635 .391234$ H $0.165443-5.0790164 .795483$ H $0.517149-4.799633 \quad 3.106012$ H $-2.973457-3.1510604 .721713$ H -1.810710 -4.118344 5.791345 H -2.443335 -2.717475 2.460869 H -1.171278 $-3.650571 \quad 1.738511$ H $-0.948922-0.8640853 .077008$ H $3.506850-0.6158503 .048871$ C $-0.892064 \quad 6.0808240 .766628$ O -1.3904854 .7873150 .367967$ C $-2.827173 \quad 4.819193 \quad 0.355050$ C $-3.2167406 .068393 \quad 1.133150$ C $-2.099817 \quad 7.0173570 .743633$ H $-3.1308274 .936503-0.693166$ C -3.4132343 .5121810 .905732$ H $-4.210043 \quad 6.4367690 .859832$ H -3.204914 5.8895722 .214805 C $-1.968896 \quad 8.2155571 .667388$ H $-2.2814267 .372332-0.279838$ C $-3.0293522 .272393 \quad 0.067713$ O -2.9948283 .3378402 .258406$ H -4.5056353 .6038210 .917393$ H $-3.2713332 .472483-0.983703$ O C -3.7728120 .9878520 .491158$ C $-3.404824-0.204144-0.412140$ H $-4.852271 \quad 1.1700820 .440590$ O -3.4557240 .6329001 .835065$ H -3.538500 $0.091662-1.458128$ H $-2.343688-0.444729-0.278043$ H -2.885491 8.8144401 .652664 H -1.786066 7.9068192 .702036 H -1.1397728 .8589641 .356372$ C $0.2227456 .503356-0.190814$ H -2.026733 3.2414472 .252516 H -1.169606 2.818091-0.202979 H $-3.743301 \quad 1.3620892 .409670$ H $0.5154587 .543069-0.006167$ H -0.134296 $6.445615-1.226900$ H $-0.5055635 .972521 \quad 1.788417$ C $-6.164270-0.995880-1.469067$ O $-5.642841-1.222356-0.157033$ C $-4.234177-1.471169-0.102709$ C $-3.876651-2.685165-0.971748$ C $-4.386022-2.531683-2.403294$ C $-5.873895-2.177278-2.403848$ H $-5.699465-0.090370-1.878768$ C $-7.660410-0.669117-1.349093$ H -4.016926 -1.745351 0.936175 O $-2.467765-2.888610-0.973359$ H -4.329672 -3.582328 -0.529871 H $-3.794954-1.790898-2.954229$ O $-4.201310-3.776041-3.081999$ H -6.440377 -3.064766 -2.100498 O $-6.284140-1.862692-3.732729$
C $-8.506308-1.799329-0.798171$

H $-7.7971580 .181585-0.670758$

H $-8.046807-0.390452-2.336360$

H -2.285136 -3.656486 -1.541486

H $-4.573682-3.676507-3.974962$

H $-5.784717-1.080301-4.022560$

O $-9.799803-1.625379-1.202046$

O $-8.098095-2.699971-0.077984$

C $-10.726419-2.613547-0.740887$

H $-10.436362-3.604176-1.103923$

H $-10.779266-2.6018920 .351874$

H -11.713110 -2.367962 -1.142675

SCF Energy (B3LYP/6-31G**//MMFF) $=-3245.92442742$

05_00278

MM̄MF Geometry

C $3.395958-1.0299713 .464294$

C $2.359450-0.1828583 .357374$

C $0.887927-0.4603713 .559934$

O $0.214023-0.1696352 .306506$

C $0.519891-1.9007873 .962002$

C $-0.973739-2.1233084 .135070$

C -1.676094 -3.075685 3.490111

C -1.675204-1.216844 5.113806

C $-1.145437-4.1226652 .554410$

C $-1.203625-3.7451631 .068724$

C $-2.633740-3.5848040 .529456$

C $-2.644489-3.703901-0.995763$

C $-4.050941-3.501610-1.573535$

O $-3.124660-2.2930290 .908296$

O $-3.923770-3.332630-2.993472$

C $-4.626784-2.189120-1.010723$

C $-4.945928-4.727354-1.358996$

O $-5.990210-2.023559-1.394307$

C $-4.485645-2.0500930 .526303$

C $-4.863743-0.6086240 .934780$

O $-5.379112-2.9625601 .167298$

C -0.1071041 .1296852 .061253$

$\begin{array}{lllll}\text { O } & 0.110932 & 2.088077 & 2.786348\end{array}$

C $-0.770409 \quad 1.1985830 .740684$

C -0.9584232 .4052050 .185645$

C -1.650643 $2.698056-1.069171$

C $-2.1953221 .542430-1.862434$

C $-4.502018-0.2234502 .373517$

C -5.0298911 .1666562 .674935$

C -4.7096553 .5580602 .019851$

O -6.0219251 .3402053 .378818$

C -4.9815083 .9429090 .597462$

C -4.4716205 .0368910 .010791$

C $-4.7192775 .404736-1.426140$

C $-3.4438055 .710909-2.185598$

C $-3.1370436 .971427-2.536392$

C $-2.5564154 .551576-2.598739$

C $-1.7836253 .989660-1.436167$

N -4.305312 2.1635452 .041403

H $3.211183-2.0625843 .748756$

H $2.564003 \quad 0.8501313 .087011$

H $\quad 0.5296730 .224787 \quad 4.338715$

H $1.013901-2.1597004 .907213$

H $0.916427-2.5787803 .199471$

H $-2.747433-3.1408653 .675421$

H -2.701008 -1.542118 5.318316

H -1.141296-1.205311 6.069638

H -1.727846 $-0.194524 \quad 4.727330$

H $-0.114270-4.3863522 .814646$

H - $-1.718259-5.0449472 .716998$

H $-0.633882-2.8216370 .905077$

H $-0.695770-4.5456350 .516297$

H $-3.266043-4.3622940 .973949$

H -2.235534 -4.669246 -1.318678

H $-1.983931-2.936383-1.421018$

H -3.499305 -4.130259-3.353427

H -4.071711 -1.366010-1.482555

H $-4.499736-5.613322-1.826204$

H $-5.920541-4.593433-1.841440$

H $-5.112497-4.958285-0.304771$

H $-6.030399-2.100747-2.363006$

H -4.3520040 .0946710 .268075$ 
H $-5.941177-0.455306 \quad 0.789065$ H -5.601265 -2.621107 2.047835 H $-1.1001330 .273704 \quad 0.282350$ H $-0.5912143 .281103 \quad 0.720286$ H -2.543960 $1.827246-2.857758$ H $-3.035257 \quad 1.079354-1.337882$ H -1.422009 $0.784622-2.023256$ H $-4.936753-0.9173383 .099929$ H -3.416763 -0.231698 2.524222 H -3.891530 4.1402652 .455326 H -5.6097053 .7001032 .625300$ H -5.6261723 .2840570 .019853$ H -3.8471505 .7096670 .594501$ H -5.381274 6.280317 -1.434112 H -5.264159 4.610965 -1.952125 H -2.237675 $7.198815-3.099116$ H -3.775646 $7.805561-2.265038$ H -1.829794 $4.880643-3.352558$ H -3.186032 $3.803319-3.088731$ H -1.312359 $4.752836-0.814552$ H -3.4791621 .9074701 .509866$ C 5.1397040 .6733922 .598892 O $4.502671 \quad 0.7846991 .313835$ C 5.2764481 .6724150 .485953 C 6.4809132 .1031861 .321342 C 6.6100100 .9561802 .310951 H $5.6365481 .078224-0.360516$ C $4.3798972 .811915-0.020729$ H 7.3771212 .2403610 .708037 H 6.2963633 .0464781 .847990 C 7.4325611 .3051783 .538116 H 7.0648830 .0982021 .797893 C $3.0829062 .322698-0.705044$ O 4.0005793 .6184501 .101627 H $4.9532173 .460576-0.693349$ H 2.4244401 .8500170 .033850 O $2.3789643 .466003-1.203558$ C $3.3175561 .350153-1.875154$ C $2.0022210 .921126-2.554444$ H $3.831490 \quad 0.460930-1.498919$ O $4.1754731 .976904-2.826578$ H $1.3481710 .479272-1.794483$ H $1.4859251 .804424-2.949378$ H $8.452447 \quad 1.5815613 .251324$ H 6.9975432 .1473574 .086712 H $7.494516 \quad 0.4533724 .222440$ C $4.849926-0.7071753 .194546$ H 3.6048784 .4354650 .754222 H $2.0376643 .956799-0.437126$ H $4.494551 \quad 1.289194-3.434878$ H $5.399690-0.8266884 .135010$ H $5.220595-1.4743422 .503237$ H 4.7300421 .4605043 .245942 C $2.532216-2.092007-2.445069$ O $3.084738-1.148172-3.369781$ C $2.209737-0.071735-3.721092$ C $0.912230-0.618824-4.333908$ C $0.259874-1.685957-3.456507$ C $1.283799-2.744640-3.046633$ H $2.272269-1.583404-1.508618$ C $3.616700-3.132777-2.146326$ H $2.720520 \quad 0.486725-4.515631$ O $-0.0029680 .444706-4.575233$ H $1.147214-1.065285-5.309130$ H $-0.219316-1.236151-2.579803$ O $-0.783322-2.307205-4.210434$ H $1.552557-3.343664-3.925592$ O $0.689885-3.631182-2.102361$ C $4.694679-2.540131-1.267880$ H $3.218291-4.002723-1.613067$ H $4.065448-3.494400-3.079505$ H $-0.8159760 .053004-4.937915$ H -1.147483 -3.025851 -3.665919 H $0.486914-3.119363-1.300980$ O $5.896571-2.573420-1.909417$ O $4.490344-2.098182-0.144455$ C $7.001240-2.037999-1.173642$ H $7.907916-2.186022-1.766191$
H $6.859511-0.965943-1.010643$

H $7.116692-2.561998-0.219804$

SCF Energy (B3LYP/6-31G**//MMFF) $=-3245.90762446$

06_00001

MM̄MF Geometry

C -2.162458 -4.072472 1.630048

C $-0.899975-3.7148071 .348668$

C $0.115524-3.3697242 .408310$

O $0.806429-2.1579172 .023250$

C $1.152412-4.5022372 .512223$

C $2.195295-4.2711293 .588960$

C $3.473714-3.9101173 .358252$

C $1.725071-4.5168214 .999804$

C $4.122974-3.6163312 .034029$

C $4.748621-2.2151042 .022863$

C $5.263039-1.8281120 .628044$

C $5.952989-0.4634210 .671358$

C $6.3645100 .025206-0.724392$

O $4.136987-1.784989-0.254921$

O $6.6933831 .417519-0.599132$

C $5.151444-0.092332-1.673473$

C $7.639675-0.674071-1.208583$

O $5.5270450 .228015-3.010552$

C $4.455298-1.467874-1.616290$

C $3.136495-1.518255-2.427800$

O $5.331021-2.451325-2.181971$

C $0.188307-0.9727582 .282107$

O $-\begin{array}{llll}0.934980 & -0.810089 & 2.730867\end{array}$

C 1.1455530 .1082341 .954069

C 0.8098111 .3899712 .162669

C 1.6764292 .5545311 .960057

C 3.0564232 .3117241 .408882

C $2.026327-0.577836-1.947726$

C $2.0064120 .774445-2.638880$

C $0.8988703 .004452-2.494128$

O $2.6671341 .029240-3.641639$

C $1.6708813 .961178-1.643422$

C $1.1012454 .967001-0.962644$

C $1.8690785 .969611-0.152455$

C 1.3813286 .0663451 .274594

C 0.5460207 .0479111 .654840

C 1.9441885 .0891492 .279825

C 1.2116713 .7756712 .298047

N $1.1273711 .653265-2.019870$

H -2.460811 -4.1141462 .676344$

H $-0.555479-3.6735100 .319584$

H $-0.365110-3.216366 \quad 3.382865$

H $1.630310-4.6528631 .538258$

H $0.643102-5.4515592 .728448$

H $4.138324-3.8066104 .215462$

H $2.530210-4.4004235 .733210$

H $0.931745-3.8131815 .269617$

H $1.336340-5.5357115 .098675$

H $4.905574-4.363627 \quad 1.856836$

H $3.413686-3.7043581 .208423$

H $3.994914-1.4863682 .346463$

H $5.572157-2.1759292 .746812$

H $5.961045-2.6012590 .285809$

H $6.820846-0.4873191 .342253$

H 5.2655540 .2744001 .104694

H $6.8816451 .756866-1.491175$

H $4.4404090 .681902-1.362242$

H $8.467817-0.475871-0.517304$

H $7.963708-0.283281-2.179359$

H $7.529885-1.757399-1.291114$

H $5.993542-0.541066-3.379952$

H $3.344354-1.385844-3.496450$

H $2.739615-2.539634-2.334998$

H $4.800350-3.219985-2.449469$

H $2.120812-0.183767 \quad 1.583448$

H -0.1835201 .6084312 .550204$

H 3.0086001 .7260410 .484916

H $3.665757 \quad 1.7691082 .139159$

H 3.5915953 .2286381 .152870

H $1.059563-1.045872-2.172175$

H $2.074877-0.434676-0.864928$

H $-0.1776683 .189020-2.446119$ 
H $\quad 1.218383 \quad 3.094176-3.537100$ H $2.7507993 .837281-1.610527$ H $0.0241825 .102928-1.019615$ H $1.7667856 .944202-0.647745$ H $2.9440555 .750451-0.157040$ H $\quad 0.2180507 .1433342 .684673$ H 0.1649137 .7725210 .943007 H 1.8658785 .5127253 .290781 H 3.0199874 .9760042 .117798 H 0.1970643 .8537422 .689496 H $0.6413601 .360172-1.177168$ C $-2.956141-4.249079-0.831118$ O $-2.814285-2.843042-1.117499$ C $-3.487373-2.549133-2.355418$ C $-3.847038-3.894247-2.974758$ C $-4.085809-4.737945-1.738797$ H -4.414654 -2.026428 -2.094794 C $-2.614700-1.654026-3.239785$ H $-4.722594-3.829051-3.627771$ H -3.018602 -4.306529 -3.562627 C -4.062840 -6.231867 -2.011604 H -5.059224 -4.464932 -1.309368 C $-2.173334-0.330412-2.584216$ O $-1.424961-2.367319-3.604015$ H -3.138600 -1.445868 -4.180037 H -1.437442 $-0.522158-1.793996$ O $-1.497340 \quad 0.438089-3.588318$ C $-3.3217790 .520708-2.011805$ C $-2.8456281 .904863-1.532306$ H -3.779560 -0.014695-1.174254 O $-4.3326420 .693764-3.004331$ H -2.012048 $1.768333-0.834878$ H $-2.4645372 .471024-2.390016$ H $-4.848528-6.504459-2.723933$ H -3.102745 -6.548225 -2.433030 H -4.230433 -6.800392 -1.091721 C -3.251381-4.446258 0.657949 H $-0.975054-2.625890-2.781176$ H $-0.764179-0.105470-3.924716$ H -3.923235 $1.139508-3.765805$ H $-3.508116-5.4955070 .844550$ H -4.131995 -3.8490630 .923710$ H -2.013713 -4.737228 -1.110092 C -3.8265311 .8430901 .360961$ O -4.6206422 .0346930 .182998$ C $-3.9694582 .738410-0.877695$ C $-3.5273054 .125119-0.391663$ C -2.6881784 .0416300 .880656$ C -3.4015683 .1991661 .938998$ H $-2.940507 \quad 1.2441371 .116779$ C $-4.680322 \quad 1.0454112 .351187$ H -4.732027 $2.899092-1.650182$ O $-2.8023904 .797097-1.415875$ H $-4.4209304 .728273-0.184238$ H $-1.686453 \quad 3.6516500 .665909$ O -2.5048835 .3667801 .383356$ H -4.273592 3.7552292 .307148 O -2.5087153 .0201693 .034431$ C -4.904337 -0.3569981 .837895$ H $-4.2017150 .950791 \quad 3.331274$ H -5.6512351 .5298782 .511155$ H -2.521745 $5.656542-1.057346$ H -1.994721 5.2941492 .208344 H -3.021889 2.6793573 .785767 O $-6.173074-0.4850091 .356838$ O $-4.041794-1.2254351 .841427$ C $-6.498832-1.7681600 .814338$ H $-5.850730-1.994636-0.036916$ H -6.408987 -2.539467 1.585096 H -7.535026 -1.737848 0.467249

SCF Energy (B3LYP/6-31G**//MMFF) $=-3245.93147247$

06_00002

MMFF Geometry

C $-0.1245120 .409924-2.839498$

C $-1.124339-0.466800-3.026800$

C -1.280163 -1.847493 -2.428242

O $-2.446155-1.823025-1.569838$
C $-0.111844-2.338422-1.554831$

C $-0.100693-3.840246-1.345383$

C $-0.599244-4.482296-0.271061$

C $0.584901-4.624842-2.435951$

C $-1.379072-3.9156270 .881441$

C $-0.576419-3.8427682 .188069$

C $0.217187-2.5353092 .322956$

C $1.029395-2.5148633 .618587$

C $1.742994-1.1696933 .823827$

O $-0.729814-1.4609442 .334205$

O $2.207564-1.1461585 .181765$

C $0.720544-0.0258333 .647638$

C $2.989729-1.0536862 .941962$

O $1.364478 \quad 1.2452383 .693068$

C $-0.146805-0.1556722 .373588$

C -1.2904940 .8855362 .406273$

O $0.668573 \quad 0.091526 \quad 1.226626$

C $-3.669769-2.048620-2.111182$

O $-3.937704-2.261979-3.281388$

C $-4.643074-1.957892-1.001278$

C $-5.938976-1.738406-1.263705$

C $-6.995861-1.561075-0.265113$

C $-6.622454-1.7344021 .184880$

C $-2.4075150 .627453 \quad 1.383593$

C -3.4186561 .7625561 .351081$

C -5.4577462 .5172580 .125701$

O -3.3757882 .7207622 .118364$

C -6.7042332 .0265780 .793691$

C $-7.8966491 .968020 \quad 0.180107$

C $-9.1610251 .506688 \quad 0.849537$

C -9.9148550 .4736130 .038984$

C $-10.9967410 .816065-0.680771$

C $-9.464247-0.9682250 .129159$

C $-8.232913-1.231349-0.691252$

N $-4.378064 \quad 1.5760230 .365120$

H $0.6998550 .171658-2.174698$

H -1.925178 -0.179678 -3.707248

H -1.442149 -2.554651 -3.251767

H $-0.136084-1.828244-0.586698$

H $\quad 0.846615-2.075689-2.016465$

H $-0.483286-5.565045-0.227811$

H $\quad 0.592737-5.701229-2.234522$

H $0.075706-4.472791-3.392899$

H $1.625417-4.302726-2.542154$

H -1.817031 -2.944733 0.637859

H $-2.233244-4.5867931 .041227$

H -1.294698 -3.899079 3.016760

H $0.092138-4.7073402 .279164$

H $\quad 0.880279-2.4322131 .457679$

H $1.750516-3.3412173 .641701$

H $0.356776-2.6826624 .471750$

H $2.593029-0.2682375 .346114$

H $0.057628-0.0580714 .523096$

H $3.681480-1.8799463 .144660$

H $3.547059-0.1379513 .165700$

H $2.758396-1.0653871 .875732$

H 1.8074021 .3841372 .838389

H -1.7571230 .8847803 .400296$

H -0.8745801 .8882792 .244940$

H $\quad 0.103867 \quad 0.3928890 .497401$

H $-4.255062-2.0297450 .008668$

H -6.250982 -1.648982 -2.303422

H $-6.256222-2.7511001 .365873$

H -7.452201 -1.571144 1.874823

H -5.839091 -1.024458 1.471625

H -1.9936240 .5054040 .378617$

H -2.945640 -0.291866 1.642299

H $-5.5773612 .602818-0.958975$

H -5.1852583 .4982840 .527284$

H $-6.627838 \quad 1.726481 \quad 1.836170$

H $-7.9773022 .299403-0.852844$

H -9.7937332 .3902031 .005954$

H $-8.959878 \quad 1.104058 \quad 1.850065$

H -11.558935 $0.077787-1.243007$

H $-11.3415381 .843720-0.728367$

H -10.252558 -1.634754 -0.245763

H -9.347781 -1.2400701 .181387$

H $-8.399605-1.126917-1.764576$ 
H $-4.353006 \quad 0.731771-0.197941$

C $0.0566292 .950388-2.604845$

O $1.3062842 .927391-1.889901$

C $1.0764953 .401744-0.548757$

C $-0.3383803 .973689-0.531577$

C -1.036048 $3.091243-1.548293$

H 1.1067652 .5213140 .101590

C $2.1732894 .385804-0.127049$

H $-0.792112 \quad 3.9256370 .463064$

H $-0.3609555 .018928-0.860153$

C $-2.333613 \quad 3.674279-2.077834$

H $-1.2438972 .125877-1.075814$

C $3.6138573 .871736-0.320274$

O $2.0497795 .590745-0.894842$

H 2.0176934 .6801810 .917358

H $3.8613033 .837658-1.388459$

$\begin{array}{lllll}\text { O } & 4.500309 & 4.834289 & 0.265306\end{array}$

C 3.9029342 .4976110 .309352

C 5.3864532 .1006340 .183045

H $3.2825591 .742740-0.183392$

O 3.5356732 .5091551 .687302

H $5.6913352 .213217-0.862926$

H 6.0012452 .7903850 .774014

H -3.037392 $3.854385-1.259585$

H -2.166236 $4.627952-2.589435$

H $-2.8063892 .988545-2.788046$

C $-0.0503221 .741933-3.539135$

H $2.1137255 .347940-1.834681$

H $4.3115595 .692905-0.150983$

H 4.0572823 .2037282 .125066

H $0.8388901 .716545-4.181572$

H $-0.9168151 .876570-4.197779$

H $0.0889023 .854054-3.228406$

C $5.036497-0.525553-1.326681$

$\begin{array}{lllll}\text { O } & 4.799598 & -0.297741 & 0.065963\end{array}$

C $5.6704880 .662503 \quad 0.672441$

C $7.131347 \quad 0.217900 \quad 0.514145$

C $7.486081-0.077836-0.942389$

C $6.464025-1.033381-1.561200$

H $4.8957370 .417273-1.869723$

C $3.940549-1.460538-1.846776$

H $\quad 5.4443180 .647574 \quad 1.745565$

$\begin{array}{lllll}\text { O } & 8.005263 & 1.211566 & 1.039058\end{array}$

H $7.288928-0.6931061 .106277$

H $7.5717180 .848134-1.523009$

O $8.777558-0.690381-0.972684$

H $6.612080-2.026929-1.121094$

O $6.731925-1.132250-2.956988$

C $4.040878-2.868922-1.311528$

H $2.952872-1.066668-1.579055$

H $3.961216-1.518293-2.941258$

H 8.9160610 .8999520 .900858

H $8.972084-0.907976-1.900562$

H $6.186211-1.854099-3.312106$

O $3.428234-2.957359-0.097639$

O $4.606860-3.780013-1.902271$

C $3.469646-4.2477830 .518564$

H $2.972555-4.988128-0.115175$

H $4.505798-4.5393410 .714622$

H $2.936842-4.1883731 .470711$

SCF Energy (B3LYP/6-31G**//MMFF) $=-3245.93896739$

06_00003

MM̄FF Geometry

C $0.424650-1.5915052 .280974$

C $1.131686-2.4897591 .577215$

C $0.701136-3.092123 \quad 0.257304$

O $-0.263152-2.230804-0.394825$

C $0.065799-4.4732280 .482356$

C $-0.266188-5.193865-0.810843$

C $-1.506240-5.334859-1.321307$

C $0.914291-5.800399-1.526627$

C $-2.799433-4.809666-0.762706$

C $-3.391410-3.724807-1.671104$

C $-4.603801-3.033326-1.030050$

C $-5.191076-1.989861-1.982514$

C $-6.324435-1.182235-1.333671$

O $-4.159140-2.401366 \quad 0.174623$
O $-6.567884-0.049074-2.181477$

C $-5.845263-0.6554210 .037193$

C $-7.631962-1.981652-1.300658$

O $-6.912784-0.0161570 .732743$

C $-5.190336-1.738820 \quad 0.917986$

C $-4.541083-1.1657162 .200958$

O $-6.199309-2.6661421 .336954$

C $0.241732-1.176055-1.094892$

O $1.418974-0.944216-1.321153$

C $-0.886189-0.308259-1.500152$

C $-0.6192760 .906015-2.004056$

C $-1.5927611 .905400-2.446744$

C $-3.0524111 .553314-2.350418$

C $-3.393568-0.1755511 .975055$

C -3.8025301 .2859602 .031493$

C -2.8386303 .5693901 .762437$

$\begin{array}{lllll}\text { O } & -4.921667 & 1.663342 & 2.365966\end{array}$

C $-3.211275 \quad 4.106793 \quad 0.417612$

C $-2.4702175 .001569-0.253682$

C $-2.8889895 .623291-1.554449$

C $-1.8552295 .468053-2.645348$

C $-0.9700716 .445482-2.904567$

C $-1.9171104 .225592-3.502303$

C $-1.1324273 .081469-2.921967$

N -2.744646 $2.124046 \quad 1.701875$

H $-0.561459-1.2989231 .932265$

H $2.108937-2.7970001 .941339$

H $1.587235-3.192498-0.381744$

H $-0.826059-4.3646381 .107614$

H $0.748629-5.1081701 .063316$

H $-1.616508-5.884929-2.255147$

H $0.616132-6.365131-2.416493$

H $1.611328-5.021688-1.851036$

H $1.446405-6.490638-0.863717$

H $-3.507369-5.643700-0.682685$

H $-2.674108-4.4234410 .250779$

H -2.618073 -2.975453 -1.879401

H $-3.681076-4.168150-2.632036$

H $-5.351397-3.796559-0.784186$

H -5.536705 -2.461059-2.911266

H $-4.398767-1.291289-2.282627$

H $-7.2304860 .510975-1.741427$

H $-5.1084830 .128311-0.171845$

H $-7.937910-2.252584-2.318480$

H $-8.454104-1.382923-0.893294$

H $-7.555821-2.904124-0.721251$

H $-7.480881-0.7108531 .107209$

H -5.311429 -0.7513822 .862340$

H -4.114481-2.016115 2.752676

H -5.881481 -3.131187 2.128613

H $-1.893750-0.678259-1.355148$

H $\quad 0.4232421 .204095-2.109399$

H $-3.3081411 .245300-1.331332$

H $-3.2925280 .735390-3.037390$

H -3.718680 $2.384799-2.589546$

H $-2.664905-0.3114522 .784702$

H -2.876035 -0.3729181 .031055$

H -1.8711513 .9454862 .107623$

H -3.599225 3.8562862 .495917

H $-4.1607283 .779988-0.000253$

H $-1.536154 \quad 5.3503520 .181021$

H -3.070452 $6.690134-1.367470$

H $-3.8499215 .221889-1.899578$

H $-0.2477226 .354336-3.709010$

H $-0.9444337 .355104-2.313673$

H $-1.4914734 .439267-4.492636$

H $-2.9603643 .968646-3.707239$

H $-0.053616 \quad 3.244759-2.942887$

H $-1.870261 \quad 1.719017 \quad 1.380229$

C $0.823377 \quad 0.616938 \quad 3.361890$

$\begin{array}{lllll}\text { O } & 1.630936 & 1.044089 & 2.249179\end{array}$

C 2.2900992 .2774812 .591208

C 1.6829882 .7303243 .914830

C 1.3411731 .4016404 .564276

H 3.3460342 .0316392 .755144

C $2.166883 \quad 3.2781331 .434533$

H $2.3806853 .331128 \quad 4.506038$

H 0.7731313 .3231333 .765924 
C 0.3426101 .5199015 .702004

H 2.2670920 .9464044 .940705

C 2.5697822 .7082450 .059968

O $\quad 0.8036293 .7078321 .324501$

H 2.7492584 .1787481 .658789

H $1.8439711 .948122-0.250532$

O $2.4826913 .764644-0.903763$

C 3.9848562 .1090560 .001932

C $4.3132581 .547114-1.391008$

H $4.063568 \quad 1.3034590 .733138$

O $4.9468223 .097506 \quad 0.360793$

H $3.5136710 .857765-1.681291$

H $4.3146542 .364698-2.122270$

H 0.7425012 .1536646 .500275

H -0.6012051 .9608435 .363355$

H $\quad 0.120817 \quad 0.537497 \quad 6.130260$

C $0.918413-0.9027753 .521682$

H 0.2585352 .9179831 .165282

H $1.5776634 .119100-0.863467$

H $4.8597843 .833829-0.268653$

H $\quad 0.310500-1.229806 \quad 4.372208$

H $1.956319-1.1914513 .723259$

H $-0.2102790 .907507 \quad 3.132737$

C $5.018114-1.316288-0.575677$

O $5.852711-0.160040-0.445773$

C $5.6825090 .833122-1.462276$

C $5.9834650 .219063-2.835416$

C $5.122103-1.014038-3.099721$

C $5.227223-1.999597-1.936002$

H $3.968759-1.025095-0.453864$

C $5.387020-2.2856910 .561016$

H $6.4461011 .597830-1.271971$

O $5.7797831 .186436-3.860066$

H $7.041481-0.071439-2.872489$

H $4.080765-0.734351-3.297319$

O $5.602690-1.639271-4.292505$

H $6.211554-2.484928-1.962669$

O $4.242893-3.021094-2.090818$

C $4.990332-1.780647 \quad 1.931619$

H $4.884563-3.2489330 .418674$

H $6.472886-2.4399400 .569516$

H $5.9563210 .749839-4.711047$

H $4.991428-2.362416-4.512061$

H $4.456596-3.517348-2.898567$

O $5.480969-2.6387342 .875458$

O $4.325503-0.7796902 .154914$

C $5.177441-2.3002424 .232523$

H $5.692935-3.0114884 .883445$

H $5.532472-1.2916904 .465480$

H $4.100695-2.3815584 .406892$

SCF Energy $\left(B 3 L Y P / 6-31 G^{* *} / / M M F F\right)=-3245.92964590$

06_00004

MM̄FF Geometry

C -1.3358803 .0453442 .400039$

C -1.7485191 .8590241 .928305$

C -2.0963540 .6943642 .819670$

O $-1.477408-0.5042752 .303192$

C -3.613866 0.4602372 .760674

C $-4.141648-0.4408393 .856620$

C $-4.431135-1.7484463 .708809$

C $-4.404180 \quad 0.246920 \quad 5.171922$

C $-4.193195-2.6292372 .513148$

C $-5.464346-2.9138681 .698667$

C $-5.740746-1.8412350 .634111$

C $-7.050999-2.115761-0.103578$

C -7.283790 -1.101482 -1.234826

O $-4.648960-1.868747-0.292875$

O $-8.350768-1.596751-2.057931$

C $-6.020367-1.040484-2.119029$

C $-7.7434990 .259993-0.699977$

O $-6.130447-0.009530-3.097910$

C $-4.705737-0.873321-1.319914$

C $-3.493809-1.082680-2.261121$

O $-4.6472750 .447640-0.779305$

C $-0.163980-0.729348 \quad 2.556314$

$\begin{array}{lllll}\text { O } & 0.619979 & 0.008410 & 3.128225\end{array}$

C $0.140465-2.0711472 .009988$
C $1.404156-2.5135731 .955727$

C $1.830357-3.8223391 .453436$

C $0.757197-4.7933251 .031453$

C $-2.141206-1.244331-1.547348$

C $-1.022061-1.493353-2.547580$

C $1.306865-2.348727-2.715259$

O $-1.112257-1.183388-3.733528$

C $1.872302-3.671957-2.310574$

C $3.162471-3.855654-1.988878$

C $3.756655-5.193358-1.650203$

C $4.563957-5.176656-0.371045$

C $5.904634-5.092426-0.402956$

C $3.825027-5.3546570 .935914$

C $3.150919-4.0916971 .393163$

N $0.093527-2.079504-1.963922$

H -1.224792 3.1562033 .477570

H -1.883872 1.7075320 .861679

H $-1.7656340 .860306 \quad 3.852277$

H -3.8680620 .0413371 .783100$

H -4.1528811 .4154892 .822960$

H $-4.853298-2.2758444 .563839$

H -4.805974 -0.4369195 .927163$

H -3.4789040 .6722195 .573064$

H -5.1302191 .0557255 .039603$

H -3.396072 -2.238815 1.874471

H $-3.811924-3.5841502 .898346$

H $-5.320024-3.876747 \quad 1.190867$

H $-6.328977-3.0242382 .364236$

H $-5.789040-0.8626201 .121199$

H -7.898418 -2.115696 0.593207

H $-7.021516-3.123510-0.541042$

H $-9.136960-1.705680-1.495804$

H $-5.968178-1.988168-2.673277$

H $-8.6741940 .152918-0.130000$

H -7.972642 $0.949305-1.520283$

H $-7.0089020 .734661-0.046127$

H -6.958472 -0.160114 -3.585403

H -3.654522 -1.991841 -2.855982

H $-3.428523-0.243312-2.965497$

H $-3.7179170 .697101-0.654629$

H $-0.697985-2.6661531 .664237$

H $2.199586-1.8590292 .307743$

H $0.128409-5.0626581 .887271$

H $1.147539-5.7244560 .616196$

H $\quad 0.120357-4.357706 \quad 0.254193$

H $-1.885727-0.342296-0.981777$

H -2.182655 -2.088359 -0.849726

H $2.001295-1.530712-2.509893$

H $1.086716-2.353989-3.787479$

H $1.198902-4.525693-2.320882$

H $3.840897-3.005867-2.015157$

H $4.399457-5.490553-2.489432$

H $2.983731-5.968945-1.581780$

H $6.494196-5.1116730 .507568$

H $6.442259-4.994571-1.340298$

H $4.526450-5.6505291 .727854$

H $3.137125-6.2005070 .850541$

H $3.851133-3.3241301 .722770$

H $0.132482-2.159426-0.952221$

C -1.1714304 .2139840 .090830$

O $-0.1405873 .366078-0.454336$

C $0.3723503 .971163-1.655556$

C $-0.5638405 .127414-1.985099$

C $-0.9944915 .566653-0.600492$

H $1.3574514 .381831-1.407149$

C $0.5078862 .927533-2.767365$

H $-0.0625275 .918171-2.551562$

H -1.432034 $4.795752-2.566385$

C $-2.2420696 .433201-0.602497$

H $-0.1695996 .122645-0.134756$

C $1.3846201 .711245-2.410605$

O $\quad-0.7933512 .429597-3.108460$

H $0.8920163 .408085-3.674772$

H $0.8722551 .072390-1.681005$

O $1.5295940 .930204-3.604229$

C $2.7898652 .058829-1.885472$

C $3.6696740 .810259-1.682043$

H $2.6949512 .588668-0.932596$ 
O $3.4366172 .944813-2.798201$

H $3.1391010 .112186-1.025638$

H $3.8128360 .305805-2.645143$ H -2.071910 7.350004-1.176556 H -3.093480 $5.909682-1.050259$ H -2.521180 6.7205260 .415851 C $-1.0285794 .292897 \quad 1.612877$ H -1.183047 $2.049691-2.302142$ H $\quad 0.6352780 .699335-3.909897$ H $3.4960332 .490294-3.656121$ H -1.688597 5.0805161 .995425 H $-0.0021994 .586187 \quad 1.861797$ H -2.138165 $3.775117-0.187639$

C $4.4797241 .202844 \quad 1.235285$

O 4.9860901 .9107220 .097876

C $5.064106 \quad 1.139557-1.104195$

C $5.963354-0.084721-0.884160$

C $5.532312-0.9032880 .330950$

C $5.371127-0.0016631 .555009$

H $3.4534790 .869277 \quad 1.037336$

C 4.4423102 .1879892 .407415

H $5.5674311 .776223-1.842698$

O $5.971342-0.904710-2.048102$

H $6.9943200 .259031-0.728186$

H $4.613221-1.4614870 .119569$

O $6.548342-1.8736630 .590808$

H $6.358697 \quad 0.338171 \quad 1.892563$

O $4.783717-0.7440252 .619992$

C 3.3612603 .2189852 .190691

H $4.222726 \quad 1.6894273 .358087$

H 5.4073182 .6958352 .524987

H $\quad 6.523792-1.681159-1.853484$

H $6.222539-2.4589161 .295024$

H $5.414579-1.4355072 .881259$

O $3.914588 \quad 4.409782 \quad 1.825178$

$\begin{array}{lllll}\text { O } & 2.166102 & 2.982152 & 2.308704\end{array}$

C 2.9864345 .4676771 .568264

H $2.336517 \quad 5.2040740 .729429$

H 23953745.6805192 .464155

H 3.5555256 .3625461 .302874

SCF Energy (B3LYP/6-31G**//MMFF) $=-3245.92276335$

06_00005

MM̄FF Geometry

C $-1.403560 \quad 3.127370 \quad 0.980217$

C $-0.875273 \quad 1.895906 \quad 0.923417$

C $-0.402023 \quad 1.1280212 .130286$

O 0.9832160 .7649291 .924113

C -1.230199 -0.1618062 .275704$

C $-0.873372-0.9737723 .506795$

C $-0.192416-2.1377763 .496012$

C $-1.369726-0.4080424 .813218$

C $0.370513-2.8644502 .307373$

C $1.902735-2.9055772 .351191$

C $2.495381-3.3769221 .014930$

C $4.017613-3.4881991 .109166$

C $4.661888-3.815969-0.245656$

O $2.132968-2.4100860 .024005$

O $6.071309-3.579142-0.105862$

C $4.119175-2.836340-1.309993$

C $4.508808-5.301245-0.592508$

O $4.590577-3.189607-2.607861$

C $2.581821-2.710267-1.302717$

C $2.060406-1.584867-2.229115$

O $2.013595-3.938868-1.772064$

C $1.917626 \quad 1.712807 \quad 2.214870$

O $1.712797 \quad 2.8421592 .631343$

C 3.2520081 .1390851 .929806

C 4.3419561 .9149662 .029361

C 5.7274131 .4999221 .795340

C 5.9682880 .0780201 .363944

C $2.506775-0.166411-1.858171$

C $3.7408230 .315726-2.601159$

C $5.3406652 .228581-2.656148$

O $4.230647-0.280673-3.555823$

C $6.5844421 .850177-1.915983$

C $7.4147762 .747026-1.362237$

C $8.6980582 .384631-0.671613$
C $8.8052022 .975496 \quad 0.716026$

C $9.4919714 .110066 \quad 0.933207$

C $8.1974612 .200861 \quad 1.862595$

C 6.7123672 .4043311 .977907

N $4.2063281 .521666-2.092346$

H -1.4925713 .6269581 .941610$

H $-0.773103 \quad 1.403379-0.040195$

H $-0.493027 \quad 1.729945 \quad 3.042873$

H $-1.123552-0.7633651 .367704$

H -2.2990770 .0875282 .329672$

H $-0.010065-2.6342224 .448421$

H $-1.161050-1.0708495 .659742$

H -0.8921160 .5534235 .024236$

H $-2.453794-0.258592 \quad 4.774637$

H $-0.017870-3.8901782 .310803$

H $0.035440-2.4198831 .368513$

H $2.281333-1.9013992 .578565$

H $2.228393-3.5689523 .161925$

H $2.052365-4.3456370 .755276$

H $4.307718-4.2300821 .863684$

H $4.426407-2.5326901 .462175$

H $6.479661-3.709333-0.979154$

H $4.559498-1.858723-1.083327$

H $4.984642-5.9221070 .176168$

H $5.020363-5.545380-1.529967$

H $3.466633-5.616816-0.674666$

H $4.064671-3.946162-2.918684$

H $2.283064-1.821295-3.276520$

H $0.963993-1.594950-2.157431$

H $1.109499-3.762538-2.081134$

H 3.3021740 .0946231 .646749

H 4.2126492 .9570932 .319266

H $5.362684-0.1669540 .485163$

H $5.712213-0.6126882 .173977$

H $7.002916-0.1236301 .079115$

H $1.7022250 .529901-2.126751$

H $2.669478-0.073028-0.780781$

H $5.124575 \quad 3.299244-2.582974$

H $5.4464981 .965910-3.713584$

H $6.8301580 .792140-1.862700$

H $7.1814023 .806271-1.443638$

H $9.5246232 .750318-1.295220$

H $8.8309121 .296984-0.616834$

H $9.599603 \quad 4.523602 \quad 1.930517$

H 9.9567094 .6534160 .117110

H 8.6424042 .5284092 .812152

H 8.4793241 .1473891 .779344

H 6.4380473 .4147472 .284649

H $3.7683461 .914781-1.264286$

C $-3.357960 \quad 4.291371-0.074522$

\begin{tabular}{llllll}
\hline & -4.194148 & 3.121419 & 0.011089
\end{tabular}

C $-5.402863 \quad 3.354314-0.736921$

C $-5.3797634 .824325-1.142838$

C $-3.8913555 .084516-1.266123$

H $-5.3346192 .739201-1.641825$

C -6.6269092 .9375650 .087676$

H $-5.9264815 .004569-2.073603$

H $-5.8146135 .468650-0.370202$

C $-3.5290796 .559111-1.244376$

H $-3.5345844 .639151-2.204658$

C -6.5755301 .4940680 .627243$

$\begin{array}{lllll}\text { O } & -6.749695 & 3.808550 & 1.220730\end{array}$

H $-7.5377573 .084927-0.503810$

H -5.7969361 .4060691 .395004$

O

C $-6.3565570 .415610-0.447914$

C $-6.392594-1.0073590 .137747$

H $-5.3896060 .579025-0.927612$

O $-7.3527640 .535600-1.461995$

H $-5.688736-1.0573120 .975464$

H $-7.392329-1.2070430 .541712$

H $-4.0027267 .083615-2.080654$

H $-3.8578157 .039564-0.316712$

H $-2.4467786 .697089-1.329808$

C $-1.893302 \quad 3.878034-0.226665$

H $-5.923356 \quad 3.745417 \quad 1.730160$

H -7.9425351 .9155391 .962917$

H -8.220089 $0.424205-1.035986$ 
H $-1.265106 \quad 4.766536-0.355942$ H $-1.7769703 .260509-1.125365$ H -3.5001344 .8540900 .857774$ C -3.654879 -2.052790 -0.735128 O $-4.812485-1.881422-1.561346$ C $-6.065882-2.100560-0.905475$ C $-6.115925-3.528726-0.348081$ C $-4.928393-3.8201940 .565544$ C -3.617307 -3.465612 -0.135407 H $-3.657356-1.3023800 .064916$ C $-2.417967-1.814368-1.617887$ H -6.834570 -2.032533 -1.685576 O $-7.336704-3.7396520 .353439$ H -6.100756 -4.238033 -1.185959 H $-5.028139-3.2944151 .522237$ O $-4.938510-5.2169680 .869971$ H -3.416143 -4.202797 -0.923605 O $-2.547624-3.5380690 .803290$ C -2.290376 -0.376500 -2.073788 H $-1.500056-2.062028-1.073421$ H $-2.487508-2.441789-2.514936$ H $-7.316013-4.6453710 .706994$ H $-4.246455-5.3780731 .533065$ H -2.461042 -4.4647711 .083139$ O $-1.341985-0.304984-3.054157$ O $-2.9193440 .568120-1.619525$ C $-1.091733 \quad 1.003816-3.575864$ H $-0.3618480 .912804-4.384746$ H -2.011697 $1.437053-3.979900$ H $-0.6716891 .644953-2.795541$

SCF Energy (B3LYP/6-31G**//MMFF) $=-3245.92865505$

\section{6_00006}

MM̄FF Geometry

C $-0.2691010 .557859-3.180213$

C $-1.240271-0.332012-3.440086$

C $-1.437665-1.702979-2.837763$

O $-2.767881-1.725543-2.260620$

C $-0.439787-2.135414-1.746435$

C $-0.406686-3.638791-1.541070$

C $-0.976389-4.296336-0.513124$

C $0.370998-4.409493-2.579486$

C $-1.822667-3.7411600 .596514$

C -1.140907 -3.8571751 .966904$

C $-0.064514-2.7858942 .199181$

C $0.628457-3.0017553 .546519$

C $1.611799-1.8700963 .879788$

O $-0.724358-1.5173732 .190473$

O $1.949119-2.0061285 .268756$

C $0.896927-0.5152643 .692269$

C $2.924722-2.0160503 .105117$

$\begin{array}{llll}\text { O } & 1.807780 & 0.566844 & 3.867150\end{array}$

C $0.141162-0.3890592 .348487$

C $-0.739150 \quad 0.882024 \quad 2.360079$

O $1.087171-0.2943701 .282721$

C $-3.594069-2.746846-2.604091$

O $-3.355253-3.643813-3.402267$

C $-4.890779-2.678020-1.892142$

C $-5.159273-1.839187-0.879205$

C $-6.425227-1.762123-0.141974$

C $-7.545652-2.671000-0.577451$

C $-1.746495 \quad 0.9555241 .204323$

C -2.6410872 .1778331 .314394$

C $-4.694674 \quad 3.168643 \quad 0.305868$

O -2.4702983 .0651912 .145906$

C -5.8165412 .7377621 .195799$

C $-7.033763 \quad 2.3953750 .748632$

C -8.139610 1.9188831 .644432

C -8.5893610 .5119861 .313749$

$\begin{array}{llll}\text { C } & -9.767279 & 0.293524 & 0.705217\end{array}$

C $-7.722318-0.6424131 .769928$

C $-6.534709-0.8810430 .874855$

N -3.651048 2.1620960 .362263

H $0.4793710 .351821-2.421785$

H -1.956978 $-0.083541-4.222277$

H $-1.379057-2.398484-3.684078$

H $-0.670883-1.621091-0.807187$

H $0.578977-1.833709-2.013704$
H $-0.856596-5.378690-0.473045$

H $0.466270-5.470786-2.326421$

H $-0.121673-4.347139-3.554392$

H $1.382954-4.006282-2.678305$

H -2.138121 -2.711801 0.404498

H $-2.746956-4.332606 \quad 0.621113$

H $-1.921393-3.7437562 .730974$

H $-0.710920-4.8589052 .090501$

H $0.666209-2.8235591 .383862$

H $1.136858-3.9737443 .571261$

H $-0.130161-3.0430514 .341172$

H $2.508535-1.2482505 .511688$

H $0.167886-0.4280104 .509598$

H $3.389305-2.9860453 .318622$

H $3.655210-1.2617063 .416622$

H $2.789852-1.9411562 .025887$

H 2.3061430 .6690503 .038681

H -1.311733 0.9129573 .296687

H $-0.1040891 .776607 \quad 2.338040$

H 0.6812290 .1744700 .536735

H $-5.625340-3.397269-2.241515$

H $-4.385912-1.149431-0.546394$

H -7.798763 -2.493910 - 1.628808

H $-8.467838-2.529139-0.010463$

H $-7.257923-3.721205-0.455632$

H -1.2293000 .9985840 .240919$

H -2.390807 $0.068674 \quad 1.207766$

H -5.007092 $3.258966-0.738885$

H $-4.298540 \quad 4.1328510 .639503$

H $-5.6086382 .690218 \quad 2.262284$

H $-7.2461902 .446177-0.316708$

H -8.976656 2.6218211 .543544

H -7.8453301 .9610842 .700588$

H $-10.117602-0.7109110 .493182$

H -10.408317 1.1149310 .402585

H -8.319345 -1.5552901 .876843$

H $-7.357908-0.4404082 .785588$

H -5.669022 -0.2663751 .120686$

H $-3.7508551 .347640-0.237036$

C $-0.0828503 .111199-3.105972$

O $1.0479863 .112602-2.214291$

C $0.627628 \quad 3.649299-0.945430$

C $-0.739672 \quad 4.282287-1.186647$

C $-1.3161713 .358130-2.241517$

H $0.5080362 .793519-0.270897$

C $1.6857834 .603342-0.380924$

H $-1.3421164 .332303-0.275799$

H $-0.6536075 .299353-1.586153$

C -2.488954 $3.951149-3.001875$

H $-1.6385512 .431645-1.753403$

C $3.1158374 .030541-0.325554$

O $1.7391745 .786983-1.188876$

H 1.3778484 .9378240 .616549

H $3.5378953 .970915-1.336465$

$\begin{array}{lllll}\text { O } & 3.929617 & 4.967540 & 0.392284\end{array}$

C 3.2402282 .6558720 .353549

C $4.7076202 .215356 \quad 0.516225$

H $2.7004351 .914362-0.243526$

O $2.621752 \quad 2.6931551 .638461$

H $5.2242222 .347389-0.440297$

H $5.2037612 .867503 \quad 1.245278$

H $-3.3079274 .196263-2.319045$

H -2.205048 $4.870160-3.525592$

H $-2.8679143 .242789-3.745209$

C $-0.0971741 .838996-3.954731$

H $1.9386145 .511630-2.100328$

H $3.8485255 .825873-0.057870$

H 3.0643533 .3854702 .158802

H $0.8628091 .762772-4.481371$

H $-0.874122 \quad 1.930350-4.723338$

H $0.0724423 .963040-3.781793$

C $4.729002-0.347266-1.140735$

O $4.142150-0.1625700 .151039$

C 4.8404070 .7543331 .000951

$\begin{array}{llll}\text { C } & 6.288700 & 0.285393 & 1.203228\end{array}$

C $7.0034660 .050180-0.125729$

C $6.168583-0.861005-1.025186$

H $4.7284080 .612781-1.671508$ 
C $3.816226-1.265815-1.959538$

H $4.347496 \quad 0.702516 \quad 1.978596$

O $7.0288361 .228146 \quad 1.971007$

H $6.287897-0.6575691 .765745$

H $7.2368570 .994619-0.631136$

$\begin{array}{llll}\text { O } & 8.255958 & -0.593046 & 0.121242\end{array}$

H $6.187430-1.877122-0.612993$

O $6.753427-0.913115-2.323595$

C $3.719142-2.663072-1.393710$

H $2.796811-0.863013-1.979959$

H $4.148669-1.317850-3.002815$

H $\quad 6.563253 \quad 1.345747 \quad 2.816677$

H $8.758747-0.0222710 .727513$

H $7.673130-1.210806-2.215555$

O $4.485572-3.519027-2.127915$

O $3.055542-2.956167-0.407981$

C $4.502213-4.874787-1.670626$

H $5.143076-5.452104-2.342256$

H $4.916249-4.927306-0.659147$

H $3.493818-5.298135-1.698061$

SCF Energy (B3LYP/6-31G**//MMFF) $=-3245.92829555$

06_00007

MMFF Geometry

C $-2.287623 \quad 4.3420801 .828192$

C $-2.7295393 .113641 \quad 1.516934$

C -3.0955652 .0728652 .544978$

O -2.5242840 .8063402 .142590$

C -4.6265581 .9178972 .590861$

C -5.1033120 .8822643 .591136$

C $-5.586675-0.334523 \quad 3.268691$

C -5.0458831 .3119345 .034548$

C $-5.715154-0.9407411 .898176$

C $-4.953379-2.2698301 .805852$

C $-4.876203-2.7872830 .361165$

C $-4.190341-4.1533510 .317525$

C $-3.960376-4.642395-1.118558$

O $-4.125967-1.834707-0.398585$

O $-3.047531-5.748605-1.041279$

C $-3.277769-3.520173-1.931812$

C $-5.250628-5.193371-1.737171$

O $-3.186859-3.911311-3.299687$

C $-3.979116-2.151782-1.790081$

C $-3.195512-0.982383-2.438666$

O $-5.254542-2.213306-2.439799$

C $-1.2448620 .544704 \quad 2.526281$

O -0.4918751 .2890393 .133001$

C $-0.925397-0.8351122 .093716$

C $0.286511-1.3579002 .333703$

C $0.717390-2.7214922 .012400$

C $-0.275122-3.6240891 .327873$

C $-1.808093-0.712739-1.848097$

C $-0.672130-1.424670-2.561035$

C $1.770077-1.881982-2.319162$

O $-0.801720-1.985240-3.645456$

C $2.030382-3.165715-1.597504$

C $3.131448-3.390140-0.864761$

C $3.433600-4.693050-0.184371$

C $3.735983-4.5336621 .288382$

C $5.001680-4.5452141 .739389$

C $2.571199-4.4790172 .248698$

C $1.961310-3.1096792 .364442$

N $0.516983-1.321445-1.852783$

H $-2.177467 \quad 4.593776 \quad 2.881757$

H -2.868330 2.8263420 .478758

H -2.7221572 .3482423 .539253$

H -5.0012071 .6868651 .588003$

H -5.0868242 .8816162 .849245$

H $-5.926863-0.9807284 .077344$

H -5.4627040 .5575055 .710403$

H $-4.011141 \quad 1.491446 \quad 5.341727$

H -5.6181852 .2341285 .179647$

H $-6.777757-1.1181341 .693388$

H $-5.359057-0.2611901 .121367$

H $-3.936699-2.1256032 .192385$

H $-5.442421-3.0163362 .444143$

H -5.893039 -2.852693 - 0.043360

H $-4.762161-4.8944790 .889887$
H -3.215201-4.083612 0.816710

H $-2.841071-6.023515-1.951301$

H $-2.250563-3.445821-1.554891$

H $-5.616108-6.048322-1.155592$

H $-5.075024-5.574229-2.749323$

H $-6.054408-4.455490-1.781136$

H $-2.616881-3.270279-3.756199$

H $-3.156931-1.104234-3.527591$

H $-3.785500-0.069033-2.273540$

H $-5.540897-1.308150-2.645970$

H $-1.712785-1.3976251 .606524$

H $1.028263-0.7418812 .839050$

H $-0.670932-3.1509320 .423052$

H $-1.108581-3.8506552 .001118$

H $\quad 0.150389-4.5757601 .002719$

H $-1.5962690 .359899-1.938677$

H $-1.781972-0.958787-0.783369$

H $2.549420-1.136787-2.132025$

H $1.718882-2.065645-3.396825$

H $1.291832-3.956160-1.707861$

H $3.878424-2.605697-0.780164$

H $4.296833-5.139540-0.695330$

H $2.613503-5.410633-0.311432$

H $5.225714-4.4684372 .798125$

H $5.842562-4.6293251 .059536$

H $2.907411-4.7646173 .255165$

H $1.843062-5.2496941 .980271$

H $2.614166-2.3830852 .850142$

H $0.510953-0.878069-0.938984$

C $-1.968098 \quad 5.139870-0.617403$

O $-0.8493104 .286493-0.931345$

C $-0.2158844 .770477-2.130686$

C -1.201576 $5.747064-2.755637$

C $-1.8128936 .369745-1.514486$

H $0.6719905 .331592-1.816865$

C $0.1956193 .592963-3.019291$

H $-0.7101046 .476133-3.406766$

H $-1.9703085 .229648-3.341287$

C $-3.1126847 .107935-1.784071$

H -1.0875317.067636-1.074599

C $1.1144972 .572913-2.312604$

$\begin{array}{lllll}\text { O } & -0.980027 & 2.894553 & -3.448073\end{array}$

H $0.6757413 .963274-3.932051$

H $0.5559852 .063762-1.519541$

O $1.4764561 .567595-3.266290$

C $2.4082153 .182793-1.745479$

C $3.3932642 .172388-1.139055$

H $2.1722433 .939526-0.994971$

O $3.0973023 .878621-2.791650$

H $3.7747381 .516127-1.933926$

H $4.2819592 .731954-0.821667$

H -2.946726 $7.935551-2.481435$

H $-3.8671326 .445819-2.222107$

H $-3.5253467 .523079-0.859367$

C -1.946660 5.4643860 .879007

H $-1.4747212 .637528-2.651100$

H $\quad 0.6513901 .189309-3.615743$

H $3.2814643 .236535-3.498725$

H -2.6456896 .2834601 .085332$

H $-0.943126 \quad 5.817240 \quad 1.150259$

H -2.881846 $4.598369-0.891720$

C $5.056616 \quad 0.6546990 .857782$

$\begin{array}{llllll}\text { O } & 3.813428 & 0.231260 & 0.283483\end{array}$

C 2.8786761 .2911870 .017709

C $2.5384221 .990467 \quad 1.339744$

C 3.7903662 .5050342 .044395

C $4.814912 \quad 1.377508 \quad 2.189701$

H 5.5816501 .3193250 .161731

C $5.924371-0.5962101 .056988$

H $1.9701310 .792936-0.332563$

$\begin{array}{lllll}\text { O } & 1.604661 & 3.041439 & 1.145636\end{array}$

H 2.0538751 .2618121 .999995

H 4.2104623 .3703341 .519046

O 3.4177522 .9654073 .345208

H 4.4542460 .6656092 .942965

$\begin{array}{lllll}\text { O } & 6.038476 & 1.918346 & 2.683523\end{array}$

C $6.397901-1.178417-0.259078$

H $6.816436-0.3519261 .643883$ 
H $\quad 5.345646-1.3701401 .574024$

H 1.4264423 .4388662 .015257

H 4.2320273 .2464553 .796783

H 6.3750422 .5475102 .022742

O $7.140655-2.296276-0.005324$

O $6.149307-0.721184-1.366147$

C $7.635473-2.980816-1.160452$

H $8.300336-2.326994-1.732944$

H $8.204248-3.850872-0.821948$

H $\quad 6.802656-3.326159-1.780651$

SCF Energy (B3LYP/6-31G**//MMFF) $=-3245.93515144$

06_00008

MM̄FF Geometry

C -2.3038082 .8046361 .916765$

C -1.5315991 .7600131 .581985$

C -0.7813530 .9247332 .586316$

O $0.610431 \quad 0.8787082 .195304$

C -1.322550 -0.514019 2.577301

C $-0.820777-1.3303263 .751572$

C $0.170782-2.2402803 .690402$

C -1.554504-1.102127 5.049005

C $1.010100-2.6116162 .499786$

C $2.502524-2.6543232 .859258$

C $3.350733-3.1803721 .692787$

C $4.809029-3.3568552 .117493$

C $5.687747-3.8282810 .948280$

O $3.272527-2.2410350 .616349$

O $7.060323-3.6643661 .336834$

C $5.443396-2.902689-0.261726$

C $5.495543-5.3211240 .656424$

O $6.132200-3.379318-1.415908$

C $3.946614-2.662035-0.577508$

C $3.817203-1.539407-1.631506$

O $3.379533-3.861590-1.107022$

C 1.3885891 .9381192 .544578

O $1.0512152 .920898 \quad 3.185037$

C 2.7333581 .7114371 .971910

C 3.5679842 .7537321 .844993

C 4.9059332 .7242251 .252170

C 5.5060151 .3776370 .951965

C $2.383973-1.051072-1.860774$

C $2.3898030 .192869-2.726231$

C $2.8216382 .648923-2.617464$

O $2.225606 \quad 0.132261-3.942502$

C $4.2840382 .972968-2.608088$

C $4.7835934 .146547-2.190812$

C $6.2520574 .468115-2.179827$

C $6.7221895 .056593-0.865794$

C $7.0829036 .348217-0.778267$

C 6.8478154 .1326470 .329148

C 5.5219533 .8980481 .001173

N $2.6336731 .348823-2.000122$

H -2.4016503 .0834812 .963137$

H -1.4330331 .4801360 .536788$

H $-0.864874 \quad 1.3519183 .593483$

H -1.066382 -1.004571 1.631282

H -2.420616 -0.5063492 .613829$

H $0.406444-2.7948764 .598209$

H -1.185474 -1.745024 5.855208

H $-1.441586-0.0651175 .379359$

H -2.621766 -1.312921 4.923456

H $0.690416-3.6032062 .157934$

H $0.865334-1.9182511 .667095$

H $2.830243-1.6427193 .131659$

H $2.652619-3.2978863 .735252$

H $2.930437-4.1398511 .368653$

H $4.888978-4.0533612 .961363$

H $5.201273-2.3968852 .482270$

H $7.209282-4.1907642 .140991$

H $5.903378-1.935277-0.016550$

H $5.718901-5.9177551 .549113$

H $\quad 6.194059-5.666079-0.113913$

H $4.480331-5.5686130 .339312$

H $5.625735-4.127825-1.774514$

H $4.425839-0.684196-1.319382$

H $4.224011-1.879429-2.592785$

H $2.581502-3.635717-1.610811$
H $2.980697 \quad 0.711558 \quad 1.634093$

H 3.2262093 .7351642 .172088

H 5.4585640 .7264081 .832229

H 6.5589881 .4220820 .666833

H $4.965670 \quad 0.898096 \quad 0.132839$

H $1.779780-1.813839-2.363151$

H $1.883291-0.816726-0.914520$

H $2.2350783 .370706-2.040483$

H $2.4497732 .630322-3.646368$

H $4.9654982 .205682-2.968911$

H $4.1013784 .925634-1.858101$

H $6.4409245 .175828-2.997394$

H $6.8547423 .579530-2.406223$

H 7.4440906 .7719110 .152996

H $7.0206097 .012545-1.633718$

H $7.5221734 .572431 \quad 1.075470$

H 7.3346663 .2102390 .002361

H 5.0099624 .8190291 .285065

H $2.7884201 .278981-0.998813$

C $-4.5764703 .466233 \quad 1.105717$

O -4.9511112 .1136920 .777654$

C $-6.1500462 .138500-0.018469$

C -6.6902033 .5596510 .079566$

C -5.4049564 .3585700 .182280$

H $-5.8437001 .951979-1.054652$

C -7.1122921 .0386630 .444372$

H -7.299635 $3.833044-0.786980$

H $-7.298542 \quad 3.7040310 .979852$

C -5.6065505 .7705410 .703375$

H $-4.9403464 .405221-0.812036$

C $-6.479983-0.3668410 .512697$

O $\quad-7.591437 \quad 1.3530981 .758653$

H $-7.996828 \quad 1.023705-0.202443$

H $-5.742168-0.4077131 .323479$

O $-7.512153-1.2982890 .860269$

C $-5.825231-0.835276-0.798469$

C $-5.231679-2.249316-0.681716$

H $-5.025898-0.142928-1.068018$

O $-6.783827-0.803418-1.853720$

H $-4.531668-2.2614740 .161133$

H $-6.032217-2.961200-0.447427$

H -6.2607296 .3379390 .033364$

H -6.0640325 .7696621 .698559$

H -4.6518746 .3013910 .772096$

C -3.0680273 .6340390 .922949$

H -6.8167681 .4384552 .340840$

H $-7.914257-0.9883871 .689979$

H -7.515320 -1.393676 -1.603402

H -2.7823314 .6828671 .059610$

H -2.786296 $3.349540-0.097298$

H -4.8577813 .6295972 .154316$

C $-2.372215-1.683746-1.631174$

O $-3.543385-1.799672-2.446457$

C $-4.527372-2.725765-1.972686$

C $-3.913494-4.128423-1.859329$

C $-2.660149-4.123042-0.989003$

C -1.686259-3.049550 -1.468234

H $-2.643217-1.271810-0.651350$

C $-1.414069-0.700689-2.322941$

H $-5.298160-2.773632-2.752353$

O $-4.850223-5.056488-1.322792$

H -3.639845 -4.488626 -2.859597

H $-2.903019-3.9886870 .071477$

O $-2.010247-5.392292-1.092312$

H -1.245709 -3.363538 -2.423479

O $-0.619613-2.931456-0.529650$

C -1.898963 $0.731973-2.326730$

H $-0.452557-0.702148-1.801720$

H -1.265358 $-0.998339-3.367633$

H -5.631649 -5.050013-1.901660

H -2.655085 -6.069082 -0.823453

H $-0.217865-3.811877-0.432660$

O $-0.9358381 .513705-2.901639$

O $-2.9688591 .122999-1.884898$

C $-1.2270222 .912648-2.972863$

H $-1.1682163 .352848-1.973828$

H -0.472458 $3.383818-3.608596$

H $-2.2128843 .084695-3.415546$ 
H -0.6514716 .8308621 .585302$

H -1.8681906 .0329510 .622531$

H $2.0055956 .396060-0.930870$

H 1.6355616 .9825430 .787884

H $0.1533115 .546273-2.264688$

H -1.398109 $5.272516-1.549922$

H $1.1031283 .460707-1.462843$

H -1.2786531 .1759652 .086346$

C $1.799254-3.7107562 .369844$

O $2.088227-2.3516721 .983414$

C $2.650111-1.6545953 .110592$

C $2.507060-2.5912714 .305497$

C $2.613649-3.9500213 .640052$

H $3.716763-1.5190932 .899757$

C $1.973152-0.2903493 .279424$

H $3.280564-2.4198965 .060358$

H $1.531739-2.4816694 .793750$

C $2.101250-5.0906344 .501439$

H $3.665765-4.1346653 .384669$

C 2.0465840 .6211542 .036970

O $0.586174-0.4918653 .585157$

H 2.3991300 .2266664 .147118

H $1.4181780 .209045 \quad 1.237932$

O $1.459052 \quad 1.8714842 .413937$

C 3.4722050 .8722231 .506779

C 3.5157341 .9368650 .395053

H $3.877558-0.0695341 .124320$

O $4.318801 \quad 1.2899802 .577264$

H $2.7799421 .671563-0.368122$

H 3.2208102 .9089560 .808345

H $2.679621-5.1620825 .428363$

H $1.049362-4.9486124 .771708$

H $2.187630-6.0466113 .975533$

C $2.158276-4.6572401 .220677$

H $0.194343-0.9961002 .851427$

H 1.2067782 .3365241 .598178

H 3.9399492 .1041522 .951083

H $2.014870-5.7007041 .522631$

H $3.225458-4.5332970 .996477$

H $\quad 0.727949-3.7639182 .600297$

C $4.7616980 .295623-1.845702$

O $5.4423950 .854651-0.714746$

C $4.9129352 .096493-0.244061$

C $4.9734953 .142411-1.365119$

C $4.2908192 .649816-2.638503$

C $4.8082791 .266072-3.033397$

H $3.7200400 .079093-1.579590$

C $5.453719-1.031856-2.170699$

H $5.5877372 .435797 \quad 0.551917$

O $4.3792304 .361346-0.931567$

H $6.0256873 .360406-1.590407$

H $3.2008732 .649350-2.525390$

O $4.581363 \quad 3.575353-3.688745$

H $5.832611 \quad 1.364579-3.415191$

O $3.9914430 .785876-4.099207$

C $5.176003-2.046234-1.087041$

H $5.097688-1.468235-3.109529$

H $6.537803-0.897310-2.268832$

H $4.4134754 .983607-1.678403$

H $4.1675493 .231588-4.499013$

H $4.4361030 .017214-4.493469$

O $6.263621-2.180413-0.276914$

O $4.106912-2.629332-0.963684$

C $6.112325-3.1019140 .807122$

H $5.301061-2.7810811 .466410$

H $5.923612-4.1093810 .424430$

H $7.044552-3.1118281 .378035$

SCF Energy (B3LYP/6-31G**//MMFF) $=-3245.92275878$

0600010

MM̄FF Geometry

C $-0.638865-2.213722 \quad 2.215412$

C $0.083323-2.5285601 .129868$

C $-0.413267-2.808595-0.263513$

O $-0.003093-1.660968-1.048033$

C $-1.934892-2.980555-0.422090$

C $-2.313359-3.622640-1.742909$ 
C $-2.775450-2.960685-2.821193$ C $-2.178845-5.124132-1.785277$ C $-2.973530-1.480213-2.990247$ C $-4.453892-1.112641-3.163310$ C $-5.260861-1.227683-1.860852$ C $-6.748307-0.987868-2.126539$ C $-7.566921-0.991674-0.828047$ O $-4.761108-0.238852-0.955824$ O $-8.865510-0.472002-1.150507$ C $-6.903327-0.0283390 .177865$ C $-7.790418-2.415845-0.306925$ O $-7.557804-0.0895241 .443551$ C $-5.378736-0.2430730 .336904$ C $-4.773905 \quad 0.926528 \quad 1.147237$ O $-5.150989-1.4710231 .028505$ C $0.668014-1.862292-2.206887$ O $0.968970-2.939727-2.701391$ C $1.039341-0.577460-2.845592$ C $0.5982590 .623266-2.436177$ C $0.9281901 .914840-3.047169$ C $2.0035191 .928362-4.103169$ C $-3.246836 \quad 0.881556 \quad 1.276621$ C -2.7220092 .1905131 .829894$ C $-2.259558 \quad 4.5430361 .189882$ O -2.4410842 .3252443 .018333$ C $-1.722508 \quad 5.212887-0.032663$ C $-0.4369655 .565313-0.180052$ C $0.1061786 .263574-1.391426$ C $1.0200685 .383920-2.213402$ C $2.3549795 .525996-2.160320$ C $0.3790614 .416109-3.181590$ C $0.2658673 .017955-2.636906$ N -2.621993 3.1782050 .861702 H - $1.720502-2.1433032 .158892$ H $1.166562-2.5582151 .231497$ H $0.104980-3.714621-0.600745$ H $-2.433880-2.011541-0.311886$ H -2.333293 -3.6175710 .378317$ H $-3.028246-3.543156-3.706782$ H -2.499092 -5.544405 -2.744587 H -1.138272 $-5.423476-1.627558$ H -2.794978 $-5.581991-1.004540$ H -2.528939 -0.902036 -2.175378 H -2.433542 -1.180591-3.897278 H $-4.497716-0.075196-3.519922$ H $-4.902967-1.742277-3.941602$ H -5.111189-2.223342 -1.429385 H -7.143619-1.729707 -2.831699 H $-6.878230-0.012000-2.615907$ H $-9.367711-0.398187-0.320636$ H $-7.0732770 .989703-0.198764$ H -8.314256 -3.018308 -1.058844 H -8.436311 $-2.418068 \quad 0.578037$ H $-6.861134-2.931688-0.056323$ H $-7.259907-0.899626 \quad 1.891383$ H $-5.055903 \quad 1.871346 \quad 0.664804$ H -5.2002280 .9438142 .158403$ H $-4.256563-1.4606841 .403472$ H $1.693614-0.679623-3.705582$ H $-0.0846760 .676101-1.590093$ H $2.911868 \quad 1.438117-3.734393$ H $2.3088832 .933434-4.401577$ H $1.6638571 .408172-5.005230$ H -2.9335910 .0802591 .953179$ H -2.763555 0.6926950 .311226 H - 1.5424014 .5425562 .017221 H $-3.164098 \quad 5.0554341 .533483$ H -2.429945 $5.426812-0.830289$ H $\quad 0.266458 \quad 5.3589990 .622704$ H $0.6441687 .157122-1.048289$ H $-0.7032596 .643031-2.027910$ H $3.0112774 .921430-2.777394$ H $2.8205886 .244170-1.493654$ H $0.9148724 .443512-4.136033$ H -0.631262 $4.763260-3.434372$ H -0.487514 2.908755-1.856362 H -3.035776 $3.002039-0.049791$ C $0.071249-0.3710813 .742478$ $\begin{array}{llll}\text { O } & 0.975181 & 0.187139 & 2.767016\end{array}$

C 1.8501131 .1249813 .417904

C 1.2243361 .3974384 .779522

C $0.6252520 .041526 \quad 5.104655$

H $2.8079430 .612763 \quad 3.567924$

C 2.0530052 .3568212 .529451

H $1.9607951 .731247 \quad 5.516579$

H 0.4349132 .1557194 .720557

C $-0.4188870 .086056 \quad 6.206305$

H $1.434699-0.6382545 .403282$

C 2.5244372 .0327351 .096530

$\begin{array}{lllll}\text { O } & 0.812803 & 3.065105 & 2.419897\end{array}$

H 2.7532623 .0496333 .009708

H 1.7320501 .5095420 .547766

$\begin{array}{lllll}\text { O } & 2.737504 & 3.273447 & 0.413571\end{array}$

C 3.8257591 .2158231 .023904

C $4.2782520 .964251-0.424975$

H 3.6734520 .2538291 .515705

$\begin{array}{llll}\text { O } & 4.858481 & 1.894498 & 1.736097\end{array}$

H $3.464780 \quad 0.461667-0.957930$

H $4.4470331 .925412-0.925189$

H $\quad 0.0229520 .448098 \quad 7.140504$

H - 1.2483620 .7523315 .946475

H $-0.832582-0.9101036 .391925$

C $-0.012274-1.884217 \quad 3.543507$

H 0.1567112 .4517052 .046496

H 1.8992223 .7644310 .441992

H 4.9796062 .7644581 .318193

H $-0.610983-2.3470334 .335775$

H $0.991416-2.322408 \quad 3.599421$

H -0.9084580 .0907703 .570381$

C $4.629710-2.081402-0.353249$

O $5.511300-1.0969790 .198676$

C $5.5794040 .135902-0.524994$

C $6.038189-0.127582-1.964766$

C $5.152432-1.157503-2.659577$

C $5.023748-2.414637-1.800602$

H $3.596188-1.715484-0.313685$

C $4.732936-3.342206 \quad 0.523244$

H $6.3666710 .728355-0.042233$

O $6.0492641 .087557-2.706929$

H $7.070035-0.502199-1.946290$

H $4.172387-0.733629-2.902203$

O $5.764051-1.496504-3.906246$

H $5.976628-2.959645-1.812940$

O $4.033554-3.272305-2.362715$

C $4.146790-3.1642701 .907899$

H $4.194101-4.1718660 .051970$

H $5.786732-3.6181090 .649429$

H $6.3211420 .870943-3.615178$

H $5.145730-2.069202-4.390477$

H $4.354921-3.566063-3.231521$

O $4.258786-4.3456242 .586335$

O $3.655413-2.1344842 .345920$

C $3.743605-4.3410893 .921588$

H $4.220310-3.5548244 .514727$

H $2.658139-4.2084253 .903086$

H $3.971072-5.3087764 .376480$

SCF Energy (B3LYP/6-31G**//MMFF) $=-3245.92708121$

06_00011

MMFF Geometry

C -0.0133890 .0128472 .953900$

C $0.972599-0.8687643 .182134$

C $1.214287-2.1904442 .492822$

O $2.325713-2.0208871 .580306$

C $0.044919-2.7319451 .649456$

C $0.085297-4.2386791 .483358$

C $0.644590-4.8956410 .449210$

C $-0.612014-5.0119172 .574471$

C $1.424656-4.333452-0.705063$

C $0.667607-4.420922-2.036601$

C $-0.319253-3.260880-2.234319$

C $-1.110987-3.439931-3.530648$

C $-2.022856-2.237454-3.811853$

O $0.449296-2.054345-2.297106$

O $-2.478603-2.365121-5.166980$

C $-1.186382-0.944178-3.709632$ 
C $-3.276993-2.268527-2.932361$

O $-2.0166540 .206722-3.841062$

C $-0.322395-0.856492-2.429026$

C $0.659980 \quad 0.334407-2.538902$

O $-1.179105-0.650993-1.304938$

C $3.595086-2.0785352 .054561$

O $3.952455-2.2343363 .209539$

C $4.484120-1.8929850 .886990$

C $5.756153-1.5090301 .061287$

C $6.722431-1.247471-0.008348$

C $6.289188-1.528458-1.424754$

C $1.7593880 .350997-1.465226$

C $2.6748471 .555731-1.615126$

C $4.6781902 .620601-0.578689$

O $2.5127022 .417680-2.475195$

C $5.9279602 .202050-1.287703$

C $7.1534172 .325801-0.753869$

C $8.4208631 .948169-1.467581$

C $9.3387571 .088766-0.624677$

C 10.3984491 .6229890 .005764

C $9.077763-0.400558-0.582769$

C $7.934232-0.7562950 .324660$

N $3.6886001 .561405-0.666188$

H $-0.749607-0.1723852 .178025$

H $1.695667-0.6283973 .960435$

H $1.482026-2.9267903 .261766$

H $\quad 0.034472-2.2482930 .666557$

H $-0.914687-2.4880122 .118723$

H $0.571646-5.9830520 .439223$

H $-0.525816-6.0952332 .439410$

H $-0.182282-4.7676363 .551101$

H -1.678357 -4.764950 2.591490

H $\quad 1.755277-3.309369-0.518983$

H $2.342578-4.929316-0.789265$

H $1.413611-4.386641-2.841528$

H $\quad 0.147491-5.383337-2.116761$

H $-0.998660-3.217578-1.376162$

H -1.694963 -4.368373 -3.506254

H $-0.412274-3.548857-4.372311$

H -2.996121 -1.569327 -5.379645

H $-0.517005-0.932089-4.580798$

H -3.844055 -3.190383 -3.109484

H -3.958268 -1.448812 -3.184578

H $-3.051321-2.218211-1.865324$

H $-2.4458480 .359452-2.982090$

H $1.1598490 .300060-3.516028$

H $0.1012981 .277954-2.493074$

H $-0.691651-0.176116-0.613722$

H 4.050642 -2.045827 -0.095071

H $6.116864-1.3480862 .076237$

H $6.054145-2.591596-1.548322$

H $7.045480-1.282455-2.172188$

H $5.400954-0.941694-1.683344$

H $\quad 1.320627 \quad 0.381167-0.462865$

H $2.376269-0.551245-1.543932$

H 4.8556032 .8111950 .483871

H $4.2803323 .531557-1.036966$

H $5.823723 \quad 1.804331-2.294377$

H 7.2562202 .7561110 .240008

H $8.9344952 .878333-1.743916$

H $8.2096841 .433577-2.413216$

H 11.0775941 .0101520 .589032

H $10.6074712 .686552-0.042910$

H $9.964821-0.929026-0.208162$

H $8.947285-0.770661-1.603212$

H $8.149934-0.5810191 .379663$

H $3.7654090 .784938-0.016079$

C 0.0271542 .5648462 .923684

O $-1.0274192 .718331 \quad 1.957008$

C -0.4653603 .2682890 .749005$

C 0.9548553 .7059451 .099531

C 1.3379402 .6791002 .147532

H -0.4152462 .4424830 .029085$

C -1.362664 4.3802320 .191081

H 1.6156823 .7056320 .228673

H 0.9787624 .7114291 .534762

C 2.5229193 .0847383 .005207

H 1.5738801 .7400881 .633699
C $-2.855468 \quad 4.0142880 .080167$

O

H $-0.9798734 .702561-0.784117$

H -3.305089 3.9734381 .080177

O $-3.5174055 .080334-0.612667$

C $-3.1491462 .697789-0.657520$

C $-4.6617982 .440053-0.793886$

H $-2.6885771 .871910-0.107396$

O $-2.5546212 .721933-1.952899$

H -5.1285942 .5682110 .188752$

H $-5.1043893 .190093-1.460778$

H 3.4134383 .2348422 .387905

H 2.3283214 .0180883 .543941

H 2.7510022 .3099953 .744206

C $-0.172976 \quad 1.2862613 .740205$

H -1.5460165 .2567611 .936079$

H -3.329069 $5.902134-0.127569$

H -2.928823 $3.479549-2.434503$

H $-1.188203 \quad 1.283708 \quad 4.156590$

H $0.517917 \quad 1.2962134 .591990$

H -0.0737153 .4177013 .608641$

C $-4.738442-0.2095270 .690355$

O $-4.299460-0.001987-0.657339$

C $-4.9862261 .039329-1.358400$

C $-6.4851930 .717139-1.423895$

C $-7.0720060 .442717-0.039793$

C -6.225844 -0.5872490 .711667$

H $-4.576280 \quad 0.710230 \quad 1.263528$

C $-3.850082-1.3111431 .285674$

H $-4.6061901 .020918-2.387091$

O $\quad-7.1883401 .784546-2.050682$

H -6.628121 -0.173397-2.050090

H $-7.175679 \quad 1.3711740 .533683$

O $-8.394905-0.072384-0.208216$

H $-6.372342-1.5694870 .245197$

O $-6.696904-0.6908362 .052349$

C $-3.770790-1.2704462 .795446$

H -4.210152 -2.2945290.960693

H -2.824836 -1.208303 0.911576

H $-8.1317491 .548095-2.055237$

H $-8.738373-0.2820570 .677232$

H $-6.556406 \quad 0.1685112 .485631$

O $-3.440016-2.5104313 .260766$

O $-3.935059-0.2696263 .479970$

C $-3.268284-2.6107884 .677672$

H $-2.452068-1.9601395 .006213$

H $-4.199400-2.3543885 .191810$

$\mathrm{H}-3.008751-3.6456734 .915879$

SCF Energy (B3LYP/6-31G**//MMFF) $=-3245.93676432$

0600012

MM̄FF Geometry

C -1.4031570 .9949582 .442600$

C $-2.333498 \quad 0.3418193 .155553$

C $-2.794967-1.0859192 .982582$

O $-2.074760-1.7746531 .939352$

C -4.273282 -1.080994 2.556505

C $-4.986871-2.3953412 .793327$

C $-5.246097-3.3161811 .844747$

C $-5.481114-2.6043964 .202077$

C $-4.802703-3.3283460 .408166$

C $-5.913816-2.934839-0.577904$

C $-5.995812-1.418057-0.808502$

C $-7.147399-1.060413-1.748273$

C $-7.1702870 .442365-2.069515$

O $-4.753388-1.011526-1.391860$

O $-8.0802580 .621556-3.165190$

C $-5.7643280 .874153-2.540695$

C $-7.7360571 .259231-0.902026$

O $-5.7030302 .285823-2.731311$

C $-4.6210710 .395373-1.614897$

C $-3.2531350 .643645-2.295871$

O $-4.671808 \quad 1.133953-0.392735$

C $-0.903050-2.3869942 .247038$

O $-0.304264-2.3563573 .308509$

C $-0.483034-3.1211721 .033408$

C $0.766674-3.5920040 .919608$

C $1.292181-4.344138-0.223120$ 
C $0.324546-4.711470-1.319986$

C $-2.070325-0.057918-1.610371$

C $-0.7600230 .201041-2.335940$

C $1.629677-0.513644-2.345478$

O $-0.6428721 .042148-3.223372$

C $2.067250-1.839140-2.880844$

C $3.260815-2.381250-2.594114$

C $3.745909-3.692021-3.143355$

C $4.314935-4.600790-2.075023$

C $5.642394-4.704291-1.893531$

C $3.351841-5.453591-1.280464$

C $2.602027-4.668477-0.239189$

N $0.264806-0.606261-1.860630$

H $-0.866173 \quad 0.4896111 .645857$

H -2.836232 0.8841673 .955361

H -2.667268 -1.6163883 .934037$

H -4.333409-0.809195 1.498721

H $-4.830330-0.3010643 .093467$

H -5.813234 -4.200130 2.135326

H $-6.015554-3.5530404 .320024$

H $-4.642845-2.6102754 .905626$

H -6.168767 -1.801196 4.486732

H -3.905646 -2.7213380 .256483$

H $-4.494700-4.3581540 .184037$

H -5.686420 -3.418761 -1.537036

H $-6.882234-3.323226-0.240480$

H $-6.133437-0.9187470 .155583$

H -8.108927 -1.378831 -1.326725

H -7.035384 -1.615133 -2.690599

H $-8.0452041 .557848-3.427228$

H $-5.6150640 .430219-3.534568$

H $-8.7541790 .928183-0.664149$

H -7.821887 2.319514-1.164271

H -7.1382691 .1771000 .008241$

H -5.629061 2.699656-1.854369

H $-3.2932830 .277238-3.330259$

H $-3.053331 \quad 1.721868-2.340821$

H -3.7866551 .1509990 .004131$

H -1.226701 -3.251599 0.254752

H $\quad 1.469977-3.407714 \quad 1.730497$

H $-0.460437-5.369978-0.932034$

H $0.789635-5.230073-2.160293$

H $-0.146275-3.815857-1.739636$

H - $-1.9554870 .296473-0.581966$

H -2.237484 -1.140801 -1.585104

H $2.242248-0.199227-1.495984$

H $1.7005050 .244028-3.131728$

H $1.389444-2.362013-3.551057$

H $3.945260-1.833738-1.951542$

H $4.518228-3.471799-3.891960$

H $2.949820-4.219368-3.683315$

H $6.061394-5.373865-1.149666$

H $\quad 6.340169-4.114738-2.478990$

H $3.897253-6.248485-0.753803$

H $2.691321-5.986936-1.969823$

H $3.225792-4.3511030 .597623$

H $0.081937-1.227591-1.078616$

C -1.1654673 .2794341 .424106$

O -0.0150013 .0382060 .583369$

C $0.5848714 .303398 \quad 0.251891$

C -0.5034175 .3412010 .483967$

C -1.170882 4.7817921 .722046

H 1.3933024 .4715640 .973632

C $1.1502124 .288978-1.169434$

H $-0.097804 \quad 6.3466860 .629962$

H $-1.2161265 .376082-0.348321$

C -2.556692 5.3507801 .975735

H -0.5339204 .9956242 .591102$

C $2.1941273 .188913-1.439490$

O $0.0767184 .102888-2.101509$

H $1.5800295 .270294-1.401665$

H $1.7080832 .208517-1.456678$

O $2.7123993 .403964-2.758607$

C $3.3741613 .146973-0.449473$

C $4.5045772 .208197-0.914069$

H 3.0067452 .8243980 .529640

O $3.9114684 .460037-0.284533$

H $4.0729111 .246395-1.204111$
H $4.9756402 .635826-1.807246$

H -2.505371 6.4334262 .130815

H -3.228133 5.1636191 .130856

H -3.0052574 .9019172 .867621$

C -1.0505732 .4353642 .695252$

H $-0.3595683 .261195-1.883011$

H $1.9564683 .398146-3.370718$

H $4.1945994 .774141-1.160502$

H -1.7028112 .8469503 .474532$

H $-0.0226252 .472853 \quad 3.072712$

H -2.0514172 .9963710 .841462$

C 4.6918270 .0317721 .237047

O $5.076870 \quad 1.407291 \quad 1.356721$

C 5.5951532 .0041020 .162043

C $6.8201231 .217491-0.327216$

C $6.502058-0.262635-0.510756$

C $5.870176-0.8270280 .758802$

H $3.850747-0.0578640 .539606$

C $4.223850-0.4418872 .622109$

H 5.9510163 .0013280 .448941

O $7.3134911 .745046-1.553848$

H 7.6285521 .3090470 .409673

H $5.859472-0.430769-1.382050$

O $7.713596-0.978348-0.761984$

H $6.633402-0.8894141 .545415$

O $5.410950-2.1529780 .507767$

C 2.9270430 .2039773 .058022

H $4.058231-1.5246752 .628651$

H $4.989415-0.1890503 .366160$

H $7.5186712 .684098-1.406069$

H $8.125710-0.580790-1.548153$

H $6.173626-2.6687410 .194604$

O $2.764632-0.0058844 .398508$

$\begin{array}{llll}\text { O } & 2.148095 & 0.792596 & 2.321976\end{array}$

C 1.5786480 .5460434 .976687

H $1.5949260 .337140 \quad 6.049618$

H 1.5544311 .6299994 .832289

H 0.6934930 .0744994 .542431

SCF Energy $(B 3 L Y P / 6-31 G * * / / M M F F)=-3245.94293699$

0600013

MMFF Geometry

C $1.791962-4.189993-1.213675$

C $0.586806-3.651935-0.971772$

C $-0.361725-3.242593-2.069215$

O $-0.943136-1.958959-1.744036$

C -1.507209-4.263586 -2.165885

C $-2.459697-3.979547-3.310190$

C $-3.689388-3.449997-3.157901$

C $-1.970822-4.386089-4.675718$

C $-4.318004-2.989605-1.871239$

C $-5.226691-1.771406-2.089722$

C $-5.864292-1.306751-0.772872$

C $-6.979317-0.294981-1.039681$

C $-7.591260 \quad 0.229110 \quad 0.266284$

$\begin{array}{lllll}\text { O } & -4.841858 & -0.704406 & 0.029898\end{array}$

O $-8.4034481 .361721-0.077108$

C $-6.451624 \quad 0.717748 \quad 1.182250$

C $-8.533177-0.7989680 .903175$

O $-6.957607 \quad 1.0972862 .460521$

C $-5.283543-0.2896881 .329401$

C $-4.105607 \quad 0.4067182 .048415$

O $-5.720333-1.3996522 .115045$

C $-0.237423-0.844147-2.070926$

O $0.894773-0.795878-2.523892$

C $-1.1098780 .323347-1.804065$

C $-0.6275551 .562432-1.977407$

C $-1.3583342 .816969-1.780154$

C $-2.8352122 .730695-1.496730$

C $-2.790680-0.3849292 .061814$

C -1.7034650 .4289722 .740610$

C -0.3516352 .5072372 .439984$

O $-1.2849950 .146033 \quad 3.860340$

C -0.8000533 .8478331 .951267$

C -0.0196354 .6859151 .252755$

C -0.4834426 .0201260 .742101$

C $-0.2870936 .180874-0.750278$

C $0.6064477 .057825-1.238208$ 
C -1.180602 $5.382731-1.679673$ C $-0.6773523 .977608-1.884416$ N -1.3002941 .5128691 .978660$ H $2.090730-4.325488-2.251983$ H $\quad 0.238418-3.5149040 .047555$ H $\quad 0.157360-3.172469-3.033538$ H -2.054247 -4.297919-1.215863 H -1.098878 -5.274411 -2.301604 H -4.311778 -3.340409-4.044895 H -2.722091 -4.214998 -5.454040 H - $-1.078038-3.816307-4.950595$ H -1.721452 -5.452241 -4.688284 H -4.916838 -3.814595-1.467382 H -3.558693 -2.735316 -1.125546 H -4.636859 -0.954590 -2.525697 H $-6.013162-2.034827-2.807998$ H $-6.265120-2.184087-0.251613$ H -7.758285 -0.729332 -1.678911 H -6.571134 $0.557175-1.601728$ H $-8.733568 \quad 1.7495980 .751576$ H -6.0587341 .6388910 .730038$ H $-9.338930-1.0598170 .206575$ H $-9.028447-0.387568 \quad 1.789751$ H -8.027971 -1.7237461 .189564$ H -7.1260570 .2831692 .965095$ H -3.9182061 .3700611 .558251$ H -4.3844850 .6300943 .086545$ H $-4.946008-1.8264192 .514518$ H $-2.1272340 .131080-1.482337$ H $\quad 0.4104791 .673144-2.286082$ H $-3.0199152 .263264-0.525804$ H -3.333452 $3.702354-1.486745$ H $-3.3406172 .138772-2.268500$ H -2.899228 -1.3284542 .606392$ H -2.460957 -0.631793 1.046806 H $\quad 0.6265152 .2319102 .039041$ H -0.3026142 .4971623 .532939$ H -1.8222184 .1407482 .180332$ H 1.0088354 .4040211 .041634 H $0.073736 \quad 6.7945421 .284834$ H -1.541111 6.1902850 .979230 H $0.7364577 .196159-2.306471$ H $1.2335407 .651683-0.581466$ H -1.219068 $5.865247-2.664885$ H -2.201634 5.419771-1.290537 H $0.3811193 .928994-2.143071$ H -1.5853861 .5514811 .005286$ C $2.534435-4.3690301 .266491$ O $2.608923-2.9462451 .491553$ C $3.418015-2.7000802 .654944$ C $3.538222-4.0390603 .370847$ C $3.575858-4.9958682 .197083$ H $4.410697-2.4069972 .292404$ C $2.822407-1.5745293 .503412$ H $4.430663-4.0950704 .001471$ H $2.664429-4.2456063 .999544$ C $3.278431-6.4362012 .577405$ H $4.571697-4.9461051 .736311$ C $2.589251-0.2518242 .746491$ O $1.553564-2.0088134 .011142$ H $3.461054-1.4050924 .378724$ H $1.747859-0.3655852 .052481$ O $2.168744 \quad 0.715306 \quad 3.719070$ C 3.8127730 .2949981 .985794 C 3.5422321 .6884271 .385923 H $4.081622-0.4048591 .189352$ O 4.9122520 .3704142 .891846 H 2.6323631 .6335630 .778986 H 3.3492582 .3956062 .201381 H $4.021462-6.8064963 .291262$ H $2.290606-6.5333443 .040252$ H $3.302904-7.0858591 .697032$ C $2.807398-4.668947-0.209767$ H $1.184702-1.2773584 .535603$ H 2.9224620 .8829664 .310631 H 5.7230120 .4663682 .364540 H $2.915009-5.751192-0.348542$ H $3.766218-4.213944-0.486150$
H $1.527108-4.6937801 .554621$

C $4.1329041 .270389-1.600197$

O $5.0784421 .358828-0.526257$

C 4.7108622 .2408260 .539516

C $4.4764873 .658987-0.002518$

C $3.4741163 .667576-1.151382$

C $3.8820692 .653113-2.215861$

H $3.1912910 .846899-1.230073$

C $4.7172670 .315560-2.653086$

H $5.5834892 .300247 \quad 1.200971$

O 4.0180904 .5330161 .023436

H $5.4267654 .067419-0.370675$

H $2.4551193 .476149-0.797966$

O $3.4572154 .964080-1.751838$

H $4.7853383 .007693-2.729011$

O $2.8488042 .554763-3.192824$

C $4.752817-1.121811-2.182379$

H $4.1198880 .334908-3.570935$

H $5.7461600 .617710-2.883898$

H 4.6805234 .5207051 .735190

H $3.2408525 .603063-1.051240$

H $2.6885713 .449980-3.537590$

O $5.546200-1.842910-3.029175$

O $4.147741-1.563417-1.216140$

C $5.674587-3.234856-2.723900$

H $4.719069-3.741296-2.886582$

H $6.418594-3.663765-3.400505$

H $6.017726-3.373865-1.694131$

SCF Energy $\left(B 3 L Y P / 6-31 G^{* *} / / M M F F\right)=-3245.93136636$

\section{4}

MMFFF Geometry

C $2.8416593 .764951-0.279402$

C $2.3543604 .704806 \quad 0.545327$

C 1.1589104 .5732311 .462520

$\begin{array}{lllll}\text { O } & 0.573495 & 3.253213 & 1.401427\end{array}$

C 0.0755955 .5878011 .049571

C -1.0750865 .6740762 .033420$

C $-2.3435145 .297327 \quad 1.778648$

C $-0.732170 \quad 6.264808 \quad 3.378641$

C $-2.922975 \quad 4.730017 \quad 0.512237$

C $-3.215556 \quad 3.238403 \quad 0.671894$

C $-3.9120882 .602464-0.541313$

C $-5.3489503 .107785-0.709623$

C $-6.1129192 .349088-1.804520$

O $-3.9048641 .189385-0.301511$

O $-7.500612 \quad 2.688102-1.657985$

C $-5.9648990 .834844-1.550025$

C $-5.7024552 .820502-3.204078$

O $-6.5681490 .081502-2.599127$

$\begin{array}{llll}C & -4.503106 & 0.392896 & -1.332074\end{array}$

C $-4.393473-1.085805-0.890900$

O $-3.7920840 .525357-2.566962$

C $1.1024112 .285413 \quad 2.199393$

$\begin{array}{lllll}\text { O } & 2.035253 & 2.399927 & 2.977740\end{array}$

C 0.3496471 .0376591 .937416

C $0.773449-0.1250572 .455205$

C $0.138445-1.4329442 .266388$

C $-1.180415-1.4627581 .540335$

C $-5.090072-1.402193 \quad 0.435110$

C $-4.671284-2.7448941 .009339$

C $-4.732980-4.012870 \quad 3.162344$

$\begin{array}{llll}\text { O } & -4.046704 & -3.587536 & 0.371678\end{array}$

C $-3.461065-3.6854613 .881539$

C $-2.438844-4.5441184 .014448$

C $-1.162676-4.2032614 .731959$

C $0.071688-4.6205023 .961933$

C $0.905390-5.5529214 .453737$

C $0.339546-3.9729992 .621204$

C $0.759693-2.5313682 .745704$

N $-5.096363-2.8852732 .323802$

H $2.3565812 .796845-0.351021$

H 2.8726845 .6608390 .598494

H 1.4946824 .7795322 .485862

H -0.2858275 .3292780 .049226$

H $0.514577 \quad 6.5906090 .958066$

H -3.0766695 .4141322 .576869$

H $-1.6208176 .440743 \quad 3.994477$ 
H $-0.073027 \quad 5.595576 \quad 3.939635$ H -0.2283447 .2288763 .253116$ H -3.845122 5.2827570 .301894 H -2.272632 $4.888394-0.351961$ H -2.273275 2.7059300 .852579 H -3.821486 3.0601411 .570901 H $-3.3190972 .809242-1.438935$ H -5.370564 4.183491 -0.918964 H -5.8895602 .9789720 .238854$ H -8.002795 2.156389-2.299416 H $-6.5474940 .614711-0.647077$ H $-5.9219943 .888309-3.324754$ H $-6.2803392 .308689-3.981392$ H -4.640105 2.676774 -3.411443 H $-5.9671160 .103636-3.363216$ H -4.769096 -1.748785 - 1.680755 H -3.328002 -1.332301-0.788459 H -3.027616 $-0.072614-2.550306$ H -0.5286841 .1072261 .306293$ H $1.679077-0.1266553 .060343$ H -1.919495 -0.8427762 .060317$ H -1.613714 -2.460396 1.461847 H -1.066607 -1.0871850 .519145$ H -4.854751 -0.630606 1.177062 H $-6.176499-1.4260480 .296440$ H -4.639521 -4.9118292 .544505$ H -5.544045 -4.154209 3.882935 H -3.377793 -2.693359 4.320088 H -2.529841 -5.5472063 .604339$ H -1.187943 -4.706515 5.707060 H -1.104585 -3.129093 4.946382 H $1.792114-5.8625333 .910318$ H $0.722015-6.0283185 .411650$ H $-0.526556-4.1126441 .969774$ H $1.155518-4.5016552 .110347$ H $1.714388-2.4023463 .257963$ H -5.554072 -2.099149 2.776315 C $5.2454603 .074733-0.706271$ O $4.9490071 .682518-0.933809$ C 5.5218130 .9134970 .141955 C 6.4100351 .8716050 .933376 C 5.6759763 .1842650 .756781 H 4.6873030 .5973820 .778945 C $6.265929-0.311466-0.398004$ H 6.5100031 .5716321 .980999 H 7.4178261 .9410700 .507714 C 6.5215484 .4078071 .062050 H 4.8078413 .1746411 .426856 C $5.428758-1.264054-1.272392$ O $7.377690 \quad 0.125209-1.192958$ H $6.701428-0.8686470 .439840$ H $5.218231-0.806235-2.246584$ O $6.245004-2.413655-1.541181$ C $4.106247-1.741412-0.642921$ C $3.476375-2.914281-1.420800$ H $3.404935-0.901847-0.602168$ O $4.335548-2.1608050 .703450$ H $3.461728-2.667510-2.486915$ H $4.113259-3.798513-1.299041$ H 6.8609844 .3906962 .102865 H 7.4078764 .4540380 .420545 H 5.9461635 .3262660 .909423 C $4.0664623 .955662-1.129222$ H $7.0254400 .684860-1.906436$ H $7.065424-2.095550-1.955747$ H $4.992941-2.8772380 .680904$ H $3.7889723 .715199-2.162946$ H $4.3794875 .006478-1.118509$ H $6.0939273 .309335-1.363195$ C $0.778819-1.995691-2.537100$ O $1.120932-2.206781-1.161328$ C $2.056962-3.262901-0.920631$ C $1.500793-4.585044-1.465043$ C $1.143478-4.473416-2.942042$ C $0.221378-3.278731-3.170857$ H $1.661732-1.653247-3.089630$ C $-0.277356-0.881656-2.589238$ H $2.123469-3.3729680 .166792$
O $2.431664-5.646786-1.285461$

H $0.594420-4.855710-0.907520$

H $2.038903-4.407328-3.571105$

O $0.450851-5.655490-3.350014$

H $-0.770484-3.501564-2.756466$

O $0.060345-3.075871-4.573850$

C $0.2834450 .482281-2.251086$

H $-0.700808-0.814475-3.597896$

H -1.078299-1.093964-1.873695

H $2.631621-5.701757-0.335226$

H $1.031787-6.411458-3.156710$

H $-0.274415-3.907854-4.950528$

O $-0.6791851 .420540-2.496162$

O $1.4074760 .699579-1.822319$

C $-0.3082982 .778717-2.244058$

H $0.655803 \quad 3.015148-2.704367$

H -1.071160 $3.426688-2.683673$

H $-0.2782282 .954190-1.166770$

SCF Energy (B3LYP/6-31G**//MMFF) $=-3245.93560023$

\section{5}

MM̄FF Geometry

C $2.430055-3.791627-0.996084$

C $1.117604-3.533524-0.886152$

C $0.208786-3.332422-2.073241$

O $-0.587892-2.144860-1.849706$

C $-0.741400-4.535699-2.204305$

C $-1.656995-4.452305-3.410836$

C $-2.973655-4.166587-3.361713$

C $-1.003010-4.757720-4.734310$

C $-3.800066-3.836228-2.150053$

C $-4.444419-2.450009-2.280336$

C $-5.127911-2.014378-0.975708$

C $-5.837531-0.672295-1.161781$

C $-6.425500-0.1400920 .152829$

O $-4.113946-1.8996430 .028521$

O $-6.7709301 .235596-0.071350$

C $-5.333059-0.1835431 .244343$

C $-7.731972-0.8567510 .512428$

O

C $-4.598343-1.5381101 .328358$

C $-3.381768-1.5150302 .287390$

O $-5.507297-2.5259871 .830603$

C $-0.033015-0.951763-2.198271$

O $1.088099-0.765991-2.643207$

C $-1.0410340 .106116-1.963978$

C $-0.7199271 .387698-2.196486$

C $-1.6103792 .544206-2.071925$

C $-3.0257772 .294022-1.625064$

C $-2.261325-0.5392661 .912234$

C -2.3769250 .8246042 .570912$

C -1.362485 3.1007712 .500049

O -3.1504421 .0638473 .493778$

C -2.0307384 .0164911 .525256$

C -1.3954355 .0270330 .913473$

C $-2.0585485 .994790-0.021422$

C $-1.3902116 .048778-1.375725$

C $-0.4715126 .987955-1.658102$

C $-1.8614305 .076130-2.431102$

C $-1.1281563 .765292-2.384273$

N -1.4803111 .7347922 .028269$

H $2.855741-3.854832-1.996334$

H $0.649039-3.4724990 .091662$

H $0.787850-3.218157-2.998278$

H $-1.322588-4.645209-1.282875$

H $-0.156416-5.462431-2.283827$

H -3.531788 -4.162617 -4.297385

H - $1.719055-4.754333-5.563135$

H $-0.232352-4.015712-4.964057$

H $-0.536957-5.748242-4.708151$

H -4.586958 -4.593446 -2.049197

H -3.209607 -3.881415 -1.232805

H -3.669478 -1.719974 -2.544769

H -5.175432 -2.461369-3.098333

H $-5.843841-2.790537-0.680555$

H $-6.617459-0.747338-1.929850$

H $-5.118840 \quad 0.065723-1.541222$

H -7.0727121 .6052240 .776492$ 
H -4.6108970 .6002830 .987494$ H -8.474666 $-0.714442-0.281867$ H -8.181113 -0.4343751 .417840$ H $-7.605307-1.9314940 .658537$ H $-6.362056-0.5919542 .855791$ H -3.718165 -1.366256 3.320567 H -2.937891 -2.521012 2.269713 H -4.990605 -3.265234 2.191921 H -2.025522 $-0.199031-1.630826$ H $\quad 0.290114 \quad 1.614958-2.535546$ H $-3.043691 \quad 1.731477-0.685784$ H $-3.5682691 .724254-2.386844$ H -3.591907 $3.209074-1.437772$ H $-1.307872-0.9603702 .255577$ H -2.192361 -0.4189220 .827465$ H -0.2955673 .3178032 .603790$ H -1.831164 3.2008413 .484049 H $-3.0901303 .858261 \quad 1.338510$ H -0.3432085 .1962541 .127371$ H $-2.025538 \quad 6.9867730 .448369$ H $-3.1243915 .766650-0.145276$ H $-0.0115107 .051057-2.638735$ H $-0.1535947 .708738-0.912029$ H -1.692253 $5.505094-3.428573$ H $-2.9472614 .959716-2.369324$ H $-0.0868283 .845122-2.698394$ H -0.8882811 .4497601 .253341$ C $2.913972-3.8509651 .550873$ O $2.657342-2.4530951 .793541$ C $3.229270-2.0864863 .062771$ C $3.524771-3.3968813 .778120$ C $3.932958-4.2752572 .610720$ H $4.181913-1.5875552 .848643$ C $2.296388-1.1263553 .807483$ H $4.309049-3.2917354 .533848$ H $2.632285-3.8046304 .266634$ C $3.910024-5.7597792 .931702$ H $4.945834-3.9924612 .293559$

C $1.893210 \quad 0.1191662 .991744$ O $1.090281-1.8173844 .157777$ H $2.756192-0.8230044 .754877$ H $1.236775-0.1712582 .162325$ O $1.1189750 .970948 \quad 3.844267$ C 3.0802540 .9314412 .445177 C 2.6273902 .1565881 .631456 H 3.6867570 .2898581 .800159 O $3.913631 \quad 1.3526053 .522679$ H 1.9625621 .8129820 .831009 H 2.0413432 .8279002 .269848 H $4.613742-5.9892873 .738494$ H $2.914607-6.0859953 .251378$ H $4.194003-6.3529692 .056949$ C $3.419798-4.0303680 .116471$ H $\quad 0.698547-2.1592853 .335592$ H $\quad 0.3652630 .4484374 .168376$ H $3.371621 \quad 1.9018254 .114902$ H $3.807047-5.048291-0.009006$ H $4.257858-3.341358-0.051116$ H $1.970552-4.3890711 .705887$ C $4.3556631 .511764-0.801292$ O $4.7852082 .145896 \quad 0.411147$ C 3.8036412 .9698231 .044052 C 3.3777294 .0905610 .087930 C $2.9105903 .538445-1.257281$ C $3.9428672 .568510-1.836986$ H $3.5054790 .856222-0.579692$ C $5.5367580 .645963-1.272544$ H $4.3090003 .443691 \quad 1.895397$ O $2.3489814 .874346 \quad 0.680750$ H $4.2329804 .757457-0.082246$ H $1.9268793 .065324-1.164591$ O $2.7423934 .636711-2.157198$ H $4.8152423 .144776-2.171060$ O $3.3687631 .951585-2.985590$ C $5.132777-0.585452-2.051752$ H $6.2235291 .242955-1.883773$ H $6.0980110 .292391-0.397818$ H 2.0816085 .5451650 .029092
H $2.4743064 .267176-3.016104$

H $4.0687721 .447273-3.432862$

O $6.261023-1.233241-2.469787$

O $3.984249-0.946598-2.261477$

C $6.038870-2.435432-3.213910$

H $5.529631-3.176407-2.590638$

H $5.456966-2.222817-4.115949$

H $7.011834-2.835951-3.510946$

SCF Energy (B3LYP/6-31G**//MMFF) $=-3245.92595782$

\section{6}

MMFF Geometry

C $-0.216051 \quad 0.700031-2.835409$

C $-1.234376-0.147019-3.054101$

C $-1.371606-1.578992-2.587223$

O $-2.525468-1.643239-1.714103$

C $-0.188157-2.140208-1.779136$

C $-0.183724-3.653892-1.691928$

C $-0.650992-4.375876-0.654939$

C $0.458064-4.352426-2.864843$

C $-1.387218-3.8974240 .564061$

C $-0.537637-3.908873 \quad 1.843301$

C $0.232613-2.5974612 .058982$

C $1.066679-2.6526463 .340461$

C $1.747492-1.3077603 .642233$

O $-0.736893-1.548205 \quad 2.163330$

O $2.224781-1.3744184 .994315$

C $0.697594-0.1787443 .560597$

C $2.981328-1.0940022 .762241$

$\begin{array}{lllll}\text { O } & 1.314117 & 1.100880 & 3.689091\end{array}$

C $-0.182840-0.2365492 .291155$

C $-1.3487150 .772767 \quad 2.414546$

$\begin{array}{lllll}\text { O } & 0.611762 & 0.112743 & 1.156096\end{array}$

C $-3.752635-1.844498-2.257247$

O $-4.032250-1.971091-3.437282$

C $-4.714042-1.850472-1.133500$

C $-6.014045-1.621540-1.365269$

C $-7.061070-1.525330-0.345262$

C $-6.669819-1.7988881 .084332$

C -2.4738540 .5682701 .388651$

C -3.4988861 .6897531 .445226$

C $-5.5385602 .524282 \quad 0.274835$

$\begin{array}{lllll}\text { O } & -3.471769 & 2.580612 & 2.290567\end{array}$

$\begin{array}{llll}\text { C } & -6.774079 & 1.987773 & 0.927798\end{array}$

C $-7.975300 \quad 1.9634350 .329263$

$\begin{array}{llll}\text { C } & -9.227905 & 1.455522 & 0.986998\end{array}$

C $-9.988706 \quad 0.4696130 .125976$

C $-11.0845910 .848686-0.553294$

C $-9.527702-0.9718410 .117789$

C $-8.304503-1.173773-0.733712$

N $-4.451947 \quad 1.5754730 .442374$

H $0.6418100 .390425-2.246742$

H -2.066489 $0.210785-3.659195$

H $-1.540872-2.206154-3.471578$

H $-0.194398-1.709428-0.772886$

H $0.765415-1.843824-2.232818$

H $-0.542834-5.459414-0.701095$

H $0.485582-5.440004-2.738832$

H $-0.096324-4.141065-3.784458$

H $1.489786-4.010977-2.996492$

H $-1.841947-2.9168170 .403986$

H $-2.230829-4.5855660 .708362$

H -1.224892 -4.0476812 .688556$

H $0.151140-4.7623001 .843384$

H $0.878904-2.4192431 .192825$

H $1.810112-3.4576983 .287276$

H $0.413564-2.9054254 .187812$

H $2.594182-0.5031375 .220031$

H $0.047286-0.2887514 .439187$

H $3.701133-1.9076162 .909843$

H $3.512113-0.1751043 .034703$

H $2.736719-1.0490741 .700274$

H 1.7377791 .3127142 .839628

H $-1.8022940 .687147 \quad 3.410808$

H $-0.956349 \begin{array}{lll}1.793788 & 2.324709\end{array}$

H 0.0333490 .4673100 .462408

H $-4.314155-1.995134-0.136177$

H $-6.337974-1.455871-2.391905$ 
H $-6.291677-2.8221651 .187735$ H -7.493569 -1.695209 1.792893 H -5.890148 -1.1031661 .413276$ H $-2.0709300 .519497 \quad 0.372938$ H $-2.997027-0.3735631 .590875$ H $-5.6724682 .676284-0.800857$ H $-5.263996 \quad 3.4796120 .733211$ H -6.6819351 .6228951 .948214$ H -8.072170 $2.358618-0.679575$ H -9.8620502 .3247301 .205956$ H $-9.0104620 .993777 \quad 1.958264$ H -11.651425 $0.142873-1.151418$ H $-11.436507 \quad 1.874780-0.530684$ H -10.316209-1.618898 -0.289171 H -9.395755 -1.308952 1.149202 H -8.483482 $-0.994740-1.795141$ H $-4.4166520 .780264-0.187567$ C $0.0563093 .195698-2.362167$ O $1.3539343 .061586-1.754319$ C $1.2599333 .475345-0.376261$ C $-0.1428354 .050090-0.190374$ C $-0.9400443 .258626-1.209106$ H 1.3511352 .5680000 .229901 C $2.3985484 .443064-0.033969$ H -0.5080403 .9235480 .833396$ H $-0.1814815 .119548-0.426982$ C -2.269107 $3.894357-1.574011$ H $-1.1192162 .259501-0.799208$ C $3.8092353 .918433-0.370281$ O $2.2109795 .662697-0.766064$ H 2.3417894 .7181301 .025536 H $3.9288303 .857781-1.459155$ O 4.7336564 .9141350 .087765 C $4.1590582 .561778 \quad 0.272870$ C $5.6011212 .116683-0.037133$ H $3.4674451 .799352-0.099026$ O 3.9776982 .6388861 .685490 H $5.7256502 .087201-1.125233$ H $\quad 6.3067902 .8611150 .349811$ H -2.894909 4.017148 -0.684626 H -2.131354 $4.883370-2.023589$ H $-2.8145063 .271266-2.289712$ C $-0.1644882 .094356-3.401388$ H $2.2044655 .439119-1.712595$ H $5.5748054 .773239-0.378498$ H 4.5676303 .3364452 .019463 H $0.6671942 .118066-4.116798$ H -1.078649 2.313532 -3.965819 H $0.0723134 .157742-2.892391$

C $4.982038-0.716650-1.052279$

O $5.012431-0.2605450 .302480$

C 5.9759230 .7537940 .586302

C 7.3826190 .2622810 .218980

C $7.454832-0.222529-1.227424$

C $6.350619-1.242557-1.508521$

H $4.6900070 .121133-1.698471$

C $3.849392-1.750027-1.168297$

H $5.9580320 .893513 \quad 1.674625$

$\begin{array}{lllll}\text { O } & 8.334978 & 1.298304 & 0.435487\end{array}$

H $7.653728-0.5680050 .884028$

H $7.4119020 .618819-1.928811$

O $8.726885-0.844222-1.428124$

H $6.612431-2.179694-1.004964$

O $6.335537-1.503790-2.910308$

C $4.025127-2.977827-0.294849$

H $2.909976-1.279031-0.853546$

H $3.743675-2.073526-2.209170$

H 9.2050970 .9524890 .172323

H $8.742832-1.180916-2.340660$

H $5.765488-2.276309-3.061461$

O $3.064531-3.892337-0.619684$

O $4.870338-3.1150640 .578371$

C $3.082406-5.1011360 .145795$

H $2.871894-4.8833761 .197033$

H $2.300938-5.760829-0.239902$

H $4.048043-5.6048430 .040448$

SCF Energy (B3LYP/6-31G **//MMFF) $=-3245.92518243$
06_00017

MM̄FF Geometry

C -1.5980513 .7614770 .446233$

C -0.9543453 .4373341 .578164$

C -1.4631312 .5490462 .691328$

O -0.5504981 .4338612 .903805$

C -2.9173882 .0376282 .586823$

C -3.4868011 .5681353 .912662$

C -3.6580050 .2814934 .273106$

C -3.9446752 .6691934 .837053$

C $-3.267912-0.9611923 .524865$

C $-4.479083-1.7240162 .973491$

C $-5.010149-1.1363371 .655958$

C $-6.294230-1.8540701 .233552$

C $-6.791204-1.383933-0.140012$

O $-3.991305-1.3159340 .664687$

O $-7.806796-2.309376-0.556378$

C $-5.621451-1.477376-1.141908$

C $-7.468979-0.011867-0.056840$

O $-5.998351-0.948156-2.411072$

C $-4.319254-0.815939-0.637047$

C -3.121856-1.106039-1.573937

O $-4.5033790 .604446-0.611306$

C -0.2994350 .5605691 .890433$

O -0.6966090 .6294160 .738314$

C $0.511201-0.5474742 .443965$

C $0.673964-1.6568081 .707959$

C $1.405993-2.8645052 .095346$

C $2.046266-2.8915863 .457199$

C $-2.683484-2.573237-1.623857$

C $-1.477905-2.778696-2.525154$

C $0.224077-4.495183-3.130129$

O $-1.084471-1.931148-3.321007$

C $1.056694-5.393817-2.273780$

C $2.293420-5.086671-1.852907$

C $3.103134-5.968589-0.946106$

C $3.338854-5.3469180 .414098$

C $4.571354-4.9895980 .811579$

C $2.154787-5.2122481 .348580$

C $1.467899-3.8804701 .209573$

N $-0.908169-4.031186-2.347538$

H $-2.593983 \quad 3.3740650 .253330$

H 0.0420893 .8439291 .739349

H -1.3760043 .1562193 .600570$

H $-2.988603 \quad 1.2405341 .841044$

H -3.5754512 .8386302 .226165$

H $-4.114803 \quad 0.0817635 .242294$

H -4.4051002 .2810825 .751964$

H -3.1016203 .2985735 .137376$

H -4.688998 3.2986964 .338100

H $-2.551884-0.7555902 .727663$

H -2.741571 -1.611604 4.235256

H $-4.161172-2.7592802 .791872$

H $-5.277276-1.7570783 .725292$

H $-5.201268-0.0660571 .788832$

H $-7.078856-1.7255311 .989798$

H -6.105509 -2.935897 1.181460

H -8.074029 -2.066536 -1.459681

H -5.442107 -2.546771 -1.304151

H $-8.327914-0.0530250 .623813$

H $-7.8740650 .290301-1.028920$

H -6.8021310 .7762990 .298929$

H $-5.9780970 .021770-2.344773$

H $-3.341748-0.736619-2.583947$

H $-2.259953-0.513833-1.236305$

H $-3.6292011 .028697-0.626130$

H $0.918959-0.4340353 .441488$

H $\quad 0.218420-1.6910010 .718308$

H $1.281630-2.8316274 .239553$

H $2.735730-2.0511663 .579467$

H $2.629789-3.7942383 .648326$

H -2.426600 -2.921284 -0.616728

H -3.486813 -3.205634 -2.014583

H $\quad 0.790883-3.638583-3.508727$

H $-0.168945-5.049031-3.988450$

H $0.608842-6.336683-1.968674$

H $2.742255-4.142446-2.153400$

H $4.057178-6.177560-1.446523$ 
H $2.620193-6.944428-0.809019$

H $4.744981-4.5669041 .795766$

H $5.431931-5.1047680 .161245$

H $2.473463-5.4064342 .377283$

H $1.425577-6.003861 \quad 1.133857$

H $\quad 0.950400-3.7535750 .259142$

H $-1.405078-4.712617-1.780261$

C $-0.940426 \quad 3.923762-1.964827$

O $0.046673 \quad 2.874801-1.898554$

C $0.7973592 .864441-3.127945$

C $0.0718543 .809613-4.079102$

C $-0.4990104 .828673-3.113979$

H $1.7820963 .288397-2.899929$

C $0.948418 \quad 1.431823-3.651060$

H $\quad 0.746250 \quad 4.249038-4.820444$

H $-0.7364653 .303269-4.619370$

C -1.616386 $5.667382-3.709367$

H $0.3117745 .491063-2.781457$

C $1.6013860 .448695-2.657418$

O $-0.3482740 .917233-3.979899$

H $1.5136681 .441163-4.590123$

H $0.9307130 .260903-1.810385$

O $1.766617-0.806253-3.329626$

C $2.977704 \quad 0.896332-2.137019$

C $3.643347-0.158670-1.234998$

H $2.8624091 .824243-1.573356$

O $3.837918 \quad 1.180797-3.240136$

H $2.946537-0.424746-0.433049$

H $3.830890-1.068739-1.817161$

H $-1.253124 \quad 6.233775-4.573233$

H -2.451992 $5.043455-4.043942$

H -2.002599 $6.382213-2.976176$

C -1.020019 4.649069-0.620946

H $-0.8839740 .929434-3.167978$

H $\quad 0.890246-1.077825-3.652685$

H $3.9142110 .368297-3.769262$

H $-1.6614235 .533215-0.707236$

H -0.021884 $4.994371-0.327439$

H -1.899926 $3.444286-2.200891$

C 4.1440831 .4885351 .295830

$\begin{array}{llllll}\text { O } & 4.862828 & 1.565004 & 0.059402\end{array}$

C $4.9885360 .326095-0.646437$

C $5.700276-0.710128 \quad 0.233714$

C $5.012913-0.871098 \quad 1.586147$

C $4.817610 \quad 0.492745 \quad 2.249655$

H 3.1081811 .1836171 .104878

C 4.1304302 .8983651 .908851

H $5.6505700 .528480-1.497929$

O $5.760999-1.974628-0.417430$

H $6.735356-0.3881410 .407831$

H $4.063463-1.4058861 .478575$

O $5.844891-1.6844522 .415963$

H $\begin{array}{llll}5.791425 & 0.882177 & 2.573648\end{array}$

$\begin{array}{lllll}\text { O } & 4.005926 & 0.346794 & 3.412130\end{array}$

C $3.251767 \quad 3.8615461 .139746$

H 3.7499252 .8723642 .935711

H 5.1498573 .3032781 .910006

H $6.218281-1.845027-1.265757$

H $5.344403-1.8871703 .224051$

H $4.499178-0.1964664 .049437$

O 3.5093125 .1334281 .566931

O $2.427317 \quad 3.5453350 .294143$

C $2.7377506 .163173 \quad 0.940313$

H $2.8247956 .103923-0.149010$

H 1.6916756 .0839831 .249919

H 3.1293597 .1291631 .269845

SCF Energy (B3LYP/6-31G*//MMFF) $=-3245.92976839$

0600018

MMFF Geometry

C $-0.8719332 .962531 \quad 1.410095$

C -0.4140402 .0431392 .273350$

C -1.1725820 .9129202 .922878$

O $-0.596740-0.3110052 .406885$

C -2.693271 0.8674902 .676427

C $-3.426513 \quad 0.036622 \quad 3.713748$

C $-3.837557-1.2350953 .545057$

C -3.7253240 .7538595 .006751$
C $-3.634428-2.1297692 .355182$

C $-4.960309-2.5282251 .691540$

C $-5.634036-1.3702710 .940189$

C $-6.976456-1.813427 \quad 0.354760$

C $-7.620331-0.710502-0.498272$

O $-4.752831-0.970998-0.112611$

O $-8.692257-1.320987-1.232399$

C $-6.578583-0.195652-1.514642$

$\begin{array}{llll}C & -8.261026 & 0.373803 & 0.375731\end{array}$

O $\begin{array}{llll}-7.091055 & 0.916173 & -2.245868\end{array}$

C $-5.2090520 .147048-0.882138$

C $-4.1664850 .402523-1.994470$

O $-5.3482021 .321567-0.081755$

C $-0.196968-1.2641753 .284600$

O $-0.228170-1.1956564 .505581$

C $0.307840-2.4694652 .585903$

C $0.283123-2.629196 \quad 1.253471$

C $0.768211-3.7970090 .512800$

C $1.453557-4.8871981 .294868$

C $-2.7376110 .602101-1.476227$

C $-1.7568170 .662249-2.626703$

C $-0.525768-0.761149-4.239748$

O $-1.3633091 .733263-3.081727$

C $-0.038640-2.172381-4.290424$

C $1.222379-2.534806-4.010955$

C $1.720322-3.949035-4.075051$

C $2.097133-4.494232-2.716149$

C $3.382408-4.670991-2.367124$

C $0.975557-4.915466-1.792955$

C $0.588656-3.829266-0.824515$

$\mathrm{N}-1.392605-0.592639-3.089774$

H $-1.9012142 .930371 \quad 1.066791$

H 0.6227052 .1220692 .595810

H $-0.978063 \quad 1.0044793 .998707$

H -2.898332 $0.489713 \quad 1.669323$

H -3.1214241 .8772572 .713082$

H $-4.368611-1.7119154 .368508$

H -4.2752700 .1250845 .715188$

H $-2.798867 \quad 1.0670785 .497508$

H -4.3352951 .6427194 .814686$

H -2.956282 -1.6937181 .616453$

H $-3.139895-3.0418422 .712432$

H $-4.741740-3.3358330 .980638$

H $-5.646010-2.9364912 .444375$

H $-5.780632-0.5314371 .629382$

H $-7.661666-2.1346821 .149335$

H $-6.822050-2.696615-0.281320$

H $-9.059083-0.648608-1.831975$

H $-6.430414-0.997135-2.251401$

H $-9.029761-0.064781 \quad 1.023374$

H $-8.7785491 .121878-0.234919$

H $-7.541830 \quad 0.888963 \quad 1.015911$

H -7.041953 $1.697409-1.668962$

H $-4.167049-0.451045-2.684663$

H $-4.4485591 .288035-2.578070$

H $-4.475104 \quad 1.7236210 .046199$

H $0.700133-3.238152 \quad 3.244338$

H $-0.137200-1.8375320 .635519$

H $2.276738-4.4783131 .890208$

H $1.894674-5.6643870 .667635$

H $\quad 0.743749-5.378698 \quad 1.969035$

H -2.646136 $1.535762-0.912453$

H $-2.436180-0.208681-0.803183$

H $0.293659-0.039010-4.175641$

H $-1.105722-0.527505-5.138685$

H $-0.760598-2.932118-4.580278$

H $1.943861-1.776045-3.720361$

H $2.586305-3.968072-4.749170$

H $0.977326-4.612157-4.535804$

H $3.651970-5.087566-1.402227$

H $4.192362-4.396328-3.034451$

H $\quad 1.247646-5.844922-1.283259$

H $0.090409-5.181455-2.384975$

H $0.078660-2.987691-1.293529$

H $-1.906760-1.401375-2.750859$

C $0.1226264 .158794-0.593313$

O $1.0193003 .110150-1.019292$

C $2.0533133 .687472-1.838860$ 
C $1.5121225 .036041-2.292925$

C $0.7516815 .475867-1.061200$

H $2.9173913 .852192-1.183386$

C $2.4495962 .750105-2.985062$

H $2.3083345 .730096-2.578595$

H $\quad 0.8257174 .936728-3.141701$

C $-0.2593686 .577282-1.332392$

H $1.4743055 .834030-0.315241$

C $2.8171011 .316201-2.557726$

O $1.3659412 .652375-3.917140$

H $3.2794743 .194996-3.546533$

H $1.9257290 .786681-2.199908$

O $3.2593830 .619958-3.731240$

C $3.9293361 .229097-1.497745$

C $4.307104-0.229414-1.176532$

H $3.5883891 .722643-0.585019$

O $5.0716621 .936655-1.974867$

H $3.413143-0.740421-0.801137$

H $4.601934-0.738700-2.101860$

H $0.2410337 .472975-1.714673$

H -1.002842 $6.265547-2.073696$

H $-0.7919316 .852544-0.416675$

C $-0.043925 \quad 4.1221150 .927717$

H $0.5857512 .341621-3.426543$

H $2.5604420 .706774-4.401837$

H $5.6603032 .091128-1.216830$

H $-0.5438655 .037717 \quad 1.266073$

H 0.9446164 .1019451 .401964

H $-0.8367303 .982890-1.095848$

C $4.254103-0.1220501 .904380$

O $5.267155 \quad 0.383578 \quad 1.026488$

C $5.471359-0.372564-0.170900$

C $5.825995-1.8278920 .168576$

C $4.792400-2.4579571 .095784$

C $4.572323-1.5667812 .315445$

H $3.277046-0.0736921 .407520$

C $4.2174790 .785146 \quad 3.146162$

H $6.3568450 .059235-0.653697$

O $5.940977-2.617714-1.009874$

H $\quad 6.802357-1.8558010 .669897$

H $3.848305-2.654650 \quad 0.575424$

O $5.274795-3.7253381 .546746$

H $5.470398-1.5889112 .946426$

O $3.496844-2.0855983 .091667$

C 3.6843212 .1722722 .859269

H 3.5703250 .3472203 .914400

H 5.2314730 .9015523 .547432

H $6.612899-2.201587-1.576372$

H $5.439369-4.2684880 .756778$

H $3.720230-3.0040733 .320269$

O 3.6890692 .8933644 .020366

O 3.3084062 .5778931 .769346

C 3.2043504 .2358713 .915224

H 3.3564594 .7283634 .879279

H 3.7590704 .7868393 .149578

H 2.1343324 .2298303 .688942

SCF Energy (B3LYP/6-31G**//MMFF) $=-3245.93667346$

0600019

MM̄FF Geometry

C $-2.096617 \quad 3.5665320 .664822$

C -1.472515 2.3890020 .818260

C -0.7777231 .9839072 .093533$

O 0.5900161 .6209961 .791696

C -1.4832190 .7588802 .702148$

C $-0.927986 \quad 0.3613304 .057538$

C $-0.119621-0.6941904 .281897$

C -1.3800851 .2201345 .211324$

C $0.431811-1.6583183 .269579$

C $1.962712-1.7306933 .334855$

C $2.541644-2.6328602 .232797$

C 4.067531-2.686965 2.332533

C $4.702630-3.4610301 .168520$

O $2.147452-2.0887890 .970004$

O $6.108291-3.1681801 .189766$

C $4.127140-2.928024-0.162316$

C $4.577701-4.9753231 .371227$

O $4.592642-3.707735-1.261007$
C $2.587575-2.836186-0.170813$

C $2.031542-2.128323-1.429834$

O $2.044593-4.162395-0.160116$

C 1.4866772 .6398391 .672990

O 1.2492013 .8362561 .728048

C 2.8296862 .0455121 .485158

C 3.8884622 .8469311 .291723

C 5.2846642 .4253081 .148389

C 5.5772320 .9492441 .183993

C $2.423363-0.655393-1.583683$

C $3.614993-0.414324-2.492988$

C $5.0745151 .439369-3.305477$

O $4.152208-1.297030-3.155178$

C $6.3246151 .474395-2.485207$

C $7.0383722 .590376-2.273009$

C $8.3274872 .623108-1.504822$

C $8.3132793 .628674-0.376565$

C $8.8829904 .837393-0.519728$

C 7.7288073 .1949070 .947694

C $6.235403 \quad 3.3740501 .014431$

N $3.985406 \quad 0.924571-2.498544$

H -2.098399 4.2787941 .486609

H -1.468464 $1.673349-0.000037$

H -0.7810862 .8060502 .820415$

H -1.434197 -0.081421 2.001311

H -2.5553570 .9683552 .821404$

H $0.186548-0.8960445 .307718$

H $-1.0046370 .855847 \quad 6.173621$

H -1.025737 2.2481445 .089231

H $-2.473453 \quad 1.232688 \quad 5.269744$

H $0.010840-2.6510893 .467219$

H $0.132266-1.3818682 .257802$

H $2.367494-0.716723 \quad 3.224926$

H $2.275612-2.1013604 .318892$

H $2.112320-3.6357852 .341948$

H $4.379175-3.1129813 .294425$

H $4.464932-1.6637922 .320890$

H $6.509154-3.5927030 .411673$

H $4.546533-1.924954-0.303151$

H $5.080402-5.2775672 .298001$

H $5.079005-5.5269770 .568423$

H $3.541932-5.3157281 .430832$

H $4.081345-4.534610-1.275809$

H $2.265762-2.710598-2.328950$

H $\quad 0.936658-2.152034-1.350647$

H $1.141040-4.130655-0.515366$

H 2.9148630 .9669541 .537874

H 3.7239263 .9232811 .257216

H 4.9602480 .4135190 .455504

H 5.3739680 .5475322 .181940

H $\begin{array}{llll}6.611991 & 0.702753 & 0.937053\end{array}$

H $1.581240-0.118655-2.038203$

H $2.613343-0.192675-0.611181$

H $4.7787842 .434276-3.653116$

H $5.2225830 .795413-4.178268$

H $6.6750640 .530653-2.073876$

H $6.6999733 .528065-2.708116$

H $9.1291102 .869884-2.213572$

H $8.5829581 .633019-1.107499$

H $8.9056665 .551408 \quad 0.296907$

H $9.3339485 .141627-1.458506$

H 8.1600793 .7997181 .757366

H $8.0497772 .173774 \quad 1.172296$

H 5.9240694 .4196600 .997977

H $3.5167941 .571641-1.870721$

C $-4.3174714 .145724-0.319989$

O $-4.9007032 .848872-0.080450$

C $-6.1275602 .743649-0.826044$

C $-6.4725394 .161039-1.262284$

C $-5.0888784 .736404-1.500268$

H $-5.9029122 .150753-1.720772$

C -7.1965712 .0330780 .011130$

H -7.106480 $4.179850-2.153811$

H $-6.9839134 .718451-0.468910$

C $-5.0633906 .253774-1.558495$

H $-4.6976394 .334144-2.444514$

C -6.7496880 .6700620 .581379$

$\begin{array}{llll}\text { O } & -7.556774 & 2.863605 & 1.122487\end{array}$ 
H $-8.110121 \quad 1.911646-0.581894$ H $\quad-5.991763 \quad 0.818376 \quad 1.360457$ O C $-6.212100-0.311563-0.473977$ C $-5.817257-1.6723510 .128288$ H $-5.3330720 .128540-0.953578$ O $-7.192011-0.509265-1.491477$ H $-5.113809-1.4990440 .950155$ H $-6.702061-2.1576600 .557902$ H -5.684172 $6.617199-2.383939$ H $-5.443166 \quad 6.697450-0.632005$ H $-4.0445276 .621413-1.714925$ C $-2.8199853 .983684-0.585352$ H -6.7462553 .0430401 .629639$ H -8.2047790 .7025351 .888576$ H -7.987142 $-0.873643-1.065871$ H -2.389159 $4.931301-0.927359$ H $-2.6597063 .242817-1.378482$ H $-4.487570 \quad 4.743126 \quad 0.585429$ C $-2.963394-1.737064-0.961798$ O $-4.167831-2.004022-1.684169$ C $-5.199174-2.631346-0.915691$ C $-4.690653-3.957678-0.334169$ C $-3.385179-3.7855580 .442974$ C $-2.355816-3.027271-0.398179$ H $-3.191606-1.059218-0.128966$ C $-2.014809-0.948833-1.876813$ H $-5.999666-2.876249-1.625284$ O $\quad-5.685523-4.5365240 .504081$ H -4.517802 -4.660884-1.159535 H $-3.562350-3.2880561 .403804$ O $-2.879166-5.0869250 .749351$ H -2.001294 -3.680762 -1.203189 O $-1.231128-2.6950240 .408516$ C -1.570936 -1.690797 -3.121485 H -2.521503 -0.039032 -2.221361 H -1.124285 -0.657033 -1.308645 H $-5.319335-5.363614 \quad 0.861272$ H -2.111047 -4.9734381 .334072$ H $-0.811125-3.5249150 .690074$ O $-0.498484-1.039402-3.662391$ O $-2.103147-2.691621-3.580115$ C $0.053966-1.630929-4.842379$ H $0.926101-1.042703-5.140165$ H $\quad 0.376984-2.656327-4.638149$ H $-0.679692-1.609869-5.653636$ SCF Energy (B3LYP/6-31G**//MMFF) $=-3245.92598453$

0600020

MM̄FF Geometry

C $0.518686-0.8417232 .453259$

C $1.054618-1.9478381 .913514$

C $0.489958-2.7270040 .747366$

O $-0.471099-1.9319750 .014993$

C $-0.202800-4.0013791 .250557$

C $-0.656531-4.908090 \quad 0.123902$

C -1.929427 -5.012486 -0.306056

C $0.422800-5.760603-0.491250$

C $-3.122211-4.2400360 .184350$

C $-3.969263-3.711010-0.981878$

C $-5.172707-2.906813-0.471689$

C $-6.135216-2.574530-1.611903$

C $-7.311397-1.715356-1.124973$

O $-4.679185-1.6957530 .111743$

O $-7.978391-1.217955-2.294624$

C $-6.753623-0.508082-0.342920$

C $-8.345344-2.552439-0.363174$

$\begin{array}{lllll}\text { O } & -7.809926 & 0.249154 & 0.243602\end{array}$

C $-5.690418-0.8826050 .719053$

C $-5.0257280 .405188 \quad 1.256032$

O $-6.328193-1.5594981 .803600$

C $0.033258-1.053472-0.895596$

O $1.203389-0.920317-1.217938$

C -1.086322 -0.228406 -1.399422

C $-0.8030590 .930848-2.012213$

C $-1.766576 \quad 1.900977-2.535454$

C $-3.2195221 .508613-2.540263$

C -3.7528530 .1734022 .081111$
C -3.1666381 .5031062 .516108$

C -2.0509173 .5390691 .594952$

O $-3.266670 \begin{array}{lll}1.905562 & 3.672603\end{array}$

C $-2.588737 \quad 4.352747 \quad 0.459836$

C $-1.8218635 .064274-0.379959$

C $-2.3685175 .876013-1.519764$

C $-1.7085495 .553285-2.843007$

C $-0.8706156 .426172-3.427816$

C $-2.0800754 .253649-3.528321$

C $-1.3007323 .083884-2.988677$

N -2.570385 2.1900391 .471016

H $-0.432153-0.4659212 .085618$

H $1.996579-2.3201032 .308650$

H $1.318140-2.9979600 .080459$

H -1.049634 -3.734559 1.893297

H $0.479794-4.5699051 .896905$

H -2.145221 -5.732173 -1.094624

H $\quad 0.032027-6.437485-1.258446$

H $1.184674-5.133859-0.964087$

H $0.904444-6.3763020 .275392$

H $-3.734393-4.9105860 .799271$

H -2.827969 -3.3997090 .818839$

H -3.343434 -3.075740 -1.622141

H $-4.318305-4.555295-1.589498$

H $-5.683556-3.4992370 .296530$

H $-6.502884-3.489105-2.093796$

H -5.596910 -2.019297-2.393045

H -8.678329 -0.610506 -1.998933

H $-6.2843450 .155060-1.082773$

H -8.726433 -3.359201-1.000870

H $-9.219697-1.950985-0.090563$

H -7.943797 -3.0069080 .544946$

H $-8.113767-0.2297731 .033458$

H $-4.766617 \quad 1.0497200 .408554$

H -5.7432620 .9666851 .868429$

H $-5.785624-1.4555472 .601402$

H -2.097956 -0.572622 -1.219092

H $\quad 0.242845 \quad 1.216204-2.121233$

H $-3.3583300 .544860-3.043381$

H $-3.8616082 .218954-3.064621$

H $-3.5962991 .425136-1.517658$

H $-3.961257-0.4183302 .978334$

H -2.995999 -0.3693351 .503905$

H -0.9598853 .4728381 .576225$

H -2.3589973 .9758252 .549732$

H $-3.668194 \quad 4.3586310 .325497$

H -0.745054 $5.081072-0.233156$

H $-2.2204906 .935150-1.272254$

H -3.452783 $5.741738-1.620688$

H $-0.4133096 .212982-4.388473$

H $-0.618377 \quad 7.370580-2.957200$

H -1.866023 $4.324338-4.602830$

$\mathrm{H}-3.1624134 .120215-3.451993$

H $-0.2224763 .252379-2.970142$

H -2.4447551 .7161410 .581474$

C 1.3555031 .4208643 .078958

O 2.1545811 .4708531 .882537

C 3.0489302 .5962431 .958688

C 2.6229853 .3950123 .186319

C 2.0823172 .3025344 .090690

H 4.0504212 .1866812 .135795

C $3.0349593 .370804 \quad 0.633514$

H 3.4569983 .9472583 .630096

H 1.8316834 .1159012 .951464

C 1.1942762 .8214935 .207787

H 2.9299831 .7563274 .525873

C $3.2261842 .492359-0.620156$

O 1.7724834 .0355510 .491916

H 3.7901634 .1643240 .661277

H $2.344357 \quad 1.853708-0.752355$

O $3.2622303 .375142-1.748123$

C $4.4998911 .627610-0.604400$

C $4.6327660 .753878-1.862266$

H 4.4765900 .9763120 .270307

O $5.6495882 .460384-0.476136$

H $3.7027490 .190270-1.989624$

H $4.7460081 .396898-2.743182$

H 1.7520663 .5048055 .856447 
H $\quad 0.328466 \quad 3.363872 \quad 4.812971$

H $\quad 0.822557 \quad 1.997836 \quad 5.825141$

C $1.182385-0.0305843 .530974$

H 1.0819353 .3509200 .478463

H $3.0993112 .843021-2.544926$

H $5.6518623 .075715-1.229209$

H $0.565996-0.0724804 .435524$

H $2.160126-0.4612013 .776424$

H $0.378935 \quad 1.8523982 .826089$

C $4.848302-1.984248-0.507901$

O $5.885871-1.004825-0.630748$

C $5.839815-0.210964-1.820705$

C $5.938687-1.121572-3.052593$

C $4.852194-2.193490-3.041733$

C $4.851247-2.940587-1.709748$

H $3.877401-1.481991-0.421877$

C $5.104450-2.7724150 .788548$

H $6.7415450 .414065-1.803730$

O $5.838331-0.367710-4.255911$

H $6.917718-1.618111-3.063175$

H $3.863628-1.771035-3.256028$

O $5.114598-3.138957-4.081991$

H $5.731032-3.595653-1.664675$

O $3.697054-3.776353-1.642499$

C $4.927561-1.9423852 .041842$

H $4.410980-3.6175090 .862535$

H $\quad 6.135413-3.1469880 .788277$

H $6.5476770 .297441-4.243351$

H $5.151352-2.644220-4.918668$

H $3.712463-4.356346-2.423090$

O $5.294815-2.694245 \quad 3.122444$

O $4.510359-0.7943632 .080772$

C $5.175273-2.0460554 .392722$

H 5.568849-2.721462 5.157069

H $5.760003-1.1213224 .407996$

H $4.123361-1.8424314 .612261$

SCF Energy (B3LYP/6-31G*//MMFF) $=-3245.93156858$

06_00021

MM̄FF Geometry

C $4.150800-2.375399-0.659864$

C $3.895507-3.200547-1.686696$

C $2.600934-3.289602-2.463552$

O $1.660058-2.265777-2.068577$

C $1.934398-4.654226-2.216934$

C $0.737907-4.899241-3.115230$

C $-0.550204-4.823862-2.725802$

C $1.072241-5.295266-4.531369$

C $-1.097669-4.455716-1.372999$

C $-2.121697-3.322024-1.487604$

C $-2.730677-2.897999-0.140691$

C $-3.623950-3.9909250 .456078$

C $-4.368680-3.5204431 .714256$

O $-3.500540-1.715722-0.400708$

O $-5.388816-4.494426 \quad 1.984557$

C $-5.063882-2.1781341 .407326$

C $-3.449500-3.5139772 .940729$

O $-5.670989-1.6412432 .580266$

C $-4.128739-1.140816 \quad 0.753195$

C -4.8869710 .1236050 .293646$

O $-3.155504-0.7196221 .714013$

C $1.786976-1.038801-2.644151$

O $2.643122-0.680543-3.436488$

C $0.685427-0.195826-2.130328$

C $0.6529381 .113668-2.417937$

C $-0.3838102 .057935-1.994328$

C $-1.623558 \quad 1.481438-1.362892$

C $-5.977228-0.126097-0.750331$

C $-6.3307441 .151130-1.486932$

C $-5.5097812 .666176-3.280343$

O -7.370007 1.765511 -1.260139

C $-4.1601993 .094982-3.760007$

C -3.670876 $4.334652-3.606148$

C $-2.3129914 .754103-4.094632$

C $-1.464146 \quad 5.377833-3.006872$

C $-1.0659146 .658226-3.096723$

C $-1.074518 \quad 4.527931-1.815289$

C $-0.180208 \quad 3.377112-2.195020$
N $-5.3539201 .525524-2.397805$

H $3.376833-1.703537-0.301343$

H $4.686987-3.866604-2.025137$

H $2.832980-3.176777-3.529982$

H $1.644988-4.737627-1.163859$

H $2.658338-5.463520-2.383237$

H $-1.316512-5.059927-3.464141$

H $\quad 0.178696-5.524127-5.122031$

H $1.604622-4.487531-5.042436$

H $1.705498-6.188444-4.536395$

H $-1.570251-5.350858-0.954847$

H $-0.311479-4.151362-0.676883$

H -1.647408 -2.447587 -1.951431

H -2.929479 -3.605925 -2.176102

H -1.915862 -2.642087 0.546027

H $-3.048157-4.8957120 .682479$

H -4.370371 -4.293201-0.292071

H -5.914989 -4.169153 2.735473

H $-5.887093-2.4040080 .718013$

H $-3.062383-4.5222103 .131404$

H $-3.996291-3.2292963 .846321$

H -2.593084 -2.846996 2.830334

H -4.966808 -1.254894 3.128199

H -5.3201630 .6418631 .159173$

H $-4.1533380 .829466-0.114147$

H $-2.799403 \quad 0.141217 \quad 1.438406$

H $-0.071556-0.677824-1.522824$

H $1.4631921 .539636-3.008004$

H -2.092126 $0.752335-2.033601$

H -2.386698 2.227376 -1.136974

H $-1.3818020 .980948-0.419563$

H -5.664564 -0.870055 -1.492088

H $-6.885634-0.512101-0.274791$

H -6.042663 $3.463206-2.751377$

H $-6.1259982 .346252-4.126813$

H $-3.5559262 .346217-4.267465$

H $-4.2794795 .092440-3.118280$

H -2.464006 $5.467038-4.915390$

H -1.770509 $3.906800-4.531896$

H $-0.4571577 .117406-2.324846$

H $-1.338807 \quad 7.277963-3.944473$

H -1.981278 $4.213090-1.293545$

H $-0.5233845 .133901-1.083487$

H $0.7581063 .689748-2.655571$

H $-4.6242230 .852751-2.617329$

C $6.139931-0.930297-0.039941$

$\begin{array}{llll}\text { O } & 5.329871 & 0.068103 & 0.614791\end{array}$

C $5.2800061 .235396-0.225759$

C $6.4217961 .080021-1.224659$

C $6.406127-0.417274-1.457028$

H $4.3284261 .190282-0.769496$

C 5.3410642 .5163800 .610490

H $6.2570521 .659522-2.138066$

H $7.383096 \quad 1.385871-0.795949$

C $7.683189-0.957136-2.076305$

H $5.566654-0.646984-2.124314$

C 4.2669782 .6373601 .709827

O $6.6193302 .601888 \quad 1.254758$

H $5.2860623 .385350-0.055811$

H 4.4944001 .9615622 .543353

O 4.3509103 .9672322 .241037

C 2.8217352 .3886811 .241325

C 1.7761392 .7963632 .298525

H 2.7048951 .3286360 .992972

O $2.568576 \quad 3.1297210 .046475$

H 2.0849652 .4113143 .275181

H 1.7538113 .8902432 .369379

H $7.854001-0.506741-3.059551$

H $8.555985-0.740142-1.451496$

H $7.622603-2.042200-2.207125$

C $5.470576-2.3047970 .056248$

H 6.7280791 .8013811 .796701

H 5.2642794 .0989482 .548531

H 2.7187224 .0700040 .244657

H $5.280733-2.5355441 .111665$

H $6.157617-3.070554-0.322520$

H $7.079395-0.9687200 .526714$

C $0.337698 \quad 0.266977 \quad 3.261549$ 
O $\quad 0.277144 \quad 0.863536 \quad 1.959044$

C 0.3606372 .2935511 .938369

C -0.7688082 .8972012 .782287$

C -0.7645002 .3454224 .202562$

C -0.7660850 .8191754 .174830$

H 1.3190170 .4581843 .711358

C $0.177031-1.2505003 .080169$

H 0.1717532 .5977150 .903453

O -0.6722084 .3165712 .828393$

H -1.7352842 .6566412 .319818$

H 0.0834442 .7288294 .782099

O

H -1.7477880 .4616493 .840399$

O

C $1.442466-1.9217182 .593024$

H $-0.082716-1.7203514 .035443$

H $-0.605827-1.4609622 .344947$

H $-0.6882604 .640068 \quad 1.911415$

H -1.952747 3.7520564 .852983

H -1.2633530 .7110636 .058957$

O $1.247572-3.2742682 .607812$

O $2.461849-1.3441262 .244093$

C $2.361446-4.0782702 .208102$

H $2430730-4.0862641 .118432$

H $\quad 3.293647-3.7179392 .653810$

H $2.183645-5.0996462 .555209$

SCF Energy (B3LYP/6-31G**//MMFF) $=-3245.93564750$

0600022

MM̄FF Geometry

C $0.0909402 .319766 \quad 1.665285$

C 1.0081403 .2059181 .251169

C $1.5658033 .192388-0.145449$

O $3.0031473 .087077-0.005254$

C $1.2359444 .455243-0.958034$

C $-0.2354074 .716129-1.199599$

C $-1.0328233 .960331-1.980451$

C $-0.7839585 .942551-0.519146$

C $-0.6609662 .708226-2.727094$

C $-1.4703031 .517412-2.197567$

C $-0.8792390 .160153-2.607561$

C $-1.864616-0.955750-2.252713$

C -1.290899-2.351398-2.514450

O $0.347033-0.016955-1.886978$

O $-2.174422-3.292621-1.886639$

C $0.081549-2.449875-1.821752$

C -1.294718 -2.690935 -4.008756

O $0.714330-3.689386-2.135875$

C $1.018682-1.258236-2.134594$

C $2.291499-1.308747-1.254998$

O $1.435767-1.352874-3.501235$

C $3.6849692 .475032-1.009895$

O $3.2347782 .071094-2.069636$

C $5.0897952 .340994-0.568584$

C $5.8977871 .477403-1.200755$

C $7.2951841 .198646-0.863924$

C $7.913926 \quad 1.9982160 .253482$

C $2.070128-0.9418520 .216480$

C $2.969090-1.7314161 .146149$

C $5.321324-2.2974131 .630104$

O $2.502043-2.4771362 .005608$

C $6.678778-1.7057841 .428053$

C $7.672571-2.3428600 .789928$

C $9.066771-1.8030080 .636708$

C $9.465654-1.615940-0.810517$

C $9.963026-2.636828-1.528806$

C $9.367128-0.224453-1.394303$

C $7.9445470 .236996-1.553305$

N $4.322359-1.5078120 .935491$

H -0.2988651 .5964630 .953325$

H 1.4059103 .9390701 .947877

H $1.2072212 .296466-0.661217$

H $\quad 1.7466414 .410654-1.929355$

H $1.6934805 .325212-0.465819$

H -2.074067 4.259950-2.094687

H $-1.854857 \quad 6.078489-0.702774$

H -0.6407125 .8729620 .563908$

H $-0.2682096 .838209-0.880845$
H $-0.8875972 .853706-3.790318$

H $0.4117582 .508772-2.676095$

H -1.514400 $1.564462-1.102364$

H $-2.5001101 .604498-2.566025$

H $-0.6710630 .168325-3.683577$

H -2.810358 $-0.828101-2.791890$

H $-2.115661-0.881556-1.185964$

H -1.775176 -4.175420 -1.973863

H $-0.113160-2.482783-0.743180$

H -2.317674 -2.658268 -4.403047

H $-0.940866-3.712752-4.184875$

H $-0.687425-2.007468-4.605859$

H $1.075510-3.617403-3.035835$

H $2.764086-2.294539-1.354098$

H $3.031428-0.605506-1.658612$

H $2.241487-0.821942-3.614097$

H 5.4085522 .9310580 .282919

H $5.4945910 .902872-2.033906$

H 7.9132453 .0658640 .006693

H 8.9481941 .7243920 .469543

H 7.3566951 .8528431 .185758

H $2.258446 \quad 0.1249160 .377745$

H $1.037559-1.1264760 .530711$

H $5.253901-3.3229211 .250173$

H $5.074728-2.3215672 .697113$

H $6.860747-0.7249731 .859871$

H $7.486697-3.3334960 .380251$

H $9.756097-2.5097001 .117582$

H $9.192433-0.8605621 .183695$

H $10.278572-2.504614-2.558243$

H $10.062383-3.629475-1.101931$

H $9.831360-0.194109-2.389205$

H $9.9679450 .457784-0.786592$

H $7.404465-0.294070-2.338380$

H $4.605991-0.9966610 .104507$

C $-0.3105130 .783667 \quad 3.583704$

O $-1.217448-0.0652102 .847881$

C $-1.904879-0.9263443 .771856$

C -1.141854 -0.821344 5.086090

C $-0.6971460 .627910 \quad 5.053480$

H $-2.906286-0.503203 \quad 3.914082$

C $-2.011437-2.3430843 .200566$

H -1.768239-1.060685 5.950747

H $-0.270226-1.4858685 .104157$

C 0.4279160 .9401636 .024656

H -1.5600371 .2667015 .285059$

C -2.702032 -2.432006 1.823493

O $-0.695321-2.8934783 .056736$

H $-2.529189-2.9900933 .918025$

H -2.062420 -1.993295 1.048843

O $-2.831856-3.8216631 .496069$

C $-4.097972-1.7828751 .759885$

C $-4.833820-2.0777500 .439008$

H -3.994703 -0.7013261 .884578$

O $-4.895994-2.2553842 .846002$

H $-4.173453-1.833622-0.397165$

H -5.048012 -3.151531 0.382374

H $\quad 0.110956 \quad 0.7475797 .054722$

H $1.3126440 .326127 \quad 5.824910$

H $\quad 0.7247641 .9911405 .953660$

C -0.4304662 .2201433 .069712$

H -0.189375 -2.298382 2.476891

H -1.939805 -4.208688 1.520628

H $-4.961622-3.2222312 .763232$

H $\quad 0.1132172 .9056853 .729257$

H -1.4832562 .5233483 .063465$

H $\quad 0.701517 \quad 0.394744 \quad 3.408214$

C $-5.3500270 .698923-0.770088$

$\begin{array}{llll}\text { O } & -5.985306 & 0.112871 & 0.373540\end{array}$

C $-6.168010-1.3070410 .310626$

C $-6.993257-1.679732-0.929466$

C $-6.389950-1.105811-2.207662$

C $-6.1476310 .393750-2.045921$

H $-4.3292050 .312406-0.861904$

C $-5.2721652 .215650-0.536389$

H $-6.773854-1.5747321 .185351$

O $-7.112724-3.094319-1.035805$

H $-8.009439-1.281680-0.809829$ 
H $-5.471544-1.636523-2.484303$

O $-7.315338-1.317697-3.276013$

H $-7.1165050 .907933-2.014829$

O $-5.4574910 .884938-3.192558$

C $-4.278217 \quad 2.593370 \quad 0.540820$

H $-4.9589942 .730653-1.451165$

H $-6.2559892 .588409-0.226722$

H -7.621778 -3.283349-1.842582

H $-6.937256-0.905754-4.071942$

H -4.598762 $0.433085-3.241750$

$\begin{array}{lllll}0 & -4.406826 & 3.927857 & 0.805254\end{array}$

O $-3.483527 \quad 1.8305131 .071167$

C -3.5301784 .4453891 .809804$

H $-3.729778 \quad 3.9621382 .770775$

H -2.487906 4.3014731 .513081

H -3.720666 5.5172941 .908897

SCF Energy (B3LYP/6-31G*//MMFF) $=-3245.93483404$

06_00023

MMFF Geometry

C $3.309602-3.6296800 .300735$

C $3.263154-3.013532-0.890215$

C $2.343810-3.344892-2.043577$

O $1.560498-2.172758-2.406326$

C $1.437535-4.585619-1.886285$

C $0.621462-4.914444-3.122938$

C $-0.723814-4.868671-3.200717$

C $1.419011-5.376200-4.317456$

C $-1.712729-4.472015-2.139243$

C $-2.633950-3.349536-2.634382$

C $-3.661072-2.933206-1.571337$

C $-4.587625-1.850385-2.121113$

C $-5.585854-1.367902-1.060534$

O $-2.959240-2.428031-0.432546$

O $-6.189427-0.158515-1.546077$

C $-4.820324-1.0070220 .231924$

C $-6.726892-2.373403-0.861678$

O $-5.743619-0.6884391 .268858$

C $-3.799963-2.0809450 .684613$

C $-2.832775-1.5569971 .775931$

O $-4.487777-3.2280521 .183712$

C $0.717177-1.601326-1.504681$

O $0.535619-1.932461-0.344391$

C $0.019144-0.492220-2.194820$

C $-0.849002 \quad 0.267159-1.510557$

C -1.672866 $1.350928-2.050149$

C $-1.5585551 .648391-3.522047$

C -3.419455-1.361529 3.178674

$\begin{array}{llll}C & -3.135906 & 0.023859 & 3.727017\end{array}$

C -3.682086 $2.424146 \quad 3.392270$

O -2.3944650 .1994384 .691192$

C -4.1045893 .2590882 .227368$

C -3.271266 4.0366321 .519254

C $-3.713124 \quad 4.8686490 .349548$

C -3.079926 $4.438071-0.954682$

C $-2.2505625 .253189-1.627136$

C $-3.4835843 .099741-1.531204$

C $-2.4991332 .008875-1.209543$

N -3.7842861 .0252933 .024273$

H $2.654172-4.4709560 .506355$

H $3.928766-2.170935-1.062399$

H $3.006926-3.515930-2.900440$

H $0.786817-4.458992-1.017400$

H $2.059331-5.461496-1.656736$

H $-1.185701-5.164181-4.142755$

H $0.780013-5.736987-5.130731$

H $2.025430-4.557826-4.716923$

H $2.083201-6.200086-4.036230$

H $-2.317388-5.352644-1.890986$

H $-1.222023-4.159481-1.215361$

H $-2.020987-2.484326-2.912487$

H $-3.160341-3.677935-3.539684$

H $-4.233698-3.821504-1.279962$

H $-5.117309-2.205533-3.013823$

H $-3.989740-0.989645-2.451630$

H $-6.621259-0.358422-2.394201$

H $-4.269724-0.0808850 .026790$

H $-7.234144-2.570578-1.813816$
H -7.497164 -1.971664 -0.193961

H $-6.386740-3.331428-0.462447$

H $-6.130816-1.5216961 .586866$

H -2.016120 -2.287972 1.871805

H -2.337932 -0.647022 1.414098

H $-3.876540-3.7238231 .753968$

H $\quad 0.211695-0.355325-3.252761$

H $-0.9870410 .065264-0.449992$

H -2.147428 $2.510700-3.840919$

H $-1.8962280 .789894-4.113063$

H $-0.521747 \quad 1.873733-3.792740$

H $-4.496979-1.5393253 .227839$

H -2.962847 -2.085988 3.864774

H -2.6582102 .6394623 .714953$

H $-4.3467872 .597876 \quad 4.244665$

H $-5.156643 \quad 3.2199111 .953984$

H -2.2191154 .0816351 .790563$

H -3.465142 5.9143310 .572168

H -4.8043354 .8408200 .235574$

H -1.819884 $4.959008-2.578669$

H $-1.9806606 .230111-1.240397$

H -3.651095 $3.199586-2.608191$

H -4.463452 2.806268 -1.132583

H $-2.5032151 .714463-0.159857$

H $-4.519976 \quad 0.7552652 .377351$

C $3.370042-2.6458192 .587972$

O $2.802637-1.3764332 .203140$

C $2.868246-0.4784223 .327428$

C $3.299478-1.3224284 .521238$

C $4.177833-2.3617353 .851604$

H $3.6631300 .243968 \quad 3.109418$

C 1.5293560 .2457963 .504733

H $3.828655-0.7329615 .276072$

H 2.443431-1.803065 5.009137

C $4.433915-3.5852474 .713919$

H $5.137480-1.8968643 .588476$

C 1.0693801 .0474692 .270054

O $0.510634-0.7206853 .794388$

H $1.5777620 .904424 \quad 4.379297$

H $\quad 0.8044670 .365816 \quad 1.453595$

O $-\begin{array}{llll}0.133292 & 1.741938 & 2.620043\end{array}$

C 2.0914952 .0795941 .763425

C $1.5365832 .948288 \quad 0.621445$

H 2.9838651 .5552961 .414900

O 2.4966242 .9268472 .838103

H $1.1020202 .291012-0.137776$

H 0.7272623 .5807871 .006040

H $4.948896-3.3029885 .638003$

H $3.499103-4.0849384 .989415$

H $5.060804-4.3109564 .186806$

C $4.202455-3.2041431 .433362$

H $\quad 0.479227-1.3462963 .050100$

H -0.7663091 .0768972 .939398$

H 1.6997253 .3658713 .182131

H $4.776974-4.0776391 .760215$

H $4.918385-2.4482481 .090574$

H $2.529411-3.3149132 .815822$

C $3.4769562 .295994-1.637073$

O $3.7401143 .126730-0.499733$

C $2.6132283 .861870-0.009217$

C $2.0806624 .780909-1.114582$

C $1.7281953 .992078-2.371947$

C $2.9123383 .125761-2.801159$

H $2.773077 \quad 1.502623-1.358417$

C $4.8046251 .638685-2.047972$

H 2.9934974 .5072380 .792495

O $0.9461255 .500142-0.647480$

H $2.8506875 .522541-1.364436$

H $0.8236053 .393926-2.219713$

O $1.4227134 .918725-3.416137$

H $3.6945253 .778400-3.208974$

O $2.5019722 .263828-3.860877$

C $5.3057340 .633508-1.033033$

H $4.6881761 .105946-2.998219$

H $5.5765072 .410501-2.155060$

H $0.6398696 .063664-1.378511$

H $1.2360374 .399563-4.217241$

H $1.8359261 .651592-3.505169$ 
O $6.542302 \quad 0.198794-1.419003$

O $4.695625 \quad 0.263972-0.040323$

C $7.155177-0.768663-0.560665$

H $7.161791-0.419870 \quad 0.476644$

H $6.629456-1.723905-0.644885$

H $8.189014-0.906732-0.888420$

SCF Energy (B3LYP/6-31G*//MMFF)= -3245.92422057

06_00024

MM̄FF Geometry

C $-2.638204-3.504512-0.260118$

C $-2.261577-4.312687 \quad 0.742326$

C $-1.375583-3.9451011 .909033$

O $-1.024756-2.5441631 .911885$

C $-0.059289-4.7390691 .851153$

C $0.789351-4.5440593 .093235$

C $1.842825-3.7073053 .176122$

C $0.402599-5.3950554 .274789$

C $2.364230-2.7770112 .115034$

C $3.665376-3.3161361 .515274$

C $4.229954-2.4036070 .416676$

C $5.452901-3.064014-0.225133$

C $6.117963-2.156008-1.264922$

O $4.590377-1.1493431 .005959$

O $7.394025-2.736037-1.573663$

C $6.364008-0.776308-0.622483$

C $5.326302-2.132197-2.577942$

O $6.8822410 .136858-1.588568$

C $5.118425-0.1851630 .080801$

C $5.533962 \quad 1.0673470 .893849$

$\begin{array}{lllll}\text { O } & 4.161048 & 0.170651 & -0.912617\end{array}$

C $-1.889806-1.6632662 .479614$

O $-3.012978-1.8926772 .896175$

C $-1.199819-0.3552772 .524405$

C -1.870306 0.7513802 .874305

C -1.2946692 .0913223 .010470$

C 0.1975232 .2262772 .852368

C 4.3841121 .8377071 .562939

C 3.8669382 .9938950 .715931

C 2.6601325 .1504570 .893025

$\begin{array}{lllll}\text { O } & 4.088428 & 3.095479 & -0.488160\end{array}$

C 1.5429515 .7054871 .716236

C 0.2975845 .8831411 .250560

C $-0.828548 \quad 6.4706372 .050732$

C -1.928002 5.4659982 .315614

C -3.0270715 .4364891 .541929$

C $-1.744667 \quad 4.5673623 .520958$

C -2.116038 3.1266263 .284181

N $3.105863 \quad 3.8892811 .458137$

H -2.271148 -2.483663 -0.303499

H $-2.650077-5.3294540 .759716$

H $-1.912058-4.1834902 .836207$

H $0.509093-4.452307 \quad 0.958515$

H $-0.265533-5.8113261 .733798$

H $2.393836-3.6661114 .114996$

H $1.065573-5.2415875 .132913$

H $-0.615714-5.1613814 .600255$

H $0.448481-6.4561594 .009193$

H $1.629622-2.6122981 .321962$

H $2.536150-1.7967832 .575846$

H $4.407679-3.4221432 .317640$

H $3.485542-4.3163591 .100618$

H $3.450689-2.234242-0.333570$

H $5.184246-4.028380-0.674697$

H $6.191936-3.2955180 .555337$

H $7.856215-2.127212-2.175370$

H $7.157744-0.9056070 .125974$

H $5.257444-3.142813-2.998258$

H $5.834812-1.528356-3.337489$

H $4.308658-1.753406-2.460391$

H $6.1393110 .437643-2.139507$

H $6.208050 \quad 0.7349791 .694860$

H $\begin{array}{llll}6.105836 & 1.756208 & 0.260807\end{array}$

H $3.4992940 .753076-0.508901$

H $-0.142647-0.3430592 .281894$

H -2.9348430 .6708653 .089243$

H $0.722354 \quad 1.5787273 .563711$

H 0.5615883 .2383413 .031079
H 0.5052001 .9545561 .837003

H $3.546314 \quad 1.1784541 .813822$

H 4.7650692 .2646432 .499360

H $2.3750124 .998263-0.152982$

H 3.5120105 .8386060 .907735

H 1.7751585 .9894772 .739784

H 0.0710105 .6103090 .223358

H -1.224276 $7.329962 \quad 1.493845$

H -0.4720206 .8823163 .003462$

H $-3.8439014 .749868 \quad 1.734964$

H -3.1373926 .1068610 .695580$

H $-2.363054 \quad 4.9567104 .340610$

H -0.7187694 .6408303 .895798$

H -3.1847522 .9299913 .376889$

H 3.1136683 .7998392 .470464

C $-4.900040-3.113681-1.333429$

O $-4.631685-1.716378-1.567107$

C $-5.356272-0.942434-0.593084$

C $-6.447104-1.866236-0.066087$

C $-5.713308-3.193305-0.037617$

H $-4.654062-0.7241050 .221349$

C $-5.852600 \quad 0.370749-1.205212$

H $-6.816455-1.5572480 .916339$

H $-7.300813-1.922524-0.751090$

C $-6.630149-4.401523 \quad 0.036253$

H $-5.057369-3.1977630 .841039$

C $-4.766628 \quad 1.190365-1.929862$

O

H $-6.3279890 .980407-0.428194$

H $-4.4870980 .695201-2.867991$

O $\begin{array}{lllll} & -5.343140 & 2.449951 & -2.298254\end{array}$

C $-3.4973151 .458717-1.102003$

C $-2.4938892 .348337-1.858161$

H $-3.0200880 .504403-0.859038$

O -3.8458492 .0814830 .132220$

H $-2.3240391 .915724-2.850188$

H $-2.928943 \quad 3.343651-2.010695$

H $-7.227938-4.3751010 .953181$

H $-7.320202-4.434931-0.813426$

H $-6.049555-5.3294560 .036948$

C $-3.590179-3.910727-1.350919$

H $-6.504273-0.510754-2.830539$

H $-6.1371802 .261286-2.827452$

H $-4.3008772 .914973-0.077539$

H -3.079801 -3.741407-2.307085

H $-3.815317-4.981996-1.291172$

H $-5.512720-3.445585-2.181479$

C $-0.1105920 .449476-1.791582$

O $-0.581112 \quad 1.270056-0.714597$

C -1.151427 2.519869-1.113751

C $-0.1109073 .340730-1.886388$

C $0.4800622 .560306-3.058449$

C $0.9702311 .189061-2.592334$

H $-0.9561490 .196160-2.441259$

C $0.420020-0.844779-1.159458$

H $-1.372853 \quad 3.064219-0.187671$

O $-0.6886274 .556407-2.348538$

H $0.7027423 .608764-1.201797$

H $-0.2409842 .472795-3.879211$

$\begin{array}{lllll}\text { O } & 1.587811 & 3.301577 & -3.575607\end{array}$

H $1.8628991 .334734-1.974060$

$\begin{array}{llll}\text { O } & 1.371197 & 0.424058 & -3.724488\end{array}$

C $0.410488-2.036777-2.089778$

H $1.436222-0.687586-0.781643$

H $-0.204492-1.111393-0.300686$

H $0.0003455 .037594-2.838158$

H $1.9862902 .769120-4.285294$

H $\quad 0.5831500 .265994-4.272164$

O $1.122931-3.049299-1.513271$

O $-0.168949-2.091825-3.165281$

C $1.188663-4.265766-2.262701$

H $0.185796-4.682695-2.394927$

H $1.666705-4.089342-3.230898$

H $1.793982-4.979512-1.697298$

SCF Energy (B3LYP/6-31G*//MMFF)= -3245.93779463

0600025

MM̄FF Geometry 
C $-1.0704584 .030844 \quad 1.356121$ C -1.1922642 .7195581 .613257$ C -1.0434342 .1413792 .995861$ O 0.0054551 .1420462 .982216 C $-2.3249431 .424893 \quad 3.457275$ C -3.5307932 .3279773 .617246$ C -4.6178272 .2877182 .821445$ C $-3.484356 \quad 3.2738484 .788361$ C -4.8196111 .4124131 .617024$ C $-5.5502200 .117256 \quad 1.987197$ C $-5.696445-0.8286270 .786624$ C $-6.443479-2.1005861 .198020$ C $-6.501708-3.133410 \quad 0.063277$ O $-4.382245-1.1543950 .323465$ O $-6.929881-4.373314 \quad 0.647327$ C $-5.075905-3.337501-0.493766$ C $-7.562191-2.762807-0.979410$ O $-5.092806-4.199262-1.629166$ C $-4.350691-2.014567-0.821096$ C $-2.871621-2.233168-1.204845$ O $-4.989599-1.401056-1.947164$ C 1.2718191 .5619463 .236070 O 1.6465402 .7200603 .361761 C 2.1890530 .4194383 .449457 C $1.951982-0.8286923 .015860$ C $2.813682-1.9933443 .248305$ C $3.920330-1.8436764 .261712$ C $-1.988512-2.805934-0.096526$ C $-0.537326-2.799536-0.531201$ C $1.084376-4.026238-1.970769$ O $0.251327-1.942511-0.140057$ C $1.882121-4.879213-1.036181$ C $3.091777-4.532154-0.572105$ C $3.907504-5.388800 \quad 0.350255$ C $4.324518-4.6648911 .610732$ C $5.603715-4.3012111 .802885$ C $3.286209-4.4567352 .690379$ C $2.567748-3.1367322 .573606$ N $-0.239211-3.823833-1.414785$ H -0.8270224 .6917512 .186832$ H -1.426199 2.0196790 .817034 H -0.7877412 .9159763 .729965$ H $-2.1270760 .937517 \quad 4.422446$ H -2.533486 0.5839062 .784150 H -5.4498932 .9541993 .042705$ H -4.4193583 .8312284 .909029$ H -3.310173 2.7207355 .717206 H -2.6803044 .0052344 .661604$ H -5.4072891 .9699560 .877154$ H $-3.862898 \quad 1.197274 \quad 1.130644$ H $-4.985405-0.3929842 .778829$ H -6.5409010 .3545522 .394739$ H $-6.238755-0.302548-0.007878$ H -7.453884 -1.860969 1.552610 H -5.929979 -2.560463 2.054601 H $-6.899467-5.052487-0.048335$ H -4.514151 $-3.871800 \quad 0.281932$ H -8.553676 -2.715712 -0.512817 H -7.637294 -3.528252 -1.759521 H -7.375887 -1.799027 -1.457600 H $-5.414962-3.683263-2.387798$ H -2.806995 -2.853168 -2.108255 H $-2.444814-1.264346-1.494201$ H $-4.367449-0.773578-2.351394$ H $3.098080 \quad 0.6723513 .986331$ H $1.050529-1.0235052 .438082$ H $4.465034-2.7695464 .455757$ H $3.516104-1.5141505 .225564$ H $4.660327-1.1108523 .923485$ H -2.066331 -2.212272 0.821236 H $-2.276040-3.8319720 .156093$ H $1.554311-3.052974-2.143541$ H $0.962731-4.534866-2.931786$ H $1.449097-5.830881-0.737786$ H $3.532591-3.592210-0.893207$ H $4.793596-5.721740-0.205763$ H $3.369505-6.3047260 .624999$ H $5.922862-3.8149692 .718229$
H $\quad 6.361476-4.4777761 .046678$

H $3.742938-4.574403 \quad 3.679152$

H $2.542231-5.2627492 .645613$

H $1.744361-3.1514171 .860283$

H $-0.947323-4.523148-1.618220$

C $-1.7964623 .925406-1.134271$

O $-0.8359192 .936950-1.548528$

C $-0.8151502 .872575-2.985937$

C -1.971569 $3.738734-3.472627$

C $-2.0579744 .779556-2.375567$

H $0.1286953 .329372-3.305541$

C $-0.8752201 .413963-3.452527$

H $-1.7781254 .169065-4.460102$

H -2.909460 $3.174946-3.533629$

C $-3.3829085 .522688-2.351530$

H $-1.2420075 .501760-2.513769$

C $0.1914250 .491354-2.828721$

O $-2.1574720 .865495-3.118808$

H $-0.810578 \quad 1.375520-4.546043$

H $-0.0269260 .322681-1.767606$

O $0.084824-0.785977-3.471026$

C $1.6393580 .991365-2.977043$

C $2.676972-0.041302-2.495116$

H $1.7599701 .917460-2.407850$

O $1.8965051 .304915-4.346361$

H $2.419773-0.356546-1.478615$

H $2.620886-0.928109-3.137223$

H -3.544485 $6.050663-3.297131$

H $-4.2241734 .837821-2.200887$

H -3.402952 $6.262641-1.545577$

C -1.2407214 .7379790 .037750$

H -2.285164 $0.984448-2.161687$

H $-0.831423-1.091650-3.358703$

H $1.7605330 .492661-4.863952$

H -1.9050375 .5909190 .221350$

H $-0.2615895 .146745-0.238481$

H $-2.7086403 .391825-0.836972$

C $4.2711221 .444026-0.319847$

O $4.2868901 .673832-1.734617$

C $4.1222520 .503562-2.544905$

C $5.204464-0.534899-2.215536$

C $5.275039-0.836008-0.722736$

C 5.3836170 .4628490 .071284

H $3.2947821 .045202-0.019460$

C 4.4792372 .7976670 .375818

H $4.3026240 .824113-3.578723$

O $4.977890-1.735129-2.946995$

H $6.177423-0.143012-2.540496$

H $4.414344-1.430995-0.398214$

O $6.435064-1.633776-0.479642$

H $\quad 6.3676470 .912716-0.110190$

O $5.313224 \quad 0.164375 \quad 1.462384$

C $3.269373 \quad 3.7016340 .274037$

H 4.6774882 .6566571 .443962

H $5.3264023 .319233-0.085735$

H $5.666279-2.370149-2.684657$

H $6.500669-1.7623920 .482181$

H $4.448599-0.2455081 .631555$

O $3.6124534 .942946 \quad 0.731241$

O $2.1648883 .365546-0.128647$

C 2.5658615 .9180540 .719375

H 2.9879366 .8735551 .042138

H $2.1651886 .036760-0.291775$

H 1.7770975 .6289631 .419545

SCF Energy (B3LYP/6-31G**//MMFF) $=-3245.94357454$

$06 \_00026$

MM̄FF Geometry

C $2.284888-3.0849742 .365504$

C $1.220672-2.4105881 .906821$

C $0.303349-1.5774782 .759148$

O $-1.054348-1.9680212 .446188$

C $0.451803-0.0924242 .386396$

C -0.2050620 .8483253 .379077$

C 0.4617961 .6683454 .214591

C -1.7114930 .8738593 .366467$

C 1.9437091 .7613174 .430898

C 2.5684803 .0827123 .963379 
C 2.6480913 .2230102 .433761

C 3.6798644 .2906132 .058456

C 3.7632824 .4932670 .541100

O $1.3606983 .603073 \quad 1.930391$

O 4.5263335 .6886650 .319405

C $2.3407384 .726392-0.003037$

C $4.5365483 .357901-0.137379$

O $2.3489414 .783785-1.428558$

C 1.2951603 .6997270 .499649

C $-0.117836 \quad 4.1856280 .090928$

O $1.5519122 .438617-0.118882$

C -1.550244 -3.111125 2.984897

O $-0.976156-3.8710853 .750832$

C -2.902954 -3.390521 2.447008

C $-3.691622-2.4153821 .969721$

C $-4.999971-2.5714071 .332077$

C $-5.489260-3.9719191 .080515$

C -1.2767693 .3911370 .701657$

C -2.6305883 .9352970 .270349$

C -5.0696193 .6209580 .710596$

O $-2.7614194 .865232-0.520909$

C $-5.6917992 .733178-0.320014$

C $-6.5995621 .788958-0.029659$

C -7.279885 $0.923745-1.052289$

C $-6.788655-0.506760-1.050218$

C $-6.257713-1.056862-2.155580$

C $-6.960019-1.3249660 .213988$

C $-5.661109-1.4531620 .966228$

$\begin{array}{llll}N & -3.675520 & 3.260116 & 0.884678\end{array}$

H $2.550169-3.0272903 .417541$

H $0.978860-2.4676170 .849108$

H $0.494687-1.7255593 .829647$

H $\quad 0.001750 \quad 0.092141 \quad 1.400795$

H $1.512297 \quad 0.1556402 .272114$

H -0.1210122 .3243954 .860471$

H $-2.115250 \quad 1.7670953 .855130$

H -2.088406 0.8683462 .339780

H -2.111549 -0.0000923 .888962$

H 2.1126501 .6720855 .512249

H 2.4823280 .9179963 .987716

H 2.0087423 .9248304 .390038

H 3.5826353 .1207834 .380903

H 2.9355812 .2550852 .007515

H 4.6688454 .0391802 .461896

H 3.4003505 .2461672 .524793

H $4.5190675 .870056-0.636408$

H $2.033908 \quad 5.725776 \quad 0.335265$

H 5.5509523 .2878340 .273237

H $4.6624263 .550318-1.208476$

H $4.0601672 .383503-0.014089$

H $2.4157553 .872088-1.760665$

H -0.2395315 .2318790 .401720$

H $-0.2130684 .158450-1.002296$

H $0.7411121 .907314-0.093600$

H -3.206876 -4.431350 2.462148

H -3.335969 -1.387967 2.022282

H $-4.779961-4.5190580 .449223$

H $-6.456847-4.0135150 .576674$

H -5.606627 -4.5126162 .026143$

H -1.226262 2.3405220 .402658

H -1.2278293 .4464121 .794566$

H -5.150239 $4.667140 \quad 0.398202$

H -5.5536113 .5178601 .687401$

H -5.405032 2.899593-1.355109

H -6.8920181 .6392091 .007334$

H $-7.1651991 .367958-2.049739$

H -8.358262 $0.928662-0.849986$

H -5.920544 -2.088317 -2.164229

H -6.143298 $-0.489201-3.072892$

H -7.389852 -2.295037 -0.050902

H $-7.701222 \quad-0.8509940 .868922$

H -5.211053 -0.491954 1.218401

H -3.468306 2.4693021 .486598

C $4.337919-3.2352110 .910711$

O $3.881036-2.228225-0.016841$

C $4.557885-2.405838-1.272294$

C $5.797316-3.227940-0.949587$

C $5.256344-4.1645360 .112101$
H $3.892150-2.998189-1.912080$

C $4.832805-1.050092-1.926889$

H $6.190566-3.751692-1.826121$

H $6.600099-2.609321-0.532375$

C $6.336726-4.8260510 .950274$

H $4.663906-4.946329-0.382388$

C $3.593273-0.146089-2.073980$

O $5.788780-0.329912-1.135804$

H $5.306248-1.201301-2.903722$

H $3.2575520 .194858-1.087169$

O $4.0016621 .028469-2.789318$

C $2.406078-0.778920-2.825085$

C $1.2469200 .219275-2.997458$

H $2.045747-1.650041-2.271356$

O $2.838393-1.237896-4.103670$

H $0.9318620 .545628-2.001825$

H $1.6120671 .107055-3.527182$

H $6.991650-5.4373370 .320822$

H $6.959060-4.0836411 .461148$

H $5.894987-5.4767851 .711420$

C $3.128332-3.9687001 .489581$

H $5.417192-0.233869-0.241915$

H $4.7769931 .393411-2.329919$

H $3.180244-0.469024-4.591445$

H $3.451746-4.8307572 .084673$

H $2.511591-4.3624040 .671709$

H $4.893128-2.7143291 .700820$

C $-1.141127-1.401101-1.958604$

O $-0.486169-1.542843-3.224472$

C $0.039747-0.336445-3.784371$

C $-1.0896500 .686618-3.970502$

C $-1.8164720 .954152-2.656812$

C $-2.271268-0.361027-2.029627$

H $-0.404725-1.117208-1.196825$

C -1.727081-2.773151-1.585821

H $0.405663-0.597371-4.785196$

O $-0.5705131 .902840-4.497970$

H $-1.8083000 .298334-4.703948$

H -1.191205 $1.535704-1.970707$

O $-2.9631571 .761085-2.931476$

H $-3.105825-0.758625-2.620439$

O $-2.780576-0.100696-0.722605$

C $-0.674143-3.785056-1.190553$

H -2.406882 -2.659693 -0.735569

H -2.282139 -3.196603 -2.430956

H -1.311953 $2.528025-4.571196$

H -3.433191 $1.885859-2.089648$

H $-2.041251 \quad 0.201329-0.168563$

O $-1.268781-4.778314-0.464867$

O $0.508569-3.730226-1.496211$

C $-0.404508-5.825586-0.013824$

H $0.246541-5.4509320 .780303$

H -1.026878 -6.6271690 .392633$

H $0.186000-6.228448-0.842374$

SCF Energy (B3LYP/6-31G**//MMFF) $=-3245.92132637$

06_00027

MM̄FF Geometry

C $1.524402-0.1883772 .779371$

C $2.661048 \quad 0.3221743 .279320$

C 3.4524771 .4959752 .751732

O 2.7627382 .1715121 .680085

C 4.7850060 .9942962 .168924

C 5.8581892 .0616572 .116711

C 6.1830262 .7806411 .024945

C 6.6275122 .2502623 .399571

C $5.5178962 .783123-0.322981$

C $6.3403052 .063870-1.401906$

C $6.1147740 .544041-1.411190$

C $7.007599-0.134831-2.450490$

C $6.711817-1.638162-2.567311$

O $4.738800 \quad 0.321356-1.736346$

O $7.376923-2.095993-3.754312$

C $5.191983-1.835630-2.758604$

C $7.314313-2.424535-1.397133$

O $4.842113-3.217444-2.717899$

C $4.334828-1.051648-1.737058$

C $2.843526-1.103674-2.150776$ 
O $4.483207-1.648950-0.447274$

C 1.8655213 .1387931 .998459

O 1.5325713 .5059483 .112476

C 1.3341933 .6563040 .719693

C 0.1816934 .3394050 .706203

C $-0.4887434 .862728-0.484920$

C $0.2299664 .736774-1.803610$

C $1.939918-0.136473-1.370687$

C $0.484596-0.250549-1.792475$

C $-1.7690640 .666551-1.246138$

O $0.094961-1.040911-2.648038$

C $-2.1703871 .869997-2.037518$

C $-3.1626382 .692718-1.663258$

C $-3.6402523 .860168-2.479623$

C $-3.8232925 .118710-1.659288$

C $-5.0408675 .492566-1.230559$

C $-2.6081605 .982300-1.405483$

C $-1.7154165 .405980-0.342717$

N $-0.3277150 .629314-1.089479$

H $1.061690 \quad 0.250262 \quad 1.900971$

H $3.093050-0.1543374 .158459$

H 3.6308482 .1963113 .577034

H 4.6115330 .5892891 .166585

H 5.1784770 .1566792 .760769

H 7.0059223 .4900371 .108886

H 7.4016323 .0200743 .312447

H 5.9548912 .5507354 .209033

H 7.1219861 .3163813 .686603

H $4.4999532 .386525-0.277961$

H $5.4070523 .834562-0.618539$

H $6.0289212 .467931-2.374438$

H $7.4067622 .290893-1.283166$

H $6.3265410 .145020-0.413711$

H $8.0678020 .031571-2.221698$

H $6.8383610 .328101-3.433095$

H $7.249468-3.057307-3.820753$

H $4.940904-1.479314-3.767573$

H $8.404150-2.304297-1.378987$

H $7.133110-3.499461-1.506858$

H $6.929213-2.109622-0.424914$

H $5.312205-3.662525-3.442549$

H $2.754304-0.848071-3.214877$

H $2.462497-2.125654-2.028790$

H $3.682550-1.4862220 .075386$

H $1.8895543 .425137-0.182439$

H -0.3344664 .5077791 .650326$

H $1.1773075 .286625-1.775901$

H $-0.3370325 .125942-2.651215$

H $0.4422233 .686682-2.031984$

H $1.994109-0.344583-0.298337$

H $2.2585610 .898751-1.537996$

H -2.195721 $0.674018-0.239988$

H -2.110151 $-0.233526-1.765255$

H -1.657279 $2.046084-2.979705$

H -3.693393 2.489071-0.736292

H $-4.5979313 .574620-2.934746$

H -2.962477 $4.066749-3.317092$

H $-5.1850086 .406515-0.664053$

H -5.920002 $4.889048-1.431385$

H -2.916004 $6.980442-1.066386$

H $-2.0889896 .160317-2.351155$

H -2.154125 5.4427980 .655621

H $\quad 0.090928 \quad 1.256312-0.409147$

C $0.925087-2.5948932 .433660$

O $-0.171255-2.5606711 .491746$

C $-0.735927-3.8843651 .400410$

C $0.270466-4.8176112 .060294$

C $0.806186-3.9325103 .164273$

H -1.651198 -3.881854 2.003491

C -1.054998 -4.252558 -0.050894

H $-0.194688-5.7361442 .431001$

H $1.074921-5.0984131 .369980$

C $2.113188-4.4311673 .757541$

H $\quad 0.051976-3.8617663 .959664$

C $-2.122802-3.371427-0.727030$

O $0.144945-4.163816-0.830298$

H -1.367302 $-5.302437-0.101213$

H - $1.731543-2.363443-0.892869$
O $-2.377346-3.924154-2.024740$

C $-3.458400-3.2784120 .034581$

C $-4.588491-2.636296-0.795085$

H -3.303405 -2.701825 0.951054

O $-3.877540-4.5862950 .430386$

H $-4.217707-1.715659-1.255284$

H -4.864159 -3.319871-1.606629

H $1.978108-5.4202854 .207213$

H $2.894849-4.5127442 .994630$

H $2.474204-3.7528744 .536835$

C $0.843022-1.3903333 .374552$

H $0.473206-3.250851-0.759029$

H -1.528240 -3.952311-2.498419

H $-3.971248-5.121844-0.376302$

H $1.307018-1.6310814 .338088$

H $-0.202952-1.129473 \quad 3.575057$

H $1.850021-2.5707211 .841904$

C $-5.391939-0.0514280 .651445$

O $-5.584097-1.3999081 .098531$

C $-5.846157-2.3521300 .059808$

C $-7.087309-1.933591-0.741712$

C $-6.984106-0.500095-1.250480$

C $-6.6311670 .444332-0.103383$

H $-4.517916-0.003166-0.006747$

C -5.1027630 .8158521 .885995$

H $-6.104591-3.2900930 .567041$

O $-7.307230-2.807098-1.843970$

H -7.972675 -2.005294 -0.096445

H $-6.258482-0.414141-2.068033$

O $-8.246753-0.099206-1.787053$

H -7.4841070 .5273950 .582255$

O $-6.3788751 .743497-0.633339$

C -3.6665380 .6611662 .341678$

H -5.2665371 .8729331 .647302$

H -5.7493090 .5459852 .728177$

H $-7.386894-3.709405-1.490060$

H -8.476487 -0.732989-2.488396

H $-7.1696182 .013122-1.131117$

$\begin{array}{lllll}\text { O } & -3.176310 & 1.874477 & 2.729627\end{array}$

O $-3.054743-0.3984702 .365421$

C -1.8105251 .8781703 .156348$

H -1.556187 2.8926693 .474109

H -1.6725321 .2005184 .004299$

H - 1.1588711 .5964062 .324401

SCF Energy $\left(B 3 L Y P / 6-31 G^{* *} / / M M F F\right)=-3245.92264524$

$06 \_00028$

MM̄FF Geometry

C -2.150474 3.1729731 .582692

C -2.9130612 .9563412 .665138$

C -3.1489171 .6323173 .358103$

O -2.5551680 .5293902 .636256$

C -4.6657481 .3741293 .446771$

C -5.0260170 .0689544 .130220$

C $-5.472378-1.0324803 .492993$

C -4.8947490 .0734785 .631184$

C $-5.649238-1.2240912 .011465$

C $-4.933043-2.4907321 .521741$

C $-4.888639-2.562581-0.012336$

C $-4.269003-3.882637-0.472392$

C $-4.085245-3.934493-1.995067$

O $-4.098232-1.461913-0.473521$

O $-3.230579-5.054540-2.273832$

C $-3.355911-2.653989-2.458853$

C $-5.410433-4.217067-2.712730$

O $-3.301094-2.625520-3.883014$

C $-3.982690-1.357157-1.900021$

C $-3.144838-0.085791-2.192735$

O $-5.266363-1.161402-2.505407$

C -1.2870330 .1607462 .968162$

O $-0.536263 \quad 0.7229443 .748653$

C -0.978583 -1.081140 2.222351

C $0.223261-1.6648782 .341400$

C $0.633544-2.9297451 .723249$

C $-0.374596-3.6450420 .861944$

C $-1.730532-0.086361-1.605692$

C $-0.669845-0.639058-2.541921$

C $1.677340-1.493030-2.510968$ 
O $-0.825131-0.721912-3.756939$

C $1.979214-2.855670-1.975979$

C $3.094937-3.155803-1.294089$

C $3.430566-4.536862-0.813225$

C $3.664782-4.6042450 .677990$

C $4.909643-4.6620151 .180229$

C $2.458364-4.7322791 .578536$

C $1.869164-3.4074151 .983717$

N $0.484549-0.989265-1.858059$ H -1.6035632 .3492541 .133018$ H -3.4250793 .8088713 .108684$ H -2.7313371 .6874464 .371126$ H -5.1020331 .4162772 .442889$ H -5.1490852 .1935223 .996826$ H -5.735787 -1.901403 4.094974 H $-5.222530-0.8699596 .080573$ H $-3.852586 \quad 0.2324525 .925011$ H -5.505161 0.8729636 .063650 H $-6.720761-1.3099781 .795190$ H $-5.282927-0.3623841 .449558$ H -3.908778 -2.4985871 .914180$ H $-5.442962-3.3754261 .922900$ H -5.910079 -2.461275 -0.397396 H $-4.867798-4.734966-0.127678$ H -3.283667-4.003712 -0.002587 H $-3.051557-5.056933-3.230117$ H $-2.322214-2.744429-2.103519$ H $-5.813900-5.186940-2.397594$ H $-5.267417-4.291098-3.796429$ H -6.174177 -3.461659-2.517030 H $-2.710743-1.899612-4.145204$ H $-3.1378800 .128362-3.267994$ H $-3.6730170 .762765-1.733888$ H $-5.505326-0.223044-2.426713$ H -1.769388 -1.503282 1.614283 H $0.970325-1.1913532 .976859$ H $-0.752551-2.9840620 .074861$ H - $-1.220146-3.986706 \quad 1.468750$ H $\quad 0.031359-4.5183740 .347417$ H -1.438591 $0.949860-1.395963$ H $-1.705705-0.625914-0.655358$ H $2.484664-0.783160-2.306507$ H $1.530784-1.540625-3.594264$ H $1.254751-3.638811-2.186410$ H $3.828104-2.375892-1.104376$ H $4.332275-4.862174-1.348871$ H $2.650988-5.257413-1.090951$ H $5.085165-4.7509162 .247240$ H $5.780997-4.6194440 .535801$ H $2.747754-5.2462382 .505963$ H $1.728207-5.4030841 .117177$ H $2.529924-2.8150302 .618521$ H $0.526138-0.843305-0.853459$ C $-2.0162704 .408036-0.593346$ O $-0.7895883 .781925-1.020662$ C $-0.1688614 .602637-2.025139$ C -1.289855 $5.467217-2.578285$ C $-2.0692395 .761056-1.308018$ H $0.5500505 .250521-1.508804$ C $0.5699573 .718824-3.034506$ H $-0.9184336 .368680-3.074077$ H $-1.9168474 .915631-3.288264$ C $-3.4795336 .264736-1.561263$ H $-1.5219756 .514601-0.725648$ C $1.5254732 .703500-2.369755$ O $-0.3851322 .974986-3.801317$ H $1.1135364 .342021-3.753025$ H $\quad 0.935124 \quad 1.974417-1.807327$ O $2.195993 \quad 1.972822-3.401946$ C $2.5891763 .338575-1.456495$ C $3.5581892 .337149-0.806827$ H $2.1132533 .928434-0.672293$ O $3.3671914 .266325-2.221272$ H $4.1252331 .822673-1.595088$ H $4.3236562 .910956-0.269357$ H -3.456965 $7.208764-2.115435$ H -4.063409 $5.545812-2.145813$ H $-4.0059996 .438361-0.617422$
C $-1.996337 \quad 4.523106 \quad 0.933293$

H $-0.9324102 .467138-3.178309$

H $1.5109251 .571274-3.963890$

H $3.7713283 .773422-2.956159$

H -2.792479 $5.197928 \quad 1.267614$

H $-1.035844 \quad 4.9422871 .257559$

H -2.838276 $3.760636-0.924866$

C $5.0127840 .549712 \quad 1.129915$

$\begin{array}{lllll}\text { O } & 3.864618 & 0.202630 & 0.347325\end{array}$

C $2.9369251 .277748 \quad 0.129120$

C 2.4098961 .7587351 .487416

C $3.5450092 .162762 \quad 2.424527$

C 4.5824761 .0417492 .518391

H 5.5919411 .3270240 .617183

C $5.893576-0.703251 \quad 1.236850$

H $2.0965670 .824700-0.400570$

$\begin{array}{lllll}\text { O } & 1.486827 & 2.825847 & 1.336493\end{array}$

H 1.8580970 .9338701 .954177

H 3.9985803 .1088232 .107576

$\begin{array}{llll}\text { O } & 2.993976 & 2.396609 & 3.722659\end{array}$

H 4.1556110 .2115103 .095069

O $5.715317 \quad 1.512153 \quad 3.245623$

C $6.509894-1.085483-0.093177$

H $6.715003-0.5316131 .940910$

H $5.289467-1.5517391 .577930$

H $1.187303 \quad 3.0816482 .225763$

H 3.7338882 .6087504 .317198

H 6.1103852 .2445002 .742289

O $7.265517-2.2103730 .078108$

O $6.351244-0.484032-1.146412$

C $7.894523-2.714310-1.104330$

H $8.591241-1.973532-1.507921$

H $8.454786-3.613315-0.834178$

H $7.139325-2.982675-1.849413$

SCF Energy (B3LYP/6-31G**//MMFF) $=-3245.93627778$

0600029

MM̄FF Geometry

C -0.429193 -3.758182 -0.213871

C $-1.134146-2.617234-0.243923$

C $-1.681861-2.041581-1.525835$

O $-1.429203-0.617424-1.543264$

C $-3.207468-2.219647-1.594543$

C $-3.715111-2.300095-3.020118$

C $-4.198584-1.263585-3.731295$

C $-3.686756-3.681366-3.624060$

C $-4.2744540 .183980-3.334045$

C $-5.7153460 .654501-3.094384$

C $-6.2583330 .233949-1.720597$

C $-7.7327640 .616176-1.580481$

$\begin{array}{llll}C & -8.269916 & 0.308438 & -0.174866\end{array}$

O

$\begin{array}{lllll}0 & -9.535783 & 0.970344 & -0.031005\end{array}$

C -7.3156620 .9261200 .867959$

C -8.537739 -1.1883520 .021585$

\begin{tabular}{lllll}
\hline & -7.691690 & 0.548104 & 2.190525
\end{tabular}

C -5.8252990 .5888650 .624160$

C $-4.9380251 .444683 \quad 1.559449$

O $-5.607119-0.7942520 .908507$

C $-0.172217-0.183062-1.813519$

O $0.810600-0.859604-2.064625$

C $-0.2078521 .291773-1.712807$

C $0.9320211 .973441-1.536502$

C $1.0469503 .423284-1.369919$

C $-0.206297 \quad 4.245370-1.526754$

$\begin{array}{llll}\text { C }-3.435597 & 1.400262 & 1.237867\end{array}$

C $-2.630377 \quad 2.221466 \quad 2.232893$

$\begin{array}{llll}\text { C } & -0.284704 & 2.803226 & 2.833698\end{array}$

O -3.1457332 .8539243 .151514$

C $0.147048 \quad 4.100856 \quad 2.226769$

C 1.4317104 .4830242 .153559

C 1.8876535 .8032051 .599171

C 3.0046595 .6609160 .588018

C 4.2873505 .8474850 .942550

C $2.6265005 .381761-0.848961$

C $2.2559253 .941780-1.071019$

N $-1.266748 \quad 2.158670 \quad 1.981113$

H $-0.282230-4.289793-1.153098$ 
H -1.325355 -2.060891 0.668701 H -1.196923 -2.496655 -2.399155 H -3.705661 -1.400933 -1.063648 H -3.516839 -3.138689-1.078873 H $-4.559342-1.462190-4.740183$ H -4.055501 -3.694032 -4.655239 H -2.664685 -4.073027 -3.635112 H $-4.314043-4.363296-3.040529$ H -3.646940 $0.409076-2.467773$ H $-3.8531730 .766809-4.163236$ H $-5.7189631 .750754-3.154393$ H $-6.3702110 .283356-3.892389$ H -6.136656 -0.848096-1.602042 H -8.339777 $0.111566-2.342407$ H $-7.8506371 .692702-1.768150$ H $-10.1268560 .642158-0.730342$ H -7.435941 2.0171980 .812263 H $-9.273000-1.544695-0.709813$ H $-8.975942-1.3852651 .006238$ H -7.641613 $-1.802777-0.086522$ H -8.6266990 .7897382 .305946$ H -5.2539112 .4944151 .493714$ H -5.0882241 .1253432 .598876$ H -4.686993 -0.922344 1.186846 H -1.179557 $1.771736-1.746445$ H $1.8660741 .416786-1.486379$ H -0.614806 4.130354 -2.536927 H $-0.0525825 .314203-1.366888$ H $-0.9678613 .933245-0.803609$ H $-3.061950 \quad 0.371449 \quad 1.268774$ H -3.2500821 .8050620 .236509$ H 0.5480392 .1048472 .942897 H -0.7198602 .9853713 .821428$ H -0.6291654 .7695191 .862918$ H 2.2016363 .8271162 .552855 H 2.2292536 .4166872 .443170 H 1.0552936 .3571361 .147789 H 5.0912635 .7715650 .217853 H 4.5624016 .0734641 .967492 H $3.4729365 .603943-1.512787$ H $1.8434236 .079623-1.157320$ H $3.1003813 .260328-0.964853$ H -0.9316051 .6440081 .172007$ C $0.379393-3.5859342 .247813$ O $1.281735-2.4867851 .999557$ C $2.249236-2.4266373 .063333$ C $1.698782-3.3045604 .180434$ C $1.005176-4.3926903 .389124$ H $3.170489-2.8792942 .679131$ C $2.521291-0.9785893 .480127$ H $2.487554-3.6848914 .836528$ H $0.971916-2.7680884 .800982$ C $0.002261-5.1913344 .203981$ H $1.768647-5.0763172 .993563$ C $2.960125-0.0344142 .343793$ O $1.330008-0.4230674 .054859$ H $3.270621-0.9665284 .280272$ H 2.1140800 .1796651 .679287 O 3.3257011 .2152682 .947963 C $4.157252-0.5251831 .508733$ C 4.6263980 .5362400 .493936 H $3.874694-1.4372610 .973548$ O $5.239910-0.8612982 .374631$ H $3.7780080 .799982-0.144943$ H $4.920226 \quad 1.4476191 .028544$ H $0.499595-5.6940305 .039901$ H -0.781974 -4.548242 4.617348 H $-0.479947-5.9570693 .588484$ C $0.210099-4.4214560 .975242$ H $0.625071-0.4882183 .387656$ H 2.6017371 .4781163 .541061 H $5.478300-0.0596052 .871224$ H $-0.367690-5.3258391 .199128$ H $1.203796-4.7523890 .648290$ H $-0.577840-3.1554932 .566238$ C $4.626820-1.163218-2.039848$ O $5.610610-1.174539-1.000030$ C $5.8290390 .086510-0.364353$
C $6.2793841 .122290-1.403859$

C $5.3093601 .211847-2.580028$

C $5.018017-0.177145-3.147325$

H $3.651309-0.891216-1.618963$

C $4.528974-2.589407-2.590699$

H $6.669251-0.0612950 .325926$

O $6.4238812 .400357-0.792528$

H $7.2685520 .834668-1.783891$

H $4.3862801 .724120-2.289675$

O $5.9155192 .019652-3.591707$

H $5.900760-0.544865-3.685891$

O $3.946333-0.088918-4.084211$

C $3.878694-3.503764-1.582105$

H $3.922166-2.634827-3.502135$

H $\quad 5.518689-2.987288-2.845895$

H $6.6853863 .027717-1.488078$

H $5.2548152 .159979-4.290541$

H $4.2531910 .447854-4.833951$

O $4.830587-4.155667-0.856370$

O $2.667237-3.603464-1.441132$

C $4.336664-5.0393520 .154308$

H $3.783686-4.4748980 .909896$

H $3.703089-5.812228-0.291247$

H $5.193118-5.5209270 .633534$

SCF Energy $\left(B 3 L Y P / 6-31 G^{* *} / / M M F F\right)=-3245.91359421$

0600030

MM̄FF Geometry

C $-0.111538-3.4176681 .386928$

C $-0.657122-2.1990351 .259028$

C $-1.294582-1.4599752 .406099$

O $-2.706407-1.3862262 .102408$

C $-0.741288-0.0295572 .512728$

C -0.9914450 .5988853 .868908$

C -1.9875951 .4614024 .150397$

C 0.0052000 .2248574 .936456

C -3.0880181 .9372863 .243306$

C -2.9839383 .4348802 .923464$

C -1.828485 3.7697581 .968756

C -1.7359865 .2781931 .735916$

C -0.6558715 .6338540 .703222$

$\begin{array}{lllll}\text { O } & -2.084038 & 3.105664 & 0.727557\end{array}$

O $-0.8701367 .006910 \quad 0.342610$

C $-0.8612944 .765441-0.557635$

C 0.7502745 .5562221 .308879

O $0.2108464 .937317-1.481844$

C -1.048135 $3.260407-0.249545$

C $-1.4916342 .514499-1.531070$

$\begin{array}{llll}\text { O } & 0.190933 & 2.717840 & 0.208495\end{array}$

C $-3.598230-1.6678403 .084699$

O $-3.342694-1.9749954 .240909$

C $-4.992690-1.5928312 .587343$

C $-5.328557-1.0984301 .385345$

C $-6.679954-1.0258010 .823863$

C $-7.810056-1.6000271 .637172$

C $-1.9160951 .057873-1.298046$

C $-2.4111380 .401386-2.575039$

C $-3.633829-1.623674-3.364782$

O $-2.2652860 .900143-3.687783$

C $-5.043462-1.160027-3.557599$

C $-6.123633-1.911350-3.295615$

C -7.533763-1.424099-3.471749

C $-8.327133-1.446680-2.182467$

C $-9.254462-2.393989-1.962926$

C $-8.111374-0.325163-1.188107$

C $-6.840355-0.481583-0.400685$

N -3.017648 -0.822104 -2.323193

H $-0.127469-3.9047522 .359228$

H $-0.672222-1.7196610 .283781$

H - $1.125656-2.0136813 .337680$

H $-1.1717690 .594147 \quad 1.722345$

H $\quad 0.342725-0.0250302 .334972$

H $-2.045893 \quad 1.8610775 .162370$

H -0.2031600 .7171395 .892337$

H $-0.007832-0.8550425 .113541$

H 1.0156120 .5150364 .630044

H -3.1452711 .3557042 .319062$

H -4.0379471 .7600973 .763638$ 
H -3.9306863 .7378062 .457323$ H -2.881988 4.0085123 .853077 H -0.8920373 .3957322 .396880$ H -1.554769 5.8082642 .679448 H -2.7030445 .6484691 .366626$ H $-0.1673147 .271061-0.274683$ H -1.768208 $5.129830-1.060376$ H $0.839135 \quad 6.2484982 .154815$ H 1.5104575 .8705900 .585557 H 1.0086844 .5578811 .668048 H $0.2297265 .873451-1.741646$ H -2.348713 $3.040372-1.972562$ H $-0.6836192 .534004-2.272488$ H 0.2111921 .7657680 .026086 H -5.739241 -1.969634 3.279122 H $-4.543497 \quad-0.712950 \quad 0.737167$ H -7.624734 -2.656176 1.862965 H -8.776420 -1.557483 1.130207 H -7.921314 -1.052850 2.579690 H -1.075079 $0.463128-0.929232$ H $-2.7214261 .014736-0.555889$ H -3.579299 -2.670132 -3.049085 H -3.072073 -1.508443 -4.297263 H -5.180405 -0.146793 -3.928672 H $-5.992123-2.928209-2.933180$ H -8.014295 -2.062373 -4.224651 H -7.557229 $-0.409389-3.888738$ H $-9.851102-2.402584-1.056981$ H $-9.435766-3.185197-2.682887$ H $-8.974837-0.226020-0.520411$ H $-8.0743070 .631030-1.725738$ H -5.960536 -0.089896 -0.910962 H -3.152724 -1.117765 -1.360701 C $1.874611-4.7367760 .608962$ O $2.838624-3.663768 \quad 0.597083$ C $4.070945-4.1590890 .035217$ C $3.892915-5.663971-0.134952$ C $2.403191-5.765812-0.388655$ H $4.169232-3.708548-0.958830$ C $5.260956-3.7692190 .918811$ H $4.498049-6.061760-0.955360$ H $4.164211-6.2101130 .776332$ C $1.853354-7.168001-0.191410$ H $2.195802-5.435701-1.415542$ C $5.463848-2.2507001 .094019$ O $5.081659-4.3376302 .222750$ H $6.177828-4.2187600 .519895$ H $4.643104-1.8187541 .679112$ O $\quad 6.655812-2.0597281 .867299$ C $5.615967-1.477874-0.228611$ C $6.007471-0.002312-0.022258$ H $4.672262-1.526521-0.778880$ O $6.606436-2.109036-1.041413$ H $\begin{array}{llll}5.305113 & 0.455663 & 0.681999\end{array}$ H 7.0042740 .0480720 .431788 H $2.334243-7.869635-0.881120$ H 2.027047 -7.527280 0.828498 H $0.775750-7.197321-0.379566$ C $0.487569-4.200743 \quad 0.252523$ H $4.241354-3.9986722 .576777$ H $6.543089-2.5516922 .698810$ H $7.443102-2.098950-0.545394$ H $-0.189945-5.0344810 .037082$ H $0.548946-3.579287-0.648409$ H $1.877185-5.1578591 .623331$ C $3.7172951 .448907-1.440671$ O $4.789704 \quad 0.717631-2.046904$ C $6.0383890 .786960-1.350980$ C $6.4876142 .247608-1.217338$ C $5.4057053 .125880-0.595418$ C $4.0781012 .935298-1.328950$ H $3.5075261 .045086-0.443055$ C $2.4749721 .244717-2.321102$ H $6.7737550 .289156-1.995740$ O $7.6839332 .325463-0.449430$ H $6.7239552 .636074-2.216759$ H 5.3046992 .9238040 .477455 O $5.8204994 .488632-0.713902$
H $4.1403613 .385823-2.327888$

O $3.0477853 .609648-0.612779$

C $1.888365-0.143814-2.168131$

H $\quad 1.6840311 .954040-2.061808$

H $2.749598 \quad 1.386605-3.373442$

H $7.9214853 .265636-0.375956$

H $5.1786475 .033800-0.228512$

H $3.2235864 .563596-0.671708$

O $1.138621-0.437489-3.271331$

O $2.039392-0.868257-1.193924$

C $0.520106-1.727909-3.267382$

H $1.283970-2.511208-3.270468$

H $-0.136414-1.834133-2.399120$

H -0.082407 -1.819330 -4.174965

SCF Energy (B3LYP/6-31G**//MMFF) $=-3245.91560300$

06_00031

MM̄FF Geometry

C -1.351475 3.0212322 .421203

C -1.7563751 .8341981 .944585$

C -2.1032540 .6653882 .831046$

O $-1.480434-0.5302222 .312208$

C -3.6201520 .4287202 .766620$

C $-4.149623-0.4806633 .854723$

C $-4.440918-1.7866173 .695387$

C -4.4127800 .1964845 .175509$

C $-4.203043-2.6572932 .492201$

C $-5.472789-2.9304371 .671432$

C $-5.740989-1.8492130 .613490$

C $-7.048857-2.114185-0.132089$

C $-7.274146-1.087819-1.255422$

O $-4.645416-1.874310-0.309241$

O $-8.363180-1.551935-2.067549$

C $-6.005035-1.018193-2.134143$

C $-7.734496 \quad 0.263622-0.698431$

O $-6.1162480 .013539-3.111930$

C $-4.691272-0.869899-1.327483$

C $-3.477814-1.078032-2.266331$

O $-4.627916 \quad 0.446684-0.776582$

C $-0.165520-0.7498162 .562409$

O $0.616238-0.0094903 .134014$

C $0.144177-2.0892102 .013138$

C $1.410022-2.5257341 .956404$

C $1.841766-3.8321601 .452467$

C $0.772600-4.8076801 .030445$

C $-2.129221-1.255384-1.548923$

C -1.006785 -1.496738 -2.547194

C $1.319865-2.356621-2.715985$

O $-1.090121-1.170978-3.729508$

C $1.891804-3.677373-2.312344$

C $3.182768-3.854620-1.990215$

C $3.783883-5.189640-1.652744$

C $4.586614-5.170975-0.370740$

C $5.926892-5.078888-0.397318$

C $3.843829-5.3546930 .933123$

C $3.163475-4.0956051 .391195$

N $0.103815-2.094657-1.966206$

H -1.2452873 .1296783 .499372$

H -1.8868491 .6849960 .876927$

H -1.7754670 .8283523 .865061$

H $-3.8705110 .015997 \quad 1.785436$

H -4.1611861 .3824942 .833846$

H -4.864342 -2.320810 4.545562

H -4.818562 -0.4925035 .923762$

H -3.4870270 .6153835 .582246$

H -5.1358921 .0087885 .048713$

H -3.402250 -2.263974 1.859751

H -3.826235 -3.617050 2.869963

H $-5.330841-3.8902821 .157259$

H $-6.340229-3.0417942 .333170$

H $-5.787800-0.8741261 .107524$

H -7.900268 -2.119460 0.559792

H -7.017327 -3.118900 -0.576087

H $-8.123500-2.421394-2.430547$

H $-5.956953-1.960113-2.697327$

H $-6.9958880 .734765-0.046552$

H -8.659351 $0.143037-0.121306$

H $-7.9779870 .962276-1.506610$ 
H $-5.9604110 .862787-2.664655$ H -3.641914 -1.980457 -2.870451 H $-3.404877-0.232334-2.962334$ H -3.697815 $0.687869-0.641587$ H $-0.691901-2.6878581 .667930$ H $2.202970-1.8681682 .308076$ H $\quad 0.145280-5.0801611 .886472$ H $\quad 1.166997-5.7367790 .614472$ H $\quad 0.133658-4.3743200 .253647$ H -1.872050 -0.361850-0.970766 H -2.177824 -2.107975 -0.862262 H $2.009610-1.534852-2.509292$ H $1.100640-2.361811-3.788395$ H $1.222996-4.534568-2.323953$ H $3.856754-3.001389-2.014976$ H $4.431205-5.480969-2.490554$ H $3.015536-5.970131-1.588671$ H $6.512976-5.0953910 .515502$ H $6.467710-4.976202-1.332302$ H $4.543688-5.6499321 .726681$ H $3.159189-6.2025980 .843306$ H $3.860787-3.3251511 .720218$ H $0.139189-2.183988-0.955061$ C -1.190874 4.1974650 .115806 O $-0.1562823 .356451-0.432657$ C $0.3446103 .962252-1.638828$ C $-0.5954065 .118630-1.959693$ C $-1.0201515 .552872-0.571799$ H $1.3303684 .376278-1.398288$ C $0.4747792 .921467-2.754902$ H $-0.0979205 .911886-2.525844$ H -1.465948 4.788271-2.538204 C $-2.2693476 .417066-0.565438$ H $-0.1940556 .109007-0.108376$ C $1.3605141 .709834-2.401528$ O $\quad-0.8360342 .428892-3.062994$ H $0.8459063 .410483-3.663923$ H $0.8497761 .062163-1.678438$ O $1.528198 \quad 0.939153-3.598787$ C $2.7602332 .067434-1.866117$ C $3.6530430 .826291-1.670240$ H $2.6565652 .586977-0.908495$ O $3.4016912 .968196-2.768370$ H $3.1289190 .118360-1.019233$ H $3.8024310 .330046-2.636540$ H $-2.1039447 .335360-1.138503$ H -3.122238 $5.893010-1.009741$ H $-2.543661 \quad 6.7020260 .454877$ C -1.048505 4.2724081 .638123 H $-0.7752061 .927808-3.893421$ H $0.6689710 .539741-3.815094$ H $3.4669442 .522769-3.630625$ H -1.712015 5.056102 2.022690 H $-0.023614 \quad 4.569698 \quad 1.888428$ H -2.155723 $3.755241-0.163891$ C 4.4618701 .1923521 .254420 O 4.9568761 .9197640 .122713 C $5.0435391 .166530-1.089498$ C $5.960598-0.048628-0.888350$ C $5.541559-0.8854560 .316465$ C $5.372679-0.0042061 .554496$ H 3.4404000 .8459041 .054464 C 4.4100272 .1739512 .429103 H $5.5374321 .820751-1.818971$ O $5.978938-0.872907-2.049141$ H $6.9891310 .298799-0.725316$ H 4.628109-1.451706 0.101047 O $6.564480-1.8509730 .571181$ H $6.3621470 .334201 \quad 1.887900$ O $4.817201-0.8074742 .592337$ C 3.3342873 .2078262 .198713 H 4.1727261 .6787253 .376218 H 5.3744732 .6790212 .561389 H $6.271382-0.320988-2.794539$ H $6.291139-2.3614361 .352755$ H $4.914614-0.3237913 .429336$ O $3.894890 \quad 4.399413 \quad 1.847516$ O 2.1370722 .9719242 .295912
C 2.9717845 .4585381 .577267

H 3.5456106 .3510641 .314195

H 2.3295075 .1935660 .732996

H 2.3724915 .6756242 .466523

SCF Energy (B3LYP/6-31G**//MMFF) $=-3245.92120769$

0600032

MM̄FF Geometry

C $0.668776-0.9108773 .772794$

C $1.715351-1.5939964 .263309$

C $2.845505-2.2033333 .466513$

O $3.005526-1.5553922 .183299$

C $2.583672-3.7016773 .240158$

C $3.761014-4.4340012 .625632$

C $3.840516-4.8254761 .338075$

C $4.881051-4.7597413 .580760$

C $2.841430-4.5937200 .238289$

C $3.478942-3.824445-0.926130$

C $2.453219-3.453821-2.007999$

C $3.138685-2.718801-3.161594$

C $2.136613-2.199300-4.203797$

O $1.466905-2.613009-1.403276$

O $2.823306-1.253042-5.038239$

C $1.012115-1.428879-3.477549$

C $1.653333-3.321136-5.130254$

O $-0.012403-1.045674-4.391798$

C $0.413985-2.196833-2.281547$

C $-0.573758-1.345687-1.449194$

O $-0.320008-3.324220-2.776120$

C $3.665893-0.3619062 .185155$

O $4.076524 \quad 0.245938 \quad 3.161744$

C 3.8225980 .0815160 .782077

C 4.4069351 .2619670 .526122

C $4.6899941 .825978-0.796106$

C $4.2538331 .037531-2.001444$

C $0.028340-0.127229-0.743919$

C $-0.1074311 .173999-1.513983$

C $0.5628563 .572927-1.376535$

O $\quad-0.783697 \quad 1.290727-2.531723$

C $1.7378993 .811180-2.269750$

C $2.6378154 .784493-2.063102$

C $3.7833525 .071987-2.989759$

C $5.1218015 .067602-2.287716$

C $5.7172496 .216612-1.925754$

C $5.8125093 .737272-2.094918$

C $5.3390053 .007305-0.868299$

N $0.5897802 .205033-0.897390$

H $0.550438-0.7948362 .698909$

H $1.797510-1.7165075 .341767$

H $3.772887-2.0776194 .039806$

H $1.689857-3.8272502 .622319$

H $2.341885-4.1875664 .195456$

H $4.728938-5.3729741 .025086$

H $5.678193-5.3409483 .105128$

H $5.332705-3.8433093 .972466$

H $4.503146-5.3495244 .422406$

H $2.485819-5.567103-0.120013$

H $1.962806-4.053600 \quad 0.597415$

H $3.937796-2.908376-0.533269$

H $4.282393-4.426102-1.369461$

H $1.974142-4.372502-2.366615$

H $3.891485-3.360106-3.637080$

H $3.692896-1.859810-2.762229$

H $3.581489-1.706369-5.445183$

H $1.458472-0.495957-3.114427$

H $2.502069-3.776314-5.655068$

H $\quad 0.994666-2.931052-5.914048$

H $1.129271-4.118929-4.600037$

H $-0.554778-1.831331-4.576026$

H - $-1.446517-1.076927-2.055589$

H $-0.960950-1.998445-0.656971$

H - $0.955199-3.602748-2.095707$

H $3.477206-0.583110-0.000142$

H 4.7332521 .8721241 .367690

H $4.8403410 .116701-2.083094$

H $4.3650801 .580552-2.942303$

H $3.1931350 .775805-1.930696$

H $-0.510596 \quad 0.0229950 .200732$ 
H $\quad 1.079650-0.294040-0.494029$ H $0.570077 \quad 4.224538-0.496798$ H $-0.3634553 .749553-1.932323$ H $1.8330473 .173867-3.145784$ H $2.5249915 .438131-1.201058$ H $3.6032766 .055545-3.443641$ H $3.8092504 .363664-3.827158$ H $6.6926786 .222199-1.450641$ H $5.2398737 .176665-2.091338$ H $6.8934553 .894822-1.977126$ H $5.7225523 .143597-3.008930$ H 5.6125363 .5053240 .063066 H $1.1604942 .001444-0.081982$ C -0.6167451 .2204394 .347153$ O -1.5419301 .3601453 .252832$ C -1.245066 2.5884072 .560209 C $-0.097974 \quad 3.2595453 .318268$ C 0.5902042 .0772253 .970628 H $-0.892503 \quad 2.3009391 .564723$ C -2.499634 $3.462208 \quad 2.436199$ H $0.560492 \quad 3.8220652 .648670$ H -0.4615973 .9564924 .081838$ C 1.4757762 .4504435 .145312 H 1.1994571 .5878243 .201181 C -3.7295152 .7774121 .809938$ O -2.8937283 .9143933 .739827$ H -2.252695 4.3685461 .870615 H -4.166182 2.0586212 .514416 O $-4.723947 \quad 3.7920991 .609521$ C -3.476009 $2.072163 \quad 0.466537$ C $-4.7839531 .651182-0.231130$ H -2.843956 1.1951410 .636986 O $-2.7596542 .944625-0.407356$ H $-5.453606 \quad 1.2001510 .507536$ H -5.281940 $2.548304-0.617775$ H 2.2774583 .1231134 .823740 H 0.9068602 .9573245 .931994 H 1.9374261 .5602405 .583888 C $-0.366018-0.2617384 .649683$ H $-3.059748 \quad 3.1265414 .285603$ H $-4.885934 \quad 4.2128332 .471431$ H $-3.292763 \quad 3.749617-0.524543$ H $-1.303627-0.8189524 .535000$ H $-0.062677-0.3503715 .700386$ H $-1.126922 \quad 1.6545025 .218169$ C $-4.922742-1.402554-0.217254$ O $-4.010454-0.579285-0.957548$ C $-4.5370390 .675616-1.404108$ C $-5.762730 \quad 0.434533-2.293248$ C $-6.815006-0.405814-1.579729$ C $-6.191624-1.690082-1.035465$ H $-5.188430-0.9083820 .724361$ C -4.183312 $-2.706148 \quad 0.122355$ H -3.764659 $1.125412-2.040300$ O $-6.3226691 .676076-2.707243$ H -5.444818 $-0.092195-3.202574$ H $-7.3090140 .169398-0.787782$ O $-7.833512-0.745501-2.523967$ H $-5.966087-2.357994-1.877136$ O $-7.170785-2.334437-0.222125$ C $-3.073873-2.5046651 .132375$ H $-4.869947-3.4419120 .552604$ H $-3.730731-3.118009-0.787991$ H $-7.1069561 .475869-3.246286$ H $-8.477880-1.309766-2.062946$ H $-6.882592-3.250861-0.076801$ O $-2.402996-3.686581 \quad 1.279644$ O $-2.822717-1.4592341 .714814$ C $-1.290504-3.6559512 .177122$ H $-0.512514-2.9994391 .778065$ H $-1.606919-3.3265883 .171333$ H $-0.887795-4.6692632 .255289$ SCF Energy (B3LYP/6-31G*//MMFF) $=-3245.92114747$

0600033

MM̄FF Geometry

C $0.671715 \quad 0.817084 \quad 3.274196$

C 1.7784880 .1340323 .604633
C $2.185108-1.2530853 .173545$

O $3.383556-1.1136352 .371307$

C $1.160439-2.0643812 .356564$

C $1.372760-3.5609342 .499678$

C $1.971479-4.3541601 .590871$

C $0.808354-4.1654303 .762103$

C $2.580330-3.966757 \quad 0.273022$

C $1.816765-4.579783-0.910260$

C $0.432511-3.948301-1.121623$

C $-0.337890-4.675039-2.224705$

C $-1.683571-3.995316-2.517094$

O $0.636833-2.581998-1.490513$

O $-2.180012-4.557973-3.740751$

C $-1.437842-2.489297-2.748631$

C $-2.725400-4.319997-1.440118$

O $-2.672782-1.797302-2.906683$

C $-0.565990-1.822564-1.657727$

C $-0.164751-0.396906-2.103929$

O $-1.312231-1.741922-0.443252$

C $4.476183-1.8420412 .713814$

O $4.591158-2.5786113 .684094$

C $5.588721-1.6469621 .755455$

C $5.453051-1.0595040 .556070$

C $6.516811-0.869754-0.434966$

C $7.910617-1.300231-0.057552$

C $0.8960450 .264663-1.211897$

C $1.4019151 .566715-1.809417$

C $3.2931573 .181634-1.612527$

O $\quad 0.826657 \quad 2.155621-2.720732$

C $4.3332602 .767477-2.603780$

C $5.6525122 .890549-2.394541$

C $6.6931492 .455221-3.385079$

C $7.6161801 .391902-2.831149$

C $8.8727481 .695669-2.464693$

C $7.117026-0.036323-2.789912$

C $6.205049-0.310914-1.623436$

N $2.5626312 .002718-1.185571$

H -0.0397560 .4025932 .568248$

H $2.4626310 .593386 \quad 4.317493$

H $2.411296-1.7861564 .105373$

H $1.198836-1.7645491 .304114$

H $0.137196-1.8576822 .689770$

H $2.045204-5.4182941 .813645$

H $0.949157-5.2507373 .803876$

H $1.290631-3.7386024 .646570$

H $-0.267654-3.9720883 .825887$

H $2.661891-2.882903 \quad 0.153228$

H $3.608936-4.3483760 .259479$

H $2.423542-4.426108-1.812008$

H $1.717693-5.662902-0.765742$

H $-0.130018-3.991071-0.182903$

H $-0.488875-5.730553-1.965975$

H $0.259399-4.675371-3.147584$

H $-2.993763-4.079400-3.975936$

H $-0.917467-2.398199-3.711872$

H $-2.886867-5.403158-1.381454$

H $-3.701150-3.886686-1.686518$

H -2.436011 -3.973222 -0.446012

H $-3.067464-1.695376-2.023737$

H $0.242356-0.445377-3.122731$

H $-1.0514180 .248140-2.137799$

H $-0.975098-1.005137 \quad 0.090212$

H $6.538818-2.0558782 .084718$

H $4.472716-0.6924610 .256921$

H $8.234873-0.7949400 .859136$

H $8.659391-1.066733-0.817342$

H $7.947624-2.3830330 .104591$

H $0.4852900 .488452-0.221724$

H $1.754169-0.404810-1.083735$

H $3.7297423 .636987-0.718112$

H $2.6062443 .899166-2.072123$

H $3.9801772 .340673-3.539752$

H $6.0073603 .325716-1.463214$

H $7.2678973 .343944-3.676252$

H $6.2347632 .085676-4.311030$

H $9.5547870 .936111-2.097992$

H $9.2474792 .712388-2.520225$

H $7.957491-0.739535-2.800354$ 
H $6.572936-0.255394-3.718006$

H $5.167475-0.032209-1.806865$

H $2.9922101 .416613-0.475501$

C $0.173264 \quad 3.309412 \quad 2.935885$

$\begin{array}{lllll}0 & -0.962886 & 3.137412 & 2.066865\end{array}$

C $-0.641786 \quad 3.7234290 .789263$

C $0.693707 \quad 4.442595 \quad 0.967021$

C 1.3625903 .5814132 .019639

H $-0.5006202 .890160 \quad 0.093016$

$\begin{array}{llll}\text { C } & -1.775756 & 4.628684 & 0.298622\end{array}$

H $1.256623 \quad 4.5067220 .032790$

H $0.559173 \quad 5.4639401 .342611$

C 2.5385524 .2522132 .704945

H 1.7045282 .6552871 .542479

C $-3.152130 \quad 3.947185 \quad 0.171573$

O $-1.926796 \quad 5.731127 \quad 1.202987$

H $-1.4942985 .070545-0.664629$

H -3.5794803 .7574281 .163820$

O $-4.032654 \quad 4.879234-0.470992$

C -3.152543 $2.638292-0.637184$

C $-4.5728712 .143991-0.975191$

H $-2.6177901 .870067-0.069718$

O $-2.4377682 .826406-1.860193$

H $-5.2003812 .212286-0.081535$

H $-5.0077842 .806536-1.732804$

H 3.3204894 .4930821 .977523

H 2.2391355 .1832473 .197661

H 2.9741843 .5941203 .463422

C $0.306795 \quad 2.1349803 .903650$

H -2.109322 5.3635092 .084963

H -4.029832 5.6937760 .060665

H -2.872465 $3.546705-2.348209$

H -0.6544801 .9896664 .412689$

H 1.03622023982564 .679418

H -0.0448574 .2055373 .533350$

$\begin{array}{llll}\text { C }-5.030078 & -0.484168 & 0.533298\end{array}$

O $-4.112036-0.240557-0.539574$

C -4.560446 $0.699919-1.523405$

C $-5.8868820 .230201-2.134718$

C $-6.938290-0.053759-1.068229$

C $-6.372937-0.995049-0.005047$

H -5.1891480 .4395791 .102438$

C $-4.385610-1.5153541 .474161$

H $-3.8183720 .676302-2.330708$

O $-6.3949151 .191822-3.053472$

H $-5.714074-0.693869-2.702410$

H $-7.3097790 .877823-0.624741$

O $-8.057135-0.677960-1.702061$

H $-6.251460-2.000194-0.429161$

O $-7.298948-1.0902831 .075717$

C $-3.379031-0.8696472 .403446$

H $-5.156043-1.9812342 .099144$

H -3.867038 -2.298934 0.912119

H $-5.7136811 .335472-3.732471$

H -8.766886 $-0.748726-1.041883$

H -8.099751 -1.5267300 .739831$

O $-3.341176-1.565003 \quad 3.577677$

O $-2.701446 \quad 0.110426 \quad 2.126321$

C $-2.477069-1.0438974 .592899$

H $-1.443488-1.3193114 .372578$

H -2.5781590 .0422834 .680669$

H -2.764081-1.495961 5.546087

SCF Energy (B3LYP/6-31G**//MMFF) $=-3245.92486323$

06_00034

MM̄FF Geometry

C $-0.522518-2.1502540 .091674$

C $0.171698-1.1676250 .688685$

C $-0.231752-0.4194251 .938628$

O $-1.632831-0.6508712 .200996$

C -0.0276691 .0915721 .733903$

C $-0.1055691 .898176 \quad 3.013842$

C -1.1390312 .6906713 .359378$

C 1.1075411 .8129133 .904375

C -2.441285 2.8869092 .633817

C $-2.541475 \quad 4.2583801 .950372$

C $-1.699795 \quad 4.363496 \quad 0.668618$

C -1.7912305 .7693230 .071033$
C $-1.051725 \quad 5.870084-1.272027$

O $-2.213034 \quad 3.407614-0.264155$

O $-1.4519857 .107935-1.878566$

C $-1.5201704 .717567-2.187312$

C $0.4659515 .960415-1.071724$

O $-0.768426 \quad 4.694996-3.398977$

C -1.493603 $3.333200-1.500425$

C $-2.1962522 .282770-2.392836$

O $-0.1335032 .947680-1.301566$

C -2.036169 -0.8995593 .470230$

O $-1.329767-0.9533264 .467019$

C $-3.499315-1.1365913 .529363$

C $-4.321807-0.9588152 .482934$

C $-5.766541-1.2005662 .459995$

C $-6.407119-1.7453233 .709128$

C $-2.4095200 .926082-1.706050$

C $-3.168396-0.051686-2.585950$

C $-4.282143-2.283072-2.476424$

O $-3.4380230 .170197-3.763153$

C $-5.727537-2.028375-2.184060$

C $-6.491018-2.832494-1.428648$

C $-7.937959-2.562202-1.127798$

C $-8.217393-2.4513820 .355957$

C $-8.797458-3.4630231 .023718$

C $-7.908996-1.140398 \quad 1.047190$

C $-6.441940-0.9526851 .317913$

N -3.495588 -1.210979-1.895358

H $-1.466683-2.4870650 .510454$

H $1.131163-0.885782 \quad 0.259027$

H $\quad 0.391780-0.7935382 .758934$

H -0.7645601 .4543491 .011255$

H $\quad 0.952523 \quad 1.2861291 .277994$

H -1.0649693 .2499614 .291547$

H 1.0280002 .4664524 .779603

H $1.2464980 .791263 \quad 4.270701$

H 2.0059792 .1097843 .355274

H -2.642392 2.0818161 .920617

H -3.240599 2.8159393 .382966

H -3.5967794 .4197541 .694101$

H -2.254622 5.0488702 .654823

H -0.6578314 .1207670 .903768$

H -1.4110106 .5184560 .776626$

H -2.847239 $6.025285-0.095664$

H $-1.0486937 .146631-2.762986$

H -2.555087 $4.942265-2.479131$

H $0.7177376 .840320-0.467471$

H $0.9852296 .097535-2.026652$

H $0.8868135 .084620-0.573184$

H $0.0974814 .298288-3.202549$

H -3.182771 $2.664203-2.687985$

H -1.618083 2.127952 -3.312282

H $-0.0708921 .980287-1.288822$

H $-3.865639-1.4688204 .495216$

H $-3.905783-0.6072251 .540291$

H -5.936662 -2.690349 4.003023

H $-7.473059-1.9540363 .595583$

H $-6.308016-1.0313054 .534038$

H - $1.4486770 .466123-1.457382$

H $-2.9785591 .057908-0.778553$

H -3.928690 -3.221768 -2.038414

H $-4.119942-2.317445-3.558292$

H $-6.166371-1.132248-2.617041$

H $-6.057682-3.735260-1.004257$

H $-8.526198-3.380469-1.563205$

H $-8.287417-1.650515-1.628352$

H $-9.030983-3.3832522 .080077$

H $-9.048350-4.3958130 .529571$

H $-8.492433-1.040898 \quad 1.969404$

H $-8.255830-0.3108150 .417553$

H $-5.888591-0.5584530 .465444$

H $-3.290957-1.266020-0.901702$

C $0.770343-4.081841-0.855470$

O $2.033531-3.662521-0.299551$

C $3.098895-4.330596-0.996855$

C $2.445344-5.510419-1.701454$

C $1.112074-4.904628-2.098832$

H $3.471155-3.631438-1.755374$

C $4.223954-4.682492-0.019308$ 
H $3.030695-5.862471-2.556006$

H $2.289439-6.355797-1.021245$

C $0.057449-5.935177-2.462406$

H $1.271234-4.238951-2.958044$

C $4.735912-3.4835180 .807541$

O $3.751744-5.6704450 .905989$

H $5.054151-5.150091-0.560715$

H $3.960375-3.1496241 .507718$

O $5.832568-3.9403201 .609429$

C $5.216301-2.286766-0.034250$

C $5.744968-1.1324490 .835923$

H $4.387062-1.913443-0.638600$

O $6.235070-2.715991-0.935317$

H $4.943332-0.8158151 .512444$

H $6.568606-1.4985721 .460332$

H $0.380370-6.528231-3.324274$

H $-0.133131-6.624340-1.632830$

H $-0.888846-5.449640-2.721113$

C $-0.069418-2.842949-1.163949$

H $2.972284-5.3017331 .356361$

H $5.513155-4.6947432 .133801$

H $6.967346-3.070337-0.402068$

H $-0.958195-3.115471-1.744297$

H $0.508872-2.142636-1.777430$

H $\quad 0.279057-4.706331-0.098254$

C $4.1774471 .233290-0.363091$

O $5.307647 \quad 0.559518-0.928159$

C 6.2656830 .0699510 .015221

C 6.7958841 .2255210 .874426

C 5.6630211 .9949711 .547660

C 4.6234022 .4161060 .510793

H 3.5849090 .5250810 .229150

C $3.3125891 .743881-1.528948$

H $7.112248-0.299179-0.577533$

O 7.7036810 .7333741 .854537

H $7.360048 \quad 1.9150250 .232797$

H 5.2116651 .4088362 .356596

O 6.2224903 .1610152 .156601

H $5.0427693 .210685-0.120206$

O 3.4825512 .9552341 .174863

C $2.5841910 .640784-2.266034$

H $2.5512582 .434707-1.151909$

H $3.9417542 .264179-2.260901$

H 7.9969861 .4928542 .386437

H 5.5183103 .5900302 .671246

H $3.759908 \quad 3.771617 \quad 1.623396$

O $1.7434291 .207515-3.182741$

O $2.732456-0.557543-2.077466$

C $0.9762290 .291799-3.970542$

H $1.632098-0.417185-4.484789$

H $0.257574-0.234938-3.336991$

H $\quad 0.4262640 .867123-4.719960$

SCF Energy (B3LYP/6-31G**//MMFF)= -3245.93302162

06_00035

MM̄FF Geometry

C $-0.327752-3.6813892 .211140$

C $0.421302-2.6758371 .732583$

C $1.211409-1.7626172 .635145$

O $0.762723-0.4023442 .432689$

$\begin{array}{lll}\text { C } 2.709219 & -1.812858 & 2.285497\end{array}$

C $3.576080-1.3337833 .433496$

C $4.066657-0.0863793 .565628$

C $3.905912-2.3852434 .462624$

C 3.8479931 .0970862 .665626

C 5.1525231 .5863002 .022062

C 5.6919310 .6330270 .944356

C $7.037267 \quad 1.1310940 .413336$

C $7.5544840 .259356-0.740724$

$\begin{array}{llll}\text { O } & 4.733336 & 0.592317 & -0.116397\end{array}$

O $8.6341100 .963510-1.373256$

C $6.4323990 .109918-1.789119$

C $8.137761-1.065760-0.236187$

O $6.819654-0.794621-2.821815$

C $5.065249-0.297747-1.189294$

C $3.964641-0.156146-2.265305$

O $5.133756-1.654437-0.750921$

C $-0.203844 \quad 0.074866 \quad 3.257646$
O $-0.818679-0.5588314 .104905$

C $-0.412693 \quad 1.5299243 .070777$

C 0.0699382 .2334322 .034609

C -0.0851713 .6762571 .823595$

C $-0.741644 \quad 4.4800572 .915331$

C $2.550873-0.485138-1.772240$

C $1.526490 \quad 0.037291-2.757999$

C $0.1236892 .017437-3.269921$

O $1.134207-0.641646-3.703711$

C $0.201458 \quad 3.485652-3.010984$

C $-0.7901314 .189299-2.444192$

C $-0.7017815 .656970-2.144796$

C $-0.8463855 .958674-0.668943$

C $-1.9548306 .550023-0.192585$

C $0.325576 \quad 5.655357 \quad 0.239956$

$\begin{array}{llll}\text { C } & 0.370782 & 4.214695 & 0.672994\end{array}$

N 1.148809 1.344889 -2.495139

H $-0.367247-3.8138193 .291840$

H $0.490162-2.4948650 .664289$

H $1.071082-2.0464503 .685979$

H $2.908147-1.2210121 .385349$

H $3.013037-2.8389642 .038384$

H 4.6883600 .1274214 .434538

H $4.538433-1.9970185 .268019$

H $2.992093-2.7751084 .921590$

H $4.442253-3.2174803 .994922$

H $3.0980750 .902647 \quad 1.894609$

H 3.4405391 .9075483 .283040

H 4.9476222 .5629491 .564528

H 5.9122221 .7450112 .797636

H $5.802707-0.3696821 .371722$

H 7.7796501 .1810831 .219695

H $6.9257622 .161007 \quad 0.045494$

H $9.3194381 .126474-0.702790$

H $6.3188911 .088933-2.275152$

H $8.953500-0.8796730 .472822$

H $8.582572-1.639552-1.056703$

H $7.400666-1.6948690 .267178$

H $6.734614-1.697658-2.471309$

H $3.9725560 .875243-2.642420$

H $4.184787-0.811335-3.117684$

H $4.232909-2.002424-0.661001$

H - -1.0040311 .9997373 .850460$

H $\quad 0.6308691 .7117501 .260999$

H $-1.772500 \quad 4.150526 \quad 3.073207$

H -0.7885725 .5499292 .702732$

H $-0.1902294 .371525 \quad 3.856044$

H $2.404879-1.566398-1.672728$

H $2.355277-0.046975-0.787417$

H $-0.8412581 .598918-2.970090$

H $0.2745881 .803115-4.332881$

H $\quad 1.1230543 .990008-3.291564$

H $-1.7132753 .684308-2.169517$

H -1.490065 6.161986-2.717956

H $0.2437336 .081662-2.504639$

H -2.0566826 .7974940 .858585$

H -2.788981 $6.794633-0.841879$

H $\quad 0.325434 \quad 6.328996 \quad 1.103987$

H $1.2615615 .891433-0.282678$

H $0.8441883 .553707-0.051957$

H $1.4163291 .752888-1.603603$

C $-1.051894-4.685981-0.077951$

O $-1.629315-3.481534-0.615726$

C $-2.403312-3.812063-1.783685$

C $-2.117347-5.277550-2.091866$

C $-1.842129-5.833751-0.711330$

H $-3.457115-3.709144-1.500628$

C $-2.083066-2.854770-2.935700$

H -2.959016 $-5.765927-2.592658$

H $-1.234849-5.398555-2.730265$

C $-1.104314-7.161826-0.731895$

H -2.800474 $-5.963056-0.190219$

C $-2.202175-1.357609-2.595226$

O $-0.736789-3.081222-3.375884$

H $-2.720516-3.093820-3.794993$

H $-1.383209-1.051670-1.932741$

O $-2.028545-0.632566-3.820534$

C $-3.543916-0.934629-1.973230$ 
C $-3.6281100 .589298-1.753408$ H -3.672005 -1.441772 -1.011923 O $-4.614974-1.353365-2.817266$ H -2.790601 $0.897100-1.117487$ H $-3.5078601 .103846-2.714299$ H -1.689666 -7.918269 -1.264776 H $-0.133955-7.073807-1.232015$ H $-0.927312-7.5280850 .283987$ C - $-1.147295-4.687912 \quad 1.449771$ H $-0.148797-2.922786-2.617136$ H -1.163222 $-0.885680-4.186162$ H $-4.487880-0.930299-3.683873$ H $-0.851374-5.6772281 .819441$ H -2.192817 -4.5383591 .745183$ H $-0.002984-4.717322-0.399716$ C $-4.491747 \quad 0.610213 \quad 1.185637$ $\begin{array}{lllll}\text { O } & -5.300943 & 0.308087 & 0.043721\end{array}$ C $-4.9701361 .042147-1.138188$ C $-5.0673212 .553196-0.873687$ C $-4.2666402 .976751 \quad 0.356259$ C -4.6261682 .0918281 .550070$ H -3.4429290 .3702450 .970592$ C $-4.965355-0.2868782 .333255$ H $-5.7478200 .798094-1.872989$ O $-4.6242753 .299485-2.002390$ H $-6.1191582 .820461-0.706258$ H -3.189499 2.9575920 .151484 $\begin{array}{lllll}\text { O } & -4.597958 & 4.333291 & 0.659686\end{array}$ H -5.6521062 .3100621 .873754$ O -3.7587312 .3845732 .639727$ C -4.533481-1.7134542.095869 H $-4.540050 \quad 0.0152423 .296789$ H $-6.056628-0.2500092 .435801$ H $-5.1674573 .029655-2.762460$ H $-4.0004004 .628736 \quad 1.367228$ H $-3.966437 \quad 3.2835332 .945527$ O $-5.604646-2.4778421 .741935$ O $-3.374415-2.0947152 .195231$ C $-5.317858-3.8550421 .481215$ H $-4.617161-3.944450 \quad 0.646749$ H -4.913811 -4.3344212 .377874$ H -6.252665 -4.351604 1.208397

SCF Energy (B3LYP/6-31G**//MMFF)= -3245.91979567

06_00036

MM̄FF Geometry

C -0.363276 -0.0377571 .985756$

C $-0.146660-1.3526841 .825083$

C $-0.426978-2.1195730 .555598$

O $-0.227887-1.217395-0.558195$

C $-1.866656-2.6498190 .526320$

C $-2.062674-3.737161-0.509817$

C $-2.609688-3.552216-1.726772$

C $-1.624420-5.114402-0.081218$

C $-3.085378-2.267243-2.346918$

C $-4.604663-2.259084-2.563733$

C -5.395971-2.057699-1.262272

C $-6.893035-2.254689-1.507796$

C $-7.718944-1.965985-0.246912$

O $-5.147130-0.723033-0.806267$

O $-9.093299-1.892831-0.654549$

C -7.309967 -0.5847780 .304453$

C -7.629993 -3.1162110 .762469$

O $-7.961795-0.3229971 .545678$

C $-5.779661-0.3895280 .435228$

C $-5.4785091 .096391 \quad 0.744242$

O $-5.298686-1.2023001 .506725$

C $0.523293-1.619521-1.610626$

O $0.969074-2.740676-1.807594$

C $0.807985-0.490652-2.528133$

C $0.2884290 .738380-2.379422$

C $0.570327 \quad 1.906018-3.218099$

C $1.6379251 .766846-4.271716$

C -3.9911641 .4724000 .702670$

C -3.7797262 .9574610 .954928$

C -1.9631504 .6642331 .073255$

O -4.6980253 .7354041 .200390$

C $-1.7006525 .278893-0.264471$
C $-0.4801565 .649129-0.680652$

C $-0.2054476 .289674-2.009688$

C $0.6598645 .430782-2.904276$

C $1.9753065 .670749-3.035768$

C $-0.0188414 .354153-3.720779$

C $-0.1163773 .045031-2.987196$

N -2.4390483 .3067440 .882912$

H -0.8479430 .5219721 .190321$

H $\quad 0.325792-1.9144292 .627373$

H $\quad 0.302577-2.937366 \quad 0.509177$

H $-2.563827-1.8230580 .348460$

H -2.141934 -3.061326 1.506701

H $-2.711726-4.420541-2.377024$

H $-1.812483-5.869979-0.851412$

H $-0.551869-5.1271760 .135999$

H $-2.166010-5.4212360 .819648$

H $-2.774971-1.386355-1.777828$

H -2.594825 -2.177844 -3.324605

H $-4.838024-1.437635-3.253450$

H -4.912509-3.191505 -3.053293

H $-5.041551-2.770131-0.509603$

H -7.098515 -3.267359 -1.876747

H -7.224397 -1.573531-2.304466

H -9.617696-1.633195 0.122585

H -7.696292 $0.163845-0.400698$

H $-7.986734-4.0488490 .308957$

H -8.280357 -2.937756 1.625905

H $-6.615111-3.2904461 .126252$

H -7.499678 -0.8300512 .234813$

H $-5.997947 \quad 1.7249450 .008466$

H -5.8776691 .3561651 .733138$

H -4.458360 -0.839545 1.827006

H $1.494063-0.727451-3.335174$

H $-0.4082580 .922205-1.563403$

H $2.5708951 .398702-3.829840$

H $1.8894322 .708097-4.764702$

H $1.3182441 .066790-5.051080$

H $-3.4344730 .921883 \quad 1.467779$

H $-3.564577 \quad 1.231367-0.277597$

H -1.057893 4.6095841 .684747

H -2.7096335 .2536491 .614812$

H -2.558139 $5.436682-0.914116$

H $0.3725815 .502064-0.022296$

H $\quad 0.286167 \quad 7.252882-1.820136$

H -1.137060 $6.538798-2.533026$

H $2.5935055 .076482-3.700525$

H $2.4640526 .461271-2.476116$

H $0.4858774 .241703-4.685880$

H -1.033354 $4.679117-3.985558$

H $-0.8653343 .042996-2.195262$

H $-1.7590582 .603050 \quad 0.611121$

C 0.9228931 .9622272 .695806

O $2.003173 \quad 1.522857 \quad 1.854672$

C 3.1253172 .4062782 .032597

C 2.6738063 .4837913 .016649

C 1.5961682 .7595293 .806542

H 3.9177381 .8113232 .501140

C 3.6000832 .9148320 .663642

H 3.4976463 .8396143 .643059

H 2.2491864 .3546142 .504222

C 0.6641343 .6892904 .561705

H 2.0819412 .0769324 .516717

C $3.8011181 .803141-0.387934$

O $2.625775 \quad 3.8212220 .129820$

H 4.5198223 .4982980 .782830

H $2.8309751 .375093-0.665877$

O $4.3290272 .404650-1.576143$

C 4.7466960 .6723750 .048930

C $4.898607-0.410993-1.033404$

H $4.3576580 .207178 \quad 0.956101$

O $6.026147 \quad 1.212806 \quad 0.371622$

H $3.900940-0.764274-1.315812$

H $5.3515880 .032256-1.928317$

H 1.2225694 .2801725 .295213

H 0.1568664 .3859363 .886465

H -0.1022593 .1219205 .098571$

C $\quad 0.107443 \quad 0.757667 \quad 3.171224$

H 1.7870723 .3359880 .044552 
H $3.7078103 .102623-1.846812$ H $6.367477 \quad 1.653865-0.425191$ H $-0.760163 \quad 1.0942053 .748687$ $\begin{array}{llll}\text { H } & 0.721237 & 0.127498 & 3.825287\end{array}$ H $\quad 0.2922842 .617647 \quad 2.082304$ C $4.100031-2.9057890 .574516$

O $5.331556-2.1777360 .651421$ C $5.777544-1.598581-0.580077$

C $5.948559-2.691383-1.643524$

C $4.682401-3.528465-1.808623$

C $4.209676-4.044398-0.449931$

H $3.284540-2.2257700 .299311$

C $3.809198-3.4753731 .973571$

H $6.775672-1.189722-0.378477$

O $6.317562-2.110606-2.890015$

H $6.771725-3.352608-1.342223$

H $3.897257-2.959724-2.320013$

O $4.994494-4.638204-2.653763$

H $4.910134-4.808382-0.088197$

O $2.935117-4.666326-0.592007$

C $3.435403-2.4111542 .982879$

H $2.972647-4.1815531 .933416$

H $4.701511-3.9895382 .350906$

H $6.394010-2.833465-3.536220$

H $4.163166-5.103814-2.845309$

H $3.049125-5.448763-1.156976$

O $3.338751-2.9887534 .217529$

O $3.235058-1.2323962 .730055$

C $2.974801-2.1048875 .282731$

H $3.687545-1.2776855 .355021$

H $1.959622-1.7284025 .126889$

H $2.999606-2.6711136 .217652$

SCF Energy (B3LYP/6-31G**//MMFF) $=-3245.92469546$

06_00037

MM̄FF Geometry

C -3.266099 $3.295183-0.117427$

C -3.110143 4.2440060 .819440

C $-1.913238 \quad 4.377222 \quad 1.733505$

O $-\begin{array}{llll}0.786343 & 3.642461 & 1.210126\end{array}$

C $-2.256413 \quad 3.7754173 .109329$

C $-1.136105 \quad 3.9082424 .122459$

C $-0.191526 \quad 2.970764 \quad 4.341931$

C -1.146666 5.1831864 .922669

C 0.0014011 .6803863 .593586

C $-0.185464 \quad 0.4470764 .483859$

C $-0.091183-0.8458623 .657780$

C $-0.352753-2.077640 \quad 4.525748$

C $-0.195625-3.3809053 .726260$

O $1.219679-0.9195853 .084339$

O $-0.161997-4.4545674 .678510$

C $1.163146-3.3515142 .991690$

C - $1.410998-3.6415052 .828505$

O $1.302693-4.4816872 .134036$

C $1.415604-2.0420132 .213470$

C $2.848168-1.9603041 .640392$

O $0.519939-1.9842491 .097257$

C $0.025676 \quad 4.240528 \quad 0.301718$

O $-0.0711355 .365680-0.156685$

C $1.0586073 .241043-0.048154$

C $1.9447863 .477510-1.025895$

C $2.9906632 .547277-1.460061$

C $3.0954921 .240959-0.718050$

C $3.966868-1.9769562 .681807$

C $5.319217-1.7285612 .040205$

C $6.779000 \quad 0.0754651 .158543$

O $6.132750-2.6353211 .878507$

C $6.568606 \quad 1.3668810 .434525$

C $7.0189971 .610391-0.806072$

C $6.8124682 .917868-1.519067$

C $6.2666872 .750182-2.922467$

C $6.9726833 .169922-3.986166$

C $4.9118112 .099212-3.104256$

C $3.7964582 .899360-2.483459$

N $5.506017-0.4062421 .665207$

H - $2.4902222 .548194-0.263623$

H -3.9065354 .9691410 .971907$

H $-1.645525 \quad 5.4359661 .836372$
H -2.522174 2.7160602 .998432

H $-3.158124 \quad 4.254873 \quad 3.513979$

H 0.5574823 .1628485 .108892

H -0.3174335 .2341335 .636127$

H -1.064775 6.0497764 .258830

H -2.0784375 .2669215 .491526$

H -0.6746021 .6029642 .737823$

H 1.0170341 .6863503 .178758

H $\quad 0.5880470 .449033 \quad 5.262437$

H -1.1610170 .4937334 .983387$

H $-0.832999-0.7879092 .852112$

H - $-1.347450-2.0275754 .986095$

H $0.362805-2.0928165 .360006$

H $0.012943-5.2769654 .189229$

H $1.932155-3.462736 \quad 3.765504$

H -2.322975 -3.7122503 .433521$

H $-1.323363-4.6021122 .308992$

H $-1.568235-2.8601782 .082440$

H $\quad 0.763003-4.3206581 .341217$

H $3.008553-2.7652710 .911223$

H $2.928764-1.0326341 .060820$

H $\quad 0.848871-1.3118200 .478177$

H 1.0497812 .3121450 .512080

H $1.8963314 .424635-1.560567$

H $2.1602950 .676718-0.800028$

H $3.301407 \quad 1.4214360 .342783$

H $3.8873870 .585149-1.081976$

H $3.803445-1.2166543 .454108$

H $4.015802-2.9461713 .189223$

H $7.229441-0.6942420 .523012$

H 7.4341170 .2327572 .021738

H 6.0288042 .1490740 .963767

H $7.5842430 .842120-1.328484$

H $7.7822983 .431355-1.549474$

H $6.1429633 .575436-0.950932$

H $6.5952783 .056458-4.997065$

H $7.9452263 .637230-3.872111$

H $4.9588871 .071671-2.735561$

H $4.6783812 .000999-4.172959$

H $3.6384843 .869270-2.957147$

H 4.8423450 .2825752 .008922

C $-5.325012 \quad 1.957120-0.575102$

O $-4.5793720 .734741-0.744209$

C $-5.475552-0.295453-1.201550$

C $-6.8769280 .309240-1.181803$

C $-6.5858121 .777683-1.416451$

H $-5.201668-0.511689-2.240408$

C $-5.326700-1.555907-0.341432$

H $-7.525307-0.128149-1.947313$

H $-7.3675270 .168295-0.211586$

C $-7.7331672 .692375-1.024835$

H $-6.3521031 .923896-2.479590$

C $-3.905966-2.154270-0.294913$

O $-5.705537-1.252006 \quad 1.008245$

H -6.036305 -2.317463 -0.685796

H $-3.233581-1.5050590 .279016$

O $-3.987789-3.389096 \quad 0.430157$

C $-3.283095-2.441159-1.673129$

C $-1.979992-3.260400-1.582447$

H -3.084749 -1.491827 -2.179569

O $-4.218402-3.158658-2.480511$

H -1.313215 $-2.789812-0.854043$

H $-2.216400-4.263817-1.209727$

H -8.629049 2.456426 -1.608571

H $-7.9856772 .587727 \quad 0.035708$

H -7.478462 $3.740768-1.208435$

C $-4.4773363 .158398-0.994009$

H $-5.130422-0.531567 \quad 1.318656$

H $-4.363875-3.1869681 .304142$

H $-4.433544-3.985937-2.016535$

H $-5.0776354 .074517-0.960176$

H $-4.1293223 .035128-2.026802$

H -5.5817602 .0292030 .490537$

C $0.218150-1.499580-2.822425$

O $-0.889315-2.125204-3.484164$

C $-1.278655-3.397414-2.952905$

C $-0.092536-4.373786-2.971510$

C $1.143592-3.789235-2.298107$ 
C $1.452702-2.408660-2.875383$ H $-0.043299-1.289578-1.779093$ C $0.484733-0.162813-3.532173$ H -2.023244 -3.800641 -3.650646 O -0.432028 - $5.605473-2.343099$ H $0.162834-4.607354-4.013544$ H $1.019214-3.753531-1.209672$ O $2.250194-4.657868-2.549574$ H $1.794603-2.521434-3.911836$ O $2.529955-1.830015-2.144311$ C $-0.5385920 .895922-3.174370$ H $1.4647680 .233724-3.247786$ H $0.451965-0.304616-4.618769$ H -1.204266 -5.968223 -2.809807 H $3.035674-4.250076-2.146239$ H $2.245620-1.736421-1.219973$ O $-0.2503712 .037750-3.867537$ O $-1.4646380 .751066-2.388760$ C -1.135599 $3.138050-3.636295$ H -2.151609 $2.877652-3.947695$ H -1.116715 $3.426120-2.581694$ H - $0.7901793 .984014-4.236392$

SCF Energy (B3LYP/6-31G**//MMFF)= -3245.92699091

06_00038

MM̄FF Geometry

C $2.029524-3.2231631 .548287$

C $1.333151-2.0924751 .357939$

C $0.648105-1.3390662 .467092$

O $-0.732579-1.1319652 .094471$

C 1.3092320 .0389842 .636560

C $0.756694 \quad 0.8159443 .814728$

C -0.1302181 .8253803 .712499$

C 1.3091570 .4244175 .160586

C -0.7804912 .3341762 .456003$

C -2.2596212 .6773752 .678237$

C -2.9002203 .2488241 .404874$

C -4.3069263 .7716921 .697503$

C -4.9925834 .2968620 .427561$

$\begin{array}{lllll}\text { O } & -2.964231 & 2.207538 & 0.423348\end{array}$

O $-6.386775 \quad 4.4722000 .722046$

C $-4.8909393 .218979-0.670718$

C -4.4590395 .6748440 .018661$

O $-5.3954773 .708364-1.911910$

C $-3.4679612 .633148-0.850359$

C $-3.5396871 .404572-1.786078$

O $-2.6263593 .622261-1.445074$

C -1.615664 -2.118354 2.404719

O $-1.371221-3.1788092 .957482$

C $-2.949886-1.6856381 .933108$

C $-3.952318-2.5760471 .899638$

C $-5.313520-2.3305141 .419606$

C $-5.700422-0.9093841 .109734$

C $-2.2377330 .604702-1.900392$

C -2.478591-0.661849-2.697755

C $-3.531367-2.919755-2.506638$

O $-2.202586-0.732453-3.893074$

C $-5.025358-2.954514-2.407197$

C $-5.713889-3.989951-1.902728$

C $-7.212244-4.015028-1.786049$

C $-7.692118-4.409778-0.404340$

C -8.294397 -5.592674 -0.195016

C $-7.541028-3.4050190 .721046$

C $-6.141855-3.3859681 .275526$

N -3.065983 -1.665226 -1.943668

H $2.098259-3.6491502 .546082$

H $1.275832-1.6618670 .361079$

H $0.700603-1.8924943 .413151$

H 1.2022190 .6214041 .713950

H $2.392501-0.0787462 .778081$

H -0.4178882 .3470984 .624198$

H 0.9131621 .0470635 .969884

H $1.056978-0.6148535 .392572$

H 2.3991550 .5286505 .168541

H -0.2443953 .2343282 .132861$

H -0.7130521 .6000121 .649499$

H -2.794482 1.7702702 .988476

H -2.345482 3.4084863 .491842
H $-2.2629484 .059487 \quad 1.031723$

H -4.2816204 .5516292 .468662$

H -4.9192322 .9586432 .112826$

H -6.4606595 .0993801 .461733$

H $-5.5646312 .402718-0.374649$

H $-4.593926 \quad 6.3951280 .834594$

H $-5.0203776 .079585-0.830705$

H $-3.3983465 .664263-0.240725$

H -4.718532 $4.290176-2.297406$

H $-4.3259810 .730984-1.427498$

H $-3.835126 \quad 1.722395-2.794586$

H $-1.8574143 .181878-1.840383$

H -3.061796 -0.6607961 .597775$

H -3.750784 -3.596725 2.223409

H $-5.163191-0.5522470 .228036$

H $-5.465424-0.2505581 .953500$

H $-6.767365-0.7806720 .916573$

H -1.464175 $1.183018-2.414835$

H $-1.8411230 .333758-0.915583$

H -3.061167 -3.725956-1.934902

H $-3.224246-3.000460-3.553643$

H $-5.567439-2.083175-2.768067$

H -5.174885 -4.872669-1.566015

H -7.590215 -4.724516 -2.533371

H -7.647442 -3.042326 -2.047392

H $-8.662186-5.8735330 .786237$

H -8.429091 -6.309281-0.998616

H -8.218805 -3.659273 1.546357

H -7.872576 -2.4292090 .356113$

H $-5.772915-4.3723781 .561562$

H $-3.242990-1.499980-0.957668$

C $4.287836-3.7832210 .657816$

O $4.646892-2.3917140 .563153$

C $5.910269-2.276970-0.117195$

C $6.430260-3.699386-0.294280$

C $5.135665-4.486151-0.398525$

H $5.696178-1.858641-1.106783$

C $6.830938-1.3281320 .660574$

H $7.066769-3.799627-1.178757$

H $7.009231-4.0336930 .574194$

C $5.309246-5.976252-0.165289$

H $4.705103-4.323084-1.395665$

C 6.1891420 .0368520 .989564

O $7.191384-1.9395221 .906508$

H $7.768793-1.1848240 .112241$

H $5.361746-0.0985471 .696864$

O 7.1680920 .8374341 .663305

C $5.6917030 .816119-0.240433$

C $5.0241892 .149447 \quad 0.144214$

H $4.9627020 .208880-0.782880$

O $6.7810951 .057009-1.128185$

H 4.1692951 .9303470 .794449

H $5.7228072 .757573 \quad 0.731655$

H $5.985298-6.405916-0.911714$

H $5.728419-6.1814860 .825487$

H $4.349779-6.497422-0.239562$

C $2.779937-3.932636 \quad 0.454739$

H $6.368581-2.1313312 .388535$

H 7.4755090 .3305792 .434371

H $7.4428881 .580313-0.644110$

H $2.494079-4.9900430 .463547$

H $2.492244-3.526279-0.522092$

H $4.571382-4.1192161 .664390$

C $2.5755981 .768476-1.652070$

O $3.8774452 .210238-2.051110$

C $4.5735852 .987880-1.073436$

C $3.7554294 .236307-0.710171$

C $2.3233223 .886947-0.306409$

C $1.6808632 .976524-1.352975$

H $2.6579831 .131302-0.762649$

C $2.0061370 .922918-2.794844$

H $5.4913073 .336860-1.563850$

O $4.397408 \quad 4.956070 \quad 0.336940$

H $3.7216544 .901499-1.582961$

H 2.2948203 .4332720 .691208

O $1.5715325 .099207-0.216206$

H $1.5022313 .554400-2.268277$

O $0.4097732 .545269-0.878348$ 
C $2.777707-0.368509-2.933087$

H $0.9589540 .653242-2.623120$

H $2.0481301 .471544-3.743641$

H 3.8395115 .7249890 .545426

H $0.6548384 .855532-0.000709$

H $0.5621381 .952795-0.123520$

O $3.549011-0.337208-4.056422$

O $2.718132-1.285583-2.124479$

C $4.347794-1.501842-4.287365$

H $4.851194-1.383128-5.250476$

H $5.105647-1.597485-3.504758$

H $3.719122-2.396575-4.328784$

SCF Energy (B3LYP/6-31G**//MMFF) $=-3245.93401844$

06_00039

MM̄FF Geometry

C $-0.619699-1.8170152 .609137$

C $0.175935-2.1992151 .599066$

C $-0.231905-2.740108 \quad 0.253577$

O $0.100318-1.690274-0.688886$

C $-1.722783-3.0889900 .085980$

C $-1.988780-3.970118-1.119398$

C $-2.478134-3.541810-2.299014$

C $-1.703143-5.436487-0.913877$

C $-2.815112-2.136278-2.712337$

C $-4.317954-1.952948-2.964869$

C $-5.148623-1.929338-1.672601$

C $-6.643617-1.884471-1.994428$

C -7.496522 -1.752284 -0.725055

O $-4.771919-0.759567-0.940048$

O $-8.828089-1.423108-1.147732$

C $-6.956936-0.5744730 .113073$

C $-7.600307-3.084938 \quad 0.025512$

O $-7.640229-0.4878261 .361818$

C $-5.424592-0.6100640 .326447$

$\begin{array}{llll}\text { C } & -4.956348 & 0.730481 & 0.938347\end{array}$

O $-5.103579-1.6788061 .216991$

C $0.769616-2.012483-1.821295$

O $1.127626-3.129656-2.166958$

C $1.060509-0.809582-2.636608$

C $0.5242630 .400246-2.407104$

C $0.7627931 .612309-3.196882$

C $1.8428941 .556254-4.246449$

C -3.4357070 .8612211 .083095$

C -3.0589662 .2798871 .457758$

C -2.8019904 .5571250 .504880$

O -2.8308862 .6032832 .620880$

C -2.252394 $5.093174-0.776772$

C $-0.9893885 .521874-0.918612$

C $-0.4342696 .085779-2.192917$

C $0.5676535 .164848-2.850923$

C $1.8873455 .406595-2.780475$

C $0.0292994 .023503-3.683235$

C $0.0153952 .709088-2.947235$

N $-3.025083 \quad 3.131396 \quad 0.363937$

H -1.699297 -1.885334 2.520288

H $1.250981-2.0830621 .729158$

H $\quad 0.387907-3.6276210 .075921$

H -2.315585 -2.1697360 .025334$

H -2.090905 -3.627209 0.969139

H -2.641279 -4.282117 -3.081814

H -1.945294 -6.036184 -1.797726

H - $0.644006-5.596022-0.690158$

H -2.297406 -5.825354 -0.080546

H -2.453580 -1.391346 -1.997961

H $-2.279368-1.935131-3.648715$

H $-4.450600-0.998923-3.491563$

H -4.680962 -2.744870 -3.631901

H -4.917740 -2.819852 -1.078160

H $-6.945785-2.768613-2.569610$

H $-6.850654-1.021370-2.643005$

H -9.359612 -1.262233 -0.349019

H $-7.2105280 .344950-0.432156$

H -8.043071 $-3.852168-0.620900$

H $-8.268483-3.002858 \quad 0.889887$

H $-6.634359-3.4588100 .371385$

H -7.281517 -1.179405 1.943717

H $\quad-5.316824 \quad 1.5518700 .305463$
H -5.4065180 .8688021 .929670$

H -4.227989-1.517724 1.601325

H $1.733348-0.985758-3.470011$

H $-0.1744110 .520711-1.580901$

H $2.7836191 .199946-3.812124$

H $2.0704102 .527487-4.690999$

H $1.5536150 .883656-5.061073$

H $-3.0552170 .195271 \quad 1.863627$

H -2.9167950 .5953500 .154936$

H $-2.1379154 .742928 \quad 1.355215$

H -3.7661505 .0259340 .726693$

H -2.930699 $5.138322-1.625351$

H $-0.3156285 .482474-0.066645$

H $0.0319807 .051107-1.955978$

H -1.235173 $6.320651-2.905429$

H $2.6050254 .766632-3.282860$

H $2.2788126 .244480-2.213470$

H $0.5849523 .963896-4.624662$

H $-0.9991474 .247716-3.994992$

H $-0.7353012 .654453-2.158423$

H $-3.3890562 .792254-0.522390$

C $-0.209175 \quad 0.298841 \quad 3.866177$

O $0.652174 \quad 0.8288852 .836634$

C 1.4343711 .9014773 .387062

C 0.7202452 .3100464 .667879

C 0.2330220 .9636195 .169822

H 2.4121801 .4792453 .648940

C 1.6120483 .0147342 .350884

H 1.3853852 .8175845 .372741

H -0.1318242 .9694614 .465879$

C -0.8672861 .0634216 .211700$

H 1.0847380 .4195335 .599975

C 2.1903772 .5450860 .998629

O 0.3407073 .6181322 .084800

H 2.2384323 .8104972 .769953

H 1.4513631 .9397360 .459555

O $2.4166593 .710340 \quad 0.196376$

C 3.5115481 .7618841 .104266

C $4.1189101 .453747-0.278402$

H 3.3250730 .8255411 .641081

O 4.4378832 .5284571 .872399

H $3.3649380 .938300-0.882442$

H $4.3554892 .394207-0.790784$

H -0.5099481 .5971497 .098407$

H -1.7392341 .6013885 .824427$

H $-1.197546 \quad 0.068327 \quad 6.525781$

C $-0.087966-1.2250633 .886116$

H $-0.2550182 .918511 \quad 1.766065$

H $1.569198 \quad 4.181148 \quad 0.126141$

H 5.1653501 .9386682 .132266

H $-0.649788-1.6470944 .726832$

H $\quad 0.962277-1.5137504 .020862$

H -1.2326300 .5971023 .609907$

C $4.439767-1.5807840 .019519$

O $5.251737-0.557170 \quad 0.601970$

C $5.4130300 .611878-0.207217$

C $6.0042190 .241542-1.576560$

C $5.214038-0.868457-2.265498$

C $5.020659-2.051529-1.318334$

H $3.432187-1.179323-0.146853$

C $4.266165-2.7099671 .045316$

H $6.162715 \quad 1.2313850 .300534$

O $6.0569501 .376470-2.434678$

H $7.036618-0.106362-1.440546$

H $4.251149-0.504101-2.639358$

O $5.941000-1.323924-3.408728$

H $5.985176-2.551860-1.173318$

O $4.137582-2.997233-1.912492$

C $5.548367-3.4323391 .403718$

H $3.867775-2.3002211 .981108$

H $3.547613-3.4403650 .655066$

H $6.5806492 .060158-1.982941$

H $\quad 6.089856-0.552394-3.982076$

H $4.510862-3.241137-2.776743$

O $5.245569-4.6934551 .832184$

O $6.669401-2.9452241 .351658$

C $6.363928-5.4952962 .225083$

H $5.988688-6.4760062 .529562$ 
H $7.049904-5.6280411 .383008$

H $6.878145-5.0365893 .075049$

SCF Energy (B3LYP/6-31G**//MMFF)= -3245.91953311

06 00040

MM̄FF Geometry

C $1.938846-4.146217-0.029145$

C $0.707774-3.6309850 .108293$

C -0.252195 -3.499663 -1.048163

O $-0.892378-2.203593-0.983538$

C $-1.351067-4.569645-0.948230$

C $-2.147271-4.706740-2.230772$

C $-3.382884-4.208264-2.428743$

C -1.480782 -5.516576 -3.314712

C $-4.212879-3.389275-1.479756$

C $-4.824451-2.171577-2.188403$

C $-5.802498-1.417378-1.277240$

C $-6.528011-0.316915-2.052918$

C $-7.4705190 .487944-1.146813$

O $-5.061816-0.840449-0.197284$

O $-7.860631 \quad 1.657285-1.882223$

C -6.6870620 .9469040 .100126$

C $-8.761343-0.283246-0.848270$

O -7.5530431 .5857061 .035800$

C $-5.875597 \quad-0.181700 \quad 0.782744$

C -4.9484820 .4317521 .855232$

O $-6.777379-1.0908311 .416347$

C $-0.179446-1.129361-1.415754$

O $0.957579-1.129384-1.858818$

C $-1.0306800 .067393-1.236592$

C $-0.4689851 .284627-1.273867$

C -1.163805 $2.562034-1.099197$

C $-2.6687262 .540450-1.090649$

C $-3.910998-0.5376892 .433074$

C $-2.9586990 .218055 \quad 3.337905$

C -1.0370451 .8095013 .250992$

O

C -1.3601143 .1999232 .798377$

C -0.4474674 .0486192 .301438$

C -0.7655115 .4399921 .833534$

C -0.2126465 .7351930 .455349$

C $0.813767 \quad 6.586497 \quad 0.289352$

C $-0.8934865 .095757-0.737719$

C $-0.4250773 .683185-0.967394$

N -1.977543 0.8955602 .630891

H $2.236691-4.499618-1.015096$

H $\quad 0.358623-3.2860851 .076848$

H $\quad 0.277508-3.587663-2.004990$

H -2.014499-4.347532 -0.104324

H $-0.907866-5.549138-0.722205$

H $-3.866438-4.419067-3.382254$

H -2.115116 -5.629063 -4.200517

H $-0.550354-5.039533-3.637216$

H -1.247763 -6.521694 -2.948315

H -5.017361-4.029028 -1.097582

H -3.633309 -3.048065 -0.617539

H -4.014586 -1.500455 -2.502853

H -5.352512 -2.499468 -3.092795

H $-6.524581-2.139073-0.877160$

H -7.080121 $-0.735556-2.903531$

H $-5.7878760 .371382-2.485500$

H -8.397533 2.212186-1.290479

H $-5.985431 \quad 1.722532-0.236738$

H -9.282573 -0.528649-1.781345

H $-9.4626940 .326362-0.267715$

H $-8.588137-1.216500-0.308362$

H $-8.050527 \quad 0.8909291 .500076$

H $-4.4148871 .283631 \quad 1.419186$

H -5.5488910 .8300902 .683616$

H -6.304445 -1.563244 2.119516

H -2.088554 - $0.087319-1.056434$

H $\quad 0.607324 \quad 1.354979-1.424687$

H -3.036267 2.021029 -0.202331

H -3.120814 $3.534134-1.094981$

H -3.053367 2.027224-1.979516

H -4.386408 -1.3300343 .020610$

H -3.331031 -1.026671 1.642168

H -0.0363531 .4981792 .940600$
H -1.1078511 .7438714 .340724$

H -2.3944403 .5246962 .886602$

H $0.590740 \quad 3.7338442 .236804$

H -0.3450296 .1406212 .566364$

H -1.847533 5.6212151 .829760

H $1.2018156 .817905-0.697213$

H 1.2939817 .0659371 .135967

H $-0.6686265 .667976-1.647327$

H -1.975236 $5.173413-0.601141$

H $0.6601303 .590238-1.012175$

H -1.9462560 .8053681 .619687$

C $2.824889-3.5020642 .322753$

O $2.904778-2.0913752 .027346$

C $3.827496-1.4742772 .942257$

C $4.011631-2.4703094 .080133$

C $3.939796-3.7857763 .333682$

H $4.779345-1.3695442 .407481$

C $3.327757-0.0959333 .383155$

H $4.958165-2.3264924 .609910$

H $3.199059-2.4076644 .813098$

C $3.668994-4.9811364 .231148$

H $4.893015-3.9422392 .810619$

C 3.0148470 .8803802 .232284

O $2.120859-0.2698164 .138199$

H 4.0574770 .3485004 .070875

H $2.109597 \quad 0.5540201 .706202$

O 2.6885502 .1417282 .832552

C 4.1516711 .0910921 .215017

C 3.8058022 .1919650 .192924

H 4.3459210 .1485360 .696422

O $5.335677 \quad 1.4519311 .924120$

H $2.8363451 .953891-0.257317$

H 3.6915963 .1469080 .719984

H $4.469998-5.0974354 .968490$

H $2.725136-4.8689344 .775211$

H $3.612178-5.9039813 .645435$

C $2.993178-4.309376 \quad 1.032357$

H 1.8293560 .6124944 .425438

H 3.4961302 .4844683 .252658

H 6.0861861 .3526421 .314638

H $3.064744-5.3775991 .267800$

H $3.946327-4.0208750 .572028$

H $1.843996-3.6846622 .777767$

C $4.1238860 .678589-2.450718$

O $5.1709011 .166144-1.602969$

C $4.8731522 .381393-0.908714$

C $4.5424243 .493051-1.915755$

C $3.4309833 .077538-2.873550$

C $3.7440471 .725008-3.506816$

H $3.2486820 .423412-1.842344$

C $4.637040-0.600715-3.130733$

H $5.8004022 .679804-0.404603$

O $4.1581934 .691549-1.249827$

H $5.4381663 .726104-2.506091$

H $2.4574403 .056319-2.374514$

O $3.3223754 .046948-3.918362$

H $4.559631 \quad 1.842951-4.231826$

O $2.597708 \quad 1.269394-4.223612$

C $4.795548-1.753613-2.164002$

H $3.943686-0.932378-3.911220$

H $5.618442-0.402257-3.578923$

H $4.8872024 .943191-0.657556$

H $3.1540814 .907405-3.497129$

H $2.3636241 .960809-4.866341$

O $5.549202-2.726871-2.756458$

O $4.304297-1.809292-1.046042$

C $5.782572-3.897250-1.966830$

H $4.850770-4.457485-1.849178$

H $6.505126-4.524866-2.495128$

H $6.200231-3.629473-0.991346$

SCF Energy $\left(B 3 L Y P / 6-31 G^{* *} / / M M F F\right)=-3245.92568753$

06_00041

MM̄MF Geometry

C $0.463142-2.3502370 .428894$

C $1.046401-1.9172171 .558474$

C $0.542149-0.8083112 .455986$

O $-0.831521-0.4741112 .159470$ 
C $\quad 1.3995590 .4495352 .235939$ C $1.090012 \quad 1.556826 \quad 3.222198$ C $0.341876 \quad 2.6422582 .943598$ C 1.7287211 .4050724 .578357 C $-0.3692542 .957622 \quad 1.656759$ C $-1.809123 \quad 3.4252661 .912169$ C -2.5250773 .7691290 .598978$ C $-3.875908 \quad 4.4316790 .870112$ C $-4.635250 \quad 4.720529-0.433444$ O $-2.7211832 .554213-0.132263$ O $-5.9915815 .044173-0.091602$ C $-4.6766513 .432879-1.280798$ C $-4.0697185 .940476-1.169855$ O $-5.2385843 .685535-2.566827$ C $-3.3016312 .737522-1.430328$ C $-3.4994921 .346282-2.073417$ O $-2.459808 \quad 3.523329-2.275244$ C $-1.794786-1.2006492 .788570$ O $-1.630205-2.0846193 .614172$ C -3.102766 -0.7629392 .254402$ C $-4.172846-1.5510112 .435209$ C $-5.519252-1.3167261 .911074$ C -5.8023250 .0201111 .281346$ C $-2.255797 \quad 0.450314-2.057636$ C $-2.628981-0.944617-2.519326$ C $-3.782917-3.015989-1.734245$ O $-2.467332-1.303515-3.683270$ C $-5.273049-2.885451-1.653804$ C $-6.052988-3.696407-0.922177$ C $-7.546917-3.554669-0.831080$ C $-8.059578-3.5770550 .594474$ C $-8.759510-4.626073 \quad 1.058934$ C $-7.825893-2.3546121 .460434$ C $-6.425498-2.3117472 .007314$ N $-3.196492-1.707157-1.509947$ H $-0.466510-1.8833780 .110592$ H $1.982358-2.363327 \quad 1.883546$ H $0.626305-1.1381763 .498932$ H 1.2717940 .8084231 .208169 H 2.4662530 .2003542 .322153 H $0.227872 \quad 3.3959403 .721762$ H 1.5096502 .2509115 .238680 H $1.3673960 .499227 \quad 5.074726$ H 2.8172041 .3380494 .479682 H $0.181986 \quad 3.757567 \quad 1.148945$ H $-0.387122 \quad 2.0988290 .980239$ H $-2.3565892 .631647 \quad 2.436950$ H -1.7949864 .3068332 .565229$ H -1.881141 4.4419670 .020233 H -3.749865 5.3532511 .451686 H $-4.4910893 .765607 \quad 1.491514$ H -5.9753305 .8149630 .501330$ H -5.361296 $2.736282-0.776343$ H -4.120869 $6.830686-0.531373$ H -4.665010 6.175779-2.059134 H -3.029163 $5.812883-1.475517$ H -6.104416 4.106207-2.427133 H $-4.3042270 .822082-1.546619$ H -3.829444 $1.459875-3.114385$ H -1.775906 $2.950934-2.657504$ H -3.138014 0.1592191 .686068 H -4.043803 -2.4833892 .983870$ H -5.4924830 .8357831 .944591$ H $-6.861376 \quad 0.1880331 .074760$ H -5.2638930 .1149750 .335603$ H - $1.4793620 .837645-2.724760$ H $-1.8159340 .383750-1.056094$ H -3.385377 -3.686001 -0.965495 H -3.493092 -3.392689-2.719716 H -5.733518 -2.084181-2.227602 H $-5.597130-4.515583-0.370404$ H $-7.992353-4.378409-1.403779$ H -7.888577 -2.631715 -1.316128 H $-9.150771-4.6407792 .070825$ H $-8.953073-5.495973 \quad 0.440037$ H $-8.514661-2.3602932 .315134$ H -8.085214 -1.4672060 .876852$ H $-6.128016-3.2319532 .512943$
H $-3.302591-1.299476-0.586055$

C $2.308648-4.074102-0.197844$

O $3.357699-3.090781-0.158241$

C $4.554996-3.647810-0.727743$

C $4.248289-5.111707-1.031425$

C $2.749802-5.079363-1.260895$

H $4.726085-3.115202-1.670690$

C $5.743515-3.423853 \quad 0.216529$

H $4.807334-5.477557-1.898347$

H $4.486892-5.766078-0.185468$

C $2.088708-6.441463-1.142194$

H $2.560948-4.673121-2.263783$

C $5.915237-1.9742290 .713076$

O $5.583678-4.244327 \quad 1.383056$

H $6.665027-3.768923-0.266129$

H $5.112734-1.7205521 .416983$

O $7.136899-1.9152561 .462071$

C $5.972389-0.906397-0.393021$

C $6.258806 \quad 0.4962250 .175976$

H $5.020261-0.892670-0.929391$

O $6.983057-1.245224-1.340992$

H $\begin{array}{llll}5.555657 & 0.692458 & 0.992527\end{array}$

H 7.2675420 .5112280 .606319

H $2.503482-7.135625-1.880372$

H $2.241489-6.876174-0.148642$

H $1.010635-6.369689-1.316313$

C $0.968694-3.405697-0.521833$

H $4.735708-4.0058501 .795634$

H $7.078813-2.5862962 .163837$

H $7.827543-1.303668-0.862057$

H $0.191904-4.175375-0.602483$

H $1.045304-2.926334-1.505688$

H $2.268360-4.5500320 .790605$

C $3.7980862 .050857-0.747012$

O $4.9064151 .638648-1.556620$

C $6.1714201 .614012-0.887460$

C $6.4992543 .013872-0.348601$

C $5.381698 \quad 3.5509190 .541135$

C $4.0354313 .453524-0.173942$

H 3.6493291 .3320630 .067420

C $2.5458842 .036122-1.638330$

H $6.9190101 .380216-1.655768$

O $7.718608 \quad 3.007901 \quad 0.385901$

H $6.6341893 .705325-1.190534$

H $5.351398 \quad 3.033887 \quad 1.507382$

$\begin{array}{lllll}\text { O } & 5.632796 & 4.927970 & 0.832206\end{array}$

H $3.996267 \quad 4.200435-0.977257$

$\begin{array}{llllll} & & 2.993313 & 3.763845 & 0.747871\end{array}$

C $2.1824530 .636578-2.082690$

H $1.6774452 .446017-1.111978$

H $2.7378192 .638113-2.535089$

H $8.4143252 .674093-0.205965$

H 6.5093684 .9791451 .250714

H $3.1805174 .645706 \quad 1.112608$

$\begin{array}{llll}\text { O } & 1.479013 & 0.700768 & -3.250712\end{array}$

O $2.460591-0.385245-1.470935$

C $1.085674-0.560960-3.799429$

H $1.969504-1.152190-4.057432$

H $0.452883-1.102966-3.091229$

H $0.510384-0.371821-4.709568$

SCF Energy (B3LYP/6-31G**//MMFF)= -3245.92681541

06_00042

MM̄FF Geometry

C $0.459072 \quad 4.171337-0.321022$

C $0.836597 \quad 3.0337340 .278761$

C $1.545798 \quad 3.0116361 .608043$

O $2.6458762 .078681 \quad 1.532450$

C 0.5911422 .4859332 .690005

C $1.0115282 .857474 \quad 4.096324$

C 1.6747052 .0469064 .943428

C 0.5754154 .2280054 .547658

C $2.2362590 .678728 \quad 4.674438$

C $1.396598-0.4515445 .285733$

C $0.197141-0.8488004 .411302$

C $-0.639472-1.9317745 .093947$

C $-1.780251-2.4217754 .190803$

O $0.717197-1.3417113 .171462$ 
O $-2.308077-3.6132654 .792572$

C -1.194176 -2.801519 2.814545

C $-2.935631-1.4148274 .144337$

O $-2.234221-3.1461391 .902783$

C $-0.271242-1.718178 \quad 2.207456$

C $0.467915-2.2913150 .975109$

O $-1.074625-0.6086791 .800481$

C 3.8027532 .4837490 .950676

O $4.0481803 .575563 \quad 0.466779$

C $4.718194 \quad 1.322263 \quad 0.985974$

C $5.808971 \quad 1.304410 \quad 0.207671$

C $6.7621690 .198037 \quad 0.096303$

C $6.538778-1.0068920 .973912$

C $1.583217-1.3953170 .413822$

C $2.226103-2.013750-0.818490$

C $3.798662-1.477318-2.676406$

O $1.975879-3.153166-1.203945$

C $5.140573-2.067312-2.374259$

C $6.266597-1.695293-3.003101$

C $7.614652-2.306934-2.741276$

C $8.695742-1.273040-2.508467$

C $9.546920-0.931155-3.490511$

C $8.840643-0.697517-1.116724$

C $7.7661430 .302767-0.799062$

N $3.114869-1.146266-1.438480$

H 0.6799925 .1223870 .158341

H $0.6024862 .078650-0.184462$

H 1.9273234 .0052841 .874412

H 0.5106921 .3991292 .599856

H -0.4246812 .8732292 .532505$

H 1.8798412 .4166565 .947636

H $0.888466 \quad 4.448345 \quad 5.573773$

H 1.0045894 .9991433 .900289

H -0.5158394 .3105844 .510648$

H $2.4020750 .508543 \quad 3.607168$

H 3.2347800 .6510695 .130283

H $2.053329-1.3249605 .393776$

H $1.059328-0.1734776 .291664$

H -0.4188810 .0369754 .224950$

H -1.034649 -1.573357 6.052707

H $0.002933-2.7897925 .337589$

H -2.981224 -3.974408 4.190252

H -0.606486 -3.717866 2.961616

H -3.329952 -1.241634 5.152934

H $-3.776879-1.8021233 .558793$

H -2.644976 -0.4469193 .730384$

H -2.649404-2.319822 1.601820

H $0.922842-3.2534791 .245085$

H $-0.255195-2.4861050 .175964$

H $-0.647234-0.1579121 .055597$

H $4.4439300 .491421 \quad 1.626771$

H $6.0093042 .166364-0.427355$

H $6.609305-0.7277362 .031105$

H $7.259847-1.8096740 .809677$

H $5.549520-1.4413340 .791742$

H $1.185767-0.4149030 .133973$

H $2.367989-1.2490711 .164065$

H $3.876131-0.554780-3.260420$

H $3.197236-2.198881-3.238741$

H $5.185622-2.854885-1.625836$

H $6.213165-0.931552-3.775857$

H $7.873011-2.923639-3.612213$

H $7.582815-2.996351-1.888446$

H $10.341502-0.211036-3.325311$

H $9.464670-1.360026-4.483753$

H $9.804844-0.181090-1.018423$

H $8.890457-1.517159-0.395066$

H $7.8378741 .215270-1.392940$

H $3.297358-0.238013-1.022531$

C -1.751810 $4.598336-1.383696$

O $-2.4889473 .400556-1.046876$

C -3.766037 $3.446491-1.714282$

C $-3.8997874 .856970-2.275199$

C $-2.456928 \quad 5.178987-2.606848$

H $-3.7110052 .748836-2.557116$

C -4.892342 $3.046694-0.755834$

H -4.561616 $4.896603-3.145775$

H $-4.2871065 .554415-1.522812$
C $-2.204288 \quad 6.660885-2.824821$

H $-2.165707 \quad 4.624123-3.508835$

C $-4.8453801 .578087-0.280595$

$\begin{array}{lllll}\text { O } & -4.842437 & 3.891136 & 0.400725\end{array}$

H -5.862527 $3.243260-1.227210$

H $-3.971167 \quad 1.404606 \quad 0.358130$

$\begin{array}{llll}\text { O } & -5.998556 & 1.353725 & 0.540674\end{array}$

C $-4.861350 \quad 0.549792-1.428083$

C $-5.088065-0.902772-0.960557$

H $-3.9130450 .610196-1.970012$

O $-5.8935420 .891905-2.356912$

H $-4.368683-1.142936-0.171140$

H $-6.091860-0.988151-0.528398$

H $-2.7964507 .031434-3.668067$

H $-2.474427 \quad 7.248398-1.940900$

H $-1.1492496 .850228-3.045125$

C $-0.284776 \quad 4.241872-1.623872$

H -3.966001 3.7781120 .807450

H $-5.9749862 .010273 \quad 1.257985$

H $-6.7389010 .873496-1.876106$

H $0.2063445 .003523-2.239703$

H $-0.2085663 .288068-2.159171$

H $-1.8550175 .284085-0.531629$

C $-2.620690-2.437415-1.972044$

O $-3.676535-1.851555-2.744068$

C $-4.971593-1.902563-2.135097$

C $-5.359902-3.352255-1.807196$

C $-4.273457-4.085696-1.029060$

C $-2.920024-3.920071-1.721332$

H $-2.523981-1.915373-1.013110$

C $-1.317835-2.247607-2.762366$

H $-5.677637-1.566107-2.904609$

O $-6.579424-3.402692-1.074294$

H $-5.530654-3.895261-2.746245$

H $-4.240428-3.7418320 .010971$

O $-4.607959-5.474500-0.984966$

H $-2.931078-4.470343-2.670387$

O $-1.904508-4.507000-0.912446$

C $-0.809145-0.821229-2.689971$

H $-0.524207-2.888420-2.366679$

H $-1.488537-2.492970-3.817444$

H $-7.259855-2.958796-1.608828$

H $-3.885268-5.931340-0.521112$

H $-1.927096-4.073656-0.042461$

O $0.187594-0.658470-3.609890$

$\begin{array}{llll}0 & -1.211944 & 0.035630 & -1.915211\end{array}$

C $\quad 0.7712890 .647323-3.657331$

H $1.5880560 .628654-4.383712$

H $0.026423 \quad 1.378875-3.984198$

H $1.1785420 .921365-2.679415$

SCF Energy (B3LYP/6-31G**//MMFF) $=-3245.94006094$

06_00043

MM̄FF Geometry

C $2.058224-3.1564111 .843479$

C $1.323382-2.0508721 .652139$

C $0.630093-1.3030482 .762258$

O $-0.754836-1.1118752 .384106$

C 1.2871850 .0752902 .944584

C $0.7791130 .826893 \quad 4.160165$

C 0.0037121 .9284284 .119306

C $1.2551540 .297399 \quad 5.490050$

C -0.5545952 .6186662 .907130$

C $-2.0609782 .866728 \quad 3.048471$

C $-2.6389593 .613561 \quad 1.835429$

$\begin{array}{llll}C & -4.134894 & 3.863732 & 2.040425\end{array}$

C $-4.790288 \quad 4.4957350 .806592$

O $-2.418207 \quad 2.806857 \quad 0.676626$

O $-6.210574 \quad 4.4111590 .997855$

C $-4.4248563 .649053-0.429768$

C $-4.459796 \quad 5.9881050 .694922$

O $-4.919354 \quad 4.260026-1.620182$

C $-2.9110193 .352679-0.552828$

C $-2.6287732 .322219-1.675489$

O $-2.233808 \quad 4.566879-0.891745$

C $-1.587671-2.1742742 .568765$

O $-1.302241-3.2483543 .075902$

C $-2.913013-1.8284772 .006038$ 
C $-3.781284-2.8213911 .757046$ C $-5.119693-2.6948881 .176169$ C $-5.686982-1.3115211 .009739$ C $-2.949697 \quad 0.881223-1.273697$ C $-2.923151-0.094674-2.434560$ C $-3.301592-2.527329-2.874415$ O $-2.816051 \quad 0.250648$-3.606979 C $-4.758406-2.868540-2.818053$ C $-5.232669-4.060789-2.426254$ C $-6.697673-4.383367-2.344946$ C $-7.111624-4.913089-0.988393$ C $-7.518081-6.185359-0.839433$ C $-7.131213-3.9495220 .182473$ C $-5.780826-3.8216330 .835802$ N -3.075268 -1.401288 -1.988533 H $2.178070-3.5511812 .849468$ H $1.202320-1.6574690 .647057$ H $0.677872-1.8667943 .701990$ H 1.1548970 .6678162 .033060 H $2.374040-0.0434663 .056129$ H -0.2572012 .4022065 .065185$ H $\quad 0.953232 \quad 0.938330 \quad 6.325354$ H $0.846088-0.6995345 .678796$ H $2.348276 \quad 0.235126 \quad 5.505290$ H -0.0367893 .5775722 .787758$ H -0.3780602 .0379981 .999678$ H -2.564868 1.8966743 .155016 H -2.2602993 .4424583 .960970$ H -2.1017804 .5619241 .717453$ H $-4.307816 \quad 4.4849862 .928237$ H -4.637605 2.9075692 .245203 H -6.637059 4.7396990 .187602 H $-4.9702512 .702522-0.332952$ H -4.8127916 .5232331 .584744$ H $-4.9787996 .447717-0.153449$ H -3.3904546 .1843420 .592631$ H -4.325864 $4.997253-1.842966$ H -3.163183 2.610197 -2.589089 H -1.567285 2.360112 -1.951340 H -1.374578 4.343706 -1.285878 H $-3.121644-0.788148 \quad 1.785600$ H -3.472917 -3.843382 1.976384 H $-5.185243-0.7886590 .192297$ H -5.566001 -0.7262921 .928242$ H $-6.758161-1.3082260 .794477$ H $-2.2304010 .538645-0.520036$ H -3.951100 $0.826770-0.837844$ H - $2.670516-3.349829-2.524400$ H -3.013526 -2.268757 -3.897749 H -5.458390 -2.087593 -3.107312 H $-4.534625-4.848763-2.153331$ H -6.917457 -5.123419 -3.125307 H -7.311248 -3.505445 -2.582911 H $-7.840420-6.5655110 .124426$ H -7.533239 -6.876658 -1.675426 H $-7.836543-4.3059480 .944466$ H -7.527326 -2.991720 -0.166654 H $-5.299959-4.780070 \quad 1.037998$ H -3.175871 -1.569002 -0.991941 C $4.257413-3.8338770 .847153$ O $4.692689-2.486196 \quad 0.572591$ C $5.834952-2.533446-0.302967$ C $6.300415-3.983881-0.308004$ C $4.981805-4.718079-0.168125$ H $5.473979-2.278736-1.306418$ C $6.893986-1.5194490 .143264$ H $6.843040-4.242776-1.222108$ H $6.950702-4.2066380 .545905$

C $5.135440-6.1645310 .268826$ H $4.459023-4.687975-1.133783$ C $6.377744-0.0725720 .277857$ O $7.408717-1.9091661 .423704$ H $7.746803-1.550046-0.544633$ H $5.686036 \quad 0.003716 \quad 1.125743$ O $7.4974990 .764223 \quad 0.594965$ C $5.6994080 .484511-0.986278$ C $5.2773461 .955154-0.824860$ H $4.815793-0.115509-1.210190$
O $\quad 6.5814790 .365027-2.100731$

H $4.6803112 .046703 \quad 0.088722$

H $6.1717722 .575423-0.691443$

H $5.718238-6.728265-0.466943$

H $5.648764-6.2401181 .233320$

H $4.158936-6.6485560 .368172$

C $2.734593-3.9133660 .734943$

H $6.657487-1.9550892 .040052$

H 7.9125270 .4009861 .396066

H $7.3861980 .870580-1.893391$

H $2.404073-4.9567540 .788573$

H $2.414615-3.521088-0.237593$

H $4.582919-4.0723931 .868094$

C $2.2816081 .752598-1.386593$

O $3.3372051 .709431-2.355053$

C $4.4893002 .498617-2.039870$

C $4.0805303 .970228-1.905817$

C $2.9721744 .147504-0.873615$

C $1.8086893 .199632-1.162124$

H $2.6277101 .312351-0.443530$

C $1.1287890 .886873-1.923439$

H $5.1562882 .426370-2.908187$

O $5.2090144 .763530-1.554326$

H $3.7227284 .332512-2.878275$

H 3.3558854 .0133750 .144437

O $2.5029955 .496557-0.947077$

H $1.2662913 .567209-2.043076$

O $0.9176163 .267586-0.051914$

C $1.487865-0.574798-2.080471$

H $\quad 0.2754240 .927080-1.239915$

H $0.825500 \quad 1.256169-2.910549$

H $4.8999305 .680293-1.454504$

H $1.7769295 .584758-0.305833$

H $0.0979002 .806465-0.293317$

O $0.441416-1.220758-2.676522$

O $2.533384-1.092197-1.716169$

C $0.615521-2.625571-2.886164$

H $-0.174536-2.968806-3.559118$

H $1.583233-2.836974-3.351753$

H $\quad 0.521033-3.153680-1.933319$

SCF Energy $(B 3 L Y P / 6-31 G * * / / M M F F)=-3245.93589624$

06_00044

MMFF Geometry

C -1.3362303 .4431490 .546474$

C -0.9031762 .1811780 .683269$

C -0.4034561 .6085211 .985267$

O 0.9712651 .1955941 .803982

C -1.2447280 .3761712 .363446$

C $-0.847201-0.2455523 .688860$

C $-0.172349-1.4048883 .826581$

C -1.2931930 .5139314 .912064$

C $0.348560-2.3009412 .738256$

C $1.880309-2.3728722 .760645$

C $2.435868-3.0340461 .490651$

C $3.956305-3.1743701 .576321$

C $4.566390-3.7014480 .269396$

O $2.081192-2.1992910 .382882$

O $5.983627-3.4859230 .353566$

C $4.027871-2.862869-0.911200$

C $4.370686-5.2156180 .132392$

O $4.464066-3.405808-2.154871$

C $2.494941-2.693217-0.896646$

C $1.982653-1.697998-1.966671$

O $1.887083-3.959277-1.177866$

C 1.9234822 .1611441 .939973

O 1.7399853 .3431372 .185544

C 3.2474401 .5316701 .735300

C 4.3493242 .2968581 .720140

C $5.7279101 .832051 \quad 1.548715$

C 5.9470820 .3595051 .326908

C $2.470938-0.253916-1.812257$

C $3.7092560 .080006-2.626339$

C $5.3621261 .917219-2.965746$

O $4.171434-0.662160-3.488071$

C $6.5974761 .630008-2.172565$

C $7.4439972 .583613-1.754873$

C $8.7182932 .304501-1.011187$ 
C 8.8302053 .0889720 .276416

C 9.5331944 .2330860 .330053

C 8.2082092 .4973601 .520137

C 6.7269032 .7381161 .601146

N $4.2131901 .332808-2.300552$

H -1.3215844 .1086381 .406201$

H $-0.9064951 .518758-0.179322$

H -0.4619622 .3503462 .791568$

H -1.188445 -0.3617391 .557879$

H -2.3059130 .6554422 .420488$

H $0.040787-1.7580844 .834856$

H -1.048175 -0.0123265 .841000$

H $-0.810940 \quad 1.4952154 .954344$

H -2.378435 0.6587174 .894383

H $-0.062596-3.3067202 .888417$

H $\quad 0.006250-1.9793821 .753187$

H $2.285421-1.3569902 .844649$

H $2.207309-2.9290443 .647862$

H $1.963628-4.0162131 .370525$

H $4.241174-3.8147372 .420493$

H $4.396178-2.1917891 .789750$

H $6.372082-3.745968-0.499672$

H $4.497719-1.876187-0.829359$

H $4.846332-5.7378740 .971428$

H $4.858063-5.599631-0.770568$

H $3.319829-5.5117610 .110894$

H $3.911762-4.181940-2.349671$

H $2.178388-2.088591-2.972373$

H $\quad 0.887819-1.664860-1.878231$

H $0.969812-3.800676-1.455147$

H $3.280858 \quad 0.456770 \quad 1.606540$

H 4.2364703 .3718011 .857147

H $5.334602-0.0012880 .494008$

H $5.684550-0.2028602 .229043$

H $\quad \begin{array}{llll}6.977617 & 0.103893 & 1.072139\end{array}$

H $1.6813110 .417066-2.175103$

H $2.647541-0.008926-0.761393$

H $5.1707852 .990844-3.059914$

H $5.4609841 .491616-3.969468$

H $6.8221350 .587193-1.960577$

H $7.2312123 .623179-1.994316$

H $9.5532982 .562036-1.676053$

H $8.8323611 .234333-0.798214$

H 9.6444894 .7852341 .257399

H $10.0081104 .645712-0.553804$

H 8.6569772 .9514452 .414315

H 8.4732201 .4386141 .590900

H 6.4684103 .7861521 .758832

H $3.7941281 .854153-1.535802$

C $-3.2844474 .522599-0.592710$

O $-4.1635613 .413586-0.321179$

C -5.368842 $3.572984-1.091740$

C $-5.3243174 .985557-1.662566$

C -3.833595 $5.183462-1.856075$

H $-5.3075612 .858178-1.920962$

C $-6.5954383 .262953-0.227107$

H $-5.8951775 .075317-2.591842$

H $-5.7156615 .724876-0.954264$

C -3.430193 $6.638251-2.020396$

H -3.516918 $4.619534-2.743748$

C -6.5509731 .8924920 .479628$

O

H -7.503716 $3.342236-0.835531$

H -5.7964641 .9007971 .275766$

O $\quad-7.8149871 .696091 \quad 1.126413$

C $-6.2908180 .698137-0.455114$

C $-6.375937-0.6533890 .278608$

H $-5.2959130 .801206-0.899491$

O $\quad-7.234514 \quad 0.712592-1.525422$

H $-5.700980-0.626738 \quad 1.140944$

H $-7.392011-0.7942430 .666273$

H -3.910874 $7.073062-2.902831$

H -3.721257 $7.236244-1.150253$

H -2.347022 $6.731438-2.145962$

C -1.850178 $4.015322-0.745186$

H $\quad-5.893984 \quad 4.2610251 .307997$

H -7.959349 2.4558421 .716397

H -8.123002 $0.650948-1.134355$
H -1.189354 $4.836984-1.043084$

H -1.808696 $3.255221-1.535359$

H $-3.360675 \quad 5.2064470 .263073$

C $-3.633053-1.823100-0.436220$

O $-4.774677-1.696002-1.288956$

C $-6.038042-1.853060-0.635282$

C $-6.109298-3.2180240 .063303$

C $-4.924387-3.4463150 .999690$

C $-3.610575-3.1888710 .261137$

H $-3.664502-1.0306520 .322136$

C $-2.376378-1.549335-1.272086$

H $-6.792980-1.862958-1.431685$

O $-7.331982-3.3371260 .783071$

H $-6.110125-4.005888-0.701468$

H $-5.008128-2.8276141 .900839$

O $-4.962559-4.8065981 .436806$

H -3.449217 -3.993940 -0.465445

O $-2.528602-3.217397 \quad 1.186500$

C $-2.222785-2.502864-2.434376$

H $-2.425042-0.541183-1.702840$

H $-1.471396-1.575396-0.654749$

H -7.327849 -4.205321 1.221444

H $-4.269476-4.9183112 .109215$

H -2.460555 -4.122115 1.534710

O $-1.360971-3.508026-2.109131$

O $-2.815913-2.378233-3.498362$

C -1.164719-4.498345 -3.123712

H $-0.533634-5.289412-2.710153$

H -2.121830 -4.936966 -3.422109

H $-0.655522-4.055407-3.984608$

SCF Energy $\left(B 3 L Y P / 6-31 G^{* *} / / M M F F\right)=-3245.92516844$

06_00045

MMFF Geometry

C $-0.928216 \quad 1.5124482 .428003$

C $-1.9634611 .007943 \quad 3.116603$

C $-2.441743-0.4239133 .150275$

O $-1.517849-1.3275292 .503359$

C -3.802063 -0.549660 2.445068

C $-4.588083-1.7755092 .870575$

C $-4.847147-2.8391742 .085774$

C $-5.171503-1.7183244 .260946$

C $-4.392484-3.0932380 .676434$

C $-5.528859-2.908392-0.341826$

C $-5.854302-1.432496-0.622544$

C $-7.049955-1.301890-1.566414$

C $-7.312160 \quad 0.160808-1.959795$

O $-4.697650-0.855042-1.230023$

O $\quad-8.238480 \quad 0.130929-3.056015$

C $-5.9925160 .790402-2.458969$

C $-7.9976430 .935290-0.827850$

O $-6.1431252 .189370-2.690049$

C $-4.7959750 .546137-1.510223$

C $-3.4762010 .962558-2.201821$

O $-4.970566 \quad 1.316997-0.321145$

C $-0.517055-1.8382773 .267483$

O $-0.272603-1.5516304 .432486$

C $0.281056-2.8525542 .542943$

C $0.085372-3.2099501 .264296$

C $0.824338-4.2539170 .547085$

C $1.873512-5.0217741 .309504$

C $-2.2140690 .595001-1.405396$

C $-0.9744300 .622089-2.280942$

C $1.302048-0.377274-2.446753$

O $-0.906773 \quad 1.270876-3.321838$

C $1.276126-1.742891-3.052847$

C $2.248778-2.645199-2.857735$

C $2.236284-4.028958-3.436834$

C $2.370939-5.095314-2.372687$

C $3.558195-5.677730-2.133354$

C $1.122006-5.544505-1.647185$

C $0.530667-4.491269-0.748720$

N $0.051570-0.146436-1.750046$

H -0.3426300 .8748881 .772423$

H $-2.520771 \quad 1.680028 \quad 3.767972$

H $-2.542850-0.7057604 .205202$

H -3.643369 -0.548209 1.362539

H -4.4275040 .3280612 .657510$ 
H $-5.458537-3.6408692 .499477$ H -5.841062 -2.560761 4.466154 H $-4.380611-1.7387645 .016385$ H -5.755138 -0.8008574 .390822$ H -3.520514 -2.489380 0.407551 H $-4.050763-4.1352210 .629272$ H -5.211485 -3.385281-1.278434 H $-6.428372-3.4342600 .001317$ H -6.064296 -0.9226670 .323502$ H -7.948523 -1.747036 -1.121386 H $-6.854381-1.876702-2.482821$ H -8.462364 $1.047930-3.287898$ H $-5.7615110 .335862-3.432535$ H -8.964823 $0.478766-0.585611$ H $-8.2195311 .964781-1.129929$ H -7.4059310 .9673150 .089383$ H $-6.8263122 .304232-3.371560$ H $-3.4213940 .462002-3.177660$ H -3.468539 $2.043803-2.388097$ H -4.1022301 .4922070 .074313$ H $1.051276-3.3199813 .149122$ H $-0.694208-2.7136750 .689335$ H $2.651137-4.3460351 .682354$ H $2.381901-5.7778300 .707522$ H $1.424169-5.5458312 .160394$ H $-2.059611 \quad 1.301533-0.583482$ H -2.309210 -0.408545 -0.976742 H $2.099574-0.276651-1.706604$ H $1.4420670 .373350-3.230203$ H $\quad 0.423763-2.000872-3.676662$ H $3.108581-2.374244-2.250013$ H $3.068064-4.100937-4.149818$ H $1.327276-4.212694-4.022984$ H $3.664594-6.465288-1.394962$ H $4.452897-5.376819-2.668520$ H $1.304539-6.464488-1.078606$ H $\quad 0.365609-5.829530-2.390307$ H $-0.239252-3.886680-1.228773$ H $-0.104956-0.656236-0.885257$ C $-0.5109323 .728241 \quad 1.265468$ O $0.576654 \quad 3.3057770 .414185$ C 1.3353344 .4651240 .022962 C 0.4105565 .6533690 .247143 C -0.3109695 .2285561 .506262$ H 2.1848174 .5352200 .713365 C $1.8450754 .339307-1.414875$ H $\quad 0.959307 \quad 6.5931320 .361128$ H $-0.3055315 .774487-0.573823$ C -1.604270 5.9880791 .751180 H 0.3597835 .3864602 .361879 C $2.6708053 .075993-1.709374$ O $0.7248554 .329448-2.311827$ H $2.4269255 .231280-1.675085$ H $2.0174932 .199207-1.696241$ O $3.1634583 .189709-3.051496$ C $3.8648172 .820193-0.770696$ C $4.7543751 .667517-1.275989$ H 3.4899492 .5889340 .230280 O $4.6582034 .000900-0.660118$ H $4.116240 \quad 0.819138-1.536991$ H $5.2602721 .985806-2.195625$ H -1.405742 7.0589191 .864814 H -2.3064945 .8627450 .920135$ H -2.096544 5.6369292 .663599 C -0.4830102 .9419802 .577501$ H $\quad 0.1556413 .580842-2.062592$ H $2.3937663 .316566-3.632553$ H $4.9505234 .244465-1.555202$ H -1.109974 3.4488063 .321002 H 0.5384932 .9305922 .975892 H -1.441296 3.5399190 .715391 C $4.535860-0.4326740 .932990$ O $5.275621 \quad 0.7940240 .984016$ C $5.832277 \quad 1.225491-0.262128$ C $6.7802160 .144681-0.803015$ C $6.075682-1.202953-0.932627$ C $5.398645-1.577563 \quad 0.383814$ H $3.643210-0.2981070 .310944$
C $4.079143-0.7573362 .364633$

H $6.4408662 .110177-0.037228$

O $7.3124910 .516542-2.069804$

H $7.6302770 .028265-0.118173$

H $5.357220-1.205042-1.760156$

O $7.040564-2.212175-1.239597$

H $6.166907-1.8537201 .117610$

O $4.575912-2.7248580 .180245$

C 3.1022240 .2615522 .907356

H $3.587600-1.7351242 .406796$

H $4.954222-0.764563 \quad 3.026308$

H $7.7626541 .371275-1.957820$

H $7.491511-1.940611-2.057549$

H $5.138359-3.424029-0.194941$

O $3.047436 \quad 0.142200 \quad 4.266828$

O 2.4581321 .0486112 .228803

C 2.1861821 .0674814 .936901

H 1.1446040 .8713884 .671090

H 2.3058370 .9239546 .014114

H 2.4622862 .0968554 .688609

SCF Energy (B3LYP/6-31G**//MMFF) $=-3245.92859197$

0600046

MMFF Geometry

C $0.776014-2.5899081 .761232$

C $-0.432298-2.9514721 .306849$

C $-0.678262-3.409941-0.107384$

O $-1.383571-2.333058-0.771689$

C -1.562113 -4.667817 -0.119839

C $-1.869875-5.175214-1.517142$

C $-3.072562-5.086843-2.121291$

C $-0.718140-5.835718-2.230509$

C $-4.331697-4.482150-1.567185$

C $-4.633253-3.124263-2.214232$

C $-5.606818-2.296364-1.363588$

C $-5.999218-1.006259-2.084346$

C $-6.871639-0.097320-1.205707$

O $-4.938558-1.984105-0.135591$

O $-6.9115391 .187596-1.845625$

C -6.1900580 .0776160 .169970$

C $-8.320659-0.594498-1.151498$

$\begin{array}{lllll}\text { O } & -7.034692 & 0.799727 & 1.062511\end{array}$

C $-5.731635-1.2509760 .806237$

C $-4.870416-1.0540382 .078604$

O $-6.888630-2.0017961 .195347$

C $-0.620150-1.314756-1.260955$

O $0.599172-1.269687-1.313855$

C $-1.527237-0.221511-1.672596$

C $-1.0094850 .982501-1.958325$

C $-1.7555942 .179754-2.349360$

C $-3.2573522 .084662-2.393064$

C $-3.574096-0.2581451 .884354$

C $-3.703527 \quad 1.2219152 .203499$

C -2.4225503 .3546702 .072999$

O $-4.686016 \quad 1.7090332 .755921$

C -2.7947914 .1105580 .836962$

C $-1.983396 \quad 4.999603 \quad 0.243722$

C $-2.3758705 .826709-0.946767$

C - $-1.3873355 .726265-2.086041$

C $-0.4082276 .634815-2.234207$

C $-1.5944734 .629659-3.104851$

C -1.063978 $3.301598-2.639211$

N $-2.5685691 .931391 \quad 1.834115$

H $1.641917-2.6780321 .110234$

H -1.302453 -2.813464 1.944546

H $\quad 0.264399-3.617608-0.628607$

H -2.484883 -4.4608980 .430375$

H - $1.066534-5.4734920 .438722$

H $-3.174065-5.485834-3.129686$

H -1.015417 -6.261106 -3.195171

H $0.082028-5.114761-2.422885$

H $-0.314075-6.652679-1.623606$

H -5.164119 -5.168799-1.765763

H $-4.287928-4.393413-0.479576$

H -3.699685 -2.561803 -2.336055

H $-5.048693-3.285936-3.216460$

H -6.493401-2.905655 -1.151863

H $-6.506553-1.228011-3.031598$ 
H -5.090466 -0.453789-2.357386 H $-7.3963351 .793708-1.259160$ H -5.3139730 .7134670 .000478$ H -8.750223 -0.625185 -2.160211 H $-8.9552270 .091448-0.579728$ H -8.415252 $-1.593269-0.720252$ H $-7.7148920 .186214 \quad 1.388843$ H $-5.483228-0.6380342 .887401$ H -4.574696 -2.054928 2.425491 H -6.623252 -2.647959 1.870809 H $-2.590325-0.426313-1.700688$ H $0.0708841 .110416-1.897744$ H -3.574067 $1.394049-3.181867$ H -3.751052 $3.040065-2.581457$ H -3.647463 $1.727766-1.434064$ H $-2.820408-0.6507432 .579010$ H $-3.181723-0.3833990 .870958$ H -1.3852703 .5323762 .370740$ H -3.073638 3.6581642 .899172 H -3.793254 3.9495750 .437197 H $-0.997170 \quad 5.1803110 .665635$ H -2.450221 $6.870726-0.614618$ H -3.378245 5.562468 -1.306004 H $0.2857276 .587758-3.066806$ H $-0.2757627 .442563-1.521860$ $\mathrm{H}-1.0662204 .878814-4.035330$ H -2.649200 4.593745 -3.391596 H $0.0241773 .272061-2.569279$ H -1.820563 1.4539331 .339572 C $0.876395-0.4147402 .953654$ O $1.896757 \quad 0.0805692 .067261$ C 2.2882031 .3963282 .502173 C 1.4180661 .7330923 .710840 C 1.0757150 .3574064 .252496 H 3.3304881 .3222102 .831214 C 2.1799902 .3872521 .336716 H 1.9471902 .3590204 .436218 H 0.5065322 .2668843 .420509 C $-0.134717 \quad 0.353847 \quad 5.169549$ H $1.946381-0.0353374 .794268$ C 2.9504981 .9737680 .066147 O 0.8032632 .5304520 .963070 H 2.5058003 .3802931 .666767 H $2.4603271 .116135-0.410705$ O $2.8557433 .057489-0.868004$ C 4.4380131 .6456230 .291790 C $5.1768411 .385728-1.034989$ H 4.5116830 .7627440 .935143 $\begin{array}{lllll}\text { O } & 5.051939 & 2.736358 & 0.975484\end{array}$ H $4.6215480 .627155-1.597622$ H $5.1736222 .302189-1.637716$ H $0.040796 \quad 0.994213 \quad 6.040142$ H -1.028976 0.7224924 .655444 H - $0.347712-0.6564335 .532124$ C $0.993139-1.9336533 .094945$ $\mathrm{H} \quad 0.473791 \quad 1.6501580 .713106$ H $1.9108853 .252539-0.990291$ H 5.9153542 .4321401 .301910 H $\quad 0.266080-2.3073623 .824824$ H $1.989880-2.2132533 .455480$ H $-0.091082-0.1529702 .503294$ C $6.261801-1.402565-0.431323$ O $6.776961-0.1570530 .053527$ C $6.645708 \quad 0.942932-0.851059$

C $7.376146 \quad 0.629522-2.164947$ C $6.913314-0.692995-2.770450$ C $6.969825-1.809684-1.729158$ H $5.181854-1.313143-0.602629$ C $6.487661-2.4490310 .664961$ H $7.1744421 .784641-0.386975$ O $7.1863901 .670597-3.117204$ H $8.4549630 .562147-1.972593$ H $5.908401-0.609275-3.200839$ O $7.782391-1.045170-3.849540$ H $8.017603-2.064295-1.523536$ O $6.342443-2.975607-2.259312$ C $5.534424-2.2354471 .815881$ H $6.316928-3.4675040 .297838$
H $7.516247-2.4159061 .044282$

H $7.5007472 .497339-2.712935$

H $7.764571-0.309832-4.486019$

H $6.795258-3.195418-3.091650$

O $6.122820-1.4708072 .778629$

O $4.395063-2.6820811 .844087$

C $5.315329-1.2013343 .928518$

H $5.857905-0.5015364 .569424$

H $4.367331-0.7430553 .634635$

H $5.138490-2.1259784 .485633$

SCF Energy $(B 3 L Y P / 6-31 G * * / / M M F F)=-3245.92359268$

06_00047

MMFF Geometry

C $-0.8949342 .618616 \quad 0.725535$

C -1.8176302 .7189021 .694970$

C -2.3723951 .6007192 .549820$

O -1.8621970 .3069892 .157407$

C -3.9029861 .5242662 .393013$

C $-4.6013400 .992123 \quad 3.628150$

C $-4.952423-0.2926813 .829214$

C -4.9452232 .0377644 .658313$

C $-4.654131-1.4849222 .965217$

C $-5.894821-2.0528822 .263658$

C $-6.315674-1.2481831 .023737$

C $-7.550226-1.8759220 .371447$

C $-7.919035-1.187356-0.951457$

O $-5.217727-1.2659680 .106154$

O $-8.855971-2.028821-1.641308$

C $-6.657878-1.096466-1.837026$

C $-8.6288470 .151543-0.717949$

O $-6.916910-0.339683-3.017318$

C $-5.428600-0.520062-1.098271$

C $-4.159143-0.677157-1.969400$

O $-5.653481 \quad 0.866329-0.836485$

C $-0.650678-0.0961512 .616029$

$\begin{array}{llll}\text { O } & 0.122630 & 0.524548 & 3.325207\end{array}$

C $-0.420603-1.4456552 .057050$

C $0.829902-1.8589341 .809991$

C $1.208988-3.1605381 .254702$

C $0.119674-4.1833491 .062095$

C $-2.855331-0.319879-1.243159$

C $-1.673172-0.493601-2.172790$

C $-0.120534-2.184184-3.088525$

O $-1.2829490 .422526-2.893293$

C $0.573987-3.411877-2.594924$

C $1.907752-3.509215-2.486500$

C $2.626476-4.761438-2.076165$

C $3.631894-4.530304-0.971200$

C $4.930104-4.325862-1.252130$

C $3.149570-4.6439430 .456129$

C $2.506184-3.3805630 .957378$

N $-1.135251-1.771566-2.137923$

H $-0.467127 \quad 1.6516830 .479468$

H -2.1990713 .7104671 .934640$

H -2.1016901 .7978773 .594767$

H $-4.1566190 .903273 \quad 1.527529$

H -4.3252552 .5145902 .175738$

H -5.484204 -0.537967 4.748175

H -5.4421521 .6115735 .536315$

H -4.0387122 .5433065 .006210$

H -5.6181942 .7870414 .228779$

H -3.865041 -1.279130 2.238507

H -4.246774 -2.262077 3.624955

H $-5.648247-3.0757631 .949080$

H $-6.731230-2.1253312 .969605$

H -6.524281 -0.2146881 .321578$

H -8.402262 -1.864940 1.062491

H -7.350791 -2.936371 0.161365

H -9.626783 -2.156841-1.062161

H $-6.415870-2.115803-2.169298$

H -9.558007 -0.001113 -0.155714

H $-8.9255600 .612520-1.666412$

H $-8.0217540 .867532-0.160040$

H -7.688033 -0.739928 -3.454494

H $-4.079683-1.718638-2.307938$

H -4.244046 -0.051819 -2.867314

H $-4.8040101 .332324-0.805135$ 
H -1.294603 -2.048167 1.837597 H $1.652230-1.1789132 .027902$ H $-0.337970-4.4413052 .023418$ H $0.470203-5.1150850 .613251$ H $-0.659855-3.7985240 .395488$ H $-2.853367 \quad 0.719088-0.899912$ H $-2.713874-0.948471-0.357168$ H $\quad 0.571952-1.353925-3.259647$ H $-0.628942-2.399146-4.034335$ H $-0.044467-4.274187-2.357494$ H $2.523117-2.655810-2.758424$ H $3.140203-5.153590-2.964052$ H $1.922755-5.547146-1.774390$ H $5.666220-4.198708-0.465520$ H $5.285275-4.277573-2.276320$ H $3.998897-4.8559431 .120254$ H $2.503338-5.5206420 .555938$ H $3.214482-2.5688051 .118429$ H -1.656971 -2.497027-1.653584 C $-0.1651053 .490098-1.518818$ O $0.9244912 .552425-1.666680$ C $1.8538993 .067764-2.634981$ C $1.0795734 .114176-3.424182$ C $0.2245894 .725912-2.333307$ H $2.6532153 .567188-2.074248$ C $2.4418931 .935372-3.481820$ H $1.7374294 .838815-3.913465$ H $0.443513 \quad 3.657257-4.191193$ C $-0.9628735 .513281-2.860232$ H $0.8533525 .392923-1.727951$ C $3.128857 \quad 0.809222-2.680987$ O $1.3961841 .337255-4.258967$ H $3.1470022 .355137-4.208795$ H $2.376473 \quad 0.221987-2.140578$ O $3.713581-0.069259-3.652371$ C $4.2203631 .291409-1.703059$ C $5.0277420 .138019-1.075076$ H $3.7518331 .877744-0.906978$ O $5.1215922 .161822-2.389192$ H $4.330866-0.585323-0.639248$ H $5.584577-0.377863-1.865932$ H $-0.6247756 .351309-3.478459$ H -1.619511 4.886771 -3.473415 H -1.558051 $5.919322-2.036324$ C $-0.3650213 .803423-0.034756$ H $\quad 0.706314 \quad 1.039385-3.641312$ H $3.930392-0.903858-3.204879$ H $5.5076611 .661443-3.128463$ H -1.041931 4.6581240 .077993 H $0.599407 \quad 4.078170 \quad 0.408016$ H -1.059792 $3.013445-1.937777$ C 4.6563090 .4563371 .956797 O 5.4158671 .2970951 .079643 C $6.0453230 .624621-0.016409$ C $6.983712-0.4719810 .507956$ C $6.260914-1.4327861 .444598$ C $5.533970-0.6593262 .540950$ H $3.8103190 .024147 \quad 1.410237$ C 4.1041021 .3416313 .084831 H $6.6804361 .373060-0.506631$ O $7.561300-1.210783-0.563118$ H $7.811279-0.0078341 .060302$ H $5.569531-2.0807530 .897023$ O $7.215817-2.2970562 .063891$ H $6.270856-0.2345573 .234864$ O $4.716630-1.5589683 .287229$ C 3.0386562 .3040242 .608187 H 3.6503460 .7285503 .871049 H 4.9211631 .9349893 .513232 H $8.028871-0.578124-1.135019$ H $7.694931-2.7546851 .351732$ H $5.293213-2.2688953 .618146$ O 2.7840783 .2019923 .606267 O 2.4860942 .2649501 .518279 C 1.7840834 .1836453 .319693 H 1.7480334 .8862984 .156393 H 2.0380944 .7365292 .410379 H 0.8067603 .7032923 .223844
SCF Energy (B3LYP/6-31G**//MMFF)= -3245.92314605

\section{6_00048}

MM̄FF Geometry

C $0.745153 \quad 0.016825-2.909444$

C $1.732003-0.789332-3.328800$

C $2.163394-2.088473-2.685066$

O $1.302919-2.380311-1.560583$

C $3.608813-1.931478-2.183506$

C $4.322581-3.229841-1.875012$

C $4.633974-3.654603-0.635028$

C $4.759045-4.026562-3.077420$

C $4.266109-3.019768 \quad 0.676370$

C $5.438270-2.2900781 .348883$

C $5.759683-0.922643 \quad 0.721595$

C $6.940839-0.2644361 .439431$

C 7.2040131 .1619210 .932403

O $4.592242-0.108053 \quad 0.850850$

$\begin{array}{lllll}\text { O } & 8.112048 & 1.774502 & 1.860702\end{array}$

C 5.8780391 .9537770 .970774

C $7.9184391 .154275-0.423586$

O $6.0400993 .250262 \quad 0.401011$

C 4.7003371 .2108600 .306028

C 3.3386381 .9184840 .519041

O $4.9256051 .161560-1.109006$

C $0.952150-3.667983-1.325150$

O $1.322274-4.653627-1.947832$

C $0.050166-3.776316-0.153994$

C $-0.642265-2.744296 \quad 0.355575$

C -1.552504-2.797405 1.505572

C $-1.633176-4.0977372 .262788$

C 2.9320962 .2287911 .962940

C 2.9416511 .0398232 .904075

C $1.914801-1.1990783 .276878$

O 3.6837541 .0071493 .883937

C $0.605264-1.3112373 .996000$

C $-0.340333-0.3676664 .127489$

C -1.649433 -0.602743 4.825558

C -2.835853 -0.3821093 .912593$

C $-3.5677120 .741367 \quad 3.998880$

C $-3.225958-1.5003372 .967654$

C -2.247976 -1.687964 1.837010

N 2.0469250 .0465542 .545446

H $0.156656-0.252542-2.037373$

H $2.296845-0.497837-4.212808$

H $2.079584-2.872834-3.446254$

H $3.599489-1.284975-1.301887$

H $4.215218-1.400470-2.930422$

H $5.192178-4.584816-0.536602$

H $5.335292-4.915859-2.800340$

H $3.891580-4.364560-3.652152$

H $5.392102-3.415490-3.729060$

H $3.398747-2.3604230 .578568$

H $3.937617-3.8284951 .342281$

H $5.161225-2.1351012 .400202$

H $6.331701-2.9265761 .341165$

H $5.991268-1.060091-0.340186$

H $7.844632-0.8792641 .345764$

H $6.727043-0.2120762 .516673$

H 8.2247102 .7030621 .593452

H 5.6505962 .1172082 .030696

H $8.8819960 .636883-0.342260$

H $8.1542532 .171832-0.753875$

H $7.3432820 .660757-1.209640$

H $6.0594803 .148334-0.565838$

H $3.3240472 .856113-0.052135$

H 2.5556221 .2932680 .068095

H $4.0690611 .054435-1.555165$

H $-0.040356-4.7795320 .250033$

H - $-0.547305-1.765459-0.110535$

H $-2.053234-4.885305 \quad 1.627644$

H -2.254120 -4.048368 3.158850

H -0.637582 -4.409727 2.598779

H 3.5855013 .0028792 .380823

H 1.9200042 .6518791 .971699

H $2.739659-1.3126233 .987109$

H $1.984317-2.0045092 .539799$

H $0.414940-2.2906364 .431934$ 
H $-0.1856150 .624956 \quad 3.712883$ H -1.697619 0.0794585 .684305 H -1.704657 -1.613693 5.248380 H -4.4433240 .8969363 .378551$ H $-3.307676 \quad 1.5328504 .693978$ H -4.200781 -1.283787 2.514490 H $-3.381177-2.4082803 .555481$ H $-2.122848-0.7972001 .219272$ H $1.457298 \quad 0.1781151 .728943$ C $0.4465042 .473205-2.584599$ O $-0.6568762 .368448-1.658543$ C - $-1.3117773 .645361-1.574857$ C $-0.3222044 .653606-2.142478$ C $0.3117703 .841727-3.253266$ H $-2.1836103 .599049-2.238074$ C -1.759462 $3.932247-0.140223$ H $-0.8127305 .563901-2.499974$ H $0.4342924 .940844-1.402680$ C $1.6280074 .414954-3.750157$ H $-0.3931353 .782610-4.093692$ C -2.7085582 .8817440 .471366$ O $-0.605557 \quad 4.012694 \quad 0.707185$ H -2.229950 4.921771 -0.097840 H -2.1620841 .9601900 .706516$ O $-3.1655503 .405518 \quad 1.725857$ C $-3.9373702 .534409-0.391581$ C $-4.995808 \quad 1.7216910 .378255$ H -3.607879 $1.972169-1.271426$ O $-4.5486883 .735901-0.865357$ H -4.4976150 .9041980 .905869$ H $\quad-5.4557122 .3691791 .133981$ H $1.4788095 .417640-4.163616$ H $2.3633074 .490614-2.941892$ H $2.0558843 .785202-4.536454$ C $0.3861401 .312995-3.579190$ H $-0.127906 \quad 3.168780 \quad 0.631000$ H -2.378219 3.5892752 .266860 H $-4.8042624 .262481-0.088604$ H $1.0591651 .504548-4.422600$ H $-0.6303461 .215124-3.979100$ H $1.3671992 .426087-1.989110$ C $-5.218723-1.051750-0.887820$ O $-5.6072340 .199029-1.470270$ C $-6.1073321 .172708-0.545889$ C -7.3253300 .6071720 .195394$ C $-7.004861-0.7155370 .882302$ C -6.381498 -1.691005 -0.114874 H $-4.366822-0.896912-0.217838$ C -4.765467 -1.974139-2.030276 H $-6.4685782 .013457-1.151092$ O H -8.135802 $0.443734-0.526968$ H $-6.360165-0.5579601 .753885$ O $-8.228052-1.2643421 .378084$ H -7.152188 -2.037297 -0.815791 O $-5.894087-2.8340120 .586151$ C -3.423134 -1.578721 -2.607664 H -4.663221 -3.004315 -1.671025 H -5.499615 -1.943244 -2.844146 H $-8.558785 \quad 1.144810 \quad 1.606267$ H -8.005054 -2.050359 1.904613 H -6.659479 -3.292694 0.971095 O $-3.133687-2.420619-3.644892$ O $-2.715990-0.667291-2.202402$ C $-1.878673-2.199525-4.294144$ H $-1.061437-2.456511-3.615211$ H -1.827486 -2.855158 -5.167421 $\mathrm{H}-1.796098-1.162128-4.631523$

SCF Energy (B3LYP/6-31G**//MMFF) $=-3245.92450286$

0600049

MM̄FF Geometry

C $2.574743-4.0916650 .439699$

C $1.365919-3.6191420 .099270$

C $0.830559-3.645977-1.306501$

O $0.400960-2.298703-1.614208$

C $-0.390246-4.577047-1.406667$

C $-0.909233-4.734244-2.824505$
C $-2.129612-4.348423-3.246539$

C $0.032877-5.403878-3.793676$

C $-3.237808-3.727960-2.444149$

C $-3.314970-2.214968-2.671352$

C $-4.511869-1.586427-1.943674$

C $-4.589520-0.085822-2.231334$

C $-5.7167110 .594973-1.442160$

O $-4.349056-1.810241-0.539286$

O $-5.4994352 .010838-1.540348$

C -5.5942340 .1957150 .045223$

C $-7.0866370 .328924-2.076434$

$\begin{array}{llll}\text { O } & -6.696869 & 0.700322 & 0.793684\end{array}$

C $-5.433478-1.3243090 .265332$

C $-5.129071-1.7026531 .736248$

O $-6.662379-1.975091-0.083231$

C $1.110131-1.592447-2.529982$

O $2.089734-1.971221-3.156590$

C $0.507466-0.260471-2.755076$

C $-0.1731180 .408399-1.810765$

C $-0.7917441 .728462-1.970010$

C $-0.6883312 .384686-3.323351$

C $-3.856159-1.0871242 .326550$

C -4.0921740 .2303973 .046851$

C -2.8713012 .2576863 .827960$

O -5.1977140 .5983913 .434595$

C -3.0427003 .2668342 .736970$

C -2.070995 4.0960332 .329255

C $-2.2323195 .087942 \quad 1.215795$

C $-1.5243884 .647139-0.044823$

C $-0.3834885 .232480-0.445379$

C $-2.1977573 .579276-0.878011$

C -1.430369 $2.282296-0.916660$

N -2.910309 $0.931762 \quad 3.239193$

H $3.185073-4.548026-0.337517$

H $0.723594-3.1642970 .847990$

H $1.613406-3.977962-1.997670$

H -1.174985 -4.197226 -0.744632

H $-0.129802-5.574806-1.028924$

H -2.382013 -4.507959 -4.294428

H $-0.453265-5.646196-4.744911$

H $0.885240-4.755831-4.018061$

H $0.406881-6.342910-3.372790$

H -4.180269-4.194689-2.757196

H -3.139562 -3.952224 -1.378309

H $-2.386580-1.759736-2.306131$

H $-3.385610-2.002089-3.745382$

H -5.424545 -2.088789 -2.285679

H $-4.7051210 .100792-3.306248$

H $-3.6389240 .384687-1.948609$

H $-6.1579952 .449744-0.974664$

H -4.7040080 .7100020 .427006$

H $-7.1086070 .706846-3.105833$

H -7.880995 $0.865701-1.546353$

H -7.346949 -0.731038 -2.107471

H -7.4617120 .1258230 .618959$

H $-6.004198-1.4988402 .365201$

H -4.996164 -2.793594 1.769174

H $-6.683801-2.8446180 .349747$

H $\quad 0.6922760 .157979-3.738490$

H $-0.293033-0.047906-0.829384$

H $-1.1134083 .389614-3.358540$

H -1.210462 $1.787647-4.079171$

H $0.3596452 .492382-3.622701$

H $-3.453615-1.7740133 .081963$

H -3.087263 -0.9629101 .556167$

H -1.9109682 .3604594 .342868$

H -3.674846 2.361904 4.563748

H $-4.014973 \quad 3.3068372 .250992$

H -1.099163 4.0621052 .814341

H -1.842457 6.0533691 .563191

H -3.2920265 .2658130 .992438$

H $\quad 0.1081814 .939299-1.366962$

H $\quad 0.085361 \quad 6.016317 \quad 0.139917$

H $-2.4035503 .979906-1.875106$

H -3.191245 $3.359499-0.466956$

H $-1.449187 \quad 1.735550 \quad 0.026918$

H -2.056382 0.5793572 .817219

C $2.528628-3.1978992 .867605$ 
O $2.480071-1.8223522 .442012$

C $2.747474-0.9712213 .569677$

C $2.764973-1.8781554 .795933$

C $3.268187-3.1806264 .204996$

H $3.754057-0.5627093 .421353$

C 1.7248600 .1683643 .629263

H $3.409898-1.4881015 .589295$

H $1.762451-2.0153365 .217581$

C $2.995928-4.3904185 .081594$

H $4.350072-3.0935524 .036393$

C 1.6155980 .9945382 .331500

O $0.433591-0.3968313 .891866$

H 1.9571490 .8202374 .480188

H $1.145840 \quad 0.400323 \quad 1.537861$

O $\quad 0.7327602 .0904652 .592577$

C 2.9474341 .5684991 .814339

C 2.7478242 .5685870 .658263

H 3.5868930 .7456611 .481703

$\begin{array}{lllll}\text { O } & 3.632483 & 2.228153 & 2.879585\end{array}$

H $2.0987902 .113274-0.096306$

H 2.2306283 .4570551 .039637

H $3.493445-4.282416 \quad 6.051046$

H $1.924176-4.5192985 .266526$

H $3.369039-5.3057364 .611582$

C $3.226297-4.0416151 .797758$

H $-0.155194 \quad 0.3303804 .155077$

H $-0.164362 \quad 1.7269222 .673982$

H 3.0552282 .9404553 .204166

H $3.339993-5.0718482 .154000$

H $4.236193-3.6406151 .644143$

H $1.493733-3.5337143 .011575$

C $4.2635461 .322347-1.690228$

O $4.824509 \quad 1.915774-0.512179$

C 4.0840733 .0178660 .025066

C $3.9434194 .122670-1.030981$

C $3.3448063 .591548-2.329367$

C $4.1264232 .367815-2.805438$

H $3.2836620 .890896-1.455939$

C $5.1987140 .185104-2.126356$

H $4.6971313 .431200 \quad 0.835722$

O $3.1511035 .190711-0.524009$

H $4.9376494 .536420-1.244764$

H $2.2789523 .368541-2.207785$

O $3.4314934 .618073-3.319273$

H $5.1190622 .690179-3.143958$

O $3.4684551 .801399-3.935679$

C $5.177388-0.981840-1.162156$

H $4.909593-0.205776-3.107725$

H $6.2272540 .562714-2.176219$

H $3.0636865 .850503-1.233223$

H $3.0871924 .247804-4.150120$

H $2.586346 \quad 1.511087-3.650345$

O $6.279428-1.758018-1.384160$

O $4.298736-1.201881-0.340550$

C $6.401517-2.913088-0.548981$

H $5.603659-3.624985-0.777820$

H $7.364372-3.385412-0.760894$

H $6.377320-2.6277370 .507183$

SCF Energy (B3LYP/6-31G**//MMFF)= -3245.92167849

06_00050

MMFF Geometry

C $0.239194-3.093200 \quad 0.478734$

C $-0.164531-1.815165 \quad 0.426597$

C $-0.444221-0.9788451 .646914$

O $-1.843042-0.6204661 .646596$

C $0.3528810 .334566 \quad 1.565916$

C 0.5952360 .9709562 .917565

C $-0.1828351 .916213 \quad 3.479023$

C 1.8428100 .5007913 .621813

C -1.490253 2.4654602 .980430

C $-1.391549 \quad 3.9176172 .496651$

C -0.9314914 .0338761 .035050$

C $-0.773547 \quad 5.504634 \quad 0.642168$

C $-0.4341335 .669866-0.845484$

$\begin{array}{lllll}\text { O } & -1.932259 & 3.414188 & 0.217073\end{array}$

O $-0.6125007 .058313-1.164285$

C $-1.4466524 .855978-1.679021$
C $1.0392275 .352266-1.123337$

O $-1.0962654 .877949-3.061335$

C $-1.6377323 .402916-1.183755$

C $-2.8280402 .749772-1.926060$

O $-0.4519652 .662426-1.470542$

C $-2.737438-1.504446 \quad 2.157908$

O $-2.508860-2.6105182 .617841$

C $-4.071129-0.8775492 .032567$

C $-5.175447-1.6324022 .116736$

C $-6.552066-1.1577441 .958736$

C $-6.7623530 .321723 \quad 1.762251$

C $-3.2708531 .392430-1.357741$

C $-4.379596 \quad 0.769116-2.192137$

C $-5.769765-1.295061-2.355357$

O $-4.868822 \quad 1.318879-3.175944$

C $-7.106888-1.014133-1.743645$

C $-7.952823-1.975434-1.341075$

C $-9.314441-1.703949-0.765225$

C $-9.564764-2.441681 \quad 0.532494$

C $-10.304920-3.5629850 .557880$

C $-9.027826-1.8338971 .809782$

C $-7.549755-2.0660111 .975446$

N $-4.755176-0.478729-1.712518$

H $\quad 0.339611-3.5810161 .445260$

H $-0.286056-1.332509-0.539984$

H $-0.199241-1.5220732 .568541$

H -0.1608001 .0447080 .907354$

H $1.3324380 .158437 \quad 1.103128$

H $\quad 0.1235502 .3201124 .443616$

H $1.987148 \quad 0.993966 \quad 4.588751$

H $1.792212-0.577343 \quad 3.805263$

H 2.7277240 .7066373 .010960

H $-1.936148 \quad 1.8337732 .209318$

H -2.1886592 .4316143 .826745$

H -2.393499 4.3592012 .581170

H -0.7275584 .4935293 .152708$

H 0.0188783 .5023990 .914840

H $-0.015877 \quad 5.9950881 .266233$

H -1.7142426 .0369360 .842689$

H $-0.4742627 .160881-2.121633$

H -2.410862 $5.377283-1.605112$

H $1.6888316 .018534-0.542946$

H $1.2937385 .529375-2.173936$

H $1.3084754 .325727-0.871210$

H $-0.3608824 .254815-3.189697$

H $-3.6952583 .421778-1.879542$

H $-2.5712452 .623676-2.985825$

H $-0.6711961 .721040-1.548321$

H $-4.1048610 .188346 \quad 1.837624$

H -5.062121 -2.7015362 .291285$

H -6.4011870 .8779802 .634430$

$\begin{array}{llll}H & -7.807713 & 0.601518 & 1.619739\end{array}$

H -6.2259880 .6759090 .875129$

H $-2.4333540 .687582-1.333442$

H $-3.6455351 .518270-0.335921$

H $-5.470796-2.340808-2.233043$

H $-5.797727-1.060306-3.424428$

H $-7.4059100 .027470-1.652514$

H $-7.666878-3.017848-1.463210$

H -10.056811 -2.008169-1.514852

H $-9.475346-0.630241-0.607467$

H -10.512062 -4.0830641 .487308$

H -10.715784 -3.989249 -0.351359

H $-9.524994-2.2830662 .680032$

H $-9.305113-0.7770961 .846780$

H -7.291243 -3.116001 2.121017

H $-4.334038-0.830413-0.858000$

C $1.889530-4.676078-0.554210$

O $2.977983-3.765651-0.312831$

C $4.164674-4.278041-0.949551$

C $3.791762-5.639068-1.532354$

C $2.305840-5.476496-1.785660$

H $4.397300-3.593564-1.773319$

C $5.329847-4.3120480 .048553$

H $4.359356-5.867282-2.440046$

H $3.969055-6.452207-0.819199$

C $1.570508-6.794752-1.950976$

H $2.170431-4.870248-2.691535$ 
C $5.612168-2.971207 \quad 0.756240$

O $5.045474-5.2768241 .071638$

H $6.235628-4.668490-0.455159$

H $4.791718-2.7286941 .442870$

O $6.778620-3.1449921 .571696$

C $5.851587-1.781787-0.190424$

C $6.226759-0.5026060 .581040$

H $4.945757-1.607040-0.775466$

O $6.896813-2.118651-1.100252$

H $5.483752-0.3428741 .370056$

H $7.196048-0.6496061 .072812$

H $1.971008-7.354076-2.802904$

H $1.668757-7.423630-1.059851$

H $0.503757-6.627854-2.129580$

C $0.580214-3.905953-0.739071$

H $4.210991-5.0154991 .497658$

H $6.608387-3.8975622 .163984$

H $6.892215-1.458765-1.813779$

H $-0.238146-4.609953-0.926185$

H $0.660882-3.249251-1.613831$

H $\quad \begin{array}{llll}1.819782 & -5.330810 & 0.325087\end{array}$

C $3.9884311 .354634-0.363762$

O $5.1512650 .962810-1.102140$

C $6.327390 \quad 0.753882-0.313299$

C $6.682323 \quad 2.0382870 .451857$

C 5.5023812 .5523901 .273541

C $4.2491892 .657202 \quad 0.405608$

H 3.7056890 .5561660 .332370

C $2.8449531 .553235-1.369288$

H $7.1430670 .572943-1.024018$

O $7.797058 \begin{array}{llll}1.829966 & 1.312110\end{array}$

H $6.9713282 .816531-0.266459$

H 5.3209411 .9142122 .145782

O $5.8158313 .844766 \quad 1.799020$

H $4.360765 \quad 3.492196-0.298155$

O 3.1265692 .9484991 .235274

C $2.341745 \quad 0.258719-1.969689$

H $1.9896912 .021216-0.875153$

H $3.1876612 .193527-2.191071$

H 8.5357861 .5161140 .762989

H 5.9961804 .4387211 .050519

H 3.3321343 .7607961 .728906

O $1.514462 \quad 0.551830-3.016812$

O $2.609525-0.862143-1.562584$

C $0.935264-0.574276-3.683277$

H $0.413729-0.210615-4.572568$

H $1.710773-1.279812-3.996801$

H $0.210156-1.062671-3.026610$

SCF Energy (B3LYP/6-31G**//MMFF) $=-3245.92346917$

0600051

MMFF Geometry

C - -1.0747413 .3111870 .663455$

C $-0.3769792 .714167 \quad 1.641369$

C -0.9073841 .7991742 .721086$

$\begin{array}{lllll} & -0.244514 & 0.526668 & 2.506930\end{array}$

C -2.431909 1.5708802 .705687

C -2.9801930 .9237863 .961389$

C $-3.408057-0.3506354 .046207$

C -3.0957711 .8362205 .156071$

C $-3.356941-1.4174392 .989693$

C $-4.739544-1.7531152 .411675$

C $-5.250852-0.7166661 .396893$

C $-6.644831-1.099740 \quad 0.893175$

C -7.132759 $-0.167287-0.226192$

O $-4.324763-0.6953530 .308669$

O -8.287852 -0.786887 -0.812421

C $-6.036823-0.081354-1.311383$

C $-7.605761 \quad 1.1802800 .329859$

$\begin{array}{llll}\text { O } & -6.386915 & 0.864682 & -2.318466\end{array}$

C $-4.6351420 .222956-0.743739$

C $-3.5104410 .111984-1.804071$

O $-4.619638 \quad 1.571223-0.259125$

C $0.253100-0.1355893 .580238$

$\begin{array}{lllll}\text { O } & 0.172756 & 0.207258 & 4.752178\end{array}$

C $0.936652-1.3900503 .184143$

C $1.209049-1.7365231 .915732$

C $1.912817-2.9499931 .484734$
C $2.363660-3.9138832 .551129$

C $-3.434247-1.189062-2.610125$

C $-3.411365-2.463110-1.788086$

C $-2.122922-3.667371-0.031445$

O $-4.299089-3.307410-1.895381$

C $-0.992595-4.564783-0.432715$

C $-0.240163-4.489471-1.541622$

C $0.919774-5.404268-1.814746$

C $2.239621-4.664305-1.830048$

C $2.878961-4.413530-2.984755$

C $2.855490-4.283160-0.497679$

C $2.148312-3.1314860 .167778$

N $-2.315659-2.560320-0.948514$

H -2.1470393 .1673930 .589803$

H 0.6968042 .8856561 .680655

H -0.5965242 .2353623 .678204$

H $-2.6957780 .976371 \quad 1.825425$

H -2.957414 2.5280572 .592452

H $-3.819663-0.6896554 .996288$

H $-3.555726 \quad 1.3389306 .016784$

H $-2.1086332 .187948 \quad 5.470555$

H -3.713742 2.7062504 .911190

H -2.648233 -1.1726382 .193050$

H -2.955192 -2.320943 3.466604

H $-4.653024-2.7242441 .906457$

H $-5.467022-1.8757563 .223688$

H -5.2759320 .2703341 .871574$

H -7.365765 -1.1181631 .720208$

H $-6.618480-2.1256530 .498856$

H $-8.558912-0.244570-1.573183$

H $-6.022830-1.057845-1.809082$

H -8.4326561 .0313101 .034702$

H -8.004590 $1.819960-0.465179$

H $-6.819971 \quad 1.7290630 .852944$

H $-6.2303181 .753684-1.956922$

H $-3.5940450 .948332-2.510722$

H $-2.546867 \quad 0.258913-1.296392$

H $-3.7025871 .889280-0.269824$

H $1.236637-2.0145554 .019924$

H $0.904966-1.0698601 .110388$

H $3.015207-3.4105903 .274592$

H $2.938225-4.7573592 .162687$

H $1.500404-4.3293973 .082201$

H $-4.280739-1.247262-3.304185$

H $-2.532210-1.177427-3.234307$

H $-3.044817-4.2505860 .056190$

H $-1.900462-3.2305610 .946810$

H $-0.757355-5.3415270 .293106$

H $-0.439272-3.727469-2.290372$

H $0.741325-5.893364-2.781010$

H $0.972316-6.216303-1.078752$

H $\quad 3.837139-3.904772-3.001648$

H $2.455158-4.704609-3.940094$

H $3.902910-3.988147-0.641983$

H $2.870202-5.1789950 .129918$

H $1.816271-2.355654-0.522574$

H -1.618522 -1.821644 -0.950474

C $-0.5341313 .524071-1.762745$

O $0.4401412 .460833-1.816191$

C $1.1263342 .523311-3.080642$

C $0.3147623 .473187-3.951628$

C $-0.1870734 .461888-2.917904$

H $2.1050422 .978557-2.888923$

C $1.304153 \quad 1.116469-3.658353$

H $0.9201523 .938785-4.735206$

H $-0.5286042 .963389-4.432182$

C $-1.3539395 .305150-3.401729$

H $\quad 0.6411425 .123459-2.630037$

C $2.064310 \quad 0.142275-2.736261$

$\begin{array}{lllll}\text { O } & 0.012594 & 0.548444 & -3.914436\end{array}$

H $1.8052041 .176242-4.631394$

H $1.462304-0.086436-1.848303$

O $2.222166-1.092633-3.445486$

C $3.4580350 .631939-2.298281$

C $4.220736-0.426856-1.483849$

H $3.347246 \quad 1.535639-1.694997$

O $4.2291840 .984695-3.445610$

H $3.573079-0.764791-0.671508$ 
H $4.428394-1.293501-2.122346$ H -1.061294 $5.903363-4.270813$ H -2.205638 $4.681792-3.694127$ H -1.692058 5.990851-2.618519 C $-0.4721244 .196666-0.391245$ H $-0.4773470 .543859-3.074034$ H $1.333240-1.400773-3.692479$ H $4.302317 \quad 0.192312-4.005007$ H -1.033039 $5.137870-0.399442$ H $0.566914 \quad 4.436719-0.139152$ H -1.517594 3.063546-1.927003 C 4.7096381 .0093031 .159998 O $5.391979 \quad 1.242771-0.077957$ C $5.5588140 .088752-0.908550$ C $6.360048-0.977661-0.150757$ C $5.710533-1.3273821 .185095$ C $5.429708-0.061681 \quad 1.994127$ H 3.6757910 .7019860 .960778 C 4.6741762 .3424221 .926345 H $6.1708770 .408764-1.760958$ O $6.495199-2.148577-0.949798$ H $7.372844-0.5972290 .035471$ H $4.801643-1.9184461 .035851$ O $6.615308-2.162147 \quad 1.911941$ H $\quad \begin{array}{llll}6.376336 & 0.338402 & 2.380123\end{array}$ O $4.614413-0.3913623 .117676$ C 3.7772723 .3748041 .277470 H 4.2996132 .1858262 .943950 H $5.6844802 .766713 \quad 1.971210$ H $\quad 6.982935-2.805035-0.423369$ H $6.153709-2.4705012 .709830$ H $5.127520-0.9848803 .691303$ O 3.8666864 .5402571 .985509 O 3.0818423 .1896240 .289367 C 3.0661575 .6234451 .501576 H 3.2361685 .7867690 .432973 H 2.0100725 .4195151 .699204 H 3.3564116 .5280202 .042689 SCF Energy (B3LYP/6-31G**//MMFF) $=-3245.92481562$

\section{2}

MM̄FF Geometry

C $-0.453200-3.0655332 .255525$

C $-0.715422-1.8828202 .833625$

C $0.276962-0.8240423 .258251$

O -0.0494580 .3485532 .471339$

C $1.761623-1.1755133 .019911$

C $2.744406-0.2843583 .753970$

C 3.4868010 .6843453 .182813

C $2.907802-0.5875295 .221911$

C 3.4521651 .1643881 .759800

C 4.7162360 .8010650 .971682

C $4.793599-0.6825990 .578995$

C $6.088452-0.957181-0.190369$

C $6.152849-2.396026-0.719773$

O $3.659371-0.973277-0.242114$

O $7.237488-2.447761-1.659136$

C $4.846183-2.699252-1.484172$

C $6.502802-3.3896770 .393629$

O $4.808574-4.064697-1.894632$

C $3.568620-2.327289-0.695237$

C $2.289329-2.463738-1.561850$

O $3.436610-3.2356550 .404666$

C 0.0401991 .5709893 .056883

O $0.289887 \quad 1.826243 \quad 4.223146$

C -0.1452022 .5705371 .985821$

C 0.6271043 .6667831 .994116

C 0.6787854 .6704700 .932056

C $-0.4936214 .742182-0.009352$

C $2.129080-1.409166-2.663446$

C $1.551433-0.092771-2.165363$

C $1.3425942 .307810-2.806954$

O $0.9128340 .007748-1.120593$

C $2.3123502 .999533-1.901743$

C $2.9409634 .147847-2.197015$

C $3.9012114 .821445-1.255790$

C $3.4763646 .221115-0.853647$

C $4.3162107 .257785-1.015845$
C $2.0979716 .442043-0.253812$

C 1.7699825 .4592930 .840039

N $1.7963160 .950370-3.049067$

H $\quad 0.570255-3.3568892 .042156$

H -1.755899-1.632644 3.034383

H $0.093519-0.6276954 .321626$

H $1.966836-1.1599931 .944322$

H $1.966479-2.2016363 .351317$

H $4.1848891 .232108 \quad 3.815154$

H 3.6602290 .0502875 .697839

H $1.964461-0.4339245 .754627$

H $3.222100-1.6269695 .362646$

H 2.5568360 .8261231 .230721

H $3.3775162 .258558 \quad 1.792472$

H $4.713647 \quad 1.4077520 .056517$

H 5.6085361 .0853851 .543163

H $4.750946-1.2973891 .484839$

H $6.965104-0.7365110 .431606$

H $6.152913-0.272296-1.047956$

H $7.240622-3.335808-2.056499$

H $4.884269-2.111524-2.408596$

H $7.477131-3.1417120 .831747$

H $6.604052-4.4073750 .000718$

H $5.770066-3.4034131 .203180$

H $4.581318-4.601324-1.116081$

H $2.269182-3.463512-2.013882$

H $1.407735-2.431338-0.907796$

H $2.510381-3.235840 \quad 0.696111$

H $-0.8234002 .326073 \quad 1.176558$

H 1.3437003 .7964692 .804071

H -1.4298254 .7912440 .557231$

H $-0.5253023 .865497-0.663001$

H $-0.4872475 .630116-0.645131$

H $3.074604-1.230247-3.182575$

H $1.419486-1.787976-3.409066$

H $0.3547272 .273572-2.336745$

H $1.2530102 .804376-3.778092$

H $2.4873162 .538776-0.932226$

H $2.7703664 .622274-3.160136$

H $4.8809844 .845900-1.750371$

H $4.0438394 .221796-0.347724$

H $4.0325758 .265890-0.732122$

H $5.3072137 .124040-1.436856$

H $1.3770956 .401788-1.075067$

H 2.0237897 .4495390 .174319

H $2.5499415 .357876 \quad 1.595832$

H $2.3904760 .789153-3.856922$

C $-1.454019-4.3710060 .367505$

O $-1.875615-3.212898-0.379362$

C $-2.717033-3.640311-1.468181$

C $-2.603932-5.159267-1.521690$

C $-2.395694-5.495976-0.058296$

H $-3.745108-3.376792-1.194218$

C $-2.315982-2.919043-2.760086$

H $-3.496674-5.626246-1.948558$

H $-1.739653-5.483416-2.112776$

C -1.839562 -6.8908970 .169004$

H $-3.356565-5.4004470 .465235$

C $-2.311647-1.380637-2.649236$

O $-0.993883-3.327044-3.133017$

H $-2.970416-3.234800-3.580459$

H -1.523251 -1.053903 -1.961724

O $-1.974335-0.845327-3.934651$

C $-3.656779-0.777511-2.209309$

C $-3.6375370 .761245-2.183060$

H $-3.904714-1.144392-1.211335$

O $-4.691155-1.221487-3.086721$

H $-2.7935331 .091137-1.567473$

H $-3.4670121 .139100-3.198175$

H $-2.527311-7.647255-0.223080$

H $-0.873554-7.024044-0.329559$

H -1.697106 -7.086257 1.236464

C -1.506709-4.065947 1.865001

H $-0.409015-3.141238-2.378847$

H -1.119425 -1.230993 -4.192597

H -4.458735 -0.930267 -3.985122

H -1.331949 -4.9810882 .441849$

H -2.501756 -3.693172 2.133172 
H $-0.424778-4.5944230 .055689$

C -4.4847851 .3038280 .715214$

O $\quad \begin{array}{lllll}-5.316278 & 0.858894 & -0.363741\end{array}$

C $-4.9603881 .363872-1.655846$

C $-4.9941712 .898483-1.648715$

C $-4.1331573 .475293-0.529843$

C -4.4985482 .8352210 .807515$

H -3.4590150 .9455800 .565466$

C -5.0340550 .6816672 .009361$

H $-5.7522921 .031806-2.338991$

O $-4.5553223 .426986-2.895491$

H $-6.0273073 .238887-1.500657$

H $-3.0662003 .352428-0.744758$

O $-4.3666394 .881672-0.431734$

H $-5.490814 \quad 3.1855991 .119557$

O -3.5664543 .2546601 .801155$

C $-4.798989-0.8116432 .087190$

H -4.5517261 .1250842 .887222$

H $-6.116017 \quad 0.8522412 .064261$

H -5.128406 $3.055503-3.587828$

H $-4.1607745 .269484-1.299701$

H -3.5800844 .2270811 .820061$

O $-5.518032-1.3184993 .132585$

O $-4.065023-1.451757 \quad 1.348236$

C $-5.385117-2.727172 \quad 3.347777$

H -5.609834 -3.281797 2.431358

H $-4.375400-2.9552883 .700783$

H -6.101967 -3.022115 4.118634

SCF Energy $\left(B 3 L Y P / 6-31 G^{* *} / / M M F F\right)=-3245.92806299$

\section{3}

MM̄FF Geometry

C $-2.7971592 .771817 \quad 1.634187$

C -1.9826092 .4057562 .636323$

C -2.1273181 .1791573 .511251$

O $-0.8773140 .436673 \quad 3.565515$

C $-3.299670 \quad 0.226878 \quad 3.190796$

C $-3.586554-0.8025154 .266394$

C $-3.398949-2.1304094 .137089$

C $-4.208640-0.2580275 .528423$

C $-2.789918-2.8887492 .991223$

C $-3.838550-3.5455382 .080946$

C $-4.222202-2.6590890 .885248$

C $-5.368353-3.2819770 .087825$

C $-5.696075-2.461570-1.168199$

O $-3.057288-2.533347 \quad 0.061447$

O $-6.577232-3.261545-1.971038$

C $-4.391938-2.241807-1.963627$

C $-6.468410-1.182674-0.826012$

O $-4.617831-1.387010-3.082707$

C $-3.217772-1.717326-1.103937$

C $-1.878061-1.740052-1.882146$

O $-3.483518-0.356107-0.745444$

C $-0.341699-0.0916282 .432669$

$\begin{array}{lllll}\text { O } & -0.726417 & 0.064238 & 1.285031\end{array}$

C $0.782392-0.9622722 .846038$

C $1.265820-1.8626201 .977426$

C $2.341482-2.8245442 .230169$

C $3.003968-2.8026893 .582341$

C $-1.337471-3.141668-2.175356$

C $-0.117139-3.114605-3.074925$

C $2.315125-2.566345-3.081891$

O $-0.155724-3.558839-4.220608$

C $3.134772-3.647274-2.451426$

C $4.218452-3.409513-1.697106$

C $5.007329-4.483369-1.006086$

C $4.993955-4.3325860 .500830$

C $6.085351-3.9134971 .162806$

C $3.743963-4.7455371 .249024$

C $2.684751-3.6753171 .240608$

N $1.000785-2.573359-2.464161$

H $-3.6566102 .158883 \quad 1.379135$

H -1.140375 3.0501572 .880390

H -2.2642971 .5676704 .527423$

H -3.113844 -0.2651072 .233207$

H $-4.2242750 .802848 \quad 3.055489$

H -3.695310 -2.767822 4.970140

H -4.512594 -1.051904 6.219272
H -3.5023080 .3853996 .061278$

H $\quad-5.1035390 .326520 \quad 5.290755$

H -2.098241 -2.268989 2.416434

H -2.170567 -3.680492 3.432733

H -3.403258 -4.476573 1.693986

H $-4.730558-3.8199492 .657133$

H -4.520642 -1.672469 1.251189

H $-6.261918-3.3974550 .713968$

H $-5.087732-4.298140-0.223117$

H $-6.727255-2.786300-2.806487$

H $-4.125307-3.217715-2.384163$

H -7.404401-1.430218 -0.310635

H $-6.761991-0.642138-1.732638$

H $-5.907030-0.499561-0.185174$

H -4.669652 -0.474079-2.751899

H - $1.973937-1.166658-2.812434$

H -1.122923 -1.203157 -1.292982

H $-2.6460250 .063075-0.486045$

H $1.153722-0.8636203 .859450$

H $\quad 0.822938-1.9108570 .983063$

H $2.286660-3.0802384 .362582$

H $3.397961-1.8072743 .810045$

H $3.848868-3.4898623 .663700$

H -1.068239 -3.657635 -1.246053

H -2.087790 -3.760658 -2.676501

H $2.747190-1.576610-2.916003$

H $2.232463-2.735143-4.159727$

H $2.803662-4.671777-2.603733$

H $4.548448-2.384916-1.547313$

H $6.036111-4.439089-1.385759$

H $4.637665-5.482347-1.269218$

H $6.092074-3.8283492 .244339$

H $6.994373-3.6392140 .637967$

H $3.993956-5.0525662 .270258$

H $3.328698-5.6514430 .788768$

H $2.128157-3.6216170 .305902$

H $0.937181-2.272856-1.496019$

C $-2.635598 \quad 3.716812-0.678472$

O $-1.4132893 .046357-1.045043$

C $-0.9591503 .572245-2.307112$

C $-2.0899034 .444532-2.839744$

C $-2.6948074 .967824-1.553206$

H $-0.0982424 .214995-2.090487$

C $-0.5467492 .431673-3.242594$

H $-1.7255475 .238306-3.499032$

H $-2.8288413 .857245-3.397360$

C $-4.0913825 .538130-1.730369$

H $-2.0357105 .744999-1.143508$

C $0.5572451 .512378-2.683742$

O $-1.6915551 .613818-3.517804$

H $-0.2326852 .843199-4.208857$

H $\quad 0.1742270 .922144-1.842770$

O $0.9008640 .576125-3.712744$

C $1.8371652 .249601-2.250082$

C $2.9686831 .288837-1.843735$

H $1.6032542 .904748-1.407430$

O $2.2956793 .083885-3.314066$

H $2.5816620 .577991-1.105768$

H $3.2822880 .713189-2.721960$

H $-4.0760026 .382493-2.427391$

H $-4.7840104 .787962-2.126574$

H $-4.4922445 .896225-0.777065$

C $-2.6301534 .023656 \quad 0.819131$

H $-2.0409801 .303194-2.664833$

H $0.0865370 .104672-3.959216$

H $2.4660912 .512408-4.082519$

H -3.461570 4.6933991 .066047

H -1.699793 4.5344611 .091284

H $-3.4604763 .037022-0.931343$

C 3.5316852 .2067821 .023277

O $3.8854112 .883105-0.189079$

C $4.2084202 .025752-1.289120$

C $5.3758321 .099826-0.915332$

C 5.1025210 .3338110 .375751

C 4.6812091 .3007881 .481990

H 2.6246691 .6111040 .864402

C 3.2300853 .2795312 .080415

H $4.5681572 .683749-2.090180$ 
O $5.6513110 .176683-1.963180$

H $6.2826031 .702392-0.772906$

H $4.352504-0.4502580 .219659$

O $6.303994-0.3313670 .770772$

H 5.5477941 .9069991 .774197

O 4.2896350 .5555252 .630852

C 1.9449054 .0260431 .792971

H 3.1230282 .8336133 .074980

H 4.0492224 .0088312 .098446

H $\quad 5.842377 \quad 0.692948-2.764758$

H $\quad 6.119537-0.785678 \quad 1.610729$

H 3.5058320 .0343032 .389642

O 1.9501905 .2042222 .483823

O 1.0378303 .6170251 .081881

C 0.7857856 .0206662 .323737

H $\quad 0.976754 \quad 6.9826972 .806556$

H $0.578692 \quad 6.196780 \quad 1.263639$

H -0.0701205 .5463022 .811963$

SCF Energy (B3LYP/6-31G**//MMFF) $=-3245.93304603$

\section{00054}

MM̄FF Geometry

C $0.7268272 .199287-1.761039$

C $\quad 0.1153691 .157002-2.344986$

C $0.712738-0.184698-2.691935$

O $0.102841-1.134549-1.785489$

C $2.246113-0.282480-2.550337$

C $2.821081-1.575189-3.097143$

C $3.232078-2.615298-2.344753$

C $2.952548-1.633968-4.597548$

C $3.187503-2.747681-0.848158$

C $4.580093-2.904277-0.221009$

C $5.522488-1.714762-0.467695$

C $6.881649-1.9645620 .191044$

C $7.825947-0.7647490 .029165$

O $4.920685-0.5388520 .080781$

O $8.931291-0.9798250 .919778$

C 7.0876540 .5121850 .484885

C $8.419629-0.701276-1.382784$

$\begin{array}{llll}\text { O } & 7.876766 & 1.671649 & 0.225511\end{array}$

C $5.6737240 .662645-0.126855$

C 4.8934091 .8395450 .507432

O $5.8070240 .949595-1.524693$

C $-0.341161-2.320995-2.273913$

O $-0.352962-2.681217-3.441763$

C $-0.727756-3.219488-1.162414$

C -1.211578 -2.748935 -0.003169

C $-1.514256-3.5319281 .196738$

C -1.170599-4.997297 1.181968

C 4.5422721 .6721551 .991188

C 3.0471041 .5392142 .204158

C $1.145868-0.0134301 .781444$

O 2.3826752 .4437162 .704271

C $0.911077-1.1287052 .748173$

C $0.100881-1.0246783 .812687$

C $-0.239527-2.1675974 .729298$

C $-1.723645-2.482138 \quad 4.722125$

C $-2.537480-1.9802895 .666238$

C -2.256353 -3.421256 3.653507

C -2.028954 -2.889519 2.265727

N $2.5527380 .322319 \quad 1.768009$

H $1.7699512 .130430-1.467587$

H $-0.9332841 .264709-2.613785$

H $0.420221-0.399747-3.727008$

H $2.527916-0.157622-1.499716$

H $2.7250510 .548044-3.085959$

H $3.637787-3.487763-2.855845$

H $3.412925-2.567258-4.938848$

H $1.971072-1.558945-5.075179$

H $3.577922-0.809436-4.955432$

H $2.658321-1.918806-0.372939$

H $2.600859-3.644099-0.610775$

H $4.438251-3.0341890 .859809$

H $5.046434-3.824113-0.596421$

H $5.650691-1.583980-1.547880$

H $7.346971-2.874804-0.207155$

H $6.736543-2.148413 \quad 1.265103$

H $9.498153-0.1901460 .882161$
H $7.0043280 .449633 \quad 1.576222$

H $8.975260-1.620753-1.603208$

H $9.1466810 .113493-1.471584$

H $7.664239-0.575325-2.161236$

H $7.8118091 .867147-0.724817$

H $\begin{array}{llll}5.474811 & 2.761681 & 0.376962\end{array}$

H $3.9727782 .004064-0.069017$

H $4.9769811 .345432-1.837990$

H $-0.583671-4.277854-1.348985$

H -1.370236 -1.675639 0.095092

H $-1.688472-5.5053950 .360736$

H -1.465134 -5.521834 2.093566

H $-0.091401-5.1384731 .058262$

H $5.032596 \quad 0.8102262 .454901$

H 4.8740762 .5579232 .546231

H $0.881459-0.3403720 .772344$

H 0.5497960 .8716202 .014903

H $1.379046-2.0842912 .523898$

H $-0.372872-0.0692734 .026305$

H $\quad 0.083602-1.8949755 .742210$

H $0.325234-3.0721574 .471532$

H -3.597218 -2.211590 5.681926

H -2.161984 -1.321327 6.442124

H -3.335263 -3.573903 3.781101

H -1.782002 -4.394659 3.808025

H $-2.281022-1.8346612 .157941$

H $3.195995-0.3567871 .371922$

C $-0.124297 \quad 3.767898 \quad 0.012886$

$\begin{array}{llll}\text { O } & -1.115859 & 2.863976 & 0.543631\end{array}$

C -2.0739763 .6182201 .309425$

C -1.4060674 .9546501 .599831$

C -0.6336745 .1791300 .315402$

H -2.9359823 .7888580 .653074$

C -2.5191682 .8325292 .548703$

H -2.131263 5.7462951 .810648

H -0.7150934 .8901542 .448260$

C $0.469075 \quad 6.216208 \quad 0.442211$

H $-1.3373255 .500407-0.464571$

C -3.0019841 .3990202 .249711$

O -1.4268792 .7356813 .470897$

H -3.3020383 .3897403 .075606$

H -2.1638380 .7848241 .899587$

O $-3.442457 \quad 0.816188 \quad 3.482621$

C $-4.158551 \quad 1.3172801 .236471$

C $-4.630871-0.1281751 .006888$

H $-3.831059 \quad 1.7351020 .282999$

O -5.2527952 .1109181 .690614$

H $-3.770075-0.7187160 .679921$

H $-4.974938-0.5527091 .957520$

H $0.051066 \quad 7.1909170 .714270$

H 1.1969245 .9359851 .211231

H $1.0069066 .331426-0.504042$

C $0.0509273 .514311-1.485568$

H -0.6691902 .3612802 .991587$

H -2.702332 0.8737294 .110995

H -5.5312861 .7594432 .553779$

H $0.6641874 .304849-1.933225$

H $-0.9264543 .544744-1.981002$

H 0.8102823 .5685200 .551810

C $-4.450568-0.300403-2.044493$

O $\quad-5.4689790 .355586-1.277859$

C $-5.778841-0.251474-0.019938$

C $-6.252419-1.692721-0.245256$

C -5.224152 -2.500581-1.029699

C $-4.828012-1.767757-2.310766$

H $-3.493237-0.242323-1.512203$

C $-4.313547 \quad 0.468729-3.370458$

H -6.6301150 .3076710 .388151$

O $-6.520595-2.3225901 .002936$

H -7.195652 -1.675929-0.806677

H $-4.349917-2.732025-0.411600$

O $-5.810356-3.758663-1.373929$

H $-5.664097-1.826414-3.019924$

O $-3.727088-2.467703-2.886788$

C $-3.8003311 .882692-3.196915$

H $-3.612397-0.043451-4.037441$

H $-5.2943170 .537089-3.856429$

H $-6.787756-3.2386580 .814800$ 
H -5.153721 -4.248889-1.898190

H -3.614502 -2.152156 -3.798691

O $-3.7487402 .484047-4.423343$

O $-3.4789362 .397505-2.136160$

C -3.274395 $3.834237-4.429026$

H -2.218954 $3.860748-4.143505$

H $-3.3729424 .224565-5.445401$

H -3.873689 $4.455362-3.756150$

SCF Energy (B3LYP/6-31G**//MMFF) $=-3245.93177000$

\section{5}

MM̄FF Geometry

C $1.842516 \quad 3.353454-0.748941$

C $0.8855664 .033365-0.100260$

C -0.0429373 .3994870 .901194$

O -1.3888683 .6010800 .409038$

C 0.0966794 .0378512 .296346

C 1.4397893 .8148052 .952345

C 1.8261612 .6513243 .510410

C 2.3455315 .0174002 .994011

C 1.0525581 .3618663 .591478

C 1.8488190 .2092742 .966453

C $1.191386-1.1582643 .215123$

C $2.202253-2.2720772 .933367$

C $1.588715-3.6624503 .125322$

O $0.053416-1.3005702 .353734$

O $2.478253-4.6249082 .537411$

C $0.266002-3.7371162 .338999$

C $1.477594-4.0297784 .609819$

O $-0.427132-4.9495242 .628267$

C $-0.677180-2.5238672 .551361$

C $-1.823150-2.5946201 .516203$

O $-1.299464-2.5567083 .838222$

C -2.3603442 .8030370 .929749$

O -2.2295491 .9661521 .808322$

C -3.6268503 .1125980 .233603$

C -4.7313942 .4194670 .548095$

C $-6.0531772 .571835-0.062139$

C $-6.1847813 .555419-1.195755$

C -2.797021 -1.4133621 .564277$

C $-4.007709-1.7091340 .698682$

C $-4.725072-1.850776-1.683940$

O $-5.041034-2.1739491 .175769$

C $-5.748463-0.779219-1.889983$

C $-7.062520-0.977696-1.702648$

C $-8.1280910 .049397-1.964079$

C $-8.8634790 .454924-0.705780$

C $-9.855626-0.302730-0.208384$

C $-8.4951231 .776085-0.068203$

C -7.0670041 .8108320 .402112$

N $-3.789209-1.461393-0.646760$

H $1.9517352 .287994-0.565734$

H $0.7672335 .098444-0.284176$

H 0.1466112 .3203070 .948034

H -0.1162825 .1141292 .229171$

H -0.6882543 .6653512 .966235$

H 2.8076512 .6156953 .982483

H 3.3192154 .7907623 .441023

H 2.5284785 .3982001 .984874

H 1.8851045 .8153963 .585817

H $0.873972 \quad 1.1494504 .652692$

H 0.0704521 .4330643 .118240

H $1.9502390 .385103 \quad 1.887480$

H 2.8595280 .2069423 .394969

H $\quad 0.860467-1.2041004 .259281$

H $3.092255-2.1610293 .565878$

H $2.560483-2.1839791 .900469$

H $3.347896-4.5286102 .962373$

H $\quad 0.526603-3.7793161 .272281$

H $2.471566-4.0562015 .072973$

H $1.061224-5.0345744 .739905$

H $0.870296-3.3248945 .181476$

H $\quad 0.185676-5.6834172 .449889$

H -1.402115 -2.653649 0.505265

H -2.397301 -3.517446 1.673642

H - $-1.026938-3.3666304 .297405$

H -3.611855 $3.885532-0.525983$

H -4.6644951 .6575151 .323958$
H $-5.9789394 .573116-0.846097$

H -7.176169 $3.571152-1.651888$

H $-5.4823363 .311356-2.000468$

H -3.151072 -1.222044 2.582966

H -2.309699 -0.5003741 .212060$

H $-5.181128-2.807454-1.406630$

H $-4.151915-1.997919-2.604803$

H $-5.3958940 .186151-2.243073$

H -7.404491 -1.954421 -1.366132

H -8.840693 -0.376116 -2.683078

H $-7.7138240 .935698-2.460327$

H -10.404341 -0.0067020 .679349$

H -10.138965 -1.238503 -0.679011

H $-9.130343 \quad 1.9700420 .806017$

H $-8.7288242 .578326-0.773100$

H -6.8655131 .1225381 .224264$

H -2.922401-1.021411-0.938098

C $4.2671293 .626352-1.454180$

O $4.4797572 .208610-1.611464$

C $5.3128131 .747830-0.530116$

C 5.9439952 .9997150 .068372

C $4.8022703 .990440-0.068180$

H $4.641785 \quad 1.3084760 .218429$

C $6.2985710 .682922-1.023495$

H $6.2630572 .850671 \quad 1.104248$

H $6.8102863 .337641-0.511767$

C 5.2259095 .4416120 .067266

H 4.0622863 .7631880 .708269

C $5.632299-0.482732-1.785702$

O $7.2316891 .309431-1.911657$

H $6.8812130 .304629-0.174994$

H $5.268463-0.134638-2.760423$

O $6.633435-1.471395-2.054106$

C $4.479222-1.161738-1.024906$

C $3.880806-2.352969-1.797519$

H $3.690154-0.425847-0.844151$

O $4.938195-1.6074420 .250194$

H $3.613044-2.018024-2.805495$

H $4.637267-3.139277-1.909131$

H 5.6546315 .6259941 .057724

H $5.9787215 .712174-0.680607$

H $4.3681636 .109869-0.059512$

C $2.7992913 .969421-1.730253$

H $7.9623230 .685490-2.058151$

H $7.251504-1.094337-2.702495$

H $5.664247-2.2360470 .097032$

H $2.5320263 .589234-2.723951$

H $2.6796685 .058925-1.753632$

H $4.8791434 .106722-2.228962$

C $1.002628-1.359136-1.815313$

O $1.690131-1.970204-0.717136$

C $2.650184-2.963354-1.089766$

C $1.957699-4.089752-1.868472$

C $1.144851-3.556636-3.046872$

C $0.222708-2.419853-2.602619$

H $1.728045-0.863516-2.471226$

C $0.094158-0.281971-1.214384$

H $3.016904-3.401245-0.153423$

O $2.905914-5.038054-2.345629$

H $1.275790-4.626881-1.196255$

H $\quad 1.795028-3.232837-3.868161$

O $0.332844-4.610286-3.570809$

H $-0.588113-2.829039-1.986468$

O $-0.372491-1.827551-3.753444$

C $-0.3974260 .717145-2.232959$

H $-0.752426-0.754929-0.710580$

H $\quad 0.6261220 .277248-0.436666$

H $3.393957-5.375691-1.575193$

H $0.928684-5.338602-3.817133$

H $-0.833793-2.533664-4.237955$

O $-1.733740 \quad 0.929593-2.062426$

O $\quad 0.315591 \quad 1.256372-3.069032$

C $-2.3301811 .850640-2.980173$

H $-1.9320012 .855934-2.813822$

H $-2.1560931 .530664-4.012065$

$\mathrm{H}-3.407521 \quad 1.863211-2.797438$

SCF Energy (B3LYP/6-31G**//MMFF) $=-3245.91784663$ 
0600056

MM̄FF Geometry

C $0.220066-2.3145472 .813466$

C $-0.503714-1.7180841 .854161$

C $-0.859645-2.3887740 .555660$

O $-0.461934-1.463230-0.483369$

C $-2.371200-2.6242270 .430432$

C $-2.687765-3.636654-0.652915$

C -3.086601-3.333876-1.903165

C $-2.557442-5.080083-0.235185$

C $-3.285721-1.974238-2.513287$

C $-4.753097-1.732811-2.896958$

C $-5.668574-1.544018-1.677747$

C -7.136674 -1.501446 -2.102846

C $-8.063835-1.232242-0.907934$

O $-5.307478-0.308688-1.052411$

O $-9.363514-0.908337-1.425080$

C $-7.5498450 .011884-0.154965$

C -8.243823 -2.477537 -0.031836

O

C $-6.030822-0.0177230 .149086$

C $-5.582881 \quad 1.374845 \quad 0.650231$

O $-5.777047-0.989240 \quad 1.164718$

C $0.555827-1.798122-1.311866$

O $1.131535-2.875596-1.367490$

C $0.939346-0.656508-2.177954$

C $0.4169800 .574634-2.056291$

C $0.7783391 .757517-2.842170$

C $1.8834921 .613104-3.854996$

C -4.0644401 .5493870 .794142$

C -3.7045342 .9783801 .167575$

C -1.7336824 .4674681 .482153$

O -4.5447663 .8433341 .402259$

C -1.405091 5.1282830 .180629

C $-0.1564465 .425124-0.209745$

C $0.1743446 .111944-1.502981$

C $1.0081225 .253103-2.427242$

C $2.3330195 .444517-2.543592$

C $0.2918124 .239392-3.291328$

C $0.1189052 .911850-2.606155$

N $-2.3313893 .172779 \quad 1.213590$

H $0.500862-3.3570532 .674241$

H $-0.811070-0.6819301 .965512$

H $-0.313530-3.3350220 .463653$

H -2.888262 -1.6773740 .237785$

H $-2.780962-2.999626 \quad 1.377530$

H -3.290561 -4.158164 -2.586097

H -2.823700 -5.772835 -1.040718

H $-1.529412-5.3050630 .064501$

H -3.220186 -5.2903940 .610724$

H -2.923483 -1.167412 -1.870032

H -2.675372 -1.929260 -3.423945

H -4.791274 -0.829157 -3.518968

H -5.113215 -2.566592 -3.512566

H -5.508040 -2.367683 -0.973994

H -7.423692 -2.432110 -2.608151

H -7.280823 -0.701418 -2.842573

H $-9.668985-1.660287-1.961000$

H $-7.7559790 .877432-0.799540$

H -8.639199 -3.309981 -0.626366

H $-8.981035-2.3005910 .759079$

H -7.313916 -2.814443 0.431014

H $-7.988690-0.4092851 .703334$

H $-5.9366492 .135249-0.058922$

H -6.0530001 .5896521 .618514$

H -4.939946 -0.7743421 .604931$

H $1.709648-0.881459-2.908645$

H $-0.3471340 .749943-1.300580$

H $2.8016501 .258436-3.374955$

H $2.1441732 .548569-4.354192$

H $1.5978380 .901017-4.636952$

H -3.670083 0.8877771 .572602

H -3.562332 $1.310814-0.150414$

H -0.8473534 .2988522 .099113$

H -2.432045 5.0919072 .048486

H $-2.2406655 .386064-0.466085$

H $\quad 0.6774445 .1821920 .444012$

H $0.7124257 .037542-1.259379$
H $-0.7337016 .434582-2.027583$

H $2.9305284 .853094-3.229310$

H $2.850306 \quad 6.191322-1.950545$

H $0.8060874 .134832-4.252376$

H $-0.7007734 .623189-3.560032$

H $-0.6634612 .912461-1.846920$

H -1.7135682 .4125440 .943402$

C $0.928768-0.1529754 .033278$

$\begin{array}{lllll}\text { O } & 1.774789 & 0.220080 & 2.927515\end{array}$

C 2.6894181 .2444343 .354803

C 2.2152651 .6864624 .735502

C 1.6174430 .4036425 .279417

H 3.6720170 .7677613 .451628

C 2.7534502 .3650672 .310985

H 3.0343372 .0736265 .349454

H 1.4462822 .4651254 .673980

C 0.6846020 .6176926 .458457

H $2.437345-0.2597765 .585848$

C 3.0699841 .8991660 .876075

O 1.4858163 .0314972 .264346

H 3.4787183 .1229252 .628212

H $2.227561 \quad 1.3266850 .469724$

O 3.1910133 .0699930 .057709

C 4.3601011 .0734210 .732112

C $4.7020550 .786009-0.741784$

H $4.2443430 .129678 \quad 1.271589$

O $5.449415 \quad 1.7702831 .335762$

H $3.8165270 .369433-1.231026$

H $4.9383631 .730193-1.246965$

H 1.2208231 .0793527 .293875

H $-0.152981 \quad 1.2718946 .193996$

H $\quad 0.271687-0.3341216 .807000$

C $0.763865-1.6763044 .064933$

H 0.8126562 .3663472 .039020

H $2.3507863 .554990 \quad 0.122239$

H $5.5313602 .630703 \quad 0.889547$

H $\quad 0.136497-1.9758224 .911844$

H $1.754283-2.1202774 .228961$

H $-0.0401220 .339176 \quad 3.881993$

C $4.708249-2.263989-0.846987$

O $5.704379-1.423302-0.252826$

C $5.906103-0.167604-0.909009$

C $6.318071-0.403230-2.369220$

C $5.318438-1.290765-3.105217$

C $5.054074-2.569756-2.310726$

H $3.730006-1.774028-0.787516$

C $4.649377-3.560559-0.024255$

H $6.7590850 .305943-0.406991$

O $6.4514930 .830990-3.066842$

H $7.299920-0.893903-2.393640$

H $4.389095-0.748742-3.313853$

O $5.879387-1.633404-4.374853$

H $5.937873-3.218866-2.360732$

O $3.967203-3.276988-2.904032$

C $4.066873-3.3439891 .355880$

H $4.024838-4.311520-0.519926$

H $5.664562-3.9576180 .097043$

H $7.1067491 .367214-2.588449$

H $5.195663-2.103692-4.880759$

H $4.249519-3.564310-3.788527$

O $4.378499-4.4169362 .141774$

O $3.402248-2.3757311 .696453$

C $3.887050-4.3641073 .484592$

H $4.192351-3.4332713 .972267$

H $2.797946-4.4631613 .485786$

H $4.316463-5.2042584 .036713$

SCF Energy $\left(B 3 L Y P / 6-31 G^{* *} / / M M F F\right)=-3245.91369213$

\section{7}

MM̄FF Geometry

C $-0.224201-3.4789751 .625328$

C $0.417705-2.3235661 .396549$

C $0.819088-1.3455902 .469578$

O $0.339176-0.0367812 .076427$

C $2.353400-1.2846792 .555092$

C $2.863339-0.3856223 .662329$

C 3.3323000 .8648003 .478962

C $2.864000-1.0011015 .037257$ 
C 3.3779491 .6523642 .199404

C 4.7997871 .9693421 .721920

C 5.6151220 .7360631 .300926

C 6.9892951 .1670650 .781163

C $7.806481-0.0203480 .251416$

$\begin{array}{lllll}\text { O } & 4.896202 & 0.048248 & 0.272837\end{array}$

O $8.9120580 .507215-0.498473$

C $6.932041-0.824149-0.735560$

C $8.413904-0.8446981 .392683$

O $7.603349-2.011919-1.152375$

C $5.522521-1.154214-0.188378$

C $4.620915-1.805801-1.269041$

O $5.659748-2.107748 \quad 0.873025$

C -0.9604850 .2697002 .323638$

O $-1.783735-0.4458552 .878328$

C -1.3145181 .6176701 .815882$

C -0.4229322 .4761011 .297876$

C $-0.694407 \quad 3.8247910 .796367$

C -2.129984 4.2569250 .669085

C $4.133662-0.870456-2.381607$

C $2.867081-0.108721-2.024602$

C $1.3245751 .627703-2.912683$

O $2.233170-0.297757-0.990354$

C $1.7075862 .964923-2.364260$

C $1.2233724 .118088-2.848856$

C $1.5549875 .474469-2.293817$

C $0.3774496 .100481-1.577716$

C $-0.569414 \quad 6.766608-2.260008$

C $\quad 0.329814 \quad 6.014299-0.068124$

C 0.3516164 .6034650 .449791

N $2.5060820 .792600-3.016212$

H -0.453913 -3.746902 2.654816

H $0.666593-2.0336260 .380420$

H $\quad 0.395113-1.6170083 .444138$

H $2.762870-0.9618431 .593009$

H $2.759123-2.2929392 .715477$

H 3.6977091 .4083294 .349380

H $3.274295-0.3256085 .795576$

H $1.845214-1.2586485 .342961$

H $3.471139-1.9122155 .045096$

H $2.817197 \quad 1.1690281 .395294$

H 2.8621472 .6030922 .385339

H 4.7067122 .6453610 .861596

H 5.3390992 .5187192 .503838

H 5.7277760 .0746972 .167219

H 7.5485231 .7023391 .558861

H $6.8580291 .886280-0.039787$

H 9.4312201 .0779390 .093723

H $6.830630-0.207748-1.636713$

H $9.062682-0.2163032 .014919$

H $9.056889-1.6427181 .005570$

H $7.662694-1.2904672 .047664$

H $7.539242-2.657382-0.428058$

H $5.162283-2.647093-1.720566$

H $3.748909-2.264523-0.783815$

H $4.798527-2.532807 \quad 1.018767$

H -2.363344 1.8773681 .912385

H 0.6200032 .1756821 .241588

H -2.606946 4.3092031 .653695

H $-2.686936 \quad 3.5512860 .042811$

H -2.2457145 .2380630 .201982$

H $4.909896-0.160648-2.681295$

H $3.888379-1.481731-3.259369$

H $0.6007901 .152533-2.242983$

H $0.8878831 .699617-3.913602$

H $2.3812172 .986248-1.510782$

H $0.5350684 .091008-3.691025$

H $\quad 1.8690146 .117268-3.126501$

H $2.4212355 .430042-1.621889$

H -1.405842 $7.237991-1.754840$

H $-0.5292136 .852143-3.340985$

H -0.5313016 .5597210 .334632$

H $1.205368 \quad 6.5385460 .337330$

H $1.3588894 .201540 \quad 0.565907$

H $3.1155860 .924128-3.818246$

C $-0.608652-4.024753-0.874361$

O $-1.447590-2.872431-1.085378$

C $-2.060418-2.970251-2.383572$
C $-1.440071-4.188035-3.060854$

C $-1.112266-5.068441-1.869371$

H $-3.124671-3.169168-2.215177$

C $-1.892715-1.651352-3.146569$

H $-2.127051-4.661337-3.769046$

H $-0.525122-3.929162-3.606296$

C $-0.109513-6.164626-2.183544$

H -2.042157 -5.522324 -1.501192

C $-2.405157-0.402880-2.397082$

O $-0.501314-1.439695-3.423012$

H $-2.383807-1.727780-4.123514$

H $-1.747484-0.171457-1.550135$

O $-2.3108730 .710597-3.295449$

C $-3.859472-0.502643-1.902343$

C $-4.3669600 .815336-1.285596$

H $-3.928260-1.298276-1.154193$

O $-4.710229-0.870317-2.987668$

H $-3.6717811 .122752-0.497265$

H $-4.3583991 .602805-2.048572$

H $-0.500246-6.832106-2.958673$

H $\quad 0.838458-5.750655-2.543019$

H $0.101600-6.767720-1.295051$

C $-0.698115-4.4684960 .589018$

H $-0.031362-1.428564-2.571217$

H -1.396655 $0.741689-3.624709$

H $-4.630477-0.180913-3.669216$

H $-0.131149-5.3960260 .729276$

H -1.745866 -4.693996 0.823065

H $0.419592-3.733895-1.126351$

C $-5.270703-0.2258081 .435151$

$\begin{array}{llll}\text { O } & -5.952237 & -0.387466 & 0.184775\end{array}$

C $-5.804500 \quad 0.703612-0.727707$

C $-6.3401351 .990861-0.087985$

C -5.7052152 .2577951 .273629$

C $-5.786021 \quad 1.0200022 .167427$

H $-4.192578-0.1366811 .257455$

C -5.515972 -1.502647 2.245428

H $-6.4554910 .476978-1.581600$

O $-6.1205573 .098188-0.955636$

H -7.4260891 .8957490 .042555$

H -4.6713472 .5996091 .161166$

O -6.4138823 .3322751 .895984$

H $\quad-6.8259870 .8800582 .489313$

$\begin{array}{llll}\text { O } & -5.001294 & 1.273845 & 3.330717\end{array}$

C $-4.778654-2.6662721 .626525$

H $-5.158350-1.4172713 .276750$

H -6.586404 -1.736540 2.292552

H $-6.451603 \quad 3.892599-0.502567$

H -6.0163303 .4703442 .772930$

H $\quad-5.2127870 .5913313 .988941$

O $-5.654824-3.505067 \quad 1.005696$

O $-3.562797-2.7991951 .673408$

C $-5.066690-4.6419020 .366025$

H $-4.375034-4.320082-0.417420$

H $-4.552166-5.2670131 .102078$

H $-5.868169-5.226829-0.092624$

SCF Energy (B3LYP/6-31G**//MMFF) $=-3245.92247487$

0600058

MM̄FF Geometry

C $1.1376130 .350782-3.692066$

C $0.109088-0.497222-3.849530$

C $-0.101813-1.825917-3.157245$

O $-1.191964-1.684150-2.212664$

C $1.118679-2.362482-2.384471$

C $0.919691-3.757219-1.819551$

C $0.766194-4.047506-0.511849$

C $0.955498-4.871797-2.834388$

C $0.716550-3.0933930 .648804$

C $-0.603005-3.2308501 .419887$

C $-0.777775-2.1137902 .459564$

C -2.061635 -2.325378 3.264065

C $-2.355947-1.1515504 .210006$

O $-0.839592-0.875470 \quad 1.746671$

O $-3.713342-1.2793304 .661470$

C -2.2694830 .1675413 .412802$

C -1.475552 -1.195911 5.464209

O $-2.389918 \quad 1.293697 \quad 4.278104$ 
C $-0.984347 \quad 0.2891892 .568752$ C -0.9997641 .5137291 .621879$ O $0.140117 \quad 0.444162 \quad 3.442277$ C $-2.461688-1.813451-2.685318$ O $-2.802153-1.977365-3.845956$ C $-3.381271-1.734510-1.527727$ C $-4.707457-1.707727-1.728066$ C $-5.740658-1.674788-0.688987$ C $-5.288202-1.6377580 .746752$ C -2.0892291 .4930040 .545431$ C -3.3339352 .2915120 .888534$ C $-5.5872252 .834691-0.036754$ O -3.4441172 .9752321 .901652$ C $-6.5995331 .907486 \quad 0.557334$ C $-7.7315261 .549696-0.067541$ C -8.7811580 .6691500 .546065$ C $-9.127084-0.522069-0.317454$ C $-10.220260-0.515364-1.099283$ C -8.262665 -1.756070 -0.197662 C -7.036516 -1.696189-1.066509 N $-4.3030782 .164188-0.098877$ H $1.9323090 .125269-2.987393$ H $-0.668219-0.224776-4.561912$ H $-0.376818-2.558704-3.926311$ H $\quad 1.376207-1.662218-1.584927$ H $1.993360-2.392063-3.047872$ H $0.663594-5.095071-0.230228$ H $0.915195-5.861400-2.366923$ H $0.105844-4.797881-3.519560$ H $1.880582-4.824762-3.418368$ H $1.552525-3.3192481 .321609$ H $\quad 0.851020-2.0586090 .328288$ H - $-1.437069-3.1935460 .707684$ H $-0.638377-4.2099351 .913618$ H $0.096246-2.1072663 .121484$ H $-2.018189-3.268753 \quad 3.822834$ H -2.908752 -2.429195 2.573255 H -3.802864 -2.141964 5.101696 H $-3.138654 \quad 0.1927832 .744315$ H -1.652270 -2.122915 6.022944 H -1.725286 -0.3802816 .151755$ H $-0.407917-1.1459755 .240806$ H $-3.218248 \quad 1.1885494 .776867$ H - 1.0116412 .4450632 .200488 H $-0.038726 \quad 1.516348 \quad 1.089601$ H $\quad 0.865631 \quad 0.8554092 .943573$ H -2.940716 -1.719566 -0.538168 H $-5.077760-1.729262-2.752267$ H -4.785690 -2.574288 1.010639 H -6.103561 -1.4941161 .458654$ H $-4.594787 \quad-0.8069320 .913870$ H -1.680590 $1.942049-0.366552$ H -2.3803140 .4690410 .296724$ H -5.847258 $3.133299-1.057227$ H $-5.503826 \quad 3.7356770 .579215$ H -6.4052281 .5386051 .561739$ H -7.936182 $1.941748-1.061382$ H $-9.676303 \quad 1.2841890 .707451$ H $-8.4809530 .322026 \quad 1.542564$ H -10.493021 -1.379154 -1.696522 H $-10.8630760 .356340-1.163662$ H -8.835525 -2.640398 -0.509360 H -8.032470 -1.9394650 .855589$ H -7.263966 -1.720330 -2.133263 H $-4.1407351 .527152-0.873530$ C $1.0914252 .899952-3.636229$ O $2.0347032 .953095-2.546047$ C $1.3390523 .410800-1.369890$ C $0.0299884 .012794-1.867941$ C $-0.2956443 .079653-3.017360$ H $1.1113662 .518573-0.776049$ C $2.2080234 .369823-0.552825$ H $-0.7417374 .036134-1.093436$ H $0.1677205 .035284-2.238639$ C $-1.3372413 .628218-3.975891$ H $-0.6615732 .133514-2.600888$ C $3.5786593 .805921-0.130498$ O $2.4511235 .556360-1.319979$
H $1.653493 \quad 4.696745 \quad 0.334707$

H $4.2416513 .726779-1.000641$

O 4.1843194 .7632770 .748708

C 3.5260182 .4464340 .591472

C 4.8954712 .0317131 .162211

H $3.1766441 .683656-0.111873$

O 2.5800182 .5008661 .658989

H 5.6506952 .1373440 .376396

H 5.1717692 .7176141 .972119

H -2.285297 $3.799645-3.455479$

H -1.018164 $4.579400-4.415101$

H -1.522869 $2.924079-4.792995$

C $1.2922361 .630766-4.467665$

H $2.8805565 .286010-2.149942$

H 4.2197465 .6111730 .273352

H 2.8607713 .2075352 .265411

H $2.3107781 .633703-4.876138$

H $0.610998 \quad 1.646009-5.326731$

H $1.3247813 .762003-4.275334$

C $5.426984-0.589904-0.312943$

O $4.490062-0.3714780 .747221$

C 4.8987980 .5915151 .724639

C 6.2335470 .1665142 .352368

C $7.307328-0.0758661 .294512$

C $6.794544-1.0389020 .223319$

H $5.5504070 .346822-0.870562$

C $4.799058-1.576034-1.308441$

H 4.1436120 .5598122 .520099

O $\quad 6.674431 \quad 1.1521193 .280338$

H $6.079676-0.7613372 .918760$

H $7.6496930 .868573 \quad 0.855542$

O $8.446280-0.6547871 .936360$

H $6.745123-2.0432780 .659463$

O $7.751004-1.078496-0.833202$

C $4.607065-2.974238-0.757908$

H $3.809926-1.215543-1.609587$

H $5.426718-1.631667-2.205193$

H 7.5289290 .8539413 .636532

H $9.105420-0.8375551 .244786$

H $7.510627-1.812876-1.422689$

O $4.689132-3.875543-1.780272$

O $4.387971-3.2420120 .415550$

C $4.549074-5.249724-1.405646$

H $3.615725-5.410111-0.858349$

H $4.525566-5.850095-2.319050$

H $5.406863-5.559745-0.801740$

SCF Energy (B3LYP/6-31G**//MMFF) $=-3245.92239783$

0600059

MM̄FF Geometry

C $-1.978420-3.9728371 .934038$

C $-0.763148-3.4705951 .666025$

C $0.167647-2.9501152 .729821$

O $0.694350-1.6693412 .317237$

C $1.359074-3.9106842 .893037$

C $2.377787-3.428743 \quad 3.907493$

C $3.595329-2.9364793 .601799$

C $1.960810-3.5651555 .349636$

C $4.200787-2.7320152 .239323$

C $4.747509-1.3069092 .069472$

C $5.490257-1.1378100 .736131$

C 6.1083580 .2567820 .624610

C $6.8145590 .458674-0.725989$

O $4.564755-1.342811-0.339070$

O $7.0752591 .862588-0.880969$

C $5.8494780 .050996-1.860709$

C $8.178370-0.240384-0.771162$

O $6.5065180 .068791-3.125070$

C $5.173012-1.319824-1.639461$

C $4.090323-1.659118-2.698847$

O $6.163225-2.354907-1.738912$

C $-0.040845-0.5593322 .593194$

O $-1.183912-0.5135153 .016239$

C 0.8312310 .6069772 .332183

C 0.3603631 .8563062 .451059

C 1.1413553 .0814892 .259565

C 2.6421982 .9432372 .201896

C $3.156519-0.538869-3.161727$ 
C $2.2117240 .012472-2.110837$

C $0.7004451 .966307-1.727347$

O $1.966895-0.559033-1.053139$

C $1.3761703 .201261-1.225236$

C $0.7790474 .401897-1.182726$

C $1.4319985 .641206-0.640164$

C $0.708393 \quad 6.196964 \quad 0.568277$

C -0.1607397 .2142130 .444406$

C 1.0593515 .6304311 .928604

C 0.4860844 .2563302 .149751

N $1.637411 \quad 1.206530-2.531276$

H -2.304735 -3.995422 2.972648

H $-0.392945-3.4390180 .645560$

H $-0.350617-2.8378393 .690479$

H $1.835149-4.0741921 .920257$

H $0.997655-4.9002993 .204420$

H $4.249074-2.6541594 .426638$

H $2.758264-3.2769226 .042783$

H $1.095860-2.9295095 .562489$

H $1.693831-4.6037205 .571290$

H $5.021347-3.4508702 .125752$

H $3.486650-2.9348961 .437413$

H $3.912988-0.5989612 .119527$

H $5.428730-1.0702752 .896520$

H $6.273998-1.9033360 .691060$

H 6.8042610 .4433561 .451979

H 5.3175651 .0141120 .720099

H $7.6210182 .150762-0.129139$

H $5.0743630 .826956-1.897710$

H $\quad 8.8343240 .1496150 .016478$

H $8.694210-0.038755-1.716463$

H $8.112292-1.322001-0.637875$

H $6.9080990 .948293-3.230709$

H $4.591576-2.046882-3.596481$

H $3.479941-2.489626-2.318279$

H $5.706047-3.198520-1.893496$

H 1.8589280 .4026712 .058344

H -0.6893911 .9973942 .700203$

H 3.1661193 .8930792 .080734

H 2.9471572 .3118291 .360856

H 3.0178902 .4917093 .127084

H $3.7355650 .278657-3.604490$

H $2.521712-0.938004-3.963933$

H $0.3547691 .358668-0.886109$

H $-0.1503602 .207430-2.369887$

H $2.3927583 .101410-0.853846$

H $-0.2400204 .502422-1.549157$

H $1.452436 \quad 6.388192-1.444377$

H $2.4831535 .460470-0.382336$

H $-0.6631017 .637418 \quad 1.307851$

H $-0.3945057 .641643-0.525068$

H $\quad 0.661910 \quad 6.2774592 .722113$

H 2.1443645 .6667492 .057113

H -0.6020834 .2485682 .214160$

H $1.9010641 .592581-3.433333$

C $-2.623079-4.454957-0.529367$

O $-2.619277-3.075890-0.947956$

C $-3.227029-2.981473-2.249227$

C $-3.412197-4.412561-2.739719$

C $-3.640049-5.149870-1.435070$

H -4.216269 -2.532672 -2.104384

C $-2.382269-2.094866-3.168401$

H $-4.248757-4.505762-3.438829$

H $-2.513057-4.791629-3.239228$

C $-3.449123-6.652379-1.548064$

H -4.660209-4.940679-1.086106

C $-2.108695-0.678842-2.623824$

O $-1.110255-2.719713-3.388574$

H -2.858410 -2.029407 -4.153621

H -1.413442 -0.723151 -1.776435

O $-1.437596 \quad 0.052143-3.658749$

C $-3.3673430 .106672-2.209940$

C $-3.0494561 .561833-1.817934$

H -3.842030 -0.397804 -1.362529

O $-4.3082320 .105631-3.282679$

H $-2.2802981 .552583-1.038757$

H -2.630320 $2.086696-2.684500$

H $-4.157257-7.076617-2.267504$
H $-2.437690-6.904497-1.883750$

H -3.615568 -7.141800 -0.583453

C $-2.975231-4.5424950 .957561$

H $-0.690867-2.844642-2.519755$

H $-0.629818-0.441147-3.883469$

H $-3.8802260 .524543-4.049017$

H $-3.133879-5.5917881 .232679$

H -3.923597 -4.019164 1.128611

H -1.619323 -4.857705 -0.715842

C $-4.287268 \quad 1.6536190 .971492$

O $-4.9814171 .680131-0.281080$

C $-4.2890172 .349249-1.338729$

C $-3.9959783 .804188-0.941482$

C -3.2751003 .8929900 .402738$

C -4.0215913 .0804951 .460671$

H $-3.340091 \quad 1.1115140 .859212$

C $-5.1619990 .882758 \quad 1.964369$

H $-4.9862892 .383737-2.185434$

O $-3.2287724 .439685-1.958286$

H $-4.9472194 .347637-0.867954$

H -2.2321703 .5655830 .321883$

O $\quad-3.2418795 .2539990 .836019$

H -4.9684933 .5795661 .703635$

O -3.2454933 .0335412 .654742$

C $-5.187311-0.5847281 .611616$

H -4.7847190 .9584902 .990275$

H -6.1881801 .2699201 .969613$

H $-3.1635995 .383065-1.734270$

H -2.6765145 .7445940 .216281$

H $-3.070898 \quad 3.9515642 .924011$

O $-6.363390-0.9004520 .999305$

O $-4.258530-1.3510571 .832455$

C $-6.499935-2.2659850 .595212$

H -7.484308 -2.3881420 .135675$

H $-5.734570-2.519090-0.143341$

$\mathrm{H}-6.433252-2.9279811 .463882$

SCF Energy $(B 3 L Y P / 6-31 G * * / / M M F F)=-3245.91393828$

0600061

MM̄FF Geometry

C $1.154478-0.6222232 .598175$

C 2.1411820 .0174153 .245099

C 2.7377021 .3648152 .913915

O $2.069148 \quad 1.9876501 .798225$

C 4.2027331 .1700072 .484310

C 5.0432732 .4250582 .589853

C 5.3524483 .2388281 .561680

C 5.6017462 .7001963 .962792

C 4.8601723 .1800040 .142590

C $5.9014312 .629412-0.843273$

C $5.8572321 .098274-0.963666$

C $6.9455550 .588811-1.909261$

C $6.840593-0.928356-2.131177$

O $4.5668840 .750064-1.477561$

O $7.677774-1.269922-3.246387$

C $5.390973-1.276327-2.530677$

C $7.371494-1.720727-0.930762$

O $5.207227-2.688251-2.609316$

C $4.323556-0.655336-1.599359$

C $2.915584-0.840238-2.217590$

O $4.368178-1.312511-0.331899$

C 1.0458682 .8432592 .043948

O 0.5441893 .1137543 .121271

C 0.6466503 .4055580 .736010

C -0.5380174 .0153980 .593673$

C $-1.0451084 .611272-0.644645$

C $-0.1137564 .656194-1.829689$

C $1.804100-0.046792-1.513621$

C $0.455034-0.233769-2.188949$

C $-1.922650 \quad 0.504717-2.016392$

O $0.284696-0.978380-3.150853$

C $-2.2364231 .763066-2.762328$

C $-3.3447322 .486524-2.540467$

C $-3.7085433 .732640-3.295609$

C $-4.1228034 .866491-2.382341$

C $-5.4206525 .128428-2.152027$

C $-3.0359265 .743026-1.802749$

C $-2.3094865 .081960-0.664909$ 
N $-0.538127 \quad 0.523139-1.581093$ H $0.663898-0.1611931 .746397$ H $2.602364-0.4834254 .095291$ H 2.6759952 .0062173 .801354 H $4.225490 \quad 0.791672 \quad 1.457637$ H 4.6869560 .3948873 .093933 H 6.0080044 .0859041 .762606 H 6.2175893 .6055883 .988944 H 4.7912292 .8335974 .686268 H $6.2285391 .865176 \quad 4.292456$ H 3.9163312 .6341920 .062963 H $4.6227464 .211413-0.149691$ H $5.6785763 .060075-1.828508$ H $6.9085112 .962286-0.564463$ H 5.9898570 .6588760 .030059 H $7.9419810 .854900-1.535442$ H $6.8474131 .088515-2.883266$ H $8.585308-0.990159-3.036468$ H $5.231621-0.886762-3.546101$ H $8.423438-1.473232-0.743918$ H $7.348210-2.798263-1.127516$ H $6.818662-1.525986-0.009395$ H $5.886522-3.035491-3.212586$ H $2.938857-0.512861-3.265496$ H $2.647170-1.904405-2.214858$ H $3.504570-1.2275540 .101087$ H $1.3446903 .289174-0.085777$ H -1.2010904 .0744181 .455641$ H $0.7686935 .263299-1.598895$ H $-0.5662595 .080830-2.727751$ H $0.217423 \quad 3.648313-2.102846$ H $1.703868-0.370009-0.473590$ H $2.0384801 .023716-1.522299$ H $-2.5367750 .399579-1.117638$ H -2.095773 $-0.358589-2.666012$ H -1.539442 $2.078375-3.534857$ H -4.054685 2.147446 -1.791151 H $-4.5365573 .481410-3.971538$ H $-2.8868984 .064658-3.942024$ H -5.727442 $5.954933-1.519746$ H $-6.2053094 .517546-2.586031$ H -3.468617 $6.674187-1.412621$ H -2.367821 $6.067767-2.605332$ H -2.909976 4.9964290 .241942 H $-0.2965611 .120460-0.796047$ C $0.988430-3.0004651 .843260$ O $-0.090029-2.958726 \quad 0.883174$ C $-0.503748-4.3098280 .600105$ C $0.638803-5.1963371 .071401$ C $1.074105-4.4522982 .317859$ H -1.377908 -4.513205 1.230195 C $-0.879558-4.467102-0.876113$ H $0.316791-6.222478 \quad 1.271850$ H $1.455926-5.2283940 .341225$ C $2.451853-4.8554952 .814757$ H $0.338379-4.6322173 .113497$ C -1.978924 -3.498442 -1.354660 O $0.281113-4.239686-1.685193$ H -1.184882 -5.501640 -1.070908 H -1.586926 -2.476088 -1.383148 O $-2.316770-3.840337-2.703264$ C -3.262407 -3.523840 -0.506899 C $-4.360685-2.603018-1.066783$ H -3.014909 -3.208586 0.510501 O $-3.766824-4.855203-0.422738$ H -3.921687 -1.624708 -1.282123 H -4.732749 -3.004477 -2.017355 H $2.467404-5.9161603 .085967$ H $3.218059-4.6934452 .049022$ H $2.733015-4.2759653 .699643$ C $0.703081-2.0083082 .974394$ H $0.606469-3.344646-1.487223$ H -1.496486 -3.803353 -3.224901 H -3.943847 -5.159989-1.329333 H $1.214174-2.3260543 .890624$ H $-0.370922-1.9729923 .193033$ H $1.903490-2.7276891 .301372$ C $-4.560776-0.830571 \quad 1.387589$
O $-5.148871-2.1291361 .233786$

C $-5.559208-2.443905-0.101782$

C $-6.610742-1.427920-0.568981$

C $-6.1191940 .011662-0.416624$

C -5.5730850 .2533040 .992888$

H -3.670914 -0.7638850 .752465$

C $-4.111097-0.7139302 .850602$

H $-6.050310-3.423792-0.047680$

O $-6.967735-1.681829-1.923396$

H -7.519791 -1.555774 0.033054

H $\quad-5.3719790 .247987-1.181519$

O $-7.2208810 .891566-0.649819$

H $-6.4135190 .259188 \quad 1.697903$

O -4.9769041 .5457041 .049338$

C $-3.0099370 .300076 \quad 3.071992$

H $-4.970554-0.4621673 .483265$

H -3.725823 -1.681110 3.196974

H $-7.617090-1.006294-2.184425$

H $-6.8982471 .799692-0.517323$

H -4.1876831 .5366940 .481521$

O -2.9379120 .5869804 .405682$

$\begin{array}{llll}\text { O } & -2.284084 & 0.758193 & 2.200227\end{array}$

C -1.9222801 .5214864 .781493$

H -2.009621 1.7051355 .855510

H -0.9331201 .1022224 .576652$

$\mathrm{H}-2.0612372 .4689414 .252065$

SCF Energy $(B 3 L Y P / 6-31 G * * / / M M F F)=-3245.93123713$

0600062

MM̄FF Geometry

C $1.807351-0.0250803 .039359$

C 3.0473370 .3808193 .354291

C 3.8848931 .4074902 .629826

O 3.1095312 .1604671 .673747

C 4.9911450 .6934281 .834409

C 6.1955831 .5686391 .557929

C 6.4203802 .2396490 .411795

C 7.2161041 .6086792 .666888

C $5.5186192 .378273-0.782491$

C $5.9735581 .537679-1.984108$

C $5.4178950 .105623-1.952817$

C $5.962225-0.716847-3.121187$

C $5.327380-2.114366-3.183017$

O $3.992528 \quad 0.201847-2.048312$

O $5.681602-2.669649-4.458496$

C $3.791266-1.966736-3.130072$

C $5.914291-3.050082-2.119684$

O $3.154407-3.239090-3.030790$

C $3.297476-1.047502-1.986752$

C $1.788582-0.755595-2.171213$

O $3.503117-1.707719-0.736663$

C 2.4248883 .2481502 .110801

O $2.3337123 .656623 \quad 3.256234$

C 1.7875243 .8535590 .921270

C 0.8048944 .7523371 .071289

C $0.0725695 .410389-0.013018$

C $0.5156535 .129388-1.426053$

C $1.2217670 .309584-1.220734$

C $-0.2632660 .538646-1.450760$

C $-2.2341291 .683866-0.444410$

O $-0.8815250 .037185-2.386575$

C $-2.4975992 .962797-1.173612$

C $-3.2476403 .956142-0.671679$

C $-3.5699085 .220021-1.417709$

C $-3.276366 \quad 6.469246-0.615996$

C $-4.260557 \quad 7.1333640 .013592$

C -1.858105 $6.994785-0.616667$

C -0.9602016 .2176920 .306761$

N $-0.818116 \quad 1.369867-0.486405$

H 1.2898720 .3993702 .184998

H $3.536675-0.0807054 .210843$

H 4.3185372 .0914753 .369925

H $4.575966 \quad 0.3202970 .892377$

H $5.345160-0.1966322 .372356$

H 7.3509782 .8000940 .324891

H 8.0726702 .2461642 .423229

H 6.7673751 .9986133 .585868

H 7.5996160 .6027932 .866688 
H $4.4749702 .171745-0.531910$

H $5.5433543 .436551-1.073732$

H $5.6020932 .033894-2.890533$

H $7.0683421 .522384-2.048805$

H $5.692454-0.363877-1.002787$

H $7.055430-0.793617-3.067719$

H $5.745533-0.196222-4.064826$

H $5.336907-3.577558-4.499372$

H $3.471915-1.531555-4.087492$

H $6.993283-3.171459-2.273226$

H $5.485709-4.055547-2.196600$

H $5.762156-2.690172-1.099986$

H $3.396234-3.752837-3.819324$

H $1.614969-0.407037-3.197950$

H $1.216393-1.682157-2.042318$

H $2.887966-1.347496-0.079579$

H $2.1340483 .523678-0.051658$

H 0.4947575 .0199222 .080423

H $1.5451375 .471818-1.578517$

H $-0.0979895 .620014-2.183891$

H $0.4691014 .056581-1.642432$

H $1.3580090 .002413-0.180144$

H $\quad 1.7346641 .265957-1.370426$

H -2.5260031 .7372950 .609205$

H $-2.7925370 .875693-0.923209$

H $-2.0840803 .062598-2.174352$

H -3.689998 3.8389350 .315290

H -4.636111 5.187276 -1.677562

H -3.034029 $5.268435-2.373804$

H -4.0631418 .0454290 .567118$

H $-5.2849416 .776313-0.005838$

H -1.842710 8.040041 -0.279491

H -1.488832 $7.031802-1.645156$

H -1.204886 6.3525681 .361483

H $-0.218010 \quad 1.7832190 .221024$

C $1.039454-2.4081763 .053424$

O $\quad 0.066977-2.3476901 .988921$

C $-0.533555-3.6535571 .844203$

C $0.180925-4.5720622 .831237$

C $0.611986-3.5982573 .908226$

H -1.580346 -3.559644 2.151862

C $-0.437606-4.1269400 .389599$

H $-0.474771-5.364418 \quad 3.205330$

H $1.054759-5.0529842 .375299$

C $1.703557-4.1477374 .810393$

H $-0.263307-3.3304444 .515187$

C -1.235017 -3.269923 -0.612721

O $0.939822-4.110267-0.010296$

H $-0.759228-5.172100 \quad 0.316003$

H $-0.854426-2.243551-0.621589$

O $-1.001820-3.805394-1.922395$

C -2.757314 -3.257189-0.365455

C $-3.528757-2.504996-1.467304$

H -2.965066 -2.784341 0.599071

O $-3.237665-4.599076-0.287523$

H -3.180054 -1.467778 -1.484500

H -3.287305 -2.938384 -2.445152

H $\quad 1.359420-5.0528575 .321833$

H $2.603080-4.4048134 .240978$

H $1.986677-3.4179615 .574948$

C $1.076005-1.0845673 .817329$

H $1.259422-3.1958640 .077300$

H - $0.039712-3.815539-2.064506$

H -3.033656 -5.032705 -1.133866

H $1.558658-1.2233694 .791493$

H $0.060568-0.7233564 .010255$

H $2.014157-2.6089382 .587581$

C $-5.330766-0.7945530 .323450$

O $-5.490534-2.182793 \quad 0.012872$

C $-5.064133-2.560805-1.298033$

C $-5.841599-1.762060-2.354552$

C $-5.746129-0.257958-2.112764$

C $-6.1116550 .076118-0.667547$

H $-4.266046-0.5367450 .297290$

C $-5.839122-0.5863121 .753245$

H $-5.353804-3.612437-1.418230$

O $-5.372154-2.054351-3.666245$

H $-6.900313-2.050332-2.320058$
H $-4.752400 \quad 0.128335-2.366726$

O $-6.6678590 .416163-2.972840$

H -7.190806 -0.062691 -0.523012

O $-5.8204781 .449094-0.415302$

C $-4.879055-1.1857832 .751539$

H $-5.9663950 .474877 \quad 1.995928$

H $-6.813445-1.0669851 .903646$

H -5.457988 -3.013776 -3.800007

H $-6.4475340 .163969-3.886076$

H $-6.2977391 .974397-1.080305$

O $-3.927108-0.2685863 .086128$

O $-4.954042-2.3350963 .165827$

C $-2.953787-0.7144014 .034025$

H $-2.274537 \quad 0.117147 \quad 4.237928$

H $-3.439390-1.0071334 .969734$

$\mathrm{H}-2.378998-1.5477563 .620648$

SCF Energy $(B 3 L Y P / 6-31 G * * / / M M F F)=-3245.92006634$

0600063

MM̄FF Geometry

C -0.8981552 .9285601 .420688$

C -0.4366502 .0078262 .280740$

C -1.1893230 .8676752 .919886$

O $-0.606057-0.3483762 .393990$

C -2.7096860 .8170792 .671807$

C $-3.441509-0.0232053 .702524$

C $-3.852152-1.2934383 .522829$

C -3.7402360 .6828265 .001699$

C -3.648617 -2.177761 2.325309

C $-4.973741-2.5632321 .652550$

C $-5.635448-1.3962110 .904756$

C $-6.974916-1.828600 \quad 0.304953$

C $-7.622749-0.701086-0.512179$

O $-4.747952-1.000247-0.142638$

O $-8.675286-1.299851-1.287182$

C $-6.581552-0.131670-1.502564$

C -8.3086750 .3225590 .399485$

O $-7.0819261 .024226-2.169013$

C $-5.1842450 .138141-0.893486$

C $-4.1492920 .334470-2.023548$

O $-5.149798 \quad 1.332639-0.113174$

C $-0.212861-1.3115693 .263699$

O $-0.257734-1.2587094 .485232$

C $0.304542-2.5057522 .555421$

C $0.293480-2.6498901 .220928$

C $0.793168-3.8060200 .471686$

C $1.480265-4.8992181 .247610$

C $-2.7286020 .580754-1.502608$

C $-1.7439960 .661358-2.648040$

C $-0.485632-0.736801-4.262388$

O $-1.361571 \quad 1.740125-3.094111$

C $0.015041-2.143054-4.321669$

C $1.276875-2.496343-4.034761$

C $1.788596-3.905257-4.106900$

C $2.156288-4.459529-2.749283$

C $3.439404-4.628860-2.389192$

C $1.029233-4.898012-1.840913$

C $0.625417-3.824691-0.867519$

N $-1.361069-0.585968-3.116708$

H -1.9254332 .8893331 .071850$

H 0.5978112 .0935682 .608748

H -0.9955120 .9512503 .996492$

H $-2.9116390 .445353 \quad 1.661827$

H -3.1420361 .8248032 .714846$

H $-4.383058-1.7777014 .342020$

H -4.2911410 .0483345 .704269$

H -2.8137070 .9907645 .495654$

H $-4.349241 \quad 1.5739954 .817427$

H -2.964887 -1.738343 1.593758

H -3.160095 -3.095455 2.676380

H $-4.756992-3.3681880 .937938$

H -5.666011 -2.971393 2.399407

H -5.779572 -0.5592431 .596417$

H -7.659662 -2.177053 1.088249

H $-6.815287-2.690608-0.358312$

H $-9.050968-0.607562-1.858093$

H -6.461193 -0.892995-2.285953

H $-9.133934-0.1508250 .945821$ 
H $-8.7636221 .132589-0.180461$ H $-7.638371 \quad 0.758841 \quad 1.142119$ H -7.015128 $1.776963-1.557854$ H -4.141317 -0.554124 -2.667802 H -4.435837 $1.186905-2.652812$ H -6.0176531 .4799040 .287596$ H $0.694633-3.2801083 .208333$ H $-0.125616-1.8533490 .608255$ H $2.295047-4.4904401 .854647$ H $1.933183-5.6659410 .615924$ H $\quad 0.768465-5.4038601 .910028$ H -2.674856 $1.520825-0.943284$ H -2.407356 $-0.215367-0.821118$ H $\quad 0.326502-0.007540-4.187075$ H -1.062096 -0.501105 -5.162912 H $-0.697435-2.906786-4.624208$ H $1.988790-1.733643-3.731173$ H $2.661509-3.910004-4.772441$ H $1.056419-4.570690-4.581434$ H $3.702958-5.051911-1.425341$ H $4.253884-4.341663-3.045810$ H $1.303026-5.830898-1.338448$ H $\quad 0.151388-5.163768-2.443813$ H $0.113412-2.981919-1.331705$ H -1.863809 -1.403607 -2.782363 C $0.094274 \quad 4.148887-0.569471$ O $1.0029413 .112090-0.999019$ C $2.0367093 .705793-1.806935$ C $1.4848355 .052075-2.254886$ C $0.7126355 .475453-1.024590$ H $2.8944203 .874234-1.144251$ C $2.4505562 .781207-2.957303$ H $2.2757895 .756002-2.530841$ H $\quad 0.8046564 .951923-3.108433$ C $-0.3079606 .568449-1.294081$ H $1.4273625 .835630-0.271876$ C $2.8280191 .347341-2.538608$ O $1.3751392 .680905-3.898605$ H $3.2805483 .238241-3.508562$ H $1.9385310 .806519-2.193220$ O $3.287514 \quad 0.665534-3.713861$ C $3.9312251 .261442-1.469134$ C $4.318901-0.196150-1.156142$ H $3.5774411 .744273-0.555564$ O $5.0716851 .983017-1.929738$ H $3.426160-0.717648-0.792453$ H $4.625529-0.695861-2.082856$ H $\quad 0.1856167 .472094-1.666388$ H -1.043515 $6.254644-2.042378$ H $-0.8490716 .831426-0.379765$ C $-0.078614 \quad 4.0991730 .950330$ H $0.5938422 .360225-3.416427$ H $2.5938810 .751520-4.390054$ H $5.6525262 .135151-1.165240$ H $-0.588147 \quad 5.0076201 .293307$ H 0.9081044 .0843811 .428556 H -0.861097 $3.967712-1.077692$ C $4.239826-0.1104031 .924550$ $\begin{array}{lllll}\text { O } & 5.256760 & 0.408247 & 1.058992\end{array}$ C $5.476218-0.337613-0.142140$ C $5.838438-1.7927260 .190173$ C $4.800875-2.4367971 .103385$ C $4.563357-1.5560452 .327340$ H $3.266539-0.0642691 .420136$ C 4.1874890 .7868113 .173249 H $6.3624010 .103996-0.614674$ O $5.969814-2.573424-0.992635$ H $6.810271-1.8171260 .700182$ H $3.862911-2.636440 \quad 0.573157$ O $5.288144-3.7039771 .549647$ H $5.455528-1.5772412 .966673$ O $3.483962-2.0875133 .089438$ C 3.6504732 .1737982 .892957 H 3.5355230 .3394213 .931762 H $5.1970990 .904743 \quad 3.584724$ H $6.643604-2.148129-1.550208$ H $5.463567-4.2401510 .757252$ H $3.710413-3.006740 \quad 3.312354$
O $3.6357422 .883055 \quad 4.061121$

O 3.2872682 .5882841 .802172

C 3.1448794 .2237763 .963302

H 3.2804884 .7068154 .934574

H 3.7073194 .7860183 .211613

H $2.0780874 .214146 \quad 3.721931$

SCF Energy $(B 3 L Y P / 6-31 G * * / / M M F F)=-3245.92960638$

0600064

MM̄FF Geometry

C $2.7511213 .827319-0.324033$

C 2.2485384 .7700950 .488093

C 1.0560534 .6301211 .408228

O $\quad 0.4980553 .2974151 .370502$

C -0.0479075 .6150890 .978983$

C -1.1983885 .6961931 .963506$

C -2.4598735 .2912831 .717621$

C -0.8646656 .3157223 .298156$

C -3.0301194 .6920790 .461879$

C -3.2883823 .1967390 .644270$

C $-3.9728442 .527998-0.557993$

C $-5.4221722 .995589-0.727550$

C $-6.1706102 .204775-1.810380$

O $-3.9302511 .118765-0.299931$

O $-7.5657122 .511063-1.663110$

C $-5.9842380 .697910-1.537910$

C $-5.7761422 .668956-3.216938$

O $-6.571348-0.082939-2.575861$

C $-4.5111420 .294860-1.318800$

C $-4.363567-1.174945-0.860013$

O $-3.806448 \quad 0.429497-2.557191$

C 1.0523942 .3529832 .179140

O 1.9845692 .4998622 .952837

C 0.3304441 .0839231 .933871

C $0.790923-0.0630152 .455517$

C $0.190126-1.3890282 .282634$

C -1.129861-1.461016 1.561604

C $-5.046960-1.4923790 .472602$

C $-4.594012-2.8182011 .059714$

C $-4.614336-4.062568 \quad 3.227117$

O $-3.953603-3.6534820 .427904$

C $-3.347517-3.6969413 .937042$

C $-2.305308-4.5301414 .076576$

C $-1.035056-4.1513844 .785529$

C $0.206385-4.5528114 .018284$

C $1.061026-5.4601194 .520995$

C $0.457463-3.9183282 .667892$

C $0.841479-2.4654012 .772186$

N $-5.008300-2.9535952 .377928$

H $2.2817232 .850786-0.383112$

H 2.7513925 .7349460 .529146

H 1.3878754 .8603462 .427641

H $-0.4058175 .330792-0.015615$

H $\quad 0.370898 \quad 6.624490 \quad 0.868556$

H -3.193846 5.4072852 .515208

H -1.7557716 .4870713 .911727$

H -0.1939225 .6675853 .869870$

H -0.3777067 .2861023 .155824$

H -3.9652685 .2204180 .246298$

H $-2.3861834 .852614-0.406833$

H -2.3338202 .6882580 .830138$

H $-3.888023 \quad 3.0179131 .547373$

H $-3.3881672 .737457-1.460434$

H $-5.4711984 .067783-0.949882$

H -5.9564562 .8652590 .224298$

H $-8.056613 \quad 1.959214-2.296212$

H $-6.5587160 .474437-0.630593$

H $-6.0221553 .729414-3.349819$

H $-6.3436892 .133681-3.986023$

H -4.711132 $2.548750-3.426148$

H $-5.973053-0.055359-3.341919$

H -4.725476 -1.856671 -1.640256

H -3.291992 -1.393860 -0.758591

H $-3.023590-0.143871-2.531869$

H $-0.555763 \quad 1.1255421 .311353$

H $1.701926-0.0354633 .051899$

H -1.884732 -0.8598342 .081187$

H -1.534134 -2.471101 1.489060 
H $-1.030301-1.0873900 .538107$ H $-4.826716-0.7069601 .204752$ H $-6.133019-1.5433430 .339264$ H $-4.502054-4.9658882 .618848$ H -5.418789 -4.2152573 .952787$ H -3.285529-2.697874 4.362872 H -2.374851 -5.540161 3.679216 H $-1.047064-4.6411805 .767823$ H -0.999912 -3.073274 4.984608 H $1.952806-5.7585393 .979794$ H $0.889861-5.9257555 .485936$ H $-0.405607-4.0883532 .019668$ H $1.285605-4.4345192 .163916$ H $1.794122-2.3058803 .279439$ H $-5.481988-2.1734932 .824377$ C $5.165563 \quad 3.167612-0.735856$ O $4.892547 \quad 1.768480-0.949223$ C 5.4812301 .0198540 .132207 C 6.3448392 .0032620 .920796 C $5.586587 \quad 3.299607 \quad 0.727544$ H 4.6522650 .6895030 .769215 C $6.254669-0.192548-0.394740$ H $6.443396 \quad 1.7144961 .971801$ H 7.3542122 .0889030 .502145 C $6.406724 \quad 4.5421151 .025000$ H 4.7155353 .2792971 .393582 C $5.437635-1.164434-1.267112$ O $7.3513450 .278746-1.190337$ H $6.700223-0.7288580 .452043$ H $5.219969-0.701590-2.237014$ O $6.288737-2.282236-1.561741$ C $4.120883-1.670689-0.645005$ C $3.535189-2.873225-1.412430$ H $3.397694-0.849157-0.629786$ O $4.338780-2.0544570 .713512$ H $3.519714-2.640649-2.481822$ H $4.203220-3.730918-1.273455$ H 6.7404304 .5422522 .067807 H $7.295644 \quad 4.5989410 .387946$ H 5.8147065 .4478410 .859702 C $3.9746094 .025154-1.174119$ H $7.837617-0.502313-1.505676$ H $6.519647-2.711832-0.720730$ H $4.952923-2.8078390 .718848$ H $3.7032853 .766661-2.205019$ H $4.2715955 .080769-1.176800$ H $6.0134293 .408908-1.390961$ C $0.813147-2.022184-2.527167$ O $1.162281-2.233271-1.152951$ C $2.125680-3.267292-0.919640$ C $1.600011-4.598478-1.473922$ C $1.231647-4.488644-2.949889$ C $0.282619-3.313503-3.167541$ H $1.687554-1.657280-3.078847$ C $-0.268817-0.932569-2.571918$ H $2.194058-3.3849430 .166973$ O $2.538437-5.649470-1.270429$ H $0.699001-4.879521-0.913440$ H $2.120849-4.397746-3.584657$ O $0.563905-5.683978-3.359309$ H $-0.702623-3.561035-2.751117$ O $0.112097-3.105997-4.568732$ C $0.2606420 .444109-2.235769$ H $-0.698752-0.874613-3.578404$ H $-1.061564-1.164147-1.853384$ H $3.239781-5.567210-1.938217$ H $1.159221-6.428716-3.167174$ H $-0.209177-3.941909-4.948444$ O $-0.7167901 .361769-2.500056$ O $1.373690 \quad 0.686242-1.792197$ C $-0.3759562 .728596-2.252896$ H $0.5878562 .981270-2.705108$ H $-1.147107 \quad 3.358300-2.704431$ H $-0.3605302 .911513-1.176435$ SCF Energy (B3LYP/6-31G**//MMFF) $=-3245.92426215$

0600065

MM̄FF Geometry
C $-1.573816 \quad 3.864410 \quad 1.305787$

C $-0.946128 \quad 2.691626 \quad 1.134311$

C $-0.530525 \quad 1.794238 \quad 2.269564$

$\begin{array}{lllll}\text { O } & 0.814054 & 1.330161 & 2.015078\end{array}$

C -1.4621520 .5732762 .308822$

C $-1.189898-0.3489933 .478608$

C $-0.549615-1.5306893 .383108$

C $-1.742013 \quad 0.1102094 .802823$

C $0.055987-2.138732 \quad 2.149196$

C $1.448615-2.7185882 .429798$

C $2.064174-3.3251351 .161050$

C $3.343630-4.0939631 .491852$

C $4.018095-4.642860 \quad 0.226078$

O $2.360729-2.2541830 .258183$

O $5.341479-5.070750 \quad 0.580953$

C $4.171109-3.494742-0.791546$

C $3.293294-5.878357-0.320934$

O $4.658777-3.980853-2.040172$

C $2.874785-2.678652-1.010870$

C $3.195860-1.418455-1.846891$

O $1.927557-3.477576-1.720965$

C 1.8347682 .1366652 .411338

O 1.7447293 .2097292 .986049

C 3.0990151 .4943881 .988292

C 4.2320392 .2120682 .005017

C 5.5515111 .7599351 .560198

C $5.712797 \quad 0.304601 \quad 1.212326$

C $2.060902-0.388806-1.916531$

C $2.5361050 .845584-2.657696$

C $4.0118282 .843261-2.375682$

O $2.269748 \quad 1.027996-3.843123$

C $5.4844772 .607903-2.237787$

C $6.3293613 .483915-1.672571$

C $7.8041303 .237075-1.518498$

C $8.3022453 .486315-0.109711$

C 9.0916584 .5382530 .166546

C 7.9472032 .4776030 .965283

C 6.5447262 .6696561 .476036

N $3.3150961 .675629-1.867179$

H -1.7881294 .2184592 .310918$

H $-0.759693 \quad 2.3298820 .125397$

H $-0.5678182 .323996 \quad 3.229561$

H -1.389332 0.0243341 .363813

H -2.5072920 .9048562 .368504$

H $-0.454195-2.1344504 .284499$

H $-1.567472-0.6187465 .601335$

H -1.2749181 .0510555 .109263$

H -2.8235330 .2653654 .730253$

H $-0.604342-2.9435461 .807760$

H $0.131502-1.4138891 .334343$

H $2.099199-1.9214482 .812517$

H $1.371557-3.4879993 .208063$

H $1.325817-3.9931890 .701632$

H $3.139021-4.9067362 .199627$

H $4.051174-3.4237982 .000281$

H $5.261727-5.7468151 .275595$

H $4.943162-2.816533-0.401127$

H $3.273152-6.6749280 .432458$

H $3.822733-6.296443-1.184206$

H $2.261097-5.676108-0.614413$

H $5.477894-4.473006-1.859647$

H $4.076387-0.924510-1.421156$

H $3.464256-1.709312-2.870976$

H $1.345034-2.894453-2.232180$

H 3.0554530 .4701281 .635780

H 4.1852283 .2473102 .341634

H $5.344750-0.3316942 .025276$

H 6.7510260 .0092201 .047113

H $\begin{array}{llll}5.156139 & 0.066259 & 0.302756\end{array}$

H $1.188593-0.788079-2.443397$

H $1.724575-0.091667-0.916736$

H $3.6748293 .703386-1.788701$

H $3.7558193 .008201-3.426543$

H $5.8745811 .668039-2.622215$

H $5.9448404 .435144-1.311463$

H $8.3252643 .896386-2.224667$

H $8.0684112 .213394-1.811890$

H 9.4721904 .7115351 .167742 
H $\quad 9.3713625 .252897-0.600303$ H 8.6282092 .5823281 .819806 H $8.124447 \quad 1.4753540 .566050$ H 6.3315143 .6943681 .785323 H $3.4543371 .435617-0.890427$ C $-3.5662764 .508728-0.042563$ O $-3.7990183 .160604-0.499944$ C $-4.7510013 .187304-1.577776$ C $-5.3581364 .584189-1.562417$ C $-4.1713205 .416252-1.112020$ H $-4.1814383 .064793-2.506465$ C $-5.7479972 .034311-1.420114$ H $-5.7448344 .880214-2.542268$ H $-6.1755944 .665012-0.836549$ C $-4.5520516 .797006-0.607635$ H $-3.4746285 .520752-1.954744$ C $-5.0892560 .641069-1.291277$ O $-6.5324552 .251055-0.240771$ H -6.456979 $2.039962-2.255763$ H $-4.5556600 .570244-0.337828$ O $-6.137458-0.333703-1.249832$ C $-4.147937 \quad 0.283737-2.452570$ C $-3.584873-1.151031-2.450537$ H -3.303834 $0.981116-2.472478$ O $-4.8496990 .463257-3.688666$ H -4.409996 -1.870334 -2.426412 H -3.108779 -1.300656 -3.428122 H $-5.0367437 .375894-1.400584$ H $-5.246040 \quad 6.7383240 .237586$ H -3.666656 $7.349981-0.278658$ C -2.0651894 .7122240 .165358$ H -5.9159802 .3565730 .504061$ H $-6.721017-0.100740-0.507314$ H $-5.617979-0.133373-3.677822$ H -1.8428615 .7610800 .390282$ H -1.521802 $4.453526-0.752059$ H $-4.106077 \quad 4.6205120 .907260$ C $-3.925616-2.7429170 .174055$ O $-3.153959-1.555018-0.038569$ C $-2.556468-1.423614-1.331543$ C $-1.644913-2.629092-1.602931$ C $-2.401496-3.944922-1.459488$ C $-3.103037-4.008293-0.105676$ H $-4.813931-2.721482-0.467376$ C $-4.387350-2.734806 \quad 1.636787$ H -1.904283 - $0.542136-1.280600$ O $-1.069017-2.533365-2.901212$ H $-0.815070-2.614690-0.885295$ H -3.115259-4.081268 -2.279823 O $-1.478293-5.032381-1.559637$ H $-2.356625-4.1538650 .684809$ O $-3.968707-5.142061-0.080021$ C $-5.444113-1.6851761 .878883$ H $-4.825537-3.6956161 .930881$ H $-3.542533-2.5531972 .312429$ H $-0.602083-3.367874-3.077647$ H $-0.813360-4.929265-0.857368$ H $-3.429067-5.924735-0.285352$ O $-4.892755-0.6027732 .495322$ O $-6.616843-1.8158701 .553240$ C $-5.783293 \quad 0.4712442 .814774$ H -6.549533 0.6075392 .046489 H -6.2494310 .2746563 .783986$ H $-5.194657 \quad 1.3900182 .884670$ SCF Energy (B3LYP/6-31G**//MMFF) $=-3245.93126692$

06_00066

MM̄FF Geometry

C $-2.6593601 .481123-2.380166$

C $-2.9432140 .455444-3.196558$

C $-2.952948-0.977516-2.741209$

O $-1.771884-1.594264-3.302500$

C $-4.197114-1.743781-3.226877$

C $-5.513462-1.224285-2.692733$

C $-5.939531-1.403209-1.427588$

C $-6.373810-0.493415-3.688957$

C $-5.212757-2.107697-0.316234$

C $-4.859753-1.1224730 .803950$
C $-4.053074-1.7912041 .924865$

C $-3.948702-0.8537363 .130015$

C $-3.085160-1.4510554 .247383$

O $-2.745751-2.0935461 .418196$

O $-2.817444-0.3932455 .180289$

C $-1.742825-1.9025313 .638165$

C $-3.839337-2.5313525 .030351$

O $-0.948346-2.5763914 .611916$

C -1.892171 -2.759627 2.358153

C $-0.521026-3.0215711 .688161$

O $-2.430846-4.0356102 .726998$

C -1.259785 -2.664261 -2.636710

O $-1.735608-3.223469-1.662025$

C $0.019850-3.019451-3.285673$

C $0.865959-3.848784-2.657299$

C $2.179782-4.271771-3.144804$

C $2.613354-3.769604-4.497797$

C $0.138431-1.7996131 .038764$

C $1.618798-2.0206690 .765614$

C $3.616424-0.803179-0.085751$

O $2.236019-3.0092801 .153435$

C $4.137963-1.704724-1.160054$

C $5.064972-2.648453-0.932459$

C $5.674089-3.523901-1.990937$

C $5.373473-4.991129-1.777781$

C $6.083654-5.724302-0.903767$

C $4.302385-5.622545-2.638146$

C $2.934844-5.065245-2.355981$

N $2.181292-0.9582710 .068947$

H -2.408980 $1.284180-1.340995$

H $-3.1693800 .652901-4.241584$

H -2.881854 -1.011506 -1.647344

H $-4.101613-2.807188-2.970654$

H -4.215194 -1.748252 -4.325919

H -6.912805 -1.001383 -1.148966

H $-7.315115-0.145610-3.250280$

H $-5.8487210 .383649-4.079512$

H $-6.623525-1.151477-4.527779$

H $-5.868012-2.8930800 .079449$

H -4.312291 -2.613797 -0.674158

H $-4.278425-0.2914500 .383685$

H $-5.786366-0.7015731 .214460$

H $-4.555588-2.7233672 .208842$

H $-4.944130-0.5964033 .513097$

H -3.4970320 .0959072 .811717$

H $-2.198213-0.7378515 .846726$

H -1.196701 -0.987135 3.391038

H -4.748532 -2.111742 5.477611

H -3.240831 -2.904694 5.868650

H $-4.135480-3.3819344 .412877$

H $-1.302746-3.4764834 .710896$

H $0.148342-3.4870642 .422701$

H $-0.643200-3.7798750 .902406$

H $-2.233714-4.6691472 .017363$

H $\quad 0.240650-2.568068-4.246059$

H $\quad 0.579397-4.246082-1.684247$

H $1.923250-4.116610-5.274908$

H $3.611140-4.100844-4.791180$

H $2.637135-2.674051-4.511807$

H $-0.365486-1.5599370 .096382$

H $0.066108-0.9214261 .686128$

H $4.090238-0.9961630 .883061$

H $3.8091720 .237683-0.359569$

H $3.767760-1.545421-2.169364$

H $5.441160-2.782100 \quad 0.079865$

H $6.761047-3.368748-1.974430$

H $5.355562-3.215553-2.994039$

H $5.892241-6.783076-0.764599$

H $6.871965-5.278784-0.305838$

H $4.256109-6.705061-2.459704$

H $4.591010-5.524620-3.688312$

H $2.536246-5.365273-1.385732$

H $1.597593-0.157883-0.156281$

C $-1.3282603 .358178-3.397067$

O $-0.288783 \quad 3.272649-2.403192$

C $0.6160334 .379199-2.584980$

C $0.1593645 .114614-3.842408$

C $-1.3245894 .813469-3.859637$ 
H $\quad 0.479540 \quad 5.040532-1.721843$

C $2.066313 \quad 3.886071-2.649896$

H $0.3800686 .185520-3.795900$

H $0.6392264 .717095-4.744275$

C -1.971886 $5.040039-5.214897$

H -1.819442 $5.447453-3.111528$

C $2.5420473 .082060-1.424743$

O $2.2257843 .042355-3.799277$

H $2.7341974 .740091-2.812543$

H $2.0554832 .099536-1.399177$

O $3.9437042 .831380-1.598009$

C $2.3379093 .785518-0.071003$

C 3.0456363 .0568281 .087662

H $1.265741 \quad 3.853488 \quad 0.136628$

O $2.8358515 .122188-0.142574$

H 2.7893111 .9944621 .047197

H 4.1305843 .1378690 .951163

H -1.860586 $6.084429-5.524200$

H $-1.516894 \quad 4.410627-5.987093$

H -3.041547 $4.811558-5.181519$

C -2.669451 2.918619-2.811331

H $1.5950832 .306821-3.712404$

H $4.0524142 .370388-2.447605$

H $3.7808875 .072695-0.367528$

H -3.471858 $3.076619-3.541587$

H -2.916416 $3.527551-1.933037$

H $-1.0375492 .692999-4.221122$

C 0.7948842 .2154012 .980884

O $1.275518 \quad 3.5438422 .735896$

C 2.6786873 .6448672 .467901

C 3.4830133 .0645983 .639207

C 3.0482641 .6427523 .978732

C $1.5332131 .585328 \quad 4.169547$

H $\quad 0.936668 \quad 1.5976702 .086601$

C $-0.711258 \quad 2.321028 \quad 3.263153$

H 2.9061654 .7176012 .428380

$\begin{array}{lllll}\text { O } & 4.875254 & 3.094336 & 3.343542\end{array}$

H 3.3302583 .6999024 .521499

H $3.3860840 .934413 \quad 3.213078$

$\begin{array}{llll}\text { O } & 3.688058 & 1.255330 & 5.196474\end{array}$

H 1.2697902 .1082135 .097474

O $1.137473 \quad 0.225646 \quad 4.332970$

C -1.5162732 .5991372 .010829$

H $-1.093164 \quad 1.3863643 .684067$

H $-0.896226 \quad 3.1388083 .969944$

H 5.3418812 .7010354 .100856

H $3.365137 \quad 0.3660135 .422697$

H $1.395125-0.259738 \quad 3.530857$

O -2.8042352 .8811442 .369161$

$\begin{array}{lllll}\text { O } & -1.084998 & 2.555066 & 0.867243\end{array}$

C $-3.7013843 .147642 \quad 1.286898$

H $-3.341113 \quad 3.991787 \quad 0.691166$

H -3.815694 2.255186 0.665275

H -4.6757773 .4072801 .709074$

SCF Energy (B3LYP/6-31G**/MMFF) $=-3245.93736749$

0600067

MM̄FF Geometry

C $4.779472-2.881923-1.430087$

C $3.499827-3.033378-1.060977$

C $2.351991-3.095377-2.033169$

O $1.367341-2.109077-1.648064$

C $1.687678-4.481059-1.972265$

C $0.538664-4.633027-2.948693$

C $-0.765673-4.596692-2.610731$

C $0.941703-4.879587-4.379818$

C $-1.364216-4.361534-1.249600$

C $-2.406944-3.241123-1.293976$

C $-2.995906-2.8885640 .081679$

C $-3.807497-4.0426440 .678194$

C $-4.527505-3.6416391 .973899$

O $-3.833969-1.743425-0.122779$

O $-5.475853-4.679702 \quad 2.264235$

C $-5.312651-2.3383401 .719790$

C $-3.559342-3.6003313 .162159$

O $-5.911733-1.8635112 .923108$

C $-4.462241-1.2331251 .060954$

C $-5.308275-0.0098970 .644146$
O $-3.490226-0.7710172 .004340$

C $1.534615-0.842553-2.116798$

O $2.470939-0.417401-2.774190$

C $0.350282-0.058082-1.702346$

C $0.2481991 .235070-2.043551$

C $-0.8618302 .130305-1.704061$

C $-2.0682451 .518315-1.042651$

C $-6.416202-0.315467-0.366271$

C $-6.8128280 .925447-1.141368$

C $-6.0647732 .381803-3.013826$

O $-7.864038 \quad 1.521276-0.920765$

C $-4.7362892 .833731-3.529788$

C $-4.3011134 .100981-3.464054$

C $-2.9638564 .544324-3.986832$

C -2.148729 $5.291637-2.952914$

C $-1.8177026 .580618-3.140037$

C $-1.7161514 .555322-1.702385$

C $-0.7441563 .443615-1.993196$

$\mathrm{N}-5.8603641 .288344-2.082642$

H $5.019220-2.813711-2.487916$

H $3.260362-3.118810-0.005055$

H $2.690881-2.896252-3.057761$

H $1.346717-4.686120-0.952429$

H $2.431627-5.260150-2.187347$

H $-1.499834-4.755923-3.400098$

H $0.077995-5.037743-5.034385$

H $1.500666-4.025920-4.774896$

H $1.573044-5.771517-4.448189$

H $-1.832488-5.297953-0.927851$

H $-0.606717-4.105721-0.504053$

H $-1.957909-2.336455-1.724474$

H $-3.221728-3.509157-1.980421$

H -2.174283 -2.6071770 .749911$

H $-3.176320-4.9198700 .861297$

H $-4.563470-4.366359-0.051158$

H $-5.988626-4.4008493 .042467$

H -6.143502 -2.599289 1.052378

H $-3.109126-4.587938 \quad 3.319985$

H $-4.083344-3.3603294 .093793$

H -2.745468 -2.884707 3.028981

H $-5.213913-1.4477793 .457687$

$\begin{array}{llll}H & -5.743587 & 0.470618 & 1.530267\end{array}$

H -4.6304860 .7433660 .223118$

H $-3.2098790 .122324 \quad 1.745943$

H $-0.409208-0.568244-1.121558$

H $1.0588021 .689426-2.611549$

H $-2.4664330 .697527-1.650081$

H $-2.8920192 .219240-0.899884$

H $-1.8087751 .128544-0.053145$

H $-6.107022-1.078900-1.089619$

H $-7.304573-0.7038510 .144349$

H $-6.619163 \quad 3.183709-2.515204$

H $-6.6786552 .001938-3.837025$

H $-4.1012672 .078917-3.988386$

H $-4.9414404 .863308-3.026453$

H $-3.1468685 .182479-4.861023$

H $-2.380795 \quad 3.691897-4.356421$

H $-1.2340187 .127535-2.406948$

H $-2.1222007 .120060-4.030828$

H -2.604540 $4.218093-1.163111$

H $-1.2133055 .246744-1.012774$

H $0.1778173 .787134-2.464621$

H $-5.1152240 .627121-2.285192$

C $6.634065-1.481471-0.374883$

$\begin{array}{lllll}\text { O } & 5.780428 & -0.503309 & 0.252245\end{array}$

C $6.0381170 .777849-0.355446$

C $7.2482790 .590536-1.270510$

C $7.095199-0.856565-1.690244$

H $5.1634191 .010850-0.973371$

C 6.2400951 .8621330 .708408

H $7.2383531 .287280-2.114407$

H $8.1929090 .732138-0.732460$

C $8.363275-1.469665-2.256295$

H $6.304922-0.901405-2.448890$

C 5.0700002 .0658931 .690421

O 7.4002641 .5450491 .490603

H 6.4687142 .8132390 .212543

H 5.0148511 .2342962 .403425 
O $5.373173 \quad 3.2344232 .466579$

C 3.6901622 .2596321 .035296

C 2.6352572 .8228052 .011050

H 3.3515411 .2999080 .629852

O $3.801373 \quad 3.163313-0.067379$

H 2.6972442 .2817262 .959623

H 2.8739583 .8731182 .214810

H $8.685123-0.928818-3.152354$

H $9.183839-1.437091-1.531672$

H $8.199216-2.515756-2.533653$

C $5.929487-2.837808-0.463532$

H 7.2548430 .6689201 .887332

H 6.2360933 .0848702 .889839

H 4.1521104 .0037750 .274311

H $5.578468-3.1159940 .538418$

H $6.652887-3.603359-0.768387$

H $7.494077-1.5985550 .298773$

C 0.4010250 .7997482 .630263

O $\quad 0.7423251 .3849551 .367741$

C 1.2099092 .7372751 .421896

C 0.1679043 .6324402 .104809

C -0.2294213 .0987273 .474888$

C $-0.6577881 .637023 \quad 3.360091$

H $1.297940 \quad 0.7163363 .255132$

C $-0.123251-0.6180472 .352679$

H 1.2760603 .0809980 .383083

O $\quad 0.6468324 .9667522 .235809$

H -0.7333343 .6781641 .479283$

H $\quad 0.5835523 .226593 \quad 4.199483$

O

H -1.617265 1.5767542 .830677

O -0.8511251 .1003054 .667444$

C $1.000599-1.6114082 .155038$

H $-0.718937-0.9681513 .203445$

H $-0.742818-0.6392501 .450793$

H $\quad 0.869814 \quad 5.2854321 .344365$

H -1.501111 3.6070274 .871404

H -1.6068121 .5628485 .066920$

O $0.549393-2.8581192 .481710$

O $2.120117-1.3313031 .750129$

C $1.503412-3.9221712 .410177$

H $1.562416-4.2868801 .382892$

H $2.489176-3.6053032 .764235$

H $1.151251-4.7353433 .050691$

SCF Energy (B3LYP/6-31G**//MMFF)= -3245.91701013

0600068

MM̄FF Geometry

C -0.5664912.0126112.037246

C -1.5031071 .7029222 .945463$

C $-1.840816 \quad 0.279781 \quad 3.293075$

O $-3.276968 \quad 0.159775 \quad 3.162061$

C $-1.436802-0.1248644 .717704$

C $0.045158-0.0491275 .015086$

C $0.968705-0.8981004 .522540$

C 0.4528791 .0473065 .963418

C $0.746667-2.0283143 .556037$

C $1.701025-1.9139602 .358075$

C $1.234561-2.7633451 .164914$

C $2.425887-3.1438770 .285440$

C $1.994069-3.904635-0.972380$

O $0.304620-1.9712450 .410579$

O $3.134877-3.955057-1.842087$

C $0.891433-3.093902-1.677337$

C $1.626144-5.358502-0.657494$

O $0.368167-3.815237-2.790979$

C $-0.248698-2.628746-0.737164$

C $-1.145030-1.627051-1.506564$

O $-1.033459-3.760471-0.358119$

C -3.770242 -1.037933 2.749907

O $-3.156015-2.0782962 .580287$

C $-5.201675-0.8324182 .451887$

C $-5.730826-1.4807801 .404461$

C $-7.080249-1.2918900 .873158$

C $-8.063677-0.5337921 .725859$

C $-2.168174-0.878196-0.646494$

C $-2.9774290 .110079-1.470953$

C $-4.5789972 .001907-1.224883$
O $-2.9625880 .125423-2.699267$

C $-6.0101121 .578392-1.117847$

C $-6.740391 \quad 1.143517-2.156634$

C $-8.1951160 .770294-2.072384$

C $-8.443012-0.697971-2.346687$

C $-8.573147-1.154617-3.603695$

C $-8.619300-1.618807-1.157278$

C $-7.352242-1.766912-0.360561$

N $-3.7288260 .962947-0.674707$

H $\quad 0.006655 \quad 1.209647 \quad 1.578448$

H -2.079282 2.4920403 .421100

H $-1.371082-0.3811402 .557615$

H -1.786144 -1.146751 4.918721

H -1.9956670 .4867075 .439973$

H $2.003811-0.7677954 .836524$

H $1.533047 \quad 1.0630286 .143565$

H 0.1722782 .0250775 .558950

H -0.0427380 .9151436 .930848$

H $0.943013-2.9737364 .076436$

H $-0.289203-2.0811583 .215464$

H $1.787591-0.8654162 .044313$

H $2.694400-2.2386682 .692170$

H $\quad 0.731075-3.6643761 .534101$

H $3.158191-3.7322050 .852211$

H $2.949070-2.229806-0.024266$

H $2.850233-4.362406-2.678379$

H $1.377482-2.203611-2.099571$

H $2.478699-5.876652-0.202060$

H $1.393311-5.915727-1.571622$

H $\quad 0.778605-5.4477130 .025282$

H $-0.236366-4.494096-2.445650$

H $-0.509351-0.871740-1.985381$

H -1.677347 -2.154381 -2.308752

H -1.915070 -3.455155 -0.091611

H -5.740306 -0.089848 3.028462

H $-5.096377-2.1633800 .840258$

H -8.135420 -0.9859902 .721533$

H -9.077192 -0.5236371 .320550$

H $-7.7537520 .510787 \quad 1.837583$

H -2.869834 -1.579537 -0.187278

H -1.654895 -0.3241890 .145135$

H -4.292348 $2.212078-2.260694$

H $-4.4145902 .911051-0.637877$

H $-6.4731011 .666336-0.138540$

H $-6.273518 \quad 1.071533-3.136621$

H $-8.7419561 .378232-2.805324$

H -8.620202 $1.043576-1.098492$

H $-8.777586-2.200155-3.808260$

H -8.474436 -0.490886 -4.456432

H -8.917971 -2.622077 -1.488049

H $-9.457930-1.255822-0.557255$

H -6.564554 -2.297261 -0.897941

H $\quad-3.699290 \quad 0.856870 \quad 0.335107$

C -0.4156663 .4885990 .045643$

O $0.6040242 .689835-0.588282$

C $1.0725333 .377083-1.762675$

C $0.1475684 .574092-1.954053$

C $-0.2379004 .893502-0.521821$

H $2.0783603 .746527-1.533327$

C $1.1294912 .409507-2.949217$

H $0.6466995 .405687-2.460816$

H -0.741971 $4.311212-2.538236$

C $-1.4737065 .768789-0.408677$

H $0.6079685 .393716-0.031356$

C $1.9852861 .148483-2.705888$

O $-0.1992161 .972446-3.263879$

H $1.4890922 .938378-3.839395$

H $1.5055550 .498962-1.964219$

O $2.0183130 .404798-3.930594$

C $3.4337011 .431452-2.266564$

C $4.3008110 .158128-2.217874$

H $3.4199641 .897579-1.276268$

O $4.0328652 .366546-3.163424$

H $3.791608-0.591845-1.604892$

H $4.391919-0.257201-3.228682$

H $-1.3089736 .731950-0.902409$

H $-2.3443775 .296031-0.875703$

H -1.7193565 .9650510 .639583$ 
C $-0.261973 \quad 3.4048341 .565140$ H $-0.5713621 .564864-2.463166$ H $1.0968120 .219456-4.181243$ H $4.0178721 .971679-4.052243$ H -0.9229874 .1290112 .053760$ H $\quad 0.7672703 .6430851 .854614$ H -1.384325 $3.072656-0.262856$ C $5.262043 \quad 0.2228480 .684147$ O $5.7042111 .058758-0.392130$ C $5.7239280 .426617-1.676454$ C $6.637583-0.807038-1.643427$ C $6.279669-1.758647-0.504636$ C $6.181104-0.9965490 .816885$ H $4.229395-0.0976070 .501774$ C 5.2763591 .0661821 .961977 H $6.189191 \quad 1.146340-2.361724$ O $6.583872-1.492895-2.889763$ H $7.674499-0.472486-1.507474$ H $5.355524-2.303475-0.726038$ O $7.310268-2.743446-0.401439$ H $7.186581-0.6802531 .121186$ O $5.698274-1.8734801 .831171$ C 4.1827472 .1065221 .919482 H 5.1041800 .4577152 .856907 H 6.2435051 .5660512 .094302 H $7.158114-2.274341-2.816660$ H $7.088002-3.3163540 .352563$ H $4.801864-2.1526351 .581506$ O $4.7087423 .325444 \quad 1.610291$ O $2.999947 \quad 1.8567732 .113182$ C 3.7675004 .3986721 .519243 H 4.3176575 .3158421 .292943 H 3.0565154 .2080600 .710732 H 3.2451184 .5286792 .471750 SCF Energy (B3LYP/6-31G**//MMFF) $=-3245.92999405$

0600069

MM̄FF Geometry

C $-0.302237 \quad 0.6613892 .780578$

C $-1.333560-0.0314773 .287269$

C -1.784694 -1.405558 2.848281

O $-0.925014-1.8942621 .794237$

C $-3.225963-1.3401512 .309416$

C -3.815406 -2.707674 2.024818

C $-3.925696-3.2598230 .800154$

C $-4.322153-3.4495113 .234750$

C -3.481417 -2.684031-0.515745

C $-4.641036-2.487007-1.502929$

C $-5.734346-1.528080-1.005744$

C $-6.840384-1.383381-2.052952$

C $-7.910406-0.373068-1.616440$

O $-5.136357-0.254987-0.748893$

O $-8.718829-0.096623-2.770742$

C $-7.2170300 .941093-1.194537$

C $-8.854721-0.967976-0.564933$

O $-8.1820861 .837582-0.647709$

C $-6.0373410 .724497-0.214589$

C -5.2256892 .0176180 .040465$

O $\quad-6.561078 \quad 0.3023291 .051156$

C $-0.456298-3.1665851 .865798$

O $-0.637171-3.971192 \quad 2.767697$

C $0.246102-3.5256600 .612970$

C $0.892709-2.614622-0.129681$

C $1.512027-2.840236-1.436907$

C $1.356519-4.200578-2.062620$

C $-4.5186332 .608114-1.184882$

C -3.011557 2.501157-1.063938

C $-1.1557830 .848675-1.171513$

O $-2.3218973 .463492-0.734581$

C $-0.6718210 .162669-2.407800$

C $0.3244610 .639221-3.169869$

C $0.913569-0.088215-4.346304$

C $2.373027-0.436745-4.124515$

C $3.350630 \quad 0.367102-4.575496$

C $2.701062-1.745375-3.428012$

C $2.144748-1.808025-2.032343$

$\mathrm{N}-2.5417571 .228961-1.343578$

H $\quad 0.2856020 .240788 \quad 1.970196$
H -1.896144 0.4036494 .111281

H -1.729157 -2.059083 3.726867

H -3.252815 -0.7154141 .409671$

H $-3.876812-0.8305143 .032706$

H -4.373658 -4.249906 0.723606

H -4.768321 -4.415253 2.974482

H -3.506671 -3.643604 3.937866

H $-5.090288-2.8599973 .746087$

H $-2.936276-1.745025-0.395638$

H $-2.765094-3.385248-0.962111$

H $-4.213245-2.095761-2.435177$

H $-5.088343-3.460902-1.738543$

H $-6.153067-1.925083-0.074519$

H -7.298383 -2.355523 -2.274369

H -6.401918 -1.036623 -2.999507

H $-9.3594200 .592383-2.523246$

H $-6.8517331 .407078-2.117547$

H -9.367814 -1.848699 -0.969672

H $-9.645826-0.259334-0.295605$

H $-8.342446-1.2740970 .349458$

H $-7.7747032 .718108-0.595560$

H -5.8876932 .7769020 .475559$

H -4.4823851 .8137850 .824309$

H $-5.8898490 .467780 \quad 1.733717$

H $0.189993-4.5722290 .334636$

H $0.943448-1.5888920 .232870$

H $1.754940-4.976703-1.399380$

H $1.895627-4.307684-3.006785$

H $0.300872-4.414906-2.261548$

H $-4.8246792 .141686-2.126186$

H $-4.7638773 .673617-1.271647$

H -1.112624 $0.155252-0.326705$

H $-0.5497881 .722328-0.917804$

H -1.115864 -0.800605 -2.646861

H $\quad 0.7692291 .599492-2.919435$

H $0.8093130 .555661-5.229197$

H $\quad 0.350967-1.001118-4.578070$

H $4.3976450 .119217-4.435722$

H $3.1245921 .296318-5.088241$

H $3.786755-1.883283-3.365510$

H $2.317465-2.552921-4.058882$

H $2.249166-0.878504-1.471156$

H $-3.2137210 .489779-1.529957$

C 0.2023843 .0505512 .184383

O 1.2450692 .7111301 .244996

C 2.0814923 .8624581 .040494

C 1.2716985 .0524341 .537902

C 0.5463784 .4425972 .718795

H 2.9588553 .7327291 .685596

C $2.5246883 .966361-0.421580$

H 1.9046945 .9015091 .812569

H $\quad 0.5484325 .3954190 .788967$

C -0.6635045 .2434693 .169082$

H 1.2513664 .3604143 .557210

C $3.2120112 .709247-0.988699$

O $1.3836584 .236674-1.246838$

H $3.1849684 .833567-0.539301$

H $2.4845851 .898360-1.111042$

O $3.6720013 .037642-2.307166$

C $4.4088232 .188886-0.169784$

C $5.2075031 .108621-0.925055$

H 4.0450041 .7848870 .779908

O $5.2862643 .271213 \quad 0.143025$

H $4.5099830 .358775-1.309521$

H $5.6959581 .571803-1.790610$

H -0.3637536 .2475423 .486563$

H -1.3965005 .3508732 .362321$

H -1.161438 4.756762 4.013540

C $0.1437512 .000213 \quad 3.295312$

H $0.7342503 .530724-1.089716$

H $2.8983453 .331090-2.818528$

H $5.5817113 .662849-0.696786$

H -0.5218822 .3413534 .096556$

H 1.1423661 .8687213 .729923

H $\quad-0.739423 \quad 3.0694571 .622004$

C $5.002387-1.4472780 .741007$

O $5.746472-0.264527 \quad 1.064512$

C $6.2957620 .440466-0.053854$ 
C $7.237108-0.486437-0.832729$

C $6.524997-1.760466-1.269887$

C $5.851796-2.434592-0.075770$

H $4.103937-1.1698770 .180663$

C $4.560029-2.0824562 .069455$

H $\quad 6.913167 \quad 1.2458020 .363126$

O $7.7692910 .191869-1.965566$

H $8.086569-0.755142-0.191170$

H $5.809127-1.553647-2.072793$

O $7.497139-2.651940-1.821739$

H $6.626913-2.8887020 .555388$

O $5.035878-3.490275-0.581875$

C $3.523369-1.2567962 .800001$

H $4.112598-3.0668271 .899257$

H $5.427794-2.1861922 .731841$

H $8.335062-0.439155-2.442706$

H $7.031915-3.470579-2.065748$

H $4.779619-4.0565260 .164656$

O $3.289837-1.8314084 .017624$

O $2.978488-0.2545302 .360877$

C $2.317106-1.1822164 .841788$

H $2.496004-0.1037224 .887829$

H $1.315289-1.3951294 .460393$

H $2.401709-1.5912005 .852173$

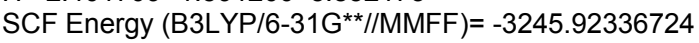

0600070

MM̄FF Geometry

C $0.795789-3.4133081 .016054$

C $0.765804-2.1729711 .525449$

C $0.390878-1.8397472 .943019$

O $-0.628139-0.8181342 .834762$

C $1.589634-1.2636863 .721363$

C $2.665633-2.2845394 .033017$

C $3.750330-2.5217513 .267411$

C $2.450064-3.0630965 .304481$

C $4.089545-1.8772651 .952999$

C $5.563322-1.4644971 .852268$

C $5.820176-0.7224330 .531936$

C $7.314096-0.5184790 .287270$

C $7.5683750 .295534-0.990596$

$\begin{array}{lllll}\text { O } & 5.166303 & 0.547633 & 0.621383\end{array}$

O $8.952310 \quad 0.675772 \quad-0.975488$

C $6.7176471 .582710-0.938066$

C $7.369202-0.554790-2.250747$

O $6.8224832 .310049-2.160139$

C $5.2339781 .340420-0.569220$

C $4.5462802 .694945-0.274113$

O $4.576686 \quad 0.703147-1.665137$

C -1.529103 -0.684695 3.837594

O $-1.568285-1.3326414 .874106$

C $-2.509607 \quad 0.388526 \quad 3.539158$

C -2.5465551 .0551632 .373877$

C -3.479409 2.124346 2.007379

C $-4.5218552 .522646 \quad 3.017473$

C 3.1236072 .5774260 .292073

C 2.5929313 .9203090 .768165

C 0.6900244 .9123182 .040130

O $3.170396 \quad 4.984047 \quad 0.559673$

C -0.3235375 .4241941 .066889$

C -1.6447795 .3982651 .295940$

C -2.674473 5.9181950 .335365

C $-3.3017714 .830369-0.508077$

C $-3.1398234 .812982-1.841970$

C -4.1722123 .7987140 .180292$

C -3.3604662 .6848710 .785350$

N $1.392396 \quad 3.7868641 .451667$

H $0.522005-4.2439071 .663608$

H $1.041769-1.3243650 .905440$

H $-0.025452-2.7190813 .450472$

H $1.234180-0.8288074 .665416$

H $2.003319-0.3980853 .188760$

H $4.446898-3.294543 \quad 3.589473$

H $3.244297-3.7953075 .484828$

H $2.423921-2.3855276 .164145$

H $1.501698-3.6082085 .261778$

H $3.880392-2.6043311 .158757$

H $3.459405-1.0057821 .760086$
H $5.818004-0.8156892 .700024$

H $6.196162-2.3585051 .911035$

H $5.376874-1.302412-0.286380$

H $7.838158-1.4812640 .239804$

H 7.7535360 .0206001 .138332

H $9.1064501 .257881-1.739511$

H $7.1658862 .224830-0.167556$

H $8.039762-1.422325-2.234441$

H $7.6318120 .006992-3.154072$

H $\quad 6.347974-0.925345-2.361412$

H $6.260505 \quad 1.865911-2.817626$

H 5.1524103 .2437670 .458994

H $4.5095783 .303143-1.186820$

H $3.6228490 .866247-1.599563$

H $-3.2119880 .596505 \quad 4.339823$

H -1.8234480 .8016881 .600204$

H -5.1295751 .6573913 .305202$

H -5.2205443 .2763572 .648991$

H -4.0484112 .9343653 .915381$

H $2.4361852 .197688-0.470422$

H $3.108517 \quad 1.8886841 .144495$

H 1.4024735 .7060052 .286800

H 0.2309134 .5581422 .968647

H 0.0487565 .8436590 .135168

H -2.013381 4.9867092 .232641

H $-2.2245856 .688868-0.304547$

H -3.4591416 .4342580 .902466$

H -3.603356 $4.048455-2.456850$

H -2.536022 $5.558230-2.348958$

H $-4.8588093 .347579-0.547874$

H -4.8043044 .3123610 .909628$

H -2.587022 2.2994630 .119209

H 0.9546022 .8720441 .512468

C $1.565030-2.667369-1.343766$

O $0.399385-1.864024-1.609040$

C $0.367757-1.525555-3.006281$

C $1.713249-1.957145-3.579073$

C $2.044675-3.154903-2.710338$

H $-0.421989-2.136286-3.459251$

C $0.042753-0.038260-3.181144$

H $1.650207-2.201966-4.643770$

H $2.476635-1.179820-3.458892$

C $3.513944-3.540254-2.746817$

H $1.437831-4.008293-3.040926$

C $-1.2367160 .433278-2.460947$

O $1.1283090 .744099-2.664159$

H $-0.0175540 .201861-4.248917$

H $-1.0802250 .439498-1.375084$

O $-1.4657421 .797197-2.839172$

C $-2.502762-0.381553-2.784639$

C $-3.7687460 .242721-2.169125$

H -2.379708 -1.400869 -2.408346

O $-2.669526-0.472887-4.199016$

H $-3.5782900 .438821-1.110965$

H $-3.9645681 .207397-2.652206$

H $3.815414-3.809891-3.764274$

H $4.153645-2.715630-2.414134$

H $3.709101-4.399748-2.098053$

C $1.196649-3.804655-0.384887$

H $1.2477510 .494581-1.731521$

H $-0.6683032 .301473-2.603063$

H -2.740363 $0.432989-4.545891$

H $2.043860-4.495175-0.299506$

H $0.359629-4.370480-0.811233$

H $2.320713-2.009494-0.895580$

C $-4.806024-1.916978-0.276841$

O $-4.857970-1.929535-1.709344$

C $-5.021743-0.646766-2.324876$

C $-6.3184680 .007019-1.827280$

C $-6.3626980 .087638-0.304654$

C $-6.073352-1.2801170 .310549$

H -3.917801-1.367176 0.056133

C $-4.675383-3.3740450 .193296$

H $-5.148387-0.834851-3.398390$

O $-6.461047 \quad 1.307025-2.390186$

H -7.172762 $-0.588300-2.175363$

H -5.6721880 .8504610 .067891$

$\begin{array}{lllll}\text { O } & -7.670802 & 0.509686 & 0.086949\end{array}$ 
H $-6.937101-1.9345530 .138792$

O $\quad-5.938331-1.1362821 .723544$

C $-3.330853-3.978449-0.148771$

H $-4.790346-3.443603 \quad 1.280322$

H -5.448580 -3.981715 -0.292598

H $-7.2774431 .692612-2.028566$

H $-7.6927550 .517796 \quad 1.059367$

H $-5.167544-0.5684591 .893341$

O $-3.401831-5.3326620 .018091$

O $-2.340786-3.348928-0.492961$

C $-2.198826-6.055728-0.259656$

H $-1.440690-5.8188840 .491927$

H -2.424541 -7.124113 -0.205699

H -1.834262 -5.826408 -1.265637

SCF Energy $\left(B 3 L Y P / 6-31 G^{* *} / / M M F F\right)=-3245.93547487$

06_00071

MM̄MF Geometry

C $\quad 0.770507 \quad 0.8189322 .956588$

C 1.7464361 .7265883 .107615

C 2.1020872 .7240732 .041197

O 3.4845612 .4686631 .697380

C 1.9724874 .1845882 .499130

C 0.5751134 .6214622 .878392

C -0.4175654 .8436361 .994963$

C 0.3472534 .8470984 .349343

C -0.3706324 .6427170 .505788$

C -1.5166853 .7288230 .050845$

C -1.282366 $3.169969-1.361107$

C $-2.6189662 .846282-2.028679$

C $-2.4302622 .192558-3.401959$

O $-0.501526 \quad 1.972662-1.224896$

O $-3.7106951 .688374-3.808797$

C -1.477079 $0.994975-3.240510$

C $-2.0120653 .214176-4.464813$

O $-1.1757460 .416141-4.508882$

C $-0.178626 \quad 1.322737-2.461354$

C $0.556315-0.004929-2.152603$

O $\quad 0.6592472 .144421-3.275508$

C 3.8701272 .7233910 .419042

O $3.2135443 .246030-0.466616$

C 5.2299152 .1691680 .261715

C $5.5668311 .632699-0.919766$

C $6.8088580 .917977-1.213079$

C $7.9304691 .034784-0.214438$

C $1.7405560 .114917-1.188117$

C $2.394402-1.233953-0.935654$

C $4.046544-2.3520400 .556955$

O $2.154164-2.229813-1.613893$

C $5.468804-2.2076100 .115446$

C $6.003992-2.883579-0.913083$

C $7.446102-2.789508-1.329961$

C $7.622020-2.190267-2.709148$

C $7.531672-2.953065-3.811526$

C $7.982425-0.722022-2.800680$

C $6.8632930 .166469-2.332763$

N $3.290271-1.1918970 .123421$

H 0.1792480 .8185462 .043742

H 2.3438211 .7293294 .015860

H $1.4814752 .538973 \quad 1.158121$

H 2.3487654 .8489781 .709382

H 2.6611524 .3654293 .336422

H -1.376136 5.1933742 .376274

H -0.6785755 .1617184 .568783$

H 0.5358373 .9261814 .910145

H 1.0199735 .6263234 .722254

H -0.4773575 .6195450 .018339$

H $0.589597 \quad 4.2414010 .175078$

H -1.630701 2.8947870 .755041

H $-2.445124 \quad 4.3134140 .082175$

H $-0.7258253 .902810-1.956808$

H -3.242047 $3.745073-2.116955$

H -3.178903 2.155147 -1.385446

H -3.584838 $1.200948-4.641212$

H -2.030643 $0.225319-2.684240$

H $-2.7662324 .005965-4.548798$

H -1.952970 $2.753165-5.456940$

H -1.053003 $3.689784-4.249266$
H $-0.5190200 .985445-4.944962$

H -0.154902 -0.711785-1.705489

H $0.909660-0.452150-3.090731$

H $1.5761542 .056906-2.970696$

H 5.8679252 .1314681 .136764

H $4.8396241 .659750-1.730330$

H $8.1666552 .088192-0.025326$

H $8.8600250 .566930-0.544027$

H $7.6545160 .562406 \quad 0.734452$

H $2.5053850 .778170-1.600427$

H $1.4021000 .520456-0.229972$

H $3.589963-3.2667120 .164891$

H $3.997924-2.3914891 .649563$

H $6.093988-1.5339520 .695501$

H $5.374931-3.566513-1.480012$

H $7.868500-3.802978-1.313211$

H $8.035227-2.220995-0.599650$

H $7.683047-2.537505-4.802184$

H $7.300616-4.011359-3.746062$

H $8.207604-0.445018-3.838922$

H $8.914829-0.557505-2.254196$

H $5.990043 \quad 0.145397-2.986590$

H $3.443451-0.3143790 .611628$

C $\quad 0.738467-1.6319953 .401568$

O $-0.207323-1.9459332 .357657$

C $-0.637377-3.3103182 .516967$

C $0.306331-3.9346993 .537670$

C $0.601492-2.7492324 .433983$

H -1.644361 -3.2754072 .948731$

C $-0.668517-4.0270641 .163670$

H $-0.153418-4.7733954 .069378$

H $1.229196-4.2966693 .069512$

C $1.830334-2.9410105 .306067$

H -0.271197-2.564762 5.074801

C -1.579032 -3.371893 0.104305

O $0.658622-4.0728630 .625066$

H $-0.966221-5.0718501 .310121$

H -1.156040 -2.416835 -0.227925

O $-1.586340-4.228877-1.044802$

C $-3.033408-3.1535010 .560463$

C $-3.977259-2.748539-0.590120$

H $-3.050856-2.3818521 .336185$

O $-3.531482-4.3542801 .154801$

H -3.529194 -1.919578-1.147282

H $-4.075659-3.593935-1.280972$

H $1.698253-3.7996395 .972489$

H $2.726904-3.1187584 .702535$

H $2.011818-2.0577635 .926091$

C $0.444165-0.2418903 .967557$

H $0.972785-3.1560660 .545401$

H $-0.662305-4.341063-1.327490$

H -3.485596 -5.0559090 .482673$

H $1.017454-0.0784994 .887024$

H $-0.618770-0.1538434 .220679$

H $1.736323-1.6563092 .943840$

C $-5.1063980 .033287 \quad 0.095437$

O $-5.342473-1.2089860 .772161$

C $-5.383886-2.365308-0.073912$

C $-6.451267-2.196726-1.164801$

C $-6.295592-0.886394-1.927678$

C $-6.2046150 .281405-0.946791$

H $-4.1251490 .011359-0.392749$

C $\quad-5.090940 \quad 1.145849 \quad 1.154189$

H -5.723752 -3.192135 0.562245

O $-6.416306-3.297606-2.066574$

H $-7.441115-2.208016-0.689283$

H -5.427781-0.921463 -2.596996

O $-7.443533-0.711238-2.760330$

H $-7.1744490 .408379-0.449650$

O $-5.950271 \quad 1.482789-1.667934$

C -3.8509861 .1056532 .023568$

H -5.1100882 .1306310 .674421$

H $-5.962067 \quad 1.0424441 .812032$

H -7.094644 -3.139401 -2.745382

H $-7.3510640 .150144-3.202394$

H $-5.1434021 .350031-2.193592$

O -3.9572602 .0750882 .981192$

$\begin{array}{llll}\text { O } & -2.908458 & 0.339282 & 1.881986\end{array}$ 
C $-2.855043 \quad 2.171858 \quad 3.888195$ H -3.032577 3.0232404 .550553 H -2.786758 1.2632024 .493059 H -1.924289 2.3439803 .341241

SCF Energy (B3LYP/6-31G**//MMFF) = -3245.94202113

06_00072

MM̄FF Geometry

C $0.829627 \quad 4.440667 \quad 1.471041$

C $-0.254681 \quad 3.752798 \quad 1.083143$

C -1.316765 3.2907182 .048815

O -1.6482841 .9153121 .754427$

C -2.5891584 .1329961 .862067$

C -3.660769 3.8186022 .887280

C -4.7680343 .0935822 .634164$

C -3.4498914 .4283554 .249072$

C $-5.133398 \quad 2.4153691 .342673$

C -5.7717231 .0406211 .589001$

C $-6.158452 \quad 0.3602490 .268602$

C $-7.005267-0.8865660 .525888$

C $-7.348926-1.615811-0.780611$

O $-4.953320-0.004652-0.413190$

O $-7.877104-2.899853-0.416414$

C $-6.047737-1.842296-1.577235$

C $-8.460013-0.896879-1.554177$

O $-6.329021-2.400929-2.858751$

C $-5.162553-0.578279-1.710147$

C $-3.788318-0.978737-2.292440$

O $-5.793630 \quad 0.345164-2.598424$

C $-0.904470 \quad 0.948676 \quad 2.354244$

O $0.059931 \quad 1.1072573 .085106$

C -1.481134 -0.3574611 .964546$

C $-0.833145-1.4862582 .287361$

C $-1.254513-2.849621 \quad 1.958047$

C $-2.623522-3.0360141 .359292$

C $-2.726074 \quad 0.128271-2.258284$

C $-1.387943-0.428902-2.705780$

C $0.417106-1.986122-1.953871$

O $-0.936234-0.207412-3.826435$

C $0.134786-3.420700-1.632952$

C $0.869769-4.144630-0.775740$

C $0.589164-5.580326-0.435092$

C $0.603235-5.8464401 .056084$

C $1.581722-6.5767441 .617347$

C $-0.551590-5.3233501 .887356$

C $-0.396363-3.8607252 .206761$

N $-0.796708-1.223767-1.737581$

H $0.940514 \quad 4.6566832 .532626$

$\begin{array}{llll}H & -0.418424 & 3.524878 & 0.034237\end{array}$

H $-0.967332 \quad 3.372964 \quad 3.085734$

H -2.979889 $3.996826 \quad 0.846637$

H -2.346532 5.2016941 .937698

H -5.495963 2.9777613 .436076

H -4.2820184 .2220104 .930577$

H -2.541101 4.0321884 .712370

H -3.354083 5.5160204 .167760

H -5.8495213 .0539900 .811972$

H -4.2618732 .2899880 .693733$

H $-5.062464 \quad 0.4083752 .138893$

H $-6.664203 \quad 1.1627952 .215420$

H $-6.7172971 .079755-0.341395$

H $-7.921033-0.6323351 .074147$

H -6.448925 -1.577013 1.175678

H -8.026257 -3.400758 -1.236894

H -5.476823 -2.608696 -1.034971

H $-9.362876-0.816562-0.936989$

H -8.756923 -1.465538 -2.442450

H $-8.1804560 .110853-1.868480$

H $-6.652635-1.683078-3.429470$

H -3.406012 -1.838593 -1.729783

H $-3.906344-1.311349-3.332066$

H $-5.1195030 .940140-2.963169$

H -2.412902 $-0.354036 \quad 1.410212$

H $0.112993-1.4070982 .821950$

H -2.665557 -2.611528 0.353074

H $-2.926248-4.0823061 .282638$

H -3.385323 -2.546168 1.976840

H -2.992322 $0.956597-2.922960$
H -2.610495 $0.545559-1.251552$

H $1.182659-1.562502-1.297961$

H $\quad 0.744743-1.891377-2.992927$

H $-0.712379-3.885220-2.132140$

H $1.726569-3.680195-0.295904$

H $1.350784-6.193616-0.933845$

H $-0.376100-5.904012-0.844164$

H $1.588685-6.7889632 .681367$

H $2.402059-6.9724551 .027697$

H $-0.606086-5.8659492 .840132$

H $-1.483874-5.5525651 .364696$

H $\quad 0.557395-3.6200192 .678427$

H $-1.219117-1.267676-0.815588$

C $1.851987 \quad 4.755867-0.896125$

O $2.2273493 .389883-1.180601$

C $3.1798833 .390462-2.260278$

C $3.0819494 .768062-2.901559$

C $2.8267055 .633419-1.686740$

H $4.1728843 .290251-1.806166$

C $2.926126 \quad 2.234740-3.230234$

H $3.9929415 .041777-3.442342$

H $2.2386534 .831777-3.599331$

C $2.2830787 .010479-2.028430$

H $3.7672995 .747283-1.130846$

C $3.0348770 .827977-2.609666$

$\begin{array}{lllll}\text { O } & 1.605655 & 2.375847 & -3.770020\end{array}$

H $3.6147322 .321114-4.079805$

H $2.1763830 .644199-1.953762$

O $2.913987-0.114291-3.684919$

C $4.3381190 .549439-1.833748$

C $4.533858-0.948695-1.528470$

H $4.3278071 .133564-0.907500$

O $5.4422200 .999206-2.621420$

H $3.584007-1.369773-1.187240$

H $4.805252-1.464498-2.457471$

H $2.9984697 .563324-2.646171$

H $1.3413096 .943840-2.583688$

H $2.0984047 .594723-1.121642$

C 1.9280295 .0089280 .612483

H $1.4564261 .627166-4.372645$

H $3.693174-0.007970-4.257548$

H $6.2250151 .027749-2.046426$

H 1.9376416 .0900510 .796418

H 2.8756634 .6081000 .993500

H $0.832744 \quad 4.900575-1.274218$

C $4.236406-1.2496631 .494843$

O $5.321059-0.629081 \quad 0.792470$

C $5.636953-1.211003-0.477593$

C $5.997589-2.692153-0.295999$

C $4.893052-3.4548580 .425493$

C $4.513065-2.7429001 .721463$

H $3.304930-1.1198200 .931101$

C $4.085037-0.5268242 .841991$

H $6.545736-0.707523-0.827587$

O $6.253300-3.315054-1.550175$

H $6.918338-2.772540 \quad 0.296390$

H $4.020772-3.595917-0.220387$

O $5.360737-4.764525 \quad 0.756420$

H $5.322308-2.8678402 .452681$

$\begin{array}{llll}\text { O } & 3.348063 & -3.356117 & 2.268857\end{array}$

C 3.5891970 .8958872 .694936

H $3.368549-1.0503453 .484401$

H $5.060307-0.4892533 .342836$

H $6.967144-2.816780-1.983655$

H $5.633330-5.191801-0.073806$

H $3.541651-4.3029732 .377223$

O $3.7575421 .539162 \quad 3.888973$

O $3.099174 \quad 1.3753001 .682254$

C 3.3245472 .9015263 .929776

H 2.2334502 .9436393 .878766

H 3.6476913 .3324764 .881178

H 3.7764943 .4761793 .116059

SCF Energy (B3LYP/6-31G**//MMFF)= -3245.92702163

06_00073

MM̄FF Geometry

C -2.972837 2.526289-1.141090

C $-2.4659192 .075715-2.298865$ 
C $-2.2759380 .612202-2.600488$ O $-0.8975820 .424712-2.999336$ C $-3.190654 \quad 0.157717-3.750751$ C $-3.308388-1.353711-3.832338$ C $-4.354386-2.060487-3.358484$ C $-2.169845-2.066712-4.515050$ C $-5.586425-1.538081-2.673148$ C $-5.398562-1.006456-1.245455$ C $-4.909534-2.038582-0.214654$ C $-4.809077-1.3793791 .166947$ C $-4.233744-2.3285582 .227990$ O $-3.627669-2.537847-0.608393$ O $-3.871563-1.544883 \quad 3.374998$ C $-2.933561-2.9531731 .679498$ C $-5.276388-3.3420292 .713010$ O $-2.427760-3.9436552 .570737$ C $-3.092782-3.5438290 .262384$ C $-1.749967-4.036143-0.324632$ O $-3.956474-4.685710 \quad 0.327244$ C $0.0204030 .338048-1.998144$ O $-0.192908 \quad 0.405355-0.797604$ C $1.3481280 .139738-2.616451$ C $2.376563-0.204500-1.828566$ C $3.761465-0.414823-2.243695$ C $4.091433-0.269383-3.704856$ C $-0.712508-2.946713-0.597319$ C $0.563745-3.557583-1.143853$ C $2.503201-4.967017-0.445277$ O $0.834398-3.526070-2.341958$ C $3.743136-4.185728-0.143507$ C $4.795336-4.139136-0.975495$ C $6.091425-3.441672-0.668070$ C $6.474551-2.432323-1.727641$ C $7.150997-2.808581-2.826117$ C $6.140893-0.979371-1.482451$ C $4.669576-0.738080-1.300529$ N $1.327071-4.167286-0.160981$ H -3.323682 $1.801217-0.410003$ H -2.116342 $2.777554-3.050707$ H -2.473734 $0.000380-1.711321$ H $-2.8124030 .531979-4.711313$ H -4.187101 $0.598495-3.627791$ H -4.334196 -3.143676 -3.471930 H -2.366275 -3.137053 -4.640299 H -2.002113 -1.647670 -5.512585 H - $-1.247519-1.971177-3.936210$ H -6.033989 -0.750852 -3.292314 H $-6.330245-2.345065-2.651832$ H $-4.708006-0.159046-1.268944$ H $-6.363759-0.606244-0.909046$ H -5.627806 -2.866565 -0.194596 H -5.785500 -0.994124 1.486359 H -4.150091 -0.5018741 .100604$ H -4.663574 -1.066183 3.674190 H -2.184535 -2.152889 1.647294 H $-6.124925-2.8250363 .177417$ H -4.862268 -3.994119 3.489942 H $-5.674393-3.967548 \quad 1.911410$ H -2.334043 -3.525925 3.444046 H -1.331061 -4.8183760 .321399$ H -1.950855 -4.540855 -1.280911 H -3.812524 -5.227377 -0.466284 H $\quad 1.4429790 .286555-3.685759$ H $2.187376-0.347512-0.765703$ H $3.901587 \quad 0.755647-4.041599$ H $3.484983-0.955543-4.306670$ H $5.135365-0.494946-3.936241$ H -1.093154 -2.235914 -1.336421 H $-0.467570-2.378507 \quad 0.304163$ H $2.472349-5.309206-1.484979$ H $2.455834-5.8431490 .209503$ H $3.798078-3.6778360 .815743$ H $4.743536-4.677330-1.919754$ H $6.875252-4.207267-0.593755$ H $6.063123-2.9570280 .315884$ H $7.452326-2.089037-3.580082$ H $7.415517-3.847121-2.996134$ H $6.541709-0.337250-2.275346$
H $6.656618-0.650452-0.570495$

H $4.337974-0.834322-0.266875$

H $1.026295-4.1031620 .807251$

C -1.843866 $4.823074-1.113975$

O $-0.6356894 .236199-0.590432$

C $0.2269725 .284005-0.112872$

C $-0.4081436 .596330-0.562530$

C $-1.8829746 .241417-0.548465$

H 0.1983615 .2395650 .982053

C $1.6601845 .057643-0.608523$

H $-0.158861 \quad 7.4263860 .105570$

H $-0.0946436 .875833-1.574969$

C $-2.7488327 .202390-1.343674$

H -2.2284096 .2231660 .493939$

C $2.2424503 .673125-0.252901$

O $1.6639435 .178021-2.036955$

H $2.3085955 .855473-0.226997$

H $1.7229722 .887956-0.814618$

O $3.6080483 .647093-0.687428$

C 2.2098943 .3297861 .249011

C 2.9533362 .0190541 .570475

H 1.1699613 .2444391 .576117

O $2.7995214 .393771 \quad 1.996409$

H $2.563322 \quad 1.2282190 .924798$

H 4.0163782 .1414121 .332057

H $-2.6788458 .214156-0.931075$

H -2.440694 $7.245598-2.393744$

H $-3.799456 \quad 6.897124-1.313126$

C $-3.0458133 .965929-0.706760$

H $2.5908965 .237549-2.323309$

H $3.6049413 .616505-1.658942$

H 3.7146844 .4996111 .684600

H -3.981476 $4.403568-1.071925$

H -3.1037353 .9587030 .389387$

H -1.740439 $4.856775-2.205669$

C 0.8054880 .2923752 .925261

O 1.4707191 .4419913 .462896

C $2.832561 \quad 1.6051593 .053771$

C 3.6524530 .3621593 .431448

C $3.020194-0.9243032 .909515$

C $1.547841-0.9938553 .311022$

H $\quad 0.747146 \quad 0.374081 \quad 1.833969$

C $-0.6227740 .282806 \quad 3.491930$

H 3.2299232 .4381463 .647323

O 4.9860320 .4605052 .941597

H $3.7177860 .294042 \quad 4.525484$

H $3.142371-1.0126221 .824438$

O $3.719797-2.0285963 .487929$

H $1.470084-1.1640284 .392660$

O $0.932914-2.1005402 .655481$

C -1.5078331 .3417032 .868864$

H -1.105248 -0.6817743 .306146$

H -0.5892050 .4680044 .572359$

H $\quad 5.372093 \quad 1.2745823 .307381$

H $3.376905-2.8408063 .079345$

H $1.332786-2.9126063 .009373$

O -2.6813241 .3984793 .566534$

O -1.2244662 .0165701 .889574$

C -3.643532 2.3409033 .084239

H -4.0057772 .0336372 .099187$

H -4.4869342 .3511143 .779850$

H -3.211328 3.3452853 .043144

SCF Energy (B3LYP/6-31G**//MMFF) $=-3245.89470875$

06_00074

MM̄FF Geometry

C $0.306764-1.7737224 .436189$

C $0.487148-0.7114183 .636900$

C -0.1555040 .6270573 .909061$

O

C $0.9051801 .741316 \quad 3.934860$

C 0.3581193 .0731094 .419022

C 0.1237604 .1479053 .639578

C 0.1062263 .1611095 .903838

C 0.3321904 .2762592 .158266

C -1.0000424 .4222561 .413382$

C $-0.8137274 .308826-0.106950$

C $-2.1440434 .515234-0.832492$ 
C $-2.0273934 .263782-2.343376$

O $-0.3069432 .998898-0.373974$

O $-3.3655144 .173565-2.856029$

C $-1.3372482 .901160-2.574387$

C $-1.3726985 .451069-3.058573$

O $-1.0882982 .687790-3.961565$

C $-0.0447862 .721182-1.753901$

C $0.5171121 .282600-1.825857$

O $\quad 0.9618543 .597602-2.273287$

C -2.3282790 .3382462 .953130$

O $-2.733511-0.363270 \quad 3.867310$

C -3.1151640 .7019691 .754198$

C -4.2807460 .0783921 .523986$

C $-5.193537 \quad 0.3189090 .404026$

C $-4.7913521 .347495-0.617922$

C $-0.3888190 .197024-1.236599$

C $-1.190868-0.577333-2.267142$

C $-2.894380-2.392909-2.416369$

O $-1.054890-0.434782-3.478629$

C $-4.216240-1.750702-2.695603$

C $-5.392758-2.298445-2.355100$

C $-6.724243-1.692737-2.694762$

C $-7.620112-1.524961-1.488733$

C $-8.587444-2.417100-1.216414$

C $-7.457464-0.273442-0.656942$

C $-6.343472-0.3854410 .347184$

N -2.059411 -1.477795 -1.663025

H $-0.309909-1.6627705 .325654$

H $1.087052-0.8105952 .736115$

H $-0.689717 \quad 0.611472 \quad 4.867185$

H $1.350921 \quad 1.8385662 .940529$

H 1.7319421 .4552254 .599178

H -0.2613565 .0504824 .112917$

H -0.1943854 .1671136 .216072$

H $-0.6904752 .474128 \quad 6.204107$

H $1.0150312 .905837 \quad 6.458757$

H 0.9465435 .1655061 .972952

H $0.8972263 .432057 \quad 1.758980$

H -1.6858613 .6357581 .752383$

H $-1.4587295 .386591 \quad 1.664527$

H $-0.0772655 .054798-0.428604$

H -2.541512 $5.519283-0.638191$

H -2.888184 $3.818666-0.423994$

H -3.304564 $3.938420-3.798012$

H -2.059388 2.132680 -2.277247

H $-1.9637416 .361687-2.903033$

H -1.345622 $5.296368-4.142757$

H $-0.3567325 .652261-2.712451$

H $-0.3087113 .214502-4.207291$

H $\quad 0.837031 \quad 1.046027-2.847405$

H $1.4356831 .272836-1.226424$

H $1.8328753 .261254-2.003865$

H -2.7177131 .4671331 .098876$

H $-4.608505-0.6836692 .230277$

H -5.450765 $1.381760-1.487672$

H $-4.7889712 .346879-0.170673$

H -3.791620 1.132161-1.007636

H $0.245791-0.536519-0.725445$

H -1.070409 $0.609188-0.487693$

H -2.993935 -3.306993 -1.822494

H $-2.398920-2.643111-3.360151$

H -4.205368 -0.806790 -3.235573

H -5.403259 -3.256874 -1.840907

H -7.208834 -2.349771 -3.429134

H -6.607620 -0.725414 -3.199276

H $-9.254941-2.286548-0.371057$

H -8.729256 -3.301077 -1.829534

H $-8.380089-0.079323-0.093026$

H -7.354784 $0.592079-1.317300$

H $-6.543574-1.1140411 .134193$

H -2.148494 -1.480002 -0.650941

C $0.310787-3.9127292 .993432$

O $1.017936-3.547898 \quad 1.796010$

C $0.140525-3.7509730 .669321$

C -1.191702 -4.256094 1.229118

C -1.165988 -3.7353642 .653924$

H $-0.013582-2.7644610 .220532$

C $0.791384-4.691065-0.355554$
H $-2.044490-3.8811670 .654373$

H -1.253869 -5.3500181 .224262$

C $-2.110518-4.4632703 .592445$

H -1.438897 -2.674546 2.619535

C $2.227730-4.306976-0.761748$

O $0.855188-6.0083790 .209734$

H $\quad 0.146425-4.766612-1.239088$

H $2.902101-4.4679550 .087864$

O $2.656601-5.230261-1.771684$

C $2.402057-2.868387-1.279414$

C $3.843470-2.596956-1.749710$

H $2.148250-2.169548-0.476483$

O $1.494403-2.624296-2.351157$

H $4.527982-2.859970-0.935974$

H $4.081618-3.253908-2.595222$

H $-3.146033-4.3594813 .252234$

H -1.879356 -5.5323973 .645127$

H -2.045299-4.053903 4.605517

C $0.867338-3.1461724 .198829$

H $1.260065-6.590401-0.456055$

H $2.046266-5.154592-2.524508$

H $1.772528-3.163546-3.110524$

H $1.959751-3.0743674 .125850$

H $0.656479-3.7330525 .102308$

H $0.531505-4.977693 \quad 3.151347$

C $4.448742-0.141806-0.031159$

O $3.649993-0.190015-1.215704$

C $4.077283-1.136979-2.198052$

C $5.522685-0.837632-2.620365$

C $6.464537-0.777626-1.417515$

C $5.9163090 .171085-0.350243$

H $4.389234-1.1153160 .471857$

C 3.8093330 .8643620 .938970

H $3.441757-0.972229-3.077275$

O $5.975747-1.818193-3.547350$

H $5.5459330 .129928-3.138490$

H $6.637011-1.776627-1.001812$

O $7.746994-0.311726-1.845132$

H $6.028571 \quad 1.202757-0.702446$

O $6.690548 \quad 0.044817 \quad 0.839993$

C 3.8407562 .2989570 .450811

H 2.7547950 .6099071 .097329

H 4.3263900 .8087951 .903966

H $6.899736-1.607687-3.765725$

H $7.6325310 .573973-2.229836$

H 7.6189610 .2101440 .601943

O $3.8609683 .140743 \quad 1.525535$

O $3.8229162 .641589-0.723806$

C 3.9021274 .5362921 .211217

H 4.8646354 .7867770 .755747

H 3.0782424 .8069260 .543459

H 3.7960825 .0979582 .143081

SCF Energy (B3LYP/6-31G**//MMFF) $=-3245.92337917$

06_00075

MM̄FF Geometry

C $-0.8075750 .554330-2.796334$

C $-1.350930-0.673654-2.782148$

C $-0.705317-1.911634-2.201861$

O $0.335240-1.516709-1.278406$

C $-0.078520-2.775497-3.305774$

C $0.106363-4.215717-2.868676$

C $1.237357-4.729842-2.347641$

C $-1.098825-5.099083-3.072513$

C $2.524205-4.014417-2.045653$

C $2.813561-4.043103-0.539463$

C $4.115703-3.315661-0.177286$

C $4.344919-3.3617061 .335758$

C $5.584945-2.5613231 .756503$

O $4.011034-1.957359-0.614280$

O $5.520341-2.4083203 .182363$

C $5.508412-1.1565401 .123289$

C $6.876354-3.3335531 .464799$

O $6.701047-0.4190811 .382065$

C $5.188730-1.172943-0.390526$

C $4.9115220 .266781-0.878689$

O $6.315895-1.695256-1.096330$

C $-0.003209-1.3867950 .032801$ 
O $-1.012164-1.7976330 .581334$

C $1.044691-0.5646590 .673491$

C $0.887890-0.151141 \quad 1.938621$

C $1.800404 \quad 0.7521242 .632364$

C 2.1189220 .3223024 .036503

C $4.3514950 .359096-2.304467$

C $4.1786211 .814782-2.687774$

C $2.7600353 .799273-2.181333$

O $5.0237962 .412551-3.350002$

C $3.2180034 .357037-0.870350$

C $2.386304 \quad 4.7782060 .094144$

C 2.8536175 .2609921 .437364

C 2.4596514 .3251892 .560558

C 1.6024494 .7113523 .520425

C 3.1177232 .9602942 .611162

C 2.2336091 .8835442 .039958

N $3.0213922 .371505-2.166621$

H $\quad 0.2057370 .700585-2.431126$

H -2.357891 $-0.807822-3.170049$

H -1.482211 -2.477047 -1.672981

H $\quad 0.877236-2.345587-3.627274$

H $-0.715309-2.774634-4.200873$

H $\quad 1.245772-5.788767-2.090633$

H $-0.924703-6.126737-2.736251$

H -1.957198 -4.711802 -2.514519

H - $-1.363767-5.139632-4.134101$

H $3.335273-4.517593-2.585370$

H $2.506752-2.981574-2.402532$

H $1.975147-3.573131-0.010900$

H $2.869712-5.082870-0.192710$

H $4.941832-3.802740-0.708659$

H $4.418431-4.3982691 .687867$

H $3.470267-2.9350771 .847492$

H $6.261138-1.8380563 .451358$

H $4.704103-0.6176911 .642357$

H $6.878258-4.2890382 .003329$

H $7.755630-2.7882591 .824869$

H $7.015389-3.5505400 .403644$

H $7.389038-0.7484480 .778951$

H $4.1897620 .742404-0.203061$

H $5.8341280 .859330-0.825320$

H $6.300133-1.367601-2.008966$

H $1.893562-0.2604940 .071863$

H $0.027433-0.4742522 .520821$

H 2.8552950 .9589284 .530741

H $2.530018-0.6940014 .038204$

H 1.2135850 .3279894 .653666

H $5.021219-0.103216-3.036511$

H $3.385019-0.151673-2.384933$

H $1.6873243 .937883-2.339278$

H $3.2999224 .277209-3.004492$

H $4.2915234 .381829-0.696429$

H $1.3129824 .749547-0.075988$

H 2.4330946 .2615291 .599761

H 3.9432805 .3900851 .453397

H $1.338274 \quad 4.0487174 .338218$

H 1.1424205 .6936893 .507122

H 3.4125402 .7488553 .643360

H 4.0561412 .9800872 .043176

H 1.9122612 .0746241 .016188

H $2.4233801 .796606-1.579996$

C -1.502560 2.850701-2.131289

O $-2.1133802 .333373-0.936309$

C $-2.8209533 .396805-0.271655$

C $-2.5290354 .669251-1.062579$

C $-2.2794134 .122249-2.456641$

H -3.888163 $3.162342-0.360886$

C -2.4235723 .4409841 .210565$

H -3.361038 $5.378954-1.022765$

H -1.635699 5.185744 -0.693251

C $-1.5379815 .087641-3.364129$

H -3.246101 $3.866354-2.910837$

C -2.4877902 .0758301 .924056$

O -1.0724583 .9070621 .324783$

H -3.0407344 .1765891 .738557$

H -1.7061701 .4160861 .529771$

O -2.1785962 .2812813 .307722$

C $-3.8460591 .361573 \quad 1.826767$
C $-3.834728 \quad 0.007173 \quad 2.554774$

H -4.0898561 .1954380 .776413$

O -4.8711842 .1896862 .369484$

H -2.976580 -0.570132 2.195459

H $-3.6853590 .172846 \quad 3.628651$

H $-2.1144936 .008698-3.498646$

H $-0.5619365 .359916-2.948598$

H $-1.3722484 .646255-4.351764$

C -1.536554 $1.793001-3.237011$

H -0.5056103 .2895380 .831334$

H $-1.308707 \quad 2.7149013 .347797$

H -4.6422802 .3702203 .297469$

H -1.060404 2.180685-4.144019

H $-2.5761131 .548775-3.484652$

H $-0.4634863 .091208-1.873745$

C $-4.629104-1.8037480 .223197$

O $\quad-5.501817-0.9666740 .990952$

C $-5.137505-0.8033392 .366378$

C $-5.114593-2.1723313 .059439$

C $-4.186069-3.1475302 .340602$

C $-4.519395-3.2024270 .850208$

H -3.640862 -1.335307 0.156118

C $-5.211405-1.909178-1.196508$

H $-5.942654-0.2177042 .827718$

O $-4.714973-2.0277824 .418131$

H $-6.130992-2.5871393 .065063$

H -3.134336 -2.889476 2.510018

O $-4.372585-4.4464532 .908099$

H $-5.465556-3.7435340 .722656$

O $-3.517464-3.962306 \quad 0.175847$

C $-5.139737-0.610387-1.970453$

H -4.661798 -2.657920 -1.777779

H $-6.267851-2.197851-1.136850$

H $-4.688429-2.9177164 .809373$

H -3.806195 -5.062452 2.412224

H -2.670229 -3.4957320 .279027$

O $-5.787299-0.772790-3.162996$

O $-4.5801950 .409606-1.596214$

C $-5.7969350 .373696-4.019457$

H $-4.7801630 .600305-4.352954$

H $-6.4093060 .139087-4.894126$

H -6.236562 $1.233629-3.504932$

SCF Energy $\left(B 3 L Y P / 6-31 G^{* *} / / M M F F\right)=-3245.93492722$

06_00076

MM̄FF Geometry

C $-0.2784680 .559207-2.966528$

C $-1.250147-0.333406-3.216454$

C -1.389342 -1.738120 -2.671592

O $-2.741562-1.843902-2.156118$

C $-0.414395-2.131386-1.546239$

C $-0.307669-3.624685-1.306386$

C $-0.798906-4.273441-0.232849$

C $0.479771-4.385356-2.344264$

C $-1.638354-3.7267780 .886567$

C $-0.855156-3.5351432 .195800$

C $-0.078667-2.2102892 .247377$

C $0.678546-2.0586013 .568295$

C $1.347065-0.6784503 .695463$

O $-1.040370-1.1593882 .130617$

O $1.739137-0.5284845 .068320$

C 0.3121670 .4224923 .371156

C $2.635111-0.6032532 .872043$

O $0.926594 \quad 1.7085313 .329042$

C $-0.487030 \quad 0.1578902 .076557$

C $-1.663273 \quad 1.1562321 .949788$

$\begin{array}{lllll}\text { O } & 0.377970 & 0.323868 & 0.951271\end{array}$

C $-3.463374-2.948106-2.475468$

O $-3.091905-3.889902-3.163545$

C $-4.824395-2.911704-1.891142$

C $-5.287823-1.930965-1.100475$

C $-6.630119-1.860465-0.511804$

C $-7.606842-2.950617-0.871712$

C -2.7416620 .6884710 .960911$

C $-3.731177 \quad 1.7601950 .546501$

C -5.2878363 .4921731 .417282$

O $-4.070281 \quad 1.882425-0.630228$

C -6.6075842 .8517981 .709350$ 
C -7.5362522 .5956840 .775897$ C -8.8574091 .9497061 .075826$ C -9.0003370 .5882420 .433852$ C $-9.8550530 .386728-0.583062$ C $-8.218514-0.5609701 .032049$ C $-6.929058-0.8262420 .303094$ N -4.2450912 .4991821 .597282$ H $\quad 0.5274610 .318015-2.281305$ H -2.017418 -0.055251 -3.938363 H -1.262095 -2.404046 -3.533753 H $-0.709938-1.619914-0.626382$ H $0.600122-1.785986-1.776219$ H $-0.613127-5.344501-0.156947$ H $0.632646-5.433382-2.065060$ H $-0.040092-4.374577-3.306906$ H $1.468982-3.936488-2.479715$ H -2.157663 -2.8087980 .594060$ H -2.434779 -4.4601241 .069639$ H -1.587150 -3.540507 3.014324 H $-0.176775-4.3802972 .363916$ H $0.617635-2.1712761 .402663$ H $1.419565-2.8588143 .686436$ H $-0.024614-2.1776284 .404872$ H 2.1005920 .3683025 .175396 H $-0.391246 \quad 0.4519974 .214389$ H $3.337390-1.3829783 .189386$ H $3.155783 \quad 0.3472323 .031356$ H $2.457763-0.7248701 .802752$ H 1.4089941 .7792252 .487586 H -2.141146 1.2686842 .930696 H -1.2893162 .1439911 .654793$ H -0.1496830 .5985020 .184568$ H -5.441334 -3.765312 -2.155153 H $-4.631480-1.099017-0.854298$ H -7.725319 -3.018106-1.959097 H $-8.608725-2.792522-0.467924$ H -7.258083 -3.918131 -0.494345 H -2.2715450 .3202870 .042222$ H $-3.316778-0.1424031 .386332$ H -5.2414613 .8987550 .401622$ H -5.1007624 .3055212 .124913$ H -6.8051662 .5857632 .745131$ H -7.340475 $2.862230-0.260201$ H -9.6471392 .6260860 .724000$ H -9.0155901 .8520442 .157467$ H $-9.983820-0.595750-1.024837$ H -10.442110 $1.199098-0.998305$ H -8.853084 -1.451463 1.084131 H -7.981107 -0.340082 2.080863 H $-6.155385-0.0843440 .498099$ H -4.0129312 .2384172 .550238$ C $0.1160483 .079912-2.739184$ O $1.3975392 .918228-2.103958$ C $1.3277873 .481756-0.779117$ C $-0.0318684 .167698-0.669851$ C $-0.8807603 .340494-1.615996$ H $1.3563482 .641269-0.077994$ C $2.5255754 .406023-0.532501$ H -0.4108524 .1705280 .356724$ H $0.0089985 .209179-1.009091$ C $-2.1430294 .051608-2.072123$ H -1.149884 2.405616-1.112095 C $3.9010343 .752834-0.772019$ O $2.4344445 .536419-1.411287$ H 2.4752714 .8120630 .484267 H $4.0366003 .534292-1.838242$ O $4.9031214 .720050-0.429192$ C $4.1610302 .476258 \quad 0.049548$ C $5.5874241 .932887-0.165069$ H $3.4386851 .705891-0.237999$ O 3.9571982 .7486341 .434682 H $5.7218521 .733086-1.233876$ H $\quad 6.3179832 .7024200 .111759$ H -2.772790 4.308850 -1.214454 H -1.910951 $4.980193-2.604618$ H $-2.7262523 .415499-2.745284$ C $-0.1810121 .893229-3.659223$ H $2.4161955 .198474-2.323211$
H $4.7299805 .516773-0.959481$

H 4.5795633 .4510981 .690323

H $0.638973 \quad 1.811536-4.384259$

H -1.095155 $2.099138-4.228658$

H $0.2061023 .975445-3.369131$

C $4.892580-0.997590-0.763029$

O $4.904454-0.344551 \quad 0.507971$

C 5.8988680 .6682930 .664413

C 7.2960760 .0819330 .413455

C $7.384070-0.618258-0.941032$

C $6.249644-1.631084-1.093577$

H $4.646776-0.257804-1.535702$

C $3.734330-2.008086-0.768637$

H 5.8617290 .9690961 .719129

O 8.2940831 .0950770 .480112

H $7.528171-0.6512161 .196902$

H $7.3825200 .101069-1.768207$

O $8.625063-1.322792-1.026605$

H $6.452131-2.490100-0.444401$

O $6.224178-2.114275-2.435084$

C $3.827314-3.0807590 .299873$

H $2.796548-1.469830-0.585945$

H $3.674144-2.495726-1.747884$

H 8.2291811 .5111391 .356718

H $9.335598-0.674090-0.884211$

H $7.104550-2.479729-2.628319$

O $2.857422-4.015097 \quad 0.073027$

O $4.622021-3.0929691 .229399$

C $2.800128-5.0851711 .021102$

H $2.008003-5.7721150 .712046$

H $3.748885-5.6300161 .037737$

H $2.559954-4.6957692 .014842$

SCF Energy (B3LYP/6-31G**//MMFF) $=-3245.92701524$

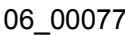

MMFF Geometry

C $0.235938 \quad 0.761045-2.415535$

C $1.2190330 .014886-2.945590$

C $1.717371-1.321099-2.444158$

O $1.105913-1.657201-1.179237$

C $3.241976-1.314798-2.240200$

C $3.838538-2.707873-2.319339$

C $4.224702-3.449757-1.263454$

C $4.039506-3.237221-3.717867$

C $4.126576-3.1138450 .197724$

C $5.508417-2.9325380 .846045$

C $6.235834-1.6610340 .381727$

C $7.621445-1.5551751 .019710$

C $8.318897-0.2333080 .659393$

O $5.439190-0.5437620 .778089$

O $9.444870-0.1099021 .541543$

C $7.3529370 .939440 \quad 0.941960$

C $8.881781-0.263934-0.766629$

O 7.8893852 .1744910 .473460

C 5.9393530 .7284510 .351215

C 4.9703591 .8093560 .887360

O $6.0055670 .821817-1.072692$

C $0.101341-2.569025-1.172326$

O $-0.331839-3.188291-2.134519$

C $-0.473983-2.7382180 .182486$

C $-0.041983-2.0842701 .272333$

C $-0.599146-2.1961172 .622949$

C $-1.779516-3.1097752 .823685$

C 3.4979091 .5410930 .544750

C 2.5611052 .4866541 .271445

C $0.1708052 .690801 \quad 1.944764$

O 2.9307603 .5414851 .779559

C 0.0864472 .1871253 .350259

C -1.0210731 .6427773 .876290$

C $-1.1012821 .112180 \quad 5.279051$

C $-1.521548-0.3412245 .326781$

C $-2.774803-0.6811665 .672598$

C $-0.474232-1.4005015 .056118$

C $-0.052408-1.4601633 .613200$

N 1.2556342 .0158621 .262070

H $-0.2672330 .437106-1.509530$

H $1.6653160 .347476-3.881611$

H $\quad 1.450937-2.061199-3.208080$ 
H $3.486069-0.846624-1.282270$ H $3.733889-0.699139-3.004928$ H $4.659129-4.429577-1.460657$ H $4.520302-4.221322-3.725824$ H $3.081534-3.337031-4.236675$ H $4.677061-2.556915-4.292281$ H $3.498649-2.2374320 .382134$ H $3.618229-3.9505130 .693564$ H $5.357121-2.8817271 .932282$ H $6.131304-3.8133910 .646699$ H $6.324328-1.677778-0.709964$ H $8.247852-2.4100580 .736130$ H $7.522607-1.6068242 .113339$ H 9.9348080 .6929181 .296136 H $7.261868 \quad 1.0321522 .033249$ H $9.621701-1.067243-0.866448$ H $9.4136120 .664183-1.003009$ H $8.114556-0.422602-1.527322$ H $8.7255412 .330643 \quad 0.943412$ H $5.055514 \quad 1.8533741 .981268$ H 5.2515092 .7956830 .497821 H $5.1370391 .084003-1.415371$ H $-1.301147-3.4388580 .230920$ H $0.792534-1.392176 \quad 1.176409$ H -2.628479 -2.772560 2.219706 H $-2.127589-3.1500663 .857941$ H $-1.526939-4.1369932 .538822$ H $3.3208391 .661879-0.529168$ H $3.2262740 .517076 \quad 0.824715$ H $-0.737828 \quad 2.472217 \quad 1.380287$ H 0.3481383 .7706301 .941157 H 0.9804792 .2716193 .963629 H -1.920989 1.5724893 .269590 H $-1.820738 \quad 1.7327335 .829017$ H -0.1465911 .2277565 .807099$ H -3.081913 -1.719380 5.740329 H -3.524146 0.0718555 .893213 H -0.812598 -2.386058 5.397115 H $0.411906-1.1899915 .668987$ H $0.798581-0.820558 \quad 3.377665$ H 1.0608151 .1030910 .860887 C - $0.6219173 .141894-2.133956$ O $-1.7101152 .768542-1.260946$ C $-2.7802023 .717186-1.413000$ C $-2.1207434 .975899-1.958476$ C $-1.0893244 .387539-2.897733$ H -3.459823 $3.303551-2.168704$ C -3.543271 $3.916291-0.097118$ H $-2.8334925 .636163-2.461343$ H -1.620307 $5.550596-1.170895$ C $0.0367305 .347179-3.244979$ H -1.597062 4.092494 -3.826211 C -3.976996 2.6158370 .607591 $\begin{array}{lllll}\text { O } & -2.731480 & 4.651938 & 0.826939\end{array}$ H -4.418357 $4.550767-0.279656$ H -3.100064 2.0656000 .967070 O -4.7214122 .9894621 .775758$ $\begin{array}{llll}\text { C }-4.852062 & 1.676752 & -0.244545\end{array}$ C -5.3838230 .4803420 .570825$ H $-4.269705 \quad 1.307825-1.093365$ O $-5.9546332 .409228-0.778402$ H $-4.531294-0.053017 \quad 1.005133$ H -5.9873100 .8591121 .404150$ H $-0.356734 \quad 6.236009-3.748937$ H $0.5721165 .678079-2.348692$ H $0.7610004 .871210-3.913587$ C $-0.3040791 .990699-3.093881$ H -1.8721174 .2041440 .885504$ H -4.148956 3.5654382 .311194 H -6.460062 $2.767229-0.028387$ H $0.4005162 .338067-3.859004$ H $-1.2268581 .693703-3.608020$ H $\quad 0.2411803 .364199-1.494897$ C $-4.557630-1.995752-1.060639$ O $-5.574530-1.036819-1.377537$ C $-6.266302-0.483034-0.254293$ C $-6.913389-1.6037630 .573449$ C $-5.896531-2.6651420 .979023$
C $-5.139130-3.161785-0.249505$

H $-3.752359-1.507770-0.497770$

C $-3.981145-2.510667-2.389249$

H $-7.0873460 .115095-0.668727$

O $-7.539385-1.0853841 .742230$

H $-7.698335-2.087442-0.022620$

H $-5.206502-2.2932701 .745185$

O $-6.582164-3.7766851 .560026$

H $-5.816264-3.755983-0.876867$

O $-4.081476-4.022422 \quad 0.165749$

C $-3.192950-1.455394-3.132961$

H $-3.304850-3.354326-2.214475$

H $-4.801668-2.831848-3.042529$

H $-8.192775-0.4250031 .454591$

H $-7.094619-3.4374282 .313975$

H $-4.477154-4.732368 \quad 0.699945$

O $-2.866314-1.939878-4.367795$

O $-2.890200-0.357149-2.689723$

C $-2.113838-1.055690-5.204137$

H $-1.092880-0.967575-4.824253$

H -2.078312 -1.486605 -6.208197

H -2.592173 - $0.073261-5.263468$

SCF Energy (B3LYP/6-31G**//MMFF)= -3245.92910859

06_00078

MM̄FF Geometry

C $1.151918-4.3341041 .581526$

C $0.288160-3.308503 \quad 1.634065$

C $-0.419520-2.8933442 .898138$

O $-1.795850-2.6238392 .530320$

C $0.178809-1.6170973 .521185$

C $1.600210-1.7749934 .021096$

C $2.672289-1.1259603 .525960$

C $1.774472-2.723300 \quad 5.181234$

C $2.709938-0.1066862 .422571$

C 3.5535331 .1109962 .828645

C 3.4400822 .2517041 .807643

C 4.4588193 .3517472 .111562

C $4.2972634 .558866 \quad 1.176567$

$\begin{array}{lllll}\text { O } & 2.112187 & 2.779190 & 1.898304\end{array}$

O $5.0758495 .627482 \quad 1.735605$

C $2.8170464 .994173 \quad 1.179074$

C $4.8841454 .280835-0.211313$

$\begin{array}{lllll}\text { O } & 2.591420 & 6.030560 & 0.225936\end{array}$

C $1.8233693 .827896 \quad 0.967410$

C $0.379897 \quad 4.3208601 .228429$

O $1.9210543 .373995-0.383955$

C $-2.744933-2.748693 \quad 3.492847$

O $-2.564799-3.1022514 .650532$

C $-4.089615-2.3722112 .995251$

C $-4.350965-2.0176261 .727286$

C $-5.652631-1.6005271 .194572$

C $-6.816343-1.5518682 .149109$

C $-0.657387 \quad 3.1962351 .357709$

C -2.063381 3.7432251 .540657

C $-4.3996772 .986401 \quad 1.970761$

O -2.3259824 .9429381 .519429$

C -5.1520412 .8438750 .685130$

C $-6.171163 \quad 1.986624 \quad 0.525136$

C $-6.9714781 .844349-0.736709$

C $-6.6358660 .596830-1.523873$

C $-6.1612410 .683227-2.778306$

C $-6.915371-0.752299-0.892151$

C $-5.732456-1.262868-0.109561$

N -2.990936 2.7294391 .735970

H $1.382549-4.8817772 .492306$

H $\quad 0.066556-2.7501230 .727795$

H $-0.406302-3.7218043 .617239$

H $-0.444627-1.3017124 .368607$

H $0.075775-0.7963542 .804002$

H $3.645737-1.3489963 .962216$

H 2.792471-2.703509 5.585198

H $1.096060-2.4523625 .996939$

H $1.562455-3.7528184 .878680$

H $3.158945-0.5743561 .540502$

H 1.7097740 .2224312 .133816

H 3.2185511 .4701583 .810792

H 4.6018210 .8026202 .928964 
H 3.6032591 .8449910 .803394 H 5.4825452 .9604692 .059271 H 4.3237063 .6953423 .147114 H 4.9191376 .4206651 .194642 H 2.6214345 .4429492 .162567 H $5.9461554 .019612-0.131126$ H $4.8441485 .173171-0.845550$ H $4.3791453 .466210-0.732506$ H $2.5608205 .620349-0.655173$ H $\quad 0.3554364 .8930342 .165361$ H 0.0743445 .0026290 .424343 H $1.0626463 .019392-0.662374$ H $-4.868148-2.393607 \quad 3.751528$ H -3.537149 -2.0145131 .004124$ H -7.005286 -2.543789 2.574751 H $-7.750261-1.2302011 .684204$ H $-6.614481-0.8510512 .966829$ H -0.6540842 .5582260 .470004$ H -0.4238542 .5705552 .227237$ H -4.531265 3.9985932 .366111 H -4.735942 2.2717462 .728904 H $-4.8575903 .485755-0.141484$ H $-6.4727361 .365867 \quad 1.364689$ H $-6.8403222 .738397-1.360296$ H -8.036766 $1.824944-0.474729$ H $-5.937633-0.205783-3.358911$ H $-5.9770171 .642678-3.250028$ H -7.133246 -1.489285-1.676782 H $-7.831477-0.687093-0.299758$ H $-4.831857-1.353394-0.715795$ H $-2.687377 \quad 1.7611081 .688647$ C $3.205096-4.2164280 .121353$ O $3.064090-2.885706-0.426562$ C $3.873405-2.786189-1.613452$ C $4.892714-3.910182-1.502514$ C $4.043501-5.004307-0.889784$ H $3.215625-2.991142-2.466594$ C $4.479842-1.386698-1.749600$ H $5.317675-4.186451-2.472009$ H $5.716411-3.647242-0.828688$ C $4.855142-6.128702-0.269346$ H $3.394458-5.423467-1.670790$ C $3.451686-0.239170-1.761450$ O $5.375684-1.152296-0.655191$ H $5.096128-1.340461-2.654977$ H $2.978946-0.143506-0.777953$ O $4.1678030 .981680-1.986754$ C $2.359552-0.363139-2.839239$ C $1.414040 \quad 0.851241-2.850004$ H $1.774598-1.266173-2.654394$ O $2.962910-0.507115-4.123370$ H $1.0115910 .980538-1.839885$ H $1.9875421 .754499-3.089866$ H $5.471211-6.622206-1.028107$ H $5.522603-5.756190 \quad 0.514997$ H $4.199238-6.882790 \quad 0.176827$ C $1.817902-4.8257390 .328824$ H $4.874654-1.2836010 .167785$ H $4.8549611 .044885-1.301215$ H $3.5108430 .280972-4.280338$ H $1.887224-5.9173140 .406093$ H $1.182366-4.609777-0.536196$ H $3.739562-4.1146191 .074269$ C -1.288189-0.521133 -2.501938 O $-0.506482-0.468825-3.700733$ C $0.262810 \quad 0.725856-3.874278$ C $-0.6737401 .941336-3.907357$ C -1.544645 $2.009165-2.657215$ C $-2.2491640 .675134-2.421118$ H -0.623396 -0.538712 -1.629751 C $-2.089382-1.833184-2.524745$ H $\quad 0.7269150 .649558-4.865446$ O $0.082595 \quad 3.140007-4.040707$ H -1.322506 $1.869418-4.790021$ H $-0.9537842 .312692-1.788219$ O $-2.5282043 .029582-2.843844$ H -3.044491 $0.563840-3.168432$ O $-2.8834560 .705403-1.143866$
C $-1.226866-3.067338-2.382325$

H $-2.801920-1.847814-1.694739$

H -2.631272 -1.920821 -3.473949

H $-0.5459653 .882318-4.035059$

H -3.100233 $3.027321-2.057128$

H $-2.1897900 .781403-0.466942$

O $-2.037463-4.167488-2.368237$

O $-0.008023-3.073964-2.294793$

C $-1.375573-5.429792-2.238536$

H $-1.026219-5.557404-1.210445$

H $-2.100210-6.217161-2.463202$

H $-0.543029-5.511833-2.944121$

SCF Energy (B3LYP/6-31G**//MMFF $)=-3245.92935350$

0600079

MM̄FF Geometry

C $4.547012-2.176221-2.452139$

C $3.271807-2.338236-2.072654$

C $2.103681-2.032142-2.971887$

O $1.274051-1.041054-2.324416$

C $1.238958-3.288715-3.172535$

C $0.165184-3.086758-4.224486$

C $-1.098434-2.691918-3.970563$

C $0.598754-3.374288-5.638732$

C $-1.701671-2.330327-2.641138$

C $-2.928347-3.194237-2.325983$

C $-3.524316-2.862727-0.949231$

C $-4.710075-3.780065-0.642172$

C $-5.384047-3.4208690 .689816$

O $-3.944599-1.493385-0.955000$

O $-6.634968-4.1243540 .729050$

C $-5.688413-1.9069690 .694722$

C $-4.570091-3.9257811 .886996$

O $-6.210535-1.4985081 .956704$

C $-4.478435-1.0341820 .294892$

C -4.8506030 .4576790 .138649$

O $-3.494243-1.1074391 .332911$

C $1.6636090 .259678-2.352274$

O $2.6731810 .722062-2.855918$

C $0.6157601 .027752-1.643837$

C $0.7816122 .333015-1.386948$

C $-0.1867483 .196194-0.704475$

C $-1.5016562 .577629-0.307441$

C $-5.8671930 .764382-0.960801$

C $-6.0843832 .259237-1.104810$

C $-5.0741434 .316348-2.053281$

O $-7.1127002 .802446-0.707096$

C $-3.6908354 .828703-2.297358$

C $-3.1856515 .924860-1.710450$

C $-1.8041776 .449345-1.986775$

C $-1.0030626 .711371-0.728135$

C $-0.5759087 .951400-0.434533$

C -0.6889885 .5496930 .191744$

C $0.1371484 .486997-0.480713$

N -5.015322 $2.907779-1.705266$

H $4.759044-1.837834-3.463562$

H $3.052498-2.670647-1.061554$

H $2.435466-1.643470-3.943154$

H $0.780982-3.589315-2.222110$

H $1.868191-4.136736-3.474511$

H $-1.778425-2.581358-4.814601$

H $-0.206532-3.211691-6.362983$

H $1.433223-2.725463-5.922660$

H $0.920902-4.416589-5.731660$

H $-0.979959-2.422530-1.825893$

H $-1.992612-1.273804-2.681685$

H -3.686553 -3.027920 -3.102556

H -2.650327 -4.255119 -2.358877

H $-2.740063-2.994495-0.195769$

H $-4.399744-4.832463-0.643362$

H -5.456777 -3.688486 -1.443876

H -7.104811 -3.844977 1.533747

H -6.496796 -1.753795 -0.030100

H -4.463136 -5.016363 1.840496

H $-5.083340-3.7177162 .832197$

H $-3.566891-3.4974231 .937635$

H $-5.467108-1.4439672 .581127$

H $\quad-5.2060590 .8589611 .096891$ 
H $-3.931247 \quad 1.015780-0.076981$ H $-2.901748-0.3416131 .251292$ H $-0.2760350 .486618-1.346070$ H $1.7063282 .814325-1.701502$ H -2.008881 2.166388 -1.187181 H -2.2010123 .2772230 .152486$ H $-1.347051 \quad 1.7702770 .416109$ H $-5.5402810 .364453-1.927333$ H $-6.8385810 .311935-0.736071$ H -5.590235 4.862390-1.256306 H $-5.6713234 .401868-2.967112$ H -3.078757 4.281028-3.010605 H -3.803769 $6.492348-1.018524$ H -1.915784 7.376671-2.563642 H -1.243335 $5.762650-2.632605$ H -0.0000568 .1547650 .462153$ H $-0.7917998 .793560-1.083674$ H -1.624862 5.1667350 .605314 H -0.1167355 .8980341 .061806$ H $1.1185944 .837738-0.803240$ H $-4.3063232 .336194-2.156349$ C $6.369102-1.135111-1.015850$ O $5.522236-0.546900-0.008217$ C $5.6189990 .887387-0.107914$ C $6.7432231 .178034-1.101413$ C $6.660049-0.022447-2.022152$ H $4.669871 \quad 1.234145-0.533363$ C 5.8321731 .5163451 .273439 H $6.5974512 .130022-1.621202$ H $7.7239151 .212094-0.613254$ C $7.911584-0.252118-2.849758$ H $5.8098520 .136629-2.695912$ C 4.7962601 .1271292 .344993 O 7.1191641 .1278291 .775656 H 5.8700172 .6073851 .173069 H 4.9410710 .0844652 .653326 O 5.0657891 .9268403 .505738 C 3.3243311 .3337201 .943602 C $2.366997 \quad 1.1442373 .137362$ H 3.0687150 .6261301 .147988 O $3.1459812 .646477 \quad 1.412522$ H 2.6083490 .1993523 .635325 H 2.5357921 .9471513 .865089 H $8.1082330 .608905-3.496885$ H $8.791211-0.402764-2.214915$ H $7.799691-1.135468-3.486407$ C $5.731958-2.417230-1.561847$ H $7.1400710 .155871 \quad 1.807375$ H 5.9924471 .7700053 .756444 H 3.4120323 .2811232 .099829 H $5.435574-3.055037-0.719442$ H $\quad 6.476345-2.979386-2.138198$ H $7.294372-1.417146-0.495451$ C $0.614933-1.1763882 .221318$ O $0.5681890 .176206 \quad 1.750370$ C 0.8793351 .1727112 .725742 C $-0.103337 \quad 1.069176 \quad 3.899111$ C $-0.125435-0.3347504 .497011$ C $-0.342192-1.3832363 .404175$ H $1.640393-1.4218912 .520645$ C $0.248173-2.0499091 .017760$ H $\quad 0.7021242 .1419792 .243762$ O 0.2208312 .0091894 .918178 H -1.114874 1.3118993 .547400 H $0.789812-0.5334595 .067231$ O $-1.205714-0.4013655 .431601$ H -1.382146 -1.336042 3.060139 O $-0.133138-2.6803593 .957441$ C $0.508307-3.5212161 .208083$ H $-0.805034-1.9081860 .751696$ H $0.810520-1.7292490 .134850$ H $\quad 0.2092572 .8936004 .514142$ H $-1.146458-1.2571235 .888501$ H $-0.834066-2.8388004 .611800$ O $1.847151-3.7490751 .323614$ O $-0.374602-4.3691261 .228861$ C $2.226607-5.118868 \quad 1.484887$ H $1.913010-5.7035040 .614754$
H $\quad 3.316167-5.1625781 .562576$

H $1.792176-5.5264712 .402712$

SCF Energy (B3LYP/6-31G**//MMFF) $=-3245.92443074$

06_00080

MM̄FF Geometry

C 0.0316430 .0885021 .429158

C $0.169746-1.1908731 .048603$

C $-0.138128-1.745833-0.322106$

O $-0.340321-0.669698-1.265473$

C $-1.426715-2.579952-0.279005$

C $-1.608258-3.433959-1.517125$

C $-2.423789-3.132597-2.546220$

C $-0.830830-4.725284-1.523378$

C $-3.264626-1.900093-2.734212$

C $-4.767434-2.198157-2.634854$

C $-5.240699-2.454560-1.195292$

C $-6.714024-2.866314-1.176181$

C $-7.242817-3.0275220 .256459$

O $-5.066318-1.239370-0.459797$

O $-8.672782-3.1149010 .167368$

C $-6.891062-1.7581741 .060889$

C $-6.770219-4.3426520 .886431$

O $-7.252579-1.9113632 .431874$

C $-5.411898-1.3249420 .927964$

C $-5.2217840 .078075 \quad 1.552589$

O $-4.590074-2.2647031 .622068$

C $0.703222-0.290289-2.044330$

O $1.799925-0.827299-2.113872$

C $0.3695700 .910247-2.846463$

C $-0.6889421 .697736-2.596409$

C -1.043955 $2.925065-3.314265$

C $-0.1862683 .323459-4.486825$

C -3.8446520 .7041381 .291656$

C $-3.7606602 .127651 \quad 1.817921$

C -2.3315974 .1670621 .657913$

O -4.6077012 .6227962 .556390$

C -2.8802394 .9950230 .538813$

C $-2.1222455 .731852-0.286948$

C $-2.6799906 .545610-1.419389$

C $-2.0979026 .158378-2.761967$

C $-1.1796116 .935222-3.361178$

C $-2.6442574 .927068-3.452591$

C $-2.1016053 .644356-2.882724$

N -2.6130342 .7716961 .376354$

H -0.3642180 .8173780 .729066$

H $\quad 0.551127-1.9113301 .768577$

H $\quad 0.708329-2.369937-0.632104$

H $-2.288344-1.917068-0.144542$

H $-1.425927-3.2437280 .596017$

H -2.488495 -3.841539 -3.371109

H -1.022199 -5.322473 -2.421268

H $\quad 0.244535-4.528192-1.483599$

H $-1.104630-5.336662-0.657321$

H -2.986072 -1.096026 -2.046983

H $-3.053541-1.514144-3.739694$

H $-5.303467-1.327648-3.035190$

H -5.018740 -3.055175 -3.272202

H $-4.623958-3.241684-0.748360$

H $-6.868832-3.789429-1.748860$

H $-7.313714-2.096717-1.682690$

H $-9.023521-3.1398151 .074397$

H -7.525955 -0.950451 0.671483

H $-7.109091-5.1956070 .285929$

H -7.207872 -4.486621 1.880340

H $-5.683928-4.4098580 .975412$

H -6.577950 -2.469584 2.854753

H $-5.9780530 .758256 \quad 1.138519$

H $-5.386766 \quad 0.0285322 .636385$

H -3.758109-1.834473 1.873944

H $1.0816841 .141048-3.632136$

H -1.354949 $1.439936-1.774546$

H $0.8477693 .493772-4.166949$

H $-0.5164174 .242538-4.975455$

H $-0.1940302 .539688-5.252463$

H $-3.058406 \quad 0.1231131 .783750$

H $-3.636510 \quad 0.725003 \quad 0.215702$

H -1.2464384 .2687391 .755808$ 
H -2.7983314 .4581542 .604030$ H $-3.959120 \quad 4.9818770 .400421$ H -1.044559 $5.755922-0.143177$ H -2.467970 $7.601240-1.205246$ H $-3.773556 \quad 6.468840-1.467196$ H $-0.7790956 .687371-4.338392$ H $-0.8045947 .835923-2.886495$ H -2.468488 $4.977580-4.533225$ H $-3.7379574 .916344-3.358405$ H -2.661929 $3.277731-2.022398$ H -2.0265092 .3094810 .688473$ C 1.3050031 .8526692 .653813 O 2.4794851 .5394651 .885852 C 3.6099362 .2294592 .448622 C 3.0560123 .1449743 .536590 C 1.8236132 .3825443 .987484 H 4.2386851 .4629372 .917058 C $4.3984262 .933043 \quad 1.335441$ H 3.7772953 .3107884 .342795 H 2.7689654 .1258563 .141302 C 0.8212693 .2359104 .744596 H 2.1435841 .5480744 .625874 C 4.7086562 .0427000 .114389 O 3.6418664 .0519630 .852002 H 5.3238823 .3523541 .745913 H $3.783057 \quad 1.830517-0.435442$ O $5.5422302 .791703-0.778877$ C $5.4176450 .718340 \quad 0.444777$ C $5.681770-0.120728-0.816949$ H $4.796137 \quad 0.137708 \quad 1.128061$ O $6.647447 \quad 0.9809111 .115690$ H $4.733276-0.247627-1.350218$ H $\quad 6.356157 \quad 0.429475-1.484204$ H 1.2737513 .6454725 .653621 H $0.468640 \quad 4.0757504 .136316$ H $-0.0513812 .644415 \quad 5.038784$ C $\quad 0.4209730 .6107072 .783459$ H $2.785336 \quad 3.7139670 .538227$ H $5.0767823 .621279-0.981640$ H 7.1949721 .5235320 .522458 H -0.4890650 .8580043 .341342$ H $0.953610-0.1663143 .343987$ H 0.7698612 .6406252 .107196 C $4.299942-2.7204200 .018191$ O $5.584398-2.2558820 .448642$ C $6.316258-1.498641-0.520533$ C $6.546706-2.352193-1.774005$ C $5.232240-2.882178-2.342356$ C $4.422218-3.580236-1.250160$ H $3.641196-1.862961-0.163828$ C $3.701637-3.5575841 .162213$ H $7.299883-1.303142-0.075189$ O $7.237245-1.595284-2.762666$ H $7.189682-3.202915-1.513200$ H $4.656621-2.081805-2.821419$ O $5.545362-3.823423-3.372084$ H $4.897786-4.539374-1.006696$ O $3.113498-3.865116-1.740201$ C $3.357659-2.7398412 .388574$ H $2.777566-4.0453950 .832530$ H $4.425452-4.3213471 .471612$ H $7.339841-2.164107-3.544884$ H $4.710825-4.078991-3.799888$ H $3.202795-4.500241-2.470295$ O $2.934031-3.5854523 .375298$ O $3.432825-1.5231922 .476002$ C $2.560586-2.9580014 .606107$ H $2.323925-3.7428095 .329250$ H $3.388048-2.3587094 .998087$ H $1.671439-2.3390434 .456147$ SCF Energy $\left(B 3 L Y P / 6-31 G^{* *} / / M M F F\right)=-3245.92792486$

06_00081

MM̄FF Geometry

C $-2.085770-3.340557-1.667275$ C $-1.243948-2.578968-0.951912$ C $-0.047158-1.873515-1.531013$ O $1.099205-2.366070-0.791486$
C $-0.121768-0.345190-1.355310$

C $-0.9182160 .351357-2.439056$

C $-0.3846820 .884688-3.556318$

C $-2.3950860 .476582-2.183145$

C $1.0599470 .892412-3.971043$

C $1.6314882 .308073-4.142680$

C $1.5071523 .193117-2.892729$

C $2.2026614 .540071-3.105637$

C $2.1520875 .416251-1.843966$

O $2.0990802 .506874-1.785018$

O $3.0868526 .488236-2.041849$

C $2.6344874 .580230-0.637061$

C $0.7781516 .073961-1.670428$

O 2.4810645 .3077730 .579042

C $1.9574703 .195377-0.535685$

C 2.5676242 .3120370 .574676

O $0.5755843 .380618-0.201659$

C $2.292901-2.415600-1.441492$

O $2.519330-2.073031-2.590838$

C $3.292813-2.978026-0.505369$

C $4.571065-3.086870-0.897943$

C $5.679936-3.641022-0.116270$

C $5.359259-4.2015601 .243690$

C 4.0433211 .9496120 .394462

C 4.5464761 .0696611 .527660

C 6.6399610 .1526012 .484384

$\begin{array}{llll}\text { O } & 3.818131 & 0.635829 & 2.416632\end{array}$

C $7.891355-0.4455021 .928849$

C $8.146616-1.7616801 .957116$

C $9.403746-2.3940611 .437482$

C $9.236522-3.0288370 .075299$

C $10.022056-2.664349-0.952785$

C $8.212633-4.133359-0.084996$

C $6.915713-3.626069-0.659281$

N $5.9099620 .822241 \quad 1.424323$

H -1.922977 $-3.459350-2.735532$

H -1.405392 -2.4701300 .117508$

H $0.068766-2.136857-2.590436$

H $0.8924390 .066593-1.313836$

H $-0.537103-0.091280-0.370227$

H $-1.054702 \quad 1.371523-4.264031$

H $-2.8968181 .099556-2.931376$

H $-2.875948-0.504155-2.199392$

H -2.570256 $0.937972-1.206565$

H $1.6903310 .334693-3.274536$

H $1.1395200 .367223-4.930749$

H $2.6909452 .201268-4.409305$

H $1.1384542 .803434-4.988614$

H $\quad 0.4422073 .354647-2.690204$

H $1.7690655 .072739-3.961270$

H $3.2569224 .366911-3.364073$

H $3.1185547 .006867-1.219440$

H $3.7137664 .442997-0.772775$

H $0.5461606 .703819-2.537729$

H $\quad 0.7654176 .744858-0.804360$

H $-0.0333985 .351973-1.559226$

H 1.5406615 .2783960 .824688

H 2.4161932 .7906251 .551214

H $1.997068 \quad 1.3737340 .622588$

H $\quad 0.2257372 .537550 \quad 0.131573$

H $2.949141-3.2946830 .472728$

H $4.837226-2.738606-1.895490$

H $4.955865-3.4191441 .896283$

H $6.224912-4.6243971 .757170$

H $4.620344-5.0067531 .163394$

H $4.1914691 .410170-0.547786$

H 4.6596232 .8541210 .380822

H $5.992726-0.5955552 .954991$

H 6.8877920 .9050343 .240326

H 8.6241920 .2330371 .499523

H $7.415757-2.4311842 .402922$

H $9.727344-3.1624612 .150864$

H $10.211199-1.6501901 .420787$

H $9.925941-3.131019-1.927682$

H $10.775541-1.891443-0.844768$

H $8.088203-4.6592230 .865190$

H $8.603153-4.892694-0.776086$

H $7.028224-3.206276-1.660524$ 
H 6.4438811 .3588890 .745934

C $-4.591280-3.593386-1.618816$

O $-4.857358-2.260903-1.135761$

C -6.223889-2.189001-0.686122

C $-6.905821-3.444507-1.214435$

C $-5.771130-4.446976-1.151421$

H $-6.197375-2.2390970 .408912$

C $-6.870599-0.871137-1.129200$

H $-7.770456-3.733487-0.609315$

H -7.243869 -3.321048 -2.249769

C $-6.009940-5.687736-1.994537$

H $-5.626388-4.747039-0.104552$

C $-6.0822620 .393836-0.737592$

O $-6.995952-0.865289-2.558000$

H -7.892764 $-0.812420-0.738114$

H $-5.1547360 .447718-1.317467$

O $-6.8601811 .535005-1.120523$

C -5.7563350 .5109900 .761007$

C $-4.978876 \quad 1.797505 \quad 1.087348$

H -5.155338 -0.3471611 .065048$

O $-6.961317 \quad 0.472215 \quad 1.522015$

H -4.0833001 .8289260 .458600$

H -5.5897602 .6688060 .821593$

H $-6.904189-6.218736-1.652246$

H $-6.153349-5.433958-3.050233$

H $-5.161876-6.376079-1.926113$

C $-3.249308-4.085126-1.073464$

H -6.105542 -0.985473 -2.930913

H $-7.0544271 .447595-2.069614$

H -7.5165411 .2155241 .230179$

H $-3.116984-5.148400-1.304496$

H $-3.238263-3.9880550 .018072$

H -4.565487 -3.536231 -2.714924

C -2.593565 0.5516502 .568372

$\begin{array}{lllll}\text { O } & -3.915530 & 0.762041 & 3.078190\end{array}$

C $-4.587891 \quad 1.9223102 .577292$

C $-3.768043 \quad 3.1815572 .895306$

C -2.3379983 .0670272 .373715$

C -1.7013791 .7664932 .856963$

H -2.640080 0.3606041 .489036

C $-2.019919-0.6962303 .261927$

H -5.5256951 .9959213 .142246$

$\begin{array}{lllll}\text { O } & -4.375510 & 4.342938 & 2.339851\end{array}$

H -3.730109 3.3265303 .982830

H -2.297124 3.142628 1.280889

O -1.5634614 .1572972 .878566$

H -1.5048191 .8395693 .934547$

O $-0.445147 \begin{array}{lll}1.589935 & 2.207422\end{array}$

C -2.708197-1.980290 2.852979

H -0.957982 -0.8118623 .018049$

H -2.134518 -0.5951284 .348028$

H -5.2779874 .3996942 .697720$

H -2.002585 4.9780052 .596158

H 0.1016362 .3647322 .423905

O $-2.191464-3.0092133 .589303$

O $-3.573866-2.0801171 .996135$

C -2.736967 -4.302901 3.312095

H $-3.827903-4.2893193 .396749$

H -2.429724 -4.6299522 .314689$

H -2.338502 -5.004482 4.049723

SCF Energy (B3LYP/6-31G**//MMFF) $=-3245.93986401$

06_00082

MM̄FF Geometry

C $0.028837 \quad 3.0601550 .376352$

C $-0.6755812 .702247 \quad 1.462329$

C $-0.298713 \quad 1.657770 \quad 2.492403$

$\begin{array}{lllll}\text { O } & 0.956159 & 1.017875 & 2.173743\end{array}$

C -1.394906 0.5754342 .518192

C $-1.224218-0.4536083 .618694$

C $-0.870081-1.7402193 .426699$

C -1.5274410 .0405865 .010234$

C $-0.544511-2.420767 \quad 2.127444$

C $0.935481-2.8155502 .070475$

C $1.370213-3.2283140 .656286$

C $2.833302-3.6744310 .661509$

C $3.367591-3.942019-0.751212$

O $1.204692-2.088063-0.191513$
O $4.796969-4.035635-0.645981$

C $3.036875-2.732897-1.653845$

C $2.886813-5.296625-1.284688$

O $3.405337-3.027509-2.999018$

C $1.559759-2.295363-1.565202$

C $1.258803-0.969778-2.307934$

O $0.742070-3.308316-2.161805$

C 2.0918211 .6039202 .643615

O 2.1713022 .6178953 .318630

C 3.2412560 .8040292 .163759

C 4.4868401 .2787652 .312859

C $5.724152 \quad 0.613219 \quad 1.898435$

C $5.606649-0.7341341 .236874$

C $2.0058660 .262190-1.785426$

C $3.309000 \quad 0.559213-2.506584$

C $5.2843082 .076885-2.386715$

$\begin{array}{llll}\text { O } & 3.642026 & 0.007045 & -3.551544\end{array}$

C $6.4354801 .320694-1.802258$

C $7.4757261 .913867-1.196676$

C $8.6718801 .173141-0.671087$

C $8.990305 \quad 1.5139420 .767178$

C 9.9289162 .4268851 .069848

C $8.290256 \quad 0.7275301 .851111$

C $6.899924 \quad 1.2340562 .129241$

N $4.0370911 .551955-1.863198$

H $0.994807 \quad 2.5952180 .197224$

H -1.6326353 .1830301 .650299$

H $-0.2361502 .143833 \quad 3.473514$

H $-1.4428100 .096266 \quad 1.535656$

H $-2.375578 \quad 1.0486602 .654671$

H $-0.797113-2.3883774 .299258$

H -1.478973 -0.7601465 .755997$

H - 0.8120610 .8119295 .310760

H -2.5363640 .4641945 .050914$

H -1.164691 -3.321088 2.039301

H $-0.801027-1.7955621 .270812$

H $1.543907-1.9623252 .395886$

H $1.122364-3.6356842 .774806$

H $\quad 0.718017-4.037147 \quad 0.306568$

H $2.968180-4.5575751 .298638$

H $3.447152-2.8863281 .116753$

H $5.151773-4.137909-1.545977$

H $3.682403-1.911205-1.322198$

H $3.249704-6.108521-0.642734$

H $3.294540-5.499122-2.281228$

H $1.798771-5.376376-1.334551$

H $3.337422-2.206096-3.513673$

H $1.391230-1.097659-3.388667$

H $0.186960-0.762476-2.173928$

H $-0.097725-2.906104-2.438552$

H $3.030702-0.1488611 .693202$

H 4.6184392 .2500412 .788585

H $4.926695-0.6859790 .379921$

H $5.226950-1.4742591 .948807$

H $6.552855-1.1145320 .846699$

H $1.3686441 .140970-1.947291$

H $2.1899340 .186482-0.709581$

H $5.3184453 .141960-2.136516$

H $5.292778 \quad 1.974512-3.476786$

H $6.431444 \quad 0.239335-1.916654$

H $7.4922262 .998329-1.113141$

H $9.5272751 .430208-1.309867$

H $8.5472800 .087808-0.770763$

H 10.1855842 .6540942 .099277

H $10.4573412 .971436 \quad 0.294312$

H 8.8541270 .8025822 .791089

H $8.316678-0.3373901 .603264$

H 6.8835182 .2145472 .607187

H $3.7076901 .913702-0.972868$

C -1.869991 $4.417022-0.738005$

O -2.644619 3.228885 -0.990789

C $-3.6527173 .522307-1.973673$

C $-3.6852295 .040158-2.098220$

C $-2.2254765 .386099-1.866334$

H $-3.299356 \quad 3.101445-2.922527$

C $-4.9767252 .861612-1.574103$

H $-4.056513 \quad 5.369379-3.073318$

H $-4.3108535 .499836-1.324446$ 
C -1.992704 6.846994-1.523192 $\mathrm{H}-1.6572445 .135044-2.772065$ C $-4.8618751 .350452-1.267611$ O $-5.4837633 .499516-0.395341$ H $-5.7272373 .033653-2.353930$ H $-4.3009801 .206672-0.338348$ O $\quad-6.182356 \quad 0.849259-1.032081$ C $-4.2319070 .525378-2.400406$ C $-4.201268-1.000426-2.184560$ H $-3.2054220 .862317-2.578806$ O $-4.955101 \quad 0.773348-3.611479$ H -5.217145 -1.367902 -2.006342 H -3.903603 -1.445367 -3.142532 H -2.324865 $7.492041-2.343185$ H -2.541309 7.140094 -0.621876 H $-0.9297977 .042093-1.349082$ C $-0.3849694 .045885-0.689336$ H -4.7964663 .4361830 .290189$ H $-6.572970 \quad 1.383033-0.319156$ H $-5.8772440 .502122-3.461718$ H $\quad 0.2256254 .948645-0.575422$ H $-0.1126703 .591627-1.651275$ H -2.2021294 .8258030 .224394$ C $-4.805594-2.0016230 .677826$ O $-3.710793-1.1870450 .241358$ C $-3.225210-1.456040-1.078020$ C $-2.798247-2.927495-1.188131$ C $-3.924181-3.872422-0.787964$ C $-4.456964-3.4938510 .591207$ H -5.688103 -1.7860380 .064705$ C $-5.118090-1.6170972 .129386$ H -2.313175 -0.856825 -1.195745 O $-2.359172-3.219465-2.510694$ H $-1.940689-3.100233-0.526357$ H -4.719672 -3.880844 -1.542282 O $-3.399515-5.200766-0.737426$ H -3.702184 -3.7487601 .345644$ $\begin{array}{llll}\text { O } & -5.610288 & -4.283076 & 0.877837\end{array}$ C $-5.850590-0.2993282 .205670$ H -5.761942 -2.356329 2.619495 H -4.197687 -1.554256 2.722396 H -2.133859 -4.165152 -2.540059 H -4.116970 $-5.783838-0.435052$ H $-6.294536-4.0610150 .223578$ O O $-7.019716-0.1529871 .873044$ C -5.5992441 .9698152 .846169$ H $-6.2294072 .234747 \quad 1.992677$ H -6.179625 2.0016013 .772392 H -4.783319 2.694475 2.914414

SCF Energy (B3LYP/6-31G**//MMFF) $=-3245.94227595$

0600083

MM̄FF Geometry

C $2.485239-1.936747-2.881805$

C $1.654706-1.132114-2.201015$

C $0.155667-1.247978-2.243143$

O $-0.3462010 .090248-2.481641$

C $-0.445445-1.732867-0.911144$

C $-0.230463-3.202234-0.628868$

C $-0.980960-4.189536-1.155116$

C $0.877357-3.5259350 .336260$

C $-2.131586-4.051793-2.113971$

C $-3.404886-4.744781-1.608685$

C $-4.037004-4.069435-0.380580$

C $-5.232527-4.8888570 .111674$

C $-5.966546-4.2021831 .270754$

O $-4.467361-2.759229-0.764064$

O $-7.213950-4.893693 \quad 1.434294$

C $-6.272225-2.743898 \quad 0.869741$

C $-5.204207-4.3560282 .592069$

O $-6.817786-2.0163461 .968801$

C $-5.046310-1.9883930 .299288$

C $-5.509484-0.631309-0.291808$

O $-4.110264-1.7589021 .351114$

C $-1.4542790 .237852-3.249530$

O $-2.049494-0.645249-3.851660$

C $-1.9327751 .641113-3.251117$
C $-1.2905172 .645805-2.635655$

C -1.732479 $4.039569-2.551115$

C $-3.0909934 .377475-3.105816$

C $-4.4019520 .198576-0.952328$

C -3.6937661 .1112390 .030010$

C -4.0390943 .1885521 .371848$

$\begin{array}{llll}\text { O } & -2.581615 & 0.837533 & 0.474603\end{array}$

C $-3.8124094 .518108 \quad 0.724845$

C -2.730739 5.2776870 .956054

C $-2.5318196 .648320 \quad 0.375107$

C $-1.2007996 .804708-0.323865$

C -0.1473807 .3501430 .307490$

C $-1.1192376 .413335-1.781640$

C $-0.9124644 .936526-1.964435$

N -4.4449162 .2249840 .366190$

H $2.075683-2.741314-3.487201$

H $2.071258-0.338293-1.585924$

H $-0.144726-1.907382-3.066902$

H $-1.521384-1.518461-0.895912$

H $-0.053652-1.127927-0.081213$

H $-0.740027-5.216730-0.884197$

H $0.958551-4.5999860 .533908$

H $1.839418-3.192345-0.058782$

H $\quad 0.702274-3.0276121 .295450$

H -2.351295 -3.008115 -2.353868

H -1.833009 -4.526650 -3.056834

H -4.130669 -4.738384 -2.432446

H $-3.183436-5.795444-1.382558$

H -3.284195 -3.9861650 .411055$

H $-4.919074-5.8994700 .402321$

H $-5.943910-5.024301-0.715531$

H -7.671908 -4.514046 2.203011

H -7.051934 -2.771675 0.095593

H $-5.104918-5.4166482 .852791$

H -5.750907 -3.893729 3.421501

H $-4.200689-3.9265332 .561244$

H -7.645575 -2.452983 2.230174

H $-6.257107-0.838013-1.068941$

H $-5.999333-0.0353960 .487172$

H -3.423848 -1.156149 1.024891

H $-2.8693371 .798859-3.776326$

H $-0.3464902 .436497-2.137128$

H $-3.1046934 .246714-4.193196$

H -3.403514 $5.403055-2.899022$

H -3.859153 $3.731899-2.664687$

H $-3.662866-0.435389-1.449244$

H -4.840479 $0.831785-1.734291$

H $-3.1539002 .827948 \quad 1.903838$

H -4.864232 3.2663562 .087013

H -4.5931814 .8923630 .066665$

H -1.9671424 .9191831 .642385$

H -2.603111 7.3705791 .199171

H -3.341672 $6.912230-0.316195$

H $0.8024117 .492490-0.197131$

H -0.2099647 .6598971 .345554$

H $-0.2683006 .920351-2.256554$

H -1.996746 $6.795278-2.310432$

H $0.0495704 .587584-1.586845$

H $-5.3617412 .345199-0.054805$

C $4.650583-2.746680-1.893626$

O $4.366328-2.313168-0.547814$

C $5.587342-2.304998 \quad 0.213241$

C $6.580754-3.127608-0.594988$

C $6.175636-2.763376-2.010685$

H $5.935196-1.2654340 .242163$

C $5.326480-2.8016791 .639467$

H $7.619120-2.871325-0.364402$

H $6.450237-4.203217-0.429258$

C $6.702252-3.726218-3.060621$

H $6.546402-1.753559-2.233180$

C $4.173412-2.0754312 .361959$

O $4.990221-4.1950131 .599216$

H $6.246521-2.7310332 .230678$

H $3.217181-2.3387901 .896081$

O $4.113271-2.5674873 .706380$

C $4.309649-0.5437662 .411178$

C $3.120230 \quad 0.120013 \quad 3.125192$

H $4.362609-0.1552221 .393193$ 
O $5.525640-0.187017 \quad 3.063321$

H $2.199536-0.1979932 .623443$

H $3.066352-0.2445204 .158238$

H $7.797089-3.737418-3.057180$

H $6.355070-4.748664-2.877506$

H $6.370551-3.432055-4.061307$

C $3.981487-1.789267-2.879362$

H $4.211129-4.2924431 .024879$

H $4.021649-3.5345823 .656619$

H $5.496415-0.5586053 .961663$

H $4.339965-1.975117-3.898268$

H $4.247685-0.755500-2.631449$

H $4.241654-3.760132-1.996762$

C $2.3471992 .136155 \quad 0.955232$

O $3.4327522 .250601 \quad 1.880872$

C 3.2034811 .6635013 .165259

C 1.9872042 .3271293 .825239

C $0.7500692 .240782 \quad 2.935330$

C 1.0632192 .7545441 .530342

H $2.184538 \quad 1.0797110 .708501$

C $2.7505902 .879065-0.330801$

H 4.0807551 .9150343 .774331

O 1.7014751 .7276655 .084800

H 2.2071283 .3859394 .014234

H $0.350586 \quad 1.2202702 .910375$

\begin{tabular}{lllll}
\hline & -0.270282 & 3.057070 & 3.516242
\end{tabular}

H 1.1531523 .8484101 .557799

O -0.0193472 .4371490 .659255$

C $3.8863882 .218987-1.082024$

H $1.8991932 .927590-1.017833$

H $3.0747603 .896627-0.081337$

H 2.4985521 .8113635 .635458

H -1.087736 2.9058183 .013353

H -0.8063322 .9094330 .976794$

O $4.1474992 .947748-2.208587$

O $4.472827 \quad 1.204076-0.736582$

C $5.2107562 .455843-3.030687$

H $4.9245361 .500971-3.479932$

H $5.3852143 .181230-3.829725$

H $6.1308382 .349805-2.447852$

SCF Energy (B3LYP/6-31G**//MMFF) $=-3245.91823536$

06_00084

MM̄FF Geometry

C - $0.463305-2.5021323 .035070$

C $0.755347-2.7444523 .542417$

C $1.934988-1.8011103 .505357$

$\begin{array}{lllll}\text { O } & 1.465027 & -0.466736 & 3.214028\end{array}$

C $2.924788-2.2338132 .407504$

C $4.327751-1.7021032 .619906$

C $4.864450-0.6497421 .971848$

C $5.156577-2.473553 \quad 3.615431$

C 4.2097410 .2603500 .970351

C $4.8426770 .150290-0.422093$

C $4.481131-1.151883-1.152743$

C $5.235427-1.240825-2.481025$

C $4.818674-2.472536-3.294881$

O $3.069670-1.138348-1.384348$

O $5.357993-2.303648-4.614671$

C $3.279833-2.488872-3.406914$

C $5.443490-3.756319-2.736948$

O $2.835237-3.680993-4.052181$

C $2.554769-2.293920-2.052932$

C $1.028011-2.102393-2.245281$

O $2.743078-3.473615-1.262597$

C 2.0688110 .5873713 .819251

$\begin{array}{lllll}\text { O } & 2.976888 & 0.566293 & 4.632666\end{array}$

C $1.448382 \quad 1.802917 \quad 3.255457$

C 2.2026382 .8962193 .075293

C 1.7644814 .1183282 .401745

C $0.282227 \quad 4.308367 \quad 2.210297$

C $0.615271-0.753731-2.847515$

C $0.4720990 .355884-1.816685$

C $0.267038 \quad 2.833900-1.649511$

O $0.3777330 .146969-0.609864$

C $1.612578 \quad 3.315967-1.209449$

C $2.0954034 .540048-1.474034$

C $3.4407235 .014495-0.999770$
C $3.3690096 .242894-0.112195$

C $4.0850897 .339966-0.412193$

$\begin{array}{llll}\text { C } 2.488715 & 6.219238 & 1.125988\end{array}$

C $2.7008784 .988317 \quad 1.968816$

N $0.4177691 .609589-2.412161$

H $-0.659466-1.5662942 .519324$

H $0.933299-3.6952834 .041282$

H $2.395406-1.8239144 .500468$

H $2.544116-1.9260541 .426562$

H $2.991241-3.3296202 .363542$

H $5.894838-0.3810682 .202810$

H $6.170622-2.0717513 .714078$

H $4.694167-2.4437994 .606896$

H $5.246304-3.5195063 .304404$

H 3.1284230 .1087540 .911080

H 4.3476891 .2885201 .327989

H $4.4840131 .002101-1.014867$

H $5.9326710 .247489-0.342639$

H $4.734380-2.006630-0.516040$

H $6.319909-1.240149-2.314456$

H $5.025466-0.342965-3.079542$

H $5.035916-3.041076-5.161124$

H $3.010567-1.668761-4.082289$

H $6.537652-3.682623-2.748324$

H $5.197544-4.621829-3.362041$

H $5.136288-3.973322-1.711765$

H $2.882329-4.403570-3.403012$

H $0.643342-2.912606-2.877634$

H $0.523006-2.235157-1.279616$

H $2.063233-3.491053-0.568826$

H $0.419716 \quad 1.7314512 .920451$

H 3.2492192 .8663773 .375520

H -0.2488664 .1638623 .158533$

H $-0.1084883 .591476 \quad 1.482268$

H $0.0127365 .309817 \quad 1.868336$

H $1.306389-0.437851-3.633986$

H $-0.371200-0.866203-3.312380$

H $-0.3574232 .632273-0.774290$

H $-0.2454273 .559370-2.288702$

H $2.2098042 .628973-0.613725$

H $1.5023995 .235034-2.063393$

H $4.0480785 .220872-1.890417$

H $3.9680694 .217977-0.459628$

H $4.048974 \quad 8.2274560 .210931$

H $4.7239507 .377895-1.288345$

H 1.4525996 .3185750 .791145

H 2.6940447 .0921101 .758445

H 3.7479894 .7770172 .190116

H $\quad 0.5655711 .692044-3.413661$

C -1.989634 -4.0838591 .810644$

\begin{tabular}{llll}
\hline & -2.417221 & -3.075986 & 0.873251
\end{tabular}

C -3.517956 -3.5948830 .102614$

C $-3.667486-5.0626390 .492494$

C $-3.159799-5.0598091 .918621$

H $-4.412459-3.050400 \quad 0.425721$

C $-3.280745-3.376209-1.395337$

H $-4.702178-5.4085930 .404978$

H -3.045882 -5.716115 - 0.130412

C $-2.777549-6.4390652 .427143$

H $-3.940996-4.6396912 .566644$

C $-3.046496-1.913928-1.818875$

O $-2.126222-4.124709-1.802829$

H $-4.121190-3.794147-1.961971$

H $-2.072922-1.563746-1.457899$

O $-2.976811-1.896004-3.251670$

C $-4.135377-0.920855-1.373674$

C $-3.9781790 .462709-2.037494$

H $-4.096850-0.813339-0.284747$

O $-5.423463-1.443777-1.699688$

H $-2.9520720 .813947-1.885907$

H $-4.1297200 .360419-3.118745$

H -3.642010 -7.110749 2.403228

H $-1.986659-6.8868301 .816027$

H $-2.418265-6.3904893 .459745$

C $-1.622410-3.4495743 .151819$

H $-1.378451-3.830241-1.255037$

H -2.278694 -2.519854 -3.515466

H $-5.450537-1.579967-2.662325$ 
H $-1.398250-4.2328103 .885419$ H -2.472975 -2.8796983 .543806$ H -1.112542 -4.577316 1.371285 C -3.6971492 .3658190 .338213$ O $-4.8830501 .685242-0.091339$ C $-4.9937781 .498847-1.505719$ C $-4.9512852 .857586-2.220571$ C $-3.7305713 .677903-1.815119$ C $-3.6271723 .760753-0.293175$ H $-2.812778 \quad 1.7798150 .059899$ C -3.7422822 .4508191 .866586$ H $-5.9934031 .081386-1.680484$ O $-4.9566852 .694853-3.634728$ H -5.851385 $3.429999-1.960671$ H -2.811250 $3.269974-2.250365$ O $-3.8640375 .005508-2.327236$ H $-4.437077 \quad 4.392107 \quad 0.094345$ O $-2.3954094 .382408 \quad 0.060687$ C -3.5617051 .0797972 .472795$ H -2.9442103 .0873152 .264996$ H -4.6928132 .8725882 .214942$ H -5.752509 2.186218 -3.866906 H $-3.9700184 .933600-3.291468$ H $-2.3679295 .247746-0.382467$ O $-4.777422 \quad 0.530768 \quad 2.754683$ O $-2.471313 \quad 0.5498702 .642040$ C - $4.746672-0.7941693 .292600$ H $-4.136585-0.8264874 .200356$ H -5.769939-1.081808 3.548092 H $-4.363574-1.4915272 .542632$

SCF Energy $\left(B 3 L Y P / 6-31 G^{* *} / / M M F F\right)=-3245.92917562$

06_00085

MMFF Geometry

C $-0.7501650 .363163 \quad 3.939876$

C $0.344766-0.3626644 .216655$

C $0.682134-1.7474693 .712035$

O $1.572065-1.6287962 .575970$

C $-0.533890-2.5901573 .280582$

C $-0.196691-4.0458683 .002558$

C $-0.181091-4.6205551 .782524$

C $0.088337-4.8838614 .223826$

C $-0.463038-3.9748920 .456467$

C $0.759674-4.022065-0.468021$

C $0.559789-3.140775-1.710682$

C $1.756512-3.257306-2.655361$

C $1.660120-2.279164-3.836109$

O $0.421766-1.792339-1.254769$

O $2.947287-2.226956-4.471047$

C $1.368854-0.863886-3.292536$

C $0.670268-2.766348-4.900646$

O $1.1233230 .051015-4.357501$

C $0.200839-0.822810-2.285915$

C $0.059370 \quad 0.551447-1.587554$

O $-1.026727-1.078736-2.977439$

C $2.892921-1.4169852 .827644$

O $3.420248-1.2710143 .918849$

C $3.600734-1.3971571 .527684$

C $4.890227-1.0311141 .476953$

C $5.733041-0.9858690 .278787$

C $5.097404-1.346972-1.037534$

C $1.2597850 .966303-0.729967$

C $2.2058021 .945495-1.401204$

C $4.3438003 .157757-0.970611$

O $2.0126082 .421527-2.515694$

C $5.4612622 .390234-1.603226$

C $6.7265782 .422570-1.158457$

C $7.8637351 .702572-1.823797$

C $8.6286270 .808201-0.874967$

C $9.7875801 .211660-0.327141$

C $8.100512-0.588472-0.640378$

C $7.029245-0.6351770 .415024$

N $3.2907932 .239028-0.584294$

H -1.502072 -0.0164273 .254669$

H $1.079403 \quad 0.0643204 .897767$

H $1.198720-2.2743334 .524340$

H -0.998535 -2.129696 2.405200

H -1.297916 -2.5687364 .069156$
H $\quad 0.055841-5.6819701 .718846$

H $0.215645-5.9441363 .980326$

H $1.005162-4.5496064 .718337$

H $-0.740193-4.8105714 .936000$

H -1.297213 -4.505031 -0.019005

H $-0.789900-2.9422290 .578408$

H $1.639495-3.6704180 .085842$

H $0.953896-5.058849-0.768932$

H $-0.363285-3.447997-2.216293$

H $1.867334-4.287413-3.016536$

H $2.678377-3.036502-2.101073$

H $3.178947-3.128250-4.753462$

H $2.282859-0.521227-2.792607$

H $0.983601-3.739438-5.297721$

H $\quad 0.647052-2.086563-5.759409$

H $-0.347176-2.879059-4.520711$

H $1.8879150 .009860-4.957052$

H $-0.2126511 .322939-2.317102$

H $-0.8010300 .480572-0.907165$

H -1.758464 -0.700395 -2.462245

H $3.043057-1.6949090 .647980$

H $5.385151-0.7466262 .404805$

H $4.814990-2.404995-1.046119$

H $5.747270-1.177091-1.898383$

H $4.201854-0.742187-1.213692$

H $0.887596 \quad 1.4658460 .170565$

H $1.8305090 .094872-0.398682$

H $4.6627113 .685517-0.066225$

H $3.9493313 .890799-1.681534$

H $5.222417 \quad 1.804207-2.487760$

H $6.9688793 .030780-0.289858$

H $8.5365352 .462255-2.243114$

H $7.517678 \quad 1.112295-2.681507$

H $\quad \begin{array}{llll}10.358535 & 0.563051 & 0.329017\end{array}$

H $10.1878802 .201991-0.518050$

H $8.916412-1.240812-0.299932$

H $7.784478-1.022812-1.592855$

H $7.395872-0.3875531 .412404$

H $3.395163 \quad 1.7456530 .297718$

C -0.8777402 .9039323 .648518$

O -1.8136502 .8507492 .555298$

C - -1.1974413 .4703831 .406622$

C 0.1322304 .0508161 .883822

C 0.4939113 .0996283 .008054

H -0.9880142 .6671670 .694468$

C -2.1416214 .4973280 .772946$

H 0.8808014 .0790521 .085978

H 0.0143865 .0711072 .267502

C 1.5527073 .6423323 .950466

H 0.8536262 .1633702 .564211

C -3.4847813 .9157820 .286404$

O -2.4312005 .5224351 .731779$

H -1.634251 $4.998904-0.059310$

H -4.0955173 .5985511 .140287$

O $-4.2079524 .971492-0.358789$

C $-3.3421832 .748182-0.706947$

C $-4.6905252 .286247-1.289149$

H $-2.8683041 .904740-0.195970$

O $-2.4795023 .131682-1.777412$

H $-5.3694772 .055387-0.461254$

H $-5.1442023 .105065-1.860521$

H 2.4840303 .8385903 .409238

H 1.2320634 .5787684 .418994

H $1.7706572 .923984 \quad 4.746961$

C $-1.0370801 .696849 \quad 4.573341$

H -2.8334625 .0936272 .506669$

H $-4.287917 \quad 5.7003970 .280311$

H -2.881219 $3.900418-2.217435$

H $-2.0734191 .661790 \quad 4.933201$

H $-0.406640 \quad 1.838413 \quad 5.459736$

H -1.1436443 .7994534 .227239$

C $-4.493888-0.667558-0.552046$

O $-3.813335-0.002579-1.623960$

C $-4.5455711 .066975-2.229589$

C $-5.8711630 .538990-2.792789$

C $-6.674542-0.224228-1.742311$

C $-5.806018-1.282315-1.058925$

H $-4.704557 \quad 0.0531220 .246911$ 
C $-3.516746-1.713691-0.004073$

H $-3.944317 \quad 1.400362-3.084945$

O $-6.644598 \quad 1.613522-3.316064$

H -5.654641 -0.137633 -3.629727

H -7.117602 $0.462210-1.011002$

O $-7.768425-0.869950-2.398874$

H -5.605802 -2.089715 -1.775139

O $-6.556907-1.8342180 .018465$

C $-3.860749-2.1633961 .395543$

H -3.463912 -2.573130 -0.683280

H $-2.500995-1.3018710 .036248$

H -7.480133 $1.238046-3.642942$

H -8.249741 -1.379251 -1.724268

H -6.132642 -2.6698620 .275926$

O $-3.879686-3.525648 \quad 1.450693$

O $-4.079448-1.4017432 .328536$

C $-4.210455-4.0881072 .724156$

H -4.101929 -5.1736742 .654495$

H -3.531732 -3.718213 3.498049

H -5.248425 -3.852942 2.977437

SCF Energy (B3LYP/6-31G**//MMFF) $=-3245.92729282$

06_00086

MMFF Geometry

C $-2.269248-3.678019-0.238670$

C $-1.857106-4.433350 \quad 0.790914$

C -1.084151 -3.958070 1.998122

O $-0.858410-2.5321791 .977281$

C $0.298089-4.6319212 .055064$

C $1.080419-4.2387993 .294998$

C $2.071420-3.324733 \quad 3.319152$

C $0.694454-4.9691564 .555179$

C $2.591293-2.4978932 .173991$

C $4.006892-2.9431741 .795850$

C $4.526932-2.2607980 .522518$

C $5.912576-2.8114250 .170630$

C $6.517319-2.114642-1.053653$

O $4.593404-0.8463050 .740936$

O $7.904644-2.479469-1.101768$

C $6.432132-0.590210-0.840627$

C $5.892875-2.626889-2.356988$

O $6.8742820 .105404-2.004775$

C $5.029667-0.100770-0.405837$

C $5.1019371 .397179-0.021730$

O $4.134674-0.254236-1.511172$

C $-1.820307-1.7197782 .489053$

O $-2.944215-2.0322162 .844572$

C - $-1.231220-0.3660242 .568355$

C -1.9943330 .6965512 .860359$

C -1.5082442 .0689603 .021143$

C -0.0188232 .2856692 .958672$

C 3.8319341 .9529040 .639985

C 3.8434183 .4749330 .671571

C $2.328327 \quad 5.4280120 .863689$

O $4.857748 \quad 4.139658 \quad 0.474139$

C 1.1701755 .7893391 .737274

C -0.0509736 .0876511 .268269$

C -1.2103176 .5048792 .126550$

C -2.248545 5.4136622 .269722

C -3.2598765 .3139501 .389870$

C -2.1146264 .5164243 .482962$

C -2.3998503 .0605093 .228553$

N 2.5940924 .0045550 .969160

H -2.019308 -2.622162 -0.273654

H -2.130714 -5.4868510 .801184$

H -1.656893 -4.2198902 .896968$

H $\quad 0.868618-4.3857101 .152239$

H $0.187910-5.7243582 .038751$

H $2.569200-3.1328184 .268981$

H $1.315554-4.6808995 .410062$

H $-0.346272-4.7580164 .819380$

H $\quad 0.806917-6.0493734 .417082$

H $1.934652-2.5515651 .302123$

H $2.599183-1.4455742 .483074$

H $4.679776-2.7171722 .633819$

H $4.022003-4.0310561 .649393$

H $3.820082-2.464235-0.289599$

H $5.872768-3.8966030 .012228$
H $6.589283-2.6601811 .023784$

H $8.315140-1.981603-1.829753$

H $7.149827-0.336927-0.048362$

H $6.059559-3.706090-2.459506$

H $\quad 6.367187-2.168573-3.231685$

H $4.816575-2.451576-2.416356$

H $6.1581920 .068258-2.661584$

H 5.9312321 .5559850 .680005

H $5.3221851 .985316-0.922148$

H $3.5044080 .481724-1.520607$

H $-0.162696-0.2852562 .399463$

H -3.0637950 .5509903 .003703$

H $0.487225 \quad 1.7152203 .745338$

H 0.2754343 .3275113 .088025

H $\quad 0.3742521 .9686351 .986959$

H $2.937701 \quad 1.624514 \quad 0.103911$

H 3.7558451 .5914691 .671766

H $2.1318565 .644097-0.192165$

H 3.2167565 .9954681 .159849

H 1.3550135 .8283792 .808037

H -0.2298386 .0543400 .196401$

H -1.662550 7.3974481 .674189

H -0.8726886 .8303063 .118715$

H -4.0360544 .5635691 .491856$

H -3.335662 5.9885540 .543086

H -2.8060214 .8758794 .256504$

H -1.121749 4.6324143 .929915

H -3.4608042 .8097823 .250564$

H 1.7948163 .3789510 .937259

C $-4.512926-3.567436-1.409689$

O $-4.416888-2.144339-1.623698$

C $-5.284250-1.481059-0.684900$

C $-6.265363-2.546200-0.209744$

C $-5.364856-3.765015-0.151882$

H $-4.656341-1.1785110 .162604$

C $-5.926165-0.239444-1.312575$

H $-6.715506-2.2971430 .755863$

H $-7.073264-2.709714-0.932084$

C $-6.116313-5.084320-0.122006$

H $-4.753751-3.6897640 .755422$

C $-4.9253110 .723507-1.981794$

O $-6.851719-0.668779-2.318953$

H $-6.5163390 .286476-0.552264$

H $-4.5441940 .281168-2.910737$

O $-5.6378321 .908237-2.359673$

C $-3.7361071 .140531-1.096474$

C $-2.8320522 .172873-1.795661$

H $-3.1452020 .253411-0.848801$

$\begin{array}{lllll}\text { O } & -4.212444 & 1.686013 & 0.132067\end{array}$

H -2.574375 $1.793171-2.790311$

H $-3.3879273 .107269-1.940914$

H $-6.752420-5.1446360 .766992$

H $-6.757449-5.201710-1.002135$

H $-5.418735-5.927478-0.097188$

C $-3.112681-4.192048-1.372075$

H $-7.4004630 .096482-2.559461$

H -6.245526 $1.668541-3.079287$

H $-4.7616162 .459466-0.082716$

H -2.586572 -3.951206 -2.304070

H -3.201905 -5.283619-1.325541

H $-5.040548-3.965698-2.286016$

C $-0.2189240 .607751-1.705101$

O $-0.8279621 .320741-0.620171$

C -1.549754 $2.495622-0.997944$

C $-0.6056183 .476903-1.701847$

C $0.1180372 .825825-2.877697$

C $\quad 0.7762741 .514692-2.445233$

H $-1.0014790 .268787-2.393365$

C $0.449631-0.628551-1.084770$

H $-1.8717642 .968923-0.063560$

O $-1.3264474 .622423-2.141298$

H $0.1436313 .824200-0.980361$

H $-0.5604532 .673966-3.725061$

O $1.1304393 .728128-3.331654$

H $1.6299881 .756867-1.802860$

O $1.2840130 .871003-3.609309$

C $0.580082-1.805193-2.025644$

H $1.438466-0.368652-0.692467$ 
H $-0.152275-0.973410-0.237018$

H $-0.6942995 .208144-2.592154$

H $1.6051983 .287514-4.057332$

H $1.8438890 .131441-3.319513$

O $1.275490-2.795721-1.393091$

O $0.122265-1.863529-3.157897$

C $1.474941-3.993196-2.149418$

H $2.053516-3.778152-3.052779$

H $2.039688-4.694908-1.529768$

H $0.511642-4.445401-2.403623$

SCF Energy (B3LYP/6-31G**//MMFF) $=-3245.92088163$

06_00087

MM̄FF Geometry

C $3.975845-2.7762492 .044136$

C $2.998235-1.9239101 .699520$

C $1.766820-1.6939082 .530559$

O $0.615693-1.6635491 .655312$

C $1.834924-0.3253323 .229432$

C $0.759668-0.1788334 .288167$

C $-0.444146 \quad 0.394434 \quad 4.094044$

C $1.124325-0.7283655 .643858$

C -1.0010910 .9809202 .825673$

C -1.421823 2.4400443 .038409

C $-2.0277803 .067408 \quad 1.773473$

C -2.3922244 .5319552 .036735$

C -3.1062105 .1775990 .841336$

O -3.1972292 .3212921 .418049$

O $-3.667336 \quad 6.4148141 .306048$

C $-4.270451 \quad 4.2606150 .408899$

C $-2.1164325 .547166-0.268346$

O $-4.8947344 .756026-0.772881$

C -3.8576812 .7831720 .231710$

C $-5.0663041 .856453-0.032124$

O $-2.9999832 .679962-0.909859$

C $0.070289-2.8426071 .260372$

O $0.480292-3.9659411 .499535$

C $-1.145238-2.5087180 .485438$

C $-1.841335-3.471450-0.136125$

C $-3.069175-3.272719-0.911870$

C $-3.636275-1.877469-0.961089$

C $-6.095777 \quad 1.799414 \quad 1.095987$

C -7.1832750 .7852450 .798872$

C -7.685094 -1.639273 0.853110

O

C $-6.926330-2.9161150 .685534$

C -7.190796 -3.818588 -0.271839

C $-6.453254-5.120722-0.409266$

C $-5.910682-5.351626-1.804152$

C $-6.328594-6.397289-2.537642$

C $-4.883467-4.383665-2.354701$

C -3.629084 -4.337969-1.522962

N $-6.756826-0.5271110 .941926$

H $3.900894-3.3283682 .977356$

H $3.083841-1.3580130 .775868$

H $1.637474-2.4938113 .270783$

H $1.754870 \quad 0.4823362 .491858$

H $2.813528-0.1895383 .709178$

H - 1.1319390 .4287934 .938688

H $0.319672-0.6013676 .375888$

H $1.343628-1.7986615 .578269$

H $2.009370-0.2154666 .034569$

H -0.2903650 .9268851 .996533$

H -1.870792 0.3804712 .533453

H -2.161438 $2.478013 \quad 3.849453$

H -0.5534093 .0289193 .359921$

H -1.293418 2.9926520 .962649

H -1.501441 5.1112532 .310572

H -3.061230 4.5869852 .907415

H $-4.183547 \quad 6.7967210 .575285$

H $-5.025677 \quad 4.330074 \quad 1.200809$

H -1.382612 $6.272848 \quad 0.102954$

H -2.622048 $6.037693-1.107046$

H -1.564186 $4.687451-0.650986$

H $-4.3217474 .533428-1.526210$

H -5.559089 2.138801 -0.971830

H $-4.6864510 .841620-0.206285$

H $-2.9936441 .754675-1.207170$
H -1.439642 -1.465486 0.451763

H -1.484449-4.498329 -0.070619

H -2.935417 -1.195445 -1.455033

H -3.830837 -1.5085070 .052109$

H -4.580625 -1.801088 -1.502263

H -5.6254621 .5370402 .050544$

H -6.5830862 .7703211 .233445$

H -8.386524 -1.455857 0.032113

H -8.254602 -1.6635121 .788276$

H $-6.136378-3.1224301 .404214$

H $-7.999175-3.629733-0.974499$

H -7.151646 $-5.922895-0.136958$

H $-5.629843-5.1862380 .312463$

H $-5.947426-6.576416-3.537458$

H -7.062494 -7.099633 -2.156138

H $-5.355472-3.405346-2.470643$

H -4.586450 -4.681969 -3.368801

H -3.131722 -5.304997 -1.433595

H $-5.863060-0.6888931 .398082$

C $6.292978-2.001381 \quad 1.437277$

O $5.868185-0.7365100 .887823$

C $6.880662-0.252915-0.009469$

C $8.153542-0.9798160 .396526$

C $7.603173-2.3540900 .727358$

H $6.595333-0.574860-1.018383$

C $6.948506 \quad 1.2745290 .047212$

H $8.899552-0.995746-0.403644$

H $8.612235-0.531807 \quad 1.285527$

C $8.544166-3.2026501 .564752$

H $7.391313-2.882010-0.212386$

C $5.5984751 .978411-0.204031$

O $7.407707 \quad 1.6790161 .343753$

H $7.6997281 .637785-0.663370$

H $4.915817 \quad 1.8018220 .636328$

O $5.8468213 .390413-0.229429$

C $4.9003971 .582767-1.519877$

C $3.6221212 .403161-1.772756$

H $4.6394590 .521453-1.487506$

O $5.8028441 .763218-2.610191$

H $2.9521022 .273630-0.915903$

H $3.8835453 .466827-1.821385$

H $9.480908-3.3828061 .027292$

H $8.789746-2.7129122 .513072$

H $8.093045-4.1733311 .793702$

C $5.192759-3.0363371 .200877$

H 6.7919201 .3109242 .000906

H $6.2809073 .623060 \quad 0.609374$

H $6.0607412 .700980-2.625564$

H $5.558405-4.043276 \quad 1.433323$

H $4.904383-3.0398260 .142983$

H $6.457941-1.8449382 .510665$

C $1.5093200 .231394-2.259096$

O $2.543640 \quad 0.646147-3.158103$

C $2.9005172 .029845-3.087955$

C $1.6721212 .899587-3.387268$

C $0.5141852 .573897-2.451488$

C $0.236714 \quad 1.072117-2.448517$

H $\quad 1.8709000 .306822-1.226255$

C $1.195642-1.244285-2.560692$

H $3.6160962 .199989-3.901974$

O $1.9870014 .284001-3.278921$

H $1.3450702 .723950-4.420503$

H $0.7010162 .953156-1.440097$

O $-0.6474643 .257984-2.928454$

H $\quad-0.2479640 .805069-3.396408$

O $-0.697508 \quad 0.772138-1.412247$

C $2.287327-2.191189-2.114655$

H $\quad 0.276567-1.536757-2.041614$

H $1.058974-1.393798-3.638014$

H $2.7137414 .467436-3.898407$

H -1.391055 2.998916 -2.357912

H $-0.2843041 .000430-0.562011$

O $1.766823-3.451531-2.028696$

O $3.448841-1.876871-1.898227$

C $2.677142-4.484275-1.638524$

H $2.210536-5.448056-1.859227$

H $3.615573-4.416490-2.197312$

H $2.861565-4.423093-0.563137$ 
H $3.070190-5.2133840 .181479$

H $1.406333-5.5803390 .573702$

H $3.693047-3.6703073 .458302$

H $4.440734-4.274357 \quad 1.877548$

H $\quad 1.314177-3.9822743 .824177$

H $0.348081-4.7105752 .588602$

H $-0.241525-2.6360901 .579765$

H -1.568332 -2.546718 -1.053244

C $0.0063975 .110442-1.738226$

O $0.6786193 .837016-1.695440$

C $1.9418024 .009714-1.022140$

C $2.0968105 .509305-0.766512$

C $0.6528705 .954956-0.642833$

H $1.8559913 .499207-0.057120$

C $3.0742173 .397245-1.853265$

H 2.6883435 .7142790 .131265

H $2.5820036 .019804-1.606532$

C $0.4539217 .449669-0.815946$

H 0.2936575 .6563920 .349536

C $2.9061161 .906985-2.214915$

O $3.1790194 .119871-3.090459$

H $4.0311733 .550779-1.341952$

H $2.0451581 .790571-2.885025$

O $4.0610851 .506232-2.966931$

C $2.7746170 .915104-1.045293$

C 3.8427301 .1079430 .048049

H $2.863740-0.098811-1.451804$

O $1.4836671 .009744-0.449855$

H $4.8142331 .295913-0.419221$

H $3.590187 \begin{array}{lll}1.993393 & 0.642355\end{array}$

H $1.0039258 .002522-0.047404$

H $0.8084007 .791696-1.794120$

H $-0.6049077 .713441-0.729602$

C -1.508204 $4.909546-1.664172$

H $2.3222294 .047130-3.545387$

H $4.1398072 .113711-3.722393$

H $0.8251170 .854152-1.148185$

H - $-1.8081614 .185030-2.432317$

H -2.016309 $5.850666-1.905449$

H $0.2384675 .538077-2.723211$

C $5.611924-1.361856-0.221938$

O $4.277596-1.3106870 .294508$

C $3.939747-0.1092480 .995032$

C 4.8913790 .0812772 .183381

C $6.3551120 .040777 \quad 1.748522$

C $6.629980-1.2111070 .914444$

H $5.757187-0.565944-0.961585$

C $5.780460-2.711990-0.935776$

H $2.936761-0.267011 \quad 1.407231$

O 4.6017841 .3078452 .844349

H $4.716685-0.7249642 .907993$

H $6.6376570 .946677 \quad 1.199334$

O $7.193064-0.0099592 .905311$

H $6.603709-2.0953021 .564410$

O $7.941372-1.1261650 .360551$

C $4.953869-2.799278-2.201688$

H $6.824211-2.879011-1.222014$

H $5.458957-3.517978-0.265945$

H $\quad 5.1262081 .333802 \quad 3.662091$

H 7.0992390 .8371823 .372571

H $8.561439-1.0077211 .100442$

O $4.782133-4.113423-2.532577$

O $4.533541-1.841531-2.835930$

C $4.019461-4.354429-3.719293$

H $3.030566-3.892785-3.640266$

H $4.555204-3.972172-4.593065$

H $3.893068-5.434698-3.828429$

SCF Energy (B3LYP/6-31G**//MMFF) $=-3245.92234428$

0600089

MMFF Geometry

C $-2.5268903 .975684-0.684345$

C $-1.3467983 .694891-0.111493$

C $-0.0404173 .757401-0.860568$

O $0.6646402 .515635-0.624790$

C $0.8332094 .905820-0.325952$

C $1.7736925 .445083-1.385785$ 
C $3.0549845 .066861-1.554012$

C $1.1768156 .500171-2.283280$

C $3.826097 \quad 4.034524-0.782411$

C $4.2544792 .882597-1.700236$

C $5.1610001 .875235-0.980849$

C $5.5634210 .744335-1.929382$

C $6.407927-0.325725-1.221081$

O $4.447777 \begin{array}{llll}1.340990 & 0.137541\end{array}$

O $6.456283-1.481318-2.072006$

C $5.689867-0.748136 \quad 0.077453$

C $7.8583810 .131833-1.028152$

O $6.499313-1.640556 \quad 0.840624$

C 5.2160490 .4422350 .946808

C $4.308177-0.0763702 .082922$

O 6.3573061 .0849151 .517239

C $0.423477 \quad 1.480465-1.472916$

O $-0.2866221 .488488-2.465424$

C $1.1681410 .308635-0.962117$

C $1.191333-0.825454-1.676431$

C $1.860872-2.058040-1.270681$

C $2.618918-2.723282-2.385323$

C 3.5998701 .0208522 .889918

C 2.7208130 .3811953 .945633

C $0.670012-1.0176964 .152754$

$\begin{array}{lllll}\text { O } & 3.083877 & 0.277649 & 5.114787\end{array}$

C $1.125686-2.4099683 .843165$

C $0.456580-3.2603913 .050597$

C $0.987042-4.6068832 .650133$

C $1.262561-4.704738 \quad 1.163207$

C $0.637734-5.618166 \quad 0.401829$

C $2.322948-3.7973590 .570153$

C $1.737295-2.536490-0.016413$

N $1.532166-0.1059763 .423407$

H -2.527411 $4.267659-1.733474$

H $-1.295243 \quad 3.4094770 .934958$

H $-0.2164193 .884239-1.936413$

H $1.392447 \quad 4.5823050 .559501$

H 0.2065395 .7419360 .013662

H $3.6282145 .548524-2.345891$

H $1.8846036 .853382-3.040736$

H $0.3016396 .106164-2.809563$

H $0.8654037 .367889-1.692422$

H $4.714177 \quad 4.518627 \quad-0.358747$

H 3.2523423 .6412870 .060949

H $3.3540772 .373061-2.065908$

H $4.7813213 .279148-2.577557$

H $6.0485272 .409422-0.621638$

H $6.0974711 .139275-2.802686$

H $4.6581050 .263029-2.325600$

H $6.841377-1.211043-2.923243$

H $4.807999-1.328801-0.223779$

H $8.3154180 .373517-1.995342$

H $8.472124-0.669149-0.601692$

H $7.9479261 .014373-0.391310$

H $7.182582-1.1119391 .286802$

H $3.539044-0.7278141 .650965$

H $4.894799-0.6935572 .775828$

H $\begin{array}{llll}6.085827 & 1.563660 & 2.315747\end{array}$

H $1.6708850 .397976-0.005462$

H $\quad 0.701319-0.865127-2.645906$

H $3.214845-3.577356-2.057850$

H $3.316205-2.015200-2.847759$

H $1.928819-3.079596-3.157932$

H $4.314408 \quad 1.6767943 .397191$

H 2.9800871 .6539202 .244867

H $-0.354947-0.831033 \quad 3.822529$

H $0.738086-0.8257875 .227840$

H $2.084805-2.712908 \quad 4.257445$

H $-0.496004-2.9592082 .623313$

H $\quad 0.255190-5.3629252 .961005$

H $1.912447-4.8416243 .191252$

H $\quad 0.852253-5.708878-0.657980$

H $-0.103156-6.2920830 .818612$

H $2.900681-4.362746-0.167150$

H $3.045771-3.5205231 .347801$

H $1.147750-1.9514020 .687882$

H $1.369332-0.0201582 .424321$

C $-4.003123 \quad 3.397744 \quad 1.373257$
O -3.7021071 .9860851 .376853$

C -4.7262691 .2934462 .111734$

C -5.4660622 .3632632 .904218$

C -5.4207713 .5278551 .938005$

H $-5.4125580 .865492 \quad 1.371197$

$\begin{array}{lll}\text { C }-4.124750 & 0.178142 & 2.970582\end{array}$

H -6.4841802 .0587293 .164925$

H -4.9404832 .6203583 .831215$

C $-5.705325 \quad 4.8698432590742$

H $-6.160144 \quad 3.3531121 .144598$

C -3.282442 -0.8620602 .206114$

$\begin{array}{lllll}\text { O } & -3.275398 & 0.757679 & 3.970846\end{array}$

H $-4.927446-0.3285443 .519455$

H $-2.325362-0.4258831 .894767$

O -2.968132 -1.9107493 .133536$

C $-3.978061-1.494307 \quad 0.987550$

C $-3.155558-2.642128 \quad 0.367569$

H $-4.149695-0.724593 \quad 0.229894$

O $-5.257736-1.9926941 .378263$

H $-2.156712-2.2669650 .120098$

H $-3.029036-3.4350441 .114140$

H -6.709162 4.8794933 .027806

H -4.988918 5.0873323 .390205

H $-5.648438 \quad 5.680777 \quad 1.857884$

C $-3.892435 \quad 3.946710-0.052069$

H $-2.591872 \quad 1.2748713 .510587$

H -2.570576-1.497108 3.918656

H -5.113749 -2.659723 2.071434

H $-4.2839224 .970569-0.079683$

H $-4.5302183 .341113-0.707731$

H -3.2826923 .8865882 .040490$

C $-2.885237-1.840002-2.594708$

O $-4.059797-2.288978-1.907038$

C $-3.831674-3.257968-0.878343$

C $-3.108614-4.484968-1.450952$

C $-1.839828-4.100568-2.205851$

C $-2.157973-3.025966-3.243473$

H $-2.217221-1.327207-1.892180$

C $-3.333789-0.831839-3.663501$

H $-4.824123-3.601597-0.560836$

O $-2.802964-5.407218-0.410712$

H $-3.786303-5.000840-2.144089$

H $-1.054363-3.772654-1.515868$

O $-1.344575-5.266770-2.865631$

H -2.777270 -3.458790 -4.039902

O $-0.949842-2.568458-3.842065$

C $-3.8442760 .461675-3.065576$

H $-2.503770-0.569236-4.328009$

H $-4.145431-1.271723-4.256079$

H $-2.329544-6.154606-0.814494$

H $-0.473595-5.045533-3.236078$

H $-0.564858-3.311542-4.336234$

O $-4.552053 \quad 1.137940-4.018724$

O $-3.6301130 .844916-1.924305$

C $-5.0973832 .395420-3.609222$

H $-5.7465692 .265498-2.738044$

H $-4.2902373 .100409-3.391353$

H $-5.6954772 .791352-4.434344$

SCF Energy (B3LYP/6-31G**//MMFF) $=-3245.92099298$

0600090

MMFF Geometry

C $2.628373 \quad 1.324922-3.172544$

C $1.5798401 .124210-2.361349$

C $0.3081690 .427245-2.752240$

O $-0.735544 \quad 1.403680-2.515049$

C $0.012073-0.799556-1.871266$

C $0.769284-2.043359-2.275759$

C $0.289018-2.979667-3.116961$

C $2.113899-2.231866-1.627455$

C $-1.033898-2.982847-3.833062$

C $-1.894274-4.197806-3.457863$

C $-2.393040-4.181172-2.003184$

C $-3.101318-5.497438-1.674689$

C $-3.700197-5.491747-0.262325$

O $-3.303077-3.085721-1.853378$

O $-4.574296-6.627628-0.182413$

C $-4.549591-4.215625-0.091014$ 
C $-2.622835-5.6914150 .810687$

O $-5.006124-4.0843031 .254382$

C $-3.818572-2.920015-0.524024$

C $-4.832628-1.746570-0.541837$

O $-2.777990-2.640707 \quad 0.410287$

C $-1.833348 \quad 1.400724-3.308964$

O $-2.0529860 .655319-4.252829$

C $-2.8169062 .414492-2.856450$

C $-2.5265633 .367346-1.956406$

C $-3.4502404 .367182-1.416933$

C $-4.9095614 .223388-1.757869$

C $-4.273246-0.403068-1.024962$

C $-3.607080 \quad 0.3994380 .076214$

C -4.1018191 .6256382 .192740$

O $\begin{array}{llll}-2.389406 & 0.558419 & 0.107238\end{array}$

C -4.6011893 .0329112 .103688$

C -3.8428774 .1062462 .375001$

C -4.3606645 .5165552 .354235$

C -3.4917606 .4541181 .546692$

C -2.6403047 .3003342 .150852$

C -3.6719976 .4709920 .044762$

C $-2.9476295 .338579-0.626586$

N $-4.504436 \quad 0.898851 \quad 1.003407$

H $2.6079760 .938498-4.187906$

H $1.6166421 .522743-1.351788$

H $\quad 0.3251200 .155981-3.815243$

H -1.064829 -1.003561 -1.881233

H $\quad 0.213779-0.569905-0.815730$

H $\quad 0.914767-3.845511-3.330372$

H $2.558385-3.204222-1.865442$

H $2.815488-1.462844-1.958688$

H $2.020002-2.175459-0.538048$

H -1.600362 -2.061567-3.674226

H -0.825682 -3.025984 -4.909461

H -2.759674 -4.211369-4.133187

H -1.324078 -5.116579-3.645000

H -1.539976 -4.036789-1.331530

H $-2.419123-6.348307-1.796834$

H -3.914822 -5.661431-2.395646

H $-4.921440-6.6793720 .724008$

H -5.446709 -4.331434 -0.715344

H $-2.121456-6.656770 \quad 0.671190$

H -3.063370 -5.7247601 .813371$

H -1.855752 -4.9142910 .799442$

H -5.556072 -4.859625 1.456440

H -5.646142 $-2.013477-1.229267$

H -5.271965 -1.624395 0.454903

H $-2.422762 \quad-1.758440 \quad 0.219717$

H -3.801855 2.326835 -3.303009

H -1.512954 $3.427773-1.563154$

H $-5.0709614 .385063-2.829182$

H $-5.5538904 .926357-1.226377$

H $-5.2693903 .221746-1.495816$

H -3.566365 - $0.537322-1.849056$

H $-5.0942670 .209292-1.419507$

H -3.0154491 .5772242 .316782$

H -4.571084 1.1236563 .044770

H $-5.6466053 .175326 \quad 1.839277$

H -2.806312 3.9627212 .672054

H -4.415012 5.8632563 .394538

H -5.3888595 .5597451 .974001$

H -2.033461 7.9952091 .579787

H -2.5262687 .3127993 .229770$

H -3.268906 $7.405164-0.369149$

H -4.740105 $6.498519-0.185536$

H -1.871423 $5.349580-0.446042$

H -5.4957210 .7145220 .879190$

C $5.0759041 .250036-2.566749$

O $4.898096 \quad 0.413181-1.405344$

C $6.0776550 .492213-0.585083$

C $7.1546381 .117915-1.462037$

C $6.3351522 .077362-2.299299$

H $5.844727 \quad 1.179906 \quad 0.236717$

C $6.437887-0.886400-0.023156$

H $7.9323191 .614674-0.874060$

H $7.6399010 .375439-2.105996$

C $7.0451482 .544529-3.558396$

H $6.0868302 .952740-1.683869$
C $5.297329-1.5969460 .730097$

O $6.830332-1.746527-1.102159$

H $7.317385-0.8001940 .625294$

H $4.517186-1.9054640 .026067$

O $5.835799-2.8060641 .283157$

C $4.660397-0.7821351 .871554$

C $3.608927-1.6006112 .643036$

H 4.1849640 .1111101 .458102

O $5.675739-0.3434572 .771779$

H $2.859501-1.9577401 .928696$

H $4.090813-2.4861353 .074251$

H $7.9675853 .077172-3.304572$

H $7.3109031 .701791-4.205242$

H $6.4103793 .224527-4.135113$

C $3.8364142 .121660-2.763938$

H $\quad 6.088245-1.786187-1.730067$

H $6.220162-3.3143150 .548380$

H $6.122811-1.1347803 .118304$

H $4.0210852 .871191-3.542240$

H $3.6238482 .669567-1.837051$

H $5.2221170 .581005-3.424377$

C 1.1534940 .2291702 .536278

O $2.310610 \quad 0.390968 \quad 3.364071$

C $2.931198-0.8235203 .794275$

C $1.924030-1.6647734 .590791$

C $0.664579-1.9429603 .776582$

C $0.088991-0.6380313 .229110$

H $1.448452-0.2169051 .578455$

C $0.578303 \quad 1.6303982 .262687$

H $3.726397-0.5294474 .490557$

O $2.501209-2.9007444 .999118$

H $1.637976-1.1256775 .503322$

H $0.861444-2.6642782 .974822$

O $-0.297553-2.5541734 .640075$

H $-0.372381-0.0753134 .051356$

O $-0.939499-0.9381982 .288662$

C 1.4608702 .4838041 .378412

H -0.3913181 .5402551 .761297$

H $0.4467492 .173127 \quad 3.206208$

H $3.291010-2.6935585 .527364$

H $-1.052887-2.8264024 .092218$

H -1.661729 -1.377603 2.767935

O 0.7560903 .5915930 .997198

O $2.6174192 .230651 \quad 1.073355$

C 1.4580384 .5201240 .164989

H $1.6278954 .081634-0.822128$

H $0.8349635 .411048 \quad 0.050454$

H 2.4056724 .8139070 .626697

SCF Energy (B3LYP/6-31G**//MMFF) $=-3245.91567279$

0600091

MM̄FF Geometry

C $-1.7362013 .607307-1.912030$

C $-1.9061412 .304968-1.639781$

C $-2.3274351 .271948-2.648441$

O $-3.5594790 .712988-2.141128$

C $-1.2755640 .152638-2.728852$

C $-1.453742-0.751917-3.930158$

C $-2.044700-1.962502-3.902974$

C $-0.870837-0.225032-5.216224$

C $-2.712603-2.642248-2.740450$

C $-1.982420-3.916409-2.295093$

C $-0.665335-3.641588-1.552453$

C $0.016833-4.957125-1.170581$

C $1.276894-4.727340-0.324608$

O $-0.978587-2.898851-0.370819$

O $1.646622-5.999615 \quad 0.228430$

C $0.930720-3.7812160 .845017$

C $2.458074-4.277020-1.188845$

O $2.102335-3.4340031 .580219$

C $0.157577-2.5132680 .412179$

C $-0.364233-1.7651721 .662309$

O $1.039861-1.659461-0.317244$

C $-4.5922390 .506324-2.993608$

O $-4.605304 \quad 0.724210-4.196870$

C $-5.782110-0.009608-2.273540$

C $-5.754540-0.402152-0.989798$

C $-6.890560-0.881440-0.199544$ 
C $-8.246330-0.894680-0.854637$ C -1.327204 -0.6121311 .346044$ C $-1.911730 \quad 0.002541 \quad 2.606030$ C -3.785623 1.4669833 .359003 $\begin{array}{lllll} & 0 & -1.450721 & -0.197864 & 3.726647\end{array}$ C $-4.855165 \quad 0.5237223 .814684$ C $-6.169523 \quad 0.7579223 .681366$ C $-7.233870-0.1991774 .139115$ C $-8.149414-0.6413333 .017432$ C $-9.385188-0.1277852 .895252$ C $-7.669930-1.7394102 .092114$ C $-6.671060-1.2501611 .080020$ N -2.998352 0.8204622 .324426 H -1.905283 $3.976359-2.919955$ H $-1.7396091 .953908-0.623957$ H -2.483270 $1.743143-3.626195$ H $-1.292498-0.438940-1.806969$ H $-0.2671040 .584854-2.780620$ H -2.089877 -2.529490 -4.832218 H $-1.004044-0.921894-6.050551$ H $-1.3495780 .718351-5.496487$ H $0.203971-0.049746-5.101815$ H -2.855009 -1.969662 -1.890230 H -3.722492 -2.921583 -3.066949 H -2.659551 -4.462090 -1.624772 H $-1.796366-4.562725-3.161862$ H $-0.008668-3.044202-2.194698$ H $0.255388-5.546437-2.064813$ H $-0.682436-5.572475-0.586588$ H $2.398642-5.8529920 .827852$ H $\quad 0.299399-4.3531831 .538709$ H $2.668410-5.020930-1.966591$ H $3.373169-4.205946-0.592624$ H $2.289561-3.317827-1.682824$ H $2.582793-2.7592881 .070443$ H $-0.899833-2.4757812 .305900$ H $0.479613-1.3735742 .243485$ H $0.734630-0.742391-0.238914$ H $-6.691183-0.046833-2.865028$ H $-4.810779-0.363014-0.448256$ H -8.510866 $0.106142-1.214018$ H $-9.050347-1.203953-0.183378$ H -8.255241 - $1.586988-1.703736$ H -0.8093350 .1842640 .803254$ H -2.155121 -0.9718580 .724638$ H -4.1970002 .3863962 .931032$ H -3.138167 1.7305214 .201580 H $-4.527042-0.4015214 .283034$ H -6.5024611 .6877313 .226104$ H $-7.8141340 .301417 \quad 4.925158$ H -6.796853 -1.085250 4.616334 H -10.060841 -0.4592152 .113808$ H -9.7478040 .6393083 .571495$ H -8.517086 -2.2310471 .600793$ H -7.203708 -2.534926 2.687712 H -5.651483 -1.2053321 .463128$ H $-3.348395 \quad 0.865626 \quad 1.371806$ C $0.0385105 .238085-1.192167$

O $1.041688 \quad 4.205787-1.265253$

C $2.1036894 .519194-0.351379$

C $2.0035476 .021741-0.126358$

C $0.4995466 .213119-0.103577$

H 1.8749904 .0033860 .588458

C $3.4470864 .027217-0.897384$

H $2.491904 \quad 6.3387520 .799843$

H $2.4398986 .589787-0.955767$

C $0.0614617 .647791-0.342655$

H $0.124275 \quad 5.889913 \quad 0.876827$

C $3.4753592 .562707-1.383054$

O $3.8217434 .841193-2.019513$

H $4.2280934 .187996-0.145603$

H $2.8730412 .477308-2.296524$

O $4.8234332 .250487-1.761974$

C $3.0242831 .476773-0.391110$

C $3.7212941 .552801 \quad 0.978559$

H $3.2424490 .502562-0.842151$

O $1.6147751 .529994-0.189842$

H $4.790907 \quad 1.7331580 .836304$
H 3.3198152 .4048901 .538109 H $\quad 0.455767 \quad 8.305870 \quad 0.438536$

H $0.4187688 .020958-1.308336$

H $-1.0302087 .727326-0.333511$

C $-1.3196874 .609446-0.871624$

H $3.1123934 .772380-2.681652$

H $5.1042922 .918489-2.410848$

H $1.1905441 .496076-1.063942$

H $-2.0875175 .389183-0.809312$

H $-1.274476 \quad 4.122157 \quad 0.110950$

H $0.0134445 .724954-2.175110$

C $5.456794-0.9569911 .088626$

O $4.026398-0.8942031 .156898$

C $3.500927 \quad 0.268031 \quad 1.810688$

C $4.032290 \quad 0.342502 \quad 3.248004$

$\begin{array}{llll}\text { C } & 5.555661 & 0.282171 & 3.292015\end{array}$

C $6.065282-0.9184952 .496491$

H $5.838821-0.1179370 .495215$

C $5.832299-2.260805 \quad 0.366522$

H $2.416963 \quad 0.116778 \quad 1.879067$

O $3.591443 \quad 1.530733 \quad 3.896166$

H $3.636155-0.5034983 .824844$

H 6.0060601 .2136092 .929171

$\begin{array}{lllll}\text { O } & 5.981191 & 0.131630 & 4.648382\end{array}$

H $5.825686-1.8435263 .036527$

O $7.485656-0.8390472 .392368$

C $5.555224-2.201817-1.121656$

H $6.902668-2.4638940 .482300$

H $5.255801-3.0961180 .780450$

H 2.6193541 .5347073 .868189

H 5.6150620 .8799615 .150473

H $7.840522-0.7930313 .296856$

O $5.938798-3.385303-1.687674$

O $5.069056-1.250444-1.716441$

C $5.738216-3.484253-3.101188$

H $6.353036-2.743994-3.621620$

H $6.047007-4.483691-3.419115$

H $4.680480-3.349647-3.346849$

SCF Energy (B3LYP/6-31G**//MMFF) $=-3245.93787763$

0600092

MMFF Geometry

C $2.471207-3.531534 \quad 0.260037$

C $2.102058-4.369314-0.720922$

C $1.248728-4.012320-1.916589$

O $1.054900-2.584200-2.029123$

C $-0.142323-4.658239-1.817455$

C $-0.936260-4.496345-3.100049$

C $-1.892769-3.568547-3.302822$

C $-0.605287-5.487674-4.186456$

C $-2.369281-2.500631-2.355448$

C $-3.819074-2.770445-1.941066$

C $-4.360385-1.733930-0.945425$

C $-5.769516-2.137775-0.502204$

C $-6.403412-1.0959470 .424386$

O $-4.382684-0.450402-1.580674$

O $-7.797641-1.420706 \quad 0.529410$

C $-6.2851220 .287131-0.244548$

C $-5.832527-1.1844791 .844474$

O

C $-4.8579880 .620141-0.747177$

C $-4.9143461 .903905-1.616081$

$\begin{array}{llllll} & \text { O } & -4.011481 & 0.819928 & 0.381852\end{array}$

C $2.040082-1.860139-2.624047$

O $3.110317-2.271103-3.041891$

C $1.580363-0.456388-2.672324$

C $2.4540830 .515367-2.971960$

C $2.1456291 .942347-3.074020$

C $0.6964452 .342368-3.013442$

C $-3.5570292 .496778-2.020230$

C $-3.0315003 .518354-1.020212$

C $-1.2645395 .221695-0.710106$

$\begin{array}{lllll}\text { O } & -3.552470 & 3.721462 & 0.073545\end{array}$

C $-0.0411635 .712873-1.412625$

C $1.2061545 .556041-0.942980$

C $2.4257136 .118568-1.616808$

C $3.4030445 .049923-2.050814$

C $4.5294874 .827762-1.351206$ 
C $3.0935824 .316357-3.339743$

C $3.1632442 .816972-3.213889$

N -1.899126 $4.172606-1.485513$

H $2.098133-2.5114330 .266252$

H $2.481441-5.388923-0.706437$

H $1.760667-4.374527-2.817272$

H $-0.693930-4.228482-0.974283$

H $-0.051274-5.729500-1.594001$

H -2.397005 -3.559206 -4.268795

H - $-1.229346-5.353959-5.076474$

H $0.438283-5.384113-4.498724$

H -0.761404 -6.510058 -3.826758

H -1.737055 -2.426058 -1.466733

H -2.297205 -1.533203 -2.866526

H -4.448300 -2.770397 -2.841375

H -3.887325 -3.771142 -1.494846

H $-3.687241-1.693674-0.083469$

H $-5.759175-3.123314-0.019615$

H $-6.410597-2.248053-1.388508$

H $-8.223971-0.7214481 .054406$

H $-6.9662840 .288372-1.106615$

H -6.021225 -2.178199 2.268601

H $-6.327456-0.4754012 .517062$

H $-4.756224-1.0060611 .886493$

H $-6.0678551 .480342 \quad 1.290119$

H $-5.4513641 .657186-2.541945$

H $-5.5003942 .678844-1.108036$

H $-3.166577 \quad 1.1853250 .075722$

H $0.535956-0.261897-2.455312$

H $3.494607 \quad 0.246422-3.148976$

H $\quad 0.110121 \quad 1.793584-3.759135$

H $0.5330923 .400682-3.219011$

H $0.2836942 .135496-2.020094$

H $-2.8057041 .713222-2.165947$

H -3.684486 $3.021711-2.975471$

H $-1.044958 \quad 4.8286230 .287715$

H -1.983131 $6.039974-0.592007$

H $-0.1952676 .255634-2.342312$

H $1.3605655 .026315-0.005622$

H $2.9057436 .808857-0.910793$

H $2.1557256 .732689-2.485284$

H $5.2608974 .092187-1.668293$

H $4.7477815 .380007-0.443013$

H $3.8157884 .633182-4.103853$

H $2.1237894 .633725-3.734779$

H $4.1805442 .424217-3.244020$

H -1.628752 4.047114 -2.457013

C $4.654002-2.9607601 .407468$

O $4.236746-1.6159921 .723294$

C $4.816859-0.7180660 .759561$

C $6.018849-1.4611610 .192156$

C $5.476275-2.8763120 .116213$

H $4.072594-0.584686-0.035640$

C $5.123890 \quad 0.6405551 .397694$

H $6.331911-1.068143-0.779741$

H $6.878588-1.4252480 .870799$

C $6.552365-3.9426590 .008104$

H $4.832402-2.943998-0.768130$

C 3.9562351 .2519072 .197680

O $6.227551 \quad 0.5034642 .302479$

H $\begin{array}{llll}5.458323 & 1.341136 & 0.623747\end{array}$

H 3.8081110 .6893993 .127998

O 4.3377302 .5775212 .587351

C 2.6201941 .3334241 .438971

C 1.5186101 .9951622 .285810

H $2.302851 \quad 0.3242671 .160560$

O 2.7922722 .0695540 .231972

H 1.4856061 .4980013 .261445

H 1.7776973 .0452572 .469068

H $7.140963-3.804066-0.904667$

H $7.239889-3.9076080 .859764$

H $6.105635-4.941516-0.024856$

C $3.430731-3.887887 \quad 1.361327$

H $5.983780-0.1674222 .963556$

H 5.1782412 .5063723 .071733

H 3.1003422 .9601970 .471574

H $2.886495-3.8081412 .310000$

H $3.765921-4.9267931 .262552$
H $\quad 5.292273-3.2773302 .242142$

C $-0.460352-0.3091142 .216027$

$\begin{array}{llll}\text { O } & -0.235211 & 0.643616 & 1.168869\end{array}$

C $\quad 0.1246761 .9533391 .619278$

C -0.9974082 .5203902 .499265$

C -1.3478941 .5817313 .652763$

C $-1.5942320 .162877 \quad 3.137457$

H $0.465789-0.4299162 .789322$

C $-0.789809-1.6467701 .537872$

H $\quad 0.1869952 .578247 \quad 0.719948$

O -0.6268923 .7989493 .003322$

H -1.8921892.664683 1.882660

H -0.5702631 .5989944 .425050$

O -2.5399982 .0651374 .276660$

H -2.5481230 .1496842 .598294$

O $-1.738316-0.7199844 .246444$

C $-0.434168-2.8596302 .368439$

H -1.856060 -1.686574 1.293143

H $-0.235784-1.7283870 .595392$

H -1.358678 4.1144493 .560906

H -2.7761341 .4335274 .977698$

H $-0.891617-0.739617 \quad 4.724431$

O $-0.970818-3.968800 \quad 1.778543$

O $0.246867-2.8486373 .384094$

C $-0.682431-5.2117652 .425448$

H $\quad 0.397982-5.3821362 .456357$

H -1.104501 -5.2191933 .434724$

H -1.146454 -6.012770 1.843932

SCF Energy (B3LYP/6-31G**//MMFF) $=-3245.93902778$

0600093

MMFF Geometry

C $-0.815402 \quad 0.967327 \quad 3.234435$

C -1.483004 2.0930093 .531269

C -1.942436 3.1335892 .542144

O -2.5168212 .5132431 .369165$

C -0.7707324 .0067062 .063293$

C -1.1981585 .4090641 .682486$

C -1.5327645 .8117670 .441623$

C -1.191082 6.3999292 .819104

C $-1.6604394 .987665-0.808562$

C $-0.5422645 .268865-1.821134$

C $0.6871134 .374404-1.602349$

C $1.8336744 .798783-2.521409$

C $3.0331823 .846258-2.414977$

O $0.2909683 .028842-1.898075$

O $3.918306 \quad 4.127644-3.509789$

C $2.5318122 .400104-2.598818$

C $3.8403684 .078262-1.132274$

O $3.5857551 .464351-2.384564$

C $1.3154502 .046393-1.709475$

C $0.747195 \quad 0.673224-2.139965$

O $1.7336521 .980312-0.347255$

C -3.7731392 .0048221 .460946$

O $-4.467746 \quad 1.9241062 .460283$

C $-4.1623391 .572506 \quad 0.100598$

C $-5.2699680 .839680-0.080499$

C $-5.7868500 .366793-1.367250$

C $-5.0600440 .800749-2.614281$

C $-0.5776040 .278195-1.471439$

C $-1.058509-1.080928-1.956162$

C $-2.759739-2.834883-1.467261$

O $-0.521649-1.692207-2.876935$

C $-3.889472-2.709907-2.439322$

C $-5.121223-3.188601-2.205644$

C $-6.248687-3.112553-3.195263$

C -7.507174 -2.512407 -2.608754

C $-8.532690-3.292702-2.227277$

C $-7.610749-1.005058-2.548613$

C $-6.881729-0.421948-1.370227$

N $-2.159853-1.532182-1.241856$

H -0.5309140 .7639052 .205550$

H -1.739869 2.2925274 .569411

H -2.7123233 .7524943 .021096$

H -0.2682813 .5262951 .215706$

H -0.0087624 .0955112 .849002$

H -1.789846 6.8615390 .301018

H -1.516692 7.3975272 .506023 
H -1.8637406 .0693553 .616989$ H $-0.1816396 .496274 \quad 3.232343$ H $-1.7288333 .918964-0.592838$ H -2.619854 $5.258302-1.268154$ H $-0.9445215 .068478-2.823062$ H $-0.2572126 .327671-1.791744$ H $0.9996044 .441985-0.555063$ H $2.1422625 .829705-2.307860$ H $1.4847064 .800921-3.563636$ H $4.1856565 .060648-3.446706$ H $2.2325852 .290892-3.650852$ H $4.2184905 .107084-1.099413$ H $4.7255893 .433633-1.101342$ H $3.2600823 .912179-0.222247$ H $4.3222611 .717742-2.966894$ H $0.5788620 .683301-3.225141$ H $1.485832-0.110500-1.934331$ H $1.130648 \quad 1.4036220 .146449$ H -3.525120 $1.879133-0.720978$ H -5.8557570 .5583300 .793725$ H $-5.1069521 .889911-2.724214$ H $-5.4666330 .369032-3.530739$ H $-4.0078800 .499438-2.576776$ H $-0.4632920 .229207-0.383726$ H -1.354803 $1.012907-1.706818$ H -3.091596 -3.212889-0.495870 H -2.004666 -3.519338 -1.866954 H $-3.677118-2.230049-3.391844$ H $-5.323710-3.695582-1.264752$ H $-6.450654-4.133285-3.545548$ H -5.959396 -2.545541-4.088939 H -9.449892 -2.868039-1.833055 H -8.475433 -4.374044 -2.295431 H -8.663555 -0.705266 -2.455629 H -7.289065 -0.582300 -3.504290 H -7.335166 $-0.680819-0.412167$ H $-2.571428-0.935341-0.530353$ C $-0.888386-1.4812963 .938938$ O $0.006105-2.0731812 .977678$ C $-0.758986-2.9306172 .110230$ C $-2.163286-3.0118552 .708527$ C $-2.294915-1.6529193 .365870$ H $-0.812404-2.4160971 .145005$ C - $0.070498-4.2889551 .934192$ H -2.928115 -3.197680 1.948985 H $-2.243592-3.8037313 .461841$ C -3.399639-1.571248 4.403186 H $-2.494019-0.9208752 .573760$ C $1.410132-4.2318151 .508024$ O $-0.122715-5.012048 \quad 3.172129$ H $-0.643144-4.8886931 .216918$ H $2.028125-3.9112032 .355398$ O $1.826716-5.5744591 .221442$ C $1.722065-3.3286200 .300581$ C $3.149092-3.543566-0.240143$ H $1.587429-2.2829960 .596131$ O $\quad 0.793097-3.585328-0.752179$ H $3.844058-3.6125920 .602064$ H $3.183935-4.502681-0.770423$ H -4.373200 -1.772749 3.944511 H -3.249950 -2.298811 5.207853 H -3.437859 -0.573907 4.852629 C $-0.441040-0.0547064 .271795$ H $0.322014-4.4707773 .846953$ H $1.280167-5.9080430 .490149$ H $0.913461-4.504291-1.044770$ H $0.649693-0.0357974 .383486$ H -0.8711150 .2260225 .241144$ H -0.779159 -2.086539 4.849251 C $4.815511-1.0540360 .366420$ O $3.714281-1.157473-0.545298$ C $3.584890-2.421234-1.209010$ C $4.866998-2.728897-1.994015$ C $6.098312-2.679362-1.097191$ C $6.145413-1.355173-0.339229$ H $4.668835-1.7451691 .204558$ C $4.826980 \quad 0.3751490 .930452$ H $2.781006-2.301736-1.945976$
O $4.792672-4.005977-2.618430$

H $4.990429-1.987329-2.794156$

H $6.130162-3.530489-0.406785$

O $7.273528-2.772509-1.905507$

H $6.390988-0.545146-1.038467$

O $7.189239-1.4104310 .631627$

C 3.6686880 .6505211 .863938

H 5.7464900 .5474361 .501062

H 4.7683501 .0999650 .111242

H $4.006631-4.005314-3.191052$

H $7.208523-3.598608-2.414900$

H $8.013311-1.6208490 .159636$

O $3.802141 \quad 1.9189792 .355764$

O $2.763105-0.1290532 .123603$

C 2.7654702 .3489063 .241494

H 3.0053883 .3593103 .582654

H 1.8117142 .3761412 .709570

H 2.7073811 .6881714 .111490

SCF Energy (B3LYP/6-31G**//MMFF)= -3245.91267166

\section{4}

MM̄FF Geometry

C -0.7176412 .5125630 .115811$

C -1.817076 2.8080940 .826163

C -2.3496432 .0344372 .011311$

O -1.6752150 .7592582 .105244$

C -3.8512561 .7556751 .819225$

C -4.5547951 .2993473 .080739$

C -4.9391050 .0324533 .331280$

C $-4.8749972 .392778 \quad 4.067415$

C $-4.694538-1.1936632 .496392$

C $-5.967372-1.7139781 .813073$

C $-6.404891-0.8660750 .607699$

C -7.699010-1.413868 0.001896

C $-8.096978-0.662733-1.277805$

O $-5.355529-0.922267-0.362432$

O $-9.121568-1.438855-1.917178$

C $-6.878483-0.609085-2.225204$

C $-8.7264700 .697754-0.956544$

O $\quad-7.1639770 .186570-3.373515$

C $-5.579096-0.132832-1.536927$

C $-4.370401-0.344140-2.480209$

O $-5.7011301 .257242-1.231286$

C -1.2309030 .3354223 .313236$

O $-1.2917450 .946346 \quad 4.371085$

C $-0.629437-1.0180293 .223393$

C $-0.673429-1.7819312 .120052$

C $-0.121091-3.1312831 .972186$

C $0.611497-3.7246373 .145972$

C $-3.009206-0.091213-1.815254$

C $-1.850815-0.483810-2.715672$

C $0.585888-1.011158-2.611263$

O $-1.975557-0.689007-3.920065$

C $0.636032-2.505014-2.533864$

C $1.549751-3.188872-1.829397$

C $1.568733-4.686953-1.726951$

C $1.504519-5.178141-0.295966$

C $2.590710-5.701510 \quad 0.297165$

C $0.168655-5.1565890 .416211$

C $-0.285866-3.7715280 .795371$

N $-0.655889-0.558731-2.011894$

H -0.1117521 .6510450 .383394$

H -2.386210 3.6924130 .544499

H -2.173662 2.6436982 .905451

H -3.9778041 .0129141 .025636$

H -4.3654292 .6578701 .461241$

H -5.470947 -0.1659434 .261222$

H $-5.4336912 .021706 \quad 4.933227$

H $-3.9566762 .854100 \quad 4.443103$

H -5.4846253 .1677063 .591111$

H $-3.897056-1.0419541 .763313$

H -4.322434 -1.974253 3.172531

H $-5.757830-2.7351491 .468246$

H $-6.784018-1.7801722 .542688$

H -6.5470260 .1707500 .931262$

H $-8.515072-1.3856380 .734613$

H -7.561359-2.475041-0.249947

H $-9.327893-1.011799-2.766413$ 
H -6.720605 -1.630189-2.598491 H $-9.6205730 .565720-0.335338$ H -9.069044 1.202010-1.866757 H -8.048874 $1.368849-0.424603$ H -7.107506 $1.120285-3.107902$ H -4.377402 -1.381009-2.841704 H $-4.4607610 .308437-3.357605$ H -4.817032 $1.652513-1.181793$ H $-0.162612-1.3657884 .139489$ H -1.171521 $-1.395171 \quad 1.232713$ H $1.427641-3.0676403 .464233$ H $1.064683-4.6944312 .928618$ H $-0.071341-3.8695503 .990278$ H -2.891695 $0.969504-1.573258$ H $-2.929826-0.667575-0.886543$ H $1.403965-0.539269-2.058881$ H $0.629866-0.687801-3.655982$ H $-0.129280-3.051774-3.080361$ H $2.321372-2.646818-1.290516$ H $2.489914-5.040042-2.208531$ H $\quad 0.745258-5.137036-2.295423$ H $2.552708-6.0874401 .310281$ H $3.545684-5.751054-0.215471$ H $\quad 0.184262-5.8091181 .297026$ H $-0.590190-5.603811-0.239175$ H $-0.835212 \quad-3.263170 \quad 0.002700$ H -0.671362 -0.442155-1.003025 C $0.9992124 .124516-0.849889$ O $2.0728173 .291253-0.373818$ C 2.8076184 .0231110 .628663 C 2.3308935 .4677170 .532041 C $0.874725 \quad 5.2693410 .154654$ H 2.4993283 .6223661 .601184 C 4.3127573 .8211930 .430472 H 2.4624716 .0124741 .471829 H $2.8578326 .018074-0.256056$ C $0.2134686 .515888-0.405516$ H $0.327796 \quad 4.9453921 .048831$

C $4.7433992 .339028 \quad 0.441637$ O $4.7002134 .381398-0.830297$ H 4.8684324 .3818571 .190366 H $4.2914301 .820345-0.407295$ O 6.1618112 .2923110 .249708 C 4.4102751 .6010151 .748176 C 4.9209020 .1503491 .835702 H 3.3287551 .5984221 .916213 O 4.9867222 .3258902 .841419 H 5.9631050 .1007541 .503139 H $4.947029-0.1193972 .899421$ H $\quad 0.223740 \quad 7.3222410 .335089$ H $\quad 0.7295386 .876454-1.301567$ H $-0.8289086 .316803-0.673373$ C $-0.2619243 .292329-1.085937$ H $4.1793613 .938616-1.521127$ H $6.3543102 .742441-0.590927$ H 5.9486392 .3528932 .698850 H $-0.0572012 .561014-1.878258$ H -1.066014 3.937390-1.459339 H $1.3290604 .519942-1.819993$ C $5.282526-1.071745-0.989429$ O $4.060413-0.705240-0.338365$ C $4.040480-0.8694871 .081729$ C $4.346944-2.3303961 .442559$

C $5.669619-2.787600 \quad 0.839681$

C $5.682240-2.517802-0.661414$ H $6.077178-0.380234-0.686483$ C $5.065050-0.928532-2.503196$ H $3.005438-0.6787041 .393908$ O $4.387793-2.5204842 .852618$ H $3.545917-2.9721631 .057947$ H $6.525334-2.3125811 .333625$ O $5.819173-4.1946371 .040908$ H $4.999749-3.219238-1.158764$ O $6.990634-2.766794-1.172305$ C $4.8335990 .507178-2.911207$ H $5.929034-1.287062-3.073763$ H $4.202052-1.528915-2.816537$ H $3.549006-2.1907313 .217136$
H $5.756299-4.3577951 .997763$

H $7.225893-3.680174-0.934943$

O $3.5808210 .643628-3.431841$

O $5.6636301 .397550-2.783861$

C $3.2243821 .958157-3.868887$

H $3.1198782 .620332-3.006494$

H $3.9682792 .348398-4.570030$

H $2.2602841 .895410-4.380631$

SCF Energy (B3LYP/6-31G**//MMFF) $=-3245.93882199$

\section{5}

MMFF Geometry

C 1.3210333 .6063830 .980720

C 0.8244772 .9859122 .061989

C 1.3193591 .6472262 .537310

$\begin{array}{lllll}\text { O } & 0.163377 & 0.803501 & 2.777759\end{array}$

C 2.0830731 .7165783 .869339

C 3.3260952 .5768873 .867207

C 4.4907832 .2356413 .282861

C 3.2072883 .8778524 .617286

C 4.7909210 .9939962 .488789

C $5.241216 \quad 1.3696341 .070971$

C $5.480074 \quad 0.139094 \quad 0.185174$

C $6.1609570 .558423-1.120048$

C $6.328454-0.620685-2.085632$

O $4.208725-0.457309-0.094157$

O $6.691692-0.069454-3.360220$

C $4.966528-1.324916-2.241208$

C $7.485672-1.533905-1.665689$

O $5.097084-2.500642-3.038407$

C $4.269975-1.646207-0.895941$

C $2.818173-2.105177-1.177302$

O $4.988643-2.687576-0.238262$

C -0.3778090 .1574801 .711618$

O $\begin{array}{llll}-0.006241 & 0.212152 & 0.551180\end{array}$

C $-1.508362-0.6538012 .212140$

C $-1.932981-1.7011611 .490884$

C $-3.017409-2.6154761 .848416$

C $-3.878412-2.2563643 .030298$

C $1.963705-2.3849210 .064233$

C $2.012021-3.8219870 .553067$

C $0.971497-5.3032162 .272160$

O $2.685016-4.6968330 .016424$

C $-0.300787-5.8725301 .726098$

C $-1.368287-6.1659572 .483881$

C $-2.648251-6.7394021 .943919$

C $-3.849074-5.8760872 .264999$

C $-4.589498-6.1113883 .361572$

C $-4.240954-4.7954371 .281695$

C -3.192447 -3.731455 1.110471

N $1.197160-4.0063361 .662598$

H $2.156135 \quad 3.141120 \quad 0.461849$

H $0.002863 \quad 3.4241182 .620459$

H 1.9505391 .1696481 .778611

H 2.3510320 .6964204 .178365

H 1.4007492 .0532204 .662929

H 5.3296042 .9249643 .373537

H 4.1310974 .4649234 .583109

H 2.4096724 .4943644 .191082

H 2.9748533 .6875365 .670271

H 5.5969680 .4478172 .993496

H 3.9348610 .3150912 .449341

H 4.4751352 .0054720 .608142

H 6.1634651 .9612181 .135870

H $6.104771-0.5760940 .732819$

H $7.1314851 .029783-0.920141$

H $5.5527111 .328728-1.615372$

H $6.724259-0.801955-3.999533$

H $4.320316-0.644960-2.813233$

H $8.424846-0.968587-1.635724$

H $7.644294-2.335165-2.395868$

H $7.339340-1.987152-0.683030$

H $5.481896-3.196162-2.478113$

H $2.317353-1.295483-1.722264$

H $2.817815-2.985098-1.831055$

H $4.421697-3.0693630 .450004$

H $-1.938264-0.3843393 .170070$

H -1.424719 -1.9263800 .555287$ 
H -3.284611 -2.248303 3.950753 H $-4.702754-2.9548613 .192582$ H $-4.327713-1.2664912 .892253$ H $2.224560-1.7145220 .889924$ H $0.917974-2.194501-0.205429$ H $0.926152-5.1505393 .355010$ H $1.807144-5.9731652 .047278$ H $-0.341331-6.0558480 .654825$ H -1.319269 -5.996393 3.557265 H -2.779085 -7.7375232 .382372$ H $-2.592144-6.9002660 .860128$ H -5.469009 -5.5183383 .589084$ H $-4.329868-6.9014164 .058629$ H -5.200352 -4.338198 1.552323 H -4.422852 -5.2602810 .303718$ H $-2.512734-3.9197090 .279024$ H $\quad 0.635667-3.2279871 .995691$ C -0.5801695 .3315390 .751001$ $\begin{array}{lllll}\text { O } & -1.526961 & 4.318378 & 0.357281\end{array}$ C $-2.6934724 .954397-0.192530$ C $-2.542799 \quad 6.4433370 .099651$ C -1.036193 6.6094810 .048575 H $-2.6533684 .803774-1.277592$ C $-3.959821 \quad 4.311590 \quad 0.380751$ H $-3.0696807 .064253-0.631344$ H -2.9203356 .7032141 .095434$ C $-0.542198 \quad 7.887246 \quad 0.703652$ H $-0.7175726 .596760-1.002521$ C -4.0552002 .7844510 .178679$ O $-4.0155304 .559291 \quad 1.792300$ H -4.844668 4.799873 -0.043585 H -3.314216 2.2681110 .801195 O $-5.3410092 .369140 \quad 0.659451$ C $-3.9069752 .318146-1.282050$ C $-4.2225640 .820391-1.462140$ H $-2.8861972 .516637-1.621830$ O $-4.7826123 .079196-2.115178$ H $-3.6166370 .244987-0.754787$ H $-5.2747080 .646733-1.208189$ H $-0.958816 \quad 8.7644180 .198097$ H -0.8342897 .9368521 .758017$ H $\quad 0.5490187 .955150 \quad 0.652981$ C $0.829864 \quad 4.8863740 .357250$ H -3.2048704 .1927642 .186029$ H -5.4126032 .6619451 .584372$ H $-5.6908362 .929313-1.801150$ H 1.5543275 .6773410 .581046 H $0.8496134 .728451-0.729255$ H -0.6618675 .4518381 .838623$ C -1.691991 $-0.425077-2.695528$ O $-2.6212090 .474406-3.316001$ C $-3.9859520 .330144-2.909447$ C $-4.467049-1.101205-3.178381$ C $-3.557478-2.129219-2.518766$ C $-2.104639-1.888680-2.923411$ H $-1.643668-0.217594-1.620942$ C $-0.313610-0.139577-3.314707$ H $-4.5703250 .985113-3.567591$ O $-5.805613-1.265464-2.722419$ H -4.474178 -1.277255 -4.262051 H $-3.680147-2.117480-1.430520$ O $-3.955737-3.430833-2.954430$ H $-1.979293-2.166742-3.977885$ O $-1.290590-2.764153-2.144728$ C $0.3407991 .105969-2.752663$ H $0.370960-0.968709-3.113406$ H $-0.403514-0.004329-4.398810$ H $-6.057174-2.190512-2.886596$ H -3.348733 -4.070385 -2.543742 H $-0.414969-2.812836-2.562502$ O $1.6411601 .139214-3.172953$ O $-0.2069011 .939126-2.045115$ C $2.4288252 .234125-2.695819$ H $1.9006333 .184479-2.818757$ H $2.6878492 .065550-1.647421$ H $3.3507812 .269375-3.282384$ SCF Energy $\left(B 3 L Y P / 6-31 G^{* *} / / M M F F\right)=-3245.93519037$
0600096

MM̄FF Geometry

C $-0.330549-4.133277-0.357709$

C $-0.601250-2.972440-0.973831$

C $-0.439658-2.753294-2.452369$

O $0.407729-1.593658-2.639560$

C $-1.779321-2.421443-3.130833$

C $-2.783053-3.552745-3.125001$

C $-3.923916-3.550575-2.408876$

C $-2.465241-4.707742-4.038521$

C $-4.401276-2.497223-1.448570$

C $-5.352940-1.512140-2.134621$

C $-6.071157-0.599237-1.130976$

C $-7.0166810 .356485-1.863674$

C $-7.7111671 .332462-0.901709$

O $\quad-5.094150 \quad 0.143814-0.391114$

O $-8.3012872 .379297-1.688563$

C $-6.6388231 .988409-0.005362$

C $-8.8630170 .662606-0.143669$

O -7.2411062 .8065320 .994912$

C -5.6591490 .9759870 .632776$

C -4.5080901 .6681541 .397530$

O $-6.3744150 .190891 \quad 1.597191$

C $1.743079-1.791477-2.778948$

O $2.337560-2.859008-2.734615$

C $2.429996-0.517073-3.085777$

C $1.9655580 .675534-2.681111$

C $2.565373 \quad 1.980158-2.973143$

C $3.7421192 .008526-3.914640$

C -3.5780452 .5466470 .556562$

C -2.5430363 .2501361 .420852$

C -0.8145045 .0111991 .341492$

O -2.4441013 .0700402 .632249$

C 0.1343015 .5950910 .347416

C 1.4609015 .3966490 .381747

C $2.4261926 .068919-0.550599$

C $3.1723795 .094872-1.429990$

C $4.4712904 .823598-1.219675$

C $2.4355204 .517253-2.615956$

C $2.0285713 .082722-2.407895$

N -1.7388134 .1096480 .680990$

H $0.022946-4.966152-0.963028$

H $-0.958733-2.118901-0.405435$

H $0.014681-3.621910-2.944358$

H -1.584705 -2.135829 -4.174179

H $-2.197130-1.508388-2.688302$

H -4.595550 -4.402496 -2.507427

H -3.270070 -5.450195 -4.064920

H -2.314036 -4.351877 -5.062876

H -1.556566 -5.220849 -3.709424

H $-4.917852-3.004521-0.624163$

H -3.562279 -1.963280 -0.990775

H $-4.773215-0.901021-2.838778$

H $-6.101761-2.060892-2.719979$

H -6.636064 -1.236629 -0.440220

H $-7.760661-0.201633-2.445779$

H $-6.4434750 .942938-2.595773$

H -8.925322 $1.970295-2.312461$

H $-6.0766032 .670412-0.653773$

H $-9.6081920 .272304-0.847635$

H $-9.396957 \quad 1.3853690 .483118$

H -8.538268 -0.1689670 .484688$

H $-7.5911282 .219216 \quad 1.686364$

H -4.9247582 .2514892 .229618$

H $-3.8922870 .891947 \quad 1.873251$

H $-5.732360-0.2041372 .210155$

H $3.353907-0.619325-3.644605$

H $\quad 1.0576270 .707179-2.080446$

H $3.4618111 .599317-4.891642$

H $4.5727861 .419620-3.513252$

H $4.1387063 .009591-4.093414$

H -3.052281 $1.939108-0.188291$

H $-4.146731 \quad 3.323247 \quad 0.036004$

H -0.3003534 .4753112 .146029$

H -1.4050555 .8095001 .804185$

H $-0.2870786 .254264-0.407813$

H $1.8854504 .752544 \quad 1.148544$

H 3.1310256 .6458060 .062423 
H $1.9194816 .808923-1.183169$ H $5.0209134 .153963-1.872587$ H $5.0135325 .262370-0.388735$ H $3.0373224 .653026-3.520141$ H $1.5265655 .101145-2.810981$ H $1.1800952 .961418-1.733519$ H $-2.0173144 .322646-0.273022$ C $-0.882302-3.2772032 .019938$ O $0.120263-2.2451751 .991853$ C $0.215456-1.6475693 .296547$ C $-0.867029-2.3003314 .151776$ C $-1.009736-3.6591943 .492674$ H $1.196874-1.9245593 .698181$ C $0.106073-0.1234753 .176795$ H $-0.577363-2.3625965 .205411$ H $-1.814177-1.7509484 .101533$ C $-2.306126-4.3684923 .842554$ H $-0.159987-4.2864423 .793921$ C $1.110813 \quad 0.5199182 .198330$ $\begin{array}{llll}\text { O } & -1.208613 & 0.216897 & 2.716869\end{array}$ H $\quad 0.2039630 .3321724 .168883$ H $\quad 0.8549250 .260992 \quad 1.163387$ $\begin{array}{lllll}\text { O } & 0.965605 & 1.941317 & 2.302648\end{array}$ C 2.5829690 .1620372 .463137 C 3.5569190 .9624351 .577568 H $2.727917-0.9075192 .287066$ $\begin{array}{lllll}\text { O } & 2.896755 & 0.406917 & 3.834544\end{array}$ H 3.2342710 .8843420 .534206 H $3.5080082 .022057 \quad 1.855672$ H -2.371346 -4.539674 4.922021 H -3.179542 -3.7797913 .542125$ H $-2.367659-5.3404473 .343327$ C $-0.452568-4.4339851 .115061$ H $-1.341197-0.2169041 .856414$ H 0.0303602 .1467102 .133260 H 2.7253201 .3478974 .011494 H - $-1.159376-5.2646621 .223846$ H $0.527350-4.7974091 .447379$ H $-1.821906-2.8363521 .661305$ C $5.039975-1.2102390 .004212$

O $5.170045-0.902577 \quad 1.398046$

C 5.0182810 .4824061 .731415 C $6.046694 \quad 1.3266350 .965795$ C $5.9890311 .065523-0.535679$ C $6.079052-0.433382-0.814108$ H $4.030013-0.957816-0.338535$ C $5.241436-2.724620-0.156950$ H $5.269456 \quad 0.5674382 .796088$ O 5.8535112 .7155801 .208720 H 7.0552621 .0768431 .320138 H $5.086057 \quad 1.495214-0.982786$ O $7.097702 \quad 1.706777-1.169238$ H $7.089966-0.788671-0.576311$ O $5.860451-0.669461-2.202207$ C $4.073957-3.5266230 .378105$ H $5.350671-2.992794-1.213384$ H $\quad 6.140314-3.032440 \quad 0.390586$ H 5.9074352 .8544682 .169638 H $7.0490092 .652549-0.947666$ H $6.513665-0.141602-2.692745$ O $4.442862-4.8398430 .458170$ O $2.974902-3.072763 \quad 0.663286$ C $3.433304-5.7369100 .930061$ H $3.878636-6.7308591 .024036$ H $3.069562-5.4224711 .913073$ H $2.611822-5.7868620 .209595$ SCF Energy (B3LYP/6-31G**//MMFF) $=-3245.92530017$

0600097

MMFF Geometry

C $-0.107687 \quad 1.177506-0.585020$

C $-0.483407 \quad 0.011050-0.037558$

C $-0.047261-0.5394371 .300750$

$\begin{array}{lllll}\text { O } & 0.940890 & 0.336495 & 1.884335\end{array}$

C $0.592589-1.924957 \quad 1.106838$

C $0.746507-2.708252 \quad 2.393760$

C $1.907871-2.880173 \quad 3.054534$

C $-0.512955-3.3641882 .898590$
C $3.254232-2.2935972 .731958$

C $4.250043-3.3437062 .219339$

C $3.975499-3.7900960 .774335$

C $4.954767-4.8878970 .352810$

C $4.780195-5.275480-1.123094$

O $4.137202-2.646236-0.069667$

O $5.921251-6.067468-1.485592$

C $4.798647-3.991718-1.980737$

C $3.553160-6.171506-1.327083$

O $4.515251-4.290421-3.345999$

C $3.860314-2.879892-1.455698$

C $4.127266-1.560807-2.220184$

O $2.505478-3.280891-1.668033$

C $0.8528310 .648458 \quad 3.199784$

$\begin{array}{lllll}\text { O } & 0.006829 & 0.256643 & 3.991296\end{array}$

C 1.9318251 .5835583 .601701

C 2.9455861 .9377942 .795527

C $4.0264482 .873668 \quad 3.115978$

C 3.9899453 .5541154 .458884

C $3.411967-0.338273-1.627813$

C $3.7778550 .946396-2.351918$

C $3.537553 \quad 3.422262-2.133165$

O $4.4164230 .970425-3.400437$

C $4.7734963 .923506-1.455071$

C $4.786914 \quad 4.943723-0.583787$

C $6.0294505 .431052 \quad 0.105574$

C 5.9311555 .3536401 .614195

C 5.7184226 .4594782 .347269

C 6.1692824 .0150182 .279759

C 4.9790613 .0991842 .186515

N $3.2856282 .063473-1.690686$

H $0.5735561 .835825-0.053854$

H $-1.176639-0.616815-0.591636$

H $-0.940546-0.6000201 .933747$

H $1.564252-1.8061850 .616584$

H $-0.010866-2.5318510 .417880$

H $1.897622-3.4930763 .955231$

H $-0.337845-3.9669773 .796179$

H $-1.264237-2.6100603 .151848$

H $-0.928683-4.0280912 .133457$

H $3.184888-1.4544272 .033672$

H $3.651062-1.8662573 .661813$

H $5.251416-2.8960932 .267084$

H $4.251970-4.2134772 .888013$

H $2.945117-4.1542150 .700766$

H $4.852521-5.7702730 .996865$

H $5.984596-4.5313470 .496978$

H $5.859186-6.254928-2.438140$

H $5.829736-3.613217-1.963587$

H $3.648252-7.087919-0.732009$

H $3.470586-6.500968-2.368704$

H $2.616446-5.687257-1.043299$

H $3.556560-4.432872-3.424625$

H $5.205165-1.351485-2.208267$

H $3.828213-1.671801-3.270200$

H $1.944753-2.492233-1.732201$

H $1.844123 \quad 1.967883 \quad 4.612878$

H 2.9968931 .5103111 .795471

H 3.0502594 .1027154 .587671

H 4.7926214 .2812184 .600067

H 4.0809562 .8176275 .264752

H $2.326444-0.457409-1.698083$

H $3.678206-0.219863-0.571152$

H $2.6540334 .016630-1.879653$

H $3.6725863 .440292-3.219053$

H $5.7052833 .415482-1.692893$

H $3.8579605 .459857-0.352435$

H $6.1988216 .468793-0.209976$

H $6.9153764 .875006-0.225781$

H 5.6775956 .4210043 .430618

H 5.5760177 .4283951 .880211

H $6.481303 \quad 4.1506093 .321410$

H 7.0269723 .5224661 .803392

H 4.9185122 .5569191 .242799

H $2.8422171 .941859-0.785125$

C -1.417914 $2.914534-1.828836$

O $-2.6593522 .598848-1.168576$

C $-3.7457693 .190969-1.903491$ 
C $-3.1069814 .194583-2.854886$ C $-1.8037683 .495248-3.189604$ H $-4.2012402 .387063-2.494280$ C $-4.7826303 .774618-0.937660$ H -3.729603 4.389149-3.733259 H -2.905608 $5.152924-2.362700$ C $-0.7527414 .415469-3.786173$ H $-2.0139302 .683560-3.899321$ C $-5.2664402 .789298 \quad 0.145885$ O $-4.2139064 .903260-0.260108$ H -5.637172 4.163616 - 1.503039 H -4.4536532 .5803650 .852378$ $\begin{array}{lllll}\text { O } & -6.297658 & 3.438508 & 0.900515\end{array}$ C $-5.824948 \quad 1.461584-0.396685$ C $-6.3162420 .536826 \quad 0.730177$ H $-5.0438780 .945397-0.957996$ O $-6.893724 \quad 1.722924-1.303507$ H -5.4904140 .3844991 .433842$ H -7.1224821 .0350351 .281808$ H -1.109065 4.846681-4.727393 H $-0.5106995 .240902-3.108441$ H $0.1723953 .868507-3.993792$ C $-0.5660441 .650457-1.936700$ H $-3.412545 \quad 4.596800 \quad 0.198628$ H $-5.927626 \quad 4.271914 \quad 1.238837$ H -7.580910 2.207758 -0.814778 H $0.3214861 .843197-2.549464$ H $-1.1395840 .861053-2.435809$ H $-0.9124183 .670880-1.214361$ C $-4.747733-2.0043470 .046652$ O $-5.921449-1.516708-0.612628$ C $-6.850796-0.8235710 .226031$ C -7.324081-1.753541 1.350176 C $-6.148581-2.3088032 .150037$ C $-5.116113-2.9353091 .213773$ H $-4.149586-1.1579860 .406238$ C $-3.917596-2.778725-0.992746$ H -7.723906 -0.605849-0.401936 O $-8.220783-1.0628862 .213798$ H -7.881969 -2.590494 0.910113 H $-5.700386-1.5351902 .784134$ O $-6.652694-3.3119593 .035405$ H $-5.511233-3.8829880 .824966$ O $-3.932389-3.236631 \quad 1.950206$ C $-3.326813-1.898646-2.073220$ H $-3.081466-3.286678-0.498475$ H $-4.550421-3.522358-1.491818$ H -8.475487 -1.679416 2.921589 H -5.920979 -3.5901413 .611424$ H -4.157508 -3.917053 2.606516 O $-2.542735-2.670167-2.884635$ O $-3.520068-0.698170-2.197822$ C $-1.900725-1.984229-3.964286$ H $-2.625135-1.401470-4.541635$ H $-1.107790-1.340440-3.574011$ H -1.452274 -2.732914 -4.622820

SCF Energy (B3LYP/6-31G**/MMFF) $=-3245.92811450$

0600098

MM̄FF Geometry

C $-3.2816954 .698775-0.691312$

C $-3.6527793 .450533-1.010999$

C $-4.690730 \quad 2.674544-0.241094$

$\begin{array}{llll}\text { O } & -4.078080 & 1.477377 & 0.291918\end{array}$

C $-5.8343052 .263416-1.185409$

C $-6.9531691 .510607-0.490853$

C $-7.172897 \quad 0.184554-0.598023$

C $-7.880354 \quad 2.362212 \quad 0.338213$

C $-6.364843-0.823419-1.367343$

C $-5.809308-1.911524-0.439180$

C $-4.790301-2.809544-1.156454$

C $-4.323025-3.935657-0.232601$

C $-3.199264-4.773776-0.860062$

O $-3.680010-1.988027-1.533074$

O $-2.607458-5.566776 \quad 0.180893$

C $-2.098844-3.825672-1.383619$

C $-3.738727-5.760015-1.902514$

O $-1.107171-4.549502-2.107393$
C $-2.643664-2.672041-2.249938$

C $-1.563636-1.622162-2.615294$

O $-3.140750-3.207099-3.482747$

C $-3.3745401 .611647 \quad 1.451326$

$\begin{array}{llll}\text { O } & -3.146748 & 2.647339 & 2.056750\end{array}$

C $-2.9413330 .262744 \quad 1.879680$

$\begin{array}{lll}\text { C }-2.219516 & 0.121989 & 3.001638\end{array}$

C $-1.759883-1.1411003 .584868$

C $-2.079132-2.4111772 .842229$

C $-0.933345-0.883784-1.429767$

C $0.338814-1.522892-0.901279$

C $1.927622-1.3685371 .013379$

O $0.968124-2.378933-1.516231$

C $1.591413-2.2774392 .150172$

C $1.949440-2.0320963 .419520$

C $1.673502-2.9668114 .560820$

C $0.910484-2.3070065 .686204$

C $1.545358-1.8540726 .780546$

C $-0.596664-2.2618185 .583784$

C - $-1.091773-1.1052354 .757175$

N $0.712187-0.9853610 .323069$

H $-3.743216 \quad 5.1887290 .162728$

H -3.187252 $2.955164-1.859852$

H $-5.0985523 .271676 \quad 0.584509$

H $-5.4296211 .674916-2.015160$

H $-6.2645253 .158590-1.655448$

H $-8.027371-0.237331-0.069917$

H -8.7089451 .7845860 .761848$

H -7.3403352 .8206941 .172149$

H $-8.3156343 .157825-0.275429$

H $-7.012077-1.286688-2.121666$

H $-5.545353-0.352286-1.913491$

H $-5.325970-1.4332750 .421675$

H $-6.636635-2.519641-0.052720$

H -5.258811 -3.220793 -2.058378

H $-5.165701-4.5762570 .055766$

H -3.946433 -3.5025590 .703225$

H $-3.307200-6.1164740 .573505$

H $-1.599743-3.404199-0.502829$

H $-4.450323-6.454671-1.439854$

H $-2.935415-6.383238-2.310440$

H $-4.251886-5.269446-2.732126$

H $-0.774949-5.250956-1.521435$

H $-0.801923-2.072656-3.263059$

H -2.053737 $-0.856480-3.233882$

H $-3.161837-2.494765-4.143011$

H $-3.247801-0.580701 \quad 1.273229$

H $-1.954571 \quad 1.0166363 .563957$

H -3.158896 -2.593695 2.848709

H $-1.596390-3.2959003 .262573$

H $-1.738546-2.3491601 .803077$

H $-0.6483550 .123402-1.760707$

H $-1.653014-0.766097-0.615193$

H $2.411749-0.4460721 .348626$

H $2.599626-1.8849540 .322390$

H $1.058959-3.1953211 .912632$

H $2.506114-1.1260793 .648773$

H $2.640234-3.3337344 .930145$

H $1.130485-3.8594384 .225862$

H $1.000457-1.4082257 .606093$

H $2.624825-1.9136646 .872328$

H -1.033308 -2.148236 6.585683

H $-0.967460-3.2294845 .234053$

H $-0.905343-0.1335685 .217182$

H $0.118824-0.2878730 .762938$

C $-0.872526 \quad 5.520802-0.811798$

O $-0.232567 \quad 4.240370-0.961903$

C $0.664153 \quad 4.043422 \quad 0.149154$

C $0.598325 \quad 5.3171210 .993204$

$\begin{array}{llll}\text { C }-0.789891 & 5.839802 & 0.677393\end{array}$

$\begin{array}{lllll}\mathrm{H} & 0.264994 & 3.201488 & 0.728144\end{array}$

C $2.0808123 .703319-0.341455$

H 0.7472495 .1117952 .057908

H $1.359656 \quad 6.0450120 .688473$

C $-0.9832387 .308487 \quad 1.007535$

H -1.5107495 .2425801 .249128$

C $2.1637932 .383263-1.138980$

O $2.5752204 .784890-1.130727$ 
H $2.735183 \quad 3.6224360 .534530$

H $1.7139061 .583753-0.539676$

O $1.4181092 .477586-2.351517$

C $3.6118091 .974213-1.482508$

C $3.6783250 .618151-2.210178$

H $4.1850721 .920405-0.550994$

O $4.2331192 .963206-2.300293$

H $3.154018-0.129689-1.607227$

H $3.1450050 .689990-3.166001$

H -0.8323567 .4855542 .077334$

H $-0.276013 \quad 7.9401200 .459507$

H $-1.996017 \quad 7.6348580 .750378$

C $-2.2617365 .495231-1.452194$

H $2.0160354 .844323-1.924405$

H $0.4919872 .655727-2.115842$

H $3.6870753 .069079-3.098151$

H -2.176058 $5.098456-2.471976$

H -2.639732 $6.520360-1.545792$

H $-0.2577676 .232180-1.380459$

C $5.632048-0.772877-0.335282$

O $5.9563710 .201226-1.333561$

C $5.126706 \quad 0.158768-2.499342$

C $5.232892-1.221030-3.164193$

C $4.951918-2.354552-2.178196$

C $5.780117-2.187370-0.902883$

H $4.603846-0.6154450 .004907$

C $6.565329-0.5436240 .857547$

H $5.551851 \quad 0.886643-3.201850$

O $4.341075-1.302939-4.270548$

H $\quad 6.249003-1.341994-3.561474$

H $3.883353-2.411907-1.943675$

O $5.279800-3.604332-2.790462$

H $6.835153-2.400387-1.116400$

O $5.338668-3.1327220 .068741$

C $6.202087 \quad 0.732017 \quad 1.580741$

H $6.523254-1.3641801 .582596$

H $7.607293-0.4535210 .527813$

H $4.416720-2.199286-4.640400$

H $6.225725-3.589944-3.015363$

H $5.405427-4.015719-0.333516$

O $5.067600 \quad 0.5435932 .314736$

O 6.8414691 .7720001 .492419

C 4.5897891 .6945623 .017291

H 3.6600601 .4218423 .523783

H 5.3211362 .0086923 .767816

H 4.3803702 .5082332 .316322

SCF Energy (B3LYP/6-31G**//MMFF) $=-3245.91811470$

0600099

MM̄FF Geometry

C $-1.241339-4.1604140 .941324$

C $-0.161322-3.3651510 .977066$

C $0.452834-2.8735302 .263723$

O $0.759658-1.4664052 .134208$

C $1.774387-3.6138572 .535069$

C $2.465861-3.1534743 .804400$

C $3.655835-2.5222623 .850200$

C $1.739128-3.4673765 .088141$

C $4.572022-2.1635112 .714019$

C $4.627011-0.6477832 .481135$

C $5.712279-0.2781351 .459557$

C 5.8485781 .2380631 .317781

C $6.902179 \quad 1.6141790 .263238$

O $5.358954-0.851318 \quad 0.196074$

O $\quad 6.7415473 .015819-0.004529$

C $6.5998590 .841802-1.040124$

C $8.327451 \quad 1.4418560 .800248$

O $7.6257491 .047797-2.007755$

C $6.347181-0.668135-0.827328$

C $5.858153-1.395106-2.106674$

O $7.579957-1.297160-0.448854$

C $-0.261159-0.5787382 .266136$

O $-1.444177-0.8255412 .432606$

C $0.326906 \quad 0.7722042 .154616$

C -0.4374891 .7929621 .742437$

C 0.0208273 .1653871 .521503

C 1.3539293 .5544672 .106484

C $4.743794-0.722600-2.911128$
C $3.462932-0.500977-2.128909$

C $1.6793221 .192268-1.753669$

O $2.972851-1.365937-1.407191$

C $1.7403882 .668764-1.527272$

C $0.8683383 .544335-2.049568$

C $0.9075195 .024150-1.788370$

C $-0.3611555 .533119-1.135888$

C $-1.3185586 .135860-1.860695$

C -0.4841195 .4060830 .369062$

C -0.7588963 .9924960 .794686$

N $2.9276670 .759555-2.348726$

H -1.674128 $-4.476676 \quad 1.889266$

H $0.314001-3.0400160 .056450$

H -0.236564 -3.013986 3.105655

H $1.587237-4.6929272 .615104$

H $2.431110-3.4897841 .668322$

H $4.043442-2.2400604 .828978$

H $2.343445-3.2427435 .973716$

H $\quad 0.817005-2.8834735 .164305$

H $1.484715-4.5315885 .130002$

H $5.573655-2.5297012 .973137$

H $4.301587-2.6729751 .785836$

H $3.647502-0.3103422 .119828$

H $4.826830-0.1327973 .429022$

H $6.659222-0.7118161 .802067$

H $\quad \begin{array}{llll}0.089576 & 1.702140 & 2.282239\end{array}$

H 4.8818211 .6635991 .012856

H $7.3514803 .249874-0.725321$

H $5.6968941 .303286-1.455413$

H 8.4765602 .0648241 .690432

H 9.0702191 .7833760 .070865

H 8.5620740 .4109301 .072992

H $8.3861380 .497134-1.754496$

H $6.711975-1.546519-2.780873$

H $5.522113-2.402250-1.823652$

H $7.499401-2.250621-0.618614$

H 1.3843720 .8785242 .367456

H $-1.479778 \quad 1.596636 \quad 1.498538$

H 1.3733193 .3470553 .182607

H 1.5874664 .6147691 .994265

H 2.1669752 .9962021 .630752

H $5.1105150 .216103-3.339609$

H $4.482903-1.362685-3.763228$

H $1.5220890 .668868-0.805606$

H $0.8765850 .908250-2.439578$

H $2.5303373 .032256-0.874195$

H $0.0788993 .183425-2.703529$

H $1.0652005 .529001-2.750361$

H $1.7693645 .298545-1.167437$

H -2.219885 $6.522779-1.396876$

H -1.224722 $6.249930-2.935594$

H -1.312871 6.0256330 .736693

H 0.4131335 .8267520 .830177

H -1.707169 3.6092150 .417312

H $3.312678 \quad 1.329336-3.096774$

C $-1.617385-4.043431-1.621696$

O $-2.016612-2.657085-1.614874$

C $-2.677374-2.354636-2.857136$

C $-2.406867-3.537514-3.778869$

C $-2.369840-4.683932-2.789591$

H $-3.751407-2.314962-2.642949$

C -2.208633 -1.003785 -3.404909

H $-3.181130-3.652979-4.543512$

H -1.440690 -3.445200 -4.288197

C $-1.711163-5.937944-3.338189$

H $-3.399586-4.921314-2.489996$

C $-2.3714840 .185050-2.437085$

O $-0.816956-1.089840-3.741560$

H $-2.730295-0.790488-4.345136$

H -1.650751 $0.107526-1.613846$

O $-2.0292991 .374383-3.162472$

C $-3.7885150 .366392-1.861985$

C $-3.9156191 .653771-1.024620$

H -4.036031 -0.496789-1.236392

O $-4.737650 \quad 0.402778-2.926680$

H $-3.1518161 .632038-0.241836$

H $-3.7035132 .523711-1.657839$

H $-2.256958-6.307756-4.212348$ 
H $-0.676852-5.747163-3.643942$ H -1.698864 -6.733763 -2.586889 C -1.956310 -4.687552 -0.274437 H - $0.336366-1.350517-2.936815$ H -1.154166 $1.230512-3.561226$ H -4.502212 1.149021 -3.504297 H $-1.772975-5.767208-0.323279$ H -3.029175 -4.554684 -0.088116 H - $0.536682-4.071058-1.808637$ C -5.0328380 .4766251 .560106$ $\begin{array}{lllll}\text { O } & -5.749032 & 0.706908 & 0.341640\end{array}$ C $-5.3170681 .847575-0.404809$ C $-5.456162 \quad 3.115276 \quad 0.451277$ C -4.7486182 .9765851 .796655$ C -5.1609611 .6831632 .495988$ H -3.9768970 .2826541 .335754$ C $-5.622031-0.7807982 .206514$ H $-6.019827 \quad 1.947412-1.241475$ O $-4.9395704 .253668-0.230403$ H $-6.520216 \quad 3.3088170 .640267$ H -3.6609303 .0267101 .685513$ O -5.1136764 .0750042 .635511$ H $-6.193911 \quad 1.7753322 .855493$ O -4.3269381 .4817313 .635358$ C $-5.248108-2.0080201 .412230$ H $-5.246440-0.932063 \quad 3.224869$ H $-6.714929-0.7181882 .274518$ H $-5.4162934 .329641-1.074630$ H -4.8720874 .8906692 .163636$ H -4.4065532 .2722484 .196509$ O $-6.271080-2.361298 \quad 0.583682$ O $-4.163477-2.5679861 .501365$ C $-6.028419-3.504559-0.241181$ H $-5.195042-3.308598-0.921010$ H $-5.824142-4.3837750 .377304$ H $-6.927339-3.693594-0.833882$ SCF Energy (B3LYP/6-31G**//MMFF) $=-3245.92221387$

0600100

MM̄FF Geometry

C -1.689121 1.4710523 .611352

C -2.8770771 .9753083 .243680$

C -3.1471113 .0203302 .185119$

O

C -1.9110683 .7728791 .655160$

C -2.2499015 .1222311 .051407$

C $-2.4466205 .363666-0.258993$

C $-2.308421 \quad 6.2591632 .040817$

C $-2.4882454 .382257-1.395660$

C $-1.2586974 .468924-2.310497$

C $-0.0714483 .644260-1.790908$

C $1.1477463 .812658-2.699645$

C $2.3087522 .898686-2.279004$

O $-0.4828892 .272801-1.778501$

O $3.2572892 .906683-3.356442$

C $1.7769981 .458002-2.121908$

C $3.0464403 .450997-1.053909$

O $2.7905360 .596734-1.611014$

C $0.4916811 .359185-1.267630$

C $-0.094056-0.069182-1.362295$

$\begin{array}{llll}\text { O } & 0.818628 & 1.638820 & 0.094860\end{array}$

C -5.0677502 .0874561 .045571$

O

C $-5.3580751 .400425-0.231590$

C $-6.4620190 .650452-0.352913$

C $-6.838751-0.131998-1.532648$

C $-5.964830-0.016405-2.755167$

C $-1.518637-0.209508-0.803159$

C $-1.992428-1.653930-0.825074$

C $-3.911161-3.083451-0.119764$

O $-1.323363-2.572135-1.291951$

C $-4.835164-3.297968-1.277612$

C $-6.105475-3.709415-1.141516$

C -7.031439-3.964498 -2.297860

C $-8.379158-3.294957-2.133676$

C $-9.454591-3.996235-1.736537$

C -8.497991-1.834323 -2.509968

C $-7.926963-0.926047-1.455896$
N -3.250233 -1.797662 -0.255054

H $-0.7802291 .792013 \quad 3.111056$

H -3.7633131 .6196633 .767360$

H -3.8630243 .7416142 .600046$

H -1.389295 3.1551090 .916678

H -1.190896 3.9561622 .462518

H -2.645683 $6.391391-0.562009$

H $-2.556713 \quad 7.2141971 .565943$

H -3.069175 6.0638852 .803227

H -1.340303 6.3799272 .538216

H $-2.6417013 .357954-1.047769$

H -3.378152 4.625257 -1.990790

H -1.552895 $4.074955-3.292363$

H $-0.9628395 .514932-2.456454$

H $0.1684133 .964706-0.771260$

H $1.4725244 .860356-2.726013$

H $0.8681613 .561426-3.732686$

H $3.9584772 .269812-3.136683$

H $1.552077 \quad 1.090774-3.132589$

H $3.4449394 .449979-1.268350$

H $3.9150842 .832786-0.800828$

H $2.4097723 .530323-0.170143$

H $2.8815790 .779508-0.660704$

H $-0.127740-0.377753-2.415506$

H $\quad 0.568723-0.770829-0.840297$

H $\quad 0.151353 \quad 1.233626 \quad 0.670251$

H -4.626463 $1.497103-1.026010$

H -7.1374890 .5777380 .498445$

H $-5.9552051 .016365-3.121326$

H -6.288902 -0.643444 -3.587605

H $-4.936066-0.315927-2.527020$

H -1.568686 0.1485810 .229999

H $-2.2179600 .382509-1.404557$

H -4.444162 -3.0745690 .836030$

H -3.158751 -3.878209-0.095134

H $-4.434474-3.131986-2.274885$

H -6.493978 -3.907153 -0.144885

H -7.164552 -5.051106 -2.381217

H -6.582890 -3.646403 -3.247085

H -10.430072 -3.530615 -1.642404

H $-9.382419-5.050668-1.491204$

H $-9.554257-1.561378-2.636718$

H $-8.052226-1.682231-3.496501$

H -8.491076 -0.941967 -0.522074

H $-3.747879-0.9728250 .065926$

C $-0.946820-0.8718674 .297197$

O $0.342920-0.7014743 .677040$

C $0.401890-1.5458082 .513154$

C $-0.734079-2.5474312 .672994$

C -1.797133-1.674324 3.312202

H $0.192452-0.9018931 .652102$

C $1.790655-2.1700932 .336869$

H -1.046137 -2.983991 1.720565

H $-0.456270-3.3658673 .347914$

C -2.923139-2.456899 3.963363

H $-2.219618-1.0198032 .540141$

C $2.899626-1.1166162 .127855$

O $2.091802-2.990153 \quad 3.463434$

H $1.759052-2.8273391 .459748$

H $2.579429-0.4189411 .345030$

O $3.101859-0.3563183 .318152$

C $4.246849-1.7290451 .696766$

C $5.313482-0.6481281 .438414$

H $4.082744-2.3134160 .785271$

O $4.732409-2.6289872 .689648$

H 4.8981440 .0997010 .754361

H $5.543473-0.1309492 .378115$

H -3.445455 -3.069404 3.221774

H $-2.548092-3.1251444 .745689$

H -3.653688 -1.780668 4.418511

C -1.5144310 .4811564 .731582$

H $2.135559-2.4093644 .242356$

H 2.2616920 .0817763 .535340

H $4.806840-2.1340803 .523485$

H -0.8204670 .9367695 .449452$

H $-2.460467 \quad 0.3249315 .263487$

H -0.766893 -1.468166 5.201684

C $5.964933-1.354305-1.443992$ 
O $6.410398-2.054083-0.276487$

C $6.630132-1.2242390 .868807$

C $7.694306-0.1658380 .546071$

C $7.347146 \quad 0.630073-0.711102$

C $7.003907-0.310623-1.867502$

H $5.009117-0.863577-1.232223$

C $5.730231-2.392653-2.544592$

H $7.048968-1.8781041 .644116$

O $7.860792 \quad 0.715051 \quad 1.652072$

H $8.657178-0.6694280 .389040$

H $6.5328221 .337388-0.514441$

O $8.4833501 .418932-1.071157$

H $7.920609-0.805674-2.211002$

O $6.508675 \quad 0.457808-2.961913$

C $4.508503-3.224268-2.232979$

H $5.584982-1.930246-3.527374$

H $6.583319-3.076136-2.631905$

H $8.527987 \quad 1.3760001 .399194$

H $8.2621841 .885259-1.895639$

H $5.6827300 .884215-2.676680$

O $3.381007-2.545246-2.590998$

O $4.551628-4.331028-1.712020$

C $2.145141-3.211636-2.316294$

H $2.138795-4.213392-2.756678$

H $1.982355-3.262589-1.235674$

H $1.338287-2.628676-2.767487$

SCF Energy (B3LYP/6-31G**//MMFF) $=-3245.92577856$

0600101

MMFF Geometry

C $-2.793854-3.686548-0.460299$

C $-1.972302-2.635600-0.321095$

C $-0.949142-2.238280-1.349638$

O $0.331187-2.106729-0.692329$

C $-1.300841-0.861829-1.941683$

C $-0.447607-0.520651-3.148193$

C $0.6957580 .191853-3.107949$

C $-0.975373-1.031075-4.464239$

C $1.387603 \quad 0.754349-1.897511$

C $1.519924 \quad 2.278843-1.986314$

C $2.1479502 .867086-0.714336$

C $2.157767 \quad 4.395666-0.777115$

C 2.8490305 .0138330 .446349

O $3.4844032 .367231-0.596849$

O $3.070226 \quad 6.4001890 .145809$

C 4.2266224 .3395030 .627150

C 1.9405314 .9826031 .680749

O 4.8610344 .7915441 .820861

C $4.1692652 .795447 \quad 0.589367$

C $5.5741802 .150722 \quad 0.582981$

O 3.5000662 .3268241 .766797

C $1.120953-3.206707-0.589950$

O $0.849742-4.347083-0.926719$

C $2.404751-2.757627-0.004323$

C $3.374517-3.6440590 .265138$

C $4.696195-3.3273230 .814954$

C $5.035731-1.8710031 .000134$

C $6.4465522 .520392-0.616700$

C $7.7180861 .694815-0.654075$

C $8.598760-0.530638-1.299611$

O $8.8092512 .176734-0.358180$

C $8.093619-1.937468-1.290084$

C $8.665639-2.928025-0.588262$

C $8.172395-4.347462-0.606110$

C $7.956977-4.9162200 .780690$

C $8.665345-5.9769111 .204028$

C $6.925855-4.2662861 .680129$

C $5.535506-4.3424251 .110057$

N $7.4990260 .381382-1.043317$

H -2.724075 -4.305437 -1.351429

H $-2.043094-2.0156190 .568584$

H $-0.880862-2.985145-2.150910$

H -1.198742 -0.083566 -1.175906

H -2.356449 $-0.836497-2.244588$

H $1.2189860 .373309-4.046084$

H $-0.327360-0.763147-5.305522$

H -1.058029 -2.122306 -4.446575

H - $-1.966352-0.610194-4.663854$
H $0.8695530 .491599-0.971286$

H $2.383306 \quad 0.298613-1.835461$

H $2.1423322 .531249-2.854808$

H $0.5313652 .725492-2.149608$

H 1.5650842 .5257840 .148414

H $1.1386724 .789015-0.880979$

H $2.6955944 .718391-1.679795$

H 3.5765076 .7840560 .882468

H $4.8520904 .695482-0.200388$

H 1.0149955 .5380861 .485786

H 2.4129525 .4816022 .533943

H 1.6611113 .9714461 .983982

H 4.4380714 .3389902 .570434

H 6.1039842 .3904991 .514216

H $5.4481901 .060740 \quad 0.601747$

H $3.745151 \quad 1.3977231 .911384$

H $2.520913-1.6950980 .180369$

H $3.188004-4.6981500 .064532$

H $4.365774-1.4098421 .734332$

H $4.939135-1.3335350 .049979$

H $6.054028-1.6944491 .349991$

H $5.9103672 .371505-1.560922$

H $6.7392593 .575083-0.576302$

H $9.387714-0.361794-0.558851$

H $9.000563-0.284729-2.288210$

H $7.226446-2.158968-1.908354$

H $9.550305-2.7170210 .008158$

H $8.914887-4.945813-1.150028$

H $7.241167-4.434733-1.179162$

H $8.521138-6.3955402 .194573$

H $9.406830-6.4524260 .570537$

H $7.244957-3.2441781 .896982$

H $6.902370-4.7708372 .655168$

H $5.189151-5.3638010 .944741$

H $6.5890880 .146977-1.431053$

C $-5.247755-3.904131-0.030734$

O $-5.508024-2.507976-0.277581$

C $-6.854267-2.2024520 .132154$

C $-7.526118-3.5416750 .411400$

C $-6.355875-4.3646670 .914681$

H $-6.778264-1.6472431 .074407$

C $-7.545571-1.338015-0.928621$

H -8.336944 -3.454512 1.141097

H $-7.940129-3.988453-0.499874$

C $-6.610609-5.8615310 .889120$

H $-6.125339-4.0555361 .943162$

C $-6.769518-0.060879-1.311491$

O $-7.714195-2.108333-2.126340$

H $-8.556246-1.078089-0.593674$

H $-5.848326-0.325634-1.844874$

O $-7.5725780 .681132-2.238085$

C $-6.4283240 .856679-0.124363$

C $-5.6942472 .135778-0.563616$

H -5.7954320 .3127530 .578863$

$\begin{array}{lllll}\text { O } & -7.622856 & 1.208070 & 0.571506\end{array}$

H $-4.8134271 .847259-1.147597$

H $-6.3456292 .718395-1.225863$

H $-7.459827-6.1178901 .530926$

H $-6.836372-6.213044-0.123270$

H $-5.736672-6.4128981 .249894$

$\begin{array}{llll}C & -3.841767 & -4.068486 & 0.547774\end{array}$

H $-6.832810-2.407401-2.409286$

H $-7.7792640 .088337-2.981066$

H $-8.206827 \quad 1.662866-0.059438$

H -3.670686 -5.1097310 .842528$

H $-3.738064-3.4527381 .448870$

H $-5.327902-4.419327-0.997157$

C $-3.199343 \quad 1.954097 \quad 1.205164$

O $-4.5179202 .337323 \quad 1.611860$

C $-5.281757 \quad 3.0369750 .623091$

$\begin{array}{llll}C & -4.547608 & 4.319287 & 0.203572\end{array}$

C $-3.1256714 .025898-0.264584$

C -2.3876853 .1844650 .774946$

H $-3.262366 \quad 1.232207 \quad 0.381700$

C -2.521162 1.2677512 .402975

H -6.2093693 .3468691 .120433$

$\begin{array}{llll}\text { O } & -5.252756 & 4.994948 & -0.832505\end{array}$

H -4.4966675 .0052931 .059121$ 
H $-3.1255813 .542526-1.248457$

O $-2.4367275 .267869-0.429485$

H $-2.1684863 .813467 \quad 1.647067$

$\begin{array}{llll}\text { O } & -1.133960 & 2.775065 & 0.236119\end{array}$

C -3.116547 -0.0842862 .731907$

H -1.457603 1.1053632 .195012

H -2.625868 1.8985103 .294003

H $-6.1466365 .186209-0.500612$

H -1.521775 $5.059343-0.685461$

H -1.310698 2.222171 -0.543906

O $-2.507356-0.5709923 .854225$

O $-3.988801-0.6511462 .090334$

C $-2.954757-1.8584074 .290949$

H -2.484615 -2.072311 5.254544

H -4.041058 -1.8661824 .422791$

$\mathrm{H}-2.646136-2.6226883 .572206$

SCF Energy (B3LYP/6-31G**//MMFF) $=-3245.92837386$

0600102

MMFF Geometry

C $-0.286197 \quad 3.7125402 .320322$

C 0.2049382 .5388531 .898518

C 0.6557121 .4296942 .809113

$\begin{array}{lllll}\text { O } & 2.020781 & 1.141129 & 2.424037\end{array}$

C $-0.2162890 .170695 \quad 2.611403$

C $-1.318326 \quad 0.021375 \quad 3.640799$

C $-1.389531-0.9717054 .549104$

C -2.4254341 .0412183 .572414$

C $-0.420709-2.0972814 .784851$

C $-1.003275-3.4601864 .384534$

C $-1.149559-3.6433652 .864909$

C $-1.842086-4.9703922 .551112$

C $-1.916812-5.2303011 .041240$

O $0.161333-3.6338602 .291371$

O $-2.302536-6.6032650 .873067$

C $-0.509955-5.052880 \quad 0.431595$

C $-3.015094-4.391402 \quad 0.376675$

O $-0.591453-5.156879-0.988672$

C $0.189824-3.7376710 .859412$

C $1.679972-3.7651240 .428661$

O $-0.471618-2.6357890 .241030$

C $2.810861 \quad 0.4922173 .316759$

$\begin{array}{lllll}\text { O } & 2.489393 & 0.098879 & 4.428936\end{array}$

C $4.154208 \quad 0.2402052 .747996$

C 4.6932901 .0157531 .794362

C 6.0085630 .8356891 .175271

C $6.719552-0.4681771 .425198$

C $2.506781-2.5612940 .900017$

C $2.603165-1.487422-0.166701$

C $3.855814-0.902974-2.243194$

O $1.822831-0.539367-0.201874$

C $5.313915-0.586894-2.339945$

C $5.7810520 .646125-2.588151$

C $7.2413110 .980162-2.692396$

C $7.6372812 .144349-1.811067$

C $7.8935513 .353675-2.338251$

C $7.8116421 .887575-0.329529$

C 6.4972191 .8290530 .403174

N $3.624658-1.726732-1.071782$

H $-0.363356 \quad 3.911493 \quad 3.386069$

H 0.2648232 .3458150 .829964

H $\quad 0.650914 \quad 1.7622993 .855199$

H $0.420956-0.7164222 .603490$

H $-0.677494 \quad 0.1702891 .613750$

H -2.246708 -0.9848195 .221963$

H -3.293218 0.7540444 .176056

H -2.078476 2.0110473 .938962

H -2.774408 1.1570652 .542483

H $0.541760-1.9361974 .293001$

H - $0.200326-2.1174765 .859807$

H $-0.328989-4.2340424 .773958$

H $-1.975253-3.6019174 .873429$

H $-1.730987-2.8099812 .456136$

H -2.844356 -5.0031122 .996345$

H -1.281238 $-5.792973 \quad 3.017473$

H -2.289516 $-6.798182-0.079916$

H $0.095211-5.9039750 .774279$

H $-3.990764-4.6203700 .822089$
H $-3.109849-4.634490-0.687407$

H $-2.851418-3.3158820 .471174$

H $0.308684-5.283465-1.331234$

H $2.141388-4.6596400 .867205$

H $1.755809-3.853691-0.660818$

H $\quad 0.120157-1.8675350 .251004$

H $4.690236-0.5958643 .184159$

H 4.1293771 .8767721 .438291

H $7.032884-0.5396592 .472287$

H $7.611692-0.6038810 .810804$

H $6.062642-1.315387 \quad 1.196858$

H $2.116773-2.1173281 .819612$

H $3.525693-2.8942051 .136519$

H $3.2445860 .002381-2.191305$

H $3.542398-1.488680-3.112924$

H $6.017013-1.406356-2.211206$

H $5.0756391 .459620-2.743533$

H $7.4540771 .214151-3.743655$

H $7.8684150 .114243-2.446321$

H $8.2024704 .187349-1.716333$

H $7.7950213 .538859-3.402679$

H $8.3975032 .698708 \quad 0.123102$

H $8.4202460 .989575-0.196759$

H 5.9001812 .7359950 .293910

H $4.141241-2.598981-1.004117$

C $-2.321054 \quad 4.7889261 .370667$

O $-2.797964 \quad 3.6034950 .699315$

C $-3.9273903 .955720-0.121619$

C $-4.282767 \quad 5.393774 \quad 0.239095$

C -2.922411 5.9596660 .597797

H $-3.5830083 .933550-1.161872$

C $-5.062877 \quad 2.9473650 .082912$

H -4.763956 $5.921718-0.589896$

H $-4.955948 \quad 5.4410201 .103205$

C -2.993870 7.2545051 .388006

H $-2.3558266 .130761-0.327509$

C $-4.6887391 .485848-0.241824$

$\begin{array}{llll}\text { O } & -5.484993 & 2.991420 & 1.452774\end{array}$

H $-5.9348583 .247551-0.509702$

H -3.9511351 .1100750 .475863$

O $-5.8664450 .687709-0.064403$

C $-4.166508 \quad 1.266360-1.673205$

C -3.939879 -0.221688-2.005270

H $-3.224604 \quad 1.806048-1.799630$

O $-5.0948631 .819795-2.606353$

H $-3.259223-0.649866-1.261447$

H $-4.892972-0.757005-1.922054$

H $-3.501784 \quad 8.0315040 .807212$

H $-3.543648 \quad 7.1226942 .325999$

H -1.991485 7.6185521 .633782

C $-0.792747 \quad 4.779516 \quad 1.390818$

H -4.7101592 .7875472 .004506$

H -6.1792040 .8321450 .845142$

H $-5.9432651 .359976-2.483386$

H $-0.4023795 .744347 \quad 1.732627$

H $-0.4063494 .611376 \quad 0.377875$

H -2.720804 4.7599882 .393333

C $-1.015094-0.283089-2.988653$

O $-2.160733 \quad 0.267952-3.649765$

C $-3.389164-0.434618-3.434024$

C $-3.248502-1.905222-3.850504$

C $-2.046289-2.574424-3.193105$

C $-0.789931-1.737801-3.424089$

H $-1.156491-0.239505-1.901677$

C $0.1923560 .590658-3.364560$

H $-4.1176960 .013805-4.121210$

O $-4.438722-2.622158-3.539721$

H $-3.123940-1.951876-4.940417$

H $-2.226770-2.741776-2.125818$

O $-1.875049-3.862450-3.786394$

H $-0.512666-1.775828-4.485410$

O $0.291100-2.289106-2.677436$

C $0.270406 \quad 1.863308-2.547444$

H $1.1201550 .040223-3.183377$

H $0.1461750 .877206-4.421326$

H -4.295423 $-3.549262-3.796419$

H $-1.204634-4.336819-3.266115$

H $0.476838-3.173184-3.035628$ 
O $1.500672 \quad 2.429439-2.732803$

O $-0.6258962 .312951-1.847830$

C $1.7403993 .642130-2.012118$

H $0.9629684 .381067-2.229563$

H $1.7887723 .434759-0.939552$

H $2.7046434 .043745-2.334988$

SCF Energy (B3LYP/6-31G**//MMFF) $=-3245.92303982$

06 00103

MM̄FF Geometry

C $-3.202634-3.3268622 .317939$

C -1.951761 -3.2023891 .848630$

C $-0.802656-2.6743922 .664596$

O $-0.141715-1.6487271 .887539$

C $0.223407-3.7910452 .923354$

C $1.343294-3.3397243 .839933$

C $2.534700-2.8681073 .421967$

C $1.048001-3.4548995 .312917$

C $2.998226-2.6592402 .005868$

C $4.321758-3.3875901 .743027$

C $4.779216-3.2614040 .280907$

C $6.131654-3.9545520 .088103$

C $6.692510-3.750830-1.327254$

O $4.880604-1.871298-0.041939$

O $8.066315-4.168867-1.327711$

C $6.646232-2.246122-1.672496$

C $5.995587-4.660251-2.344548$

O $7.026934-2.021995-3.027603$

C $5.271645-1.599696-1.395336$

C $5.295563-0.064532-1.581536$

O $4.313247-2.120022-2.324048$

C $-0.544474-0.3604972 .043461$

O $\begin{array}{lllll} & -1.460201 & 0.050494 & 2.736425\end{array}$

C $\quad 0.3586490 .474114 \quad 1.217782$

C $0.246020 \quad 1.810268 \quad 1.234747$

C 1.0727232 .7533720 .475620

C $2.1974232 .181482-0.345857$

C $6.1869380 .689018-0.591873$

C $6.0113202 .194923-0.697701$

C 6.4239094 .2831020 .610460

O $5.5015252 .746297-1.669212$

C 5.1399504 .5960451 .314749

C 4.3568745 .6387780 .998215

C 3.0774035 .9607771 .718341

C 1.9372696 .2886880 .777722

C 1.3704757 .5073140 .783175

C $1.4511425 .214632-0.171366$

C 0.7840834 .0699030 .545274

N 6.5207792 .8480910 .415519

H -3.393752 -3.043748 3.351665

H $-1.719544-3.4840370 .825711$

H -1.149855 -2.257647 3.618339

H $0.634849-4.1540911 .974026$

H $-0.271375-4.6607483 .376201$

H $3.257678-2.5661454 .179022$

H $1.887037-3.1226235 .933461$

H $\quad 0.179482-2.8443165 .578987$

H $0.835391-4.4961565 .576331$

H $2.254659-2.9849791 .273688$

H $3.133981-1.5814561 .855194$

H $5.089058-2.9607272 .402634$

H $4.222785-4.4492212 .002280$

H $4.016918-3.721674-0.358909$

H $6.058482-5.0239600 .322326$

H $\quad 6.852176-3.5418380 .808198$

H $8.547949-3.634388-0.673702$

H $7.402366-1.749894-1.051668$

H $\quad 6.157810-5.713934-2.086851$

H $6.417675-4.529734-3.347118$

H $4.917215-4.498238-2.400327$

H $7.891706-2.446804-3.160487$

H $5.5752100 .186586-2.612779$

H $4.2700080 .311163-1.464096$

H $3.567696-1.499663-2.379910$

H $1.102933-0.0399100 .619301$

H -0.5254962 .2586821 .858409$

H $1.8084401 .504992-1.115020$

H 2.8928041 .6260620 .293464
H $2.7903232 .935773-0.864646$

H $5.9487660 .383676 \quad 0.433328$

H $7.2431730 .470183-0.780298$

H $6.4956294 .786359-0.359167$

H 7.2730394 .5804451 .233226

H 4.8471193 .9445582 .135058

H 4.6645736 .3118070 .201203

H 3.2778616 .8140442 .379337

H 2.7749955 .1349392 .373712

H 0.5524837 .7541960 .114472

H $1.709858 \quad 8.287028 \quad 1.457037$

H $2.2837194 .899848-0.804318$

H $0.7088475 .633242-0.864205$

H -0.0573074 .3800021 .165833$

H 6.8676032 .2950851 .194077

C $-4.256877-4.0250610 .059192$

O $-4.000901-2.758259-0.578576$

C $-4.704385-2.716484-1.833775$

C $-5.280047-4.112006-2.049296$

C $-5.511760-4.568697-0.621775$

H $-5.535272-2.013504-1.707265$

C $-3.770757-2.227374-2.946142$

H $-6.195057-4.093711-2.649226$

H -4.564806 -4.774719 -2.550191

C $-5.692516-6.070679-0.488257$

H $-6.403230-4.060792-0.229886$

C -3.112532 -0.857956-2.675571

O $-2.715053-3.180739-3.127636$

H $-4.314994-2.198225-3.897137$

H $-2.378427-0.940921-1.864772$

O $-2.375670-0.492742-3.849699$

C $-4.1043050 .274407-2.352295$

C $-3.4223831 .652460-2.249175$

H $-4.6049700 .049774-1.405412$

O $\quad-5.1142980 .328867-3.359762$

H $-2.598611 \quad 1.584368-1.530382$

H -2.984605 $1.915954-3.219460$

H $-6.563290-6.405105-1.061597$

H $-4.817145-6.614802-0.858553$

H $-5.848186-6.3546510 .557027$

C $-4.414855-3.8193361 .568036$

H -2.254491 -3.273872 -2.275844

H $-1.750169-1.214304-4.034744$

H $-4.6717780 .491849-4.210388$

H -4.734241 -4.757159 2.036989

H $-5.212067-3.0859061 .740439$

H -3.402733 -4.680306 -0.155370

C -4.3201442 .4832490 .548921$

O $-5.1162932 .464808-0.641940$

C $-4.4056442 .774913-1.844795$

C $-3.7697474 .168591-1.735351$

C $-2.9195454 .308746-0.475790$

C -3.7109963 .8729990 .756490$

H -3.5241881 .7318620 .474758$

C $-5.2320712 .095573 \quad 1.716518$

H $-5.1617182 .831667-2.638015$

O $-2.9669814 .456979-2.874854$

H $-4.5632254 .926608-1.700897$

H -1.987358 $3.740246-0.562499$

O $-2.5447605 .677473-0.307965$

H -4.5008304 .6065370 .962863$

O -2.8463393 .8453391 .888594$

C $-5.6008790 .633641 \quad 1.630259$

H -4.7459582 .2455692 .686845$

H -6.1469162 .7003941 .715952$

H $-3.5355094 .377863-3.659884$

H -2.060984 5.944151-1.108583

H $-2.454444 \quad 4.7313001 .975987$

O -6.9025630 .4994941 .248956$

O $-4.815655-0.278571 \quad 1.853771$

C $-7.368428-0.8462611 .112832$

H -7.297681-1.370925 2.070317

H $-8.417533-0.8139870 .807180$

H $-6.795014-1.3689730 .342305$

SCF Energy $\left(B 3 L Y P / 6-31 G^{* *} / / M M F F\right)=-3245.92480441$

0600104

MM̄MF Geometry 
C $-2.119822 \quad 3.210077 \quad 1.525508$

C -2.8915973 .0177452 .606031$

C $-3.136327 \quad 1.7087843 .324080$

O $-2.555636 \quad 0.5892042 .617613$

C -4.6555361 .4669013 .421084$

C -5.0278570 .1732084 .119301$

C -5.491191 -0.9289793 .495419$

C -4.8882110 .1909115 .619411$

C -5.677822 -1.133289 2.016790

C $-4.976810-2.4116921 .536375$

C $-4.933802-2.4969950 .002948$

C $-4.327086-3.827597-0.444094$

C $-4.139068-3.895616-1.965411$

O $-4.133244-1.407909-0.468119$

O $-3.294206-5.026239-2.231059$

C $-3.396166-2.626521-2.438920$

C $-5.464868-4.172453-2.684178$

O $-3.336774-2.612841-3.863116$

C $-4.013542-1.318831-1.895340$

C $-3.165096-0.057266-2.200224$

O $-5.294247-1.119560-2.505844$

C $-1.295538 \quad 0.2031342 .959751$

O $-\begin{array}{llll}0.540481 & 0.757915 & 3.741257\end{array}$

C -1.003489-1.048013 2.223070

C $0.190334-1.6479772 .342160$

C $0.576227-2.924672 \quad 1.732438$

C $-0.443045-3.6221790 .869470$

C $-1.757281-0.052242-1.597549$

C $-0.685285-0.626852-2.506997$

C $1.680502-1.425243-2.435362$

O $-0.841020-0.782565-3.714682$

C $1.919237-2.814879-1.938430$

C $3.015846-3.178387-1.256769$

C $3.291439-4.581797-0.803570$

C $3.548609-4.6830450 .682447$

C $4.798657-4.8000011 .161508$

C $2.354249-4.7733811 .602912$

C $1.799918-3.4288401 .999204$

N $0.480769-0.904972-1.809133$

H -1.567802 2.3770841 .099799

H -3.4070903 .8802043 .025974$

H $-2.715141 \quad 1.7778864 .334643$

H -5.0950121 .5029352 .418356$

H -5.128236 2.2974163 .963653

H -5.761788 -1.7885384 .107569$

H $-5.224654-0.7440956 .079881$

H -3.842546 0.3401715 .905798

H -5.4864101 .0020416 .047234$

H $-6.751243-1.2105831 .806776$

H $-5.306279-0.2806831 .444338$

H $-3.952306-2.4279731 .928211$

H $-5.496420-3.2871901 .945200$

H -5.954123 -2.389342 -0.383069

H $-4.935851-4.670366-0.093392$

H -3.344805 $-3.955310 \quad 0.029633$

H -3.112700 -5.039532 -3.186785

H -2.364511 -2.722086 -2.079024

H $-5.877475-5.136402-2.362423$

H -5.320056-4.256094 -3.766839

H -6.222747 -3.409109-2.496057

H $-2.735076-1.898264-4.130223$

H -3.144916 $0.138910-3.278742$

H -3.693909 $0.801598-1.761739$

H -5.527060 $-0.178922-2.436478$

H -1.802026 -1.464494 1.621056

H $0.946824-1.1814352 .971432$

H $-0.808747-2.9538970 .082486$

H $-1.294500-3.950286 \quad 1.475317$

H $-0.051032-4.5010780 .353612$

H -1.464797 $0.987264-1.405583$

H $-1.744839-0.573540-0.636840$

H $2.501269-0.748948-2.176717$

H $1.570933-1.431576-3.524044$

H $1.164382-3.561068-2.174725$

H $3.778032-2.433646-1.043813$

H $4.167241-4.941714-1.359479$

H $2.472563-5.258803-1.077345$

H $4.988910-4.9124262 .223765$
H $\quad 5.659892-4.7835370 .502449$

H $2.645405-5.2814812 .532892$

H $1.603192-5.4342181 .161132$

H $2.472832-2.8487262 .632226$

H $0.510994-0.732270-0.808375$

C $-1.9742374 .390720-0.679127$

O $-0.7395723 .763031-1.081420$

C $-0.0996764 .583968-2.073028$

C $-1.2185145 .419458-2.671973$

C $-2.0324685 .726393-1.426364$

H $0.5886125 .251552-1.540110$

C $0.6924103 .705017-3.045258$

H $-0.8487336 .316715-3.176542$

H -1.819602 $4.843376-3.384896$

C $-3.4449986 .199463-1.722534$

H $-1.5124956 .502723-0.848746$

C $1.6353492 .707231-2.336734$

O $-0.217826 \begin{array}{lll}2.942932 & -3.847512\end{array}$

H $1.2561274 .333007-3.743964$

H $1.0332711 .955719-1.818168$

O $2.3825632 .007295-3.336924$

C $2.6257213 .358736-1.354039$

C $3.5920702 .378063-0.667877$

H $2.0809183 .914549-0.591056$

O $3.4122554 .325626-2.058491$

H $4.1807291 .862218-1.439074$

H $4.3382732 .962501-0.114597$

H $-3.4260887 .132256-2.295548$

H $-4.0025875 .458470-2.305188$

H $-3.9961546 .382743-0.794631$

C -1.9646054 .5442820 .844449$

H $-0.7815352 .427826-3.245465$

H $1.7416411 .595428-3.941679$

H $3.8770393 .859212-2.774573$

H $-2.7652295 .224747 \quad 1.156195$

H $-1.0075114 .974147 \quad 1.164757$

H -2.788961 $3.728793-0.999757$

C 5.0249930 .4383221 .131606

$\begin{array}{lllll}\text { O } & 3.820853 & 0.190584 & 0.396651\end{array}$

C $2.9525341 .327840 \quad 0.264920$

C 2.5303881 .7979241 .665617

C 3.7299772 .0601152 .576370

C 4.6860630 .8662042 .564168

H 5.6121251 .2200920 .634312

C $5.848850-0.8565711 .120449$

H $2.0562740 .945414-0.228625$

O $1.6836342 .937211 \quad 1.609596$

H 1.9317971 .0014242 .123904

H 4.2451342 .9853492 .293544

O $3.2496922 .259398 \quad 3.907727$

H 4.2223830 .0329953 .107353

O 5.8754861 .2128553 .269867

C $6.380602-1.184509-0.259630$

H $6.711599-0.7670091 .789272$

H $5.220913-1.6950201 .442465$

H 2.2433583 .7297801 .553633

H 4.0261282 .3791594 .481099

H 6.3047581 .9428382 .791943

O $7.044044-2.377191-0.204521$

O $6.235406-0.492351-1.257655$

C $7.580867-2.840457-1.447373$

H $8.320709-2.130535-1.828791$

H $8.073913-3.799518-1.267947$

H $6.776581-2.990476-2.174085$

SCF Energy (B3LYP/6-31G**//MMFF) $=-3245.92677635$

0600105

MM̄MF Geometry

C $0.936786-3.853725-2.324184$

C $0.325705-3.267560-1.284987$

C $-0.797978-2.279461-1.441564$

O $-0.390011-1.018801-0.853899$

C $-2.065886-2.703411-0.684260$

C $-2.818898-3.858122-1.302673$

C $-3.786095-3.715214-2.229235$

C $-2.483908-5.224159-0.763821$

C $-4.264487-2.442949-2.875616$

C $-5.680691-2.063192-2.418971$ 
C $-5.698870-1.374553-1.044993$

C $-7.127723-1.268169-0.511232$

C $-7.176479-0.5074380 .820303$

O $-5.139605-0.066120-1.212744$

O $-8.557445-0.225182 \quad 1.090609$

C -6.4392330 .8350280 .644849$

C $-6.676216-1.3696701 .984941$

O $-6.353383 \quad 1.5291851 .888217$

C $-5.0411690 .706810-0.009850$

C $-4.5332702 .118354-0.390990$

$\begin{array}{llll}0 & -4.143493 & 0.111353 & 0.924846\end{array}$

C $0.437423-0.217255-1.575030$

O $0.921455-0.448574-2.670751$

C $0.6431411 .015740-0.785869$

C $1.5317391 .931964-1.193948$

C $1.8109893 .208518-0.533853$

C $0.9346913 .600762 \quad 0.628128$

C -3.197456 2.150321-1.147522

C $-2.9071603 .536053-1.706054$

C $-1.286378 \quad 4.757017-3.152560$

O $-3.6373564 .504806-1.511024$

C $-0.4341195 .586099-2.243502$

C $0.7671836 .071362-2.593681$

C $1.6120706 .935702-1.700322$

C $3.0255836 .417250-1.546803$

C $4.040735 \quad 6.963755-2.237164$

C $3.2727505 .321093-0.533632$

C $2.8083683 .976560-1.019606$

$\mathrm{N}-1.7389143 .565624-2.455523$

H $0.599678-3.630934-3.333611$

H $0.666422-3.484106-0.276059$

H $-1.045370-2.107918-2.497175$

H -2.726811 -1.832146 -0.602983

H -1.824913 -2.9355190 .362258$

H $-4.291608-4.612294-2.585205$

H -3.118402 -6.006942 -1.193438

H $-1.446158-5.487335-0.985448$

H -2.625106 -5.2488450 .321756$

H $-3.570003-1.611216-2.720491$

H $-4.283082-2.619088-3.958628$

H -6.100119-1.375880 -3.165193

H $-6.317067-2.956897-2.406115$

H -5.083089-1.947163 -0.344377

H $-7.578760-2.262303-0.400185$

H -7.752724 -0.735623 -1.241992

H $-8.594217 \quad 0.3265591 .890965$

H -7.069886 $1.458762-0.003514$

H -7.280260 -2.280895 2.070566

H $-6.787621-0.8476482 .941717$

H -5.632159-1.671232 1.877846

H $-5.643156 \quad 1.1162032 .408394$

H -5.291546 2.597651-1.024500

H $-4.427732 \quad 2.733557 \quad 0.511724$

H -3.232933 0.3341430 .675170

H $0.047578 \quad 1.1429870 .110281$

H $2.1095181 .731945-2.095347$

H $\quad-0.123047 \quad 3.5659250 .343955$

H 1.1166954 .6144850 .990135

H 1.0891022 .9235641 .474513

H -2.375827 $1.875791-0.478411$

H $-3.2145991 .445720-1.986320$

H $-2.1582505 .338444-3.469716$

H $-0.7424514 .426446-4.042952$

H $-0.8345265 .821461-1.260173$

H $1.1546725 .862027-3.588227$

H $1.1591047 .049026-0.707434$

H $1.6294277 .943816-2.134820$

H $5.0609366 .616062-2.113435$

H $3.873217 \quad 7.768145-2.945921$

H 2.8257355 .6091820 .421610

H $4.3475625 .235366-0.324082$

H $3.3800053 .604852-1.871406$

H -1.163432 $2.730960-2.515510$

C $3.454952-4.165815-2.458798$

O $3.799958-3.268479-1.383898$

C $4.477789-2.125904-1.940513$

C $4.775591-2.471068-3.398609$

C $3.597716-3.358173-3.748461$
H $3.761663-1.296094-1.914573$

C $5.718176-1.768613-1.114103$

H $4.844488-1.578711-4.028246$

H $5.712927-3.029032-3.504880$

C $3.813515-4.208998-4.986948$

H $2.728726-2.709015-3.908965$

C $5.458559-1.5541050 .390160$

O $6.664744-2.838504-1.239402$

H $6.195289-0.881121-1.547841$

H $5.249271-2.5125400 .881310$

O $6.674536-1.0609170 .969776$

C $4.329560-0.5599630 .724415$

C $4.289447-0.2056672 .224522$

H $3.370082-0.9913760 .420952$

$\begin{array}{lllll}\text { O } & 4.509081 & 0.641346 & -0.025699\end{array}$

H $4.323097-1.1315262 .807634$

H $\quad \begin{array}{llll}5.187688 & 0.370220 & 2.478037\end{array}$

H $3.981021-3.575700-5.864388$

H $4.682406-4.866364-4.876756$

H $2.938454-4.836197-5.184532$

C $2.089710-4.806648-2.191866$

H $7.520324-2.513512-0.912368$

H $7.321328-1.7862120 .955725$

H $5.3757291 .012141 \quad 0.213440$

H $2.090813-5.241210-1.184175$

H $1.927670-5.634418-2.892318$

H $4.210604-4.962563-2.433010$

C $1.547566-1.166388 \quad 3.182143$

O $1.822814-0.0494132 .330374$

C $3.050670 \quad 0.634038 \quad 2.611006$

C 3.0555541 .1303024 .064198

C $2.789060-0.0020175 .052650$

C $1.517536-0.7534124 .659799$

H $2.327751-1.9221143 .030677$

C $0.245496-1.8267302 .716184$

H $3.062097 \quad 1.5241131 .971077$

$\begin{array}{lllll}\text { O } & 4.296113 & 1.760800 & 4.364402\end{array}$

H 2.2734091 .8921414 .180189

H $3.651190-0.6750675 .124561$

$\begin{array}{lllll}O & 2.613406 & 0.566926 & 6.351904\end{array}$

H $0.658753-0.1031424 .863551$

O $1.371599-1.9046195 .487561$

C $-0.974567-0.959282 \quad 2.925748$

H $0.294776-2.0459341 .645197$

H $0.099494-2.7876993 .223662$

H 4.2622862 .0404295 .295219

H $2.396415-0.1608106 .959576$

H $2.130710-2.4896455 .322482$

O $-1.722746-1.4522363 .953937$

$\begin{array}{lllll}\text { O } & -1.223347 & 0.042701 & 2.268614\end{array}$

C $-2.896978-0.7038254 .283817$

H -3.297818 -1.0976915 .221518$

H -2.6578220 .3547984 .424645$

H -3.648789 -0.8302473 .500588$

SCF Energy (B3LYP/6-31G**//MMFF) $=-3245.92738921$

06_00106

MM̄FF Geometry

C $0.811992 \quad 0.147626 \quad 3.393822$

C $2.012608-0.4243693 .581598$

C $2.540432-1.7091732 .990095$

O $3.704298-1.3728112 .195188$

C $1.567931-2.5023952 .099447$

C $1.962372-3.9587591 .938435$

C $2.514192-4.4966150 .833987$

C $1.640195-4.843803 \quad 3.117410$

C $2.915116-3.808046-0.439605$

C $2.022028-4.215798-1.620758$

C $0.639791-3.545843-1.587525$

C $-0.250344-4.076313-2.712664$

C $-1.594065-3.334866-2.775661$

O $0.847816-2.141544-1.756126$

O $-2.210090-3.690255-4.022606$

C $-1.324495-1.815181-2.792475$

C $-2.549846-3.806563-1.674925$

O $-2.544881-1.082316-2.725551$

C $-0.340198-1.345324-1.696434$

$\begin{array}{llll}\text { C } & 0.068377 & 0.124058 & -1.952045\end{array}$ 
O $-0.978354-1.441053-0.422888$

C $4.846862-2.0727392 .414890$

O $5.028566-2.924757 \quad 3.274957$

C $5.921758-1.6967581 .468471$

C $5.744920-0.930126 \quad 0.380794$

C $6.782432-0.572577-0.593066$

C $8.184354-1.061070-0.332986$

C $1.2423000 .602684-1.086569$

C $1.6996741 .997980-1.473609$

C $3.5666113 .601941-1.063875$

O $1.0831652 .716445-2.255570$

C $4.5974423 .376783-2.122563$

C $5.9181783 .459102-1.904168$

C $6.950066 \quad 3.194235-2.960981$

C $7.857878 \quad 2.035967-2.608320$

C $9.126906 \quad 2.251207-2.222610$

C $7.3277940 .630234-2.794105$

C $6.4425970 .175971-1.663776$

N $2.875327 \quad 2.348823-0.824323$

H $0.092741-0.2838572 .704600$

H $2.6937950 .049540 \quad 4.287730$

H $2.830684-2.3229053 .851522$

H $1.477933-2.0142771 .123289$

H $0.564388-2.4939072 .537709$

H $2.725742-5.5655690 .839534$

H $1.881069-5.8952322 .927255$

H $2.205660-4.5339234 .001061$

H $0.571914-4.7910533 .351503$

H $2.945934-2.720004-0.332856$

H $3.945686-4.112625-0.662459$

H $2.538642-3.918239-2.542687$

H $1.912664-5.307097-1.647856$

H $0.170445-3.731242-0.615176$

H $-0.411618-5.156266-2.606274$

H $\quad 0.263121-3.944633-3.675604$

H -3.024862 -3.165786 -4.108747

H $-0.888634-1.582899-3.773797$

H -2.732484 -4.884154-1.764214

H $-3.530930-3.329770-1.772933$

H -2.170980 -3.616019-0.669179

H $-2.850480-1.098928-1.802359$

H $\quad 0.3665800 .234354-3.003108$

H $-0.7906160 .786169-1.785949$

H $-0.583897-0.793396 \quad 0.181962$

H $6.889110-2.1294081 .705127$

H $4.752406-0.5373940 .167464$

H $8.542031-0.7046950 .639558$

H $8.910566-0.716869-1.072017$

H $8.217714-2.156126-0.341919$

H $0.9594660 .620266-0.029287$

H $2.098212-0.072413-1.200997$

H $4.0087003 .917896-0.114022$

H $2.8549424 .366519-1.390466$

H $4.2362623 .120044-3.115794$

H $6.2807373 .721182-0.912946$

H $7.5362574 .112981-3.092244$

H $6.4845303 .000699-3.935490$

H $9.7985501 .428930-2.000998$

H $9.5230763 .255790-2.117324$

H $8.151976-0.074760-2.951976$

H $6.7527940 .581900-3.728023$

H $5.4022200 .482373-1.770529$

H $3.337666 \quad 1.662278-0.235125$

C $0.120708 \quad 2.616371 \quad 3.326013$

O $\begin{array}{llll}-1.041573 & 2.476115 & 2.486857\end{array}$

C -0.8379383 .2752071 .303185$

C $0.5046953 .987871 \quad 1.480865$

C 1.2515023 .0391412 .394810

H $-0.765672 \quad 2.575974 \quad 0.463356$

C -2.004216 4.2446591 .085724

H 1.0067974 .1623830 .526176

H $\quad 0.3873914 .9642991 .965552$

C $2.436613 \quad 3.672822 \quad 3.100273$

H $1.6002912 .189273 \quad 1.797628$

C -3.397366 $3.604416 \quad 0.951365$

O $-2.0661715 .159922 \quad 2.190120$

H -1.799247 4.8613590 .202311

H -3.7359803 .2104091 .917320$ $\begin{array}{llll}\text { O } & -4.307128 & 4.664363 & 0.616138\end{array}$

C $-3.5237512 .497911-0.115432$

C $-4.995796 \quad 2.185352-0.452735$

H $-3.017093 \quad 1.5948150 .240881$

O $-2.8628922 .902971-1.315469$

H -5.5548482 .0659280 .480373$

H $-5.4211813 .048783-0.978155$

H 3.1714494 .0309612 .372330

H 2.1293124 .5257263 .714750

H 2.9341952 .9488923 .753165

C $0.3566751 .359534 \quad 4.162651$

H $-2.191757 \quad 4.634417 \quad 2.999077$

H -4.2342875 .3397451 .312341$

H $-3.275728 \quad 3.731050-1.615139$

H -0.5796581 .0882614 .666614$

H $1.080174 \quad 1.5947304 .953132$

H $-0.1123293 .434722 \quad 4.021568$

C $-5.687243-0.671890 \quad 0.373512$

O $-4.803946-0.271452-0.681304$

C $-5.1638710 .940937-1.352798$

C $-6.5670250 .796778-1.958529$

C $-7.5969660 .427736-0.897009$

C $-7.134880-0.806749-0.125537$

H $-5.633413 \quad 0.052691 \quad 1.194328$

C $-5.196102-2.026746 \quad 0.905832$

H $-4.4645981 .049543-2.190636$

O $-6.969427 \quad 1.998392-2.607903$

H $-6.5550600 .008190-2.722315$

H $-7.795407 \quad 1.272581-0.226971$

O $-8.8308670 .134353-1.556683$

H $-7.226432-1.690172-0.770957$

O $-7.990582-1.0091590 .998100$

C $-3.858575-1.9436821 .606886$

H $-5.914340-2.4309851 .628134$

H $-5.085725-2.7310060 .073203$

H $-6.3017092 .202056-3.284977$

H $-9.5079960 .008032-0.870882$

H $-8.878798-1.2122140 .659536$

O $-3.524888-3.1964782 .038401$

O $-3.192154-0.9289021 .750448$

C $-2.259068-3.3261552 .690484$

H $-1.477543-3.4072491 .931550$

H -2.061683 -2.486219 3.363403

H -2.272787 -4.246808 3.279987

SCF Energy (B3LYP/6-31G**//MMFF) $=-3245.92387971$

06_00107

MM̄FF Geometry

C $-4.574180-3.3263170 .421175$

C $-3.234841-3.3138710 .366799$

C $-2.349923-3.3905751 .580370$

O $-1.413801-2.2900891 .534823$

C $-1.530765-4.6942681 .573852$

C $-0.602235-4.8134392 .767680$

C $0.743202-4.8368072 .695025$

C $-1.277979-4.9211784 .111378$

C $1.616916-4.8129891 .471559$

C $2.174527-3.4155221 .190139$

C $3.256732-3.400396 \quad 0.096429$

C $4.561592-4.0463870 .578375$

C $5.694796-3.917078-0.448779$

O $3.488009-2.024522-0.244802$

$\begin{array}{lllll}\text { O } & 6.913549 & -4.264626 & 0.226458\end{array}$

C $5.798790-2.442228-0.886340$

C $5.534935-4.928240-1.589734$

O $6.760793-2.291168-1.927661$

C $4.443910-1.827744-1.296964$

C $4.558704-0.312453-1.579635$

O $4.006146-2.445824-2.513682$

C $-1.754245-1.1452642 .184010$

O $-2.823200-0.8851702 .711580$

C $-0.562193-0.268486 \quad 2.175144$

C $-0.579700 \quad 0.893728 \quad 2.843899$

C $0.528198 \quad 1.8472802 .942983$

C 1.8058611 .5132122 .217641

C $4.9166340 .556409-0.372088$

C $4.8743462 .036330-0.718010$

$\begin{array}{llll}\text { C } 4.943222 & 4.283278 & 0.341096\end{array}$ 
O $4.8155042 .454405-1.871684$

C 4.2831284 .8453031 .558565

C 3.1018265 .4807301 .533060

C 2.4336646 .0620582 .745792

C $1.1665675 .318148 \quad 3.108761$

C -0.0321845 .7983972 .734904$

C 1.3477944 .0654443 .943658

C 0.3501632 .9715793 .668577

N 4.9185222 .8344300 .416238

H $-5.061024-3.4186811 .388320$

H $-2.746128-3.242833-0.599632$

H $-2.948600-3.3380152 .498102$

H $-0.961337-4.7478430 .641031$

H -2.205394 -5.5603001 .568481$

H $1.301712-4.9055543 .628418$

H $-0.571108-5.1591074 .913639$

H -1.768812 -3.9797914 .375820$

H -2.030249 -5.7166984 .095193$

H $2.430201-5.5243491 .651654$

H $1.092745-5.1882540 .587177$

H $1.351870-2.7579420 .880673$

H $2.577139-2.9649332 .107154$

H $2.865334-3.919332-0.786400$

H $4.416689-5.1014200 .836187$

H $4.882612-3.5556531 .508443$

H $7.647055-4.115752-0.394948$

H $6.196450-1.890039-0.026279$

H $5.554170-5.951736-1.195681$

H $6.370160-4.866453-2.295922$

H $4.602654-4.806031-2.144857$

H $6.352914-2.599354-2.754836$

H $5.272668-0.138805-2.395409$

H $3.5962210 .041379-1.969019$

H $3.353936-1.864321-2.938285$

H $0.308921-0.6126621 .630067$

H - $-1.488031 \quad 1.1744723 .375382$

H 2.5593652 .2995502 .282348

H 2.2552080 .6026932 .628668

H $1.6180921 .359744 \quad 1.149935$

H $4.212751 \quad 0.3710580 .446773$

H $5.9282930 .331058-0.019454$

H $4.4577004 .613786-0.583294$

H 5.9910314 .5974060 .301493

H 4.8069234 .7284832 .504079

H 2.5819505 .5988100 .585198

H 2.2134097 .1170702 .536984

H 3.1097416 .0716983 .610149

H -0.9600335 .3057923 .002692$

H -0.1142006 .7083572 .148952$

H 1.2737074 .3443335 .002958

H 2.3673423 .6837813 .829613

H -0.6141623 .1284014 .153459$

H $5.1299472 .397411 \quad 1.309201$

C $-6.318719-1.989952-0.877274$

O $-5.493004-0.830318-1.107699$

C $-6.0599630 .279843-0.385201$

C -7.426640 -0.1834520 .118345$

C -7.184523 -1.6620650 .338550$

H -5.4091410 .4504290 .480238$

C $-6.1215191 .540722-1.254609$

H -7.7315110 .3432561 .027929$

H -8.211611 -0.035966 -0.632616

C $-8.456361-2.4857540 .432866$

H -6.618313 -1.770903 1.270749

C $-4.7762242 .008284-1.844345$

O $-7.010813 \quad 1.287169-2.350743$

H -6.575499 2.351624 -0.671164

H -4.468135 $1.352195-2.667775$

O $-4.9939693 .303324-2.422862$

C $-3.6188682 .121248-0.834098$

C $-2.4228062 .928383-1.381866$

H -3.294316 $1.113775-0.551636$

O $-4.076712 \quad 2.7633790 .358535$

H -2.219370 $2.617033-2.410387$

H -2.700226 $3.988576-1.408032$

H $-9.068891-2.1523691 .276944$

H -9.060884 -2.397471 -0.475980

H $-8.223585-3.5448870 .581869$
C $-5.461880-3.256345-0.790024$

H $-7.2164672 .141033-2.766696$

H $-5.5517853 .183338-3.209649$

H -4.4158533 .6404150 .110056$

H -4.849959-3.326564 -1.698390

H $-6.115368-4.136652-0.779736$

H -6.955355 -2.080913 -1.767971

C $0.0540561 .175743-1.872065$

O $-0.6083221 .444365-0.630287$

C $-1.1657112 .758097-0.500230$

C $-0.0788263 .821366-0.707927$

C $0.6562983 .628568-2.028333$

C $1.1699182 .195797-2.129363$

H $-0.6705141 .202518-2.694085$

C $0.635525-0.244301-1.793321$

H -1.4921062 .8517490 .542682$

O $\quad-0.6491975 .123132-0.641279$

H $\quad 0.646422 \quad 3.746070 \quad 0.112483$

H $0.0228683 .882378-2.886366$

O $1.7842014 .504261-2.078007$

H $1.9816592 .049195-1.406787$

O $1.7107651 .986207-3.432284$

C $-0.410390-1.317378-1.996591$

H $1.377578-0.380325-2.587522$

H $1.108218-0.418174-0.820915$

H $0.0809545 .764276-0.621178$

H $1.4470735 .415012-2.116303$

H $2.4181572 .641454-3.560317$

O $0.225834-2.487973-2.298508$

O $-1.617533-1.152030-1.896729$

C $-0.593857-3.620872-2.605310$

H $-0.066216-4.227856-3.346045$

H $-0.731129-4.216479-1.700515$

H -1.562795 -3.331911-3.023299

SCF Energy $\left(B 3 L Y P / 6-31 G^{* *} / / M M F F\right)=-3245.91533978$

0600108

MM̄FF Geometry

C $\quad 0.8989890 .6649973 .423924$

C $\quad 0.3375641 .6919184 .081194$

C -0.6495332 .6877883 .518969$

O -1.4371112 .1129602 .449402$

C 0.0835413 .9305352 .986691

C -0.8381125 .1143692 .758214$

C -1.2651985 .5486861 .556023$

C -1.2448965 .8567524 .007162$

C $-0.971286 \quad 4.968610 \quad 0.201648$

C $-2.2603594 .499647-0.485472$

C $-1.9841003 .842231-1.845906$

C $-3.2903333 .377129-2.492458$

C $-3.0524452 .577926-3.783126$

O $-1.1212892 .726213-1.618904$

O $-4.2770531 .906196-4.117898$

C $-1.9987161 .482103-3.511663$

C $-2.7243673 .496113-4.966470$

O $-1.6326150 .817472-4.718075$

C $-0.7385782 .011497-2.800272$

C $0.2239860 .886165-2.354449$

O $\quad-0.0123172 .856245-3.700988$

C $-2.435463 \quad 1.2624142 .825140$

O $-2.6973080 .899700 \quad 3.962097$

C $-3.173776 \quad 0.8442201 .612780$

C $-4.123077-0.098378 \quad 1.720339$

C $-4.989583-0.5953620 .648842$

C $-4.807010-0.013104-0.726135$

C $-0.336990-0.082815-1.308055$

C $-0.885583-1.376438-1.882959$

C $-1.991360-3.489506-1.151703$

O $-0.775153-1.694410-3.063406$

C $-3.436986-3.409213-1.524588$

C $-4.408278-4.060980-0.867562$

C $-5.851462-4.040946-1.280382$

C $-6.776301-3.607440-0.166482$

C $-7.473305-4.5096560 .545036$

C $-6.967689-2.1247330 .054145$

C $-5.916237-1.5294550 .950078$

N $-1.480388-2.156694-0.899452$

$\mathrm{H} \quad 0.7010170 .5256722 .364634$ 
H $0.573526 \quad 1.8267225 .135225$ H -1.330541 2.9787314 .328960 H 0.6222493 .6720782 .069194 H $\quad 0.8573104 .2449843 .700052$ H -1.906840 6.4289521 .530418 H -1.851179 6.7419563 .786896 H -1.834367 5.2125354 .666376 H $-0.358096 \quad 6.1955824 .552876$ H $-0.4928345 .742936-0.409936$ H $-0.2615354 .140586 \quad 0.262885$ H $-2.763726 \quad 3.7784880 .171079$ H -2.942188 $5.349007-0.618758$ H $-1.4730294 .568974-2.488357$ H -3.953542 4.230001-2.683798 H -3.831938 $2.734112-1.786825$ H -4.971208 $2.580190-4.217051$ H -2.476458 $0.728916-2.874556$ H $-3.5538314 .188318-5.155133$ H -2.599276 $2.921158-5.890537$ H -1.826249 $4.096301-4.807080$ H $-2.4502640 .481738-5.123649$ H $0.6277620 .359971-3.227478$ H $1.0884591 .379252-1.889655$ H $\quad 0.9119462 .902971-3.405739$ H -2.934881 1.3357220 .677770 H $-4.302023-0.5427032 .699003$ H $-5.1105541 .038530-0.734816$ H -5.383976 -0.526217 -1.498333 H $-3.760700-0.081479-1.038917$ H $0.479950-0.372485-0.635012$ H - $-1.1059880 .394458-0.693848$ H -1.828170 -4.077330 -0.242628 H -1.424385 -3.948261 -1.968202 H $-3.691508-2.814783-2.399085$ H -4.148734 -4.677432 -0.009824 H $-6.114881-5.054489-1.610782$ H -6.009462 -3.397902 -2.155241 H $-8.163704-4.2031081 .324022$ H $-7.364399-5.5745330 .368448$ H -7.939221 -1.9452850 .535067$ H -7.045633 $-1.617898-0.911776$ H -5.970821 -1.891302 1.977982 H -1.578292 -1.783077 0.040272 C $1.432642-1.8047753 .803366$ O $1.961321-2.1846842 .520279$ C $1.045941-3.1074501 .898598$ C $0.004425-3.4623542 .957379$ C $-0.047854-2.1835633 .773269$ H $0.554016-2.5494931 .094623$ C $1.804665-4.3001971 .300080$ H $-0.959871-3.7297522 .514273$ H $0.327194-4.2984093 .587942$ C $-0.669953-2.3507445 .147259$ H $-0.633173-1.4520803 .203106$ C $3.031094-3.919280 \quad 0.445632$ O $2.280220-5.1437652 .357581$ H $1.112516-4.9166980 .715211$ H $3.839809-3.5620131 .095392$ O $3.520161-5.107702-0.186784$ C $2.754045-2.868244-0.639378$ C $4.004388-2.544998-1.476809$ H $2.397173-1.952879-0.159966$ O $1.715662-3.324118-1.502920$ H $4.852898-2.391458-0.801428$ H $4.251347-3.400563-2.117285$ H -1.708106 -2.687717 5.060695 H $-0.126258-3.0877735 .747614$ H $-0.666776-1.4018415 .693099$ C $1.786108-0.3429224 .102184$ H $2.843717-4.6010392 .935733$ H $3.693296-5.7588090 .514806$ H $2.007963-4.163359-1.898122$ H $2.817520-0.1506293 .783556$ H $1.748192-0.1955065 .188674$ H $1.963246-2.4313964 .533098$ C $4.2588520 .383115-0.696319$ O $3.324501-0.172442-1.631574$ C $3.806579-1.299404-2.370999$
C $5.047839-0.893127-3.175019$

C $6.112615-0.242499-2.292962$

C $5.5076540 .876535-1.441053$

H $4.536856-0.3853310 .033587$

C 3.5200701 .5060570 .049460

H $3.018094-1.552019-3.091404$

O $5.590826-2.026537-3.843525$

H $4.747457-0.176184-3.950356$

H $6.616137-0.990529-1.669255$

$\begin{array}{llll}\text { O } & 7.119716 & 0.310704 & -3.144204\end{array}$

H $5.2597291 .721721-2.096140$

O $6.504697 \quad 1.318020-0.525052$

$\begin{array}{llll}\text { C } & 4.079504 & 1.804967 & 1.422758\end{array}$

H $3.5290842 .421894-0.553244$

H 2.4707351 .2212820 .195848

H $6.383339-1.729355-4.322645$

H $7.7724930 .749205-2.571544$

H $6.1918832 .145975-0.123762$

O 3.5405852 .9758331 .875081

$\begin{array}{lllll}\text { O } & 4.867507 & 1.097294 & 2.034498\end{array}$

C $3.9715613 .388643 \quad 3.175700$

H 3.4953534 .3457663 .403753

H 3.6637902 .6549513 .926572

H 5.0570633 .5254953 .190832

SCF Energy (B3LYP/6-31G**//MMFF)= -3245.91809802

0600109

MM̄FF Geometry

C $0.429540 \quad 4.416327 \quad 1.625764$

C $-0.4994693 .450703 \quad 1.561463$

C -0.8945432 .5811442 .723660$

O $-0.882111 \quad 1.2038182 .279908$

C -2.329409 2.9208313 .168621

C $-2.8549551 .981522 \quad 4.237492$

$\begin{array}{llll}C & -3.585739 & 0.876550 & 3.982947\end{array}$

C $-2.504602 \quad 2.3584445 .651672$

C $-3.978714 \quad 0.3469762 .630785$

C $-5.491430 \quad 0.1322102 .509389$

$\begin{array}{llll}C & -5.935414 & 0.032576 & 1.041707\end{array}$

C $-7.404204-0.3791410 .939856$

C $-7.852047-0.519833-0.523181$

O $-5.118736-0.929046 \quad 0.368280$

O $-9.101949-1.227282-0.537925$

C $-6.832722-1.396057-1.286736$

C $-8.1253530 .848910-1.158856$

O $-7.112423-1.400986-2.684517$

C $-5.355380-0.999553-1.045519$

C $-4.407548-2.067207-1.631085$

$\begin{array}{llll}\text { O } & -5.026565 & 0.228228 & -1.701191\end{array}$

C $0.2844110 .511897 \quad 2.359074$

$\begin{array}{lllll}\text { O } & 1.386549 & 0.951888 & 2.641226\end{array}$

C $-0.040290-0.9092882 .093494$

C $0.907146-1.8485962 .225939$

C $0.717996-3.2955192 .088413$

C $-0.683282-3.7984151 .858726$

C $-2.922717-1.779401-1.362095$

C $-2.014024-2.764740-2.078246$

C $0.393196-3.349540-2.349108$

O $-2.431515-3.626699-2.847463$

C $0.583461-4.603095-1.554369$

C $1.769119-5.006071-1.072999$

C $1.967420-6.296533-0.331253$

C $2.668422-6.1169090 .995988$

C $3.950530-6.4862421 .155959$

C $1.855135-5.6024872 .162341$

C $1.795504-4.0997772 .204880$

N $-0.675804-2.557290-1.769743$

H $0.920767 \quad 4.5960922 .580224$

H - $1.017698 \quad 3.252567 \quad 0.627531$

H -0.2090512 .7002593 .572435$

H -3.0048932 .9176142 .304768$

H -2.365912 3.9517123 .546316

$\begin{array}{llll}H & -3.907908 & 0.264425 & 4.823906\end{array}$

H $-2.903463 \quad 1.6471206 .382693$

H -1.4180202 .3898235 .780139$

H -2.912791 3.3452235 .893447

H -3.638051 1.0003391 .823496

H -3.469349 -0.6130752 .481595$ 
H $-5.750628-0.7916013 .042619$ H -6.0407090 .9501182 .992262$ H -5.7795321 .0162430 .582283$ H $-8.0476770 .331757 \quad 1.473161$ H -7.546861 -1.3474981 .439809$ H $-9.734430-0.7307730 .009247$ H $-6.967468-2.430117-0.939169$ H $-8.9345751 .363378-0.626453$ H -8.468639 $0.744362-2.193914$ H $-7.2553131 .508696-1.145528$ H $-8.036981-1.682411-2.793810$ H -4.646742 -3.050033 -1.204724 H $-4.560922-2.134589-2.715724$ H -5.774698 $0.492947-2.259668$ H -1.066608 -1.1498061 .840750$ H $1.915550-1.5320242 .484941$ H -1.323567 -3.548035 2.711898 H $-0.743959-4.8792521 .719141$ H -1.114411 -3.3510000 .958656$ H -2.658259 -0.774396-1.710294 H -2.720086 -1.835402 -0.286853 H $1.292676-2.727981-2.360197$ H $\quad 0.129471-3.604276-3.380683$ H -0.291983 -5.228771-1.396250 H $2.651957-4.399238-1.256584$ H $2.554350-6.961506-0.978500$ H $1.013295-6.812511-0.165486$ H $4.450406-6.3898162 .114109$ H $4.531341-6.8856020 .331230$ H $2.309618-5.9349073 .105784$ H $0.868963-6.0738932 .151923$ H $2.766392-3.6373072 .385075$ H $-0.427154-1.860054-1.073832$ C $0.4190394 .932331-0.909843$ O $0.9555123 .643798-1.269722$ C $1.3869043 .682113-2.641374$ C $0.8580434 .991295-3.215160$ C $0.9056095 .891978-1.995531$ H $2.4822163 .726162-2.626409$ C $0.9225082 .418900-3.374512$ H $1.4695825 .357439-4.045228$ H $-0.1728754 .890970-3.574349$ C $0.0649587 .148849-2.135985$ H $1.9500866 .175328-1.807746$ C $1.3790491 .096550-2.720430$ O $\quad-0.5101402 .403405-3.424419$ H $1.2586472 .457373-4.417088$ H $\quad 0.852070 \quad 0.949639-1.770040$ O $\quad 0.954340 \quad 0.020127-3.565836$ C $2.896956 \quad 0.990637-2.477931$ C $3.327033-0.403033-1.983959$ H $3.1893521 .739262-1.736589$ O $3.6007001 .301900-3.680387$ H $2.716970-0.675483-1.116044$ H $3.124655-1.138919-2.770832$ H $0.4206837 .757530-2.973704$ H -0.988009 $6.909378-2.318079$ H $0.1196957 .759003-1.228986$ C 0.8922345 .3049570 .497879 H $-0.8358542 .424228-2.507898$ H $1.3594270 .147910-4.440113$ H $3.4117080 .602405-4.328286$ H 0.5845596 .3301670 .733455 H 1.9885935 .2861890 .511120 H $-0.6750064 .854002-0.944706$ C 4.7217960 .2220660 .679250 O $5.2035830 .491500-0.642946$ C $4.831170-0.471652-1.634472$

C $5.323686-1.866857-1.228384$

C $4.864033-2.2487770 .175402$

C $5.224860-1.1435271 .166871$ H 3.6254020 .2421270 .686373 C 5.2360371 .3443421 .594400 H $5.379043-0.197247-2.544870$ O $4.883331-2.838132-2.171437$ H $6.421393-1.873979-1.252746$ H $3.790760-2.4697550 .191277$ O $5.535221-3.4542110 .545989$
H $6.313723-1.1168001 .303284$

O $4.640963-1.4302062 .434408$

C 4.5627772 .6726381 .323037

H 5.0526981 .1055912 .647523

H $\quad 6.313535 \quad 1.474870 \quad 1.435813$

H $5.192547-3.706334-1.860693$

H $5.166779-3.7452621 .396947$

H $5.060077-2.2371802 .777237$

O 5.2937263 .6653331 .912375

O 3.5195552 .8224250 .702963

C 4.7741754 .9898171 .760725

H 4.6386065 .2309520 .701968

H 3.8300585 .0843552 .304879

H 5.4972885 .6895452 .188269

SCF Energy (B3LYP/6-31G**//MMFF)= -3245.91685599

06_00110

MM̄FF Geometry

C $2.016147-3.907240 \quad 0.774328$

C $0.750350-3.5673070 .485943$

C $0.181023-3.575790-0.910323$

O $-0.561245-2.344859-1.098681$

C $-0.787175-4.757778-1.079995$

C -1.308546-4.900771-2.497606

C $-2.552353-4.573592-2.901007$

C $-0.330930-5.488875-3.483741$

C $-3.666055-3.989957-2.078119$

C $-4.044685-2.588197-2.572335$

C $-5.081467-1.924241-1.655060$

C $-5.502126-0.562376-2.208700$

C $-6.4554520 .176818-1.258366$

O $-4.483956-1.762625-0.364192$

O $-6.525306 \quad 1.537329-1.712489$

C $-5.8472340 .180708 \quad 0.162091$

C $-7.880838-0.379685-1.351481$

$\begin{array}{llll}\text { O } & -6.767436 & 0.731708 & 1.100916\end{array}$

C $-5.356433-1.2059230 .628103$

C $-4.570901-1.1610471 .962044$

O $-6.494361-2.0507930 .842462$

C $0.162363-1.221558-1.360487$

O $1.374204-1.144200-1.487186$

C $-0.776466-0.080069-1.448688$

C $-0.2802301 .153779-1.621660$

C -1.037459 2.400198 -1.742420

C $-2.5387622 .315771-1.699038$

C $-3.300760-0.3025001 .956240$

C -3.5085111 .1120982 .471253$

C -2.2870343 .2639322 .767341$

O -4.5570361 .4989682 .979361$

C -2.4356014 .1706111 .587348$

C -1.4900095 .0382421 .196245$

C $-1.665706 \quad 6.0169440 .072379$

C $-0.6111705 .879542-1.000603$

C $0.4938966 .644407-0.988491$

C $-0.8912674 .933902-2.144684$

C $-0.3521883 .551573-1.904156$

N -2.365132 1.8854412 .324798

H $2.651700-4.256699-0.036789$

H $0.079601-3.2352531 .272994$

H $\quad 0.980145-3.634378-1.659868$

H $-1.612702-4.658818-0.367900$

H $-0.282619-5.694811-0.807792$

H -2.811915 -4.739551 -3.946041

H $-0.776791-5.639019-4.472855$

H $0.533743-4.830508-3.610896$

H $0.021958-6.464183-3.132642$

H $-4.537362-4.651467-2.158227$

H -3.410034 -3.952102 -1.017333

H $-3.141147-1.967346-2.609117$

H -4.439013 -2.652845 -3.594183

H $-5.948534-2.590368-1.572710$

H -5.954875 -0.667977 -3.202539

H $-4.608670 \quad 0.059018-2.354337$

H -7.065103 $2.033762-1.073339$

H -4.9957200 .8700010 .130222$

H $-8.260827-0.282667-2.375662$

H -8.572823 $0.191739-0.723016$

H -7.948971 -1.432811 -1.070862 
H $-7.430503 \quad 0.0493951 .301793$ H $-5.241382-0.8850372 .784992$ H -4.252098 -2.1901902 .182427$ H -6.232251 -2.776911 1.432425 H -1.837337 -0.282054-1.367185 H $\quad 0.799430 \quad 1.266075-1.698493$ H $-2.8714441 .797885-0.793542$ H $-2.9137761 .773374-2.573095$ H -3.029595 $3.291273-1.686307$ H -2.567984 -0.7661692 .629452$ H $-2.851417 \quad-0.2660870 .959115$ H -1.325243 3.3923503 .272183 H -3.080759 3.4707793 .492312 H -3.3843354 .1391841 .056713$ H -0.5528345 .0858311 .746271$ H -1.625227 7.0264910 .503005 H -2.662824 $5.935206-0.377603$ H $1.2370776 .574471-1.775902$ H $0.6822987 .351421-0.187329$ H -0.410052 $5.312636-3.057259$ H $-1.9588194 .940387-2.382318$ H $0.7380503 .507252-1.915754$ H -1.5495191 .4879441 .867605$ C $2.062690-2.8662423 .132235$ O $2.138016-1.5257872 .607147$ C $2.561598-0.6342203 .653967$

C $2.435518-1.4238264 .950293$ C $2.789617-2.8219474 .476800$ H $3.622999-0.4222803 .479029$ C $1.753798 \quad 0.6666813 .590907$ H $3.104947-1.0510505 .731290$ H $1.411948-1.4030975 .341841$ C $2.371071-3.9162225 .442836$ H $3.875386-2.8763694 .320569$ C 1.7828941 .3589532 .211665 O $\quad 0.382956 \quad 0.3822393 .901595$ H 2.1016861 .3572644 .367480 H $1.2311950 .750591 \quad 1.484237$ O 1.0621982 .5880002 .347211 C 3.1981481 .6598221 .682824 C 3.1815642 .3912550 .329871 H 3.7424230 .7182651 .567001 O 3.9123682 .4446552 .635550 H $2.5767371 .805267-0.367029$ H $2.695548 \quad 3.3678650 .440935$ H $2.876749-3.792621 \quad 6.406070$ H $1.291365-3.8991445 .625610$ H $2.630566-4.9039045 .049316$

C $2.687468-3.8339342 .122668$ H $0.064533-0.271198 \quad 3.255242$ H 0.8099412 .8822331 .455602 H 3.4131723 .2684052 .770427 H $2.732711-4.8458092 .540544$ H $3.721612-3.5146171 .936587$ H $1.002041-3.0952103 .292134$ C $4.9681230 .417590-1.160681$ O $5.3941591 .445634-0.255152$ C $4.5897882 .627829-0.259258$

C $4.5814833 .246230-1.663391$

C $4.1655852 .233109-2.728369$

C $4.9826150 .945094-2.603461$

H $3.9560950 .097647-0.888260$

C $5.926469-0.765974-0.948641$

H 5.0919113 .3402930 .407326

O $3.7140314 .375034-1.696621$

H $5.5907613 .609518-1.896934$

H $3.0898022 .029379-2.679368$

O $4.4017832 .814446-4.013231$

H $6.0103261 .149337-2.930297$

O $4.427962-0.012192-3.500453$

C $5.332887-2.117546-1.280885$

H $6.838102-0.622997-1.540366$ H $6.223524-0.8042910 .107406$ H $3.7157974 .716696-2.607233$ H $4.1641032 .147031-4.679722$ H $5.039019-0.766457-3.543974$ O $6.296823-3.072466-1.117945$ O $4.178720-2.320647-1.628606$
C $5.885757-4.416102-1.389451$

H $5.133106-4.732343-0.661504$

H $5.497673-4.499488-2.409220$

H $6.760635-5.064796-1.294111$

SCF Energy (B3LYP/6-31G**//MMFF) $=-3245.92034243$

0600111

MM̄FF Geometry

C $2.177984-3.3261950 .592785$

C $0.895024-3.6615490 .391199$

C $0.217376-3.547340-0.951904$

O $-0.660738-2.398952-0.938843$

C $-0.625395-4.807069-1.221867$

C -1.247726 -4.831106-2.604911

C $-2.548582-4.597259-2.870863$

C $-0.304432-5.189697-3.725076$

C $-3.637543-4.224454-1.902928$

C $-4.293703-2.895531-2.300168$

C $-5.319469-2.421107-1.259227$

C $-5.978684-1.119459-1.718717$

C $-6.901308-0.522173-0.648446$

O $-4.625592-2.215295-0.025676$

O $-7.1945660 .823899-1.054350$

C $-6.141558-0.4571250 .695156$

C $-8.246205-1.256611-0.594947$

O $-7.031912-0.0353901 .725289$

C $-5.450454-1.7867401 .067167$

C $-4.533817-1.6815412 .311274$

O $-6.454195-2.7638341 .368345$

C $-0.108272-1.207228-1.303008$

O $1.062179-0.994746-1.580418$

C -1.181459-0.188339-1.326155

C $-0.8654681 .082790-1.615063$

C $-1.7839712 .219194-1.700828$

C $-3.2514591 .951056-1.507173$

C $-3.340986-0.7305112 .174163$

C -3.5901970 .6767742 .688165$

C -2.4580112 .8978662 .836191$

O -4.5957311 .0008743 .312766$

C -2.8975963 .7993801 .727425$

C -2.1190164 .7540951 .195167$

C -2.5886715 .7253190 .151112$

C $-1.7113905 .745926-1.078836$

C $-0.7683056 .689448-1.240560$

C $-1.9997784 .738142-2.167103$

C $-1.2701043 .439403-1.961940$

N $-2.526143 \quad 1.5189372 .394558$

H $2.741090-2.908500-0.237623$

H $\quad 0.308066-4.0443901 .222413$

H $0.961941-3.439058-1.750906$

H $-1.397189-4.904976-0.450875$

H $0.001869-5.702824-1.114513$

H $-2.879826-4.675668-3.905927$

H $-0.813604-5.258636-4.692378$

H $0.483061-4.436279-3.822491$

H $\quad 0.163739-6.160433-3.531396$

H -4.393695 -5.018512 -1.907213

H -3.262468 -4.155322 -0.879696

H -3.510430 -2.134905 -2.409705

H -4.781467 -3.003403 -3.277162

H $-6.071123-3.207574-1.123729$

H -6.525461 -1.270845 -2.657964

H $-5.197973-0.382931-1.947896$

H $-7.7249871 .234470-0.349776$

H -5.3859770 .3304680 .587711$

H $-8.766586-1.167308-1.556186$

H -8.914590 -0.8089830 .148847$

H $-8.145248-2.320763-0.371671$

H -6.5020810 .1599582 .516060$

H -5.126516 -1.463771 3.207586

H $-4.112203-2.6825872 .484274$

H -6.052064 -3.465735 1.906159

H -2.193969-0.509784-1.117024

H $\quad 0.1778091 .321871-1.815896$

H $-3.4313481 .429583-0.561619$

H $-3.8599502 .857118-1.471755$

H -3.632365 $1.334569-2.327674$

H $-2.516760-1.1302012 .778288$ 
H $-2.990673-0.6828131 .139499$ H -1.424755 $3.090733 \quad 3.140978$ H -3.105786 3.0402923 .707093 H -3.921634 3.6894011 .377964 H -1.104266 4.8803101 .565899 H -2.604460 6.7221430 .611692 H -3.626329 $5.525191-0.143952$ H $-0.1597316 .731036-2.137732$ H $-0.5794697 .436512-0.476713$ H -1.676400 $5.140260-3.137200$ H $-3.0805694 .609571-2.274525$ H $-0.1897443 .529752-2.084175$ H -1.7530591 .1642871 .839025$ C $4.418518-3.5527031 .791670$ O $4.921346-2.2850591 .316017$ C $5.766930-2.5318170 .181153$ C $6.237671-3.971674 \quad 0.343223$ C $4.972885-4.6342090 .858083$ H $5.128323-2.466717-0.708303$ C $6.878754-1.4857930 .075346$ H $6.596317-4.402933-0.596045$ H $7.036376-4.0567361 .089059$ C $5.213760-5.9667561 .546532$ H $4.303529-4.8024390 .005914$ C $6.407692-0.0180220 .147550$ O $7.807729-1.6882561 .148029$ H $7.441644-1.659806-0.849773$ H $\quad \begin{array}{llll}6.170136 & 0.239736 & 1.186828\end{array}$ O $7.5294960 .806108-0.199498$ C $5.2013660 .350666-0.738491$ C $4.9832551 .874186-0.836948$ H $4.305037-0.127398-0.330012$ O $5.382422-0.169194-2.056554$ H 5.1215682 .3206800 .152278 H $5.7516572 .296377-1.495284$ H $5.655851-6.6857270 .849078$ H $5.895123-5.8622972 .397588$ H $4.273067-6.3869841 .916495$

C $2.889530-3.4866001 .908647$ H $8.511522-1.0239161 .051700$ H $7.7770740 .602467-1.117220$ H $6.1640710 .258619-2.444615$ H $2.635514-2.6226002 .532074$ H $2.529026-4.3876612 .418548$ H $4.833298-3.6745932 .800326$

C 2.4522292 .6536600 .698885

O $2.5348721 .867724-0.497347$ C $3.5878532 .231554-1.399922$

C $3.4279823 .699088-1.818085$

C $3.3563434 .624784-0.607796$

C 2.2844164 .1428000 .367398

H 3.3524742 .5013961 .305540

C 1.2489802 .1528701 .511907

H $3.4410211 .626303-2.303227$

O $4.4975054 .088899-2.672457$

H $2.5020423 .801266-2.398912$

H $4.3312774 .702497-0.113144$

O $3.0335495 .947639-1.042916$

H $1.2912584 .320846-0.062865$

O $2.3689884 .906024 \quad 1.569104$

C 1.4654680 .7715522 .089537

H 1.0613002 .8284362 .353896

H 0.3575562 .1099540 .878478

H $4.3717805 .029384-2.886075$

H $2.1770755 .912218-1.501789$

H 2.2791445 .8430641 .324492

O $\quad 0.415817 \quad 0.4752012 .911619$

O $2.419476 \quad 0.0452301 .851145$

C $0.463620-0.8009653 .555281$

H $-0.329220-0.8310964 .307479$

H $0.281123-1.5907312 .821761$

H $1.424588-0.9483594 .057365$

SCF Energy (B3LYP/6-31G**//MMFF) $=-3245.92261768$

06 00112

MM̄MF Geometry

C $1.545371-4.463636-0.122245$

C $0.715764-3.5850570 .458554$
C $-0.032765-3.8640731 .734990$

O $-1.401412-3.4735981 .465220$

C $0.498585-3.0542702 .930471$

C $1.962852-3.2888063 .239561$

C $2.942199-2.3911993 .012680$

C $2.284069-4.6189673 .868949$

C $2.794325-1.0294252 .396963$

C 3.0636310 .0783633 .422886

C 2.5079521 .4273312 .948577

C 2.9734092 .5617403 .861144

C 2.3206613 .8991703 .485077

O $1.080617 \quad 1.3263012 .989802$

O 2.5876604 .8119524 .560478

C 0.7913163 .7032473 .394533

C 2.9643424 .5078852 .232760

O 0.1835304 .9028932 .919671

C 0.3795022 .4871502 .530856

C -1.1347012 .2021152 .676872$

O 0.6706002 .7705361 .164263

C $-2.395931-4.0095212 .213743$

O $-2.267748-4.7704803 .162295$

C $-3.724432-3.5399681 .750294$

C $-3.919277-2.9602320 .554864$

C $-5.194885-2.4880840 .009344$

C $-6.398233-2.5265690 .912726$

C -1.5944310 .8949382 .013895$

C -3.0306970 .5686832 .371730$

C -5.3721541 .3574592 .030490$

O $-3.310284-0.3833713 .097066$

C -6.1547651 .2010860 .760661$

C $-5.6894511 .203140-0.498725$

C $-6.5676201 .034641-1.705677$

C $-6.139710-0.121220-2.581129$

C $-5.6224320 .089216-3.803397$

C $-6.380628-1.524779-2.067149$

C $-5.217700-2.042488-1.264368$

N -3.9423381 .4407331 .801156$

H $1.728018-5.4214360 .359105$

H $\quad 0.543707-2.622666-0.016595$

H $-0.011497-4.9386161 .957771$

H $-0.086391-3.3058963 .825318$

H $\quad 0.276271-1.9928482 .778861$

H $3.962404-2.6567773 .285360$

H $3.345182-4.7125434 .123829$

H $1.711394-4.7495564 .793079$

H $2.036423-5.4382123 .187040$

H $3.516251-0.9413501 .578506$

H $1.814017-0.8998991 .931508$

H $2.592802-0.1854134 .379330$

H 4.1445800 .1513293 .595666

H 2.8334051 .6029851 .916647

H 4.0669722 .6499093 .847264

H 2.7060902 .3259064 .901040

H 2.1229915 .6434624 .362957

H $0.426623 \quad 3.5511734 .420258$

H 4.0303964 .6971472 .407292

H 2.5231685 .4818031 .993164

H 2.8819133 .8676621 .351726

H -0.7738954 .8317413 .068937$

H $-1.3881102 .142913 \quad 3.743588$

H -1.7097253 .0332282 .251794$

H 0.0984182 .2278350 .601024

H -4.543206 -3.732643 2.435648

H $-3.065334-2.826879-0.107646$

H $-6.661018-3.5616681 .156928$

H $-7.286016-2.0648470 .476305$

H -6.198072 -1.987142 1.845320

H -1.5126170 .9428020 .923407$

H -0.9773660 .0522762 .342798$

H -5.6719512 .2833022 .531791$

H -5.5944360 .5203982 .699543$

H -7.225121 1.0673990 .908823

H $-4.6299801 .343931-0.690764$

H $-6.5364911 .974158-2.272302$

H $-7.6173350 .893393-1.419326$

H $-5.334727-0.738313-4.443773$

H $-5.467806 \quad 1.091769-4.187805$

H $-6.520295-2.207439-2.916237$ 
H -7.328734 -1.553588 -1.523888 H -4.287177 -2.070741 -1.832085 H -3.5985812 .2209911 .248997$ C $3.614506-3.621253-1.291017$ O $3.488946-2.180884-1.223659$ C $4.381196-1.596405-2.191660$

C $5.401227-2.679886-2.508785$ C $4.521297-3.911410-2.489831$ H $3.789062-1.397713-3.092888$ C $4.991930-0.294317-1.667041$ H $5.896728-2.518781-3.470855$ H $6.172250-2.750415-1.732627$ C $5.298163-5.211680-2.371298$ H $3.927746-3.933800-3.414016$ C $3.9698210 .812702-1.337664$ O $5.743352-0.569390-0.478747$ H $5.7188250 .086510-2.394151$ H $3.359606 \quad 0.529368-0.472334$ O $4.7092751 .976368-0.944124$ C $3.0454201 .197052-2.511016$ C $2.2313872 .476677-2.239601$ H $2.3640940 .365775-2.714461$ O $3.824617 \quad 1.397426-3.691767$ H $1.7447962 .383272-1.265469$ H $2.9177613 .329676-2.181480$ H $5.965946-5.340216-3.229407$ H $5.910185-5.232074-1.463170$ H $4.619621-6.069982-2.338938$

C $2.224526-4.241271-1.443232$ H $5.157295-1.0414920 .136270$ H $5.2723451 .720834-0.193258$ H $4.4634592 .106882-3.505496$ H $2.291442-5.219307-1.934657$ H $1.596623-3.608328-2.081880$ H $4.094649-3.942525-0.357757$ C $-0.7030761 .616108-2.368340$ O $0.2156751 .721025-3.463306$ C $1.1879062 .765755-3.344067$ C $0.4807434 .121455-3.207935$ C $-0.5166574 .125309-2.055257$ C -1.464703 $2.933305-2.165090$ H $-0.1583831 .349766-1.455930$ C $-1.6792290 .472938-2.689698$ H $1.7302392 .786927-4.297511$ O $1.4198725 .173942-3.016466$ H $-0.0629294 .341691-4.135880$ H $-0.0080484 .138886-1.085301$ O $-1.2977535 .321549-2.112075$ H -2.159657 $3.097385-2.998794$ O $-2.2429132 .848043-0.973322$ C - $-1.012234-0.885352-2.655783$ H -2.496074 $0.447005-1.960470$ H $-2.0921560 .616508-3.695387$ H $2.0350985 .156981-3.769308$ H $-0.6790686 .071526-2.081940$ H -2.700205 $3.699394-0.863616$ O $-1.886079-1.817251-3.138864$ O $0.117825-1.108103-2.245484$ C -1.410130 -3.166443 -3.146168 H $-1.235419-3.508352-2.122081$ H $-2.181306-3.795838-3.598333$ H -0.497091-3.246291-3.743696 SCF Energy (B3LYP/6-31G**//MMFF) $=-3245.92897874$

\section{6_00113}

MM̄FF Geometry

C -3.0048031 .8205372 .462249$

C -2.0247541 .0520941 .962285$

C -1.110956 0.2092362 .818882

O $0.261041 \quad 0.4865572 .445842$

C $-1.394800-1.2836782 .570641$

C $-0.840518-2.1900603 .652496$

C $0.201500-3.032293 \quad 3.509914$

C -1.595844 -2.159788 4.959540

C $1.077191-3.2542202 .308071$

C $2.556746-3.0842902 .674926$

C $3.501485-3.4729381 .526138$

C $4.956356-3.3416341 .987471$
C $5.953404-3.6039470 .852393$

O $3.244251-2.6074990 .417586$

O $7.239637-3.1647401 .315236$

C $5.557623-2.729587-0.354511$

C $6.092039-5.1012920 .557456$

O $6.389986-3.012063-1.477262$

C $4.064071-2.843975-0.736191$

C $3.668843-1.810122-1.818003$

O $3.827804-4.147046-1.280728$

C $0.793714 \quad 1.6492992 .915338$

O $0.2614672 .445943 \quad 3.673538$

C 2.1314031 .8279002 .308543

C 2.6101873 .0766482 .194989

C 3.8886963 .4786931 .606361

C 4.9099192 .4053861 .345524

C $3.701885-0.360042-1.335515$

C $3.1741360 .611088-2.376050$

C $2.3632662 .968624-2.547841$

O $3.2319690 .392281-3.582709$

C $3.5290803 .902333-2.443100$

C $3.4691365 .119778-1.882870$

C $4.6624326 .021460-1.741727$

C $4.8950016 .478853-0.317120$

C 4.8154917 .7801070 .010020

C 5.2930545 .4420790 .716880

C 4.0952564 .7895161 .358214

N $2.6923551 .774648-1.792477$

H -3.1621071 .8526953 .537629$

H -1.8705421 .0149170 .887529$

H -1.248772 0.4488653 .880171

H $-1.013574-1.5736691 .587406$

H -2.479210 -1.456938 2.525294

H $\quad 0.461929-3.6587204 .363192$

H -1.229680 -2.908565 5.670285

H -1.502794 -1.181564 5.439974

H $-2.657840-2.3653504 .789440$

H $\quad 0.904030-4.2737551 .943549$

H $\quad 0.828697-2.5756601 .490971$

H $2.726362-2.0346102 .950400$

H $2.793506-3.6954473 .555357$

H $3.285261-4.5061001 .229633$

H $5.157318-4.0082902 .835687$

H $5.125226-2.3229042 .365058$

H $7.865359-3.2560560 .575998$

H $5.777962-1.693548-0.070600$

H $6.447435-5.6326331 .448714$

H $6.843787-5.285700-0.217779$

H $5.154426-5.5667910 .246912$

H $6.084905-3.848051-1.868947$

H $4.298274-1.934857-2.708253$

H $2.649739-2.027931-2.160953$

H $3.040332-4.109682-1.848275$

H $2.656600 \quad 0.951507 \quad 1.948182$

H 1.9874843 .9008442 .542903

H $4.601576 \quad 1.7717650 .511441$

H 5.0423051 .7739782 .231658

H 5.9001232 .8022281 .108871

H $3.115660-0.254982-0.416642$

H $4.730071-0.054765-1.115416$

H $1.4519473 .390809-2.113134$

H $2.1737102 .714966-3.595009$

H $4.4790493 .545722-2.835418$

H $2.5188985 .487038-1.502312$

H $4.5045556 .885722-2.399641$

H $5.5742995 .529502-2.103337$

H 5.0041288 .1191631 .023380

H $4.5565788 .534660-0.725251$

H 5.8773965 .9207711 .513337

H 5.9640274 .7199630 .242349

H 3.3083835 .4930861 .635265

H $2.715628 \quad 1.863782-0.781465$

C -5.3473982 .0601321 .624786$

O -5.3260420 .7933050 .939416$

C -6.4266510 .7390680 .013509$

C $-7.3697391 .856593 \quad 0.433488$

C -6.3768622 .9144220 .884222$

H $-6.0208200 .983513-0.975249$

C $-7.014634-0.676631-0.014309$ 
H -8.012761 2.190501 -0.386185 H $-8.010027 \quad 1.5572611 .271132$ C -6.9965854 .0085291 .735524$ H $-5.918473 \quad 3.368528-0.004805$ C $-5.950661-1.784453-0.175123$ O $\quad-7.706525-0.926700 \quad 1.215657$ H -7.768294 -0.752905 -0.805838 H $-5.336609-1.8390630 .732515$ O $-6.621733-3.044803-0.282440$ C $-5.037605-1.612525-1.401524$ C $-3.910279-2.654833-1.434755$ H $-4.578092-0.625128-1.371406$ O $-5.809317-1.682533-2.596355$ H -3.340583 -2.564604 -0.503045 H -4.342478 -3.662890 -1.449393 H $\quad-7.7659564 .545270 \quad 1.171109$ H -7.4650793 .5990032 .636782$ H -6.2395334 .7339282 .049528$ C -3.9365252 .6501131 .621643$ H -7.070578 $-0.802887 \quad 1.941317$ H $-7.198366-3.1328660 .495946$ H -6.237402 -2.555596-2.617682 H -3.9472013 .6696182 .022685$ H -3.5586672 .7079960 .594041$ H -5.6796461 .8606912 .652024$ C $-1.623796-0.646771-1.902015$ O $-2.522639-1.177305-2.883319$ C $-2.966470-2.518681-2.652659$ C $-1.757945-3.462399-2.595673$ C $-0.748760-3.005517-1.546159$ C $-0.380096-1.538703-1.763004$ H -2.141562 -0.567663 -0.939157 C $-1.2185320 .766383-2.362003$ H -3.556393 -2.797085 -3.535137 O $-2.186086-4.791163-2.317407$ H -1.268326 -3.479597 -3.577978 H -1.126813 -3.179038 -0.532057 O $0.427851-3.807533-1.669680$ H $\quad 0.249179-1.462392-2.659060$ O $0.402366-1.118637-0.648715$ C $-2.3426651 .777271-2.290502$ H $-0.4146821 .153732-1.728193$ H $-0.875350 \quad 0.734987-3.402942$ H -1.390037 -5.347134 -2.261247 H $1.046595-3.513399-0.978901$ H $\quad 0.797750-0.258387-0.862959$ O $-1.8373053 .016042-2.571609$ O $-3.5101251 .527004-2.032215$ C -2.785775 $4.087352-2.544320$ H -3.568563 $3.919953-3.290306$ H -3.219087 4.184600 -1.544400 H -2.259443 $5.013522-2.789819$ SCF Energy (B3LYP/6-31G**//MMFF) $=-3245.93805101$

06_00114

MM̄FF Geometry

C -0.5963422 .4856451 .239366$

C $-1.180076 \quad 1.7169242 .173592$

C -0.5287350 .5843802 .939230$

O 0.8976990 .6044342 .713646

C $-1.110309-0.7550612 .449449$

C $-0.725146-1.9412603 .309218$

C $0.157683-2.8974722 .959122$

C $-1.448860-2.0360944 .628805$

C $0.962169-3.0032641 .693967$

C $2.465738-3.0344141 .993856$

C $3.300211-3.1913550 .715567$

C $4.790821-3.2662491 .051367$

C $5.657380-3.345572-0.213394$

O $3.049243-2.062982-0.128646$

O $7.012331-3.0989380 .191503$

C $5.234937-2.217739-1.178119$

C $5.639014-4.753475-0.819661$

O $5.923170-2.331907-2.421814$

C $3.705652-2.125810-1.401254$

C $3.374576-0.833765-2.179630$

O $3.275860-3.253497-2.164844$

C 1.7271910 .4553043 .776820
O 1.4048320 .1655204 .920962

C 3.1374560 .7411263 .414306

C 3.5326501 .0865582 .179101

C 4.8868041 .4726211 .771567

C 5.9686871 .4631132 .817302

C $1.876915-0.528497-2.311113$

C $1.6974610 .840568-2.936014$

C $1.975438 \quad 3.256453-2.386222$

O $1.5415950 .984261-4.146084$

C $3.4491303 .511403-2.478073$

C $4.1387464 .253243-1.597998$

C $5.6338094 .401364-1.633421$

C $6.3055003 .859934-0.386242$

C 6.9231484 .6839500 .476915

C $6.3305292 .358855-0.172694$

C 5.0777701 .8577290 .492886

N $1.7984651 .865646-2.007453$

H 0.4497222 .3110590 .997786

H -2.2270671 .8756152 .418053$

H $-0.7500830 .747903 \quad 4.000530$

H $-0.813824-0.9178891 .408004$

H -2.207823 -0.7016692 .429156$

H $\quad 0.325280-3.7150773 .659750$

H -1.182409-2.940483 5.186072

H -1.210563 -1.178218 5.264493

H -2.531501 -2.058751 4.466321

H $0.675851-3.9318291 .185931$

H $0.745615-2.1880150 .999841$

H $2.744323-2.1035452 .500680$

H $2.690383-3.8602252 .680615$

H $2.975153-4.1051170 .204228$

H $5.000465-4.1147291 .714676$

H $5.081451-2.3678641 .614695$

H $7.559499-3.070460-0.612322$

H $5.577598-1.274269-0.731364$

H $6.006130-5.486248-0.091101$

H $6.315003-4.824840-1.679025$

H $4.643380-5.070249-1.137199$

H $5.497271-3.042282-2.931264$

H $3.8534500 .013558-1.675489$

H $3.804168-0.885525-3.188559$

H $2.424190-3.046046-2.580435$

H $3.836403 \quad 0.6894134 .243095$

H $2.7904601 .125014 \quad 1.383661$

H 5.7105132 .1315443 .646219

H $6.939117 \quad 1.7948502 .441937$

H 6.1098410 .4509203 .212396

H $1.370166-1.261523-2.946918$

H $1.379046-0.545403-1.335316$

H $1.4942913 .870812-1.619050$

H $1.4957293 .449933-3.350328$

H $3.9814143 .014994-3.286504$

H $3.6110904 .746120-0.784790$

H $5.8607485 .467587-1.760601$

H $6.0619493 .898459-2.509817$

H $7.4269974 .302248 \quad 1.358768$

H $6.9350975 .758016 \quad 0.324443$

H 7.2259532 .0706680 .387637

H $6.4392701 .854157-1.141405$

H $4.2212141 .821039-0.176485$

H $1.985106 \quad 1.625475-1.038261$

C -2.7170533 .8672370 .645443$

O $-3.513608 \quad 2.7042910 .359498$

C $-4.7817083 .122939-0.176847$

C $-4.7593674 .649683-0.211448$

C $-3.2741144 .949411-0.276745$

H $-4.8252502 .744091-1.204259$

C -5.9285062 .5203470 .645792$

H $-5.3135195 .047880-1.067313$

H -5.1969405 .0879270 .692570$

C $-2.924596 \quad 6.3672770 .139952$

H -2.930219 $4.778950-1.305794$

C $-5.8434290 .996121 \quad 0.861047$

O $-5.947155 \quad 3.123171 \quad 1.947898$

H -6.8870622 .7818790 .182967$

H $\quad-5.0274590 .757506 \quad 1.554664$

O -7.0534960 .5839461 .511042$

C $-5.6733420 .165157-0.422643$ 
C $-5.734750-1.349229-0.144174$ H $-4.7115440 .411535-0.882790$ O $-6.6902140 .511695-1.361144$ H -5.026871 -1.5862460 .657605$ H $-6.735180-1.6119250 .220656$ H -3.424334 $7.093152-0.509965$ H -3.2335286 .5701651 .170903$ H $-1.846333 \quad 6.5413970 .071074$ C - -1.2346663 .5685850 .405487$ H -5.0829042 .9556552 .361606$ H -7.1377591 .1085542 .325681$ H $-7.5496810 .329309-0.944007$ H $-0.653007 \quad 4.4851270 .558782$ H -1.098788 $3.275903-0.643504$ H -2.8858644 .1331241 .697304$ C $-3.031846-2.184651-1.262432$ O $-4.202196-1.854810-2.018812$ C $-5.439523-2.207810-1.394405$ C -5.476984 -3.720768 -1.141546 C $-4.259751-4.198263-0.351284$ C $-2.968121-3.692854-0.995975$ H -3.038845 -1.635956 -0.312656 C -1.819260 -1.714838 -2.071952 H $-6.226583-1.982515-2.125052$ O $-6.674213-4.073828-0.456773$ H -5.495419 -4.240430 -2.108611 H -4.329346 -3.893191 0.699515 O $-4.263169-5.627747-0.357116$ H -2.790109-4.235030 -1.933686 O $-1.870178-3.960144-0.127322$ C $-1.758078-0.207086-2.089886$ H - $0.876571-2.070270-1.641435$ H -1.864566 -2.086689-3.102692 H $-6.647785-5.033510-0.301008$ H $-3.548447 \quad-5.925730 \quad 0.230224$ H $-1.775738-4.924853-0.057948$ O $-2.159280 \quad 0.270071-3.301175$ O $-1.4180750 .466464-1.126344$ C $-2.1810101 .695780-3.422178$ H -2.900686 2.122862 -2.718169 H -1.183163 2.109661 -3.250705 H -2.494041 $1.945918-4.439188$ SCF Energy (B3LYP/6-31G**//MMFF) $=-3245.92100727$

06_00115

MM̄FF Geometry

C -0.994716 -3.886596 0.816287

C $-0.242892-3.780403-0.290382$

C $-0.722718-3.414312-1.678557$

O $-0.020591-2.231245-2.158064$

C $-2.244954-3.239012-1.880147$

C $-2.680100-3.320638-3.331312$

C $-3.032186-2.270688-4.098508$

C $-2.777397-4.719143-3.889431$

C $-2.991877-0.806571-3.763031$

C $-4.386634-0.202591-3.552685$

C $-4.918783-0.414489-2.126509$

C $-6.362700 \quad 0.078225-2.012806$

C $-6.889207-0.014475-0.572203$

O

O

$\begin{array}{llll}\text { C } & -5.875764 & 0.674767 & 0.366024\end{array}$

C -7.246962 -1.454071 -0.185736

$\begin{array}{lllll}\text { O } & -6.253723 & 0.517727 & 1.731713\end{array}$

C -4.4161150 .2152340 .148725$

C -3.4059571 .0639330 .956882$

O $-4.282842-1.1377690 .601616$

C $-0.095022-1.071702-1.448992$

O $-0.626662-0.902163-0.363526$

C $0.547766-0.008542-2.251276$

C $0.4683251 .259081-1.821356$

C $1.0183792 .441966-2.485385$

C $1.7209512 .241467-3.801829$

C -3.3665232 .5527810 .597567$

C $-2.269043 \quad 3.289913 \quad 1.347617$

C $-1.4225845 .597421 \quad 1.779972$

O -1.4295622 .7186682 .037332$

C $-0.544534 \quad 6.2023430 .731773$
C $0.784140 \quad 6.0222390 .675641$

C $1.6653276 .647484-0.367999$

C $2.2626305 .630775-1.314401$

C $3.5767385 .351496-1.303625$

C $1.3403214 .990596-2.330134$

C $0.8600243 .637948-1.881100$

N $-2.3355754 .661406 \quad 1.148979$

H $-2.065330-3.7113540 .768802$

H $\quad 0.827759-3.956911-0.205734$

H $-0.377112-4.228315-2.327007$

H $-2.578441-2.294651-1.440412$

H $-2.790898-4.024919-1.342540$

H $-3.367101-2.474893-5.115480$

H $-3.151714-4.732290-4.918701$

H -1.796341 -5.203471 -3.892416

H $-3.461949-5.322829-3.284471$

H $-2.351836-0.600350-2.904030$

H -2.518298 $-0.298152-4.612841$

H $-4.310096 \quad 0.876762-3.739665$

H -5.090667 -0.610422 -4.288349

H $-4.863565-1.479644-1.877353$

H -7.017607 -0.476185 -2.696600

H $-6.4161391 .128213-2.333603$

H $-8.7335860 .359924-1.145459$

H $-5.948504 \quad 1.7484090 .155513$

H $-8.006587-1.855518-0.867324$

H -7.693100 -1.495345 0.814062

H $-6.391087-2.131599-0.211800$

H $-6.025296-0.3886841 .999673$

H -3.593665 0.9441992 .032072

H -2.4021120 .6504360 .794833$

H -3.343904 -1.310387 0.783262

H $1.041832-0.291191-3.173103$

H $-0.0535001 .456285-0.885038$

H $2.1104893 .163318-4.236763$

H $1.0335241 .812403-4.539627$

H $2.5746341 .566161-3.688254$

H $-3.1834242 .682509-0.475011$

H -4.3198553 .0286440 .849597$

H -0.8447495 .0934962 .561480$

H -2.0273476 .3781242 .251646$

H -1.028289 $6.831821-0.011606$

H 1.2707875 .3959851 .419854

H 2.4570177 .2023050 .151948

H $1.1164977 .397446-0.951821$

H $4.0059734 .638934-2.000682$

H $4.2499895 .822371-0.595247$

H $1.8478014 .955588-3.298225$

H $\quad 0.4660015 .633148-2.493043$

H $0.3105453 .661316-0.939676$

H -3.0626975 .0401660 .548903$

C $-0.723093-3.0899303 .170548$

O $0.087942-1.9435392 .838063$

C $0.685647-1.4328454 .043685$

C $-0.068862-2.0861265 .194106$

C $-0.362228-3.4539454 .611228$

H $1.721263-1.7931334 .058639$

C 0.6681710 .0992404 .050533

H $0.525471-2.1268466 .111802$

H -1.004491 -1.560744 5.418221

C -1.453887 -4.207609 5.350838

H $0.560590-4.0495964 .628848$

C $1.328146 \quad 0.7576872 .821385$

O $-\begin{array}{llll}0.689396 & 0.554835 & 4.107283\end{array}$

H 1.1442580 .4649274 .967859

H $\quad 0.700426 \quad 0.619610 \quad 1.932514$

O 1.3846302 .1695143 .061423

C 2.7520460 .2606922 .519807

C 3.4249481 .0431461 .377024

H $2.706630-0.7994612 .251994$

O $3.5583390 .365066 \quad 3.692794$

H $2.748537 \quad 1.059868 \quad 0.516214$

H 3.5805892 .0837841 .686216

H $-1.163237-4.3808906 .392136$

H -2.396091 -3.649105 5.353608

H - $1.640681-5.1806464 .886060$

C $-0.447837-4.2200752 .176407$

H $-1.1475860 .211326 \quad 3.320773$ 
H $\quad 0.4729922 .472573 \quad 3.215062$

H $3.570773 \quad 1.3008173 .958052$

H $-0.926080-5.1461062 .514503$

H $\quad 0.631431-4.4074702 .115638$

H $-1.770382-2.7656603 .108424$

C $4.009902-1.278539-0.514678$

O $4.707945-0.9556490 .692188$

C 4.7941860 .4449880 .977996

C $5.4905821 .176403-0.177586$

C $4.8248560 .883336-1.519873$

C $4.679618-0.625082-1.730778$

H $2.979408-0.912693-0.433743$

C $3.889627-2.806216-0.607297$

H $5.4456660 .537196 \quad 1.856266$

O 5.5021642 .5787440 .067827

H $6.5376880 .849668-0.224464$

H $3.8589851 .393766-1.593081$

O $5.6445821 .425940-2.557157$

H $5.675496-1.044780-1.914451$

O $3.901468-0.841191-2.904103$

C $5.210412-3.526084-0.782577$

H $3.440738-3.1976340 .313476$

H $3.231285-3.065489-1.444304$

H $5.9235073 .004625-0.698362$

H $5.2215371 .204463-3.404465$

H $3.953058-1.786357-3.124092$

O $4.989800-4.717651-1.412801$

O $6.293177-3.116413-0.386981$

C $6.152740-5.522508-1.631489$

H $6.607981-5.797619-0.675276$

H $5.842600-6.434930-2.147613$

H $6.869943-4.988823-2.262420$

SCF Energy (B3LYP/6-31G**//MMFF) $=-3245.92655428$

06_00116

MMFF Geometry

C $1.659078-3.1159302 .861602$

C $0.720349-2.2831562 .389637$

C $0.123221-1.1506983 .179318$

O $-1.307238-1.2043582 .967168$

C 0.6174730 .2007632 .631929

C 0.3904641 .3576623 .588635

C 1.3658732 .1084684 .136435

C -1.046583 1.6930343 .897310

C 2.8522121 .9411744 .021943

C 3.5587603 .1056723 .315242

C 3.4016303 .0939431 .785033

C 4.4488944 .0162011 .151602

C $4.3071654 .083585-0.373746$

O 2.0863463 .5454541 .436235

O $5.1034415 .181117-0.844944$

C $2.8413994 .407339-0.717265$

C $4.8548202 .824667-1.055192$

O $2.623834 \quad 4.334645-2.125404$

C 1.8145103 .5169070 .025164

C $0.3922854 .077329-0.228118$

O $1.8941942 .188764-0.490602$

C $-2.111111-1.736103 \quad 3.920987$

O $-1.764962-2.2034474 .995835$

C -3.536567 -1.630306 3.517994

C $-3.900697-1.4638812 .237210$

C $-5.256368-1.3233751 .704611$

C $-6.387808-1.2049092 .687474$

C -0.7218943 .3833550 .559119$

C $-2.090177 \quad 3.983888 \quad 0.278442$

C -4.5002083 .6288880 .832724$

O $-2.2529265 .019977-0.359935$

C $-5.1961362 .931559-0.292415$

C $-6.1400511 .995519-0.109252$

C $-6.9147031 .352066-1.222843$

C $-6.474552-0.061145-1.526032$

C $-5.991784-0.386535-2.737724$

C $-6.653917-1.116878-0.453370$

C $-5.404411-1.2910900 .363846$

N $-3.104988 \quad 3.2374380 .860884$

H $2.039447-2.9811143 .870807$

H $\quad 0.360289-2.4173841 .372610$

H $0.364593-1.2410434 .245819$
H $\quad 0.0946780 .434773 \quad 1.694695$

H $1.676930 \quad 0.1256772 .368063$

H 1.0682222 .9283654 .790096

H -1.160194 2.7097954 .288426

H -1.661955 1.6240682 .996760

H -1.446085 1.0024324 .645698

H 3.2406791 .8858595 .047544

H $3.137590 \quad 0.9937173 .556277$

H 3.1978634 .0586873 .722969

H 4.6254293 .0300673 .562683

H 3.5386302 .0658541 .429875

H 5.4638013 .6997031 .423778

H 4.3327585 .0311131 .557443

H $6.0230115 .024396-0.569661$

H $2.6616565 .453026-0.430087$

H $5.9191352 .695592-0.824749$

H $4.7925542 .905461-2.145932$

H $4.3389971 .913920-0.745966$

H $3.2771374 .916702-2.550086$

H $\quad 0.376218 \quad 5.1420150 .039821$

H $0.1524064 .007045-1.296876$

H $1.0977721 .702080-0.229079$

H -4.260356-1.719161 4.320588

H $-3.119844-1.4251511 .481572$

H -6.454993 -2.106195 3.306755

H $-7.363148-1.0780522 .213043$

H $-6.238140-0.3397723 .342986$

H -0.7618572 .3187410 .314886$

H -0.5345653 .4900101 .631977$

H -4.5804224 .7129890 .703652$

H -4.9281553 .3673391 .805996$

H $-4.9387373 .242008-1.302162$

H $-6.403610 \quad 1.6990330 .903421$

H $-6.8514671 .975049-2.124905$

H -7.977409 $1.344040-0.949607$

H $-5.692458-1.403225-2.970507$

H $-5.8777870 .355578-3.520847$

H $-6.880985-2.081577-0.925558$

H -7.532051 -0.8704480 .149323$

H -4.510436 -1.417588 -0.247708

H -2.8773432 .3514271 .301102$

C $3.608543-3.9347881 .503947$

O $3.472664-2.9750180 .432851$

C $4.181016-3.462148-0.721250$

C $5.143555-4.515670-0.192587$

C $4.300947-5.1556930 .892645$

H $3.440197-3.953993-1.363143$

C $4.839395-2.306930-1.482191$

H $5.456732-5.221502-0.967685$

H $6.042296-4.064323 \quad 0.243287$

C $5.108395-5.9678801 .890666$

H $3.557911-5.8113790 .418644$

C $3.877821-1.168455-1.878053$

O $5.867408-1.730342-0.665084$

H $5.348506-2.694250-2.372015$

H $3.566372-0.619697-0.980438$

O $4.611491-0.238092-2.684768$

C $2.626167-1.606564-2.661796$

C $1.734720-0.404433-3.024844$

H $2.044506-2.310355-2.058240$

O $2.999993-2.269378-3.867193$

H $1.5193480 .150621-2.107634$

H $2.2815480 .272684-3.692273$

H $5.621919-6.7940551 .388137$

H $5.866682-5.3535932 .387773$

H $4.459721-6.3928752 .663162$

C $2.224608-4.2608392 .068146$

H $5.452305-1.4342660 .163390$

H $5.397636 \quad 0.028688-2.178059$

H $3.444189-3.099279-3.626443$

H $2.276678-5.1336662 .729127$

H $1.540908-4.5177851 .248843$

H $4.243383-3.4703272 .269297$

C $-0.833218-1.450434-1.764514$

O $-0.277752-1.842322-3.022032$

C $0.413314-0.803317-3.720661$

C $-0.5394120 .373308-3.978629$

C $-1.1987640 .871653-2.692259$ 
C $-1.825787-0.291071-1.920765$ H $-0.018208-1.133463-1.101179$ C -1.437349 -2.697083-1.102204 H $0.687152-1.221659-4.697539$ O $\quad 0.1628801 .438842-4.610452$ H -1.324184 $0.047922-4.673914$ H $-0.4812811 .422188-2.074704$ O $-2.2201101 .806448-3.048304$ H -2.730692 -0.618258 -2.444839 O $-2.2185860 .156102-0.626238$ C $-2.618527-3.293438-1.839630$ H - $0.677274-3.485175-1.041136$ H -1.751985 -2.443975 -0.085134 H $-0.4667042 .169949-4.733160$ H -2.568650 $2.188775-2.225425$ H $-2.9334180 .804479-0.742029$ O $-3.368746-4.022626-0.961018$ O $-2.840627-3.167922-3.036033$ C $-4.524372-4.657880-1.516407$ H -4.221462 $-5.425181-2.234817$ H $-5.074162-5.135644-0.701068$ H $-5.176601-3.919376-1.992078$

SCF Energy (B3LYP/6-31G**//MMFF)= -3245.90470359

06_00117

MM̄FF Geometry

C - $-1.519539-3.2538720 .352018$

C $-0.565978-3.351857-0.585888$

C $-0.433930-2.498264-1.830665$

O $0.790541-1.729230-1.727716$

C $-1.592538-1.506797-2.070083$

C $-1.488903-0.736224-3.372683$

C $-1.1817440 .572770-3.473185$

C -1.811314 -1.529663 -4.613272

C $-0.8375331 .529088-2.365130$

C $0.5619092 .124353-2.565306$

C $1.0351982 .907048-1.331087$

C $2.3903943 .561906-1.602510$

C $2.9747614 .229176-0.350820$

O $1.1443841 .977435-0.249580$

O $4.3508404 .522652-0.638335$

C 2.9390383 .2229260 .820492

C $2.2991945 .575569-0.064737$

O 3.3502693 .8732922 .020477

C 1.5630192 .5456020 .999078

C 1.5733911 .3943482 .034775

O $\quad 0.6200693 .519567 \quad 1.461687$

C $1.947345-2.330695-2.120142$

O $2.084487-3.480838-2.505779$

C $3.035210-1.332926-2.009264$

C $4.306487-1.705122-2.221275$

C $5.482729-0.831374-2.189588$

C $5.2663070 .621618-1.859274$

C $2.478821 \quad 0.208106 \quad 1.689178$

C 3.8599250 .2637682 .316695

C $6.018029-0.9892932 .337704$

O 4.2012921 .1188863 .128180

C $6.968103-0.364217 \quad 1.366077$

C $7.945122-1.0437270 .746729$

C $8.942382-0.413127-0.181398$

C $9.004180-1.095431-1.528719$

C $9.963660-1.994792-1.805028$

C $8.016135-0.660567-2.586256$

C $6.690669-1.363824-2.471258$

N $4.658337-0.7846691 .877473$

H -2.286474 -2.4918080 .259759$

H $0.188562-4.127403-0.468364$ H $-0.370622-3.174931-2.692292$ H -1.661683 -0.816096 -1.224255 H -2.544825 -2.051979 -2.084662 H $-1.1689471 .019681-4.466832$ H -1.807281 -0.910083 -5.516481 H -1.079090 -2.329105 -4.760910 H -2.806703 -1.978385 -4.529050 H -1.576381 2.339416 -2.363297 H $-0.8998701 .053374-1.384619$ H $1.2694221 .311015-2.769109$ H $\quad 0.5568702 .780547-3.444642$
H $\quad 0.2821063 .664090-1.082867$

H $2.3154524 .284455-2.424795$

H $3.0986142 .796412-1.945770$

H 4.7497284 .8845370 .171768

H 3.6961292 .4609910 .598593

H $2.4539896 .264503-0.904010$

H 2.7428356 .0652890 .809035

H 1.2228555 .4893990 .098156

H 3.4803533 .1909312 .699934

H 1.7739651 .7821993 .040368

H 0.5495400 .9987852 .081482

H -0.1233453 .0545431 .879899$

H $2.758200-0.312979-1.771999$

H $4.508550-2.748828-2.458809$

H $4.7181270 .727856-0.917341$

H $6.1937451 .183463-1.730227$

H $4.6962051 .110592-2.656089$

H $2.010228-0.7066982 .070814$

H 2.5802840 .0919940 .607009

H $6.170375-2.0693132 .429880$

H $6.145997-0.534293 \quad 3.325108$

H $6.8657780 .703866 \quad 1.188925$

H $8.061735-2.1065800 .946860$

H $9.925272-0.4622250 .305778$

H $8.7388710 .655654-0.322532$

H $10.034285-2.463856-2.780955$

H $10.696242-2.286177-1.059613$

H $8.415942-0.890712-3.583417$

H $7.9259530 .429358-2.575774$

H $6.746591-2.430125-2.695212$

H $4.304300-1.4108161 .159799$

C -1.502990 -3.408004 2.887584

O $-2.383974-2.2618672 .921545$

C -1.583060 -1.100445 3.200924

C $-0.389686-1.6340573 .978261$

C $-0.090083-2.8921673 .185364$

H -1.228228 -0.7180062 .236142$

C $-2.391542-0.0161573 .911452$

H $0.446808-0.9305974 .005213$

H $-0.660693-1.8900055 .009347$

C $0.783878-3.888673 \quad 3.926931$

H $\quad 0.420047-2.6078202 .256868$

C $-3.6485770 .451328 \quad 3.147642$

O $-2.810397-0.5156985 .186346$

H $-1.7370590 .839745 \quad 4.117591$

H $-4.426349-0.3193873 .205782$

O -4.1791431 .5832323 .850040$

C -3.4197320 .8211001 .668125$

C $-4.6121591 .569081 \quad 1.039741$

H -3.222003 -0.095936 1.102962

O -2.2563671 .6428101 .546283$

H -5.5401461 .0564721 .309352$

H $-4.6578662 .577349 \quad 1.467224$

H $1.761000-3.4492504 .152642$

H $0.327569-4.1973624 .873366$

H $0.947405-4.786403 \quad 3.322193$

C -1.649231-4.149034 1.553634

H $-3.3211070 .188873 \quad 5.620749$

H -3.5099732 .2882143 .830433$

H -2.4294282 .4785052 .011523$

H $-2.653339-4.5898621 .509534$

H $-0.936135-4.9802281 .508406$

H -1.838515 -4.073579 3.692743

C $-5.921070-0.149102-1.147405$

O $-4.597840 \quad 0.403692-1.136158$

C $-4.4737551 .680273-0.496622$

C $-5.4132422 .698930-1.156172$

C $-6.8539332 .201376-1.186952$

C $-6.9125500 .811362-1.816361$

H $-6.246704-0.353529-0.121059$

C $-5.859058-1.481892-1.911429$

H -3.452949 $2.025012-0.700149$

O $-5.3309823 .947520-0.478119$

H -5.076963 $2.871086-2.187240$

H -7.303086 2.199992 -0.186666

O $-7.6448103 .080974-1.988561$

H $\quad-6.7008900 .885478-2.890909$

O $\quad-8.234440 \quad 0.293310-1.674990$ 
C $-5.279397-2.604648-1.077217$

H $-6.864072-1.802041-2.207724$

H $-5.234028-1.370103-2.805260$

H -5.831282 $4.599158-0.997218$

H $-7.7009423 .931918-1.521955$

H $-8.8414410 .931010-2.088315$

O $-5.202801-3.727713-1.851754$

O $-4.948507-2.516846 \quad 0.096684$

C $-4.703627-4.905547-1.209763$

H $-3.613068-4.859547-1.161400$

H $-5.130746-5.027030-0.209392$

H $-4.991315-5.768808-1.815832$

SCF Energy (B3LYP/6-31G**//MMFF)= -3245.92165699

06_00118

MM̄FF Geometry

C $0.996971-2.592291-0.818939$

C $0.144099-1.696764-1.344458$

C $0.492809-0.608048-2.337383$

O $1.927851-0.474844-2.420405$

C $-0.0810800 .744675-1.882236$

C $-0.2335541 .740367-3.013593$

C $0.6039762 .767899-3.254855$

C $-1.4602411 .548455-3.869087$

C $1.8718443 .121191-2.527043$

C $1.7582844 .438710-1.746064$

C $0.8294604 .345483-0.526021$

C 0.7156025 .6980840 .179742

C $-0.1307155 .603631 \quad 1.459631$

O $1.381696 \quad 3.378293 \quad 0.372499$

O $0.0778596 .805452 \quad 2.216834$

C 0.3896594 .4318762 .319764

C $-1.6315525 .541841 \quad 1.148951$

O -0.4584664 .2088383 .444226$

C 0.5734653 .1172231 .526663

C 1.3054962 .0686122 .399284

O -0.7109262 .6106431 .165253$

C $2.519237-0.362147-3.633872$

O $1.964160-0.345170-4.722958$

C $3.995222-0.295629-3.497101$

C $4.614788-0.122294-2.318456$

C $6.060139-0.090942-2.086382$

C $6.966249-0.333539-3.263940$

C $1.756671 \quad 0.8157551 .634323$

C $2.558460-0.1309042 .510869$

C $4.076353-2.1048802 .342247$

O $2.619251-0.0270643 .732960$

C $5.468223-1.5537822 .342423$

C $6.487858-2.0916601 .655795$

C $7.879873-1.5248261 .654351$

C $8.367115-1.1658070 .266366$

C $9.233545-1.959708-0.385210$

C $7.9218050 .152101-0.331394$

C $6.5017930 .114141-0.827584$

N $3.183955-1.118348 \quad 1.761256$

H $2.043378-2.553010-1.114291$

H - $0.909762-1.752935-1.087029$

H $\quad 0.071535-0.910655-3.303555$

H $\quad 0.548746 \quad 1.161737-1.091175$

H -1.069539 $0.609758-1.427440$

H $0.3711353 .432556-4.086136$

H -1.440941 $0.569897-4.358295$

H $-2.3646401 .611816-3.254846$

H -1.542584 $2.306902-4.654861$

H $2.2094772 .317644-1.866530$

H $2.661323 \quad 3.232890-3.281055$

H $2.7673184 .707990-1.407682$

H $1.4169695 .238184-2.415374$

H $-0.1597614 .011825-0.858036$

H $0.3049486 .457485-0.497076$

H 1.7192756 .0520540 .454271

H -0.1760957 .5596741 .657720$

H 1.3672924 .7308902 .723305

H -1.945558 6.4379750 .600230

H -2.225375 5.5319842 .069611

H -1.9116964 .6739160 .548377$

H $-0.5343305 .052923 \quad 3.921422$

H 2.2019442 .5291532 .835295
H $\quad 0.659388 \quad 1.761511 \quad 3.231462$

H -0.6425481 .6552951 .012478$

H $4.545893-0.398458-4.426301$

H $4.010621-0.000146-1.420811$

H $\quad 6.738914-1.297187-3.733492$

H $8.025208-0.361842-2.998445$

H $6.8447960 .458779-4.010666$

H $\quad 0.8934490 .259340 \quad 1.256800$

H $2.382906 \quad 1.1021760 .781675$

H $3.990866-3.0163851 .742612$

H $3.762754-2.3273913 .367086$

H $5.643664-0.6629372 .941526$

H $\quad 6.318716-2.9896851 .066037$

H $8.543721-2.2741742 .105092$

H $7.952817-0.6413462 .300703$

H $9.612577-1.697498-1.367234$

H $9.580342-2.8924040 .047266$

H $8.6056990 .469355-1.126502$

H 8.0061530 .9392140 .429056

H $5.7643070 .280851-0.042180$

H $3.129964-1.0750830 .747814$

C $-0.678488-3.6770630 .835341$

O $-0.677056-2.6300071 .830164$

C -1.261521 -3.146315 3.033688

C -1.969493 -4.435792 2.645509

C $-1.020934-4.9655891 .586811$

H $-0.421357-3.4172813 .688961$

C $-2.107104-2.1114973 .779521$

H -2.096038 -5.1205153 .489296$

H -2.955274 -4.236523 2.209914

C $-1.629622-6.0519670 .717308$

H $-0.123301-5.3621772 .080446$

C $-3.333906-1.5186633 .050723$

O $-2.561697-2.7384454 .986433$

H -1.450421 -1.288192 4.088459

H $-4.088307-2.2959762 .887068$

O $-3.945374-0.5883643 .959992$

C $-3.044184-0.7978691 .718817$

C $-4.218877 \quad 0.072775 \quad 1.226570$

H -2.807435 -1.542790 0.954248

O

H $-5.119947-0.5466531 .172085$

H -4.4081970 .8625121 .962863$

H -1.897226 -6.9234441 .323710$

H $-2.537400-5.7033480 .214545$

H $-0.923331-6.379830-0.051557$

C $0.681669-3.716595 \quad 0.137046$

H -3.078209 -2.078217 5.479651

H -3.2966810 .1087854 .155836$

H -2.1048650 .7246072 .517621$

H $0.784314-4.652871-0.423958$

H $1.466842-3.7122010 .903995$

H -1.480188 -3.451083 0.126700

C $-4.883463-0.914283-1.613627$

O $-3.715317-0.209403-1.177007$

C $-3.9304740 .746997-0.134800$

C $-4.9771631 .781693-0.570066$

C $-6.2700831 .118866-1.029395$

C $-5.9704880 .059644-2.087129$

H $-5.270701-1.529078-0.792685$

C $-4.451734-1.837941-2.763639$

H -2.986686 $1.293757-0.025378$

O -5.2649032 .6959210 .482429$

H -4.576513 2.371670 -1.405069

H $-6.8255350 .689730-0.187261$

O $-7.1226882 .105449-1.614975$

H $-5.6588750 .550493-3.018208$

O $-7.163225-0.670852-2.367078$

C $-3.673567-3.039252-2.273253$

H $-5.318412-2.227955-3.307901$

H $-3.815383-1.276568-3.459013$

H -4.4267853 .1152650 .742169$

H -7.270044 $2.794200-0.944114$

H -7.839529 -0.027673 -2.641084

O $-2.907983-3.523777-3.295006$

O $-3.758306-3.517760-1.150835$

C $-2.149349-4.699782-2.996742$

H $-2.809036-5.506629-2.663447$ 
H $-1.642163-5.017900-3.911547$ H $-1.395367-4.478802-2.237065$

SCF Energy (B3LYP/6-31G**//MMFF)= -3245.92466273

06_00119

MM̄̄FF Geometry

C $-0.504034-3.7442290 .247271$

C $-1.187850-2.6102870 .031722$

C $-1.644036-2.182248-1.339968$

O $-1.356305-0.774289-1.504968$

C $-3.167069-2.338412-1.473153$

C $-3.611623-2.502917-2.912194$

C $-4.055916-1.508914-3.704931$

C $-3.571924-3.918836-3.428386$

C $-4.133268-0.038292-3.402943$

C $-5.5757710 .450762-3.215833$

C $-6.1168840 .173408-1.805422$

C $-7.5944570 .550671-1.707738$

C $-8.1339600 .354720-0.284202$

O $-5.358206 \quad 0.971783-0.892551$

O $-9.412010 \quad 1.008532-0.235586$

C $-7.194143 \quad 1.0686510 .713604$

C $-8.395353-1.1264270 .013935$

$\begin{array}{lllll}\text { O } & -7.542094 & 0.759783 & 2.061251\end{array}$

C $-5.686908 \quad 0.7817420 .487885$

C $-4.839015 \quad 1.785047 \quad 1.300250$

O $\quad-5.317764-0.520210 \quad 0.945027$

C $-0.075657-0.396856-1.747170$

O $0.900368-1.117758-1.866014$

C $-0.0774491 .080577-1.813000$

C $1.0721941 .755689-1.677301$

C $1.2184933 .212490-1.691769$

C $-0.0092834 .034099-1.989386$

C -3.3238011 .6506111 .081596$

C -2.5517302 .6363371 .944777$

C -0.2323763 .2982442 .560482$

O -3.1006713 .4482302 .686353$

C 0.2369784 .4575191 .738897

C 1.5241704 .8313071 .670684

C 2.0183506 .0194760 .896058

C $3.1685645 .678908-0.026164$

C 4.4420925 .8794050 .352946

C $2.8371495 .187948-1.416650$

C $2.4328413 .740523-1.433679$

N -1.177744 2.5019551 .798210

$\mathrm{H}-0.300916-4.388507-0.606750$

H -1.431058 -1.9473080 .856639$

H -1.123125 -2.746812 -2.123821

H -3.670226 -1.477122 -1.020721

H -3.518594 -3.215521 -0.913160

H $-4.377016-1.767421-4.713759$

H -3.898180 -3.992345 -4.471274

H -2.554074 -4.317985 -3.373351

H -4.229170 -4.559060 -2.830751

H $-3.5160030 .240552-2.544982$

H $-3.6979340 .487510-4.262572$

H $-5.5825351 .535121-3.388237$

H $-6.228917-0.002644-3.971487$

H $-5.978316-0.886459-1.568174$

H -8.193103 -0.023030 -2.426458

H $-7.7213361 .606302-1.986641$

H $-9.796950 \quad 0.8548320 .643607$

H -7.3563632 .1487280 .589673$

H $-9.165345-1.519734-0.660893$

H -8.786875 -1.2650531 .027648$

H -7.506991 -1.751075 -0.101437

H $-8.454268 \quad 1.0613882 .207432$

H -5.1295052 .8088551 .029999$

H -5.0476331 .6585482 .370596$

H $-6.114821-0.992448 \quad 1.231064$

H $-1.0346591 .573325-1.942055$

H $1.9905571 .190813-1.528511$

H $-0.3846563 .810663-2.994130$

H $0.1630755 .111206-1.945321$

H $-0.8029173 .822166-1.264274$

H -2.9909990 .6382821 .336957$

H -3.071553 1.8450950 .033213

H 0.5906062 .6378072 .844623
H $-0.715673 \quad 3.6653903 .471523$

H -0.5139735 .0329801 .203248$

H 2.2656804 .2769332 .240819

H $2.3391906 .778818 \quad 1.621361$

H 1.2108856 .4860260 .318355

H $5.2710535 .663261-0.312722$

H 4.6837596 .2582191 .340530

H $3.7148295 .286074-2.070079$

H $2.0880485 .846720-1.864301$

H $3.2576613 .061949-1.214670$

H $-0.809396 \quad 1.8369001 .124510$

C $0.170852-3.2438362 .698803$

O $1.111657-2.2133842 .331360$

C $2.024628-2.0014123 .423009$

C $1.397840-2.6869434 .630835$

C $0.721744-3.8755903 .979430$

H $2.954012-2.5222663 .164067$

C $2.298417-0.5066143 .615439$

H $2.142875-2.9719235 .379677$

H $0.652350-2.0497985 .120454$

C $-0.337239-4.5269404 .851986$

H $1.489343-4.6214153 .732406$

C 2.8272630 .2257112 .365724

O $1.081614 \quad 0.1416684 .011560$

H $2.995370-0.3680384 .450130$

H 2.0296390 .3044861 .616432

O $3.131727 \quad 1.5637812 .781238$

C $4.083442-0.4038531 .731781$

C $4.6664440 .453013 \quad 0.592977$

H $3.828539-1.3938341 .344985$

O $5.085426-0.5900252 .731058$

H $3.8615260 .695387-0.106695$

H 5.0353621 .3991951 .006440

H $0.107176-4.9041155 .778831$

H -1.126634 -3.817513 5.122231

H $-0.805103-5.3702014 .334353$

C $0.040936-4.2534431 .554802$

H $0.424641-0.0144913 .311317$

H $3.1867772 .113917 \quad 1.981798$

H 5.2854810 .2819893 .112786

H $\quad-0.581177 \quad-5.0990541 .870353$

H $1.039354-4.6550301 .340425$

H $-0.788515-2.7522052 .901001$

C $4.553095-1.475641-1.772152$

O $5.482296-1.508694-0.682800$

C $5.837415-0.229238-0.149816$

C $6.4621150 .635722-1.252948$

C $5.5469550 .751550-2.468949$

C $5.097259-0.632441-2.934590$

H $3.597549-1.066134-1.426040$

C $4.324673-2.925733-2.227591$

H $6.620525-0.4149240 .595910$

O $6.7680751 .931273-0.747269$

H $7.4111180 .181033-1.566052$

H $4.6904051 .400094-2.256920$

O $6.2829901 .387482-3.516477$

H $5.941537-1.148051-3.410286$

O $4.072239-0.489334-3.916300$

C $3.572414-3.748429-1.204187$

H $3.741006-2.957017-3.154012$

H $5.295761-3.407390-2.396129$

H $7.1391822 .449934-1.481586$

H $5.667326 \quad 1.551012-4.250643$

H $4.465718-0.057681-4.693114$

O $3.718471-5.072305-1.508360$

O $2.919826-3.294086-0.275504$

C $3.053494-5.987371-0.632042$

H $3.334219-5.7998900 .408994$

H $1.970866-5.905925-0.762527$

H $3.364183-7.000701-0.899848$

SCF Energy (B3LYP/6-31G**//MMFF) $=-3245.91161893$

06_00120

MM̄FF Geometry

C $0.746164-0.4346472 .614610$

C $0.409070-1.3422251 .686013$

C $-0.463279-1.0320660 .498731$

O $-1.646474-1.8555520 .573639$ 
C $0.291648-1.368108-0.801741$

C $-0.312523-0.709043-2.026023$

C $0.193120 \quad 0.390915-2.619370$

C -1.533592 -1.379283 -2.597774

C $1.4048891 .181819-2.209732$

C $1.2657942 .016809-0.928757$

C $0.203836 \quad 3.128497-0.981317$

C 0.1724303 .8811410 .354409

C $-0.933160 \quad 4.946434 \quad 0.400426$

O $-1.0796672 .552812-1.240642$

O

C $-2.2635944 .305388-0.053479$

C $-0.523566 \quad 6.210293-0.364214$

O $-3.2928895 .286521-0.150488$

C $-2.1395143 .508944-1.369487$

C $-3.4364132 .751583-1.732517$

O $-1.8850374 .424484-2.442776$

C $-2.695612-1.3537831 .281416$

O $-2.727265-0.3074951 .910197$

C $-3.827017-2.2947041 .137024$

C $-5.054926-1.8923071 .495628$

C $-6.283782-2.6818021 .419407$

C -6.177937 -4.1046770 .937475$

C $-3.874213 \quad 1.676784-0.736116$

C $-5.0730680 .916697-1.272323$

C $-7.4956001 .208170-1.797580$

O $-4.968021-0.218295-1.731084$

C -8.371376 $0.615845-0.738910$

C $-9.002526-0.559229-0.886611$

C $-9.959442-1.1375650 .117803$

C $-9.589044-2.5429790 .535010$

C $-10.004049-3.604824-0.176554$

C $-8.812975-2.7237351 .818230$

C $-7.446893-2.0992721 .777042$

N $-6.2444411 .654428-1.215327$

H 0.3486330 .5748332 .542908

H $0.793618-2.3559601 .763696$

H -0.7515400 .0266480 .488890$

H $0.308679-2.454233-0.963253$

H $1.339792-1.063873-0.704822$

H $-0.3163600 .778860-3.500441$

H -1.321285 -2.430150 -2.819569

H -2.366872 -1.332993 -1.892131

H $-1.867898-0.909234-3.528889$

H $2.2574730 .500886-2.103415$

H $1.6715351 .849906-3.038627$

H $1.0561401 .349791-0.087479$

H $2.2399772 .476757-0.720760$

H $0.4658023 .808552-1.800324$

H $1.149324 \quad 4.3314040 .571227$

H $-0.009799 \quad 3.1632141 .167116$

H -1.825937 5.9599231 .825208

H -2.569936 3.6241860 .749831

H $0.382494 \quad 6.6438310 .076322$

H -1.291680 $6.987683-0.288146$

H $-0.3209126 .026205-1.421129$

H -3.147220 $5.788201-0.970646$

H -4.248147 $3.471443-1.899348$

H -3.289089 2.260924 -2.705600

H -2.110401 $3.986831-3.280438$

H -3.622281 -3.2775260 .728654$

H $-5.177905-0.8800711 .879946$

H $-5.536619-4.6904021 .605291$

H $-5.756967-4.138002-0.073580$

H -7.140941 -4.618296 0.888735

H $-3.0669400 .958084-0.565663$

H $-4.1404632 .108567 \quad 0.234070$

H -7.289310 $0.500682-2.607610$

H $-7.9820962 .091337-2.223593$

H -8.530223 1.1979210 .165621

H -8.863679 -1.119529-1.808852

H -10.959016 - $1.144119-0.337007$

H -10.046595 -0.4970651 .004129$

H $-9.768788-4.6188380 .129120$

H - $-10.586275-3.481870-1.083933$

H -8.742483 -3.782201 2.095310

H $-9.377890-2.2624642 .639026$

H $-7.433190-1.0570242 .097543$
H -6.232676 $2.567380-0.770096$

C $2.7870240 .324622 \quad 3.853733$

$\begin{array}{lllll}\text { O } & 3.570689 & 0.330311 & 2.645900\end{array}$

C 4.9516350 .5603852 .985579

C 4.9878540 .8370864 .486253

C $3.785094 \quad 0.0555904 .977363$

H $5.482712-0.3778022 .787047$

$\begin{array}{llll}\text { C } 5.530667 & 1.684028 & 2.116143\end{array}$

H $5.9275770 .510826 \quad 4.942528$

H 4.8644341 .9031264 .708834

C $3.3045680 .482247 \quad 6.353191$

H $4.047103-1.0108585 .000198$

C 5.3490871 .4802070 .599107

O 4.8857442 .9203152 .454255

H 6.5915621 .8244722 .352311

H $4.288387 \quad 1.562687 \quad 0.334555$

O $6.015757 \quad 2.557812-0.070934$

C 5.9061540 .1535300 .055359

C $5.7593600 .048421-1.473115$

H $5.375245-0.6770810 .524432$

$\begin{array}{lllll}\text { O } & 7.281899 & 0.024195 & 0.408408\end{array}$

H $4.7186120 .261437-1.737793$

H $6.3783340 .817694-1.950672$

$\begin{array}{llll}\text { H } & 4.093449 & 0.336062 & 7.098243\end{array}$

H $3.0190981 .539476 \quad 6.368647$

H $2.435201-0.1058556 .663459$

C $1.675845-0.7229073 .759733$

H 3.9309192 .8021892 .310396

H $\quad \begin{array}{llll}5.646797 & 3.387489 & 0.277569\end{array}$

H 7.7550580 .7850620 .030054

H $1.092513-0.7298804 .687102$

H $2.118735-1.7189823 .639589$

H 2.3521051 .3278933 .956465

C $4.104168-2.510881-1.684136$

O $5.504639-2.416823-1.396971$

C $6.182943-1.328230-2.032855$

C $6.040728-1.448229-3.556938$

C $4.577079-1.543870-3.979409$

C $3.864332-2.642940-3.193923$

H $3.591831-1.622402-1.298836$

C $3.561972-3.745349-0.944997$

H $7.247018-1.452089-1.795683$

O $6.645161-0.340775-4.216364$

H $6.560846-2.351461-3.901311$

H $4.060748-0.583330-3.867957$

O $4.509604-1.874229-5.368780$

H $4.211369-3.623284-3.545259$

O $2.463520-2.569355-3.452626$

C $3.525753-3.5618780 .557207$

H $2.538076-3.969075-1.263221$

H $4.204913-4.607642-1.160093$

H $7.577248-0.310218-3.940617$

H $4.999956-1.187547-5.852689$

H $2.342224-2.627115-4.415902$

O $3.289016-4.7721051 .145262$

O $3.661974-2.497895 \quad 1.143421$

C $3.217252-4.759607 \quad 2.574707$

H $4.121435-4.3153583 .002523$

H $2.327095-4.2132512 .898861$

H $3.139428-5.7939482 .919915$

SCF Energy (B3LYP/6-31G**//MMFF) $=-3245.92265735$

06_00121

MM̄FF Geometry

C $-0.474276-1.2449882 .307829$

C $0.286710-1.7509631 .325324$

C $-0.161370-2.3050880 .001548$

O $0.041826-1.223468-0.940547$

C -1.630312 - $2.752223-0.079020$

C $-1.896256-3.636260-1.282025$

C $-2.504654-3.236371-2.415197$

C $-1.463868-5.073259-1.130224$

C $-3.013165-1.865638-2.766366$

C $-4.544468-1.838571-2.875134$

C $-5.243305-1.908950-1.508454$

C $-6.750238-2.101881-1.681003$

C $-7.481504-2.087207-0.330325$

O $-4.984598-0.676419-0.828549$ 
O $-8.887965-1.970572-0.593956$ C -7.056954 -0.8238550 .445812$ C $-7.291470-3.4005550 .437398$ O $-7.609577-0.824546 \quad 1.760920$ C $-5.524789-0.599942 \quad 0.496097$ C -5.2346820 .8167491 .045109$ O $-4.938911-1.5735291 .360672$ C $0.738454-1.453966-2.078006$ O $1.201041-2.522945-2.449883$ C $0.920787-0.205761-2.857387$ C $0.3218770 .958139-2.556455$ C $0.467945 \quad 2.220643-3.286742$ C $1.4933442 .271286-4.389664$ C -3.7597891 .2385820 .990576$ C -3.5840542 .7008101 .366617$ C $-1.854788 \quad 4.492101 \quad 1.437327$ O $-4.508900 \quad 3.4114721 .752087$ C -1.810375 5.1879890 .112987 C $-0.6968565 .724013-0.408138$ C $-0.6415106 .427465-1.731917$ C $0.2444495 .716120-2.730124$ C $1.5170846 .101080-2.924387$ C $-0.3754974 .627336-3.575790$ C $-0.3064723 .268819-2.932543$ N -2.267972 3.1172491 .224435 H $-1.554929-1.2200722 .214058$ H $1.366946-1.7127351 .450182$ H $0.503604-3.147387-0.224491$ H -2.287793 -1.876091-0.094307 H -1.905864 -3.320481 0.819055 H -2.651676 -3.975284 -3.202495 H - $1.729942-5.682446-2.000728$ H $-0.379139-5.140549-1.003304$ H $-1.945525-5.523621-0.256160$ H -2.663448 -1.096475 -2.071755 H -2.589448 -1.600138 -3.743229 H $-4.827637-0.902971-3.374547$ H - $-4.881916-2.663494-3.515039$ H $-4.824584-2.738133-0.928477$ H -6.964682 -3.031717 -2.222341 H $-7.154093-1.291787-2.304436$ H $-9.154044-2.728127-1.142830$ H $-7.5143430 .031543-0.070377$ H $-7.642160-4.248841-0.162568$ H -7.894236 -3.4138521 .352185$ H $-6.250727-3.5935710 .705844$ H -7.085397-1.439655 2.301514 H -5.8112971 .5443380 .458259$ H -5.5784200 .8915492 .084703$ H -4.081683 -1.243528 1.672065 H $1.580538-0.303572-3.713817$ H $-0.3517531 .000512-1.702106$ H $2.4717861 .941126-4.021834$ H $1.6484833 .273611-4.794583$ H $1.1941811 .625715-5.222515$ H -3.163071 0.6400171 .685816 H -3.359138 $1.099772-0.019972$ H $-0.8789134 .468514 \quad 1.930574$ H -2.564544 4.9983992 .099187 H -2.743259 $5.255880-0.441983$ H $0.231965 \quad 5.6740180 .153548$ H $-0.2669407 .444261-1.554641$ H -1.643949 $6.559359-2.157769$ H $2.146857 \quad 5.616954-3.663317$ H $1.9586386 .904359-2.344060$ H $0.0765654 .624902-4.573225$ H $-1.4304954 .865989-3.763378$ H $-1.013132 \quad 3.135952-2.113084$ H $-1.5824702 .470796 \quad 0.844115$ C $0.383473 \quad 0.878912 \quad 3.316454$ $\begin{array}{lllll}\text { O } & 1.353360 & 1.036946 & 2.261267\end{array}$ C 2.3662591 .9669902 .678083 C 1.7783312 .6958953 .878772 C $0.977238 \quad 1.584917 \quad 4.535073$ H 3.2255881 .3730573 .011413 C 2.7759502 .8520901 .495228 H 2.5506453 .1131064 .531690 H 1.1123493 .5116183 .574993
C $-0.0640932 .085207 \quad 5.520559$

H 1.6720800 .9136095 .057588

$\begin{array}{llll}\text { C } 3.145327 & 2.070018 & 0.216462\end{array}$

$\begin{array}{lllll}\text { O } & 1.682442 & 3.713370 & 1.161544\end{array}$

H 3.5998263 .5111801 .790843

H $2.2533751 .561969-0.169337$

O $3.5248003 .036399-0.770970$

C 4.2874781 .0553530 .405403

C $4.6172200 .283260-0.882996$

H 4.0006760 .3358921 .173780

O $5.454044 \quad 1.7279790 .873716$

H $3.702568-0.205409-1.234619$

H $4.9284940 .988837-1.662049$

H 0.4132362 .6309326 .340880

H -0.7802472 .7600405 .039511$

H -0.6254991 .2499015 .951204$

C $0.120712-0.6131993 .538017$

H $0.929733 \quad 3.1498010 .912620$

H $3.4608982 .609365-1.641799$

H 5.6921302 .3984100 .210550

H $-0.564104-0.7648684 .379660$

H $1.057669-1.1240243 .791275$

H -0.5328851 .3818882 .983390$

C $4.453902-2.581492 \quad 0.208632$

O $5.526961-1.6503780 .387263$

C $5.749187-0.755840-0.707612$

C $6.054743-1.555310-1.982221$

C $4.956721-2.571547-2.284221$

C $4.675980-3.431118-1.053133$

H $3.504385-2.0374570 .141277$

C $4.409624-3.4904441 .449375$

H $6.656345-0.191583-0.457120$

O $6.211643-0.691852-3.103267$

H $7.002188-2.095261-1.855866$

H $4.044331-2.082443-2.643221$

O $5.391087-3.430841-3.341095$

H $5.523749-4.111018-0.899733$

O $3.533671-4.249427-1.297741$

C $4.001949-2.7699232 .717108$

H $3.687894-4.3000831 .293269$

H $5.403492-3.9188761 .627257$

H $6.927894-0.068455-2.893224$

H $5.601983-2.866139-4.104368$

H $2.772256-3.663036-1.446567$

O $3.945205-3.677191 \quad 3.737923$

O $3.767393-1.5743562 .812669$

C $3.579345-3.1461125 .015479$

H $4.268959-2.3495475 .311127$

H 2.550351-2.776554 4.987607

H $3.642299-3.9536505 .749649$

SCF Energy (B3LYP/6-31G**//MMFF) $=-3245.91839868$

06_00122

MM̄FF Geometry

C $0.216659-2.4609421 .688925$

C $0.785181-1.6343212 .581294$

C $0.325728-0.2422742 .950542$

$\begin{array}{lllll}\text { O } & -0.990606 & 0.040900 & 2.425214\end{array}$

C 1.2974090 .8027592 .382720

C 1.0742172 .1852132 .962940

C 0.4379313 .1912352 .331635

C 1.6726742 .4111214 .327994

$\begin{array}{lllll}\text { C } & -0.211597 & 3.162767 & 0.976308\end{array}$

C $-1.615148 \quad 3.782417 \quad 1.013476$

C $-2.2572043 .808854-0.381315$

C $-3.5563164 .615886-0.361889$

C $-4.2551054 .600361-1.728294$

O $-2.5292802 .459705-0.774966$

O $-5.5780115 .120029-1.527700$

C $-4.382803 \quad 3.138112-2.201151$

C $-3.5688415 .542003-2.724354$

O $-4.912112 \quad 3.077551-3.523999$

C $-3.0677572 .328309-2.097406$

C $-3.365305 \quad 0.834116-2.354071$

O $-2.152802 \quad 2.794950-3.089950$

C $-2.054502-0.3533293 .176536$

O $-2.023780-0.914000 \quad 4.260536$

C $-3.282788-0.015606 \quad 2.425269$ 
C $-4.435864-0.6094742 .767063$

C $-5.728092-0.4381392 .101544$

C $-5.849100 \quad 0.6647901 .085609$

C -2.207244 -0.117163 -2.029135

C $-2.695022-1.549511-2.117449$

C $-4.063229-3.222609-0.864949$

O $-2.542059-2.220265-3.135363$

C $-5.534756-2.943447-0.891004$

C $-6.404403-3.452390-0.004633$

C $-7.880167-3.167000-0.028804$

C $-8.427567-2.7539531 .321886$

C $-9.227455-3.5756292 .022476$

C $-8.111178-1.3604521 .827557$

C -6.733405 -1.277166 2.427104

N $-3.354253-1.960276-0.968579$

H $-0.655935-2.1395161 .127784$

H $1.658019-1.9866813 .128544$

H $\quad 0.304410-0.1718944 .045275$

H $1.2091390 .826217 \quad 1.291937$

H 2.3367300 .5109212 .586059

H 0.3820114 .1541372 .838294

H 1.5332463 .4391684 .678909

H 1.2118871 .7473575 .065806

H 2.7495442 .2148274 .307827

H $\quad 0.416465 \quad 3.7430470 .290722$

H -0.2782192 .1478720 .575787$

H -2.244040 3.2021891 .700904

H -1.5506514 .8049791 .406254$

H -1.542469 $4.253862-1.083764$

H -3.371411 $5.647902-0.038468$

H $-4.240053 \quad 4.1862900 .384188$

H $-6.0559945 .043205-2.371483$

H $-5.1343872 .663038-1.555731$

H $-3.5639556 .567893-2.336697$

H -4.116904 $5.585104-3.672040$

H $-2.5351005 .261643-2.937052$

H -4.194736 $3.304081-4.140130$

H $-4.2305800 .535733-1.752631$

H $-3.6505120 .685550-3.403923$

H -1.553883 2.071149 -3.332222

H $-3.197217 \quad 0.6726661 .592584$

H $-4.428772-1.3219083 .591451$

H -5.4846341 .6135201 .495842$

H -6.8761010 .8516770 .765316$

H -5.2663620 .4226260 .194409$

H - $-1.376206 \quad 0.008523-2.730837$

H -1.808359 $0.057587-1.023476$

H $-3.750087-3.6951280 .071203$

H -3.792672 -3.874195 -1.701185

H -5.903505 -2.293996-1.681782

H $-6.041209-4.1252330 .769112$

H -8.386690 -4.076698 -0.377038

H -8.122312 -2.388491 - 0.763065

H $-9.643703-3.2785012 .979387$

H -9.480428 -4.5657501 .657933$

H -8.827890 -1.070561 2.606949

H -8.266220 -0.6541571 .007681$

H -6.550365 -2.023937 3.201408

H -3.439929-1.311561 -0.192136

C $1.337900-4.1205290 .092253$

O $2.549933-3.358903-0.045709$

C $2.778412-3.125787-1.453257$

C $1.615121-3.780453-2.200761$

C $0.519161-3.792631-1.149777$

H $2.726213-2.043191-1.606263$

C $4.164129-3.658270-1.849205$

H $1.338138-3.219346-3.098985$

H $1.858106-4.801915-2.516114$

C $-0.588234-4.789707-1.438805$

H $0.096239-2.782780-1.084141$

C $5.326338-3.042735-1.042595$

O $4.192246-5.076603-1.636293$

H $4.330395-3.509712-2.922081$

H $5.201821-3.2612650 .024670$

O $6.542390-3.683498-1.450190$

C $5.498025-1.527115-1.250373$

C $6.615908-0.941602-0.369446$

H $4.565937-1.018255-1.000261$
O $5.766744-1.260960-2.624990$

H $6.308642-1.0456450 .677549$

H $7.529861-1.536383-0.488250$

H - $1.079232-4.557093-2.388818$

H $-0.201048-5.812089-1.504259$

H $-1.346523-4.768164-0.650258$

C $0.690715-3.8682421 .453784$

H $4.024240-5.234886-0.691480$

H $6.420488-4.640598-1.326714$

H $\quad 6.584765-1.731944-2.859437$

H $1.414131-4.1418502 .233010$

H $-0.167734-4.5389021 .579527$

H $1.637059-5.177790 \quad 0.065322$

C $5.095613 \quad 1.634478 \quad 0.363200$

O $5.823217 \quad 1.365642-0.839331$

C $6.9703260 .528202-0.687140$

C $7.953757 \quad 1.1585780 .309017$

C 7.2791601 .4759841 .641532

C 6.0023182 .2837981 .417107

H 4.6705650 .7020330 .754722

C $3.9466942 .584670-0.009871$

H $7.4668470 .520061-1.665979$

O 9.0661590 .3012350 .541741

H $8.3485782 .093099-0.110447$

H 7.0715910 .5663082 .216720

O 8.1671482 .2598752 .442357

H 6.2688113 .3029521 .108835

O 5.2874512 .3844402 .647332

C $2.9279651 .934680-0.921126$

H 3.4131332 .9306180 .880951

H $4.3608213 .454909-0.534514$

H $9.4858980 .127902-0.318219$

H 8.9894201 .7503502 .544333

H 5.8894022 .7793443 .301286

O $2.2908162 .901490-1.644566$

O $2.6929840 .735582-0.970444$

C $1.2723952 .431057-2.532171$

H $1.7134251 .805399-3.313619$

H $0.510463 \quad 1.875527-1.977283$

H $0.8019203 .299853-2.999841$

SCF Energy (B3LYP/6-31G**//MMFF) $=-3245.93680147$

06_00123

MMFF Geometry

C $0.880170-2.3289833 .098018$

C $-0.119074-1.6912843 .727157$

C $-0.312381-0.1990563 .861702$

O -1.3346090 .2114522 .921587$

C 0.9385190 .6521513 .580798

C 0.7682622 .0999183 .998473

C 0.5472823 .1217473 .148779

C 0.9294532 .3682115 .473021

C 0.3829563 .0536841 .656139

C $-0.757163 \quad 3.963491 \quad 1.176728$

C $-0.8643303 .967494-0.354120$

C $-1.8648335 .022425-0.827360$

C $-2.0305185 .000177-2.353532$

O $-1.2941622 .669092-0.776042$

O $\quad-3.183517 \quad 5.798050-2.660725$

C $-2.3184803 .551688-2.799492$

C $-0.8449885 .667009-3.060381$

O $-2.3498953 .456313-4.221952$

C $-1.3490522 .504142-2.198962$

C $-1.8774411 .085350-2.503931$

O $-0.0613202 .658664-2.797456$

C -2.6345970 .0861703 .297016$

O $-3.059027-0.3494994 .354931$

C -3.4665410 .5581442 .168156$

C -4.7677310 .2356282 .133970$

C $-5.7172450 .585327 \quad 1.075524$

C $-5.266181 \quad 1.5718320 .032128$

C $-1.144228-0.042700-1.769716$

C $-1.870011-1.350718-2.010248$

C $-3.874991-2.637224-1.256285$

O $-1.545159-2.108820-2.920993$

C $-5.189715-2.087034-1.715632$

C $-6.360209-2.361971-1.119547$

C $-7.679049-1.807879-1.581015$ 
C $-8.490353-1.194608-0.458922$

C $-9.608819-1.790001-0.011030$

C -8.0499410 .1466550 .091976$

C $-6.936251 \quad 0.006821 \quad 1.090897$

N $-2.932646-1.539365-1.139844$

H $1.656674-1.7655262 .589569$

H $-0.885134-2.2959624 .210311$

H $-0.651928-0.000994 \quad 4.886176$

H 1.1940920 .5950382 .517368

H $1.803907 \quad 0.2458124 .120998$

H 0.4960824 .1267193 .566156

H 0.8623563 .4350485 .711847

H $\quad 0.152474 \quad 1.8544596 .046970$

H 1.9075582 .0165645 .817523

H 1.3225363 .3823531 .196510

H $\quad 0.1922892 .0328581 .313644$

H -1.7009813 .6147841 .615540$

H -0.5830984 .9866201 .533062$

H $0.1296454 .173596-0.768666$

H -1.568762 $6.023224-0.489171$

H $-2.8439814 .827966-0.367017$

H -3.343719 $5.728146-3.617693$

H $-3.3364963 .314796-2.460541$

H $-0.7366596 .705406-2.724603$

H -1.006883 5.720016-4.142656

H $\quad 0.103798 \quad 5.157717-2.879026$

H -1.430526 $3.459346-4.538675$

H $-2.9384351 .034922-2.234216$

H $-1.8191170 .890092-3.582855$

H $0.4291721 .826356-2.706042$

$\mathrm{H}-2.980044 \quad 1.1270411 .383831$

H -5.173581 -0.3714592 .942513$

H $-6.0660851 .899909-0.634450$

H $-4.4829881 .134089-0.591207$

H -4.8721132 .4800360 .502332$

H $-0.114901-0.150647-2.125142$

H $-1.0932230 .142812-0.690938$

H -3.946471 -3.109131 -0.271447

H $-3.507373-3.373629-1.977131$

H -5.178212 -1.435031 -2.586303

H $-6.376235-3.036361-0.266171$

H -8.239142 -2.630880 -2.043756

H -7.540949 -1.057876 -2.369856

H -10.209382 $-1.344800 \quad 0.775457$

H -9.946709 -2.737893 -0.416419

H -8.8895120 .6375520 .601409$

H -7.796829 $0.799092-0.747743$

H -7.172343 -0.673663 1.910736

H -3.114468 -0.840968 -0.425549

C $0.718213-4.460476 \quad 1.706592$

O $1.626339-3.9724210 .698819$

C $0.888230-3.802105-0.528661$

C $-0.444508-4.514040-0.318643$

C $-0.689351-4.2420501 .152110$

H $0.699785-2.726948-0.632761$

C $1.696759-4.305388-1.727772$

H -1.232794 -4.128867 -0.968638

H $-0.362716-5.593101-0.494796$

C - $-1.748113-5.1349911 .772914$

H $-0.996664-3.1944651 .260517$

C $3.102017-3.687897-1.866519$

O $1.864643-5.725532-1.622528$

H $1.125088-4.139807-2.648450$

H $3.763234-4.070446-1.079348$

O $3.653270-4.143220-3.109217$

C $3.136070-2.148932-1.853097$

C $4.539200-1.591680-2.158413$

H $2.809478-1.794351-0.870366$

O $2.214435-1.636206-2.814214$

H $5.265098-2.095238-1.511138$

H $4.807739-1.831919-3.194339$

H -2.713396 -4.988207 1.277445

H -1.483142 -6.1939861 .684695$

H -1.875776 -4.9060872 .835466$

C $1.017222-3.8261743 .065598$

H $2.307713-5.906546-0.775729$

H $3.631308-5.115595-3.096958$

H $2.471319-1.986667-3.684351$
H $2.052933-4.0578353 .344742$

H $0.378460-4.2862283 .829198$

H $0.917126-5.5376461 .790624$

C $5.083052-0.0028440 .391124$

O $4.2022020 .359545-0.677373$

C $4.629013-0.059057-1.977954$

C $6.0124550 .529030-2.288545$

C $7.0320800 .187948-1.204081$

C 6.4931690 .5652260 .176497

H $5.141043-1.0973100 .442390$

C $4.4328340 .432418 \quad 1.712423$

H $3.9216230 .384874-2.689707$

O $6.4694650 .063295-3.553800$

H $5.9254431 .621106-2.360537$

H $7.317121-0.869706-1.248728$

O $8.2247540 .934078-1.459878$

H $6.503763 \quad 1.6579980 .262476$

O 7.3885310 .0505161 .159071

C 4.3286251 .9335221 .886910

H $3.4133920 .036513 \quad 1.769359$

H 5.0053870 .0172762 .549584

H $7.3537020 .441150-3.700415$

H $8.8495870 .734647-0.741576$

H 7.1387700 .4335242 .016692

O $4.3883642 .237103 \quad 3.216690$

O $4.1881002 .737054 \quad 0.975022$

C 4.3240463 .6301993 .538819

H 5.2564674 .1200913 .243870

H 3.4692964 .1071683 .050434

H 4.2033193 .7230664 .621356

SCF Energy $\left(B 3 L Y P / 6-31 G^{* *} / / M M F F\right)=-3245.93060647$

06_00124

MMFF Geometry

C $-0.901434-2.930096-2.069917$

C $-1.956826-3.112616-2.879324$

C $-3.031058-2.113508-3.231865$

O $-2.870516-0.837046-2.575894$

C $-4.462985-2.629595-2.955371$

C $-4.856048-2.866906-1.507740$

C $-5.801980-2.171235-0.846265$

C $-4.184366-4.015656-0.799381$

C $-6.661264-1.044104-1.341047$

C $-6.1124520 .363773-1.065957$

C $-5.7335520 .623270 \quad 0.402193$

C -5.5491182 .1212490 .653079$

C -5.0492102 .4149232 .075019$

O $-4.505246-0.0628240 .664626$

O -4.6198053 .7852222 .091634$

C -3.8189991 .5280832 .367024$

C -6.1833012 .3084343 .100826$

O -3.4037661 .6700413 .722877$

C -4.0326330 .0416602 .013195$

C $-2.736420-0.8005492 .115844$

O $-4.974483-0.5238552 .932836$

C $-2.0649480 .079185-3.183188$

O $-1.384557-0.088553-4.183074$

C $-2.162172 \quad 1.345040-2.422170$

C $-1.3615202 .374559-2.736713$

C $-1.3617503 .696691-2.105225$

C $-2.3571803 .945022-1.003280$

C -1.603902 -0.3777811 .174893$

C -0.6233410 .6099331 .782902$

C 1.2510002 .1235511 .140758

O -0.5359550 .8132972 .990687$

C 0.7722103 .5296910 .971775

C 1.4466694 .4500070 .265693

C 1.0112495 .8778740 .107881

C $0.9429066 .307271-1.340983$

C $1.9641186 .964929-1.915797$

C $-0.3428796 .052604-2.093467$

C $-0.4828684 .623721-2.540968$

N $0.182481 \quad 1.198147 \quad 0.817053$

H $-0.736228-1.970514-1.589653$

H $-2.076077-4.087434-3.350119$

H -2.973624 -1.956161 -4.317924

H -4.622962 -3.565309 -3.508136

H $-5.149133-1.905131-3.407558$ 
H $-6.002108-2.4474750 .189195$ H $-4.755143-4.3509800 .073985$ H -4.089212 $-4.879812-1.464789$ H -3.192906 -3.723866 -0.445233 H $-6.867175-1.145584-2.411714$ H $-7.641188-1.139505-0.854990$ H $-5.2370970 .526751-1.705882$ H $-6.8795561 .082687-1.379904$ H -6.5239350 .2236541 .048317$ H -6.4795572 .6676330 .454094$ H -4.815532 $2.516377-0.061072$ H -4.2294403 .9607432 .965217$ H -3.0019971 .9275121 .755859$ H -6.982691 3.0194702 .859221 H -5.8382452 .5795984 .104582$ H $-6.628366 \quad 1.3122643 .145851$ H $-4.011304 \quad 1.1489604 .275085$ H -2.399548 -0.8509423 .158307$ H -2.998486 -1.833698 1.845170 H -4.865876 -1.489462 2.929619 H $-2.9006091 .399996-1.631318$ H -0.640148 $2.244270-3.542456$ H $-2.2667403 .183612-0.221692$ H -3.376909 $3.924390-1.401718$ H -2.224828 $4.905452-0.500982$ H $-1.013725-1.2674090 .923015$ H $-2.002214 \quad 0.0213740 .238585$ H 2.0830091 .8945820 .469299 H 1.5763931 .9637092 .173473 H $-0.1528243 .809791 \quad 1.469849$ H $2.387748 \quad 4.172341-0.202808$ H 1.7311646 .5070640 .647738 H 0.0427816 .0584870 .590209 H $1.9114937 .302215-2.945697$ H $2.8780297 .175438-1.370199$ H $-0.3791956 .676941-2.996687$ H -1.189134 6.396317-1.491710 H $0.2076904 .349919-3.339858$ H $0.0181820 .990499-0.163630$ C $0.187808-4.300623-0.269546$ O $1.023037-3.3045750 .360193$ C $1.894997-3.9612081 .301857$ C $1.367223-5.3827051 .447608$ C $0.838040-5.6457670 .053381$ H $2.887024-4.0060370 .838789$ C $1.963812-3.1803282 .617525$ H $2.147845-6.0864571 .752328$ H $0.554706-5.4403992 .181489$ C $-0.114791-6.827041-0.017173$ H $1.687162-5.824834-0.619975$ C $2.488326-1.7378782 .482298$ O $0.652149-3.1144853 .192592$ H $2.579983-3.7292223 .339123$ H $1.769151-1.1231761 .930580$ O $2.568208-1.1807113 .800344$ C $3.872891-1.6172851 .817321$ C $4.437355-0.1861161 .886135$ H $3.790309-1.9267010 .770930$ O $4.793326-2.5071842 .447564$ H 3.6779450 .5062411 .515040 H 4.6329480 .0792372 .932224 H $\quad 0.390398-7.7470710 .294843$ H $-0.982473-6.6822030 .635384$ H $-0.482717-6.973951-1.037242$ C $0.090484-4.022640-1.771124$ H $0.068586-2.6853762 .543839$ H $1.680457-1.2401724 .193667$ H $4.841719-2.2624293 .387619$ H $-0.197261-4.937547-2.301974$ H $1.065253-3.712591-2.161356$ H $-0.799791-4.2338680 .205171$ C $4.7905090 .355114-1.090325$ O $5.631611-0.457209-0.263011$ C $5.751381-0.0124861 .092057$ C 6.3221731 .4128441 .123783 C 5.5147372 .3720010 .250721 C $5.3256601 .790237-1.150579$ H $3.7686540 .350275-0.692432$
C $4.760509-0.282586-2.482311$

H $6.493559-0.6682971 .564444$

O $6.369591 \quad 1.8929192 .463155$

H 7.3561521 .3875970 .755498

H 4.5541032 .6142180 .719239

O $\quad 6.232546 \quad 3.6040820 .152611$

H $6.287327 \quad 1.813417-1.678109$

O $4.4288652 .619900-1.885646$

C $3.965889-1.566220-2.451289$

H $4.2851650 .369116-3.223710$

H $5.775711-0.493023-2.839541$

H 6.7171352 .8009612 .434548

H $5.7253464 .187792-0.437618$

H $3.5551602 .563787-1.462960$

O $4.802962-2.638659-2.533017$

O $2.747121-1.605900-2.342017$

C $4.169009-3.920463-2.491795$

H $3.650873-4.054753-1.538252$

H $3.473081-4.027250-3.329253$

H $4.943890-4.686376-2.580131$

SCF Energy $(B 3 L Y P / 6-31 G * * / / M M F F)=-3245.92149370$

0600125

MMFF Geometry

C $0.791028-2.1592770 .727399$

C $-0.060490-2.121865-0.308913$

C $0.168397-1.477594-1.657045$

O $1.526219-1.008239-1.800517$

C $-0.739903-0.246255-1.822326$

C $-1.0688340 .052505-3.271322$

C $-0.4016460 .918204-4.058959$

C $-2.274490-0.675330-3.809381$

C $0.8437521 .700338-3.747731$

C $0.5818363 .203960-3.582324$

C $0.0902653 .566365-2.173393$

C $-0.2563955 .052613-2.078301$

C $-0.6422735 .460006-0.647716$

O $1.1495203 .259197-1.259550$

O $-0.647356 \quad 6.895258-0.616479$

C 0.4474874 .9598920 .326463

C $-2.0659005 .009330-0.298281$

O $0.0622595 .170337 \quad 1.683219$

C 0.8260623 .4754660 .117328

C 2.0692793 .1201040 .968102

O -0.2650592 .6546750 .536269$

C $2.475803-1.879330-2.226842$

O $2.333005-3.057429-2.506812$

C $3.750557-1.130120-2.249973$

C $4.913793-1.792387-2.185532$

C $6.244034-1.182133-2.124795$

C $6.3295050 .318124-2.241314$

C $2.719547 \quad 1.7764710 .603488$

C 3.8160881 .3920881 .584341

C $5.377539-0.4786752 .121128$

O 4.1858282 .1247162 .498272

C $6.709370-0.2269481 .485857$

C $7.625151-1.1870991 .282821$

C $8.979310-0.9370970 .680024$

C $9.314035-1.897903-0.441003$

C $10.137626-2.939484-0.234592$

C $8.761453-1.603500-1.818703$

C $7.311575-1.987285-1.944715$

N $4.333341 \quad 0.131486 \quad 1.317387$

H $1.763372-1.6828930 .645027$

H -1.025607 -2.608068 -0.196491

H $-0.046094-2.221332-2.434808$

H $-0.2665890 .627473-1.361139$

H -1.687831 $-0.385192-1.286329$

H $-0.766367 \quad 1.064397-5.075351$

H -2.430784 -0.491199-4.877618

H $-2.161642-1.756115-3.676947$

H -3.177444 -0.351734 -3.283267

H $1.3719381 .303673-2.877541$

H $1.5267951 .560200-4.595527$

H $1.5298313 .726029-3.767670$

H $-0.1335523 .550792-4.337902$

H $-0.7884182 .957152-1.935789$

H $-1.0583895 .311904-2.780741$ 
H $0.6142985 .649339-2.385340$ H $-0.944304 \quad 7.1773950 .264999$ H 1.3437835 .5729850 .157431 H -2.790429 $5.479517-0.973939$ H $-2.346694 \quad 5.3285590 .711574$ H -2.202214 $3.928118-0.366436$ H -0.0637416 .1256051 .809641$ H 2.8349653 .8964110 .838968 H 1.7921053 .1105922 .030189 H $\quad 0.073966 \quad 1.777547 \quad 0.772557$ H $3.692257-0.047632-2.267545$ H $4.890563-2.880363-2.139427$ H $5.9305440 .649406-3.206529$ H $7.3470150 .706881-2.171217$ H $5.7583090 .802846-1.442002$ H 1.9744820 .9754590 .593810 H $3.1673051 .836271-0.395196$ H $5.149094-1.5467042 .192230$ H $5.361131-0.0479713 .127405$ H $6.941961 \quad 0.796717 \quad 1.201834$ H $7.404347-2.2047431 .597391$ H $9.721260-1.0312481 .483921$ H 9.0694050 .0925310 .312085 H $10.404565-3.617286-1.038678$ H $\quad 10.559087-3.1410690 .744673$ H $9.314724-2.173132-2.577289$ H $8.953341-0.555572-2.063432$ H $7.141540-3.062423-1.869451$ H $3.999575-0.3805550 .506679$ C $0.109415-1.9371833 .184454$ O $-1.204460-1.3894732 .948431$ C -1.159709 0.0227853 .210696 C 0.0789650 .2448274 .071033 C $1.040160-0.7537943 .458171$ H $-1.007708 \quad 0.5091952 .241507$ C $-2.468500 \quad 0.5205343 .829539$ H $0.441074 \quad 1.276178 \quad 4.021376$ H $-0.104320-0.0047105 .122391$ C $2.225025-1.0951654 .343544$ H $1.414721-0.3243402 .524186$ C $-3.753622 \quad 0.1433313 .065176$ O $-2.599678-0.0035495 .157554$ H -2.415286 1.6096153 .946169 H -4.015212 -0.9051703 .254503$ O C $-3.7109890 .375751 \quad 1.545235$ C $-5.098473 \quad 0.2667390 .882027$ H $-3.024691-0.3504481 .096803$ O $\quad-3.1867341 .6747031 .265071$ H -5.651725 -0.5615921 .333646$ H $-5.659973 \quad 1.1859621 .086035$ H $2.823888-0.2011724 .546432$ H $1.902598-1.5084135 .305058$ H $2.871456-1.8332123 .858420$ C $0.504331-2.8615342 .028080$ H -2.568173 -0.9737565 .093943$ H -4.8474450 .7458644 .573340$ H $-3.7494212 .325344 \quad 1.718729$ H $-0.297457-3.5946541 .876886$ H $1.400197-3.4297212 .305785$ H $0.011445-2.5515364 .088963$ C $-5.329178-2.327994-0.739562$ O $-4.445216-1.222386-0.967652$ $\begin{array}{llll}\text { C } & -4.979927 & 0.069050 & -0.645947\end{array}$ C $-6.2731350 .317856-1.432627$ C $-7.285668-0.803581-1.234065$ C $-6.635406-2.154566-1.525527$ H $-5.553190-2.4105690 .329913$ C $-4.595046-3.605534-1.175899$ H -4.247763 $0.802404-1.007683$ O $-6.8435231 .568081-1.060831$ H -6.027766 $0.385220-2.500840$ H -7.716967 -0.770890 -0.226699 O $-8.367756-0.599153-2.144744$ H -6.442070 -2.234641 -2.602586 O $-7.553364-3.193605-1.189040$ C -3.570292-4.060644 -0.157831 H $-5.305423-4.431068-1.295974$
H $-4.071747-3.433003-2.123667$

H $-7.6666131 .674458-1.567745$

H $-8.980422-1.347158-2.038561$

H $-7.727938-3.140454-0.233815$

O $-2.983867-5.207835-0.613221$

O $-3.317988-3.4939820 .896309$

C $-2.000696-5.7976820 .243729$

H -1.847649 -6.831305 -0.078332

H -1.056999 -5.2558690 .143283$

H -2.333660 -5.805494 1.286114

SCF Energy $(B 3 L Y P / 6-31 G * * / / M M F F)=-3245.91904740$

06_00126

MM̄FF Geometry

C -2.5160643 .5925162 .348226$

C -2.4945822 .3040711 .973869$

C -2.6224051 .1437912 .930317$

O $-1.3430960 .501376 \quad 3.184278$

C -3.6625770 .1187222 .432818$

C $-4.221601-0.7635313 .530660$

C $-3.901563-2.0580653 .722249$

C $-5.266674-0.1138424 .403185$

C $-2.888871-2.8912732 .988134$

C $-3.534229-3.9240332 .053876$

C $-3.942117-3.3319680 .695174$

C $-4.705700-4.366087-0.134733$

C $-5.028364-3.846680-1.544647$

O $-2.739901-2.947270 \quad 0.017447$

O $-5.459913-4.964961-2.335594$

C $-3.726823-3.314994-2.181588$

C $-6.192382-2.849471-1.534057$

O $-3.984296-2.711981-3.447455$

C $-2.939665-2.348077-1.269069$

C -1.545919-2.000394-1.842541

O $-3.666241-1.118742-1.154323$

C $-0.583801 \quad 0.0091842 .169923$

$\begin{array}{llll}\text { O } & -0.779122 & 0.121140 & 0.971384\end{array}$

C $0.517669-0.7676882 .780693$

C $1.178599-1.6638092 .031984$

C $2.240041-2.5618592 .493581$

C $2.697489-2.4253333 .922442$

C $-0.616097-3.198470-2.053764$

C $0.817704-2.779072-2.333794$

C $3.010454-3.741763-3.043075$

O $1.223470-1.629921-2.187793$

C $3.780035-4.476930-1.992601$

C $4.661165-3.894814-1.165334$

C $5.434398-4.643720-0.119125$

C $5.137738-4.1717661 .287009$

C $6.075463-3.5481732 .019673$

C $3.791012-4.5150281 .883037$

C $2.747774-3.4619471 .625089$

N $1.595182-3.853423-2.741442$

H -2.5895803 .8185453 .410459$

H -2.422167 2.0504660 .921206

H -2.9386191 .5346853 .904527$

H -3.235178 -0.4971211 .636421$

H -4.5105300 .6415581 .969159$

H $-4.407988-2.5933534 .525023$

H -5.687655 -0.809133 5.137370

H $-4.8399980 .727594 \quad 4.957250$

H -6.0953880 .2569343 .790990$

H -2.167388 -2.2779602 .445087$

H $-2.306024-3.4276823 .748068$

H -2.796169-4.718603 1.881395

H $-4.400077-4.3862452 .543850$

H -4.566062 -2.447202 0.860536

H $-5.624373-4.675240 \quad 0.379379$

H $-4.097224-5.276395-0.232493$

H $-6.238236-5.355880-1.902758$

H $-3.107438-4.196092-2.388419$

H -7.091220 -3.315542 -1.112606

H $-6.462988-2.547793-2.551840$

H $-5.983317-1.951005-0.949851$

H $-4.372345-1.835423-3.284278$

H - $1.652916-1.445680-2.783705$

H $-1.059580-1.301478-1.151351$

H $-3.046107-0.418396-0.890620$ 
H $\quad 0.716350-0.6190653 .835813$ H $0.899574-1.7728090 .984372$ H $3.555453-3.0546884 .167505$ H $1.889827-2.6970314 .610906$ H $3.006596-1.3957494 .133982$ H -0.600031 -3.834304 -1.161321 H $-0.968395-3.797548-2.900589$ H $3.301846-2.688669-3.109807$ H $3.176030-4.208604-4.018962$ H $3.606010-5.547845-1.915483$ H $4.844199-2.826721-1.246524$ H $6.501601-4.524034-0.346496$ H $5.239150-5.721988-0.178305$ H $5.880769-3.2387373 .040906$ H $7.056986-3.3276901 .613460$ H $3.896227-4.7230752 .953134$ H $3.435412-5.4638351 .460571$ H $2.347632-3.4797660 .611456$ H $1.167738-4.772113-2.817334$ C $-2.6028124 .558155-0.053356$ O $-1.4430253 .873047-0.561547$ C $-1.1918194 .319788-1.907037$ C $-2.3004805 .310432-2.251684$ C $-2.6889085 .836869-0.883228$ H $-0.2376004 .857734-1.886581$ C - $-1.0945603 .117470-2.854248$ H -1.953758 $6.095865-2.930513$ H $-3.1551304 .816617-2.728522$ C $-4.0509416 .508571-0.862356$ H -1.924918 $6.552496-0.551205$ C $-0.0532612 .055396-2.445966$ O $-2.3675212 .459363-2.917238$ H $-0.8886403 .469264-3.871839$ H $-0.3944341 .508690-1.558698$ O $0.020578 \quad 1.094077-3.506649$ C $1.3590942 .609754-2.191168$ C $2.3958381 .499211-1.939264$ H $1.3282093 .276663-1.324120$ O $1.7765823 .392282-3.309570$ H $2.0255480 .846583-1.141776$ H $2.4984330 .885771-2.842424$ H -4.068689 $7.362189-1.547955$ H -4.844045 5.816997-1.165959 H $-4.291403 \quad 6.8777090 .139237$ C $-2.455786 \quad 4.804214 \quad 1.451213$ H $-2.5986852 .180799-2.014403$ H $-0.8780680 .750399-3.649023$ H $1.7745252 .812328-4.090464$ H -3.255322 5.4793021 .778795 H -1.504052 5.3106281 .650264 H $-3.475507 \quad 3.921313-0.251644$ C 3.4454952 .3522430 .793836 O $3.7349282 .964139-0.468649$ C $3.7895082 .058071-1.575561$

C $4.8594540 .985592-1.327498$

C 4.6772850 .2904410 .019496

C $4.5263391 .322247 \quad 1.137587$ H $2.462394 \quad 1.8675590 .754279$

C 3.3838303 .4656261 .842901

H $4.1256982 .649490-2.436657$

O $4.8485230 .026436-2.379419$

H $5.8469941 .465523-1.343429$

H $3.828432-0.402834-0.006780$

O $5.838177-0.503058 \quad 0.272792$

H 5.4900421 .8225621 .294402

O $4.2035690 .652611 \quad 2.352979$

C 2.1424854 .3033401 .645648

H 3.3414983 .0683642 .863063

H 4.2695254 .1096951 .782162

H $5.533994-0.633419-2.178231$

H $5.738622-0.8807301 .163197$

H $3.333986 \quad 0.2341232 .238573$

O $2.4765555 .526343 \quad 1.145218$

O 1.0100323 .9065611 .889105

C 1.3799906 .4115260 .899020

H $\quad 0.842381 \quad 6.617756 \quad 1.829343$

H 1.7816727 .3511520 .510356

H $\quad 0.7081015 .9818920 .151166$
SCF Energy (B3LYP/6-31G**//MMFF) $=-3245.92974868$

06_00127

MMFF Geometry

C $-0.652338-1.8001612 .594917$

C $0.151372-2.1899371 .594179$

C $-0.246559-2.7319150 .246161$

O $0.093146-1.683522-0.695084$

C -1.736335 -3.0809180 .069272$

C $-1.996125-3.966421-1.134126$

C $-2.484745-3.543193-2.315878$

C $-1.706814-5.431492-0.923812$

C $-2.824639-2.139907-2.733692$

C $-4.329430-1.957613-2.975762$

C $-5.149072-1.918796-1.676837$

C $-6.646375-1.868014-1.987328$

C $-7.487763-1.758841-0.707880$

O $-4.766617-0.740653-0.963071$

O $-8.818303-1.396100-1.113809$

C $-6.935348-0.6070970 .160516$

C $-7.621026-3.117345-0.010611$

O $-7.578150-0.5661791 .431864$

C $-5.395212-0.5866750 .314790$

C $-4.9496890 .790396 \quad 0.856212$

O $-4.928044-1.5541161 .253862$

C $0.762121-2.009199-1.826810$

O $1.113002-3.128457-2.173039$

C $1.062956-0.807620-2.640463$

C $0.5367850 .406456-2.410072$

C $0.7867851 .617277-3.198577$

C $1.8664741 .552024-4.248036$

C -3.4327030 .9107671 .041557$

C $-3.0544272 .326121 \quad 1.425470$

C -2.7733234 .6047940 .481048$

O -2.8353562 .6444132 .591832$

C $-2.2074165 .138202-0.794593$

C $-0.9380955 .551365-0.925763$

C $-0.3658706 .109032-2.195120$

C $0.6257905 .173823-2.848516$

C $1.9482855 .397706-2.773637$

C $0.0749404 .038505-3.680896$

C $0.0499292 .720911-2.947806$

N -3.0061683 .1810090 .335083$

H -1.731395 -1.861020 2.494791

H $1.225776-2.0804331 .734904$

H $\quad 0.374241-3.6198160 .073974$

H -2.327620 -2.161178 0.001589

H -2.110626 -3.615509 0.952042

H -2.644545 -4.286198 -3.096670

H -1.947467 -6.034671-1.805699

H $-0.647479-5.587792-0.699239$

H -2.300556 -5.819284 -0.089478

H $-2.456749-1.391442-2.026134$

H -2.295866 -1.943746 -3.675194

H -4.465469 -1.009047 -3.511579

H $-4.698717-2.756154-3.631191$

H -4.915232 -2.802125 -1.073271

H $-6.953929-2.742552-2.574218$

H $-6.857881-0.993704-2.619063$

H $-9.344938-1.258520-0.307542$

H $-7.2306550 .322009-0.347003$

H -8.159650 -3.822358 -0.655954

H -8.216925 -3.0394160 .905093$

H $-6.661014-3.5738560 .236192$

H -7.191109-1.263331 1.987359

H $-5.287566 \quad 1.575506 \quad 0.167614$

H -5.4265100 .9856901 .825464$

H -5.511901 -2.325043 1.237775

H $1.735095-0.988341-3.473327$

H $-0.1613340 .532178-1.584183$

H $2.8038861 .186672-3.813809$

H $2.1027412 .521214-4.691989$

H $1.5710160 .882568-5.063030$

H $-3.0825990 .237381 \quad 1.830725$

H -2.8924840 .6391380 .127087$

H $-2.1158684 .784189 \quad 1.337878$

H -3.7360725 .0806750 .694257$

H $-2.8782415 .193140-1.648524$ 
H $-0.271445 \quad 5.502173-0.068727$

H $\quad 0.1135857 .066547-1.953350$

H -1.158270 $6.357562-2.912491$

H $2.6592004 .747523-3.272722$

H $2.3492686 .231075-2.206436$

H $0.6281623 .974753-4.623366$

H $-0.9519024 .272713-3.990585$

H $-0.7012482 .672847-2.159163$

H -3.360934 $2.845841-0.556473$

C $-0.241976 \quad 0.314772 \quad 3.853761$

O 0.6314350 .8383692 .831104

C 1.4175951 .9046293 .387889

C 0.6956472 .3197724 .662191

C $0.193506 \quad 0.9776005 .160818$

H 2.3894921 .4744983 .658359

C 1.6134233 .0159542 .352911

H 1.3587812 .8225715 .372319

H -0.1494222 .9857204 .452365$

C $-0.915530 \quad 1.0868396 .192591$

H $\quad \begin{array}{llll}1.036974 & 0.427521 & 5.599517\end{array}$

C 2.1972582 .5406371 .005124

O 0.3500313 .6313672 .077198

H 2.2440283 .8058482 .776729

H 1.4562101 .9427910 .460497

O 2.4407173 .7038680 .204966

C 3.5099351 .7445411 .119878

C $4.1237871 .431787-0.258882$

H 3.3107920 .8096121 .654604

O 4.4380152 .5019481 .894966

H $3.3695230 .922291-0.867769$

H $4.3707842 .370431-0.769462$

H -0.5625211 .6190687 .081930$

H -1.7798471 .6305035 .796551$

H -1.255934 $0.094606 \quad 6.504889$

C $-0.129908-1.2098383 .876481$

H -0.2501432 .9372361 .754525$

H 1.5982924 .1826400 .128276

H $5.157798 \quad 1.9048892 .159952$

H $-0.702440-1.6277194 .712029$

H $0.917171-1.5046624 .022119$

H -1.2613370 .6189103 .588249$

C $4.420266-1.6061120 .036810$

O $5.235456-0.5890330 .626169$

C $5.4106630 .580007-0.180134$

C $6.0081510 .207641-1.546189$

C $5.213742-0.894762-2.242422$

C $5.005995-2.078229-1.298802$

H $3.416387-1.197514-0.134483$

C $4.233662-2.7363021 .059061$

H 6.1615001 .1932320 .333506

O $6.075737 \quad 1.343702-2.401927$

H $7.036892-0.148503-1.403923$

H $4.255938-0.522395-2.621417$

O $5.944536-1.353317-3.382094$

H $5.966007-2.585804-1.149134$

O $4.119590-3.016389-1.899925$

C $5.509250-3.4681291 .421857$

H $3.833638-2.3260481 .993933$

H $3.512120-3.4608580 .663476$

H $6.6024682 .022003-1.945617$

H $6.102985-0.581575-3.952579$

H $4.496760-3.262282-2.761920$

O $5.196748-4.730003 \quad 1.841349$

O $6.633346-2.9871531 .379686$

C $6.308215-5.5397442 .237591$

H $5.926226-6.5206802 .532438$

H $7.000501-5.6698331 .400279$

H $6.817586-5.0891703 .094795$

SCF Energy (B3LYP/6-31G**//MMFF) $=-3245.91322160$

0600128

MM̄FF Geometry

C $2.024829-3.5884120 .206456$

C $0.961262-4.092479-0.436630$

C $-0.097857-3.218925-1.049315$

O $-1.344466-3.515169-0.375489$

C $-0.300656-3.475699-2.553130$

C $0.895080-3.150085-3.417952$
C $1.215301-1.910265-3.836260$

C $1.713672-4.334664-3.861942$

C $0.507547-0.616805-3.530517$

C $1.4918130 .426082-2.983510$

C $0.8674611 .827401-2.880054$

C $1.9506312 .853343-2.542167$

C $1.3706164 .259498-2.351378$

O $-0.1276351 .813058-1.853454$

O $2.3758075 .072909-1.726149$

C $0.1830244 .183907-1.370571$

C $1.0534864 .923779-3.696317$

O $-0.4923745 .437488-1.294115$

C $-0.8314343 .057480-1.687993$

C -1.801212 $2.882458-0.495730$

O $-1.6284663 .367558-2.831073$

C $-2.213558-2.478243-0.237422$

O $-2.024251-1.320769-0.574751$

C $-3.457919-2.9839720 .380930$

C $-4.402403-2.0966180 .727979$

C $-5.716794-2.3888181 .299113$

C $-6.101263-3.8333371 .473969$

C $-2.8707521 .806400-0.720953$

C $-4.1831042 .324765-1.287476$

C $-6.4272081 .479064-2.002876$

O $-4.4264513 .515468-1.458567$

C $-7.3421981 .295071-0.832173$

C $-8.2418540 .303837-0.737101$

C -9.1555540 .1112530 .441023$

C $-8.983598-1.2418311 .099282$

C $-9.804153-2.2627020 .798765$

C $-7.918503-1.3907312 .164358$

C $-6.525310-1.3517391 .602739$

N $-5.061393 \quad 1.281390-1.553872$

H $2.113522-2.5096940 .312088$

H $\quad 0.851800-5.168789-0.540112$

H $\quad 0.167464-2.166827-0.889799$

H -0.603006 -4.520435 -2.711660

H -1.169268 -2.903015 -2.905308

H $2.078944-1.804482-4.492292$

H $2.582534-4.040230-4.460148$

H $2.085391-4.895822-2.999906$

H $1.102564-5.007026-4.473089$

H $0.067664-0.248404-4.465148$

H $-0.315655-0.752708-2.823686$

H $1.8376340 .099264-1.993741$

H $2.3709680 .474241-3.638867$

H $0.3941852 .072926-3.837969$

H $2.7319432 .864228-3.312557$

H $2.4536972 .554521-1.614246$

H $3.1681785 .056913-2.289925$

H $0.598066 \quad 3.995677-0.370845$

H $1.9674405 .037938-4.291637$

H $0.6603695 .936507-3.555809$

H $0.3415544 .354145-4.297444$

H $0.1714216 .110561-1.065000$

H $-1.2122942 .583690 \quad 0.380490$

H -2.280672 $3.836946-0.250197$

H -1.410011 $4.263961-3.131118$

H $-3.568901-4.0533740 .514413$

H $-4.199450-1.0383250 .569290$

H $-6.034289-4.3687270 .520099$

H -7.124215 -3.9691481 .832385$

H -5.439022 -4.3211632 .197559$

H $-2.4959531 .017527-1.379605$

H $-3.111127 \quad 1.3524850 .248825$

H $-6.6178780 .747851-2.794648$

H $-6.5477182 .484064-2.418742$

H -7.255813 $2.015632-0.022109$

H -8.334816 -0.409237-1.553306

H $-10.187761 \quad 0.2250250 .084358$

H $-9.0158520 .900553 \quad 1.190100$

H -9.707928 -3.227958 1.284594

H $-10.588004-2.1566610 .056095$

H $-8.077962-2.3006202 .753622$

H $-8.022990-0.5737882 .890203$

H $-6.153520-0.3402721 .437026$

H $-4.7726260 .330443-1.343244$

C $4.525693-3.9751340 .341710$ 
O $4.777985-2.613690 \quad 0.749057$

C $5.355791-1.904313-0.363582$

C $5.869333-2.979878-1.312361$

C $4.783952-4.030053-1.165448$

H $4.536999-1.367046-0.857659$

C $6.408184-0.9054290 .125260$

H $5.978811-2.614001-2.337536$

H $6.834934-3.383438-0.986158$

C $5.182807-5.403920-1.672820$

H $3.904790-3.688859-1.724494$

C $5.896008 \quad 0.087272 \quad 1.190007$

O $7.508524-1.622410 \quad 0.699315$

H $6.820697-0.357135-0.729388$

H $5.707289-0.4363942 .135062$

O $\quad 6.946247 \quad 1.026393 \quad 1.452900$

C $4.6352520 .870140 \quad 0.779777$

C $4.217906 \quad 1.9123461 .834803$

H 3.8110870 .1660440 .629801

O $4.861232 \quad 1.525636-0.467111$

H 4.1233751 .4104972 .803881

H 5.0076862 .6663331 .938341

H $5.411212-5.367021-2.742893$

H $6.068897-5.782942-1.152789$

H $4.370599-6.122825-1.524960$

C $3.130559-4.4105990 .804012$

H $7.156215-2.1720991 .420467$

H 7.7319280 .5161601 .714651

H $5.6120642 .132256-0.347885$

H $3.068970-4.2910321 .892836$

H $2.986873-5.4745940 .583322$

H $5.267156-4.5879350 .870948$

C $1.353091 \quad 0.9621592 .241623$

$\begin{array}{lllll}\text { O } & 1.847873 & 1.732851 & 1.140263\end{array}$

C 2.9022452 .6410601 .474611

C 2.4155523 .6375272 .535935

C 1.8295882 .9286923 .756425

C $\quad 0.793746 \quad 1.886745 \quad 3.329842$

H 2.1675290 .3536462 .651840

C $0.287194 \quad 0.0138301 .679615$

H 3.1085013 .2204770 .566840

$\begin{array}{lllll}\text { O } & 3.486651 & 4.490419 & 2.924656\end{array}$

H 1.6396354 .2747332 .091744

H 2.6135592 .4697104 .370281

$\begin{array}{lllll}\text { O } & 1.172190 & 3.885577 & 4.590492\end{array}$

H -0.1059432 .3987542 .964668$

O 0.4149251 .1148444 .466191

C $0.034129-1.1738592 .577085$

H -0.6469930 .5573531 .499514$

H $0.597638-0.377210 \quad 0.703722$

H 3.1185325 .1863943 .494477

H 1.8532434 .4790194 .949347

H $0.085291 \quad 1.7332095 .140667$

O $-1.304062-1.3096202 .800572$

O $0.914215-1.9006393 .019696$

C -1.678671-2.408197 3.637223

H $-2.765278-2.3906433 .755808$

H $-1.391240-3.3543543 .168773$

H $-1.216957-2.3088274 .624249$

SCF Energy (B3LYP/6-31G**//MMFF) $=-3245.92443002$

06_00129

MMFF Geometry

C $-0.800023-3.600478-0.322993$

C $-0.136442-2.462530-0.061491$

C $0.358720-2.1146591 .318672$

$\begin{array}{llll}\text { O } & 0.161125 & -0.694486 & 1.513287\end{array}$

C $1.863844-2.4146841 .439692$

C $2.330019-2.5123192 .878824$

C $3.051438-1.5754153 .523939$

C $1.974147-3.7986963 .581576$

C $3.517151-0.2398073 .013964$

C $5.043754-0.1924982 .855835$

C $5.540246-0.9652501 .624096$

C $7.064650-1.0854841 .637844$

C $7.589685-1.7732840 .369974$

O $5.118677-0.240362 \quad 0.463919$

$\begin{array}{llll}\text { O } & 9.011629 & -1.579266 & 0.347245\end{array}$

C $6.990228-1.062351-0.860439$
C $7.362213-3.288607 \quad 0.412817$

O $7.350641-1.740587-2.062165$

C $5.457341-0.857880-0.783607$

C $5.0113810 .091043-1.920957$

O $4.809991-2.121003-0.938751$

C $-0.271942-0.269940 \quad 2.727679$

O $-0.637213-0.9729553 .660566$

C $-0.249005 \quad 1.2076382 .837083$

C 0.3144652 .0186281 .928222

C $0.3830943 .481397 \quad 1.997162$

C $-0.192124 \quad 4.151767 \quad 3.217518$

C $3.5429990 .532020-1.850223$

C $3.2267151 .591551-2.894491$

C $1.3938673 .136858-3.587104$

$\begin{array}{lllll}\text { O } & 4.032924 & 1.963608 & -3.742919\end{array}$

C $1.5187094 .423633-2.835344$

C $0.4717475 .101315-2.341136$

C $0.5958666 .392809-1.587785$

C $0.1723786 .265636-0.141780$

C $-0.997330 \quad 6.770225 \quad 0.285345$

C 1.1487675 .6355390 .827143

C 0.9483594 .1508430 .969856

N $1.9308202 .067752-2.765859$

H $-0.966350-4.295778 \quad 0.495028$

H $0.083312-1.767008-0.866749$

H $-0.220421-2.6834992 .055631$

H $2.436167-1.6538610 .897332$

H $2.104179-3.3683850 .950410$

H $3.341552-1.7771774 .554751$

H $2.390802-3.8496714 .593249$

H $\quad 0.888948-3.9059343 .669209$

H $2.364786-4.6560323 .023543$

H 3.0241880 .0489512 .081340

H 3.2199520 .5134933 .754422

H $5.3373910 .860903 \quad 2.759464$

H $5.520302-0.5813653 .764424$

H $5.086651-1.9619401 .613924$

H $7.406506-1.6197742 .532996$

H $7.509342-0.0820741 .700124$

H $9.345042-1.941305-0.491981$

H $7.469306-0.075412-0.918388$

H $7.858977-3.723498 \quad 1.288514$

H $7.808852-3.781559-0.457603$

H $6.306087-3.5627350 .458995$

H $6.785802-2.528518-2.137545$

H $5.6318970 .996598-1.886427$

H $5.185532-0.385651-2.894065$

H $3.899077-1.973849-1.237885$

H $-0.7084601 .596700 \quad 3.740202$

H $0.7772701 .578643 \quad 1.046713$

H -1.2515803 .9018493 .333547$

H $-0.138738 \quad 5.241695 \quad 3.185655$

H 0.3444283 .8332604 .118198

H $2.874814-0.318881-2.020397$

H $3.3194010 .955347-0.864204$

H $0.3520242 .889953-3.811202$

H $\quad 1.943740 \quad 3.200127-4.531332$

H $2.5231624 .815283-2.693487$

H $-0.5330884 .712909-2.486853$

H $-0.0217037 .140125-2.102850$

H $1.6206516 .782746-1.630593$

H $-1.2917196 .707948 \quad 1.327577$

H $-1.689937 \quad 7.249480-0.398482$

H 1.0984626 .1478281 .793495

H 2.1758385 .8147990 .483725

H $1.334913 \quad 3.5740390 .129764$

H $1.3574131 .731957-1.997552$

C $-2.748403-4.473385-1.740665$

O $-3.626076-3.422188-1.289917$

C $-4.513013-3.969774-0.300019$

C $-4.535196-5.470218-0.563134$

C $-3.083586-5.723195-0.919201$

H $-4.042434-3.7882250 .674262$

C $-5.885505-3.290419-0.334001$

H $-4.870757-6.0423680 .306854$

H $-5.179603-5.725825-1.412028$

C $-2.854827-7.025640-1.667657$

H $-2.506424-5.7548260 .011275$ 
C $-5.870348-1.749309-0.302829$

O $-6.584548-3.677293-1.524448$

H $-6.489582-3.6711370 .498271$

H -5.626870 -1.353362 -1.296707

O $-7.206506-1.312979-0.018832$

C $-4.919333-1.1158560 .725268$

$\begin{array}{lllll}C & -5.139387 & 0.401716 & 0.885577\end{array}$

H $-3.887745-1.3147300 .417979$

O $-5.096126-1.7297132 .003199$

H $-5.4157570 .834908-0.079899$

H $-5.978593 \quad 0.5708371 .570257$

H -3.161638 -7.880063 -1.055584

H -3.426862 -7.059085 -2.600996

H -1.795996 -7.149624 -1.915960

C -1.285477 -4.012263-1.686514

H -6.031991 -3.424234 -2.284041

H -7.787603 -1.700733 -0.695677

H $-6.032332-1.6342382 .249261$

H -1.168092 $-3.171006-2.377868$

H - $0.641644-4.820871-2.051600$

H $-3.007734-4.644467-2.793586$

C $-2.9440311 .857476-0.659163$

O -2.7899391 .0357310 .504659$

C -3.8760901 .0963961 .439284$

C -4.0880942 .5419281 .906883$

C $-4.246533 \quad 3.5101080 .739474$

C $-3.1063723 .330061-0.262031$

H $-3.8177231 .532628-1.235662$

C - $1.7027821 .659565-1.540622$

H -3.5573340 .5216062 .318339$

O $\quad-5.2239042 .6419672 .759605$

H -3.219781 2.8567242 .498042

H $-5.225198 \quad 3.3894880 .259914$

O $-4.208470 \quad 4.8413651 .259422$

H $-2.173003 \quad 3.7079090 .171474$

O $-3.3816454 .100295-1.430245$

C - $-1.7245630 .341367-2.285392$

H -1.656222 $2.449257-2.297750$

H $-0.7923631 .682353-0.933722$

H -5.0851192 .0316153 .503763$

H -4.4101845 .4484930 .527782$

H -3.340730 $5.038630-1.180359$

O $-0.7349630 .358335-3.225408$

O $-2.495384-0.581712-2.066252$

C $-0.605672-0.796338-4.061593$

H $-0.194219-0.472828-5.021578$

H $0.095713-1.494942-3.598515$

$\mathrm{H}-1.568648-1.282381-4.244606$

SCF Energy (B3LYP/6-31G**//MMFF) $=-3245.91401715$

0600130

MM̄FF Geometry

C $-1.449346-4.7390821 .261200$

C $-0.301386-4.0730411 .061681$

C $0.585764-3.5980952 .184155$

O $1.073588-2.2717321 .869187$

C $1.799030-4.5352502 .314606$

C $2.731928-4.1575933 .448999$

C $3.930883-3.5613963 .291842$

C $2.252392-4.5359154 .826885$

C $4.569176-3.1072122 .008327$

C $4.971826-1.6279702 .079184$

C $5.486412-1.1143510 .726380$

C $5.9790290 .327758 \quad 0.848364$

C $6.3923920 .911267-0.509220$

O $4.405395-1.184632-0.209384$

O $6.5320932 .329263-0.328312$

C $5.2569920 .672435-1.529593$

C $7.7693180 .397194-0.946393$

O $5.6907491 .078634-2.825430$

C $4.744044-0.784860-1.545750$

C $3.477740-0.991631-2.416250$

O $5.765737-1.630230-2.089401$

C $0.256018-1.2178052 .139627$

O $-0.892690-1.2635762 .548904$

C $1.027848 \quad 0.0206381 .885675$

C $\quad 0.47085912191372 .115857$

C 1.1374982 .5201112 .000146
C 2.5677992 .5343091 .529541

C $2.245732-0.181333-1.999329$

C $2.1321541 .171132-2.681235$

C $0.845543 \quad 3.303127-2.551723$

O $2.7961491 .485282-3.664936$

C $1.2721264 .303297-1.527017$

C $0.4152295 .095942-0.865707$

C $0.8434396 .158787 \quad 0.102434$

C 0.2457335 .9787901 .477589

C $-0.819744 \quad 6.698180 \quad 1.868444$

C $0.951903 \quad 5.0479242 .435520$

C 0.4559203 .6301302 .353319

N $1.172591 \quad 1.972151-2.078935$

H -1.745098 -4.953799 2.286828

H $0.041791-3.8587260 .053864$

H $0.036941-3.5651243 .133772$

H $2.341813-4.5696611 .363762$

H $1.455656-5.5653992 .482680$

H $4.528151-3.3709704 .182920$

H $2.987492-4.2982045 .603147$

H $1.330113-4.0013935 .073789$

H $2.056567-5.6119094 .878810$

H $5.462977-3.7171821 .830599$

H $3.908451-3.2607411 .152305$

H 4.101751-1.0351712.387356

H $5.745891-1.4959562 .845501$

H $6.298210-1.7709370 .391761$

H $\quad \begin{array}{llll}6.806850 & 0.397781 & 1.565063\end{array}$

H $5.1754890 .951411 \quad 1.261866$

H $6.7277412 .718877-1.197946$

H $4.4394411 .344896-1.242581$

H $8.5329370 .685550-0.213790$

H $8.0799830 .849634-1.894615$

H $7.807641-0.688762-1.054528$

H $4.9214411 .057518-3.418658$

H $3.716415-0.845343-3.476414$

H $3.196853-2.051370-2.328987$

H $5.352083-2.450091-2.406774$

H $2.053791-0.0893601 .555704$

H -0.5619451 .2519852 .458330$

H $2.670661 \quad 1.9985720 .580219$

H 3.2168872 .0591642 .272767

H 2.9579833 .5382311 .349649

H $1.346920-0.741449-2.288416$

H $2.213728-0.051738-0.914208$

H $-0.2327003 .331942-2.727769$

H $1.3494763 .506955-3.501574$

H $2.3402304 .403563-1.350037$

H $-0.6511395 .007590-1.061011$

H $0.5380017 .127826-0.314093$

H $1.936426 \quad 6.2098790 .183782$

H -1.230938 6.6000222 .867698

H -1.3093267 .3944951 .195629$

H $\quad 0.7807725 .3875113 .466527$

H 2.0338715 .1311672 .300767

H -0.5754413 .5187162 .687629$

H $0.6634471 .618279-1.273821$

C $-2.216741-4.720227-1.210756$

O $-2.454733-3.299635-1.216275$

C $-3.350704-2.975587-2.290536$

C $-3.327557-4.181035-3.217791$

C $-3.208454-5.313286-2.214784$

H -4.350858 -2.879844 -1.849659

C $-2.966936-1.640883-2.941321$

H $-4.225941-4.248213-3.838459$

H $-2.453649-4.164679-3.879524$

C $-2.754047-6.625888-2.828572$

H $-4.185762-5.462244-1.736075$

C $-2.994958-0.463758-1.939623$

O $-1.675544-1.753916-3.535705$

H $-3.678320-1.438690-3.750460$

H -3.935375 -0.510210 -1.383368

O $-1.922589-0.585908-1.004285$

C $-2.8920790 .914949-2.611381$

C $-2.8981392 .111774-1.637828$

H $-3.711607 \quad 1.031182-3.329616$

O $-1.6823430 .987763-3.370607$

H $\quad-2.1692401 .944040-0.837259$ 
H $-2.5195612 .977081-2.195406$ H $-3.475211-6.970220-3.577014$ H -1.781522 -6.523238 -3.321644 H -2.663493 -7.403095 -2.063275 C $-2.404614-5.2511520 .213007$ H -1.039962 -1.922854 -2.819018 H -2.059563 -1.408787 -0.504809 H $-0.945566 \quad 0.799948-2.764273$ H -2.346352 -6.3457020 .212109$ H -3.412743 -4.9826570 .553088$ H -1.188835 -4.880983 -1.557942 C -4.1842351 .3888191 .112313$ O $-4.8095861 .468716-0.174894$ C $-4.2892622 .460175-1.065497$ C $-4.3578893 .847468-0.407645$ C $-3.647103 \quad 3.8627630 .940137$ C -4.2008132 .7500841 .824037$ H $-3.153856 \quad 1.0342140 .994879$ C $-4.976517 \quad 0.3688291 .947942$ H -4.975266 2.486609-1.922028 O $-3.8009674 .836135-1.267470$ H -5.411523 4.116019-0.255593 H -2.5607123 .7846460 .818206$ O -3.8971455 .1225851 .566303$ H -5.2272553 .0101162 .113047$ O $-3.4411522 .684776 \quad 3.028724$ C $-4.751056-1.0614241 .512413$ H -4.6996080 .4147303 .007080$ H -6.0473610 .5941361 .865372$ H -3.834124 $5.685379-0.794567$ H -3.4794295 .0964172 .444354$ H -2.5444792 .3897632 .798611$ O $-5.928335-1.748786 \quad 1.581501$ O $-3.665996-1.5303311 .198953$ C $-5.851704-3.1406261 .258859$ H $-6.849796-3.5715081 .372963$ H -5.529932 -3.2721550 .222505$ H -5.169241-3.651494 1.944651 SCF Energy (B3LYP/6-31G**//MMFF) $=-3245.93588848$

\section{1}

MM̄FF Geometry

C $0.574878 \quad 4.254978-3.147696$

C $1.1552023 .920471-1.984584$

C $2.6454353 .820973-1.809316$

O $2.9319052 .587002-1.106065$

C $3.3001934 .999483-1.051848$

C 2.5137365 .6557450 .068614

C $2.5791875 .299174 \quad 1.366327$

C $1.7009046 .862353-0.331988$

C 3.3344624 .1425591 .955568

C 2.6708473 .5573303 .213691

C 1.2124293 .1055573 .029158

C 0.6616092 .5465114 .344168

C -0.7734682 .0225634 .192933$

O 1.1618272 .1030232 .009994

O -1.0609831 .2644885 .378156$

C -0.8258391 .0641742 .984157$

C -1.792946 3.1670204 .149426

O

C -0.1659311 .6428901 .712366$

C -0.0527360 .5990330 .578567$

O $-0.9839112 .712661 \quad 1.219563$

C $4.0716041 .927592-1.457474$

O $4.8353152 .215136-2.366389$

C $4.2638490 .797900-0.519916$

C $5.205140-0.118785-0.792106$

C $5.572418-1.2721430 .033180$

C $4.986418-1.3557811 .416883$

C $0.759490-0.6498330 .914665$

C $1.000977-1.522864-0.306698$

C $2.028665-3.697664-0.944843$

O $0.601774-1.239755-1.432308$

C $3.213990-4.471297-0.464609$

C $4.330059-4.654175-1.185927$

C $5.518581-5.424558-0.685997$

C $6.808023-4.636211-0.758939$

C $7.813589-5.029207-1.559225$
C $6.964377-3.4355580 .152835$

C $6.439429-2.172800-0.476338$

N $1.731330-2.6564610 .019946$

H $1.2218564 .468321-3.997428$

H $0.5489113 .719646-1.108721$

H $3.1081783 .768824-2.803898$

H $3.5354525 .781131-1.787701$

H $4.2756704 .681912-0.660444$

H 2.0338785 .9064582 .087879

H 1.1852397 .3185490 .519996

H $2.3500017 .627591-0.770540$

H $0.9373556 .593946-1.067158$

H 3.4669213 .3425841 .225055

H 4.3363864 .4915982 .233185

H 3.2778882 .6998733 .532865

H 2.7189044 .2998194 .020616

H 0.6142923 .9676072 .714510

H $0.710483 \quad 3.3008765 .139537$

H 1.2992751 .7163864 .680255

H -1.9845000 .9653125 .329230$

H $-0.2967300 .150375 \quad 3.281150$

H -1.752952 3.7472705 .079190

H -2.816972 2.7835094 .079790

H -1.628939 3.8581173 .320130

H -2.532867 0.2465123 .476131

H -1.0528370 .2953110 .255476$

H $0.4036631 .087426-0.293518$

H -0.8920652 .7584270 .254503$

H 3.6466450 .7642730 .369241

H $5.768433-0.020158-1.719694$

H $3.905628-1.5094331 .371493$

H $5.182981-0.4323081 .973655$

H $5.401318-2.1675762 .017612$

H $1.731436-0.3703611 .332645$

H $0.228433-1.2561211 .655302$

H $2.189239-3.246139-1.929407$

H $1.152930-4.350671-1.005874$

H $3.149190-4.9065340 .530217$

H $4.391627-4.239215-2.189367$

H $5.596488-6.340029-1.286470$

H $5.368583-5.7556610 .349348$

H $8.750234-4.482705-1.597623$

H $7.722396-5.902417-2.196731$

H $8.028114-3.2761770 .373436$

H $\quad 6.496807-3.6633231 .114390$

H $6.835927-1.991353-1.476848$

H $1.850820-2.8766431 .005093$

C $-1.8856553 .907297-2.382571$

O $-1.8213712 .467408-2.308986$

C $-3.1475891 .931160-2.499044$

C $-4.0875823 .071543-2.153568$

C $-3.3371504 .252028-2.735143$

H $-3.2201681 .678074-3.564701$

C $-3.3117780 .673764-1.641521$

H $-5.0851852 .946042-2.584447$

H $-4.1890843 .177711-1.066344$

C $-3.7976625 .593433-2.190320$

H $-3.4710364 .248459-3.825448$

C $-4.7297000 .081986-1.652881$

O $-2.405322-0.316893-2.135902$

H $-3.0155930 .898104-0.612018$

H $-5.4298120 .782710-1.183557$

O $-5.165048-0.059761-3.011828$

C $-4.862789-1.298174-0.978646$

C $-4.360123-1.3325210 .475332$

H $-4.348678-2.058856-1.576835$

O $-6.251207-1.650804-0.976972$

H -3.298374 -1.071427 0.501769

H $-4.896292-0.570241 \quad 1.054003$

H $-4.8514865 .765677-2.432379$

H $-3.6907575 .642302-1.101446$

H -3.212632 $6.411076-2.623410$

C $-0.8951804 .404964-3.440914$

H $-1.5148580 .074535-2.134626$

H $-4.532970-0.646289-3.462164$

H $-6.556641-1.627649-1.900184$

H -1.078725 $5.467133-3.642506$

H $-1.0840293 .861624-4.376296$ 
H -1.634709 $4.299264-1.390299$

C $-2.598580-3.8346790 .346727$

O $-4.029824-3.7768620 .369978$

C $-4.574238-2.7073751 .150180$

C $-4.076590-2.7996622 .599972$

C $-2.557920-2.9353272 .690456$

C -2.060599-4.045685 1.765206

H $-2.196345-2.902091-0.067346$

C $-2.202308-4.984698-0.583985$

H $-5.658224-2.8757181 .180518$

O $-4.511057-1.6648803 .342073$

H -4.533088 -3.6811693 .069417$

H -2.066328 -1.984025 2.463499

O $-2.221171-3.253214 \quad 4.041917$

H -2.378894 -5.021910 2.152453

O $-0.636684-4.0342561 .734132$

C -2.455253 -4.611141-2.025227

H -1.145216 $-5.255302-0.487914$

H -2.787611 -5.886133 -0.366347

H $-4.170866-1.7623584 .248009$

H $-1.252396-3.2277854 .116841$

H -0.320295 -4.283889 2.618536

O $-1.481758-3.761472-2.462110$

O $-3.409301-5.014554-2.677320$

C $-1.624469-3.301136-3.808979$

H $-1.548593-4.142141-4.504529$

H $-2.578612-2.780198-3.934389$

$\mathrm{H}-0.812491-2.598981-4.015750$

SCF Energy (B3LYP/6-31G**//MMFF) $=-3245.92003318$

0600132

MM̄FF Geometry

C $-2.195567 \quad 4.831490 \quad 0.600994$

C -3.1214163 .8616410 .628304$

C -3.5438233 .1358081 .879016$

O $-3.179607 \quad 1.7425421 .731701$

C -5.0705353 .2350442 .043964$

C -5.6016772 .4823873 .248357$

C -6.2625151 .3083263 .190679$

C -5.3726843 .1553394 .577057$

C $-6.574550 \quad 0.4887251 .968710$

C $-5.919049-0.8962372 .047921$

C $-5.972367-1.6313960 .700104$

C $-5.412751-3.0473570 .838356$

C $-5.322808-3.768590-0.512870$

O $-5.185030-0.882619-0.232358$

O $-4.509880-4.935011-0.309696$

C $-4.597823-2.856019-1.527239$

C $-6.695364-4.276661-0.970331$

O $-4.638834-3.455764-2.819894$

C $-5.157963-1.417262-1.563767$

C $-4.314171-0.443412-2.425957$

O $-6.473615-1.445250-2.131010$

C $-1.907574 \quad 1.3983302 .080259$

O -1.0320982 .1432672 .491903$

C -1.756554 $-0.059766 \quad 1.874257$

C $-0.572764-0.6469952 .105982$

C $-0.267540-2.0731951 .961849$

C -1.368064 -2.977397 1.474207

C $-2.864171-0.241419-1.972228$

C $-1.862515-1.166459-2.642268$

C $0.543016-1.820323-2.528657$

O $-2.141502-1.882085-3.600022$

C $0.742056-3.013174-1.648709$

C $1.885980-3.269998-0.996299$

C $2.112138-4.493903-0.157664$

C $2.557549-4.1695571 .249938$

C $3.858592-4.1990741 .585321$

C $1.489415-3.9199532 .289997$

C $0.971225-2.5061502 .278031$

N $-0.605835-1.068451-2.060676$

H -1.710407 5.1380031 .523927

H -3.584642 $3.547436-0.304787$

H -3.055181 3.5551552 .767523

H $-5.5615002 .890470 \quad 1.128099$

H -5.3617854 .2903162 .138495$

H $-6.6163620 .872274 \quad 4.124190$

H $\quad-5.8251482 .6023045 .407052$
H $-4.3016653 .241574 \quad 4.784887$

H $-5.809557 \quad 4.1594124 .574658$

H $-7.662450 \quad 0.370658 \quad 1.896028$

H -6.2614240 .9926661 .052420$

H $-4.872144-0.7808292 .353927$

H -6.420888 -1.492112 2.820539

H -7.013006 -1.658995 0.356328

H $-6.008454-3.6340971 .548794$

H -4.404488 -2.996167 1.269321

H $-4.389423-5.363876-1.174511$

H $-3.544673-2.830677-1.223024$

H -7.099169-4.989490 -0.241244

H $-6.621229-4.826216-1.915229$

H -7.429364 -3.477564-1.093416

H $-4.050910-2.947923-3.403541$

H $-4.371265-0.723285-3.484599$

H $-4.7987190 .541934-2.363776$

H $-6.687348-0.556605-2.460314$

H $-2.625622-0.6132301 .540337$

H $0.255269-0.0270582 .447471$

H $-1.791131-2.6016300 .536510$

H -2.165966 -3.041153 2.221411

H -1.035610 -3.996293 1.264428

H -2.558348 $0.779250-2.236977$

H -2.777946 -0.335974 -0.886745

H $1.402777-1.144069-2.510579$

H $0.379893-2.145085-3.561058$

H $-0.086063-3.713307-1.568265$

H $2.723476-2.584030-1.100805$

H $2.877673-5.101253-0.658568$

H $1.216230-5.126068-0.120989$

H $4.184971-4.0018192 .601093$

H $4.626993-4.4106300 .849094$

H $1.900070-4.0996853 .293202$

H $0.696811-4.6663802 .185002$

H $1.703706-1.7730932 .617896$

H $-0.479016-0.472593-1.247580$

C $-0.8402934 .612681-1.499109$

O $\quad 0.3646314 .318827-0.771534$

C $1.4311054 .102189-1.715630$

C $0.8413504 .356355-3.103229$

C $-0.3522265 .242071-2.798134$

H $2.2011854 .853015-1.502206$

C $2.0207572 .693100-1.543113$

H $1.5641314 .831692-3.774056$

H $0.5181093 .423782-3.581095$

C -1.386649 5.266011-3.909410

H $0.0065626 .262794-2.610465$

C $2.7080152 .470854-0.176898$

O $0.9859891 .730018-1.737464$

H $2.7622062 .528375-2.333955$

H $3.4868963 .232453-0.049302$

O $1.7763292 .628961 \quad 0.892617$

C $3.3565371 .077788-0.047050$

C 4.1702180 .9370851 .253319

H $4.0142810 .915622-0.907212$

O $2.3587890 .061859-0.083158$

H 4.9653791 .6909111 .248315

H 3.5278341 .1583752 .114459

H $-0.9449255 .643207-4.837649$

$\mathrm{H}-1.7848824 .265337-4.108770$

H -2.226742 $5.916806-3.648255$

C $-1.7486705 .512832-0.663035$

H $0.3495911 .836836-1.009503$

H $1.4259703 .534650 \quad 0.850676$

H 1.7282340 .2472820 .632808

H -2.626950 $5.820301-1.242278$

H -1.214710 $6.428234-0.379600$

H $-1.3386563 .655456-1.704725$

C $6.638344-0.294941-0.044381$

O $5.418443-0.9671680 .283817$

C $4.762683-0.4756161 .453724$

C $5.687072-0.6133002 .671289$

C 7.0511680 .0340452 .429133

C $7.645630-0.4419111 .101306$

H $6.4365080 .766793-0.233089$

C $7.166925-0.914662-1.341080$

H $3.910216-1.1426741 .624984$ 
O $5.063770-0.044048 \quad 3.817251$

H $5.836622-1.6805062 .881269$

H $\quad \begin{array}{llll}6.985373 & 1.127961 & 2.458997\end{array}$

O $7.947804-0.3451723 .475621$

H $7.952004-1.4906291 .202170$

O $8.823348 \quad 0.3103030 .816349$

C $6.289608-0.518069-2.505100$

H $8.182549-0.574173-1.571586$

H $7.201347-2.008967-1.279095$

H $5.613117-0.2584014 .589692$

H 7.6232160 .0545034 .299934

H 8.5662381 .2430620 .719307

O $5.316918-1.453856-2.698961$

O $6.431536 \quad 0.518969-3.140366$

C $4.383871-1.164063-3.744251$

H $4.904642-1.046847-4.699412$

H $3.691150-2.005959-3.824457$

H $3.814421-0.262047-3.500148$

SCF Energy (B3LYP/6-31G**//MMFF) $=-3245.92324402$

0600133

MM̄FF Geometry

C $0.148479-2.5481070 .655049$

C $0.744323-2.1960601 .806114$

C $0.264887-1.1290912 .767791$

O $-1.035214-0.6286052 .385158$

C $1.253751 \quad 0.0496342 .749013$

C 0.8935771 .1427153 .734087

C 0.2928772 .3014853 .398369

C 1.2945300 .8795665 .161787

C -0.1825892 .7184082 .033882$

C - -1.5881943 .3328922 .087804$

C $-2.069963 \quad 3.7386710 .687775$

C -3.3499214 .5694720 .770674$

C $-3.8854664 .927793-0.623083$

O $-2.3183312 .543587-0.060649$

O $-5.2140725 .437110-0.433179$

C $-3.9771423 .639339-1.466897$

C $-3.0722866 .054608-1.270947$

O $-4.3143883 .933652-2.820930$

C $-2.6935132 .772593-1.425202$

C $-2.9826491 .404352-2.084551$

O $-1.652913 \quad 3.428627-2.151216$

C -2.124936-1.254801 2.905284

O $-2.135164-2.1690603 .713465$

C $-3.320400-0.6630682 .265485$

C $-4.485245-1.3246672 .326343$

C $-5.737543-0.9327791 .677361$

C -5.7960490 .4244651 .029681$

C $-1.8700690 .363325-1.917495$

C $-2.350933-0.988095-2.408404$

C -3.871193 -2.866670 -1.772978

O $-2.059946-1.409626-3.525139$

C $-5.335999-2.561190-1.843903$

C $-6.276009-3.265244-1.194347$

C $-7.743913-2.945819-1.252962$

C $-8.393537-2.886840 \quad 0.114642$

C $-9.259227-3.8365730 .507905$

C $-8.097729-1.691100 \quad 0.999047$

C $-6.761143-1.8118971 .679681$

N $-3.162001-1.632760-1.487315$

H -0.776195 -2.050549 0.372852

H $1.671666-2.6821172 .097894$

H $0.222124-1.5584073 .776279$

H 1.3219560 .4620861 .735875

H $2.267425-0.3063272 .979011$

H 0.1227123 .0353264 .185057

H $1.046791 \quad 1.7156305 .824423$

H $0.782654-0.0077945 .546866$

H 2.3749480 .7152065 .228789

H 0.5159053 .4663051 .642887

H -0.1864211 .8811441 .330676$

H -2.284186 2.6050732 .524130

H -1.572855 4.212745 2.743145

H -1.2738044 .3139390 .200650$

H -3.1889165 .4786911 .363333$

H -4.121362 3.9963691 .304427

H $-5.5559285 .721057-1.297640$
H $-4.8078383 .044770-1.061097$

H -3.111570 $6.957807-0.650463$

H -3.493515 $6.339459-2.241506$

H -2.021433 $5.796004-1.416878$

H $-5.1782144 .378849-2.820196$

H $-3.9040670 .993039-1.657505$

H -3.167884 $1.539054-3.158295$

H $-0.9962692 .768604-2.424118$

H -3.1966980 .2625631 .715460$

H -4.517052 -2.270734 2.865747

H $-5.468096 \quad 1.2024671 .728519$

$\begin{array}{llll}H & -6.800344 & 0.711704 & 0.711327\end{array}$

H $\quad-5.151847 \quad 0.453006 \quad 0.147411$

H $-0.9822320 .639462-2.494734$

H -1.560012 $0.266404-0.871163$

H $-3.634958-3.570376-0.968664$

H -3.529637 -3.285463 -2.724182

H -5.638254 -1.718031-2.461183

H $-5.978210-4.125514-0.599082$

H $-8.225926-3.718837-1.865378$

H $-7.923183-1.995678-1.771559$

H -9.747086 -3.790692 1.475840

H -9.496194 -4.684065 -0.126611

H -8.861478 -1.6029711 .782403$

H $-8.189113-0.7861080 .392464$

H $-6.626137-2.7576592 .207169$

H -3.337486 -1.188760 -0.591040

C $1.994568-4.193804-0.133831$

O $3.027325-3.190532-0.120697$

C $4.208985-3.724272-0.745044$

C $3.904727-5.182778-1.076834$

C $2.398873-5.160441-1.246079$

H $4.346081-3.169217-1.680103$

C $5.427808-3.5242230 .163842$

H $4.432587-5.518618-1.974891$

H $4.184939-5.856255-0.258833$

C $1.757798-6.533399-1.143800$

H $2.165167-4.724419-2.226766$

C $5.660501-2.0748550 .636019$

O $5.275727-4.3292201 .342008$

H $6.325053-3.901398-0.340485$

H $4.891327-1.7852901 .362910$

O $6.907070-2.051211 \quad 1.345251$

C $5.713997-1.022271-0.486973$

C 6.1535020 .3574190 .042593

H $4.724384-0.950813-0.950679$

O $6.633038-1.459767-1.486261$

H 5.5448560 .6042320 .919378

H $7.1960680 .298137 \quad 0.378348$

H $2.150589-7.199719-1.918885$

H $1.954358-6.996513-0.170994$

H $0.673058-6.469750-1.273339$

C $0.629150-3.543150-0.369153$

H $4.448585-4.0590191 .776941$

H $6.849543-2.7140032 .054721$

H $6.504709-0.901296-2.271266$

H $-0.136617-4.322850-0.456002$

H $0.654544-3.011701-1.329175$

H $2.015541-4.6999820 .840083$

C $3.7200452 .020471-0.715798$

O $4.753717 \quad 1.568099-1.598210$

C $6.0538851 .486556-1.008210$

C $6.4791322 .868666-0.490002$

C 5.4390383 .4653120 .457422

C $4.0485373 .417984-0.177419$

H 3.6084491 .3120820 .114347

C $2.4098032 .028273-1.509282$

H $6.7438251 .226984-1.820487$

O 7.7430692 .7748680 .157647

H $6.6043503 .543376-1.347144$

H $5.4444342 .958943 \quad 1.429757$

O $5.7610734 .834200 \quad 0.713593$

H $4.0027044 .154107-0.989878$

O 3.0785383 .8086840 .791494

C $1.9825880 .614784-1.818651$

H $1.5927692 .497332-0.949429$

H $2.5178482 .584568-2.448108$

H $8.040243 \quad 3.6781380 .358597$ 
H $6.5910324 .851057 \quad 1.219037$

H $3.0972743 .157448 \quad 1.513638$

O $2.3015010 .294950-3.104217$

O $1.455525-0.126865-1.000358$

C $1.980056-1.041501-3.501768$

H $2.267764-1.163285-4.549270$

H $2.541390-1.760177-2.897776$

H $0.904294-1.217106-3.410684$

SCF Energy (B3LYP/6-31G*//MMFF) $=-3245.90838589$

\section{4}

MMTFF Geometry

C $0.850646-2.5870291 .722572$

C $-0.347643-2.951837 \quad 1.245122$

C $-0.571542-3.373832-0.184051$

O $-1.290513-2.290786-0.823218$

C $-1.431142-4.646848-0.243233$

C $-1.703980-5.123721-1.658536$

C $-2.896803-5.042132-2.282858$

C $-0.527074-5.744259-2.367112$

C $-4.177592-4.477145-1.736805$

C $-4.491825-3.105964-2.348837$

C $-5.502544-2.325581-1.496791$

C $-5.903505-1.019872-2.184177$

C $-6.816480-0.159121-1.298242$

O $-4.871112-2.041326-0.242778$

O $-6.867678 \quad 1.145656-1.895764$

C -6.173361 -0.0171190 .099680$

C $-8.255629-0.687268-1.297960$

O -7.0553240 .6566580 .993910$

C $-5.702504-1.3569240 .702670$

C $-4.876844-1.1860232 .001966$

O $-6.852386-2.1438331 .037386$

C $-0.541516-1.244917-1.274602$

O $0.677168-1.179207-1.320033$

C $-1.464227-0.152077-1.651878$

C $-0.9645641 .071393-1.881105$

C -1.725542 2.271784-2.231367

C $-3.2239292 .151809-2.309277$

C $-3.594113-0.3566961 .867304$

C $-3.763095 \quad 1.107242 \quad 2.238135$

C $-2.522529 \quad 3.267557 \quad 2.227637$

$\begin{array}{lllll}\text { O } & -4.770942 & 1.553870 & 2.778974\end{array}$

C $-2.8760104 .064276 \quad 1.011781$

C $-2.066178 \quad 4.9900940 .475627$

C $-2.4418735 .855858-0.692581$

C $-1.4240775 .812646-1.809343$

C $-0.4534876 .738040-1.896905$

C $-1.5917454 .755381-2.875738$

C -1.049284 $3.417612-2.456986$

N -2.6328151 .8523131 .929480$

H $1.725447-2.6477081 .080055$

H - $1.226786-2.8414971 .876013$

H $0.380138-3.548024-0.701343$

H -2.367233 -4.4703860 .295160$

H $-0.930368-5.457297 \quad 0.303467$

H -2.972029 $-5.416518-3.303030$

H $-0.799225-6.151239-3.346899$

H $0.262198-5.003718-2.527437$

H $-0.118085-6.568168-1.773219$

H $-4.993054-5.172553-1.972383$

H -4.156601 -4.420856 -0.646358

H $-3.567676-2.521079-2.430123$

H $-4.879887-3.244075-3.365568$

H -6.381589-2.958654-1.327695

H -6.382276 -1.219589 -3.150996

H $-4.999893-0.440338-2.415369$

H $-7.3791961 .721577-1.301623$

H $-5.3070370 .641879-0.025869$

H -8.658844 $-0.692863-2.317724$

H $-8.918819-0.033968-0.720278$

H -8.339657-1.701656 -0.902459

H $-7.730186 \quad 0.018635 \quad 1.281755$

H -5.518192 -0.8115062 .808912$

H $-4.567702-2.1916672 .321702$

H -6.590412 -2.806047 1.698331

H -2.523136 $-0.374006-1.700353$

H $0.1124551 .214780-1.801966$
H $-3.6257281 .748646-1.373723$

H $-3.5128301 .488802-3.131726$

H $-3.731493 \quad 3.105398-2.468311$

H $-2.847766-0.7596442 .564113$

H -3.176286 -0.4351480 .859285$

H $-1.497573 \quad 3.4533272 .561336$

H -3.2021643 .5256893 .046204$

H $-3.860168 \quad 3.9009840 .578856$

H -1.0951145 .1720210 .930869$

H $-2.5402846 .885577-0.323750$

H $-3.4310885 .591053-1.086231$

H $0.2614006 .732131-2.712937$

H $-0.3489827 .518163-1.150104$

H $-1.0498835 .052091-3.784183$

H $-2.6396904 .713414-3.185594$

H $0.0377843 .404518-2.364186$

H -1.8615411 .4090121 .438895$

C $0.873059-0.4433042 .981789$

$\begin{array}{llllll}\text { O } & 1.845588 & 0.112409 & 2.077934\end{array}$

C 2.2393141 .4114762 .557666

C 1.3878811 .6929113 .793498

C $1.088505 \quad 0.293515 \quad 4.298646$

H 3.2875821 .3274852 .866008

C 2.1025582 .4477561 .433819

H $1.9179532 .308944 \quad 4.526463$

H 0.4578842 .2124673 .537984

$\begin{array}{llll}\text { C } & -0.098019 & 0.232882 & 5.244495\end{array}$

H $1.980908-0.0961424 .806414$

C 2.8510722 .0900210 .133175

$\begin{array}{lllll}\mathrm{O} & 0.716602 & 2.592516 & 1.095828\end{array}$

H 2.4270903 .4297321 .796914

H $2.3639941 .233234-0.348344$

O $2.6882223 .203713-0.754248$

C $4.3514221 .800027 \quad 0.322481$

C $5.0846301 .542186-1.006538$

H 4.4649600 .9244550 .966449

$\begin{array}{lllll}\text { O } & 4.971870 & 2.895559 & 0.994394\end{array}$

H $4.529400 \quad 0.787422-1.573367$

H $5.0888432 .462311-1.602729$

H $0.0855500 .847888 \quad 6.131555$

H - -1.0125510 .5983814 .764972$

H $-0.280105-0.7935425 .577677$

C $1.044603-1.9614893 .074158$

H $\quad 0.390743 \quad 1.7206910 .813881$

H $2.8971152 .897875-1.652919$

H $4.836413 \quad 3.6894140 .449053$

H $\quad 0.334783-2.383792 \quad 3.794597$

H $2.051844-2.2146023 .424323$

H $-0.116480-0.2021772 .569973$

C $6.170876-1.280637-0.536551$

O $6.659244-0.0596690 .035115$

C $6.550608 \quad 1.094193-0.804266$

C $7.3241850 .862772-2.108206$

C $6.880057-0.417501-2.808778$

C $6.914224-1.601178-1.841840$

H $5.095492-1.193843-0.732326$

C $6.386690-2.3825030 .513501$

H $7.0579901 .907973-0.270981$

$\begin{array}{llll}\text { O } & 7.165467 & 1.978321 & -2.978262\end{array}$

H $8.3947430 .786036-1.877119$

H $5.887665-0.296212-3.258822$

O $7.780887-0.671453-3.889809$

H $7.960690-1.862633-1.638136$

O $6.309018-2.713957-2.497417$

C $5.484686-2.2092281 .717188$

H $6.176465-3.3753910 .104537$

H $7.427440-2.3508890 .858825$

H $7.6513811 .779583-3.797124$

H $7.505219-1.506986-4.304817$

H $6.527780-3.513939-1.991468$

O $6.049732-2.8250272 .796925$

O $4.403089-1.6384981 .705142$

C $5.291402-2.7583914 .008995$

H $4.368963-3.3370023 .904431$

H $5.892228-3.1966294 .810270$

H $5.068737-1.7183654 .267251$

SCF Energy (B3LYP/6-31G**//MMFF) $=-3245.92500716$ 
0600135

MM̄FF Geometry

C $0.195157-3.3808770 .848257$

C $0.209766-3.846029-0.411212$

C $-0.625261-3.361707-1.571058$

O $-1.104822-2.015604-1.361229$

C $-1.837389-4.286615-1.763533$

C $-2.598104-4.006846-3.044546$

C $-3.780823-3.364073-3.101424$

C $-1.973381-4.556602-4.300705$

C $-4.532826-2.753268-1.951544$

C $-5.235453-1.452549-2.366151$

C $-5.995920-0.828931-1.188107$

C $-6.8933510 .313352-1.664589$

C $-7.6144840 .993710-0.491268$

O $-5.032343-0.331317-0.253101$

O

C -6.5704431 .3639750 .581701$

C $-8.786796 \quad 0.151753 \quad 0.025339$

O

C $-5.601876 \quad 0.2108220 .944900$

C $-4.454960 \quad 0.766547 \quad 1.819510$

O $-6.314447-0.7819311 .684696$

C $-0.263597-1.012501-1.733312$

O $0.871119-1.134551-2.167040$

C $-0.9615590 .276245-1.535501$

C $-0.2537701 .411400-1.634548$

C $-0.7648682 .775937-1.493763$

C $-2.2572122 .960325-1.416025$

C $-3.284745-0.2022592 .041225$

C -2.2152290 .4500362 .898353$

C -0.5610082 .3152632 .875424$

O $\begin{array}{llll}-1.990941 & 0.080320 & 4.048123\end{array}$

C -0.6690783 .7048082 .333200$

C 0.3513684 .3663951 .766972

C 0.2366575 .7598531 .217954

C $0.7226975 .870632-0.211093$

C $1.8476216 .542929-0.508799$

C $-0.1333125 .263460-1.304266$

C $0.1262193 .789361-1.468757$

N -1.5781691 .4947952 .249403$

H $-0.488207-2.5845081 .128296$

H $\quad 0.878197-4.672306-0.647295$

H $-0.000294-3.387704-2.472860$

H -2.504888 -4.206024-0.897230

H -1.512587 -5.336039-1.782345

H -4.263995 -3.269469-4.072884

H -2.594528 -4.380555 -5.185379

H -1.001415 -4.088858 -4.484472

H -1.828415 -5.638262 -4.211133

H -5.285448 -3.474404 -1.611197

H -3.869546 -2.544661-1.106797

H -4.486632 $-0.742876-2.741964$

H -5.933968 -1.664810 -3.185352

H -6.597724 -1.610363 -0.709267

H -7.620483 -0.043507 -2.404710

H $-6.2826171 .066263-2.182423$

H -8.788810 2.005724 -1.701714

H -5.9789872 .1940740 .170974$

H -9.501563 $-0.045963-0.782465$

H -9.3502330 .6900480 .795622$

H -8.475387 -0.8116630 .434118$

H $-7.571221 \quad 1.1263732 .244564$

H -4.0603971 .6724451 .343212$

H $-4.847386 \quad 1.0688172 .799315$

H -5.686286 -1.280275 2.230915

H -2.023431 $0.256715-1.320474$

H $0.8161861 .338676-1.828380$

H -2.749036 $2.472462-2.265457$

H -2.568108 $4.006707-1.445908$

H $-2.6564932 .531725-0.492888$

H -3.615181-1.114371 2.548620

H -2.830781 -0.5056541 .091379$

H 0.4046661 .8592492 .648853

H -0.7018192 .3168373 .960726$

H -1.637886 4.1924692 .414986

H 1.3256473 .8885291 .702071

H 0.8216226 .4218971 .869460
H $-0.796955 \quad 6.1242201 .270217$

H $2.1896996 .644900-1.533401$

H 2.4549567 .0009320 .264895

H $0.0892135 .747829-2.264086$

H -1.180733 5.494445-1.092982

H $1.1846053 .544251-1.574167$

H $-1.7020591 .583746 \quad 1.245927$

C $1.491760-2.9530132 .991135$

O $2.220159-1.8555602 .411941$

C $3.299602-1.4915723 .295088$

C $3.131606-2.3305644 .559237$

C $2.432795-3.5639444 .029886$

H $4.226835-1.7868222 .790388$

C 3.3040160 .0205183 .553982

H $4.091638-2.5524755 .035706$

H $2.502247-1.8311085 .304521$

C $1.730701-4.3722465 .107698$

H $3.179675-4.1984933 .533901$

C $3.301508 \quad 0.9019132 .291763$

O 2.1372950 .3714654 .312154

H 4.1594130 .2802804 .188390

H 2.3393490 .8161421 .773125

O 3.4123492 .2664532 .719502

C 4.4374650 .6183501 .295527

C $4.4289921 .612708 \quad 0.118824$

H $4.333142-0.3995370 .912385$

O $5.6957290 .687981 \quad 1.963764$

H $3.4254661 .625805-0.320361$

H 4.6284222 .6228140 .496825

H $2.448220-4.7130585 .861236$

H $0.963459-3.7798365 .617457$

H $1.245895-5.2557824 .681294$

C $1.077581-3.9634251 .918250$

H 1.3615990 .0466183 .823364

H 2.6974852 .4297793 .358443

H 5.7794081 .5808602 .340214

H $0.533944-4.7875262 .394665$

H $1.978684-4.3836031 .453525$

H $0.602100-2.5267053 .473183$

C $4.206192-0.318651-2.230165$

O $5.375906-0.054509-1.446550$

C $5.4854591 .286834-0.960472$

C $5.4979722 .271184-2.139277$

C $4.2878552 .075456-3.050797$

C $4.1551210 .606675-3.452512$

H $3.312042-0.171626-1.614357$

C $4.265171-1.792104-2.662490$

H $6.4661991 .355890-0.472960$

O $5.5404923 .608680-1.653839$

H $6.4125402 .110196-2.724967$

H $3.3657422 .437115-2.581153$

O $4.4613342 .843985-4.243343$

H $4.9572930 .347191-4.155459$

O $2.9150380 .419040-4.131507$

C $4.045341-2.740436-1.502687$

H $3.496213-2.018055-3.408743$

H $5.252754-2.001623-3.091408$

H $5.6743654 .195866-2.416522$

H $4.4270893 .782965-3.994488$

H $2.9002911 .034116-4.884776$

O $4.587959-3.955865-1.807912$

O $3.446090-2.464240-0.473077$

C $4.450722-4.962208-0.799418$

H $4.856131-4.6101720 .154335$

H $3.399257-5.242778-0.692441$

H $5.018191-5.841050-1.117100$

SCF Energy (B3LYP/6-31G**//MMFF) $=-3245.92202581$

0600136

MM̄FF Geometry

C $-1.963041-3.074041-2.096740$

C $-3.218523-3.114042-2.569959$

C $-4.262452-2.023294-2.492376$

O $-3.742872-0.805900-1.914456$

C $-5.431806-2.508614-1.614405$

C $-6.582151-1.523909-1.528145$

C $-6.867894-0.766185-0.449880$

C $-7.462963-1.459370-2.749356$ 
C $-6.105784-0.683837 \quad 0.844347$ C $-5.718277 \quad 0.7659361 .166704$ C $-4.742371 \quad 0.8520742 .350351$ C -4.4395372 .3131632 .686783$ C -3.354351 2.4489093 .762754 O O $-2.938916 \quad 3.8238153 .764971$ C $-2.137096 \quad 1.586276 \quad 3.362437$ C $-3.912660 \quad 2.167170 \quad 5.162617$ O -1.1779821 .5969644 .416969$ C -2.5166700 .1415852 .970892$ C -1.336051 -0.6696002 .379285$ O $-2.962592-0.5553444 .140171$ C $-3.1520810 .083399-2.760287$ O $-2.910069-0.077699-3.945655$ C $-2.871688 \quad 1.315107-1.988944$ C $-2.2618232 .354537-2.578015$ C $-1.9758133 .651831-1.958503$ C $-2.346673 \quad 3.831535-0.509881$ C $-0.758067-0.121596 \quad 1.071422$ $\begin{array}{llll}\text { C } & 0.424777 & 0.814400 & 1.247310\end{array}$ C $1.8999622 .325924-0.082405$ $\begin{array}{lllll}\text { O } & 0.999618 & 0.983231 & 2.318669\end{array}$ C $1.3876273 .729932-0.092511$ C $1.6278154 .590927-1.092955$ C $1.1590126 .016395-1.101087$ C $0.359688 \quad 6.360587-2.337148$ C $0.9275467 .005164-3.370649$ C -1.118210 $6.045110-2.332682$ C -1.416752 $4.620705-2.714124$ N 0.7841101 .4101950 .045827 H - $1.594251-2.181274-1.601053$ H -3.551838-4.026655-3.062295 H $-4.628700-1.821098-3.506795$ H $-5.057938-2.753838-0.614520$ H $-5.824139-3.456176-2.009099$ H $-7.744791-0.121241-0.495800$ H -8.314982 -0.784854 -2.613141 H $-6.895864-1.100829-3.613970$ H -7.863768 -2.451375 -2.981926 H $-6.738776-1.0761501 .649161$ H $-5.207544-1.3042290 .821439$ H $-5.251274 \quad 1.2148620 .281141$ H $-6.623282 \quad 1.345857 \quad 1.386944$ H -5.1875190 .3410703 .211962$ H -5.3528842 .8391752 .992032$ H -4.0912772 .8278261 .781553$ H -2.203384 3.9076634 .396083 H -1.6712042 .0875742 .505340$ H -4.7041252 .8856885 .407701$ H -3.142939 2.2974335 .931317 H $-4.333691 \quad 1.1647135 .262905$ H $-0.361447 \begin{array}{lll}1.190663 & 4.081721\end{array}$ H $-0.557508-0.8251413 .135295$ H -1.718238-1.675490 2.151471 H $-2.875164-1.5102413 .984159$ H -3.203294 $1.344505-0.958170$ H $-1.9638692 .258958-3.621405$ H -1.9102603 .0366380 .104240$ H -3.435191 $3.810594-0.390913$ H $-1.9895184 .769805-0.080535$ H $-0.395544-0.9677600 .475138$ $\begin{array}{llll}\mathrm{H} & -1.529809 & 0.379737 & 0.482107\end{array}$ H $2.4159032 .074503-1.013529$ H 2.5950542 .1882920 .751630 H 0.8149334 .0585740 .771339 H $2.222695 \quad 4.263733-1.943007$ H $2.0476256 .658486-1.040329$ H $0.5671076 .249362-0.207217$ H $0.3524357 .284106-4.247260$ H $1.9813817 .262864-3.365061$ H -1.635700 $6.685640-3.060203$ H - $1.5482166 .328676-1.367868$ H -1.180477 4.393861-3.754795 H $0.2215841 .235582-0.781898$ C $-0.488801-4.650213-0.823031$ O $0.609527-3.790216-0.449524$ C $1.670825-4.6010110 .092327$
C $1.053381-5.9656510 .361958$

C $0.070403-6.072256-0.785519$

H $2.429462-4.700755-0.694595$

C $2.299375-3.9429191 .328352$

H $1.798635-6.7668010 .367347$

H $0.525598-5.984406 \quad 1.323126$

C $-0.983879-7.146418-0.580516$

H $0.625689-6.282781-1.709662$

C $3.093672-2.661526 \quad 0.990034$

$\begin{array}{lllll}\text { O } & 1.274973 & -3.668849 & 2.281597\end{array}$

H $2.987539-4.6596551 .792066$

H $3.828235-2.921605 \quad 0.221745$

O $2.232103-1.6692300 .444683$

C $3.831090-2.0516462 .194710$

C $4.649072-0.7788891 .893183$

H $4.499905-2.8068662 .623388$

O $2.894915-1.710026 \quad 3.221245$

H $3.9778160 .005661 \quad 1.528301$

H $5.016422-0.4082082 .858666$

H $-0.516074-8.132777-0.496490$

H $-1.563312-6.9708990 .332125$

H $-1.682730-7.175004-1.422241$

C $-1.012731-4.237500-2.200224$

H $\quad 0.675868-3.014368 \quad 1.883008$

H $1.815688-2.044322-0.348865$

H $2.253352-1.0877282 .837212$

H $-1.517298-5.084542-2.679146$

H $-0.179079-3.940183-2.847818$

H $-1.264443-4.527952-0.055258$

C $4.931593-0.011653-1.083759$

O $5.441870-1.178410-0.427527$

C $5.840591-0.998258 \quad 0.935679$

C 6.9163450 .0946031 .012235

C 6.4357041 .3998240 .387173

C $5.9390651 .144941-1.034564$

H $3.9957530 .290655-0.603630$

C $4.628013-0.397371-2.538161$

H $6.320933-1.9362691 .242491$

$\begin{array}{lllll}\text { O } & 7.308553 & 0.309720 & 2.363728\end{array}$

H $7.809337-0.2479710 .472964$

H 5.6651481 .8743801 .006328

$\begin{array}{lllllll}\text { O } & 7.541084 & 2.304671 & 0.345807\end{array}$

H $6.7946120 .926021-1.686698$

O $5.3094732 .324142-1.532845$

C $3.292337-1.092183-2.654816$

H $4.6051100 .476611-3.199769$

H $5.390718-1.075307-2.939678$

H 7.9682551 .0242472 .364883

H 7.2045283 .1657380 .046000

H $5.9903963 .013619-1.606062$

O $2.310155-0.169998-2.869141$

O $3.138924-2.301935-2.551618$

C $0.985630-0.691259-3.014435$

H $0.957772-1.474058-3.778453$

H $0.630528-1.074521-2.055086$

H $0.331726 \quad 0.126591-3.328467$

SCF Energy (B3LYP/6-31G**//MMFF)= -3245.93037808

0600137

MM̄FF Geometry

C $-0.476165-3.505606 \quad 0.704340$

C $0.323176-3.276363-0.349354$

C $-0.109184-2.954367-1.762628$

O $0.390761-1.637434-2.136466$

C $-1.614914-3.082293-2.078429$

C $-1.936226-3.107787-3.559666$

C $-2.526597-2.106835-4.241211$

C $-1.623766-4.406129-4.261486$

C $-2.906940-0.735491-3.757719$

C $-4.414505-0.584564-3.511984$

C $-4.835427-1.010360-2.095852$

C $-6.359048-0.992827-1.960741$

C $-6.808270-1.308222-0.527660$

O $-4.245094-0.082355-1.177109$

O $-8.207768-0.997224-0.450725$

C $-6.056638-0.3692220 .438550$

C $-6.682148-2.802291-0.209820$

O $-6.651976-0.6980021 .794757$ 
C $-4.527385-0.3295320 .205275$

C $-3.8505720 .789307 \quad 1.038073$

O $-3.968434-1.578137 \quad 0.628914$

C $-0.012989-0.539780-1.439455$

O $-0.774372-0.508516-0.485304$

C $0.6270580 .649163-2.044164$

C $0.204591 \quad 1.866974-1.672211$

C $0.7431123 .153650-2.115923$

C $1.9779723 .136561-2.976070$

C -4.1516662 .2088850 .551686$

C -3.7060763 .2670841 .541749$

C -1.679304 4.3944072 .450276

O $-4.520778 \quad 3.970570 \quad 2.136125$

C -1.277020 5.4966311 .521094

C -0.0030525 .8355441 .271488$

C 0.3994856 .9106640 .303250

C $1.2245466 .376070-0.848395$

C $2.5556876 .555590-0.885355$

C $0.4991495 .710122-1.998399$

C $0.1297904 .282141-1.701805$

N -2.3300383 .3557161 .670634$ H - 1.555648 -3.485532 0.589123 H $1.399532-3.280804-0.190662$ H $\quad 0.439582-3.656823-2.400989$ H -2.171481 -2.280226 -1.587820 H $-2.006285-4.014731-1.650398$ H -2.746452 -2.272047 -5.295947 H - $1.958692-4.407185-5.304321$ H $-0.546026-4.594600-4.264693$ H -2.122324 -5.240542 -3.757500 H $-2.336748-0.438670-2.875714$ H -2.612685 -0.030093 -4.545750 H $-4.6656090 .476280-3.643814$ H $-4.979590-1.147467-4.264983$ H -4.454419 -2.016245 -1.893472 H $-6.821335-1.691365-2.669546$ H $-6.737950 \quad 0.002622-2.232724$ H $-8.485492-1.1163360 .473948$ H -6.4738020 .6313920 .278791$ H -7.289680 -3.391847 -0.907013 H -7.070885 -3.0296220 .788951$ H -5.655764 -3.169680 -0.271511 H -5.836834 -1.489722 2.025778 H -4.1136490 .6789582 .097950$ H -2.7622330 .6482201 .003188$ H $-3.018613-1.4504640 .788750$ H $1.4258410 .491466-2.758890$ H $-0.6178851 .933959-0.960682$ H $1.7761172 .639950-3.931221$ H $2.7897612 .606221-2.467812$ H $2.3609744 .132521-3.208565$ H -3.656652 $2.402368-0.407395$ H $-5.223528 \quad 2.3567700 .389528$ H -0.8199763 .9411472 .951909$ H -2.364038 4.7790373 .212450 H -2.0782676 .0302671 .014806$ H $\quad 0.799493 \quad 5.308987 \quad 1.782039$ H $\quad 0.9690597 .6656570 .860687$ H $-0.4758217 .438896-0.095067$ H $3.1508406 .200642-1.720238$ H $3.0808097 .056276-0.078777$ H $1.0889235 .784422-2.918479$ H $-0.4187276 .269730-2.220408$ H -0.761736 4.183890 -1.082956 H -1.741454 2.7757921 .079952 C $-0.159811-2.4715492 .947050$ O $0.698461-1.4303232 .440245$ C $1.318283-0.7496483 .546370$ C $0.572981-1.2126274 .791486$ C $0.228536-2.6424574 .414417$ H $2.350751-1.1140933 .603664$ C $\quad 1.314891 \quad 0.761993 \quad 3.292588$ H $1.187354-1.1413305 .694113$ H $-0.342350-0.6330564 .957930$ C $-0.863296-3.252725 \quad 5.275238$ H $1.135915-3.2562644 .494337$ C 1.9104831 .1676531 .928199 O $-0.036739 \begin{array}{lll}1.238613 & 3.326336\end{array}$
H $1.838846 \quad 1.2790554 .104177$

H $1.256576 \quad 0.818181 \quad 1.121864$

O 1.9168222 .5968431 .848948

C 3.3414430 .6622151 .677922

C 3.8720631 .0839450 .298223

H $3.352221-0.4265451 .736734$

O $4.212447 \quad 1.1474642 .696434$

H $3.1529110 .757949-0.459143$

H 3.9244542 .1781160 .245350

H $-0.551163-3.2879216 .324114$

H -1.790154 -2.671760 5.219595

H -1.087044 -4.275056 4.954760

C $0.030980-3.7317592 .102079$

H -0.5370050 .7537182 .647402$

H 0.9980752 .8933491 .962823

H 4.1790262 .1192882 .672233

H -0.514823 -4.576116 2.537142

H $1.090898-4.0086022 .075412$

H -1.191141-2.103546 2.862226

C $4.603154-1.681911-0.781694$

O $5.356964-0.9027300 .153712$

C $5.2722260 .514732-0.024246$

C $5.7811910 .888819-1.422930$

C $5.0313020 .130802-2.515443$

C $5.026706-1.369707-2.225353$

H $3.532458-1.485129-0.647246$

C $4.867966-3.165802-0.469624$

H 5.9658540 .9543750 .703382

O $5.6552762 .288633-1.652558$

H $6.8485910 .644077-1.499882$

H $4.0133340 .515640-2.636547$

O $5.698867 \quad 0.367544-3.757703$

H $6.027960-1.776845-2.417997$

O $4.121747-2.019681-3.116932$

C $4.297967-3.6142270 .859023$

H $4.419602-3.800918-1.241878$

H $5.949737-3.343610-0.436058$

H $6.1523762 .745166-0.952536$

H $5.156832-0.025279-4.462315$

H $4.466671-1.910578-4.018993$

O $4.657390-4.9151231 .074951$

O $3.621346-2.9278361 .610246$

C $4.190578-5.4877652 .300717$

H $4.605150-6.4958382 .384422$

H $4.533434-4.8961973 .155111$

H $3.099001-5.5567802 .291032$

SCF Energy $\left(B 3 L Y P / 6-31 G^{* *} / / M M F F\right)=-3245.92069350$

0600138

MM̄FF Geometry

C $-1.810189-2.614253-3.611562$

C $-2.184131-1.832223-2.587736$

C $-3.462417-2.039653-1.818779$

O $-4.066855-0.733374-1.676081$

C $-3.171022-2.604308-0.418941$

C $-4.377510-3.2693710 .213299$

C $-5.133577-2.7325331 .190599$

C $-4.679570-4.657929-0.291706$

C $-5.006134-1.3761181 .825822$

C $-4.540393-1.4591263 .286943$

C $-3.062245-1.8536333 .428253$

C -2.677759-2.013048 4.900342

C $-1.178363-2.3050015 .068647$

O $-2.280268-0.8146442 .832865$

O $-0.846752-2.1273526 .454287$

C $-0.373245-1.2566364 .273066$

C $-0.835257-3.7588264 .723142$

O $1.017346-1.5715304 .274518$

C $-0.870081-1.0639382 .821191$

C -0.1804710 .1676662 .190306$

O $-0.543122-2.2262982 .056579$

C $-5.409550-0.613473-1.820013$

O $-6.202895-1.509364-2.072908$

C $-5.8429110 .795739-1.662784$

C $-5.0478831 .767674-1.187833$

C $-5.4015923 .181082-1.032047$

C $-6.7625803 .619633-1.505039$

C -0.7913190 .6184130 .857206$ 
C $-0.050892 \quad 1.8074720 .269354$ C $-0.1436493 .375996-1.666795$ O 0.9208852 .3304680 .807743 C $-0.8599694 .585680-1.154190$ C $-1.7323185 .303358-1.877956$ C $-2.4644816 .501320-1.343982$ C $-3.9679206 .325630-1.363820$ C $-4.7215676 .952079-2.283117$ C $-4.6070205 .499925-0.267343$ C $-4.4847454 .019921-0.505328$ N $-0.6119042 .214445-0.933681$ H -2.439832 $-3.452980-3.899588$ H - $1.548389-1.001502-2.290067$ H -4.123291 -2.703666 -2.388746 H $-2.790048-1.8068490 .226867$ H $-2.369293-3.353490-0.468351$ H $-5.960845-3.3252281 .579636$ H $-5.525410-5.116610 \quad 0.231600$ H $-4.926181-4.637103-1.357447$ H -3.812270 -5.310719 -0.147901 H $-4.356885-0.707597 \quad 1.253150$ H -5.999862 -0.9110081 .804277$ H -4.690206 -0.4693333 .737531$ H $-5.172421-2.1659073 .838876$ H -2.888681 -2.792790 2.891821 H $-3.277541-2.7983375 .376779$ H -2.912950 -1.085911 5.441750 H -1.400183 -2.734550 6.974839 H $-0.474685-0.2988614 .802591$ H -1.392620 -4.4474475 .369422$ H $0.224491-3.9685794 .906162$ H -1.065121 -4.019836 3.687903 H $1.293211-1.6621335 .202659$ H -0.2508661 .0161762 .882982$ H $0.884120-0.0452532 .040945$ H $-0.415246-1.9748681 .128709$ H $-6.8701390 .988544-1.955195$ H $-4.0332521 .515353-0.883950$ H $-6.9022753 .369444-2.562710$ H -6.927155 4.695841-1.419170 H -7.548502 $3.128912-0.920352$ H $-0.765900-0.1930460 .124208$ H $-1.8369220 .914071 \quad 1.002727$ H $-0.3402913 .195045-2.728171$ H $0.9357013 .491723-1.524679$ H $-0.6525314 .880509-0.128072$ H -1.936510 $5.016190-2.906789$ H $-2.1747937 .367047-1.953706$ H -2.145402 $6.742203-0.322106$ H $-5.8023456 .858851-2.287443$ H -4.274479 $7.569797-3.054973$ H -5.654149 $5.789825-0.125788$ H -4.1289245 .7400570 .691148$ H -3.527296 $3.605047-0.190604$ H -1.466566 $1.768556-1.254606$ C $0.540444-3.385995-4.025696$ O $1.131868-2.940700-2.784747$ C $2.548446-2.788049-2.980771$

C $2.882393-3.691991-4.157033$

C $1.680818-3.442199-5.047702$ H $2.721179-1.749599-3.285371$ C $3.312066-3.084951-1.688746$ H $3.832107-3.428936-4.631862$ H $2.923298-4.746667-3.861362$ C $1.488035-4.499224-6.121573$ H $1.799955-2.463524-5.532392$ C $2.872978-2.262456-0.458039$ O $3.142596-4.470562-1.356631$ H $4.386562-2.952360-1.858668$ H $1.868260-2.582209-0.153399$ O $3.746798-2.5913830 .629961$ C $2.885130-0.728836-0.601890$ C $4.216953-0.162130-1.126144$ H $2.690558-0.2953140 .384965$ O $1.833277-0.299833-1.461238$ H $5.042780-0.579407-0.541489$ H $4.358958-0.483859-2.163452$ H $2.351063-4.525483-6.794849$
H $1.371382-5.497298-5.686025$ H $0.597056-4.286748-6.720873$

C $-0.564419-2.406565-4.425459$

H $2.188526-4.634807-1.262182$

H $3.720238-3.5572260 .741275$

H $\quad 1.012927-0.726077-1.162002$

H $-0.831836-2.541251-5.480079$

H $-0.210670-1.374057-4.310155$

H $\quad 0.132795-4.388228-3.842889$

C 5.1918361 .6755891 .122269

O $4.0888321 .899606 \quad 0.235476$

C $4.2567781 .383670-1.089535$

C $5.5054461 .997719-1.737370$

C $6.7456371 .801412-0.871914$

C 6.4804992 .2834650 .552750

H 5.3273120 .5992441 .281076

C 4.8371542 .3254972 .469530

H $3.3935341 .739328-1.666062$

O $5.7415191 .441227-3.026254$

H $5.3485843 .075322-1.877194$

H $7.0810620 .757600-0.878142$

O $7.8220342 .572490-1.410688$

H 6.4165183 .3792640 .561502

O 7.5800991 .9131001 .382576

C 3.7897761 .5459143 .235980

H 5.7200282 .3853623 .115340

H 4.4444143 .3347542 .296592

H $4.9420481 .591029-3.559506$

H $7.9520592 .283219-2.330232$

H 8.3831442 .2923360 .986028

O 3.3727042 .2970524 .298602

O $3.404420 \quad 0.4185092 .960847$

C 2.3973341 .6820355 .145882

H 1.4557591 .5615994 .604051

H 2.7598050 .7171975 .513272

H 2.2274892 .3402576 .002026

SCF Energy $(B 3 L Y P / 6-31 G * * / / M M F F)=-3245.91179130$

0600139

MM̄FF Geometry

C $0.781167-3.3453650 .807176$

C $0.108099-2.1856180 .753057$

C $-0.383818-1.4610141 .979072$

O $-1.816726-1.6439252 .015715$

C -0.0816080 .0437891 .877604$

C $-0.0719920 .739497 \quad 3.222933$

C -1.1079501 .4178773 .753028$

C 1.2370940 .6606163 .966931

C -2.4940061 .5827443 .195643$

C -2.8551663 .0500262 .927403$

C -2.1659413 .6320501 .683845$

C -2.5344625 .1081671 .511089$

C $-1.9757095 .695200 \quad 0.206179$

O $-2.6139242 .881923 \quad 0.550918$

O $-2.6311426 .950643-0.030023$

C $-2.3539174 .761965-0.963089$

C -0.4773326 .0020790 .314355$

O $-1.7337775 .186668-2.174919$

C $-2.0269673 .275722-0.694370$

C $-2.6343452 .389617-1.806832$

O $-0.6100023 .109472-0.687765$

C -2.391639-2.000596 3.191681

O $-1.825514-2.1675794 .263282$

C $-3.850690-2.2127243 .040709$

C $-4.542835-1.8835571 .938732$

C $-5.975334-2.1009231 .718754$

C $-6.752200-2.8064612 .799326$

C $-2.5635250 .882101-1.521045$

C $-3.1782470 .065608-2.644932$

C $-3.998217-2.235496-3.145539$

O $-3.462280 \quad 0.538868-3.742234$

C $-5.480888-2.125551-2.974045$

C $-6.249852-3.086105-2.439580$

C -7.733672 -2.946973-2.250303

C $-8.151279-3.043980-0.798377$

C $-8.764097-4.144951-0.331637$

C -7.941132 -1.835795 0.090180

C $-6.517512-1.6920240 .552450$ 
N -3.354566 -1.262842 -2.280761 H $\quad 0.966002-3.804973 \quad 1.775407$ H $-0.110366-1.738578-0.214053$ H $0.089659-1.8916342 .870024$ H $-0.796976 \quad 0.529876 \quad 1.204088$ H $\quad 0.904561 \quad 0.1952551 .420882$ H -0.9644331 .8872784 .725804$ H $1.501644-0.3824274 .166942$ H 2.0387441 .1148843 .375488 H 1.2020781 .1816894 .929594 H -2.6565800 .9828802 .296300$ H $-3.191840 \quad 1.1907783 .946719$ H -3.9427363 .0965232 .782995$ H -2.620236 3.6605263 .808031 H -1.080852 3.5197251 .788539 H -2.195909 5.6962702 .373275 H -3.628906 5.2095871 .491958 H -2.4637687 .5250910 .736753$ H -3.437755 4.855055-1.120088 H $-0.292732 \quad 6.7310331 .112529$ H $-0.100595 \quad 6.463616-0.605097$ H 0.1266935 .1189810 .530369 H -1.973436 $6.119343-2.311416$ H -3.693525 $2.649132-1.936572$ H -2.132189 2.597363 -2.758964 H $-0.4001842 .187377-0.897298$ H -4.331829 -2.660521 3.904465 H $-4.017494-1.4170361 .107344$ H $-6.746672-2.2169683 .722801$ H -6.317340 -3.790532 3.006833 H $-7.798912-2.9794392 .540604$ H -1.526409 $0.551108-1.407096$ H $-3.1009590 .652699-0.593697$ H -3.619442 -3.223896 -2.867499 H -3.727422 -2.035243 -4.187141 H $-5.944534-1.198283-3.303132$ H -5.793422 -4.018434 -2.115738 H $-8.219212-3.735494-2.839735$ H -8.102538 -2.000596 -2.665483 H $-9.096279-4.2138220 .698753$ H -8.944816 $-5.004513-0.968598$ H -8.629708 $-1.857018 \quad 0.942347$ H -8.221490 -0.928793 -0.460903 H -5.873176 -1.186526 -0.166554 H -3.161040 -1.541532 -1.323195 C $2.689638-4.646092-0.181256$ O $3.635935-3.573325-0.016050$ C $4.852016-3.912692-0.711058$

C $4.706358-5.367236-1.144059$

C $3.208897-5.469852-1.359110$

H $4.883258-3.283916-1.608820$

C $6.073207-3.6187020 .170390$

H $5.286829-5.591332-2.044241$

H $5.024781-6.061870-0.358370$

C $2.698131-6.899813-1.392041$

H $2.955214-4.973970-2.305687$

C $6.109780-2.1925370 .756137$

O $6.089631-4.5302561 .277128$

H $6.991081-3.816786-0.394841$

H $5.329394-2.0815161 .519079$

O $7.361002-2.0221961 .432513$

C $5.964332-1.073393-0.287285$

C 6.0379710 .3296620 .342059

H $5.001488-1.186848-0.793054$

O $6.978293-1.200462-1.282020$

H $\quad \begin{array}{lll}5.336346 & 0.372575 & 1.182428\end{array}$

H $7.0411090 .501614 \quad 0.751127$

H $3.165485-7.455904-2.211278$

H $2.920338-7.426978-0.458250$

H $1.614523-6.924704-1.543318$

C $1.284446-4.080923-0.404178$

H $5.251090-4.4230771 .758278$

H $7.435789-2.7372852 .087498$

H $7.839060-1.138248-0.833293$

H $\quad 0.589177-4.900727-0.616503$

H $1.289548-3.409658-1.271937$

H $2.718488-5.2404680 .741862$

C $3.3281531 .263240-0.668438$
O $4.5393191 .193244-1.425079$

C $5.7268581 .455703-0.671385$

C $5.6497782 .858270-0.053435$

C 4.3711033 .0605950 .761261

C $3.1384392 .654161-0.051473$

H 3.3694710 .5216810 .139578

C $2.1729520 .807551-1.570704$

H $6.552118 \quad 1.461863-1.394708$

$\begin{array}{lllll}\text { O } & 6.791914 & 3.093591 & 0.763832\end{array}$

H $5.6734793 .601837-0.860857$

H 4.4210442 .5148401 .710867

O 4.2785994 .4468191 .099129

H $2.9563363 .407578-0.825914$

O $1.9972742 .631173 \quad 0.800325$

C $1.9174311 .692895-2.772740$

H $2.396698-0.191788-1.963704$

H $1.2544360 .746730-0.978993$

H 6.6966653 .9824781 .146922

H 3.5190324 .5541251 .695898

H 1.8383403 .5388851 .108230

O $0.6859331 .395113-3.284926$

O $2.7005392 .514416-3.228343$

C $0.3071932 .138757-4.447385$

H $-0.6639421 .768391-4.786223$

H $0.2121743 .199998-4.199246$

H $1.0366001 .991957-5.249824$

SCF Energy $(B 3 L Y P / 6-31 G * * / / M M F F)=-3245.91854388$

0600140

MM̄FF Geometry

C 2.6922481 .3741463 .505598

C $1.4106191 .203498 \quad 3.147551$

C $0.600943-0.0113913 .515793$

O $-0.018560-0.4749092 .291481$

C $-0.5086200 .354366 \quad 4.514677$

C $-1.064778-0.8623355 .229102$

C $-2.246887-1.4504224 .962811$

C $-0.199654-1.3996546 .343166$

C $-3.269506-1.0708983 .930670$

C $-3.362796-2.1363442 .832953$

C $-4.548112-1.8939461 .889372$

C $-4.569967-2.9410050 .774259$

C $-5.705761-2.686690-0.228341$

O $-4.426862-0.5852621 .324848$

O $-5.451217-3.488287-1.392275$

C $-5.657297-1.210132-0.674663$

C -7.061682 $-3.142347 \quad 0.323526$

O $-6.776525-0.890473-1.498621$

C $-5.533733-0.2050190 .496923$

C $-5.256873 \quad 1.208233-0.059024$

O $-6.762258-0.186613 \quad 1.225622$

C $0.548835-1.5221631 .640646$

O $1.452279-2.2364412 .052733$

C $-0.062591-1.7290890 .305717$

C $-0.763262-0.780921-0.337049$

C $-1.344791-0.885953-1.679982$

C -1.221196 -2.205164 -2.393936

C -4.9394712 .2701671 .003522$

C -4.5783383 .5697170 .312075$

C $-2.7817584 .541271-1.112873$

O -5.4079664 .4564690 .125407$

C $-3.0104173 .935714-2.463806$

C $-2.0300603 .453697-3.241866$

C $-2.2798382 .731926-4.534599$

C $-1.8703671 .273929-4.476977$

C $-0.9127030 .790365-5.285869$

C -2.626952 $0.352960-3.541442$

C $-1.9484070 .201309-2.205090$

N $-3.2632933 .587640-0.129128$

H 3.1564480 .6153714 .133155

H 0.9101531 .9468432 .533531

H $1.252190-0.7869733 .935060$

H -1.302874 0.9069164 .001751

H -0.1197931 .0390125 .280690$

H $-2.530394-2.3151685 .562578$

H $-0.669889-2.2375556 .868983$

H $0.759171-1.7549235 .954508$

H $-0.006509-0.6166327 .083839$ 
H $-4.238098-0.9753724 .436747$ H -3.060121 -0.0959363 .483170$ H $-2.428183-2.1268342 .260185$ H -3.457848 -3.134091 3.279892 H -5.468736 -1.946266 2.482876 H $-4.644429-3.953247 \quad 1.190734$ H $-3.615892-2.9116360 .229374$ H $-5.407536-4.418563-1.112212$ H -4.772863 -1.102856 -1.316948 H -7.032752 -4.2089170 .577619$ H $-7.850450-3.038145-0.429686$ H -7.363753 -2.597586 1.220438 H -7.549218 $-0.781351-0.918616$ H -4.407802 $1.154240-0.750425$ H $-6.1197951 .553949-0.642986$ H $-6.8212690 .642857 \quad 1.724824$ H $0.151795-2.690621-0.148948$ H -0.9105090 .1812390 .151304$ H -0.167559 -2.456916 -2.551538 H -1.691638 -2.216774 -3.378893 H -1.692068 $-3.004146-1.810630$ H -5.7974372 .4599921 .656215$ H -4.1062601 .9599641 .643907$ H -1.720901 4.717805 -0.910071 H -3.324956 5.486745-1.023042 H $-4.0439173 .840994-2.789712$ H $-0.9982253 .541742-2.913823$ H $-1.7307603 .258843-5.325130$ H $-3.3388622 .785889-4.817556$ H $-0.635420-0.258617-5.265614$ H $-0.380749 \quad 1.431746-5.980452$ H -2.791559-0.611322 -4.031684 H $-3.6372820 .748200-3.374218$ H -1.978995 $1.104737-1.597440$ H -2.6888942 .7650600 .031134$ C 3.1638543 .3506791 .905321 O 3.0226482 .5096130 .743172 C $3.6080553 .170254-0.391655$ C 3.8689424 .6069470 .045423 C 4.1847784 .4210481 .516665 H $4.5694322 .678012-0.580770$ C $2.6992183 .025287-1.616285$ H $4.6868725 .068876-0.515724$ H $2.9804555 .237691-0.073689$ C 4.0801395 .7002752 .328314 H 5.2038204 .0212311 .607125 C $2.3075911 .574636-1.962700$ O $1.4813853 .748064-1.387036$ H $3.1714543 .502809-2.482542$ H $1.6171281 .178500-1.208611$ O $1.579391 \quad 1.610072-3.196145$ C $3.4884410 .602444-2.131051$ C $3.035345-0.783604-2.628992$ H $4.0038250 .493377-1.173380$ O $4.4302801 .146121-3.055156$ H $2.237159-1.147775-1.974448$ H $2.609144-0.682632-3.634322$ H 4.7899376 .4483151 .960010 H 3.0754386 .1323832 .268823 H 4.3042035 .5126703 .383080 C 3.6060742 .5020793 .101325 H $1.0695113 .382028-0.585339$ H $0.8244582 .208696-3.068417$ H $3.9676681 .283075-3.899774$ H 3.7689143 .1413413 .976439 H 4.5725512 .0439732 .855666 H 2.1860773 .8107582 .095390 C $4.063060-2.643222-0.431766$ O $4.843751-1.982200-1.434432$ C $4.189345-1.809399-2.695360$ C $3.780108-3.176573-3.261766$ C $2.935061-3.972026-2.271203$ C $3.624230-4.034530-0.908768$ H $3.184692-2.034520-0.190330$ C $4.934252-2.7597040 .829117$ H $4.943557-1.394444-3.375671$ O $3.058703-3.028552-4.480552$ H $4.682793-3.757981-3.491394$
H $1.926295-3.553488-2.189473$

O $2.781377-5.298219-2.782425$

H $4.493325-4.702202-0.970678$

O $2.724473-4.5880590 .049402$

C $5.182784-1.4217051 .490754$

H $4.455241-3.4007881 .576943$

H $5.907884-3.1856380 .557440$

H $3.626479-2.536602-5.097947$

H $2.151865-5.763830-2.206655$

H $2.551089-5.509466-0.206600$

O $6.210071-1.5538682 .381663$

O $4.546191-0.3999491 .277414$

C $6.562276-0.3684043 .101654$

H 6.7625990 .4576962 .412348

H $5.762308-0.1061473 .799518$

H $7.471766-0.5750043 .671943$

SCF Energy (B3LYP/6-31G**//MMFF) $=-3245.91262937$

0600141

MM̄FF Geometry

C 4.6338071 .1431122 .758062

C 3.4229791 .5036472 .305134

C 2.1962071 .5144883 .177569

$\begin{array}{llll}\text { O } & 1.143584 & 0.763449 & 2.532849\end{array}$

C 1.6784972 .9519123 .364455

C 0.4691933 .0132884 .279458

C -0.8091793 .0799633 .855781$

C 0.7801953 .0148395 .753608

C - -1.3074553 .0750762 .436092$

C -1.9615004 .4146972 .085190$

C -2.2864924 .5371810 .589278$

C $-2.929197 \quad 5.897573 \quad 0.303884$

C $-3.3507466 .040726-1.164303$

$\begin{array}{lllll}\text { O } & -3.177228 & 3.479499 & 0.217092\end{array}$

O $-4.2215947 .177738-1.266040$

C $-4.1770674 .802256-1.564583$

C $-2.1528966 .329280-2.076936$

O $-4.4722694 .819700-2.959788$

C $-3.5081973 .460367-1.180012$

C $-4.4997982 .298015-1.429569$

O $-2.3494413 .274938-1.997028$

C $1.048262-0.5617972 .816450$

O $1.891267-1.266733 \quad 3.345672$

C $-0.324471-0.9787112 .450845$

C $-0.751814-2.2150892 .745396$

C $-2.115162-2.7209932 .562040$

C $-3.159839-1.7417212 .097707$

C $-4.0266590 .934163-0.909243$

C $-4.944747-0.187029-1.371944$

C $-4.938114-2.647444-1.807047$

O $-6.1171050 .000071-1.687438$

C $-5.235269-3.503463-0.617572$

C $-4.602044-4.656808-0.353911$

C $-4.965863-5.5711780 .779210$

C $-3.842905-5.7590451 .772281$

C $-3.075698-6.8624421 .744337$

C $-3.699521-4.7224372 .863900$

C $-2.372544-4.0122202 .857163$

N $-4.307568-1.418772-1.366861$

H 4.7183950 .8409053 .800968

H $3.290537 \quad 1.817179 \quad 1.274479$

H 2.4090731 .0751334 .160950

H 1.4382713 .3948322 .390809

H 2.4711283 .5863103 .783165

H -1.597283 3.1278214 .606232

H -0.1227343 .0863496 .369295$

H 1.3000912 .0947766 .037658

H 1.4188833 .8677496 .005455

H $-0.5084202 .853778 \quad 1.724512$

H -2.0396682 .2651332 .332040$

H -2.8842804 .5152282 .672058$

H -1.298585 5.2398862 .375592

H -1.351069 4.4318290 .026964

$\begin{array}{llll}H & -2.254914 & 6.714474 & 0.590384\end{array}$

H $-3.823527 \quad 6.0153880 .932002$

H $-3.7367197 .956521-0.943014$

H -5.142321 4.868491-1.042915

H $-1.6640067 .265563-1.781787$ 
H $-2.4724146 .473296-3.114860$ H -1.395608 $5.542860-2.054079$ H $-4.9018945 .670288-3.154400$ H -5.455999 $2.524775-0.939812$ H -4.697814 2.213139-2.505920 H -2.181946 2.327864 -2.115985 H $-0.970232-0.2294922 .006581$ H $-0.049228-2.9153093 .195309$ H -3.288126 -0.939448 2.832716 H $-4.141560-2.1907641 .938637$ H $-2.865977-1.2951961 .143308$ H $-3.0134400 .722105-1.258180$ H -4.0145540 .9276440 .186271$ H -4.247957 -3.135349 -2.501962 H $-5.864047-2.427691-2.347884$ H $-6.041935-3.1758350 .033880$ H -3.808665 -4.992194 -1.017452 H $-5.249011-6.5390540 .344623$ H $-5.862660 \quad-5.219778 \quad 1.304890$ H -2.298395 -7.0296612 .482474$ H $-3.207692-7.6229830 .981854$ H $-3.804380-5.2248403 .835475$ H $-4.538016-4.0207292 .833375$ H -1.543271 -4.638064 3.189862 H -3.337802 -1.474281-1.072415 C 5.9031951 .4972860 .520290 O $5.1676250 .504536-0.221044$ C $5.9421830 .103605-1.364605$ C $6.9948771 .186752-1.549532$ C $7.2997871 .542853-0.108049$ H $6.443278-0.833354-1.092203$ C $5.029695-0.132661-2.571429$ H $7.8701050 .828219-2.099518$ H $6.5945142 .060128-2.076922$ C 8.0043982 .8792410 .051826 H 7.9317110 .7534540 .321163 C $3.839778-1.075366-2.293063$ O $4.487176 \quad 1.118876-3.011192$ H $5.622398-0.510560-3.412328$ H $3.122909-0.575294-1.635139$ O $3.172771-1.300817-3.540593$ C $4.229638-2.438872-1.703015$ C $3.079417-3.453922-1.555858$ H $4.698952-2.302735-0.723210$ O $5.220868-3.036408-2.547773$ H $2.544947-3.544841-2.507455$ H $3.541651-4.437002-1.396871$ H $8.9698012 .868991-0.464815$ H $7.4093043 .698926-0.364640$ H 8.1886053 .0994241 .108006 C 5.9358531 .1074862 .001327 H $4.040527 \quad 1.527147-2.250405$ H $2.901197-0.432245-3.884351$ H $4.825016-3.146758-3.429623$ H 6.6350581 .7654452 .531170 H 6.3238490 .0843892 .090198 H $5.3900812 .456626 \quad 0.378837$ C $0.320511-2.073533-1.608262$ O $1.313323-1.988261-0.580245$ C $2.108616-3.161480-0.389098$ C $1.193970-4.345684-0.042140$ C $0.140201-4.563386-1.122788$ C $-0.620619-3.267723-1.391690$ H $0.812016-2.153007-2.583971$ C $-0.490921-0.771470-1.583894$ H $2.730441-2.9692490 .494579$ O $1.961159-5.5303840 .141409$ H $0.692390-4.1423830 .911484$ H $0.591354-4.953379-2.042105$ O $-0.782772-5.564849-0.688434$ H -1.297224 -3.064902 -0.552227 O $-1.430393-3.433775-2.554272$ C $0.3436260 .437511-1.930569$ H -1.318450 -0.801402 -2.301278 H $-0.930708-0.622475-0.590787$ H $1.338854-6.2549410 .324998$ H -1.198355 -5.2528960 .133477$ H -1.975944 -4.227194-2.418548
O $0.408697 \quad 1.281522-0.860906$

O $0.8677370 .614973-3.022353$

C $1.1531162 .485031-1.069353$

H $1.0235353 .123790-0.192319$

H $2.2152012 .251180-1.176592$

H $\quad 0.7828083 .021801-1.948002$

SCF Energy (B3LYP/6-31G**//MMFF) $=-3245.92122032$

0600142

MM̄FF Geometry

C $3.305652-3.539311-1.740509$

C $2.007916-3.581898-1.404926$

C $0.902361-3.130089-2.320372$

O $0.267313-1.976390-1.724903$

C $-0.162198-4.232483-2.460569$

C $-1.221804-3.881708-3.487493$

C $-2.399481-3.290146-3.204713$

C $-0.879701-4.248959-4.908140$

C $-2.895411-2.830822-1.861400$

C $-4.272130-3.421534-1.538035$

C $-4.747010-3.050898-0.123351$

C $-6.147400-3.6170450 .129393$

C $-6.714569-3.1785701 .488396$

O $-4.757380-1.624648-0.008563$

O $-8.112965-3.504611 \quad 1.512261$

C $-6.570748-1.6455191 .616466$

C $-6.096700-3.9822762 .637412$

O $-6.973307-1.2028732 .910211$

C $-5.153226-1.1322061 .278857$

C $-5.082146 \quad 0.411786 \quad 1.240620$

O $-4.248249-1.5675242 .300411$

C $0.732676-0.748382-2.078117$

O $1.689728-0.498898-2.792748$

C $-0.1534350 .254858-1.447146$

C $0.0841391 .562483-1.626657$

C $-0.6915692 .665927-1.055407$

C $-1.9365762 .311018-0.288322$

C $-5.899476 \quad 1.0669370 .125156$

C $-5.6437002 .562676 \quad 0.037157$

C $-5.8561054 .468972-1.563755$

O -5.1603803 .2167430 .957256$

C $-4.5314404 .609487-2.248097$

C $-3.6896695 .632295-2.033040$

C $-2.3709055 .779672-2.739689$

C $-1.2404226 .167357-1.809899$

C $-0.6022787 .340970-1.958253$

C $-0.8460855 .207906-0.708069$

C $-0.2529103 .929388-1.237628$

N $-6.0493103 .081266-1.184081$

H $3.594123-3.155373-2.716119$

H $1.723075-3.948369-0.421452$

H $1.293421-2.874164-3.313498$

H $-0.629447-4.433105-1.489105$

H $0.311712-5.178612-2.755071$

H -3.077732 -3.079422-4.030766

H -1.674153 -3.978082 -5.611500

H $0.032632-3.735588-5.227900$

H $-0.719113-5.328863-4.992069$

H $-2.199681-3.086781-1.059719$

H -2.959968 -1.736265 -1.884394

H $-4.992916-3.044855-2.275873$

H -4.244034 -4.513997 -1.637052

H -4.030942 -3.4615290 .598412$

H $-6.146107-4.7112650 .048590$

H $-6.825875-3.262441-0.659384$

H $-8.549115-3.0313080 .783478$

H -7.285016 -1.203325 0.911614

H $-6.304562-5.0515602 .508510$

H $-6.543827-3.7073073 .598968$

H -5.013748 -3.864735 2.712551

H $-6.256901-1.4153573 .532445$

H $-5.3676490 .826297 \quad 2.216127$

H $-4.0323520 .703736 \quad 1.102924$

H $-3.464631-0.9933312 .284565$

H $-0.977570-0.112097-0.845783$

H $\quad 0.937665 \quad 1.857711-2.235500$

H -1.684632 1.7342030 .607810

H -2.615428 1.717501 -0.911134 
H $-2.507340 \quad 3.1785660 .044512$ H $-5.6460680 .612539-0.839367$ H -6.9716710 .9300920 .299736$ H $-5.9301585 .103058-0.674352$ H $-6.6625044 .727147-2.256662$ H $-4.2577513 .842348-2.969139$ H $-3.9757626 .419314-1.339047$ H -2.498471 $6.545184-3.516144$ H -2.101044 $4.856569-3.267178$ H $0.2091267 .631535-1.299312$ H $-0.8754738 .039285-2.742438$ H -1.705686 $5.043798-0.054073$ H $-0.0862415 .667572-0.061503$ H $0.6672704 .080452-1.803959$ H -6.384846 2.445011-1.901470 C $5.091212-2.817613-0.136304$ O $5.727862-1.952890-1.099495$ C $7.041218-1.621694-0.624033$ C $7.078803-2.0174810 .845850$ C $6.187880-3.2450440 .840332$ H $7.732967-2.281469-1.166233$ C $7.419807-0.171725-0.952246$ H $8.092935-2.2252731 .199676$ H $6.645048-1.2442021 .488439$ C $5.672544-3.6254852 .217999$ H $6.754302-4.0884190 .423286$ C $6.7431130 .950597-0.136470$ O $8.837706-0.043245-0.771782$ H $7.2334890 .010500-2.017633$ H 7.0937290 .9246890 .901928

O $7.1948102 .204403-0.671024$

C $5.2056850 .954296-0.159210$ C 4.6108692 .1308670 .637669 H 4.8385930 .0241830 .276653 O $4.740021 \quad 1.005998-1.505407$ H 5.0068882 .0911321 .658311 H 4.9444593 .0795950 .199944 H $6.505594-3.8625502 .887800$ H $5.099063-2.8097792 .671238$ H $5.022460-4.5041842 .163315$

C $4.418012-3.995491-0.839865$ H $9.031618-0.1741980 .172046$ H $8.1670622 .196496-0.646236$ H $5.0901521 .820027-1.906302$ H $4.033887-4.705649-0.098246$ H $5.145953-4.536783-1.456546$ H $4.336045-2.2139800 .379784$ C 2.7050190 .4923552 .387782 O 2.5182450 .8615381 .019152 C 3.0651542 .1332340 .659376

C 2.4517423 .2352161 .535231

C 2.6155612 .9355973 .023970

C 2.1062161 .5300363 .345558

H 3.7817580 .4136312 .587333

C $2.148381-0.9262822 .583883$

H $2.7443922 .323095-0.371720$

O $3.034127 \quad 4.5006891 .243851$

H $1.378973 \quad 3.3170411 .321036$

H 3.6545383 .0615443 .349699

O 1.8440603 .8726573 .779637

H 1.0124581 .5316623 .295591

O $2.465407 \quad 1.1919334 .683627$

C $0.696308-1.1099722 .188915$

H $2.729320-1.6030171 .946206$

H $2.265407-1.2460263 .624706$

H 2.8880074 .6775910 .298703

H 2.1463194 .7628853 .530078

H 2.1020541 .8824215 .264442

O $0.428603-2.4487532 .120454$

O $-0.106210-0.2113921 .983266$

C $-0.910936-2.8049651 .762766$

H -1.306668 -2.149319 0.981650

H $-0.899888-3.8296781 .381787$

$\mathrm{H}-1.544896-2.7649172 .652472$

SCF Energy (B3LYP/6-31G**/MMFF) $=-3245.91590984$

0600143

MM̄MF Geometry
C $-1.611934-3.163531-2.424464$

C $-0.777549-2.405298-1.698402$

C $0.252930-1.479651-2.281753$

O $1.521192-1.900962-1.721486$

C $0.006277-0.013330-1.876334$

C $-0.8423790 .753345-2.866717$

C $-0.3469421 .556189-3.829572$

C $-2.3307220 .616204-2.692216$

C $1.097238 \quad 1.844416-4.135982$

C $1.4358593 .339980-4.073307$

C $1.2027143 .982699-2.696521$

C $1.5543505 .472024-2.743664$

C $1.3890936 .142858-1.372600$

O $2.0214513 .316823-1.726514$

O $2.0355547 .423871-1.429090$

C $2.1373855 .302070-0.318169$

C $-0.0838946 .416279-1.045696$

O 1.9012505 .8084530 .994572

C $1.8247993 .786603-0.385140$

C 2.7851023 .0244940 .560339

$\begin{array}{llllllllllllllll}\text { O } & 0.478948 & 3.578724 & 0.053466\end{array}$

C $2.642325-1.662745-2.452059$

O $2.710825-1.163006-3.562901$

C $3.796589-2.094442-1.639179$

C $5.018277-1.626927-1.930802$

C $6.217281-1.940712-1.164407$

C $7.026149-0.728289-0.794846$

C 2.7517131 .4973350 .416302

C $3.4330440 .801441 \quad 1.583575$

C $3.680860-1.4565872 .611167$

$\begin{array}{lllll}\text { O } & 4.058320 & 1.401903 & 2.453604\end{array}$

C $5.128517-1.8013342 .452263$

C $5.592301-3.0602202 .423862$

C $7.052084-3.4119482 .360394$

C $7.387840-4.3352981 .211249$

C $7.445679-5.6661961 .387018$

C $7.745976-3.706767-0.116126$

C $6.536914-3.214180-0.861304$

N $3.253677-0.5746701 .541134$

H - $1.571171-3.118434-3.509663$

H $-0.823069-2.459458-0.614208$

H $0.288535-1.588634-3.373550$

H $0.9657740 .494727-1.742690$

H $-0.4534420 .044173-0.879874$

H - $1.0525712 .065870-4.484634$

H $-2.891613 \quad 1.273397-3.365240$

H $-2.653720-0.408594-2.891946$

H -2.612920 $0.881486-1.668430$

H $1.7808891 .297509-3.483647$

H $1.3009731 .486270-5.152767$

H $2.4932043 .448219-4.348027$

H $0.8508863 .876206-4.831302$

H $0.1484193 .858047-2.425448$

H $0.9512735 .991401-3.498908$

H $2.5996815 .589543-3.062934$

H $1.6191067 .938809-2.141381$

H $3.2111905 .441055-0.504628$

H - $0.5350837 .052582-1.816745$

H $-0.1837196 .971587-0.106543$

H $-0.6843445 .506945-0.976299$

H 1.0136025 .5198251 .267578

H 3.8182883 .3442860 .370962

H 2.5490063 .2940411 .597801

H 0.3934992 .6834210 .416026

H $3.616018-2.755100-0.797533$

H $5.160684-0.928460-2.752490$

H $7.486129-0.289503-1.686916$

H $7.822601-0.941360-0.078490$

H $6.3864700 .031171-0.329955$

H $1.721157 \quad 1.1344460 .376675$

H $3.2563081 .196070-0.508696$

H $3.037747-2.3420582 .580622$

H $3.528122-0.9487133 .569393$

H $5.834614-0.9755892 .407411$

H $4.889276-3.8865962 .500987$

H $7.318273-3.8936293 .310747$

H $7.678981-2.5133932 .303579$

H $7.715623-6.3328980 .574832$ 
H $7.218726-6.1185742 .346743$ H $8.246222-4.442483-0.759958$ H $8.493766-2.9247800 .045559$ H $5.874736-4.010677-1.202655$ H $2.719719-0.9809390 .778851$ C -4.050509 -3.641476 -2.071593 O $-4.310017-2.439445-1.317740$ C $-5.599454-2.552333-0.686930$ C $-6.281093-3.751982-1.333361$ C $-5.096020-4.655582-1.607115$ H $-5.414915-2.7807410 .369416$ C $-6.373306-1.235476-0.809335$ H -7.028396 -4.208144 -0.677063 H $-6.776790-3.482146-2.272993$ C $-5.388303-5.750599-2.618334$ H $-4.779655-5.115442-0.660994$ C $-5.6262180 .000702-0.271443$ O $-6.663563-0.988152-2.192117$ H -7.345053 -1.331133 -0.311345 H $-4.7736990 .234992-0.917635$ O $-6.516947 \quad 1.120468-0.362116$ C $-5.145166-0.1202821 .185534$ C $-4.489776 \quad 1.1765561 .695403$ H -4.421951 -0.935318 1.260216 O $-6.247741-0.4572732 .025915$ H -3.6726981 .4444441 .016786$ H -5.2245711 .9894221 .654947$ H -6.189764 -6.403308 -2.257175 H $-5.702480-5.334028-3.581259$ H -4.502166 -6.368861 -2.792191 C $-2.615699-4.104995-1.819724$ H -5.816403 -0.964916 -2.669736 H $-6.8046201 .187147-1.288908$ H $-6.910290 \quad 0.2494741 .938273$ H -2.454051 -5.096361 -2.257970 H $-2.442763-4.195647-0.740545$ H -4.197038 -3.392163 -3.130724 C $-1.763807 \quad 0.1797892 .716318$ O $-3.046183-0.0076153 .327541$ C -3.9734601 .0688023 .148356$ C -3.3804682 .3751363 .696846$ C -2.0138042 .6721183 .088850$ C $-1.098691 \quad 1.4603213 .239817$ H -1.877586 0.2273081 .626169 C $-0.896273-1.0429593 .060760$ H $-4.841444 \quad 0.827416 \quad 3.774603$ O -4.2490293 .4765303 .454182$ H -3.2640782 .2919014 .785381$ H -2.093731 2.9776132 .039013 O -1.4178243 .7706883 .782489$ H $-0.830296 \quad 1.338484 \quad 4.297411$ O $\quad 0.1079591 .6933132 .520253$ C - $-1.352512-2.3118132 .373568$ H $0.140169-0.8662332 .751645$ H -0.927123 -1.2280294 .141089$ H $-5.105616 \quad 3.2688043 .864877$ H -2.031280 4.5224153 .713748 H $0.5001762 .512122 \quad 2.869341$ O $-0.472148-3.3129352 .674995$ O $-2.345622-2.4207401 .669204$ C $-0.765247-4.5907592 .101144$ H -1.791991 -4.892751 2.329373 H $-0.602168-4.5584521 .020457$ H -0.081148 -5.323459 2.537566 SCF Energy (B3LYP/6-31G**//MMFF) $=-3245.92738233$

$06 \_00144$

MM̄FF Geometry

C $0.556307-2.2222631 .003911$

C $-0.234061-1.7143850 .043789$

C $0.128507-1.511508-1.410529$

O $1.541774-1.739745-1.591729$

C $-0.178712-0.062717-1.829027$

C $-0.2193950 .136891-3.329563$

C $0.7595120 .710080-4.056668$

C $-1.492736-0.312703-3.999429$

C $2.1021911 .195129-3.585018$

C $2.2341642 .723753-3.640144$
C $1.4298403 .446322-2.548032$

C $1.5604414 .963993-2.694449$

C $0.8627575 .712809-1.548168$

O $1.9517783 .035976-1.280712$

O $1.3010777 .079648-1.582034$

C $1.3344085 .120661-0.202951$

C $-0.6599195 .740928-1.727856$

O 0.6048585 .6832960 .885608

C $1.2603953 .577114-0.148044$

C 1.9631163 .0573521 .129099

O $-0.1098473 .178302-0.124140$

C $1.965037-2.476329-2.646827$

O $1.267841-2.993123-3.508411$

C $3.441180-2.623354-2.634258$

C $4.243128-1.944872-1.797805$

C $5.700439-2.057292-1.703191$

C $6.385574-3.067299-2.585013$

C 2.1451941 .5330911 .168398

C 2.9643911 .0895422 .368460

C $4.234511-0.8823453 .221966$

O $3.202277 \quad 1.8199613 .326651$

C $5.661259-0.6901262 .813000$

C $6.487330-1.6951832 .484526$

C $7.915772-1.4938642 .064373$

C $8.207359-2.0550010 .688764$

C $8.862117-3.2196430 .545825$

C $7.822081-1.227962-0.519067$

C $6.347851-1.276823-0.812301$

N $3.379490-0.2296442 .247876$

H $1.560939-2.5454110 .740095$

H $-1.247309-1.4178820 .297941$

H -0.463454 -2.224342 -1.995719

H $\quad 0.5565970 .609464-1.375549$

H -1.150386 $0.254174-1.428010$

H $0.5999890 .823788-5.128309$

H $-1.506348-0.076622-5.068693$

H -1.616174 -1.395129 -3.900768

H $-2.3580030 .180072-3.543896$

H $2.3503770 .824973-2.586235$

H $2.8561770 .759473-4.253341$

H $3.2979632 .964876-3.515337$

H $1.9338703 .087939-4.630508$

H $0.3781713 .148727-2.622517$

H $1.1701155 .296504-3.664464$

H $2.6241795 .240499-2.689516$

H $1.0759137 .444924-2.454861$

H $2.3821225 .421578-0.062368$

H $-0.9250286 .241411-2.666996$

H -1.140885 $6.322052-0.933308$

H -1.108602 $4.745676-1.747945$

H $\quad 0.688121 \quad 6.650156 \quad 0.820799$

H 2.9603163 .5121521 .198317

H 1.4000483 .3687622 .018067

H -0.1827442 .3079150 .297547$

H $3.834431-3.326270-3.361379$

H $3.797595-1.236179-1.101572$

H $5.978765-4.069767-2.411459$

H $7.461594-3.137090-2.411199$

H $6.248650-2.809888-3.641001$

H $\quad \begin{array}{llll}1.175137 & 1.029710 & 1.227787\end{array}$

H 2.6561921 .1907840 .261368

H $3.949377-1.9386703 .250357$

H $4.067613-0.4469504 .212282$

H 6.0318520 .3321412 .786099

H $6.122672-2.7191112 .522845$

H $8.553762-1.9804442 .813768$

H $8.193942-0.4327372 .086248$

H $9.103636-3.616179-0.434593$

H $9.167832-3.8054151 .406426$

H $8.406560-1.526174-1.396696$

H $8.108274-0.182425-0.346181$

H $5.761992-0.575223-0.218110$

H $3.190120-0.7282381 .383475$

C $-1.075370-1.7598512 .959584$

O $-0.953274-0.3227412 .887250$

C -1.4918070 .2450144 .088913$

C $-2.256607-0.8714864 .785983$

C $-1.405833-2.0738654 .419257$ 
H $-0.627343 \quad 0.497050 \quad 4.719501$

C -2.268252 $1.540673 \quad 3.829561$ H -2.338007 -0.7164095 .865973$ H $-3.266894-0.9842294 .377657$ C $-2.115082-3.4017904 .620847$ H -0.490095 -2.057978 5.025239 C -3.561834 1.4464692 .989120 O -2.6109752 .0814415 .112485$ H -1.5907962 .2633383 .356999$ H -4.320992 $0.877218 \quad 3.536418$ O $\begin{array}{llll}-4.095174 & 2.775320 & 2.877890\end{array}$ C -3.397575 $0.857875 \quad 1.577077$ $\begin{array}{llll}C & -4.679031 & 0.945248 & 0.726561\end{array}$ H -3.119641 -0.1934721 .666082$ $\begin{array}{lllll}\text { O } & -2.333626 & 1.519775 & 0.899084\end{array}$ H -5.4843240 .4256151 .257224$ H $-4.983628 \quad 1.9937530 .624638$ H -2.386562 -3.536501 5.673071 H -3.033382 -3.4608724 .026779$ H $-1.470845-4.2370184 .328814$ C $0.217685-2.4098452 .463034$ H $-3.078747 \quad 2.920088 \quad 4.958170$ H -3.429253 3.3287082 .435756 H $-2.610202 \quad 2.435432 \quad 0.725717$ H $0.189588-3.4879682 .658730$ H $1.056749-1.9985103 .038415$ H -1.920171 -2.052747 2.326273 C $-4.770511-1.980016-0.193740$ O $-3.913255-0.943103-0.675961$ C $-4.4970590 .361726-0.694674$ C $-5.7721790 .359392-1.547807$ C $-6.758289-0.720662-1.105598$ C $-6.060621-2.079410-1.018092$ H $-5.034732-1.7640850 .849681$ C -3.968497-3.290200 -0.153276 H $-3.7736631 .009285-1.206407$ O $-6.3943371 .639144-1.499232$ H $-5.4960960 .176327-2.594645$ H $-7.235318-0.452256-0.155601$ O $-7.804301-0.787517-2.077637$ H $-5.853654-2.441435-2.031227$ O $-6.931582-3.022852-0.398214$ C $-3.341751-3.696163-1.472494$ H $-3.140650-3.1632070 .554125$ H $-4.602102-4.1118630 .197574$ H -7.203454 $1.589551-2.036471$ H -8.476797 -1.404624 -1.743696 H $-7.694314-3.151230-0.986729$ O $-2.615525-4.837963-1.277945$ O $-3.456635-3.097060-2.532036$ C $-1.939785-5.340999-2.434705$ H $-1.231715-4.598584-2.814562$ $\mathrm{H}-1.384709-6.236088-2.141803$ H -2.665076 -5.614083 -3.206996 SCF Energy (B3LYP/6-31G*//MMFF)= -3245.91598355

06_00145

MM̄FF Geometry

C $-0.801724 \quad 4.2737512 .350260$

C $-1.553599 \quad 3.2027952 .051530$

C -1.9238152 .1373583 .053207$

O

C -3.3993712 .3128193 .457999$

C -3.8935601 .2666944 .438702$

C $-4.734147 \quad 0.257841 \quad 4.130659$

C $-3.407678 \quad 1.4364915 .855297$

C $-5.315348-0.0777842 .784487$

C $-4.941978-1.5043982 .359071$

C $-5.265012-1.765460 \quad 0.879563$

C $-4.967548-3.220807 \quad 0.515918$

C $-5.124942-3.489102-0.986623$

O $-4.447505-0.8851680 .102601$

O $-4.525266-4.767883-1.246968$

C $-4.331872-2.423935-1.775840$

C $-6.600615-3.618646-1.381984$

O $-4.580960-2.580156-3.170688$

C $-4.629089-0.980239-1.316877$

C $-3.6965340 .077336-1.960115$
O $-5.970350-0.640522-1.687137$

C $-0.5539490 .248817 \quad 2.480703$

$\begin{array}{lllll}\text { O } & 0.470967 & 0.716640 & 2.949035\end{array}$

C $-0.688058-1.0976681 .879484$

C $0.378481-1.9027511 .761332$

C $0.370452-3.2640201 .214818$

C $-0.925860-3.7859770 .653952$

C $-2.209074-0.073190-1.627081$

C $-1.410216-0.866313-2.645762$

C $0.875797-1.809018-2.989222$

O $-1.855628-1.205945-3.737789$

C $0.930839-3.241339-2.566541$

C $2.047986-3.838904-2.125192$

C $2.121762-5.290809-1.752459$

C $2.682710-5.515084-0.366937$

C $3.961248-5.890691-0.194340$

C $1.735398-5.4045840 .806321$

C $1.511793-3.9830791 .244903$

$\mathrm{N}-0.120116-1.114661-2.196430$

H $-0.4263604 .366083 \quad 3.368131$

H $-1.962563 \quad 3.0802921 .052854$

H -1.2870172 .1920063 .945031$

H -4.0246072 .3252022 .559259$

H $-3.540422 \quad 3.3030533 .912863$

H $-5.040405-0.4156884 .930392$

H -3.8476780 .7008606 .537154$

H -2.320711 1.3213595 .905503

H -3.671294 2.4305896 .230906

H -6.4071390 .0072252 .843800$

H -4.9931270 .6297872 .018400$

H $-3.868113-1.6569912 .524533$

H -5.474966 -2.224705 2.992088

H $-6.320972-1.5302670 .701442$

H $-5.599237-3.9038201 .097750$

H -3.934724 -3.4598180 .800685$

H $-4.558906-4.918281-2.207483$

H -3.269971 - $2.647630-1.617477$

H -7.063852 -4.459392 -0.851599

H $-6.708163-3.843171-2.448821$

H -7.184984 - $2.723891-1.157507$

H $-3.950948-2.017576-3.651094$

H $-3.8690170 .135299-3.041209$

H $-4.0040051 .058015-1.568311$

H $-6.0484870 .327457-1.714021$

H -1.678256 -1.401453 1.561373

H $1.345147-1.5415262 .109800$

H - $-1.340856-3.087610-0.080274$

H -1.655894 -3.9282601 .457588$

H $-0.824197-4.7403050 .132796$

H $-1.7582420 .926153-1.598534$

H $-2.073160-0.516451-0.637727$

H $\quad 1.829979-1.296689-2.833237$

H $0.616391-1.744382-4.050570$

H $0.014782-3.820666-2.655292$

H $2.969327-3.264130-2.061365$

H $2.754679-5.791624-2.496908$

H $\quad 1.140059-5.774839-1.828747$

H $4.362152-6.0932330 .792774$

H $4.636267-6.002541-1.036270$

H $2.148727-5.9441381 .669561$

H $0.806352-5.9324680 .573573$

H $2.398924-3.5217031 .675482$

H $0.140468-0.831053-1.256477$

C $-0.8369205 .303838-0.029858$

O $-0.0205814 .299529-0.663900$

C $0.4551504 .810071-1.923629$

C $-0.4090346 .024675-2.228400$

C $-0.6114186 .586687-0.833196$

H $1.4836605 .152170-1.760624$

C $0.4323023 .699899-2.978810$

H $0.0824256 .726262-2.908900$

H $-1.3716705 .738750-2.667983$

C $-1.7539007 .583303-0.740746$

H $0.3180257 .074799-0.510136$

C $1.2299292 .442487-2.568326$

O $-0.924595 \quad 3.299939-3.207612$

H $0.7995964 .084361-3.936851$

H $0.7582251 .975196-1.697350$ 
O $\quad 1.1460391 .494089-3.638098$

C $2.7181662 .713300-2.293162$

C $3.5351171 .479003-1.884298$

H $2.8374163 .479601-1.526370$

O $3.3180763 .260504-3.474273$

H $3.5190720 .740558-2.697810$

H $4.5865901 .788070-1.846690$

H - $1.5621278 .448297-1.384029$

H -2.703307 $7.135994-1.053802$

H -1.8743637 .9453900 .285076$

C $-0.430628 \quad 5.4209641 .442252$

H $-1.3081943 .060315-2.346576$

H $0.2022271 .342006-3.817096$

H $3.1950192 .614449-4.191078$

H -0.8744316 .3268091 .871877$

H 0.6596525 .5339821 .501094

H -1.882673 $4.987581-0.127694$

C $5.312526-0.207832-0.128833$

O $3.922099-0.429138-0.396250$

C $3.1400290 .767367-0.568782$

C $3.2270941 .584307 \quad 0.726784$

C 4.6707191 .9764991 .027624

C 5.5485360 .7237401 .074050

H $5.7669010 .238375-1.021567$

C $5.999153-1.5757110 .024494$

H $2.1042790 .429517-0.680771$

$\begin{array}{lllll}\text { O } & 2.392440 & 2.730205 & 0.674862\end{array}$

H 2.8563210 .9668761 .555186

H 5.0425252 .7108190 .304221

$\begin{array}{lllll}\text { O } & 4.709556 & 2.617877 & 2.304573\end{array}$

H 5.3411650 .2063162 .016195

O $6.921591 \quad 1.1077241 .126483$

C $5.562694-2.3651901 .241892$

H $5.775141-2.197049-0.850761$

H $7.083467-1.4257720 .083114$

H 2.4979373 .2075711 .515546

H 5.6416142 .8151602 .500766

H 7.1274691 .5914460 .308324

O $6.467747-3.3616771 .473948$

O $4.554410-2.1504711 .899538$

C $6.205491-4.1791532 .619438$

H $5.180325-4.5609722 .602011$

H $6.894528-5.0275542 .596389$

H $6.384579-3.6033913 .532150$

SCF Energy (B3LYP/6-31G**/MMFF) $=-3245.93148499$

06_00146

MM̄FF Geometry

C -4.430009 -1.928200 2.486579

C $-3.136336-1.8999352 .134977$

C $-2.077716-1.1810092 .928072$

O $-1.487090-0.1711542 .077357$

C $-0.963502-2.1582903 .335660$

C $-0.003703-1.5600714 .343851$

C $1.203944-1.0427974 .045584$

C $-0.472718-1.6037915 .775867$

C $1.843079-0.8955672 .692572$

C $3.121146-1.7390262 .591973$

C $3.825880-1.5571731 .239355$

C $5.054710-2.4637981 .141783$

C $5.853071-2.216864-0.148422$

O $4.224336-0.1880931 .134986$

O $7.134450-2.848326-0.003053$

C $6.111946-0.701172-0.298009$

C $5.183711-2.870371-1.363538$

O $6.719082-0.407376-1.553957$

C $4.843424 \quad 0.158437-0.110405$

C $5.2059631 .663320-0.055793$

O $3.966640-0.061332-1.217844$

C -2.099856 1.0419482 .017438

O $-3.112991 \quad 1.3871982 .603310$

C -1.3137441 .8847901 .088973$

C -1.7415993 .1167910 .772198$

C $-1.0849294 .044843-0.154627$

C $0.1922923 .578406-0.803231$

C $4.051523 \quad 2.5317190 .459961$

C $4.318906 \quad 4.022366 \quad 0.356031$

$\begin{array}{llll}\text { C } 3.248528 & 6.196925 & 0.954024\end{array}$
O $5.3284744 .500459-0.150672$

C $1.8372426 .643781 \quad 1.165008$

C 1.1794527 .4827730 .350908

C $-0.2509457 .886467 \quad 0.576530$

C $-1.1261267 .697532-0.645718$

C $-1.839704 \quad 8.725829-1.135907$

C $-1.1818246 .336838-1.312483$

C $-1.6578475 .244931-0.389433$

$\begin{array}{llll}\mathrm{N} & 3.260811 & 4.748261 & 0.886544\end{array}$

H $-4.746808-1.4212263 .394785$

H -2.824062 -2.3897191 .216783$

H -2.506347 -0.7087163 .820855$

H $-0.417773-2.4885642 .446139$

H $-1.396013-3.0691173 .771080$

H $1.807634-0.6527774 .864662$

H $0.266593-1.1889786 .469274$

H $-1.395692-1.0285245 .897017$

H -0.664730 -2.637882 6.080159

H $1.161455-1.1635691 .880460$

H 2.0815930 .1657942 .552590

H $3.804621-1.4429543 .399107$

H $2.876518-2.7974612 .743107$

H $3.112970-1.7876400 .439305$

H $4.765669-3.5194831 .219567$

H $5.716837-2.2716721 .997888$

H $6.984719-3.7938950 .168500$

H $6.840609-0.4150340 .473481$

H $5.110099-3.955528-1.222951$

H $5.781394-2.725928-2.270198$

H $4.175719-2.495981-1.550461$

H $7.514911-0.961455-1.627488$

H 6.0586691 .8162970 .618162

H $5.5103752 .008440-1.051712$

H $3.4334760 .733686-1.372765$

H $-0.409128 \quad 1.4551380 .672789$

H $-2.6649793 .479374 \quad 1.221931$

H $0.0039632 .706718-1.439651$

H $0.9251763 .299872-0.040396$

H $0.6720614 .330145-1.431887$

H $3.1364912 .325037-0.104279$

H 3.8582662 .2988821 .514195

H $3.6912586 .603762 \quad 0.038994$

H $3.8727206 .496118 \quad 1.801844$

H 1.3237316 .2463192 .038102

H $1.6895617 .901671-0.513114$

H $-0.250316 \quad 8.9397180 .885379$

H -0.6893427 .3259221 .411662$

H -2.470259 $8.611741-2.011469$

H $-1.8112469 .706907-0.673414$

H $-0.2005456 .130032-1.745933$

H $-1.8772696 .362985-2.161764$

H -2.598982 5.4742550 .112302

H 2.5670894 .2442451 .432799

C $-6.170850-1.7061430 .639706$

O $-5.252701-1.464517-0.446439$

C $-5.268485-0.060434-0.755583$

C $-6.5623430 .477561-0.154327$

C $-6.650735-0.3347801 .123551$

H $-4.4199420 .385422-0.222106$

C $-5.1029980 .175967-2.260364$

H -6.5216391 .5563380 .023542$

H $-7.4284130 .268316-0.792399$

C -8.035673 -0.3569861 .745881$

H -5.9497410 .1006921 .845399$

C $-3.917640-0.568827-2.909254$

O $-6.297203-0.259562-2.923019$

H $-5.0241301 .254405-2.445187$

H $-4.162236-1.631190-3.034252$

O $-3.740956-0.038422-4.229679$

C $-2.577923-0.450562-2.160305$

C $-1.396804-1.018585-2.969712$

H -2.655287 - $0.973812-1.201986$

O $-2.3084870 .920809-1.872574$

H $-1.708487-1.951153-3.449758$

H $-1.140456-0.311574-3.767805$

H -8.350233 0.6551252 .020635

H -8.780824 -0.7638161 .054156$

H -8.043591 -0.9721572 .651348$ 
C $-5.505285-2.6072251 .685696$ H $-6.2680850 .076760-3.834301$ H $-4.514387-0.298698-4.757552$ H -2.295684 $1.404152-2.716358$ H -5.079621 -3.486221 1.184795 H -6.263241 -2.980066 2.384685 H -7.007711 $-2.261810 \quad 0.197163$ C $-0.498821-3.598906-1.589743$ O $-0.372017-2.260697-1.092286$ C $-0.145376-1.258262-2.092199$ C $1.120528-1.598303-2.887730$ C $1.046587-2.992618-3.500955$ C $0.719026-4.026802-2.424306$ H -1.394581 -3.653427 -2.220134 C $-0.791018-4.529075-0.401174$ H $0.053295-0.323579-1.552380$ O $1.340436-0.624274-3.901693$ H $1.982121-1.558056-2.211525$ H $\quad 0.325117-3.025216-4.325753$ O $2.321558-3.300370-4.070223$ H $1.609647-4.163133-1.801145$ O $0.461302-5.271654-3.070811$ C $0.308622-4.6002820 .637929$ H $-1.686307-4.1668280 .116948$ H $-0.988250-5.543851-0.763432$ H $2.141005-0.886845-4.387591$ H $2.270770-4.206899-4.419181$ H $0.440416-5.961823-2.386837$ O $-0.026429-5.5424091 .569598$ O $1.318418-3.9115750 .653963$ C $0.911143-5.7091922 .637996$ H $1.884258-6.0176662 .244364$ H $1.002292-4.7810963 .210170$ H $\quad 0.535680-6.4937863 .300209$ SCF Energy (B3LYP/6-31G**//MMFF)= -3245.91287701

0600147

MM̄MF Geometry

C $0.4658552 .567091-1.811510$

C $1.6663783 .146056-1.656683$

C $2.2768323 .635525-0.363704$

$\begin{array}{lllll}\text { O } & 3.188509 & 2.619141 & 0.114572\end{array}$

C 1.2699753 .8910990 .773458

C 1.7994054 .8244961 .843747

C 2.3489904 .4348393 .010050

C 1.6192046 .2923321 .549731

C 2.6640763 .0405283 .473435

C 1.7016642 .5333474 .555948

C 0.4060491 .9437253 .977270

C -0.5406941 .5122065 .098768$

C $-1.790400 \quad 0.8075524 .552101$

$\begin{array}{lllll}\text { O } & 0.772849 & 0.809696 & 3.184229\end{array}$

$\begin{array}{lllll}\text { O } & -2.447554 & 0.192567 & 5.670404\end{array}$

C -1.347249 -0.3109513 .585327$

C -2.7904681 .8101693 .964196$

O $-2.478634-0.9086582 .957706$

C -0.3163250 .1511252 .529988$

C $0.262959-1.0748051 .783109$

O $\quad-0.9747071 .0066191 .594274$

C $4.4301892 .536830-0.424625$

O $4.9129103 .243298-1.293008$

C 5.0908591 .3838470 .224900

C $6.1499470 .805225-0.357448$

C $6.859610-0.3756990 .138802$

C $6.420113-0.9503891 .461121$

C $1.455112-0.756606 \quad 0.865145$

C $1.964835-1.9982550 .150714$

C $3.536807-2.703259-1.650516$

O $1.545297-3.1280250 .388050$

C $4.766667-3.270350-1.013128$

C $5.925084-3.441977-1.669369$

C $7.150562-4.061761-1.057828$

C $8.404328-3.246090-1.290293$

C $9.269320-3.572219-2.265601$

C $8.696394-2.106618-0.339367$

C $7.845973-0.897923-0.619472$

N $2.947180-1.698173-0.783715$

H $-0.1590722 .375853-0.945041$
H $2.2794313 .303284-2.542523$

H $2.8317654 .558245-0.575654$

H $\quad 0.9768982 .937810 \quad 1.224563$

H $\quad 0.347441 \quad 4.3373530 .379538$

H $2.6520475 .209518 \quad 3.713949$

H $2.008128 \quad 6.9294952 .351121$

H $2.144106 \quad 6.5657240 .629043$

H 0.5568056 .5276411 .427405

H 2.7201452 .3349422 .640577

H 3.6759013 .0696093 .898323

H $2.223811 \quad 1.744598 \quad 5.113873$

H 1.4713543 .3353195 .267978

H -0.0775682 .6936693 .342492$

H -0.8238852 .3698955 .721679$

H $-0.0171830 .816295 \quad 5.769831$

H $-3.197449-0.3216635 .324904$

H $-0.889974-1.0998444 .198083$

H -3.1001172 .5316374 .729932$

H -3.7092971 .3102653 .637993$

H -2.387076 2.3741153 .120440

H $-2.776772-0.3101872 .251655$

H $0.601162-1.8164182 .518794$

H $-0.525391-1.5462431 .185407$

H -0.5805010 .8869170 .716882$

H 4.6560251 .0152341 .147262

H $6.5148281 .215207-1.298263$

H $6.562942-0.2170972 .262646$

H $6.966631-1.8490261 .752908$

H $5.361743-1.2321551 .429192$

H $1.170688-0.0216460 .105196$

H $2.285215-0.344337 \quad 1.449543$

H $3.753103-2.219407-2.608248$

H $2.808569-3.503370-1.818737$

H $4.692985-3.5861910 .024885$

H $5.985868-3.158949-2.717944$

H $7.271505-5.059711-1.499500$

H $7.020423-4.2282250 .018732$

H $10.184127-3.010270-2.421646$

H $9.078865-4.405761-2.933552$

H $\quad 9.744900-1.793667-0.434020$

H $8.607367-2.4662990 .689123$

H $8.084378-0.411282-1.566375$

H $3.274853-0.740936-0.873019$

C -1.492290 $2.751330-3.345380$

O $-2.4452562 .026440-2.537850$

C $-3.6539901 .844736-3.300452$

C $-3.5061902 .715005-4.543018$

C $-2.0101092 .643838-4.778041$

H $-3.6738180 .796439-3.618977$

C $-4.8830912 .163685-2.442288$

H $-4.0931612 .337651-5.386096$

H -3.815399 $3.749543-4.352719$

C $-1.4928513 .726652-5.709015$

H $-1.7626581 .658185-5.194860$

C $-5.0283551 .268745-1.191780$

O $-4.783573 \quad 3.525992-2.010364$

H -5.786774 2.098416 -3.060403

H $-4.2175231 .468647-0.480443$

O $-6.2479541 .630672-0.532176$

C $-5.077530-0.240294-1.504079$

C $-5.419593-1.095919-0.267938$

H -4.108234 -0.548812 -1.906174

O $-6.058811-0.463530-2.517352$

H $-4.723769-0.8421130 .538966$

H $-6.428372-0.8417700 .078597$

H $-1.9585843 .639327-6.696067$

H -1.711765 4.727360 -5.321559

H $-0.409273 \quad 3.646212-5.839781$

C $-0.0981242 .156108-3.144041$

H $-5.6496223 .783366-1.652164$

H $-6.1223662 .515490-0.150187$

H -5.889981-1.338875 -2.904464

H $\quad 0.5706432 .476664-3.950609$

H $-0.1515801 .062058-3.167188$

H -1.518729 $3.797001-3.010186$

C $-3.024007-3.016949-0.197379$

O $-4.125550-3.022639-1.113771$

C $-5.378686-2.614393-0.556323$ 
C $-5.749915-3.5060920 .637722$

C $-4.625685-3.5900851 .664697$

C $-3.307493-3.952830 \quad 0.983714$

H -2.855119-1.998951 0.172500

C -1.780886 -3.472787 -0.976926

H -6.131904 -2.808703 -1.329689

O $-6.933286-3.040781 \quad 1.277620$

H -5.959654 -4.521420 0.276165

H -4.532761 -2.658069 2.233457

O $-4.933137-4.611936 \quad 2.615737$

H $-3.346010-4.9947670 .640772$

O $\quad-2.247187-3.853467 \quad 1.931605$

C $-1.215517-2.378331-1.859170$

H $-0.979424-3.770920-0.294248$

H -2.042128 -4.323661 -1.617471

H -7.637684 -3.0165690 .607550$

H $-5.791478-4.3866803 .014054$

H -2.473772 -4.428520 2.682554

O $-0.321173-2.930979-2.731655$

O $-1.493032-1.189858-1.778606$

C $0.315683-2.011235-3.623982$

H $1.003206-2.575153-4.259730$

H $-0.429314-1.521852-4.258613$

H $0.888817-1.270311-3.059228$

SCF Energy (B3LYP/6-31G $\left.{ }^{* *} / / M M F F\right)=-3245.92295180$

06_00148

MM̄FF Geometry

C $0.1312174 .341826-0.328086$

C 0.5648543 .4126680 .535995

C 1.1989443 .7566091 .860951

O 2.3801722 .9320532 .035590

C 0.2895033 .4437193 .061912

C -1.053162 4.1420213.059971

C -2.2170983 .5229572 .778693$

C -1.0302915 .5956643 .445997$

C -2.372088 2.0979442 .332693

C $-2.796161 \quad 1.1622953 .471130$

C $-2.493217-0.2979673 .107271$

C $-3.140407-1.2697634 .091544$

C $-2.763766-2.7265203 .772078$

O $-1.069440-0.454363 \quad 3.150724$

O $-3.143878-3.5350004 .896088$

C -1.228361 -2.829962 3.635890

C $-3.546499-3.2637132 .568272$

O $-0.841318-4.1306393 .196188$

C $-0.598296-1.7399322 .733988$

C $0.945149-1.7637162 .889081$

O $-0.941490-2.0042041 .376731$

C 3.4813293 .2166591 .292273

O 3.6278594 .1414470 .510319

C 4.4752202 .1566231 .571585

C 5.4726581 .9400690 .702276

C $6.501704 \quad 0.902686 \quad 0.809136$

C 6.4897550 .0317212 .038290

C $1.684555-0.5964362 .221449$

C $2.061633-0.8709030 .780549$

C $3.659112-2.153379-0.624819$

O $1.403296-0.416880-0.151879$

C $5.123959-2.442892-0.543410$

C $6.016178-2.010785-1.447950$

C $7.483163-2.330347-1.387604$

C $8.362329-1.107388-1.532203$

C $9.037276-0.872282-2.670289$

C $8.526033-0.202917-0.329782$

C $7.3923890 .777837-0.196967$

N $3.189475-1.6669740 .659376$

H $0.2128965 .392822-0.060078$

H 0.4781922 .3601970 .278385

H 1.5031364 .8104511 .901055

H 0.8238703 .7156943 .983412

H $\quad 0.1703872 .3578153 .158729$

H -3.142681 4.0923932 .837304

H -2.032855 6.0361523 .463273

H $-0.5985925 .717246 \quad 4.444717$

H $-0.429837 \quad 6.1712822 .734472$

H -3.132307 2.0675761 .545184

H -1.4559681 .7383961 .854901$
H $-2.255696 \quad 1.425480 \quad 4.389594$

H $-3.867278 \quad 1.295717 \quad 3.664619$

H -2.853320 -0.487394 2.088952

H $-4.230609-1.1481814 .100800$

H -2.802866 -1.038052 5.111435

H -4.099937 -3.424228 5.035504

H $-0.815022-2.7205524 .647978$

H -4.625634 -3.198482 2.751973

H -3.336916 -4.3259912.401007

H -3.335497 -2.719162 1.646739

H -1.013369 -4.182843 2.240555

H $1.177607-1.7037113 .960411$

H $1.340489-2.7165612 .517161$

H $-0.353534-1.4875800 .803918$

H $4.334686 \quad 1.5598812 .465447$

H $5.5308082 .568019-0.186030$

H $5.534044-0.4944812 .131843$

H 6.6436920 .6385032 .937677

H $7.265029-0.7366022 .039549$

H 1.1106060 .3334022 .260279

H $2.613275-0.4016662 .773254$

H $3.420148-1.420810-1.402665$

H $3.111768-3.076103-0.840426$

H $5.465855-3.0570650 .286513$

H $5.671053-1.422801-2.295578$

H $7.698680-3.043721-2.193715$

H $7.740601-2.848034-0.455212$

H $9.691105-0.012388-2.771849$

H $8.947178-1.530758-3.527786$

H $9.4506060 .382274-0.425700$

H $8.675878-0.817407 \quad 0.561497$

H $7.3155741 .465473-1.040882$

H $3.550080-2.1167831 .496336$

C -1.975894 $4.151082-1.675405$

O $-2.5393652 .849112-1.393541$

C $-3.6090732 .598506-2.327813$

C $-3.9407763 .948087-2.948122$

C $-2.5628334 .573065-3.022942$

H $-3.203107 \quad 1.950002-3.112435$

C $-4.7884911 .914395-1.630347$

H $-4.4239903 .850731-3.924892$

H -4.596371 $4.541395-2.299668$

C $-2.5899646 .076320-3.239394$

H -2.003405 $4.101000-3.842024$

C $-4.4555430 .528576-1.038640$

O $-5.2536402 .754256-0.566774$

H $-5.6289871 .823018-2.327951$

H $-3.710240 \quad 0.619967-0.241236$

O $-5.6451780 .015285-0.426440$

C $-3.969124-0.495679-2.081886$

C $-3.773572-1.905735-1.495220$

H -3.021753 -0.154997-2.505097

O $-4.906565-0.563269-3.156977$

H $-3.093263-1.836454-0.640766$

H -4.734011 -2.277268 -1.118904

H -3.073676 $6.318711-4.191274$

H -3.141539 $6.586680-2.442675$

H $-1.5754696 .486473-3.261877$

C $-0.4496674 .047247-1.681748$

H -4.4921892 .9543450 .003603$

H -5.9389800 .6714180 .228633$

H -5.760956 -0.840718 -2.783907

H $-0.0141454 .773559-2.377926$

H $-0.1369603 .051929-2.018868$

H $-2.3270614 .824943-0.882817$

C $-0.866058-2.523209-2.262529$

O $-2.006009-2.485402-3.129542$

C $-3.236927-2.915236-2.537095$

C $-3.100175-4.352666-2.017391$

C $-1.905995-4.506029-1.081096$

C $-0.640032-3.942844-1.724309$

H -1.016499-1.828268 -1.429003

C $0.349038-2.050687-3.078628$

H $-3.970687-2.945711-3.352542$

O $-4.294785-4.753661-1.355226$

H $-2.967593-5.025698-2.874488$

H -2.101688 -4.027144 -0.116914

O $-1.708351-5.891690-0.789192$ 
H $-0.314623-4.604789-2.536851$

O $0.401678-3.917091-0.750395$

C $0.415238-0.542679-3.206896$

H $1.274820-2.366918-2.586520$

H $\quad 0.318143-2.469241-4.090979$

H $-4.152383-5.654262-1.016714$

H $-1.534640-6.353939-1.626875$

H $\quad 0.499868-4.820926-0.404862$

O $1.641479-0.196078-3.700929$

O $-0.482873 \quad 0.234400-2.916137$

C $1.8746031 .208916-3.840240$

H $1.1078991 .665473-4.473655$

H $1.8957301 .682791-2.854425$

H $2.8491441 .346134-4.316417$

SCF Energy (B3LYP/6-31G**//MMFF) $=-3245.93000492$

06_00149

MM̄FF Geometry

C 0.0695793 .2977082 .164756

C -0.6148862 .2204841 .748020$

C -1.1523661 .1908502 .711217$

O $-0.549031-0.0840232 .392920$

C -2.6790001 .0636072 .557704$

C $-3.342246 \quad 0.412867 \quad 3.755323$

C $-3.811351-0.8493913 .787785$

C -3.5117591 .3061184 .958667$

C $-3.750313-1.8950882 .709219$

C $-5.137553-2.1914922 .121452$

C $-5.628924-1.0915061 .167656$

C $-7.087602-1.3245610 .773734$

C $-7.570463-0.294663-0.257877$

O $-4.799103-1.1329520 .002564$

O $-8.825663-0.775853-0.761171$

C $-6.563761-0.254007-1.426925$

C -7.8500591 .0673730 .388176$

O $-6.8783880 .794827-2.340710$

C $-5.088542-0.125218-0.973527$

C $-4.157062-0.361479-2.186364$

O $-4.8670031 .187364-0.456306$

C $-0.148009-0.8583503 .434612$

O $-0.165301-0.5542024 .620509$

C $0.335438-2.1859492 .995660$

C $0.241960-2.6612151 .744122$

C $0.698047-3.9826191 .298200$

C $1.400089-4.8573872 .305561$

C $-2.665921-0.472825-1.837718$

C $-1.839881-0.874933-3.049282$

C $0.461481-1.602379-3.671466$

O $-2.302208-0.945120-4.184977$

C $0.602403-3.083830-3.540389$

C $1.719148-3.678474-3.094404$

C $1.872340-5.162966-2.950482$

C $2.051452-5.587220-1.510528$

C $3.254317-5.964241-1.045808$

C $0.818072-5.663644-0.638237$

C $0.477780-4.3508630 .018631$

$\mathrm{N}-0.520566-1.136760-2.710719$

H $\quad 0.273103 \quad 3.3880413 .231157$

H -0.8363982 .0786760 .694557$

H $-0.894560 \quad 1.4935203 .732990$

H $-2.909650 \quad 0.5101951 .640830$

H -3.133074 2.0554492 .426521

H -4.291057 -1.191133 4.704595

H -4.0530640 .8109645 .771990$

H -2.538919 1.6138565 .353552

H -4.0774572 .2037284 .687915$

H -3.041212 -1.638180 1.917025

H $-3.362621-2.8133543 .168316$

H -5.070592 -3.138306 1.569888

H -5.859767 -2.338671 2.934085

H $-5.524083-0.1165461 .654983$

H -7.737260 -1.313789 1.657983

H $-7.191340-2.3285210 .338368$

H -9.178020 -0.113295 -1.378945

H $-6.673847-1.195570-1.983326$

H $-8.6274380 .972207 \quad 1.155598$

H -8.239448 $1.781492-0.345969$

H $\quad-6.968974 \quad 1.5078980 .859673$
H $-7.7671450 .622127-2.693813$

H -4.452070 -1.297507 -2.679399

H $-4.2857050 .446041-2.918395$

H $-3.923973 \quad 1.401897-0.530937$

H $\quad 0.766972-2.781107 \quad 3.795011$

H $-0.216811-2.0414530 .975982$

H $2.264913-4.3380862 .732792$

H $1.785396-5.7880691 .884118$

H $\quad 0.717449-5.1321873 .117107$

H $-2.2810650 .484603-1.470729$

H $-2.512578-1.228402-1.058741$

H $1.399845-1.089619-3.451560$

H $\quad 0.152601-1.341368-4.688304$

H $-0.252717-3.691842-3.825159$

H $2.574104-3.067037-2.814996$

H $2.739081-5.469347-3.550529$

H $1.015995-5.697045-3.380924$

H $3.385049-6.300809-0.022966$

H $4.137053-5.938739-1.676166$

H $0.926652-6.4643580 .101336$

H $-0.039968-5.979017-1.245899$

H $-0.037440-3.650320-0.637888$

H $-0.241445-1.091820-1.734742$

C $0.0962304 .589489-0.091478$

O $0.7426203 .600625-0.917288$

C $1.3965374 .266650-2.011596$

C $0.6859205 .603365-2.150246$

C $0.4581945 .953740-0.694329$

H $2.4327414 .445843-1.697817$

C $1.3909923 .391845-3.267673$

H $1.2884856 .344966-2.682956$

H $-0.2749935 .503945-2.667912$

C $-0.6055797 .018333-0.484785$

H $1.405526 \quad 6.312988-0.269564$

C $1.8837291 .949763-3.045839$

O $0.056843 \quad 3.304879-3.785618$

H $1.9839273 .876439-4.051912$

H $1.1213781 .376842-2.504438$

O $2.017911 \quad 1.336371-4.333729$

C $3.2257121 .826956-2.305425$

C $3.6613280 .360293-2.130981$

H $3.1326802 .286671-1.317292$

O $4.2353812 .544815-3.011022$

H $2.839152-0.194269-1.665806$

H $3.840618-0.089921-3.114808$

H $-0.3154687 .953375-0.975251$

H -1.571035 $6.707627-0.898089$

H -0.7460887 .2247090 .580858$

C 0.5868064 .4554651 .354138

H $-0.5083392 .939869-3.082858$

H $1.155127 \quad 1.403657-4.778044$

H $4.3005362 .160189-3.902068$

H 0.3048755 .3639691 .901333

H 1.6826114 .3977711 .367085

H $-0.9842564 .413285-0.158369$

C 3.9756310 .3875740 .912372

O $4.8712230 .938998-0.056961$

C $4.9459630 .208309-1.286125$

C $5.352696-1.249450-1.018602$

C $4.467844-1.9151230 .036667$

C $4.390661-1.0388101 .288228$

H 2.9628980 .3663860 .489444

C 3.8943991 .3465672 .108694

H $5.7562500 .669379-1.865034$

O $5.316251-1.998674-2.229020$

H $6.391737-1.264674-0.664278$

H $3.470949-2.132330-0.364451$

O $5.051104-3.1748590 .375234$

H $5.365915-1.0447021 .788726$

O $3.439912-1.5782872 .197848$

C 5.1917061 .5040462 .874139

H $3.6139862 .347478 \quad 1.759613$

H 3.1195020 .9927872 .798960

H $5.550945-2.916803-2.010142$

H $4.424349-3.6411030 .953998$

H $3.779085-2.4359362 .504109$

O 4.9108321 .8965704 .152024

O 6.3104151 .3407002 .406682 
C 6.0476682 .1054834 .995830

H 6.6234071 .1799265 .092590

H 6.6722322 .9100114 .596469

H 5.6873012 .3991225 .985273

SCF Energy (B3LYP/6-31G**//MMFF) $=-3245.91263374$

06_00150

MM̄FF Geometry

C -1.433943 4.6605930 .187769

C -0.9478263 .6243520 .886080$

C $0.3884692 .987846 \quad 0.612027$

$\begin{array}{lllll}\text { O } & 0.083573 & 1.674297 & 0.075193\end{array}$

C 1.2368372 .8157201 .888117

C 2.0416004 .0379142 .288357

C 3.3887784 .0891052 .308432

C 1.2534455 .2456752 .724211

C 4.3538352 .9865971 .978062

C 4.7616293 .0252650 .503085

C 5.7538021 .9099990 .145746

C $6.1203281 .976703-1.339339$

C $7.0350180 .818030-1.762287$

$\begin{array}{lllll}\text { O } & 5.144762 & 0.649428 & 0.447314\end{array}$

O $7.049707 \quad 0.799215-3.198089$

C $6.411211-0.508467-1.275399$

C $8.4841191 .056993-1.323564$

O $7.282885-1.605597-1.536109$

C $5.992855-0.4823650 .211593$

C $5.224251-1.7534120 .637059$

O $7.173064-0.4219281 .022997$

C $0.8856211 .186950-0.907846$

O $1.9111441 .688304-1.338932$

C $0.264721-0.055394-1.418891$

C $0.691174-0.563613-2.584626$

C $0.155680-1.750311-3.246704$

C $1.205760-2.570398-3.947002$

C $3.902248-1.993132-0.094677$

C $3.174932-3.2189580 .433876$

C $1.113247-4.5533580 .053669$

O $3.580850-3.8919111 .377199$

C $0.346947-4.947073-1.167398$

C $-0.982055-4.812452-1.284200$

C $-1.764248-5.227472-2.495080$

C -2.574354 -4.094787 -3.084059

C $-3.897464-4.004794-2.866463$

C $-1.868788-3.127251-4.006876$

C $-1.165928-2.017059-3.272157$

N $2.019333-3.475382-0.289807$

H $-0.8379635 .090051-0.613445$

H - 1.5539513 .1905921 .677853

H $0.9414253 .570598-0.136028$

H 1.8946091 .9536791 .739285

H 0.5994132 .5274682 .734407

H 3.8647225 .0229922 .604923

H 1.8823595 .9923733 .221452

H 0.7871655 .7334531 .864002

H 0.4736924 .9579333 .436950

H 5.2433013 .1100382 .608681

H 3.9395122 .0102042 .247297

H $3.8608172 .921452-0.112881$

H $5.204924 \quad 4.0004560 .265259$

H 6.6478362 .0353720 .768077

H $6.5832062 .941015-1.583665$

H $5.2012941 .926770-1.940823$

H $7.5656170 .023076-3.476988$

H $5.522561-0.674428-1.896116$

H $8.8711051 .978024-1.775998$

H $9.1428140 .254521-1.673564$

H $8.595961 \quad 1.148545-0.241373$

H $7.988597-1.588316-0.867354$

H $5.873961-2.6325790 .534360$

H $5.007236-1.6805441 .712361$

H $6.947423-0.7250531 .918207$

H $-0.543727-0.501041-0.850106$

H $1.508075-0.080460-3.118015$

H $\quad 0.858285-3.569789-4.219896$

H $1.537688-2.064283-4.859884$

H $2.079467-2.715629-3.301433$

H $3.239817-1.1283060 .022885$
H $4.080615-2.152006-1.163204$

H $\quad 0.462439-4.1957380 .858121$

H $1.684638-5.4060620 .434740$

H $\quad 0.914969-5.375309-1.989704$

H -1.545604 -4.398631 -0.453461

H -2.428887 -6.048282 -2.195228

H $-1.110581-5.646993-3.269903$

H $-4.490741-3.213584-3.312819$

H $-4.415543-4.715918-2.231886$

H $-2.600400-2.655489-4.677194$

H -1.202735 -3.681290 -4.675059

H -1.844129 -1.337446 -2.756566

H $1.678082-2.751792-0.915271$

C $-3.8691074 .836610-0.530928$

O $-4.2158113 .457819-0.290496$

C $-4.4985602 .829932-1.555971$

C $-4.5488203 .952041-2.591744$

C $-3.5492304 .938474-2.021555$

H $-3.6420042 .183463-1.781142$

C $-5.7795061 .991879-1.476473$

H $-4.2835893 .601030-3.593819$

H -5.542359 $4.411248-2.650438$

C $-3.6791736 .341776-2.585898$

H -2.543502 $4.558103-2.237384$

C $-5.792610 \quad 0.929088-0.359303$

O $-6.8818002 .878825-1.243355$

H -5.961870 $1.520721-2.450209$

H $-5.884515 \quad 1.408406 \quad 0.623151$

O $-6.9748630 .136005-0.533982$

C $-4.577069-0.017853-0.347715$

C $-4.763255-1.2029790 .621563$

H -3.683688 $0.550146-0.067552$

O $-4.355549-0.530755-1.661452$

H $-5.079521-0.8166841 .596016$

H $-5.568752-1.8467440 .248371$

H $-3.5108176 .338451-3.667772$

H -4.675265 $6.758127-2.402331$

H $-2.9435407 .012832-2.131281$

C -2.7763945 .2848230 .443438$

H -7.699559 2.382547 -1.415311

H $-7.7384110 .695262-0.313037$

H -5.160595 -1.004421 -1.932547

H $-3.0971855 .052700 \quad 1.467127$

H -2.659133 6.3736520 .390581

H -4.775955 $5.413999-0.305692$

C $-2.458396-0.9210122 .631830$

O $-2.380137-1.2935351 .253114$

C $-3.486151-2.0605180 .767961$

C $-3.669687-3.3291201 .615369$

C $-3.780725-3.0131993 .103559$

C $-2.605201-2.1435583 .544943$

H -3.326333 -0.264190 2.770095

C -1.235139-0.061007 2.976396

H -3.207963 -2.388099 -0.240482

O $-4.819833-4.0591041 .202973$

H $-2.805003-3.9891241 .474283$

H $-4.737716-2.5365493 .345692$

O $-3.737727-4.232442 \quad 3.848397$

H -1.689409 -2.746736 3.539201

O $-2.818721-1.7062274 .884900$

C $0.095558-0.7612032 .793118$

H -1.2175010 .8156602 .322697$

H -1.314761 0.2877044 .012387

H -4.708862 -4.2688010 .259856$

H -4.463449 -4.793674 3.525146

H -2.939536 -2.500556 5.433161

O $1.019214-0.1966573 .625427$

O $0.312706-1.6590311 .991418$

C $2.346981-0.7192753 .516104$

H $2.352207-1.7969223 .705638$

H $2.754506-0.4980122 .525687$

H $2.969199-0.2272084 .268156$

SCF Energy (B3LYP/6-31G**//MMFF) $=-3245.91983618$

0600151

MM̄MF Geometry

C $1.747639-0.5414812 .977048$

C $3.000424-1.0093113 .094117$ 
C $4.138729-0.8142092 .122426$

$\begin{array}{lllll}\text { O } & 3.894225 & 0.286462 & 1.222196\end{array}$

C $4.288424-2.0737691 .250863$

C $5.688974-2.2622350 .707042$

C $6.104493-1.880221-0.515774$

C $6.634061-2.9860111 .632310$

C $5.350147-1.104403-1.558452$

C $4.921376-1.964450-2.755407$

C $3.566348-2.656519-2.539318$

C $3.228146-3.562528-3.724152$

C $1.821133-4.163083-3.601875$

O $2.579653-1.626870-2.415032$

O $1.500834-4.740617-4.876668$

C $0.813370-3.026488-3.326962$

C $1.783151-5.311948-2.586844$

O $-0.477586-3.581774-3.085544$

C $1.245033-2.088191-2.173541$

C $0.329790-0.839904-2.125901$

O $1.144108-2.797544-0.937492$

C 4.2230701 .5386991 .630989

O $4.664708 \quad 1.8727502 .717518$

C 3.9305042 .4438210 .498600

C $3.844423 \quad 3.7657680 .701532$

C $3.5202884 .768584-0.315641$

C $3.3639814 .295222-1.738015$

C $0.8185770 .264594-1.176971$

C $-0.1721241 .412315-1.080962$

C $-0.610303 \quad 3.4950990 .220397$

O $-1.1687321 .498526-1.793574$

C $-0.1890884 .660422-0.618359$

C $0.0403195 .884839-0.118951$

C $0.4252537 .071480-0.957413$

C $1.6406907 .800587-0.425712$

C 1.5116528 .9459770 .265104

C $3.0068157 .246066-0.766276$

C 3.3610536 .0504460 .074686

N $0.1941432 .332713-0.107576$

H 1.4620490 .0592632 .119987

H $3.237086-1.6312963 .956245$

H $5.056904-0.6232082 .691725$

H $3.568702-2.0359060 .425294$

H $4.031050-2.975721 \quad 1.822845$

H $7.130807-2.112256-0.799505$

H $7.634069-3.1014811 .200902$

H $6.743188-2.4369512 .572934$

H $6.253364-3.9878201 .856484$

H $4.495792-0.571024-1.134686$

H $\quad 6.030377-0.323086-1.922064$

H $4.834782-1.296977-3.623303$

H $5.695424-2.704493-2.992380$

H $3.604915-3.240812-1.613922$

H $3.977258-4.356653-3.833898$

H $3.274762-2.978430-4.654268$

H $0.584830-5.064930-4.830862$

H $0.734058-2.439431-4.252756$

H $2.460791-6.116228-2.897793$

H $\quad 0.786412-5.763879-2.535744$

H $2.073066-5.005189-1.579604$

H -1.124192 -2.856773 -3.102296

H $0.261031-0.403317-3.131022$

H $-0.683884-1.132864-1.830977$

H $0.984464-2.169416-0.216317$

H $3.7638691 .989645-0.471682$

H 4.0010344 .1485201 .709168

H $4.3001163 .854514-2.098551$

H $3.0983195 .089062-2.438454$

H $2.5721703 .541950-1.812307$

H $0.968625-0.134489-0.170340$

H $1.7716840 .672944-1.531084$

H $-0.4848883 .687218 \quad 1.290580$

H $-1.660548 \quad 3.2658370 .026072$

H $-0.0950444 .498246-1.689477$

H $-0.087047 \quad 6.0541220 .948022$

H $-0.4356577 .752210-0.982018$

H $0.6050286 .782745-2.000563$

H 2.3786319 .4891400 .626416

H $\quad 0.5357659 .3634290 .489575$

H $3.7785978 .007409-0.590282$
H $3.0536217 .046743-1.840030$

H 3.4939626 .2899021 .130959

H 1.0665312 .2035650 .395710

C - $-0.023559-2.1720853 .650467$

O $-0.900169-2.0065272 .517546$

C $-2.043631-2.8685772 .698563$

C $-1.795966-3.6567403 .981344$

C $-0.918967-2.7016154 .765979$

H -2.909534 -2.2178412.858439

C $-2.250265-3.7453921 .457671$

H $-2.727900-3.9074324 .497661$

H -1.263337 -4.594534 3.782899

C $-0.171514-3.3719825 .905573$

H -1.543341 -1.891080 5.165019

C $-2.556162-2.9567210 .168412$

O $-1.064409-4.5183661 .230175$

H -3.050446 -4.470541 1.644982

H -1.695320 -2.341262 -0.112599

O $-2.746462-3.903425-0.889561$

C $-3.814421-2.075386 \quad 0.259217$

C $-4.185266-1.403617-1.077266$

H -3.650599-1.298666 1.011143

O $-4.916922-2.8651670 .706662$

H -3.329246 -0.816736-1.427241

H $-4.380961-2.172863-1.833755$

H $-0.875040-3.8106286 .620728$

H $0.480831-4.1728545 .541548$

H $0.450174-2.6512626 .445334$

C $0.671036-0.8510823 .981335$

H $-0.330333-3.8954791 .090591$

H $-1.935311-4.437089-0.949375$

H -5.052601 -3.574443 0.055000

H $1.114061-0.8901364 .983152$

H $-0.049794-0.0246843 .979730$

H $\quad 0.716521-2.935403 \quad 3.372603$

C $-4.4146551 .478263-0.076495$

$\begin{array}{llll}\text { O } & -5.347767 & 0.411085 & 0.128942\end{array}$

C $-5.444968-0.514162-0.958098$

C $-5.8461290 .216435-2.248051$

C $-4.9529001 .423241-2.532395$

C $-4.8366972 .309350-1.290908$

H $-3.4129781 .066366-0.243111$

C -4.3871902 .3109581 .210820$

H $-6.275950-1.186220-0.708036$

O $-5.820420-0.689251-3.345839$

H $-6.8819450 .565606-2.143218$

H $-3.9618981 .114649-2.885634$

O $-5.5315692 .205776-3.579142$

H $-5.7976102 .803536-1.098731$

O $-3.8705023 .328085-1.532604$

C -3.6155861 .5884892 .295569$

H -3.899559 3.2805911 .068010

H -5.4111152 .4964981 .557090$

H $-6.198307-0.232041-4.115616$

H $-5.4972631 .676886-4.393932$

H $-4.1483693 .808924-2.330940$

O $\quad-4.349797 \quad 1.5484813 .443932$

O -2.4912951 .1259672 .147472$

C $-3.725650 \quad 0.8837894 .547221$

H -2.7866461 .3808214 .809373$

H -4.4024850 .9381995 .403971$

H -3.550912 -0.168431 4.304044

SCF Energy (B3LYP/6-31G**//MMFF) $=-3245.93011066$

06_00152

MM̄MF Geometry

C -0.559746 -2.061723 -0.119851

C $0.128007-1.1635500 .603607$

C $-0.316688-0.5379201 .906868$

O $-1.717169-0.8182892 .121457$

C -0.1411690 .9888701 .842333$

C $-0.2923281 .682608 \quad 3.180541$

C -1.3532432 .4309803 .540565$

C 0.8785801 .5389074 .118611

C -2.6213612 .6751042 .770830$

C -2.6989074 .0942162 .189304$

C -1.820117 4.2924280 .943618

C -1.8885415 .7416190 .456496$ 
C $-1.1246385 .941696-0.862020$ O $-2.3125993 .414325-0.073035$ O $-1.5030357 .216864-1.402388$ C -1.583907 4.869278-1.872349 C $0.392302 \quad 5.996167-0.642559$ O $-0.8056824 .925559-3.065588$ C $-1.5673503 .434881-1.296712$ C $-2.2563002 .458969-2.281058$ O $-0.2129713 .027640-1.100685$ C $-2.133162-1.1966373 .354160$ O $-1.438794-1.3387434 .350849$ C $-3.593396-1.4554923 .371426$ C $-4.406446-1.1975852 .334470$ C $-5.848630-1.4497322 .276696$ C $-6.495139-2.1027313 .469866$ C $-2.4801351 .051818-1.708711$ C $-3.246966 \quad 0.155424-2.665531$ C $-4.347882-2.083403-2.750117$ O $-3.5281730 .481194-3.815440$ C $-5.794321-1.866271-2.434553$ C $-6.550575-2.741242-1.754555$ C $-7.999598-2.511597-1.431756$ C $-8.279081-2.5321480 .055777$ C $-8.850280-3.6025940 .633190$ C $-7.982186-1.2830270 .857361$ C $-6.517184-1.1114731 .153822$ N -3.566867 -1.061374 -2.078402 H $-1.528878-2.4136310 .222508$ H $1.110025-0.8613940 .244805$ H $0.299747-0.9712702 .703165$ H $-0.855011 \quad 1.396951 \quad 1.120410$ H $0.852303 \quad 1.2404481 .449055$ H -1.3320282 .9108844 .518557$ H $1.032248 \quad 0.489782 \quad 4.387976$ H 1.7918011 .9147173 .647836 H 0.7368462 .0979295 .049787 H -2.782633 1.9243911 .991282 H -3.453782 2.5448263 .474428 H -3.7459314 .2778251 .914289$ H -2.432855 4.8293942 .958952 H -0.7860374 .0252681 .186936$ H $-1.5141996 .428367 \quad 1.225829$ H $-2.938820 \quad 6.0212830 .291687$ H $-1.2870607 .897899-0.742467$ H -2.615132 5.113679-2.163662 H $0.652597 \quad 6.8277200 .023412$ H $0.9208036 .189364-1.582496$ H $0.7964625 .082988-0.200719$ H $-0.8484205 .839031-3.396495$ H -3.237755 2.864808 -2.560102 H $-1.6633852 .375788-3.200624$ H $-0.1535992 .062225-1.165380$ H -3.967125 -1.873781 4.300531 H $-3.984630-0.7624871 .430021$ H -6.408834 -1.460041 4.352828 H $-6.019209-3.065553 \quad 3.687217$ H -7.558308 -2.309492 3.329848 H $-1.521706 \quad 0.566617-1.501229$ H -3.047583 $1.109211-0.772685$ H -3.986640 -3.054549 -2.397542 H $-4.187774-2.021087-3.831048$ H $-6.240549-0.938283-2.784997$ H -6.109806 -3.675123 -1.413290 H -8.580266 -3.294694 -1.936483 H -8.358111 -1.563469-1.851719 H $-9.084526-3.616577 \quad 1.692305$ H $-9.093442-4.4913030 .060221$ H $-8.572526-1.2650271 .780373$ H -8.329736 -0.4046690 .298022$ H -5.960522 -0.6431000 .341989$ H $-3.355263-1.204165-1.095098$ C $0.575328-4.007892-1.210055$ O $1.855297-3.820745-0.569923$ C $2.861095-4.509972-1.332087$ C $2.103253-5.540489-2.154424$ C $0.860338-4.749082-2.518503$ H $3.303323-3.775281-2.016245$ C $3.943378-5.063011-0.401081$
H $2.669710-5.878743-3.027091$

H $1.832650-6.418564-1.556767$

C $-0.294362-5.611671-2.997737$

H $1.119110-4.031005-3.308507$

C $4.561174-4.0064570 .540336$

O $3.380570-6.0940700 .419509$

H $4.729475-5.545339-0.993035$

H $3.826938-3.6987701 .294787$

O $5.636469-4.6277371 .254544$

C $5.108886-2.759848-0.177594$

C $5.759529-1.7549910 .790706$

H $4.289213-2.259479-0.701620$

O $6.061126-3.154405-1.163730$

H $5.029621-1.4958151 .565561$

H $6.611014-2.2280751 .294823$

H $-0.017477-6.153778-3.907825$

H $-0.584611-6.349617-2.242320$

H - $1.171855-4.997304-3.222920$

C $-0.071408-2.638948-1.419987$

H $2.634941-5.7057390 .908775$

H $5.272792-5.4120371 .700367$

H $6.780340-3.622689-0.705924$

H $-0.928318-2.718490-2.098497$

H $0.645950-1.950455-1.884853$

H $-0.030353-4.628471-0.537346$

C $4.1880370 .727284-0.082857$

O $5.2759740 .103921-0.769773$

C $6.268379-0.4786180 .081176$

C $6.844067 \quad 0.583551 \quad 1.028554$

C 5.7510381 .3203851 .802690

C 4.6805801 .8451850 .844208

H $3.668496-0.0312930 .516430$

C $3.1625231 .201473-1.123710$

H $7.085381-0.795331-0.579663$

O $7.764558-0.0164711 .934182$

H 7.4084991 .3154370 .435794

H 5.3162530 .6784352 .577761

O 6.3579582 .4211942 .483170

H 5.0977032 .6781710 .266646

O 3.5758402 .3509551 .587236

C $3.6676262 .289906-2.048431$

H $2.8662740 .360141-1.761448$

H $2.2715361 .570139-0.603761$

H 8.0923430 .6851192 .522637

H 5.6800532 .8255263 .050395

H 3.8853423 .1241092 .088072

O $2.604875 \quad 3.012302-2.511155$

O $4.8388692 .466220-2.354716$

C $2.9284614 .078132-3.409201$

H $1.9967424 .560741-3.714843$

H $3.5578314 .817891-2.905509$

H $3.4282993 .686095-4.300000$

SCF Energy (B3LYP/6-31G**//MMFF)= -3245.91757624

06_00153

MMFF Geometry

C $0.143245 \quad 3.017447 \quad 0.420243$

C -1.107822 3.0881490 .897855

C -1.9205501 .8536681 .185886$

$\begin{array}{lllll}\text { O } & -3.237440 & 1.989236 & 0.596308\end{array}$

C -2.1461691 .6204132 .689874$

C $-0.894518 \quad 1.388727 \quad 3.507528$

C $-0.152070 \quad 0.2652053 .463310$

C $-0.518753 \quad 2.511227 \quad 4.437756$

C $-0.386191-0.9470212 .604935$

C $0.766189-1.1280501 .610125$

C $0.512638-2.2737010 .622176$

C $1.754562-2.514741-0.237414$

C $1.517653-3.594859-1.299017$

O $-0.588646-1.904142-0.215214$

O $2.619210-3.524839-2.216959$

C $0.227549-3.252720-2.071247$

C $1.553460-5.000516-0.689338$

O $-0.105982-4.297114-2.983411$

C $-0.978559-2.910469-1.160387$

C $-2.125744-2.339286-2.031936$

O $-1.417512-4.100373-0.507651$

C $-3.3603741 .788648-0.740143$ 
O $-2.4483851 .627162-1.539918$ C $-4.7816861 .749639-1.158793$ C $-5.8109621 .724947-0.297232$ C -7.230406 $1.610568-0.644180$ C $-7.5798621 .420883-2.096949$ C $-3.326133-1.771543-1.263693$ C $-4.410665-2.780155-0.928531$ C $-6.661617-2.8995890 .141736$ O $-4.319907-3.978174-1.178599$ C -7.894394 -2.140335 -0.234444 C $-8.796916-1.6976120 .654155$ C $-10.062356-0.9813260 .276264$ C $-10.203395 \quad 0.3604170 .959775$ C -10.880044 0.4790392 .114826 C -9.6398231 .5754950 .256972$ C -8.1410901 .6564300 .351336$ N -5.499583 -2.166249 -0.322288 H $\quad 0.5880602 .0371770 .267878$ H -1.570371 4.0552961 .073758 H -1.4497340 .9658490 .747478$ H $-2.824951 \quad 0.7662442 .821978$ H -2.712745 2.4651533 .106846 H $\quad 0.723270 \quad 0.200137 \quad 4.108384$ H $0.4050272 .304747 \quad 4.988627$ H $-0.368727 \quad 3.4391993 .877141$ H -1.3136342 .6748065 .172748$ H - $0.446251-1.8266253 .257362$ H -1.343381 -0.8929382 .078797$ H $\quad 0.909202-0.1959551 .050021$ H $1.690310-1.3186452 .168092$ H $\quad 0.253313-3.1750051 .189870$ H $2.616619-2.7778430 .384758$ H $2.028732-1.580440-0.747466$ H $2.441280-4.151989-2.939149$ H $0.454122-2.374242-2.690992$ H $2.521228-5.180784-0.205707$ H $1.459163-5.771152-1.462364$ H $\quad 0.773110-5.1638380 .057027$ H -0.508912 -5.019030 -2.471506 H -1.716030 -1.504685-2.616793 H -2.470936 -3.097921 -2.744570 H -2.321356 -3.961549 -0.184686 H $-4.9335151 .688566-2.231684$ H -5.6049831 .7747590 .770645$ H -7.296618 2.305148 -2.678301 H $-8.6433131 .248656-2.273015$ H -7.058910 $0.548229-2.507193$ H -3.010695 -1.270165 -0.342865 H -3.801069-1.020583 -1.907387 H $-6.559780-3.0156961 .225588$ H $-6.684102-3.896423-0.309647$ H -8.064076 -1.970240 -1.295175 H $-8.636972-1.8900141 .712687$ H $-10.906093-1.6275240 .552991$ H -10.139843 -0.849462 -0.810224 H $-11.012681 \quad 1.4417882 .597039$ H -11.312156 -0.386516 2.606289 H -10.041674 2.491946 0.709868 H $-10.0048311 .591451-0.773368$ H $-7.783373 \quad 1.790596 \quad 1.373469$ H $-5.421443-1.189173-0.054564$ C $1.5544854 .064119-1.350747$ O $2.4360812 .923702-1.421896$ C $3.5922813 .271569-2.203496$

C $3.2771074 .616480-2.848698$

C $2.3968385 .258643-1.793935$

H $4.4209233 .406326-1.498467$

C $3.9211092 .147267-3.191767$

H $4.1810515 .194020-3.065089$

H $2.7215524 .497811-3.786212$

C $1.5842916 .431904-2.313239$

H $3.0326485 .598712-0.965333$

C $4.1377650 .761475-2.547537$

O $2.8451982 .017578-4.131044$

H $4.8018172 .424297-3.782751$

H $3.1900870 .367568-2.160608$

O $4.550328-0.136231-3.586622$

C $5.1964590 .730501-1.429589$
C $5.525574-0.699921-0.958309$

H $4.8365981 .318856-0.580756$

O $6.3967431 .351893-1.890742$

H $4.592567-1.217158-0.714921$

H $5.996761-1.248246-1.782709$

H $2.2447217 .224495-2.679897$

H $0.9286026 .132426-3.137768$

H $0.9579756 .854325-1.521268$

C 1.0018894 .1978530 .069994

H $2.0348551 .834986-3.624739$

H $3.864149-0.115925-4.275745$

H $6.7049810 .854898-2.667875$

H $\quad 0.4344315 .1296690 .170244$

H 1.8313834 .2323570 .785052

H $0.7383883 .880998-2.062586$

C $4.859240-0.6598242 .038510$

O $5.954824-0.0131721 .377862$

C $6.487831-0.7198550 .251431$

C $6.951340-2.1214810 .674125$

C $5.855588-2.8931051 .403083$

C $5.282859-2.0470502 .539419$

H $4.014351-0.7510951 .346851$

C 4.4300250 .2340093 .211465

H $7.387884-0.170807-0.052736$

O $7.397250-2.856976-0.460268$

H $7.813750-2.0183831 .346022$

H $5.074429-3.2186530 .706646$

O $6.430260-4.0824421 .948570$

H $6.042260-1.9473693 .325194$

O $4.174278-2.7275203 .122076$

C 3.7703381 .5162132 .750315

H $3.708575-0.2815353 .854262$

H 5.3138090 .5029523 .802706

H $7.661361-3.739885-0.149042$

H $5.730951-4.5390022 .447234$

H $3.499907-2.8481742 .433253$

O 3.7635932 .4071303 .785907

O 3.2985251 .7071121 .638254

C 3.1723123 .6782293 .501760

H 3.7339784 .1879672 .713246

H 2.1257323 .5533403 .211658

H 3.2138144 .2839724 .410847

SCF Energy (B3LYP/6-31G**//MMFF) $=-3245.93749710$

06_00154

MM̄FF Geometry

C $4.502774-0.4144512 .022106$

C $3.365696-0.9031862 .541168$

C $2.439017-0.2199953 .518367$

O $1.161182-0.0853652 .848147$

C 2.8705821 .1901203 .978512

C 1.9094951 .8444704 .957251

C 1.2342602 .9865384 .712690

C 1.7506711 .1487936 .284373

C 1.3092013 .8459973 .479751

C 0.3258673 .3782362 .408960

C 0.4983244 .0912301 .062632

C 0.0436265 .5509491 .105544

C $0.0955526 .212073-0.283358$

O $-0.293873 \quad 3.3590300 .118127$

O $-0.6485647 .438694-0.213093$

C $-0.6281845 .301266-1.300659$

C $1.5272026 .598353-0.673062$

O $-0.4941225 .804775-2.627411$

C $-0.1812993 .825607-1.232209$

C $-1.0371822 .894088-2.118333$

O $1.1679603 .722329-1.702504$

C $0.120773-0.8548053 .263024$

O $0.142642-1.7404574 .101652$

C -1.081885 -0.392948 2.531894

C $-2.295494-0.8331962 .896158$

C -3.573839-0.435022 2.300355

C $-3.5391420 .626226 \quad 1.234066$

C $-2.5345062 .900340-1.805832$

C $-3.2658011 .751471-2.480667$

C $-5.5044010 .661232-2.548723$

O $-2.7411501 .012017-3.308433$

C $-6.4399870 .270543-1.449649$ 
C $-6.485511-0.957787-0.912038$

C $-7.436829-1.356578 \quad 0.179458$

C $-6.724753-1.8234931 .430222$

C $-6.699449-3.1280731 .754014$

C $-6.129372-0.7540182 .323632$

C $-4.705731-1.0244072 .740134$

N $-4.5788951 .656517-2.041624$

H 4.8544740 .5734692 .304061

H $3.049313-1.8986052 .234423$

H $2.353572-0.8730844 .395000$

H $2.988148 \quad 1.822607 \quad 3.092293$

H 3.8569891 .1387264 .457371

H 0.5592403 .3619845 .480497

H 1.1621961 .7399796 .994372

H 1.2450120 .1867986 .160787

H $2.7306880 .973586 \quad 6.740328$

H 1.0850344 .8790313 .771130

H 2.3307023 .8666723 .084771

H 0.4583322 .3053522 .235235

H -0.7078593 .4877832 .763155$

H 1.5517664 .0202950 .767133

H $\quad 0.638270 \quad 6.130347 \quad 1.821484$

H $-0.9921195 .597088 \quad 1.470406$

H -0.2475107 .9938110 .477524$

H -1.696954 5.362371-1.061062

H 1.9505357 .2911830 .064181

H $1.5461257 .133843-1.628694$

H $2.1995675 .740887-0.741642$

H $0.4034465 .592761-2.935288$

H $-0.8772363 .127577-3.178920$

H $-0.6649301 .868908-1.984109$

H $1.3459692 .791677-1.916551$

H $-0.937023 \quad 0.318510 \quad 1.726704$

H -2.364369 -1.5489503 .714271$

H $-4.527046 \quad 0.9038760 .865087$

H -2.9633350 .2817340 .368356$

H -3.080751 1.5445921 .618095

H -2.695926 2.819617 -0.725402

H -2.989805 $3.833750-2.154498$

H -4.949211-0.194208 -2.946661

H $-6.0624041 .116820-3.372872$

H -7.118980 $1.037426-1.084253$

H -5.815798 -1.727337 -1.287041

H -8.080313 -2.153764 -0.214710

H -8.114366 -0.5335890 .440070$

H $-6.229641-3.4800972 .665999$

H -7.153825 -3.877076 1.113719

H $-6.745081-0.6786613 .229870$

H -6.2214530 .2281401 .850640$

H -4.619969 -1.782532 3.519849

H $-4.9538852 .407954-1.469092$

C $5.374258-0.442679-0.322509$

O $4.078485-0.558904-0.946548$

C $4.259734-0.858819-2.343568$

C $5.739023-0.635424-2.634563$

C $6.364959-1.047299-1.316563$

H $4.043028-1.926319-2.467492$

C $3.300269-0.028091-3.204168$

H $6.089699-1.231028-3.482829$

H $5.958570 \quad 0.417244-2.846986$

C $7.792804-0.559089-1.144335$

H $6.347626-2.143248-1.245305$

C $1.812683-0.181026-2.822356$

O $3.6495631 .354138-3.056563$

H $3.450972-0.277876-4.261460$

H $1.6218240 .286196-1.848351$

O $1.033126 \quad 0.539510-3.785408$

C $1.309985-1.635604-2.804311$

C $-0.186167-1.743796-2.456293$

H $1.878083-2.204172-2.066412$

O $1.551222-2.237981-4.075134$

H $-0.352880-1.274023-1.480435$

H $-0.771671-1.179355-3.191457$

H $8.436981-0.970953-1.928050$

H $7.8529970 .533061-1.199556$

H $8.198281-0.870142-0.176582$

C $5.359993-1.1535721 .031554$

H $3.210371 \quad 1.846050-3.770375$
H $1.2433841 .483198-3.691266$

H $1.059748-1.725365-4.739832$

H $6.374710-1.2123581 .441030$

H $4.997822-2.1806200 .909070$

H $5.5696620 .630045-0.192494$

C $-0.049333-3.855335-0.214279$

O $0.092992-4.061848-1.624653$

C $-0.699416-3.202237-2.450698$

C $-2.185997-3.346442-2.098601$

C $-2.441740-3.151039-0.606825$

C $-1.511765-4.0475950 .208959$

H $0.288919-2.8454750 .048738$

C $0.853892-4.8774090 .496133$

H $-0.581118-3.576919-3.475454$

O $-2.962338-2.422714-2.853832$

H $-2.519880-4.352802-2.383850$

H $-2.336419-2.097480-0.325293$

O $-3.797660-3.511204-0.339396$

H $-1.807640-5.0963380 .076518$

O $-1.640875-3.738196 \quad 1.593034$

C $2.327530-4.5713640 .336726$

H $0.649498-4.8932751 .572076$

H $\quad 0.673219-5.8749310 .077734$

H -3.891246 -2.541236 -2.591462

H -3.988304 -3.266016 0.581543

H $-2.547817-3.9603731 .862632$

O $3.052949-5.6390590 .784577$

O $2.790243-3.527227-0.098808$

C $4.474527-5.4894980 .715352$

H $4.802245-4.7100871 .409425$

H $4.930802-6.4377621 .011485$

H $4.787474-5.254339-0.306644$

SCF Energy $\left(B 3 L Y P / 6-31 G^{* *} / / M M F F\right)=-3245.91289343$

06_00155

MM̄FF Geometry

C -2.482684 -0.192328 3.237486

C -2.1441451 .0674213 .555711$

C -2.5128642 .3332372 .814282$

O -3.3310862 .0617341 .656237$

C -1.2441043 .0290122 .290784$

C -1.409094 4.5263112 .124614

C -1.7005465 .1534980 .968366$

C -1.1627235 .3308193 .375946$

C $-2.0298714 .548833-0.368098$

C $-0.8923404 .689680-1.390586$

C $0.1680993 .588582-1.248459$

C $1.3333463 .810590-2.213690$

C $2.3489052 .658130-2.149844$

O $-0.4715702 .341115-1.543403$

O $3.2266102 .777501-3.279593$

C $1.5967891 .319062-2.301474$

C $3.2348802 .744190-0.901631$

O $2.4741520 .214520-2.094493$

C $0.3638511 .191404-1.376933$

C $-0.457029-0.061074-1.767868$

O $0.8078581 .057160-0.026687$

C $-4.6793202 .012358 \quad 1.803029$

O $\quad-5.3207702 .1311482 .832901$

C $-5.236722 \quad 1.7549590 .457484$

C $-6.469173 \quad 1.2451610 .327993$

C $-7.1200440 .892858-0.936199$

C $-6.3916621 .226323-2.213115$

C $-1.840100-0.150047-1.104288$

C $-2.550687-1.449256-1.451069$

C $-4.626969-2.729237-0.922182$

O $-2.075057-2.293313-2.206095$

C $-5.717891-2.460967-1.910532$

C $-7.013770-2.710366-1.665044$

C $-8.114034-2.481174-2.662840$

C $-9.278729-1.702955-2.088862$

C $-10.405280-2.328006-1.706773$

C $-9.169633-0.194859-2.037748$

C $-8.3196670 .276807-0.891500$

N -3.776894 -1.557880 -0.808768

H $-3.090738-0.3823502 .358953$

H -1.5524051 .2257324 .456343$

H -3.0545972 .9911123 .505190$ 
H -0.9507442 .5799191 .336978$ H -0.3967802 .8616912 .969245$ H $-1.750117 \quad 6.2421150 .979307$ H -1.286413 $6.406563 \quad 3.211813$ H -1.862444 5.0359564 .164358 H $-0.1421595 .167276 \quad 3.737510$ H -2.345331 $3.506123-0.278041$ H $-2.9065975 .086622-0.751895$ H -1.338132 4.618923 -2.391409 H $-0.4284195 .680163-1.310139$ H $0.5339083 .580266-0.216297$ H $1.8275604 .769565-2.014321$ H $0.9497023 .878136-3.241442$ H $3.6568473 .648470-3.234164$ H $1.2552731 .253069-3.344188$ H $3.7974633 .685455-0.894378$ H $3.9850631 .947367-0.898334$ H 2.6708492 .6891670 .031689 H $3.2175800 .314812-2.713687$ H $-0.617880-0.063520-2.854121$ H $\quad 0.116319-0.964668-1.524362$ H $\quad 0.1142010 .6243580 .494324$ H $-4.5949971 .957065-0.392967$ H -7.044849 1.0410041 .229689 H $-6.2356582 .307949-2.292531$ H $-6.9227120 .916860-3.115229$ H $-5.4160430 .729292-2.245173$ H -1.751823 $-0.096034-0.014792$ H -2.472043 $0.680288-1.439235$ H $-5.018943-2.9411400 .077619$ H -4.030551 -3.585614 -1.252412 H -5.426851 -2.067112 -2.881527 H $-7.298292-3.135354-0.704856$ H -8.458525 -3.465237 -3.006832 H -7.741874 -1.967310-3.557865 H -11.255418 -1.775583 -1.320353 H -10.500742 -3.407142 -1.766571 H -10.164795 $0.251385-1.908201$ H -8.826579 $0.172266-3.008634$ H -8.762322 0.0763320 .085416 H $-4.100229-0.793424-0.223877$ C -1.247096 -2.433753 3.352281 O $0.000012-1.8590292 .917351$ C $0.333011-2.4113931 .629535$ C $-0.617809-3.5831311 .410475$ C $-1.863874-3.0919312 .120403$ H $0.112142-1.6329030 .891690$ C $1.820315-2.7786421 .538110$ H $-0.781326-3.7923300 .348877$ H $-0.244878-4.4993651 .883265$ C $-2.863567-4.1898982 .434240$ H -2.346225 $-2.344278 \quad 1.483516$ C $2.759084-1.5641071 .699382$ O $2.125102-3.7704942 .516569$ H $1.998673-3.2314840 .555544$ H $2.429910-0.7708681 .019120$ O $2.687036-1.0452453 .026325$ C $4.230702-1.8847791 .370217$ C $5.130673-0.6393651 .478977$ H $4.275044-2.2843290 .351895$ O $4.734038-2.8958522 .239457$ H $4.6826990 .170421 \quad 0.893477$ H $5.161781-0.3027732 .522494$ H -3.207771 -4.671173 1.512870 H -2.424137 -4.963134 3.073186 H $-3.739248-3.7830322 .949579$ C -2.101287 -1.386444 4.072507 H $1.982005-3.3693373 .391064$ H $1.767406-0.7746113 .187621$ H $4.624677-2.5788503 .152313$ H -1.549177 -1.0476264 .958567$ H -3.022195 -1.856667 4.437077 H $-0.980948-3.2077774 .085067$ C $6.228123-0.605957-1.357171$ O $6.633617-1.479598-0.297478$ C $6.579868-0.9027181 .010440$ C 7.4973190 .3251241 .069907 C $7.1812811 .330445-0.036267$
C $7.1254410 .637093-1.399226$

H $5.183231-0.308872-1.210217$

C $6.313766-1.399607-2.665939$

H $6.999863-1.6525561 .692654$

O $7.3947990 .951823 \quad 2.344137$

H $8.538224-0.0058440 .957747$

H $6.251116 \quad 1.8683150 .179339$

O $8.2195512 .313245-0.048268$

H $8.1403240 .358680-1.710683$

O $6.6094131 .545955-2.369653$

C $5.160990-2.369271-2.780289$

H $6.261995-0.741934-3.541154$

H $7.252948-1.960527-2.740889$

H 7.9765531 .7311662 .332425

H $7.9623983 .004451-0.681273$

H $7.2499472 .270661-2.465088$

O $5.437245-3.511769-2.089857$

O $4.129215-2.128089-3.393626$

C $4.396643-4.493907-2.084643$

H $3.484849-4.079451-1.643545$

H $4.207670-4.848976-3.102125$

H $4.728393-5.337506-1.473604$

SCF Energy (B3LYP/6-31G**//MMFF)= -3245.91786061

06_00156

MM̄MF Geometry

C -0.8640882 .9449832 .198120$

C -1.5412162 .7141543 .333067$

C -1.9028181 .3615443 .900435$

O -1.4533500 .2821003 .050413$

C -3.4352521 .2495934 .024273$

C $-3.893373-0.1089784 .519034$

C $-4.277886-1.1197053 .712906$

C $-3.899419-0.2790896 .014477$

C $-4.289211-1.1212472 .208850$

C $-5.675514-1.4515351 .644992$

C $-5.802330-1.0477140 .167838$

C $-7.118012-1.554566-0.426028$

C $-7.239347-1.220322-1.920610$

O $-4.695709-1.588447-0.560890$

O $-8.320274-2.009739-2.439889$

C $-5.942938-1.655087-2.639867$

C $-7.6380620 .244674-2.134671$

O $-5.958680-1.239841-4.003978$

C $-4.650933-1.178605-1.934346$

C $-3.414255-1.828855-2.596567$

O $-4.554070 \quad 0.241992-2.066338$

C $-0.230066-0.2599053 .292468$

$\begin{array}{lllll}\text { O } & 0.629768 & 0.166010 & 4.045753\end{array}$

C $-0.149885-1.5155112 .510248$

C $0.945307-2.2857882 .586257$

C $1.115986-3.6053201 .970503$

C $-0.064104-4.1961211 .243533$

C $-2.096931-1.603785-1.838388$

C $-0.909509-2.188462-2.585568$

C $1.562950-2.439607-2.413961$

O $-1.013231-2.771032-3.661939$

C $1.828044-3.824091-1.910929$

C $2.969781-4.182468-1.304829$

C $3.274072-5.576642-0.841918$

C $3.734507-5.6276740 .597051$

C $5.036924-5.7565510 .902725$

C $2.677492-5.6268481 .676511$

C $2.297841-4.2395722 .120041$

N $0.284487-1.975319-1.909026$

H $-0.5072402 .109957 \quad 1.602116$

H -1.869266 3.5687553 .922211

$\mathrm{H}-1.452246 \quad 1.2617224 .896189$

H $-3.906071 \quad 1.4766473 .060381$

H -3.8117982 .0232644 .707170$

H $-4.585413-2.0582444 .171464$

H -4.238632 -1.274928 6.318586

H -2.892504 -0.1356136 .419059$

H $-4.568697 \quad 0.453631 \quad 6.477073$

H -3.944133 -0.169332 1.797234

H $-3.572937-1.877826 \quad 1.865412$

H $-5.841014-2.5315081 .752938$

H $-6.460084-0.9446552 .220831$ 
H $-5.758741 \quad 0.0472730 .119943$ H $-7.976445-1.1565620 .129232$ H -7.169340 -2.646729 -0.311986 H -8.358287 -1.861315 -3.400548 H $-5.942185-2.753506-2.659779$ H $-8.6004420 .451442-1.651120$ H $-7.7868210 .465047-3.197519$ H $-6.9077410 .950840-1.734413$ H -5.753946 $-0.289500-4.026273$ H -3.570669 -2.913202 -2.669566 H $-3.304383-1.446047-3.619363$ H $-3.6258440 .509546-1.985411$ H -1.023026 -1.801143 1.934651 H $1.786453-1.9361933 .183793$ H $-0.891533-4.3745981 .939124$ H $\quad 0.156287-5.1462140 .752619$ H $-0.412241-3.5222420 .455027$ H -1.904370 -0.535391 -1.696822 H -2.146278 -2.077891 -0.851582 H $2.322064-1.727368-2.079647$ H $1.540803-2.442137-3.508434$ H $1.057997-4.573229-2.079027$ H $3.751446-3.441338-1.167338$ H $4.056976-5.979559-1.497741$ H $2.410050-6.239988-0.971713$ H $5.370536-5.8251631 .932881$ H $5.798223-5.7885160 .130308$ H $3.056319-6.1559402 .562023$ H $1.819879-6.2235521 .353393$ H $3.091386-3.7303062 .668872$ H $0.264613-1.547239-0.987826$ C -0.8854944 .4553750 .199800$ O $0.0222613 .643253-0.576185$ C $0.5542354 .436703-1.650491$ C $-0.3771765 .634202-1.776454$ C $-0.7249615 .884247-0.322643$ H $1.5399774 .790783-1.325729$ C $0.6948783 .595252-2.921285$ H $0.1064396 .489802-2.257203$ H -1.281152 $5.388277-2.345666$ C $-1.9572476 .751569-0.132165$ H $0.131556 \quad 6.3690250 .165059$ C $1.5528262 .323258-2.763318$ O $-0.6059423 .181785-3.360583$ H $1.0989344 .217064-3.728591$ H $1.0214481 .575699-2.161486$ O $1.7084131 .747377-4.067464$ C $2.9522372 .556008-2.161268$ C $3.8459791 .305003-2.247880$ H $2.8459302 .858780-1.115179$ O $3.5997373 .628941-2.845917$ H $3.2820750 .445782-1.874100$ H $4.0821861 .108992-3.300543$ H -1.803289 $7.741048-0.575169$ H -2.838950 $6.305523-0.604507$ H $-2.177902 \quad 6.8880500 .931241$ C $-0.540075 \quad 4.3204801 .684177$ H - $-1.0169112 .685103-2.632057$ H $0.8166491 .590854-4.422995$ H $3.6538783 .387636-3.786654$ H -1.076222 5.0799442 .264675 H 0.5339834 .4869171 .826841 H -1.8978974 .0804750 .001217$ C 4.4562380 .5134490 .640666 O $4.9622141 .653744-0.065680$ C $5.1726311 .459993-1.469231$ C $6.1647740 .309622-1.689511$ C $5.693639-0.968445-1.005579$ C $5.380434-0.6998890 .464109$ H $3.447026 \quad 0.2753850 .284114$ C 4.3629800 .8927332 .127211 H $5.6558822 .374098-1.835688$ O $6.3547280 .054731-3.077063$ H $7.1434270 .585705-1.275771$ H $4.829312-1.398675-1.521533$ O $6.729947-1.950308-1.069227$ H $6.319472-0.5396481 .009522$ O $4.752519-1.8487151 .029263$
C 3.3119051 .9461702 .399379

H 4.1065760 .0182072 .734964

H 5.3288091 .2940892 .458444

H $6.6624190 .881613-3.486383$

H $6.944999-2.082476-2.008605$

H $5.344007-2.6064010 .880923$

O 3.4837852 .4170743 .670503

O 2.4441642 .2980361 .613271

C 2.5665293 .4319444 .088504

H 2.8900493 .8003385 .065707

H 2.5686274 .2679823 .382904

H 1.5637803 .0089764 .188616

SCF Energy (B3LYP/6-31G**//MMFF)= -3245.93528889

06_00157

MM̄FF Geometry

C $-0.510885-0.057118-2.015756$

C $-1.3587950 .549787-1.171597$

C -1.031222 1.0902300 .201557

O $0.394712 \quad 1.1331790 .432166$

C $-1.6669990 .176561 \quad 1.264779$

C -1.4835610 .6714392 .685622$

C -0.6417310 .1289903 .588386$

C -2.3580581 .8350313 .075954$

C $0.305827-1.0216453 .390180$

C $1.758471-0.5818083 .612720$

C $2.760461-1.6662173 .188837$

C $4.191300-1.2202213 .496549$

C $5.237887-2.2072912 .961510$

O $2.600624-1.8764101 .782005$

O $6.508291-1.5402583 .018403$

C $4.927487-2.5113811 .479396$

C $5.359468-3.4383113 .866652$

O $5.798076-3.5236210 .980187$

C $3.452842-2.8848061 .224144$

C $3.109573-3.002095-0.281370$

O $3.190926-4.1638601 .814830$

C $1.0609892 .232509-0.019145$

O $\quad 0.5872713 .183431-0.620755$

C 2.4873792 .0756950 .343918

C $3.3844192 .982596-0.071379$

C 4.8188662 .9829910 .228217

C 5.3625871 .8443341 .049410

C $3.254702-1.711021-1.093213$

C $4.592976-1.550910-1.792662$

C $5.8770030 .106545-3.144936$

O $5.431341-2.444694-1.861988$

C $6.8426580 .830121-2.260988$

C $7.3016222 .063454-2.523038$

C $8.3207742 .773012-1.679380$

C $7.8699454 .149348-1.246496$

C $8.2702075 .248215-1.908575$

C 7.0383014 .2568630 .010576

C $5.5754014 .001068-0.233154$

N $4.718454-0.293335-2.369649$

H $\quad 0.529697-0.191960-1.737307$

H -2.399404 $0.642784-1.472051$

H -1.446309 2.1012770 .288616

H -1.272434 -0.8385611 .152302$

H -2.7436270 .0823681 .077196$

H $-0.616831 \quad 0.5601204 .588645$

H -2.246506 2.1040824 .131715

H -2.106083 2.7198692 .484002

H -3.4124351 .5912912 .910226$

H $0.051539-1.8106844 .108168$

H $0.201969-1.4656402 .398378$

H 1.9468590 .3293363 .031569

H $1.906340-0.3297874 .670296$

H $2.521813-2.5935473 .722672$

H $4.326132-1.0618624 .573958$

H $4.371077-0.2428423 .030099$

H $7.167753-2.1278882 .610799$

H $5.168409-1.6001650 .919864$

H $5.656163-3.1382464 .878919$

H $6.146403-4.1144533 .514963$

H $4.428786-4.0036193 .948648$

H $5.475198-4.3804431 .307554$

H $3.666481-3.831163-0.734174$ 
H $2.051915-3.294701-0.347864$

H $2.403759-4.5443311 .391019$

H $2.760555 \quad 1.2212250 .951024$

H $3.0359113 .817123-0.678570$

H 6.4523821 .8363851 .118311

H 4.9705541 .8928022 .070677

H 5.0837440 .8809950 .609312

H $2.502472-1.722743-1.891463$

H $3.058431-0.830702-0.474412$

H $\quad 5.5166340 .734246-3.966079$

H $6.358453-0.779991-3.569940$

H $7.206457 \quad 0.300827-1.383404$

H $6.9577702 .578386-3.417420$

H $9.2421512 .853629-2.271316$

H $8.5911922 .182317-0.795309$

H $7.9775966 .241455-1.584419$

H $8.8949925 .178194-2.792986$

H 7.1203055 .2711770 .424804

H 7.4630193 .6127110 .785729

H $5.0918114 .778724-0.826174$

H $3.9925220 .400043-2.213104$

C -1.429773 -2.055923 -3.301739

O $-2.709059-2.097060-2.634120$

C $-2.681816-3.154632-1.660728$

C -1.545911 -4.075650 -2.091833

C $-0.527574-3.068130-2.590102$

H -2.414417 -2.691973 -0.702681

C $-4.051800-3.828964-1.536452$

H -1.173366 -4.691902 -1.268123

H -1.847356 -4.741111 -2.908845

C $0.544187-3.666626-3.484124$

H - $0.043537-2.619801-1.714806$

C $-5.234377-2.866119-1.298050$

O $-4.316190-4.544390-2.749964$

H -4.007266 -4.580999-0.739038

H $-5.495242-2.348260-2.229510$

O $-6.374733-3.661066-0.946315$

C $-5.016634-1.819098-0.189511$

C -6.306246-1.052850 0.167602

H -4.249581-1.111283 -0.517995

O $-4.524955-2.457610 \quad 0.989482$

H $-6.796888-0.730940-0.756289$

H $-6.992884-1.7347300 .683025$

H $1.129264-4.412627-2.936226$

H $0.108444-4.158653-4.360071$

H $1.230854-2.891731-3.839335$

C $-0.920715-0.610920-3.352790$

H $-5.076973-5.127073-2.589053$

H $-6.632286-4.170577-1.732860$

H -5.179121 -3.1257601 .257051$

H -1.701739 $0.030114-3.781513$

H - $0.057869-0.547757-4.026451$

H - $-1.624613-2.384369-4.330867$

C $-5.8271691 .888891-0.594876$

O $-5.200204 \quad 1.1356750 .447333$

C $-6.0332790 .161031 \quad 1.085496$

C $-7.296017 \quad 0.8293871 .648399$

C -8.0438051 .6234200 .582160$

C -7.095986 $2.601178-0.109632$

H $-6.0970191 .206020-1.410177$

C $-4.7908342 .851751-1.195686$

H -5.462127 -0.2117571 .945079$

O $-8.176002-0.1349892 .216315$

H -7.011946 1.5180032 .455069

H -8.529822 $0.963160-0.145808$

O

H -6.8507143 .4131330 .584002$

O $-7.7595333 .188076-1.227482$

C $-4.1040833 .758707-0.194263$

H -4.002568 2.251854-1.663298

H $-5.2514633 .475821-1.969016$

H -7.680697 -0.613270 2.903102

H -9.6576101 .7473121 .675050$

H -8.574129 $3.603337-0.895614$

O $-3.114634 \quad 4.448737-0.837755$

O -4.3841753 .8546960 .991943$

C -2.350492 $5.338486-0.017706$

H $-2.996428 \quad 6.1161880 .400627$
H $-1.847702 \quad 4.782611 \quad 0.779327$

H -1.591599 $5.812256-0.646139$

SCF Energy (B3LYP/6-31G**//MMFF) $=-3245.91672637$

06_00158

MM̄FF Geometry

C 0.4752472 .3166672 .686785

C 1.6417562 .9758662 .615097

C 2.3192593 .3101111 .316107

O 3.4477702 .4090601 .208568

C 2.8365754 .7580131 .256407

C 1.7693055 .8309991 .308742

C 1.0005736 .1882820 .260903

C 1.6398896 .5510142 .625011

C $0.9943395 .564632-1.106588$

C $-0.4244175 .137754-1.518578$

C $-0.4067003 .891043-2.413054$

C $-1.7760603 .648531-3.042291$

C $-1.7991362 .346678-3.853717$

O $-0.0663842 .782865-1.575099$

O $-3.1739652 .077483-4.163138$

C $-1.2647541 .192746-2.974516$

C $-1.0818332 .510251-5.199207$

O $-1.1546990 .001364-3.748176$

C $0.0603061 .521473-2.250652$

C $0.3959580 .503168-1.135898$

O $1.1314301 .555409-3.193376$

C $3.8374412 .035837-0.039815$

O $3.4028272 .445903-1.103926$

C 4.8744660 .9926120 .113173

C $5.1427010 .174442-0.915680$

C $6.130819-0.907664-0.923950$

C $6.972090-1.0872080 .313538$

C $0.750296-0.910900-1.591422$

C $1.367331-1.735295-0.471875$

C $2.287906-4.016923-0.046167$

O $1.551648-1.3067120 .663835$

C $3.776871-3.925927-0.154891$

C $4.514772-4.752883-0.910538$

C $6.014133-4.715458-0.989390$

C $6.520740-4.164140-2.303334$

C $6.443825-4.883022-3.436070$

C $7.196451-2.811720-2.280519$

C $6.235459-1.681698-2.025173$

N $1.682306-3.016053-0.902052$

H -0.0205972 .0008881 .772578$

H 2.1487193 .2649863 .532496

H 1.6276993 .1209010 .488478

H $3.435027 \quad 4.892427 \quad 0.345215$

H 3.5658064 .9191162 .062947

H 0.2918127 .0045320 .394133

H $0.868027 \quad 7.3276062 .600577$

H 1.3750145 .8498213 .422159

H 2.5865167 .0346582 .887290

H $1.3661566 .301684-1.828720$

H $1.6817444 .716838-1.160746$

H -1.027108 4.924614-0.626602

H $-0.9027155 .974292-2.043043$

H $0.3593114 .018106-3.186978$

H -2.075059 $4.497789-3.669179$

H $-2.5357963 .579135-2.250946$

H -3.214039 $1.210250-4.601825$

H -2.027185 $0.989848-2.213042$

H $-1.5393803 .321081-5.778824$

H - $-1.1844051 .610531-5.816088$

H $-0.0184172 .735033-5.092899$

H $-0.3466330 .070979-4.284595$

H $1.2426140 .901619-0.564302$

H $-0.4296990 .461181-0.413007$

H $1.9663191 .459666-2.704734$

H 5.3706750 .9150861 .073697

H $4.5821710 .302208-1.841031$

H $7.562453-0.1855210 .510998$

H $7.676696-1.9184560 .249138$

H $6.337171-1.2899241 .183343$

H $-0.150499-1.433202-1.930881$

H $1.470958-0.881193-2.416106$

H $\quad 1.912137-4.993459-0.368290$ 
H $\quad 1.980647-3.856374 \quad 0.991873$ H $4.267839-3.1602350 .440103$ H $4.014471-5.520380-1.497201$ H $6.392316-5.737272-0.853455$ H $6.432765-4.144249-0.152002$ H $6.832200-4.503606-4.375333$ H $5.989405-5.868319-3.447713$ H $7.679748-2.611862-3.246419$ H $8.019672-2.837851-1.560782$ H $5.571504-1.479403-2.866780$ H $1.556553-3.251400-1.882282$ C $\quad 0.0333420 .501793 \quad 4.355609$ O $-0.668003-0.372148 \quad 3.446597$ C -1.222248 -1.469463 4.198775 C $-0.664261-1.3490235 .613231$ C $-0.490531 \quad 0.148857 \quad 5.746515$ H -2.305816 -1.309421 4.237271 C $-0.906160-2.8092593 .525512$ H -1.341154 -1.772422 6.361883 H $\quad 0.302947-1.8556405 .712933$ C $0.434471 \quad 0.549935 \quad 6.882813$ H -1.476929 0.6057085 .903962 C -1.453202 -2.971393 2.094353 O $0.516564-2.9797923 .464668$ H -1.277952 -3.627825 4.153058 H $-0.907252-2.324996 \quad 1.398959$ O $-1.186722-4.3192791 .683805$ C $-2.961635-2.7067381 .946446$ C $-3.510692-3.1404620 .573456$ H -3.151080 -1.640201 2.100276 O $-3.677709-3.4089002 .963847$ H -2.846040 -2.766908 -0.211938 H $-3.501424-4.2354270 .516196$ H 0.0410340 .1959497 .841419 H $1.436100 \quad 0.126028 \quad 6.753491$ H $\quad 0.534681 \quad 1.638033 \quad 6.941924$ C -0.2025191 .9646993 .979207$ H $\quad 0.878817-2.2357072 .952967$ H $-0.231814-4.4706281 .788690$ H -3.479216 -4.355827 2.863470 H 0.1591962 .6231174 .777382 H -1.275320 2.1597323 .861680 H $\quad 1.1003600 .2535224 .276128$ C $-4.450629-0.577373-0.804639$ $\begin{array}{llll}\text { O } & -5.037490 & -1.215358 & 0.337444\end{array}$ C $-4.956988-2.6458790 .344160$ C $-5.635762-3.217783-0.907223$ C $-5.076784-2.604892-2.186050$ C $-5.106182-1.079796-2.098325$ H -3.373596 -0.780300 -0.830974 C $-4.6500010 .937344-0.638396$ H -5.549355 -2.981246 1.204798 O $-5.496659-4.634090-0.940018$ H -6.711418 -3.003742 -0.854738 H -4.066635 -2.978034 -2.392105 O $-5.893117-3.026308-3.280866$ H $-6.147626-0.738453-2.150547$ O $-4.434945-0.541370-3.234430$ C -3.7119371 .5349660 .390690$ H -4.454533 $1.452630-1.584829$ H $-5.676007 \quad 1.147915-0.314936$ H -5.922756 -4.949217 -1.755547 H -5.552657 -2.589222 -4.080359 H -3.508077 -0.834200 -3.201002 O O C $-3.0871443 .599248 \quad 1.360747$ H $-2.0318703 .460810 \quad 1.110238$ H -3.3284844 .6625241 .282852$ H -3.2895713 .2661172 .383049$ SCF Energy $\left(B 3 L Y P / 6-31 G^{* *} / / M M F F\right)=-3245.94663014$

06_00159

MM̄FF Geometry

C $-0.311615-3.7306171 .607492$

C $0.308490-2.5600911 .399277$

C $0.648254-1.5783392 .485847$

O $-0.010426-0.3445682 .120582$
C $2.165840-1.3275652 .512686$

C $2.578769-0.3001703 .546401$

C $2.8368870 .994878 \quad 3.274612$

C $2.711455-0.8232734 .952064$

C 2.7355771 .6954421 .946126

C 4.0808452 .2476701 .457180

C 5.1429101 .1671401 .202841

C 6.4568301 .8062390 .747550

C 7.5208140 .7500030 .418578

$\begin{array}{lllll}\text { O } & 4.652992 & 0.277568 & 0.193679\end{array}$

O $8.5828511 .427255-0.270780$

C $6.906498-0.283505-0.549821$

C 8.1435910 .1601861 .689029

O $7.815339-1.357032-0.785003$

C $5.527136-0.820388-0.095898$

C $4.864665-1.712820-1.176555$

O $5.719020-1.6438111 .062338$

C $-0.7615070 .308507 \quad 3.041951$

O $-1.029325-0.0675244 .173931$

C $-1.179145 \quad 1.6361772 .529645$

C -1.1128451 .9578891 .228981$

C -1.4084963 .2575680 .629473$

C -1.7741604 .4000121 .536240$

C $4.442447-0.996636-2.463110$

C $3.098892-0.294224-2.359852$

C $1.7458931 .483469-3.464731$

O $2.219970-0.644820-1.576090$

C $1.6519092 .555680-2.428024$

C $1.3573013 .837690-2.695780$

C $1.1846744 .875757-1.621661$

C $-0.2021745 .492425-1.590018$

C $-0.3422936 .829343-1.577436$

C $-1.4358144 .608119-1.552992$

C -1.283481 $3.377502-0.707348$

N $2.973076 \quad 0.730847 \quad-3.288828$

H $-0.560426-4.0069962 .630390$

H $0.569281-2.2546010 .389857$

H $\quad 0.299389-1.9401523 .460259$

H $2.508822-1.0171041 .519110$

H $2.696014-2.2676922 .716004$

H 3.1345461 .6421034 .098671

H $3.029114-0.0473875 .656732$

H $1.754196-1.2179285 .306483$

H $3.454645-1.6264644 .988946$

H 2.2927861 .0628551 .171703

H 2.0439752 .5384852 .068729

H 3.8914102 .7948510 .524274

H 4.4624052 .9747462 .185081

H 5.3041530 .6067382 .130123

H 6.8333812 .5067541 .503562

H $6.2749362 .408439-0.153966$

H $9.2273680 .754932-0.551706$

H $6.7960050 .229538-1.511662$

H 8.6092350 .9525552 .287242

H 8.949311-0.541692 1.447113

H $7.419248-0.3528522 .324926$

H $7.790875-1.940584-0.007552$

H $5.554335-2.527321-1.432814$

H $3.988100-2.210050-0.740162$

H $4.937017-2.2101601 .169719$

H $-1.5264862 .336872 \quad 3.281279$

H -0.7812591 .2000240 .520275$

H -2.700287 4.1804062 .078222

H -1.9360245 .3377420 .998143$

H -0.9765914 .5853822 .264155$

H $5.210774-0.299296-2.806042$

H $4.323206-1.749424-3.252931$

H $\quad 0.8999330 .798829-3.356105$

H $1.7465331 .886024-4.482298$

H $1.7760442 .250519-1.391100$

H $1.2138284 .154601-3.725611$

H $1.9456465 .649314-1.787415$

H $1.4005504 .452743-0.631903$

H -1.321903 $7.294992-1.549852$

H $0.5180497 .490204-1.594710$

H $-1.6569174 .284437-2.578744$

H -2.321481 $5.170125-1.231477$

H -1.022132 2.489396-1.283299 
H $3.7659230 .976909-3.873885$ C $-0.569243-4.314676-0.906220$ O $-1.353485-3.139782-1.192461$ C -1.954754 -3.278621 -2.491102 C $-1.339228-4.526327-3.116075$ C $-1.057461-5.375439-1.892322$ H $-3.023090-3.458446-2.323188$ C $-1.763825-1.996043-3.308800$ H -2.016010 -5.008575 -3.828041 H $-0.405168-4.300899-3.643473$ C $-0.061403-6.493573-2.146085$ H -2.004093 -5.808333 -1.541647 C $-2.251147-0.702710-2.623207$ O $-0.368947-1.821362-3.594945$ H -2.255580 -2.108768 -4.282116 H - $-1.577033-0.430158-1.801675$ O $\quad-2.156627 \quad 0.356336-3.586309$ C $-3.698053-0.750165-2.099789$ C $-4.1883190 .624293-1.604939$ H -3.760971 -1.475702 -1.283679 O $-4.568707-1.206352-3.135252$ H $-3.4491601 .030783-0.908343$ H $-4.2456601 .311823-2.457058$ H $-0.438272-7.177091-2.913925$ H $\quad 0.902547-6.102383-2.488479$ H $0.115151-7.074325-1.235323$ C $-0.752782-4.7212130 .559994$ H $\quad 0.102026-1.777157-2.744675$ H -1.249168 $0.353035-3.934855$ H -4.485586 $-0.588979-3.882174$ H $-0.223151-5.6621780 .748436$ H -1.818899 $-4.910366 \quad 0.737990$ H $0.480302-4.063987-1.107608$ C $-4.873946 \quad 0.146804 \quad 1.336600$ O $-5.602272-0.3177240 .193378$ C $-5.5807980 .559861-0.937489$ C $-6.1556301 .929445-0.548168$ C -5.4431562 .5206350 .664152$ C -5.4092921 .5063611 .807002$ H -3.8093590 .2266301 .089056$ C $-5.032383-0.9062922 .444173$ H $-6.2672070 .124254-1.674183$ O $-6.0716652 .845966-1.634775$ H -7.220232 $1.818746-0.303725$ H $-4.437347 \quad 2.8623130 .397975$ O -6.1656053 .6799561 .084795$ H -6.4198881 .3897242 .219358$ O -4.5739651 .9966482 .852762$ C -4.308298 -2.196465 2.124584 H $-4.628663-0.540643 \quad 3.394317$ H $-6.096840-1.1398752 .569788$ H $-6.5490962 .453259-2.385506$ H -5.6399664 .1184891 .774851$ H -4.987683 2.8007023 .209024 O $-4.794918-3.1870092 .929557$ O $-3.414890-2.317773 \quad 1.298541$ C -4.202983 -4.477328 2.751011 H -4.275644 -4.792756 1.705679 H -3.160262 -4.4577143 .079451$ H $-4.752467-5.1916383 .369811$

SCF Energy (B3LYP/6-31G**//MMFF) $=-3245.92789628$

0600160

MM̄FF Geometry

C $-1.874786-1.692280-1.448798$

C $-0.745678-1.953973-0.774325$

C $-0.443196-1.3557090 .576582$

O $0.838521-0.6860340 .526125$

C $-0.375082-2.4716701 .633743$

C $-0.226380-1.9486123 .050145$

C $0.908514-1.9944943 .776474$

C -1.485011-1.383375 3.658765

C $2.249423-2.5308603 .359480$

C $3.318000-1.4307283 .379326$

C $4.644646-1.9107792 .770979$

C $5.706084-0.8133682 .864327$

C $7.005941-1.1968182 .142668$

O $4.396675-2.2417371 .400934$
O 7.7841230 .0037332 .018204

C $6.663428-1.6898940 .719303$

C $7.845718-2.1663692 .982029$

O $7.829261-2.1805760 .062165$

C $5.535039-2.7411930 .687880$

C $5.066592-3.086558-0.747304$

O $6.018378-3.9554721 .275587$

C $0.8670940 .541500-0.065117$

O $\quad-0.0613051 .112047-0.616151$

C 2.2328851 .0913630 .084448

C $2.5237552 .290414-0.442648$

C $3.8078292 .990308-0.355877$

C 4.9371162 .2941950 .354872

C $4.446883-1.927545-1.535664$

C $5.420615-1.190024-2.438587$

C $5.5391480 .805769-3.930328$

O $6.560419-1.583739-2.668449$

C $6.180406 \quad 1.936772-3.190504$

C $5.9643603 .227947-3.484811$

C $6.6497264 .369437-2.790925$

C $5.6797765 .394519-2.249040$

C $5.4166986 .524060-2.928007$

C $5.0920805 .154570-0.877631$

C $3.9124344 .218843-0.904921$

N $4.843651-0.053777-2.991447$

H -2.604903 -1.013446 -1.015071

H $-0.006815-2.627598-1.201054$

H $-1.210927-0.6282500 .863999$

H $\quad 0.437717-3.1612301 .384278$

H -1.290026 -3.078918 1.592414

H $0.881586-1.6047464 .793520$

H -1.336741 -1.054702 4.692909

H -1.837285 -0.5192673 .088902$

H -2.275747 -2.1408233 .664699$

H $2.536343-3.3274144 .056724$

H $2.210616-2.9911252 .370199$

H $2.950067-0.5680812 .809842$

H $3.480763-1.0950924 .411065$

H $4.971166-2.8097823 .306958$

H $5.908281-0.5556923 .911498$

H $\begin{array}{llll}5.315722 & 0.103814 & 2.405088\end{array}$

H $8.575710-0.2115211 .495332$

H $6.345587-0.8065350 .153732$

H $8.107672-1.7081703 .943488$

H $8.798946-2.3937912 .492382$

H $7.331915-3.1065793 .192406$

H $8.016287-3.0678170 .413153$

H $5.878057-3.569614-1.304721$

H $4.283667-3.853223-0.654612$

H $5.462392-4.6902920 .967804$

H 2.9563880 .5041010 .636509

H $1.7408872 .824820-0.979602$

H 5.8961662 .8077190 .260110

H 4.7158712 .2084711 .423754

H $5.0931571 .291096-0.055843$

H $3.672989-2.337364-2.197873$

H $3.957328-1.213286-0.867571$

H $4.8008861 .153574-4.659964$

H $\quad 6.3050800 .228646-4.458197$

H $6.8797021 .680140-2.398178$

H $5.2866383 .480660-4.297166$

H $7.3228694 .842944-3.517900$

H $7.2972974 .014986-1.979356$

H $4.7464537 .279148-2.530718$

H $5.8614186 .715608-3.898964$

H $4.7388396 .104127-0.452797$

H $5.8819054 .828683-0.194876$

H $3.0387074 .638956-1.405309$

H $3.9108690 .222811-2.699096$

C $-3.492483-3.112948-2.790480$

O $-4.614251-2.323691-2.343161$

C $-5.388118-3.114728-1.420660$

C $-4.943959-4.556305-1.638512$

C $-3.463666-4.363413-1.909476$

H $-5.085540-2.810650-0.411337$

C $-6.886286-2.860241-1.611776$

H $-5.143620-5.189815-0.769113$

H $-5.434438-5.005989-2.509643$ 
C $-2.798267-5.561884-2.562508$ H $-2.963457-4.157509-0.955001$ C $-7.294398-1.376878-1.484933$ O $-7.253995-3.309867-2.921235$ H -7.453344 -3.473474 -0.901042 H $-6.909872-0.802722-2.336927$ O $-8.723681-1.305807-1.557746$ C $-6.846993-0.704475-0.172302$ C $-7.3851550 .731999-0.025316$ H -5.753405 -0.679054 -0.138664 O $-7.285520-1.4843620 .939620$ H -7.098661 $1.305058-0.913812$ H -8.4810970 .7108720 .006252$ H -2.867027 -6.440714 -1.913266 H -3.270505 -5.812718 -3.518300 H - $1.738588-5.362913-2.751293$ C $-2.215262-2.267458-2.793993$ H -8.224799 -3.339059-2.959589 H -8.982219 -1.531011 -2.467265 H $-8.255813-1.5372830 .896301$ H -2.355794 -1.420590 -3.477303 H -1.381934 -2.861190 -3.187314 H -3.717304 -3.394035 -3.827703 C -4.7424672 .2010310 .455922$ O C $-6.8820971 .433978 \quad 1.257509$ C -7.372903 2.8806091 .420567 C -6.6583853 .8388920 .469722$ C -5.1427063 .6748840 .582610$ H $-4.9661251 .858577-0.562620$ C -3.2396541 .9536120 .647136$ H $-7.282523 \quad 0.8626212 .104561$ O H -7.1838313 .2127662 .449794$ H $-6.991623 \quad 3.701591-0.565640$ O -6.9889865 .1844490 .821767$ H -4.8087814 .0921161 .539380$ O $-4.5080224 .423826-0.451121$ C -2.6984432 .3111842 .016687$ H -3.0393630 .8858690 .507529$ H $-2.6788912 .517336-0.107023$ H -9.2085432 .3680431 .834353$ H $-7.957875 \quad 5.2624960 .785584$ H $-4.8067105 .345650-0.366445$ O $-1.3331252 .327597 \quad 1.957458$ O -3.3719832 .5146423 .016781$ C -0.6609982 .6131783 .187739$ H -0.9052813 .6251813 .523910$ H -0.9311241 .8765043 .950233$ H 0.4156982 .5517073 .008149

SCF Energy $\left(\mathrm{B} 3 \mathrm{LYP} / 6-31 \mathrm{G}^{* *} / / \mathrm{MMFF}\right)=-3245.91744823$

0600161

MM̄FF Geometry

C $-1.2417951 .548198 \quad 3.884127$

C -0.0058251 .3840244 .380503$

C 0.8735680 .1622154 .275946

O $1.843551 \quad 0.4601713 .243942$

C $0.146863-1.1532993 .930410$

C $0.924670-2.3941844 .331738$

C $1.552160-3.2319403 .483423$

C $0.917186-2.7025325 .809492$

C $1.663995-3.1310451 .989798$

C $3.123428-3.2388221 .527473$

C $3.239937-3.227258-0.003156$

C $4.703841-3.322001-0.436735$

C $4.857974-3.217174-1.961382$

O $2.670838-2.007971-0.485494$

O $6.251499-2.997677-2.227031$

C $4.077027-1.982193-2.460879$

C $4.491208-4.535605-2.652703$

O $4.083782-1.921559-3.885573$

C $2.633070-1.898772-1.913928$

C $2.014423-0.528614-2.265014$

O $1.846682-2.931596-2.508471$

C 3.1444710 .1463983 .461538

O $3.606032-0.4239454 .440505$

C $4.004140 \quad 0.572393 \quad 2.331097$
C $3.590798 \quad 1.3790231 .341148$

C $4.401527 \quad 1.8551390 .216473$

C 5.7893751 .2907870 .063431

C $0.627128-0.293459-1.653302$

C $0.3051911 .184138-1.632885$

C $-0.2160753 .101521-3.129075$

O $0.3673451 .838820-0.594868$

C $1.1090773 .711212-3.463381$

C $1.7234384 .629855-2.702582$

C $3.0749775 .201697-3.023431$

C $4.1164004 .857942-1.980381$

C $4.7174825 .815507-1.254411$

C $4.5117313 .401616-1.829044$

C $3.8613142 .753167-0.634536$

N $-0.0165281 .685766-2.883343$

H -1.7108720 .7566313 .307065$

H 0.4352222 .2109594 .935690

H 1.3726440 .0702495 .248393

H $-0.095081-1.1720672 .862844$

H $-0.816341-1.2019044 .455899$

H $2.040134-4.1096663 .906418$

H $1.419379-3.6482056 .040589$

H $1.427416-1.9164066 .373661$

H $-0.111799-2.7820936 .175394$

H $1.090328-3.9554351 .554053$

H $1.230007-2.202177 \quad 1.611842$

H $3.689303-2.3986421 .946445$

H $3.573288-4.1600421 .918404$

H $2.665895-4.074542-0.396728$

H $5.162060-4.247221-0.065747$

H $5.272140-2.5019500 .024799$

H $6.349914-2.855210-3.184297$

H $4.629501-1.095999-2.119770$

H $5.132341-5.347503-2.288367$

H $4.667199-4.480467-3.732669$

H $3.453413-4.832120-2.486803$

H $3.435993-2.569094-4.212116$

H $2.6859290 .262699-1.910846$

H $1.936570-0.420410-3.354086$

H $\quad 0.907723-2.712804-2.397318$

H 5.0237970 .2029442 .377100

H 2.5671691 .7491041 .357702

H $6.435806 \quad 1.6339850 .878309$

H $6.2693751 .571203-0.877060$

H $5.7639600 .195013 \quad 0.071645$

H $-0.149278-0.821025-2.218093$

H $0.574602-0.652655-0.619803$

H $-0.6740153 .562991-2.248707$

H $-0.9028383 .201772-3.974736$

H $1.5936273 .362736-4.372584$

H $1.2410534 .980859-1.793440$

H $2.9649026 .289419-3.117293$

H $3.4296724 .851271-4.001020$

H $5.4815865 .570741-0.523880$

H $4.4581226 .862269-1.371936$

H $5.6040993 .350850-1.770738$

H $4.2280902 .846430-2.731300$

H $2.8326263 .066183-0.457708$

H $0.038828 \quad 1.071430-3.690422$

C -2.2628753 .6611122 .870645$

O $-\begin{array}{llll}-3.007810 & 2.955214 & 1.860792\end{array}$

C -2.5313243 .3838310 .567704$

C -1.5728364 .5442970 .821704$

C -1.0020814 .1703802 .176254$

H -1.9613922 .5467330 .155963$

C $-3.7070513 .718193-0.354988$

H -0.8095004 .6313160 .043152$

H -2.1023495 .5024430 .880403$

C -0.3179075 .3214322 .890675$

H -0.2801093 .3571132 .030650$

C $-4.6875532 .548579-0.574943$

$\begin{array}{lllll}\text { O } & -4.447751 & 4.808223 & 0.208525\end{array}$

H $-3.3314474 .075517-1.320626$

H -5.1981252 .2997930 .363116$

O $-5.7028342 .992970-1.483826$

C $-4.0364251 .281903-1.161534$

C $-5.0598340 .162627-1.424781$

H $-3.2793020 .908514-0.465703$ 
O $-3.3671201 .607353-2.378505$ H $-5.569282-0.068231-0.482705$ H -5.825231 $0.520250-2.124083$ H $0.526217 \quad 5.6926142 .300349$ H -1.005595 6.1575593 .056326 H 0.0662235 .0022753 .864591 C -2.060249 2.7897434 .110655 H -4.7439224 .5349681 .093827$ H $-6.1017073 .794075-1.102929$ H -4.030436 $1.970423-2.990178$ H -3.041391 2.4627004 .477955 H -1.6165263 .3979884 .908363$ H -2.890681 4.5136903 .164468 C -3.555504 -2.092770 -0.002068 O $-3.276464-1.551006-1.295828$ C $-4.426468-1.113623-2.024782$ C $-5.413943-2.276345-2.203558$ C $-5.775541-2.930350-0.870661$ C $-4.508170-3.291639-0.093592$ H -4.013062 -1.311665 0.618255 C $-2.218400-2.4313880 .669464$ H $-4.064689-0.842180-3.024643$ O $-6.605110-1.836354-2.847999$ H -4.960953 -3.039014 -2.850488 H $-6.438408-2.286278-0.281225$ O $-6.513168-4.123496-1.147489$ H $-4.020379-4.138936-0.590480$ O $-4.854411-3.7058991 .225169$ C $-1.470093-3.531255-0.048144$ H $-1.559821-1.5543360 .674198$ H $-2.369228-2.7138381 .717857$ H $-6.348777-1.448808-3.702317$ H $-6.829793-4.476372-0.299069$ H $-5.368465-4.527377 \quad 1.150924$ O $-1.536995-4.6884470 .670071$ O $-0.915578-3.386735-1.129832$ C $-0.901211-5.8215810 .069972$ H $0.152710-5.609024-0.133899$ H $-0.960146-6.6562180 .773530$ H - $1.423561-6.099182-0.850432$

SCF Energy (B3LYP/6-31G**//MMFF) $=-3245.92247080$

06_00162

MM̄FF Geometry

C -2.952711 -1.731270 -2.869663

C $-2.134956-1.325807-1.886966$

C $-0.836858-0.594859-2.081542$

O $0.138720-1.372858-1.340829$

C $-0.8662360 .829732-1.503708$

C $-1.6091341 .835388-2.355721$

C $-1.0401832 .565669-3.334737$

C $-3.0532612 .059648-1.998757$

C $0.3871252 .516192-3.802769$

C $1.1512433 .809189-3.485256$

C $1.3688754 .042946-1.981193$

C $2.0524595 .391137-1.741984$

C $2.3556345 .620933-0.254872$

$\begin{array}{lllll}\text { O } & 2.186425 & 2.980037 & -1.479307\end{array}$

O $3.2382826 .750781-0.178080$

C 3.1088924 .3888620 .289819

$\begin{array}{llll}\text { C } & 1.094822 & 6.008433 & 0.526337\end{array}$

O 3.3013754 .4970681 .699126

C $2.4388373 .040653-0.069914$

C $3.3312501 .834861 \quad 0.311335$

O 1.2242642 .9258920 .681838

C $1.440318-1.331057-1.726226$

O $1.929286-0.650510-2.611923$

C $2.167821-2.311732-0.888854$

C $3.443076-2.620305-1.166444$

C $4.241158-3.643236-0.486505$

C $3.630908-4.3322970 .706869$

C $4.5779891 .659925-0.561452$

C 5.6304890 .7831610 .095895

C $8.054704 \quad 0.230159-0.102611$

$\begin{array}{lllll}\text { O } & 5.430578 & 0.138317 & 1.121181\end{array}$

C $8.161238-1.191697-0.551725$

C $8.339141-2.2117910 .301140$

C $8.534156-3.640390-0.116085$
C $7.468594-4.557700 \quad 0.437031$

C $7.528265-5.0034511 .703448$

C $6.389700-5.033239-0.504580$

C $5.467990-3.937309-0.965340$

N $6.8297090 .821195-0.603328$

H $-2.714423-1.501530-3.904201$

H $-2.403376-1.562098-0.861109$

H $-0.556488-0.585213-3.142582$

H $\quad 0.162034 \quad 1.172596-1.346962$

H $-1.2866280 .821159-0.488869$

H $-1.6606313 .284697-3.868027$

H $-3.5176962 .841802-2.608673$

H $-3.636121 \quad 1.147366-2.141863$

H $-3.1356192 .370424-0.952437$

H $0.9238531 .654500-3.398810$

H $0.3749722 .376255-4.890840$

H $2.1275523 .746134-3.983040$

H $0.6200904 .664616-3.921362$

H $0.3991354 .022449-1.472404$

H $1.4444206 .213151-2.140016$

H $2.9996215 .422920-2.298862$

H 3.4985316 .8586010 .753040

H $4.1120164 .424281-0.149549$

H $0.656260 \quad 6.923714 \quad 0.110532$

H 1.3306276 .2400551 .570888

H $0.324547 \quad 5.234691 \quad 0.510716$

H $2.456612 \quad 4.282400 \quad 2.130234$

H 3.5959681 .9037751 .374096

H 2.7445260 .9102200 .224757

H 0.9790521 .9873460 .731099

H $1.612248-2.796144-0.093487$

H $3.935525-2.112575-1.994731$

H $2.767462-4.9347290 .404845$

H $3.304765-3.5968661 .451319$

H $4.326467-4.9971391 .224727$

H $4.3012581 .204384-1.519459$

H $5.0450032 .626473-0.772443$

H 8.0736600 .3220540 .988762

H $8.8875800 .810243-0.512185$

H $8.132335-1.384123-1.621167$

H $8.390271-2.0042001 .367927$

H $9.518423-3.9682510 .243844$

H $8.580070-3.732909-1.208212$

H $6.785393-5.6881662 .098815$

H $8.322228-4.6904212 .373579$

H $5.819606-5.859861-0.064622$

H $6.864816-5.475196-1.390572$

H $5.842321-3.378948-1.823748$

H $\quad 6.880973 \quad 1.347422-1.470801$

C $-5.454858-1.700052-2.497947$

O $-5.361313-0.852442-1.335243$

C $-6.542738-1.016106-0.532478$

C $-7.579817-1.633075-1.460166$

C $-6.712861-2.550569-2.300260$

H $-6.291654-1.7380120 .254426$

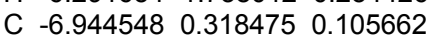

H $-8.365100-2.163664-0.913485$

H -8.057152 -0.880784 -2.098465

C $-7.367029-2.988188-3.599342$

H $-6.474161-3.443178-1.705960$

C -5.7947501 .0569780 .818965$

$\begin{array}{lllll}\text { O } & -7.438024 & 1.199369 & -0.913448\end{array}$

H $-7.7806780 .161213 \quad 0.796266$

H $-5.0735551 .417524 \quad 0.078377$

$\begin{array}{llll}\text { O } & -6.343076 & 2.224965 & 1.443748\end{array}$

C -5.0542830 .2364291 .890056$

C $-3.901794 \quad 1.0364152 .523245$

H $-4.640569-0.6653371 .434329$

O $-5.972136-0.1794142 .897084$

H -3.201353 1.3090921 .725733

H -4.2950181 .9737002 .935629$

H -8.286282 $-3.547955-3.397704$

H $-7.627448-2.129088-4.226711$

H $-6.696591-3.634607-4.174498$

C $-4.188015-2.549447-2.608069$

H $-6.7337621 .305215-1.576133$

H -6.7990192 .7358840 .752934$

H $-6.3578520 .621818 \quad 3.291041$ 
H -4.289776 -3.282097 -3.417095

H $-4.050287-3.121591-1.681803$

H -5.555964 -1.039568 -3.368226

C $-1.691128-1.0879472 .331219$

O $-2.742146-1.0226373 .300609$

C $-3.1612130 .296398 \quad 3.660373$

C $-1.971140 \quad 1.082615 \quad 4.227928$

C -0.7976631 .1054853 .251275$

C $-0.451392-0.3146472 .807905$

H -2.050107 -0.6820351 .378891$

C $-1.333993-2.5710002 .128187$

H $-3.885074 \quad 0.1727294 .475860$

O -2.3678012 .4123654 .546725$

H -1.6454030 .6108425 .164256$

H $-1.008997 \quad 1.7549592 .393979$

O $\quad 0.327498 \quad 1.678824 \quad 3.921176$

H $\quad 0.022225-0.8479213 .642584$

O $0.496659-0.2580851 .744197$

C -2.428674 -3.373500 1.457468

H $-0.440801-2.6533881 .499093$

H -1.137451 -3.0433973 .098106$

H - 1.5813792 .8786634 .878474

H 1.0389291 .7793703 .266398

H $1.328914 \quad 0.0847742 .111054$

O $-1.937368-4.6109271 .147939$

O $-3.567133-2.9839721 .243107$

C -2.861767 -5.5014790 .515352$

H $-2.369311-6.4695300 .390179$

H -3.750055 -5.6388721 .139498$

$\mathrm{H}-3.137274-5.117874-0.471118$

SCF Energy $\left(B 3 L Y P / 6-31 G^{* *} / / M M F F\right)=-3245.93134764$

06_00163

MM̄FF Geometry

C $-0.000131-4.3215561 .622223$

C $0.705481-3.1968091 .427236$

C $1.340446-2.3932232 .537225$

O $0.659329-1.1204572 .728704$

C $2.852795-2.1942662 .304040$

C $3.622422-1.8887993 .573810$

C $4.056798-0.6679003 .941782$

C $3.952950-3.0900004 .424008$

C $3.8230040 .648623 \quad 3.257597$

C 5.1005401 .2503612 .659738

C $5.5644380 .555477 \quad 1.368587$

C 6.8584561 .1990370 .862095

C $7.2981480 .631431-0.495541$

O $4.524693 \quad 0.701307 \quad 0.395228$

O $8.3114161 .499504-1.026961$

C $6.0979680 .689294-1.464520$

C $7.945504-0.750612-0.351768$

O $6.4161120 .067267-2.707524$

C $4.7993720 .095906-0.874458$

C $3.5769570 .339822-1.791161$

O $4.955426-1.322338-0.746835$

C $0.555967-0.2211401 .714413$

O $0.937022-0.3558420 .562774$

C $-0.0745391 .005576 \quad 2.252750$

C $-0.0141742 .123897 \quad 1.514826$

C $-0.573590 \quad 3.434318 \quad 1.849013$

C -1.3157953 .5772393 .149059$

C $3.1761561 .809016-1.952449$

C $1.8716481 .968730-2.716291$

C $0.2109603 .710860-3.366019$

O $1.3168491 .043022-3.300904$

C $-0.2972864 .954707-2.709703$

C -1.476515 $5.039920-2.074150$

C $-1.9382196 .273308-1.349447$

C -2.150160 6.0417840 .133600

C -3.3817436 .0847890 .668713$

C -0.9326955 .8425331 .014354$

C $-0.413277 \quad 4.4338650 .957533$

N $1.4103413 .277316-2.669661$

H -0.139222 -4.6720422 .643430$

H $\quad 0.867867-2.8368310 .416764$

H $1.191603-2.9317503 .480701$

H $3.030610-1.4121891 .560603$

H $3.285633-3.1054311 .868389$
H $4.630112-0.5877034 .864996$

H $4.544279-2.8257935 .307177$

H $3.038743-3.5777294 .775917$

H $4.533764-3.8159713 .845521$

H 3.0455680 .5860502 .494877

H $3.436607 \quad 1.3419724 .015680$

H 4.8880572 .3039212 .434602

H 5.9056651 .2357473 .404848

H $5.722861-0.5098871 .570279$

H 7.6611911 .0899931 .602226

H 6.7050372 .2815380 .747572

H $9.0437831 .532366-0.387813$

H $5.935131 \quad 1.749767-1.692231$

H $8.823265-0.6953360 .303556$

H $8.317086-1.115438-1.315589$

H $7.270616-1.4998570 .066782$

H $6.380686-0.895251-2.574590$

H $3.747222-0.116643-2.774937$

H $2.717634-0.201015-1.373498$

H $4.073621-1.728340-0.709496$

H -0.5484730 .9594613 .225786$

H 0.4959112 .0775480 .552319

H -1.757412 4.5659663 .290753

H -0.641640 3.4023603 .994666

H -2.1394012 .8605773 .207142$

H $3.0519922 .276329-0.968763$

H $3.9475412 .358017-2.502331$

H $-0.5255312 .900997-3.357681$

H $0.4833823 .914327-4.406469$

H $0.3558175 .824278-2.734440$

H $-2.1336644 .173597-2.047124$

H -2.870412 $6.607591-1.822731$

H -1.226635 $7.098597-1.478343$

H $-3.5400535 .946357 \quad 1.733138$

H $-4.260168 \quad 6.2541450 .054956$

H $-1.150616 \quad 6.1462752 .043779$

H -0.1357706 .5241650 .690632$

H $\quad 0.166787 \quad 4.2231810 .060039$

H $2.0358723 .992403-2.308227$

C $-0.505086-4.738258-0.888110$

O $-1.193955-3.489698-1.092766$

C $-1.802423-3.499324-2.397310$

C $-1.346237-4.786202-3.077518$

C $-1.134083-5.705186-1.890241$

H $-2.885211-3.553257-2.238501$

C $-1.453334-2.214820-3.157552$

H $-2.089817-5.164221-3.785969$

H $-0.406453-4.647892-3.624629$

C -0.277735 -6.917812 -2.210639

H -2.114414 -6.040298 -1.525666

C $-1.791728-0.908801-2.411260$

O $-0.045044-2.193961-3.429190$

H -1.946735 -2.224053 -4.136503

H -1.104236 - $0.762352-1.569312$

O $-1.5581830 .176731-3.317585$

C $-3.241906-0.808925-1.908813$

C $-3.5489420 .563911-1.280722$

H -3.418369 -1.591287-1.164325

O $-4.142007-1.045088-2.990388$

H $-2.8194660 .755203-0.485982$

H $-3.4167241 .349744-2.034351$

H $-0.746575-7.523262-2.993354$

H $0.717105-6.624905-2.563033$

H $-0.149066-7.550999-1.327456$

C $-0.650567-5.1852140 .569698$

H $0.419341-2.232360-2.575250$

H $-0.6314470 .117367-3.607372$

H -3.967776 $-0.370700-3.669350$

H $-0.237882-6.1948700 .679971$

H $-1.716838-5.2450700 .819603$

H $0.552080-4.574975-1.135786$

C $-4.631899-0.3861341 .408982$

O $-5.314743-0.411807 \quad 0.150535$

C $-4.9893470 .666886-0.731516$

C $-5.3134352 .010058-0.062646$

C $-4.6513922 .138217 \quad 1.307280$

C -4.9544230 .9085092 .163563$

H $-3.550486-0.4642201 .242077$ 
C $-5.084782-1.6146012 .203307$

H $-5.6613930 .568324-1.593320$

O $-4.911753 \quad 3.085980-0.903713$

H -6.4012732 .0893280 .062692$

H -3.5731172 .3018331 .205030$

O

H -6.0131350 .9264002 .450297$

O -4.1963160 .9758353 .368882$

C $-4.466985-2.8672461 .630771$

H $-4.777238-1.5624783 .253669$

H -6.177192 -1.710785 2.191503

H $-5.1294073 .912699-0.440331$

H -4.7639123 .3497352 .834398$

H $-3.2579710 .870788 \quad 3.136848$

O $\quad-5.398819 \quad-3.577440 \quad 0.935079$

O $\quad-3.287743-3.1649301 .771067$

C $-4.927226-4.7880430 .335960$

H $-4.565684-5.476271 \quad 1.105961$

H $-5.763802-5.256490-0.189191$

H $-4.137308-4.569660-0.387522$

SCF Energy (B3LYP/6-31G**//MMFF)= -3245.93438899

0600164

MM̄FF Geometry

C $-3.344807-1.215878-3.110395$

C $-2.372706-1.046277-2.201322$

C - $-1.033406-0.423977-2.474675$

O $-0.063643-1.397552-2.014986$

C $-0.8291650 .888150-1.692826$

C $-1.4353372 .093462-2.381591$

C $-0.7772662 .877818-3.258267$

C $-2.8507302 .430951-1.994814$

C $0.6475372 .745844-3.719820$

C $1.4790464 .005216-3.431548$

C $1.5366354 .385255-1.943697$

C $2.3647485 .655671-1.739283$

C $2.4735786 .028422-0.253384$

O $2.1180263 .297673-1.215055$

O $3.4956227 .031357-0.148181$

C 2.9408954 .7854990 .534848

C 1.1841896 .6765570 .263806

O 2.9288995 .0404031 .937626

C 2.1432043 .5013000 .203988

C 2.7553492 .2465470 .865960

O $0.815143 \quad 3.6395830 .727770$

C $1.143549-1.439829-2.636395$

O $1.540878-0.711589-3.530659$

C $1.908155-2.553323-2.032716$

C $3.209686-2.698622-2.319742$

C $4.095229-3.745621-1.804153$

C $3.474564-4.824931-0.955974$

C $4.141257 \quad 1.8426970 .360759$

C 4.7906390 .8814521 .335200

C $4.772865-1.4933642 .079552$

O 5.6110861 .2674062 .165756

C $6.047291-2.0746301 .553642$

C $6.166508-3.3467111 .147019$

C $7.440546-3.9537680 .637010$

C $7.567974-3.908578-0.869536$

C $8.618838-3.306022-1.451960$

C $6.519516-4.614179-1.703963$

C $5.407536-3.685267-2.113388$

N $4.333245-0.4193251 .208057$

H -3.202018 -0.868168 -4.129589

H -2.545865 -1.389927 -1.184288

H $-0.901067-0.264941-3.552701$

H $0.2409921 .053435-1.520960$

H $-1.2366930 .798038-0.676272$

H -1.311049 $3.722150-3.692975$

H -3.216912 $3.331529-2.499345$

H $-3.5332371 .617275-2.249797$

H -2.909942 $2.615666-0.917843$

H $\quad 1.1459481 .879202-3.280026$

H $0.6389402 .573839-4.803015$

H $2.4964163 .820670-3.799824$

H $1.0755194 .846897-4.008632$

H $0.5144774 .552738-1.586957$

H $1.9471396 .491815-2.314013$
H $3.3782255 .497189-2.133958$

H $3.629346 \quad 7.2177120 .797192$

H $3.9930894 .637137 \quad 0.267987$

H $0.9560207 .581436-0.312287$

H 1.2948327 .0059051 .302857

H 0.3165416 .0166620 .202547

H 2.0058784 .9904942 .239460

H 2.7662452 .3791001 .955876

H 2.0828921 .3953860 .690780

H $\quad 0.4193332 .7552860 .800982$

H $1.377998-3.221021-1.363101$

H $3.677784-1.976928-2.988199$

H $2.688458-5.346541-1.513609$

H $3.034814-4.396583-0.048154$

H $4.183959-5.588411-0.631247$

H $4.0739591 .376491-0.628967$

H 4.8152642 .6979680 .262021

H $3.965103-2.2310402 .121054$

H $4.934470-1.0984803 .087896$

H $6.916212-1.4216371 .518534$

H $5.301294-4.0020471 .196229$

H $7.486696-4.9989020 .968118$

H $8.297091-3.4587391 .113331$

H $8.730319-3.286787-2.531247$

H $9.388401-2.815920-0.864951$

H $6.169176-5.502486-1.172153$

H $6.980119-5.000816-2.623234$

H $5.747371-2.863650-2.746327$

H $3.698346-0.6530480 .450211$

C $-5.689151-0.930003-2.223352$

O $-5.214371-0.395806-0.971958$

C $-6.248743-0.5093910 .018493$

C $-7.533738-0.756529-0.759199$

C $-7.024338-1.606922-1.908044$

H $-6.017663-1.3979120 .617481$

C -6.2469820 .7277660 .920720$

H -8.295482 -1.259263 -0.155826

H $-7.9645440 .175075-1.143564$

C -7.975997 -1.669060 -3.089964

H $-6.851240-2.626072-1.536758$

C $-4.8615461 .095367 \quad 1.489767$

O

H $-6.9691620 .595216 \quad 1.734148$

H -4.2101811 .4504100 .683673$

O -5.0382302 .2059692 .379544$

C $-4.145136-0.0321852 .256720$

C -2.7784670 .4306892 .797400$

H $-3.988157-0.8872881 .592317$

O $-4.968381-0.4772533 .331605$

H $-2.156906 \quad 0.724120 \quad 1.943844$

H $-2.916043 \quad 1.327448 \quad 3.413555$

H -8.931682 -2.111939-2.791447

H $-8.179295-0.671719-3.494245$

H -7.556970 -2.280092 -3.895561

C $-4.646702-1.896985-2.786848$

H $-6.1155151 .962366-0.597581$

H -5.4794342 .9123131 .876988$

H $-5.1287550 .285516 \quad 3.913506$

H -5.023535 -2.376087 -3.697833

H -4.456078 -2.701210-2.065535

H $-5.830083-0.078263-2.900821$

C -1.082593 -1.959175 1.893748

O $-1.952689-1.8985503 .028470$

C $-2.048806-0.6215403 .660964$

C $-0.660861-0.1720014 .140762$

C $0.354981-0.1757453 .002558$

C $0.338829-1.5187602 .273974$

H -1.480322 -1.322516 1.094642

C -1.069784 -3.412865 1.397848

H -2.664738 -0.7729024 .556388$

O $-0.716264 \quad 1.1298194 .714451$

H -0.307664 -0.855258 4.924213

H $\quad 0.1785010 .6579602 .313510$

O 1.6549230 .0342453 .559677

H $0.804645-2.2743292 .919279$

O $1.148263-1.4214311 .103886$

C $-2.347316-3.8029230 .691743$

H $-0.254632-3.5707410 .681974$ 
H $-0.915616-4.1172412 .224320$ H -1.351912 $1.098008 \quad 5.449771$ H $2.296172-0.0288152 .831402$ H $\quad 0.736075-0.772556 \quad 0.508220$ O $-3.385473-3.8432501 .573225$ O $-2.405390-4.070986-0.501643$ C $-4.649129-4.2298411 .025454$ H $-4.616949-5.278630 \quad 0.716225$ H $-5.407078-4.1146231 .804764$ H -4.915815 -3.587535 0.181874

SCF Energy (B3LYP/6-31G**//MMFF) $=-3245.92243836$

06_00165

MM̄FF Geometry

C $0.0703273 .562258-1.826380$

C $0.7833944 .631695-1.438549$

C $1.7529804 .674121-0.284091$

O $2.3362413 .376757-0.031483$

C 1.0133725 .1341620 .987259

C 1.8829765 .1261822 .229146

C 1.9671174 .0846983 .082236

C 2.6625926 .3884152 .477910

C 1.2961502 .7479822 .928920

C 0.3288562 .4357294 .075694

C $-0.6455341 .311606 \quad 3.689724$

C -1.4845350 .8644464 .886952$

C $-2.418908-0.3032774 .525060$

O $0.107026 \quad 0.2056703 .182599$

O $-2.904252-0.8744575 .749806$

C $-1.600690-1.3998703 .805684$

C -3.6536590 .1805093 .754819$

O $-2.451750-2.438573 \quad 3.324632$

C $-0.702249-0.8589332 .668476$

C $0.239725-1.9709422 .154898$

O $-1.539089-0.4245601 .594004$

C $3.4407933 .037089-0.748364$

O $3.8911993 .617103-1.723093$

C $4.0555911 .864596-0.083216$

C $5.2419571 .410097-0.513703$

C 6.0401410 .3439910 .097860

C $5.531410-0.2631741 .378711$

C $1.321353-1.4776341 .181629$

C $2.103661-2.6311240 .577378$

C $3.639962-3.154631-1.314124$

O $2.058890-3.7763381 .018917$

C $5.006100-3.316081-0.728017$

C $6.138085-3.128407-1.423199$

C $7.508980-3.350880-0.853063$

C $8.410971-2.147403-1.000641$

C $9.397859-2.129002-1.912662$

C $8.228527-1.004995-0.027266$

C $7.201371-0.007584-0.493664$

N $2.868155-2.227374-0.508604$

H $0.1692152 .619288-1.295599$

H $0.6376815 .574786-1.961546$

H $2.5553765 .387111-0.513766$

H 0.1285524 .5074581 .156441

H $\quad 0.611521 \quad 6.145508 \quad 0.836870$

H 2.6116904 .1768763 .954635

H $3.275694 \quad 6.3267413 .383077$

H 3.3333566 .5929841 .637448

H 1.9821337 .2379332 .595725

H $\quad 0.7654312 .669527 \quad 1.976184$

H 2.0793571 .9800582 .898170

H 0.9178352 .1385924 .952803

H -0.2458373 .3272174 .355670$

H -1.294017 1.6969332 .893231

H -2.0586121 .7047945 .296683$

H -0.8173790 .5342935 .695471$

H -3.360232 -0.1741046 .247340$

H $-0.956098-1.8660374 .563530$

H -4.2156030 .9095714 .351006$

H -4.348782 -0.6438473 .561531$

H -3.405186 0.6558532 .804082

H -2.891373 -2.112764 2.520924

H $0.750548-2.4385413 .006964$

H $-0.354063-2.7509661 .663907$

H -1.050794 -0.4770640 .758445$
H $3.545715 \quad 1.4476410 .777606$

H $5.6937851 .885567-1.383641$

H 5.4966170 .4917212 .171972

H $6.144928-1.0879261 .746093$

H $4.525252-0.6699231 .243130$

H $0.876612-0.9028660 .363631$

H $2.031218-0.8276691 .705265$

H $3.669296-2.755166-2.332700$

H $3.130364-4.123618-1.334088$

H $5.067049-3.6357620 .309751$

H $6.078709-2.836061-2.469087$

H $7.945964-4.214583-1.371393$

H $7.460370-3.6361820 .205279$

H $10.067888-1.280276-2.001826$

H $9.554224-2.959063-2.593671$

H $9.177063-0.4621460 .085592$

H $8.021720-1.4055880 .968757$

H $7.4854770 .503969-1.414823$

H $2.902968-1.242412-0.754958$

C $-2.3468343 .756849-2.436681$

O $-2.8020202 .514446-1.860179$

C $-4.1822322 .317885-2.226649$

C $-4.6415483 .615173-2.882757$

C $-3.3591154 .094870-3.529331$

H $-4.198328 \quad 1.525219-2.983087$

C $-5.0175731 .910311-1.008314$

H -5.451379 $3.452212-3.600468$

H $-4.9915964 .342523-2.140490$

C $-3.3924755 .563501-3.916601$

H $-3.1643103 .488010-4.423777$

C $-4.5973970 .583854-0.343688$

O $-4.9330352 .940175-0.014612$

H $-6.0750191 .856308-1.292893$

H -3.6328920 .6984310 .163422$

$\begin{array}{lllll}\text { O } & -5.559022 & 0.292054 & 0.678732\end{array}$

C $-4.531131-0.615726-1.305689$

C $-4.382672-1.970974-0.585896$

H -3.691146 -0.472440 -1.992105

O $-5.718222-0.655649-2.101647$

H $-3.575273-1.9021030 .148938$

H -5.307893 -2.186860 -0.039076

H -4.191898 $5.751596-4.640720$

H -3.569976 $6.204185-3.046267$

H -2.446476 $5.870702-4.372896$

C $-0.9175723 .605397-2.958416$

H -3.9935603 .0517860 .211815$

H -5.5809131 .0550051 .281701$

H -6.475206 $-0.739061-1.496533$

H -0.669168 $4.435094-3.630393$

H $-0.8158692 .679597-3.536900$

H $-2.3874744 .511142-1.638988$

C -1.698330 -3.167923 -1.502288

O $-2.877731-2.933774-2.283110$

C $-4.115848-3.119140-1.585629$

C $-4.194801-4.532078-0.989698$

C $-2.961714-4.881674-0.165164$

C -1.694582 -4.605696 -0.971354

H -1.663327 -2.468442 -0.659247

C $-0.485391-2.895357-2.405239$

H $-4.902271-3.063801-2.348874$

O $-5.357522-4.687695-0.183338$

H -4.275376 -5.260432 -1.807519

H $-2.947459-4.3401280 .788280$

O $-2.992314-6.2733230 .158853$

H -1.621393 -5.318396 -1.802743

O $-0.559743-4.804106-0.134535$

C $-0.182988-1.414530-2.523393$

H $0.408955-3.369830-1.987789$

H $-0.662129-3.282130-3.415164$

H $-6.128179-4.482127-0.739567$

H $-3.821610-6.4394590 .639283$

H $-0.608109-5.7115210 .212179$

O $1.031453-1.259654-3.130634$

O $-0.908951-0.509207-2.136945$

C $1.4849490 .090747-3.266718$

H $2.4788420 .071234-3.721561$

H $\quad 0.8096180 .651428-3.919378$

H $1.5602930 .566309-2.284262$ 
H $\quad 1.3986185 .6490622 .002531$

06_00166

MM̄FF Geometry

C $-3.340227-0.724678-2.888891$

C $-2.263169-1.444455-2.536508$

C $-0.819471-1.092045-2.801336$

O $-0.7334860 .304186-3.175301$

C $-0.198352-1.964185-3.908356$

C $0.076276-3.398740-3.502669$

C $1.206896-3.791697-2.881591$

C $-0.965412-4.410857-3.898945$

C $2.328113-2.883221-2.457491$

C $3.362699-3.600390-1.579650$

C $4.492272-2.654182-1.143174$

C $5.561641-3.422128-0.363682$

C $6.668286-2.4939720 .155130$

O $3.931379-1.626250-0.317714$

O $7.432752-3.2466181 .108952$

C $6.014748-1.3087710 .895891$

C $7.642221-2.100430-0.961410$

O $6.999120-0.3535331 .286504$

C $4.871437-0.6292310 .103877$

C $4.142453 \quad 0.382449 \quad 1.021150$

O $5.4318720 .070829-1.008592$

C $0.4403880 .948961-2.942380$

O $1.497243 \quad 0.451403-2.590397$

C $0.1960462 .396594-3.124814$

C $1.1159013 .270478-2.689445$

C $0.9879624 .728680-2.672705$

C $-0.2416405 .325698-3.304910$

C 2.8529580 .9694010 .432544

C 2.1900971 .9520651 .385020

C $\quad 0.1013443 .2871781 .657277$

O 2.7301132 .3567372 .411345

C 0.0185624 .5530090 .864475

C 0.7194575 .6604131 .152744

C 0.6239486 .9477350 .380932

C $1.9068767 .285267-0.349323$

C 2.9047357 .9370430 .271156

C $1.9991066 .931274-1.819599$

C $1.9481505 .446911-2.052258$

N 0.9312922 .3355170 .942265

H -3.219936 $0.197587-3.450531$

H -2.419570 -2.365382 -1.981607

H $-0.282072-1.221098-1.852920$

H $0.737144-1.506906-4.255758$

H $-0.838023-1.944856-4.801557$

H $1.335130-4.850337-2.663761$

H $-0.676817-5.431297-3.624905$

H - $-1.921028-4.200846-3.411543$

H -1.117196 -4.392781-4.983122

H $2.830654-2.493694-3.350695$

H $1.918264-2.037105-1.898660$

H $2.858951-4.006852-0.693347$

H $3.786569-4.447418-2.133675$

H $4.926200-2.198816-2.041195$

H $5.990386-4.225487-0.975760$

H $5.094711-3.9202240 .497908$

H $8.089029-2.6449631 .501183$

H $5.598036-1.7097451 .830082$

H $8.105892-2.994902-1.394632$

H $8.469242-1.496011-0.572472$

H $7.163847-1.548223-1.773008$

H 7.2386580 .1630220 .498366

H $3.877627-0.1130691 .963815$

H 4.8229811 .2071021 .269252

H $4.8086130 .755268-1.298070$

H $-0.7558242 .701758-3.543672$

H $2.0361192 .882606-2.255326$

H $-0.3190665 .021225-4.354814$

H $-0.2526406 .417029-3.299599$

H -1.143699 4.997574 -2.776531

H $3.0658951 .509027-0.494743$

H $2.1400320 .167480 \quad 0.212677$

H $0.500823 \quad 3.4598452 .661861$

H -0.8950832 .8482081 .760318$

H -0.6688024 .5590210 .022286$

H 0.3803417 .7500391 .089852

H $-0.2127476 .926209-0.328368$

H $3.8171938 .205322-0.250609$

H $2.830458 \quad 8.214011 \quad 1.317784$

H $2.9425267 .297912-2.244687$

H $1.2133827 .472353-2.353004$

H $2.7983774 .914252-1.623183$

H 0.5726751 .9515850 .072758

C $-5.431195-0.040236-1.694779$

O $-4.7279450 .114525-0.447747$

C $-5.6851050 .280836 \quad 0.614489$

C $-7.0427100 .453318-0.057950$

C $-6.869398-0.383520-1.311206$

H $-5.690050-0.6584611 .179793$

C $-5.260097 \quad 1.436179 \quad 1.529081$

H -7.8654610 .1115850 .577496$

H $-7.2374901 .498241-0.324770$

C -7.890141 -0.077518 -2.393145

H $-6.942396-1.444496-1.036460$

C $-3.806763 \quad 1.3427512 .036804$

O $\quad-5.3762562 .6729920 .811730$

H -5.9532541 .5157592 .373924$

H -3.1138831 .5050111 .201809$

O -3.5896142 .4233412 .952901$

C -3.4441960 .0240742 .743913$

C $-1.978421 \quad 0.015141 \quad 3.210923$

H -3.599494 -0.8147742 .062238$

O $-4.283643-0.1756743 .878711$

H $-1.339786 \quad 0.2422242 .350411$

H -1.8283680 .8147753 .946327$

H -8.904363 $-0.277293-2.032244$

H $-7.8442120 .971914-2.703059$

H -7.718790 -0.697941-3.278259

C $-4.750201-1.116924-2.540560$

H -4.8191952 .6052130 .017105$

H -3.808665 3.2478272 .485586

H $-5.190098-0.3154183 .557757$

H $-5.306499-1.273034-3.471421$

H $-4.751127-2.067738-1.994825$

H $-5.3892710 .930208-2.206936$

C $-0.983631-2.5348671 .842683$

O $-1.798668-2.4541393 .017585$

C -1.549071 -1.321830 3.857772

C $-0.088195-1.3373074 .328105$

C $0.875821-1.3938003 .148193$

C $\quad 0.507457-2.5395772 .209852$

H -1.210641 -1.692232 1.177971

C -1.343544 -3.840137 1.111494

H -2.176106-1.459482 4.747440

O $0.208420-0.1899595 .116932$

H $0.083496-2.2176134 .960977$

H $0.908033-0.4410812 .609386$

O $2.200262-1.6242703 .633618$

H $\quad 0.774252-3.4931742 .683580$

O $1.275825-2.4295511 .014689$

C $-2.731298-3.8307450 .507813$

H $-0.638605-4.0165860 .290907$

H -1.300808 -4.680673 1.814491

H $-0.414121-0.1803975 .863998$

H $2.412526-0.8996334 .246958$

H $2.213653-2.4022581 .271360$

O $-2.929466-5.013231-0.148595$

O $-3.537332-2.9157710 .590918$

C $-4.202103-5.163439-0.785681$

H $-4.282821-6.193325-1.143319$

H -5.014947 -4.974892 -0.077706

$\mathrm{H}-4.270086-4.487910-1.642747$

SCF Energy $(B 3 L Y P / 6-31 G * * / / M M F F)=-3245.93021908$

0600167

MM̄FF Geometry

C -0.8138532 .0192982 .097257$

C -1.807978 1.6436912 .917096

C -2.3916760 .2583653 .070691$

O $-1.756817-0.6905942 .185812$

C -3.8899110 .2603832 .715347$

C $-4.675207-0.7803753 .488709$ 
C $-4.978451-2.014426 \quad 3.042277$

C $-5.163518-0.3272954 .841273$

C $-4.555540-2.6679661 .757389$

C $-5.722934-2.887466 \quad 0.785314$

C $-6.170205-1.6016210 .072789$

C $-7.341681-1.886641-0.869344$

C $-7.728384-0.655148-1.702658$

O $-5.053147-1.116059-0.676417$

O $-8.598687-1.121185-2.745067$

C $-6.456533-0.075025-2.360592$

C $-8.5366610 .351898-0.875884$

O $-6.7343521 .158979-3.018802$

C $-5.2859730 .115940-1.368015$

C $-3.9886040 .467763-2.134500$

O $-5.607700 \quad 1.179622-0.469779$

C $-0.663888-1.3650282 .627798$

O $-0.101635-1.2438693 .703146$

C $-0.268478-2.2903461 .544016$

C $0.863990-3.0007971 .641219$

C $1.322981-3.9616300 .641480$

C $1.900345-5.2170881 .237215$

C $-2.7289120 .468137-1.258219$

C $-1.503900 \quad 0.788566-2.087286$

C $0.104365-0.197053-3.692304$

O $-1.0522941 .929360-2.151320$

C $0.696472-1.545748-3.939834$

C $1.940502-1.895683-3.577662$

C $2.535452-3.245884-3.859255$

C $2.826476-4.040303-2.605637$

C $4.086339-4.336108-2.245871$

C $1.651337-4.582530-1.823275$

C $1.256290-3.691307-0.678269$

N $-0.994399-0.315603-2.752903$

H -0.3467881 .2940341 .437531$

H $-2.2339702 .393517 \quad 3.582251$

H $-2.248483-0.0541374 .112865$

H $-4.0193190 .102777 \quad 1.639402$

H $-4.341842 \quad 1.2394002 .924550$

H $-5.576019-2.6575453 .687674$

H -5.721917 -1.109136 5.366990

H -4.319264 -0.042005 5.477050

H $-5.826630 \quad 0.5375854 .735024$

H -3.745406 -2.124559 1.264620

H -4.135423 -3.6478602 .018227$

H $-5.385437-3.6094790 .030069$

H $-6.571808-3.3424121 .310735$

H $-6.456883-0.8571300 .823860$

H -8.208508 $-2.260656-0.310264$

H -7.062332 -2.694905 -1.560252

H -8.904310 -0.349789 -3.251353

H $-6.138870-0.781187-3.140482$

H -9.469512 $-0.105693-0.525268$

H -8.835257 $1.214138-1.482286$

H -7.9975350 .7184820 .000267$

H $-7.3964980 .983451-3.708112$

H $-3.839572-0.261747-2.941203$

H -4.087990 $1.453665-2.606101$

H $-4.790323 \quad 1.597827-0.158444$

H $-0.926620-2.3750880 .684960$

H $1.492581-2.9068352 .523783$

H $2.632765-4.9785112 .016867$

H $2.421060-5.8429880 .509200$

H $1.105605-5.8203461 .689286$

H $-2.7925031 .214699-0.460253$

H -2.581794 -0.503713 -0.773368

H $0.8360800 .515396-3.300631$

H $-0.2960130 .215215-4.624376$

H $0.071082-2.267457-4.460103$

H $2.572003-1.174383-3.064042$

H $\quad 3.455197-3.089244-4.437597$

H $1.878075-3.839058-4.507720$

H $4.294780-4.935093-1.365410$

H $4.936973-3.982138-2.818890$

H $\quad 1.869598-5.603089-1.492507$

H $\quad 0.785581-4.691612-2.489244$

H $\quad 0.842278-2.731763-0.986297$

H -1.549635 -1.166796 -2.768837

C $-0.129003 \quad 3.9712080 .654163$
O $\quad 0.9305513 .280474-0.043351$

C $1.8268494 .253054-0.608801$

C $1.0443285 .558048-0.642497$

C $0.2510025 .454647 \quad 0.643212$

H 2.6632614 .3572150 .093008

C $2.3496653 .790775-1.971715$

H $1.6974286 .435298-0.676657$

H $0.3652625 .604078-1.501802$

C -0.9411326 .3946340 .699855$

H 0.9199595 .6779041 .485575

C $3.0193782 .403022-1.971071$

O $1.264323 \quad 3.742863-2.906335$

H $3.0464194 .538830-2.367445$

H $2.2736861 .619606-1.793199$

O $3.5353002 .176036-3.289653$

C $4.1697242 .237649-0.960800$

C $4.9502730 .926132-1.173026$

H 3.7623082 .2634880 .054501

O $5.0750163 .335668-1.077840$

H $4.2381580 .096345-1.227188$

H $5.4686290 .973925-2.138083$

H -0.6123837 .4369620 .634415$

H -1.637561 $6.209528-0.124878$

H -1.490600 6.2716821 .638416

C -0.2532743 .4151602 .074028$

H $0.5946183 .136050-2.546316$

H $2.7917262 .265302-3.910358$

H $5.4125163 .342857-1.990083$

H -0.8780144 .0817622 .679830$

H $\quad 0.739483 \quad 3.3803792 .538523$

H -1.0524813 .7998300 .086815$

C $4.635246-0.6459231 .432366$

O $5.4089820 .543026 \quad 1.227225$

C $6.0017690 .669698-0.069819$

C $6.917417-0.530192-0.342576$

C $6.172284-1.850756-0.182582$

C $5.478757-1.9033511 .177385$

H $3.761690-0.638350 \quad 0.769514$

C $4.137655-0.6246972 .886436$

H $6.6452151 .557298-0.027730$

O $7.475192-0.436844-1.649134$

H $7.755705-0.5072440 .365881$

H $5.462090-1.999133-1.003125$

O $7.125173-2.911901-0.270502$

H $6.233695-2.0163181 .966353$

O $4.629180-3.0461751 .230260$

C $3.1096260 .457743 \quad 3.135147$

H $3.670557-1.5784613 .153233$

H $4.986664-0.4385693 .555563$

H $8.025598-1.226749-1.787169$

H $6.631981-3.749321-0.279455$

H $5.191995-3.8368321 .178386$

O $2.9513680 .611024 \quad 4.483566$

O 2.5089691 .0741842 .266695

C 2.0114371 .6103324 .889187

H 2.2747452 .5813214 .459181

H 1.0009701 .3156754 .594598

H 2.0493331 .6896355 .978848

SCF Energy $\left(B 3 L Y P / 6-31 G^{* *} / / M M F F\right)=-3245.92194333$

0600168

MM̄FF Geometry

C $-3.487657-2.381281-1.438734$

C $-2.833986-1.609900-2.320994$

C $-1.335072-1.527319-2.406141$

O $-1.026655-0.113789-2.337771$

C $-0.768369-2.109433-3.711357$

C $-0.811960-3.622328-3.765739$

C $0.109553-4.430215-3.203799$

C $-1.969512-4.212522-4.528359$

C $1.349521-4.030444-2.452348$

C $1.189199-4.232734-0.941234$

C $2.501771-4.012893-0.172944$

C $2.249764-4.0579811 .336195$

C $3.518872-3.7572972 .146574$

O $3.046579-2.740546-0.535187$

O $3.123638-3.4858623 .500271$

C $4.170009-2.4690011 .600033$ 
C $4.457372-4.9681142 .203388$

O $5.434422-2.2352322 .216315$

C $4.320191-2.4599430 .061797$

C $4.780769-1.064890-0.423510$

O $5.307508-3.425982-0.312017$

C $0.157913 \quad 0.249299-1.781044$

O $1.060295-0.488859-1.423262$

C $0.1397311 .721994-1.636330$

C $1.1380242 .338109-0.986848$

C $1.2208363 .770390-0.694286$

C $0.1078704 .654501-1.193482$

C $4.690251-0.881848-1.945170$

C $5.5830580 .242694-2.428728$

C $5.8706482 .701297-2.388104$

O $6.548580 \quad 0.026575-3.158613$

C $5.6450173 .772851-1.372081$

C $5.0691304 .951612-1.652258$

C $4.854236 \quad 6.038372-0.637973$

C $3.3980446 .423803-0.500475$

C $2.9027947 .485504-1.158743$

C 2.5393395 .6363590 .463532

C 2.2761014 .2271560 .011682

N $5.1959791 .489274-1.963274$

H $-2.904201-3.032604-0.790513$

H -3.388618 $-0.961321-2.993298$

H $-0.891521-2.022494-1.536032$

H $\quad 0.262612-1.763328-3.854467$

H -1.301639-1.686494 -4.573974

H $-0.024328-5.507058-3.300118$

H -1.936735 -5.306966 -4.552470

H $-2.922750-3.923587-4.077142$

H -1.956315 -3.860955 -5.565303

H $2.179654-4.645748-2.821007$

H $1.625199-2.993417-2.664423$

H $\quad 0.433366-3.529849-0.572905$

H $\quad 0.814115-5.242240-0.730293$

H $3.203184-4.800990-0.471668$

H $1.821579-5.0234931 .632599$

H $1.496192-3.3022581 .596418$

H $2.643428-4.263133 \quad 3.833737$

H $3.525718-1.6277601 .889317$

H $3.953510-5.8207822 .674377$

H $5.335239-4.7594282 .824613$

H $4.799380-5.2920721 .218336$

H $5.292223-2.2374373 .178461$

H $4.154116-0.2914410 .039597$

H $5.812784-0.885594-0.095602$

H $5.768571-3.126571-1.111066$

H $-0.7183042 .254887-2.030399$

H $1.9606381 .735311-0.604359$

H $0.0114884 .574840-2.282024$

H $0.2638285 .713245-0.974716$

H $-0.8449324 .369967-0.733301$

H $4.993178-1.789139-2.478575$

H $3.659860-0.668313-2.250191$

H $5.4753702 .968390-3.373944$

H $6.9422572 .502121-2.494057$

H $5.9836013 .572318-0.358275$

H $4.7467505 .157310-2.670517$

H $5.444536 \quad 6.909383-0.951807$

H 5.2506615 .7550720 .344991

H $1.8680967 .790365-1.044577$

H $3.5215318 .071657-1.830331$

H $1.5962806 .157087 \quad 0.667432$

H 3.0434445 .5970851 .438068

H 3.0357313 .5140600 .333287

H $4.2810381 .578660-1.529963$

C $-5.763317-1.182303-1.661246$

O $-5.222412-0.009970-1.019270$

C $-6.2865240 .709194-0.377629$

C $-7.5585990 .237564-1.067023$

C -7.244206 -1.233830 -1.272699

H -6.3082830 .3749620 .667198$

C $-6.0154592 .217060-0.422714$

H $-8.4514910 .405884-0.457901$

H -7.704576 $0.729873-2.035289$

C -8.126514 -1.909179 -2.308164

H $-7.364754-1.753638-0.312735$
C $-4.6020112 .619146 \quad 0.055244$

O $-6.1579642 .663807-1.776501$

H -6.7833862 .7407720 .159322$

H -3.856847 $2.342057-0.701221$

O $-4.558314 \quad 4.0470780 .162209$

C -4.1885812 .0213001 .412569$

C -2.8176982 .5335611 .894898$

H $-4.1511210 .931374 \quad 1.324746$

O -5.1750642 .3304872 .396227$

H -2.0933732 .4239371 .080774$

H -2.8880083 .6034372 .125014$

H -9.176568 -1.874386 -2.000329

H -8.046233 -1.419901 -3.284702

H -7.844464 -2.959514 -2.433113

C $-4.975384-2.414107-1.198225$

H $-6.1802213 .635285-1.765057$

H $-4.6210594 .411171-0.736729$

H -5.2441133 .2992162 .448646$

H -5.385510 -3.321902 -1.654825

H $-5.105109-2.513379-0.112039$

H $-5.657985-1.036520-2.743098$

C -1.296031-0.110939 2.092773

O $-2.308301 \quad 0.3713672 .981525$

C -2.3224451 .7927843 .159615$

C -0.9599702 .2729583 .682408$

C 0.1917431 .7925782 .802340

C 0.1034350 .2829072 .579376

H -1.4643130 .3219331 .098715$

C -1.485150 -1.623282 1.910354

H $-3.058074 \quad 1.9925203 .948933$

O $-0.9183553 .693493 \quad 3.769330$

H -0.8034391 .8835284 .696808$

H $\quad 0.2108392 .329003 \quad 1.848957$

O 1.4336052 .0789463 .448504

H $0.352976-0.2283123 .515945$

O $1.069371-0.1137901 .611341$

C -1.265661 -2.443549 3.164306

H -2.511467 -1.825922 1.582841

H $-0.795946-1.9783611 .135813$

H -1.6481643 .9716954 .348592$

H 1.4603053 .0377213 .609711

H $1.9349110 .210277 \quad 1.913958$

O $-0.985739-3.7322972 .807550$

O $-1.359604-2.0236034 .309312$

C $-0.745502-4.6339353 .892358$

H $\quad 0.093543-4.2824804 .500614$

H -1.648147 -4.739822 4.501337

$\mathrm{H}-0.487795-5.6096543 .472065$

SCF Energy (B3LYP/6-31G**//MMFF) $=-3245.90398880$

0600169

MM̄FF Geometry

C -1.2395282 .5962552 .252803$

C $-0.507608 \quad 1.6123372 .798913$

C -0.9590980 .2141093 .150095$

O $-0.148760-0.6868992 .355287$

C -2.444505 -0.097530 2.868759

C $-2.934311-1.3789203 .515212$

C $-3.143144-2.5410372 .866298$

C $-3.242634-1.2752664 .987405$

C $-2.882132-2.8582091 .420516$

C $-4.175061-3.0905930 .627987$

C $-4.951663-1.7972910 .335235$

C $-6.273835-2.115944-0.366945$

C $-7.027179-0.843419-0.778874$

O $-4.129448-0.984196-0.506207$

O $-8.081448-1.249657-1.664557$

C $-6.0632240 .065503-1.570151$

C -7.707933 -0.1772750 .422084$

O $-6.6776221 .317761-1.867975$

C $-4.7001940 .277621-0.870476$

C $-3.6856790 .994468-1.792486$

O $-4.897632 \quad 1.1054830 .281045$

C $0.331815-1.8113642 .950455$

O $0.264966-2.1154754 .130051$

C $0.892130-2.6628441 .881273$

C $0.942971-3.9904802 .059424$

C $1.344493-4.9458941 .032323$ 
C $0.406421-6.117090 \quad 0.919440$ C $-3.273440 \quad 0.190292-3.033533$ C $-1.776924-0.044056-3.084173$ C $0.064221-1.198838-1.861999$ O $-1.0678390 .513053-3.918463$ C $0.314882-2.670158-1.951495$ C $1.334408-3.188814-2.652723$ C $1.639345-4.656261-2.756740$ C $2.982052-5.009422-2.153160$ C $4.120468-4.814497-2.840436$ C $2.997515-5.660683-0.787365$ C $2.455320-4.7658120 .290751$ N $-1.338225-0.926475-2.109699$ H $-2.2795432 .427197 \quad 1.991845$ H $0.528847 \quad 1.8277773 .053106$ H $-0.741596 \quad 0.0801634 .216941$ H -2.610330 -0.1305991 .786387$ H -3.081591 0.7126193 .246549 H -3.523919 -3.3849213 .440839$ H $-3.635501-2.211708 \quad 5.397788$ H -2.341090 -1.022453 5.553602 H -3.994018 -0.4987565 .164208$ H -2.261595 -2.100867 0.933129 H -2.292932 $-3.783402 \quad 1.393894$ H -3.895728 $-3.559610-0.324809$ H -4.816352 -3.8017911 .163312$ H $-5.141222-1.2685541 .275548$ H -6.908095 -2.7492940 .265851$ H $-6.071714-2.706400-1.272035$ H $-8.517480-0.444259-1.992507$ H -5.900854 -0.421751-2.539156 H $-8.418125-0.8703990 .889180$ H -8.300219 0.6904970 .111780 H $-7.003007 \quad 0.143996 \quad 1.191571$ H -6.661889 $1.855160-1.057598$ H $-4.1008141 .961115-2.106207$ H -2.794319 $1.250953-1.203374$ H $-4.052281 \quad 1.526630 \quad 0.507851$ H $1.183118-2.1910060 .950439$ H $\quad 0.594257-4.4307552 .991367$ H $-0.627096-5.769410 \quad 0.807116$ H $0.463123-6.7396701 .819172$ H $0.610480-6.7588600 .060069$ H $-3.765905-0.786184-3.093999$ H -3.557803 $0.741356-3.937843$ H $0.281498-0.842963-0.850574$ H $0.686008-0.638151-2.565541$ H $-0.346806-3.331307-1.397566$ H $1.995323-2.516329-3.196186$ H $1.632135-4.931263-3.819617$ H $0.849232-5.261442-2.295583$ H $5.085531-5.090075-2.428994$ H $4.110745-4.372324-3.831419$ H $4.024252-5.926618-0.502914$ H $2.463400-6.612862-0.850897$ H $3.066370-3.8850920 .480493$ H $-2.012770-1.296765-1.445969$ C $-0.805517 \quad 4.3591710 .515007$

O $0.141573 \quad 3.577776-0.239455$ C $0.8175024 .439101-1.175707$ C $0.0330605 .744928-1.190356$ $\begin{array}{lllll}\text { C } & -0.444376 & 5.820039 & 0.245907\end{array}$ H $1.8140584 .633246-0.761851$ C $0.943793 \quad 3.749444-2.538080$ H $0.6523246 .597812-1.484252$ H $-0.8239115 .699012-1.872212$ C -1.594231 $6.790667 \quad 0.452717$ H $0.399493 \quad 6.1220500 .880852$ C $1.5988042 .356140-2.482297$ O $-0.3642293 .583901-3.102530$ H $1.4916864 .396285-3.232635$ H $\quad 0.940216 \quad 1.659392-1.952067$ O $1.714393 \quad 1.870303-3.825248$ C $2.9945422 .324383-1.837761$ C $3.6071480 .911828-1.846609$ H $2.9230042 .670565-0.805194$ O $3.860907 \quad 3.226116-2.523572$ H $2.9074980 .225914-1.357022$
H $3.7207910 .572935-2.883319$ H $-1.293257 \quad 7.8070640 .178566$ H -2.461963 $6.520802-0.158575$ H $-1.9112526 .803723 \quad 1.500113$ C -0.7190623 .9840351 .994532$ H $-0.8867903 .047463-2.481640$ H $0.8199401 .863352-4.207669$ H $3.9052362 .940741-3.452411$ H $-1.317468 \quad 4.6806402 .592752$ H 0.3183784 .0684322 .337449 H $-1.801377 \quad 4.1280890 .114423$ C $4.2699290 .577919 \quad 1.131129$ $\begin{array}{lllll}\text { O } & 4.972909 & 1.372631 & 0.168531\end{array}$ C $4.990996 \quad 0.844622-1.162017$

C $5.623971-0.554766-1.168707$

C $4.940583-1.487035-0.171683$

C $4.871975-0.831066 \quad 1.205978$

H 3.2080290 .5213500 .861727

C 4.3916401 .2828472 .492639

H $5.6578991 .498305-1.738407$

O $5.568755-1.107065-2.479535$

H $6.684606-0.464596-0.900216$

H $3.942782-1.782782-0.515720$

O $5.702124-2.689789-0.046119$

H $5.880813-0.7863231 .636825$

O $4.075989-1.6365292 .071657$

C 3.5952792 .5677192 .567435

H 4.0233280 .6339953 .294934

H 5.4426581 .5335072 .681055

H $6.080353-1.933284-2.474526$

H $5.649861-3.159651-0.895059$

H $4.471647-2.5249822 .087490$

O $3.888056 \quad 3.199988 \quad 3.742836$

$\begin{array}{lllll}\text { O } & 2.805673 & 2.962403 & 1.721998\end{array}$

C 3.1960534 .4320443 .969778

H 2.1306994 .2376374 .123481

H 3.6038754 .8888204 .875384

H 3.3475335 .1203073 .132485

SCF Energy (B3LYP/6-31G**//MMFF)= -3245.92876690

0600170

MMFF Geometry

C $3.519696-3.5109421 .866905$

C $2.423997-2.8968961 .395719$

C $1.150959-2.713812 \quad 2.174414$

$\begin{array}{llll}\text { O } & 0.063568 & -2.915338 & 1.242345\end{array}$

C $1.097848-1.2736962 .718828$

C $-0.130727-0.961863 \quad 3.548093$

C $-1.0250820 .001353 \quad 3.250823$

C $-0.269490-1.7365704 .834303$

C $-1.003177 \quad 0.9625732 .093369$

C $-0.3806542 .288800 \quad 2.543992$

C $-0.4322823 .388601 \quad 1.474127$

C 0.2852094 .6373931 .997552

C 0.1460195 .8315291 .048302

$\begin{array}{lllll}\text { O } & -1.806004 & 3.678024 & 1.203637\end{array}$

$\begin{array}{lllll}O & 0.574971 & 6.995175 & 1.771204\end{array}$

$\begin{array}{llll}\text { C } & -1.346529 & 6.010569 & 0.701648\end{array}$

C $1.0880955 .700939-0.153873$

O $-1.5112197 .048554-0.263351$

$\begin{array}{llll}\text { C }-2.035302 & 4.705566 & 0.232965\end{array}$

C -3.5650024 .9390740 .117797$

O $-1.5081014 .357817-1.044548$

C $-0.961196-3.7278221 .612685$

O $-1.083833-4.3398692 .661135$

C - $-1.941448-3.7407320 .505665$

C $-3.202563-4.1293840 .744034$

C $-4.289011-4.191975-0.236899$

C $-3.955385-3.859637-1.668119$

C $-4.3917013 .772914-0.440723$

C -4.506427 2.5726850 .482715

C $-4.858916 \quad 0.1079150 .425672$

$\begin{array}{lllll}\text { O } & -4.422569 & 2.658670 & 1.704928\end{array}$

C $-5.716249-0.791211-0.405939$

C $-6.829581-1.388370 \quad 0.045188$

C $-7.702020-2.273154-0.798798$

C $-7.838311-3.670761-0.237129$

C $-8.904595-4.0171520 .503534$ 
C $-6.784747-4.689358-0.613471$

C $-5.521774-4.5376130 .190958$

N $-4.780502 \quad 1.406160-0.218737$

H $3.516699-3.9273462 .870547$

H $2.456420-2.4515420 .405000$

H $1.110981-3.4427942 .992253$

H $1.184678-0.5743331 .879965$

H $1.979729-1.0888783 .347707$

H -1.8541130 .1607963 .939673$

H $-1.106690-1.3814675 .444958$

H $-0.435963-2.7985594 .641563$

H $0.639342-1.6326575 .436359$

H $-0.4650490 .560372 \quad 1.229825$

H -2.0350651 .1152361 .759984$

H $-0.9141942 .639386 \quad 3.438300$

H 0.6631402 .1157922 .836783

H $0.047935 \quad 3.0211280 .560631$

H 1.3435464 .4234142 .194131

H $-0.144194 \quad 4.9210022 .969189$

H 0.4213347 .7685941 .201504

H $-1.850533 \quad 6.3626371 .611994$

H $2.131288 \quad 5.6626790 .182986$

H $1.0194626 .574833-0.810592$

H $0.9014284 .804964-0.748647$

H - $1.2631616 .687124-1.131335$

H -3.9617395 .2083191 .104911$

H -3.743932 $5.791157-0.550205$

H -2.023879 $3.620756-1.405188$

H $-1.600963-3.420241-0.470525$

H -3.471640 -4.421408 1.758593

H -3.217491-4.566553 -2.063241

H $-3.545093-2.846873-1.744735$

H $-4.816954-3.891515-2.337488$

H $-5.418354 \quad 4.128015-0.600382$

H $-4.017333 \quad 3.467536-1.422267$

H -5.2382680 .2269041 .445946$

H $-3.842056-0.2931540 .484903$

H -5.399901 -0.964599-1.431861

H $-7.149493-1.2132221 .069917$

H -7.335436 -2.329595 -1.831406

H -8.690128 $-1.799122-0.866187$

H $-9.025022-5.0258070 .884481$

H $-9.678281-3.2974220 .750193$

H $-6.614876-4.647644-1.692688$

H -7.162754 -5.704585 -0.431466

H $-5.657664-4.757787 \quad 1.250937$

H $-4.711323 \quad 1.424695-1.232210$

C $5.705736-2.4077141 .332437$

O $5.078111-1.227516 \quad 0.788335$

C $6.028989-0.519999-0.024204$

C $7.392492-1.057170 \quad 0.386637$

C $7.064188-2.5173890 .637810$

H $5.836201-0.808574-1.064649$

C 5.8381780 .9909230 .138600

H $8.148905-0.913250-0.390512$

H $7.754589-0.5866901 .308032$

C $8.115545-3.2470271 .455566$

H $6.951510-3.023186-0.330785$

C $4.3972841 .481386-0.123017$

O 6.1809151 .3675051 .478829

H $6.5407051 .524591-0.511685$

H 3.7293951 .1308350 .673642

O $4.4014242 .912472-0.039964$

C $3.8172621 .070363-1.490246$

C $2.4234291 .677946-1.730712$

H $3.750341-0.019749-1.532977$

O $4.712468 \quad 1.494059-2.515775$

H $1.781977 \quad 1.403603-0.885956$

H $2.5025832 .771779-1.734643$

H $9.077826-3.2461980 .933244$

H $8.263084-2.7748452 .432561$

H $7.823538-4.2881231 .625213$

C $4.799036-3.6144181 .082942$

H 5.6128980 .8567892 .081212

H 4.7649803 .1511350 .829989

H $4.4620391 .034513-3.334851$

H $5.305610-4.5440711 .365616$

H $4.565827-3.6882040 .014073$
H $5.831188-2.2339862 .408916$

C $0.827618-0.858527-2.334955$

O $1.711146-0.173464-3.228968$

C $1.7682161 .245191-3.063605$

C $0.3726771 .840513-3.287879$

C $-0.6521301 .220824-2.343265$

C $-0.604479-0.303346-2.428589$

H $1.206655-0.773899-1.309110$

C $0.830098-2.345028-2.733489$

H $2.4085501 .621627-3.870599$

O $0.3902473 .251921-3.111458$

H $\quad 0.054517 \quad 1.649103-4.320982$

H $-0.5050681 .567898-1.315190$

O $-1.951532 \quad 1.670420-2.735734$

H -1.067763 -0.624693 -3.370538

O $-1.378510-0.852881-1.364197$

C $2.156141-3.036764-2.500254$

H $0.074439-2.884148-2.152290$

H $0.601674-2.444618-3.801503$

H $1.0456363 .616897-3.730026$

H $-2.5853271 .346771-2.073956$

H $-2.304645-0.595725-1.507513$

O $1.985399-4.380109-2.685768$

O $3.206480-2.481539-2.212973$

C $3.155138-5.187991-2.521196$

H $3.398777-5.269976-1.458649$

H $2.934723-6.186401-2.908367$

H $3.999584-4.776189-3.082286$

SCF Energy (B3LYP/6-31G**//MMFF)= -3245.91496173

0600171

MMFF Geometry

C $3.0583873 .625161-0.218947$

C 2.6145564 .5730530 .620821

C 1.4091184 .4813811 .530093

$\begin{array}{lllll}\text { O } & 0.767502 & 3.188731 & 1.447294\end{array}$

C $0.372827 \quad 5.548013 \quad 1.127410$

C $-0.783830 \quad 5.659848 \quad 2.101631$

C $-2.061000 \quad 5.325244 \quad 1.832126$

C $-0.4337816 .225135 \quad 3.455917$

C $-2.6477104 .787454 \quad 0.555972$

C $-3.0049893 .308492 \quad 0.705141$

C $-3.7241772 .709310-0.513798$

C $-5.1427723 .266548-0.671966$

C $-5.9170442 .572349-1.803489$

O -3.776279 1.296373 -0.283061

O $-7.3034732 .935455-1.679998$

C $-5.810408 \quad 1.040989-1.622506$

C $-5.495772 \quad 3.115849-3.172601$

O $-6.3886350 .344733-2.722414$

C $-4.3810640 .528316-1.330690$

C $-4.376915-0.944060-0.855053$

O $-3.5628160 .519488-2.503662$

C $1.2497942 .188412 \quad 2.234801$

$\begin{array}{lllll}\text { O } & 2.183890 & 2.251539 & 3.017496\end{array}$

$\begin{array}{lllll}C & 0.441604 & 0.979484 & 1.956947\end{array}$

C $0.799428-0.2042572 .476633$

C $0.100279-1.4771072 .276871$

C $-1.215059-1.4359701 .544799$

C $-5.102604-1.1935230 .469982$

C $-4.769652-2.5505601 .066779$

C $-4.891438-3.773610 \quad 3.241939$

$\begin{array}{llll}\text { O } & -4.212636 & -3.446390 & 0.438711\end{array}$

C -3.589089 -3.524597 3.937917

C $-2.619890-4.4447084 .055989$

C $-1.314334-4.1842274 .754046$

C $-0.118855-4.668168 \quad 3.961824$

C $0.663903-5.6540104 .432719$

C $0.169367-4.0239972 .623489$

C $0.662521-2.6080922 .753587$

N $-5.187323-2.6382032 .387962$

H $2.5252962 .684322-0.308824$

H 3.1777145 .5019760 .693090

H 1.7471914 .6585032 .558026

H 0.0111775 .3270010 .118305

H 0.8538046 .5332991 .061416

H -2.7968615 .4578122 .625380$

H -1.321244 6.4166724 .068799 
H 0.2043925 .5322384 .012410 H 0.0955917 .1771283 .345091 H -3.542639 5.3796140 .336583 H $-1.9803154 .920973-0.299250$ H -2.0866982 .7357700 .885029$ H -3.620540 3.1503821 .601501 H -3.123593 $2.898514-1.410124$ H $-5.1314474 .349107-0.842064$ H -5.6925323 .1186970 .268090$ H -7.615299 2.658176 -0.801790 H -6.442018 $0.799324-0.759258$ H -5.777414 4.172563-3.263816 H $-6.0172472 .599641-3.985521$ H $-4.4205313 .056435-3.351258$ H $-5.7624500 .377321-3.464598$ H $-4.788855-1.597084-1.635597$ H $-3.330554-1.259360-0.741670$ H -3.869748 $1.197566-3.120361$ H $-0.423651 \quad 1.093807 \quad 1.314722$ H $1.695931-0.2533833 .093256$ H -1.922861 -0.7790582 .063161$ H -1.700158 -2.409030 1.462298 H $-1.077528-1.0646680 .524861$ H $-4.826665-0.4259351 .201873$ H $-6.187450-1.1546490 .323356$ H $-4.871026-4.6874482 .639568$ H -5.699472 -3.845519 3.976082 H $-3.437510-2.5390094 .372600$ H $-2.779334-5.4406863 .649199$ H $-1.353639-4.6917655 .726571$ H $-1.189837-3.1169314 .974432$ H $1.522286-6.0113373 .873504$ H $0.465808-6.1266965 .388927$ H $-0.709734-4.1142421 .980815$ H $0.952897-4.5882152 .099826$ H $1.620428-2.5304203 .269980$ H $-5.583243-1.8146662 .831906$ C $5.4142052 .800834-0.668851$ O $5.026907 \quad 1.437218-0.932172$ C $5.5385380 .605895 \quad 0.127565$ C 6.4951631 .4793210 .936854 C 5.8539862 .8438110 .794858 H $4.6821050 .341507 \quad 0.759003$ C $6.188124-0.661019-0.436755$ H $6.577652 \quad 1.148066 \quad 1.976545$ H 7.5034681 .4880880 .506857 C 6.7832113 .9976191 .126823 H 4.9883632 .8788951 .467329 C $5.280766-1.532020-1.326422$ O $7.328800-0.294183-1.225839$ H $6.581289-1.2649810 .389661$ H $5.104889-1.042273-2.292033$ O $6.006984-2.735233-1.616932$ C $3.926233-1.918569-0.702753$ C $3.207253-3.026011-1.499248$ H $3.291378-1.028589-0.645629$ O $4.123955-2.378080 \quad 0.635613$ H $3.212902-2.762158-2.561351$ H $3.772219-3.959524-1.391177$ H 7.1216823 .9319902 .166023 H 7.6700303 .9971770 .484162 H $6.273234 \quad 4.9573880 .997611$ C $4.2935463 .767180-1.062939$ H $7.0189260 .303164-1.928192$ H $6.848965-2.473106-2.027659$ H $4.724494-3.1421360 .599179$ H $4.0029733 .576492-2.103321$ H $4.6713024 .795623-1.019154$ H $6.2744482 .997300-1.322797$ C $0.589597-1.880124-2.600791$ O $0.917266-2.135821-1.229285$ C $1.764233-3.267869-1.004352$ C $1.103816-4.533289-1.566238$ C $0.751877-4.372200-3.039958$ C $-0.072743-3.104654-3.247865$ H $1.496147-1.605097-3.152715$ C $-0.370887-0.681804-2.634024$ H $1.820410-3.3982940 .081400$
O $1.947884-5.667975-1.405551$

H $\quad 0.180280-4.739334-1.009056$

H $1.647960-4.368451-3.671540$

O $-0.033833-5.489338-3.461677$

H - $1.077513-3.252497-2.830775$

O $-0.223026-2.870907-4.647240$

C $0.3066290 .627613-2.295854$

H $-0.799287-0.573249-3.636754$

H $-1.177361-0.829532-1.908667$

H $2.144965-5.752849-0.457020$

H $0.485659-6.291747-3.281639$

H $-0.627759-3.667142-5.032145$

O $-0.5723071 .646110-2.534411$

O $1.4465090 .746166-1.870266$

C $-0.0884052 .964805-2.267022$

H $0.8790203 .132535-2.749960$

H $-0.8090583 .680214-2.671913$

H $-0.0157253 .114140-1.187827$

SCF Energy (B3LYP/6-31G*//MMFF) $=-3245.92045143$

\section{2}

MM̄FF Geometry

C $-3.656194 \quad 3.624606 \quad 0.370405$

C $-2.378814 \quad 3.3040050 .629799$

C $-1.2478563 .686829-0.293275$

O $-0.345766 \quad 2.568193-0.472642$

C $-0.436345 \quad 4.846531 \quad 0.313725$

C $0.3240465 .637642-0.732752$

C $1.6476265 .555205-0.968296$

C $-0.5123016 .619608-1.517457$

C $2.6723974 .677132-0.306147$

C $3.3462413 .760770-1.335106$

C $4.5965693 .062218-0.778469$

C $5.2031962 .144984-1.843811$

C $6.3926881 .335068-1.312200$

$\begin{array}{lllll}\text { O } & 4.227750 & 2.307600 & 0.379484\end{array}$

O $6.6511520 .295044-2.268196$

C $5.987100 \quad 0.6656620 .018713$

C $7.6658412 .186898-1.252567$

$\begin{array}{lllll}\text { O } & 7.107044 & 0.013660 & 0.612573\end{array}$

C 5.3259431 .6375411 .018643

C 4.7799690 .9246492 .278859

O 6.3111892 .5767501 .468836

C $-0.7516561 .557673-1.288822$

\begin{tabular}{lllll}
\hline & -1.844847 & 1.436132 & -1.818689
\end{tabular}

$\begin{array}{llll}\text { C } & 0.389610 & 0.627526 & -1.456358\end{array}$

C $0.218082-0.507213-2.150835$

C $1.243728-1.504471-2.467597$

C $2.665290-1.189749-2.089802$

C $3.660005-0.0877172 .031712$

C $4.114016-1.5315311 .908762$

C $3.175731-3.8098841 .507835$

O $5.271431-1.8970872 .087768$

C $3.347842-4.195701 \quad 0.073455$

C $2.485174-4.980384-0.589642$

C $2.696348-5.457335-1.996029$

C $1.591185-5.036618-2.935989$

C $0.559165-5.856080-3.200606$

C $1.749968-3.722126-3.663184$

C $0.872623-2.627827-3.117037$

N $3.047181-2.3702991 .610831$

H $-3.8661724 .201534-0.526487$

H $-2.1293752 .773814 \quad 1.542743$

H $-1.6401773 .971601-1.277682$

H 0.2438294 .4651301 .082297

H -1.1021805 .5489030 .833590$

H $2.0638256 .209002-1.735020$

H $0.0848797 .204123-2.225554$

H $-1.2843816 .099965-2.092713$

H $-1.0011207 .327283-0.839757$

H 3.4235745 .3285350 .156626

H 2.2424924 .0716560 .495732

H $2.6143903 .009018-1.656899$

H $3.6299214 .336257-2.225771$

H $5.3176323 .835033-0.486312$

H $5.4959342 .720113-2.731278$

H $4.435527 \quad 1.441238-2.189807$

H $7.353589-0.271188-1.904194$ 
H $5.272180-0.126278-0.236059$ H $7.9242312 .554649-2.253158$ H $8.5263161 .594246-0.923226$ H $7.5711613 .054366-0.596228$ H $7.660076 \quad 0.6955291 .030629$ H 5.6039370 .4843772 .853305 H 4.3578401 .7033562 .930598 H 6.0090672 .9629772 .307504 H $1.3411720 .911927-1.022459$ H $-0.773178-0.723631-2.547795$ H $3.361520-2.006958-2.288998$ H $3.018831-0.319087-2.650364$ H $2.745131-0.976372-1.019787$ H $2.989173-0.0645892 .899639$ H 3.0709720 .1834981 .150002 H $2.275305-4.2474651 .948713$ H $4.040870-4.1471882 .087601$ H $4.248155-3.848219-0.427675$ H $1.600190-5.348934-0.076191$ H $2.760296-6.553296-1.967358$ H $3.664537-5.124844-2.390323$ H $-0.214509-5.577482-3.908598$ H $\quad 0.463081-6.819479-2.711075$ H $1.472786-3.864092-4.717241$ H $2.804612-3.434611-3.704148$ H $-0.185284-2.766413-3.344873$ H $2.132786-1.9644711 .431805$ C -5.9822652 .5587620 .607501$ $\begin{array}{lllll}\text { O } & -5.629049 & 1.181394 & 0.376986\end{array}$ C $-6.1834530 .773103-0.889108$ C $-7.1703151 .865606-1.295163$ C $-6.4994693 .099854-0.726538$ H $-5.347606 \quad 0.772763-1.599888$ C $-6.788089-0.636263-0.818828$ H $-7.3130201 .913044-2.378980$ H $-8.1533631 .726963-0.831058$ C -7.423450 4.296732 -0.589887 H $-5.6752813 .361626-1.399241$ C $-5.891847-1.708334-0.169375$ O $-7.997462-0.575769-0.050826$ H -7.081910 $-0.945541-1.829592$ H -5.915854 -1.601711 0.922670 O $-6.456324-2.993036-0.461853$ C $-4.427500-1.711821-0.633686$ C -3.634931 -2.905147 -0.063369 H $-3.961279-0.772052-0.324054$ O $-4.359217-1.757004-2.058535$ H $-3.986628-3.1235670 .949702$ H -3.824747 $-3.797802-0.671373$ H -7.816688 4.592847 -1.568000 H -8.2755874 .0743570 .061001$ H $-6.8894195 .153230-0.166622$ C $-4.822928 \quad 3.3182931 .268357$ H -8.471565 -1.414226 -0.179714 H $-7.308318-3.0533300 .001684$ H -4.842731 -2.548295 -2.351832 H -4.4769692 .7445772 .137397$ H -5.2034264 .2752641 .645714$ H -6.8069642 .5273491 .332626$ C -2.003651 -1.569449 2.124591 O $-1.813417-1.4396390 .712043$ C $-2.117993-2.619305-0.043112$ C $-1.249200-3.7855300 .444076$ C -1.375208 -4.0028021 .950582$ C -1.135234 -2.692404 2.702821 H -3.057084 -1.801437 2.323714 C $-1.773910-0.2016872 .781434$ H -1.829479 -2.409782 -1.079277 O $-1.585946-4.990272-0.234041$ H $-0.198389-3.5684630 .215367$ H $-2.348787-4.4329962 .213581$ O $-0.392458-4.9495102 .374831$ H $-0.071465-2.4364632 .645997$ O $-1.454917-2.8731154 .080209$ C $-0.401963 \quad 0.3923172 .549511$ H -2.4963950 .5085942 .362375$ H -1.942539 -0.275435 3.861464 H -1.478206 -4.827951 -1.186827
H $-0.524229-5.7578371 .850055$

H $-0.916235-3.6134414 .408073$

O $-0.288257 \quad 1.5339213 .290789$

O $0.450029-0.0665611 .803303$

C 0.9392622 .2590143 .159632

H 1.6852091 .8303583 .833773

H 1.3042542 .2540712 .128114

H $\quad 0.753073 \quad 3.2951543 .455051$

SCF Energy (B3LYP/6-31G**//MMFF) $=-3245.91641708$

0600173

MMFF Geometry

C $0.9317132 .097918-2.854225$

C $-0.1251721 .458135-3.378538$

C $-0.340309-0.035017-3.469312$

O $-1.477775-0.363105-2.634117$

C $0.839424-0.902201-2.997845$

C $0.608955-2.386050-3.205281$

C $0.294276-3.250564-2.220726$

C $0.816690-2.881914-4.612372$

C $0.053192-2.928435-0.771544$

C $-1.117263-3.743884-0.204168$

C $-1.369412-3.4118181 .273119$

C -2.354487 -4.405986 1.889325

C $-2.671994-4.057123 \quad 3.349627$

O $-1.906614-2.0859911 .348305$

O $-3.802723-4.853513 \quad 3.733798$

C $-3.084815-2.5733683 .423836$

C $-1.526724-4.4544204 .287867$

O $-3.266844-2.1664374 .778576$

C $-2.119512-1.6132322 .685184$

C $-2.761581-0.2096432 .612192$

O $-0.894101-1.5277023 .413748$

C $-2.710146-0.361416-3.207190$

O $-2.985860-0.093053-4.365246$

C $-3.685181-0.728352-2.156674$

C $-4.991781-0.516225-2.371336$

C $-6.075587-0.770545-1.420248$

C $-5.737244-1.519577-0.159256$

C -2.0172510 .7988391 .730261$

C -2.8531652 .0573601 .601662$

C $-4.8011552 .990386 \quad 0.348345$

O -2.7209373 .0004362 .378081$

C $-6.1268592 .427194 \quad 0.759111$

C $-7.2254722 .474212-0.010350$

C -8.5523261 .8971910 .396853$

C $-9.1607771 .003998-0.664709$

C $-10.247490 \quad 1.392507-1.353319$

C $-8.556368-0.369881-0.877079$

C $-7.309808-0.313972-1.718780$

N -3.7829781 .9810710 .576663$

H $1.7569381 .536827-2.426018$

H $-0.9297382 .061329-3.796833$

H $-0.559199-0.277652-4.516825$

H $1.045317-0.700605-1.940714$

H $1.752566-0.621679-3.539216$

H $0.207508-4.305513-2.477460$

H $0.703981-3.968444-4.691186$

H $0.090548-2.425881-5.292258$

H $1.824454-2.630714-4.958949$

H $0.964477-3.168738-0.210878$

H $-0.152060-1.864453-0.623243$

H -2.020882 -3.533062 -0.790846

H $-0.894857-4.813296-0.308861$

H $-0.411420-3.4460901 .805255$

H $-1.973464-5.432271 \quad 1.815777$

H -3.292059 -4.392797 1.315334

H -4.060915 -4.584786 4.632387

H -4.074846 -2.498259 2.952902

H -1.326477 -5.5298664 .209967$

H $-1.792427-4.2769195 .335834$

H $-0.595040-3.9258944 .075015$

H -2.385875 -2.027344 5.166265

H $-3.784260-0.3064602 .229559$

H -2.8439510 .2136553 .621963$

H $-0.442754-0.7033803 .172375$

H -3.299496 -1.126180 -1.224873

H $-5.298024-0.079645-3.321401$ 
H $-6.613200-1.8101560 .424089$ H $-5.103011-0.9086040 .487455$ H -5.207052 -2.450379-0.391508 H -1.0489291 .0744082 .160888$ H -1.821073 0.3936900 .731298 H $-4.7726673 .244963-0.715653$ H -4.5788623 .8872290 .934274$ H -6.1859031 .9592121 .739212$ H -7.174339 $2.966057-0.979146$ H -9.2254432 .7368730 .613172$ H -8.4688651 .3300511 .332393$ H $-10.7038110 .749216-2.098419$ H -10.702441 2.364041-1.191615 H -9.273232 -1.021250 -1.393788 H -8.394822 -0.8295690 .101418$ H $-7.4541190 .191905-2.674917$ H -3.8216641 .1409640 .008199$ C $1.0934014 .196060-1.422014$ O $2.2241133 .706726-0.674670$ C 1.8240633 .5474360 .701749 C $0.447747 \quad 4.1952940 .825530$ C $-0.1334143 .932738-0.550366$ H 1.7180662 .4702740 .875989 C 2.8852414 .1306591 .641062 H -0.1409303 .7572351 .636139$ H 0.5196235 .2737441 .008374 C - $1.3286204 .805966-0.887387$ H $-0.4321182 .878293-0.600862$ C 4.2980663 .5396591 .461895 O $2.9809695 .544441 \quad 1.421034$ H 2.5612614 .0108372 .681415 H 4.7204873 .8492830 .498109 O 5.1390704 .1109272 .472624 C 4.3682772 .0066201 .582782 C 5.8135041 .4751861 .528361 H 3.7909961 .5583510 .768220 O 3.7617921 .5922882 .806234 H $\quad 6.288747 \quad 1.8502000 .615449$ H 6.3834761 .8743982 .376012 H -2.136934 4.649549 -0.166667 H -1.067194 $5.869360-0.869688$ H -1.713924 4.569591-1.884290 C $1.0715323 .594787-2.828776$ H 3.2052445 .6809930 .484445 H 5.0866455 .0778332 .380483 H 4.2523022 .0123183 .533774 H $2.0166073 .836760-3.331120$ H $0.2727714 .067393-3.412672$ H $1.2483245 .278876-1.524143$ C $5.466416-0.543398-0.740584$ O $5.039019-0.6886550 .617248$ C $5.890650-0.0679551 .584460$ C $7.313391-0.6338261 .474326$ C $7.860005-0.5368490 .051279$ C $6.867611-1.133328-0.948243$ H $5.484851 \quad 0.524471-0.992347$ C $4.389913-1.159288-1.643854$ H $5.502328-0.3651492 .566890$ O 8.1806010 .0420952 .378602 H $7.297700-1.6899431 .773920$ H $\quad 8.1121590 .499034-0.204139$ O $9.084202-1.273057-0.009287$ H $6.865924-2.223086-0.825949$ O $7.335295-0.848614-2.264072$ C $4.264068-2.662156-1.510638$ H $3.410105-0.739518-1.391957$ H $4.603770-0.906396-2.688615$ H $9.070432-0.3331522 .263102$ H $9.400056-1.232741-0.928393$ H $\quad 6.797293-1.364936-2.887414$ O $4.027956-3.210100-2.737999$ O $4.337916-3.275989-0.454609$ C $3.876986-4.633056-2.759238$ H $4.817330-5.113580-2.473428$ H $3.066813-4.943305-2.092590$ H $3.624165-4.932576-3.779755$ SCF Energy (B3LYP/6-31G**//MMFF) $=-3245.93075175$

\section{6_00174}

MMFF Geometry

C $-0.357992-1.8568431 .405678$

C $0.797631-1.6318520 .762610$

C $1.007765-1.863977-0.710482$

O $2.387642-2.276393-0.850703$

C $0.807564-0.561271-1.500040$

C $0.777325-0.756258-3.003043$

C $1.758135-0.381652-3.847536$

C $-0.486775-1.363872-3.554046$

C $3.0886400 .233157-3.515609$

C $3.1564801 .721601-3.890387$

C $2.3668382 .627633-2.931813$

C $2.4164464 .085653-3.392916$

C $1.7368545 .027442-2.386619$

O $2.9683662 .508656-1.640615$

O $2.1011926 .366215-2.754475$

C $2.3063324 .747891-0.978310$

C $0.2090794 .953245-2.491988$

O $1.6170715 .511498 \quad 0.009119$

C $2.3178813 .249160-0.601932$

C 3.1257843 .0316530 .699889

O $0.9713562 .815318-0.398794$

C $2.702512-3.269297-1.716380$

O $1.927760-3.905793-2.416290$

C $4.161809-3.536110-1.710592$

C $5.053755-2.745826-1.091508$

C $6.501992-2.953577-1.020188$

C $7.064055-4.200447-1.650376$

C 3.4031551 .5543181 .012452

C 4.2571061 .3723652 .254463

C 5.444094-0.413996 3.531956

O 4.6099002 .3033252 .972861

C $6.848334-0.4641153 .016195$

C $7.572119-1.5903032 .926775$

C $8.979601-1.6355022 .402889$

C $9.133581-2.5576591 .212425$

C $9.680622-3.7769101 .352790$

C $8.739651-2.036192-0.152814$

C $7.251287-2.037087-0.371679$

N 4.5714920 .0354502 .462835

H -1.222344 -2.2096390.849879

H $1.651172-1.2718441 .332275$

H $0.332345-2.656111-1.054291$

H $1.5951790 .146860-1.225294$

H $-0.135077-0.083578-1.204563$

H $1.604001-0.545290-4.913694$

H $-0.442242-1.505853-4.639191$

H $-0.678090-2.343700-3.107131$

H $-1.338331-0.711128-3.345132$

H $3.3654540 .081594-2.468013$

H $3.848039-0.305118-4.097243$

H $4.2135462 .017305-3.866829$

H $2.8032321 .864299-4.919212$

H $1.3283392 .282487-2.883916$

H $1.9665834 .195622-4.387481$

H $3.4649264 .396624-3.503501$

H $1.7382896 .966157-2.080153$

H $3.3412925 .116061-0.975104$

H $-0.1163195 .226829-3.502938$

H $-0.2709035 .673518-1.820301$

H $-0.1882153 .960001-2.273199$

H 0.7621675 .0785850 .174305

H 4.0953173 .5391460 .611336

H 2.5969623 .4832891 .548547

H $0.9652482 .067567 \quad 0.218422$

H $4.464223-4.423876-2.256555$

H $4.698558-1.854237-0.577107$

H $6.592284-5.093540-1.225410$

H $8.138977-4.318228-1.497559$

H $6.893531-4.193530-2.732501$

H 2.4650981 .0159371 .172140

H $3.9306691 .082840 \quad 0.175140$

H $5.085408-1.3961843 .855006$

H 5.3815260 .2781594 .377495

H 7.2911020 .4783772 .701940

H $7.135859-2.5316703 .252932$

H $9.628634-1.9678003 .223693$ 
H $9.338323-0.6345822 .132102$ H $9.824477-4.4338470 .501469$ H $9.993181-4.1475692 .323431$ H $9.251607-2.595433-0.943970$ H $9.108645-1.008678-0.266841$ H $6.750341-1.1667630 .052475$ H $4.287449-0.6509251 .769533$ C $-1.273858-0.4298683 .324695$ O $-2.628017-0.4348092 .839698$ C -3.0549970 .9371662 .684858$ C $-1.8837601 .815383 \quad 3.126195$ C -0.6932280 .9080042 .879440$ H -3.2224001 .0957931 .615033$ C -4.3512931 .1794793 .471604$ H -1.8343182 .7494002 .557584$ H -1.952711 2.0801824 .187695 C $0.555172 \quad 1.3268003 .634908$ H -0.4843200 .9027531 .802732$ C $-5.525754 \quad 0.2722383 .051425$ O H -4.6452132 .2321623 .389841$ H $-5.281378-0.778303 \quad 3.248799$ O $-6.652116 \quad 0.590764 \quad 3.878414$ C $-5.9499860 .441700 \quad 1.582208$ C -7.121764 -0.479794 1.196104 H -5.0993190 .2146810 .935719$ O $\quad-6.3090551 .8006901 .339061$ H $-6.813789-1.5176931 .366147$ H $-7.977591-0.2904231 .855399$ H $0.8837192 .320325 \quad 3.313113$ H $\quad 0.3772891 .3652374 .714781$ H 1.3745220 .6244003 .455643 C $-0.522725-1.6890562 .889867$ H -3.8040640 .0250314 .958618$ H -6.3695390 .4894864 .803533$ H -7.0483182 .0186681 .932295$ H -1.068359 -2.569735 3.252077 H $0.457608-1.7104973 .381921$ H -1.344014 -0.453163 4.421306 C $-5.815036-1.471861-1.372137$ O $-6.511529-0.234295-1.191803$ C -7.596134 -0.289025 -0.262690 C $-8.631716-1.321268-0.731524$ C $-7.997060-2.684067-1.008303$ C $-6.770783-2.540537-1.911254$ H $-5.387335-1.801429-0.417106$ C -4.660079-1.205036 -2.341549 H $-8.0795650 .695365-0.304836$ O $-9.669679-1.4762980 .230220$ H -9.101322 -0.966776 -1.658449 H $-7.736986-3.201487-0.077437$ O $-8.947568-3.517398-1.676023$ H -7.092943 -2.283846 -2.928628 O $-6.088090-3.790079-1.979262$ C -3.642949 -0.286324 -1.704094 H -4.136554 -2.125742 -2.621445 H -5.027137 -0.746777 -3.267960 H -10.067317 -0.6006910 .374294$ H -9.734469 -3.571262 -1.106614 H -6.726098 -4.452086 -2.296506 O $-3.703161 \quad 0.950089-2.275271$ O $-2.898429-0.624086-0.792767$ C $-2.799507 \quad 1.923853-1.742529$ H -2.962192 $2.050439-0.668328$ H -1.766018 $1.629863-1.946074$ H -2.995896 $2.877382-2.239822$

SCF Energy (B3LYP/6-31G**//MMFF) $=-3245.93010893$

0600175

MM̄FF Geometry

C $-1.474550-4.1441331 .365049$

C $-0.265508-3.5895271 .186554$

C $0.559728-3.0290472 .317044$

O $1.063755-1.728607 \quad 1.934545$

C $1.767320-3.9447732 .576029$

C $2.613475-3.4937693 .750025$

C $3.810222-2.8855763 .630891$

C $2.059082-3.8208445 .111976$
C $4.492791-2.4885082 .350753$

C $5.296876-1.1906882 .514677$

C $5.977650-0.7886481 .198951$

C 6.9907090 .3326191 .432004

C 7.6646380 .7621110 .121673

$\begin{array}{lllll}\text { O } & 4.963812 & -0.336807 & 0.294979\end{array}$

$\begin{array}{lllll}\text { O } & 8.339897 & 2.002960 & 0.388153\end{array}$

C $6.5741911 .041294-0.934878$

C $8.759092-0.225966-0.296342$

O $7.1475411 .281381-2.217162$

C $5.448224-0.022254-1.016795$

C $4.2621870 .563145-1.814938$

O $5.854361-1.195225-1.721134$

C $0.250746-0.6583332 .138261$

O $-0.903678-0.6782452 .534515$

C 1.0264320 .5605701 .810950

C 0.4141841 .7527731 .850314

C 1.0164973 .0574581 .569952

C 2.5069583 .1141441 .365395

C $3.030534-0.350443-1.878165$

C $1.9335700 .299920-2.701218$

C $0.3921492 .260928-2.700066$

O $1.604314-0.135072-3.801938$

C $0.6396743 .678307-2.293014$

C $-0.2831894 .460113-1.712503$

C $-0.0263825 .882226-1.302322$

C $-0.357180 \quad 6.1470290 .150549$

C -1.3939336 .9311550 .491264$

C 0.5577135 .5630051 .209203

C 0.2064994 .1359521 .529523

N $1.4019901 .421662-2.086709$

H -1.876998 -4.176371 2.376335

H $\quad 0.181005-3.5471090 .197612$

H $-0.036560-2.9355213 .233549$

H $2.381325-4.0136441 .669814$

H $1.424838-4.9701042 .771792$

H $4.359248-2.6540804 .542429$

H $2.739431-3.5283085 .918828$

H $1.111192-3.2997705 .277083$

H $1.885190-4.8980215 .202852$

H $5.174315-3.2967702 .059978$

H $3.770437-2.3563871 .539568$

H $4.624485-0.3885592 .845804$

H $6.054944-1.3341223 .294810$

H $6.473506-1.6710990 .778165$

H 7.7466590 .0322732 .168333

H $6.477856 \quad 1.2031231 .865205$

H $8.7141122 .321113-0.451770$

H $6.1094221 .992760-0.640018$

H $9.554907-0.2505520 .458444$

H $9.244050 \quad 0.086086-1.227536$

H $8.396772-1.248070-0.418667$

H $7.3967930 .423960-2.600695$

H $3.9661671 .517530-1.362974$

H $4.5789760 .782828-2.843037$

H $\quad 6.801997-1.336769-1.586941$

H 2.0729260 .4453571 .552399

H -0.6442491 .7794342 .101690$

H 3.0287942 .6489632 .209629

H 2.8965164 .1317001 .292676

H 2.7952382 .5911710 .449949

H $3.281409-1.311108-2.341333$

H $2.638114-0.563423-0.877673$

H $-0.5803251 .899215-2.359273$

H $0.4410692 .164184-3.789083$

H $1.6297814 .082706-2.491107$

H -1.279755 $4.063873-1.533417$

H $-0.630036 \quad 6.528301-1.952809$

H $1.0177186 .166062-1.484368$

H $-1.623541 \quad 7.1432231 .530270$

H -2.040782 $7.372518-0.259597$

H $0.469876 \quad 6.1414542 .138099$

H $1.5925705 .686796 \quad 0.878972$

H -0.8533493 .9887921 .742474$

H $1.6091961 .576130-1.105060$

C $-1.906874-4.685799-1.135695$

O $-2.019036-3.319412-1.584584$

C $-2.760736-3.296199-2.816675$ 
C $-2.678266-4.711643-3.366168$

C $-2.778131-5.516048-2.084595$

H $-3.805059-3.085365-2.555240$

C $-2.231197-2.198940-3.743145$

H -3.480103 -4.932172 -4.076984

H -1.718094 -4.901383 -3.859849

C $-2.323960-6.957884-2.234594$

H -3.822198 -5.505831 -1.743242

C $-2.178827-0.796771-3.102452$

O $-0.900948-2.530087-4.160600$

H -2.833951 -2.169762 -4.658170

H $-1.371149-0.749535-2.361618$

O $-1.8390240 .138200-4.133467$

C -3.496407 -0.344339-2.448380

C $-3.4214151 .087571-1.888237$

H $-3.741781-1.029548-1.631341$

O $-4.557432-0.423410-3.398878$

H $-2.5544601 .156775-1.222771$

H -3.259184 $1.795691-2.709686$

H -2.949638 -7.484137 -2.962873

H -1.285819 -7.018023 -2.578265

H -2.394442 -7.490072 -1.280907

C $-2.363771-4.7744830 .323800$

H $-0.354525-2.616650-3.360432$

H $-0.991064-0.148080-4.514966$

H $-4.325486 \quad 0.154393-4.146070$

H -2.487673 -5.8281320 .601687$

H -3.347812 -4.2966630 .416086$

H -0.855419 -4.979136 -1.240228

C $-4.220801 \quad 0.4920901 .001628$

O $-5.0821830 .542888-0.141078$

C $-4.7055301 .502298-1.134916$

C $-4.6490322 .908254-0.519218$

C -3.7704652 .9516750 .729944$

C $-4.181676 \quad 1.8520361 .709973$

H -3.2083220 .2254150 .673912$

C -4.682494 -0.6666701 .891949$

H $-5.5157691 .506891-1.874888$

O $-4.1689693 .860886-1.461911$

H $-5.6630903 .219607-0.236532$

H -2.7079032 .8713560 .474428$

O

H -5.1618422 .1113262 .128843$

O $-3.246425 \quad 1.8279872 .783387$

C -6.043811-0.430655 2.502955

H -4.756339 -1.592007 1.307016

H -3.953400 -0.8625552 .686610$

H $-4.7548663 .825375-2.237294$

H -3.6979714 .9027740 .727700$

H -3.597172 1.2353213 .469181

O $-5.913982-0.128143 \quad 3.826428$

O $-7.093300-0.4870431 .875410$

C $-7.136787 \quad 0.1451194 .517272$

H $-6.8953800 .369438 \quad 5.559620$

H -7.633698 1.0148744 .076955

H -7.792197 -0.7306194 .489061$

SCF Energy (B3LYP/6-31G**//MMFF) $=-3245.92703262$

0600176

MM̄FF Geometry

C $4.459634-3.118144-0.372229$

C $3.119056-3.125625-0.328860$

C $2.265198-3.312524-1.556354$

O $1.353980-2.194698-1.646757$

C $1.417385-4.594550-1.463586$

C $0.639618-4.878597-2.738618$

C $-0.704709-4.898609-2.836034$

C $1.473633-5.175192-3.960225$

C $-1.718777-4.714122-1.743223$

C $-2.194711-3.264551-1.635516$

C $-3.268285-3.068225-0.554545$

C $-4.627522-3.637737-0.971959$

C $-5.719609-3.3476990 .070107$

O $-3.383949-1.656015-0.320734$

O $-6.980613-3.630993-0.555759$

C $-5.689669-1.8435780 .419242$

C $-5.615766-4.2972031 .269551$

O $-6.599901-1.5478371 .475142$
C $-4.276982-1.3187740 .751108$

C $-4.235430 \quad 0.2114480 .954961$

O $-3.846481-1.902781 \quad 1.987908$

C $1.792077-1.075002-2.281043$

O $2.899306-0.878620-2.754717$

C $0.664477-0.118771-2.301000$

C $0.8300561 .099282-2.835919$

C $-0.1854842 .151393-2.898096$

C $-1.5646311 .826638-2.384740$

C $-4.7139531 .054414-0.228814$

C -4.5728152 .5430100 .048664$

C $-5.1362864 .763683-0.929697$

O -4.0538092 .9899861 .068376$

C $-4.0648135 .311992-1.816617$

C $-2.9238985 .847900-1.356970$

C $-1.8662246 .455054-2.233893$

C $-0.6578565 .556131-2.377591$

C $0.3816425 .680019-1.533922$

C $-0.7137084 .574819-3.532704$

C $0.1643493 .360929-3.383123$

N $-5.1045913 .314231-0.975667$

H $4.947702-3.304083-1.325759$

H $2.609248-2.9646770 .615978$

H $2.893057-3.348767-2.454936$

H $\quad 0.737990-4.506041-0.609155$

H $2.063390-5.457770-1.256961$

H -1.143526 -5.096285 -3.813578

H $0.870154-5.552805-4.792765$

H $1.985870-4.274002-4.309947$

H $2.223587-5.939711-3.731962$

H $-2.563380-5.376057-1.966657$

H -1.326494 -5.056742 -0.780821

H $-1.339031-2.621341-1.398017$

H $-2.570593-2.905908-2.602600$

H -2.916510 -3.547306 0.365346

H $-4.569336-4.715663-1.161451$

H $-4.930789-3.183574-1.925787$

H -7.682508 -3.3820080 .070092$

H $-6.069566-1.317616-0.464824$

H $-5.726701-5.3377300 .940969$

H $-6.426942-4.1237461 .985113$

H $-4.664545-4.2181471 .799603$

H $-6.192764-1.8413682 .307994$

H -4.8061390 .4796801 .854064$

H -3.1976090 .4956221 .176535$

H $-3.113660-1.3703992 .339403$

H -0.272459-0.438885 -1.859711

H $1.8031111 .360344-3.249885$

H $-2.2496162 .676487-2.408816$

H $-2.0191611 .030266-2.984351$

H $-1.5234981 .498478-1.340670$

H $-4.1360770 .814170-1.127228$

H $-5.7718730 .857795-0.431720$

H $-5.0254375 .107397 \quad 0.104245$

H $-6.1182005 .086904-1.289588$

H $-4.2516015 .289817-2.887523$

H $-2.7396795 .867432-0.285162$

H -1.575231 $7.419994-1.798464$

H $-2.2650916 .697635-3.227153$

H $1.2729805 .068576-1.617150$

H $0.365316 \quad 6.408798-0.729606$

H -0.422049 5.103309-4.449534

H -1.749019 $4.262412-3.706529$

H $1.1908473 .515782-3.716405$

H $-5.4765782 .855478-1.802364$

C $6.223443-1.6262450 .728079$

O $5.418198-0.4455040 .923619$

C $5.8585380 .567501-0.001023$

C $7.2085570 .094057-0.534906$

C $7.005625-1.407105-0.568982$

H $5.1333300 .564224-0.824243$

C 5.8856381 .9601970 .644109

H $7.4389740 .520459-1.516019$

H 8.0310600 .3444480 .144645

C $8.296151-2.202789-0.650610$

H $6.393642-1.633645-1.449161$

C 4.6092002 .3668941 .406080

O 6.9696512 .0062751 .581891 
H $6.1185592 .702083-0.129830$

H 4.5870321 .8741402 .386381

O 4.6814813 .7752901 .664328

C 3.2855392 .0817640 .679820

C 2.0697612 .6810191 .417758

H 3.1686960 .9983160 .582136

O $3.3225002 .614576-0.644001$

H 2.2342602 .6250092 .498274

H 1.9721633 .7425611 .160162

H $8.843699-1.954893-1.565786$

H $8.952515-1.9927490 .200456$

H $8.089291-3.277618-0.660581$

C $5.358802-2.8928310 .811386$

H 7.1075862 .9360641 .828592

H 5.4104893 .9234972 .289611

H $3.4937123 .569372-0.573272$

H $4.757112-2.8494211 .727984$

H $6.014572-3.7672800 .899908$

H $6.918978-1.6454111 .577988$

C $0.829758 \quad 0.2391652 .743603$

O $0.842323 \quad 0.545871 \quad 1.345914$

C 0.7643091 .9464021 .045078

C -0.5135472 .5436831 .647222$

C -0.6507122 .2343343 .137280$

C -0.4604240 .7386543 .401428$

H $1.685298 \quad 0.728307 \quad 3.224790$

C $1.086241-1.2609532 .931162$

H $0.6698842 .024192-0.041858$

O -0.5507043 .9496741 .431696$

H $-1.3745942 .118041 \quad 1.118526$

H 0.0592182 .8269583 .725507

O -1.9499232 .6282103 .584922$

H $-1.329427 \quad 0.1901573 .020663$

O $-0.394000 \quad 0.5118654 .806494$

C $0.020262-2.1620222 .348825$

H $2.029481-1.5340192 .444838$

H $1.181154-1.4756954 .002129$

H -1.3794004 .2811871 .818106$

H -2.609580 2.1426963 .060441

H -1.204095 0.8802995 .198862

O $0.021687-3.345873 \quad 3.030132$

O $-0.695188-1.8818741 .397697$

C $-0.924162-4.3212392 .583796$

H -0.741344 -4.575000 1.536499

H $-0.794839-5.2222223 .189264$

H -1.943182 -3.949586 2.722415

SCF Energy (B3LYP/6-31G**//MMFF) $=-3245.91951260$

0600177

MM̄FF Geometry

C $3.350980-3.5966010 .005953$

C $2.040040-3.3107640 .048014$

C $1.140251-3.429886-1.155375$

O $0.116403-2.409552-1.095412$

C $0.435370-4.798161-1.145310$

C $-0.374868-5.060797-2.400387$

C -1.720023 -5.015817 -2.473656

C $0.431490-5.443896-3.615493$

C $-2.692862-4.659753-1.383740$

C $-3.683691-3.585622-1.853554$

C $-4.680148-3.203383-0.749037$

C $-5.701886-2.193688-1.273753$

C $-6.645633-1.700586-0.169374$

O $-3.942582-2.6373290 .338646$

O $-7.332517-0.551513-0.689740$

C $-5.806651-1.2426351 .044215$

C $-7.728330-2.7384640 .149832$

O $-6.676327-0.918918 \quad 2.126307$

C $-4.741320-2.2747611 .475596$

C $-3.772106-1.7513572 .565804$

O $-5.402720-3.4218462 .024318$

C $0.418154-1.179571-1.590811$

O $1.506681-0.789664-1.980643$

C $-0.839835-0.396422-1.618437$

C $-0.8459110 .843011-2.131132$

C $-2.0161281 .710180-2.295993$

C -3.359086 $1.159302-1.898095$

C $-2.935804-0.5277622 .179127$
C -3.5814960 .8037822 .524022$

C -3.3275503 .2472472 .077366$

$\begin{array}{lllll}\text { O } & -4.536417 & 0.909896 & 3.288294\end{array}$

C -3.8310043 .7742200 .771817$

C -3.2216834 .7525140 .085311$

C $-3.7550445 .342879-1.186183$

C $-2.7938985 .212345-2.344230$

C $-1.9614026 .216148-2.669706$

C $-2.8777923 .963657-3.191263$

C $-1.8383932 .937015-2.828812$

N $-2.9351301 .862727 \quad 1.904071$

H $3.770397-3.935211-0.936940$

H $1.580733-3.0203980 .988175$

H $1.716932-3.311633-2.081400$

H $-0.195488-4.881292-0.253309$

H $1.178021-5.601902-1.048393$

H -2.186044 -5.265872 -3.426298

H $-0.201692-5.690728-4.474468$

H $1.087835-4.622332-3.918010$

H $1.048675-6.322428-3.400342$

H $-3.245290-5.565525-1.106376$

H -2.182386 -4.308394 -0.483765

H -3.120856 -2.696473 -2.164163

H $-4.232142-3.948882-2.731922$

H $-5.183977-4.115397-0.407426$

H $-6.274720-2.616520-2.108573$

H -5.173273 -1.325008 -1.687429

H -7.884681 -0.1902990 .025224$

H $-5.312384-0.3109410 .742825$

H -8.338240 -2.937477 -0.739760

H $-8.423507-2.3669560 .910785$

H -7.321377 -3.6923180 .491907$

H $-6.145529-0.4929962 .819740$

H -4.309439 -1.590417 3.508090

H -3.058435 -2.560904 2.777624

H $-4.770989-3.9063442 .581085$

H -1.736818 $-0.876797-1.245522$

H $\quad 0.094487 \quad 1.258262-2.491097$

H $-3.3490880 .818445-0.858388$

H $-3.625767 \quad 0.314117-2.541359$

H $-4.168708 \quad 1.888627-1.969234$

H -1.996606 -0.5605032 .745610$

H -2.675107 -0.5517261 .116810$

H -2.446370 3.7922792 .428504

H -4.1078713 .3327892 .839779$

H -4.7578103 .3509200 .392253$

H -2.307232 5.1875150 .481259

H -3.965023 $6.403959-0.996285$

H $-4.7223204 .902015-1.457557$

H -1.295412 $6.141687-3.523015$

H $-1.9226647 .129607-2.085668$

H -2.714524 $4.231889-4.244447$

H $-3.8954663 .563442-3.172651$

H $-0.8255813 .240296-3.096471$

H -2.1509211 .6715491 .287866$

C $5.600512-2.8506140 .996574$

O $5.398946-1.4456680 .752288$

C $6.326769-1.024269-0.264860$

C $7.348058-2.150112-0.403943$

C $6.487221-3.367804-0.137283$

H $5.751514-0.954529-1.195853$

C $6.9401660 .340516 \quad 0.066754$

H $7.819050-2.162861-1.391615$

H $8.142277-2.0785230 .348072$

C $7.279754-4.6135920 .218176$

H $5.903335-3.566897-1.042457$

C 5.9379451 .4670360 .383398

O $7.799787 \quad 0.213495 \quad 1.207991$

H $7.5916600 .648452-0.759843$

H 5.5373221 .3442521 .397544

O 6.6719622 .6998150 .394566

C $4.7628671 .602411-0.598986$

C $3.9775802 .913850-0.395209$

H $4.1061480 .733887-0.481307$

O $5.2675121 .580733-1.935523$

H 3.9875263 .1832250 .665165

H $4.4849353 .718425-0.940904$

H $7.948654-4.891119-0.603018$ 
H $7.892686-4.4586831 .112481$

H $6.610888-5.4585540 .409455$

C $4.261958-3.5690001 .202856$

H $7.264077-0.1377871 .940080$

H 7.3955232 .6002111 .036970

H $4.5068801 .575366-2.540182$

H $3.742427-3.0929382 .043084$

H $4.461234-4.6069231 .495322$

H $6.153536-2.9069431 .944157$

C 1.4676762 .2559921 .226589

O $1.7749231 .849764-0.114230$

C $2.5194432 .796621-0.890715$

C $1.7604684 .126067-0.966195$

C $1.394874 \quad 4.6509200 .415757$

C 0.6757993 .5719141 .224505

H $2.3939192 .387073 \quad 1.798224$

C 0.6713031 .1114891 .873091

H $2.5565612 .395197-1.910740$

O $2.5280535 .093383-1.673935$

H $0.8373543 .974319-1.537586$

H 2.2813075 .0241510 .942299

O $\quad 0.523094 \quad 5.772000 \quad 0.251637$

H $-0.325773 \quad 3.4192520 .804364$

O $\quad 0.5252984 .0665342 .553910$

C $1.573554-0.0093232 .345458$

H $\quad 0.128847 \quad 1.4763142 .750789$

H -0.0447340 .6802051 .166694$

H $2.0183145 .921690-1.678930$

H $0.263967 \quad 6.069068 \quad 1.140768$

H $-0.1303313 .513548 \quad 3.009314$

O $0.959790-0.670968 \quad 3.370339$

O $2.665076-0.2803161 .865220$

C $1.699454-1.7362633 .976340$

H $2.759812-1.4843474 .075787$

H $\quad 1.289840-1.9053514 .975915$

H $1.573244-2.6480023 .387817$

SCF Energy (B3LYP/6-31G**//MMFF) $=-3245.91646580$

0600178

MM̄FF Geometry

C $-0.9339193 .147036 \quad 1.571721$

C -0.1296212 .2172892 .110648$

C $-0.535017 \quad 0.977718 \quad 2.872505$

O $-0.102872-0.1235822 .033082$

C -2.0470290 .8241303 .123628$

C $-2.410547-0.2220814 .156882$

C -2.890167 -1.449082 3.875138

C $-2.268117 \quad 0.220490 \quad 5.590688$

C -3.044906 -2.096301 2.528599

C $-4.501007-2.1890412 .051097$

C $-5.012524-0.8994751 .387160$

C $-6.458421-1.0741440 .914458$

C $-6.957274 \quad 0.1363130 .109854$

O $-4.160906-0.632180 \quad 0.270315$

O $-8.182366-0.260420-0.525635$

C $-5.9283880 .454319-0.998257$

C -7.3123321 .3120961 .026548$

O $-6.284706 \quad 1.641926-1.701184$

C $-4.4792780 .543837-0.478556$

C $-3.4288200 .672956-1.610151$

O $-4.355641 \quad 1.7153030 .337277$

C $0.547087-1.1740812 .594736$

O $0.826063-1.3326943 .771046$

C $0.856127-2.1175861 .497420$

C $1.384561-3.3202451 .764455$

C $1.704110-4.3176630 .746419$

C $1.221444-5.6991111 .089850$

C $-3.465713-0.377575-2.724210$

C $-3.422153-1.819860-2.259593$

C $-2.041943-3.460572-0.996967$

O $-4.349467-2.592180-2.496790$

C $-0.996135-4.250949-1.721739$

C $-0.085791-3.798107-2.598081$

C $0.933211-4.684145-3.257384$

C $2.349414-4.355260-2.840783$

C $3.178414-3.694425-3.666202$

C $2.828956-4.868893-1.498490$

C $2.397744-3.985152-0.360687$
N - $-2.262925-2.149729-1.577649$

H -2.0105783 .0782251 .684164$

H 0.9429092 .3185751 .952140

H 0.0174400 .9816683 .819768

H -2.546963 0.6011022 .175980

H -2.472546 1.7734663 .473830

H -3.161186 -2.095904 4.709044

H -2.576335 -0.5557926 .299102$

H -1.2264030 .4720205 .812668$

H -2.8875041 .1033295 .779796$

H $-2.415882-1.6160601 .774132$

H -2.651229 -3.116976 2.623812

H $-4.544925-2.9988871 .310501$

H -5.158694 -2.475406 2.880804

H -4.942038 -0.0746062 .104616$

H $-7.123541-1.2731301 .763956$

H $-6.524190-1.9633370 .271269$

H $-8.4665470 .471420-1.100083$

H -6.001810 -0.360661-1.727840

H $-8.103516 \quad 1.021348 \quad 1.728307$

H -7.7173702 .1539090 .454345$

H -6.4645591 .6697651 .614425$

H $-6.0541202 .400071-1.137617$

H $-3.5137341 .665626-2.071908$

H -2.430535 $0.652773-1.150997$

H $-3.424142 \quad 1.989070 \quad 0.340137$

H $0.631804-1.8063270 .481664$

H $1.572693-3.6234502 .791854$

H $\quad 0.139925-5.6909451 .267849$

H $1.715686-6.0624311 .997750$

H $1.406643-6.4334080 .303379$

H $-4.366000-0.242961-3.334782$

H -2.616912 -0.220698 -3.400891

H -2.982071 -4.021790 -0.996613

H -1.735142 -3.316064 0.042511

H $-0.983270-5.311300-1.474367$

H $-0.055908-2.744649-2.862308$

H $0.818671-4.572536-4.343472$

H $0.735171-5.743528-3.051096$

H $4.203275-3.478340-3.383020$

H $2.848421-3.346964-4.639538$

H $3.926377-4.904968-1.484703$

H $2.506047-5.906843-1.380537$

H $2.719320-2.950435-0.470633$

H -1.545882 -1.443600 -1.440993

C $-0.6152743 .966840-0.754448$

O $0.2891102 .900129-1.105166$

C $0.9403693 .222962-2.345694$

C $0.1423184 .372795-2.944354$

C $-0.2722395 .123793-1.691362$

H $1.9422103 .588145-2.093671$

C $1.0559431 .965888-3.212110$

H $0.7382504 .979193-3.632818$

H $-0.7431494 .016323-3.483363$

C -1.417858 $6.095091-1.914922$

H $0.5960645 .675404-1.306891$

C $1.7051440 .766631-2.488267$

O $-0.2534621 .557471-3.627011$

H $1.6053552 .196219-4.131822$

H $1.0421070 .400120-1.694742$

O $1.826701-0.302617-3.434838$

C $3.0992311 .056545-1.898810$

C $3.711188-0.182402-1.221965$

H $3.0229291 .856591-1.156705$

O $3.9674571 .519107-2.930998$

H $3.033032-0.500564-0.423141$

H $3.766002-1.003493-1.946009$

H -1.133566 $6.864656-2.640011$

H -2.307785 $5.584727-2.298071$

H -1.692443 $6.595550-0.980956$

C $-0.440874 \quad 4.2922720 .729781$

H $-0.7834551 .416442-2.823908$

H $0.938483-0.477245-3.790540$

H $4.0246470 .817272-3.602188$

H -1.0057215 .1912010 .999954$

H $0.614114 \quad 4.491657 \quad 0.949919$

H -1.631355 $3.600464-0.952919$

C $4.536176 \quad 1.096244 \quad 1.438005$ 
$\begin{array}{llll}\text { O } & 5.217016 & 1.199195 & 0.181741\end{array}$

C $5.132186 \quad 0.050184-0.662426$

C $5.725334-1.1646770 .060543$

C $5.053052-1.3921891 .410208$

C $5.050184-0.1099292 .241039$

H $3.456621 \quad 1.0062021 .264565$

C 4.7995872 .4057852 .195693

H $5.7784110 .257331-1.524846$

O $5.603431-2.326738-0.752367$

H $6.798008-0.9963450 .222407$

H $4.039264-1.7832231 .283076$

O $5.782451-2.4028452 .110632$

H 6.0685490 .0754382 .606741

$\begin{array}{lllll}\text { O } & 4.217263 & -0.337268 & 3.377337\end{array}$

C $4.026888 \quad 3.569249 \quad 1.620194$

H 4.5003142 .3259553 .246286

H 5.8644222 .6684682 .185876

H $5.948948-3.077927-0.240420$

H $5.355011-2.5189362 .976748$

H 4.3858890 .3710314 .020221

O $4.5740253 .963663 \quad 0.436236$

O 3.0617814 .0796812 .174215

C $3.9425375 .079927-0.198088$

H $2.8593144 .939494-0.249556$

H 4.1847115 .9978650 .344979

H $4.3322565 .157466-1.216515$

SCF Energy (B3LYP/6-31G*//MMFF)= -3245.91617671

0600179

MMFF Geometry

C 2.3881862 .0006652 .368796

C 1.6659950 .8866492 .566052

C 0.1883480 .7237732 .311694

O $-0.286432 \quad 1.700257 \quad 1.353693$

C -0.6429170 .9222993 .591820$

C $-0.545145-0.2122914 .588426$

C $-1.304231-1.3245994 .537585$

C $0.431169-0.0116665 .717406$

C $-2.335826-1.673123 \quad 3.500542$

C $-2.052419-3.0446922 .873509$

C $-3.098290-3.405968 \quad 1.809044$

C $-2.882239-4.8315001 .298601$

C $-3.854527-5.1846460 .164236$

$\begin{array}{llll}O & -2.965419 & -2.475871 & 0.730344\end{array}$

O $-3.373978-6.396967-0.436011$

C -3.800995 -4.070457 -0.902297

C $-5.255837-5.497193 \quad 0.701290$

O $-4.780494-4.291858-1.914880$

C $-3.923119-2.640635-0.322411$

C $-3.601972-1.603100-1.429514$

O $-5.257038-2.449194 \quad 0.145773$

C $-0.172059 \begin{array}{lll}1.396689 & 0.035111\end{array}$

O $0.3256860 .384835-0.440119$

C $-0.7638792 .446937-0.824829$

C $-1.2608013 .605554-0.364495$

C $-1.8911064 .654268-1.171460$

C $-2.1304204 .362053-2.630049$

C $-3.480624-0.149100-0.950098$

C $-4.8009530 .592275-0.833723$

C -5.7593442 .7075830 .083866$

O $-5.8525800 .165767-1.302406$

C $-5.5779343 .932819-0.755480$

C $-5.5500935 .175841-0.250687$

C $-5.4038146 .417263-1.083800$

C $-4.265107 \quad 7.299311-0.621303$

C $-4.487670 \quad 8.3410190 .198073$

C -2.885485 $7.025261-1.177358$

C $-2.2456825 .807680-0.566805$

N $-4.6554651 .798742-0.163247$

H 1.9021482 .9056252 .014518

H 2.1694170 .0022032 .948292

H $\quad 0.004450-0.2804181 .908300$

H -1.694837 1.0793073 .317420

H $-0.365717 \quad 1.8689774 .076568$

H - $-1.183344-2.0665645 .325905$

H $0.416856-0.8423906 .431111$

H 1.4530860 .0753055 .338110

H $\quad 0.1872550 .900843 \quad 6.271551$
H -3.317905 -1.692309 3.988070

H -2.389271 -0.9161492 .712942$

H -1.053930 -3.026768 2.417589

H $-2.044175-3.8120553 .657686$

H $-4.095677-3.3057632 .253194$

H -2.968392 -5.5566022 .117535$

H $-1.855326-4.9313410 .919783$

H -3.939095 -6.589596-1.204045

H $-2.828318-4.159937-1.402476$

H $-5.214808-6.336671 \quad 1.405807$

H $-5.927323-5.817182-0.103106$

H $-5.715212-4.6518681 .218090$

H $-5.642603-4.019400-1.556702$

H $-2.628250-1.864028-1.865202$

H $-4.345926-1.667318-2.232323$

H $-5.410220-1.5000330 .269733$

H $-0.7767462 .202993-1.882587$

H -1.2098563 .8147610 .702496$

H $-1.1785384 .275281-3.165113$

H -2.718638 $5.128055-3.139019$

H -2.687803 $3.425691-2.749232$

H $-2.942603-0.0938200 .002006$

H -2.891553 $0.402626-1.693362$

H $-5.754710 \quad 2.936158 \quad 1.154324$

H $-6.7095452 .224723-0.164038$

H $-5.4863203 .791149-1.829712$

H -5.6680365 .3144280 .821783$

H -6.349975 $6.971036-1.024653$

H $-5.270336 \quad 6.175158-2.145495$

H -3.6821028 .9962900 .512221$

H -5.4809198 .5583610 .577097$

H -2.221181 $7.875708-0.972606$

H -2.944808 $6.975938-2.267932$

H $-2.036814 \quad 5.9192330 .498291$

H -3.7379822 .0681830 .179205$

C 4.6267922 .6428901 .426811

$\begin{array}{lllll}\text { O } & 4.433777 & 1.794491 & 0.280524\end{array}$

C $5.6769791 .685524-0.437930$

C 6.6354532 .6808250 .208015

C 6.1382372 .7009791 .640121

H $6.0504280 .669968-0.261084$

C $5.441703 \quad 1.897499-1.938783$

H 7.6797122 .3655560 .119473

H $6.5501703 .679466-0.235201$

C 6.6086073 .9094912 .430308

H 6.4827961 .7864882 .141588

C $4.3131991 .034634-2.536735$

O $5.0897293 .268135-2.173862$

H $6.3762661 .730116-2.486116$

H $3.3413761 .375012-2.157854$

O $4.2923551 .258511-3.952391$

C $4.455659-0.476454-2.286362$

C $3.315667-1.274590-2.940280$

H $4.449083-0.664930-1.211176$

O $5.709196-0.935329-2.786493$

H $2.363115-0.847287-2.608679$

H $3.363859-1.149878-4.028900$

H 7.7019473 .9318202 .484302

H $6.2754954 .845127 \quad 1.968698$

H 6.2213063 .8822423 .453522

C 3.8671722 .0816112 .629688

H $4.2971843 .462604-1.644340$

H $4.1968722 .216186-4.092715$

H $5.734048-0.740732-3.739205$

H 4.0292132 .7239193 .502466

H 4.2572101 .0869782 .876888

H 4.2352963 .6335191 .159550

C $2.236699-2.735197-0.488253$

O $3.418962-3.068953-1.224303$

C $3.362170-2.787531-2.627229$

C $2.211747-3.578363-3.263291$

C $0.882088-3.272127-2.579765$

C $1.006147-3.451096-1.067564$

H $2.092420-1.649295-0.506644$

C $2.454180-3.1679000 .971618$

H $4.297204-3.171157-3.054300$

O $2.125327-3.286367-4.653941$

H $2.421354-4.651965-3.171693$ 
H $\quad 0.524795-2.269011-2.839638$

O $-0.097977-4.184480-3.079749$

H $1.059103-4.524310-0.845135$

O $-0.177742-2.960105-0.442872$

C $3.508232-2.351757 \quad 1.688255$

H $1.523784-3.0602571 .540628$

H $2.777945-4.2153910 .997854$

H $1.370858-3.786768-5.009365$

H $-0.932212-3.991334-2.619613$

H $-0.230870-2.003380-0.610207$

O $3.682502-2.8659952 .942581$

O $4.096225-1.3856201 .224989$

C $4.652364-2.1982183 .755899$

H $4.295422-1.1980094 .016508$

H $4.779665-2.775798 \quad 4.675291$

H $5.616397-2.1437083 .241172$

SCF Energy (B3LYP/6-31G*//MMFF)= -3245.94259011

06_00180

MM̄FF Geometry

C $4.141871-1.934777-1.045012$

C $4.085714-2.462875-2.277567$

C $2.894113-2.417314-3.208467$

O $1.905919-1.464002-2.755052$

C $2.224776-3.801347-3.278919$

C $1.151783-3.892383-4.346706$

C $-0.170872-4.000849-4.111942$

C $1.656941-3.888115-5.768421$

C $-0.894414-4.063252-2.794667$

C $-1.662185-2.766656-2.533081$

C $-2.520682-2.797897-1.258450$

C $-3.733692-3.722546-1.410461$

C $-4.696915-3.630340-0.218082$

O $-2.950639-1.448209-1.032655$

O $-5.915185-4.280025-0.612828$

C $-5.016380-2.1441360 .047053$

C $-4.167426-4.4116170 .990201$

O $-5.820711-1.9998031 .215195$

C $-3.757288-1.2587330 .136982$

$\begin{array}{llll}C & -4.099654 & 0.244491 & 0.240637\end{array}$

O $-3.035928-1.6035301 .322223$

C $2.116441-0.153139-3.059859$

O $3.0385680 .314834-3.708166$

C $1.0329710 .628044-2.425044$

C $1.1245691 .964364-2.350998$

C $0.1450922 .855989-1.724313$

C -1.141112 2.237338-1.246216

C $-4.8126820 .821822-0.984023$

C $-4.8386202 .341091-0.965824$

C -5.130919 4.281175 -2.509730

O $-4.616507 \quad 3.0062040 .042159$

C -3.760306 $4.647892-2.989360$

C -3.071606 $5.707906-2.539669$

C $-1.6983616 .070730-3.031774$

C $-0.7292796 .366499-1.906787$

C $-0.2018017 .593936-1.760155$

C $-0.3724655 .251283-0.947799$

C $0.4443284 .166210-1.599940$

N $-5.1563422 .860550-2.213125$

H $3.261802-1.458302-0.622009$

H $4.973198-2.947825-2.679012$

H $3.250690-2.120671-4.202362$

H $1.812011-4.046736-2.295608$

H $2.977308-4.573793-3.486969$

H $-0.834674-4.061038-4.974532$

H $0.859366-4.081003-6.494071$

H $2.100482-2.920279-6.019785$

H $2.414898-4.666595-5.904418$

H -1.582869 -4.914202 -2.842134

H - $0.220733-4.264991-1.957684$

H $-0.947711-1.938183-2.454786$

H -2.298250 -2.515450 -3.392743

H $-1.891835-3.110982-0.417043$

H $-3.424241-4.763431-1.559574$

H $-4.286153-3.444674-2.319325$

H -6.557585 -4.1643320 .108609$

H -5.636483-1.802717 -0.790990

H $-4.055218-5.4728760 .736568$
H $-4.874790-4.3792061 .826088$

H $-3.198222-4.0531291 .342872$

H $-5.241163-2.1044891 .988968$

H -4.6881020 .4389061 .146659$

H -3.1627850 .7984910 .386939$

H -2.411108 -0.8859491 .517415$

H $0.1981260 .077695-2.008023$

H $2.0063462 .451399-2.765007$

H $-1.6672351 .754514-2.077524$

H $-1.8383192 .954963-0.813006$

H $-0.942057 \quad 1.486326-0.474747$

H $-4.3060820 .499372-1.900626$

H $-5.8499690 .472551-1.022861$

H $-5.422246 \quad 4.845430-1.618039$

H $-5.8640914 .460351-3.301822$

H $-3.3155144 .013456-3.752878$

H $-3.524726 \quad 6.361134-1.797477$

H $-1.8031176 .949459-3.681072$

H $-1.2824265 .276886-3.664230$

H $0.4928337 .819431-0.957745$

H $-0.451458 \quad 8.402488-2.439069$

H $-1.2854804 .879248-0.477109$

H $0.2281075 .646694-0.117337$

H $1.4089494 .513199-1.973577$

H $-5.2614592 .223732-2.997512$

$\begin{array}{llll}\text { C } 5.819627 & -0.523645 & 0.222818\end{array}$

O $4.840302 \quad 0.0734551 .098400$

C 4.6200911 .4319900 .678490

C $5.7966931 .785889-0.225675$

C $6.0414490 .464541-0.925217$

H 3.7009501 .4293500 .080224

C 4.4506652 .3598391 .885089

H $5.5591402 .600912-0.916023$

H 6.6826072 .0762670 .350756

C $7.4100990 .354238-1.573421$

H $5.2757790 .349352-1.701332$

C 3.3676391 .9371442 .897775

O 5.6915502 .4382782 .600119

H 4.2496743 .3768931 .528303

H 3.7139731 .0700593 .472882

O 3.2387592 .9995153 .853800

C 1.9845371 .6137012 .299686

C 0.8899431 .5016813 .379572

H $2.0529040 .676568 \quad 1.737819$

O 1.6060102 .6277961 .369203

H 1.2655070 .8932844 .208135

H 0.6890802 .5039903 .774576

H 7.534628 1.123865 -2.342168

H $8.2136120 .479316-0.839958$

H $7.536366-0.623384-2.049300$

C $5.374380-1.932827-0.184647$

H $5.932111 \quad 1.5350962 .869809$

H 2.9578163 .7986333 .376873

H 1.5138433 .4649861 .854385

H $5.146999-2.5112660 .718811$

H $6.201468-2.439080-0.696024$

H $6.734874-0.6153850 .821862$

C $-0.062142-1.4072653 .426069$

$\begin{array}{llll}\text { O } & -0.267435 & -0.441393 & 2.385799\end{array}$

C $-0.428600 \quad 0.912305 \quad 2.831286$

$\begin{array}{llll}C & -1.621017 & 1.004193 & 3.794492\end{array}$

C $-1.4856280 .030691 \quad 4.961422$

C $-1.214331-1.3795694 .442782$

H $0.888266-1.2045653 .933523$

C $0.033154-2.7935452 .767534$

H $-0.695406 \quad 1.4987091 .944135$

O $-1.796505 \quad 2.333876 \quad 4.272356$

H $-2.5371140 .755993 \quad 3.244028$

H -0.7062730 .3490505 .663496$

$\begin{array}{llll} & -2.709184 & 0.009826 & 5.699530\end{array}$

H $-2.130257-1.7718193 .984317$

O $-0.910761-2.232207 \quad 5.546430$

C $1.359310-3.0267262 .077092$

H $-0.069408-3.5761273 .527826$

H $-0.753340-2.9146652 .015349$

H -1.135061 2.5005124 .964680

H -2.8940570 .9224445 .980424$

H $-0.098556-1.9006525 .965900$ 
O $1.421982-4.3360261 .690216$

O $2.222659-2.1804451 .895584$

C $2.632041-4.7420611 .044290$

H $3.508183-4.4205891 .615521$

H $2.636088-5.8337380 .986746$

H $2.659712-4.3380880 .030137$

SCF Energy (B3LYP/6-31G**//MMFF)= -3245.92393491

0600181

MM̄FF Geometry

C $-0.5981641 .855790-1.964589$

C $-1.3409702 .957000-1.773435$

C $-1.2876633 .866862-0.567014$

O $-0.2552343 .407719 \quad 0.336115$

C -2.6169703 .8856100 .210680$

C $-3.7916644 .438112-0.564159$

C $-4.8021853 .693597-1.053265$

C $-3.7996295 .934553-0.739507$

C $-4.9678372 .199653-0.990743$

C $-6.1156251 .822148-0.049536$

C $-6.4362390 .320823-0.089579$

C -7.7196360 .0430040 .698390$

C -8.053401 -1.4521770 .741955$

O $-5.339500-0.4058770 .480633$

O $-9.075828-1.6247311 .734693$

C $-6.801059-2.2189931 .208205$

C $-8.654666-1.935738-0.582595$

O $-7.022953-3.6269451 .148025$

C $-5.505912-1.8308550 .449518$

C $-4.296136-2.5008631 .150205$

O $-5.599255-2.320951-0.891056$

C 0.3816914 .3170951 .113417

O 0.1807155 .5231341 .141821

C 1.3707543 .6615492 .002136

C 1.7461282 .3792711 .870103

C $2.670651 \quad 1.6481612 .739631$

C 3.2658242 .3911093 .906091

C $-2.914394-2.0867500 .631768$

C $-2.363212-0.8293031 .276759$

C $-1.498937 \quad 0.050511 \quad 3.448845$

$\begin{array}{lllll}\text { O } & -2.193083 & 0.202342 & 0.632367\end{array}$

C $-0.200978-0.3531014 .086733$

C $0.522593-1.4591643 .846892$

C $1.816818-1.7789904 .543966$

C $2.980978-1.8917003 .583072$

C $3.365316-3.0847563 .099169$

C $3.746115-0.6285313 .245144$

C 2.9141430 .3493012 .465101

N -2.057897 -0.9980792 .616896$

H $0.117298 \quad 1.546698-1.208157$

H -2.033799 $3.251928-2.558770$

H -1.030118 $4.871598-0.924512$

H $-2.4933354 .488367 \quad 1.120921$

H -2.826203 2.8829520 .600700

H -5.606152 4.206821-1.580015

H $-4.7012116 .288858-1.250683$

H -3.7583386 .4314580 .235268$

H $-2.9380216 .257387-1.331878$

H -5.189071 $1.845108-2.005130$

H -4.048112 $1.693016-0.689113$

H $-5.844351 \quad 2.1140880 .973589$

H -7.012463 2.391034 -0.327017

H -6.560892 $0.021562-1.136955$

H -8.5631930 .6099150 .284758$

H -7.596472 0.4022801 .730102

H $-9.240099-2.5792601 .825400$

H -6.662448 -1.982617 2.272289

H -9.571255 -1.378151 -0.809999

H -8.954316 -2.987729 -0.522228

H -7.976053 -1.818936-1.430124

H $-6.949015-3.8980380 .217109$

H -4.350972 -2.299712 2.226599

H $-4.378019-3.5875831 .017925$

H -4.707851 -2.479181 -1.238445

H 1.7723254 .2996232 .782549

H 1.3227891 .7882741 .059821

H 3.8094783 .2773333 .559616

H 3.9822331 .8024754 .481975
H 2.4800532 .7090524 .600219

H -2.202748 -2.8919830 .854133$

H $-2.900794-1.958256-0.454273$

H $-2.236651 \quad 0.255638 \quad 4.231194$

H -1.3555530 .9651442 .864862$

H $\quad 0.1777010 .3556564 .821263$

H $0.177879-2.1895893 .119416$

H $1.681876-2.7269405 .081149$

H $2.052158-1.0361975 .316443$

H $4.214879-3.1778662 .430903$

H $2.834812-3.9954143 .357032$

H $4.627929-0.8690572 .640538$

H $4.129681-0.2102344 .179249$

H $2.452905-0.0850901 .576440$

H -2.269423-1.886465 3.062186

C $-0.936868-0.464276-2.841850$

O $0.219672-1.024598-2.181621$

C $0.569495-2.259680-2.828394$

C $-0.667725-2.675803-3.613227$

C $-1.187215-1.331229-4.076962$

H $1.377167-2.030604-3.533732$

C $1.045101-3.298435-1.809505$

H $-0.429360-3.353651-4.438441$

H $-1.409770-3.166536-2.972412$

C $-2.640799-1.361258-4.518974$

H $-0.566048-0.984043-4.913680$

C $2.234778-2.869170-0.928943$

O $-0.037924-3.621397-0.927321$

H $1.291178-4.230691-2.331670$

H $1.917839-2.120706-0.192370$

O $2.635974-4.023303-0.176641$

C $3.460584-2.335524-1.694294$

C $4.708813-2.217610-0.797669$

H $3.216002-1.358397-2.123233$

O $3.767018-3.207451-2.783539$

H $4.430179-1.7141580 .132427$

H $5.048653-3.226100-0.533114$

H -2.770352 -2.039362 -5.368796

H -3.298469 -1.703357 -3.712538

H $-2.975737-0.365536-4.826388$

C $-0.6686940 .999436-3.196168$

H -0.309422 -2.795606 -0.490855

H $1.859892-4.3249260 .326217$

H $3.942383-4.090004-2.414088$

H -1.446562 $1.370135-3.873456$

H $\quad 0.293399 \quad 1.084690-3.716071$

H -1.774927 -0.542534-2.138796

C $5.4468610 .738401-0.613005$

O $5.557765-0.105135-1.766103$

C $5.874310-1.474332-1.490403$

C $7.207661-1.552921-0.736183$

C $7.184017-0.7157730 .537798$

C 6.7328830 .7105640 .227123

H $4.5951080 .416879-0.004953$

C $5.1680432 .166440-1.107697$

H $6.030664-1.955849-2.463708$

O $7.518296-2.907702-0.427046$

H $8.008273-1.182299-1.389668$

H $6.554125-1.1836431 .302007$

O $8.509694-0.6856081 .071786$

H $7.537096 \quad 1.237878-0.302562$

O $\quad 6.4968671 .4071821 .449850$

C $3.7781702 .328708-1.683626$

H $5.2549612 .881726-0.282245$

H $5.8859822 .428503-1.894231$

H $8.355109-2.9080850 .068434$

H $8.468866-0.2392771 .934286$

H $7.346520 \quad 1.4826701 .915454$

O $3.6709763 .585770-2.208891$

O $2.8988851 .479459-1.664039$

C $2.4043373 .917061-2.784763$

H $1.6667134 .055901-1.990643$

H $2.5145534 .858732-3.329000$

H $2.0806253 .144620-3.488753$

SCF Energy (B3LYP/6-31G**//MMFF) $=-3245.92363590$

0600182

MM̄MF Geometry 
C $0.967636 \quad 1.619527 \quad 3.383765$

C 1.1394740 .7821314 .418272

C $1.064119-0.7238854 .360671$

O $1.483050-1.2261933 .070610$

C $-0.374024-1.2014954 .613967$

C $-0.481279-2.7089864 .736973$

C $-1.006679-3.5051003 .784612$

C $0.018155-3.2970136 .030119$

C $-1.556046-3.0465152 .461087$

C $-2.151399-4.2103221 .655946$

C $-2.789970-3.731050 \quad 0.345169$

C $-3.490212-4.890916-0.366517$

C $-4.077906-4.461606-1.718124$

O $-1.758706-3.195978-0.492664$

O $-4.426418-5.662048-2.423709$

C $-2.983079-3.734006-2.526085$

C $-5.381367-3.674161-1.540302$

O $-3.517549-3.199872-3.735550$

C $-2.239844-2.640148-1.723074$

C $-1.035798-2.119235-2.542534$

O $-3.137720-1.552235-1.493121$

C $2.823789-1.3202582 .855752$

O $3.713873-0.9372813 .598829$

C $3.028509-1.9970711 .555479$

C $4.274550-2.1105951 .072177$

C $4.673194-2.770909-0.172627$

C $3.617131-3.529495-0.931050$

C $-0.019976-1.300642-1.732548$

C $1.000300-0.623890-2.633688$

C $3.196824 \quad 0.550547-2.566256$

O $0.843532-0.498442-3.846075$

C $4.137591-0.442807-3.174131$

C $5.463551-0.442495-2.968563$

C $6.400575-1.419375-3.621325$

C $7.269942-2.161398-2.630740$

C $8.589817-1.921798-2.552939$

C $6.622895-3.239954-1.787443$

C $5.963072-2.676313-0.558578$

N $2.094863-0.145669-1.925363$

H $\quad 0.704463 \quad 1.222648 \quad 2.406851$

H 1.3715011 .1987735 .396409

H $1.731594-1.1305095 .130868$

H - $1.014404-0.8439803 .800199$

H $-0.771071-0.7484905 .531858$

H -1.038972 -4.5767053 .973472$

H $-0.162186-4.3755766 .092254$

H $1.095270-3.1377956 .137479$

H -0.490032 -2.830569 6.880402

H -2.342653 -2.302606 2.632852

H $-0.756296-2.5731371 .880113$

H $-1.354409-4.9325541 .435558$

H -2.906537 -4.7258972 .262771$

H -3.510257 -2.939281 0.583076

H $-4.271033-5.3258370 .269715$

H -2.765518 -5.698257 -0.543939

H -4.728569 -5.404610 -3.311837

H -2.253494 -4.496766 -2.830758

H $-6.124669-4.283380-1.011996$

H $-5.832392-3.428933-2.508288$

H $-5.250220-2.748083-0.976510$

H $-4.006340-2.389899-3.510629$

H $-0.488948-2.968064-2.973866$

H $-1.408985-1.517778-3.381275$

H $-2.623440-0.734614-1.402025$

H $2.158522-2.3908781 .042975$

H $5.092272-1.6719961 .643306$

H $2.830561-2.855027-1.279911$

H $3.161491-4.294302-0.291942$

H $3.998810-4.049964-1.811404$

H $-0.514843-0.516533-1.153063$

H $0.504615-1.958166-1.030547$

H $3.6817361 .169663-1.805591$

H $2.7964851 .202077-3.349587$

H $3.709583-1.186143-3.842975$

H $5.9036630 .314844-2.324221$

H $7.025975-0.855906-4.325982$

H $5.852776-2.150434-4.229221$

H $9.223330-2.469640-1.863032$
H $9.066127-1.169532-3.172979$

H $7.383896-3.956255-1.450052$

H $5.943413-3.820270-2.416877$

H $6.651346-2.1217550 .081656$

H $2.170999-0.343357-0.932001$

C 1.9741223 .7364952 .385408

$\begin{array}{lllll}\text { O } & 1.213864 & 3.777968 & 1.163986\end{array}$

C 2.1040353 .5324880 .057723

C 3.5191803 .5785490 .628736

C 3.2946423 .0520792 .034809

H $1.8954372 .511469-0.282688$

C $1.8226474 .521203-1.081555$

H 4.2200532 .9707540 .048533

H 3.9129854 .6004100 .667389

C 4.4247863 .3626042 .998735

H 3.1715061 .9641111 .968831

C $0.333738 \quad 4.643997-1.467057$

O $2.2644485 .829864-0.696474$

H $2.4203834 .250063-1.959161$

H $-0.2158335 .162639-0.671690$

O $0.2419515 .478057-2.628081$

C $-0.358493 \quad 3.306645-1.772742$

C $-1.834843 \quad 3.486505-2.170362$

H $-0.3024492 .665782-0.888370$

O $0.332367 \quad 2.636172-2.823634$

H $-2.3238664 .126105-1.427766$

H $-1.8968534 .003858-3.135377$

H 5.3581302 .9030322 .657781

H 4.5935864 .4410613 .086376

H $4.2010282 .973643 \quad 3.997238$

C 1.1322493 .1093613 .501270

H 1.7978876 .0683090 .123162

H $0.6838376 .318394-2.416624$

H $0.3204823 .218082-3.602858$

H $0.1306063 .556122 \quad 3.483812$

H 1.5867103 .3611434 .467098

H 2.1570104 .7858952 .653353

C -3.061056 1.8069910 .057075

O $-2.411647 \quad 1.324379-1.124075$

C $-2.5813802 .138774-2.287935$

C $-4.073898 \quad 2.265666-2.618295$

C $-4.8853572 .753994-1.419571$

C $-4.5741351 .916292-0.177309$

H -2.6486702 .7881820 .319065$

C $-2.715096 \quad 0.824040 \quad 1.184851$

H $-2.1115681 .590224-3.114431$

O $-4.257277 \quad 3.142966-3.724091$

H $-4.452963 \quad 1.281142-2.922639$

H $-4.7118983 .820981-1.237022$

O $-6.2734862 .623093-1.735355$

H $-5.0084940 .917290-0.308364$

$\begin{array}{lllll}\text { O } & -5.215699 & 2.502473 & 0.951907\end{array}$

C -2.9164551 .4109602 .562128$

H -3.310248 -0.0904821 .074423$

H $-1.6642810 .516523 \quad 1.116809$

H $-5.213722 \quad 3.207630-3.888352$

H $-6.7760622 .896625-0.948877$

H -4.8293983 .3841001 .090695$

$\begin{array}{lllll}\text { O } & -3.429377 & 0.459896 & 3.394525\end{array}$

$\begin{array}{lllll}\text { O } & -2.631087 & 2.559657 & 2.873957\end{array}$

C -3.643532 0.8762654 .746546

H -2.705495 1.2203875 .192819

H -4.4009321 .6650374 .781762$

H -4.0052630 .0148175 .313930$

SCF Energy (B3LYP/6-31G**//MMFF)= -3245.94506196

06_00183

MM̄FF Geometry

C $-0.8861702 .869437 \quad 1.494552$

C $-0.416698 \quad 1.9261562 .325200$

$\begin{array}{llll}\text { C } & -1.166730 & 0.774487 & 2.946553\end{array}$

O $-0.588358-0.4306682 .391266$

C -2.6884780 .7314892 .706276$

C $-3.414301-0.1429263 .712254$

C $-3.825898-1.4063893 .491665$

C $-3.705796 \quad 0.5186125 .036252$

C -3.627989 -2.249059 2.263436

C $-4.956135-2.6107801 .583711$ 
C $-5.620385-1.419105 \quad 0.878035$

C $-6.963639-1.8310520 .272338$

C $-7.615126-0.677790-0.504590$

O $-4.738226-0.989962-0.160763$

O $-8.672788-1.250871-1.292118$

C $-6.579098-0.078114-1.482299$

C -8.2948610 .3169680 .442981$

O $-7.0822321 .098454-2.109104$

C $-5.1781820 .171530-0.873101$

C $-4.1502290 .402079-2.003090$

O $-5.138295 \quad 1.341067-0.056099$

C $-0.190224-1.4127713 .237255$

O $-0.229453-1.3879104 .459773$

C $0.326803-2.5888552 .499050$

C $0.306928-2.7020961 .161683$

C $0.808146-3.8370890 .382148$

C $1.511928-4.941361 \quad 1.127007$

C $-2.7271060 .639074-1.484486$

C $-1.7487150 .737241-2.634128$

C $-0.497497-0.637866-4.273032$

O $-1.374147 \begin{array}{lll}1.822472 & -3.070811\end{array}$

C $-0.006589-2.045956-4.364282$

C $1.255690-2.412851-4.096086$

C $1.759568-3.822084-4.205502$

C $2.144911-4.407018-2.865650$

C $3.432766-4.575950-2.522700$

C $1.030106-4.875510-1.957312$

C $0.627674-3.826907-0.955394$

N -1.364707 $-0.503697-3.119176$

H -1.9189242 .8439521 .161266$

H 0.6231431 .9980612 .639224

H -0.9661780 .8351204 .023461$

H -2.896163 0.3936741 .685603

H -3.120184 1.7373362 .784939

H -4.352405 -1.918424 4.296549

H $-4.252366-0.1395975 .720141$

H -2.7763590 .8100375 .534960$

H -4.3159251 .4153274 .885691$

H -2.947336 -1.785031 1.544316

H -3.138292 -3.178419 2.580536

H $-4.742876-3.3914010 .841731$

H -5.645448 -3.0432552 .319687$

H -5.759569 -0.6047771 .597341$

H -7.644142 -2.203612 1.048059

H $-6.808416-2.671454-0.418944$

H $-9.050746-0.540631-1.838969$

H $-6.463726-0.814498-2.290042$

H -9.117739 -0.1727360 .978415$

H -8.751956 $1.145571-0.108132$

H -7.6199810 .7287561 .195391$

H -7.009654 $1.831753-1.475695$

H $-4.143871-0.468570-2.671413$

H -4.442995 $1.270437-2.607215$

H -6.0032391 .4757740 .355005$

H $0.725225-3.3759413 .131386$

H $-0.120923-1.8939530 .570639$

H $2.326872-4.5387041 .738084$

H $1.968119-5.6880340 .473850$

H $\quad 0.810197-5.468741 \quad 1.782251$

H -2.670269 $1.569990-0.910354$

H $-2.403028-0.168021-0.817397$

H $0.3195920 .084387-4.187396$

H $-1.077203-0.379912-5.165336$

H $-0.726066-2.798621-4.677444$

H $1.974580-1.660848-3.782765$

H $2.623006-3.816302-4.883282$

H $1.017940-4.474174-4.683867$

H $3.709013-5.020673-1.572366$

H $4.238388-4.266617-3.180190$

H $1.316293-5.817179-1.478701$

H $0.148676-5.134921-2.557740$

H $0.104408-2.978229-1.395892$

H $-1.861998-1.326934-2.790154$

C $0.070206 \quad 4.133948-0.484114$

O $0.967396 \quad 3.107801-0.962593$

C $1.9912573 .726126-1.765353$

C $1.4238545 .077598-2.175677$

C $0.6789305 .471994-0.918729$
H $2.8485643 .891806-1.100627$

C $2.4236982 .824827-2.928096$

H $2.2043755 .790871-2.456895$

H $0.718646 \quad 4.993268-3.009495$

C $-0.3470286 .570899-1.139410$

H $1.4096475 .813888-0.173018$

C $2.8091751 .389790-2.520492$

O $1.3537582 .701424-3.871976$

H $3.2573503 .290865-3.467968$

H $1.9202700 .838236-2.190603$

O $3.284895 \quad 0.725312-3.700183$

C $3.9044931 .296832-1.443242$

C $4.301285-0.163080-1.151220$

H $3.5394051 .761550-0.524620$

O $5.0422702 .035115-1.883234$

H $3.412184-0.693493-0.791529$

H $4.606047-0.648911-2.085779$

H $0.1385437 .483428-1.500404$

H $-1.0990346 .275153-1.878805$

H $-0.8679826 .811414-0.207258$

C $-0.067528 \quad 4.045562 \quad 1.037526$

H $1.3319703 .515006-4.403202$

H $2.5925810 .806838-4.378277$

H $5.6160082 .178987-1.111804$

H $-0.5622184 .948126 \quad 1.415932$

H $0.929517 \quad 4.010587 \quad 1.492246$

H $-0.8969483 .966532-0.974454$

C $4.237757-0.1217201 .930321$

O $5.249466 \quad 0.411862 \quad 1.067721$

C $5.464139-0.315148-0.145652$

C $5.831909-1.774097 \quad 0.162897$

C $4.799042-2.4347061 .069427$

C $4.563159-1.5739542 .307717$

H $3.261485-0.0666391 .432400$

C $4.1925010 .754218 \quad 3.194309$

H $6.3464400 .136004-0.616433$

O $5.960780-2.536215-1.032240$

H $6.805684-1.8035780 .669134$

H $3.860011-2.6285250 .539131$

O $5.291076-3.7073801 .494316$

H $5.456629-1.6048092 .944839$

O $3.485932-2.1192423 .063279$

C 3.6592402 .1475462 .940044

H 3.5414120 .2944953 .946462

H 5.2033640 .8626013 .605334

H $6.630853-2.100269-1.586054$

H $5.464714-4.2309640 .693037$

H $3.711763-3.0430063 .266789$

O 3.6099622 .8219184 .127872

O $3.3287552 .594407 \quad 1.851640$

C 3.1212894 .1651884 .056035

H 3.2345344 .6208525 .043269

H 3.7005944 .7480873 .333452

H 2.0604334 .1626273 .790033

SCF Energy (B3LYP/6-31G**//MMFF)= -3245.91134587

0600184

MM̄FF Geometry

C -2.259713 2.7498512 .785997

C -2.367521 1.5498572 .195160

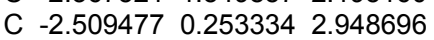

O $-1.668159-0.7397672 .323802$

C $-3.954744-0.2443642 .789776$

C $-4.322839-1.3915253 .705531$

C $-4.352336-2.6893313 .343419$

C $-4.741451-0.9842795 .094762$

C $-3.914285-3.3086062 .044486$

C $-5.076087-3.6734221 .107819$

C $-5.457528-2.5258110 .160343$

C $-6.656871-2.898804-0.710376$

C $-6.987312-1.787082-1.718051$

O $-4.320611-2.258950-0.670135$

O $-7.918842-2.340159-2.659713$

C $-5.700387-1.410440-2.483090$

C $-7.702500-0.609229-1.046065$

O $-5.929262-0.296441-3.343380$

C $-4.476337-1.156738-1.569696$

C $-3.197423-1.054438-2.436918$ 
$\begin{array}{llll}\text { O } & -4.663229 & 0.074581 & -0.870639\end{array}$

C $-0.371531-0.8468602 .703441$

O $0.232196-0.1427463 .493835$

C $0.174331-2.0146941 .974767$

C $1.484512-2.2951362 .000235$

C $2.123813-3.4355771 .338577$

C $1.226392-4.4478250 .671977$

C $-1.874075-1.068584-1.653340$

C $-0.679428-1.050738-2.597168$

C $1.782870-1.432099-2.653865$

O $-0.776507-0.750165-3.784885$

C $2.465047-2.732021-2.368109$

C $3.733920-2.827742-1.940829$

C $4.442164-4.134657-1.722040$

C $5.140747-4.218701-0.382830$

C $6.466680-4.025068-0.284495$

C $4.328391-4.6395360 .819833$

C $3.469497-3.5313901 .367480$

N $0.506050-1.393088-1.960916$ H -2.231964 2.7857753 .873650 H -2.410723 1.4745821 .112734 H -2.2437870 .3630684 .006948$ H -4.110563 -0.5403361 .748689$ H $-4.664013 \quad 0.5743982 .972774$ H -4.685967 -3.4161284 .083770$ H $-5.018055-1.8430065 .715681$ H $-3.924730-0.4614145 .602216$ H -5.607942 -0.3164285 .050339$ H $-3.175749-2.6876071 .530880$ H -3.380849 -4.2326942 .304390$ H $-4.751357-4.5285230 .500041$ H -5.948828 -3.9994321 .686240$ H -5.697606 -1.6406600 .756387$ H $-7.532973-3.129654-0.091681$ H -6.432556 -3.819112 -1.267813 H $-8.074874-1.670613-3.347973$ H $-5.469303-2.257019-3.144184$ H -8.627476 -0.949675 -0.565392 H $-8.009272 \quad 0.141363-1.782992$ H -7.094011 -0.115279-0.285514 H $-5.9368790 .505396-2.793147$ H $-3.170915-1.904413-3.132132$ H $-3.238773-0.140912-3.044130$ H $-3.7950120 .435047-0.630739$ H $-0.535348-2.6267521 .428386$ H $2.149498-1.6378362 .557339$ H $0.574078-4.9258881 .411233$ H $1.768273-5.2452600 .159851$ H $0.600471-3.971080-0.090151$ H -1.797091 -0.193536 -0.999926 H -1.812513 - $1.970214-1.033798$ H $2.370316-0.576890-2.309465$ H $1.627951-1.328562-3.732286$ H $1.898716-3.640763-2.558712$ H $4.311066-1.919382-1.785888$ H $5.176870-4.249089-2.530085$ H $3.755425-4.983951-1.825569$ H $6.984320-4.1181970 .664390$ H $7.064082-3.766185-1.152058$ H $4.996377-4.9571941 .632255$ H $3.758601-5.5395750 .571944$ H $4.036352-2.7436321 .865653$ H $0.507268-1.520543-0.953246$ C -2.3618654 .1064690 .585018$ O $-1.140508 \quad 3.6110440 .003991$ C $-0.7808154 .447887-1.110414$ C -2.012219 5.288257-1.414866 C $-2.5674415 .497089-0.017242$ H $0.0126415 .118465-0.760236$ C $-0.261808 \quad 3.583466-2.262927$ H $-1.7631406 .223863-1.924215$ H -2.733415 4.743277-2.034793 C $-4.0102435 .971321-0.003888$ H -1.9413836 .2312610 .507454$ C $0.9012612 .650585-1.859451$ O $-1.327757 \quad 2.759098-2.751606$ H $0.037065 \quad 4.220374-3.103047$ H $0.5269821 .875432-1.181828$
O $1.3568721 .975450-3.037140$

C $2.0990593 .377423-1.224107$

C $3.2974932 .475750-0.893312$

H $1.7865103 .901798-0.318795$

O $2.5466924 .388219-2.135606$

H $3.7234062 .084255-1.827845$

H $4.0940593 .118790-0.499117$

H -4.101269 $6.938909-0.508163$

H $-4.670605 \quad 5.262720-0.515033$

H -4.373092 6.0900121 .021766

C -2.2272364 .1000702 .110755$

H -1.651098 $2.224045-2.006412$

H $0.5876001 .531871-3.433887$

H $3.2670714 .877064-1.702423$

H $-3.040577 \quad 4.6928062 .545767$

H -1.2832904 .5824262 .394244$

H $-3.173337 \quad 3.4429290 .259848$

C $5.3685850 .947240 \quad 0.664464$

$\begin{array}{llllll}\text { O } & 4.184351 & 0.409962 & 0.061980\end{array}$

C $3.050342 \quad 1.294008 \quad 0.068412$

C 2.6939631 .6272081 .523076

C 3.8829862 .2241092 .271702

C 5.1056351 .3146162 .130015

H $5.700015 \quad 1.8348130 .112486$

C $6.471871-0.1144490 .545057$

H $2.2232310 .706229-0.341072$

O 1.5675732 .4869351 .592536

H 2.4090830 .6967942 .027011

H $4.106423 \quad 3.2413531 .929729$

$\begin{array}{llll}\text { O } & 3.569388 & 2.322760 & 3.662475\end{array}$

H 4.9535060 .4037422 .723590

O $\quad 6.2486921 .9835342 .659180$

C $6.970507-0.255842-0.878848$

H 7.3303190 .1671051 .165019

H $6.096473-1.0925900 .864773$

H 1.2797272 .5259492 .520012

H 2.8594342 .9800853 .756636

H 6.0453322 .2203663 .580429

O $8.115311-1.001076-0.876261$

O $6.423966 \quad 0.211153-1.868755$

C $8.702261-1.229963-2.161342$

H $9.008506-0.280663-2.610895$

H $9.588080-1.855606-2.023566$

H $7.998402-1.757458-2.812527$

SCF Energy (B3LYP/6-31G**//MMFF)= -3245.92113325

06_00185

MM̄FF Geometry

C -1.817659 -4.0049660 .734578$

C $-1.098439-4.0282731 .868403$

C $-0.848269-2.8810622 .820061$

O $-1.563735-1.692350 \quad 2.422333$

C $0.653884-2.5459782 .810991$

C $1.053564-1.5340423 .864678$

C $1.354365-0.2457413 .609699$

C $1.165465-2.0743985 .266798$

C $1.290206 \quad 0.4626742 .284085$

C 0.7335801 .8841832 .444414

C 0.6734842 .6287491 .103546

$\begin{array}{llll}\text { C } & 0.333849 & 4.103580 & 1.328093\end{array}$

$\begin{array}{llll}\text { C } & 0.178566 & 4.862692 & 0.003119\end{array}$

$\begin{array}{lllll}\text { O } & -0.336045 & 2.012711 & 0.295744\end{array}$

$\begin{array}{lllll}0 & -0.437514 & 6.127677 & 0.288085\end{array}$

C $-0.7912344 .080627-0.904333$

C $1.5336665 .174685-0.641500$

O $-0.8558384 .666724-2.203043$

C $-0.4592412 .572705-1.018959$

C $-1.6127691 .850182-1.750803$

O $0.7420432 .412050-1.771674$

C -2.804314 -1.503496 2.945601

O $-3.401267-2.239940 \quad 3.714155$

C $-3.330215-0.2373772 .389462$

C $-4.640592 \quad 0.0279172 .494730$

C $-5.332551 \quad 1.1994061 .953723$

C -4.4882282 .3104841 .390120$

C $-1.5049420 .322363-1.766518$

C $-2.759015-0.264759-2.382660$

C $-5.096305-0.929298-1.816984$ 
O $-2.849473-0.463224-3.592152$

C $-6.0240180 .242700-1.724374$

C $-7.2049810 .207375-1.087733$

C $-8.1308721 .387094-0.984776$

C -8.6012621 .6497710 .430976$

C $-9.875708 \quad 1.4175190 .788628$

C -7.6155932 .2511351 .413049$

C -6.6816391 .2145851 .978458$

N $-3.761309-0.490930-1.452919$

H -2.326392 -3.0970540 .425684$

H $-0.595826-4.9567872 .136388$

H -1.154315 -3.190222 3.826683

H $0.941693-2.1901091 .815680$

H $1.246057-3.4569772 .973946$

H 1.6864340 .3724674 .442970

H $1.520490-1.3195305 .976596$

H $0.192311-2.4266795 .622273$

H $1.871359-2.9108345 .296717$

H 2.3051190 .5190831 .875562

H $\quad 0.674458-0.083050 \quad 1.563517$

H -0.2726841 .8268432 .880017$

H 1.3670092 .4426923 .145215

H 1.6460622 .5325570 .606402

H 1.0909284 .5862991 .958697

H $-0.6115994 .179103 \quad 1.883867$

H $\quad 0.131236 \quad 6.6027620 .917981$

H -1.796017 4.180095 -0.469616

H 2.1487095 .7824890 .032908

H $1.4110775 .769660-1.553266$

H $2.1038314 .278194-0.889047$

H -1.079759 $5.605958-2.086211$

H -2.562390 2.119323 -1.274359

H -1.670790 2.201017 -2.789519

H $\quad 0.7731741 .512265-2.132399$

H $-2.6353730 .418784 \quad 1.877468$

H $-5.278967-0.7003552 .993836$

H -3.7258772 .6228262 .112947$

H $-5.056793 \quad 3.2096871 .144771$

H -3.9888411 .9815610 .475682$

H $-0.648357-0.014778-2.359127$

H -1.374504 -0.080922 -0.756011

H -5.374150 -1.731313-1.125968

H $-5.093978-1.324578-2.837289$

H $-5.7101351 .165360-2.207575$

H -7.532729 -0.722458 -0.628295

H $-8.9914601 .189409-1.636891$

H -7.659550 2.297212 -1.376424

H $-10.227043 \quad 1.6252901 .793924$

H -10.595375 1.0099600 .086502

H -8.1527812 .7051752 .255925$

H $-7.096313 \quad 3.0759780 .918447$

H -7.197622 0.3686492 .435960

H -3.595204 -0.253932 -0.479950

C $-1.135178-4.973350-1.511417$

O $0.178240-4.439196-1.229173$

C $0.307762-3.192738-1.935058$

C $-0.583698-3.361298-3.151734$

C $-1.795722-4.011718-2.506578$

H $-0.110202-2.409248-1.288583$

C $1.772155-2.853264-2.224828$

H $-0.813489-2.412947-3.643929$

H $-0.130897-4.035987-3.887994$

C $-2.721515-4.694896-3.497699$

H -2.367853 -3.243253 -1.973250

C $2.564670-2.582959-0.928913$

O $2.374379-3.900424-2.977722$

H $1.798444-1.950392-2.846296$

H $2.000940-1.860739-0.325576$

O $2.693584-3.775461-0.156020$

C $3.966470-1.993676-1.168604$

C $4.651883-1.6363230 .162080$

H $3.866078-1.098064-1.786853$

O $4.786437-2.909516-1.887994$

H $3.943291-1.0751280 .779773$

H $4.890237-2.5599260 .703682$

H $-3.130977-3.966587-4.205309$

H -2.196780 -5.464724 -4.073254

H $-3.559286-5.172341-2.979478$
C $-1.900205-5.186559-0.197610$

H $2.364483-4.699770-2.423737$

H $1.798277-4.0837170 .063940$

H $4.812310-3.740268-1.383081$

H -1.476408 -6.0675220 .301856$

H $-2.951261-5.420581-0.400818$

H $-0.975479-5.950788-1.983603$

C $4.9632991 .381052-0.156057$

O $5.7587820 .369248-0.785869$

C $5.952825-0.823397-0.017197$

C $6.646648-0.4741641 .306184$

C 5.8652420 .5834192 .083278

C 5.5634831 .7913561 .196804

H $3.9391621 .013456-0.020675$

C $4.9210632 .592164-1.103576$

H $6.643519-1.451590-0.593534$

O $6.808243-1.6441112 .100652$

H $7.651934-0.0900111 .090426$

H 4.9433840 .1648072 .501944

O 6.6379811 .0098443 .208543

H 6.4860842 .3640851 .037073

O 4.6437142 .6480251 .872854

C $4.0818812 .351032-2.340192$

H $4.4914793 .458246-0.588330$

H $5.9362792 .835833-1.438978$

H $7.220790-1.3727992 .938477$

H 7.4737391 .3802562 .876789

H 5.0343782 .8712822 .735112

O $4.0830843 .487861-3.099160$

O $3.497603 \quad 1.312112-2.611349$

C $3.3208963 .422554-4.308526$

H $3.4291234 .377160-4.830158$

H $3.6999672 .624958-4.954606$

H $2.2625863 .265795-4.080442$

SCF Energy $\left(B 3 L Y P / 6-31 G^{* *} / / M M F F\right)=-3245.92849583$

06 00186

MM̄FF Geometry

C $0.1653473 .286068-2.165902$

C $-0.9497873 .860217-2.644302$

C $-2.3788673 .457057-2.364996$

O $-2.4842812 .586011-1.216619$

C $-2.9780212 .721613-3.574591$

C $-4.4898722 .812346-3.614616$

C $-5.3366171 .844092-3.216070$

C $-5.0349404 .090465-4.202656$

C $-5.0103860 .505892-2.611827$

C $-5.8434360 .264754-1.343719$

C $-5.753720-1.189297-0.859442$

C $-6.716967-1.4264990 .305503$

C $-6.608924-2.8574420 .851000$

O $-4.411426-1.453361-0.440953$

O $-7.292278-2.9022042 .112733$

C $-5.125491-3.1715261 .128489$

C $-7.309783-3.873141-0.058865$

O $-4.954441-4.5390991 .494888$

C $-4.181277-2.814444-0.045248$

C $-2.714216-2.9430720 .422544$

O $-4.404472-3.721199-1.126130$

C -2.4411623 .1779530 .008486$

O $-2.307122 \quad 4.3680000 .247039$

C -2.5599702 .1146001 .028262$

C -2.1378812 .3691892 .275489$

C -2.1628441 .4402143 .406070$

C -2.9463210 .1665993 .240874$

C $-1.680990-2.416760-0.577768$

C $-0.320201-2.3476580 .083663$

C $1.066431-0.8958761 .561543$

O $0.469769-3.2874520 .031842$

C $0.742221-1.1133613 .006120$

C $1.082263-0.2535653 .979065$

C $0.781767-0.4844505 .433197$

C $0.1294980 .706323 \quad 6.102446$

C $0.8020551 .449846 \quad 6.997231$

C -1.3265630 .9876405 .795598$

C $-1.490721 \quad 1.7764464 .526458$

$\mathrm{N}-0.115493-1.1570010 .763763$

H $0.0997072 .401192-1.539564$ 
H $-0.8452274 .736955-3.281563$ H -2.948807 4.373176 -2.163455 H $-2.6584661 .673860-3.573551$ H -2.594596 $3.145016-4.512896$ H $-6.4034822 .025502-3.345574$ H $-6.1296434 .100331-4.237441$ H $-4.7184204 .954150-3.609905$ H -4.672406 $4.220898-5.227635$ H -5.246269 -0.263452 -3.356663 H -3.948032 $0.407458-2.371214$ H $-5.4897640 .943367-0.556595$ H $-6.8951830 .509532-1.540282$ H $-6.007140-1.846117-1.700084$ H -7.750484 -1.207793 0.009093 H -6.483478 -0.7255031 .119473$ H -8.215551-2.635555 1.963012 H -4.825886 -2.577178 2.003513 H $-8.372579-3.623619-0.163529$ H -7.280188 -4.8786300 .375503$ H -6.881373 -3.917939-1.062211 H $-5.550759-4.7154322 .242856$ H -2.591670 -2.396061 1.363757 H -2.480172 -3.9937910 .637386$ H -3.614481 -3.740923 -1.688820 H $-2.963998 \quad 1.1565860 .721978$ H $-1.705897 \quad 3.3468292 .487753$ H -3.9720250 .3819682 .919964$ H -3.029823 -0.4197944 .157685$ H -2.474400 -0.4727762 .492051$ H -1.602259-3.067378-1.455117 H - $1.950172-1.419631-0.941801$ H 1.3712090 .1338871 .357662 H $1.869258-1.5732671 .261265$ H $0.220905-2.0311933 .267786$ H $1.6328950 .648643 \quad 3.721896$ H $1.730403-0.7223755 .931841$ H $0.141666-1.3646915 .572106$ H 0.3330012 .2891737 .500182 H 1.8377631 .2400657 .243251 H -1.7744831 .5735916 .609141$ H -1.873582 0.0413925 .789857 H -0.9728912 .7369604 .546964$ H $-0.852811-0.4587990 .774535$ C $2.3853724 .076996-1.174621$ O $3.0439712 .862710-0.771535$ C 3.2652832 .9157600 .651886 C $2.6987224 .251251 \quad 1.140891$ C 1.6688194 .5780620 .076953 H 2.6784752 .0995721 .086075 C $4.7520142 .716073 \quad 0.976017$ H 2.2653644 .1676312 .142787 H 3.4645005 .0338141 .182562 C 1.2822606 .0448890 .024934 H $\quad 0.7769103 .9766090 .289756$ C 5.4085861 .4731640 .345756 O 5.4953333 .8545050 .515463 H 4.8852832 .6964712 .064011 H $5.5320721 .615581-0.735029$ $\begin{array}{lllll}\text { O } & 6.733817 & 1.372950 & 0.887266\end{array}$ C 4.6792670 .1415290 .596866 C $5.511614-1.0693450 .131474$ H 3.7195590 .1543210 .072068 O $4.397374 \quad 0.000778 \quad 1.988954$ H $5.875753-0.877528-0.882911$ H $\quad 6.390539-1.1646050 .780280$ H 0.8414556 .3600110 .976466 H $2.1510686 .682218-0.170590$ H $0.5463916 .226983-0.764540$ C $1.5403323 .834684-2.431335$ H $5.3521573 .930016-0.443674$ H 7.1868492 .2132970 .701446 H 5.2445790 .0316892 .465854 H $2.063193 \quad 3.131674-3.091156$ H $1.4558054 .784758-2.973814$ H $3.1919594 .771406-1.448106$ C $3.869094-2.389123-2.084604$ O $3.582196-2.388552-0.681230$ C $4.726261-2.3987820 .179786$
C $5.580786-3.640110-0.109321$

C $5.971690-3.718689-1.582206$

C $4.733962-3.598387-2.469575$

H $4.377734-1.457122-2.358413$

C $2.527468-2.442444-2.832646$

H $4.341661-2.5062991 .201623$

O $\quad 6.746012-3.6388310 .708184$

H $5.004599-4.5361510 .155379$

H $6.712513-2.951962-1.835365$

O $6.610299-4.972592-1.835646$

H $4.142206-4.519935-2.395277$

O $5.145308-3.466265-3.828970$

C $1.724307-1.165597-2.707834$

H $2.695879-2.609632-3.902442$

H $1.915118-3.258495-2.431171$

H $7.270347-4.4223870 .469307$

H $5.982059-5.680885-1.613405$

H $5.708772-4.231062-4.037644$

O $0.524055-1.347985-3.335964$

O $2.094727-0.142374-2.150560$

C $-0.355699-0.221144-3.331705$

H $\quad 0.0684600 .586223-3.935563$

H $-1.305607-0.530893-3.775344$

H $-0.5429200 .116704-2.308695$

SCF Energy (B3LYP/6-31G**//MMFF) $=-3245.92170759$

0600187

MM̄FF Geometry

C $1.0456073 .586443-0.813203$

C $1.4908152 .389021-1.220943$

C $1.6650412 .027414-2.674853$

O $1.1020310 .710453-2.897103$

C $3.1491511 .919295-3.074590$

C $3.9903613 .147270-2.797749$

C $4.8562573 .247310-1.768702$

C $3.8363134 .272252-3.784463$

C $5.0704662 .227343-0.685470$

C $6.3476051 .405495-0.897852$

C $6.3076890 .112928-0.069014$

C $7.659374-0.599269-0.083277$

C $7.597544-1.9448690 .655890$

O $5.312044-0.732966-0.657535$

O $8.803414-2.654217 \quad 0.333942$

C $6.404312-2.7603310 .111473$

C $7.604927-1.7524472 .176849$

O $6.229971-3.9631220 .857378$

C $5.079541-1.9618830 .039017$

C $4.025956-2.776250-0.756783$

O $4.602875-1.7344721 .364365$

C $-0.2494900 .576190-2.905586$

O $-1.083561 \quad 1.460419-2.806146$

C $-0.531667-0.872103-3.012579$

C $-1.729014-1.342187-2.636391$

C $-2.138461-2.748663-2.625893$

C $-1.159231-3.768810-3.143427$

C $2.744623-2.013532-1.119336$

C $1.704280-2.025237-0.019452$

C $0.083346-3.5105941 .135778$

O $1.519726-1.0456100 .698862$

C $-0.935940-4.4925780 .653403$

C $-2.260018-4.3058410 .768210$

C $-3.282782-5.3110710 .319909$

C $-4.359818-4.711876-0.557487$

C $-5.607357-4.528517-0.092628$

C $-4.012186-4.401705-1.997917$

C $-3.361283-3.051545-2.144551$

N $1.027108-3.2309750 .070401$

H $0.7911534 .320976-1.575711$

H $1.7652741 .628799-0.496342$

H $1.1597182 .742320-3.336552$

H $3.2047951 .687868-4.147812$

H $3.5909211 .029761-2.608782$

H $5.4459404 .156454-1.666984$

H $4.4851145 .122597-3.549018$

H $4.0908703 .930823-4.793142$

H $2.8041574 .636624-3.790396$

H 5.1323272 .7512560 .276806

H $4.2025431 .568474-0.594133$ 
H $6.4483861 .148140-1.960394$ H $7.2169662 .013119-0.619006$ H $\quad \begin{array}{llll}6.015157 & 0.362241 & 0.957807\end{array}$ H $8.442286 \quad 0.0388810 .344767$ H $7.961789-0.786995-1.123250$ H $8.741302-3.5381390 .735638$ H $6.675642-3.074841-0.905682$ H $6.740882-1.1936252 .542373$ H $8.507686-1.2129942 .487922$ H $7.639654-2.7152262 .698778$ H $5.797324-3.7301621 .696490$ H $4.480859-3.079610-1.708959$ H $\quad 3.772035-3.689869-0.206204$ H $3.682314-1.433577 \quad 1.313943$ H $0.269296-1.515802-3.357472$ H -2.473554 -0.632894 -2.277033 H $-0.920668-3.570212-4.194343$ H $-1.528543-4.794840-3.093809$ H -0.230378 -3.744259-2.564449 H $2.948801-0.975856-1.398120$ H $2.291914-2.480464-2.003629$ H $-0.367995-2.5754101 .477229$ H $0.651703-3.939751 \quad 1.967140$ H $-0.567079-5.4143600 .209406$ H -2.630877 $-3.397811 \quad 1.238124$ H $-3.733066-5.7470351 .221332$ H -2.812724 -6.147230 -0.212755 H $-6.391171-4.123019-0.724015$ H $-5.871450-4.7712090 .931268$ H -4.926045 -4.389030 -2.607165 H -3.412888 -5.219987 -2.404964 H -3.992616 -2.230139-1.808296 H $1.369005-4.016374-0.476332$ C 1.1690513 .0926751 .736800 O $\quad 0.1057592 .117907 \quad 1.816768$ C $-0.3533912 .045483 \quad 3.179049$ C 0.7502512 .6731654 .019466 C $1.2143003 .784733 \quad 3.103083$ H -1.247036 2.6779663 .244883 C $-0.703383 \quad 0.6092293 .577344$ H $0.384386 \quad 3.0350784 .984979$ H $1.5696681 .969447 \quad 4.206527$ C 2.5813954 .3399113 .466420 H 0.4778554 .5990603 .138044 C -1.769275 -0.0679222 .693981$ O $0.487385-0.1853593 .512602$ H -1.021774 0.5958654 .626829 H $-1.351311-0.2778781 .702358$ O $-2.052901-1.3486043 .274919$ C -3.0838250 .7187562 .531054$ C $-4.187469-0.1112461 .847133$ H -2.8853181 .6193401 .942701$ O $-3.551840 \quad 1.1530343 .808263$ H -3.781054 -0.552774 0.931216 H -4.473296 -0.9389592 .507228$ H 2.5667844 .7685854 .473808 H 3.3501373 .5601673 .444150 H 2.8814015 .1277072 .768349 C 0.8833814 .0792950 .599633 H $0.243724-1.0952663 .754374$ H $-2.396082-1.2014414 .172334$ H -3.789860 0.3651064 .325086 H $1.540654 \quad 4.9508890 .704832$ H -0.1477604 .4400770 .697383$ H 2.1028612 .5442121 .563142 C $-4.8444011 .462945-0.684795$ O $\quad-5.1808951 .8212590 .662328$ C $-5.452794 \quad 0.721731 \quad 1.536896$ C $-6.627936-0.0975570 .987735$ C $-6.368010-0.553624-0.442153$ C $-5.9663570 .628639-1.323593$ H $-3.9033450 .900823-0.694184$ C $-4.6286962 .773046-1.458656$ H -5.7869381 .1611002 .485015$ O $-6.882403-1.235377 \quad 1.804937$ H -7.5378350 .5165730 .997356$ H -5.612701-1.345043 -0.463107 O $-7.568469-1.133050-0.959072$
H $-6.8534781 .249655-1.503923$

O $-5.542210 \quad 0.108104-2.582182$

C $-3.4284113 .551781-0.963596$

H $-4.4648942 .582765-2.523970$

H $-5.5140173 .409582-1.338504$

H $-7.061583-0.9141052 .705071$

H -7.386412 -1.403076 -1.875634

H $-5.4906230 .847562-3.210157$

O $-3.5410634 .847573-1.381607$

O $-2.5000943 .083065-0.320344$

C $-2.4735795 .718592-0.996725$

H $-1.5514645 .425457-1.505725$

H $-2.736194 \quad 6.734277-1.304495$

H -2.340019 5.7064430 .089226

SCF Energy (B3LYP/6-31G**//MMFF) $=-3245.91284099$

0600188

MM̄FF Geometry

C -0.278828 $3.051079-0.659668$

C $1.0069263 .153879-1.029754$

C $1.9534792 .000853-1.268046$

O $1.242060 \quad 0.747802-1.124024$

C $3.1164151 .979037-0.258127$

C $4.1746553 .033350-0.489399$

C $5.1358172 .936384-1.428681$

C 4.1313284 .2178890 .438295

C $5.3310361 .816502-2.413603$

C $6.7557751 .248414-2.397914$

C $7.1409750 .536384-1.090215$

C $8.5913890 .051508-1.177422$

C $9.004124-0.7625670 .054625$

O $6.257975-0.575147-0.899706$

O $10.236334-1.433253-0.250926$

C $7.945040-1.8545170 .301554$

C $9.2871770 .135076 \quad 1.264923$

O $8.215225-2.5500291 .517587$

C $6.491307-1.3222290 .305805$

C $5.507842-2.5222720 .318290$

O $6.293340-0.517521 \quad 1.466169$

C $1.469135-0.245639-2.019197$

O $2.215926-0.207012-2.986568$

C $0.732748-1.475516-1.642298$

C $-0.297680-1.474530-0.782385$

C $-1.003327-2.648832-0.267124$

C $-0.537132-4.004584-0.725628$

C $4.021943-2.1487640 .405421$

C $3.519289-1.9656031 .830085$

C $1.525755-1.1670883 .100211$

O $4.149732-2.3360002 .817034$

C $0.564711-2.3016723 .278874$

C $-0.737743-2.1357653 .555975$

C -1.705662 -3.273567 3.726421

C $-2.928901-3.1425772 .842767$

C $-4.119168-2.7938433 .359660$

C $-2.791393-3.4885491 .372986$

C $-2.005152-2.4547490 .615649$

N $2.267336-1.3686711 .868028$

H $-0.7162622 .071149-0.493631$

H $1.4141074 .145818-1.212379$

H $2.3299272 .095092-2.293579$

H $3.5940570 .992677-0.271146$

H $2.7225942 .057576 \quad 0.764878$

H $5.8544073 .750068-1.516345$

H 4.9294864 .9383130 .229975

H $3.178570 \quad 4.7474750 .347801$

H 4.2474253 .8886061 .476244

H $4.6160211 .003147-2.265023$

H $5.1334452 .219072-3.414864$

H $6.8294460 .533560-3.228071$

H $7.4717932 .054139-2.604387$

H $7.0252801 .235142-0.254206$

H $9.2754470 .896692-1.325060$

H $8.708920-0.585592-2.065538$

H $10.895921-0.757287-0.483233$

H $8.038632-2.594453-0.506032$

H $\quad \begin{array}{llll}10.101030 & 0.834941 & 1.040014\end{array}$

H $9.628649-0.4543042 .122914$

H $8.4221790 .725731 \quad 1.573390$ 
H $9.129155-2.8785091 .466709$ H $5.644887-3.079373-0.618257$ H $5.756897-3.2058151 .138153$ H $5.351799-0.2924841 .525877$ H $1.104783-2.387905-2.096328$ H $-0.639765-0.523865-0.376553$ H $-0.567799-4.072251-1.819007$ H -1.156114 -4.825339-0.356678 H $0.488377-4.192617-0.389334$ H $3.811632-1.254995-0.187166$ H $3.432524-2.971046-0.019954$ H $1.015916-0.2020223 .018712$ H $2.219616-1.1313013 .945781$ H $0.961199-3.3099083 .181072$ H -1.131341 -1.129034 3.677971 H -2.004596 -3.298954 4.782328 H $-1.224610-4.2397303 .529025$ H $-5.006295-2.7220912 .738936$ H $-4.233249-2.5666974 .414363$ H -3.781701 -3.560589 0.908491 H -2.349096 -4.4863731 .304564$ H -2.292236 -1.428322 0.850101 H $1.804994-1.1331150 .996153$ C -1.861930 4.2167230 .896003 O -2.7504403 .0835681 .005248$ C -4.0119723 .5296611 .530825$ C $-3.754124 \quad 4.913522 \quad 2.113226$ C -2.725543 5.4551121 .142447 H $-4.693016 \quad 3.6273910 .677044$ C -4.5722052 .5171712 .533151$ H -4.6631295 .5205402 .161118$ H -3.3294194 .8578653 .122384$ C -1.9538006 .6490541 .677495$ H -3.2381815 .7469630 .215771$ C $-4.749241 \quad 1.0834131 .994502$ O -3.6958332 .4394413 .666038$ H -5.5288102 .8849812 .922658$ H -3.7744440 .5954641 .873476$ $\begin{array}{llll}\text { O } & -5.446110 & 0.339440 & 3.003935\end{array}$ C $-5.5373590 .971673 \quad 0.675475$ C $-5.923033-0.481978 \quad 0.336823$ H $-4.9367141 .393401-0.136633$ $\begin{array}{llllllll} & \mathrm{O} & -6.932071 & 1.750151 & 0.762268\end{array}$ H $-5.037648-1.1165540 .436943$ H -6.659289 -0.8310951 .070402$ H -2.6326697 .4811011 .891130$ H -1.423382 6.4020012 .603166 $\mathrm{H}-1.214696 \quad 6.9949320 .948096$ C -1.202022 $4.222909-0.484080$ H -2.8161902 .1840243 .338426$ H -4.9203850 .3966063 .820324$ H -7.2528181 .4080271 .509208$ H $-0.6599305 .163004-0.636909$ H - $1.9730024 .154550-1.261322$ H -1.1063914 .1058081 .684687$ C $-4.524318-1.211016-2.286899$ O $-5.601622-0.282288-2.108567$ C $-6.535475-0.621773-1.076296$ C $-7.153763-1.996863-1.363448$ C $-6.085278-3.069263-1.540881$ C $-5.055806-2.622392-2.576659$ H -3.897605 -1.220582 -1.389154 C $-3.669297-0.714607-3.462927$ H $-7.3490350 .110857-1.147016$ O $-8.047732-2.363450-0.317948$ H $-7.747969-1.933290-2.284580$ H -5.616309 -3.317043 -0.582801 O $-6.719262-4.265815-1.997980$ H $-5.519718-2.650943-3.570660$ O $-3.978873-3.557792-2.597438$ C $-2.8799980 .533016-3.130480$ H -2.943363 -1.480622 -3.757170 H $-4.317710-0.479569-4.315482$ H $-8.397550-3.245696-0.530600$ H $-6.019492-4.924569-2.148020$ H -3.557534 -3.547642 -1.721343 O $-2.2335490 .950838-4.259572$ O $\quad-2.8235391 .064092-2.030808$
C -1.423396 $2.121595-4.119584$

H -1.132693 $2.454602-5.119536$

H -1.980809 $2.927585-3.633265$ H $-0.5199101 .876483-3.555806$ SCF Energy $(B 3 L Y P / 6-31 G * * / / M M F F)=-3245.92521907$

0600189

MM̄FF Geometry

C -0.602895 1.6786202 .378834

C $-1.622111 \quad 1.356467 \quad 3.189413$

C -2.138561 -0.0342663 .483515$

O $-1.347962-1.0108492 .768472$

C $-3.598154-0.122527 \quad 3.001996$

C $-4.388908-1.2856653 .561012$

C $-4.784357-2.3555402 .844119$

C $-4.800398-1.1505265 .004433$

C $-4.458530-2.6790521 .413286$

C $-5.632297-2.4326670 .454151$

C $-5.858761-0.947840 \quad 0.121469$

C $-7.056844-0.782535-0.816945$

C $-7.2318250 .671987-1.279841$

O $-4.670627-0.465257-0.510359$

O $-8.1744250 .655091-2.362773$

C $-5.881997 \quad 1.181001-1.831799$

C $-7.8556091 .541310-0.182557$

O $-5.9566762 .563653-2.170123$

C $-4.6923910 .915724-0.885526$

C $-3.3222991 .248822-1.528296$

$\begin{array}{llll}\text { O } & -4.824764 & 1.755398 & 0.268996\end{array}$

C -1.112019-2.209078 3.357115

O $-1.506749-2.5764734 .455146$

C $-0.325712-3.1002562 .472769$

C $0.422375-2.6674821 .444668$

C $1.216304-3.5109620 .543195$

C $1.057966-5.0039830 .678750$

C $-3.0025890 .582290-2.870026$

C $-3.111186-0.929893-2.878157$

C $-2.185133-2.987087-1.822947$

O $-3.920903-1.507761-3.600890$

C $-0.929377-3.612144-2.348955$

C $0.080859-3.010185-2.996132$

C $1.319987-3.733923-3.440734$

C $2.577388-3.171448-2.814365$

C $3.419042-2.408089-3.531041$

C $2.908791-3.575505-1.392453$

C $2.018685-2.918716-0.367898$

N -2.222386 -1.550436 -2.017199

H $-0.0368990 .898554 \quad 1.877861$

H -2.158992 $2.163563 \quad 3.685675$

H -2.056541 -0.1910444 .565341$

H $-3.599175-0.153327 \quad 1.908831$

H $-4.1430220 .793468 \quad 3.269679$

H $-5.390549-3.1110353 .342667$

H $-5.432377-1.9813335 .336162$

H $-3.921855-1.1271915 .656101$

H $-5.368844-0.2265945 .152844$

H -3.559060 -2.157442 1.073120

H $-4.202688-3.7460911 .376456$

H $-5.406748-2.968993-0.477259$

H $-6.550650-2.8704150 .864534$

H $-6.027349-0.3929171 .050619$

H -7.976081 -1.148692 -0.342606

H -6.910321 -1.411505 -1.706669

H $-8.233818 \quad 1.559701-2.715610$

H $-5.7187570 .648803-2.776135$

H -8.8412991 .1502940 .097607$

H -8.030861 $2.562959-0.537015$

H $-7.249171 \quad 1.5896080 .724286$

H -5.917409 $3.073302-1.342912$

H $-3.2355772 .336249-1.654018$

H $-2.5345410 .977062-0.812094$

H $-3.9455701 .879732 \quad 0.663414$

H $-0.371579-4.1506002 .742522$

H $0.471005-1.5993321 .241433$

H $1.448918-5.3444991 .643916$

H $1.575539-5.574211-0.094345$

H $\quad 0.001625-5.287657 \quad 0.607284$

H $-3.6573520 .978126-3.654486$ 
H $-1.9810450 .843388-3.171367$ H $-3.055458-3.454559-2.293813$ H $-2.245303-3.162200-0.744730$ H $-0.843601-4.678946-2.148261$ H $\quad 0.033967-1.947932-3.220493$ H $1.373801-3.660999-4.534786$ H $1.259739-4.807007-3.219537$ H $4.344578-2.035011-3.106892$ H $3.202884-2.140060-4.559953$ H $3.939734-3.288966-1.152489$ H $2.899363-4.666458-1.333220$ H $2.067298-1.828805-0.383027$ H -1.578192 -0.984516-1.472965 C -0.2160213 .3783710 .589371$ O $0.8643462 .670995-0.055028$ C $1.4830063 .544909-1.017158$ C $0.5524524 .743027-1.159443$ C $-0.008681 \quad 4.8517980 .243922$ H $2.4258693 .886757-0.575157$ C $1.7581882 .794911-2.323315$ H $1.0824125 .644032-1.482872$ H $-0.2530734 .548782-1.877268$ C -1.2725875 .6902710 .326788$ H 0.7582135 .2877050 .898400 C $2.6416391 .539628-2.175495$ O $0.5120102 .376723-2.895977$ H $2.2074103 .480033-3.051643$ H $2.0934130 .744422-1.655605$ O $2.9078011 .046891-3.495292$ C $3.9870011 .779932-1.464151$ C $4.9157580 .552547-1.525541$ H $3.7947652 .038701-0.418095$ O $4.6539272 .893726-2.057567$ H $4.361704-0.322832-1.171843$ H $5.1951880 .363189-2.568688$ H $-1.0755036 .715889-0.002286$ H $-2.0677775 .281365-0.305786$ H -1.6478285 .7319631 .353895$ C -0.1911133 .0962522 .092179$ H $\quad 0.060367 \quad 1.821033-2.237667$ H $2.0491130 .875283-3.918791$ H $4.7955562 .685113-2.997017$ H -0.8512473 .7966442 .616061$ H 0.8206683 .2405012 .484986 H -1.1495263 .0098940 .144199$ C 5.3653630 .0160171 .435048 O 5.9546851 .0669500 .658598 C $6.2143760 .736688-0.708083$ C $7.179147-0.453705-0.774552$ C $6.668166-1.6423520 .036087$ C $6.278489-1.2177221 .453172$ H $4.385444-0.2471201 .018266$ C 5.1425820 .5738402 .844383 H $6.7391841 .599201-1.137613$ O $7.390879-0.839806-2.128231$ H $8.152348-0.142432-0.373386$ H $5.825844-2.122443-0.471572$ O $7.692256-2.6381120 .106002$ H $7.190114-1.0141422 .029366$ O $5.611569-2.3172242 .069905$ C 4.0861331 .6524282 .818599 H $4.797450-0.1934693 .545201$ H 6.0701170 .9905123 .255254 H $7.979472-1.614059-2.121862$ H $8.461143-2.2478120 .555491$ H $5.549505-2.1333633 .021827$ O 4.6724982 .8826282 .847341 O 2.8857421 .4235382 .748991 C 3.7761553 .9958812 .787606 H 3.0866413 .9749043 .636703 H 4.3687624 .9129092 .839989 H 3.2273553 .9871041 .841719 SCF Energy (B3LYP/6-31G**//MMFF)= -3245.91882337

\section{6_00190}

MM̄FF Geometry

C -1.487945 2.4788482 .629626

C $-0.8759481 .418293 \quad 3.178606$
C $-1.4095320 .016012 \quad 3.326097$

O $-0.509093-0.8490032 .591856$

C $-2.829658-0.2332642 .783369$

C $-3.397002-1.5748663 .208133$

C $-3.375311-2.6952872 .459840$

C $-4.053812-1.5872084 .564636$

C $-2.741022-2.8938251 .112782$

C $-3.756313-3.2030390 .006591$

C $-4.680992-2.025353-0.342596$

C $-5.598356-2.407396-1.506078$

C $-6.450663-1.224135-1.983415$

O $-3.860256-0.914313-0.705947$

O $-7.026106-1.581737-3.250337$

C $-5.528306-0.010346-2.231312$

C $-7.625917-0.950623-1.036896$

O $-6.322206 \quad 1.132908-2.544172$

C $-4.5690980 .281807-1.050760$

C $-3.5195831 .370973-1.397632$

O $\quad-5.338397 \quad 0.7669100 .055350$

C $0.211694-1.7719253 .280284$

O $0.293206-1.8988144 .490724$

C $0.849397-2.6550082 .279961$

C $1.448328-3.7871822 .675442$

C $2.041603-4.7660671 .771384$

C $3.396831-5.2522152 .203388$

C $-2.4034670 .911982-2.343638$

C $-1.2554550 .220420-1.622957$

C $0.766437-1.163177-2.059697$

O $\quad-1.0598940 .328888-0.415084$

C $0.530427-2.638068-2.001186$

C $1.380850-3.525269-2.539281$

C $1.204903-5.015647-2.488118$

C $2.248221-5.690777-1.624016$

C $3.513750-5.840309-2.050801$

C $1.804285-6.260770-0.296819$

C $1.375964-5.2024610 .683207$

N $-0.440683-0.487467-2.494373$

H -2.4845582 .3807702 .210236$

H 0.1141981 .5660443 .605429

H -1.388616 -0.2199724 .397210$

H $-2.829393-0.1548161 .691584$

H $-3.5177680 .544067 \quad 3.139413$

H -3.836462 -3.593185 2.869609

H -4.459319 -2.571308 4.822658

H -3.333354 -1.314530 5.342002

H $-4.882500-0.8721074 .591359$

H -2.116033 -2.046830 0.818812

H $-2.056909-3.7477201 .197271$

H $-3.181702-3.480950-0.887070$

H $-4.358020-4.0771670 .284937$

H $-5.273994-1.7617460 .540375$

H -6.236730 -3.257331 -1.234168

H $-4.988030-2.752542-2.352741$

H -7.555220 -2.387759 -3.122596

H $-4.942450-0.239323-3.130550$

H $-8.277270-1.830651-0.971622$

H -8.257690 -0.139187-1.414750

H -7.310795 -0.700188 -0.021896

H $-5.745473 \quad 1.790899-2.966126$

H $-4.0259182 .243114-1.827341$

H -3.068662 $1.747184-0.469871$

H $-4.741607 \quad 1.2224170 .671771$

H $0.773401-2.3803911 .233044$

H $1.518797-4.0333173 .732684$

H $3.920045-5.8091641 .421913$

H $3.307852-5.9014123 .081060$

H $4.043884-4.4075442 .466612$

H $-2.791910 \quad 0.264159-3.134874$

H -1.970484 $1.796113-2.827400$

H $1.062017-0.798594-1.071493$

H $1.549011-0.905248-2.779341$

H $-0.350640-2.990575-1.470861$

H $2.266444-3.159910-3.055224$

H $1.270566-5.406572-3.511852$

H $\quad 0.198093-5.280516-2.141669$

H $4.259558-6.344691-1.445510$

H $3.831596-5.458125-3.015461$

H $2.582152-6.9018620 .132185$ 
H $\quad 0.954995-6.934843-0.469205$ H $0.385466-4.7939730 .482286$ H $-0.681102-0.530666-3.480263$ C $-0.941898 \quad 4.496716 \quad 1.214481$ $\begin{array}{llllll}0 & -0.008278 & 3.849154 & 0.326248\end{array}$ C $0.6591014 .856675-0.460068$ C $-0.0781656 .165156-0.196760$ C -0.5384065 .9720361 .231650$ H $1.6769664 .942609-0.062023$ C $0.7036154 .460860-1.939279$ H $0.5690917 .038043-0.326523$ H $-0.9419416 .287734-0.860642$ C - $1.6544976 .918741 \quad 1.639153$ H $\quad 0.3209396 .1190361 .899945$ C $1.3944993 .115792-2.227228$ O $-0.6445864 .367473-2.420343$ H $1.1812335 .261310-2.517320$ H $0.7861782 .298604-1.824401$ O $1.3964672 .958596-3.652585$ C $2.836998 \quad 3.003422-1.696025$ C $3.5438591 .723091-2.174247$ H $2.8132333 .021999-0.603442$ O $3.6002534 .133747-2.116052$ H $2.8673970 .878723-2.024043$ H $3.7397261 .798960-3.250543$ H $-1.324715 \quad 7.959527 \quad 1.556607$ H -2.537787 6.7948121 .003619 H $-1.958595 \quad 6.7436702 .675670$ C $-0.886567 \quad 3.8575682 .603268$ H $-0.5996914 .123455-3.360763$ H $1.5546882 .019048-3.845441$ H $3.5829474 .156287-3.088237$ H - -1.4512864 .4717503 .314119$ H 0.1531513 .8242992 .950589 H $-1.939825 \quad 4.376407 \quad 0.772337$ $\begin{array}{llll}\text { C } & 4.007843 & 0.243936 & 0.443187\end{array}$

O $4.738637 \quad 1.381712-0.034659$ C $4.8854051 .459075-1.455807$

C $5.6266410 .214165-1.960630$

C $4.924105-1.068986-1.527358$

C $4.669078-1.063173-0.020497$

H 2.9739830 .2910040 .082750

C 3.9823680 .3399731 .976772

H $5.5291562 .325301-1.653504$

O $5.742760 \quad 0.253753-3.378934$

H $6.6463460 .215920-1.553981$

H $3.996417-1.217976-2.090862$

O $5.767110-2.177082-1.853496$

H $5.623005-1.2189840 .499783$

O $3.828437-2.1751910 .277478$

$\begin{array}{llll}\text { C } 3.203121 & 1.546080 & 2.456641\end{array}$

H $3.517783-0.5413892 .429139$

H 5.0114780 .4300632 .346155

H $6.194032-0.561929-3.656364$

H $5.316278-2.982488-1.545913$

H $3.819678-2.2912151 .241783$

O 3.6695651 .9243643 .682652

$\begin{array}{lllll}\text { O } & 2.283142 & 2.072021 & 1.845906\end{array}$

C 3.0363383 .0691504 .263264

H 2.0317102 .8038874 .603038

H 3.6280103 .3819585 .127775

H 2.9971593 .8983323 .549925

SCF Energy (B3LYP/6-31G**//MMFF) $=-3245.90157354$

06_00191

MM̄FF Geometry

C -1.941027 3.211609-2.708210

C $-2.2145762 .038871-2.117498$

C $-3.0253020 .945191-2.758834$

O $-4.065107 \quad 0.616757-1.808870$

C $-2.158867-0.303042-2.988960$

C $-2.784450-1.292770-3.950686$

C $-3.405540-2.432681-3.589665$

C $-2.629711-0.949659-5.410442$

C $-3.677041-2.943017-2.201624$

C $-2.860018-4.199199-1.866604$

C $-1.369255-3.907973-1.636319$

C $-0.592543-5.203820-1.391513$
C $0.880783-4.929021-1.053852$

O $-1.265950-3.055948-0.491393$

O $1.434768-6.156400-0.557713$

C $0.941110-3.8847820 .081596$

C $1.683992-4.569356-2.309319$

O $2.287588-3.5114110 .358193$

C $0.067607-2.636965-0.178582$

C $0.009306-1.7574691 .092755$

O $0.649594-1.880110-1.241565$

C $-5.3249370 .399334-2.258149$

$\begin{array}{lllll}\text { O } & -5.707441 & 0.443077 & -3.418971\end{array}$

C $-6.2516780 .116424-1.135209$

C $-5.842296-0.103787 \quad 0.124457$

C $-6.697753-0.3550651 .287028$

C $-8.189198-0.3131651 .083141$

C $-1.046881-0.645808 \quad 1.032125$

$\begin{array}{llll}C & -1.078064 & 0.188108 & 2.300647\end{array}$

C -2.441579 1.9582503 .411478

O $-0.270297 \quad 0.054987 \quad 3.215814$

C -3.398069 1.2297714 .303025

C $-4.662144 \quad 1.6206824 .524823$

C $-5.620316 \quad 0.870440 \quad 5.405702$

C $-6.8606090 .413103 \quad 4.667656$

C -8.0326331 .0492864 .832460$

C $-6.766590-0.831663 \quad 3.810969$

C $-6.109734-0.5774002 .481499$

N -2.120716 $1.105621 \quad 2.281471$

H -2.301096 $3.402888-3.715739$

H $-1.852306 \quad 1.857628-1.108257$

H $-3.4602701 .308682-3.697445$

H $-1.950715-0.786982-2.029240$

H $-1.178516-0.016540-3.393621$

H -3.791922 -3.075848 -4.379775

H $-3.061956-1.713226-6.066042$

H $-3.126940-0.001961-5.638748$

H $-1.569522-0.857422-5.667921$

H $-3.525969-2.173743-1.438870$

H -4.742867 -3.200164 -2.152689

H $-3.283638-4.632802-0.951345$

H $-2.979030-4.943830-2.663466$

H $-0.967461-3.388519-2.512910$

H $-0.670304-5.874979-2.256005$

H $-1.045665-5.746836-0.549922$

H $2.345106-5.972587-0.268167$

H $0.575270-4.3822220 .990242$

H $1.655296-5.396667-3.028682$

H $2.741927-4.415189-2.074936$

H $1.312851-3.676332-2.816494$

H $2.572493-2.892361-0.335750$

H $-0.228217-2.3889751 .959004$

H $0.993006-1.3081631 .277975$

H $\quad 0.357470-0.958667-1.169817$

H $-7.3018960 .096441-1.407791$

H $-4.775010-0.0942480 .339616$

H -8.494517 0.6537430 .667895

H $-8.758057-0.4456272 .005928$

H -8.504849 -1.1065170 .396791$

H -0.8499460 .0304070 .195910$

H -2.042889 -1.0819020 .891897$

H $-2.868122 \quad 2.883986 \quad 3.012724$

H -1.5276832 .2010633 .962827$

H -3.028531 $0.328600 \quad 4.787423$

H $-5.031808 \quad 2.5264374 .049837$

H $-5.896421 \quad 1.5331936 .236310$

H -5.1390830 .0012965 .871376$

H -8.9347040 .7116534 .333181$

H -8.1160591 .9249575 .467480$

H $-7.754803-1.2860543 .678594$

H $-6.187748-1.5954304 .346269$

H $-5.020716-0.5893772 .526172$

H -2.7794481 .0849941 .508097$

C $0.176505 \quad 4.570952-2.704627$

O $1.0503123 .449506-2.451733$

C $2.2420653 .930214-1.808158$

C $2.3375915 .397868-2.199368$

C $0.8800985 .806293-2.130573$

H $2.0671553 .866190-0.728405$

C $3.4570573 .080725-2.190017$ 
H $2.9774765 .970785-1.521762$

H $2.7160095 .524306-3.220166$

C $0.5699207 .090429-2.880944$

H $0.6051015 .940446-1.075441$

C $3.3086401 .559644-1.977571$

O $3.7470843 .281759-3.581569$

H $4.3392393 .445285-1.651439$

H $2.5949631 .163438-2.711188$

O $4.571676 \quad 0.948377-2.276184$

C $2.8981161 .079427-0.574224$

C 3.7389621 .6834690 .565149

H $2.999973-0.012165-0.545906$

O $1.5261631 .377642-0.328673$

H 4.7967891 .6673050 .287806

H 3.4543302 .7323710 .698620

H $1.1317087 .929085-2.456850$

H $0.8349337 .009963-3.940546$

H $-0.496327 \quad 7.330057-2.817195$

C -1.178135 4.319399-2.038654

H $2.9535683 .031449-4.085570$

H $4.8246421 .228284-3.172736$

H $1.0133691 .078193-1.098064$

H -1.793179 $5.225832-2.083078$

H -1.028577 4.080931-0.977762

H $0.0675634 .650694-3.793388$

C $5.421659-0.5558321 .799181$

O $3.995657-0.4128141 .848389$

C $3.5192470 .938107 \quad 1.902877$

C 4.1035611 .6510663 .129644

C 5.6253461 .5728693 .154794

C 6.0820360 .1235083 .006786

H $5.807245-0.1181580 .870936$

C $5.737680-2.0601361 .785681$

H 2.4355540 .8739442 .061377

O 3.7029263 .0165683 .168799

H $3.717678 \quad 1.1781014 .042213$

H 6.0749702 .2086342 .382957

O 6.1015872 .0646344 .409603

H $5.847295-0.4295573 .925579$

O $7.499017 \quad 0.0972052 .842636$

C $5.478261-2.6986940 .437329$

H $6.796568-2.2243782 .014934$

H $5.119767-2.5831882 .524772$

H 2.7306183 .0352993 .163988

H 5.7660192 .9720704 .510098

H 7.8881770 .5519193 .609405

O $5.923474-3.990308 \quad 0.474549$

O $4.949443-2.148819-0.518557$

C $5.761097-4.744780-0.730510$

H $6.098981-4.175183-1.601776$

H $6.370672-5.648722-0.649188$

H $4.713889-5.036712-0.840621$

SCF Energy (B3LYP/6-31G**//MMFF) $=-3245.93133675$

06_00192

MM̄FF Geometry

C $3.310609-4.095409-0.945788$

C $2.145124-3.432098-0.931166$

C $1.394006-3.051624-2.180407$

O $1.308984-1.607514-2.243826$

C $-0.039340-3.601548-2.131443$

C $-0.798154-3.346222-3.417142$

C $-1.715283-2.374446-3.589600$

C $-0.480270-4.291694-4.547581$

C $-2.183766-1.342831-2.598377$

C $-3.695315-1.473016-2.377074$

C $-4.252403-0.446221-1.378373$

C $-5.767943-0.631730-1.245744$

C $-6.4196850 .451420-0.378150$

O $-3.9512940 .863696-1.866877$

$\begin{array}{lllll}0 & -7.836271 & 0.367360 & -0.595987\end{array}$

C $-5.9443551 .834050-0.871974$

C $-6.199670 \quad 0.178491 \quad 1.114023$

O $-6.4366302 .865067-0.017993$

C $-4.4099461 .937487-1.032881$

C $-4.0399403 .264852-1.739096$

O -3.8137291 .9015530 .260896$

C $2.397774-0.941249-2.711950$
O $3.458146-1.420910-3.080249$

C $2.0902510 .502816-2.675334$

C $3.0942921 .361856-2.447379$

C $2.9892252 .819269-2.356800$

C $1.7097253 .454725-2.828917$

C $-2.5307253 .512648-1.876704$

C $-1.9700814 .260101-0.678552$

C $-0.1684284 .178981 \quad 1.036700$

O $-2.4517905 .324196-0.294077$

C 0.8884065 .1223990 .553820

C 2.1914485 .0083830 .853899

C 3.2238916 .0169990 .438618

C $4.4027955 .405387-0.281544$

C 5.6048785 .3150850 .313422

C $4.2156354 .993481-1.725105$

C $4.0481433 .506423-1.880792$

N $-0.8812803 .627772-0.099901$

H $3.735940-4.402763-1.898302$

H $1.733322-3.1014190 .018107$

H $1.904993-3.424628-3.076972$

H $-0.571045-3.160175-1.283318$

H $-0.025428-4.682952-1.941189$

H -2.188391 -2.292886 -4.568068

H -1.090915 -4.099022 -5.436093

H $0.568882-4.200722-4.844696$

H $-0.667059-5.325940-4.239864$

H -1.663535 -1.413233 -1.639705

H -1.948402 -0.353133 -3.007541

H $-4.198503-1.339902-3.344547$

H -3.931739 -2.486035 -2.027213

H $-3.757379-0.590282-0.411092$

H -6.003793 -1.630739 -0.857333

H $-6.225280-0.588296-2.244781$

H -8.252920 $1.098506-0.108161$

H -6.409912 2.005814-1.852003

H $-6.640577-0.786012 \quad 1.393462$

H -6.7043550 .9268361 .734823$

H $-5.145190 \quad 0.152025 \quad 1.393077$

H -5.8858622 .8737640 .783344$

H $-4.4609903 .241196-2.752881$

H $-4.5015284 .108958-1.213667$

H -2.863711 2.0737710 .167589

H $1.0577810 .806724-2.798565$

H $4.0928720 .956641-2.283857$

H $1.5467923 .238726-3.890687$

H $1.6924554 .540474-2.717371$

H $0.8557473 .071724-2.264592$

H $-1.9885772 .579506-2.060845$

H -2.348240 $4.158506-2.744573$

H 0.2497553 .3422051 .601431

H $-0.8742254 .714417 \quad 1.680102$

H $0.5552845 .970939-0.039746$

H 2.5269754 .1813611 .474928

H 3.5642456 .5307131 .347509

H $2.785806 \quad 6.799293-0.194141$

H $6.4638084 .910027-0.211564$

H 5.7557025 .6395091 .337633

H $5.1044435 .286762-2.300369$

H $3.3984795 .566057-2.171513$

H $4.9305662 .940164-1.577389$

H $-0.5231412 .769086-0.506385$

C $5.044363-3.2659470 .733291$

O $4.256020-2.2248351 .346936$

C $4.603667-0.9697800 .740948$

C $5.995168-1.1772810 .156639$

C $5.890437-2.600452-0.358346$

H $3.889752-0.810022-0.075152$

C 4.4810680 .1809521 .743319

H $6.229645-0.452261-0.628626$

H $6.774370-1.1151610 .924599$

C $7.233013-3.274846-0.582052$

H $5.357651-2.570295-1.314970$

C 3.1736480 .2074882 .559511

O 5.5582930 .1115972 .687773

H 4.6134131 .1330821 .215621

H $3.198066-0.5689253 .334246$

O $3.132147 \quad 1.4615563 .255386$

C 1.8749370 .0572531 .746401 
C $0.623256 \quad 0.2810102 .615563$

H $1.837833-0.943047 \quad 1.305220$

$\begin{array}{lllll}\mathrm{O} & 1.864649 & 0.992573 & 0.670970\end{array}$

H $0.718095-0.3179603 .527060$

H $\quad 0.586292 \quad 1.3324602 .922294$

H $7.815685-2.732404-1.333755$

H $7.823857-3.3098430 .339317$

H $7.095859-4.301955-0.935046$

C $4.122315-4.406930 \quad 0.280711$

H $5.512049-0.757473 \quad 3.122421$

H 3.9485801 .5290733 .779813

H 2.0053751 .8773151 .048080

H $3.443823-4.6736351 .101091$

H $4.724555-5.298175 \quad 0.067353$

H $5.691575-3.6493021 .532317$

C $-0.909565-2.3743532 .556583$

O $-0.752894-1.4370251 .485638$

C $-0.692926-0.0618221 .881497$

C -1.955486 0.3081592 .670300

C $-2.159331-0.6072663 .873968$

C $-2.134066-2.0702193 .433264$

H $-0.008367-2.3412953 .181560$

C $-0.943152-3.7915731 .960864$

H -0.7073380 .5253520 .954746$

O -1.8957791 .6670063 .087490$

H -2.8226700 .2106232 .008198$

H -1.410287 -0.4151574 .650090$

O $-3.424259-0.3214354 .476594$

H $-3.063908-2.2948262 .899760$

O $-2.102170-2.9096314 .586081$

C $-2.067055-4.0389640 .973542$

H $-0.007898-3.966037 \quad 1.417338$

H $-1.022387-4.5308882 .765658$

H -2.703411 1.8510523 .597012

H $-4.116157-0.4660623 .808675$

H $-2.866796-2.6735045 .138839$

O $-2.025590-5.3473590 .580096$

O $-2.864097-3.201328 \quad 0.575635$

C $-3.011263-5.728802-0.384934$

H $-4.015209-5.6093650 .033256$

H $-2.899729-5.135656-1.297628$

H $-2.855933-6.782554-0.631038$

SCF Energy (B3LYP/6-31G**/MMFF) $=-3245.93374567$

06_00193

MM̄FF Geometry

C -1.909059 3.3337252 .191021

C - -1.0914482 .3279421 .847229$

C $-0.648558 \quad 1.239397 \quad 2.788372$

O 0.7782191 .1077882 .598200

C $-1.357640-0.0694292 .401560$

C $-1.126977-1.2147073 .365326$

C $-0.359490-2.2925703 .108932$

C $-1.881865-1.1197854 .666629$

C $0.438959-2.5743631 .868337$

C $1.901082-2.8998272 .196809$

C $2.725980-3.105797 \quad 0.919025$

C $4.151620-3.542148 \quad 1.256771$

C $5.021062-3.666345-0.003243$

$\begin{array}{llll}\text { O } & 2.754650 & -1.863472 & 0.208192\end{array}$

O $6.391260-3.7632740 .413676$

C $4.890004-2.370520-0.829474$

C $4.713167-4.947450-0.787059$

O $5.562574-2.494551-2.080770$

C $3.426540-1.921612-1.056421$

C $3.411613-0.502384-1.667585$

O $2.783853-2.825071-1.955571$

C 1.5587670 .8511293 .677158

$\begin{array}{llllll}\text { O } & 1.180677 & 0.623677 & 4.818005\end{array}$

C $2.997904 \quad 0.8662493 .321127$

C $3.467521 \quad 1.4072342 .186214$

C 4.8737781 .5108791 .784784

C 5.9012540 .8429152 .658365

C $2.0152320 .117152-1.822694$

C $2.1111421 .622010-1.984804$

C $3.1222063 .367408-3.447645$

O $1.6954612 .390836-1.120628$

C $4.5651343 .507707-3.072437$
C $5.0131254 .308135-2.093402$

C $6.4587864 .399936-1.694098$

C $6.7050243 .936458-0.273868$

C $7.144944 \quad 4.7867550 .668997$

C 6.5111782 .4652900 .037503

C 5.1652092 .1899230 .655095

N $2.7196222 .000357-3.171462$

H -2.283063 3.4015753 .209182

H $-0.743522 \quad 2.2578590 .818427$

H $-0.873755 \quad 1.525928 \quad 3.822538$

H $-1.060633-0.3504851 .386637$

H -2.4412450 .1022602 .344610$

H $-0.302378-3.0718043 .868093$

H $-1.723781-1.9960785 .304267$

H $-1.566096-0.2402255 .235208$

H $-2.957307-1.0427364 .475543$

H $-0.013422-3.4323201 .357538$

H $0.407812-1.737634 \quad 1.167509$

H $2.329621-2.0766232 .781137$

H $1.945919-3.8031402 .817812$

H $2.227506-3.8641990 .303492$

H $4.148940-4.4858821 .816223$

H $4.612130-2.7993851 .923625$

H $6.473271-4.5378640 .996115$

H $5.411541-1.576931-0.275493$

H $4.886811-5.830563-0.160570$

H $5.383180-5.054836-1.647228$

H $3.682452-4.994487-1.144660$

H $6.478692-2.760914-1.891748$

H $4.0087930 .159598-1.030800$

H $3.894238-0.520988-2.652651$

H $2.028214-2.374921-2.364562$

H 3.6589420 .4502844 .074416

H 2.7594391 .8463351 .485755

H $5.941574 \quad 1.324575 \quad 3.641360$

H $\quad 6.9109410 .8711892 .244188$

H $5.658183-0.2171362 .795707$

H $1.495224-0.295241-2.693263$

H $1.391089-0.080193-0.944150$

H $2.4810154 .062297-2.895947$

H $2.9935263 .543842-4.519629$

H $5.2759842 .900173-3.628229$

H $4.3060494 .918199-1.536157$

H $6.7747335 .443388-1.820163$

H $7.0933873 .812378-2.369792$

H 7.3454184 .4494711 .680783

H 7.3110425 .8366220 .452246

H 7.3354432 .1325960 .675090

H $6.5998261 .878686-0.885236$

H 4.3316182 .6045880 .088857

H $3.0544941 .279683-3.804025$

C $-3.823992 \quad 4.105386 \quad 0.783870$

$\begin{array}{lllllll} & \text { O } & -3.871007 & 2.878025 & 0.026870\end{array}$

C $-4.6763343 .080721-1.147345$

C $-5.4227784 .389021-0.919484$

C $-4.3940605 .183447-0.137816$

H $-3.983473 \quad 3.213531-1.986629$

C $-5.566627 \quad 1.858987-1.395875$

H $-5.7160164 .869183-1.857840$

H $-6.3263994 .243058-0.316310$

C $-4.976826 \quad 6.3783730 .596339$

H -3.618667 $5.532956-0.832706$

C $-4.8056460 .516559-1.472162$

$\begin{array}{llll}\text { O } & -6.520329 & 1.748430 & -0.330974\end{array}$

H $-6.1544882 .010176-2.308442$

H $-4.4197900 .258627-0.481826$

O $-5.753865-0.498162-1.824086$

C $-3.669056 \quad 0.484372-2.505286$

C $-2.993493-0.886626-2.713343$

H $-2.897757 \quad 1.213096-2.234543$

O $-4.1908500 .899113-3.773607$

H -3.754764 -1.648105 -2.914086

H -2.411678 $-0.815619-3.641338$

H $-5.423748 \quad 7.083894-0.111691$

H -5.7551246 .0740321 .304218$

H -4.2002956 .9083351 .156758$

C -2.382536 4.3764251 .216954

H -6.0227841 .6701390 .501521$ 
H -6.490316 -0.440050 -1.192538 H $-4.8923220 .271789-4.020066$ H -2.3012305 .3591181 .694722$ H -1.726502 4.3885610 .337318 H $-4.4701213 .963573 \quad 1.660232$ C $-3.517313-2.837146-0.361094$ O $-2.754197-1.625573-0.351846$ C $-2.058481-1.314995-1.561978$ C -1.108992 -2.466387 -1.926227 C $-1.848997-3.796441-2.012532$ C $-2.647245-4.044511-0.735962$ H -4.349884 -2.739262 -1.066800 C $-4.091531-3.0303921 .048736$ H -1.426606 -0.446225 -1.336325 O $-0.447289-2.197255-3.157515$ H $-0.329838-2.540725-1.157448$ H -2.497686 -3.830658 -2.895158 O $-0.900586-4.852804-2.184332$ H -1.958753 -4.2797890 .085033$ O $-3.485740-5.183501-0.923311$ C $-5.192471-2.0460891 .359317$ H -4.496580 -4.039357 1.190995 H -3.312730 -2.896754 1.808804 H $0.082683-2.981172-3.382361$ H $-0.297190-4.842845-1.421597$ H -2.913623 -5.926212 -1.182178 O $-6.380093-2.5108120 .877191$ O $-5.018517-0.997407 \quad 1.966097$ C -7.528860 -1.709399 1.172595 H -7.803860 -1.837814 2.223340 H -7.344231 -0.6542360 .952856$ H -8.354918 -2.056257 0.546173

SCF Energy (B3LYP/6-31G**//MMFF)= -3245.93140662

06_00194

MM̄FF Geometry

C $-0.519292-1.9528903 .076183$

C $0.591288-1.7936163 .810889$

C $1.461457-0.5696443 .736654$

O $2.705489-1.0006443 .134128$

C 1.7612350 .0282405 .122279

C 0.5720060 .6745085 .798475

C 0.1104451 .9037815 .495365

C $-0.066561-0.129556 \quad 6.899221$

C 0.6291092 .8242564 .425259

C $-0.492473 \quad 3.230523 \quad 3.461130$

C 0.0548753 .9389412 .212377

C -1.077265 4.6585591 .476849

C $-0.6018495 .288871 \quad 0.162314$

O $0.6397252 .938036 \quad 1.368282$

O $-1.7760465 .677624-0.566045$

C $0.1345864 .212028-0.659216$

C $0.194513 \quad 6.5751920 .408612$

O $0.7034574 .780696-1.837552$

C 1.2034053 .4355070 .147981

C $1.7526792 .235580-0.662541$

O $2.302917 \quad 4.3144830 .411393$

C $3.422746-0.0701092 .449905$

O $3.180077 \quad 1.1212842 .349440$

C $4.552912-0.7678771 .800894$

C $5.237149-0.1414770 .832393$

C $6.340609-0.7030410 .051727$

C $6.835335-2.0738970 .434013$

C $0.7828491 .059843-0.801097$

C $1.1246430 .208994-2.006477$

C $2.669779-1.528739-2.840276$

O $0.439217 \quad 0.244443-3.027060$

C $4.125102-1.838071-2.699285$

C $5.048694-1.468063-3.600009$

C $6.505257-1.832718-3.524749$

C $7.401207-0.628665-3.332392$

C $7.7747230 .130599-4.376200$

C $7.925675-0.356748-1.939495$

C $6.8311200 .019165-0.977859$

N $2.264367-0.563042-1.836076$

H $-0.827913-1.1630942 .395757$

H $\quad 0.911239-2.5933154 .474794$

H 0.9916890 .1779503 .084789
H $2.5711910 .765176 \quad 5.040297$

H $2.189727-0.7452065 .775116$

H -0.7311572 .2943476 .065527$

H $-0.9092010 .394864 \quad 7.362261$

H $-0.444313-1.0806136 .511836$

H $0.663948-0.3405547 .687168$

H 1.0349713 .7227694 .905573

H 1.4528422 .3735883 .868721

H -1.0525962 .3372283 .153641$

H -1.188869 3.8900643 .994352

H 0.8285464 .6538262 .516098

H -1.537543 5.4195942 .119699

H $-1.8755593 .938606 \quad 1.252261$

H -1.488386 $6.006555-1.435180$

H $-0.6308163 .511549-1.011108$

H -0.4214547 .3069960 .945285$

H $0.4729027 .056942-0.535187$

H 1.1022396 .4131780 .993444

H $1.5114355 .254877-1.576632$

H $2.0930612 .581854-1.647122$

H $2.6605391 .856646-0.176244$

H 3.0819913 .7754910 .627116

H $4.764580-1.7848672 .110005$

H $4.9454880 .872558 \quad 0.561760$

H $7.189468-2.0779011 .470899$

H $7.664059-2.429633-0.180838$

H $\quad 6.034167-2.814567 \quad 0.333538$

H 0.8017460 .4345620 .099447

H $-0.252437 \quad 1.392741-0.926515$

H $2.424750-1.142237-3.835613$

H $2.079495-2.437488-2.683605$

H $4.422836-2.430129-1.837916$

H $4.736894-0.891232-4.468220$

H $6.774541-2.343734-4.458768$

H $6.690484-2.568398-2.732502$

H $8.4383870 .979502-4.250877$

H $7.421491-0.080613-5.380329$

H $8.644350 \quad 0.473366-1.955014$

H $8.503785-1.222981-1.606728$

H $6.3961050 .999193-1.179325$

H $2.653853-0.649320-0.901454$

C -1.123242 -4.062392 1.880671

O $-1.533966-3.371096 \quad 0.685611$

C $-2.087763-4.329528-0.236681$

C $-1.900163-5.7035450 .399914$

C $-1.917085-5.3659251 .878154$

H -3.160918 -4.119503 -0.302622

C $-1.439784-4.172124-1.617056$

H $-2.690123-6.4033200 .110103$

H $-0.940172-6.1539030 .122370$

C $-1.328676-6.4579112 .754217$

H $-2.954545-5.1749512 .183880$

C -1.530338 -2.751599-2.209554

O $-0.047921-4.506756-1.526251$

H -1.877331 -4.896460 -2.313552

H $-0.890613-2.064639-1.643433$

O $-0.994395-2.801389-3.538057$

C $-2.956040-2.176591-2.282088$

C $-3.007714-0.818507-3.006975$

H $-3.345237-2.056056-1.266465$

O $-3.811577-3.100091-2.954263$

H -2.300309 -0.137080 -2.523197

H -2.677240 -0.945923 -4.044696

H $-1.895234-7.3881132 .641253$

H $-0.285984-6.6653532 .491110$

H $-1.358926-6.1720083 .810075$

C $-1.373368-3.1885813 .109786$

H $0.354844-3.911087-0.871043$

H $-0.096378-3.169056-3.475224$

H $-3.454396-3.232837-3.849218$

H $-1.176106-3.7591174 .024549$

H -2.422422 -2.872023 3.146781

H $-0.049243-4.2695691 .777011$

C $-4.3775490 .864595-0.860728$

O $-4.991263-0.107984-1.714669$

C $-4.423552-0.200604-3.025515$

C $-4.5103821 .157997-3.735960$

C $-3.9055532 .281625-2.897469$ 
C $-4.4869862 .260794-1.484029$

H $-3.3235900 .604735-0.703143$

C -5.0909250 .8059890 .493099$

H $-5.062528-0.894843-3.585702$

O $-3.8665391 .091967-5.003928$

H -5.566673 $1.390029-3.925155$

H -2.811140 2.217116 -2.883654

O $-4.2262913 .528772-3.517209$

H $-5.5368732 .576977-1.524175$

O $-3.7959823 .212448-0.680776$

C $-4.701996-0.447567 \quad 1.239713$

H $-4.823701 \quad 1.6524221 .135687$

H -6.1800620 .8288610 .366577$

H -3.925676 $1.975360-5.406506$

H -3.871502 $4.233568-2.948409$

H $-2.8659552 .934729-0.626928$

O $-5.713125-1.3597691 .187476$

O $-3.617289-0.6018821 .786296$

C $-5.458360-2.5980121 .857364$

H $-4.591130-3.0964171 .415990$

H $-5.301031-2.4247722 .926144$

H $-6.333745-3.2402911 .730692$

SCF Energy (B3LYP/6-31G**//MMFF) $=-3245.93034358$

06_00195

MMFF Geometry

C -2.753014 3.086412 1.671633

C $-1.8303702 .195377 \quad 1.280396$

C -0.8459921 .5310372 .204559$

$\begin{array}{lllll}\text { O } & 0.453439 & 1.770099 & 1.617477\end{array}$

C $-1.134395 \quad 0.0186402 .266626$

C $-0.628277-0.6530843 .528651$

C $0.386158-1.5380713 .585295$

C $-1.400990-0.3249564 .782816$

C $1.256652-2.0212792 .460415$

C $2.738467-2.0548592 .864916$

C $3.603759-2.7107841 .779618$

C $5.065741-2.7943982 .218064$

C $5.956342-3.3895821 .115117$

O $3.503710-1.9237470 .591208$

O $7.324109-3.138367 \quad 1.472764$

C $5.686839-2.639393-0.207433$

C $5.805294-4.9127851 .023725$

O $6.382233-3.251443-1.292070$

C $4.184298-2.479055-0.542278$

C $4.006663-1.491585-1.717696$

O $3.652040-3.752660-0.909258$

C 1.5329681 .8478542 .435859

O $1.572263 \quad 1.6496503 .638706$

C 2.6806172 .2525301 .598095

C 3.9316252 .0632442 .037879

C $5.1172712 .453131 \quad 1.286547$

C 6.1041581 .3402761 .081342

C $2.541910-1.206835-2.069565$

C $2.4263810 .042517-2.916539$

C $2.4191672 .530193-2.733652$

O $2.353583-0.010418-4.141637$

C $3.8158843 .067389-2.744286$

C $4.1571004 .258634-2.230089$

C $5.5538414 .809443-2.267898$

C $6.0289815 .308554-0.921388$

C $6.1432856 .624869-0.674701$

C 6.4549884 .2858530 .111382

C $5.292342 \quad 3.7282120 .885769$

N $2.4383331 .200795-2.155275$

H -2.8167293 .3711472 .718533$

H $-1.777356 \quad 1.9146060 .232348$

H -0.8953392 .0024903 .193078$

H $-0.731000-0.4681941 .372351$

H -2.218768 -0.1588092 .230208$

H $0.612467-1.9861034 .552393$

H $-1.066695-0.9134845 .643957$

H -1.286342 0.7307715 .045329

H -2.466043 -0.5346094 .638831$

H $0.931960-3.0342392 .194747$

H $1.147958-1.3972751 .569409$

H $3.078830-1.0267763 .041734$

H $2.857588-2.6074953 .805146$
H $3.205499-3.7138191 .585095$

H $5.163235-3.3740003 .144447$

H $5.433728-1.7861632 .454853$

H $7.487788-3.5488292 .339182$

H $6.127069-1.640103-0.099337$

H $6.045640-5.3797641 .986489$

H $6.512548-5.3374330 .302749$

H $4.796914-5.2271050 .746531$

H $5.890919-4.051731-1.544481$

H $4.499128-0.544924-1.462432$

H $4.502883-1.879040-2.616302$

H $2.821249-3.619637-1.391951$

H 2.4741502 .7079950 .635210

H $4.116631 \quad 1.5711132 .989729$

H 6.2769720 .7920052 .014483

H 7.0824721 .6901640 .742681

H $5.721700 \quad 0.6375410 .335642$

H $2.099438-2.039363-2.627450$

H $1.933862-1.069353-1.168735$

H $1.7447543 .140252-2.124616$

H $2.0287692 .485448-3.755072$

H $4.5803192 .453678-3.215550$

H $3.3875674 .886015-1.785658$

H $5.5651575 .629811-2.997303$

H $6.2662404 .065246-2.645399$

H $6.509876 \quad 6.9911210 .278554$

H $5.8679877 .365865-1.417765$

H 7.1411874 .7512810 .830876

H $7.0294443 .503713-0.395201$

H 4.5321854 .4612641 .158573

H $2.5638111 .127183-1.150148$

C $-5.164914 \quad 3.266695 \quad 1.019512$

O \begin{tabular}{lllll}
\hline & 5.279690 & 1.853146 & 0.762510
\end{tabular}

C $-6.511896 \quad 1.601450 \quad 0.062785$

C $-7.3125492 .896203 \quad 0.136620$

C $-6.2072543 .933842 \quad 0.122907$

H $-6.245708 \quad 1.411370-0.983793$

$\begin{array}{llll}\text { C }-7.213762 & 0.373498 & 0.653024\end{array}$

H $-8.0110292 .999805-0.699111$

H $-7.8878082 .968468 \quad 1.066830$

C $-6.652806 \quad 5.3035310 .604449$

H $-5.821745 \quad 4.021013-0.901918$

C -6.333201 -0.8902760 .729624$

$\begin{array}{llll}0 & -7.642216 & 0.672338 & 1.988815\end{array}$

H -8.1272110 .1617360 .085588$

H $-5.543402-0.7552301 .478737$

O $-7.155225-1.9657501 .201989$

C $-5.700490-1.314827-0.607865$

C $-4.901611-2.624152-0.482673$

H $-5.032811-0.524341-0.956864$

O $-6.719326-1.471972-1.593568$

H $-4.162292-2.502826 \quad 0.316445$

H $-5.578194-3.431461-0.177681$

H $-7.4463535 .697845-0.038673$

H $-7.039360 \quad 5.262876 \quad 1.628464$

H -5.8201196 .0134840 .586823$

C $-3.735753 \quad 3.724442 \quad 0.729160$

H -6.8548380 .9300452 .498681$

H -7.542014 -1.683985 2.048836

H -7.336965 - $2.150778-1.271551$

H $-3.655627 \quad 4.8122520 .833118$

H $-3.4746753 .477116-0.307006$

H -5.4100333 .4194902 .078736$

C $-2.177526-1.758870-1.580354$

O $-3.361140-2.032861-2.338385$

C $-4.210032-3.050950-1.797686$

C $-3.432525-4.370020-1.685069$

C $-2.157305-4.197664-0.866137$

C $-1.333581-3.031334-1.405628$

H $-2.454098-1.344167-0.603257$

C $-1.365707-0.698343-2.342224$

H -5.005076 -3.208069-2.537176

O $-4.232637-5.388473-1.094369$

H -3.156856 -4.718452 -2.688835

H $-2.374665-4.0659520 .200193$

O $-1.367903-5.385242-0.968819$

H $-0.887825-3.319368-2.366733$

O $-0.261105-2.763090-0.505848$ 
C $-2.0287350 .660576-2.390147$ H $-0.395949-0.559768-1.854066$ H $-1.210439-1.020891-3.378644$ H -5.031549 -5.485346 -1.640365 H $-1.918970-6.125398-0.661184$ H $\quad 0.246847-3.586216-0.403868$ O $-1.167347 \quad 1.542976-2.980583$ O $-3.1470690 .922659-1.972519$ C -1.640491 2.887924 -3.100737 H $-1.7022783 .349721-2.111571$ H $-0.9211853 .447595-3.704618$ H $-2.6128372 .913325-3.602127$

SCF Energy (B3LYP/6-31G**/MMFF) $=-3245.91630877$

0600196

MM̄FF Geometry

C -1.226812 -3.492472 -0.797996

C $-0.328542-3.275641-1.769510$

C $-0.278093-2.078716-2.693137$

O $0.877301-1.286581-2.328262$

C $-1.508247-1.152539-2.638536$

C $-1.486887-0.067982-3.696416$

C $-1.1985721 .225863-3.455470$

C -1.882485 -0.510471 -5.081390

C $-0.7930761 .842476-2.144982$

C $0.3219222 .879746-2.336730$

C $0.7014383 .551296-1.009276$

C $1.5921944 .769010-1.260464$

C 2.0402525 .4238960 .052951

O $1.3995212 .594577-0.203229$

O $3.0965496 .347068-0.251906$

C 2.6379574 .3368790 .966680

C 0.9183326 .2447380 .699411

O 2.9376054 .8660032 .256973

C 1.7511673 .0738351 .102975

C 2.5564511 .9715831 .828059

$\begin{array}{lllll}\text { O } & 0.591619 & 3.389529 & 1.874317\end{array}$

C $2.064110-1.584510-2.919765$

O $2.282826-2.467192-3.733652$

C $3.073725-0.648992-2.377722$

C $4.375738-0.914202-2.558577$

C $5.497753-0.126034-2.045291$

C $5.1848651 .204809-1.416019$

C $1.874206 \quad 0.6020391 .886922$

C $2.849211-0.4127852 .449004$

C $4.627443-2.0205091 .765144$

O $3.000916-0.5536503 .660747$

C $5.935311-1.3090381 .601758$

C $6.969670-1.8045690 .904970$

C $8.280805-1.0881760 .739692$

C $8.744899-1.023994-0.700624$

C $9.798761-1.745537-1.118736$

C $8.036904-0.066937-1.638454$

C $6.741755-0.637801-2.152220$

N $3.551892-1.0919681 .466603$

H $-2.000683-2.755888-0.608805$

H $0.442735-4.025576-1.934211$

H - $0.167861-2.455089-3.717959$

H -1.595205 -0.702825 -1.642839

H -2.423149 -1.740699-2.782243

H $-1.2696231 .925468-4.287384$

H -1.912577 $0.322626-5.791676$

H -1.171376 -1.246201 -5.469075

H -2.879166 $-0.963898-5.064795$

H -1.672555 2.338906-1.719200

H $-0.4593501 .089296-1.425437$

H $\quad 1.2020272 .386569-2.769323$

H - $0.0158623 .642370-3.049889$

H -0.219232 $3.854168-0.496705$

H $1.0816495 .502303-1.897233$

H $2.4868944 .458085-1.818404$

H $2.7507046 .999309-0.885091$

H $3.599017 \quad 4.0355970 .525915$

H 0.5785637 .0327870 .016560

H $1.2742406 .760867 \quad 1.598021$

H 0.0473565 .6438890 .969309

H 3.5070695 .6435572 .126317

H 3.5232501 .8479981 .326163
H 2.7745522 .2884052 .856485

H 0.2180722 .5673642 .228993

H $2.7198000 .208022-1.815803$

H $4.650916-1.815180-3.105667$

H $4.5821691 .821076-2.092915$

H $6.071127 \quad 1.795693-1.177301$

H $4.6298531 .062858-0.485533$

H 0.9960790 .6178762 .540048

H 1.5343890 .2785330 .897089

H $4.527713-2.8659041 .077182$

H $4.526769-2.3846082 .792280$

H $6.039062-0.3408902 .086767$

H $6.878055-2.7855300 .443850$

H $9.024440-1.6154851 .351165$

H $8.229901-0.068577 \quad 1.141778$

H $10.153018-1.687100-2.142607$

H $10.328019-2.414914-0.448627$

H $8.6713800 .145861-2.508823$

H $7.9137190 .891237-1.127202$

H $\quad 6.860626-1.602139-2.649068$

H $3.395913-0.8534850 .492217$

C -1.012781-4.419441 1.554628

O $-1.864039-3.3440392 .013598$

C -1.025284-2.352001 2.632526

C $0.206533-3.1180713 .089584$

C $0.427810-4.0161341 .888301$

H $-0.726966-1.6475381 .846162$

C $-1.769021-1.6013473 .736643$

H $1.055439-2.4661463 .302905$

H $\quad 0.000675-3.7201473 .982456$

C $1.349520-5.1908802 .164462$

H $0.861218-3.4151681 .078669$

C $-3.064219-0.8940353 .285380$

O $-2.115478-2.5157034 .783532$

H -1.092179 -0.869051 4.192886

H -3.864852 -1.626319 3.124330

O $-3.496160-0.0636954 .371673$

C $-2.923103-0.0190212 .025022$

C -4.1375890 .8998331 .785089$

H $-2.775227-0.6693171 .156155$

O -1.7594620 .8038292 .136045$

H -5.0575130 .3244551 .923214$

H -4.1305311 .6948052 .539969$

H $2.346200-4.8380582 .449366$

H $0.969562-5.8176752 .978098$

H $1.454493-5.8195391 .274441$

C -1.277378 -4.7146870 .075126$

H $-2.657560-3.2205424 .389205$

H -3.595246 -0.636109 5.151846

H $-1.857904 \quad 1.3464072 .937213$

H $-2.289352-5.128136-0.022221$

H $-0.585532-5.486321-0.281755$

H $-1.298791-5.3029482 .139538$

C $-5.6374010 .062631-0.763270$

O $-4.3001420 .569235-0.654936$

C $-4.097207 \quad 1.5415080 .378572$

C -5.0479232 .7285530 .181890$

C -6.5017882 .2838760 .076460$

C $-6.6416881 .200492-0.991230$

H $-5.904374-0.4794830 .151090$

C $-5.661952-0.928867-1.938234$

H $-3.082216 \quad 1.9317510 .236254$

O -4.8945513 .6639231 .244056$

H $-4.7717963 .250933-0.743689$

H -6.8814481 .9456631 .047944$

O $-7.2857183 .419267-0.295924$

H $-6.4939041 .641048-1.985723$

O $-7.9635990 .666446-0.946903$

C $-5.081772-2.278417-1.570524$

H $-6.692138-1.111732-2.263893$

H $-5.079895-0.530473-2.777499$

H -5.5271894 .3862271 .089144$

H $-8.2205593 .155975-0.256754$

H $-8.5755841 .372270-1.215362$

O $-5.134390-3.089215-2.669233$

O $-4.647879-2.586962-0.469717$

C -4.649195 -4.423982 -2.491468

H $-5.017330-5.031188-3.322782$ 
H $-3.556781-4.421716-2.516370$

H $-5.014049-4.859243-1.556095$

SCF Energy (B3LYP/6-31G**//MMFF)= -3245.92578215

06_00197

MM̄FF Geometry

C $0.422553-3.748667-0.133919$

C $0.476467-3.1673821 .074232$

C $-0.696854-2.4552971 .692927$

O $-0.260845-1.1085991 .990688$

C $-1.162596-3.1350462 .994173$

C -1.747013 -4.516602 2.797640

C $-2.970634-4.7533492 .285858$

C $-0.870984-5.6595703 .237333$

C $-3.974023-3.7377751 .812111$

C $-4.186818-3.8448020 .297425$

C $-5.251161-2.860643-0.208598$

C $-5.573755-3.141759-1.678796$

C $-6.594795-2.144963-2.242667$

O $-4.755295-1.521773-0.060557$

O $-6.592284-2.306270-3.669476$

C $-6.111045-0.715110-1.923705$

C $-8.018979-2.469934-1.778373$

O $-7.086627 \quad 0.247786-2.316104$

C $-5.699606-0.511065-0.445239$

C $-5.0749240 .884751-0.202311$

O $-6.879549-0.577536 \quad 0.368198$

C -1.229391 -0.1589622 .098453$

O $-2.434133-0.3217271 .990169$

C -0.5663801 .1316152 .380596$

C -1.3147432 .2361722 .519378$

C -0.8225613 .5819832 .817159$

C 0.6662873 .7605222 .959970

C $-3.7531621 .162235-0.923571$

C $-3.3693642 .634182-0.870097$

C $-1.7442394 .217805-1.898533$

O $-4.0550533 .492083-0.320491$

C $-1.1029894 .896172-0.730014$

C -1.527448 $6.072875-0.244595$

C $-0.859801 \quad 6.8241240 .871997$

C -1.7385976 .9487042 .096065$

C -2.6927657 .8919132 .171691$

C -1.4540976 .0295303 .261746$

C -1.718343 4.5844892 .937645

N -2.179818 $2.879250-1.544690$

H $-0.513657-3.690280-0.687112$

H $1.388964-3.2009361 .661941$

H -1.518171 -2.400631 0.968976

H -1.899955 -2.503259 3.505908

H $-0.326225-3.1778213 .705831$

H $-3.299414-5.7877612 .195024$

H -1.343660 -6.633668 3.072488

H $0.074272-5.6552092 .685805$

H -0.649924 -5.5764314 .306457$

H -4.921267 -3.929764 2.331032

H $-3.680619-2.7195672 .079688$

H -3.235828 -3.650336 -0.214519

H $-4.489291-4.8698640 .047898$

H -6.150206 -2.9827570 .406950$

H -5.932159-4.170315 -1.810952

H -4.651270 -3.068396 -2.272361

H -7.179096 -1.626600 -4.043694

H -5.241902 -0.540438 -2.567630

H -8.303864 -3.477882 -2.103386

H -8.749473 -1.792994 -2.234958

H -8.138726 -2.425492 -0.694070

H -7.791554 $0.244296-1.646308$

H $-5.8171741 .653223-0.455095$

H -4.8930401 .0097220 .874611$

H -6.690130 -0.148687 1.219206

H $\quad 0.513394 \quad 1.1350332 .475027$

H -2.395373 2.1505072 .411240

H 1.0355353 .2128333 .833808

H 0.9748974 .8010233 .077058

H 1.1842163 .3889842 .069614

H $-2.9476070 .570976-0.478229$

H -3.823266 $0.896994-1.982406$

H $\quad-2.608974 \quad 4.774877-2.275682$
H $-1.0168264 .120523-2.710798$

H $-0.2313704 .419381-0.291439$

H $-2.3915596 .550841-0.701564$

H -0.6049187 .8258590 .501638$

H $0.099615 \quad 6.3659221 .141740$

H $-3.3093118 .004748 \quad 3.057089$

H -2.879082 8.571015 1.346000

H -2.0899096 .2904304 .118319$

H $-0.431814 \quad 6.2055573 .608031$

H -2.7768034 .3549742 .806242$

H $-1.7102182 .102499-2.000964$

C $2.821506-4.732736-0.091049$

O $3.538078-3.4870810 .038672$

C $4.919204-3.708531-0.297082$

C $5.112346-5.219546-0.313782$

C $3.766914-5.683272-0.829298$

H $5.060474-3.326116-1.314656$

C $5.844627-2.9714310 .674794$

H $5.947275-5.524732-0.951770$

H $5.291274-5.6184330 .691577$

C $3.493959-7.155107-0.569545$

H $3.721428-5.495210-1.910624$

C $5.628295-1.4479750 .767016$

O $5.669415-3.507417 \quad 1.993378$

H $6.887654-3.1775740 .406631$

H $4.704055-1.2228621 .313180$

O $6.698742-0.9164541 .560555$

C $5.607072-0.709613-0.584383$

C $5.7164860 .820332-0.432039$

H $4.683702-0.965924-1.114625$

O $6.694925-1.157404-1.396321$

H $5.034376 \quad 1.1518230 .356564$

H $6.7346891 .068693-0.110056$

H $4.242979-7.778626-1.068544$

H $3.523728-7.3859110 .500645$

H $2.508829-7.443590-0.948739$

C $1.510278-4.504417-0.847935$

H $4.730666-3.4061832 .227975$

H $6.685359-1.3824052 .414172$

H $7.518555-0.978060-0.910921$

H $1.086612-5.476168-1.128931$

H $1.720490-3.961161-1.778210$

H $2.627844-5.1046310 .923198$

C $3.1020032 .131960-1.346448$

O $4.0487171 .417615-2.152549$

C $5.4196611 .558771-1.757836$

C $5.8201723 .039856-1.765334$

C $4.8856913 .880679-0.904805$

C $3.4347043 .631160-1.310281$

H $3.0978981 .724245-0.329007$

C $1.7102771 .908462-1.957485$

H $6.0145541 .069798-2.539408$

O $7.1647623 .189899-1.322385$

H $5.7811343 .412091-2.797468$

H 5.0481833 .6844420 .161376

O $5.1991085 .259192-1.114083$

H $3.2586184 .078994-2.296550$

O $2.5765004 .303991-0.391754$

C $1.1766230 .510973-1.724828$

H $0.9873472 .597963-1.510884$

H $1.7503852 .073417-3.040764$

H $7.3666294 .141319-1.323204$

H $4.5634125 .782252-0.595966$

H 2.7447743 .9389340 .493453

O $-0.0312340 .399689-2.355647$

O $1.729758-0.362426-1.071979$

C $-0.689109-0.863099-2.221339$

H $-1.601137-0.837335-2.823705$

H $-0.048384-1.669555-2.589738$

H $-0.965527-1.029585-1.177057$

SCF Energy (B3LYP/6-31G**//MMFF) = -3245.93249518

06_00198

MM̄MF Geometry

C $-0.8227530 .544699-2.797764$

C $-1.359772-0.686150-2.781304$

C $-0.706063-1.919928-2.201391$

O $0.329353-1.518715-1.275185$ 
C $-0.069262-2.776134-3.305545$

C $0.137251-4.212859-2.867326$

C $1.277190-4.709606-2.348934$

C $-1.055310-5.113858-3.067482$

C $2.554242-3.975460-2.049806$

C $2.856271-4.015566-0.546368$

C $4.157326-3.284239-0.188987$

C $4.402858-3.3482221 .319421$

C $5.659507-2.5680471 .723365$

O $4.036942-1.919879-0.595804$

O $5.622993-2.426543 \quad 3.152412$

C $5.598321-1.1521091 .108473$

C $6.931283-3.3639791 .407001$

O $6.833356-0.4772991 .335119$

C $5.219139-1.132859-0.394524$

C $4.8932760 .304856-0.848161$

O $6.296767-1.577313-1.220923$

C $-0.011873-1.3935320 .035555$

O $-1.020135-1.809408 \quad 0.581562$

C $1.032295-0.568887 \quad 0.679105$

C $0.871895-0.155358 \quad 1.943779$

C 1.7808850 .7507292 .638888

C 2.0972070 .3228634 .044121

C $4.3833650 .395489-2.292728$

C $4.1916451 .848832-2.674775$

C $2.740543 \quad 3.810197-2.172350$

O $5.0327952 .461162-3.328586$

C $3.1916364 .369025-0.859210$

C $2.355151 \quad 4.7799440 .105567$

C $2.816698 \quad 5.2636661 .450443$

C 2.4272084 .3233982 .571601

C 1.5683314 .7031353 .532533

C 3.0913562 .9613372 .619101

C 2.2116321 .8831202 .046547

N $3.0208472 .386201-2.163170$

H $0.1902960 .696463-2.434076$

H -2.366197 -0.826299-3.168199

H -1.479371 -2.492794 -1.675282

H $0.879814-2.333265-3.629235$

H $-0.707745-2.785004-4.199392$

H $1.301905-5.768258-2.092276$

H $-0.864570-6.139141-2.733001$

H $-1.917189-4.739831-2.505667$

H - $-1.323836-5.157137-4.128164$

H $3.368694-4.459944-2.601399$

H $2.517294-2.939017-2.394553$

H $2.019539-3.555418-0.006615$

H $2.921838-5.058148-0.209869$

H $4.980530-3.754443-0.739349$

H $4.469472-4.3885891 .661541$

H $3.541390-2.9144801 .847286$

H $6.385547-1.8820093 .413818$

H $4.831373-0.5990851 .669201$

H $6.949528-4.2964111 .984433$

H $7.830661-2.8126741 .702472$

H $7.019062-3.6299060 .351416$

H $6.6974970 .466261 \quad 1.147386$

H $4.1346230 .735074-0.183579$

H $5.7876170 .935165-0.763604$

H $7.052369-1.805146-0.657129$

H $1.881529-0.2628270 .078951$

H $0.011018-0.4805492 .524196$

H 2.8264730 .9651504 .541562

H $2.515867-0.6902364 .046922$

H 1.1894490 .3213774 .657742

H $5.096785-0.049408-2.995022$

H $3.433416-0.137574-2.413791$

H $1.6660603 .935209-2.329593$

H $3.2741724 .297929-2.993844$

H $4.2646014 .403795-0.684200$

H $1.2823704 .741842-0.066114$

H 2.3889816 .2609231 .613550

H 3.9054285 .4001431 .468502

H 1.3073354 .0372094 .348671

H $1.1039415 .683563 \quad 3.521941$

H 3.3888232 .7493113 .650311

H 4.0289072 .9859412 .049679

H 1.8913392 .0726751 .022163
H $2.4260861 .799967-1.584512$

C -1.527146 $2.839435-2.137368$

$\begin{array}{lllll}\text { O } & -2.134799 & 2.322641 & -0.940544\end{array}$

C $-2.8473983 .384411-0.278826$

C $-2.5602934 .656191-1.072579$

C $-2.3093894 .106860-2.465495$

H $-3.9136313 .145284-0.368595$

C -2.451332 $3.433276 \quad 1.203392$

H -3.395006 $5.362778-1.034090$

H $-1.6688035 .176649-0.704660$

C $-1.571895 \quad 5.072715-3.375861$

H $-3.2753443 .846060-2.918460$

C $-2.5106192 .069242 \quad 1.919448$

O $-1.102136 \quad 3.9048191 .317848$

H $-3.071854 \quad 4.1674691 .729406$

H $-1.725948 \quad 1.4119451 .527140$

O -2.2035042 .2788563 .302953$

C -3.8657791 .3492971 .822309$

C $-3.849757-0.0037982 .552650$

H $-4.1081601 .180240 \quad 0.772197$

$\begin{array}{llll}\text { O } & -4.894680 & 2.174483 & 2.362594\end{array}$

H $-2.989725-0.5788242 .194184$

H -3.7007610 .1644193 .626183$

H $-2.152000 \quad 5.991222-3.512352$

H $-0.5966705 .349652-2.961489$

H $-1.4049904 .629414-4.362440$

C $-1.558177 \quad 1.778770-3.240630$

H $-0.532438 \quad 3.2883890 .826316$

H $-1.3356002 .716435 \quad 3.343043$

H $-4.6672102 .357497 \quad 3.290446$

H -1.084946 2.166490 -4.149270

H $-2.596893 \quad 1.529237-3.486065$

H $-0.488768 \quad 3.084768-1.881571$

C $-4.639195-1.8217330 .224477$

O $-5.514168-0.9856630 .990941$

C $-5.150088-0.8188652 .366012$

C $-5.122942-2.1863693 .061630$

C $-4.191904-3.1604342 .344083$

C $-4.526172-3.2191300 .854147$

H -3.652139 -1.3507240 .156289$

C $-5.221784-1.931612-1.194772$

H $-5.957056-0.2349562 .826360$

O $-4.723036-2.0380904 .419836$

H -6.138034 -2.604086 3.068414

H -3.140916 -2.899101 2.512445

O $-4.374631-4.4587912 .914354$

H $-5.471127-3.7627140 .728199$

O $-3.522615-3.977650 \quad 0.180463$

C $-5.153744-0.634409-1.971703$

H $-4.670730-2.680546-1.774611$

H $-6.277490-2.222769-1.134086$

H $-4.694542-2.9272144 .813025$

H $-3.806927-5.0742872 .419357$

H -2.676836 -3.5081320 .281271$

O $-5.800588-0.801426-3.163883$

O $-4.597023 \quad 0.388090-1.599776$

C $-5.813205 \quad 0.342978-4.023085$

H $-4.7968400 .573668-4.354694$

H $-6.4223800 .103774-4.898742$

H -6.257962 $1.202040-3.511309$

SCF Energy (B3LYP/6-31G**//MMFF) $=-3245.92808647$

06_00199

MM̄FF Geometry

C 0.1058530 .0109701 .508008

C $0.315622-1.2464421 .087672$

C $-0.042886-1.795722-0.272758$

O $-0.287224-0.712537-1.197908$

C $-1.321079-2.643801-0.186097$

C $-1.562622-3.466161-1.434732$

C $-2.369902-3.101293-2.449945$

C $-0.848050-4.792076-1.473203$

C $-3.128618-1.813173-2.610912$

C $-4.649930-2.009961-2.604635$

C $-5.223998-2.352892-1.220167$

C $-6.739763-2.549995-1.310614$

C $-7.376837-2.772595 \quad 0.067750$

O $-4.924106-1.266203-0.338730$ 
O - $-8.795694-2.632042-0.098839$

C $-6.896701-1.658907 \quad 1.022507$

C $-7.142898-4.2023600 .568882$

O $-7.368743-1.8940582 .347728$

C $-5.364392-1.4519521 .013200$

C $-5.002548-0.1712571 .802715$

O $-4.742741-2.5821601 .625171$

C $0.737526-0.289911-1.979634$

O $1.854364-0.783098-2.053201$

C $0.354710 \quad 0.896440-2.780730$

C $-0.756593 \quad 1.616862-2.559735$

C $-1.1664152 .821303-3.287502$

C $-0.287738 \quad 3.288876-4.418361$

C $-3.503815 \quad 0.144932 \quad 1.826041$

C $-3.255216 \quad 1.5445872 .348703$

C $-2.9977793 .897848 \quad 1.612952$

O -3.0695551 .7669773 .542865$

C -3.490653 4.7227910 .469019

C $-2.6970735 .488601-0.294778$

C -3.195532 $6.300336-1.455695$

C $-2.4903385 .964586-2.752330$

C $-1.5829416 .805475-3.277312$

C $-2.8997664 .705968-3.486862$

C $-2.291314 \quad 3.459792-2.900936$

N $-3.298875 \quad 2.503753 \quad 1.349019$

H -0.4017950 .7200230 .859415$

H $0.801845-1.9487201 .760760$

H $0.793512-2.411207-0.625105$

H -2.184206 $-1.998606 \quad 0.006525$

H $-1.269470-3.3268060 .672579$

H -2.479582 -3.788539 -3.288072

H $-1.060518-5.353656-2.389144$

H $0.235224-4.645519-1.420767$

H $-1.156416-5.413675-0.626248$

H -2.844162 -1.064880 -1.866447

H $-2.840779-1.387462-3.580781$

H $-5.099979-1.070189-2.951201$

H $-4.928470-2.786473-3.327873$

H $-4.742703-3.264229-0.848173$

H $-6.985068-3.378860-1.986377$

H $-7.195429-1.655793-1.759694$

H $-9.203699-2.6978720 .781807$

H -7.375644 -0.7265290 .693561$

H -7.573716 -4.926683 -0.133060

H -7.650016 -4.3768101 .524141$

H $-6.085417-4.4470130 .689551$

H -6.812818 -2.588356 2.740741

H -5.5332500 .6813691 .358882$

H -5.345053 -0.2594092 .841352$

H -3.831396 -2.351382 1.862887

H $1.0714101 .172198-3.547657$

H $-1.4297601 .315023-1.759105$

H $0.719343 \quad 3.519413-4.053395$

H $-0.6543874 .192231-4.910279$

H $-0.2142302 .516651-5.192197$

H $-2.960595-0.5532352 .472087$

H $-3.0669150 .059763 \quad 0.825377$

H -1.915051 3.9811051 .755959

H -3.488497 4.2008522 .543645

H $-4.556652 \quad 4.6830600 .256511$

H $-1.6332005 .539984-0.074762$

H -3.047733 7.359451-1.207512

H $-4.2770526 .177690-1.594762$

H -1.094639 $6.593291-4.222358$

H $-1.3051807 .724737-2.772306$

H -2.667330 $4.787689-4.554809$

H -3.992880 4.609264 -3.456601

H -2.861764 $3.046041-2.069373$

H $-3.2946352 .191007 \quad 0.381941$

C 1.3430631 .8358752 .676578

$\begin{array}{lllll}\text { O } & 2.487595 & 1.606347 & 1.836127\end{array}$

C 3.6120902 .3363672 .358635

C 3.0642463 .2226843 .472706

C 1.9026412 .3900283 .983958

H 4.2875221 .5932192 .798901

C 4.3295653 .0732991 .219842

H 3.8146953 .4319084 .241175

H 2.7003004 .1832573 .091104
C $\quad 0.8954723 .1820014 .799148$

H 2.3024451 .5726554 .599037

C $4.6293172 .196034-0.013610$

O $3.510598 \quad 4.161049 \quad 0.768624$

H 5.2523043 .5289561 .596572

H $3.692506 \quad 1.943227-0.525997$

$\begin{array}{llll}\text { O } & 5.393567 & 2.978628 & -0.939331\end{array}$

$\begin{array}{llll}\text { C } 5.407820 & 0.904179 & 0.288735\end{array}$

C $5.6491520 .068802-0.979745$

H $4.842597 \quad 0.3015851 .001101$

$\begin{array}{lllll} & \text { O } & 6.654107 & 1.222087 & 0.902654\end{array}$

H $4.682268-0.108451-1.463085$

H $\quad 6.2609070 .645752-1.683914$

H $1.371795 \quad 3.6126195 .685867$

H $0.464374 \quad 4.0032084 .216520$

H $0.0743272 .540598 \quad 5.134519$

C $0.555254 \quad 0.535283 \quad 2.843090$

H 2.6568113 .7890800 .487508

H $4.8856893 .787635-1.122413$

H 7.1514931 .7831970 .283208

H -0.3252860 .7096313 .470382$

H $1.178050-0.2125473 .347383$

H 0.7242032 .5899662 .171759

C $4.446079-2.585890-0.037826$

O $5.729499-2.054192 \quad 0.309672$

C $6.364255-1.274702-0.708919$

C $6.560452-2.131328-1.966240$

C $5.241785-2.731394-2.448700$

C $4.533894-3.453976-1.302938$

H $3.736466-1.763423-0.187188$

C $3.959877-3.4375241 .148021$

H $7.362299-1.027345-0.325503$

O $7.152059-1.353890-3.001999$

H $7.259071-2.946523-1.736997$

H $4.600154-1.965841-2.900002$

O $5.538274-3.669390-3.486424$

H $5.069430-4.386108-1.079968$

O $3.213209-3.807654-1.709885$

C $3.646122-2.6231322 .384551$

H $3.044360-3.9758590 .878204$

H $4.739019-4.1593821 .421573$

H $7.235177-1.926552-3.783728$

H $4.693070-3.970640-3.860139$

H $3.289247-4.446351-2.438496$

O $3.319515-3.476394 \quad 3.401121$

O $3.667932-1.4033812 .457081$

C $2.985797-2.853138 \quad 4.645400$

H $3.805973-2.2139824 .986405$

H $2.063132-2.2755174 .539462$

H $2.824055-3.6399215 .386951$

SCF Energy (B3LYP/6-31G**//MMFF) $=-3245.92871589$

06_00200

MM̄FF Geometry

C $-3.016136-0.912925-3.047556$

C $-2.000781-0.839973-2.173547$

C $-0.704625-0.124250-2.433607$

O $0.350575-1.050194-2.065964$

C $-0.5482941 .140299-1.567091$

C -1.186492 $2.375761-2.166879$

C $-0.5693053 .201390-3.035666$

C -2.578777 $2.689677-1.690939$

C $0.8199663 .063803-3.592448$

C $1.7292724 .256245-3.265089$

C $1.9047764 .520468-1.761483$

C $2.9075545 .652780-1.526869$

C $3.1519195 .901856-0.029898$

O $2.3673643 .320322-1.131970$

$\begin{array}{lllll}\text { O } & 4.321525 & 6.726292 & 0.092565\end{array}$

C 3.4729064 .5539070 .653495

$\begin{array}{llll}\text { C } 2.002188 & 6.688599 & 0.610542\end{array}$

$\begin{array}{lllll}\text { O } & 3.566990 & 4.710518 & 2.067843\end{array}$

C 2.4797223 .4234810 .293090

C $2.9234252 .053910 \quad 0.852727$

$\begin{array}{lllll}\text { O } & 1.211209 & 3.721318 & 0.893295\end{array}$

C $1.502822-1.009601-2.786324$

O $1.742983-0.303015-3.752330$

C $2.445864-1.982123-2.193262$ 
C $3.700678-2.035301-2.662970$

C $4.739485-2.944983-2.195175$

C $6.080530-2.285686-2.023287$

C $4.175963 \quad 1.445773 \quad 0.216353$

C 4.7776690 .4027161 .138666

C $4.295152-1.8021792 .191380$

O $5.826953 \quad 0.6101401 .744805$

C $5.195262-2.8213601 .571698$

C $4.869333-4.1171541 .449848$

C $5.800458-5.1670380 .916603$

C $5.231390-5.910411-0.269305$

C $4.606532-7.089699-0.113230$

C $5.478355-5.333678-1.643240$

C $4.493714-4.257091-2.009341$

N 4.009399-0.745069 1.240149

H -2.934054 -0.418827 -4.011945

H -2.105125 -1.326814 -1.208070

H -0.621156 $0.119681-3.500292$

H $0.5159491 .330125-1.385414$

H $-0.9509610 .968172-0.559216$

H -1.114984 4.071757 -3.397376

H -2.979081 $3.602094-2.145597$

H -3.265967 $1.875944-1.934178$

H -2.578063 $2.837590-0.606480$

H $1.3051442 .146477-3.253768$

H $0.7396262 .976579-4.682919$

H $2.7098874 .049598-3.713260$

H $1.3403765 .159990-3.750960$

H $0.9293574 .794369-1.343712$

H $2.5763816 .574948-2.020386$

H $3.8674145 .391322-1.994127$

H $4.1605417 .550324-0.398358$

H $4.477063 \quad 4.2701090 .317991$

H 1.8683107 .6516090 .102940

H 2.2238426 .9323561 .655430

H 1.0468116 .1615190 .572230

H 2.6628864 .7788582 .418974

H 3.0399792 .1284971 .941972

H 2.1047391 .3363410 .706676

H 0.6853052 .9050660 .924428

H $2.095732-2.626696-1.394467$

H $4.019603-1.351210-3.447139$

H $6.804071-2.912070-1.496326$

H $5.982990-1.365372-1.435596$

H $6.506652-2.025470-2.998279$

H $3.9384080 .984057-0.748842$

H $4.9487012 .196798 \quad 0.029806$

H $3.336577-2.2309342 .499977$

H $4.779091-1.3726543 .074870$

H $6.179080-2.4861681 .252710$

H $3.894754-4.4545581 .795773$

H $6.007055-5.8724181 .732434$

H $6.774912-4.7399750 .648865$

H $4.225536-7.644319-0.964257$

H $4.457365-7.5233860 .870187$

H $5.389688-6.124100-2.401062$

H $6.517606-4.999469-1.719096$

H $3.483871-4.628063-2.187014$

H $3.185557-0.8465170 .654621$

C $-5.409052-0.704729-2.311514$

O $-5.061432-0.146112-1.028702$

C $-6.187709-0.260795-0.141055$

C $-7.386802-0.540054-1.036029$

C $-6.753078-1.406496-2.109056$

H $-6.006243-1.1412850 .487085$

C -6.2910110 .9868120 .744278$

H -8.198615 $-1.041492-0.500907$

H $-7.7873150 .377990-1.480755$

C $-7.586354-1.516784-3.373823$

H -6.593585 -2.412482 -1.697648

C $-4.968962 \quad 1.377626 \quad 1.436646$

O

H -7.084230 0.8508921 .487990

H -4.2516891 .7284340 .686890$

O -5.2267522 .4929552 .297833$

$\begin{array}{llll}\text { C }-4.323120 & 0.258496 & 2.271025\end{array}$

$\begin{array}{llll}C & -2.968126 & 0.686627 & 2.858838\end{array}$

H $-4.159525-0.6133111 .637035$
O $-5.206794-0.1382313 .315363$

H -2.3161060 .9837882 .029644$

H -3.1075701 .5730243 .489746$

H $-8.557050-1.974114-3.156098$

H $-7.770420-0.533680-3.820017$

H $-7.080538-2.136437-4.121037$

C $-4.296327-1.650586-2.764914$

H $-6.0338022 .207013-0.768952$

H $-5.6334113 .190101 \quad 1.754914$

H $-5.3736150 .642734 \quad 3.870709$

H $-4.593417-2.184250-3.674824$

H $-4.118372-2.409724-1.993829$

H $-5.5093210 .136447-3.009436$

C $-1.353754-1.7525501 .934251$

O $-2.227734-1.6803893 .065560$

C $-2.283248-0.4050623 .713365$

C $-0.883504-0.0110774 .205011$

C $0.138209-0.0327713 .070660$

C $0.079839-1.3647522 .325225$

H $-1.725480-1.0888701 .145501$

C $-1.375813-3.1994241 .410532$

H -2.911538 -0.5445904 .602224$

O $-0.918888 \quad 1.279602 \quad 4.804857$

H $-0.566624-0.7189184 .982000$

H -0.0048410 .8155782 .391648$

$\begin{array}{lllll} & 1.440083 & 0.127249 & 3.639485\end{array}$

H $0.517388-2.1430382 .963275$

O $0.894000-1.284087 \quad 1.157396$

C $-2.698660-3.6029620 .795539$

H $-0.610140-3.3280490 .636817$

H $-1.177745-3.8931092 .236559$

H -0.0120001 .4964495 .081763$

H 2.0850140 .0439292 .916517

H $0.505924-0.6165430 .567002$

$\begin{array}{lllll} & 0 & -2.589387 & -4.878567 & 0.316682\end{array}$

O $-3.700054-2.904827 \quad 0.739826$

C -3.769936 $-5.410283-0.293167$

H $-4.599801-5.4121240 .420143$

H $-4.027607-4.831282-1.184785$

H -3.562902 -6.441224-0.592137

SCF Energy (B3LYP/6-31G**//MMFF) $=-3245.92402573$

06_00201

MMFF Geometry

C -2.101969 4.032167-1.214712

C $-2.6557762 .930609-0.686297$

C $-3.1822092 .849108 \quad 0.719562$

\begin{tabular}{llll}
\hline & -2.383403 & 1.855986 & 1.405342
\end{tabular}

C $-4.638476 \quad 2.363188 \quad 0.722634$

C $-5.2986842 .516992 \quad 2.077624$

C $-5.486673 \quad 1.5156562 .958910$

C $-5.804733 \quad 3.9018632 .390672$

$\begin{array}{llll}C & -5.050099 & 0.081499 & 2.840550\end{array}$

C $-6.236111-0.8743072 .649107$

C $-6.847170-0.8064081 .240541$

C $-8.097839-1.6828591 .151385$

C $-8.670839-1.714286-0.272741$

O $-5.858643-1.2738050 .317285$

O $-9.637303-2.774999-0.311417$

C $-7.535060-2.066824-1.256198$

C $-9.432962-0.425697-0.602119$

O $-7.991052-1.987662-2.605112$

C $-6.253011-1.222796-1.059124$

C $-5.103454-1.821789-1.903479$

O $-6.5080830 .114990-1.489265$

$\begin{array}{llll}\text { C } & -1.389792 & 2.283424 & 2.223457\end{array}$

$\begin{array}{lllll}\text { O } & -1.124176 & 3.443330 & 2.508097\end{array}$

C $-0.611708 \quad 1.1489832 .774238$

C $-0.677980-0.102666 \quad 2.293311$

C $0.104196-1.2518532 .758851$

C $1.031196-1.0411103 .927525$

C $-3.727233-1.188047-1.650520$

C $-2.624487-1.938603-2.379230$

C $-0.144869-2.158049-2.456212$

O $-2.846456-2.810548-3.215684$

C $0.185571-3.280858-1.522715$

C $1.324218-3.359942-0.818409$

$\begin{array}{lll}\text { C } 1.639786 & -4.478378 & 0.132075\end{array}$ 
C $1.861525-3.9964641 .549670$ C $3.103316-3.8855722 .051125$ C $0.647853-3.7390882 .416702$ C $-0.030582-2.4304732 .114289$ N -1.360529 -1.512860 -1.991678 H $-2.0460404 .942075-0.623410$ H -2.685890 $2.018291-1.277234$ H $-3.1078703 .822588 \quad 1.218464$ H -4.6787111 .3181600 .397427$ H $-5.2299292 .925425-0.012591$ H $-5.999567 \quad 1.7458763 .892156$ H -6.3114083 .9508123 .360500$ H -4.9781254 .6183432 .416151$ H $-6.521974 \quad 4.2246181 .628964$ H -4.307185 -0.064441 2.051133 H -4.540192 -0.1807683 .776231$ H -5.870939-1.893770 2.829409 H -7.005015 -0.666804 3.403665 H $-7.099827 \quad 0.2334611 .007051$ H -8.861166 -1.3481401 .864927$ H -7.843852 -2.710955 1.445918 H -9.950826 -2.854579-1.228759 H -7.285095 -3.123004-1.085483 H -10.254951 -0.2775600 .108613$ H -9.901760 -0.483292 -1.590569 H $-8.802426 \quad 0.465456-0.571233$ H -8.026489 -1.047404 -2.850512 H - $-5.021973-2.893666-1.678305$ H $-5.338871-1.730498-2.971539$ H $-5.6683710 .536185-1.730478$ H $0.065561 \quad 1.416363 \quad 3.579029$ H -1.349668 -0.3118371 .462307$ H $1.761184-0.2547213 .706547$ H $1.608366-1.9299704 .191126$ H $\quad 0.463687-0.752598 \quad 4.819050$ H -3.710472 -0.150936 -1.999564 H -3.495209 -1.197357 -0.579402 H $0.641025-1.397596-2.489913$ H $-0.298201-2.548273-3.467332$ H -0.557195 -4.068591 -1.417430 H $2.077343-2.585437-0.934276$ H $2.539369-4.986316-0.239884$ H $0.850950-5.2408470 .129628$ H $3.274301-3.5729273 .075620$ H $3.978320-4.0967021 .445127$ H $0.910474-3.8051593 .478616$ H - $0.077555-4.5497872 .269344$ H $-0.725002-2.4844681 .275729$ H -1.275932 -0.835604 -1.238933 C $0.0234593 .828689-2.586974$ O $0.3040562 .540511-1.998645$ C $1.2541222 .726999-0.928670$ C $1.9988093 .998214-1.295667$ C $0.8616954 .856761-1.820169$ H $0.6602642 .881208-0.018813$ C $2.1052251 .464340-0.790260$ H $2.5136674 .452380-0.444295$ H $2.7337763 .804762-2.086609$ C $1.3221866 .025436-2.674459$ H $\quad 0.3068735 .253238-0.961385$ C $3.273477 \quad 1.5977780 .208855$ O $1.2095930 .428093-0.379241$ H $2.4895011 .175188-1.776116$ H $3.9308772 .413644-0.114408$ O 2.7794541 .9335281 .505487 C 4.1275380 .3191610 .323638 C $\quad 5.360574 \quad 0.518418 \quad 1.224141$ H $4.4503370 .029387-0.681449$ $\begin{array}{llll}\text { O } & 3.349437 & -0.755975 & 0.840897\end{array}$ H 5.9472931 .3569530 .833321 H $\begin{array}{llll}5.036427 & 0.797936 & 2.234085\end{array}$ H $1.9528736 .703773-2.090594$ H $1.9035085 .687029-3.538756$ H $0.4644816 .595136-3.046146$ C -1.492026 $4.066801-2.589919$ H $1.714235-0.400702-0.338446$ H 2.3924852 .8235291 .456951 H $3.012624-0.4822751 .710850$
H -1.966244 $3.294495-3.209633$ H -1.722952 $5.030575-3.057689$ H $\quad 0.3637173 .765298-3.628259$ C $7.416421-0.538199-0.757116$

O $6.534317-1.3196750 .056402$

C $6.237271-0.750141 \quad 1.334127$

C $7.533372-0.5598542 .135364$

C 8.5836150 .2216561 .346350

C $8.766769-0.366696-0.054733$

H $6.9695840 .444600-0.952233$

C $7.565040-1.263698-2.097774$

H $5.637680-1.4987301 .867616$

O $7.2783880 .107415 \quad 3.366544$

H $7.949083-1.5451892 .383283$

H 8.3191651 .2838451 .289282

O 9.8326270 .1721952 .040709

H $9.282165-1.3330320 .014898$

O $9.5906790 .505549-0.824416$

C $6.292375-1.138160-2.902562$

H $8.371776-0.838725-2.705697$

H $7.794839-2.326697-1.957219$

H $6.622340-0.4193693 .854049$

H $10.097160-0.7603422 .117903$

H $10.4240880 .623195-0.337036$

O $5.482443-2.210288-2.670865$

O $6.035710-0.181161-3.621890$

C $4.219408-2.184129-3.343253$

H $4.362517-2.099594-4.424756$

H $3.702865-3.124023-3.131944$

H $3.611444-1.355798-2.967059$

SCF Energy $($ B3LYP/6-31G**//MMFF $)=-3245.91920196$

06_00202

MMFF Geometry

C $0.314731-1.4630182 .136177$

C $0.873905-2.4678971 .443659$

C $0.400293-2.9732330 .099384$

O $-0.354065-1.955475-0.599018$

C $-0.501072-4.2054110 .280706$

C $-0.988514-4.781367-1.034644$

C $-2.255361-4.706715-1.489802$

C $0.059031-5.500151-1.847273$

C $-3.449755-4.066412-0.837075$

C $-3.964803-2.873865-1.653926$

C $-5.254078-2.288068-1.059542$

C $-5.791200-1.151452-1.928450$

C -7.049839-0.516407-1.316148

O $-4.965226-1.7927290 .251114$

O $-7.3006030 .715062-2.012358$

C $-6.767961-0.1542400 .159745$

C $-8.291652-1.391645-1.526867$

$\begin{array}{lllll}\text { O } & -7.972056 & 0.282103 & 0.787057\end{array}$

C $-6.107787-1.2981570 .963876$

C $-5.638997-0.8831182 .384850$

O $-7.064791-2.3523851 .146651$

C $0.361316-1.023094-1.286065$

O $1.574757-0.962424-1.401205$

C $-0.594428-0.049830-1.853273$

C $-0.1491651 .161064-2.218271$

C $-0.9641392 .255967-2.745490$

C $-2.3681431 .927880-3.182839$

C -4.9772880 .4859562 .568927$

C -3.6512530 .6991241 .861887$

C -2.0127412 .5227211 .373242$

O $-3.055954-0.179516 \quad 1.248338$

C -2.4335113 .4189690 .251900$

C -1.898689 $4.627210 \quad 0.020076$

C $-2.3210735 .522865-1.110456$

C $-1.2106405 .766580-2.109719$

C $-0.4398576 .864407-2.032708$

C $-1.0521324 .777142-3.245724$

C $-0.4188603 .489609-2.795359$

N -3.2043572 .0060962 .020129$

H $-0.578149-0.9806501 .750111$

H $1.753262-2.962307 \quad 1.849526$

H $1.280385-3.243016-0.497878$

H -1.345973 -3.940663 0.924289

H $0.043213-4.9896310 .823859$ 
H $-2.477251-5.170350-2.450776$ H $-0.361155-5.977371-2.739118$ H $0.833685-4.803727-2.182529$ H $\quad 0.530867-6.285911-1.248309$ H $-4.237867-4.826255-0.763821$ H $-3.240228-3.7466070 .186234$ H -3.186965 -2.101874 -1.674767 H $-4.148617-3.184858-2.689843$ H $-5.991668-3.096160-0.988541$ H $-5.995719-1.501660-2.947839$ H -5.021940 -0.372526 -2.027205 H -7.413792 $0.508082-2.955915$ H -6.0922430 .7097440 .135942$ H -8.463574 -1.560688 -2.596735 H -9.193956 -0.893422 -1.155143 H $-8.214539-2.369366-1.047134$ H -7.7563680 .5713061 .688761$ H -6.505406 -0.9123123 .060013$ H -4.948853 -1.652235 2.758669 H $-6.761687-2.9140381 .879767$ H -1.636617 $-0.336734-1.914652$ H $0.9074881 .389078-2.085482$ H $-2.9068482 .780686-3.599843$ H -2.964931 $1.560825-2.341199$ H $-2.3535531 .155207-3.960001$ H $-5.667449 \quad 1.2842982 .275651$ H -4.7832940 .6202893 .641375$ H -1.4100571 .6963240 .984509$ H -1.436418 3.0577962 .134188 H -3.210803 $3.050105-0.412915$ H -1.1191195 .0004130 .680110$ H -2.651921 $6.475569-0.676544$ H -3.198792 5.121433-1.632290 H $0.3402527 .060147-2.760804$ H $-0.5687797 .589129-1.235464$ H $-0.4100145 .199970-4.029768$ H -2.023356 $4.631643-3.725973$ H $0.6104853 .613221-2.455260$ H -3.7764652 .6634672 .542638$ C 1.0114420 .6021843 .350287 O $1.913461 \quad 0.952602 \quad 2.285121$ C 2.7416722 .0502342 .711741 C 2.1687072 .5229524 .043610 C 1.6045391 .2317794 .608062 H 3.7415851 .6361872 .887333 C 2.8100593 .1153451 .609499 H 2.9312672 .9756684 .684566 H 1.3670503 .2575953 .906270 C 0.6000961 .4447425 .726532 H 2.4361730 .6182674 .979883 C 3.1681412 .5595140 .216446 O $1.5322953 .754940 \quad 1.489892$ H 3.5150153 .9032421 .897653 H $2.3449231 .939413-0.156972$ O $3.2810363 .664720-0.688211$ C 4.4758131 .7513740 .164938 C $4.7669701 .216281-1.246731$ H 4.4037770 .9067870 .851790 O 5.5636402 .5600010 .606306 H $3.8866240 .665918-1.594749$ H $4.9120132 .059581-1.932863$ H 1.0670781 .9658646 .568652 H -0.2529012 .0444575 .391240$ H $\quad 0.215476 \quad 0.488037 \quad 6.093265$ C $0.859658-0.9186333 .426801$ H 0.8815043 .0678321 .266043 H $2.4436074 .157897-0.647790$ H 5.6099013 .3347260 .020121 H $0.173321-1.1848174 .238101$ H $1.829952-1.3779493 .647152$ H 0.0444751 .0638353 .112428 C $5.025732-1.761193-0.578111$ O $6.008655-0.743814-0.354504$ C $6.021450 \quad 0.315349-1.317864$ C $6.283988-0.261388-2.716070$ C $5.268967-1.344018-3.073201$ C $5.194906-2.394278-1.966942$ H $4.024190-1.329025-0.476793$
C $5.204858-2.8304550 .513341$

H $6.8791440 .949295-1.060337$

O $6.2441890 .760128-3.706806$

H $7.288643-0.702578-2.747144$

H $4.279523-0.919641-3.278965$

O $5.680770-1.995058-4.277850$

H $6.105475-3.007044-1.990324$

O $4.092043-3.263752-2.219998$

C $4.806460-2.3499891 .892259$

H $4.587466-3.7082560 .291827$

H $6.258960-3.1297530 .560886$

H $6.9048551 .430318-3.461608$

H $5.760546-1.309505-4.963268$

H $4.211919-3.632199-3.112199$

O $5.122800-3.3179572 .803612$

O $4.274364-1.2791222 .145025$

C $4.793899-3.0152534 .163217$

H $3.707907-2.9650324 .282712$

H $5.179545-3.8212124 .793306$

H $5.261527-2.0753194 .471991$

SCF Energy (B3LYP/6-31G**//MMFF) $=-3245.91139005$

06_00203

MMFF Geometry

C -1.5599853 .8520421 .288000$

C $-0.9544732 .667473 \quad 1.119381$

C -0.5367161 .7751652 .257579$

O 0.8088291 .3142422 .002467

C -1.465260 0.5517952 .305663

C $-1.179089-0.3650613 .476512$

C $-0.521133-1.5369383 .381560$

C -1.7354110 .0884764 .800918$

C $0.088087-2.1370162 .145653$

C $1.485999-2.7076182 .418853$

C $2.094611-3.3117611 .145494$

C $3.376236-4.0810071 .465840$

C $4.038752-4.6304330 .193879$

O $2.384861-2.2393720 .242284$

O $5.364630-5.060380 \quad 0.537177$

C $4.184265-3.482194-0.824708$

C $3.307714-5.864542-0.347941$

O $4.659781-3.968658-2.077990$

C $2.887704-2.662439-1.031832$

C $3.206025-1.401408-1.868019$

O $1.932631-3.457727-1.735471$

C 1.8279252 .1198172 .404736

O $1.735823 \quad 3.1905102 .983527$

C 3.0937641 .4798491 .982953

C 4.2275722 .1956862 .012536

C 5.5488621 .7459341 .570377

C $5.7101300 .293667 \quad 1.209906$

C $2.073065-0.369173-1.929196$

C $2.5500170 .870525-2.660517$

C $4.0285662 .863966-2.362040$

O $2.283887 \quad 1.062799-3.844435$

C $5.5006842 .627882-2.219782$

C $6.3429323 .498855-1.642730$

C $7.8170133 .250581-1.484434$

C $8.3090813 .485079-0.070998$

C 9.0977194 .5337560 .219509

C 7.9486762 .4659010 .992275

C 6.5434632 .6551151 .499396

N $3.3301951 .693116-1.863406$

H -1.754864 4.2187442 .292550

H -0.7857182 .2941090 .111587$

H -0.5740612 .3085813 .215661$

H -1.397395 -0.000816 1.362309

H -2.5114390 .8795632 .371538$

H - $0.414785-2.1385104 .283192$

H $-1.545211-0.6345065 .601283$

H -1.2846491 .0388505 .102740$

H -2.819680 0.2235974 .730846

H $-0.566107-2.946667 \quad 1.805447$

H $0.154815-1.4105411 .331486$

H $2.134298-1.9063612 .796531$

H $1.418302-3.4768673 .198008$

H $1.353732-3.9795880 .689823$

H $3.177182-4.8939762 .175127$ 
H $4.088335-3.4112581 .968205$ H $5.289898-5.7367921 .232038$ H $4.961238-2.805864-0.440846$ H $3.291627-6.6612340 .405567$ H $3.830251-6.283704-1.214888$ H $2.273844-5.660154-0.633951$ H $5.479895-4.462037-1.904958$ H $4.090502-0.910151-1.447135$ H $3.467200-1.691181-2.894276$ H $1.343973-2.872297-2.236735$ H 3.0511460 .4589101 .620820 H 4.1803003 .2277892 .358280 H $5.336426-0.3498432 .014689$ H $6.748988-0.0017141 .048021$ H $\quad \begin{array}{llll}5.158344 & 0.064584 & 0.295004\end{array}$ H $1.200574-0.763039-2.459404$ H $1.736722-0.079461-0.927229$ H $3.6891963 .720205-1.770945$ H $3.7764483 .035384-3.412805$ H $5.8927001 .691710-2.611208$ H $5.9567424 .446588-1.274289$ H $8.3412883 .917126-2.181427$ H $8.0827742 .229977-1.787066$ H $9.473856 \quad 4.696653 \quad 1.224212$ H $9.3812195 .256103-0.538823$ H 8.6266102 .5609471 .850364 H $8.125903 \quad 1.4676370 .583439$ H $6.330017 \quad 3.6770701 .817174$ H $3.4680021 .445629-0.888422$ C $-3.5571254 .522579-0.037494$ O $-3.8199403 .179262-0.493042$ C $-4.8126403 .222265-1.533224$ C $-5.3840524 .634662-1.508944$ C $-4.1655885 .439674-1.095768$ H $-4.2839613 .078618-2.482591$ C $-5.8326712 .097689-1.324844$ H $-5.7900474 .934856-2.479678$ H $-6.1798654 .739454-0.762382$ C $-4.4991536 .832004-0.590108$ H -3.488749 $5.521388-1.956857$ C $-5.2111700 .684735-1.217909$ O $-6.5570102 .346106-0.112772$ H -6.577577 2.121143 -2.128585 H $-4.6407480 .608751-0.285962$ O $-6.307155-0.230908-1.114214$ C $-4.3388710 .297325-2.424936$ C $-3.751520-1.128432-2.424918$ H $-3.5032120 .999281-2.514145$ O $-5.1147940 .441178-3.620351$ H $-4.560026-1.862635-2.354384$ H -3.318125 -1.283845 -3.421172 H $-4.9922417 .417362-1.373067$ H $-5.170163 \quad 6.7951110 .274674$ H -3.592064 $7.365981-0.290128$ C -2.050263 4.6995490 .147004 H -5.9074592 .4079160 .608487$ H -5.954095 -1.083370 -0.813658 H $-5.880194-0.154491-3.544967$ H $-1.804692 \quad 5.745577 \quad 0.360645$ H -1.524801 4.423129 -0.775790 H -4.0799414 .6422000 .920836$ C -3.873204 -2.823210 0.160918 O $-3.214026-1.568084-0.044508$ C $-2.667348-1.356017-1.348759$ C $-1.672563-2.477813-1.680953$ C $-2.314569-3.854206-1.548770$ C $-2.958391-4.007748-0.173560$ H $-4.776951-2.869461-0.456337$ C $-4.296904-2.8809351 .633487$ H -2.081049 -0.429973 -1.288416 O $-1.149155-2.303068-2.993041$ H $-0.823369-2.413453-0.988687$ H -3.045217 -4.026705 -2.347278 O $-1.311564-4.860311-1.711087$ H -2.175832 -4.1074170 .587572$ O $-3.725040-5.210547-0.146879$ C $-5.403890-1.896788 \quad 1.921788$ H $-4.671492-3.8728381 .911028$
H $-3.449422-2.6662232 .295788$

H $-0.612723-3.086749-3.202424$

H $-0.631169-4.720839-1.030234$

H $-3.129137-5.940483-0.387802$

$\begin{array}{llll}\text { O } & -4.889591 & -0.767775 & 2.485505\end{array}$

O $-6.581739-2.1041951 .659941$

C $-5.835787 \quad 0.2552132 .810154$

H $-6.375989-0.0217863 .719657$

H $-5.284627 \quad 1.1813292 .993505$

H -6.5348040 .4234881 .985862$

SCF Energy (B3LYP/6-31G**//MMFF) $=-3245.92156160$

06_00204

MMFF Geometry

C $-2.153058 \quad 4.702456-0.779324$

C $-2.7895873 .565867-0.462773$

C $-3.462594 \quad 3.314442 \quad 0.859335$

O $-2.859912 \quad 2.127901 \quad 1.428423$

C -4.9636203 .0509090 .645596$

C $-5.7383302 .766361 \quad 1.921305$

$\begin{array}{llll}\text { C }-6.487828 & 1.663361 & 2.123551\end{array}$

C $-5.662033 \quad 3.8236692 .992158$

C $-6.7309480 .547116 \quad 1.148258$

C $-5.797220-0.6405631 .406599$

C $-5.682152-1.547106 \quad 0.172995$

C $-4.903724-2.8180670 .513041$

C $-4.611266-3.670653-0.728341$

O $-4.992786-0.793489-0.830300$

O $-3.628217-4.644773-0.344818$

C $-3.988412-2.772466-1.819575$

C $-5.847498-4.461876-1.171162$

O $-3.828328-3.522279-3.021088$

C $-4.789785-1.476653-2.073854$

C $-4.078687-0.478839-3.024316$

O $-6.041552-1.815343-2.682479$

$\begin{array}{llll}\text { C } & -1.783500 & 2.308189 & 2.238857\end{array}$

O $-1.288210 \quad 3.376792 \quad 2.570844$

C -1.2669991 .0210512 .752900$

C $-1.418496-0.1477922 .110960$

C $-0.935443-1.4508822 .578246$

C $-0.309502-1.5173373 .947380$

C $-2.724433 \quad 0.047111-2.535910$

C $-1.547862-0.815333-2.952764$

C $0.745807-1.458107-2.231963$

O $-1.567640-1.535102-3.948130$

C $0.524025-2.775113-1.556818$

C $1.196460-3.178859-0.469730$

$\begin{array}{lllll}\text { C } & 0.967094 & -4.499275 & 0.205543\end{array}$

C $0.658847-4.3582931 .679880$

C $1.580339-4.6736752 .605578$

C $-0.741283-3.9593682 .088097$

C $-1.082978-2.5276561 .778282$

N $-0.461028-0.663113-2.102634$

H $-2.1357835 .521373-0.065642$

H -2.798871 $2.748700-1.178910$

H -3.3319324 .1709941 .531805$

H $-5.0625682 .218531-0.058790$

H -5.4223843 .9220570 .159324$

H $-6.996554 \quad 1.548163 \quad 3.079277$

H -6.3730893 .6421053 .805518$

H $-4.6610593 .856788 \quad 3.432872$

H $-5.894051 \quad 4.807474 \quad 2.571326$

H -7.7722970 .2159201 .250280$

H -6.6501630 .9046970 .117508$

H $-4.796727-0.2709261 .667761$

H $-6.170633-1.2111382 .265783$

H $-6.688150-1.795215-0.185171$

H $-5.435315-3.4088101 .269535$

H $-3.948287-2.5405470 .975373$

H $-3.379157-5.141723-1.143281$

H $-2.981718-2.517867-1.467358$

H $-6.163346-5.149069-0.376959$

H $-5.626298-5.091154-2.040184$

H $-6.700259-3.824856-1.415025$

H $-3.293870-2.992058-3.635435$

H $-4.008396-0.900022-4.034255$

H $-4.7347230 .398193-3.123170$

H $-6.387590-1.029218-3.136437$ 
H $-0.711522 \quad 1.096902 \quad 3.682067$ H -1.943381 -0.1591301 .157098$ H -1.016149-1.174974 4.711290 H $0.588061-0.8905403 .990342$ H $0.001199-2.5241944 .234409$ H $-2.5467291 .030284-2.989627$ H $-2.7301860 .186527-1.449837$ H $1.565963-0.890572-1.780968$ H $0.968855-1.615735-3.291940$ H $-0.228500-3.430753-1.989176$ H $1.955706-2.528170-0.045490$ H $1.872158-5.1044490 .064874$ H $0.157092-5.060481-0.277171$ H $1.361828-4.6139323 .666360$ H $2.576534-4.9987132 .324589$ H $-0.915838-4.1693003 .150270$ H -1.458551 -4.605499 1.565616 H -1.532551 -2.386452 0.795516 H -0.562891 -0.101083 -1.262394 C $0.1007234 .852395-1.934469$ O $0.5037193 .583314-1.376011$ C $1.4550783 .830829-0.318828$ C $1.9775725 .237310-0.570156$ C $0.7234795 .937043-1.054269$ H 0.8806513 .7992610 .615808 C $2.5229072 .730920-0.324167$ H 2.4024215 .6985970 .326255 H $2.7437175 .234346-1.355421$ C $0.9935617 .240333-1.785664$ H $0.0928456 .148019-0.182556$ C 3.6975763 .0027430 .631472

O 1.8902961 .4980140 .036108 H $2.8914642 .598363-1.347258$ H 4.2338023 .9037330 .310552 O 3.1600693 .2915331 .929179 C 4.6974121 .8420080 .787407

C $5.2546681 .332594-0.552062$ H $4.226006 \quad 1.0292811 .349010$

O $\quad 5.7725242 .3315801 .596245$ H $4.4355090 .901689-1.136397$ H $5.6418192 .185545-1.123600$ H $0.0585697 .693012-2.130662$ H $1.4926667 .956109-1.124328$ H $1.6364107 .085704-2.658761$ C -1.423852 $4.900095-2.079675$ H 1.5930681 .5780250 .958926 H 3.9113883 .4740582 .519390 H 6.2740371 .5618991 .914748 H -1.728616 4.118489 -2.787755 H -1.731301 $5.857063-2.516347$ H $0.5403024 .891321-2.939665$

C $5.048547-1.6575610 .059667$ O $6.055175-0.7468420 .516273$ C $6.388622 \quad 0.293847-0.405274$

C $6.873085-0.307171-1.731342$

C $5.882279-1.320721-2.300257$

C $5.495684-2.347554-1.235220$

H $4.111351-1.115630-0.114993$

C $4.826070-2.6814521 .182262$

H 7.2448240 .8244440 .029986

O $7.1125650 .728121-2.678984$

H $7.832738-0.812244-1.559378$

H $4.998987-0.818971-2.712467$

O $6.514680-1.988576-3.394433$

H $6.348297-3.009448-1.036039$

O $4.426889-3.154342-1.723913$

C 4.083565 -2.076504 2.355606

H $4.236360-3.5375940 .839607$

H $5.798131-3.0484141 .534490$

H $7.3996310 .304442-3.506051$

H $5.847585-2.554775-3.817967$

H $4.765747-3.671384-2.473738$

O $4.546313-2.621353 \quad 3.518342$

O $3.181303-1.2550362 .260498$

C $3.914011-2.1487464 .712027$

H $2.850308-2.4042924 .703717$

H 4.386612 -2.642465 5.565242

H $4.050943-1.0676514 .811906$
SCF Energy (B3LYP/6-31G**//MMFF) $=-3245.92965040$

06_00205

MMFF Geometry

C $0.097880-1.8720881 .617193$

C -1.126611-1.804362 1.075922

C - $1.448551-2.284877-0.315014$

O $-1.641036-1.107934-1.133797$

C $-2.776442-3.057087-0.315340$

C $-2.987846-3.885491-1.565204$

C $-3.681477-3.486166-2.649134$

C $-2.396219-5.270673-1.514241$

C $-4.291327-2.139265-2.921446$

C $-5.823942-2.139778-2.853964$

C $-6.365478-2.056444-1.418262$

C $-7.895253-2.103541-1.418314$

C $-8.476633-1.902989-0.011572$

O $-5.915724-0.817645-0.856620$

O $-9.882313-1.659151-0.170528$

C $-7.841891-0.6422630 .613721$

C $-8.354558-3.1764680 .833081$

O $-8.259820-0.4836681 .967848$

C $-6.298970-0.6051190 .506572$

C -5.7739460 .7844710 .942465$

O $-5.755471-1.6021791 .374875$

C $-0.539857-0.470161-1.609372$

O $0.621477-0.834971-1.534255$

C $-1.0069030 .813363-2.174363$

C $-0.1438481 .833750-2.276094$

C $-0.4691113 .194394-2.706524$

C $-1.8884033 .472387-3.128987$

C -4.2879231 .0286390 .634578$

C $-3.7991692 .347875 \quad 1.211077$

C -1.722442 3.6922851 .515741

O $-4.537775 \quad 3.141140 \quad 1.789567$

C $-1.687844 \quad 4.7652380 .472245$

C $-0.589618 \quad 5.4842880 .191825$

C $-0.5503396 .596666-0.818613$

C $0.6028516 .469685-1.791042$

C $1.7337807 .174349-1.617132$

C $0.4127245 .588315-3.006159$

C $0.5044424 .127474-2.666184$

N -2.4374462 .5338641 .009292$

H $0.908971-2.3254311 .053696$

H -1.921268 -1.311302 1.630373

H $-0.640311-2.901070-0.728152$

H -3.608941 -2.355673 -0.195232

H $-2.827693-3.729800 \quad 0.551317$

H -3.794596 -4.193413 -3.470251

H -2.572142 -5.833796 -2.436839

H $-1.313485-5.217770-1.362457$

H -2.835821 $-5.841189-0.689579$

H $-3.880890-1.362072-2.272548$

H -3.992485 -1.856364 -3.939309

H -6.174872 -1.260607 -3.410499

H $-6.225784-3.025392-3.361094$

H -5.959769 -2.890231 -0.834892

H $-8.256277-3.043812-1.853515$

H -8.282954 -1.306259-2.068475

H -10.247253 -1.455526 0.708044

H -8.2491050 .2215340 .070422$

H -7.320404 -3.4914830.987206

H -8.886690 -4.005235 0.350431

H -8.827922 -3.0503291 .812946$

H -7.754315 -1.113751 2.509163

H -6.3462541 .5672080 .427304$

H -5.9417670 .9148522 .019295$

H -4.872883 -1.326932 1.667774

H $-2.0588240 .902995-2.420299$

H $0.8913831 .670352-1.978790$

H $-2.1544592 .862317-3.999382$

H -2.068056 $4.512699-3.406202$

H $-2.586028 \quad 3.244837-2.315157$

H -3.6712000 .2314061 .058365$

H -4.125485 $1.050321-0.449101$

H $-0.7195003 .357073 \quad 1.796057$

H -2.231380 4.0653432 .410268

H $-2.6130934 .982939-0.055920$ 
H $\quad 0.325050 \quad 5.2911550 .747664$ H $-0.4663747 .542184-0.266753$ H -1.491669 $6.662815-1.378212$ H $2.5550267 .109389-2.323067$ H $1.8656827 .827964-0.761075$ H $1.1910745 .797519-3.752113$ H $-0.5251825 .857929-3.498833$ H $1.4993703 .823118-2.338902$ H -1.914798 1.8493690 .470820 C $0.859854 \quad 0.2048102 .764561$ O $2.042381 \quad 0.291991 \quad 1.948532$ C 2.8431731 .3965142 .409983 C 2.0574352 .0646793 .535314 C 1.2226240 .9138244 .064667 H $3.759130 \quad 0.9668832 .830174$ C 3.1931482 .3194091 .236616 H 2.7154532 .5004984 .293605 H 1.4106032 .8662963 .161420 C 0.0250331 .3620374 .883830 H 1.8633810 .2676514 .679431 C 3.8614381 .6124660 .040082 O 1.9959342 .9372630 .746277 H 3.8294083 .1385571 .590716 H $3.1365110 .969867-0.474443$ O $4.2409512 .623780-0.902632$ C 5.1105250 .7867530 .397477 C $5.8144820 .217415-0.848769$ H $4.818307-0.0389101 .053514$ O $\quad 6.0306041 .5984551 .125926$ H $5.080708-0.341627-1.439487$ H $6.1715461 .043955-1.474938$ H $\quad 0.3501901 .9369685 .757187$ H -0.6490431 .9948684 .296608$ H -0.5483640 .5014635 .241768$ C $0.447724-1.2574722 .942621$ H 1.3834972 .2278130 .486367 H $3.4425033 .137911-1.112633$ H $\quad 6.2666382 .3540950 .560907$ H -0.403775 -1.3334063 .628479$ H $1.268110-1.8378263 .380833$ H $\quad 0.069061 \quad 0.7577882 .239203$

C $5.837800-2.747152-0.119975$ O $6.687858-1.7240030 .413263$ C $7.025278-0.678111-0.501388$

C $7.744012-1.267140-1.722894$

C $6.931313-2.382392-2.373658$

C $6.498555-3.416528-1.333151$

H $4.873614-2.311748-0.409728$

C $5.587453-3.7520361 .009803$

H $7.749792-0.0399010 .019991$

O $8.015914-0.258044-2.689701$

H $8.711124-1.681786-1.409611$

H $6.069188-1.976081-2.915842$

O $7.752145-3.024723-3.353003$

H $7.376666-3.998656-1.024587$

O $5.585234-4.311172-1.965440$

C $4.681192-3.1572972 .061029$

H $5.095530-4.6630160 .653218$

H $6.527715-4.0615531 .482113$

H $8.5516250 .426828-2.254132$

H $7.229230-3.749132-3.737629$

H $5.486331-5.090606-1.394027$

O $5.418339-2.669003 \quad 3.098179$

O $3.461963-3.1156691 .958988$

C $4.665750-2.0745524 .159982$

H $5.370017-1.6572354 .884359$

H $4.036383-1.2662683 .778041$

H $4.055943-2.8341234 .658221$

SCF Energy (B3LYP/6-31G**//MMFF) $=-3245.92320723$

0600206

MMFF Geometry

C $-2.0712243 .077131-1.207709$

C - $1.7015362 .469741-2.345381$

C $-1.9622491 .011880-2.625244$

O $-0.6991180 .416428-3.000163$

C $-2.9536290 .838485-3.787968$

C $-3.574110-0.546768-3.808007$
C $-4.810700-0.828631-3.350797$

C $-2.722215-1.641198-4.396261$

C $-5.8018990 .120613-2.735592$

C $-5.4651750 .624311-1.324682$

C $-5.420393-0.458110-0.233548$

C $-5.0797510 .175626 \quad 1.121144$

C $-4.947215-0.8716382 .238027$

O $-4.432350-1.440553-0.564434$

O $-4.304202-0.2463323 .359208$

C $-4.010605-2.0000311 .754885$

C $-6.316058-1.3463642 .739019$

O $-3.968649-3.0692332 .695683$

C $-4.377631-2.5382770 .357100$

C $-3.367068-3.577645-0.175488$

O $-5.642337-3.2116870 .432512$

C $0.1100420 .014552-1.982820$

$\begin{array}{lllll}\text { O } & -0.144751 & 0.036077 & -0.788997\end{array}$

C $1.388820-0.432224-2.576089$

C $2.370016-0.830277-1.755028$

C $3.704866-1.280207-2.140395$

C $4.038233-1.346458-3.605897$

C $-1.914556-3.112827-0.318894$

C $-1.032447-4.250980-0.811203$

C $1.313531-4.961599-1.090467$

O $-1.489566-5.287544-1.289982$

C $2.657973-4.648978-0.515170$

C $3.794852-4.848724-1.201063$

C $5.176820-4.593148-0.664345$

C $5.982818-3.683054-1.568068$

C $6.745098-4.191400-2.551216$

C $5.951536-2.192635-1.310117$

C $4.560671-1.643278-1.163686$

N $0.324034-3.989854-0.659416$

H $-2.6333022 .503899-0.473542$

H -1.151008 $3.020333-3.102886$

H $-2.3505570 .505563-1.732413$

H $-2.4534241 .013535-4.749610$

H $-3.744518 \quad 1.594509-3.718715$

H -5.158265 -1.859136 -3.412703

H -3.267912 -2.586303 -4.489398

H $-2.380951-1.361569-5.398225$

H -1.847167 -1.830723 -3.767953

H $-5.9352860 .981617-3.401978$

H $-6.779491-0.377472-2.708559$

H $-4.5096731 .153055-1.352661$

H $-6.2201451 .370434-1.045314$

H -6.406638 $-0.935652-0.196548$

H $-5.8224850 .935676 \quad 1.394123$

H $-4.122527 \quad 0.7097761 .038273$

H -4.8337140 .5281453 .615631$

H $-2.999463-1.5767541 .717506$

H -6.883736 -0.5049313 .154595$

H $-6.209519-2.0677833 .556664$

H $-6.928114-1.7984381 .956048$

H -3.727600 -2.687581 3.557187

H $-3.404462-4.4756470 .456267$

H -3.711083 -3.909590 -1.165956

H $-5.723560-3.801955-0.334948$

H $1.494467-0.403112-3.654133$

H $2.175356-0.832415-0.683875$

H $3.967485-0.352700-4.061485$

H $3.351221-2.022361-4.126962$

H $5.049479-1.713069-3.800436$

H -1.846111 -2.289778 -1.037263

H -1.523263 -2.773635 0.644103

H $1.321361-4.958212-2.186034$

H $0.989084-5.951927-0.752348$

H $2.705642-4.2765880 .504833$

H $3.733328-5.252171-2.209944$

H $5.684339-5.562269-0.570360$

H $5.146038-4.1773280 .350434$

H $7.340570-3.551707-3.194034$

H $6.788103-5.259778-2.736041$

H $6.495628-1.636596-2.082979$

H $6.494084-1.993030-0.376627$

H $4.232629-1.572041-0.127295$

H $0.622582-3.046292-0.427835$

C $-0.3725145 .003066-1.244124$ 
O $0.657510 \quad 4.149745-0.704566$

C $1.7307494 .967495-0.204689$

C $1.4575816 .379254-0.712570$

C $-0.058127 \quad 6.406374-0.724811$

H 1.6442404 .9659890 .888392

C $3.0865604 .386590-0.621586$

H $1.8967787 .144502-0.065273$

H $1.8473226 .531242-1.725702$

C $-0.641073 \quad 7.524610-1.570901$

H $-0.413848 \quad 6.5151920 .308680$

C $3.3019562 .915698-0.209270$

O $3.1831694 .457111-2.050087$

H $3.8910065 .016943-0.223752$

H $2.6366212 .260163-0.784035$

O $4.6408182 .551812-0.570561$

C 3.1169432 .6330851 .294765

C 3.5206911 .1956201 .671970

H 2.0708022 .8002171 .565284

O 3.8999523 .5498032 .058587

H 3.0145030 .5040380 .994765

H 4.5993781 .0730011 .517280

H $-0.3268248 .500805-1.187370$

H $-0.3134977 .451912-2.613433$

H $-1.7350577 .493359-1.558616$

C $-1.7460124 .489618-0.801822$

H $4.1091664 .285021-2.289886$

H $4.6761062 .492677-1.540218$

H 4.8285573 .4361901 .792261

H $-2.5426705 .151783-1.158494$

H -1.7800284 .5138870 .294982$

H $-0.2761694 .975258-2.336330$

C 0.9349280 .0416712 .825324

$\begin{array}{lllll}\text { O } & 1.808583 & 0.991194 & 3.448029\end{array}$

C 3.1970880 .8338953 .138301

C $3.668195-0.5679753 .553168$

C $2.804102-1.6647512 .937455$

C $1.324711-1.3936563 .205364$

H $\quad 0.9736790 .163578 \quad 1.737116$

C $-0.4942220 .351935 \quad 3.297898$

H $3.734168 \quad 1.5534803 .768674$

O $5.026933-0.7798303 .183022$

H $3.615051-0.6586254 .646128$

H $3.001705-1.7680621 .865501$

O $3.171461-2.9098853 .536158$

H $1.106376-1.5753124 .265579$

O $0.534816-2.3022582 .441174$

C -1.031598 1.6439702 .720960

H $-1.185249-0.4425582 .998147$

H $-0.501614 \quad 0.443500 \quad 4.390845$

H $5.556534-0.0815233 .604274$

H $2.699674-3.6148033 .061716$

H $\quad 0.710461-3.1986532 .772869$

O $-2.137398 \quad 2.0175693 .430406$

O $-0.5634412 .236614 \quad 1.759400$

C -2.780344 3.2182122 .992252

H -3.571232 3.4598823 .707219

H -2.068593 4.0488002 .963826

H -3.233020 3.0612582 .009412

SCF Energy (B3LYP/6-31G**//MMFF) $=-3245.89929422$

0600207

MMFF Geometry

C $-0.120758-0.6690672 .596858$

C $-1.075801-1.6055002 .714461$

C $-1.532847-2.5526551 .627557$

O $-0.919381-2.2042390 .366375$

C $-3.056705-2.4864221 .433440$

C $-3.589417-3.719438 \quad 0.728330$

C $-3.886565-3.795091-0.583306$

C $-3.816677-4.9150251 .618997$

C $-3.739984-2.728146-1.630994$

C $-5.093326-2.306464-2.222036$

C $-5.998045-1.574094-1.218453$

C $-7.343725-1.225231-1.857228$

C $-8.226810-0.396011-0.913825$

O $-5.321882-0.380973-0.814692$

O $-9.3113330 .118588-1.701920$

C $-7.4070380 .803531-0.390436$
C $-8.863708-1.265004 \quad 0.176726$

$\begin{array}{lllll}\text { O } & -8.161990 & 1.515656 & 0.586646\end{array}$

C -6.0209620 .4020240 .163057$

C $-5.1214301 .617420 \quad 0.484233$

$\begin{array}{lllll}\text { O } & -6.194027 & -0.331413 & 1.380501\end{array}$

C $0.117167-2.951207-0.087576$

O $0.555477-3.9777490 .412493$

C $0.728819-2.345848-1.295470$

C $0.315782-1.190080-1.840184$

C $0.916928-0.506709-2.988417$

C $2.131592-1.133280-3.618755$

C $-4.8655452 .543371-0.705938$

C $-3.7466453 .523197-0.422205$

C $-1.2622603 .683528-0.307385$

O $-3.970868 \quad 4.708632-0.188931$

C $-0.7078543 .972156-1.667656$

C $0.5406233 .653190-2.039655$

C $1.1008173 .907951-3.408480$

C $1.7475872 .678532-4.011494$

C $3.0813662 .607845-4.161064$

C $0.8494461 .564518-4.506279$

C $0.380798 \quad 0.663036-3.395786$

N -2.493544 $2.932197-0.460313$

H $0.380906-0.5264651 .644285$

H $-1.531023-1.7589713 .691217$

H $-1.238199-3.5632711 .935110$

H $-3.324743-1.5784280 .881841$

H -3.567690 -2.404744 2.402081

H $-4.274059-4.739147-0.965189$

H $-4.228928-5.769844 \quad 1.072174$

H $-2.877661-5.2406292 .076714$

H $-4.523598-4.6636582 .416558$

H -3.199904 -1.851554-1.262401

H $-3.121624-3.140228-2.438389$

H $-4.887699-1.642048-3.071407$

H $-5.614033-3.186897-2.618962$

H $-6.154438-2.214631-0.343596$

H -7.870476 -2.132809 -2.177668

H $-7.171543-0.641145-2.772465$

H $-9.8397780 .701641-1.129962$

H $-7.2797321 .481661-1.243537$

H $-9.498119-2.037169-0.274976$

H $-9.525051-0.6734700 .819588$

H $-8.129667-1.7678570 .809631$

H -7.6996752 .3504380 .769961$

H $-5.5393432 .193679 \quad 1.319565$

H $-4.159096 \quad 1.2392120 .856326$

H $-5.368528-0.2828401 .890406$

H $1.573163-2.896771-1.696999$

H $-0.532581-0.671583-1.396665$

H $2.941223-1.208398-2.886247$

H $2.527245-0.566720-4.464034$

H $1.899073-2.135924-3.993933$

H $-4.6020571 .975767-1.606090$

H $-5.763507 \quad 3.123677-0.945202$

$\begin{array}{llll}H & -0.586497 & 3.069338 & 0.295069\end{array}$

H $-1.459098 \quad 4.619862 \quad 0.223470$

H $-1.3654744 .464748-2.380079$

H $1.2004893 .177101-1.320713$

H $1.8365574 .717786-3.320165$

H $0.3272314 .276388-4.093641$

H $3.5551961 .743738-4.614252$

H $3.7312863 .410616-3.828817$

H $1.3467140 .988262-5.294588$

H $-0.0286971 .998434-5.001566$

H $-0.4992491 .038181-2.873195$

H $-2.4167741 .953280-0.721630$

C 0.6280191 .6186113 .368632

$\begin{array}{llll}\text { O } & 1.665384 & 1.697842 & 2.367411\end{array}$

C 2.6809152 .6131522 .811932

C 2.0168623 .4636363 .886327

C $1.1213912 .444774 \quad 4.560396$

H 3.4735912 .0076903 .268298

C $3.2523183 .406401 \quad 1.631628$

H 2.7437403 .9159124 .567773

H 1.4096084 .2674223 .454602

C $0.002814 \quad 3.064018 \quad 5.380939$

H 1.7405691 .8218775 .220185 
C $3.7329052 .548298 \quad 0.443871$

$\begin{array}{lllll}\text { O } & 2.248764 & 4.299807 & 1.132286\end{array}$

H 4.0681524 .0482141 .983347

H $2.8804892 .064932-0.047452$

O $4.3031213 .438976-0.525176$

C 4.7818651 .4774860 .796919

C $5.363564 \quad 0.794917-0.457715$

H 4.3245250 .7242001 .444501

O $5.8458182 .076303 \quad 1.536828$

H $4.5364620 .424822-1.072497$

H $5.9002181 .544309-1.051622$

H $\quad 0.413146 \quad 3.682312 \quad 6.185878$

H $-0.642353 \quad 3.6992794 .764591$

H -0.62253422887425 .834851$

C $\quad 0.3862390 .154577 \quad 3.747585$

H $1.461738 \quad 3.7671590 .929131$

H $3.6200484 .092051-0.755500$

H 6.2380032 .7699090 .978759

H $-0.3098550 .099793 \quad 4.592633$

H $1.335229-0.2919604 .069532$

H -0.2754562 .0577122 .927304$

C $4.783281-2.198192-0.096995$

O $5.730566-1.400108 \quad 0.626341$

C $6.348011-0.348954-0.123059$

C $7.072630-0.933671-1.342404$

C $6.139327-1.782677-2.197399$

C $5.435752-2.830690-1.336688$

H $3.929437-1.578175-0.394322$

C $4.279066-3.2843940 .866967$

H 7.1212280 .0767960 .528478

O $7.6420100 .110062-2.125388$

H $7.903263-1.561740-0.994607$

H $5.422034-1.155374-2.738674$

O $6.922351-2.445725-3.193175$

H $\quad 6.168542-3.592635-1.040674$

O $4.452812-3.471270-2.147219$

C $3.466733-2.719466 \quad 2.011845$

H $3.636084-4.0013430 .346882$

H $5.137834-3.812681 \quad 1.298709$

H $8.067118-0.303594-2.896181$

H $6.318661-3.009606-3.706544$

H $4.166464-4.278500-1.688745$

O $3.260051-3.7071892 .932668$

O $3.050388-1.5722902 .083609$

C $2.498919-3.334524 \quad 4.085362$

H $2.906125-2.4290454 .545323$

H $1.451931-3.1910733 .805891$

H $2.559544-4.1506134 .810365$

SCF Energy (B3LYP/6-31G**/MMFF)= -3245.92895204

0600208

MMFF Geometry

C $0.448172-0.7915882 .505879$

C $1.123742-1.7795691 .898204$

C $0.662948-2.549998 \quad 0.682045$

O $-0.366697-1.823595-0.027444$

C $0.101432-3.9149701 .105497$

C $-0.268673-4.791864-0.074090$

C $-1.529174-4.996427-0.504889$

C $0.885105-5.492098-0.743425$

C $-2.786182-4.374496 \quad 0.037555$

C $-3.719532-3.909552-1.089857$

C $-4.982567-3.245927-0.524481$

C $-6.010555-2.993282-1.626386$

C $-7.260324-2.292832-1.072819$

O $-4.600581-1.9985120 .061865$

O -8.027814 -1.811609-2.187551

C $-6.825655-1.053443-0.260513$

C $-8.168223-3.270298-0.316780$

O $-7.932697-0.4755420 .426488$

C $-5.664960-1.3118330 .733690$

C $-5.105174 \quad 0.035497 \quad 1.235876$

O $-6.098131-2.0228021 .894262$

C $0.052246-0.873840-0.908894$

O $1.205226-0.605164-1.207709$

C $-1.144083-0.172227-1.422680$

C $-0.980188 \quad 1.000239-2.053407$

C $-2.0398261 .862454-2.578924$
C $-3.4461991 .327205-2.572196$

C $-3.849138-0.0981332 .105615$

C $-3.338840 \quad 1.2731432 .503945$

C -2.2631403 .3129091 .539981$

O $-3.495479 \quad 1.7155293 .639382$

C -2.9462734 .1121050 .474184$

C $-2.2984024 .907632-0.390585$

C $-2.9833895 .696582-1.470751$

C $-2.3592005 .494142-2.835711$

C $-1.6637406 .478937-3.429496$

C $-2.6020294 .176648-3.545502$

C $-1.7007993 .086531-3.035123$

N -2.732185 1.9424791 .453172

H $-0.541529-0.5159812 .152922$

H $2.104284-2.0533772 .280877$

H $1.524382-2.6979810 .018976$

H $-0.764513-3.7722821 .762334$

H $0.839816-4.4509761 .717386$

H -1.680513 -5.685927-1.334503

H $0.557420-6.155194-1.551131$

H $1.576641-4.763731-1.177378$

H $1.431979-6.103008-0.017805$

H -3.304376 -5.124904 0.646566

H -2.566895 -3.5239030 .688821$

H -3.182967 -3.198708-1.731701

H $-3.998597-4.772172-1.707803$

H -5.402072 -3.8990240 .249698$

H $-6.287136-3.929636-2.126757$

H $-5.566678-2.355175-2.403441$

H -8.240495 -2.571562 -2.756049

H $-6.486347-0.301056-0.986932$

H -8.516629 -4.065314 -0.987253

H $-9.072122-2.7705280 .048045$

H -7.671832 -3.7511680 .528692$

H -8.623953 $-0.298802-0.234627$

H -4.8669830 .6686780 .374464$

H $-5.8716080 .565838 \quad 1.816135$

H -7.037792 -2.241816 1.796371

H -2.116133 -0.612686-1.233636

H $\quad 0.031281 \quad 1.388909-2.169731$

H -3.486987 $0.337311-3.040878$

H $-4.1515411 .950338-3.125967$

H $-3.817710 \quad 1.244307-1.547833$

H $-4.063116-0.6658573 .017768$

H -3.049894 -0.627456 1.574762

H $-1.1781843 .291411 \quad 1.405255$

H -2.489575 3.7289222 .526022

H -4.0293204 .0319820 .411743$

H -1.218596 $5.008826-0.313123$

H -2.935826 $6.756005-1.187367$

H $-4.0506605 .448649-1.528292$

H -1.233252 $6.350224-4.417088$

H -1.504487 $7.433904-2.939921$

H -2.410765 4.287979-4.620662

H -3.663632 $3.930531-3.457575$

H $-0.6454283 .364530-3.027158$

H -2.594132 1.4537850 .573763

C 0.9829021 .5034543 .325252

O 1.7815521 .7504152 .152870

C 2.5874162 .9219602 .369139

C 2.0344453 .5853653 .625410

C 1.5770272 .3799324 .425537

H 3.6033412 .5650552 .577672

C 2.6021713 .7986941 .111190

H 2.7881254 .1856524 .143751

H 1.1800374 .2349353 .403459

C 0.5989082 .7207675 .536059

H $2.460096 \quad 1.8927204 .860367$

C $2.9083073 .031635-0.191934$

O 1.3105524 .3998130 .955780

H 3.3092544 .6251531 .249364

H $2.0628072 .376092-0.435779$

O $2.9787284 .009616-1.237235$

C $4.2151712 .217038-0.159338$

C $4.5087521 .509047-1.494255$

H $4.145600 \quad 1.4638620 .631193$

O $5.306677 \quad 3.073536 \quad 0.171784$

H $3.6314870 .915410-1.772189$ 
H $4.6570952 .257046-2.282026$ H 1.0591083 .4033226 .257961 H -0.3018873 .2042315 .142978$ H 0.2895241 .8188016 .073338 C 0.9962150 .0093543 .654074 H 1.3435424 .9551330 .157982 H $2.8844463 .542844-2.084595$ H $5.3521843 .767474-0.508309$ H $0.386829-0.1852254 .543404$ H $2.021389-0.3107193 .877640$ H -0.0380251 .8264483 .087403$ C $4.811589-1.339612-0.414774$ O $5.767186-0.279790-0.332331$ C $5.7703270 .616018-1.447353$ C $6.035419-0.159411-2.746835$ C $5.048667-1.312513-2.930970$ C $5.010993-2.186837-1.677062$ H $3.806111-0.902530-0.431660$ C $4.881824-2.1590230 .882503$ H $6.623291 \quad 1.289242-1.293313$ O $5.973005 \quad 0.727047-3.858882$ H $7.054304-0.567064-2.714024$ H $4.045758-0.950524-3.185943$ O $5.471426-2.130771-4.024091$ H 5.941327 -2.763292 -1.618795 O $3.940154-3.122314-1.785054$ C $6.210385-2.8474741 .121450$ H $4.714968-1.4980551 .741335$ H $4.093598-2.9204370 .869720$ H $6.2726680 .238058-4.643535$ H $5.384937-1.603094-4.835794$ H $4.073190-3.622360-2.608468$ O $6.024306-3.8781051 .998918$ O $7.279983-2.5099190 .633107$ C $7.201715-4.6171862 .338794$ H $7.637172-5.0701381 .442978$ H $7.927357-3.9654232 .834700$ H $6.915284-5.4136793 .030633$

SCF Energy (B3LYP/6-31G**//MMFF)= -3245.91965405

0600209

MMFF Geometry

C $-2.085636-3.427839-0.200565$

C -1.569257 -4.139506 0.812602

C $-0.856469-3.5905822 .027449$

O $-0.963754-2.1500672 .101483$

C $0.632788-3.9724621 .973086$

C $1.407128-3.6134073 .226823$

C $2.415746-2.7206073 .267110$

C $1.035567-4.3834594 .467673$

C $2.925872-1.8708662 .138520$

C $4.123679-2.5214691 .436610$

C $4.598567-1.6939960 .234625$

C $5.743653-2.395256-0.497757$

C $6.276388-1.548808-1.664579$

O $5.036489-0.4166120 .706082$

O $7.522803-2.133603-2.071286$

C $6.564960-0.118375-1.155506$

C $5.347761-1.628779-2.882692$

O $\quad 6.932711 \quad 0.740729-2.232356$

C $5.4040610 .494752-0.337717$

C 5.8444061 .8409960 .300559

O $4.314404 \quad 0.743593-1.231137$

C $-2.008673-1.6503882 .818459$

O $-2.881546-2.2895063 .386059$

C $-1.910216-0.1741702 .820870$

C $-2.552531 \quad 0.503023 \quad 3.783741$

C -2.5329921 .9494673 .961039$

C -2.1461702 .3700665 .352687$

C 5.1018942 .2335931 .587173

C 3.6017222 .3654771 .416484

C 1.4600451 .7608832 .522561

O 3.0930352 .8402550 .403923

C 0.8197623 .0435892 .940828

C 0.1169153 .8123112 .096543

C -0.6033195 .0660552 .493141$

C -2.069282 5.0224572 .120142

C -2.5259375 .6797881 .040707$
C $-3.0164384 .288884 \quad 3.043573$

C -2.886906 2.7892922 .967791

N $2.900277 \begin{array}{lll}1.907793 & 2.521169\end{array}$

H -1.990007 -2.346085 -0.208210

H -1.672833 -5.2229070 .788839$

H -1.314202 -4.045555 2.914269

H $1.089882-3.5076501 .094536$

H $\quad 0.732911-5.055378 \quad 1.817554$

H $2.934264-2.5686994 .212733$

H $1.721145-4.1868905 .299083$

H $0.029436-4.1140474 .802595$

H $1.062631-5.4600394 .270062$

H $2.130863-1.6485941 .419393$

H $3.224190-0.9041502 .558480$

H $4.942958-2.6254462 .159891$

H $3.852278-3.5296201 .099239$

H $3.747706-1.553831-0.439408$

H $5.432192-3.385617-0.852755$

H $6.568645-2.5749280 .205895$

H $7.904255-1.563654-2.761208$

H $7.449460-0.185397-0.507363$

H $5.253431-2.667726-3.220984$

H $5.760341-1.075573-3.733492$

H $4.341794-1.253722-2.682579$

H $6.1202970 .979940-2.710188$

H 6.9053591 .7792410 .575349

H $5.7545822 .648689-0.436755$

H $3.7954841 .492668-0.899586$

H -1.304443 0.3127692 .065520

H -3.111456 -0.033797 4.548394

H -2.9517102 .1421526 .058976$

H -1.916123 3.4348965 .433642

H $-1.244323 \quad 1.8376725 .677218$

H 5.3337361 .5073062 .374835

H 5.4731053 .2050621 .934997

H $1.2051990 .967116 \quad 3.231727$

H 1.1341991 .4435841 .525933

H $0.899236 \quad 3.3241673 .987774$

H 0.0511203 .5194181 .052337

H -0.1106155 .9088981 .991340$

H -0.5079165 .2615443 .568460$

H -3.578768 5.6759820 .778905

H -1.855011 6.2287420 .388225

H -4.0559124 .5313802 .783975$

H -2.8863234 .6734184 .059358$

H -3.1660432 .3632322 .004576$

H 3.4073141 .5799223 .337782

C $-4.239958-3.514447-1.542185$

O $-4.206342-2.110211-1.864751$

C $-5.186791-1.432118-1.057861$

C $-6.137289-2.517257-0.568193$

C $-5.170013-3.663313-0.335069$

H $-4.651418-1.018747-0.193820$

C $-5.837836-0.292875-1.848033$

H $-6.679272-2.2206300 .334638$

H $-6.871351-2.792749-1.334008$

C $-5.838582-5.023311-0.240429$

H $-4.633680-3.4696430 .601962$

C $-4.8370990 .670019-2.519581$

O $-6.660061-0.841582-2.885923$

H $-6.5164220 .266047-1.193387$

H $-4.3552350 .175673-3.372258$

O $-5.5805531 .768643-3.061075$

C $-3.7538381 .228151-1.580588$

C $-2.8357412 .243654-2.284360$

H $-3.1475780 .399509-1.202334$

O $-4.3667361 .850473-0.453023$

H -2.477882 $1.804246-3.221548$

H -3.413556 $3.138306-2.546881$

H $-6.535230-5.0515960 .603780$

H $-6.402690-5.258136-1.149210$

H -5.094080 -5.811978 -0.091809

C $-2.812573-4.042735-1.364945$

H $-6.094271-1.403817-3.442626$

H $-6.2550381 .396995-3.655291$

H $-4.9316922 .569352-0.784668$

H -2.233172 -3.810153 -2.267191

H $-2.840474-5.134628-1.270793$ 
H $-4.679973-4.010123-2.417318$

C $-0.1741200 .821109-1.869891$

O $-0.9058831 .563464-0.886569$

C -1.640929 2.677931-1.405370

C $-0.6824343 .662321-2.089831$

C $0.1831712 .976119-3.145306$

C $0.8506031 .728461-2.564045$

H $-0.8803840 .420352-2.605367$

C $0.492465-0.359473-1.147321$

H $-2.0671373 .189488-0.535315$

O $-1.4147174 .732828-2.676456$

H $-0.0245614 .104362-1.330825$

H $-0.4029742 .737543-4.040265$

O $1.1944093 .896433-3.562309$

H $1.6180272 .045510-1.848368$

O $1.5210421 .025602-3.605605$

C $0.715621-1.563953-2.037054$

H $1.452294-0.047600-0.719655$

H $-0.143440-0.686989-0.316650$

H $-0.7726615 .320078-3.110802$

H $\quad 1.765916 \quad 3.430362-4.196583$

H $0.8493950 .715026-4.236627$

O $1.457463-2.484153-1.355119$

O $0.282434-1.698112-3.172979$

C $1.751030-3.692728-2.062070$

H $\quad 0.825572-4.226468-2.297624$

H $2.314882-3.472010-2.973458$

H $2.365592-4.323306-1.414003$

SCF Energy (B3LYP/6-31G**//MMFF) $=-3245.94009300$

0600210

MM̄FF Geometry

C $-3.623944-1.977429-2.371151$

C $-2.506177-2.573945-1.928130$

C $-1.099533-2.134808-2.245092$

O $-1.110638-0.700549-2.440113$

C $-0.502121-2.807696-3.489682$

C $-0.236390-4.287098-3.321124$

C $0.863938-4.799262-2.735931$

C $-1.289058-5.204963-3.886422$

C $2.029864-4.055122-2.144501$

C $2.054985-4.188455-0.617215$

C $3.376863-3.700399-0.005123$

C $3.336218-3.8315661 .520209$

C $4.606796-3.2814522 .182954$

O $3.587870-2.334443-0.377341$

O $4.324917-3.1511953 .584672$

C $4.895997-1.8725151 .622908$

C $5.769788-4.2741372 .070133$

O $6.144897-1.3825362 .107163$

C $4.836318-1.7940000 .078990$

C $4.925240-0.318805-0.378964$

O $5.953490-2.511563-0.455472$

C $-0.0527730 .003560-1.962378$

O $0.995814-0.445763-1.530819$

C $-0.4314131 .433139-1.981777$

C $0.2916732 .307074-1.266845$

C $0.0035913 .730830-1.094472$

C -1.149495 $4.314306-1.867378$

C $4.630939-0.111915-1.870185$

C $5.1846021 .209565-2.362189$

C $4.8651293 .657977-2.261960$

O $6.1424001 .256767-3.131808$

C $4.3020674 .645806-1.292533$

C $3.5309975 .680902-1.658850$

C $2.9821476 .699777-0.700485$

C $1.4751976 .811807-0.774104$

C $0.8918447 .744676-1.545629$

C 0.6471615 .9013700 .103962

C $0.7799164 .448742-0.256461$

N $4.5193482 .311439-1.846736$

H -3.550438 -1.156143 -3.079735

H -2.594274 -3.406353 -1.233874

H $-0.493528-2.361226-1.359022$

H $0.426028-2.295104-3.772660$

H - $1.158718-2.640983-4.354684$

H $\quad 0.952965-5.883499-2.674837$

H - $-1.028717-6.261754-3.763547$
H $-2.250998-5.045215-3.391112$

H -1.415282 $-5.020655-4.958393$

H $2.947511-4.479885-2.569835$

H $2.022923-2.997587-2.423733$

H $1.222279-3.605091-0.205539$

H $1.892233-5.234304-0.327010$

H $4.187038-4.312927-0.418590$

H $3.170096-4.8749441 .816575$

H $2.474061-3.2735731 .910120$

H $5.098064-2.7350114 .003216$

H $4.133231-1.2008952 .036913$

H $5.507194-5.2239442 .551673$

H $6.657217-3.9091522 .598693$

H $6.048257-4.4921871 .036995$

H $6.850557-1.8221551 .602841$

H $4.202901 \quad 0.2840910 .186639$

H $5.924090 \quad 0.074417-0.149575$

H $6.224888-2.110754-1.295718$

H $-1.3249481 .714364-2.527272$

H $\quad 1.162167 \quad 1.940147-0.724663$

H -1.011174 $4.154207-2.942661$

H -1.263544 $5.391745-1.727120$

H -2.093122 $3.849844-1.562276$

H $5.075705-0.900661-2.485887$

H $3.553228-0.137531-2.061155$

H $4.4781993 .799658-3.276905$

H $5.9560563 .747438-2.298551$

H $4.5569364 .515008-0.243547$

H $3.2977855 .821298-2.712143$

H $3.4399057 .667718-0.943696$

H 3.2830486 .4839860 .332240

H $-0.1868787 .853349-1.585084$

H $1.4811848 .418939-2.158402$

H $-0.407486 \quad 6.2010590 .103708$

H $0.967667 \quad 6.0285871 .146409$

H $1.598358 \quad 3.9445190 .258207$

H $3.6296872 .163708-1.377961$

C $-5.773323-1.069609-1.484557$

O $-5.025455-0.312537-0.516663$

C $-5.945798 \quad 0.3484540 .371641$

C $-7.351156-0.026775-0.098564$

C $-7.112787-1.345980-0.810321$

H $-5.779899-0.0809091 .366408$

C -5.6573381 .8564340 .400911$

H -8.052704 -0.1134560 .737178$

H $-7.7626840 .712851-0.794535$

C -8.225572 -1.730674 -1.768624

H $-6.996909-2.133601-0.053691$

C -4.1833122 .2222360 .672900$

O $-5.9989582 .409079-0.878582$

H -6.3159692 .3413371 .131244$

H -3.570584 $1.933385-0.190475$

O $-4.124603 \quad 3.6524760 .753289$

C -3.5993291 .6127001 .960216$

C -2.1482072 .0541752 .222492$

H $-3.6297250 .524017 \quad 1.883581$

O -4.4113991 .9753383 .075664$

H $-1.560717 \quad 1.8763301 .316195$

H -2.127184 3.1323102 .420119

H -9.174666 -1.836705 -1.233101

H $-8.362265-0.974735-2.549222$

H -8.006679 -2.684599 -2.258343

C $-5.002385-2.330475-1.885899$

H $-5.8225973 .364751-0.836940$

H -3.1934493 .9159400 .662063$

H -4.4221402 .9465793 .127832$

H -5.538882 -2.857763 -2.682027

H -4.931851 -3.008316 -1.026524

H $-5.908527-0.421666-2.361376$

C $-0.716747-0.6480142 .286980$

O $-1.553421-0.0930763 .309062$

C -1.504205 1.3331373 .429081

C $-0.067423 \quad 1.782713 \quad 3.729709$

C 0.9173031 .2534852 .692179

C $0.749355-0.2540212 .517131$

H - $1.055061-0.3003951 .303387$

C $-0.869075-2.1770752 .338712$

H $-2.110472 \quad 1.586014 \quad 4.308035$ 
O $\quad 0.0014593 .203777 \quad 3.787340$

H 0.2234861 .4063214 .719086

H $0.805486 \quad 1.7840091 .741521$

O $2.244707 \quad 1.5267903 .146272$

H $1.138851-0.7587403 .410226$

O $1.547321-0.6848031 .418940$

C -2.239946 -2.650280 1.905731

H $-0.141064-2.6575491 .676933$

H $-0.709489-2.5234173 .367084$

H $\quad 0.9294513 .443183 \quad 3.953659$

H $2.857768 \quad 1.1419402 .496359$

H $1.209387-0.2561590 .614671$

O $-2.350784-3.9857032 .172958$

O $-3.099012-1.9525691 .386863$

C $-3.603919-4.5824541 .823853$

H -3.732787 -4.576602 0.737888

H -3.594579 -5.619366 2.170309

H -4.429185 -4.057192 2.314289

SCF Energy (B3LYP/6-31G**//MMFF) $=-3245.92854544$

\section{1}

MM̄FF Geometry

C $-0.288906-2.583963-3.189318$

C $0.703019-3.425770-3.521419$

C $1.938407-3.736536-2.710840$

O $1.829564-3.260777-1.350755$

C $3.174575-3.077410-3.345815$

C $4.466430-3.461491-2.647741$

C $5.144292-2.647132-1.814094$

C $4.983488-4.843671-2.948181$

C $4.746796-1.245829-1.440248$

C $5.866478-0.504225-0.695457$

C $5.4874240 .958359-0.425686$

C 6.6407981 .7100870 .240420

C 6.2543473 .1583540 .579889

O $4.3393590 .977062 \quad 0.429847$

O 7.2464743 .6818401 .476086

C 4.9129203 .1539311 .342993

C $6.2718074 .060388-0.660132$

O 4.4334714 .4820111 .541798

C 3.8186722 .2902010 .671946

C 2.5916112 .1753921 .607510

O $3.4033562 .921143-0.542272$

C $1.204489-4.078609-0.460540$

O $0.624474-5.124442-0.706825$

C $1.370633-3.4890440 .886757$

C $0.741515-4.0549041 .927348$

C $0.816052-3.6321013 .327402$

C $1.811440-2.5594283 .681529$

C 1.6163641 .0525771 .228315

C 0.3166001 .1371732 .010252

C -1.720370 -0.1905762 .552849$

O -0.0143732 .1278392 .657165$

C -1.491055 -0.647933 3.959706

C $-2.116843-1.6962224 .516159$

C $-1.911218-2.1196285 .943238$

C -1.532388 -3.577476 6.081525

C $-2.393887-4.470510 \quad 6.597382$

C $-0.125584-3.9843675 .697418$

C $0.004193-4.2367794 .219814$

N $-0.445947-0.016276 \quad 1.880476$

H $-0.234257-2.010567-2.268298$

H $\quad 0.623420-3.974533-4.458323$

H $2.071585-4.825778-2.700750$

H $3.044458-1.989707-3.336180$

H $3.253700-3.356947-4.404684$

H $\quad 6.054093-3.026659-1.352281$

H $5.949880-5.037941-2.470887$

H $4.282531-5.604835-2.592426$

H $5.119339-4.971442-4.027081$

H $4.501080-0.677977-2.344409$

H $3.853264-1.285933-0.806838$

H $6.059918-1.0182820 .255064$

H $6.790587-0.540242-1.285858$

H $5.2326901 .425686-1.384345$

H $7.5368371 .690406-0.392317$

H $\quad 6.9198741 .1980351 .172053$

H 8.1106213 .6232131 .033854
H $\quad 5.1093432 .7426752 .343257$

H $7.2705424 .068826-1.113129$

H $6.0555765 .101035-0.394771$

H $5.5639323 .745916-1.429722$

H 5.1429504 .9835941 .979062

H 2.9261081 .9742542 .633580

H 2.0621253 .1364591 .624276

H $2.5088222 .618245-0.763545$

H $2.011713-2.6208160 .986938$

H $\quad 0.100612-4.9156051 .738492$

H $2.821362-2.8592123 .379561$

H $1.860068-2.3422664 .750102$

H $1.563844-1.6198863 .180050$

H 1.3623331 .0956530 .166838

H $2.0837870 .080621 \quad 1.422774$

H $-2.301773-0.911947 \quad 1.970913$

H $-2.247707 \quad 0.7674122 .564508$

H $-0.794651-0.0695794 .562715$

H -2.836857 -2.260828 3.928405

H -2.845000 -1.919050 6.484518

H $-1.145515-1.5058296 .434173$

H -2.118744 -5.5124226 .723640$

H -3.394790 -4.181552 6.900209

H $\quad 0.148582-4.9136856 .214162$

H $0.574493-3.2333326 .071944$

H $-0.664139-5.0201903 .857912$

H -0.064843 -0.810362 1.374675

C $-2.838426-2.629953-3.291965$

O $-3.191754-1.474098-2.510465$

C $-3.979681-1.910727-1.384330$

C $-4.114976-3.431349-1.499945$

C $-2.896507-3.805899-2.320124$

H $-3.397759-1.670917-0.486949$

C $-5.326504-1.175319-1.350289$

H $-4.134084-3.914868-0.518002$

H $-5.031878-3.723969-2.024026$

C $-3.001288-5.161912-2.993896$

H $-2.031828-3.803805-1.645368$

C $-5.2432970 .362778-1.372101$

O $-6.108238-1.566569-2.488791$

H $-5.896177-1.503714-0.473075$

H $-4.9198480 .713534-2.359907$

O $-6.575230 \quad 0.863891-1.187533$

C $-4.3383160 .984415-0.294488$

C $-4.4923262 .516021-0.216584$

H $-3.2967560 .724022-0.506247$

$\begin{array}{lllll}\text { O } & -4.660261 & 0.429071 & 0.981102\end{array}$

H $-4.4735812 .927528-1.230606$

H -5.4737652 .7513340 .212295$

H -3.116559 -5.954393 -2.247241

H $-3.861951-5.208973-3.669548$

H -2.101619 $-5.377461-3.578759$

C $-1.518385-2.395053-4.034367$

H -5.601991 -1.333956 -3.286130

H $-7.1299520 .475593-1.885995$

H $-5.5989380 .614631 \quad 1.155466$

H -1.501412 -1.371272 -4.427501

H -1.481658 -3.073020 -4.896260

H -3.629840 -2.728674 -4.047795

C $-1.8255903 .716013-1.101624$

O $-2.0896562 .995700 \quad 0.109538$

C -3.4021533 .1788170 .654800$

C -3.6435924 .6662830 .943291$

C $-3.4129605 .522515-0.297641$

C $-2.0429835 .223415-0.903159$

H $-2.4744953 .344113-1.903314$

C $-0.3680753 .439191-1.500670$

H -3.4096072 .6639901 .622896$

O -4.9629154 .8653741 .438803$

H $-2.952964 \quad 4.989787 \quad 1.733077$

H $-4.2097165 .371295-1.034737$

O $\quad-3.475097 \quad 6.9045710 .063054$

H -1.258541 $5.632502-0.253356$

O $-1.9380815 .882388-2.164087$

C $-0.1593932 .040882-2.042037$

H $-0.0560644 .135622-2.287201$

H $0.2875043 .557656-0.630258$

H -5.0805025 .8206461 .578725$ 
H -2.782502 7.0747130 .724057

H -2.102897 $6.828764-2.011908$

O $1.114631 \quad 1.952454-2.529895$

O $-0.9849341 .139276-2.023658$

C $1.4958940 .674032-3.044571$

H $\quad 0.817070 \quad 0.365534-3.844973$

H $2.5050640 .761072-3.455771$

H $1.509514-0.063916-2.237325$

SCF Energy (B3LYP/6-31G**//MMFF) $=-3245.92638844$

0600212

MM̄FF Geometry

C - $-0.152437-2.646489-0.865537$

C $-0.691810-2.428805-2.075051$

C $-0.388797-1.246349-2.966971$

O $0.859181-0.622913-2.589887$

C -1.512217 -0.208642 -2.808866

C $-1.3403901 .001154-3.701924$

C $-0.8735422 .194783-3.284953$

C $-1.7782460 .811678-5.130356$

C $-0.3831462 .555224-1.908611$

C $0.9422183 .326970-1.972185$

C $1.4487073 .695601-0.570060$

C $2.6186464 .676420-0.661173$

C 3.1857615 .0129930 .724392

O 1.8730092 .4942680 .085223

O $4.4345195 .688174 \quad 0.512684$

C 3.4754223 .6958591 .471863

C 2.2862435 .9980181 .479623

O 3.8851463 .9555582 .813030

C 2.3035022 .6834551 .440568

C 2.7938831 .3252721 .992820

O 1.2430523 .1675212 .265551

C $1.995768-1.128053-3.140724$

O $2.079319-2.019302-3.970518$

C $3.140707-0.435027-2.510050$

C $4.369197-0.956067-2.644758$

C $5.595718-0.455052-2.022625$

C $5.5286330 .866600-1.306275$

C 1.7893110 .1772911 .854690

C $2.446227-1.1401672 .214980$

C $4.104100-2.7958411 .350569$

O $2.284927-1.6602693 .316536$

C $5.526267-2.3272901 .397535$

C $6.514193-2.8736910 .671992$

C $7.937164-2.3893620 .705056$

C $8.521953-2.170848-0.675561$

C $9.478104-2.984237-1.155675$

C $8.052172-0.969292-1.472644$

C $6.711006-1.209403-2.110360$

N $3.245860-1.6354001 .196570$

H $0.579252-1.949778-0.466719$

H $-1.450103-3.115797-2.443114$

H $-0.335898-1.585990-4.008516$

H -1.579045 $0.103842-1.760170$

H $-2.483741-0.671120-3.031119$

H $-0.8372253 .008512-4.008361$

H -1.668429 $1.725385-5.724110$

H -1.182087 $0.031614-5.613891$

H $-2.8326030 .518582-5.168433$

H $-1.1424653 .185363-1.430643$

H $-0.2513411 .672180-1.277433$

H $1.6922502 .712991-2.487169$

H $0.7984444 .240106-2.563623$

H $0.6202504 .142050-0.007535$

H $2.3213925 .593538-1.185197$

H $3.4200924 .231208-1.267746$

H 4.8399535 .8382411 .384144

H 4.3426093 .2369730 .977341

H $2.169640 \quad 6.924676 \quad 0.904767$

H $2.735793 \quad 6.2947372 .433668$

H 1.2883305 .6016191 .678374

H 3.0890374 .1698843 .328813

H 3.7149151 .0426051 .471573

H 3.0525861 .4258303 .055056

H 0.6620832 .4251852 .495962

H $2.9360230 .444970-1.911441$

H $4.485107-1.865921-3.232756$
H $4.9228520 .778132-0.401180$

H $5.0859081 .635022-1.950447$

H $6.506840 \quad 1.251047-1.010005$

H 0.9386410 .3230722 .526632

H 1.3935920 .1062530 .835738

H $3.915108-3.4549680 .497606$

H $3.854552-3.3269812 .273971$

H $5.754608-1.4964482 .061680$

H $6.293240-3.7227360 .029036$

H $8.528060-3.1368271 .250109$

H $8.026346-1.4582801 .278624$

H $9.919069-2.822367-2.133721$

H $9.838405-3.833234-0.584366$

H $8.766400-0.746286-2.275821$

H $8.063463-0.096857-0.813832$

H $6.661402-2.136684-2.683619$

H $3.313192-1.1152410 .326890$

C $-1.604067-3.2967011 .043318$

O $-1.070310-2.2333961 .861270$

C - $-1.515133-2.4301953 .209084$

C $-2.596773-3.499803 \quad 3.156333$

C $-2.078863-4.3681682 .023125$

H $-0.658227-2.8569003 .749340$

C -1.889428 -1.116628 3.900753

H -2.700368 -4.041910 4.100910

H $-3.571502-3.0723232 .894837$

C $-3.117634-5.3231681 .465062$

H -1.219365 -4.947814 2.386412

C -3.070475 -0.299604 3.332353

O $-2.209922-1.4181395 .266833$

H $-1.007415-0.4645893 .928029$

H -4.015431 -0.8298843 .498234$

O $\quad-3.154130 \quad 0.897925 \quad 4.125131$

C -2.9805940 .1197761 .850162$

C $-4.010527 \quad 1.2065411 .474820$

H $-3.136793-0.7533621 .210651$

$\begin{array}{llll}\mathrm{O} & -1.683311 & 0.643670 & 1.569744\end{array}$

H $-5.0099790 .862040 \quad 1.759813$

H -3.796202 2.1113732 .055713

H -3.438080 -6.032726 2.235041

H $-4.006279-4.7909281 .113428$

H -2.712046 -5.8969220 .626136$

C $-0.550432-3.7733250 .044983$

H -1.429610 -1.840747 5.664911

H -3.2497240 .6240885 .053582$

H $-1.072329-0.1107581 .523589$

H -0.932042 -4.619624 -0.537383

H $\quad 0.349164-4.1171690 .569415$

H -2.460132 -2.878243 0.500972

C $-5.6644600 .076988-0.863618$

O $-4.2883540 .474183-0.870718$

C $-3.9654841 .581861-0.024892$

C $-4.8138752 .803483-0.403278$

C $-6.3039082 .482674-0.394969$

C $-6.5784451 .248014-1.250880$

H $-5.934828-0.2916980 .132798$

C $-5.810920-1.072795-1.872118$

H $-2.9266641 .845493-0.254167$

O -4.5392963 .8875950 .477276$

H -4.529183 $3.132068-1.411646$

H -6.6747412 .3564720 .628959$

O $-7.0077193 .598514-0.944663$

H $-6.4327111 .507657-2.307011$

O $-7.946450 \quad 0.871996-1.101343$

C $-5.204218-2.361219-1.362676$

H $-6.864856-1.282891-2.083167$

H -5.304435 -0.801622 -2.806648

H -5.1110094 .6287110 .212999$

H -7.949963 $3.359099-0.976303$

H $-8.0903130 .632404-0.169863$

O $-4.946271-3.183386-2.421967$

O $-5.018833-2.629746-0.183906$

C $-4.411629-4.468362-2.090458$

H $-4.233383-5.013112-3.021351$

H $-3.461468-4.359879-1.561030$

$\mathrm{H}-5.128782-5.031088-1.485407$

SCF Energy (B3LYP/6-31G**//MMFF) $=-3245.93579875$ 
0600213

MM̄FF Geometry

C -2.127715 -3.000160 -2.276682

C -1.608322 -1.842049-2.711307

C $-0.144243-1.505693-2.651723$

O $-0.055790-0.230634-1.968656$

C $0.484271-1.326602-4.042640$

C $1.050415-2.616247-4.597515$

C $2.278015-3.087032-4.298281$

C $0.149203-3.373368-5.535069$

C $3.296575-2.453772-3.389634$

C $3.407815-3.209288-2.060151$

C $4.442488-2.577693-1.117166$

C $4.493274-3.3408440 .208985$

C $5.456412-2.6897281 .211914$

O $4.073009-1.214413-0.878400$

O $5.187347-3.2781712 .493432$

C $5.138468-1.1819271 .303865$

C $6.916151-3.0244720 .884501$

O $6.094300-0.5116892 .122445$

C $5.009650-0.488803-0.072370$

C 4.4912870 .9579770 .110661

O $6.302717-0.435050-0.682751$

C $0.484150-0.193077-0.725466$

O $1.006517-1.120449-0.122494$

C $0.3652771 .155825-0.124489$

C $-0.3739862 .141593-0.655695$

C $-0.5901533 .469978-0.079535$

C $\quad 0.1444843 .8152041 .188874$

C $4.0272561 .623749-1.192984$

C $3.8698153 .129560-1.052749$

C $3.0933415 .108707-2.355864$

O $4.3636553 .773554-0.130733$

C $2.0803795 .753287-1.463429$

C $1.0312046 .456036-1.917380$

C $0.0794917 .206475-1.029693$

C -1.357041 $6.770047-1.200074$

C $-2.1692937 .386358-2.075406$

C -1.874195 $5.685650-0.284022$

C -1.437819 4.310642 -0.708953

N $3.1619043 .678760-2.114407$

H -1.447181 -3.769820 -1.917361

H -2.262644 -1.059431 -3.085277

H $0.397609-2.270724-2.086293$

H $\quad 1.273190-0.565299-3.999368$

H $-0.244187-0.904103-4.748010$

H $2.593749-4.028919-4.744466$

H $\quad 0.618524-4.287026-5.915107$

H -0.775861 -3.664769 -5.029146

H $-0.107343-2.751100-6.398651$

H $4.266455-2.464689-3.901887$

H $3.066077-1.401333-3.203180$

H $2.427333-3.211729-1.569274$

H $3.675997-4.256452-2.248810$

H $5.418689-2.604538-1.615787$

H $4.761561-4.3918650 .044657$

H $3.488863-3.3558660 .656259$

H $5.738270-2.8184063 .150272$

H $4.177884-1.0905831 .827830$

H $7.071174-4.1100730 .904754$

H $7.596927-2.6136291 .638073$

H $7.229060-2.663191-0.097237$

H $\quad 6.905026-0.3975491 .597610$

H $3.634886 \quad 0.9618630 .797484$

H 5.2802841 .5615250 .576324

H $6.3436920 .328621-1.279594$

H $\quad 0.889176 \quad 1.2796970 .817190$

H $-0.9040721 .957499-1.588523$

H -0.2347803 .2187032 .024765$

H 0.0528504 .8663391 .471046

H 1.2184183 .6264181 .080240

H $4.7523991 .457253-1.996806$

H $3.0739721 .189017-1.511577$

H $2.8576115 .249032-3.415735$

H $4.0798205 .543519-2.162266$

H $2.2529315 .692121-0.391754$

H $\quad 0.8763236 .545329-2.990287$

H $0.1710938 .273777-1.272050$
H $\quad 0.3673607 .1247860 .025706$

H -3.210472 $7.099802-2.179518$

H -1.808191 $8.188019-2.711149$

H -2.972786 $5.688346-0.280450$

H -1.5977685 .9181340 .748633$

H $-1.9183663 .972090-1.627931$

H $2.8135093 .063967-2.844134$

C $-4.563349-2.160229-2.127637$

O $-4.217479-1.295167-1.028309$

C $-5.420058-0.856925-0.373095$

C $-6.573444-1.296599-1.268477$

C $-6.015374-2.567895-1.880990$

H $-5.479216-1.4059060 .574119$

C $-5.3474810 .649621-0.097859$

H -7.496362 -1.460301 -0.703944

H $-6.785633-0.560447-2.052232$

C $-6.752940-3.018045-3.129549$

H $-6.060613-3.366692-1.128568$

C $-4.081501 \quad 1.1036470 .658450$

O $-5.3696151 .357982-1.345181$

H -6.2447660 .9694460 .444182$

H -3.2010210 .9999290 .013398$

O -4.2167852 .5045440 .931203$

C -3.8240800 .3719091 .988245$

C -2.6224970 .9561812 .755033$

H $-3.642059-0.6867921 .786580$

O $-4.987450 \quad 0.4479752 .811113$

H -1.7604410 .9881232 .082547$

H -2.8483331 .9897213 .043314$

H -7.802482 -3.229818 -2.900598

H $-6.727430-2.249841-3.909725$

H -6.306123 -3.929267-3.539110

C $-3.591941-3.344700-2.171335$

H $-4.6153151 .044475-1.873649$

H -4.3533762 .9532720 .079070$

H $-5.175547 \quad 1.3894862 .967073$

H -3.851980 -4.021181-2.993108

H -3.708650 -3.913504 -1.240255

H -4.489181-1.565113 -3.046551

C $-0.785326-1.4789753 .061271$

O $-2.007204-1.2202143 .764004$

C $-2.273601 \quad 0.1611304 .033614$

C -1.1270060 .7668644 .856280$

C 0.2293510 .5460394 .192798

C $0.415151-0.9287673 .843294$

H $-0.831043-1.0203312 .067342$

C -0.659098 -3.000592 2.889285

H -3.1669900 .1800894 .670280$

O -1.3499412 .1578345 .062249$

H -1.1156050 .2945145 .847310$

H $\quad 0.345830 \quad 1.185795 \quad 3.311668$

O 1.2499050 .9415765 .111992

H $0.552474-1.4978594 .771262$

O $1.612158-1.0820493 .083962$

C $-1.662530-3.5612781 .905146$

H $\quad 0.335299-3.2683292 .515932$

H -0.827973 -3.490249 3.855964

H -0.5927082 .5022735 .565951$

H $2.106534 \quad 0.7517414 .692118$

H $1.492712-0.6182282 .237927$

O $-1.684072-4.9220232 .024050$

O $-2.323381-2.9004401 .117063$

C $-2.580775-5.6070001 .144162$

H $-3.592017-5.1963281 .224479$

H -2.215088 -5.5388550 .115663$

H -2.607342 -6.659549 1.438307

SCF Energy (B3LYP/6-31G**//MMFF) $=-3245.93213083$

\section{4}

MM̄̄FF Geometry

C $1.4617173 .561280-0.892904$

C $0.3218194 .125545-0.464880$

C $-0.7033353 .380140 \quad 0.346387$

O $-1.9275903 .375892-0.424406$

C -0.9861964 .0322021 .711198$

C 0.1941164 .0145392 .659061

C 0.5910022 .9334253 .359425

C 0.9211435 .3250342 .807620 
C $-0.054751 \quad 1.575808 \quad 3.385693$

C 0.7736950 .5300262 .630639

C $0.199161-0.8881752 .778654$

C $0.944653-1.8648141 .865212$

C $0.347082-3.2789151 .915467$

O $-1.190811-0.8665112 .436618$

O $0.891698-4.0092320 .806360$

C $-1.181571-3.182706 \quad 1.715311$

C $0.793192-4.0271643 .176772$

O $-1.794837-4.4587391 .885118$

C $-1.855403-2.1244172 .617261$

C $-3.341479-1.9478682 .224140$

O $-1.790854-2.5740893 .974556$

C $-2.6788022 .241850-0.383425$

O $-2.460586 \quad 1.2375320 .275052$

C $-3.8094422 .416942-1.320990$

C $-4.5868341 .363083-1.611500$

C $-5.7190541 .351075-2.538736$

C $-6.1022322 .649711-3.198097$

C $-4.013482-0.7382302 .888761$

C $-5.517319-0.9043752 .960789$

C -7.582106 -0.9170341 .604555$

O $-6.093466-1.0597004 .036120$

C -7.966565 -1.315543 0.216853

C $-8.786439-0.590063-0.558977$

C $-9.216420-0.998693-1.939427$

C $-8.8696940 .036795-2.986090$

C $-9.7601030 .976433-3.346716$

C -7.527439-0.066622 -3.673764

C $-6.3672650 .186962-2.750874$

N $-6.136553-0.8717641 .720569$

H $1.6586842 .519033-0.653854$

H $0.1119545 .164687-0.705110$

H -0.3658252 .3488890 .489699$

H $-1.846603 \quad 3.5389402 .181431$

H -1.3323015 .0646241 .562479$

H 1.4593933 .0304524 .009519

H 1.8103745 .2391003 .441141

H 1.2464815 .7017721 .833985

H 0.2601116 .0714403 .260322

H $-0.161011 \quad 1.273394 \quad 4.434923$

H -1.069353 1.6142482 .977798

H 0.8018210 .8012921 .568906

H 1.8118070 .5374442 .986385

H $\quad 0.303663-1.1852233 .828965$

H $2.013604-1.8880042 .110153$

H $0.881915-1.5037060 .829130$

H $0.461242-4.8812840 .786921$

H -1.350484 -2.903859 0.666199

H $1.886176-4.1158973 .198703$

H $0.411952-5.0541463 .186002$

H $0.482475-3.5347614 .100482$

H -1.841286 -4.639976 2.839350

H -3.420704 -1.813250 1.137611

H -3.896340 -2.859896 2.479206

H $-2.560218-2.2426584 .463369$

H -3.954286 $3.395630-1.763741$

H $-4.3660030 .407621-1.136804$

H $-6.3192043 .415112-2.444386$

H $-6.9950982 .571676-3.822627$

H $-5.2907993 .008634-3.840647$

H -3.655159 -0.586890 3.912666

H $-3.785234 \quad 0.1825772 .341512$

H $-7.964770 \quad 0.072499 \quad 1.877127$

H -7.975340 -1.642447 2.324849

H -7.572016 -2.257202 -0.157377

H $-9.1969970 .340109-0.172282$

H -10.302744 -1.156274 -1.918122

H -8.786139 -1.966851 -2.224308

H $-9.5330431 .705957-4.116799$

H $-10.735606 \quad 1.038884-2.875846$

H $-7.4778880 .594920-4.546313$

H -7.420446 -1.076654 -4.090887

H $-6.025923-0.703751-2.222587$

H $-5.599384-0.5466600 .921303$

C $3.9044714 .233250-1.070342$

O $4.4123382 .882949-1.049606$

C 5.1117722 .6787170 .193136
C $5.2768734 .059858 \quad 0.822424$

C 4.0099994 .7510390 .363396

H 4.4508102 .0786180 .829614

C $6.4331821 .940149-0.037009$

H 5.3701244 .0100821 .911607

H 6.1573014 .5836840 .432413

C 4.0601396 .2642030 .472204

H 3.1833074 .3752250 .976863

C $6.3145330 .572811-0.737936$

O $7.2956162 .757145-0.841071$

H 6.9505291 .8172030 .921808

H $6.0630020 .704385-1.797261$

O $7.617179-0.028487-0.710015$

C $5.311313-0.406494-0.098978$

C $5.461430-1.842889-0.639917$

H $4.293951-0.044779-0.279873$

O $5.499964-0.4350651 .317227$

H $5.505526-1.809212-1.732909$

H $6.414356-2.253500-0.285765$

H 4.2011856 .5699741 .514037

H $4.8844046 .682324-0.115356$

H 3.1282246 .7112660 .111900

C $2.5089954 .273066-1.700909$

H $6.8293082 .942902-1.674372$

H $8.2323270 .593732-1.134964$

H $6.417589-0.709388 \quad 1.486525$

H $2.5500623 .784669-2.682331$

H $2.2184225 .316710-1.869636$

H $4.5858114 .796564-1.722045$

C $2.879368-2.613342-2.110888$

O $3.053755-2.380931-0.708166$

C $4.320788-2.772715-0.169614$

C $4.566920-4.263880-0.436351$

C $4.442829-4.597638-1.917758$

C $3.109807-4.090077-2.460360$

H $3.572296-1.981229-2.678669$

C $1.444267-2.198844-2.474164$

H $4.239347-2.6575020 .918275$

O $5.851620-4.6640890 .028423$

H $3.827442-4.8585530 .115845$

H $5.283306-4.194711-2.494924$

O $4.481443-6.016607-2.085742$

H $2.294377-4.704136-2.056145$

O $3.095772-4.243129-3.878757$

C $1.253560-0.697832-2.482212$

H $1.194527-2.558511-3.478786$

H $\quad 0.733549-2.616589-1.752107$

H $5.898605-4.4506860 .976055$

H $5.315503-6.330411-1.696099$

H $3.262833-5.181987-4.070542$

O $0.022090-0.418775-3.005424$

O $2.0654350 .122525-2.078644$

C $-0.3134710 .968651-3.087754$

H -1.290755 $1.055738-3.569852$

H $-0.3784021 .392688-2.083869$

H $0.4248891 .507132-3.689349$

SCF Energy (B3LYP/6-31G**//MMFF) $=-3245.93376278$

\section{5}

MM̄FF Geometry

C $2.142200-2.6498503 .094483$

C $1.270825-1.7605782 .594691$

C $0.819984-0.5229583 .323985$

O $-0.610605-0.4355413 .127961$

C 1.5204220 .7268952 .744481

C 2.4990721 .3705773 .710635

C 2.4645822 .6601784 .099862

C 3.6316910 .4929544 .183957

C 1.5038373 .7419493 .689430

C 2.1745614 .7472082 .739394

C 2.4112564 .1685891 .335387

C 3.3328525 .0701050 .514823

C $3.5252244 .524713-0.907497$

O $1.137557 \quad 4.0613330 .691998$

O $4.1634095 .560654-1.668832$

C $2.1374634 .261083-1.525869$

C $4.4768723 .322993-0.932591$

O $2.2617853 .627621-2.798167$ 
C $1.1771263 .462908-0.608476$ C $-0.2483673 .520285-1.201780$ O $1.6119222 .104879-0.548390$ C $-1.3139240 .349083 \quad 3.983196$ O $\quad-0.850770 \quad 1.0707794 .856170$ C $-2.775230 \quad 0.2099883 .780058$ C $-3.331910-0.5666992 .837099$ C $-4.766374-0.8022952 .646692$ C $-5.720418-0.1076003 .582876$ C $-1.3530542 .942652-0.304222$ C $-2.7241943 .164446-0.922852$ C $-5.1376702 .880117-0.492949$ O $-2.8868993 .725785-2.004221$ C -6.0067971 .8485720 .147038$ C $-6.6273850 .881003-0.544751$ C $-7.582540-0.1084310 .055915$ C $-7.057070-1.524526 \quad 0.028151$ C $-7.122589-2.269147-1.088561$ C $-6.546136-2.1034041 .326286$ C $-5.156887-1.6392431 .662600$ N $-3.7500902 .655345-0.136931$ H $2.542034-2.5078104 .094811$ H $0.899651-1.8953071 .580982$ H $1.002983-0.6378784 .400001$ H $\quad 0.767717 \quad 1.4449192 .406112$ H $2.088306 \quad 0.4686041 .839598$ H 3.2424243 .0057784 .780601 H 4.4144631 .0642494 .694847 H $3.270080-0.2632994 .886141$ H $4.104748-0.0098203 .333852$ H 0.5851243 .3480223 .246853 H 1.1934284 .2708204 .599139 H 1.5223605 .6265612 .662719 H 3.1241825 .0823273 .175263 H 2.8572673 .1732401 .422732 H 4.3035715 .1938431 .011050 H $2.897700 \quad 6.077267 \quad 0.449334$ H $4.2155435 .258486-2.592042$ H $1.6908185 .245252-1.723508$ H $5.4462883 .595334-0.498317$ H $4.6898883 .009791-1.960525$ H $4.0951712 .460101-0.382479$ H $2.4623452 .688655-2.643020$ H -0.502080 $4.570403-1.400184$ H $-0.2691272 .995108-2.165131$ H $0.8594531 .543364-0.304686$ H -3.379722 0.7679264 .488146 H -2.684055 -1.1086112 .150320$ H -5.569446 -0.453724 4.611131 H $-6.771195-0.2757373 .338249$ H -5.5704850 .9773553 .552055$ H -1.212163 $1.866727-0.158134$ H -1.3435663 .4306660 .676641$ H -5.237754 2.880659-1.584068 H $-5.4156643 .877504-0.136321$ H $-6.170103 \quad 1.933178 \quad 1.217857$ H -6.470653 $0.815859-1.619430$ H -8.523409 $-0.057443-0.507990$ H $-7.849880 \quad 0.174614 \quad 1.081286$ H -6.774936 -3.296708 -1.104748 H -7.521996 -1.863925 -2.012526 H -6.513184 -3.199695 1.261460 H -7.265488 -1.901197 2.125610 H -4.390734 -2.091931 1.033342 H $-3.5354042 .363360 \quad 0.812767$ C $3.876990-3.4126531 .468708$ O $3.422622-2.594936 \quad 0.369223$ C $4.082957-3.019970-0.836367$ C $5.230939-3.912760-0.385735$ C $4.615336-4.586380 \quad 0.827482$ H $3.365952-3.634848-1.392878$ C $4.485058-1.799360-1.670670$ H $5.537926-4.619967-1.162055$ H $6.109609-3.326743-0.091803$ C $5.635347-5.2466161 .738256$ H $3.895057-5.3417550 .485297$ C $3.308535-0.863344-2.025303$ O $5.445333-1.026800-0.938586$
H $\quad 4.993777-2.125057-2.585068$

H $2.925115-0.379981-1.118847$

O $3.814306 \quad 0.185663-2.859815$

C $2.152234-1.556800-2.767954$

C $1.029140-0.584231-3.171093$

H $1.729428-2.331882-2.126194$

O $2.655423-2.207452-3.934173$

H $0.689867-0.051631-2.276646$

H $1.4290580 .165764-3.863989$

H $6.175344-6.0344021 .202905$

H $6.372185-4.5245932 .105972$

H $5.147447-5.7013922 .605921$

C $2.672184-3.8230932 .317675$

H $5.034937-0.773184-0.093841$

H $4.5598900 .595474-2.388736$

H $3.058978-1.526038-4.498942$

H $2.949059-4.6081163 .030162$

H $1.879156-4.2302171 .680904$

H $4.572309-2.7983032 .056202$

C $-1.387251-1.956363-1.882924$

O $-0.696566-2.363683-3.069376$

C $-0.160217-1.296521-3.858662$

C $-1.290195-0.357099-4.296084$

C $-2.0960960 .151270-3.105151$

C -2.547861 -1.010185 -2.222043

H $-0.686571-1.463965-1.197357$

C -1.923262 -3.227210 -1.201970

H $\quad 0.236428-1.762215-4.769754$

O $-0.7584020 .740824-5.030229$

H -1.962557 -0.900529 -4.972676

H $-1.5210870 .886742-2.534434$

O $-3.2449760 .835497-3.609934$

H -3.348807 -1.563146 -2.729651

O $-3.085384-0.495234-1.005867$

C $-0.830693-4.085351-0.602505$

H $-2.604088-2.965374-0.385572$

H -2.457526 -3.838966 -1.938902

H $-1.5019591 .322990-5.262526$

H $-3.7059091 .235982-2.854055$

H $-3.8864550 .008388-1.226448$

O $-1.384524-5.249364-0.148998$

O $0.349504-3.777531-0.524051$

C $-0.474547-6.1705450 .460440$

H $-0.120370-5.7671941 .413355$

H -1.012449 -7.102910 0.651914

H $0.366424-6.383325-0.206796$

SCF Energy (B3LYP/6-31G**//MMFF) $=-3245.93100153$

0600216

MM̄FF Geometry

C -0.5686174 .1710300 .919948$

C -0.603495 2.9351721 .439293

C -0.3486062 .6515562 .896838$

O $\quad 0.847514 \quad 1.8466963 .009553$

C -1.4989601 .8411303 .521795$

C -2.7921672 .6173173 .641453$

C -3.887026 2.4193402 .882534

C -2.8156073 .6608494 .730241$

C -4.0915621 .4386901 .760300$

C $-5.191150 \quad 0.4350562 .127642$

C $-5.632618-0.4241290 .934176$

C $-6.815123-1.3095811 .339228$

C $-7.251977-2.2436520 .204468$

O $-4.528443-1.2313880 .512910$

O $-8.143581-3.211510 \quad 0.778513$

C $-6.008998-2.991932-0.319057$

C $-8.060839-1.496505-0.861973$

O $-6.340678-3.779961-1.461067$

C $-4.807072-2.063699-0.621100$

C $-3.531702-2.872129-0.965111$

O $-5.127555-1.272571-1.773013$

C 2.0320312 .4969313 .156311

O $2.2053003 .708198 \quad 3.158864$

C 3.1626811 .5509303 .297989

C 2.9991300 .2383543 .520433

C $4.059809-0.7641883 .633896$

C $5.487643-0.3062863 .504896$

C $-2.918728-3.6631950 .192733$ 
C $-1.860717-2.870730 \quad 0.936366$

C $0.527453-2.170823 \quad 0.782028$

O $-2.080753-2.3667202 .035008$

C $1.330224-3.2109661 .498597$

C $2.579232-3.5609761 .154841$

C $3.406281-4.5662891 .908867$

C $4.664826-3.9602712 .497730$

C $5.845398-4.0796651 .867448$

C $4.569927-3.2749523 .846651$

C $3.697812-2.0519663 .805876$

N $-0.664081-2.7925720 .238830$

H -0.3088334 .9956231 .582127$

H -0.8357302 .0811780 .810228$

H -0.2126203 .5781093 .467975$

H -1.196082 1.5093834 .524795

H -1.634424 0.9026122 .971009

H -4.7559793 .0483563 .076385$

H -3.799560 4.1301324 .836507

H -2.564220 3.2083175 .695147

H -2.094682 4.4564094 .519511

H -4.3907942 .0063080 .870536$

H $-3.173400 \quad 0.908575 \quad 1.493760$

H -4.816452 -0.2132962 .930802$

H $-6.063703 \quad 0.9722982 .521581$

H $-5.921258 \quad 0.2452630 .115217$

H $-7.661709-0.6992211 .678402$

H $-6.527160-1.9252872 .203325$

H -8.365206 -3.8559960 .084213$

H $-5.727433 \quad-3.7067420 .462317$

H $-8.957910-1.050817-0.415457$

H -8.423544 -2.180542 -1.637136

H -7.498312 -0.695052 -1.345172

H -6.410179 -3.180566 -2.223600

H $-3.758098-3.557976-1.791334$

H $-2.777828-2.182749-1.362709$

H $-4.300989-0.932263-2.153528$

H 4.1467192 .0011563 .217940

H $1.987845-0.1553333 .609969$

H $6.205246-1.1298793 .482202$

H $5.757560 \quad 0.335533 \quad 4.350799$

H 5.6375420 .2595222 .580178

H $-3.662011-4.0131580 .914093$

H -2.435456 -4.565152 -0.204593

H $\quad 0.244778-1.373347 \quad 1.476962$

H $1.077538-1.727396-0.052812$

H $0.871436-3.6709112 .370982$

H $3.039400-3.0967690 .286947$

H $3.666634-5.3743861 .212908$

H $2.826094-5.0433602 .708639$

H $6.756222-3.6678522 .289391$

H $5.925896-4.5857750 .911102$

H $5.563702-3.0330134 .239077$

H $4.147218-3.9809554 .573164$

H $2.636836-2.2800733 .909958$

H $-0.568569-3.302493-0.634618$

C $-1.6536643 .599805-1.363881$

O $-0.8215412 .490950-1.759860$

C $-1.081223 \quad 2.182652-3.141979$

C $-2.2969203 .008671-3.546417$

C -2.139860 4.231799-2.669971

H $-0.2121822 .533646-3.710316$

C $-1.2689010 .675283-3.341072$

H -2.300903 $3.242459-4.615547$

H -3.236331 2.493608 -3.313844

C -3.418972 $5.040426-2.532591$

H $-1.3531564 .869649-3.094833$

C $-0.091376-0.206228-2.883681$

O $-2.427566 \quad 0.248054-2.612150$

H $-1.4897640 .473790-4.396028$

H $-0.043259-0.239181-1.788933$

O $-0.368320-1.546501-3.313185$

C $1.2839340 .204884-3.438962$

C $2.367756-0.861306-3.186854$

H $1.5811801 .153641-2.981800$

O $1.1901140 .430054-4.846582$

H $2.307747-1.192843-2.146029$

H $2.163533-1.733872-3.818877$

H $-3.7553785 .396206-3.511906$
H $-4.2265744 .442543-2.096706$

H $-3.2649885 .914511-1.892468$

C $-0.8596184 .582885-0.497944$

H $-2.2881320 .480226-1.677497$

H $-1.246250-1.786122-2.971710$

H $0.880569-0.395617-5.257185$

H $-1.4214465 .522705-0.434227$

H $0.0980324 .812767-0.979994$

H $-2.5015463 .186059-0.802564$

C $4.3681190 .459669-1.301609$

$\begin{array}{lllll}\text { O } & 4.151118 & 0.771157 & -2.684137\end{array}$

C $3.788069-0.343080-3.508160$

C $4.883003-1.416485-3.448058$

C $5.189529-1.831157-2.013206$

C $5.469737-0.599122-1.154640$

H $3.4362790 .099077-0.850476$

C $4.7693301 .761453-0.592552$

H $3.7662670 .031901-4.539063$

O $4.501457-2.552694-4.216045$

H $5.798010-1.015186-3.903146$

H $4.376427-2.436749-1.598976$

O $6.347900-2.668235-2.022706$

H $6.439061-0.176597-1.447813$

O $5.588659-1.0012350 .206533$

C $3.6294122 .755813-0.522262$

H 5.0899891 .5667250 .435965

H $5.5932682 .231410-1.143420$

H $5.211889-3.211616-4.133188$

H $6.556109-2.883925-1.097356$

H $4.725149-1.3419590 .495712$

O $4.1431403 .999595-0.287062$

O $2.4433652 .478816-0.634245$

C $3.1860405 .059903-0.211227$

H 2.4975194 .8904670 .620566

H $3.7261545 .993673-0.033923$

H $2.6404595 .144827-1.155666$

SCF Energy (B3LYP/6-31G**//MMFF) $=-3245.93844058$

0600217

MM̄FF Geometry

C $-4.239404-3.036434-1.606069$

C $-3.202538-2.380737-1.061825$

C $-1.865181-2.227530-1.733334$

O $-0.863673-2.463858-0.717154$

C $-1.713720-0.791057-2.275969$

C $-0.687562-0.678425-3.385907$

C $0.528669-0.110661-3.272618$

C $-1.127233-1.233203-4.719363$

C $1.1666830 .523934-2.067902$

C $1.5393741 .979948-2.375452$

C $2.2613892 .674509-1.211556$

C $2.577606 \quad 4.125766-1.588530$

C $3.4120764 .841644-0.518364$

O $3.4686751 .955382-0.938592$

O $3.9079786 .050560-1.114171$

C $4.6241513 .954614-0.163646$

C 2.5490165 .2755490 .670830

O $5.367325 \quad 4.520418 \quad 0.913338$

C 4.2465932 .4885810 .141506

C 5.4907991 .5904800 .326560

$\begin{array}{lllll}\text { O } & 3.516516 & 2.446741 & 1.373174\end{array}$

C $0.322470-3.002859-1.102300$

O $0.646443-3.360127-2.223252$

C $1.189483-3.0581130 .094150$

C $2.519837-3.113568-0.064271$

C $3.516920-3.1505391 .007745$

C $3.017466-3.3218282 .418470$

C $6.371018 \quad 1.430932-0.916275$

C $7.4978160 .443949-0.662376$

C $8.893499-1.265644-1.810856$

$\begin{array}{llll}\text { O } & 7.974826 & 0.254586 & 0.454335\end{array}$

C $8.173241-2.526131-1.444782$

C $8.424155-3.229285-0.329984$

C $7.620374-4.4272560 .090622$

C $6.918792-4.2176591 .419322$

C $7.104687-5.0786452 .434051$

C $6.017416-3.0102811 .588510$

C $4.820494-3.0404410 .676501$ 
N $7.928029-0.180121-1.825846$ H -4.129693 -3.503235 -2.581473 H -3.331653 -1.891304 -0.100663 H -1.773655 -2.978228 -2.527304 H -1.478311 -0.108604 -1.452110 H -2.670336 - $0.437136-2.685148$ H $1.165600-0.105287-4.157516$ H $-0.388408-1.056545-5.508421$ H -1.287759 -2.313439-4.658376 H -2.062164 -0.759670 -5.037034 H $0.5228290 .488798-1.185863$ H $2.067709-0.050713-1.822901$ H $2.1916431 .995293-3.259201$ H $0.6323702 .542896-2.630714$ H $1.6140282 .638827-0.328634$ H $1.6549824 .683650-1.793022$ H $3.1455244 .138146-2.529921$ H $4.5000526 .475259-0.469491$ H $5.2900803 .978787-1.034683$ H $1.758997 \quad 5.9597730 .338478$ H 3.1372645 .8352871 .406207 H 2.0686394 .4382201 .178301 H 4.8811724 .3395161 .735829 H 6.0881191 .9539591 .172879 H 5.1532370 .5871810 .624894 H $3.575316 \quad 1.5477371 .736961$ H $0.711662-3.0215011 .066468$ H $2.922088-3.109953-1.076896$ H $2.349786-4.1880932 .489220$ H $2.470380-2.4297572 .742547$ H $3.814119-3.498108 \quad 3.144234$ H $5.7650921 .082293-1.759605$ H $6.8339212 .382792-1.195260$ H $9.691824-1.024062-1.101391$ H $9.322851-1.348955-2.813410$ H $7.375511-2.848046-2.110268$ H $9.218999-2.9069960 .338766$ H $8.302419-5.2852040 .143617$ H $6.871261-4.684075-0.668511$ H $6.613096-4.9395973 .391363$ H $7.750432-5.9443492 .331203$ H $6.587404-2.0984581 .373003$ H $5.722360-2.8990632 .636396$ H $5.073038-2.916458-0.377290$ H $7.417984-0.020305-2.689536$ C $-6.537206-2.060128-1.522767$ O $-6.037608-0.755800-1.165518$ C $-7.1158400 .036099-0.638931$ C $-8.390829-0.679788-1.060576$ C -7.955264 -2.131118 -0.955786 H -7.036868 -0.0070220 .453971$ C -6.970264 $1.487860-1.108037$ H $-9.238983-0.436805-0.413714$ H -8.669342 -0.442076 -2.093706 C -8.865831-3.096177 -1.694074 H $-7.921775-2.4116820 .105752$ C -5.569839 $2.088724-0.855309$ O $-7.2142881 .555081-2.519014$ H -7.740969 2.109649 -0.639047 H $-4.8310301 .599050-1.501676$ O $-5.5988763 .465899-1.248503$ C -5.1003212 .0113730 .608279$ C -3.6884392 .5887330 .804194$ H $-5.0892260 .968150 \quad 0.926384$ O $-6.0260792 .698107 \quad 1.446809$ H -3.0000932 .0435610 .148478$ H -3.675808 3.6374450 .483579 H -9.879140 -3.061227 -1.280809 H -8.929149 -2.852556 -2.759955 H -8.499173 -4.123512 -1.604217 C $-5.594080-3.123629-0.958562$ H -6.581084 $0.959058-2.955298$ H $-5.8999313 .496496-2.172935$ H $-6.0560753 .624747 \quad 1.152558$ H -5.998494 -4.127910-1.126518 H $-5.492013-2.9946130 .125224$ H -6.555351 -2.104342 -2.619626 C -2.4547730 .2273092 .328764$
O -3.3299021 .2300372 .856718$

C -3.1965112 .5289812 .269104$

C $-1.763840 \quad 3.0423862 .462311$

C $-0.7338562 .055846 \quad 1.920996$

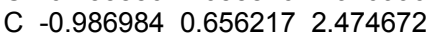

H $-2.698700 \quad 0.047556 \quad 1.275273$

C $-2.695449-1.0724903 .116484$

H $-3.855048 \quad 3.1904992 .846174$

$\begin{array}{lllll}O & -1.604223 & 4.308617 & 1.830904\end{array}$

H -1.5818063 .1930413 .534482$

H $-0.718108 \quad 2.060726 \quad 0.825020$

$\begin{array}{lllll}\text { O } & 0.559928 & 2.489594 & 2.344620\end{array}$

H -0.6976200 .6410903 .533127$

O $-0.134864-0.2699131 .803263$

C $-4.063882-1.6779612 .889595$

H $-1.956384-1.8274912 .824608$

H $-2.605634-0.8727654 .191098$

H -0.6790394 .5813451 .955822$

H $1.2043301 .835428 \quad 2.024052$

H $-0.407942-0.3051350 .870659$

O $-4.151515-2.841926 \quad 3.600877$

O $-4.945605-1.202347 \quad 2.190028$

C $-5.399716-3.534557 \quad 3.499374$

H $-5.364417-4.398026 \quad 4.168897$

H $-6.224579-2.885313 \quad 3.808883$

H $-5.549778-3.8899512 .476077$

SCF Energy (B3LYP/6-31G**//MMFF) $=-3245.93780871$

0600218

MM̄FF Geometry

C $0.0190142 .370677 \quad 0.846192$

C $1.1027942 .687514 \quad 0.120749$

C $1.3753682 .181658-1.276292$

O $0.8246930 .852200-1.414341$

C $2.8864502 .085129-1.547170$

C $3.2140041 .942108-3.020640$

C $3.614990 \quad 0.801067-3.614092$

C $3.1193513 .213334-3.825925$

C $3.772143-0.561113-2.998154$

C $5.245909-0.972067-2.867015$

C $5.985085-0.221815-1.747729$

C $7.471252-0.583230-1.740126$

C $8.210506 \quad 0.075024-0.565865$

O $5.386431-0.603790-0.506056$

O $9.498120-0.553138-0.477352$

C $7.440879-0.2274360 .737432$

C $8.474218 \quad 1.562070-0.828414$

$\begin{array}{llll}\text { O } & 8.020015 & 0.466532 & 1.840505\end{array}$

C $5.9227830 .056367 \quad 0.646459$

C $5.210093-0.5206801 .891751$

$\begin{array}{llll}\text { O } & 5.715780 & 1.468361 & 0.595406\end{array}$

C $0.018507 \quad 0.578168-2.468249$

$\begin{array}{lllll}0 & -0.339377 & 1.357999 & -3.339749\end{array}$

C $-0.416429-0.839586-2.458530$

C $0.033202-1.751306-1.581045$

C $-0.354192-3.163225-1.519350$

C $-1.384683-3.649705-2.504269$

C $3.675677-0.4750921 .826012$

C $3.046814-1.2487742 .972936$

C $0.870136-2.2130923 .700793$

O $3.691514-1.6748833 .927926$

C $0.812349-3.6070373 .157759$

C $-0.324346-4.2200392 .795862$

C $-0.383265-5.614796 \quad 2.243247$

C $-0.958127-5.665405 \quad 0.844193$

C $-2.240550-6.0098760 .637965$

C $-0.025161-5.410332-0.319674$

C $0.208365-3.946003-0.573925$

N $1.676831-1.4015772 .807246$

H -0.7245321 .6966330 .429654$

H 1.8087043 .4053970 .532629

H $\quad 0.9054152 .886299-1.972510$

H $3.3038931 .248962-0.977141$

H $3.4016032 .981998-1.177999$

H $3.8484390 .833422-4.677795$

H $3.432998 \quad 3.071782-4.865767$

H $2.090825 \quad 3.585753-3.842520$

H $3.7630023 .985850-3.392384$ 
H $3.260950-0.643624-2.034686$ H $3.268904-1.277694-3.659794$ H $5.270598-2.048115-2.650966$ H $5.759978-0.821341-3.824430$ H $5.8620890 .856515-1.894496$ H $7.944188-0.311932-2.692269$ H $7.580697-1.673412-1.652009$ H $9.945473-0.1983690 .310150$ H $7.582834-1.2964360 .947739$ H $9.0706321 .687527-1.740051$ H $9.0665822 .008582-0.022007$ H $7.5588782 .145669-0.946857$ H 7.7354751 .3949821 .787834 H $5.507401-1.5711162 .011056$ H 5.5374130 .0146572 .792186 H $4.825305 \quad 1.6703260 .922768$ H -1.130643-1.097118-3.234063 H $0.758138-1.445651-0.828365$ H -2.299887 -3.052501-2.432071 H $-1.685138-4.687310-2.344372$ H - $0.999852-3.579936-3.527470$ H $3.3157590 .557667 \quad 1.879810$ H $3.321723-0.9158530 .887160$ H $-0.118379-1.7499683 .764787$ H $1.316850-2.2214984 .699946$ H $1.756712-4.1372053 .056802$ H -1.269891-3.698089 2.914165 H -0.997921 -6.217242 2.924893 H $0.605882-6.0893522 .246967$ H -2.653952 -6.078679 -0.362721 H $-2.908414-6.222677 \quad 1.466155$ H $-0.390642-5.914022-1.221129$ H $0.942715-5.888144-0.120031$ H $0.941332-3.4990190 .097852$ H $1.241795-1.0692961 .950976$ C -1.407771 3.9556332 .228550 O -2.6110653 .4057871 .647613$ C $-3.057307 \quad 4.3002800 .614441$ C -2.474397 5.6577830 .984484 C $-1.098207 \quad 5.253493 \quad 1.472520$ H $-2.5936533 .959528-0.319423$ C $-4.580186 \quad 4.2821910 .463993$ H -2.443841 6.3461790 .134771 H $-3.036775 \quad 6.134676 \quad 1.795495$ C $-0.415267 \quad 6.309153 \quad 2.325247$ H -0.4702135 .0582450 .595504$ C $-5.219750 \quad 2.896393 \quad 0.247787$ O -5.1831654 .8469841 .635833$ H $-4.8617194 .947194-0.361392$ H -5.2796272 .3520651 .198560$ O $-6.5753683 .116720-0.167432$ C $-4.5289751 .996302-0.792310$ C $-5.3907050 .783551-1.199697$ H $-3.5713451 .659336-0.381381$ O $-4.235242 \quad 2.744178-1.973901$ H $-5.9374820 .417259-0.326172$ H -6.135865 $1.108462-1.935403$ H $-0.2662367 .229748 \quad 1.751575$ H -1.010638 $6.557903 \quad 3.210188$ H $\quad 0.564697 \quad 5.9590532 .664731$ $\begin{array}{llll}C & -0.277766 & 2.917745 & 2.214933\end{array}$ H -4.8838094 .3246272 .399946$ H -7.0029503 .6606530 .516418$ H -5.070831 $3.119134-2.301535$ H $-0.566000 \quad 2.067207 \quad 2.844421$ H 0.6242163 .3495702 .664433 H -1.654951 4.179646 3.274019 C $-4.292483-1.7026190 .178438$ O $-3.649371-0.923424-0.839074$ C $-4.533733-0.348541-1.810551$ C $-5.336420-1.457416-2.505040$ C $-6.057667-2.353248-1.502975$ C $-5.081988-2.859703-0.442693$ H $-4.963972-1.0657190 .765157$ C -3.207565 -2.224069 1.122379 H -3.894339 $0.110346-2.575472$ O $-6.286472-0.911085-3.413486$ H $-4.653301-2.081987-3.095316$
H $-6.907182-1.836543-1.040749$

O $-6.598268-3.485271-2.188147$

H $-4.395984-3.588712-0.892638$

O $-5.811135-3.5340190 .580735$

C $-2.616419-1.0987531 .933534$

H $-3.590401-2.979647 \quad 1.817747$

H $-2.391583-2.6895240 .562285$

H $-5.799587-0.355716-4.046151$

H $-7.185851-3.146759-2.885639$

H $-6.318818-4.2454490 .153804$

O $-3.426784-0.8144672 .992343$

O $-1.568573-0.5343811 .649887$

C $-2.9886110 .251444 \quad 3.839622$

H $-3.641104 \quad 0.2785604 .716323$

H $-1.9606080 .084474 \quad 4.174860$

H -3.0733821 .2036313 .309713$

SCF Energy (B3LYP/6-31G*//MMFF) $=-3245.93037645$

0600219

MM̄FF Geometry

C $0.3375051 .602407 \quad 3.319659$

C 1.3626690 .8780113 .793703

C $1.658120-0.5835443 .571386$

O $2.936745-0.6544072 .890603$

C $0.647401-1.3818912 .726812$

C $0.808499-2.8777722 .929336$

C $1.527848-3.6989332 .140431$

C $0.091589-3.4364034 .133807$

C $2.362712-3.3452640 .941963$

C $2.035878-4.213785-0.280771$

C $0.704801-3.850563-0.955525$

C $0.411607-4.823956-2.100698$

C $-0.840644-4.425040-2.892799$

O $0.831066-2.520111-1.464581$

O $-0.840626-5.204733-4.098095$

C $-0.725507-2.937978-3.286503$

C $-2.122060-4.797397-2.140645$

O $-1.924577-2.488729-3.914785$

C $-0.341400-2.009850-2.110570$

C $0.004967-0.604580-2.650905$

O $-1.443907-1.918255-1.210653$

C $4.041909-0.9575503 .616046$

O $4.089869-1.1739704 .819501$

C $5.256979-1.0122582 .768976$

C $5.276106-0.7392491 .454464$

C $6.452381-0.7867700 .579970$

C $7.772362-1.1579851 .205190$

C $0.4409530 .390186-1.571019$

C $1.1107031 .587421-2.213658$

C $3.3358172 .556730-2.727154$

O $0.4588342 .512564-2.692587$

C $4.6194531 .974714-3.222405$

C $5.8199582 .260777-2.696754$

C $7.1074241 .678889-3.203546$

C $7.8508810 .891218-2.147846$

C $8.9745991 .374118-1.591432$

C $7.348069-0.494551-1.808159$

C $6.300672-0.489408-0.728208$

N $2.4924421 .482908-2.238026$

H $-0.406496 \quad 1.1508792 .670895$

H 2.0787591 .3813664 .442943

H $1.729280-1.0420264 .565658$

H $0.747114-1.1247111 .665986$

H $-0.381309-1.1224653 .005851$

H $1.567212-4.7538302 .411186$

H $0.156010-4.5283364 .188547$

H $0.521636-3.0330345 .055916$

H $-0.969380-3.1726454 .106667$

H $2.295745-2.2859120 .680474$

H $3.409801-3.5280821 .214449$

H $2.849299-4.075835-1.005437$

H $2.035872-5.273194 \quad 0.004709$

H $-0.099147-3.880102-0.211795$

H $0.317483-5.850422-1.724433$

H $1.266212-4.838082-2.792169$

H - $1.589983-4.906262-4.641895$

H $0.058851-2.871058-4.052811$

H $-2.156043-5.877842-1.955619$ 
H -3.013962 -4.568688 -2.734029 H -2.209105 -4.292766 -1.177631 H $-2.584539-2.336123-3.216990$ H $0.814086-0.697978-3.387551$ H $-0.860117-0.177426-3.173835$ H $-1.334596-1.131943-0.653629$ H $6.158559-1.2962943 .303039$ H $4.348128-0.4558630 .960907$ H $8.024922-0.4618382 .012885$ H $8.610414-1.1324320 .505875$ H $7.731275-2.1733111 .614782$ H $-0.4195140 .749403-0.997307$ H $1.127202-0.070896-0.852432$ H $3.4866503 .257326-1.898977$ H $2.8241893 .089693-3.535098$ H $4.5557601 .286984-4.062550$ H $5.8862642 .958193-1.864836$ H $7.7270452 .509494-3.565637$ H $6.9387381 .036136-4.076747$ H $9.533748 \quad 0.801947-0.858938$ H $9.3593742 .354242-1.853213$ H $8.187041-1.148488-1.546216$ H $\quad 6.919277-0.958672-2.705875$ H $5.304773-0.229215-1.086491$ H $2.9331210 .751372-1.687465$ C 0.1616754 .0061072 .473636 O $-0.9983693 .803343 \quad 1.644043$ C -0.6020273 .9596760 .267784$ C $0.804306 \quad 4.5518130 .291936$ C 1.3710283 .9111511 .543952 H $-0.5501892 .950213-0.149398$ C -1.632433 $4.785793-0.506259$ H $1.3737844 .313703-0.610694$ H $0.785158 \quad 5.642548 \quad 0.399578$ C 2.6173104 .5948422 .075132 H 1.6083582 .8652821 .311789 C $-3.0760504 .251768-0.424416$ O -1.6426356 .1248270 .006251$ H -1.324242 $4.868717-1.554762$ H $-3.474566 \quad 4.393610 \quad 0.587531$ O $-3.8851485 .058155-1.291116$ C $-3.2446992 .776875-0.835610$ C $-4.7222712 .344097-0.848265$ H -2.690705 2.142977 -0.136186 O $-2.6842032 .567697-2.130266$ H -5.1601342 .5810220 .127462$ H -5.267851 $2.930647-1.597446$ H 3.4220744 .5565541 .333718 H 2.4285175 .6471552 .312281 H 2.9726614 .1020262 .985751 C 0.1374733 .0531133 .669759 H -1.848085 6.0741190 .955653 H $-3.7719975 .983829-1.014582$ H -3.142299 $3.162110-2.748951$ H -0.8364453 .1340404 .168760$ H $0.893603 \quad 3.3770534 .395225$ H 0.0714405 .0300032 .861188 C $-4.559325-0.0659511 .023573$ O $-4.135764-0.007445-0.341320$ C $-4.9192640 .847051-1.179180$ C $-6.3876180 .396297-1.160142$ $\begin{array}{llll}\text { C } & -6.940045 & 0.329661 & 0.262297\end{array}$ C $-6.023326-0.5088001 .152415$ H -4.4468330 .9314761 .467163$ C $-3.581729-0.9701401 .790711$ H $-4.5466880 .696869-2.200199$ O $-7.200027 \quad 1.272128-1.934631$ H -6.468411 $-0.601581-1.610560$ H -7.091264 1.3297660 .684881 O $-8.227646-0.291276 \quad 0.238529$ H $-6.141450-1.5637640 .881826$ O $-6.431768-0.3759762 .512303$ C $-3.586097-2.4186971 .343926$ H -2.558639 -0.6019181 .648475$ H -3.821060 -0.9351822 .859689$ H $-6.8372021 .285463-2.836816$ H $-8.787508 \quad 0.228579-0.363510$ H $-7.369181-0.6308342 .559803$
O $-3.005864-3.1834742 .314464$

O $-4.011693-2.8271190 .272463$

C $-2.912611-4.5837872 .032800$

H -2.622288 -5.0963082 .953735$

H -3.876930-4.981891 1.702506

H -2.141979-4.757344 1.276950

SCF Energy $(B 3 L Y P / 6-31 G * * / / M M F F)=-3245.92658101$

0600220

MM̄MF Geometry

C $-0.779003-3.280809-2.112155$

C $-0.734272-2.072656-2.694216$

C $0.496355-1.207614-2.707516$

O $\quad 0.1172420 .094670-2.200244$

C $1.073564-1.002416-4.116831$

C $1.707607-2.241272-4.715791$

C $2.887600-2.757485-4.316300$

C $0.930001-2.889810-5.829503$

C $3.778458-2.229227-3.225045$

C $3.706887-3.123636-1.980955$

C $4.423071-2.515043-0.766304$

C $4.223126-3.4096210 .461454$

C $4.833208-2.8007721 .731139$

O $3.879541-1.212628-0.512622$

O $4.304943-3.5380822 .844167$

C $4.359026-1.3378621 .862029$

C $6.353093-2.9964381 .769177$

O $5.006746-0.6931072 .956534$

C $4.520567-0.5150410 .562926$

C 3.8520190 .8705390 .731089

O $5.916171-0.3203140 .313660$

C $0.1654630 .273834-0.854373$

O $0.551975-0.538715-0.024879$

C $-0.3321801 .609388-0.457800$

C $-0.7456212 .542537-1.327924$

C $-1.2633483 .869439-0.988126$

C $-1.362418 \quad 4.2291110 .471761$

C $3.6997221 .664558-0.574911$

C $3.4231623 .140124-0.324503$

C $3.2382485 .285858-1.576186$

O $3.432164 \quad 3.647377 \quad 0.794128$

C $1.9267795 .874409-1.160768$

C $1.2280026 .726021-1.927558$

C $-0.0216287 .433477-1.483440$

C $-1.2116437 .140883-2.368420$

C $-1.4354997 .851670-3.486543$

C $-2.1867536 .083593-1.904713$

C $-1.6150644 .696682-1.994662$

N $3.2095793 .835516-1.508714$

H $\quad 0.137250-3.676470-1.678939$

H - $1.624779-1.648070-3.148219$

H $\quad 1.270622-1.618477-2.055230$

H $1.814673-0.192112-4.091365$

H $0.291134-0.616987-4.785283$

H $\quad 3.256372-3.654918-4.810372$

H $1.428523-3.783319-6.219836$

H $-0.061876-3.191797-5.478908$

H $0.806414-2.189408-6.662082$

H $4.809760-2.198042-3.596788$

H $3.517646-1.196539-2.975744$

H $2.656285-3.299958-1.722075$

H $4.141137-4.105626-2.208658$

H $5.487817-2.419643-1.009836$

H $4.627751-4.4136230 .282267$

H $3.146177-3.5498820 .633579$

H $4.624436-3.1117303 .658100$

H $3.292178-1.3695962 .120200$

H $6.599513-4.0649601 .746652$

H $6.780414-2.6132952 .702300$

H $6.867418-2.5201900 .931978$

H $5.908685-0.4650282 .673953$

H $2.8469720 .747594 \quad 1.152674$

H $4.434876 \quad 1.4569391 .452381$

H $6.0427670 .504278-0.181040$

H $-0.346177 \quad 1.7842080 .612628$

H $-0.7094432 .323232-2.393387$

H -2.072424 3.5684550 .981216

H -1.6965805 .2531910 .648969$ 
H -0.3852874 .1386050 .959635$ H $4.6151991 .608211-1.173583$ H $2.8841391 .246838-1.174194$ H $3.5043945 .557197-2.603000$ H $4.0276935 .650181-0.909729$ H $1.5707075 .645260-0.159632$ H $1.6065136 .979681-2.915441$ H $\quad 0.1832648 .512447-1.493674$ H $-0.2652147 .197236-0.440346$ H $-2.3036177 .667541-4.110622$ H $-0.7515408 .632112-3.803645$ H -3.093193 $6.102826-2.524601$ H $-2.5341636 .334101-0.898368$ H -1.497271 $4.346063-3.021050$ H $3.2505923 .327671-2.387613$ C $-3.350757-3.486919-2.112820$ O $-3.485441-2.426530-1.146129$ C $-4.823667-2.436575-0.619647$ C $-5.610455-3.422130-1.476947$ C $-4.533575-4.420170-1.857640$ H $-4.757871-2.8323750 .400484$ C $-5.387689-1.012089-0.593897$ H -6.442612 -3.874557 -0.929135 H $-6.020220-2.944994-2.374772$ C $-4.903668-5.291684-3.044668$ H $-4.327825-5.061269-0.989930$ C -4.5181210 .0108030 .167031$ O $-5.523754-0.535727-1.940028$ H $-6.401287-1.024667-0.176887$ H $-3.5896300 .206296-0.383510$ O $-5.236431 \quad 1.251528 \quad 0.193519$ C $-4.176836-0.3850201 .615846$ C -3.4657560 .7455862 .383063$ H $-3.537974-1.2724211 .605020$ O $-5.373343-0.7374282 .310266$ H -2.6071571 .0831651 .794557$ H $-4.149874 \quad 1.5971612 .478541$ H $-5.798097-5.883093-2.823219$ H $-5.111140-4.689613-3.935695$ H $-4.092520-5.984884-3.288025$ C $-1.988273-4.163031-1.939537$ H $-4.644866-0.575812-2.355104$ H $-5.431043 \quad 1.492797-0.728363$ H $-5.960970 \quad 0.0375622 .295043$ H -1.896021 -5.011278 -2.626941 H - $-1.933058-4.566768-0.920169$ H -3.436474 -3.034851 -3.108989 C $-0.814798-0.5198413 .265043$ O $-2.122299-0.7947643 .783063$ C $-3.017770 \quad 0.323587 \quad 3.801489$ C -2.4176921 .4653604 .634201$ C -1.023229 1.8471474 .149083 C -0.1380390 .6058644 .061399$ H $-0.894702-0.2370342 .210631$ C $0.009610-1.8133443 .362871$ H $-3.917173-0.0154654 .330606$ O -3.2748472 .6015674 .603311$ H -2.3517111 .1429935 .681736$ H -1.070106 2.3785443 .191786 O $-0.4546652 .762118 \quad 5.088325$ H 0.0916460 .2610525 .077478 O $1.103107 \quad 0.9613653 .455413$ C $-0.379749-2.8460462 .326327$ H $1.071954-1.5966623 .206741$ H $-0.127152-2.2681134 .350978$ H -2.846642 3.3030015 .123673 H 0.4499232 .9583994 .789430 H $0.918041 \quad 1.2641702 .550513$ O $0.426290-3.9395132 .479041$ O $\quad-1.262869-2.7175251 .490665$ C $\quad 0.196404-5.014226 \quad 1.563047$ H $-0.859310-5.3026031 .558783$ H $\quad 0.523099-4.7224760 .561538$ H $\quad 0.790894-5.8715031 .889764$ SCF Energy (B3LYP/6-31G**//MMFF) $=-3245.92695170$

0600221

MM̄MF Geometry
C $1.393917 \quad 1.6242322 .446343$

C 0.6705370 .6377552 .997473

C $0.935619-0.8485412 .922702$

O $2.090366-1.1512992 .110812$

C $-0.248743-1.5806842 .265957$

C $-0.433212-2.9889672 .794301$

C $0.079406-4.1020092 .234803$

C $-1.301582-3.0931864 .022279$

C $0.999289-4.2115791 .051114$

C $0.320297-4.825873-0.180020$

C $-0.566697-3.824098-0.933515$

C $-1.280079-4.503076-2.102833$

C $-2.097012-3.498320-2.928711$

O $0.282158-2.782802-1.428112$

O $-2.467211-4.137015-4.160308$

C $-1.197544-2.298087-3.294382$

C $-3.405327-3.118239-2.228306$

O $-1.952950-1.265168-3.923601$

C $-0.406357-1.719803-2.096824$

C $0.654595-0.712784-2.606430$

O $-1.308619-1.043401-1.220215$

C $3.315835-1.1927182 .690988$

O $3.603070-0.9385173 .847965$

C $4.262321-1.5904011 .627184$

C $5.559537-1.2688951 .723075$

C $6.582461-1.5524670 .713547$

C $6.179115-2.392212-0.471779$

C $1.697745-0.300891-1.555308$

C $2.7238480 .665537-2.127302$

C $4.8468121 .831364-1.522006$

O $2.6131591 .180062-3.237248$

C $6.0702951 .071071-1.928031$

C $7.2913611 .327294-1.432668$

C $8.5382250 .601317-1.854092$

C $9.3787110 .142501-0.681116$

C $10.4603910 .839211-0.293006$

C $9.014986-1.165351-0.013439$

C $7.816705-1.0349570 .884326$

N $3.7655670 .903670-1.240709$

H 2.2794931 .3823281 .867453

H -0.1914890 .9175923 .598159$

H $1.094794-1.2169783 .944076$

H $-0.115374-1.6058301 .179199$

H $-1.187425-1.0391112 .432292$

H $-0.155521-5.0594882 .698775$

H -1.390931 -4.1227694 .384220$

H -0.884505 -2.493033 4.837283

H $-2.311333-2.7307083 .804606$

H $1.456332-3.2532830 .794403$

H $1.829862-4.8638711 .349727$

H $\quad 1.114277-5.170830-0.855450$

H -0.264068 -5.707239 0.111357

H $-1.298233-3.400340-0.236648$

H -1.920846 -5.319675 -1.747570

H $-0.536495-4.970827-2.763373$

H $-2.976510-4.936397-3.941793$

H -0.475651 -2.652995 -4.043483

H -4.016425 -4.010226 -2.045412

H -4.014637 -2.465460 -2.862581

H -3.247956 -2.624151-1.267505

H $-2.421828-1.665934-4.675463$

H $1.201198-1.160945-3.446787$

H $\quad 0.1556350 .187527-2.987487$

H $-0.839296-0.322146-0.772307$

H $3.850024-2.1032790 .765398$

H $5.897128-0.7227382 .602782$

H $5.845547-3.382613-0.142210$

H $6.984493-2.553861-1.190382$

H $5.363502-1.915318-1.026475$

H $1.2158580 .188599-0.702671$

H $2.231931-1.184839-1.189467$

H $5.0135372 .415191-0.611599$

H $4.5489552 .511373-2.326244$

H $5.9526550 .294136-2.679677$

H $7.4093072 .129364-0.707053$

H $9.1230051 .286042-2.482414$

H $8.303539-0.260165-2.491415$

H $\quad \begin{array}{lll}11.084467 & 0.503618 & 0.528658\end{array}$ 
H $10.7429291 .763926-0.785246$

H $9.849507-1.5186660 .607226$

H $8.895027-1.936895-0.778707$

H $8.008050-0.4334321 .774177$

H $3.7765370 .421608-0.347102$

C $0.807008 \quad 3.8755201 .363702$

$\begin{array}{lllll}\text { O } & -0.434524 & 3.457046 & 0.766625\end{array}$

C $-0.3023033 .544954-0.665601$

C $1.0451174 .211595-0.943042$

C $1.858209 \quad 3.789113 \quad 0.261311$

H $-0.2701442 .514095-1.036722$

C -1.489819 4.292131-1.284843

H $1.4794463 .881509-1.892016$

H $0.9632015 .303823-0.979452$

C 3.0833614 .6515480 .506542

H $2.1730552 .753790 \quad 0.102697$

C $-2.8853993 .731378-0.951134$

O $-1.4695605 .658472-0.846750$

H $-1.3572014 .328841-2.372775$

H $-3.149474 \quad 3.9756700 .084674$

O $-3.8327594 .442921-1.761258$

C -3.062862 2.216652 -1.162765

C -4.544537 $1.788589-1.172228$

H $-2.5235061 .686374-0.370564$

O $-2.4708331 .822959-2.402341$

H $-5.0696102 .291949-0.355328$

H $-4.9993212 .126249-2.110861$

H $3.7502484 .630559-0.361323$

H 2.8092235 .6954820 .691729

H $3.6449524 .290977 \quad 1.374029$

C 1.0904593 .0860962 .645101

H $-1.519150 \quad 5.655440 \quad 0.124680$

H -3.626906 4.258788 -2.693402

H -2.947718 2.272208 -3.120353

H $0.2285013 .194756 \quad 3.315719$

H 1.9488993 .5355633 .158725

H 0.6589494 .9262421 .648661

C -5.2504710 .1013361 .292749$

O $-4.333261-0.222295 \quad 0.238749$

C $-4.7022160 .256405-1.061003$

C $-6.086824-0.278083-1.447152$

C $-7.1366710 .049116-0.392581$

C $-6.660584-0.4208190 .980426$

H -5.2864721 .1879941 .432514$

C $-4.710676-0.5299932 .586443$

H -3.989972 $-0.186462-1.766547$

O $-6.480241 \quad 0.241122-2.712966$

H -6.027270 -1.368853 - 1.555964

H $-7.3768441 .118886-0.393862$

O -8.340123 $-0.640195-0.737371$

H $-6.673943-1.5177381 .019625$

$\begin{array}{lllll}\text { O } & -7.561968 & 0.058264 & 1.977006\end{array}$

C $-3.6390660 .322023 \quad 3.234312$

H $-5.520915-0.6335613 .317347$

H $-4.277970-1.5172582 .389890$

H -7.366992 $-0.107590-2.907463$

H $-9.033856-0.343778-0.124622$

H $-8.417537-0.3807351 .836037$

O $-3.542204-0.024767 \quad 4.551692$

O $-2.962611 \quad 1.1610812 .655857$

C -2.5930450 .6915245 .349774$

H -2.987782 0.7551556 .367447

H -1.6547420 .1318415 .373303$

H -2.423104 1.7076934 .981566

SCF Energy (B3LYP/6-31G**/MMFF) $=-3245.90459226$

0600222

MM̄FF Geometry

C $0.110293-3.5398381 .150490$

C $-0.544912-2.3711651 .087266$

C $-1.206882-1.7314012 .278622$

O $-2.629059-1.7388862 .012942$

C $-0.748036-0.272392 \quad 2.436627$

C $-1.078753 \quad 0.307703 \quad 3.796643$

C -2.1464831 .0843684 .065404$

C $-0.083098-0.0092644 .882716$

C -3.2592221 .4835693 .136607$

C -3.2852992 .9904572 .849589$
C $-2.1364663 .459517 \quad 1.943838$

C $-2.208114 \quad 4.972172 \quad 1.726273$

C $-1.144552 \quad 5.463872 \quad 0.733579$

O -2.2635202 .7831180 .689206$

$\begin{array}{lllll}0 & -1.505967 & 6.806809 & 0.377021\end{array}$

C $-1.2076754 .592951-0.540024$

C 0.2407605 .5430051 .385769

O $-0.1350344 .904123-1.427123$

C $-1.2189403 .073409-0.248461$

C $-1.5280262 .293653-1.548567$

$\begin{array}{lllll}\text { O } & 0.059886 & 2.685463 & 0.251970\end{array}$

C $-3.463058-2.2006072 .978008$

O $-3.141948-2.6420584 .073202$

C $-4.882959-2.1262072 .558658$

C $-5.309242-1.5199151 .439163$

C $-6.702455-1.4107000 .993595$

C $-7.756824-2.1022371 .818354$

C $-1.6272220 .776295-1.362817$

C $-2.127296 \quad 0.115813-2.630053$

C $-4.200417-0.584333-3.792363$

O $-1.357184-0.301919-3.491523$

C $-5.662954-0.300250-3.685490$

C $-6.585811-1.242831-3.441568$

C $-8.051987-0.954366-3.299728$

C $-8.595634-1.353506-1.944804$

C $-9.379944-2.436243-1.809392$

C $-8.309855-0.448654-0.765713$

C $-6.970783-0.714951-0.131884$

N $-3.5103790 .080346-2.703766$

H $\quad 0.164481-4.065677 \quad 2.100980$

H $-0.627589-1.8573300 .133070$

H $-0.972248-2.3066553 .182382$

H - $-1.1931890 .344141 \quad 1.649445$

H $\quad 0.337933-0.1970182 .290095$

H -2.262351 1.4595995 .081665

H -0.3543920 .4439855 .842106$

H $-0.014982-1.0905125 .037549$

H 0.9084540 .3665494 .609562

$\begin{array}{llll}H & -3.238648 & 0.922648 & 2.198624\end{array}$

H -4.2033051 .2093883 .624498$

H -4.2404183 .2120572 .355601$

H $-3.271602 \quad 3.5503903 .792943$

H -1.1821993 .1880492 .408616$

H -2.1199615 .5076232 .679996$

H -3.198816 5.2344031 .327970

H $-0.8164877 .160034-0.209868$

H -2.135926 4.850418 -1.069253

H 0.2243616 .2419492 .230599

H 0.9857855 .9376750 .686273

H 0.5954314 .5799711 .758916

H $-0.2228585 .838204-1.680451$

H -2.479208 $2.654330-1.961542$

H $-0.7548522 .494243-2.300772$

H $\quad 0.171767 \quad 1.7291650 .140082$

H -5.576800 -2.591956 3.251616

H $-4.578774-1.0475350 .784700$

H -7.530559 -3.1700371 .916361$

H -8.758093 -2.0408121 .386901$

H -7.812381 -1.6604482 .819282$

H $-0.6536970 .338149-1.124754$

H -2.301292 $0.519872-0.537932$

H -3.974841 $-1.654426-3.728048$

H $-3.803206-0.212214-4.742508$

H $-5.9743820 .735067-3.801981$

H $-6.273894-2.278924-3.331598$

H $-8.576693-1.497873-4.096114$

H $-8.2694610 .106722-3.474785$

H $-9.801745-2.712441-0.848877$

H $-9.615061-3.073463-2.655370$

H $-9.116767-0.517323-0.027651$

H $-8.3381510 .597859-1.095797$

H $-6.138954-0.249946-0.659939$

H $-4.0442290 .328136-1.875109$

C $2.173987-4.674090 \quad 0.285786$

$\begin{array}{llllll}\text { O } & 3.062208 & -3.540726 & 0.369858\end{array}$

C $4.338748-3.916098-0.187233$

C $4.253230-5.406417-0.501537$

C $2.775932-5.574838-0.790572$ 
H $4.445172-3.369809-1.130939$

C $5.475667-3.5503380 .774510$

H $4.891560-5.687956-1.344864$

H $4.547890-6.0196150 .358264$

C $2.312103-7.019922-0.730140$

H $2.561501-5.165648-1.787098$

C $5.596587-2.045848 \quad 1.091165$

O $5.279135-4.2416762 .015158$

H 6.427625 -3.918489 0.374367

H $4.733258-1.7062241 .675843$

O $6.747088-1.8701451 .927891$

C $5.761503-1.152465-0.151088$

C 6.0689740 .3170200 .196855

H $4.842368-1.198228-0.742269$

O $6.812953-1.661833-0.972521$

H 5.3120770 .6810020 .899613

H 7.0392340 .3777440 .704455

H $2.847034-7.626091-1.468526$

H $2.491324-7.4580750 .257577$

H $\quad 1.241770-7.098022-0.943589$

C $0.751312-4.211334-0.031667$

H $4.411472-3.9745382 .364640$

H $6.627997-2.4400312 .707116$

H $7.628507-1.655560-0.442447$

H $\quad 0.130029-5.074409-0.295617$

H $0.763708-3.532591-0.892497$

H $2.205187-5.1783551 .261206$

C $3.7666511 .676339-1.269561$

O $4.9398681 .098811-1.854436$

C $6.1234981 .217029-1.059484$

C $6.4247142 .695593-0.781096$

C $5.2192683 .434635-0.203191$

C $3.9730013 .179130-1.053154$

H $3.5515501 .193105-0.308963$

C $2.5997811 .389895-2.221801$

H $6.9454070 .838294-1.680220$

O 7.5384242 .8123720 .098070

H $6.7110513 .178381-1.724775$

H $5.0539033 .153640 \quad 0.843713$

O $5.5159134 .832708-0.205598$

H $4.0707603 .692315-2.018344$

O $2.8340093 .716097-0.388648$

C $2.218242-0.073995-2.166643$

H $1.7053151 .963979-1.963007$

H $2.883017 \quad 1.651264-3.248444$

H 7.6840663 .7604110 .258590

H 4.7910305 .2870950 .255772

H $2.9265904 .683424-0.378563$

O $2.169923-0.592852-3.426312$

O $1.983504-0.683060-1.130386$

C $1.838025-1.982741-3.504410$

H $2.582502-2.580721-2.969932$

H $0.837860-2.157188-3.097156$

H $1.843837-2.275177-4.557650$

SCF Energy (B3LYP/6-31G**//MMFF)= -3245.91722903

0600223

MM̄FF Geometry

C $3.017207-3.947657-1.686278$

C $1.867539-3.271223-1.546571$

C $1.183453-2.587838-2.701279$

O $0.949903-1.196733-2.375526$

C $-0.182788-3.235753-2.984592$

C $-0.843691-2.655316-4.221408$

C $-1.810497-1.715863-4.212036$

C $-0.343346-3.207055-5.532170$

C $-2.443931-1.056657-3.017915$

C $-3.974070-1.059731-3.137391$

C $-4.666945-0.684799-1.819862$

C $-6.181100-0.579011-2.017039$

C $-6.898338-0.139958-0.731937$

O $-4.145010 \quad 0.563855-1.355805$

O $-8.232608 \quad 0.236872-1.104323$

C $-6.190328 \quad 1.110941-0.166188$

C -7.041483 -1.3058910 .253671$

O -6.7403821 .4749201 .098105$

C $-4.6533380 .963131-0.075863$

C -4.0082592 .3125650 .314915$ $\begin{array}{llll}\text { O } & -4.348371 & 0.006487 & 0.943317\end{array}$

C $1.994657-0.338424-2.525477$

O $3.162085-0.630987-2.730097$

C $1.4538371 .039595-2.469610$

C $2.2967182 .079283-2.561762$

C $1.9304483 .496172-2.642679$

C $0.4694543 .832376-2.781587$

C -2.4857882 .3542210 .117363$

C -1.8392803 .4965220 .884492$

C $0.4079344 .351749 \quad 1.552427$

O -2.4823334 .3691571 .461958$

C 0.7720345 .4797020 .640958

C 2.0364325 .8439870 .379264

C $2.3988547 .014613-0.487850$

C $3.3619636 .654561-1.595329$

C $4.6436737 .057103-1.556397$

C $2.8162605 .909770-2.793055$

C $2.9192194 .415018-2.639116$

N $-0.454033 \quad 3.4224610 .848212$

H $3.467740-4.021763-2.673727$

H $1.410324-3.182724-0.566377$

H $1.804371-2.638056-3.605216$

H $-0.831815-3.125087-2.110265$

H $-0.066558-4.318612-3.125835$

H -2.186453 -1.363001 -5.171918

H $-0.875048-2.785115-6.391788$

H $0.720388-2.987014-5.663801$

H $-0.480812-4.292958-5.562849$

H -2.152405 -1.539524 -2.083217

H -2.085414 -0.021552 -2.965242

H $-4.258385-0.344314-3.920442$

H -4.329246 -2.047822 -3.456814

H $-4.434130-1.465318-1.086960$

H $-6.594328-1.526943-2.383800$

H $-6.3932480 .162091-2.800622$

H $-8.6722380 .586815-0.310216$

H -6.419956 $1.943001-0.845636$

H -7.616773 -2.120763 -0.202393

H -7.605418 -1.0068631 .144120$

H $-6.082184-1.7176630 .574407$

H -6.3821240 .8631581 .763513$

H $-4.4364543 .119702-0.293645$

H $-4.2506802 .532783 \quad 1.362409$

H $\quad-3.4439870 .156058 \quad 1.259909$

H $0.3780641 .158073-2.410325$

H $3.3659821 .875371-2.609956$

H -0.106935 $3.416197-1.951263$

H $0.0718633 .423611-3.717210$

H $\quad 0.2650744 .904660-2.786728$

H -2.0228691 .4194790 .448535$

H $-2.2544032 .481612-0.946436$

H $1.2849573 .789943 \quad 1.889431$

H -0.1166404 .7403182 .431303$

H -0.0459246 .0445610 .199649$

H 2.8551475 .2978900 .842486

H $2.841078 \quad 7.7815690 .161458$

H $1.5061207 .477418-0.926810$

H $5.3312216 .828207-2.364065$

H $5.0352957 .619266-0.715201$

H $3.3929266 .183785-3.687210$

H $1.8005856 .254324-3.004137$

H $3.9480804 .061632-2.552516$

H -0.0104312 .7064140 .280458$

C $4.915312-3.8061700 .011885$

O $4.390667-2.7791270 .876836$

C $5.060142-1.5419800 .581224$

C $6.330339-1.928804-0.168838$

C $5.841837-3.107858-0.987966$

H $4.397893-0.988270-0.095710$

C $5.282568-0.7161181 .852731$

H $6.717434-1.109364-0.781805$

H $7.126580-2.2518250 .511188$

C $6.953578-3.998216-1.514114$

H $5.282365-2.706090-1.840357$

C $4.059235-0.5865342 .780984$

O $6.331565-1.3061672 .633268$

H 5.6487290 .2789901 .573844

H $3.914152-1.5183253 .342040$ 
$\begin{array}{llll}\text { O } & 4.365376 & 0.419266 & 3.756471\end{array}$

C $2.735503-0.2140842 .092173$

C $1.616798 \quad 0.075624 \quad 3.112213$

H $2.430485-1.0342861 .434782$

$\begin{array}{llll}\text { O } & 2.918890 & 0.936595 & 1.267687\end{array}$

H $1.634237-0.6940883 .890421$

H 1.8179841 .0378763 .597916

H $7.616915-3.434304-2.177872$

H $7.561672-4.405983-0.699614$

H $6.540807-4.838808-2.081030$

C $3.758520-4.627412-0.570486$

H $6.069622-2.2214842 .833067$

H 5.1903870 .1533994 .197630

H 3.2399631 .6581591 .835322

H $3.055824-4.8822880 .233108$

H $4.148396-5.574947-0.961544$

H $5.499872-4.4651010 .667235$

C $-0.437434-2.176376 \quad 2.723047$

O $-0.125109-1.1063861 .825802$

C 0.2229450 .1336812 .451904

C $-0.901908 \quad 0.5942493 .389515$

C -1.280086 -0.4865494 .398849$

C $-1.575695-1.8040583 .682328$

H $0.458319-2.4184553 .308412$

C $-0.739617-3.437266 \quad 1.896947$

H 0.2913300 .8683001 .643185

O $\quad-0.522533 \quad 1.7878554 .065652$

H $-1.786640 \quad 0.8370912 .789571$

H $-0.493864-0.6160255 .151091$

O $-2.443778-0.0732485 .119297$

H $-2.526101-1.7146883 .143851$

O $-1.732712-2.8406844 .648440$

C $-1.899855-3.2887690 .934757$

H $0.137683-3.690891 \quad 1.292576$

H $-0.948127-4.2753852 .571378$

H -1.252982 2.0312204 .659768

H $-3.163642 \quad 0.0587744 .479040$

H $-2.432150-2.5608685 .263774$

O $-2.297917-4.5269420 .518092$

O $-2.380190-2.2256640 .568587$

C $-3.390855-4.541018-0.406290$

H $-4.272897-4.0722730 .040913$

H $-3.110969-4.030566-1.332422$

H -3.626704 -5.582971 - 0.637755

SCF Energy (B3LYP/6-31G**/MMFF) $=-3245.92771242$

06_00224

MMFFF Geometry

C $-0.483397-3.758735-0.592984$

C $-0.475976-2.966568-1.675963$

C $0.668726-2.062529-2.040078$

O $0.121882-0.732845-2.228235$

C $1.330472-2.444491-3.374514$

C $2.006188-3.796718-3.395215$

C $3.228400-4.041199-2.884230$

C $1.241599-4.892618-4.090446$

C $4.128285-3.074750-2.164370$

C $4.521047-3.628129-0.788070$

C $5.417709-2.656197-0.008406$

C $5.961601-3.3316801 .252558$

C $6.782837-2.3629142 .112917$

$\begin{array}{lllll}\text { O } & 4.627563 & -1.517240 & 0.348057\end{array}$

O $6.983088-2.998696 \quad 3.384270$

C $5.952444-1.0841732 .344549$

C $8.178770-2.1305341 .523978$

O $6.722268-0.103742 \quad 3.038109$

C $5.342569-0.4908601 .050677$

C 4.3300610 .6199551 .426504

$\begin{array}{lllll}\text { O } & 6.391313 & 0.058018 & 0.255118\end{array}$

C $0.085707 \quad 0.102284-1.157741$

O $0.478839-0.124623-0.025435$

C $-0.5204631 .370869-1.616753$

C $-0.2546802 .503790-0.951933$

C $-0.7669843 .835600-1.273820$

C $-1.8225653 .942399-2.342360$

C $3.574095 \quad 1.242727 \quad 0.245380$

C $4.2650352 .441389-0.382380$

C $3.9257024 .135224-2.183434$
O $5.3214732 .907614 \quad 0.034245$

C $3.1841495 .297689-1.601296$

C $2.3217216 .053318-2.297619$

C $1.5708777 .217984-1.716078$

C $0.0712847 .061814-1.846107$

C $-0.5882957 .613097-2.878987$

C $-0.6748506 .340489-0.744995$

C $-0.2804424 .897486-0.598331$

N $3.5452232 .934852-1.462461$

H $\quad 0.399975-3.7690430 .043096$

H $-1.335362-2.935792-2.339217$

H $1.427795-2.039302-1.248583$

H $2.059360-1.667757-3.644268$

H $0.588944-2.387974-4.184219$

H $3.635690-5.046092-2.989576$

H $1.782970-5.844652-4.087061$

H $0.277691-5.061829-3.601482$

H $1.057865-4.621511-5.135323$

H $5.031563-2.930430-2.769359$

H $3.664402-2.090786-2.052168$

H $3.608398-3.827041-0.210343$

H $5.043118-4.584425-0.920151$

H $6.240981-2.338051-0.658698$

H $6.558408-4.2158780 .995489$

H $5.121483-3.7045901 .855733$

H $7.436074-2.3619733 .963858$

H $5.131948-1.3561933 .022570$

H $8.722320-3.0799791 .447437$

H $8.784682-1.4933702 .177608$

H $8.155222-1.6802620 .529301$

H 7.3244260 .3081512 .395215

H 3.5703910 .1722182 .081421

H 4.8298491 .4085922 .001080

H $6.0003080 .638649-0.415927$

H $-1.148998 \quad 1.338462-2.499334$

H $0.4149572 .456499-0.094963$

H $-1.424843 \quad 3.618353-3.310312$

H $-2.1978234 .959157-2.479188$

H $-2.6887823 .320035-2.090707$

H $3.3641950 .499497-0.531019$

H 2.6125491 .6115560 .622985

H $3.6815603 .973081-3.237861$

H $5.0043524 .297809-2.093931$

H $3.3695895 .528750-0.554827$

H $2.1485335 .830340-3.348037$

H $1.8993378 .124083-2.242104$

H $1.8310137 .379641-0.662596$

H - $1.6664277 .533468-2.970087$

H $-0.0633888 .154284-3.659391$

H $-1.7603886 .420702-0.878879$

H -0.4759576 .8514880 .206281$

$\begin{array}{lllll}H & 0.487266 & 4.729046 & 0.157464\end{array}$

H $2.6478332 .516085-1.689129$

C $-2.957851-4.471709-0.825527$

O $-3.456638-3.139565-0.594503$

C $-4.884300-3.197882-0.422232$

C $-5.294038-4.637469-0.715902$

C $-4.050561-5.400896-0.300890$

H $-5.083373-2.9872730 .634552$

C $-5.564376-2.143467-1.302676$

H $-6.187326-4.934559-0.157989$

H $-5.499080-4.793267-1.781424$

C $-3.990416-6.811465-0.859925$

H $-4.014257-5.4441300 .795958$

C -5.069152 -0.698991-1.076965

O $-5.334486-2.462509-2.681704$

H $-6.650236-2.191700-1.161191$

H $-4.043507-0.584353-1.448581$

O $-5.8850690 .167119-1.876553$

C $-5.137990-0.220016 \quad 0.384297$

C $-4.803191 \quad 1.2767150 .538985$

H -4.438652 -0.8101020 .984660$

$\begin{array}{lllll}\text { O } & -6.445248 & -0.460029 & 0.905640\end{array}$

H $-3.837943 \quad 1.4739880 .060382$

H $-5.555543 \quad 1.8740620 .010142$

H $-4.843551-7.400774-0.508241$

H $-4.010914-6.810663-1.954933$

H $-3.075598-7.320027-0.540604$ 
C $-1.608634-4.645976-0.123508$ H -4.371899 -2.489605 -2.819660 H -5.823739 -0.144751 -2.795858 H -7.0759710 .0366840 .356449$ H -1.274339 $-5.685802-0.214200$ H -1.742766 -4.443096 0.946294 H -2.847901 -4.598843 -1.910281 C -2.5010391 .1288682 .541808$ O -3.8957650 .9339062 .811618$ C -4.7807581 .7295552 .017133$ C -4.4766903 .2190262 .226199$ C -3.0017243 .5370661 .999502$ C -2.1144432 .5888872 .807244$ H -2.284759 0.8679511 .498680 C $-1.729800 \quad 0.158178 \quad 3.441112$ H -5.7881831 .5532792 .414739$ O $\quad-5.286904 \quad 4.011744 \quad 1.364928$ H -4.7408003 .4925123 .256168$ H $-2.759612 \quad 3.5007510 .932427$ O -2.7598634 .8809122 .420955$ H -2.2135152 .8303413 .873260$ O -0.7606412 .8268782 .433370$ C $-1.975679-1.2672993 .004096$ H -0.6478300 .3194203 .397185$ H $-2.033728 \quad 0.273416 \quad 4.488433$ H -5.0447624 .9420251 .512899$ H -1.812077 5.0572872 .291577 H -0.1889792 .3875883 .084702$ O $-2.754039-1.9119933 .918438$ O $-1.543560-1.7416921 .961556$ C $-3.075967-3.270028 \quad 3.603329$ H -3.627873 -3.320177 2.660543 H $-2.164200-3.8724593 .550288$ H $-3.710514-3.6634824 .401847$ SCF Energy (B3LYP/6-31G**//MMFF) $=-3245.93112908$

0600225

MM̄MF Geometry

C -1.081716 3.4085172496834

C -1.4250052 .2257701 .963202$

C -1.7833361 .0245402 .802739$

O $-0.922648-0.0566752 .377173$

C $-3.247248 \quad 0.6200812 .555811$

C -3.809032 -0.2742383 .642841$

C $-3.979782-1.6063613 .536815$

C $-4.2347810 .439636 \quad 4.900968$

C -3.614747 -2.498929 2.384349

C $-4.847378-3.0971801 .692292$

C -5.670195 -2.064226 0.906137

C $-6.904082-2.7209830 .282251$

C $-7.686897-1.744475-0.609077$

O $-4.827685-1.525696-0.116985$

O $-8.619668-2.509311-1.388219$

C $-6.705096-1.084483-1.600474$

C $-8.519583-0.7560650 .215219$

O $-7.350922-0.067640-2.362326$

C $-5.441403-0.511500-0.923367$

C $-4.382506-0.035370-1.944832$

O $\quad-5.8061050 .625105-0.131784$

C $-0.412366-0.8853883 .323275$

O $-0.580106-0.8093184 .532630$

C $0.449876-1.9284632 .720714$

C $0.455774-2.2200141 .410370$

C $1.322213-3.1973970 .746208$

C $2.395622-3.8564911 .571875$

C $-3.863038-1.129293-2.879591$

C -2.673444 -0.651931-3.687002

C $-0.227720-0.205687-3.505740$

O $-2.774101-0.371088-4.879139$

C $0.547033-1.452225-3.794391$

C $1.775762-1.688683-3.310446$

C $2.540975-2.954768-3.564372$

C $2.968079-3.645235-2.286589$

C $4.262186-3.686372-1.928000$

C $1.912772-4.359374-1.469508$

C $1.144238-3.425459-0.571920$

N -1.510461 $-0.574831-2.937542$

H -1.0572173 .4880823 .582847$
H -1.4663152 .0992840 .885283$

H -1.6113631 .2551083 .860943$

H $-3.3344750 .137214 \quad 1.576570$

H $-3.884247 \quad 1.5134502 .500632$

H $-4.416067-2.1346304 .383926$

H $-4.672147-0.2414205 .638759$

H -3.3794510 .9305335 .375000$

H $-4.987936 \quad 1.199874 \quad 4.668975$

H -2.975386 -1.995567 1.653580

H -3.010135 -3.321684 2.786600

H $-4.490572-3.8707480 .999713$

H $-5.484123-3.5959832 .433641$

H $-5.976175-1.2593961 .583444$

H -7.556431 -3.139112 1.058934

H $-6.589546-3.574321-0.335100$

H $-9.197372-2.993589-0.773660$

H -6.407346 -1.864288 -2.312427

H $-9.247826-1.2931690 .834841$

H $-9.107022-0.097366-0.433911$

H -7.916283 -0.1360490 .881443$

H $-8.136061-0.468113-2.773578$

H $-4.7763370 .801635-2.536003$

H $-3.5342750 .384673-1.386576$

H $-5.0197431 .181702-0.007479$

H $1.088030-2.4507653 .426135$

H $-0.227434-1.6880070 .750144$

H $1.949661-4.4833162 .351689$

H $3.033112-3.1026952 .047454$

H $3.061526-4.4966450 .989434$

H -3.563039 -2.023416 -2.321039

H $-4.639589-1.438566-3.587560$

H $0.2743430 .427409-2.770135$

H $-0.3761180 .367032-4.426199$

H $0.070420-2.199131-4.424968$

H $2.259733-0.936119-2.693027$

H $3.419347-2.696174-4.169796$

H $1.957334-3.658875-4.170544$

H $4.584957-4.202996-1.030507$

H $5.028711-3.199875-2.521943$

H $2.360453-5.178355-0.895566$

H $1.206513-4.858336-2.145659$

H $\quad 0.339895-2.895005-1.081577$

H - $1.523643-0.888458-1.971145$

C $-0.730240 \quad 4.6809880 .252959$

O $0.3790283 .895675-0.227983$

C $1.1153134 .667066-1.194938$

C $0.1717845 .775660-1.637356$

C $-0.5412346 .083795-0.336282$

H $1.9624485 .115560-0.661849$

C $1.6337283 .768331-2.322258$

H $0.7047556 .636752-2.051237$

H $-0.5469585 .425755-2.387297$

C $-1.8378976 .853260-0.522176$

H $\quad 0.1330196 .672074 \quad 0.300910$

C $2.4194072 .532947-1.837578$

O $0.5241513 .291418-3.094658$

H $2.2466404 .358035-3.013334$

H $1.730197 \quad 1.836427-1.348605$

O $2.9349651 .856526-2.990773$

C $3.5996482 .848086-0.904299$

C $4.4436071 .626798-0.502647$

H $3.2442783 .347998-0.000826$

O $4.4714983 .785243-1.547025$

H $4.9649931 .238958-1.389200$

H 5.2601901 .9853530 .136476

H $-1.6458297 .821789-0.995323$

H -2.543068 $6.304157-1.155172$

H $-2.323658 \quad 7.0382650 .441016$

C $-0.7288734 .688524 \quad 1.785270$

H $-0.0856972 .841136-2.485009$

H $2.1873091 .690840-3.590202$

H $4.7692383 .383721-2.381574$

H -1.432013 5.4521282 .139494

H 0.2692484 .9802522 .137140

H -1.647538 $4.224045-0.137858$

C $5.663263-0.5926651 .141507$

O $4.554344-0.7017540 .241414$

C 3.7051670 .4560160 .180401 
C $3.1256110 .715181 \quad 1.575763$

C 4.2223560 .8765232 .624474

C $5.181005-0.3151372 .571961$

H $6.332764 \quad 0.210497 \quad 0.811339$

C $6.439551-1.9169591 .072498$

H $2.8763610 .164970-0.473188$

O 2.2600761 .8393821 .579509

H $2.514174-0.1476691 .857836$

H 4.7507751 .8287132 .499798

O 3.5995550 .9224583 .910573

H $4.679448-1.2033292 .977547$

O $6.313700-0.0482563 .396303$

C $7.150022-2.098614-0.252850$

H 7.203445-1.955566 1.856639

H $5.746220-2.7574351 .195800$

H 1.9280061 .9507862 .486744

H 4.2897941 .1251154 .564094

H 6.0025040 .0108064 .315138

O $7.720645-3.339971-0.280109$

O $7.206466-1.265220-1.146431$

C $8.435734-3.662153-1.477026$

H $8.829616-4.676798-1.374856$

H $7.764254-3.631832-2.340431$

H $9.273643-2.972133-1.614265$

SCF Energy (B3LYP/6-31G**//MMFF) $=-3245.91314112$

0600226

MM̄FF Geometry

C $0.084910 \quad 1.1230063 .696930$

C 0.7349300 .3598094 .589852

C $1.336159-1.0007244 .328164$

O $1.939204-1.0684993 .015773$

C $0.254230-2.0897654 .437432$

C $0.807326-3.5013524 .394017$

C $0.720585-4.3341323 .337325$

C $1.452068-3.9750145 .672067$

C $0.126291-4.0529231 .984753$

C $1.154559-4.3010620 .872544$

C $0.628965-3.882504-0.509375$

C $1.676399-4.173034-1.586686$

C $1.261709-3.637307-2.965766$

O $0.349959-2.482489-0.454513$

O $2.420690-3.665288-3.813499$

C $0.843452-2.158562-2.819536$

C $0.215643-4.538000-3.632309$

O $0.327256-1.650396-4.047746$

C $-0.154817-1.914510-1.668278$

C $-0.384275-0.409611-1.399140$

O $-1.418955-2.487137-2.020559$

C $3.173219-0.5028902 .886551$

O $3.778850 \quad 0.1343853 .734580$

C $3.683847-0.8124481 .532251$

C $4.858775-0.2984921 .137168$

C $5.528128-0.532482-0.145713$

C $4.818650-1.392785-1.156876$

C $0.8503120 .344705-0.896851$

C $1.4783491 .269766-1.922389$

C $3.3679642 .882074-2.153356$

O $1.0691191 .388207-3.073161$

C $4.5165782 .144317-2.765497$

C $5.8022442 .459149-2.547400$

C $6.9547391 .748974-3.196482$

C $7.9528651 .213722-2.195239$

C $9.1099691 .855940-1.961291$

C $7.651606-0.114181-1.537006$

C $6.7428740 .021033-0.346987$

N $2.563217 \quad 1.952263-1.385762$

H -0.0639240 .7574562 .684868$

H $\quad 0.835261 \quad 0.7226345 .610867$

H $2.114219-1.1825345 .080543$

H $-0.490842-1.9460213 .648676$

H $-0.299577-1.9715595 .378956$

H $1.127907-5.338973 \quad 3.445081$

H $1.774580-5.0202175 .613424$

H $2.335128-3.3724755 .904890$

H $0.746308-3.8962586 .505438$

H $-0.735648-4.7142591 .837143$

H $-0.248142-3.0294921 .915083$
H $2.063439-3.7294391 .100860$

H $1.432502-5.3623580 .856861$

H $\quad-0.299764-4.427504-0.715069$

H $1.889834-5.247980-1.639460$

H $2.625602-3.696488-1.306292$

H $2.740268-4.582847-3.854884$

H $1.761473-1.592025-2.624225$

H $\quad 0.607579-5.555148-3.752928$

H $-0.020487-4.188795-4.643456$

H $-0.713458-4.609416-3.062980$

H $-0.580463-1.984185-4.148705$

H $-0.8408690 .065886-2.274219$

H -1.138495 -0.332793 -0.605982

H -2.117629 -2.021747 -1.531800

H $3.093158-1.4718560 .908427$

H $\begin{array}{llll}5.398066 & 0.349508 & 1.827292\end{array}$

H $4.706534-2.414080-0.777600$

H $5.336378-1.461433-2.115435$

H $3.826038-0.988509-1.377228$

H $0.5605940 .960291-0.038482$

H $1.620407-0.345042-0.543947$

H $3.6974483 .670136-1.468850$

H $2.7561073 .335022-2.940018$

H $4.2762051 .325729-3.440040$

H $6.0418403 .292911-1.891256$

H $7.4463592 .462639-3.870556$

H $6.6099790 .928629-3.838202$

H $9.8447551 .461477-1.267235$

H $9.3454912 .793396-2.454171$

H $8.584954-0.570170-1.179741$

H $7.274295-0.810748-2.290198$

H 7.1732930 .6238190 .454328

H $2.8689361 .731727-0.442174$

C -0.4728683 .5051872 .933678$

O -1.4358353 .1736401 .914101$

C -0.9390263 .6609820 .651722$

C 0.3474244 .4285850 .953758

C $0.846623 \quad 3.7171982 .197635$

H $-0.684707 \quad 2.781173 \quad 0.055692$

C $-2.0108664 .479063-0.075130$

H $1.056898 \quad 4.3881780 .121457$

H $\quad 0.1479835 .4846001 .170389$

C 1.8779034 .5046352 .984341

H $1.2833802 .758708 \quad 1.890051$

C $-3.3268653 .723444-0.358318$

O -2.3357315 .6341300 .708673$

H -1.598550 $4.863137-1.015765$

H -3.890782 3.5722590 .570063

O $-4.1359604 .571496-1.184472$

C $-3.1512912 .369435-1.071742$

C $-4.4785401 .776951-1.586658$

H $-2.6809101 .663735-0.379573$

O $-2.2654242 .527535-2.181696$

H $-5.2165991 .799375-0.778761$

H $-4.8586752 .410808-2.396347$

H 2.7665524 .6925042 .372759

H 1.4824355 .4729153 .309151

H 2.1919293 .9520503 .875513

C $-0.4947642 .464190 \quad 4.054451$

H -2.6470515 .3217831 .575659$

H $-4.2434905 .416251-0.714604$

H -2.660341 $3.181555-2.783567$

H -1.5367892 .3036554 .354928$

H $0.0356422 .875457 \quad 4.922297$

H $\quad-0.8142374 .4573293 .363439$

C $-4.886873-0.901503-0.128295$

O $-3.889089-0.572276-1.104201$

C $-4.3038270 .338170-2.127914$

C $-5.523801-0.223561-2.868012$

C $-6.650414-0.589367-1.909739$

C $-6.131429-1.499478-0.797335$

H $\quad-5.1676190 .0021380 .425912$

C $-4.238113-1.8883660 .850981$

H -3.484711 $0.369451-2.857090$

O $\quad-5.986250 \quad 0.711391-3.836701$

H -5.220119 -1.125984 -3.415034

H $-7.1285530 .309989-1.503447$

O $-7.659239-1.283119-2.647995$ 
H $-5.911201-2.489503-1.216949$

O $-7.181703-1.6524630 .156225$

C $-3.431446-1.1397641 .884022$

H -4.972666 -2.499804 1.385396

H -3.562993 -2.584202 0.339220

H $-6.7685420 .323468-4.265053$

H -8.353720 -1.539458 -2.017018

H $-6.959559-2.4042170 .729929$

O $-4.225067-0.8336712 .950565$

O $-2.254729-0.835291 \quad 1.745831$

C $-3.599844-0.0739203 .987967$

H -3.3576650 .9254883 .616995$

H -4.3084580 .0209714 .814846$

H $-2.703219-0.5833604 .352866$

SCF Energy $\left(B 3 L Y P / 6-31 G^{* *} / / M M F F\right)=-3245.92515504$

06_00227

MM̄MF Geometry

C $0.037141-3.2858552 .081151$

C $-0.381045-2.046677 \quad 1.785223$

C $-1.040111-1.1441382 .795320$

O $-2.316976-0.7254342 .261261$

C $-0.2117590 .133862 \quad 3.011916$

C -0.3660140 .6915984 .412586$

C -1.2981371 .5859654 .794597$

C 0.6487000 .1869715 .406548

C -2.4220452 .1630473 .981016$

C $-2.2651413 .666743 \quad 3.726707$

C -1.234995 3.9883432 .633417

C -1.0798215 .5032542 .479458$

C -0.1497375 .8704801 .315509$

O -1.7076143 .4152241 .407996$

O $-\begin{array}{llll}0.319876 & 7.274651 & 1.070522\end{array}$

C -0.6108275 .1073480 .056072$

C 1.3238155 .6751481 .690040

O $0.2875365 .331980-1.026899$

C $-0.829517 \quad 3.5935150 .290570$

C -1.497374 $2.962605-0.954815$

O 0.4373092 .9691210 .507455

C -3.368545 -1.579866 2.336066

O $-3.388890-2.6951242 .828489$

C $-4.497453-0.9114561 .654768$

C $-5.488804-1.6476871 .134654$

C $-6.616322-1.1334920 .354601$

C -6.7310690 .3608490 .195140$

C $-1.9962671 .522876-0.754715$

C $-2.5501690 .935363-2.043692$

C $-3.688838-1.098636-2.929031$

O $-2.4750391 .508235-3.127957$

C $-5.146352-0.786409-3.070721$

C $-6.091501-1.727764-3.221888$

C -7.552563 -1.425317 -3.408804

C $-8.446528-2.239205-2.497261$

C $-9.091945-3.325836-2.953845$

C $-8.655713-1.747224-1.081431$

C $-7.466452-2.019871-0.203924$

N -3.119859-0.315401-1.846268

H $-0.092286-3.6600373 .094336$

H $-0.267073-1.6654950 .773972$

H -1.201251 -1.666477 3.747350

H -0.4896970 .8983352 .276928$

H $0.856034-0.0619112 .844660$

H -1.285516 1.9235935 .830491

H $\quad 0.489990 \quad 0.596008 \quad 6.409915$

H $0.595762-0.9039875 .482801$

H 1.6595570 .4654405 .091226

H -2.563059 1.6361633 .035332

H -3.3463192 .0033034 .550847$

H -3.244742 4.0494403 .411224

H -2.001541 4.1811094 .659097

H -0.2748703 .5328442 .900321$

H -0.7239445 .9556203 .413687$

H -2.065228 5.9515042 .288395

H 0.2081497 .5026180 .285945

H -1.567293 $5.551623-0.252042$

H 1.5824006 .2998462 .553671

H 1.9864965 .9996580 .880172

H 1.5690034 .6416471 .943458
H $1.0913304 .812212-0.856241$

H -2.365521 $3.568933-1.245901$

H $-0.7931432 .984067-1.795391$

H 0.3928052 .0432920 .222197

H -4.4478100 .1662521 .547199$

H -5.451572 -2.729598 1.255713

H -6.8444910 .8417631 .173217$

H $-7.5839010 .674175-0.409556$

H $-5.8390840 .768169-0.293750$

H $-1.184450 \quad 0.870171-0.417793$

H $-2.789368 \quad 1.5052370 .000943$

H -3.514925 -2.153247 -2.693491

H -3.167666 -0.854460 -3.860461

H $-5.4362800 .261807-3.078326$

H -5.795979 -2.774289-3.249467

H -7.801909 -1.636254 -4.457001

H -7.762060 -0.358405 -3.262389

H $-9.755333-3.899789-2.315316$

H -8.967095 -3.668991-3.975657

H $-9.517341-2.254175-0.626852$

H $-8.936736-0.691248-1.109870$

H -7.292534 -3.083199-0.031920

H -3.177902 -0.693002 -0.905370

C $2.083871-4.5981531 .484679$

O $2.969734-3.517848 \quad 1.119146$

C $4.162344-4.0779230 .535395$

C $4.109627-5.574506 \quad 0.817224$

C $2.618112-5.831840 \quad 0.758234$

H $4.083646-3.931064-0.547866$

C $5.413506-3.3786831 .076396$

H $4.676857-6.1552220 .083540$

H $4.500044-5.8139561 .813272$

C $2.207383-7.1511001 .389278$

H $2.297435-5.818237-0.292330$

C $5.470576-1.8644750 .783665$

O $5.448374-3.5617992 .496917$

H $6.307525-3.8733160 .677322$

H $4.697073-1.3348001 .353161$

O $6.731520-1.3752651 .257748$

C $5.342890-1.505005-0.710404$

C $5.627357-0.015988-0.987145$

H $4.334619-1.759029-1.049902$

O $6.265522-2.303301-1.452660$

H $5.0232660 .591010-0.304437$

H $6.6809090 .192947-0.765999$

H $2.680187-7.9899220 .868088$

H $2.502286-7.2011252 .442840$

H $1.123105-7.2899211 .336540$

C $0.650256-4.238691 \quad 1.094846$

H $6.334753-3.3069062 .803432$

H $6.711581-1.4073872 .228912$

H $5.995628-2.276969-2.385858$

H $\quad 0.023678-5.1376531 .078563$

H $0.633010-3.8083490 .087123$

H $2.173085-4.7302632 .571247$

C $3.0065110 .955754-2.260193$

O $4.0047330 .110528-2.848474$

C $5.3516760 .390671-2.452566$

C $5.7122221 .844769-2.786977$

C $4.7012962 .829117-2.210016$

C $3.2821472 .426444-2.605636$

H $3.0025810 .823871-1.171559$

C $1.6445730 .499072-2.807504$

H $5.990828-0.238514-3.083552$

O $7.0119372 .175306-2.309356$

H $5.7305921 .970386-3.877613$

H $4.7975922 .914012-1.120956$

O $4.9626754 .131249-2.739871$

H $3.1485092 .604203-3.680675$

O $2.3766493 .276401-1.908708$

C $1.229534-0.854772-2.271173$

H $\quad 0.8513201 .199571-2.531405$

H $\quad 1.6996530 .427640-3.900488$

H $7.6378861 .545582-2.705838$

H $5.8800264 .357773-2.509427$

H $1.5175163 .223716-2.359397$

O $\quad 0.230242-1.355823-3.056233$

O $1.696281-1.392630-1.277021$ 
C $-0.273637-2.637820-2.669218$

H $-0.980465-2.969986-3.434167$

H $\quad 0.539013-3.367834-2.604623$

H -0.800409 -2.558896 -1.713875

SCF Energy (B3LYP/6-31G**//MMFF)= -3245.91479092

06_00228

MM̄FF Geometry

C -0.265610 -3.382350 1.056678

C $-0.764764-2.1405730 .964720$

C $-1.141500-1.2996122 .156018$

O $-2.575318-1.1261262 .072280$

C -0.4797750 .0867762 .086179$

C $-0.596488 \quad 0.866734 \quad 3.381134$

C -1.4941201 .8435803 .616750$

C 0.4144120 .5114014 .440504

C -2.598882 2.3293722 .720917

C -2.3623873 .7596842 .216436$

C -1.285978 3.8449801 .123742

C -1.0494405 .2986410 .709600$

C $-0.0624015 .407419-0.463815$

O $-1.7536153 .091640 \quad 0.001189$

O $-0.1479986 .741781-0.987502$

C $-0.5163264 .449236-1.586349$

C $1.3875675 .214935-0.004867$

O $0.433324 \quad 4.421456-2.649504$

C $-0.8332293 .017143-1.093326$

C -1.510235 2.207291-2.224671

O $\quad 0.3844202 .373594-0.717159$

C $-3.322542-1.2883623 .191527$

O $-2.914714-1.562338 \quad 4.311611$

C $-4.766912-1.1144232 .904242$

C $-5.244160-0.6909801 .722927$

C $-6.654966-0.5255941 .364051$

C -7.689516 -0.9057872 .390304$

C $-2.0567630 .844085-1.776635$

C $-2.8311650 .147152-2.881776$

C $-4.392585-1.764685-3.242161$

O $-2.826614 \quad 0.527759-4.049401$

C $-5.759269-1.154625-3.231600$

C $-6.847966-1.761617-2.735423$

C $-8.213418-1.135061-2.722245$

C $-8.783650-0.999402-1.326637$

C $-9.737701-1.838370-0.889088$

C $-8.3165260 .159198-0.471812$

C $-6.955531-0.0723790 .128868$

$\mathrm{N}-3.515488-0.964349-2.406813$

H $-0.098715-3.8180882 .038768$

H - $0.957623-1.719737-0.018559$

H $-0.863729-1.8196973 .080618$

H $-0.917217 \quad 0.6610201 .262425$

H $0.586160-0.0129361 .842371$

H -1.4587012 .3464344 .582840$

H $\quad 0.2550011 .0656945 .371766$

H $0.362403-0.5540854 .683588$

H 1.4243390 .7429224 .090324

H -2.781544 1.6522021 .881847

H -3.522862 2.320269 3.313102

H -3.3135784 .1239081 .806337$

H -2.099829 4.4145253 .056600

H -0.3568943 .4020001 .499122$

H -0.7002495 .8942541 .562336$

H -2.0031465 .7495250 .400751$

H $0.0650027 .360699-0.268112$

H -1.432875 4.876854 -2.015818

H 1.6518925 .9615600 .753563

H $2.0874085 .372162-0.832758$

H 1.5733654 .2290780 .426188

H $1.1750443 .859295-2.369824$

H -2.352039 $2.787682-2.625091$

H $-0.8011392 .050063-3.047318$

H $0.2756891 .412833-0.789546$

H -5.424886 -1.356862 3.732596

H $-4.541090-0.444428 \quad 0.929092$

H -7.565501-1.951411 2.693733

H $-8.715915-0.8089492 .030015$

H -7.602009 -0.268945 3.277498

H $-1.2375270 .181186-1.482201$
H $-2.7267640 .968347-0.918221$

H $-4.388497-2.782670-2.840412$

H $-4.003616-1.789143-4.265134$

H -5.855609 -0.158243 -3.657080

H $-6.758354-2.762559-2.319802$

H -8.871002 -1.759605 -3.340867

H -8.206122 -0.149476 -3.204342

H - $10.176022-1.7314130 .097434$

H $-10.099530-2.654136-1.506202$

H -9.0535630 .3898210 .305458$

H $-8.2785001 .068303-1.085838$

H $-6.1350020 .177331-0.543797$

H -3.515918-1.154077-1.408685

C $1.462888-4.772528-0.131797$

O $2.362113-3.699670-0.475733$

C $3.364811-4.204523-1.379969$

C $3.203346-5.719686-1.393235$

C $1.711581-5.864045-1.172511$

H $3.114898-3.817058-2.374378$

C $4.756622-3.718265-0.961671$

H $3.545057-6.164592-2.332801$

H $3.756380-6.195911-0.575300$

C $1.299111-7.255850-0.724497$

H $1.191703-5.617369-2.108000$

C $4.882601-2.184689-0.851224$

O $5.085666-4.2743490 .317948$

H $5.509213-4.107713-1.656447$

H $4.244114-1.821357-0.042056$

O $6.234456-1.889085-0.480095$

C $4.564567-1.428687-2.151103$

C $4.8375860 .088097-2.118716$

H $3.519969-1.591759-2.434987$

O $5.361320-1.981846-3.206351$

H $5.8233570 .279084-1.681403$

H $4.9188970 .424508-3.160532$

H $1.571041-7.999171-1.481170$

H $\quad 1.788785-7.5368170 .213980$

H $0.217295-7.313171-0.569960$

C $0.022368-4.259840-0.130502$

H $4.397023-3.9962390 .944702$

H $6.411303-2.3498330 .358106$

H $6.295093-1.859761-2.962021$

H $-0.673627-5.105415-0.087957$

H $-0.178063-3.714936-1.061380$

H $1.750820-5.1246860 .867545$

C 4.7047801 .1196590 .800335

O $3.6264140 .646429-0.014935$

C $3.7432260 .917447-1.413026$

C $3.8786682 .429952-1.637098$

C $5.0502113 .012459-0.854815$

C 4.9320652 .6276220 .617958

H 5.6187450 .5687550 .551437

C 4.3388640 .8239672 .261778

H $2.7886090 .614627-1.862415$

O $4.0178132 .718092-3.023759$

H $2.9583532 .920108-1.302415$

H $6.0090492 .696770-1.281904$

O $4.9991414 .435693-0.976042$

H 4.1060313 .1894511 .073371

O 6.1281432 .9971881 .301651

C $4.335804-0.6576902 .557072$

H 5.0465391 .2836112 .961007

H $3.348107 \quad 1.2357772 .491262$

H $4.1174303 .681539-3.112055$

H $5.8017104 .794735-0.561841$

H 6.1931233 .9666661 .279738

O $3.070582-1.0690372 .851960$

O $5.336634-1.3615932 .528951$

C $2.915318-2.4476523 .201142$

H $3.701527-2.7756423 .888012$

H $1.949243-2.5637333 .699670$

H $2.916279-3.0555882 .294378$

SCF Energy (B3LYP/6-31G**//MMFF)= -3245.92411631

06_00229

MM̄MF Geometry

C $1.372661-3.0309210 .649549$

C $0.557540-2.384127 \quad 1.497015$ 
C $0.937908-1.3904672 .566093$

O $0.341336-0.1306622 .171472$

C $2.447904-1.1598472 .756280$

C $2.798414-0.4212444 .032823$

C 3.2239380 .8553274 .093695

C $2.711343-1.2427215 .294552$

C 3.3703391 .8471092 .973228

C 4.8379772 .0606062 .571238

C 5.3357881 .0061541 .571126

C 6.8399941 .1349051 .334096

C 7.3284340 .1488490 .260306

$\begin{array}{lllll}O & 4.632823 & 1.226819 & 0.344495\end{array}$

O $8.6615030 .530499-0.111857$

C $6.442898 \quad 0.303288-0.994309$

C $7.421330-1.2834510 .799370$

O $6.772371-0.676777-1.976491$

C $4.923908 \quad 0.289888-0.697504$

C $4.1446780 .731426-1.959487$

O $4.527426-1.034604-0.342549$

C $-0.372765 \quad 0.562893 \quad 3.091484$

$\begin{array}{lllll}\text { O } & -0.620206 & 0.219731 & 4.239190\end{array}$

C -0.8564221 .8487032 .543029$

C -0.4268082 .3822351 .389252$

C $-0.859097 \quad 3.6854230 .898751$

C $0.2768114 .630786 \quad 0.623230$

C $2.6468040 .985752-1.728303$

C $2.0170601 .658522-2.937231$

C $0.090873 \quad 3.076352-3.600843$

O $2.4813031 .561060-4.070670$

C $-0.5897384 .240828-2.958348$

C $-1.9204974 .351944-2.832582$

C $-2.6072455 .552682-2.251370$

C $-3.4489075 .220840-1.041411$

C $-4.7677324 .990916-1.158170$

C $-2.782936 \quad 5.270360 \quad 0.313782$

C $-2.1657593 .960698 \quad 0.716665$

N $0.874076 \quad 2.371279-2.604899$

H $2.443373-2.8567120 .670799$

H $-0.510589-2.5822241 .426949$

H $\quad 0.491612-1.7566003 .498337$

H $2.836854-0.6215251 .886305$

H $2.980512-2.1191052 .785916$

H 3.4829051 .2593685 .072138

H $3.050553-0.688767 \quad 6.176455$

H $1.679916-1.5557375 .482257$

H $3.337218-2.1372045 .209659$

H $2.756154 \quad 1.5829872 .107035$

H 2.9676732 .8011503 .337388

H 4.9131753 .0511342 .103474

H 5.4761342 .0729643 .463310

H $5.1038190 .007414 \quad 1.954347$

H 7.3958320 .9903302 .268853

H 7.0729872 .1559851 .000734

H 9.2152350 .5031930 .687259

H $6.6988631 .273372-1.442148$

H $8.087965-1.3225921 .669196$

H $7.862163-1.9570390 .056064$

H $6.455007-1.6903711 .104852$

H $6.378690-1.520493-1.695918$

H $4.5836971 .665360-2.335378$

H $4.256182-0.021159-2.750353$

H $3.585101-1.144276-0.545736$

H -1.5701742 .3773313 .169324$

H $\quad 0.327917 \quad 1.8850550 .786189$

H $0.758544 \quad 4.9277181 .561252$

H -0.0360105 .5414650 .107128$

H $1.0325844 .155448-0.012096$

H $2.1127370 .046290-1.551027$

H $2.5018081 .632092-0.855629$

H $-0.6181172 .364942-4.031726$

H $0.7455473 .424157-4.406732$

H $0.0480095 .048599-2.607625$

H -2.559218 $3.554377-3.203674$

H -3.240326 $5.985655-3.037224$

H -1.887902 $6.340224-1.994560$

H $-5.3849084 .792286-0.288315$

H $-5.2574394 .991640-2.126258$

H -3.5286315 .5234891 .080088$
H $-2.069805 \quad 6.099876 \quad 0.346304$

H $-2.893387 \quad 3.1763160 .926559$

H $0.4621912 .228931-1.687106$

C $0.835880-3.411379-1.767607$

O $-0.201165-2.409769-1.798697$

C -1.057395-2.657262 -2.926155

C $-0.256478-3.558293-3.855054$

C $0.475608-4.428917-2.852140$

H $-1.922238-3.216934-2.551947$

C $-1.532431-1.337344-3.534932$

H $-0.895149-4.128952-4.535864$

H $0.462469-2.989619-4.456020$

C $1.675860-5.154110-3.435444$

H $-0.228742-5.171458-2.453384$

C -2.147006 $-0.359937-2.511753$

O $-0.407528-0.683161-4.134559$

H $-2.239220-1.543671-4.347453$

H $-1.3616860 .012640-1.842853$

$\begin{array}{lllll}0 & -2.617627 & 0.784146 & -3.234797\end{array}$

C $-3.305459-0.932581-1.672444$

C $-3.8743600 .110035-0.690616$

H $-2.947707-1.791636-1.096848$

O $-4.333715-1.408888-2.537404$

H -3.0825040 .3753710 .019157$

H $-4.129604 \quad 1.027179-1.235490$

H $1.364453-5.827570-4.240605$

H $2.407308-4.451549-3.849056$

H $2.179463-5.752284-2.669466$

C $0.889436-4.025401-0.369282$

H $-0.7371440 .126713-4.559335$

H -3.261676 $0.478140-3.895361$

H $-4.747224-0.638833-2.962849$

H $1.566114-4.887435-0.354496$

H $-0.104116-4.392495-0.085717$

H $1.778592-2.902309-2.006077$

C $-4.110078-1.7292161 .759712$

O $-5.000618-1.6530100 .641790$

C $-5.141826-0.3572110 .057589$

C $-5.6483790 .640631 \quad 1.107983$

C -4.7608800 .6488552 .348720$

C $-4.558917-0.7712722 .872048$

H $-3.090147-1.4871811 .435662$

C $-4.129789-3.1787782 .265208$

H $-5.931416-0.452159-0.698598$

O

H -6.6662740 .3643131 .412094$

H -3.7989051 .1330352 .151839$

O $-5.392417 \quad 1.418622 \quad 3.374156$

H $-5.495082-1.1284653 .320572$

O $-3.572938-0.755475 \quad 3.901717$

C $-3.383177-4.122181 \quad 1.351547$

H $-3.655339-3.255270 \quad 3.250561$

H $-5.154958-3.5537802 .371854$

H $-6.294341 \quad 1.928817-0.206397$

H -5.544463 2.3093163 .013760

H $-3.866763-0.1197304 .576440$

O $-4.074578-4.3068940 .191894$

O $-2.321268-4.6537051 .649852$

C $-3.483902-5.211723-0.746116$

H $-3.586208-6.238238-0.382523$

H $-4.020039-5.116673-1.694065$

H -2.431431 -4.968032 -0.915409

SCF Energy (B3LYP/6-31G**//MMFF) $=-3245.90486623$

06_00230

MM̄FF Geometry

C $-0.6242083 .007008 \quad 1.839701$

C $-1.512098 \quad 3.165264 \quad 2.833872$

C $-2.3475132 .098356 \quad 3.500575$

O $-2.0666850 .777420 \quad 2.994124$

C -3.8402912 .3829693 .244454$

$\begin{array}{llll}\text { C } & -4.765070 & 1.345551 & 3.852347\end{array}$

C -5.5257470 .4816893 .150189$

C -4.8169641 .3346835 .359215$

C $-5.6544340 .343610 \quad 1.657979$

C $-5.140491-1.0172481 .168864$

C $-5.371889-1.200511-0.337996$

C $-4.974641-2.606048-0.788518$ 
C $-5.141223-2.781574-2.305337$

O $-4.582036-0.231246-1.033793$

O $-4.477506-4.005173-2.658419$

C $-4.419969-1.622220-3.028329$

C $-6.612435-2.963485-2.697067$

O $-4.688639-1.683486-4.427042$

C $-4.765148-0.227232-2.456955$

C $-3.8945920 .917015-3.040036$

O $-6.1257890 .088191-2.782677$

C - -1.0954550 .0542073 .613216$

O $-\begin{array}{llll}0.306686 & 0.438841 & 4.460310\end{array}$

C -1.176861-1.319530 3.076244

C $-0.114521-2.1348483 .131876$

C $-0.066544-3.4894362 .576249$

C -1.379905 -4.146665 2.234351

C $-2.3841420 .678896-3.116198$

C $-1.7261930 .418210-1.774169$

C $0.017344-1.009694-0.706364$

O $-2.0312041 .038209-0.758765$

C $0.135391-2.499245-0.763145$

C $1.301797-3.162470-0.770592$

C $1.406861-4.662734-0.784260$

C $2.179265-5.2114180 .398823$

C $3.455027-5.6099420 .261456$

C $1.448061-5.3779151 .717270$

C $1.139690-4.0587672 .373265$

N $-0.751486-0.565453-1.852412$

H $-0.4295882 .020976 \quad 1.428555$

H -1.667197 4.1695663 .225893

H -2.149612 2.1301124 .579414

H -4.0032272 .4629672 .165212$

H -4.107229 3.3670493 .652883

H $-6.153148-0.2119613 .709548$

H -5.5778260 .6474025 .744462$

H $-3.854201 \quad 1.0248565 .776789$

H -5.0577992 .3334945 .737896$

H -6.7175290 .4427791 .405742$

H $-5.141141 \quad 1.1441691 .120824$

H $-4.067905-1.0869601 .385184$

H $-5.643777-1.8222231 .718725$

H $-6.433335-1.019755-0.544234$

H -5.550802 -3.368019-0.249012

H -3.921535 -2.787374 -0.530123

H -4.518371 -4.093454 -3.626343

H -3.347131 -1.813738 -2.906383

H $-7.030886-3.848675-2.203014$

H -6.717173 -3.144612 -3.772605

H -7.237101 -2.108318 -2.431122

H -4.139334 -1.014452 -4.867808

H -4.248051 $1.157735-4.051551$

H -4.071962 $1.822899-2.443800$

H $-6.2411211 .050526-2.710565$

H -2.109353 -1.612678 2.608811

H $\quad 0.811317-1.7622393 .567409$

H -1.282191 -5.1942001 .944049$

H -1.873731 -3.6286231 .405752$

H -2.050175 -4.132210 3.101636

H -2.168715 $-0.131551-3.820322$

H -1.908238 $1.575893-3.529194$

H $-0.484370-0.7057380 .216122$

H $0.992154-0.515247-0.744428$

H $-0.794508-3.063268-0.769364$

H $2.232405-2.600757-0.766526$

H $1.898948-4.952162-1.722058$

H $0.415843-5.132666-0.814182$

H $4.009838-6.0292921 .094125$

H $3.976853-5.518010-0.685481$

H $2.058174-5.9579562 .422038$

H $0.557431-5.9869731 .542701$

H $2.028649-3.5015072 .670224$

H $-0.471353-0.909035-2.766596$

C $-0.0279274 .270755-0.259433$

O $0.7774923 .269543-0.922163$

C $1.5986463 .919711-1.909659$

C $0.9237215 .256313-2.183078$

C $0.4473245 .625015-0.794848$

H $2.5713224 .107628-1.438726$

C $1.7846593 .042601-3.150888$
H $1.6111615 .991490-2.612026$

H $0.0684685 .150266-2.860536$

C $-0.6270346 .699379-0.786313$

H $1.3083855 .979008-0.211596$

C $2.3754371 .647891-2.868889$

O $0.5058772 .862335-3.769864$

H $2.4079493 .576583-3.878750$

H $1.6405261 .025125-2.350752$

O $2.6206681 .016403-4.132334$

C $3.6917321 .652628-2.067815$

C $4.3493130 .259899-1.999022$

H $3.4946392 .010932-1.052795$

O $4.6097122 .568376-2.666780$

H $3.611664-0.462116-1.634118$

H $4.634161-0.047777-3.012082$

H $-0.2465717 .627128-1.226012$

H -1.507038 $6.391356-1.360926$

H -0.9521496 .9181320 .235742$

C $0.1528894 .156087 \quad 1.256467$

H $0.6575502 .496225-4.657193$

H $1.7578000 .811554-4.530178$

H $4.7666842 .272266-3.579813$

H -0.1544475 .0922911 .737714$

H 1.2121644 .0018431 .490355

H -1.070507 $4.083810-0.546810$

C $4.608634-0.3868250 .997240$

$\begin{array}{lllll}\text { O } & 5.332114 & 0.596034 & 0.246013\end{array}$

C $5.6156320 .240645-1.112642$

C $6.405526-1.075140-1.165954$

C $5.703550-2.191127-0.400711$

C $5.366463-1.7217191 .012169$

H $3.611293-0.5235620 .562585$

C 4.4467620 .1455492 .429443

H $6.2841181 .021073-1.496944$

O $6.622036-1.467642-2.517140$

H $7.395122-0.910629-0.719591$

H $4.812691-2.537975-0.936699$

O $6.588761-3.310419-0.323811$

H $6.297508-1.6207761 .584228$

O $4.587664-2.7209571 .663473$

C 3.4699051 .2970452 .522582

H $4.071542-0.6406513 .094017$

H 5.4170070 .5001722 .797706

H $7.106609-2.310776-2.500838$

H $\quad 6.148615-3.990876 \quad 0.214145$

H $3.744574-2.7955161 .185536$

O 3.5582151 .8420923 .772780

O $2.715396 \quad 1.659249 \quad 1.631498$

C 2.6855602 .9447634 .034212

H 1.6426042 .6255383 .960562

H 2.8734803 .2923815 .053600

H 2.8930133 .7654143 .341468

SCF Energy (B3LYP/6-31G**//MMFF) $=-3245.91268096$

06_00231

MM̄FF Geometry

C -1.734790 $3.632337-1.885685$

C $-1.9011322 .327317-1.623756$

C $-2.3343601 .305064-2.638313$

O $-3.5616080 .741819-2.123879$

C $-1.2846330 .185758-2.743092$

C -1.474161 -0.700509-3.956235

C $-2.068947-1.909336-3.942652$

C $-0.899053-0.156450-5.238537$

C $-2.730887-2.603790-2.785293$

C $-2.003061-3.887691-2.365837$

C $-0.680105-3.630242-1.627210$

C $-0.000857-4.954350-1.272741$

C $1.261877-4.748467-0.424422$

O $-0.982774-2.907488-0.430761$

O $1.618949-6.0374450 .097923$

C $0.925021-3.8222010 .764192$

C $2.444189-4.281711-1.279046$

O $2.099772-3.4673501 .490494$

C $0.160918-2.5415240 .351058$

C $-0.350380-1.8058551 .613464$

O $1.043780-1.682457-0.372050$

C $-4.6043930 .548523-2.967404$ 
O $-4.6303310 .781885-4.167551$

C -5.787795 $0.026831-2.240855$

C $-5.747667-0.382427-0.962781$

C $-6.876911-0.868294-0.166669$

C $-8.239192-0.870502-0.808011$

C $-1.309323-0.6441341 .317365$

C -1.877620 -0.0381952 .588952$

C $-3.734008 \quad 1.4313393 .374333$

O $-1.407764-0.2534613 .703120$

$\begin{array}{llll}\text { C } & -4.802108 & 0.488732 & 3.834400\end{array}$

$\begin{array}{llll}\text { C } & -6.117065 & 0.728418 & 3.716642\end{array}$

C -7.179698 -0.2290294 .177634$

C $-8.106472-0.6614973 .061275$

C $-9.343210-0.1467832 .956030$

C $-7.636452-1.7514662 .121464$

C $-6.645638-1.252527 \quad 1.106346$

N -2.961223 0.7900122 .325895

H -1.916027 $4.010524-2.888204$

H $-1.722757 \quad 1.966230-0.613473$

H -2.500267 $1.786784-3.609371$

H $-1.294668-0.419315-1.829907$

H $-0.2759000 .617331-2.796983$

H -2.122997-2.462469 -4.879855

H $-1.041273-0.840112-6.082245$

H $-1.3761640 .792856-5.501013$

H $0.1772590 .013070-5.129722$

H -2.863175 -1.943736 -1.923694

H -3.744748 -2.873404-3.107969

H -2.678045 -4.441742 -1.700068

H -1.825393 -4.520510 -3.244371

H $-0.025664-3.024294-2.263507$

H $0.234079-5.526461-2.179141$

H $-0.701744-5.578828-0.700302$

H $2.447858-5.9429950 .596695$

H $0.291666-4.3952911 .455893$

H $2.670185-5.021832-2.056014$

H $3.352741-4.197419-0.674597$

H $2.267448-3.324138-1.773071$

H $2.500530-4.2872571 .824181$

H $-0.886223-2.5212442 .251534$

H $0.498674-1.4259672 .194636$

H $0.753677-0.763100-0.267450$

H -6.703426 $0.000163-2.822721$

H $-4.797926-0.353210-0.430954$

H -8.505482 $0.135446-1.151429$

H $-9.037038-1.187369-0.132942$

H -8.258018 -1.551437 -1.665970

H -0.7924580 .1543680 .776920$

H -2.145475 -0.9932740 .700789$

H -4.1464552 .3558942 .958643$

H -3.0760201 .6851264 .211597$

H $-4.472469-0.4413444 .292228$

H $-6.451544 \quad 1.662706 \quad 3.271555$

H -7.7515310 .2677804 .972350$

H -6.740631-1.119489 4.644933

H - $10.026777-0.4713252 .178588$

H -9.699046 0.6143453 .642536

H -8.488371 -2.237566 1.632875

H -7.165785 -2.552865 2.705381

H -5.622069 -1.215029 1.479466

H -3.3199030 .8475251 .377055$

C $0.0455375 .260073-1.173546$

O $1.0509864 .230801-1.259008$

C $2.1124234 .537016-0.342203$

C $2.016706 \quad 6.039286-0.114103$

C $0.5131576 .233498-0.086030$

H $1.879847 \quad 4.0198380 .595928$

C $3.4554084 .041942-0.886140$

H $2.508694 \quad 6.3537390 .811047$

H $2.4513786 .607639-0.944183$

C $0.0769667 .669327-0.322243$

H $\quad 0.140637 \quad 5.910525 \quad 0.895611$

C $3.4811332 .577778-1.373312$

O $3.8349144 .856269-2.006382$

H $4.2351794 .199621-0.132567$

H $2.8832062 .495450-2.289943$

O $4.8301942 .261921-1.746029$

C $3.0215111 .492371-0.384876$
C 3.7086001 .5668110 .990108

H $3.2414510 .517673-0.834241$

$\begin{array}{lllll} & & 1.610599 & 1.547907 & -0.194354\end{array}$

H $4.7804451 .739608 \quad 0.855476$

H 3.3086912 .4225631 .544781

H $0.475173 \quad 8.3258930 .458243$

H $\quad 0.431605 \quad 8.042497-1.288793$

H $-1.0146437 .750529-0.309425$

C -1.307392 $4.626027-0.841739$

H $3.1270054 .790086-2.670328$

H $5.1161432 .929660-2.392796$

H $1.1929501 .507297-1.071223$

H -2.075945 $5.403495-0.763729$

H $-1.249407 \quad 4.130426 \quad 0.135994$

H $0.009748 \quad 5.749755-2.154647$

C $5.427714-0.954478 \quad 1.122624$

O $3.997291-0.8811651 .176131$

C $3.4723340 .285127 \quad 1.822628$

C 3.9877860 .3594203 .266161

C $5.510643 \quad 0.2831043 .327456$

C $6.021368-0.9218162 .536974$

H $5.822459-0.1174690 .534675$

C $5.800407-2.2603800 .402627$

H 2.3866320 .1405331 .880195

$\begin{array}{lllll}\text { O } & 3.549893 & 1.553620 & 3.905069\end{array}$

H $3.574147-0.4810133 .838839$

H 5.9654331 .2108502 .961849

$\begin{array}{lllll}\text { O } & 5.926153 & 0.158071 & 4.689824\end{array}$

H $5.769066-1.8464333 .071908$

O $7.443053-0.8521822 .449092$

C $5.552635-2.192061-1.090311$

H $6.865874-2.4768970 .537683$

H $5.206045-3.0904130 .801645$

H $2.578360 \quad 1.567358 \quad 3.863951$

H $5.527953-0.6513785 .052853$

H $7.787933-0.8009603 .357048$

O $5.948296-3.371711-1.656256$

O $5.077386-1.237601-1.688759$

C $5.776793-3.461561-3.074199$

H $4.724532-3.324190-3.340939$

H $6.403111-2.718652-3.577058$

H $6.090992-4.459259-3.391948$

SCF Energy (B3LYP/6-31G**//MMFF) = -3245.91335790

06_00232

MM̄FF Geometry

C -0.101806 -0.031221 -3.261567

C $-1.021044-0.994486-3.431544$

C $-1.202409-2.268414-2.641408$

O $-2.547074-2.224541-2.100084$

C $-0.225157-2.509087-1.474738$

C $-0.111720-3.971324-1.084733$

C $-0.675733-4.533708 \quad 0.001183$

C $0.752330-4.809386-1.994761$

C -1.594789 -3.904996 1.009332

C $-0.937103-3.7546192 .388901$

C $-0.016828-2.5286532 .482617$

C $0.677408-2.4705243 .843683$

C $1.505528-1.1874724 .010729$

O $-0.841511-1.3728582 .317632$

O $1.844122-1.0886005 .402629$

C $0.630258 \quad 0.030686 \quad 3.643079$

C $2.832581-1.2725503 .253022$

$\begin{array}{lllll}\text { O } & 1.398865 & 1.230746 & 3.646074\end{array}$

C $-0.138090-0.1271652 .309693$

C -1.1850521 .0056352 .161722$

O $0.782859-0.0483801 .219752$

C $-3.337688-3.316036-2.254781$

O $-3.042944-4.357179-2.826902$

C $-4.671479-3.125433-1.638973$

C $-5.032849-2.052313-0.917214$

C $-6.342109-1.842248-0.288277$

C $-7.406060-2.882156-0.533345$

C $-2.2861620 .687544 \quad 1.138992$

C -3.2316091 .8396950 .850889$

C $-4.712006 \quad 3.533847 \quad 1.905642$

O $-3.564457 \quad 2.110678-0.302115$

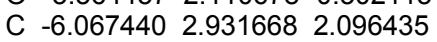


C $-6.965212 \quad 2.787674 \quad 1.110082$

C -8.3264712 .1881321 .308382$

C -8.4788740 .8532690 .616106$

C $-9.2773430 .712208-0.455253$

C $-7.771581-0.3373491 .224980$

C $-6.535520-0.7367340 .462730$

N $-3.7217092 .476107 \quad 1.977937$

H $0.619399-0.091914-2.452965$

H -1.716097 -0.886709 -4.263980

H -1.115827 $-3.080127-3.374234$

H $-0.521413-1.901943-0.612849$

H $0.785656-2.182395-1.742823$

H $-0.488912-5.5923680 .180069$

H $\quad 0.886980-5.829299-1.619114$

H $\quad 0.305317-4.882528-2.990804$

H $1.746994-4.364913-2.094378$

H -2.000084 -2.950219 0.661836

H -2.460559 -4.571895 1.111379

H -1.743626 -3.646279 3.126347

H $-0.383459-4.6659402 .646120$

H $0.724234-2.5699791 .676863$

H $1.304389-3.3571753 .999806$

H -0.080559-2.497792 4.639423

H $2.300786-0.2400785 .535347$

H $-0.103191 \quad 0.1424114 .453322$

H $3.401224-2.1544243 .571230$

H $3.470817-0.4115833 .477557$

H $2.697434-1.3324802 .172892$

H 1.9134921 .2543542 .821119

H -1.667207 $1.163128 \quad 3.133737$

H -0.6902861 .9463411 .889459$

H $\quad 0.3127590 .2700890 .432451$

H -5.355883 -3.951386-1.806715

H $-4.309749-1.254310-0.758626$

H -7.562708 -3.026881-1.608394

H -8.381720 -2.617865 -0.120978

H -7.116517 -3.839830 -0.087087

H $-1.832500 \quad 0.3950670 .184894$

H -2.900344 -0.1540121 .480223$

H -4.6251974 .0582650 .948201$

H -4.5015304 .2460392 .709231$

H -6.3202222 .6004363 .100946$

H -6.7127593 .1173360 .104971$

H -9.0665482 .9060010 .931984$

H -8.554525 2.0649072 .374728

H $-9.413407-0.252108-0.933589$

H -9.810367 $1.556850-0.878895$

H -8.479921 -1.164655 1.332363

H -7.472703 -0.105298 2.255403

H -5.708108 -0.0361950 .574604$

H -3.523345 2.0901322 .895156

C $-0.0914462 .514128-3.494430$

O $1.0116082 .720485-2.592051$

C $0.5158283 .371039-1.406050$

C $-0.8955463 .843614-1.739572$

C $-1.3611282 .748645-2.679810$

H $0.4534912 .598167-0.630465$

C $1.4748034 .478202-0.955708$

H -1.519305 $3.945940-0.847151$

H $-0.8918394 .808858-2.258923$

C $-2.5688383 .129015-3.517659$

H -1.604197 $1.862790-2.081986$

C $2.9460514 .040799-0.813120$

O $1.4454425 .550525-1.907197$

H $1.1181864 .907539-0.012115$

H $3.3920613 .886856-1.803576$

O $3.6661215 .125710-0.214107$

C 3.1638262 .7797950 .039682

C $4.6566812 .472200 \quad 0.259615$

H $2.6872531 .928403-0.456021$

O 2.5282542 .9385561 .306749

H $5.1696782 .500852-0.707471$

H 5.1016633 .2548610 .886036

H -3.425568 $3.362703-2.877525$

H -2.365990 4.007661 -4.139187

H $-2.8562332 .306479-4.180222$

C $\quad 0.028447 \quad 1.153249-4.183308$

H $1.6857005 .180482-2.774194$
H $3.5251035 .909845-0.771958$

H 2.9152753 .7218081 .734389

H $1.0171571 .084531-4.654497$

H $-0.7132861 .094724-4.989011$

H $0.0058963 .291106-4.264555$

C $4.833720-0.299170-1.007312$

$\begin{array}{lllll}\text { O } & 4.241994 & 0.035720 & 0.251501\end{array}$

C 4.8846091 .1088880 .949035

C $6.3603860 .765217 \quad 1.198115$

C $7.0827420 .379190-0.091241$

C $6.302006-0.703724-0.836004$

H $4.7735100 .572601-1.670651$

C $3.974345-1.378305-1.674308$

H $4.398698 \quad 1.171747 \quad 1.929979$

O 7.0435321 .8555401 .807331

H $6.421581-0.0817521 .894020$

H $7.2555701 .250971-0.733116$

O $8.373522-0.1435130 .231463$

H $6.384337-1.644871-0.279231$

O $6.884136-0.911541-2.119967$

C $3.965640-2.685653-0.917353$

H $2.932147-1.044127-1.735974$

H $4.302510-1.557194-2.704929$

H 6.5760062 .0682522 .633121

H 8.8426530 .5401320 .740080

H $7.820521-1.135171-1.980977$

O $4.782916-3.587189-1.532515$

O $3.322688-2.8784370 .106062$

C $4.882166-4.862966-0.891965$

H $5.550573-5.490876-1.486984$

H $5.306379-4.7499320 .110303$

H $3.900058-5.342581-0.844319$

SCF Energy (B3LYP/6-31G**//MMFF) $=-3245.92824282$

06_00233

MM̄FF Geometry

C -3.020377 -3.365564 -0.070106

C $-2.650005-4.1536100 .950267$

C -1.657635 -3.816948 2.037587

O $-1.158713-2.4656691 .932107$

C $-0.439553-4.7545431 .950653$

C $0.512463-4.5749943 .117618$

C $1.655434-3.8617053 .076747$

C $0.111270-5.2855364 .384949$

C $2.222997-3.0795231 .923279$

C $3.570170-3.6663341 .484295$

C $4.177868-2.9162310 .289836$

C $5.493290-3.575425-0.135158$

C $6.190781-2.802626-1.263351$

O $4.413242-1.5586200 .678385$

O $7.529379-3.313303-1.356088$

C $6.275743-1.313704-0.866276$

C $5.532314-3.076812-2.620420$

O $6.802172-0.533226-1.937351$

C $4.934303-0.729514-0.369200$

C 5.1526510 .6936110 .197523

O $4.035066-0.646760-1.477350$

C -1.865908 -1.468894 2.527780

O $-2.974605-1.5455323 .030116$

C -1.015191 -0.2587322 .487832$

C -1.4969880 .9302772 .878231$

C -0.7330222 .1794052 .936825$

C 0.7364312 .1102692 .612527

C 3.9613801 .2305600 .999177

C 4.1431272 .7097331 .279328

C 3.4854104 .9653470 .431025

O 4.8639283 .1106312 .190329

C 2.4717755 .4671331 .411721

C 1.2891805 .9939601 .059472

C 0.2875876 .5499282 .031077

C -0.8767775 .6117892 .266537$

C -1.9651905 .6666541 .479631$

C -0.7535054 .6879803 .461450$

C -1.365032 3.3206953 .282429

N 3.4519963 .5152640 .388941

H $-2.575079-2.382320-0.188014$

H -3.117967 -5.1324131 .040952$

H $-2.154844-3.9447943 .007497$ 
H $\quad 0.086265-4.5927531 .002269$ H $-0.767256-5.8025301 .926234$ H $2.257342-3.8119363 .983761$ H $0.846303-5.1550875 .186305$ H -0.846558 -4.905452 4.753180 H $\quad 0.012278-6.3604734 .201871$ H $1.539855-3.0450391 .070126$ H $2.356010-2.0418002 .252199$ H $4.265866-3.6218042 .332863$ H $3.441172-4.7240881 .221994$ H $3.455106-2.935629-0.533981$ H $5.328729-4.619528-0.429838$ H $6.173702-3.6138570 .727538$ H $8.003052-2.779459-2.016989$ H $7.009605-1.241530-0.052065$ H $5.577871-4.147000-2.855734$ H $\quad 6.067989-2.571508-3.431629$ H $4.484010-2.774015-2.657855$ H $6.093224-0.412292-2.591672$ H $\quad \begin{array}{lll}6.021046 & 0.692699 & 0.869291\end{array}$ H $5.3823961 .385738-0.622783$ H $3.4352900 .102207-1.342869$ H $0.008310-0.3877492 .152609$ H -2.537247 0.9960193 .193257 H $1.251907 \quad 1.4258023 .295263$ H 1.2449313 .0717612 .689547 H $\quad 0.8863801 .7629841 .585367$ H 3.0169511 .0688600 .470388 H $3.8718720 .713511 \quad 1.961737$ H $3.2865965 .325924-0.583393$ H 4.4853285 .2999520 .726000 H 2.7381835 .4137642 .464527 H 1.0264076 .0502200 .005800 H -0.0743327 .5080971 .635160$ H $\quad 0.762628 \quad 6.8007942 .987993$ H -2.8273805 .0303371 .645922$ H -2.019286 6.3583600 .645070 H -1.234706 5.1714414 .321866 H $0.297416 \quad 4.592138 \quad 3.754438$ H -2.434695 3.2823593 .490570 H $2.8424413 .084204-0.298999$ C $-5.300212-2.866861-1.067904$ O $-4.943541-1.500914-1.360757$ C $-5.608315-0.644017-0.413595$ C $-6.737856-1.4801140 .175768$ C $-6.076910-2.8431470 .251088$ H -4.882717 -0.4307630 .381175$ C $-6.0435110 .666465-1.075965$ H -7.068181 -1.107132 1.149956 H -7.607972 -1.518223 -0.489725 C $-7.056092-3.9930150 .407758$ H $-5.394381-2.8431231 .109775$ C $-4.9116001 .405896-1.818894$ O $\quad-7.0714210 .371920-2.029522$ H $-6.497673 \quad 1.319937-0.321193$ H $-4.655500 \quad 0.873282-2.743211$ O $-5.4104992 .689378-2.215096$ C $-3.6331071 .621253-0.988195$ C -2.588647 2.479159-1.726859 H $-3.1927420 .647859-0.750010$ $\begin{array}{llll}\text { O } & -3.962654 & 2.248632 & 0.250863\end{array}$ H -2.418881 $2.051203-2.720587$ H $-2.9853623 .491254-1.871128$ H -7.625072 $-3.889573 \quad 1.337471$ H -7.770922 -4.028157 -0.421113 H $-6.527761-4.9512710 .439071$ C $-4.052239-3.754771-1.093041$ H $-7.4788681 .214260-2.292278$ H $-6.0847082 .543842-2.899823$ H -4.3759163 .1050080 .045972$ H -3.578035 -3.672641 -2.078392 H $-4.348367-4.802933-0.967511$ H $-5.961080-3.184776-1.884818$ C $-0.1971850 .563386-1.744647$ O $-0.712831 \quad 1.303192-0.631224$ C $-1.2586742 .585631-0.950629$ C $-0.1945203 .455362-1.627056$ C $0.4364682 .756418-2.827003$
C $\quad 0.9169901 .357227-2.440338$

H $-1.0091370 .363438-2.453300$

C $0.288619-0.776526-1.179279$

H $-1.494906 \quad 3.061050 \quad 0.007646$

O $-0.754824 \quad 4.702311-2.022161$

H $0.5930533 .674464-0.896279$

H $-0.2576102 .723144-3.674954$

O $1.5584393 .533168-3.254486$

H $1.7865841 .461854-1.781493$

$\begin{array}{llll}\text { O } & 1.345718 & 0.697229 & -3.626966\end{array}$

C $0.418592-1.861609-2.217090$

H $1.247773-0.658776-0.661985$

H $-0.403421-1.142931-0.412725$

H $-0.0491355 .214690-2.452768$

H $1.9706553 .059809-3.997688$

H $1.884185-0.067250-3.361986$

O $-0.812693-2.223111-2.676462$

O $1.489564-2.337280-2.571238$

C $-0.819324-3.249430-3.673077$

H $-0.209325-2.953097-4.531902$

H $-0.456406-4.188915-3.245884$

H -1.849874 -3.390589-4.009422

SCF Energy (B3LYP/6-31G**//MMFF)= -3245.92341198

06_00234

MMFF Geometry

C -2.688356 -3.084041 -2.628991

C $-1.748848-2.282965-2.105142$

C $-0.749480-1.491395-2.908716$

O $0.586151-1.973868-2.581114$

C $-0.8766320 .038221-2.715541$

C $-1.8431760 .726685-3.662666$

C $-1.5265641 .762829-4.464536$

C $-3.275140 \quad 0.257959-3.617633$

C $-0.206265 \quad 2.463786-4.629414$

C $-0.2244893 .854173-3.974774$

C $-0.143193 \quad 3.792891-2.440949$

C $-0.4298195 .161696-1.822345$

C $-0.2574205 .140054-0.296003$

O $1.1806723 .368969-2.098258$

$\begin{array}{llllll}0 & -0.244344 & 6.499245 & 0.165612\end{array}$

C $1.121978 \quad 4.536037 \quad 0.034546$

C -1.432889 4.4520260 .409287

$\begin{array}{lllll}\text { O } & 1.284029 & 4.373387 & 1.441232\end{array}$

C $1.407918 \quad 3.200230-0.693285$

C $2.8918682 .814808-0.490790$

O $0.5785332 .173986-0.151473$

C $1.128879-1.735872-1.356910$

O $0.672947-1.036615-0.467347$

C $2.381277-2.518995-1.264075$

C $3.173438-2.370660-0.191773$

C $4.407446-3.1088030 .084362$

C $4.789344-4.207780-0.872689$

C $3.3449961 .581884-1.283085$

C $4.8526541 .400259-1.224213$

C $6.7050060 .166708-2.340122$

O $5.5716102 .016109-0.441242$

C $7.050874-1.098950-1.624569$

C $7.786422-1.139318-0.503545$

C $8.203319-2.4065100 .185603$

C $7.597929-2.5499891 .563118$

C $8.177249-1.9857612 .636354$

C $6.376072-3.4283241 .704267$

C $5.124194-2.7799791 .179856$

N $5.2990780 .463986-2.146516$

H $-2.760044-3.200530-3.706847$

H $-1.711599-2.165963-1.025301$

H $-0.864737-1.720552-3.975408$

H $0.1210410 .479845-2.798545$

H $-1.2036780 .263697-1.691273$

H -2.320568 2.189427 -5.077814

H $-3.9573540 .956241-4.114951$

H $-3.379685-0.708538-4.117235$

H $-3.6146810 .166974-2.581329$

H $0.636657 \quad 1.872903-4.261708$

H $-0.0340212 .586981-5.706085$

H $0.637258 \quad 4.416425-4.357150$

H $-1.1283454 .393814-4.284450$ 
H $-0.8665823 .061071-2.067430$ H -1.436637 5.506801-2.088520 H $\quad 0.2631475 .905055-2.240956$ H -1.083466 $6.913631-0.099537$ H $1.8800035 .267382-0.279735$ H -2.3726804 .9674890 .178199$ H -1.3242474 .5027331 .498408$ H $-1.553133 \quad 3.4049160 .123688$ H 1.1030645 .2342851 .855795 H $3.5198623 .661498-0.799014$ H 3.0893252 .6330630 .573393 H $0.9028521 .317193-0.471472$ H $2.602192-3.213432-2.066577$ H $2.888438-1.6442540 .568282$ H $4.024991-4.992819-0.878846$ H $5.739643-4.687842-0.630825$ H $4.896069-3.815589-1.889997$ H $2.8865800 .678527-0.872658$ H $3.0508561 .674649-2.334377$ H $7.3128561 .010387-1.996018$ H $6.8738690 .044182-3.414452$ H $6.710436-2.026977-2.075511$ H $8.137268-0.206623-0.067068$ H $9.298742-2.4034480 .263585$ H $7.962349-3.287570-0.421732$ H $7.766530-2.1107103 .632700$ H $9.073782-1.3821622 .539719$ H $6.198601-3.6608912 .763096$ H $6.575109-4.3992551 .241349$ H $4.767243-1.9617501 .807221$ H $4.622731-0.042373-2.710615$ C $-4.954266-2.962535-1.585969$ O $-4.629894-1.852825-0.722668$ C $-5.683591-1.6829500 .240878$ C $-6.840053-2.545155-0.248965$ C $-6.095881-3.701148-0.890906$ H $-5.317341-2.0953511 .188327$ C $-6.005813-0.1942780 .405354$ H -7.500134 -2.857069 0.566066 H -7.448587 -2.022493 -0.996216 C $-6.955300-4.532555-1.827017$ H -5.697530 -4.347978 -0.097551 C $-4.795404 \quad 0.6762350 .810117$ $\begin{array}{lllll}\text { O } & -6.512619 & 0.315527 & -0.834876\end{array}$ H -6.815945 -0.0724561 .133407$ H $-4.069808 \quad 0.712281-0.010909$ O C -4.0808300 .2221442 .098776$ C -2.9881611 .2079282 .554573$ H -3.626146 -0.7570651 .927021$ O H -2.2783971 .3492031 .732539$ H -3.4482492 .1822182 .757479$ H -7.792226 -4.982201-1.282793 H -7.369825 -3.924209-2.637911 H $-6.371864-5.341646-2.277465$ C -3.702478 -3.814964 -1.792915 H $-5.8319430 .166422-1.513260$ H -5.951546 2.0199231 .655688 H $-5.3892710 .935973 \quad 3.370007$ H -3.950991 -4.755379 -2.297315 H -3.261199 -4.075594 -0.822877 H -5.294684 -2.536907 -2.539269 C $-0.482916-0.5607062 .827102$ O $-1.634586-0.5364313 .679820$ C $-2.254836 \quad 0.745222 \quad 3.835182$ C $-1.243751 \quad 1.7614574 .383719$ C $0.0152941 .821723 \quad 3.527306$ C $0.586426 \quad 0.419893 \quad 3.330892$ H $-0.780868-0.3027361 .803878$ C $0.069148-1.9959942 .829412$ H -3.0213610 .6185344 .609932$ O $-1.839100 \quad 3.0517694 .470773$ H -0.9639961 .4686165 .404085$ H -0.1880992 .3120222 .569681$ O 0.9811362 .6311564 .200471 H $\quad 0.9992390 .068007 \quad 4.284741$ O 1.6731540 .4833612 .409612
C $-0.754418-2.9413051 .981230$

H $1.086614-2.0014152 .422851$

H $0.089219-2.4012923 .847414$

H -1.156204 3.6658204 .791303

H 1.7821492 .6426603 .648580

H $1.3215120 .786827 \quad 1.555773$

O $0.004714-4.035627 \quad 1.675899$

O $-1.916179-2.7595961 .646136$

C $-0.634162-5.0312410 .871229$

H $-1.593664-5.3269631 .306426$

H $-0.769773-4.653852-0.145871$

H $0.017638-5.908148 \quad 0.835501$

SCF Energy $\left(B 3 L Y P / 6-31 G^{* *} / / M M F F\right)=-3245.92935415$

06_00235

MM̄F Geometry

C -0.1289510 .8754092 .798226$

C -0.5810622 .0757842 .401652$

C -0.0948292 .8562231 .201135$

O 0.7519692 .0330110 .368154

C 0.7230104 .0702621 .668783

C 1.2160614 .9284890 .521139

C 2.4765414 .9058150 .043875

C $0.1985415 .875790-0.058180$

C 3.5995824 .0109560 .493126

C $4.3847053 .446402-0.699867$

C $5.4803442 .475425-0.236683$

C $6.4182582 .120548-1.390504$

C $7.4777371 .094432-0.962402$

O $4.843264 \quad 1.288656 \quad 0.250633$

O $8.1085240 .590437-2.149434$

C $6.770574-0.096257-0.284664$

C $8.5871991 .731384-0.117545$

O $7.718957-1.0087110 .264402$

C $5.7447440 .317607 \quad 0.798781$

C $4.924760-0.9226361 .225411$

O $\quad 6.436622 \quad 0.8301861 .938221$

C $0.1834351 .441626-0.715947$

O $-0.9642761 .568750-1.109954$

C $1.2029270 .561177-1.326834$

C $0.807630-0.390905-2.184482$

C $1.662130-1.397692-2.815389$

C $3.152137-1.240003-2.674903$

C $3.702931-0.6153812 .100033$

C $2.875484-1.8722262 .296197$

C $1.342407-3.4106961 .054711$

O $2.934907-2.5292383 .332544$

C $1.882739-4.180849-0.110882$

C $1.116940-4.684533-1.090822$

C $1.660996-5.439750-2.270805$

C $1.147045-4.915545-3.595941$

C $0.259186-5.619338-4.318747$

C $1.717071-3.612168-4.120190$

C $1.070578-2.415149-3.475486$

N $2.111378-2.185978 \quad 1.183229$

H $\quad 0.6641940 .3854582 .242577$

H -1.369126 2.5532392 .980592

H -0.9701353 .1993620 .636081$

H 1.5677643 .7350322 .282235

H 0.1163784 .6956272 .337826

H $2.7340745 .599838-0.755103$

H $0.6169026 .497936-0.856536$

H $-0.6449025 .321298-0.481351$

H $-0.180807 \quad 6.5476350 .718689$

H 4.2775204 .5997361 .122452

H $3.2335773 .179298 \quad 1.101327$

H $3.6925302 .927142-1.375299$

H $4.8352924 .276095-1.258820$

H 6.0415222 .9478050 .578332

H $6.8987103 .020213-1.794726$

H $5.8342251 .694003-2.218276$

H $8.499734 \quad 1.344008-2.623814$

H $\quad 6.235399-0.644670-1.072885$

H $9.0832352 .532717-0.678406$

H $9.370687 \quad 1.002840 \quad 0.118946$

H 8.2218702 .1604020 .817722

H $8.331624-1.254497-0.449866$

H $4.578461-1.4438340 .326095$ 
H $5.568783-1.6276891 .767185$ H $5.868621 \quad 0.7361362 .719129$ H $2.2334790 .682038-1.014513$ H -0.253282 -0.471051 -2.416316 H $3.463201-0.225151-2.947907$ H $3.722574-1.911312-3.320201$ H $3.460290-1.434543-1.644763$ H $4.001609-0.2436593 .085751$ H 3.0684520 .1527481 .644956 H $\quad 0.296263-3.1237200 .909252$ H $1.423813-4.0055891 .969212$ H $2.959672-4.328710-0.151640$ H $0.038172-4.562496-1.036645$ H $1.378215-6.493372-2.147997$ H $2.758006-5.423230-2.282754$ H $-0.096323-5.262658-5.279797$ H $-0.137011-6.564813-3.963622$ H $1.548306-3.535577-5.202233$ H $2.802581-3.635355-3.992840$ H - $0.018085-2.418357-3.546785$ H $2.108428-1.5471980 .394190$ C -1.235571-1.219958 3.602486 O $-2.395486-1.0067332 .776209$ C $-3.498318-1.7571683 .312482$ C $-2.868236-2.8188004 .202831$ C $-1.709672-2.0430654 .802058$ H -4.072086 -1.064636 3.941003 C $-4.393368-2.2730222 .180250$ H -3.565140 -3.201359 4.954369 H -2.489710 -3.667778 3.622356 C $-0.627866-2.9259545 .400532$ H -2.100365 -1.378698 5.584655 C $-4.739603-1.2033631 .120107$ O $-3.711545-3.3382491 .507382$ H -5.304446 -2.711892 2.603573 H $-3.831893-0.9663330 .555972$ O $-5.651948-1.8126620 .200557$ C -5.3739010 .0718351 .700343$ C -5.7038731 .1876880 .688536$ H -4.7101920 .5060472 .455020$ O $-6.582248-0.2776342 .382011$ H -6.410486 $0.813351-0.058499$ H -6.2579041 .9564441 .242026$ H -1.033163 -3.530278 6.218526 H $-0.208341-3.6086734 .653675$ H $0.191674-2.3202635 .800358$ C $-0.645730 \quad 0.134628 \quad 3.999931$ H $-4.316155-3.6813280 .827308$ H -5.728131 -1.229750 -0.572137 H -7.175602 -0.6910381 .731494$ H $0.184910-0.0084014 .700343$ H -1.408354 0.7358024 .510657 H $-0.518101-1.7902723 .000217$ C $-4.6755410 .968197-2.223870$ O $-3.9128551 .004983-1.012433$ C $-4.448677 \quad 1.8122500 .039695$ C $-4.6349993 .253534-0.455723$ C $-5.4946343 .306850-1.712400$ C $-4.9174142 .377854-2.776713$ H $-5.6347140 .471208-2.040112$ C $-3.8864150 .128029-3.235466$ H -3.675293 1.8510520 .817575 O H -3.653170 $3.686125-0.689606$ H -6.541282 $3.061036-1.497466$ O $-5.4884684 .638259-2.231942$ H $-3.9785302 .800012-3.158086$ O $-5.8262092 .299724-3.873347$ C $-3.904889-1.334402-2.862717$ H $-4.308417 \quad 0.202988-4.244292$ H -2.844376 $0.464201-3.302932$ H -4.6439124 .0267231 .334395$ H $-5.8145545 .224450-1.527532$ H $-5.9777493 .207048-4.189487$ O $-2.775217-1.653355-2.171758$ O $-4.823438-2.093813-3.142413$ C $-2.650639-3.016344-1.754364$ H -3.608965 -3.428592 -1.425636
H -2.243415 -3.610622 -2.576609

H -1.953083 -3.047760 -0.913485

SCF Energy (B3LYP/6-31G**//MMFF)= -3245.91885232

06_00236

MMFF Geometry

C -1.013398 $1.981457-2.161312$

C $-1.7529273 .085286-1.974005$

C -1.728409 $3.971222-0.749854$

O -0.6329493 .5640110 .104874$

C -3.0312963 .8601170 .066079$

C $-4.2782794 .324261-0.659000$

C $-5.1729383 .502690-1.245172$

C $-4.4944995 .814535-0.667180$

C $-5.0855822 .006577-1.363180$

C $-6.1786531 .299791-0.552269$

C $-5.895899-0.205718-0.436490$

C $-7.056205-0.9340740 .242277$

C $-6.737845-2.4160670 .499069$

O $-4.706453-0.3452350 .345501$

$\begin{array}{llll}\text { O } & -7.753230 & -2.905350 & 1.388517\end{array}$

C $-5.375462-2.5145351 .222108$

C $-6.843741-3.247728-0.784490$

O $-4.953386-3.8692651 .355352$

C $-4.262747-1.6932820 .536600$

C $-2.966194-1.6111671 .376308$

O $-3.927410-2.305883-0.712756$

C 0.0087744 .5018800 .841386

O -0.2635325 .6928040 .902760$

C 1.1415193 .9127501 .598132

C 1.4672862 .6099171 .567205

C 2.5661221 .9686112 .297130

C 3.4552772 .8521903 .131832

C $-3.165935-1.0630642 .793108$

C $-1.897729-0.481713 \quad 3.394485$

C -0.9439460 .2024835 .600653$

O $-0.958913-0.0722472 .717030$

C $0.295769-0.6355525 .596761$

C $1.488343-0.1641345 .203546$

C $2.738588-0.9887325 .127875$

C $3.116656-1.3358803 .704172$

C $2.967487-2.5868813 .236396$

C $3.742098-0.2555842 .847269$

$\begin{array}{llll}C & 2.715418 & 0.630708 & 2.192074\end{array}$

N -1.963980 -0.4281194 .781771$

H $-0.3061401 .665985-1.399530$

H -2.446473 $3.380571-2.758571$

H $-1.5636605 .002078-1.086578$

H $-2.926951 \quad 4.4475470 .988792$

H -3.1456362 .8333720 .436817$

H $-6.0467583 .944047-1.722209$

H $-5.4159006 .098474-1.186663$

H -4.562805 6.1940920 .357553

H -3.663685 $6.318911-1.170452$

H $-5.1909671 .737165-2.421659$

H $-4.0993911 .642527-1.065038$

H $-6.220822 \quad 1.736970 \quad 0.454279$

H -7.152544 $1.467673-1.028004$

H $-5.720728-0.610546-1.440244$

H -7.976082 -0.835342 -0.347762

H $-7.269561-0.4547041 .208384$

H -7.605602 -3.8567011 .521653$

H $-5.530368-2.1335802 .239538$

H $-7.864951-3.205150-1.182067$

H $-6.642161-4.306596-0.588266$

H $-6.167264-2.910043-1.572544$

H -5.627628 -4.339146 1.874009

H -2.484767 -2.596010 1.431428

H $-2.257879-0.9716490 .832040$

H -3.013443 -2.068931 -0.938954

H $1.700697 \quad 4.6257802 .195628$

H 0.8718531 .9325940 .957676

H 3.9330673 .6149522 .506606

H 4.2608562 .3151333 .635584

H 2.8743393 .3518683 .915025

H $-3.906275-0.2545122 .796952$

H -3.538282 -1.865363 3.440664

H $-1.3382870 .284414 \quad 6.617922$ 
H $-0.765910 \quad 1.209950 \quad 5.209506$ H $0.202155-1.6740985 .903727$ H $1.5692780 .878898 \quad 4.909891$ H 2.619264 -1.903976 5.722782 H $3.560424-0.4392725 .602902$ H $3.260713-2.8504382 .225672$ H $2.543570-3.3738073 .851254$ H $4.327944-0.7186502 .044904$ H 4.4712330 .2946113 .446787 H 2.0233910 .0846811 .548563 H -2.809134 -0.743597 5.248499 C - $-1.445228-0.332621-2.975344$ O $-0.300963-0.931226-2.328111$ C $-0.035038-2.203455-2.941278$ C $-1.313259-2.579314-3.678500$ C $-1.774988-1.224123-4.173356$ H $0.760527-2.040628-3.678228$ C $0.419291-3.227399-1.898273$ H -1.132769 -3.293717 -4.487269 H -2.059776 -3.012182 -3.002976 C $-3.240304-1.190828-4.574132$ H -1.160657 -0.936659 -5.037326 C $1.672544-2.832851-1.090193$ O $-0.642644-3.447477-0.960533$ H $0.587663-4.193423-2.388949$ H $1.434450-2.035803-0.375047$ O $2.044605-3.974215-0.304739$ C $2.886972-2.404321-1.936483$ C $4.188083-2.324056-1.113750$ H $2.676427-1.432303-2.394128$ O $3.085351-3.335722-3.001797$ H $3.988826-1.778603-0.187186$ H $4.491918-3.340191-0.835817$ H $-3.429646-1.887192-5.397560$ H -3.891590 -1.471950 -3.739580 H $-3.531731-0.188793-4.904503$ C $-1.1093101 .104894-3.376606$ H $-0.832041-2.595589-0.531155$ H $1.276643-4.2107140 .243392$ H $3.227657-4.212016-2.604301$ H $-1.868716 \quad 1.491053-4.066098$ H $-0.1421041 .135019-3.892703$ H $-2.265880-0.345433-2.247186$ C $5.1044820 .591732-1.050982$ O $5.081127-0.281963-2.186588$ C $5.343322-1.660883-1.899425$ C $6.720905-1.799210-1.239369$ C $6.841630-0.9284750 .006162$ C $6.4506850 .513163-0.315274$ H $4.2889780 .328840-0.369492$ C $4.8595532 .021314-1.558976$ H $5.405763-2.169437-2.869529$ O $6.974979-3.161313-0.912195$ H $7.491493-1.494941-1.959938$ H $\quad 6.245953-1.3381390 .828977$ O $8.203650-0.9624270 .439179$ H $7.2376390 .975266-0.925282$ O $6.356078 \quad 1.2582800 .897825$

C $3.4324992 .250730-2.007603$ H $5.0621262 .748940-0.765060$ H $5.5137462 .224550-2.415409$ H $7.845126-3.198858-0.479315$ H $8.253262-0.493510 \quad 1.289144$ H 7.2440821 .2964381 .290994 O $3.3396513 .512533-2.524684$ O $2.5184131 .444741-1.908490$ C $2.0431373 .904011-2.984325$ H $1.6457663 .171344-3.692937$ H $1.3714394 .027219-2.131054$ H $2.1398004 .865665-3.495384$ SCF Energy (B3LYP/6-31G**//MMFF) $=-3245.91799452$

06_00237

MMFF Geometry

C $-1.175796 \quad 3.787870 \quad 1.104844$

C -0.6618572 .5515921 .030705$

C -0.3712671 .6920372 .233688$

O 0.9231581 .0743182 .044408

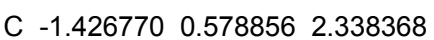

C $-1.234752-0.3342823 .535330$

C $-0.837748-1.621558 \quad 3.469744$

C $-1.548595 \quad 0.284335 \quad 4.873414$

C $-0.484107-2.411822 \quad 2.242011$

C $1.015237-2.7273752 .195343$

C $1.461349-3.1678360 .792994$

C $2.936612-3.5691370 .802667$

C $3.464215-3.872022-0.605759$

O $1.260611-2.056039-0.086099$

O $4.896328-3.926056-0.511930$

C $3.093881-2.703587-1.546147$

C $3.012751-5.255381-1.088983$

O $3.454921-3.036438-2.884396$

C $1.607821-2.295803-1.456510$

C $1.272873-0.999531-2.237131$

O $0.805101-3.342723-2.013999$

C 2.0202191 .8178232 .354213

O 2.0371562 .9549822 .797436

C 3.2150661 .0038382 .037253

C 4.4370251 .5483752 .132726

C $5.707176 \quad 0.877740 \quad 1.844301$

C $5.650388-0.542498 \quad 1.346873$

C $2.0098240 .260192-1.768320$

C $3.2942000 .549969-2.526396$

C $5.2671982 .074482-2.485412$

O $3.612444-0.032038-3.559586$

C $6.4242211 .368149-1.852162$

C $7.4632442 .009236-1.295266$

C $8.6594771 .315542-0.708905$

$\begin{array}{llll}\text { C } & 8.972723 & 1.774206 & 0.697814\end{array}$

C 9.9084732 .7110870 .926776

C $8.2672801 .081201 \quad 1.841068$

C 6.8552501 .5627362 .028299

N $4.025414 \quad 1.569283-1.930268$

H -1.4120374 .2135502 .076727$

H -0.4429712 .1242610 .054597$

H $-0.3640132 .288988 \quad 3.154017$

H $-1.4264250 .007746 \quad 1.406210$

H -2.428945 1.0232332 .404977

H $-0.744557-2.1792164 .400822$

H $-1.506275-0.4453025 .689208$

H $-0.834763 \quad 1.0800395 .107019$

H -2.556278 $0.710758 \quad 4.869938$

H -1.054556 -3.348682 2.250403

H $-0.783882-1.8923291 .332107$

H $1.580769-1.8324732 .484119$

H $1.245547-3.5098852 .928858$

H $0.832609-4.0052110 .468399$

H $3.102872-4.4250521 .468876$

H $3.530864-2.7473661 .223571$

H $5.245430-4.049512-1.411500$

H $3.724184-1.856556-1.250227$

H $3.396286-6.035455-0.420300$

H $3.421853-5.483659-2.079368$

H $1.926661-5.361374-1.132078$

H $3.359154-2.236309-3.427372$

H $1.389444-1.161485-3.315118$

H $0.200730-0.803752-2.092697$

H $-0.050272-2.968341-2.282304$

H $3.056219-0.0208241 .722229$

H 4.5200802 .5856832 .454365

H $5.001724-0.6173700 .467492$

H $5.263367-1.2047532 .128459$

H $6.620071-0.9378151 .037897$

H $1.356626 \quad 1.125637-1.939441$

H $2.2186650 .217256-0.695705$

H $5.2893693 .153332-2.301131$

H $5.2792921 .904755-3.566908$

H $6.4227890 .280971-1.882404$

H $7.4758033 .097063-1.295296$

H $9.5162091 .520662-1.364466$

H $8.5362080 .225435-0.718926$

H $10.160903 \quad 3.024210 \quad 1.934476$

H 10.4392653 .1898500 .110522

H $8.799878 \quad 1.2729862 .782482$

H $8.335226-0.0015791 .704270$

H 6.7935002 .5924652 .383441 
H $3.7090001 .958230-1.046721$

C $-2.9452704 .648386-0.447254$

O $-3.3080953 .359873-0.987157$

C $-3.9995803 .545939-2.233375$

C $-4.4737084 .993619-2.205396$

C $-3.3021485 .672251-1.525371$

H $-3.2651803 .426078-3.038870$

C $-5.1365692 .531128-2.396422$

H $-4.6763925 .386120-3.206354$

H -5.383077 5.104759-1.602349

C -3.629764 $7.051252-0.979680$

H $-2.4796085 .761079-2.248033$

C $-4.7436261 .067178-2.692946$

O $-5.9758622 .561930-1.240645$

H -5.753335 $2.869236-3.239173$

H $-5.6795860 .493285-2.696315$

O $\quad-4.214540 \quad 0.973570-4.015683$

C $-3.794080 \quad 0.373269-1.696879$

C $-3.727338-1.148380-1.942563$

H $-4.1432660 .561646-0.678648$

O $-2.4753120 .905612-1.800096$

H -4.745949 -1.549192 -1.970960

H $-3.278489-1.334926-2.925774$

H -3.929148 $7.724204-1.789810$

H -4.450328 $7.011783-0.255401$

H $-2.7600607 .489374-0.479868$

C -1.456423 $4.644456-0.097743$

H $-5.4136352 .408132-0.462182$

H $-3.391717 \quad 1.488605-4.049234$

H -2.112721 $0.650195-2.664896$

H -1.111130 5.6609670 .122924

H $-0.8736244 .283864-0.954963$

H $-3.556646 \quad 4.8007590 .451360$

C $-4.634410-2.230654 \quad 0.772264$

O $-3.375394-1.6301880 .453662$

C $-2.894430-1.884333-0.869694$

C $-2.751348-3.394978-1.097430$

C $-4.043953-4.143733-0.779340$

C $-4.557986-3.7536070 .606921$

H -5.415646 -1.821456 0.120866

C $-4.967222-1.8548612 .224346$

H -1.883735 -1.460957 -0.911869

O $-2.350973-3.645785-2.440032$

H -1.953396 -3.775625 -0.446457

H $-4.808207-3.968904-1.545789$

O $-3.787849-5.549897-0.775848$

H $-3.892230-4.181808 \quad 1.366986$

O $-5.846146-4.3314310 .810345$

C $-5.301149-0.3857082 .376611$

H -5.832884 -2.418502 2.588215

H $-4.101880-2.0735782 .861432$

H $-2.139100-4.591580-2.513280$

H $-3.570910-5.811222-1.686579$

H $-6.449075-3.9596250 .144163$

O $-5.167382-0.0306613 .688550$

O $-5.663251 \quad 0.3523361 .470721$

C $-5.4619851 .337758 \quad 3.986835$

H -5.2971751 .4954035 .055980$

H -4.7940602 .0004263 .428051$

H -6.5083711 .5582643 .755991$

SCF Energy (B3LYP/6-31G**//MMFF) $=-3245.92689779$

06_00238

MM̄FF Geometry

C $0.350127 \quad 3.419077 \quad 1.874185$

C 0.6759122 .1533301 .572352

C 1.0849121 .1425112 .613419

O $2.355024 \quad 0.5773292 .213371$

C $0.087703-0.0261742 .676393$

C $0.037262-0.6704584 .047414$

C $0.797621-1.7079194 .448346$

C $-0.983694-0.0789134 .985164$

C $1.895502-2.4046513 .695135$

C $1.548616-3.8557583 .339036$

C $0.562229-3.9664112 .167342$

C $0.214685-5.4302751 .892232$

C $-0.688452-5.5802790 .659261$

O $1.190280-3.3936021 .015297$
O $-0.699671-6.9668190 .286177$

C $-0.063660-4.801136-0.517452$

C $-2.141638-5.2046270 .969098$

O $-0.942776-4.782087-1.640069$

C $0.364431-3.359247-0.154191$

C $1.199827-2.754011-1.308763$

O $\quad-0.803571-2.5628680 .049053$

C 3.4871151 .2947072 .423378

O 3.5907452 .3823052 .964298

C 4.5871460 .5205141 .810249

C 5.6933601 .1548031 .398788

C 6.8140030 .5410000 .683540

C $6.771620-0.9499520 .467632$

C $1.899820-1.429319-0.965879$

C $2.655853-0.866777-2.159654$

C $4.1403591 .027831-2.812143$

O $2.612420-1.371868-3.278895$

C $5.5503760 .523866-2.847881$

C $6.6212231 .332409-2.890907$

C $8.0395850 .840445-2.978288$

C $8.9622601 .513222-1.983883$

C $9.7650072 .522791-2.360936$

C $9.0128730 .962768-0.575262$

C $7.798511 \quad 1.3425410 .227088$

N $3.3713560 .278355-1.833578$

H $\quad 0.348152 \quad 3.7321532 .915748$

H $0.6870691 .833808 \quad 0.533120$

H $\quad 1.194543 \quad 1.6122403 .599461$

H $\quad 0.337819-0.7810501 .922627$

H $-0.924270 \quad 0.316147 \quad 2.427564$

H $0.644330-2.0914445 .456739$

H -0.945412 -0.5293925 .982585$

H $-0.8158310 .996553 \quad 5.103489$

H -1.992962 -0.2332754 .590856$

H $2.192748-1.8593402 .796945$

H $2.779790-2.4101894 .345312$

H $2.484834-4.3580583 .061918$

H $1.150375-4.3750024 .219615$

H $-0.343770-3.3993472 .408511$

H $-0.253895-5.8911692 .770661$

H $1.139164-5.9975531 .713977$

H -1.023145 -7.479303 1.046885

H $0.830820-5.355388-0.834836$

H $-2.535537-5.8325381 .777246$

H $-2.789425-5.3906850 .105458$

H $-2.258803-4.1630401 .274707$

H -1.169902 -5.705409-1.845096

H $1.981620-3.467979-1.600470$

H $\quad 0.558542-2.602126-2.185617$

H $-0.608067-1.640221-0.176810$

H $4.424312-0.5398461 .652683$

H 5.7675592 .2293161 .561549

H $6.749994-1.4746621 .429282$

H $7.630144-1.339011-0.082769$

H $5.882118-1.233078-0.106113$

H $1.171635-0.675216-0.649839$

H $2.614872-1.582708-0.149822$

H $4.0868572 .083519-2.527872$

H $3.6814150 .904156-3.798379$

H $5.698362-0.553143-2.872160$

H $6.4699772 .409658-2.904534$

H $8.3937701 .033166-3.999570$

H $8.094604-0.247139-2.845019$

H $10.4487402 .993302-1.662269$

H $9.7526282 .905028-3.376408$

H $9.8889091 .357929-0.043800$

H $9.178444-0.116745-0.621145$

H 7.7320882 .4123450 .431070

H $3.3901360 .595990-0.869215$

C -1.4277214 .9190750 .927141$

O -2.2414693 .9513790 .230256$

C $-3.1206264 .650075-0.672252$

C $-3.1256916 .097952-0.203416$

C -1.6843656 .2599330 .235303$

H $-2.6544014 .609425-1.663753$

C $-4.4968853 .979873-0.716161$

H -3.415415 $6.792603-0.997366$

H $-3.801114 \quad 6.248778 \quad 0.646707$ 
C $-1.454144 \quad 7.467473 \quad 1.127948$ H -1.051791 $6.349586-0.658293$ C $-4.4438212 .484296-1.097338$ O -5.0919894 .0937670 .580676$ H -5.150148 4.528484 -1.405062 H $-3.923171 \quad 1.923717-0.316326$ O $-5.7882491 .993686-1.140536$ C $-3.7932472 .212520-2.464884$ C $-3.8489220 .752796-2.955584$ H $-2.7463192 .532126-2.452198$ O $-4.4499523 .017593-3.452132$ H $-4.8728230 .373101-2.873715$ H $-3.6376420 .769157-4.032736$ H -1.7088978 .3911910 .598182$ H -2.067666 7.4197202 .033913 H -0.4055127 .5322231 .434719$ C 0.0364934 .4835770 .860982 H -6.0258093 .8369310 .498220$ H $-6.1119241 .946959-0.225097$ H $-5.3866752 .755881-3.466370$ H 0.6953875 .3339081 .071814 H $0.277174 \quad 4.128011-0.148718$ H -1.784892 4.9484951 .964690 C $-4.289792-1.214553-0.614207$ O $-3.131221-0.414993-0.880212$ C $-2.840248-0.187282-2.261240$ C $-2.653440-1.531730-2.979741$ C $-3.870982-2.432275-2.801052$ C $-4.207087-2.574951-1.319071$ H -5.187310 -0.677132 -0.940427 C $-4.370131-1.4150350 .904916$ H $-1.8685270 .322603-2.288119$ O $-2.392301-1.325814-4.363438$ H -1.774003 -2.036149-2.563365 H -4.730172 -2.053309-3.365620 O $-3.587442-3.724515-3.343049$ H $-3.446681-3.198250-0.835728$ O $-5.456056-3.249788-1.183309$ C $-4.754471-0.1389881 .614055$ H -5.120823 -2.165708 1.177657 H -3.411093 -1.767712 1.304007 H -2.308481 -2.201893 -4.777456 H -2.818428 -4.086804 -2.870280 H -5.380812 -4.097407 -1.654299 O O C -3.8551551 .6476332 .902147$ H -4.1035191 .4063983 .939174$ H -2.9199582 .2138742 .884767$ H -4.6420992 .2630182 .459167$

SCF Energy (B3LYP/6-31G**//MMFF) $=-3245.93100123$

06_00239

MM̄FF Geometry

C $1.130493-3.7190720 .642183$

C $0.635172-2.4799000 .773034$

C $0.101086-1.9035332 .055324$

O $-1.266216-1.4923671 .816634$

C $0.910165-0.6556952 .454419$

C 0.3392840 .0491893 .670863

C -0.5363731 .0743293 .616794$

C $0.810855-0.4829554 .997653$

C $-1.122524 \quad 1.6959872 .378817$

C -1.069454 3.2291932 .412797

C $-1.1222313 .830978 \quad 1.000577$

C -1.2718015 .3514951 .048273$

C -1.362935 $5.956001-0.361699$

O $-2.217978 \quad 3.2543050 .283457$

O $-1.8199007 .308212-0.208828$

C $-2.4258005 .181748-1.174317$

C $0.018586 \quad 6.042222-1.022387$

O $-2.4444695 .621017-2.530741$

C $-2.2702673 .644058-1.095674$

C $-3.4835512 .949518-1.755495$

O $-1.0855793 .267298-1.803616$

C $-2.243846-2.423521 \quad 1.974395$

O $-2.098601-3.6073282 .232537$

C $-3.542300-1.7344751 .795502$
C $-4.680912-2.4408151 .849631$

C $-6.040217-1.9068991 .729928$

C $-6.197104-0.4130691 .618143$

C $-3.5609411 .436831-1.496200$

C $-4.6703270 .779681-2.301070$

C $-5.769093-1.429221-2.657468$

O $\quad-5.3520681 .384668-3.124591$

C $-7.080391-1.343976-1.940781$

C -7.778826 -2.416196 -1.537097

C $-9.119870-2.328325-0.865468$

C $-9.184325-3.1022110 .431988$

C $-9.855409-4.2637940 .512240$

C $-8.546059-2.4856851 .657295$

C -7.072396-2.776328 1.740816

N $-4.797335-0.571220-2.003989$

H $1.170543-4.3763181 .506798$

H $0.581374-1.838406-0.101975$

H $0.131162-2.6353102 .872700$

H 0.9675980 .0406321 .611297

H $1.952674-0.9366252 .656252$

H $-0.898801 \quad 1.4928644 .554555$

H 0.3679460 .0543845 .842840

H $0.544844-1.5396115 .101890$

H $1.898672-0.3876215 .078837$

H $-0.625876 \quad 1.335072 \quad 1.475016$

H -2.1707561 .3825632 .301724$

H -1.9221253 .5872843 .004124$

H -0.1597503 .5807822 .915243$

H -0.1885473 .5624390 .494806$

H -0.4483885 .8084751 .611112$

H -2.1889935 .6092541 .596106$

H -1.951260 $7.677308-1.099360$

H -3.405286 $5.452552-0.756690$

H $0.6919546 .660379-0.416229$

H $-0.0382346 .535218-1.999037$

H $0.4933925 .067829-1.154986$

H -1.682812 $5.219133-2.982326$

H $-4.4121693 .393727-1.373748$

H -3.458538 $3.128810-2.837962$

H -1.168096 2.348568 -2.102685

H $-3.526597-0.6622031 .638634$

H -4.615762 -3.517565 2.001801

H -5.8014440 .0806982 .512686$

H $-7.233203-0.0877301 .508490$

H -5.661172 -0.0303990 .744293$

H $-2.6223100 .943184-1.768397$

H $-3.7485891 .251085-0.432582$

H $-5.361069-2.444823-2.657143$

H -5.895094 -1.100601 -3.694260

H -7.491531 -0.351151-1.772396

H -7.385643 -3.411479-1.731241

H -9.867709 -2.714469-1.570460

H $-9.403211-1.285616-0.674880$

H -9.929949 -4.809416 1.447101

H -10.340495 -4.697844 -0.355689

H $-9.008743-2.8971782 .564532$

H -8.780050 -1.418405 1.685764

H $-6.848761-3.8400761 .837583$

H $-4.239594-0.970166-1.254442$

C $3.066895-4.734017-0.608046$

O $3.929976-3.601773-0.385345$

C $5.086070-3.719797-1.236216$

C $5.037890-5.123442-1.830681$

C $3.545260-5.366155-1.915289$

H $4.953601-2.991291-2.045081$

C $6.367970-3.403639-0.456611$

H $5.539050-5.178554-2.802027$

H $5.504127-5.864417-1.171291$

C $3.176387-6.831704-2.067460$

H $3.148366-4.804998-2.772062$

C $6.347104-2.0508730 .281134$

O $6.559259-4.4285210 .527525$

H $7.228586-3.458622-1.134319$

H $5.639185-2.0889491 .118540$

O $7.644391-1.8444910 .855297$

C $6.018352-0.836216-0.607327$

C $6.123608 \quad 0.4882060 .169230$

H $5.004507-0.941820-1.000418$ 
O $6.902970-0.800194-1.725450$

H 5.5123340 .4047311 .074127

H 7.1613080 .6360910 .491812

H $3.607259-7.243096-2.986107$

H $3.546106-7.427906-1.226440$

H $2.090451-6.958488-2.118446$

C $1.609362-4.276278-0.669808$

H $7.469249-4.3497230 .859927$

H $7.750206-2.4856901 .578104$

H $7.810196-0.738311-1.380395$

H $0.963866-5.122194-0.932442$

H $1.496116-3.523386-1.459718$

H $3.218430-5.4221450 .233917$

C $3.3213621 .621717-0.211542$

O $4.3743651 .616934-1.183620$

C $5.6989401 .724483-0.656384$

C 5.8309853 .0477940 .105172

C $4.7965213 .143803 \quad 1.220316$

C 3.3905782 .8711570 .686753

H 3.3798160 .7106630 .396171

C $1.9947821 .600695-0.992245$

H $6.3713511 .777995-1.521617$

O 7.1431753 .1752110 .642085

H $5.684833 \quad 3.882522-0.592705$

H 5.0481062 .4721632 .049460

$\begin{array}{llll}\text { O } & 4.840087 & 4.468025 & 1.759638\end{array}$

H $3.0448503 .756408 \quad 0.137238$

O 2.5364322 .7117251 .819348

C $1.7636740 .317379-1.760799$

H $1.1484891 .713995-0.309416$

H $1.9850612 .421129-1.720094$

H 7.1734804 .0104291 .139717

H 4.1555224 .5219392 .448415

H 1.6234692 .6350151 .501040

O $0.5674390 .412921-2.415878$

O $2.518997-0.643278-1.786161$

C $0.198667-0.723212-3.203714$

H $-0.735304-0.487869-3.721242$

H $0.969325-0.937018-3.950471$

H $0.032984-1.590958-2.559271$

SCF Energy (B3LYP/6-31G**//MMFF) $=-3245.92317952$

06_00240

MM̄FF Geometry

C $0.461823-3.6946740 .127941$

C $0.987947-2.4681310 .267515$

C $1.560076-1.9571581 .564094$

O $1.124484-0.586245 \quad 1.707678$

C $3.097307-1.9754061 .490497$

C $3.774670-1.8179772 .836237$

C $4.390696-0.6975943 .261055$

C $3.778850-3.0533343 .700025$

C $4.482703 \quad 0.6320132 .565217$

C 5.8991520 .9354792 .057169

C $6.284966 \quad 0.125006 \quad 0.809511$

$\begin{array}{llll}\text { C } 7.732157 & 0.413194 & 0.404421\end{array}$

C $8.112401-0.296050-0.903540$

O $5.3996160 .509564-0.246778$

O $9.355014 \quad 0.273190-1.342335$

C $7.0407490 .018196-1.969289$

C $8.379806-1.788383-0.676548$

O $7.285166-0.718704-3.165533$

C $5.590523-0.199310-1.477096$

C $4.5932740 .374468-2.512151$

O $5.359106-1.600660-1.325463$

C $0.809658-0.1324962 .946446$

O $0.750083-0.7928193 .974616$

$\begin{array}{lllll}\text { C } & 0.537952 & 1.324671 & 2.939247\end{array}$

C $0.764243 \quad 2.1183131 .880491$

C 0.5354243 .5641551 .812822

C -0.0252444 .2451903 .033551$

C $3.1328770 .393014-2.037524$

C $2.2225631 .099774-3.027673$

C $-0.0731032 .058780-3.205875$

O $2.5807411 .424338-4.156653$

C $0.100593 \quad 3.514644-2.904214$

C $-0.8037214 .258125-2.248971$

C $-0.6033215 .710280-1.921265$
C $-0.5933395 .980940-0.431383$

C $-1.645838 \quad 6.561133 \quad 0.169576$

$\begin{array}{llll}\text { C } & 0.668750 & 5.671010 & 0.345906\end{array}$

C $0.819822 \quad 4.206316 \quad 0.660126$

N $0.9548451 .312902-2.501303$

H $\quad 0.444408-4.3593620 .987731$

H $1.042720-1.809446-0.595447$

H $1.188999-2.5684052 .395221$

H $3.434766-1.1948180 .801011$

H $3.445057-2.9222651 .054987$

H $4.870851-0.7180704 .238829$

H $4.330295-2.9058474 .634818$

H $2.757417-3.3431183 .964553$

H $4.250749-3.8864093 .168891$

H 3.7544390 .7276041 .754862

H 4.2061951 .4003473 .298654

H 5.9368232 .0047301 .810417

H $\quad 6.6277690 .7621172 .858849$

H $6.157706-0.9421681 .020759$

H $8.4251410 .134724 \quad 1.208208$

H 7.8610651 .4954540 .261045

H $9.567263-0.114982-2.208714$

H $7.1616501 .076416-2.238499$

H $9.193224-1.9233080 .046569$

H $8.717500-2.275902-1.597757$

H $7.508187-2.327979-0.300275$

H $6.990268-1.633003-3.015017$

H $4.8797681 .408273-2.746653$

H $4.652112-0.200059-3.445288$

H $4.408430-1.776764-1.399768$

H $\quad 0.160648 \quad 1.718144 \quad 3.877586$

H 1.1617301 .6765790 .968272

H $-0.9690743 .780893 \quad 3.339710$

$\begin{array}{llll}\text { H } & -0.241501 & 5.304527 & 2.878828\end{array}$

H $0.6831614 .179853 \quad 3.866757$

H $2.756413-0.627006-1.913722$

H $3.0541110 .910180-1.074429$

H -1.042836 $1.677352-2.871120$

H $\quad 0.0208791 .887023-4.282823$

H $1.0279503 .977710-3.234192$

H $-1.7365333 .801991-1.928133$

H $-1.4114646 .274313-2.404935$

H $0.3259556 .094011-2.360633$

H $-1.636796 \quad 6.788644 \quad 1.230211$

H $-2.5427236 .817425-0.384429$

H $0.718228 \quad 6.276240 \quad 1.257941$

H $1.5400675 .993132-0.238892$

H $1.2296613 .624134-0.164955$

H $0.7790881 .076434-1.528962$

C $-1.491799-4.712711-1.186683$

O $-2.393062-3.595168-1.096647$

C $-3.647664-4.077543-0.563811$

C $-3.449967-5.561546-0.243002$

C $-1.944083-5.665886-0.087334$

H $-3.825575-3.5347540 .369111$

C $-4.783431-3.806273-1.559832$

H $-3.993183-5.8593360 .659553$

H $-3.799384-6.204758-1.059126$

C $-1.411474-7.080001-0.228828$

H $-1.681570-5.2752260 .903233$

C $-4.969607-2.319516-1.920223$

O $-4.513148-4.517239-2.776318$

H $-5.723014-4.217630-1.173871$

H $-4.066484-1.929274-2.404363$

O $-6.020163-2.237160-2.893832$

C $-5.353604-1.418630-0.729643$

C $-5.562094 \quad 0.044657-1.163410$

H $-4.565275-1.4518110 .028165$

O $-6.543409-1.916248-0.120720$

H $-4.6021310 .428432-1.526984$

H $-6.2564050 .080826-2.011570$

H $-1.844935-7.7326590 .535995$

H $-1.653448-7.504229-1.209104$

H $-0.323403-7.099968-0.112070$

C $-0.033208-4.250990-1.176114$

H $-3.678487-4.173383-3.138065$

H $-5.768991-2.808501-3.639907$

H $-7.244347-1.905158-0.795042$ 
H $\quad 0.098758-3.502699-1.968182$ H $0.608580-5.101809-1.435319$ H $-1.674808-5.172524-2.168284$ C $-4.103952 \quad 1.3765831 .181318$ $\begin{array}{llll}\text { O } & -5.434788 & 0.849805 & 1.180868\end{array}$ C $-6.1339060 .961664-0.059600$ C $-6.2342392 .436199-0.472359$ C $-4.8592303 .095494-0.524755$ C $-4.104452 \quad 2.859166 \quad 0.782041$ H -3.4803440 .7972870 .489582$ C $-3.549514 \quad 1.2002372 .600488$ H -7.1560600 .6134510 .135943$ O $-6.8804352 .550384-1.735587$ H -6.8569142 .9698780 .257454$ H -4.284275 $2.745452-1.389944$ O $-5.0414284 .501231-0.711163$ H -4.5642303 .4719431 .568345$ O -2.7682943 .3178260 .603704$ C -3.503106 $-0.247573 \quad 3.024018$ H $-2.542103 \quad 1.6141402 .705747$ H $-4.188346 \quad 1.7237083 .323094$ H $-6.8992473 .494718-1.968021$ H $-4.1578264 .907651-0.715779$ H $-2.360547 \quad 3.3888741 .481964$ O $-2.527078-0.9098862 .341623$ O $-4.239201-0.7261943 .877674$ C -2.363757 -2.286696 2.694238 H $-3.324380-2.8109772 .687612$ H -1.711740 -2.7519561 .953209$ H -1.891156 -2.362419 3.677647

SCF Energy (B3LYP/6-31G**//MMFF) $=-3245.92619807$

06_00241

MMFF Geometry

C $-0.436978 \quad 1.0078793 .856275$

C $0.6488531 .592783 \quad 3.330601$

C $0.571706 \quad 2.7083842 .319790$

O $0.9728062 .141381 \quad 1.048315$

C 1.5417043 .8427522 .686778

C 1.5162444 .9975681 .701082

C 2.5006895 .2857390 .825402

C $0.2859915 .866876 \quad 1.758840$

C 3.7969674 .5506110 .632893

C $3.7804573 .712189-0.651842$

C $4.8565952 .617753-0.625980$

C $4.9112771 .874912-1.960483$

C $5.886526 \quad 0.690003-1.918993$

O $4.510231 \quad 1.706717 \quad 0.423012$

O $5.629065-0.102852-3.088508$

C $5.579790-0.179190-0.678435$

C $7.342743 \quad 1.157792-2.029567$

O $6.574493-1.192152-0.548782$

C 5.4457600 .6386820 .624829

C $4.928054-0.1875791 .829339$

O 6.7341301 .1490520 .988891

$\begin{array}{llll}\text { C } & 0.017610 & 1.451075 & 0.363193\end{array}$

O $\begin{array}{llll}0.1 .167610 & 1.362843 & 0.643902\end{array}$

C $0.6734900 .762029-0.768358$

C $-0.001299-0.194122-1.423122$

C $0.482827-0.985212-2.554567$

C $1.898232-0.755832-3.011444$

C $3.552872-0.8395641 .642795$

C $3.610264-2.2764221 .153400$

C $2.129735-4.1253410 .383582$

O $4.651248-2.924617 \quad 1.088747$

C $2.109849-4.163127-1.111809$

C $1.106346-4.700942-1.821853$

C $1.107595-4.815577-3.319372$

C $-0.133105-4.230024-3.954216$

C -1.184035 -5.007535 -4.264944

C $-0.116025-2.761805-4.311661$

C $-0.358074-1.875533-3.121220$

N $2.350537-2.7638620 .832242$

H $-1.424844 \quad 1.3840723 .601249$

H $1.632323 \quad 1.1826463 .547928$

H -0.4478543 .1066572 .244135$

H 2.5515743 .4321092 .779454

H 1.2942804 .2337253 .682973
H 2.3689656 .1407690 .163640

H $\quad 0.3591246 .735203 \quad 1.095257$

H -0.6018845 .3008351 .461268$

H 0.1354886 .2444962 .775588

H 4.6089945 .2861830 .572783

H 4.0370753 .9307791 .499402

H $2.7966443 .241953-0.769062$

H $3.9303864 .371266-1.515745$

H $5.8240013 .082043-0.401404$

H $5.1718092 .559347-2.777367$

H $3.9113651 .491394-2.203202$

H $6.193664-0.893436-3.038056$

H $4.633171-0.691740-0.885992$

H $7.5003441 .690362-2.975232$

H $8.0332640 .307388-2.047784$

H $7.6411801 .825978-1.219057$

H $6.288078-1.8051380 .148652$

H $5.683545-0.9176722 .142850$

H 4.8318950 .5065922 .676947

H 6.7257691 .3605661 .937050

H $1.6893301 .042162-1.017021$

H $-1.016791-0.422004-1.102548$

H $2.595839-0.889602-2.177829$

H $2.0129640 .258520-3.408444$

H $2.224456-1.447068-3.791058$

H $3.052050-0.8738462 .618872$

H $2.923051-0.2478330 .972289$

H $1.185914-4.4648220 .819876$

H $2.933195-4.7687810 .756441$

H $2.977991-3.765640-1.632168$

H $0.253578-5.125318-1.296288$

H $1.182429-5.881998-3.570312$

H $1.998665-4.348686-3.756882$

H $-2.066102-4.600089-4.747821$

H $-1.187763-6.068311-4.036984$

H $-0.908360-2.544315-5.040925$

H $0.813554-2.529250-4.838485$

H - $1.363352-1.973499-2.708788$

H $1.550263-2.1391070 .865289$

C $-0.473082-1.476327 \quad 3.774905$

O $-1.695075-1.4442593 .015867$

C -2.186578 -2.7879602 .860339$

C $-1.134506-3.7064953 .476242$

C $-0.500465-2.8057034 .520700$

H -3.103534-2.854336 3.457365

C $-2.507935-3.0606341 .383914$

H $-1.577305-4.6118403 .902845$

H $-0.385132-4.0206022 .741616$

C $0.864799-3.2829994 .984051$

H $-1.175849-2.7393215 .384120$

C -3.399716 -1.993238 0.712215

O $-1.284845-3.113606 \quad 0.636420$

H $-2.962379-4.0528281 .283766$

H -2.818496 -1.0730820 .593540$

O $-3.689866-2.474517-0.604688$

C $-4.716540-1.7070331 .452879$

C $-5.665143-0.6842230 .792036$

H -4.494977 -1.343409 2.461913

O $-5.439556-2.9310011 .614284$

H $-6.001620-1.069103-0.175736$

H $-6.569163-0.6522741 .412983$

H $\quad 0.790677-4.2755105 .440377$

H $1.571759-3.3480364 .149858$

H $1.285226-2.5998715 .728703$

C $-0.386583-0.2457004 .682111$

H $-0.830973-2.2616480 .754421$

H $-4.036915-1.729669-1.120862$

H $-5.610886-3.2886320 .726088$

H $0.531489-0.2750835 .279838$

H $-1.230601-0.2232035 .381711$

H $0.350681-1.4646903 .048281$

C $-4.735310 \quad 0.873906-1.725054$

$\begin{array}{lllll}\text { O } & -4.142710 & 0.835728 & -0.423743\end{array}$

$\begin{array}{llll}\text { C } & -5.057923 & 0.731249 & 0.671351\end{array}$

C -6.0865501 .8730230 .612895$

C $-6.7932361 .940592-0.736453$

C $-5.7611262 .004402-1.861924$

H $-5.246207-0.078405-1.909933$ 
C -3.609692 $0.942044-2.768101$ H -4.4651990 .8971941 .580364$ O H -5.5709522 .8285310 .777839$ H $-7.4806361 .096006-0.863924$ O $-7.5929713 .124570-0.771551$ H $-5.2744802 .985179-1.835164$ O $-6.4273471 .911755-3.119546$ C -2.676302 2.128262 -2.625878 H -2.989326 $0.044654-2.664011$ H $-4.0358380 .955991-3.777330$ H $-6.577048 \quad 1.7263912 .495116$ H $-7.9989193 .173533-1.654024$ H $-6.8804801 .052050-3.156054$ O $-1.7594632 .085973-3.639216$ O $-2.7238182 .972821-1.743009$ C $-0.786043 \quad 3.135146-3.634195$ H -0.249365 $3.159042-2.680949$ H $-0.067766 \quad 2.935651-4.433936$ H -1.271414 4.096321-3.827566

SCF Energy (B3LYP/6-31G**//MMFF) $=-3245.91570656$

\section{6_00242}

MMFF Geometry

C $1.637355-3.9215210 .355009$

C $1.444385-4.0198791 .679821$

C $2.029542-3.0956142 .720989$

$\begin{array}{llll}\text { O } & 2.175387 & -1.754211 & 2.198051\end{array}$

C $3.404379-3.6129063 .175013$

C $4.023166-2.7829114 .282884$

C $5.019470-1.8911854 .110123$

C $3.469472-3.0386415 .660953$

C $5.685851-1.4980752 .820737$

C 5.5602310 .0103422 .569181

C $6.058021 \quad 0.397417 \quad 1.168803$

$\begin{array}{llll}\text { C } & 6.014850 & 1.914698 & 0.980744\end{array}$

C $6.3969882 .330695-0.447078$

$\begin{array}{llllll}\text { O } & 5.205917 & -0.239143 & 0.209835\end{array}$

O $6.0205893 .708669-0.595046$

C $5.5658891 .500775-1.450530$

C $7.9142402 .275940-0.659301$

O $5.988007 \quad 1.759004-2.787173$

C $5.575745-0.014093-1.157255$

C $4.589060-0.814206-2.044336$

O $6.887057-0.527515-1.424637$

C $1.043874-0.9941522 .151011$

O $-0.085548-1.3418092 .459416$

$\begin{array}{lllll}\text { C } & 1.415689 & 0.354907 & 1.670684\end{array}$

C 0.4640091 .2897541 .529299

C 0.6666652 .6821001 .121940

C 2.0627223 .1113850 .758949

C $3.112165-0.429962-1.905096$

C $2.6383150 .601226-2.915089$

C $0.6650102 .034791-3.435660$

O $3.2986460 .939708-3.893168$

C $0.638957 \quad 3.353430-2.732141$

C $-0.493792 \quad 3.972830-2.367661$

C $-0.5364365 .329157-1.727068$

C $-1.2495445 .325948-0.395158$

C -2.536775 $5.699391-0.296953$

C -0.4398414 .9855350 .834199$

C $-0.399325 \quad 3.509107 \quad 1.113801$

N $1.3673051 .069639-2.611867$

H $2.297973-3.152644-0.037397$

H $\quad 0.800381-4.8089622 .061810$

H $1.349118-3.0641193 .581662$

H $4.079331-3.6690752 .314346$

H $3.312448-4.6487073 .530015$

H $5.402242-1.3737874 .989086$

H $3.982861-2.4531856 .431106$

H $2.407677-2.7773445 .703475$

H $3.579398-4.0957345 .924158$

H $6.746929-1.7683472 .880720$

H $5.274172-2.0449981 .969938$

H 4.5087810 .3040142 .674919

H $\begin{array}{llll}6.129816 & 0.556494 & 3.331362\end{array}$

H 7.0817550 .0239201 .048134

H $\quad 6.6592342 .4169291 .713125$
H $4.9978042 .275068 \quad 1.184639$

H $6.1866863 .961632-1.519474$

H $4.5372031 .872645-1.378608$

H 8.4212092 .9452020 .046420

H $8.1894332 .633831-1.657451$

H $8.3302191 .275457-0.524243$

H $6.8118311 .265077-2.938541$

H $4.916139-0.787550-3.090787$

H $4.667357-1.869075-1.743206$

H $6.819761-1.486568-1.564977$

H 2.4617810 .5504331 .470354

H -0.5664711 .0194491 .757933$

H 2.7099553 .0870681 .641979

H 2.1138924 .1203770 .344220

H $2.4850622 .450212-0.005213$

H $2.503619-1.325771-2.081918$

H $2.891964-0.080619-0.892784$

H $-0.340717 \quad 1.643053-3.612393$

H $1.1639052 .141531-4.403866$

H $1.5962033 .834473-2.545819$

H $-1.4500423 .498412-2.576999$

H $-1.0478026 .008224-2.422033$

H $0.4709445 .745398-1.602337$

H $-3.044598 \quad 5.729352 \quad 0.661288$

H -3.112999 $5.975756-1.173773$

H $-0.887993 \quad 5.4672861 .714221$

H $0.554547 \quad 5.4339550 .756278$

H $-1.369953 \quad 3.098862 \quad 1.392515$

H $0.9044160 .742328-1.768503$

C $0.273090-4.002615-1.745047$

O $-0.653335-3.067278-1.165250$

C $-1.829587-2.994422-1.990641$

C $-1.531433-3.823417-3.238123$

C $-0.556062-4.853983-2.703568$

H -2.635083 -3.481316 -1.430273

C $-2.190291-1.529693-2.265914$

H $-2.436783-4.268426-3.662411$

H $-1.056203-3.226564-4.024838$

C $0.258122-5.541665-3.785112$

H $-1.121992-5.609836-2.142807$

C $-2.290954-0.620174-1.022050$

O $-1.183031-0.960525-3.115699$

H $-3.120798-1.481461-2.842446$

H $-1.294962-0.513698-0.574266$

O $-2.6778450 .689800-1.460311$

C $-3.287178-1.0375230 .073409$

C $-4.698339-1.345182-0.463854$

H $-3.346540-0.2122490 .792017$

O $-2.771692-2.1648190 .773492$

H $-4.994041-0.561075-1.168357$

H $-4.672743-2.288469-1.020927$

H $-0.398637-6.074069-4.480828$

H $0.847029-4.821525-4.362894$

H $0.949362-6.269861-3.349396$

C $0.958769-4.815564-0.644900$

H $-0.328837-1.049859-2.659061$

H -2.033417 $0.972491-2.130965$

H $-3.282342-2.2654941 .594042$

H $1.706268-5.483172-1.087735$

H $\quad 0.216542-5.443361-0.136159$

H $1.017443-3.412669-2.295961$

C -6.3538610 .8622410 .848959$

$\begin{array}{lllll}\text { O } & -5.813286 & -0.304663 & 1.482233\end{array}$

C $-5.757921-1.471760 \quad 0.654663$

C $-7.166090-1.8267930 .154972$

C $-7.845411-0.642542-0.526753$

C $-7.7843490 .596438 \quad 0.365256$

H $-5.723914 \quad 1.149411-0.001213$

C $-6.3239482 .000013 \quad 1.881297$

H $-5.437402-2.2936751 .306181$

O $-7.127009-2.926543-0.748325$

H $-7.785243-2.1342041 .007928$

H -7.407212 -0.439297-1.511012

O $-9.220396-0.958787-0.757321$

H $-8.4558490 .463631 \quad 1.223262$

O $-8.248262 \quad 1.725905-0.371334$

C $-4.9164702 .493963 \quad 2.142297$

H -6.9038902 .8632051 .538062$ 
H $-6.742091 \quad 1.6398452 .829140$

H $-6.706628-3.670584-0.284379$

H $-9.243809-1.770206-1.293410$

H $-9.1445501 .517986-0.686899$

O $-4.877203 \quad 3.1200013 .355309$

O $-\begin{array}{llll}-3.977231 & 2.377470 & 1.367392\end{array}$

C -3.6037153 .6550853 .729804$

H -3.6988354 .0929894 .727049$

H -2.852435 2.8602683 .766386

$\mathrm{H}-3.3033934 .4395923 .029109$

SCF Energy (B3LYP/6-31G**//MMFF) $=-3245.91981718$

06_00243

MMFF Geometry

C $2.634458-0.4139642 .914802$

C $1.804915-0.1867313 .943639$

C $0.337880-0.5170223 .911500$

O $-\begin{array}{lllll}0.350717 & 0.753937 & 3.839569\end{array}$

C $-0.126001-1.2684365 .171453$

C $0.393494-2.6852595 .273404$

C $-0.146154-3.7346134 .623132$

C $1.560349-2.8863476 .203860$

C $-1.311172-3.7171343 .672109$

C $-0.902283-4.2510572 .293826$

C $-2.079285-4.2521351 .307087$

C $-1.712083-5.0359610 .045105$

C $-2.835476-5.000720-0.999026$

O $-2.388716-2.893160 \quad 0.971030$

O $-2.281690-5.499256-2.226342$

C $-3.252796-3.533273-1.225146$

C $-3.980337-5.950268-0.628717$

O $-4.385357-3.458562-2.089056$

C $-3.505804-2.7464810 .083440$

C $-3.724366-1.238580-0.193377$

O $-4.708169-3.2432290 .685267$

C $-1.5784010 .769153 \quad 3.255788$

O $-2.221436-0.1910542 .865064$

C -1.9958232 .1829903 .140128$

C -3.0911202 .4938532 .431252$

C -3.6094373 .8427142 .194404$

C -2.8439464 .9950342 .792344$

C $-2.480694-0.473900-0.657671$

C $-2.8188070 .897650-1.220480$

C -1.695330 $2.900692-2.186758$

O $-3.9621051 .247091-1.501972$

C $-2.2517494 .023196-1.368288$

C -3.357150 $4.699163-1.718514$

C $-3.9267225 .864663-0.960620$

C $-5.3067995 .577527-0.410149$

C $-6.3966185 .670176-1.190861$

C -5.4273485 .2579601 .062872$

C -4.720555 3.9847431 .441096

N -1.679998 $1.667556-1.421757$

H $2.247271-0.8663412 .005162$

H 2.1897560 .2928394 .840615

H $0.106906-1.0984063 .009386$

H -1.222701 -1.279410 5.214497

H $0.155211-0.700026 \quad 6.069022$

H $\quad 0.288495-4.7197774 .787772$

H $\quad 1.874897-3.9341176 .255837$

H $2.423534-2.3008735 .873757$

H $1.294061-2.5717267 .218320$

H -2.100978 -4.3530284 .090218$

H -1.739624 -2.717698 3.568793

H -0.088968 -3.630324 1.895039

H $-0.514962-5.2716922 .405962$

H -2.945993 -4.709165 1.799056

H -1.453626 -6.0735590 .291394$

H - $0.806252-4.602076-0.400020$

H -2.962650 -5.408579 -2.915116

H $-2.432420-3.061890-1.775337$

H -3.609009-6.978672 -0.543567

H -4.745273 -5.974836 -1.412827

H $-4.463455-5.692350 \quad 0.316026$

H -5.172036 -3.688474 -1.565760

H -4.548095 -1.122254 -0.909076

H $-4.080887-0.7523170 .725329$

H $\quad-5.043077-2.5716971 .302428$
H -1.3756312 .9309713 .621145$

H $-3.6587501 .687657 \quad 1.968198$

H -2.8393814 .9262003 .885870$

H -3.2528155 .9746062 .538537$

H -1.8078254 .9948482 .435034$

H $-1.780810-0.3570040 .176571$

H -1.963538 -1.014247-1.455503

H -2.273691 2.730511 -3.101568

H $-0.6637263 .126669-2.471201$

H -1.707206 4.306976-0.471585

H $-3.8808534 .413226-2.628663$

H -3.977838 $6.722334-1.644281$

H $-3.256076 \quad 6.180323-0.152323$

H -7.392019 $5.491296-0.798472$

H $-6.3153965 .925917-2.242261$

H -6.4820865 .1377601 .343768$

H $-5.0805006 .121247 \quad 1.637289$

H -5.1967713 .0880131 .041878$

H $-0.7736691 .270513-1.192913$

C 4.3170201 .3427852 .322918

O $3.846607 \quad 1.3864210 .964421$

C 4.6915462 .2751110 .208354

C 5.7175162 .8411051 .187519

C 5.7844281 .7530182 .242737

H $5.2139121 .657708-0.530611$

C $3.8369373 .326741-0.510160$

H 6.6823753 .0310330 .706767

H 5.3815453 .7843391 .633220

C 6.3746312 .2225113 .560720

H 6.3862890 .9221041 .851367

C $2.6777082 .746515-1.345015$

O $3.254858 \quad 4.2088380 .459680$

H $4.4779223 .954660-1.139502$

H $1.9070262 .331373-0.683701$

O $2.0612423 .834630-2.045861$

C $3.0948941 .679799-2.372922$

C $1.9019971 .192965-3.215200$

H $3.5321310 .824648-1.848904$

O $4.1022862 .207988-3.232954$

H $1.1266730 .826987-2.534216$

H $1.4742612 .038742-3.766973$

H 7.3993242 .5808303 .417065

H 5.7897453 .0409523 .993508

H 6.4025821 .4066144 .289435

C $4.091507-0.0468082 .918154$

H 2.7244203 .6669151 .069020

H $1.7900604 .490771-1.380967$

H $3.7271592 .985022-3.682001$

H $4.488781-0.0894913 .938941$

H $4.628771-0.8040402 .334837$

H 3.7404862 .0895492 .885833

C $2.221095-1.811904-2.759459$

O $2.993116-0.987672-3.639943$

C $2.2766910 .097368-4.236872$

C $1.082668-0.438279-5.040659$

C $0.189489-1.342787-4.194595$

C $1.026077-2.418916-3.504273$

H $1.872614-1.213979-1.908181$

C $3.145031-2.912584-2.227969$

H $2.9666060 .559832-4.954111$

O $0.3219660 .642827 \quad-5.569572$

H $1.460315-1.014121-5.895617$

H $-0.390693-0.757840-3.471946$

O $-0.762350-1.970864-5.055902$

H $1.375035-3.135938-4.257902$

O $0.199947-3.141061-2.595388$

C $4.087502-2.368960-1.182070$

H $2.580316-3.721760-1.750490$

H $3.733712-3.368305-3.033278$

H $-0.4320770 .260030-6.050026$

H -1.294494 -2.573304 -4.507970

H $-0.073841-2.530030-1.890234$

O $5.241426-1.939859-1.766266$

O $3.819417-2.3217700 .011586$

C $6.228237-1.412727-0.874184$

H $5.788051-0.685414-0.186915$

H $6.695132-2.230445-0.317833$

H $6.992846-0.909184-1.471598$ 
H $\quad 0.0346884 .201649-3.356389$

H $-0.9884026 .572256-3.210364$

06_00244

MM̄FF Geometry

C $-0.354460-3.283702-1.460099$

C $-0.163772-2.953381-0.174066$

C $0.992311-2.1964350 .425722$

O $0.525646-0.8307700 .578865$

C $2.322931-2.156926-0.352714$

C $3.133616-3.432280-0.257510$

C $4.016112-3.6947490 .726804$

C $2.953110-4.423140-1.376637$

C $4.353539-2.8396341 .916903$

C $5.851629-2.5125381 .993582$

C $6.334394-1.5614330 .886500$

C $7.854074-1.3941160 .959382$

C $8.367339-0.370015-0.061931$

O $5.692771-0.2965321 .079509$

O $9.723407-0.045792 \quad 0.280824$

C 7.5431710 .9252770 .078805

C $8.404421-0.946248-1.482349$

O $7.8883661 .858362-0.943494$

C 6.0130610 .6919220 .089763

C 5.2961022 .0088480 .483595

O $5.5921140 .287714-1.211789$

C $0.830298-0.1671991 .721492$

O $1.443626-0.6104952 .683009$

C 0.3547251 .2358321 .684596

C $-0.402658 \quad 1.749040 \quad 0.702649$

C -0.8878253 .1300990 .620561$

C -0.3683154 .1135831 .635746$

C 3.7710451 .9020810 .606228

C $3.0555392 .180571-0.701776$

C $2.5207714 .039444-2.282636$

O $2.5505951 .278044-1.365052$

C $1.4058114 .987657-1.974132$

C $0.2145224 .952792-2.590391$

C $-0.8906905 .933711-2.322615$

C $-2.2123235 .262735-2.023913$

C $-3.1756385 .187544-2.957813$

C $-2.4490374 .758644-0.617780$

C - $1.7677433 .444625-0.352799$

N $3.0623533 .523473-1.039768$

H $0.374095-2.999966-2.213101$

H $-0.928798-3.2415770 .544166$

H $1.173550-2.6347441 .415996$

H $2.928676-1.3214530 .019985$

H $2.158045-1.885498-1.402842$

H $4.552136-4.6427870 .693176$

H $3.706153-5.218509-1.351044$

H $1.972417-4.901683-1.314292$

H $3.043897-3.926063-2.347965$

H $3.768935-1.9169011 .948746$

H $4.078265-3.4048492 .816128$

H $6.037684-2.0475132 .970577$

H $6.430922-3.4438741 .963913$

H $6.046672-1.968152-0.089162$

H $8.358751-2.3585640 .821583$

H $8.135407-1.0503761 .964944$

H $10.240716-0.8691870 .267845$

H $7.827341 \quad 1.3921821 .032476$

H $9.061701-1.823120-1.522470$

H $8.825670-0.224245-2.190587$

H $7.422261-1.254417-1.846545$

H $8.8519921 .984921-0.906633$

H 5.6717732 .3175291 .468070

H $5.5551572 .799008-0.230836$

H $4.6231620 .315308-1.244607$

H 0.6674441 .8357822 .533465

H $-0.7130191 .102659-0.116446$

H -0.7177893 .8500872 .640016$

H -0.6794695 .1425941 .446822$

H $\quad 0.727744 \quad 4.123076 \quad 1.635597$

H 3.4619860 .9229480 .982425

H 3.4142752 .6391381 .337260

H $2.1998483 .215373-2.927497$

H $3.3318464 .577312-2.783639$

H $1.5963505 .757933-1.230328$

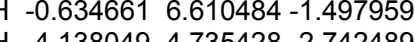

H -3.022132 $5.572968-3.960365$

H $-3.5238454 .602301-0.453995$

H -2.1658515 .5410760 .090765$

H $-2.0799902 .654947-1.036659$

H $3.5289524 .182806-0.423306$

C $-2.382872-3.219913-2.935245$

O $-2.970395-2.096149-2.250599$

C $-4.329296-1.932216-2.699224$

C $-4.495797-2.858803-3.898058$

C $-3.556544-3.992201-3.535472$

H $-4.973478-2.289660-1.887216$

C $-4.619133-0.454122-2.983856$

H -5.532482 -3.183649-4.029399

H $-4.172427-2.382252-4.830671$

C -3.181928 -4.871322 -4.715836

H $-4.034518-4.610146-2.763353$

C $-4.2981740 .493903-1.811615$

O $-3.829878-0.027111-4.102764$

H $-5.664525-0.333643-3.290217$

H $-3.2148040 .529975-1.645662$

O $-4.6929601 .816617-2.197145$

C $-5.0053480 .144134-0.490686$

C -4.6904101 .1586610 .622208$

H $-4.688877-0.848457-0.165140$

O $-6.4152490 .088422-0.698345$

H $-3.603546 \quad 1.238748 \quad 0.716710$

H $-5.0660382 .146247 \quad 0.328764$

H $-4.073894-5.339707-5.144577$

H -2.692413 -4.293681 -5.507144

H $-2.497701-5.668143-4.408228$

C $-1.554346-4.046711-1.951944$

H $-2.896014-0.183142-3.879395$

H -4.223565 $2.029522-3.022060$

H $-6.6993870 .962054-1.017998$

H -1.197943 -4.963082 -2.435699

H -2.181916 -4.348506 -1.104956

H $-1.743260-2.811929-3.729002$

C $-3.574561-0.6829322 .795380$

O $-4.948722-0.5256432 .421790$

C $-\begin{array}{llll}-5.315628 & 0.787217 & 1.985117\end{array}$

C -5.0275691 .8044743 .097002$

C -3.5760831 .7350863 .563871$

C -3.1985050 .2970463 .916809$

H $-2.932480-0.5235551 .920565$

C $-3.387913-2.1327713 .274436$

H $\quad-6.403008 \quad 0.768171 \quad 1.839897$

O $\quad-5.334470 \quad 3.1209502 .649571$

H -5.6849391 .5940583 .950802$

H -2.9009892 .1556002 .811103$

O $-3.4451962 .557574 \quad 4.725457$

H $-3.7008310 .009708 \quad 4.849574$

O $-1.793521 \quad 0.2187134 .145945$

C -3.513991-3.149463 2.159972

H $-2.392915-2.2624313 .714332$

H $-4.151597-2.3717044 .024401$

H -5.1143003 .7325663 .373036$

H -2.5007002 .5979824 .951463$

H -1.591279 0.7561224 .929963

O $-3.414929-4.4027542 .695993$

O $-3.667047-2.8898750 .975402$

C $-3.504664-5.4831961 .761841$

H $-3.536108-6.4187932 .326619$

H -4.418894 -5.404156 1.165663

$\mathrm{H}-2.621214-5.4917911 .117153$

SCF Energy (B3LYP/6-31G**//MMFF) $=-3245.92197428$

0600245

MM̄FF Geometry

C $-0.156728-3.6637052 .228519$

C -1.024133 -3.335310 3.198430

C $-1.065968-2.0515313 .994601$

O $-2.355338-1.4114863 .785489$

C $0.108280-1.0631663 .816805$

C -0.0556330 .2391084 .580586$ 
C $-0.112302 \quad 1.456623 \quad 4.002445$ C $-0.1430830 .113966 \quad 6.079230$ C 0.0300821 .7632632 .536834 C 0.6386713 .1510522 .292118 C 1.1494903 .2963760 .852240 C 1.6806344 .7077450 .597913 C $2.1538734 .883672-0.852951$ O $0.0810173 .004932-0.055059$ O $2.3218646 .292562-1.071481$ C $1.0456994 .382109-1.805693$ C $3.5329894 .248582-1.071709$ O $1.497768 \quad 4.399513-3.158237$ C $0.4876812 .988718-1.429779$ C $-0.7383502 .651653-2.308479$ O $1.5031962 .013789-1.670014$ C $-2.670531-0.8777302 .575419$ O $-1.972968-0.8378991 .575309$ C $-4.048851-0.3480742 .674430$ C $-4.5462290 .383837 \quad 1.667123$ C -5.9020370 .9284541 .579981$ C -6.8614800 .6075382 .695696$ C -1.526485 $1.414773-1.851108$ C $-2.6219831 .048765-2.839660$ C $-4.606181-0.442271-3.084214$ O $-2.722791 \quad 1.561841-3.951327$ C $-5.7590850 .496387-2.912322$ C $-6.9207020 .165621-2.328258$ C $-8.0584751 .131108-2.146005$ C $-8.4313291 .331330-0.692046$ C $-9.5380540 .764378-0.182874$ C -7.5716892 .2515520 .149874$ C -6.2362391 .6505650 .490516$ N $-3.4650320 .064341-2.341833$ H $\quad 0.636007-2.9783931 .944888$ H -1.808471 -4.049096 3.446347 H -1.074736 -2.361231 5.047357 H $0.245814-0.8632552 .752413$ H $1.038329-1.5441784 .147318$ H -0.2686112 .3218244 .645199$ H -0.1234951 .0886796 .578683$ H -1.069820 $-0.387700 \quad 6.373042$ H $0.705253-0.4639236 .460536$ H 0.6657551 .0199082 .046975 H -0.9600491 .7217902 .069182$ H -0.1308983 .9071122 .494804$ H 1.4680673 .3359872 .985409 H 1.9483752 .5599400 .708497 H $2.4882734 .954171 \quad 1.298513$ H 0.8816865 .4368330 .793190 H $2.550096 \quad 6.419893-2.008517$ H $0.2249385 .110300-1.752857$ H $4.2734764 .709388-0.406813$ H $3.8963974 .427574-2.089559$ H $3.5458143 .172800-0.885231$ H $2.0744713 .627217-3.286821$ H -1.434485 $3.500765-2.305681$ H $-0.4093472 .505197-3.345318$ H $1.0907471 .147360-1.810370$ H -4.620835 -0.5904873 .562758$ H $-3.904914 \quad 0.5936500 .812363$ H $-6.978170-0.4765152 .804185$ H -7.862646 1.0145382 .537834 H -6.4985011 .0190723 .643855$ H $-0.8717740 .542822-1.752911$ H -1.987048 $1.608703-0.875440$ H -4.825496 -1.445383 -2.705272 H -4.342785 -0.516701-4.144247 H $-5.6280391 .508444-3.289275$ H -7.064204 -0.846914 -1.958639 H $-8.917327 \quad 0.742208-2.708212$ H -7.830952 2.106564 -2.593850 H -9.8320980 .9237190 .849085$ H -10.174064 $0.124870-0.785986$ H -8.099582 2.5597911 .059683 H -7.404831 $3.187381-0.399059$ H $-5.4709871 .834030-0.263838$ H -3.341106 -0.258320 -1.386753 C $-0.584577-4.798687-0.010434$
O $0.432100-4.053422-0.706737$ C $-0.206357-3.223650-1.698891$ C $-1.669344-3.657796-1.746876$ C $-1.904509-4.091419-0.313164$ H $-0.158775-2.194457-1.323643$ C $0.533582-3.327499-3.037814$ H -2.330712 -2.846495 -2.065372 H $-1.823425-4.502131-2.428472$ C $-3.133208-4.963399-0.128853$ H $-2.014186-3.1894240 .298439$ C $2.045067-3.032820-2.960132$ O $0.387160-4.657087-3.555199$ H $\quad 0.059797-2.666409-3.772608$ H $2.557307-3.829869-2.407600$

O $2.567002-3.068934-4.294749$ C $2.399156-1.670672-2.338718$ C $3.913420-1.390301-2.369091$ H $2.049705-1.648156-1.302064$ O $1.713894-0.634140-3.038764$ H $4.435004-2.248697-1.932297$ H $4.249507-1.304840-3.409626$ H $-4.036227-4.423908-0.432684$ H $-3.070511-5.877961-0.727927$ H $-3.250317-5.2539030 .919872$ C $-0.217577-4.960188 \quad 1.467829$ H $\quad 0.741659-5.271629-2.889757$ H $2.337508-3.935569-4.671878$ H $1.992340-0.673773-3.969810$ H $\quad 0.773771-5.4246921 .540720$ H $-0.926234-5.6501861 .941368$ H $-0.587482-5.798289-0.465703$ C $4.350057-0.9600840 .616511$ O $3.767463-0.032040-0.303081$ C $4.305786-0.090551-1.628646$ C $5.8178320 .176934-1.590345$ C $6.539023-0.763755-0.626098$ C $5.863450-0.7479930 .745468$ H $4.162086-1.9783990 .253211$ C $3.596740-0.8629121 .950759$ H $3.8471330 .736313-2.183845$

O $6.3915420 .045056-2.886532$

H $5.9950881 .209679-1.262622$

H $6.594815-1.783704-1.024175$

O $7.889737-0.324739-0.463028$

H 6.0863840 .2055681 .237421

O $6.413575-1.7824291 .557476$

C 3.7782810 .4498162 .684313

H $2.521755-0.9779481 .773235$

H $3.928180-1.6769002 .606247$

H $5.9285310 .665541-3.475069$

H $8.293867-0.299792-1.347450$

H $7.375361-1.6417881 .593979$

O 3.5718150 .2454024 .019058

O 4.0384701 .5225332 .157015

C 3.7184511 .3994064 .852102

H 3.4792151 .1095815 .878782

H 4.7518151 .7573104 .818662

H 3.0272472 .1875954 .541426

SCF Energy (B3LYP/6-31G**//MMFF) $=-3245.93461196$

0600246

MM̄FF Geometry

C $1.177317-1.2100541 .052521$

C $-0.049244-1.0030730 .552607$

C $-0.331369-0.845518-0.918113$

O $-0.810710 \quad 0.505058-1.097504$

C -1.434239-1.825887 -1.351700

C $-1.528174-2.002112-2.852875$

C $-2.452248-1.418383-3.640636$

C $-0.517179-2.947757-3.449195$

C $-3.515754-0.430926-3.247705$

C $-4.932765-1.010457-3.356492$

C $-5.269746-2.012300-2.240916$

C $-6.671478-2.592512-2.443231$

C $-7.085194-3.510927-1.283906$

O $-5.210361-1.310751-0.995327$

O $-8.495339-3.744024-1.417384$

C $-6.848759-2.7680400 .048818$ 
C $-6.411317-4.883969-1.387509$

O $-7.100962-3.6268321 .158928$

C $-5.447856-2.1232110 .160462$

C $-5.387815-1.2056721 .405533$

O $-4.473021-3.159666 \quad 0.291660$

C $-0.3835111 .206860-2.177012$

O $\quad 0.377794 \quad 0.816201-3.051234$

C $-0.9300202 .584304-2.175174$

C -1.894920 $2.996504-1.338180$

C $-2.4434294 .351232-1.245378$

C $-1.8504855 .411219-2.135478$

C $-4.117536-0.3475341 .490004$

C -4.1318810 .5846322 .689136$

C $-2.8713732 .503613 \quad 3.665178$

O -4.9870380 .5362903 .569110$

C -3.6442713 .7189683 .259277$

C -3.0828574 .8973692 .949320$

C $-3.871748 \quad 6.1082242 .538238$

C -3.5096476 .6033981 .154522$

C -2.7414547 .6940230 .993443$

C $-4.1078485 .890819-0.039874$

C $-3.4198144 .588510-0.344533$

N $-3.061076 \quad 1.4692992 .664290$

H $2.025376-1.2476000 .373744$

H $-0.894519-0.943326 \quad 1.232304$

H $0.589694-1.010145-1.490207$

H -2.397209 -1.494108 -0.949660

H -1.256785 -2.815709 -0.909336

H -2.433321 -1.646836 -4.705849

H $-0.645538-3.067379-4.530249$

H $0.499793-2.581938-3.278262$

H $-0.611470-3.939646-2.995003$

H -3.348238 -0.014709 -2.250267

H $-3.4371040 .419702-3.936887$

H -5.635567 -0.168697 -3.300691

H $-5.069218-1.481048-4.338099$

H $-4.523759-2.814559-2.242475$

H $-6.738345-3.126213-3.399546$

H -7.398652 -1.770718 -2.509059

H -8.782579 -4.262683 -0.646008

H $-7.603493-1.9718680 .107205$

H $-6.681351-5.369803-2.333034$

H $-6.759685-5.558121-0.597257$

H -5.321565 -4.831566 -1.341044

H $-6.336324-4.2198701 .253802$

H $-6.247888-0.5231241 .390471$

H -5.467543 -1.811033 2.317381

H -3.690078 -2.8073660 .742010$

H $-0.4841813 .249658-2.907425$

H $-2.3216392 .282826-0.635441$

H $-0.7715385 .498701-1.964550$

H -2.271129 $6.405087-1.968239$

H -2.021219 5.166581 -3.189576

H -3.232710 -0.983933 1.582077

H $-4.012597 \quad 0.263126 \quad 0.586079$

H $-1.7969672 .698715 \quad 3.736452$

H -3.227128 2.1437844 .635914

H -4.7269473 .6216363 .221439$

H -2.001620 5.0029442 .996449

H -3.685835 $6.893708 \quad 3.282439$

H -4.9511725 .9153082 .579401$

H -2.501086 8.0759590 .006965

H -2.332032 8.2275791 .844735

H -4.114277 $6.544924-0.919012$

H -5.1691805 .6888430 .154358$

H $-3.783391 \quad 3.7540140 .255074$

H -2.448840 1.4811491 .853560

C $2.354393-2.6062232 .817824$

O $3.652511-2.4169342 .218176$

C $3.985145-3.5997121 .469678$

C $3.130897-4.7065582 .072306$

C $1.832977-3.9590992 .323617$

H $3.657151-3.4248280 .437252$

C $5.497322-3.8385181 .485593$

H $3.012578-5.5588251 .396836$

H $3.545907-5.0710673 .019056$

C $0.904862-4.6510613 .306593$

H $1.309086-3.8528801 .365784$
C $6.339426-2.6072331 .089899$

O $5.904990-4.2270862 .803393$

H $5.741420-4.6862920 .835277$

H $6.316126-1.8634831 .896076$

O $7.704121-3.0280280 .972672$

C $5.919830-1.933923-0.229672$

C $6.838425-0.755689-0.601994$

H $4.893168-1.568279-0.133113$

O $5.927836-2.891528-1.286407$

H $6.919623-0.0893550 .263631$

H $7.846878-1.130674-0.815530$

H $0.605811-5.6334352 .926993$

H $1.386956-4.7992894 .278631$

H $-0.001876-4.0587413 .465811$

C $1.468542-1.3916532 .516530$

H $5.636572-3.5193903 .414664$

H $7.952888-3.4419951 .817001$

H $6.830547-3.247625-1.353139$

H $1.986928-0.4871202 .857859$

H $0.536928-1.4714103 .089093$

H $2.529435-2.6419883 .900685$

C $4.7106051 .420605-0.730567$

O $4.9711470 .425048-1.728517$

C $6.3433170 .031067-1.837970$

C $7.2033101 .252801-2.187926$

C $6.9942012 .401504-1.201328$

C $5.5029692 .700746-1.030706$

H $4.999646 \quad 1.0222710 .248496$

C $3.1927991 .668366-0.726450$

H $6.395646-0.658642-2.689938$

O $8.5788610 .887603-2.229089$

H $6.9329591 .598804-3.194195$

H $7.4720632 .184801-0.238748$

O $7.6515043 .557402-1.726311$

H $5.1239013 .172760-1.946379$

O 5.3226453 .6266120 .036613

C 2.6506692 .1100480 .616498

H $2.9315512 .418180-1.482506$

H $2.6692180 .743131-0.993354$

H $9.0867151 .691053-2.435250$

H $7.6068184 .253538-1.049420$

H $5.7346884 .466263-0.227579$

O 1.3647602 .5418770 .455503

O 3.2570062 .0561791 .677475

C 0.6975772 .9523411 .652942

H 0.6073742 .1080272 .342906

H 1.2352503 .7811602 .123071

$\mathrm{H}-0.3054163 .293687 \quad 1.385173$

SCF Energy (B3LYP/6-31G**//MMFF) $=-3245.92276163$

0600247

MMFF Geometry

C $2.764099-1.603241-2.839336$

C $2.055249-1.075240-1.830592$

C $0.708409-1.557923-1.372068$

O $-0.147004-0.385997-1.416802$

C $0.739199-2.0756660 .080937$

C $1.075976-3.5497200 .216496$

C $0.204487-4.4984500 .615757$

C $2.497790-3.940949-0.090628$

C $-1.230136-4.3099431 .017533$

C $-2.180309-4.553076-0.160013$

C $-3.623860-4.1575110 .179998$

C $-4.554688-4.414232-1.006440$

C $-5.986547-3.924708-0.731502$

O $-3.630332-2.763651 \quad 0.503398$

O $-6.689004-3.895460-1.983619$

C $-5.929573-2.471775-0.209528$

C $-6.753380-4.8982660 .171360$

O $-7.221479-2.0194730 .189603$

C $-4.902336-2.2612570 .925905$

C $-4.742780-0.7539861 .233049$

O $-5.369736-2.9222672 .104677$

C -1.445814 -0.546914-1.780365

O $-2.014501-1.591357-2.050843$

C $-2.0616360 .797160-1.821230$

C $-3.3207660 .940625-2.259128$

C $-4.026326 \quad 2.214255-2.368415$ 
C $-5.4638012 .145302-1.930775$

C -3.508092 -0.4312862 .084804$

C $-3.545826 \quad 0.9776242 .642114$

C -3.4002423 .3630321 .993886$

O

C $-4.021542 \quad 4.1881760 .914737$

C $-3.337348 \quad 5.053670 \quad 0.151396$

C $-3.9753495 .942337-0.877063$

C $-3.4081025 .743209-2.263317$

C $-2.4531176 .557132-2.744286$

C $-4.0211854 .664213-3.126353$

C $-3.4229543 .309346-2.872757$

N -3.5318361 .9578351 .662467$

H $2.375788-2.457325-3.386878$

H $2.473931-0.230809-1.289906$

H $0.320560-2.324769-2.054247$

H $-0.229965-1.8587670 .541419$

H $1.452162-1.4941490 .681855$

H $\quad 0.551605-5.5295670 .669447$

H $2.735637-4.9483810 .268022$

H $2.677959-3.927966-1.168294$

H $3.197973-3.2553240 .395232$

H -1.460232 -5.0186691 .823033$

H -1.384002 -3.319435 1.455025

H -1.838031 -3.968384-1.021903

H $-2.142506-5.610038-0.451988$

H $-3.943738-4.7355261 .055023$

H -4.556148 -5.477424 -1.277084

H $-4.173418-3.881984-1.889381$

H $-6.667833-4.791963-2.360419$

H -5.639523 -1.839806 -1.060321

H -6.795149 $-5.893593-0.287499$

H $-7.795654-4.5848130 .295993$

H $-6.303830-5.0104031 .160084$

H -7.421587 -2.419841 1.052754

H $-4.646348-0.2040320 .290281$

H $-5.642741-0.3783871 .736319$

H $-5.024959-2.4637752 .887110$

H -1.462837 $1.645228-1.506224$

H -3.900203 $0.067369-2.552140$

H -6.040965 $1.509034-2.610602$

H $-5.9529603 .121254-1.893887$

H $-5.537776 \quad 1.724492-0.922030$

H -3.426192 -1.116320 2.935639

H -2.590022 $-0.544218 \quad 1.496172$

H -2.3324093 .5724202 .116013$

H -3.893422 3.5641382 .950628

H -5.0966694 .0941130 .781722$

H -2.265423 5.1622670 .300642

H -3.821644 $6.981778-0.557718$

H $-5.0636745 .805777-0.904404$

H -2.065382 $6.440140-3.750756$

H -2.033900 7.354397 -2.139430

H -3.854391 4.897628 -4.186937

H -5.108808 $4.677717-3.011056$

H $-2.3809953 .230828-3.185107$

H -3.316203 1.6785950 .710010

C $5.253633-1.747755-2.506789$

O $5.157368-1.429133-1.103113$

C $6.420402-0.917994-0.646865$

C $7.437664-1.382408-1.678887$

C $6.631277-1.256090-2.957824$

H $\quad 6.3522660 .176318-0.682179$

C $6.684834-1.3706470 .792651$

H $8.343137-0.768442-1.678131$

H $7.731564-2.426411-1.521099$

C $7.209738-2.038345-4.123975$

H $6.579917-0.194127-3.234048$

C $5.526728-1.0866891 .771310$

O $6.912135-2.7865460 .807096$

H $7.610903-0.9141031 .160020$

H $4.670169-1.7272521 .532144$

O $5.958446-1.4695083 .083666$

C 5.0640010 .3809721 .821642

C 3.9064840 .5830332 .815236

H 4.7279020 .6880220 .829169

O $\quad 6.160177 \quad 1.2195822 .176264$

H $3.080062-0.0687452 .510294$
H $4.2227710 .253123 \quad 3.812168$

H $\quad 8.213599-1.676748-4.369570$

H $7.284438-3.106354-3.893240$

H $6.583131-1.929333-5.014833$

C $4.106150-1.067370-3.255818$

H $6.126383-3.213860 \quad 0.424557$

H $6.239661-2.3993473 .036043$

H 6.4770350 .9338813 .050405

H $4.216033-1.208046-4.337083$

H $4.1344710 .013603-3.071736$

H $5.177405-2.839218-2.591552$

C 1.9796202 .1206470 .995501

O 3.1252732 .6496561 .670752

C 3.4224242 .0464762 .933710

C 2.2289272 .2216763 .882970

C 0.9471701 .6545823 .278993

C 0.7245942 .2185271 .877292

H $2.171553 \quad 1.0782210 .718285$

C $1.7718172 .938517-0.291163$

H $4.2576592 .619903 \quad 3.355171$

O $2.4989391 .595367 \quad 5.132557$

H 2.0852883 .2914674 .083169

H $\quad 0.9615300 .5586813 .275975$

O -0.1492162 .0353434 .113859$

H $0.412298 \quad 3.266597 \quad 1.967220$

O -0.3546571 .5194441 .261100$

C $2.8687372 .748741-1.316852$

H $0.8289972 .645934-0.766834$

H $1.7386674 .007872-0.050510$

H $\quad 1.711011 \quad 1.7098005 .691149$

H -0.9613791 .7014723 .695914$

H -0.0929080 .5879331 .166510$

O $2.5004873 .379657-2.472166$

O $3.9156902 .144726-1.135670$

C $3.4453483 .308549-3.544520$

H $3.079123 \quad 3.935918-4.361584$

H $4.4209863 .686395-3.224151$

H $3.5273892 .278581-3.902539$

SCF Energy $\left(B 3 L Y P / 6-31 G^{* *} / / M M F F\right)=-3245.92196565$

\section{8}

MM̄FF Geometry

C -0.5446392 .8604402 .715877$

C -0.5272751 .5565612 .398761$

C -0.0501930 .4794923 .337673$

O $0.997457-0.2443872 .650267$

C -1.175151-0.518213 3.673085

C -2.3231940 .0873524 .448801$

C $-3.5207300 .406383 \quad 3.920782$

C -2.0612050 .3023635 .916887$

C -3.9627370 .2875552 .488222$

C $-5.100219-0.7296712 .347012$

C $-5.669667-0.7599910 .921545$

C $-6.899497-1.668906 \quad 0.859735$

C $-7.460445-1.776704-0.564271$

O $-4.653911-1.2428050 .034470$

O $-8.402821-2.859959-0.561797$

C $-6.307458-2.149370-1.519729$

C $-8.250646-0.523162-0.956696$

O $-6.751070-2.133156-2.875296$

C $-5.044564-1.271294-1.344167$

C $-3.855316-1.796970-2.186043$

O $-5.3408990 .048866-1.819567$

C $1.938197-0.8632823 .414305$

O $2.059751-0.8081254 .627081$

C $2.775599-1.6738362 .506138$

C $3.732132-2.4754142 .997668$

C $4.546342-3.3523142 .164328$

C $6.025497-3.2248182 .385346$

C $-3.277551-3.146532-1.752905$

C $-2.148179-3.005646-0.750233$

C $0.277999-2.438652-0.606367$

O $-2.301023-3.2737280 .438980$

C $1.126386-3.631808-0.916416$

C $2.328706-3.561906-1.509583$

C $3.190989-4.761120-1.792016$

C $4.532184-4.702375-1.089682$

C $5.639522-4.325372-1.750487$ 
C $4.609978-5.166157 \quad 0.350169$ C $3.958845-4.2033951 .300617$ N $-0.971825-2.557336-1.331737$ H -0.1776643 .1515413 .698879$ H $-0.883662 \quad 1.2222691 .429010$ H $0.3569370 .927922 \quad 4.252432$ H $-0.759699-1.3495524 .258916$ H -1.521057 -1.007235 2.753817 H -4.2747430 .8280864 .584590$ H -2.935991 0.7002576 .442142 H -1.792029 -0.6442396 .397006$ H -1.240601 1.0119466 .061155 H $-4.310501 \quad 1.2758832 .162803$ H -3.1394670 .0201381 .820540$ H -4.719146 -1.724272 2.613764 H $-5.902548-0.4833323 .054028$ H -5.9391890 .2639750 .637039$ H -7.677374 -1.321902 1.551359 H $-6.623749-2.6764941 .202065$ H -8.706373 -2.988278 -1.477073 H $-6.056012-3.193520-1.302742$ H $-9.084558-0.366396-0.261725$ H $-8.706404-0.634812-1.946725$ H $-7.6442090 .384727-0.957133$ H -6.804874 -1.204162 -3.157497 H $-4.167510-1.860764-3.236372$ H -3.055965 -1.046450 -2.169056 H -4.502248 $0.511811-1.981907$ H $2.566935-1.6227891 .442004$ H $3.948814-2.5012114 .062540$ H $6.307004-3.6810473 .340600$ H $6.326064-2.1722892 .410794$ H $6.617035-3.6981631 .597516$ H $-4.030224-3.820779-1.336082$ H $-2.869301-3.659683-2.633344$ H $0.084467-2.3862020 .469507$ H $0.752092-1.505535-0.920597$ H $0.741807-4.601286-0.608056$ H $2.716517-2.593044-1.814758$ H $3.337309-4.816031-2.878735$ H $2.681221-5.693302-1.518117$ H $6.609449-4.306874-1.264784$ H $5.599789-4.021252-2.791131$ H $5.648773-5.3628710 .637102$ H $4.107344-6.1377330 .440297$ H $2.870075-4.2340941 .279887$ H -0.943436 -2.413812 -2.337046 C -1.663653 3.6789010 .518712 O $-0.6950643 .066196-0.347953$ C -0.931963 $3.494405-1.701291$ C $-2.1700534 .386474-1.666015$ C -2.143004 4.913110 -0.243939 H $-0.0661544 .100982-1.990144$ C -1.033811 2.278042 -2.630895 H -2.124716 5.180968 -2.417450 H -3.092031 $3.821328-1.842433$ C -3.4824115 .4546680 .225170$ H $-1.3890635 .709479-0.181867$ C $0.0964391 .245918-2.444589$ O $-2.2708391 .605163-2.364323$ H $-1.0836192 .618688-3.672054$ H $-0.0149170 .742624-1.476465$ O $-0.058743 \quad 0.234376-3.447823$

C $1.5199521 .821634-2.556535$ C $2.5979910 .727827-2.432097$ H $1.6750422 .564373-1.768145$ O $1.6664042 .495635-3.804976$ H $2.4499420 .201631-1.483065$ H $2.464816-0.008491-3.233740$ H -3.807056 $6.282910-0.413300$ H -4.2591254 .6830840 .196233$ H -3.416416 5.8274821 .251780 C $-1.0232884 .012314 \quad 1.869155$ H -2.451410 $1.021597-3.120384$ H $-0.876253-0.252034-3.248643$ H $1.5094251 .844480-4.510175$ H -1.743839 4.5767292 .473120 H -0.1602474 .6689441 .705600$
H -2.4867212 .9642660 .650846$

C $4.3191531 .953424-0.220752$

O $4.2766302 .342402-1.598150$

C $4.0383811 .276702-2.521417$

$\begin{array}{llll}\text { C } 5.127571 & 0.205229 & -2.377867\end{array}$

C $5.265967-0.279916-0.936614$

C $5.4165570 .908916 \quad 0.011590$

H 3.3434931 .5508670 .079420

$\begin{array}{llll}\text { C } & 4.593539 & 3.216580 & 0.602477\end{array}$

H $4.147047 \quad 1.711379-3.523073$

O $4.851742-0.893677-3.239819$

H $6.0867130 .631867-2.699785$

H $4.421347-0.917809-0.651370$

O $6.435649-1.096911-0.856588$

H $6.4050231 .365203-0.128708$

$\begin{array}{lllll}\text { O } & 5.340430 & 0.451859 & 1.359154\end{array}$

C 3.3658624 .0901140 .675397

H 4.8708842 .9773291 .635567

H 5.4196093 .8010120 .179371

H $5.560548-1.546967-3.111884$

H $6.459336-1.487522 \quad 0.032829$

H $6.135279-0.0794031 .532309$

O $3.3695254 .993454-0.344988$

$\begin{array}{lllll}\text { O } & 2.500518 & 3.970029 & 1.532824\end{array}$

C $2.2542785 .888838-0.378579$

H $1.3138505 .331998-0.391786$

H $2.290096 \quad 6.562167 \quad 0.482920$

H $2.3215806 .482665-1.293842$

SCF Energy (B3LYP/6-31G**/MMFF)= -3245.90896886

\section{6_00249}

MMFF Geometry

C $-0.883553-2.0177712 .643055$

C $-0.105172-2.9639182 .095432$

C $-0.296276-3.5144270 .698072$

O $-0.907475-2.508351-0.148708$

C $-1.197312-4.759266 \quad 0.727704$

C $-1.339522-5.425265-0.627845$

C $-2.427654-5.342156-1.419832$

C $-0.155210-6.250692-1.062508$

C $-3.697724-4.578235-1.167127$

C $-3.858496-3.428985-2.170083$

C $-5.054859-2.527969-1.829227$

C $-5.202938-1.416473-2.869135$

C $-6.306682-0.417253-2.496416$

O $-4.820693-1.958865-0.537754$

O $-6.1410990 .725010-3.351103$

C $-6.1003200 .047782-1.037375$

C -7.699666 -0.982138 -2.799934

O $-7.202114 \quad 0.857612-0.634189$

C $-5.877747-1.119000-0.051747$

C $-5.469421-0.6592561 .369216$

$\begin{array}{lllll} & -5.469421 & -1.059478 & -1.855701 & 0.080772\end{array}$

C $-0.082469-1.528575-0.615605$

O $1.130566-1.473541-0.488671$

C $-0.911718-0.493278-1.270233$

C $-0.3409700 .672145-1.611107$

C $-0.9916051 .805501-2.271012$

C $-2.4598151 .688579-2.578435$

C $-4.1599210 .131383 \quad 1.457169$

$\begin{array}{llll}C & -4.334377 & 1.639828 & 1.456261\end{array}$

C -2.9837993 .7360151 .494662$

$\begin{array}{llll}\text { O } & -5.427862 & 2.194216 & 1.519082\end{array}$

C $-2.912680 \quad 4.3066890 .114374$

C $-1.8910645 .058167-0.323202$

C $-1.8551235 .713088-1.673308$

C $-0.6161135 .366351-2.465042$

C $0.4494316 .185213-2.477244$

C $-0.654347 \quad 4.124655-3.324484$

C $-0.2433902 .885962-2.577491$

$\begin{array}{llll}\mathrm{N} & -3.109895 & 2.294037 & 1.418568\end{array}$

H -1.751704 -1.665463 2.092106

H $0.756321-3.3327222 .646233$

H $0.686779-3.7767090 .287390$

H $-2.178505-4.4893061 .131126$

H $-0.790359-5.4966971 .432890$

H $-2.412786-5.877374-2.368690$

H $-0.329392-6.758616-2.017157$ 
H $\quad 0.730973-5.620231-1.183719$ H $0.064303-7.021629-0.316669$ H -4.543614 -5.269322 -1.266321 H -3.741009-4.192525 -0.146539 H -2.941588 -2.827296 -2.168931 H -3.977628 -3.837184 -3.181640 H $-5.958393-3.147832-1.793593$ H -5.384734 -1.837267 -3.865995 H -4.254634 -0.868440 -2.947892 H -6.793969 1.392957 -3.079172 H $-5.2171340 .697114-1.039527$ H -7.796933 -1.198944-3.870581 H -8.483483 -0.251647 -2.571123 H -7.917137 -1.902893 -2.254609 H $-6.978456 \quad 1.2617430 .220712$ H $-6.298227-0.1285841 .852498$ H $-5.324086-1.5687151 .970500$ H -7.071600 -2.353734 0.914554 H -1.958709 -0.710239-1.442359 H $\quad 0.7179140 .812546-1.395976$ H $-3.0219921 .426640-1.676375$ H -2.630337 $0.917641-3.336975$ H $-2.9028712 .615227-2.949819$ H $-3.682184-0.1119392 .414920$ H -3.465616 -0.1563870 .661381$ H -2.0860693 .9539432 .081246$ H -3.848493 4.1525422 .021346 H $-3.7632164 .129544-0.539740$ H -1.055273 5.2582710 .343672 H -1.901087 6.798871-1.515178 H -2.747502 $5.467310-2.262087$ H $1.3286745 .959119-3.071232$ H $0.4679587 .096304-1.888351$ H $\quad 0.0421454 .238033-4.166877$ H -1.636304 $4.032587-3.797435$ H $\quad 0.8133512 .877519-2.306370$ H -2.2599431 .7513891 .297074$ C $-0.4541680 .161115 \quad 3.728811$ $\begin{array}{lllll}\text { O } & 0.774701 & 0.403062 & 3.016396\end{array}$ C 1.3498351 .6357073 .490576 C 0.3365582 .2377084 .459403 C -0.3486970 .9954304 .999866$ H 2.2507151 .3666284 .053416 C 1.7182202 .5298022 .300533 H 0.8162562 .8374995 .239051 H -0.3869232 .8795043 .943121$ C -1.683163 1.2830705 .665384 H 0.3206100 .5048535 .719022

C 2.6669981 .8625541 .282508

O $0.5226642 .891327 \quad 1.596657$ H 2.1515763 .4697082 .660472 H 2.1472521 .0368990 .785111 O $2.9690542 .824260 \quad 0.264274$ C $3.992807 \quad 1.3640691 .882599$ C $5.0141700 .843493 \quad 0.858039$ H 3.7992260 .5847022 .622584 O 4.6166942 .4346382 .602017 H 5.3609281 .6817180 .237251 H 5.9143720 .5463451 .410476 H -1.549603 1.9540076 .520259 H $-2.383838 \quad 1.7588384 .970848$ $\mathrm{H}-2.1451640 .360373 \quad 6.029934$ C $-0.624858-1.3412293 .958871$ H $\quad 0.0980832 .0691591 .296797$ H $2.1227173 .132207-0.102921$ H 4.7581963 .1653041 .975563 H -1.475812 -1.534211 4.620595 H $\quad 0.273337-1.755724 \quad 4.431895$ H -1.267042 0.5435053 .098145 C $6.773557-0.963725-0.790156$

O $5.488067-0.451421-1.160449$ C $4.559512-0.299076-0.073718$ C $4.321867-1.6723590 .568156$ C $5.629269-2.3205241 .018963$ C $6.637492-2.343538-0.131893$ H $7.268148-0.268427-0.101338$ C $7.620316-1.045010-2.069937$ H $3.6221260 .015921-0.543940$
O $3.415482-1.5823831 .657677$

H $3.852366-2.327773-0.177527$

H $6.035570-1.8134531 .901829$

O $5.341693-3.6625181 .419456$

H $6.322819-3.085368-0.877288$

O $7.912378-2.7464440 .363611$

C $7.9811660 .324285-2.608776$

H $8.559374-1.574221-1.874986$

H $7.058991-1.571353-2.851083$

H $3.306698-2.4774612 .022302$

H $6.154289-4.0356941 .800522$

H $7.832687-3.665048 \quad 0.670551$

O $8.650960 \quad 0.170503-3.789897$

O $7.7201771 .389815-2.067712$

C $9.0642211 .384622-4.424860$

H $9.755393 \quad 1.933850-3.778406$

H $9.5823661 .124338-5.351610$

H $8.1928501 .999138-4.670536$

SCF Energy (B3LYP/6-31G**//MMFF) $=-3245.92492532$

0600250

MMFF Geometry

C $0.777322-0.493907-1.980184$

C $-0.346678-0.684620-1.274874$

C $-0.374348-1.2478350 .120169$

O $-1.594094-2.0159580 .216948$

C $-0.432600-0.0956131 .137090$

C $-0.198916-0.5279222 .568904$

C $-1.156116-0.6038633 .513864$

C $1.234570-0.8278402 .922900$

C $-2.631914-0.3611393 .360185$

C -3.0912200 .9367844 .040259$

C -2.6670162 .2082373 .286818$

C -3.0973083 .4594994 .054595$

C -2.7938004 .7441573 .270578$

O -3.3023312 .1858022 .006004$

O -3.4961965 .8102623 .927308$

C -3.3623024 .6085151 .841256$

C $-1.3048175 .105513 \quad 3.335992$

O -2.9713375 .7366361 .061352$

C -2.9668463 .2834181 .146327$

C $-3.7670153 .101114-0.166817$

O -1.5708443 .3159920 .844640$

C $-1.587773-3.1865550 .898565$

O $-0.630041-3.7095531 .450124$

C $-2.930480-3.8167130 .883754$

C $-4.037192-3.1804380 .467277$

C $-5.390056-3.7372420 .398133$

C $-5.573270-5.184900 \quad 0.769821$

C $-3.6195571 .708886-0.797222$

C $-4.5062731 .530181-2.017036$

C $-5.304749-0.243450-3.580746$

O $-5.1447252 .447364-2.525609$

C $-6.613745-0.729664-3.041733$

C $-7.045841-1.994993-3.154261$

C $-8.360587-2.476745-2.609133$

C $-8.206015-3.615290-1.623530$

C $-8.458971-4.882714-1.991200$

C $-7.847369-3.279502-0.191886$

C $-6.392566-2.937337-0.022683$

N $-4.4862690 .218884-2.474965$

H $1.734716-0.771465-1.548438$

H -1.296515 -0.380460 -1.707404

H $0.501019-1.8885310 .278638$

H $-1.3964790 .414773 \quad 1.046791$

H $\quad 0.3241300 .6626490 .892630$

H -0.858979 -0.897062 4.520329

H $1.362364-1.0603123 .985382$

H $1.595652-1.6881232 .353913$

H 1.8720590 .0328912 .695840

H -2.949908 - 0.3825212 .313639

H -3.149298-1.203105 3.837816

H -4.1874450 .9068914 .095044$

H -2.7165560 .9690285 .070897$

H -1.5798052 .2011743 .154134$

H -2.624648 3.4920525 .044121

H -4.179602 3.4169284 .242442

H $\quad-3.3639976 .617746 \quad 3.400974$ 
H $-4.457128 \quad 4.6514591 .928043$ H $-0.998197 \quad 5.2677564 .376382$ H -1.101480 6.0473072 .814438 H -0.6565914 .3333792 .916118$ H -3.493172 5.7284260 .241823 H $-4.833374 \quad 3.2635120 .037852$ H $-3.4561303 .851420-0.904097$ H -1.393103 2.7339820 .089920 H -2.958619 -4.8387751 .247426$ H -3.955779 $-2.146230 \quad 0.136384$ H $-4.931197-5.8250210 .154382$ H $-6.594437-5.5445340 .626422$ H $-5.322757-5.3449391 .824113$ H -2.586093 $1.543769-1.114475$ H $-3.8870410 .932727-0.071255$ H -4.746705 -1.034155 -4.091924 H $-5.473447 \quad 0.578197-4.284015$ H -7.239732 $0.001833-2.535412$ H $-6.428402-2.724841-3.672880$ H -8.975723 -2.796260 -3.460544 H $-8.919568-1.661543-2.132861$ H -8.381472 -5.702903-1.285302 H -8.745318 $-5.129535-3.008316$ H -8.140590 -4.0916380 .482662$ H -8.448995 -2.4229430 .139049$ H -6.158513 -1.901687 -0.270037 H -3.980419 -0.481996-1.940981 C $1.3628191 .562326-3.342286$ O $2.7179861 .567339-2.850062$ C $2.8700572 .678235-1.944929$

C $1.6837713 .597460-2.211839$ C $0.5935592 .583017-2.499450$ H $2.7752892 .273488-0.929848$ C $4.2463903 .327117-2.116802$ H $1.4568084 .242948-1.358089$ H $1.8505014 .234340-3.088206$ C $-0.6249793 .166131-3.192324$ H $0.2793372 .146200-1.544406$

C $5.4333442 .351272-1.981992$

O $4.3276293 .923887-3.417643$

H $4.3564794 .150675-1.401913$

H $5.4645511 .672757-2.843162$

O $6.6398513 .123423-2.034531$

C $5.4399971 .525723-0.682220$

C $6.6980950 .647234-0.550481$

H $4.5536120 .884150-0.661794$

O 5.3546772 .3984440 .443065 H $6.7901510 .033930-1.453534$ H $7.5879401 .286636-0.502402$ H -1.096951 $3.927755-2.563058$ H $-0.3592863 .634883-4.145699$ H -1.367432 2.387691 -3.394128 C $0.8042350 .135834-3.344299$ H $4.1651333 .223124-4.072522$ H $6.6212673 .633699-2.862431$ H 6.1255402 .9909570 .414035 H $1.435619-0.496683-3.980619$ H $-0.197176 \quad 0.135573-3.790941$

H $1.4194501 .907181-4.383186$

C $5.330833-2.057658-0.136834$

O $5.475220-1.0074970 .824306$

C $6.682418-0.248198 \quad 0.710117$

C $7.899513-1.1747760 .838020$

C $7.839890-2.334636-0.154009$

C $6.494249-3.055644-0.058762$

H $5.307473-1.614238-1.140388$

C $3.947641-2.6947610 .061298$

H 6.6996960 .4277391 .574466

O $9.100538-0.4330820 .651919$

H $7.928555-1.5830051 .856682$

H $8.039061-1.990535-1.175674$

O $8.886446-3.2540260 .168569$

H $\quad 6.474414-3.6254330 .877274$

O $6.415226-3.991904-1.130913$

C $3.768019-3.4123931 .382946$

H $3.179598-1.9136650 .029765$

H $3.753697-3.401980-0.752818$

H $9.842063-1.0588370 .720605$
H $8.811915-4.003636-0.446797$

H $5.645640-4.563075-0.968786$

O $2.751669-4.3172011 .262178$

O $4.402878-3.1824732 .402901$

C $2.435546-5.0492312 .450557$

H $2.088557-4.3669443 .232451$

H $1.630689-5.7501522 .213912$

H $3.306853-5.6171392 .790524$

SCF Energy $(B 3 L Y P / 6-31 G * * / / M M F F)=-3245.91785418$

\section{1}

MMFF Geometry

C $-1.016132-2.0853071 .384163$

C $-0.350657-1.3237712 .267063$

C $-0.7447110 .046168 \quad 2.770279$

$\begin{array}{llll}\text { O } & -2.065383 & 0.423007 & 2.324560\end{array}$

C $0.2205461 .109372 \quad 2.217858$

C $0.3981612 .291171 \quad 3.148351$

C $-0.298783 \quad 3.4423093 .084341$

C 1.4887382 .1244374 .175824

C $-1.443695 \quad 3.792187 \quad 2.174299$

C -1.0638324 .8278401 .106607$

C $-0.3230914 .203777-0.084609$

C $0.1457475 .279151-1.065691$

C $0.8079874 .662871-2.307529$

O $-1.2355553 .315233-0.741627$

O $\quad 0.936919 \quad 5.712504-3.277958$

C $-0.132913 \quad 3.586098-2.891000$

C $2.2317454 .180639-2.003732$

O $0.496538 \quad 2.893512-3.967312$

C $-0.6679522 .589052-1.836273$

C $-1.7709611 .701150-2.459587$

O $0.4085181 .755467-1.409867$

C -3.1400890 .0069603 .041621$

O $-3.142197-0.6854754 .045103$

$\begin{array}{llll}\text { C } & -4.334997 & 0.527646 & 2.343524\end{array}$

C $-5.522300-0.0684012 .518570$

C $-6.7694020 .293712 \quad 1.840891$

C $-6.748474 \quad 1.5007530 .938525$

C $-2.5578510 .858291-1.444246$

C $-3.537813-0.082495-2.128155$

C $-5.165524-1.903001-1.616452$

O $-3.698289-0.115371-3.345483$

C $-6.551361-1.343267-1.537298$

C $-7.571131-1.977144-0.937267$

C $-8.975316-1.442899-0.887689$

C $-9.569314-1.470427 \quad 0.504432$

C $-10.431775-2.434678 \quad 0.867998$

C $-9.229354-0.3349881 .444843$

C $-7.856349-0.4804942 .040299$

N $-4.206965-0.888375-1.216560$

H $-1.943779-1.728250 \quad 0.948188$

H $0.576016-1.7132432 .686002$

H -0.7195410 .0322793 .867302$

H -0.1318411 .4570251 .241248$

H 1.2133740 .6782382 .035208

H -0.0469994 .2294213 .794628$

H 1.2868481 .2541084 .808559

H 2.4557251 .9791083 .682592

H 1.5801712 .9956564 .832986

H -1.8853072 .9073521 .709500$

H -2.234032 4.2195382 .804748

H $-1.993668 \quad 5.2846610 .743147$

H $-0.4611345 .627575 \quad 1.554234$

H 0.5358223 .6364490 .289675

H $0.8290875 .984051-0.576006$

H $-0.7173895 .876398-1.392084$

H $1.2870685 .316889-4.094915$

H $-0.9876314 .116177-3.332788$

H $2.8497055 .015809-1.652493$

H $2.7255423 .805119-2.906776$

H $2.2667293 .399337-1.241768$

H $1.1268982 .260340-3.583260$

H $-2.4948432 .337058-2.986104$

H $-1.3218561 .033097-3.205882$

H $0.0468460 .928994-1.054808$

H -4.1914021 .3602601 .664095$

H -5.581683 -0.9198693 .195363$ 
H -6.4926542 .4002581 .509685$ H $-7.703311 \quad 1.6993960 .448424$ H $-6.012003 \quad 1.3740020 .137484$ H -1.879587 $0.252291-0.835794$ H $-3.127731 \quad 1.512559-0.774793$ H -5.027267 -2.759891-0.949538 H $-4.953697-2.219606-2.642736$ H $-6.731467-0.384617-2.018365$ H -7.396776 -2.951555 -0.485990 H -9.585960 -2.055607 -1.563933 H -9.028665 -0.421059 -1.283450 H -10.883546 -2.446713 1.854397 H -10.701166 -3.236954 0.189183 H $-9.943848-0.3081272 .278534$ H $-9.374657 \quad 0.6154990 .924702$ H -7.766118-1.336174 2.711246 H $-4.042977-0.756799-0.223165$ C $-0.035060-3.537434-0.469322$ O $1.115193-2.685303-0.627328$ C $1.079299-2.110258-1.949220$ C $-0.022439-2.845718-2.705403$ C $-1.000582-3.145952-1.584463$ H $\quad 0.773967-1.065901-1.828974$ C $2.468917-2.176486-2.591258$ H $-0.456278-2.234797-3.502932$ H $\quad 0.342841-3.777282-3.153079$ C -2.020612 -4.215149-1.929224 H $-1.525879-2.218348-1.333748$ C $3.579866-1.517596-1.743689$ O $2.829558-3.548395-2.793755$ H $2.439026-1.725706-3.589488$ H $3.711896-2.075524-0.813016$ O $4.808495-1.627772-2.470982$

C $3.333363-0.032352-1.435403$

C $4.4734110 .696832-0.699386$

H $2.4183750 .077040-0.846327$

O $3.1100730 .658609-2.670566$ H $5.4328510 .455935-1.168694$ H $4.3342001 .771952-0.870393$ H -2.630471 -3.903129 -2.783472 H $-1.537438-5.162868-2.189256$ H -2.691980 -4.398857-1.084566 C $-0.558708-3.4561750 .965827$ H $2.802880-3.990183-1.928592$ H $4.961139-2.573816-2.638436$ H $3.9127330 .557320-3.210948$ H $\quad 0.237468-3.7935301 .642185$ H -1.394345 -4.153637 1.095872 H $0.322983-4.562325-0.636967$

C $6.198167-1.2934870 .912287$

O $4.842081-0.9105461 .166699$

C 4.5070320 .4347150 .822278

C 5.4117621 .4045571 .595991

C $6.885594 \quad 1.1205201 .327367$

C $7.193463-0.3498001 .605294$

H $6.376078-1.304578-0.169120$

C $6.382691-2.7211381 .446346$

H $3.4860440 .596993 \quad 1.188037$

O $5.1205102 .756921 \quad 1.258685$

H 5.2261561 .2963942 .672770

H 7.1650671 .4067640 .306665

O $7.663770 \quad 1.9369192 .205826$

H $7.177695-0.5208352 .689675$

O $8.508878-0.655277 \quad 1.145654$

C $5.533535-3.7191980 .695470$

H $7.422807-3.0558241 .361834$

H $6.119342-2.7635302 .510938$

H 4.1795892 .9098441 .451135

H 8.5968401 .8317271 .955018

H $9.135140-0.1339921 .675416$

O $4.550511-4.1971781 .511036$

O $5.717602-4.030108-0.473987$

C $3.675757-5.1690120 .930551$

H $3.035323-5.5657951 .722834$

H $3.044595-4.6958750 .175120$

H $4.247424-5.9949390 .496348$

SCF Energy (B3LYP/6-31G**//MMFF) $=-3245.92518348$
0600252

MMFF Geometry

C 0.4368913 .7679691 .731545

C 0.8953812 .5522481 .397732

C $1.336481 \quad 1.5360512 .418671$

O 2.7257361 .2551612 .133346

C 0.5349550 .2309922 .283451

C $0.550549-0.5958393 .553670$

C $1.366044-1.6422033 .788274$

C $-0.463770-0.1848164 .590963$

C $2.465254-2.1774322 .914045$

C $2.218857-3.6244962 .466507$

C $1.100745-3.7584971 .422212$

C $0.891392-5.2269431 .044219$

C $-0.137105-5.390776-0.084649$

O $1.493376-3.0113580 .266376$

O $-0.017471-6.737380-0.567652$

C $0.233027-4.434728-1.239434$

C -1.570992 -5.2492810 .437370$

O $-0.763677-4.461572-2.257933$

C $0.515777-2.985323-0.779687$

C $1.086917-2.160123-1.956205$

O $-0.703900-2.389328-0.342723$

C 3.6119331 .2292613 .159367

O $3.367638 \quad 1.407467 \quad 4.344601$

C 4.9906040 .9848252 .672209

C 5.2739900 .5882151 .421436

C 6.6092620 .3655530 .861874

C 7.7968840 .6577141 .740471

C $1.619581-0.776354-1.556419$

C $2.240413-0.048983-2.736871$

C $3.7536671 .862378-3.262039$

O $2.067393-0.392898-3.903278$

C $5.0851761 .219322-3.494999$

C $6.2576361 .769143-3.144391$

C $7.5851861 .102293-3.369623$

C $8.3501250 .867252-2.084274$

C $9.4018331 .638069-1.759388$

C $7.954707-0.311590-1.219936$

C $6.708027-0.052312-0.417600$

N $2.993837 \quad 1.045419-2.332640$

H $0.370175 \quad 4.0380302 .783278$

H 0.9781112 .2781040 .348817

H 1.2271661 .9582363 .425040

H $0.913329-0.3593841 .441911$

H -0.5130790 .4519862 .040905$

H $1.265443-2.1630054 .740047$

H $-0.449069-0.8377085 .470280$

H $-0.2682790 .835558 \quad 4.934956$

H -1.474485 -0.220770 4.172521

H $2.653921-1.5412552 .045269$

H $3.389478-2.1546713 .505482$

H $3.156610-3.9958992 .032843$

H $1.995177-4.2523273 .337956$

H $\quad 0.176417-3.3368571 .832840$

H $0.597645-5.8171541 .921267$

H $\quad \begin{array}{llll}1.845997 & -5.653427 & 0.704658\end{array}$

H $-0.609033-6.828802-1.334614$

H $1.145410-4.834458-1.702786$

H $-1.768809-5.9954221 .216404$

H $-2.304401-5.443778-0.352959$

H -1.774164 -4.2647690 .861326$

H -1.541608 -3.984433 -1.923640

H $1.918214-2.713996-2.412509$

H $\quad 0.317467-2.033959-2.728421$

H $-0.615180-1.423876-0.369864$

H $5.768150 \quad 1.1477103 .411499$

H 4.4538540 .4154540 .726599

H $7.772577 \quad 1.6961972 .089068$

H 8.7555860 .5240241 .234588

H $7.801680-0.0061062 .612055$

H $\quad 0.812745-0.144492-1.170683$

H $2.381368-0.879478-0.775227$

H $3.8451962 .860515-2.822657$

H $3.2086921 .943039-4.207897$

H $5.0811300 .244636-3.977763$

H $6.2684052 .748068-2.670845$

H $8.167276 \quad 1.741624-4.046077$ 
H $7.4686220 .146843-3.896289$ H $9.9763921 .459862-0.856541$ H $\quad 9.7100232 .467547-2.387077$ H $8.783144-0.610619-0.567977$ H $7.784323-1.186968-1.859958$ H $5.788492-0.239399-0.971978$ H $3.1385471 .205718-1.339959$ C -1.4385125 .2120980 .917072$ $\begin{array}{llll}\text { O } & -2.269239 & 4.200266 & 0.309925\end{array}$ C $-3.3230964 .849331-0.428814$ C $-3.2970336 .311616-0.004236$ C $-1.813616 \quad 6.5223320 .224714$ H -3.048204 4.793229-1.488400 C $-4.6582744 .137000-0.192191$ H $-3.7139146 .974176-0.768762$ H $-3.854103 \quad 6.474390 \quad 0.925845$ C -1.498137 7.7660441 .037554 H -1.311045 $6.590396-0.749592$ C $-4.6532172 .641121-0.574628$ O -4.9981264 .2284531 .197640$ H $-5.4576544 .657881-0.731535$ H -3.999139 2.0933920 .110535 O $-5.9782272 .136786-0.371673$ C $-4.2513432 .366123-2.034241$ C $-4.3829740 .906406-2.512507$ H $-3.2172372 .685831-2.199219$ O $-5.0658683 .169677-2.896659$ H $-5.4057300 .552912-2.344410$ H $-4.2655560 .917911-3.603848$ H -1.853402 8.6633100 .520131 H -1.976883 7.7342532 .022062 H -0.4195747 .8702671 .191015$ C 0.0328234 .8307060 .748372 H $-4.2709753 .827313 \quad 1.704273$ H $-6.2158172 .311646 \quad 0.555215$ H $-5.9921562 .912974-2.746232$ H $0.672748 \quad 5.701970 \quad 0.928352$ H $0.2181454 .491114-0.278040$ H -1.7125235 .2539541 .979714$ C $-4.654355-1.040520-0.134346$ O $-3.521212-0.246137-0.494285$ C -3.340961-0.052532-1.899809 C $-3.237262-1.410620-2.613938$ C $-4.392560-2.346107-2.266577$ C $-4.565576-2.440511-0.749729$ H $-5.563581-0.550424-0.501455$ C $-4.788778-1.0469101 .394844$ H $-2.3654220 .437520-2.015293$ O $-3.171407-1.248475-4.027344$ H -2.303196 -1.896504 -2.315359 H $-5.318607-2.023743-2.757546$ O $-4.084764-3.642272-2.784929$ H $-3.723955-3.000039-0.325418$ O $-5.758317-3.158536-0.447111$ C -3.660506-1.760207 2.106512 H -4.801384 -0.020257 1.779731 H $-5.741421-1.5137471 .671658$ H -2.404432 -0.682751 -4.220890 H $-4.868766-4.202644-2.658774$ H $-5.637483-4.073089-0.753342$ O $-4.167797-2.5745113 .076360$ O $-2.474105-1.5977931 .856926$ C $-3.200446-3.2940343 .847812$ H $-2.577502-2.5972974 .415860$ H -3.736900 -3.937389 4.550235 H -2.583698 $-3.922583 \quad 3.199177$

SCF Energy (B3LYP/6-31G**//MMFF) $=-3245.92130628$

0600253

MMFF Geometry

C -1.820996 -3.728337 -0.694917

C $-1.046868-2.800753-0.111646$

C $0.141771-2.162874-0.778933$

O $1.293813-2.509615 \quad 0.028818$

C $0.032682-0.626616-0.824480$

C $-0.667594-0.104220-2.060605$

C $-0.0368920 .428870-3.125779$

C $-2.171658-0.155436-2.032400$
C $1.4431930 .579091-3.348713$

C $1.8850962 .049256-3.386168$

C $1.7576642 .761824-2.030754$

C $2.1134384 .244725-2.162938$

C $2.0829844 .959799-0.802976$

O $2.6487922 .123822-1.109088$

O $2.687086 \quad 6.252423-0.964201$

C 2.9324464 .1517210 .203690

C $0.6480245 .241508-0.343955$

O 2.8227814 .6950341 .516802

C 2.5940162 .6435590 .224247

C 3.5610531 .8236691 .116986

O $1.2848462 .473847 \quad 0.781962$

C $2.484345-2.652908-0.613880$

O $2.691203-2.545928-1.812058$

C $3.512605-2.9551550 .407495$

C $4.788049-3.117640 \quad 0.025140$

C $5.929677-3.3919390 .898462$

C $5.675324-3.4925702 .379108$

C 5.0430611 .8466920 .733075

C $5.4023790 .971360-0.455476$

C $7.3233680 .476140-1.971414$

O $4.6589400 .103239-0.902610$

C $8.313331-0.515002-1.429275$

C $8.739224-0.637528-0.161289$

C $9.742227-1.6640580 .288753$

C $9.213713-2.5401631 .404145$

C $9.469710-2.2453072 .689961$

C $8.459147-3.7967801 .031051$

C $7.151656-3.5177950 .340306$

N $6.6796581 .247036-0.924453$

H -1.592507 -4.053413 -1.707014

H -1.265995 -2.488821 0.906532

H $\quad 0.270037-2.572254-1.789438$

H $1.033989-0.189515-0.745561$

H $-0.481829-0.2479220 .069763$

H $-0.6473120 .792639-3.951785$

H $-2.6218840 .398015-2.863598$

H -2.524202 -1.187904 -2.098523

H -2.551536 $0.287167-1.106857$

H $2.0343490 .026891-2.613690$

H $1.6773390 .122383-4.318589$

H $2.9352952 .070457-3.705227$

H $1.3044302 .587178-4.145980$

H $0.7285922 .657269-1.670531$

H $1.4446884 .745994-2.873664$

H $3.1236604 .337759-2.585545$

H $3.5943536 .126337-1.290592$

H $3.9784714 .278199-0.097694$

H $0.1421815 .901494-1.059155$

H 0.6350515 .7731230 .613936

H $0.0417044 .339185-0.245059$

H 3.0408495 .6410921 .458742

H 3.4647862 .1765022 .152392

H $3.2215140 .778709 \quad 1.135779$

H 1.1983051 .5590401 .097011

H $3.195845-3.0223181 .442004$

H $5.026956-3.031423-1.034444$

H $6.583182-3.6552632 .964481$

H $5.000433-4.3274562 .597301$

H $5.224517-2.5676582 .756053$

H 5.3975962 .8669680 .565120

H 5.6168241 .4497151 .581031

H $7.8347641 .182694-2.632284$

H $6.561926-0.053502-2.553213$

H $8.716011-1.201166-2.172441$

H 8.3722530 .0406950 .604531

H $10.638513-1.1284910 .628520$

H $10.075642-2.294690-0.544774$

H $9.119545-2.8800183 .497139$

H $10.032332-1.3589062 .964173$

H $8.302625-4.4396371 .905059$

H $9.084145-4.397448 \quad 0.357123$

H $7.242881-3.418622-0.741837$

H $7.2352161 .957026-0.456135$

C $-4.286958-4.187219-0.829453$

O $-4.684183-2.801339-0.804443$

C $-6.113635-2.728912-0.636539$ 
C $-6.634930-4.152516-0.796856$

C $-5.473376-4.962113-0.257248$

H $-6.293244-2.4055470 .395367$

C $-6.720450-1.718197-1.616968$

H $-7.566600-4.316399-0.246940$

H $-6.817233-4.404081-1.848110$

C $-5.515277-6.425234-0.662929$

H $-5.475135-4.8914420 .838875$

C $-6.123860-0.299426-1.530995$

O $-6.516754-2.185207-2.957724$

H $-7.806596-1.674782-1.476123$

H $-5.088748-0.304605-1.889043$

O $-6.8564740 .538955-2.433944$

C -6.179443 $0.334070-0.130185$

C $-5.646257 \quad 1.778325-0.115668$

H $-5.586558-0.269794 \quad 0.559800$

$\begin{array}{llll}\text { O } & -7.522945 & 0.320672 & 0.350002\end{array}$

H -4.642212 $1.785172-0.552807$

H $-6.2813142 .405355-0.752902$

H -6.424805 -6.902965 -0.284329

H $-5.503720-6.540290-1.752007$

H $-4.656048-6.968736-0.257907$

C -2.999464 -4.378618 -0.025620

H $-5.558925-2.295559-3.087049$

H $-6.8096460 .127083-3.313785$

H $-8.0635310 .831375-0.276816$

H -2.778078 -5.4469080 .076958$

H -3.131546 -3.972591 0.984009

H $-4.125988-4.454102-1.882394$

C -3.4860811 .5499282 .047778$

O $-4.904598 \quad 1.5918072 .240786$

C -5.6216792 .3983811 .300196$

C -5.1038303 .8424201 .343636$

C $-3.591283 \quad 3.9114041 .147262$

C -2.8885202 .9615762 .116303$

H -3.2585441 .0954341 .076759$

C -2.8946540 .6588143 .152330$

H -6.661335 2.4216771 .650709

O $-5.762135 \quad 4.630422 \quad 0.357282$

H $-5.352798 \quad 4.278196 \quad 2.320221$

H -3.318317 3.6995790 .106833

O $-3.170731 \quad 5.251838 \quad 1.410456$

H -2.967760 3.3560053 .137622

O $-1.5045152 .891131 \quad 1.787371$

C $-3.213968-0.8075062 .953494$

H $-1.802697 \quad 0.7432453 .177265$

H -3.3043510 .9632114 .123134$

H -5.3901945 .5277550 .406324$

H $-2.227024 \quad 5.3126951 .185307$

H -1.1144203 .7645321 .959379$

O $-2.900217-1.4878474 .095947$

O $-3.653529-1.3022951 .925524$

C -3.127359-2.900231 4.055623

H $-2.965941-3.2990155 .060612$

H $-4.156515-3.118627 \quad 3.754207$

H -2.415279 -3.371381 3.371987

SCF Energy (B3LYP/6-31G*//MMFF) $=-3245.91993069$

0600254

MM̄FF Geometry

C $0.179529-3.0301430 .348110$

C $-0.276664-1.7689150 .345100$

C $-0.575402-0.9869211 .596288$

O $-2.002656-0.773195 \quad 1.662824$

C $0.092897 \quad 0.396332 \quad 1.518534$

C 0.2618791 .0488962 .873965

C -0.5868191 .9478313 .408789$

C 1.5142100 .6554283 .615388

C $-1.903493 \quad 2.4235982 .861134$

C -1.864954 3.8833782 .390355

C $-1.244024 \quad 4.0510850 .994951$

C -1.165074 5.5321700 .618565

C $-0.6589615 .734265-0.818043$

$\begin{array}{lllll}0 & -2.081995 & 3.356611 & 0.063826\end{array}$

O $-0.9185857 .097347-1.187769$

C $-1.4870514 .841045-1.766754$

C $0.8589055 .540798-0.916340$

O $-0.9716174 .895138-3.095670$
C $-1.6148143 .375119-1.289167$

C -2.643161 $2.621191-2.164597$

O $-0.3440972 .740642-1.419736$

C $-2.755890-1.710746 \quad 2.295259$

O $-2.365807-2.7372462 .827026$

C $-4.165025-1.2696872 .208420$

C -5.144069-2.094958 2.606096

C $-6.573092-1.7967562 .549564$

C $-7.352149-2.3658943 .704239$

C $-3.0195521 .226300-1.641387$

C $-4.0152320 .531914-2.556181$

C $-5.423188-1.521079-2.697329$

O $-4.3801011 .000514-3.631472$

C $-6.791963-1.116839-2.246600$

C $-7.661362-1.951325-1.656512$

C $-9.024286-1.531838-1.183886$

C $-9.227665-1.761074 \quad 0.298877$

C $-10.036514-2.7387330 .741004$

C $-8.561776-0.8078681 .268664$

C $-7.115513-1.1413851 .503660$

N $-4.440143-0.682636-2.033606$

H $\quad 0.312272-3.5460641 .295963$

H -0.433955 - $1.261927-0.603703$

H $-0.230371-1.5243722 .488570$

H -0.4846081 .0516940 .857059$

H $1.0877510 .316161 \quad 1.060887$

H $-0.335260 \quad 2.370654 \quad 4.380874$

H 1.6113581 .1746724 .574768

H $1.514437-0.4197623 .820606$

H $2.4016380 .893663 \quad 3.020522$

H -2.280612 1.7740852 .066916

H -2.634245 2.3438993 .676361

H -2.901536 4.2439852 .357729

H -1.3295484 .5019363 .121200$

H -0.2439363 .6034630 .990792$

H -0.5341226 .0807201 .329086$

H $-2.164800 \quad 5.9821350 .698063$

H -0.461657 $7.673226-0.550955$

H $-2.4940135 .277400-1.817181$

H $1.3783786 .245253-0.255552$

H $1.2214015 .758548-1.926935$

H $1.1806924 .535009-0.641062$

H $-0.1729824 .341045-3.126000$

H $-3.567768 \quad 3.210672-2.223228$

H -2.253978 $2.523488-3.186221$

H $-0.4698421 .785875-1.532577$

H $-4.367277-0.2831151 .804426$

H -4.895478 -3.0730473 .013722$

H -7.212573 -3.4512293 .765730$

H -8.427403 -2.189062 3.633358

H $-7.011830-1.9216464 .646366$

H $-2.1352040 .585080-1.568216$

H $-3.4698631 .307062-0.645329$

H $-5.191665-2.560125-2.442945$

H -5.337469 -1.396614 -3.781547

H $-7.081146-0.081570-2.412981$

H -7.381923 - $2.990811-1.500765$

H $-9.765204-2.099114-1.761973$

H $-9.217574-0.475847-1.410536$

H -10.210045 -2.892291 1.800870

H -10.540066 -3.4103880 .053589$

H $-9.116498-0.7707402 .212606$

H -8.6260140 .2147510 .875153$

H $-6.453279-0.7977800 .710512$

H $-4.142259-0.951289-1.100147$

C $1.834241-4.573060-0.739291$

O $2.924549-3.684803-0.434417$

C $4.120373-4.181337-1.066825$

C $3.744360-5.503343-1.732141$

C $2.265389-5.310471-2.004176$

H $4.385029-3.457256-1.845850$

C $5.257101-4.287588-0.041371$

H $4.327120-5.686670-2.640277$

H $3.899989-6.357215-1.062889$

C $1.520003-6.609671-2.254423$

H $2.153664-4.654810-2.878184$

C $5.538838-2.9914520 .744831$

$\begin{array}{lllll}\text { O } & 4.931463 & -5.301240 & 0.920311\end{array}$ 
H $6.171328-4.630320-0.539526$

H $4.704500-2.7714491 .422244$

O $6.681894-3.226073 \quad 1.577820$

C $5.823544-1.759614-0.130931$

C $6.191623-0.5201760 .705492$

H $4.939862-1.536070-0.733170$

O $6.886124-2.048558-1.038231$

H $5.416341-0.3662961 .463884$

H $7.132734-0.7076311 .236456$

H $1.932063-7.127301-3.126943$

H $1.593484-7.286101-1.396224$

H $\quad 0.458905-6.421360-2.445004$

C $0.532315-3.785826-0.902769$

H $4.089470-5.0501831 .337640$

H $6.484276-4.0033582 .128202$

H $7.666633-2.288260-0.509560$

H $-0.286898-4.475830-1.133732$

H $\quad 0.628142-3.087637-1.743197$

H $1.746734-5.272720 \quad 0.103040$

C $4.0560501 .458316-0.233936$

O $5.214901 \quad 1.029589-0.958128$

C $6.3655280 .752952-0.152695$

C 6.7575062 .0020380 .649738

C $5.5850762 .546737 \quad 1.461097$

C 4.3598862 .7306980 .568120

H 3.7229850 .6607780 .440996

C $2.9454421 .724863-1.259773$

H $7.1856020 .551815-0.853472$

O $7.844793 \quad 1.7277051 .526770$

H $7.0924892 .786302-0.041710$

H 5.3533301 .9033432 .318086

O 5.9391503 .8221902 .000839

H $4.5292113 .574535-0.113373$

O 3.2326493 .0577141 .378332

C $2.3851930 .457918-1.870152$

H $2.1084812 .241355-0.782700$

H $3.3397752 .345272-2.073619$

H 8.5813851 .3977290 .984271

H 6.7429853 .6967482 .533815

H $3.4633483 .852176 \quad 1.889759$

O $1.618070 \quad 0.790536-2.950611$

O $2.565610-0.673300-1.442900$

C $0.987312-0.303339-3.623920$

H $\quad 0.206251-0.730225-2.988912$

H $\quad 0.5251560 .081484-4.536833$

H $\quad 1.722920-1.065660-3.898617$

SCF Energy (B3LYP/6-31G**//MMFF)= -3245.92476057

0600255

MM̄FF Geometry

C $4.000585-2.7070641 .739449$

C $2.752059-3.0690212 .072008$

C $1.947296-2.5905253 .256702$

O $0.672866-2.1082642 .763313$

C $2.577562-1.4328014 .058178$

C $1.685972-0.9151295 .172499$

C $\quad 0.912953 \quad 0.1854235 .067482$

C $1.735476-1.6980466 .456287$

C $0.726766 \quad 1.035443 \quad 3.840282$

C 1.5336482 .3345623 .918442

C 1.6362823 .0166922 .546609

C 2.4735344 .2934892 .638663

C 2.5527825 .0178641 .287880

O $0.317895 \quad 3.3260552 .081326$

O $3.083865 \quad 6.326517 \quad 1.543739$

C 1.1234465 .1830490 .729491

C $3.542368 \quad 4.329670 \quad 0.340029$

O $1.1572435 .734509-0.585562$

C 0.2851273 .8814550 .759004

C -1.1833424 .2046430 .396471$

O $0.8163282 .974830-0.209447$

C $-0.355804-2.9881442 .654626$

O $-0.303838-4.2033992 .743381$

C - $1.584650-2.1858962 .465285$

C $-2.759073-2.7914622 .238944$

C $-4.055535-2.1213702 .110933$

C -4.112112 -0.653471 2.445920

C -2.1641423 .0373510 .576189$
C $-3.5651113 .409874 \quad 0.113825$

C $-5.7645282 .405946-0.419818$

O $-3.8844394 .550028-0.213729$

C $-6.591317 \quad 1.2741970 .097771$

C $-6.9038040 .191204-0.629438$

C $-7.780481-0.927220-0.146462$

C $-6.999924-2.1888420 .146437$

C $-6.790911-3.101588-0.818249$

C $-6.553225-2.3933521 .579355$

C $-5.125426-2.8471231 .724286$

N -4.4242672 .3185450 .126926$

H $4.552358-2.0101692 .363373$

H $2.246554-3.7911241 .434559$

H $1.779422-3.4407003 .930147$

H $2.830057-0.606773 \quad 3.381752$

H $3.531945-1.7545594 .495225$

H 0.3301160 .4979095 .932180

H $1.089061-1.2691657 .229379$

H $1.408872-2.7290156 .287877$

H $2.756448-1.7159326 .851385$

H $\quad 0.9946780 .4848752 .933435$

H -0.3410621 .2657393 .738621$

H 1.0480213 .0068724 .637394

H 2.5449712 .1287344 .291469

H 2.1036882 .3071241 .853057

H 3.4804104 .0753853 .016416

H 2.0193794 .9740443 .372713

H 3.0682806 .8197190 .705378

H $0.6217245 .932498 \quad 1.356806$

H 4.5453054 .3129860 .783415

H $3.6425394 .882189-0.600614$

H $3.266223 \quad 3.2994070 .107409$

H $1.4031015 .021146-1.199096$

H -1.536051 5.0310131 .027938

H -1.234297 $4.546991-0.645338$

H $\quad 0.1320452 .333298-0.453859$

H -1.492005 -1.108951 2.552051

H -2.778149 -3.876409 2.145862

H -3.829845 -0.4892493 .491824$

H $-5.100478-0.2117982 .310501$

H $-3.428735-0.0836571 .808551$

H -1.838436 2.1649110 .001698

H -2.2222912 .7501681 .632191$

H -5.677951 2.389855 -1.511472

H $-6.2141693 .364308-0.139052$

H -6.9700341 .3644051 .112769$

H $-6.5258730 .107423-1.645708$

H -8.534972 -1.123213 -0.919865

H $-8.354700-0.628650 \quad 0.739651$

H -6.268733 -4.031475 -0.621870

H -7.145502 -2.939449-1.831093

H -7.204676 -3.147559 2.040449

H -6.731550-1.485192 2.163255

H -4.979310 -3.906068 1.507694

H -4.0127021 .3942920 .216466$

C $5.284054-2.075660-0.316654$

O $4.207539-1.352610-0.949098$

C $4.620043-0.983763-2.279939$

C $6.095055-1.356126-2.393240$

C $6.182216-2.544980-1.458873$

H $4.049211-1.612443-2.972536$

C $4.3321260 .498195-2.545808$

H $6.383137-1.594635-3.421750$

H $6.746167-0.546433-2.043178$

C $7.602979-2.879982-1.039035$

H $5.735709-3.417277-1.955071$

C $2.8487780 .905332-2.434900$

O $5.0693131 .295164-1.608772$

H $4.7143800 .772226-3.536203$

H $2.5179510 .861995-1.390292$

O $2.7502982 .280176-2.831540$

C $1.8901050 .075310-3.309628$

C $0.4694030 .671105-3.353560$

H $1.856816-0.947614-2.922033$

O $2.413111 \quad 0.020213-4.637263$

H $\quad 0.1401220 .872540-2.329281$

H $\quad 0.5002161 .631488-3.881999$

H $8.213670-3.127829-1.913480$ 
H $8.078624-2.038123-0.524740$

H $7.619384-3.740156-0.362779$

C $4.726150-3.2228920 .527348$

H $4.7875951 .032497-0.715583$

H $3.3743472 .785922-2.283325$

H $1.956980-0.698697-5.105780$

H $5.544190-3.8633970 .875374$

H $4.056076-3.840335-0.082813$

H $5.820462-1.3576010 .318280$

C -1.227871 -1.583391 -2.177927

O $-0.620407-1.543034-3.475615$

C $-0.549413-0.243294-4.070567$

C $-1.9573860 .354151-4.200558$

C $-2.7040830 .347736-2.871459$

C $-2.660903-1.041314-2.239167$

H $-0.634604-0.991973-1.470261$

C -1.216756 -3.047453-1.713496

H $-0.180209-0.394921-5.091953$

O $-1.9060091 .686267-4.700020$

H -2.535290 $-0.237206-4.923004$

H $-2.3081681 .104769-2.185376$

O $-4.0717210 .689799-3.103281$

H $-3.299926-1.725063-2.812823$

O $-3.190073-0.966993-0.918907$

C $0.183511-3.536799-1.409703$

$\mathrm{H}-1.808872-3.182456-0.802945$

H -1.635613 -3.678719 -2.506888

H $-1.439607 \quad 1.660799-5.553115$

H $-4.0825861 .563618-3.530671$

H $-4.101770-0.636649-0.989947$

O $0.227720-4.892506-1.565981$

O $1.112141-2.825534-1.051638$

C $1.497027-5.505994-1.319354$

H $1.705990-5.504491-0.246312$

H $\quad 1.446009-6.541811-1.665454$

H $2.292103-4.993678-1.870096$

SCF Energy (B3LYP/6-31G**//MMFF)= -3245.92606165

0600256

MM̄FF Geometry

C $1.553819-3.2825052 .504442$

C $1.327207-2.0003622 .179710$

C $0.820499-0.9618553 .149879$

O $-0.493618-0.5420052 .711941$

C 1.7662530 .2510073 .125944

C 1.4386761 .3062844 .161701

C 0.9410032 .5279383 .886182

C 1.7688700 .9346535 .585296

C 0.5552583 .0949742 .547418

C -0.8830203 .6263022 .578649$

C -1.3193024 .2065661 .224930$

C -2.725134 4.8014101 .342224

C $-3.2655085 .282613-0.009607$

$\begin{array}{lllll}0 & -1.301574 & 3.144167 & 0.268803\end{array}$

O -4.6668365 .5398850 .167385$

C $-3.1167744 .136276-1.030777$

C $-2.6339386 .614865-0.428041$

O $-3.5021894 .570688-2.333713$

C $-1.7019243 .510541-1.056814$

C $-1.6624472 .232797-1.932286$

O $-0.7972674 .459994-1.628684$

C $-1.537163-1.3272573 .102719$

O $-1.483648-2.3016013 .838295$

C $-2.769463-0.8318512 .448133$

C $-3.834521-1.6474452 .398180$

C $-5.129857-1.3713721 .773865$

C -5.4031740 .0311751 .304636$

C $-2.3177171 .019147-1.271192$

C $-2.539948-0.144052-2.219441$

C $-3.406457-2.489027-2.177539$

O $-2.420690-0.056450-3.437485$

C $-4.900841-2.496357-2.082838$

C $-5.607072-3.447972-1.453904$

C $-7.104881-3.427954-1.339567$

C -7.590157 -3.5418770 .090128$

C $-8.253439-4.6333550 .508541$

C -7.378097 -2.359690 1.016326

C $-6.018461-2.3819811 .665138$
N - $2.928294-1.283898-1.527557$

H $1.346275-3.5975533 .525579$

H $1.507474-1.6533461 .167200$

H $\quad 0.765099-1.3668284 .167670$

H $2.798887-0.0814673 .300558$

H 1.7688180 .6858222 .122395

H $\quad 0.7897213 .2133254 .719861$

H 1.6131251 .7672216 .279743

H 1.1412250 .1048795 .923485

H 2.8187190 .6341355 .666111

H 1.2423803 .9153832 .308449

H 0.6498422 .3579851 .746917

H -1.5548392 .8022102 .854354$

H $-0.973771 \quad 4.397607 \quad 3.353981$

H -0.5990954 .9749980 .920482$

H -2.7424825 .6178662 .075140$

H -3.410739 4.0363581 .734348

H $-5.0360485 .770592-0.702555$

H -3.846676 $3.369779-0.745506$

H $-2.846401 \quad 7.3878210 .320537$

H $-3.0660256 .982300-1.365277$

H -1.549983 $6.557822-0.546503$

H $-2.7726155 .101473-2.696412$

H -2.103482 $2.441859-2.914504$

H $-0.6184021 .965591-2.142985$

H $-0.0194543 .982201-1.959977$

H -2.755970 0.1569622 .005371

H -3.744124 -2.640507 2.838358

H -5.1146030 .7639072 .066757$

H -6.4606380 .2168411 .101435$

H -4.8482320 .2407600 .387687$

H -1.694950 $0.675513-0.436692$

H $-3.299206 \quad 1.288074-0.873259$

H -2.953477 -3.340610 -1.660502

H -3.094233 -2.503625 -3.226041

H $-5.426651-1.666551-2.550018$

H -5.085195 -4.287377 -1.000123

H -7.494025 -4.259827 -1.940633

H -7.524695 -2.516077 -1.782627

H -8.626550 -4.711905 1.524338

H $-8.433999-5.474312-0.152668$

H $-8.127847-2.377306 \quad 1.817893$

H -7.564676 -1.441754 0.451349

H $-5.744052-3.3542572 .077567$

H -3.021755 -1.234687 -0.517565

C $2.602346-3.9396920 .233739$

O $1.500349-3.567509-0.621621$

C $1.730462-4.113320-1.927676$

C $3.181538-4.570538-1.952107$

C $3.352679-5.039804-0.519277$

H $1.101404-5.012287-1.995539$

C $1.294281-3.163502-3.046815$

H $3.363793-5.360813-2.686331$

H $3.861629-3.737930-2.164282$

C $4.804341-5.209020-0.107105$

H $2.831500-5.998887-0.395848$

C $2.044606-1.822008-3.206060$

O $1.398600-3.878558-4.286820$

H $\quad 0.226596-2.945428-2.918988$

H $3.079631-1.992557-3.522940$

O $1.411675-1.126690-4.294074$

C $2.031373-0.888325-1.978940$

C $2.5213840 .539379-2.305149$

H $2.662233-1.311473-1.193058$

O $0.703414-0.806394-1.463605$

H $3.5291330 .477175-2.729197$

H $1.8694160 .969077-3.074390$

H $5.288859-5.977199-0.718798$

H $5.369088-4.280018-0.230756$

H $4.882357-5.5143470 .940836$

C $2.080447-4.3740771 .605939$

H $2.342415-4.024148-4.469536$

H $1.438663-1.714053-5.068943$

H $\quad 0.137008-0.469063-2.178143$

H $2.890306-4.8700472 .154076$

H $1.276273-5.109207 \quad 1.477423$

H $3.244918-3.0587180 .334885$

C $4.7562361 .173583-0.269089$ 
O $3.351538 \quad 1.032728-0.029077$

C $2.4975411 .486142-1.082645$

C $2.7878142 .958282-1.413370$

C $4.265376 \quad 3.192694-1.708637$

C $5.1207492 .630407-0.577297$

H $5.0510430 .532729-1.108427$

C $5.482731 \quad 0.701562 \quad 1.000587$

H $1.4780951 .457035-0.678281$

O $1.994196 \quad 3.377342-2.517932$

H $2.4949003 .576472-0.554379$

H $4.5587202 .763720-2.674164$

O $4.5173224 .596967-1.793217$

H 4.9947103 .2503470 .319772

O $6.4949792 .698250-0.954608$

C $5.473853-0.8036491 .140229$

H 6.5344391 .0074510 .995709

H 4.9900481 .1326611 .880829

H $2.1292814 .332701-2.633851$

H $4.0769664 .926663-2.594572$

H $6.6925953 .627424-1.163257$

O $5.640647-1.1327152 .454740$

O $5.379318-1.5911520 .209299$

C $5.705454-2.5334702 .738119$

H $4.755994-3.0122402 .486961$

H $6.529658-2.9966712 .187297$

H $5.886960-2.6556253 .809203$

SCF Energy (B3LYP/6-31G**/MMFF) $=-3245.93420477$

0600257

MM̄FF Geometry

C $-1.2791290 .912527 \quad 2.483831$

C $-2.1882850 .257648 \quad 3.222614$

C $-2.684590-1.1595573 .037188$

O $-1.976913-1.8604471 .992234$

C $-4.158663-1.1001442 .595748$

C $-4.943145-2.3703952 .845423$

C $-5.252155-3.2870641 .907634$

C $-5.456824-2.5310564 .253677$

C $-4.791375-3.354404 \quad 0.478594$

C $-5.836574-2.872480-0.542031$

C $-5.715041-1.374783-0.864346$

C $-6.743373-0.936489-1.907842$

C $-6.5409570 .531325-2.325706$

O $-4.397216-1.161864-1.377367$

O $-7.315638 \quad 0.762725-3.512233$

C $-5.058686 \quad 0.752015-2.704331$

C $-7.071017 \quad 1.503907-1.265167$

O $-4.7894572 .129554-2.952662$

C $-4.067864 \quad 0.202620-1.655187$

C $-2.617568 \quad 0.231756-2.201348$

O $-4.129273 \quad 1.013311-0.479398$

C $-0.795505-2.461787 \quad 2.282725$

O $-0.202968-2.460743 \quad 3.347909$

C $-0.343300-3.124468 \quad 1.039035$

C $0.954545-3.399606 \quad 0.846407$

C $1.523508-4.057988-0.334032$

C $0.572216-4.488960-1.420889$

C $-1.662877-0.665801-1.400324$

C $-0.198457-0.437871-1.716009$

C $1.455296-0.505801-3.580688$

O $0.605370-0.132612-0.837175$

C $2.080097-1.857077-3.728989$

C $3.300120-2.165024-3.262721$

C $3.971450-3.486261-3.503244$

C $4.540756-4.107884-2.248971$

C $5.839446-3.961534-1.936694$

C $3.642758-5.003883-1.429527$

C $2.856534-4.261733-0.385817$

N $0.114884-0.650228-3.046617$

H $-0.7793490 .412827 \quad 1.660578$

H -2.661561 0.7966704 .042527

H -2.578972 -1.703086 3.983736

H $-4.195460-0.8444891 .533087$

H $-4.685427-0.2853013 .111414$

H -5.873498 -4.1309312 .206776$

H -6.042615 -3.447452 4.382245

H $-4.624014-2.5718254 .962534$

H $-6.101557-1.6877004 .522179$
H -3.833945 -2.842826 0.344378

H $-4.578570-4.4120100 .272035$

H -5.667140 -3.436686 - 1.468976

H $-6.851715-3.108982-0.201316$

H $-5.852683-0.8029190 .058550$

H $-7.764169-1.095842-1.539371$

H $-6.646124-1.565927-2.803594$

H $-8.2444790 .558976-3.308049$

H $-4.8842980 .226056-3.653529$

H -8.142236 $1.340183-1.097190$

H $-6.9763912 .543002-1.599658$

H $-6.5676541 .404119-0.301365$

H -5.427439 $2.433383-3.621102$

H -2.615378 $-0.121859-3.239790$

H -2.238112 $1.261019-2.207707$

H $-3.2753330 .973036-0.020436$

H -1.104315 -3.3568060 .302286$

H $1.665027-3.1232241 .624204$

H $-0.114467-5.256656-1.047755$

H $1.071291-4.901741-2.300094$

H $-0.015244-3.637824-1.781336$

H $-1.793061-0.491920-0.329126$

H -1.884609-1.723636 -1.583886

H $2.0436630 .151584-2.934276$

H $1.360363-0.033852-4.563634$

H $1.521086-2.603380-4.288627$

H $3.874001-1.404556-2.738934$

H $4.780048-3.316087-4.226759$

H $3.291896-4.197916-3.988407$

H $6.267388-4.439886-1.061897$

H $6.501539-3.353125-2.544077$

H $4.252470-5.749766-0.900442$

H $3.011175-5.601543-2.093245$

H $3.468081-3.9061790 .444243$

H $-0.603214-0.997592-3.675038$

C $-1.197633 \quad 3.201887 \quad 1.469821$

$\begin{array}{lllll}\text { O } & -0.127861 & 3.017886 & 0.517071\end{array}$

C 0.3399204 .3127960 .093140

C $-0.781103 \quad 5.280727 \quad 0.437143$

C $-1.2517274 .704271 \quad 1.758500$

H 1.2086234 .5566270 .716736

C $0.7566634 .284953-1.381241$

H -0.4319576 .3143780 .517757$

H $-1.5894475 .246331-0.302486$

C -2.624222 5.1986352 .182205

H -0.5219744 .9637602 .537492$

C $1.7867533 .189435-1.723942$

O $-0.4002544 .056782-2.195470$

H $1.1430195 .267695-1.674776$

H $1.3116452 .206477-1.662230$

O $2.1844603 .357834-3.089051$

C $3.0457683 .204661-0.841933$

C $4.0435292 .089413-1.204388$

H 2.7484903 .0773230 .202546

O $3.6895384 .473081-0.944063$

H $3.5192491 .128024-1.176763$

H $4.4029822 .230955-2.230794$

H -2.615474 6.2846132 .321420

H $-3.3855184 .962097 \quad 1.431244$

H $-2.9298234 .738899 \quad 3.127403$

C $-0.9191182 .355968 \quad 2.716217$

H $-0.8038723 .221416-1.903234$

H $1.376263 \quad 3.340505-3.630034$

H $3.9307774 .604736-1.877131$

H -1.4859632 .7482153 .568556$

H 0.1452182 .4044492 .976199

H -2.121945 2.8773790 .975001

C 4.2294550 .9475521 .612073

$\begin{array}{lllll}\text { O } & 4.894810 & 2.118404 & 1.120817\end{array}$

C $5.2680352 .056113-0.260183$

C $6.2180530 .873045-0.490661$

C $5.657455-0.4379770 .058921$

C $5.161380-0.2649101 .495243$

H $3.3181200 .778707 \quad 1.026554$

C 3.8212501 .2411803 .062799

H $5.8388422 .971758-0.460881$

$\begin{array}{llll}\text { O } & 6.503891 & 0.736733 & -1.878899\end{array}$

H 7.1702531 .0849420 .013342 
H $4.865630-0.828201-0.589646$

O $6.705463-1.409906 \quad 0.044165$

H $6.028263-0.1407182 .155924$

O $4.496076-1.4565671 .902269$

C 2.6732200 .3884653 .554380

H 4.6834671 .1069033 .726609

H 3.5019372 .2873643 .150014

H $7.085967-0.035692-1.981357$

H $6.353687-2.220093 \quad 0.451591$

H $3.702174-1.5578191 .349622$

$\begin{array}{llll}\text { O } & 2.577071 & 0.513075 & 4.911181\end{array}$

O $1.936364-0.2798312 .842935$

C $1.519549-0.2310645 .524095$

H $1.554883-0.0472536 .601253$

H 0.5503340 .1034505 .143433

H $1.657821-1.3016605 .346497$

SCF Energy (B3LYP/6-31G*//MMFF) $=-3245.92100956$

06_00258

MM̄FF Geometry

C -1.016999 -3.387759 1.167262

C $-1.254595-2.1059031 .479247$

C -1.716497-1.667324 2.846347

O $-2.982310-0.9796062 .691190$

C $-0.755545-0.6612453 .501953$

C $0.688456-1.1095723 .583612$

C $1.678333-0.5757032 .840308$

C $0.982561-2.1828194 .595818$

C $1.516066 \quad 0.4752491 .778858$

C $1.764407 \quad 1.8843992 .327098$

C 1.2009022 .9662391 .393786

C 1.5985904 .3578951 .885783

C 0.9486615 .4709621 .052361

O $-0.2252392 .838006 \quad 1.398015$

O 1.1134316 .7057271 .767722

$\begin{array}{llll}\text { C } & -0.567809 & 5.203539 & 0.952566\end{array}$

C $1.6462175 .658756-0.299876$

$\begin{array}{lllll}\text { O } & -1.161142 & 6.150922 & 0.066059\end{array}$

C $-0.916438 \quad 3.7519910 .534908$

C -2.434616 3.4988910 .722324

O $-0.5569763 .559428-0.831019$

C $-4.097906-1.7398182 .528159$

O $-4.183843-2.9548072 .599298$

C $-5.196693-0.8199252 .164661$

C $-6.234813-1.2926971 .460196$

C $-7.341781-0.5021700 .917564$

C $-7.3481610 .977011 \quad 1.202563$

C -2.9075442 .0669090 .424411$

C $-3.3828621 .860787-1.005101$

C $-4.5720720 .198775-2.434972$

O $-3.2384342 .699282-1.890568$

C $-6.0150480 .599883-2.460220$

C $-7.015881-0.237796-2.773475$

C $-8.4605580 .174858-2.832109$

C $-9.371737-0.743827-2.046387$

C $-10.137295-1.655454-2.670026$

C $-9.456191-0.550905-0.547004$

C $-8.268746-1.1328070 .166624$

N $-3.9793230 .618164-1.177500$

H $-1.165786-4.1508081 .928134$

H - $1.132352-1.3371160 .720855$

H -1.853251 -2.521398 3.522054

H $-1.115145-0.446546 \quad 4.518128$

H $-0.846326 \quad 0.3077902 .995635$

H $2.696050-0.9331812 .985484$

H $2.049003-2.4277874 .642254$

H $0.674596-1.8574615 .594760$

H $0.444231-3.1027384 .347500$

H $2.2312210 .265017 \quad 0.976748$

H 0.5305550 .4020831 .309085

H $1.286761 \quad 1.9828113 .310965$

H 2.8424622 .0300562 .469254

H 1.5761972 .7900570 .380632

H $2.689637 \quad 4.4716201 .895362$

H 1.2784314 .4789802 .930540

H 2.0662446 .8532551 .895522

H -0.9919715 .3964531 .948222$

H $2.7022465 .915141-0.153658$
H $1.211956 \quad 6.496697-0.856304$

H $1.6052544 .768218-0.929841$

H -2.121591 6.1368690 .211499

H $-2.676693 \quad 3.6961341 .775403$

H -3.016809 4.2035640 .118497

H $-1.0110602 .770003-1.164957$

H -5.0841490 .2270072 .420502$

H $-6.256145-2.3548521 .218573$

H $-7.385222 \quad 1.1586542282466$

H $-8.199821 \quad 1.5041580 .769036$

H $-6.447788 \quad 1.4519710 .797764$

H -2.1313701 .3283990 .649679$

H -3.765499 $1.857627 \quad 1.074692$

H $-4.441226-0.885030-2.512148$

H $-4.0414930 .678811-3.263476$

H $-6.2462951 .637809-2.231473$

H $-6.784036-1.268298-3.032680$

H -8.759184 $0.181564-3.888595$

H $-8.596718 \quad 1.204432-2.478461$

H -10.812715 -2.299889-2.117288

H $-10.101657-1.781431-3.747098$

H -10.351794 - $1.048876-0.152083$

H $-9.6066660 .510885-0.336834$

H $-8.171558-2.210408 \quad 0.025030$

H $-4.1034540 .012256-0.372202$

C $0.688787-4.705281-0.097622$

O $1.840307-3.838778-0.004883$

C $2.920112-4.434747-0.754328$

C $2.432866-5.811934-1.192365$

C $0.942396-5.577864-1.324344$

H $3.062883-3.820980-1.650201$

C $4.201574-4.4752490 .084995$

H $2.901971-6.139254-2.125261$

H $2.637009-6.573205-0.429774$

C $0.133563-6.863325-1.356649$

H $0.751844-5.003981-2.241193$

C $4.785796-3.0918240 .436432$

O $3.932742-5.167587 \quad 1.311094$

H $4.962966-5.069725-0.433421$

H $4.108011-2.543668 \quad 1.101432$

O $5.993822-3.3090191 .177182$

C $5.122428-2.224286-0.791875$

C $5.916322-0.951993-0.439534$

H $4.193824-1.941908-1.293386$

O $5.882462-2.993220-1.726290$

H $5.387422-0.4108170 .351655$

H $6.896191-1.239576-0.040205$

H $0.427283-7.480233-2.212323$

H $0.284578-7.455533-0.447832$

H $-0.936431-6.652303-1.446508$

C $-0.588869-3.870325-0.189587$

H $3.222404-4.685724 \quad 1.768880$

H $5.766125-3.8583611 .946936$

H $6.699138-3.274915-1.279262$

H $-1.409428-4.476536-0.589000$

H $-0.438065-3.023230-0.869777$

H $\quad 0.683570-5.3267820 .808386$

C $4.1551221 .323217-1.494978$

$\begin{array}{lllll}\text { O } & 4.906631 & 0.377161 & -2.264568\end{array}$

C $6.140486-0.043082-1.671350$

C $7.0419551 .170842-1.413892$

C $6.3316222 .246327-0.599169$

C $4.9833472 .588033-1.232488$

H $3.8646480 .872688-0.540163$

C $2.877197 \quad 1.650100-2.285668$

H $6.648416-0.654164-2.427944$

$\begin{array}{llll}\text { O } & 8.237040 & 0.765329 & -0.754698\end{array}$

H $7.3399531 .600071-2.379585$

H $6.218068 \quad 1.936342 \quad 0.446454$

O $7.1587663 .411934-0.589611$

H $5.1433773 .136266-2.169802$

O $4.2595343 .440446-0.349409$

C $1.8278990 .562949-2.166841$

H $2.4248972 .571867-1.905437$

H $3.1070791 .774091-3.349988$

H $8.767617 \quad 1.564961-0.596498$

H $6.753795 \quad 4.054496 \quad 0.016773$

H $4.7366654 .285579-0.297585$ 
O $0.647796 \quad 1.028938-2.673779$

O $2.011736-0.552578-1.700699$

C $-0.4486980 .110891-2.619583$

H - $0.726040-0.077891-1.578506$

H $-1.2980390 .567060-3.134327$

H $-0.193963-0.824113-3.127885$

SCF Energy (B3LYP/6-31G**//MMFF) $=-3245.91511663$

0600259

MM̄FF Geometry

C 0.0891890 .5032293 .131279

C 1.1404450 .0424062 .438281

C $2.543089-0.0266482 .975530$

O $3.286152 \quad 0.9787802 .250964$

C $3.157168-1.4038702 .679013$

C $4.449000-1.6601423 .425802$

C $5.680428-1.5551052 .889360$

C $4.283380-2.1072894 .855269$

C $6.053510-1.0962531 .506726$

C $6.611787-2.2300430 .635831$

C $5.530151-3.1950440 .124747$

C $6.162498-4.340067-0.668952$

C $5.105306-5.258733-1.299339$

O $4.647858-2.449225-0.719521$

O $5.794257-6.086830-2.248463$

C $4.084094-4.391569-2.067262$

C $4.488285-6.203381-0.261251$

O $2.994089-5.179137-2.541711$

C $3.544682-3.199566-1.241927$

C $2.723002-2.257060-2.155200$

O $2.702089-3.698574-0.200947$

C 4.1276781 .7900552 .937095

O 4.3798131 .7414944 .132939

C 4.7224222 .8205852 .052232

C 4.5353472 .8581880 .723463

C $5.0563133 .870345-0.198685$

C 5.8372195 .0173410 .386396

C $2.337717-0.925554-1.495519$

C $1.540982-0.029890-2.428124$

C $0.6092732 .280187-2.564110$

O $1.166017-0.382124-3.543166$

C $1.6181703 .062641-3.344371$

C $1.9229194 .346123-3.100050$

C $2.9472045 .117965-3.882549$

C $4.0827205 .627043-3.020730$

C $4.1537716 .922632-2.671545$

C $5.1815384 .658090-2.638729$

C $4.7835563 .742719-1.514362$

N $1.2908451 .211292-1.856969$

H $\quad 0.222050 \quad 0.8413094 .155364$

H $0.991371-0.2698461 .408350$

H 2.5416280 .1828544 .051828

H $3.320200-1.5070281 .600838$

H $2.448373-2.1995912 .945733$

H $6.535190-1.7950953 .521036$

H $5.243173-2.3099985 .342046$

H $3.773811-1.3370655 .442284$

H $3.691341-3.0272734 .899145$

H $5.225959-0.5933810 .998121$

H $6.833790-0.3328201 .621003$

H $7.107343-1.766159-0.227083$

H $7.379144-2.7838581 .190685$

H $4.969456-3.5901960 .978910$

H $6.847480-4.919887-0.037543$

H $\quad 6.782126-3.923004-1.475674$

H $5.154202-6.716549-2.620969$

H $4.593704-3.995114-2.956723$

H $5.261571-6.8480620 .173680$

H $3.760048-6.879060-0.723276$

H $3.995521-5.6769980 .558985$

H $3.355110-5.850044-3.144987$

H $3.310196-2.021954-3.052837$

H $1.810042-2.766824-2.487934$

H $2.049458-3.0222420 .036916$

H 5.3243733 .5634392 .565749

H 3.9330312 .0820940 .254248

H $5.237617 \quad 5.551378 \quad 1.132089$

H $6.1407305 .760114-0.354450$
H $\quad 6.752573 \quad 4.6533410 .865720$

H $1.724565-1.102492-0.608418$

H $3.238511-0.380845-1.189711$

H $0.0971162 .894706-1.818002$

H $-0.1384621 .855067-3.241099$

H $2.1248962 .541537-4.153416$

H $1.4112294 .873401-2.298161$

H $2.4316695 .958080-4.366093$

H $3.3608124 .516897-4.701996$

H $4.9794537 .304823-2.080624$

H $3.3856037 .630266-2.965477$

H $6.1059835 .195576-2.399380$

H $5.4433774 .046244-3.511672$

H $4.2110712 .874538-1.840893$

H $1.7069091 .433492-0.956982$

C -1.7764322 .0303572 .331624$

O $\quad-3.1568911 .9573001 .906103$

C -3.3255982 .7552950 .722159$

C $-2.1344403 .698648 \quad 0.695400$

C -1.032081 2.8099951 .241209

H $-3.2564922 .076772-0.136283$

C $-4.6901303 .452070 \quad 0.736161$

H -1.923868 $4.075159-0.310279$

H -2.286635 4.5605491 .355580

C 0.1654623 .6137561 .724221

H -0.7081592 .1244040 .447631$

C -5.8955492 .4966650 .849539$

O -4.7458434 .3502091 .852545$

H $-4.7944004 .077942-0.157621$

H $\quad-5.9031502 .009511 \quad 1.832074$

O

C $-5.9663481 .426811-0.255623$

C $-7.2430150 .570076-0.165978$

H $-5.0938390 .773835-0.180196$

O $-5.9095482 .055773-1.535498$

H -7.3021020 .1361810 .837868$

H $-8.1210631 .214682-0.292656$

H $\quad 0.5730974 .2198580 .907902$

H $-0.109816 \quad 4.2911122 .539620$

H 0.9678672 .9659972 .083714

C -1.2887650 .5958572 .552600$

H -4.591039 3.8266002 .657659

H -7.0291073 .9593611 .487352$

H $-6.6689802 .660212-1.599233$

H $-1.3524660 .044212 \quad 1.606767$

H $-1.983570 \quad 0.078823 \quad 3.226875$

H -1.771844 2.5814773 .280357

C $-6.040172-2.247400-0.088252$

O $-6.148999-1.383840-1.225751$

C $-7.304335-0.539935-1.240214$

C $-8.577155-1.394916-1.188173$

C $-8.569838-2.353809-0.000584$

C $-7.270535-3.1588330 .025331$

H -5.934737 -1.646306 0.822968

C $-4.763993-3.085681-0.271232$

H $-7.299901-0.035221-2.214653$

O $-9.728452-0.559061-1.135426$

H -8.648508 -1.980283 -2.114261

H -8.728792 -1.815681 0.941237

O $-9.676433-3.246686-0.147850$

H $-7.276532-3.887326-0.795771$

O $-7.191704-3.8890971 .247731$

C $-3.502678-2.261588-0.124443$

H $-4.709598-3.8864980 .473883$

H -4.763557 -3.527309-1.275410

H -10.507157 -1.139499-1.083848

H -9.728966 -3.7851190.659806

H -7.909402 -4.544606 1.246613

O $-2.456196-2.946023-0.674086$

O $-3.434124-1.1732130 .428490$

C - $-1.183096-2.296843-0.595902$

H $-0.851666-2.2494400 .445398$

H - $0.464332-2.891135-1.165853$

H $-1.233057-1.294603-1.032629$

SCF Energy (B3LYP/6-31G**//MMFF) $=-3245.91748611$

0600260

MM̄MF Geometry 
C -1.705842 2.690191 1.591272 C - -1.0475572 .8084302 .753927$ C -0.0294091 .8255693 .286378$ O 1.2622572 .1610482 .724198 C $-0.321581 \quad 0.3600012 .903402$ C $0.386356-0.6585123 .777102$ C $-0.236852-1.4916494 .633718$ C $1.879381-0.7524753 .601925$ C -1.693242 -1.520130 4.997058 C $-2.464580-2.7345624 .461591$ C -2.689234 -2.706632 2.939737 C $-3.863718-3.6132652 .563377$ C $-4.090265-3.6557051 .047297$ O $-1.500257-3.1687242 .288363$ O $\quad-5.009245-4.7301390 .799422$ C $-2.753714-3.9931630 .358281$ C $-4.764376-2.3769070 .538870$ O $-2.864768-3.883047-1.059922$ C -1.560272 -3.1384970 .854136$ C $-0.246996-3.7449940 .297667$ O $-1.709315-1.803661 \quad 0.366829$ C 1.9478943 .2213223 .219720 O 1.5866433 .9804054 .106886 C 3.2215353 .4014762 .483915 C 3.8559052 .3705461 .903805 C 5.0986522 .4241321 .131602 C 5.6416883 .7845950 .785802 C $1.034740-3.1343970 .873826$ C $2.286185-3.8033120 .325381$ C $4.768273-3.7904250 .619616$ O $2.260392-4.686330-0.527612$ C $5.385690-2.955985-0.457242$ C $6.361008-2.062060-0.234656$

C $7.025411-1.250261-1.310042$

C $6.6120260 .204858-1.307940$

C $6.0568620 .767371-2.394756$

C $6.9006891 .033386-0.073589$ C 5.6615661 .2574940 .751362 N $3.436569-3.2960340 .912746$ H -1.4942991 .8395850 .948566$ H -1.2271603 .6863293 .370471$ H $0.009302 \quad 1.913848 \quad 4.380039$ H -0.0093130 .1752921 .866439$ H -1.401095 0.1839912 .925859 H $0.369906-2.2128305 .180300$ H $2.293666-1.6631244 .047759$ H $2.142637-0.7628632 .540123$ H 2.3724980 .1019724 .075011 H -1.746004 -1.543888 6.093732 H -2.207870 -0.597572 4.710363 H -1.941736 -3.6558444 .748229$ H -3.437412 -2.740808 4.969377 H $-2.898966-1.6763902 .631947$ H -4.781864 -3.296607 3.074280 H -3.661780 -4.634774 2.916111 H $-5.206101-4.746924-0.152287$ H -2.535501 -5.0487940 .572867$ H $-5.736813-2.2379001 .026286$ H $-4.974826-2.441920-0.534054$ H -4.170571 -1.4783110 .718831$ H $-3.544227-4.512904-1.353197$ H $-0.227031-4.8197730 .522379$ H $-0.226163-3.642061-0.794595$ H $-0.833238-1.401310 \quad 0.263258$ H 3.6193914 .4101042 .474587 H 3.4246901 .3755141 .997603 H $4.884477 \quad 4.378567 \quad 0.261354$ H $6.516373 \quad 3.7540880 .133600$ H 5.9404764 .3201741 .693668 H $1.091818-2.0677780 .639245$ H $1.046085-3.2524091 .962892$ H $4.715643-4.8352550 .297238$ H $5.341983-3.7477041 .551077$ H $5.027934-3.115209-1.471044$ H $6.720873-1.915240 \quad 0.781234$ H $\quad 6.830267-1.703304-2.290886$ H $8.111975-1.310295-1.169183$ H $5.779075 \quad 1.816357-2.405815$
H $5.8631890 .192714-3.294350$

H $7.3777731 .968677-0.379857$

H 7.6467960 .5252870 .549764

H $\quad \begin{array}{llll}5.181019 & 0.332363 & 1.073059\end{array}$

H $3.357865-2.5204011 .563469$

C -3.9883753 .0482050 .588418$

O $\quad-3.6924892 .047842-0.405522$

C $-4.4396752 .328403-1.598333$

C $-5.6034223 .196152-1.141628$

C $-4.9336874 .051195-0.081834$

H $-3.7831562 .916767-2.251692$

C $-4.8192891 .021066-2.301251$

H $-6.0334263 .782280-1.959043$

H $-6.4046652 .601118-0.688925$

C -5.9082934 .7086190 .879932$

H $-4.3545024 .836215-0.586582$

C $-3.6547790 .021764-2.460112$

O $-5.842001 \quad 0.355399-1.547675$

H $-5.2661551 .243129-3.276954$

H -3.378096 -0.386489-1.481342

O $-4.134236-1.085776-3.234415$

C $-2.395750 \quad 0.585486-3.144927$

C $-1.298683-0.485494-3.288931$

H $-2.0001601 .417617-2.557139$

O $-2.740227 \quad 1.100321-4.428801$

H $-1.055426-0.863484-2.290225$

H -1.696259 -1.330325 -3.864018

H $-6.586166 \quad 5.379418 \quad 0.342247$

H -6.5172613 .9649121 .404832$

H -5.3737775 .2980601 .631743$

C -2.6850933 .6995491 .060638$

H -5.501388 $0.220588-0.646590$

H -4.926024 -1.430769-2.787415

H -3.114072 $0.367621-4.947969$

H -2.903972 4.4668441 .812015

H $-2.205126 \quad 4.201210 \quad 0.212720$

H -4.4805202 .5312691 .421604$

C $1.1450110 .966090-2.104644$

O $0.5451451 .175765-3.387818$

C $-0.0211510 .013244-4.000460$

C $1.053339-1.072290-4.163830$

C $1.719885-1.399796-2.830509$

C $2.227134-0.120087-2.173097$

H $\quad 0.3704770 .692192-1.377371$

C $1.7864172 .293337-1.664543$

H $-0.3225640 .318653-5.010382$

O $0.479614-2.244565-4.731628$

H $1.817592-0.713670-4.865751$

H $1.045554-1.944855-2.161278$

O $2.842893-2.253078-3.057801$

H $3.0911530 .251936-2.737631$

O $2.676510-0.411590-0.851953$

C $0.7782993 .358390-1.298909$

H $2.4102562 .124119-0.780679$

H $2.4084072 .699212-2.471070$

H $1.203462-2.863445-4.925593$

H $2.501203-3.117275-3.343091$

H $3.359792-1.099083-0.924505$

O $1.4296614 .368804-0.649207$

O $-0.4138323 .323974-1.565681$

C $0.6307765 .485865-0.246708$

H $\quad 0.1044295 .2419300 .679394$

H $1.3004316 .328968-0.056857$

$\mathrm{H}-0.0770375 .774890-1.029625$

SCF Energy $\left(B 3 L Y P / 6-31 G^{* *} / / M M F F\right)=-3245.91597845$

06_00261

MM̄FF Geometry

C $2.7632063 .203391-1.688344$

C $1.7324223 .981720-2.051821$

C $0.2866173 .784525-1.662631$

O $0.1789292 .951229-0.484324$

C $-0.4950813 .113051-2.804212$

C - $-1.9877493 .368954-2.712825$

C $-2.8947692 .518909-2.193252$

C $-2.4420344 .684412-3.295123$

C $-2.6526031 .173051-1.573153$

C $-3.0080991 .124827-0.081590$ 
C $-4.498573 \quad 1.3134360 .239554$

C $-4.739844 \quad 1.131248 \quad 1.740814$

C -6.2291491 .2201032 .098798$

O

O $-6.3645120 .736063 \quad 3.444180$

C $-7.022627 \quad 0.274023 \quad 1.171168$

C -6.7216602 .6718522 .114755$

O $-8.4203130 .439975 \quad 1.396330$

C $-6.6739190 .448619-0.325554$

C $-7.347499-0.607959-1.237592$

O $-7.1508951 .728116-0.765065$

C 0.2435003 .5725450 .723168

O $0.345546 \quad 4.776100 \quad 0.920583$

C 0.1408872 .6114681 .843445

C 0.4126891 .3018121 .731973

C 0.3367580 .3165812 .815974

C $-0.2804290 .758758 \quad 4.117166$

C $-7.069110-2.079139-0.923879$

C $-5.644946-2.481681-1.246419$

C $-3.463903-3.010188-0.221570$

O $-5.240144-2.552781-2.405190$

C $-2.932083-3.4253451 .110518$

C $-2.066317-2.6864551 .820527$

C -1.496361 -3.100776 3.146079

C $0.006995-3.2614173 .097311$

C $0.560845-4.4323262 .739137$

C $0.859833-2.0933533 .542019$

C $0.800462-0.9307472 .591693$

$\mathrm{N}-4.883123-2.738723-0.117091$

H $2.5838212 .311493-1.094356$

H $1.9314714 .858528-2.664996$

H $-0.1401474 .772584-1.449419$

H $-0.1521363 .491328-3.776948$

H $-0.2851072 .037583-2.820834$

H -3.941742 $2.817968-2.220965$

H $-3.5261964 .821584-3.221232$

H -1.969383 $5.521432-2.771951$

H $-2.1749194 .741714-4.355496$

H $-3.2375100 .427135-2.123342$

H -1.607292 $0.869975-1.675899$

H -2.6870330 .1462550 .292406$

H -2.427017 1.8791110 .460185

H $-4.7983782 .320908-0.068694$

H $-4.160377 \quad 1.8609082 .320557$

H -4.3692810 .1434702 .050453$

H $-7.3131910 .726378 \quad 3.659248$

H $-6.773019-0.7446491 .490693$

H -6.1716053 .2512842 .866200$

H -7.7762582 .7300262 .405921$

H $-6.6015913 .177574 \quad 1.154508$

H $-8.882996-0.2862400 .946067$

H -8.433476 -0.448822 -1.230209

H -7.034080 -0.415592 -2.273452

H -7.221182 $1.713639-1.734111$

H $-0.136080 \quad 3.0575702 .793494$

H $\quad 0.7357320 .9112620 .768335$

H 0.3591531 .4957364 .614860

H $-0.443754-0.0578134 .823257$

H $-1.265540 \quad 1.207130 \quad 3.943459$

H -7.329245 -2.330042 0.108380

H -7.715760 -2.706192 -1.550900

H -2.979955 -2.104915 -0.601313

H -3.308002 -3.804114 -0.959554

H -3.255147 $-4.389788 \quad 1.493987$

H -1.751960 -1.723954 1.427609

H -1.954523 -4.039609 3.484786

H -1.768883 -2.354830 3.902416

H $1.636418-4.5715912 .728849$

H $-0.052159-5.2782482 .445738$

H $1.912292-2.3994663 .611928$

H $0.578484-1.8216174 .563060$

H $1.219150-1.1524311 .611255$

H $\quad-5.251348-2.4585700 .787555$

C $5.1392453 .479158-0.835140$

O $5.3003192 .125181-0.371175$

C 5.2823212 .1250731 .068613

C 5.4632453 .5796291 .490961

C 4.7218364 .3050300 .383301
H $\quad 4.280314 \quad 1.7924951 .366946$

C 6.3298211 .1486331 .615786

H 5.0520003 .7772442 .485496

H 6.5179983 .8770821 .494866

C 5.0665985 .7793010 .272427

H $3.647143 \quad 4.2072880 .578832$

C $6.287813-0.2569170 .982505$

O $7.6421601 .675811 \quad 1.378709$

H 6.2300751 .0758062 .704805

H $6.656247-0.212883-0.049942$

O $7.207341-1.0893961 .700494$

C $4.905619-0.9317260 .997717$

C $4.947761-2.3496760 .397580$

H $4.201943-0.3218910 .423263$

O $4.413223-0.9951672 .334503$

H $5.416685-2.296291-0.591046$

H $5.580195-2.9935481 .020819$

H 4.8045106 .3061621 .195754

H $6.136375 \quad 5.9294480 .092161$

H $4.5165236 .245358-0.551331$

C $4.1934853 .505130-2.041003$

H $7.732847 \quad 1.8184740 .420710$

H $8.075469-0.6514191 .674540$

H $5.052671-1.5065092 .859279$

H $4.5203082 .749641-2.765996$

H $4.2672304 .483326-2.530684$

H $6.1339063 .793545-1.178120$

C $2.816749-1.970884-1.760617$

O $2.602580-2.152818-0.359112$

C $3.551529-3.0012930 .292634$

C $3.550831-4.389529-0.363067$

C $3.765663-4.306724-1.873824$

C $2.786238-3.313413-2.500088$

H $3.793239-1.494111-1.912281$

C $1.779012-0.965420-2.274199$

H $3.191316-3.1284561 .320335$

O $4.553501-5.2239130 .206853$

H $2.584130-4.876325-0.178938$

H $4.801313-4.045260-2.120239$

O $3.528592-5.592134-2.452851$

H $1.779695-3.748247-2.476793$

O $3.130177-3.102633-3.866800$

C $0.363909-1.486249-2.179116$

H $1.812400-0.044043-1.680682$

H $1.996727-0.679241-3.309640$

H $4.381593-5.2748761 .162680$

H $4.128963-6.220584-2.016101$

H $3.132594-3.972253-4.302577$

O $-0.111195-1.774756-3.424066$

O $-0.236800-1.639811-1.123776$

C $-1.429775-2.328147-3.468881$

H $-2.155507-1.609798-3.078250$

H $-1.674156-2.540289-4.513084$

$\mathrm{H}-1.467907-3.263879-2.903384$

SCF Energy (B3LYP/6-31G**//MMFF)= -3245.92098382

06_00262

MM̄MF Geometry

C -0.8190914 .6418861 .611002$

C -1.6797473 .6165671 .519250$

C -2.0004042 .6913262 .666454$

O -1.9286561 .3301122 .177636$

C $-3.4299612 .953643 \quad 3.169753$

C -3.7960582 .1318584 .391945$

C $-4.6341021 .075768 \quad 4.391737$

C -3.1736312 .5936275 .685551$

C $-5.357198 \quad 0.472870 \quad 3.220775$

C $-4.861662-0.9507712 .936978$

C $-5.423968-1.4939081 .615747$

C $-4.988697-2.9424761 .396210$

C $-5.429936-3.4748390 .025876$

O $-4.919861-0.6692870 .560592$

O $-4.713764-4.699867-0.195654$

C $-5.005249-2.471566-1.070183$

C $-6.919111-3.8408020 .018867$

O $-5.543431-2.881868-2.324914$

C $-5.395217-1.012510-0.748506$

C $-4.7919940 .024510-1.728991$ 
O - $-6.820121-0.883917-0.826151$ C $-0.689105 \quad 0.7740972 .089488$ $\begin{array}{lllll}\text { O } & 0.369700 & 1.292173 & 2.407491\end{array}$ C $-0.836953-0.5839891 .519114$ C $0.263091-1.2881351 .215203$ C $0.312805-2.6436940 .664490$ C $-0.992786-3.3407160 .394656$ C $-3.2606740 .058138-1.797032$ C $-2.671661-0.795210-2.907224$ C $-0.478367-1.562412-3.804414$ O $-3.351936-1.337978-3.773319$ C $-0.084296-2.891880-3.245088$ C $1.190458-3.272690-3.070697$ C $1.596069-4.640527-2.603683$ C $2.496107-4.609737-1.390629$ C $3.831538-4.691593-1.517087$ C $1.841419-4.591664-0.029280$ C $1.522077-3.1990720 .441271$ $\mathrm{N}-1.286563-0.850081-2.834316$ H -0.3488174 .8335162 .573899$ H -2.188444 3.4099470 .582270 H -1.2866342 .8227373 .489055$ H - 4.1391112 .7797562 .354402 H $-3.543663 \quad 4.015344 \quad 3.428606$ H $-4.8286180 .575472 \quad 5.339828$ $\mathrm{H}-3.5326042 .0213136 .547686$ H -2.085386 2.4859395 .651207 H -3.414781 3.6458395 .868588 H -6.4290120 .4444953 .452289$ H $-5.259911 \quad 1.0887032 .324498$ H $-3.765765-0.9447802 .888153$ H $-5.148583-1.6103623 .765377$ H $-6.517345-1.4193551 .644761$ H $-5.363892-3.5887622 .199436$ H -3.893794 -3.0029081 .454167$ H $-4.933486-5.011132-1.090847$ H -3.914766 -2.548474 -1.154003 H $-7.122694-4.6202310 .763041$ H $-7.220008-4.263924-0.945783$ H -7.570138 -2.992632 0.240756 H $-5.149540-2.323768-3.015842$ H $-5.239873-0.085781-2.723754$ H $-5.1103171 .018139-1.380923$ H $-7.0391430 .052836-0.961558$ H $-1.838985-0.9658911 .366123$ H $1.233803-0.8322791 .400433$ H -1.634064 -2.725941 - 0.245531 H $-1.518954-3.5394481 .334049$ H $-0.877840-4.295725-0.122689$ H -2.946987 $1.088918-2.007410$ H $-2.815723-0.229709-0.839954$ H $0.387564-0.935695-4.032053$ H - $-1.046102-1.706352-4.729372$ H $-0.886390-3.589583-3.016409$ H $1.990804-2.581913-3.326789$ H $2.115300-5.133399-3.436593$ H $0.721003-5.268513-2.395062$ H $4.480771-4.707600-0.648537$ H $4.304608-4.739364-2.492244$ H $2.520504-5.0346790 .712450$ H $0.970845-5.253691-0.029234$ H $2.409757-2.6064280 .662889$ H $-0.809050-0.397641-2.059887$ C $-0.5512415 .070771-0.923856$ O $0.409927 \quad 4.014229-1.133967$ C $1.0756854 .235532-2.391501$ C $0.232097 \quad 5.263825-3.132431$ C $-0.2411606 .131257-1.982583$ H $2.0483004 .686030-2.161404$ C $1.2739252 .910582-3.133593$ H $0.8095575 .816061-3.879847$ H $-0.623144 \quad 4.799296-3.637059$ C -1.423802 $7.016335-2.336210$ H $0.5934776 .763298-1.650336$ C $2.0881561 .860214-2.347857$ O $-0.008872 \quad 2.340861-3.425239$ H $1.745503 \quad 3.099836-4.104569$ H $1.5006961 .519314-1.488323$ $\begin{array}{llll}\text { O } & 2.253070 & 0.715798 & -3.193705\end{array}$

C $3.4782712 .333938-1.884429$

C $4.3711221 .223916-1.302557$

H $3.3720193 .145520-1.160675$

O $4.164502 \quad 2.887882-3.013907$

H $4.6252900 .514447-2.102877$

H $5.338997 \quad 1.670499-1.042498$

H -1.157666 $7.709812-3.140615$

H -2.281681 $6.424634-2.672953$

H $-1.7410327 .607909-1.471725$

C -0.4212565 .5921410 .510685$

H $-0.4697252 .206960-2.579172$

H $2.7949680 .987858-3.954281$

H $5.012673 \quad 3.242412-2.696928$

H $-1.003656 \quad 6.5120830 .636123$

H 0.6313745 .8509150 .686268

H $-1.5454434 .647391-1.108152$

C $5.889823-0.619907 \quad 0.553592$

$\begin{array}{llllll}\text { O } & 4.553155 & -0.785652 & 0.073529\end{array}$

C $3.8134770 .433937-0.100966$

C 3.7419641 .1789561 .239320

C 5.1311291 .4394191 .818660

$\begin{array}{llll}\text { C } & 5.928602 & 0.135576 & 1.888884\end{array}$

H $6.461875-0.054435-0.192776$

C $6.555516-2.0034290 .615718$

H $2.7993880 .114472-0.359920$

$\begin{array}{lllll}\text { O } & 3.026776 & 2.398560 & 1.107134\end{array}$

H 3.1830250 .5607031 .953515

H $5.6604242 .208297 \quad 1.244490$

O 4.9773151 .9622843 .140399

H $5.515754-0.4766932 .697676$

$\begin{array}{llll}\text { O } & 7.278157 & 0.425812 & 2.246970\end{array}$

C $5.877287-2.9975861 .537790$

H $6.535157-2.448701-0.386752$

H $7.599320-1.8950870 .931176$

H 3.0032592 .8198901 .983420

H 5.8689282 .0769113 .511665

H 7.6610670 .9799561 .545639

O $6.656697-4.1171721 .624177$

O $4.798818-2.8366112 .090950$

C $6.131350-5.1691252 .439931$

H $5.171902-5.5139902 .042415$

H $6.839256-6.0018862 .418400$

H $6.021894-4.8277563 .473739$

SCF Energy (B3LYP/6-31G**//MMFF) $=-3245.92420181$

06_00263

MM̄FF Geometry

C $-4.567930-2.8527811 .275719$

C $-3.245814-2.6541731 .174188$

C $-2.340975-2.4173112 .350568$

O $-1.622282-1.180903 \quad 2.147200$

C -1.290112 -3.536262 2.449030

C $-0.401333-3.3798613 .667419$

C $0.849396-2.8783423 .646142$

C $-1.000891-3.8594484 .964291$

C $1.623107-2.3501642 .467358$

C $2.778843-3.2969942 .130335$

C $3.663603-2.790240 \quad 0.980485$

C $4.727274-3.8462630 .660189$

C $5.735982-3.363542-0.386811$

O $4.281278-1.5649001 .390627$

O $6.834132-4.288201-0.369300$

C $6.269327-1.9840780 .047751$

C $5.147418-3.422888-1.801081$

O $7.131494-1.446305-0.954004$

C $5.154374-0.9755370 .414103$

$\begin{array}{llll}\text { C } & 5.789306 & 0.288211 & 1.048621\end{array}$

O $4.456132-0.625452-0.776319$

C -2.217151-0.025871 2.542072

$\begin{array}{llll}\text { O } & -3.361385 & 0.121908 & 2.937439\end{array}$

C -1.195003 1.0372502 .431936

C -1.5423192 .3244332 .569546$

C $-0.632105 \quad 3.468182 \quad 2.482814$

C $0.841695 \quad 3.177235 \quad 2.376513$

C 4.8109851 .4247651 .385062

C $4.643608 \quad 2.432549 \quad 0.255646$

C $3.8134424 .712795-0.220311$ 
O $5.033128 \quad 2.230318-0.891674$

C $2.945560 \quad 5.744726 \quad 0.423301$

C $1.7473516 .114482-0.053634$

C 0.8985727 .1915640 .559123

C $-0.425126 \quad 6.6670991 .068247$

C $-1.548320 \quad 6.832410 \quad 0.347978$

C $-0.424678 \quad 6.0279772 .441616$

C -1.152981 4.7127292 .502819

N $3.983147 \quad 3.5815250 .672204$

H -5.034466 -2.844540 2.257586

H $-2.784540-2.653646 \quad 0.192676$

H -2.913994 -2.360025 3.284727

H -0.681156 -3.559486 1.537228

H $-1.783621-4.5160742 .496367$

H $1.393434-2.8270084 .589037$

H $-0.304264-3.7672495 .804465$

H $-1.894577-3.2795825 .213998$

H - $1.281142-4.9148864 .884819$

H $\quad 0.990718-2.2055571 .586693$

H $2.009438-1.3587802 .732759$

H $3.399037-3.4272913 .027599$

H $2.372208-4.2828661 .869819$

H $3.032334-2.5986630 .105138$

H $4.258352-4.7844530 .337391$

H $5.279173-4.0928511 .578852$

H $7.513866-3.949153-0.977039$

H $6.899352-2.1444420 .933344$

H $4.870063-4.453386-2.054295$

H $5.885189-3.120769-2.552293$

H $4.257178-2.802852-1.921985$

H $6.572232-1.093705-1.667205$

H $6.265564-0.0153571 .990697$

H 6.5815110 .6797550 .399624

H $3.9267200 .168260-0.605755$

H -0.1752020 .7272692 .230709$

H -2.5893052 .5661482 .747124$

H $1.463168 \quad 4.0725262 .414143$

H 1.1718902 .5404023 .204880

H 1.0665402 .6700461 .431999

H $3.825274 \quad 1.0372321 .664120$

H 5.2120521 .9717072 .247909

H $3.4046484 .351065-1.169785$

H $4.8047305 .132024-0.424016$

H $3.336643 \quad 6.2306751 .314000$

H $1.3628835 .640086-0.953517$

H $0.7335527 .961825-0.205632$

H $1.424867 \quad 7.7014781 .375865$

H -2.513703 6.4973050 .711430

H - $-1.5282717 .314232-0.624187$

H $-0.899626 \quad 6.7212103 .148493$

H 0.5999085 .9220442 .811363

H -2.235911 4.8183722 .579935

H 3.8452413 .7247921 .668860

C $-6.195247-1.871052-0.426241$

O $-5.248806-0.982125-1.054164$

C $-5.5304670 .360441-0.622854$

C $-6.9469670 .326248-0.058683$

C -6.963431 -1.0387040 .602386$

H $-4.834156 \quad 0.5758820 .196857$

C $-5.3104661 .364069-1.759367$

H $-7.136406 \quad 1.1457820 .640982$

H $-7.7036570 .370840-0.850293$

C $-8.357118-1.5636400 .897879$

H $-6.408610-0.9625071 .545084$

C -3.937040 $1.267601-2.457069$

O $-6.317294 \quad 1.149547-2.756390$

H $-5.4701202 .378545-1.373676$

H $-3.9158770 .407634-3.138301$

O $-3.7836532 .434421-3.276083$

C $-2.7282861 .182489-1.508772$

C $-1.379723 \quad 1.359209-2.234497$

H -2.746070 $0.216462-0.993080$

O $-2.8315712 .197158-0.507862$

H $-1.4033900 .810613-3.180246$

H -1.246002 $2.420684-2.473335$

H $-8.880464-0.8967681 .590841$

H -8.959734 -1.640230 -0.013412

H $-8.308513-2.5571381 .355007$
C $-5.469164-3.111797 \quad 0.100218$

H $-6.2908861 .902891-3.369559$

H $-4.4310172 .374211-3.998365$

H $-2.8753193 .056704-0.960872$

H -4.880765 $-3.549251-0.716439$

H $-6.203663-3.8697660 .397384$

H $-6.873461-2.191525-1.227932$

C $0.201897-1.271363-2.397635$

O $-0.184073-0.546792-1.220791$

C $-0.2005790 .880652-1.357804$

C $1.1778441 .386622-1.797252$

C $1.6560860 .692881-3.065207$

C $1.580126-0.821894-2.897696$

H $-0.540053-1.116547-3.188900$

C $0.224548-2.765699-2.033620$

H $-0.368299 \quad 1.281729-0.352800$

O $1.163503 \quad 2.794123-2.006888$

H $1.9039551 .191476-0.998310$

H $1.087597 \quad 1.021787-3.943121$

O $3.0191401 .046616-3.309860$

H $2.353794-1.154028-2.197432$

O $1.849668-1.443167-4.153533$

C $-1.154336-3.390393-2.038570$

H $\quad 0.820649-3.330093-2.759348$

H $0.650899-2.898918-1.032335$

H $0.8637393 .208670-1.179698$

H $3.0630222 .017340-3.353617$

H $2.722315-1.131442-4.449267$

O $-1.046488-4.686027-1.616855$

O $-2.186403-2.829776-2.379562$

C $-2.259515-5.445617-1.602393$

H $-1.995992-6.501810-1.500819$

H $-2.867246-5.152134-0.743018$

H $-2.818070-5.315367-2.534349$

SCF Energy (B3LYP/6-31G**//MMFF) = -3245.91984427

0600264

MM̄FF Geometry

C $-0.1123193 .038990-0.093949$

C $0.8956413 .378015-0.911198$

C $1.6109612 .377333-1.779925$

O $3.0267862 .556017-1.532191$

C $1.3225312 .580795-3.279031$

C $-0.1245422 .435367-3.693618$

C $-0.8143761 .278100-3.684698$

C $-0.778767 \quad 3.702721-4.177188$

C $-0.341779-0.080786-3.240951$

C $-1.203586-0.571647-2.072530$

C $-0.765297-1.936360-1.523270$

C $-1.847954-2.470869-0.581446$

C $-1.452022-3.7991810 .066652$

O $0.469038-1.775741-0.808435$

O $-2.389565-4.0420491 .126357$

C $-0.055009-3.6352950 .691166$

C -1.594728 $-4.969741-0.911799$

$\begin{array}{lllll}\text { O } & 0.416024 & -4.880968 & 1.202370\end{array}$

C $0.989221-2.997602-0.260715$

C $2.271187-2.687183 \quad 0.549347$

O $1.307180-3.938428-1.289482$

C $3.8561471 .540137-1.893153$

O $3.5507700 .509669-2.471724$

C $5.2165721 .888540-1.431383$

C $6.1900600 .969589-1.509676$

C $7.5745501 .137303-1.065621$

C $7.9621342 .473454-0.487154$

C $3.316289-1.839955-0.183658$

C $4.655033-1.9558390 .521425$

C $5.790122-1.3420662 .650384$

O $5.534407-2.7113810 .112724$

C $7.016772-0.5896012 .239729$

C $8.211822-1.1784012 .074778$

C $9.486188-0.4515971 .748042$

C $10.083669-0.8863560 .427865$

C $10.843143-1.9914680 .341513$

C $9.8624900 .005543-0.773112$

C $8.4153510 .087133-1.173396$

N $4.732854-1.1826181 .669424$

H $-0.4435542 .004547-0.072137$ 
H $\quad 1.226544 \quad 4.412374-0.957831$ H $1.3430161 .364397-1.459074$ H $1.9214361 .882515-3.877182$ H $1.7008123 .567303-3.582622$ H $-1.846898 \quad 1.298770-4.032743$ H -1.831749 $3.555926-4.439720$ H $-0.7364474 .473296-3.400932$ H $-0.2632954 .078277-5.067284$ H $-0.445728-0.772712-4.085583$ H $0.716587-0.085309-2.970033$ H - $-1.1724100 .167554-1.263799$ H $-2.243763-0.634699-2.414944$ H $-0.611871-2.622906-2.363836$ H -2.804067 -2.578411 - 1.105281 H -2.024832 -1.7350880 .215375$ H -2.101372 $-4.843251 \quad 1.596846$ H $-0.173452-2.9786421 .564173$ H $-2.630110-5.045632-1.265627$ H $-1.376975-5.925264-0.422227$ H - $0.950251-4.876792-1.788550$ H $0.722457-5.4129380 .448269$ H $1.998491-2.1453881 .464726$ H $2.735418-3.6313470 .863777$ H $2.207805-3.770272-1.608071$ H $5.3727432 .879085-1.019957$ H $5.950288-0.008916-1.924461$ H $7.8377823 .265262-1.234238$ H $8.9979742 .521475-0.146096$ H 7.3405762 .7107260 .383688 H $3.457173-2.160225-1.220514$ H $3.003914-0.790812-0.206628$ H $5.987061-2.4122462 .777933$ H $5.417172-0.9464123 .600392$ H 6.9260010 .4864992 .118546 H $8.292816-2.2534332 .222115$ H $10.203940-0.6492552 .555251$ H $9.338667 \quad 0.6351191 .754165$ H $11.296775-2.295655-0.595759$ H $11.025339-2.6180591 .208479$ H $10.420089-0.372591-1.640119$ H $10.2971450 .987409-0.566336$ H $8.033865-0.843498-1.596098$ H $3.937136-0.6072891 .929184$ C -0.9805713 .4259692 .218926$ O $\begin{array}{llll}-1.849930 & 2.273338 & 2.190791\end{array}$ C $-2.764814 \quad 2.349035 \quad 3.298284$ C $-2.226267 \quad 3.442773 \quad 4.212267$ C -1.6214464 .4011413 .205152$ H -3.728729 2.6756532 .890778 C -2.913443 0.9760423 .961708 H -3.0121103 .8983184 .822352$ H -1.4510583 .0628484 .887975$ C - $0.646646 \quad 5.3912543 .818500$ H $-2.433726 \quad 4.953508 \quad 2.713650$ C -3.373234 -0.1532723 .015817$ $\begin{array}{lllll}0 & -1.650157 & 0.582814 & 4.515249\end{array}$ H -3.601672 1.0514744 .811593 H $-2.578744-0.3985242 .300858$ O $-3.571787-1.3284963 .812816$ C $-4.676714 \quad 0.1404052 .249516$ C $-5.205425-1.0858421 .479848$ H $-4.5010330 .961167 \quad 1.548483$ $\begin{array}{llllll} & \text { O } & -5.681539 & 0.579058 & 3.164445\end{array}$ H $-4.398373-1.4931930 .864290$ H $-5.491199-1.8635102 .198094$ H -1.1507216 .0156754 .563356$ H 0.1848884 .8804014 .315537 H $-0.226844 \quad 6.051437 \quad 3.053232$ C $-0.842453 \quad 3.9953050 .805145$ H -1.0033050 .5707273 .788767$ H -2.736114 -1.507872 4.277166 H -5.819097 -0.1327593 .812935$ H -0.3258384 .9611040 .835154$ H $-1.838374 \quad 4.160347 \quad 0.379935$ H $-0.006883 \quad 3.0822192 .592251$ C $-5.296957-0.107220-1.426256$ O $-6.1633590 .275113-0.350109$ C $-6.436951-0.7563640 .605537$
C $-7.063044-1.972537-0.092315$

C $-6.218217-2.456206-1.267348$

C $-5.895859-1.289489-2.200057$

H $-4.312996-0.377468-1.027348$

C $-5.1294521 .112363-2.344538$

H $-7.202444-0.3508741 .279084$

O $-7.256833-3.030598 \quad 0.840203$

H -8.056221 -1.690989-0.466097

H $-5.309027-2.958629-0.917831$

O $-6.968643-3.436872-1.986908$

H $-6.816576-0.977719-2.709121$

O $-4.994484-1.729791-3.212688$

C $-4.3273742 .219039-1.693980$

H $-4.6077340 .841623-3.268644$

H $-6.1194801 .513177-2.594304$

H $-7.634577-3.7825510 .352529$

H $-6.435774-3.701323-2.756368$

H $-4.176541-2.025030-2.779317$

O $-4.590961 \quad 3.394563-2.337678$

O $-3.5492602 .070641-0.762183$

C $-3.906507 \quad 4.543401-1.829998$

H $-4.1847794 .719383-0.786614$

H -2.825286 $4.412210-1.925151$

H $-4.2081335 .409679-2.424699$

SCF Energy (B3LYP/6-31G**//MMFF)= -3245.92832494

0600265

MM̄FF Geometry

C $-4.708346-2.7441101 .464502$

C $-3.386011-2.7729981 .245250$

C $-2.363902-2.651022 \quad 2.343724$

O $-1.476490-1.5590752 .017024$

C $-1.507925-3.9270292 .427477$

C $-0.492970-3.8600193 .553074$

C $0.789694-3.4694613 .409228$

C $-1.014262-4.2659474 .907075$

C $1.478511-3.0170262 .149920$

C $2.793069-3.7765331 .935218$

C $3.405510-3.5092590 .552076$

C $4.747251-4.2347260 .414517$

C $5.433913-3.932417-0.925324$

O $3.583144-2.097960 \quad 0.384254$

O $6.790557-4.388969-0.813085$

C $5.462540-2.401915-1.130813$

C $4.806675-4.734725-2.071170$

O $5.983136-2.071544-2.415848$

C $4.092141-1.726379-0.905295$

C $4.164915-0.184289-0.968498$

O $3.204454-2.139609-1.952964$

C $-1.799642-0.310628 \quad 2.443559$

\begin{tabular}{llll}
\hline & -2.811732 & 0.034420 & 3.029692
\end{tabular}

C $-0.666491 \quad 0.567526 \quad 2.075843$

$\begin{array}{llll}C & -0.682507 & 1.871583 & 2.385007\end{array}$

C 0.3848832 .8276842 .086242

C 1.5929592 .3253791 .335968

C 5.0834370 .4831450 .057349

C $5.0880731 .996578-0.095110$

C $6.223253 \quad 4.0321070 .790909$

O $4.3704712 .594089-0.893384$

C 5.4542964 .6327731 .924234

C 4.3775465 .4156541 .756507

C 3.6186506 .0527702 .885961

C 2.4054405 .2469353 .301976

C 2.3967964 .6279954 .496184

C 1.2487865 .2215582 .318282

C 0.2598924 .1022302 .513018

N $5.9971682 .599231 \quad 0.763965$

H $-5.070240-2.6485902 .485243$

H $-3.014690-2.8819310 .229142$

H $-2.841600-2.4629543 .313633$

H $-1.002430-4.1054301 .471285$

H -2.151883 -4.803161 2.581597

H $1.418162-3.4449804 .298856$

H $-0.245072-4.2073145 .684348$

H $-1.840408-3.6156535 .211262$

H $-1.376444-5.2988354 .879251$

H $0.842484-3.135777 \quad 1.269622$

H $1.689355-1.9448762 .243683$ 
H $3.501041-3.4690392 .716132$

H $2.628331-4.8553182 .051801$

H $2.699697-3.872003-0.203583$

H $4.620844-5.3166820 .545817$

H $5.416607-3.9128651 .224967$

H $7.254720-4.136036-1.629735$

H $6.182002-2.009242-0.402463$

H $4.904711-5.810219-1.879672$

H $5.329486-4.552685-3.016501$

H $3.745621-4.521258-2.216286$

H $5.284077-2.239646-3.070539$

H $4.4462260 .129227-1.982597$

H $3.1521210 .212467-0.812954$

H $2.502416-1.475643-2.046480$

H $0.1683830 .107231 \quad 1.558877$

H -1.5470192 .2749832 .909767$

H 2.0969601 .5334101 .901252

H 2.3397953 .1005621 .152738

H 1.3058311 .9293020 .356658

H 4.7563510 .2411991 .074608

H $6.1146170 .138935-0.070464$

H $5.9411434 .468398-0.173212$

H 7.2949254 .1970250 .939346

H 5.8136864 .4270622 .929591

H $4.0196485 .618240 \quad 0.749659$

H 3.3008597 .0575832 .581020

H 4.2918876 .2093223 .739358

H 1.5456804 .0570094 .849216

H 3.2507094 .6839865 .164023

H 1.6226865 .1830491 .288752

H $\quad 0.7123106 .176308 \quad 2.391569$

H -0.6338454 .3890753 .067143$

H 6.5078912 .0252281 .428750

C $-6.564045-1.598891 \quad 0.134351$

O $-5.752912-0.594597-0.506813$

C $-6.1567080 .698694-0.015191$

C -7.4229770 .4728610 .809640$

C -7.187784 -0.9216601 .353817$

H -5.3573101 .0390640 .653542$

C -6.333265 $1.695197-1.167067$

H -7.5445711 .2262861 .593897$

H $-8.325516 \quad 0.4891720 .187875$

C -8.444829 -1.6033651 .863469$

H $-6.466071-0.8396872 .175098$

C -5.117528 $1.853769-2.100620$

O $-7.4389501 .284866-1.983694$

H -6.613953 $2.672835-0.757762$

H $-5.0133800 .971633-2.744188$

O $-5.3922672 .955784-2.976410$

C -3.778983 2.117558-1.390145

C $-2.6561652 .514049-2.370173$

H -3.490391 $1.216290-0.839079$

O $-3.9354843 .163039-0.430113$

H -2.668174 1.830148 -3.224868

H $-2.8532123 .521734-2.755523$

H $-8.886128-1.0312722 .686130$

H -9.199832 -1.695349 1.075451

H -8.218966 -2.608362 2.233465

C $-5.747685-2.8704920 .386305$

H $-7.2503850 .385008-2.301561$

H $-6.2312612 .763550-3.429275$

H $-4.2411743 .955107-0.904595$

H $-5.267884-3.172233-0.553508$

H $-6.425628-3.6842070 .670931$

H -7.350362 -1.848925 -0.590984

C $-0.6740590 .201570-2.127361$

O $-0.9493411 .220368-1.156471$

C $-1.2678772 .507528-1.694270$

C $-0.1172813 .008247-2.576634$

C $0.2814621 .983121-3.635952$

C $0.5191510 .613147-2.998975$

H $-1.5609560 .042467-2.751314$

C $-0.416473-1.086987-1.336666$

H $-1.3308623 .190537-0.837766$

O $-0.4702354 .240695-3.194691$

H $0.7518703 .209951-1.936716$

H $-0.4752991 .920912-4.426306$

O $1.4828962 .415722-4.279623$
H $1.4309900 .651885-2.392306$

O $0.730406-0.348961-4.026491$

C $-0.507855-2.353030-2.155257$

H $0.561594-1.028870-0.844852$

H $-1.165438-1.178000-0.544037$

H $0.2750574 .507398-3.759639$

H $2.1790612 .482272-3.603840$

H $1.470256-0.033788-4.573517$

O $0.219043-3.332412-1.542127$

O $-1.164790-2.481730-3.179020$

C $0.216148-4.605328-2.194820$

H $0.834144-5.289528-1.607234$

H $-0.801557-5.004151-2.242465$

H $0.645752-4.517491-3.197207$

SCF Energy (B3LYP/6-31G**//MMFF)= -3245.93203210

0600266

MM̄FF Geometry

C $0.622704 \quad 4.271553-0.610687$

C -0.1409854 .9930590 .224121$

C -0.9340734 .4732351 .400135$

O $-0.804998 \quad 3.043812 \quad 1.559866$

C -2.4308814 .7723841 .202114$

C $-3.264683 \quad 4.4203962 .420429$

C $-4.006103 \quad 3.3008962 .539260$

C $-3.255566 \quad 5.4449373 .524590$

C $-4.1152352 .172608 \quad 1.551703$

C -5.4468742 .2263560 .797968$

C $-5.5186241 .177910-0.321436$

C $-6.8279481 .324945-1.099615$

C $-6.9761840 .246116-2.179184$

O $-5.435136-0.1246090 .266011$

O $-8.3389830 .287315-2.628341$

C $-6.727682-1.132598-1.534856$

C $-6.1096660 .553854-3.405903$

O $-6.736384-2.157378-2.527440$

C $-5.436235-1.202642-0.682640$

C $-5.422432-2.5325220 .112067$

O $-4.307269-1.140645-1.551422$

C $0.2425102 .569807 \quad 2.283823$

$\begin{array}{lllll}\text { O } & 1.185399 & 3.206508 & 2.723691\end{array}$

C $0.011028 \quad 1.1208872 .476567$

$\begin{array}{lllll}\text { C } & 0.946077 & 0.364846 & 3.069721\end{array}$

C $0.815309-1.0555443 .404686$

C $-0.523574-1.7016253 .167613$

C $-4.191656-2.7626341 .001923$

C $-3.100385-3.5729140 .317490$

C $-1.173746-5.048106 \quad 0.797664$

O $-3.008326-3.680147-0.902854$

C $-0.219668-5.2972341 .920030$

C $1.074081-4.9439101 .888438$

C $2.054294-5.2574232 .981229$

C $2.756226-4.0285933 .512458$

C $4.055348-3.8090613 .245570$

C $1.967937-3.1184014 .428272$

C $1.878601-1.6963363 .933276$

N $-2.217005-4.1377251 .228963$

H $0.7000043 .196438-0.488439$

$\begin{array}{llll}H & -0.183480 & 6.071278 & 0.079529\end{array}$

H $-0.5748624 .975706 \quad 2.307084$

H $-2.802576 \quad 4.234766 \quad 0.321205$

H $-2.578758 \quad 5.836898 \quad 0.976314$

H $-4.5891213 .160842 \quad 3.448323$

H -3.9026795 .1604254 .361107$

H -2.2437025 .5739753 .921064$

H -3.6085626 .4109643 .149098$

H -3.2845412 .1802920 .841241$

H $-4.030295 \quad 1.2269132 .100717$

H -6.2633882 .0603931 .512912$

H $-5.586063 \quad 3.2257030 .366183$

H $-4.6615101 .326677-0.987606$

H $-6.9084232 .325202-1.543548$

H $-7.6750701 .238512-0.404364$

H $-8.463746-0.443263-3.258444$

H $-7.586434-1.339943-0.881637$

H $-6.3905851 .522522-3.836621$

H $-6.270579-0.182527-4.201006$

H $-5.0419970 .588630-3.179321$ 
H $-5.880073-2.130346-2.987611$ H $-6.297943-2.5318820 .775529$ H -5.537403 -3.382032 -0.571780 H -3.521476 -1.431121-1.062294 H $-0.943956 \quad 0.7214442 .152827$ H $1.887461 \quad 0.8308173 .357860$ H -1.311100 -1.172080 3.715683 H $-0.568227-2.7421483 .491566$ H $-0.770237-1.6888272 .100731$ H -3.760602 -1.818920 1.352409 H $-4.517074-3.3274561 .884740$ H $-0.683222-4.629669-0.086649$ H - $-1.651812-5.9868010 .498053$ H -0.606693 -5.8265752 .787594$ H $1.461164-4.4299571 .013682$ H $2.786830-5.9663032 .573743$ H $1.568835-5.7801943 .814960$ H $4.580615-2.9523123 .654329$ H $4.624518-4.4815602 .612311$ H $2.457226-3.1045625 .411800$ H $0.978713-3.5417164 .625951$ H $2.803272-1.1326954 .066663$ H $-2.464852-4.1231592 .214574$ C $2.9405724 .783845-1.506188$ O $3.3872953 .413871-1.537077$ C $4.4669533 .266272-0.592663$ C $4.8040854 .670719-0.093091$ C $3.4624655 .365592-0.193132$ H 4.0731372 .6775780 .243998 C $5.6561912 .545017-1.234708$ H 5.2082594 .6600050 .923992 H $5.5383085 .168490-0.737138$ C $3.5527396 .880949-0.180149$ H $2.8513185 .036106 \quad 0.655931$ C $5.353174 \quad 1.147274-1.806025$ O $6.1621263 .344446-2.313807$ H $6.4743282 .475437-0.507988$ H $4.7258421 .225967-2.702134$ O $\quad 6.603081 \quad 0.592457-2.242241$ C $4.7018330 .161138-0.816476$ C $4.701387-1.285096-1.349983$ H $3.6760230 .482608-0.611871$ O $5.405604 \quad 0.1891190 .426088$ H 4.298336-1.286118-2.367675 H $5.738084-1.637171-1.410113$ H 4.0056547 .2311830 .753131 H $4.1621697 .254229-1.010040$ H $2.5585417 .330931-0.262600$ C $1.4279494 .868701-1.729839$ H $5.4344433 .477898-2.945324$ H $6.9905831 .217099-2.879274$ H $\quad 6.328743-0.059576 \quad 0.247437$ H $1.1672924 .326665-2.647261$ H $1.1448855 .916321-1.886933$ H $3.4336305 .277748-2.354643$ C $1.776509-2.159493-1.590642$ O $2.519363-1.937778-0.383662$ C $3.911507-2.261911-0.449601$ C $4.083860-3.737989-0.828711$ C $3.363784-4.067434-2.130252$ C $1.905830-3.615566-2.066605$ H $2.138788-1.482325-2.373552$ C $0.312375-1.800290-1.285116$ H $4.302325-2.1467450 .568500$ O $5.466255-4.061593-0.935805$ H $3.673568-4.364688-0.027673$ H $3.883901-3.629067-2.990139$ O $3.404151-5.484505-2.317815$ H $1.354929-4.292249-1.403247$ O $1.356370-3.758284-3.376370$ C $0.086881-0.303973-1.308924$ H - $0.350996-2.241708-2.035754$ H $0.013667-2.162111-0.295619$ H $5.526482-4.994767-1.202856$ H $2.910745-5.681971-3.132494$ H $0.388437-3.724433-3.301690$ O $-1.194032-0.048493-1.706807$ O $0.9231780 .533738-1.000598$
C $-1.5408221 .333851-1.826529$

H $-1.5833981 .792061-0.836118$

H $-0.8242501 .858440-2.465922$

H -2.529034 $1.398116-2.288609$

SCF Energy $\left(B 3 L Y P / 6-31 G^{* *} / / M M F F\right)=-3245.93124496$

06 00267

MM̄FF Geometry

C $0.1304551 .914563-2.840677$

C $0.6558810 .832055-3.436225$

C $0.134940-0.587512-3.379449$

O $-1.065850-0.697315-2.582800$

C $1.206619-1.493299-2.745627$

C $0.851961-2.966749-2.760024$

C $0.464424-3.676134-1.681278$

C $1.013059-3.650790-4.093135$

C $0.241950-3.179437-0.278667$

C $-1.163653-3.549640 \quad 0.212251$

C -1.475194 -2.963112 1.597916

C $-2.872079-3.3968532 .048484$

C $-3.303374-2.7184973 .354291$

O $-1.404905-1.5384281 .496753$

O $-4.714262-2.9464813 .497034$

C $-3.079962-1.1960443 .227171$

C $-2.637958-3.3707254 .571880$

O $-3.351440-0.5695774 .478640$

C - $-1.670361-0.8304142 .716491$

C -1.5021630 .6775692 .409392$

O $-0.709362-1.1642873 .725131$

C $-2.255515-0.496653-3.214194$

O $-2.423410-0.156138-4.374354$

C $-3.334567-0.780258-2.240550$

C $-4.615054-0.581563-2.588061$

C $-5.793347-0.871313-1.765902$

C $-5.564610-1.434060-0.388395$

C $-2.382127 \quad 1.2207511 .280149$

C $-3.676107 \quad 1.8665541 .740219$

C -5.7308832 .9340360 .810220$

O -3.9664822 .0365222 .920394$

C $-6.833166 \quad 1.923507 \quad 0.785227$

C $-7.8415401 .951050-0.099410$

C $-8.9881920 .982672-0.087864$

C $-9.1821880 .285149-1.414345$

C $-10.119210 \quad 0.700031-2.283787$

C $-8.361597-0.954238-1.686325$

C $-7.016956-0.646141-2.289390$

N $-4.456637 \quad 2.259003 \quad 0.660111$

H $-0.777276 \quad 1.820044-2.253524$

H $1.5599850 .962175-4.029173$

H $-0.067618-0.923108-4.404132$

H $1.402105-1.158924-1.722808$

H $2.161491-1.369915-3.274906$

H $0.277606-4.741522-1.812717$

H $\quad 0.808638-4.725302-4.037475$

H $\quad 0.326033-3.224230-4.830234$

H $2.037647-3.530541-4.460266$

H $\quad 0.989887-3.639737 \quad 0.377691$

H $\quad 0.378856-2.098299-0.205875$

H $-1.898093-3.175257-0.512125$

H -1.266395 -4.641664 0.243797

H $-0.714752-3.3113512 .306668$

H -2.927592 -4.488602 2.144867

H $-3.599737-3.133148 \quad 1.269794$

H -5.011392 -2.463762 4.287626

H $-3.832630-0.8280012 .519044$

H -2.931777 -4.4247094 .646295$

H -2.969341-2.901707 5.504911

H -1.547174 -3.333846 4.535887

H $-3.3793460 .390506 \quad 4.331462$

H $-1.599615 \quad 1.2712473 .325865$

H -0.4624180 .8238382 .087895$

H $\quad 0.092619-0.6359393 .578545$

H -3.046299-1.170257 -1.271869

H $-4.827334-0.189537-3.582066$

H $-4.884987-0.7949910 .184762$

H $-5.130911-2.437073-0.456777$

H $-6.475633-1.5128100 .208513$

H -1.8219652 .0065410 .760984$ 
H -2.6083170 .4445920 .543687$ H -5.813122 $3.657783-0.006833$ H -5.7492693 .4781831 .760037$ H -6.8178391 .1533451 .552867$ H $-7.8688052 .738449-0.849455$ H $-9.894108 \quad 1.5458740 .172374$ H -8.872251 0.2332960 .704993 H -10.286810 $0.182825-3.222740$ H -10.734945 $1.569451-2.078351$ H -8.892860 -1.597887 -2.401208 H $-8.296094-1.557701-0.776773$ H -7.080006 -0.234615 -3.298047 H $-4.1580872 .018657-0.280932$

C $1.1519193 .889190-1.589857$ O $2.0256992 .980143-0.889385$ C 1.5270942 .8161340 .450900 C 0.6675194 .0445650 .714498 C $0.001948 \quad 4.226164-0.636548$ H $\quad 0.880843 \quad 1.931002 \quad 0.440017$ C 2.6784422 .6025221 .437947 H $-0.0465513 .888623 \quad 1.528649$ H 1.2777984 .9224670 .955916 C $-0.5838905 .611968-0.847047$ H $-0.8065023 .493070-0.728440$ C 3.6291771 .4428481 .067332 O 3.4631343 .7988221 .509930 H 2.2754282 .4486732 .445482 H 4.2092481 .7170330 .179996

O $4.565692 \quad 1.2843502 .138585$

C 2.9239250 .0967630 .842422

C $3.851936-1.1074490 .589501$

H $2.2332550 .186243-0.001911$

O $2.132640-0.198214 \quad 1.998174$

H $4.513342-1.2448681 .451164$ H $3.213981-2.0001800 .578904$ H $-1.3641205 .815636-0.106246$ H $0.1806956 .390375-0.752410$ H -1.031287 $5.697173-1.842399$

C $0.740944 \quad 3.288911-2.939780$

H 3.7608064 .0079460 .607711

H 4.9919142 .1463482 .283005

H $1.619198-1.0013281 .805457$

H $1.6338393 .242234-3.576572$

H $0.030375 \quad 3.956129-3.440907$

H $1.7471794 .790897-1.784163$

C $6.854896-0.5405550 .140724$

O $5.750108-0.122324-0.672047$

C $4.641932-1.024433-0.734502$

C $5.119749-2.382703-1.264186$

C $6.243603-2.945337-0.404176$

C $7.373063-1.925079-0.281201$

H $6.549068-0.5581981 .193042$

C $7.9548710 .516701-0.023458$

H $3.957245-0.615287-1.487637$

O $4.034973-3.302230-1.321011$

H $5.485493-2.257266-2.291747$

H $5.871538-3.2570720 .578922$

O $6.747007-4.124037-1.037427$

H $7.898247-1.863399-1.243366$

O $8.300084-2.421116 \quad 0.683635$

C 7.5830861 .8154930 .650419

H 8.9034250 .1985870 .422089

H $8.1549170 .711369-1.084558$

H $4.388533-4.150575-1.639221$

H $7.486703-4.448831-0.495667$

H $9.115597-1.8979140 .612844$

O $7.1305432 .709293-0.273607$

O 7.6828772 .0064811 .855504

C $6.780316 \quad 4.005010 \quad 0.223750$

H $6.1372904 .489858-0.515672$

H 6.2345553 .9389571 .169130

H 7.6878734 .6018690 .349610

SCF Energy (B3LYP/6-31G**//MMFF) $=-3245.93053148$

06_00268

MM̄FF Geometry

C $0.951652-4.370600-1.915452$

C $-0.048057-3.481343-1.816994$
C $-0.603333-2.722165-2.991618$

O $-0.806431-1.338548-2.618749$

C $-1.948655-3.281347-3.506765$

C $-3.024628-3.504475-2.459038$

C $-3.854318-2.551047-1.990037$

C $-3.154308-4.921924-1.958238$

C $-3.903329-1.102740-2.394792$

C $-5.332219-0.537702-2.472172$

C $-5.990268-0.339999-1.096636$

C $-7.399754 \quad 0.236087-1.238875$

$\begin{array}{llll}C & -8.040035 & 0.523209 & 0.128706\end{array}$

O $-5.1760020 .563560-0.342959$

O $-9.1988591 .332607-0.122766$

C $-7.051832 \quad 1.3471790 .984375$

C $-8.541635-0.762062 \quad 0.797878$

\begin{tabular}{lllll}
\hline & -7.527439 & 1.497398 & 2.320092
\end{tabular}

C $-5.6173580 .766942 \quad 1.004168$

C $-4.652891 \quad 1.7691401 .682061$

O $-5.613953-0.4524781 .750331$

C $0.271016-0.509578-2.606228$

O $1.434542-0.808877-2.818997$

C $-0.2293870 .854156-2.314233$

C $0.6171351 .894070-2.337257$

C $0.2662063 .302627-2.135269$

C $-1.1751213 .629973-1.845342$

C $-3.164121 \quad 1.440374 \quad 1.491513$

C -2.273495 2.4349012 .217382

C $0.093146 \quad 3.1977342 .352254$

O $-2.692168 \quad 3.2027303 .080290$

C 0.1234514 .4943331 .605645

C 1.2372975 .0182011 .072228

C 1.2783926 .3494550 .379207

C $1.9332256 .289814-0.981885$

C $3.1597676 .801234-1.181495$

C $1.1284865 .726508-2.131461$

C $1.2419794 .229468-2.237858$

N -0.9487102 .3453451 .810332$

H $1.379354-4.554443-2.899597$

H $-0.506106-3.277618-0.853736$

H $0.100094-2.744655-3.834925$

H $-1.759248-4.234400-4.018915$

H -2.337482 -2.612427 -4.284434

H $-4.564044-2.844555-1.219538$

H $-3.954115-5.031129-1.217924$

H $-3.384608-5.595493-2.790411$

H $-2.226361-5.258759-1.487920$

H $-3.314594-0.519936-1.678112$

H -3.442422 -0.959962 -3.377192

H $-5.268797 \quad 0.437026-2.973437$

H $-5.957226-1.185520-3.098980$

H $-6.033612-1.304560-0.580989$

H -8.037813 $-0.433949-1.828605$

H $-7.3521801 .177994-1.803488$

H $-9.651723 \quad 1.4838750 .723999$

H -7.009848 2.3567720 .552218

H $-7.746844-1.4879490 .982479$

H $-9.298223-1.2477840 .170040$

H -9.037371 -0.5454601 .750595$

H $-8.379621 \quad 1.962733 \quad 2.279537$

H $-4.8187862 .771711 \quad 1.265946$

H $-4.876144 \quad 1.8228392 .755272$

H $-4.724077-0.6059102 .104563$

H -1.289919 $0.970298-2.122300$

H $1.6675541 .700766-2.547742$

H $-1.8042833 .383865-2.707727$

$\mathrm{H}-1.3461744 .682320-1.610574$

H $-1.5347373 .064864-0.979998$

H $-2.9290080 .444555 \quad 1.881221$

$\begin{array}{llll}\text { H } & -2.912215 & 1.459288 & 0.425228\end{array}$

H 1.0374292 .6536422 .268927

H -0.1132553 .3861153 .410687$

H -0.8100555 .0472791 .530209$

H 2.1776934 .4830951 .174452

H 1.8281607 .0422391 .029738

H $0.2729516 .775990 \quad 0.274315$

H $3.6231736 .790193-2.162493$

H $3.7313147 .235020-0.367756$

H $1.4953436 .144865-3.078729$ 
H $0.0959816 .077972-2.057343$

H $2.253797 \quad 3.889292-2.462244$

H $-0.7038601 .727197 \quad 1.042221$

C $1.109484-4.8637180 .622778$

O $1.529230-3.5335200 .986916$

C $1.976221-3.5432942 .355222$

C $1.613718-4.9149962 .914101$

C $1.724898-5.7839121 .676814$

H $3.068461-3.4585742 .330486$

C $1.379278-2.360248 \quad 3.124840$

H $2.286848-5.2246053 .719552$

H $0.589882-4.9386793 .305065$

C $1.027702-7.1261081 .815311$

H $2.788242-5.9499781 .457760$

C $1.669530-0.9802322 .499107$

$\begin{array}{lllll} & \text { O } & -0.043421 & -2.527441 & 3.173027\end{array}$

H $1.731293-2.3888144 .163224$

H $1.102783-0.8554801 .567934$

$\begin{array}{lllll}\text { O } & 1.184459 & 0.016803 & 3.407389\end{array}$

C $3.160632-0.7001802 .229170$

$\begin{array}{lllll}\text { C } 3.419067 & 0.749773 & 1.776498\end{array}$

H $3.518412-1.3880201 .456642$

O $3.919655-0.9600543 .409651$

H 2.7651970 .9748870 .927111

H 3.1473971 .4355592 .587662

H $1.465874-7.7035702 .636023$

H $-0.040678-7.0039172 .023434$

H $1.127015-7.7152520 .898502$

C $1.568651-5.179409-0.803206$

H $-0.391103-1.8961173 .825052$

H $0.213199-0.015247 \quad 3.387146$

H $3.584476-0.3735304 .109501$

H $1.376801-6.235813-1.023750$

H $2.653107-5.030089-0.866219$

H $0.014712-4.893900 \quad 0.693982$

C $4.8320360 .248930-0.878736$

$\begin{array}{lllll}\text { O } & 5.388018 & 0.089975 & 0.431537\end{array}$

C 4.8975441 .0112921 .409039

C $5.1924992 .448491 \quad 0.961136$

C $4.6538182 .730993-0.439704$

C $5.1224271 .654174-1.419309$

H $3.7497750 .072924-0.844967$

C $5.459284-0.824234-1.773884$

H $\begin{array}{llll}5.484820 & 0.832732 & 2.318474\end{array}$

$\begin{array}{lllll}\text { O } & 4.647381 & 3.374802 & 1.894336\end{array}$

H $6.2798192 .601207 \quad 0.957177$

H $3.5614222 .816695-0.429499$

O $5.1577604 .000934-0.857410$

H $6.1980541 .772092-1.603881$

O $4.4520671 .819327-2.666005$

C $4.972905-2.195625-1.372245$

H $5.194927-0.684565-2.828107$

H $6.553992-0.800701-1.712149$

H 4.8313124 .2687061 .558097

H $4.7326684 .221715-1.703070$

H $4.7333672 .669730-3.042683$

O $5.911164-2.819610-0.605203$

O $3.877031-2.644115-1.681840$

C $5.555842-4.123421-0.135999$

H $6.396120-4.5181030 .441275$

H $4.680375-4.0633700 .516119$

H $5.364790-4.792771-0.980111$

SCF Energy (B3LYP/6-31G**//MMFF) $=-3245.90619950$

06_00269

MM̄FF Geometry

C -0.798794 -3.368342 2.396010

C $-1.451959-2.5425271 .565860$

C $-1.569257-2.763737 \quad 0.083254$

O $-0.872212-1.656715-0.533757$

C $-3.043187-2.718991-0.349862$

C $-3.268807-3.234647-1.755887$

C $-3.428553-2.457212-2.844722$

C $-3.353706-4.734157-1.879627$

C $-3.343569-0.958875-2.934031$

C $-4.690097-0.304981-3.271633$

C $-5.687813-0.315363-2.102520$

C $-7.0152280 .318559-2.526509$
C $-7.9979730 .436760-1.352626$

O $\quad-5.110250 \quad 0.436112-1.030788$

$\begin{array}{llll}0 & -9.057005 & 1.306801 & -1.779406\end{array}$

C $-7.2767551 .110817-0.165563$

C -8.652864 -0.911678 -1.031811

$\begin{array}{lllll}\text { O } & -8.113594 & 1.130695 & 0.989022\end{array}$

C $-5.902107 \quad 0.4827090 .162644$

C $-5.151697 \quad 1.3640401 .189876$

O $-6.111287-0.8179210 .714590$

C - $0.024534-1.905143-1.561650$

O $0.225816-2.994765-2.057656$

C $0.611838-0.658154-2.051477$

C $0.3174780 .564545-1.581154$

C $0.903741 \quad 1.832295-2.026420$

C $1.987138 \quad 1.772643-3.070366$

C $-3.7143050 .908097 \quad 1.470163$

C -2.9872591 .8862892 .370205$

C $-1.943837 \quad 4.137992 \quad 2.391021$

O $-2.794923 \quad 1.648400 \quad 3.560419$

C $-1.2052624 .968687 \quad 1.392479$

$\begin{array}{llll}\text { C } & 0.132600 & 5.048679 & 1.342039\end{array}$

C $0.8867195 .872737 \quad 0.340175$

C $1.6527815 .025749-0.651499$

C $2.9891404 .906548-0.581297$

C $0.874844 \quad 4.390550-1.782825$

C $0.4526432 .978999-1.474985$

$\mathrm{N}-2.5832353 .0322451 .703879$

H $-0.340667-4.2723262 .002944$

H $-1.884498-1.6291231 .966076$

H -1.106458 $-3.719544-0.190209$

H $-3.423223-1.695990-0.260049$

H $-3.657162-3.320760 \quad 0.333958$

H -3.610707 -2.947728 -3.800350

H $-3.543581-5.057208-2.908728$

H $-2.417651-5.199864-1.556500$

H $-4.167844-5.120810-1.257772$

H $-2.919407-0.511351-2.030952$

H -2.635320 -0.722995 -3.738686

H $-4.485170 \quad 0.736510-3.552535$

H $-5.133652-0.794314-4.147795$

H $-5.847221-1.349090-1.776427$

H $-7.468678-0.242813-3.353003$

H $-6.8260831 .327416-2.920353$

H $-9.6471001 .447666-1.018955$

H -7.121382 $2.162423-0.442487$

H $-9.198122-1.287047-1.906192$

H $-9.399785-0.811554-0.236699$

H $-7.933760-1.678040-0.735459$

H $-8.1038810 .238918 \quad 1.376580$

H -5.1222532 .3956850 .816876$

H -5.6989581 .3816192 .140805$

H $-5.331507-1.0754921 .229887$

H $1.342575-0.805780-2.840352$

H $-0.4306240 .660598-0.795913$

H $2.8014891 .121359-2.739792$

H $2.4442172 .740599-3.284724$

H $1.5896941 .386206-4.015028$

H $-3.704275-0.0666941 .967330$

H -3.1393520 .8022940 .542979$

H -1.289082 3.7452053 .176461

H -2.727068 4.7323072 .872609

H -1.801514 5.5273870 .675000

H $0.723438 \quad 4.4921462 .064151$

H 1.5714106 .5282280 .893776

H $0.2152286 .548046-0.204917$

H $3.5422174 .322565-1.309659$

H 3.5584525 .3825540 .210100

H $1.4536984 .454570-2.709400$

H $-0.0268364 .981868-1.987870$

H $-0.3357142 .911980-0.725397$

H -2.9234783 .1880910 .758992$

C $0.661491-2.3087234 .190805$

O $0.559838-0.973206 \quad 3.655473$

C $1.724404-0.7012092 .843694$

C $2.783268-1.6506253 .390572$

C $1.965214-2.8999533 .655228$

H $1.447843-0.9664721 .815697$

$\begin{array}{llll}\text { C } 2.068706 & 0.792291 & 2.924478\end{array}$ 
H $3.605577-1.8515792 .702626$

H $3.204170-1.2636614 .326793$

C $2.629383-3.8784714 .608190$

H $1.806584-3.4064742 .696557$

C $2.9314491 .343707 \quad 1.771492$

O $\quad 0.838417 \quad 1.5375822 .857166$

H $2.513367 \quad 1.0387533 .895657$

H 2.3162331 .4119370 .865929

O 3.3009872 .6882442 .105756

C 4.2124760 .5732441 .437632

C $4.999578 \quad 1.2130940 .280095$

H $3.944760-0.4423991 .148514$

O $5.047461 \quad 0.4926632 .589434$

H $4.3105851 .405887-0.549242$

H 5.4007012 .1819460 .600967

H $3.578982-4.2335024 .194642$

H $2.837930-3.4158845 .578636$

H $1.987513-4.7486324 .779038$

C $-0.615634-3.0936863 .863742$

H $\quad 0.2595381 .203778 \quad 3.564004$

H 2.4831193 .1694852 .315265

H 5.2443231 .4018062 .873776

H -1.483286 -2.5327694 .234252$

H $-0.609080-4.0520724 .396027$

H $\quad 0.718488-2.1877085 .279922$

C $4.944022-1.123800-1.689797$

O $5.763347-1.006204-0.520147$

C $6.1739170 .328407-0.198069$

C $6.9691250 .924518-1.367645$

C $6.1904420 .847436-2.678337$

C $5.680007-0.572809-2.918027$

H $4.006088-0.577985-1.538386$

C $4.610932-2.613525-1.878266$

H $\quad \begin{array}{lllll}\text { H } & 864111 & 0.236947 & 0.650067\end{array}$

O $7.3170582 .280893-1.111073$

H $7.9078320 .368586-1.489848$

H $5.3665591 .570062-2.699853$

O $7.0565051 .198172-3.760579$

H $6.525296-1.224791-3.173668$

O $4.792020-0.568477-4.034305$

C $3.627892-3.130370-0.848158$

H $4.158965-2.788324-2.860647$

H $5.530161-3.205270-1.791037$

H $7.8220622 .300424-0.280320$

H $7.4039322 .087631-3.575372$

H $5.276860-0.192667-4.789184$

O $3.590346-4.494466-0.914000$

O $2.958083-2.435476-0.097835$

C $2.690580-5.1287830 .000860$

H $3.028005-4.967776 \quad 1.028887$

H $1.672664-4.751023-0.135656$

H $2.695799-6.202119-0.206528$

SCF Energy (B3LYP/6-31G**//MMFF) $=-3245.93329629$

06_00270

MM̄FF Geometry

C -3.170398 4.699315-0.510786

C $-3.6049553 .464111-0.799654$

C -4.6269532 .7388020 .040358$

O -4.0465341 .4997540 .511189$

C $-5.8656792 .409940-0.811656$

C $-7.0012781 .791790-0.016276$

C $-7.3819290 .500129-0.084325$

C -7.753282 2.7465370 .876648

C $-6.776963-0.597325-0.913431$

C $-6.132373-1.668577-0.024913$

C $-5.268440-2.641837-0.839865$

C $-4.705737-3.7439150 .058941$

C $-3.714178-4.651313-0.684727$

O $-4.201028-1.882635-1.415815$

$\begin{array}{llll}0 & -3.000625 & -5.420920 & 0.295837\end{array}$

C -2.673323 -3.770148 -1.408890

C $-4.432836-5.659703-1.588481$

O $-1.822291-4.561799-2.233477$

C $-3.303599-2.635503-2.241051$

C $-2.258065-1.640643-2.804471$

O $-3.991360-3.202866-3.362402$

C -3.2286111 .5810991 .598458$
O $\quad-2.9052072 .5938002 .199439$

C -2.7909780 .2105931 .945719$

C -1.9088210 .0318082 .940703$

C -1.396227 -1.250800 3.429257

C $-1.877852-2.5023632 .745256$

C - $-1.444562-0.878087-1.752857$

C $-0.122109-1.533191-1.395370$

C $1.739276-1.3600580 .248978$

O $0.396384-2.416827-2.072193$

C $1.588578-2.3264101 .378089$

C $2.173245-2.1498222 .572719$

C $2.088044-3.1342743 .702186$

C $1.577652-2.5110404 .981371$

C $2.426493-2.1217195 .947512$

C $0.080855-2.4244035 .174581$

C $-0.529374-1.2471514 .464042$

N $0.435856-0.975419-0.252907$

H -3.5755315 .2107710 .359484$

H -3.206432 $2.942763-1.666476$

H -4.9277973 .3468610 .902684$

H $-5.5702981 .760478-1.641512$

H -6.244603 $3.326948-1.283751$

H -8.2249680 .1810140 .527607$

H -8.6169592 .2743321 .357028$

H $-7.104116 \quad 3.126128 \quad 1.671212$

H -8.1270393 .5957740 .295160$

H -7.570120 -1.055926 -1.516571

H $-6.046544-0.208985-1.625660$

H -5.504999 -1.177064 0.729185

H $-6.915326-2.2194190 .510779$

H $-5.881324-3.071720-1.640906$

H -5.516482 -4.3382260 .498641$

H $-4.181357-3.2849480 .907069$

H -3.651904 -5.9251770 .813052$

H -2.036456 -3.330466 -0.632247

H $-5.089968-6.305841-0.993794$

H -3.718864 -6.330239-2.079139

H $-5.047139-5.185531-2.356621$

H -1.429998 $-5.249885-1.669207$

H -1.611030 -2.143805 -3.532967

H $-2.809499-0.883829-3.381092$

H -4.083996 -2.519518 -4.046844

H $-3.212836-0.6128541 .382238$

H $-1.529427 \quad 0.9103243 .461339$

H -2.947359 -2.648582 2.929697

H $-1.364218-3.4072263 .076717$

H -1.711653 -2.441289 1.664464

H $-1.1895230 .108729-2.160675$

H -2.032453 -0.711092 -0.845842

H $2.245004-0.4415460 .561434$

H $2.317645-1.825491-0.554575$

H $1.004765-3.2250171 .194585$

H $2.780108-1.2627122 .740236$

H $3.094420-3.5433453 .862475$

H $1.460195-3.9943693 .438647$

H $2.066419-1.7007816 .880455$

H $3.500956-2.2090095 .823606$

H $-0.151497-2.3107576 .242297$

H $-0.377381-3.3775504 .895816$

H $-0.235385-0.2865404 .889832$

H $-0.078345-0.2643220 .258745$

C $-0.7669895 .539611-0.686119$

O $-0.0728574 .288336-0.842206$

C $0.8444994 .133328 \quad 0.258470$

C 0.7223185 .3969251 .112636

C -0.6872235 .8610970 .803292$

H $0.4945003 .267690 \quad 0.834301$

C $2.2754393 .870030-0.239748$

H $0.881317 \quad 5.1896382 .175603$

H $1.449474 \quad 6.1614270 .815255$

C $-0.9408387 .320107 \quad 1.135983$

H -1.376459 5.2330731 .380795

C $2.4181512 .589761-1.091275$

O $2.7294115 .000351-0.985046$

H $2.9276713 .782577 \quad 0.637575$

H $1.9491071 .754475-0.558816$

O $1.7360192 .737016-2.335541$

C $3.8902112 .215854-1.371833$ 
C $4.0248700 .943408-2.228415$

H $4.3977932 .073032-0.413294$

O $4.5624153 .281897-2.040539$

H $3.3916930 .158931-1.804056$

H $3.6502441 .151653-3.237782$

H -0.7927627 .5021802 .205436$

H $-0.263008 \quad 7.9811950 .585554$

H - -1.9673837 .6041310 .883622$

C $-2.1592865 .459438-1.319158$

H $2.1689185 .070000-1.776846$

H $0.798326 \quad 2.902917-2.139628$

H $4.0663193 .477163-2.853995$

H -2.073136 $5.011966-2.317473$

H -2.549119 $6.474685-1.461546$

H $-0.1853796 .275750-1.258124$

C $5.545187-0.982865-0.393871$

O $6.0682380 .175300-1.055463$

C $5.4893640 .455717-2.336889$

C $5.688531-0.742550-3.275024$

C $5.161382-2.036292-2.660507$

C $5.733048-2.236488-1.257361$

H $4.481788-0.833880-0.190366$

C $6.272261-1.1269870 .953162$

H $6.0693211 .287108-2.757014$

O $5.054123-0.500278-4.525869$

H $6.761389-0.857094-3.477020$

H $4.065567-2.044368-2.642052$

O $5.543253-3.140462-3.484538$

H $6.798476-2.490564-1.325154$

O $5.067952-3.336554-0.638742$

C $5.734678-0.1778902 .004707$

H $6.136635-2.1409881 .346106$

H $7.342429-0.9226920 .834833$

H $5.178390-1.293760-5.074232$

H $6.514464-3.162320-3.527361$

H $5.173619-4.106483-1.223544$

O $6.260925-0.5199453 .218651$

O $4.959004 \quad 0.7445951 .797741$

C 5.8232290 .2766354 .324190

H $6.290815-0.1148075 .231507$

H 6.1356661 .3161754 .187895

H 4.7363530 .2098704 .433521

SCF Energy (B3LYP/6-31G*//MMFF) $=-3245.92174570$

06_00271

MMFF Geometry

C -2.357707 -3.133370 -0.812435

C $-1.279489-3.815279-0.398595$

C $-0.175593-3.190786 \quad 0.409760$

O $1.011008-3.221865-0.415855$

C $0.112498-3.954348 \quad 1.715502$

C $-1.005645-3.8789572 .734516$

C $-1.193163-2.8475603 .581801$

C $-1.922163-5.0738362 .783537$

C $-0.348771-1.6114603 .717330$

C $-1.034962-0.3849393 .107564$

C -0.2398840 .9094263 .341738$

C $-0.8661212 .063698 \quad 2.556831$

C $-0.050518 \quad 3.3593312 .665176$

O 1.1094870 .7053032 .910951

O $-0.5336164 .245058 \quad 1.643456$

C 1.4307353 .0450492 .360368

C -0.3040484 .0670254 .000749$

$\begin{array}{lllll}\text { O } & 2.247697 & 4.192508 & 2.580664\end{array}$

C 1.9730391 .8259653 .139362

C $3.388844 \quad 1.4527102 .640908$

O $2.0517812 .166520 \quad 4.527119$

C $1.865367-2.167419-0.317265$

O $1.772544-1.210636 \quad 0.434453$

C $2.931657-2.364373-1.323969$

C $3.830431-1.391285-1.535291$

C $4.900854-1.408841-2.534028$

C $5.037113-2.636483-3.396334$

C $3.8796570 .092343 \quad 3.156323$

C $5.391576 \quad 0.0040093 .153673$

C $7.367316-0.1443971 .675260$

O $6.030972-0.071348 \quad 4.201364$

C 7.7460820 .3980590 .335963
C $8.368638-0.326417-0.605940$

C $8.7823230 .217081-1.943766$

C $8.177490-0.555099-3.094838$

C $8.855731-1.554230-3.684124$

C $6.829322-0.115264-3.618502$

C $5.708082-0.332737-2.638875$

N 5.9415180 .0250551 .881621

H $-2.436005-2.074345-0.578394$

H $-1.184944-4.870218-0.643890$

H $-0.433062-2.1501750 .633551$

H $1.046031-3.5828362 .156345$

H $\quad 0.340160-5.0054661 .489399$

H $-2.033147-2.8943434 .273448$

H $-2.727901-4.9507173 .515036$

H -2.388459 -5.2502621 .810752$

H $-1.356800-5.9694693 .061438$

H $-0.167243-1.4397004 .785485$

H $0.635956-1.754742 \quad 3.262777$

H $-1.150834-0.5486252 .029560$

H -2.044875 -0.2686813 .520899$

H $-0.244634 \quad 1.1226854 .417187$

H $-1.9056782 .231607 \quad 2.865013$

H -0.9097621 .7811891 .497731$

H $\quad 0.0262125 .0404341 .654240$

H 1.4978062 .8287771 .285357

H $-1.3662294 .321576 \quad 4.100477$

H $0.235700 \quad 5.0188834 .055321$

H -0.0263243 .4614624 .865981$

H 2.3724654 .2869543 .540390

H 3.3929251 .4100991 .543943

H 4.0986112 .2355572 .937736

H 2.7782631 .6741454 .940501

H $2.925355-3.289431-1.889022$

H $3.764845-0.482760-0.937736$

H $5.192976-3.526682-2.776633$

H $5.882119-2.589778-4.086759$

H $4.136505-2.782681-4.003038$

H $3.546019-0.0950554 .182709$

H $3.477199-0.7226862 .544332$

H $7.588542-1.2126881 .772708$

H $7.910800 \quad 0.3876962 .463134$

H 7.5141951 .4422510 .139626

H $8.617473-1.364912-0.398774$

H $9.8778970 .171450-1.998724$

H $8.5285791 .279893-2.042119$

H $8.445703-2.095416-4.530293$

H $9.836165-1.855927-3.330331$

H $6.599808-0.598540-4.575623$

H $6.8751990 .955213-3.858455$

H $5.5431990 .510990-1.968120$

H $5.322460-0.1063181 .086274$

C $-4.870079-3.441985-1.002741$

O $-5.131420-2.024532-1.052427$

C $-5.696206-1.6195230 .208871$

C $-6.179098-2.9024720 .874550$

C $-5.092385-3.8721800 .448490$

H $-4.873495-1.2056700 .804926$

C $-6.767086-0.545984-0.003972$

H $-6.265187-2.8019921 .960519$

H -7.150207 -3.225110 0.481757

C $-5.473240-5.3331820 .605799$

H $-4.202683-3.6712071 .056876$

C $-6.2968740 .665063-0.836508$

O $-7.888075-1.123295-0.685474$

H -7.147965 -0.2087990 .966829$

H $-6.1614800 .372094-1.884853$

O

C $-5.007761 \quad 1.332572-0.324586$

C $-4.6297582 .583865-1.139388$

H $-4.1860060 .612003-0.379279$

O $-5.162571 \quad 1.690891 \quad 1.047576$

H $-4.5829432 .309461-2.198931$

H $-5.4162493 .341127-1.035764$

H $-5.676017-5.5663701 .656185$

H $-6.370178-5.5793600 .027748$

H $-4.662146-5.9844350 .264999$

C $-3.488617-3.738139-1.595635$

H $-7.562796-1.493427-1.524244$ 
H -8.151654 $1.201539-1.155000$ H $-5.9138042 .305747 \quad 1.107171$ H -3.443293 -3.316122 -2.607304 H -3.358248 -4.822679-1.687464 H -5.625038 -3.906559-1.650501 C -1.784739 $1.764932-1.895472$ O $-2.2332052 .257179-0.629301$ C $-3.2931813 .213364-0.687122$ C $-2.8530834 .428498-1.513882$ C $-2.3369804 .024441-2.893272$ C $-1.2846742 .918898-2.775897$ H $-2.6099211 .243160-2.395052$ C $-0.6827950 .739123-1.597065$ H -3.4436753 .5621300 .342441$ O $-3.9242815 .354231-1.665677$ H $-2.0469684 .953248-0.984543$ H -3.162867 $3.722105-3.547945$ O $-1.7432065 .175903-3.498402$ H $-0.3614133 .339364-2.356941$ O $-0.9810982 .422791-4.076798$ C $-0.423811-0.185108-2.761066$ H $0.241573 \quad 1.244212-1.291091$ H $-0.9624740 .109528-0.743239$ H -4.215343 $5.612152-0.774321$ H $-1.5209934 .943967-4.415795$ H $-0.5517453 .139560-4.573273$ O $0.864141-0.051188-3.186843$ O $-1.267942-0.929922-3.242320$ C $1.223836-0.855277-4.314594$ H $\quad 1.134921-1.917227-4.066339$ H $\quad 0.595095-0.605387-5.174543$ H $2.264812-0.639232-4.568685$ SCF Energy (B3LYP/6-31G**//MMFF) $=-3245.92175126$

06_00272

MM̄FF Geometry

C -2.287307 3.0256661 .953313 C -3.1948482 .7662062 .907400$ C $-3.636562 \quad 1.403358 \quad 3.392674$ O -3.0436630 .3358492 .616520$ C $-5.168170 \quad 1.2901393 .277494$ C $-5.709843-0.0476653 .743919$ C $-6.139611-1.0257382 .921534$ C $-5.780122-0.2271235 .237737$ C $-6.126677-1.0183021 .418209$ C $-5.433137-2.2638610 .848895$ C $-5.221372-2.135189-0.666885$ C $-4.667548-3.431576-1.257623$ C $-4.355867-3.285074-2.753900$ O $-4.294408-1.065169-0.883662$ O $-3.584071-4.434387-3.134980$ C $-3.475012-2.032263-2.960079$ C $-5.630996-3.323782-3.604882$ O $-3.281761-1.806135-4.353688$ C $-4.028660-0.769414-2.262373$ C $-3.0527350 .436903-2.283637$ O $-5.223212-0.353735-2.935981$ C -1.863741 $-0.177818 \quad 3.063194$ O $-1.2063820 .218125 \quad 4.012390$ C -1.505620 -1.327734 2.197728 C $-0.351723-1.9754632 .418770$ C $0.156156-3.1536971 .710801$ C $-0.770470-3.8373220 .742377$ C $-1.6866240 .192106-1.632793$ C $-0.652354-0.351256-2.602315$ C $1.366118-1.791654-2.701607$ O $-0.645284-0.061493-3.796338$ C $1.715097-3.087422-2.045151$ C $2.868752-3.305883-1.398393$ C $3.244405-4.621317-0.785107$ C $3.350310-4.551480 \quad 0.719253$ C $4.538255-4.3554271 .316036$ C $2.103425-4.8309191 .527731$ C $1.402211-3.5877442 .000886$ N $0.268535-1.179748-1.979461$ H -1.7781112 .2091321 .449938$ H -3.665246 3.6106173 .409665 H $-3.351357 \quad 1.3041504 .447607$
H $-5.475528 \quad 1.4951792 .246710$

H -5.6449892 .0814313 .872541$

H $-6.540631-1.9349303 .367527$

H -6.232963 -1.183307 5.521161

H $-4.777823-0.1956665 .675800$

H -6.3822590 .5682935 .688661$

H -7.162996 -0.9879321 .060924$

H $-5.634261-0.1256431 .026585$

H -4.462695 -2.395557 1.342724

H $-6.037759-3.1515411 .070721$

H $-6.182229-1.882873-1.130528$

H -5.359968 -4.265959-1.089019

H -3.739472 -3.702593 -0.734683

H $-3.329235-4.321325-4.067079$

H -2.493855 -2.281911 -2.539247

H $-6.146739-4.282839-3.472936$

H $-5.397923-3.254533-4.673161$

H $-6.338928-2.530255-3.356666$

H -2.606414 -1.114892-4.453199

H -2.946962 $0.819169-3.305781$

H $-3.5287561 .250361-1.716847$

H $-5.3692800 .589605-2.756003$

H -2.200017 -1.614756 1.418058

H $0.287732-1.6165953 .222816$

H -1.103205 -3.143501-0.036817

H $-1.652615-4.2221451 .266104$

H $-0.309442-4.6807870 .224222$

H -1.287533 $1.147492-1.271550$

H -1.783744 -0.465151-0.763771

H $2.200251-1.083645-2.700673$

H $1.075277-1.965948-3.742893$

H $0.988984-3.892757-2.127926$

H $3.601664-2.505811-1.329628$

H $4.207509-4.927888-1.215649$

H $2.539965-5.412704-1.069885$

H $4.631512-4.3345992 .396820$

H $5.440647-4.1950530 .735726$

H $2.383629-5.3997922 .425323$

H $1.443831-5.5061600 .976106$

H $1.989782-3.0164472 .720916$

H $0.296394-1.200901-0.963963$

C -1.8131854 .5552790 .015742$

O $-0.6386473 .851248-0.448399$

C $0.2088764 .783959-1.136141$

C $-0.7282585 .876602-1.625869$

C $-1.6480456 .013618-0.428971$

H $0.8860395 .212585-0.386903$

C $1.0210224 .090688-2.232322$

H $-0.1999836 .803337-1.869059$

H -1.296579 $5.559132-2.507718$

C $-2.960306 \quad 6.710730-0.745157$

H -1.125428 $6.586348 \quad 0.349486$

C $1.8921002 .909871-1.753805$

O $0.1332153 .590204-3.239697$

H $1.6529044 .832383-2.734801$

H $1.2619362 .035210-1.556681$

O $2.7530612 .547237-2.841475$

C $2.7643633 .206587-0.521194$

C $3.8654232 .177619-0.223713$

H 2.1249103 .3155610 .359624

O $3.4168914 .471428-0.689006$

H $4.6586002 .275106-0.978727$

H $4.3609002 .487788 \quad 0.704924$

H $-2.7785537 .735854-1.084437$

H -3.512264 $6.188952-1.534039$

H -3.6005906 .7556010 .141629$

C - $-1.9037334 .419511 \quad 1.538847$

H $-0.4901532 .982355-2.806018$

H $2.1852372 .339610-3.603454$

H $3.9424034 .424207-1.506048$

H -2.6264765 .1434601 .933908$

H -0.9267484 .6416791 .985806$

H -2.679259 $4.090573-0.471692$

C $5.439549-0.0708301 .027809$

O $4.642086-0.130183-0.162039$

C $3.4628860 .693121-0.142965$

C 2.5664600 .2680971 .026655

C 3.3236300 .2877512 .353601 
C $4.614221-0.5259442 .236177$

H 5.7922900 .9538201 .192433

C $6.670403-0.9613310 .803355$

H $2.9314970 .452633-1.068506$

$\begin{array}{lllll}\text { O } & 1.403253 & 1.078960 & 1.103152\end{array}$

H $2.221900-0.7569130 .839789$

H 3.5240601 .3162772 .674813

O $2.496532-0.2916153 .361712$

H $4.356687-1.5864312 .142773$

O $5.369318-0.3864313 .436707$

C $7.668105-0.317039-0.139163$

H $7.191054-1.1399711 .750641$

H $6.369453-1.9171630 .361454$

H $\quad 0.8840570 .7755911 .867456$

H $3.029214-0.3648174 .171930$

H 5.5977570 .5529183 .539723

O $8.748390-1.142831-0.274719$

O $7.526890 \quad 0.769237-0.684828$

C $9.791081-0.653129-1.124530$

H $10.2417270 .242346-0.685743$

H $10.557367-1.428370-1.204886$

H $9.402485-0.439439-2.124942$

SCF Energy (B3LYP/6-31G**//MMFF) $=-3245.93632164$

06_00273

MM̄FF Geometry

C $-4.354890-2.9060831 .679235$

C $-3.238699-3.0931010 .958796$

C $-1.851744-2.9434991 .520927$

O $-1.187382-2.0063350 .639093$

C $-1.084212-4.276096 \quad 1.482913$

C -0.031802 -4.365962 2.570672

C $1.266011-4.0348682 .427611$

C $-0.534679-4.885705 \quad 3.893943$

C $1.946191-3.4613801 .217706$

C $3.133055-4.3228930 .767922$

C $3.897238-3.663549-0.390180$

C $5.018444-4.574287-0.894514$

C $5.862140-3.896450-1.985741$

O $4.447526-2.4327040 .092936$

O $7.041356-4.696666-2.159916$

C $6.301397-2.503988-1.481251$

C $5.139938-3.898074-3.337739$

O $6.980167-1.780638-2.504733$

C $5.142978-1.666536-0.898727$

C $5.630812-0.365611-0.223893$

O $4.261204-1.281552-1.960413$

C $-0.482300-0.9776151 .171380$

O $-0.305601-0.7277052 .351537$

C $0.061161-0.2042050 .031400$

$\begin{array}{llll}\text { C } & 1.040688 & 0.687428 & 0.236149\end{array}$

C $1.6758951 .521366-0.787792$

C $1.1364601 .434546-2.191613$

C $6.664140-0.5579010 .890409$

C $6.724922 \quad 0.6510851 .804545$

C $7.124415 \quad 3.1112821 .784813$

O 6.3988330 .5801472 .987786

C 5.7399923 .6559521 .623815

C $5.402164 \quad 4.615786 \quad 0.749622$

C $3.9838495 .067103 \quad 0.536997$

C $3.4507164 .686682-0.831337$

C $2.9258465 .619084-1.644151$

C $3.5324123 .236863-1.270935$

C $2.6992622 .319189-0.418279$

N 7.1649421 .7986311 .163397

H $-4.252956-2.6965332 .742499$

H -3.304477 -3.312949 -0.103091

H -1.904891 -2.541543 2.540356

H $-0.627924-4.4290830 .497667$

H -1.774012 -5.1199441 .620020$

H $1.918365-4.1471373 .293093$

H $\quad 0.251690-4.9196144 .655478$

H $-1.336146-4.2456154 .276441$

H $-0.926496-5.9014943 .777936$

H $1.257412-3.3385210 .377460$

H $2.296550-2.4572641 .485159$

H $3.813928-4.4670451 .617178$

H $2.771999-5.3125120 .461813$
H $3.185784-3.449708-1.196801$

H $4.613421-5.526861-1.258391$

H $5.680544-4.832636-0.055991$

H $7.618155-4.237198-2.794370$

H $7.045154-2.682749-0.694805$

H $4.925416-4.926479-3.652911$

H $5.770815-3.471619-4.125439$

H $4.194065-3.352857-3.320206$

H $6.310048-1.446124-3.124896$

H $6.0249970 .319736-0.985236$

H 4.7566020 .1452450 .202376

H $3.749466-0.508669-1.669237$

H $-0.353741-0.402116-0.950635$

H $1.4200940 .816061 \quad 1.249105$

H $1.5880822 .150754-2.880420$

H $0.0601391 .638665-2.206021$

H $1.3095940 .435624-2.606703$

H $6.411966-1.4257161 .510506$

H $7.662723-0.7315820 .474203$

H 7.8764863 .7346361 .291547

H 7.3754943 .0271812 .846801

H $4.965603 \quad 3.2023702 .238546$

H 6.1678635 .0680570 .124195

H 3.9539156 .1549100 .678656

H 3.3200964 .6480761 .303610

H $2.5417395 .362779-2.625983$

H $2.8627316 .660128-1.345035$

H $4.5746342 .899060-1.217522$

H $3.2742413 .152478-2.330166$

H 3.0133652 .2894880 .625583

H 7.3518531 .7634040 .165970

C $-5.947138-2.732167-0.348972$

O $-5.310156-1.516697-0.789035$

C $-6.135235-0.882634-1.781243$

C $-7.251864-1.871201-2.102693$

C $-7.406190-2.601635-0.782455$

H $-6.5725030 .003116-1.305495$

C $-5.283488-0.462036-2.984580$

H $-8.168441-1.368138-2.425660$

H -6.961074 - $2.573517-2.892256$

C $-8.138731-3.926531-0.901537$

H $-7.950073-1.950475-0.084999$

C -4.058756 $0.411554-2.641025$

O $-4.790612-1.635935-3.645940$

H $-5.9147330 .051353-3.719017$

H $-3.301637-0.184044-2.115989$

O $-3.4595610 .817690-3.879148$

C $-4.372131 \quad 1.673294-1.816297$

C $-3.1473462 .597344-1.666081$

H $-4.7274701 .374823-0.825929$

O $-5.4296412 .403211-2.438568$

H -2.299169 $2.002625-1.312761$

H $-2.8778002 .996946-2.650954$

H $-9.153259-3.772023-1.283199$

H -7.623261 -4.610237-1.584398

H $-8.216275-4.4185820 .073041$

C $-5.771814-2.8869781 .165215$

H $-4.280258-2.146805-2.994104$

H $-3.2580480 .009730-4.381686$

H $-5.1375832 .635522-3.336832$

H $-6.278083-3.7943631 .513239$

H $-6.264600-2.0369781 .654488$

H $-5.466607-3.563811-0.879977$

C -2.7086412 .7554001 .357084$

O $-3.768092 \quad 3.3579170 .603600$

C $-3.4079923 .786624-0.713763$

C $-2.2580964 .801689-0.637901$

C $-1.0702294 .258274 \quad 0.149636$

C $-1.528928 \quad 3.725678 \quad 1.506817$

H $-2.377003 \quad 1.8359500 .861037$

C -3.2789732 .3800822 .733450$

H $-4.2797254 .323693-1.108082$

O $-1.8236315 .179329-1.940471$

H $-2.6133845 .714134-0.141314$

H $-0.542237 \quad 3.491613-0.425834$

O $-0.141272 \quad 5.3253250 .351543$

H $-1.8139024 .567868 \quad 2.150596$

O -0.4482223 .0472982 .141920$ 
C -4.2866371 .2536172 .651761$

H -2.4848642 .0509613 .412076$

H -3.7835803 .2535023 .164633$

H -2.593884 $5.540449-2.411702$

H $\quad 0.6669694 .9422300 .732029$

H $\quad 0.2515453 .6998632 .312437$

O

O $-4.3892090 .466596 \quad 1.721405$

C -6.0368590 .2258023 .870173$

H $-5.563861-0.7563123 .956594$

H $-6.6374790 .406176 \quad 4.765731$

H -6.6935610 .2650152 .995703$

SCF Energy (B3LYP/6-31G**//MMFF)= -3245.91570371

06_00274

MM̄FF Geometry

C -1.173126 $4.417993 \quad 0.520692$

C -0.4372603 .3488890 .186147$

C 0.5923282 .7118581 .078254

O 1.8446832 .8062560 .354551

C $0.276217 \quad 1.2268381 .342412$

C -0.5854620 .9893192 .566024$

C -0.1394470 .4758393 .730323$

C -2.0496541 .3021742 .403846$

C 1.2611120 .0568884 .081216

C $1.389884-1.4507684 .349241$

C $0.910034-2.3414513 .192044$

C $1.185188-3.8181353 .486557$

C $0.775806-4.7221122 .312582$

O $1.591823-1.9472231 .997020$

O $1.360510-6.0113042 .554425$

C $1.388775-4.1578111 .010771$

C $-0.740864-4.9425272 .273506$

O $0.928910-4.885746-0.124936$

C $1.151377-2.642678 \quad 0.823082$

C $1.910065-2.065516-0.392900$

O $-0.243431-2.419088 \quad 0.585228$

C $2.9952782 .761672 \quad 1.077687$

O 3.1005422 .6273482 .286097

C 4.1222712 .9105860 .129687

C 5.3774612 .7105650 .558456

C $6.5985292 .839659-0.241920$

C $6.4561043 .374408-1.642561$

C $3.434919-2.157638-0.313436$

C $4.106465-1.519050-1.518147$

C $6.335645-1.267590-2.578512$

O $3.486873-0.949183-2.412342$

C $7.723281-1.025685-2.078733$

C $8.337610 \quad 0.162728-2.169774$

C $9.7347610 .433650-1.695253$

C $9.7853031 .154551-0.366795$

C $10.427334 \quad 0.614873 \quad 0.683514$

C $9.1601172 .530789-0.269713$

C $7.7742462 .487058 \quad 0.319616$

N $5.487873-1.660377-1.468638$

H $-1.042726 \quad 4.8771191 .497302$

H $-0.5754122 .905452-0.796628$

H 0.6843693 .2703452 .019001

H 1.2125950 .6669691 .417931

H $-0.216636 \quad 0.7783870 .470097$

H $-0.858840 \quad 0.3324004 .535736$

H -2.652728 0.9084203 .229171

H -2.213292 2.3821692 .365363

H -2.432920 0.8529861 .481814

H $1.985918 \quad 0.351127 \quad 3.318279$

H 1.5574180 .5974854 .988853

H $2.449067-1.6559044 .551715$

H $0.835560-1.7104805 .259923$

H -0.166892 -2.185396 3.062146

H $0.680990-4.1331534 .408726$

H $2.259978-3.9569583 .670990$

H $1.172218-6.5698971 .780565$

H $2.466979-4.3469451 .074138$

H -1.081871 -5.404435 3.207908

H -1.019845 -5.6423161 .478153$

H -1.306681 -4.018961 2.135222

H $0.026013-4.583879-0.323455$

H $1.552090-2.542376-1.314650$
H $1.642462-1.004784-0.496910$

H $-0.349493-1.5523020 .160415$

H $3.8876073 .182529-0.893072$

H 5.5304042 .4197811 .597131

H $5.8471302 .697997-2.252625$

H $7.4081933 .497257-2.162410$

H $5.9789344 .360898-1.630648$

H $3.800575-1.6505550 .586531$

H $3.751510-3.205040-0.277311$

H $5.907455-0.386641-3.068679$

H $6.334893-2.089482-3.302056$

H $8.247813-1.866176-1.631024$

H $7.8125720 .993331-2.633364$

H $10.2488601 .040944-2.450874$

H $10.296672-0.508313-1.644591$

H $10.490546 \quad 1.136196 \quad 1.633190$

H $10.900853-0.3590300 .619617$

H $9.1977343 .020535-1.246238$

H 9.7757143 .1639480 .383627

H 7.7601112 .1237041 .348764

H $5.879870-2.287031-0.770845$

C -3.6012564 .9304860 .160700$

O -4.0355493 .5537370 .138134$

C $-5.4146123 .510543-0.274778$

C $-5.8990524 .956993-0.308928$

C $-4.6292905 .697476-0.668743$

H $-5.4271673 .111114-1.295217$

C -6.2352832 .6155800 .658512$

H -6.704775 $5.105647-1.034479$

H -6.2669615 .2852730 .670342$

C $-4.6897657 .184830-0.365781$

H -4.431269 $5.554984-1.739708$

C $-5.799103 \quad 1.1385920 .718098$

$\begin{array}{lllll}\text { O } & -6.161321 & 3.139521 & 1.992071\end{array}$

H -7.2933172 .6706340 .375942$

H -4.8386621 .0400901 .234910$

O $\quad-6.7601470 .4525161 .534403$

C $-5.7193620 .424199-0.646330$

C $-5.616618-1.109436-0.511216$

H $-4.8568770 .804896-1.203299$

O $-6.8853610 .733999-1.413103$

H -4.798491 -1.3516330 .174146$

H $-6.543637-1.479360-0.058198$

H $-5.4957907 .660206-0.934241$

H $-4.873621 \quad 7.3704740 .697693$

H $-3.7511197 .678291-0.636249$

C $-2.1838115 .041533-0.399428$

H -5.2224223 .1604552 .246169$

H -6.7829180 .9035492 .395911$

H $-7.6590700 .428588-0.908877$

H -1.909862 $6.094042-0.533421$

H -2.139568 4.565279 -1.387108

H -3.6295785 .2576911 .208535$

C -3.029331-2.185020 -1.800004

O $-4.139816-1.548797-2.446177$

C $-5.423832-1.817918-1.872654$

C $-5.684482-3.330907-1.841161$

C $-4.568860-4.081063-1.125066$

C $-3.220091-3.708143-1.735198$

H $-2.909264-1.779376-0.788397$

C $-1.768679-1.845341-2.612722$

H -6.159859 -1.392569 -2.565699

O $-6.928796-3.624460-1.214263$

H $-5.749745-3.707945-2.870329$

H -4.592116 -3.890283 -0.045541

O $-4.791607-5.482439-1.297441$

H -3.143886 -4.138593 -2.742209

O $-2.175989-4.275113-0.947695$

C $-1.304671-0.418519-2.413836$

H $-0.939308-2.494175-2.310235$

H $-1.963739-1.982477-3.682880$

H -7.621405 -3.147019-1.702018

H $-4.148539-5.952564-0.740632$

H $-2.244845-5.242078-1.018558$

O $-0.117825-0.254909-3.071689$

O $-1.8963810 .437376-1.771715$

C $0.4798031 .041552-2.978699$

H $0.900451 \quad 1.179962-1.979426$ 
H $\quad 1.2907901 .094372-3.710010$ H -0.247275 $1.826446-3.207988$

SCF Energy (B3LYP/6-31G**//MMFF) $=-3245.92389646$

$06 \_00275$

MM̄FF Geometry

C $-3.405870 \quad 4.4314470 .177824$

C $-2.244553 \quad 3.8964900 .581777$

C $-0.9546644 .073665-0.180436$

O $-0.4825732 .774909-0.613284$

C $0.130897 \quad 4.6840560 .723041$

C $1.1620875 .469022-0.061818$

C $2.4162455 .060603-0.333633$

C $0.7164936 .845608-0.494788$

C 3.0872173 .7591300 .015929

C $3.8825453 .212188-1.181722$

C $5.0119262 .256918-0.760898$

C $5.9187131 .958828-1.959573$

C $7.0444510 .981007-1.599383$

O $4.4492121 .041097-0.252067$

O $7.6350600 .549534-2.834912$

C $6.417196-0.249590-0.915208$

C $8.1625181 .667799-0.807482$

O $7.426612-1.142759-0.451117$

C $5.432447 \quad 0.1039820 .224312$

C $4.698248-1.1455410 .768038$

O $\quad 6.1747190 .6564391 .319708$

C $-1.1197792 .199488-1.669046$

O $-2.0358122 .670910-2.324025$

C $-0.5172110 .866641-1.883061$

C $-1.154734-0.022330-2.659539$

C $-0.695563-1.375156-2.980785$

C $0.675094-1.767227-2.504456$

C $3.907274-1.936531-0.274186$

C $3.097708-3.0675850 .340012$

C $1.811433-5.095939-0.322444$

O $2.910526-3.1871381 .547251$

C $1.000819-5.478010-1.519211$

C $-0.336950-5.392985-1.574308$

C $-1.150523-5.792261-2.771588$

C $-1.980618-4.651396-3.317423$

C $-3.312048-4.625490-3.132939$

C $-1.261075-3.602454-4.140898$

C -1.512811-2.190500 -3.679691

N 2.584714 -3.910175-0.637701

H $-3.4215175 .045545-0.719559$

H -2.225697 3.2788161 .474695

H -1.113086 $4.700307-1.066918$

H $0.613823 \quad 3.899822 \quad 1.317527$

H $\quad-0.3180695 .3734691 .450944$

H $3.0628345 .763778-0.858994$

H $1.5087587 .397884-1.011287$

H $-0.1352116 .781517-1.178401$

H $\quad 0.418804 \quad 7.4388580 .376234$

H 3.7681863 .9586190 .851607

H 2.3723093 .0017890 .347906

H $3.1870962 .698134-1.857772$

H $4.3257694 .044811-1.742790$

H $5.5918022 .743746 \quad 0.032232$

H $6.3387652 .884562-2.372924$

H $5.317376 \quad 1.517235-2.767082$

H $8.297070-0.131032-2.622990$

H $5.877168-0.787218-1.703510$

H $8.5937832 .488834-1.393042$

H $8.9898550 .977510-0.609105$

H 7.8241792 .0821180 .144303

H $7.799666-0.7687750 .365414$

H $5.415319-1.8115681 .266296$

H $4.009199-0.8247371 .559053$

H 5.6562040 .5409792 .132991

H $0.4238630 .652574-1.389273$

H -2.110995 $0.260565-3.098092$

H $0.989358-2.751941-2.851658$

H $1.429208-1.059110-2.865925$

H $0.703814-1.784641-1.409671$

H $3.211830-1.275920-0.802783$

H $4.591373-2.379021-1.005934$

H $1.192197-4.9035370 .558423$
H $2.513851-5.896921-0.070205$

H $1.546460-5.850137-2.383186$

H $-0.883455-5.030077-0.708218$

H -1.798385 -6.625630 -2.470400

H $-0.514799-6.188004-3.573791$

H $-3.928323-3.836072-3.549589$

H $-3.818326-5.397011-2.562080$

H -1.596550 -3.687948 -5.183208

H $-0.189891-3.821394-4.181947$

H -2.489714 -1.806255 -3.976473

H $2.930028-3.807953-1.588163$

C -5.6234573 .1857880 .183437$

$\begin{array}{llll}\text { O } & -5.128924 & 1.855243 & 0.433877\end{array}$

C $-5.3691751 .046818-0.734545$

C $-6.2307531 .888949-1.675515$

C $-5.7831863 .295604-1.332815$

H $-4.3913230 .881303-1.202223$

C $-5.994864-0.300222-0.350940$

H $-6.0663761 .629543-2.725943$

H $-7.3007511 .773374-1.468771$

C $-6.7545164 .376059-1.772998$

H -4.817509 $3.462639-1.823736$

C $-5.235869-1.0863610 .735328$

O $-7.317655-0.0558020 .147044$

H -6.112273 -0.912018 -1.253896

H $-5.358110-0.5980561 .710245$

O $-5.855691-2.3750710 .848636$

C $-3.734994-1.2999460 .463919$

C $-3.095636-2.2532481 .492231$

H -3.224682 -0.3319220 .489640$

O $-3.552083-1.840118-0.842966$

H -3.381002 -1.925967 2.497674

H -3.504802 -3.2614301 .353153$

H $-6.8818224 .359132-2.860239$

H -7.740974 $4.239657-1.317193$

H $-6.3864935 .367545-1.490751$

C -4.7235834 .2221070 .866526$

H -7.785928 -0.9072170 .167074$

H $-6.733882-2.244346 \quad 1.244207$

H -4.037921 -2.681512 -0.885085

H $-4.5514653 .917743 \quad 1.906624$

H -5.2404425 .1885500 .900566$

H -6.6092293 .2290750 .665996$

C $-0.990883-0.4437622 .747779$

O $-0.931752-1.0550631 .455050$

C $-1.557772-2.3399611 .373728$

C $-0.912730-3.3008632 .385142$

C $-0.920573-2.7324253 .802589$

C $-0.328990-1.3241733 .813168$

H -2.042947 -0.2806413 .011866$

C -0.3752380 .9545422 .647805$

H -1.337596 -2.724067 0.370147

O $-1.571502-4.5626362 .389099$

H $\quad 0.129451-3.4813302 .097139$

H -1.927619 -2.739868 4.235552

O $-0.115758-3.5601134 .645775$

H $\quad 0.752487-1.3944143 .644518$

O $-0.524532-0.7349115 .095916$

C 1.1179160 .9351122 .423721

H -0.8087691 .4970081 .803491$

H -0.6021801 .5317763 .551660$

H -1.540656 -4.912404 1.482736

H $-0.473777-4.4629544 .590631$

H $-0.123839-1.3302415 .752554$

O $1.749413 \quad 1.523626 \quad 3.479051$

O 1.6546640 .4466531 .438329

C 3.1782401 .5453243 .422749

H 3.5273251 .9288122 .460376

H 3.5393972 .2092274 .212613

H 3.5682730 .5399473 .603345

SCF Energy (B3LYP/6-31G**//MMFF) $=-3245.91143618$

06_00276

MMFF Geometry

C -1.504589 -0.0175663 .077462$

C -1.5075801 .2397873 .548235$

C -2.007350 2.4808902 .847593

O -2.7698842 .1607821 .664232$ 
C $-0.824665 \quad 3.3540132 .395534$

C -1.140484 4.8348772 .431785

C $-1.5823505 .559744 \quad 1.386484$

C -0.8801105 .5034993 .757684$

C -1.9493095 .0804520 .010902$

C $-0.9359075 .508587-1.058007$

C $0.2928654 .587919-1.123141$

C $1.3045495 .118055-2.141905$

C $2.4843804 .155910-2.335757$

O $-0.1682633 .289621-1.514267$

O $3.1937844 .593729-3.504398$

C $1.9279582 .744994-2.616309$

C $3.4790664 .239650-1.172545$

O $2.9862041 .793738-2.699925$

C $0.8516642 .289214-1.602823$

C $0.1900000 .983038-2.099962$

O $1.4783572 .052315-0.340952$

C -4.0833761 .8482091 .812607$

O -4.7169121 .7774642 .852460$

C -4.6109751 .5719850 .459788$

C $\begin{array}{llll}-5.715686 & 0.826353 & 0.319142\end{array}$

C $-6.3217260 .421642-0.950937$

C $-5.7156750 .966234-2.218560$

C $-1.0408250 .535820-1.297431$

C $-1.567667-0.803787-1.788066$

C $-3.153238-2.621943-1.162101$

O $-1.149536-1.357608-2.802166$

C $-4.311574-2.519165-2.104812$

C $-5.517418-3.055900-1.860628$

C $-6.666372-3.002038-2.828571$

C $-7.948195-2.500658-2.199101$

C $-8.917928-3.355366-1.831828$

C $-8.139692-1.004986-2.074746$

C -7.369704 -0.427652 -0.918474

N -2.560224 -1.311947 -0.960867

H -1.870818 -0.2315762 .078160$

H -1.127793 1.4130694 .554155

H -2.6468683 .0332303 .548222$

H -0.5086153 .0618001 .388139$

H 0.0509693 .1899153 .036675

H -1.7396236 .6274861 .536950$

H -1.128298 6.5701123 .743495

H -1.480274 5.0361094 .544905

H 0.1774685 .4135954 .026886

H -2.110948 4.000111-0.019888

H $-2.9205515 .529446-0.234121$

H -1.449221 $5.474858-2.028045$

H $-0.6252796 .547508-0.891988$

H $0.7523614 .527538-0.130609$

H $1.6636546 .113605-1.852091$

H $0.8058915 .252605-3.112429$

H $3.8978283 .946026-3.681424$

H $1.4734082 .775714-3.615991$

H $3.8746015 .258529-1.083267$

H $4.3491833 .597375-1.347812$

H $3.0389933 .969426-0.210371$

H $3.2575931 .572005-1.792979$

H $-0.1280451 .114384-3.142861$

H $0.928366 \quad 0.174688-2.086114$

H $\quad 0.980517 \quad 1.3774330 .145683$

H $-4.0505391 .957286-0.384059$

H $-6.2071540 .452223 \quad 1.216393$

H -4.660833 $0.681576-2.298559$

H -5.784532 $2.059636-2.235548$

H $-6.2006530 .605168-3.127295$

H $-0.7949330 .435112-0.235675$

H -1.845061 $1.272399-1.398028$

H -3.450642 -2.999632 -0.178824

H -2.396782 -3.293834 -1.581386

H -4.141927 -2.007392 -3.049250

H $-5.678651-3.597507-0.931243$

H -6.816740 -4.016977-3.219305

H $-6.425801-2.381839-3.701111$

H -9.851120 -3.002401-1.405455

H -8.798089 -4.427862 -1.944141

H $-9.199783-0.772558-1.906087$

H -7.901095 -0.533029 -3.031519

H $-7.731289-0.7661320 .053733$
H $-2.886009-0.758223-0.174294$

C $0.214617-1.8543713 .334985$

O $-0.120623-2.6419382 .167429$

C $0.222166-4.0072362 .443558$

C $1.352880-3.935278 \quad 3.455774$

C $0.839427-2.8244254 .349196$

H $-0.653646-4.4474582 .942715$

C $0.486892-4.8286551 .180919$

H $1.507647-4.8760823 .991401$

H $2.297468-3.6366422 .988364$

C $1.906954-2.1988345 .230148$

H $\quad 0.056107-3.2379974 .999711$

C $1.799407-4.5813180 .410275$

O $0.467304-6.2132921 .565175$

H $-0.355970-4.6991390 .491151$

H $2.664283-4.8717091 .017988$

O $1.790622-5.479855-0.712912$

C $2.006125-3.153289-0.130442$

C $3.229125-3.062644-1.071645$

H $2.137480-2.4580280 .703678$

O $0.837072-2.745808-0.837272$

H $4.124567-3.342680-0.506258$

H $\quad 3.114337-3.798865-1.875398$

H $2.313715-2.9410705 .924882$

H $2.739128-1.8055194 .639597$

H $1.492343-1.3748375 .819450$

C -1.045013-1.190269 3.898845

H $1.233659-6.3741702 .141699$

H $1.680742-6.380612-0.362890$

H $\quad 0.702428-3.369876-1.571136$

H $-0.858827-0.8666674 .930361$

H -1.867877 -1.915290 3.931810

H $0.939056-1.1002323 .011887$

C $4.820081-0.611515-0.089369$

O $3.564717-0.628660-0.779376$

C $3.408309-1.679940-1.735014$

C $4.529445-1.619858-2.783662$

C $5.910686-1.621274-2.139180$

C $5.993326-0.529420-1.075588$

H $4.911244-1.515650 \quad 0.524619$

C 4.8172730 .6147660 .836738

H $2.477195-1.470483-2.273265$

O $4.439195-2.707886-3.697430$

H $4.425173-0.699411-3.372602$

H $6.158181-2.603427-1.719922$

O $6.895885-1.349448-3.138376$

H $6.0016210 .450107-1.570525$

O $7.229424-0.649573-0.373582$

C $3.8547010 .452247 \quad 1.989672$

H 5.8086520 .7849991 .269673

H $4.516640 \quad 1.5008210 .265806$

H $3.559084-2.670304-4.109671$

H $6.799881-2.026371-3.830260$

H $7.239967-1.5164490 .066845$

O 3.5824931 .6780522 .525212

O $3.421404-0.6183952 .390887$

C 2.6960821 .6732473 .648097

H 2.5565102 .7066663 .976199

H 1.7247141 .2624233 .360049

H 3.1298361 .0982004 .471646

SCF Energy (B3LYP/6-31G**//MMFF) $=-3245.92667437$

06_00277

MM̄MF Geometry

C $0.388106 \quad 0.035158 \quad 3.717985$

C $-0.2253930 .974396 \quad 4.455201$

C -1.0691972 .1145853 .936577$

O $-1.724571 \quad 1.7688652 .693107$

C -0.2059853 .3682663 .716140$

C -1.0287814 .6280423 .515402$

C -1.2278635 .2469822 .334790$

C -1.614869 5.2162094 .774938

C -0.7249524 .8372020 .980330$

C -1.8852394 .5561700 .017686$

C $-1.3892694 .020257-1.333536$

C $-2.5660153 .770109-2.276408$

C $-2.1204723 .118313-3.592214$

O $-0.6992872 .795099-1.084349$ 
O $-3.3082382 .643208-4.246121$ C -1.232011 $1.891202-3.280577$ C $-1.498894 \quad 4.152848-4.537934$ O $-0.6778551 .378548-4.489180$ C $-0.122617 \quad 2.176856-2.242617$ C $0.6033220 .908535-1.743384$ O $0.9150242 .992823-2.799510$ C $-2.812462 \quad 0.951228 \quad 2.790837$ O -3.2517880 .4369293 .808326$ C $-3.3970410 .789404 \quad 1.440954$ C $-4.410797-0.072175 \quad 1.265040$ C $-5.141930-0.3198710 .019806$ C $-4.7040320 .428187-1.210144$ C $-0.267835-0.106254-0.999673$ C $-0.786052-1.240311-1.865343$ C $-2.179328-3.302476-1.703182$ O $-0.479962-1.387013-3.044980$ C $-3.528995-3.003428-2.272651$ C $-4.651592-3.624922-1.881317$ C $-5.998949-3.376011-2.494485$ C $-7.045258-2.988059-1.475586$ C $-7.929706-3.888586-1.014500$ C $-7.128280-1.534918-1.068376$ C $-6.177396-1.1854880 .041682$ $\mathrm{N}-1.613219-2.091269-1.143933$ H 0.3530350 .0883382 .633154 H $-0.153907 \quad 0.914572 \quad 5.539702$ H - -1.8429272 .3273264 .685155$ H $0.469766 \quad 3.2029332 .870532$ H 0.4495453 .5305134 .582799 H -1.821789 6.1604702 .330250 H -2.140113 6.1592064 .589041 H -2.332816 4.5257435 .227315 H -0.8224185 .4214695 .502203$ H $-0.111737 \quad 5.6529440 .579274$ H -0.0753783 .9616041 .040545$ H -2.554554 3.8185560 .478132 H -2.467846 $5.472726-0.138334$ H $-0.691445 \quad 4.747355-1.765095$ H -3.113153 4.700631-2.472920 H -3.285326 $3.101189-1.786425$ H -3.032431 2.173724 -5.052257 H - $-1.9030841 .117577-2.889095$ H -2.240488 $4.914153-4.808903$ H $-1.1852453 .693014-5.481374$ H $-0.6413994 .670298-4.101969$ H $-0.2618730 .523810-4.286765$ H $1.1700500 .441026-2.557366$ H $1.3618691 .246337-1.025593$ H $0.742394 \quad 3.114774-3.746612$ H -2.9964351 .3969200 .638877$ H $-4.759338-0.6435372 .124862$ H $-5.1949470 .089948-2.125054$ H $-4.9170331 .496247-1.099477$ H $-3.6308070 .298179-1.381072$ H $0.345151-0.570509-0.217149$ H $-1.1111600 .381094-0.503745$ H $-2.222462-4.042767-0.898038$ H -1.521216 -3.684192 -2.490575 H -3.578820 -2.263259 -3.067975 H $-4.596077-4.383531-1.103807$ H -6.303141 -4.296653 -3.009955 H -5.948335 -2.606836 -3.275289 H -8.704057 -3.609036 - 0.307776 H -7.895319 -4.926445 -1.328968 H $-8.141390-1.310227-0.707455$ H -7.007506 $-0.900833-1.951038$ H $-6.418048-1.6800860 .983882$ H $-1.857656-1.853309-0.186915$ C $0.723512-2.4999713 .755833$ O $1.456297-2.7470572 .541812$ C $0.607622-3.4707441 .629709$ C $-0.624418-3.8867432 .429988$ C $-0.747445-2.735873 \quad 3.412328$ H $0.299688-2.7448120 .870245$ C $1.380908-4.6056540 .944647$ H $-1.508198-4.009207 \quad 1.796577$ H $-0.468122-4.8226182 .976554$
C -1.621099 -3.038652 4.615599

H $-1.171334-1.8829882 .868787$

C $2.765101-4.1957590 .402649$

O $1.624397-5.6626321 .881742$

H $0.775168-5.0369730 .138140$

H $3.459375-4.0404161 .238195$

O $3.286544-5.298814-0.350064$

C $2.770437-2.948877-0.497227$

C $4.174757-2.639523-1.047556$

H $2.411944-2.0908730 .078336$

O $1.869182-3.133591-1.585981$

H $4.889700-2.670185-0.218341$

H $4.469923-3.421231-1.758190$

H -2.644726 -3.2651574 .299863$

H $-1.245030-3.8989605 .179235$

H $-1.658568-2.1805855 .294336$

C $1.110651-1.1322484 .332811$

H $0.806299-6.1823091 .958053$

H $3.294503-6.0726710 .239195$

H $2.159352-3.920290-2.078787$

H $2.186912-0.9727624 .193463$

H $\quad 0.922323-1.1518085 .413550$

H $1.069001-3.2676054 .461735$

C $4.562174 \quad 0.1249990 .175270$

O $3.751099-0.204426-0.955075$

C $4.251696-1.272400-1.765381$

C $5.652390-0.917998-2.286384$

C $6.607157-0.557343-1.147210$

C $5.9858970 .510358-0.244464$

H $4.611025-0.7470360 .839691$

C $3.842261 \quad 1.2128660 .988068$

H $3.585049-1.334938-2.634839$

O $6.173953-2.004714-3.043410$

H $5.570676-0.059161-2.965431$

H $6.895024-1.441298-0.566340$

O $7.816231-0.022692-1.691048$

H $5.9948181 .468569-0.775175$

O $6.7892810 .668750 \quad 0.923118$

C 3.6371702 .5237390 .255129

H 2.8439170 .8505501 .262743

H 4.4068751 .4149721 .905325

H $6.995378-1.702955-3.466250$

H $8.262965-0.738971-2.172875$

H 7.6909870 .8828030 .627732

O $3.219563 \quad 3.468067 \quad 1.150516$

O $3.7933802 .699564-0.945043$

C $2.973252 \quad 4.766210 \quad 0.601307$

H $2.2447004 .707024-0.213200$

H $2.5628865 .398041 \quad 1.393441$

H $3.9104495 .206946 \quad 0.248817$

SCF Energy (B3LYP/6-31G**//MMFF)= -3245.92074908

06_00278

MM̄FF Geometry

C $-4.4400632 .001227 \quad 1.926230$

C $-3.162713 \quad 1.7624891 .593459$

C $-1.9974661 .912972 \quad 2.529813$

$\begin{array}{llll}\text { O } & -0.910595 & 2.543457 & 1.816213\end{array}$

C -1.5225190 .5213652 .984776$

C $-0.260403 \quad 0.551679 \quad 3.823519$

C $0.882614-0.0826613 .496590$

C $-0.3550451 .306555 \quad 5.124270$

C $1.159140-0.915308 \quad 2.274768$

C $0.890775-2.3951342 .565468$

C $1.323044-3.3191851 .417446$

C $0.907409-4.7585401 .735248$

C $1.440801-5.7598750 .705464$

O $2.744997-3.2258581 .293410$

$\begin{array}{llll}\text { O } & 1.260667 & -7.070666 & 1.262275\end{array}$

C $2.954793-5.5219830 .528064$

C $0.616942-5.725195-0.586876$

O $3.473467-6.363423-0.500735$

C $3.323945-4.040494 \quad 0.268810$

C $4.865741-3.8739910 .319862$

O $2.849205-3.681454-1.026255$

C $-0.910663 \quad 3.901066 \quad 1.740845$

O $-1.7712414 .664606 \quad 2.146516$

C 0.3497374 .2986011 .080042 
C $0.5907125 .586856 \quad 0.799448$

C 1.8207406 .0690210 .179684

C $2.4150297 .258197 \quad 0.882604$

C $5.408909-2.4718350 .011894$

C $5.153296-1.4357111 .092571$

C 4.9561241 .0211231 .419339

O $4.976731-1.7262112 .272579$

C 5.4972592 .2340810 .733312

C $4.752932 \quad 3.302101 \quad 0.408552$

C $5.2992684 .512744-0.295558$

C $4.6191624 .763817-1.623673$

C $4.9835674 .086111-2.725241$

C $3.5678705 .850306-1.682792$

C $2.3244305 .482439-0.924911$

N $5.214783-0.1421860 .591349$

H $-4.6844572 .332156 \quad 2.931727$

H -2.936834 1.4018130 .593554

H -2.2679602 .5189123 .403733$

H $-1.381888-0.1151942 .103984$

H -2.315268 0.0389903 .572438

H $1.722038-0.0180254 .188110$

H $0.532643 \quad 1.1674755 .750648$

H -0.4620152 .3800784 .940970$

H -1.2190900 .9615735 .701711$

H $0.571271-0.5839081 .413076$

H $2.207199-0.7638841 .994510$

H $1.433564-2.6804143 .477015$

H $-0.179203-2.5345602 .766002$

H $0.855281-2.9773810 .487197$

H $-0.183945-4.8381061 .819647$

H $1.301463-5.0394682 .722517$

H $1.664621-7.708900 \quad 0.649235$

H $3.443833-5.8453571 .457144$

H $-0.429458-5.978103-0.377501$

H $0.967117-6.477163-1.302294$

H $0.629225-4.749685-1.076812$

H $3.221749-5.974352-1.355617$

H $5.226939-4.1791271 .310283$

H $5.318322-4.550193-0.416486$

H $3.211325-2.814246-1.262432$

H 1.0672193 .5197690 .845065

H $-0.130115 \quad 6.355187 \quad 1.069131$

H 1.7542628 .1269860 .788095

H 3.3951047 .5468910 .497718

H 2.5491147 .0432831 .949134

H $6.500505-2.543552-0.081172$

H $5.041822-2.114154-0.954279$

H 5.4397390 .8834222 .391832

H 3.8747231 .0799531 .580322

H $6.557603 \quad 2.2310550 .491371$

H $3.697223 \quad 3.3087970 .666558$

H $6.3807594 .412261-0.457121$

H $5.182722 \quad 5.3859260 .357680$

H $4.5178244 .275148-3.686676$

H $5.7552303 .324028-2.687446$

H $3.2724126 .039237-2.723173$

H $4.0115806 .788710-1.338264$

H $1.7857524 .638339-1.355516$

H $5.237932-0.012896-0.416226$

C $-6.060306 \quad 0.347721 \quad 0.957563$

O $-5.013174-0.4832150 .415202$

C $-5.558515-1.313461-0.621809$

C $-7.062551-1.312280-0.388660$

C -7.2819770 .1193020 .065703$

H -5.350602 -0.811467 - 1.574565

C $-4.883958-2.687950-0.603737$

H -7.625230 -1.568836 -1.291105

H -7.352442 -2.009757 0.405785

C $-8.607922 \quad 0.3388330 .773113$

H $-7.2343850 .776954-0.812893$

C $-3.341754-2.627646-0.665056$

O $-5.248181-3.3516220 .612732$

H $-5.278875-3.300287-1.423124$

H $-2.939240-2.2135980 .267893$

O $-2.854481-3.972949-0.754137$

C $-2.778556-1.831432-1.859983$

C $-1.243441-1.915548-1.947377$

H $-3.070807-0.781504-1.768217$
O $-3.348754-2.325441-3.071246$

H $-0.823910-1.558750-1.000609$

H $-0.949103-2.965625-2.057551$

H -9.4434780 .1109540 .103244$

H -8.701891 -0.3006371 .657134$

H $-8.708176 \quad 1.379421 \quad 1.097576$

C $-5.583407 \quad 1.8012880 .969305$

H $-4.990571-4.2847510 .526145$

H $-3.014996-4.4000760 .104106$

H -3.109632 -3.265304 -3.146229

H $-6.4000562 .472597 \quad 1.258141$

H $-5.2667282 .098261-0.038350$

H $-6.250334-0.0052921 .979218$

C $-0.3722661 .026982-2.068785$

O $-0.9861610 .261874-3.112582$

C $-0.649453-1.127886-3.138406$

C $0.867635-1.289396-3.291272$

C $1.614837-0.565245-2.176683$

C $1.160106 \quad 0.890219-2.103509$

H $-0.7667090 .702143-1.098175$

C $-0.7619782 .498153-2.287151$

H $-1.105685-1.535541-4.049064$

O $1.212683-2.669086-3.309566$

H $1.176761-0.869566-4.257372$

H $1.489281-1.075039-1.215192$

O $3.012692-0.603194-2.470481$

H $1.562567 \quad 1.426246-2.972535$

O $1.7347631 .497257-0.947702$

C $-2.2170492 .783029-1.987360$

H $-0.1603513 .134317-1.630605$

H $-0.5832572 .793586-3.327551$

H $2.180397-2.726327-3.385827$

H $3.464390-0.075667-1.789716$

H $1.3740411 .049805-0.163585$

O $-2.3584094 .115009-1.718893$

O $-3.1174841 .956184-2.015396$

C $-3.6870694 .563308-1.433496$

H $-4.4045204 .162023-2.155651$

H $-3.959797 \quad 4.274256-0.415410$

H -3.698286 $5.654352-1.502792$

SCF Energy (B3LYP/6-31G**//MMFF) $=-3245.91965338$

06_00279

MMFF Geometry

C $0.419383-3.3130361 .188187$

C $-0.780538-3.3985280 .596956$

C $-0.992888-3.426138-0.894247$

O $-1.607762-2.170548-1.262313$

C $-1.947018-4.578342-1.258581$

C $-2.355104-4.599926-2.718775$

C $-3.574364-4.251802-3.179227$

C $-1.290277-5.068052-3.675980$

C $-4.748578-3.757099-2.379220$

C $-5.017030-2.271204-2.649785$

C $-5.818960-1.614330-1.515652$

C $-6.184039-0.176305-1.885304$

C $-6.8536420 .570214-0.724487$

O $-4.993214-1.626685-0.344794$

O $-6.8583381 .963768-1.072255$

C $-5.9943680 .401852 \quad 0.548070$

$\begin{array}{llll}C & -8.323335 & 0.161977 & -0.568425\end{array}$

$\begin{array}{llll}\text { O } & -6.677891 & 0.971280 & 1.662019\end{array}$

C $-5.598724-1.0637240 .828091$

C $-4.580084-1.2189021 .986954$

O $-6.771226-1.7981581 .200983$

C $-0.769413-1.142708-1.574831$

O $0.449706-1.177965-1.632665$

C $-1.5977750 .062533-1.800052$

C $-1.002932 \quad 1.246730-2.005284$

C $-1.6771482 .534542-2.182596$

C $-3.1801042 .555176-2.097579$

C $-3.225448-0.5338111 .777522$

C $-3.136166 \quad 0.8770202 .332255$

C -1.628747 2.8650202 .340696

O $\begin{array}{lllll}-3.978587 & 1.359093 & 3.084203\end{array}$

C $-2.126775 \quad 3.853658 \quad 1.332792$

C $-1.357992 \quad 4.816344 \quad 0.800401$

C $-1.8573975 .853148-0.166056$ 
C -1.014214 $5.945430-1.419052$ C $-0.0236946 .847930-1.519866$ C $-1.3775325 .053523-2.584791$ C $-0.9221443 .634236-2.385039$ N $-1.976524 \quad 1.5208331 .917861$ H $\quad 1.318347-3.2647260 .579365$ H -1.672287 -3.420152 1.220250 H $-0.045164-3.558633-1.430804$ H $-2.830467-4.533200-0.614568$ H -1.471177 -5.537959-1.014382 H $-3.756502-4.311396-4.251537$ H -1.653528 -5.122377 -4.707861 H $-0.435347-4.385085-3.664016$ H $-0.942174-6.068014-3.397224$ H -5.633834 -4.340913 -2.660486 H -4.613234 -3.933004 -1.310421 H $-4.062409-1.742833-2.760571$ H $-5.555020-2.170930-3.600648$ H -6.721533 -2.208121 -1.330106 H $-6.822920-0.154024-2.777034$ H $-5.2713250 .366531-2.162805$ H $-7.2194812 .454761-0.314072$ H $-5.089131 \quad 1.0005970 .392689$ H -8.883763 $0.403317-1.479822$ H $-8.808500 \quad 0.7233510 .237685$ H -8.453875 $-0.904388-0.372752$ H -6.0584520 .9989692 .410170$ H -5.037601 -0.9264092 .939434$ H $-4.370772-2.2939782 .087849$ H $-6.496331-2.5936881 .686139$ H $-2.674083-0.049234-1.755788$ H $0.0852331 .283520-2.031378$ H $-3.5197232 .108207-1.157302$ H -3.615272 $1.996120-2.932773$ H -3.607763 $3.559253-2.124904$ H -2.462124 -1.113102 2.312150 H -2.951392 -0.5292830 .720067$ H -0.5402412 .8989012 .451941$ H -2.086334 3.0695013 .313922 H -3.1762383 .7961281 .053554$ H $-0.3177084 .892661 \quad 1.108745$ H -1.851645 6.8205980 .353396 H -2.904213 $5.674167-0.440924$ H $0.567337 \quad 6.940755-2.424893$ H $\quad 0.2221107 .510037-0.696072$ H $-0.8998025 .419389-3.503703$ H -2.450005 5.135301-2.781909 H $0.1613623 .518466-2.427494$ H -1.360386 1.0591751 .254911 C $0.856308-1.8395403 .172755$ O $2.123823-1.3733612 .673649$ C $2.868687-0.788823 \quad 3.757128$ C $1.862197-0.5562484 .876378$ C $0.939778-1.7455144 .697112$ H $3.592230-1.5461844 .082490$ C 3.6150900 .4624193 .279978 H $2.336393-0.5230025 .861816$ H $1.302903 \quad 0.376046 \quad 4.737691$ C $-0.409463-1.5758995 .375092$ H $1.435344-2.6378025 .102969$ C $4.4281680 .266673 \quad 1.982087$ O $2.669344 \quad 1.5105423 .032283$ H 4.2644190 .8315434 .081923 H $3.739648 \quad 0.1702261 .136636$ O $5.1909831 .457778 \quad 1.757821$ C $5.399867-0.9220062 .012248$ C $6.289076-1.0874150 .763611$ H $4.842892-1.8537692 .155844$ O $6.263913-0.7883803 .145721$ H $6.886984-0.1821810 .615747$ H $7.017902-1.8730361 .000042$ H -0.283946 -1.452190 6.455716 H $-0.941655-0.6969494 .995461$ H -1.043988 -2.4516185 .206498$ C $0.596514-3.2639802 .679705$ H 2.0238131 .1760172 .385968 H 4.5722512 .2075641 .770710 H 6.7480280 .0494823 .046899
H $-0.289506-3.6757573 .177420$

H $1.441131-3.9152732 .936315$

H $0.092588-1.1530692 .785179$

C $5.5595890 .650119-1.686514$

O $4.772866-0.376717-1.069941$

C $5.507162-1.471621-0.512100$

C $6.365698-2.126824-1.603746$

C $7.286099-1.114031-2.274107$

C $6.4707180 .073693-2.779359$

H $6.1623201 .155975-0.923599$

C $4.5878841 .670339-2.293477$

H $4.761259-2.218368-0.210530$

O $7.125932-3.200260-1.059565$

H $5.702745-2.560544-2.364143$

H $8.087033-0.797994-1.595669$

O $7.922977-1.749429-3.384633$

H $5.869194-0.249427-3.638457$

O $7.3610371 .082066-3.255041$

C $4.0032162 .573520-1.234981$

H $5.0843422 .327109-3.017395$

H $3.7729261 .167628-2.828766$

H $7.669595-3.565357-1.778786$

H $8.467744-1.077135-3.828926$

H $7.8966481 .386862-2.502598$

O $2.7235242 .201551-0.951376$

O $4.6171843 .500356-0.721737$

C $2.0388663 .018030 \quad 0.003504$

H $1.7577153 .966872-0.461207$

H 1.1328662 .4910750 .313556

H 2.6566273 .1997990 .886793

SCF Energy (B3LYP/6-31G**//MMFF) = -3245.93244285

06_00280

MMFF Geometry

C $1.175559-3.8401052 .959154$

C $0.239707-2.9332552 .643641$

C $-0.358406-1.9531043 .615641$

O $-1.777199-1.8922663 .332749$

C $0.248091-0.5351933 .520646$

C $0.389217 \quad 0.0449702 .124786$

C 1.5520000 .4489931 .577382

C $-0.8879540 .221418 \quad 1.344563$

$\begin{array}{llll}\text { C } 2.932357 & 0.400747 & 2.165760\end{array}$

C 3.4709981 .7646642 .625824

C 3.5305062 .8228381 .508814

C 4.5247693 .9289611 .869446

C $4.5464695 .042758 \quad 0.812974$

$\begin{array}{lllllllll}\text { O } & 2.222185 & 3.388041 & 1.357477\end{array}$

$\begin{array}{lllll}\text { O } & 5.259831 & 6.160086 & 1.364009\end{array}$

C 3.1003315 .5108940 .557149

C $5.3068124 .621839-0.450241$

O $3.0506006 .443512-0.520850$

C $2.1052544 .353297 \quad 0.302638$

C $0.659748 \quad 4.9117360 .317700$

O $2.376274 \quad 3.771659-0.972958$

C $-2.622989-1.4982944 .316701$

O $-2.337115-1.308454 \quad 5.490777$

C $-3.990401-1.2591103 .792358$

C $-4.317945-1.3788602 .495740$

C $-5.619290-1.0827041 .890118$

C $-6.716847-0.5909662 .795541$

C $-0.439046 \quad 3.8460690 .219974$

C $-1.830961 \quad 4.4316170 .396095$

C $-4.217096 \quad 3.747248 \quad 0.519439$

$\begin{array}{lllll}0 & -2.031326 & 5.611817 & 0.670790\end{array}$

C $-5.091795 \quad 3.054055-0.475402$

C $-6.000258 \quad 2.122582-0.145925$

C $-6.9471701 .490072-1.125666$

C $-6.6496370 .033951-1.402660$

C $-6.210469-0.361203-2.609967$

C $-6.948721-0.978872-0.316678$

C $-5.751576-1.243048 \quad 0.556623$

N -2.8231303 .4727290 .222369$

H $1.572725-3.8739043 .970326$

H $-0.138018-2.9079271 .624482$

H $-0.226254-2.3287864 .638873$

H $1.215106-0.5547604 .032497$

H -0.3721260 .1633774 .097461$ 
H $1.5186050 .861697 \quad 0.569627$ H $-1.625426 \quad 0.772731 \quad 1.936642$ H -0.7309040 .7795600 .416380$ H $-1.310063-0.7467061 .063120$ H $2.991341-0.3023133 .001859$ H $3.611883-0.0046001 .407226$ H 2.8521232 .1317543 .454657 H 4.4797231 .5933093 .022006 H 3.8301672 .3352550 .573518 H 5.5322863 .5175862 .009191 H 4.2443544 .3707552 .835990 H 6.1549625 .8590411 .596526 H 2.7720566 .0566411 .453049 H $6.342724 \quad 4.359417-0.203721$ H $5.3702525 .448339-1.166630$ H $4.8601763 .762150-0.954141$ H $3.6761257 .158984-0.314043$ H 0.5074395 .4667451 .252896 H $0.5247455 .621636-0.508195$ H $1.5947863 .279033-1.267770$ H $-4.710349-0.9327874 .535896$ H -3.555644 -1.703715 1.789754 H $-6.946783-1.3400993 .561366$ H $-7.652217-0.3795382 .273823$ H -6.418119 0.3389513 .292103 H $-0.4015213 .346773-0.752493$ H $-0.3006223 .098011 \quad 1.006436$ H $-4.4029314 .825680 \quad 0.487241$ H $-4.401250 \quad 3.4036951 .542817$ H -5.000488 $3.366168-1.513038$ H -6.1050101 .8332600 .896406$ H -6.947157 2.061207-2.063668 H $-7.9684311 .580185-0.734547$ H $-6.022123-1.407555-2.826527$ H $-6.0201140 .352261-3.404789$ H $-7.237285-1.934142-0.775429$ H $-7.830615-0.6574740 .243336$ H $-4.892883-1.620327-0.000290$ H $-2.545198 \quad 2.500033 \quad 0.126047$ C $3.048957-4.4845861 .399674$ O $2.876946-3.4146430 .445332$ C $3.631451-3.721704-0.743330$ C $4.559989-4.868012-0.365150$ C $3.702243-5.6297320 .624129$ H $2.915707-4.080606-1.491006$ C $4.343735-2.471953-1.268065$ H $4.853938-5.467579-1.232102$ H $5.473299-4.5086700 .123105$ C $4.490775-6.594094 \quad 1.493547$ H $2.939158-6.1907320 .067835$ C $3.412908-1.276692-1.545786$ O $5.312155-2.045269-0.299911$ H $4.911798-2.720720-2.171744$ H $2.986764-0.909771-0.608546$ O $4.217441-0.210932-2.067411$ C $2.268946-1.554636-2.538144$ C $1.443642-0.286311-2.829530$ H $1.608715-2.323391-2.125367$ O $2.804463-2.066576-3.756358$ H $1.0481820 .093704-1.881205$ H $2.1043200 .488861-3.235641$ H $4.985661-7.3511590 .876500$ H $5.262643-6.0743172 .071055$ H $3.833521-7.1112222 .199456$ C $1.691025-4.8667921 .988781$ H $4.843025-1.9006240 .539931$ H $4.919168-0.033334-1.417654$ H $3.411599-1.396540-4.114670$ H $1.761532-5.8205712 .524291$ H $0.962956-5.0094331 .180711$ H $3.723062-4.1115902 .181597$ C -1.358798 -1.413692 -2.337142 O $-0.540566-1.609583-3.495417$ C $0.298991-0.505365-3.842078$ C $-0.5621530 .740359-4.098183$ C $-1.4767261 .051420-2.915460$ C -2.269818 $-0.191058-2.514442$ H $-0.719670-1.288104-1.454819$
C $-2.198417-2.683471-2.147782$

H $0.771446-0.769944-4.796463$

O $0.249056 \quad 1.868593-4.409775$

H $-1.1894360 .557946-4.980332$

H $-0.9043261 .446013-2.069482$

O $-2.381802 \quad 2.084439-3.309024$

H $-3.031358-0.398406-3.277050$

O $-2.9467160 .061551-1.285949$

C $-1.372749-3.824058-1.603517$

H -3.014264 -2.519013-1.434953

H -2.658030 -3.008510 -3.088982

H $0.6578352 .187398-3.587554$

H $-2.8789292 .355705-2.519293$

H $-3.6056560 .756067-1.451046$

O $-0.638940-4.392883-2.601107$

O $-1.385896-4.169578-0.429266$

C $0.178592-5.501306-2.213511$

H $0.784276-5.251935-1.338019$

H $-0.451817-6.371256-2.008691$

H $0.848494-5.736957-3.044646$

SCF Energy (B3LYP/6-31G**//MMFF) $=-3245.91085732$

06_00281

MMFF Geometry

C $-0.554187 \quad 3.436372-2.734672$

C $0.0518512 .919268-1.656093$

C $0.7473193 .771145-0.624253$

$\begin{array}{lllll}\text { O } & 1.974879 & 3.129346 & -0.209397\end{array}$

C $-0.142483 \quad 3.870498 \quad 0.627015$

C 0.1525675 .0758901 .494120

C $0.890915 \quad 5.0554382 .620665$

C $-0.510661 \quad 6.354388 \quad 1.049005$

C $1.675208 \quad 3.9114343 .198805$

C $1.0124803 .269244 \quad 4.426931$

C $-0.064105 \quad 2.2328454 .066190$

C $-0.670903 \quad 1.6129415 .326634$

C $-1.6434370 .467894 \quad 4.999598$

O $0.5710541 .215454 \quad 3.287479$

O $-1.893624-0.2232366 .232878$

C $-0.954719-0.5168024 .027209$

C $-2.997351 \quad 1.0011634 .514745$

O $-1.867990-1.511741 \quad 3.573241$

C -0.3007510 .1828752 .815328$

C $0.564838-0.8132232 .004571$

O -1.3309830 .6974021 .967516$

C $3.0234473 .093580-1.070022$

O $3.0772003 .560478-2.195104$

C $4.1031932 .345538-0.388126$

C $5.1065701 .814714-1.100773$

C $6.2280091 .041002-0.561196$

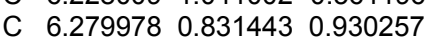

C $1.481221-0.122120 \quad 0.985184$

C $2.135154-1.1122450 .045821$

C $3.873227-2.891951-0.040003$

O $1.741689-1.252955-1.109747$

C $5.349176-2.7275150 .137076$

C $6.222744-2.753806-0.881462$

C $7.712172-2.670582-0.706302$

C $8.357140-1.634058-1.598367$

C $8.947680-1.987152-2.752890$

C $8.406345-0.206709-1.104121$

C $7.1508650 .558789-1.419343$

N $3.181138-1.8065950 .630172$

H $-0.5331954 .512278-2.892346$

H $0.0038611 .847893-1.479448$

H $0.9837264 .766299-1.020722$

H $-0.0399792 .954933 \quad 1.216433$

H -1.2033313 .9213730 .344892$

H $0.9881045 .983466 \quad 3.183289$

H -0.2832357 .1953411 .712736$

H -0.1763796 .6295760 .043790$

H $-1.5986756 .232051 \quad 1.032163$

H 1.9132723 .1588802 .441870

H 2.6451284 .3213533 .509973

H 1.8055042 .7619404 .992634

H 0.5918024 .0410945 .082863

H $-0.8428002 .720146 \quad 3.469839$

H -1.1676342 .3784135 .935861$ 
H $\quad 0.1351831 .209165 \quad 5.955641$ H -2.555262 -0.9148726 .063017$ H $-0.171217-1.0430484 .590034$ H -3.469830 1.6066885 .297595 H -3.695119 0.1823354 .306905 H -2.919088 1.6205823 .618833 H -2.181566 -2.003456 4.350471 H $1.199664-1.3905652 .688486$ H $-0.082210-1.5265151 .482189$ H -1.038176 $0.665188 \quad 1.043327$ $\begin{array}{llll}\text { H } & 4.027724 & 2.234478 & 0.687828\end{array}$ H $5.1066101 .954416-2.180977$ H 6.4089951 .7887491 .447016 H 7.0943320 .1789621 .251724 H 5.3569300 .3619181 .288150 H $\quad 0.9223440 .5876870 .369799$ H 2.2659580 .4490951 .494434 H $3.582961-2.933447-1.094807$ H $3.549394-3.8211910 .439593$ H $5.720290-2.6214681 .153778$ H $5.851555-2.893345-1.894406$ H $8.126503-3.661876-0.933216$ H $7.982234-2.4716630 .338163$ H $9.441460-1.253973-3.382059$ H $8.944836-3.016079-3.097168$ H $9.2372610 .325269-1.587794$ H $8.661106-0.195686-0.040667$ H $7.0207420 .757110-2.484246$ H $3.368397-1.6669931 .619062$ C -2.818481 $2.638848-3.372395$ O $-3.0599951 .656060-2.341257$ C $-4.3223101 .012198-2.605692$ C $-4.9724641 .803585-3.733952$ C $-3.7539492 .258057-4.515343$ H $-4.0964580 .008979-2.982820$ C $-5.151168 \quad 0.930517-1.318914$ H $-5.6610631 .193084-4.325891$ H -5.529369 $2.667336-3.351430$ C $-4.0459093 .390725-5.483830$ H -3.344693 $1.402533-5.068888$ C -4.511085 $0.063959-0.211208$ O $-5.3326152 .253539-0.798850$ H -6.155619 $0.555680-1.546011$ H $-3.5660390 .507000 \quad 0.123513$ $\begin{array}{llllll}\text { O } & -5.389507 & 0.073028 & 0.920317\end{array}$ C -4.282062 -1.399674 -0.630735 C -3.758642 -2.2962130 .508364$ H -3.562731 -1.424749-1.452883 O $-5.504240-1.945883-1.129568$ H -2.832893 -1.863207 0.900897 H $-4.485560-2.3113121 .329141$ H -4.781304 $3.076473-6.231803$ H -4.447468 $4.268407-4.966252$ H -3.138233 $3.697906-6.012279$ C $-1.333714 \quad 2.629615-3.735217$ H $-4.4493252 .626787-0.634476$ H -5.525792 1.0024911 .172647 H -6.157806 -1.905161 -0.410170 H $-1.167423 \quad 3.074192-4.722546$ H $-0.9534721 .601553-3.770645$ H $-3.1124293 .613275-2.958332$ C $-1.324675-3.497837-0.912984$ O $-2.705200-3.810626-1.132266$ C $-3.524663-3.751800 \quad 0.039513$ C $-2.989472-4.7085531 .113237$ C $-1.496645-4.5234061 .375176$ C $-0.713155-4.5088350 .061552$ H $-1.232809-2.487177-0.498784$ C $-0.629480-3.526391-2.280787$ H $-4.506016-4.140973-0.260481$ O $-3.722275-4.5480672 .323500$ H -3.156797 -5.7395620 .774347$ H $-1.313944-3.6147661 .957911$ O $-1.051004-5.6157152 .181946$ H $-0.712750-5.512887-0.381602$ O $0.639167-4.1486200 .323616$ C $-0.992321-2.304790-3.100832$ H $0.461054-3.533669-2.186808$
H $-0.936350-4.425953-2.828135$

H $-3.344231-5.1622582 .976035$

H $-0.130568-5.4358992 .437001$

H $1.038936-4.8627890 .847584$

O $-1.149020-2.656889-4.409671$

O $-1.097764-1.172014-2.648494$

C $-1.495090-1.585417-5.293463$

H $-2.450107-1.142138-4.995238$

H $-0.703952-0.829563-5.301139$

H $-1.598448-1.995771-6.301438$

SCF Energy (B3LYP/6-31G**//MMFF) $=-3245.91302058$

06_00282

MMFF Geometry

C $-3.228945-3.251300-1.905708$

C $-2.390460-2.240667-1.631998$

C $-1.120019-1.961752-2.386318$

O $-0.082348-1.660317-1.418380$

C $-1.199412-0.799291-3.401067$

C -2.063951 $0.386418-3.022373$

C $-1.6593341 .428553-2.272558$

C $-3.4562260 .378342-3.603734$

C $-0.3074541 .655917-1.659998$

C $0.2346013 .036855-2.050700$

C $1.5632963 .356944-1.353869$

C $2.0042344 .785845-1.680761$

C $3.3721005 .119160-1.070405$

O $2.5488542 .423363-1.808379$

O $3.8199596 .335956-1.686433$

C $4.3665564 .000449-1.443444$

C 3.2628895 .4130140 .429892

O $5.618434 \quad 4.198611-0.789767$

C $3.8278812 .574275-1.179588$

C $4.7994821 .532643-1.782569$

O 3.7482032 .3670530 .233478

C $0.535551-2.721328-0.828657$

O $0.320446-3.904638-1.041555$

C $1.529859-2.198740 \quad 0.135316$

C $2.358191-3.0638200 .739348$

C $3.412629-2.7452151 .702471$

C $3.514731-1.3318782 .206824$

C $4.2368420 .105824-1.849378$

C $5.315487-0.911734-2.182382$

C $5.685087-3.369672-2.340584$

O $6.477004-0.599975-2.432687$

C $5.942524-3.992976-1.004310$

C $6.910150-3.586537-0.167966$

C $7.180046-4.1975721 .179107$

C $6.725478-3.3069852 .316352$

C $7.480980-2.2749122 .728472$

C $5.426773-3.6456533 .016969$

C $4.252775-3.7316662 .078157$

N $4.827461-2.211622-2.164130$

H $-3.016804-3.913039-2.741182$

H -2.613052 -1.582938 -0.795298

H $-0.807175-2.849404-2.952609$

H $-1.585074-1.203127-4.347514$

H $-0.185414-0.451594-3.636015$

H $-2.3820122 .218243-2.068666$

H $-4.036396 \quad 1.259777-3.311331$

H $-3.4064430 .369603-4.697867$

H $-4.011841-0.504565-3.278524$

H $-0.4156371 .599888-0.572300$

H $0.4106800 .885153-1.950921$

H $0.3737183 .064476-3.139602$

H $-0.5029163 .807879-1.793606$

H $1.4239933 .239576-0.273112$

H $1.2504625 .512015-1.351261$

H $2.0739424 .902838-2.771593$

H $4.7207636 .514781-1.365330$

H $4.5696344 .101072-2.518412$

H 2.5765156 .2501770 .605395

H 4.2271905 .7270650 .844421

H 2.9014644 .5605101 .007426

H 5.5167723 .9180560 .135609

H $5.0625161 .824543-2.807747$

H $5.7287461 .529689-1.198271$

H 3.8589981 .4227740 .423706 
H $1.561252-1.1301060 .310175$ H $2.272513-4.1208510 .488766$ H $3.693893-0.6380161 .381533$ H $4.330982-1.1847522 .918372$ H $2.591589-1.0394712 .717815$ H $3.791851-0.179826-0.893146$ H $3.4580340 .045348-2.618439$ H $6.619629-3.080907-2.832794$ H $5.161836-4.077697-2.990508$ H $5.298892-4.820496-0.717810$ H $7.548518-2.756546-0.463507$ H $8.259306-4.3819421 .261625$ H $6.713840-5.1869731 .266201$ H $7.174389-1.6430283 .555221$ H $8.423366-2.0389922 .245054$ H $5.216612-2.9448253 .833123$ H $5.541448-4.6213163 .507189$ H $4.107887-4.7272201 .656584$ H $3.857337-2.373388-1.910084$ C $-5.621267-2.610633-1.389736$ O $-5.314885-1.297450-0.877683$ C $-6.439858-0.805892-0.130455$ C $-7.613323-1.692015-0.524364$ C $-6.922785-3.030109-0.703613$ H $-6.213037-0.9693080 .929913$ C $-6.6363100 .688859-0.397075$ H -8.396413 -1.7100340 .239651$ H $-8.067318-1.371106-1.469118$ C -7.740728 -4.035171-1.495807 H $-6.706363-3.4484290 .288795$ C $-5.3860791 .553954-0.136022$ O $\quad-7.005290 \quad 0.876844-1.770190$ H -7.4806241 .0620630 .193745$ H $-4.6104171 .330803-0.876450$ O $\quad-5.7572752 .923107-0.343697$ C -4.7889931 .4200011 .277399$ C -3.6259212 .4019791 .516243$ H -4.4287520 .3982701 .422863$ $\begin{array}{lllll}\text { O } & -5.809091 & 1.649561 & 2.248351\end{array}$ H -2.8692682 .2418580 .740948$ H -3.9958413 .4278251 .401735$ H -8.683966 -4.252093 -0.983897 H -7.980111 -3.658351-2.495999 H -7.195271 -4.976868 -1.612487 C $-4.455769-3.554137-1.089184$ H $-6.3035630 .485397-2.318363$ H $-6.1180232 .991573-1.244429$ H -6.1515302 .5488722 .107189$ H -4.746266 -4.591698 -1.291008 H -4.195570 -3.500151 -0.024755 H -5.767275 -2.506836 -2.472517 C $-1.333446 \quad 0.5785442 .457315$ O -2.5043220 .9582693 .190060$ C -2.9966652 .2747272 .922243$ C -1.9060043 .3132803 .214877$ C -0.6210783 .0144672 .449429$ C -0.1848091 .5716862 .697033$ H $-1.5715180 .523875 \quad 1.388164$ C $-0.925594-0.8243052 .938379$ H -3.8020772 .4508893 .646210$ O -2.3723054 .6204962 .897141$ H -1.687327 3.3044964 .290743 H $-0.7403583 .224351 \quad 1.380449$ O $\quad 0.3921553 .9030052 .925321$ H $\quad 0.1835801 .4756443 .726708$ O $0.896678 \quad 1.2487181 .825019$ C -1.821553 -1.917912 2.399165 H $0.092292-1.0480812 .603741$ H $-0.962640-0.8807914 .032496$ H -1.6450205 .2404593 .077841$ H 1.1818793 .7686962 .375500 H 1.6537711 .8056502 .072067 O $-1.188415-3.1193082 .551169$ O $-2.937190-1.7501151 .928309$ C $-1.909814-4.2665402 .092538$ H -2.914943 -4.292655 2.524424 H -1.957202 -4.261484 1.000477 H $-1.367825-5.1593202 .415461$
SCF Energy (B3LYP/6-31G**//MMFF)= -3245.92594420

0600283

MMFF Geometry

C 4.2258350 .0347742 .173752

C $3.590457-0.405077 \quad 3.270437$

C $2.137098-0.1706143 .601425$

O $1.410143-1.2444072 .957281$

C 1.5611101 .1654343 .099082

C 0.1509151 .4300393 .591650

C $-0.965071 \quad 1.3031492 .845555$

C 0.0501721 .9024575 .019771

C $-1.0795770 .840747 \quad 1.418357$

C $-1.890774-0.4545351 .280899$

C $-3.400850-0.292997 \quad 1.507475$

C $-4.101486-1.6463951 .360196$

C $-5.625497-1.5192591 .482652$

O -3.9056720 .6296890 .537575$

O $-6.184827-2.7644001 .036061$

C $-6.110967-0.4089930 .525639$

C $-6.064849-1.3511562 .941692$

$\begin{array}{lllll}\text { O } & -7.504807 & -0.181387 & 0.719174\end{array}$

C -5.3080560 .9067330 .653736$

C $-5.6801811 .955941-0.424631$

O $\begin{array}{llll}-5.599972 & 1.505240 & 1.924087\end{array}$

C $0.938848-2.2677503 .710958$

O $0.968442-2.357094 \quad 4.929930$

C $0.330690-3.3175822 .858669$

C $0.604900-3.4422391 .549987$

C $0.059165-4.4503510 .637948$

C $-1.047437-5.3306431 .155296$

C $-5.5607791 .524728-1.887953$

C $-4.1238501 .314149-2.318663$

C $-2.465093-0.427960-2.875470$

O $-3.3341352 .251452-2.413481$

C $-2.447276-1.880580-3.219895$

C $-1.888748-2.817178-2.438581$

C $-1.835098-4.276234-2.788551$

C $-0.415747-4.783294-2.912577$

C $0.256256-4.676994-4.071417$

C $0.191137-5.480227-1.715485$

C $0.559362-4.523194-0.613742$

N $-3.820013-0.014295-2.572027$

H $3.6950110 .644804 \quad 1.449148$

H $4.129286-1.0536953 .958287$

H $2.032056-0.2202024 .691755$

H 2.2018041 .9928413 .431516

H 1.5840921 .1967722 .006073

H -1.9152841 .5602583 .311525$

H $-0.9753212 .165718 \quad 5.299987$

H 0.3884301 .1218495 .707889

H 0.6696502 .7918395 .172010

H -1.5317291 .6420790 .822840$

H -0.0964590 .6554320 .978146$

H $-1.725919-0.8335030 .266126$

H $-1.498863-1.2075681 .972226$

H -3.5686640 .1099402 .511827$

H $-3.715960-2.3672632 .092171$

H $-3.868275-2.0707960 .373191$

H -7.153198 -2.672290 1.046651

H $-5.998389-0.812091-0.487521$

H $-5.759504-2.2226863 .533258$

H $-7.156317-1.3016833 .024204$

H $-5.643645-0.4637043 .418412$

H $-7.8180700 .383762-0.006549$

H $-6.7052902 .306198-0.247387$

H $-5.0425232 .839036-0.277064$

H $-5.3659582 .447332 \quad 1.880490$

H $-0.326941-4.0069343 .377632$

H $1.309527-2.7489001 .094340$

H $-0.674107-5.9948311 .942164$

H $-1.498751-5.9608690 .386286$

H $-1.862662-4.7230781 .565352$

H $-6.1729130 .643137-2.099394$

H $-5.9613672 .320932-2.528339$

H - $-1.842885-0.198741-2.005577$

H $-2.0927080 .167683-3.715318$

H $-2.892434-2.168806-4.168866$ 
H $-1.449887-2.519159-1.490275$ H $-2.374688-4.468176-3.725474$ H $-2.371863-4.847379-2.021460$ H $1.266230-5.058753-4.177344$ H $-0.188842-4.199708-4.938324$ H $1.112325-6.000809-2.009882$ H $-0.484254-6.273963-1.384797$ H $1.354063-3.830600-0.892945$ H $-4.487710-0.726760-2.291621$ C $5.726621-1.3495740 .672959$ O $4.979142-0.889498-0.475048$ C $4.028732-1.910773-0.826992$ C $4.639196-3.206332-0.313354$ C $5.194013-2.7463181 .020711$ H $3.112289-1.701181-0.262232$ C $3.716080-1.890462-2.323853$ H $3.903264-4.009681-0.217361$ H $5.451711-3.556566-0.960339$ C $6.248635-3.6747351 .598187$ H $4.362528-2.6848651 .732407$

C $3.251506-0.527130-2.872862$

O $4.884682-2.272512-3.060185$ H $2.962429-2.654719-2.547002$ H $4.1026520 .160971-2.946863$ O $2.793388-0.740963-4.214893$ C $2.1241050 .154707-2.073839$ C $1.5732961 .401932-2.791977$ H $2.508050 \quad 0.439093-1.088802$ O $1.055354-0.765611-1.858096$ H $2.4160382 .022957-3.113129$ H $1.0360381 .088591-3.695383$ H $5.827207-4.6684871 .781656$ H $7.098259-3.7911060 .917128$ H $6.628191-3.2866092 .548799$ C $5.640813-0.3220121 .810912$ H $5.596039-1.652469-2.823842$ H $3.523039-1.154321-4.707930$ H $\quad 0.747350-1.069092-2.729292$ H 6.1341230 .6049591 .493108 H $6.189916-0.6900462 .685378$ H $6.771961-1.4156010 .346230$

C $2.2173513 .652134-0.816622$ O $1.2115512 .643775-0.684953$ C $0.6057692 .226712-1.912956$ C $-0.0147943 .434993-2.629430$

C $1.0032464 .551764-2.843215$

C $1.6854804 .906074-1.523262$ H $3.0519473 .239663-1.397470$ C 2.7936163 .9489840 .576333 H $-0.217606 \quad 1.558915-1.634504$ O $-0.5616323 .057269-3.888144$ H $-0.8404273 .832122-2.024629$ H $1.7408014 .285703-3.609295$ O $0.3296865 .717581-3.323780$ H $0.9699325 .440191-0.887881$ O $2.7708235 .796038-1.775761$ C 1.7916544 .5224301 .558463 H 3.1727363 .0227501 .021105 H 3.6288954 .6520680 .478175 H -1.248056 $2.390193-3.717268$ H $-0.1393285 .464850-4.137597$ H $2.4108836 .564782-2.250759$ O 2.4670515 .1603732 .560010 O 0.5767324 .4027251 .480373 C 1.6485185 .7561453 .571214 H 1.0152365 .0007624 .044829 H 2.3060096 .1881234 .330284 H 1.0385926 .5546753 .138323

SCF Energy (B3LYP/6-31G**//MMFF) $=-3245.92315320$

0600284

MM̄FF Geometry

C 1.2234912 .6729302 .062186

C 0.5108833 .0041560 .975065

C $1.0244723 .207393-0.432868$

O $0.3310702 .221519-1.239475$

C $2.5435503 .016088-0.614401$

C $3.0867063 .512449-1.938839$
C $3.4740512 .719715-2.956785$

C $3.2452425 .007750-2.047467$

C $3.3697981 .224455-3.056453$

C $4.7298320 .519667-2.951411$

C $5.2674740 .429023-1.513298$

C $6.636011-0.257055-1.494229$

C $7.144996-0.495716-0.064477$

O $4.325775-0.332543-0.754461$

O $8.263008-1.391190-0.162950$

C $6.036094-1.2060060 .742748$

C 7.6839580 .7942060 .563603

O $6.410509-1.3476002 .110891$

C $4.658492-0.5220510 .624048$

C $3.514058-1.3426811 .271132$

O $4.709421 \quad 0.735054 \quad 1.310038$

C $-0.1515792 .593313-2.451125$

O $-0.0245333 .686744-2.984956$

C $-0.8817641 .484686-3.111338$

C $-1.2701450 .364223-2.481604$

C $-2.024796-0.745699-3.074269$

C $-2.391585-0.641892-4.531226$

C $3.362485-2.8010360 .825969$

C $3.268335-3.014258-0.672309$

C $1.907885-2.435765-2.675588$

O $4.110182-3.676287-1.276604$

C $0.732116-3.290291-3.038433$

C $-0.026909-4.034029-2.218207$

C -1.227051 -4.809557-2.682665

C -2.513503 -4.294104 -2.075110

C $-3.133669-4.969304-1.092889$

C $-3.122141-3.035500-2.662170$

C $-2.362581-1.789590-2.287870$

N $2.165714-2.407714-1.248559$

H $2.2960402 .528747 \quad 1.991551$

H $-0.5622243 .140787 \quad 1.095013$

H $0.7269494 .219969-0.731431$

H $2.7894741 .958450-0.476300$

H 3.0898953 .5575290 .168870

H $3.8871213 .194269-3.846270$

H $3.6909425 .310949-3.000821$

H $2.2740685 .505413-1.967297$

H $3.8945425 .380125-1.248373$

H $2.6602250 .813840-2.332367$

H $2.9409740 .998661-4.041487$

H $4.597842-0.498865-3.340272$

H $5.463106 \quad 1.018744-3.596888$

H $5.3418431 .437632-1.092232$

H $7.3685030 .320143-2.072283$

H $6.560137-1.230941-1.998897$

H $8.544780-1.6112940 .741759$

H $5.971317-2.2242530 .342232$

H $8.5151331 .188750-0.033251$

H 8.0964760 .6078381 .561318

H $6.931106 \quad 1.581097 \quad 0.642737$

H $6.299151-0.4819822 .539461$

H $3.628846-1.3271442 .363105$

H $2.566582-0.8222841 .073504$

H 3.8069210 .9765501 .573538

H $-1.1200271 .664156-4.154840$

H $-1.0288380 .243673-1.426747$

H $-2.9797960 .263999-4.716607$

H -2.997228 -1.476071-4.890907

H -1.489463 -0.610014 -5.152111

H $4.201692-3.3966111 .203404$

H $2.460745-3.2283971 .281401$

H $2.796335-2.783495-3.211937$

H $1.706651-1.404524-2.980716$

H $0.468395-3.263938-4.094586$

H $0.199811-4.086408-1.156712$

H $-1.070422-5.862183-2.414017$

H - $-1.315867-4.794925-3.776267$

H $-4.069540-4.619748-0.669847$

H $-2.716166-5.883904-0.684938$

H $-4.150800-2.914313-2.298990$

H $-3.196698-3.173800-3.744223$

H $-2.069677-1.753006-1.238392$

H $1.509503-1.903172-0.660113$

C 0.7349031 .0060923 .850894 
$\begin{array}{llll}\text { O } & -0.277847 & 0.245465 & 3.157408\end{array}$

C -0.913631 -0.6409124 .097316$

C $-0.022485-0.6508795 .332218$

C $\quad 0.478891 \quad 0.7792605 .340163$

H $-1.873038-0.1840534 .366525$

C $-1.143526-2.0161543 .465731$

H $-0.571362-0.9206436 .239420$

H $0.816018-1.3485755 .221648$

C 1.7022050 .9875946 .216037

H $-0.329651 \quad 1.4362415 .687869$

C -2.013724 -1.999205 2.192727

O $0.123221-2.5898463 .116840$

H -1.585435 -2.692311 4.206800

H $-1.473758-1.5202931 .366864$

O $-2.225698-3.3608131 .799197$

C $-3.387347-1.3222542 .366492$

C $-4.295268-1.4985551 .136028$

H -3.236060 -0.254578 2.553867

O $-4.050113-1.8614443 .509821$

H -3.742491-1.181312 0.249192

H $-4.534950-2.5609111 .010226$

H 1.4739430 .7449307 .259126

H 2.5362820 .3527695 .898238

H $2.0382252 .028417 \quad 6.177433$

C $0.638575 \quad 2.473325 \quad 3.432347$

H $0.565883-1.9733702 .508278$

H -1.350331 -3.764111 1.667942

H -4.155814 -2.817399 3.364485

H $1.198218 \quad 3.1086824 .128133$

H $-0.407342 \quad 2.803183 \quad 3.453349$

H 1.7052740 .5794303 .563104

C $-4.8299591 .415745 \quad 0.405851$

O -5.4073230 .6805481 .494990$

C $-5.620569-0.7117941 .242096$

C $-6.551801-0.8851580 .035232$

C $-6.045182-0.126805-1.188128$

C $-5.733899 \quad 1.327892-0.834428$

H -3.839854 1.0032720 .180035

C -4.6473042 .8582620 .906464$

H $-6.152245-1.1042682 .117957$

O $-6.702988-2.266715-0.274041$

H $-7.547435-0.504708 \quad 0.298583$

H -5.174352 -0.625220 - 1.626856

O $-7.066897-0.157195-2.188022$

H $-6.679975 \quad 1.856985-0.662015$

O $-5.107376 \quad 1.923383-1.966709$

C $-3.571874 \quad 3.633082 \quad 0.178347$

H $-5.5955823 .403165 \quad 0.830807$

H $-4.3630172 .840943 \quad 1.966363$

H -7.275450 -2.328503 -1.057766

H $-6.7418980 .355653-2.947962$

H $-5.0855442 .884014-1.822102$

O -3.6004874 .9331310 .596361$

O $-2.795838 \quad 3.164179-0.641805$

C $-2.6318995 .794778-0.010286$

H $-1.621402 \quad 5.4769250 .262229$

H -2.754887 $5.801685-1.097731$

H -2.794868 6.8077810 .367011

SCF Energy (B3LYP/6-31G**/MMFF) $=-3245.92397666$

0600285

MMTFF Geometry

C $0.884777 \quad 0.889138 \quad 3.302826$

C 1.9560831 .6937343 .361807

C 2.3200912 .6412822 .252681

$\begin{array}{llll}\text { O } & 3.660040 & 2.289684 & 1.835372\end{array}$

C 2.3077734 .1147272 .690273

C $0.9572554 .644103 \quad 3.119719$

C -0.0476194 .9357052 .270788$

C 0.7983674 .8840504 .597559

C $-0.058570 \quad 4.7450120 .779470$

C $-1.296876 \quad 3.951642 \quad 0.339711$

C $-1.1591373 .433162-1.099880$

C $-2.5384953 .240486-1.731307$

C -2.439777 2.644195-3.140238

O $-0.4676432 .175938-1.042251$

O $-3.7659142 .260601-3.529751$

C $-1.5759151 .372047-3.066470$
C $-1.9758883 .682940-4.167214$

O $-1.359221 \quad 0.833532-4.369304$

C $-0.2323841 .564309-2.317645$

C $0.4117140 .173652-2.096141$

O $0.6386602 .360697-3.122242$

C 3.9962462 .5448480 .542629

O $3.3270993 .122201-0.298966$

C 5.3159861 .9258230 .305289

C $5.5938121 .462761-0.921956$

C $6.7914020 .715973-1.306113$

C $7.9404680 .696204-0.332205$

C $1.6286150 .157309-1.163788$

C $2.207702-1.242345-1.024608$

C $3.909068-2.5295930 .264152$

O $1.833182-2.191735-1.708605$

C $5.326188-2.420530-0.202317$

C $5.802816-3.041369-1.292678$

C $7.238587-2.988872-1.738124$

C $7.415677-2.292010-3.070497$

C $7.266087-2.960707-4.226234$

C $7.844348-0.839513-3.056195$

C $6.7827250 .058339-2.484613$

N $3.200912-1.304148-0.057147$

H $\quad 0.236147 \quad 0.9346342 .430749$

H 2.6102591 .6589234 .229098

H 1.6396122 .4815991 .408875

H 2.6921014 .7431001 .875594

H 3.0412994 .2620253 .495421

H $-0.966325 \quad 5.3500802 .684124$

H $-0.194515 \quad 5.269473 \quad 4.852700$

H 0.9430103 .9526825 .154139

H $1.5364315 .614754 \quad 4.944170$

H $-0.080905 \quad 5.7329300 .303330$

H $\quad 0.848975 \quad 4.254184 \quad 0.422124$

H $-1.458173 \quad 3.103904 \quad 1.017981$

H -2.168758 4.6125800 .426252

H $-0.5689674 .146403-1.686942$

H -3.093985 4.186329-1.756981

H $-3.1302472 .561918-1.101437$

H $-3.7014081 .802290-4.385511$

H $-2.1687230 .620448-2.526989$

H $-2.668873 \quad 4.532692-4.188293$

H $-1.9826983 .268385-5.181441$

H $-0.9768974 .073378-3.962716$

H $-0.6754551 .372818-4.801967$

H $-0.337552-0.502313-1.664403$

H $0.705612-0.248658-3.065792$

H $1.5559982 .188720-2.857284$

H 5.9760521 .7941381 .154501

H $4.8478391 .586186-1.706228$

H $8.2380491 .717969-0.070594$

H $8.8351700 .206148-0.720722$

H 7.6609830 .1674270 .585381

H $2.4196250 .802715-1.555016$

H $1.3435790 .513446-0.168105$

H $3.400449-3.389387-0.183786$

H $3.884067-2.6513601 .351670$

H $5.997972-1.8264140 .411224$

H $5.126995-3.646479-1.893207$

H $7.606634-4.020767-1.811199$

H $7.872979-2.510978-0.981438$

H $7.417233-2.476057-5.185023$

H $6.985581-4.008967-4.237521$

H $8.056170-0.490563-4.075278$

H $8.797261-0.762582-2.525983$

H $5.8948800 .133001-3.114421$

H $3.446014-0.4639190 .458204$

C $0.572338-1.5290063 .838059$

O $-0.381880-1.7139582 .773818$

C $-0.853422-3.0756312 .807389$

C $-0.136370-3.7571073 .972216$

C $0.205642-2.5870404 .873283$

H $-1.926486-3.0350243 .024194$

C $-0.604938-3.7494821 .452470$

H $-0.769873-4.5031664 .462561$

H $0.776255-4.2688043 .644536$

C $1.308200-2.8956065 .870929$

H $-0.700903-2.2847415 .414426$ 
C -1.303036 -3.072943 0.254864 O $0.804432-3.7509751 .187840$ H $-0.898835-4.8041331 .505102$ H $-0.904936-2.0643750 .097174$ O $-0.965523-3.828861-0.916412$ C $-2.839689-3.0083880 .362928$ C $-3.514597-2.555408-0.949913$ H -3.116535 -2.320259 1.168446 O $-3.340918-4.2971190 .721474$ H -3.129217 -1.566859-1.221422 H -3.232992 -3.245074 -1.754098 H $1.014094-3.7229636 .525151$ H $2.237754-3.1799415 .366186$ H $1.520011-2.0271486 .502126$ C $0.504796-0.0971504 .369434$ H $1.099160-2.8242301 .172696$ H $\quad 0.004733-3.832767-0.987993$ H -3.083722 -4.9179860 .018116$ H 1.1596460 .0113655 .241314 H -0.5141490 .1371524 .697505$ H $1.567914-1.7280283 .418106$ C $-5.396644-0.290924-0.043500$ O $-5.526623-1.6974530 .197296$ C $-5.057870-2.539547-0.859947$ C $-5.775643-2.204283-2.176353$ C $-5.714175-0.716061-2.504919$ C $-6.1762440 .104953-1.302044$ H $-4.336583-0.033886-0.160162$ C $-5.9375810 .436579 \quad 1.190477$ H -5.366716 -3.558169 -0.593322 O $-5.226972-2.965527-3.246916$ H -6.829558 -2.498335 -2.084702 H $-4.707653-0.427984-2.829512$ O $-6.584274-0.461207-3.609075$ H -7.250530 -0.057235 -1.148902 O $-6.0023791 .491158-1.578725$ C $-4.987730 \quad 0.2819882 .352399$ H -6.0832001 .5076431 .010491$ H -6.9074990 .0285851 .499235$ H -5.697867 -2.708320 -4.058120 H $-6.566166 \quad 0.496918-3.775845$ H $-5.0643631 .638215-1.785841$ O -4.0111631 .2315912 .281222$ O $-5.088249-0.5909523 .204771$ C $-3.027291 \quad 1.1702683 .316316$ H -2.2928531 .9596933 .137876$ H -3.492571 1.3382184 .292207 H -2.518720 0.2026423 .292256

SCF Energy $\left(B 3 L Y P / 6-31 G^{* *} / / M M F F\right)=-3245.93968454$

0600286

MM̄FF Geometry

C 0.0109022 .8558431 .291508

C - $-1.2798352 .840061 \quad 1.656341$

C -2.1083731 .5844301 .667918$

O $-3.276006 \quad 1.7730990 .830877$

C -2.6432471 .2437283 .070295$

C -1.5885310 .9216114 .104829$

C $-0.902817-0.2368644 .151095$

C $-1.350571 \quad 1.9875445 .141166$

C $-1.008612-1.3913023 .195121$

C $0.307288-1.5688122 .428570$

C $0.209078-2.6662561 .361271$

C $1.586542-2.9362190 .757788$

C $1.522406-3.954150-0.386271$

O $-0.687513-2.2155430 .340964$

O $2.786355-3.899334-1.065839$

C $0.427537-3.520313-1.382750$

C $1.378733-5.386306 \quad 0.141342$

O $0.260958-4.530672-2.375603$

C $-0.923219-3.166725-0.708014$

C $-1.869136-2.506596-1.744572$

O $-1.520458-4.361735-0.208424$

C $-3.1241191 .649991-0.511864$

O $-2.0701821 .503379-1.116151$

C $-4.4291461 .676405-1.214304$

C $-5.6153551 .687171-0.585951$

C $-6.9346971 .638489-1.222769$
C $-6.9804261 .466363-2.718604$

C $-3.153373-1.888279-1.175065$

C $-4.327187-2.843255-1.048481$

C $-6.717178-2.868579-0.324044$

O $-4.267256-4.030412-1.353583$

C -7.816381 -2.075395 -0.957467

C $-8.865309-1.584803-0.279605$

C $-9.996441-0.829183-0.917872$

C $-10.2286040 .524117-0.283572$

C $-11.1334840 .680540 \quad 0.697646$

C $-9.4776711 .709265-0.848533$

C $-8.0303351 .723379-0.438977$

N $-5.455661-2.194246-0.564356$

H $\quad 0.491749 \quad 1.9259770 .996904$

H -1.7680833 .7646971 .953379$

H -1.5415620 .7312981 .276506$

H -3.3426750 .3998392 .991356$

H -3.277909 2.0662633 .429601

H $-0.175431-0.3748204 .949991$

H $-0.5793181 .700693 \quad 5.863903$

H -1.0259712 .9186014 .666319$

H -2.271107 2.1844405 .700319

H -1.223797 -2.299805 3.770505

H -1.841646-1.266212 2.497563

H $0.573286-0.6188661 .946666$

H $1.108072-1.8117343 .138668$

H $-0.193748-3.5735931 .826165$

H $2.286453-3.2719761 .532335$

H $2.001453-1.9975830 .364302$

H $2.732972-4.493021-1.834621$

H $0.807573-2.629594-1.902993$

H $2.233873-5.6445690 .777744$

H $1.385677-6.114116-0.677551$

H $\quad 0.470086-5.537707 \quad 0.727773$

H $-0.257498-4.151579-3.104508$

H -1.319746 -1.683572 -2.221086

H -2.130629 -3.224115 -2.530703

H -2.466915 -4.202434 -0.069310

H $-4.3573961 .638389-2.296763$

H $-5.634873 \quad 1.7173090 .501957$

H $-6.5455582 .338701-3.218641$

H -7.989827 $1.340219-3.114569$

H $-6.4218220 .572858-3.020341$

H $-2.973031-1.414501-0.204818$

H -3.477095 -1.105696-1.872800

H $-6.837743-2.9509080 .760979$

H -6.694612 -3.878021 -0.745956

H -7.756709-1.919212 -2.032038

H -8.934937 -1.763502 0.791156

H -10.902204 -1.442599-0.822272

H $-9.838942-0.706099-1.996609$

H -11.328491 1.6525241 .138358

H $-11.695960-0.1624861 .085421$

H $-9.9247192 .645113-0.487147$

H $-9.6176661 .733870-1.932453$

H $-7.888713 \quad 1.8404370 .636643$

H $-5.371343-1.229948-0.256015$

C $1.3045784 .432719-0.157066$

O $2.2323543 .437557-0.639029$

C $3.2980614 .099992-1.344851$

C $2.8626485 .551909-1.509357$

C $2.0451315 .765684-0.252505$

H $4.1762244 .070624-0.689396$

C $3.6001273 .385417-2.666245$

H $3.7150756 .233344-1.590017$

H $2.2365365 .690185-2.398591$

C $1.1341516 .979201-0.322175$

H 2.7303625 .8773000 .598532

C $4.0067811 .903734-2.533473$

O $2.4383343 .438191-3.505349$

H $4.3819203 .933259-3.205468$

H $3.1451181 .293490-2.236640$

O $4.3892711 .448306-3.838656$

C $5.1741981 .639937-1.564535$

C $5.7300020 .205372-1.668788$

H $4.8363111 .831251-0.541439$

O $6.2366372 .557619-1.831759$

H $4.897192-0.504219-1.643896$ 
H $6.2305320 .089109-2.637315$ H $1.7210507 .892464-0.464676$ H $0.425596 \quad 6.901989-1.153710$ H 0.5579617 .0899540 .601649 C $0.863308 \quad 4.0915551 .266596$ H $1.7074273 .018332-3.019721$ H $3.6373611 .611604-4.433892$ H $6.5162252 .423501-2.753812$ H $0.3176414 .935248 \quad 1.704119$ H 1.7408113 .9062281 .897433 H $0.4434844 .422230-0.838571$ C $5.243421-1.1271281 .050549$ O $6.143762-0.0518070 .751508$ C $6.747512-0.107129-0.547030$ C $7.511322-1.427203-0.721765$ C $6.638491-2.639075-0.414776$ C $5.971286-2.4741340 .950039$ H $4.396669-1.1076020 .355274$ C $4.702056-0.8989112 .469644$ H $7.4987100 .692471-0.567253$ O $8.037973-1.522754-2.040911$ H $8.368884-1.430092-0.036066$ H $5.900200-2.803201-1.208553$

O $7.472068-3.799525-0.391372$ H $6.735982-2.5490821 .733283$ O $5.060196-3.5497331 .156855$ C 3.7553670 .2812052 .545320 H $4.146883-1.7766532 .817226$ H $5.537006-0.7015643 .152611$ H $8.495247-2.378349-2.110254$ H $6.909118-4.554811-0.148935$ H $4.408314-3.5299770 .435591$ O 3.4719930 .5335543 .858185 O $3.308672 \quad 0.894351 \quad 1.586154$ C $2.585648 \quad 1.630691 \quad 4.097555$ H 3.0630052 .5688763 .799969 H 1.6458771 .4878383 .558045 H 2.3709651 .6682815 .168809 SCF Energy (B3LYP/6-31G**//MMFF) $=-3245.94294533$

\section{7}

MMFF Geometry

C -0.448995 -3.426209 0.122918

C $-0.615397-2.438595-0.769280$

C $-0.302030-2.545547-2.235353$

O $0.489925-1.379546-2.575571$

C $-1.574678-2.478757-3.094940$

C $-2.370928-3.764756-3.116275$

C $-3.515394-3.965136-2.435359$

C $-1.830398-4.841890-4.020739$

C $-4.185491-3.025065-1.474661$

C $-5.339066-2.267128-2.137743$

C $-6.175149-1.487570-1.112853$

C $-7.307803-0.728524-1.808545$

C $-8.1236850 .118523-0.821167$

O $-5.319587-0.571293-0.418636$

O $-8.929967 \quad 1.012390-1.603932$

C $\quad \begin{array}{llll}-7.150128 & 0.970400 & 0.022619\end{array}$

C $-9.101794-0.743117-0.014531$

O

C -5.9799000 .1586830 .624461$

C -4.9382061 .0556411 .330796$

O $-6.502094-0.7273371 .624210$

C $1.840253-1.468847-2.476477$

O $2.493826-2.471213-2.223827$

C $2.479100-0.160146-2.743597$

C $1.8423811 .008809-2.566837$

C $2.4058652 .344524-2.779356$

C $3.8172112 .427371-3.300877$

C $-4.197628 \quad 2.050206 \quad 0.432657$

C $-3.2859442 .959197 \quad 1.241473$

C -1.8714694 .9724851 .033211$

O -3.1641772 .8752522 .461239$

C $-0.983567 \quad 5.6048750 .011279$

C 0.3454195 .7147530 .159206

C $1.2382056 .455203-0.792880$

C $2.3103545 .581651-1.399597$

C $3.5387835 .516981-0.858194$
C $1.9920814 .874046-2.695709$

C $1.6422403 .423273-2.501330$

N $-2.626313 \quad 3.8851400 .440150$

H $-0.095091-4.391834-0.232443$

H $-0.981331-1.468848-0.443118$

H $0.264635-3.455118-2.466536$

H - $1.295852-2.228254-4.128257$

H -2.195200 -1.628604 -2.782918

H $-4.026634-4.919368-2.556395$

H -2.471582 -5.729569-4.040006

H $-1.751437-4.471321-5.047993$

H $-0.837838-5.161422-3.688753$

H $-4.563391-3.619174-0.633060$

H $-3.471349-2.317627-1.043465$

H -4.919762 -1.572463 -2.877195

H $-5.989122-2.966715-2.678182$

H $-6.585116-2.208553-0.395314$

H $-7.962925-1.418754-2.354664$

H $-6.880891-0.058513-2.568487$

H $-9.3947091 .605102-0.988264$

H $-6.7480451 .733064-0.654218$

H $-9.801287-1.254548-0.686928$

H $-9.721770-0.1273630 .646389$

H $-8.605883-1.505808 \quad 0.589125$

H -8.0554081 .0512301 .753325$

H $-5.421017 \quad 1.5839792 .163740$

H -4.1824440 .4101321 .801416$

H -5.773433 -0.996311 2.208081

H $3.520791-0.211012-3.040975$

H $\quad 0.8098300 .996370-2.220685$

H $4.1739443 .448824-3.444919$

H $3.9004361 .924052-4.270455$

H $4.5122691 .956628-2.597642$

H $-3.5893711 .514649-0.304744$

H $-4.9050552 .693416-0.099210$

H -1.3134254 .5957321 .897113$

H -2.594015 5.7089401 .401563

H -1.462004 $6.042832-0.861244$

H $0.815731 \quad 5.302296 \quad 1.049147$

H $1.7040797 .279134-0.235778$

H $0.6599796 .940403-1.588898$

H $4.3254514 .922658-1.310659$

H 3.7826326 .0530650 .053056

H $2.8199505 .007218-3.399772$

H $1.1434825 .363720-3.190508$

H $0.6362103 .264080-2.111778$

H $-2.9339083 .986850-0.523235$

C $-0.809061-1.9176082 .184558$

O $0.406083-1.1862881 .934592$

C $0.651951-0.294563 \quad 3.036906$

C $-0.534343-0.4453203 .986651$

C $-0.991862-1.864276 \quad 3.698415$

H $1.553337-0.6586933 .542131$

C $\quad 0.8880391 .1270652 .513224$

H $-0.246749-0.2919975 .031397$

H $-1.3349940 .267462 \quad 3.756926$

C -2.409195 -2.149909 4.162879

H $-0.300126-2.5631664 .187167$

C $1.9865881 .231441 \quad 1.435447$

$\begin{array}{llll}\text { O } & -0.332059 & 1.591864 & 1.924078\end{array}$

H 1.1059651 .7970843 .353399

H $1.650300 \quad 0.751908 \quad 0.508478$

$\begin{array}{llll}\text { O } & 2.172346 & 2.619038 & 1.130985\end{array}$

C 3.3493500 .6425721 .842336

$\begin{array}{llll}\text { C } & 4.437432 & 0.907829 & 0.784285\end{array}$

H $3.243108-0.4365451 .989680$

$\begin{array}{lllll}\text { O } & 3.762819 & 1.199921 & 3.088768\end{array}$

H $4.0702610 .564903-0.187755$

H 4.6103201 .9877310 .701260

H -2.492819-2.016443 5.246392

H -3.131559-1.479620 3.684546

H -2.697730 -3.1789503 .926758$

C $-0.677114-3.3326401 .612550$

H -0.2304852 .5429091 .753761$

H 1.3976152 .9119250 .622459

H 3.8259382 .1636392 .972972

H $-1.567081-3.9225601 .858541$

H $0.178549-3.8235372 .093100$ 
H $-1.629043-1.3774911 .693347$

C $5.304750-1.9570030 .217251$

O $5.643028-1.1726771 .366983$

C $5.785210 \quad 0.2294991 .117370$

C 6.8825890 .4643750 .069640

C $6.641020-0.345189-1.202358$

C $6.380691-1.812985-0.865454$

H $4.331879-1.634926-0.172189$

C $5.177234-3.4131130 .673720$

H 6.1372770 .6728752 .057335

$\begin{array}{lllll} & 0 & 6.973648 & 1.850282 & -0.243695\end{array}$

H $7.849186 \quad 0.1679220 .497648$

H $5.8204340 .081298-1.788866$

O $7.807828-0.252383-2.022629$

H $7.318483-2.273328-0.530076$

O $5.980883-2.499165-2.050088$

C $3.914456-3.6044401 .478129$

H $5.120873-4.105381-0.173846$

H $6.040058-3.716167 \quad 1.279065$

H $7.6629711 .950149-0.922526$

H $7.655299-0.808060-2.806153$

H $5.129096-2.128434-2.337793$

O $4.204146-3.6391602 .809228$

O $2.800323-3.6896990 .978514$

C $3.080133-3.8085093 .677866$

H $2.586187-4.7638193 .476189$

H $3.443864-3.8116094 .708777$

H $2.378876-2.9787273 .556265$

SCF Energy (B3LYP/6-31G**//MMFF) $=-3245.92034877$

0600288

MMFF Geometry

C -2.601024 -2.757954 -2.734940

C $-1.777702-2.146310-1.871046$

C $-0.573122-1.340010-2.267626$

O $0.539162-1.927733-1.546179$

C $-0.6919390 .138900-1.858313$

C -1.474507 $0.984121-2.839158$

C $-0.916588 \quad 1.674241-3.853699$

C $-2.956198 \quad 1.077571-2.589984$

C $0.538192 \quad 1.738250-4.232298$

C $1.0985623 .166765-4.176718$

C $1.096146 \quad 3.781489-2.767894$

C $1.5622575 .238539-2.820720$

C $1.6259245 .866632-1.421772$

$\begin{array}{llll}\text { O } & 1.976126 & 3.017770 & -1.933027\end{array}$

O $2.3470357 .101161-1.549491$

C $2.4354604 .933587-0.498319$

C $0.2306816 .235476-0.904425$

O $2.412478 \quad 5.408486 \quad 0.846402$

C $1.998078 \quad 3.449997-0.565159$

C $3.013267 \quad 2.581538 \quad 0.216412$

$\begin{array}{lllllllllll}\text { O } & 0.711875 & 3.320884 & 0.046791\end{array}$

C $1.764225-1.899421-2.133338$

O $2.054534-1.400689-3.208341$

C $2.705365-2.603249-1.232422$

C $3.994220-2.716589-1.586107$

C $5.049915-3.391366-0.826119$

C $4.656767-4.064575 \quad 0.461691$

C $2.8690211 .073239-0.015328$

C $3.5573290 .281801 \quad 1.079395$

C $5.775816-0.1450132 .107482$

O $2.912710-0.374856 \quad 1.894832$

C $7.065439-0.6214781 .521745$

C $7.443820-1.9077101 .542878$

C $8.733489-2.4237250 .977030$

C $8.610149-2.929042-0.443216$

C $9.448592-2.493660-1.399422$

C $7.563255-3.979627-0.751770$

C $6.305381-3.373994-1.321561$

$\begin{array}{llll}\mathrm{N} & 4.938363 & 0.390308 & 1.050081\end{array}$

H -2.430415 -2.663224 -3.803874

H - $1.970023-2.246867-0.806237$

H $-0.394728-1.432529-3.346774$

H $0.309550 \quad 0.561845-1.729843$

H $-1.1342110 .227413-0.857231$

H -1.571600 $2.262868-4.495003$

H $-3.4479411 .784264-3.267078$
H -3.437045 $0.106520-2.727702$

H $-3.1452551 .421620-1.567989$

H $1.1582151 .078940-3.620562$

H $0.6308411 .369308-5.261233$

H $2.1290073 .133041-4.553825$

H $0.5253543 .806321-4.859864$

H $0.079893 \quad 3.731969-2.362087$

H $0.9147135 .832888-3.477442$

H $2.5650965 .283757-3.268749$

H $2.4596897 .470309-0.656531$

H $3.4832835 .000154-0.822014$

H $-0.254816 \quad 6.940816-1.589654$

H $0.288657 \quad 6.7510380 .060662$

H $-0.428553 \quad 5.372057-0.794137$

H 1.5489605 .1745761 .227689

H $4.0319912 .862026-0.080662$

H 2.9220932 .7924751 .289994

H 0.6199012 .4270250 .412903

H $2.311471-3.012319-0.308843$

H $4.313016-2.272634-2.528479$

H $4.268627-3.3292101 .175081$

H $5.483276-4.5788970 .955599$

H $3.884124-4.8202750 .281419$

H $1.8189420 .768194-0.020064$

H $3.2913820 .786140-0.985071$

H $5.242196-0.9399542 .639254$

H 5.9654520 .6658592 .818348

H $7.716807 \quad 0.121773 \quad 1.068883$

H $6.790670-2.6437752 .004820$

H $9.087551-3.2434191 .614846$

H $9.501608-1.6423551 .046578$

H $9.383314-2.864249-2.417187$

H $10.216754-1.757318-1.187914$

H $7.384814-4.5927570 .135540$

H $7.959509-4.679243-1.500396$

H $6.471346-2.865155-2.272944$

H $5.361308 \quad 1.045153 \quad 0.398662$

C $-5.094428-2.872005-2.424842$

O $-5.146697-1.808547-1.449561$

C $-6.372183-1.917023-0.706233$

C $-7.286772-2.792113-1.552073$

C $-6.292828-3.778435-2.130407$

H $-6.132592-2.4478020 .223316$

C $-6.933886-0.530720-0.380182$

H -8.072333 $-3.269744-0.958931$

H $-7.765342-2.222970-2.357356$

C $-6.812053-4.524919-3.347177$

H $-6.031098-4.507574-1.351590$

$\begin{array}{llll}\text { C } & -5.954840 & 0.417691 & 0.339898\end{array}$

O

H $-7.849831 \quad-0.6392800 .212383$

H $-5.177757 \quad 0.757398-0.354017$

O $-6.692883 \quad 1.5888140 .714986$

C $-5.285841-0.1628601 .600027$

C -4.4802990 .8983642 .374954$

H $-4.621905-0.9828631 .307471$

O $-6.280762-0.7120662 .463231$

H -3.8028651 .4024641 .677666$

H -5.1693581 .6578702 .763385$

H -7.698343 -5.113426 -3.087998

H $-7.089484-3.836149-4.152219$

H $-6.052956-5.210548-3.736834$

C -3.760832 -3.608545 -2.298996

H $-6.5493040 .162325-2.173451$

H $-7.0925161 .951049-0.094474$

H $-6.8955320 .004602 \quad 2.696875$

H $-3.765727-4.515303-2.914789$

H $-3.612581-3.928922-1.259503$

H $-5.190027-2.403983-3.412710$

C -1.657306 -0.2882102 .412722$

O $-2.791230-0.7295353 .166806$

C $-3.691105 \quad 0.3099233 .567991$

C $-2.943277 \quad 1.3606104 .401769$

C $-1.717400 \quad 1.901078 \quad 3.667194$

C $-0.8329130 .750256 \quad 3.187069$

H -2.015296 0.1624991 .479086

C $-0.852641-1.5244771 .984765$

H $-4.426174-0.1617504 .232104$ 
O $-3.8178992 .429428 \quad 4.746342$

H $-2.617604 \quad 0.8974345 .342285$

H -2.0113052 .5459952 .831444$

O $-0.967523 \quad 2.735384 \quad 4.554215$

H -0.3439130 .3030284 .060042$

$\begin{array}{lllll}\text { O } & 0.189260 & 1.288329 & 2.354523\end{array}$

C $-0.161975-2.2493743 .120148$

H -1.517941 -2.255847 1.512733

H $-0.097132-1.2217211 .250762$

H -3.297769 3.0819715 .245946

H -0.6885572 .1944995 .312623$

H $0.851798 \quad 0.590570 \quad 2.216834$

O $0.961586-2.8613632 .641855$

O $-0.572043-2.3028114 .271537$

C $1.721179-3.595874 \quad 3.606434$

H $2.091356-2.9235494 .386179$

H $1.113074-4.395177 \quad 4.040460$

H $2.576610-4.0450833 .095244$

SCF Energy (B3LYP/6-31G**/MMFF) $=-3245.93498519$

\section{6_00289}

MM̄FF Geometry

C $-1.958045 \quad 3.387464 \quad 1.881112$

C -0.9400292 .7471062 .477004$

C -1.039382 1.6500743 .511592

O -0.4735300 .4519602 .924638$

C $-2.472088 \quad 1.2911903 .956758$

C -2.5432820 .0846324 .875000$

C $-2.903145-1.1468694 .456604$

C $-2.225656 \quad 0.3469696 .320961$

C $-3.198310-1.5438573 .035124$

C $-4.691233-1.4413862 .704149$

C $-4.924769-1.3099121 .192015$

C $-6.416540-1.1868270 .881543$

C $-6.674877-1.097124-0.629003$

O $-4.381343-2.459725 \quad 0.536200$

O $-8.083270-1.293903-0.824882$

C $-5.928963-2.252544-1.329943$

C $-6.3559360 .301128-1.172514$

O $-6.032914-2.131165-2.747463$

C $-4.448372-2.398566-0.897031$

C $-3.876290-3.722099-1.464423$

O $-3.708924-1.292594-1.411759$

C $0.846204 \quad 0.1910283 .104858$

$\begin{array}{llll}\text { O } & 1.686148 & 0.922926 & 3.600596\end{array}$

C $1.075559-1.1861552 .611668$

C $2.319075-1.6832182 .545779$

C $2.682909-3.0324932 .104451$

C $1.564597-4.0183951 .896359$

C -2.445703 -4.066295-1.026137

C $-1.359451-3.485073-1.916952$

C $1.111235-3.432286-2.165326$

O $-1.586036-2.723342-2.852594$

C $2.248906-4.344197-1.838134$

C $3.446273-3.912278-1.413337$

C $4.595699-4.831430-1.113865$

C $5.337942-4.4758220 .155816$

C $6.632061-4.1143670 .117335$

C $4.612414-4.6172261 .476649$

C $3.987698-3.3219331 .920545$

$\mathrm{N}-0.099345-3.924237-1.535101$

H -2.984892 3.1549902 .146225

H 0.0732783 .0063932 .176740

H $-0.456747 \quad 1.9442874 .393659$

H -3.097336 $1.102403 \quad 3.076124$

H -2.9325252 .1506304 .461481$

H -2.966059 -1.952765 5.185281

H $-2.325498-0.5530226 .937006$

H $-1.197930 \quad 0.708016 \quad 6.427513$

H -2.904331 1.1036686 .727918

H $-2.611253-0.9365142 .338329$

H -2.852705 -2.573556 2.879807

H $-5.195853-2.3368573 .088850$

H $-5.137434-0.5741853 .206857$

H $-4.392486-0.4149600 .847479$

H -6.854141 -0.3232691 .397683$

H $-6.943185-2.0683621 .273691$

H $-8.247008-1.315852-1.783552$
H -6.459013 -3.178606 -1.068032

H $-6.963770 \quad 1.056229-0.659357$

H $-6.6143360 .382994-2.233847$

H $-5.3072220 .580643-1.051055$

H -5.400686 -1.449484 -3.033031

H $-4.515483-4.541791-1.109739$

H $-3.930552-3.717227-2.559569$

H -2.762942 -1.500060 -1.359012

H $0.202501-1.7615722 .324286$

H $3.150358-1.0436392 .835004$

H $1.033254-4.1960162 .838115$

H $1.897467-4.9911201 .529785$

H $0.848864-3.6379151 .162692$

H $-2.260450-3.7560320 .007942$

H $-2.333574-5.157714-1.068994$

H $1.279162-2.413440-1.801842$

H $\quad 0.953360-3.392982-3.247970$

H $2.088051-5.410709-1.977182$

H $3.616606-2.844207-1.307846$

H $5.278813-4.792015-1.972205$

H $4.256895-5.872516-1.040263$

H $7.179398-3.883307 \quad 1.025341$

H $7.171236-4.034353-0.820577$

H $5.324870-4.9223272 .255123$

H $3.899797-5.4437701 .414449$

H $4.719063-2.5369372 .117347$

H $-0.014274-4.459563-0.676245$

C -2.195291 3.825504-0.556301

O $-1.176182 \quad 2.902985-0.997816$

C $-0.9400403 .110493-2.403186$

C $-2.0772013 .997689-2.892967$

C $-2.3213414 .862100-1.670851$

H $-0.0033243 .673502-2.488265$

C $-0.8133151 .765870-3.126976$

H $-1.8017914 .573996-3.781265$

H $-2.9736433 .413311-3.132148$

C $-3.6575785 .583634-1.695628$

H -1.512317 $5.600977-1.591830$

C $0.301896 \quad 0.848562-2.582061$

O $-2.0526851 .054117-3.014817$

H $-0.6615801 .937323-4.198796$

H $0.0467010 .494635-1.575681$

O $0.357800-0.311855-3.420304$

C $1.6985151 .494359-2.564508$

C $2.8028080 .529864-2.092154$

H $1.6826062 .360383-1.900503$

O $2.0187851 .974797-3.869911$

H $2.5195730 .123073-1.115210$

H $2.872031-0.314388-2.788716$

H $-3.7148446 .257786-2.556467$

H $-4.4931404 .878841-1.764798$

H $-3.7958106 .181025-0.789015$

C - $1.794294 \quad 4.4157750 .796550$

H $-2.2408380 .939530-2.067187$

H - $0.524474-0.721217-3.407631$

H $2.0138341 .211220-4.472304$

H $-2.4270295 .274512 \quad 1.047099$

H -0.7587154 .7717830 .762279$

H $-3.126496 \quad 3.249649-0.467144$

C $4.0158562 .172766 \quad 0.198912$

O $4.1762002 .392813-1.208799$

C $4.1899671 .210446-2.015296$

C $5.3284920 .286789-1.566948$

C $5.254796-0.016161-0.074262$

$\begin{array}{llll}\text { C } & 5.144210 & 1.277447 & 0.732184\end{array}$

H 3.0424351 .7062660 .393331

C 4.0372013 .5535370 .874872

H $4.4329451 .541339-3.033126$

O $5.306674-0.921215-2.318542$

H $6.2873880 .777586-1.779127$

H $4.426991-0.6986230 .146839$

$\begin{array}{lllll}\text { O } & 6.452668 & -0.698483 & 0.302388\end{array}$

H $\quad 6.107416 \quad 1.802743 \quad 0.691177$

$\begin{array}{lllll} & \text { O } & 4.902469 & 0.926016 & 2.092057\end{array}$

C 2.8361854 .3959540 .501801

H 4.0336533 .4648411 .965566

H 4.9397494 .0920050 .560787

H $6.011958-1.493911-1.971068$ 
H $\quad 6.406871-0.855411 \quad 1.261151$ H 5.0510671 .7168992 .636510 O $3.132561 \quad 5.7147700 .695389$ O 1.7616583 .9541130 .120155 C $2.078152 \quad 6.6334200 .390087$ H $1.7473146 .506503-0.645465$ H 1.2436996 .4900251 .082879 H 2.4661357 .6484210 .511607 SCF Energy (B3LYP/6-31G**//MMFF) $=-3245.94382253$

0600290

MM̄FF Geometry

C 0.0634872 .0197052 .254007

C -0.9740331 .6943063 .041220$

C $-1.511566 \quad 0.299172 \quad 3.268619$

O $-0.797657-0.6441142 .437252$

C -3.0070490 .2232962 .910652$

C $-3.638498-1.0970253 .306741$

C $-3.819408-2.1433152 .476287$

C $-4.084401-1.1853914 .743023$

C $-3.426648-2.2493481 .028561$

C $-4.594737-2.6405210 .114391$

C $-5.771603-1.6531050 .129026$

C $-6.854663-2.113120-0.850583$

C $-8.016337-1.115163-0.933230$

O $-5.285759-0.359401-0.240590$

O $-8.803106-1.482402-2.076756$

C $-7.4389760 .293752-1.184384$

C $-8.945545-1.2305230 .280588$

O $-8.474517 \quad 1.273997-1.140066$

C $-6.2811430 .671112-0.227536$

C $-5.6177022 .009651-0.638765$

O $-6.819526 \quad 0.852927 \quad 1.087869$

C $-0.268612-1.7538523 .010799$

O $-0.296985-2.054128 \quad 4.195921$

C $0.315467-2.653161 \quad 1.988432$

C $0.659257-2.245327 \quad 0.756775$

C $1.177867-3.090959-0.320181$

C $1.238194-4.577444-0.082979$

C $-4.7482211 .948234-1.899601$

C $-3.2994221 .578379-1.622072$

C $-1.1511091 .153775-2.803006$

O $-2.8278161 .495875-0.491573$

C $-0.880252-0.286756-3.095129$

C $-0.023192-0.681798-4.048832$

C $0.331967-2.113103-4.336470$

C $1.772769-2.422083-3.987141$

C $2.776192-2.064039-4.806459$

C $2.047689-3.203991-2.718729$

C $1.564217-2.507995-1.474437$

N $-2.5798961 .395798-2.794293$

H $\quad 0.582891 \quad 1.2430001 .699542$

H -1.458890 2.4831373 .613299

H $-1.3666310 .069734 \quad 4.331315$

H $-3.143290 \quad 0.398967 \quad 1.839477$

H $-3.5570801 .033740 \quad 3.407331$

H -4.280169 -3.043580 2.881273

H -4.542777 -2.151789 4.978456

H -3.233518 -1.051646 5.418168

H -4.825267 -0.4083864 .957935$

H $-2.962085-1.3342750 .652144$

H -2.659963 -3.029749 0.948821

H -4.196888 $-2.713368-0.906399$

H $-4.954019-3.6404790 .388776$

H $-6.180358-1.606743 \quad 1.144677$

H -7.224313 -3.111178 -0.583138

H -6.416613 -2.216725 -1.853607

H -9.499831 $-0.811810-2.182689$

H $-7.068550 \quad 0.299481-2.216297$

H $-9.359600-2.2438580 .347394$

H $-9.808957-0.5628950 .185029$

H -8.445416 -1.014060 1.226885

H -8.688794 $1.435442-0.205306$

H $-6.4016902 .764730-0.779989$

H $-5.0158982 .388610 \quad 0.197659$

H -6.1719601 .3459841 .618490$

H $\quad 0.447328-3.6806982 .311268$

H $0.538001-1.1947170 .497932$
H $\quad 1.855249-4.8052340 .793077$

H $1.680090-5.130971-0.915304$

H $0.233136-4.9816380 .079465$

H $-5.1610941 .261641-2.644265$

H $-4.7271852 .948776-2.350377$

H $-0.7227881 .416692-1.830491$

H $-0.7232951 .814286-3.563540$

H -1.367540 -1.029561 -2.468569

H $0.4719810 .071959-4.657792$

H $0.163396-2.301903-5.404591$

H $-0.338976-2.802743-3.809345$

H $3.809628-2.298441-4.573457$

H $2.584858-1.523706-5.727802$

H $3.124684-3.381117-2.610658$

H $1.568944-4.182860-2.833519$

H $1.539179-1.420297-1.533735$

H -3.055412 $1.470085-3.688954$

C 0.8009033 .8495230 .678060

O $1.7192402 .969571-0.001005$

C $2.6559693 .754516-0.760689$

C $2.0936475 .170836-0.787370$

C 1.4048825 .2503110 .559781

H $3.5960013 .753026-0.196975$

C $2.8853043 .129803-2.141824$

H $2.8763295 .924633-0.915714$

H $1.3615035 .306970-1.591379$

C $0.385717 \quad 6.372348 \quad 0.656687$

H $2.1711935 .395867 \quad 1.333127$

C $3.2352311 .628462-2.122727$

O $1.6961593 .273961-2.928725$

H $3.6620343 .690450-2.674348$

H $2.3686841 .041229-1.795611$

O $3.4991791 .231626-3.475422$

C $4.4574811 .259726-1.260412$

C $4.841641-0.225480-1.405177$

H $4.2369491 .475907-0.210383$

O $5.5697192 .071685-1.633404$

H $3.967363-0.832879-1.155724$

H $5.093129-0.435203-2.451744$

H $\quad 0.866975 \quad 7.3434340 .501162$

H $-0.4030856 .263693-0.095276$

H -0.0888446 .3851591 .642871$

C 0.6286793 .4073872 .133555

H $0.9594112 .916536-2.405422$

H $2.7074041 .444625-3.998747$

H $5.7539281 .903403-2.573457$

H $-0.002244 \quad 4.1268992 .667834$

H 1.6070793 .4005672 .628751

H $-0.155530 \quad 3.786537 \quad 0.143275$

C $4.868109-1.0863711 .529717$

O $5.865304-0.3166750 .845964$

C $6.047612-0.640577-0.533956$

C $6.442416-2.116776-0.673056$

C $5.443189-3.0370000 .021122$

C $5.197644-2.5842401 .459803$

H $3.884708-0.8932711 .083820$

C $4.841858-0.5925052 .980645$

H $6.903881-0.046371-0.877162$

O $6.556281-2.467488-2.048178$

H $7.431867-2.264000-0.220866$

H $4.507124-3.096809-0.543690$

O $5.984732-4.3601130 .033201$

H $6.087748-2.8133482 .059945$

O $4.115981-3.3558861 .977808$

C 4.2534250 .7942043 .065867

H $4.228084-1.2330753 .622670$

H $5.848098-0.5758253 .416733$

H $6.779659-3.413189-2.089443$

H $5.349238-4.9272610 .502919$

H $4.096131-3.2355802 .941626$

O 5.2367901 .7309932 .952223

O 3.0566731 .0136033 .197531

C 4.7986063 .0919322 .998830

H 4.1318733 .3020662 .158355

H 4.2983383 .2986323 .949766

H 5.6783743 .7357312 .917488

SCF Energy (B3LYP/6-31G**//MMFF) $=-3245.92974448$ 
0600291

MM̄FF Geometry

C $-3.224167 \quad 0.446832 \quad 3.562629$

C $-2.142480 \quad 0.6488112 .794609$

C $-0.849133-0.1036512 .945505$

O $0.191607 \quad 0.9031492 .973518$

C $-0.567549-1.0366551 .752460$

C $-1.383568-2.310021 \quad 1.750807$

C -0.999739 -3.455787 2.346041

C $-2.678826-2.2639250 .986014$

C $0.248758-3.7057993 .145391$

C $1.074704-4.8719132 .585114$

C $1.742314-4.5683971 .233789$

C 2.433209-5.825025 0.697020

C $3.211337-5.547159-0.595325$

O $2.704346-3.5287331 .440637$

O $4.046389-6.689587-0.835392$

C $4.126731-4.326811-0.366375$

C $2.273968-5.437812-1.803792$

O $4.783527-3.958339-1.577882$

C $3.398201-3.1115550 .256684$

C $4.438278-2.0376190 .665075$

O $2.506307-2.567929-0.712803$

C $1.305725 \quad 0.6607243 .706932$

O $1.517908-0.315746 \quad 4.412585$

C $2.314601 \quad 1.732773 \quad 3.530914$

C 2.1035642 .8426042 .805995

C 3.0695083 .9165982 .563436

C 4.4863273 .6989913 .024962

C $3.849302-0.7907631 .337554$

C 3.4042730 .2587410 .336816

C $4.3080851 .879882-1.330084$

O 2.2157690 .4488450 .089294

C $4.7628213 .228684-0.872297$

C $4.0597534 .352267-1.081943$

C $4.5379635 .721332-0.691582$

C $3.507674 \quad 6.503010 \quad 0.091377$

C $2.7452747 .434239-0.506641$

C $3.4235326 .266176 \quad 1.582138$

C 2.6453655 .0272861 .924558

N $4.4668630 .923361-0.252128$

H -3.194086 -0.3066524 .345524$

H $-2.190514 \quad 1.389811 \quad 1.999264$

H $-0.843818-0.6656743 .887932$

H $\quad 0.500163-1.2860271 .728876$

H $-0.718652-0.4964400 .808448$

H -1.664544 -4.316034 2.275191

H -3.125049 -3.256674 0.865276

H -3.409900 -1.640687 1.502685

H -2.518976 -1.854923 -0.015221

H $0.874260-2.8139413 .237175$

H $-0.061882-3.9642444 .165467$

H $1.853273-5.1071783 .322585$

H $\quad 0.437356-5.7606872 .494799$

H $0.982589-4.2222800 .524155$

H $1.706830-6.6325580 .541032$

H $3.139025-6.2014431 .451218$

H $4.599666-6.492393-1.610978$

H $4.921392-4.6461950 .321687$

H $\quad 1.705087-6.366982-1.929363$

H $2.838054-5.306653-2.733698$

H $1.556722-4.619302-1.717718$

H $4.140125-3.481742-2.129652$

H $5.129920-2.4917551 .386724$

H $5.025163-1.738960-0.211294$

H $2.142549-1.740239-0.361088$

H 3.2610861 .5479954 .029026

H 1.1311452 .9904142 .339281

H 4.5336373 .6727374 .118925

H 5.1784274 .4736502 .688918

H 4.8785412 .7531942 .633437

H $3.008753-1.0438321 .989944$

H $4.611291-0.3304501 .979420$

H $3.2698981 .887808-1.671777$

H $4.9425231 .538365-2.154101$

H $5.7308783 .290812-0.380704$

H $3.1073754 .292250-1.603509$

H $4.7889056 .259650-1.615197$
H $5.4708335 .671368-0.116354$

H 2.0236118 .0192050 .053932

H $2.8216397 .628061-1.571454$

H 2.9151547 .1109682 .066472

H 4.4318606 .2703502 .004703

H 1.5972945 .0829201 .625945

H 5.4139340 .6791950 .022631

C -5.4336880 .4802972 .384955$

O -4.8844330 .6405481 .060124$

C -5.9327321 .0397910 .161512$

C -7.2332430 .7268650 .886781$

C -6.8485141 .0561582 .318457$

H -5.8547142 .1273550 .044683$

C $-5.7450580 .358708-1.197536$

H $-8.071625 \quad 1.324900 \quad 0.517583$

H $-7.502780-0.3320920 .799520$

C $-7.7985370 .477148 \quad 3.352068$

H -6.8157362 .1480552 .433205$

C $-4.3488160 .574982-1.824125$

O $-5.933909-1.054316-1.048096$

H $-6.5250400 .694390-1.890473$

H -3.597823 $0.021882-1.251585$

O $-4.3696660 .005168-3.137599$

C $-3.9344332 .048042-1.953567$

C $-2.5798312 .297458-2.645269$

H $-3.9000702 .515991-0.964522$

O $-4.9378622 .747696-2.697654$

H $-2.570708 \quad 1.809958-3.625609$

H -2.531152 $3.372434-2.860034$

H -8.8067290 .8822623 .217021$

H $-7.861390-0.613393 \quad 3.273130$

H $-7.4680780 .722976 \quad 4.366118$

C $-4.520401 \quad 1.1904293 .384962$

H -5.292258 -1.367935 -0.387737

H -4.597772 -0.935467 -3.041070

H $-4.9955582 .329730-3.574190$

H $-5.006093 \quad 1.2689114 .364106$

H -4.3115832 .2127793 .045136$

H -5.468286 -0.5978262 .588964$

C $-0.621719-0.088742-3.004268$

O $-1.1343430 .470430-1.788053$

C $-1.361871 \quad 1.883022-1.789429$

C $-0.0648382 .621213-2.153697$

C $0.4643402 .154186-3.504188$

C $0.6449160 .639395-3.480549$

H $-1.393035-0.038652-3.780878$

C $-0.286298-1.563806-2.717292$

H $-1.5938792 .155712-0.751865$

O $-0.2850494 .027056-2.160037$

H $0.6897712 .419643-1.382413$

H $-0.1913692 .461212-4.327518$

O $1.7377462 .749037-3.756719$

H $1.4911360 .385383-2.829139$

O $0.9728660 .187118-4.793972$

C $-1.496364-2.470446-2.703474$

H $\quad 0.391788-1.971308-3.475093$

H $\quad 0.194297-1.632026-1.734647$

H $0.5800574 .460599-2.250714$

H $1.5975623 .704151-3.870249$

H $1.7665780 .672513-5.077799$

O $-1.193101-3.601619-1.999148$

O $-2.554483-2.245164-3.272376$

C $-2.227145-4.588529-1.934062$

H $-1.918550-5.355861-1.219026$

H $-3.166499-4.146435-1.588463$

$\mathrm{H}-2.358425-5.052407-2.915887$

SCF Energy (B3LYP/6-31G**//MMFF)= -3245.93739356

0600292

MM̄FF Geometry

C $0.782899-0.0758382 .663700$

C $0.447560-1.0725681 .831136$

C $-0.428846-0.8847580 .622018$

O $-1.587573-1.7340120 .759656$

C $0.343945-1.302222-0.643792$

C $-0.265558-0.753214-1.918447$

C $\quad 0.216618 \quad 0.312765-2.588199$

C $-1.461918-1.498588-2.447854$ 
C $1.4027091 .165299-2.228627$

C $1.2250252 .093079-1.017957$

C $0.1314103 .165203-1.165754$

C $0.060867 \quad 4.0157890 .108275$

C $-1.077156 \quad 5.046526 \quad 0.062580$

O $-1.1309622 .532373-1.393134$

O -1.2511245 .5376801 .400713$

C $-2.3817294 .331562-0.354112$

C $-0.6968976 .261260-0.791410$

O $-3.439590 \quad 5.269594-0.535197$

C -2.217543 3.442499-1.604761

C $-3.4862212 .619908-1.922294$

O $-1.9775284 .281656-2.742046$

C -2.655325 -1.207450 1.419788

O $-2.721808-0.1147191 .960493$

C $-3.757333-2.1904941 .347910$

C $-5.000803-1.7947761 .656890$

C $-6.205146-2.6246381 .637402$

C $-6.050868-4.0810961 .287167$

C $-3.9043651 .611308-0.851053$

C $-5.071866 \quad 0.774495-1.340124$

C $-7.4940680 .947292-1.915968$

O $-4.926155-0.389790-1.704696$

C $-8.3666430 .417049-0.822318$

C $-8.957400-0.786748-0.878099$

C $-9.910677-1.3099510 .159171$

C $-9.500418-2.660710 \quad 0.701004$

C $-9.867763-3.7937560 .078428$

C $-8.740145-2.7032132 .005531$

C $-7.389980-2.0477481 .926721$

N $-6.265256 \quad 1.478146-1.357594$

H 0.3770710 .9188842 .496208

H $0.843835-2.0700232 .002605$

H $-0.747770 \quad 0.1607820 .526088$

H $0.387281-2.396851-0.720946$

H $1.384406-0.966857-0.565143$

H - $0.2940470 .619655-3.500128$

H -1.216039-2.555125 -2.595712

H $-2.300259-1.429888-1.750233$

H -1.804778 -1.103897-3.410324

H $2.2717590 .518010-2.061052$

H $1.6618281 .774383-3.104059$

H $1.0248961 .486972-0.129835$

H $2.1833652 .596191-0.836389$

H $\quad 0.383166 \quad 3.788859-2.031644$

H 1.0209454 .5116940 .299627

H -0.1089953 .3562950 .971603$

H -2.018350 6.1357531 .397281

H -2.676565 3.7034720 .495585

H $0.1896876 .755407-0.375766$

H - $-1.4894637 .017488-0.782577$

H $-0.4753216 .004366-1.829271$

H -3.300158 $5.711546-1.390065$

H -4.316995 $3.299460-2.152444$

H -3.311838 $2.061091-2.853236$

H $-2.1800303 .775630-3.546371$

H -3.519292 $-3.198773 \quad 1.029922$

H $-5.157959-0.7567101 .948722$

H $-5.400740-4.5842152 .011422$

H -5.616417 -4.1923680 .287233$

H $-6.997292-4.626726 \quad 1.275975$

H -3.077665 $0.933775-0.616515$

H -4.197322 2.1075410 .079815

H $-7.2553500 .182663-2.662547$

H -8.000640 $1.777157-2.418819$

H -8.558135 1.0672780 .027576

H -8.785987 -1.417961 -1.747667

H -10.901797 -1.390403-0.307167

H -10.034067 -0.5992880 .985695$

H $-9.603638-4.7687900 .473822$

H -10.438838 $-3.769716-0.844044$

H -8.644217 -3.7300892 .377277$

H $-9.330712-2.1876782 .774334$

H $-7.411280-0.9810562 .152343$

H $-6.2874802 .423325-0.986123$

C $2.8613900 .790486 \quad 3.752901$

$\begin{array}{lllll}\text { O } & 3.616686 & 0.625514 & 2.538030\end{array}$

C 5.0063010 .8886462 .812524
C 5.0814611 .3476824 .267059

C $3.8823710 .650003 \quad 4.879097$

H $5.529342-0.0702182 .720634$

C 5.5695861 .8918091 .796658

H 6.0281111 .0661184 .738764

H 4.9768772 .4347404 .359705

C 3.4393591 .2518756 .200964

H $4.133213-0.4087645 .029339$

C $5.3517291 .491896 \quad 0.323230$

$\begin{array}{lllll}\text { O } & 4.917010 & 3.151748 & 2.002769\end{array}$

H 6.6352572 .0554651 .997438

H 4.2848711 .5405230 .074268

O $6.0064252 .464035-0.501447$

C $5.8970520 .102205-0.051627$

C $5.733836-0.200509-1.552222$

H $5.368657-0.6580340 .526858$

$\begin{array}{lllll}\text { O } & 7.276622 & 0.012233 & 0.299934\end{array}$

H $4.691754-0.018017-1.833133$

H $6.3519590 .495998-2.131681$

H $4.244191 \quad 1.1893876 .940658$

H 3.1657482 .3065426 .090156

H $2.571524 \quad 0.719064 \quad 6.601822$

C $1.731946-0.2396013 .817215$

H 5.4226823 .8262781 .519141

H $5.5073723 .294684-0.426893$

H $7.7488540 .713056-0.181718$

H $1.170157-0.1204274 .750120$

H $2.155671-1.2511283 .812695$

H $2.445131 \quad 1.8067903 .734489$

C $4.058178-2.751537-1.432082$

O $5.459442-2.632745-1.158788$

C $6.146420-1.641318-1.929898$

C $6.003642-1.956364-3.425933$

C $4.539717-2.094625-3.836195$

C $3.818544-3.076460-2.913865$

H $3.553204-1.816532-1.165011$

C $3.505492-3.875298-0.540632$

H $7.209580-1.742391-1.678259$

O $6.617870-0.948886-4.222214$

H $6.516590-2.900787-3.649308$

H $4.033052-1.122673-3.852113$

O $4.472676-2.602234-5.170959$

H $4.168407-4.092589-3.135554$

O $2.421123-3.049592-3.199162$

C $3.483946-3.5038010 .926718$

H $2.476475-4.122152-0.822856$

H $4.135447-4.766710-0.648934$

H $7.550709-0.892459-3.953173$

H $4.968611-1.987778-5.738801$

H $2.099229-2.148706-3.026067$

O $3.223547-4.624507 \quad 1.663408$

O $3.650031-2.3783601 .374273$

C $3.165177-4.4312293 .080295$

H $4.083355-3.9591943 .443397$

H $2.290995-3.8270213 .338983$

H $3.066363-5.4120373 .553111$

SCF Energy (B3LYP/6-31G**//MMFF) $=-3245.91070920$

\section{3}

MM̄FF Geometry

C $-4.643141-2.724091-1.047145$

C $-3.342377-2.614763-1.359493$

C $-2.319628-3.627145-0.902476$

O $-1.245547-2.959770-0.195382$

C $-1.712953-4.355750-2.115382$

C $-1.047892-5.667644-1.741159$

C $0.280928-5.875465-1.660324$

C $-1.997063-6.815760-1.496633$

C $1.395540-4.893039-1.882546$

C $2.277938-4.771003-0.633077$

C $3.488114-3.853564-0.864787$

C $4.323981-3.7362900 .411809$

C $5.472400-2.726990 \quad 0.267518$

O $2.999856-2.569158-1.256782$

O $5.965159-2.463037 \quad 1.590247$

C $4.910371-1.405647-0.301934$

C $6.646532-3.325043-0.515763$

O $5.963375-0.485085-0.576212$ 
C $4.020392-1.604703-1.546812$

C $3.308562-0.306573-1.992439$

O $4.840603-2.032742-2.640603$

C -1.499814 -2.564524 1.083286

O $-2.569074-2.626478 \quad 1.670157$

C $-0.233657-2.0726071 .674395$

C $-0.252340-1.5314912 .902324$

C $0.904713-1.0829293 .682258$

C 2.271801-1.287440 3.088571

C $2.3322860 .278326-0.968658$

C $2.8885951 .433723-0.154633$

C 2.2139692 .9761201 .690468

O $3.9838331 .946363-0.362439$

C 2.7156622 .4807973 .008454

C 2.0622362 .6654994 .165408

C 2.5990802 .2443305 .501565

C 1.6582891 .3336296 .254656

C $0.877548 \quad 1.8072097 .240811$

C $1.704439-0.1433385 .939604$

C $0.690313-0.5550714 .906035$

N $1.9835801 .846641 \quad 0.813637$

H $-4.968759-3.576778-0.454811$

H $-2.994876-1.775687-1.955879$

H $-2.779454-4.355546-0.222726$

H -1.009784 -3.693507 -2.631995

H $-2.496259-4.583326-2.851591$

H $0.622691-6.879505-1.409356$

H -1.471726 -7.754975 -1.292727

H $-2.642931-6.609962-0.637949$

H -2.628801-6.979098 -2.375981

H $2.001197-5.249350-2.724552$

H $1.018235-3.906207-2.161858$

H $1.665605-4.3719510 .185917$

H $2.629621-5.762880-0.322327$

H $4.089463-4.273439-1.679758$

H $4.709459-4.7176380 .715833$

H $3.677852-3.4053751 .235325$

H $6.642927-1.7688751 .518746$

H $4.312781-0.9519120 .497505$

H $7.033246-4.213426-0.001761$

H $7.486843-2.624287-0.569970$

H $6.377309-3.621680-1.531632$

H $6.387794-0.758681-1.407104$

H $4.0426890 .433908-2.331640$

H $2.716041-0.554130-2.885173$

H $4.376600-1.834597-3.470932$

H $\quad 0.677583-2.2076971 .104179$

H -1.213029 -1.429396 3.406407

H $2.335694-0.8345952 .095188$

H $3.075331-0.8376693 .675333$

H $2.489735-2.3567513 .002280$

H $1.4701080 .678609-1.512208$

H $1.951766-0.492340-0.291655$

H 1.2638343 .5118831 .784212

H 2.9492793 .6520121 .242786

H 3.6804681 .9792373 .013523

H 1.1087623 .1888994 .159978

H 2.7833243 .1559866 .085500

H 3.5779741 .7582025 .405155

H $0.227406 \quad 1.1508197 .809750$

H 0.8670512 .8615797 .496585

H $1.485386-0.7152996 .852115$

H $2.725514-0.4350105 .678272$

H $-0.340026-0.453077 \quad 5.250269$

H $1.131180 \quad 1.3126630 .951893$

C $-6.188856-0.812250-0.358707$

O $\quad-5.1976410 .213093-0.160016$

C -4.9922540 .3911521 .252568$

C $-6.210714-0.2297831 .925380$

C $-6.469762-1.4194441 .018611$

H $-4.103601-0.1988891 .509461$

C -4.7241561 .8631491 .585933$

H $-6.014030-0.5138382 .963390$

H -7.0747210 .4440861 .912458$

C -7.862934 -2.007637 1.154015

H $-5.735684-2.1930631 .271746$

C $-3.7294882 .558814 \quad 0.633611$

O $\quad-5.9570792 .590327 \quad 1.508232$
H $-4.389942 \quad 1.9431142 .627191$

H $-4.2396692 .753746-0.315233$

O $-3.427305 \quad 3.847514 \quad 1.183156$

C $-2.418591 \quad 1.7984080 .379094$

C $-1.3932122 .561339-0.474485$

H $-2.6266800 .831880-0.087393$

O

H -0.9781993 .3883350 .118881$

H $-0.5368411 .895247-0.625649$

H -8.028753 -2.373486 2.172682

H $-8.636371-1.2640650 .934603$

H -7.997069 -2.848056 0.465646

C $-5.722499-1.766403-1.464787$

H $-5.7627793 .515657 \quad 1.736610$

H -2.923877 3.7048962 .003254

H -1.0165000 .9810051 .474335$

H -5.376134 -1.177709-2.323715

H $-6.576157-2.360763-1.812286$

H -7.081201-0.286722 -0.723295

C $0.341693 \quad 3.464570-2.768928$

O $-0.8941554 .052120-2.344890$

C $-1.8638253 .120543-1.834107$

C $-2.2059042 .104228-2.933406$

C $-0.9578631 .413077-3.476460$

C $0.0920612 .446270-3.887472$

H $0.8303462 .974794-1.920187$

C $1.2578364 .602438-3.242221$

H $-2.7597653 .722113-1.649143$

O $-3.1256981 .119174-2.487410$

H -2.687638 $2.631656-3.767550$

H $-0.5503510 .698678-2.752797$

O $-1.307727 \quad 0.650299-4.634208$

H $\quad-0.2404202 .961330-4.797909$

O $\quad 1.3126221 .777762-4.197227$

C $1.7551405 .450224-2.088798$

H $2.1403094 .194376-3.747045$

H $0.7135805 .261285-3.928778$

H $-3.950067 \quad 1.574667-2.246339$

H -2.004583 $0.025797-4.368259$

H $1.1165161 .119571-4.885905$

O $2.5870436 .417141-2.578753$

O $1.4574655 .291430-0.912942$

C $3.1451287 .300347-1.600726$

H $3.7933118 .013376-2.116997$

H $2.3494297 .853141-1.092241$

H $3.7462736 .737175-0.880493$

SCF Energy $(B 3 L Y P / 6-31 G * * / / M M F F)=-3245.92345384$

0600294

MM̄FF Geometry

C $3.706283-3.1025572 .111627$

C $2.763356-2.2229041 .742594$

C $1.600519-1.8301562 .612351$

O $0.407412-1.8403211 .795462$

C $1.787712-0.3977723 .139377$

C $0.758236-0.0383994 .191884$

C $-0.325176 \quad 0.733744 \quad 3.981758$

C $1.031939-0.5880685 .569644$

C -0.7784791 .3856682 .704093$

C -0.7560362 .9122592 .850913$

C -1.3251103 .6420311 .624365$

C -1.2454535 .1566831 .838594$

C -1.9324595 .9462890 .716710$

O $-2.6878193 .239562 \quad 1.463293$

O -2.0711117 .2954521 .187328$

C -3.3460645 .3669460 .493063$

C $-1.0561486 .010000-0.539274$

O $-3.9704755 .967568-0.639814$

C $-3.3599903 .828370 \quad 0.341244$

C $-4.818993 \quad 3.3087390 .349843$

O $-2.7361423 .481578-0.894194$

C $-0.293357-3.0009811 .696676$

O $-0.045998-4.0642112 .240532$

C $-1.423509-2.7434120 .775968$

C $-2.272013-3.7282610 .445922$

C $-3.406706-3.606829-0.475137$

C -3.589703 -2.281019-1.168021

C $-4.933864 \quad 1.7832680 .240397$ 
C $-6.334627 \quad 1.2692790 .530886$ C -7.612043 -0.8749390 .570267$ O C -7.225525 $-2.295745 \quad 0.830144$ C -7.621268 -3.3342540 .078985$ C -7.187964 -4.7492830 .340996$ C $-6.600707-5.426226-0.880645$ C $-7.133695-6.567612-1.349439$ C $-5.406034-4.794317-1.569435$ C $-4.202124-4.680173-0.671486$ N $-6.397176-0.1079360 .374293$ H $3.655573-3.5643003 .094263$ H $2.835568-1.7483670 .767538$ H $1.486639-2.5307283 .448533$ H $1.753801 \quad 0.3147412 .307144$ H $2.786007-0.2841823 .583208$ H -0.9867530 .9195544 .827862$ H $0.293953-0.252687 \quad 6.306243$ H $1.012466-1.6819635 .561283$ H $2.016089-0.2598645 .920009$ H $-0.171521 \quad 1.0886831 .844612$ H -1.7980801 .0396302 .496766$ H -1.347025 $3.187983 \quad 3.735012$ H 0.2737923 .2460343 .031348 H $-0.752393 \quad 3.3428350 .738730$ H $-0.2023815 .478251 \quad 1.952085$ H -1.738399 5.4128222 .787550 H -2.448911 7.8290210 .468053 H -3.9568685 .6354181 .366483$ H $-0.1186606 .536498-0.323253$ H -1.544296 $6.580681-1.336730$ H $-0.7982705 .023867-0.929114$ H -4.049472 $6.919965-0.462928$ H $-5.300093 \quad 3.6287281 .283168$ H $-5.3847293 .752213-0.478953$ H -2.985392 2.576518 -1.134809 H -1.516072 -1.7391510 .377725$ H $-2.123738-4.7137870 .884620$ H $-3.701777-1.476170-0.434818$ H -4.465302 -2.237667 -1.817595 H -2.720992 -2.055730 -1.796800 H $-4.6633511 .458046-0.770568$ H -4.254183 1.2975400 .950155 H -8.224639 -0.762939-0.330160 H -8.166713 $-0.463767 \quad 1.419507$ H $-6.571465-2.4763511 .680633$ H -8.288726 -3.163112 -0.762105 H $-8.066616-5.302071 \quad 0.697843$ H $-6.454000-4.791517 \quad 1.155330$ H -6.727493 -7.065410 -2.223750 H -7.988771 -7.032383 -0.870021 H -5.720804 -3.831161 -1.978121 H $-5.107059-5.400416-2.434677$ H -3.958337 -5.604769 -0.145950 H -5.624587 -0.566870 -0.097199 C $6.081097-2.6087821 .469735$ O $5.819558-1.3024450 .911985$ C $6.885348-0.9610260 .009805$ C $8.058758-1.8306920 .434339$ C $7.342061-3.1248920 .770468$ H $6.566484-1.271596-0.992832$ C 7.1400490 .5489920 .022428 H $8.803960-1.944734-0.358444$ H $8.559430-1.4328201 .324418$ C $8.167183-4.0760171 .619934$ H $7.072019-3.630388-0.166801$ C $5.8795791 .399377-0.247616$ O $7.6463320 .910594 \quad 1.312546$ H $7.9282460 .791162-0.700655$ H 5.2001491 .3514110 .612444 O $6.2867682 .767664-0.368519$ C $5.1104321 .011713-1.525069$ C $3.9264931 .953807-1.814171$ H $4.732847-0.009814-1.418285$ O $5.9985991 .018834-2.641829$ H $3.2980142 .011206-0.918839$ H $4.3027892 .965070-2.010383$ H $9.077486-4.3742581 .089645$
H $8.466113-3.6125992 .566162$

H $7.598917-4.9820141 .853153$

C $4.860278-3.4996471 .233832$

H $7.9907381 .817131 \quad 1.249528$

H $\quad 6.582878 \quad 3.0642280 .508384$

H $6.3648411 .916449-2.719967$

H $5.101998-4.5475911 .445023$

H $4.553911-3.4459810 .181119$

H $6.260903-2.4673112 .543021$

C $1.685422-0.115028-1.935832$

O $2.6701110 .140619-2.940501$

C $3.0882951 .506202-3.034975$

C $1.8782562 .403115-3.330906$

C $0.7540352 .203655-2.316515$

C $0.4191120 .720311-2.162142$

H $2.1101400 .136058-0.955599$

C $1.421831-1.626511-1.892759$

H $3.7509331 .560868-3.907895$

O $2.252053 \quad 3.776889-3.345933$

H $1.4876472 .165073-4.328977$

H $0.9990472 .654763-1.347834$

O $-0.4178022 .873640-2.787339$

H $-0.1155570 .384723-3.058215$

O $-0.460796 \quad 0.545355-1.054093$

C $0.729136-2.175292-3.121826$

H $2.367216-2.172587-1.794261$

H $\quad 0.806921-1.850477-1.016083$

H $2.9526083 .881565-4.012282$

H -0.178995 $3.804666-2.936037$

H -1.232714 $1.117646-1.202397$

O $-0.099635-3.195912-2.752959$

O $0.913664-1.778683-4.264456$

C $-0.829868-3.814490-3.816590$

H -1.470355 -3.079672 -4.313673

H $-0.140734-4.274482-4.531122$

$\mathrm{H}-1.462215-4.595175-3.385344$

SCF Energy $\left(B 3 L Y P / 6-31 G^{* *} / / M M F F\right)=-3245.90996820$

0600295

MM̄FF Geometry

C $3.1393780 .537112-3.100784$

C $2.0714610 .499974-2.289904$

C $0.800313-0.247200-2.582860$

O $-0.2604370 .734523-2.442775$

C $0.532479-1.378907-1.576864$

C $1.284739-2.662892-1.851201$

C $0.804769-3.682132-2.591573$

C $2.617824-2.797182-1.167044$

C $-0.503901-3.744829-3.328297$

C $-1.469564-4.794935-2.757950$

C $-1.927375-4.505552-1.319535$

C $-2.929945-5.559117-0.841509$

C $-3.466581-5.2451710 .564680$

O $-2.538045-3.212269-1.296190$

O $-4.601968-6.0973990 .778778$

C $-3.957394-3.7797640 .599070$

C $-2.446339-5.6118411 .648962$

O $-4.329959-3.3987271 .921750$

C $-2.939447-2.7786210 .008865$

C $-3.522563-1.355187-0.127784$

O $-1.818089-2.6895150 .897264$

C $-1.3782110 .596999-3.198386$

O $-1.608079-0.290631-4.007708$

C $-2.3339671 .706061-2.964687$

C $-2.2051622 .606111-1.977358$

C $-3.0829453 .752496-1.725217$

C $-4.2539373 .953937-2.650710$

C $-4.679072-1.216513-1.123067$

C $-5.867541-0.538617-0.475778$

C -6.5699611 .5901050 .566777$

O $-6.890444-1.157850-0.193068$

C -6.3465613 .0444080 .311750$

C $-5.7832903 .874916 \quad 1.201732$

C $-5.624621 \quad 5.3511200 .985294$

C -4.1809805 .7959031 .002114$

C -3.5964576 .2072642 .140174$

C $-3.4536565 .870972-0.319732$

C $-2.7849694 .579085-0.700297$ 
N $-5.6499660 .811261-0.236962$

H $3.131214-0.026400-4.029926$

H $2.0982041 .065708-1.362463$

H $0.808548-0.629277-3.611459$

H $-0.542005-1.583457-1.554974$

H $0.745302-1.038813-0.554248$

H $1.419027-4.575365-2.697299$

H $3.079463-3.775278-1.339964$

H $3.317375-2.039384-1.526891$

H $2.496977-2.680367-0.085425$

H $-0.997368-2.770601-3.368054$

H $-0.285099-4.006369-4.371269$

H -2.347942 -4.820078 -3.415966

H -1.005211 $-5.787973-2.803769$

H -1.047182 -4.502997 -0.666823

H -2.484642 -6.561540 -0.867965

H -3.781186 -5.591501-1.536347

H -4.995604 -5.854359 1.634650

H -4.881433 -3.747529 0.009829

H -2.202994 -6.679998 1.596109

H -2.856950 -5.4503132 .651672$

H -1.511267 -5.054285 1.565197

H $-3.513328-3.2328992 .423120$

H -3.791440 -0.9788990 .867735$

H -2.721982 $-0.689866-0.478355$

H $-1.367795-1.8424000 .744149$

H -3.161481 $1.741899-3.665980$

H -1.362987 2.517141-1.294617

H -3.907473 4.168085 -3.667678

H -4.908482 4.775280 -2.351296

H $-4.8843653 .058004-2.676065$

H -4.366289 -0.615485 -1.985438

H -5.011818 -2.177362 -1.527740

H -6.3978411 .3216281 .614934$

H $-7.599834 \quad 1.3135370 .317545$

H $-6.698393 \quad 3.436719-0.638974$

H -5.4489943 .4801072 .158644$

H -6.1795765 .8663261 .780537$

H -6.1002645 .6712450 .049926$

H -2.571713 6.5636112 .152015

H -4.1289096 .1908553 .085400$

H -2.665233 $6.634392-0.261592$

H -4.129367 6.244641-1.094589

H -1.918784 4.343804 -0.079922

H $-4.7072081 .171366-0.358632$

C $5.5329980 .514164-2.307036$

O $5.214716-0.008610-1.001258$

C $6.3041860 .269210-0.103474$

C $7.4958880 .588844-0.994634$

C $6.818601 \quad 1.327174-2.132754$

H 6.0290151 .1714290 .456410

C $6.506317-0.8968540 .870805$

H $8.251811 \quad 1.189765-0.480311$

H $7.980208-0.319366-1.371205$

C $7.6650731 .415604-3.390937$

H $6.5773292 .344062-1.794156$

C $5.219426-1.3612241 .581300$

O $7.022008-2.0271810 .154519$

H $7.272659-0.6339931 .608790$

H $4.553211-1.8422820 .857582$

O $5.577480-2.3744482 .529580$

C $4.451622-0.2524932 .321897$

C $3.156494-0.7806672 .961761$

H $4.1892710 .537387 \quad 1.616530$

O $5.286950 \quad 0.329733 \quad 3.318395$

H $2.534962-1.2064562 .166173$

H $3.398815-1.5985473 .651038$

H $8.593713 \quad 1.960487-3.192101$

H $7.932240 \quad 0.421704-3.765434$

H $7.1277931 .942157-4.185923$

C $4.3641731 .360990-2.811439$

H $\quad 6.388256-2.241327-0.551767$

H $6.056805-3.0683142 .044872$

H $5.540413-0.3763983 .937335$

H $4.6441761 .885254-3.732406$

H $4.1212692 .130725-2.068825$

H $5.700234-0.348304-2.964658$

C 1.2287021 .3686611 .904645
O $2.135044 \quad 1.4866513 .007936$

C 2.3622090 .2852873 .750341

C $1.033374-0.2345034 .314882$

C $0.000630-0.4339463 .209564$

C -0.1367540 .8361522 .371984$

H $1.659146 \quad 0.7016501 .148399$

C 1.0719972 .7707611 .289261

H 2.9873090 .5710764 .605701

O $1.241670-1.4556525 .016340$

H 0.6433610 .4914395 .040185

H $0.250021-1.3009632 .587404$

O $-1.259116-0.7304033 .817412$

H $-0.661966 \quad 1.5935202 .968583$

O $\quad-0.957887 \quad 0.5287251 .247774$

C $2.333198 \quad 3.2923290 .633641$

H $\quad 0.2976712 .7579470 .516262$

H 0.7930503 .4879052 .070557

H $\quad 0.373827-1.7617005 .331195$

H -1.909443 -0.8290093 .100553$

H $-1.270706 \quad 1.366540 \quad 0.868271$

$\begin{array}{lllll}\text { O } & 2.058337 & 4.500457 & 0.055852\end{array}$

O $3.4171092 .728106 \quad 0.625059$

C $3.1584925 .129989-0.608310$

H $2.8220126 .103197-0.975369$

H 3.9872485 .2860710 .089037

H $3.4791794 .524969-1.461251$

SCF Energy $(B 3 L Y P / 6-31 G * * / / M M F F)=-3245.92907828$

0600296

MM̄FF Geometry

C -0.0338132 .9845820 .599798$

C $0.8978583 .625359-0.121273$

C $1.4607213 .052388-1.393441$

O $2.8978633 .008379-1.225424$

C $1.1118343 .877220-2.643176$

C $-0.3594463 .917501-2.999716$

C $-1.0754452 .858754-3.429033$

C $-1.0076165 .270901-2.875953$

C $-0.5984131 .442436-3.609065$

C $-1.3596380 .499996-2.668055$

C $-0.666881-0.860717-2.488763$

C -1.630489-1.837094 -1.810781

C $-0.972126-3.180625-1.486533$

O $0.489449-0.657716-1.665502$

O $-1.872936-3.889880-0.622396$

C $0.324611-2.905775-0.701827$

C $-0.806749-4.046386-2.739705$

O $1.040699-4.118891-0.475269$

C $1.237117-1.843495-1.362433$

C $2.407488-1.456770-0.421670$

O $1.805103-2.407491-2.549715$

C $3.5711522 .075903-1.952336$

O $3.1186181 .359553-2.831343$

C $4.9549652 .017686-1.435001$

C $5.7004980 .945242-1.738413$

C $7.0315020 .634090-1.217739$

C $7.7103941 .671535-0.364780$

C $2.005047-0.5628830 .754456$

C $2.961538-0.6243441 .931601$

C $5.302489-0.1654472 .611851$

O $2.546710-0.8245993 .072629$

C $6.621439-0.7839792 .264150$

C $6.847941-1.8342671 .458896$

C $8.224102-2.3905181 .208512$

C $8.579062-2.482595-0.259891$

C $8.730750-3.675061-0.860569$

C $8.832375-1.193314-1.015292$

C $7.550724-0.578594-1.500841$

N $4.284908-0.3826941 .599661$

H -0.4267942 .0410020 .228770$

H $1.299078 \quad 4.5722420 .229632$

H $1.1071572 .022260-1.497764$

H $1.6676033 .497036-3.510335$

H $1.4968744 .898780-2.516842$

H -2.125709 $3.013620-3.673484$

H $-2.0752075 .246468-3.118640$

H $-0.9100185 .647424-1.852580$

H $-0.5281165 .983320-3.555338$ 
H $-0.783912 \quad 1.141049-4.647307$ H $\quad 0.479970 \quad 1.354740-3.463439$ H -1.467356 $0.972180-1.683420$ H -2.370103 $0.356092-3.070399$ H -0.354158 -1.239865 -3.468389 H -2.527565 -1.990078 -2.423006 H $-1.980501-1.392480-0.869583$ H $-1.428819-4.708831-0.342360$ H $0.019383-2.5540950 .290550$ H -1.783668 -4.240177 -3.199341 H $-0.394822-5.030520-2.490876$ H $-0.168776-3.587106-3.497673$ H $1.490005-4.356042-1.304392$ H $2.919129-2.362242-0.071485$ H $3.163866-0.915289-1.004078$ H $2.591977-1.890451-2.789113$ H $5.2878702 .802879-0.766706$ H $5.2708010 .184356-2.389624$ H $7.8076662 .614202-0.915355$ H $8.7188071 .389762-0.055722$ H 7.1357251 .8590110 .548615 H 1.9372390 .4812430 .430934 H $1.017422-0.8394461 .137466$ H $4.965313-0.5283153 .587960$ H 5.4253600 .9207902 .684859 H $7.477887-0.3175042 .748506$ H $6.014108-2.3437090 .982930$ H $8.265387-3.3850221 .671332$ H $8.991787-1.7936681 .717153$ H $9.008837-3.748651-1.906845$ H $8.575903-4.604162-0.322034$ H $\quad 9.460442-1.389807-1.894227$ H $9.420911-0.523065-0.383595$ H $6.974943-1.246835-2.143727$ H $4.499958-0.0837540 .654035$ C -0.4119032 .3687572 .983888$ O $-1.244170 \quad 1.2374332 .650960$ C $-1.903276 \quad 0.774230 \quad 3.842483$ C -1.2134171 .4786165 .005122$ C -0.8561342 .8113684 .376887$ H $-2.939664 \quad 1.1276103 .786662$ C -1.875499 -0.7562753 .911235$ H $-1.866426 \quad 1.5750405 .877919$ H -0.3050590 .9516985 .319605$ C 0.2015263 .5820515 .147703 H $-1.766313 \quad 3.4220604 .305375$ C -2.484092 -1.460764 2.680723 O $-0.509881-1.1749374 .024045$ H -2.379054 -1.087731 4.827498 H -1.840060 -1.320903 1.803757 O $-2.501643-2.8687182 .949568$ C -3.919374 -1.019348 2.331940 C $-4.560604-1.9000771 .243200$ H $-3.9011240 .021171 \quad 1.996187$ O $-4.736821-1.0679143 .501691$ H -3.866563 -1.9846150 .402945$ H -4.710254 -2.909885 1.643458 H -0.1498893 .8099996 .159204$ H $1.130823 \quad 3.0092485 .235834$ H 0.4352684 .5285694 .650310 C $-0.574036 \quad 3.455777 \quad 1.918561$ H $-0.509787-2.1152914 .269258$ H -1.581696 -3.181819 2.928391 H $-4.725920-1.9834243 .830123$ H $-0.067956 \quad 4.3747102 .234449$ H $-1.636526 \quad 3.6878821 .785145$ H 0.6242072 .0054283 .006500 C $-5.1444200 .066990-1.029876$ O $-5.833677-0.037101 \quad 0.222154$ C $-5.926305-1.3621580 .758263$ C $-6.641798-2.282897-0.241080$ C $-5.967363-2.258098-1.610397$ C $-5.808995-0.817403-2.092974$ H -4.097474 -0.225484 -0.898014 C $-5.1803021 .541875-1.460916$ H $-6.570853-1.2891821 .643181$ O $-6.684859-3.611807 \quad 0.266785$ H -7.680961 -1.945876 -0.351351
H $-5.001358-2.776271-1.596103$

O $-6.784601-2.950785-2.556248$

H $-6.794929-0.412259-2.355571$

O $-5.015497-0.805732-3.277873$

C $-4.306147 \quad 2.426515-0.598216$

H $-4.8243221 .651687-2.491129$

H $-6.2080851 .917697-1.389009$

H $-7.250163-4.136161-0.324721$

H $-6.785894-3.890880-2.309257$

H $-5.447798-1.391802-3.922384$

O $-4.549876 \quad 3.731698-0.921349$

O $\begin{array}{lllll}-3.503941 & 2.032756 & 0.236299\end{array}$

C $-3.796313 \quad 4.700022-0.186333$

H $-4.024964 \quad 4.6272830 .881235$

H -2.726816 $4.559579-0.363307$

H $-4.0821685 .693994-0.540134$

SCF Energy (B3LYP/6-31G**//MMFF)= -3245.92165577

06_00297

MM̄FF Geometry

C $-0.294356 \quad 4.880239 \quad 1.751002$

C $-1.1352693 .883357 \quad 1.433413$

C $-1.834258 \quad 3.026552 \quad 2.455829$

$\begin{array}{llll}\text { O } & -1.794446 & 1.652613 & 2.006485\end{array}$

C $-3.311753 \quad 3.4413292 .550030$

C -4.0857972 .6424873 .579178$

C $-4.947347 \quad 1.652340 \quad 3.273177$

C -3.8788473 .0577855 .011809$

C $-5.250732 \quad 1.1205791 .898656$

C $-5.577722-0.379074 \quad 1.929605$

C $-5.880536-0.914062 \quad 0.522642$

C $-6.524255-2.2988840 .600369$

C $-6.785139-2.879181-0.796014$

O $-4.647870-0.989365-0.205223$

O $-7.100096-4.271473-0.643458$

C $-5.482502-2.796061-1.614163$

C $-7.997809-2.230604-1.473639$

O $-5.705129-3.187980-2.967370$

C $-4.798069-1.406353-1.569851$

C $-3.393891-1.522073-2.207711$

O $-5.575403-0.471886-2.319703$

C $-\begin{array}{llll}0.802379 & 0.854790 & 2.479758\end{array}$

$\begin{array}{llll}\text { O } & 0.134566 & 1.184633 & 3.187921\end{array}$

C $-1.079740-0.5195522 .002044$

C $-0.168892-1.4881262 .177459$

C $-0.320657-2.8951961 .796536$

C $-1.687223-3.352717 \quad 1.355145$

C $-2.488913-0.294666-2.033341$

C $-1.134219-0.565557-2.664112$

C $0.883765-2.012481-2.393639$

O $-0.798476-0.049088-3.727008$

C $0.966502-3.443047-1.963929$

C $1.937976-3.935695-1.181399$

C $2.002674-5.369981-0.741579$

C $2.066348-5.5265650 .762552$

C $3.155059-6.0414701 .358465$

C $0.833155-5.1789851 .571787$

C $0.749665-3.7112571 .899899$

N $-0.385232-1.477530-1.939356$

H $-0.096767 \quad 5.0663732 .805420$

H $-1.374333 \quad 3.6764830 .394448$

H -1.3584663 .1189143 .439953$

H $-3.787115 \quad 3.3493181 .565442$

H -3.3868854 .5064162 .808188$

H $-5.492715 \quad 1.1788824 .088244$

H -4.5037022 .4845145 .704903$

H -2.836324 2.9077415 .308667

H -4.1292604 .1158145 .140940$

H -6.1156311 .6664421 .503036$

H -4.4129781 .2852991 .214324$

H $-4.729847-0.9264722 .361608$

H $-6.445693-0.5406012 .581221$

H $-6.555711-0.2110830 .020374$

H $-7.455877-2.265348 \quad 1.178991$

H $-5.856919-2.9823091 .144275$

H $-7.881793-4.340598-0.068727$

H $-4.787906-3.534748-1.189502$

H $-8.897890-2.370856-0.863135$ 
H $-8.212725-2.706453-2.436821$ H -7.874768 -1.158255-1.639145 H $-6.118578-4.068155-2.949964$ H -2.881026 -2.386778 -1.768527 H -3.491888 -1.727071 -3.281944 H $-4.9999940 .251250-2.615115$ H -2.037965 -0.7001821 .527597$ H $0.781243-1.2307022 .643414$ H -2.442973 -3.083745 2.102240 H -1.764680 -4.433872 1.224260 H -1.962813 -2.8916220 .402912$ H -2.917951 $0.589989-2.514672$ H $-2.343033-0.050986-0.975606$ H $1.677173-1.401958-1.956235$ H $0.949434-1.938304-3.483546$ H $\quad 0.179756-4.107322-2.315064$ H $2.733904-3.278096-0.844242$ H $2.887822-5.819827-1.209422$ H $1.142922-5.938371-1.118179$ H $3.196426-6.1845492 .433193$ H $4.029464-6.3320340 .786515$ H $\quad 0.848800-5.7270082 .523118$ H $-0.043724-5.5465721 .031894$ H $1.683612-3.2888692 .274422$ H $-0.680696-1.703457-0.994746$ C $0.2584635 .546696-0.690603$ O $\quad 0.9980804 .353337-1.015607$ C $1.7802624 .597388-2.198792$ C $1.1984435 .856075-2.825258$ C $0.8355416 .648426-1.582600$ H $2.7996794 .823104-1.864225$ C $1.7909193 .352663-3.091173$ H $1.9160876 .371160-3.470803$ H $0.3002255 .639792-3.415241$ C $-0.125617 \quad 7.793195-1.851734$ H $1.7560517 .049775-1.137558$ C $2.2617732 .077467-2.357293$ O $0.456953 \quad 3.126401-3.561398$ H $2.4081393 .539148-3.977941$ H $1.5081891 .780595-1.619366$ O $2.3425371 .015079-3.313776$ C $3.6395842 .213851-1.684170$ C $4.1769070 .929083-1.036369$ H $3.6173013 .003763-0.931378$ O $4.5986702 .636711-2.661178$ H $4.3584000 .178222-1.818219$ H $5.1817371 .149054-0.654848$ H $0.3210688 .515423-2.542868$ H -1.060636 $7.436336-2.296787$ H $-0.373396 \quad 8.320881-0.925525$ C 0.4036345 .8287990 .807378 H $\quad 0.5013202 .451178-4.259034$ H $1.4359860 .799411-3.590249$ H $4.6094851 .969189-3.368735$ H $0.0312876 .836196 \quad 1.027337$ H 1.4694615 .8113931 .069400 H $-0.7897685 .367191-0.959265$ C $5.074717-1.0978070 .996701$ O $3.794516-1.0408440 .355127$ C $3.317310 \quad 0.285166 \quad 0.071614$ C 3.1806471 .0494681 .393916 C 4.4925421 .0661282 .175187 C $5.035250-0.3557842 .338518$ H $5.839095-0.6535480 .348108$ C $5.427583-2.5802041 .189784$ H $2.3086830 .149745-0.331205$ O 2.7052422 .3696231 .184416 H 2.4277160 .5386912 .005175 H 5.2302251 .7165351 .691894 O 4.2653911 .6305153 .469205 H $4.410369-0.9068173 .053090$ O $6.352262-0.2947702 .881084$ C $5.754268-3.263819-0.122497$ H $6.307125-2.6779411 .835519$ H $4.581623-3.1123061 .639469$ H 2.6405352 .7998302 .054181 H 3.5979801 .0869003 .921757 H 6.3007410 .2061373 .713300
O $\quad 6.159060-4.5428540 .134204$

O $5.660961-2.752320-1.229913$

C $6.485808-5.326656-1.017724$

H $5.615404-5.416444-1.674694$

H $7.329871-4.880219-1.551865$

H $6.772958-6.325567-0.678569$

SCF Energy (B3LYP/6-31G**//MMFF) $=-3245.92542961$

0600298

MM̄FF Geometry

C $-2.054780-3.4221862 .401664$

C $-0.940618-2.7108882 .171758$

C $-0.226356-1.9223203 .240286$

O $-0.109105-0.5476492 .812706$

C $1.196723-2.4821503 .435930$

C $2.057314-1.7076424 .419540$

C $3.327982-1.3230464 .181319$

C $1.430051-1.4031075 .756312$

C $4.172795-1.6112182 .971831$

C $4.517348-0.3376952 .188569$

C $5.336447-0.6705950 .931951$

C 5.8763550 .5960680 .267422

C $6.6035800 .286758-1.050849$

O $4.473875-1.3713340 .029186$

O $6.7820691 .538581-1.731419$

C $5.695039-0.605661-1.926294$

C $8.010071-0.268734-0.800474$

O $6.374987-1.026230-3.105998$

C $5.107099-1.819433-1.174618$

C $4.062395-2.611540-2.004639$

O $6.165941-2.738074-0.871026$

C $-1.156878 \quad 0.277513 \quad 3.067458$

O $-2.252553-0.047167 \quad 3.505370$

C -0.8291071 .6881102 .758344$

C 0.4171782 .1260012 .522860

C 0.8115153 .5068772 .237270

C -0.2665444 .5548732 .161636$

C $2.970161-1.805243-2.711926$

C $2.089538-0.996754-1.777555$

C $1.0408561 .257129-1.576772$

O $1.728353-1.413343-0.679846$

C $2.0745082 .196616-1.042499$

C $2.1264103 .505050-1.332078$

C $3.1842774 .436376-0.807135$

C 2.6338725 .4304750 .191102

C $2.1209546 .603635-0.216831$

C 2.7356655 .0969651 .664502

C 2.1163993 .7742962 .023177

N $1.727597 \quad 0.224015-2.329059$

H -2.466983 -3.414726 3.409501

H $-0.498624-2.6874621 .180049$

H -0.770121 -1.967917 4.192403

H $1.679991-2.5109822 .453989$

H $1.132162-3.5213393 .784527$

H $3.849902-0.7704584 .962007$

H $2.154366-1.0029046 .474020$

H $\quad 0.631132-0.6627975 .650655$

H $\quad 1.010037-2.3147406 .193962$

H $5.102704-2.0784553 .320664$

H $3.706732-2.3472562 .312266$

H $3.5878040 .169151 \quad 1.898743$

H 5.0830440 .3453882 .833996

H $6.166531-1.3256601 .222438$

H $6.537801 \quad 1.1427050 .951180$

H 5.0445091 .2789070 .052830

H $7.1743761 .347896-2.600944$

H $4.8753050 .040141-2.262875$

H $8.6093060 .454705-0.234520$

H $8.547507-0.427818-1.741737$

H $8.007935-1.206708-0.241506$

H $6.986414-1.739990-2.856466$

H $4.587351-3.204394-2.765696$

H $3.578407-3.342611-1.342164$

H $5.772074-3.608177-0.691620$

H -1.677249 2.3631522 .779852

H 1.2379941 .4110552 .542327

H -0.8387344 .5927023 .095065$

H -0.9495904 .3381021 .333961$ 
H $\quad 0.1217735 .5627441 .995452$

H $3.419986-1.163719-3.476864$

H $2.307659-2.498200-3.245134$

H $\quad 0.4768920 .810877-0.751972$

H $\quad 0.341944 \quad 1.752029-2.258013$

H $2.8163191 .772661-0.371498$

H $1.3793053 .933764-1.995928$

H $3.6213924 .967879-1.662599$

H $4.0180443 .882278-0.357585$

H 1.7441947 .3338220 .491609

H $2.0647586 .857953-1.270210$

H 2.3097235 .8918482 .288766

H 3.7985425 .0614781 .937700

H 2.8360112 .9571432 .085050

H $2.1041530 .488002-3.234688$

C $-2.268870-4.3883120 .002749$

O $-2.362100-3.120395-0.675189$

C $-2.787404-3.344539-2.031718$

C $-2.734837-4.851629-2.253311$

C $-3.053555-5.372100-0.866677$

H -3.831886 -3.017265 -2.091750

C - $-1.925791-2.530847-3.002254$

H -3.449321 -5.183583 -3.012793

H $-1.737589-5.184348-2.563520$

C $-2.667703-6.827332-0.663987$

H $-4.130898-5.256273-0.687293$

C -1.879904-1.017592 -2.713187

O $-0.576444-3.014013-2.952680$

H -2.267505 -2.703829 -4.029261

H -1.308576 -0.822688 -1.797933

O $-1.154978-0.399281-3.784346$

C $-3.256766-0.339660-2.605301$

C $-3.1538521 .193571-2.487361$

H $-3.783901-0.734300-1.732301$

O $-4.041287-0.667135-3.752552$

H $-2.4688151 .441070-1.669612$

H -2.718799 $1.594722-3.410346$

H -3.208384 -7.469436 -1.366960

H $-1.595375-6.984045-0.822151$

H -2.910257 -7.1596170 .350146$

C $-2.830765-4.2629591 .421464$

H $-0.270108-2.933903-2.032748$

H $-0.279458-0.821628-3.822135$

H -3.562198 $-0.348137-4.536682$

H $-2.919397-5.2636401 .860641$

H $-3.842366-3.8432101 .368671$

H -1.207926 -4.6687940 .028694$

C $-4.5942841 .828140 \quad 0.152930$

O $\quad-5.1765841 .386380-1.080003$

C $-4.5287791 .863423-2.265501$

C $-4.4971813 .398655-2.277037$

C -3.898294 $3.967904-0.995155$

C $-4.593938 \quad 3.3613150 .221967$

H $-3.569014 \quad 1.4481250 .236246$

C -5.4263991 .2353051 .300119$

H $-5.1676021 .557592-3.103618$

O $-3.7720123 .869481-3.408005$

H $-5.5250643 .770793-2.379865$

H -2.813814 $3.809499-0.961629$

O $-4.1000115 .382695-0.993629$

H $-5.623167 \quad 3.7384490 .269975$

O -3.9348363 .8036191 .405065$

C $-5.222062-0.2561991 .460931$

H -5.1519981 .6958122 .255388$

H $-6.4917781 .408731 \quad 1.105545$

H $-3.7633504 .841041-3.363886$

H -3.754613 $5.722856-0.150455$

H $-3.0253513 .463696 \quad 1.378362$

O $-6.159184-0.7346302 .332998$

O $-4.346340-0.914517 \quad 0.917822$

C $-6.087517-2.1362152 .610041$

H -6.174126 -2.713738 1.684709

H $-5.152072-2.368143 \quad 3.127294$

H $-6.923648-2.3966413 .264205$

SCF Energy (B3LYP/6-31G**//MMFF) $=-3245.93285023$

0600299

MM̄MF Geometry
C $-4.250721-3.1866070 .079551$

C $-3.112612-2.487823 \quad 0.204024$

C $-1.797488-2.909551-0.390458$

O $-0.783643-2.7144150 .619657$

C $-1.458415-2.001341-1.586257$

C $-0.254401-2.478802-2.375409$

C $0.971708-1.919472-2.341083$

C $-0.515784-3.650129-3.289491$

C $1.439025-0.751074-1.518482$

C $2.522529-1.130558-0.499091$

C $3.870210-1.532971-1.116189$

C $4.880562-1.870525-0.016550$

C $6.267569-2.194154-0.592479$

O $4.352906-0.441044-1.906601$

O $7.209729-2.1736430 .491304$

C $6.675601-1.067908-1.566494$

C $6.326636-3.604864-1.189296$

O $7.890823-1.385674-2.239462$

C $5.580481-0.713335-2.596419$

C $5.9410610 .518610-3.467702$

O $5.413865-1.812637-3.503137$

C $-0.387813-3.7855511 .354236$

O $-0.771643-4.9404571 .239652$

C $0.663709-3.3919252 .319703$

C $0.724386-2.1545982 .836010$

C $1.749660-1.6371903 .744297$

C $2.937406-2.5175874 .028353$

C $6.3860811 .785894-2.733958$

C $5.3028412 .412041-1.876689$

C 4.9592843 .5382560 .320278

O $4.1258382 .455577-2.227001$

C 4.4581062 .4438721 .207613

C 4.7267762 .3480172 .518126

C 4.2297381 .2204443 .379023

C 3.1380651 .5748094 .372960

C 2.7020872 .8207214 .628512

C $2.5338180 .394988 \quad 5.112915$

C $1.601967-0.386798 \quad 4.229670$

N $5.8064102 .952365-0.701760$

H -4.249628 -4.127729 -0.464176

H $-3.130241-1.5339400 .725160$

H -1.832128 -3.958376 -0.707699

H -1.313283 -0.972961 -1.238014

H -2.313834 -1.953924 -2.274597

H $1.737030-2.343520-2.989791$

H $\quad 0.361544-3.914857-3.889406$

H $-0.796335-4.536049-2.712003$

H -1.328532 -3.415104 -3.984637

H $1.8111150 .027162-2.194909$

H $\quad 0.610908-0.297764-0.969469$

H $2.677377-0.2607290 .149304$

H $2.157463-1.9440430 .137030$

H $3.715650-2.405823-1.759386$

H $4.519049-2.7006870 .602940$

H $4.980626-1.0121320 .662544$

H $6.919934-2.8253401 .152561$

H $6.881816-0.185576-0.947953$

H $6.102974-4.354706-0.420880$

H $7.333990-3.838665-1.550813$

H $5.621702-3.755199-2.009220$

H $8.552524-1.596886-1.558659$

H $6.7374630 .236055-4.169093$

H $5.0720550 .765540-4.093174$

H $4.978805-1.482389-4.306912$

H $1.365092-4.1727292 .591942$

H $-0.045849-1.4373782 .556271$

H $3.711998-2.0274674 .621495$

H $3.417820-2.8299133 .094194$

H $2.628104-3.4119334 .580258$

H $7.2903701 .591329-2.149237$

H $6.6625442 .543110-3.478591$

H $4.1173384 .052242-0.153472$

H 5.5598404 .2735250 .864726

H $3.829317 \quad 1.6906040 .740802$

H 5.3524323 .0989552 .994205

H 5.0860430 .8117443 .930179

H 3.8695730 .4114052 .731100

H 1.9155293 .0009495 .354716 
H $3.106023 \quad 3.6943774 .130333$ H 1.9540240 .7399905 .979181 H $3.337950-0.2136755 .535836$ H $\quad 0.7041070 .1697823 .959010$ H $6.7824402 .801591-0.464957$ C $-6.398330-2.064250-0.509625$ O $-5.719884-0.881013-0.976701$ C $-6.6776470 .178808-1.143700$ C -8.047137 -0.483589-1.069136 C -7.783096 -1.594384-0.068007 H $-6.5654660 .845653-0.280794$ C $-6.3781050 .947454-2.435572$ H $-8.8278990 .211211-0.745535$ H -8.348478 $-0.906826-2.034278$ C -8.837852 -2.686467 -0.076978 H $-7.728323-1.1555960 .937452$ C $-4.9162981 .432636-2.553118$ O $-6.636257 \quad 0.096336-3.560178$ H -7.067370 $1.793644-2.533827$ H $-4.2430910 .573853-2.665781$ O $-4.7997852 .196827-3.759736$ C $-4.4411082 .306464-1.378750$ C $-2.9809152 .765824-1.537957$ H $-4.5234891 .737989-0.451328$ O $-5.2935003 .442864-1.253111$ H $-2.3469341 .876954-1.631531$ H -2.879527 $3.334844-2.469873$ H $-9.818317-2.276260 \quad 0.186127$ H -8.923113 -3.154061 -1.063664 H $-8.593978-3.4700440 .647221$ C $-5.573436-2.7035150 .608099$ H $-6.079379-0.695341-3.462840$ H $-5.1043281 .631821-4.490471$ H $-5.2408723 .941693-2.086388$ H -6.107188 -3.553295 1.047536 H -5.407017 -1.975677 1.411273 H -6.476581 -2.745772 -1.366952 C $-1.8763891 .940571 \quad 1.217878$ O -2.7082063 .0658680 .911070$ C $-2.4902973 .658137-0.374260$ C $-1.0296894 .111929-0.507841$ C $-0.0562392 .975724-0.205126$ C $-0.393061 \quad 2.330317 \quad 1.137273$ H -2.0932071 .1197880 .523102$ C $-2.223414 \quad 1.4795722 .643967$ H $-3.1071504 .565488-0.396485$ O $-0.7898574 .627303-1.812699$ H -0.8487864 .9322110 .198965$ H $-0.0556182 .232017-1.009637$ O $1.2747173 .493955-0.156959$ H -0.1442993 .0256651 .949402$ O $\quad 0.4115821 .1686341 .312013$ C -3.5926780 .8442642 .757044$ H -1.499435 0.7315542 .983272 H -2.204629 2.3390523 .324756 H $0.1515214 .866930-1.863016$ H 1.3159304 .1545410 .555424 H $1.341457 \quad 1.4491591 .261129$ O $-3.787898 \quad 0.4512574 .051591$ O C $-5.045320-0.1764154 .322239$ H -5.105798 -0.3661865 .397141$ H $-5.872056 \quad 0.4807654 .035682$ H -5.108515 -1.131497 3.792878 SCF Energy (B3LYP/6-31G**//MMFF)= -3245.92167315

06_00300

MM̄MF Geometry

C $3.600997-3.669502-1.526463$

C $2.299983-3.377028-1.377308$

C $1.359410-3.166354-2.533277$

O $0.818999-1.828383-2.453324$

C $0.173761-4.142884-2.451513$

C $-0.784946-3.982588-3.615930$

C $-1.959468-3.323623-3.555614$

C $-0.356158-4.649255-4.897391$

C -2.553154 -2.596858 -2.378507

C $-3.763239-3.363483-1.836212$
C $-4.386935-2.696886-0.600795$

C $-5.521011-3.574724-0.062289$

C $-6.255476-2.9183361 .111579$

O $-4.890995-1.410603-0.979174$

O $-7.461411-3.6675431 .323870$

C $-6.650821-1.4851700 .703440$

C $-5.448166-3.0266912 .410506$

O $-7.234445-0.7964951 .808369$

C $-5.485337-0.6665550 .096718$

C $-6.0397120 .658969-0.486922$

O $-4.538788-0.3896451 .124751$

C $1.530621-0.824671-3.029239$

O $2.647483-0.893001-3.515399$

C $0.6841870 .387064-2.980329$

C $1.2144221 .582707-3.274432$

C $0.5006792 .861272-3.270602$

C $-0.9939442 .816133-3.110046$

C $-4.9862581 .632057-1.035083$

C -4.4303442 .5853430 .013682$

C $-2.7299964 .361670 \quad 0.310355$

O -4.7610932 .5584241 .196203$

C -2.061765 $5.398234-0.533231$

C $-0.7351635 .602664-0.552529$

C $-0.0785916 .694406-1.348720$

C $1.0611316 .200932-2.210552$

C $2.3335656 .522071-1.918215$

C $0.7146505 .418557-3.458839$

C $1.2116783 .998820-3.412006$

N -3.510248 $3.472186-0.529333$

H $4.013426-3.772795-2.526865$

H $1.889513-3.264677-0.376426$

H $1.876845-3.296963-3.492292$

H $-0.358893-4.006137-1.502656$

H $0.539108-5.178273-2.433766$

H -2.572659 -3.286771 -4.455419

H $-1.101925-4.541646-5.692196$

H $0.579010-4.214899-5.263548$

H $-0.202204-5.721019-4.734156$

H -1.821680 -2.439999-1.580274

H -2.857683 -1.597462 -2.712003

H $-4.518372-3.435478-2.630474$

H -3.459495 -4.386822 -1.580357

H $-3.606162-2.5682500 .158111$

H $-5.144554-4.5642360 .226559$

H -6.249226 -3.757669 -0.865491

H $-7.971253-3.2142862 .017382$

H -7.444700 -1.571537-0.051171

H $-5.272522-4.0798662 .661827$

H -6.000984 -2.607412 3.258407

H -4.475601 -2.533284 2.354555

H $-6.512502-0.5131982 .395138$

H $-6.7076350 .404057-1.320553$

H $-6.647187 \quad 1.177586 \quad 0.264293$

H -3.8817510 .2314070 .773052$

H $-0.3543980 .260498-2.696052$

H $2.273309 \quad 1.636103-3.524395$

H -1.469742 $3.796594-3.165080$

H -1.448349 2.204301 -3.897419

H - $1.2567162 .386765-2.137954$

H $-4.1584301 .091475-1.506879$

H $-5.4609722 .254808-1.803924$

H -2.010929 3.7500930 .865083

H $-3.3980194 .837231 \quad 1.036337$

H $-2.7073016 .035132-1.133480$

H -0.0912364 .9846420 .069161$

H $0.2855507 .446526-0.636610$

H $-0.8051057 .215213-1.985120$

H $3.1556006 .205121-2.551654$

H $2.5823887 .108880-1.040292$

H $1.1781305 .910948-4.324667$

H $-0.3594435 .476815-3.656600$

H $2.2941083 .916069-3.521536$

H -3.211765 3.327151-1.488974

C $5.451482-2.666960-0.096929$

O $4.645760-1.5375730 .288570$

C $5.187049-0.352297-0.327442$

C $6.574133-0.739242-0.827205$

C $6.342296-2.182790-1.240357$ 
H $4.555614-0.135565-1.197412$

C 5.1326690 .8247010 .654057

H $6.911410-0.104140-1.651792$

H $7.324572-0.689513-0.029832$

C $7.619820-2.985713-1.407320$

H $5.795365-2.182060-2.191176$

C 3.7370191 .0192631 .287322

O $6.066756 \quad 0.5757031 .710109$

H $5.466417 \quad 1.740977 \quad 0.153134$

H 3.4995600 .1501161 .904652

$\begin{array}{lllll}\text { O } & 3.788856 & 2.149197 & 2.163863\end{array}$

C $2.618242 \quad 1.260197 \quad 0.262080$

C 1.2104891 .4662540 .852768

H $2.5702730 .427281-0.444345$

O $2.9464672 .425586-0.502430$

H $1.2566072 .194966 \quad 1.668781$

H $0.597908 \quad 1.9438160 .077407$

H $8.246441-2.552210-2.193595$

H $8.206491-3.004704-0.482820$

H $7.394779-4.020153-1.685600$

C $4.549340-3.871199-0.377736$

H $6.154253 \quad 1.396606 \quad 2.223185$

H 4.3203661 .8944582 .937024

H $2.991233 \quad 3.1770220 .113751$

H $3.972258-4.0932650 .529605$

H $5.161538-4.756807-0.583518$

H $6.070895-2.9068160 .777623$

C 1.0030610 .2345523 .670849

O $1.145038-0.4635452 .428161$

C $\quad 0.523600 \quad 0.154521 \quad 1.299131$

C -0.9802780 .3044321 .567649$

C $-1.230753 \quad 1.1130152 .836145$

C -0.4731410 .4909964 .006715$

H $1.551028 \quad 1.182516 \quad 3.620531$

C $1.641942-0.6330234 .763757$

H $0.628726-0.553366 \quad 0.467055$

$\begin{array}{lllll} & 0 & -1.635391 & 0.922284 & 0.467505\end{array}$

H -1.432166 -0.6883661 .692287$

H $-0.960742 \quad 2.1673982 .706514$

O $-2.623948 \quad 1.0896213 .149992$

H $-0.962078-0.4501414 .290976$

O $-0.548892 \quad 1.363207 \quad 5.132953$

C $3.135078-0.7584424 .577717$

H $1.477518-0.2140005 .763057$

H $1.200040-1.6374084 .764399$

H - $-1.4687730 .373956-0.318199$

H -3.094281 1.5028972 .405981

H -1.4911771 .5237995 .312567$

O $3.443085-2.0044804 .117065$

O $3.928753 \quad 0.146107 \quad 4.803067$

C $4.834790-2.2630803 .910638$

H $5.256167-1.5462683 .201685$

H $5.369912-2.2226644 .863858$

H $4.935485-3.2681393 .492282$

SCF Energy (B3LYP/6-31G**//MMFF) $=-3245.94159040$

06_00301

MM̄FF Geometry

C $0.783405-4.5471330 .688468$

C $0.040247-3.7714901 .492032$

C $0.490805-3.026007 \quad 2.723504$

O $0.479740-1.6176032 .381823$

C $1.907989-3.3609553 .235764$

C $2.316212-2.5518774 .455447$

C $3.357350-1.6948594 .483948$

C $1.484748-2.7635615 .694118$

C $4.335873-1.4039993 .378636$

C $3.800627-0.3767832 .381160$

C $4.781276-0.0708861 .240017$

C $5.954945 \quad 0.7972201 .701044$

C 6.8582231 .2225110 .530752

$\begin{array}{lllll}\text { O } & 4.028006 & 0.617899 & 0.232927\end{array}$

$\begin{array}{llllll}\text { O } & 7.709986 & 2.281254 & 0.996377\end{array}$

C $5.9767551 .811810-0.592427$

C $7.786826 \quad 0.0848350 .090194$

O $6.7523412 .104053-1.752217$

C $4.7690220 .922469-0.955913$

C $3.8019171 .605792-1.947819$
O $5.241869-0.271644-1.591071$

C $-0.635291-0.8876142 .643605$

O $-1.683285-1.284599 \quad 3.124788$

C -0.3396210 .5074122 .243062$

C -1.2300451 .4800112 .486940$

C -1.066021 2.9016062 .167716

C $0.2301713 .321968 \quad 1.529512$

C $3.1756132 .908526-1.445685$

C $2.0225863 .354470-2.327965$

C $0.0802384 .896053-2.354594$

O $1.8142662 .893353-3.447203$

C $-0.662825 \quad 5.742302-1.372731$

C $-1.9042705 .476466-0.937556$

C $-2.654980 \quad 6.376135 \quad 0.002144$

C -3.1563325 .6674851 .239639$

C $-4.4741795 .509978 \quad 1.452586$

C $-2.136061 \quad 5.243862 \quad 2.274910$

C -2.0721543 .7508552 .463729$

N $1.2551304 .335006-1.717184$

H $1.834132-4.7171280 .902012$

H $-1.002689-3.6135741 .224588$

H $-0.233800-3.2303363 .521243$

H $2.626234-3.1938292 .426309$

H $1.968603-4.4268793 .490437$

H $3.551739-1.1514845 .407580$

H $1.918439-2.2743676 .572914$

H $\quad 0.476749-2.3601425 .558234$

H $1.407605-3.8318255 .921423$

H $5.259579-1.0363643 .840442$

H $4.613359-2.3300982 .861919$

H $2.870773-0.7493861 .936958$

H 3.5237550 .5529702 .895673

H $5.137511-1.0199070 .822488$

H $6.548903 \quad 0.2868452 .468108$

H $5.565587 \quad 1.7060172 .181387$

H $8.218967 \quad 1.9457951 .754240$

H $5.6104922 .777756-0.223364$

H $8.422095-0.2353770 .924980$

H $8.4743150 .417025-0.695382$

H $7.248087-0.794673-0.267931$

H $6.9334721 .263845-2.206973$

H $4.3041201 .777238-2.908841$

H $2.9889410 .899287-2.167773$

H $4.510666-0.660991-2.098961$

H $\quad 0.6155120 .698344 \quad 1.766419$

H $-2.167168 \quad 1.2151292 .973894$

H $\quad 0.284050 \quad 4.389513 \quad 1.310551$

H 0.3715542 .7969520 .578811

H 1.0765543 .0922412 .186505

H $2.7981382 .777570-0.425430$

H $3.9187123 .713164-1.439090$

H $-0.5299324 .075564-2.747800$

H $0.4128295 .503529-3.202757$

H $-0.1579516 .639555-1.021831$

H -2.421303 $4.591805-1.301547$

H $-3.4962536 .806139-0.556892$

H -2.037956 $7.229167 \quad 0.312181$

H -4.8475485 .0449592 .358875$

H $-5.209570 \quad 5.843620 \quad 0.728243$

H -2.409276 $5.692427 \quad 3.239941$

H -1.1595915 .6766822 .040568$

H $-2.966997 \quad 3.3375722 .931653$

H $1.5949504 .756381-0.856813$

C $0.716340-4.383560-1.797454$

O $0.028306-3.114422-1.829606$

C $-0.383259-2.841239-3.181802$

C $0.339268-3.860450-4.054579$

C $0.389558-5.059941-3.127554$

H $-1.458839-3.046607-3.232847$

C $-0.111196-1.375666-3.538950$

H $-0.194637-4.059940-4.988615$

H $1.354002-3.531959-4.308118$

C $1.398087-6.113067-3.551747$

H $-0.609588-5.514065-3.083257$

C $-0.797048-0.360158-2.599320$

O $1.303133-1.154622-3.473356$

H $-0.407567-1.192494-4.578941$

H $-0.349366-0.402452-1.598720$ 
O $-0.5398590 .954112-3.107007$

C $-2.323425-0.532455-2.489378$

C $-2.9925610 .574279-1.652766$

H -2.542557-1.500780 -2.035354

O $-2.897509-0.543239-3.796746$

H -2.503667 $0.619008-0.673764$

H $-2.8319371 .543235-2.140470$

H $1.146835-6.509126-4.541099$

H $2.411453-5.701022-3.602252$

H $1.409614-6.949672-2.846289$

C $0.257749-5.176353-0.572757$

H $1.486557-0.302566-3.903635$

H $0.4088331 .131397-2.993211$

H $-2.6828230 .306809-4.218023$

H $0.623273-6.208158-0.618699$

H $-0.837233-5.219624-0.544554$

H $1.790144-4.162096-1.734667$

C $-4.488528-1.0592220 .465586$

O $-4.834109-0.912362-0.916826$

C $-4.5159780 .359858-1.491792$

C $-5.2329791 .475980-0.722106$

C $-4.931267 \quad 1.4173320 .771293$

C -5.1859190 .0127741 .315009$

H -3.400574 -0.9889810 .584642$

C $-4.934930-2.4625030 .909746$

H $-4.9416670 .350498-2.503112$

O $-4.866403 \quad 2.748290-1.245883$

H $-6.3158001 .369066-0.868578$

H -3.9076551 .7477610 .973149$

$\begin{array}{llll}\text { O } & -5.795811 & 2.341905 & 1.434013\end{array}$

H $-6.267076-0.175166 \quad 1.341893$

$\begin{array}{llll}\text { O } & -4.703540 & -0.070578 & 2.654138\end{array}$

C $-4.101104-3.5713610 .304389$

H $-4.851813-2.5589891 .998121$

H $-5.975330-2.6316570 .607279$

H $-5.3192473 .423894-0.712735$

H -5.5149472 .3916812 .363218$

H -5.2340050 .5355423 .197670$

O $-4.582094-4.7721110 .745655$

O $-3.151624-3.415722-0.449456$

C $-3.888631-5.929526 \quad 0.268636$

H $-2.894088-5.9779830 .721091$

H $-4.455659-6.8137690 .571425$

H $-3.817615-5.917212-0.823388$

SCF Energy (B3LYP/6-31G**/MMFF) $=-3245.92292834$

06_00302

MM̄FF Geometry

C $0.851972-1.3189682 .675995$

C $1.985051-0.9096393 .267901$

C $2.6736630 .425186 \quad 3.098527$

$\begin{array}{lllll}\text { O } & 1.829447 & 1.315439 & 2.334752\end{array}$

$\begin{array}{lll}\text { C } 4.011228 & 0.217590 & 2.356137\end{array}$

C 5.1502661 .0857272 .852095

C 5.7266002 .0800802 .148635

C $5.696393 \quad 0.7147754 .208343$

C $5.3429372 .606963 \quad 0.794417$

C $6.3307932 .191585-0.305991$

C $6.1932870 .719340-0.726196$

C $7.222550 \quad 0.360831-1.799827$

C $7.028238-1.071013-2.326034$

O $4.8737320 .544962-1.247026$

O $7.804794-1.206772-3.526175$

C $5.546897-1.262685-2.719721$

C $7.567403-2.115065-1.341013$

O $5.287775-2.614529-3.092726$

C $4.550306-0.795458-1.633409$

C $3.110773-0.778419-2.200501$

O $4.611229-1.700651-0.529993$

C 1.9981652 .6520092 .501848

$\begin{array}{lllll}\text { O } & 2.788417 & 3.215818 & 3.241111\end{array}$

C 1.0511343 .3333821 .597725

C 1.3783924 .5357101 .106227

C $0.543231 \quad 5.306096 \quad 0.194641$

C $1.2610385 .742796-1.051696$

C $2.083017-0.124601-1.264636$

C $0.727640 \quad 0.040594-1.927265$

C $-1.4972141 .095050-1.529542$
O $0.448724-0.447657-3.018736$

C -1.599966 $2.537629-1.910630$

C $-2.5699723 .341024-1.449405$

C $-2.7672994 .764109-1.884024$

C $-2.9164955 .715150-0.718996$

C $-4.1188865 .942656-0.163026$

C $-1.6899906 .469934-0.264031$

C -0.7307635 .6106490 .511629$

N $-0.1361050 .788333-1.138815$

H $\quad 0.317448-0.6606851 .997029$

H $2.492327-1.600783 \quad 3.939725$

H 2.8138550 .8405114 .103596

H 3.8501820 .3662041 .284921

H $4.352429-0.8222762 .456448$

H 6.5686062 .6032052 .600956

H $6.575661 \quad 1.308896 \quad 4.479619$

H 4.9433770 .8715264 .986309

H $5.996835-0.3381204 .221496$

H 4.3191052 .3359330 .520288

H $5.342977 \quad 3.7025270 .862855$

H $6.1327022 .827689-1.178640$

H 7.3593382 .3959120 .016459

H $\quad \begin{array}{llll}6.326163 & 0.080537 & 0.153801\end{array}$

H $8.2431370 .496659-1.420906$

H $7.1233021 .055025-2.646282$

H $8.732887-1.017153-3.306073$

H $5.378605-0.663939-3.625316$

H $8.633168-1.943785-1.147210$

H $7.500151-3.125205-1.759597$

H $7.051796-2.102936-0.378482$

H $5.233378-3.141345-2.277209$

H $3.107302-0.218118-3.144775$

H $2.782417-1.801034-2.425729$

H $3.761600-1.694441-0.062650$

H $\quad 0.1256382 .8305641 .341009$

H $2.3386204 .986793 \quad 1.347984$

H $2.0250946 .489959-0.811596$

H $0.5972176 .172442-1.805119$

H $1.7549744 .887058-1.527146$

H $1.940515-0.732992-0.365548$

H $2.4264430 .869634-0.957143$

H -2.137926 $0.846384-0.677987$

H -1.788802 $0.474957-2.382488$

H $-0.8823912 .922162-2.631201$

H $-3.3033432 .933181-0.758464$

H $-3.6748504 .802325-2.501223$

H -1.953311 $5.100146-2.538005$

H $-4.241374 \quad 6.6467850 .653189$

H $-5.0067115 .424225-0.510067$

H $-1.981603 \quad 7.3008780 .392457$

H -1.224785 $6.949174-1.130363$

H -1.128790 5.2491181 .460574

H $\quad 0.190157 \quad 1.139026-0.243491$

C $0.281336-3.5101531 .601461$

O $-0.741733-3.0339820 .701786$

C $-1.388042-4.1698160 .093437$

C $-0.545956-5.3859110 .459041$

C $-0.027774-4.989921 \quad 1.826043$

H $-2.368880-4.2701530 .571171$

C -1.557722 -3.952481-1.413037

H $-1.133962-6.3087250 .471475$

H $0.287866-5.527340-0.238693$

C $1.165909-5.8125272 .280029$

H $-0.840382-5.0978692 .557137$

C $-2.375239-2.702773-1.791647$

O $-0.262894-3.820657-2.015510$

H -2.010598 -4.842138 -1.865639

H -1.818738 -1.798671 -1.526734

O $-2.513728-2.690129-3.217883$

C $-3.779820-2.634331-1.164144$

C $-4.618953-1.464708-1.714721$

H -3.678774 -2.528774 -0.079543

O $-4.475190-3.857196-1.404900$

H $-4.041220-0.540635-1.619077$

H $-4.803571-1.620926-2.784321$

H $0.898397-6.8721452 .348386$

H $2.004528-5.7220571 .581461$

H $1.513081-5.4888013 .266125$ 
C $0.266810-2.6882982 .891268$ H $0.181893-3.065265-1.593743$ H -1.617293 -2.705277 -3.595394 H $-4.531488-3.978183-2.368403$ H $0.824649-3.212946 \quad 3.675484$ H $-0.760237-2.5652833 .251442$ H $1.242109-3.3959821 .081285$ C $-5.265745-0.017453 \quad 0.893128$ O $-5.854545-1.2325740 .414744$ C $-5.985110-1.317429-1.007759$ C $-6.842438-0.152514-1.525621$ C $-6.3207461 .200248-1.045182$ C $-6.112076 \quad 1.186697 \quad 0.468744$ H -4.2484910 .0816310 .496028$ C $-5.171597-0.1233352 .418060$ H $-6.543750-2.240550-1.207349$ O $-6.900476-0.151687-2.947937$ H $-7.871483-0.272346-1.162179$ H $-5.3983911 .482528-1.565983$ O $-7.2813672 .212376-1.354541$ H -7.0867141 .1616930 .972690$ O -5.4559842 .3880990 .865806$ C $-4.112423-1.1272272 .807493$ H $-4.893940 \quad 0.8297562 .881594$ H $-6.133861-0.4253122 .848883$ H - $-7.251516-1.014740-3.226278$ H $-7.4317272 .183403-2.315113$ H -5.9965513 .1323750 .549909$ O $-4.694564-2.2554043 .303575$ O $-2.911449-0.9361982 .664931$ C $-3.791771-3.2950313 .692221$ H -3.128516 -2.943403 4.488152 H $-4.381599-4.1334554 .071714$ H $-3.213320-3.6357482 .829059$ SCF Energy (B3LYP/6-31G*//MMFF) $=-3245.91666714$

0600303

MM̄FF Geometry

C $-2.032600-2.848591-1.948456$

C $-1.303492-2.211079-1.020086$

C $-0.078529-1.390591-1.319727$

O $0.979571-1.959874-0.508215$

C $-0.2492800 .088390-0.935043$

C $-1.1781850 .861069-1.844190$

C $-0.8092011 .406200-3.019503$

C $-2.5755171 .060891-1.327329$

C $0.547108 \quad 1.350337-3.664728$

C $1.2012442 .734698-3.775098$

C $1.5236753 .375452-2.415292$

C $2.1100724 .775820-2.608249$

C $2.5084135 .415962-1.271174$

O $2.4642152 .536888-1.734773$

O $3.3007306 .572404-1.581190$

C $3.3981394 .421836-0.494771$

C $1.2848685 .928779-0.502831$

O 3.6891814 .9180690 .810365

C $2.8132592 .989992-0.420320$

C $3.822711 \quad 1.9850010 .186248$

$\begin{array}{lllll}\text { O } & 1.668423 & 3.013870 & 0.442395\end{array}$

C $2.262658-1.819938-0.934916$

O $2.653192-1.210190-1.916424$

C $3.119070-2.585797-0.001479$

C $4.414648-2.778818-0.290108$

C $5.355175-3.5872140 .488591$

C $4.875386-4.1565891 .798576$

C $5.0267901 .662028-0.704376$

C $6.1802091 .060556 \quad 0.082111$

C $8.6441870 .715846-0.092591$

$\begin{array}{lllll}\text { O } & 6.070170 & 0.647169 & 1.233084\end{array}$

C $8.926108-0.740774-0.273995$

C $9.250312-1.5515110 .744709$

C $9.630192-2.9955470 .589620$

C $8.713140-3.9239671 .351112$

C $8.877862-4.1329672 .668402$

C $7.668132-4.6808180 .568285$

C $6.593987-3.797412-0.003662$

N $7.3531851 .050404-0.660641$

H $-1.754893-2.768066-2.996397$
H $-1.579508-2.3061430 .026668$

H $\quad 0.192019-1.490617-2.378644$

H $\quad 0.7324460 .574954-0.919338$

H -0.5897330 .1686990 .106592$

H -1.554853 $1.961441-3.587205$

H $-3.0496770 .101473-1.104718$

H $-2.5502231 .658336-0.410623$

H $-3.2149101 .585074-2.045419$

H $1.2216150 .663878-3.147438$

H $0.4203080 .941960-4.674956$

H $2.1310212 .615246-4.346229$

H $0.5495993 .401665-4.353528$

H $0.6031883 .437049-1.824691$

H $1.4072235 .422735-3.148020$

H $3.0051914 .711378-3.243126$

H $3.6236326 .941985-0.741144$

H $4.3581624 .395031-1.022213$

H $0.7439606 .671591-1.101496$

H $1.581736 \quad 6.4467230 .415833$

H $0.5795875 .137914-0.239472$

H 2.9017554 .7756961 .363037

H 4.1461632 .3507381 .169162

H 3.3071521 .0366400 .392674

H 1.5088022 .1140360 .771398

H $2.646311-3.0125800 .876177$

H $4.817491-2.339578-1.201898$

H $4.087567-4.8987801 .630717$

H $4.480269-3.3623442 .442217$

H $5.665776-4.6449862 .373490$

H $4.7356160 .949866-1.484927$

H $5.4000462 .564670-1.196854$

H 8.6593121 .0119730 .961914

H $9.3960241 .305783-0.626318$

H $8.907623-1.133208-1.287622$

H $9.290833-1.1422441 .752091$

H $10.657697-3.1171300 .957418$

H $9.665307-3.286534-0.467707$

H $8.244292-4.8223943 .216388$

H $9.650214-3.6155543 .228042$

H $7.231088-5.4853661 .170889$

H $8.159640-5.203443-0.263043$

H $6.864737-3.334125-0.952774$

H $7.3314431 .366002-1.625926$

C $-4.510649-3.245567-2.247512$

O $-4.944455-2.031536-1.600183$

C $-6.342293-2.149204-1.272704$

C $-6.860491-3.335212-2.075440$

C $-5.652727-4.248461-2.066275$

H $-6.395331-2.395089-0.205343$

C $-7.080383-0.831826-1.537529$

H $-7.747230-3.788463-1.621843$

H -7.112635 -3.053850 -3.104368

C $-5.701092-5.329144-3.132931$

H $-5.581986-4.724428-1.078776$

C $-6.4604690 .404588-0.860111$

O $-7.095699-0.575951-2.948941$

H $-8.130567-0.933673-1.240587$

H $-5.5067590 .651204-1.338734$

O $-7.328993 \quad 1.518842-1.104246$

C $-6.252767 \quad 0.2759780 .659376$

C $-5.6703851 .561406 \quad 1.271142$

H $-5.569428-0.5513010 .858355$

$\begin{array}{llll}\text { O } & -7.490733 & -0.037040 & 1.292799\end{array}$

H $-4.753592 \quad 1.8138700 .729217$

H $-6.3736462 .388357 \quad 1.114471$

H $-6.572515-5.975532-2.985928$

H $-5.767897-4.898291-4.137603$

H $-4.805329-5.956715-3.094538$

C $-3.203080-3.733767-1.620959$

H $-6.171784-0.551055-3.252423$

H $-7.4436291 .591044-2.067465$

H $-8.1135130 .682497 \quad 1.091899$

H $-2.967444-4.739573-1.987978$

H $-3.324385-3.807455-0.533986$

H $-4.367332-3.007539-3.309260$

C -3.228791 0.3696572 .681450

$\begin{array}{llll}\text { O } & -4.585380 & 0.325659 & 3.136339\end{array}$

$\begin{array}{llll}\text { C } & -5.384249 & 1.459808 & 2.786867\end{array}$ 
C $-4.7582142 .729693 \quad 3.377576$

C -3.3100872 .9028262 .924823$

C -2.5103981 .6284743 .194143$

H -3.2103980 .3385511 .586246$

C $-2.519662-0.8884843 .212313$

H -6.3531531 .3143483 .280965$

O

H -4.7833852 .6644684 .473147$

H -3.257314 3.1959581 .869966

O -2.7416793 .9828793 .669623$

H $-2.327307 \quad 1.5400974 .272954$

O $-1.239372 \quad 1.7238682 .552266$

C $-3.030741-2.1722502 .595205$

H -1.446016 -0.8344682 .999330$

H -2.672321 -0.9651534 .295626$

H -5.0817794 .6521663 .383354$

H -1.8557004 .1547943 .308693$

H -0.7527702 .4557392 .966969$

O $-2.367017-3.2245553 .161125$

O $-3.888727-2.2518821 .728434$

C - $-2.733801-4.5204602 .677237$

H -3.815450 -4.669382 2.750381

H $-2.394952-4.6412201 .644494$

H $-2.235826-5.2686503 .299586$

SCF Energy (B3LYP/6-31G**//MMFF) $=-3245.92877564$

0600304

MM̄FF Geometry

C -3.629340 -3.557442 -0.493016

C $-2.697008-2.649317-0.166681$

C $-1.460043-2.398495-0.987006$

O $-0.336634-2.520222-0.085080$

C $-1.496157-0.971239-1.569588$

C $-0.617313-0.806055-2.793266$

C $0.593450-0.215492-2.808660$

C $-1.208685-1.335634-4.076232$

C $1.3620190 .368965-1.656435$

C $1.6154081 .864659-1.878690$

C $2.4136062 .504531-0.733477$

C $2.6234463 .997082-1.006173$

C 3.4954264 .6639300 .066190

O $3.6773641 .838799-0.631788$

O $3.8732835 .952106-0.442790$

C 4.7800993 .8270280 .243964

C 2.7047814 .9269571 .352606

O 5.5730944 .3373231 .313119

C 4.5113082 .3172340 .432703

C 5.8156581 .4880490 .433332

O 3.8871062 .1146221 .706711

C $0.824819-3.025579-0.577546$

O $1.040452-3.417090-1.713043$

C $1.816834-3.0023960 .519989$

C $3.122015-3.097700 \quad 0.226326$

C $4.228156-3.086781 \quad 1.186672$

C $3.879151-3.1660462 .649735$

C $6.5959331 .499275-0.884678$

C $7.8029280 .580401-0.805965$

C $9.139637-1.016945-2.166239$

$\begin{array}{lllll}\text { O } & 8.427157 & 0.397614 & 0.237040\end{array}$

C $8.538333-2.324933-1.754889$

C $8.953497-3.033900-0.694024$

C $8.264915-4.279726-0.212174$

C $7.699764-4.126121 \quad 1.187868$

C $8.022486-5.0020602 .154363$

C $6.780833-2.955814 \quad 1.483225$

C $5.489468-3.0170870 .711357$

N $8.1208410 .010550-2.031581$

H $-3.494059-4.168058-1.382437$

H -2.838703 -2.0305770 .715609$

H -1.389929 -3.153891 -1.779112

H -1.222406 $-0.245829-0.796087$

H -2.520952 -0.707521-1.866319

H $\quad 1.118258-0.158749-3.762086$

H $-0.555464-1.164255-4.938435$

H -1.382404 -2.413684 -4.006197

H $-2.163720-0.842295-4.284916$

H $\quad 0.8546440 .217554-0.700362$

H $2.317475-0.164975-1.587413$
H $2.1693801 .990605-2.818645$

H $0.6569512 .387294-1.991475$

H 1.8576972 .3582130 .199205

H $1.6627284 .518206-1.099124$

H $3.1209004 .119923-1.978978$

H $4.4879526 .352455 \quad 0.196148$

H $5.3724283 .974440-0.667051$

H 1.8589725 .5946301 .148719

H 3.3185965 .4452972 .097371

H 2.3063394 .0169891 .806110

H 5.1652634 .0494992 .147621

H 6.4596971 .8113131 .261511

H 5.5628310 .4421830 .659964

H 4.0111291 .1865451 .966128

H $1.447544-2.8883021 .532632$

H $3.413836-3.175100-0.820508$

H $3.217333-4.0181282 .843092$

H $3.374113-2.2492822 .973155$

H $4.745508-3.305723 \quad 3.299138$

H $5.943893 \quad 1.182399-1.705857$

H $6.9713062 .501753-1.112849$

H $10.003972-0.746506-1.550584$

H $9.447775-1.051521-3.215169$

H $7.687024-2.678041-2.332349$

H $9.801095-2.680086-0.111745$

H $8.993225-5.099668-0.248594$

H $7.452802-4.566936-0.891433$

H $7.631163-4.9031493 .161491$

H $8.683291-5.8404491 .960848$

H $7.290548-2.0204741 .223989$

H $6.606300-2.8778732 .560194$

H $5.623749-2.951738-0.368970$

H $7.4977570 .155674-2.820474$

C $-6.105678-3.272000-0.521702$

O $-6.000771-1.846539-0.701663$

C $-7.293891-1.248693-0.497412$

C $-8.288308-2.402190-0.465434$

C $-7.449590-3.4982140 .167912$

H $-7.272056-0.7818220 .494489$

C $-7.545329-0.178676-1.566597$

H $-9.186849-2.1603420 .110054$

H -8.601612 -2.699221-1.472930

C $-8.019603-4.891559-0.029346$

H -7.359082 -3.294585 1.243470

C $-6.3987590 .845927-1.708436$

O $-7.707105-0.813974-2.841522$

H $-8.4914640 .335371-1.363758$

H $\quad-5.5087280 .355979-2.122471$

O $-6.7980941 .832195-2.667531$

C $-6.0259791 .564293-0.400250$

C $-4.8558872 .545470-0.581924$

H $\quad-5.738757 \quad 0.8241430 .347036$

O -7.1635892 .2562010 .109385$

H $-4.0033071 .993989-0.993936$

H $-5.1328713 .309877-1.317935$

H $-9.010560-4.9701350 .429605$

H -8.121054 -5.137024 -1.091851

H -7.373666 -5.6459960 .430568$

C $-4.901862-3.7676520 .280427$

H $-6.901866-1.331089-3.016229$

H $-7.0319861 .361633-3.486073$

H -7.442396 $2.898582-0.565570$

H $-5.002270-4.8357510 .502241$

H -4.848768 -3.239193 1.239367

H $-6.108078-3.719952-1.524386$

C -3.0347191 .5438821 .686507$

O -4.2116982 .3527791 .809150$

C $-4.4505403 .259613 \quad 0.727710$

C -3.2591174 .2139120 .571130$

C -1.9461833 .4533030 .419478$

C $-1.7864132 .428031 \quad 1.541253$

H -3.1328280 .8792350 .819564$

C -2.9399010 .6800682 .956808$

H -5.3116593 .8689381 .030173$

O $-3.4366385 .072671-0.550449$

H -3.1878474 .8550631 .459517$

H -1.870933 $2.982278-0.567722$

O -0.8724154 .3942960 .496169$ 
H -1.5805902 .9621522 .478129$

O -0.6443451 .6296021 .240847$

C $-4.042709-0.351153 \quad 3.066305$

H -1.993861 0.1297322 .979273

H -3.0058531 .3261063 .840695$

H -4.268281 $5.558842-0.417166$

H -0.0436353 .8874480 .461404$

H $-0.387817 \quad 1.1565192 .049672$

O $-3.955987-0.9619504 .285844$

O $-4.864171-0.6071642 .198723$

C $-4.931865-1.9789904 .534085$

H -5.942098 -1.5655194 .456859$

H $-4.798534-2.8079363 .832723$

H $-4.783020-2.3518155 .550910$

SCF Energy (B3LYP/6-31G**//MMFF) $=-3245.92955425$

06_00305

MM̄FF Geometry

C 2.4639013 .8633871 .630705

C 1.1678883 .6626661 .346525

C $0.127746 \quad 3.3706452 .397037$

O -0.5821272 .1713982 .010163$

C -0.8847974 .5274062 .471141$

C -1.879720 4.3756433 .607013

C -3.1667544 .0009493 .464703$

C -1.3444314 .7127604 .975911$

C -3.8868053 .6308392 .198506$

C -4.3384842 .1653812 .229519$

C $-4.951391 \quad 1.7243870 .892956$

C -5.4111860 .2665420 .962147$

C $-5.939268-0.234272-0.390484$

O $-3.9544601 .872547-0.124718$

O $-6.040871-1.663267-0.296214$

C $-4.9018150 .098650-1.486720$

C $-7.3587420 .276836-0.662427$

O $-5.409229-0.230861-2.776795$

C $-4.4123671 .562106-1.448467$

C $-3.2451721 .849717-2.425686$

O $-5.4927452 .422589-1.832601$

C $-0.120604 \quad 0.9903992 .503315$

O $0.873420 \quad 0.815994 \quad 3.190051$

C - $1.071113-0.0672572 .099251$

C -1.093661 -1.216267 2.789135

C -2.031455 -2.311712 2.567932

C $-2.577963-2.8855143 .848132$

C $-1.9888800 .995724-2.227063$

C -1.976865 -0.267768 -3.071236

C $-0.932885-2.526205-3.174500$

O $-2.655065-0.404157-4.085964$

C -1.892658 -3.462107 -2.513400

C $-1.518871-4.441019-1.676403$

C $-2.483700-5.366808-0.997566$

C $-2.557242-5.1245930 .492384$

C $-2.074329-6.0210641 .368659$

C $-3.267495-3.874910 \quad 0.963332$

C $-2.314729-2.768917 \quad 1.331662$

N -1.086530 -1.211654 -2.581464

H 2.7738683 .8020542 .672751

H $0.8137113 .719168 \quad 0.321458$

H 0.5949913 .2312893 .379723

H -1.4021034 .6238281 .510984$

H -0.3539365 .4793772 .609267$

H -3.7856533 .9461484 .359673$

H -2.116066 4.653136 5.750963

H -0.5429714 .0234935 .258094$

H -0.9471895 .7329924 .987388$

H -4.7647154 .2803382 .096546$

H -3.2701443 .8108841 .315581$

H -3.473388 1.5332912 .458455

H -5.0676012 .0208313 .036576$

H -5.7974752 .3849150 .669486$

H -6.1685840 .1326881 .744489$

H $-4.563340-0.367126 \quad 1.257451$

H -6.294621 -1.999095 -1.173238

H -4.049627 -0.569430 - 1.312957

H $-8.042840-0.0653080 .123511$

H -7.755173 -0.130470 -1.598944

H $-7.4223371 .366043-0.705198$
H $-6.0309150 .469456-3.038758$

H $-3.603698 \quad 1.802270-3.461146$

H -2.943462 $2.895727-2.270912$

H $-5.1213003 .272673-2.122051$

H $-1.762250 \quad 0.1380491 .289651$

H $-0.412889-1.3585553 .626213$

H -1.761778 -3.1995504 .508933$

H -3.212759 -3.761318 3.700456

H -3.176185 -2.133393 4.374651

H $-1.1150611 .577641-2.546593$

H -1.846176 $0.748991-1.170121$

H $0.106386-2.832947-3.031411$

H -1.135216 -2.478225 -4.249065

H $-2.948707-3.320362-2.731444$

H $-0.464767-4.583704-1.454583$

H $-2.167508-6.396416-1.208365$

H -3.490436 -5.275279-1.424896

H -2.152729 -5.8617722 .438832$

H -1.587092 -6.9314631 .035821$

H -3.945220 -4.125919 1.785688

H $-3.931079-3.5054450 .171360$

H -1.830898 -2.2928470 .479444$

H $-0.613287-1.036239-1.699805$

C $3.2249054 .264621-0.817117$

O $2.9245912 .940517-1.304321$

C $3.6587022 .714978-2.520547$

C $4.0935734 .091801-3.000979$

C $4.3811634 .777764-1.680223$

H $4.5535932 .142273-2.248242$

C $2.8162961 .915521-3.518136$

H $4.9639234 .047462-3.662477$

H $3.2870404 .611767-3.530881$

C $4.4492966 .291647-1.784505$

H $5.3372114 .403226-1.290125$

C $2.2651130 .584062-2.969619$

O $1.6929222 .704603-3.930814$

H $3.4022031 .730662-4.426108$

H $1.4622970 .775253-2.247039$

O $1.663352-0.118398-4.064114$

C $3.320607-0.330056-2.321964$

C $2.747390-1.702128-1.924499$

H $3.7201850 .165213-1.431717$

O $4.410527-0.524063-3.222230$

H $1.836148-1.546287-1.337663$

H $2.465463-2.249639-2.831363$

H $5.2561816 .594476-2.459973$

H $3.5136636 .710097-2.170450$

H $4.6412556 .742375-0.805768$

C 3.5790434 .1912090 .671361

H $1.1932722 .944310-3.131295$

H $0.9827190 .464067-4.443085$

H $4.054068-0.925219-4.033499$

H $4.0035385 .151086 \quad 0.989121$

H 4.3559973 .4291640 .814805

H $2.3330274 .882398-0.979331$

C $3.370667-1.6568461 .070785$

O $4.290988-1.869545-0.005368$

C $3.752209-2.568422-1.132778$

C $3.206406-3.935591-0.691878$

C $2.213457-3.8110150 .463610$

C $2.829995-2.9921211 .599343$

H $2.534007-1.0466790 .707791$

C $4.073127-0.8016412 .132002$

H $4.598128-2.761046-1.804792$

O $2.617888-4.629021-1.786547$

H $4.048083-4.553921-0.353955$

H $1.264528-3.3760960 .128569$

O $1.907857-5.1223630 .941865$

H $3.625519-3.5892502 .061455$

O $1.828404-2.7704662 .585843$

C $5.199092-1.5219892 .835286$

H 4.4873920 .1083531 .679935

H $3.362991-0.4681712 .896753$

H $1.765586-4.209452-1.988949$

H $1.296652-5.0207611 .691699$

H $2.260383-2.3845713 .366395$

O $6.321601-1.5092032 .062076$

O $5.087225-2.0433473 .937589$ 
C $7.456363-2.1836572 .613996$

H $7.228389-3.2421762 .770901$

H $\quad 8.279677-2.1044681 .899022$

H $7.759250-1.7095123 .552463$

SCF Energy (B3LYP/6-31G**//MMFF) $=-3245.92129387$

0600306

MM̄MF Geometry

C -4.103899 1.208809 -3.237460

C $-3.0687331 .785072-2.608409$

C $-1.7100301 .949707-3.236065$

O $-0.7675621 .158102-2.478813$

C $-1.2578333 .418550-3.155189$

C $0.0441773 .660058-3.893226$

C $1.2649763 .644506-3.322285$

C $-0.0997243 .937752-5.367208$

C $1.6069853 .363481-1.884274$

C $2.4438454 .497675-1.280719$

C 2.7434104 .2747090 .210431

C 3.6284655 .4037310 .746602

C 4.0528215 .1695082 .203171

O 3.4032043 .0133310 .354606

O 5.1102626 .0987692 .485684

C 4.6285853 .7421162 .326839

C 2.9187215 .5025843 .178899

O 4.9299193 .4337613 .685680

C 3.7202272 .6579251 .707211

C 4.3951381 .2670521 .687364

O 2.5343432 .5377162 .502107

C $-0.497262-0.098510-2.921344$

O $-1.023167-0.683368-3.854087$

C $0.589350-0.635740-2.072543$

C $1.054133-1.875703-2.285351$

C $2.130587-2.528995-1.536564$

C $2.877632-1.693608-0.530988$

C $5.632901 \quad 1.1660940 .793179$

C $6.129894-0.2647490 .668764$

C $7.543900-1.696342-0.812158$

O $5.821173-1.1543491 .456982$

C $6.611823-2.360643-1.777944$

C $6.322418-3.670722-1.747909$

C $5.400368-4.336379-2.731084$

C $4.420009-5.284557-2.073549$

C $4.454022-6.601163-2.341370$

C $3.404181-4.719195-1.104767$

C $2.392777-3.830476-1.779656$

N $6.982741-0.418016-0.414895$

H $-3.9425780 .831079-4.246032$

H -3.181345 2.159433-1.595319

H -1.718705 $1.628757-4.285552$

H -1.160027 $3.727052-2.107642$

H -2.029433 $4.074406-3.580335$

H $2.1291333 .824258-3.961152$

H $0.8656304 .109036-5.855283$

H $-0.5787363 .092956-5.871767$

H $-0.7126004 .831146-5.526132$

H $0.7168963 .204776-1.269749$

H $2.1785892 .427988-1.854741$

H $3.3892524 .564132-1.835743$

H $1.9232245 .455235-1.407606$

H 1.7905454 .2431360 .752062

H 3.1266416 .3739630 .643303

H 4.5372715 .4729730 .131819

H 5.4373435 .9090483 .381881

H 5.5900003 .7487991 .799006

H 2.6265496 .5548033 .076718

H 3.2413035 .3834884 .219033

H 2.0251854 .8948533 .022371

H 4.0922743 .2313794 .136184

H 4.6376540 .9508402 .710067

H 3.6601660 .5339761 .328340

H 2.1307001 .6718192 .325738

H $0.984450 \quad 0.011140-1.297205$

H $0.600083-2.475554-3.072851$

H $2.208305-1.3665410 .272223$

H $3.308786-0.808346-1.012273$

H $3.707012-2.218772-0.056095$

H $5.4008391 .541871-0.209785$
H $\quad 6.454864 \quad 1.761977 \quad 1.203185$

H $7.719968-2.3083630 .078358$

H $8.500962-1.489002-1.300333$

H $6.165026-1.738190-2.550106$

H $6.794544-4.302216-0.998785$

H $6.025479-4.880974-3.450527$

H $4.844160-3.593401-3.315725$

H $3.758034-7.291721-1.876659$

H $5.178321-7.020353-3.031947$

H $3.929861-4.225907-0.283863$

H $2.844221-5.534817-0.627369$

H $1.788892-4.348783-2.525955$

H $7.1280050 .368525-1.041236$

C $-5.8688351 .697914-1.400590$

O $-5.1871181 .065875-0.298904$

$\begin{array}{llll}\text { C } & -6.084320 & 0.994444 & 0.824907\end{array}$

C $-7.327695 \quad 1.7872770 .436243$

C $-7.357148 \quad 1.594121-1.065398$

H $-6.362341-0.0596530 .938042$

C $-5.397114 \quad 1.4997712 .098072$

H -8.2274491 .4154650 .935935$

H -7.2257812 .8509860 .681212$

C $-8.2324482 .605704-1.785271$

H -7.721668 $0.580931-1.282320$

C $-4.103467 \quad 0.7558832 .484508$

O -5.0597582 .8842151 .933730$

H -6.1063371 .4630542 .933385$

H $-3.294848 \quad 1.003744 \quad 1.786174$

O $-3.692246 \quad 1.2522743 .765688$

C $-4.242823-0.7743992 .577069$

C $-3.014211-1.4480043 .218993$

H -4.401991-1.177656 1.572200

O $-5.397984-1.1070023 .349370$

H -2.106019-1.056606 2.750031

H -2.975707 -1.179261 4.281352

H $-9.2686322 .532261-1.438928$

H -7.890593 $3.630812-1.607084$

H $-8.2265242 .431981-2.865576$

C $-5.5027141 .006365-2.716910$

H -4.4832892 .9541791 .153264$

H -3.5971092 .2170583 .685186$

H -5.287061 -0.7103124 .230550$

H $-6.1828301 .357145-3.502217$

H -5.657488 -0.074742 -2.614730

H $-5.5575812 .750633-1.417548$

C $-1.815826-3.2428831 .048565$

O $-3.059611-3.4230131 .737903$

C $-3.066969-2.9882493 .102394$

C $-1.979676-3.7251803 .896719$

C $-0.608576-3.5955403 .242511$

C $-0.686447-3.9918301 .768539$

H -1.573588 -2.175343 0.987147

C $-2.002104-3.775709-0.380501$

H $-4.031033-3.3067963 .518010$

O $-1.909986-3.2444565 .235203$

H $-2.235071-4.7916843 .952983$

H -0.207296 -2.582532 3.366283

O $0.292315-4.4754523 .918988$

H $-0.841032-5.0755291 .687019$

O $0.551856-3.6839241 .136195$

C $-2.889210-2.880746-1.221338$

H -1.040429 -3.849292 -0.898530

H -2.467910 -4.767879 -0.340586

H $-2.789741-3.3599145 .633026$

H $1.184375-4.3051363 .572570$

H $1.231907-4.2643531 .517274$

O $-3.228288-3.548124-2.364440$

O $-3.226713-1.741777-0.930111$

C $-4.076881-2.833369-3.267564$

H $-3.575426-1.929293-3.623222$

H $-4.282266-3.479898-4.124829$

H -5.024658 -2.585475 -2.780630

SCF Energy (B3LYP/6-31G**//MMFF) $=-3245.92792077$

0600307

MM̄FF Geometry

C -0.666210 $3.796002-0.032878$

C -1.0909292 .5457820 .205848$ 
C -1.6952502 .1072391 .512447$ O -1.1418110 .8020131 .801104$ C -3.2195651 .9672541 .375983$ C $-3.933847 \quad 1.871547 \quad 2.708827$ $\begin{array}{llll}C & -4.441887 & 0.738242 & 3.231065\end{array}$ C -4.1080893 .1795753 .437701$ C $-4.364063-0.6563312 .675440$ C $-5.723118-1.1681812 .176702$ C $-6.172561-0.5179270 .858661$ C $-7.561927-1.0156250 .455103$ C $-7.988122-0.471215-0.917052$ O $-5.219385-0.874578-0.146334$ O $-9.130347-1.222939-1.354949$ C $-6.856758-0.735385-1.933080$ C $-8.4367550 .992780-0.836701$ O $-7.143326-0.120438-3.187237$ C $-5.461070-0.294195-1.434058$ C $-4.367374-0.817526-2.396321$ O $-5.4082531 .132508-1.391988$ C $-0.7381350 .523132 \quad 3.064369$ $\begin{array}{lllll}0 & -0.736203 & 1.288387 & 4.018494\end{array}$ C $-0.270251-0.8793003 .184569$ C $-0.405799-1.7942412 .211248$ C $0.022799-3.1945872 .266234$ C $0.715972-3.669655 \quad 3.515374$ C $-2.933044-0.618227-1.884494$ C $-1.910009-1.289653-2.784127$ C $0.478709-2.003590-2.803247$ O $-2.179431-1.725362-3.900391$ C $0.448890-3.447200-2.409657$ C $1.404667-4.044697-1.682442$ C $1.354847-5.487091-1.267567$ C $1.396016-5.6668640 .235259$ C $2.512850-6.0996310 .844515$ C $0.122492-5.4387601 .021195$ C $-0.211056-3.982037 \quad 1.195161$ N $-0.653539-1.330196-2.193870$ H -0.7852294 .5499630 .740055$ H $-0.9903861 .792279-0.571549$ H -1.427458 2.8234392 .298219 H $-3.450707 \quad 1.0953560 .756066$ H $-3.634327 \quad 2.8302920 .837411$ H $-4.961210 \quad 0.804267 \quad 4.186665$ H -4.6791203 .0665454 .365392$ H -3.136239 3.6074373 .701324 H -4.644824 3.8965452 .807706 H -3.606792 -0.7495911 .891844$ H $-4.025143-1.3107463 .488828$ H $-5.629505-2.2513972 .024177$ H $-6.485549-1.0148012 .950525$ H -6.1828810 .5705900 .980825$ H -8.305324 -0.7564851 .219195$ H -7.555422 -2.113516 0.403965 H $-9.828750-1.124012-0.685324$ H -6.830949-1.818589-2.118131 H $-9.2874391 .095085-0.152141$ H -8.790972 $1.351269-1.809508$ H -7.650103 $1.664218-0.486566$ H -8.019749 $-0.433439-3.469838$ H $-4.517228-1.893860-2.554028$ H $-4.463625-0.328260-3.373778$ H $-4.4868211 .421238-1.480954$ H $0.178702-1.125340 \quad 4.141534$ H $-0.880915-1.500807 \quad 1.276674$ H $1.579379-3.0366953 .745405$ H $1.097903-4.6899993 .438377$ H $\quad 0.027666-3.6443654 .367135$ H -2.684890 $0.446769-1.840740$ H -2.828458 -1.039226 -0.878106 H $1.383003-1.498221-2.454360$ H $\quad 0.419980-1.910982-3.892139$ H $-0.410120-4.029030-2.736257$ H $2.269145-3.469006-1.364498$ H $2.208516-5.995927-1.733935$ H $0.461725-5.986458-1.663288$ H $2.546136-6.2619601 .916584$ H $3.422501-6.2938450 .285908$ H $\quad 0.168355-5.9480531 .990480$
H $-0.711982-5.9254850 .499739$ H $-0.726233-3.546940 \quad 0.338911$ H $-0.547045-1.006233-1.236810$ C $1.3824624 .788737-1.168153$ $\begin{array}{lllll}\text { O } & 2.212619 & 3.856517 & -0.448634\end{array}$ C 2.9536174 .5745950 .554508 C $2.902236 \quad 6.0339130 .115805$ C $1.4924376 .125483-0.434321$ H $2.412245 \quad 4.468676 \quad 1.503067$ C $4.376618 \quad 4.027250 \quad 0.699338$ H 3.0863846 .7252250 .943592 H $3.6365596 .238997-0.672802$ C $1.2609327 .335939-1.321989$ $\begin{array}{llll}\text { H } & 0.794090 & 6.168896 & 0.409788\end{array}$ C $4.5162302 .678102 \quad 1.436858$ O $4.9746713 .932626-0.593165$ H $4.951994 \quad 4.770081 \quad 1.267447$ H 5.5848122 .4258131 .446233 O 4.1105712 .8306262 .797191

C $3.768507 \quad 1.4789230 .823654$ C 4.2366020 .1477151 .443885 H $3.943867 \quad 1.459991-0.256338$ $\begin{array}{llll}\text { O } & 2.363513 & 1.599275 & 1.033722\end{array}$ H $\begin{array}{llll}5.322399 & 0.065739 & 1.322787\end{array}$

H 4.0340260 .1575032 .522075

H $1.4160868 .261556-0.757909$

H $1.9459217 .345069-2.176489$

H $0.237347 \quad 7.343753-1.709754$

C $-0.032013 \quad 4.222857-1.328451$

H $5.8956913 .645655-0.472578$

H 4.6448873 .5435583 .186301

H 2.2142001 .6864321 .990730

H $0.0209363 .359761-2.004297$

H $-0.6768124 .964729-1.812845$

H $1.8263614 .880965-2.168179$

C $4.884440-1.302924-1.157143$

O $3.584862-1.079814-0.599395$

C $3.535326-1.0832970 .829643$

C $4.034857-2.4293051 .369626$

C $5.403054-2.8061720 .805673$

C $5.419039-2.670382-0.717503$

H $5.567640-0.509452-0.830294$

C $4.753965-1.215616-2.680676$

H $2.474293-1.0047161 .096109$

O $4.087278-2.3999662 .791658$

H $3.313021-3.2060871 .091749$

H $6.194986-2.2059821 .268803$

O $5.673689-4.1649121 .157475$

H $4.813963-3.473945-1.154949$

O $6.752937-2.851749-1.187926$

$\begin{array}{llll}\text { C } & 4.546234 & 0.218088 & -3.109109\end{array}$

H $5.657803-1.572537-3.187314$

H $3.919172-1.822077-3.051081$

H $4.402954-3.2707233 .088444$

H $6.525650-4.4042310 .753700$

H $7.299631-2.136509-0.820075$

$\begin{array}{lllll}\text { O } & 3.215107 & 0.509174 & -3.142838\end{array}$

O $5.4619990 .993218-3.353452$

C $2.8862011 .856955-3.492015$

H $3.3763532 .559460-2.811680$

H $3.1760232 .057067-4.527667$

H $1.8037751 .977048-3.399380$

SCF Energy (B3LYP/6-31G**//MMFF) $=-3245.91009399$

0700001

MM̄FF Geometry

C -2.331731 -3.709290 1.671119

C $-1.243897-3.1628252 .235406$

C $-1.206855-2.1467673 .350591$

O $-0.624472-0.9428552 .792893$

C -2.576359-1.752989 3.946309

C $-2.501290-0.6099054 .943543$

$\begin{array}{llll}C & -3.090507 & 0.589788 & 4.759421\end{array}$

C $-1.717298-0.883412 \quad 6.199842$

C $-3.959467 \quad 1.008462 \quad 3.603111$

C $-3.145681 \quad 1.4562002 .388439$

C $-3.994442 \quad 1.9804231 .219838$

C -4.9340870 .9152930 .651098$ 
C $-5.689206 \quad 1.417773-0.587261$

O

O $-6.2847620 .263157-1.200446$

C $-4.6805532 .022824-1.587032$

C $-6.8467932 .342584-0.192250$

O $-5.3496922 .673400-2.664818$

C -3.660422 $2.996019-0.946982$

C $-2.5171393 .279671-1.946100$

O $-4.2478154 .258435-0.630026$

C $0.687129-0.6775013 .024679$

O $1.501178-1.3908143 .586324$

C 0.9472290 .6773412 .488262

C 2.1509961 .2490382 .638784

C 2.5278952 .5852892 .166553

C 1.4511403 .4180171 .520092

C $-1.4324204 .208353-1.385390$

C $-0.2228444 .293745-2.300430$

C $2.0618575 .289604-2.392315$

O $-0.1352813 .680241-3.360701$

C $3.0116514 .336024-1.737801$

C $4.1698014 .704753-1.169806$

C $5.1030293 .736382-0.499619$

C 5.5003034 .1690280 .896448

C 6.7854604 .4249111 .194715

C 4.4189454 .3139471 .947152

C 3.8028702 .9963072 .332273

N $0.7549945 .131208-1.779877$

H $-3.323979-3.4566832 .031898$

H $-0.268796-3.4391301 .838609$

H $-0.572039-2.5454014 .151396$

H -3.030131 -2.621918 4.440048

H -3.250864 -1.478599 3.128655

H $-2.961793 \quad 1.3523515 .525994$

H -1.800708 $-0.067746 \quad 6.925923$

H -2.086215 -1.792352 6.685965

H $-0.654914-1.0141915 .973848$

H -4.6432550 .1933983 .345289$

H $-4.598576 \quad 1.834767 \quad 3.938682$

H -2.437072 2.2412392 .686314

H $-2.523171 \quad 0.6316372 .022661$

H -4.5538182 .8584331 .562494$

H -5.6520860 .5694521 .403195$

H $-4.347214 \quad 0.0305980 .367030$

H $-6.8111650 .562527-1.960750$

H -4.120795 $1.186548-2.027753$

H $-7.582576 \quad 1.7952270 .409592$

H -7.389116 2.700575 -1.074207

H -6.5235823 .2077840 .390542$

H -5.888708 2.007108 -3.122982

H -2.052782 2.328907 -2.236013

H $-2.9184173 .736433-2.859802$

H $-5.1942984 .226788-0.838559$

H 0.1271221 .1764821 .983858

H 2.9345390 .6899813 .148346

H 0.6090383 .5606162 .206769

H 1.0845152 .9282500 .611579

H $1.7811784 .415743 \quad 1.227380$

H -1.835782 $5.218255-1.249764$

H -1.085428 $3.843226-0.412447$

H $2.362126 \quad 6.333231-2.256701$

H $1.9975705 .075549-3.463650$

H $2.7289703 .285727-1.730219$

H $4.4682525 .750005-1.194216$

H $5.9923263 .639931-1.135680$

H $4.6581562 .736246-0.450892$

H 7.0845214 .7368452 .189946

H $7.569607 \quad 4.327748 \quad 0.451290$

H 3.6831475 .0457761 .605389

H 4.8391274 .7497942 .863649

H 4.5060942 .3161072 .815062

H $0.6141765 .539146-0.860087$

C -2.484012 -3.965814-0.850799

O $-1.361482-3.106455-1.143047$

C $-1.856600-1.886173-1.724860$

C $-3.304834-2.166654-2.114359$

C $-3.736079-3.099902-1.000345$

H -1.845060 -1.131657 -0.928906

C $-0.964930-1.431515-2.885362$
H -3.905285 -1.254746 -2.168857

H $-3.375614-2.676204-3.082348$

C $-4.996828-3.886103-1.310864$

H -3.908457 -2.494974 -0.102203

C $0.531242-1.270361-2.543810$

O $-1.054766-2.385700-3.952315$

H $-1.354407-0.491727-3.294531$

H $\quad 0.992762-2.255289-2.410789$

O $1.169360-0.678955-3.683848$

C $0.826461-0.391998-1.316932$

C $2.3039710 .004496-1.143368$

H $0.484334-0.906537-0.412564$

O $0.0731980 .820869-1.400717$

H $2.6160990 .662810-1.964431$

H $2.3569530 .629810-0.245794$

H $-5.841660-3.208851-1.473328$

H -4.877999-4.497073 -2.212006

H $-5.252553-4.553297-0.481644$

C $-2.284656-4.6549470 .503717$

H $-0.782332-3.249041-3.596552$

H $0.983149-1.250285-4.448735$

H $0.3370961 .275255-2.219393$

H -1.323733 -5.182672 0.500856

H $-3.058339-5.4179910 .648811$

H -2.474111 -4.741575 -1.627922

C $3.625682-3.2165820 .233321$

O $2.836463-2.0369420 .019621$

C $3.290154-1.168298-1.022058$

C $4.719611-0.676737-0.731430$

C $5.674261-1.847724-0.485511$

C $5.092015-2.8500430 .517548$

H $3.214532-3.6684011 .145209$

C $3.472888-4.231330-0.911871$

H $3.284164-1.711431-1.972270$

$\begin{array}{lllll}\text { O } & 4.710047 & 0.162266 & 0.421962\end{array}$

H $5.066236-0.073515-1.579077$

H $6.626669-1.461392-0.102291$

O $5.935034-2.490905-1.728930$

H $5.699293-3.7618880 .535157$

O $5.169113-2.2887901 .831413$

C $2.070007-4.788960-0.999755$

H $3.707377-3.784084-1.881105$

H $4.146188-5.079746-0.744320$

H 5.6125710 .5020610 .546666

H $\quad 6.559852-3.216244-1.558495$

H $4.569642-1.5232051 .863783$

O $1.957282-5.532778-2.139684$

O $1.188513-4.609763-0.172036$

C $0.677269-6.133383-2.360145$

H $-0.067410-5.358819-2.562157$

H $0.753361-6.783901-3.235429$

H $0.381301-6.739632-1.498543$

SCF Energy $\left(B 3 L Y P / 6-31 G^{* *} / / M M F F\right)=-3245.92254002$

07_00002

MM̄FF Geometry

C $-2.561678-1.766572-2.253602$

C $-1.434363-2.197031-1.668440$

C $-0.470063-3.152996-2.314017$

O $0.830027-2.515736-2.322977$

C $-0.342082-4.462637-1.508984$

C $-1.588953-5.323990-1.488340$

C $-2.434440-5.444558-0.444240$

C $-1.835393-6.111774-2.749776$

C $-2.377145-4.7280410 .877783$

C $-3.629171-3.8602591 .080613$

C -3.447515 -2.799349 2.177950

C $-4.711080-1.9441952 .309489$

C $-4.516371-0.7537863 .261733$

O $-2.342712-1.9791861 .794453$

O $-5.604566 \quad 0.1600703 .054175$

C -3.221910-0.004964 2.877182

C $-4.586863-1.1868184 .730716$

O $-2.928724 \quad 1.024243 \quad 3.819351$

C $-2.001572-0.9380792 .715040$

C $-0.809681-0.1598182 .102695$

O $-1.634017-1.457403 \quad 3.991015$

C $1.657684-2.811805-3.358105$ 
O $1.386170-3.497351-4.335332$

C $2.995588-2.200136-3.195103$

C $3.457059-1.687939-2.043379$

C $4.798312-1.132801-1.827930$

C $5.743315-1.114641-3.001808$

C $0.414324-1.0288301 .773479$

C $1.423132-1.1531272 .902022$

C $3.546850-2.3429863 .461097$

O $1.336134-0.5354383 .958979$

C $4.702814-1.4658843 .100457$

C $5.831875-1.9211082 .536883$

C $6.998067-1.0464012 .180533$

C $7.261459-1.0047600 .691624$

C $8.265746-1.7101390 .144961$

C $6.422859-0.067975-0.148394$

C $5.126504-0.686028-0.597221$

N $2.437670-2.0429972 .576110$

H -2.794170 -2.089914 -3.265342

H $-1.212894-1.887888-0.652053$

H $-0.788337-3.364334-3.342233$

H $0.010625-4.235337-0.496055$

H $0.479198-5.059711-1.929724$

H -3.283483 -6.119334-0.548237

H $-2.720642-6.752244-2.674973$

H - $1.987929-5.438595-3.599055$

H $-0.978989-6.758569-2.967155$

H -2.317977 -5.4714701 .681602$

H $-1.474306-4.1198630 .958963$

H $-3.865773-3.3541130 .137253$

H $-4.485436-4.5028761 .320821$

H $-3.215075-3.3029453 .123475$

H -5.562586 -2.558696 2.627565

H -4.986124 -1.547222 1.322177

H $-6.434310-0.3193673 .219771$

H -3.404584 0.4977461 .916665

H -5.567238 -1.625042 4.953865

H $-4.484026-0.3272935 .402103$

H -3.828954 -1.926908 4.995868

H $-3.716631 \quad 1.5908103 .883809$

H -1.1467750 .2759881 .152819$

H -0.5174690 .6693452 .757154$

H $-0.738217-1.8237473 .933888$

H $3.614695-2.244312-4.085767$

H $2.809030-1.689740-1.168552$

H $5.891280-2.127438-3.393122$

H $6.737694-0.735066-2.759027$

H $5.346760-0.481046-3.802970$

H $0.115219-2.032907 \quad 1.455058$

H $0.945223-0.5702480 .932852$

H $3.780844-3.4054613 .340508$

H $3.254602-2.1683484 .501405$

H $4.604657-0.4054583 .320232$

H $5.930546-2.9829672 .323356$

H $7.878451-1.4316212 .711281$

H $6.858808-0.0237372 .552840$

H $8.479345-1.657468-0.917502$

H $8.892929-2.3596300 .746468$

H $7.0076260 .305432-0.995272$

H $6.1970320 .833060 \quad 0.434604$

H $4.372842-0.7441210 .187538$

H $2.457954-2.4476051 .644262$

C $-3.935626 \quad 0.364736-2.262149$

O $-3.0329651 .402472-1.812254$

C $-3.8171812 .508191-1.328843$

C $-5.1851322 .349454-1.976445$

C $-5.3505800 .848427-1.921983$

H $-3.9227342 .371706-0.245902$

C $-3.1547093 .855811-1.625135$

H $-5.9687932 .889215-1.436455$

H -5.184858 2.693716-3.017289

C -6.421441 $0.319476-2.861316$

H $-5.6093580 .562898-0.892862$

C $-1.7542924 .063656-1.016852$

O $-3.0374314 .022234-3.044608$

H -3.820451 4.659186-1.287309

H - $1.0190513 .488558-1.589692$

O $-1.4125815 .442972-1.217435$

$\begin{array}{llll}\text { C } & -1.621021 & 3.722800 & 0.479027\end{array}$
C $-0.348902 \quad 4.2642851 .159834$

H -1.6681142 .6351740 .600491$

$\begin{array}{llll} & -2.740441 & 4.272434 & 1.181264\end{array}$

H $-0.431115 \quad 5.3534491 .271936$

H -0.3392223 .8645322 .182969$

H $-7.4004460 .735088-2.601272$

H $-6.2092250 .585790-3.902231$

H $-6.489772-0.771283-2.800284$

C $-3.608040-0.949543-1.551468$

H $-2.5081803 .279704-3.383264$

H $-2.0180265 .977326-0.675174$

H -2.6780793 .9792642 .106528$

H $-4.505530-1.578590-1.506745$

H $-3.303095-0.743659-0.519074$

H $-3.8051980 .277941-3.347904$

C $2.2534621 .999861-0.275681$

$\begin{array}{lllll}\text { O } & 1.098889 & 2.493849 & 0.418903\end{array}$

C $0.967605 \quad 3.916296 \quad 0.453047$

C 2.1755384 .5467551 .163370

C $3.487300 \quad 4.1140370 .504694$

$\begin{array}{lllllllll}\text { C } 3.546889 & 2.596822 & 0.303168\end{array}$

H $2.2703810 .924173-0.067385$

C $2.1229972 .168784-1.797284$

H $0.9255554 .301858-0.569466$

$\begin{array}{lllll}\text { O } & 2.191060 & 4.182148 & 2.541986\end{array}$

H 2.0838055 .6383441 .119872

H 4.3257054 .4376601 .133435

O $3.6045594 .773737-0.752498$

H $4.3904172 .330504-0.342585$

$\begin{array}{lllll}\text { O } & 3.770158 & 1.970926 & 1.570627\end{array}$

C $0.976511 \quad 1.354032-2.360289$

H $1.951233 \quad 3.210423-2.079977$

H $3.0426731 .818965-2.280252$

H 2.1873303 .2108062 .593228

H $4.4551954 .511057-1.143625$

H 4.6190812 .2979831 .914030

O $\quad 0.954601 \quad 1.497334-3.718475$

O $0.1926080 .686930-1.699834$

C $-0.1005450 .803512-4.391768$

H - $-0.0089881 .002993-5.462707$

H $-0.012156-0.274054-4.227449$

H - $-1.0730891 .168860-4.049281$

SCF Energy (B3LYP/6-31G**//MMFF) $=-3245.90932451$

07_00003

MM̄FF Geometry

C $0.2583690 .803307 \quad 3.548418$

$\begin{array}{llll}\text { C } & -0.393323 & 0.122674 & 4.504349\end{array}$

C -1.388241 -0.9941354 .302204$

O $-2.038207-0.9343603 .014365$

C $-0.668459-2.3494334 .406289$

C $-1.605910-3.536350 \quad 4.300098$

C $-1.767284-4.275868 \quad 3.184430$

C $-2.339527-3.8938775 .565329$

C $-1.125987-4.0315101 .844558$

C $-2.070745-4.3798090 .686227$

C $-1.593490-3.893085-0.693239$

C $-0.161433-4.316405-1.030837$

C $0.300458-3.763530-2.388992$

O $-1.708521-2.464573-0.725505$

O $1.727028-3.915092-2.464814$

C $0.007215-2.247584-2.437172$

C $-0.281240-4.564304-3.559404$

O $0.271705-1.715681-3.733552$

C -1.431613 -1.889158 -2.010925

C $-1.656361-0.365157-1.912889$

O $-2.347926-2.375976-3.000885$

C $-3.079208-0.0693712 .882306$

$\begin{array}{llll}\text { O } & -3.411518 & 0.810160 & 3.660518\end{array}$

C -3.786244 -0.4135601 .630033$

C $-4.6125280 .479725 \quad 1.067445$

C $-5.4130720 .267752-0.138973$

C $-5.564246-1.145903-0.638144$

C $-0.7626200 .372603-0.913359$

C $-1.3273201 .741409-0.579439$

C $-2.1128823 .854831-1.644279$

O

C $-3.5062773 .762661-2.197141$ 
C $-3.9924512 .809788-3.008960$ C $-5.4027142 .790441-3.528378$ C $-6.1059871 .481472-3.243468$ C $-6.1111430 .492814-4.153741$ C $-6.8711051 .347727-1.946337$ C $-5.9884281 .335123-0.730603$ N -1.424651 $2.577306-1.680208$ H 0.1040520 .5526522 .502757 H $-0.192437 \quad 0.3704315 .545276$ H -2.155261 -0.9238425 .084307$ H $-0.126489-2.4157735 .359363$ H $0.105236-2.4148133 .632058$ H -2.426336 -5.141447 3.232856 H $-2.973703-4.7782605 .442241$ H -1.628095 -4.107393 6.369742 H -2.984519 -3.067943 5.880816 H $-0.807605-2.9903961 .743368$ H $-0.234206-4.6640721 .780889$ H -2.205444 -5.4685230 .653617$ H -3.056697 -3.9386860 .884048$ H - $-2.290971-4.311712-1.428632$ H - $0.061730-5.408381-1.001865$ H $\quad 0.529658-3.930136-0.271785$ H $1.934878-4.858372-2.350917$ H $\quad 0.713044-1.762189-1.750774$ H $\quad 0.037387-5.612088-3.501295$ H $0.093235-4.191184-4.518920$ H -1.372654 -4.554721 -3.586801 H $1.161120-2.009071-3.994014$ H -1.552737 $0.081845-2.909863$ H $-2.702345-0.188662-1.630948$ H $-3.175878-1.872318-2.931373$ H -3.613557 -1.398038 1.211758 H -4.7056971 .4639291 .524868$ H $-5.985838-1.7854840 .145562$ H $-4.597237-1.559068-0.940933$ H -6.225730 -1.231559-1.503015 H $-0.680770-0.1891360 .023822$ H $0.2484250 .500487-1.310089$ H -1.526364 $4.571605-2.226763$ H -2.163076 $4.205792-0.608473$ H -4.171182 4.569901-1.895681 H -3.344522 2.007301 -3.351504 H -5.361386 $2.960373-4.612361$ H -5.993755 $3.620759-3.122604$ H $-6.636151-0.439422-3.974595$ H -5.585963 $0.592918-5.097876$ H -7.515489 $0.460602-1.955933$ H -7.569380 2.190413 -1.858720 H $-5.8197972 .323431-0.301903$ H -1.096143 2.248019-2.582922 C 0.9609923 .1668983 .045894 O $1.500361 \quad 3.003017 \quad 1.716147$ C $2.2802234 .168687 \quad 1.384354$ C 1.8538095 .2428012 .374006 C 1.6402904 .4114913 .622792 H 3.3294433 .9145951 .576416 C $2.0927814 .538551-0.090361$ H 2.6128606 .0208962 .498363 H 0.9160415 .7243612 .073430 C 0.8231225 .1192444 .690168 H 2.6206604 .1450514 .040628 C $2.4266663 .394673-1.070937$ O $0.7279874 .913058-0.306137$ H $2.6890185 .425945-0.331256$ H $\quad 1.7096412 .577757-0.939294$ O $2.2438443 .881668-2.405224$ C $3.8633172 .860153-0.941744$ C $4.2485261 .799765-1.989536$ H 4.0081192 .4414140 .058957 O $4.7738983 .956183-1.066657$ H $4.2351212 .246207-2.992041$ H $5.2995231 .533479-1.821173$ H 1.3321366 .0296125 .023246 H -0.1652985 .4050904 .314959$ H 0.6780044 .4746415 .562726 C $\quad 1.2391921 .9019913 .861488$ H 0.1731434 .1769340 .004370
H $\quad 1.3350104 .222900-2.466265$

H $5.6713133 .611116-0.921267$

H 1.2024312 .1349614 .932117

H 2.2461641 .5294303 .637978

H -0.1181373 .3349632 .935527$

C $4.299120-0.513125-0.007883$

O $3.1452380 .051658-0.637550$

C $3.3723170 .531505-1.966653$

C $3.887255-0.607111-2.859788$

C $5.098296-1.316554-2.255251$

C $4.820168-1.713286-0.804836$

H 5.0853900 .2501070 .045831

C $3.945213-0.8375661 .446835$

H $2.3914920 .824393-2.359453$

O $4.225421-0.125363-4.155903$

H $3.091601-1.349562-2.990695$

H $6.002754-0.701124-2.326492$

O $5.364665-2.507317-3.000157$

H $4.093243-2.534654-0.793721$

O $6.020252-2.191512-0.204113$

C $2.958295-1.9734871 .577166$

H $4.852631-1.0774932 .013510$

H 3.4915390 .0329641 .935596

H $3.4335110 .302235-4.524408$

H $5.498626-2.244109-3.926833$

H $6.344110-2.928434-0.750196$

O $3.580719-3.0634642 .110540$

O $1.786273-1.9051481 .232006$

C $2.767526-4.2288612 .269223$

H $2.375771-4.5531281 .300947$

H $3.393357-5.0269322 .677275$

H $1.954340-4.0283292 .972258$

SCF Energy $(B 3 L Y P / 6-31 G * * / / M M F F)=-3245.90962545$

0700004

MM̄FF Geometry

C -3.116760 -2.515910 -2.770803

C $-2.251260-2.770815-1.779268$

C $-0.763172-2.895020-1.982286$

O $-0.099632-1.867662-1.207260$

C $-0.285618-4.264967-1.467563$

C $1.184068-4.537371-1.721888$

C $2.109947-4.708482-0.757925$

C $1.588024-4.648769-3.170083$

C $1.917767-4.6728460 .732009$

C $2.158418-3.2991801 .376723$

C $3.590149-2.7647391 .206318$

C $3.817523-1.5325282 .084652$

C $5.212941-0.9237551 .871356$

O $3.779540-2.408122-0.165385$

O 5.2199350 .3809972 .471559

C $5.452452-0.725350 \quad 0.358597$

C $6.302977-1.7339922 .582723$

O $6.795438-0.3226700 .103360$

C $5.105663-1.967471-0.487670$

C $5.140050-1.695296-2.011878$

O $6.066180-2.997678-0.223911$

C $-0.121026-0.599700-1.702734$

O $-0.653859-0.219964-2.733505$

C $0.6181140 .267108-0.759465$

C $0.4616811 .597392-0.827956$

C 1.1221772 .5898640 .021084

C 2.2581572 .1229830 .891255

C $4.171226-0.616443-2.510034$

C $4.7968780 .761547-2.652883$

C $4.1885693 .119508-3.189093$

O $6.0030910 .969312-2.556041$

C $4.0837183 .895313-1.914280$

C $3.3619685 .020103-1.793359$

C $3.2880205 .837082-0.535153$

C $1.8665386 .095026-0.088046$

C $1.2513437 .254970-0.374163$

C $1.1921135 .044740 \quad 0.764412$

C $0.7041103 .871332-0.037234$

N $3.845678 \quad 1.731072-2.943199$

H $-2.747270-2.408178-3.787812$

H $-2.619576-2.864832-0.762715$

H $-0.499465-2.775225-3.040047$ 
H $-0.864610-5.065014-1.948142$ H $-0.508550-4.331892-0.397178$ H $3.136035-4.906760-1.066594$ H $2.618207-5.001963-3.287896$ H $0.938961-5.359130-3.692490$ H $1.517189-3.676713-3.667438$ H $0.915802-5.0219741 .002970$ H $2.603232-5.4043861 .178902$ H $1.443604-2.5821690 .956755$ H $1.934056-3.3914192 .446667$ H $4.291337-3.5622081 .478811$ H $3.662342-1.7734153 .143671$ H $3.067248-0.7694901 .838760$ H $4.998747 \quad 0.2791553 .413079$ H 4.8130680 .1067650 .043775 H $6.106024-1.7765373 .660753$ H $7.283180-1.2561092 .477042$ H $6.378567-2.7614762 .221049$ H $6.964790 \quad 0.474870 \quad 0.633460$ H $\quad 6.168818-1.498722-2.337062$ H $4.852315-2.631089-2.512936$ H $6.063071-3.620543-0.969589$ H $1.263589-0.211844-0.032722$ H $-0.2314192 .002257-1.564642$ H 1.8865871 .5137031 .719385 H $2.8408782 .940657 \quad 1.321075$ H 2.9677391 .5274930 .307943 H $3.827731-0.898302-3.513784$ H $3.287237-0.552909-1.868658$ H $3.5041603 .491306-3.958183$ H $5.211803 \quad 3.179955-3.573987$ H $4.6508973 .531753-1.060717$ H $2.8190895 .396978-2.657393$ H $3.7958716 .791145-0.728685$ H 3.8478195 .3665120 .282659 H $0.2421457 .459239-0.032400$ H $1.7418998 .023997-0.961682$ H $\quad 0.315375 \quad 5.4754251 .267385$ H 1.8613834 .7554381 .579753 H $-0.1121164 .120500-0.715794$ H $2.862027 \quad 1.477274-2.951559$ C $-4.961075-0.848475-2.752109$ O $-4.463259-0.083670-1.634944$ C $-5.3894000 .986985-1.358397$ C $-6.4727450 .905974-2.430495$ C $-6.460078-0.571309-2.775557$ H $-5.8445040 .771967-0.385828$ C $-4.6371612 .322171-1.319621$ $\mathrm{H}-7.4432881 .252767-2.062403$ H $-6.2169571 .508948-3.309998$ C -7.133298 $-0.887686-4.099385$ H -6.958716 -1.127291-1.970393 C $-3.5621742 .403079-0.214693$ O $-3.9858762 .518383-2.582715$ H -5.346366 $3.150564-1.213074$ H $-2.811257 \quad 1.619476-0.362248$ O $-2.8802003 .655805-0.360887$ C -4.1304572 .3400691 .217278$ C -3.0619032 .4943292 .318119$ H $-4.6724591 .402897 \quad 1.371176$ O $\quad-5.085175 \quad 3.393327 \quad 1.383954$ H -2.381762 3.3174202 .069080 H -3.5725432 .7929343 .243864$ H -8.185549 -0.585899 -4.077046 H -6.650662 $-0.362825-4.930677$ H -7.095640 -1.960602 -4.311340 C -4.593139 -2.319534 -2.566708 H $-3.3714351 .775533-2.713754$ H -2.537002 $3.693521-1.270141$ H $-4.6210904 .234798 \quad 1.232653$ H -5.122573 -2.946742 -3.291960 H -4.885839 -2.656253 -1.565408 H -4.485842 $-0.444957-3.657045$ C -2.632775 -1.007182 3.529186 $\begin{array}{llll}\text { O } & -3.168448 & 0.282028 & 3.205505\end{array}$ C -2.2694181 .2004462 .570545$ C -1.038720 1.4473393 .456173 C $-0.362007 \quad 0.129247 \quad 3.842918$
C $-1.368829-0.8833834 .394496$

H -3.407861 -1.483716 4.144165

C $-2.438257-1.8643992 .273960$

H -1.9465720 .7867801 .610490$

O -1.4289042 .1509004 .633680$

H -0.3358192 .0869082 .909789$

H $\quad 0.4106910 .3296934 .594923$

O $0.278656-0.4127592 .693449$

H $-0.904434-1.8704744 .502395$

O $-1.779373-0.4728525 .701440$

C $-3.783068-2.2986031 .737975$

H -1.919378 -1.347924 1.461973

H -1.847907 -2.758933 2.505968

$\mathrm{H}-0.6254302 .3202115 .154196$

H $0.728956-1.2294292 .967782$

H $-0.984149-0.4312656 .259344$

O $\quad-3.980457-3.6241941 .988390$

O $-4.570182-1.5452831 .179741$

C $-5.232258-4.1554831 .543194$

H $-5.287554-5.2017491 .855563$

H $-6.063433-3.6092661 .999581$

H -5.292948 -4.111430 0.452068

SCF Energy (B3LYP/6-31G**//MMFF) $=-3245.90313803$

$07 \quad 00005$

MM̄FF Geometry

C -4.327438 -1.370149 2.274202

C $-3.061629-1.6628202 .610812$

C $-2.154248-0.8736593 .522386$

O $-0.959502-0.5353152 .774233$

C $-2.7330520 .453679 \quad 4.058774$

C -1.7606971 .2291954 .930121$

C $-1.2684592 .446476 \quad 4.624226$

C -1.356082 0.5580926 .217349

C -1.5940153 .2984753 .427844$

C -0.6581603 .0176462 .252159$

C -0.7897284 .0245901 .099616$

C $-2.171373 \quad 3.9947420 .444692$

C -2.249906 $4.937652-0.764907$

O 0.2023213 .6634820 .132409

O $-3.4530294 .596722-1.472477$

C -1.052735 $4.669168-1.703744$

C $-2.410316 \quad 6.396065-0.318558$

O $-0.9712825 .655154-2.730856$

C $0.3122294 .562999-0.979221$

C $1.3695973 .966555-1.935186$

O $0.8033755 .842465-0.575933$

C $0.137374-1.3250452 .922472$

O $0.197186-2.4111573 .474906$

C $1.285116-0.5831502 .352861$

C 2.536771-1.012419 2.572954

C $3.767193-0.3261142 .165568$

C 3.6193961 .0054451 .476621

C $2.7594143 .829576-1.299796$

C $3.7116243 .027509-2.170924$

C $6.0140392 .066905-2.137033$

O $3.3938902 .554491-3.258713$

C $5.9182180 .704152-1.525858$

C $6.9093230 .118713-0.837146$

C $6.784166-1.234831-0.195614$

C $7.152641-1.2217361 .272907$

C $8.187723-1.9487731 .726878$

C $6.339635-0.3593982 .215349$

C $4.954824-0.9007062 .453122$

N $4.9603182 .895008-1.577418$

H $-4.809045-0.4864432 .680664$

H -2.617005 -2.564684 2.195248

H -1.897317 -1.5187264 .371799$

H $-3.638297 \quad 0.2573094 .647583$

H $-3.0437051 .067407 \quad 3.206415$

H $-0.5599772 .902400 \quad 5.314863$

H $-0.770736 \quad 1.220475 \quad 6.864280$

H $-2.243576 \quad 0.2531796 .781432$

H $-0.746156-0.3281706 .017844$

H -2.640212 3.1673143 .135743

H -1.5013984 .3493153 .729879$

H 0.3853963 .0191092 .596180

H -0.8342622 .0061771 .865731$ 
H $-0.560067 \quad 5.023751 \quad 1.487391$ H -2.9603834 .2481691 .162099$ H -2.391734 2.9724850 .106709 H -3.551807 $5.215002-2.215952$ H -1.247645 $3.713654-2.208547$ H $-3.3503846 .524197 \quad 0.231738$ H -2.473456 $7.071269-1.178926$ H $-1.600348 \quad 6.7366580 .330171$ H -1.796382 $5.617278-3.242881$ H $\quad 1.0364102 .974957-2.265199$ H $1.4655034 .592855-2.831198$ H $0.1360386 .515345-0.780442$ H $\quad \begin{array}{llll}1.069958 & 0.332057 & 1.813470\end{array}$ H $2.679264-1.9373873 .130167$ H 3.0822331 .7138732 .117217 H 3.0658810 .8928540 .538204 H 4.5700381 .4762941 .222369 H $3.1960114 .820975-1.133278$ H $2.6861003 .320025-0.332902$ H $6.9675382 .555686-1.914714$ H $5.8956091 .998902-3.222879$ H $4.9772240 .175191-1.653528$ H $7.8590780 .636383-0.724718$ H $7.436554-1.923848-0.747114$ H $5.768375-1.631611-0.302903$ H $8.464897-1.9452782 .775872$ H $8.781709-2.5658341 .060977$ H $\quad 6.339840 \quad 0.671774 \quad 1.854462$ H $6.834715-0.3072893 .194687$ H $4.950870-1.8661832 .961176$ H $5.1006973 .263533-0.641074$ C $-5.331875-1.598089-0.049894$ O $-4.048242-1.494812-0.699839$ C $-3.952601-0.198858-1.316333$ C $-5.3819690 .321486-1.408107$ C $-5.966035-0.206758-0.111879$ $\mathrm{H}-3.3915130 .438816-0.622416$ C $-3.213425-0.286162-2.653997$ H $-5.4238931 .411961-1.486598$ H -5.916056 -0.104198 -2.265416 C $-7.484189-0.223000-0.083850$ H $-5.6058790 .429406 \quad 0.705236$ C -1.816663 -0.938674 -2.585088 O $-3.995526-1.050739-3.580637$ H $-3.1332130 .716783-3.089865$ H - $-1.915715-2.021258-2.448129$ O $-1.193037-0.750324-3.862237$ C $-0.887531-0.364206-1.502148$ C $0.590216-0.780346-1.616833$ H -1.262244 -0.650030 -0.513201 O $-0.9240111 .063958-1.552005$ H $1.037399-0.334494-2.514713$ H $\quad 1.107530-0.310995-0.770698$ H $-7.8818540 .791176-0.193927$ H -7.894773 $-0.834254-0.894425$ H $-7.850410-0.629414 \quad 0.864232$ C -5.163179 -2.2076671 .345052$ H -4.146307 -1.926063 -3.184128 H - $1.785843-1.131251-4.532775$ H $-0.6591861 .331180-2.448895$ H -4.709471 -3.2010841 .242775$ H -6.146288 -2.359691 1.805291 H -5.918471 -2.299902 -0.657321

C $0.381080-4.251141-0.247522$

O $0.281307-2.829096-0.405792$

C $0.840178-2.296393-1.609212$

C $2.337386-2.638222-1.716833$

C $2.584213-4.139692-1.552230$

C $1.846748-4.707835-0.333633$ H $0.031279-4.444650 \quad 0.775014$ C $-0.542892-5.008298-1.215932$ H $\quad 0.320559-2.730405-2.468934$ O $3.084868-1.960021-0.712298$ H $2.715018-2.308316-2.692520$ H $3.661976-4.312462-1.441747$

O $2.159846-4.805063-2.737665$ H $1.886562-5.803210-0.335821$ O $2.502114-4.269475 \quad 0.859961$
C $-2.006648-4.736358-0.948111$

H - $0.348555-4.734552-2.255887$

H $-0.388667-6.087444-1.101502$

H $3.133744-1.022484-0.961414$

H $2.346617-5.752671-2.624893$

H $3.424746-4.5733670 .817908$

O $-2.753225-5.316948-1.933617$

O $-2.443891-4.1060390 .003355$

C $-4.169161-5.144133-1.818251$

H $-4.430050-4.095123-1.982018$

H $-4.649347-5.749911-2.591258$

H $-4.521857-5.482769-0.839260$

SCF Energy (B3LYP/6-31G**//MMFF) $=-3245.90582847$

07 00006

MM̄FF Geometry

C -3.9549841 .7379191 .570595$

C -3.1329472 .2394270 .638169$

C -2.900826 3.7087720 .424038

O -1.4850203 .9267770 .640140$

C $-3.2433204 .152403-1.009605$

C $-4.7072274 .019093-1.374181$

C $-5.2236783 .043339-2.148019$

C $-5.6010185 .101471-0.826419$

C $-4.4968451 .878224-2.759928$

C $-5.0751070 .543838-2.266260$

C $-4.164212-0.638076-2.626648$

C $-4.808968-1.964900-2.226471$

C $-3.870975-3.152079-2.492340$

O $-2.939777-0.470436-1.907831$

O $-4.401495-4.297486-1.807104$

C -2.489982 -2.856570 -1.866290

C $-3.835242-3.518651-3.980747$

O $-1.538225-3.851256-2.235783$

C $-1.934165-1.449483-2.194657$

C $-0.721075-1.137052-1.286567$

O $-1.486527-1.360031-3.545934$

C $-1.0963075 .141479 \quad 1.101898$

O $-1.825181 \quad 6.0896241 .361611$

C 0.3716025 .2150641 .289685

C 1.2367164 .2746420 .877017

C 2.6945874 .3220731 .029587

C 3.2805705 .4839261 .789899

C $-0.1473010 .277774-1.448417$

C $0.8316860 .440181-2.597787$

C $2.2139012 .198995-3.709120$

O $1.247560-0.495387-3.274018$

C $3.5529282 .123790-3.048305$

C $4.2877873 .192966-2.709003$

C $5.6096873 .091112-2.004228$

C $5.6261043 .829993-0.683216$

C $6.3052004 .982213-0.552760$

C 4.9394843 .1926320 .506629

C $3.440713 \quad 3.3411750 .478555$

N $1.2077201 .768372-2.755717$

H -4.5040332 .4137182 .221462$

H -2.581247 1.5550880 .001459

H -3.4761944 .2904631 .155240$

H -2.596340 $3.625439-1.720506$

H $-2.9492115 .203226-1.139415$

H $-6.2917543 .067860-2.360590$

H -6.641660 $4.984443-1.147093$

H -5.5912555 .0897910 .267975$

H $-5.2600306 .084442-1.167446$

H $-4.5998431 .935027-3.850302$

H $-3.4255861 .918844-2.552036$

H $-5.1981990 .583106-1.176694$

H $-6.0712240 .396872-2.702148$

H $-3.962641-0.610759-3.703810$

H -5.765455 -2.106492 -2.744847

H $-5.048642-1.946382-1.153776$

H -5.305472 -4.449524 -2.132159

H -2.608451 -2.929118 -0.775903

H $-4.832956-3.816332-4.325600$

H $-3.186914-4.383169-4.160741$

H $-3.502140-2.695525-4.616507$

H $-1.906109-4.714028-1.978420$

H $-1.044026-1.230196-0.241277$ 
H $\quad 0.072011-1.874334-1.453862$ H -1.551332 -2.236944-3.956184 H $0.710856 \quad 6.122246 \quad 1.780270$ H $\quad 0.854377 \quad 3.391270 \quad 0.368568$ H 3.0569356 .4283971 .281665 H 4.3662085 .4335311 .894196 H 2.8719905 .5241172 .805775 H $-0.9466411 .016878-1.560185$ H $\quad 0.4065170 .527485-0.534034$ H $1.9598543 .217985-4.017065$ H $2.1930041 .549813-4.590061$ H $3.9344541 .130890-2.828264$ H $3.9189044 .190279-2.936447$ H $6.3769753 .495532-2.677027$ H $5.8908162 .044635-1.829989$ H 6.3561735 .5057550 .395859 H $6.8254095 .430391-1.392845$ H 5.3472463 .5828061 .446389 H 5.1839812 .1235240 .531905 H $2.9308122 .538103-0.053792$ H $0.8763072 .455245-2.084278$ C $-3.498620-0.1953663 .077806$ O $-2.068590-0.2299702 .885630$ C -1.539526 -1.385723 3.563607 C $-2.691745-1.9727004 .371418$ C $-3.881004-1.6104753 .503479$ H -1.263085 -2.107897 2.788178 C $-0.310709-1.0001384 .393650$ H -2.582736 -3.050773 4.524285 H $-2.778088-1.5002645 .357036$ C $-5.211128-1.7042634 .229989$ H -3.897048 -2.275343 2.629308 C $0.848066-0.4117513 .559997$ O $-0.713673-0.0194645 .357103$ H $\quad 0.035458-1.8717474 .962172$ H $0.559901 \quad 0.5544863 .128351$ O $1.942278-0.1528894 .448354$ C $1.351930-1.3470412 .443186$ C $2.615269-0.8126341 .737933$ H $0.564293-1.4743151 .694239$ O $1.616734-2.6347182 .998764$ H $2.4338910 .217606 \quad 1.409937$ H $3.443896-0.8046212 .456766$ H $-5.388459-2.7277584 .576136$ H $-5.238624-1.0445605 .103773$ H $-6.038063-1.4239593 .570368$ C -4.1709640 .2657841 .786660$ H 0.0056750 .0667226 .005096 H 1.6891290 .5968485 .012967 H $2.324895-2.5307373 .657282$ H $-5.2510670 .087128 \quad 1.826402$ H -3.776611 -0.3044930 .936426$ H $-3.6959580 .514073 \quad 3.892948$ C $3.770611-3.916286-0.008297$ O $3.418056-2.9598820 .997639$ C $3.026993-1.6692070 .521812$ C $4.176273-1.028231-0.270789$ C $4.669785-1.954305-1.390124$ C $4.913292-3.381042-0.888284$ H $4.149217-4.7812610 .551151$ C $2.548767-4.380792-0.809758$ H $2.152357-1.772152-0.127901$ O $5.256322-0.7342780 .613350$ H $3.825853-0.077865-0.688342$ H $5.603325-1.558160-1.808170$ O $3.711002-2.002732-2.441055$ H $5.075990-4.055636-1.737134$ O $6.118825-3.376350-0.118778$ C $1.551938-5.0767770 .086986$ H $2.040862-3.562272-1.327253$ H $2.841974-5.097848-1.585755$ H $5.956000-0.3074490 .090250$ H $3.568117-1.095036-2.756774$ H $\quad 6.300967-4.2935470 .147937$ O $0.476196-4.268180 \quad 0.306266$ O $1.719313-6.197466 \quad 0.550617$ C $-0.545224-4.8136731 .146357$ H -1.356544 -4.084396 1.212278
H $-0.940450 \quad-5.737430 \quad 0.713315$

H $-0.151722-4.9938022 .151118$

SCF Energy (B3LYP/6-31G**//MMFF) $=-3245.89627559$

07_00007

MM̄FF Geometry

C $4.434301-2.0351241 .998344$

C $3.104282-2.0984641 .830210$

C $2.125345-1.8824512 .953264$

O $1.104337-0.952042 \quad 2.528427$

C $1.420059-3.2034013 .316206$

C $0.417869-3.0519924 .447063$

C $-0.901783-3.3032124 .337651$

C $0.982478-2.6002235 .770045$

C $-1.646476-3.8345013 .145479$

C $-2.220320-2.7186442 .270698$

C $-3.257175-3.2179261 .250962$

C $-2.677802-4.2341180 .264611$

C $-3.703266-4.643381-0.803344$

O $-3.730176-2.0665110 .538813$

O $-2.999687-5.330977-1.849150$

C $-4.310051-3.372485-1.427665$

C $-4.738884-5.631275-0.253001$

O $-5.372713-3.699829-2.320453$

C $-4.792972-2.334794-0.386540$

C $-5.153043-1.016182-1.111625$

$\begin{array}{llll}\text { O } & -5.955301 & -2.831248 & 0.277859\end{array}$

C 1.3534380 .3740092 .694275

$\begin{array}{lllll}\text { O } & 2.415399 & 0.893083 & 2.996269\end{array}$

C 0.0690581 .0847562 .502465

$\begin{array}{llll}\text { C } & 0.009815 & 2.415237 & 2.660137\end{array}$

C - $1.197852 \quad 3.2418972 .572847$

C -2.5096522 .5353502 .346299$

C $-5.4209420 .175794-0.182959$

C $-5.5125851 .477743-0.962603$

C $-5.531616 \quad 3.944596-0.620024$

O $-5.5138101 .526394-2.190138$

C $-4.1196954 .430717-0.512909$

C -3.7777715 .6079070 .031763$

C $-2.3555006 .073733 \quad 0.169085$

C -2.0417996 .5836951 .560323$

C -1.6810117 .8631401 .757603$

C -2.1573925 .6229202 .724428$

C -1.0749324 .5781882 .719299$

N $-5.5880272 .580872-0.123085$

H $4.837339-1.8217662 .985500$

H $2.695743-2.3246600 .848297$

H $2.637938-1.4837883 .837788$

H $2.163960-3.9549323 .611500$

H $0.928565-3.5935842 .418258$

H $-1.529157-3.1374455 .212826$

H $\quad 0.250365-2.6770926 .581219$

H $1.840713-3.2207736 .048208$

H $1.305717-1.5561455 .718173$

H - $1.007814-4.4989272 .555258$

H -2.462059-4.465995 3.520372

H $-2.695980-1.9524572 .898270$

H -1.408387 -2.198691 1.745741

H $-4.093664-3.6558041 .808315$

H -2.307502 -5.1271320 .780963$

H $-1.805708-3.793995-0.238922$

H -2.552716 -6.099637 -1.455030

H -3.524966 - $2.906298-2.039671$

H $-4.244824-6.5383730 .115548$

H -5.424847 $-5.963626-1.039982$

H $-5.329011-5.2201700 .568576$

H $-5.023581-4.339802-2.964415$

H $-4.325249-0.743146-1.776683$

H $-6.035961-1.167311-1.746102$

H $-6.456030-2.0819430 .637165$

H -0.8049920 .4853152 .273999$

H 0.9288962 .9526002 .890582

H -2.505455 2.0151311 .382889

H -3.372063 3.2034912 .335478

H -2.694885 1.8025843 .139584

H -6.3611090 .0370680 .361177$

H -4.6099510 .2805920 .546485$

H -6.221773 $4.541148-0.015622$ 
H $-5.8571573 .972211-1.664415$ H $-3.3378623 .779880-0.897643$ H $-4.557907 \quad 6.2739510 .393214$ H -2.193635 $6.866987-0.572046$ H -1.650742 $5.272290-0.085149$ H -1.4569848 .2416542 .749452$ H -1.599585 8.5624380 .932026 H -3.163495 5.1971352 .745794 H -2.069904 6.1695523 .673354 H -0.0781654 .9903282 .883903$ H -5.5224192 .4422730 .881276$ C $6.364462-1.0704970 .708512$ $\begin{array}{llll}\text { O } & 5.603122 & 0.060920 & 0.238340\end{array}$ C $6.1537210 .495589-1.014771$ C $7.5997290 .026697-0.992122$ C $7.443311-1.337562-0.348379$ H $5.617597-0.050125-1.800036$ C $5.9450241 .997041-1.225039$ H $8.042601-0.017674-1.991378$ H $8.2267020 .669164-0.363348$ C $8.735333-1.8942400 .224996$ H $7.064590-2.038560-1.104749$ C $4.5060972 .491818-0.971468$ O $6.8064202 .715774-0.333302$ H $6.2678882 .263374-2.238950$ H 4.3336712 .5648800 .110039 O $4.4430283 .830087-1.481351$ C $3.3976891 .634289-1.609720$ C $2.0003242 .260276-1.450770$ H $3.4059040 .642332-1.145119$ O $3.6832531 .464241-2.997578$ H $1.9211832 .752200-0.473786$ H $1.8643893 .017879-2.232275$ H $9.482073-2.024898-0.564875$ H $9.159630-1.2256540 .981440$ H $8.564123-2.8683350 .694011$ C $5.431634-2.2697530 .900036$ H $6.6702863 .664174-0.501082$ H $3.6284604 .239491-1.144517$ H $2.9926620 .889763-3.370157$ H $6.018961-3.1589341 .156627$ H $4.906186-2.483165-0.038522$ H $6.815064-0.7743831 .663971$ C -0.007877 -0.492510 -3.028890 O $0.9484840 .557262-2.815702$ C $0.8731971 .215032-1.547746$ C $-0.5095121 .855543-1.357389$ C $-1.6189660 .817865-1.531979$ C -1.444231 $0.017995-2.826380$ H $\quad 0.103126-0.757380-4.088563$ C $0.310036-1.739796-2.192569$ H $1.0438310 .480576-0.753027$ O $-0.7007562 .924840-2.280779$ H $-0.5652272 .290442-0.355203$ H -2.586946 $1.330337-1.540357$ O $-1.592049-0.060172-0.411727$ H -2.136850 -0.830388 -2.855762 O $-1.767170 \quad 0.854940-3.939993$ C $1.459367-2.507413-2.798776$ H $\quad 0.551820-1.529109-1.147363$ H $-0.547477-2.423699-2.180851$ H $-0.5714942 .571092-3.177498$ H $-2.293347-0.721403-0.534839$ H -2.690851 $1.140269-3.833457$ O $2.647804-1.963938-2.411096$ O $1.313368-3.468308-3.543766$ C $3.810069-2.601322-2.949145$ H $3.799243-2.551255-4.042145$ H $4.691839-2.069803-2.585231$ H $\quad 3.863221-3.639954-2.609007$ SCF Energy (B3LYP/6-31G**//MMFF) $=-3245.92369601$

\section{00008}

MM̄̄FF Geometry

C 2.8377730 .3233843 .966082

C $2.144450-0.5625393 .234733$

C $0.673584-0.8330403 .423514$

O $-0.005887-0.4931182 .192253$
C $0.441790-2.3299883 .695998$

C $-1.009967-2.6806523 .962510$

C $-1.758641-3.4882963 .185662$

C -1.609672 -2.082574 5.209713

C $-1.349668-4.2133881 .934747$

C $-1.617617-3.4502060 .629205$

C $-3.101008-3.1560630 .356862$

C $-3.289291-2.617460-1.063848$

C $-4.750842-2.237054-1.345586$

O $-3.554946-2.180258 \quad 1.298177$

O $-4.755379-1.467450-2.557934$

C $-5.265606-1.330955-0.204690$

C $-5.608518-3.477256-1.622511$

O $-6.660588-1.078532-0.351328$

C $-4.953150-1.8748121 .205126$

C $-5.283577-0.8702442 .335872$

O $\quad-5.750953-3.0424341 .439231$

C $-0.2987890 .819117 \quad 1.983083$

O -0.0858971 .7530662 .739414$

C -0.8986430 .9302740 .636426$

C -0.9285822 .1249120 .028185$

C $-1.4326132 .392378-1.319714$

C $-2.1871541 .288699-2.012313$

C -4.5482010 .4722712 .260125$

C $-5.341216 \quad 1.5726831 .573664$

C -5.1029323 .9140200 .753044$

O $\quad-6.530377 \quad 1.468127 \quad 1.286086$

C $-4.8259873 .901389-0.717247$

C $-4.2495844 .925219-1.365083$

C $-3.9997614 .947622-2.845991$

C $-2.5585115 .252807-3.188508$

C $-2.1745536 .498596-3.514674$

C $-1.5828584 .098597-3.233736$

C -1.203245 $3.605683-1.863921$

N $-4.5697662 .706602 \quad 1.356171$

H 2.3378590 .8742804 .758653

H $2.644725-1.0949822 .428658$

H $0.267161-0.2335494 .247031$

H $1.033397-2.6455584 .565619$

H $0.824331-2.9034622 .844582$

H $-2.791756-3.6670753 .482578$

H -2.599161 -2.495711 5.434098

H $-0.969350-2.2849776 .074483$

H -1.724388 -0.9997305 .103204$

H $-0.289755-4.4861401 .975433$

H $-1.886954-5.1702281 .907793$

H -1.048989-2.513102 0.641536

H -1.223714 -4.058405 -0.193478

H -3.666331 -4.0857720 .490746$

H -2.931955 -3.341246-1.807011

H $-2.665365-1.723209-1.197017$

H -5.664603 -1.154080 -2.704395

H $-4.767311-0.363408-0.332490$

H $-5.212766-4.028980-2.483750$

H $-6.633149-3.199684-1.893480$

H $-5.650140-4.167093-0.777020$

H -7.138450 -1.868232 -0.045732

H $-6.368882-0.7335002 .412979$

H $-4.983117-1.3408823 .283270$

H -5.809154 -3.187709 2.398246

H -1.2589010 .0224330 .166911$

H $-0.5108382 .985210 \quad 0.549884$

H $-2.9886060 .911226-1.368378$

H $-1.5154460 .460063-2.260576$

H $-2.6716901 .604708-2.938267$

H $-4.364706 \quad 0.8247893 .283251$

H -3.5751120 .3608891 .773206$

H -4.6305614 .7635641 .256323$

H -6.1830623 .9627520 .924254$

H $-5.135813 \quad 3.022427-1.277684$

H $-3.9669255 .814243-0.805381$

H $-4.6558085 .711984-3.282727$

H $-4.2945594 .001885-3.317483$

H -1.148696 $6.721290-3.788595$

H $-2.8775327 .325048-3.509931$

H $-0.6542094 .409299-3.731287$

H $-1.9910283 .311617-3.873723$

H $-0.6465984 .339005-1.278545$ 
H -3.579693 2.6868511 .582926

C 4.4412872 .0253703 .083482

O $3.837762 \quad 2.004257 \quad 1.773034$

C $4.808723 \quad 2.4373140 .806402$

C $5.814168 \quad 3.255398 \quad 1.600648$

C 5.9036812 .4304302 .872840

H $5.310307 \quad 1.5342860 .438020$

C $4.1214353 .137119-0.370690$

H $6.773332 \quad 3.3574411 .085118$

H $5.435776 \quad 4.256792 \quad 1.833347$

C 6.5058003 .1817864 .047433

H 6.5158341 .5408632 .671241

C $2.9425192 .337081-0.965437$

O $3.581033 \quad 4.392884 \quad 0.056512$

H $4.8543153 .363041-1.154653$

H $2.0930202 .364075-0.272981$

O $2.508858 \quad 3.005202-2.156311$

C $3.2632900 .872446-1.312525$

C $2.0767910 .162845-1.992782$

H $3.5218260 .334566-0.393969$

$\begin{array}{llll}\text { O } & 4.403789 & 0.843092 & -2.168690\end{array}$

H $1.1535630 .413641-1.462546$

H $1.9933760 .522019-3.026060$

H $7.5332293 .486603 \quad 3.823198$

H 5.9316374 .0840074 .283651

H 6.5266402 .5514684 .942092

C $4.2866690 .644758 \quad 3.726019$

H 4.3218695 .0148720 .151784

H $2.280833 \quad 3.917799-1.908687$

H $4.611106-0.088807-2.351892$

H 4.8165970 .6074284 .684434

H $4.733231-0.1189173 .076996$

H 3.9052692 .7849493 .666587

C $3.673270-3.087917-2.924669$

O $3.336386-1.697023-2.851104$

C $2.235889-1.369898-1.999253$

C $0.959843-2.078468-2.479002$

C $1.176990-3.589624-2.611312$

C $2.461989-3.920609-3.378981$

H $4.438436-3.146574-3.709122$

C $4.304708-3.591002-1.621253$

H $2.461011-1.689705-0.975728$

O $0.537507-1.545907-3.732706$

H $0.154621-1.882493-1.761154$

H $\quad 0.315425-4.028581-3.129417$

O $1.237435-4.155460-1.305630$

H $2.691048-4.989082-3.290828$

O $2.222287-3.655988-4.764310$

C $5.590926-2.852729-1.331395$

H $3.637883-3.486033-0.760853$

H $4.550758-4.656855-1.692477$

H $1.268892-1.656399-4.364227$

H $1.329473-5.118184-1.406895$

H $3.014847-3.928872-5.257402$

O $5.430880-2.043864-0.245209$

O $6.609118-2.968487-2.001318$

C $6.580681-1.279240 \quad 0.129238$

H $7.435425-1.9369620 .313862$

H $6.815151-0.548996-0.650892$

H $6.346612-0.7443881 .053248$

SCF Energy (B3LYP/6-31G**//MMFF) $=-3245.91583751$

07_00009

MM̄FF Geometry

C -2.243587 -3.938639 0.980219

C $-1.110033-3.481866 \quad 1.535120$

C $-0.976911-2.6613042 .794500$

O $-0.438934-1.3773892 .393768$

C $-2.292020-2.3848723 .556392$

C $-2.135080-1.4204534 .719025$

C $-2.736560-0.2144424 .783043$

C -1.253554 -1.887821 5.846949

C $-3.696854 \quad 0.376307 \quad 3.784751$

C $-2.983613 \quad 1.0342302 .603115$

C -3.9255441 .7050361 .591527$

C -4.8668590 .7068990 .915722$

C $-5.7202351 .374690-0.171349$

$\begin{array}{llll}\text { O } & -3.081880 & 2.302402 & 0.600853\end{array}$
O $\begin{array}{llll}-6.327908 & 0.333571 & -0.953875\end{array}$

C $-4.8038012 .173337-1.122670$

C -6.8736372 .1803050 .439795$

O $-5.5974872 .938483-2.027153$

C $-3.7653023 .069028-0.400651$

C $-2.6886873 .548924-1.399462$

O $\begin{array}{llll}\text { C } & -362446 & 4.243320 & 0.149610\end{array}$

C $0.872575-1.1099532 .624265$

O $1.723757-1.8741053 .046467$

C 1.0762110 .3196942 .298797

C 2.2674530 .9027632 .497596

C 2.5818382 .3119392 .238406

C 1.4514293 .1976871 .782257

C $-1.586288 \quad 4.395132-0.748815$

C $-0.4379044 .672574-1.703911$

C $1.8133185 .745436-1.753898$

O $-0.401442 \quad 4.247873-2.855555$

C $2.8248644 .733540-1.314975$

C $3.9955055 .046790-0.739101$

C $4.9912734 .016877-0.286407$

C 5.4362274 .2135431 .147835

C $6.724714 \quad 4.463464 \quad 1.436638$

C 4.3993204 .1304732 .248715

C 3.8485842 .7402852 .422343

N $0.5500355 .443588-1.105578$

H $-3.202781-3.7535131 .453490$

H $-0.172012-3.682517 \quad 1.019797$

H $-0.280204-3.1807293 .463654$

H $-2.706474-3.3281093 .934887$

H -3.029784 -1.9874202 .851890$

H -2.5459400 .4135755 .652054$

H $-1.275172-1.2022826 .700911$

H -1.585749 -2.867413 6.205910

H $-0.213264-1.9704115 .518602$

H $-4.396274-0.395722 \quad 3.449026$

H -4.3104461 .1244184 .302344$

H -2.272618 1.7858162 .972908

H -2.370979 0.2981172 .070372

H -4.4867092 .4933672 .106372$

H $-5.520016 \quad 0.213673 \quad 1.644044$

H $-4.274755-0.0936770 .451122$

H $-6.843191-0.232099-0.353661$

H $-4.2618351 .435324-1.730100$

H -7.5396381 .5220831 .011089$

H $-7.4981002 .632946-0.338064$

H -6.5343922 .9672601 .116772$

H $-5.021176 \quad 3.242445-2.747768$

H -2.228279 $2.674206-1.875404$

H -3.146493 $4.149672-2.194889$

H $-5.3193994 .212040-0.004580$

H $0.2228630 .864183 \quad 1.909605$

H 3.0873520 .2983942 .882856

H 0.6356903 .1906462 .514048

H 1.0617522 .8521240 .818597

H 1.7356684 .2425241 .649640

H $-1.997823 \quad 5.353964-0.413738$

H -1.171705 $3.876077 \quad 0.122290$

H $2.0932106 .763604-1.466693$

H $1.6923275 .704804-2.840831$

H $2.5783503 .686557-1.478688$

H $4.2568246 .092251-0.592877$

H $5.8534174 .070363-0.963426$

H $4.5832153 .006499-0.398328$

H 7.0581264 .6081422 .458954

H 7.4774334 .5284820 .657914

H 3.6241134 .8789552 .068150

H 4.8456154 .4206513 .209610

H 4.5943982 .0177302 .756943

H $0.4555135 .688536-0.124088$

C -2.553193 -3.792387-1.535586

O $-1.440496-2.895895-1.733104$

C $-1.951852-1.603307-2.107126$

C $-3.418024-1.821932-2.466407$

C $-3.803275-2.914047-1.486997$

H $-1.899468-0.971909-1.211826$

C $-1.102794-0.994401-3.227416$

H $-4.014173-0.912644-2.353845$

H $-3.536229-2.177966-3.496418$ 
C $-5.084582-3.641651-1.851506$ H $-3.924745-2.455828-0.498265$ C $0.404195-0.877398-2.912897$ O $-1.230285-1.800936-4.406353$ H -1.503875 -0.009755 -3.495186 H $\quad 0.865567-1.871531-2.905686$

O $1.012813-0.159838-3.994781$

C $0.733501-0.145112-1.601003$

C $2.2158750 .227350-1.422831$

H $0.410499-0.756387-0.751863$

O $-0.0161021 .071729-1.531238$

H $2.5233640 .929834-2.208407$

H $2.2857730 .798783-0.490439$

H $-5.930307-2.946260-1.865855$

H -5.015362 -4.106891 -2.840447

H $-5.305139-4.427991-1.122841$

C $-2.293452-4.688694-0.321180$

H $-0.943979-2.701867-4.176808$

H $\quad 0.802698-0.639348-4.814578$

H $\quad 0.234730 \quad 1.616799-2.296955$

H - $1.346879-5.220528-0.468521$

H -3.073620 -5.455384 -0.249207

H $-2.582611-4.432500-2.427456$

C $3.593050-3.008895-0.127894$

O $2.779559-1.843366-0.328981$

C $3.192447-0.960669-1.377854$

C $4.639582-0.480062-1.160275$

C $5.597651-1.653945-0.951776$

C $5.065096-2.6269910 .102882$

H $3.214125-3.4509970 .802412$

C $3.418064-4.035161-1.254532$

H $3.132667-1.489185-2.334196$

O $4.6951250 .374694-0.020695$

H $4.9481840 .110853-2.031226$

H $6.574332-1.269541-0.633157$

O $5.777009-2.323594-2.195517$

H $5.684800-3.5308940 .127008$

O $5.185802-2.0079831 .386187$

C $2.148778-4.827731-1.064748$

H $3.372768-3.587051-2.250641$

H $4.249870-4.750232-1.274493$

H $5.6105430 .689740 \quad 0.068354$

H $6.404463-3.051772-2.048475$

H $4.879424-2.6466962 .052325$

O $2.378835-5.869979-0.215620$

O $1.084173-4.545008-1.596861$

C $1.261940-6.7235510 .052724$

H $\quad 1.641467-7.6482780 .495604$

H $0.595906-6.2385680 .770943$

H $0.724451-6.974602-0.866826$

SCF Energy $\left(B 3 L Y P / 6-31 G^{* *} / / M M F F\right)=-3245.91245931$

07 00010

MM̄FF Geometry

C $3.389092-4.2134800 .432548$

C $2.364226-3.777485-0.315415$

C $0.914029-3.9752240 .046506$

O $0.252417-2.6901020 .024390$

C $0.241755-4.869858-1.011453$

C $-1.222127-5.152854-0.736089$

C $-2.238484-4.767944-1.533058$

C $-1.511393-5.946070 \quad 0.512424$

C $-2.165927-4.008780-2.828672$

C $-2.319499-2.486182-2.688813$

C $-3.695356-2.040230-2.167231$

C $-3.852450-0.523569-2.282163$

C $-5.189119-0.039381-1.698527$

O $-3.807977-2.420971-0.794603$

O $\quad-5.1124461 .388120-1.556469$

C $-5.370102-0.626878-0.280766$

C $-6.358617-0.317175-2.651233$

O $-6.677831-0.3267090 .201571$

C $-5.085307-2.143484-0.202200$

C $-5.035750-2.6927921 .245282$

O $-6.130186-2.849812-0.882507$

C $0.337337-1.9172351 .140215$

O $0.922143-2.1805232 .178276$

C $-0.393698-0.6613580 .862221$
C $-0.1765990 .414388 \quad 1.632710$

C $-0.760247 \quad 1.7423321 .433945$

C -1.9138241 .8604790 .474608$

C -3.980538 -2.054689 2.156019

C $-4.509062-0.9167303 .013635$

C -3.7311750 .8188864 .625490$

O $-5.700593-0.6323073 .097870$

C -3.5743872 .1201473 .904273$

C -2.7663413 .1044354 .326962$

C -2.6311934 .4315763 .637155$

C -1.1956484 .7750203 .308055$

C -0.4827045 .5962984 .097367$

C -0.6178834 .2371272 .019184$

C -0.2384492 .7848022 .113351$

N -3.489164 -0.275979 3.704618

H $3.188464-4.7378841 .363427$

H $2.570729-3.257189-1.248247$

H $0.813870-4.4275881 .040641$

H $0.768804-5.831474-1.072909$

H $\quad 0.361723-4.398001-1.992595$

H $-3.251014-5.036199-1.232974$

H $-2.563219-6.2446080 .580670$

H $-0.911170-6.8616410 .528799$

H -1.279960 -5.3585321 .406019$

H -1.228641 -4.223855 -3.352372

H $-2.953363-4.391644-3.490718$

H -1.530594 -2.107599-2.028375

H $-2.151980-2.043744-3.678669$

H -4.468092 -2.550152 -2.754370

H -3.749857 -0.197905 -3.324778

H $-3.037423-0.032606-1.733975$

H $-4.9289381 .767121-2.433076$

H $-4.666871-0.0918280 .367112$

H $-6.1919480 .176701-3.616147$

H $-7.2939760 .099041-2.261096$

H $-6.508478-1.380862-2.847085$

H -6.714788 -0.5762161 .139964$

H $-6.033906-2.6644861 .698024$

H -4.783698 -3.7610991 .174783$

H $-6.143680-3.766324-0.560281$

H -1.052752 -0.6412650 .001940$

H 0.5349910 .3333062 .453466

H -2.6811521 .1154540 .708555$

H -1.577372 $1.705483-0.554979$

H -2.416207 $2.829250 \quad 0.514092$

H $-3.620198-2.8200472 .855538$

H -3.118464 -1.707686 1.578550

H $-3.0186130 .713340 \quad 5.449665$

H -4.7457600 .7407245 .029069$

H -4.1721702 .2690943 .008100$

H -2.1904082 .9628005 .238856$

H -3.054185 5.1961534 .302064

H -3.2342254 .4714662 .721519$

H 0.5381645 .8706093 .852815

H -0.9028476 .0082935 .008940$

H 0.2952294 .7892911 .758336

H -1.309346 4.4463111 .198025

H 0.5963032 .5970522 .790111

H -2.524895 -0.5439493 .530180$

C $5.590026-3.1560531 .032919$

O $5.073333-1.8123890 .960602$

C $6.170209-0.8962720 .795784$

C $7.411602-1.6645841 .225692$

C $7.084035-3.0536940 .713304$

H $6.241448-0.681089-0.277543$

C 5.9009540 .4040651 .560555

H $8.328315-1.2511460 .794692$

H $7.524163-1.6828672 .315643$

C $7.924199-4.1503441 .344605$

H $7.237910-3.070120-0.374242$

C 4.5149501 .0229881 .289660

O $5.9819450 .152438 \quad 2.969777$

H 6.6904051 .1315971 .340916

H $3.7358960 .400478 \quad 1.747745$

O 4.4578342 .2892371 .958336

C $4.1808871 .236009-0.198812$

C $2.7910261 .861969-0.370016$

H $4.2126840 .269321-0.708742$ 
$\begin{array}{llll}\text { O } & 5.171337 & 2.073767 & -0.786617\end{array}$

H $2.061601 \quad 1.2733120 .193934$

H 2.7763292 .8617510 .085113

H $8.985889-3.9953841 .126412$

H $7.804477-4.1711812 .433142$

H $7.638948-5.1327290 .955400$

C $4.832288-4.0442970 .044892$

H $5.333165-0.540526 \quad 3.182441$

H 4.6640622 .1314622 .895634

H $5.0179412 .087483-1.746035$

H $5.292107-5.0383650 .004760$

H $4.899206-3.614211-0.962498$

H $5.434386-3.5019852 .062780$

C $0.3138152 .877296-2.942592$

O $0.9646082 .547906-1.710163$

C $2.2960002 .021130-1.818381$

C $2.2852610 .724452-2.643350$

C $1.6252590 .947572-4.009004$

C $0.2775901 .664211-3.884899$

H $-0.7210223 .109367-2.658236$

C $0.914306 \quad 4.134627-3.580384$

H $2.9312572 .764965-2.310279$

O $1.566438-0.301705-1.962972$

H $3.3109340 .370418-2.795708$

H $1.469320-0.020208-4.501663$

O $2.485637 \quad 1.726579-4.835070$

H $-0.0731841 .980162-4.873793$

O $-0.6942190 .738633-3.388834$

C $0.6006095 .351205-2.741849$

H $1.9976414 .084150-3.721091$

H $0.4805834 .307845-4.572559$

H $2.081256-0.561727-1.181078$

H $3.3180941 .234738-4.938361$

H $-0.428645 \quad 0.480727-2.489334$

O $1.5131525 .473681-1.736030$

O $-0.355036 \quad 6.089038-2.944358$

C $1.2997086 .568658-0.840439$

H $1.4141857 .517364-1.372857$

H $0.3084416 .499788-0.382030$

H $2.0541376 .514135-0.051167$

SCF Energy (B3LYP/6-31G**//MMFF) $=-3245.91349382$

07_00011

MM̄FF Geometry

C 0.2917503 .9502841 .011766

C $-0.4935892 .871437 \quad 1.146488$

C -1.3404892 .5914952 .359800$

$\begin{array}{llll}\text { O } & -2.703277 & 2.409406 & 1.892319\end{array}$

C $-0.946378 \quad 1.287778 \quad 3.067771$

C $0.255541 \quad 1.409442 \quad 3.975854$

C 1.4725110 .9003203 .707466

C $0.002947 \quad 2.100976 \quad 5.291238$

C 1.9246690 .1979272 .459909

C $2.181903-1.2999142 .672749$

C $0.899871-2.1240492 .868536$

C $1.239373-3.5967343 .108223$

C $-0.023526-4.4636293 .216146$

O $0.107380-1.9991051 .684068$

O $0.400372-5.8327613 .134076$

C $-0.933401-4.1724912 .003682$

C $-0.699244-4.3043244 .582936$

O $-2.177874-4.8578722 .128529$

C $-1.160817-2.6647831 .742993$

C $-1.861523-2.469728 \quad 0.377533$

O $-1.991470-2.1389932 .779554$

C -3.480291 3.5145381 .761184

O -3.1716264 .6613892 .056800$

C $-4.797654 \quad 3.195167 \quad 1.163107$

C -5.1893251 .9604160 .810559$

C -6.4593731 .6086340 .166562$

C $-7.4153022 .732853-0.140001$

C $-1.985255-1.004936-0.067510$

C $-2.502077-0.884135-1.490341$

C $-3.1927780 .818285-3.168980$

O $-2.751694-1.855936-2.198872$

C $-4.6777040 .955008-3.042048$

C $-5.541835-0.003437-3.408862$

C $-7.0338460 .095214-3.260615$
C $-7.564426-0.847657-2.201094$

C $-7.795808-2.141372-2.481863$

C $-7.891433-0.275064-0.837896$

C $-6.6899870 .315491-0.147635$

N -2.646446 $0.442303-1.878195$

H $\quad 0.3751874 .654187 \quad 1.835858$

H $-0.5956382 .182408 \quad 0.311204$

H $-1.3156073 .425648 \quad 3.070745$

H -0.8122010 .4939872 .327008$

H $-1.7942390 .936815 \quad 3.672913$

H 2.2543081 .0193274 .456894

H 0.8825802 .0882465 .943744

H -0.2706473 .1482535 .131144$

H $-0.8115431 .606187 \quad 5.830511$

H 1.2429710 .3555311 .619037

H 2.8676300 .6703492 .164401

H $2.710397-1.6719991 .786253$

H $2.852074-1.4429193 .529644$

H $\quad 0.344237-1.7199563 .721824$

H $1.863213-3.7131844 .003148$

H $1.845461-3.9709242 .270643$

H $-0.398213-6.3881963 .124410$

H $-0.439758-4.6079051 .125231$

H $-0.005918-4.5847935 .385187$

H -1.557132 -4.978390 4.682951

H $-1.037680-3.2845204 .777683$

H -2.724130 -4.365051 2.764369

H -1.294391 -3.010856 -0.390471

H -2.866948 -2.9088190 .406273$

H -2.448028 -1.348694 2.452283

H -5.4343744 .0600581 .004748$

H -4.5180341 .1232810 .989113$

H $-8.3230302 .413206-0.654368$

H $-6.9394783 .475266-0.790701$

H $-7.734515 \quad 3.2271890 .784279$

H -2.674463 -0.4655760 .589600$

H -1.010903 -0.507138 -0.026987

H $-2.9146830 .067439-3.916358$

H -2.748862 $1.774709-3.460577$

H $-5.0523951 .884084-2.620671$

H $-5.155065-0.929112-3.829909$

H -7.490671 -0.141748 -4.230432

H -7.345796 $1.123060-3.038336$

H -8.197752 -2.820068 -1.737146

H -7.585377 -2.550147 -3.464859

H $-8.290308-1.058301-0.179835$

H $-8.7077770 .442243-0.954019$

H $-5.930763-0.4245540 .109641$

H $-2.4931071 .178061-1.194378$

C $2.4965004 .290228-0.287134$

O $2.9948832 .940631-0.329401$

C $4.3377742 .950738 \quad 0.202692$

C 4.6212784 .3849640 .661566

C 3.2266254 .9406060 .879499

H 4.3326752 .3019511 .084280

C $5.3420862 .432495-0.839456$

H 5.2364214 .4098021 .566867

H $5.1508404 .960317-0.107099$

C 3.1673836 .4570300 .886952

H 2.8533814 .5546801 .836482

C $5.1035590 .980769-1.309971$

O $5.2754803 .286287-1.989033$

H $6.3586762 .532148-0.439154$

H $4.1956120 .926254-1.921605$

O $6.194050 \quad 0.613035-2.164006$

C $5.024823-0.045891-0.168183$

C $5.102384-1.521566-0.595657$

H $4.0968690 .116947 \quad 0.384936$

O $6.103257 \quad 0.1901320 .743115$

H $6.099280-1.743681-0.997639$

H $5.023785-2.1153180 .325083$

H 3.7847576 .8621691 .695357

H $3.5305216 .877914-0.056685$

H $2.1412376 .806447 \quad 1.038880$

C $0.9690154 .323719-0.276164$

H $6.0496033 .091875-2.543759$

H $6.1070681 .123596-2.986411$

H $5.982227-0.4059231 .501820$ 
H $\quad 0.6003343 .676638-1.079582$

H $0.6401975 .340739-0.525270$

H $2.8187864 .756156-1.229441$

C $1.609630-1.995067-1.848597$

O $2.744036-1.651285-1.042814$

C $4.023325-1.975818-1.592227$

C $4.129198-3.484690-1.882065$

C $2.962951-3.975723-2.743342$

C $1.615988-3.492609-2.194847$

H $\quad 0.744145-1.806937-1.204049$

C $1.479047-1.095493-3.086621$

H 4.163992 -1.419882 -2.524588

O $4.141691-4.210326-0.652933$

H $5.082438-3.678671-2.388697$

H $2.974631-5.072080-2.772260$

O $3.149494-3.507062-4.074901$

H $\quad 0.810349-3.704775-2.907298$

O $1.313415-4.211246-0.996493$

C $1.144400 \quad 0.337442-2.734228$

H $2.399287-1.072890-3.675568$

H $0.663615-1.469539-3.717076$

H $4.252502-5.151924-0.868529$

H $2.409450-3.841372-4.609996$

H $1.272409-5.155892-1.222623$

O $0.9503901 .034063-3.893947$

O $1.071404 \quad 0.791865-1.601674$

C $0.6422062 .421832-3.740818$

H $\quad 1.4986912 .948907-3.310168$

H $\quad 0.4375872 .835967-4.731520$

H $-0.2482022 .549931-3.118418$

SCF Energy $\left(B 3 L Y P / 6-31 G^{* *} / / M M F F\right)=-3245.90105432$

07 00012

MM̄MF Geometry

C $-0.254906-4.1509721 .222182$

C $-0.057957-3.3568902 .285702$

C $1.120341-2.4313992 .419858$

O $0.589460-1.1293162 .766730$

C $2.090722-2.8647893 .533262$

C $2.863800-4.1298923 .232045$

C $3.957268-4.1791992 .445964$

C $2.363100-5.3755053 .914749$

C $4.604735-3.0403111 .704581$

C $4.643097-3.3227640 .196788$

C $5.179288-2.168638-0.667066$

C $6.590720-1.728796-0.280428$

C $7.077650-0.564202-1.155243$

O $4.279059-1.062732-0.560013$

O $8.254258-0.014570-0.541917$

C $6.0069070 .550282-1.157633$

C $7.498881-1.036690-2.552444$

O $6.3697141 .570650-2.084447$

C $4.5774610 .036376-1.443828$

C $3.4917821 .098746-1.153958$

O $4.469896-0.366385-2.810564$

C $1.292680-0.0426482 .351277$

O $2.322082-0.0408741 .696877$

C $0.593617 \quad 1.1650342 .838715$

C 0.9213312 .3629452 .331531

C $0.345603 \quad 3.6531932 .715441$

C -0.6114013 .6672623 .879094$

C $3.5114672 .350757-2.032107$

C $2.1996783 .116874-1.969736$

C $1.0834325 .120897-2.939004$

O $1.2246612 .738553-1.327107$

C $0.9627416 .093689-1.808374$

C $-0.1523716 .230858-1.074431$

C $-0.324213 \quad 7.2585570 .005850$

C $-0.7725956 .663803 \quad 1.321319$

C -2.0710576 .6562891 .667741$

C $0.290028 \quad 6.1783842 .279452$

C $0.704861 \quad 4.7555142 .023247$

N $2.2403334 .270182-2.739509$

H $0.474402-4.1478530 .415758$

H $-0.769241-3.3782003 .107799$

H $1.646095-2.3644281 .459614$

H $1.543442-2.9759484 .479913$

H $2.797419-2.0510443 .743027$
H $4.454314-5.1400502 .318355$

H $2.968598-6.2550053 .671653$

H $2.389763-5.2473285 .001894$

H $1.333512-5.5938663 .616615$

H $4.096805-2.0916121 .892501$

H $5.624686-2.9349502 .090791$

H $5.250329-4.2179220 .010543$

H $3.622427-3.552001-0.138781$

H $5.168163-2.526628-1.703527$

H $7.290626-2.571756-0.332774$

H $6.605268-1.3866160 .762580$

H $8.914528-0.725145-0.470664$

H $6.009338 \quad 1.016037-0.162320$

H $8.293839-1.788580-2.478587$

H $7.921461-0.212658-3.137880$

H $6.678461-1.478436-3.121670$

H $7.2611271 .876746-1.844771$

H $2.5159430 .606252-1.258889$

H $3.5467031 .402294-0.100911$

H $3.529398-0.365903-3.054828$

H $\quad-0.170421 \quad 1.0334983 .596056$

H 1.6849772 .4077701 .556165

H -1.460412 3.0021053 .691123

H -1.036028 4.6517384 .084784

H $-0.105844 \quad 3.3378774 .793565$

H $4.3115083 .023949-1.705875$

H $3.6840062 .084131-3.080668$

H $0.1928554 .490518-3.037059$

H $1.2325275 .660806-3.879322$

H $1.8152596 .739703-1.613179$

H -1.011135 $5.601273-1.294852$

H -1.070746 $7.983876-0.344355$

H $0.594866 \quad 7.837795 \quad 0.158114$

H -2.398691 6.2649102 .624848

H $-2.836387 \quad 7.034680 \quad 0.998017$

H $-0.040311 \quad 6.319417 \quad 3.314288$

H $1.178473 \quad 6.8176902 .196394$

H 1.3988824 .6432791 .189754

H $3.1070474 .529807-3.201522$

C $-2.030741-5.089012-0.303590$

O $-2.803246-3.884991-0.504684$

C $-4.046991-4.237039-1.142037$

C $-3.895813-5.682590-1.602068$

C $-3.003294-6.249361-0.520897$

H -4.819216 -4.197654 -0.365291

C $-4.392329-3.272638-2.282546$

H $-4.859228-6.195996-1.679247$

H -3.400065 -5.748329 -2.577776

C $-2.335768-7.556484-0.913853$

H $-3.605259-6.4102390 .383790$

C $-4.579085-1.795329-1.883534$

O $-3.353711-3.322633-3.270467$

H $-5.299090-3.625966-2.788426$

H -3.608017 -1.342238 -1.658231

O $-5.084293-1.107059-3.036536$

C $-5.546570-1.554070-0.711891$

C $-6.015042-0.096094-0.536521$

H $-5.074485-1.8992630 .215272$

O $-6.718819-2.352773-0.906720$

H $-6.7024580 .171721-1.349613$

H $-6.623283-0.0701500 .377609$

H -3.087934 -8.322771-1.127993

H -1.714347 -7.436795 -1.807829

H -1.696571 -7.928683 -0.107523

C $-1.408368-5.1045271 .095846$

H $-2.520160-3.083257-2.829971$

H -4.453976 -1.256242 -3.762067

H -7.284337 -2.240185 -0.123853

H $-1.023723-6.1072401 .314551$

H -2.175771 -4.8702771 .843930$

H $-1.249777-5.096958-1.075364$

C $-2.884709 \quad 1.431893 \quad 0.820458$

$\begin{array}{lllll}\text { O } & -4.025231 & 0.583177 & 0.628933\end{array}$

C $-4.8938160 .951365-0.444131$

C $-5.4540452 .367201-0.221741$

C -4.3340173 .3866510 .005078$

C -3.3170872 .8920451 .039970$

H -2.4248371 .0759631 .751113$ 
C $-1.851646 \quad 1.277027-0.306593$ H $-4.3338240 .936694-1.383944$ O -6.3368292 .3849590 .899288$ H -6.044869 2.655782 -1.098964 H $-4.776073 \quad 4.3312560 .344928$ O $-3.6842573 .634387-1.237586$ H -2.4304243 .5353931 .047096$ O -3.9056702 .9733922 .341013$ C -1.254824 -0.112244-0.360421 H $-2.2862321 .478378-1.288838$ H -1.029838 1.979947 -0.136584 H -5.8440702 .0559811 .670593$ H -2.994499 4.300969-1.079231 H -4.116178 3.9079492 .508481 O $-0.359961-0.167769-1.390869$ O $-1.537460-1.031674 \quad 0.394080$ C $0.282484-1.430906-1.588912$ H $-0.456147-2.233598-1.672260$ H $0.849672-1.380258-2.522206$ H $\quad 0.977471-1.626069-0.768342$

SCF Energy $\left(B 3 L Y P / 6-31 G^{* *} / / M M F F\right)=-3245.91201526$

\section{00013}

MM̄MF Geometry

C $-5.2144642 .642040 \quad 0.775639$

C -3.8967752 .8599370 .647671$

C -2.9930953 .2253241 .797371$

O -1.8649212 .3213021 .801523$

C -2.4490754 .6528541 .604540$

C $-1.526048 \quad 5.1009012 .722067$

C -0.2153045 .3810162 .577641$

C -2.174735 5.2550104 .074999

C 0.6215055 .3490721 .328221

C $1.607854 \quad 4.179561 \quad 1.344287$

C 2.6520314 .2448260 .218182

C $2.0300654 .109866-1.174704$

C $3.0950764 .030136-2.281845$

O 3.5764873 .1723330 .452574

O $2.4500693 .592149-3.488116$

C $4.1607012 .979864-1.898231$

C $3.6627435 .415803-2.609192$

O $5.2493232 .993695-2.818273$

C $4.6861203 .141264-0.456964$

C $5.6192621 .985240-0.027411$

O $5.4557014 .347513-0.371264$

C -2.0086181 .1386412 .457675$

O $-3.003612 \quad 0.7283303 .032584$

C $-0.727198 \quad 0.4100252 .332352$

C $-0.679093-0.9006442 .611359$

C $0.488751-1.7638452 .440057$

C $1.824507-1.1042462 .218645$

C $4.9936570 .588151-0.059475$

C $5.898624-0.4557100 .576071$

C $5.996109-2.8791281 .108820$

O $7.007658-0.196703 \quad 1.035625$

C $4.988034-3.9343451 .427787$

C $4.565944-4.2055492 .672261$

C $3.527134-5.2415492 .997356$

C $2.196451-4.6121413 .358568$

C $1.813207-4.5216294 .643480$

C $1.363081-4.1449562 .182531$

C $\quad 0.322124-3.1026612 .478765$

N $5.311329-1.7137720 .580269$

H -5.678141 2.7308021 .754990

H -3.435963 $2.779090-0.334159$

H $-3.528516 \quad 3.1567492 .752374$

H $-3.284250 \quad 5.363310 \quad 1.543077$

H -1.9400104 .7066850 .637129$ H 0.3375165 .6918893 .463999 H -1.505557 5.7205134 .806566 H -3.0644365 .8887063 .999715$ H -2.471150 4.2809804 .475573 H $\quad 0.0060245 .3113710 .427107$ H 1.1745786 .2952111 .275510 H 2.1392424 .1528202 .305678 H 1.0640833 .2275611 .287950 H 3.1885225 .1967630 .308103 H $1.3407004 .936211-1.383998$
H $1.4169203 .200222-1.208010$

H $2.0488922 .722706-3.319317$

H $3.6906291 .995705-1.995639$

H $2.8659376 .078856-2.967872$

H $4.3946215 .362639-3.422744$

H $4.1338025 .900975-1.751895$

H $4.8766322 .890238-3.710657$

H $6.5332821 .996058-0.635732$

H 5.9540902 .1856261 .000517

H $\quad 6.045728 \quad 4.2835370 .398010$

H $\quad 0.1302670 .9597651 .961414$

H -1.590507 -1.401741 2.934754

H $2.018463-0.3552552 .994784$

H $1.858009-0.6132061 .243180$

H $2.660209-1.8059772 .259128$

H 4.0438450 .5864240 .486194

H $4.8038010 .279889-1.093105$

H $6.581497-2.5861291 .987224$

H $6.694388-3.2376660 .345639$

H $4.577570-4.4933490 .590767$

H $4.969892-3.6379883 .507600$

H $3.905699-5.8556383 .824453$

H $3.382570-5.9358702 .159784$

H $0.859198-4.0960524 .933724$

H $2.450503-4.8811065 .445045$

H $0.860883-5.0189111 .747852$

H $2.013933-3.7693841 .384139$

H $-0.672725-3.5066302 .665762$

H $4.470348-1.8576310 .028255$

C $-6.8145790 .951148-0.174476$

O $-5.835653-0.105971-0.213836$

C -6.291289-1.133610-1.112531

C $-7.780699-0.883416-1.301866$

C $-7.8254940 .632151-1.279116$

H $-5.781180-0.962859-2.068587$

C $-5.919833-2.520587-0.575785$

H -8.160394 -1.311807 -2.234265

H -8.372335 -1.289852 -0.473751

C $-9.2127001 .196751-1.026247$

H $-7.4586711 .007288-2.244101$

C $-4.442316-2.666184-0.159613$

O $-6.714562-2.8113650 .581517$

H $-6.180704-3.284343-1.317297$

H -4.255306 -2.089828 0.755214

O $-4.210388-4.0394450 .176957$

C $-3.426469-2.249173-1.237746$

C -1.981017 -2.415695 -0.753746

H $-3.595958-1.200133-1.493124$

O $-3.626521-3.013849-2.422721$

H $-1.855922-1.8827730 .194722$

H - $-1.780991-3.471497-0.525768$

H -9.903344 $0.888955-1.818130$

H $-9.6199260 .848283-0.071155$

H -9.190261 2.290891-1.003816

C $-6.1197332 .299759-0.375467$

H $-6.552343-2.1078891 .233699$

H $-4.854444-4.2815020 .864522$

H -3.513453 -3.951357 -2.189395

H $-6.8679173 .095504-0.466167$

H $-5.5451212 .282240-1.310122$

H $-7.2917570 .905700 \quad 0.812925$

C $1.535728-1.785321-1.778787$

O $0.349083-2.118785-1.050670$

C $-0.896111-1.954118-1.746299$

C $-1.063944-0.503115-2.226866$

C $0.151326-0.032548-3.031149$

C $1.463294-0.346329-2.309432$

H $2.339699-1.823105-1.032805$

C $1.850261-2.823180-2.860321$

H $-0.911689-2.631435-2.606928$

O $-1.2499370 .350633-1.099419$

H -1.961976 -0.430937 -2.850827

H $0.075756 \quad 1.049174-3.196547$

O $0.129556-0.671977-4.303549$

H $2.315759-0.156533-2.971125$

O $1.6031410 .546027-1.201841$

C $2.357369-4.101252-2.236002$

H $0.997583-3.069269-3.499336$ 
H $2.643968-2.459080-3.523805$

H $-1.4021611 .250832-1.433839$

H $\quad 0.891820-0.343881-4.810178$

H $\quad 0.8645580 .382466-0.590695$

O $1.310418-4.858287-1.801137$

O $3.544498-4.383658-2.136596$

C $1.663228-6.096681-1.177364$

H $2.098736-6.775689-1.916361$

H $2.360286-5.927916-0.351118$

H $\quad 0.751082-6.547908-0.777754$

SCF Energy $\left(B 3 L Y P / 6-31 G^{* *} / / M M F F\right)=-3245.92112114$

07 00014

MM̄FF Geometry

C $1.795762 \quad 1.2142832 .847614$

C 2.1305880 .1749943 .628344

C $1.411873-1.1536793 .704069$

O $0.222028-1.0973032 .880258$

C $2.268702-2.3350443 .210442$

C $3.636944-2.4682813 .845714$

C $4.793097-2.278173 \quad 3.178165$

C $3.646078-2.8872195 .290888$

C $4.923186-1.8529271 .741936$

C $5.029608-3.0622660 .805192$

C $4.655262-2.759911-0.653697$

C $5.542624-1.693080-1.293657$

C $5.106228-1.389033-2.736231$

O $3.286801-2.339938-0.674085$

O $5.768343-0.183602-3.149312$

C $3.589583-1.100961-2.747235$

C $5.552702-2.484651-3.711101$

O $3.111689-0.939541-4.081575$

C $2.740475-2.148160-1.984135$

C $1.294565-1.615238-1.822951$

O $2.726871-3.363696-2.730204$

C $-0.826025-1.9016883 .191843$

O $-0.904572-2.6889294 .119885$

C $-1.870783-1.6560132 .171892$

C $-2.998486-2.3809232 .171450$

C $-4.096034-2.2610831 .207598$

C $-3.911868-1.3018360 .060265$

C $0.368810-2.456677-0.933984$

C $-0.370493-3.565161-1.662573$

C -2.041853 -5.370306-1.247206

O $-0.251108-3.778647-2.865558$

C $-3.409038-4.831724-1.531541$

C $-4.528984-5.321474-0.978347$

C $-5.906332-4.808233-1.287866$

C $-6.687210-4.436836-0.046675$

C $-7.619757-5.2635410 .456032$

C $-6.449086-3.0707580 .558299$

C -5.197297-3.019650 1.389115

N -1.187361 $-4.285979-0.800907$

H $\quad 0.944774 \quad 1.1370072 .176547$

H 2.9737990 .2940894 .305994

H $1.114949-1.3070914 .749443$

H $2.334883-2.2885792 .117128$

H $1.719457-3.2700363 .387871$

H $5.734251-2.4302653 .702833$

H $4.661823-3.0307685 .674448$

H $3.162860-2.1282605 .913940$

H $3.109752-3.8336055 .415439$

H $4.085919-1.2072061 .461498$

H $5.818982-1.2276111 .644470$

H $6.046862-3.4706640 .850294$

H $4.353536-3.8518851 .160033$

H $4.741039-3.703216-1.206083$

H $\quad 6.596956-1.994218-1.263827$

H $5.479688-0.758716-0.719840$

H $6.727415-0.329375-3.079428$

H $3.448984-0.127227-2.261744$

H $6.641423-2.610943-3.672538$

H $5.321825-2.210207-4.746338$

H $5.099795-3.455890-3.501256$

H $3.030912-1.824841-4.475631$

H $\quad 1.355038-0.628315-1.347144$

H $\quad 0.833972-1.477432-2.808100$

H $1.985357-3.908213-2.423335$
H -1.674925 -0.885029 1.434613

H $-3.132223-3.1357202 .945241$

H $-3.796261-0.2783250 .431863$

H $-4.746308-1.291657-0.643127$

H -3.021731 -1.564112 -0.522149

H $\quad 0.909270-2.883011-0.082545$

H $-0.402454-1.787435-0.533688$

H -2.050348 -6.125314 -0.454855

H $-1.624108-5.818460-2.154408$

H -3.482291 -4.013042 -2.243921

H $-4.457472-6.157755-0.286530$

H $-6.436462-5.594858-1.840847$

H -5.869894 -3.945179 -1.964370

H -8.204426 -4.9923941 .328882$

H -7.815360 -6.232711 0.009184

H -7.286154 -2.801993 1.216756

H -6.468569-2.321262 -0.237015

H $-5.229650-3.6828082 .255078$

H -1.269371 -3.9890590 .167166$

C 3.0010223 .0098791 .500971

O 1.9051513 .1181550 .568297

C $2.3609822 .656724-0.718922$

C $3.8807022 .579896-0.619583$

C 4.0478152 .1308870 .819565

H $1.9733411 .640243-0.844401$

C $1.8341103 .561189-1.835515$

H $4.3151821 .884462-1.342401$

H $4.3463263 .561150-0.770804$

C 5.4560142 .3026681 .357489

H $3.766007 \quad 1.073367 \quad 0.885373$

C $0.2936563 .635233-1.929611$

O $2.3241254 .892298-1.630342$

H $2.2465003 .235417-2.797524$

H $-0.1082974 .178009-1.066833$

O $-0.0316444 .417541-3.085817$

C $-0.4037082 .268390-2.057705$

C $-1.8945982 .324730-2.440986$

H $-0.2948221 .716259-1.117338$

O $0.2664941 .509527-3.070132$

H -1.999739 $2.694613-3.469129$

H $-2.2510581 .285913-2.467776$

H $\quad \begin{array}{llll}6.164610 & 1.703862 & 0.775812\end{array}$

H $5.780078 \quad 3.347641 \quad 1.309957$

H 5.5164281 .9768302 .400614

C 2.5035182 .5401512 .869186

H $2.0240495 .182462-0.751651$

H $0.4187815 .274406-2.991744$

H $-0.1714460 .643614-3.128283$

H 1.7831383 .2751523 .247812

H 3.3409702 .5243303 .576348

H 3.3958424 .0279581 .619782

C $-3.383907 \quad 3.299940 \quad 0.845337$

O $-2.6444772 .618883-0.178549$

C $-2.7815133 .148962-1.499369$

C $-4.2545003 .118254-1.944215$

C $-5.1665883 .812368-0.927539$

C $-4.884763 \quad 3.3444010 .503542$

H -3.2608032 .6771171 .740570$

C -2.8011934 .6884671 .154238$

H -2.427939 $4.184379-1.512391$

O $-4.694941 \quad 1.772943-2.125228$

H -4.338522 $3.613411-2.918482$

H $-6.2143453 .599322-1.172947$

O $-4.9803045 .222524-0.998985$

H -5.4109843 .9877821 .218556$

O -5.4252952 .0281980 .649123$

C -1.409314 4.6169861 .744105

H $-2.7399935 .309886 \quad 0.257285$

H $-3.4366405 .194723 \quad 1.890002$

H $-4.5794171 .306805-1.279922$

H $-5.1993705 .502228-1.904158$

H -5.299659 1.7587921 .574972

O -0.9305005 .8886291 .888955$

O -0.8205823 .5888952 .046055$

C 0.3838645 .9992362 .442877

H 1.1192015 .5989621 .739922

H 0.5957677 .0593672 .605071

H 0.4415495 .4808343 .404640 


\section{MM̄FF Geometry}

C $1.605182-0.8390183 .150724$

C $1.256742-2.0988153 .456130$

C $0.033476-2.8411842 .972273$

O $-0.940258-1.8964612 .462272$

C $0.332686-3.8616631 .857854$

C $1.439037-4.8559602 .140983$

C $2.577414-4.9325361 .422550$

C $1.171414-5.8314173 .255382$

C $2.968631-4.0294240 .286615$

C $2.505531-4.579049-1.067920$

C $2.439827-3.518124-2.178512$

C $3.794839-2.873507-2.469586$

C $3.683655-1.772237-3.534545$

O $1.494508-2.522743-1.772228$

O $4.922050-1.045927-3.503774$

C $2.548928-0.805532-3.134209$

C $3.554515-2.357581-4.945170$

O $2.301478 \quad 0.148650-4.164775$

C $1.226898-1.513096-2.752707$

C $0.255935-0.483509-2.115126$

O $0.645294-2.069023-3.930059$

C $-2.250611-2.2596322 .504359$

O $-2.714134-3.2902892 .965350$

C $-3.033615-1.1793581 .864035$

C $-4.359705-1.317247 \quad 1.718125$

C $-5.268408-0.3542761 .091326$

C -4.6646770 .8818510 .479501$

C $-1.049989-1.064726-1.553220$

C $-2.155335-1.236672-2.580841$

C $-4.431819-2.224027-2.833404$

O $-2.080314-0.804998-3.727803$

C $-5.460595-1.164223-2.594552$

C $-6.714882-1.427457-2.197188$

C $-7.765174-0.373379-1.992712$

C $-8.416789-0.451435-0.629897$

C $-9.622133-1.024148-0.471962$

C -7.7112580 .2123740 .531410$

C $-6.589958-0.6278231 .081466$

N $-3.243043-1.923203-2.057869$

H $1.004028-0.2627892 .453141$

H $1.881558-2.6478994 .158540$

H $-0.396773-3.3479813 .845891$

H $\quad 0.507670-3.3147440 .924325$

H $-0.582609-4.4317571 .646692$

H $3.302771-5.7056941 .668480$

H $1.966733-6.5782173 .352549$

H $1.091530-5.3094034 .213824$

H $\quad 0.235512-6.3694103 .072469$

H $2.576255-3.0222290 .454596$

H $4.060258-3.9231050 .290706$

H $3.164586-5.400035-1.375843$

H $1.498293-5.002662-0.957073$

H $2.058322-4.018212-3.076664$

H $4.529671-3.630130-2.770804$

H $4.193991-2.414927-1.555022$

H $4.889884-0.365242-4.197036$

H $2.899387-0.240468-2.261690$

H $4.433560-2.967257-5.186226$

H $3.522732-1.565417-5.701493$

H $2.669784-2.985615-5.069692$

H $3.1065910 .682527-4.271341$

H $0.772609-0.019319-1.264726$

H $\quad 0.0256290 .313284-2.832184$

H $-0.279143-2.293637-3.743776$

H $-2.495019-0.3046401 .519426$

H -4.831327 -2.224822 2.093189

H -4.2531191 .5305941 .258900$

H $-5.3769151 .477573-0.095024$

H -3.862838 $0.618777-0.218887$

H $-0.870668-2.018413-1.045922$

H $-1.437303-0.361926-0.805390$

H $-4.778669-3.215199-2.524502$

H -4.176739 -2.258599 -3.897410

H $-5.166197-0.133898-2.780068$
H $-8.522876-0.503999-2.776568$

H $-7.3568380 .633889-2.142053$

H -10.109074 -1.058503 0.497024

H -10.146224 -1.476739-1.307255

H -8.420335 $0.379875 \quad 1.353648$

H $-7.393126 \quad 1.2154790 .234233$

H -6.930116 -1.554712 1.545632

H $-3.242071-2.179907-1.075040$

C $3.903707 \quad 0.248467 \quad 2.832799$

$\begin{array}{llll}\text { O } & 3.441313 & 1.147740 & 1.809394\end{array}$

C $4.220080 \quad 0.912467 \quad 0.615403$

C $5.278624-0.1257840 .982938$

C $4.584220-0.8950202 .090394$

H $3.5400330 .467026-0.116836$

C 4.7872992 .2373040 .088666

H $5.552816-0.7539110 .129588$

H 6.1952250 .3441491 .358276

C $5.524247-1.7267652 .942534$

H $3.836166-1.5510111 .634779$

C $3.7064763 .285628-0.250790$

O $5.645796 \quad 2.802153 \quad 1.088521$

H $5.4211122 .053465-0.786001$

H 3.1412473 .5345440 .653291

O $4.3619644 .490206-0.665467$

C $2.7563582 .850669-1.381069$

C $1.6906443 .895935-1.758363$

H $2.2509331 .921542-1.107530$

O $3.5246512 .567528-2.554174$

H $2.1700294 .845053-2.026906$

H $1.1963563 .540435-2.672694$

H $6.038605-2.4740862 .329368$

H $6.285801-1.1048193 .424677$

H $4.974014-2.2560163 .726796$

C $2.769405-0.1277623 .785170$

H 5.1130162 .9356721 .891427

H $4.9744404 .746106 \quad 0.045542$

H $4.0003483 .380831-2.795825$

H $2.3720750 .792414 \quad 4.231793$

H $3.176355-0.7223174 .611867$

H 4.6511670 .8073043 .412959

C - -0.9821812 .8758600 .626220$

$\begin{array}{lllll}\text { O } & -0.034134 & 2.874989 & -0.450346\end{array}$

C $0.6430974 .119163-0.660823$

C $-0.3729815 .205569-1.042507$

$\begin{array}{llll}C & -1.470871 & 5.331378 & 0.018402\end{array}$

C $-2.049145 \quad 3.9658250 .415132$

H -1.4777251 .8999050 .548653$

C $-0.2526892 .926302 \quad 1.976996$

H $1.152370 \quad 4.415728 \quad 0.261706$

O $-0.9656374 .911138-2.305882$

H $0.1515606 .162485-1.147591$

H -2.271131 $5.970392-0.374581$

O $-0.923296 \quad 5.980248 \quad 1.162100$

H -2.669625 4.0564661 .313170

O $-2.904620 \quad 3.513965-0.640754$

C $-1.050913 \quad 2.303716 \quad 3.101314$

H $0.6850082 .363497 \quad 1.884913$

H $0.0075113 .947246 \quad 2.267513$

H $-1.3701884 .028678-2.246267$

H -1.6394906 .0837701 .811646$

H $-3.6231914 .162503-0.733577$

O -0.2132292 .0339714 .145529$

$\begin{array}{llll}0 & -2.255642 & 2.095224 & 3.081368\end{array}$

C $-0.824152 \quad 1.410093 \quad 5.279244$

H -1.5711592 .0768435 .720244$

H $-1.277863 \quad 0.457152 \quad 4.989830$

H -0.0446231 .2154496 .020698$

SCF Energy (B3LYP/6-31G**//MMFF) $=-3245.92950872$

0700016

MM̄FF Geometry

C -2.104897 $-3.020306 \quad 1.335087$

C $-1.318487-1.9331141 .333926$

C $-0.752485-1.2982372 .578809$

O $0.686916-1.2565942 .441872$

C - $-1.264057 \quad 0.1451302 .711703$

C $-0.7606410 .843232 \quad 3.959207$ 
C $\quad 0.210453 \quad 1.7778503 .976558$

C $-1.463208 \quad 0.4599145 .236242$

C 1.0168832 .2871992 .812660

C 2.5198842 .3012063 .135279

C 3.4209662 .6677281 .941802

C 3.1366074 .0697941 .394855

C $3.9606254 .384706 \quad 0.137307$

O 3.2334691 .6730040 .931293

O $3.3915955 .548858-0.482763$

C $3.801160 \quad 3.220602-0.863450$

C 5.4114364 .7426420 .478636

O $4.6495053 .401639-1.995142$

C $4.0540561 .835028-0.233376$

C $3.7143810 .675221-1.197526$

$\begin{array}{lllll}\text { O } & 5.444806 & 1.725634 & 0.093371\end{array}$

C $1.361458-2.4094422 .698925$

O $0.897979-3.4662613 .097571$

C $2.776366-2.1845222 .343512$

C $3.473867-3.2188521 .852462$

C $4.849996-3.1754031 .363686$

C $5.676814-1.9627351 .694336$

C $2.2367700 .570046-1.583075$

C $1.966569-0.692434-2.377867$

C $1.883797-3.177170-2.135506$

O $1.750077-0.660942-3.586833$

C $3.214081-3.862145-2.272102$

C $4.419622-3.279097-2.379102$

C $5.716848-4.036378-2.472674$

C $6.698501-3.631779-1.391288$

C $7.638607-2.701981-1.631583$

C $6.634577-4.341353-0.054486$

C $5.298569-4.2076860 .620245$

N $2.011288-1.836219-1.597072$

H -2.370907 -3.483993 2.281096

H $-1.042342-1.4764800 .386473$

H $-1.019596-1.8710073 .475711$

H $-2.361456 \quad 0.1471102 .737344$

H - $0.995556 \quad 0.7190091 .817763$

H $0.4569812 .239448 \quad 4.932153$

H $-1.104304 \quad 1.0340106 .097093$

H -2.539303 0.6409005 .146418

H $-1.305370-0.5995505 .459619$

H 0.8494281 .6904571 .912622

H 0.6737003 .3048962 .597205

H 2.7131122 .9970623 .961424

H 2.8075251 .3000853 .483850

H 4.4584102 .6104802 .293089

H 3.3077484 .8277242 .169375

H 2.0786564 .1592121 .122902

H 3.4119016 .2736980 .165423

H $2.7726853 .260193-1.240422$

H 5.4454305 .6355921 .114603

H $5.9786754 .996251-0.423485$

H 5.9382203 .9452001 .006474

H $4.4589524 .281930-2.362195$

H $4.3342500 .737818-2.101290$

H $4.014285-0.264545-0.715798$

H 5.7021670 .7899350 .071186

H $3.173068-1.1799282 .426929$

H $2.972172-4.1805561 .747146$

H $5.728792-1.8197352 .779521$

H $5.242418-1.0650431 .246037$

H $6.706910-2.0319061 .336411$

H $1.5945290 .559863-0.695145$

H $1.9300571 .422896-2.197209$

H $1.369603-3.155683-3.101350$

H $\quad 1.264615-3.734025-1.424639$

H $3.168640-4.949589-2.268020$

H $4.495875-2.195729-2.410417$

H $6.149148-3.838707-3.462223$

H $5.555534-5.120682-2.431790$

H $8.360663-2.417603-0.873588$

H $7.704162-2.201254-2.591896$

H $7.432409-4.0053560 .618642$

H $6.833514-5.408806-0.216823$

H $4.625458-5.0433750 .425431$

H $2.223665-1.749382-0.607627$

C $-4.120316-3.467429-0.161116$
O $-4.427573-2.059090-0.215718$

C $-5.593421-1.8195350 .593067$

C $-6.270789-3.1748020 .743852$

C $-5.061173-4.081700 \quad 0.877188$

H $-5.233674-1.5018231 .579277$

C $-6.463005-0.717061-0.017377$

H $-6.937017-3.2153311 .610646$

H $-6.849233-3.440877-0.148360$

C $-5.367718-5.5495490 .636563$

H $-4.654911-3.9678871 .889487$

C $-5.7210670 .611288-0.279970$

O $-6.996562-1.167679-1.268218$

H $-7.330010-0.5369580 .628780$

H $-5.0542160 .499990-1.142224$

O $-6.697201 \quad 1.592632-0.648221$

C $-4.927872 \quad 1.143214 \quad 0.924810$

C $-4.358926 \quad 2.5631390 .769823$

H -4.0995010 .4601631 .136832$

O

H $-5.174504 \quad 3.2978150 .764440$

H -3.7894862 .7694001 .686162$

H -6.090946 -5.9169001 .371954$

H $-5.790154-5.713903-0.360322$

H $-4.460015-6.1552030 .722625$

C -2.622487 -3.671094 0.081135

H $-6.245474-1.403664-1.839294$

H $-7.185423 \quad 1.242176-1.413051$

H $-6.5301081 .710071 \quad 1.896579$

H -2.074548 $-3.274741-0.782495$

H $-2.394587-4.7425870 .124023$

H $-4.359439-3.865739-1.155880$

C $-1.5270521 .811781-1.564069$

O $-2.4432441 .774709-0.460238$

C $-3.4627492 .777458-0.461319$

C $-2.8418544 .185530-0.467418$

C $-1.8424844 .354524-1.614076$

C $-0.8509373 .187937-1.673631$

H $-0.7504031 .083360-1.302702$

C $-2.183327 \quad 1.359515-2.878505$

H $-4.0826902 .657702-1.355334$

$\begin{array}{llllll} & \text { O } & -2.180737 & 4.447015 & 0.770445\end{array}$

H $-3.6447214 .925626-0.563948$

H $-1.2964645 .295527-1.474203$

O $-2.562846 \quad 4.447125-2.838819$

H $-0.2567393 .230843-2.593698$

O $0.063174 \quad 3.300612-0.580390$

C $-2.521018-0.115413-2.873589$

H $-3.1066401 .910181-3.075206$

H $-1.494453 \quad 1.521495-3.715553$

H $-1.526198 \quad 3.7417720 .913120$

H $-1.9142704 .577291-3.551537$

H $\quad 0.519013 \quad 4.154972-0.668942$

O $-3.394746-0.376743-3.890172$

O $-2.056751-0.940805-2.100484$

C $-3.784256-1.746596-4.029976$

H $-2.906998-2.375955-4.207739$

H $-4.323485-2.078413-3.138666$

H $-4.451692-1.823977-4.892315$

SCF Energy (B3LYP/6-31G**//MMFF) $=-3245.92043312$

0700017

MMFF Geometry

C $2.928779-3.862368-2.455689$

C $2.064767-2.872380-2.724932$

C $0.567025-3.038179-2.706806$

O $0.024886-2.117763-1.732062$

C $-0.012230-2.671913-4.084899$

C $-1.516656-2.837239-4.180499$

C $-2.381357-1.831227-4.417687$

C $-2.034025-4.243714-4.019848$

C $-2.069860-0.378458-4.643830$

C $-2.0648910 .483957-3.372561$

C $-3.4295430 .579420-2.671423$

C $-3.3931211 .627456-1.556510$

C $-4.7189941 .690324-0.782399$

O $-3.745471-0.697494-2.111058$

$\begin{array}{lllll}0 & -4.470647 & 2.453022 & 0.408698\end{array}$

C $-5.1253440 .261501-0.356610$ 
C $-5.7889612 .458205-1.567403$

$\begin{array}{lllll}\text { O } & -6.423846 & 0.259461 & 0.230584\end{array}$

C $-5.040418-0.771272-1.499482$

C $-5.243130-2.229975-1.022945$

O $-6.075444-0.496211-2.451914$

C $0.059012-2.499739-0.426522$

O $0.463559-3.5601930 .022256$

C $-0.453455-1.3705810 .378857$

C $-0.209846-1.3385511 .697257$

C $-0.591995-0.2650302 .616552$

C -1.5242150 .7970342 .097113$

C $-4.246327-2.7228100 .031886$

C $-4.751888-2.6049811 .460601$

C -3.996610 -2.901224 3.817051

O $-5.912689-2.3226001 .744831$

C $-3.642865-1.5648474 .388871$

C -2.830752 -1.409059 5.445450

C $-2.496906-0.0790186 .057775$

C $-1.006557 \quad 0.1546796 .165478$

C $-0.352565-0.072897 \quad 7.317129$

C $-0.293040 \quad 0.731697 \quad 4.964028$

C $-0.090900-0.2810543 .869359$

N $-3.757559-2.8943192 .385698$

H $2.552910-4.854718-2.220496$

H $2.447076-1.877985-2.945260$

H $0.286791-4.064995-2.442064$

H $\quad 0.448815-3.299227-4.859470$

H $\quad 0.274529-1.641100-4.320090$

H -3.442979 -2.070883 -4.468036

H -3.101497 -4.322494 -4.252657

H -1.502793 -4.923571 -4.693935

H -1.897282 -4.590999 -2.991272

H -1.106959 $-0.266351-5.153885$

H $-2.8103540 .021936-5.348158$

H -1.316614 $0.088979-2.675618$

H $-1.7403091 .491370-3.659435$

H $-4.1829670 .846884-3.421548$

H $-3.1392312 .616137-1.958601$

H $-2.5892441 .377464-0.850827$

H -5.2815742 .4334260 .945501$

H -4.432590 -0.0306050 .440188$

H -5.448967 $3.480138-1.774305$

H $-6.7100922 .564571-0.984028$

H -6.037254 $1.991067-2.522702$

H $-7.0750160 .313808-0.489411$

H -6.280874 -2.380587 -0.701771

H -5.116286 -2.877068 -1.903013

H -6.235561 -1.300745 -2.972768

H $-0.978850-0.577783-0.140601$

H $\quad 0.358106-2.1547412 .142049$

H -2.4142320 .3393491 .652794$

H $-1.026317 \quad 1.408623 \quad 1.337022$

H -1.8912201 .4735312 .871507$

H -4.063770 -3.791894 -0.137459

H -3.285664 -2.208140 -0.062228

H $-3.391206-3.7075264 .243094$

H -5.052335 -3.117345 4.010242

H -4.094516 -0.6884773 .929912$

H -2.403206 -2.290014 5.919422

H -2.951684 -0.0488337 .056600$

H -2.9559430 .7467185 .499839$

H $\quad 0.7111630 .1185227 .411199$

H $-0.866319-0.4610818 .190521$

H $\quad 0.702878 \quad 1.092710 \quad 5.254747$

H -0.8248721 .6261724 .628480$

H $0.579299-1.0952874 .148892$

H -2.810326 -3.066996 2.061683

C $4.970890-3.766223-1.012066$

O $4.469460-2.657914-0.236063$

C $5.568616-2.0277280 .443636$

C $6.705733-3.0395990 .410653$

C $6.496575-3.667062-0.953791$

H $5.854260-1.156831-0.158971$

C $5.142959-1.5687431 .841802$

H $7.686715-2.5681630 .522346$

H $6.599722-3.7978401 .195043$

C $7.206636-4.998301-1.128058$

H $6.858182-2.967681-1.719539$
C $3.871027-0.6962961 .872598$

O $4.888135-2.7175822 .661133$

H $5.973298-1.0381992 .321900$

H $2.989792-1.3119481 .654953$

O $3.702942-0.2198293 .213894$

C 3.8889710 .5115280 .916254

C 2.6583891 .4093791 .107766

H $3.9237710 .139104-0.111814$

O 5.0731901 .2706511 .147972

H 1.7501810 .7991951 .084398

H 2.6854501 .8626442 .108136

H $8.289008-4.873892-1.019433$

H $6.876003-5.730143-0.383228$

H $7.012125-5.416590-2.120705$

C $4.420121-3.677340-2.436378$

H $4.198344-3.2441882 .221572$

H $3.671214-0.9984193 .795909$

H 5.1566221 .9170150 .426972

H $4.871972-4.449677-3.068916$

H $4.680693-2.705197-2.873788$

H $4.621353-4.682443-0.519024$

C $1.0620204 .466818-0.267833$

O 1.3358423 .2783970 .486699

C 2.5152132 .5595490 .094445

C $2.3784932 .091159-1.362944$

C $2.0637693 .258089-2.303863$

C $0.9362764 .147982-1.767053$

H 0.0762394 .7911250 .089945

C 2.0731705 .5691400 .079781

H $3.379417 \quad 3.2248270 .196706$

O $1.3519691 .106374-1.472122$

H $3.3132581 .618661-1.682980$

H $1.7794332 .855066-3.283839$

O $3.2494694 .027237-2.481981$

H $0.8750575 .079244-2.340556$

O $-0.3080023 .464412-1.947468$

C $1.5840926 .957416-0.270885$

H 2.2637795 .5517321 .159955

H $3.0302325 .402560-0.422021$

H $0.5276121 .496899-1.134091$

H $3.0436934 .743432-3.106707$

H $-0.4306433 .330000-2.902592$

O $2.5887007 .852304-0.032166$

O $0.4673997 .230350-0.688589$

C $2.2738589 .219894-0.312558$

H $2.0260459 .342274-1.371277$

H 1.4463629 .5551980 .319974

H $3.1558199 .825036-0.086655$

SCF Energy (B3LYP/6-31G**//MMFF)= -3245.91879627

\section{8}

MM̄FF Geometry

C 1.5429201 .7894512 .462008

C 1.6063990 .9234433 .484959

C $0.728683-0.2907193 .688104$

O $-0.320675-0.3030912 .688956$

C $1.496082-1.6221903 .570248$

C $2.748689-1.7441264 .411854$

C $3.992954-1.8068093 .896045$

C $2.527329-1.8501895 .896399$

C $4.352582-1.7019922 .439750$

C $4.453106-3.0823281 .780465$

C $4.342598-3.0547580 .247791$

C $5.440309-2.228311-0.420868$

C $5.264369-2.180921-1.947029$

O $3.055600-2.525993-0.088712$

O $6.144679-1.154016-2.429035$

C $3.813046-1.764068-2.271644$

C $5.713592-3.488211-2.610044$

O $3.551960-1.856765-3.670675$

C $2.743184-2.562393-1.486768$

C $1.357228-1.894707-1.682475$

O $2.708577-3.900167-1.980797$

C $-1.486518-0.9321562 .990417$

O $-1.790966-1.4522474 .051180$

C $-2.325859-0.9033321 .771051$

C $-3.506013-1.5399721 .756667$

C $-4.424179-1.6339340 .618450$ 
C $-3.970202-1.051863-0.694576$

C $0.227021-2.434351-0.794811$

C $-0.485738-3.656825-1.345329$

C $-2.288843-5.275484-0.749696$

O $-0.242142-4.139978-2.447248$

C $-3.574190-4.788860-1.342311$

C $-4.782892-5.086810-0.841352$

C $-6.075731-4.631676-1.456189$

C $-6.979105-3.924350-0.470510$

C $-8.027388-4.5571530 .083082$

C $-6.719854-2.459237-0.196468$

C $-5.611338-2.2499730 .799671$

N -1.438093 -4.137432 -0.455901

H $\quad 0.821098 \quad 1.634173 \quad 1.664923$

H $2.327057 \quad 1.1183004 .276980$

H $0.274136-0.1993584 .682864$

H $1.703350-1.8155042 .511012$

H $0.821163-2.4444683 .845794$

H $4.836266-1.9323564 .572402$

H $3.463105-1.9998496 .445387$

H $2.061751-0.9377136 .281545$

H $1.872581-2.6979286 .123221$

H $3.630089-1.0696411 .916416$

H $5.313219-1.1793692 .357339$

H $5.394933-3.5611782 .075920$

H $3.640206-3.7179902 .157068$

H $4.394004-4.095929-0.092257$

H $6.433230-2.613435-0.158232$

H $5.410337-1.195960-0.047217$

H $6.088162-1.138043-3.399259$

H $3.710952-0.703610-2.007993$

H $6.773153-3.677097-2.399845$

H $5.630770-3.428071-3.700953$

H $5.146876-4.358009-2.271301$

H $4.159668-1.249944-4.125488$

H $1.459272-0.832848-1.425782$

H $1.057910-1.948793-2.735839$

H $1.868001-4.307166-1.720576$

H $-1.938868-0.372630 \quad 0.908574$

H -3.832617 -2.048188 2.663221

H $-3.8201140 .027590-0.603927$

H $-4.676146-1.207388-1.512260$

H -3.026966 -1.507708 -1.012879

H $\quad 0.583518-2.6482850 .218080$

H $-0.534037-1.648626-0.714830$

H - $2.444005-5.8168430 .188922$

H $-1.782634-5.939368-1.457777$

H -3.506633 -4.179628 -2.240725

H $-4.851091-5.7177360 .041912$

H -6.582312 -5.519581-1.856670

H $-5.900313-3.977749-2.319460$

H -8.697284 $-4.048370 \quad 0.768358$

H -8.235718 -5.600005 -0.132056

H $-7.622258-1.9920620 .220803$

H -6.553394 -1.941316 -1.144465

H $-5.843226-2.6414611 .791478$

H $-1.617629-3.623700 \quad 0.401944$

C 3.1899223 .1389951 .062938

O $2.3011193 .144891-0.072053$

C $2.8824462 .327613-1.108141$

C $4.3414692 .135840-0.711845$

C $4.211456 \quad 2.0353330 .796530$

H $2.3791801 .355833-1.060527$

C $2.6504352 .964964-2.480030$

H 4.788731 1.248431-1.166701

H $4.9544223 .003119-0.983853$

C $\quad 5.5226192 .205637 \quad 1.540671$

H 3.7893951 .0536971 .038595

C $1.1672523 .268046-2.782222$

O $3.3678864 .204079-2.547077$

H $3.0738092 .328057-3.264811$

H $0.8070124 .044190-2.098635$

O $1.0896943 .824925-4.100420$

C $0.242660 \quad 2.036002-2.726444$

C - $-1.2250252 .332216-3.094281$

H $\quad 0.2707551 .588723-1.728124$

O $\quad 0.731738 \quad 1.043298-3.632590$

H $-1.2731702 .835677-4.067174$
H $-1.7341971 .368105-3.228214$

H $6.232201 \quad 1.423192 \quad 1.252057$

H 5.9843623 .1748061 .324966

$\begin{array}{llll}\text { H } & 5.367217 & 2.138126 & 2.622078\end{array}$

C 2.3894533 .0284272 .361844

H $3.0522324 .765935-1.818500$

H $1.6823134 .595880-4.124416$

H $\quad 0.731407 \quad 1.434209-4.523174$

H 1.7041593 .8837992 .420473

H 3.0704443 .1058913 .217359

H 3.6909724 .1163271 .057102

C -2.649738 $2.983560 \quad 0.283250$

O $-2.0719812 .337514-0.857688$

C $-1.9601153 .156819-2.026566$

C $-3.3576643 .593266-2.488312$

C $-4.1033534 .324817-1.367276$

C $-4.0409203 .561392-0.037278$

H -2.7896082 .1733791 .009660$

C -1.6603513 .9893700 .880056$

H -1.369309 $4.047199-1.789708$

O $-4.1178352 .463405-2.911096$

H $-3.2546754 .255708-3.355744$

H -5.149701 $4.460058-1.667370$

O $-3.527895 \quad 5.618844-1.211992$

H $-4.374020 \quad 4.196740 \quad 0.791067$

O $-4.9511842 .458529-0.105035$

C $-1.917865 \quad 4.216920 \quad 2.348584$

H $-0.627787 \quad 3.6370600 .765612$

H -1.6903604 .9572820 .371270$

H $-4.1627841 .839574-2.166663$

H $-4.031067 \quad 6.082713-0.521192$

H $-5.8424942 .821674-0.243791$

O -1.4853503 .1427353 .068438$

O $-2.446713 \quad 5.2246172 .799187$

C $-1.679622 \quad 3.226037 \quad 4.483134$

H -1.1031634 .0599964 .894017$

H -2.7429843 .3384414 .714828$

$\mathrm{H}-1.3201052 .2956994 .930687$

SCF Energy (B3LYP/6-31G**//MMFF) = -3245.92385422

\section{9}

MMFF Geometry

C $0.6362796 .039847 \quad 0.371958$

C $1.2143335 .151634-0.451060$

C $2.3993824 .297002-0.082264$

$\begin{array}{lllll}\text { O } & 1.946072 & 2.924639 & 0.004595\end{array}$

C $3.465756 \quad 4.380352-1.189773$

C $4.671843 \quad 3.492933-0.950379$

C $5.0342662 .468486-1.748208$

C 5.5018923 .8297040 .261252

C $4.3658442 .000486-3.011975$

C $3.293268 \quad 0.919129-2.808532$

C $3.847517-0.433326-2.331477$

C $2.746736-1.496956-2.327405$

C $3.248002-2.842744-1.780844$

O $4.360505-0.276742-1.007276$

O $2.086382-3.648102-1.527598$

C $3.968053-2.606392-0.433909$

C $4.073278-3.598949-2.828445$

O $4.582089-3.806011 \quad 0.029845$

C $4.987923-1.449552-0.472154$

C $5.539624-1.077298 \quad 0.925029$

O $6.108133-1.845315-1.273755$

C $1.3705772 .531351 \quad 1.173897$

O $1.218580 \quad 3.2022842 .182530$

$\begin{array}{lllll}\text { C } & 0.918432 & 1.132843 & 1.005484\end{array}$

C -0.0012600 .6377851 .847332$

C $-0.585461-0.7040111 .800699$

C $0.027645-1.6985010 .852272$

C $4.489153-0.6282301 .947551$

C $4.042212-1.7264682 .898932$

C $2.442945-2.1361534 .767233$

O $4.574717-2.8314032 .954150$

C $1.284478-2.9195484 .236513$

C $0.070684-2.9190674 .808467$

C $-1.084402-3.7458484 .321626$

C $-2.319836-2.9200124 .044255$

C $-3.323732-2.8529304 .934885$ 
C $-2.429850-2.2503112 .694584$ C -1.631125 -0.9794112 .607929$ N $2.997786-1.3077903 .713306$ H 1.0379066 .1930461 .369918 H $\quad 0.7760874 .983085-1.432748$ H 2.8317984 .6092220 .876352 H $3.8134475 .417190-1.290374$ H $2.9947084 .128155-2.146021$ H $5.9228471 .899373-1.477417$ H $6.427343 \quad 3.2453880 .306142$ H 5.7842574 .8874580 .245986 H 4.9431653 .6289271 .180374 H $3.9200432 .847538-3.544579$ H $5.1419781 .619654-3.688220$ H $2.5452331 .285933-2.096105$ H $2.7801500 .773159-3.767307$ H $4.660079-0.727717-3.005977$ H $2.324067-1.625117-3.331782$ H $1.915353-1.154279-1.696471$ H $2.385085-4.472200-1.105722$ H $3.187278-2.3675810 .295137$ H $3.472778-3.779105-3.728276$ H $4.366281-4.589655-2.464068$ H $4.974582-3.062197-3.132143$ H $5.402829-3.933477-0.475630$ H $6.164439-1.8907581 .312870$ H $6.225010-0.2287720 .785063$ H $\quad 6.868414-1.288943-1.035800$ H 1.3256090 .5647490 .177565 H $-0.389363 \quad 1.2864982 .631906$ H $-0.134438-1.394628-0.186105$ H $-0.368363-2.7101790 .959482$ H $1.104365-1.7755731 .030567$ H $4.933048 \quad 0.1548362 .575985$ H $3.614565-0.1935791 .454147$ H $2.152499-1.4691775 .585116$ H $3.213511-2.8218495 .134164$ H $1.461113-3.5406183 .361579$ H $-0.095762-2.3186105 .700127$ H -1.302956 -4.496895 5.092187 H $-0.818546-4.3160313 .422827$ H $-4.226324-2.2876284 .727550$ H -3.262987 -3.357917 5.893281 H -3.476011-1.986518 2.491252 H -2.170701 -2.971949 1.916497 H $-2.000615-0.1901593 .264314$ H $2.579960-0.3948023 .557966$ C -1.857802 6.0334970 .461617 O $-1.9537924 .814705-0.300771$ C -3.312665 4.618082 -0.723409 C -4.1396445 .6069570 .087051$ C -3.171835 6.7709450 .214095 H $-3.3563314 .894180-1.784818$ C $-3.7133543 .143198-0.568244$ H $-5.0741005 .876773-0.413994$ H $-4.387914 \quad 5.207917 \quad 1.077507$ C -3.5429687 .7552251 .308942$ H $-3.1304077 .300505-0.747213$ C -2.854585 2.191505-1.435878 O -3.6222142 .7789060 .808356$ H $-4.7643373 .034394-0.860224$ H -2.895631 $2.535128-2.477178$ O $-1.4862342 .242269-1.030634$ C $-3.311648 \quad 0.717470-1.399493$ C $-2.411098-0.164279-2.290169$ H $-4.3501000 .658833-1.745977$ O $\quad-3.274655 \quad 0.245282-0.057417$ H $-2.3438750 .289053-3.286523$ H -1.404347 $-0.181622-1.855541$ H $-4.520774 \quad 8.204498 \quad 1.106817$ H $-3.593511 \quad 7.2665512 .287771$ H -2.806612 8.5625811 .373007 C $-0.612457 \quad 6.799360 \quad 0.010945$ H -2.683147 2.8232161 .058804 H -1.171476 $3.152843-1.157833$ H $-3.592646-0.672804-0.054987$ H -0.5708297 .7869110 .483544$ H $-0.6424206 .960441-1.074155$
H -1.7700405 .7437711 .517164$

C $-3.269678-3.609910-1.096519$

O $-2.807786-2.254750-1.157226$

C $-2.907703-1.616779-2.434019$

C $-2.084581-2.388319-3.479754$

C -2.486532 -3.865205 -3.525847

C $-2.536643-4.484694-2.125471$

H $-2.979682-3.960757-0.098412$

C $-4.796011-3.696746-1.195470$

H $-3.957987-1.585325-2.743204$

O $-0.697282-2.282788-3.163489$

H -2.236470 -1.924565 -4.461901

H $-1.765521-4.415428-4.142586$

O $-3.762658-3.967539-4.150532$

H -3.007883 -5.473074 -2.165439

O $-1.197884-4.694348-1.664924$

C $-5.440618-3.0657970 .016014$

H $-5.200691-3.222339-2.093875$

H $-5.125228-4.742318-1.225926$

H $-0.200775-2.751287-3.855845$

H $-3.991996-4.911411-4.196045$

H $-0.780074-3.822805-1.554540$

O $-5.801167-1.780952-0.263338$

O $-5.581452-3.6392911 .088365$

C $-6.403983-1.0602770 .816013$

H $-7.338899-1.5424171 .116896$

H -5.713085 -0.9937811 .661634$

H $-6.626224-0.0486330 .466026$

SCF Energy (B3LYP/6-31G**//MMFF) $=-3245.92041412$

\section{0}

MM̄FF Geometry

C -1.204095 2.2178821 .825927

C -0.7905933 .4955991 .836018$

C $0.632498 \quad 3.9780921 .693527$

O 1.5371472 .8764001 .937204

C 0.9094154 .5216340 .273728

C $0.0873755 .724704-0.137822$

C $-0.8561135 .724872-1.101436$

C $0.407976 \quad 6.9998990 .599503$

C $-1.3163104 .573542-1.955009$

C $-2.6946864 .086650-1.498717$

C $-3.2067792 .870162-2.286104$

C $-4.5433662 .407070-1.691318$

C $-5.0463121 .089515-2.295611$

O $-2.225579 \begin{array}{lll}1.829859 & -2.207279\end{array}$

O $-6.0604520 .564085-1.424088$

C $-3.8886430 .069727-2.303852$

C $-5.7209211 .317180-3.653355$

O $-4.279276-1.132216-2.965179$

C $-2.5797820 .630716-2.906929$

C $-1.432692-0.395167-2.728692$

O $-2.7903220 .863082-4.302640$

C 2.8128053 .1716612 .298447

O 3.2841844 .2707472 .538193

C 3.5655261 .9022642 .263647

C 4.8491141 .9304601 .878925

C 5.6793140 .7558291 .615065

C $5.178580-0.5770332 .104529$

C $-0.0518040 .074284-3.212329$

C $0.8234230 .704730-2.141104$

C $3.1731661 .524971-1.835374$

O $0.4482750 .912806-0.992074$

C $4.1620810 .427752-1.588468$

C $5.4155800 .418906-2.068501$

C $6.404323-0.687693-1.814820$

C $7.681121-0.198929-1.159955$

C $8.7619730 .105891-1.897590$

C $7.726033-0.1351580 .354338$

C 6.8071330 .9237940 .894035

N $2.0874350 .997152-2.641082$

H -0.4853551 .4152691 .701974$

H -1.5384384 .2803061 .934990$

H 0.8046304 .7477922 .455697

H $0.8075763 .696911-0.439201$

H 1.9694394 .7973450 .192509

H -1.376210 $6.660157-1.306301$

H -0.1465497 .8587720 .206691$ 
H $\quad 0.157626 \quad 6.905127 \quad 1.660557$ H $1.474564 \quad 7.231162 \quad 0.511677$ H - $1.3722254 .914133-2.996086$ H $-0.5966413 .751885-1.946352$ H -2.633469 $3.821293-0.437296$ H -3.422813 $4.903982-1.579500$ H -3.332928 $3.161432-3.335657$ H -5.302195 $3.193057-1.795759$ H $-4.4280582 .259464-0.609717$ H -6.761614 $1.232942-1.341873$ H -3.697155 - $0.210319-1.258946$ H -6.583892 $1.985437-3.544256$ H -6.120390 $0.381789-4.060010$ H $-5.0540291 .764046-4.393498$ H $-4.260027-0.962971-3.922442$ H $-1.367310-0.688536-1.675231$ H -1.684199 -1.295977 -3.303541 H -1.980181 $0.668555-4.797599$ H 3.0186390 .9835572 .439330 H 5.3051422 .8932061 .651476 H $4.296516-0.8863851 .535725$ H $4.909450-0.5220873 .165653$ H $5.917412-1.3768392 .022773$ H $0.489943-0.808284-3.576070$ H $-0.1264500 .778231-4.046953$ H $2.7885541 .904926-0.884644$ H $3.6143862 .358564-2.390495$ H $3.831314-0.396574-0.962719$ H $5.7485371 .242071-2.696524$ H $6.637473-1.153333-2.781363$ H $5.963987-1.485791-1.203909$ H $9.6881580 .432722-1.436753$ H $8.7425380 .039188-2.980464$ H 8.7407740 .0983650 .701460 H $7.507318-1.1317110 .746654$ H 7.0826501 .9344320 .588391 H $2.2966170 .766666-3.608334$ C -2.919336 0.7759522 .995782 O $-2.545722-0.5345142 .504616$ C $-3.718914-1.3651922 .503015$ C $-4.610301-0.7796883 .587055$ C -4.4127390 .7002183 .342075$ H -4.205691 -1.225786 1.529864 C -3.378732 -2.844515 2.702612 H -5.653089-1.095809 3.489602 H $-4.259101-1.0472264 .590418$ C -4.8086451 .5724394 .521509$ H -5.0170400 .9924142 .472003$ C $-2.389050-3.4506201 .687569$ O $-2.817202-3.0325814 .008406$ H -4.308428 -3.4262032 .695223$ H -1.368964 -3.133555 1.930048 O $-2.418506-4.8735671 .866055$ C $-2.682150-3.1371240 .210765$ C $-1.909659-3.995292-0.809767$ H $-2.469733-2.0779280 .028823$ O $-4.076932-3.333563-0.041734$ H $-2.326814-5.010826-0.826375$ H $-2.126909-3.576269-1.801475$ H -5.8726301 .4516914 .749419$ H -4.2404791 .3128145 .421089$ H -4.6268192 .6293404 .301311$ C -2.6503941 .8223121 .910482$ H -2.032627 -2.461359 4.073922 H $-2.210864-5.0528672 .799162$ H $-4.253269-3.043101-0.953152$ H -3.265138 2.7124592 .090698 H -2.934174 1.4124740 .934409 H -2.314852 0.9670903 .890910 C $1.542558-2.633457-0.342315$ O $0.134067-2.741571-0.594110$ C $-0.390834-4.070131-0.598490$ C $0.295578-4.913100-1.685474$ C $1.819569-4.892104-1.524624$ C $2.353084-3.467684-1.350413$ H $1.776521-1.578542-0.521471$ C $1.889185-2.9425611 .123258$ H $-0.205969-4.5360540 .373692$
O $-0.050336-4.431721-2.983200$

H $-0.070859-5.943979-1.618780$

H $2.285754-5.343695-2.409014$

O $2.194174-5.665907-0.388923$

H $3.407512-3.493858-1.051097$

O $2.296642-2.823052-2.626186$

C $1.333403-1.8971032 .067147$

H $1.495863-3.9108411 .442199$

H $2.977762-2.946752 \quad 1.247041$

H $\quad 0.200464-3.493118-3.028754$

H $1.873383-6.572644-0.532717$

H $2.696080-1.942071-2.529742$

O $1.664399-2.2319313 .349019$

O $\quad 0.691322-0.9127501 .730203$

C $1.191301-1.3328974 .357112$

H $\quad 0.100798-1.251923 \quad 4.315425$

H $1.475515-1.7360325 .332709$

H $1.655993-0.3503024 .233880$

SCF Energy (B3LYP/6-31G*//MMFF) $=-3245.90594160$

\section{1}

MM̄FF Geometry

C $3.161296-3.0765811 .821459$

C $2.107448-3.115528 \quad 0.994403$

C $0.966576-4.0857651 .113810$

O $-0.198744-3.2451791 .295512$

C $0.780580-4.937891-0.156247$

C $2.014268-5.694879-0.603354$

C $2.755369-5.384916-1.686846$

C $2.383126-6.8777810 .253517$

C $2.539908-4.246552-2.644611$

C $3.665912-3.206962-2.549071$

C $3.231315-1.856679-3.136444$

C $4.402632-0.876537-3.182505$

C $3.9593520 .507038-3.681329$

O $2.208055-1.333754-2.283822$

O $5.0153191 .438805-3.398505$

C $2.7287330 .966771-2.869488$

C $3.766415 \quad 0.522955-5.202258$

O $2.1774182 .165110-3.410201$

C $1.619999-0.107107-2.730563$

C $0.6012320 .334326-1.652454$

O $0.899337-0.286196-3.947840$

C -1.344279 -3.790624 1.772324

O $-1.540582-4.9490482 .095973$

C $-2.341729-2.6988431 .795731$

C $-3.650963-2.9837101 .770704$

C $-4.736129-2.0011721 .719020$

C $-4.358942-0.5432051 .774796$

C $-0.499530-0.688203-1.333475$

C $-1.698587-0.637270-2.265598$

C $-3.882703-1.771443-2.676462$

O $-1.8181930 .183093-3.170835$

C $-4.979057-1.062218-1.943308$

C $-6.168414-1.623549-1.674282$

C $-7.296316-0.909227-0.983493$

C $-7.908469-1.7194130 .139342$

C $-9.032970-2.429168-0.055804$

C $-7.267794-1.637104 \quad 1.506974$

C $-6.003964-2.4457191 .596366$

N $-2.634798-1.614807-1.951526$

H $3.242455-3.8011692 .627388$

H $2.030653-2.3704030 .206710$

H $1.087452-4.723767 \quad 1.998224$

H $\quad 0.375220-4.305417-0.952781$

H $-0.023590-5.6662110 .015051$

H $3.620141-6.006387-1.915063$

H $3.254421-7.416584-0.133781$

H $2.621591-6.5552701 .271874$

H $1.550538-7.5875540 .298733$

H $2.509418-4.650468-3.664194$

H $1.570048-3.769316-2.490844$

H $3.949504-3.064626-1.498755$

H $4.549927-3.584348-3.077755$

H $2.822243-2.023682-4.139651$

H $5.215617-1.268114-3.806485$

H $4.825430-0.763193-2.174113$

H $5.8223861 .120090-3.837840$ 
H $3.0859611 .217363-1.860720$ H $4.7092780 .286157-5.710103$ H $3.479411 \quad 1.519529-5.554920$ H $3.020290-0.196219-5.546887$ H $2.8914902 .824795-3.439675$ H $1.1487720 .509777-0.716911$ H $0.1401761 .283097-1.948055$ H $1.2237690 .352850-4.601643$ H -1.963753 -1.6824131 .783758$ H -3.956179 -4.029062 1.755817 H $-3.866818-0.3099282 .725447$ H -5.2078630 .1363841 .680647$ H -3.675850 -0.2937960 .956402$ H $-0.100045-1.707318-1.319954$ H $-0.881596-0.469438-0.328043$ H -4.073181 -2.845577 -2.765267 H $-3.777646-1.348971-3.680876$ H -4.797811 -0.031728 -1.646467 H -6.358318 -2.642642 -2.004299 H -8.061797 -0.690640 -1.739710 H $-6.9753770 .065783-0.596218$ H -9.495145 -2.9899790 .749736$ H -9.513652 -2.469300 - 1.027688 H -7.954874 -2.024979 2.271000 H -7.124917 -0.5864011 .773681$ H $-6.174213-3.5223891 .553248$ H -2.476750 -2.213379-1.146180 C $4.132877-1.0458202 .885714$ O $2.902311-0.3019262 .776245$ C 3.1365221 .0509683 .210656 C 4.5675091 .0916873 .738850 C 5.2334110 .0107412 .907806 H 3.0863341 .6799632 .315131 C 2.0532091 .4773694 .208928 H 5.0265032 .0767133 .609388 H 4.6226410 .8367854 .802442 C $6.551078-0.4738623 .485862$ H 5.4025040 .3997621 .894617 C 0.6156121 .3880483 .651974 O $2.096887 \quad 0.618327 \quad 5.355939$ H 2.2473262 .4947304 .569546 H 0.3393560 .3419363 .472246 O $-0.272611 \quad 1.869730 \quad 4.670083$ C 0.3728522 .2135362 .374293 C -1.111032 2.2257891 .949275 H $\quad 0.967047 \quad 1.7973941 .554326$ O 0.8278993 .5485312 .589220 H -1.4693131 .1921341 .876591$ H -1.695419 2.739171 2.722941 H 7.2696320 .3500413 .548215 H $6.421208-0.8835024 .493231$ H $6.989172-1.2561272 .858290$ C $4.239346-2.0328311 .723959$ H 2.8454990 .8983195 .908380 H -0.1173451 .3326915 .465968$ H $\quad 0.2935703 .9291913 .307367$ H $5.211775-2.5368151 .731974$ H $4.157984-1.4950240 .770843$ H 4.098402 -1.584399 3.842618 C $-1.1380715 .139759-0.397717$ O $-1.046612 \quad 4.3300090 .779929$ C -1.344849 2.9430600 .603338 C -2.7865722 .7701900 .099432$ C $-3.0535263 .611998-1.154738$ C -2.549354 5.050903-1.003089 H $-0.9832576 .166322-0.041758$ C $-0.0226174 .824502-1.401708$ H $-0.6519542 .517699-0.127001$ O -3.6976293 .1496541 .129286$ H -2.957241 $1.709770-0.117228$ H $-4.1309763 .632185-1.359930$ O $-2.4124963 .018071-2.278430$ H -2.570411 $5.560995-1.973376$ O $-3.4584975 .743971-0.143178$ C $1.3388465 .056048-0.788827$ H $-0.0676753 .797512-1.773658$ H $-0.0983315 .471903-2.283191$ H -4.5979242 .9917350 .797779$
H $-2.7736442 .121471-2.384462$

H -3.169828 $6.671366-0.096586$

O $1.9714323 .863525-0.594726$

O $1.7796806 .162161-0.504494$

C $3.2770843 .942972-0.015436$

H 3.6647462 .9263090 .089365

H $3.9472604 .507907-0.670073$

H 3.2255084 .4026890 .976055

SCF Energy (B3LYP/6-31G**//MMFF) $=-3245.90158960$

0700022

MMFF Geometry

C $-2.918037-3.4696881 .198293$

C $-2.075475-3.0298942 .145870$

C $-0.605910-2.8165251 .908029$

O $-0.279264-1.5251392 .482621$

C $0.283704-3.8725302 .586348$

C $0.212961-5.2576231 .980881$

C $0.926960-5.646088 \quad 0.905518$

C $-0.661541-6.243942 \quad 2.708585$

C $1.836254-4.7934860 .065003$

C $3.306796-4.9590650 .462539$

C $4.248016-4.150737-0.445884$

C $5.697512-4.3019690 .016314$

C $6.653068-3.426542-0.806531$

O $3.864038-2.775697-0.383714$

O $7.924339-3.406226-0.139463$

C $6.111987-1.978521-0.858959$

C $6.929342-4.044616-2.182495$

O $6.903781-1.198276-1.750852$

C $4.616777-1.895084-1.238995$

C $4.004895-0.495134-1.000702$

O $4.445705-2.241684-2.614210$

C $0.737184-0.8251151 .912260$

O $1.385370-1.143714 \quad 0.929026$

C 0.9341450 .4115292 .701320

C 1.8147921 .3291942 .275050

C 2.1196312 .6095112 .916950

C 1.4193102 .9207844 .213831

C $4.6023510 .661150-1.803133$

C $3.7319831 .906958-1.743483$

C $3.7113334 .298326-2.435610$

O $2.6163411 .928234-1.231729$

C $4.0379845 .088033-1.207003$

C $3.1091795 .613960-0.393397$

C $3.435567 \quad 6.453297 \quad 0.807930$

C 2.8058005 .9370222 .082136

C 1.7344726 .5468722 .617055

C 3.4776094 .7839952 .793013

C 2.9950203 .4424822 .314318

N $4.3416872 .994519-2.351590$

H -2.524258 -3.7318530 .219744$

H $-2.460097-2.794256 \quad 3.135194$

H $-0.414483-2.786630 \quad 0.829189$

H $\quad 0.050092-3.921273 \quad 3.659022$

H $1.327014-3.5295082 .568437$

H $\quad 0.843420-6.6799050 .573608$

H $-0.648972-7.2342252 .240990$

H $-0.318925-6.3631803 .741852$

H -1.700472 -5.9029162 .725454$

H $1.700846-5.082471-0.984550$

H $1.546881-3.7411360 .123922$

H $3.426384-4.6229531 .500966$

H $3.587571-6.0191940 .428806$

H $4.128077-4.512241-1.473893$

H $6.011709-5.352569-0.017569$

H $5.773689-4.0024701 .071074$

H $7.793843-3.0408090 .752166$

H $6.234941-1.5359660 .139014$

H $7.375128-5.040421-2.070164$

H $7.661104-3.453066-2.743879$

H $6.030857-4.151062-2.793935$

H $7.828372-1.270260-1.457763$

H $2.932417-0.562672-1.231939$

H $4.051826-0.2532960 .069408$

H $3.603086-1.868281-2.921734$

H $\quad 0.3402100 .538542 \quad 3.599066$

H 2.3647611 .1306661 .357400 
H $\quad 0.332172 \quad 2.9113934 .077444$

H 1.6689873 .9028894 .620497

H 1.6834702 .1824424 .978951

H $5.5889780 .920721-1.404821$

H $4.7092720 .384030-2.857700$

H $2.6317394 .168739-2.567391$

H $4.1132274 .801510-3.320358$

H $5.0909055 .256652-0.993507$

H $2.0560445 .464940-0.620976$

H 3.0835627 .4728140 .602303

H 4.5197306 .5413290 .951764

H 1.2976586 .2063953 .549825

H 1.2660357 .3963152 .131235

H 3.3597184 .8883503 .877039

H 4.5627454 .8386592 .636687

H 3.4432293 .1291611 .371430

H $5.2959112 .905118-2.688513$

C $-5.208859-3.238158 \quad 0.176583$

O $-5.101070-1.820753-0.074491$

C $-6.395875-1.319632-0.460289$

C $-7.289032-2.541235-0.656840$

C $-6.703045-3.5140620 .342707$

H $-6.774109-0.7440530 .392145$

C $-6.307341-0.425780-1.704304$

H -8.343811 -2.316005 -0.471302

H -7.207552 -2.945303 -1.672804

C -7.103903 -4.9570490 .087620$

H $-7.030020-3.2215161 .349676$

C $-5.4083210 .821007-1.580377$

O $-5.810262-1.200212-2.805000$

H $-7.318381-0.114866-1.993924$

H $-4.3543850 .525861-1.602184$

O $\quad-5.6238781 .617585-2.754110$

C $-5.6736901 .697079-0.345319$

C $-5.0297453 .097493-0.381694$

H $-5.331925 \quad 1.1632020 .549011$

O

H $-5.5531173 .728838-1.111673$

H -5.2324803 .5574820 .594817$

H -8.191655 -5.0690860 .142853$

H $-6.781231-5.293926-0.903268$

H $-6.659159-5.6249950 .831625$

C -4.394535 -3.640733 1.410896

H $-4.941843-1.552944-2.545053$

H $-5.4378981 .053899-3.524578$

H -7.2365262 .3803330 .617499$

H -4.579403 -4.696043 1.641421

H -4.716932 -3.0453422 .274017$

H -4.822995 -3.747634 -0.716616

C -1.4500492 .1431790 .121234$

O $\begin{array}{llll}\text { O } & -8.870498 & 2.270109 & 0.274302\end{array}$

C $-3.5188273 .127889-0.665415$

C $-2.9495624 .555655-0.575429$

C -1.422473 $4.567297-0.709424$

C $-0.765503 \quad 3.5184250 .193845$

H -1.1325991 .5651410 .996126$

C -1.066135 $1.346425-1.135566$

H -3.354439 2.743611 -1.676750

O -3.3103035 .1602750 .666137$

H -3.398270 5.165071-1.368340

H -1.040845 $5.560572-0.442641$

O $-1.0536684 .310203-2.060595$

H $\quad 0.297563 \quad 3.410907-0.050628$

O -0.8427953 .9616301 .551231$

C -1.522869 -0.093259-1.070420

H -1.496711 $1.778864-2.041984$

H $0.0242621 .333506-1.233927$

H $-2.983394 \quad 4.5876581 .381250$

H -1.455065 5.003271 -2.611871

H -0.3522044 .7986961 .612985$

O $-1.096100-0.741929-2.194032$

O $-2.175544-0.584878-0.161520$

C - $-1.470823-2.118898-2.298977$

H -2.541471 -2.248632 -2.113574

H -1.251707 -2.453157 -3.316496

H $-0.880557-2.716195-1.599042$

SCF Energy (B3LYP/6-31G**//MMFF) $=-3245.91916212$
0700023

MMFF Geometry

C $2.9636901 .498597 \quad 3.720688$

C $2.1329200 .525423 \quad 3.317396$

C 0.6380500 .5793913 .497938

O 0.0206230 .5085562 .190420

C $0.151559-0.6214444 .327950$

C $-1.357608-0.6924114 .479335$

C $-2.098658-1.7695994 .152114$

C -2.0285810 .5289745 .052976$

C $-1.603882-3.0982493 .657149$

C -1.546567 -3.238532 2.130351

C $-2.925847-3.1982061 .454488$

C $-2.844642-3.7774840 .040394$

C $-4.194478-3.702928-0.685393$

O $-3.363424-1.8359831 .386330$

O $-3.962271-3.974095-2.075998$

C $-4.726452-2.260388-0.587762$

C $-5.174618-4.772510-0.190935$

O $-6.048540-2.166812-1.113883$

C $-4.680764-1.6722510 .843962$

C $-5.002766-0.1621930 .780776$

O $-5.664557-2.320921 \quad 1.652305$

C -0.0602301 .6735381 .490838$

O 0.3649182 .7657331 .834251

C -0.7691491 .3971290 .221766$

C $-0.8851842 .385077-0.678299$

C $-1.5801872 .321929-1.964468$

C $-2.1644111 .001537-2.387275$

C -4.7097770 .6154042 .068313$

C -5.1772542 .0497211 .914460$

C -4.6575474 .1491590 .664953$

O -6.2166562 .4470152 .435756$

C $-4.8976694 .112076-0.813415$

C $-4.3313754 .966143-1.679772$

C $-4.5481034 .912326-3.166595$

C $-3.2524914 .932112-3.952174$

C $-2.8868876 .020450-4.650686$

C $-2.4130753 .669087-3.985836$

C $-1.6668903 .450960-2.698581$

N -4.3394842 .8035721 .107881$

H 2.5626612 .3689264 .234000

H $2.535726-0.3337882 .785798$

H $\quad 0.338463 \quad 1.510323 \quad 3.996054$

H $0.597292-0.5796415 .330426$

H $0.530611-1.5361263 .859358$

H -3.178237-1.713191 4.285092

H $-3.084490 \quad 0.3517635 .284392$

H -1.537238 0.8290935 .984157

H $-1.984366 \quad 1.3638814 .346954$

H $-0.613675-3.3171564 .072033$

H -2.257048 -3.8785984 .068701$

H $-0.901707-2.4531201 .715875$

H $-1.068259-4.2022431 .915620$

H -3.629878 -3.7794012.061288

H $-2.478691-4.8115730 .059545$

H -2.108868 -3.207315 -0.542349

H $-3.558945-4.856374-2.145734$

H -4.092102 -1.638628 -1.235261

H $-4.754337-5.774462-0.339221$

H $-6.106227-4.750747-0.767294$

H -5.422069 -4.671722 0.867872

H -6.028146 -2.530841 -2.015558

H $-4.4112330 .295054-0.019933$

H $-6.058492-0.0210130 .514515$

H $-5.926222-1.7248552 .371697$

H -1.1836300 .4070690 .071398$

H $-0.4442763 .353167-0.442440$

H $-3.0089040 .733752-1.747311$

H $-1.4125840 .207287-2.327231$

H $-2.5204960 .996853-3.419894$

H -5.2244950 .1810172 .931170$

H -3.6378330 .6135232 .294262$

H $-3.8082114 .787107 \quad 0.928733$

H $-5.5510234 .514593 \quad 1.179942$

H $-5.5673253 .341765-1.189568$

H -3.681922 $5.752626-1.301665$

H $-5.1687685 .774732-3.442235$ 
H $-5.1241774 .023569-3.453122$ H -1.972161 $6.036836-5.233982$ H -3.491088 $6.921532-4.651399$ H -1.672197 $3.729195-4.793161$ H $-3.0718512 .833365-4.238779$ H -1.167389 4.346896 -2.326113 H $-3.4972642 .374827 \quad 0.736286$ C 4.8057732 .5407102 .399985 O 4.1756722 .1920591 .149981 C 5.1670042 .1955770 .110374 C 6.3158673 .0321120 .652376 C 6.3071152 .6159542 .112191 H $5.5097591 .160512-0.002821$ C $4.5544622 .673961-1.208912$ H 7.2632802 .8175720 .149169 H 6.1145384 .1058410 .564117 C 7.0596233 .5693593 .023726 H 6.7565291 .6170672 .194246 C $3.2578351 .938852-1.611406$ O $4.2400384 .068647-1.111561$ H $5.2982152 .590627-2.009737$ H $2.4282342 .264177-0.971957$ O $2.9181102 .342990-2.943173$ C $3.3504480 .402986-1.574456$ C $2.070793-0.276339-2.098335$ H $3.5218270 .079056-0.542495$ O $4.472208-0.026668-2.343656$ H $\quad 1.1967670 .217506-1.663054$ H $2.026015-0.160577-3.188291$ H $8.115623 \quad 3.6207732 .739074$ H 6.6479994 .5829482 .972104 H 7.0053193 .2361304 .064955 C 4.4428281 .4912363 .453472 H $3.6328144 .180844-0.359876$ H $2.8491683 .313243-2.942485$ H $4.336557 \quad 0.277918-3.257210$ H $4.9704101 .692164 \quad 4.392421$ H 4.7540740 .4966373 .109689 H 4.4149913 .5254322 .686933 C $3.205710-3.839046-2.196682$ O $3.061013-2.439081-2.464619$ C $2.017721-1.775957-1.746592$ C $0.654890-2.397550-2.089305$ C $0.660801-3.911812-1.854306$ C $1.889969-4.584414-2.477012$ H $3.950749-4.187046-2.923840$ C $3.765170-4.095516-0.792536$ H $2.197254-1.874395-0.670192$ O $0.338907-2.128185-3.453622$ H $-0.114945-1.921509-1.470216$ H $-0.250374-4.345819-2.283942$ O $0.636674-4.151122-0.450664$ H $1.975186-5.618464-2.124822$ O $1.705699-4.653195-3.894330$ C $5.130758-3.467380-0.636521$ H $3.110188-3.713954-0.004127$ H $3.877711-5.170033-0.607059$ H $-0.547485-2.489084-3.625738$ H $0.599315-5.113602-0.317256$ H $1.698725-3.743115-4.237624$ O $5.075945-2.4509010 .271068$ O $6.122895-3.828877-1.256113$ C $6.310100-1.7662930 .505752$ H $6.138475-1.0124671 .278653$ H $7.072351-2.4656100 .862408$ H $6.642193-1.264659-0.407864$ SCF Energy (B3LYP/6-31G**//MMFF)= -3245.91529305

\section{4}

MM̄FF Geometry

C 4.0188210 .5754893 .637900

C $3.075953-0.2853473 .224144$

C $1.627335-0.2155643 .634742$

$\begin{array}{lllll}\text { O } & 0.833497 & 0.021777 & 2.447957\end{array}$

C $1.187080-1.5638554 .233363$

C $-0.263299-1.5886694 .676952$

C -1.214671 -2.379768 4.142520

C $-0.614526-0.663575 \quad 5.814228$
C $-1.070429-3.3926193 .041185$

C $-1.383690-2.8636721 .633789$

C $-2.843405-2.431640 \quad 1.426914$

C $-3.110729-2.136009-0.050155$

C $-4.539335-1.620963-0.283915$

O $-3.085559-1.2531882 .196383$

O $-4.592519-1.068258-1.608871$

C $-4.836291-0.4679190 .701729$

C $-5.564561-2.761129-0.249700$

O $-6.211083-0.1007440 .612094$

C $-4.443267-0.7896442 .160751$

C $-4.5239660 .429728 \quad 3.112114$

O $-5.338560-1.7823652 .676933$

C 0.7041421 .3080532 .023703

O 1.1767752 .3075672 .540391

C $-0.096717 \quad 1.285550 \quad 0.780260$

C $-0.0517202 .334667-0.054093$

C $-0.7249022 .432332-1.350805$

C $-1.7178751 .357204-1.702132$

C -3.6445451 .6244422 .726826$

C -4.3679432 .6880021 .918054$

C -3.9431714 .8124450 .687453$

O -5.5824072 .6873691 .736228$

C $-3.8407864 .508924-0.773748$

C $-3.2045355 .303939-1.647109$

C $-3.1293955 .036311-3.122755$

C $-1.7093105 .033122-3.641883$

C $-1.1881296 .119449-4.236915$

C $-0.9262183 .743554-3.548533$

C $-0.4221043 .469392-2.159322$

N $-3.4965293 .663266 \quad 1.452059$

H 3.7548331 .3752874 .324752

H $3.347704-1.0651182 .516190$

H 1.4614880 .5901594 .360190

H $1.815544-1.8081395 .100197$

H $1.373187-2.3509383 .494603$

H -2.223079 -2.309284 4.549181

H -1.631381 -0.8297526 .185812$

H $\quad 0.069009-0.8188076 .655351$

H -0.5492810 .3815685 .496908$

H $-0.063344-3.8230193 .040765$

H -1.737224 -4.234957 3.266075

H -0.710682 -2.028402 1.407872

H -1.155842 -3.6651250 .922846$

H -3.494758 -3.240415 1.778645

H $-2.919953-3.023339-0.666320$

H -2.403579 -1.372775 -0.401519

H $-4.340525-1.768019-2.235750$

H $-4.2579130 .394648 \quad 0.352107$

H -5.328519 -3.511314-1.014211

H $-6.569518-2.396919-0.490015$

H $-5.603413-3.2735880 .713804$

H -6.3380610 .7112151 .130366$

H $-5.5685980 .726166 \quad 3.263644$

H -4.1765220 .0906544 .099075$

H $-5.316888-1.742743 \quad 3.647483$

H $-0.661306 \quad 0.3866240 .561643$

H $0.570578 \quad 3.187200 \quad 0.215585$

H $-2.4509761 .238625-0.897986$

H $-1.2094390 .400129-1.859435$

H $-2.295867 \quad 1.572757-2.602959$

H -3.310130 2.1187373 .648120

H $-2.750074 \quad 1.2985672 .187584$

H -3.3160845 .6605820 .979985$

H -4.9823345 .0411030 .944794$

H $-4.3342073 .609086-1.133586$

H -2.735794 $6.217743-1.288264$

H $-3.7108195 .816077-3.632181$

H $-3.6156014 .088266-3.384370$

H $-0.1798156 .116120-4.637561$

H $-1.7569507 .038516-4.331687$

H $-0.0467513 .790533-4.205064$

H -1.530487 2.927408 -3.954127

H $0.3112254 .199081-1.812820$

H -2.4978423 .5522991 .602279$

C 5.6787791 .5858942 .070058

O $4.855637 \quad 1.2673020 .932407$

C $5.6392101 .369996-0.269091$ 
C $6.893442 \quad 2.136957 \quad 0.125965$

C 7.1126741 .6285521 .541651

H $5.9261080 .348370-0.546076$

C $4.7834891 .983437-1.384564$

H $7.7355881 .930310-0.540897$

H $6.724227 \quad 3.2196650 .136321$

C $8.046107 \quad 2.4985872 .364621$

H 7.5243890 .6117001 .489064

C $3.3906191 .329632-1.517325$

O $4.5730773 .373936-1.105241$

H $5.3208531 .943708-2.338274$

H $2.809167 \quad 1.532970-0.610923$

O $2.6924831 .964525-2.594556$

C $3.415928-0.187413-1.779849$

C $2.005770-0.791743-1.695767$

H $4.048030-0.676407-1.034208$

O $3.993002-0.417623-3.061830$

H $1.599000-0.620414-0.691697$

H $1.332447-0.255037-2.377724$

H 9.0400032 .5363361 .906853

H 7.6731323 .5253262 .443108

H 8.1553942 .1013803 .378737

$\begin{array}{llll}\text { C } 5.444226 & 0.532953 & 3.154637\end{array}$

H $4.1398873 .437528-0.236427$

H $2.6853292 .919820-2.412044$

H $4.127814-1.373803-3.165632$

H 6.1105740 .7010414 .007649

H $5.669636-0.4654072 .758716$

H 5.3652772 .5772062 .422762

C $0.156286-3.987690-2.112821$

O $0.538644-2.659001-1.735016$

C $1.891449-2.287116-2.041617$

C $2.858699-3.211116-1.288705$

C $2.592772-4.675815-1.657307$

C $1.111532-5.040235-1.523123$

H $-0.828319-4.136400-1.651124$

C $-0.023661-4.103246-3.630023$

H $2.052985-2.385723-3.120443$

O $2.703363-3.059964 \quad 0.120526$

H $3.895454-2.963658-1.540434$

H $3.182463-5.329792-1.003099$

O $3.002413-4.914206-3.001072$

H $0.917705-6.011474-1.992295$

O $0.806757-5.199652-0.134383$

C $-1.184531-3.248194-4.082116$

H $0.864362-3.815284-4.199822$

H $-0.252182-5.136792-3.916158$

H $2.978182-2.1591250 .358394$

H $3.952984-4.717246-3.057499$

H $\quad 0.979023-4.3501330 .306744$

O $-0.751983-1.986623-4.367232$

O $-2.340671-3.646532-4.141918$

C - $1.769557-1.059539-4.757420$

H $-2.226533-1.376551-5.699499$

H $-2.525400-0.970315-3.971420$

H - $1.301088-0.082527-4.904076$

SCF Energy (B3LYP/6-31G*//MMFF) $=-3245.91408254$

0700025

MM̄FF Geometry

C $0.309493-3.0928510 .040472$

C $-0.380500-3.823108 \quad 0.930793$

C $-1.042921-3.3178282 .193151$

O $-0.904514-1.8825062 .286541$

C -2.545683 -3.660057 2.141580

C $-3.322297-3.2210873 .367565$

C $-4.076675-2.1050353 .426934$

C $-3.233993-4.1471264 .550900$

C $-4.222092-1.0623692 .354782$

C $-5.621437-1.0493811 .732315$

C $-5.640788-0.198716 \quad 0.452810$

C $-7.0726680 .025268-0.031587$

C $-7.1076020 .775426-1.370801$

O $-4.893493-0.910662-0.540538$

O $-8.4399180 .679018-1.896441$

C $-6.1748350 .056365-2.366586$

C -6.814544 2.270648 -1.198333

O $-6.0676220 .793011-3.583334$
C $-4.764860-0.237773-1.799629$

C $-4.011567-1.192737-2.761535$

O $-4.0451390 .986931-1.671441$

C $-0.474192-1.339775 \quad 3.453234$

O $-0.095597-1.945140 \quad 4.446666$

C -0.5169910 .1423953 .414266$

C -1.1058640 .8382182 .429368$

C -1.2262332 .2943092 .335031$

C -0.5633593 .1256873 .400553$

C $-2.613588-1.618698-2.290072$

C $-1.528125-0.724941-2.860839$

C $0.2809630 .850720-2.194434$

O $-1.368203-0.592416-4.072568$

C $-0.3293092 .215587-2.249399$

C $-0.1399243 .143629-1.300957$

C $-0.8017404 .489930-1.292290$

C $-2.1253374 .487097-0.555196$

C $-3.2752904 .722676-1.209755$

C -2.1202114 .2633590 .946115$

C -1.928498 2.8111991 .304949

N $-0.766299-0.105316-1.885149$

H $0.451502-2.0295380 .199656$

H $-0.502426-4.8875370 .736299$

H $-0.568028-3.817777 \quad 3.045571$

H -2.675609 -4.7444292 .020681$

H -2.992059 -3.223204 1.239617

H $-4.617508-1.8906604 .347275$

H -3.826507 -3.792729 5.401072

H $-3.603198-5.1424114 .282755$

H $-2.196826-4.2396484 .887703$

H $-4.024009-0.0815392 .804386$

H -3.466339-1.187563 1.575439

H -5.923249 -2.076861 1.490284

H $-6.337156-0.6548492 .463793$

H -5.1501230 .7604200 .657350$

H -7.6602950 .5613150 .724188$

H -7.570074 $-0.945910-0.164402$

H $-9.0482391 .066074-1.243690$

H $-6.649163-0.900858-2.625387$

H $-7.5535752 .731167-0.531784$

H $-6.8995372 .802737-2.152270$

H $-5.8256002 .468229-0.779769$

H $-6.9707650 .943654-3.911343$

H $-4.605818-2.111016-2.858769$

H $-3.944022-0.738927-3.757320$

H $-3.1029270 .785001-1.562019$

H -0.0657540 .6334254 .270254$

H -1.5555000 .2948361 .600668$

H $-0.698455 \quad 4.200343 \quad 3.259191$

H 0.5144822 .9368313 .427762

H -0.9821592 .8885204 .385000$

H -2.554867 -1.677616 -1.198737

H -2.396933 -2.626806 -2.665952

H $\quad 0.7428190 .604538-3.155690$

H $1.0396610 .765544-1.410600$

H $-0.9792692 .437292-3.092382$

H $0.5005752 .909776-0.456722$

H $-0.9346334 .837820-2.325100$

H $-0.126575 \quad 5.217161-0.824874$

H $-4.2281164 .738044-0.691205$

H $-3.2915314 .898641-2.280267$

H $-3.0746534 .592254 \quad 1.376413$

H -1.3456424 .9034891 .379691$

H -2.4236282 .1168430 .623869$

H $-1.022356-0.214904-0.908487$

C $2.363120-3.894009-1.207672$

O $3.085966-2.643696-1.163540$

C $4.111816-2.667324-2.172826$

C $4.265825-4.127795-2.580860$

C $2.842111-4.621162-2.467960$

H $3.721278-2.100650-3.026321$

C $5.420011-2.036467-1.682177$

H $4.680972-4.236313-3.587522$

H $4.911197-4.679676-1.887782$

C $2.729732-6.134537-2.388878$

H $2.280389-4.267434-3.343201$

C $5.333752-0.586451-1.170095$

O $5.956163-2.827641-0.612020$ 
H $\quad 6.162393-2.091689-2.487788$

H $4.879615-0.568825-0.173755$

O $6.681815-0.122050-1.003608$

C $4.593618 \quad 0.401378-2.087446$

C $4.8183861 .893295-1.766559$

H $3.5204420 .184373-2.051070$

O $5.002400 \quad 0.201196-3.444444$

H $5.8341692 .184738-2.065004$

H $4.1471062 .461533-2.424564$

H $3.144293-6.598188-3.290095$

H $3.273880-6.532558-1.525649$

H $1.684370-6.445684-2.300006$

C $0.852852-3.649450-1.243841$

H $5.281084-2.8697230 .086731$

H $7.128390-0.740217-0.399799$

H $5.9650790 .334577-3.484512$

H $0.331389-4.583166-1.486741$

H $0.610321-2.936497-2.041516$

H $2.642164-4.453560-0.305827$

C 2.8623982 .2352551 .400067

O 3.2309441 .9818160 .036765

C $4.5797552 .300327-0.305313$

C $4.8469153 .796555-0.076630$

C 4.5076504 .2062261 .360491

C 3.1249023 .7031921 .784935

H 1.7798662 .0682891 .428445

C 3.5072671 .2292782 .367411

H $5.260691 \quad 1.7232110 .326875$

O $4.0833094 .584615-0.987940$

H $5.9036884 .003837-0.281905$

H 4.5320655 .2999421 .443050

O $5.483776 \quad 3.6788182 .253763$

H 2.9924833 .8349952 .865197

O $\quad 2.149541 \quad 4.527622 \quad 1.140707$

C $3.052486-0.1885082 .096419$

H $4.597778 \quad 1.2399232 .295987$

H 3.2170791 .4759503 .395107

H $3.1456214 .356903-0.865323$

H 6.3482124 .0367401 .988804

H $1.273903 \quad 4.258110 \quad 1.465369$

O $3.684922-1.0340562 .962251$

O $2.251398-0.5173671 .233366$

C $3.374008-2.4219162 .802532$

H $3.643624-2.7588781 .797572$

H $3.960843-2.9877663 .530752$

H $2.312211-2.5977002 .994864$

SCF Energy (B3LYP/6-31G**//MMFF) $=-3245.91856180$

0700026

MM̄FF Geometry

C $-2.220379-2.4465942 .203865$

C -1.219480 -2.483302 3.095719

C $-0.452484-1.2928663 .624980$

O $0.782473-1.2629782 .872802$

C -1.1812980 .0588353 .476771$

C -0.6168821 .1702374 .339249$

C 0.0944442 .2177913 .878963

C -0.9638431 .0848695 .804410$

C 0.5341322 .4831462 .465358

C 2.0086082 .9132002 .422861

C 2.5787053 .0935631 .006725

C $1.876177 \quad 4.2069380 .225181$

C $2.3971204 .322175-1.215711$

$\begin{array}{lllll}\text { O } & 2.471758 & 1.842509 & 0.319878\end{array}$

O $1.4707705 .133039-1.956114$

C $2.3823962 .920025-1.860569$

C $3.7488975 .041655-1.280645$

O $2.9810402 .945491-3.153694$

C $3.0554161 .837982-0.990180$

C $2.8867950 .417735-1.569511$

O $4.4637012 .095557-0.927900$

C $1.934465-0.9484423 .519233$

O $2.068734-0.6332454 .689723$

C $3.028065-1.0728692 .533254$

C $4.273040-0.6894142 .846830$

C $5.404299-0.7959441 .932908$

C 6.2050520 .4689881 .807819

C $1.441276-0.046171-1.757261$
C $1.439489-1.505194-2.158162$

C $1.741783-3.775040-1.187553$

O $1.496897-1.848042-3.336871$

C $3.177550-3.985361-0.812400$

C $4.204991-3.703029-1.628747$

C $5.652200-3.882541-1.267822$

C $6.363028-2.566672-1.027336$

C $6.637353-1.724559-2.038107$

C $6.824332-2.2651680 .381513$

C $5.680966-1.9588011 .309538$

N $1.439889-2.361242-1.067746$

H -2.530365 -1.495301 1.781555

H $-0.909717-3.4500673 .487905$

H $-0.248000-1.4992464 .682809$

H -2.236342 -0.0562993 .760193$

H -1.1876190 .3595732 .423378$

H 0.3975022 .9802054 .595976

H -0.5969851 .9479566 .370060$

H -2.050095 1.0450405 .935849

H -0.5255340 .1891116 .254232$

H $\quad 0.396037 \quad 1.606076 \quad 1.827750$

H -0.0994593 .2825542 .066371$

H 2.1385973 .8466872 .984993

H 2.6065842 .1478352 .934745

H 3.6418653 .3369471 .121642

H 1.9716365 .1665980 .748220

H 0.8005364 .0034730 .172128

H $1.4043945 .994962-1.510649$

H $1.3294012 .651890-2.010551$

H $3.6586616 .061433-0.887373$

H $4.0896995 .149546-2.316180$

H $4.5315234 .538688-0.709202$

H $2.5232523 .629958-3.671270$

H $3.4263910 .336286-2.522645$

H $3.393252-0.288890-0.896724$

H $4.923517 \quad 1.262496-0.732462$

H $2.791765-1.4861951 .559294$

H $4.490527-0.245473 \quad 3.815250$

H 6.8186150 .6213462 .702129

H 5.5436631 .3360381 .697253

H 6.8651960 .4722950 .936952

H $0.8560770 .085733-0.840483$

H $0.9388260 .516808-2.550474$

H $1.541260-4.126009-2.204999$

H $1.087294-4.316836-0.498079$

H $3.374029-4.3757790 .182749$

H $3.996542-3.311169-2.621985$

H $6.150763-4.421636-2.083762$

H $5.753292-4.532278-0.389489$

H $7.166371-0.792400-1.870092$

H $6.335437-1.948749-3.055855$

H $7.564500-1.4583560 .378035$

H $7.364181-3.1377460 .771534$

H $5.038095-2.8154801 .512895$

H $1.418173-1.965835-0.132966$

C $-2.647080-4.0169600 .283928$

O $-2.805337-2.875654-0.575597$

C $-3.409957-3.291426-1.812524$

C $-3.457675-4.816267-1.777017$

C $-3.568390-5.092065-0.290432$

H $-4.433658-2.898413-1.802096$

C -2.642154 -2.696915 -3.000038

H $-4.298869-5.215521-2.351891$

H -2.541563 -5.267090 -2.174580

C $-3.182914-6.5087960 .097418$

H $-4.605140-4.9042440 .019624$

C $-2.343213-1.188730-2.880059$

O $-1.377865-3.362011-3.128246$

H $-3.182165-2.905780-3.930500$

H -1.603160 -1.027444 -2.088863

O $-1.723655-0.769646-4.102706$

C $-3.572115-0.297106-2.627799$

C $-3.2788801 .214650-2.695037$

H $-4.007340-0.533981-1.653011$

O $-4.576158-0.588462-3.603750$

H $\quad-2.905750 \quad 1.462814-3.696335$

H $-4.2352521 .745084-2.615642$

H $-3.835407-7.234490-0.398775$ 
H $-2.149819-6.735682-0.187046$ H $-3.274662-6.6562651 .178152$ C $-2.989029-3.6456801 .727659$ H -0.904505 -3.255036 -2.285372 H $-0.937519-1.327176-4.234955$ H $-4.190923-0.419071-4.480703$ H $-2.816557-4.5057572 .385059$ H -4.054295 -3.395736 1.804633 H -1.595195 -4.323565 0.213820 C -3.8754562 .2636490 .122660$ O $-2.759047 \quad 1.464848-0.289525$ C $-2.2926951 .714552-1.620127$ C $-1.8917973 .186321-1.768591$ C $-3.0255814 .126010-1.376821$ C -3.5542023 .7619720 .009396$ H -4.750229 $2.018992-0.491707$ C -4.1950921 .9019031 .582834$ H $-1.3709191 .135458-1.733746$ O $-1.4699003 .450339-3.101931$ H -1.032191 3.382767 -1.117162 H $-3.8231484 .118307-2.128807$ O $-2.5050245 .457078-1.349449$ H $-2.811907 \quad 4.0441300 .767676$ $\begin{array}{llll}\text { O } & -4.740821 & 4.509438 & 0.270419\end{array}$ C $-4.838076 \quad 0.539431 \quad 1.730588$ H -3.2750491 .9008622 .177694$ H -4.8968632 .6304212 .004470$ $\mathrm{H}-1.2241744 .390258-3.147992$ H -3.252069 $6.061329-1.202515$ H -4.4938625 .4482940 .316805$ O $-\begin{array}{llll}-5.190912 & 0.364511 & 3.039424\end{array}$ $\begin{array}{lllll}\text { O } & -5.007661 & -0.268331 & 0.828639\end{array}$ C $-5.839950-0.8722713 .350224$ H $-6.225019-0.8059424 .371315$ H $-6.679900-1.0528342 .672454$ H $-5.119148-1.6919833 .300109$ SCF Energy (B3LYP/6-31G**//MMFF) $=-3245.90972594$

07_00027

MM̄FF Geometry

C $1.830329-2.2413312 .468193$

C $1.474377-1.2486723 .298187$

C $2.286699-0.0155953 .626230$

O 3.5336930 .0090352 .889452

C 1.5415921 .2764913 .248987

C 0.4004641 .6371324 .174531

C -0.9048421 .5257773 .861614$

C 0.8087162 .2103655 .507456

C - 1.5090610 .9808562 .598251

C -2.1658872 .0750721 .748380$

C -1.1601003 .0098121 .059049$

C -1.894546 4.1010770 .278209

C $-0.9185565 .006752-0.484605$

$\begin{array}{lllll}\text { O } & -0.372271 & 2.225173 & 0.159527\end{array}$

O $-1.6774525 .784064-1.424038$

C $0.0433234 .123683-1.308379$

C -0.2326396 .0103830 .449710$

O $1.0744904 .909979-1.900877$

C $0.6666272 .952086-0.511137$

C $1.3624481 .970295-1.477256$

O 1.6690023 .4069780 .399790

C $4.639776-0.4964023 .492484$

O $4.697392-1.0029974 .605017$

C $5.833287-0.4015922 .620080$

$\begin{array}{llll}\text { C } & 5.866821 & 0.263241 & 1.454127\end{array}$

C 7.0211190 .3692360 .555151

C $8.272845-0.3751750 .944495$

C $1.996448 \quad 0.760577-0.777087$

C $2.663150-0.174469-1.770127$

C $3.998939-2.272893-1.872061$

O $2.6546550 .017862-2.983347$

C $5.440578-1.877094-1.918303$

C $6.001404-1.253746-2.965458$

C $7.436357-0.813258-3.021907$

C $7.570404 \quad 0.693944-2.971226$

C $7.4331681 .436534-4.082768$

C $7.9319691 .331654-1.646654$

C $6.8981611 .094443-0.577579$
N $3.259261-1.259860-1.141805$

H $2.791120-2.2075651 .963298$

H $0.519644-1.3315413 .813955$

H $2.500037-0.0134974 .701570$

H $1.211928 \quad 1.2134102 .207013$

H 2.2540392 .1136683 .245158

H - 1.6378771 .8460424 .601128

H -0.0524182 .5275526 .105388$

H 1.3601151 .4697556 .094406

H 1.4481563 .0875705 .363749

H -0.7902840 .4115532 .001701$

H -2.281956 0.2586652 .891164

H $-2.762621 \quad 1.5719960 .980792$

H -2.8598522 .6594202 .365290$

H $-0.5083403 .457348 \quad 1.816931$

H -2.5322934 .6963330 .943310$

H -2.571197 $3.635822-0.451437$

H $-2.3490976 .282712-0.927858$

H $-0.5381343 .698033-2.138554$

H -0.9758906 .6691330 .915110$

H $\quad 0.444651 \quad 6.669027-0.104697$

H 0.3288695 .5321851 .255140

H $0.6426235 .612863-2.416041$

H $\quad 0.6323431 .601542-2.209767$

H $2.1490532 .494692-2.034917$

H 1.7791994 .3647650 .292111

H $6.707646-0.9155983 .006868$

H $4.9708950 .779615 \quad 1.115546$

H 8.6806860 .0235761 .879910

H $9.069869-0.3174460 .201141$

H $8.059792-1.4418241 .078974$

H $2.7553951 .093486-0.060796$

H $1.2337670 .191138-0.234302$

H $3.575344-2.384586-2.875940$

H $3.884450-3.224068-1.343655$

H $6.043191-2.108934-1.044342$

H $5.387859-1.027181-3.834926$

H $7.875086-1.194444-3.953239$

H $8.026287-1.269416-2.217351$

H $7.5503932 .514892-4.062515$

H $7.1976170 .980242-5.038673$

H $8.0333972 .419002-1.761594$

H $8.9269220 .984111-1.356567$

H $5.9463521 .589010-0.776936$

H $3.293412-1.285402-0.127201$

C $0.860382-3.7562030 .714049$

O $-0.025245-2.8037950 .088190$

C $-0.856160-3.503612-0.859919$

C $-0.285793-4.912562-0.972735$

C $0.256639-5.129605 \quad 0.423779$

H $-1.855018-3.568553-0.415969$

C $-0.918927-2.753406-2.194533$

H $-1.046340-5.645616-1.258553$

H $0.524766-4.964455-1.709506$

C $1.247618-6.2776230 .513141$

H $-0.584272-5.3228801 .103481$

C $-1.507907-1.330396-2.110012$

O $0.402579-2.652845-2.737401$

H -1.492119 -3.345952 -2.917328

H $-0.826133-0.672390-1.559966$

O $-1.562841-0.808078-3.444267$

C $-2.913145-1.256220-1.480461$

C $-3.5663130 .129237-1.655728$

H -2.838379 -1.496221 -0.413987

O $-3.735103-2.249326-2.094485$

H $-2.8388850 .909266-1.402200$

H $-3.8493540 .250797-2.708799$

H $\quad 0.773238-7.2184200 .215366$

H $2.110588-6.114393-0.141321$

H $1.618584-6.3969991 .535699$

C $0.988175-3.4601912 .209592$

H $0.957028-2.212977-2.071007$

H -2.195674 -1.350228 -3.946004

H $-4.596774-2.238372-1.644832$

H $1.471901-4.3038522 .714589$

H $-0.008804-3.3344182 .649369$

H $1.834369-3.6603310 .217396$

C $-7.024784-0.644478-0.437437$ 
O $-5.813436-0.629470-1.204374$

C $-4.8235620 .311325-0.780501$

C $-5.3851601 .738802-0.871957$

C $-6.694158 \quad 1.876403-0.088766$

C $-7.6786700 .747036-0.416639$

H -7.692264 -1.320862 -0.987110

C $-6.808713-1.2365930 .959880$

$\begin{array}{llll}\mathrm{H} & -4.542616 & 0.094415 & 0.255589\end{array}$

O $-5.606332 \quad 2.099733-2.233993$

H -4.645267 $2.439659-0.471753$

H -7.150619 2.844820 -0.327055

O -6.3914441 .8696061 .303285$

H -8.5166370 .7449310 .290087$

O $-8.228600 \quad 0.977515-1.717382$

C -6.356203 -2.6748850 .864652$

H $-6.078934-0.6758291 .550765$

H -7.741402 -1.228047 1.535981

H -6.205258 $1.439606-2.623184$

H $-7.227501 \quad 1.9871181 .785738$

H -8.688717 $1.833949-1.693512$

$\begin{array}{llll}\text { O } & -5.040463 & -2.774472 & 1.208227\end{array}$

O $-7.080902-3.594583 \quad 0.507397$

C $-4.484418-4.0914981 .147147$

H -3.459727 -4.0452111 .525237$

H $-5.057680-4.7795941 .775771$

H $-4.462488-4.4408880 .110671$

SCF Energy (B3LYP/6-31G**/MMFF)= -3245.91857061

0700028

MM̄FF Geometry

C $3.677435-4.593460-0.073031$

C $2.429384-4.141966-0.267647$

C $1.304038-4.3403640 .715761$

O $\quad 0.825965-3.040675 \quad 1.134673$

C $0.141589-5.087062 \quad 0.034144$

C $-1.094808-5.269427 \quad 0.897877$

C $-2.347508-4.9539240 .508594$

C $-0.869202-5.8779252 .258202$

C $-2.787337-4.425182-0.828378$

C $-3.077591-2.923979-0.811362$

C $-4.344489-2.491449-0.051538$

C $-5.618675-3.029204-0.709617$

C $-6.890210-2.458479-0.066662$

O $-4.347838-1.059669-0.086058$

O $-7.996455-2.753420-0.934115$

C -6.773681-0.921752 0.000455

C $-7.196587-3.142644 \quad 1.271011$

O $-7.865559-0.3614760 .726265$

C $-5.437831-0.4244890 .596350$

C -5.2760331 .0947090 .357807$

O $\begin{array}{llll}-5.376479 & -0.637317 & 2.007068\end{array}$

C $1.580653-2.3682902 .047275$

O $2.639510-2.7272432 .537505$

C $0.888992-1.0982902 .353033$

C $1.552118-0.1224832 .992376$

$\begin{array}{lllll}\text { C } & 1.005073 & 1.180576 & 3.377627\end{array}$

C $-0.479274 \quad 1.369805 \quad 3.218479$

C -4.0341881 .6710651 .047311$

C -3.6430713 .0483090 .538586$

C -1.894477 4.7951470 .965250

O $-4.158027 \quad 3.585872-0.436421$

C $-0.4326694 .520927 \quad 1.128152$

C $0.378728 \quad 5.177138 \quad 1.970353$

C $1.831216 \quad 4.828265 \quad 2.142627$

C $2.214636 \quad 4.5940093 .589360$

C 3.1586355 .3489704 .176697

C $1.505376 \quad 3.5020264 .364772$

C 1.8412612 .1193193 .869887

N -2.601138 $3.570343 \quad 1.289376$

H $3.912026-5.1575190 .826066$

H $2.207575-3.559029-1.159001$

H $1.641505-4.9034821 .595012$

H $0.484884-6.080764-0.282571$

H $-0.113712-4.540689-0.879815$

H -3.162040 -5.1230981 .211640$

H $-1.808561-6.1365512 .758601$

H -0.284233 -6.799269 2.168971

H $-0.331825-5.1817032 .909519$
H -2.035286 -4.626016 -1.597660

H $-3.667761-4.990029-1.153161$

H -2.213256 -2.387102 -0.397104

H $-3.156212-2.572717-1.849319$

H $-4.270988-2.8154860 .992148$

H $-5.649929-4.124289-0.681157$

H $-5.619753-2.757494-1.774602$

H -8.033262 -3.717553-1.056772

H $-6.854788-0.540782-1.027311$

H $-7.383064-4.212873 \quad 1.119998$

H -8.110322 -2.7409631 .722034$

H -6.382166 -3.052963 1.993214

H -8.684393 -0.6799990 .309126$

H $-5.2169561 .281813-0.722012$

H $-6.150741 \quad 1.6381640 .736187$

H $-6.237690-0.9647332 .311683$

H $-0.142865-0.9996452 .035523$

H $2.597445-0.2847143 .251857$

H $-1.0288340 .579466 \quad 3.742677$

H $-0.754141 \quad 1.3427082 .158575$

H -0.8450132 .3134443 .624914$

H $-4.210824 \quad 1.7335952 .127784$

H $-3.170094 \quad 1.0159260 .883340$

H $-2.251668 \quad 5.572634 \quad 1.647468$

H -2.114744 $5.097626-0.062770$

H $-0.020242 \quad 3.7144490 .527807$

H -0.0160505 .9954042 .567327$

H 2.4212935 .6506381 .718343

H 2.0939043 .9366681 .560559

H 3.4423155 .1962365 .212759

H 3.6718166 .1369503 .635442

H 0.4326593 .7084584 .366209

H 1.7993713 .5414345 .422362

H 2.9007981 .8728253 .954263

H $-2.361257 \quad 3.1114512 .162244$

C $5.773628-3.283748-0.431690$

O $5.083710-2.024636-0.285146$

C $5.865815-0.988753-0.901304$

C $7.274840-1.554119-1.005648$

C $6.979127-3.005721-1.332755$

H $5.465392-0.850030-1.913147$

C $5.7366230 .319238-0.114810$

H $7.870474-1.052383-1.773969$

H $7.813550-1.483858-0.053656$

C $8.153738-3.937736-1.091116$

H $6.683692-3.073210-2.388523$

C $4.2843020 .766972 \quad 0.153659$

$\begin{array}{lllll}\text { O } & 6.377092 & 0.173910 & 1.159396\end{array}$

H $6.283758 \quad 1.114008-0.634828$

H $3.826404 \quad 0.1203370 .910896$

$\begin{array}{lllll} & \text { O } & 4.332316 & 2.080359 & 0.724796\end{array}$

C $3.3778640 .803225-1.090049$

C $1.9959841 .404121-0.795982$

H $3.258294-0.216666-1.465904$

O $4.004197 \quad 1.563981-2.122038$

H 1.5460380 .9162030 .075446

H $2.1078582 .460824-0.518266$

H $9.002124-3.659539-1.725051$

H $8.487215-3.901262-0.048559$

H $7.883096-4.972809-1.322470$

C $4.811756-4.319039-1.019665$

H $5.953782-0.5729151 .616999$

H 4.8816712 .0277551 .525666

H $4.1499482 .461984-1.777811$

H $5.336200-5.260014-1.219322$

H $4.417053-3.956859-1.977435$

H $6.098451-3.5852910 .572336$

C - $1.1643272 .246531-2.562322$

O $-0.1453932 .079317-1.570423$

C $1.0184321 .350767-1.985081$

C $0.625157-0.073544-2.408216$

C $-0.477975-0.052613-3.472524$

C $-1.6310060 .882129-3.091299$

H $-2.0045682 .702614-2.024983$

C $-0.7383453 .218894-3.666754$

H $1.480713 \quad 1.874173-2.828117$

O $0.173504-0.798943-1.266478$

H $1.505338-0.591725-2.804586$ 
H - $-0.863902-1.070265-3.609826$

O $0.0966830 .366140-4.707449$

H $-2.297531 \quad 1.030597-3.948189$

O $-2.4153490 .254366-2.074207$

C $-0.5863974 .617364-3.116752$

H $0.1958262 .940629-4.162591$

H -1.500855 $3.264198-4.453696$

H $-0.018765-1.708040-1.553076$

H $-0.6061350 .347887-5.379219$

H -1.863406 $0.174655-1.277366$

O $0.6731034 .790929-2.624140$

O $-1.4857775 .447885-3.112757$

C $0.9494346 .078175-2.063427$

H $0.1736556 .370142-1.349454$

H $1.9047936 .020108-1.535012$

H $1.0323436 .818668-2.864174$

SCF Energy (B3LYP/6-31G**//MMFF) $=-3245.92324019$

0700029

MM̄FF Geometry

C $2.921767-0.0693873 .734767$

C $2.238002-0.8797542 .912454$

C $0.778573-1.2106913 .096050$

O $0.061267-0.7503051 .926971$

C $0.586443-2.7342643 .199196$

C $-0.843187-3.1438123 .500746$

C $-1.629831-3.8580682 .672021$

C $-1.372693-2.7246584 .848966$

C $-1.294504-4.4103531 .315871$

C $-1.669226-3.5014280 .135989$

C $-3.168892-3.1856350 .023193$

C $-3.472631-2.506547-1.315033$

C $-4.948435-2.097064-1.431737$

O $-3.533223-2.3102821 .093803$

O $-5.047376-1.209069-2.555913$

C $-5.354580-1.309503-0.166107$

C $-5.839480-3.301234-1.757474$

O $-6.753968-1.039494-0.167936$

C $-4.931396-1.9949431 .150176$

C $-5.156752-1.1103202 .400856$

O $-5.718169-3.1792671 .331049$

C -0.2544650 .5720021 .869343$

O -0.0270351 .4233872 .713904$

C $-0.9052880 .816400 \quad 0.564822$

C $-0.971475 \quad 2.0681120 .088536$

C $-1.5370082 .469620-1.200410$

C $-2.317488 \quad 1.436643-1.968942$

C $-4.418398 \quad 0.2325832 .400257$

C -5.2514491 .3953541 .886069$

C -5.0506853 .7988001 .256632$

O -6.4615531 .3236371 .690506$

C $-4.8683953 .921883-0.223515$

C -4.332199 $5.002988-0.809915$

C $-4.1747125 .161347-2.295319$

C $-2.7573915 .505673-2.696446$

C $-2.3937186 .778957-2.924521$

C $-1.7868554 .366368-2.912762$

C -1.339969 $3.735771-1.623394$

N $-4.4865602 .540727 \quad 1.709224$

H 2.4229920 .3641104 .597953

H $2.733959-1.2954282 .038068$

H $0.376663-0.7188803 .989986$

H $1.229171-3.1382713 .992608$

H $0.929309-3.194746 \quad 2.266180$

H -2.640746 -4.091844 3.005282

H $-2.340386-3.1847915 .076453$

H $-0.677402-3.0232325 .640314$

H - $-1.508947-1.6398184 .891762$

H $-0.229263-4.6560641 .249921$

H -1.813653 -5.371142 1.205903

H $-1.098598-2.5688270 .212502$

H $-1.345963-4.005774-0.782525$

H -3.727461 -4.1249300 .110368$

H -3.187412 -3.152769 -2.154745

H -2.852617 -1.604999 -1.411347

H $-5.962527-0.881547-2.592974$

H $-4.857103-0.335680-0.238088$

H -5.524689 -3.763356-2.701041
H $-6.880627-2.995649-1.909758$

H $-5.815825-4.073320-0.985742$

H -7.213630 -1.854558 0.096162

H $-6.230621-0.9810762 .582107$

H -4.782634 -1.674995 3.267054

H -5.696889-3.421685 2.271681

H $-1.276589-0.0412420 .016590$

H -0.5406672 .8742060 .681335$

H $-3.0906530 .991224-1.333726$

H $-1.6547050 .641548-2.327332$

H $-2.8406191 .840781-2.837868$

H -4.1545530 .4835193 .435594$

H -3.4862970 .1673291 .831830$

H -4.5414814 .5979291 .804386$

H -6.1172473 .8318421 .501014$

H $-5.2142173 .096356-0.841227$

H $-4.0134665 .838206-0.189957$

H -4.856802 $5.958379-2.619507$

H $-4.4973854 .260520-2.831995$

H -1.386732 $7.032403-3.239116$

H $-3.0944937 .597371-2.796699$

H $-0.884864 \quad 4.730789-3.422754$

H -2.230233 $3.649341-3.609046$

H $-0.7597574 .408736-0.990343$

H -3.481896 2.4960751 .853136

C 4.4531451 .7863203 .059318

O $3.8252791 .917681 \quad 1.766620$

C 4.7773412 .4698510 .842841

C $5.7660313 .226827 \quad 1.714029$

C 5.8980822 .2650042 .882934

H 5.2997401 .6213700 .382989

C $4.0620873 .265711-0.254793$

H 6.7148303 .4184401 .204868

H $5.357563 \quad 4.1816972 .062910$

C 6.4888612 .8971414 .131187

H 6.5377501 .4261642 .576184

C $2.8959342 .497542-0.915172$

O $3.497705 \quad 4.4607900 .296203$

H $4.7817003 .583655-1.018731$

H $2.0493972 .446434-0.220293$

O $2.4424423 .254369-2.043210$

C $3.2484891 .076377-1.384762$

C $2.0777450 .385984-2.111197$

H $3.5331140 .474004-0.515259$

$\begin{array}{llll}\text { O } & 4.380462 & 1.146271 & -2.250669\end{array}$

H $1.1403020 .611482-1.594931$

H $2.0140380 .773332-3.135342$

H 7.5032223 .2598793 .935482

H 5.8883423 .7466024 .473611

H 6.5403062 .1696674 .947583

C $4.3547730 .327192 \quad 3.512436$

H $4.2263895 .083762 \quad 0.455942$

H $2.1998134 .138644-1.718958$

H $4.6112150 .237690-2.508364$

H 4.9081890 .1786634 .446367

H $4.806790-0.3272442 .757212$

H $3.9040572 .442557 \quad 3.746285$

C $3.795454-2.813979-2.983801$

O $3.400694-1.436624-2.954943$

C $2.259367-1.142944-2.145873$

C $1.026867-1.874855-2.694101$

C $1.290114-3.380304-2.821586$

C $2.638134-3.696620-3.483238$

H $4.601008-2.861457-3.727961$

C $4.377976-3.269189-1.636163$

H $2.442548-1.476316-1.118319$

O $0.640341-1.346283-3.959624$

H $0.184451-1.707628-2.012263$

H $0.478154-3.837637-3.400168$

O $1.266941-3.946365-1.512629$

H $2.894574-4.750531-3.326691$

O $2.505257-3.528414-4.897485$

C $5.552516-2.412244-1.211408$

H $3.624943-3.222161-0.844802$

H $4.730152-4.304241-1.706727$

H $1.444471-1.149157-4.467119$

H $1.369098-4.908753-1.607153$

H $2.600319-2.584226-5.103658$ 
O $5.685898-2.471066 \quad 0.146489$

O $6.274989-1.785837-1.975012$

C $6.758665-1.6971870 .692424$

H $6.762113-1.8377491 .776645$

H $7.717158-2.040376 \quad 0.291444$

H $6.606549-0.6355340 .476581$

SCF Energy (B3LYP/6-31G*//MMFF)= -3245.91159201

07_00030

MM̄FF Geometry

C $4.157564-2.9266741 .683516$

C $2.833184-2.8561651 .485881$

C $1.849962-2.6495622 .606424$

O $1.090010-1.4423892 .359361$

C $0.853598-3.8186912 .674330$

C $-0.140146-3.663770 \quad 3.809739$

C $-1.421536-3.2733053 .660034$

C $0.388131-4.0091145 .178429$

C $-2.134064-2.8949182 .388357$

C $-3.201654-3.9425572 .054340$

C $-4.006990-3.6730210 .774585$

C $-3.160433-3.787783-0.494816$

C $-3.996119-3.520848-1.756246$

O $-4.605500-2.3749950 .859731$

O $-3.071570-3.361410-2.843127$

C $-4.751748-2.187074-1.569338$

C $-4.877332-4.720756-2.119894$

O $-5.635053-1.944792-2.661117$

C $-5.502211-2.077048-0.222111$

C $-6.088504-0.6657040 .015901$

O $-6.609009-2.988266-0.235368$

C $1.689324-0.2486982 .616399$

O $2.859370-0.0520132 .900917$

C $0.642147 \quad 0.7916412 .528827$

C $0.978932 \quad 2.0889412 .485593$

C $0.039765 \quad 3.212128 \quad 2.447253$

C - 1.4186732 .9026912 .664862

$\begin{array}{llll}\text { C } & -5.064479 & 0.471182 & 0.024295\end{array}$

C $-5.6955891 .815246 \quad 0.346894$

C $-5.110847 \quad 4.2030390 .666984$

O -6.8899511 .9593010 .593335$

C -4.0148915 .1249370 .239068$

C -3.262851 5.8235611 .102615

C $-2.152043 \quad 6.750360 \quad 0.701304$

C -0.7869196 .1000220 .784396$

C $-0.0684225 .904160-0.334895$

C -0.2696415 .7440912 .165567$

C 0.5149804 .4567862 .229935

$\begin{array}{llll}N & -4.759608 & 2.841463 & 0.311521\end{array}$

H $4.548988-2.8667452 .696466$

H $2.438449-2.8992870 .475079$

H $2.365878-2.5478233 .569858$

H $1.393865-4.7656952 .806003$

H $\quad 0.327693-3.915617 \quad 1.717417$

H -2.043083 -3.207124 4.552640

H $-0.383503-3.9378615 .952500$

H $0.769881-5.0353525 .189696$

H $1.199835-3.3320605 .461288$

H -2.603063 -1.915308 2.539804

H -1.437563 -2.772309 1.556170

H $-2.740926-4.9364511 .984707$

H -3.910675 -3.981704 2.892862

H $-4.805154-4.4248020 .751976$

H $-2.675750-4.769943-0.555105$

H $-2.346720-3.051623-0.469036$

H $-3.582817-3.105073-3.630057$

H -3.991917 -1.400215 -1.617466

H $-4.257590-5.609385-2.290862$

H $-5.414705-4.547796-3.058976$

H -5.607303 -4.969343 -1.346995

H $-6.417758-2.508755-2.539132$

H $-6.868333-0.457634-0.728677$

H $-6.606155-0.6660160 .985693$

H -7.255610 -2.696136 0.428535

H $-0.3890350 .455948 \quad 2.518130$

H 2.0345392 .3559652 .475757

H -1.560729 2.341155 3.595181

H -1.8156242 .3115391 .832869$
H -2.041595 3.7932832 .750591

H -4.2822910 .2730270 .765561$

H -4.599282 $0.564571-0.961679$

H $-5.280806 \quad 4.228578 \quad 1.748918$

H $-6.050697 \quad 4.4708680 .172825$

H $-3.8322355 .219955-0.828560$

H $-3.453753 \quad 5.7263562 .168806$

H -2.171996 7.6325191 .353244

H $-2.3392517 .132169-0.311119$

H $0.9275465 .477410-0.307118$

H $-0.4549096 .179454-1.310856$

H $-1.089570 \quad 5.710182 \quad 2.890694$

H $0.372606 \quad 6.5625842 .516242$

H $1.586570 \quad 4.5863302 .074678$

H -3.7746552 .5928410 .275094$

C $5.905148-1.6810450 .452046$

O $5.010780-0.695341-0.101762$

C $5.7896750 .260751-0.849912$

C $7.244662-0.192151-0.747259$

C $7.097127-1.682785-0.495665$

H $5.4738980 .182286-1.894718$

C $5.5158661 .670914-0.312599$

H $7.8103670 .034270-1.656337$

H $7.7603930 .292148 \quad 0.090469$

C $8.349182-2.3254770 .073753$

H $6.828295-2.176823-1.438670$

C $4.0352872 .096117-0.420497$

O 5.8870301 .7173401 .071859

H $6.1554392 .399716-0.822634$

H 3.4043041 .4078610 .151890

$\begin{array}{lllll} & & 3.898300 & 3.382155 & 0.198937\end{array}$

C $3.5244672 .209999-1.870858$

C $2.0618752 .682657-1.975109$

H $3.6293861 .253153-2.389684$

O $4.341358 \quad 3.148020-2.578164$

H $1.8925813 .519451-1.289601$

H $1.9047463 .080933-2.986735$

H $9.189032-2.210841-0.619295$

H $8.635858-1.8703551 .027768$

H $8.197182-3.3957690 .243649$

C $5.173149-3.0152670 .579231$

H 5.3401941 .0660001 .544158

H 4.2243713 .2994781 .111512

H $4.2715544 .001953-2.117442$

H $5.872549-3.8230180 .819733$

H $4.682548-3.272095-0.365889$

H $6.212786-1.3202621 .443729$

C $0.232012-0.457931-2.803987$

O $1.1053650 .677379-2.839236$

C $1.0484961 .557356-1.708159$

C $-0.3739392 .104249-1.513722$

C $-1.4004520 .971408-1.442561$

C -1.225659 -0.022016-2.591913

H $0.298504-0.891787-3.810443$

C $0.716088-1.511709-1.798127$

H $1.3484421 .011386-0.808956$

O $-0.7024112 .979156-2.591341$

H $-0.4010132 .695162-0.591773$

H -2.407298 $1.401666-1.481006$

O $-1.2566350 .295762-0.198019$

H $-1.844774-0.907546-2.423760$

O $-1.7025650 .576185-3.800414$

C $1.787508-2.381948-2.414797$

H $1.114262-1.093362-0.870530$

H $-0.106039-2.183303-1.523572$

H $-1.5817153 .351799-2.408247$

H $-1.930785-0.404251-0.162533$

H -1.116252 $1.321897-4.015126$

O $2.917133-1.654280-2.644494$

O $1.632553-3.570444-2.666020$

C $3.974016-2.356150-3.306198$

H $4.830219-1.682665-3.393069$

H $4.275047-3.234181-2.727733$

H $3.656683-2.649179-4.311428$

SCF Energy (B3LYP/6-31G*//MMFF) $=-3245.92649448$

0700031

MM̄FF Geometry 
C $-1.505613 \quad 4.831487 \quad 0.459299$ C $-0.7316934 .129395-0.381972$ C $0.744567 \quad 3.908677-0.171030$ O $1.0111832 .488524-0.251457$ C $1.5410554 .605160-1.288771$ C $3.0448644 .526833-1.106823$ C $3.8875373 .902965-1.953255$ C $3.596306 \quad 5.2370190 .103304$ C $3.5551023 .175127-3.225083$ C $3.3084931 .668011-3.057741$ C $4.5207560 .885384-2.526874$ C $4.281876-0.622127-2.638538$ C $5.439185-1.435147-2.038917$ O $4.7185111 .232600-1.153627$ O $4.977038-2.789100-1.915114$ C $5.744861-0.905104-0.620240$ C $6.643742-1.478363-2.986156$ O $6.907428-1.532992-0.086286$ C $5.8712910 .631071-0.549200$ C 5.9669491 .1701950 .898943 O $7.071607 \quad 1.029731-1.223272$ C $0.701433 \quad 1.728480 \quad 0.833725$ $\begin{array}{llll}\text { O } & 0.241783 & 2.112905 & 1.896888\end{array}$ C 0.9941090 .3204180 .487269 C $0.480920-0.664191 \quad 1.238886$ C $0.649256-2.1006591 .012554$ C $1.661454-2.530592-0.015474$ C $4.7776070 .835081 \quad 1.805938$ C $4.983506-0.4038112 .662182$ C $3.759662-1.9016774 .234016$ O $6.065551-0.9729472 .776200$ C $3.288922-3.1172753 .499822$ C $2.278281-3.8889373 .928273$ C $1.818368-5.1351543 .227254$ C $0.334563-5.1244002 .934745$ C $-0.534586-5.7502973 .746305$ C $-0.129582-4.4603171 .658382$ C $-0.095007-2.9594141 .740402$ N $3.818555-0.7764543 .319840$ H -1.060272 $5.296837 \quad 1.335031$ H -1.179752 $3.662550-1.256266$ H 1.0601824 .2820750 .810659 H $1.2626895 .666452-1.338106$ H $1.2471664 .169619-2.249934$ H $4.9513443 .918484-1.717632$ H 4.6905185 .2868910 .092586 H 3.2244406 .2660790 .142743 H 3.3017624 .7214551 .022386 H $2.6845153 .626449-3.713007$ H $4.3839983 .327342-3.928281$ H $2.4527161 .521893-2.388616$ H $3.0242601 .268331-4.039116$ H $5.4024501 .176397-3.109985$ H $4.109288-0.914459-3.681794$ H $3.360659-0.882167-2.100020$ H $5.672906-3.297692-1.464068$ H $4.909741-1.2243860 .013329$ H $6.357060-1.924601-3.946184$ H $7.439873-2.117174-2.588116$ H $7.063382-0.491286-3.191207$ H $7.684396-1.115207-0.495118$ H 6.9192210 .8683881 .351294 H $\begin{array}{llll}6.012792 & 2.266930 & 0.832529\end{array}$ H $7.3226141 .913585-0.907354$ H $1.6017850 .132331-0.389966$ H $-0.145441-0.3968642 .088668$ H $2.624481-2.0420870 .167300$ H $1.320343-2.271240-1.022712$ H $1.865267-3.603353-0.005069$ H 4.6319861 .6668142 .507180 H $3.8562810 .732111 \quad 1.225272$ H $3.084065-1.6223685 .048696$ H $4.754247-2.0858854 .652630$ H $3.818798-3.3892832 .589933$ H $1.769437-3.6290584 .854052$ H $2.064444-5.9893853 .871553$ H $2.372945-5.2996192 .295040$ H $-1.597485-5.7706573 .529380$
H $-0.202532-6.2516644 .649409$ H $-1.167937-4.7453341 .441433$ H $0.448162-4.8568340 .818770$ H $-0.797257-2.5487592 .467375$ H $2.955932-0.2755413 .127960$ C $-3.759745 \quad 4.379428 \quad 1.446485$ O $-3.502230 \quad 2.9623601 .495207$ C $-4.7479592 .262273 \quad 1.655186$ C $-5.761102 \quad 3.3149582 .088040$ C $-5.276243 \quad 4.525726 \quad 1.313679$ H $-5.032693 \quad 1.8928700 .663488$ C -4.5751881 .0846362 .619731$ H -6.7882403 .0291521 .842364$ H $-5.711756 \quad 3.5118413 .165081$ C -5.8170975 .8435351 .840280$ H $-5.575227 \quad 4.4110120 .262997$ C $-3.408184 \quad 0.127526 \quad 2.293718$ O $-4.337401 \quad 1.596253 \quad 3.940153$ H -5.5133390 .5225892687065$ H -2.4598850 .6537742 .459308$ O $-3.438890-0.9496583 .239658$ C $-3.389567-0.5005450 .888286$ C $-4.705034-1.2083700 .510668$ H $-2.593694-1.2543870 .863355$ O $-3.0626710 .479123-0.089489$ H $-4.993152-1.890367 \quad 1.318940$ H $-5.494797-0.456910 \quad 0.395619$ H $-6.9097895 .864662 \quad 1.774074$ H $-5.540775 \quad 6.000722 \quad 2.888431$ H -5.4268536 .6848101 .259180$ C $-2.9885694 .998996 \quad 0.279319$ H $-3.5447182 .158596 \quad 3.898153$ H $-3.427549-0.5548994 .128470$ H -2.2185240 .8863290 .168915$ H -3.212288 6.0688870 .204328 H $-3.3051414 .530976-0.661322$ H -3.4114804 .7972922 .400039$ C $-4.321834-1.634141-3.189658$ O $-4.495510-1.070258-1.883763$ C $-4.588439-2.005618-0.804902$ C $-5.791088-2.938702-1.013505$ C $-5.722632-3.629181-2.379030$ C $-5.449200-2.631004-3.509266$ H $-4.425500-0.785874-3.878759$ C $-2.914231-2.221970-3.376382$ H $-3.671439-2.602833-0.758771$ O $-6.998497-2.185980-0.915428$ H $-5.801114-3.687774-0.212405$ H $-6.671561-4.145249-2.569232$ O $-4.691888-4.611728-2.338365$ H $-5.213084-3.164626-4.436658$ O $-6.645789-1.888944-3.765891$ C $-1.840465-1.152580-3.363295$ H -2.662684 -2.943800 -2.595563 H -2.860594 -2.721989-4.350649 H $-7.739981-2.807064-1.015523$ H $-4.677479-5.057919-3.202120$ H $-6.834060-1.347322-2.980178$ O $-0.635797-1.735348-3.639843$ O $-2.024758 \quad 0.035979-3.141723$ C $0.481813-0.843245-3.681269$ H $1.381830-1.435118-3.866484$ H $0.360334-0.123703-4.496339$ H $0.592016-0.326126-2.723979$ SCF Energy (B3LYP/6-31G**//MMFF)= -3245.91343169

0700032

MM̄FF Geometry

C -2.959124 -0.5345732 .245691$

C $-1.781461-0.7845112 .837068$

C $-0.892098-1.9314622 .435481$

O $0.474934-1.4587262 .540902$

C $-1.058130-3.162473 \quad 3.345780$

C $-2.364199-3.9052193 .171217$

C $-2.610818-4.7790712 .175162$

C $-3.393293-3.6703784 .245952$

C $-1.697532-5.163247 \quad 1.040323$

C $-2.428076-5.220800-0.309257$ 
C $-2.819003-3.841820-0.871908$ C $-3.936799-4.013769-1.904899$ C $-4.314847-2.687340-2.570601$ O $-1.659741-3.254863-1.486255$ O $-5.138476-2.982966-3.708665$ C -3.029120 -2.031154 -3.100551 C $-5.151763-1.795396-1.648087$ O $-3.301042-0.734300-3.626984$ C $-1.882429-1.956620-2.059160$ C $-0.598236-1.488896-2.787552$ O $-2.220459-0.994373-1.060232$

C $1.416915-2.1260331 .821704$ O $1.232000-3.0589181 .056433$ C $2.736398-1.5359492 .130330$ C $3.830225-2.1036491 .601613$ C $5.210261-1.6392231 .739482$

C $5.453171-0.3929192 .546663$ C $0.705976-1.570637-1.983165$ C $1.870108-1.023168-2.799518$ C $4.329976-0.995172-2.969600$ O $1.710937-0.343165-3.811898$ C $5.524948-1.184589-2.090405$ C $6.310945-0.172267-1.689416$ C $7.572291-0.223493-0.864158$ C $8.033073-1.585953-0.393563$ C $8.834779-2.346768-1.157734$ C $7.641344-2.0181121 .003896$ C $6.177190-2.3295971 .100550$ N $3.110841-1.342578-2.259875$ H $-3.310145-1.2122521 .471814$ H $-1.436928-0.1361873 .638520$ H $-1.087804-2.1886411 .387269$ H $-0.931393-2.8645624 .396117$ H $-0.231161-3.8651503 .179194$ H -3.569949 -5.295642 2.179012 H $-4.314182-4.2368444 .071190$ H -2.997678 -3.9758495 .220222$ H $-3.665139-2.6125014 .300317$ H $-0.834658-4.5012280 .959158$ H -1.308657 -6.165525 1.258550 H -1.770428 $-5.727233-1.027567$ H $-3.320927-5.848743-0.194231$ H $-3.158650-3.195017-0.056645$ H $-4.822083-4.473471-1.447238$ H $-3.606623-4.713887-2.685461$ H $-5.921985-3.465907-3.394289$ H -2.682648 -2.639310 -3.948233 H $-6.075245-2.308286-1.353714$ H $-5.468722-0.881890-2.162814$ H $-4.626372-1.513795-0.734334$ H $-4.014617-0.829763-4.280782$ H $-0.455570-2.100540-3.688796$ H $-0.738202-0.452186-3.121417$ H $-1.404898-0.655668-0.658693$ H $2.771256-0.6538542 .758037$ H $3.704372-2.9905310 .981092$ H 4.9141250 .4549862 .113854 H $6.503845-0.1010952 .596787$ H $5.116347-0.5327433 .579784$ H $0.639126-0.980015-1.064129$ H $0.916329-2.612285-1.718244$ H $4.2428300 .034631-3.334165$ H $4.404261-1.649640-3.844845$ H $5.766132-2.208856-1.823914$ H $\quad 6.0347870 .833756-2.002833$ H 7.4459790 .4358490 .003817 H $8.3720800 .236494-1.460355$ H $9.196529-3.310360-0.814754$ H $9.142528-2.022883-2.146588$ H $7.963780-1.2535011 .715886$ H $8.190044-2.9240321 .293452$ H $5.892943-3.2242530 .544015$ H $3.156009-2.047768-1.529442$ C $-4.371251 \quad 1.295051 \quad 1.312988$ O -3.2807731 .9157610 .608709$ C -3.790221 $3.061394-0.106004$ C $-5.281230 \quad 3.1474710 .216902$ C -5.3644872 .4263451 .549740$
H -3.2896223 .9425730 .308398$

C $-3.4746492 .913156-1.600267$

H $-5.630145 \quad 4.1835610 .268934$

H $-5.8898772 .634977 \quad-0.537559$

C $-6.768196 \quad 1.965418 \quad 1.899573$

H -4.9920873 .0954082 .336961$

C -1.965558 $2.802134-1.906772$

O $-4.1146301 .727465-2.087289$

H $-3.9134503 .745238-2.161706$

H $-1.5369281 .943318-1.379350$

O $-1.8129722 .535198-3.306909$

C $-1.1762394 .084410-1.578072$

C $0.3252493 .995028-1.911687$

H $-1.2937354 .343355-0.522088$

O $-1.7270635 .173725-2.325471$

H $0.4652363 .566260-2.911014$

H $0.7173485 .019935-1.964101$

H -7.4489152 .8207951 .962619$

H $-7.163570 \quad 1.275629 \quad 1.146358$

H $-6.782526 \quad 1.4526992 .866221$

$\begin{array}{lll}\text { C }-3.859443 & 0.619801 & 2.584737\end{array}$

H $-3.7292230 .969873-1.614155$

H -2.317394 $1.726951-3.503470$

H $-1.6493194 .949504-3.268777$

H -4.7032850 .2347283 .167259$

H -3.3240281 .3472733 .204605$

$\begin{array}{llll}H & -4.808565 & 0.545791 & 0.639469\end{array}$

C 1.8044743 .4052821 .460961

$\begin{array}{lllllllll}\text { O } & 1.109867 & 3.967487 & 0.341362\end{array}$

C $1.1258143 .197882-0.867925$

C $2.5687682 .939605-1.330035$

C $3.4026022 .311558-0.209696$

C $3.2640123 .086427 \quad 1.101926$

H 1.8187424 .2075742 .210593

C 1.0429712 .2160732 .059655

H $0.6279682 .239194-0.693110$

O $3.1689444 .165161-1.743546$

H $2.5440852 .275325-2.202316$

H $4.4586092 .289552-0.503360$

$\begin{array}{lllll}\text { O } & 2.978981 & 0.971095 & 0.006273\end{array}$

H 3.7264672 .5345051 .927672

$\begin{array}{llllll}\text { O } & 3.963194 & 4.328996 & 0.990788\end{array}$

C $-0.1490542 .711344 \quad 2.845857$

H $\quad 0.6675021 .5087091 .315367$

H 1.6930221 .6508342 .737588

H $4.0659723 .961362-2.058156$

H $3.1330120 .479204-0.817332$

H $4.895627 \quad 4.125002 \quad 0.805527$

$\begin{array}{lllll}\text { O } & 0.061322 & 2.523183 & 4.179927\end{array}$

O $\begin{array}{llll}0.1 .136474 & 3.225576 & 2.337429\end{array}$

C -0.9798882 .9917085 .042390$

H -1.1691734 .0558334 .871852$

H $-1.8901202 .406842 \quad 4.885185$

H $-0.650612 \quad 2.856747 \quad 6.076153$

SCF Energy (B3LYP/6-31G**//MMFF)= -3245.91914506

0700033

MM̄FF Geometry

C -0.0485921 .1797553 .365092$

C $-0.6558310 .521144 \quad 4.365394$

C $-1.552952-0.6852414 .240162$

O $-2.177463-0.7635382 .941439$

C $-0.734940-1.969998 \quad 4.449585$

C $-1.580728-3.2270424 .389180$

C -1.649804 -4.040760 3.316457

C $-2.334434-3.5594195 .649332$

C $-0.970392-3.8354431 .988988$

$\begin{array}{llll}C & -1.829176 & -4.334643 & 0.819644\end{array}$

C $-1.282756-3.975652-0.574242$

C $0.185625-4.365695-0.773407$

C $0.715035-3.946361-2.154301$

O $-1.456621-2.566196-0.782995$

O $2.146660-4.064613-2.137392$

C $0.357917-2.462491-2.398813$

C $0.247065-4.906289-3.252739$

O $0.697714-2.067537-3.725259$

C -1.125079 -2.137459-2.113872

C $-1.439027-0.631016-2.237908$ 
O $-1.943458-2.800283-3.088184$ C $-3.354709-0.1025802 .779328$ $\begin{array}{llll}\text { O } & -3.866429 & 0.692655 & 3.550927\end{array}$ C -3.939674 -0.5497361 .496863$ C $-4.9850870 .112101 \quad 0.981087$ C $-5.665134-0.206213-0.273601$ C $-5.398009-1.549150-0.900178$ C $-0.6773120 .291665-1.289613$ C -1.131375 $1.720041-1.517259$ C $-2.897023 \quad 3.350955-0.845227$ O $-0.592047 \quad 2.442127-2.352524$ C $-4.1617343 .263262-1.648854$ C $-4.4249232 .403243-2.646225$ C $-5.7364622 .346206-3.381162$ C $-6.3734790 .973184-3.313724$ C $-6.2233220 .097450-4.321727$ C $-7.2332330 .634409-2.113318$ C $-6.4895450 .716596-0.810787$ N $-2.2238362 .069465-0.743271$ H $-0.167266 \quad 0.840498 \quad 2.339761$ H -0.4879420 .8601055 .386269$ H -2.332005 -0.6203205 .010751$ H $-0.221975-1.9366295 .420160$ H $0.066515-2.0224913 .703403$ H $-2.249269-4.9463473 .396920$ H $-2.896568-4.4952355 .562733$ H $-1.640729-3.6678126 .489470$ H -3.050069 -2.766715 5.888491 H $-0.727941-2.7819451 .825029$ H $-0.032610-4.3997692 .010664$ H -1.924828 -5.4258010 .891556$ H -2.839566 -3.9156720 .914304$ H - $1.911889-4.509278-1.296867$ H $0.329024-5.441225-0.613272$ H $\quad 0.805322-3.863594-0.020810$ H $2.491441-3.489003-1.433711$ H $0.986136-1.866432-1.725416$ H $0.624762-5.917789-3.059910$ H $0.651237-4.617981-4.229510$ H $-0.839637-4.972158-3.335007$ H $1.628025-2.309460-3.872084$ H $-1.281263-0.305919-3.275272$ H $-2.512608-0.484999-2.060467$ H -2.806453 -2.355415-3.122004 H -3.489648 -1.405614 1.007846 H -5.3603260 .9885611 .507747$ H -5.608932 -2.353429 -0.186131 H $-4.354778-1.631653-1.217359$ H -6.016229 -1.744392 -1.779767 H $-0.8417410 .010284-0.243607$ H $0.4010140 .256546-1.468333$ H -2.230914 4.105643 -1.273689 H $-3.142340 \quad 3.6476840 .179857$ H $-4.9348163 .975197-1.365822$ H -3.663450 $1.697697-2.967259$ H -5.546313 2.620762 -4.426929 H -6.444389 $3.093986-3.002913$ H $-6.691383-0.880955-4.296211$ H $-5.6246430 .338082-5.194150$ H -7.699949 $-0.352517-2.217746$ H -8.073157 $1.340491-2.076439$ H $-6.6262091 .663513-0.287678$ H $-2.634693 \quad 1.373224-0.129866$ C 0.5414003 .5204872 .636500 O $1.211137 \quad 3.2662891 .381221$ C 1.9846134 .4291761 .025573 C $1.387545 \quad 5.5728501 .830965$ C $1.074498 \quad 4.867214 \quad 3.134539$ H $3.008416 \quad 4.2502631 .375356$ C $1.9824554 .640402-0.491290$ H 2.0833386 .4087181 .949422 H 0.4664025 .9542511 .375242 C $0.096374 \quad 5.6251174 .015916$ H 2.0118974 .7191753 .687776 C $2.4884893 .424220-1.293487$ O $0.6455904 .923803-0.920529$ H $2.5723645 .528524-0.744317$ H $1.7987942 .585306-1.158373$
O $2.4529393 .762490-2.684609$

C $3.9172422 .979186-0.939747$

C $4.4620931 .843373-1.826950$

H 3.9538092 .6652520 .108127

O $4.7936544 .101807-1.067179$

H $4.5464602 .191550-2.864331$

H $5.4964021 .646577-1.518201$

H 0.5089306 .5989814 .299101

H -0.8546875 .7999013 .501721$

H -0.1148675 .0672254 .933675$

C $\quad 0.838407 \quad 2.3725393 .605786$

H $0.0887374 .172650-0.653610$

H $1.5399764 .024615-2.894679$

H $5.680426 \quad 3.816042-0.788727$

H 0.7132702 .7187714 .638528

H 1.8794042 .0481563 .487167

H -0.5321683 .5866922 .418383$

C $4.422112-0.2862600 .341706$

O $3.3069470 .163498-0.432999$

C $3.6347770 .542605-1.773360$

C $4.270015-0.642183-2.515737$

C $5.453882-1.236876-1.753431$

C $5.071472-1.518389-0.298354$

H $5.1634630 .520404 \quad 0.401206$

C $3.948476-0.511503 \quad 1.781455$

H $2.6829350 .755701-2.274259$

O $4.696617-0.253770-3.817519$

H $3.517316-1.429465-2.646278$

H $6.331561-0.582902-1.817385$

O $5.818037-2.464483-2.389367$

H $4.389378-2.376577-0.270195$

O $6.235514-1.866287 \quad 0.445976$

C $3.009903-1.6871051 .915795$

H $4.808611-0.6550402 .446027$

H 3.4089210 .3684912 .151916

H $3.9209760 .099203-4.286077$

H $6.634646-2.782282-1.968958$

H $6.574543-2.7042010 .088836$

O $3.629578-2.6945862 .594717$

O $1.875803-1.7130841 .456633$

C $2.865708-3.8907772 .767797$

H $1.995387-3.6921333 .398976$

H $2.560869-4.292548 \quad 1.797091$

H $3.498795-4.6289823 .267138$

SCF Energy (B3LYP/6-31G**//MMFF)= -3245.90621998

0700034

MM̄FF Geometry

C -3.233700 $1.868722 \quad 2.683453$

C $-2.0829841 .936126 \quad 3.369112$

C $-0.872832 \quad 1.1057603 .040873$

$\begin{array}{lllll}\text { O } & 0.215497 & 2.043042 & 2.843486\end{array}$

C $-0.4858320 .142957 \quad 4.178513$

C $-1.444945-1.0079804 .398167$

C -1.369373 -2.183709 3.743350

C $-2.472711-0.8090895 .480838$

C $-0.409511-2.5469872 .645715$

C $0.800857-3.317908 \quad 3.181247$

C $1.758863-3.7499152 .059520$

C $2.962953-4.4874682 .646054$

C $3.996634-4.8442351 .569811$

O $2.198670-2.5794361 .368338$

O $5.208039-5.2356432 .234186$

C $4.323980-3.5831100 .739677$

C $3.550556-6.0512740 .734626$

O $5.163779-3.930913-0.358293$

C $3.069982-2.826657 \quad 0.249345$

C $3.391055-1.424213-0.317318$

O $2.408834-3.591100-0.760312$

C 1.2482401 .6510222 .050505

$\begin{array}{llll}\text { O } & 1.361566 & 0.585972 & 1.466568\end{array}$

C 2.2353972 .7538472 .020813

C $3.3365182 .639127 \quad 1.262659$

C 4.4126803 .6256891 .138635

C $4.2994014 .883477 \quad 1.960574$

C $4.274834-1.379992-1.563384$

C $4.234238-0.025434-2.253350$

C $5.3428831 .242891-4.089738$ 
O $3.4499220 .868207-1.947168$

C $6.3362502 .149226-3.433009$

C $6.0755453 .425584-3.111082$

C $7.0844884 .351582-2.496376$

C $6.5928074 .998530-1.221050$

C $6.1870066 .279664-1.211687$

C $6.670177 \quad 4.1989070 .059518$

C 5.4417253 .3636240 .304226

N $5.1693320 .055102-3.275923$

H -3.3165291 .1698861 .854343$

H -2.000304 2.6244934 .206949

H -1.0415020 .5516232 .109384$

H $-0.358927 \quad 0.7043485 .114865$

H $0.518381-0.2572203 .986681$

H -2.075329 -2.970172 4.005241

H -3.093974 -1.698612 5.631440

H $-1.981200-0.5874306 .433891$

H $-3.142871 \quad 0.0191695 .235080$

H $-0.945477-3.1661961 .916584$

H -0.085555 -1.654724 2.102485

H $1.338766-2.6748803 .890158$

H $0.464061-4.2029623 .735606$

H $1.209236-4.3943811 .363340$

H $2.643154-5.3871383 .186379$

H $3.452842-3.8484343 .394308$

H $5.001779-5.9881582 .814814$

H $4.906516-2.9041051 .377787$

H $3.396436-6.9258771 .378107$

H $4.323950-6.3431120 .015577$

H $2.620691-5.875186 \quad 0.189825$

H $5.946302-4.3796680 .005605$

H $2.434348-0.936817-0.546537$

H $3.842376-0.8046020 .469088$

H $1.899581-2.984233-1.322444$

H 2.0312003 .6276722 .629095

H $3.470583 \quad 1.7312040 .677767$

H 3.3812585 .4259991 .708659

H 5.1223875 .5836361 .804757

H 4.2845744 .6440653 .029593

H $5.314173-1.588282-1.287571$

H $3.949843-2.127464-2.295658$

H $4.371419 \quad 1.728077-4.232273$

H $5.7194960 .920733-5.065263$

H $7.3245101 .740745-3.235718$

H $5.0941293 .839606-3.330812$

H $7.3203125 .123285-3.241000$

H $8.0324423 .836190-2.297927$

H $5.8678226 .765204-0.295747$

H $6.161076 \quad 6.871255-2.120848$

H $6.877826 \quad 4.8611190 .907278$

H 7.5424243 .5332690 .023770

H $5.4307992 .428138-0.255491$

H $5.821384-0.711808-3.412783$

C -4.9696543 .5165001 .819577$

O

C $-4.7686703 .170775-0.482954$

C $-4.4900274 .638927-0.193684$

C $-4.002415 \quad 4.553122 \quad 1.242681$

H $-3.8083052 .663341-0.634482$

C $-5.6864822 .880055-1.673230$

H $-3.7484355 .066445-0.874937$

H $-5.4017585 .244729-0.250111$

C $-4.0181045 .882302 \quad 1.976557$

H -2.9753884 .1671021 .235567$

C $-6.0838651 .392912-1.800830$

O $-6.8898543 .641467-1.523277$

H $-5.2110173 .232938-2.596115$

H -6.789078 1.135068 -1.001766

O $-6.806803 \quad 1.232545-3.026967$

C $-4.8996640 .411031-1.777157$

C $-5.344496-1.053539-1.952990$

H $-4.3725320 .506918-0.822075$

O $-3.9904220 .773377-2.814889$

H -6.220094 -1.244031 -1.321393

H $-5.624761-1.220066-3.000324$

H $-3.3535006 .600566 \quad 1.485430$

H -5.0230876 .3166902 .000201$

H -3.6790625 .7587463 .009965$
C -4.4512772 .6918923 .002222$

H -7.458727 $3.436733-2.285256$

H $-6.1839691 .392961-3.756686$

H $-3.2290230 .171344-2.763764$

H -5.2374721 .9910963 .310554$

H -4.2604693 .3561573 .853361$

H -5.9010274 .0079172 .129767$

C $-2.002412-2.709519-2.256188$

O $-3.171031-1.921846-2.520163$

C $-4.224756-2.037602-1.559943$

C $-4.719968-3.488270-1.493600$

C $-3.563611-4.449084-1.192464$

C $-2.359036-4.198272-2.106055$

H $-1.387829-2.605823-3.160024$

C $-1.201490-2.144886-1.077288$

H $-3.853226-1.742727-0.572565$

O $-5.344186-3.856934-2.721224$

H $-5.481616-3.568688-0.709470$

H -3.906201 -5.482972-1.321609

O $-3.149233-4.2924380 .161823$

H $-1.480937-4.748480-1.748162$

O $-2.660993-4.695583-3.413553$

C $-0.627907-0.793518-1.435380$

H $-1.798129-2.045588-0.166898$

H $-0.358863-2.797488-0.825193$

H $-4.700405-3.716305-3.436434$

H -3.916233 -4.483689 0.727950

H $-2.828528-5.650087-3.332249$

O $-1.2773930 .188853-0.748973$

O $0.270226-0.631549-2.251789$

C $-0.8509141 .526741-1.026601$

H -1.276382 2.184048 -0.263705

H $0.2394931 .609307-0.988276$

H -1.224520 $1.835266-2.007005$

SCF Energy $\left(B 3 L Y P / 6-31 G^{* *} / / M M F F\right)=-3245.92918113$

0700035

MM̄FF Geometry

C -1.924573 $5.358906-0.567485$

C $-0.8397374 .630396-0.869512$

C $0.4846554 .753619-0.161309$

O 0.8093923 .4649790 .410436

C $1.5862545 .120704-1.173058$

C $2.9869795 .130933-0.587828$

C $3.9983214 .346540-1.013477$

C 3.2207816 .1141510 .529922

C $3.9797053 .355021-2.143945$

C $3.7604211 .914747-1.676583$

C $4.9019581 .293441-0.853450$

C $6.1933741 .164075-1.667223$

C $7.2850730 .386394-0.917497$

O $4.440519-0.004637-0.450766$

O $8.3079810 .042546-1.865934$

C $6.688378-0.937499-0.397336$

C $7.962525 \quad 1.248378 \quad 0.154727$

O $7.647789-1.6154640 .410624$

C $5.359497-0.745840 \quad 0.364731$

C $4.677223-2.0850660 .725086$

O $5.618627-0.0625361 .596455$

C 0.2185933 .1376601 .592365

O -0.5698023 .8104512 .236506$

C 0.6913021 .7856821 .960136

C 0.0518121 .0901732 .912611

C $0.378662-0.2695373 .349168$

C $1.604710-0.9032682 .751249$

C $4.303245-2.965501-0.468246$

C $3.387062-4.098991-0.045617$

C $1.027611-4.5993960 .555458$

O $3.789465-5.2564080 .044731$

C $-0.075673-3.857547 \quad 1.239596$

C $-0.633848-4.2391422 .398353$

C -1.757455-3.488491 3.057095

C $-1.477710-3.1466974 .505760$

C $-2.266835-3.6205865 .484931$

C $-0.292961-2.2598164 .830321$

C $-0.424334-0.8778534 .247410$

N $2.096428-3.672577 \quad 0.229927$

H $-1.868588 \quad 6.1089760 .216986$ 
H $-0.9121293 .868404-1.642734$ H $0.444012 \quad 5.508787 \quad 0.633624$ H $1.380046 \quad 6.112687-1.596463$ H $1.5282164 .419097-2.011227$ H $4.9614934 .432889-0.512061$ H 4.2766536 .1754810 .815007 H 2.9069737 .1176390 .224044 H 2.6561605 .8268721 .422204 H $3.2056233 .608006-2.875045$ H $4.9192353 .443835-2.700483$ H $2.831947 \quad 1.858028-1.092835$ H $3.5788331 .284039-2.557330$ H 5.0657841 .8984870 .044993 H $6.5773922 .147488-1.961580$ H $5.9772060 .634695-2.605865$ H $8.6450390 .867202-2.256170$ H $6.516833-1.569147-1.278426$ H $8.4408782 .122638-0.303092$ H 8.7654790 .6972110 .656324 H 7.2678201 .6156380 .912851 H $7.329204-2.5222410 .553660$ H $5.299120-2.6554061 .426843$ H $3.765663-1.8563151 .288176$ H $4.886614-0.2403882 .209761$ H $1.539707 \quad 1.3894121 .413629$ H $-0.805401 \quad 1.5469293 .405479$ H $2.491765-0.2963492 .964700$ H $1.494133-0.9941441 .665543$ H $1.817267-1.9042533 .129574$ H $3.797138-2.388388-1.250418$ H $5.197835-3.406005-0.921398$ H $1.429091-5.4141831 .167160$ H $0.666604-5.023783-0.386847$ H $-0.447318-2.9617070 .748316$ H $-0.286469-5.1466702 .886146$ H -2.657957 -4.111484 2.980143 H - $1.986529-2.5661732 .511537$ H $-2.080293-3.383886 \quad 6.527236$ H $-3.119010-4.2560565 .268169$ H $0.621894-2.7696454 .519756$ H $-0.195310-2.1406775 .917664$ H -1.286218 -0.3271154 .626761$ H $1.836748-2.731601-0.052909$ C $-4.2549374 .512483-0.273408$ O -3.7906143 .1935040 .075116$ C -4.9041042 .2823510 .065869$ C $-6.1547203 .148039-0.044452$ C $-5.6441684 .310840-0.874589$ H $-4.8156901 .682409-0.846476$ C $-4.8479021 .375221 \quad 1.299398$ H $-6.9866102 .615494-0.515226$ H $-6.4943903 .502726 \quad 0.935474$ C $-6.5312985 .541636-0.809106$ H -5.558434 $3.984179-1.919675$ C -3.5133000 .6254691 .506296$ O H -5.6796840 .6627261 .274715$ H $-2.7246101 .358022 \quad 1.713636$ O $-3.637994-0.1823682 .684517$ C $-3.043891-0.3008340 .369369$ C $-4.122794-1.307272-0.075647$ H -2.173791 -0.8615880 .732537$ O $-2.6007240 .489703-0.727321$ H $-4.585388-1.7597230 .809321$ H -4.897322 -0.765215 -0.631158 H -7.531909 5.315198 -1.191613 H $-6.6384415 .904990 \quad 0.218491$ H -6.115412 6.354795-1.412215 C $-3.2585255 .163401-1.233642$ H -4.3686692 .8607752 .488781$ H -3.8974280 .4068993 .413496$ H -2.269344 -0.114162 -1.413118 H -3.628122 6.139019-1.567836 H -3.141344 $4.536542-2.126728$ H -4.3171315 .0858240 .661062$ C $-2.585701-2.771500-3.166581$ O $-3.155866-1.862222-2.214936$ C $-3.571697-2.436139-0.971999$
C $-4.627950-3.529127-1.206843$

C $-4.132442-4.577917-2.205772$

C $-3.551942-3.929518-3.466696$

H $-2.476380-2.180436-4.085198$

C $-1.191535-3.247077-2.744233$

H $-2.703927-2.874458-0.468572$

O $-5.827697-2.933361-1.697617$

H -4.863475 -4.004398 -0.246835

H $-4.966746-5.233469-2.483545$

O $-3.141993-5.378224-1.567241$

H $-3.041020-4.682482-4.077074$

O $-4.626407-3.425533-4.265534$

C $-0.193825-2.117826-2.824534$

H -1.160941 -3.666056-1.735623

H $-0.831354-4.036220-3.415258$

H $-6.487840-3.640805-1.795034$

H $-2.854629-6.053650-2.205037$

H $-5.050553-2.702686-3.772033$

O $-0.140996-1.455991-1.634748$

O $0.445164-1.845214-3.832522$

C $0.760723-0.347676-1.589905$

H $0.7052610 .091964-0.592183$

H $1.785434-0.686092-1.768344$

H $\quad 0.4690250 .410502-2.322799$

SCF Energy $\left(B 3 L Y P / 6-31 G^{* *} / / M M F F\right)=-3245.91278696$

0700036

MM̄FF Geometry

C - $-0.1537264 .952531-1.264384$

C $-1.1530614 .637014-0.427208$

C $-2.3504463 .820156-0.841780$

O $-2.3117042 .571688-0.108902$

C $-3.6519024 .552680-0.472597$

C $-4.9025923 .842811-0.955451$

C $-5.8458473 .315978-0.150173$

C $-5.0763383 .768613-2.451368$

C -5.8899333 .3129321 .351677$

C -5.2605252 .0787272 .016069$

C $-5.917257 \quad 0.744093 \quad 1.625834$

C $-5.418035-0.3863452 .528276$

C $-5.983558-1.7504332 .107317$

$\begin{array}{lllll}\text { O } & -5.576055 & 0.450804 & 0.268355\end{array}$

O $-5.224181-2.7498252 .805337$

C $-5.755480-1.9517940 .592264$

C -7.437735 -1.920438 2.564157

O $-6.417348-3.1395350 .164186$

C $-6.187178-0.736588-0.257681$

C $-5.769099-0.836779-1.746033$

O $-7.616058-0.632487-0.231858$

C $-1.4321101 .627330-0.542390$

O $-0.7044941 .692634-1.520607$

C -1.4804660 .4943230 .406095$

C $-0.473022-0.3905560 .413709$

C $-0.361378-1.5715721 .270951$

C $-1.548276-1.9297792 .123996$

C $-4.264931-0.984884-1.999815$

C $-3.802910-2.423265-2.165315$

C $-1.726296-3.777611-2.425070$

O $-4.576058-3.371834-2.267501$

C $-1.338266-4.353542-1.099570$

C $-0.100367-4.792644-0.825792$

C $0.306374-5.407373 \quad 0.482555$

C $1.469980-4.6860361 .125479$

C $2.731276-5.1201060 .962649$

C $1.159299-3.5060242 .018504$

C $0.785903-2.2799051 .235302$

N -2.418678 -2.518309 -2.223241

H $-0.2118604 .650153-2.307070$

H -1.0774604 .9111490 .622558$

H -2.328398 $3.609955-1.917921$

H $-3.6466965 .562372-0.904498$

H -3.682771 4.6877690 .614002

H $-6.7024212 .831119-0.617761$

H $-6.0646493 .391721-2.736525$

H $-4.9669774 .762814-2.896802$

H $-4.3319533 .100958-2.895420$

H -5.4165814 .2146911 .754729$

H -6.9418763 .3851691 .657230$ 
H -4.1927992 .0505651 .770339$ H -5.3373662 .2137143 .102219$ H -7.0042360 .8537321 .715892$ H -5.656614 -0.181205 3.579393 H -4.321994 -0.4337682 .476230$ H -5.526292 -3.6201892 .493342$ H -4.680667 -2.1249710 .461503$ H -7.504272 -1.8471713 .656383$ H -7.821267 -2.914326 2.308365 H -8.110806 -1.172595 2.139951 H $-6.151331-3.316233-0.753430$ H $-6.344558-1.621626-2.250965$ H $-6.0778830 .101277-2.230041$ H -7.900611-0.105024-0.996593 H -2.3326090 .4245371 .071154$ H $\quad 0.362297-0.231147-0.267350$ H -2.445494 -2.033997 1.504463 H - $1.728706-1.1554362 .877334$ H -1.435478 -2.876037 2.656409 H -4.021231 -0.477104-2.942001 H $-3.684627-0.502606-1.208776$ H - $0.856865-3.571384-3.057295$ H -2.384825 -4.475706 -2.951731 H -2.112836 -4.434769 -0.340701 H $0.663815-4.735944-1.597739$ H $0.577492-6.4536990 .289988$ H $-0.534328-5.4462131 .186358$ H $3.567738-4.6200251 .439592$ H $2.955766-5.9805390 .341144$ H $2.040539-3.2483192 .621221$ H $\quad 0.397313-3.8015692 .744381$ H $1.582229-1.9329050 .576355$ H -1.855547 -1.685831 -2.075883 C $2.2503584 .617154-0.729955$ O $1.999147 \quad 3.732550 \quad 0.383635$ C 3.2160813 .5773991 .143214 C 4.3249514 .1083390 .249226 C $3.6158755 .245723-0.460025$ H 3.0989634 .2174062 .027182 C 3.3544492 .1099201 .565186 H 5.2024224 .4371710 .813612 H $4.6440713 .344938-0.471502$ C $4.3338895 .723487-1.710084$ H 3.5116326 .0867900 .238546 C 4.7104331 .7710132 .211279 O $2.310379 \begin{array}{lll}1.824227 & 2.503152\end{array}$ H $3.173614 \quad 1.4637160 .699633$ H 5.5131471 .8940311 .475404 O 4.9612362 .7323143 .245098 C 4.7855930 .3684462 .843726 C $4.393598-0.7955801 .924904$ H $4.141341 \quad 0.331835 \quad 3.731232$ O $\quad \begin{array}{lll}6.111992 & 0.137514 & 3.324147\end{array}$ H $4.562587-1.7426222 .455239$ H $3.310103-0.757168 \quad 1.762310$ H $5.3287956 .105783-1.459448$ H $4.4585714 .912889-2.435927$ H $3.7752646 .528792-2.197048$ C $1.1123615 .635816-0.831198$ H 1.4653962 .0329812 .068456 H 5.8959382 .6527323 .499240 H 6.2698350 .7515184 .060811 H $1.3525686 .415584-1.562063$ H $0.961783 \quad 6.1281010 .137621$ H $2.2886653 .989423-1.630249$ C $4.907117-2.270448-1.452396$ O $4.497080-2.025650-0.101099$ C $5.073786-0.8773240 .543010$ C $6.605439-0.9926850 .568350$ C $7.165071-1.229654-0.837406$ C $6.437337-2.368035-1.557655$ H $4.501160-3.261662-1.692936$ C $4.289156-1.255765-2.428489$ H $4.7909810 .012528-0.025752$ O $7.016630-2.0690181 .406833$ H $7.042177-0.0699310 .965724$ H $8.233160-1.467200-0.758884$ O $7.041576-0.023198-1.585629$
H $\quad 6.728138-2.405122-2.613824$

O $6.827572-3.615791-0.976514$

C $2.797607-1.462900-2.600889$

H $4.440841-0.225858-2.095752$

H $4.741545-1.378943-3.419360$

H $6.823383-1.8178052 .325814$

H $7.425519-0.182899-2.464631$

H $7.792076-3.694360-1.069963$

O $2.305489-0.483669-3.416962$

O $2.147359-2.372566-2.106236$

C $0.907098-0.571469-3.709705$

H $0.321173-0.643801-2.789388$

H $0.6126010 .337605-4.240688$

H $\quad 0.716734-1.434608-4.354027$

SCF Energy (B3LYP/6-31G**//MMFF)= -3245.91152497

0700037

MM̄FF Geometry

C -2.214780 2.1746862 .663379

C -1.9436281 .1422273 .477512$

C $-2.739797-0.1386983 .599235$

O $-3.860940-0.1521072 .680906$

C $-1.890945-1.3781693 .263663$

C $-0.879780-1.758744 \quad 4.323707$

C $0.454848-1.6246784 .198240$

C $-1.456360-2.3843325 .568437$

C $1.224925-1.0362493 .049439$

C $2.031203-2.0944592 .283515$

C $1.159387-3.0534501 .455807$

C $2.033080-4.0975830 .757047$

C $1.216804-5.005812-0.171541$

O $\quad 0.451375-2.278346 \quad 0.483928$

O $2.155824-5.719677-0.989823$

C $0.355304-4.121007-1.096462$

C $0.441721-6.0662660 .618495$

O $-0.509696-4.923566-1.897536$

C $-0.443837-3.029774-0.345365$

C $-1.074593-2.054214-1.366346$

O $-1.475821-3.6573560 .415869$

C $-5.0598420 .293527 \quad 3.137204$

O $\begin{array}{llll}-5.302490 & 0.712378 & 4.261311\end{array}$

C -6.0982050 .2629952 .081070$

C $-5.937786-0.3063370 .876013$

C $-6.933439-0.338143-0.200905$

C $-8.2288730 .396857 \quad 0.032508$

C $-1.826179-0.869948-0.742516$

C $-2.2588480 .139270-1.791382$

C $-3.4527952 .311364-2.019668$

O $-2.077435-0.023010-2.995222$

C $-4.8850231 .981133-2.298390$

C $-5.3043571 .433533-3.449006$

C $-6.7274021 .048708-3.736852$

C $-6.907599-0.453715-3.792118$

C $-6.618508-1.142395-4.909233$

C $-7.486947-1.146636-2.577166$

C $-6.634202-0.990803-1.344970$

N $-2.878577 \quad 1.244015-1.221230$

H -3.079786 2.137214 2.007656

H - $-1.083941 \quad 1.2242824 .139618$

H -3.113143 -0.2207454 .626627$

H - $1.418962-1.2401182 .285969$

H -2.559549 -2.237133 3.110008

H $1.082146-1.9627785 .022506$

H $-0.679427-2.7293536 .258943$

H -2.079662 -1.666281 6.109688

H $-2.070468-3.2526235 .307731$

H $0.585468-0.4702352 .366457$

H $1.926372-0.3030833 .467703$

H $2.716686-1.5630541 .610873$

H $2.651558-2.6667852 .984549$

H $0.440028-3.5466952 .118645$

H $2.589854-4.6950411 .489629$

H $2.791755-3.5858690 .150823$

H $1.647475-6.242370-1.633804$

H $1.042798-3.627665-1.797409$

H $1.133684-6.6916961 .195362$

H -0.089635 -6.751371-0.051304

H $\quad-0.279651-5.6381701 .317652$ 
H -1.252965 -5.206385 -1.337959 H $-0.277138-1.651791-2.005053$ H -1.772092 $-2.595751-2.017897$ H -2.157325 -3.0013890.627990 H $-7.030017 \quad 0.743817 \quad 2.362254$ H -4.993746 -0.7953020 .645770$ H -8.787702 -0.0630460 .854895$ H -8.891864 $0.409160-0.834351$ H -8.0359501 .4467610 .280899$ H -2.726980 -1.219357 -0.228316 H -1.194881 $-0.351688-0.014556$ H -2.874048 $2.430832-2.941754$ H $-3.3833213 .240848-1.447087$ H $-5.6020712 .190703-1.509188$ H -4.576435 $1.227120-4.230842$ H $-7.0151081 .493034-4.698489$ H -7.415503 $1.482485-3.000805$ H $-6.765747-2.215550-4.967968$ H -6.225333 -0.645672 -5.790333 H -7.587630 -2.223919-2.764646 H $-8.509163-0.787815-2.431607$ H -5.665155 -1.485844 -1.422070 H $-3.0639791 .245543-0.222596$ C -0.9610203 .7620501 .180500$ O 0.0444842 .8214580 .755038 C 1.0852403 .5328050 .058834 C $0.5428764 .935050-0.186720$ C $-0.3199735 .141641 \quad 1.041178$ H 1.9377923 .5918180 .746803 C $1.5118872 .797846-1.221139$ H $1.3401855 .678205-0.283116$ H $-0.0696014 .976045-1.095414$ C -1.3176926 .2775670 .895283$ H $\quad 0.3329345 .342418 \quad 1.901343$ C $2.1820211 .435509-0.938615$ O $\quad 0.3768012 .636354-2.068391$ H $2.2275383 .429126-1.761177$ H $3.0103071 .625523-0.248380$ O $1.2570720 .585492-0.269919$ C $2.7153970 .733544-2.205072$ C $3.470714-0.586971-1.941703$ H $3.3747061 .427599-2.739778$ O $1.6239480 .436279-3.078726$ H $2.783564-1.341288-1.538914$ H $3.787215-0.969910-2.920881$ H $-0.797784 \quad 7.2261110 .725679$ H -1.995494 6.1103650 .051298 H -1.9253586 .3833541 .799188$ C -1.402403 3.4395872 .608721 H $-0.2539772 .064074-1.599162$ H $1.734921-0.2057730 .026752$ H $1.9940820 .070297-3.899960$ H -2.0262544 .2518362 .998668$ H -0.5230343 .3539893 .258778$ H -1.8046053 .6637540 .485683$ C $6.7382910 .859359-0.870048$ O $5.5775820 .508381-1.634006$ C $4.695645-0.439726-1.026051$

C $5.430003-1.767091-0.781811$ C $6.695959-1.5417750 .051575$ C $7.550281-0.393630-0.499072$ H $7.3522581 .462326-1.551693$ C 6.3783201 .7385470 .337927 H $4.353873-0.056469-0.060624$ O $5.802757-2.375722-2.015200$ H $4.766668-2.460021-0.253326$ H $7.286967-2.4660460 .059420$ O $6.309633-1.258627 \quad 1.393664$ H $8.330103-0.122406 \quad 0.221257$

O $8.229184-0.850130-1.673516$

C $5.7827603 .067576-0.081995$

H $5.6542661 .246747 \quad 0.992714$ H 7.2834811 .9559210 .916226 H $4.997604-2.738416-2.421361$ H $7.122640-1.1527851 .916520$ H $7.556625-1.047692-2.347888$ O 5.3481953 .7299841 .031051 O $5.7162093 .481412-1.231244$
C 4.7706845 .0184280 .796503

H 4.3103825 .3593041 .727638

H $5.5525995 .727070 \quad 0.508313$

H 3.9973074 .9648210 .024448

SCF Energy (B3LYP/6-31G**//MMFF)= -3245.91133967

0700038

MM̄FF Geometry

C -1.057243 -2.810118 -0.114231

C $-0.436127-3.2099911 .007719$

C $-0.653027-2.5833032 .364079$

O $-0.793270-1.1510002 .222496$

C $-1.933364-3.1414723 .013471$

C $-2.316734-2.4364364 .300221$

C $-3.167697-1.3908654 .357276$

C $-1.696029-2.9941905 .551240$

C $-3.807440-0.6982453 .184618$

C $-5.324191-0.9126113 .129944$

C $-5.911419-0.7586281 .713455$

C $-5.652741-2.0146020 .871934$

C $-6.144620-1.858310-0.571150$

$\begin{array}{lllll}\text { O } & -5.337689 & 0.399845 & 1.098898\end{array}$

O $-5.583945-2.946324-1.323081$

C $-5.574815-0.544315-1.141474$

C $-7.666368-2.005748-0.673563$

O $-6.101286-0.321215-2.447271$

C $-5.8155210 .678393-0.224145$

C $-5.0918571 .950562-0.727715$

O $-7.2175760 .973669-0.208130$

C $0.337937-0.3994972 .204615$

O $1.489793-0.8006202 .235814$

C -0.0829971 .0196362 .164036$

C 0.8479031 .9841772 .159052

C 0.6053353 .4283302 .166016

C -0.8227303 .8976122 .256240$

C $-3.5622091 .869470-0.752669$

C $-2.9385763 .076832-1.433445$

C $-0.7277353 .957533-2.172103$

O $-3.5986294 .000608-1.902155$

C $-0.3883135 .087900-1.253309$

C $0.8642885 .515807-1.034119$

C $1.2001806 .702084-0.177658$

C 2.2064396 .3843990 .903841

C 3.4981446 .7304850 .771472

C 1.6952185 .7559292 .179657

C 1.6699514 .2559462 .113155

N -1.553291 $2.987296-1.478307$

H -1.791815 -2.012682 -0.045211

H $\quad 0.270373-4.034804 \quad 0.964225$

H $\quad 0.207110-2.7998803 .010256$

H $-1.809831-4.2149773 .209911$

H -2.772500 -3.085074 2.309299

H -3.401329 -0.9596855 .329044$

H $-2.003967-2.4458836 .447777$

H -1.987650 -4.040917 5.685872

H $-0.604318-2.9417765 .489692$

H -3.5983190 .3759353 .269070$

H -3.345771 -1.006307 2.244412

H -5.606034 -1.892007 3.536119

H $-5.787751-0.1599293 .781407$

H $-6.991570-0.6041271 .821698$

H -6.111201-2.895181 1.339568

H -4.576597 -2.226855 0.838046

H $-5.826382-2.814470-2.256019$

H $-4.497945-0.709112-1.262420$

H $-7.974404-3.004951-0.342720$

H -8.004555 -1.917148 -1.711888

H -8.211095 -1.276188 -0.070853

H $-5.5642420 .369351-2.870471$

H $-5.4796052 .225017-1.716964$

H $-5.3649182 .791939-0.074705$

H -7.335772 1.8973820 .068982

H $-1.146243 \quad 1.2312282 .160847$

H 1.8962091 .6896822 .164723

H -1.2684063 .5852063 .207089$

H -1.420632 3.4820181 .438962

H -0.9309214 .9820282 .190185$

H $\quad-3.1709891 .8013890 .268434$ 
H $-3.2305100 .989132-1.308916$ H -1.278464 4.341773 -3.037148 H $0.1616313 .432266-2.533206$ H -1.216826 $5.607240-0.777152$ H $1.6916635 .019182-1.535371$ H $\quad 0.3008397 .1337710 .279155$ H $1.5971297 .484153-0.838433$ H 4.2186116 .5339601 .558713 H $3.8650017 .211773-0.129084$ H $\quad 0.726641 \quad 6.1908252 .440898$ H 2.3528486 .0323413 .015277 H 2.6651363 .8126182 .049883 H -1.087440 2.221565 -1.000090 C $-0.488204-2.396476-2.562774$ O $0.696279-1.650688-2.215709$ C $0.500389-0.277380-2.605381$ C $-0.727693-0.265756-3.510610$ C $-1.566539-1.361298-2.884061$ H $0.2645670 .277199-1.689693$ C $1.7648930 .293667-3.254964$ H -1.227582 $0.707293-3.521687$ H $-0.473202-0.526826-4.544510$ C $-2.675853-1.875317-3.783968$ H -2.012702 -0.963123 -1.966771 C $3.0336870 .239339-2.379103$ O $2.044352-0.431820-4.459686$ H $1.5776051 .327744-3.567184$ H $3.384055-0.795066-2.290747$

O $4.064348 \quad 0.956557-3.070874$

C $2.8740840 .855414-0.979062$

C $4.1858831 .072938-0.203023$

H $2.2089680 .222632-0.382760$

O $2.2336912 .129945-1.086350$

H $4.7881241 .847975-0.694519$ H 3.9081751 .4982630 .770076 H -3.374325 -1.069193 -4.031167 H -2.278929 -2.275958 -4.722681 H $-3.241238-2.671001-3.289275$ C $-0.829016-3.416149-1.470124$ H $2.145804-1.369064-4.220049$

H $4.1608470 .549205-3.948882$ H $2.7938772 .694321-1.646370$ H $-0.010357-4.143623-1.408741$ H -1.728729 -3.971522 -1.758730 H $-0.235897-2.954946-3.474331$

C $4.849739-2.4432850 .882257$

O $4.224841-1.173746 \quad 0.648370$

C $5.037422-0.1891610 .004894$

C $6.294317 \quad 0.107427 \quad 0.841463$

C $7.064908-1.1760181 .164174$

C $6.140205-2.2741571 .701572$

H $4.133706-2.9997511 .500971$

C $5.062282-3.230477-0.420515$

H $5.343096-0.559478-0.978539$

O 5.9178090 .7487302 .059372

H 6.9329590 .8036260 .284512

H $7.837881-0.9539291 .910083$

O $7.718971-1.622162-0.019677$

H $6.674030-3.2302551 .742632$

O $5.779337-1.9555563 .048855$

C $3.755323-3.612325-1.080685$

H $5.638629-2.656495-1.150447$

H $5.598067-4.161363-0.201837$

H $\quad 6.7340710 .9631452 .542590$

H $8.222971-2.4221710 .207327$

H $5.234480-1.1499293 .028024$

O $4.024737-4.214279-2.277053$

O $2.643668-3.416356-0.611296$

C $2.882434-4.635334-3.028939$

H $3.235702-5.200218-3.895653$

H $2.241050-5.285409-2.426193$

H $2.327278-3.762157-3.382021$

SCF Energy (B3LYP/6-31G**//MMFF) $=-3245.91055476$

\section{00039}

MM̄MF Geometry

C $0.4021555 .077377-1.395315$

C $0.1461684 .255248-0.366367$
C $-0.847625 \quad 4.543076 \quad 0.727003$

O -1.7823413 .4417850 .757189$

C -0.1290364 .5872372 .089713$

C -1.075121 4.7574583 .263012

C -1.2695603 .8307374 .222993$

C $-1.816093 \quad 6.068013 \quad 3.320027$

C -0.5908912 .4949224 .364527$

C -1.2896581 .4048423 .552270$

C $-0.812127-0.0203033 .868360$

C $0.678561-0.2240663 .583385$

C $1.107848-1.6889263 .764719$

O $-1.597551-0.8947473 .044972$

O $2.407789-1.8452603 .175453$

C $0.142299-2.5923612 .971446$

C $1.257753-2.0624045 .243849$

O $0.413809-3.9707003 .210381$

C $-1.346345-2.2923533 .246984$

C $-2.296322-3.0750202 .308713$

O $-1.667448-2.6813514 .587314$

C -2.9418053 .5583750 .059316$

O $-3.3126454 .508045-0.609852$

C -3.6806332 .2893900 .245071$

C $-4.8244942 .065406-0.418024$

C $-5.6155900 .832343-0.364374$

C $-5.111387-0.273180 \quad 0.526718$

C $-2.058275-2.8360520 .816972$

C $-3.164419-3.400276-0.057932$

C $-3.828125-3.530776-2.465309$

$\begin{array}{llll}\text { O } & -4.132760 & -4.010646 & 0.384821\end{array}$

C $-4.614009-2.336816-2.908769$

C $-5.945035-2.342643-3.080719$

C $-6.719295-1.155209-3.580250$

C $-7.907218-0.805262-2.708248$

C $-9.153625-0.822165-3.211029$

C $-7.665928-0.428301-1.260031$

C $-6.7337790 .745795-1.115096$

N -2.927184 -3.135098 -1.399552

H $-0.1414316 .018874-1.449448$

H $0.6668423 .305145-0.285943$

H -1.3726495 .4894130 .549386$

H 0.5956505 .4120482 .100674

H 0.4592863 .6701462 .209109

H -1.987939 4.050940 5.011673

H -2.3841256 .1834004 .249386$

H -1.1118976 .9045623 .262420$

H -2.5245806 .1487942 .490091$

H 0.4652532 .5733644 .087048

H -0.5994672 .2251255 .428107$

H -2.3747831 .4505903 .718265$

H -1.1532201 .5923552 .480572$

H $-1.035483-0.2305294 .920877$

H 1.2957640 .4235974 .216826

H 0.8946380 .0758782 .548530

H $3.010722-1.2151073 .605726$

H $\quad 0.351609-2.4146691 .910188$

H $2.022289-1.4372695 .720759$

H $1.603123-3.0959285 .357373$

H $\quad 0.334047-1.9418355 .813285$

H $1.354810-4.1185363 .013386$

H -2.240372 -4.149773 2.525375

H $-3.328648-2.7822122 .547517$

H -2.627193 -2.820753 4.646160

H -3.2402301 .5521240 .907359$

H $-5.2072302 .843680-1.076601$

H -5.0381380 .0715501 .564298$

H $-4.121891-0.6020120 .196316$

H -5.749828 -1.1577310 .542389$

H -1.984705 -1.762278 0.614882

H -1.122851 -3.3130660 .507441$

H -4.471906 -4.347964 -2.124872

H $-3.208002-3.891458-3.291788$

H -4.055224 -1.429835 -3.128893

H $-6.503530-3.256454-2.891229$

H -7.054269 -1.389283 -4.599263

H $-6.072064-0.274582-3.675112$

H - $10.014357-0.572158-2.599764$

H -9.343603 -1.087347 -4.245829

H $-7.311995-1.318914-0.735534$ 
H -8.612090 -0.153548 -0.776055

H -7.026896 $1.613916-1.707136$

H -2.110716 -2.587441 -1.655884

C $2.1991553 .552489-2.466722$

O $3.0899103 .590117-1.337183$

C $4.2996132 .874377-1.668917$

C $4.1100202 .349109-3.087025$

C $3.1156043 .337342-3.667833$

H $5.1059833 .616675-1.636135$

C $4.5168671 .785001-0.612147$

H $5.0506162 .307467-3.644536$

H $3.6811631 .338923-3.076490$

C $2.4079842 .820579-4.908146$

H $3.6447604 .268658-3.910086$

C $5.7173250 .861207-0.886474$

O 4.6861372 .4180340 .661020

H $3.5976671 .194960-0.530877$

H $5.5864970 .342102-1.841691$

O $6.8980481 .656911-1.045774$

C $5.978318-0.1741360 .226329$

C $4.767532-1.0433700 .598681$

H 6.3240330 .3380241 .132741

O $7.055094-1.023709-0.170756$

H $5.069246-1.7666661 .367287$

H $4.017771-0.411298 \quad 1.092147$

H $3.1312722 .603709-5.701007$

H $1.8523731 .900019-4.698943$

H $1.6999183 .561245-5.292268$

C $1.3837554 .847929-2.519823$

H 5.4266463 .0435900 .589528

H $7.0967212 .073400-0.190178$

H $7.808758-0.452353-0.397679$

H $0.8184454 .884197-3.458196$

H $2.0721365 .702702-2.520824$

H $1.5375002 .685000-2.341348$

C $1.970720-2.931160-0.970448$

O $2.832792-2.272708-0.034092$

C $4.082656-1.800275-0.558515$

C $4.895322-2.977803-1.120915$

C $4.082614-3.802579-2.126642$

C $2.684650-4.139152-1.598023$

H $1.139256-3.311925-0.364928$

C $1.384864-1.965912-2.013694$

H $3.864934-1.089005-1.358806$

O $5.331981-3.830139-0.063811$

H $5.792096-2.596214-1.618587$

H $4.617080-4.734679-2.347877$

O $3.951692-3.078035-3.346340$

H $2.061180-4.557731-2.396603$

O $2.797798-5.141746-0.584096$

C $0.460178-0.937558-1.398037$

H $2.152414-1.412904-2.560342$

H $\quad 0.799884-2.549353-2.735210$

H $4.544486-4.1218530 .426806$

H $4.847270-2.907414-3.684559$

H $3.204263-5.925724-0.991430$

O $-0.394286-0.478354-2.360221$

O $0.498686-0.559613-0.235643$

C -1.331582 $0.511644-1.928616$

H $-1.9599310 .781975-2.781367$

H -1.969121 $0.106033-1.138610$

H -0.805302 $1.405206-1.582062$

SCF Energy (B3LYP/6-31G**//MMFF) $=-3245.90722812$

07 00040

MM̄MF Geometry

C -1.511922 5.271497-0.827080

C $-0.3644234 .576428-0.831301$

C $0.707313 \quad 4.755413 \quad 0.211475$

O $0.9300793 .472807 \quad 0.843682$

C $2.0349275 .200510-0.427397$

C 3.1108935 .4978000 .601754

C 4.2673764 .8169250 .723049

C 2.8346986 .6604121 .522090

C $4.7764523 .688706-0.128520$

C 4.3376452 .3189750 .390254

C $5.1850331 .154709-0.147993$

C $5.1980791 .081646-1.676492$
C $5.964262-0.149435-2.184056$

O $4.624742-0.0470830 .397492$

O $5.651272-0.321862-3.575607$

C $5.444386-1.404418-1.450739$

C $7.4813190 .060494-2.108001$

O $6.230815-2.549101-1.769439$

C $5.356033-1.2422850 .084538$

C $4.632626-2.4236960 .770205$

O $6.657190-1.2186200 .684231$

C 0.0903703 .1117981 .851682

O $\begin{array}{llll}-0.843936 & 3.755227 & 2.301353\end{array}$

C 0.5085151 .7686182 .309878

C $-0.191168 \quad 1.1343203 .262346$

C $0.099599-0.1986703 .797125$

C $1.259010-0.9440353 .190551$

C $3.198928-2.6595060 .295958$

C $2.456117-3.6864931 .134056$

C $0.182201-4.7263531 .305913$

O $2.961188-4.2846172 .078875$

C $-0.821887-3.8909822 .035077$

C $-1.242063-4.1395523 .284485$

C $-2.265953-3.2941373 .988922$

C $-1.823076-2.8492385 .366754$

C $-2.529862-3.1929246 .456893$

C $-0.568194-2.0104635 .493420$

C $-0.678628-0.6859004 .786398$

N $1.153338-3.8455670 .683926$

H -1.670967 $6.035626-0.069948$

H $-0.2102273 .798795-1.575579$

H 0.3917785 .4855390 .966382

H $1.8768866 .104744-1.029517$

H $2.3718774 .422554-1.121678$

H 4.9540415 .1139211 .515343

H 3.7140906 .9401162 .112476

H $2.540397 \quad 7.5418690 .943102$

H 2.0322266 .4181372 .225223

H $4.4687623 .820896-1.171159$

H $5.8723113 .750362-0.132561$

H $4.3811642 .293613 \quad 1.487698$

H 3.2827462 .1485410 .139961

H $6.204348 \quad 1.2743520 .238006$

H $5.6281151 .990053-2.114625$

H $4.166531 \quad 1.031200-2.048685$

H $5.8939950 .496435-4.041897$

H $4.439538-1.597718-1.845842$

H $7.7844000 .898452-2.747483$

H $8.022028-0.814084-2.485691$

H $7.8336210 .278185-1.097468$

H $6.249714-2.627087-2.738723$

H $5.218126-3.3447900 .650294$

H $4.615154-2.2306111 .852376$

H $7.314116-1.4553530 .009943$

H 1.3843741 .3331951 .842385

H -1.0600871 .6322613 .690590$

H $2.195491-0.397343 \quad 3.347683$

H $1.101654-1.0703262 .114301$

H $1.407472-1.9431603 .602320$

H $2.634453-1.7229050 .339086$

H $3.196163-3.019290-0.738667$

H $\quad 0.688387-5.4385591 .964889$

H $-0.312045-5.2770870 .499882$

H -1.237331 -3.040090 1.501263

H $-0.852567-5.0031023 .818148$

H $-3.188049-3.8855684 .058882$

H -2.522020 -2.409176 3.393738

H $-2.225363-2.8820407 .450803$

H -3.430498 -3.793334 6.382157

H $0.285141-2.6043315 .158043$

H $-0.364685-1.7920116 .550258$

H $-1.491119-0.0609635 .160018$

H $0.830239-3.262856-0.082559$

C $-3.8463274 .438899-1.004749$

O $-3.489173 \quad 3.149689-0.468351$

C $-4.5897532 .241853-0.651525$

C $-5.7762223 .102850-1.069216$

C $-5.0839904 .195145-1.864984$

H -4.328005 $1.589488-1.491780$

C -4.7928831 .4068710 .617251$ 
H $-6.5099772 .541871-1.655884$

H $-6.2928913 .533056-0.203530$

C $-5.9419905 .429368-2.078907$

H $-4.7895643 .786885-2.841150$

C $-3.5335550 .667297 \quad 1.125962$

O

H $-5.611071 \quad 0.6943180 .465737$

H $-2.799958 \quad 1.408145 \quad 1.466473$

O $-3.901735-0.0972182 .281085$

C $-2.844334-0.2948900 .140216$

C $-3.806521-1.336501-0.465339$

H $-2.059233-0.8305430 .688231$

O $-2.1997840 .463687-0.875124$

H -4.360608 -1.824537 0.344885

H -4.525602 -0.817884 -1.110360

H $-6.8453545 .176526-2.643540$

H $-6.2538595 .871600-1.126734$

H $-5.3946596 .192246-2.641380$

C $-2.6533125 .012844-1.771082$

H -4.544342 2.9567091 .794050

H $-4.307830 \quad 0.5165532 .916949$

H -1.751939 -0.157986 -1.472588

H $-2.9260495 .954893-2.258772$

H -2.341691 4.312115 -2.555480

H $-4.0954475 .080573-0.148912$

C $-1.899045-2.656279-3.408183$

O $-2.617909-1.802405-2.507750$

C $-3.092450-2.416450-1.304857$

C $-4.036966-3.584731-1.633245$

C $-3.376164-4.584968-2.585198$

C $-2.739882-3.885206-3.790524$

H -1.760262 -2.051695-4.313932

C $-0.512094-3.019661-2.868137$

H $-2.239141-2.789661-0.729960$

O $-5.230768-3.077842-2.227458$

H -4.318884 -4.083938 -0.698214

H -4.129686 -5.301094 -2.934967

O $-2.386027-5.310990-1.862698$

H $-2.118984-4.591517-4.352814$

O $-3.776718-3.463390-4.681399$

C $0.385818-1.806300-2.848092$

H $-0.539061-3.453493-1.865830$

H $-0.026465-3.763226-3.511416$

H -5.823356 -3.832606 -2.384724

H -1.994578 -5.959379-2.472551

H $-4.299108-2.779572-4.227917$

O $0.330358-1.201858-1.627817$

O $1.036372-1.426782-3.813442$

C $1.110372-0.009115-1.499687$

H $1.0311630 .338644-0.467364$

H $2.160398-0.217490-1.719813$

H $0.7220030 .768495-2.163848$

SCF Energy (B3LYP/6-31G**//MMFF)= -3245.92223981

07 00041

MM̄FF Geometry

C 2.0617113 .7105861 .965170

C 1.0770052 .9825802 .512878

C $1.215817 \quad 1.773783 \quad 3.403555$

O 0.6565060 .6375522 .698430

C 2.6564861 .3903353 .796731

C 2.7119950 .1688704 .696997

C $2.962553-1.0830744 .262741$

C $2.487108 \quad 0.437166 \quad 6.161229$

C $3.180003-1.5225382 .839958$

C $4.625180-1.9802132 .615795$

C $5.018929-2.1273931 .136251$

C $5.221916-0.7652830 .465760$

C $5.598447-0.913809-1.013046$

O $4.009977-2.8753380 .453980$

O $5.4688820 .387427-1.608836$

C $4.575211-1.849364-1.694772$

C $7.068978-1.311800-1.181306$

O $4.985860-2.118459-3.032843$

C $4.311874-3.167523-0.919289$

C $3.124555-3.977284-1.489364$

O $5.431639-4.057136-1.018905$

C $-0.663970 \quad 0.3631132 .858841$
O

C $-0.888956-0.9967842 .318251$

C $-2.099883-1.5626172 .422960$

C -2.451799-2.929249 2.030016

C $-1.334942-3.8379101 .591162$

C $1.766817-3.275348-1.425151$

C $0.665091-4.075500-2.101369$

C $-1.738557-3.867621-2.751569$

O $0.844906-5.179516-2.607394$

C $-2.539309-4.717776-1.817304$

C $-3.800064-4.437169-1.452257$

C $-4.648802-5.354976-0.620709$

C $-5.208609-4.6952980 .617334$

C $-6.474543-4.2444320 .649047$

C $-4.339527-4.6649251 .853009$

C $-3.743027-3.3103382 .113872$

N $-0.542408-3.390172-2.087058$

H 3.1004503 .4572112 .153184

H 0.0506303 .2729292 .299426

H $\quad 0.640329 \quad 1.958775 \quad 4.319817$

H 3.1415042 .2291794 .312951

H 3.2581421 .2120442 .897789

H $2.995246-1.8884684 .994994$

H $2.559134-0.4726586 .766437$

H $3.234837 \quad 1.1432756 .536885$

H 1.4924440 .8634576 .324420

H $2.495956-2.3548462 .632137$

H $2.908258-0.7363732 .133225$

H $5.331045-1.3004263 .109689$

H $4.741579-2.9587003 .101074$

H $5.956505-2.6954331 .111855$

H $5.981467-0.178248 \quad 0.997305$

H $4.297819-0.1754430 .521255$

H $5.6375430 .292888-2.562228$

H $3.645184-1.272742-1.762965$

H $7.722899-0.529005-0.777809$

H $7.336205-1.408261-2.239325$

H $7.322856-2.244897-0.674019$

H $4.237714-2.530055-3.496596$

H $3.345422-4.282920-2.520261$

H $3.044233-4.918797-0.927028$

H $\quad 6.088529-3.667694-1.617497$

H $-0.036938-1.5148251 .892333$

H -2.908889 -0.9798632 .860943$

H -1.672772 -4.828810 1.281650

H $-0.616259-3.9828722 .405434$

H -0.807074 -3.4102170 .734193$

H $1.479475-3.100083-0.382531$

H $1.815655-2.311347-1.938166$

H -1.453848 -4.455677 -3.630418

H $-2.295552-2.988660-3.090857$

H -2.077539 -5.639297-1.470884

H $-4.272045-3.530784-1.824513$

H $-4.098424-6.260052-0.334755$

H -5.471574 -5.706841 -1.257498

H $-6.894005-3.8067301 .548998$

H -7.116047 -4.298364 -0.224331

H $-3.585753-5.4559871 .805856$

H $-4.951044-4.9290842 .727119$

H -4.476434 -2.574259 2.447067

H $-0.600529-2.505581-1.591310$

C $1.9952664 .590509-0.420031$

O $0.9760173 .669779-0.861360$

C $1.5745152 .729798-1.772463$

C $2.9354283 .310419-2.144282$

C $3.3327713 .984955-0.845945$

H $1.7323191 .803232-1.207765$

C $0.6521672 .465241-2.966231$

H $3.6421812 .539152-2.464412$

H $2.8563594 .051625-2.948033$

C $4.4493745 .001944-0.998443$

H $3.6554113 .204912-0.145810$

C $-0.762828 \quad 1.965730-2.606461$

O $0.4975343 .674161-3.721849$

H $\quad 1.1337601 .751027-3.644509$

H $-1.3557192 .786691-2.188058$

O $-1.4054521 .589609-3.832108$

C $-0.8013840 .760834-1.650572$ 
C $-2.1634480 .050079-1.543262$ H $-0.4857531 .081947-0.651662$ O $0.148532-0.217457-2.081274$ H -2.398611 -0.448859-2.492085 H -2.039086 $-0.759023-0.811639$ H $5.3590674 .520995-1.372546$ H $4.1764715 .796436-1.701286$ H $4.6840705 .468122-0.036401$ C 1.8211704 .8950581 .070419 H $0.1398554 .350782-3.121520$ H -1.386863 2.365524 -4.418416 H $-0.091952-0.483797-2.985363$ H $0.8063885 .277353 \quad 1.236843$ H 2.5089085 .6953381 .368179 H $1.8164655 .520547-0.976001$ C -3.9903502 .4515880 .658647$ O -3.0172981 .5372420 .135128$ C $-3.3369900 .949303-1.128154$ C $-4.6543770 .158858-1.044624$ C $-5.7902751 .020579-0.486282$ C $\quad \begin{array}{llll}-5.365219 & 1.771450 & 0.780966\end{array}$ H -3.641043 2.6842651 .672940 C $-4.0380613 .765319-0.138687$ H -3.446004 $1.739202-1.877453$ O $-4.474664-0.980510-0.207521$ H -4.911306 $-0.205023-2.046786$ H $-6.6499020 .378721-0.257703$ O $-6.1941771 .944771-1.491805$ H -6.1196172 .5203111 .047176$ O C $-2.7377574 .535182-0.061294$ H -4.245765 3.588021-1.196919 H -4.8232724 .4118680 .269790$ H -5.305076 -1.486191 -0.215882 H $-6.9314372 .466562-1.131607$ H $-4.6117760 .200305 \quad 1.677353$ O $-2.8200635 .626533-0.878856$ O $\quad \begin{array}{llll}-1.774987 & 4.227478 & 0.626027\end{array}$ C -1.654451 $6.455841-0.919400$ H -1.886872 $7.339577-1.519465$ H $-1.376187 \quad 6.7802480 .087983$ H -0.830093 5.916539-1.393859

SCF Energy (B3LYP/6-31G**//MMFF) $=-3245.92262527$

07_00042

MM̄FF Geometry

C -5.3967452 .3995460 .697192$

C -4.0765412 .6276520 .623324$

C -3.221524 2.9949021 .809337

O -2.0771872 .1124411 .848250$

C -2.6972754 .4340771 .647615$

C -1.808984 4.8853142 .791428

C -0.5015455 .1944662 .680066$

C -2.4913345 .0069064 .131049$

C $0.364005 \quad 5.195748 \quad 1.449725$

C 1.3822364 .0542041 .481080

C 2.4459604 .1513900 .375486

C $1.8555443 .997115-1.029402$

C $2.9430893 .941163-2.115902$

$\begin{array}{lllll}\text { O } & 3.397690 & 3.107956 & 0.630371\end{array}$

O $2.3334603 .481959-3.332518$

C $4.0287312 .920875-1.707487$

C $3.4796295 .340374-2.438569$

O $5.1323412 .957904-2.608682$

C $4.5238213 .105453-0.258876$

C 5.4826251 .9800320 .193734

O $5.255846 \quad 4.334248-0.166397$

C -2.2236220 .9171522 .480479$

O $-3.236402 \quad 0.472245 \quad 2.995475$

C -0.9166350 .2268422 .418162$

C $-0.847969-1.0904362 .659153$

C $0.354818-1.9139032 .541603$

C $1.682775-1.2097552 .441870$

C $4.897824 \quad 0.565462 \quad 0.162751$

C $5.813379-0.4424650 .839332$

C $5.943062-2.8417421 .459409$

O $6.913848-0.1517671 .300518$

C $4.962670-3.9407701 .708022$
C $4.481625-4.2454022 .923022$

C $3.461362-5.3198373 .174141$

C $2.092743-4.7390833 .470555$

C $1.629048-4.6984944 .731272$

C $1.319831-4.2583162 .258858$

C $0.227267-3.2574952 .520381$

N $5.247124-1.7090350 .877060$

H $-5.8994722 .475771 \quad 1.658274$

H $-3.5778122 .561749-0.340937$

H -3.7894572 .9080192 .743731$

H $-3.5431585 .130667 \quad 1.573393$

H $-2.166176 \quad 4.5083600 .693494$

H 0.0244795 .5051883 .582757

H -1.848189 5.4740484 .884591

H -3.3912845 .6246104 .044111$

H -2.7777914 .0213814 .510466$

H -0.2290655 .1473720 .534326$

H $0.8916206 .157188 \quad 1.416496$

H 1.8954804 .0393432 .452533

H $0.866423 \quad 3.0874821 .412008$

H 2.9516375 .1191380 .474716

H $1.1497654 .805220-1.254117$

H $1.2658693 .072610-1.072552$

H $1.9588172 .599788-3.170050$

H $3.5865331 .923739-1.805678$

H $2.6729505 .979514-2.817987$

H $4.2302425 .303347-3.235920$

H $3.9182455 .842796-1.574026$

H $4.7781902 .837931-3.506532$

H $6.4059062 .012532-0.399645$

H 5.7945502 .1978631 .225312

H 5.8339924 .2915030 .613299

H -0.0529570 .8118922 .122753$

H -1.763637 -1.626965 2.904193

H $1.796019-0.4829873 .254262$

H $1.774053-0.6843821 .488461$

H $2.534242-1.8887492 .522096$

H 3.9333070 .5438590 .681540

H $4.7463140 .237690-0.871421$

H $6.446178-2.5174922 .377056$

H $6.713705-3.1688020 .754221$

H $4.619856-4.5007920 .841251$

H $4.816534-3.6762863 .787208$

H $3.814010-5.9359974 .011095$

H $3.385236-6.0031092 .318658$

H $0.647206-4.3082294 .974074$

H $2.226221-5.0650835 .560181$

H $\quad 0.876579-5.1320361 .763576$

H $2.006598-3.8345831 .516486$

H -0.765419-3.696392 2.620604

H $4.413116-1.8841690 .322957$

C $-6.9576690 .720237-0.341183$

O $-5.978749-0.337514-0.356123$

C $-6.382752-1.336264-1.309912$

C $-7.864736-1.091642-1.554988$

C $-7.9219680 .422302-1.492828$

H $-5.832212-1.126816-2.235593$

C $-6.020760-2.738502-0.807750$

H $-8.201726-1.496676-2.513863$

H $-8.487054-1.524237-0.763282$

C $-9.3223160 .970908-1.279715$

H -7.521625 $0.825824-2.432710$

C $-4.564928-2.883700-0.321633$

O $-6.869298-3.0830940 .295307$

H $-6.233425-3.475746-1.590349$

H $-4.434175-2.344366 \quad 0.624987$

O $-4.331762-4.267175-0.030371$

C $-3.499560-2.409182-1.325643$

C $-2.080188-2.577156-0.770230$

H $-3.670787-1.352664-1.546735$

O $-3.623667-3.126528-2.549834$

H -2.015428 -2.0842360 .206189$

H -1.876978 -3.638973 -0.575980

H $-9.9787400 .681295-2.106781$

H $-9.7645130 .593074-0.351724$

H $-9.3086202 .063987-1.225667$

C $-6.2541892 .070643-0.493743$

H $-6.749501-2.4040270 .981496$ 
H $-5.007360-4.5456350 .611636$ H -3.511225 -4.071483 -2.348915 H $-6.9979922 .867863-0.605159$ H -5.641684 2.065101-1.404168 H -7.4752840 .6606360 .624751$ C $1.470420-1.867326-1.578412$ O $0.253818-2.226603-0.915740$ C $-0.949733-2.060703-1.680896$ C $-1.114833-0.594283-2.113897$ C $0.131691-0.072681-2.834943$ C $1.415422-0.414027-2.073910$ H $2.235843-1.919470-0.793750$ C $1.827406-2.906286-2.643892$ H -0.894964 -2.705181 -2.565940 O $-1.3702620 .210996-0.964594$ H $-1.981249-0.508880-2.779500$ H $0.052566 \quad 1.015917-2.943959$ O $0.169995-0.636080-4.141862$ H $2.291655-0.197142-2.693220$ O $1.5060220 .444062-0.933195$ C $3.265849-2.788781-3.080051$ H $1.662230-3.921391-2.260478$ H $\quad 1.191506-2.836185-3.531087$ H -1.520241 $1.121703-1.270349$ H $\quad 0.954219-0.279667-4.592997$ H $\quad 0.740234 \quad 0.262967-0.361767$ O $4.084883-3.360546-2.151934$ O $3.619008-2.238712-4.115120$ C $5.483795-3.299373-2.446999$ H $6.016838-3.880867-1.690587$ H $5.689647-3.735043-3.429501$ H $5.829139-2.262233-2.403235$ SCF Energy (B3LYP/6-31G**//MMFF) $=-3245.92341976$

\section{7 _00043}

MM̄FF Geometry

C $3.1663770 .861583-3.296819$

C $2.0192771 .182028-2.681008$

C $\quad 0.737281 \quad 1.508043-3.396070$

$\begin{array}{llll}\text { O } & -0.261157 & 0.622468 & -2.833015\end{array}$

C $0.3008052 .964084-3.147778$

C $1.2563074 .017507-3.670412$

C $2.0341594 .785728-2.880960$

C $1.2531764 .211855-5.162707$

C $2.1617424 .673069-1.387930$

C $1.1571955 .563919-0.646023$

C 0.8602615 .0922200 .787211

C 2.1040285 .0687691 .677079

C 1.7951924 .5105773 .075659

O $0.286673 \quad 3.7837020 .694794$

O 3.0464414 .2256983 .720555

C 1.0503363 .1674752 .918869

C 1.0796675 .5415683 .955923

O $\quad 0.6395132 .662577 \quad 4.187977$

C $-0.1475253 .225808 \quad 1.938745$

C $-0.658413 \quad 1.7888601 .663529$

O -1.1876704 .0029832 .528298$

C -1.394352 $0.399050-3.548571$

O $-1.6716100 .842960-4.650602$

C $-2.261336-0.474987-2.727111$

C $-3.540714-0.668272-3.079410$

C $-4.520823-1.480987-2.354567$

C $-4.073962-2.131648-1.071760$

C -1.7259801 .6554330 .567965$

C -3.1500441 .8890721 .040828$

C -5.4731232 .1050780 .157836$

O -3.4501862 .0438312 .221276$

C -6.1698850 .7815230 .201865$

C -7.209258 $0.468456-0.586880$

C $-7.948035-0.837805-0.519830$

C $-8.067238-1.514252-1.867596$

C $-9.189279-1.404173-2.598832$

C $-6.922527-2.390096-2.324077$

C $-5.766308-1.594910-2.861192$

N $-4.0486321 .893290-0.017818$

H $3.2036390 .826785-4.382522$

H $1.9937521 .213903-1.593575$

H $0.832696 \quad 1.303698-4.469842$
H $\quad 0.1011893 .101029-2.078334$

H $-0.6806393 .134399-3.610169$

H $2.6594505 .547974-3.341819$

H $1.9168825 .024506-5.476642$

H $1.5864273 .300512-5.668779$

H $\quad 0.2447614 .456023-5.512402$

H $2.0617913 .627412-1.082239$

H $3.1831224 .958828-1.107301$

H $1.5245166 .597373-0.630654$

H $0.2062975 .571375-1.195369$

H 0.1102995 .7749691 .204275

H 2.5486406 .0690841 .748491

H 2.8754334 .4296861 .227268

H 3.5576285 .0522153 .761805

H 1.7801952 .4436152 .530677

H 1.6908126 .4463884 .058647

H 0.9375975 .1615304 .973630

H 0.1081415 .8438633 .559691

H $-0.1459343 .164316 \quad 4.465624$

H $\quad 0.197242 \quad 1.187824 \quad 1.328217$

H $-1.030563 \quad 1.3452582 .593734$

H -2.014555 3.8268082 .053590

H -1.824644 -0.922472 -1.841119

H $-3.910879-0.181915-3.981080$

H -3.300935 -2.881302 -1.273720

H $-4.878223-2.635213-0.531746$

H -3.667920 -1.384841-0.380249

H $-1.5105492 .317543-0.276634$

H -1.6961130 .6242350 .194353$

H $-5.8139542 .724572-0.677754$

H -5.6520682 .6482141 .091180$

H -5.8205470 .0584800 .935232$

H $-7.574977 \quad 1.205185-1.298771$

H -8.948888 $-0.632291-0.117759$

H -7.479703 -1.527230 0.193603

H $-9.294825-1.910182-3.552792$

H -10.025763 -0.800670 -2.261903

H -7.258330 -3.056979 -3.129810

H -6.638385 -3.063872 -1.511039

H -5.995152 -1.077944 -3.794283

H $-3.7125691 .692842-0.955442$

C $4.871791-0.894293-2.747239$

O $3.904885-1.771752-2.134551$

C $4.600737-2.770852-1.368851$

C $6.059956-2.691859-1.800951$

C $6.208321-1.209063-2.073953$

H $4.529585-2.465696-0.318493$

C $3.953155-4.146320-1.550494$

H $6.742286-3.058185-1.027966$

H $6.246661-3.264984-2.716431$

C $7.422762-0.864550-2.918644$

H $6.279257-0.681955-1.112794$

C $2.440511-4.191551-1.253438$

O $4.135425-4.562150-2.910616$

H $4.485475-4.879776-0.932894$

H $1.890814-3.682188-2.054009$

O $2.037135-5.566793-1.321626$

C $2.017131-3.6052250 .106863$

C $0.535459-3.894650 \quad 0.421921$

H $2.190404-2.5234250 .101956$

O $2.843255-4.1762311 .120809$

H -0.074053 -3.691616 -0.466912

H $0.439062-4.9589910 .669941$

H $8.343351-1.172049-2.411922$

H $7.390618-1.367020-3.891246$

H $7.4803340 .213577-3.098245$

C $4.4397340 .559547-2.558278$

H $3.725733-5.439558-3.002557$

H $2.460903-6.033036-0.580426$

H $2.602300-3.7600861 .965641$

H $5.2174541 .236782-2.928794$

H $4.3101150 .769938-1.489166$

H $4.904447-1.151566-3.813967$

C $0.401904-2.6147843 .940452$

O $0.742280-3.3644002 .767425$

C $-0.016124-3.0586661 .595385$

C -1.506751 -3.3419911 .842469$

C $-2.020135-2.6037073 .083531$ 
C $-1.088094-2.7864944 .286596$ H $0.994807-3.0706974 .743506$ C $0.829323-1.1480523 .823631$ H $0.118676-2.001397 \quad 1.341281$ O $-1.703607-4.7450072 .010595$ H -2.073619 -3.0349690 .957109$ H $-3.017263-2.9777483 .346663$ O $-2.144150-1.2148972 .796362$ H $-1.367053-2.0906675 .086658$ O $-1.285877-4.1097474 .793619$ C $2.320379-1.0310923 .612248$ H $0.320668-0.6366483 .004418$ H $0.582359-0.5926744 .735891$ H $-2.656170-4.8956562 .134268$ H -2.774472 -1.120830 2.062052 H $-0.744075-4.2012495 .595890$ O $2.576324-0.5574812 .359089$ O $3.155455-1.3376834 .453124$ C $3.959283-0.4117192 .023667$ H $4.022727-0.0669130 .988317$ H 4.4274250 .3344762 .672413 H $4.474193-1.3738902 .104827$

SCF Energy (B3LYP/6-31G**//MMFF)= -3245.91493909

07 00044

MM̄FF Geometry

C -1.074501 -2.164362 2.598466

C $-1.766193-1.4247173 .479368$

C $-1.560500 \quad 0.0487043 .754846$

O $-0.483387 \quad 0.5330112 .916140$

C -2.8128680 .8942813 .449720$

C $-4.099147 \quad 0.440334 \quad 4.108765$

C $-5.1768350 .004795 \quad 3.424940$

C $-4.140068 \quad 0.5559605 .608758$

C $-5.275826-0.1483521 .931431$

C $-5.725677 \quad 1.1598091 .269406$

C $-5.466814 \quad 1.228748-0.242139$

C $-6.2695880 .202384-1.038677$

C $-5.960593 \quad 0.296870-2.542864$

O $-4.066903 \quad 1.032359-0.461253$

O $-6.499805-0.874465-3.175011$

C $-4.429510 \quad 0.255572-2.739411$

C $-6.6532961 .498371-3.196045$

O $-4.0799990 .472742-4.104752$

C -3.644666 $1.220843-1.815382$

C $-2.1257010 .915400-1.909047$

O $-3.8760632 .561960-2.254427$

C 0.1960671 .6464913 .286963

O $\quad 0.0253842 .3198594 .288907$

C 1.1902891 .9073172 .221008

$\begin{array}{llll}\text { C } & 1.891150 & 3.049879 & 2.216782\end{array}$

C 2.8822803 .4454821 .212075

C $3.1436982 .487878 \quad 0.078718$

C $-1.2865951 .338528-0.696097$

C -1.229394 $2.834562-0.478203$

C $-0.2996464 .922550-1.471398$

O $-1.860363 \quad 3.377993 \quad 0.425271$

C $1.1271055 .282994-1.739503$

C $1.7970626 .217555-1.047804$

C $3.2227376 .598359-1.329484$

C $4.0894646 .601347-0.089223$

C $4.5088167 .754980 \quad 0.457897$

C 4.5392205 .2678610 .468022

C 3.4988924 .6376321 .352497

N $-0.4180283 .477890-1.397706$

H $-0.285098-1.7075582 .007627$

H -2.531291 -1.919426 4.074038

H -1.2693650 .1562824 .807231$

H $-2.924016 \quad 0.9705152 .362139$

H -2.6246901 .9315203 .758634$

H $-6.073645-0.268403 \quad 3.977653$

H -5.1209310 .2920526 .018030$

H -3.402160 -0.1098306 .066696$

H -3.920557 1.5835985 .916300

H $-4.320654-0.4968451 .525762$

H $-5.996258-0.9449331 .710141$

H $-6.7906151 .328991 \quad 1.472218$

H -5.1814451 .9957441 .729306$
H $-5.7352722 .243155-0.560978$

H $-7.3448620 .321372-0.857524$

H $-6.016823-0.812435-0.703246$

H $-7.455829-0.897239-2.998218$

H -4.116204 $-0.771174-2.510590$

H $-7.737866 \quad 1.445067-3.042365$

H $-6.5062081 .496180-4.281825$

H $-6.3102692 .457461-2.802694$

H $-4.1690641 .424163-4.284827$

H - $1.991656-0.168608-1.999463$

H $-1.7138941 .362325-2.822328$

H $-3.1146283 .111533-2.012147$

H $1.304661 \quad 1.151343 \quad 1.451595$

H 1.7183483 .7684933 .016703

H 3.5218781 .5353850 .465547

H $3.8788222 .849331-0.642310$

H $2.2251512 .294657-0.485745$

H -1.6537300 .8633910 .219949$

H $-0.258750 \quad 0.977183-0.824908$

H $-0.6796965 .378827-0.551757$

H $-0.9280595 .251533-2.305337$

H $1.6294474 .763541-2.552334$

H $1.2867906 .758968-0.254339$

H $3.2140957 .597701-1.783804$

H $3.6724465 .934065-2.078031$

H 5.1526647 .7647501 .331160

H 4.2134158 .7153980 .048556

H 5.4431125 .4042351 .076828

H $4.8514964 .624707-0.358577$

H 3.2423535 .2501782 .218471

H $-0.0279812 .935384-2.163351$

C $-1.556427-3.991016 \quad 0.905410$

O $-0.426890-3.6144330 .087716$

C $-0.927278-3.035297-1.132161$

C $-2.397528-3.429553-1.204265$

C $-2.784002-3.3480710 .259483$

H $-0.865954-1.949140-1.010217$

C $-0.089940-3.479107-2.333326$

H $-2.978581-2.759250-1.842794$

H $-2.523780-4.452676-1.577883$

C $-4.095169-4.036795 \quad 0.589637$

H $-2.860404-2.288906 \quad 0.535490$

C $1.389230-3.038030-2.284639$

O $-0.120966-4.908938-2.421777$

H $-0.550339-3.111770-3.257971$

H $1.915473-3.578354-1.489720$

O $1.999251-3.438446-3.518404$

C $1.596480-1.521130-2.111961$

C $3.032433-1.024198-2.362968$

H $1.282915-1.226053-1.104298$

O $0.743243-0.834466-3.034359$

H $3.291932-1.154215-3.421496$

H $3.0234530 .061647-2.196952$

H $-4.921355-3.5719090 .041619$

H $-4.069986-5.0985940 .323226$

H $-4.315109-3.9613031 .659150$

C $-1.300038-3.6321792 .370758$

H $0.226118-5.261404-1.584254$

H $1.857971-4.395981-3.613895$

H $\quad 0.8439300 .119469-2.875500$

H $-0.393978-4.1505832 .706027$

H -2.127722 -4.0008082 .987772$

H $-1.627713-5.0843420 .832068$

C $4.632598-2.0066590 .868337$

O $3.751646-1.440038-0.111208$

C $4.109286-1.670440-1.476144$

C $5.507814-1.102621-1.777539$

C $6.555220-1.616375-0.783935$

C $6.071724-1.4995690 .665175$

H $4.270691-1.617527 \quad 1.828600$

C $4.537758-3.5400710 .916585$

H $4.114593-2.747090-1.671257$

O $5.4892360 .323478-1.740556$

H $5.789998-1.388311-2.797543$

H $7.480614-1.038548-0.898694$

O $6.855819-2.979918-1.064026$

H $6.754888-2.0375861 .332946$

O $6.123209-0.1185751 .035454$ 
C $3.179636-4.0254241 .375849$

H $4.725258-3.988775-0.062159$

H $\quad 5.276260-3.9268941 .628369$

H $5.2288350 .593283-0.843794$

H $7.195326-3.024870-1.974123$

H $5.874517-0.0558501 .973523$

O $3.165660-5.3911081 .327823$

O $2.255066-3.3097771 .733300$

C $1.945341-6.0156181 .737000$

H $2.104946-7.0970341 .749296$

H $1.667011-5.6925792 .744480$

H $1.151114-5.7871581 .021239$

SCF Energy (B3LYP/6-31G**//MMFF) $=-3245.90736530$

07 00045

MM̄FF Geometry

C $0.1536252 .016428-2.220047$

C $-0.7440451 .254415-2.864064$

C $-0.595332-0.210003-3.214110$

O $0.649750-0.756838-2.725174$

C - $-1.742212-0.991565-2.546246$

C -1.634144 -2.492855 -2.716738

C $-1.213268-3.328972-1.746334$

C -2.071359-3.025784 -4.054142

C $-0.722637-2.933158-0.379058$

C $0.434569-3.8196350 .099597$

C $1.157286-3.2941161 .353466$

C $0.208233-2.9419482 .503779$

C $0.956176-2.3667673 .716453$

O $1.929075-2.1453330 .974552$

O $-0.032121-1.7840854 .580077$

C $1.887788-1.2344103 .230373$

C $1.637686-3.4702894 .532750$

O $2.692044-0.7456854 .300928$

C $2.765127-1.6362582 .024971$

C $3.580751-0.4509171 .466850$

O $3.716355-2.6208882 .451280$

C $1.743782-0.662230-3.525814$

O $1.821811-0.109161-4.610558$

C $2.856325-1.342124-2.828497$

C $4.116727-0.967947-3.090390$

C $5.324784-1.516815-2.472760$

C $5.187772-2.788016-1.676193$

C $2.759071 \quad 0.7278990 .945164$

C $3.651071 \quad 1.6714590 .163646$

C 5.5856383 .2059610 .432006

O $3.6194381 .723091-1.063661$

C 6.8133692 .3498720 .387352

C $7.2918991 .792858-0.735667$

C $8.5050570 .908719-0.776250$

C $8.156149-0.564181-0.787113$

C $8.195222-1.2832490 .347677$

C $7.847983-1.224772-2.112942$

C $6.491744-0.861810-2.647665$

N 4.5006992 .4096410 .973852

H $1.1136541 .601219-1.933920$

H -1.696763 $1.702985-3.140830$

H $-0.655405-0.331053-4.302855$

H -2.707023 -0.658182 -2.952888

H -1.787204 -0.738389-1.479912

H -1.217363 -4.398647 -1.949471

H -2.019352 -4.118575 -4.102584

H -3.106400 -2.735235 -4.260635

H -1.433500 -2.629264 -4.850282

H $-0.396276-1.889341-0.360824$

H -1.562076 -3.0355660 .316871$

H $0.047461-4.8256230 .306094$

H $1.171142-3.916045-0.709099$

H $1.849973-4.0842571 .667144$

H $-0.391080-3.8136052 .794377$

H $-0.509322-2.1793312 .174757$

H $\quad 0.436635-1.3485705 .312835$

H $1.237883-0.4009332 .936150$

H $0.891893-4.1846584 .902426$

H $2.124833-3.0614905 .424765$

H $2.378862-4.0328413 .961593$

H $3.402004-1.3916764 .456540$

H $4.295012-0.1056512 .225946$
H $4.205546-0.8180370 .641684$

H $4.450520-2.6359311 .814903$

H $2.604356-2.103112-2.099241$

H $4.280766-0.159982-3.802789$

H $4.524309-2.643454-0.817921$

H $6.136409-3.155496-1.279319$

H $4.776237-3.587805-2.302271$

H 1.9566920 .3907240 .280030

H 2.2939331 .2823881 .766211

H 5.7441234 .0612451 .095653

H $5.3104633 .580725-0.559495$

H 7.3201322 .1677141 .332047

H $6.7785151 .973734-1.676566$

H 9.1554911 .1326350 .080196

H $9.1041231 .157742-1.660602$

H $7.977390-2.3459400 .352907$

H $8.441837-0.8233291 .299043$

H $7.955648-2.313279-2.042251$

H $8.606320-0.925280-2.847907$

H $6.4899320 .052311-3.242099$

H 4.4942752 .2435111 .975954

C $0.1107843 .610618-0.292296$

O -0.6939492 .6623280 .434869$

C -1.420467 3.3522661 .463304

C -0.7056534 .6843281 .644856$

C $-0.295464 \quad 4.994740 \quad 0.217414$

H -2.424115 3.5388591 .063344

C -1.5349642 .4824022 .719801$

H -1.353803 5.4491862 .082725

H 0.1841854 .5876802 .277363

C 0.8060826 .0347530 .109144

H -1.178702 $5.353788-0.327781$

C -1.9822631 .0285832 .462397$

O $-0.2635112 .417223 \quad 3.378431$

H -2.212634 $2.964598 \quad 3.433724$

H -1.1554160 .4529152 .027659$

$\begin{array}{llll}\text { O } & -2.260641 & 0.428330 & 3.733609\end{array}$

C $-3.2232280 .872573 \quad 1.565976$

C $-3.693417-0.5927081 .479478$

H -2.9860151 .2305010 .558682$

O -4.2823491 .6869292 .064820$

H -2.821871 -1.245659 1.361308

H $-4.197882-0.8582162 .416724$

H $\quad 0.479067 \quad 6.987780 \quad 0.537638$

H 1.7098555 .7191490 .641374

H $1.0749266 .208636-0.937478$

C $-0.1079473 .434998-1.798564$

H 0.3773352 .0585802 .741357

H -1.4509610 .4952794 .268657$

H $-4.478771 \quad 1.3928942 .970755$

H $0.5614344 .102551-2.352316$

H -1.136344 $3.713807-2.057787$

H $1.1552373 .394773-0.035938$

C $-6.817694-0.141025-0.534696$

O $-5.843690-0.0767650 .515390$

C $-4.654918-0.8402280 .300948$

C $-4.998395-2.3302330 .149132$

C $-6.036276-2.549003-0.956848$

C $-7.228514-1.596206-0.816813$

H $-7.6945060 .383073-0.132593$

C $-6.3556190 .613706-1.786723$

H -4.160792 -0.488432 -0.610582

O $-5.503815-2.8308291 .385140$

H $-4.080759-2.883990-0.079544$

H $-6.397425-3.584027-0.921232$

O $-5.429728-2.347768-2.229844$

H -7.854931 -1.629107 -1.716041

O $-8.042914-2.0283430 .277223$

C $-6.1811352 .083684-1.486676$

H $-5.4196260 .224729-2.197568$

H $-7.0997700 .533654-2.587925$

H -5.685091 -3.778665 1.266340

H -4.696447 -2.981561 -2.308373

H $-8.348213-2.9296420 .077185$

O $-4.8554752 .402181-1.462373$

O $-7.1121962 .851478-1.279861$

C $-4.5584893 .770820-1.170901$

H $-5.0426914 .430848-1.896977$ 
H $-4.8759744 .015513-0.152979$

H $-3.4770083 .906572-1.243747$

SCF Energy (B3LYP/6-31G**//MMFF)= -3245.90204832

07 00046

MM̄FF Geometry

C $2.6493192 .676581-1.951132$

C $2.0019213 .849873-2.040077$

C $0.6011984 .134140-1.553286$

O $-0.0828242 .893157-1.268738$

C $0.5156705 .095690-0.346485$

C 1.5639164 .9178760 .733189

C 1.4833854 .0467241 .756616

C 2.7407955 .8584830 .644324

C 0.3711633 .0938182 .086060

C 0.8179131 .6346821 .939682

C -0.1205590 .6693512 .678453$

C $0.315554-0.7779062 .454763$

C $-0.625614-1.7744093 .143487$

O -1.4564040 .8496532 .198662$

O $-0.317578-3.0730432 .615793$

C $-2.080309-1.4355842 .756707$

C $-0.358170-1.8480084 .650776$

O $-2.999233-2.2450333 .487941$

C -2.4338130 .0652692 .898509$

C $-3.818230 \quad 0.3335612 .265826$

O -2.4844650 .3970054 .287547$

C $-1.4265492 .863726-1.480968$

O $-2.1504283 .812967-1.736966$

C $-1.8621361 .451225-1.408783$

C $-3.1283571 .144803-1.727540$

C $-3.715446-0.193637-1.768062$

C $-2.800855-1.371760-1.568888$

C -4.2198801 .8135822 .217967$

C -5.4767112 .0142251 .392677$

C $-7.896178 \quad 1.4226541 .319954$

O $\begin{array}{lllll}-5.454571 & 2.610568 & 0.318281\end{array}$

C -8.0100880 .1267270 .579678$

C $-8.2055770 .037712-0.744513$

C $-8.305732-1.265613-1.484118$

C $-7.235286-1.424177-2.541984$

C $-7.549872-1.466934-3.847640$

C $-5.804863-1.614140-2.079123$

C $-5.040976-0.317360-1.990216$

N $-6.604517 \quad 1.463273 \quad 1.979526$

H $2.1706521 .825601-1.474257$

H $2.5056524 .686369-2.520625$

H $0.0842794 .609236-2.398662$

H -0.4813995 .0414680 .106532$

H $0.5964086 .123804-0.726018$

H 2.3026534 .0302732 .475082

H 3.4940655 .6591961 .413955

H $3.2361595 .777290-0.326927$

H $2.404214 \quad 6.8929120 .771344$

H $\quad 0.0732193 .287578 \quad 3.124209$

H $-0.5187703 .272879 \quad 1.475673$

H $\quad 0.846273 \quad 1.3841690 .871943$

H 1.8365231 .5045792 .327281

H $-0.0851180 .919157 \quad 3.745530$

H $1.348619-0.9325472 .787862$

H $0.308899-0.9872551 .377130$

H $-0.957019-3.7032732 .990054$

H $-2.202993-1.7229851 .704740$

H $\quad 0.680717-2.1453064 .838652$

H $-0.977508-2.6158005 .127258$

H $-0.530958-0.8990335 .162903$

H -3.062180 -1.881271 4.387539

H $-3.823220-0.0491971 .238526$

H -4.584720 -0.2236492 .818077$

H $-2.983804 \quad 1.2214094 .395821$

H $-1.1259740 .704982-1.133055$

H $-3.8078601 .953816-1.993184$

H $-2.287027-1.305966-0.605081$

H -2.048282 -1.410651 -2.364162

H $-3.327330-2.330260-1.576859$

H $-4.4012912 .212733 \quad 3.221577$

H -3.426504 2.4189481 .765348

H -7.9971682 .2885460 .657671$
H $-8.666051 \quad 1.4806012 .095192$

H -7.925365 -0.7848251 .167034$

H $-8.3015260 .947789-1.332243$

H $-9.303948-1.315155-1.937810$

H -8.249301 -2.117542 -0.794843

H $-6.786728-1.606766-4.606257$

H $-8.574480-1.358716-4.187137$

H $-5.295150-2.290368-2.777746$

H $-5.805673-2.111634-1.101642$

H $\quad-5.6276280 .592054-2.117697$

H -6.5102480 .9570302 .854897$

C $5.0887932 .110107-1.459922$

O $4.7872280 .872368-0.789897$

C 5.2947840 .9520640 .560146

C 6.0287342 .2874910 .673001

C $5.2936173 .133122-0.348158$

H $4.422413 \quad 0.975748 \quad 1.222857$

C $6.166510-0.2714930 .869743$

H 5.9802132 .6988921 .686078

H 7.0871912 .1955950 .403038

C $6.0543614 .374570-0.775805$

H 4.3322553 .4270660 .087410

C $5.447421-1.6227370 .681510$

O $7.305442-0.264729-0.002369$

H $6.566796-0.1967111 .887071$

H $5.195768-1.757958-0.375773$

O $6.373460-2.6655051 .012321$

C $4.191725-1.7980141 .555289$

C $3.547938-3.1950591 .450641$

H $3.450293-1.0363621 .293501$

O $4.554121-1.5767842 .921012$

H $4.284573-3.9695681 .695665$

H $2.775737-3.2621862 .228755$

H 6.2324565 .0307360 .082538

H $7.0260994 .119933-1.211844$

H $5.4884854 .941155-1.521780$

C $4.0244192 .437991-2.508971$

H $\quad 6.975016-0.282220-0.916953$

H $7.165091-2.5352590 .462673$

H $3.741741-1.6280743 .452961$

H $3.9442501 .593830-3.205571$

H $4.3534633 .302730-3.097604$

H $6.0368471 .943822-1.989926$

C $1.191308-2.567563-1.356945$

O $1.850872-2.528273-0.087167$

C $2.914835-3.469650 \quad 0.079676$

C $2.371422-4.900949-0.035357$

C $1.628427-5.104403-1.361092$

C $0.632286-3.973334-1.643950$

H $0.340625-1.884191-1.241144$

C $2.103590-1.994165-2.444846$

H $3.665102-3.311495-0.701500$

O $1.493715-5.1911601 .050408$

H $3.208871-5.6047310 .033954$

H $1.086502-6.057950-1.330876$

O $2.566667-5.173260-2.430216$

H $0.275351-4.035522-2.678014$

O $-0.501254-4.185903-0.795113$

C $1.306439-1.456791-3.606544$

H $2.720115-1.179997-2.042114$

H $2.819818-2.725926-2.829479$

H $\quad 0.785799-4.5239371 .047690$

H $3.161141-5.921798-2.251741$

H $-1.174468-3.527562-1.034242$

O $0.946291-0.163726-3.365811$

O $1.021166-2.114130-4.598988$

C $0.1828660 .465898-4.399225$

H $-0.799150-0.009104-4.480680$

H $0.045592 \quad 1.516278-4.129550$

H $0.7162240 .418705-5.353580$

SCF Energy (B3LYP/6-31G**//MMFF) $=-3245.91612043$

07 00047

MM̄FF Geometry

C $-4.197172-2.910824-2.150127$

C $-2.867358-2.920152-1.970674$

C $-1.893644-2.743984-3.106493$

O $-1.090608-1.567866-2.843506$ 
C $-0.938418-3.945338-3.219684$

C $0.000692-3.835566-4.408159$

C $1.343506-3.763570-4.323583$

C $-0.656499-3.827117-5.765952$

C $2.207012-3.845338-3.097338$

C $2.539908-2.470518-2.513761$

C $3.788007-2.477950-1.614903$

C $3.680693-3.459564-0.446614$

C $4.911301-3.3886220 .470498$

O $3.955884-1.144761-1.113264$

O $4.597440-4.088547 \quad 1.684337$

C $5.169768-1.9184950 .850963$

C $6.122987-4.101640-0.141660$

O $6.391132-1.7853921 .575452$

C $5.156603-0.946207-0.351442$

C 5.1583890 .5114750 .171065

O $6.324775-1.167609-1.142714$

C $-1.642596-0.356373-3.126679$

O $-2.772500-0.137265-3.532794$

C $-0.6106920 .671817-2.869139$

C $-0.9251951 .971567-2.979434$

C $-0.0063083 .099144-2.802456$

C $1.4372842 .769940-2.528959$

C $4.9896721 .576671-0.918281$

C $4.8461042 .961755-0.314672$

C 3.2251864 .3957870 .935198

O $5.758716 \quad 3.783219-0.357036$

C 1.7952474 .6896010 .609519

C 1.3676145 .8452430 .079954

C $-0.0686046 .100962-0.281424$

C $-0.2417946 .554332-1.715951$

C $-0.8208497 .734754-1.994584$

C $0.2597535 .657415-2.829288$

C $-0.4905264 .354325-2.915700$

N $3.602622 \quad 3.1678890 .258371$

H -4.598939 -2.824052 -3.157067

H -2.459516 -2.979355 -0.964718

H $-2.430420-2.606895-4.053207$

H -1.514473 -4.874461 -3.319961

H $-0.369823-4.032239-2.287221$

H $1.908088-3.664370-5.250443$

H $0.069156-3.940810-6.578738$

H -1.367254 -4.655929 -5.849154

H -1.191021 -2.887317 -5.933629

H $1.745046-4.481005-2.334894$

H $3.132680-4.358721-3.388064$

H $2.704687-1.741368-3.318677$

H $1.679181-2.087247-1.949974$

H $4.649005-2.727903-2.246360$

H $3.543056-4.488521-0.798692$

H $2.786473-3.2221870 .145606$

H $4.365800-5.0038501 .450855$

H $4.372163-1.6188211 .540498$

H $5.890024-5.155757-0.334268$

H $\quad 6.971216-4.1052650 .551762$

H $6.449852-3.658102-1.084474$

H $6.349808-2.3990852 .328860$

H 4.3427550 .6253490 .895911

H $\quad \begin{array}{llll}6.093502 & 0.716074 & 0.707916\end{array}$

H $6.506924-0.373416-1.668448$

H $0.3849440 .328596-2.611881$

H -1.949926 $2.239847-3.233454$

H $1.5256882 .187557-1.605544$

H $2.0749143 .646991-2.409492$

H $1.8617362 .184913-3.352719$

H $5.8528881 .594383-1.591918$

H $4.1065541 .377446-1.535686$

H 3.8979645 .2078680 .641865

H 3.3371374 .2231272 .009977

H 1.0714083 .9023610 .807470

H $2.0803716 .644864-0.107189$

H -0.4534156 .8615920 .409856$

H $-0.6809275 .206254-0.116832$

H $-0.9494058 .075347-3.016687$

H -1.181443 8.388753 -1.207513

H $1.3383315 .522938-2.719100$

H $\quad 0.137766 \quad 6.158444-3.799119$

H $-1.5537284 .480331-3.126509$
H $\quad 2.9710692 .376067 \quad 0.340967$

C $-5.829834-1.527513-0.901183$

O $-4.820933-0.598799-0.453184$

C $\quad-5.3643470 .2062660 .605980$

C -6.8727070 .0182120 .532740$

C $-6.958766-1.4427870 .125256$

H $-5.010775-0.2303211 .546691$

C -4.8556451 .6457630 .490496$

H -7.3664780 .2292051 .486012$

H $\quad-7.3250880 .654833-0.236492$

C $-8.318209-1.844903-0.418578$

H $-6.722352-2.0674780 .996985$

C $-3.317173 \quad 1.7588510 .394609$

O $\quad-5.4089962 .233911-0.692816$

H -5.2326642 .2369451 .333640$

H $-2.9877341 .407098-0.590192$

O -2.9984813 .1538920 .453724$

C -2.5580401 .0025121 .501041$

C -1.0343661 .2302221 .457895$

H -2.757468 -0.0697111 .397657$

O -3.0718361 .4195222 .765902$

H -0.6828651 .1716490 .421337$

H -0.8174422 .2334951 .844648$

H -9.092980 -1.706255 0.342548

H -8.594557 -1.245247 -1.292368

H $-8.322306-2.897887-0.717507$

C $-5.200238-2.916196-1.030556$

H $-5.0716893 .144481-0.747927$

H -2.0737723 .2569580 .172523$

H -2.632681 0.8896553 .452318

H $-5.964283-3.670501-1.247214$

H -4.712939-3.197684 -0.088901

H -6.176862 -1.169266 -1.879699

C $-0.094961-0.6021584 .587161$

O $-0.655870 \quad 0.343545 \quad 3.667718$

C $-0.268577 \quad 0.1874732 .299935$

C 1.2537630 .3356942 .163627

C $1.991914-0.6280043 .097553$

C 1.441071-0.579699 4.527461

H $-0.397171-0.2389225 .578067$

C $-0.691300-2.0020664 .399995$

H $-0.568124-0.8068051 .950303$

O $1.649444 \quad 1.6722812 .460558$

H 1.5404450 .1373921 .124011

H $3.056588-0.3659223 .107190$

O $1.868354-1.9476912 .576493$

H $1.841306-1.4062915 .125969$

O $1.873626 \quad 0.634670 \quad 5.148492$

C $-2.178440-1.9904054 .667767$

H $-0.516521-2.4015223 .397075$

H $-0.242142-2.7155325 .100621$

H $1.329128 \quad 1.887338 \quad 3.353253$

H $2.385079-2.5392123 .149782$

H 2.8460330 .6277425 .160031

O $-2.861548-2.2713723 .521663$

O $-2.672908-1.7496465 .761589$

C $-4.286876-2.2914463 .643890$

H $-4.655327-1.2977893 .915417$

H -4.706479 -2.570093 2.673758

H -4.595694 -3.034795 4.385063

SCF Energy (B3LYP/6-31G**//MMFF) $=-3245.91965659$

$07 \_00048$

MM̄MF Geometry

C $-1.436343-3.4435221 .545912$

C $-0.908209-2.2135631 .452617$

C $-0.762568-1.2681382 .618518$

O $0.550478-0.6644342 .572932$

C $-1.806477-0.1489432 .480304$

C -1.6910320 .9349363 .530908$

C -1.1915622 .1643293 .292649$

C -2.2288710 .5766994 .890217$

C -0.6118212 .6606601 .995737$

C 0.6569083 .4964882 .214695

C 1.4661583 .7481250 .929809

C $0.6353684 .375381-0.194593$

C $1.4453124 .533118-1.491431$

O 2.0146152 .4925470 .509356 
O $0.530414 \quad 4.845657-2.553751$

C $2.1240903 .184956-1.821661$

C $2.4004485 .728514-1.419631$

O $2.995756 \quad 3.312623-2.941971$

C $2.8879082 .578371-0.625576$

C $3.4120511 .156204-0.920989$

O $4.0309733 .391754-0.334993$

C $1.600374-1.4167612 .995226$

O $1.572548-2.5725113 .386489$

C $2.815719-0.5837162 .879220$

C $3.999344-1.1878432 .702386$

C $5.293880-0.5240792 .550445$

C 5.3666900 .9493872 .854147

C $2.3349450 .128136-1.272868$

C $2.858839-1.285551-1.112914$

C $4.611779-2.836480-1.969290$

O $2.424852-2.037749-0.243545$

C $5.966588-2.633397-1.351262$

C $6.624111-1.471128-1.205501$

C $7.990311-1.349775-0.589396$

C $8.034395-0.3296950 .527822$

C $8.373948 \quad 0.946588 \quad 0.279620$

C $7.766249-0.8015561 .939058$

C $6.351442-1.2597352 .149556$

N $3.848678-1.603814-2.027874$

$\mathrm{H}-1.768850-3.7847292 .524609$

H $-0.571970-1.8317290 .493168$

H $-0.891586-1.7836523 .578667$

H $-2.813015-0.5792202 .534705$

H $-1.744640 \quad 0.2883501 .478318$

H -1.1983302 .8899874 .104484$

H -2.1683251 .4132215 .594353$

H -3.281116 0.2826784 .817026

H $-1.663427-0.2564655 .318707$

H -0.3742191 .8325251 .322816$

H $-1.3717453 .278248 \quad 1.503926$

H $0.381173 \quad 4.4585792 .664362$

H 1.3064122 .9772852 .932061

H 2.2948654 .4127211 .201595

H $0.216759 \quad 5.338787 \quad 0.121327$

H $-0.2259003 .734227-0.422329$

H $-0.1131034 .120242-2.626018$

H $1.3301242 .489869-2.123288$

H $1.8359386 .658606-1.280176$

H $2.9516845 .851518-2.358549$

H $3.1218295 .656700-0.603249$

H $2.479403 \quad 3.701723-3.668560$

H $4.165702 \quad 1.198522-1.717824$

H $3.9533120 .796935-0.035921$

H 4.6841812 .8512650 .139352

H 2.6969450 .4929082 .910254

H $4.021998-2.2751902 .636127$

H 5.0527271 .1428513 .886027

H $4.718787 \quad 1.5192102 .180594$

H 6.3712141 .3645692 .746093

H $1.4597910 .231743-0.621433$

H $1.9932570 .260866-2.304690$

H $4.720308-3.214588-2.990333$

H $4.055637-3.575806-1.383704$

H $6.448045-3.543902-0.999418$

H $6.182311-0.549116-1.574363$

H $8.689795-1.057315-1.383759$

H $8.353105-2.316597-0.219203$

H $8.4364561 .681237 \quad 1.075479$

H $8.5930281 .286184-0.727416$

H $8.030892-0.0306562 .672238$

H $8.439989-1.6380072 .166671$

H $6.201614-2.3200821 .943099$

H $4.125762-0.911585-2.717576$

C -1.149816 -4.033924 -0.960292

O $-1.870565-2.885063-1.435578$

C -1.915144 -2.923903 -2.874764

C $-1.264038-4.241207-3.295941$

C $-1.381930-5.086671-2.039376$

H -2.974831 -2.943356 -3.152689

C $-1.249077-1.660872-3.444054$

H $-1.770809-4.688788-4.157011$

H $-0.212161-4.110245-3.573758$
C $-0.404614-6.248365-2.001080$

H $-2.408438-5.471541-1.972893$

C $-1.754357-0.343597-2.816087$

O $0.163230-1.724368-3.206220$

H $-1.367750-1.635864-4.532921$

H $-1.454948-0.303754-1.762841$

O $-1.084767 \quad 0.745846-3.463031$

C $-3.271005-0.123774-2.943697$

C $-3.755371 \quad 1.221981-2.370637$

H $-3.808129-0.940381-2.451392$

O $-3.621166-0.172950-4.329394$

H $-3.2368862 .047427-2.874064$

H $-4.8087011 .347186-2.649149$

H $-0.568913-6.917115-2.852434$

H $0.633204-5.900838-2.041350$

H $-0.528147-6.834276-1.085144$

C $-1.660697-4.4315050 .427054$

H $0.294570-1.800230-2.245620$

H $-0.1291640 .578788-3.397590$

H $-4.588239-0.093638-4.391414$

H $-1.189938-5.3749130 .726724$

H -2.742239 -4.6104090 .374460$

H $-0.085696-3.767120-0.910204$

C $-5.554128 \quad 0.115515-0.177235$

O $-4.126066 \quad 0.211395-0.146844$

C $-3.5909241 .344799-0.840646$

C $-4.1609602 .640985-0.246741$

C $-5.6882982 .632769-0.214519$

C -6.2052861 .3541110 .450790$

H $-5.8815530 .049389-1.222772$

C $-5.959323-1.2294120 .443295$

H $-2.513727 \quad 1.350822-0.637507$

O $-3.709985 \quad 3.774594-0.980355$

H -3.7971082 .7573410 .782345$

H $-6.1058442 .757902-1.220437$

$\begin{array}{lllll} & \text { O } & -6.128231 & 3.762496 & 0.544007\end{array}$

H -5.9971551 .4264281 .522781$

$\begin{array}{llll}\text { O } & -7.620002 & 1.306674 & 0.288363\end{array}$

C $-5.530968-1.4388651 .881724$

H $-7.042013-1.3829120 .388294$

H $-5.483987-2.018668-0.154897$

H $-2.7378243 .767913-0.959856$

H -7.0991323 .7223340 .585099$

H -7.9589370 .5982750 .860975$

O $-5.194218-2.7507042 .064884$

O $-5.530062-0.5792532 .751101$

C $-4.810112-3.109843 \quad 3.396086$

H $-4.518528-4.163538 \quad 3.392689$

H $-3.955025-2.5112603 .723745$

H -5.655484-2.981011 4.078423

SCF Energy (B3LYP/6-31G**//MMFF) $=-3245.90393753$

07_00049

MM̄FF Geometry

C 3.2880010 .9055292 .294460

$\begin{array}{llll}\text { C } 2.097493 & 1.093780 & 2.884558\end{array}$

C 1.0601502 .0755702 .413044

$\begin{array}{lllll}\text { O } & -0.219261 & 1.417809 & 2.596966\end{array}$

C 1.0792733 .3825733 .228412

C 2.2936384 .2519602 .990008

C 2.4372315 .0832051 .939214

C 3.3510874 .1995714 .062298

C $1.4778875 .315436 \quad 0.801115$

C $2.1784835 .275867-0.565566$

C $2.4417523 .850690-1.083333$

C $3.5320863 .884446-2.156861$

C $3.7596312 .508658-2.791496$

O $1.214387 \quad 3.352582-1.636356$

O $4.5663512 .693474-3.964891$

C $2.4011121 .958371-3.257718$

C $4.5413011 .564859-1.872950$

O $2.5335990 .622576-3.740715$

C $1.2891512 .026393-2.179629$

C $-0.0640931 .695727-2.858115$

O $1.561167 \quad 1.060123-1.164417$

C $-1.292003 \quad 1.926104 \quad 1.937195$

O $-1.3030932 .876538 \quad 1.171895$

C $-2.477143 \quad 1.1321142 .326524$ 
C -3.6954361 .6069712 .030755$

C $-4.977306 \quad 0.9580452 .308686$

C $-4.949928-0.4101012 .936106$

C $-1.3126171 .875288-1.985481$

C $-2.5774751 .535874-2.761972$

C $-5.0360151 .397550-2.552763$

O $-2.5677851 .195648-3.943109$

C $-6.0520691 .245672-1.466122$

C $-6.6508060 .080293-1.171530$

C $-7.714770-0.198721-0.140224$

C -8.1721170 .9676940 .708174$

C $-9.175578 \quad 1.7645870 .303752$

C -7.5296671 .1458672 .068036$

C -6.1056551 .6103901 .960341$

N $-3.7226681 .634998-1.981498$

H 3.5621141 .5075881 .433571

H 1.8510590 .5100283 .769252

H 1.1936472 .2741881 .344002

H 0.9868903 .1512954 .299027

H 0.1778283 .9720863 .018795

H 3.3375485 .6955901 .898685

H 4.2008684 .8543453 .841335

H 2.9305514 .5182555 .022013

H 3.7419633 .1850374 .177370

H $0.637778 \quad 4.617726 \quad 0.811129$

H 1.0557496 .3189760 .937337

H $1.5385595 .801610-1.286312$

H $3.1185395 .838555-0.502598$

H $2.7531273 .207843-0.253910$

H $4.4718484 .272328-1.743725$

H $3.2412394 .588939-2.949186$

H $5.4030503 .106806-3.690954$

H $2.0846522 .566040-4.117399$

H $5.5084952 .004809-1.602228$

H $4.7728810 .625364-2.385536$

H $4.0129521 .331749-0.947556$

H $3.2264150 .628174-4.423302$

H $-0.1851892 .344489-3.736264$

H $-0.0451780 .660370-3.222289$

H $\quad 0.7303210 .815448-0.727912$

H -2.3168050 .1923372 .841598$

H -3.7654892 .5689931 .523753$

H -4.381808 -1.1101752 .315569$

H -5.939901-0.850084 3.070123

H -4.486039 -0.3668653 .927820$

H $-1.2692251 .216938-1.112450$

H -1.391075 2.912828 -1.643694

H $-4.9849270 .513613-3.198551$

H -5.287914 2.255064 -3.185757

H $-6.3221242 .153629-0.936236$

H $-6.356217-0.797120-1.745744$

H -7.359838 -1.004357 0.514956

H -8.581758 $-0.618023-0.668638$

H -9.5324272 .5829130 .920025$

H -9.658989 $1.617443-0.656511$

H -7.6368230 .2158092 .632480$

H -8.074727 1.8988092 .652306

H -6.0116312 .6063601 .524541$

H -3.657683 2.096086 -1.077924

C $5.029953-0.8251391 .671967$

O $4.104626-1.3816870 .721846$

C $4.498976-2.7289730 .412003$

C $5.904779-2.9025910 .977249$

C $5.860017-2.0007242 .192622$

H $3.809056-3.3823220 .959031$

C $4.374218-3.009591-1.090636$

H $6.126151-3.9458371 .222896$

H $6.675663-2.5516120 .282346$

C $7.231368-1.6059992 .712566$

H $5.314483-2.5249352 .988912$

C $3.063032-2.540773-1.748828$

O $5.440609-2.356586-1.794028$

H $4.520881-4.081522-1.268254$

H $3.077332-1.451909-1.877208$

O $3.026762-3.095209-3.071463$

C $1.768097-2.934680-1.018049$

C $0.517598-2.616518-1.861222$

H $1.714016-2.393639-0.067819$
O $1.792139-4.327462-0.711134$

H $\quad 0.642437-1.642509-2.348884$

H $0.421710-3.379805-2.643416$

H $7.799516-2.4930313 .011210$

H $7.811925-1.0756861 .950211$

H $7.143862-0.9513133 .585423$

C $4.288649-0.0956872 .796432$

H $5.406821-1.412331-1.564665$

H $3.834479-2.804824-3.529083$

H $1.877881-4.813010-1.549375$

H 5.0191780 .4242613 .427363

H $3.776330-0.8288843 .431366$

H $5.658414-0.1163971 .117487$

C -2.118134-3.955361 0.444460

O $-0.976239-3.852903-0.417395$

C $-0.781273-2.578760-1.033387$

C $-1.993426-2.220643-1.907568$

C $-3.293710-2.278229-1.101780$

C $-3.410178-3.579781-0.301757$

H $-2.176929-5.0210310 .701470$

C $-1.921379-3.1714441 .746974$

H $-0.653839-1.816571-0.256466$

O $-2.075868-3.124409-3.007905$

H -1.844691 -1.217315 -2.319100

H -4.150494 -2.195557 -1.781044

O $-3.345769-1.178002-0.201458$

H $-4.238286-3.5176660 .414113$

O $-3.712173-4.652225-1.198971$

C $-0.781112-3.7519562 .548407$

H -1.729060 -2.107589 1.582754

H $-2.817801-3.2280562 .375715$

H -2.825871 -2.846107 -3.560544

H $-3.324206-0.362745-0.730300$

H -4.554805 -4.440752 -1.635571

O $0.325310-2.9654842 .423487$

O $-0.859797-4.7925093 .188695$

C $1.488218-3.4282333 .115571$

H $2.289974-2.7050652 .951562$

H $1.291010-3.4949874 .189678$

H $1.803803-4.3973232 .717668$

SCF Energy $($ B3LYP/6-31G**//MMFF $)=-3245.90709082$

07_00050

MMFF Geometry

C $2.4763492 .197276-2.166240$

C $1.3652692 .485494-1.472906$

C $0.3583813 .509367-1.922665$

O $-0.9251632 .841826-2.001511$

C $0.2283774 .677169-0.925970$

C $1.4565705 .560179-0.825520$

C 2.3239195 .5709420 .207104

C $1.6592816 .502054-1.985235$

C 2.2977624 .7090651 .438158

C 3.5191613 .7786971 .488245

C 3.3135752 .6106632 .463693

C 4.5551711 .7186602 .513134

C 4.3240080 .4551893 .356909

O $2.197372 \quad 1.8585101 .984290$

O $5.394528-0.4612403 .081313$

C $3.018086-0.2267902 .895279$

C 4.3839440 .7552334 .859064

O $2.688957-1.3259903 .741479$

C 1.8190280 .7414492 .794186

C 0.6331370 .0482062 .078526

O $1.428278 \quad 1.1440954 .104621$

C $-1.7865033 .271534-2.959571$

O $-1.5729884 .141989-3.793763$

C $-3.0769572 .548267-2.918135$

C $-3.4897951 .786168-1.893439$

C $-4.7847791 .101069-1.805128$

C $-5.7134341 .207736-2.987828$

C -0.5822890 .9524591 .823622$

C -1.6229440 .9298202 .929229$

C -3.8388761 .9235093 .505634$

$\begin{array}{lllll}0 & -1.527504 & 0.233884 & 3.935745\end{array}$

C -4.8850480 .9566603 .050390$

C -6.0374901 .3258392 .471651$

C -7.0850870 .3553212 .010092$ 
C -7.3072190 .4065810 .514669$

C $-8.3776611 .031318-0.004098$

C $-6.343112-0.349793-0.371757$

C $-5.0912490 .428537-0.675762$

N -2.6770791 .7884902 .647909$

H $2.6634792 .716985-3.103476$

H $1.1742441 .976609-0.533418$

H $0.6358563 .886182-2.914959$

H -0.0798784 .2880130 .050695$

H $-0.6216015 .307196-1.223300$

H $3.161246 \quad 6.2668830 .167610$

H $2.5219537 .161103-1.840621$

H $1.8252105 .942895-2.911141$

H $0.7781747 .138981-2.115613$

H 2.3018195 .3580212 .322359

H $1.374074 \quad 4.1302141 .497651$

H $3.705443 \quad 3.3742440 .485795$

H 4.4073604 .3560041 .773635

H 3.0823573 .0142643 .456425

H 5.4214892 .2790042 .885849

H 4.8195861 .4052651 .493169

H $6.232229-0.0174693 .298743$

H $3.205843-0.6487251 .899015$

H $5.365340 \quad 1.1632825 .129044$

H $4.267775-0.1597405 .450207$

H 3.6287941 .4745695 .182742

H $3.473716-1.8981573 .791814$

H $0.986294-0.2738991 .090803$

H $\quad 0.326445-0.8493892 .627198$

H $\quad 0.535199 \quad 1.5194174 .063775$

H $-3.7065572 .720730-3.785788$

H -2.837875 $1.664289-1.030193$

H -5.960471 2.255953 -3.190290

H $-6.6649900 .691612-2.846530$

H $-5.2484940 .775400-3.880720$

H $-0.278283 \quad 1.989227 \quad 1.644915$

H -1.0858150 .6015650 .915452$

H -4.1766322 .9624223 .436852$

H -3.562592 1.7148644 .544038

H -4.680402 -0.1000453 .205793$

H -6.2444922 .3828472 .321513$

H -8.016302 0.5924812 .540900

H $-6.835646-0.6720322 .304020$

H -8.561835 $1.043280-1.073082$

H -9.0913371 .5487970 .628442$

H $-6.843976-0.676091-1.289432$

H $-6.061907-1.2873870 .123026$

H -4.3563240 .4110530 .128346$

H -2.700807 2.2601141 .748205

C $3.6876680 .062380-2.709955$

O $2.998249-1.088992-2.183954$

C $3.915706-1.828596-1.358481$

C $5.325693-1.393919-1.756460$

C $5.135290-0.395768-2.882839$

H $3.746140-1.519577-0.321765$

C $3.669462-3.338569-1.484489$

H $5.794324-0.894915-0.898365$

H $5.983205-2.229550-2.018456$

C $5.353379-1.043202-4.251506$

H $5.8463960 .434066-2.802331$

C $2.275922-3.812437-1.020184$

O $3.836990-3.731878-2.850455$

H $4.441553-3.878404-0.923645$

H $1.511525-3.438031-1.710008$

O $2.247129-5.241171-1.137120$

C $1.915780-3.4212310 .425103$

C $0.685091-4.1399961 .006675$

H $1.764031-2.3395830 .473205$

O $3.027139-3.7274081 .273657$

H $0.893761-5.2134271 .098811$

H $0.558904-3.7764702 .035678$

H $\quad 6.363417-1.459252-4.327348$

H $4.638130-1.845606-4.450085$

H $5.239677-0.297512-5.045948$

C $3.5421011 .233801-1.721036$

H $3.154021-3.275633-3.371362$

H $2.897136-5.600355-0.508930$

H $2.810794-3.4080942 .166291$
H $4.4732601 .810579-1.670223$

H $3.3278600 .873367-0.708305$

H $3.2376660 .314412-3.677009$

C -2.087961-2.124046-0.471007

O $-0.911587-2.5243880 .244293$

C $-0.615481-3.9230320 .223244$

C $-1.777476-4.7286040 .827424$

C $-3.101100-4.4019020 .133029$

C -3.324770 -2.890490 0.024812

H $-2.229674-1.068860-0.209524$

C $-1.891659-2.200428-1.992193$

H $-0.460076-4.245685-0.810478$

O $-1.898331-4.4635812 .223971$

H -1.559277 -5.7978850 .721963$

H -3.922577 -4.857519 0.699016

O $-3.090106-4.985088-1.166466$

H $-4.173582-2.675900-0.633663$

O $-3.653609-2.3748991 .318453$

C $-0.881074-1.186196-2.486529$

H -1.546538 -3.184647 -2.318072

H $-2.845469-1.978293-2.485361$

H -2.007736 -3.503616 2.336409

H -3.947392 -4.791478 -1.582328

H -4.469303 -2.815766 1.611110

O $-0.954421-1.118676-3.848886$

O $-0.112844-0.551201-1.777947$

C $-0.037380-0.216017-4.474531$

H $-0.1200910 .784591-4.041053$

H $0.982323-0.598068-4.376819$

H $-0.289862-0.157243-5.536447$

SCF Energy $($ B3LYP/6-31G**//MMFF $)=-3245.90797387$

07 00051

MMFF Geometry

C $0.675508 \quad 2.2354750 .099710$

C 1.5901572 .5085701 .042717

C 1.9463161 .6434222 .228839

O 1.1720290 .4203592 .208037

C 3.4334201 .2395982 .196375

C 4.3871892 .3060432 .686345

C 5.1640683 .0683451 .892599

C 4.4760172 .4417894 .184990

C 5.2000903 .1072100 .390333

C $6.5013722 .541995-0.192803$

C $6.5817561 .007521-0.142544$

C $7.9170450 .528250-0.717832$

C $7.989550-1.002438-0.812088$

O $5.4985830 .489076-0.921187$

O $9.109353-1.341499-1.644314$

C $6.723483-1.517549-1.527606$

C $8.253711-1.6463900 .554019$

O $6.678386-2.942990-1.516140$

C $5.409525-0.941472-0.949655$

C $4.222997-1.316157-1.871401$

O $5.198049-1.4884040 .352283$

C $0.819814-0.1249493 .400941$

O $1.0188110 .350978 \quad 4.510156$

C $0.109261-1.4109933 .219868$

C $0.248057-2.1736762 .124417$

C $-0.398275-3.4659151 .879933$

C $-1.340154-3.9893692 .932596$

C $2.877009-0.705221-1.454932$

C $1.783142-0.990694-2.471881$

C $-0.703257-1.132738-2.662007$

O $2.015018-1.264559-3.646595$

C $-0.921262-2.611509-2.717944$

C -1.761139-3.274867 -1.909791

C $-1.934038-4.765667-1.934755$

C $-0.804790-5.507531-1.250764$

C $0.047965-6.264494-1.962208$

C $-0.697162-5.4308220 .260167$

C $-0.111967-4.1268290 .738246$

N $0.515715-0.867773-1.919555$

H $\quad 0.095094 \quad 1.321517 \quad 0.151092$

H 2.1440983 .4416270 .959349

H 1.7077172 .2141673 .134174

H 3.6985290 .9018541 .188522

H 3.5851210 .3436722 .814342 
H 5.8379593 .7799042 .368815 H 5.2100023 .1943444 .492217 H 3.5087532 .7368974 .602825 H 4.7733091 .4891104 .635596 H $4.3346082 .613056-0.059419$ H 5.1201004 .1613810 .094918 H $6.5532402 .862837-1.241704$ H 7.3651202 .9811190 .321938 H $6.473157 \quad 0.6757580 .895954$ H $\quad 8.7562240 .917594-0.127884$ H $8.0467880 .940614-1.728457$ H $9.907853-0.956475-1.243939$ H $\quad 6.797868-1.218295-2.582563$ H $9.207521-1.2940260 .964917$ H $8.349588-2.7342590 .466338$ H $7.477922-1.4257181 .290022$ H $7.514715-3.262052-1.896541$ H $4.451020-0.978419-2.890765$ H $4.106071-2.406702-1.907830$ H $4.258797-1.4205880 .580687$ H $-0.497485-1.7206584 .064247$ H $0.903110-1.8284161 .326723$ H $-1.766128-4.9651812 .688314$ H -2.177524 -3.300414 3.081935 H $-0.816123-4.1131473 .887095$ H $2.566533-1.104521-0.484316$ H $2.9591070 .383546-1.366912$ H $-0.611141-0.727755-3.674861$ H -1.520494 -0.613533 -2.150992 H -0.341592 -3.166200 -3.452081 H $-2.333178-2.724676-1.169123$ H -2.041480 -5.095194 -2.976826 H $-2.883947-5.026525-1.452702$ H $0.848664-6.817479-1.482525$ H -0.031504 -6.344017 -3.041368 H $-0.052779-6.2392040 .628672$ H -1.692971 -5.611805 0.676485 H $0.630730-3.6931680 .066800$ H $\quad 0.427300-0.731520-0.917230$ C $-0.7938834 .001257-0.953206$ O $-2.0049923 .214355-0.951664$ C $-2.9481013 .816697-1.856823$ C $-2.4172605 .215054-2.151621$ C $-0.9243144 .980813-2.122051$ H $-2.9150403 .225302-2.779338$ C $-4.3702463 .816693-1.285269$ H -2.774195 5.600717 -3.111607 H -2.701849 5.932165 -1.373035 C $-0.1141816 .255519-1.955653$ H $-0.6293214 .489739-3.059467$ C $-4.9507012 .443781-0.895869$ O $-4.4076624 .637746-0.109207$ H -5.041024 4.302212 -2.004504 H -4.5039052 .1016350 .043807$ O $-6.3465822 .641787-0.626804$ C $-4.8159821 .340151-1.958696$ C $-5.6994310 .096731-1.733129$ H -3.767192 $1.028434-2.017269$ O $\quad-5.156779 \quad 1.864192-3.246385$ H $-6.7476400 .345908-1.944724$ H -5.413790 $-0.634925-2.501108$ H $-0.3007866 .940821-2.788927$ H $-0.3725756 .776687-1.027591$ H $0.9581206 .038001-1.931802$ C $0.4363673 .104624-1.100329$ H $-3.756573 \quad 4.2793300 .518146$ H -6.414464 3.3219360 .065248 H $-6.0691312 .198015-3.196224$ H $1.3250763 .718754-1.290093$ H $0.3181082 .443481-1.968068$ H -0.7655334 .5402050 .002544$ C -3.959052 -1.471469 1.178838 O $-4.238506-0.911466-0.112490$ C $-5.598378-0.541706-0.341924$ C $-6.515200-1.768890-0.212513$ C $-6.328191-2.4607991 .141600$ C $-4.849419-2.6960231 .460761$ H $-2.921886-1.8190791 .108916$
C $-4.017903-0.4123562 .291541$

H -5.9018440 .2019250 .400733$

O $-6.253628-2.700373-1.260760$

H -7.556878 -1.446488 -0.323860

H $-6.851080-3.4252441 .133734$

O $-6.900424-1.6619602 .172913$

H -4.735941-3.007481 2.505640

O $-4.398496-3.7839200 .649559$

C -2.9593080 .6536892 .114090$

H -4.9866900 .0926412 .320859$

H $-3.838690-0.8919113 .260739$

H -5.312266 -2.941351-1.217030

H -7.845308 -1.553784 1.970296

H -3.477249 -3.9721410 .895461$

O $-3.090326 \quad 1.583123 \quad 3.105570$

O $-2.1250470 .673396 \quad 1.220575$

C -2.1616232 .6709203 .055160$

H -2.2217513 .1827872 .090166$

H -2.425959 3.3801883 .843788

$\mathrm{H}-1.1469322 .3064203 .236394$

SCF Energy $(B 3 L Y P / 6-31 G * * / / M M F F)=-3245.91514254$

07 00052

MMFF Geometry

C $3.111137-2.5734052 .752257$

C $2.516064-1.3867752 .945000$

C $1.153215-1.1290563 .541423$

O $0.327925-0.5602772 .493484$

C $0.419237-2.3698034 .087921$

C $-0.940867-2.0570134 .687623$

C $-2.122435-2.4325664 .156475$

C $-0.913231-1.2894145 .984768$

C $-2.359573-3.2170882 .895998$

C -2.948662 -2.333303 1.795144

C $-2.965193-3.0148720 .418740$

C $-1.559261-3.124701-0.185717$

C -1.592447 -3.682564 -1.616016

O $-3.793986-2.209558-0.430504$

O $-0.298070-3.491729-2.204930$

C $-2.621406-2.883652-2.442771$

C $-1.811281-5.199657-1.620217$

O $-2.780744-3.460096-3.737518$

C $-3.996722-2.745508-1.745152$

C $-4.884603-1.756324-2.540697$

O $-4.627453-4.024881-1.707395$

C 0.3125860 .7938512 .364960

O $\quad 0.961711 \quad 1.6030743 .008123$

C -0.6684311 .1295251 .309289$

C -1.0785582 .3989501 .176756$

C -2.0720582 .8993360 .222834$

C $-2.7066481 .905094-0.712907$

C $-6.330359-1.604306-2.056000$

C $-6.475796-1.001850-0.674006$

C $-6.312571 \quad 1.144308 \quad 0.567404$

O $-6.843398-1.6730960 .287755$

C -6.3828872 .5886500 .189171$

C -5.7111703 .5645750 .818661$

C $-5.7706485 .002506 \quad 0.385968$

C $-4.4117745 .658167 \quad 0.267862$

C $-4.168954 \quad 6.838728 \quad 0.863674$

C $-3.3524534 .990468-0.585989$

C $-2.368467 \quad 4.2157230 .249055$

N $-6.1849910 .351185-0.642152$

H $2.614994-3.4911283 .052150$

H $3.048120-0.4928672 .626270$

H $1.277674-0.4065344 .357438$

H $1.030077-2.8518764 .862572$

H $0.321246-3.104746 \quad 3.284317$

H -3.034114 -2.149319 4.681264

H - $1.908851-1.1910246 .430818$

H $-0.277945-1.8008046 .715314$

H -0.523172 -0.2792765 .828151$

H -1.450186 -3.711080 2.553499

H -3.063203 -4.027809 3.122900

H -3.974435 -2.050961 2.069397

H $-2.401193-1.3862681 .722513$

H $-3.425786-4.0035760 .527152$

H $-0.896350-3.7368330 .435894$ 
H $-1.100241-2.126635-0.213057$ H $-0.103951-2.539136-2.212536$ H -2.214273 -1.875045 -2.600105 H -0.990724 -5.703604 -1.094942 H -1.801393 $-5.598592-2.640663$ H $-2.743978-5.502735-1.140604$ H -1.896685 $-3.522081-4.138202$ H $-4.405329-0.769226-2.539641$ H -4.943928 -2.088444 -3.584844 H -5.524473 $-3.915672-1.355067$ H -1.0430620 .3241340 .689179$ H -0.6569873 .1495911 .844784$ H $-3.2009591 .106207-0.149774$ H -1.951524 $1.452881-1.364471$ H -3.463305 2.343197-1.366456 H $-6.869940-0.953051-2.756544$ H $-6.864130-2.560109-2.079565$ H $\quad-5.453776 \quad 0.9181981 .207835$ H -7.2264840 .8422891 .089280$ H -7.017378 $2.847455-0.656052$ H -5.1001273 .3213331 .684377$ H -6.3920975 .5415691 .112260$ H $-6.2762985 .093517-0.583960$ H -3.206121 7.3301670 .768284 H $-4.924631 \quad 7.3398251 .459377$ H -2.789218 $5.759515-1.131713$ H -3.829404 $4.387354-1.363072$ H -1.8313134 .8432460 .962840$ H $-5.7543750 .777961-1.457810$ C $4.392427-3.1013470 .621692$ O $3.862927-1.996205-0.140050$ C $2.977065-2.517368-1.147776$ C $3.232723-4.021258-1.198234$ C $3.545002-4.3198930 .254467$ H $1.957283-2.344573-0.784376$ C $3.186307-1.799224-2.484869$ H $2.369451-4.577413-1.575737$ H $4.094784-4.267028-1.829014$ C $4.249190-5.6470490 .472194$ H $2.597530-4.3255500 .806749$ C $3.029630-0.265116-2.441424$ O $4.508562-2.077417-2.966572$ H $2.503506-2.218578-3.233213$ H $3.8810630 .181671-1.915857$ O $3.099461 \quad 0.209655-3.792958$ C $1.7168740 .238074-1.817298$ C $1.4234451 .731981-2.052418$ H $1.7274910 .030635-0.741263$ O $0.625374-0.500723-2.374070$ H $1.2274771 .910192-3.117679$ H $0.475714 \quad 1.950262-1.546637$ H $3.622646-6.476107 \quad 0.127358$ H $5.197451-5.693230-0.073819$ H $4.463124-5.8035211 .534219$ C $4.459801-2.7354022 .107760$ H $5.136535-1.779951-2.286046$ H $3.938668-0.107620-4.168484$ H $-0.185006-0.213027-1.919899$ H $5.032515-1.8069172 .221267$ H $5.009567-3.5098632 .655338$ H $5.420818-3.2428380 .263314$ C 3.7366953 .2438470 .464531 O $2.7222752 .446720-0.163086$ C $2.5069762 .700019-1.553291$ C $2.1046264 .168253-1.783017$ C $3.1016075 .137564-1.142611$ C 3.4308624 .7419960 .301944 H 3.6614163 .0026651 .532861 C $5.1497912 .863839-0.008314$ H $3.4298132 .494655-2.104175$ O $0.8055084 .421695-1.251345$ H $2.0488024 .353447-2.862119$ H $2.675406 \quad 6.148139-1.159337$ O $4.2891095 .155202-1.928297$ H 4.2754205 .3291810 .680586 O $2.3062125 .040151 \quad 1.134684$ C 5.5266881 .4506800 .377547 H $5.2455392 .938262-1.094465$
H 5.8833713 .5328510 .455906

H $0.8244724 .200417-0.304544$

H $4.8996735 .794258-1.522857$

H 2.1386925 .9957931 .069524

O $6.7294111 .141588-0.191313$

O $4.8610790 .713047 \quad 1.089330$

C $7.220028-0.1752390 .079441$

H $6.582805-0.917168-0.409224$

H $8.229263-0.253215-0.333416$

H $7.268533-0.3556321 .157578$

SCF Energy (B3LYP/6-31G**//MMFF) $=-3245.91946973$

07_00053

MMFF Geometry

C $1.582328-0.5317433 .180453$

C $1.279745-1.7739123 .590044$

C $0.082467-2.5968823 .176333$

O $-0.910965-1.7400442 .560385$

C $0.424447-3.7191512 .177983$

C $1.530792-4.6671772 .590354$

C $2.691244-4.8007061 .916978$

C $1.238227-5.5321853 .786804$

C $3.110301-4.0043470 .713644$

C $2.690006-4.676778-0.598681$

C $2.664878-3.722015-1.803675$

C $4.033014-3.114997-2.112597$

C $3.963045-2.116532-3.277965$

O $1.717680-2.686948-1.519193$

O $5.206899-1.398954-3.276417$

C $2.825478-1.108492-3.004726$

C $3.870785-2.830562-4.631383$

O $2.614857-0.256274-4.128867$

C $1.486320-1.771451-2.596542$

C $0.501823-0.684157-2.087194$

O $0.936759-2.432382-3.734610$

C $-2.209087-2.1420002 .621432$

O $-2.645827-3.1355373 .180008$

C $-3.016898-1.1631131 .860300$

C $-4.327121-1.3875941 .680639$

C $-5.261343-0.5353500 .942143$

C -4.7056630 .6901420 .268040$

C $-0.820901-1.206747-1.504645$

C $-1.887432-1.507892-2.543482$

C $-4.102058-2.628330-2.785533$

O $-1.794296-1.165666-3.719148$

C $-5.198852-1.617042-2.673042$

C $-6.455750-1.927248-2.319948$

C $-7.574395-0.927606-2.244760$

C $-8.298945-0.957109-0.917313$

C $-9.474282-1.595084-0.785519$

C $-7.704457-0.1736830 .231415$

C $-6.561677-0.8938590 .894265$

N $-2.962761-2.195480-1.997598$

H $0.958967-0.0352322 .442395$

H $1.925955-2.2411284 .331253$

H $-0.344113-3.0209244 .094573$

H $0.621366-3.2652761 .200007$

H $-0.477958-4.3197181 .998139$

H $3.415464-5.5376632 .258732$

H $2.035693-6.2579583 .978541$

H $1.127305-4.9198644 .686944$

H $\quad 0.311787-6.0946643 .631456$

H $2.705142-2.9908840 .777222$

H $4.200448-3.8875100 .739703$

H $3.355326-5.523406-0.807868$

H $1.677971-5.087198-0.482043$

H $2.305195-4.300447-2.662825$

H $4.769785-3.900677-2.320651$

H $4.409377-2.576420-1.232887$

H $5.210194-0.796837-4.039328$

H $3.156080-0.461870-2.182539$

H $4.751394-3.465066-4.787797$

H $3.868295-2.112544-5.459031$

H $2.984758-3.462332-4.723598$

H $3.4440950 .221863-4.296633$

H $0.997608-0.140153-1.272821$

H $\quad 0.292603 \quad 0.038752-2.884516$

H $\quad 0.010149-2.648804-3.550481$ 
H $-2.509690-0.2933331 .459515$ H $-4.763967-2.2859442 .115295$ H -4.3711431 .4147791 .016991$ H -5.425243 $1.198835-0.376694$ H $-3.8574630 .431799-0.375337$ H $-0.652221-2.092738-0.883332$ $\mathrm{H}-1.239134-0.426140-0.856385$ H -4.403757 -3.610003 -2.406789 H $-3.803948-2.734766-3.833573$ H $-4.953778-0.586310-2.918373$ H $-6.705242-2.963486-2.103026$ H -8.276591 -1.154462 -3.057817 H $-7.2158250 .090666-2.440150$ H -10.014422 -1.596646 0.155476 H -9.919821 -2.134888 -1.614574 H $-8.467222-0.0032441 .003324$ H -7.436446 $0.828050-0.115636$ H -6.866971 $-1.805246 \quad 1.410454$ H -2.982450 -2.375409-0.998055 C 3.8312800 .6162202 .747178 O 3.3291021 .4614901 .696163 C 4.0633561 .1647860 .488339 C 5.1550550 .1690670 .874839 C $4.510105-0.5522192 .041905$ H $3.3607560 .664829-0.184886$ C $4.5861132 .458553-0.147192$ H $5.416083-0.4977160 .047338$ H $\begin{array}{llll}6.072336 & 0.675427 & 1.197096\end{array}$

C $5.489641-1.3246232 .905148$ H $3.760919-1.2417341 .642022$ C $3.4803493 .475144-0.499413$ O 5.4858903 .0942730 .770906 H $5.1793252 .225034-1.038330$ H 2.9772103 .7996940 .417399 O $4.1071514 .640370-1.050260$ C $2.4529412 .954131-1.521912$ C $1.3899453 .994469-1.926067$ H $1.9531732 .065478-1.126324$ O $3.1541162 .551813-2.701364$ H $1.8756454 .921671-2.252387$ H $0.8601523 .604554-2.805458$ H $5.997451-2.0945522 .315110$ H $6.255072-0.6673323 .330976$ H $4.972540-1.8209943 .732415$ C $2.7262270 .267332 \quad 3.743834$ H 4.9928973 .2699811 .590803 H $4.7647814 .949060-0.403589$ H $2.5054762 .152574-3.305878$ H $2.299847 \quad 1.2006014 .133059$ H $3.169277-0.2540354 .601070$ H 4.5834661 .2132583 .281148 C -1.307438 3.1178350 .483518 O $-0.3851873 .080301-0.613411$ C $0.3765404 .278143-0.805774$ C $-0.5625735 .447881-1.135576$ C $-1.6150265 .623515-0.035831$ C $-2.2888874 .296546 \quad 0.338415$ H $-1.876527 \quad 2.185000 \quad 0.385613$ C -0.5468343 .0644931 .817255$ H $0.920446 \quad 4.5123250 .114810$ O $-1.2320275 .226075-2.374169$ H $0.0236856 .369904-1.232346$ H $-2.3758336 .333483-0.383632$ O -0.9846526 .1921851 .108041$ H $-2.872156 \quad 4.4180841 .257733$ O $-3.2169153 .983231-0.705652$ C - -1.3635342 .4547612 .935332$ H 0.3469812 .4420421 .682512 H -0.2108294 .0533012 .139418$ H $-0.5773025 .319065-3.086403$ H -1.6719416 .3343041 .781092$ H -3.707534 $3.189439-0.435467$ O $-0.5280772 .112351 \quad 3.959847$ O -2.5778742 .3098082 .925549$ C $-1.155837 \quad 1.4919515 .086122$ H -1.6563290 .5682054 .780385$ H -0.3787951 .2444555 .814158$ H -1.8663562 .1823735 .550380$
SCF Energy (B3LYP/6-31G**//MMFF) $=-3245.91136761$

07 00054

MMFF Geometry

C $0.661612-2.500551-2.053383$

C $-0.377751-3.346490-2.007318$

C $-1.321999-3.441335-0.841165$

O $-2.604135-2.969332-1.319889$

C $-1.482773-4.889520-0.342040$

C $-0.268185-5.4569600 .358276$

C $-0.055932-5.3605851 .685155$

C $0.686457-6.239904-0.504975$

C $-0.906528-4.6326512 .691997$

C $-0.065023-3.9613693 .787130$

C $0.748176-2.7539883 .290450$

C $1.827063-2.4030794 .319066$

C $2.611454-1.1480923 .922363$

O $-0.151597-1.6500843 .115933$

O $3.381379-0.7292415 .059219$

C $1.605768-0.0240763 .617760$

C $3.613721-1.4246642 .796110$

O $2.277780 \quad 1.129406 \quad 3.113004$

C $0.471870-0.4449582 .648829$

C -0.5942140 .6774062 .622626$

O $1.026877-0.613821 \quad 1.343759$

C $-3.478323-2.503773-0.388604$

O $-3.311150-2.4630260 .819706$

C $-4.690805-2.039288-1.096201$

C $-5.671837-1.464325-0.385562$

C $-6.926852-0.928993-0.909614$

C $-7.195063-1.064651-2.384499$

C -1.8566430 .3826441 .800669$

C -2.6865451 .6468531 .625347$

C -4.2761882 .7393000 .051592$

O -2.6093782 .6018902 .395496$

C -5.7104132 .6125420 .457730$

C $-6.7289332 .814497-0.393075$

C $-8.1821912 .742896-0.012378$

C $-8.9517791 .753968-0.861344$

C $-9.5460672 .143416-2.002053$

C $-9.0883170 .331651-0.364419$

C $-7.769908-0.318286-0.051969$

N -3.5215881 .5897450 .518732$

H $0.830636-1.820280-1.223366$

H $-0.558370-4.013879-2.846738$

H $-0.980783-2.789365-0.025965$

H -1.767465 -5.543597-1.178195

H $-2.349536-4.9520480 .328600$

H $0.818111-5.8622192 .098396$

H $1.487727-6.7051960 .079294$

H $0.155746-7.042476-1.027498$

H $1.160218-5.593657-1.248762$

H $-1.548580-3.8843432 .220347$

H -1.562032 -5.3702343 .170345$

H $-0.747732-3.6322904 .581348$

H $0.604135-4.711149 \quad 4.228224$

H $1.211664-2.9942092 .327334$

H $2.511817-3.2474334 .468219$

H $1.355451-2.2243795 .295851$

H $3.970358-1.4618995 .308753$

H 1.1511660 .2758234 .572443

H $4.337889-2.1877043 .105758$

H $4.201891-0.5303002 .564527$

H $3.140601-1.7742431 .876220$

H $2.968468 \quad 1.3664893 .755264$

H -0.9178830 .8875093 .651012$

H $-0.136076 \quad 1.5969362 .240227$

H $\quad 0.343897-0.4433040 .678003$

H $-4.738362-2.170328-2.170898$

H $-5.540416-1.3602170 .691163$

H $-6.426593-0.542141-2.965012$

H -8.158349 -0.646687 -2.687513

H -7.201919-2.119796 -2.679018

H $-1.599547-0.0063610 .811347$

H -2.475991 -0.3605422 .313395$

H $-4.1580272 .778813-1.036297$

H -3.853048 3.6545410 .478057

H -5.9212652 .3764971 .497735$ 
H $-6.5084373 .078114-1.425474$ H -8.610764 $3.746455-0.134921$ H -8.3095712 .4975321 .049215$ H -10.118481 $1.449058-2.608218$ H $-9.4701713 .166137-2.356322$ H $-9.656417-0.288766-1.068206$ H $-9.690106 \quad 0.3420150 .553801$ H -7.489694 -0.2515820 .999905$ H $-3.5462080 .745777-0.045854$ C $3.071315-2.526646-2.690725$ O $3.450007-1.268577-2.091568$ C $4.809967-0.970296-2.461912$ C $5.350938-2.224778-3.138241$ C $4.099535-2.776897-3.790849$ H $4.769494-0.165255-3.204091$ C $5.606933-0.526153-1.232339$ H $6.146772-1.998723-3.854508$ H $5.748946-2.938690-2.407180$

C $4.225983-4.233395-4.203142$ H $3.862267-2.168691-4.674054$ C $5.0882670 .767538-0.567582$ O $5.568515-1.570923-0.251135$ H $6.663261-0.405827-1.498627$ H $4.0916670 .602316-0.143002$ O $5.956144 \quad 1.0587480 .536203$ C $5.0709321 .994345-1.502930$ C $4.8355743 .342003-0.795369$ H $4.3124811 .857060-2.280280$ O $6.3319842 .088660-2.175701$ H 5.5778073 .4767050 .001094 H 5.041954 4.135076-1.527506 H $5.030171-4.356611-4.936030$ H $4.452034-4.875290-3.345043$ H $3.297966-4.593995-4.657525$ C $1.632423-2.434113-3.198993$ H $4.633358-1.750119-0.052840$ H $5.975874 \quad 0.2704881 .105423$ H $7.0201472 .189371-1.495533$ H $1.429227-3.243334-3.909381$ H $1.478888-1.482696-3.721798$ H $\quad 3.157605-3.293173-1.908617$ C $1.1445593 .839645-1.057151$ O $2.5313593 .636582-1.359838$ C $3.4153183 .523654-0.237629$ C 3.3251844 .7692520 .660379 C 1.8788975 .0566781 .071089 C $0.9432085 .057275-0.139696$ H $0.679604 \quad 4.074808-2.023649$ C $0.4955312 .562610-0.515454$ H 3.1460672 .6407220 .349358 O $3.8597675 .898438-0.028143$ H 3.9451644 .6043701 .549867 H 1.8249256 .0326001 .568497 O $1.433964 \quad 4.0712461 .997768$ H $-0.103150 \quad 5.099360 \quad 0.185044$ O $1.182414 \quad 6.230242-0.921891$ C $0.1966401 .601846-1.641374$ H 1.1182082 .0265880 .204167 H $-0.4467782 .801022-0.009714$ H 3.8162976 .6587020 .576241 H 2.0344804 .0917702 .762180 H $1.0153237 .000877-0.353060$ O $-1.1493261 .536625-1.848695$ O $1.0566330 .996738-2.267233$ C $-1.5831070 .668298-2.899491$ H $-2.6408120 .869184-3.089778$ H -1.021037 $0.854685-3.819605$ $\mathrm{H}-1.476617-0.372184-2.584220$

SCF Energy $\left(B 3 L Y P / 6-31 G^{* *} / / M M F F\right)=-3245.91055533$

\section{00055}

MM̄FF Geometry

C $1.879867-0.442016-3.603817$

C $2.7210120 .480517-4.096946$

C $2.9040451 .889463-3.586787$

O $1.9309282 .218204-2.572128$

C $4.3008722 .030907-2.953474$

C $4.5482823 .412570-2.379923$
C $4.4178783 .729036-1.075793$

C $4.9772894 .454920-3.378067$

C 3.9761622 .8253260 .044621

C 5.0992912 .6355341 .070607

C 4.8159431 .5634892 .136671

C 4.9429000 .1444421 .572098

C $4.623974-0.9216352 .630900$

O $3.510618 \quad 1.7787162 .682953$

O $4.458864-2.1784531 .957461$

C $3.266955-0.5758523 .275943$

C $5.774924-1.1138233 .624499$

O $2.971191-1.4632414 .351503$

C 3.1667590 .8902173 .756727

C 1.7430491 .2537174 .241873

O $4.045761 \quad 1.0749334 .873680$

C $0.7800682 .825069-2.965396$

O $0.4033323 .028682-4.107553$

C $0.0643923 .228740-1.734798$

C $-1.1583993 .774306-1.811977$

C $-1.9517234 .261665-0.679473$

C -1.2936204 .2476970 .676387$

C $0.649847 \quad 1.1521123 .177000$

C $-0.724836 \quad 1.4971593 .725897$

C -3.1296321 .4138903 .074305$

O $-0.911678 \quad 1.9344994 .857415$

C -3.8459991 .7617721 .809897$

C -4.7882402 .7120161 .715881$

C -5.4881053 .0436430 .427422$

C $-5.4969154 .525510 \quad 0.113401$

C $-6.6553565 .162003-0.130520$

C -4.1836775 .2810800 .082474$

C $-3.2030784 .713259-0.908982$

N $-1.715526 \quad 1.2674692 .781417$

H $1.235922-0.200475-2.762959$

H $3.3581500 .205363-4.935503$

H $2.8131002 .585323-4.430205$

H $5.0756391 .811682-3.700574$

H $4.4363561 .270364-2.175033$

H $4.6266294 .753810-0.771461$

H $5.1617185 .427827-2.910198$

H $5.9033794 .146611-3.874162$

H $4.2045044 .596803-4.139896$

H $3.109043 \quad 3.2894070 .531019$

H $3.6279801 .860247-0.328181$

H 6.0470132 .4036890 .568037

H $5.2442303 .595507 \quad 1.584773$

H 5.5574431 .7069692 .932008

H $5.943935-0.0190261 .153966$

H 4.2449930 .0080820 .735869

H $5.282943-2.3723911 .478883$

H $2.505417-0.7549222 .507629$

H $6.682302-1.4375383 .100242$

H $5.545952-1.9073934 .344332$

H $6.023007-0.2064054 .178444$

H $3.034289-2.3698414 .005026$

H 1.4798790 .6375815 .111771

H 1.7550712 .2879734 .614204

H 3.7457641 .8493675 .377923

H $0.5798903 .082811-0.791873$

H -1.621394 $3.881556-2.791801$

H -0.4079814 .8931730 .677209$

H -0.9830243 .2325500 .940666$

H -1.9365054 .5950231 .486403$

H $\quad 0.865072 \quad 1.8339092 .346442$

H 0.5947360 .1341442 .781742

H -3.2698032 .1664623 .856837$

H -3.4800660 .4487193 .452634$

H $-3.577709 \quad 1.2003350 .918252$

H -5.0832053 .2639872 .604885$

H -6.5156152 .6656880 .505522$

H $-5.0318062 .508403-0.414953$

H $-6.6825876 .223199-0.355384$

H -7.604996 4.637536 -0.112009

H -3.7859595 .3230691 .099142$

H $-4.3563916 .327592-0.201790$

H $-3.5784024 .698389-1.933283$

H $-1.460713 \quad 0.7642801 .935635$

C $2.141203-2.919888-3.115580$ 
O $1.169986-2.962011-2.052428$

C $1.844567-3.323775-0.830604$

C $3.282853-3.666984-1.218501$

C $3.497762-2.780271-2.428582$

H $1.850230-2.428699-0.197610$

C $1.102887-4.465542-0.127377$

H $3.989866-3.467580-0.408898$

H $3.389434-4.721887-1.497234$

C $4.675896-3.193372-3.291930$

H $3.655764-1.754935-2.071722$

C $-0.375153-4.1802020 .211942$

O $1.128563-5.627306-0.969833$

H $1.644134-4.7476610 .783181$

H $-0.964892-4.127573-0.710010$

O $-0.867791-5.3158940 .937906$

C $-0.623371-2.9171801 .060835$

C $-2.027713-2.8422781 .696922$

H $-0.442757-2.0249720 .450640$

O $0.329302-2.897703 \quad 2.129653$

H -2.148474 -3.679369 2.396352

H -2.062094 -1.935762 2.316929

H $5.607295-3.149370-2.718026$

H $4.560307-4.215696-3.667079$

H $4.778807-2.527137-4.154304$

C $1.778441-1.842068-4.140169$

H $0.705779-5.387243-1.812426$

H $-0.713992-6.1017320 .385684$

H $0.170948-2.0944112 .653329$

H $0.743637-1.993861-4.469488$

H $2.415435-1.959701-5.025003$

H $2.076504-3.894345-3.618958$

C $-4.204627-1.329837-0.940804$

O $-3.185131-1.5397210 .047894$

C $-3.191759-2.8172520 .692368$

C $-4.539502-3.0588421 .391746$

C $-5.707237-2.9063640 .414523$

C $-5.611517-1.600785-0.379560$

H $-4.144365-0.259114-1.175510$

C $-3.908320-2.109878-2.225743$

H -3.040118 -3.601154-0.054496

O $-4.705573-2.1551892 .482169$

H $-4.542711-4.0718251 .811207$

H $-6.648013-2.9296350 .977879$

O $-5.703765-4.017258-0.477002$

H -6.337904 -1.592856 -1.200506

O $-5.949822-0.5107170 .481619$

C -2.687661 -1.543387 -2.907839

H $-3.719679-3.172960-2.051240$

H $-4.748232-2.054105-2.928416$

H -4.646452 -1.250971 2.129659

H $-6.468366-3.920208-1.069900$

H $-6.859235-0.6539850 .794364$

O $-3.033282-0.459681-3.659859$

O $-1.559554-1.995044-2.765252$

C $-1.9487210 .199998-4.319530$

H $-1.480315-0.473445-5.043192$

H -2.349786 $1.066171-4.852426$

H $-1.2176000 .548795-3.585141$

SCF Energy (B3LYP/6-31G**//MMFF) $=-3245.91774218$

\section{$07 \_00056$}

MM̄FF Geometry

C - $1.281472 \quad 2.0802371 .905393$

C $-0.874194 \quad 3.355787 \quad 2.006429$

C $0.5455653 .856027 \quad 1.896742$

$\begin{array}{lllll}\text { O } & 1.459969 & 2.748206 & 2.066068\end{array}$

C $0.820461 \quad 4.495636 \quad 0.517258$

C $-0.032944 \quad 5.6967150 .170715$

C $-0.970608 \quad 5.724934-0.797878$

C 0.2463796 .9346140 .983880

C $-1.3922784 .611504-1.718800$

C $-2.7696424 .076136-1.317011$

C $-3.2415752 .899800-2.185189$

C $-4.5901552 .390074-1.663154$

C $-5.0779401 .140731-2.407915$

O $-2.2594841 .860163-2.120927$

O $-6.1037390 .524705-1.609100$

C $-3.9212040 .120793-2.497898$
C $-5.7479831 .514075-3.735223$

O $-4.280866-0.992118-3.312834$

C $-2.5604610 .719792-2.934841$

C $-1.438370-0.324838-2.716860$

O $-2.532977 \quad 1.036946-4.327520$

C 2.7340433 .0332842 .441913

$\begin{array}{lllll}\text { O } & 3.190760 & 4.117970 & 2.762503\end{array}$

C 3.5068181 .7831032 .303952

C $4.7880041 .865487 \quad 1.918317$

C 5.6434390 .7330111 .565692

C $5.161983-0.6450611 .933176$

C $-0.0474170 .111931-3.198459$

C $0.8168930 .781358-2.143132$

C $3.1481551 .659388-1.866868$

O $0.4552720 .967613-0.986049$

C $4.1703860 .582078-1.672313$

C $5.4269370 .639448-2.140883$

C $6.445910-0.451095-1.945084$

C $7.6993950 .032045-1.243160$

C $8.7755760 .424640-1.945275$

C $7.728752-0.0124640 .271902$

C 6.7769490 .9818110 .877510

N $2.060646 \quad 1.120976-2.663124$

H -0.5590891 .2929381 .720334$

H -1.626136 4.1270712 .163766

H 0.7114084 .5751092 .708422

H $0.7520813 .714483-0.246896$

H 1.8726604 .8067490 .466563

H -1.516195 $6.655280-0.952859$

H $-0.335376 \quad 7.797370 \quad 0.641980$

H -0.0011886 .7680902 .036720$

H $1.305174 \quad 7.2046150 .912624$

H -1.434672 $5.005632-2.741461$

H -0.658972 $3.802202-1.739093$

H -2.725172 $3.751352-0.271499$

H $-3.5101684 .884839-1.367675$

H -3.331571 $3.244658-3.222043$

H $-5.3465053 .183999-1.709245$

H $-4.4968372 .137298-0.598935$

H $-6.7973751 .186805-1.446483$

H $-3.791470-0.287042-1.485327$

H $-6.6619702 .092708-3.551757$

H $-6.0677490 .623664-4.286533$

H -5.112663 2.121744 -4.382291

H -4.207363 -0.722578 -4.243295

H $-1.394588-0.605794-1.658906$

H -1.694728 -1.228348 -3.285099

H $-3.434673 \quad 1.163865-4.651792$

H 2.9772650 .8440972 .411639

H 5.2244112 .8519971 .766200

H $4.282994-0.9121221 .338761$

H $4.893940-0.6900872 .994940$

H $5.909391-1.4252851 .776835$

H $\quad 0.495217-0.789840-3.510042$

H $-0.1099250 .768964-4.072326$

H $2.7739822 .002018-0.897736$

H $3.5540612 .519036-2.409045$

H $3.859701-0.288270-1.100953$

H $5.739373 \quad 1.507929-2.716208$

H $6.704564-0.846636-2.936169$

H $6.023130-1.299893-1.392936$

H $9.6861480 .748232-1.452169$

H $8.7677150 .435026-3.030197$

H 8.7337350 .2226860 .645613

H $7.535617-1.0401630 .590340$

H 7.0357032 .0193090 .660387

H $2.2617760 .899000-3.634069$

C -2.9939090 .5766813 .000465$

O $-2.589993-0.7049172 .461500$

C $-3.749138-1.5526472 .399411$

C $-4.669928-1.0304893 .491210$

C -4.4913940 .4621043 .317814$

H $-4.219398-1.3770941 .423878$

C $-3.386938-3.0337222 .536828$

H $-5.705618-1.3576103 .359327$

H $-4.333763-1.3379214 .488273$

C -4.9202361 .2730674 .529141$

H $\quad-5.0861840 .7850512 .452198$ 
C $-2.353593-3.569443 \quad 1.525716$ O $-2.861571-3.2765373 .848626$ H -4.304345 -3.630913 2.471573 H -1.348511 -3.2500571 .821492$ O $-2.363890-5.000186 \quad 1.626141$ C $-2.599953-3.1807300 .058602$ C $-1.761748-3.957398-0.975712$ H -2.412821 -2.107452 -0.056182 O $-3.977505-3.401123-0.259148$ H -2.149529 -4.980340 -1.069631 H -1.947998 -3.486652 -1.950039 H $-5.985626 \quad 1.125514 \quad 4.733494$ H -4.362403 0.9809925 .425232 H -4.7519682 .3414424 .360595$ C -2.7258201 .6727941 .965006$ H $-2.090235-2.6956593 .966788$ H -2.186096 -5.226692 2.555138 H -4.125990 -3.071872 -1.162197 H -3.347477 2.5496592 .182224 H $-3.002804 \quad 1.3068570 .969615$ H -2.407182 $0.740613 \quad 3.912642$ C $1.628569-2.546495-0.249564$ O $0.236643-2.669222-0.578845$ C $-0.251084-4.006871-0.699569$ C $0.508384-4.754547-1.807208$ C $2.021394-4.711129-1.568114$ C $2.508555-3.289968-1.269651$ H $1.845354-1.476058-0.346938$ C $1.910723-2.9497621 .207454$ H $-0.097764-4.5355570 .245505$ O $0.214945-4.188927-3.083480$ H $\quad 0.166110-5.795544-1.831909$ H $2.541681-5.092406-2.455188$ O $2.359327-5.551676-0.468805$ H $3.545684-3.303770-0.914311$ O $2.493230-2.531045-2.481905$ C $1.312277-1.9652082 .189189$ H $1.507432-3.9355451 .450653$ H $2.992915-2.9619871 .377252$ H $0.432966-3.241929-3.045836$ H $2.066782-6.452943-0.687205$ H $3.100489-2.963757-3.106052$ O $1.689917-2.3104593 .455307$ O $0.597628-1.0189001 .891239$ C $1.182890-1.4670584 .494505$ H $1.554664-0.4457464 .368097$ H $0.088699-1.4823294 .496396$ H $1.540713-1.8547465 .451974$

SCF Energy (B3LYP/6-31G**//MMFF) $=-3245.89758746$

\section{7}

MM̄FF Geometry

C $3.5350982 .815564-1.868332$

C $2.4741702 .999749-1.070431$

C $1.4192294 .051109-1.278890$

O $0.2002953 .297160-1.495914$

C $1.2467134 .963851-0.047198$

C 2.5296465 .5424730 .514148

C 3.0926335 .1696351 .681751

C $3.1665496 .618021-0.326561$

C 2.5981734 .1220242 .639996

C 3.5868712 .9538322 .771933

C 2.9034041 .7221783 .382963

C 3.9169550 .6276473 .711556

C $3.223172-0.6359324 .245138$

O $1.976755 \quad 1.2300292 .410331$

O $4.180520-1.7067844 .227975$

C $2.087363-1.0373443 .277755$

C $2.791487-0.4682955 .706624$

O $1.302653-2.0982693 .815879$

C 1.1683980 .1342592 .850340

C $0.282566-0.3014931 .659148$

O 0.2805210 .5210943 .899456

C $-0.9137903 .930059-1.937912$

O $-1.0379105 .107586-2.227293$

C $-1.9846872 .909666-1.972444$

C $-3.2691973 .279789-1.875243$

C $-4.4222442 .375175-1.854461$
C $-4.1526240 .899460-1.991617$

C -0.4548530 .8373050 .946256$

C -1.5924351 .4379951 .743223$

C -3.9084660 .9540502 .525120$

O -1.4896632 .5375882 .282002$

C -5.1117790 .6535181 .687903$

C -6.0972891 .5364721 .463416$

C $-7.337926 \quad 1.2180610 .678725$

C -7.672718 2.268357 -0.356865

C $-8.6235583 .189147-0.123656$

C $-6.9798722 .190372-1.698178$

C $-5.6554372 .902310-1.707140$

N -2.7134080 .6266051 .771481$

H $3.6863433 .466557-2.725089$

H $2.3330542 .329275-0.226430$

H $1.6311444 .645367-2.176430$

H 0.6719224 .4271010 .714446

H $0.5864065 .800446-0.312048$

H 4.0128795 .6634271 .991063

H 4.0701037 .0279670 .136881

H $3.4500376 .222643-1.306990$

H $2.4670097 .447162-0.474805$

H 2.4621854 .5886463 .623561

H 1.6142393 .7453262 .354024

H 3.9835492 .6917801 .782640

H 4.4334183 .2682133 .394422

H 2.3600432 .0312064 .283593

H 4.6632450 .9887424 .429936

H 4.4763700 .3611302 .803787

H $4.943642-1.4357064 .766595$

H $2.568854-1.4354612 .373979$

H $3.665120-0.2907696 .345546$

H $2.322831-1.3817906 .088735$

H 2.1023940 .3653265 .857259

H $1.909862-2.8268724 .031253$

H $0.920458-0.7754220 .902041$

H $-0.442718-1.0496431 .998283$

H $\quad 0.438243-0.0461284 .670353$

H -1.677204 $1.871969-2.040000$

H -3.498572 $4.340953-1.787643$

H $-3.7240790 .676787-2.974921$

H $-5.0427800 .277461-1.878625$

H $-3.4505920 .566184-1.220614$

H 0.2315581 .6418650 .670657

H -0.8696010 .4572720 .004000$

H $-3.876861 \quad 1.9991812 .848929$

H -3.910082 0.3178743 .415987

H $-5.191390-0.3486331 .272898$

H $-6.0319782 .527717 \quad 1.906673$

H -8.165835 1.1282421 .394492

H -7.2621860 .2381890 .190913$

H -8.892863 $3.925329-0.873957$

H $-9.1493193 .231150 \quad 0.824405$

H -7.606168 $2.664340-2.466455$

H $-6.9089861 .145702-2.013426$

H $-5.7460593 .985026-1.607078$

H -2.644543 -0.3098221 .384172$

C $4.4404210 .663872-2.759181$

O $3.168002-0.011676-2.683824$

C $3.357625-1.397893-3.026589$

C $4.819198-1.545060-3.443204$

C $5.484648-0.442774-2.643523$

H $3.197263-1.973829-2.108840$

C $2.352764-1.825671-4.102675$

H $5.213850-2.539844-3.214367$

H $4.955594-1.373568-4.517281$

C $6.858732-0.061238-3.165216$

H $5.571401-0.770566-1.598875$

C $0.873260-1.643729-3.703773$

O $2.593665-1.037040-5.275380$

H $2.543703-2.868319-4.384839$

H $\quad 0.627707-0.578530-3.612965$

O $0.069424-2.156516-4.774871$

C $0.465446-2.376121-2.409415$

C $-1.058791-2.341203-2.167564$

H $0.970343-1.910964-1.556086$

O $0.910728-3.730092-2.476727$

H - $-1.408876-1.304448-2.239051$ 
H -1.551856 -2.925379-2.954630 H $7.535478-0.921357-3.130057$ H $6.8117430 .287312-4.202409$ H $7.2987650 .738048-2.561061$ C $4.5364081 .714864-1.653742$ H $2.116252-1.454412-6.012035$ H $0.158710-1.545654-5.525644$ H $0.461857-4.150521-3.230189$ H $5.5370382 .160607-1.634004$ H $4.3718201 .241057-0.677676$ H $4.492708 \quad 1.141545-3.746894$ C -1.402111 -4.9948540 .455179$ O $-1.145517-4.312976-0.778678$ C -1.471784 -2.921252 -0.798943 C $-2.971938-2.728193-0.525747$ C $-3.397836-3.4149730 .776013$ C $-2.880036-4.8552370 .859661$ H -1.207792 -6.0519750 .233276$ C $-0.430047-4.5672351 .560350$ H $-0.891572-2.404439-0.029527$ O $-3.726302-3.256304-1.614951$ H -3.184348 -1.655688 -0.469154 H $-4.493054-3.4165480 .838508$ O $-2.904061-2.653658 \quad 1.873577$ H -3.028060 -5.252056 1.870955 O $-3.671705-5.655543-0.023168$ C $0.994686-4.893527 \quad 1.179984$ H $-0.494788-3.5014961 .793204$ H $-0.645800-5.0971672 .495612$ H -4.665989 -3.088420 -1.430097 H -3.209193 -3.083595 2.690533 H $-3.379933-6.5781150 .074227$ O $1.660648-3.7524190 .844304$ O $1.453396-6.0284551 .166261$ C $3.028345-3.9241770 .461899$ H $3.452193-2.9359470 .264818$ H $3.594418-4.3916021 .273152$ H $3.092331-4.523162-0.451314$ SCF Energy $\left(B 3 L Y P / 6-31 G^{* *} / / M M F F\right)=-3245.89129883$

\section{8}

MM̄FF Geometry

C $-2.702723-2.5861031 .828023$

C $-2.794949-2.3117443 .137720$

C $-1.880262-1.3971823 .914704$

O $-0.783155-0.9447973 .091650$

C $-2.663274-0.1466164 .358946$

C $-1.807700 \quad 0.8511795 .114564$

C -1.240104 1.9409864 .558798

C -1.6286880 .5615026 .580842$

C -1.2810142 .3591813 .111876$

C -2.0764853 .6558422 .935576$

C -2.2362994 .1204691 .477961$

C $-3.1695793 .213852 \quad 0.669224$

C $-3.3030893 .690384-0.786243$

O $-0.945377 \quad 4.191657 \quad 0.859483$

O $-3.9148542 .633823-1.542232$

C $-1.8886183 .896677-1.365648$

C $-4.2289114 .905002-0.915580$

O $-1.9456004 .456944-2.674212$

C $-0.9666344 .748753-0.465832$

C $0.4906254 .808039-0.985261$

O $-1.4466726 .099268-0.441426$

C $0.476659-1.3455813 .404753$

O $0.807467-2.2153664 .192309$

C $1.394907-0.4540372 .661337$

C $2.709536-0.4486262 .928437$

C 3.6877560 .4815782 .354838

C 3.1503951 .5723241 .466100

C $1.1437553 .451749-1.255943$

C $2.6265703 .569923-1.575244$

C $4.5370902 .169333-2.394882$

O $3.2706224 .602522-1.414955$

C $5.0933021 .086597-1.526071$

C $6.2464571 .170818-0.845805$

C 6.7595370 .0626400 .032438

C 7.1669650 .5325041 .413248

C 8.4203540 .3380651 .857707
C $6.142776 \quad 1.2344462 .279219$

C 4.9919280 .3464872 .675954

N $3.1422912 .367988-2.038430$

H -1.902214 -2.146536 1.239978

H -3.605958 -2.760090 3.708184

H $-1.510888-1.9379454 .794164$

H -3.511721 -0.4400014 .991832$

H $-3.1160800 .338888 \quad 3.485263$

H $-0.6534942 .598490 \quad 5.199058$

H $-1.016103 \quad 1.3179317 .082639$

H -2.6006300 .5364457 .084337$

H -1.138009 -0.4067086 .722094$

H -0.2479082 .5108392 .776222$

H -1.6905421 .5698242 .478449$

H -3.0692663 .5565263 .392773$

H $-1.5527704 .448546 \quad 3.487177$

H $-2.6593245 .131427 \quad 1.522515$

H -4.1563473 .1478821 .143699$

H -2.7763212 .1892800 .651148$

H $-4.7862882 .452823-1.150318$

H $-1.4519962 .896264-1.470853$

H -5.235113 $4.658777-0.555465$

H $-4.3498175 .198432-1.964207$

H -3.882357 $5.773403-0.352031$

H -2.523842 $3.886286-3.208942$

H $0.5366635 .418819-1.896782$

H $1.0945355 .347158-0.242143$

H $-0.7155886 .680177-0.172613$

H 0.9449590 .2297451 .949452

H $3.100321-1.1570233 .656949$

H 2.4200462 .1863362 .005354

H $2.662993 \quad 1.1353080 .588205$

H 3.9130002 .2572691 .093979

H $1.0372092 .798602-0.383298$

H $0.6583562 .969936-2.111470$

H $5.0928623 .107069-2.299784$

H $4.5579731 .850683-3.441552$

H $4.5057790 .176193-1.448427$

H $6.8546992 .067836-0.931850$

H $7.615232-0.394061-0.481523$

H $6.012038-0.7323920 .139587$

H 8.7303130 .6711692 .842801

H $9.167022-0.1599231 .248018$

H $5.8215772 .151761 \quad 1.779862$

H $6.6113081 .579638 \quad 3.211013$

H $5.280692-0.4733593 .334813$

H $2.5143131 .577160-2.151990$

C $-4.361159-2.764673-0.080486$

O $-3.391547-2.324726-1.051010$

C $-3.822612-1.056618-1.588782$

C $-5.249384-0.849123-1.088839$

C $-5.193619-1.5319860 .265414$

H -3.180668 -0.289418 -1.140978

C $-3.664080-1.055467-3.113764$

H $-5.5194570 .208612-1.025599$

H $-5.983047-1.344892-1.735235$

C $-6.558593-1.8500540 .848252$

H $-4.652337-0.8754500 .958263$

C $-2.228578-1.371282-3.588182$

O $-4.536128-2.048948-3.669279$

H -3.997998 -0.095972 -3.524162

H -1.960687 -2.381909-3.264795

O $-2.232129-1.406891-5.021335$

C $-1.166834-0.355859-3.121449$

C $0.256817-0.671796-3.624150$

H -1.157654 -0.290919 -2.029016

O $-1.5417180 .932715-3.616796$

H $\quad 0.251298-0.789586-4.714492$

H $0.8850140 .205649-3.420969$

H $-7.134567-0.9316881 .001373$

H $-7.136610-2.5026880 .185525$

H $-6.460821-2.3526741 .815704$

C $-3.663623-3.4699531 .084998$

H $-4.281599-2.907956-3.290511$

H $-2.448114-0.511892-5.335140$

H $-0.874710 \quad 1.571333-3.314037$

H -3.088314 -4.3151280 .689030$

H $-4.416873-3.885176 \quad 1.764599$ 
H $-4.989243-3.504570-0.594825$

C $1.518374-2.682983-0.755691$

O $1.046150-1.599209-1.565230$

C $0.876284-1.908206-2.952417$

C $2.230733-2.286901-3.566965$

C $2.861829-3.462234-2.812325$

C $2.843777-3.255694-1.291997$

H $1.718952-2.2164120 .215544$

C $0.411283-3.728341-0.558131$

H $0.189281-2.754307-3.053851$

O $3.103259-1.160728-3.526630$

H $2.080155-2.553165-4.620142$

H $3.897389-3.590263-3.150901$

O $2.148613-4.648155-3.151816$

H $3.079534-4.196788-0.782333$

O $3.893148-2.339415-0.970598$

C $0.553995-4.4862590 .744243$

H -0.560016 -3.220275 -0.540255

H $0.390116-4.451617-1.377588$

H $3.945843-1.426323-3.932683$

H $2.582193-5.391176-2.698506$

H $3.928302-2.258287-0.002125$

O $-0.514159-5.3252510 .886633$

O $1.477042-4.364331 \quad 1.537550$

C $-0.540364-6.0873072 .097443$

H $-0.554849-5.4207722 .965333$

H -1.454591 -6.686687 2.101573

H $0.321834-6.7595442 .139474$

SCF Energy (B3LYP/6-31G**//MMFF) $=-3245.91754563$

\section{9}

MM̄FF Geometry

C $-1.562323-2.9813982 .078697$

C $-0.619328-2.4800872 .887551$

C $-0.717082-1.241756 \quad 3.745382$

O $0.425460-0.3749583 .464923$

C $-2.008398-0.3871843 .737403$

C $-3.220801-0.9833344 .428190$

C $-4.428435-1.146577 \quad 3.849596$

C $-3.044960-1.3190605 .887170$

C $-4.811273-0.8631082 .423555$

C $-5.551666 \quad 0.4705012 .277788$

C -5.7333320 .9184030 .816763$

C $-6.599366-0.0454220 .003173$

C $-6.707658 \quad 0.387348-1.466198$

O $-4.432993 \quad 1.034590 \quad 0.229800$

O $-7.270612-0.719482-2.187184$

C -5.285952 $0.637440-2.012922$

C $-7.6847721 .555033-1.641187$

O $-5.339651 \quad 1.149937-3.342669$

C $-4.418257 \quad 1.545744-1.108211$

C $-2.9509151 .527033-1.610365$

O $-4.9317572 .875182-1.167627$

C $0.566350 \quad 0.194563 \quad 2.237312$

O $-0.1529250 .044022 \quad 1.263463$

C 1.7636941 .0634052 .289916

C 2.0392951 .8769971 .260031

C 3.1764592 .7938151 .163564

C $4.138118 \quad 2.8447352 .321431$

C -1.932106 $2.215339-0.690403$

C $-1.8179893 .716763-0.884397$

C $-0.720840 \quad 5.717863 \quad 0.124506$

O $-2.354575 \quad 4.319179-1.809825$

C $0.607125 \quad 5.937217-0.527805$

C $1.668417 \quad 6.4559120 .107780$

C $3.0041406 .681567-0.541010$

C $4.119075 \quad 5.934902 \quad 0.157757$

C $4.876686 \quad 6.543207 \quad 1.086083$

C $4.4122274 .516622-0.277319$

C 3.3083993 .5494300 .053255

$\begin{array}{lllll}\mathrm{N} & -1.027182 & 4.300089 & 0.096141\end{array}$

H -2.529461 -2.504286 2.024140

H $0.337812-2.9961592 .938562$

H $-0.537712-1.5741454 .774458$

H -2.240170 -0.0820532 .712809$

H -1.7865430 .5620714 .246791$

H $-5.241698-1.5411804 .457434$

H -3.995112 -1.560879 6.375674
H -2.386234 -2.184512 6.006946

H $-2.610699-0.4695036 .424271$

H $-3.938148-0.8766431 .768362$

H $-5.450077-1.6861732 .082107$

H -6.5291290 .4113182 .772228$

H -4.9742411 .2472512 .797137$

H -6.1971251 .9116890 .841074$

H -7.595365 -0.1475590 .451461$

H $-6.157988-1.0506550 .020351$

H -7.270246 -0.486455 -3.131662

H $-4.799214-0.344283-2.088996$

H $-8.6827091 .272272-1.284931$

H $-7.8088261 .813715-2.698558$

H $-7.3815292 .453548-1.099929$

H $-5.5725432 .092491-3.286918$

H -2.635132 $0.478839-1.683972$

H $-2.8912951 .957234-2.617075$

H $-4.2568493 .484732-0.831652$

H $2.3803231 .016597 \quad 3.180338$

H 1.3704471 .8731840 .400390

H 3.6187663 .1445813 .238451

H 4.9526893 .5579092 .178118

H 4.5988361 .8649222 .483584

H $-2.142506 \quad 1.9989210 .362280$

H $-0.9399681 .804746-0.916551$

H -0.7240546 .0300381 .173642$

H $-1.4954506 .277937-0.408623$

H $0.6959345 .660243-1.575686$

H $1.5732016 .745914 \quad 1.151775$

H $3.2070917 .760393-0.522832$

H $2.9879526 .404450-1.602412$

H 5.6984546 .0285651 .572755

H 4.6891647 .5699631 .382816

H 5.3647974 .1646330 .136235

H $4.5668744 .504950-1.364311$

H $2.5590553 .455545-0.733282$

H -0.5676903 .7051350 .779775$

C -1.481655 -3.926190 -0.284458

O $-0.356382-3.144156-0.734361$

C $-0.797428-2.299404-1.817397$

C $-2.226994-2.728349-2.145170$

C $-2.721224-3.187855-0.788326$

H $-0.820411-1.276499-1.425637$

C $0.162224-2.388812-3.008751$

H -2.818044 -1.910365 -2.567024

H $-2.249170-3.559204-2.860211$

C $-3.976414-4.038036-0.845632$

H $-2.921664-2.292527-0.188072$

C $1.619900-1.981144-2.712652$

O $0.184071-3.738428-3.493508$

H $-0.226706-1.782053-3.835123$

H $2.100117-2.739181-2.084073$

O $2.327156-1.987589-3.960321$

C $1.784122-0.593299-2.069062$

C $3.224589-0.047232-2.066490$

H $1.404978-0.628155-1.042068$

$\begin{array}{llll}O & 0.973267 & 0.346723 & -2.781848\end{array}$

H $3.5438990 .166214-3.094696$

H $3.1912110 .929077-1.568034$

H $-4.802240-3.473743-1.291532$

H $-3.823409-4.941208-1.445544$

H $-4.282765-4.346747 \quad 0.158856$

C $-1.390043-4.2007651 .220347$

H $0.464776-4.309011-2.757580$

H $2.217519-2.871531-4.350930$

H $1.0405021 .200790-2.321836$

H $-0.422121-4.6680881 .435732$

H $-2.162803-4.9251101 .503646$

H $-1.396935-4.893146-0.799627$

C $4.653870-2.055768 \quad 0.743551$

O $3.821313-1.186857-0.037056$

C $4.261663-0.945397-1.375328$

C $5.660491-0.304401-1.376836$

C $6.660477-1.129558-0.560270$

C $6.095276-1.5192350 .809524$

H $4.230867-2.0138161 .755497$

C $4.581961-3.514658 \quad 0.264540$

H $4.306820-1.894494-1.917759$ 
O $5.6062201 .018968-0.847072$

H $6.007301-0.217475-2.412984$

H $7.579518-0.548224-0.414451$

O $7.003090-2.313327-1.273970$

H $\quad 6.747525-2.2596641 .287468$

O $6.108882-0.3530791 .637221$

C $3.203095-4.1148310 .431847$

H $4.847058-3.607854-0.791618$

H $5.274239-4.125386 \quad 0.855656$

H 5.2509190 .9621490 .056200

H $7.391874-2.042226-2.123036$

H $5.807798-0.6172922 .523280$

O $3.212382-5.380971-0.081817$

O $2.245123-3.555225 \quad 0.945065$

C $1.973027-6.090979-0.004790$

H $1.222457-5.602163-0.631694$

H $2.137767-7.103985-0.381308$

H $1.632355-6.1552471 .032827$

SCF Energy (B3LYP/6-31G*//MMFF) $=-3245.91769445$

0700060

MM̄FF Geometry

C $3.165214-4.3304850 .448872$

C $2.046927-3.892455-0.149002$

C $0.691047-3.8895100 .509888$

$\begin{array}{llllll}\text { O } & 0.108331 & -2.573009 & 0.366715\end{array}$

C $-0.232290-4.906722-0.185828$

C $-1.663175-4.9009170 .320984$

C $-2.741358-4.793271-0.480744$

C $-1.847439-5.0495941 .808762$

C $-2.751649-4.734597-1.981663$

C $-2.676324-3.321219-2.573917$

C $-3.923708-2.468411-2.296487$

C $-3.955288-1.255873-3.230352$

C $-5.151137-0.339301-2.938559$

O $-3.883242-2.025363-0.935349$

O $-4.931848 \quad 0.903305-3.623452$

C -5.177849 -0.025934-1.430300

C $-6.462307-0.913575-3.487594$

O $-6.3551430 .698715-1.080122$

C $-5.037302-1.277468-0.530019$

C $-4.843819-0.828543 \quad 0.935711$

O $-6.229094-2.060936-0.618625$

C $0.458445-1.6427821 .296456$

O $1.261141-1.7829762 .206046$

C $-0.317199-0.4107101 .024367$

C $-0.062280 \quad 0.6858141 .753938$

C $-0.716701 \quad 1.9901361 .647019$

C -1.790506 $2.168996 \quad 0.609394$

C -4.393301-1.933819 1.896299

C $-4.376515-1.4066953 .318353$

C -3.1519090 .1864654 .803170$

O $-5.252943-1.7074224 .125590$

C $-3.295383 \quad 1.6478104 .507213$

C -2.4313212 .5809664 .935990$

C -2.5645204 .0464504 .628048$

C -1.2897174 .6530544 .077829$

C -0.5460175 .4892284 .821820$

C $-0.914240 \quad 4.3488712 .640907$

C -0.3307022 .9695652 .491339$

N $-3.313688-0.5514803 .563306$

H $3.113341-4.7168611 .463667$ H $2.100027-3.511923-1.166475$ H $0.773294-4.1436411 .574491$ H $0.174512-5.918021-0.053953$ H $-0.211375-4.703411-1.262297$ H -3.728960 -4.784349-0.021722 H $-2.897770-5.1951152 .083587$ H -1.291711 -5.9193112 .174228$ H $-1.491945-4.1586062 .335313$ H $-1.930895-5.335890-2.389395$ H -3.664076 -5.230665 -2.336795 H $-1.782287-2.812311-2.191536$ H $-2.550115-3.431305-3.658261$ H $-4.813705-3.089331-2.452872$ H -3.965517 -1.571861 -4.281016 H -3.032023 $-0.675058-3.101049$ H $-4.8423820 .710364-4.572515$
H $-4.3303800 .643311-1.223930$

H $-6.393659-1.054568-4.572923$

H $-7.294645-0.219123-3.328569$

H -6.727077 -1.876555 -3.046219

H $-6.3957351 .481493-1.655936$

H $-4.084404-0.0406970 .966622$

H $-5.775572-0.3876941 .313406$

H $-6.329094-2.5787880 .195696$

H $-1.068098-0.4424460 .243630$

H 0.7112100 .6251932 .519250

H -2.006389 3.2183410 .393800

H -2.7187521 .6983930 .941402$

H -1.496559 $1.730103-0.348856$

H $-5.064301-2.7980061 .863297$

H -3.390095 -2.291716 1.640158

H -2.161364 -0.0582795 .198937$

H $-3.910337-0.1239065 .528092$

H -4.1534191 .9531073 .912338$

H -1.5879942 .2792255 .553290$

H -2.8523994 .5549245 .557309$

H -3.3813204 .2315913 .919242$

H $\quad 0.3556725 .946188 \quad 4.427651$

H -0.8188445 .7321195 .843376$

H -0.1638095 .0663802 .284873$

H -1.798862 4.5076912 .017866

H 0.4847622 .7618823 .186036

H $-2.633558-0.3876212 .827364$

C $5.518929-3.4669630 .555864$

O $5.096456-2.0903530 .513555$

C $6.222133-1.2630350 .169255$

C $7.454611-2.1352930 .366949$

C $6.923103-3.493012-0.050755$

H $6.123906-1.028798-0.897832$

C $6.1963820 .035296 \quad 0.983595$

H $8.303042-1.800022-0.237049$

H $7.773170-2.1618731 .415295$

C $7.778652-4.6545380 .423949$

H $6.859035-3.521251-1.146921$

C $4.8430350 .774087 \quad 0.956933$

$\begin{array}{lllll}\text { O } & 6.488397 & -0.259460 & 2.356153\end{array}$

H 6.9977870 .7007120 .643052

H $4.0967110 .206369 \quad 1.526814$

O $5.008372 \quad 2.021144 \quad 1.643437$

C $4.2948221 .061438-0.452223$

C $2.9496441 .796424-0.401927$

H $4.1588040 .113852-0.980317$

O $5.2362251 .831679-1.193825$

H 2.2432601 .2134230 .197656

H $3.0643592 .751796 \quad 0.127777$

H $8.787662-4.5855100 .004499$

H $7.868681-4.6669941 .515372$

H $7.347080-5.6103020 .110593$

C $4.516733-4.332091-0.210758$

H $5.827105-0.9024922 .665569$

H 5.3457951 .8190952 .532972

H $5.3812382 .662679-0.709717$

H $4.873540-5.367062-0.258730$

H $4.429011-3.967811-1.242102$

H $5.548285-3.7582451 .613909$

C $0.3083263 .189828-2.612790$

O $1.0586332 .729318-1.483115$

C $2.3227552 .114488-1.772066$

C $2.1234290 .889897-2.677403$

C $1.3375591 .255785-3.941164$

C $0.0701032 .051303-3.618112$

H $-0.665213 \quad 3.482547-2.198686$

C $0.938150 \quad 4.439426-3.237842$

H $2.9659582 .844688-2.274837$

O $1.432838-0.126074-1.954061$

H $3.1021200 .488128-2.963309$

H $1.0657320 .335502-4.472764$

O $2.1849052 .017978-4.796220$

H $-0.3684942 .453509-4.538838$

O $-0.885770 \quad 1.143260-3.063823$

C $0.8210785 .612836-2.293747$

H $1.9913564 .316220-3.504899$

H $0.4141394 .715308-4.160790$

H $1.343635-0.896433-2.540539$ 
H $\quad 1.6835272 .216372-5.605310$

H -1.711906 $1.635939-2.920564$

O $1.8607435 .607938-1.411433$

O $\quad-0.099438 \quad 6.419537-2.321924$

C $1.8373086 .645034-0.426149$

H $\quad 0.930444 \quad 6.5702870 .181718$

H $2.7063056 .515456 \quad 0.224344$

H $1.9012577 .625467-0.907519$

SCF Energy (B3LYP/6-31G**//MMFF) $=-3245.90948722$

\section{1}

MM̄FF Geometry

C -1.349847 3.3332450 .120293

C $-0.3693963 .851573 \quad 0.874527$

C 0.5896973 .0161341 .679019

O 1.9184813 .4260371 .278145

C 0.4114613 .2368883 .193675

C -0.9564762 .8582513 .721967$

C -1.3625521 .5940633 .949738$

C -1.8783064 .0128204 .019032$

C $-0.580605 \quad 0.323867 \quad 3.754595$

C $-1.189972-0.5367502 .638867$

C $-0.567796-1.9374862 .497547$

C $-0.951957-2.8589783 .658251$

C $-0.290284-4.2390023 .536318$

O $0.852673-1.8066252 .409474$

O $-0.437647-4.9115414 .796619$

C $1.220973-4.0431053 .302721$

C $-0.984107-5.1205822 .492295$

O $1.859336-5.2929853 .047386$

C $1.553891-3.0375702 .173387$

C $3.072379-2.7264092 .195587$

O $1.199280-3.611794 \quad 0.916524$

C 2.9357352 .5510071 .496680

O 2.8751881 .4867102 .090186

C 4.1425573 .0952790 .834830

C $5.1973512 .291850 \quad 0.631692$

C $6.4410422 .648419-0.053426$

C $6.6082034 .074332-0.508251$

C $3.530722-1.6682151 .183850$

C $3.973248-2.224749-0.159140$

C $4.828824-1.435932-2.371438$

O $4.065601-3.424938-0.397964$

C $6.297076-1.152857-2.313838$

C $6.888953-0.152537-2.983862$

C $8.3587890 .149031-2.907410$

C $8.6376811 .559803-2.433978$

C $8.9006742 .539445-3.315418$

C $8.6971951 .819355-0.943986$

C $7.3637181 .685670-0.261930$

N $4.272178-1.204864-1.052367$

H -1.459834 2.2542240 .059756

H -0.2650634 .9317570 .946569$

H $\quad 0.4619201 .9560041 .426740$

H 0.6290864 .2860313 .438540

H 1.1801232 .6820013 .745840

H -2.369137 1.4418874 .337858

H -2.8517513 .6818654 .396600$

H -1.433925 4.6639514 .778874

H -2.0612514 .6063943 .118578$

H $0.471167 \quad 0.5272313 .532661$

H -0.596389 -0.2155444 .707502$

H $-2.273926-0.6367002 .777936$

H -1.044511 -0.0032641 .690384$

H -0.940805 -2.358639 1.556607

H $-2.042076-2.9602803 .729015$

H -0.630175 -2.418559 4.610383

H -1.388162 -4.975522 4.992521

H $1.650196-3.6624094 .240312$

H -2.039718 -5.2640962 .752335$

H $-0.543245-6.1233312 .465991$

H - $0.946702-4.7012371 .485800$

H $1.637127-5.8876563 .784231$

H $3.317920-2.3338153 .191610$

H $3.650278-3.6476552 .058570$

H $1.624926-3.0998370 .210961$

H 4.1134494 .1249980 .497679

H 5.1402041 .2621820 .981606
H $5.8272144 .342808-1.228193$

H $7.5651624 .263340-0.999804$

H $\quad \begin{array}{llll}0.550884 & 4.759113 & 0.345182\end{array}$

H $2.752824-0.9167911 .019979$

H $4.405495-1.1542511 .602586$

H $4.306385-0.771363-3.066504$

H $4.656812-2.471328-2.680698$

H $\quad 6.899481-1.800523-1.680979$

H $6.2867080 .487641-3.624614$

H $8.782960-0.001535-3.908805$

H $8.882093-0.562456-2.256590$

H $9.1318413 .547900-2.989271$

H $8.8869992 .355231-4.384578$

H $9.1389452 .799949-0.730949$

H $9.3916851 .102244-0.487017$

H 7.1536090 .6767010 .094479

H $4.196385-0.241085-0.740542$

C $-3.764738 \quad 3.906356-0.099738$

O $-4.2180452 .607382-0.532314$

C $-5.6227692 .687746-0.846622$

C $-6.0787144 .079173-0.420037$

C $-4.8116744 .888416-0.618035$

H $-5.7068832 .615804-1.936818$

C $-6.3817641 .535157-0.180041$

H $-6.9187274 .441254-1.020677$

H -6.3862374 .1013360 .632119$

C $-4.827983 \quad 6.222106 \quad 0.108644$

H $-4.6659045 .061483-1.692773$

C $-5.8742940 .135150-0.587192$

O -6.2316431 .6603701 .240169$

H $-7.454271 \quad 1.629416-0.387250$

H $-4.8511600 .003096-0.219468$

O $-6.670112-0.8344750 .106863$

C -5.942508 -0.153055 -2.099158

C $-5.586209-1.602163-2.484831$

H $-5.2849840 .537139-2.637768$

O $\quad-7.277390 \quad 0.104046-2.545772$

H $-6.260964-2.302510-1.977477$

H -5.794370 -1.715109 -3.557396

H $-5.6515636 .846599-0.252948$

H -4.9558656 .0894351 .188332$

H -3.894334 $6.769062-0.054550$

C $-2.3540044 .161402-0.631662$

H -6.7088450 .9175001 .648059$

H -7.579092 -0.763773 -0.232394

H $-7.291015-0.009536-3.511420$

H -2.103592 $5.224558-0.546042$

H $-2.2925763 .889872-1.692337$

H -3.7658943 .8969980 .998821$

C $-1.891038-1.260859-2.805165$

O $-3.302481-1.115082-3.007472$

C $-4.121977-1.974156-2.206642$

C $-3.826045-3.445449-2.538440$

C $-2.335568-3.758946-2.369781$

C $-1.447640-2.713169-3.056142$

H $-1.434834-0.634427-3.582743$

C -1.488809-0.686415-1.444665

H $-3.907100-1.798460-1.147715$

O $-4.220821-3.719890-3.881260$

H $-4.424747-4.084848-1.878533$

H $-2.126075-4.748830-2.793377$

O $-2.040379-3.812955-0.977760$

H $-0.405221-2.842494-2.747202$

O $-1.478244-2.948581-4.468429$

C $-0.017458-0.366319-1.391647$

H $-2.0558330 .228567-1.232723$

H -1.722910 -1.358202 -0.614614

H -4.058347 -4.664161 -4.047139

H - $-1.095535-4.024012-0.885665$

H $-2.385044-2.781003-4.777906$

O $0.2096230 .877528-1.900078$

O $0.830512-1.142164-0.971874$

C $1.5767121 .299421-1.916268$

H $1.5995362 .373031-2.120497$

H $2.0525721 .119119-0.948614$

H $2.1135740 .775707-2.712034$

SCF Energy (B3LYP/6-31G**//MMFF) $=-3245.92276101$ 


\section{2}

MMFF Geometry

C -3.5430813 .0349910 .080214$

C -3.3890614 .0929210 .891403$

C -2.2046624 .3451951 .801111$

O -1.3320463 .1938261 .871576$

C -1.3777515 .5352271 .284069$

C $-0.272705 \quad 5.9504772 .237613$

C 1.0483785 .8238632 .001938

C $-0.738634 \quad 6.5803113 .527303$

C 1.7425065 .2785430 .784840

C 2.4665113 .9661301 .094385

C $3.4385173 .523465-0.011440$

C $2.7369463 .257647-1.344412$

C $3.6930802 .692831-2.406742$

O 4.0734902 .3236990 .454881

O $2.8731682 .197487-3.476543$

C $4.4643471 .501230-1.801693$

C $4.5776793 .790680-3.008911$

O $5.4765091 .033095-2.688985$

C $5.0870761 .812356-0.423496$

C $5.703154 \quad 0.562122 \quad 0.248903$

O $6.1480292 .761472-0.587083$

C -1.717699 2.172236 2.685454

O $-2.7003012 .133867 \quad 3.408564$

C -0.7543951 .0681812 .496201$

C -1.138191 -0.1875852 .769769$

C $-0.318294-1.3880122 .607801$

C $1.140209-1.1949362 .287922$

C $4.743280-0.6173220 .412984$

C $5.300314-1.7098251 .310727$

C $4.730250-3.9826012 .154000$

O $6.362958-1.6150421 .917415$

C $3.462152-4.7515342 .342140$

C $2.775133-4.7811813 .494102$

C $1.477074-5.5155983 .677083$

C $0.311998-4.5653023 .868339$

C $-0.182695-4.3385235 .097454$

C $-0.249708-3.9473012 .602506$

C $-0.892197-2.5985372 .773618$

N 4.441251 -2.797985 1.368635

H -2.751359 $2.295806 \quad 0.007352$

H $-4.189671 \quad 4.8274470 .950196$

H -2.589914 4.5647902 .804270

H -2.030492 6.4032901 .120797

H $-0.970083 \quad 5.2817890 .300339$

H 1.7351826 .1703782 .774301

H $0.0907396 .989147 \quad 4.114394$

H -1.426949 7.4063793 .319750

H $-1.252150 \quad 5.8456254 .154498$

H $1.0509875 .134205-0.047755$

H 2.4692936 .0302570 .453003

H 3.0345884 .0605032 .030197

H 1.7336863 .1704711 .281629

H $4.1995954 .304343-0.125830$

H $2.2498964 .161235-1.729077$

H $1.9318172 .530766-1.177986$

H $3.4584581 .885734-4.187198$

H $3.7411740 .684072-1.692041$

H $3.9592574 .545948-3.508567$

H $5.2401513 .386470-3.782271$

H $5.1908644 .303991-2.265285$

H $5.0414230 .748879-3.510153$

H $6.5932780 .235967-0.305089$

H 6.0699570 .8574651 .241893

H 6.7398112 .6965240 .180760

H $\quad 0.225157 \quad 1.3147332 .104204$

H -2.154834 -0.3581973 .122078$

H $1.615315-0.5431933 .030027$

H $1.264353-0.7448981 .298091$

H $1.707031-2.1268702 .295547$

H $3.798436-0.2779470 .851907$

H $4.531062-1.064538-0.564635$

H $5.182332-3.6800393 .104663$

H $5.464461-4.5834531 .607851$

H $3.082530-5.2941661 .479783$

H $3.153255-4.2316204 .353355$

H $1.582203-6.1790514 .545003$
H $1.267406-6.1759332 .826035$

H -1.030217 -3.6839335 .266883$

H $\quad 0.249079-4.8137235 .972583$

H $-0.992105-4.6368312 .179944$

H $\quad 0.531673-3.879798 \quad 1.839225$

H $-1.951128-2.6455833 .028973$

H $3.674680-2.8382600 .703052$

C $-5.4493691 .474650-0.247086$

O $-4.640848 \quad 0.329894-0.590711$

C $-5.511675-0.775457-0.901561$

C $-6.933513-0.290560-0.631098$

C $-6.8082721 .199595-0.882538$

H $-5.408241-0.971887-1.974332$

C $-5.110178-2.009619-0.085823$

H -7.665853 -0.776410-1.283278

H $-7.233998-0.4816000 .406189$

C -7.950138 $2.008364-0.292470$

H -6.759607 1.373437 -1.965900

C $-3.707359-2.570686-0.408340$

O $-5.138656-1.672797 \quad 1.308282$

H $-5.859004-2.799366-0.217028$

H -2.936286 -1.846339 -0.122777

O $-3.507339-3.7222410 .423002$

C $-3.514029-2.996664-1.878930$

C $-2.258641-3.853388-2.139885$

H $-3.495092-2.107164-2.517768$

O $-4.648181-3.775044-2.277701$

H $-2.289261-4.753029-1.512718$

H -2.317162 -4.216163 -3.175419

H $-8.9054601 .699645-0.729343$

H -8.0180051 .8746970 .792523$

H -7.818619 $3.076195-0.492300$

C $-4.7743972 .757790-0.735524$

H $-4.509607-0.9436001 .445791$

H -3.648255 -3.442964 1.343818

H $-4.557520-3.961087-3.227822$

H -5.469556 $3.601897-0.671195$

H -4.472103 2.653766 -1.784385

H -5.5390391 .4863110 .848087$

C $0.336900-1.301250-2.958036$

O $-0.836703-2.125207-2.977831$

C $-0.930779-3.107408-1.940294$

C $0.270218-4.064955-1.998484$

C $1.593444-3.294888-1.951841$

C $1.619341-2.148524-2.965400$

H $\quad 0.302570-0.746524-3.905097$

C $0.280631-0.282887-1.816225$

H $-0.944442-2.611445-0.965383$

O $0.209257-4.839689-3.194239$

H $\quad 0.206380-4.759767-1.152239$

H $2.424485-3.979614-2.159858$

O $1.790219-2.758954-0.648718$

H $2.485262-1.499269-2.790066$

O $1.765531-2.691710-4.280660$

C $-0.7759670 .753580-2.111218$

H $0.035455-0.723895-0.847002$

H $1.2442720 .223081-1.693450$

H $0.963342-5.453405-3.185489$

H $1.762813-3.501605-0.022373$

H $2.601651-3.187705-4.301968$

O $-0.2058851 .856663-2.670913$

O $-1.9688960 .583771-1.898995$

C $-1.0983982 .920034-3.017107$

H $-0.5593513 .616337-3.665100$

H $-1.9704222 .543054-3.560203$

$\mathrm{H}-1.4048693 .450170-2.111745$

SCF Energy (B3LYP/6-31G**//MMFF) $=-3245.89717888$

0700063

MM̄̄FF Geometry

C $-0.027044-1.0345951 .052488$

C $0.535282-0.1618220 .201551$

C $0.0879060 .134295-1.210355$

O $-0.985028-0.749316-1.615535$

C $-0.4442281 .571272-1.339817$

C $0.6352012 .631760-1.369494$

C $0.9481333 .439790-0.338199$

C $1.3484302 .783501-2.688786$ 
C 0.3810453 .4279351 .053663 C -0.4540124 .6772431 .361990$ C $-1.808344 \quad 4.7028670 .635700$ C $-2.555718 \quad 6.001512 \quad 0.947766$ C $-3.963166 \quad 6.015044 \quad 0.334283$ O $-2.569278 \quad 3.5788591 .089167$ O $-4.6946067 .097165 \quad 0.930550$ C $-4.687963 \quad 4.7106590 .724864$ C $-3.9276316 .293733-1.172956$ O $-5.947650 \quad 4.612002 \quad 0.063424$ C $-3.852110 \quad 3.434906 \quad 0.465836$ C $-4.553170 \quad 2.215418 \quad 1.111148$ O $-3.7388713 .231476-0.942752$ C $-0.640713-1.914999-2.221618$ O $0.493304-2.291618-2.486373$ C $-1.822591-2.752779-2.527342$ C $-3.093156-2.395760-2.282454$ C $-4.280558-3.215744-2.544964$ C $-4.061057-4.596542-3.108944$ C -3.7590620 .9026581 .035937$ C $-4.450034-0.2153641 .799869$ C $-4.431515-2.6932032 .065453$ O $-5.373074-0.0199702 .586810$ C $-5.568091-3.1500501 .204759$ C $-5.638677-4.359900 \quad 0.627886$ C $-6.742192-4.856417-0.271662$ C $-7.455825-3.793678-1.083428$ C $-8.638430-3.296953-0.683130$ C $-6.840212-3.376446-2.403798$ C $-5.497430-2.714517-2.242351$ N $-3.899170-1.4583751 .516116$ H $-0.900894-1.6028910 .751764$ H 1.4174070 .3820800 .532210 H $0.941057-0.002771-1.885541$ H $-1.175795 \quad 1.766031-0.548261$ H $-1.0338831 .655959-2.263600$ H $1.7255574 .187095-0.491514$ H $2.0554913 .619483-2.686101$ H $1.9120091 .876483-2.928388$ H $0.6275992 .969015-3.491776$ H $-0.1977372 .523517 \quad 1.260500$ H 1.2298423 .3984931 .748934 H -0.6318254 .6928342 .445384$ H $0.120697 \quad 5.578915 \quad 1.115930$ H -1.639165 $4.614296-0.443028$ H -1.980663 6.8723990 .609072 H -2.653327 6.1132892 .037004 H $-4.207477 \quad 7.9206220 .755138$ H -4.9070844 .7677351 .800489$ H -3.464953 7.268260 -1.370939 H $-4.9398416 .353645-1.587638$ H -3.367570 $5.543700-1.735314$ H $-6.443961 \quad 5.424244 \quad 0.263148$ H $-4.738168 \quad 2.4384552 .170324$ H -5.529722 2.0523720 .637577 $\mathrm{H}-3.5451142 .297940-1.116872$ H -1.579224 -3.713627 -2.970386 H -3.292751 -1.420888 -1.841792 H $-4.978576-5.177069-3.221814$ H -3.409509-5.182059-2.450254 H $-3.598926-4.536805-4.100511$ H $-3.6427370 .586584-0.005769$ H -2.762201 1.0306751 .472079 H $-4.786330-2.5122523 .085125$ H -3.612895 -3.418701 2.097163 H $-6.375128-2.4369111 .057476$ H $-4.838632-5.0727180 .817615$ H $-7.456778-5.4171700 .344672$ H $-6.319806-5.593127-0.966899$ H $-9.171750-2.558651-1.272611$ H $-9.096934-3.615290 \quad 0.247279$ H -7.494340 -2.662265 -2.921376 H $-6.801672-4.247324-3.064302$ H $-5.556993-1.708738-1.824376$ H $-3.209627-1.5333590 .774670$ C $1.383007-2.5415062 .529273$ O $2.516450-2.3911701 .647647$ C $2.601882-3.563700 \quad 0.820260$
C $1.889994-4.6540441 .607620$

C $0.714399-3.8716912 .165959$

H $2.023844-3.356716-0.088706$

C $4.055356-3.8578710 .444134$

H $1.586297-5.4955780 .978345$

H $2.513227-5.0374482 .423854$

C $0.025010-4.5493023 .337299$

H $-0.020004-3.7358751 .362593$

C $4.794611-2.676672-0.222707$

O $4.789108-4.217787 \quad 1.620744$

H $4.092024-4.736670-0.210100$

H $5.022519-1.9110100 .527212$

O $6.055456-3.164675-0.696787$

C $4.045831-2.036755-1.404838$

C $4.855771-1.023404-2.232127$

H $3.143350-1.539388-1.034756$

O $3.607845-3.061549-2.302779$

H $5.641414-1.544010-2.794871$

H $4.170666-0.623162-2.991824$

H $-0.387574-5.5168563 .033295$

H $0.719588-4.7256524 .165372$

H $-0.798290-3.9324463 .711801$

C $0.493499-1.2974872 .438720$

H $4.714477-3.4798542 .249899$

H $6.517292-3.5591910 .062956$

H $4.396328-3.544572-2.604332$

H $1.068318-0.4267382 .777199$

H $-0.357657-1.3919133 .122863$

H $1.796033-2.5948113 .544583$

C 4.8599371 .8806390 .133783

$\begin{array}{llllll}\text { O } & 4.436798 & 0.796091 & -0.706763\end{array}$

C $5.4764890 .131092-1.429856$

C $6.2136981 .120985-2.347840$

C $6.7282772 .333297-1.566176$

C $5.6413582 .935259-0.671802$

H 3.9285212 .3478420 .477344

C $5.626676 \quad 1.386291 \quad 1.371874$

H $6.193129-0.295773-0.721853$

$\begin{array}{lllll}\text { O } & 5.353317 & 1.567622 & -3.394885\end{array}$

H $7.0535910 .602631-2.824854$

H $7.0774683 .099469-2.269352$

O $7.8340351 .948653-0.755017$

H $\quad 6.082338 \quad 3.6731920 .008701$

O $4.7227153 .638667-1.512166$

C $4.743196 \quad 0.610625 \quad 2.324703$

H 6.4615650 .7376791 .094941

H 6.0201722 .2428911 .931187

H $4.5680311 .968280-2.984093$

H $8.5242481 .601075-1.345071$

H $4.0866894 .093528-0.934459$

O $5.530574-0.032859 \quad 3.236949$

O $3.521347 \quad 0.5901842 .294250$

C $4.831841-0.7918454 .228507$

H $4.303483-1.6260883 .759505$

H $5.567827-1.1931364 .930047$

H $4.134694-0.1532084 .779327$

SCF Energy (B3LYP/6-31G**//MMFF)= -3245.91880587

\section{4}

MM̄FF Geometry

C $2.3506892 .661021-4.058503$

C $1.7849842 .852732-2.857429$

C $0.9002954 .016337-2.499976$

O $-0.231024 \quad 3.475542-1.780459$

C $1.6496284 .969203-1.549675$

C $0.7815556 .089100-1.007577$

C $0.467945 \quad 6.242582 \quad 0.294938$

C $0.2674377 .070556-2.028198$

C 0.9281995 .3980541 .452506

C 0.0161224 .1927061 .680256

C 0.3537353 .3793452 .938608

C 1.7492302 .7511012 .877735

C $2.0203261 .822564 \quad 4.071921$

O $\begin{array}{llll}0.644194 & 2.351650 & 3.029990\end{array}$

O 3.1908651 .0473243 .771319

C 0.8456640 .8327314 .207834

C 2.3412702 .6095305 .347762

$\begin{array}{lllll}\text { O } & 0.979902 & 0.032973 & 5.379459\end{array}$ 
C $-0.537093 \quad 1.5178594 .192487$ C -1.7053190 .5050264 .147675$ O $\begin{array}{llll}-0.697860 & 2.287477 & 5.389942\end{array}$ C -1.371933 $3.200635-2.462779$ O $-1.5912203 .385222-3.647909$ C -2.312186 2.601483-1.488705 C $-3.5348072 .205862-1.871715$ C $-4.5390171 .581220-1.004533$ C $-4.144503 \quad 1.319128 \quad 0.425996$ C -1.665863 -0.4727322 .971263$ C $-2.967262-1.2418752 .808792$ C $-4.015706-2.945297 \quad 1.303259$ O $-3.931044-1.1064963 .556660$ C $-4.545578-2.441061-0.002533$ C $-5.850058-2.273089-0.268641$ C $-6.362493-1.800524-1.600671$ C $-7.355209-0.661993-1.491252$ C -8.602747 $-0.794697-1.973304$ C $-6.9181650 .629460-0.831129$ C $-5.7445651 .268092-1.524433$ N -2.912456 -2.098547 1.719607 H $2.1753103 .403408-4.834712$ H $1.9490082 .135383-2.058332$ H $0.5678804 .555498-3.395028$ H $2.5085215 .412205-2.070670$ H $2.0709394 .383143-0.725036$ H $-0.179849 \quad 7.0719880 .575273$ H - $0.2481327 .918709-1.564812$ H $1.0973987 .475233-2.616778$ H $-0.4407506 .587227-2.707845$ H 1.9663935 .0860971 .303813 H 0.9352356 .0284922 .350474 H $-1.028740 \quad 4.525327 \quad 1.749312$ H 0.0490943 .5254900 .810998 H 0.2632954 .0392633 .809357 H 2.5297593 .5186672 .822519 H 1.8408832 .1629451 .953661 H 3.9218311 .6639023 .594468 H $0.913783 \quad 0.147633 \quad 3.354036$ H 3.2391993 .2225825 .203329 H 2.5700631 .9355686 .180690 H $1.534627 \quad 3.2780185 .655137$ H $1.856579-0.3867765 .344833$ H -1.755066 -0.0552005 .090456$ H -2.644337 1.0741314 .096633 H -1.6475572 .4237445 .543282$ H -1.960903 2.490246 -0.468615 H -3.828192 2.346201-2.911019 H -3.9277342 .2595620 .944798$ H -3.2525640 .6853630 .460001$ H -4.9091290 .8071861 .011935$ H -1.471336 0.0646812 .036433 H $-0.865411-1.2060953 .120371$ H $-4.785380-2.9726232 .080938$ H -3.611915 -3.9538901 .172158$ H -3.818082 - $2.223053-0.781055$ H $-6.586686-2.5172730 .493062$ H $-6.828766-2.662423-2.095529$ H -5.536514 -1.491507 -2.253110 H $-9.3258640 .011185-1.903440$ H $-8.931525-1.712523-2.449461$ H -6.7340050 .4318240 .227343$ H -7.738036 $1.359919-0.848031$ H -5.934373 $1.508145-2.571529$ H -2.063034 -2.126444 1.162866 C $3.3348170 .337626-3.483568$ O $4.0333340 .749276-2.296786$ C $4.579945-0.427027-1.660863$

C $4.435270-1.565453-2.671863$

C $4.160662-0.842805-3.980243$

H $5.634276-0.204938-1.462364$

C $3.810975-0.662699-0.351087$ H $5.332175-2.191485-2.714233$

H $3.592994-2.219405-2.414747$

C $3.458134-1.712823-5.007996$

H $5.115059-0.487292-4.390805$

C $4.260043-1.9139910 .425428$

$\begin{array}{llll}\text { O } & 3.959270 & 0.489333 & 0.485415\end{array}$
H $2.743411-0.721686-0.586952$

H $4.208068-2.802308-0.212021$

O $5.643579-1.778087 \quad 0.771407$

C $3.454402-2.1706541 .714935$

C $1.934401-2.2928201 .517739$

H $3.646396-1.3698962 .438220$

O $3.932189-3.3679632 .331782$

H $1.468736-2.5818392 .469174$

H $1.525312-1.298277 \quad 1.295582$

H $4.065026-2.592550-5.245835$

H $2.487607-2.063063-4.640745$

H $3.287280-1.161920-5.937829$

C $3.233604 \quad 1.505742-4.468754$

H 44.9100370 .6613380 .592349

H $5.719042-1.0644401 .427144$

H $4.892416-3.2707452 .451237$

H $2.8472701 .132345-5.424366$

H $4.2343591 .913524-4.658046$

H $2.3314690 .002592-3.187346$

C $-0.583039-3.783990-0.788335$

$\begin{array}{lllll}\text { O } & 0.046690 & -3.057855 & 0.276539\end{array}$

C $1.460882-3.2622120 .414229$

C $1.750292-4.7442460 .695503$

C $1.116167-5.653483-0.364117$

C $-0.349158-5.294426-0.626962$

H $-1.656251-3.602126-0.659581$

C $-0.188788-3.246637-2.173211$

H $1.936075-2.969890-0.524844$

O $1.260172-5.108096 \quad 1.984140$

H $2.831942-4.910708 \quad 0.701415$

H $1.177326-6.696903-0.031365$

O $1.841873-5.543991-1.585470$

H $-0.721810-5.818488-1.514983$

O $-1.140854-5.7310800 .481845$

C $-0.655639-1.822749-2.395051$

H $0.892674-3.257570-2.329139$

H $-0.660055-3.868554-2.943418$

H $0.310142-4.9014372 .010324$

H $2.759905-5.810239-1.406886$

H $-1.036855-6.6952810 .554352$

O $-0.389103-1.472953-3.689169$

O $-1.174497-1.105411-1.551140$

C $-0.760984-0.141363-4.054900$

H $-0.4376990 .032615-5.084611$

H $-1.847469-0.025374-4.000755$

H $-0.2615620 .581471-3.405053$

SCF Energy (B3LYP/6-31G**//MMFF) $=-3245.91873549$

0700065

MM̄FF Geometry

C $2.8121691 .006197-3.064785$

C $1.6232841 .184930-2.469838$

C $0.304581 \quad 1.009521-3.175735$

O $-0.456708 \quad 0.039279-2.416366$

C $-0.5006412 .323619-3.215772$

C $0.1926643 .487225-3.893757$

C $0.660026 \quad 4.567652-3.236365$

C $0.288710 \quad 3.401582-5.392944$

C $0.6565734 .766828-1.746120$

C $-0.6107015 .481753-1.261211$

$\begin{array}{llll}C & -0.902718 & 5.279045 & 0.234938\end{array}$

C $0.2145345 .803301 \quad 1.138586$

C $-0.073896 \quad 5.5181092 .621720$

$\begin{array}{lllll}\text { O } & -1.101802 & 3.877170 & 0.450797\end{array}$

$\begin{array}{lrlll}\text { O } & 1.126980 & 5.778382 & 3.364857\end{array}$

$\begin{array}{llll}\text { C }-0.431522 & 4.023076 & 2.783493\end{array}$

C $-1.114288 \quad 6.490008 \quad 3.188234$

$\begin{array}{lllll}\text { O } & -0.825091 & 3.738243 & 4.124289\end{array}$

C -1.5008373 .5240351 .780289$

C -1.6026671 .9773501 .856381$

O $-2.756424 \quad 4.1072352 .123792$

C $-1.462705-0.609218-3.063611$

O $-1.755172-0.518943-4.245211$

C $-2.180935-1.431670-2.064552$

C $-3.329909-2.034206-2.404591$

C $-4.173080-2.853894-1.531032$

C $-3.746893-3.020117-0.096400$

C -2.4734831 .3163510 .776612$ 
C $-3.961578 \quad 1.3096301 .078888$

C -6.1567950 .8167650 .001327$

O $-4.426593 \quad 1.6222502 .171485$

C $-6.571598-0.5845670 .321981$

C $-7.409369-1.301265-0.442814$

C $-7.866680-2.691653-0.105630$

C -7.649786 -3.671828 -1.236944

C $-8.661109-4.025560-2.048053$

C -6.284832 -4.308721 -1.370036

C $-5.289865-3.407633-2.048578$

N $-4.7082340 .898245-0.016978$

H $2.8435260 .696973-4.106924$

H $1.5904021 .504886-1.431096$

H $\quad 0.4671200 .625748-4.190912$

H $-0.8113182 .581761-2.196060$

H -1.454404 2.147491-3.731328

H $1.1086205 .377022-3.809148$

H $0.7448234 .295736-5.831024$

H $0.8976242 .542127-5.690051$

H $-0.7081293 .290851-5.832098$

H $0.7862683 .807064-1.237115$

H $1.5400015 .359145-1.477559$

H $-0.5348476 .553221-1.483458$

H -1.475134 $5.100129-1.821015$

H -1.8410055 .8032860 .451966$

H $\quad 0.377643 \quad 6.8756080 .975058$

H $1.162434 \quad 5.313728 \quad 0.879801$

H 1.8254615 .1902673 .031064

H $\quad 0.4915493 .4523092 .614239$

H -0.7639007 .5244213 .087291$

H -1.2616586 .3303954 .262182$

H -2.084477 6.4202552 .692355

H -1.7365634 .0573254 .239009$

H -0.5924411 .5661541 .727455$

H -1.9548411 .6738242 .849436$

H -3.4609613 .6174351 .672725$

H -1.756455 -1.500190 -1.070456

H -3.696636 -1.913012 -3.423226

H $-2.839609-3.629257-0.039148$

H $-4.501336-3.501408 \quad 0.529585$

H -3.549406 -2.0486210 .369300$

H -2.300682 $1.775048-0.202440$

H -2.1767920 .2631490 .701043$

H $-6.5121341 .138928-0.982590$

H $-6.553426 \quad 1.5019810 .757324$

H $-6.184825-1.0200141 .240376$

H -7.817546 -0.853380 -1.346195

H $-8.935269-2.6380310 .141037$

H -7.373844 -3.067433 0.799770

H -8.520987 -4.750311 -2.843243

H -9.650468 -3.593824 -1.938512

H -6.355962 -5.222908 -1.974936

H $-5.949638-4.655330-0.388472$

H -5.520599 -3.223403 -3.098864

H $-4.2282470 .591512-0.858156$

C $5.0453670 .060192-2.353692$

O $4.719150-0.737543-1.192109$

C $5.924251-0.968232-0.439905$

C $7.064577-0.730165-1.419117$

C $6.5221010 .442182-2.206831$

H $5.963956-0.1965880 .338106$

C $5.936825-2.3580570 .204020$

H $8.009904-0.509826-0.914496$

H $7.219952-1.591736-2.078987$

C $7.2333230 .665458-3.530912$

H $6.616778 \quad 1.349832-1.594991$

C $4.770926-2.6587231 .167531$

O $5.909884-3.355796-0.825475$

H $6.888266-2.5004850 .730334$

H $3.855452-2.8399960 .595713$

O $5.075184-3.8915751 .834404$

C $4.508840-1.5812782 .233736$

C $3.558300-2.0028933 .371689$

H $4.109707-0.6854661 .743973$

O $5.754599-1.2112102 .833532$

H $4.061578-2.7264714 .026312$

H $3.397077-1.1119663 .993395$

H $8.2933730 .884105-3.365703$
H $7.169038-0.218175-4.174917$

H $6.7942751 .509136-4.072552$

C $4.1350741 .290867-2.410879$

H $5.110620-3.203677-1.358745$

H $5.225672-4.563076 \quad 1.147097$

H $5.576660-0.4848073 .455012$

H $4.6118612 .080120-3.004785$

H $3.983471 \quad 1.690709-1.400547$

H $4.899551-0.582990-3.230814$

C $0.328453-2.0221631 .488645$

O $1.577728-1.6125462 .064004$

C $2.204809-2.5709632 .918883$

C $1.292086-2.9052694 .109878$

C $-0.088990-3.3681653 .632240$

C $-0.676436-2.4169722 .584199$

H $-0.057027-1.1233750 .990336$

C $0.519060-3.1078330 .415632$

H $2.397264-3.4891632 .356117$

O $1.146720-1.7547274 .940212$

H $1.768635-3.6884974 .711347$

H $-0.772742-3.4267444 .487694$

O $0.009239-4.6720123 .066966$

H -1.564894 -2.860052 2.120884

O $-1.099276-1.2132013 .230208$

C $1.262691-2.580479-0.791000$

H $1.078291-3.9698200 .786547$

H -0.462938 -3.4494190.072406

H $0.588992-2.0060825 .695813$

H $\quad 0.360037-5.2644313 .753514$

H -1.776351 -1.453527 3.885326

O $1.188831-3.495006-1.801649$

O $1.851705-1.510264-0.837996$

C $1.870204-3.130318-3.006369$

H $2.932770-2.959958-2.807263$

H $1.774868-3.957710-3.714479$

H $1.411271-2.239121-3.444280$

SCF Energy $\left(B 3 L Y P / 6-31 G^{* *} / / M M F F\right)=-3245.89963686$

0700066

MM̄FF Geometry

C $3.834018-2.477691-3.002310$

C $2.803495-1.622664-3.086642$

C $1.366228-2.059472-3.204580$

O $0.658385-1.582260-2.034252$

C $0.711013-1.444726-4.453239$

C $-0.771991-1.746701-4.575910$

C $-1.724440-0.801772-4.704430$

C $-1.159584-3.202662-4.566320$

C $-1.5200940 .680615-4.825559$

C $-1.5123721 .445741-3.496019$

C $-2.8644151 .432841-2.766192$

C $-2.9087262 .544466-1.714827$

C $-4.2178712 .527648-0.915636$

$\begin{array}{llll}\text { O } & -3.031740 & 0.161356 & -2.128374\end{array}$

$\begin{array}{lllll}\text { O } & -4.046227 & 3.375423 & 0.230373\end{array}$

C $-4.4571421 .099947-0.391141$

C $-5.3877343 .110797-1.716540$

$\begin{array}{llll}\text { O } & -5.736091 & 0.992208 & 0.230275\end{array}$

C $-4.2959640 .001217-1.470330$

C $-4.325248-1.384320-0.787580$

O $-5.3828830 .084145-2.394327$

C $0.742559-2.354385-0.916030$

O $1.363438-3.399224-0.793044$

C $-0.053368-1.7093740 .152082$

C $-0.014197-2.2362091 .385290$

C $-0.745838-1.7725352 .563685$

C -1.549054 -0.506010 2.449334

C $-3.897500-2.556832-1.676679$

C $-4.111276-3.860079-0.930873$

C $-3.263624-5.1386691 .039838$

O $-5.063960-4.595305-1.179934$

C $-3.590885-4.5200482 .364983$

C $-2.930051-4.7977523 .499388$

C $-3.237211-4.1539894 .823033$

C $-2.005430-3.5973655 .508327$

C $-1.487428-4.2019736 .591003$

C -1.412644 -2.303068 4.983351

C $-0.665729-2.5066313 .693214$ 
N -3.162157 -4.075873 0.055981 H $3.650773-3.547749-3.058733$ H $2.988978-0.554476-2.997883$ H $1.293830-3.153182-3.259929$ H $1.216423-1.816533-5.354063$ H $0.878924-0.362406-4.430697$ H -2.764041-1.120271 -4.767098 H $-2.216167-3.352103-4.814150$ H - $0.570498-3.756332-5.304718$ H $-0.990032-3.643581-3.579346$ H $-0.5891370 .892530-5.364136$ H $-2.3123601 .081958-5.470853$ H $-0.729757 \quad 1.038346-2.843463$ H -1.238008 $2.482357-3.726502$ H $-3.6618871 .579516-3.504027$ H $-2.7671313 .526256-2.182108$ H -2.070591 2.417220-1.015285 H -3.812421 $4.264713-0.086567$ H -3.7144990 .9185640 .397988$ H -5.174615 4.146858 -2.006212 H $-6.2996903 .151592-1.110727$ H -5.604123 2.550971-2.628809 H $-5.786470 \quad 1.6849130 .911137$ H $-3.652951-1.3692770 .077111$ H -5.333820 -1.582912 -0.401643 H $-5.524279-0.787424-2.796158$ H $-0.647641-0.839920-0.103447$ H $0.604336-3.1181371 .549806$ H -2.446681 -0.6760811 .850435$ H -0.9606920 .2921831 .983440$ H $-1.866149-0.111278 \quad 3.417873$ H -4.477120 -2.597564 -2.604208 H -2.841467 -2.474385 -1.955781 H -2.301824 -5.660605 1.055208 H -4.048532 -5.8443320 .752099$ H $-4.409262-3.8038782 .390860$ H -2.128375 -5.5327643 .479821$ H -3.710214 -4.913656 5.458737 H $-3.976689-3.3510064 .713398$ H $-0.619162-3.799497 \quad 7.102230$ H - $-1.916743 \quad-5.115860 \quad 6.988147$ H $-0.709013-1.8850175 .714394$ H -2.224331 -1.575601 4.888425 H $-0.013737-3.3816293 .694969$ H $-2.415340-3.3985070 .175462$ C $5.620674-2.317241-1.281317$

O $4.793528-1.498212-0.431397$ C $5.608498-0.9048260 .593779$ C $6.927613-1.6646330 .563135$ C $7.061329-1.958267-0.921613$ H $\quad \begin{array}{llll}5.796314 & 0.131349 & 0.287486\end{array}$ C $4.850941-0.9193981 .926325$ H $7.757735-1.0739640 .961961$ H $6.872098-2.6006871 .130724$ C $8.066874-3.050811-1.239050$ H $7.358631-1.035288-1.437349$ C $3.423792-0.3350841 .837321$ O $4.728593-2.2720712 .385143$ H $5.431344-0.3922442 .691700$ H $2.790733-0.9957741 .234774$ O $2.861623-0.3275553 .154447$ C 3.3554051 .0929021 .268309 C 1.9174931 .6255651 .179004 H 3.7867111 .0963870 .263576 O 4.1440371 .9665272 .072781 H 1.3205100 .9707010 .532849 H 1.4389051 .5781042 .166295 H $9.068158-2.760993-0.904027$ H $7.806285-3.992262-0.744066$ H $\quad 8.111790-3.237835-2.316618$ C $5.251129-2.045524-2.741067$ H $4.264588-2.7756951 .694200$ H $2.892934-1.2409733 .487362$ H 3.7767541 .9461932 .973245 H $5.917461-2.590820-3.418396$ H $5.361950-0.976643-2.962062$ H $5.407524-3.362358-1.019894$ C 0.0453304 .7105540 .258459
O $\quad 0.396825 \quad 3.366095 \quad 0.611133$

C 1.8011903 .0843430 .693205

C $2.4712633 .350861-0.661972$

C $2.1969864 .779867-1.140320$

C $0.7104875 .134717-1.063413$

H -1.0393294 .6826250 .092750$

C 0.3145575 .6882051 .412952

H $2.246778 \quad 3.7276181 .459438$

O $1.9853022 .412764-1.619876$

H $3.5531823 .203634-0.569500$

H $2.5450644 .883571-2.175451$

O $2.9538015 .679942-0.335509$

H $0.5715326 .210802-1.220973$

O $0.0499844 .469576-2.142536$

C -0.5581885 .4042052 .618686$

H 1.3546505 .6454391 .746160

H 0.0906936 .7095411 .083693

H $2.4366112 .592092-2.462097$

H $2.7960396 .578338-0.672204$

H $-0.8664994 .792542-2.165665$

O -0.2477356 .2959273 .606636$

O -1.4032904 .5229432 .690486$

C -0.9935726 .1537384 .819713$

H -0.8152815 .1682975 .260612$

H -0.6508136 .9190715 .521046$

$\mathrm{H}-2.0602486 .3049894 .628405$

SCF Energy $\left(B 3 L Y P / 6-31 G^{* *} / / M M F F\right)=-3245.90624365$

\section{7}

MM̄̄F Geometry

C $-0.0802404 .384064-0.765016$

C 0.6931884 .6410750 .301406

C 1.9358583 .8725640 .698472

O $2.0344292 .620265-0.019144$

C 3.1984424 .6980600 .397133

C 4.4638254 .0924490 .975791

C 5.4895903 .6095990 .247862

C 4.5496424 .0751102 .481448

C $5.6304763 .564769-1.246743$

C $5.1441082 .265384-1.906254$

C $5.8569990 .994190-1.414408$

C $5.518935-0.193534-2.319554$

C $6.130057-1.506239-1.805281$

O $5.420650 \quad 0.716284-0.079979$

O $5.501767-2.589974-2.507530$

C $5.779617-1.674481-0.310914$

C $7.628020-1.598175-2.119387$

O $6.461945-2.7937720 .247872$

C $6.060245-0.4132520 .531346$

C $5.529223-0.5197611 .983111$

O $7.476554-0.2135610 .620603$

C $1.243778 \quad 1.596558 \quad 0.406121$

O $0.493277 \quad 1.595150 \quad 1.368981$

C $1.4148180 .475815-0.542589$

C $0.469032-0.471810-0.609176$

C $0.482736-1.648266-1.479744$

C $1.748257-1.935759-2.242062$

C $4.018804-0.7399162 .119945$

C $3.610221-2.1962632 .273994$

C $1.579114-3.6398022 .385057$

O $4.412521-3.1054302 .466925$

C $1.364502-4.2807121 .049884$

C $0.183045-4.7775260 .652016$

C $-0.035093-5.467613-0.665141$

C $-1.170051-4.862963-1.461714$

C $-2.395281-5.414717-1.448378$

C $-0.862434-3.666180-2.334610$

C $-0.622751-2.419256-1.532043$

N $2.231378-2.3548492 .210152$

H $0.2071513 .605537-1.466909$

H 0.3898895 .4265930 .990559

H 1.8706563 .6608461 .772667

H 3.0923535 .7118190 .805889

H $3.2881024 .816510-0.688147$

H $6.345833 \quad 3.2001330 .783444$

H 5.5422413 .7783002 .837497

H 4.3442525 .0716442 .885850

H 3.8275993 .3693802 .902938 
H $5.1156834 .413167-1.710306$ H $6.6913033 .711063-1.487967$ H $4.0648752 .169540-1.742401$ H $5.2969262 .368819-2.987926$ H $6.9375551 .179314-1.415929$ H $5.840647-0.000868-3.350684$ H $4.428506-0.314276-2.366556$ H $5.649890-2.456201-3.459533$ H $4.708370-1.903411-0.260403$ H $7.795703-1.548593-3.202057$ H $8.041339-2.560154-1.797077$ H $8.212719-0.798855-1.659539$ H $\quad 6.240096-3.569252-0.295424$ H $6.099781-1.2734322 .539163$ H 5.7529280 .4363442 .478248 H $7.660826 \quad 0.3469501 .392534$ H $2.3015550 .468988-1.164313$ H $-0.407895-0.3728510 .028719$ H $2.600741-1.993732-1.556954$ H $1.941127-1.148633-2.978890$ H $1.729046-2.883645-2.783111$ H $3.676190-0.2315133 .030488$ H $3.482794-0.2970801 .277115$ H $0.637662-3.4571122 .912733$ H $2.208459-4.2853703 .006148$ H $2.226600-4.366651 \quad 0.392572$ H -0.671091 $-4.716171 \quad 1.322835$ H $-0.248945-6.523949-0.455316$ H $\quad 0.877373-5.467161-1.274267$ H -3.208709 -5.001617 -2.035483 H $-2.614743-6.287542-0.842376$ H -1.706784 -3.461455 -3.006164 H $-0.028436-3.918413-2.994515$ H -1.483965 -2.121200 -0.935528 H $1.646350-1.5502832 .004565$ C $-2.5355204 .067387-0.834748$ O $-2.4967733 .060459-1.865393$ C $-3.8514432 .689182-2.207126$ C $-4.7521563 .432696-1.229820$ C -3.931709 $4.671995-0.924525$ H $-4.0123753 .060485-3.226863$ C $-3.9598501 .158279-2.169400$ H $-5.7328233 .662452-1.657088$ H $-4.9107632 .845391-0.317313$ C -4.3785735 .3937050 .335040$ H -3.991425 5.357527 -1.780144 C $-5.3941310 .613016-2.259977$ O H $-3.4764740 .794381-1.262263$ H $-5.9774160 .931464-1.389316$ O $-6.0163591 .217228-3.403086$ C $-5.489418-0.914483-2.429240$ C $-4.837801-1.789107-1.338067$ H -5.042702 -1.191416 -3.391791 O $-6.869711-1.284060-2.548719$ H -4.985002 -2.827696 -1.658093 H -3.760322 -1.612353-1.344604 H $-5.416055 \quad 5.7300230 .237340$ H $-4.317774 \quad 4.7425841 .213816$ H $-3.755891 \quad 6.273483 \quad 0.524115$ C $-1.3932035 .064060-1.039144$ H -3.610411 $0.912486-4.081632$ H $-6.9223950 .868325-3.458968$ H -7.365659 -0.849398 -1.835063 H -1.520203 $5.929921-0.379982$ H -1.381270 $5.430170-2.072331$ H -2.4110573 .5484190 .124941$ C $-3.574758-0.5302051 .216362$ O $-4.925515-0.4542860 .746677$ C $-5.419899-1.6259170 .088496$ C $-5.248313-2.8564310 .997083$ C -3.811529-3.021567 1.478617 C $-3.336457-1.7300932 .139146$ H -2.909994 -0.6283710 .352174$ C $-3.2062530 .812703 \quad 1.863088$ H $-6.500662-1.479516-0.013581$ O H $-5.898828-2.737708 \quad 1.873758$
H $-3.153394-3.3211680 .656483$

O $-3.771974-4.0802642 .436848$

H $-3.864786-1.6071793 .090809$

O $-1.948959-1.8419062 .450993$

C $-4.063149 \quad 1.2064473 .048292$

H $-2.1587380 .792127 \quad 2.183103$

H -3.3292941 .6070701 .119980$

H -5.530670 -4.7843190.923899

H $-2.858137-4.1396372 .764456$

H -1.464998 -1.988508 1.620420

O -3.5051352 .3018153 .646186$

$\begin{array}{llll}\text { O } & -5.097753 & 0.653744 & 3.393769\end{array}$

C -4.2171222 .8157864 .775957$

H -4.2755892 .0587675 .563851$

H -3.667706 3.6796385 .159633

H -5.2179093 .1412954 .476383$

SCF Energy (B3LYP/6-31G**//MMFF) $=-3245.90656998$

0700068

MM̄FF Geometry

C $3.696155-2.229052-2.548843$

C $2.755887-1.676485-1.769072$

C $1.412359-1.209799-2.251918$

O $0.420400-1.720677-1.332170$

C $1.3387020 .325360-2.203112$

C $0.0791120 .870091-2.846813$

C $-1.0151451 .265745-2.167441$

C $0.1258900 .993056-4.347357$

C $-1.2461981 .189965-0.684951$

C -1.0813022 .5343560 .031330$

C $-2.0622763 .631273-0.413019$

C -1.822563 4.9027770 .405752

C -2.8493825 .9974880 .086468$

O $-3.3938513 .153886-0.202790$

O

C -4.2645325 .3897440 .178877$

C $-2.5367456 .699470-1.240250$

O $-5.2474556 .340311-0.228606$

C $-4.4289594 .059923-0.599544$

C $-5.8047303 .430049-0.250690$

O $-4.3752664 .343118-1.997947$

C $-0.099701-2.953671-1.560269$

O $0.161730-3.709496-2.480901$

C $-1.064467-3.218411-0.469474$

C $-1.892722-4.269875-0.546256$

C $-2.924315-4.6190460 .433900$

C $-2.922615-3.8700931 .741729$

C $-6.1471732 .105398-0.945916$

C $-5.3819500 .898611-0.432164$

C $-4.764991-1.423642-1.106717$

$\begin{array}{lllll}\text { O } & -4.814568 & 0.871797 & 0.656232\end{array}$

C $-5.625051-2.328827-0.283640$

C $-6.143709-3.479977-0.737815$

C $-6.919247-4.4393010 .120116$

C $-6.315792-5.8297360 .133651$

C $-6.944267-6.860127-0.457207$

C $-5.018720-6.0402020 .891872$

C $-3.824650-5.5699700 .106638$

N $-5.445914-0.163476-1.324248$

H $3.512291-2.353575-3.612306$

H $2.962990-1.545313-0.710795$

H $1.206555-1.570770-3.267179$

H $2.2061520 .763032-2.715631$

H $1.4071800 .671132-1.164708$

H -1.856376 $1.662708-2.734105$

H $-0.7949501 .419955-4.758787$

H $0.9536851 .643241-4.649082$

H $\quad 0.2682000 .011302-4.809546$

H $-2.2540770 .795725-0.515667$

H $-0.5737980 .465292-0.215208$

H -1.2338212 .3439611 .100861$

H $-0.0501692 .889230-0.089819$

H -1.914705 $3.834944-1.479359$

H $-0.801977 \quad 5.2761360 .253848$

H -1.8959344 .6654291 .476716$

H -1.8412427 .3395561 .112305$

H -4.459832 5.1929751 .242024

H -1.536148 $7.147364-1.209458$ 
H $-3.2280737 .529842-1.420997$ H -2.569268 $6.026784-2.099716$ H $-5.2323546 .383048-1.199849$ H -5.8650053 .2778950 .834438$ H $-6.5951054 .139902-0.526231$ H $-4.6390503 .549005-2.487374$ H $-1.086217-2.5189980 .358810$ H -1.838406 -4.913319-1.423154 H -3.142643 -2.809006 1.583152 H -1.944042 -3.9536402 .228627$ H -3.653483 -4.2447052 .460817$ H $-7.2080741 .890305-0.763195$ H $-6.0265232 .188780-2.030142$ H -3.820145 -1.239745 -0.585357 H $-4.538377-1.848274-2.089928$ H $-5.776379-2.0507840 .756768$ H -5.987044 -3.761311-1.776756 H $-7.947222-4.472808-0.262833$ H $-6.995418-4.0771111 .153113$ H -6.530770 -7.862956-0.433134 H $-7.883899-6.723819-0.982105$ H $-4.874390-7.107787 \quad 1.103568$ H -5.107534 -5.561306 1.870239 H -3.722395 -6.064116 -0.860987 H $-6.019880-0.077551-2.157933$ C $6.078426-1.610544-1.877005$ O $5.650468-0.635839-0.904443$ C $5.9650560 .678100-1.403216$ C $6.954030 \quad 0.462538-2.542532$ C $6.427831-0.828804-3.143187$ H $5.0389321 .090394-1.821092$ C $6.4653601 .567804-0.261859$ H $6.960606 \quad 1.295769-3.251417$ H $7.9759020 .321440-2.171691$ C $7.422604-1.534522-4.047413$ H $5.524634-0.596574-3.721056$ C $5.478247 \quad 1.6875180 .920508$ $\begin{array}{llll}\text { O } & 7.691201 & 1.028267 & 0.249437\end{array}$ H $6.7129922 .563587-0.646684$ H 5.3912710 .7199291 .427013 O 6.0449112 .5931181 .875485 C 4.0812152 .2053880 .530217 C 3.1499572 .4885251 .725032 H $3.5985911 .487996-0.141030$ O $4.2372873 .423925-0.203071$ H 3.6068203 .2318742 .389786 H 2.2457762 .9668041 .324522 H $7.683974-0.900157-4.900557$ H $8.347800-1.778327-3.514466$ H $7.000864-2.467145-4.435636$ C $5.019228-2.706469-2.017180$ H 7.5118690 .1177880 .541008 H 6.9288432 .2575402 .103951 H $3.3556183 .698224-0.508318$ H $4.854218-3.166870-1.033897$ H $5.390364-3.502624-2.672961$ H 6.985668 -2.068779-1.461102 C $1.660679-0.8935512 .181347$ $\begin{array}{llll}\text { O } & 2.047870 & 0.368959 & 1.626441\end{array}$ C 2.7538501 .2352962 .519884 C 1.8706661 .5801843 .730360 C $1.351807 \quad 0.317374 \quad 4.427850$ C $0.784215-0.7012553 .431777$ H $1.036203-1.3495151 .404031$ C $2.884332-1.7927162 .385827$ H 3.6602490 .7314762 .871496 O 0.7616402 .3799553 .324806 H 2.4562832 .1819784 .434868 H $\quad 0.565457 \quad 0.593270 \quad 5.141406$ O $2.408602-0.2907285 .163493$ H $0.619384-1.6629683 .930272$ O $-0.497958-0.2238893 .008618$ C $2.500191-3.2511462 .394964$ H $3.623885-1.6347651 .591143$ H $3.420944-1.5736633 .313084$ H 0.2470651 .8625722 .682539 H 2.7226660 .3570095 .817222 H $-0.892573-0.9059162 .438641$
O $\quad 2.347625-3.7007651 .116801$

O $2.337478-3.9062743 .416116$

C $1.958918-5.0713790 .986647$

H $2.720151-5.7240861 .424315$

H $\quad 0.987501-5.2363651 .462465$

H $1.871264-5.299839-0.078814$

SCF Energy (B3LYP/6-31G**//MMFF) $=-3245.90936388$

0700069

MM̄FF Geometry

C 0.1406022 .5736322 .471127

C 1.0132041 .6460342 .896532

$\begin{array}{llllll}\text { C } & 0.708224 & 0.213777 & 3.278242\end{array}$

O $-0.679668-0.0778642 .999171$

C $1.614645-0.7304002 .461252$

C $1.712481-2.1385333 .015107$

C $1.185776-3.2373142 .439296$

C $2.529479-2.2752114 .276062$

C $0.373540-3.3179551 .177719$

C $-0.910535-4.135787 \quad 1.385408$

C $-1.863848-4.117800 \quad 0.177737$

C $-1.241146-4.745103-1.073076$

C $-2.163316-4.634678-2.295345$

O $-2.231804-2.757210-0.060743$

O $-1.375350-4.975317-3.446380$

C $-2.603212-3.162529-2.439348$

C $-3.303551-5.656613-2.239230$

O $-3.541691-3.015757-3.502519$

C $-3.159739-2.557174-1.132778$

C $-3.398075-1.036944-1.253140$

O $-4.422335-3.166513-0.840915$

C -1.339914 $-0.924147 \quad 3.829103$

O $-0.868133-1.5528184 .766080$

C $-2.766435-1.0420333 .453277$

C -3.447271 -0.0398972 .876086$

C $-4.875535-0.0428132 .548288$

C $-5.617589-1.3473322 .675669$

C $-2.130454-0.206717-1.471819$

C $-2.4344791 .253693-1.226238$

C $-3.6239623 .188493-2.227735$

O $-2.2172291 .781234-0.138139$

C $-5.0672112 .994151-1.884402$

C $-5.5623163 .146647-0.647186$

C $-6.9915642 .866361-0.288456$

C -7.1690201 .5054650 .350105$

C $-7.6328180 .466515-0.365117$

C $-6.899734 \quad 1.366859 \quad 1.830951$

C $-5.450462 \quad 1.1111532 .147213$

N -3.017499 $1.873673-2.320687$

H -0.9162882 .3397352 .391323$

H 2.0610751 .9315772 .978930

H 0.9047090 .1239654 .353351

H $2.638048-0.3309852 .431020$

H $1.279761-0.7390611 .418439$

H $1.366463-4.1993282 .917722$

H $2.649264-3.3197374 .583144$

H $3.532461-1.8625234 .125262$

H $2.055755-1.7428375 .105859$

H $0.113948-2.3263100 .800686$

H $0.997227-3.8001110 .417311$

H $-0.654471-5.1736501 .632632$

H $-1.448124-3.7239982 .249464$

H -2.763361 -4.6744610 .466384$

H $-0.967233-5.791109-0.887413$

H $-0.304207-4.231102-1.323423$

H $-1.927560-4.831483-4.234359$

H $-1.712135-2.600312-2.744803$

H $-2.899190-6.675687-2.212189$

H -3.925694 $-5.607192-3.139572$

H $-3.948791-5.533967-1.366952$

H $-4.401585-3.340484-3.185191$

H $-4.130496-0.829178-2.043923$

H $-3.876802-0.689437-0.327526$

H $-4.931172-2.567334-0.270225$

H -3.247001 -1.9664493 .755800$

H -2.9247660 .8893372 .656708$

H $-5.092949-2.1448232 .137175$

H $-6.625747-1.3147772 .256792$ 
H $-5.708632-1.6360563 .728300$ H -1.329832 -0.497201-0.782484 H - $-1.742555-0.331745-2.488472$ H -3.525327 $3.680232-3.199888$ H -3.098172 $3.787835-1.477162$ H $-5.7244512 .670433-2.687944$ H -4.8999583 .4591560 .155781$ H $-7.6229972 .945987-1.183807$ H -7.3559413 .6490840 .387640$ H -7.793174 -0.5056390 .088748$ H $-7.8565490 .563804-1.422332$ H -7.5426610 .5912962 .261378$ H -7.210332 2.286243 2.344204 H -4.8257482 .0020302 .082625$ H -3.231183 $1.316762-3.143149$ C 0.1677614 .2564970 .605008 O $0.7071053 .242356-0.264118$ C $1.4246203 .872194-1.336230$ C $0.9198355 .308082-1.373955$ C 0.7264655 .5908070 .103869 H $2.4817683 .871162-1.045114$ C $1.2621243 .077274-2.636327$ H $1.6306625 .987796-1.853069$ H -0.039055 $5.391384-1.897965$ C -0.1771996 .7782050 .387118$ H 1.7112845 .7804180 .551576 C $1.4621991 .554289-2.493471$ O $-0.0557183 .277696-3.162812$ H $1.9450493 .478276-3.394118$ H $0.599797 \quad 1.109413-1.983565$ O $1.4776430 .992399-3.812060$ C $2.7473691 .126208-1.763410$ C $2.941966-0.403719-1.784681$ H $2.7016921 .463218-0.722563$ O $3.8713561 .765243-2.365353$ H $1.994561-0.893999-1.529260$ H $3.224039-0.707049-2.800582$ H $0.2463867 .694025-0.037917$ H -1.173611 $6.636443-0.045025$ H $-0.295403 \quad 6.9280921 .464945$ C 0.5558033 .9586862 .056576 H $-0.6889213 .013083-2.474044$ H $0.6503191 .257698-4.249212$ H $3.9075011 .483022-3.295405$ H 0.0828224 .6869712 .724907 H 1.6407094 .0685332 .176250 H -0.9229294 .2215840 .490882$ C $6.387739-0.618523-0.333378$ O $5.280346-0.324955-1.194684$ C $4.026273-0.884445-0.799682$ C $4.118094-2.418105-0.756968$ C $5.276475-2.8839590 .132021$ C $6.577233-2.137546-0.184794$ H $7.263827-0.220535-0.862016$ C 6.2784920 .1159881 .007873 H $3.761266-0.5089020 .193741$ O $4.302599-2.920859-2.079125$ H $\quad 3.168711-2.817715-0.383121$ H $5.437318-3.959823-0.008817$ O $4.945793-2.6743831 .501146$ H $7.331989-2.3357410 .585229$ O $7.103049-2.624829-1.422812$ C 6.2887291 .6118590 .798406 H $5.379175-0.1541501 .568616$ H $7.128583-0.1266591 .656211$ H $4.321141-3.891346-2.024496$ H $4.150280-3.1991411 .693397$ H $7.274237-3.575904-1.314588$ O 5.0477342 .1252601 .034534 O 7.2770302 .2439790 .448268 C 4.9221243 .5383460 .851631 H 5.6168654 .0717881 .507411 H $5.1001543 .799542-0.195707$ H 3.9016353 .8253821 .116597 SCF Energy (B3LYP/6-31G**//MMFF) $=-3245.90334256$

0700070

MM̄FF Geometry
C $2.776118-3.703867-1.326305$

C $1.920471-2.864856-0.724691$

C $0.637762-2.387959-1.347137$

O $-0.416633-2.582322-0.378077$

C $0.730100-0.882255-1.647781$

C $-0.472058-0.358831-2.408768$

C $-1.4805970 .337486-1.849118$

C $-0.462471-0.622283-3.891616$

C $-1.6550590 .666237-0.394062$

C $-1.1678092 .070532-0.024815$

C $-1.9268383 .218779-0.709429$

C $-1.3752044 .563841-0.226812$

C $-2.1767145 .748463-0.781258$

O $-3.3141203 .105584-0.378464$

O $-1.790006 \quad 6.927183-0.058548$

C $-3.6790645 .499525-0.530149$

C -1.815736 $6.035336-2.242749$

O $-4.4661226 .511602-1.155337$

C $-4.1585874 .096426-0.978250$

C $-5.614553 \quad 3.871210-0.487710$

O $-4.1209194 .029269-2.403271$

C $-1.207929-3.679557-0.502366$

O $-1.114849-4.569564-1.331597$

C -2.241996 -3.5910230 .553870$

C $-3.297234-4.4184410 .523244$

C $-4.424282-4.4150171 .460276$

C $-4.338266-3.4922482 .648233$

C $-6.2886052 .562919-0.921776$

C $-5.7437121 .315101-0.251361$

C $-5.580311-1.151452-0.598391$

O $-5.177540 \quad 1.3312080 .838101$

C $-6.612318-1.7695560 .289939$

C $-7.331869-2.851358-0.044888$

C $-8.300062-3.5271590 .884144$

C $-7.976960-4.9920611 .098157$

C $-8.768426-5.9540090 .594406$

C $-6.776653-5.341517 \quad 1.956773$

C $-5.479114-5.2157241 .198175$

N $-6.0103010 .173987-0.995598$

H $2.540982-4.087117-2.315732$

H $2.160182-2.4765990 .261791$

H $0.421210-2.939741-2.269966$

H $1.629551-0.672328-2.242072$

H $\quad 0.859591-0.322409-0.713087$

H $-2.2838090 .693254-2.492703$

H $-1.328530-0.182208-4.397538$

H $\quad 0.436823-0.195000-4.347385$

H $-0.476835-1.697953-4.091621$

H $-2.7138980 .554391-0.139236$

H $-1.132853-0.0608250 .236578$

H -1.2756042 .1721861 .063041$

H -0.098082 2.154144 -0.251628

H - $1.8067703 .128174-1.794654$

H $-0.3134594 .664573-0.484492$

H -1.419182 4.5986360 .870956

H $-1.977306 \quad 6.7812130 .884309$

H -3.856538 5.5923990 .550443

H $-0.752526 \quad 6.290937-2.329016$

H -2.363197 $6.904874-2.622920$

H $-2.0061215 .190363-2.907594$

H $-4.1365567 .370928-0.840859$

H -5.6367793 .9292040 .608131$

H $-6.2453234 .683546-0.870785$

H -4.510765 $3.186480-2.682287$

H -2.122982 -2.822084 1.309035

H $-3.361503-5.148413-0.282725$

H -4.282988 -2.447072 2.325043

H -3.447888 -3.720407 3.245031

H $-5.191066-3.5682443 .325233$

H $-7.3493002 .616111-0.643817$

H $-6.2586862 .452841-2.010114$

H -4.628125 -1.081405 -0.063056

H $-5.419446-1.731339-1.512842$

H $-6.731016-1.3366001 .280343$

H -7.209056 -3.287334 -1.033683

H $-9.304786-3.4164620 .456613$

H -8.332562 -3.025299 1.859300

H $-8.557144-7.0049210 .762242$ 
H -9.642667 -5.713058 -0.001304 H $-6.852465-6.3804242 .304260$ H $-6.807793-4.7333162 .864151$ H $-5.431900-5.856340 \quad 0.315952$ H $-6.5735460 .250635-1.837460$ C $5.299222-3.398530-1.245393$ O $5.196733-1.994041-0.927764$ C $5.539169-1.236008-2.101271$ C $6.299437-2.199746-3.004644$ C $5.543021-3.490014-2.753408$ H $4.594255-0.969109-2.590019$ C $6.3063950 .036705-1.730233$ H $6.283404-1.888658-4.053359$ H $7.345466-2.307895-2.695159$ C $6.298497-4.737052-3.178025$ H $4.598967-3.444360-3.309277$ C $5.5945010 .957826-0.715995$ O $7.575180-0.322221-1.167592$ H $6.5354870 .597201-2.644145$ H $\quad \begin{array}{llll}5.645220 & 0.507384 & 0.281217\end{array}$ O $6.3528942 .171914-0.634672$

C $4.1319521 .298105-1.053621$ C $3.5170602 .432758-0.212241$ H $3.5218190 .394910-0.945651$ O $4.0572181 .695275-2.426159$ H $3.9980033 .385946-0.467335$ H $2.4730592 .536855-0.537030$ H $6.497464-4.718214-4.254530$ H $7.260084-4.820403-2.660468$ H $5.716788-5.637497-2.956752$ C $4.074447-4.148383-0.711601$ H $7.403332-0.878354-0.388403$ H $6.2865672 .617477-1.496773$ H $3.1180721 .825663-2.642848$ H $4.024873-4.0112080 .375861$ H $4.190763-5.224533-0.884247$ H $6.181986-3.762543-0.703943$ C 2.8741800 .6032012 .983041 O $2.889654 \quad 0.989541 \quad 1.601423$ C 3.5658232 .2146931 .307950 C 2.9266743 .3870902 .072522 C 2.8324553 .0988153 .574508 C 2.2500101 .7093853 .852477 H $2.209425-0.2692083 .021893$ C 4.2615990 .1599873 .477079 H 4.6140692 .1296871 .609264 O $1.6216973 .662148 \quad 1.567692$ H 3.5283664 .2875411 .902790 H 2.1956023 .8542604 .051320 O 4.1261553 .1855044 .163478 H $2.365197 \quad 1.4597704 .913852$ O $0.845727 \quad 1.7601693 .582502$ C $4.747655-1.0938082 .783162$ H 5.0150950 .9341353 .312306 H $4.214881-0.0625914 .549325$ H 1.0827772 .8624481 .691053 H 4.4612734 .0844574 .005534 H $0.4685250 .896498 \quad 3.821986$ O $6.039627-1.3345353 .155742$ O $4.082756-1.7817002 .022258$ C $6.642099-2.4971682 .578371$ H $6.032958-3.3851412 .772599$ H $\quad 6.779978-2.3501541 .503810$ H $7.622138-2.6375823 .041937$ SCF Energy (B3LYP/6-31G**//MMFF) $=-3245.91002891$

0700071

MM̄FF Geometry

C $0.221133-1.7921030 .990269$

C $1.090619-1.919126-0.023643$

C $0.806432-1.623009-1.479705$

O $-0.607695-1.449693-1.723994$

C $1.517411-0.316619-1.873196$

C $1.3281520 .052590-3.329863$

C $0.4895231 .012821-3.767771$

C $2.194136-0.703946-4.302675$

C $-0.4402081 .857812-2.938241$

C $-1.8290441 .968283-3.583757$
C $-2.8799532 .681826-2.713984$

C $-2.4638074 .102197-2.318129$

C $-3.4962604 .770454-1.396760$

O $-3.1177391 .881486-1.548968$

O $-2.9037515 .958796-0.849280$

C $-3.8138373 .808939-0.229992$

C $-4.7211095 .257911-2.177245$

O -4.8642294 .3246950 .583560$

C $-4.1604982 .378075-0.695987$

C $-4.317211 \quad 1.3927740 .484348$

O $-5.4189012 .409176-1.382454$

C $-1.357160-2.575503-1.867959$

O $-0.971025-3.732440-1.823060$

C $-2.761378-2.150890-2.042846$

C $-3.737942-2.968695-1.624523$

C $-5.172820-2.691282-1.671406$

C $-5.635002-1.511423-2.483964$

C $-3.056331 \quad 1.1620501 .319722$

C -3.3160260 .1323652 .403049$

C $-3.733932-2.3104432 .722490$

O -3.4437850 .4537813 .581958$

C $-5.118569-2.8171492 .439236$

C $-6.155574-2.0998281 .976647$

C -7.507453 -2.679518 1.663440

C -7.930724 -2.405009 0.234906

C $-8.718499-1.356044-0.056899$

C $-7.499682-3.374863-0.845229$

C $-6.006896-3.492903-0.977294$

N -3.408436 -1.156578 1.906132

H $-0.791935-1.4565560 .793945$

H $2.107732-2.2305810 .202484$

H $1.180858-2.451522-2.092993$

H $2.593044-0.399142-1.674722$

H $1.1751210 .499304-1.225739$

H $0.4684891 .225202-4.835857$

H $2.043999-0.374739-5.336273$

H $3.252189-0.558341-4.061123$

H $1.971835-1.774811-4.263400$

H $-0.551276 \quad 1.462927-1.925029$

H $0.0087272 .853738-2.856337$

H -1.743062 $2.492640-4.543936$

H $-2.1948740 .956029-3.801319$

H $-3.8042542 .714614-3.303158$

H -2.283190 $4.717727-3.207775$

H -1.510541 $4.071415-1.775135$

H $-2.1037375 .703354-0.359286$

H $-2.920244 \quad 3.7687510 .404711$

H -4.424775 $6.010490-2.918191$

H $-5.4388865 .755894-1.516074$

H $-5.2403384 .459267-2.710516$

H -4.6090805 .2239820 .851772$

H $-5.141475 \quad 1.7144181 .134700$

H -4.6413450 .4253840 .078519$

H $-5.8258031 .528837-1.332754$

H -2.946940 -1.168599-2.460490

H $-3.460435-3.918100-1.167524$

H -5.307822 -1.611904 -3.524836

H $-5.228447-0.579521-2.080098$

H -6.721771 -1.400667 -2.504960

H -2.2238130 .8160240 .696684$

H -2.7316252 .0840231 .812570$

H -3.621819 -2.080691 3.786406

H -3.007365 -3.085216 2.456718

H $-5.265417-3.8802502 .619324$

H -6.042342 -1.031533 1.812151

H $-8.231505-2.2369632 .359902$

H -7.537501 -3.759808 1.851801

H -9.047671 -1.159988-1.071895

H $-9.048660-0.6668420 .713449$

H -7.944139 -3.118963 -1.814623

H $-7.904162-4.366732-0.604904$

H -5.583040 -4.322591-0.410589

H -3.298311 -1.308215 0.907871

C $0.660997-0.7035913 .176591$

O $1.822554 \quad 0.0097642 .708959$

C 2.4595710 .6526373 .827985

C 1.5074750 .4913945 .007652

C $0.849734-0.8382694 .684221$ 
H 3.3744430 .0868414 .038506

C $2.809216 \quad 2.0979903 .457497$

H 2.0333130 .4894915 .967217

H 0.7573321 .2901825 .037265

C $-0.439139-1.0811255 .449709$

H $1.559834-1.6467644 .904123$

C 3.6581102 .2260222 .172849

O 1.5965212 .8335713 .245520

H 3.3153452 .5876824 .296898

H 3.0657281 .9096021 .306848

O 3.9498763 .6150131 .975200

C 4.9882901 .4513742 .211920

C 5.8865941 .6812240 .981358

H $4.791078 \quad 0.3800292 .320063$

O $5.715282 \quad 1.8607373 .374040$

H $6.0948612 .752663 \quad 0.872572$

H $\quad 6.864187 \quad 1.2299831 .189792$

H -0.246566 -1.108008 6.527104

H $-1.173374-0.2912685 .259841$

H $-0.890400-2.0361115 .163217$

C $0.566094-2.0344002 .432941$

H 1.0979562 .3814242 .543140

H 3.1013374 .0898691 .959415

H $6.5194191 .316536 \quad 3.424803$

H $-0.211071-2.6697972 .871331$

H $1.516864-2.5764592 .507883$

H $-0.211253-0.0738122 .956132$

C $6.036436-1.173408-0.081456$

O $4.938464-0.264961-0.208274$

C $5.3099021 .112850-0.332534$

C $6.223072 \quad 1.301823-1.552314$

C $7.4390800 .378433-1.505031$

C $7.001977-1.068460-1.270262$

H $6.581734-0.933868 \quad 0.840139$

C $5.479269-2.5882630 .137559$

H $4.384668 \quad 1.663267-0.540610$

O $\quad 6.6429782 .659286-1.641170$

H $5.6484851 .082752-2.462084$

H $8.1568970 .710963-0.746349$

O $8.1173230 .463985-2.760475$

H $6.537745-1.439113-2.189877$

O $8.152602-1.878236-1.038930$

C $4.655538-3.141586-1.007885$

H $6.307168-3.2803010 .329038$

H $4.820910-2.5751041 .015067$

H $7.2295662 .731976-2.413557$

H $8.860046-0.163280-2.731295$

H $8.586812-1.557095-0.230294$

O $4.360602-4.449750-0.742534$

O $4.291779-2.516059-1.993228$

C $3.558890-5.111665-1.726129$

H $2.574794-4.638456-1.791287$

H $4.060318-5.097862-2.698576$

H $3.428030-6.151185-1.414105$

SCF Energy (B3LYP/6-31G**/MMFF) $=-3245.91153949$

07_00072

MM̄FF Geometry

C $2.5115043 .587491-0.302716$

C $1.7347862 .510267-0.118206$

C $0.8610622 .284203 \quad 1.086796$

O $-0.4645191 .948108 \quad 0.617220$

C $1.414873 \quad 1.0978291 .894751$

C 0.5578820 .7116213 .081854

C $-0.255461-0.3627383 .121846$

C 0.6882581 .6089174 .284809

C $-0.524889-1.3550472 .022866$

C $-2.010112-1.3708801 .657435$

C $-2.350742-2.289776 \quad 0.474165$

C $-1.769298-1.788668-0.852132$

C $-2.247859-2.621315-2.051639$

O $-3.782127-2.317498 \quad 0.393935$

O $-1.938262-1.889925-3.248374$

C $-3.782797-2.753116-1.990462$

C $-1.499538-3.955255-2.155116$

O $-4.259627-3.638981-3.001081$

C $-4.306747-3.196631-0.606037$

C $-5.853452-3.102718-0.573253$
O $-3.918959-4.553556-0.375222$

C $-1.3131392 .981173 \quad 0.359729$

O $\begin{array}{lllll} & -1.080712 & 4.172913 & 0.484621\end{array}$

C $-2.5914702 .403954-0.113844$

C $-3.6065253 .211301-0.453447$

C $-4.9250372 .790880-0.938622$

C $-5.1923341 .312231-1.045355$

C $-6.474565-3.2820280 .819414$

C $-6.507095-2.0017501 .635115$

C $-7.731608 \quad 0.141072 \quad 1.873449$

O $-5.798659-1.8472332 .627304$

C $-8.511662 \quad 1.0585870 .988625$

$\begin{array}{llll}C & -8.282851 & 2.376919 & 0.886937\end{array}$

C $-9.090376 \quad 3.276051-0.005964$

C $-8.2474204 .208307-0.846660$

C $-8.4494425 .536999-0.808634$

C $-7.2237783 .605270-1.785760$

C $-5.8199493 .748921-1.258683$

N $-7.411884-1.0728341 .143499$

H $2.516966 \quad 4.3760870 .444368$

H $1.7309791 .725343-0.870602$

H $\quad 0.8186403 .174750 \quad 1.726136$

H 2.4191691 .3463782 .262768

H $1.562740 \quad 0.237549 \quad 1.233963$

H $-0.804362-0.5549234 .043140$

H $0.0847801 .261874 \quad 5.130420$

H 1.7304721 .6476724 .618445

H 0.3615272 .6250124 .042922

H $0.071780-1.1421281 .135753$

H $-0.224081-2.3495372 .372690$

H -2.594501-1.693002 2.530663

H -2.361909 -0.3546541 .441301$

H -1.987541 -3.298542 0.702966

H $-0.674645-1.766957-0.829610$

H -2.082318 $-0.749613-1.014765$

H $-0.979992-1.723911-3.258754$

H $-4.206155-1.765303-2.220233$

H $-0.427809-3.781229-2.306729$

H $-1.832732-4.530088-3.026132$

H $-1.610338-4.579030-1.265559$

H -3.917232 $-3.317494-3.852763$

H $-6.166543-2.134377-0.981957$

H $-6.266842-3.882950-1.224892$

H $-4.572890-4.9831170 .197807$

H -2.664676 $1.326354-0.174764$

H $-3.4606804 .287758-0.371212$

H $-4.5017170 .848320-1.758218$

H $-6.2021061 .074404-1.385366$

H $-5.0674320 .826230-0.071311$

H $-7.512741-3.620480 \quad 0.707225$

H $-5.964632-4.0538201 .404610$

H -6.8028700 .5930312 .237650$

H $-8.336631-0.1499782 .738469$

H -9.3213510 .6199110 .409407$

H -7.500191 2.8326391 .488589

H $-9.771418 \quad 3.8519100 .633796$

H -9.725256 $2.687053-0.680142$

H $-7.8687906 .212689-1.428268$

H $-9.1975245 .979134-0.158931$

H $-7.271776 \quad 4.127520-2.751096$

H -7.492962 $2.570968-2.015643$

H $-5.509924 \quad 4.790115-1.151959$

H $-8.065533-1.3834860 .429842$

C $4.891703 \quad 3.626375-1.205350$

O $5.1665422 .338327-0.614268$

C 6.0441392 .5339150 .508993

C 6.6640513 .9117590 .310947

C $5.4868744 .675579-0.264300$

H 5.4082912 .5671741 .402056

C 7.0511921 .3873390 .634612

H 7.0390244 .3390961 .245737

H $7.4893683 .885842-0.410049$

C $5.8765045 .970896-0.955019$

H $4.794372 \quad 4.9067760 .553943$

C $6.421997-0.0185700 .744559$

$\begin{array}{lllll}\text { O } & 7.900310 & 1.394370 & -0.519701\end{array}$

H 7.7041941 .5768201 .495353

H $6.039718-0.321751-0.235955$ 
O $7.479127-0.936627 \quad 1.052545$

C $5.310879-0.1530691 .799730$

C $4.876446-1.5946252 .119127$

H 4.4395980 .4213221 .469438

$\begin{array}{lllll}\text { O } & 5.765153 & 0.435403 & 3.022706\end{array}$

H $5.684649-2.1170152 .647408$

H $4.054430-1.5213302 .844124$

H $6.3538936 .655807-0.246484$

H $6.579658 \quad 5.793281-1.775550$

H $4.9951686 .471474-1.368055$

C $3.3960603 .764039-1.507213$

H $8.5339920 .663626-0.417671$

H $7.800965-0.724227 \quad 1.945655$

H $5.021643 \quad 0.4192023 .649508$

H $3.128433 \quad 3.016038-2.264575$

H $3.1924574 .744397-1.952678$

H $5.432323 \quad 3.639374-2.160947$

C $2.844872-2.351655-0.927609$

O $3.359145-1.731026 \quad 0.259644$

C $4.425445-2.4262150 .910171$

C $3.970070-3.8340241 .330974$

C $3.399087-4.616785 \quad 0.145552$

C $2.375701-3.789298-0.640210$

H $1.959406-1.759326-1.192260$

C $3.837916-2.265651-2.098238$

H $5.271647-2.516506 \quad 0.222216$

O $2.983002-3.7532102 .358485$

H $4.827261-4.3728641 .751390$

H $2.923813-5.5317210 .519980$

O $4.473019-5.002825-0.706163$

H $\quad 2.120550-4.284091-1.584409$

O $1.171176-3.701720 \quad 0.125526$

C $4.116131-0.837070-2.513492$

H $4.796793-2.723831-1.842747$

H $3.425155-2.782611-2.972078$

H $2.252781-3.2028942 .026709$

H $4.095933-5.520528-1.437707$

H $0.837714-4.6061450 .253506$

O $5.179718-0.822641-3.370539$

O $3.479094 \quad 0.140094-2.147652$

C $5.5717660 .468518-3.847550$

H $6.0064251 .049726-3.029886$

H $6.3317070 .328360-4.620810$

H $4.7192080 .995335-4.287233$

SCF Energy (B3LYP/6-31G*//MMFF)= -3245.90869805

07_00073

MM̄FF Geometry

C $3.149460-0.985504-2.171365$

C $2.192494-1.790131-2.655933$

C $1.479807-2.831424-1.836600$

O $0.073140-2.685678-2.152268$

C $1.927928-4.262085-2.182112$

C $3.342568-4.592780-1.762732$

C $3.696160-4.958056-0.514920$

C $4.371142-4.562377-2.863029$

C $2.811166-5.0749890 .698734$

C $3.453686-4.449752 \quad 1.946687$

C $3.427969-2.9110761 .951874$

C $4.464846-2.3801542 .944258$

C $4.424618-0.851593 \quad 3.053684$

O $2.113752-2.492748 \quad 2.345640$

O $5.207499-0.4737804 .196559$

C $2.973010-0.4216773 .333088$

C $5.070922-0.1720351 .842906$

$\begin{array}{lllll}\text { O } & 2.852770 & 1.000424 & 3.314189\end{array}$

C $1.923202-1.0716932 .394325$

C $0.512105-0.7835932 .963235$

O $2.038185-0.4821161 .100022$

C $-0.831721-3.198486-1.279258$

O $-0.599527-3.798783-0.242856$

C $-2.178129-2.894611-1.806376$

C $-3.252507-3.401730-1.187449$

C $-4.635123-3.149207-1.574096$

C $-5.491871-4.385559-1.568322$

C $-0.644242-1.5237792 .277906$

C $-1.972301-1.2001402 .948143$

C $-4.389890-1.7065772 .861009$
O $-2.059541-0.5014793 .955834$

C $-5.411470-1.9866051 .807305$

C $-6.250193-1.0577631 .322783$

C $-7.330177-1.3322190 .313119$

C $-7.084926-0.638464-1.009468$

C $-7.4615430 .636740-1.202530$

C $-6.472424-1.451563-2.129736$

C $-5.072266-1.895977-1.811232$

N $-3.053648-1.7818362 .297737$

H $3.473462-1.111474-1.143034$

H $1.895613-1.690947-3.697413$

H $1.617516-2.624626-0.768722$

H $1.804089-4.439041-3.259781$

H $1.243867-4.988887-1.725682$

H $4.738532-5.223057-0.341006$

H $5.371521-4.827829-2.504923$

H $4.103339-5.275085-3.650019$

H $4.436924-3.565190-3.307158$

H $1.820613-4.643394 \quad 0.537223$

H $2.664050-6.1447650 .891986$

H $2.909729-4.8189962 .825854$

H $4.485932-4.8124902 .032677$

H $3.641556-2.5349760 .946023$

H $5.473236-2.7147002 .669460$

H $4.269558-2.8058073 .938814$

H $6.113774-0.7993844 .060188$

H $2.749044-0.7268554 .364518$

H $6.099219-0.527327 \quad 1.706218$

H 5.1489360 .9096991 .994599

H $4.530930-0.3550600 .913101$

H $2.846374 \quad 1.2833262 .383664$

H $\quad 0.496749-1.0677604 .024128$

H 0.3106250 .2947252 .913660

H $1.184558-0.5468190 .643702$

H -2.262019 -2.254440 -2.677993

H $-3.130076-4.062377-0.331577$

H $-5.469521-4.865607-0.583298$

H -5.122861 -5.104379-2.308382

H $-6.540506-4.192747-1.802370$

H $-0.712090-1.2438541 .222918$

H $-0.491804-2.6065742 .342858$

H $-4.533305-0.7239293 .323324$

H $-4.457965-2.4566593 .656123$

H $-5.488542-3.0125901 .457234$

H $-6.178768-0.0372371 .692854$

H $-8.283423-0.9899270 .737103$

H $-7.459223-2.4095350 .150742$

H $-7.3123891 .133815-2.155251$

H $-7.9232831 .213618-0.407891$

H $-6.426492-0.860189-3.053614$

H $-7.137996-2.288624-2.358915$

H $-4.358015-1.074977-1.752331$

H $-2.865814-2.4924171 .596275$

C $4.2046571 .294946-2.135646$

O $3.0283321 .989553-1.674595$

C $3.3476483 .392158-1.553279$

C $4.8001383 .551704-2.004134$

C $4.9981532 .348546-2.901742$

H $2.6997893 .927860-2.255252$

C $3.1148463 .883019-0.118701$

H $4.9661854 .502510-2.520626$

H $5.4928983 .516652-1.154660$

C $6.4596591 .998845-3.123263$

H $4.5201422 .549469-3.869822$

C $1.6446583 .868606 \quad 0.348153$

$\begin{array}{lllll}\text { O } & 3.871747 & 3.049216 & 0.768154\end{array}$

H $3.5264114 .893992-0.010357$

H 1.2808742 .8366940 .395924

$\begin{array}{lllll}\text { O } & 1.631461 & 4.350323 & 1.700243\end{array}$

C $0.6882094 .732940-0.502320$

C $-0.660493 \quad 5.0540210 .173173$

H $0.518126 \quad 4.243692-1.467860$

O $1.328076 \quad 5.985452-0.772998$

H $-0.4799965 .594787 \quad 1.110638$

H $-1.1942145 .763219-0.474718$

H $6.9894282 .837652-3.586698$

H $6.9632331 .762989-2.179733$

H $6.5605291 .132606-3.784283$ 
C $3.8279020 .079414-2.985165$ H 3.7244793 .3782751 .671472 H 1.9260305 .2773201 .684605 H $0.7546666 .483144-1.380506$ H $4.733872-0.357794-3.419224$ H $3.1730590 .392529-3.807557$ H $4.7612970 .974432-1.244755$ C $-2.8388992 .200761-0.825465$ O $-2.0115923 .371103-0.847804$ C $-1.549703 \quad 3.8296340 .426288$ C -2.7444954 .1739051 .329792$ C -3.7019472 .9867301 .461127$ C -4.0545412 .3839760 .098544$ H -3.217583 2.110608 -1.850970 C $-2.0164670 .945738-0.510464$ H -0.9539553 .0466050 .904375$ O H -2.3687284 .4548052 .320745$ H -4.6182243 .3211801 .963110$ O -3.0873232 .0008542 .283535$ H $-4.567591 \quad 1.4251370 .229269$ O $-4.9797033 .269300-0.540459$ C $-0.9274540 .721908-1.533582$ H -1.5280321 .0163650 .465336$ H $-2.6485500 .052399-0.486691$ H $-3.7373295 .087212-0.087510$ H $-3.718576 \quad 1.2685782 .383912$ H $-5.2632222 .849508-1.370374$ O $-1.4613780 .622906-2.782649$ O $\quad 0.2574990 .625674-1.243221$ C $-0.543080 \quad 0.476790-3.871425$ H $-0.9945940 .934094-4.756033$ H $\quad 0.4114430 .975080-3.676271$ $\mathrm{H}-0.388295-0.586339-4.068702$ SCF Energy (B3LYP/6-31G**//MMFF) $=-3245.91413357$

0700074

MM̄FF Geometry

C -3.111069 2.755910-1.821247

C $-4.3517752 .938119-1.343321$

C $-5.1168911 .997362-0.441658$

$\begin{array}{lllll}\text { O } & -4.565217 & 0.661493 & -0.440779\end{array}$

C -5.0752622 .5299641 .000259$

C -5.9639381 .7518051 .949076$

C -5.5175880 .8434012 .838795$

C -7.4294122 .0963451 .887260$

C $-4.100461 \quad 0.370782 \quad 3.028047$

C $-4.053693-1.1432903 .278749$

C $-2.639567-1.7436933 .341831$

C -1.745704-1.079186 4.390656

C $-0.336608-1.6883304 .405865$

O $-2.042895-1.6361112 .044955$

O $0.489907-0.8117325 .188157$

C $0.218124-1.7010542 .964801$

C $-0.313763-3.0463605 .116940$

O $1.460921-2.3999302 .942324$

C $-0.767842-2.2881591 .930157$

C $-0.285655-2.1031650 .474775$

O $-0.887610-3.6983372 .157507$

C $-4.854493-0.127839-1.510467$

O $-5.4669830 .188662-2.518078$

C -4.288820 -1.467129-1.248299

C $-3.933189-2.233059-2.289751$

C $-3.377380-3.584395-2.218728$

C $-3.514705-4.327835-0.916009$

C $-0.114482-0.651606 \quad 0.019777$

C $0.072329-0.605588-1.482554$

C $1.645116-1.333846-3.267247$

O $-0.824995-0.232589-2.234312$

C $1.485282-2.804891-3.491508$

C $0.417562-3.355313-4.088920$

C $0.239710-4.834695-4.275733$

C $-0.670367-5.448151-3.233275$

C $-0.157030-6.040615-2.141452$

C -2.161281 $-5.452578-3.489041$

C $-2.790486-4.097699-3.320070$

N $1.319561-1.061289-1.880772$

H $-2.5515781 .864073-1.555211$
H $-4.8818323 .850253-1.611838$

H $-6.1557241 .947837-0.791887$

H -5.3878853 .5829031 .022792$

H -4.0404172 .5293581 .363967$

H -6.2454970 .3863643 .508166$

H -8.0184241 .5433772 .626689$

H -7.577320 3.1638522 .080546

H -7.8383291 .8612690 .899830$

H -3.4767990 .6096872 .162062$

H -3.6874610 .9003853 .893410$

H $-4.576989-1.3700524 .216664$

H -4.604112 -1.647970 2.473392

H -2.765431 -2.807439 3.576635

H -2.203018-1.135875 5.386065

H -1.638685 -0.009513 4.167159

H $1.401542-1.1481315 .142367$

H $0.436410-0.6584462 .706004$

H $-0.648000-2.9371846 .155746$

H $0.703883-3.4487695 .171923$

H $-0.952437-3.7925054 .639858$

H $1.859484-2.2776882 .065040$

H $0.641051-2.6674940 .311691$

H -1.016762 -2.576739 -0.193666

H -1.174328 -4.1241181 .332930$

H $-4.171467-1.773893-0.215990$

H $-4.038825-1.824872-3.294575$

H -3.011235 -3.796416 -0.103695

H -3.086686 -5.332110 -0.942385

H $-4.572393-4.438719-0.651852$

H $-0.995442-0.0493330 .267801$

H $\quad 0.749699-0.1797230 .497738$

H $2.683823-1.036379-3.437541$

H $1.001078-0.744345-3.927769$

H $2.280786-3.446970-3.120906$

H $-0.377909-2.709218-4.451050$

H $1.218546-5.332855-4.255270$

H $-0.156456-5.027475-5.280153$

H $-0.794153-6.509597-1.399210$

H $0.912952-6.064953-1.963207$

H -2.664438 -6.193882 -2.857758

H $-2.348337-5.798400-4.514020$

H -2.744800 -3.481434 -4.218701

H $1.968857-1.392988-1.173927$

C -1.211540 $4.427592-2.114520$

O $-0.1517493 .483150-1.881192$

C $0.5941163 .913006-0.724728$

C $0.0043105 .257432-0.300772$

C $-1.4286655 .135621-0.780359$

H 0.4020543 .1718330 .061022

C $2.1009433 .965224-1.022218$

H 0.0836615 .4200870 .778661

H $0.5057156 .094793-0.800504$

C $-2.1527386 .464919-0.893080$

H $-1.9673964 .489688-0.075968$

C $2.6979742 .578564-1.347163$

O $2.3230604 .876019-2.097003$

H $2.6075914 .369884-0.137239$

H $2.3973321 .893376-0.547963$

O $2.1666702 .070905-2.569590$

C $4.2374762 .582999-1.452380$

C $4.8581041 .191496-1.690317$

H $4.6591243 .016695-0.538772$

O $4.6521453 .425398-2.530286$

H $4.4683460 .762099-2.621390$

H $5.9338041 .338674-1.856062$

H -2.2024356 .9594440 .082513$

H -1.644065 $7.141842-1.587696$

H $-3.1769316 .321285-1.251310$

C $-2.4274473 .735939-2.732956$

H $3.2838374 .967454-2.212463$

H $1.2002952 .022102-2.476223$

H $4.2428863 .082293-3.343383$

H $-2.1085373 .181471-3.624420$

H $-3.1383294 .498858-3.072913$

H $-0.8217955 .140135-2.854616$

C 5.0782080 .0510911 .852352

$\begin{array}{lllll}\text { O } & 5.264162 & 0.779514 & 0.632742\end{array}$

C $4.6431590 .214728-0.525207$ 
C $5.219498-1.178801-0.808026$

C $5.062893-2.0865130 .418131$

C $5.553250-1.4055111 .702482$

H 5.7370950 .5448492 .578462

C 3.6378020 .1770192 .369200

H $3.5696480 .117104-0.350539$

O $6.597708-1.065987-1.152581$

H $4.696716-1.611246-1.669422$

H $5.630370-3.0122940 .263634$

O $3.692176-2.4418160 .576990$

H $5.245257-1.9800682 .584024$

O $6.984185-1.3850291 .695081$

C 3.2515611 .6128752 .658601

H $2.924404-0.2122181 .639651$

H $3.526775-0.3857173 .302656$

H $6.927446-1.960509-1.343044$

H $3.421553-2.927861-0.220681$

H $7.290794-2.3072731 .663142$

O 1.8953771 .6857672 .803978

$\begin{array}{lllll}\text { O } & 4.033884 & 2.547095 & 2.764318\end{array}$

C 1.3716282 .9847023 .096139

H 1.7178623 .7163612 .360181

H 0.2807252 .9296583 .046917

H 1.6652173 .2850024 .106101

SCF Energy (B3LYP/6-31G**/MMFF) $=-3245.90353053$

0700075

MM̄FF Geometry

C $1.348909-1.7610492 .506870$

C $0.843140-0.6644573 .093146$

C $1.572106 \quad 0.640411 \quad 3.327877$

$\begin{array}{lllll}\text { O } & 2.923855 & 0.547875 & 2.814248\end{array}$

C 0.8730881 .8182532 .623407

C -0.4923622 .1903043 .156205$

C -1.617776 2.2075462 .416417

C -0.5315572 .6413444 .593380$

C $-1.784882 \quad 1.817433 \quad 0.974892$

C $-1.889568 \quad 3.0322470 .041932$

C $-0.549073 \quad 3.745513-0.200018$

C $-0.7510554 .974454-1.089246$

C $0.5815845 .652163-1.443237$

O $0.3298562 .818114-0.844581$

O $0.332866 \quad 6.578944-2.511425$

C $1.5528554 .582743-1.986143$

C $1.1282576 .477226-0.272419$

$\begin{array}{llll}\text { O } & 2.846628 & 5.138917 & -2.212941\end{array}$

C $1.6487353 .318543-1.099069$

C $2.4368782 .215468-1.845268$

$\begin{array}{llllll}\text { O } & 2.335678 & 3.654195 & 0.107368\end{array}$

C 3.8505401 .4138503 .298415

O 3.6709232 .2775644 .146025

C $5.158247 \quad 1.235676 \quad 2.628008$

C 5.5109480 .1068041 .994017

C $6.794476-0.1463121 .336022$

C 7.7228051 .0226121 .134874

C $2.4476610 .851165-1.139243$

C $3.097817-0.221428-1.997517$

C $3.463345-2.684721-2.072680$

O $3.657656 \quad 0.018439-3.064062$

C $4.783416-3.065387-1.484687$

C $5.950308-2.914906-2.129157$

C $7.279148-3.355030-1.584936$

C $8.164029-2.194201-1.190469$

C $8.838126-1.488453-2.113927$

C $8.332841-1.9102010 .284277$

C $7.073845-1.4020070 .929510$

N $2.975489-1.480924-1.427940$

H $2.374203-1.7603732 .147353$

H $-0.178643-0.7070123 .465365$

H 1.6246280 .8008514 .411505

H $0.8490701 .607043 \quad 1.550156$

H 1.5031692 .7139232 .700463

H -2.540502 2.5335882 .895480

H -1.5092643 .0468534 .875430$

H $-0.317323 \quad 1.8052995 .265991$

H $0.209903 \quad 3.4285334 .765414$

H $-1.005961 \quad 1.1278580 .636039$

H $-2.719129 \begin{array}{lll}1.248146 & 0.907577\end{array}$
H -2.272856 $2.675596-0.921955$

H $-2.6264893 .742146 \quad 0.437960$

H $-0.121065 \quad 4.0443260 .762553$

H $-1.4320715 .691231-0.613541$

H -1.242771 $4.670972-2.024433$

H $-0.3348347 .216683-2.205786$

H $1.1770204 .284663-2.974596$

H $\quad 0.4030427 .2433550 .027349$

H $2.0336857 .022058-0.561727$

H 1.3532285 .8737900 .609463

H $3.2856035 .228229-1.349986$

H $1.9900062 .073219-2.838301$

H $3.4750172 .536860-1.997778$

H $2.7608812 .859471 \quad 0.465047$

H 5.8393322 .0749562 .726031

H $4.809890-0.7258501 .972265$

H 7.1942381 .8594190 .664142

H $8.5674370 .795561 \quad 0.480031$

H 8.1304301 .3593032 .094011

H $3.0029040 .906825-0.197449$

H $1.4232820 .529854-0.918239$

H $3.527215-2.524548-3.154118$

H $2.735213-3.481024-1.891020$

H $4.770524-3.516887-0.495866$

H $5.953137-2.469474-3.121793$

H $7.782807-3.955200-2.354175$

H $7.148353-4.034605-0.733386$

H $9.496296-0.672858-1.833913$

H $8.743018-1.710693-3.171758$

H $9.171778-1.2277600 .456622$

H $8.628155-2.8384800 .790925$

H $6.334834-2.1819341 .114794$

H $2.552621-1.568527-0.508838$

C $0.594626-3.5114520 .877236$

O $-0.295547-2.6841150 .097524$

C $-1.094489-3.536916-0.744087$

C $-0.413783-4.898666-0.727200$

C $0.100386-4.9471220 .696738$

H $-2.071389-3.634573-0.258259$

C $-1.265650-2.932960-2.140585$

H $-1.105215-5.713025-0.963872$

H $0.421084-4.943179-1.436500$

C $1.170844-6.0023530 .917137$

H $-0.743573-5.1428711 .371940$

C $-1.938944-1.545981-2.167628$

O $0.019397-2.806348-2.760579$

H $-1.832488-3.630343-2.768633$

H $-1.253958-0.789418-1.766185$

O $-2.143940-1.194756-3.542755$

C $-3.283029-1.464510-1.418721$

C $-4.018613-0.137096-1.682769$

H $-3.097274-1.577010-0.344758$

O $-4.106403-2.553121-1.836055$

H $-3.3016170 .689014-1.640027$

H $-4.449035-0.168477-2.691531$

H $\quad 0.774977-7.001104 \quad 0.705971$

H $\quad 2.035779-5.838836 \quad 0.265177$

H $1.523563-5.9912751 .953080$

C $0.592025-3.0498072 .335257$

H $0.554151-2.212557-2.205966$

H $-2.790914-1.820307-3.912145$

H $-4.929075-2.515016-1.319400$

H $1.077933-3.8026622 .966382$

H $-0.440268-2.9361722 .688000$

H $1.595553-3.4044170 .442243$

C $-7.240244-0.8172860 .098730$

O $-6.148989-0.875738-0.829456$

C $-5.1453720 .128714-0.665100$

C $-5.7599501 .525495-0.843485$

C $-6.952946 \quad 1.734262 \quad 0.094215$

$\begin{array}{llll}\text { C } & -7.932939 & 0.554949 & 0.046263\end{array}$

H $-7.953935-1.567996-0.264621$

C $-6.810080-1.2284831 .511625$

H -4.7167450 .0464640 .338942$

O $-6.1790861 .714770-2.193714$

H $-4.9929472 .280649-0.636929$

H $-7.4720172 .657207-0.191629$

O -6.4615851 .9054291 .420394$ 
H $-8.6642050 .624790 \quad 0.859913$

O $-8.6655460 .613799-1.181458$

C $-6.302788-2.6517371 .522772$

H $-6.036127-0.5751451 .924065$

H -7.656276 -1.179681 2.207084

H $-6.8048141 .003467-2.413417$

H -7.2267812 .0673991 .998151$

H $-9.1474001 .458308-1.194997$

O $-4.957941-2.6664741 .747663$

O $-7.014183-3.6305761 .336492$

C $-4.350307-3.9616051 .772243$

H -3.292034 -3.835487 2.015631

H $-4.814055-4.5867372 .541282$

H $-4.431901-4.4335440 .788540$

SCF Energy (B3LYP/6-31G*//MMFF)= -3245.91406125

0700076

MM̄FF Geometry

C -1.525155 3.4838291 .496416

C $-0.774928 \quad 3.5115012 .606609$

C $-0.224570 \quad 2.261846 \quad 3.236355$

$\begin{array}{lllll}\text { O } & 1.220583 & 2.323197 & 3.189891\end{array}$

C -0.6200392 .1202254 .715048$

C -2.0828451 .8075984 .940264$

C -2.621721 0.5809964 .795058

C $-2.934224 \quad 2.964676 \quad 5.390324$

C -1.919977 -0.6728554 .347017$

C $-2.639547-1.2996753 .144966$

C -1.882034 -2.453030 2.467068

C -1.429193 -3.5404403 .439241$

C $-0.654573-4.6551902 .723065$

O $-0.748737-1.907352 \quad 1.785114$

O $-0.029668-5.442563 \quad 3.748836$

C $0.458540-4.0243351 .852928$

C $-1.598985-5.5972641 .965003$

O $1.073731-5.0307231 .052445$

C $-0.037086-2.8564910 .966031$

C $1.115277-2.0526750 .316466$

O $-0.874630-3.368211-0.071613$

C $1.821491 \quad 1.7745242 .099308$

$\begin{array}{llll}\text { O } & 1.273502 & 1.298209 & 1.117640\end{array}$

C 3.2837561 .8237022 .317009

C $4.0635670 .993058 \quad 1.609945$

C $5.5208600 .884007 \quad 1.679040$

C 6.2546151 .8775352 .539873

C $2.008093-2.824341-0.657505$

C $2.914928-1.915213-1.471227$

C $4.739403-2.026200-3.171810$

O $2.882490-0.690143-1.402947$

C $6.067312-2.004938-2.481653$

C $6.791173-0.890808-2.292698$

C $8.138952-0.875521-1.629476$

C $8.2043170 .059964-0.442014$

C $8.8590041 .230814-0.519635$

C $7.592411-0.4000620 .862441$

$\begin{array}{llll}C & 6.125068 & -0.081592 & 0.954435\end{array}$

N $3.759244-2.643774-2.297589$

H $-1.7424702 .523878 \quad 1.033427$

H -0.5421174 .4625803 .078184$

H -0.5635841 .3699052 .694730$

H $-0.337895 \quad 3.0269505 .268064$

H $-0.006197 \quad 1.3354515 .178394$

H -3.681355 0.4554295 .014108

H -3.9781002 .6749665 .551112$

H -2.554662 3.3699566 .333981

$\mathrm{H}-2.9269143 .7622804 .641293$

H $-0.871868-0.4874514 .096785$

H $-1.926783-1.3757655 .188162$

H -3.623228 -1.6628913 .469268$

H -2.816537 -0.5202712 .391953$

H - $2.564113-2.8750891 .720468$

H -2.283686 -3.9534683 .989186$

H $-0.760747-3.1104164 .196923$

H $0.417591-6.1936173 .323793$

H $1.234233-3.6368732 .527861$

H $-2.313520-6.0582382 .657603$

H -1.049548 $-6.427211 \quad 1.507132$

H -2.170956 -5.0934201 .183466$
H $1.490818-5.6719411 .651839$

H $0.668346-1.201486-0.216422$

H $1.736215-1.6027041 .102320$

H $-0.931967-2.700892-0.775463$

H 3.6690332 .5158303 .056260

H 3.5843930 .3086960 .912962

H 5.9970192 .9026322 .250359

H $7.341273 \quad 1.8022152 .461237$

H 5.9963121 .7355113 .594866

H $2.651264-3.516551-0.103952$

H $1.398089-3.398434-1.363813$

H $4.401622-1.022805-3.450929$

H $4.801160-2.638638-4.076695$

H $6.460706-2.958895-2.138026$

H $6.4079780 .060691-2.654503$

H $8.875732-0.575868-2.386277$

H $8.437969-1.881684-1.309987$

H $8.936633 \quad 1.8917850 .337161$

H $9.327647 \quad 1.556855-1.442175$

H $8.147787 \quad 0.0173601 .709070$

H $7.721413-1.4853930 .964226$

H $5.498151-0.7492230 .363197$

H $3.749631-3.658217-2.242191$

C $-1.528875 \quad 4.970699-0.554266$

O $-1.629016 \quad 3.799328-1.390647$

C $-0.3815623 .633497-2.090618$

C $0.3347524 .973429-1.980065$

C $-0.058963 \quad 5.393275-0.577144$

H $0.1987282 .884954-1.538513$

C $-0.6300613 .160999-3.524137$

H $1.4165904 .880384-2.114325$

H $-0.0412575 .694638-2.715447$

C $0.1641306 .868360-0.295026$

H 0.5272024 .8026050 .138109

C $-1.323806 \quad 1.785225-3.633968$

\begin{tabular}{lllll}
\hline & -1.450076 & 4.123705 & -4.198763
\end{tabular}

H $\quad 0.3177893 .138922-4.074560$

H -2.359221 $1.855157-3.281800$

O $-1.393786 \quad 1.452692-5.027020$

C $-0.5959420 .644305-2.895070$

C $-1.054306-0.774265-3.278884$

H $-0.7007020 .785576-1.813375$

O $0.8006460 .730038-3.198786$

H $-0.850886-0.947996-4.343283$

H -0.407351 -1.480190 -2.740737

H $1.2246807 .122269-0.392853$

H $-0.3978827 .500330-0.990885$

H -0.1518267 .1223210 .721746$

C $-2.116733 \quad 4.691708 \quad 0.830935$

H -2.276442 $4.209330-3.692864$

H $-1.8529422 .182941-5.476312$

H $1.2619960 .079270-2.643061$

H -3.1921134 .5012890 .725357$

H -2.015108 $5.580087 \quad 1.464884$

H $-2.1435605 .741675-1.037340$

C $-4.006982-1.340019-1.053914$

O $-2.686298-1.062707-1.542180$

C $-2.524910-1.087729-2.964535$

C $-2.933826-2.455755-3.536681$

C $-4.353031-2.835056-3.105821$

C $-4.543898-2.676908-1.596742$

H $-3.882709-1.4468140 .030780$

C $-4.962664-0.168231-1.306853$

H $-3.154050-0.313712-3.413643$

O $-2.014749-3.454047-3.097315$

H $-2.871152-2.411488-4.630593$

H $-4.556785-3.876139-3.384785$

O $-5.297327-2.009623-3.781010$

H $-5.605029-2.782450-1.341882$

O $-3.854965-3.748802-0.949197$

$\begin{array}{lll}\text { C }-4.649610 & 0.981242 & -0.380846\end{array}$

H $-4.9148890 .217368-2.328995$

H $-6.005249-0.461476-1.134297$

H $-2.290860-4.300488-3.488043$

H $-5.176467-2.145868-4.736202$

H $-4.044723-3.6878990 .002357$

$\begin{array}{lllll}\text { O } & -5.270157 & 0.793775 & 0.819268\end{array}$

O $-3.9284361 .921605-0.684709$ 
C -5.0535101 .8168301 .795799$ H -5.6819031 .5977862 .663110$ H -4.0076971 .8138282 .112090$ H -5.335069 2.7961531 .397145 SCF Energy (B3LYP/6-31G**//MMFF) $=-3245.90966526$

07 00077

MM̄FF Geometry

C -2.790697 -3.177742 0.959351

C $-1.865224-2.2088370 .883928$

C $-1.075341-1.6995432 .061574$

O $0.313706-1.7727351 .667934$

C $-1.458728-0.2371662 .344800$

C -0.9516430 .2953663 .668803$

C 0.0689101 .1638143 .809360

C $-1.721272-0.1648964 .880821$

C 0.9522081 .7268452 .729684

C 2.4307881 .6534753 .139962

C 3.4197632 .0623162 .036578

C 3.2611853 .5237901 .609978

C 4.2094473 .8941160 .458846

$\begin{array}{lllll}\text { O } & 3.233359 & 1.185273 & 0.921187\end{array}$

O $3.7701115 .147474-0.088257$

C $4.0666312 .840460-0.660133$

C 5.6454534 .1149670 .947298

O $5.0250263 .058688-1.692698$

C $4.1640531 .387309-0.150488$

C $3.8434290 .352835-1.249959$

$\begin{array}{llllll}\text { O } & 5.507363 & 1.133347 & 0.280391\end{array}$

C $1.236019-2.1070362 .607814$

O $1.046197-2.312506 \quad 3.795209$

C $2.541785-2.1956951 .921299$

C $3.667442-2.3868742 .621731$

C $4.990113-2.4950082 .017176$

C $6.018883-1.6032042 .653594$

C $2.4269410 .421299-1.828999$

C $2.224330-0.730465-2.792283$

C $2.035284-3.217180-2.785393$

O $2.377150-0.595028-4.003795$

C $3.312486-3.881805-2.361803$

C $4.534770-3.367563-2.572567$

C $5.817822-4.012924-2.134975$

C $6.564058-3.184443-1.110350$

C $7.296270-2.119309-1.478346$

C $6.534759-3.6459160 .328788$

C $5.224415-3.3580491 .007728$

N $1.936003-1.920139-2.142893$

H $-3.000070-3.6447011 .917897$

H -1.664014 -1.742285 -0.078259

H -1.258988 -2.3374662 .934546$

H $-2.551856-0.1411842 .356905$

H -1.118947 0.3987991 .520313

H $\quad 0.3042331 .5138374 .813908$

H -1.351180 0.2893565 .806223

H $-2.778570 \quad 0.1028054 .783448$

H -1.646228 -1.2502974 .996268$

H $\quad 0.814121 \quad 1.2004011 .781825$

H 0.6619132 .7710182 .571209

H 2.6001062 .2784544 .025976

H 2.6583370 .6202303 .432488

H 4.4268651 .9021312 .440109

H 3.4114174 .1949362 .464615

H 2.2358173 .7049441 .265977

H 3.7808495 .8065570 .626897

H $3.0815602 .992844-1.116519$

H 5.6795324 .9332131 .676875

H 6.3001524 .4210010 .123992

H $6.0780713 .233076 \quad 1.423888$

H $4.9283263 .980278-1.988115$

H $4.5800050 .427363-2.061097$

H $3.993993-0.649388-0.825671$

H $5.665130 \quad 0.1750490 .256154$

H $2.558342-2.1033200 .842573$

H $3.642205-2.4431133 .707383$

H $6.300119-1.9879543 .639554$

H $5.622836-0.5890822 .782345$

H $6.928039-1.5035242 .055504$

H $1.6713630 .367929-1.037701$
H $2.261302 \quad 1.352272-2.379967$

H $1.985787-3.120776-3.874415$

H $1.180135-3.809862-2.446363$

H $3.217857-4.833956-1.845818$

H $4.626390-2.422398-3.103000$

H $6.450431-4.152973-3.021180$

H $5.638534-5.021943-1.743637$

H $7.855099-1.538626-0.751887$

H $7.347024-1.802033-2.514783$

H $7.375303-3.2159250 .883817$

H $6.711021-4.7289030 .360668$

H $4.396582-3.9700350 .648977$

H $1.824142-1.910661-1.134824$

C $-5.016362-3.189834-0.282272$

O $-5.054926-1.748657-0.286574$

C $-6.092358-1.3178080 .612132$

C $-6.972791-2.5396880 .840138$

C $-5.929868-3.6431290 .856490$

H $-5.603684-1.0653671 .560816$

C $-6.810016-0.0853310 .053498$

H -7.545131 -2.474097 1.770171

H -7.676619 -2.692461 0.013735

C $-6.507964-5.0341190 .666258$

H $-5.403137-3.6062701 .818054$

C $-5.8811581 .109058-0.262973$

O $-7.487204-0.439313-1.158538$

H $-7.593630 \quad 0.2292970 .752505$

H $-5.292750 \quad 0.896709-1.162430$

O $-6.7084322 .234177-0.581105$

C -4.9421291 .5006470 .888958$

C -4.1792972 .8214730 .698736$

H -4.2213060 .6932681 .047606$

O -5.7118531 .6180822 .090058$

H -4.8836253 .6632830 .700521$

H -3.5639232 .9563921 .598633$

H $-7.203206-5.2757421 .476852$

H -7.053813 -5.116812 -0.279614

H $-5.714388-5.7880310 .664986$

C $-3.565560-3.674544-0.230605$

H $-6.820620-0.788684-1.774935$

H $-7.2940541 .965930-1.309964$

H -5.0917421 .7785402 .821818$

H -3.062169 -3.345502 -1.147589

H $-3.535348-4.770108-0.237131$

H $-5.443239-3.504742-1.243861$

C $-1.4510911 .695150-1.612213$

O $-2.3839941 .780498-0.524391$

C $-3.2838312 .893145-0.549774$

C $-2.5136034 .226323-0.600029$

C $-1.4925514 .248658-1.738457$

C $-0.6261582 .986147-1.737071$

H $-0.7642670 .890015-1.321463$

C $-2.1312341 .294235-2.926626$

H $-3.9217412 .816236-1.435577$

O -1.8384904 .4649020 .635135$

H $-3.2327255 .043155-0.732403$

H -0.854636 $5.133993-1.627931$

O $-2.189034 \quad 4.362707-2.975145$

H $-0.0089942 .940256-2.641868$

O $0.2695823 .031776-0.624464$

C $-2.390196-0.191698-2.960053$

H -3.089898 $1.792069-3.094588$

H -1.499633 $1.540241-3.789164$

H -1.262776 $3.701060 \quad 0.811891$

H -1.524189 $4.399182-3.683814$

H $0.8095373 .835280-0.717124$

O $-1.335126-0.833359-3.538415$

O $-3.399223-0.717204-2.510691$

C $-1.454017-2.254584-3.651927$

H -1.258725 -2.715796 -2.680437

H -2.442010 -2.541994 -4.024722

H -0.701304-2.601603 -4.364730

SCF Energy (B3LYP/6-31G**//MMFF) $=-3245.89970831$

0700078

MM̄MF Geometry

C 0.4489532 .5071282 .636033

C -0.3209502 .3736303 .728313$ 
C -1.3175931 .2641353 .965857$ O -2.0199621 .0023742 .730703$ C -0.5716810 .0054944 .445558$ C -1.466586 -1.090416 4.983249 C $-1.765267-2.2237974 .318829$ C $-1.980808-0.8728816 .382570$ C -1.349494 -2.592574 2.920846 C $-2.511430-3.2322582 .148630$ C $-2.236726-3.4456200 .651608$ C $-1.009547-4.3223360 .385929$ C $-0.729551-4.476018-1.117007$ O $-2.079868-2.1607510 .036793$ O $0.588372-5.026198-1.266930$ C $-0.707894-3.071172-1.756016$ C $-1.687522-5.469013-1.784176$ O $-0.575932-3.155618-3.172478$ C $-1.937351-2.210789-1.389934$ C $-1.824546-0.761431-1.908615$ O $-3.101984-2.777258-2.004946$ C $-3.338246 \quad 0.6761252 .808850$ $\begin{array}{lllll}\text { O } & -3.999626 & 0.500025 & 3.819629\end{array}$ C $-3.860787 \quad 0.590379 \quad 1.430251$ C $-5.0738150 .066166 \quad 1.207465$ C $-5.688293-0.028920-0.110304$ C $-6.151968-1.412655-0.466093$ C $-0.6335240 .040261-1.382108$ C $-0.6880121 .432715-1.973712$ C $-1.6609413 .679521-1.568610$ O $-0.3395091 .647926-3.133403$ C $-3.1283253 .604267-1.861905$ C $-3.6265523 .158839-3.025546$ C $-5.0921263 .031619-3.327226$ C $-5.5655621 .592626-3.318342$ C $-5.2140140 .734473-4.290995$ C $-6.5146621 .173043-2.218109$ C $-5.8411801 .068708-0.877905$ N $-1.2274672 .372881-1.111081$ H $\quad 0.371206 \quad 1.778212 \quad 1.833408$ H -0.1955433 .0774314 .548353$ H -2.0237361 .6215334 .725488$ H $\quad 0.134126 \quad 0.275188 \quad 5.243620$ H $0.051990-0.3814683 .631746$ H -2.375666 -2.969002 4.827412 H -2.591755 -1.710394 6.736119 H -1.145131 -0.7569447 .080430$ H $-2.6004640 .027716 \quad 6.429739$ H - $0.989188-1.7218142 .366534$ H $-0.520058-3.3046082 .995693$ H -2.766806 -4.1956402 .608178$ H -3.395114 -2.587676 2.244659 H -3.132398 -3.9216450 .234566$ H -1.122990 -5.3044410 .860871$ H $-0.119116-3.8682630 .840170$ H $\quad 0.614397-5.875928-0.794250$ H $0.196569-2.572612-1.387348$ H - $1.581871-6.463419-1.333660$ H -1.448826 -5.594578 -2.846060 H -2.736908 -5.180734 -1.697609 H $\quad 0.208215-3.699947-3.358942$ H -1.815506 -0.764158 -3.007074 H $-2.742343-0.221287-1.637151$ H -3.771471 -2.080353 -2.103584 H -3.2465630 .9688320 .622410$ H -5.661260 -0.3436892 .025746$ H -7.064352 -1.6624680 .085824$ H $-5.386110-2.154321-0.211052$ H -6.354219 -1.534022 -1.533032 H $-0.6365990 .085017-0.287827$ H $0.316052-0.403547-1.688381$ H -1.089374 $3.981964-2.452027$ H $-1.4717274 .400797-0.768523$ H -3.805476 $3.909786-1.068647$ H -2.939138 $2.849351-3.809758$ H $-5.2857303 .473537-4.313325$ H -5.684551 $3.628544-2.622555$ H $-5.571568-0.289789-4.298198$ H $-4.557748 \quad 1.038032-5.100074$ H $\quad-7.0210470 .240140-2.486573$
H $-7.3195061 .915757-2.143631$ H $-5.4897112 .021844-0.482808$ H -1.515348 $2.084756-0.181385$ C 1.5199074 .2015891 .100130 O $2.0363803 .241351 \quad 0.151703$ C $3.1193993 .845167-0.577712$ C $2.9400535 .346949-0.401353$ C 2.4503645 .4159451 .029287 H $4.0465153 .537277-0.079084$ C $3.1364673 .379415-2.036678$ H $3.8696735 .899998-0.565663$ H $2.1784855 .749462-1.079330$ C 1.7712746 .7297851 .377045 H 3.3089285 .2721351 .699278

C $3.1868281 .850215-2.224382$

O $1.9457383 .860665-2.673230$

H $3.9772863 .853402-2.557571$

H $2.2313331 .410946-1.921424$

O $3.3328541 .590217-3.626421$

C $4.3323871 .133567-1.484828$

C $4.539756-0.340294-1.891365$

H $4.1551751 .184204-0.406971$

O $5.5638221 .820603-1.726000$

H $4.905325-0.377077-2.925431$

H $5.365815-0.738538-1.289702$

H $2.4682897 .565897 \quad 1.259183$

H 0.9076906 .9185190 .730465

H 1.4208416 .7253032 .413918

C 1.4930483 .5801142 .497910

H $2.0496733 .728871-3.630426$

H $2.487371 \quad 1.808440-4.053638$

H $5.7126951 .827455-2.687317$

H $1.3254434 .362833 \quad 3.247144$

H $2.467533 \quad 3.1233042 .708231$

H 0.5071224 .4643490 .772204

C $3.516626-1.8825640 .571144$

O $2.748651-1.191032-0.420996$

C $3.289325-1.228670-1.744956$

C $3.478467-2.678834-2.208989$

C $4.285485-3.501628-1.211840$

C $3.698729-3.3585050 .190971$

H $4.499303-1.4054240 .672692$

C $2.765951-1.7517921 .908155$

H $2.523294-0.802605-2.402393$

O $4.102432-2.708948-3.488541$

H $2.491191-3.141684-2.328348$

H $5.344915-3.220361-1.236117$

O $4.219414-4.871490-1.614704$

H $2.736602-3.8831050 .245336$

O $4.579770-3.972647 \quad 1.130157$

C $3.091802-0.4508422 .610037$

H $1.680976-1.7887981 .761253$

H $3.057652-2.5655082 .581944$

H $4.214810-3.642910-3.735204$

H $4.820168-5.375736-1.040752$

H $4.582739-4.9277420 .950227$

O $2.990674-0.6292793 .960147$

O 3.3827760 .5945672 .045960

C 3.3201110 .5086224 .762900

H 4.3291960 .8624394 .530406

H 2.5871701 .3039974 .608140

H 3.2895590 .2038065 .812300

SCF Energy (B3LYP/6-31G**//MMFF) $=-3245.89804192$

$07 \quad 00079$

MM̄FF Geometry

C $4.551634-2.7642691 .235723$

C $3.219791-2.8240111 .093000$

C $2.271415-2.8243832 .264038$

O $1.319560-1.7437782 .106851$

C $1.479023-4.1425012 .320620$

C $0.583428-4.2530933 .541401$

C $-0.761881-4.3285993 .505668$

C $1.294868-4.3040274 .870283$

C $-1.661667-4.3623682 .301984$

C $-2.237605-2.9815731 .988916$

C $-3.369596-3.0028170 .949438$

C $-2.911559-3.545616-0.405952$ 
C $-4.005354-3.429077-1.476443$

O $-3.817124-1.647803 \quad 0.813134$

O $-3.392468-3.642674-2.757397$

C $-4.559779-1.991804-1.476970$

C $-5.073906-4.517303-1.317463$

O $-5.680223-1.882027-2.353505$

C $-4.932729-1.467655-0.070311$

C $-5.2169570 .051802-0.146733$

O $-6.094686-2.1539230 .393300$

C $1.752507-0.4800782 .368544$

O $2.891421-0.1316042 .636033$

C $0.577071 \quad 0.4168602 .305653$

C 0.7478841 .7453162 .380823

C -0.3154422 .7528752 .412027$

C -1.738462 2.2608622 .420370

C -5.6712090 .6922121 .172847$

C $-5.3877242 .183448 \quad 1.295634$

C -5.2082004 .3173730 .014364$

O -5.1208142 .6819842 .389399$

C $-3.7334684 .489151-0.190720$

C $-3.035008 \quad 5.5174090 .315845$

C $-1.5538535 .691404 \quad 0.122664$

C $-0.843564 \quad 6.1518821 .378032$

C -0.2147787 .3391891 .413611$

C -0.8716875 .2559642 .596433$

C 0.0285124 .0573922 .463987

N $-5.5157342 .901154 \quad 0.120037$

H $4.979132-2.7550932 .235958$

H $2.789081-2.8152100 .096755$

H $2.818785-2.6763643 .203126$

H $2.170366-4.9955092 .325811$

H $\quad 0.889079-4.2324731 .403838$

H -1.295076 -4.391863 4.453902

H $0.618149-4.5567505 .693874$

H $2.080750-5.0663064 .851691$

H $1.749248-3.3370295 .104955$

H -1.140639 -4.770475 1.431763

H -2.478184 -5.0636682 .515397$

H -2.622652 -2.517145 2.907288

H -1.437820 -2.314344 1.642369

H -4.192324 -3.607062 1.349287

H $-2.583891-4.588373-0.325282$

H -2.032474 -2.982056 -0.745058

H -2.981494 -4.524151 -2.750343

H -3.782291 -1.338934-1.893575

H -4.621302 -5.513059-1.397279

H -5.817302 -4.462092 -2.120230

H $-5.594975-4.468140-0.358947$

H -5.399525 -2.209102 -3.225389

H $-4.3045480 .556240-0.485739$

H $-5.9975820 .241037-0.894024$

H -6.497508 -1.6426511 .112491$

H $-0.401210-0.0435972 .231033$

H $\quad \begin{array}{llll}1.762571 & 2.135728 & 2.448384\end{array}$

H -1.957240 1.6908681 .511859

H -2.4730833 .0651342 .462763$

H -1.921624 1.6201043 .290105

H -6.7475540 .5463331 .316923$

H -5.1551090 .2106502 .012055$

H $-5.558001 \quad 4.8284090 .917297$

H $-5.7543354 .699293-0.853477$

H -3.215447 $3.740105-0.785061$

H -3.559116 6.2855890 .880027

H -1.410109 $6.424489-0.681617$

H -1.090381 $4.762252-0.225666$

H $0.294177 \quad 7.6831722 .308085$

H -0.1919597 .9944910 .549281$

H $-1.905017 \quad 4.9908402 .833396$

H -0.5277875 .8140643 .478135$

H 1.0889994 .3133612 .452668

H $-5.7360752 .417323-0.743769$

C $6.112892-1.2268840 .093463$

O $5.114048-0.295020-0.366194$

C $5.7816770 .807903-1.013217$

C $7.2775410 .502015-0.955740$

C $7.288616-1.013637-0.851103$

H $5.4668920 .797895-2.061842$

C $5.3666392 .120702-0.336408$
H $7.8069490 .873525-1.838712$

H $7.7493220 .954094-0.075320$

C $8.606884-1.573383-0.347335$

H $7.062101-1.439581-1.837555$

C $3.8471902 .391075-0.410606$

O 5.7399502 .0455901 .045591

H $5.9282182 .958259-0.766016$

H $3.303925 \quad 1.572348 \quad 0.072236$

O $3.5691923 .562778 \quad 0.368157$

C $3.3259092 .613852-1.843654$

C $1.8156172 .908712-1.909164$

H $3.5575691 .748562-2.471528$

O $4.025563 \quad 3.732115-2.398906$

H $1.5349813 .625328-1.128128$

H $1.6101023 .408703-2.865101$

H $9.422739-1.305113-1.026465$

H $8.854810-1.1846080 .646069$

H $8.569094-2.665060-0.281721$

C $5.522577-2.6354830 .094629$

H 5.4434732 .8685691 .470746

H $4.0062524 .314360-0.068239$

H $3.7552753 .814704-3.329433$

H $\quad 6.306593-3.3885290 .226031$

H $5.020759-2.838279-0.859322$

H $6.391675-0.9281531 .114002$

C $0.481546-0.364959-3.094509$

O $1.188171 \quad 0.877650-2.998415$

C $0.9504051 .640182-1.809510$

C $-0.5412311 .987895-1.693694$

C $-1.4212860 .740795-1.772777$

C $-1.036253-0.160825-2.950000$

H $0.675728-0.700332-4.121419$

C $1.076584-1.410295-2.140226$

H $1.2620031 .052866-0.939206$

O $-0.9247312 .899565-2.721925$

H $-0.7096192 .494405-0.737942$

H -2.466198 $1.055377-1.876946$

O $-1.2993540 .025242-0.549644$

H -1.532322 -1.134010 -2.870690

O $-1.4999830 .444578-4.161341$

C $0.688879-2.821485-2.529240$

H $2.171469-1.351540-2.157726$

H $0.766594-1.221211-1.108667$

H $-0.7158282 .487178-3.577526$

H -1.929066 -0.713793 -0.576387

H $-2.4676670 .517930-4.099946$

O $0.731376-3.627130-1.428915$

O $0.410762-3.181180-3.665551$

C $0.419851-5.004156-1.663622$

H $1.175785-5.453940-2.314078$

H $\quad 0.428828-5.525312-0.702912$

H $-0.576707-5.101598-2.104566$

SCF Energy (B3LYP/6-31G**//MMFF) $=-3245.91981206$

$07 \_00080$

MM̄FF Geometry

C $0.7281724 .503025-0.802934$

C $0.0788193 .399150-0.407370$

C -1.159285 3.4326790 .446030

O $-2.1694852 .709708-0.299705$

C -0.9741102 .7383901 .807096$

C 0.1995163 .2235002 .633228

C 1.2232472 .4333593 .014758

C 0.1378644 .6589203 .083006

C 1.3967400 .9782422 .676304

C 0.6863400 .0858403 .702325

C $0.451916-1.3579743 .236184$

C $1.747954-2.1167462 .956702$

C $1.469061-3.5409752 .452101$

O $-0.360555-1.3139252 .059021$

O $2.712830-4.0522151 .952350$

C $0.471927-3.4622241 .276027$

C $1.041420-4.4745983 .590597$

O $0.068867-4.7640180 .856423$

C $-0.777120-2.5966031 .572791$

C $-1.574937-2.3650610 .261963$

O $-1.592095-3.2777192 .524601$

C $-3.4746812 .990462-0.050009$ 
O $-3.9156513 .814246 \quad 0.734262$

C $-4.2829672 .094886-0.908203$

C $-5.6218592 .157853-0.867138$

C $-6.5486461 .328827-1.642118$

C $-5.9580630 .253979-2.517537$

C $-2.784668-1.4247700 .386015$

C $-4.056967-2.1069850 .861342$

C $-6.369112-1.5871601 .636463$

O $-4.170302-3.3263640 .954854$

C -7.309552 -1.6917630 .477220$

C $-8.502737-1.0793960 .435469$

C $-9.466084-1.203974-0.710414$

C $-9.919790 \quad 0.137315-1.243010$

C -11.126043 $0.635764-0.923166$

C $-9.0105560 .859193-2.212933$

C $-7.872927 \quad 1.559747-1.521101$

N -5.057263 -1.1911491 .157569$

H $0.3661615 .476531-0.480949$

H $0.4448782 .425002-0.718256$

H -1.491905 4.4687940 .590166

H -0.9373271 .6549981 .644788$

H -1.887543 2.8780002 .401295

H 2.0085672 .8574593 .637677

H 0.9690954 .9212003 .746455

H $\quad 0.176793 \quad 5.3375972 .226105$

H -0.7915784 .8440883 .631329$

H $1.0498690 .779864 \quad 1.656711$

H 2.4691660 .7517172 .671358

H 1.2550940 .0831974 .640717

H $-0.297307 \quad 0.518070 \quad 3.930572$

H $-0.112344-1.8583674 .032130$

H $2.387608-2.1407863 .847462$

H $2.326225-1.5923102 .184721$

H $2.576264-4.9780581 .689402$

H $1.004468-3.0135750 .429535$

H $1.834741-4.5426864 .344612$

H $0.884856-5.4962093 .227090$

H $\quad 0.130220-4.1483004 .095840$

H $0.865893-5.2429530 .573652$

H $-0.896167-1.903053-0.467476$

H -1.898047 -3.325316 -0.156827

H $-2.458900-2.8448712 .556665$

H $-3.7509791 .400678-1.549117$

H $-6.0877222 .884717-0.202899$

H $-5.3612500 .699436-3.320969$

H $-6.705546-0.386109-2.989922$

H -5.315036 -0.412170 -1.931789

H -2.557401 -0.5754361 .039042$

H -3.007895 -1.022838 -0.610431

H $-6.690125-0.8347802 .363653$

H -6.297094 -2.555228 2.142235

H -7.003690 -2.317679 -0.357845

H -8.819593 -0.4761311 .283398$

H -10.332002 -1.777506 -0.354562

H -9.038090 -1.792794 -1.531231

H $-11.4701701 .583801-1.322928$

H -11.794304 $0.109494-0.249600$

H $-9.5770791 .626427-2.757805$

H -8.681328 $0.157538-2.983891$

H $-8.1985202 .362820-0.857942$

H $-4.894527-0.2037600 .984294$

C $3.2799114 .405311-0.989292$

O $3.5754813 .034202-0.665471$

C 4.4739783 .0208980 .461926

C 4.6780764 .4788240 .884894

C 3.4487465 .1650340 .321842

H 3.9609932 .4827801 .265854

C 5.7809292 .3057680 .094288

H 4.7599904 .5789221 .972027

H 5.5895774 .9068230 .451723

C 3.6061246 .6652830 .153908

H 2.6146994 .9623481 .004027

C $5.6248330 .854740-0.404244$

O $6.4429193 .041659-0.945648$

H 6.4628432 .3297650 .952490

H $5.1413380 .845886-1.387906$

O $6.947926 \quad 0.336187-0.605058$

C $4.870089-0.0895970 .551569$
C $5.062963-1.5879240 .244410$

H $3.803650 \quad 0.1617490 .541993$

O $5.3439330 .132758 \quad 1.883836$

H $6.128207-1.8394660 .323573$

H $4.571631-2.1552521 .045595$

H 3.8017837 .1420861 .119922

H $4.4376246 .909174-0.515859$

H $2.6965037 .108473-0.263348$

C $1.9299704 .506114-1.701195$

H $5.8490483 .059567-1.715678$

H $7.4047830 .937085-1.218372$

H $4.805343-0.4129562 .482062$

H $1.8400593 .692788-2.432130$

H $1.8995325 .440626-2.275500$

H $4.0531114 .716405-1.705506$

C $2.366334-2.414393-2.204499$

O $3.080560-2.007381-1.028542$

C $4.508719-2.040847-1.115582$

C $5.001297-3.452766-1.475587$

C $4.321906-3.976600-2.743168$

C $2.803196-3.811057-2.677887$

H $1.320991-2.490769-1.880042$

C $2.435892-1.346751-3.300866$

H $4.839935-1.345265-1.891740$

O $4.763572-4.357764-0.399775$

H $6.086736-3.417820-1.626234$

H $4.561705-5.038893-2.874388$

O $4.812966-3.270971-3.878889$

H $2.359026-4.029763-3.656138$

O $2.291949-4.783081-1.760679$

C $1.568710-0.171598-2.919684$

H $3.445647-0.965513-3.475722$

H $2.076539-1.735979-4.261021$

H $3.812607-4.334451-0.197114$

H $\quad 5.774077-3.413579-3.919916$

H $1.321483-4.723366-1.780341$

O $0.280831-0.403695-3.305093$

O $1.9815330 .810984-2.318595$

C $-0.6703000 .602854-2.945771$

H $\quad-0.8425270 .575622-1.866263$

H $-0.3289361 .593199-3.261593$

H -1.611269 $0.379245-3.455557$

SCF Energy (B3LYP/6-31G**//MMFF)= -3245.91619378

07_00081

MM̄MF Geometry

C $1.532727 \quad 1.7520872 .500725$

C 1.6121050 .8684703 .507509

C $0.751169-0.3607473 .690961$

O $-0.301532-0.3673152 .695135$

C $1.534882-1.6796563 .545147$

C $2.792692-1.8007114 .378905$

C $4.035137-1.8390783 .856372$

C $2.579826-1.9345195 .862348$

C $4.386425-1.7045902 .400583$

C $4.497606-3.0716981 .715934$

C $4.380883-3.0171110 .184445$

C $5.467679-2.167679-0.472916$

C $5.285642-2.094455-1.997341$

O $3.087465-2.494887-0.137781$

O $6.153555-1.050046-2.463664$

C $3.828808-1.686713-2.309557$

C $5.746001-3.384726-2.685575$

O $3.563666-1.757972-3.709082$

C $2.769859-2.509627-1.535064$

C $1.376719-1.851942-1.713673$

O $2.746097-3.838655-2.052496$

C $-1.459753-1.0136402 .988457$

O $-1.755132-1.5582484 .039318$

C $-2.304120-0.9670841 .773116$

C $-3.476807-1.6168501 .748153$

C $-4.397486-1.6958750 .610831$

C $-3.952071-1.082789-0.690849$

C $0.255191-2.420323-0.832963$

C $-0.447189-3.638626-1.405621$

C $-2.230735-5.288041-0.836044$

O $-0.203770-4.096359-2.518379$

C $-3.522269-4.802738-1.416158$ 
C $-4.726630-5.121831-0.918041$

C $-6.025180-4.667028-1.520725$

C $-6.932844-3.988392-0.518797$

C $-7.974494-4.641898 \quad 0.023386$

C $-6.686476-2.527156-0.214901$

C $-5.578008-2.3268540 .782247$

N $-1.390665-4.147580-0.522523$

H 0.8100571 .6011591 .703573

H 2.3333931 .0583804 .300159

H $0.298866-0.2942244 .688709$

H $1.739807-1.8508772 .481661$

H $0.871876-2.5154223 .808358$

H $4.883285-1.9659224 .526411$

H $3.520009-2.0818026 .404405$

H $2.104744-1.0345816 .264992$

H $1.936670-2.7940146 .077809$

H $3.654729-1.0704371 .892437$

H $5.341248-1.1707002 .322954$

H $5.445270-3.5465761 .998868$

H $3.692641-3.7220962 .084032$

H $4.441279-4.051301-0.174879$

H $6.465402-2.547489-0.220520$

H $5.428440-1.142620-0.080517$

H $6.092455-1.016336-3.433155$

H $3.717107-0.632094-2.027443$

H $6.807892-3.567244-2.481060$

H $5.660041-3.305101-3.774992$

H $5.188450-4.266147-2.362004$

H $4.164791-1.138804-4.155716$

H $1.468866-0.794163-1.437160$

H $1.074429-1.888890-2.767034$

H $1.912371-4.259625-1.792469$

H $-1.926129-0.412450 \quad 0.921651$

H -3.794718 -2.148859 2.644084

H -3.805999 -0.005227 -0.576626

H $-4.661245-1.222709-1.508692$

H -3.008416 -1.527373 -1.023515

H $\quad 0.617620-2.6511100 .174107$

H -0.513388 -1.644065 -0.734580

H -2.378044 -5.8488320 .092257$

H -1.719674 $-5.932975-1.558017$

H -3.462967 -4.176124 -2.303003

H $-4.786430-5.770240-0.046788$

H -6.524220 -5.551614 -1.937679

H -5.858331 -3.994231 -2.371098

H $-8.647473-4.1532590 .720205$

H -8.174160 -5.681788 -0.213231

H $-7.591955-2.0770260 .214022$

H $-6.527100-1.988037-1.152354$

H $-5.803447-2.7409991 .766420$

H $-1.571600-3.653890 \quad 0.346721$

C 3.1548103 .1471131 .118159

O $2.2603073 .169308-0.012271$

C $2.8442592 .380579-1.068809$

C $4.3063262 .190290-0.681977$

C 4.1835322 .0566080 .824326

H $2.3474921 .404648-1.042686$

C $2.6050323 .047867-2.425249$

H $4.7581311 .316210-1.157673$

H $4.9124183 .067313-0.937196$

C 5.4965992 .2206421 .566515

H 3.7696711 .0668541 .046687

C $1.1201063 .350065-2.718611$

O $3.3157344 .291998-2.466172$

H $3.0305572 .431140-3.224983$

H $0.7575574 .111737-2.020286$

O $1.0398913 .931990-4.026114$

C $0.1994912 .113854-2.685618$

C -1.266960 $2.411922-3.058107$

H $0.2238471 .652254-1.693628$

O $\quad 0.696747 \quad 1.136580-3.603848$

H -1.308686 2.941974 -4.017073

H -1.769425 $1.449326-3.223702$

H 6.2106151 .4499511 .257903

H 5.9503903 .1976081 .369956

H 5.3464212 .1283522 .646829

C 2.3624253 .0036012 .418704

H $2.9987394 .835993-1.724603$
H $1.6291144 .705734-4.035505$

H $0.701598 \quad 1.541323-4.488328$

H 1.6666613 .8488682 .495793

H 3.0468783 .0742003 .272176

H 3.6481404 .1283121 .129847

C $-2.7365092 .962156 \quad 0.319657$

O $-2.1712392 .340627-0.841231$

C $-2.013453 \quad 3.205107-1.972148$

C $-3.3947963 .682492-2.440770$

C $-4.1416654 .393807-1.307022$

C -4.0987443 .6149940 .015035$

H -2.9161792 .1309731 .012667$

C $-1.714378 \quad 3.9072840 .960628$

H -1.413738 $4.074675-1.685957$

O $-4.1788672 .592512-2.920141$

H $-3.2643124 .374856-3.280959$

H $-5.1844764 .550751-1.608492$

O $-3.5450875 .676405-1.122794$

H $-4.379806 \quad 4.2709940 .846303$

O $-5.0975582 .591370-0.023237$

C -1.9568834 .0686382 .440637$

H -0.6925513 .5332890 .822109$

H -1.723742 4.9003590 .500817

H -3.930751 $1.794082-2.425409$

H $-4.0547896 .141348-0.437525$

H $-4.8685391 .969086-0.731338$

O -1.5707972 .9401403 .101572$

O -2.4360965 .0750022 .946368$

C -1.754402 2.9601664 .520098

H -1.1338713 .7413214 .969068$

$\mathrm{H}-2.8094943 .1145654 .765044$

H -1.441201 1.9909084 .917001

SCF Energy $\left(B 3 L Y P / 6-31 G^{* *} / / M M F F\right)=-3245.91851717$

0700082

MM̄FF Geometry

C -2.928581-1.946227 -1.996687

C $-2.017727-1.683197-2.945075$

C $-0.796400-0.842520-2.692807$

O $0.345223-1.725190-2.827197$

C $-0.6325950 .302091-3.708766$

C $-1.6336471 .429988-3.579433$

C $-1.4491582 .507634-2.791436$

C $-2.8524901 .334293-4.457951$

C $-0.3152352 .739312-1.834541$

C $0.7845543 .617672-2.437466$

C $1.8875563 .929145-1.413986$

C $2.9955774 .765043-2.052769$

C $4.1529695 .014871-1.076222$

O $2.4277922 .692010-0.946196$

O $5.2656985 .518744-1.831044$

C $4.5990243 .671494-0.455535$

C $3.8012706 .097131-0.046949$

O 5.5605913 .9093890 .569572

C 3.4268392 .8190910 .081794

C 3.8346451 .3656770 .417407

O 2.8826103 .4253421 .255445

C $1.437420-1.452370-2.062703$

O $1.559023-0.548767-1.251776$

C $2.480056-2.450680-2.392494$

C $3.664102-2.401043-1.763210$

C $4.802530-3.297715-1.977436$

C $4.646689-4.383936-3.009755$

C 4.8883631 .1899341 .510336

C $4.991364-0.2508101 .985918$

C $6.420719-1.7241353 .399535$

O $4.186542-1.1249501 .676672$

C $7.337147-2.4546012 .468574$

C $7.078238-3.6770031 .979294$

C $8.017275-4.4310371 .082888$

C $7.368327-4.885119-0.205416$

C $7.021648-6.170767-0.386306$

C $7.215559-3.875827-1.320689$

C $5.921394-3.111508-1.244414$

N $6.082646-0.4369182 .823182$

H $-2.774040-1.553242-0.994413$

H $-2.149293-2.091199-3.944080$

H $-0.828079-0.440435-1.672751$ 
H $-0.658593-0.099614-4.731497$ H $\quad 0.383654 \quad 0.709528-3.624767$ H -2.202755 $3.293445-2.800254$ H -3.498344 $2.214613-4.368343$ H -2.558040 $1.249115-5.509070$ H -3.454643 $0.460731-4.192878$ H $-0.7203763 .225282-0.939360$ H $0.101840 \quad 1.787313-1.493098$ H $1.2205383 .092610-3.297218$ H $0.3551584 .555692-2.810784$ H $\quad 1.4333114 .465325-0.572402$ H $2.5986735 .715093-2.431581$ H $3.3930054 .235694-2.930187$ H $4.9784566 .331035-2.282509$ H $5.1105943 .094886-1.238734$ H $3.5474707 .037230-0.551478$ H 4.6585196 .3262660 .595672 H 2.9559995 .8249530 .588582 H 6.2858854 .4222330 .173725 H $2.924570 \quad 0.8285760 .717751$ H $4.1740720 .860327 \quad-0.496744$ H 2.4027822 .7450981 .756483 H $2.246591-3.193452-3.146564$ H $3.822359-1.623259-1.018627$ H $3.798646-5.031972-2.761024$ H $5.517809-5.036890-3.092387$ H $4.478867-3.949895-4.001613$ H 5.8705821 .4894241 .129529 H 4.6463011 .8063692 .383121 H $5.501231-2.2846153 .598657$ H $6.928280-1.5353174 .350442$ H $8.269673-1.9614682 .204239$ H $6.155898-4.1790592 .262703$ H $8.383010-5.3001991 .645382$ H $8.909334-3.8373460 .847540$ H $6.589065-6.513611-1.320064$ H $7.158672-6.9091060 .396833$ H $7.334272-4.366581-2.292897$ H $8.045194-3.158072-1.282246$ H $5.934667-2.300838-0.515598$ H $\quad 6.731417 \quad 0.3312072 .969254$ C $-5.445648-1.992587-1.937052$ O $-5.455063-1.529263-0.571229$ C $-6.559228-2.1353290 .114862$ C $-7.589816-2.379957-0.975436$ C $-6.699932-2.861144-2.107869$ H $-6.201489-3.1001200 .497439$ C -7.018451 -1.2720131 .294973$ H -8.346251 -3.113804 -0.682363 H -8.099001 -1.454781 -1.268689 C $-7.339442-2.739497-3.480641$ H $-6.454153-3.917124-1.931226$ C $-5.874865-0.7445802 .189124$ O $-7.723249-0.129700 \quad 0.792822$ H -7.745531-1.837475 1.890068 H -5.3865380 .0892641 .675360$ O $-6.466963-0.1809703 .366092$ C $-4.822565-1.7862412 .601032$ C $-3.768814-1.2834543 .605230$ H $-4.303931-2.1542301 .709122$ O $\quad-5.491784-2.906188 \quad 3.186493$ H -4.220591-1.172594 4.599601 H -3.032458 -2.090932 3.712858 H -8.247335 -3.348812 -3.537843 H $-7.614885-1.703518-3.704521$ H $-6.651239-3.081588-4.260204$ C -4.158045 -2.783404 -2.202492 H $-8.009540 \quad 0.395165 \quad 1.559963$ H $-6.860183-0.911423 \quad 3.873991$ H $-4.818047-3.577720 \quad 3.388677$ H -4.180867 -3.196318 -3.217802 H -4.092131 -3.629138-1.506306 H -5.485606 -1.101356 -2.575081 C -1.9593131 .0908821 .330733$ O $-2.593050-0.0819631 .865575$ C $-3.057316 \quad 0.0261323 .214316$ C -1.888806 0.3485194 .161589 C -1.1149571 .5880313 .699055$
C -0.7702881 .5205492 .207446$

H $-1.5532180 .771096 \quad 0.363482$

C $-2.9640912 .228472 \quad 1.075509$

H -3.7946320 .8324453 .277049$

O $-0.990716-0.757594 \quad 4.242611$

H -2.285692 0.5116025 .170212

H -0.1878801 .6760614 .278682$

$\begin{array}{lllll}\text { O } & -1.891344 & 2.757736 & 3.939742\end{array}$

H -0.3873502 .4859001 .855950$

$\begin{array}{llll}\text { O } & 0.272435 & 0.561014 & 2.010881\end{array}$

C $-3.9104501 .916678-0.063865$

H -3.5723452 .4362661 .959039$

H -2.4280203 .1435570 .800181$

H $-0.691916-0.9644613 .340461$

H -2.074158 2.7978814 .893857

H 1.0417220 .8527742 .528994

O $-4.910805 \quad 2.846231-0.082804$

O $-3.7844500 .991919-0.852989$

C $-5.8780592 .679376-1.124396$

H $-6.6503183 .442563-0.996927$

H $-5.4051212 .816634-2.101516$

H -6.346038 $1.692435-1.057804$

SCF Energy (B3LYP/6-31G**//MMFF)= -3245.91293391

07_00083

MM̄FF Geometry

C $1.2675075 .636600 \quad 0.342436$

C $1.8296754 .766547-0.509657$

C $2.9764673 .859160-0.142676$

O $2.5473752 .488264-0.311789$

C $4.1587104 .112385-1.096176$

C $5.4033983 .315306-0.758126$

C $5.9572412 .383762-1.559069$

C $6.0524753 .643540 \quad 0.562298$

C $5.4957121 .931538-2.916196$

C $4.5506360 .719763-2.902527$

C $5.174134-0.559295-2.319881$

C $4.259604-1.764053-2.554255$

C $4.810299-3.041743-1.899924$

O $5.360183-0.372174-0.914845$

O $3.756113-4.016944-1.881399$

C $5.172832-2.742043-0.428477$

C $5.951647-3.654388-2.720477$

$\begin{array}{llll}\text { O } & 5.842776 & -3.849951 & 0.166788\end{array}$

C $6.015073-1.462226-0.252294$

C $6.204166-1.0500571 .228110$

O $7.323245-1.681290-0.794418$

C 1.8139061 .9364780 .693408

O 1.4876432 .4657361 .743908

C 1.4493640 .5630540 .280701

C $0.484438-0.0895840 .945582$

C $-0.009089-1.436327 \quad 0.651810$

C $0.756827-2.253224-0.354312$

C $4.912028-0.7791982 .006886$

C $4.418039-1.9696442 .812246$

C $2.487286-2.7041034 .204131$

O $5.078816-2.9903492 .983341$

C $1.596297-3.5686683 .369456$

C $0.306192-3.791563 \quad 3.661976$

C $-0.592215-4.6883172 .859978$

C -1.861234 -3.994916 2.419499

C $-3.008862-4.1520593 .100912$

C $-1.819466-3.190667 \quad 1.140764$

C $-1.109381-1.8746921 .298606$

N $3.160540-1.7398683 .354564$

H 1.6449325 .7120621 .359118

H $1.4391904 .684624-1.521537$

H 3.2892794 .0217490 .895921

H $4.4263295 .177674-1.080601$

H $3.8332443 .897598-2.119816$

H $6.8590701 .882216-1.208926$

H 7.0258603 .1549620 .679407

H 6.2178344 .7225390 .647289

H 5.4215213 .3181051 .394921

H $5.0125592 .753684-3.454807$

H $6.3848191 .687015-3.511530$

H $3.6476870 .981095-2.338185$

H $4.2403870 .528384-3.937403$ 
H $6.146242-0.719127-2.801079$ H $4.093846-1.924317-3.626956$ H $3.269178-1.555433-2.127879$ H $3.463999-4.159973-2.797897$ H $4.226859-2.6215620 .112551$ H $5.603718-3.910020-3.728618$ H $6.297280-4.594607-2.276783$ H $6.808980-2.986798-2.827948$ H $5.269620-4.6280470 .057197$ H $6.843502-1.7771391 .743020$ H $6.775390-0.1101231 .230742$ H $7.933067-1.036716-0.398632$ H $1.9677730 .136779-0.570150$ H -0.0122010 .4180031 .771716$ H $0.664810-1.816444-1.354531$ H $0.423954-3.290632-0.421916$ H $1.817267-2.294729-0.083742$ H 5.1078390 .0188012 .734760 H $4.116257-0.4278181 .343143$ H $1.929441-2.1392444 .957572$ H $3.232012-3.3249154 .712620$ H $2.037593-4.0528892 .501444$ H $-0.124411-3.3274774 .546698$ H $-0.839271-5.5568223 .484340$ H $-0.074711-5.0939441 .981625$ H -3.930040 -3.681084 2.773719 H $-3.051335-4.7518614 .003930$ H -2.840293 -2.961458 0.815290 H $-1.403823-3.8120550 .343414$ H -1.594190 -1.203029 2.008613 H $2.662355-0.8872833 .115895$ C -1.179933 6.0649210 .647540 O $-1.596144 \quad 4.8150570 .058350$ C -2.988365 $4.900104-0.288107$ C $-3.529638 \quad 6.0932830 .486970$ C $-2.343404 \quad 7.0354350 .439609$ H -3.033778 $5.116190-1.363370$ C $-3.7172453 .578640-0.008684$ H -4.4312516 .5128010 .030733$ H $-3.763655 \quad 5.8273691 .524572$ C $-2.408408 \quad 8.1488811 .470662$ H -2.288089 $7.480132-0.563221$ C $-3.1791042 .399073-0.847481$ O $-3.638242 \quad 3.273751 \quad 1.382611$ H $-4.7791883 .716468-0.244003$ H -3.169517 2.707417 -1.899794 O $-1.8286412 .109067-0.493050$ C $-4.0296051 .116435-0.732316$ C -3.491231 -0.005508 -1.640866 H -5.063611 $1.347866-1.013253$ $\begin{array}{llll}\text { O } & -4.035994 & 0.640325 & 0.611415\end{array}$ H -3.246105 $0.407861-2.626550$ H -2.570530 -0.403333 -1.197003 H -3.291539 $8.774641 \quad 1.305406$ H -2.465139 7.7498482 .489110 H $-1.523303 \quad 8.7897351 .408802$ C $0.1151396 .530682-0.019603$ H -2.697011 3.2591441 .626795 H -1.8355521 .7354430 .404680$ H $-4.449034 \quad 1.323737 \quad 1.165433$ H 0.3630067 .5502750 .296259 H -0.014701 6.550289-1.109018 H -1.019747 5.8750121 .716755 C -5.709074 -2.825711 - 0.544655 O $-4.740877-1.769086-0.558831$ C $-4.501167-1.158379-1.827988$ C -3.990884 -2.203962 -2.831735 C $-4.957422-3.388066-2.932302$ C $-5.341969-3.929235-1.550621$ H $-5.636472-3.2546890 .463467$ C $-7.133958-2.289951-0.720485$ H $-5.434719-0.722781-2.200915$ O - $-2.704352-2.666260-2.424981$ H $-3.876921-1.726670-3.812640$ H -4.488082 -4.184599-3.522222 O $-6.124463-2.963115-3.630301$ H $-6.174346-4.637997-1.630997$ O $-4.232774-4.652156-1.009484$
C $-7.521192-1.428650 \quad 0.458165$

H $-7.269362-1.708958-1.637279$

H -7.856451 -3.113310 -0.769431

H $-2.397394-3.304763-3.090761$

H -6.716913 -3.730609-3.705875

H $-4.029029-5.379497-1.621718$

O $-7.298836-0.115043 \quad 0.169060$

O $-7.942503-1.874368 \quad 1.517645$

C -7.5814590 .8064391 .226503$

H -7.3324901 .8117700 .876319$

H $-8.6450300 .774978 \quad 1.480791$

H -6.9665850 .5790422 .102696$

SCF Energy (B3LYP/6-31G**//MMFF) $=-3245.89702673$

07_00084

MM̄FF Geometry

C $2.982683-0.8441813 .705254$

C $1.678146-0.7737103 .403657$

C $0.7240740 .177827 \quad 4.078916$

$\begin{array}{lllll}\text { O } & 0.218851 & 1.108773 & 3.097327\end{array}$

C $-0.474587-0.5968164 .652943$

C $-1.527713 \quad 0.302279 \quad 5.274938$

C $-2.715745 \quad 0.567584 \quad 4.694262$

C $-1.188453 \quad 0.871066 \quad 6.625717$

C $-3.150095 \quad 0.052879 \quad 3.348810$

$\begin{array}{lll}\text { C }-4.420812 & 0.735436 & 2.822937\end{array}$

C -4.7639650 .3787751 .363369$

C $-4.841134-1.1317881 .119293$

C $-5.082687-1.482592-0.354807$

O $\begin{array}{llll} & -3.773370 & 0.978825 & 0.518292\end{array}$

O $-4.806279-2.885491-0.494731$

C $-4.064277-0.705499-1.216546$

C $-6.550805-1.288686-0.748863$

O $-4.330772-0.887171-2.605285$

C $-4.0132190 .803412-0.884890$

C $-2.8979971 .532704-1.668610$

O $-5.255270 \quad 1.402351-1.277416$

C 0.8783122 .2903592 .966160

O 1.9363852 .6056363 .486451

C 0.0628793 .1591902 .088658

C 0.5733414 .3213421 .657646

C $-0.089590 \quad 5.287276 \quad 0.781535$

C -1.529192 5.0456850 .416431

C $-1.465631 \quad 1.212962-1.231605$

C $-0.4498911 .881944-2.144438$

C $1.9423042 .553821-2.263340$

O $-0.7238552 .253255-3.283739$

C $2.0098474 .024074-1.996487$

C $1.5080784 .953693-2.824135$

C $1.5837916 .436664-2.587117$

C $0.2760727 .007855-2.081641$

C $-0.7623917 .196294-2.913597$

C $0.1827347 .410878-0.625048$

$\begin{array}{llll}\text { C } & 0.627270 & 6.333041 & 0.321666\end{array}$

N $0.8077331 .977578-1.564734$

H $3.391794-0.1694314 .453875$

H $1.269630-1.4407712 .648287$

H 1.2198040 .7212574 .893435

H $-0.130016-1.3148575 .409072$

H $-0.925131-1.2041703 .859491$

H $-3.425842 \quad 1.197917 \quad 5.225681$

H -2.011655 1.4582137 .046502

H -0.9640950 .0650257 .331797$

H -0.3161001 .5284556 .558252$

H $-2.3431650 .192106 \quad 2.621073$

H $-3.341907-1.0205803 .451637$

H $-5.2663190 .464587 \quad 3.467782$

H $-4.294264 \quad 1.8237932 .892569$

H -5.7317970 .8443191 .141282$

H -5.604621 -1.590785 1.759463

H $-3.891285-1.5986491 .403203$

H $-4.993442-3.139426-1.414117$

H -3.077063 -1.144286 -1.026061

H $-7.192081-1.962744-0.168206$

H $-6.716828-1.548420-1.800098$

H $-6.909455-0.270463-0.585039$

H $-4.214972-1.830775-2.807255$

H $-3.0284721 .348479-2.742642$ 
H -3.036000 $2.617020-1.553568$ H $-5.1229842 .360607-1.366104$ H -0.9311462 .8194951 .821928$ H 1.5870424 .5852981 .956863 H -2.1498044 .9808831 .316976$ H -1.630382 4.113911-0.147446 H -1.955795 $5.838396-0.203286$ H -1.307197 $1.552658-0.203820$ H -1.273703 $0.137702-1.262789$ H $1.8583162 .340166-3.334413$ H $2.8477402 .069751-1.887616$ H $2.5158294 .333710-1.085979$ H $1.0063624 .633669-3.734965$ H $1.8567096 .923275-3.532807$ H $2.4000886 .682740-1.896816$ H -1.697714 $7.621148-2.564771$ H $-0.6987326 .928678-3.963250$ H $-0.8268247 .749885-0.363519$ H $0.8268918 .286254-0.468903$ H 1.6700396 .4259270 .627301 H $0.9358651 .694678-0.597679$ C $4.859988-1.1830842 .070008$ O $4.209864-1.2098380 .778452$

C $5.148862-1.699373-0.199735$

C $6.519823-1.5808670 .450108$

C $6.184374-1.9268541 .886209$

H $4.928468-2.762637-0.349901$

C $5.005406-0.939852-1.521406$

H $7.253432-2.2587100 .003318$

H $\quad 6.912547-0.559424 \quad 0.384570$

C $7.262517-1.5188152 .875541$

H $6.020762-3.0105981 .959922$

C $3.611942-1.054720-2.174735$

O $5.2682650 .450280-1.291654$

H $5.773173-1.277458-2.227246$

H $2.869770-0.538539-1.555802$

O $3.656080-0.357743-3.426498$

C $3.156597-2.499755-2.448416$

C $1.853235-2.612237-3.261887$

H $3.037642-3.032247-1.499494$

O $4.187191-3.173463-3.177041$

H $1.994593-2.157823-4.250731$

H $1.679291-3.674850-3.471663$

H $8.200682-2.0399192 .658004$

H $7.458758-0.4421432 .833952$

H $\quad 6.967439-1.7675073 .899754$

C $3.944010-1.8333703 .108293$

H $4.643690 \quad 0.758037-0.612436$

H $4.0069680 .532346-3.253330$

H $3.913132-4.100073-3.285881$

H $4.532682-2.2437213 .937382$

H $3.395276-2.6700872 .658132$

H $5.046623-0.1279842 .309415$

C $0.136925-3.801219-1.055107$

O $0.493713-2.424647-1.208436$

C $0.620165-1.999146-2.569899$

C $-0.700599-2.227017-3.321634$

C $-1.211588-3.660799-3.181000$

C $-1.217196-4.092701-1.712403$

H $0.906771-4.420738-1.531953$

C $0.199166-4.1629410 .434336$

H $\quad 0.786260-0.916565-2.538432$

O $-0.558420-1.911893-4.703031$

H -1.465434 -1.552366 -2.920274

H $-0.622650-4.349551-3.798144$

O $-2.548006-3.705845-3.687810$

H -2.024819 -3.566593 -1.190491

O $-1.484521-5.489373-1.623536$

C $-0.912290-3.554626 \quad 1.260701$

H $\quad 0.161377-5.2542640 .536000$

H $\quad 1.144339-3.8229130 .873793$

H $-0.265260-0.986439-4.762562$

H -2.826933 -4.636812 -3.697372

H $-2.388338-5.638005-1.948517$

O $-1.434684-4.5081142 .085712$

O $-1.262080-2.3837851 .197652$

C -2.511404-4.087779 2.928009

H $-2.809889-4.9382513 .546628$
H $-2.187061-3.2764323 .584642$

H $-3.367153-3.7811602 .320569$

SCF Energy $\left(B 3 L Y P / 6-31 G^{* *} / / M M F F\right)=-3245.90610865$

07 00085

MM̄FF Geometry

C $1.296091-2.6822902 .122368$

C $0.305355-2.4977293 .005835$

C $-0.384209-1.1697873 .177326$

O $-1.807392-1.3668992 .994136$

C $-0.160500-0.5703654 .575929$

C $1.276314-0.1673014 .848684$

C 1.8705180 .9276124 .332411

C $2.040412-1.0819975 .769130$

C 1.2454421 .9515803 .425667

C 1.7881191 .8459311 .992187

C 0.9997412 .6742940 .962686

C 1.1678264 .1762871 .178503

C 0.3243834 .9849600 .185236

O -0.3761462 .3057851 .061430$

$\begin{array}{lllll}\text { O } & 0.307627 & 6.349599 & 0.632296\end{array}$

C - $-1.132101 \quad 4.4765290 .229159$

C $0.9410164 .996039-1.218453$

O $-1.8924765 .117412-0.792745$

C -1.2642312 .9384610 .123950$

C -2.6701362 .4352930 .533579$

O $-0.9811842 .514570-1.209022$

C $-2.247642-1.4248251 .704861$

O $-1.571940-1.3078560 .693774$

C $-3.706229-1.6746891 .727065$

C $-4.343502-1.8887920 .565944$

C $-5.771071-2.1653250 .386800$

C $-6.642237-2.1550441 .614110$

C $-3.8489122 .889143-0.327129$

C $-5.1647262 .309406 \quad 0.169140$

C $-7.6229952 .509517-0.189225$

O $-5.2387141 .488742 \quad 1.079645$

C $-8.1563411 .528933-1.183269$

C $-8.4997760 .270758-0.869151$

C $-9.113078-0.697544-1.837527$

C $-8.147419-1.752493-2.323836$

C $-7.830420-1.846717-3.626720$

C $-7.614494-2.752323-1.320034$

C $-6.219915-2.414489-0.861566$

N $-6.2492452 .833145-0.520831$

H $1.633815-1.8357481 .528918$

H $-0.043859-3.3304513 .610435$

H $-0.044104-0.4484692 .426664$

H $-0.506267-1.2774825 .342605$

H -0.8192090 .2979974 .708794$

H $2.911130 \quad 1.1227694 .586859$

H $3.070859-0.7466275 .927771$

H $1.552785-1.1249386 .748732$

H $2.083808-2.0951545 .358416$

H $0.155548 \quad 1.860633 \quad 3.431958$

H 1.4676792 .9441873 .834318

H 2.8452842 .1366311 .971496

H 1.7469630 .7960181 .675523

H $1.3675012 .394164-0.030381$

H 2.2246714 .4628421 .110724

H 0.8466574 .4491552 .192297

H 1.2272866 .6639810 .668952

H -1.5654504 .7926631 .188360$

H $1.9566805 .407708-1.186071$

H $0.3775245 .649499-1.893586$

H $0.9963414 .002860-1.668522$

H -1.802137 $6.077138-0.663453$

H -2.651666 1.3368620 .528212

H -2.8553512 .7075801 .581976$

H -1.406453 $1.652541-1.351330$

H $-4.207901-1.6859412 .687475$

H -3.760338 -1.868874 -0.354366

H $-6.325267-2.9330592 .317205$

H -7.698177 -2.332439 1.401200

H -6.585588 -1.183019 2.117058

H $-3.9488603 .977801-0.292008$

H -3.706998 $2.579232-1.367936$

H $-8.2013563 .437554-0.238167$ 
H -7.6781752 .1280820 .835815$ H -8.298944 $1.883987-2.201023$ H -8.368012 -0.0749200 .152962$ H -9.542258 -0.149791-2.686995 H $-9.964776-1.188519-1.350062$ H -7.160898 $-2.620258-3.988705$ H $-8.228919-1.152617-4.358988$ H -7.574854 -3.745409-1.788149 H $-8.324485-2.861310-0.495946$ H $-5.493123-2.406247-1.675861$ H $-6.0853013 .478480-1.288375$ C $1.825479-4.4841360 .429618$ O $2.266830-3.467872-0.496424$ C $1.256330-3.307639-1.508179$ C $0.445674-4.595590-1.474840$ C $0.399429-4.8613400 .017985$ H $0.609259-2.482147-1.188190$ C $1.892709-2.978141-2.859409$ H -0.545969-4.477379-1.921502 H $0.962591-5.412409-1.992234$ C $0.013625-6.2862180 .374389$ H $-0.330282-4.1771370 .468316$ C $2.741339-1.687721-2.870617$ O $2.741847-4.062339-3.256324$ H $1.112572-2.912816-3.626724$ H $3.662068-1.841084-2.296202$

O $3.146696-1.450984-4.225077$ C $2.009753-0.434380-2.354437$ C $2.7302190 .897633-2.634207$ H $1.836837-0.533968-1.276653$ O $\quad 0.723322-0.362737-2.978489$ H $2.7691291 .080956-3.715690$ H $2.0915731 .693578-2.230340$ H $-0.990772-6.5163600 .004514$ H $\quad 0.707064-7.012059-0.063515$ H $0.015858-6.4292661 .459599$ C $1.986133-3.9918871 .871969$ H $3.405783-4.185403-2.556050$ H $3.606170-2.250691-4.533790$ H $0.2479850 .388964-2.585343$ H $3.052148-3.8381472 .077077$ H $1.636388-4.7626422 .568267$ H $2.496013-5.3404490 .281840$ C 5.2772220 .8347090 .101854 O $4.039994 \quad 0.798775-0.623441$ C $4.1396401 .010949-2.033996$ C $4.7720132 .381811-2.330868$ C $6.1088822 .558255-1.603980$ C $6.0079592 .169821-0.125896$ H $4.983946 \quad 0.789207 \quad 1.158360$ C $6.156807-0.390743-0.194089$ H $4.7645230 .228219-2.474348$ O $3.8902603 .433522-1.942608$ H $4.9208852 .474883-3.412816$ H $6.4276713 .605509-1.676502$ O $7.105799 \begin{array}{lll}1.755145 & -2.227864\end{array}$ H 7.0085702 .1288530 .320414 O 5.2878413 .2035910 .552293 C $5.529164-1.6794150 .290535$ H $6.345604-0.504964-1.264482$ H $7.116959-0.2849710 .323943$ H $3.6918563 .321970-0.997017$ H $7.1764702 .041782-3.154262$ H 5.2847102 .9865521 .500054 O $6.314989-2.725906-0.101337$ O $4.492616-1.7609980 .933512$ C $5.858095-4.0260710 .284123$ H $5.645304-4.0623131 .356923$ H $4.971569-4.293320-0.296839$ H $\quad 6.652133-4.7440280 .062475$

SCF Energy (B3LYP/6-31G**//MMFF) $=-3245.91760924$

\section{7_00086}

MM̄FF Geometry

C 2.7313072 .1167082 .531698

C 3.1579521 .4046643 .584906

C 2.5078160 .1544544 .129990

$\begin{array}{lllllll}\text { O } & 1.373707 & -0.241647 & 3.326558\end{array}$
C $3.521108-1.0086334 .076509$

C $2.946549-2.3364604 .532631$

C $2.788145-3.4163453 .740666$

C $2.546260-2.4054505 .984169$

C $3.167900-3.5749092 .293023$

C $1.969440-3.3689881 .364976$

C $2.247142-3.750613-0.097976$

C $3.286333-2.839041-0.754666$

C $3.493542-3.181432-2.238648$

O $1.000629-3.632494-0.796571$

O $4.194169-2.083902-2.849126$

C $2.119153-3.271447-2.937747$

C $4.403122-4.404174-2.407689$

O $2.247772-3.750928-4.273799$

C $1.043201-4.069531-2.162212$

C $-0.351061-3.787375-2.771201$

O $1.232817-5.481084-2.258754$

C $0.123125-0.0175183 .809502$

$\begin{array}{lllll}\text { O } & -0.186426 & 0.623098 & 4.799818\end{array}$

C $-0.809860-0.7619762 .933602$

C $-2.105151-0.8860143 .259471$

C -3.092858 -1.691592 2.532526

C $-2.583646-2.5255211 .385351$

C -1.487666 -4.517901 -2.046943

C $-2.850436-4.076749-2.546172$

C $-4.450382-2.161702-2.433157$

O $-3.551865-4.805710-3.243008$

C $-4.871001-1.347914-1.251189$

C $-6.004478-1.545945-0.561526$

C $-6.381864-0.7399240 .649763$

C $-6.654987-1.5967761 .867714$

C -7.863382 -1.585999 2.455705

C $-5.540482-2.4707102 .405055$

C $-4.378528-1.6791592 .944761$

N -3.191154 -2.807293 -2.110528

H 1.8120421 .8334202 .027402

H $4.076320 \quad 1.712948 \quad 4.081067$

H 2.2070590 .3402805 .167827

H $4.393148-0.773158 \quad 4.700947$

H $3.900854-1.0890643 .052192$

H $2.345573-4.3102824 .179111$

H $2.250292-3.4159106 .285925$

H $3.383863-2.1066516 .622877$

H $1.699396-1.7421636 .184528$

H $3.986540-2.9028262 .022835$

H $3.563936-4.5898762 .162390$

H $1.114697-3.9596791 .721934$

H $1.639683-2.3237461 .408812$

H $2.570433-4.797585-0.125702$

H $4.246217-2.880330-0.227700$

H $2.949390-1.796530-0.682249$

H $5.025335-1.949080-2.362362$

H $1.758506-2.237825-3.032213$

H $5.403150-4.193478-2.008440$

H $4.552481-4.646868-3.465165$

H $4.033993-5.292239-1.891917$

H $2.374187-4.713711-4.238157$

H $-0.543776-2.708861-2.750100$

H $-0.370594-4.093706-3.825110$

H $2.173252-5.672622-2.377014$

H $-0.387221-1.2402152 .057653$

H -2.471188 -0.3816894 .152430$

H -1.812161 -3.2239421 .729207$

H -2.152927 -1.884191 0.609474

H $-3.353158-3.1293480 .903010$

H - $1.405261-5.600944-2.194123$

H -1.449731-4.340371-0.965879

H -5.195384 -2.913988 -2.711378

H $-4.276490-1.510903-3.295673$

H $-4.192936-0.559182-0.935438$

H $-6.693677-2.325518-0.876950$

H -7.268238 -0.1469850 .390395$

H -5.598920 -0.0159480 .901152$

H $-8.074299-2.1957303 .327915$

H $-8.670817-0.9667002 .079218$

H $-5.248083-3.1860291 .632800$

H -5.914089 -3.090080 3.231662

H $-4.641111-1.0507763 .796963$ 
H $-2.478738-2.245280-1.653737$

C $3.938388 \quad 3.0330750 .500841$

$\begin{array}{lllll}\text { O } & 2.804490 & 2.699622 & -0.328923\end{array}$

C $3.0311091 .395775-0.889176$

C $4.5435491 .285246-0.989670$

C $4.957907 \quad 1.8958170 .337833$

H $2.6700820 .663553-0.156278$

C $2.2571991 .214185-2.195639$

H $4.8840610 .252918-1.109305$

H $4.939026 \quad 1.881523-1.820023$

C 6.4020462 .3670540 .369056

H 4.8285691 .1378791 .119712

C $0.7575481 .568845-2.108135$

O $2.8334632 .038054-3.215900$

H $2.3763370 .183464-2.546497$

H $0.6400422 .658181-2.098275$

O $\quad 0.1312241 .107095-3.311342$

C $0.0107310 .960174-0.908136$

C $-1.5175321 .142688-0.931475$

H $0.396038 \quad 1.394842 \quad 0.019513$

$\begin{array}{lllll} & 0 & 0.274440 & -0.442927 & -0.837782\end{array}$

H $-1.9541890 .537408-1.736268$

H -1.9004070 .7077680 .001185$

H $7.084604 \quad 1.5263970 .207147$

H $6.598136 \quad 3.113523-0.407987$

H 6.6410952 .8167451 .338076

C 3.4713363 .2867381 .942966

H $2.8026702 .958112-2.902680$

H $0.6070351 .509183-4.058386$

H - $0.002953-0.833065-1.683616$

H 2.7834304 .1403121 .951388

H 4.3322513 .5613972 .563485

H 4.3432503 .9700310 .099107

C -1.694832 $4.770766-0.018439$

O $-1.3944993 .367348-0.014474$

C $-1.9924112 .599830-1.063571$

C $-3.5243172 .708924-1.005054$

C $-3.9742284 .172370-1.033047$

C $-3.2151365 .016235-0.004415$

H -1.2902705 .1457170 .930143$

C $-0.9782195 .507027-1.162649$

H $-1.6491132 .979951-2.030600$

$\begin{array}{lllll} & \mathrm{H} & -3.999883 & 2.088865 & 0.187297\end{array}$

H $-3.9515882 .165215-1.856271$

H -5.049748 4.221491-0.823202

O $-3.7633324 .689224-2.343396$

H -3.421456 $6.080830-0.164797$

O $-3.7202264 .686931 \quad 1.292913$

C $0.5230465 .545445-0.968924$

H -1.173991 $5.035663-2.129044$

H -1.318635 $6.547978-1.204511$

H -4.9685872 .1720810 .194745$

H -4.077991 $5.609284-2.349845$

H -3.2780495 .2657641 .937113$

O $1.1086975 .980289-2.124098$

$\begin{array}{lllll}\text { O } & 1.108567 & 5.258086 & 0.065401\end{array}$

C $2.5341796 .099781-2.085562$

H $2.8686456 .490364-3.050271$

H $2.8368616 .798988-1.300217$

H $2.9913625 .119521-1.926946$

SCF Energy (B3LYP/6-31G**//MMFF) $=-3245.90138602$

07_00087

MM̄FF Geometry

C $-0.322312-0.2000421 .846147$

C $-0.496509-1.3874841 .242746$

C $-0.036036-1.741163-0.151810$

O $0.069242-0.514475-0.910714$

C $1.334273-2.437914-0.178190$

C $1.472014-3.6354020 .732118$

C $2.144626-3.6250291 .900275$

C $0.820513-4.8976120 .234122$

C $2.810221-2.4490672 .562484$

C $4.335587-2.5780182 .649888$

C $5.049704-2.6056731 .288573$

C $6.565566-2.6853891 .491801$

C $7.330278-2.6234680 .162287$

O $4.712825-1.4147190 .568319$
O $8.713440-2.376624 \quad 0.459462$

C $6.820690-1.413431-0.649016$

C $7.281478-3.960156-0.587514$

O $7.396349-1.402301-1.954049$

C $5.277743-1.342365-0.747622$

C $4.801905-0.031374-1.426440$

O $4.821597-2.426179-1.567674$

C $-0.066301-0.542711-2.256612$

O $-0.269134-1.527219-2.951864$

C $0.0815100 .820616-2.824885$

C $0.137776 \quad 1.923230-2.060212$

C $0.2662303 .306766-2.517178$

C $0.4589573 .545223-3.990309$

C $4.9774121 .246922-0.599385$

C 3.7961541 .5575820 .306512

C 3.0633933 .2044582 .030431

$\begin{array}{lllll}\text { O } & 2.733277 & 0.945668 & 0.263226\end{array}$

C 2.4479704 .4549601 .469713

C 2.8291965 .1373980 .377161

C $2.1619596 .400512-0.093640$

C $1.7020026 .318499-1.533222$

C $2.4707206 .789129-2.529905$

C $0.3191535 .773428-1.810839$

C $0.2236204 .289903-1.593426$

N 4.0608772 .6300271 .146945

H 0.2331110 .5822951 .338657

H -1.037766 -2.169470 1.770600

H $-0.805415-2.375383-0.609707$

H $2.114165-1.6970730 .022846$

H $1.553672-2.754323-1.207107$

H $2.200647-4.5515362 .470189$

H $0.972283-5.7408810 .916270$

H $-0.258828-4.752640 \quad 0.123869$

H $1.235204-5.180769-0.738842$

H $2.547742-1.4995102 .088405$

H $2.412390-2.3810013 .583182$

H $4.699903-1.7180653 .227070$

H $4.596061-3.4789123 .219633$

H $4.699052-3.4783270 .726173$

H $6.836257-3.5915172 .048330$

H $6.893453-1.8421872 .116269$

H $9.028648-3.0934581 .036302$

H $7.188175-0.511711-0.143995$

H $7.733336-4.7550700 .018033$

H $7.869695-3.917390-1.510829$

H $6.266910-4.276037-0.838311$

H $8.362016-1.439669-1.845205$

H $5.3343730 .084458-2.379333$

H $3.746096-0.133744-1.709974$

H $3.927200-2.219253-1.884635$

H $0.1178600 .868091-3.908036$

H $0.0694551 .816668-0.978996$

H $1.3234132 .983736-4.362095$

H $0.6442594 .592831-4.238777$

H $-0.4299353 .232305-4.548944$

H $5.894198 \quad 1.221587-0.003899$

H $5.0596682 .093881-1.292652$

H 3.5573403 .4319612 .980152

H 2.2771942 .4661812 .218653

H 1.6008594 .8336922 .039128

H $3.6833064 .800745-0.204471$

H $2.884705 \quad 7.219617 \quad 0.018287$

H 1.3126356 .6696050 .546740

H $2.1382396 .761822-3.562137$

H $3.4526757 .207630-2.335360$

H $-0.0124376 .043617-2.819603$

H -0.400749 $6.268949-1.146486$

H $0.0836404 .013988-0.547528$

H 4.9576713 .1022901 .077800

C -1.8441381 .3400073 .159864$

O -2.7961251 .1769272 .086564$

C -2.815499 2.3905091 .312982

C -2.3034643 .4689782 .254599$

C -1.1900642 .7109102 .956331$

H -2.0817702 .2700390 .507103$

C -4.2015382 .6291320 .711370$

H -1.949371 4.3568931 .722770

H -3.0711533 .7759722 .974364$ 
C $-0.720774 \quad 3.366536 \quad 4.243215$

H -0.3366692 .6349872 .271552$

C $-4.6987011 .466864-0.178619$

O $\quad-5.1400362 .8063331 .777544$

H -4.1973243 .5678440 .144701$

H -4.9374010 .6051960 .452808$

O $\quad-5.9357961 .870279-0.776991$

C $-3.7138181 .055193-1.285996$

C $-4.2433240 .018744-2.289565$

H $-2.7984470 .661517-0.831968$

O $-3.3500392 .227424-2.022504$

H $-5.0217970 .466690-2.920825$

H -3.412050 -0.199062 -2.973208

H -0.3164644 .3637654 .041047$

H -1.5407313 .4757544 .960840$

H 0.0666522 .7720184 .716965

C $-0.883006 \quad 0.144693 \quad 3.198144$

H -6.0150702 .9389591 .374210$

H $-5.7416572 .605495-1.383550$

H $-2.7149811 .961137-2.708631$

H $-1.426725-0.7211643 .595448$

H $-0.059840 \quad 0.3441563 .893249$

H -2.427495 1.3427244 .089601

C $-4.165395-2.981995-0.043073$

O $-3.803612-1.778231-0.736831$

C $-4.766583-1.288971-1.674876$

C $-5.052967-2.343901-2.759211$

C $-5.437223-3.696108-2.150946$

C $-4.470169-4.114994-1.039578$

H -3.264553 -3.264105 0.515771

C $-5.302413-2.7552730 .967793$

H -5.695526 -1.057083 -1.144570

O $-3.910742-2.523004-3.596980$

H -5.863219 -1.979076 -3.400951

H $-5.432370-4.463266-2.935213$

O $-6.756026-3.626725-1.618024$

H -4.868066 -4.985594 -0.504951

O $-3.243405-4.523192-1.651984$

C $-4.876574-1.8833002 .128478$

H $-6.168193-2.2843830 .495209$

H -5.624572 -3.711785 1.395010

H -3.161325 -2.771937 -3.029043

H -7.355755 -3.394386-2.347209

H -2.655475 $-4.844898-0.947424$

O $-5.977310-1.2797632 .666052$

O $-3.729878-1.7742222 .538284$

C $-5.730466-0.4426803 .799999$

H $-5.264772-1.0187924 .605157$

H -5.0989040 .4029723 .514856$

H $-6.690778-0.0586464 .154124$

SCF Energy (B3LYP/6-31G**//MMFF) $=-3245.91487439$

0700088

MM̄̄FF Geometry

C $3.387221-2.7863692 .369446$

C $2.154351-2.3356332 .096291$

C $1.265314-1.6650283 .112243$

O $0.852761-0.3773112 .597641$

C $0.012038-2.5332173 .338414$

C $-0.965197-2.0321024 .387677$

C $-2.303405-2.1569164 .272991$

C $-0.382440-1.4151255 .632133$

C $-3.062201-2.831173 \quad 3.163206$

C $-3.732618-1.8034392 .254175$

C $-4.433997-2.4273061 .037624$

C $-3.426867-2.9527850 .009273$

C $-4.102742-3.416653-1.288866$

O $-5.232472-1.386217 \quad 0.462623$

O $-3.082136-3.580840-2.285901$

C $-5.039086-2.296602-1.785053$

C $-4.771199-4.786252-1.126920$

O $-5.790737-2.723248-2.918399$

C $-5.989371-1.764516-0.691642$

C $-6.802554-0.531049-1.161454$

O $-6.939189-2.786107-0.362180$

C 1.7483510 .6420382 .724409

O 2.8759860 .5710083 .189109

C 1.1197371 .8802012 .212646
C 1.8329483 .0160322 .218547

C 1.3575174 .3174061 .764505

C $2.3607975 .067570 \quad 0.929151$

C $-6.0037190 .707762-1.576294$

C $-5.2974031 .356898-0.402671$

C $-3.039944 \quad 1.718810 \quad 0.584997$

$\begin{array}{llll}\text { O } & -5.921503 & 1.917213 & 0.496201\end{array}$

C $-2.6942413 .142674 \quad 0.288133$

C -3.0342084 .1669661 .083910$

C -2.7061845 .6008320 .786880$

C -1.8935676 .2473551 .885332$

C -2.4914556 .9418402 .868331$

C -0.3846726 .1686101 .792448$

C 0.1683924 .8168282 .158904

N $-3.9200551 .217656-0.453474$

H $3.789798-2.6689083 .372510$

H $1.755458-2.4503841 .093283$

H $1.797339-1.5284984 .062062$

H $\quad 0.325389-3.5421123 .638754$

H $-0.500688-2.6261222 .373297$

H $-2.931043-1.7671095 .073289$

H -1.142987 -1.226491 6.397708

H $\quad 0.362380-2.0849546 .074395$

H $0.094126-0.4569665 .403422$

H -2.415143 -3.502833 2.593835

H $-3.828476-3.4743663 .613731$

H $-4.468143-1.2300042 .835154$

H $-2.997773-1.0640041 .909121$

H $-5.097298-3.2286711 .382996$

H -2.818548 -3.7647280 .423368$

H $-2.720159-2.150241-0.241227$

H -2.427751 -4.214245 -1.944253

H $-4.394687-1.478791-2.129879$

H $-4.025553-5.547158-0.866143$

H -5.222251 -5.119686 -2.067927

H $-5.538618-4.800818-0.350324$

H $-5.157230-3.050078-3.580064$

H -7.453694 -0.825758 -1.994718

H $-7.482552-0.237843-0.349264$

H $-7.711624-2.3668830 .052070$

H $\quad 0.097314 \quad 1.836793 \quad 1.854517$

H 2.8647553 .0086062 .565175

H $2.5475904 .540216-0.010978$

H 3.3137305 .1608691 .462882

H 2.0426386 .0823140 .677594

H $-5.3044480 .482140-2.386683$

H $-6.6924851 .460545-1.980009$

H $-2.135375 \quad 1.1031710 .581034$

H $-3.5319681 .606817 \quad 1.557022$

H -2.132926 $3.338565-0.621162$

H $-3.6035003 .969187 \quad 1.989503$

H -3.653406 $6.140790 \quad 0.657189$

H $-2.1790405 .702659-0.169874$

H -1.9153547 .4354353 .643882$

H -3.5717837 .0271602 .921595$

H 0.0666096 .9078462 .466690

H $-0.0955996 .454057 \quad 0.774630$

H -0.4431614 .2240272 .838636$

H -3.493616 $0.760858-1.253419$

C $5.466654-2.5930620 .995366$

O $5.013557-1.4804260 .196899$

C $5.929007-1.290503-0.897783$

C $7.147355-2.149672-0.583923$

C $6.508080-3.3160170 .142512$

H $5.439509-1.691662-1.792958$

C $6.2298430 .199114-1.084347$

H $7.688325-2.448352-1.486927$

H $7.852105-1.6304830 .075944$

C $7.495761-4.1470200 .942933$

H $6.009704-3.959955-0.594827$

C $4.9829811 .075050-1.325171$

O $\quad 6.8854150 .6965810 .090007$

H $6.9439450 .332604-1.904989$

H $4.3756651 .116981-0.413665$

O $5.4357242 .412746-1.571171$

C $4.1111050 .632320-2.515792$

C $3.0122161 .634199-2.913828$

H $3.650253-0.334002-2.293619$ 
O $4.9412820 .432495-3.664236$

H $3.4828192 .576981-3.218239$

H $2.5299601 .256451-3.823612$

H $8.255181-4.5811340 .284426$

H $8.010906-3.5422341 .696804$

H $6.988039-4.9677081 .459286$

C $4.270752-3.4690791 .363768$

H $6.2965200 .531007 \quad 0.846527$

H $5.9755732 .680592-0.807648$

H $5.3929191 .273757-3.849833$

H $4.608612-4.415612 \quad 1.800215$

H $3.697076-3.7121830 .461003$

H $5.932837-2.1718951 .895986$

C $0.4316550 .090511-2.326634$

O $1.3241170 .689246-1.378884$

C $1.9622761 .893440-1.817555$

C $0.9043822 .941817-2.189852$

C $-0.0826912 .410787-3.224668$

C $-0.662846 \quad 1.077677-2.758782$

H $0.997332-0.247477-3.203165$

C $-0.204235-1.134669-1.647932$

H $2.5000372 .287218-0.948524$

O $1.5128414 .143092-2.652676$

H $0.345648 \quad 3.210129-1.284494$

H $0.3849942 .321110-4.212030$

O $-1.1386883 .363944-3.361382$

H -1.348366 $1.255838-1.923972$

O $-1.4199010 .496050-3.818590$

C $0.715981-2.335408-1.605418$

H $-0.481834-0.894490-0.614807$

H $-1.098339-1.444201-2.200693$

H $1.8182053 .999737-3.564154$

H $-1.7085843 .069217-4.091545$

H $-2.183406 \quad 1.073397-3.986875$

O $-0.002462-3.419540-1.184156$

O $1.904885-2.330853-1.890010$

C $0.716249-4.654768-1.116561$

H $1.473821-4.606008-0.330204$

H $0.004737-5.447153-0.869654$

H $1.175378-4.885366-2.082635$

SCF Energy (B3LYP/6-31G**//MMFF) $=-3245.90516976$

07_00089

MM̄FF Geometry

C 3.4389420 .7218693 .708143

C $2.596230-0.0915853 .053910$

C $1.0977490 .019223 \quad 3.130034$

$\begin{array}{lllll}\text { O } & 0.604348 & 0.294209 & 1.799249\end{array}$

C $0.487087-1.3144493 .588805$

C -1.016694 -1.241085 3.774684

C $-1.927062-1.6791632 .881353$

C $-1.469590-0.6650225 .091873$

C $-1.670163-2.2660241 .519618$

C $-2.454673-1.594414 \quad 0.377216$

C $-3.930541-2.0200580 .299195$

C $-4.542926-1.598710-1.038325$

C $-6.037600-1.947517-1.117985$

O $-4.642956-1.3914821 .364880$

O $-6.587873-1.255451-2.250268$

C $-6.748890-1.3995750 .138474$

C $-6.264517-3.442429-1.373057$

O $-8.104188-1.8413130 .164061$

C $-6.026539-1.7544051 .459250$

C $-6.614985-1.0305392 .697591$

O $-6.161226-3.1608851 .702494$

C $0.555051 \quad 1.593997 \quad 1.403139$

$\begin{array}{lllll}\text { O } & 0.926761 & 2.572681 & 2.029759\end{array}$

C $-0.061704 \quad 1.6156770 .059486$

C $-0.3417932 .786483-0.530178$

C $-0.997708 \quad 2.960123-1.828468$

C $-1.3369601 .717028-2.610117$

C $-6.766752 \quad 0.4901432 .607319$

C $-5.450178 \quad 1.2074952 .379703$

C $-4.3003242 .755136 \quad 0.815750$

O -4.5082751 .1012893 .162151$

C $-4.3629412 .991880-0.658854$

C $-4.4385324 .208978-1.218423$

C $-4.4761974 .446783-2.702317$
C -3.334535 $5.318395-3.181904$

C $-3.5127896 .632696-3.397858$

C $-2.0089344 .650243-3.484025$

C $-1.291902 \quad 4.213257-2.234750$

N -5.4416021 .9537881 .211812$

H 3.0442551 .5045384 .350627

H $3.000346-0.8585832 .396530$

H $\quad 0.7917620 .821237 \quad 3.813564$

H $0.939918-1.6254454 .540082$

H $0.741906-2.1084332 .876787$

H -2.973873 -1.618259 3.168549

H $-2.560335-0.6591285 .191482$

H $-1.066022-1.2533545 .922417$

H -1.1253430 .3684695 .197241$

H $-0.609642-2.1906451 .262691$

H $-1.905091-3.3365621 .548788$

H -2.384346 -0.5042640 .477396$

H $-1.961572-1.870070-0.563259$

H $-3.985006-3.1089460 .413266$

H $-4.000179-2.053003-1.876711$

H $-4.432098-0.512499-1.163485$

H $-6.102347-1.542862-3.042364$

H $-6.779049-0.3106730 .016741$

H $-5.784330-3.747846-2.310549$

H -7.330274 -3.664425 -1.497305

H $-5.869594-4.078751-0.578603$

H $-8.560455-1.363558 \quad 0.876642$

H -7.595042 -1.461806 2.939338

H $-5.974701-1.2574963 .561876$

H -6.007781 -3.3243882 .647969$

H $-0.2916720 .661659-0.399792$

H $-0.0935303 .706660-0.002924$

H $-1.9899581 .057390-2.027854$

H $-0.4245411 .168294-2.867573$

H $-1.8587161 .916295-3.547666$

H -7.502782 0.7553501 .841764

H $-7.1714970 .868457 \quad 3.554255$

H $-3.378128 \quad 2.2255381 .076718$

H -4.3337553 .6857831 .391774$

H $-4.3208062 .114185-1.299412$

H $-4.4817935 .086797-0.577601$

H $-5.4370144 .922011-2.939749$

H $-4.469747 \quad 3.502015-3.260188$

H -2.707663 $7.260072-3.765526$

H $-4.4675147 .111440-3.206731$

H $-1.3447485 .345598-4.014273$

H $-2.1811243 .831935-4.187696$

H $-1.0024665 .046390-1.592229$

H -6.3227152 .1194820 .733857$

C 5.4792091 .7976182 .734188

O 4.9208751 .7263591 .405600

C $5.9930101 .679570 \quad 0.452336$

C 7.1823622 .3020841 .166792

C 7.0010071 .7400042 .564859

H $6.2051850 .620147 \quad 0.265478$

C $5.5763102 .349228-0.859985$

H 8.1373642 .0234400 .711638

H 7.1167853 .3958821 .189628

C 7.7639722 .5022873 .634376

H 7.3411940 .6955092 .567049

C $4.2224531 .868689-1.424383$

O $5.4736703 .765396-0.664327$

H $6.3661692 .209005-1.606909$

H $3.3990742 .290591-0.834372$

O $4.0835552 .407718-2.744963$

C $4.0528730 .340610-1.499628$

C $2.737890-0.066120-2.192501$

H $4.060366-0.071155-0.484917$

O $5.158152-0.231802-2.195662$

H $1.9256130 .568386-1.824643$

H $2.8398480 .097649-3.272507$

H $8.8406722 .464498 \quad 3.439758$

H 7.4650533 .5553013 .666839

H 7.5828202 .0694474 .623417

C 4.9331290 .6382993 .572020

H 4.8283003 .9174870 .047506

H $4.1763433 .373411-2.674452$

H $5.1761050 .154251-3.088182$ 
H $5.379306 \quad 0.6479174 .572935$

H $5.206470-0.3165463 .106225$

H 5.1654882 .7600403 .157731

C $3.196938-3.774854-2.383228$

O $3.351213-2.362801-2.572422$

C $2.368110-1.540736-1.938353$

C $0.965379-1.877782-2.469135$

C $0.661101-3.372460-2.327460$

C $1.809011-4.241224-2.853792$

H $3.944951-4.228153-3.046510$

C $3.526690-4.195880-0.946854$

H $2.394014-1.711329-0.856325$

O $0.873282-1.500408-3.841519$

H $\quad 0.225977-1.289132-1.913969$

H $-0.257207-3.606252-2.879749$

O $0.426406-3.658916-0.952352$

H $1.657571-5.286477-2.561749$

O $1.786724-4.213877-4.284257$

C $4.976904-3.913618-0.632020$

H $2.900350-3.696912-0.201932$

H $3.370109-5.272282-0.809253$

H - $0.036102-1.681007-4.134721$

H $0.201751-4.602343-0.882757$

H $1.992386-3.306307-4.567390$

O $5.073771-2.855600 \quad 0.222583$

O $5.911547-4.546726-1.106379$

C $6.405405-2.4794970 .585782$

H $\quad 6.342594-1.6875591 .335890$

H $6.939769-3.3293971 .020983$

H $6.937382-2.094232-0.289093$

SCF Energy $\left(\mathrm{B} 3 \mathrm{LYP} / 6-31 \mathrm{G}^{* *} / / \mathrm{MMFF}\right)=-3245.90650088$

07 00090

MM̄MF Geometry

C $0.897268-3.1669301 .209735$

C $-0.147224-3.2729782 .044043$

C $-0.896468-2.0765252 .565155$

O $-2.303809-2.3319852 .336497$

C - $0.668022-1.8523924 .072249$

C $0.762839-1.5515974 .465515$

C $1.384935-0.3783024 .237188$

C $1.482155-2.6648795 .180512$

C $0.8220530 .832528 \quad 3.544171$

C 1.6033511 .1250972 .256516

C 0.9733342 .1888181 .345500

C 0.8385533 .5507972 .022456

C 0.1771904 .5769551 .091464

O $-0.310144 \quad 1.718761 \quad 0.927270$

O -0.1883775 .7180051 .882958$

C -1.1314983 .9796820 .526787$

C 1.1498745 .0866530 .020367

O $-1.6793064 .855276-0.455851$

C $-0.9671262 .552658-0.045660$

C $-2.321277 \quad 1.857411-0.322110$

O $-0.2201822 .598234-1.262463$

C $-3.122657-1.2489742 .251276$

O $-2.801127-0.0765282 .355565$

C $-4.500108-1.7362812 .020003$

C $-5.455198-0.8661431 .657569$

C $-6.872195-1.1727601 .449828$

C $-7.331814-2.5765391 .746982$

C $-3.2026412 .492049-1.397630$

C $-4.3072311 .558613-1.867849$

C $-6.2289221 .499221-3.457536$

O $-4.421270 \quad 0.396087-1.489990$

C $-7.4632181 .556712-2.613662$

C $-8.2316340 .484164-2.368971$

C $-9.5162810 .534302-1.593491$

C $-9.621715-0.562472-0.558206$

C $-10.176864-1.746583-0.868519$

C $-9.189681-0.2545200 .855303$

C -7.696889 -0.1901411 .028022$

N $-5.1407762 .182041-2.785592$

H $1.226350-2.1774240 .901479$

H $-0.472369-4.2549502 .378078$

H $-0.606313-1.1876191 .991895$

H -1.031743 -2.728270 4.627933

H -1.313262 -1.038845 4.428890
H $2.413649-0.2655124 .577508$

H $2.512181-2.3971685 .439048$

H $0.961946-2.9148366 .111066$

H $1.522708-3.5609964 .553582$

H $-0.2424990 .714482 \quad 3.327365$

H $\quad 0.905874 \quad 1.682476 \quad 4.231033$

H 2.6231421 .4331222 .516243

H 1.6871670 .1958941 .677395

H 1.6195912 .2703150 .464066

H 1.8150313 .9133882 .366907

H 0.2136013 .4639492 .920943

H 0.6192886 .0647162 .299395

H -1.8594383 .9365661 .349007$

H 2.0360815 .5315110 .488630

H $0.6962165 .884486-0.578145$

H $1.4922244 .301375-0.656348$

H -1.787863 $5.727397-0.039232$

H $-2.1074910 .816386-0.603221$

H $-2.895016 \quad 1.7906530 .610272$

H $-0.4348591 .802667-1.777050$

H $-4.691617-2.7925432 .169919$

H -5.1799130 .1779221 .512111$

H $-6.803784-3.2962461 .111385$

H -8.397737 -2.732698 1.568411

H -7.144470 -2.829275 2.796442

H $-3.6741733 .399541-1.004900$

H -2.606202 $2.760301-2.276754$

H $-5.9239750 .469742-3.673505$

H -6.402202 $2.020672-4.403989$

H -7.758962 2.526748 -2.221505

H $-7.949300-0.475609-2.796602$

H $-10.3416790 .436334-2.311240$

H $-9.657870 \quad 1.510999-1.114508$

H -10.295566 -2.529027 -0.126720

H -10.522610 -1.960748 -1.874519

H $-9.627195-0.9700991 .561590$

H $-9.616070 \quad 0.7111391 .157458$

H -7.2750720 .7936990 .820412$

H $-5.0003333 .166448-2.993613$

C $1.824723-4.343103-0.838274$

O $2.440172-3.121355-1.297406$

C $1.742967-2.674924-2.475382$

C $0.925267-3.870057-2.951318$

C $0.523706-4.491715-1.626747$

H $1.048039-1.889775-2.154557$

C $2.725504-2.115609-3.507140$

H $\quad 0.069207-3.571517-3.563942$

H $1.534564-4.571484-3.533427$

C $0.034389-5.924048-1.746027$

H $-0.272196-3.879664-1.183780$

C $3.559995-0.915703-3.006575$

O $3.633565-3.159152-3.880834$

H $2.179920-1.842264-4.418388$

H $4.276373-1.258344-2.252200$

O $4.346053-0.450688-4.111991$

C $2.7310740 .257622-2.452058$

C $3.5193951 .557995-2.210695$

H $2.245271-0.047676-1.518481$

O $1.6909330 .556223-3.389605$

H $3.8402361 .982795-3.170670$

H $2.8074362 .284555-1.797910$

H $-0.858538-5.973828-2.377872$

H $\quad 0.796233-6.573548-2.189861$

H $-0.224020-6.330004-0.762994$

C $1.688524-4.3312070 .686496$

H $4.252275-2.785337-4.531534$

H $3.732902-0.097049-4.779318$

H $1.1770011 .300332-3.034109$

H $2.690561-4.2589651 .126080$

H $1.258842-5.2809811 .025224$

H $2.517509-5.150293-1.110480$

C 5.2970570 .6444940 .953548

O $4.2792820 .870756-0.032727$

C $4.7281141 .429032-1.270030$

C $5.4052072 .790200-1.032616$

C 6.5235972 .6887140 .008727

C 6.0618151 .9428541 .264702

H 4.7511550 .3577751 .861452 
C $6.218824-0.5246110 .572778$

H $5.4488410 .748360-1.733422$

$\begin{array}{lllll}\text { O } & 4.449286 & 3.755922 & -0.596484\end{array}$

H $5.8133523 .152677-1.983123$

H 6.8526813 .6966540 .289321

O $7.6419012 .006124-0.549676$

H $6.9158241 .716417 \quad 1.913584$

O 5.1809862 .7884962 .010694

C $5.485170-1.848360 \quad 0.552112$

H $6.663840-0.383522-0.415395$

H $7.023086-0.6138881 .312120$

H 4.0159683 .4064340 .201039

H $7.9362552 .510797-1.326993$

H $5.679144 \quad 3.587003 \quad 2.255211$

O $6.328872-2.8111270 .075167$

O $4.330121-2.020670 \quad 0.913942$

C $5.783554-4.131394-0.006754$

H $5.040358-4.175680-0.807244$

H $6.597788-4.820542-0.246347$

H $5.343765-4.4307870 .949511$

SCF Energy (B3LYP/6-31G**//MMFF) $=-3245.91636374$

\section{7_00091}

MMFF Geometry

C -3.312256-1.011553-2.443913

C $-2.757620-1.694742-3.456726$

C $-1.284614-1.980991-3.619835$

O $-0.536463-1.308503-2.581669$

C $-0.936357-3.487236-3.627586$

C $-1.681086-4.357183-2.631602$

C $-1.308521-4.570097-1.355730$

C $-2.894903-5.072785-3.174985$

C $-0.111545-4.037374-0.624734$

C $-0.526857-3.1120500 .526246$

C $0.567854-3.0056111 .596709$

C $0.153301-2.0388362 .704433$

C $1.244272-1.9057113 .776730$

O $1.780152-2.548875 \quad 0.991214$

O $0.899480-0.769044 \quad 4.582989$

C $2.594080-1.6114653 .087705$

C $1.255497-3.1147724 .719302$

O $3.661358-1.6352984 .033882$

C $2.903134-2.5387931 .885505$

C $4.130308-1.999757 \quad 1.115853$

O $3.199971-3.847468 \quad 2.377307$

C $0.699712-0.836745-2.892260$

O $1.309998-1.001955-3.935998$

C $1.173935-0.045955-1.733711$

C $2.370868 \quad 0.556063-1.790347$

C $2.972726 \quad 1.387705-0.747991$

C 2.1755521 .6433380 .503287

C $4.451380-2.758261-0.178976$

C $5.464370-2.002404-1.017011$

C $7.809148-1.163160-0.981323$

O $5.160053-1.495849-2.094732$

C $7.7692110 .192833-0.348790$

C $7.5988381 .333377-1.034372$

C $7.5320092 .687308-0.387295$

C $6.2075093 .382453-0.617192$

C $6.1380254 .539258-1.297269$

C $4.969752 \quad 2.771500 \quad 0.008698$

C $4.212728 \quad 1.883554-0.943487$

N $6.721023-1.952921-0.435171$

H -2.680278 $-0.589762-1.666848$

H $-3.405390-2.091716-4.235260$

H $-0.990646-1.555113-4.588356$

H $\quad 0.143326-3.618556-3.486126$

H $-1.139862-3.879461-4.633512$

H $-1.925977-5.236127-0.752846$

H -3.379205 -5.703753 -2.421836

H $-3.645531-4.363673-3.532965$

H -2.607288 -5.721559-4.009019

H $0.435977-4.901958-0.229053$

H $0.582431-3.514483-1.288933$

H $-0.748328-2.1197540 .114022$

H -1.448136 -3.4747990 .999937$

H $\quad 0.726897-4.0098342 .007797$

H $-0.796948-2.3457493 .158571$
H $-0.027451-1.0465322 .267510$

H $1.621531-0.6293715 .219757$

H $2.544748-0.5774002 .725729$

H $\quad 0.277633-3.2307505 .201860$

H $1.974196-2.9760245 .534642$

H $1.489544-4.0521544 .210166$

H $3.866316-2.5666454 .224514$

H $3.957227-0.9485710 .857156$

H $5.011904-2.0272421 .768281$

H $3.680156-4.3385661 .692365$

H $\quad 0.5184590 .032319-0.873645$

H $2.9727200 .431891-2.689752$

H 1.8471850 .7029720 .955508

H 1.2912122 .2424580 .273561

H $2.7404532 .177297 \quad 1.272388$

H $4.851027-3.7561460 .031235$

H $3.551705-2.890323-0.790289$

H $7.716713-1.115181-2.071158$

H $8.746275-1.668308-0.728988$

H $7.8786250 .233700 \quad 0.732621$

H $7.4966611 .296432-2.116431$

H $8.3581013 .289414-0.786454$

H 7.7074292 .6170270 .693712

H $5.1922195 .051257-1.441311$

H $7.0223174 .997615-1.727321$

H $4.315473 \quad 3.5818500 .355392$

H 5.2590352 .1974610 .897385

H $4.7271681 .631921-1.870685$

H $6.858119-2.3674750 .481777$

C $-5.309621-1.183527-0.899931$

$\begin{array}{llll}\text { O } & -4.709696 & -0.345403 & 0.107104\end{array}$

C $-4.445427-1.1460001 .277333$

C $-5.166432-2.4716271 .053697$

C $-5.054154-2.621762-0.452783$

H -3.365917 -1.335283 1.293616

C $-4.865575-0.3785822 .536400$

H $-4.700225-3.2944271 .604084$

H $-6.219500-2.4181101 .353427$

C $-6.018265-3.633675-1.043824$

H -4.027666 -2.927932 -0.688013

C $-4.205170 \quad 1.0118692 .662847$

O $-6.285729-0.1819982 .506866$

H $-4.661631-0.9789863 .429732$

H -4.5270011 .6420781 .827343$

O $-4.702071 \quad 1.637705 \quad 3.852587$

C -2.6674310 .9742152 .747006$

C -2.0159732 .3638712 .897102$

H -2.2529750 .4792231 .863309$

$\begin{array}{llll}\text { O } & -2.303208 & 0.182624 & 3.881490\end{array}$

H -2.4382142 .8844773 .764981$

H -0.9533652 .2087303 .128395$

H $-5.824588-4.631822-0.637625$

H -7.058766 -3.375656 -0.820154

H $-5.909635-3.684871-2.131817$

C $-4.789019-0.789828-2.285442$

H -6.4980310 .3036781 .691273$

H $-5.673071 \quad 1.6365893 .796824$

H -1.3323040 .1591643 .927993$

H $-4.9757500 .279868-2.441295$

H $-5.348473-1.335136-3.054262$

H $-6.386097-0.968435-0.868645$

C $-1.499127 \quad 3.203268-0.696909$

$\begin{array}{lllll}\text { O } & -1.415478 & 2.577547 & 0.589434\end{array}$

C -2.1401503 .2334241 .634906$

C -1.5798494 .6469501 .846674$

C -1.6225625 .4559660 .544876$

C $-1.0451914 .674515-0.641159$

H $-0.7743622 .653896-1.310176$

C $-2.8885332 .983848-1.303641$

H $-3.195125 \quad 3.3079881 .353520$

\begin{tabular}{llll}
\hline & -0.238041 & 4.584220 & 2.325907
\end{tabular}

H -2.172733 5.1522262 .617648

H -1.0509116 .3833760 .674314$

$\begin{array}{lllll} & \text { O } & -2.969207 & 5.814069 & 0.249055\end{array}$

H $-1.2921875 .181295-1.581043$

O $0.3808144 .702459-0.516679$

C $-2.8715823 .112571-2.806342$

H $-3.2688771 .986536-1.051298$ 
H -3.631078 $3.682031-0.906420$ H 0.2879214 .0895311 .674594 H -3.3026206 .3437620 .993117$ H $0.7536414 .277199-1.307946$ O $-2.2393552 .038714-3.359988$ O $-3.3607464 .055680-3.414159$ C -2.149299 $2.041489-4.787648$ H $-1.6240202 .936332-5.134941$ H -1.579157 $1.160329-5.093916$ H -3.149372 $1.983798-5.227376$

SCF Energy (B3LYP/6-31G**//MMFF) $=-3245.92224474$

07_00092

MM̄FF Geometry

C -3.328171-1.349652 -3.212215

C $-2.220210-1.197554-2.470606$

C $-0.896889-0.738588-3.019871$

O $-0.6581310 .539997-2.384449$

C $0.266059-1.692178-2.684328$

C $-0.042466-3.160874-2.864263$

C $-0.195417-4.031069-1.846853$

C $-0.155087-3.626516-4.291718$

C $-0.093491-3.744966-0.372919$

C $1.047796-4.5196850 .297536$

C $2.438176-4.253910-0.305372$

C $3.476852-5.151196 \quad 0.372855$

C $4.887106-4.893682-0.170527$

O $2.781245-2.872811-0.123864$

O $5.824975-5.5617370 .686656$

C $5.169376-3.380183-0.112209$

C $5.087272-5.520567-1.554434$

O $6.414018-3.075950-0.739730$

C $4.038767-2.503274-0.713705$

C $4.338716-1.023579-0.373705$

O $4.022809-2.669722-2.131479$

C $0.2681491 .374327-2.919231$

O $\quad 0.978090 \quad 1.171772-3.889490$

C $0.251466 \quad 2.602752-2.095382$

C $1.1621363 .565785-2.293153$

C $1.2308874 .835443-1.566515$

C $0.1936435 .086101-0.501266$

C $3.299350 \quad 0.008717-0.829951$

C $3.7851161 .397750-0.450739$

C 4.3309242 .7533821 .566298

O $4.3037362 .148866-1.273573$

C 3.5959154 .0456621 .404149

C 4.2035725 .1740151 .004633

C 3.5446686 .5219140 .940465

C $3.6446657 .160044-0.426403$

C $4.7429287 .841611-0.794303$

C $2.4341857 .086820-1.325944$

C $2.2073595 .710536-1.885254$

N 3.6368411 .6676140 .901240

H -3.295863 -1.151308 -4.280070

H -2.272286 -1.373353 -1.397760

H $-0.964385-0.590661-4.105462$

H $0.625902-1.475522-1.673742$

H $1.128067-1.439978-3.316469$

H $-0.416340-5.069949-2.088262$

H $-0.350308-4.701665-4.363731$

H $-0.971614-3.108571-4.804074$

H $0.776695-3.423809-4.829768$

H $0.012123-2.679904-0.153230$

H - $-1.039797-4.046807 \quad 0.093411$

H $1.053434-4.2486891 .361038$

H $0.827368-5.5936050 .242302$

H $2.406202-4.476106-1.377216$

H $3.213559-6.2104550 .261075$

H $3.474314-4.9544681 .454101$

H $5.716664-5.2126881 .587656$

H $5.283873-3.1085240 .946513$

H $4.930992-6.605161-1.507483$

H $6.115802-5.382340-1.906282$

H $4.409352-5.121119-2.311475$

H $7.092548-3.636945-0.326411$

H $4.449108-0.9340120 .715155$

H $5.303303-0.739130-0.814709$

H $3.536162-1.931510-2.529399$
H $-0.5281602 .686161-1.346717$

H $1.9199433 .418030-3.061149$

H $0.334393 \quad 6.0263680 .035090$

H $\quad 0.222071 \quad 4.2956210 .257528$

H $-0.8095145 .115819-0.940484$

H $3.159116-0.009116-1.915082$

H $2.322514-0.172796-0.372041$

H 5.3519532 .8054321 .172432

H 4.3841452 .4964462 .629293

H 2.5457364 .0680681 .682606

H 5.2641495 .1416470 .763165

H 4.0360007 .1704571 .678197

H 2.4959596 .4692461 .256294

H $4.8099638 .329018-1.761119$

H $5.6014367 .923057-0.135689$

H $2.553917 \quad 7.766498-2.180581$

H $1.5628487 .474271-0.789764$

H $2.9220285 .433268-2.661280$

H $3.2361130 .949136 \quad 1.496082$

C -5.556532 -0.520406 -2.441658

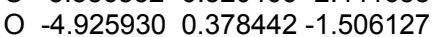

C $-5.8177730 .605405-0.405329$

C $-7.2041180 .343179-0.971005$

C $-6.925597-0.867016-1.846466$

H $-5.595301-0.1586620 .349150$

$\begin{array}{llll}\text { C } & -5.577140 & 1.992690 & 0.195917\end{array}$

H $-7.9457180 .147834-0.191089$

H $-7.5581291 .179393-1.584893$

C $-7.996930-1.125552-2.891530$

H $-6.842588-1.752490-1.201852$

C $-4.092978 \quad 2.2855750 .513203$

O $-6.0260612 .978725-0.740628$

H -6.1955212 .1122821 .093599$

H $-3.5374922 .460950-0.416832$

O $\begin{array}{llll}-4.030381 & 3.507558 & 1.258310\end{array}$

C -3.3811691 .1880531 .327352$

C $-1.9364821 .575447 \quad 1.700630$

H -3.3508640 .2632490 .741824$

O $-4.131148 \quad 0.915174 \quad 2.509710$

H -1.4240621 .9562420 .810259$

H -1.9677262 .3762482 .449877$

H $-8.960736-1.327074-2.412933$

H -8.125186 $-0.264726-3.556355$

H -7.736419-1.991796 -3.507894

C $-4.656821-1.744269-2.629566$

H $-6.0431803 .834853-0.281119$

H $-4.298273 \quad 4.2282790 .663849$

H $-4.172113 \quad 1.737018 \quad 3.028211$

H -5.133205 -2.473425 -3.294358

H $-4.499009-2.242496-1.664832$

H $-5.6635250 .028355-3.385725$

C -1.131933 -1.123509 4.164094

$\begin{array}{llll}\text { O } & -1.719381 & 0.011888 & 3.517469\end{array}$

$\begin{array}{llll}\text { C }-1.132718 & 0.388607 & 2.269749\end{array}$

C 0.3503790 .7428302 .463726

C $1.114826-0.3773783 .180172$

C $0.366864-0.8867294 .416227$

H $-1.635523-1.1798205 .137609$

C -1.425777 -2.427096 3.412212

H $-1.214816-0.4446361 .562824$

$\begin{array}{lllll}\text { O } & 0.478948 & 1.951075 & 3.211638\end{array}$

H $0.794946 \quad 0.929345 \quad 1.480013$

H $2.100716-0.0075083 .487787$

O $1.317290-1.4699692 .291250$

H $0.832927-1.8079184 .785400$

$\begin{array}{lllll} & 0 & 0.504343 & 0.096911 & 5.445872\end{array}$

C -2.913116 -2.684664 3.348994

H $-1.027203-2.4256062 .394262$

H $-0.968042-3.2834493 .920930$

H 0.0332641 .8199164 .066105

H $1.858386-1.1518681 .550000$

H $0.086035-0.2577696 .248855$

O $-3.356584-2.5489912 .066466$

O $-3.599642-2.9575944 .325138$

C $-4.759095-2.7558351 .875388$

H $-5.329015-1.9934252 .414607$

H $-4.973668-2.665210 \quad 0.807598$

H $-5.045347-3.7591082 .205535$ 
O $4.620119-5.8399561 .657085$

C $4.508714-3.4840641 .225053$

C $3.221623-5.237957-0.173094$

O $5.479519-3.4987920 .181461$

C $3.613602-2.2260351 .122852$

C $4.367348-0.9179471 .483107$

O $3.078242-2.126401-0.192806$

C 0.7069762 .0589131 .999488

O $1.056704 \quad 0.9749501 .560929$

C 1.4170933 .3386401 .778075

C 2.5526613 .3421231 .064241

C 3.3618204 .5127480 .717898

C 2.8591595 .8623061 .158422

C $5.519568-0.4675020 .583911$

C $5.0972150 .130487-0.747366$

C $6.0755930 .923668-2.902397$

O $3.945406 \quad 0.473148-0.998695$

C $6.5206012 .345763-2.764098$

C $5.7459543 .404007-3.047296$

C $6.2043544 .827642-2.908970$

C $5.3484625 .633368-1.956382$

C $4.5544346 .619874-2.405665$

C $5.4776785 .356754-0.474200$

C 4.4930184 .3244610 .005551

N $6.1776820 .279094-1.606046$ H -3.653461 0.6586921 .855035 H -2.721674 2.5638814 .065213 H -1.3176630 .5518622 .168468$ H -0.7577951 .1142725 .152045$ H $\quad 0.3216790 .230846 \quad 4.140010$ H -1.877260 -2.859527 4.223862 H -2.964387 -1.717528 5.904629 H $-1.956033-0.469615 \quad 6.643826$ H -3.250737 -0.020489 5.513429 H $-0.942237-2.5121351 .902774$ H $0.194868-1.3245592 .510800$ H $1.122475-3.1552144 .062842$ H $0.100986-4.3039073 .182595$ H $1.243117-3.4976721 .027281$ H $1.950432-5.6276122 .182327$ H $3.002253-4.7073513 .239182$ H $4.119489-6.6716741 .716796$ H $5.064890-3.4341682 .171383$ H $2.710929-6.206801-0.115795$ H $4.057354-5.382147-0.866709$ H $2.525000-4.523272-0.616519$ H $6.030803-4.2899410 .308426$ H $3.634044-0.1019511 .538948$ H $4.752307-1.0197912 .507215$ H $2.775467-1.212748-0.326817$ H 0.9796854 .2396792 .192374 H 2.9276292 .3928620 .685336 H 1.8505436 .0432110 .770164 H 3.4753506 .6933840 .809332 H 2.8307985 .9227452 .251986 H 6.0586570 .3319531 .109970 H $6.241908-1.2705360 .423205$ H $5.0494950 .844545-3.275825$ H $6.7403220 .390309-3.588647$ H $7.5384362 .510360-2.417675$

H $6.1901565 .279029-3.909467$

H $7.2488464 .878619-2.576547$

H $3.9615117 .221187-1.724583$

H $4.4763696 .846126-3.463835$

H 5.3918416 .2909580 .090337

H $6.4921035 .000642-0.253175$

H $4.7658093 .304671-0.265548$

H $7.1064520 .031766-1.276698$

C -5.7690112 .6125251 .641830$

O

C $-5.3868492 .294294-0.640936$

C $-5.5092103 .788471-0.378356$

C -5.0733813 .8522511 .075170$

H $-4.3225992 .054458-0.749212$

C $-6.1543881 .761674-1.852840$

H $-4.8765884 .383464-1.043644$

H $-6.5437004 .135563-0.482248$

C -5.4557375 .1452721 .773339$

H -3.9826513 .7383561 .115449$

C $-6.1003170 .224275-1.993864$

O $-7.5287572 .143643-1.729301$

H $-5.7801512 .243915-2.763720$

H $-6.704783-0.237911-1.204360$

O $-6.735711-0.126025-3.229257$

C $-4.679661-0.367784-1.968419$

C $-4.668376-1.891832-2.194731$

H $-4.217324-0.151299-0.999481$

O $\quad-3.905701 \quad 0.279913-2.977139$

H -5.444486 -2.355992 -1.574811

H $-4.893789-2.096615-3.248611$

H -4.9763426 .0010341 .286864$

H -6.5383215 .3094891 .750831$

H -5.1383345 .1297972 .820796$

C -5.1152951 .9774612 .872376$

H $-7.9985071 .789266-2.503706$

H $-6.1823640 .219257-3.951010$

H -2.998459 -0.064147 -2.919535

H -5.7355361 .1328363 .198591$

H -5.1027692 .7009143 .695852$

H -6.8094662 .8540541 .895798$

C $-0.984587-2.458482-2.540809$

O $-2.334361-2.035898-2.771072$

C $-3.304178-2.516813-1.836075$

C $-3.344266-4.051430-1.858268$

C $-1.952649-4.645876-1.610575$

C $-0.886291-3.992851-2.496919$

H $-0.430762-2.113655-3.423722$

C $-0.379272-1.773557-1.311504$

H $-3.036623-2.179911-0.828587$

O $-3.846054-4.516848-3.109410$

H -4.039024 -4.399663 -1.085301

H -1.976886 -5.724205 -1.810099

O $-1.586536-4.463889-0.245591$

H $\quad 0.118859-4.284617-2.170029$

O $-1.045663-4.467041-3.837417$

C $-0.219878-0.290610-1.549131$

H $-0.968394-1.921357-0.403403$

H $0.616936-2.173314-1.100317$

H $-3.281235-4.150766-3.811349$

H $-2.257408-4.9075370 .300736$

H -0.925241 -5.431846 -3.822891

O $-1.210648 \quad 0.394437-0.910638$

$\begin{array}{lllll}\text { O } & 0.663677 & 0.194537 & -2.244021\end{array}$

C $-1.1966791 .816083-1.077730$

H $-0.1846322 .220021-0.979499$

H -1.617188 $2.072448-2.053933$

$\mathrm{H}-1.8221462 .255648-0.296423$

SCF Energy $($ B3LYP/6-31G**//MMFF $)=-3245.93637745$

0700094

MM̄FF Geometry

C $-2.903867-3.4807321 .191677$

C -2.062984 -3.040596 2.140586

C $-0.593786-2.8229561 .903715$

O $-0.271057-1.5305932 .478324$

C $0.298859-3.8761912 .582358$

C $0.235469-5.2608381 .974811$ 
C $0.954943-5.6450510 .901446$

C $-0.638157-6.2512912 .697938$

C $1.863336-4.7873660 .065138$

C $3.333314-4.944816 \quad 0.468403$

C $4.273504-4.132602-0.437617$

C $5.721920-4.2728570 .031378$

C $6.675046-3.392928-0.789667$

O $3.880101-2.759992-0.380906$

O $7.943347-3.362166-0.117153$

C $6.124445-1.948633-0.848313$

C $6.961488-4.012601-2.162831$

O $6.914713-1.165496-1.739026$

C $4.630631-1.876719-1.235384$

C $4.007853-0.480343-1.003795$

O $4.467987-2.228364-2.610341$

C $0.740057-0.8253851 .904640$

O $1.384976-1.1397500 .917777$

C 0.9355510 .4110522 .694387

C 1.8098811 .3331322 .264342

C 2.1129172 .6140672 .905635

C 1.4212212 .9200564 .208273

C $4.5991590 .677779-1.807939$

C $3.7193311 .917327-1.754417$

C $3.6840874 .307479-2.450145$

O $2.6016531 .931412-1.246392$

C $4.0058955 .102115-1.223440$

C $3.0742355 .622163-0.409319$

C 3.3961256 .4659330 .790114

C 2.7742955 .9459692 .066598

C 1.7009626 .5491482 .605299

C 3.4555484 .7970972 .775210

C 2.9789373 .4523492 .296864

N $4.3230943 .007825-2.362964$

H $-2.508712 \quad-3.7397260 .212837$

H -2.448593 -2.807875 3.130216

H $-0.401671-2.7929760 .825101$

H $0.063271-3.9274173 .654587$

H $1.340803-3.5286042 .566943$

H $0.876848-6.6786920 .567994$

H $-0.620746-7.2407302 .228714$

H $-0.298605-6.3711383 .732040$

H -1.678441 -5.913956 2.711879

H $\quad 1.733826-5.076588-0.985198$

H $1.567603-3.7366440 .123381$

H $3.447248-4.6068701 .506765$

H $3.619905-6.0034620 .437072$

H $4.160655-4.497323-1.465293$

H $6.043406-5.3213690 .001409$

H $5.791230-3.9699741 .085645$

H $7.806202-2.9961040 .773213$

H $6.240243-1.5025620 .148951$

H $7.413955-5.004915-2.046393$

H $7.691189-3.417182-2.722805$

H $6.066350-4.127103-2.777713$

H $7.838594-1.230856-1.442171$

H $2.936730-0.556633-1.238093$

H $4.049584-0.2349570 .065825$

H $3.623915-1.861928-2.922537$

H $0.346106 \quad 0.5343563 .595603$

H $2.355357 \quad 1.138047 \quad 1.343278$

H 0.3330742 .9040084 .080855

H 1.6681753 .9031614 .613914

H 1.6961612 .1825984 .970587

H $5.582720 \quad 0.945878-1.407697$

H $4.7110750 .398513-2.861421$

H $2.6052294 .170749-2.581560$

H $4.0826484 .811154-3.335998$

H $5.0577395 .279460-1.011710$

H $2.0218125 .464572-0.634881$

H 3.0348427 .4821870 .583898

H 4.4799446 .5635480 .931090

H 1.2697236 .2058623 .539632

H 1.2254467 .3956982 .121309

H 3.3422224 .9019373 .859676

H 4.5396114 .8575692 .613664

H $3.4215173 .142007 \quad 1.350107$

H $5.2791502 .924682-2.696278$

C $-5.194772 \quad-3.2551460 .168579$
O $-5.094465-1.836458-0.078314$

C $-6.391698-1.341210-0.463536$

C $-7.278061-2.566827-0.665020$

C $-6.687832-3.5398860 .331954$

H $-6.773866-0.7705580 .390584$

C $-6.307099-0.442890-1.704604$

H -8.334191 -2.347895 -0.479628

H $-7.193549-2.967182-1.682098$

C -7.080249-4.984050 0.071065

H -7.017770 -3.252779 1.339412

C $-5.4156050 .808936-1.575963$

O $-5.804238-1.211061-2.807060$

H -7.319573 -0.136935 -1.994498

H $-4.3600050 .519786-1.597637$

O $-5.6345661 .607904-2.747317$

C $-5.6874591 .679341-0.338311$

C $-5.0506203 .083367-0.368514$

H $\quad-5.3436801 .1441080 .554436$

O $-7.1010411 .858200-0.199484$

H $-5.5779293 .715765-1.094738$

H -5.2551703 .5367370 .610597$

H $-8.167518-5.1024060 .124582$

H $-6.754676-5.315379-0.920745$

H $-6.632576-5.6524120 .812935$

C $-4.380116-3.6563801 .402956$

H $-4.934200-1.559741-2.547059$

H $-5.4477301 .046908-3.519539$

H -7.2550512 .3511660 .624543$

H -4.561839 -4.7124191 .632162$

H $-4.704941-3.0628782 .266468$

H $-4.804809-3.760003-0.725341$

C -1.467225 2.1361110 .116476

$\begin{array}{lllll}\text { O } & -2.886959 & 2.258665 & 0.274967\end{array}$

C $-3.5402333 .123233-0.654593$

C $-2.9760644 .553461-0.553479$

C $-1.4462224 .582675-0.667987$

C $-0.784671 \quad 3.5108200 .204783$

H -1.1475431 .5505520 .985788$

C $-1.0826571 .347583-1.144937$

H $-3.3796612 .745721-1.668546$

O -3.3514905 .1446100 .691366$

H -3.419726 $5.167736-1.345480$

H -1.089102 $5.568224-0.345299$

O $-1.0316744 .426074-2.021596$

H $0.2779153 .410648-0.044404$

O -0.8591523 .9267341 .572399$

C -1.519451-0.098511-1.078085

H -1.526983 $1.771484-2.047660$

H $0.0068591 .349899-1.252345$

H -3.017991 4.5726061 .403785

H -1.664846 $3.854106-2.483788$

H $-0.3594094 .756821 \quad 1.652306$

O $-1.078445-0.744237-2.198011$

O $-2.171171-0.596496-0.171835$

C -1.436698 -2.125622 -2.302435

H $-2.507064-2.267248-2.123632$

H -1.207454 -2.459199 -3.318052

H $-0.844003-2.714883-1.597757$

SCF Energy (B3LYP/6-31G**//MMFF) $=-3245.91660356$

0700095

MM̄FF Geometry

C $-0.771450-0.2482613 .083190$

C -1.483505 0.8549813 .354942

C -1.3388092 .1452892 .593696$

O $-\begin{array}{llll}-2.611377 & 2.361449 & 1.938417\end{array}$

C -1.001395 3.3268573 .521946

C 0.3593083 .2499724 .183383

C 1.5216893 .5362313 .563248

C 0.3492492 .8806705 .643160

C 1.7122873 .9021992 .116437

C 2.8539443 .0902411 .485183

C $2.8430003 .048778-0.052419$

C $2.7024374 .425931-0.705199$

C $2.6090484 .334757-2.237149$

O $1.7688642 .190270-0.453983$

O $2.1651665 .620320-2.698633$

C $1.5295423 .291914-2.608889$ 
C $3.9833684 .095614-2.873113$

O $1.4992243 .060570-4.015164$

C $1.7035431 .949883-1.867603$

C $0.5355540 .971725-2.123972$

O $2.8935641 .306513-2.338448$

C -2.664087 3.2429090 .908030

$\begin{array}{lllll}O & -1.796826 & 4.042320 & 0.582457\end{array}$

C -3.9194223 .1068900 .134083$

C -4.7650192 .0747400 .278531$

C $-5.9648631 .818195-0.521043$

C $-6.2590942 .759663-1.659201$

C $-0.8347671 .471943-1.669231$

C $-1.8195540 .328150-1.529293$

C $-3.080806-1.354282-2.870635$

O $-2.184443-0.075703-0.427608$

C $-4.498763-0.921335-3.077913$

C $-5.541478-1.543519-2.505253$

C $-6.979311-1.197526-2.775135$

C -7.809952 -1.098724-1.514524

C $-8.441920-2.175830-1.018010$

C $-7.9776640 .263763-0.881484$

C $-6.718840 \quad 0.741790-0.214854$

N $-2.218565-0.193951-2.746779$

H $-0.047838-0.2273442 .271780$

H -2.2081150 .8362674 .165649$

H -0.5749732 .0294561 .815730$

H -1.784953 3.4270064 .286164

H -1.0659194 .2727642 .970325$

H 2.4393203 .4970124 .148716

H 1.3563192 .8549666 .072826

H -0.2355823 .6089446 .214469$

H -0.0933411 .8901855 .785733$

H 0.7933153 .7591181 .544557

H 1.9575804 .9695822 .063180

H 3.8101893 .5078751 .825304

H 2.8072202 .0559561 .851100

H $3.7862292 .582601-0.360434$

H $3.5281535 .083021-0.405476$

H $1.7858824 .915736-0.350766$

H $2.1542015 .605397-3.670457$

H $0.5569873 .724382-2.340460$

H $4.6549684 .934231-2.653053$

H $3.9117374 .047393-3.965305$

H $4.4685473 .183307-2.520208$

H $1.2869003 .904005-4.448674$

H $\quad 0.5039220 .687262-3.183390$

H $0.7524080 .042667-1.583070$

H $2.8424560 .361655-2.117471$

H $-4.0729203 .872415-0.619477$

H -4.5512031 .3209441 .034546$

H $-6.4540853 .769275-1.281335$

H -7.126604 $2.470655-2.255250$

H $-5.4113772 .800845-2.352668$

H $-0.7569121 .955045-0.692448$

H -1.241204 $2.210733-2.368445$

H $-2.969349-1.987738-1.984319$

H -2.735439 -1.911630 -3.747124

H -4.681126 $-0.099022-3.765494$

H -5.352494 -2.392999-1.852084

H -7.394302 -1.982967 -3.420989

H $-7.066649-0.266868-3.349484$

H -9.063313 -2.109306 -0.131188

H -8.347332 -3.150127 -1.486240

H $-8.7600020 .230837-0.111480$

H -8.355402 $0.958674-1.635991$

H -6.4141810 .1189020 .627708$

H -1.901992 $0.251064-3.603280$

C $-1.328175-2.731668 \quad 3.030037$

O $-0.411709-2.969287 \quad 1.943179$

C $-1.172742-3.3655380 .786463$

C $-2.581929-3.6619901 .286872$

C -2.717042 -2.639988 2.400181

H -1.221814 $-2.493736 \quad 0.126717$

C $-0.487837-4.530210 \quad 0.066674$

H $-3.334850-3.5450780 .501421$

H $-2.662690-4.679417 \quad 1.687388$

C -3.855555 -2.929711 3.361521

H $-2.874948-1.6533141 .947226$
C $0.929095-4.211169-0.461905$

O $-0.382934-5.6414210 .964914$

H $-1.119713-4.873238-0.760937$

H $1.628594-4.0982150 .374252$

O $1.376216-5.348055-1.210946$

C $0.996017-2.968567-1.368962$

C $2.304001-2.802931-2.161690$

H $0.820381-2.076297-0.759918$

O $-0.067940-3.044224-2.324567$

H $2.447507-3.668027-2.821464$

H $2.164253-1.944884-2.833530$

H -4.814993 -2.922472 2.834044

H -3.740951 -3.909711 3.836840

H $-3.899210-2.1740744 .152114$

C $-0.878855-1.5288293 .861530$

H $0.121223-5.3430121 .741521$

H $1.321571-6.121316-0.623551$

H $-0.081027-2.205480-2.816597$

H $\quad 0.117622-1.7382614 .269675$

H -1.550745 -1.406655 4.719277

H -1.271870 -3.622136 3.670645

C $4.477029-0.951578 \quad 0.255224$

O $3.387164-1.349215-0.589179$

C $3.560587-2.575436-1.307958$

C $4.821523-2.521429-2.189363$

C $6.057509-2.130356-1.375644$

C $5.799612-0.883399-0.528319$

H 4.2232930 .0683880 .570777

C $4.573184-1.8260741 .510408$

H $3.655290-3.400998-0.596093$

O $4.629993-1.580091-3.244600$

H $4.968138-3.505154-2.651468$

H $6.897585-1.933768-2.053002$

O $6.430226-3.205852-0.519644$

H $6.634495-0.7232490 .164078$

O $5.7615750 .248511-1.400888$

C $3.478155-1.4700812 .484652$

H $4.486439-2.8965931 .304969$

H $5.534688-1.6847722 .018636$

H $5.432076-1.586752-3.794132$

H $6.614934-3.977826-1.081339$

H $5.6576101 .042422-0.849464$

O $3.908755-0.4916403 .331521$

O $2.369045-1.9861162 .477957$

C $2.958206-0.0581984 .308718$

H $2.648490-0.8974474 .938459$

H $3.4371380 .695844 \quad 4.938902$

H 2.0948670 .3951883 .815908

SCF Energy (B3LYP/6-31G**//MMFF) $=-3245.90528432$

0700096

MM̄FF Geometry

C $-2.775017-1.616773-2.763374$

C $-2.018668-2.583793-3.304391$

C $-0.581718-2.887845-2.944144$

O $-0.034308-1.878738-2.065619$

C $-0.513709-4.243292-2.216314$

C $0.876406-4.618500-1.739681$

C $1.184105-4.901287-0.458308$

C $1.944888-4.684615-2.800068$

C $0.245790-4.9674060 .713059$

C $0.149718-3.6756281 .536650$

C $1.465686-3.2966412 .234260$

C $1.200403-2.3470763 .404239$

C $2.504289-1.9178044 .090243$

O $2.316793-2.6498921 .280791$

O $2.201683-0.8208684 .965520$

C $3.472821-1.3873243 .016436$

C $3.087420-3.0289974 .970753$

O $4.755210-1.1141593 .577855$

C $3.617616-2.3165881 .785043$

C $4.390603-1.5637280 .678300$

O $4.350662-3.4845822 .155243$

C $0.594881-0.825722-2.654270$

O $0.739443-0.634276-3.851676$

C $1.0815300 .070689-1.581190$

C $1.7724001 .167836-1.924388$

C $2.3475642 .160008-1.017461$ 
C 2.0609762 .0249900 .453306

C $4.493702-2.297095-0.664520$

C $4.946864-1.346710-1.758989$

C $6.8168160 .199848-2.353918$

O $4.222588-1.067380-2.711537$

C $6.7468331 .483324-1.583664$

C $6.3412932 .651371-2.105104$

C $6.3185803 .937947-1.327468$

C $5.0426614 .729578-1.514889$

C $5.0350925 .862482-2.238129$

C $3.7919904 .260341-0.798870$

C $3.0803743 .163051-1.544760$

N $6.222655-0.851401-1.548886$

H -2.353073 -0.959099 -2.008162

H -2.464164 -3.236706 -4.052333

H $0.003429-2.941379-3.870506$

H $-0.877492-5.038325-2.880534$

H - $-1.210817-4.208530-1.372208$

H $2.218133-5.146043-0.219066$

H $2.883352-5.100477-2.417646$

H $1.618493-5.323220-3.627386$

H $2.164667-3.687109-3.192436$

H $-0.759478-5.2584140 .389561$

H $0.575906-5.787400 \quad 1.364226$

H $-0.185418-2.8544900 .890350$

H $-0.629511-3.8349822 .292611$

H $1.948719-4.2125952 .594236$

H $0.519135-2.8030884 .133317$

H $0.689756-1.4467713 .035089$

H $1.535364-1.1236985 .606039$

H $3.075414-0.4221302 .673813$

H $2.364518-3.3272035 .739672$

H $3.973062-2.6789865 .512683$

H $3.359807-3.9242984 .408180$

H $4.619768-0.5143684 .331418$

H $3.897158-0.6036690 .490848$

H $5.403632-1.3389021 .034159$

H $4.719318-3.8887551 .353736$

H $0.871518-0.198979-0.552697$

H $1.9521861 .355534-2.982638$

H 2.5695091 .1526640 .869388

H $\quad 0.985667 \quad 1.9142260 .628478$

H $2.3755082 .896077 \quad 1.033760$

H $5.197651-3.134292-0.611593$

H $3.521237-2.700951-0.963088$

H $6.3065540 .267160-3.319854$

H $7.862663-0.075023-2.521021$

H $7.0714981 .454761-0.545627$

H $6.0504762 .693830-3.152137$

H $7.1791494 .535268-1.656455$

H $6.4716793 .757774-0.256045$

H $4.1313116 .451299-2.356095$

H $5.9301506 .223867-2.733386$

H $3.0928525 .099164-0.687933$

H $4.072123 \quad 3.951993 \quad 0.213894$

H $3.1817623 .210724-2.629792$

H $6.742856-1.160173-0.733232$

C $-5.167533-1.632896-1.935966$

O $-4.786330-0.830943-0.797242$

C $-4.745279-1.682130 \quad 0.361919$

C -5.602642 -2.889916 0.007365

C $-5.231461-3.083878-1.449951$

H $-3.706106-2.014293 \quad 0.475837$

C $-5.187010-0.9269031 .616462$

H $-5.374882-3.7611050 .628823$

H -6.672461 -2.669975 0.101007

C -6.213337-3.947033 -2.222824

H $-4.241841-3.555106-1.492209$

C -4.3622230 .3401791 .927800$

O $-6.553966-0.5289541 .451752$

H -5.168466 -1.610130 2.474265

H $-4.611047 \quad 1.1219271 .201648$

O $-4.806484 \quad 0.831418 \quad 3.200016$

C -2.835748 0.1349621 .970515

C $-2.041403 \quad 1.3043042 .584983$

H $-2.469465-0.0634190 .956653$

O $-2.554754-1.0288462 .755052$

H -2.2733701 .3845873 .654783$
H -0.9786581 .0300012 .535261$

H $-6.259452-4.953950-1.795276$

H -7.223919 $-3.525820-2.199436$

H $-5.909477-4.037864-3.270578$

C $-4.217176-1.375225-3.110561$

H $-6.816000-0.0524742 .258137$

H -4.5523280 .1758853 .872196$

H - $1.599935-1.1996572 .692372$

H $-4.303051-0.325472-3.415090$

H $-4.521836-1.981142-3.971782$

H $-6.169945-1.291157-2.224255$

C $-1.9980423 .709368-0.274580$

O -1.8303662 .5409230 .539830$

C $-2.256705 \quad 2.663591 \quad 1.898853$

C -1.488152 3.7903602 .609600

C $-1.5999585 .109313 \quad 1.839503$

C $-1.292878 \quad 4.926514 \quad 0.348739$

H $-1.4836103 .471987-1.215002$

C $-3.4766963 .973863-0.599673$

H -3.3248252 .8988191 .923849$

O -0.1156393 .4286862 .741699$

H $-1.8958983 .909372 \quad 3.620813$

H -0.9030885 .8375662 .271927$

O $-2.918525 \quad 5.6223002 .006818$

H $-1.5623365 .828314-0.213225$

$\begin{array}{lllll} & 0 & 0.114635 & 4.740413 & 0.182177\end{array}$

C $-4.0854842 .879664-1.450376$

H -4.0842144 .0543390 .305244$

H $-3.5610364 .908873-1.165623$

H $\quad 0.333224 \quad 4.146243 \quad 3.220037$

H $-2.966466 \quad 6.469308 \quad 1.531591$

H $0.560107 \quad 5.5301390 .532978$

O $-5.3914503 .195065-1.699419$

O $-3.5001551 .881199-1.844343$

C $-6.1183142 .253936-2.495116$

H $-6.2994941 .344053-1.917245$

H $-7.0811052 .701661-2.755234$

H $-5.5787872 .026793-3.419675$

SCF Energy (B3LYP/6-31G**//MMFF) $=-3245.89361742$

\section{7}

MMFF Geometry

C $1.520675-2.275020-2.113908$

C $0.651246-3.285659-2.264222$

C $-0.312976-3.713425-1.189220$

O $-1.660888-3.411859-1.628814$

C $-0.273813-5.228570-0.930939$

C $0.987316-5.723897-0.261664$

C $1.149950-5.8004781 .073086$

C $2.069493-6.222949-1.183611$

C $0.181796-5.3714252 .143893$

C $0.888858-4.617146 \quad 3.279590$

C $1.261935-3.1707112 .912158$

C $2.330788-2.6523363 .876547$

C $2.658592-1.1764353 .625305$

O $0.072601-2.3781803 .019121$

O $3.471341-0.7103754 .713043$

C $1.342030-0.3757933 .622085$

C $3.519029-0.9950692 .371657$

$\begin{array}{llllll}\text { O } & 1.584049 & 0.987260 & 3.277552\end{array}$

C $0.238427-0.9844872 .720211$

C $-1.104219-0.2750593 .026862$

O $\quad 0.596200-0.779566 \quad 1.355117$

C $-2.080435-2.126023-1.487631$

O $-1.425983-1.185650-1.068648$

C $-3.489389-2.054372-1.929316$

C $-4.125784-0.873523-1.907201$

C $-5.514840-0.631629-2.302773$

C $-6.343923-1.827615-2.693136$

C $-2.306971-0.786822 \quad 2.225494$

C $-3.625539-0.2516752 .759717$

C $-6.073402-0.4061442 .386925$

$\begin{array}{lllll}\text { O } & -3.706894 & 0.473003 & 3.747998\end{array}$

C $-6.952228-0.399158 \quad 1.177528$

C $-7.6339230 .682496 \quad 0.770739$

C $-8.5639510 .704484-0.408994$

C $-8.1213751 .678549-1.478097$

C $-8.4666432 .975803-1.417106$ 
C $-7.3509861 .129315-2.657625$ C $-5.9806110 .635181-2.280889$ N $-4.703819-0.6788571 .993242$ H $1.527155-1.717864-1.180579$ H $0.618931-3.827376-3.206411$ H $-0.123553-3.187688-0.244527$ H $-0.434583-5.775141-1.870850$ H $-1.147081-5.506777-0.325377$ H $2.079334-6.2290531 .446395$ H $2.916959-6.649731-0.636491$ H $1.677489-7.006293-1.840506$ H $2.458870-5.411003-1.803815$ H $-0.636236-4.761587 \quad 1.748400$ H $-0.266730-6.2800222 .563710$ H $\quad 0.214199-4.6034764 .145634$ H $1.783650-5.1773803 .579209$ H $1.629745-3.1319141 .880979$ H $3.241933-3.2605123 .813263$ H $1.970727-2.7616304 .909335$ H $2.970700-0.8248095 .538653$ H $0.972077-0.3618604 .656320$ H $4.451307-1.5656122 .461599$ H 3.8213180 .0503402 .255671 H $3.021667-1.3151111 .453568$ H 1.7103601 .0306282 .314366 H $-1.324590-0.4006104 .095270$ H -1.0105190 .8014672 .839029$ H $-0.197855-0.8613920 .804397$ H -3.962883 -2.972099 -2.258589 H -3.579316 $0.006382-1.569662$ H $-5.947914-2.287833-3.605001$ H -7.392856 -1.591362 -2.882166 H $-6.341758-2.575511-1.891972$ H $-2.223787-0.4598731 .185967$ H $-2.351130-1.8810542 .250108$ H -6.1151900 .5406912 .935959$ H -6.384342 -1.203319 3.070192 H $-7.052916-1.3330140 .630207$ H $-7.541433 \quad 1.608068 \quad 1.335295$ H $-9.5619900 .984760-0.046797$ H -8.684035 $-0.295950-0.842721$ H -8.177716 $3.676399-2.193440$ H $-9.044353 \quad 3.365898-0.585529$ H $-7.2135531 .909190-3.418512$ H $-7.9578320 .363781-3.148767$ H $-5.3023211 .432514-1.973895$ H $-4.543220-1.4007661 .295870$ C $3.862962-1.524996-2.588435$ O $3.798739-0.285188-1.849968$ C $4.9766370 .492507-2.137693$

C $5.922137-0.438464-2.888510$

C $4.944072-1.313159-3.646423$ H $4.669725 \quad 1.298158-2.814411$

C $5.5628051 .074161-0.847657$

H $6.6081810 .107683-3.543155$

H $6.523789-1.045301-2.201591$

C $5.561002-2.599359-4.168099$

H $4.537700-0.739071-4.490087$

C $4.6158962 .039735-0.100834$

O $5.874244-0.0188980 .025600$

H $6.5135951 .573658-1.069584$

H 3.7212561 .5049920 .236494

O $5.2971612 .485545 \quad 1.079505$

C $4.206502 \quad 3.287249-0.914404$

C $3.4937594 .374942-0.083695$

H $3.5730142 .989577-1.756750$

O $5.391387 \quad 3.872999-1.464594$

H 4.0965164 .6175990 .799794

H $3.4689225 .295069-0.683788$

H $6.375345-2.379285-4.866266$

H $5.971266-3.206671-3.354269$

H $4.817710-3.203854-4.697074$

C $2.487037-1.838319-3.179435$

H $\quad 6.4421040 .3259650 .734962$

H 5.3630161 .7282091 .684897

H $5.1157904 .603396-2.044359$

H $2.575003-2.617615-3.944770$

H $2.079660-0.940228-3.658569$
H $4.166900-2.305872-1.878733$

C $-0.1414673 .797845-0.686603$

O $1.2494654 .107110-0.848795$

C $2.0600604 .003976 \quad 0.327792$

C $1.5283004 .938714 \quad 1.425911$

C 0.0456364 .6819221 .711505

C $-0.7817884 .645694 \quad 0.424101$

H $-0.6026224 .097025-1.637209$

C $-0.3616892 .292595-0.514308$

H 2.0419962 .9733150 .693291

$\begin{array}{llll}\text { O } & 1.710154 & 6.303319 & 1.051402\end{array}$

H $2.113994 \quad 4.7782732 .338530$

H -0.3408335 .4712612 .367505$

O $-0.108644 \quad 3.4386632 .387206$

H -1.7943014 .2768910 .626325$

O $-0.9149905 .978007-0.080268$

C $0.0009131 .578593-1.793174$

H 0.2423361 .8546540 .284585

H $-1.4073432 .067528-0.273383$

H $1.2599686 .440570 \quad 0.200259$

H 0.4113813 .4812513 .207767

H $-1.3683926 .504562 \quad 0.599973$

O $-1.0903521 .491425-2.605992$

O $1.128627 \quad 1.179653-2.050674$

C $-0.8684630 .856165-3.868687$

H $-1.8148550 .848612-4.415836$

H $-0.130323 \quad 1.416829-4.449944$

H $-0.540867-0.177106-3.722296$

SCF Energy (B3LYP/6-31G**//MMFF)= -3245.92409077

\section{8}

MMFF Geometry

C $-2.147285-1.832623-3.020668$

C $-1.635029-1.941094-1.785142$

C $-0.174389-1.773882-1.465626$

O $-0.028171-0.617357-0.603992$

C $0.386955-2.977108-0.687780$

C $0.690366-4.179319-1.556064$

C $1.871447-4.371278-2.176575$

C $-0.399812-5.212557-1.653836$

C $3.050109-3.437907-2.167271$

C $4.208173-4.015970-1.344677$

C $5.332425-2.989057-1.150432$

C $6.541567-3.631083-0.468637$

C $7.635005-2.599497-0.152199$

O $4.814649-1.936944-0.329710$

O $8.567861-3.2085840 .753512$

C $6.997094-1.4069160 .590962$

C $8.433691-2.211776-1.402189$

O $7.945343-0.358322 \quad 0.778135$

C $5.725126-0.855410-0.096834$

C $5.022967 \quad 0.1594290 .841023$

O $6.095084-0.206294-1.311788$

C $-0.0212210 .606610-1.200034$

$\begin{array}{llll}\text { O } & -0.137465 & 0.848413 & -2.390680\end{array}$

C $0.1470621 .618742-0.135639$

C $-0.0321022 .917193-0.417898$

C $0.093605 \quad 4.0320330 .524359$

C $0.550028 \quad 3.712913 \quad 1.924031$

C 3.6638830 .6693280 .342210

C $3.7831911 .885937-0.554555$

C $4.3333414 .316192-0.497530$

O $3.6580561 .799951-1.773939$

C $3.675635 \quad 5.414502 \quad 0.275747$

C $2.9108526 .361355-0.288820$

$\begin{array}{llll}\text { C } 2.255994 & 7.471828 & 0.482098\end{array}$

C $0.772997 \quad 7.584890 \quad 0.203877$

C $0.283775 \quad 8.595755-0.534284$

C $-0.151043 \quad 6.573243 \quad 0.847783$

C $-0.197416 \quad 5.2756390 .088273$

N $4.0501153 .048308 \quad 0.150019$

H -1.490727 -1.623689-3.861151

H -2.302857 -2.129982 -0.947895

H $0.423457-1.613664-2.372189$

H $-0.298124-3.2647410 .121316$

H $1.295232-2.669294-0.152070$

H $2.012034-5.279378-2.760590$

H $-0.091784-6.085873-2.238747$ 
H $-0.676798-5.567655-0.655733$ H -1.289349 -4.794611 -2.133426 H $3.378666-3.284420-3.202597$ H $2.771075-2.447918-1.794287$ H $3.830352-4.326519-0.361390$ H $4.598566-4.910306-1.846059$ H $5.611975-2.583563-2.129983$ H $6.947277-4.445422-1.081550$ H $6.223179-4.0969400 .474796$ H $8.924847-4.0053440 .324926$ H $6.721487-1.7578321 .595522$ H $8.916668-3.095219-1.836946$ H $9.245411-1.519282-1.153390$ H $7.817927-1.756920-2.180909$ H $8.720798-0.744671 \quad 1.220123$ H $4.836056-0.3394791 .801106$ H $5.6898691 .007917 \quad 1.033851$ H $5.3329410 .297353-1.637349$ H $\quad 0.4059291 .265950 \quad 0.855243$ H $-0.3061463 .193925-1.435196$ H 0.7011924 .5958642 .547664 H 1.5082573 .1822221 .907130 H -0.1889353 .0848892 .433794$ H $3.097840-0.106039-0.184502$ H $3.052570 \quad 0.957928 \quad 1.207052$ H $4.0061634 .286694-1.541778$ H $5.4197344 .448821-0.479977$ H 3.8452055 .4403161 .349635 H $2.7621246 .351906-1.366363$ H 2.7627998 .4069110 .210594 H 2.4089317 .3521791 .561979 H $-0.7808608 .698533-0.716533$ H $0.9367259 .340195-0.977536$ H -1.175207 6.9689080 .876081 H $\quad 0.1338346 .4508551 .895780$ H $-0.5310495 .393381-0.944084$ H 4.2420512 .9770661 .145280 C $-4.252163-0.593805-3.600688$ O $-4.122065 \quad 0.241879-2.430778$ C $-5.4343600 .528018-1.926997$ C $-6.3217580 .500861-3.159781$ C $-5.756044-0.705533-3.888915$ H -5.711609-0.301805-1.266059 C $-5.4389741 .828756-1.120086$ H $-7.3820550 .394540-2.913073$ H $-6.1952831 .402102-3.770506$ C -6.088708 $-0.733994-5.370973$ H $-6.162726-1.614385-3.424910$ C $-4.3188701 .934539-0.063554$ O $-5.2922332 .946281-2.004851$ H $-6.4178271 .954968-0.643272$ H $-3.3649112 .163896-0.554682$ O $-4.613268 \quad 3.056293 \quad 0.777821$ C -4.1335840 .6910670 .824294$ C $-3.0882180 .917099 \quad 1.933015$ H $-3.814263-0.1483440 .198537$ O H -2.2677131 .5274951 .542432$ H -3.5568091 .4627312 .761337$ H $-7.172229-0.779862-5.521587$ H $\quad-5.7153180 .159125-5.883204$ H -5.641626 -1.610205 -5.851328 C -3.615202 -1.958057-3.314594 H -4.461643 $2.824562-2.496425$ H $-4.705113 \quad 3.8343030 .201271$ H -5.6939751 .0843811 .933853$ H -3.746265 -2.625453 -4.174024 H -4.118297 -2.430521 -2.461903 H -3.723814 -0.084709-4.415807 C $-3.176804-2.4343943 .607575$ O $-3.545450-1.143108 \quad 3.102840$ C $-2.501668-0.4075112 .459166$ C $-1.333831-0.1669503 .427296$ C $-0.828360-1.4880634 .014107$ C -1.977996 -2.335753 4.566601 H $-4.045218-2.7602744 .195167$ C -2.967222 -3.447690 2.475929 H $-2.138012-0.976328 \quad 1.596425$
O

H $-0.524407 \quad 0.3383542 .888929$

H $-0.112278-1.2826184 .818949$

O $-0.148545-2.2322163 .006778$

H $-1.626553-3.3442004 .814384$

O $-2.449694-1.7482995 .782631$

C $-4.291618-3.8333641 .862638$

H $-2.306839-3.0906161 .681548$

H -2.517792 -4.371986 2.858604

H -0.9894100 .8499025 .059285$

H $0.582950-1.6827772 .677240$

H -1.705497 -1.731616 6.408139

O $-4.571820-3.027806 \quad 0.799211$

O $-5.003568-4.7303282 .296490$

C $-5.820802-3.2879050 .151900$

H $-5.908337-2.617347-0.705766$

H $-5.856169-4.320453-0.208772$

H $-6.649021-3.0885270 .838454$

SCF Energy $\left(B 3 L Y P / 6-31 G^{* *} / / M M F F\right)=-3245.90110313$

\section{9}

MM̄FF Geometry

C $-5.342373-2.291403-1.574156$

C $-4.065689-2.581693-1.278216$

C $-2.995725-2.760977-2.325641$

O $-1.941475-1.802288-2.070656$

C $-2.379975-4.168223-2.239079$

C $-1.436360-4.466811-3.389269$

C $-0.099435-4.603011-3.288735$

C $-2.100070-4.656567-4.731907$

C $0.778076-4.516769-2.070930$

C $1.738853-3.326626-2.151995$

C $2.962771-3.461408-1.232256$

C $2.594984-3.568552 \quad 0.249115$

C $3.844336-3.6124921 .146043$

O $3.777050-2.299611-1.450314$

O $3.430463-3.4049702 .504514$

C $4.762165-2.431134 \quad 0.772214$

C $4.526143-4.9851381 .113354$

O $5.998441-2.4974321 .478001$

C $5.027224-2.315418-0.743977$

C $5.794942-1.025639-1.116457$

O $5.838791-3.418040-1.166807$

C $-2.119617-0.537914-2.542344$

O $-3.076001-0.112619-3.170291$

C $-0.9433180 .259416-2.130990$

C $-0.9909741 .597555-2.211405$

C $0.0600492 .523335-1.785629$

C $1.3843931 .935862-1.373039$

C $5.1238750 .276575-0.675087$

C $5.7935061 .503616-1.270266$

C $5.6491113 .985773-1.243267$

O $6.745467 \quad 1.447187-2.043463$

C $4.5179984 .955564-1.129156$

C $3.8445345 .433443-2.186242$

C $2.6951926 .394970-2.085337$

C $1.3794835 .740494-2.452195$

C $0.8710055 .891595-3.687412$

C $0.6979994 .968572-1.338599$

C $-0.2030873 .847440-1.787567$

N $5.1807412 .673092-0.842027$

H -5.641552 -2.203115 -2.615854

H $-3.759800-2.654202-0.237249$

H $-3.406605-2.592612-3.328428$

H $-3.174347-4.926488-2.246183$

H $-1.868958-4.279878-1.277320$

H $\quad 0.452447-4.829502-4.201478$

H $-1.399501-5.008718-5.496822$

H -2.899918 $-5.400798-4.658497$

H $-2.528894-3.716246-5.090410$

H $0.198571-4.456941-1.146609$

H $1.350439-5.451158-2.016655$

H $2.103385-3.198967-3.180674$

H $1.200695-2.398270-1.921673$

H $3.522927-4.347912-1.552643$

H $1.966140-4.4448390 .443772$

H $1.992790-2.6946950 .531955$

H $2.763067-4.0784382 .721249$ 
H $4.255173-1.5213111 .117221$ H $3.838333-5.7615961 .469419$ H $5.386850-5.0146311 .790594$ H $4.861591-5.2764450 .116146$ H $5.788068-2.5631902 .425297$ H $6.816479-1.067336-0.716046$ H $5.911501-1.003765-2.209241$ H $6.279512-3.175344-1.997993$ H $-0.088887-0.272706-1.729223$ H -1.898388 2.064556 -2.592302 H $1.7638691 .254982-2.143504$ H $1.2851181 .382837-0.432832$ H $2.1622872 .688473-1.230680$ H $4.0723070 .285180-0.982803$ H 5.1685210 .3716810 .415263 H $6.0513003 .933761-2.260833$ H $6.4658264 .273429-0.573493$ H $4.2374665 .272629-0.127769$ H $4.1258895 .110177-3.186174$ H $2.9026537 .243614-2.749783$ H $2.6195876 .824312-1.078353$ H -0.079395 $5.459089-3.979246$ H $1.3976176 .464908-4.443919$ H $0.1014695 .674458-0.746111$ H $1.4483284 .583812-0.640977$ H -1.190008 4.181797 -2.109778 H $4.5170352 .615025-0.074836$ C $-6.780378-0.540109-0.537729$ O $-5.6476530 .218231-0.072936$ C -6.0886631 .1804810 .901900$ C $\begin{array}{llll}-7.600322 & 1.257948 & 0.744537\end{array}$ C $-7.928900-0.187300 \quad 0.407347$ H -5.8649140 .7552961 .887753$ C -5.3113182 .4902360 .719899$ H $-8.096773 \quad 1.6120301 .652830$ H $-7.891514 \quad 1.915080-0.082869$ C $-9.307059-0.372559-0.202483$ H $-7.859011-0.787506 \quad 1.324479$ C -3.7847322 .2850400 .609027$ O $-5.7428593 .132066-0.486703$ H -5.5474193 .1843781 .533867$ H $-3.5552731 .750314-0.320461$ $\begin{array}{lllll}\text { O } & -3.157837 & 3.567560 & 0.499884\end{array}$ C -3.1592781 .5367101 .799285$ C -1.6862031 .1957811 .549536$ H $-3.697503 \quad 0.5998141 .960827$ O -3.2913822 .3131822 .985631$ H - 1.6139630 .5630270 .657437 H -1.124190 2.108471 1.310411 H $-10.084103-0.0498200 .498123$ H $-9.4208270 .210722-1.122430$ H -9.486865 -1.424428 -0.446184 C $-6.408243-2.023967-0.546138$ H -5.593982 $2.510422-1.220006$ H -3.557658 $4.020857-0.262226$ H -2.8289973 .1558872 .836654$ H -7.283322 -2.638753 -0.783618 H $-6.053685-2.3256180 .447413$ H $-6.999697-0.192347-1.555847$ C $1.220912-0.5109353 .098997$ O $0.310626 \quad 0.0974412 .176953$ C $-0.954794 \quad 0.5091322 .715094$ C $-1.695553-0.6980663 .308403$ C $-0.830172-1.4233584 .343672$ C $0.574050-1.7124573 .807079$ H $2.037134-0.8907112 .471782$ C 1.8166670 .5229994 .058651 H $-0.785106 \quad 1.2585843 .496034$ O $-2.047768-1.5964282 .257922$ H -2.622544 -0.3589713 .784531$ H $-1.317027-2.3665934 .619808$ O $-0.749211-0.6157085 .514047$ H $1.228151-2.0537034 .617178$ O $0.484163-2.7953822 .876436$ C 2.6233801 .5423113 .288562 H 1.0608321 .0487314 .649360 H 2.4932070 .0455424 .777123 H $-2.548356-2.3294662 .654947$
H $-0.219466-1.100806 \quad 6.169347$

H -0.023810 -2.485452 2.106986

O $1.8667762 .652570 \quad 3.053832$

O 3.7732291 .3576762 .911173

C 2.4996193 .6786372 .284121

H 1.8012324 .5145072 .191044

H 3.4048734 .0307072 .787724

H 2.7336723 .3052951 .283879

SCF Energy (B3LYP/6-31G**//MMFF) $=-3245.92575175$

\section{0}

MM̄FF Geometry

C $2.558815-3.3100740 .760195$

C $1.887746-4.154355-0.037097$

C $0.689116-3.780841-0.878226$

O $0.003359-2.634669-0.320558$

C $1.139860-3.437159-2.309149$

C $0.010277-2.996003-3.220249$

C $-0.008232-1.824056-3.885452$

C $-1.132014-3.965849-3.378413$

C $1.068680-0.777662-3.921853$

C $0.907567 \quad 0.353333-2.897212$

C $-0.3578291 .199106-3.109072$

C $-0.210712 \quad 2.560989-2.427056$

C $-1.4825373 .407978-2.568716$

O $-1.4726450 .498787-2.544705$

O $-1.379790 \quad 4.521815-1.669488$

C $-2.6872642 .564914-2.110021$

C $-1.6244124 .002055-3.974786$

O $-3.912283 \quad 3.252637-2.356388$

C $-2.7356801 .150173-2.738010$

C $-3.8282250 .318373-2.027242$

O $-3.0613581 .261932-4.124186$

C $-0.967070-2.8956390 .598348$

O $-1.305654-3.9939051 .012155$

C $-1.563396-1.608707 \quad 1.023404$

C $-2.674615-1.6398831 .774656$

C $-3.427218-0.4939112 .282663$

C $-2.828066 \quad 0.876556 \quad 2.120305$

C $-3.890105-1.157558-2.439593$

C $-4.850027-1.926337-1.550244$

C $-7.249098-1.997760-0.863679$

O $-4.458685-2.801151-0.781313$

C $-7.515876-0.961548 \quad 0.184415$

C $-7.609038-1.2282511 .495933$

C $-7.902809-0.1822832 .534228$

C $-6.935853-0.209673 \quad 3.697853$

C $-7.331943-0.6216794 .914392$

C -5.5319340 .3170643 .477396$

C -4.604651-0.720496 2.902558

N $-6.167083-1.528030-1.708174$

H $2.246853-2.2702160 .818317$

H $2.219374-5.187493-0.109939$

H $0.009028-4.641335-0.907872$

H $1.628764-4.309857-2.761891$

H $1.905715-2.656573-2.245485$

H $-0.875841-1.596388-4.503334$

H $-1.835829-3.654235-4.157940$

H $-0.753731-4.954625-3.657360$

H $-1.696848-4.056455-2.445543$

H $2.057059-1.233323-3.796075$

H $1.087264-0.348388-4.932073$

H $0.914592-0.068453-1.884022$

H $1.7916470 .995482-2.989836$

H $-0.516093 \quad 1.331876-4.185631$

H $0.6543343 .105957-2.824967$

H $-0.0076292 .410613-1.358016$

H $-0.5647965 .006356-1.885730$

H -2.609435 $2.455413-1.019465$

H $-0.7607764 .635308-4.211162$

H $-2.5010784 .655894-4.040288$

H $-1.701128 \quad 3.243775-4.756549$

H $-3.8439494 .125940-1.933749$

H $-3.6559340 .349691-0.944917$

H $-4.8069460 .779210-2.208084$

H $-3.4009720 .408786-4.436763$

H $-1.090102-0.6890810 .700183$

H -3.096937 -2.611299 2.030899 
H $-2.689263 \quad 1.1234921 .064133$ H $-1.856277 \quad 0.9232262 .622335$ H $-3.440793 \quad 1.6693802 .557434$ H -4.212583 -1.274412 -3.479487 H -2.905162 -1.626027 -2.345835 H $-6.989475-2.968973-0.430680$ H -8.130511 -2.116690 -1.501049 H $-7.6569220 .061449-0.157798$ H -7.498236 -2.2541461 .839644$ H $-8.926600-0.3525502 .892034$ H $-7.9028490 .823822 \quad 2.096507$ H -6.652066 -0.619369 5.760089 H -8.342191 -0.9754125 .091252$ H -5.1131890 .6565414 .433614$ H -5.5911091 .1978232 .827629$ H -4.933460 -1.753457 3.020234 H $-6.382849-0.789604-2.371209$ C $5.061351-3.1004301 .127352$ O $4.947074-1.6737910 .947216$ C $5.610161-1.316987-0.280576$ C $6.478389-2.514501-0.646363$ C $5.593124-3.659074-0.192792$ H $4.831052-1.212569-1.044892$ C $6.372999-0.000525-0.121532$ H $6.712912-2.548470-1.714492$ H $7.422152-2.515718-0.088132$ C $6.327295-4.980952-0.054327$ H $4.780293-3.778040-0.920267$ C $5.4793721 .211558 \quad 0.222088$ O $7.334267-0.1581430 .928927$ H $6.9464690 .201590-1.034238$ H $5.076110 \quad 1.096774 \quad 1.234177$ O 6.3280462 .3665220 .265441

C $4.3336081 .465241-0.777149$

C $3.6367442 .829525-0.629032$

H $3.5916600 .663281-0.693888$

O $4.8707471 .405078-2.103445$ H $4.3562833 .636102-0.819303$ H $2.8995332 .899967-1.440094$ H $6.744807-5.291453-1.017696$ H $7.153015-4.9092410 .661569$ H $5.649756-5.7686970 .289662$ C $3.735576-3.6927911 .610820$ H 7.8014880 .6896291 .023466 H $6.6675442 .514759-0.633955$ H $4.1287831 .487930-2.726178$ H $3.530360-3.3176472 .621483$ H $3.821934-4.7822911 .695587$ H $5.808610-3.2481381 .918169$ C 1.1263412 .1154712 .046426 O 1.9210282 .0545240 .854321 C $2.932508 \quad 3.0575070 .717597$ C 2.3308864 .4729720 .797709 C 1.4693394 .6489142 .049414 C $0.473797 \quad 3.4969372 .212887$ H $\quad 0.323752 \quad 1.389161 \quad 1.872647$ C 1.9138351 .6759873 .286102 H 3.6659272 .9389221 .520771

O $1.5401384 .729848-0.360951$ H 3.1518395 .2004460 .800510 H 0.9248225 .5984441 .981262 O $2.326170 \quad 4.720030 \quad 3.184938$ H $-0.0194283 .550843 \quad 3.190485$ O $-0.553227 \quad 3.610971 \quad 1.225842$ C 2.0242310 .1726923 .345157 H 2.9304972 .0767023 .319708 H 1.4202942 .0041044 .209112 H $1.2117905 .642716-0.295936$ H 1.7652084 .8503903 .968450 H -0.9853204 .4732531 .348065$ O $0.898688-0.3526083 .909055$ O $2.989946-0.4552592 .933410$ C $0.882359-1.7759324 .051222$ H - $0.031611-2.0537044 .583017$ H $\quad 0.872925-2.2511923 .067669$ H $1.744258-2.1128094 .635105$

SCF Energy (B3LYP/6-31G**//MMFF) $=-3245.89290776$
0700101

MMFF Geometry

C $0.967588-3.799247-1.755498$

C $-0.024889-2.921336-1.548589$

C $-1.053314-2.559980-2.588882$

O $-2.332202-2.715686-1.926362$

C $-0.935745-1.106121-3.077909$

C $0.444046-0.706163-3.545268$

C $1.2899910 .056134-2.825762$

C $0.834422-1.204698-4.910256$

C $1.0668750 .600798-1.442914$

C $1.0427502 .133269-1.402271$

C $-0.1466572 .756623-2.147745$

C $-0.1255224 .279703-1.999743$

C -1.353181 $4.937066-2.647596$

O $-1.3504622 .226185-1.585769$

O $-1.4151716 .297231-2.190357$

C $-2.6253974 .227339-2.134533$

C -1.224482 $5.001054-4.174145$

O $-3.7835944 .714213-2.810133$

C $-2.5516752 .682497-2.215779$

C $-3.7604942 .006642-1.519512$

O $-2.5917192 .297889-3.595828$

C $-3.452179-2.861726-2.673478$

O $-3.513239-2.923652-3.893181$

C $-4.662324-2.918759-1.815898$

C $-4.616993-2.908434-0.473703$

C $-5.759659-2.9499660 .441365$

C $-7.140681-2.920251-0.157323$

C -3.7736152 .0962700 .010208$

C -2.9928160 .9877960 .700570$

C -2.2959640 .2590022 .981534$

O $-2.580736-0.0121910 .120077$

C -3.358134 -0.530849 3.692686

C $-4.684487-0.3182513 .674956$

C $-5.676901-1.1539634 .434898$

C $-6.760765-1.7247163 .545988$

C $-7.940191-1.0960423 .407233$

C $-6.513986-3.0645092 .890552$

C $-5.517509-2.9931781 .768665$

N $-2.8532001 .231728 \quad 2.060429$

H $1.061951-4.271862-2.730041$

H $-0.127351-2.455078-0.571136$

H $-1.000757-3.256341-3.435538$

H -1.308618 -0.431240 -2.300960

H $-1.643083-0.945691-3.902917$

H $2.2616270 .295961-3.254842$

H $\quad 0.835875-2.299066-4.932717$

H $0.127205-0.841780-5.663211$

H $1.833708-0.867727-5.205420$

H $\quad 0.1644190 .203977-0.970943$

H $1.8880730 .241140-0.815749$

H $0.9887102 .431688-0.348962$

H $1.9839312 .532121-1.800948$

H $-0.0876752 .478254-3.205852$

H $0.7994984 .698242-2.415689$

H $-0.1180334 .541899-0.931990$

H $-0.584967 \quad 6.736690-2.442908$

H -2.745515 $4.525856-1.086624$

H $-0.3255155 .561009-4.458854$

H $-2.0656715 .543748-4.619368$

H -1.160050 4.016256 -4.641336

H $-3.8037484 .307292-3.693067$

H $-4.6859172 .446992-1.912464$

H $-3.8048890 .950437-1.816639$

H $-2.8432331 .361041-3.647540$

H -5.601596-2.970905 -2.356722

H $-3.647100-2.8680180 .019409$

H -7.260975 -2.044850 -0.805550

H -7.935633 -2.858619 0.589397

H $-7.322056-3.823919-0.749445$

H -3.4144453 .0679210 .360712$

H $-4.8121351 .990500 \quad 0.349672$

H -1.6925170 .8055013 .712604$

H -1.641221 -0.4258272 .432784$

H $-2.980458-1.3619274 .285746$

H -5.0948920 .5144513 .109689$

H $-6.127890-0.5154865 .206118$ 
H $-5.183768-1.9681124 .980032$ H -8.734645 -1.511535 2.796497 H $-8.135306-0.148173 \quad 3.898066$ H -7.454926 -3.512619 2.552338 H $-6.135640-3.7669203 .644779$ H -4.480807 -3.003308 2.106482 H -3.2787172 .0642112 .457871$

C $3.384383-3.805420-0.870629$

O $3.564980-2.419966-0.521233$

C $4.754485-1.946026-1.191355$

C $5.249016-3.094923-2.076984$

C $4.000044-3.937016-2.257375$

H $4.444460-1.116412-1.835627$

C $5.803806-1.472239-0.171904$

H $5.657561-2.731301-3.025381$

H $6.039193-3.675183-1.585832$

C $4.283184-5.366380-2.681473$

H $3.370702-3.448564-3.011968$

C $5.361405-0.2959790 .724590$

O $6.131078-2.5744360 .685241$

H $6.728488-1.210953-0.700736$

H $4.567109-0.6304531 .399401$

O 6.4715350 .0497491 .563729

C $4.9137010 .956566-0.043718$

C $4.6956702 .215740 \quad 0.813874$

H $3.9952280 .733119-0.589874$

O $5.9066551 .271959-1.026015$

H 5.6614382 .6015291 .165476

H $4.3117692 .986140 \quad 0.131696$

H $4.804029-5.385767-3.644360$

H $4.910732-5.885653-1.949352$

H $3.352991-5.932948-2.789511$

C $1.929491-4.244943-0.691919$

H $6.795327-2.2590821 .321854$

H 7.1778300 .3923320 .989166

H $5.5727942 .019005-1.551576$

H $1.575181-3.9052990 .288265$

H $1.894884-5.341473-0.669737$

H $3.981760-4.376609-0.145822$

C 1.5250551 .2123762 .546087

O 2.5310851 .4520101 .552403

C 3.7388452 .0597492 .010985

C 3.4441063 .4181772 .673860

C 2.3728803 .3013453 .762685

C 1.1523112 .5127093 .278566

H $\quad 0.645707 \quad 0.885042 \quad 1.977554$

C 1.9172680 .0783703 .504990

H 4.2158611 .3983412 .741452

O 3.0088824 .3670841 .699667

H 4.3733043 .8089233 .104375

H 2.0506014 .3052094 .066036

O 2.9192162 .6548634 .907829

H 0.4917082 .2888614 .124232

O $\quad 0.413434 \quad 3.345798 \quad 2.380275$

C $2.001245-1.2620112 .808866$

H 2.8885870 .2671183 .969513

H $1.163196-0.0184154 .294574$

H 2.2202494 .0034331 .261754

H 3.6694113 .1902885 .217812

H -0.3874362 .8597742 .118857$

O $2.621438-2.1504493 .641055$

O $1.566140-1.5059031 .692534$

C $2.783919-3.4748013 .125559$

H $1.815179-3.8985952 .844886$

H $3.466098-3.4614952 .271299$

H $3.221085-4.0945453 .913028$

SCF Energy (B3LYP/6-31G**//MMFF)= -3245.91609351

\section{2}

MM̄FF Geometry

C $0.157849-3.7375751 .491344$

C $0.224175-2.7966912 .445544$

C $1.307379-1.7538042 .504521$

O $0.647331-0.4732342 .647036$

C $2.243667-1.9549413 .710423$

C $3.097247-3.2009413 .648598$

C $4.231821-3.3016322 .929376$

C $2.639492-4.3473984 .511403$
C $4.838749-2.2587442 .028567$

C $5.229308-2.8715560 .678293$

C $5.764476-1.881989-0.370420$

C $6.830233-0.9243700 .167688$

C $7.3361190 .043052-0.914512$

O $4.666655-1.137580-0.922981$

O $8.061866 \quad 1.078241-0.232451$

C $6.1173720 .690430-1.612302$

C $8.335738-0.635279-1.858453$

O $6.522236 \quad 1.475152-2.731002$

C $5.049047-0.334890-2.054434$

C $3.7887210 .329979-2.653623$

O $5.598078-1.150183-3.102001$

C 1.3403400 .6316492 .261232

O $2.460408 \quad 0.667190 \quad 1.777950$

C 0.4944981 .8114432 .542640

C 0.9303763 .0305912 .192630

C 0.2134344 .2930302 .379583

C $-1.0923514 .247053 \quad 3.127676$

C $3.0272481 .297591-1.746289$

C $1.8658741 .947137-2.485678$

C $0.1370693 .706626-2.257744$

O $1.5148071 .609916-3.613944$

C $-0.0412694 .957090-1.457459$

C -1.207895 $5.320447-0.900863$

C $-1.4951446 .553484-0.082350$

C $-0.295808 \quad 7.2620030 .511280$

C $0.2695278 .307854-0.114915$

C $0.1844626 .813397 \quad 1.876425$

C 0.7460565 .4178991 .857402

N $1.2698012 .957339-1.743564$

H $0.922559-3.7631970 .718686$

H $-0.520933-2.7845793 .237307$

H $1.874865-1.7571321 .565831$

H $1.655598-1.9491324 .639068$

H $2.899008-1.0807723 .820788$

H $4.797495-4.2303912 .995939$

H $3.305451-5.2138984 .439714$

H $2.608831-4.0412565 .562185$

H $1.639021-4.6778824 .217372$

H $4.166614-1.4136241 .866545$

H $5.732678-1.8724452 .531440$

H $5.998815-3.6353530 .853611$

H $4.356577-3.3870910 .255414$

H $\quad 6.191782-2.501109-1.168587$

H $7.666168-1.4796390 .610744$

H $6.405557-0.3163550 .977633$

H $8.4362401 .675906-0.901495$

H $5.6693411 .381591-0.887903$

H $9.216796-0.973025-1.299513$

H $8.709787 \quad 0.067497-2.611137$

H $7.920335-1.502486-2.375367$

H $7.1102692 .175980-2.403203$

H $4.0609100 .838812-3.588462$

H $3.090773-0.464649-2.954181$

H $4.864869-1.566436-3.584851$

H -0.4639321 .6431803 .020069$

H 1.9006953 .1154511 .704702

H $-0.929472 \quad 3.9127194 .158225$

H -1.7894153 .5563832 .644483$

H -1.6029205 .2102563 .181815$

H $2.6283090 .765316-0.877997$

H $3.6844282 .099637-1.396535$

H $-0.7400013 .052740-2.221260$

H $\quad 0.3294703 .957008-3.306435$

H $0.8336755 .592602-1.353241$

H -2.070094 $4.675436-1.058883$

H $-2.1740096 .275746 \quad 0.734319$

H $-2.0691407 .246400-0.711462$

H $\quad \begin{array}{llll}1.108779 & 8.841317 & 0.318519\end{array}$

H $-0.0930748 .650039-1.078583$

H -0.6330846 .9288682 .593534$

H 0.9761937 .4804302 .242256

H 1.6987075 .3451291 .330675

H $1.5078853 .045253-0.759568$

C - $-1.482723-5.0093020 .059667$

O $-2.430888-3.957884-0.228346$

C $-3.603194-4.546246-0.824770$ 
C $-3.224074-5.978138-1.184130$ C $-2.261648-6.319777-0.068751$ H $-4.371345-4.576016-0.043710$ C $-4.097491-3.731314-2.024944$ H $-4.094008-6.640952-1.221332$ H $-2.716799-6.032168-2.154617$ C $-1.395712-7.531536-0.369874$ H $-2.836931-6.5069980 .848026$ C $-4.527630-2.282332-1.722198$ O $-3.061708-3.676049-3.014979$ H $-4.931934-4.262191-2.499122$ H $-3.647725-1.659984-1.528050$ O $-5.131236-1.762868-2.915589$ C $-5.534162-2.132522-0.567921$ C $-6.249494-0.771093-0.484102$ H $-5.021212-2.3257820 .380695$ O $-6.555745-3.129207-0.687024$ H $-6.951705-0.668559-1.321820$ H $-6.876813-0.804290 \quad 0.416926$ H -2.017565 -8.419630 -0.522827 H $-0.796332-7.381620-1.274281$ H $-0.710130-7.7393780 .457333$ C $-0.881489-4.821587 \quad 1.455298$ H $-2.279807-3.275740-2.597678$ H $-4.477985-1.848969-3.630991$ H $-6.990853-3.002774-1.547745$ H $-0.387504-5.7478231 .771085$ H - $-1.679595-4.6070252 .176685$ H $-0.703237-4.953231-0.711463$ C $-3.483802 \quad 1.3610580 .849107$ \begin{tabular}{lllll}
\hline & -4.452076 & 0.315486 & 0.684743
\end{tabular} C $-5.3297510 .457402-0.433745$ C $-6.1390821 .760937-0.322144$ C $-5.2191652 .972749-0.146772$ C -4.1701552 .7367540 .943814$ H -3.0072601 .1513601 .815254$ C -2.389044 $1.312293-0.228050$ H $-4.7394070 .482725-1.354921$ O $\quad-7.030313 \quad 1.678097 \quad 0.788726$ H -6.749793 $1.875173-1.225878$ H -5.8168103 .8531620 .119464$ O $-4.558473 \quad 3.255246-1.376302$ H -3.417815 3.5323490 .910145 O $-4.825392 \quad 2.832926 \quad 2.211558$ C -1.606780 $0.017738-0.206802$ H $-2.8082541 .418322-1.231878$ H $-1.6765342 .126134-0.055152$ H -7.543921 2.5032300 .815751 H -5.242862 $3.434188-2.043500$ H -4.147202 2.7407062 .902012 O $-0.735626 \quad 0.013661-1.258567$ O $-1.739327-0.8734520 .619466$ C $0.065726-1.162912-1.401438$ H $0.545621-1.129600-2.382997$ H $0.840926-1.176492-0.631597$ H $-0.549849-2.065130-1.348401$

SCF Energy (B3LYP/6-31G**/MMFF) $=-3245.89985577$

0700103

MM̄FF Geometry

C $-4.690286 \quad 3.4841720 .201069$

C $-3.4141303 .537961-0.208018$

C -2.3310054 .2673870 .542994$

O $-1.324185 \quad 3.297662 \quad 0.917219$

C $-1.662616 \quad 5.320795-0.357323$

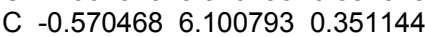

C $0.740965 \quad 6.048918 \quad 0.043646$

C -1.0380757 .0057361 .463660$

C $1.4173515 .269230-1.049699$

C $2.1716954 .057899-0.497292$

C $3.0854303 .383599-1.532892$

C $2.3077812 .782516-2.707263$

C $3.2254632 .018585-3.675541$

O $3.8070732 .351469-0.842939$

O $2.394631 \quad 1.235259-4.545967$

C $4.0898391 .031279-2.864938$

C $4.0208102 .967070-4.580421$

O $5.0541850 .390840-3.695558$
C $4.7869161 .680337-1.650325$

C $5.512190 \quad 0.647457-0.756571$

O $5.7851152 .598239-2.113206$

C -1.536685 2.581761 2.055166

$\begin{array}{llll}\text { O } & -2.482830 & 2.674789 & 2.820367\end{array}$

C $-0.410291 \quad 1.6334752 .195618$

C -0.4404630 .6960113 .154718$

C $0.578023-0.3314893 .380133$

C $1.809612-0.2881922 .516223$

C $4.628372-0.478985-0.222926$

C $5.367599-1.312807 \quad 0.804070$

C $6.058727-1.3195463 .201427$

O $5.865926-2.3985450 .515903$

C $5.069230-2.1929613 .907015$

C $4.518199-1.8887365 .091795$

C $3.549506-2.7764915 .820307$

C $2.163569-2.1733385 .896242$

C $1.770407-1.5093676 .997140$

C $1.259137-2.4185674 .705191$

C $0.371244-1.257698 \quad 4.339575$

N $5.422586-0.7086192 .049457$

H $-4.987345 \quad 4.017302 \quad 1.100929$

H -3.115029 $2.986324-1.096458$

H $-2.733813 \quad 4.7483121 .442798$

H $-2.4157486 .032727-0.720321$

H $-1.2671534 .823031-1.248262$

H 1.4267156 .6541710 .636350

H -0.2362967 .6500551 .840072$

H -1.8407057 .6604921 .108546$

H -1.4116886 .4201692 .308988$

H $0.7079234 .957022-1.819062$

H $2.1228205 .944495-1.550098$

H 2.7873404 .3599020 .361275

H $1.4615193 .322072-0.099452$

H $3.8012714 .133609-1.889474$

H $1.7530073 .552549-3.255234$

H $1.5515862 .084498-2.321309$

H $1.7855221 .839828-5.003606$

H $3.4142340 .244634-2.507868$

H $3.3406603 .575868-5.188115$

H $4.6385392 .408279-5.292112$

H $4.6656663 .651486-4.025855$

H $4.574702-0.008367-4.441669$

H $6.3651040 .216803-1.297773$

H 5.9549841 .1826610 .094941

H $6.4282492 .740871-1.398899$

H 0.4042941 .7152001 .485258

H -1.2995420 .6593153 .823130$

H 2.3344300 .6654842 .641228

H $1.544639-0.4070611 .461162$

H $2.529724-1.0728712 .748679$

H $3.714435-0.0830000 .231259$

H $4.321826-1.151149-1.030758$

H $6.423580-0.5095653 .840928$

H $6.916471-1.9167732 .875571$

H $4.806205-3.1301293 .422052$

H $4.785664-0.9531265 .578011$

H $3.945884-2.9505886 .829246$

H $3.489914-3.7696565 .357317$

H $0.772535-1.0957917 .092692$

H 2.441595-1.369504 7.838462

H $\quad 0.623510-3.2861564 .925766$

H $1.851772-2.7272473 .837582$

H $-0.549174-1.2072884 .922225$

H 4.9142020 .1578872 .199667

C $-6.129097 \quad 1.4636350 .385229$

$\begin{array}{llll}\text { O } & -5.004545 & 0.565358 & 0.470484\end{array}$

C $-5.483318-0.7910510 .412340$

C $-7.003216-0.7099450 .498548$

C $-7.2730720 .622847-0.175663$

H $-5.211512-1.179031-0.575775$

C $-4.811316-1.6278241 .506735$

H -7.491542 -1.551838 -0.001479

H $-7.351293-0.688864 \quad 1.537804$

C $-8.654764 \quad 1.1814850 .114833$

H $-7.1529930 .499643-1.260405$

C $-3.268685-1.5923131 .469040$

O $-5.220000-1.1369292 .790507$ 
H $-5.165694-2.6633701 .453452$ H -2.909372 -0.5891121 .725354$ O $-2.782285-2.4688962 .493146$ C $-2.655390-2.0292310 .126385$ C -1.114668 $-2.062076 \quad 0.166730$ H $-2.965922-1.332660-0.659353$ O $-3.161586-3.315619-0.223947$ H $-0.752028-1.1000000 .543463$ H $-0.792597-2.8498330 .858914$ H $-9.4296360 .497685-0.246826$ H $-8.810841 \quad 1.3284411 .188848$ H $-8.7982112 .146347-0.381327$ C $-5.7512692 .665812-0.481144$ H $-4.965108-0.1994652 .840818$ H -3.165369 -2.169804 3.335608 H $-2.886045-3.9388230 .469919$ H $-6.6233573 .304871-0.657117$ H $-5.3917972 .322721-1.459140$ H $-6.358327 \quad 1.7865521 .409866$ C $-0.349995-4.069077-2.897423$ O $-0.800490-3.668582-1.597808$ C $-0.486149-2.326438-1.216235$ C $1.037764-2.123077-1.200694$ C $1.662445-2.514629-2.543747$ C $1.170479-3.883061-3.028032$ H $-0.561726-5.145035-2.946721$ C -1.146563 -3.383666 -4.013156 H $-0.937666-1.629259-1.930514$ O $1.610748-2.910788-0.158668$ H $1.252588-1.070687-0.978666$ H $2.753991-2.535470-2.441743$

O $1.333431-1.514039-3.502050$ H $1.469511-4.046390-4.069407$ O $1.815846-4.901396-2.257336$ C -2.604283 -3.775076-3.944837 H $-1.072868-2.293074-3.979416$ H $-0.776057-3.686059-4.999755$ H $2.566929-2.734786-0.148296$ H $1.773835-1.749928-4.336176$ H $\quad 1.510358-4.816876-1.337834$ O $-3.350206-2.723955-3.499698$ O $-3.025013-4.887576-4.234656$ C $-4.753709-2.973085-3.374840$ H -5.233433 -2.051639-3.034419 H $-5.177853-3.251635-4.344153$ H $-4.934065-3.757593-2.633844$

SCF Energy (B3LYP/6-31G**//MMFF) $=-3245.91695046$

0700104

MM̄FF Geometry

C $0.254839-1.5136133 .046467$

C $-0.701446-0.8889903 .748685$

C $-1.158341 \quad 0.5146843 .451397$

$\begin{array}{lllll}\text { O } & -2.579652 & 0.506811 & 3.159981\end{array}$

C -0.9718571 .4369834 .669606$

C 0.3663112 .1439234 .703273

C 0.5527873 .3969674 .243193

C 1.4952351 .3935645 .355508

C -0.4792114 .2489853 .557074$

C 0.1031165 .1871112 .491616

C 0.8765374 .4871021 .362277

C $1.298638 \quad 5.519470 \quad 0.311276$

C $1.9982414 .873253-0.889994$

$\begin{array}{lllll}\text { O } & 0.037898 & 3.487392 & 0.773429\end{array}$

O $2.0564375 .867225-1.924754$

C $1.1257933 .707185-1.398349$

C $3.4506984 .508503-0.566959$

O $1.7936592 .998053-2.439088$

C $0.6658292 .742229-0.279294$

C $-0.339211 \quad 1.693287-0.809065$

O 1.8092832 .0241570 .201575

C -2.984596 -0.000443 1.965088

O $-2.295299-0.5490871 .121134$

$\begin{array}{llll}\text { C } & -4.437828 & 0.243649 & 1.850981\end{array}$

C $-5.149242-0.4221320 .929910$

C $-6.582531-0.2656970 .675353$

C $-7.2757310 .906438 \quad 1.320114$

C $-1.7343922 .233831-1.136763$
C $-2.5596521 .245533-1.946864$

C $-4.6498251 .156548-3.296812$

O $-2.1551720 .128970-2.259380$

C $-6.0769601 .191938-2.850213$

C $-6.9429490 .206915-3.135333$

C $-8.3914190 .208023-2.735284$

C $-8.804002-1.082695-2.060666$

C $-9.360147-2.082418-2.765877$

C $-8.636364-1.187187-0.560616$

C -7.192971 -1.151782 -0.138956

N $-3.7983621 .772037-2.293581$

H $0.743982-0.9870622 .230160$

H -1.196846 -1.413176 4.562204

H -0.6418270 .9376872 .579935$

H -1.113471 0.8805835 .606475

H -1.7801872 .1799074 .691766$

H 1.5335203 .8529624 .366810

H $2.404078 \quad 1.9997155 .434673$

H 1.2157021 .0887276 .369284

H $\quad \begin{array}{llll}1.748097 & 0.499588 & 4.778786\end{array}$

H -1.2547683 .6334153 .088581$

H -0.9736014 .8654254 .317699$

H -0.7398185 .7431062 .059905$

H 0.7557195 .9263552 .973418

H 1.7619414 .0033861 .790151

H 1.9379246 .2912590 .758425

H $0.4068996 .048156-0.054747$

H $2.4273425 .444255-2.718395$

H $0.2441324 .160981-1.865913$

H $4.0123245 .404299-0.275244$

H $3.9640394 .111734-1.449544$

H 3.5414513 .7827520 .243643

H $2.4725492 .436592-2.026995$

H $0.0963801 .186289-1.678401$

H $-0.4602140 .903002-0.057483$

H $1.504148 \quad 1.2168370 .647602$

H -4.8811970 .9655192 .527443$

H -4.639610 -1.155549 0.306078

H -8.2996821 .0560170 .973264$

H -6.7424931 .8383141 .098935$

H -7.3189680 .7759632 .406855$

H -2.275077 2.460061 -0.210526

H -1.664584 3.154369-1.724511

H $-4.3091130 .137546-3.506901$

H $-4.5354671 .753616-4.207677$

H $-6.4209562 .061307-2.295550$

H -6.597434 -0.643297 -3.720274

H $-8.9892170 .357027-3.644151$

H -8.628122 $1.056215-2.081104$

H -9.681100 -3.002416 -2.288803

H $-9.503201-2.003911-3.838637$

H -9.058097 -2.132830 -0.195532

H $-9.238016-0.404454-0.091816$

H $-6.598692-1.968186-0.552518$

H $-3.9935132 .739407-2.051147$

C $0.420211-3.8857952 .162574$

O $1.045037-3.4261260 .946864$

C $0.119259-3.611720-0.140243$

C $-0.960698-4.5503600 .385826$

C $-1.056188-4.1056461 .832192$

H $-0.336420-2.634358-0.338819$

C $0.848782-4.115537-1.388780$

H -1.903468 -4.446665 -0.159799

H $-0.648495-5.5997820 .330015$

C $-1.748813-5.1088652 .737209$

H $-1.609245-3.1596441 .865091$

C $1.979066-3.192939-1.893349$

O $1.429629-5.396305-1.109436$

H $0.123833-4.285959-2.193317$

H $2.825727-3.226805-1.198672$

O $2.454181-3.731753-3.133975$

C $1.556679-1.731750-2.127949$

C $2.578984-0.872911-2.895539$

H $1.339399-1.259270-1.163186$

O $0.340868-1.725371-2.883186$

H $2.648874-1.219921-3.934598$

H $2.1588250 .139296-2.955791$

H $\quad-2.779889-5.2751772 .408533$ 
H -1.234948 -6.0759072 .733031$ H -1.777747 -4.746135 3.769556 C $0.710735-2.9194573 .313797$ H $2.027826-5.286881-0.350271$ H $2.715497-4.653852-2.967862$ H $\quad 0.044109-0.802659-2.958752$ H $1.793563-2.8792613 .480357$ H $\quad 0.264184-3.3054534 .237633$ H $0.893362-4.8478522 .399595$ C $5.076617-0.258508-0.179719$ O $3.856695-0.331334-0.931666$

C $3.981764-0.814874-2.271288$

C $4.9281900 .084460-3.084259$

C $6.2855040 .231332-2.389610$

C $6.1280500 .595212-0.909701$

H 4.8052220 .2678960 .744623

C 5.598751-1.651968 0.205805

H $4.388425-1.830428-2.253071$

O $4.3407801 .373036-3.251587$

H $5.057097-0.351212-4.082310$

H $6.871764 \quad 1.011196-2.890691$

O $7.013607-0.988507-2.492020$

H $7.0897020 .509974-0.390034$

O $5.7180891 .960641-0.804795$

C $4.672940-2.3710731 .162641$

H $5.722239-2.294525-0.669707$

H $6.567305-1.5523810 .709432$

H $4.9465341 .904390-3.795552$

H $7.122958-1.187158-3.437611$

H $6.4047992 .506906-1.223360$

O $5.163126-3.6269561 .385182$

O $3.659300-1.8976671 .655766$

C $4.399781-4.4448482 .276956$

H $3.461233-4.7366331 .798565$

H $4.979532-5.3467622 .490322$

H $4.210271-3.9216623 .219100$

SCF Energy (B3LYP/6-31G**//MMFF)= -3245.91507297

\section{5}

MM̄̄F Geometry

C $0.9547430 .607596-2.488789$

C $1.336968-0.677570-2.569029$

C $0.787168-1.837176-1.771814$

O $0.021045-1.329856-0.658543$

C $-0.106047-2.720320-2.657239$

C $-0.353137-4.097075-2.073944$

C $-1.506213-4.506289-1.510111$

C $0.800786-5.060787-2.201211$

C $-2.769027-3.719351-1.289551$

C $-3.170589-3.7462950 .191230$

C $-4.469136-2.9929560 .518940$

C $-5.705957-3.685961-0.057645$

C $-6.991684-2.9070800 .252462$

O $-4.366633-1.6526720 .026966$

O $-8.027641-3.467102-0.569633$

C $-6.790528-1.436518-0.171430$

C -7.443343 -3.106181 1.703718

O $-7.924767-0.665270 \quad 0.217544$

C $-5.480703-0.814500 \quad 0.369403$

C $-5.2039240 .592896-0.203946$

O $\begin{array}{llll}-5.589790 & -0.666389 & 1.791253\end{array}$

C $0.223780-1.8715310 .568367$

O $0.887916-2.8651010 .829526$

C $-0.447520-1.0806461 .628713$

C -1.0383830 .1032861 .403576$

C -1.6385630 .9868202 .407429$

C -1.6542190 .5149103 .836022$

C $-5.0256840 .667259-1.722111$

C $-4.8623172 .112657-2.148474$

C $-3.2685294 .020093-1.998065$

O $-5.7995082 .755860-2.616076$

C $-3.4701124 .624420-0.643052$

C -2.4669365 .0470220 .140441$

C -2.6590595 .5497261 .541862$

C -2.0643604 .6131752 .573574$

C -0.9988504 .9726273 .308928$

C -2.7511283 .2797862 .800306$

C -2.1277042 .1746301 .993255$
N -3.591330 $2.607285-1.903886$

H $0.1340520 .889186-1.836498$

H $2.150251-0.935317-3.245185$

H $1.655665-2.396705-1.404155$

H $\quad 0.359316-2.859279-3.642880$

H -1.051822 -2.204156 -2.854132

H $-1.560958-5.537328-1.161051$

H $\quad 0.554409-6.058677-1.822799$

H $1.088828-5.171070-3.251875$

H $1.667871-4.703106-1.638320$

H $-2.667988-2.682433-1.619073$

H $-3.553526-4.175636-1.902420$

H -3.257671 -4.782852 0.541503

H -2.360094 -3.2880210 .770743$

H $-4.542189-2.9593231 .612787$

H $-5.782685-4.7168100 .309903$

H -5.616446 -3.762884 -1.148982

H $-8.830597-2.934874-0.434227$

H -6.772205 -1.436062 -1.267977

H $-7.649970-4.1660601 .895325$

H $-8.382343-2.5779251 .902629$

H -6.702673 -2.776313 2.434896

H $-7.8701180 .193113-0.234555$

H -5.9893611 .2893370 .117680$

H -4.2861050 .9764470 .260683$

H $-4.937733-0.0080072 .083754$

H -0.375988 -1.504162 2.625187

H -1.0615860 .4920870 .387362$

H $-0.632491 \quad 0.3883194 .210409$

H -2.179620 -0.443372 3.917547

H $-2.1599901 .201305 \quad 4.517673$

H $-4.1528490 .089877-2.047020$

H $-5.8953040 .269838-2.253762$

H $-3.9185274 .509093-2.730100$

H $-2.2322774 .097682-2.338834$

H $-4.4917834 .674111-0.273467$

H -1.445401 $4.989628-0.224552$

H -3.7225545 .6976081 .768081$

H -2.199266 6.5438661 .609400

H -0.5863664 .3097974 .062700$

H $-0.5131055 .932598 \quad 3.170012$

H -3.8097203 .3604372 .523310$

H -2.749661 3.0600163 .871799

H -2.0772932 .3898380 .926514$

H -2.909459 2.003316-1.453852

C $2.1549222 .844929-2.337820$

O $2.8351272 .301276-1.186160$

C $2.3001402 .933403-0.008720$

C $1.7103654 .247002-0.496193$

C $1.0821013 .799061-1.803347$

H 1.4787132 .3014550 .350263

C 3.3695303 .0575631 .078850

H $0.9886524 .668678 \quad 0.208833$

H $2.4892374 .995353-0.683818$

C $0.7581934 .945214-2.746420$

H $\quad 0.157140 \quad 3.252917-1.579694$

C 4.0019651 .7132811 .500508

O 4.4220003 .9114280 .616726

H $2.9427603 .557277 \quad 1.956362$

H $4.6525001 .339900 \quad 0.702066$

O 4.8477381 .9602012 .629494

C 2.9775580 .6345351 .889239

C $3.560977-0.6436962 .511984$

H 2.3979810 .3524851 .004171

O $2.046513 \quad 1.1790802 .830265$

H $3.979823-0.4254963 .502894$

H $2.709583-1.3101782 .702543$

H $0.0384025 .630795-2.287654$

H $1.6538875 .521892-3.000660$

H $0.3222884 .570526-3.677948$

C $1.6393511 .717169-3.241976$

H $4.7745633 .524307-0.202544$

H 5.4873592 .6443952 .367359

H $2.552597 \quad 1.4738413 .606761$

H $2.496641 \quad 1.305120-3.789493$

H $0.9538282 .122810-3.994353$

H $2.9085193 .414983-2.896456$

C $4.969971-2.175430-0.604425$ 
O $4.089682-1.544324 \quad 0.337167$

C $4.613605-1.3685611 .657048$

C $5.004859-2.7231392 .275688$

C $5.945225-3.5091071 .358289$

C $5.427700-3.548443-0.083646$

H $4.352133-2.350553-1.494310$

C $6.142970-1.265077-1.005392$

H $5.499705-0.7278411 .607509$

O $3.830119-3.4982752 .513037$

H $5.483235-2.5425693 .245895$

H $6.044778-4.5337041 .737078$

O $7.233180-2.9030081 .401623$

H $6.198442-3.952670-0.749480$

O $4.319284-4.450212-0.155266$

C $5.695118-0.065916-1.811996$

H $6.678241-0.891657-0.128766$

H $6.848762-1.821682-1.632675$

H $4.105513-4.3240412 .946431$

H $7.823419-3.4273510 .833957$

H $3.589072-4.0665180 .360586$

O $6.7018460 .856870-1.837446$

O $4.616070 \quad 0.039541-2.376400$

C $6.4337212 .038963-2.598054$

H $7.2964722 .704257-2.507888$

H $6.2943491 .784588-3.653006$

H $5.5522332 .553657-2.206144$

SCF Energy (B3LYP/6-31G*//MMFF) $=-3245.91370747$

0700106

MM̄FF Geometry

C $-3.470969-0.979285-3.189009$

C $-2.478449-1.404783-2.392928$

C $-1.019049-1.251564-2.732508$

O $-0.316284-0.671869-1.609570$

C $-0.405323-2.635803-3.009950$

C $1.071904-2.596874-3.353723$

C $2.029930-3.266691-2.683234$

C $1.448329-1.763569-4.552500$

C $1.865418-4.200626-1.518069$

C $2.222286-3.589658-0.154856$

C $3.695375-3.164268-0.042387$

C $4.131740-3.0826561 .421793$

C $5.584849-2.6037511 .554079$

O $3.838857-1.876018-0.652210$

O $5.814511-2.2673912 .930797$

C $5.754711-1.3081000 .736930$

C $6.589742-3.7069841 .202707$

O $7.121020-0.9018690 .699968$

C $5.189022-1.396863-0.701070$

C $5.1859180 .013161-1.334569$

O $6.020211-2.253793-1.486096$

C $-0.4217280 .673962-1.443768$

O $-1.148347 \quad 1.435154-2.062807$

C $0.543743 \quad 1.075590-0.396127$

C 0.5662692 .3532850 .010407

C 1.5007112 .9506400 .966677

C $2.5831532 .071267 \quad 1.532935$

C $4.3763810 .132296-2.630810$

C $4.465077 \quad 1.548874-3.164294$

C $3.6692043 .870019-2.691141$

O $5.2102681 .839963-4.096850$

C $4.0880954 .547668-1.422903$

C $3.4249215 .577671-0.875189$

C $3.8357316 .244520 \quad 0.407838$

C 2.6964886 .3733221 .398018

C $2.152322 \quad 7.5713361 .671354$

C 2.2348555 .1258202 .125684

C $1.361658 \quad 4.2606201 .259540$

N $3.664813 \quad 2.436300-2.462317$

H -3.231581 -0.492380 -4.131030

H $-2.718697-1.896186-1.454044$

H $-0.890026-0.605771-3.610342$

H $-0.936361-3.114668-3.843323$

H $-0.579726-3.267253-2.132646$

H $3.059210-3.161234-3.024831$

H $2.492142-1.911355-4.850013$

H $\quad 0.824545-2.030962-5.411813$

H $1.316884-0.698058-4.340088$
H $\quad 0.848248-4.601075-1.468078$

H $2.502926-5.076177-1.697395$

H $1.565850-2.7316480 .036299$

H $2.001407-4.3486110 .605954$

H $4.313439-3.894776-0.577391$

H $4.002072-4.0494781 .923859$

H $3.484444-2.3751451 .957236$

H $5.644825-3.0618263 .465616$

H $5.211527-0.5160201 .271945$

H $6.446126-4.5772501 .854277$

H $7.618923-3.3721151 .373610$

H $6.506478-4.0505710 .169624$

H $7.431565-0.8445961 .619877$

H $4.7692850 .726440-0.614854$

H $6.2173550 .332910-1.532768$

H $5.927820-2.012525-2.421369$

H $1.2282320 .323995-0.020068$

H $-0.1583883 .044684-0.418878$

H 3.1991292 .5654942 .286384

H 3.2545811 .7363050 .737905

H $2.155732 \quad 1.1894422 .021077$

H $4.749700-0.547356-3.403657$

H $3.322074-0.115293-2.463738$

H $2.6565334 .153121-2.994919$

H $4.3647714 .121730-3.497159$

H $4.9824024 .171540-0.930916$

H $2.5456365 .971799-1.379996$

H 4.2280617 .2376420 .153417

H 4.6639115 .7080610 .887605

H 1.3517127 .6762802 .396086

H 2.4913608 .4737841 .173492

H 1.6503555 .4016313 .013070

H 3.1173554 .6036382 .504349

H $\quad 0.525577 \quad 4.8012180 .812464$

H $3.0539592 .078398-1.734164$

C $-5.6541920 .177032-2.713909$

O $-5.1343150 .866965-1.557444$

C $-6.1942411 .010051-0.601027$

C $-7.4617631 .052144-1.439436$

C $-7.156894-0.018625-2.468840$

H -6.1964460 .0983990 .009851$

$\begin{array}{llll}\text { C } & -5.964923 & 2.224308 & 0.301641\end{array}$

H $-8.3607740 .840678-0.853194$

H -7.590463 $2.021753-1.933916$

C $-7.9970600 .089924-3.730156$

H $-7.340482-1.001022-2.012887$

C $-4.565844 \quad 2.3098950 .944066$

O $-6.155513 \quad 3.428916-0.451887$

H $-6.7348102 .240293 \quad 1.082097$

H -3.8331922 .6646930 .208114$

O $-4.622612 \quad 3.3164751 .964117$

C $-4.0437781 .004951 \quad 1.571279$

C $-2.758176 \quad 1.2251832 .390577$

H -3.8499960 .2779320 .775168$

$\begin{array}{lllll}\text { O } & -5.044523 & 0.444041 & 2.421113\end{array}$

H -2.1284001 .9801721 .904877$

H -3.0370881 .6039993 .381373$

H -9.060692 $-0.020525-3.495013$

H $-7.860083 \quad 1.059196-4.221289$

H $-7.724450-0.692475-4.445630$

C $-4.927144-1.160920-2.870629$

H $-5.5345943 .407805-1.200454$

H $-4.929496 \quad 4.137987 \quad 1.543301$

H -5.2632591 .1075943 .097725$

H $-5.384573-1.741588-3.680097$

H $-5.034530-1.745703-1.948606$

H $-5.4715820 .823774-3.580586$

C $-2.112330-2.3753913 .237053$

O $-2.740438-1.0925443 .102344$

C $-1.921833-0.0600782 .549741$

$\begin{array}{llll}\text { C } & -0.686284 & 0.174437 & 3.432668\end{array}$

C $0.088383-1.1269683 .656025$

C $-0.835013-2.2699234 .091400$

H $-2.838549-2.9857983 .789081$

C $-1.870133-3.0530601 .877241$

H $-1.601792-0.3593631 .546014$

O $-1.091750 \quad 0.710224 \quad 4.690900$

H -0.0454100 .9201142 .951177$ 
H $\quad 0.854131-0.9632304 .424004$ O $\quad 0.753605-1.4705102 .445083$ H $-0.292105-3.221973 \quad 4.068930$ O $-1.211313-2.0384385 .451757$ C $-3.161924-3.455505 \quad 1.197005$ H $-1.325319-2.4142861 .177856$ H $-1.288782-3.9688472 .039521$ H $-0.288205 \quad 0.873528 \quad 5.213408$ H $1.229742-2.3040042 .599205$ H $-1.755036-2.7914115 .739443$ O $-2.873759-4.332144 \quad 0.188871$ O $-4.275406-3.045018 \quad 1.492617$ C $-3.999495-4.813505-0.551533$ H $-4.548321-3.980329-0.999138$ H $-3.628584-5.458461-1.352496$ H $-4.653646-5.401106 \quad 0.099518$ SCF Energy (B3LYP/6-31G*//MMFF) $=-3245.89581492$

07_00107

MM̄FF Geometry

C $3.8730313 .796018-0.955307$

C $2.6590213 .733465-0.389375$

C $1.3854834 .112955-1.098619$

O $0.4719772 .994414-1.011417$

C $0.7416495 .323489-0.401820$

C $-0.5242135 .804299-1.084822$

C $-1.7750425 .597546-0.626507$

C $-0.3085136 .594558-2.350452$

C $-2.196841 \quad 4.844336 \quad 0.605312$

C $-3.057921 \quad 3.6344240 .238798$

C $-3.5053662 .803247 \quad 1.450758$

C -2.3345302 .1258292 .170826$

C -2.8065861 .1813503 .286773$

$\begin{array}{lllll}0 & -4.405276 & 1.808188 & 0.944420\end{array}$

$\begin{array}{lllll}O & -1.693646 & 0.360318 & 3.672137\end{array}$

$\begin{array}{llll}\text { C } & -3.889260 & 0.240734 & 2.720634\end{array}$

C $-3.227726 \quad 1.9517524 .543927$

O $-4.457661-0.5616253 .752991$

$\begin{array}{llll}C & -5.003546 & 0.981426 & 1.948847\end{array}$

C $-5.930822-0.0418201 .245719$

O -5.7788321 .7480082 .874613$

C $0.6404021 .987973-1.913198$

O $1.4822791 .924140-2.794970$

C $-0.3858770 .958715-1.635906$

C $-0.404985-0.169423-2.359700$

C $-1.350405-1.279232-2.212694$

C $-2.468966-1.115768-1.217639$

C -6.9316970 .5810320 .261816$

C $-6.3720860 .740960-1.140285$

C $-5.888041-0.538170-3.211600$

O $-6.0926871 .843176-1.605860$

C $-5.471003-1.933552-3.546149$

C $-4.400876-2.236907-4.296815$

C $-4.001134-3.650970-4.610084$

C -2.532407 -3.928691-4.380382

C $-1.775017-4.460960-5.355436$

C $-1.952322-3.660132-3.007618$

C $-1.161618-2.378998-2.971657$

N $-6.231725-0.469444-1.802219$

H $3.9706204 .154157-1.976963$

H 2.5650593 .3772720 .634045

H $1.5740444 .350782-2.153005$

H $1.455074 \quad 6.158615-0.374791$

H 0.5470925 .0813890 .648104

H -2.603913 6.012310 -1.199371

H - $1.2465896 .974373-2.769303$

H $\quad 0.3354417 .458047-2.153639$

H $0.1650975 .973778-3.117003$ H -1.3358564 .5236231 .192154$ H -2.773651 5.5236721 .244316 H $-3.9530373 .972370-0.301701$ H -2.525908 $2.988649-0.470206$ H $-4.053226 \quad 3.4612642 .135402$ H -1.637444 2.8632362 .584178 H $-1.753904 \quad 1.538602 \quad 1.447043$ H $-0.9696840 .946948 \quad 3.950874$ H -3.392964 -0.452210 2.027653 H -2.3830002 .5283334 .940112$
H $-3.524588 \quad 1.267333 \quad 5.346288$ H -4.0466002 .6515264 .365015$ H $-3.724608-1.0126804 .205908$ H $-5.319128-0.7821190 .715854$ H -6.505902 -0.5780392 .011394$ H -6.6918841 .8159302 .554872$ H $-1.0954081 .152273-0.841882$ H $0.350647-0.300918-3.133482$ H $-2.068236-0.993274-0.205664$ H -3.152126 -1.966675 -1.185117 H $-3.074098-0.235389-1.460717$ H $-7.812920-0.0692760 .187709$ H -7.3004771 .5543150 .600741$ H $-5.1080570 .199468-3.427839$ H $-6.785963-0.268563-3.777349$ H $-6.086551-2.742336-3.158211$ H $-3.798015-1.436436-4.718488$ H $-4.266656-3.845527-5.657057$ H $-4.580972-4.361424-4.007133$ H $-0.726308-4.687872-5.193023$ H $-2.184211-4.681433-6.335611$ H $-1.270586-4.478490-2.738454$ H $-2.744281-3.698840-2.254884$ H $-0.304653-2.388992-3.647750$ H $-6.657721-1.289892-1.379532$ C $5.8683562 .275856-0.992227$ O $5.0706091 .073551-0.997165$ C $5.928226-0.053205-0.739294$ C $7.359027 \quad 0.468967-0.821045$ C $7.1912041 .891205-0.329850$ H $5.730056-0.3665310 .291887$ C $5.629505-1.208586-1.699643$ H $8.049711-0.119352-0.209041$

H $7.7367800 .465561-1.850095$

C $8.354408 \quad 2.798750-0.690504$

H 7.0741501 .8726240 .762238

C $4.168616-1.701790-1.702906$

O $5.942671-0.785833-3.033753$

H $6.307618-2.041569-1.477175$

H $3.529308-0.970005-2.211145$

O $4.127242-2.882202-2.518733$

C $3.572258-2.020150-0.318657$

C $2.244403-2.796708-0.421603$

H $3.420273-1.081626 \quad 0.225377$

O $4.514480-2.8034860 .414488$

H $1.636252-2.385150-1.234826$

H $2.477480-3.840714-0.665232$

H $9.2835812 .427773-0.245735$

H $8.4993522 .853568-1.774686$

H $8.1866873 .815273-0.321366$

C $5.1359853 .394314-0.249131$

H $5.758761-1.535114-3.625957$

H $4.636278-3.573054-2.060536$

H $4.145891-2.9505071 .302122$

H $5.7793924 .278137-0.173889$

H 4.9069923 .0699870 .773182

H $6.0290112 .554919-2.042055$

C $1.587885-3.2085763 .249076$

O $2.204668-3.251144 \quad 1.954754$

C $1.409060-2.7585890 .874604$

C $0.121997-3.5907590 .748939$

C $-0.649648-3.6367302 .070110$

C $0.262031-3.986624 \quad 3.252107$

H $2.288887-3.7382273 .907279$

C $1.450218-1.7740443 .771521$

H $1.151601-1.7109621 .065737$

$\begin{array}{lllll}\text { O } & 0.425834 & -4.921969 & 0.335372\end{array}$

H $-0.510015-3.153361-0.028870$

H -1.450937 -4.3812011 .988450$

O $-1.256680-2.367107 \quad 2.282404$

H $-0.255689-3.8203904 .203941$

O $0.586106-5.378943 \quad 3.189670$

C $2.810738-1.1492303 .968992$

H $0.861222-1.1314693 .110945$

H $0.943568-1.7593634 .743839$

H $1.050528-5.2977880 .979012$

H $-1.761645-2.4160453 .111851$

H $-0.247683-5.8765423 .242129$ 
O $3.075493-0.2773502 .955067$

O $3.557172-1.4231664 .899963$

C 4.3533340 .3632203 .010969

H $5.152214-0.3809692 .940064$

H 4.4292171 .0415052 .157772

H 4.4469440 .9462393 .932164

SCF Energy (B3LYP/6-31G**//MMFF) $=-3245.91321114$

0700108

MM̄FF Geometry

C -2.543339 $1.841015-1.937652$

C $-1.3594792 .137858-2.494893$

C $-0.2139572 .773429-1.753843$

O $0.9718072 .027006-2.123972$

C $-0.0238354 .251661-2.143486$

C $-1.1035345 .177097-1.627949$

C $-1.1289445 .688975-0.381611$

C $-2.1539635 .575753-2.631551$

C $-0.154673 \quad 5.4372720 .739543$

C $-0.861483 \quad 5.1579682 .074118$

$\begin{array}{llll}C & -1.490635 & 3.756140 & 2.161098\end{array}$

C $-2.547392 \quad 3.7358453 .267469$

C $-3.1528492 .341623 \quad 3.453887$

O $-0.448343 \quad 2.8161072 .462138$

O $-3.901012 \quad 2.3479554 .679691$

C -2.007288 1.3291443 .627595

C $-4.146251 \quad 1.9887902 .343375$

O -2.510282 -0.0062483 .652572$

C $-0.882677 \quad 1.451860 \quad 2.566274$

C $0.3148940 .582345 \quad 3.025649$

$\begin{array}{llll}\text { O } & -1.365928 & 0.960606 & 1.316344\end{array}$

C $2.0651512 .147772-1.325915$

O $2.1699382 .827130-0.317637$

C $3.1399421 .309754-1.897265$

C $4.3991501 .507565-1.480998$

C $5.585527 \quad 0.769853-1.915350$

C $5.372186-0.432266-2.796729$

C $1.606167 \quad 0.7284202 .208828$

C $2.710678-0.1543792 .775809$

C $5.099607-0.7331892 .508874$

O $2.556251-0.8550503 .774249$

C $5.998540-1.0561671 .358818$

C $7.286762-0.6845731 .300814$

C $8.229629-1.0823940 .199200$

C $8.8325190 .107653-0.512669$

C $9.9780280 .663656-0.083484$

C $8.1452770 .600120-1.767036$

C $6.7924651 .190530-1.482046$

N $3.889212-0.0790552 .041917$

H -2.713166 $2.086162-0.893506$

H -1.207583 $1.931239-3.551931$

H $-0.3634962 .669647-0.673064$

H $0.0565084 .340007-3.236080$

H $0.9498014 .613442-1.789628$

H $-1.9293796 .386236-0.135927$

H -2.893296 $6.264049-2.208611$

H -1.688380 $6.077586-3.486326$

H -2.695730 $4.699672-2.998287$

H 0.5392094 .6253010 .514593

H $\quad 0.447890 \quad 6.346248 \quad 0.857311$

H $-0.1208235 .267546 \quad 2.876976$

H -1.626302 5.9282982 .235830

H -1.946389 $3.495581 \quad 1.200035$

H -3.338313 4.4689183 .064158

H -2.088471 4.0468194 .216757

H -4.5884793 .0323044 .607577$

H -1.5658751 .5106384 .617664$

H $-4.9327012 .749788 \quad 2.274892$

H -4.6613401 .0489582 .566949$

H -3.679496 1.9052321 .360697

H -3.193736 -0.0455254 .343440$

H $\quad 0.5588760 .841965 \quad 4.064770$

H $0.019552-0.4751573 .017499$

H -0.6108270 .6761540 .778185$

H $2.8697640 .567408-2.638964$

H $4.5859302 .293101-0.749225$

H $4.672983-1.134080-2.328848$

H $6.284463-0.998382-2.993841$
H $4.966383-0.128385-3.767750$

H $1.4399260 .441393 \quad 1.166429$

H $1.955191 \quad 1.7660032 .237938$

H $4.832119-1.6567173 .033495$

H $5.573644-0.0601133 .231177$

H $5.588232-1.6688600 .560452$

H $7.705558-0.0973342 .115239$

H $7.739829-1.739808-0.530013$

H $9.029157-1.6882300 .645654$

H $10.4311391 .500780-0.603777$

H $10.4861430 .294586 \quad 0.801539$

H $8.109919-0.219325-2.489620$

H $8.7465641 .382103-2.249282$

H $6.8340542 .076074-0.845808$

H 3.9783850 .6542631 .344096

C $-4.6545710 .461436-1.819006$

O $-4.030403-0.728859-1.300134$

C $-5.026317-1.771165-1.212075$

C $-6.350442-1.146168-1.644377$

C $-5.890856-0.035074-2.564897$

H $-4.744795-2.539855-1.939923$

C $-5.061394-2.3604750 .204103$

H -7.004623 -1.870785 -2.139479

H $-6.902458-0.733138-0.791865$

C $-6.950541 \quad 1.027497-2.800211$

H $-5.597574-0.475184-3.527472$

C $-3.733231-2.9896750 .667206$

O $-5.389510-1.315323 \quad 1.129186$

H $-5.869975-3.0959030 .282337$

H -2.958641 -2.216968 0.716177

O $-3.917074-3.4654652 .007102$

C $-3.258118-4.173912-0.196814$

C $-2.026123-4.9150020 .361730$

H $-3.036877-3.827522-1.211101$

O $-4.317514-5.128311-0.315099$

H $-2.236426-5.2886131 .371155$

H $-1.870775-5.805691-0.261811$

H $-7.8438860 .585409-3.253310$

H $-7.251432 \quad 1.508444-1.863398$

H $-6.5828331 .806350-3.475244$

C $-3.6836991 .239200-2.710900$

H $-4.707457-0.6278451 .040113$

H $-4.212662-2.7100952 .543934$

H $-4.546466-5.4244260 .582699$

H $-4.2218302 .058977-3.200387$

H -3.289431 $0.577106-3.491384$

H $-4.950174 \quad 1.072947-0.955655$

C $0.591530-2.763655-1.141836$

O $-0.414890-3.772117-0.993871$

C $-0.757150-4.056593 \quad 0.364164$

C $0.443922-4.731961 \quad 1.048480$

C $1.769623-4.0489590 .674405$

C $1.526246-2.6668610 .073506$

H $1.180736-3.050902-2.022454$

C $-0.090674-1.425226-1.431317$

H $-0.966430-3.1139560 .882886$

$\begin{array}{lllll}\text { O } & 0.527302 & -6.102210 & 0.653051\end{array}$

H $0.281416-4.7308862 .132870$

H $2.357971-4.657871-0.023077$

O $2.567375-3.899623 \quad 1.849584$

H $1.106955-2.0025990 .838531$

O $2.757429-2.087797-0.348145$

C $-0.636789-1.409362-2.838155$

H $-0.929849-1.239436-0.751739$

H $0.605030-0.588361-1.315720$

H $1.272984-6.5001851 .134018$

H $2.720279-4.7887012 .212789$

H $3.340099-2.0604800 .429497$

O $0.274891-0.850869-3.684355$

O $-1.728624-1.865839-3.151118$

C $-0.118188-0.791002-5.058486$

H $0.669170-0.275598-5.614824$

H $-0.230472-1.801733-5.461787$

H $-1.049492-0.226491-5.165694$

SCF Energy (B3LYP/6-31G*//MMFF)= -3245.91469538

0700109

MM̄FF Geometry 
C $-6.509564-0.0859470 .541759$

C -5.6648220 .9169680 .258574$

C -5.0702551 .8261421 .302478$

O

C $-5.281873 \quad 3.2979660 .910721$

C -4.7242064 .2726461 .930075$

C -3.6420225 .0543991 .742692$

C -5.4930924 .3589203 .224695$

C -2.7542385 .1491620 .532371$

C -1.3317934 .6832510 .849834$

C $-0.3451314 .895850-0.309068$

C $-0.6700754 .028220-1.528670$

C $0.3954254 .156059-2.629563$

O $\quad 0.955767 \quad 4.562275 \quad 0.195951$

O $0.1901793 .088210-3.568083$

C $1.7873273 .941987-2.002171$

C $0.2487435 .461129-3.420169$

O $2.823204 \quad 4.182670-2.950371$

C $2.0275284 .785817-0.732807$

C $3.3480764 .420194-0.013835$

O $2.1154496 .169633-1.090905$

C $-3.243843 \quad 0.5208782 .132041$

O $-3.951171-0.1936452 .825203$

C $-1.779116 \quad 0.3734181 .978659$

C $-1.171438-0.6974442 .512715$

C $0.256142-1.0154722 .439642$

C $1.158312-0.0174311 .764638$

C 3.4836382 .9401770 .344925

C $4.6051642 .663074 \quad 1.330746$

C 5.7113290 .7523232 .483576

O 5.2914263 .5394261 .847062

C $5.333873-0.6605532 .791534$

C $4.751416-1.0388503 .938668$

C $4.327980-2.4485314 .237845$

C $2.820932-2.5757234 .326469$

C $2.214624-2.5890665 .526312$

C $2.089617-2.7252973 .005483$

C $0.688882-2.1726212 .984667$

N $4.721122 \quad 1.3031341 .579733$

H $-6.819370-0.2539711 .570144$

H $-5.3309271 .055908-0.767350$

H -5.5179821 .6401542 .286642$

H -6.3552583 .4994470 .792603$

H -4.843400 $3.474694-0.076416$

H -3.3521125 .7230252 .553107$

H -5.1158105 .1505913 .881062$

H -6.547936 4.5754953 .026140

H -5.4276153 .4169113 .777313$

H -3.152080 $4.579440-0.308582$

H $-2.732937 \quad 6.1979950 .211584$

H $-0.953570 \quad 5.2217331 .729803$

H -1.335964 $3.624787 \quad 1.139907$

H $-0.3565855 .959026-0.576685$

H -1.656417 4.270244 -1.940168

H $-0.7244342 .974774-1.219223$

H $-0.7169183 .161044-3.911334$

H $1.8465942 .879487-1.738214$

H $\quad-0.7332675 .505634-3.906404$

H $0.9856235 .517890-4.228949$

H $0.347936 \quad 6.353289-2.798511$

H $2.6357023 .632511-3.730191$

H $4.2088514 .731338-0.619987$

H 3.4085655 .0154980 .907867

H $2.5934136 .639565-0.387404$

H -1.248082 1.1309111 .414586

H -1.779302 -1.4254923 .048866$

H $\quad 0.9008490 .0820710 .704791$

H $2.214003-0.2879021 .813882$

H 1.0702390 .9652822 .241394

H 2.5541862 .5738630 .795158

H $3.6826752 .356826-0.561090$

H 5.7629081 .3791103 .380448

H $6.6871150 .790242 \quad 1.989054$

H 5.532221-1.402329 2.021839

H $4.549498-0.2924764 .703824$

H $4.797045-2.7503905 .183142$

H $4.707251-3.1507323 .484470$

H $1.141840-2.7050945 .630928$
H $2.785388-2.4901096 .444206$

H $2.042763-3.7937112 .760238$

H $2.680817-2.2792202 .200699$

H $-0.043863-2.8300423 .454252$

H $4.226240 \quad 0.661485 \quad 0.965517$

C $-6.152475-2.333881-0.460215$

O $-4.841884-2.025020-0.978229$

C $-4.418156-3.083184-1.854757$

C $-5.400911-4.224705-1.628346$

C $-6.681502-3.462438-1.341203$

H $-4.530438-2.711244-2.881080$

C $-2.947420-3.443375-1.600496$

H -5.478536 -4.885262 -2.496951

H $-5.115167-4.834118-0.762484$

C $-7.757560-4.308084-0.683540$

H $-7.068922-3.054049-2.284276$

C $-1.974163-2.304313-1.983058$

O $-2.782139-3.814642-0.233735$

H -2.694644 -4.325261 -2.201074

H -2.140470 -2.066499-3.040875

O $-2.270919-1.117503-1.249160$

C $-0.485588-2.659856-1.792211$

C $0.430558-1.542235-2.330710$

H $-0.275589-3.597809-2.319486$

O $-0.226469-2.867473-0.407154$

H $\quad 0.095579-1.261310-3.336395$

H $0.338183-0.664741-1.678714$

H -8.046865 -5.137926 -1.336587

H -7.411179 -4.7322380 .265035$

H -8.652072 -3.711490 -0.478955

C $-7.007425-1.066129-0.484151$

H -3.072349 -3.0641840 .312298$

H -2.062097 -1.293870 -0.316384

H $0.712159-3.100186-0.311150$

H -8.054908 -1.297180 -0.262126

H $-6.977357-0.609683-1.481447$

H -6.013092 -2.686256 0.570643

C $3.768582-2.522672-0.942336$

O $2.408731-2.084792-1.064187$

C $1.912245-1.963251-2.400462$

C $2.752316-0.947592-3.189141$

C $4.237573-1.321629-3.160395$

C $4.720623-1.613123-1.736710$

H $4.004920-2.4025360 .122351$

C $3.920126-4.007916-1.287785$

H $1.963031-2.938507-2.897088$

O $2.5720660 .352260-2.632158$

H $2.390587-0.916638-4.223899$

H $4.830786-0.500659-3.580937$

O $4.459200-2.474123-3.967761$

H $5.721255-2.060702-1.753530$

O $4.827188-0.379612-1.023173$

C $3.205497-4.864481-0.269942$

H $3.545685-4.262553-2.283452$

H $4.975348-4.305853-1.271317$

H $3.0946110 .976066-3.164304$

H $4.172754-2.259285-4.871838$

H $5.4719560 .177140-1.491863$

O $1.964118-5.193807-0.726930$

O $3.685734-5.1800120 .811019$

C $1.170223-5.989150 \quad 0.159028$

H $0.212197-6.186669-0.329313$

H $\quad 1.666435-6.9432670 .360247$

H $\quad 0.982756-5.4460161 .089877$

SCF Energy (B3LYP/6-31G**//MMFF) $=-3245.91046285$

0700110

MM̄MF Geometry

C $0.439336-1.9657450 .394482$

C $1.261424-2.352205-0.593472$

C $1.553696-1.573344-1.858082$

O $0.953846-0.259370-1.756307$

C $3.073151-1.384557-2.044131$

C $3.805913-2.603728-2.557975$

C $4.646150-3.359343-1.824676$

C $3.596552-2.909306-4.019051$

C $4.973873-3.229318-0.363100$

C $6.387156-2.684870-0.117572$ 
C $6.514674-1.173220-0.368917$

C $7.963798-0.722212-0.171277$

C $8.1129610 .801133-0.289639$

O $5.661302-0.503770 \quad 0.565005$

$\begin{array}{lllll}\text { O } & 9.409068 & 1.158536 & 0.213953\end{array}$

C $7.070787 \quad 1.4745280 .626555$

C $8.0699961 .268467-1.749142$

$\begin{array}{lllll}\text { O } & 7.077975 & 2.889694 & 0.448830\end{array}$

C 5.6364520 .9248050 .442889

C $4.722823 \quad 1.470293 \quad 1.567455$

O $5.137002 \quad 1.342392-0.827291$

C $0.725218 \quad 0.442347-2.894589$

O $0.9576830 .077811-4.038297$

C $0.193587 \quad 1.790458-2.584765$

C $-0.4314792 .069101-1.430376$

C $-0.9980993 .358241-1.032129$

C $-0.6913754 .555290-1.890860$

C 3.2835990 .9338081 .540489

C $2.515237 \quad 1.2957942 .802365$

C $0.208668 \quad 1.234957 \quad 3.754234$

O 3.0477691 .7651523 .804534

C -0.2523472 .6569363 .700770$

C -1.495435 3.0245463 .357050

C $-1.951673 \quad 4.4527273 .281240$

C $-1.669618 \quad 5.0991141 .941643$

C $-0.8249396 .139747 \quad 1.844690$

C $-2.413363 \quad 4.593155 \quad 0.725456$

C -1.736731 3.4064730 .095655

$\begin{array}{llll}\mathrm{N} & 1.163567 & 1.002729 & 2.685558\end{array}$

H $-0.119887-1.0384850 .307843$

H $1.788944-3.296515-0.479048$

H $1.102398-2.106455-2.702936$

H $3.505189-1.022290-1.105329$

H $3.266140-0.562211-2.745947$

H $5.147362-4.191681-2.317415$

H $4.209089-3.750816-4.360139$

H $2.550673-3.164914-4.214180$

H $3.862603-2.040908-4.630709$

H $4.231839-2.6349320 .177723$

H $4.911557-4.2357220 .070736$

H $6.638204-2.8899920 .931477$

H $7.110151-3.231539-0.735682$

H $6.183915-0.948051-1.388622$

H $8.629161-1.229781-0.881018$

H $8.306850-1.0252380 .828089$

H $10.0758750 .680823-0.308646$

H $7.380710 \quad 1.2914841 .665152$

H $8.8824980 .806391-2.322986$

H $8.2296902 .349872-1.822627$

H $7.1320061 .022689-2.251368$

H 7.9916843 .1939660 .584867

H 5.1706391 .2065462 .534555

H 4.6771522 .5655151 .517756

H $4.1680761 .311246-0.815351$

H $\quad 0.3082572 .527755-3.372188$

H $-0.5642431 .270187-0.702493$

H -1.040780 $5.497238-1.462175$

H $-1.1617184 .453550-2.874673$

H $0.3904174 .663374-2.028848$

H 2.7436461 .3424490 .679336

H $3.281725-0.1591491 .461628$

H $\quad 0.6842081 .033462 \quad 4.719443$

H $-0.614413 \quad 0.5262903 .616697$

H 0.4762273 .4231843 .955561

H -2.226589 2.2616933 .099901

H -1.4849325 .0253094 .093722$

H -3.029519 4.4922843 .482564

H $-0.633837 \quad 6.6250230 .893492$

H $-0.302133 \quad 6.5274212 .712671$

H -2.556885 5.4023500 .001530

H $-3.4311974 .305814 \quad 1.017511$

H $-1.9025392 .473900 \quad 0.635021$

H $\begin{array}{llll}0.799816 & 0.698955 & 1.787197\end{array}$

C -1.008357 -3.5086451 .801000$

O $-2.164413-2.6764371 .575377$

C -3.051051-2.796970 2.698132

C $-2.667117-4.1025263 .384648$

C $-1.165644-4.1073253 .204071$
H -2.819750 -1.955925 3.363379

C $-4.524129-2.7221682 .279260$

H $-2.974773-4.1269594 .434421$

H $-3.102092-4.9743882 .883221$

C $-0.535125-5.4812253 .358098$

H $-0.728262-3.4348793 .954401$

C $-4.915302-1.5781461 .325426$

O -4.900669 -3.942230 1.622394

H $-5.143428-2.6779733 .183289$

H $-4.611505-1.8332470 .304060$

O $-6.350011-1.5211361 .312507$

C $-4.380019-0.1795921 .673446$

C -5.0514400 .9789230 .904298$

H $-3.300369-0.151147 \quad 1.488773$

$\begin{array}{llll}\text { O } & -4.560978 & 0.075990 & 3.068838\end{array}$

H $-6.064904 \quad 1.1387811 .295374$

H $-4.495477 \quad 1.8919201 .153871$

H $-0.721443-5.8799994 .360631$

H $-0.942239-6.1929402 .632176$

H $0.548240-5.4311403 .209900$

C $0.283914-2.6952121 .694875$

H $-4.313766-4.0549910 .854946$

H $-6.672420-2.4006181 .050383$

H $-5.511144-0.0017603 .261424$

H $1.145992-3.3509441 .868154$

H $\quad 0.308540-1.9353312 .486380$

H $-1.028239-4.2896031 .031000$

C $-3.674800 \quad 0.444407-2.533681$

O $-3.7834660 .691374-1.123563$

C $-5.1164820 .801242-0.620817$

C $-5.8552881 .962322-1.304112$

C $-5.8284821 .811432-2.829030$

C $-4.4180881 .519147-3.347991$

H $-2.606423 \quad 0.543190-2.755877$

C $-4.085128-0.992011-2.896482$

H $-5.661291-0.122046-0.836372$

$\begin{array}{llll}\text { O } & -5.274160 & 3.210907 & -0.937341\end{array}$

H $-6.893218 \quad 1.977427-0.951794$

H $-6.1977702 .734054-3.293576$

O $-6.691010 \quad 0.747834-3.221830$

H $-4.4591151 .225412-4.403410$

O $-3.6700942 .736838-3.282016$

C $-3.140380-2.015092-2.300857$

H $-5.091630-1.229163-2.542725$

H $-4.053952-1.117977-3.984773$

H $-4.3432343 .198510-1.217680$

H $-7.5867570 .967489-2.913689$

H $-2.7990882 .568624-3.680279$

O $-3.608424-3.268189-2.576709$

O $-2.120244-1.755162-1.678765$

C $-2.809461-4.343522-2.073356$

H $-2.763720-4.301721-0.981513$

H $-3.280690-5.284658-2.368807$

H -1.804126 -4.303749 -2.503719

SCF Energy (B3LYP/6-31G**//MMFF)= -3245.91424322

0700111

MM̄FF Geometry

C -1.618395 -2.719821 1.891259

C $-1.983364-1.9554242 .932023$

C -1.344212 -0.6503903 .353019$

O $-0.374042-0.2414152 .356019$

$\begin{array}{lllll}\text { C }-2.372895 & 0.491707 & 3.489343\end{array}$

C $-3.515256 \quad 0.239032 \quad 4.451521$

C $-4.7990520 .090584 \quad 4.066796$

C -3.1453830 .2138995 .909939$

C $-5.302110 \quad 0.085516 \quad 2.649855$

C -5.7132601 .4923322 .197106$

C $-5.757937 \quad 1.6716250 .672361$

C $-6.7591510 .741463-0.011349$

C $-6.7264080 .893990-1.539808$

$\begin{array}{llll}\text { O } & -4.437888 & 1.437729 & 0.170409\end{array}$

O $-7.456841-0.218877-2.078407$

C $-5.2622620 .787550-2.020481$

C $-7.4585402 .160207-1.998901$

O $-5.1570051 .068018-3.414455$

C $-4.2789111 .686636-1.231008$

C $-2.8183501 .328593-1.615977$ 
O $-4.5427413 .050562-1.554606$

C 0.6251360 .5956432 .740190

O $0.854631 \quad 0.9982703 .868955$

C 1.3789030 .9711131 .521540

C 2.3319101 .9120211 .596076

C 3.1465442 .4150390 .487481

C $2.8497071 .898026-0.895386$

C $-1.7242961 .986350-0.760315$

C -1.357063 $3.399409-1.178467$

C $-0.0246825 .364830-0.413672$

O $-1.7577553 .918356-2.216677$

C $1.3205615 .334488-1.067900$

C $2.4066125 .933296-0.555439$

C $3.7503045 .943357-1.226778$

C $4.8626915 .461158-0.322323$

C 5.6861606 .3318110 .285451

C $5.0752663 .969469-0.195345$

C 4.1056203 .3256670 .756460

N $-0.5155924 .008321-0.257009$

H $-0.774747-2.423901 \quad 1.274396$

H $-2.804508-2.3068563 .553455$

H $-0.831827-0.8230294 .307747$

H $-2.738371 \quad 0.7555402 .489887$

H $-1.855048 \quad 1.4038513 .816069$

H -5.562888 -0.0505544 .829050$

H $-4.021422 \quad 0.103696 \quad 6.557916$

H -2.472220 -0.6227276 .121178$

H $-2.643148 \quad 1.1453046 .190707$

H $-4.551140-0.3475701 .980897$

H -6.165292 -0.5888492 .592083$

H $-6.689616 \quad 1.7445912 .629218$

H -4.9927212 .2213032 .591957$

H -6.0249952 .7179210 .481948$

H -7.7727810 .9107300 .372462$

H $-6.519830-0.3031900 .226996$

H -7.497094 -0.115604 -3.043966

H $-4.954594-0.257510-1.888277$

H -8.511949 2.121310-1.696665

$\mathrm{H}-7.4631672 .241057-3.091504$

H -7.029267 $3.076840-1.589325$

H $-5.6936340 .411742-3.889357$

H -2.692400 $0.246260-1.485676$

H -2.642609 $1.553288-2.674618$

H -3.783526 $3.588296-1.282616$

H 1.1194840 .4720140 .595135

H 2.5373482 .3645892 .565493

H $3.1346350 .845277-0.977327$

H $3.3759552 .438214-1.685009$

H $1.7836101 .992552-1.128162$

H $-1.996675 \quad 1.9783130 .300133$

H $-0.809303 \quad 1.390009-0.867335$

H $0.0101595 .814618 \quad 0.583747$

H $-0.7232525 .938147-1.031472$

H $1.3986414 .816531-2.020938$

H 2.3204416 .4748360 .384038

H $3.9517076 .973499-1.549031$

H $3.7439555 .342905-2.144954$

H 6.5046375 .9930160 .911980

H $5.5566467 .403340 \quad 0.174647$

H 6.0847823 .7661400 .186775

H $5.0609213 .518372-1.191141$

H 4.2411383 .6450021 .790945

H -0.1798903 .4731580 .538450$

C -2.675101-4.100114 0.030392

O $-1.471527-4.069205-0.766303$

C $-1.609046-3.021400-1.741071$

C -3.109283 -2.885478 -1.947054

C $-3.593036-2.996662-0.510595$

H -1.231783 -2.101244 -1.276386

C $-0.779886-3.321360-2.990704$

H -3.383019-1.943548 -2.427861

H -3.513463 -3.710060 -2.545684

C $-5.071949-3.316320-0.382230$

H -3.399846 -2.041545 -0.006337

C $0.687600-3.709184-2.707834$

O $-1.375966-4.403530-3.715723$

H -0.815842 -2.457423 -3.664480

H $0.736529-4.744363-2.347170$
O $1.392923-3.688665-3.954721$

C $1.422619-2.799327-1.705315$

C $2.910442-3.158862-1.582086$

H $0.933713-2.889396-0.731915$

O $1.298035-1.443416-2.129617$

H $3.035207-4.248267-1.540880$

H $3.440191-2.833968-2.488393$

H $-5.678017-2.529411-0.841758$

H $-5.323082-4.261946-0.874132$

H $-5.360361-3.3954270 .670723$

C $-2.307758-4.0046861 .516114$

H $-1.423754-5.167961-3.115973$

H $0.920213-4.280394-4.564928$

H $1.612987-0.878119-1.405411$

H -1.615852 -4.821578 1.756531

H -3.210405 -4.151824 2.120641

H -3.124060 -5.085447 -0.148055

C $5.900339-2.3178610 .466534$

O $5.013332-2.855953-0.521693$

C $3.626463-2.521883-0.372673$

C $3.102068-3.0114680 .989110$

C $3.985239-2.5433782 .151299$

C $5.474325-2.7678311 .873584$

H $6.873999-2.7745690 .247149$

C $6.060332-0.7945840 .337289$

H $3.521173-1.436042-0.441464$

O $3.025584-4.4362271 .005121$

H $2.084785-2.6354401 .142148$

H $3.696338-3.0895423 .057869$

O $3.739616-1.1604802 .381614$

H $6.081644-2.2554262 .628738$

O $5.737200-4.1679572 .003475$

C $6.698027-0.389580-0.975352$

H $5.100017-0.2780580 .401443$

H $6.709904-0.4303751 .141827$

H $3.916800-4.7833340 .828486$

H $4.274064-0.8898393 .147565$

H $6.693521-4.2998051 .886739$

O $6.8135820 .971562-1.006786$

O $7.049143-1.157867-1.859901$

C $7.3988131 .512001-2.195683$

H $7.4792622 .595372-2.073525$

H $8.4019271 .102004-2.347857$

H $6.7593811 .301812-3.058112$

SCF Energy (B3LYP/6-31G**//MMFF) $=-3245.90654044$

0700112

MM̄FF Geometry

C -1.015937 -2.388329 2.185856

C $-1.871070-1.7840643 .027483$

C $-1.928904-0.2991373 .312297$

$\begin{array}{lllll}\text { O } & -0.735628 & 0.329540 & 2.782167\end{array}$

C -3.1602850 .3426452 .646634$

C -4.4900680 .0700313 .313727$

C $-5.447161-0.7393022 .817956$

C -4.7369330 .8428934 .583446$

C $-5.366688-1.6189751 .603417$

C $-6.231875-1.1571370 .423228$

C $-5.7587300 .145904-0.242622$

C $-6.5830320 .436093-1.498860$

C $-6.0674791 .672603-2.253080$

O $-4.385331-0.005401-0.606028$

O $-6.6886901 .692250-3.547609$

C $-4.5350901 .560005-2.451666$

C $-6.5277222 .965508-1.568384$

O $-4.0272002 .766284-3.014940$

C $-3.7775091 .175169-1.161359$

C $-2.2989260 .789248-1.401043$

O $-3.8201252 .267670-0.240749$

C $-0.3988531 .560326 \quad 3.247122$

O -0.9924732 .2248724 .080572$

C 0.8297711 .9743352 .536249

C 1.1145553 .2794432 .420762

C 2.2847203 .8524651 .750998

C 3.3724002 .9032701 .325905

C $-1.3920561 .900490-1.933610$

C $0.083818 \quad 1.594087-1.731755$

C $2.3361242 .343839-2.497927$ 
O $0.4908850 .713811-0.978662$

C $3.0258823 .644122-2.210529$

C $2.4688054 .825373-1.897544$

C $3.2709976 .071182-1.644450$

C $2.9583296 .720011-0.314786$

C $2.3603277 .922192-0.255704$

C 3.4200846 .0186360 .944395

C 2.3322595 .1887431 .569393

N $0.8905472 .453480-2.464489$

H $-0.282662-1.7983941 .642332$

H -2.598495 -2.394842 3.557888

H -1.935241 -0.1606924 .400104$

H $-3.1763960 .047450 \quad 1.594998$

H -3.034524 1.4321772 .601349

H $-6.385377-0.8210823 .365148$

H -5.7352780 .6595564 .995001$

H $-4.0089610 .562940 \quad 5.351024$

H -4.650372 1.9183404 .397090

H -4.334133 -1.759309 1.277591

H -5.709804-2.616665 1.906452

H $-6.207625-1.963142-0.322152$

H -7.275775 -1.0501930 .742984$

H $-5.856951 \quad 0.9632340 .480635$

H -7.645779 $0.549834-1.251353$

H -6.526146 $-0.428471-2.175109$

H $-6.438450 \quad 0.878515-4.017271$

H $-4.3566850 .776047-3.199746$

H -7.622720 $2.998035-1.512612$

H -6.235669 $3.848014-2.148235$

H $-6.1416993 .074544-0.552941$

H $-3.9780963 .427799-2.303921$

H -1.895691 $0.426589-0.445406$

H -2.245934 -0.078217 -2.072741

H -3.0646592 .1912250 .364933$

H $1.449878 \quad 1.1941652 .110820$

H 0.4176424 .0014282 .845098

H 3.7657632 .3622782 .193874

H $4.222883 \quad 3.3942470 .849467$

H 2.9889942 .1736240 .605650

H -1.574904 2.040136-3.004575

H -1.592153 2.847798 -1.420652

H $2.6045032 .005117-3.503932$

H $2.6730991 .585705-1.784977$

H $4.1121613 .592326-2.267410$

H $1.3882624 .923999-1.839233$

H $3.0630156 .769380-2.465687$

H $4.3482445 .867395-1.692375$

H 2.1615348 .4060940 .694961

H $2.0528478 .446881-1.154007$

H $3.7276576 .768348 \quad 1.686334$

H 4.3297735 .4495200 .735445

H 1.4907585 .7846921 .926991

H $\quad 0.4530093 .098414-3.116315$

C -1.182112 $-4.256340 \quad 0.462451$

O $-0.109581-3.741377-0.349485$

C $-0.641392-3.400626-1.647690$

C -2.085259-3.895662 -1.667431

C $-2.468441-3.778999-0.204888$

H $-0.648061-2.306371-1.707341$

C $0.245316-3.987227-2.753356$

H $-2.718664-3.293875-2.326658$

H -2.154365 -4.938412 -1.998416

C $-3.696352-4.5887880 .169534$

H -2.648211 -2.7198280 .013045$

C $1.723375-3.552753-2.672757$

O $0.212345-5.418622-2.666609$

H -0.168141 -3.735486 -3.736442

H $2.171968-3.954898-1.758149$

O $2.424265-4.159414-3.765684$

C $1.937537-2.029965-2.747728$

C $3.419216-1.606170-2.744221$

H $1.423666-1.547684-1.912270$

O $1.334019-1.542958-3.948705$

H $3.964803-2.135396-3.534656$

H $3.464486-0.542755-3.012223$

H -4.571232 -4.240769-0.389084

H -3.558541 -5.652684 -0.050912

H $\quad-3.916038-4.4926851 .237239$
C $-0.984617-3.8691161 .931500$

H $0.522820-5.666042-1.778615$

H $2.267039-5.117861-3.714598$

H $1.402469-0.572922-3.938621$

H $-0.001450-4.2293472 .259652$

H -1.735069-4.381123 2.545132

H $-1.112622-5.3505820 .393691$

C $3.886987-1.1032460 .912344$

O $3.452013-0.955968-0.444233$

C $4.098720-1.821258-1.384879$

C $5.600784-1.509652-1.429700$

C $6.223703-1.643276-0.034802$

C $5.408676-0.9024051 .032617$

H $3.401817-0.2769331 .445587$

C $3.357900-2.4158561 .498200$

H $3.959688-2.862341-1.076717$

O $5.797663-0.184390-1.918249$

H $6.086411-2.198591-2.131014$

H $7.243638-1.239580-0.047841$

O $\quad 6.308010-3.0201850 .320465$

H $5.742231-1.1970152 .033431$

O 5.6825930 .4978520 .911837

C $3.267077-2.3568543 .002305$

H $2.359141-2.6429631 .103980$

H $3.974788-3.2767871 .224186$

H $6.756361-0.027579-1.961499$

H $6.858667-3.463157-0.347453$

H 5.3312070 .7964760 .055278

O $2.165283-1.6448283 .374792$

O $4.083517-2.8724203 .754498$

C $1.967231-1.5077064 .784966$

H $1.836888-2.4912775 .246108$

H $2.810972-0.9769765 .235813$

H $1.058533-0.9221064 .946177$

SCF Energy $(B 3 L Y P / 6-31 G * * / / M M F F)=-3245.91830217$

0700113

MM̄FF Geometry

C -3.161798 -1.070018 -3.169281

C $-2.262495-1.371589-2.220492$

C $-0.785296-1.108374-2.331939$

O $-0.452958-0.075433-1.369373$

C $0.038433-2.356421-1.960985$

C $0.391228-3.228997-3.148920$

C $1.631442-3.326825-3.667960$

C $-0.729211-4.062772-3.711296$

C $2.855956-2.578993-3.221733$

C $3.741029-3.458499-2.330081$

C $4.869507-2.651733-1.675000$

C $5.828242-3.574455-0.920419$

C $6.898150-2.784406-0.151634$

O $4.262658-1.732116-0.761560$

O $7.536437-3.6841010 .767421$

C $6.204259-1.6944920 .692390$

C $7.999483-2.260822-1.081115$

O $7.163805-0.8405121 .311635$

C $5.180198-0.850897-0.104578$

C 4.3736460 .0458190 .869169

O $5.884043-0.030539-1.035753$

C $-0.591706 \quad 1.217996-1.772902$

O $-0.949146 \quad 1.612117-2.871655$

C $-0.2454592 .086675-0.626545$

C $-0.3614243 .417878-0.738179$

C -0.0764514 .4050830 .307185$

C 0.3461613 .8900961 .658158

C 3.1322860 .7198600 .270194

C $3.4432811 .934830-0.578829$

C $4.2633954 .283002-0.420522$

O $3.3621201 .898364-1.804403$

C 3.6990995 .4214060 .368608

C $3.0374156 .450361-0.182378$

C 2.4844727 .6011380 .608804

C 1.0238797 .8685180 .319428

C $0.6434828 .962887-0.361543$

C -0.0016716 .9121160 .890165$

C -0.2173525 .7135640 .007209$

$\begin{array}{llll}\text { N } 3.820041 & 3.034908 & 0.172467\end{array}$

H $-2.837777-0.608380-4.097692$ 
H $-2.610578-1.809898-1.287620$ H $-0.504809-0.766684-3.336579$ H $-0.491777-2.969261-1.219806$ H $0.945867-2.042324-1.432873$ H $1.794359-4.007784-4.501900$ H $-0.374602-4.786835-4.452841$ H -1.221729 $-4.629088-2.914102$ H -1.472240 -3.428744 -4.202884 H $3.421107-2.274742-4.111488$ H $2.587059-1.646363-2.715723$ H $3.122535-3.912285-1.544074$ H $4.161902-4.274731-2.930480$ H $5.402624-2.095031-2.454578$ H $6.297963-4.291825-1.604956$ H $5.260169-4.178196-0.198595$ H $7.916201-4.4176030 .253898$ H $5.672095-2.2041141 .507932$ H $8.498312-3.094069-1.590643$ H $8.782644-1.745144-0.514481$ H $7.625314-1.582141-1.850294$ H $7.779698-1.4078121 .806406$ H $4.008568-0.5834731 .691686$ H 5.0343340 .8049581 .304246 H $5.2748730 .636601-1.386178$ H $0.090026 \quad 1.6059650 .283734$ H $-0.7016353 .830223-1.687400$ H -0.4455283 .2725902 .096626$ H 0.5652424 .6792292 .379564 H 1.2548023 .2855631 .576781 H $2.5328200 .019284-0.320820$ H 2.4844371 .0528891 .090701 H $3.9742434 .322388-1.475504$ H $5.3563264 .295999-0.361510$ H 3.8536605 .4058951 .445058 H $2.9047216 .481256-1.261411$ H $3.085657 \quad 8.4873790 .367312$ H 2.6097437 .4401081 .686869 H $-0.4039709 .174869-0.549558$ H $1.3700139 .668269-0.750896$ H -0.9692407 .4222310 .989442$ H $\quad 0.2862986 .6513841 .911694$ H $-0.5470145 .976702-0.999279$ H 3.9526932 .9169461 .172940 C -5.401123 -0.051597-2.638698 O $-4.8835940 .478197-1.399677$ C $-5.9159480 .410489-0.406260$ C -7.205569 $0.543795-1.199101$ C $-6.893321-0.327088-2.403348$ H $-5.866277-0.5903370 .039912$ C $-5.689041 \quad 1.4563470 .688711$ H $-8.0809490 .202866-0.638661$ H -7.379960 $1.577102-1.520165$ C -7.762566 $-0.029483-3.613481$ H -7.043642 -1.377372 -2.119498 C $-4.253817 \quad 1.498297 \quad 1.253433$ O H -6.4081131 .2932441 .499875$ H -3.5852621 .9853950 .532284$ O -4.2644562 .3376372 .415218$ C $-3.656400 \quad 0.133597 \quad 1.642093$ C -2.2686750 .2793832 .295332$ H -3.564024 $-0.484980 \quad 0.744260$ O $-4.536559-0.5468652 .533192$ H -1.7018581 .0500501 .764441$ H $-2.3959210 .603418 \quad 3.335841$ H $-8.817830-0.208098-3.383045$ H -7.660101 $1.012469-3.934542$ H -7.485448 -0.671195-4.455965 C $-4.632666-1.327175-3.001278$ H $-5.3982712 .909548-0.594495$ H -4.6249293 .2000142 .145943$ H -4.6542170 .0153353 .317949$ H -5.025128 -1.754880 -3.931050 H -4.779155 -2.082305 -2.218867 H $-5.248410 \quad 0.719763-3.403038$ C - $-1.498144-3.274376 \quad 3.186322$ O $-2.100405-1.9742423 .128960$ C $-1.458824-1.0316682 .267087$
C $-0.002566-0.8058562 .703415$

C $0.763754-2.1291182 .788417$

C $-0.014345-3.1831263 .582360$

H -2.028143 -3.7895813 .998140$

C -1.735218 -4.066399 1.895834

H -1.469418-1.413242 1.240243

O $0.020512-0.154527 \quad 3.971765$

H $0.480789-0.1374801 .982622$

H $1.735065-1.9607203 .269216$

O $1.012073-2.6265821 .477121$

H $0.452487-4.1690423 .472217$

O $0.036502-2.8499694 .972697$

C $-3.198960-4.4054561 .747525$

H -1.404847 -3.5391640 .997330$

H -1.188132 -5.016492 1.914101

H 0.9514690 .0003344 .205656

H $1.541420-1.9611401 .005586$

H $0.971702-2.8436005 .238644$

O $-3.801341-3.5263220 .897161$

O $-3.745426-5.3275002 .339648$

C $-5.202841-3.7320990 .696987$

H $-5.548657-3.019505-0.055170$

H $-5.393183-4.7451770 .329650$

H $-5.745290-3.5496141 .629341$

SCF Energy $\left(B 3 L Y P / 6-31 G^{* *} / / M M F F\right)=-3245.90052872$

0700114

MM̄FF Geometry

C -3.500029 -0.6810102 .685274$

C $-2.169225-0.5535962 .576593$

C $-1.200761-1.0886323 .599757$

O $-0.251824-1.9679002 .951739$

C $-0.383400 \quad 0.050542 \quad 4.247825$

C -1.206985 1.0522435 .033617

C -1.2678722 .3720824 .766874$

C -1.9789100 .4919996 .201401$

C $-0.517667 \quad 3.142214 \quad 3.716447$

C -1.2466123 .1617472 .369805$

C $-0.6655004 .196707 \quad 1.394731$

C -1.322823 4.0596530 .017940

C $-0.7011025 .004223-1.021701$

O $0.7458003 .987606 \quad 1.290608$

O $-1.1625624 .561969-2.307275$

C $0.8360154 .847837-0.980516$

C $-1.2065876 .441201-0.850346$

O $1.4753185 .808361-1.817103$

C 1.4180994 .9339260 .447316

C 2.9305794 .6183310 .498407

O $1.257364 \quad 6.270446 \quad 0.939484$

C $-0.570224-3.2858272 .877021$

O $-1.620161-3.8034723 .234583$

C $0.522296-4.0857742 .275915$

C $1.757292-3.6168632 .036719$

C $2.864129-4.3684831 .435242$

C $2.580382-5.7761890 .977855$

C 3.3133203 .2142320 .028539

C 4.7725382 .8932530 .304635

C 6.3685110 .9998740 .297789

O $5.571796 \quad 3.7127120 .749224$

C $6.547908-0.271978-0.465849$

C $6.638242-1.4776380 .115858$

C $6.883275-2.755373-0.634110$

C $5.704920-3.701038-0.599119$

C $5.053859-4.034760-1.726424$

C $5.335775-4.3291730 .727136$

C $4.063241-3.7619501 .300711$

N $5.078468 \quad 1.577856-0.021815$

H $-3.913078-1.2488373 .515707$

H -1.7544340 .0099641 .745823$

H $-1.720217-1.645024 \quad 4.389834$

H $\quad 0.2156870 .5403993 .470583$

H $0.360103-0.3927124 .924488$

H -1.910492 2.9910025 .392165

H -2.427854 1.2783256 .817876

H -2.789690 -0.1571205 .857720$

H - $1.315345-0.0897276 .849876$

H -0.3921574 .1688754 .083192$

H 0.4967322 .7444483 .599085 
H $-1.1577262 .166814 \quad 1.921947$ H -2.316242 3.3599152 .512875 H -0.8490775 .1941921 .811426$ H -2.406084 4.2210370 .085528 H -1.198565 $3.027281-0.339980$ H $-0.8241255 .177779-2.978712$ H $1.0662593 .861537-1.401433$ H -2.292062 6.482973-1.001908 H -0.772837 7.106721-1.604709 H $-0.994954 \quad 6.8565770 .137034$ H $1.1654835 .660853-2.726300$ H $3.4921825 .370276-0.071299$ H 3.2677164 .7328021 .538664 H 1.8921356 .4125081 .661076 H $\quad 0.249486-5.1117642 .049097$ H $1.989741-2.5866872 .300829$ H $1.738114-5.7918420 .276744$ H $2.337732-6.415617 \quad 1.833515$ H $3.416440-6.2446090 .454981$ H 2.6988172 .4667600 .542146 H $3.1540763 .118786-1.050100$ H $6.400180 \quad 0.8435881 .381520$ H 7.1575001 .7138520 .038992 H $6.634888-0.190760-1.546617$ H $\quad 6.565422-1.5494801 .198637$ H $7.756139-3.256637-0.197176$ H $7.163651-2.532668-1.672087$ H $4.227525-4.737770-1.713243$ H $5.329358-3.610780-2.686180$ H $6.140266-4.1635901 .455368$ H $5.295508-5.4167470 .612882$ H $4.165205-2.7344511 .652412$ H $4.3085010 .942710-0.213672$ C -5.159982 -0.8999560 .777239$ O $-4.347164-0.931629-0.419720$ C $-5.106217-0.350700-1.492988$ C $-6.551624-0.656176-1.139226$ C $-6.537170-0.3696250 .348358$ H $-4.9483870 .734375-1.445994$ C -4.651652 -0.869121 -2.859609 H -7.261272 $-0.031863-1.689907$ H $-6.800469-1.708422-1.318919$ C $-7.692412-1.0052311 .103540$ H $-6.584373 \quad 0.7186200 .492014$ C $-3.142660-0.734165-3.145209$ O $-4.984040-2.258099-2.980473$ H -5.222968 -0.358752 -3.644105 H $-2.598923-1.517659-2.608572$ O $-2.940658-0.992030-4.540679$ C $-2.5313940 .636694-2.812031$ C $-1.0807060 .841756-3.286752$ H $-2.5642070 .791973-1.727886$ O $-3.3245041 .674315-3.394948$ H -1.055879 $0.980515-4.375364$ H $-0.7489411 .799793-2.866251$ H $-8.649811-0.6191760 .738936$ H -7.700615 -2.093756 0.982180 H -7.623069 -0.7856012 .173840$ C -4.4941060 .0209651 .804055$ H -4.555210 -2.726455 -2.243793 H $-3.316053-1.869233-4.730240$ H $-3.3502901 .519590-4.354917$ H -5.2513460 .4463602 .474811$ H -4.0115380 .8655951 .295456$ H $-5.228915-1.9305281 .145915$ C $0.585927-1.576182-0.965248$ O $-0.218811-0.508800-1.485988$ C $-0.099134-0.273807-2.891360$ C $1.3510920 .078291-3.262852$ C $2.325963-0.989892-2.759068$ C $2.069783-1.344792-1.291030$ H $0.471654-1.5032240 .123140$ C $0.081053-2.959854-1.407964$ H $-0.390118-1.178579-3.433770$ O $1.6982921 .338684-2.693845$ H $1.4235350 .175161-4.352860$ H $3.353868-0.623611-2.871576$ O $2.183713-2.150256-3.573022$
H $2.646478-2.231999-1.013716$

O $2.545575-0.280445-0.465041$

C $-1.229063-3.328005-0.746731$

H $-0.073722-3.021441-2.487589$

H $\quad 0.813994-3.720165-1.115179$

H $2.6014621 .552520-2.983878$

H $2.819709-2.812707-3.254201$

H $1.9846150 .496276-0.632892$

O $-1.525948-4.628692-1.039102$

O $-1.912557-2.575854-0.067491$

C $-2.745605-5.118892-0.473502$

H -2.693446 -5.090977 0.618997

H -3.595410 -4.532081 -0.834805

H $-2.875241-6.156548-0.792171$

SCF Energy (B3LYP/6-31G**//MMFF)= -3245.91741345

0700115

MM̄̄F Geometry

C 1.0219101 .5554912 .575755

C 0.0113071 .0141913 .273928

C $-0.221677-0.4462333 .594324$

O $-1.437306-0.8596942 .920464$

C $0.906176-1.3970683 .156411$

C $0.612964-2.8592123 .433614$

C $0.260107-3.7523092 .486729$

C $0.786502-3.2935764 .864503$

C $0.038418-3.4725581 .025287$

C $-1.224626-4.1604380 .485994$

C $-1.692242-3.612920-0.875427$

C $-0.592956-3.648344-1.942319$

C $-1.035399-2.996129-3.259661$

O $-2.152210-2.272869-0.661865$

O $0.149592-2.799437-4.047105$

C $-1.629244-1.607033-2.943189$

C $-1.940918-3.926620-4.072831$

O $-2.148963-0.997293-4.121877$

C $-2.691030-1.632213-1.824931$

C $-3.142969-0.214136-1.412643$

O $-3.851313-2.321554-2.305744$

C $-2.620807-0.5461303 .511660$

O -2.7906390 .0477724 .564767$

C $-3.702520-1.0190612 .626098$

C $-4.809969-0.2701952 .526272$

C $-5.970987-0.5505561 .683310$

C $-6.089892-1.9309201 .093486$

C $-2.0432190 .679669-0.833050$

C $-2.6441991 .856542-0.090050$

C $-4.0862643 .857538-0.428534$

O -2.5686441 .9538381 .132560$

C $-5.5486033 .510389-0.392963$

C $-6.1666662 .549922-1.100228$

C -7.642206 2.269825-1.030657

C $-7.9454010 .811475-0.761259$

C $-8.113320-0.054165-1.775427$

C -8.1369510 .3732640 .672903$

C -6.8706510 .4344041 .479990$

N -3.279362 $2.759230-0.927010$

H 1.8093250 .9300902 .166636

H -0.7454561 .6907193 .668590$

H $-0.356907-0.5422214 .679425$

H $1.840981-1.1259683 .665024$

H $1.105725-1.2560982 .091170$

H $0.116129-4.7898982 .784022$

H $0.615552-4.3672904 .996332$

H $1.803975-3.0777815 .206215$

H $0.081070-2.7660735 .513977$

H $-0.038420-2.3995940 .832787$

H $0.913829-3.8414180 .478246$

H $-1.039740-5.2385440 .401541$

H $-2.041121-4.0265711 .207811$

H $-2.546129-4.222807-1.193591$

H $-0.250530-4.676538-2.112528$

H $0.286402-3.095920-1.588816$

H $-0.107149-2.311041-4.848287$

H $-0.792436-0.977945-2.620174$

H - $1.408236-4.852506-4.321739$

H -2.216009 -3.474837 -5.032173

H $-2.856248-4.203884-3.546010$ 
H -3.002069 -1.419069-4.321571 H -3.623818 $0.283013-2.264648$ H $-3.934672-0.311322-0.658517$ H -4.624211 -2.022202 -1.799535 H -3.537994 -1.926965 2.059061 H $-4.8593340 .659203 \quad 3.093427$ H -5.241757 -2.1497010 .437351$ H $-6.991911-2.0696360 .493469$ H -6.117075 -2.684430 1.888634 H $-1.4180780 .127190-0.123059$ H $-1.3862641 .059905-1.621985$ H -3.921316 4.722014 -1.078556 H -3.7521124 .1148250 .581887$ H $-6.148176 \quad 4.1233210 .277277$ H -5.597676 $1.945662-1.801837$ H -8.083845 2.564554 -1.991861 H -8.135618 $2.894741-0.276194$ H $-8.358889-1.095656-1.596538$ H -8.004158 $0.259866-2.808341$ H -8.579260 -0.6284120 .724507$ H $-8.882281 \quad 1.0251001 .146989$ H -6.6928661 .4063841 .941569$ H $-3.2920402 .581855-1.926920$ C 1.1458033 .3863050 .837063 O 2.3614992 .9054610 .224576 C $3.1828794 .040070-0.088606$ C $2.1791545 .124993-0.442543$ C 1.1252664 .9094440 .628382 H 3.7102154 .3129310 .835129 C $4.2212103 .715747-1.168637$ H $2.6158446 .127646-0.417918$ H $1.7390674 .962387-1.433047$ C -0.2475025 .4401730 .245807$ H 1.4496545 .4220421 .543957 C $4.9465812 .366513-0.989492$ O $3.5770573 .681228-2.448009$ H $4.9444004 .538499-1.221802$ H $4.2715021 .565018-1.302976$ O $6.0399962 .336787-1.915726$ C 5.4725722 .0720440 .423603 C 6.3256700 .7944480 .539453 H $4.630021 \quad 1.993676 \quad 1.119208$ O $\quad 6.2698293 .1772350 .856009$ H 7.3099750 .9594320 .082190 H $6.5249190 .647987 \quad 1.609347$ H $-0.210164 \quad 6.5214910 .078645$ H $-0.6182274 .969882-0.671292$ H -0.9734745 .2435531 .041627$ C 1.1502473 .0332552 .330895 H $4.2599433 .484944-3.112301$ H $6.6876133 .001751-1.624931$ H 6.5494342 .9973251 .769801 H 0.3386673 .5725262 .834597 H 2.0928003 .3595292 .788286 H $\quad 0.3107582 .8953910 .324308$ C $3.617915-1.736394-0.029339$ O $4.370834-0.6249220 .479839$ C $5.689588-0.475818-0.054002$ C $6.532925-1.7280620 .237873$ C $5.838396-2.998855-0.263091$ C $4.373258-3.0596650 .181105$ H $2.720078-1.7735620 .595687$ C $3.170286-1.517069-1.483368$ H $5.625500-0.341356-1.138318$ O $6.779272-1.849978 \quad 1.637947$ H $7.508483-1.619867-0.249996$ H $6.368201-3.8789830 .121163$ O $5.890018-3.046365-1.685762$ H $3.845394-3.867124-0.339915$ O $4.321663-3.3602301 .579130$ C $2.165671-0.389644-1.606616$ H $4.020915-1.283901-2.129070$ H $2.684901-2.418813-1.872725$ H $5.918780-1.8652982 .091014$ H $6.826690-3.037757-1.946414$ H $4.745739-4.225721 \quad 1.708154$ O $2.243702 \quad 0.142081-2.861478$ O $1.391396-0.043296-0.725416$
C $1.3735321 .242033-3.151491$

H $1.8413081 .844228-3.934839$

H $0.4216020 .860340-3.527915$

H $\quad 1.210821 \quad 1.878493-2.276689$

SCF Energy (B3LYP/6-31G**//MMFF) $=-3245.91527920$

0700116

MM̄̄FF Geometry

C $2.030281-1.647826-3.242168$

C $2.052255-2.092435-1.974983$

C $0.997547-2.971429-1.347647$

O $-0.291074-2.609346-1.899180$

C $1.247400-4.470617-1.589572$

C 2.504764-5.019364-0.956061

C $2.592716-5.4106920 .329733$

C $3.683502-5.180835-1.880044$

C $1.526800-5.3318531 .391123$

C $2.070930-4.7457772 .702509$

C $2.355412-3.2352162 .628406$

C $3.340846-2.8394613 .730136$

C $3.598391-1.3296463 .745407$

O $1.113113-2.5400212 .805001$

O $4.295836-1.0118674 .959062$

C $2.242080-0.6059933 .798371$

C $4.505581-0.8880312 .593511$

O 2.4140470 .8052463 .672952

C $1.212599-1.1091152 .753062$

C $-0.170470-0.5139673 .114254$

O $1.606183-0.6559851 .457319$

C $-1.395159-2.896620-1.161436$

O $-1.435561-3.473795-0.087135$

C $-2.565259-2.365902-1.891977$

C $-3.796629-2.588542-1.412087$

C $-5.047942-2.091518-1.984408$

C $-4.955246-1.160909-3.164964$

C $-1.362497-1.0254762 .293116$

C $-2.654991-0.3882332 .785976$

C -4.9090890 .3063562 .081309$

$\begin{array}{llll}\text { O } & -2.768852 & 0.089580 & 3.913969\end{array}$

C $-5.978698-0.1472201 .141238$

C -6.5319590 .6524280 .214894$

C $-7.6554210 .345393-0.742562$

C $-8.187015-1.071245-0.746593$

C $-9.203114-1.4309880 .055691$

C $-7.604597-2.038472-1.754834$

C $-6.206987-2.455103-1.397704$

N -3.679622 -0.4303051 .847944$

H $\quad 1.210127-1.965075-3.883507$

H $2.872679-1.818230-1.318061$

H $\quad 0.981457-2.748246-0.274528$

H $1.255968-4.672405-2.669955$

H $\quad 0.386597-5.051371-1.233637$

H $3.535116-5.8418570 .666195$

H $4.550073-5.621413-1.375438$

H $3.421989-5.836782-2.716924$

H $3.996598-4.213004-2.282131$

H $\quad 0.654633-4.7591191 .066635$

H $1.181483-6.3538301 .588783$

H $1.332006-4.9358673 .491547$

H $2.982795-5.2909262 .978264$

H $2.770306-2.9843741 .646588$

H $4.287169-3.3842813 .620909$

H $2.935481-3.1352974 .708094$

H $5.125245-1.5197194 .969913$

H $1.826099-0.7770564 .801445$

H $5.468353-1.4106782 .640660$

H 4.7409730 .1790182 .667411

H $4.072321-1.0820731 .610322$

H 3.0504871 .0812654 .354648

H $-0.378191-0.7386654 .169169$

H -0.1338720 .5790123 .018215$

H $\quad 0.818919-0.5702700 .897342$

H -2.382645 -1.795807 -2.794852

H $-3.901840-3.180215-0.503316$

H $-4.339737-0.288021-2.919138$

H $-5.921465-0.772620-3.492778$

H -4.510372 -1.674195 -4.024594

H $-1.227089-0.7931631 .233193$ 
H -1.466727 -2.110202 2.400504 H -4.6799621 .3714861 .967734$ H $-5.231570 \quad 0.147025 \quad 3.116075$ H -6.335081 -1.1638671 .276017$ H -6.1542311 .6712190 .136286$ H $-7.3298970 .609794-1.756344$ H -8.477294 $1.037906-0.515770$ H $-9.612627-2.4354170 .034972$ H $-9.643874-0.7281750 .755138$ H -7.673561 -1.601369-2.755126 H $-8.215028-2.949913-1.806721$ H $-6.165714-3.126556-0.538665$ H -3.445730 -0.679439 0.891135 C $4.061039-0.053282-3.032069$ O $3.406980 \quad 0.925845-2.198462$ C $4.2585252 .082734-2.083358$ C $5.5743551 .714509-2.762153$ C $5.1113390 .730714-3.817135$ H $3.7828262 .888337-2.653299$ C $4.4142722 .480184-0.612552$ H $6.0814672 .588822-3.181565$ H $6.2672381 .229896-2.063873$ C $6.234466-0.122400-4.381178$ H $4.6336871 .288168-4.634055$ C 3.1113792 .9403180 .077092 O $4.926049 \begin{array}{lll}1.356192 & 0.113837\end{array}$ H $5.1742213 .264727-0.519694$ H 2.3953842 .1129050 .134524 O 3.4445193 .2800321 .429961 C $2.4482414 .168668-0.581306$ C 1.3748824 .8677470 .274816 H $2.0156153 .879737-1.544830$ O $3.4522995 .150777-0.865589$ H 1.8083075 .1667421 .237412 H $1.1164725 .807109-0.233952$ H $6.992160 \quad 0.507270-4.858904$ H $6.728203-0.705747-3.596708$ H $5.855825-0.821898-5.132873$ C $3.026670-0.745131-3.922459$ H $4.2972720 .623770-0.005928$ H 3.8360422 .4886101 .838250 H $3.8632935 .402934-0.020698$ H $3.535164-1.341746-4.688423$ H $2.446396 \quad 0.026290-4.445505$ H $4.555834-0.774735-2.369852$ C $-1.8044253 .215083-0.766722$ O $-0.5713393 .946694-0.761493$ C 0.0971914 .0454810 .500337 C -0.8158814 .7137301 .542650$ C -2.1608763 .9910691 .649826$ C $-2.792414 \quad 3.7646470 .273874$ H -2.232242 $3.404532-1.759600$ C $-1.5628451 .708156-0.636657$ H $\quad 0.3705643 .0440150 .847128$ O -1.0351586 .0774351 .185699$ H -0.3041284 .7066192 .512603$ H $-2.847845 \quad 4.5816782 .268069$ O $\begin{array}{llll}-1.977330 & 2.732886 & 2.287630\end{array}$ H -3.651324 3.0880430 .350783 O $-3.2876895 .011247-0.223426$ C $-0.792266 \quad 1.188877-1.824353$ H $-0.988243 \quad 1.4512980 .257179$ H $-2.507557 \quad 1.155777-0.576553$ H -1.585964 6.4777551 .879762 H -1.606206 2.9000573 .170629 H -3.9502405 .3369680 .409420$ O $-1.6019801 .131313-2.919259$ O $0.3925250 .886448-1.782425$ C $-0.974152 \quad 0.711594-4.134401$ H $-1.6819080 .867309-4.952888$ H $-0.075698 \quad 1.304326-4.332290$ H -0.731368 -0.352452 -4.078962

SCF Energy $\left(B 3 L Y P / 6-31 G^{* *} / / M M F F\right)=-3245.91148741$

\section{7_00117}

MM̄FF Geometry

C $2.956406-1.0902513 .713940$

C $2.174026-1.6163082 .759317$
C $0.678785-1.7669072 .885564$

O $0.060045-0.9742361 .844090$

C $0.297911-3.2431092 .665123$

C $-1.115977-3.5804223 .096403$

C $-2.144479-3.8335952 .263107$

C $-1.325584-3.6719734 .587866$

C $-2.152845-3.8130090 .759572$

C $-2.783687-2.5352140 .201810$

C $-4.283135-2.3675250 .491743$

C $-5.146167-3.294952-0.367560$

C $-6.648492-3.020994-0.183933$

O $-4.602446-1.0016110 .199302$

O $-7.350101-3.671311-1.254880$

C $-6.909745-1.505508-0.329359$

C -7.180661 -3.634606 1.116915

O $-8.263527-1.188778-0.013802$

C $-5.952437-0.6349780 .513767$

C -6.1282270 .8605920 .161588$

O $-6.250926-0.8250291 .898881$

C -0.0709150 .3621522 .075598$

$\begin{array}{llll}\text { O } & 0.190244 & 0.960822 & 3.107095\end{array}$

C $-0.523396 \quad 0.9955640 .817083$

C -0.2113682 .2809930 .591240$

C $-0.4579863 .023277-0.647595$

C $-1.4144032 .423922-1.643571$

C -5.0517241 .7665350 .777043$

C -5.2506653 .2274620 .417566$

C $-4.1751595 .454441 \quad 0.733635$

O $-6.2113903 .639291-0.226178$

C $-3.4147755 .760091-0.517212$

C $-2.3142796 .526164-0.546485$

C $-1.5846586 .884264-1.807682$

C $-0.1266146 .491685-1.771674$

C $0.8332727 .410038-1.568418$

C $0.2239035 .051796-2.070165$

C $0.2092204 .180150-0.844348$

N -4.226708 4.0182420 .922486

H $2.513001-0.7413874 .642821$

H $2.624325-1.9487651 .826223$

H $0.329362-1.4243413 .866821$

H $0.976791-3.894013 \quad 3.233478$

H $0.456812-3.5026511 .613053$

H -3.106057 -4.087419 2.707782

H -2.337768 -4.000638 4.846992

H $-0.626841-4.3921265 .025980$

H - $1.166611-2.6991575 .062410$

H -1.140770 $-3.905938 \quad 0.357975$

H -2.690573 -4.696952 0.398945

H -2.248494 -1.666422 0.602613

H -2.617746 -2.495603 -0.882956

H $-4.467358-2.5414101 .556523$

H -4.925582 -4.348235 -0.158250

H $-4.897674-3.142387-1.427379$

H -7.135981 -4.619463 -1.221536

H $-6.769386-1.247754-1.388549$

H $-7.039436-4.7222681 .112779$

H -8.259636 -3.474038 1.217665

H -6.687345 -3.243442 2.009156

H -8.829589-1.748330 -0.572580

H $-6.0833590 .985014-0.928416$

H -7.1164901 .2064650 .489879$

H -5.980115 -0.0401492 .399049$

H $-1.016374 \quad 0.372357 \quad 0.080645$

H $\quad 0.3422362 .8235531 .356995$

H -2.347788 2.128051-1.153389

H $-0.9741401 .540488-2.117867$

H -1.701835 $3.116369-2.437663$

H $-5.0612001 .694678 \quad 1.869641$

H $-4.059854 \quad 1.463267 \quad 0.424731$

H -3.7028285 .8836661 .622791$

H -5.1929435 .8498340 .653846$

H -3.807204 5.356171-1.447537

H -1.935265 6.9501180 .380600

H $-1.6791247 .969003-1.948400$

H $-2.0595276 .432477-2.687589$

H $1.8839067 .138781-1.573980$

H $0.5899138 .451446-1.386063$

H $1.2401995 .003143-2.484794$ 
H $-0.4206374 .680912-2.871475$

H $0.8931624 .518188-0.064035$

H -3.434454 3.5728651 .376835

C $4.8558610 .507186 \quad 3.320323$

O $4.320990 \quad 0.9325562 .049182$

C 5.4123521 .2893141 .187273

C 6.5223371 .7168602 .132958

C $6.3782570 .674195 \quad 3.226765$

H 5.7188520 .3746360 .665361

C 4.9713202 .3309330 .155683

H 7.5071491 .7066551 .657100

H 6.3448242 .7179892 .542158

C 7.0281101 .0736324 .540580

H $6.839361-0.2602652 .879417$

C $3.6679321 .977635-0.589333$

O 4.7590793 .5910510 .804621

H $5.7824292 .499092-0.562133$

H 2.8103362 .1261830 .077087

O $3.5073332 .912269-1.663116$

C $3.6105370 .552327-1.167022$

C $2.3226370 .306300-1.975107$

H $3.653781-0.170491-0.345761$

O $4.749366 \quad 0.321358-1.993234$

H $1.4715920 .722865-1.429270$

H $2.4013340 .823814-2.939158$

H 8.1057351 .2155984 .409598

H 6.6099792 .0092624 .926877

H $\quad 6.8776470 .2983645 .298807$

C $4.445712-0.9463693 .573680$

H 4.0886213 .4560371 .496428

H $3.5157993 .803872-1.274627$

H $4.7313690 .978915-2.709645$

H $4.918061-1.3194714 .489713$

H $4.797957-1.5795942 .749789$

H 4.4285421 .1692814 .083356

C $3.012339-3.087296-3.389288$

O $3.074173-1.685952-3.096200$

C $2.057455-1.190765-2.222308$

C $0.664698-1.428552-2.827586$

C $0.461346-2.903408-3.187522$

C $1.643973-3.466482-3.982684$

H $3.772150-3.241615-4.166063$

C $3.406385-3.939461-2.177164$

H $2.122845-1.707663-1.258411$

O $0.508965-0.622471-3.993544$

H $-0.094239-1.110710-2.102354$

H $-0.457846-3.005492-3.777314$

O $0.288433-3.643239-1.983144$

H $\quad 1.561957-4.556957-4.060219$

O $1.563365-2.946563-5.313024$

C $4.850248-3.695504-1.806551$

H $2.780971-3.751986-1.299730$

H $3.307998-5.007972-2.402326$

H $-0.388888-0.771327-4.335698$

H $\quad 0.125915-4.570070-2.228366$

H $2.280302-3.350420-5.830971$

O $4.922992-2.937376-0.675705$

O $5.798968-4.111883-2.459019$

C $6.246212-2.622376-0.232123$

H $6.733991-1.959621-0.952848$

H $\quad 6.169779-2.1037300 .726490$

H $6.831300-3.535672-0.087776$

SCF Energy (B3LYP/6-31G**//MMFF) $=-3245.90316737$

\section{7_00118}

MM̄FF Geometry

C $0.780919-4.1587450 .225977$

C $0.080518-3.0237470 .080691$

C -1.129168 -2.929143 -0.811860

O $-2.231777-2.5399320 .044941$

C $-0.993887-1.874967-1.926593$

C $0.251249-1.968460-2.784920$

C $1.123209-0.952978-2.948215$

C $0.437788-3.256992-3.541241$

C $1.0307230 .408912-2.317249$

C $0.1778381 .355336-3.172202$

C $-0.3034672 .614366-2.437335$

C $0.8420733 .498473-1.946233$
C $0.3206834 .719121-1.172081$

O $-1.1152142 .194899-1.335899$

O $1.4545515 .311639-0.523635$

C $-0.6611764 .230212-0.083704$

C $-0.2457585 .789491-2.112540$

O -1.2815205 .3312130 .577153$

C -1.746635 $3.263611-0.618901$

C -2.5220042 .6377110 .570041$

O $-2.6451493 .994313-1.451414$

C $-3.488367-2.886809-0.340224$

O $-3.806264-3.523661-1.331309$

C $-4.424511-2.3383510 .667103$

C $-5.738973-2.5808300 .556590$

C $-6.786498-2.0983141 .460294$

C $-6.375751-1.1628342 .567410$

C -3.5608121 .5711760 .187062$

C $-4.9216472 .134047-0.189376$

C $-7.1192861 .433586-1.136362$

O $-5.2266803 .313687-0.036444$

C $-8.0632351 .137309-0.013824$

C $-9.1365810 .342329-0.140691$

C -10.1048120 .0627790 .973035$

C $-10.319376-1.4166341 .205116$

C -11.424653 -2.033596 0.753985

C $-9.294143-2.1603912 .032515$

C $-8.056375-2.5023901 .246223$

N $-5.7610441 .156622-0.706244$

H $\quad 0.467765-5.043000-0.324981$

H $\quad 0.373906-2.1339870 .631891$

H -1.347179 -3.910635 -1.252916

$\mathrm{H}-1.105161-0.881423-1.477723$

H -1.865406 -1.956839-2.590756

H $1.972690-1.093793-3.614250$

H $1.306266-3.224214-4.207839$

H $0.583711-4.096044-2.855993$

H $-0.443030-3.462787-4.158535$

H $\quad 0.6510300 .332259-1.293158$

H $2.0431520 .816103-2.224256$

H $0.740396 \quad 1.644298-4.068916$

H $-0.7150070 .817344-3.518114$

H $-0.9320743 .170608-3.142701$

H $1.4791283 .813751-2.781667$

H $1.4917252 .924775-1.271959$

H $1.1569976 .115710-0.065900$

H -0.0722053 .7000350 .677182$

H $0.5346326 .147169-2.794881$

H $-0.5818946 .669869-1.553519$

H -1.079089 $5.432216-2.720768$

H -0.5805745 .8545971 .000400$

H -1.7918122 .1406911 .222937$

H -3.0047293 .4248381 .161226$

H -3.427846 $3.446975-1.617955$

H $-4.005185-1.7454761 .472318$

H $-6.085832-3.194060-0.274199$

H -5.718767-1.675716 3.278355

H $-7.216481-0.7634673 .137734$

H $-5.844317-0.2946142 .162573$

H -3.191032 $0.933958-0.623295$

H $-3.7276980 .928821 \quad 1.060902$

H -7.314216 $0.812672-2.016486$

H $-7.2051122 .486329-1.423620$

H -7.8656601 .6152000 .942858$

H -9.348718 - $0.113534-1.105312$

H -11.0571550 .5428580 .712779$

H $-9.783127 \quad 0.532478 \quad 1.910985$

H -11.599432 -3.088035 0.940046

H -12.177116 -1.499077 0.183623

H -9.718845 -3.107384 2.392120

H $-9.084093-1.5873742 .939376$

H $-8.248450-3.1822240 .414568$

H $-5.4440960 .191955-0.730920$

C $3.317910-4.4072410 .423849$

O $3.767062-3.0879270 .060739$

C $4.545839-3.187790-1.147214$

C $4.667467-4.677898-1.469473$

C $3.400775-5.237724-0.854232$

H $3.957060-2.703147-1.934408$

C $5.896647-2.477137-0.989171$ 
H $4.738817-4.860376-2.546293$

H $5.548529-5.129274-0.999149$

C $3.442274-6.736216-0.616501$

H $2.572492-5.002878-1.532959$

C $5.830839-1.006645-0.529726$

O $6.691685-3.179554-0.022567$

H $6.450932-2.544554-1.932852$

H $5.575070-0.9620410 .534976$

O $7.153876-0.465099-0.645983$

C $4.866363-0.112423-1.325231$

C $5.0387881 .400920-1.086040$

H $3.837831-0.406030-1.088972$

O $5.061080-0.348709-2.722887$

H $5.9926511 .734263-1.514961$

H $4.2621301 .908301-1.673755$

H $3.577412-7.272077-1.561698$

H $4.266622-7.0147890 .048399$

H $2.509563-7.083879-0.161166$

C $1.962629-4.3357211 .133579$

H $6.190655-3.1979160 .810966$

H $7.746631-1.034087-0.125367$

H $4.3952530 .171686-3.204156$

H $1.982661-3.5274551 .873527$

H $1.804754-5.2664911 .692480$

H $4.052473-4.7775291 .151864$

C 3.4251051 .7553522 .267249

$\begin{array}{lllll}\text { O } & 3.661820 & 1.465536 & 0.882001\end{array}$

C $4.946727 \quad 1.8430840 .382632$

C 5.1609603 .3578590 .535813

C 4.9336663 .8138851 .980915

C 3.6322263 .2524872 .562124

H 2.3636451 .5243372 .423588

C 4.2440010 .8460683 .197458

H 5.7237101 .3214330 .949020

O $4.2859604 .076426-0.330427$

H $\quad 6.183776 \quad 3.6017510 .226298$

H 4.8976434 .9098252 .016193

O $6.022228 \quad 3.387092 \quad 2.794774$

H 3.5991653 .4278073 .643911

O 2.5449713 .9817551 .988589

C $3.846609-0.6090203 .079275$

H 5.3145380 .9065402 .986498

H 4.0727081 .1449254 .238236

H $3.3724043 .850822-0.085314$

H 6.8328583 .7836602 .432594

H 1.7273623 .6755282 .416500

O $4.630936-1.3460833 .920745$

O $2.968759-1.0453612 .348368$

C $4.387974-2.7556983 .921075$

H $3.330256-2.9670904 .104973$

H $4.711268-3.1860342 .969906$

H $4.977710-3.2018274 .726361$

SCF Energy (B3LYP/6-31G**//MMFF) $=-3245.90914841$

0700119

MM̄FF Geometry

C -3.460172 4.376112 -0.610655

C $-2.4528813 .813018-1.294500$

C -1.027093 4.296916-1.220158

O $-0.2067693 .199108-0.752004$

C $-0.5333324 .702402-2.619497$

C $0.7777945 .463211-2.583685$

C $1.9853024 .947513-2.886121$

C $0.6598776 .918960-2.205047$

C $2.3278863 .540963-3.293885$

C $3.1834702 .863310-2.223376$

C $3.7670041 .506138-2.641368$

C $2.7040130 .479131-3.031537$

C $3.321582-0.899074-3.318141$

O $4.5016591 .029402-1.508142$

O $2.253950-1.856988-3.366719$

C $4.235251-1.300994-2.141879$

C $4.003746-0.942904-4.691194$

O $4.922223-2.510511-2.457268$

C $5.226579-0.188733-1.723592$

C $5.910067-0.552987-0.384539$

O $6.223600-0.038211-2.734062$

C -0.1818852 .9672210 .590185$
O $\quad-0.732917 \quad 3.621752 \quad 1.460852$

C $\quad 0.608814 \quad 1.7370520 .817955$

C 0.5908491 .1486002 .023258

C $1.260030-0.1059332 .376613$

C $2.260076-0.6598321 .398541$

C 6.7979600 .5625290 .183419

C $6.994577 \quad 0.3952891 .675862$

C 5.8304220 .7393643 .854941

O $8.027095-0.0786422 .143574$

C $5.167882-0.5432464 .248092$

C $4.132354-0.6145925 .098554$

C $3.522301-1.9106725 .547903$

C $2.014888-1.9260435 .447964$

C $1.249009-1.9335266 .552733$

C $1.394804-2.0341954 .073322$

C $0.930940-0.7043393 .540092$

N 5.8954610 .8195662 .408916

H -3.2620385 .2411090 .017459$

H -2.648093 $2.939055-1.911540$

H $-0.9342455 .142727-0.527935$

H $-1.2792965 .341760-3.111332$

H $-0.4484053 .813265-3.253457$

H $2.8477355 .612026-2.835984$

H $1.6233317 .438648-2.241953$

H $-0.0173787 .435831-2.892940$

H $0.2700747 .024287-1.188182$

H $1.4323472 .945807-3.483742$

H $2.8822413 .585490-4.238883$

H $4.0204203 .518671-1.943632$

H $2.6012522 .744399-1.301858$

H $4.4621821 .676718-3.471732$

H $2.1260390 .813362-3.900704$

H $1.9804920 .372220-2.211641$

H $1.626591-1.574186-4.054029$

H $3.577490-1.528715-1.291800$

H $3.282822-0.710270-5.484226$

H $4.378768-1.947835-4.914380$

H $4.831831-0.236922-4.781256$

H $5.316039-2.853166-1.637934$

H $5.135976-0.8047490 .349812$

H $6.534830-1.446178-0.506405$

H $7.0075750 .379658-2.343869$

H $1.1456621 .319185-0.025087$

H $-0.006897 \quad 1.5989772 .814734$

H $1.755588-1.0239250 .497821$

H $2.851745-1.4846391 .800867$

H 2.9786260 .1137451 .106707

H $7.7827040 .555752-0.297445$

H $\quad 6.371093 \quad 1.556767 \quad 0.010064$

H 5.2773491 .6160404 .206027

H 6.8435890 .7736924 .268080

H $5.585968-1.4618923 .842649$

H $3.735750 \quad 0.2998845 .533405$

H $3.831645-2.0758836 .588180$

H $3.924749-2.7582434 .978934$

H $0.166679-1.9795366 .486998$

H $1.684960-1.8904007 .545143$

H $\quad 0.512427-2.686628 \quad 4.129029$

H $2.080538-2.5541123 .398963$

H $\quad 0.183270-0.2205384 .170682$

H $5.066344 \quad 1.1406701 .917686$

C -5.2303843 .2506330 .735340$

O -4.4558092 .0512750 .936956$

C -5.3043121 .0356181 .502957$

C $-6.588048 \quad 1.7426831 .918317$

C -6.6908032 .8189230 .853444$

H $\quad-5.539778 \quad 0.3350810 .693760$

C $-4.566117 \quad 0.3022842 .627647$

H -7.4484471 .0668211 .932349$

H -6.4992202 .1979092 .911561$

C -7.6423833 .9448631 .217585$

H -7.023083 2.355131-0.085038

C $-3.199513-0.2798782 .208643$

O $-4.329754 \quad 1.214153 \quad 3.708445$

H $-5.204015-0.4896683 .036174$

H -2.4916790 .5336502 .011116$

O $-2.671431-1.0111963 .322102$

C $-3.252610-1.2180400 .989431$ 
C $-1.873741-1.826698 \quad 0.668594$ H -3.604509 -0.6583810 .116143$ O $-4.198017-2.2519801 .255233$ H $-1.129821-1.0238320 .644574$ H $-1.603040-2.5307911 .465213$ H $-8.658233 \quad 3.5600791 .354684$ H -7.3420774 .4395242 .147418$ H -7.671933 4.7019260 .427704 C $-4.872053 \quad 3.859389-0.620801$ H $-3.8126191 .959037 \quad 3.356224$ H -2.634169 -0.4004374 .077974$ H $-4.241019-2.8227840 .469788$ H $-5.5422334 .693199-0.857358$ H $-4.9917593 .107032-1.410267$ H -4.9678193 .9402181 .548713$ C $-2.788958-4.523650-1.758206$ O $-2.659240-3.733670-0.569680$ C $-1.844011-2.564537-0.684314$ C $-0.412167-2.945614-1.092937$ C $-0.405771-3.798263-2.365358$ C $-1.409689-4.953801-2.283740$ H $-3.321026-5.427575-1.434186$ C $-3.653415-3.826287-2.814556$ H $-2.270744-1.898179-1.442077$ O $0.212665-3.663267-0.030848$ H $0.163128-2.025803-1.253349$ H $0.603060-4.198576-2.523407$ O $-0.718060-2.960757-3.474914$ H $-1.527710-5.433225-3.262452$ O $-0.902511-5.948796-1.389824$ C $-5.070251-3.660478-2.317873$ H -3.267718 -2.845319-3.105934 H $-3.702061-4.422034-3.733812$ H $1.128890-3.846448-0.300178$ H $-0.679014-3.509865-4.276485$ H $-0.049513-6.253504-1.743259$ O $-5.279248-2.372348-1.922339$ O $-5.884880-4.572996-2.266870$ C $-6.586560-2.087593-1.415348$ H -7.346412 -2.318632 -2.168024 H $-6.766029-2.655039-0.497279$ H -6.636421 -1.020323-1.184446 SCF Energy (B3LYP/6-31G*//MMFF) $=-3245.90531646$

07_00120

MMMFF Geometry

C $4.507506-2.421196-0.229106$

C $3.910623-2.709634-1.394443$

C $2.425634-2.593739-1.589330$

O $2.224895-1.363458-2.330209$

C $1.820143-3.735301-2.420624$

C $1.689831-5.053488-1.690723$

C $0.613189-5.393411-0.954613$

C $2.817262-6.029858-1.890781$

C $-0.566657-4.518405-0.638171$

C $-1.757908-4.781534-1.563680$

C $-2.994379-3.963422-1.155440$

C $-4.143153-4.202467-2.134819$

C $-5.367495-3.339697-1.798904$

O $-2.648152-2.575559-1.152534$

O $-6.257056-3.379446-2.925491$

C $-4.926185-1.868893-1.623232$

C $-6.150284-3.908382-0.608141$

O $-6.022128-1.087772-1.153935$

C $-3.698182-1.698518-0.700714$

C $-3.094097-0.274588-0.754370$

O $-4.061990-1.987788 \quad 0.650585$

C $1.185464-0.565208-1.969924$

O $0.374148-0.765704-1.081123$

C $1.2076800 .617367-2.860376$

C $0.2377551 .538841-2.764368$

C $0.1136312 .755560-3.570850$

C $1.197958 \quad 3.035108-4.578934$

C $-3.995198 \quad 0.859223-0.262267$

C $-3.221124 \quad 2.132593 \quad 0.041825$

C $-3.5894414 .545527 \quad 0.548428$

$\begin{array}{lllll}\text { O } & -1.996807 & 2.178596 & 0.121981\end{array}$

C $-3.4088365 .298377-0.731656$
C $-2.2451555 .846451-1.113610$

C $-2.0722446 .631106-2.381805$

C $-0.9877056 .074502-3.276633$

C $0.1879786 .710733-3.413663$

C $-1.2999584 .836824-4.087247$

C $-0.949156 \quad 3.562857-3.366414$

$\begin{array}{llll}\mathrm{N} & -4.070543 & 3.210295 & 0.249942\end{array}$

H $3.894516-2.1395300 .624122$

H $4.518393-2.955815-2.261380$

H $1.926229-2.530792-0.614742$

H $2.396886-3.876934-3.345226$

H $0.831986-3.422914-2.784853$

H $0.580999-6.383679-0.503632$

H $2.634703-6.983459-1.383829$

H $2.950197-6.244039-2.956267$

H $3.753638-5.623687-1.497557$

H $-0.856449-4.701390 \quad 0.403916$

H -0.283209-3.464252 -0.691535

H $-1.467981-4.518355-2.589167$

H $-2.008848-5.849543-1.559964$

H $-3.281273-4.264660-0.141024$

H $-4.416621-5.264539-2.163923$

H -3.813164 -3.950524 -3.152597

H $-6.491218-4.309016-3.089864$

H $-4.664662-1.477662-2.616270$

H $-6.482942-4.931385-0.822086$

H -7.062905 $-3.331462-0.422059$

H $-5.566106-3.9376810 .313880$

H $-6.755073-1.209521-1.781565$

H $-2.177062-0.281048-0.152543$

H $-2.757237-0.054805-1.776262$

H $-3.397693-1.5874571 .236111$

H $2.0199810 .694423-3.574322$

H $-0.5489681 .387117-2.028156$

H $2.1773313 .077404-4.088775$

H $1.0759643 .986875-5.099784$

H $1.2211352 .252078-5.344876$

H -4.746095 $1.089565-1.025810$

$\begin{array}{llll}\mathrm{H} & -4.511828 & 0.571286 & 0.660091\end{array}$

H $-2.6599284 .476373 \quad 1.123291$

H $-4.3465505 .037697 \quad 1.166351$

H $-4.2850815 .412976-1.365469$

H $-1.3734375 .748157-0.470542$

H $-1.8363927 .665865-2.100288$

H $-3.0104246 .687083-2.947960$

H $0.9581276 .337064-4.079859$

H $0.4083467 .617316-2.859991$

H $-0.8088204 .893395-5.064624$

H $-2.3708524 .815567-4.327871$

H $-1.6784083 .275806-2.608856$

H $-5.069208 \quad 3.0889480 .108537$

C $6.566744-0.953546-0.035852$

O $5.794267-0.0940930 .831365$

C $5.2999621 .004674 \quad 0.043625$

C $6.3226531 .167782-1.069530$

C $6.588233-0.285398-1.418032$

H $4.3481290 .682664-0.398019$

C $5.056105 \quad 2.244157 \quad 0.905763$

H $5.9368491 .747393-1.913227$

H $7.2415541 .644988-0.709904$

C $7.885059-0.503764-2.179041$

H $5.760535-0.646140-2.039672$

C $4.167847 \quad 1.9924822 .141591$

O 6.3175012 .7382611 .371070

H 4.6274253 .0379390 .282149

H 4.7418351 .4394472 .894878

$\begin{array}{lllll}\text { O } & 3.878902 & 3.266723 & 2.730802\end{array}$

C 2.8483671 .2463521 .861989

C $1.9752601 .139093 \quad 3.121145$

H 3.0889910 .2481831 .486988

$\begin{array}{llll}\text { O } & 2.131256 & 1.936247 & 0.840721\end{array}$

H $2.5825090 .796676 \quad 3.969051$

H 1.6126252 .1374973 .401909

H $7.8641840 .028340-3.135721$

H $8.749607-0.141829-1.612454$

H $8.038169-1.567742-2.386107$

C $5.994909-2.380195-0.017773$

H 6.1381533 .5248741 .914411 
H 3.3066963 .7521142 .111678 H 1.3860021 .3742600 .569521 H $\quad 6.189179-2.8280080 .964862$ H $6.515882-3.002261-0.754845$ H $7.579524-0.9816530 .384737$ C $-1.155722-0.4451864 .361933$ $\begin{array}{lllll}\text { O } & 0.062989 & 0.300172 & 4.248092\end{array}$ C 0.7378850 .2280322 .983306 C $1.079163-1.2305572 .636960$ C $-0.153235-2.1364732 .727228$ C $-0.920232-1.9287094 .034453$ H -1.426538 -0.3799525 .423906$ C $-2.2949280 .188074 \quad 3.546775$ H 0.0827950 .6495112 .214021 O $2.087968-1.7080943 .524672$ H $1.488580-1.2724641 .622137$ H $0.157626-3.1853822 .651817$ O $-1.031575-1.8668591 .641781$ H -1.883834 -2.450695 4.001379 O $-0.173292-2.4957425 .113618$ C $-2.7318641 .522724 \quad 4.114058$ H -2.0141290 .3595602 .505311$ H $-3.165584-0.4777353 .574151$ H $2.312076-2.6147593 .254299$ H $-0.511693-1.8977020 .821551$ H $-0.059654-3.4429174 .925701$ O $\quad-3.890806 \quad 1.9056373 .499828$ O -2.1343502 .1569814 .972352$ C -4.4318993 .1569453 .934479$ H -4.7005823 .1042094 .993763$ H -3.7145583 .9639923 .757362$ H -5.3355263 .3569863 .352458$

SCF Energy (B3LYP/6-31G**//MMFF) $=-3245.91572838$

0700121

MM̄FF Geometry

C -3.352960 -2.846053 -2.393098

C $-2.414170-1.902490-2.560389$

C $-0.919136-2.094047-2.653015$

O $-0.309665-1.379461-1.548161$

C $-0.423734-3.552535-2.587970$

C $1.084531-3.697868-2.698825$

C $1.851397-4.349153-1.801991$

C $1.724777-3.085463-3.918373$

C $1.400588-5.095872-0.579705$

C $1.498666-4.3020760 .730211$

C $2.939793-3.9345421 .119264$

C $3.029365-3.6244502 .615137$

C $4.447578-3.2008713 .022305$

O $3.333538-2.779840 \quad 0.368666$

O $4.379997-2.6469494 .345011$

C $4.915430-2.0735722 .082220$

C $5.405929-4.395148 \quad 3.099547$

O $6.283507-1.7473232 .320011$

C $4.694101-2.3772670 .579161$

C $4.955115-1.091498-0.239200$

O $5.610575-3.3899320 .161962$

C $0.024695-0.077460-1.757166$

O $\quad-0.2008760 .585998-2.757179$

C $0.7483460 .403691-0.558787$

C $1.3190141 .616473-0.600939$

C 2.1066032 .2533760 .452923

C 2.2599951 .5281181 .762692

C $4.626170-1.194369-1.733672$

C $4.6341020 .178299-2.381545$

C $6.1218402 .141432-2.786835$

O $3.6116610 .680270-2.842353$

C $6.1989002 .992308-1.556357$

C $5.5109674 .131716-1.387079$

C $5.6191594 .987977-0.156583$

C 4.2756545 .3605520 .431597

C 3.8276396 .6267460 .382900

C 3.4828694 .2859001 .148086

C $2.637247 \quad 3.470446 \quad 0.210334$

N $5.8870530 .768500-2.380487$

H -3.069759 -3.890738 -2.310999

H -2.743377 -0.869336 -2.649603

H $-0.595885-1.657951-3.606638$
H $-0.878229-4.134613-3.399971$

H $-0.775886-3.987946-1.647462$

H $2.926193-4.385477-1.976018$

H $2.774248-3.379635-4.027807$

H $1.201081-3.407826-4.824259$

H $1.695637-1.992970-3.864676$

H $0.373927-5.457881-0.698706$

H $2.008932-6.006054-0.496822$

H $\quad 0.886109-3.3947820 .652125$

H $1.058263-4.9273821 .516905$

H $3.596841-4.7756570 .868890$

H $2.703950-4.4849193 .213271$

H $2.336161-2.8075342 .860815$

H $4.018365-3.3286594 .936996$

H $4.336573-1.1764952 .340833$

H $5.038905-5.1343803 .821600$

H $6.392393-4.0866263 .462926$

H $5.535330-4.9043102 .142335$

H $6.372855-1.5466083 .267471$

H $4.353173-0.274510 \quad 0.175348$

H $6.006988-0.800705-0.128779$

H $5.703242-3.349691-0.802963$

H $0.814406-0.2524990 .301004$

H $1.2155472 .203954-1.512864$

H $2.7761770 .574691 \quad 1.621842$

H 1.2805441 .3263362 .208279

H 2.8271272 .0961412 .504849

H $5.342049-1.834025-2.260343$

H $3.629544-1.622016-1.883479$

H $5.3310272 .469159-3.469008$

H $7.0796452 .164564-3.315161$

H $6.8719022 .659899-0.768755$

H $4.8596594 .483786-2.183772$

H $6.1728525 .894322-0.434303$

H 6.2205074 .4958060 .618152

H $2.876757 \quad 6.9061740 .824697$

H $4.3977327 .410961-0.104159$

H 2.8198434 .7517381 .888584

H 4.1823013 .6520281 .704860

H $2.4290883 .939182-0.752031$

H $6.6683450 .266027-1.969943$

C $-5.414777-2.636525-0.912485$

O $-4.856227-1.605540-0.072995$

C $-4.632268-2.156481 \quad 1.238127$

C $-5.372282-3.4908981 .269670$

C $-5.212308-3.954137-0.164823$

H -3.555986 -2.351222 1.315623

C $-5.065522-1.1700632 .327299$

H $-4.942736-4.1860111 .997544$

H $-6.434026-3.3624511 .510657$

C $-6.183990-5.048349-0.568757$

H $-4.187579-4.324688-0.287026$

C $-4.393706 \quad 0.218078 \quad 2.263112$

O $-6.482285-0.9652092 .240920$

H -4.890599-1.621054 3.311221

H -4.8042730 .7839611 .419837$

O

C -2.8572350 .1998412 .173333$

C -2.178285 1.5588072 .429626

H -2.558113 -0.179935 1.189599

O $-2.351816-0.7176453 .149019$

H -2.309916 1.8466473 .480705

H -1.100142 1.3988682 .304501

H -6.037628 -5.939963 0.049457

H -7.224119-4.725964 -0.451874

H $-6.034643-5.333837-1.614970$

C -4.826149-2.549883 -2.322989

H $-6.674961-0.6244311 .350475$

H $-4.397211 \quad 0.4776634 .206651$

H -1.388637 -0.7742593 .026415$

H $-5.015915-1.545682-2.721987$

H $-5.347823-3.251617-2.984441$

H $-6.487908-2.413653-0.983070$

C $-2.8013933 .251611-0.841818$

O -2.3937362 .3116350 .161771$

C -2.6379772 .7040241 .514278$

C -1.910512 4.0203731 .841627

C -2.2522675 .1179270 .829965$ 
C $-2.1322094 .618209-0.614637$

H -2.414272 2.841727 -1.783783

C $-4.331713 \quad 3.344070-0.960526$

H -3.7125572 .8500101 .659259$

O $-0.5018473 .803267 \quad 1.844438$

H -2.195833 4.3363742 .852313

H -1.576191 5.9682460 .981125

O -3.5780065 .5727671 .083280$

H -2.556461 $5.350166-1.311705$

O $-0.7485714 .478852-0.947841$

C $-4.9567602 .040934-1.407423$

H -4.795753 $3.612751-0.008208$

H -4.593846 $4.103335-1.706487$

H -0.0735194 .6418802 .086175$

H -3.771817 6.2819920 .446884

H $-0.3293385 .349030-0.837353$

O $-6.3156812 .180739-1.411615$

O $-4.3404231 .030985-1.714262$

C $-7.0541691 .019420-1.803707$

H $-8.1108141 .293108-1.864384$

H -6.726887 $0.667975-2.787000$

H $-6.9378880 .233314-1.052681$

SCF Energy (B3LYP/6-31G**//MMFF) $=-3245.88565725$

07_00122

MM̄FF Geometry

C $-4.015223-4.381246-0.123243$

C $-2.740962-4.0292910 .103039$

C $-1.618120-4.264310-0.874530$

O $-1.052285-2.983258-1.237800$

C $-0.516431-5.107961-0.204754$

C $0.726179-5.321321-1.051780$

C $1.982091-5.032489-0.651604$

C $0.498003-5.927449-2.412624$

C $2.420659-4.4942790 .683128$

C $2.735126-2.9978490 .634097$

C $4.119919-2.6421650 .065386$

C $5.239635-2.9967981 .048533$

C $6.612160-2.5105180 .562804$

O $4.114053-1.227314-0.156782$

O $7.524520-2.5909281 .669969$

C $6.514929-1.0196570 .170403$

C $7.175611-3.431732-0.526570$

O $7.739607-0.601552-0.428381$

C $5.311219-0.697440-0.747773$

C $5.1010180 .829286-0.875356$

O $5.514533-1.195430-2.071673$

C $-1.749060-2.224539-2.129266$

O $-2.824919-2.487760-2.642205$

C $-0.963517-0.997529-2.378556$

C $-1.5264490 .041564-3.015291$

C $-0.8616801 .305940-3.340796$

C $0.6167651 .380289-3.078342$

C $3.9395221 .179326-1.809850$

C $3.6255872 .662053-1.812660$

C $2.2951374 .374450-0.573060$

O $3.992994 \quad 3.398262-2.725190$

C $0.831898 \quad 4.267708-0.872635$

C $0.1641255 .039211-1.743698$

C -1.312642 $4.890962-1.993109$

C $-1.6712104 .808356-3.462333$

C $-2.480146 \quad 5.725592-4.019680$

C $-1.0999543 .678986-4.293592$

C - $1.5854032 .320603-3.858843$

N $2.8859103 .054131-0.709186$

H $-4.278876-4.888109-1.047867$

H -2.491147-3.499982 1.020310

H -1.976851 -4.771701-1.778806

H $-0.923200-6.0933850 .058919$

H $-0.252749-4.6235070 .740606$

H $2.799299-5.223067-1.346015$

H $1.435663-6.202445-2.907450$

H $-0.103508-6.838311-2.325795$

H - $0.024007-5.222888-3.067293$

H $1.657302-4.6674551 .447271$

H $3.288701-5.0700761 .021392$

H $1.959676-2.4713940 .061189$

H $2.660080-2.5941151 .652092$
H $4.257958-3.147995-0.896261$

H $5.272620-4.0743401 .245539$

H $5.033640-2.5234662 .019091$

H $7.539536-3.5123731 .981192$

H $6.408259-0.4487601 .103644$

H $7.321964-4.445102-0.133057$

H $8.163610-3.095573-0.859040$

H $6.522434-3.512555-1.398194$

H $7.7479240 .369757-0.445742$

H $4.9103721 .256796 \quad 0.117093$

H $6.0027661 .310861-1.271349$

H $6.420248-1.536966-2.141698$

H $0.060810-0.983456-2.023221$

H $-2.569236-0.032643-3.320626$

H $1.1450730 .575755-3.602377$

H $0.8073001 .287801-2.004224$

H $1.0785002 .312365-3.405129$

H $4.1668010 .884031-2.840878$

H $3.0342470 .634455-1.520897$

H $2.8024555 .084309-1.233530$

H $2.438743 \quad 4.6881700 .465108$

H $0.2795783 .506513-0.329369$

H $0.6915255 .823410-2.280572$

H $-1.8101725 .752676-1.529344$

H $-1.7106464 .005337-1.482981$

H -2.746578 $5.682266-5.070566$

H $-2.8963606 .541974-3.438722$

H $-0.0106093 .761609-4.301189$

H $-1.393223 \quad 3.798248-5.345482$

H -2.654641 $2.168844-4.012280$

H $2.6050882 .347323-0.035676$

C $-5.989361-2.909074 \quad 0.286635$

O $-5.182668-1.7144850 .215728$

C $-5.881419-0.6388840 .862759$

C -7.336266 -1.0813700 .929190$

C $-7.175063-2.5668931 .190962$

H $-5.485493-0.5740961 .883766$

C -5.6288840 .6810380 .128237$

H $-7.894548-0.5637311 .714816$

H -7.854886 $-0.919973-0.022871$

C $-8.425114-3.3780450 .897952$

H $-6.898239-2.7084262 .244442$

C -4.139838 $1.017557-0.098191$

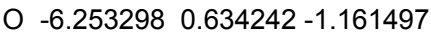

H -6.1198581 .4992440 .667606$

H $-3.7188980 .360930-0.868170$

$\begin{array}{llll}\text { O } & -4.072198 & 2.348268 & -0.626107\end{array}$

C $-3.2587020 .942243 \quad 1.162329$

C $-1.832967 \quad 1.4581550 .917796$

H -3.217540 -0.0969861 .500692$

$\begin{array}{lllll} & \text { O } & -3.852790 & 1.705542 & 2.211773\end{array}$

H -1.3968970 .9728720 .037261$

H -1.8699402 .5293170 .677531$

H $-9.252697-3.0534891 .537153$

H -8.741480 $-3.265457-0.144497$

H $-8.250917-4.442527 \quad 1.084188$

C $-5.137141-4.0608860 .823581$

H $-5.882867-0.128015-1.639080$

H $-4.6053082 .364531-1.439661$

H -3.928571 $2.623947 \quad 1.900428$

H $-5.747977-4.9601300 .960687$

H $-4.728485-3.7931031 .806332$

H -6.327557 $-3.123507-0.735415$

C 1.3171792 .0621352 .802674

$\begin{array}{lllll}\text { O } & 0.321808 & 1.986374 & 1.775970\end{array}$

C $-0.887497 \quad 1.299172 \quad 2.125633$

C $-0.574382-0.1607612 .487257$

C $0.501089-0.2442043 .577221$

$\begin{array}{llll}\text { C } & 1.705937 & 0.654456 & 3.275314\end{array}$

H 2.1940232 .5032582 .311693

C 0.9025493 .0033433 .938253

H -1.3448151 .8040702 .982422$

O $-0.128050-0.851694 \quad 1.322529$

H $-1.488954-0.6531662 .835732$

H $0.836767-1.2843283 .671407$

$\begin{array}{lllll}H & 0.0867147 & 0.138854 & 4.817560\end{array}$

H 2.3522720 .7304114 .156813

$\begin{array}{llll}\text { O } & 2.493513 & 0.045489 & 2.248914\end{array}$ 
C $0.835417 \quad 4.429763 \quad 3.445268$

H -0.0591372 .7511634 .393323$

H 1.6420282 .9765274 .747698

H $\quad 0.002370-1.7839461 .566369$

H $\quad 0.594420 \quad 0.050273 \quad 5.505441$

H $1.9588030 .020591 \quad 1.436630$

O $\quad-0.3798874 .6706442 .875930$

O 1.7610035 .2256223 .537810

C -0.5627315 .9840662 .338640$

H -1.5126256 .0001151 .797577$

$\mathrm{H}-0.605857 \quad 6.715336 \quad 3.150910$

H 0.2412826 .2321591 .639150

SCF Energy (B3LYP/6-31G**//MMFF $)=-3245.91853866$

07 00123

MM̄FF Geometry

C -2.318479 -3.644249 0.853298

C $-1.450491-2.9340061 .589898$

C $-0.052523-2.6103781 .136385$

O $0.126137-1.1828621 .306708$

C $1.018534-3.3254811 .981000$

C $1.196901-4.7895241 .633733$

C $1.989369-5.2319210 .636641$

C $0.467188-5.7673862 .515511$

C $2.792741-4.387295-0.312311$

C $4.298188-4.597203-0.109354$

C $5.124534-3.535631-0.851059$

C $6.615976-3.869871-0.779717$

C $7.486109-2.760036-1.388095$

O $4.867123-2.278853-0.215438$

O $8.847602-3.006248-1.003633$

C $7.076343-1.412224-0.759026$

C $7.457510-2.782638-2.920462$

O $7.760575-0.327072-1.380802$

C $5.553853-1.158994-0.787231$

C 5.1538740 .0937170 .027405

O $5.136820-0.937874-2.138668$

C $0.846951-0.5224780 .361090$

O $1.346200-0.993136-0.648654$

C 0.9277550 .8989520 .765328

C $1.4402451 .791002-0.095304$

C 1.6062223 .2281130 .130625

C $1.272707 \quad 3.7602371 .497897$

C 5.4634710 .0041421 .522256

C 4.7970951 .1269022 .290154

C 5.0595383 .5350422 .869439

O 3.7765920 .9366002 .948030

C 4.8210624 .7077741 .967091

C 4.9884774 .7779680 .636720

C $4.7122936 .023707-0.157929$

C $3.7321975 .801082-1.287528$

C $4.1056685 .962032-2.568419$

C $2.2981325 .470497-0.931196$

C $2.0535263 .986722-0.891912$

N 5.4534322 .3387352 .150653

H $-2.005014-4.020427-0.117613$

H -1.748102 -2.581437 2.574450

H $0.061260-2.8724020 .077128$

H $\quad 0.791139-3.2189643 .050723$

H $1.979356-2.8046271 .872404$

H $2.070211-6.3053560 .473281$

H $\quad 0.670591-6.8072942 .238597$

H $\quad 0.775330-5.6392563 .558419$

H $-0.613940-5.6174602 .452339$

H $2.521901-4.661709-1.339108$

H $2.542673-3.329271-0.208706$

H $4.527930-4.5444450 .963352$

H $4.573182-5.600807-0.456897$

H $4.789679-3.489481-1.893947$

H $6.822260-4.830648-1.267777$

H $6.909674-4.0007350 .271510$

H $9.095593-3.889236-1.327635$

H $7.415534-1.4354790 .283959$

H $7.836972-3.740629-3.295980$

H $8.117394-2.014821-3.338948$

H $6.456858-2.641368-3.333962$

H $8.712295-0.524183-1.341811$

H $5.6157930 .990042-0.405882$
H $4.0725210 .246779-0.084985$

H $4.290385-0.460821-2.125667$

H $\quad 0.5630541 .170157 \quad 1.749120$

H $1.7686051 .436778-1.071999$

H $\quad 0.2122983 .6077651 .720024$

H 1.4712404 .8273351 .613048

H 1.8633173 .2477992 .264885

H $5.104953-0.9409501 .945652$

H 6.5424160 .0479341 .706866

H 5.8695053 .7710493 .567336

H 4.1562973 .3381183 .455362

H 4.4640105 .5980232 .482525

H 5.3550253 .9181780 .082746

H $5.6716746 .385450-0.549663$

H 4.3288346 .8266680 .484162

H $3.3997195 .829589-3.381902$

H $5.1243446 .225211-2.833076$

H $1.6263715 .892795-1.690870$

H $2.0271665 .980153-0.002920$

H $2.2541123 .493518-1.844857$

H 6.3321672 .3638331 .641289

C $-4.749745-3.858513 \quad 0.217273$

O $-5.086093-2.4647890 .039619$

C $-6.517361-2.349292-0.082445$

C $-7.044578-3.767049-0.272464$

C $-6.063732-4.5637360 .558369$

H -6.883705 -1.970663 0.878736

C $-6.919014-1.396459-1.213388$

H -8.080449 -3.8730640 .064025$

H -6.997378-4.080636 -1.322105

C $-6.073545-6.0498210 .241614$

H $-6.303356-4.4201171 .620648$

C $-6.4363340 .059807-1.060232$

O $-6.388288-1.904483-2.444774$

H -8.010711 -1.414204-1.321695

H $-5.3598540 .109569-1.251661$

O $-7.0544200 .818256-2.109970$

C -6.7570420 .7190660 .292816$

C -6.5991502 .2513010 .330105$

H $-6.1266320 .267725 \quad 1.067612$

$\begin{array}{lllll}\text { O } & -8.116383 & 0.427587 & 0.635283\end{array}$

H $-7.3992672 .720039-0.257671$

H -6.7830442 .5599641 .367974$

H -7.062492 -6.4774240 .436411$

H $-5.827297-6.237793-0.808962$

H $-5.347232-6.5876570 .858618$

C -3.696355 -4.022469 1.316992

H $-6.661918-1.295081-3.151673$

H -8.011026 $0.836503-1.934708$

H -8.2779370 .7857301 .524993$

H $-3.651391-5.072607 \quad 1.628187$

H -3.978795 -3.425927 2.192969

H $-4.365486-4.217202-0.746724$

C -2.8703322 .5216630 .313664$

O -4.2189992 .1721620 .658258$

C $-5.2363142 .785963-0.135693$

C $-5.1582194 .318484-0.022210$

C $-3.7538794 .837690-0.349966$

C $-2.668243 \quad 4.0463150 .386337$

H -2.2530302 .0613391 .095537$

C $-2.4437051 .918629-1.034760$

H $-5.0981582 .502546-1.183273$

O

H $-5.8886684 .761275-0.709258$

H $-3.6804915 .894503-0.064677$

O $-3.5223364 .748795-1.752201$

H -1.679513 $4.311106-0.005753$

O -2.6984974 .4446901 .760330$

C $-2.4473150 .405569-1.014503$

H -3.107159 2.230023 -1.845496

H $-1.4249842 .242246-1.274303$

H -4.916572 4.3048031 .919987

H -4.200872 $5.284952-2.196822$

H -1.9961793 .9602862 .225621$

O $-2.140129-0.052792-2.263993$

O $-2.690732-0.286848-0.036596$

C $-2.138440-1.475209-2.416774$

H $-3.102399-1.895270-2.114472$ 
H $-1.975221-1.702941-3.473563$

H $-1.323846-1.913146-1.834281$

SCF Energy (B3LYP/6-31G**//MMFF)= -3245.91360424

\section{4}

MM̄FF Geometry

C $1.218819-3.4495161 .051021$

C $0.305275-2.6196140 .525101$

C $-0.556730-1.7032731 .351109$

O $-0.444017-0.3915030 .749360$

C -2.046182 -2.090776 1.322484

C $-2.349837-3.5110931 .735877$

C $-2.671475-4.4960740 .874414$

C $-2.299854-3.7748633 .216841$

C $-2.736753-4.416391-0.626856$

C $-4.130498-4.722592-1.185670$

C $-5.212831-3.694216-0.812559$

C $-6.560861-4.149015-1.383043$

C -7.669405 -3.118975 -1.138863

O $-4.848450-2.417758-1.354995$

O $-8.803904-3.484617-1.939325$

C $-7.173973-1.736264-1.607037$

C $-8.167772-3.1596240 .309663$

O $-8.123116-0.725158-1.271978$

C $-5.775294-1.358640-1.057971$

C $-5.284630-0.068992-1.770401$

O $-5.876039-1.129195 \quad 0.345534$

C $-0.5571050 .695674 \quad 1.554721$

$\begin{array}{lllll}\text { O } & -0.649107 & 0.709514 & 2.770868\end{array}$

C -0.5884381 .8830250 .671595$

C $-0.6655053 .110801 \quad 1.203854$

C -0.7713614 .3667190 .457854$

C $-0.9118904 .277540-1.039323$

C $-3.9541420 .504222-1.267559$

C $-4.1444551 .443998-0.089698$

C -5.0277593 .7069840 .483177$

O -3.9279631 .0826281 .064425$

C -4.4935685 .0401490 .062675$

C -4.0205835 .9488200 .929760$

C -3.4849047 .2922010 .521368$

C -2.1524347 .6167661 .163227$

C -2.0617228 .5386052 .136768$

C -0.9150806 .9287420 .626499$

C -0.7751095 .5252031 .149601$

N $-4.6010522 .696573-0.469335$

H $1.343122-3.4857962 .130583$

H $\quad 0.160287-2.599622-0.552505$

H $-0.193122-1.6725272 .385639$

H $-2.455143-1.8757090 .331293$

H -2.604410 -1.407161 1.976849

H $-2.884028-5.4840851 .280898$

H $-2.555018-4.8108793 .463426$

H -1.295825 -3.580682 3.606906

H $-3.008870-3.1263683 .741776$

H -2.386174 -3.457391-1.016807

H $-2.037857-5.165099-1.022009$

H -4.040226 -4.762805 -2.279213

H -4.442015 -5.721115 -0.853381

H $-5.271093-3.6166360 .278636$

H $-6.848830-5.125147-0.972791$

H $-6.459942-4.300614-2.467051$

H -8.528091 -3.495836 -2.871512

H -7.116895 -1.755738 -2.704381

H $-8.583659-4.1478090 .541435$

H -8.985477 -2.447578 0.467487

H -7.384296 -2.9522791 .041386$

H -8.981104 -0.999417 -1.638578

H $-5.155588-0.300166-2.836005$

H $-6.0546920 .707908-1.696083$

H $-5.023784-0.7889060 .660728$

H $-0.5769531 .710814-0.398982$

H -0.6666913 .2080042 .288708$

H $-1.0550005 .243847-1.525848$

H -1.778532 $3.664359-1.310026$

H $-0.0172713 .828736-1.482224$

H $-3.244970-0.281452-0.997135$

H -3.479627 $1.077919-2.074054$

H -4.7086123 .4320181 .493469$
H -6.1218943 .7218620 .454963$

H $-4.5052195 .277342-0.998579$

H $-4.0390675 .725124 \quad 1.994151$

H -4.2311398 .0457200 .805106$

H -3.382765 $7.365576-0.568600$

H $-1.107703 \quad 8.7947732 .585601$

H -2.939866 9.054546 2.511081

H -0.0157697 .4802350 .931684$

H $-0.9232466 .985606-0.464890$

H -0.6663815 .4780232 .234446$

H $-4.8387722 .848013-1.445560$

C $3.532816-4.3718590 .664191$

O $4.180707-3.1609550 .215420$

C $5.474532-3.501720-0.319724$

C $5.731814-4.9513190 .078271$

C $4.331593-5.5227860 .050651$

H $5.383727-3.454609-1.410839$

C $6.564722-2.5369500 .163229$

H $6.416436-5.455059-0.611113$

H $6.153766-5.0271771 .087268$

C $4.202982-6.8390680 .798607$

H $4.033044-5.672346-0.995763$

C $6.366701-1.058420-0.228468$

O $6.623447-2.6098321 .593771$

H $7.537308-2.888358-0.203726$

H $5.568983-0.6137790 .375293$

O $7.571482-0.3590690 .112618$

C $6.083963-0.814866-1.721745$

C $6.2206850 .646061-2.193557$

H $5.074972-1.171159-1.957441$

O $6.986108-1.593599-2.515680$

H $7.2782160 .941032-2.191606$

H $5.9183870 .661120-3.249432$

H $4.855976-7.5980630 .355267$

H $4.481181-6.7306231 .852318$

H $3.175823-7.2143780 .758865$

C $2.062354-4.3879030 .236048$

H $7.441667-2.1690791 .877757$

H $7.627376-0.3263161 .082356$

H $7.890966-1.323857-2.281974$

H $1.650294-5.3942870 .373284$

H $1.984949-4.138012-0.829302$

H $3.618050-4.3899491 .758628$

C $3.1286602 .101669-0.679960$

O $4.0311201 .273038-1.427255$

C $5.4015831 .674891-1.401348$

C $5.5561303 .091812-1.981310$

C $4.6323994 .092752-1.278888$

C $3.1994663 .560695-1.163119$

H $2.1307301 .716029-0.923855$

C 3.3311331 .9579850 .837260

H $5.7595041 .673836-0.367238$

O $5.2679953 .093912-3.379019$

H $6.6000373 .407208-1.870774$

H $4.6218015 .034412-1.841340$

O $5.128924 \quad 4.3734790 .025994$

H $2.6053874 .195240-0.495064$

O $2.5824173 .612282-2.452772$

C 3.0713640 .5488491 .321524

H $4.3503792 .216077 \quad 1.135514$

H 2.6338382 .6207751 .362337

H $4.3705742 .738537-3.497761$

H $6.0244114 .739205-0.072577$

H $2.5669864 .543449-2.732506$

O 3.3167430 .4902932 .663824

O $2.708623-0.3825950 .617895$

C $3.146590-0.7958603 .267187$

H $3.741815-1.5502112 .743798$

H $3.494494-0.7341944 .301713$

H $2.088157-1.0689633 .268858$

SCF Energy (B3LYP/6-31G**//MMFF) $=-3245.90842753$

07 00125

MM̄MF Geometry

C -0.4663992 .9948901 .432898$

C 0.4116872 .6646052 .392503

C $0.362074 \quad 1.4346893 .272284$

O -0.7721850 .5940042 .964034$ 
C $\quad \begin{array}{lll}1.636922 & 0.607857 \quad 3.020024\end{array}$

C $1.675107-0.6996203 .783211$

C $1.425249-1.9019773 .227078$

C $2.068135-0.5915995 .231756$

C $1.007990-2.1618901 .803887$

C $-0.006665-3.310326 \quad 1.708903$

C $-0.649795-3.4770530 .320478$

C $0.377379-3.604540-0.807899$

C $-0.283763-3.700572-2.190666$

O $-1.511316-2.3543770 .089289$

O $0.749593-3.488401-3.164962$

C $-1.307898-2.552680-2.328401$

C $-0.834964-5.106087-2.453844$

O $-2.035193-2.667237-3.549044$

C $-2.269769-2.442509-1.126058$

C $-3.185697-1.204347-1.216951$

O $-3.130602-3.589211-1.115587$

C -1.9556370 .8765613 .569637$

O $-2.197637 \quad 1.801168 \quad 4.328044$

C $-2.931747-0.1371383 .117200$

C $-4.2292990 .193356 \quad 3.051317$

C $-5.318570-0.6755432 .603727$

C -5.025169-2.146016 2.459279

C $-2.4711040 .147505-1.217347$

C $-3.4849441 .255020-1.013947$

C $-5.4262412 .351299-2.106119$

O -3.6167691 .8194940 .069538$

C $-6.6099101 .483017-1.810278$

C $-7.1886241 .399365-0.602475$

C $-8.3523310 .501010-0.296625$

C $-7.933853-0.7889520 .375806$

C $-7.781036-1.914791-0.342156$

C $-7.777171-0.8022141 .880512$

C $-6.521831-0.1202992 .347684$

N $-4.247636 \quad 1.506129-2.144637$

H -1.3386782 .3749161 .258350$

H 1.2778723 .3068092 .541990

H 0.3229771 .7413324 .324725

H 2.52270611 .1984553 .291540

H 1.7463740 .4168561 .945033

H $1.531593-2.7887533 .849993$

H $2.113167-1.5689305 .723955$

H $3.056522-0.1298045 .323534$

H 1.3449890 .0203565 .779737

H $\quad 0.573240-1.2683881 .346911$

H $1.908150-2.4232951 .236878$

H $\quad 0.489053-4.2488521 .988075$

H $-0.807507-3.1378272 .440148$

H $-1.268479-4.381216 \quad 0.367919$

H $1.043867-4.458357-0.634750$

H $1.021826-2.717967-0.818414$

H $\quad 0.328541-3.467269-4.041665$

H -0.728632 -1.624551-2.408637

H $-0.025230-5.844858-2.414867$

H $-1.260040-5.184207-3.460486$

H $-1.595636-5.412004-1.732707$

H $-2.696377-3.370884-3.434654$

H $-3.834324-1.288420-2.098767$

H $-3.874672-1.219942-0.361771$

H -3.921028 $-3.377363-0.591798$

H -2.550717 - 1.1068012 .819277

H $-4.5204201 .207863 \quad 3.321969$

H -4.248801 -2.318862 1.707555

H $-5.892917-2.7336602 .152080$

H -4.683076 -2.5618873 .413638$

H $-1.7336650 .210707-0.409555$

H - $-1.9409820 .319292-2.159826$

H -5.538216 $2.824460-3.086239$

H $-5.2948453 .135747-1.353522$

H -6.993145 $0.879297-2.629479$

H $-6.797622 \quad 1.9999750 .214677$

H -8.902825 $0.278588-1.220966$

H -9.0675191 .0391540 .337314$

H -7.510566 -2.854119 0.128038

H -7.918327 -1.920565 -1.418420

H -7.827667 -1.825709 2.269539

H -8.638964 -0.2974672 .336022$

H -6.6358310 .9558402 .481480$
H $-4.1088780 .930100-2.969858$

C $-0.2752493 .677848-0.957619$

O $0.7294782 .659781-1.125666$

C $1.5395312 .980895-2.263794$

C $0.7297143 .985517-3.070142$

C $0.0640564 .780788-1.961371$

H $2.4387363 .471778-1.871246$

C $1.972396 \quad 1.713332-3.012937$

H $1.3562024 .599276-3.723923$

H $-0.0303643 .492193-3.686865$

C $-1.1412255 .582166-2.420976$

H $0.8056655 .467396-1.531431$

C $2.5735430 .643248-2.073400$

O $0.867358 \quad 1.159813-3.725463$

H $2.7180612 .009269-3.759971$

H $3.2016811 .138965-1.326502$

O $1.532961-0.036131-1.370780$

C $3.441047-0.392178-2.811071$

C $3.930169-1.576544-1.957785$

H $4.3130850 .125206-3.228154$

O $2.730221-0.937150-3.925035$

H $3.112009-2.290477-1.806104$

H $4.667036-2.112980-2.570585$

H $-0.8485606 .318095-3.176847$

H $-1.9090814 .936185-2.860052$

H $-1.5933236 .119873-1.581554$

C $-0.282151 \quad 4.156866 \quad 0.497230$

H $0.2067120 .883355-3.067679$

H $1.0464460 .627108-0.852335$

H $1.908718-1.331324-3.584015$

H -1.0930094 .8771090 .651758$

H $\quad 0.660677 \quad 4.669546 \quad 0.723033$

H $-1.2364803 .211879-1.205932$

$\begin{array}{llll}\text { C } & 6.216971 & 0.242175 & 0.389466\end{array}$

O $5.569405-0.237825-0.798475$

C $4.544816-1.214220-0.594796$

C $5.104582-2.4557190 .121616$

C $5.823801-2.0733421 .417828$

C $6.813930-0.9243151 .196790$

H 7.0516200 .8557450 .026368

C $5.297576 \quad 1.142248 \quad 1.232279$

H $3.753746-0.7796890 .024157$

O $6.014184-3.138078-0.739727$

H $4.276345-3.1418470 .336610$

H $6.358475-2.9497721 .803788$

O $4.849244-1.7005882 .387031$

H $7.187273-0.5485062 .156611$

O $7.946714-1.4125600 .472987$

C 5.0118302 .4745890 .571734

H $4.3331510 .670517 \quad 1.431827$

H 5.7882361 .3607832 .188326

H $6.315624-3.937052-0.275025$

H $5.320050-1.4789293 .208357$

H $8.347428-2.1245111 .000353$

$\begin{array}{lllll}\text { O } & 4.279743 & 3.240839 & 1.435374\end{array}$

O $5.3735842 .804273-0.548838$

C $3.9443894 .551608 \quad 0.970266$

H 4.8504455 .1122830 .721750

H 3.2807094 .4861080 .104684

H 3.4194665 .0733151 .774856

SCF Energy (B3LYP/6-31G**//MMFF) $=-3245.90861569$

07_00126

MM̄FF Geometry

C -1.900668 $3.150090 \quad 0.971436$

C -1.5459592 .4254582 .043466$

C $-2.188482 \quad 1.1047242 .371417$

$\begin{array}{lllll}\text { O } & -1.129579 & 0.140538 & 2.582639\end{array}$

C -3.053969 1.1690103 .643702

C $-4.2639502 .073677 \quad 3.549844$

C $-5.365652 \quad 1.7957872 .824865$

C $-4.186678 \quad 3.338656 \quad 4.362485$

C $-5.6021290 .588011 \quad 1.959836$

C -5.7933600 .9999790 .494093$

C $-5.670794-0.146188-0.522894$

C $-6.559181-1.347730-0.195244$

C $-6.375861-2.486207-1.210975$

O $-4.295153-0.547206-0.586237$ 
O - $-6.971796-3.670681-0.659111$

C $-4.867445-2.773670-1.371202$

C $-7.112703-2.204761-2.525721$

O $-4.640849-3.725108-2.409487$

C $-4.015712-1.505155-1.618458$

C $-2.510606-1.866474-1.567722$

O $-4.320502-0.988791-2.916457$

C -1.437922 -1.160706 2.328028

O $-2.509471-1.6034521 .944188$

C $-0.235278-1.9793482 .579481$

C $-0.097255-3.1226121 .892913$

C $1.011727-4.0697301 .985228$

C $1.975469-3.9136903 .131389$

C $-1.565091-0.657841-1.596322$

C $-0.107265-1.080133-1.548813$

C $1.654356-2.221277-2.906562$

O $0.605624-0.834725-0.578750$

C $1.736814-3.658899-2.492986$

C $2.807345-4.176571-1.871368$

C $2.950208-5.618373-1.472362$

C $3.265557-5.786127-0.001307$

C $4.530087-5.7044310 .446307$

C $2.131320-6.1268270 .937714$

C $1.107740-5.032216 \quad 1.044334$

N $0.303925-1.730991-2.699890$

H -2.680662 2.7715610 .314447

H -0.7854912 .8000262 .722757$

H -2.787461 0.7812081 .512861

H $-2.425857 \quad 1.469448 \quad 4.494263$

H $-3.388510 \quad 0.159168 \quad 3.916367$

H -6.1887982 .5087082 .842574$

H $-5.084007 \quad 3.9574174 .256758$

H -4.0725353 .0993755 .424907$

H -3.3301863 .9435314 .048323$

H -4.791397 -0.1382412 .050243$

H -6.5070770 .0846602 .320721$

H -6.7796521 .4696150 .386323$

H $-5.046699 \begin{array}{llll}1.761894 & 0.234118\end{array}$

H $-5.9510830 .272952-1.496810$

H -7.612612 -1.047952 -0.135189

H $-6.301218-1.7451100 .795285$

H -7.908059 -3.478686 -0.478834

H $-4.521976-3.243621-0.439516$

H -8.187757 -2.086850 - 2.343769

H -7.017729 -3.047092 -3.219837

H $-6.764058-1.301126-3.029406$

H -5.181452 -4.508016 -2.207609

H -2.301802 -2.428074 - 0.649398

H $-2.272105-2.531972-2.406162$

H -3.581346 - $0.442155-3.224301$

H $0.496183-1.6132173 .288813$

H $-0.866768-3.3860831 .167889$

H $2.438234-2.9217463 .124626$

H $2.795358-4.6354923 .107733$

H $1.455047-4.0462644 .086336$

H $-1.707706-0.051878-2.497152$

H -1.752256 $-0.003291-0.738164$

H $2.357172-1.592410-2.350341$

H $1.864664-2.130721-3.976638$

H $0.896504-4.305728-2.733220$

H $3.658669-3.531427-1.669862$

H $3.761649-6.054566-2.069959$

H $2.053864-6.195906-1.730105$

H $4.770386-5.8477651 .494402$

H $5.352359-5.485448-0.227011$

H $2.507932-6.4038991 .928759$

H $1.630382-7.0326370 .571715$

H $0.364218-5.0557420 .246546$

H $-0.371511-1.903346-3.438486$

C $-0.815768 \quad 4.505594-0.824978$

O $0.3998893 .737397-0.930469$

C $1.2228784 .328604-1.959345$

C $0.4439165 .521301-2.509829$

C $-0.4404305 .892635-1.336595$

H $2.1295794 .699086-1.469346$

C $1.5700193 .280705-3.023348$

H $1.1056646 .335117-2.822436$

H $-0.1643945 .240067-3.377815$
C -1.623615 $6.760630-1.727885$

H $0.1671796 .418408-0.587838$

C $2.4391432 .115761-2.507384$

O $\quad 0.3521062 .727307-3.541534$

H $2.0658653 .762369-3.873721$

H $1.8948821 .558566-1.737748$

O $2.6380121 .209022-3.600114$

C $3.8238052 .545615-1.976759$

C $4.7825661 .370903-1.699176$

H $3.7028103 .144770-1.068124$

O $4.4427753 .386136-2.955762$

H $4.8712560 .748374-2.597820$

H $5.7851631 .784955-1.525789$

H $-1.2802817 .698722-2.176117$

H $-2.2680976 .256307-2.455885$

H $-2.2338377 .009980-0.854417$

C $-1.328405 \quad 4.491636 \quad 0.613846$

H $-0.1201792 .313076-2.798901$

H $1.7586570 .940458-3.917225$

H $5.2769713 .712962-2.577789$

H $-2.127126 \quad 5.2319210 .734597$

H -0.5227414 .7602561 .304265$

H -1.551527 $4.041547-1.496632$

C 4.2898520 .6776891 .932263

$\begin{array}{lllll}\text { O } & 4.643913 & 1.288962 & 0.684329\end{array}$

C $4.3677550 .519664-0.491365$

C $5.128709-0.811882-0.446518$

C $4.807352-1.5922410 .830154$

C $4.920486-0.7179672 .081631$

H 4.7543991 .3210002 .691901

$\begin{array}{llll}\text { C } & 2.772277 & 0.714008 & 2.159345\end{array}$

H $3.2962710 .316449-0.550747$

O $6.534612-0.581631-0.523280$

H $4.853792-1.407835-1.323662$

H $5.509700-2.4301260 .914947$

O $3.504254-2.1558950 .745501$

H $4.487792-1.2265032 .950759$

O $6.310155-0.5174782 .368684$

C 2.3624822 .0608282 .709869

H 2.1790860 .5300191 .261038

H $2.477517-0.0315612 .906501$

H $6.780504 \quad 0.005423 \quad 0.212150$

H $2.858850-1.4496150 .586960$

H $6.703702-1.3911362 .535400$

O $2.5051543 .021603 \quad 1.753238$

$\begin{array}{lllll}\text { O } & 1.983232 & 2.236037 & 3.860687\end{array}$

C 2.2633904 .3657132 .179980

H 3.1153484 .7202362 .767058

H $2.1635474 .992727 \quad 1.290175$

H $1.341843 \quad 4.4377942 .764935$

SCF Energy (B3LYP/6-31G**//MMFF) $=-3245.90958245$

07_00127

MM̄FF Geometry

C $2.491556 \quad 0.4474452 .179046$

C $1.745904 \quad 1.2819262 .916824$

C $0.8373792323176 \quad 2.323953$

$\begin{array}{lllll}\text { O } & -0.512088 & 1.883726 & 2.607677\end{array}$

C 1.0604343 .7143252 .943948

C 2.3926134 .3438802 .605123

C 2.6255665 .0638551 .490772

C 3.4737744 .1866643 .642823

C 1.6735615 .3478640 .358532

C $2.3400255 .148856-1.010963$

C $2.4928063 .669950-1.409252$

C $3.6016893 .529934-2.455401$

C $3.7283022 .092270-2.971108$

O $1.2382193 .233466-1.952130$

O $4.5737162 .115033-4.131401$

C $2.3361891 .623457-3.430861$

C $4.4127561 .173764-1.952607$

$\begin{array}{llll}\text { O } & 2.369557 & 0.253416 & -3.825731\end{array}$

C $1.2117191 .868879-2.392080$

C $-0.1530641 .593561-3.068514$

O $1.3866890 .961814-1.304052$

C $-1.5036582 .377804 \quad 1.820385$

$\begin{array}{lllll}\text { O } & -1.392893 & 3.200492 & 0.925658\end{array}$

$\begin{array}{llll}\text { C }-2.766633 & 1.728804 & 2.226499\end{array}$ 
C $-3.9188472 .170377 \quad 1.703989$ C -5.2323861 .6007951 .974785$ C -6.2932602 .6344352 .234686$ C -1.389452 $1.958082-2.236505$ C $-2.6720351 .600833-2.974165$ C $-5.1370891 .604497-2.736366$ O $-2.682844 \quad 1.149718-4.117327$ C $-6.0997021 .353975-1.620016$ C $-6.6631610 .158924-1.383082$ C -7.695901-0.097911-0.321010 C $-7.217720-1.0585430 .745903$ C -7.286558 -2.387959 0.563022 C $-6.734133-0.4719262 .054376$ C $-5.439480 \quad 0.2715491 .894070$ N -3.805901 $1.830285-2.204843$ H 2.4313640 .5052251 .096657 H 1.7747171 .2046164 .001290 H 0.9727322 .3642101 .235908 H 0.9399963 .6591204 .035135 H $\quad 0.2531914 .3916372 .637169$ H 3.6102295 .5163531 .377142 H 4.3924904 .7168063 .369707 H 3.1376744 .5884584 .604510 H 3.7336063 .1330403 .778191 H $\quad 0.762091 \quad 4.7478390 .416873$ H $\quad 1.371121 \quad 6.3989140 .442275$ H $1.7259835 .660897-1.763246$ H $3.3183555 .645964-1.004562$ H $2.7313433 .068556-0.525450$ H $4.5622813 .874403-2.051531$ H $3.3834694 .187993-3.308641$ H $5.4369922 .475980-3.866395$ H $2.0962122 .190586-4.340760$ H $5.4064291 .559870-1.695567$ H $4.5817350 .176277-2.372307$ H $3.8497921 .069075-1.023486$ H $2.373555-0.283988-3.016175$ H $-0.2086432 .167167-4.003591$ H $-0.2158280 .531076-3.336678$ H $\quad 0.5364810 .836525-0.854650$ H $-2.724968 \quad 0.9007872 .925791$ H -3.922029 3.0218351 .026355 H -6.0596173 .1938763 .147372$ H -7.292235 2.2124712 .361264 H $-6.352618 \quad 3.3454591 .402869$ H $-1.3867231 .419410-1.284433$ H -1.403764 $3.033689-2.029177$ H $-5.1082720 .771135-3.447012$ H $-5.4310282 .502238-3.290159$ H $-6.3766372 .207852-1.007547$ H $-6.392817-0.685767-2.013285$ H -8.587961 -0.510551-0.810907 H -8.0307890 .8383340 .142351$ H $-6.969209-3.082971 \quad 1.333254$ H -7.658235 -2.809675 -0.365123 H $-6.566284-1.2670152 .792648$ H -7.528289 0.1475362 .480970 H $-4.590890-0.373507 \quad 1.669633$ H $-3.7106132 .364842-1.346108$ C $4.647584-0.8325311 .918972$

O $4.247590-1.3915410 .648161$ C $4.571512-2.7882630 .665775$ C $5.885733-2.8281191 .423831$ C $5.587245-1.8667752 .564744$ H $3.797503-3.2974231 .255072$ C $4.580880-3.372026-0.747260$ H $\quad 6.144105-3.8331641 .769560$ H $6.713553-2.444613 \quad 0.816317$ C $6.835141-1.2457983 .171498$ H $5.059546-2.4150733 .356543$ C $3.331138-3.029544-1.586592$ O $5.722178-2.871580-1.453289$ H $4.715631-4.458543-0.683434$ H $3.419133-2.003635-1.960313$ O $3.350872-3.866553-2.749815$ C $1.978046-3.181648-0.868213$ C $0.784998-2.987405-1.823869$ H $1.919008-2.451673-0.053626$
O $1.910507-4.485095-0.290094$

H $1.012470-2.203907-2.556019$

H $0.620246-3.926303-2.366764$

H $7.486403-2.0198223 .590224$

H $7.410370-0.6894332 .423813$

H $6.568766-0.5531313 .976522$

C $3.386663-0.6062142 .765553$

H $5.706599-3.262021-2.343943$

H $3.198494-4.780801-2.454728$

H $1.067544-4.5482520 .190718$

H $3.671899-0.3358143 .789084$

H $2.808498-1.5374522 .806921$

H $\begin{array}{llll}5.156277 & 0.116786 & 1.714273\end{array}$

C $-1.943528-3.3136240 .730369$

O $-0.799505-3.575975-0.094009$

C $-0.508352-2.587173-1.084304$

C -1.694141 -2.428293 -2.049522

C $-3.000355-2.158791-1.297361$

C $-3.204991-3.132996-0.134040$

H -2.072661 -4.224955 1.328305

C $-1.707306-2.1452081 .702671$

H $-0.314494-1.630092-0.587781$

O $-1.846031-3.600183-2.848945$

H -1.484636 -1.599402-2.735279

H -3.839215 -2.240040 -1.999743

O $-2.968926-0.826977-0.800000$

H -4.041440 -2.802010 0.492248

O $-3.569791-4.402405-0.681888$

C $-0.687054-2.4784362 .770917$

H $-1.359762-1.2401511 .199260$

H $-2.650199-1.9215612 .215746$

H -1.969639-4.355513 -2.248822

H $-3.855111-0.624114-0.456892$

H $-3.763417-4.9988070 .061200$

O $-0.778983-1.5503963 .769996$

O $0.095159-3.4178602 .734101$

C $0.096305-1.7482474 .884280$

H $-0.002729-0.8873915 .550767$

H -0.193658 -2.651436 5.429062

H $1.136159-1.8163164 .554222$

SCF Energy (B3LYP/6-31G**//MMFF) $=-3245.91554453$

0700128

MMFF Geometry

C -1.919074 -2.788471 -2.022742

C $-0.761743-2.265857-1.589902$

C $0.164238-1.464187-2.464838$

O $0.463386-0.248672-1.737006$

C $1.492403-2.194216-2.738592$

C $1.356131-3.557332-3.384624$

C $1.646399-4.715626-2.758514$

C $0.918823-3.554461-4.824581$

C $2.080545-4.863798-1.326706$

C $3.608650-4.846096-1.195841$

C $4.112285-4.5188750 .218520$

C $3.653205-5.5315221 .267961$

C $4.121787-5.1359782 .678320$

O $3.642861-3.2083770 .553611$

O $3.426728-5.9628503 .624508$

C $3.732359-3.6633212 .940646$

C $5.606970-5.4506572 .886050$

O $4.256266-3.2143434 .188725$

C $4.132281-2.6959601 .798238$

C $3.477169-1.3098362 .037531$

O $5.552440-2.5619341 .788885$

C $0.8501420 .839966-2.452665$

O $0.9098540 .941054-3.667103$

C $1.2293511 .889821-1.480860$

C $1.7874143 .029106-1.915151$

C 2.255066 4.136168 -1.077507

C 2.1753453 .9627710 .416452

C $3.594621-0.3102160 .876187$

C 4.8998930 .4653740 .833976

C $6.1527082 .073831-0.604893$

O $5.740684 \quad 0.416314 \quad 1.727372$

C $5.8717923 .467425-0.137458$

C $5.9743094 .548625-0.925615$

C $5.7351225 .953569-0.450719$ 
C $4.7464706 .706248-1.313450$

C $5.1658947 .593912-2.231167$

C $3.2719786 .496186-1.050005$

C $2.7492725 .236618-1.682421$

N $5.0062981 .230902-0.319892$

H -2.212292 -2.640672 -3.059275

H $-0.451306-2.433551-0.561231$

H $-0.333249-1.208747-3.408705$

H $2.070224-2.244750-1.807946$

H $2.117497-1.567790-3.389567$

H $\quad 1.555863-5.648317-3.312144$

H $\quad 0.903797-4.561152-5.255384$

H $-0.089784-3.140824-4.920019$

H $1.602488-2.948072-5.427839$

H $1.618986-4.088265-0.707126$

H $1.687405-5.813757-0.944335$

H $4.015382-5.812011-1.520193$

H $4.017088-4.084828-1.874333$

H $5.207480-4.4969160 .169760$

H $3.999472-6.5410761 .014370$

H $2.556733-5.5844771 .278446$

H $2.472369-5.8083773 .521044$

H $2.639480-3.6355573 .047076$

H $5.794630-6.5205512 .733400$

H $5.916136-5.2394123 .915783$

H $6.263056-4.8999792 .208886$

H $5.203278-3.0324894 .063588$

H $2.401719-1.4686462 .193891$

H $3.877026-0.8589542 .953693$

H $5.787475-1.7733321 .276437$

H $1.0607221 .685289-0.429771$

H $1.9254913 .164171-2.987189$

H 1.1326573 .8758560 .735272

H 2.6148944 .7889160 .978478

H 2.7114863 .0609460 .730663

H $3.433895-0.804475-0.087608$

H $2.800870 \quad 0.4377330 .996100$

H $6.3300622 .027026-1.683913$

H $7.0339971 .680992-0.087810$

H 5.5949423 .5949250 .906559

H $6.2788184 .422900-1.962225$

H $6.7026406 .472711-0.453108$

H 5.3930555 .9714340 .591643

H $4.4626188 .159258-2.833491$

H $6.2222277 .770950-2.404494$

H $2.6942317 .331633-1.468524$

H 3.0877806 .5464520 .026493

H $2.7703015 .261395-2.773037$

H $4.2246061 .261949-0.967846$

C $-4.294911-3.220896-1.300366$

O $-4.544735-2.004846-0.561079$

C $-5.764846-2.1644710 .188231$

C $-6.442118-3.412690-0.365337$

C $-5.245844-4.268135-0.717316$

H -5.472211 $-2.359126 \quad 1.226349$

C $-6.644969-0.9119740 .112029$

H -7.108183 -3.8822290 .364994$

H -7.029068 -3.191124 -1.264498

C $-5.576780-5.405837-1.668629$

H $-4.825647-4.684046 \quad 0.208511$

C $-6.0232990 .382741 \quad 0.672454$

O $-6.993129-0.664187-1.257028$

H -7.592986 -1.108261 0.627494

H $-5.2567730 .758449-0.013176$

O

C -5.4356860 .2621642 .089303$

C -5.1475531 .6000782 .797137$

H -4.515634 -0.331983 2.042952

O $-6.358315-0.4588092 .913494$

H -6.0943702 .0860253 .066909$

H $-4.666791 \quad 1.3513933 .753027$

H -6.314775 -6.078958 -1.220212

H -5.991541 -5.032891 -2.611267

H $-4.683903-5.993397-1.903229$

C -2.825630 -3.633141-1.173983

H $-6.164108-0.560983-1.755048$

H -7.411035 $1.446588-0.209278$

H $-5.938061-0.5885063 .780746$
H $-2.704072-4.669856-1.508468$

H -2.516176 -3.584000 -0.122682

H $-4.552707-3.013469-2.347323$

C -2.1077102 .7031350 .921920$

O -3.0329341 .9362581 .705828$

C -4.2667342 .5872012 .016208$

C -4.0114233 .8744042 .819268$

C -3.0302424 .7985052 .091230$

C -1.7875534 .0420701 .610751$

H -1.1880132 .1043970 .908648$

C $-2.5797962 .872318-0.530861$

H -4.7828122 .8461751 .086762$

O -3.4801353 .5414594 .101035$

H -4.9680224 .3855132 .981187$

H -2.716721 5.6064862 .763585

O $-3.674196 \quad 5.3934740 .968876$

H -1.207392 4.6739250 .928879

O -0.9451693 .7773952 .736596$

C -2.701355 $1.550593-1.256692$

H $-3.5569203 .358895-0.584734$

H $-1.852413 \quad 3.481849-1.079532$

H -3.369685 4.370436 4.597180

H -4.4393185 .8939371 .300067$

H -1.416624 3.1601193 .322126

O $-3.1477331 .785422-2.526056$

O $-2.4466460 .455462-0.776777$

C $-3.342310 \quad 0.625272-3.340362$

H $-3.964586-0.112397-2.825141$

H $-3.8555190 .934526-4.254728$

H $-2.3742550 .195753-3.609799$

SCF Energy $($ B3LYP/6-31G**//MMFF $)=-3245.91646886$

07_00129

MMFF Geometry

C $-2.089203 \quad 1.2837332 .661145$

C -3.0585960 .3575582 .612471$

C $-2.842601-1.1345522 .518309$

O $-1.429589-1.4314262 .618012$

C $-3.572946-1.9339653 .610262$

C $-5.081930-1.8644773 .567080$

C $-5.848429-2.5144772 .669903$

C $-5.720293-1.0445894 .657592$

C $-5.397890-3.3793901 .521783$

C $-6.092408-2.9943610 .206751$

C $-5.661696-1.626455-0.356617$

C $-6.759614-1.086161-1.277403$

C $-6.357740 \quad 0.238070-1.934385$

O $-4.438743-1.802763-1.089111$

O $-7.305261 \quad 0.517306-2.976104$

C $-4.9874860 .041041-2.605398$

C $-6.4301711 .414038-0.952974$

O $-4.5139401 .270598-3.152326$

C $-3.918141-0.603457-1.683750$

C $-2.686058-0.966895-2.547213$

O $-3.5340520 .351330-0.695512$

C $-0.966722-2.5298861 .966352$

O $-1.616584-3.3277611 .310821$

C $0.493975-2.5905782 .188905$

C $1.194849-3.6001891 .654242$

C $2.638607-3.8024851 .761475$

C $3.441684-2.7829802 .523710$

C -1.560685-1.735034-1.838999

C $-0.399688-1.979849-2.794298$

C $1.899092-2.863390-2.925083$

O $-0.416228-1.616779-3.969166$

C $2.681943-3.984151-2.320792$

C $3.982283-3.878666-2.006222$

C $4.826124-4.999272-1.466329$

C $5.363179-4.702854-0.083480$

C $6.492887-3.9946430 .081409$

C $4.627256-5.2793381 .104638$

C $3.181394-4.8754701 .151558$

N $0.658683-2.655736-2.198373$

H -1.0453870 .9817922 .681506$

H -4.089456 0.6985532 .544821

H $-3.192107-1.4337191 .524864$

H $-3.210538-1.6127474 .596812$

H $-3.271379-2.9888943 .556570$ 
H $-6.929962-2.4243002 .766763$ H $-6.812543-1.0252044 .579623$ H $-5.465788-1.4575205 .639295$ H $-5.369435-0.0089204 .615402$ H $-4.314757-3.3700151 .385613$ H $-5.671939-4.4139281 .762693$ H $-5.875230-3.775191-0.533428$ H $-7.176966-3.0073800 .376249$ H -5.487369 -0.9236950 .464423$ H -7.702572 $-0.965179-0.729044$ H -6.966268 -1.820588 -2.068979 H $-8.1881890 .571287-2.572026$ H $-5.148084-0.624726-3.464784$ H -7.442698 $1.506629-0.541918$ H -6.226962 2.364123-1.459101 H $-5.7408251 .316223-0.111596$ H $-4.211327 \quad 1.825847-2.414147$ H $-3.018976-1.583864-3.392918$ H -2.258507 -0.047709 -2.968214 H -2.637956 $0.147411-0.386395$ H $\quad 0.951439-1.8046702 .778931$ H $0.661997-4.3525131 .073461$ H $3.285691-1.7810562 .109058$ H $4.518058-2.9681242 .490045$ H $3.148799-2.7750983 .579303$ H -1.179278 -1.170869 -0.982542 H -1.927950 -2.704679 -1.486342 H $2.448250-1.915579-2.910907$ H $1.663817-3.101425-3.968206$ H $2.170752-4.932386-2.174778$ H $4.491096-2.934303-2.183728$ H $5.662814-5.156574-2.159891$ H $4.275258-5.948016-1.458589$ H $6.896649-3.7934561 .067964$ H $7.035861-3.595104-0.769007$ H 5.125718-5.025318 2.047444 H $4.675953-6.374671 \quad 1.047537$ H $2.524154-5.5558100 .608604$ H $\quad 0.669281-2.744467-1.186210$ C $-2.350776 \quad 3.249311 \quad 1.148987$ O -1.1467842 .8405470 .476448$ C $-0.7648723 .861466-0.461788$ C $-1.8629394 .921587-0.419184$ C -2.3970714 .7658290 .993393$ H $0.1640084 .300411-0.082063$ C $-0.5136153 .236540-1.839787$ H -1.474659 $5.924510-0.622441$ H $-2.6553274 .719919-1.148874$ C $-3.7762075 .368412 \quad 1.192912$ H $-1.6899715 .237576 \quad 1.688899$ C $0.4358912 .019539-1.832966$ O $-1.7594512 .790474-2.391263$ H $-0.1401754 .003492-2.527931$ H $-0.0655241 .152993-1.385025$ O $0.6988021 .664568-3.196605$ C $1.7780622 .247726-1.115741$ C $2.7430691 .059060-1.300714$ H $1.5915932 .402942-0.047546$ O $2.3721863 .438190-1.631354$ H $2.1982980 .119118-1.151499$ H $3.1240191 .078637-2.329364$ H -3.758886 6.4442070 .990544 H -4.514216 4.9120270 .524527 H -4.119726 5.2242732 .222062 C -2.3350052 .7648192 .599739$ H -2.142389 2.152254 -1.765176 H -0.159329 $1.501114-3.624209$ H $3.1945513 .595039-1.137297$ H -3.276553 3.0220823 .098256 H -1.530601 3.2583543 .158798 H -3.1887862 .7857210 .611728$ C $5.7949322 .513927 \quad 0.312407$ O $4.6833692 .288576-0.564109$ C $3.9355111 .094425-0.323495$ C $4.842841-0.136064-0.482637$ C $6.089725-0.0332470 .401346$ C $6.776741 \quad 1.331306 \quad 0.267434$ H $6.3055913 .390474-0.107187$
C 5.3395972 .8741541 .730991

H $3.530163 \quad 1.1196640 .693941$

O $5.244106-0.281548-1.843994$

H $4.273386-1.034187-0.217474$

H $6.791729-0.8267030 .118625$

O $5.708595-0.2528081 .755608$

H 7.5383951 .4606421 .045129

O $7.4518381 .387016-0.992855$

C 4.5414954 .1569291 .724772

H 4.7385362 .0876622 .196343

H 6.2016703 .0318702 .389649

H $5.6809080 .543822-2.115517$

H $6.516419-0.2253452 .296008$

H $8.1098890 .671207-1.005069$

O 3.2241043 .8884221 .951747

O 5.0287325 .2615831 .522277

C 2.3546615 .0247461 .956738

H 1.3461034 .6796792 .199730

H 2.6710365 .7443982 .717844

H 2.3390085 .4898780 .966556

SCF Energy (B3LYP/6-31G**//MMFF) $=-3245.92769864$

0700130

MMFF Geometry

C $-0.290711-3.7045461 .396376$

C $-0.945733-3.8439652 .558064$

C $-1.265825-2.7338473 .533887$

O $-1.016427-1.4347802 .950310$

C $-2.767272-2.7894623 .887376$

C $-3.228900-1.6624514 .792667$

C $-4.131257-0.7251074 .437898$

C $-2.616916-1.6379736 .168827$

C $-4.876715-0.6208833 .134766$

C -4.1341390 .2596822 .130028$

C $-4.857770 \quad 0.4134510 .784148$

C $-4.889142-0.895616-0.010691$

C $-5.527805-0.710932-1.396244$

O $-4.126886 \quad 1.4007550 .045449$

O $-5.211012-1.870323-2.185373$

C $-4.8692350 .497002-2.098580$

C $-7.057909-0.674289-1.306011$

O $\quad-5.531017 \quad 0.809034-3.321523$

C $-4.7131281 .757366-1.213491$

C $-3.7423462 .754547-1.889081$

O $-5.9434992 .460805-1.043694$

C $0.147588-0.7948713 .239839$

O $1.093987-1.2289903 .874509$

C 0.0414010 .5649432 .661505

C 1.0313671 .4580402 .809724

C 0.9981822 .8519692 .350185

C -0.2769313 .3313631 .705031$

C $-3.5280804 .035719-1.072900$

C $-2.5153654 .955151-1.727285$

C $-0.0777285 .182821-2.176957$

O $-2.8501685 .994719-2.290014$

C $1.1621264 .691183-1.504060$

C $2.0356345 .471660-0.850664$

C $3.2515464 .926440-0.155248$

C 3.3767635 .3867601 .281631

C 4.4718076 .0498241 .691302

C 2.2440085 .0998232 .245241

C 2.0807363 .6335842 .552015

N -1.216421 $4.493996-1.598275$

H $\quad 0.103477-2.7317991 .117105$

H -1.301335 -4.834968 2.834277

H $-0.665894-2.8745714 .440977$

H -2.998862 -3.744356 4.377649

H -3.339634 -2.787259 2.953172

H -4.3787370 .0524205 .159398$

H -3.073683 -0.8784596 .812398$

H $-2.751675-2.606796 \quad 6.660789$

H -1.546170 -1.419784 6.112519

H -5.074003 -1.614970 2.725209

H $-5.864288-0.1890253 .340350$

H $-3.976341 \quad 1.2572152 .562913$

H $-3.129013-0.1377191 .947133$

$\begin{array}{llll}H & -5.870716 & 0.787454 & 0.971974\end{array}$

H $-5.416055-1.6854050 .536261$ 
H $-3.861508-1.260956-0.148368$ H -5.519014 -2.657002 -1.703479 H $-3.8605810 .168241-2.386667$ H -7.441070 -1.633851 -0.937152 H $-7.512092-0.534180-2.292697$ H $-7.4333360 .098549-0.633010$ H $-6.350737 \quad 1.285261-3.108747$ H -2.774295 2.263232 -2.048249 H $-4.1248143 .041274-2.877125$ H $-6.6826321 .841691-1.114995$ H -0.8822910 .8093062 .149090$ H 1.9370611 .1534153 .331859 H -1.115328 3.2550112 .406329 H -0.5061252 .7291650 .819554$ H $-0.241074 \quad 4.369461 \quad 1.372327$ H $-4.4671684 .590844-0.967227$ H $-3.1779103 .805423-0.059768$ H $-0.2175866 .263545-2.070741$ H -0.050965 4.942136 -3.244432 H $1.3462673 .621569-1.549501$ H $1.8730026 .545983-0.813450$ H $4.1306175 .236931-0.734331$ H $3.2495563 .831476-0.172409$ H 4.5776066 .3901342 .716105 H 5.2900406 .2665591 .012679 H 1.3290695 .5624731 .868176 H 2.4327455 .6028273 .203515 H 2.9527053 .1917673 .036298 H -1.076659 $3.546210-1.260308$ C $-0.389422-4.427446-1.030419$ O $0.424447-3.301130-1.425625$ C $-0.439587-2.290637-1.972734$ C $-1.677577-3.041261-2.438329$ C -1.845932 -4.032500-1.300639 H $-0.725588-1.629027-1.145761$ C $0.285016-1.481646-3.049131$ H $-2.539829-2.382301-2.568486$ H -1.499898 -3.570791 -3.381559 C -2.747294 -5.206381-1.639438 H $-2.272940-3.501977-0.440373$ C $1.597355-0.818933-2.575456$ O $0.604750-2.337322-4.152601$ H $-0.392305-0.718347-3.449736$ H $2.376602-1.579245-2.450167$ O $2.0456270 .048496-3.624316$ C $1.4682490 .007957-1.283547$ C $2.685160 \quad 0.886013-0.943336$ H $1.272148-0.662930-0.440257$ O $\quad 0.3337920 .875975-1.382989$ H $2.7989411 .674841-1.697280$ H $2.4417681 .407840-0.009951$ H -3.759002 -4.858929-1.873422 H $-2.374424-5.762645-2.505914$ H -2.813788 $-5.900327-0.795408$ C $-0.061946-4.8215140 .415526$ H $\quad 1.149517-3.066605-3.809790$ H $2.129868-0.490594-4.429568$ H $0.4610491 .435635-2.168311$ H $0.997699-5.0940290 .474219$ H $-0.641181-5.7102400 .691763$ H $-0.095608-5.255905-1.687394$ C $4.990801-1.7192990 .434681$ O $3.845565-0.8874930 .197943$ C $4.0168650 .135729-0.785782$ C $5.1560161 .086612-0.378276$ C $6.4522750 .317181-0.110030$ C $6.218969-0.8762460 .822138$ H $4.717379-2.3306271 .304178$ C $5.263229-2.670468-0.742297$ H $4.261138-0.324121-1.748259$ O 4.7828301 .8035850 .797254 H $5.3088031 .819336-1.179851$ H 7.1852260 .9960320 .343129 O $6.984141-0.127286-1.354181$ H $7.112034-1.5116050 .851255$ O $6.026314-0.3691372 .145898$ C $4.147675-3.674138-0.933205$ H $5.377270-2.126402-1.683263$
H $\quad 6.180301-3.239347-0.551064$

H 5.4995422 .4279761 .001295

H $7.818674-0.590313-1.167843$

H $5.932570-1.1309542 .742897$

O $4.371717-4.373984-2.084360$

O $3.207289-3.834440-0.168529$

C $3.396528-5.372411-2.401350$

H $3.767773-5.951612-3.251061$

H $3.245558-6.050647$-1.555952

H $2.455720-4.894446-2.686858$

SCF Energy $(B 3 L Y P / 6-31 G * * / / M M F F)=-3245.90346637$

07_00131

MMFF Geometry

C $1.816661-3.4233960 .837836$

C $1.798842-2.929216-0.408121$

C $0.537663-2.651212-1.178742$

O $0.662591-1.289705-1.659235$

C $0.373021-3.582332-2.393256$

C $0.109460-5.031242-2.045881$

C $-1.091746-5.512281-1.669406$

C $1.287350-5.958653-2.189123$

C $-2.368313-4.736793-1.485783$

C $-2.884071-4.873536-0.047359$

C $-4.073806-3.9644880 .300704$

C $-5.283356-4.171334-0.609448$

C $-6.436146-3.227441-0.236451$

O $-3.637270-2.6049590 .225011$

O $-7.387583-3.286265-1.310283$

C $-5.898979-1.779390-0.153499$

C $-7.174709-3.712601 \quad 1.017096$

O $-6.921927-0.9101680 .325576$

C $-4.605561-1.6415020 .681137$

C $-3.924515-0.2658740 .502875$

O $-4.902632-1.8335662 .065453$

C $-0.481473-0.598796-1.900477$

O $-1.625300-0.998586-1.764388$

C $-0.0959860 .750080-2.366636$

C $-0.9987581 .742038-2.347253$

C $-0.7696543 .121771-2.781882$

C $0.5638353 .438897-3.408932$

C $-4.6848440 .946597 \quad 1.039933$

C -3.7863722 .1638051 .189816$

C $-3.8749904 .552987 \quad 1.897277$

O -2.5762672 .1405540 .984620$

C -3.7764965 .3648860 .644268$

C $-2.6430915 .962616 \quad 0.246098$

C $-2.5372506 .839407-0.967094$

C $-1.4204706 .423967-1.897551$

C $-0.1931796 .958933-1.780775$

C $-1.7513165 .475227-3.025668$

C $-1.7504684 .032391-2.603516$

N -4.5005863 .2774491 .606973$

H $0.858185-3.6520461 .302416$

H $2.723065-2.681281-0.915939$

H $-0.331562-2.717203-0.512123$

H $1.259932-3.507649-3.038276$

H $-0.437522-3.212647-3.034923$

H $-1.178633-6.579328-1.468861$

H $1.032499-6.995627-1.945814$

H $1.657737-5.943662-3.219469$

H $2.100677-5.656495-1.523420$

H -2.240972 -3.681648 -1.738784

H $-3.107796-5.141615-2.186141$

H $-3.164139-5.9179610 .139790$

H $-2.062940-4.6330630 .641726$

H -4.341708 -4.187189 1.340495

H $-5.615446-5.216530-0.587733$

H $-5.007854-3.963217-1.651749$

H -8.085490 -2.635385 -1.121552

H $-5.682059-1.456430-1.180692$

H -7.558145 -4.7285740 .863710$

H -8.053521 -3.0925391 .225787$

H $-6.543673-3.7257121 .908211$

H -6.985499-1.030355 1.288424

H $-2.943735-0.3178920 .993984$

H $-3.701538-0.101911-0.558429$

H -4.187529 -1.433782 2.587762 
H $0.9260080 .890740-2.699826$ H -1.999446 $1.529084-1.973581$ H $1.3796863 .219929-2.711551$ H $0.676314 \quad 4.487208-3.691744$ H $0.7107652 .845781-4.318308$ H $-5.500491 \quad 1.208470 \quad 0.357580$ H $-5.109750 \quad 0.7321812 .026645$ H -2.894471 4.3746042 .351880 H -4.5104535 .0678442 .624327$ H -4.6833645 .4948870 .058682$ H -1.747831 5.8566610 .854859 H -2.361625 $7.866480-0.620221$ H -3.484736 6.877132 -1.518688 $\mathrm{H} \quad 0.6064746 .696569-2.464937$ H $0.0402557 .667572-0.992879$ H -1.076116 $5.638430-3.873162$ H $-2.7431005 .718778-3.428424$ H -2.676615 $3.714559-2.123717$ H $-5.511013 \quad 3.2153231 .692011$ C $4.430018-3.4317881 .233153$ O $4.597508-2.0663560 .815390$

C $5.513939-2.039868-0.299056$ C $6.101115-3.445058-0.404761$ C $4.946948-4.3003460 .089556$ H $4.917088-1.845503-1.197259$ C $6.538460-0.916498-0.110039$ H $6.409430-3.688591-1.426097$ H $6.973366-3.570400 \quad 0.247421$

C $5.362740-5.6989490 .511557$

H $4.206515-4.382466-0.712965$

C $5.908677 \quad 0.4725130 .130703$

O $7.360376-1.2185001 .025568$

H $7.216211-0.880424-0.970347$

H 5.3983340 .4761431 .099723

$\begin{array}{lllll}\text { O } & 6.974078 & 1.425632 & 0.234791\end{array}$

C $4.9452650 .940527-0.977771$

C $4.4135482 .374942-0.779411$

H $4.0990050 .249444-1.051874$

$\begin{array}{llll}\text { O } & 5.638665 & 0.891258 & -2.227668\end{array}$

H $5.252747 \quad 3.072337-0.670245$

H $3.8968372 .671148-1.702316$

H $5.799931-6.240927-0.333386$

H $6.106647-5.6726661 .314773$

H $4.500199-6.2703120 .868626$

C $3.002023-3.6891651 .727452$

H $6.773525-1.3155421 .795327$

H $7.575817 \quad 1.1128150 .932071$

H 5.002476 1.122184 -2.925740

H $2.848877-3.061746 \quad 2.616167$

H $2.933323-4.7293932 .068300$

H $5.095045-3.5570872 .099339$

C 1.2916341 .6804131 .124614

$\begin{array}{lllll}\text { O } & 2.269464 & 1.758574 & 0.080132\end{array}$

C 3.4485182 .5028770 .409050

C 3.0810173 .9639200 .706823

C 2.0398904 .0452151 .828703

C $0.867892 \quad 3.0841151 .593648$

H $0.419317 \quad 1.213247 \quad 0.651319$

C $1.779943 \quad 0.745613 \quad 2.234694$

H 3.9149122 .0682091 .298451

O $2.5663674 .576437-0.473253$

H 3.9893874 .5069370 .994860

H 1.6613135 .0728231 .892823

$\begin{array}{lllll}\text { O } & 2.680997 & 3.742467 & 3.063999\end{array}$

H $0.255558 \quad 3.0022102 .497851$

$\begin{array}{lllll}\text { O } & 0.025329 & 3.651958 & 0.586260\end{array}$

C $0.632462 \quad 0.169756 \quad 3.025159$

H $2.358011-0.0874661 .814522$

H $2.462076 \quad 1.2410682 .931713$

H $2.3651495 .503334-0.259386$

H 2.0141703 .8203973 .767440

H $\quad 0.5364903 .694672 \quad-0.239847$

O $0.056546-0.8537392 .332206$

O $0.287418 \quad 0.583932 \quad 4.124058$

C -1.037311 -1.5033002 .986549$

H $-0.683165-2.0197623 .883468$

H $-1.817438-0.7793933 .240780$

H -1.455102 -2.242264 2.297574
SCF Energy (B3LYP/6-31G**//MMFF)= -3245.91565096

07_00132

MMFF Geometry

C -1.1855504 .8894420 .632031$

C $-0.4617814 .210089-0.270184$

C $1.0261453 .993403-0.164938$

O $1.2852452 .573874-0.274033$

C $1.7363364 .697543-1.335092$

C $3.2481174 .586911-1.289804$

C $3.9979733 .967003-2.222395$

C $3.9191155 .256309-0.117789$

C $3.5358723 .276453-3.474838$

C $3.2663461 .771847-3.317820$

C $4.5065320 .946459-2.937585$

C $4.211260-0.551932-3.039441$

C $5.404822-1.406338-2.584634$

O $4.8705891 .267080-1.592491$

O $4.919550-2.748035-2.421988$

C $5.888327-0.904737-1.205185$

C $6.490452-1.471493-3.665169$

O $7.085529-1.572949-0.816454$

C $6.0679880 .625994-1.133426$

C $6.347594 \quad 1.143523 \quad 0.298565$

O $7.1929110 .999904-1.939085$

C $1.087441 \quad 1.811462 \quad 0.835274$

$\begin{array}{lllll}\text { O } & 0.747311 & 2.195043 & 1.942772\end{array}$

C 1.3233180 .4017760 .451540

C $0.901326-0.5811461 .260284$

C $1.014733-2.0184211 .004820$

C $1.872613-2.453773-0.153083$

C $5.264744 \quad 0.826999 \quad 1.336219$

C $5.541679-0.4239742 .154006$

C $4.482229-1.9038263 .855967$

O $6.614092-1.0215972 .131338$

C $3.908217-3.1050683 .173440$

C $2.942149-3.8642363 .712205$

C $2.381704-5.0960023 .060778$

C $0.874828-5.0554372 .940660$

C $0.092427-5.673488 \quad 3.841581$

C $0.280224-4.3701221 .731392$

C $0.355901-2.8712651 .817235$

N $4.456766-0.7729142 .947362$

H $-0.6913745 .338807 \quad 1.489603$

H $-0.9630163 .759875-1.124349$

H 1.4118844 .3644820 .792276

H $1.4746415 .764197-1.340276$

H $1.3504734 .287244-2.274594$

H $5.0786773 .956617-2.083831$

H $5.0094695 .278299-0.220483$

H $3.5799416 .293358-0.026697$

H 3.6893824 .7283750 .812685

H $2.6370633 .759541-3.872897$

H $4.3020823 .426021-4.246380$

H $2.4811551 .627904-2.566548$

H $2.8705131 .404455-4.272905$

H $5.3241561 .221233-3.614404$

H $3.913655-0.823185-4.060079$

H $3.348505-0.794134-2.404072$

H $5.647187-3.282455-2.059663$

H $5.123083-1.208886-0.482224$

H $6.081851-1.896516-4.589913$

H $7.307357-2.138395-3.368105$

H $6.913302-0.494349-3.907314$

H $7.822150-1.171776-1.308202$

H 7.3382050 .8119800 .632182

H $6.4145802 .239507 \quad 0.237974$

H $7.5045991 .872253-1.646058$

H $1.8066680 .211480-0.499547$

H $\quad 0.394511-0.3114732 .185904$

H $2.864146-1.993178-0.088432$

H $1.411248-2.165100-1.103490$

H $2.045612-3.531319-0.187875$

H $5.221802 \quad 1.6553442 .055055$

H 4.2792480 .7496020 .867325

H $3.909868-1.6190524 .744480$

H $5.514901-2.1085324 .156053$

H $4.322549-3.3769912 .205431$ 
H $2.550048-3.6051364 .693242$

H $2.683824-5.9599523 .667341$

H $2.822342-5.2625532 .070067$

H - $0.988434-5.6722713 .748060$

H $0.516039-6.1901594 .696593$

H $-0.781910-4.632471 \quad 1.636072$

H $\quad 0.748056-4.7709200 .828055$

H $-0.238550-2.4548612 .631799$

H $3.589512-0.2504312 .864081$

C $-3.3858774 .391309 \quad 1.722883$

O

C $-4.3278242 .250376 \quad 1.898050$

C -5.3443353 .2676242 .401309$

C $-4.909077 \quad 4.5130571 .653297$

H $-4.635624 \quad 1.9129660 .901522$

C -4.1067161 .0403912 .811158$

H -6.3741132 .9705962 .180843$

H -5.2609923 .4292743 .482013$

C -5.4515115 .8021372 .245315$

H -5.2466894 .4295020 .611519$

C $-2.929471 \quad 0.1201782 .422264$

O -3.8471221 .5043874 .145105$

H -5.0317980 .4572162 .879625$

H -1.9878750 .6537942 .602537$

O $-2.931282-1.0027043 .314326$

C $-2.913796-0.4391520 .988159$

C $-4.210092-1.1536880 .586168$

H -2.097677 -1.165870 0.926836

O -2.6275360 .5889520 .046891$

H -4.448153 -1.947850 1.304835

H $-5.051855-0.4513560 .637855$

H -6.5462075 .8062542 .219826$

H -5.1391605 .9278493 .287370$

H $-5.0967826 .669703 \quad 1.680137$

C $-2.677385 \quad 5.0524380 .538907$

H -3.067201 $2.084576 \quad 4.107120$

H -2.914834 -0.652091 4.221490

H -1.8042681 .0247380 .325618$

H -2.9110696 .1226240 .509386$

H -3.041120 4.613407 -0.398769

H -3.005462 4.7948382 .670076

C $-5.756698-2.809508-2.360543$

O $-5.500048-2.297353-1.047357$

C $-4.199312-1.727281-0.844421$

C $-3.111243-2.783581-1.091741$

C $-3.268573-3.432949-2.471361$

C $-4.712463-3.870876-2.741323$

H $-6.730589-3.309791-2.279075$

C -5.895155 -1.687302 -3.394971

H $-4.068408-0.893310-1.541831$

O $-3.188078-3.785192-0.079781$

H -2.124040 -2.313050 -1.020837

H $-2.605444-4.304353-2.535428$

O $-2.851022-2.496185-3.460390$

H -4.835467 -4.141376 -3.796063

O $-4.980769-5.056183-1.985798$

C -7.075090 -0.804119 -3.064747

H -5.004495 -1.057712 -3.477067

H -6.068078 -2.102613 -4.395044

H -2.466639 -4.417624 -0.238270

H $-2.926771-2.931004-4.326852$

H -4.942900 -4.822224 -1.042414

O $-6.6688680 .246545-2.295779$

O $-8.222042-1.037196-3.423350$

C $-7.7025861 .141645-1.875595$

H $-8.1729911 .611026-2.744764$

H $-8.4450880 .607903-1.274693$

H $-7.2469011 .920833-1.258553$

SCF Energy (B3LYP/6-31G**//MMFF) $=-3245.92136189$

0700133

MM̄FF Geometry

C $-2.800909-1.8112893 .761989$

C -1.992066 -0.7996863 .412242$

C $-0.491528-0.8509443 .538359$

O $0.079094-0.6686202 .220193$

C $0.013897 \quad 0.284770 \quad 4.445165$

C 1.5271340 .3630724 .544282
C $2.244275 \quad 1.4714174 .272539$

C $2.231494-0.8903144 .995890$

C 1.7163402 .8274603 .902086

C 1.5816313 .0793172 .394707

C 2.9263463 .1189561 .652618

C 2.7616653 .7865310 .286944

C $4.0876863 .826115-0.482292$

O 3.3852221 .7782251 .460853

O $3.7930464 .176236-1.844697$

C $4.6988022 .408715-0.505213$

C 5.0180434 .9225500 .050520

O $6.0166272 .459399-1.047130$

C 4.6880481 .6813880 .866899

C 4.9924840 .1828190 .663100

O $5.7016312 .177700 \quad 1.742974$

C $0.157029-1.7752301 .431393$

O $-0.234466-2.8991921 .705284$

C $0.813338-1.3881630 .162832$

C $0.923913-2.305480-0.809616$

C $1.568703-2.130428-2.111143$

C $2.080387-0.761918-2.470142$

C $4.847723-0.6692971 .928361$

C $5.327135-2.0802541 .649753$

C $4.750571-4.1085960 .313372$

O $6.416680-2.4811002 .052754$

C $4.958284-3.943472-1.161447$

C $4.390189-4.734165-2.084901$

C $4.572939-4.551390-3.566120$

C $3.261646-4.544643-4.325661$

C $2.909976-5.583803-5.101898$

C $2.387974-3.307075-4.241888$

C $1.674669-3.205570-2.919841$

N 4.435461-2.808106 0.877111

H -2.375570 -2.715389 4.190584

H -2.4197580 .0944162 .964188$

H $-0.167192-1.815263 \quad 3.949942$

H $-0.3925870 .155688 \quad 5.456735$

H $-0.392373 \quad 1.2296314 .067646$

H 3.3287951 .4191934 .358823

H $3.294070-0.7182855 .198964$

H $1.780213-1.2664385 .919839$

H $2.166879-1.6694984 .230259$

H 0.7461173 .0032064 .380395

H 2.3825773 .5827414 .338690

H 0.9306192 .3140581 .953290

H 1.0767624 .0462692 .276566

H $3.649303 \quad 3.6705932 .264553$

H 2.3507734 .7985850 .389623

H $2.0322413 .222034-0.309517$

H $3.3380125 .035936-1.842610$

H $4.0935051 .822700-1.211529$

H $4.5485105 .907918-0.056985$

H $5.9467754 .970692-0.528496$

H 5.2716374 .7960641 .105004

H $6.2879761 .551274-1.260871$

H $4.315706-0.222876-0.096115$

H 6.0142040 .0591340 .282209

H 6.1322152 .9402671 .327018

H $1.195109-0.3779490 .070321$

H $0.520193-3.301409-0.628510$

H $1.308941-0.001966-2.305749$

H $2.366493-0.671280-3.520819$

H $2.955471-0.508742-1.866715$

H $5.442918-0.2620262 .752845$

H $3.805453-0.7059352 .263042$

H $3.910909-4.7731600 .539281$

H $5.657660-4.5085640 .776027$

H $5.604703-3.130672-1.485522$

H $3.765286-5.563488-1.760820$

H $5.211034-5.369588-3.924354$

H $5.117671-3.625536-3.789030$

H $1.983896-5.579683-5.667024$

H $3.537684-6.464741-5.186489$

H $1.625837-3.328014-5.031189$

H $3.017473-2.436303-4.447617$

H $1.225048-4.143930-2.591149$

H $3.561660-2.3749380 .594491$

C $-4.669250-2.7650702 .414899$ 
O $-4.084724-2.302500 \quad 1.180274$

C $-5.097399-2.2826750 .160928$

C $-6.216154-3.1724150 .681894$

C $-6.176153-2.8446132 .163743$

H $-5.468050-1.2524880 .104303$

C $-4.496489-2.683181-1.189739$

H $-7.180306-2.946810 \quad 0.216760$

H $-5.998582-4.2349250 .523242$

C -6.891771 -3.8627593 .033865$

H -6.635707-1.8587342.317219

C $-3.230011-1.889864-1.577836$

O $-4.137568-4.070165-1.159065$

H -5.256373 -2.587439-1.973719

H -2.385066 -2.204091 - 0.953097

O $-2.884430-2.239217-2.923308$

C $-3.381414-0.360883-1.492988$

C $-2.1216560 .382897-1.975310$

H -3.576477 $-0.075959-0.453751$

O $-4.509763 \quad 0.049614-2.262271$

H -1.239666 - $0.074833-1.517455$

H $-2.0394450 .280441-3.064517$

H -7.953572 -3.9139062 .772047$

H -6.466766 -4.864690 2.911518

H $-6.816670-3.5917414 .091721$

C $-4.287981-1.8011113 .540571$

H $-3.511983-4.195467-0.424574$

H -2.781517 -3.205871 -2.953968

H $-4.350042-0.216549-3.184007$

H $-4.783970-2.0861374 .474849$

H -4.620871 -0.7861813 .289236$

H $-4.254962-3.7634922 .606424$

C -3.397084 $3.895677-2.113215$

O $-3.1777132 .505653-2.382705$

C $-2.1508381 .879801-1.609293$

C $-0.7984932 .560329-1.869384$

C $-0.8819604 .070468-1.616743$

C -2.098984 $4.699277-2.304442$

H $-4.1096904 .220294-2.882476$

C $-4.0545114 .112191-0.745146$

H -2.394864 $1.962003-0.544280$

O $-0.3980182 .323300-3.217419$

H $-0.0442622 .105857-1.216174$

H $0.0290184 .556255-1.987311$

O $-0.9684714 .319293-0.216916$

H -2.247899 $5.723512-1.944213$

O $-1.8341774 .796470-3.707263$

C $-5.397230 \quad 3.421413-0.679852$

H $-3.433368 \quad 3.7515860 .079673$

H -4.227067 $5.178561-0.558776$

H $0.4778622 .728023-3.338228$

H $-0.193038 \quad 3.9122390 .203587$

H $-1.7691303 .892609-4.060743$

$\begin{array}{lllll}\text { O } & -5.354129 & 2.402602 & 0.225901\end{array}$

O $-6.363093 \quad 3.741194-1.360851$

C $-6.567720 \quad 1.6603450 .379005$

H $-6.413246 \quad 0.915327 \quad 1.164111$

H $-7.384515 \quad 2.3226320 .681125$

H -6.813164 $1.143548-0.553472$

SCF Energy (B3LYP/6-31G**//MMFF) $=-3245.91200922$

\section{$07 \_00134$}

MM̄FF Geometry

C $-2.5530563 .635919-1.201738$

C $-2.0706752 .481784-1.684189$

C $-1.5923452 .302384-3.101425$

O $-0.226748 \quad 1.813366-3.056848$

C $-2.4276001 .254573-3.859180$

C $-3.874508 \quad 1.652526-4.077957$

C $-4.908753 \quad 1.230394-3.321866$

C $-4.1154262 .566333-5.250412$

C $-4.8355390 .354904-2.102618$

C $-5.551085-0.988532-2.300330$

C $-5.003623-2.106066-1.394834$

C $-5.075275-1.761780 \quad 0.096156$

C $-4.403594-2.832450 \quad 0.969850$

O $-3.650648-2.333337-1.801030$

O $-4.257578-2.2703332 .283057$

C $-2.990050-3.1087440 .411866$
C $-5.295004-4.0684671 .130787$

O $-2.365651-4.1769351 .120386$

C $-2.972366-3.382765-1.105664$

C $-1.538735-3.449321-1.676410$

O $-3.591567-4.649115-1.356661$

C $0.7447802 .721477-2.763183$

O $0.598508 \quad 3.922168-2.593584$

C $2.0291711 .996599-2.639519$

C $3.0632842 .618844-2.054966$

C $4.3973132 .067187-1.833374$

C $5.4825173 .109041-1.909609$

C $-0.727194-2.156506-1.533489$

C $0.395570-2.111163-2.551431$

C $2.467877-3.318093-3.235829$

O $0.381633-1.315622-3.488107$

C $3.770260-3.086321-2.540623$

C $4.757172-2.338559-3.056841$

C $6.091904-2.153365-2.396542$

C $6.498226-0.701750-2.286793$

C $7.425766-0.182124-3.108819$

C $5.9109960 .117315-1.161802$

C $4.6045080 .769496-1.529960$

N $1.378237-3.056756-2.314889$

H -2.620369 $4.503690-1.852900$

H $-1.9897101 .621251-1.023871$

H $-1.6083363 .247775-3.658835$

H $-2.3522680 .281346-3.359494$

H $-1.959911 \quad 1.069748-4.836487$

H $-5.9114731 .568901-3.578125$

H $-5.1758112 .808520-5.378442$

H $-3.5759323 .509368-5.117893$

H -3.770739 $2.094725-6.176469$

H -3.799217 $0.187653-1.802464$

H $-5.3033990 .893394-1.269093$

H $-5.431197-1.311800-3.343109$

H $-6.625709-0.859411-2.123521$

H $-5.585419-3.011981-1.602018$

H $-6.115469-1.6034370 .406533$

H $-4.560598-0.811030 \quad 0.288523$

H -3.758761 -2.907598 2.822886

H $-2.390546-2.2141700 .621530$

H $-6.246545-3.7918761 .600933$

H $-4.836107-4.8048171 .799609$

H $-5.524793-4.5585730 .182621$

H -2.757357 -5.0096080 .806397$

H $-0.993966-4.288590-1.225457$

H $-1.609643-3.694796-2.745912$

H $-3.288022-4.975150-2.220123$

H $2.0894880 .979955-3.010220$

H $2.9399613 .641455-1.700519$

H $6.4905612 .689774-1.886632$

H $5.396173 \quad 3.812811-1.074258$

H $5.4036583 .674409-2.845564$

H $-1.352449-1.272343-1.697789$

H $-0.304310-2.064370-0.528198$

H $2.360728-2.703205-4.135040$

H $2.391084-4.370377-3.527665$

H $3.923109-3.585007-1.586366$

H $4.615328-1.867604-4.027093$

H $6.832885-2.704888-2.990152$

H $6.112570-2.611250-1.399666$

H $7.7520360 .847923-3.014443$

H $7.877487-0.774429-3.897709$

H $6.6404690 .856675-0.812528$

H $5.745928-0.525656-0.288070$

H $3.7475150 .098998-1.481459$

H $1.286036-3.671132-1.510837$

C -1.9739014 .6278531 .011334$

O $\begin{array}{llll}-0.777576 & 3.839030 & 1.181077\end{array}$

C -0.3342983 .9529302 .544510$

C -1.1063175 .1232413 .138882$

C $-2.436325 \quad 4.9889202 .422563$

H $-0.645607 \quad 3.0353393 .056049$

C 1.1895934 .0858862 .597568

H -1.1939795 .0540074 .227265$

H $-0.638604 \quad 6.0843432 .895609$

C -3.292937 6.2408592 .491180

H -2.990516 4.149263 2.863392 
C 1.9541842 .9436291 .896279

O $1.578680 \quad 5.313426 \quad 1.967515$

H 1.5159474 .1681353 .640719

H $1.8300423 .017090 \quad 0.809510$

$\begin{array}{llllllll}\text { O } & 3.351464 & 3.136878 & 2.152157\end{array}$

C 1.5604371 .5262722 .355012

C 2.5030310 .4489391 .783360

H 0.5331501 .3187172 .036072

O $1.591676 \quad 1.4863843 .781012$

H 2.6542290 .6345010 .715150

H 3.4718190 .5333162 .291041

H -3.537248 6.4828913 .530670

H -2.777515 7.1043712 .057418

H $-4.232366 \quad 6.101257 \quad 1.947200$

C $-3.0005893 .817754 \quad 0.221096$

H $1.244120 \quad 5.2931261 .054280$

H 3.5822724 .0282851 .838993

H 1.2860750 .6062774 .058148

H $-3.970985 \quad 4.3262840 .212836$

H -3.149645 2.8391870 .694885

H -1.6838415 .5311890 .458456$

C $1.266823-2.5217503 .695821$

O $1.822917-1.2462563 .355753$

C $1.971480-0.9887431 .957918$

C $2.910471-2.0274541 .320697$

C $2.464165-3.4618731 .627719$

C $2.126633-3.654513 \quad 3.111466$

H $1.335369-2.5743384 .790015$

C $-0.213254-2.6224843 .312198$

H $0.990083-1.0392451 .474419$

O $4.235008-1.8363501 .816086$

H $2.935271-1.859613 \quad 0.238010$

H $3.265632-4.1550171 .344185$

O $1.327531-3.768571 \quad 0.828210$

H $1.618010-4.613303 \quad 3.263198$

O $3.347314-3.7205303 .854757$

C $-1.029325-1.5640974 .015870$

H $-0.371323-2.5253832 .235158$

H $-0.625841-3.597243 \quad 3.597279$

H $4.816297-2.4576051 .345227$

H $1.071661-4.6870511 .019227$

H $3.783721-2.8537203 .787452$

O $-1.461540-0.6351653 .115745$

O $-1.242814-1.5560015 .221182$

C -2.2431410 .4337193 .656962$

H $-2.521599 \quad 1.1000532 .836130$

H $-3.155523 \quad 0.041246 \quad 4.115850$

H -1.6560491 .0002104 .385980$

SCF Energy (B3LYP/6-31G**//MMFF) $=-3245.92201022$

\section{5}

MM̄FF Geometry

C $-3.183781-2.920389-1.757130$

C $-2.431699-2.419484-0.765352$

C $-0.931143-2.327458-0.796644$

O $-0.651344-0.909663-0.903687$

C $-0.284848-2.875080 \quad 0.492553$

C $-0.132766-4.384218 \quad 0.532190$

C $1.048653-5.0328630 .502487$

C $-1.405365-5.181990 \quad 0.656291$

C $2.424399-4.4315820 .460209$

C $2.958742-4.342444-0.973563$

C $4.275036-3.557701-1.101540$

C $5.439263-4.246641-0.385956$

C $6.728734-3.416261-0.460079$

O $4.058625-2.245959-0.573227$

O $7.665444-3.971430 \quad 0.476420$

C $6.414731-1.9816170 .012932$

C $7.392687-3.503150-1.838769$

O $7.547444-1.131825-0.159460$

C $5.178737-1.360470-0.678224$

C $4.777211-0.013339-0.032056$

O $5.500842-1.099056-2.049134$

C $0.504012-0.524350-1.505975$

O $1.417711-1.238987-1.881335$

C $0.4384440 .944987-1.665272$

C $1.4873161 .628797-2.144593$

C $1.528614 \quad 3.073317-2.390000$
C $0.2576093 .852789-2.164762$

C $4.131209-0.1309761 .351871$

C $4.119108 \quad 1.2062042 .064143$

C 3.1470443 .4838901 .985186

O 4.8067051 .4070003 .063301

C 2.7368624 .3988370 .878314

C $3.5935685 .210592 \quad 0.241190$

C $3.2047456 .159818-0.854888$

C $3.8173205 .788700-2.186946$

C $5.0682896 .164961-2.502071$

C $2.9445725 .063324-3.185633$

C $2.6854753 .628244-2.809796$

N 3.2908252 .1393791 .462325

H $-2.710380-3.301067-2.657819$

H $-2.925352-2.0226020 .119135$

H $-0.530315-2.841185-1.680027$

H $-0.859571-2.5578181 .371817$

H $0.684003-2.3834230 .624958$

H $1.039530-6.1219380 .527515$

H $-1.214017-6.2227930 .939935$

H -2.053156 -4.7543321 .428768$

H $-1.946724-5.197739-0.293209$

H $2.440250-3.4530930 .948561$

H $3.080951-5.0690321 .064287$

H $3.088663-5.350245-1.387188$

H $2.205674-3.840180-1.594325$

H $4.494698-3.470349-2.172357$

H $5.603880-5.252351-0.791983$

H $5.197093-4.3874690 .675395$

H $7.813351-4.902204 \quad 0.236138$

H $6.233233-2.0344521 .093218$

H $7.652795-4.542494-2.072986$

H $8.336422-2.946972-1.858004$

H $6.757736-3.133905-2.646557$

H $8.297768-1.5534660 .293512$

H $5.6448550 .658566-0.002050$

H $4.0566340 .491746-0.688093$

H $4.875154-0.440546-2.393649$

H $-0.494567 \quad 1.427136-1.401275$

H $2.3963841 .082962-2.391869$

H $-0.0788283 .750082-1.127168$

H $-0.5364603 .493163-2.828601$

H $0.3617314 .924274-2.345506$

H $3.101580-0.499417 \quad 1.271705$

H $4.672993-0.8340261 .991595$

H 4.0884773 .7976872 .449487

H 2.3822583 .4668652 .767265

H 1.6856824 .4026990 .603961

H 4.6421595 .2037750 .531660

H $3.5348637 .166208-0.565399$

H $2.1145146 .232000-0.950567$

H $5.5046635 .929829-3.467109$

H $5.6848316 .714299-1.797967$

H $3.4261855 .054866-4.172892$

H $2.0237395 .633994-3.336636$

H $3.5563592 .983043-2.932763$

H 2.6320651 .8228100 .756004

C $-5.350815-1.945545-2.595303$

O $-4.950993-0.621645-2.181634$

C $-6.1130050 .087570-1.727798$

C $-7.259665-0.523122-2.517827$

C $-6.881512-1.992501-2.485666$

H $-6.236241-0.153834-0.663880$

C $-5.9325591 .601344-1.880307$

H $-8.235419-0.321055-2.066266$

H $-7.276446-0.164802-3.553463$

C $-7.542319-2.817466-3.577152$

H $-7.178413-2.404932-1.511893$

C $-4.5922562 .147864-1.346326$

O $-6.003067 \quad 1.940991-3.271100$

H $-6.7761282 .111945-1.400506$

H -3.792048 $1.895219-2.052899$

O $-4.675318 \quad 3.579143-1.352287$

C -4.1895191 .6684210 .058555$

$\begin{array}{llll}\text { C }-2.908610 & 2.366557 & 0.557887\end{array}$

H -4.0337320 .5845000 .035582$

$\begin{array}{lllll}\text { O } & -5.260693 & 1.931998 & 0.962682\end{array}$

H $-2.2145792 .501216-0.277910$ 
H -3.1732513 .3568020 .949050$ H -8.632055 -2.789245 -3.474746 H $-7.288325-2.441191-4.573830$ H -7.222706 -3.862876 -3.519413 C $-4.683330-2.983163-1.686990$ H $-5.9025572 .905965-3.339460$ H -5.330148 $3.836636-0.680546$ H -4.9960471 .5966641 .836335$ H -5.005227 -3.992870 -1.967221 H $-5.005228-2.822589-0.650647$ H $-5.017081-2.069713-3.632480$ C -2.5921910 .6946703 .886949$ O -3.0593661 .4993612 .796604$ C -2.198547 1.5539601 .656656 C -0.8405732 .1587932 .043150$ C $-0.208306 \quad 1.3832893 .203393$ C $-1.200911 \quad 1.1598904 .351561$ H -3.3003100 .8870864 .703203$ C -2.652991 -0.8038713 .556718$ H -2.042789 0.5411671 .269498 O $-1.017196 \quad 3.5236372 .415476$ H $-0.1780352 .136827 \quad 1.170477$ H 0.6589511 .9385483 .578876 $\begin{array}{lllll}O & 0.258833 & 0.132102 & 2.707039\end{array}$ H -0.7947410 .4364015 .067206$ O -1.3565902 .3904645 .065754$ C $-4.061466-1.2729153 .257382$ H -2.039476 -1.040963 2.685400 H -2.287201-1.385070 4.410641 H $-0.139373 \quad 3.8878092 .620837$ H $0.688128-0.3343913 .444366$ H -1.780282 3.0298694 .467597 O $-4.008496-2.5543292 .785977$ O $-5.077541-0.6126743 .423655$ C $-5.269845-3.1390822 .447544$ H $-5.079474-4.1038201 .969462$ H $-5.859265-3.3038273 .354099$ H -5.814242 -2.501881 1.743756

SCF Energy (B3LYP/6-31G**//MMFF) $=-3245.91271336$

\section{6}

MM̄FF Geometry

C $-0.718308-0.3308630 .655292$

C $0.016617-0.695991-0.407318$

C $-0.379254-1.672075-1.491236$

O $-1.686299-2.239789-1.236523$

C $-0.478681-0.995927-2.871397$

C $0.824946-0.456230-3.420195$

C $1.1442910 .850609-3.505603$

C $1.788251-1.501554-3.920886$

C $0.3324352 .031954-3.050269$

C $1.0470252 .774485-1.914786$

C $0.1854793 .892428-1.310559$

C $0.962808 \quad 4.620443-0.211524$

C 0.1023615 .6709470 .505726

$\begin{array}{lllll}\text { O } & -0.991773 & 3.293412 & -0.760348\end{array}$

$\begin{array}{lllll}\mathrm{O} & 0.791743 & 6.059147 & 1.704001\end{array}$

C -1.2255405 .0142240 .938355$

C $-0.056644 \quad 6.943057-0.334652$

O -2.1113205 .9815021 .498800$

C $-1.9250484 .221113-0.190753$

C -3.0963113 .3964130 .393389$

O $-2.4304215 .136923-1.163050$

C $-1.775291-3.360225-0.475981$

O $-0.847172-3.979346 \quad 0.025030$

C $-3.191615-3.741396-0.252813$

C $-4.212059-3.217755-0.950910$

C $-5.638633-3.492898-0.758890$

C $-6.021948-4.4373950 .349048$

C $-3.8432242 .541687-0.644104$

C $-4.1647401 .152126-0.129701$

C $-5.237045-0.0572231 .766403$

O $-3.791340 \quad 0.147438-0.731045$

C $-6.544774-0.6698551 .364121$

C $-7.370565-0.2884220 .376895$

C $-8.657931-0.9963610 .056076$

C -8.669488-1.581285-1.339455

C $-9.294104-0.949915-2.347798$
C $-8.027392-2.938357-1.545619$

C $-6.524450-2.864654-1.561522$

N $-4.912996 \quad 1.152898 \quad 1.035420$

H -1.715562 -0.7353360 .795091$

H $1.020883-0.287432-0.495844$

H $\quad 0.361729-2.479851-1.531645$

H $-1.259572-0.227947-2.840195$

H $-0.875457-1.726695-3.590735$

H 2.108007 1.114219-3.939329

H $2.669712-1.060725-4.397169$

H $2.137552-2.128981-3.095164$

H $1.302677-2.141699-4.664832$

H $0.1960492 .709934-3.901312$

H $-0.6691701 .737022-2.730136$

H $1.2981802 .055061-1.127165$

H $1.9912973 .192659-2.285659$

H $-0.1006034 .586312-2.109655$

H $1.8725675 .080068-0.617642$

H 1.3064993 .8903510 .535297

H 1.6633236 .4074671 .449104

H $-0.986342 \quad 4.3174201 .753726$

H $\quad 0.9247127 .376808-0.561702$

H -0.6025227 .7160890 .217414$

H $-0.5670436 .768312-1.284084$

H $-2.4959776 .490587 \quad 0.765020$

H -2.7011372 .7478131 .184254$

H -3.8268174 .0693850 .859796$

H -3.168343 $4.722147-1.637505$

H $-3.347795-4.4789460 .527477$

H -3.992074 - $2.499421-1.739001$

H $-5.655296-4.0684931 .313546$

H $-5.597190-5.431270 \quad 0.169182$

H $-7.099660-4.5720800 .457529$

H $-4.7810723 .027868-0.936891$

H -3.250186 2.415871-1.557292

H -5.2722240 .2116972 .826974$

H -4.433637 $-0.788198 \quad 1.624580$

H $-6.827953-1.5390831 .955875$

H $-7.1281030 .574431-0.237255$

H $-9.474101-0.2707100 .168681$

H $-8.871479-1.7906830 .782379$

H $-9.330613-1.375093-3.345354$

H $-9.7785070 .009513-2.199220$

H $-8.342372-3.361039-2.508922$

H -8.416312 -3.626319-0.790715

H $-6.135983-2.204531-2.339008$

H -5.2493992 .0350651 .409662$

C $0.421673-0.0773382 .912252$

O $1.600248-0.7823142 .469285$

C $1.516924-2.1390522 .935427$

C $0.596723-2.0828744 .146443$

C $-0.447762-1.094313 \quad 3.659270$

H $1.022818-2.7181342 .145394$

C $2.912748-2.7122143 .188528$

H $0.177585-3.0607754 .400276$

H $1.111220-1.6859255 .029267$

C -1.283263 -0.4824664 .770003$

H $-1.119844-1.6202722 .970328$

C $3.885203-2.5900231 .994327$

O $3.512348-2.0340164 .299649$

H $2.825748-3.7618293 .492492$

H $4.218879-1.5507221 .896805$

O $5.053743-3.3584052 .306723$

C $3.324121-3.0774990 .646822$

C $4.359939-3.195085-0.486556$

H $2.521157-2.4049890 .327443$

$\begin{array}{lllll}\text { O } & 2.738847 & -4.370028 & 0.831249\end{array}$

H $5.051491-4.020157-0.272176$

H $3.809807-3.502062-1.386335$

H $-1.842777-1.2584515 .302541$

H -0.6585870 .0421915 .500590$

H $-2.0026710 .235323 \quad 4.362795$

C $-0.2386090 .619134 \quad 1.718029$

H $3.534612-1.0851924 .086616$

H $5.395914-3.0327013 .157007$

H $2.313759-4.621686-0.006441$

H $\quad 0.474110 \quad 1.323818 \quad 1.273816$

H -1.0897131 .2160252 .063347$ 
H $\quad 0.7774050 .695372 \quad 3.605390$

C $4.8089980 .423296-1.334764$

O $4.229770-0.865712-1.082548$

C $5.155466-1.912738-0.779220$

C $6.142828-2.111686-1.941272$

C $6.842565-0.800222-2.311605$

C $5.846570 \quad 0.352192-2.470103$

H $3.9728501 .041746-1.684684$

C $5.3658301 .067683-0.053842$

H $5.719211-1.6481230 .120349$

O $5.468322-2.625962-3.088458$

H $6.887549-2.860471-1.647763$

H $7.389725-0.932004-3.253347$

O $7.787169-0.457783-1.302105$

H $6.3842441 .304692-2.544426$

O $5.1546090 .166164-3.707866$

C 4.2750941 .4045800 .938659

H 6.0770600 .4119190 .454426

H $5.8703722 .007068-0.307668$

H $4.764348-1.998254-3.325026$

H $8.428732-1.185951-1.241037$

H $4.5810560 .939628-3.842613$

O 4.8447441 .8931642 .079557

$\begin{array}{llllll}\text { O } & 3.076741 & 1.266422 & 0.742101\end{array}$

C $3.926726 \quad 2.2600883 .114560$

H 4.4975502 .7093083 .931534

H 3.2055192 .9958752 .745523

H 3.4138091 .3709633 .492121

SCF Energy (B3LYP/6-31G**//MMFF) $=-3245.90926222$

\section{7}

MM̄FF Geometry

C -3.287025 $0.137745 \quad 2.437124$

C $-2.313241-0.6380172 .936685$

C $-1.989265-2.0112592 .411255$

O $-0.546859-2.145202 \quad 2.496210$

C $-2.639707-3.138140 \quad 3.233944$

C $-4.138027-3.2576943 .066325$

C $-4.737901-3.8595632 .020027$

C $-4.963405-2.7154694 .203860$

C $-4.083136-4.4840510 .815269$

C $-4.780140-4.096978-0.497846$

C -4.542363-2.637333 -0.924143

C $-5.616332-2.213152-1.929896$

C $-5.386763-0.789397-2.450103$

O $-3.240306-2.546622-1.524582$

O $-6.246164-0.591397-3.583002$

C $-3.936659-0.688597-2.955328$

C $-5.780855 \quad 0.271299-1.416134$

O $-3.6302450 .648984-3.345786$

C $-2.878587-1.226466-1.957689$

C $-1.510229-1.282792-2.680805$

O $-2.786858-0.324484-0.856365$

C $0.047091-3.0841581 .713783$

O $-0.495848-3.8213950 .907042$

C $1.491961-3.0815192 .022568$

C $2.269709-4.0275651 .478040$

C $3.705228-4.1674061 .692691$

C $4.141871-5.5986441 .852762$

C $-0.370662-1.961684-1.909524$

C $0.927124-1.916722-2.705490$

C $3.194844-2.880007-2.828457$

O $1.056849-1.254829-3.733469$

C $4.215970-3.472389-1.911987$

C $5.378852-2.871030-1.615604$

C $6.453351-3.473927-0.753060$

C $6.716637-2.6666810 .500064$

C $7.592473-1.6475350 .492880$

C $6.026907-3.0910151 .779050$

C $4.527883-3.1000241 .656511$

N $1.936355-2.681389-2.130838$

H -3.920329 -0.2521061 .645435$

H $-1.700459-0.2602183 .751940$

H -2.278868 -2.0758081 .355871$

H -2.388429 -3.012139 4.296386

H -2.181015 -4.1018082 .976824$

H -5.824944 -3.931806 2.031302

H $-6.038386-2.8330894 .031161$
H -4.718532 -3.242460 5.132063

H $-4.770349-1.6491654 .351120$

H -3.020299 -4.2458760 .749184$

H $-4.156875-5.5724420 .930925$

H -4.409964 -4.765302 -1.286045

H $-5.855493-4.289360-0.392435$

H $-4.578337-1.988652-0.043072$

H $-6.617689-2.298462-1.489109$

H $-5.606627-2.902911-2.785688$

H $-7.164032-0.724073-3.289972$

H $-3.879732-1.288038-3.874334$

H $-6.8335320 .156406-1.131561$

H $-5.6935651 .281123-1.831772$

H $-5.1852530 .220351-0.503238$

H $-3.4917801 .167807-2.535220$

H $-1.629795-1.828447-3.626557$

H $-1.196517-0.262098-2.935625$

H -1.910919-0.408849-0.448780

H $1.880169-2.3212552 .691311$

H $1.831628-4.7869640 .832881$

H $3.873796-6.1832640 .965309$

H $3.650088-6.0495432 .722276$

H $5.217050-5.7173101 .997894$

H $-0.190174-1.460122-0.953829$

H $-0.625150-3.009241-1.716899$

H $3.520468-1.922541-3.250104$

H $3.004904-3.562903-3.663608$

H $4.005740-4.458901-1.506812$

H $5.591677-1.894684-2.045445$

H $7.371408-3.532960-1.352651$

H $6.217765-4.511208-0.484811$

H $7.808960-1.0798791 .391597$

H $8.108982-1.355644-0.415549$

H $6.281874-2.4066152 .598656$

H $6.428715-4.0629342 .078127$

H $4.091937-2.1140031 .498579$

H $1.683922-3.333968-1.393898$

C $-3.7764412 .488816 \quad 1.725113$

$\begin{array}{lllll}\text { O } & -2.596502 & 2.526994 & 0.902308\end{array}$

C -2.409164 $3.869802 \quad 0.416564$

$\begin{array}{llll}\text { C } & -3.627038 & 4.669037 & 0.871858\end{array}$

C $-4.023130 \quad 3.936958 \quad 2.139751$

H -1.5221904 .2621760 .926562$

C $-2.170993 \quad 3.862432-1.098512$

H -3.3878905 .7234391 .041432$

H -4.4418514 .6273100 .140041$

C $-5.445862 \quad 4.2227592 .586955$

H -3.3276844 .2228852 .940291$

C $-1.0650092 .893608-1.561879$

O $-3.3844413 .464273-1.750276$

H $-1.9591264 .882795-1.439194$

H $-1.409523 \quad 1.862509-1.438593$

O $-0.887878 \quad 3.076638-2.972467$

C $0.2895143 .062287-0.854478$

C $1.3522402 .101085-1.423016$

H $\quad 0.1677212 .8688770 .216708$

O $0.711754 \quad 4.415216-1.010048$

H $0.9288161 .091418-1.479208$

H $1.6132512 .425098-2.438160$

H -5.5770645 .2900422 .793105$

H -6.1728223 .9366251 .819414$

H $-5.686453 \quad 3.6710703 .500994$

C $-3.567777 \quad 1.5378182 .905826$

H $-3.2184223 .485751-2.708462$

H $-0.5180223 .966034-3.107847$

H $1.5524304 .518768-0.533262$

H $-4.4678301 .521640 \quad 3.530312$

H -2.736436 1.8959563 .525519

H -4.5963042 .1273301 .090310$

C $4.4644243 .493396 \quad 0.116577$

O $3.274247 \quad 3.331075-0.665028$

C $2.6328212 .056659-0.566936$

C $3.5873190 .942566-1.023958$

C $4.9171351 .000195-0.267701$

C $5.5020362 .416481-0.237909$

H $4.8699284 .465225-0.194067$

C $4.156105 \quad 3.573693 \quad 1.615797$

H 2.3391311 .8775860 .473265 
O $3.8347851 .042353-2.424975$

H $3.107133-0.027798-0.851657$

H $5.627780 \quad 0.321077-0.751561$

$\begin{array}{lllll}\text { O } & 4.703761 & 0.538010 & 1.062092\end{array}$

H 6.3418312 .4747730 .464231

O $6.0180572 .731371-1.534624$

C $3.315738 \quad 4.7911331 .921634$

H 3.6398862 .6869141 .994375

H $5.079616 \quad 3.6637412 .199846$

H $4.1915861 .929635-2.601553$

H $5.5642840 .537782 \quad 1.514715$

H $6.7089652 .079106-1.741409$

O 2.0252984 .4204272 .159276

O $3.750977 \quad 5.9354501 .925501$

C 1.1196645 .4891812 .450939

H $\quad 0.1478895 .0530542 .696972$

H 1.4717116 .0656123 .311790

H 1.0049786 .1333481 .574291

SCF Energy (B3LYP/6-31G*//MMFF) $=-3245.92410418$

0700138

MM̄FF Geometry

C $2.634146 \quad 0.4954552 .276214$

C 1.8307511 .1848673 .098206

C 0.8025572 .1668772 .607810

$\begin{array}{llll}\text { O } & -0.482050 & 1.547699 & 2.854957\end{array}$

C $0.869586 \quad 3.5128383 .350021$

C 2.1143234 .3218153 .064141

C 2.2484495 .1565642 .015389

C 3.2188274 .2040484 .082152

$\begin{array}{llll}\text { C } & 1.257489 & 5.421858 & 0.912275\end{array}$

C $1.9277685 .414435-0.470074$

C $2.2432704 .000372-0.990007$

C $3.3660904 .070062-2.028164$

C $3.6479562 .703766-2.661260$

O $1.0464953 .478456-1.585696$

O $4.4931102 .911814-3.802791$

C $2.3187412 .129090-3.180499$

C $4.4155491 .774595-1.715174$

$\begin{array}{llll}\text { O } & 2.497718 & 0.801318 & -3.670868\end{array}$

C $1.1712222 .161281-2.140028$

C $-0.1528441 .805295-2.858443$

O $1.4352881 .189165-1.129434$

C $-1.534786 \quad 1.9749182 .109411$

O

$\begin{array}{llll}\text { C } & -2.700218 & 1.135916 & 2.460434\end{array}$

C $-3.919826 \quad 1.5163392 .054179$

$\begin{array}{llll}C & -5.171180 & 0.788094 & 2.265667\end{array}$

C $-5.085293-0.5885732 .869690$

C -1.427086 $1.955074-2.016498$

C $-2.654773 \quad 1.526128-2.807241$

C $-5.0956031 .200176-2.628180$

O $-2.6079351 .196772-3.990569$

C $-6.1159410 .980779-1.557899$

C $-6.635000-0.223076-1.267342$

C $-7.702771-0.569445-0.260687$

C $-8.283144 \quad 0.573210 \quad 0.543663$

C -9.3243561 .2851940 .080536$

C $-7.716705 \quad 0.822364 \quad 1.925315$

C $-6.321593 \quad 1.3728581 .872727$

$\mathrm{N}-3.8123661 .535663-2.039281$

H $2.537301 \quad 0.6448831 .205520$

H 1.8901721 .0135604 .170281

H 0.9161652 .3219491 .527479

H 0.7716053 .3468194 .431981

H $-0.015664 \quad 4.1143693 .107124$

H 3.1720495 .7295151 .939894

H 4.0675184 .8571763 .852305

H 2.8506804 .4826495 .074954

H 3.5987923 .1795694 .127213

H 0.4211114 .7184310 .921868

H 0.8378956 .4207221 .083740

H $1.2522495 .914215-1.176515$

H $2.8435616 .017401-0.422537$

H $2.5414533 .354918-0.156846$

H $4.2821374 .478434-1.582768$

H $3.082793 \quad 4.771791-2.825659$

H $5.3089743 .343845-3.496742$
H $2.0180002 .738289-4.044728$

H $5.3733002 .225023-1.428192$

H $4.6656140 .830403-2.210142$

H $3.8694661 .550239-0.797354$

H $3.2149070 .828392-4.327117$

H $-0.2655372 .454697-3.737238$

H $-0.0983640 .772332-3.225517$

H $0.5991660 .918032-0.719916$

H -2.5223250 .2294303 .027423$

H $-4.0144522 .451781 \quad 1.503523$

H $-4.413278-1.2262402 .285158$

H $-6.043700-1.1098982 .908226$

H $-4.709919-0.5331053 .897376$

H $-1.371024 \quad 1.334750-1.117115$

H $-1.5628282 .998991-1.713657$

H $-4.9700310 .318703-3.267113$

H $-5.3991892 .033173-3.271153$

H $-6.4621691 .870049-1.040564$

H $-6.264524-1.080869-1.827264$

H $-7.302741-1.3287320 .423072$

H -8.515188 $-1.070901-0.804031$

H $-9.768277 \quad 2.0848050 .664114$

H $-9.7525021 .085423-0.896309$

H $-7.790500-0.0988962 .509574$

H $-8.335143 \quad 1.5510442 .465837$

H -6.2722222 .3772471 .449331$

H -3.792721 $1.993756-1.131955$

C $4.825125-0.6550391 .792541$

$\begin{array}{llll}\text { O } & 4.345857 & -1.100658 & 0.505462\end{array}$

C $4.750750-2.4640060 .327437$

C $6.106975-2.5204591 .006319$

C $5.826894-1.7204072 .269699$

H $4.040990-3.0905230 .881032$

C $4.707192-2.854374-1.150663$

H $6.433721-3.543747 \quad 1.212519$

H $6.878810-2.0215340 .409376$

C $7.076565-1.1299222 .901715$

H $5.351508-2.3836853 .004922$

C $3.393976-2.477490-1.870582$

O $5.777859-2.212446-1.853067$

H $4.893387-3.930336-1.247058$

H $3.376648-1.400029-2.072746$

O $3.396038-3.122948-3.149760$

C $2.101313-2.863698-1.127353$

C $0.838965-2.575770-1.963338$

H $2.041501-2.299566-0.190879$

O $2.141403-4.247947-0.784667$

H $0.946529-1.606686-2.465173$

H $0.741579-3.352174-2.732040$

H $7.771142-1.9233443 .196419$

H $7.600538-0.4639802 .207859$

H $6.820916-0.5522423 .795994$

C $3.626848-0.5295202 .744587$

H $5.686855-1.254071-1.715253$

H $4.206923-2.848062-3.611333$

H $2.227138-4.753624-1.611039$

H $3.980090-0.2867493 .753625$

H $3.100591-1.4905392 .789624$

H 5.3028640 .3200291 .641273

C -1.777107 $-3.946538 \quad 0.353594$

O $-0.640175-3.826221-0.513497$

C $-0.447425-2.541565-1.111331$

C $-1.672354-2.151094-1.953437$

C $-2.961211-2.227933-1.130323$

C $-3.072688-3.555818-0.377511$

H $-1.837487-5.0183410 .584238$

C $-1.573688-3.1911611 .673598$

H $-0.300264-1.795245-0.322859$

O $-1.774200-3.016958-3.082007$

H $-1.525744-1.135203-2.333437$

H $-3.827777-2.115696-1.792560$

O $-2.992839-1.163743-0.185866$

H $-3.900177-3.5209430 .341070$

O $-3.372525-4.599325-1.308305$

C $-0.608427-3.9336522 .565714$

H $-1.210355-2.1673541 .550666$

H -2.518192 -3.1205072 .226672$

H -2.532460 -2.719017 -3.612888 
H $-3.020744-0.330286-0.684636$

H $-4.214288-4.374364-1.739808$

O $0.676440-3.614725 \quad 2.240154$

O $-0.958929-4.719543 \quad 3.437016$

C $1.682279-4.2899913 .001210$

H $2.660117-3.9975812 .612327$

H $1.620494-3.9942594 .052771$

H $1.576653-5.3741472 .896607$

SCF Energy (B3LYP/6-31G**//MMFF) $=-3245.90604071$

\section{9}

MM̄FF Geometry

C -1.916997 5.367365 -0.566076

C $-0.8354474 .635277-0.871223$

C $0.4908964 .752933-0.166159$

O $0.812711 \quad 3.4623100 .402759$

C $1.5915185 .117442-1.179901$

C $2.9936515 .119244-0.597966$

C $3.9992684 .328273-1.025004$

C 3.2356476 .1016150 .518919

C $3.9725783 .335494-2.154070$

C $3.7544161 .895784-1.684127$

C $4.8978661 .275977-0.863083$

C $6.184627 \quad 1.139569-1.681374$

C $7.281940 \quad 0.386310-0.916097$

O $4.439612-0.021407-0.458805$

O $8.3085630 .030551-1.856549$

C $6.698937-0.934391-0.368564$

C 7.9515341 .2843410 .131335

O $7.656424-1.5760570 .470035$

C $5.351815-0.7561270 .370574$

C $4.671096-2.0975270 .713804$

O $5.532232-0.107771 \quad 1.635372$

C $\quad 0.2258203 .1354191 .586753$

O -0.5581973 .8096662 .234703$

C 0.6970801 .7821251 .951128

C 0.0619111 .0874102 .907068

C $0.388655-0.2736363 .339861$

C $1.609775-0.9088552 .733497$

C $4.297149-2.967512-0.486922$

C $3.379669-4.103026-0.072620$

C $1.021523-4.6011140 .537500$

O $3.779315-5.262300 \quad 0.005289$

C $-0.077337-3.8586261 .228037$

C $-0.632283-4.2416142 .388000$

C $-1.751019-3.4891903 .052754$

C -1.464109-3.150418 4.500832

C $-2.249046-3.6257615 .482662$

C $-0.276768-2.2656024 .821534$

C $-0.410529-0.8820794 .241412$

N 2.091063 -3.6756540 .211309$

H -1.856750 6.1165540 .218921

H $-0.9125343 .874122-1.644956$

H 0.4550065 .5071880 .629869

H $1.3888606 .111368-1.600192$

H $1.5281474 .418548-2.019949$

H $4.9638634 .409446-0.525462$

H $4.292341 \quad 6.1566790 .801844$

H 2.9273107 .1068420 .213186

H $2.6710785 .818177 \quad 1.412461$

H $3.1946293 .588188-2.881155$

H $4.9090033 .422657-2.716077$

H $2.8275871 .840368-1.097646$

H $3.5701361 .263880-2.563582$

H 5.0642651 .8817430 .034280

H $6.5618122 .118813-1.997299$

H $5.9690060 .588466-2.607557$

H $8.6318930 .848091-2.272293$

H $6.551448-1.589830-1.236408$

H $8.4431792 .136796-0.353220$

H 8.7428550 .7480870 .666259

H $7.247514 \quad 1.6879710 .862171$

H $7.350976-2.4845950 .629602$

H $5.292670-2.6761131 .409384$

H $3.762179-1.8723951 .282789$

H $6.483201-0.0406531 .817631$

H 1.5413251 .3841601 .399240

H -0.7910051 .5456563 .405750$
H $2.499993-0.3042112 .941188$

H $1.491766-0.9982961 .648454$

H $1.822586-1.9107723 .109201$

H $3.791566-2.384313-1.264907$

H $5.191713-3.405107-0.943174$

H $1.423341-5.4187271 .145196$

H $0.656218-5.021692-0.404856$

H $-0.448422-2.9601260 .740990$

H $-0.285455-5.1512052 .872331$

H -2.653487 -4.109667 2.978552

H -1.980352 -2.565332 2.509919

H -2.057443 -3.391496 6.524495

H -3.102714 -4.260219 5.268328

H $0.635983-2.7761724 .505685$

H $-0.173970-2.1488665 .908662$

H $-1.269186-0.3304634 .626474$

H $1.833136-2.731250-0.061530$

C $-4.2480064 .525269-0.267270$

O -3.7839983 .2053310 .078025$

C -4.8991072 .2961230 .073762$

C $-6.1486653 .163609-0.032969$

C $-5.6390794 .325653-0.864940$

H $-4.8150301 .695057-0.838253$

C $-4.8395991 .390452 \quad 1.308207$

H $-6.9829012 .632110-0.500999$

H $-6.484743 \quad 3.5191600 .947883$

C -6.524654 $5.557339-0.797495$

H $-5.5565163 .998540-1.910140$

C -3.5057320 .6381131 .510403$

O -5.0465422 .1953772 .479751$

H $-5.6729960 .679705 \quad 1.287974$

H -2.714210 1.3692001 .712045

O $-3.626560-0.1666292 .691021$

C $-3.044148-0.2919140 .373177$

C $-4.127868-1.296970-0.063526$

H $-2.173580-0.8539160 .733281$

O $-2.604990 \quad 0.494840-0.727709$

H -4.587665 -1.745049 0.824993

H $-4.903386-0.754680-0.617625$

H -7.526535 $5.331841-1.177220$

H -6.6287455 .9213040 .230200$

H -6.109527 $6.369845-1.402007$

C $-3.2533955 .176768-1.228969$

H -4.3516972 .8760662 .493670$

H -3.8807970 .4249843 .419931$

H $-2.277927-0.111573-1.413331$

H -3.622036 $6.154000-1.559488$

H $-3.1402904 .551927-2.123870$

H -4.3072715 .0973670 .668152$

C $-2.608823-2.776481-3.155873$

O $-3.172155-1.862113-2.205036$

C $-3.584047-2.430025-0.958151$

C $-4.644524-3.520881-1.184365$

C $-4.156385-4.574794-2.181529$

C $-3.579744-3.932473-3.447447$

H $-2.501840-2.188964-4.077156$

C -1.214232 -3.254903 -2.738167

H $-2.715298-2.868915-0.456914$

O $-5.844746-2.923436-1.671923$

H $-4.877117-3.991795-0.221499$

H $-4.993655-5.229002-2.453084$

O $-3.165218-5.375639-1.544568$

H -3.073629 -4.689146 -4.057243

O $-4.656392-3.428252-4.243189$

C $-0.213144-2.129385-2.826559$

H $-1.180583-3.670517-1.728371$

H -0.859705 -4.047501-3.408129

H -6.507189 -3.629404-1.764308

H $-2.883633-6.054916-2.180697$

H $-5.076240-2.702458-3.750339$

O $-0.159627-1.458088-1.642232$

O $0.428398-1.867151-3.835800$

C $0.746099-0.352767-1.604837$

H $0.6856430 .099277-0.612966$

H $1.770467-0.697805-1.772223$

H $\quad 0.4626020 .397700-2.348802$

SCF Energy (B3LYP/6-31G**//MMFF) = -3245.91618674 
0700140

MM̄FF Geometry

C $4.277674-1.9471522 .670535$

C $2.986640-2.1353362 .362273$

C $1.852943-1.5652293 .171985$

O $1.222576-0.5525902 .351597$

C $0.809665-2.6431943 .510657$

C $-0.258510-2.1593614 .475097$

C -1.581896-2.124923 4.223662

C $0.238102-1.7414215 .838476$

C $-2.328864-2.5516902 .991584$

C $-3.111181-1.3876942 .376289$

C $-4.216773-1.8445541 .411242$

C $-3.671409-2.5830440 .187644$

C $-4.775441-2.921038-0.827174$

O $-4.923726-0.6649991 .004062$

O $-4.141855-3.290850-2.061988$

C $-5.604342-1.650347-1.110091$

C $-5.601552-4.135025-0.386526$

O $-6.732036-1.944622-1.930398$

C $-6.062093-0.9183860 .169117$

C $-6.7624410 .432927-0.121956$

O $-7.022254-1.7307130 .858633$

C 1.6313730 .7324632 .539960

O $2.398418 \quad 1.1420693 .397537$

C 1.0089541 .5714351 .491288

C 1.0984972 .9063891 .591484

C 0.5876723 .8919320 .635917

C $0.0921583 .378664-0.688902$

C $-6.0257701 .422095-1.026012$

C $-4.6558771 .823690-0.524729$

C $-3.494348 \quad 3.101537 \quad 1.263748$

O $-3.6331581 .485744-1.116989$

C -3.0901774 .3770930 .593812$

C -2.7846935 .5061121 .249766$

C $-2.365637 \quad 6.7717320 .558606$

C -0.9395997 .1669330 .869536$

C $-0.6693378 .220476 \quad 1.658715$

C $\quad 0.172831 \quad 6.396153 \quad 0.188859$

C 0.6119015 .1958360 .984839

N -4.6947242 .5905040 .628689$

H 4.536942 -1.394307 3.570544

H $2.732152-2.6653981 .450409$

H $2.240926-1.1262014 .098354$

H $1.307161-3.5082933 .969072$

H $\quad 0.361713-3.0068272 .581759$

H -2.234826 -1.770573 5.021978

H $-0.578396-1.6136196 .557738$

H $0.911769-2.5013536 .247989$

H $0.774504-0.7895675 .781015$

H -1.670366 -2.988934 2.238876

H -3.025753 -3.344059 3.292161

H $-3.579727-0.784863 \quad 3.166353$

H -2.423156 -0.7031701 .861750$

H -4.905142 -2.489558 1.970197

H -3.142525 -3.4976950 .479416$

H -2.925494 -1.951772 -0.310208

H -3.546681 -4.038524 -1.881148

H -4.960387 -0.981810 -1.694532

H -4.960644 -5.019964 -0.292339

H -6.357519 -4.392306 -1.136633

H -6.101568 -3.988985 0.572996

H -6.401756 $-2.398421-2.724711$

H $-7.7487620 .237839-0.563719$

H $-6.965791 \quad 0.9264460 .838372$

H $-7.535123-1.1601131 .455104$

H $\quad 0.513030 \quad 1.0719370 .667558$

H 1.5946693 .3277112 .465285

H $0.8877892 .832595-1.206401$

H $-0.2406464 .165556-1.368176$

H $-0.7580422 .704132-0.549472$

H -5.922275 $1.019912-2.039747$

H $-6.6267492 .334619-1.128048$

H $-2.6951272 .359813 \quad 1.163168$

H -3.7163273 .2409002 .326093$

H -3.016282 4.364851 -0.491506

H -2.841331 5.5282182 .335365

H -3.0593037 .5645750 .867108$
H $-2.4832436 .688496-0.529295$

H 0.3519998 .5267551 .859981

H -1.4619508 .7961672 .124925$

H 1.0505097 .0457990 .069102

H $-0.1321356 .148532-0.831213$

H 1.0100115 .4562561 .967104

H -5.5918982 .9037120 .987615$

C $6.051467-1.1609191 .132169$

O $5.168183-0.6647880 .104312$

C $5.968006-0.089315-0.949763$

C $7.423202-0.217874-0.505231$

C $7.366990-1.4273480 .410542$

H $5.820197-0.712255-1.837965$

C $5.5110701 .350948-1.216089$

H $8.102103-0.352834-1.352927$

H 7.7559690 .6692940 .046992

C $8.571396-1.5456561 .327408$

H $7.283547-2.334131-0.203139$

C $4.0519701 .461638-1.710933$

O 5.6240632 .1015970 .000629

H $6.1858601 .832972-1.932239$

H $3.3700221 .044210-0.962346$

O $3.7288902 .853568-1.825270$

C $3.8114490 .804556-3.085148$

C $2.3837750 .990705-3.636170$

H $4.038132-0.264299-3.036734$

O $4.719817 \quad 1.368607-4.036787$

H $2.0957052 .047590-3.587999$

H $2.4050600 .733456-4.703912$

H $9.491590-1.6443820 .742341$

H $8.674742-0.6649521 .970341$

H $8.487377-2.4253851 .972783$

C $5.421718-2.3841361 .797997$

H 5.0502401 .6767690 .661376

H $3.8759833 .257126-0.952545$

H $4.5452802 .324801-4.076396$

H $6.150801-2.9057412 .426973$

H $5.072669-3.0917911 .036185$

H $6.181460-0.3516741 .864606$

C $0.822702-2.223104-2.589644$

O $1.648889-1.246917-3.235701$

C $1.3406850 .116963-2.922581$

C $-0.0913780 .445392-3.371862$

C $-1.097016-0.521363-2.738053$

C $-0.666746-1.982876-2.897945$

H $1.113289-3.175428-3.051558$

C $1.167354-2.305728-1.100849$

H $1.4255020 .267941-1.841361$

O $-0.176948 \quad 0.366862-4.793024$

H $-0.326247 \quad 1.476933-3.085164$

H $-2.082262-0.387470-3.201726$

O $-1.223910-0.225605-1.351616$

H $-1.290740-2.626883-2.269700$

O $-0.915032-2.362066-4.255586$

C $0.779355-3.642694-0.521417$

H $2.244467-2.155957-0.952110$

H $0.691148-1.523457-0.503520$

H -1.089029 $0.590486-5.044951$

H -1.606929 $0.664831-1.276835$

H $-0.713672-3.309739-4.337670$

O $1.832167-4.505935-0.595810$

O $-0.331024-3.895719-0.073395$

C $1.592246-5.812556-0.064698$

H $2.511233-6.395554-0.167961$

H $1.334725-5.7486540 .996948$

H $0.795818-6.309216-0.627106$

SCF Energy (B3LYP/6-31G**//MMFF) $=-3245.92387282$

$07 \quad 00141$

MM̄̄FF Geometry

C $-3.554019 \quad 1.7780842 .184538$

C -2.8361182 .0686451 .090402$

C -2.6270803 .4617160 .565457$

O $-1.193913 \quad 3.6705340 .541365$

C $-3.1809893 .616949-0.862962$

C $-4.6739443 .874422-0.925863$

C $-5.5983482 .942976-1.235378$

C $-5.0882145 .301342-0.678438$ 
C $-5.3507611 .479359-1.460610$ C $-5.0611251 .142990-2.927940$ C $-4.340260-0.203479-3.112469$ C $-5.166694-1.395241-2.626903$ C $-4.387348-2.715453-2.733741$ O $-3.106302-0.127943-2.391376$ O $-5.126146-3.690248-1.981569$ C $-3.007998-2.534593-2.062798$ C $-4.334573-3.226916-4.177833$ O $-2.185217-3.682867-2.259128$ C $-2.252897-1.266853-2.531984$ C $-1.008002-1.043915-1.633788$ O $-1.844352-1.448743-3.886361$ C $-0.748230 \quad 4.945406 \quad 0.679730$ $\begin{array}{lllll}\text { O } & -1.438916 & 5.952092 & 0.767552\end{array}$ $\begin{array}{llll}\text { C } & 0.729134 & 5.008883 & 0.763540\end{array}$

C $1.5501803 .980740 \quad 0.496873$ C 3.0135084 .0061490 .589871 C 3.6585725 .2471831 .150922 C $-0.3191440 .321422-1.770842$ C $0.6492230 .433632-2.933151$ C $2.2430942 .050228-3.963559$ O $0.912465-0.499177-3.686199$

C $3.563848 \quad 1.775945-3.318347$

C $4.457562 \quad 2.725947-3.007677$

C $5.7569712 .440571-2.311977$

C $5.9124173 .228630-1.029095$ C $6.7248774 .297946-0.978706$ C $5.2053592 .730638 \quad 0.213347$ C $3.713733 \quad 2.928187 \quad 0.178324$ N $1.1963701 .706796-3.018814$ H -4.0191702 .5805892 .751272$ H -2.369077 1.2581680 .535343 H $-3.0833914 .190578 \quad 1.246917$ H -2.906112 2.744185-1.467872 H -2.671056 $4.448600-1.367965$ H $-6.6422023 .244979-1.300273$ H -6.170519 $5.441647-0.768859$ H $-4.798922 \quad 5.6157780 .329110$ H -4.607483 $5.967588-1.402191$ H $-4.5388331 .136506-0.815702$ H $-6.2378850 .927853-1.125231$ H $-5.995702 \quad 1.149963-3.501758$ H $-4.4182401 .924305-3.354379$ H $-4.118182-0.308153-4.181027$ H $-6.114515-1.459986-3.175403$ H $-5.440256-1.254774-1.572741$ H $-4.681577-4.548956-2.080367$ H $-3.179719-2.455064-0.980011$ H $-5.348008-3.418164-4.550827$ H $-3.806426-4.184795-4.240025$ H -3.855404 -2.526160 -4.864553 H - $2.632116-4.440494-1.845995$ H $-1.328944-1.114926-0.585830$ H $-0.277600-1.841053-1.807938$ H -1.127592 -0.826306 -4.082738 H 1.1146745 .9805291 .056812 H 1.1225243 .0313090 .179819 H 3.4579896 .1098070 .505953 H 4.7437745 .1692651 .243175 H 3.2752315 .4601842 .155142 H -1.052022 $1.131382-1.836782$ H $0.2723280 .491518-0.861056$ H $2.1177193 .106061-4.222809$ H $2.1368191 .448968-4.871801$ H $3.7889070 .738990-3.082460$ H $4.2420723 .763084-3.253779$ H $6.5689532 .685694-3.008945$ H $5.8686381 .371343-2.091593$ H $6.8745694 .853367-0.059101$ H $7.2585514 .644204-1.857762$ H 5.6272653 .1888021 .115906 H $5.411101 \quad 1.6594670 .333972$ H $3.1656782 .074148-0.219613$ H $\quad 0.9767572 .385798-2.295639$ C -2.948147 0.1084633 .941038 O $-1.554939-0.0029543 .580537$ C $-0.975722-1.1042954 .304020$
C $-2.002241-1.5074065 .355410$

C $-3.299717-1.2084044 .631396$

H $-0.867392-1.9295963 .591948$

C $0.392629-0.7109224 .867170$

H -1.904816 -2.556789 5.649653

H $-1.919606-0.8935096 .259941$

C $-4.503161-1.1235445 .553878$

H $-3.474216-1.9917863 .881499$

C $1.422598-0.2693423 .805337$

$\begin{array}{lllll}\text { O } & 0.223258 & 0.373823 & 5.789666\end{array}$

H $0.799779-1.5417975 .455175$

H 1.1290920 .6985143 .380206

O $2.654000-0.0485054 .506424$

C $1.648797-1.2933832 .672969$

C $2.842542-0.928144 \quad 1.768138$

H $0.740094-1.3583092 .064199$

O $1.869207-2.573895 \quad 3.264368$

H 2.7696010 .1271151 .481596

H $3.765426-1.0682252 .344394$

H -4.659181-2.076174 6.070771

H $-4.372019-0.3463416 .314285$

H $-5.412511-0.8930064 .990278$

C -3.7738600 .3780342 .684776$

H $-0.1878901 .109707 \quad 5.304302$

H 3.2325260 .4781153 .929568

H $1.933875-3.2243692 .545138$

H $-4.843186 \quad 0.2592592 .892650$

H $-3.512564-0.3463181 .902762$

H -3.0300050 .9452814 .647337$

C $3.164046-4.103356-0.193990$

O $3.114110-3.1507290 .875086$

C $2.931226-1.7867600 .488291$

C $4.088845-1.336680-0.418787$

C $4.279277-2.279967-1.610317$

C $4.287775-3.752704-1.181928$

H $3.425354-5.052400 \quad 0.291786$

C $1.799443-4.285113-0.866642$

H $1.982520-1.687249-0.050388$

$\begin{array}{llll}\text { O } & 5.306112 & -1.272076 & 0.323306\end{array}$

H $3.880125-0.323613-0.778777$

H $5.225239-2.034969-2.108634$

O $3.228295-2.052783-2.542081$

H $4.222659-4.411516-2.055645$

O $5.530233-4.038319-0.532603$

C $0.765861-4.766402 \quad 0.124307$

H $1.436567-3.366255-1.333095$

H $1.855792-5.031442-1.667699$

H $5.465170-2.150163 \quad 0.710359$

H $3.382386-2.631902-3.307746$

H $6.241758-3.865335-1.172575$

O $-0.104645-3.758261 \quad 0.415861$

$\begin{array}{lllll}\text { O } & 0.746126 & -5.896248 & 0.594923\end{array}$

C $-1.142127-4.083350 \quad 1.345372$

H $-0.710857-4.3425612 .316902$

H $-1.777457-3.2018661 .466396$

H $-1.753245-4.9051080 .960269$

SCF Energy (B3LYP/6-31G**//MMFF)= -3245.91098142

0700142

MM̄FF Geometry

C $-3.301957-1.203378-3.530646$

C $-2.255761-1.675010-2.837263$

C $-0.822746-1.323357-3.144115$

O $-0.277651-0.585120-2.023129$

C $0.003304-2.609296-3.331986$

C $1.468879-2.384223-3.658119$

C $2.484276-3.029108-3.049462$

C $1.770461-1.402849-4.761298$

C $2.395416-4.097307-1.996008$

C $2.569313-3.589040-0.558290$

C $3.979013-3.056284-0.250604$

C $4.158345-2.8731851 .259299$

C $5.508591-2.2348051 .612738$

O $4.141736-1.802258-0.919599$

O $5.450054-1.8096862 .983328$

C $5.696125-0.9660630 .755029$

C $6.659486-3.243193 \quad 1.521209$

O $6.998103-0.4151450 .941627$ 
C $5.429241-1.197828-0.749996$

C $5.4500790 .125231-1.553162$

O $6.467065-2.030438-1.280515$

C $-0.6284110 .728766-1.932761$

O $-1.374151 \quad 1.341223-2.681974$

C $0.0572261 .314832-0.759882$

C $-0.2862192 .549934-0.363656$

C $0.315030 \quad 3.323718 \quad 0.724834$

C 1.4191752 .6792911 .518180

C $4.2528841 .046587-1.305819$

C $4.4439122 .424982-1.918068$

C $3.2126984 .580888-2.211633$

O $5.516907 \quad 2.821745-2.363886$

C $3.1308465 .370830-0.941831$

C $2.1610386 .259525-0.677027$

C $2.068094 \quad 7.029548 \quad 0.610154$

C $0.711555 \quad 6.910016 \quad 1.272894$

C $-0.123827 \quad 7.9610991 .329110$

C $0.352263 \quad 5.5950331 .936437$

C -0.1389294 .5760240 .943038$

N $3.2761853 .172813-1.864476$

H -3.130810 - $0.549712-4.382370$

H -2.436238 -2.305547 -1.972550

H $-0.759365-0.707676-4.050198$

H $-0.433134-3.204626-4.144960$

H $-0.090975-3.205732-2.417452$

H $3.500810-2.795311-3.363425$

H $2.823280-1.427572-5.063094$

H $1.173027-1.634566-5.649220$

H $1.546382-0.380539-4.441326$

H $1.443034-4.633757-2.068882$

H $3.162317-4.852606-2.210233$

H $1.825230-2.805877-0.364070$

H $2.346513-4.4253230 .116127$

H $4.715973-3.771298-0.634688$

H $4.037651-3.8289971 .784374$

H $3.363220-2.2217711 .642503$

H $5.252035-2.5889853 .530711$

H $4.988471-0.2184791 .133305$

H $6.496520-4.0738722 .218464$

H $7.609987-2.7862231 .817968$

H $6.777701-3.6710750 .523617$

H $7.121523-0.2800921 .896762$

H $6.3966700 .648545-1.368294$

H $5.461254-0.107333-2.627419$

H $6.489673-1.919715-2.245319$

H $0.8364560 .734715-0.281183$

H -1.079594 $3.063443-0.906361$

H 1.0840551 .7261351 .936689

H $1.757728 \quad 3.2795582 .364733$

H 2.2925552 .4932010 .886707

H $3.3463100 .596368-1.727150$

H $4.092782 \quad 1.196892-0.234086$

H $2.3300624 .721077-2.843307$

H $4.1035744 .870848-2.776715$

H $3.9078895 .196962-0.200695$

H $1.3953466 .450338-1.425479$

H 2.2872018 .0809900 .383422

H $2.839203 \quad 6.7091451 .321745$

H -1.0874317 .8909421 .822840$

H 0.1340348 .9138930 .878648

H -0.4496875 .7520312 .669658$

H 1.2141595 .2527792 .514735

H -0.9631514 .9383510 .325990$

H $2.4438262 .753864-1.462379$

C $-5.314738-0.256196-2.428211$

O $-4.722686-0.153318-1.116289$

C $-5.6982600 .406868-0.213695$

C $-6.9476380 .684000-1.046750$

C -6.813833 -0.332036 -2.166205

H $-5.934339-0.3680330 .523068$

C -5.1126451 .6443320 .476776$

H -7.865804 $0.561263-0.463982$

H $-6.946356 \quad 1.703801-1.449635$

C $-7.677046-0.015532-3.374478$

H $-7.078750-1.324226-1.777360$

C -3.8675241 .3521521 .341606$

O $-4.7364272 .597665-0.526920$
H -5.8820152 .1342121 .083938$

H -3.060861 0.9571460 .715100

O $-3.3989942 .602563 \quad 1.864944$

C -4.1339130 .4054002 .531441$

C -2.934024 $0.242602 \quad 3.485696$

H -4.440485 -0.5802012 .168288$

$\begin{array}{lllll}\text { O } & -5.228601 & 0.917211 & 3.298092\end{array}$

H -2.5374381 .2295943 .752339$

H $-3.303300-0.195073 \quad 4.423245$

H $-8.7361200 .000847-3.096381$

H $-7.4266300 .961118-3.802157$

H -7.546550 -0.769059-4.157205

C $-4.733984-1.466311-3.157586$

H $-4.0697992 .176318-1.096493$

H $-3.2296883 .188427 \quad 1.107155$

H $-4.976972 \quad 1.8027133 .612591$

H $-5.293863-1.675528-4.075315$

H $-4.800459-2.357686-2.524220$

H $-5.0690360 .668846-2.968126$

C -1.398202 -3.001485 2.467483

O $-2.283133-2.0014732 .988823$

C $-1.821690-0.6473552 .909130$

C $-0.507912-0.490498 \quad 3.685364$

C $0.551737-1.4730313 .178452$

C $0.010771-2.9012603 .081825$

H $-1.832651-3.9514002 .806216$

C -1.416139 -3.0080100 .934202$

H $-1.657817-0.3783041 .862305$

O $-0.749431-0.7140785 .073378$

H $-0.1513540 .539718 \quad 3.575453$

H $1.402992-1.4636633 .869848$

O $1.047456-1.0482651 .913917$

H $0.711465-3.5323162 .522656$

O $-0.044744-3.4256164 .414073$

C -2.619881 $-3.775760 \quad 0.438020$

H $-1.444103-2.0128540 .483300$

H $-0.532882-3.519936 \quad 0.535175$

H $0.093051-0.5872045 .542087$

H $0.317882-1.0399261 .275495$

H $-0.299136-4.3620594 .351464$

O $-3.748884-3.0260720 .586951$

O $-2.561243-4.913539-0.010507$

C $-4.970270-3.670402 \quad 0.212460$

H $-5.768639-2.9242360 .228999$

H $-4.899443-4.084114-0.797819$

H $-5.208534-4.4575860 .933701$

SCF Energy (B3LYP/6-31G**//MMFF) $=-3245.90713128$

0700143

MM̄FF Geometry

C $1.965379-2.4007192 .936709$

C $1.140819-2.7160413 .947868$

C $-0.166597-2.0258754 .271929$

O $-0.402260-0.962933 \quad 3.321550$

C $-1.313714-3.0482144 .167763$

C $-2.690206-2.4691704 .426060$

C $-3.683813-2.4180793 .516236$

C $-2.931654-1.966148 \quad 5.826498$

C $-3.678505-2.936788 \quad 2.105164$

C $-3.702573-1.8019681 .080143$

C $-3.738548-2.320174-0.366099$

C $-2.390426-2.888573-0.817494$

C $-2.408674-3.301866-2.297669$

O $-4.088805-1.210903-1.201239$

O $-1.041894-3.511299-2.682552$

C $-2.976558-2.139145-3.141834$

C $-3.125594-4.643047-2.495626$

O $-3.166855-2.536471-4.498077$

C $-4.293977-1.551262-2.578029$

C $-4.667230-0.262352-3.355644$

O $-5.337726-2.512968-2.740271$

C $-0.5021740 .309046 \quad 3.789782$

$\begin{array}{llllll}\text { O } & -0.529888 & 0.669602 & 4.955271\end{array}$

C $-0.526947 \quad 1.2115302 .616038$

C - $0.6939762 .529812 \quad 2.800040$

C -0.6674423 .5615331 .759871$

C $-0.321316 \quad 3.123246 \quad 0.362113$

C $-5.9061520 .496174-2.852958$ 
C $-5.754106 \quad 1.081777-1.462484$

C $-4.3727722 .658558-0.139559$

O $-6.4142920 .666533-0.512400$

C $-3.6722113 .952665-0.396343$

C -3.8787805 .0687620 .317570$

C -3.1665226 .3626220 .049962$

C $-2.2388726 .764434 \quad 1.173638$

C -2.5630887 .7585392 .017539$

C -0.8919636 .0783721 .255771$

C -0.9217754 .8396122 .112473$

N $-4.8167342 .100176-1.403059$

H $1.733915-1.5604822 .287601$

H $1.399814-3.5584194 .586396$

H $-0.095691-1.6391745 .295096$

H -1.146676 -3.8640834 .884267$

H -1.267150 -3.514232 3.178968

H $-4.633126-1.9783453 .820649$

H -3.971912 -1.662483 5.985658

H -2.706812 -2.7526816 .554398$

H $-2.301553-1.0999806 .045158$

H $-2.833649-3.6010101 .917261$

H -4.574286 -3.558049 1.977351

H -4.585001-1.171092 1.254033

H $-2.837683-1.1394441 .216641$

H -4.526930 -3.078343 -0.438816

H $-2.082550-3.737478-0.196375$

H -1.612483 -2.123669 -0.681815

H -1.030442 -3.826216 -3.601947

H $-2.221738-1.341143-3.153703$

H -2.611047 -5.435374 -1.938661

H $-3.103781-4.953300-3.546153$

H -4.165962 -4.626338 -2.164237

H -2.300175 -2.793162 -4.854968

H $-3.8095350 .421064-3.339152$

H -4.855693 -0.520602 -4.405441

H $-6.194004-2.069190-2.642362$

H -0.3734290 .7692101 .638144$

H -0.8496572 .8968723 .813856$

H 0.6872442 .6984950 .338805

H $-0.3454583 .933391-0.368801$

H -1.0252792 .3639520 .004915$

H $-6.1177721 .326822-3.538499$

H $-6.797325-0.139237-2.867579$

H -3.6794191 .9376990 .306388$

H -5.2324472 .7684770 .529696$

H $-2.9383273 .970664-1.199224$

H -4.6011225 .0591001 .130323$

H -3.929416 $7.135361-0.111380$

H -2.597054 $6.317394-0.887143$

H -1.886345 8.0725572 .805482

H $-3.513658 \quad 8.276122 \quad 1.943157$

H -0.1591676 .7637351 .703709$

H -0.5137425 .8944340 .246603$

H -1.152037 5.0473403 .158679

H $-4.2403002 .268149-2.222548$

C $3.181484-3.7548631 .179501$

O $3.090102-2.7008290 .197780$

C $2.152610-3.102810-0.818645$

C $1.945505-4.601994-0.627855$

C $2.033956-4.7215510 .880732$

H $1.209059-2.589916-0.596871$

C $2.648931-2.703578-2.210962$

H $0.987681-4.944454-1.030780$

H $2.742449-5.186376-1.102462$

C $2.277571-6.1374961 .371327$

H $1.090857-4.3570741 .306066$

C $2.928327-1.196373-2.392539$

O $3.870450-3.405955-2.476180$

H $1.929196-3.047547-2.963570$

H $3.829368-0.923382-1.832235$

O $3.250235-0.990155-3.775056$

C $1.767793-0.263207-2.001338$

C $1.9248891 .195916-2.470420$

H $1.635131-0.287355-0.913564$

O $0.560988-0.765884-2.582577$

H $\quad 1.880683 \quad 1.240407-3.566227$

H $\quad 1.0353011 .737753-2.122445$

H $\quad 1.457073-6.7960101 .068099$
H $3.206832-6.5498740 .964041$

H $2.346320-6.1638462 .463348$

C $3.211502-3.1677212 .595105$

H $4.168470-3.143508-3.364128$

H $2.449530-1.180100-4.293835$

H - $0.172965-0.221605-2.249955$

H $4.055155-2.4729702 .677482$

H $3.385200-3.9715113 .319802$

H $4.143755-4.2512560 .997206$

C 4.3067452 .4736510 .111406

O $3.1772631 .876398-0.539682$

C $3.1922611 .907793-1.968459$

C $3.2708653 .356837-2.481818$

C $4.4404764 .117977-1.851782$

C $4.4827593 .936165-0.329685$

H $4.0460402 .477057 \quad 1.177796$

C $5.5817741 .633245-0.060244$

H $4.0647391 .356585-2.332087$

O $2.0572724 .054017-2.206075$

H $3.3859273 .337597-3.571994$

H $4.3421335 .183574-2.092658$

O $5.6537743 .651851-2.433756$

H 5.4198234 .3299520 .080801

$\begin{array}{llllll}\text { O } & 3.418189 & 4.689744 & 0.258773\end{array}$

C 5.4752220 .2797120 .608995

H $5.810245 \quad 1.454571-1.113955$

H 6.4275322 .1569910 .399994

H $1.9332664 .061582-1.241845$

H $6.3826084 .161985-2.041165$

H 3.5542645 .6230860 .022300

O $6.618171-0.4248360 .355365$

O $4.522276-0.1104451 .268102$

C $6.675827-1.739400 \quad 0.917057$

H $5.959703-2.3909500 .409380$

H $7.682897-2.1332610 .756529$

H $6.480545-1.7098291 .993284$

SCF Energy $(B 3 L Y P / 6-31 G * * / / M M F F)=-3245.90749314$

0700144

MM̄̄FF Geometry

C $4.748174-2.0220131 .862866$

C $3.417821-2.0359961 .693216$

C $2.434517-1.8044452 .810147$

O $1.574630-0.6902052 .472033$

C $1.549123-3.0513992 .996239$

C $0.537995-2.9320414 .121755$

C $-0.782499-3.1704133 .995112$

C $1.092673-2.5543575 .472553$

C -1.539601-3.642589 2.785130

C $-2.417495-2.5341542 .201999$

C $-3.443527-3.0500081 .180770$

C $-2.791928-3.616309-0.082168$

C $-3.832928-4.020682-1.138912$

O $-4.281871-1.937620 \quad 0.839152$

O $-3.142634-4.219676-2.382637$

C $-4.814163-2.848654-1.351029$

C $-4.503867-5.356178-0.797766$

O $-5.887963-3.224145-2.209441$

C $-5.372814-2.269220-0.034257$

C $-6.200643-0.979612-0.245960$

O $-6.244191-3.2285240 .576067$

C 2.0886550 .5590582 .642542

O 3.2371470 .8455922 .942461

C 0.9940751 .5340412 .443462

C 1.2676792 .8451692 .513474

C 0.2838973 .9166362 .404222

C 0.7050455 .0363051 .494119

C $-5.4778580 .115216-1.031699$

C $-6.0755941 .497254-0.830077$

C $-5.7023073 .845292-1.583675$

O

C $-4.5216534 .348398-0.812799$

C -4.6008164 .9101270 .401973$

C -3.3958625 .3364591 .192564$

C -3.1798224 .6041192 .504789$

C -4.0323343 .7164423 .047759$

C -1.9029404 .9649823 .235522$

C -0.8521613 .8935993 .129069$ 
N $-5.5440602 .410342-1.732394$ H $5.159939-1.8443072 .853366$ H $3.009232-2.2000100 .701670$ H $2.960948-1.5807083 .746565$ H $2.183270-3.9236463 .203285$ H $1.042276-3.2524642 .046543$ H -1.408029 -3.046366 4.879095 H $\quad 0.355730-2.6767396 .273701$ H $1.949692-3.1887245 .721795$ H $1.413939-1.5082035 .480473$ H $-0.872765-4.0435102 .017937$ H -2.170602 -4.4828583 .101473$ H -2.962853 -2.024908 3.008606 H -1.790325 -1.7585131 .742084$ H $-4.056737-3.8138151 .673380$ H $-2.145123-4.4703330 .149607$ H -2.136394 -2.852524 -0.519425 H $-2.462801-4.900947-2.242467$ H -4.254916 -2.065195 -1.877606 H -3.756200 -6.156574 -0.742007 H $-5.206242-5.657520-1.582612$ H $-5.036796-5.3377960 .155069$ H -5.496197 -3.578420 -3.025997 H $-7.150875-1.215115-0.742712$ H $-6.476873-0.5940730 .744877$ H $-6.839100-2.760007 \quad 1.184821$ H -0.0099421 .1597082 .274304$ H 2.2913373 .1840152 .660800 H 1.0031604 .6424550 .515628 H 1.5573955 .5766611 .920048 H $-0.091377 \quad 5.7608821 .308445$ H $-4.4295670 .178023-0.716685$ H $-5.500960-0.123330-2.101277$ H $-6.6507774 .064303-1.083088$ H $-5.7169194 .287232-2.584112$ H $-3.5442824 .213823-1.269806$ H -5.5770305 .0599490 .856944$ H -3.4934886 .4086561 .403488$ H -2.4930605 .2174120 .579960$ H $-3.812727 \quad 3.2436194 .000215$ H -4.9637093 .4310682 .572881$ H -2.125236 5.1122864 .301368 H -1.5339105 .9397382 .899499$ H -1.037465 3.0326463 .772366 H $-4.8338762 .088788-2.383618$ C $6.413713-0.8932120 .390847$

O $5.446368-0.022026-0.229347$ C $6.0548600 .618567-1.365046$

C $7.5486870 .342862-1.252772$

C $7.547086-1.037106-0.623194$

H $5.672258 \quad 0.109956-2.257685$

C $5.6671842 .099709-1.398372$

H $8.0508120 .372916-2.224493$

H $8.0468241 .061850-0.592038$

C $8.880459-1.427088-0.009862$

H $7.274097-1.774132-1.390496$

C $4.1443082 .351690-1.391000$

O $6.2168032 .755998-0.248161$

H $6.1250542 .584548-2.268018$

H $3.7254242 .059397-0.421300$

O $3.9348443 .764239-1.511856$

C $3.3785801 .649101-2.527904$

C $1.8962902 .046649-2.643203$

H $3.4458800 .565484-2.402705$

O $4.0037151 .953589-3.778492$

H $1.8287833 .125449-2.829225$

H $1.4954361 .580189-3.551213$

H $9.663837-1.448217-0.774648$

H $9.187951-0.718460 \quad 0.766513$

H $8.825362-2.4211940 .444482$

C $5.736580-2.215178 \quad 0.747451$

H 5.8740822 .3011490 .540629

H $4.4190964 .191579-0.784515$

H $3.9895092 .920243-3.885831$

H $6.477505-2.9548961 .069891$

H $5.230895-2.621955-0.136115$

H $6.769908-0.3830811 .295676$

C $0.444342-0.597231-2.060223$
O $1.1144580 .260467-1.125676$

C $1.0413451 .661836-1.420444$

C $-0.4259882 .105948-1.508566$

C -1.207201 $1.260125-2.508982$

C $-1.039181-0.220252-2.181726$

H $0.928271-0.519975-3.041454$

C $0.588887-2.043287-1.557068$

H $1.4722092 .180734-0.557943$

O $-0.5288503 .487466-1.835948$

H $-0.8901291 .989104-0.520706$

H $-0.9054111 .468991-3.541916$

O $-2.5923111 .587366-2.423607$

H -1.567535 -0.451108-1.247652

O $-1.639813-0.996212-3.216839$

C $1.949275-2.638994-1.849923$

H $0.433592-2.086381-0.472679$

H $-0.144982-2.687291-2.054754$

H $-0.3344743 .592436-2.782512$

H $-2.6740472 .534032-2.630257$

H $-2.571564-0.724400-3.279369$

O $1.910225-3.975381-1.564427$

O $2.922544-2.022415-2.258683$

C $3.122346-4.698004-1.801182$

H $2.908704-5.764410-1.689653$

H $3.488558-4.520389-2.816975$

H $3.875563-4.412193-1.062517$

SCF Energy (B3LYP/6-31G**//MMFF) $=-3245.91744268$

0700145

MM̄FF Geometry

C $-2.115656-4.267089-1.632645$

C $-0.943323-4.386301-0.992143$

C $0.345406-3.777080-1.484478$

O $0.811055-2.824914-0.501762$

C $1.409534-4.881751-1.625391$

C $2.733422-4.397748-2.183351$

C $3.904303-4.437074-1.517380$

C $2.696447-3.874510-3.596542$

C $4.165179-4.944273-0.126371$

C $4.089714-3.8703580 .970616$

C $5.156537-2.7701710 .846586$

C $5.151111-1.8655172 .081091$

C $6.150514-0.7051291 .948132$

O $4.867432-1.987205-0.313584$

O 5.8406230 .2334952 .989789

C 5.9299500 .0002920 .590704

C $7.587691-1.1722892 .206355$

O $6.9350240 .984743 \quad 0.363565$

C $5.838670-0.973542-0.602605$

C $5.407633-0.290191-1.922334$

O $7.130917-1.548810-0.832692$

C $0.292996-1.567597-0.559746$

O $-0.529442-1.145887-1.357311$

C $0.888965-0.7852910 .546112$

C 0.3468450 .3945940 .881962

C 0.7815731 .2661311 .974196

C 2.1182490 .9779132 .603365

C $4.0458360 .412581-1.884893$

C $4.1331951 .903330-1.603971$

C $2.6888423 .883840-1.168047$

O $5.1910572 .526553-1.587904$

C 2.6219194 .1318310 .305330

C 1.6432324 .8423050 .885171

C 1.5750725 .1360992 .355355

C 0.2695004 .6917222 .974426

C -0.7435405 .5571293 .148637$

C 0.1725473 .2657723 .466871

C -0.0266202 .2790732 .350564$

N $2.8789892 .466705-1.411016$

H $-2.167418-3.690001-2.552469$

H $-0.899632-4.955273-0.066155$

H $\quad 0.198621-3.275518-2.448884$

H $1.034480-5.674746-2.286432$

H $1.556142-5.349796-0.646043$

H $4.793412-4.069013-2.028500$

H $3.698892-3.686835-3.996271$

H $2.212675-4.600075-4.258438$

H $2.143490-2.931444-3.645113$ 
H $3.476429-5.7577940 .124729$

H $5.162023-5.403479-0.113787$

H $3.090069-3.4201870 .958485$

H $4.205997-4.3735391 .938560$

H $6.134657-3.2514450 .730263$

H $5.354853-2.4442142 .990612$

H $4.145621-1.4439172 .215865$

H 6.4156461 .0086892 .868288

H $4.984540 \quad 0.5473270 .678620$

H $7.673256-1.6019843 .211736$

H $8.289375-0.3312172 .182311$

H $7.927895-1.9259381 .493096$

H 7.7434280 .5235540 .081874

H $\quad 6.200648 \quad 0.379272-2.276565$

H $5.333602-1.079143-2.684984$

H $7.159527-1.886372-1.743425$

H $1.732907-1.2142081 .073556$

H -0.5202860 .7459070 .325249$

H 2.8900890 .8801741 .832538

H 2.0797130 .0488803 .181861

H 2.4635731 .7682883 .273083

H $3.5797270 .317054-2.874116$

H $3.374948-0.059909-1.160455$

H $1.7684294 .178221-1.680258$

H $3.5237304 .445226-1.599273$

H 3.4249393 .7302320 .918954

H $\quad 0.858837 \quad 5.267293 \quad 0.264315$

H 1.6992116 .2191992 .485232

H 2.4121014 .6774012 .896286

H -1.674032 5.2522833 .616054

H $-0.667505 \quad 6.5883882 .819750$

H -0.6852403 .1613334 .144940$

H 1.0448393 .0399474 .086429

H -0.9765442 .4032281 .829585$

H $2.0600761 .865808-1.387363$

C $-4.348361-3.889259-0.550579$

O $-3.853045-3.498588 \quad 0.750001$

C $-4.910328-3.6491891 .708787$

C $-6.200975-3.6819840 .904864$

C $-5.752513-4.456071-0.319654$

H $-4.771432-4.6398812 .164284$

C -4.827200 -2.604959 2.827645

H -7.021110 -4.167912 1.441399

H $-6.525887-2.6767640 .616025$

C $-6.680517-4.297657-1.511997$

H -5.689314 -5.520953 -0.057484

C -5.160954-1.141420 2.468406

O $-5.720710-3.0136553 .872960$

H -3.821062 -2.635357 3.263226

H -6.229561 -1.0413882 .244425$

O $-4.930466-0.348967 \quad 3.643194$

C $-4.337746-0.542423 \quad 1.315327$

C $-4.6531730 .943267 \quad 1.087485$

H -4.560932 -1.098283 0.402032

O $-2.951938-0.7008791 .605499$

H $-5.729867 \quad 1.0695610 .914436$

H -4.434586 1.5133862 .000539

H -7.681161 -4.672634 -1.272980

H $-6.777086-3.247643-1.808352$

H $-6.306143-4.858045-2.374401$

C $-3.391917-4.910126-1.167489$

H -6.628568 -2.956702 3.529570

H $-5.460677-0.7344394 .361692$

H -2.461610 -0.5635770 .778013$

H -3.856610 -5.393504 -2.034577

H $-3.168810-5.699383-0.438392$

H -4.394036 -2.979684 -1.163027

C -3.672582 $3.809385-1.062758$

O $-4.3508962 .962559-0.125597$

C $-3.8629341 .614548-0.051975$

C $-4.0139640 .923695-1.415841$

C $-3.3492251 .731662-2.535193$

C $-3.7302603 .215522-2.479873$

H -4.259952 4.737057-1.067967

C $-2.2688684 .151732-0.555547$

H $-2.806700 \quad 1.643793 \quad 0.233794$

O $\quad-5.394724 \quad 0.735518-1.720319$

H $-3.554553-0.069961-1.374495$
H $-3.6496741 .309905-3.502168$

O $-1.9366761 .586759-2.421576$

H -3.098532 $3.802667-3.154887$

O $-5.0773793 .359899-2.942988$

C $-1.7390095 .407653-1.198870$

H -2.2907034 .3276420 .527240$

H -1.552758 $3.335992-0.691088$

H $-5.8243761 .607855-1.700881$

H $-1.5305492 .081172-3.153818$

H $-5.108533 \quad 3.035286-3.859183$

O $-0.8842565 .088633-2.211487$

O $-2.0617526 .537098-0.854112$

C $-0.3165126 .200768-2.909860$

H $0.3445095 .811055-3.688488$

H $-1.1050046 .794004-3.382749$

H $0.2737926 .816276-2.224480$

SCF Energy $(B 3 L Y P / 6-31 G * * / / M M F F)=-3245.90739259$

0700146

MM̄FF Geometry

C $0.7524795 .460408 \quad 0.448330$

C $1.666034 \quad 4.725318-0.203346$

C 2.6457983 .8097070 .486423

O $2.6326582 .526349-0.181664$

C $4.0662204 .391251 \quad 0.364418$

C $5.1201393 .598174 \quad 1.112787$

C $6.1638312 .972870 \quad 0.533598$

C 4.9689783 .5603272 .612312

C $6.5208692 .916281-0.925179$

C $5.9731071 .690814-1.673265$

C $6.4971580 .345466-1.144761$

C $6.121554-0.794128-2.094531$

C $6.555356-2.164372-1.549061$

$\begin{array}{llll}\text { O } & 5.912279 & 0.104225 & 0.137666\end{array}$

O $5.888023-3.176295-2.319539$

C $6.062720-2.308895-0.092492$

C $8.059114-2.400566-1.734105$

O $6.588483-3.4895450 .507967$

C $6.381372-1.0841030 .789181$

C $5.705812-1.1400492 .181536$

O $7.795463-1.0216881 .013291$

C $1.6267851 .662928 \quad 0.123970$

$\begin{array}{lllllll}\text { O } & 0.715518 & 1.841494 & 0.915856\end{array}$

C $1.8206790 .449398-0.701280$

C $0.812741-0.422224-0.853449$

C $0.850620-1.650621-1.651023$

C $2.191404-2.111632-2.157282$

C $4.175075-1.2242532 .169264$

C $3.634345-2.6404322 .280841$

C $1.494084-3.9129522 .196902$

O $4.338299-3.6136252 .535470$

C $1.307425-4.4927820 .830640$

C $0.110080-4.8493970 .341954$

C $-0.095522-5.472216-1.008388$

C $-1.071104-4.696257-1.863940$

C $-2.363213-5.056987-1.942213$

C $-0.527658-3.554262-2.692223$

C $-0.304571-2.309127-1.879589$

$\begin{array}{llll}\text { N } & 2.258263 & -2.683580 & 2.099119\end{array}$

H 0.7075925 .4158161 .533788

H $1.7189894 .777792-1.288388$

H 2.3788163 .6741691 .541459

H 4.0815555 .4191170 .751379

H $4.3224234 .464184-0.697996$

H 6.8607532 .4400171 .179983

H 5.8392623 .1146453 .106370

H 4.8540724 .5743693 .008949

H 4.0932532 .9698462 .898164

H $6.1917553 .824163-1.441588$

H $7.6155872 .926281-1.005410$

H $4.8778811 .706489-1.625037$

H $6.2521071 .795583-2.729111$

H $7.5867980 .414535-1.044718$

H $6.548056-0.627190-3.091565$

H $5.032217-0.805234-2.234125$

H $6.132008-3.051847-3.252777$

H $4.975413-2.439604-0.138460$

H $8.326040-2.352762-2.796751$ 
H $8.346074-3.403484-1.399219$

H $8.676160-1.670197-1.206584$

H $6.344776-4.238234-0.062847$

H $6.150808-1.9408102 .784454$

H $5.964880-0.2073372 .703333$

H $7.958301-0.4877621 .808536$

H $2.7841910 .316998-1.179771$

H $-0.138500-0.207708-0.368636$

H $2.567940-1.428420-2.925986$

H $2.173469-3.113662-2.590710$

H $2.915933-2.152802-1.337157$

H $3.792044-0.6833573 .044160$

H $3.759920-0.7420401 .279100$

H $0.542009-3.6658552 .676474$

H $2.028328-4.6278012 .830891$

H $2.198330-4.6499360 .227111$

H $-0.774569-4.7168290 .960403$

H $-0.467636-6.493093-0.851148$

H $0.853590-5.585407-1.546908$

H -3.063465 -4.520014-2.573507

H $-2.751698-5.892308-1.368837$

H $-1.241143-3.296059-3.486423$

H $0.368217-3.888970-3.222225$

H $-1.224281-1.895854-1.467383$

H $1.762277-1.8353011 .840898$

C $-1.671645 \quad 5.9809030 .098720$

O $-1.9605284 .680715-0.447109$

C $-3.3020604 .672872-0.967638$

C $-3.925358 \quad 6.000822-0.548708$

C $-2.7153146 .913114-0.510362$

H -3.216995 4.640130-2.061045

C $-4.0781543 .439719-0.479002$

H -4.694944 6.336081-1.250728

H $-4.384921 \quad 5.9340550 .444597$

C $-2.943242 \quad 8.1909110 .277947$

H $-2.4358737 .169100-1.541210$

C $-3.5046072 .097426-0.978219$

$\begin{array}{lllll}\text { O } & -4.123227 & 3.438127 & 0.948586\end{array}$

H $-5.1120393 .534310-0.827709$

H -3.573109 2.061332 -2.071982

O $-2.1070072 .015632-0.675243$

C $-4.1723880 .842547-0.380632$

C $-5.6863760 .757414-0.638207$

H $-3.970304 \quad 0.783748 \quad 0.695276$

O $-3.549435-0.298629-0.979425$

H $-6.1776611 .678175-0.309071$

H -5.844441 $0.648765-1.718512$

H -3.753641 $8.777672-0.167014$

H -3.214037 7.9784311 .317651

H -2.042186 8.8120080 .284842

C $-0.2313516 .369434-0.235384$

H -3.207517 $3.482781 \quad 1.272690$

H -2.007436 $2.042490 \quad 0.291371$

H -2.595306 $-0.225893-0.807610$

H $-0.033172 \quad 7.3958330 .092594$

H $-0.0815016 .335654-1.321677$

H -1.8067905 .9095621 .186236$

C $-6.288190-2.8482850 .201587$

O $-5.824808-1.647600-0.428361$

C $-6.353799-0.427108 \quad 0.095125$

C $-7.883132-0.405934-0.059744$

C -8.522932 -1.644915 0.575732

C $-7.821572-2.9380910 .141406$

H $-5.878319-3.660610-0.412839$

C $-5.724957-3.0066981 .618327$

H $-6.095630-0.3462431 .156798$

O $-8.219530-0.350986-1.444717$

H -8.2738150 .5037850 .412219$

H $-9.581183-1.6874270 .290655$

O $-8.463425-1.5097461 .992555$

H $-8.159803-3.7849330 .749956$

O $-8.179187-3.228194-1.213056$

C $-4.219727-3.1288991 .587380$

H $-5.993947-2.1808942 .283226$

H $-6.113335-3.9167302 .091122$

H $-9.188490-0.305939-1.509980$

H $-8.893727-2.2907432 .380430$

H -9.145395 -3.331831-1.245762
O $-3.648322-1.9359171 .918173$

O $-3.626294-4.1566351 .286944$

C -2.217979 -1.9101291 .909779$

H $-1.894691-0.8807442 .086286$

H -1.828768 -2.5454122 .710472$

$\mathrm{H}-1.834641-2.2337580 .938715$

SCF Energy (B3LYP/6-31G**//MMFF) $=-3245.89056123$

0700147

MM̄FF Geometry

C $-0.369237-2.1132642 .482225$

C $0.681582-2.9099832 .724085$

C $1.635652-3.3575221 .651157$

O $2.917094-2.7640101 .966989$

C $1.792046-4.8877741 .609029$

C $0.582881-5.6272291 .079599$

C $0.380797-5.886945-0.226999$

C $-0.376363-6.1512972 .115281$

C $1.245932-5.440553-1.374998$

C $0.449174-5.205563-2.665448$

C $-0.534448-4.024823-2.596845$

C $-1.457088-4.064400-3.819969$

C $-2.403693-2.861137-3.870068$

O $0.216681-2.801270-2.573759$

O $-2.996046-2.818594-5.177181$

C $-1.565141-1.580832-3.712154$

C $-3.560871-2.993014-2.872640$

O $-2.409361-0.434946-3.632850$

C $-0.593857-1.617085-2.506290$

C $0.330301-0.375294-2.568798$

O $-1.354302-1.571384-1.297291$

C $3.774189-2.5655100 .929885$

O $3.604280-2.885029-0.235940$

C $4.960262-1.8527451 .449478$

C $5.828240-1.3346330 .568616$

C $7.018811-0.5478970 .880435$

C $7.399581-0.3772282 .326433$

C $1.480780-0.357125-1.551269$

C $2.1494241 .010516-1.503251$

C $3.6725822 .390954-0.094725$

$\begin{array}{lllll}\text { O } & 1.920485 & 1.895207 & -2.325198\end{array}$

C $5.0461972 .452468-0.686931$

C $6.1065992 .922768-0.010759$

C $7.4928823 .053838-0.580987$

$\begin{array}{llll}\text { C } 8.535371 & 2.356475 & 0.267724\end{array}$

C 9.1679223 .0126971 .255306

C $8.8908040 .921884-0.057846$

C $7.6950120 .016646-0.140988$

N $3.028287 \quad 1.133815-0.435520$

H $-0.531576-1.747234 \quad 1.472631$

H $0.862188-3.266948 \quad 3.735113$

H $1.298928-2.9817750 .675947$

H $2.054044-5.2666292 .606840$

H $2.668974-5.1494991 .002134$

H -0.487435 -6.484021 -0.501944

H $-1.186054-6.7397761 .670561$

H $0.148004-6.7997752 .824886$

H $-0.837582-5.3297582 .670146$

H $1.802033-4.532610-1.130601$

H $1.980544-6.231324-1.569923$

H $1.170492-5.023965-3.472980$

H $-0.091497-6.126732-2.918565$

H $-1.127350-4.097319-1.678514$

H $-2.029979-4.999956-3.844452$

H $-0.847501-4.064025-4.734891$

H $-3.465951-3.657981-5.320603$

H $-0.970801-1.462845-4.629258$

H $-4.156503-3.886699-3.094185$

H $-4.250960-2.145834-2.954013$

H -3.229246 -3.069066 -1.834996

H $-2.990796-0.447588-4.412291$

H $0.779315-0.306092-3.568508$

H $-0.2788410 .526282-2.425223$

H $-0.891232-1.027033-0.642470$

H $5.065595-1.7416082 .522103$

H $5.632046-1.470257-0.494663$

H 6.6153600 .1609102 .870258

H 8.3263550 .1856042 .463335 
H $7.551835-1.3525482 .801731$ H $1.118002-0.593757-0.546119$ H $2.237207-1.098637-1.828764$ H 3.6928102 .4556920 .997972 H $3.0721213 .219925-0.481695$ H $5.1692762 .133450-1.718853$ H 5.9660053 .2698331 .011091 H $7.7278834 .124427-0.645937$ H $7.5426412 .679166-1.610819$ H 9.9308612 .5306571 .857443 H 8.9337184 .0470881 .485183 H 9.6208950 .5144840 .651903 H $9.3977760 .904992-1.031426$ H $7.340115-0.147114-1.159143$ H $3.1915980 .335547 \quad 0.170733$ C $-2.788509-2.0406383 .110991$ O $-3.166542-1.2516921 .962798$ C $-4.414489-0.5958672 .240653$ C -5.046432 -1.395628 3.369308 C $-3.821392-1.7322274 .198324$ H $-4.169956 \quad 0.4051822 .614789$ C -5.254643 -0.4705790 .967156$ H $-5.795245-0.8203883 .921770$ H $-5.518834-2.3140713 .002247$ C $-4.041301-2.8734805 .176199$ H $-3.520628-0.8368184 .759311$ C $-4.5175600 .173918-0.225007$ O $\quad \begin{array}{llll}-5.683781 & -1.772466 & 0.548492\end{array}$ H -6.1707440 .0894301 .188072$ H -3.793707 -0.533643 -0.644737 O $-5.4824820 .414785-1.256703$ C $-3.800548 \quad 1.498180 \quad 0.094810$ C $-3.1978702 .154868-1.161646$ H -2.9955841 .3071120 .812285$ O $-4.716390 \quad 2.3981090 .715852$ H -2.672383 $1.393823-1.747157$ H $-4.0123482 .562020-1.773415$ H -4.823892 -2.6163995 .897493$ H $-4.347132-3.7901504 .660791$ H -3.124814 -3.090401 5.734003 C $-1.359522-1.6788373 .524776$ H $-4.887211-2.3125920 .406787$ H -5.906854 -0.436500 -1.460090 H -5.4452472 .5541450 .091056$ H -1.122538 -2.135905 4.492321 H $-1.267897-0.5917913 .637408$ H -2.847393 -3.093283 2.805708 C -2.124297 5.4402320 .276536 O $-2.919268 \quad 4.335357-0.172167$ C $-2.2046653 .284950-0.827498$ C $-1.5086093 .822069-2.088203$ C $-0.6109545 .019581-1.757523$ C $-1.3357196 .056143-0.892008$ H $-2.852678 \quad 6.1864260 .619785$ C -1.2445465 .0596691 .472649$ H -1.451635 2.875775 -0.144797 O $-2.4927744 .215792-3.042975$ H $-0.9169663 .016166-2.537796$ H $-0.2806635 .499914-2.686587$ O $0.5491494 .570308-1.064431$ H $-0.6196336 .788893-0.503041$ O $-2.2520246 .782691-1.716970$ C $-2.096606 \quad 4.6495182 .651032$ H $-0.5459124 .249877 \quad 1.243422$ H $-0.6338105 .911017 \quad 1.795267$ H -2.024081 $4.514049-3.840933$ H $1.0092173 .938324-1.642955$ H $-2.9373286 .161543-2.018100$ O -1.9734043 .3098452 .873528$ O -2.8001455 .4249463 .285775$ C $-2.7439112 .792923 \quad 3.962570$ H -2.5005521 .7335324 .078042$ H -2.4908193 .3125174 .891678$ H -3.8119052 .8875093 .744631$

SCF Energy (B3LYP/6-31G**//MMFF) $=-3245.92973090$

0700148

MM̄MF Geometry
C $3.814696-0.369143 \quad 3.566998$

C $2.934570-0.7329122 .621332$

C $1.450731-0.5008452 .721301$

O $1.113813 \quad 0.3950091 .636730$

C $0.694202-1.8307732 .537898$

C $-0.736368-1.7806733 .036201$

C -1.823671-1.642143 2.252920

C $-0.898524-1.9347394 .527318$

C -1.871821-1.4478990.763504

C $-2.437016-2.6555800 .002649$

C $-3.869367-3.0462840 .400251$

C $-4.394097-4.169577-0.497475$

C $-5.857061-4.519055-0.181291$

O $-4.704344-1.8920340 .272657$

O $-6.362724-5.319616-1.260609$

C $-6.689829-3.219286-0.154420$

C $-5.978010-5.3741751 .085897$

O $-8.022003-3.4759810 .284780$

C $-6.057198-2.0962750 .700768$

C $-6.831632-0.7725280 .494124$

O $-6.126874-2.4646282 .080236$

C $0.120077 \quad 1.300981 \quad 1.833854$

O $-\begin{array}{lllll}0.539620 & 1.474417 & 2.845227\end{array}$

C -0.0489102 .0421030 .565961$

C -1.2140052 .6488610 .297868$

C $-1.5312303 .396759-0.921728$

C $-0.4111493 .639348-1.900462$

C -6.1351870 .4596741 .089442$

C -6.9529951 .7266010 .901636$

C -6.8110184 .1904951 .276578$

O -8.0739471 .7386590 .400335$

C -6.430176 $4.766687-0.049990$

C $-5.5448865 .762302-0.206943$

C $-5.1583166 .323278-1.544767$

C $-3.7197826 .027641-1.906112$

C $-2.8050147 .008763-1.980882$

C $-3.3611964 .601965-2.265195$

C $-2.7946083 .838158-1.097805$

N $-6.2774552 .844359 \quad 1.373308$

H 3.4634430 .1196184 .472031

H $3.298071-1.2065101 .711524$

H $1.213868-0.0348023 .685608$

H $1.205849-2.6340413 .085729$

H $\quad 0.721943-2.133357 \quad 1.484131$

H -2.799179 -1.626918 2.737406

H $-1.949323-1.9227324 .835768$

H -0.469548 -2.885843 4.859264

H $-0.392319-1.1214385 .056270$

H $-2.483736-0.5613070 .556324$

H $-0.882501-1.2163630 .358491$

H $-2.420198-2.399013-1.064822$

H -1.772339 -3.5183180 .134996$

H $-3.863767-3.3762481 .445072$

H -3.759167 -5.060701-0.417542

H -4.335622 -3.853227 -1.548439

H $-5.801278-6.110216-1.335981$

H -6.768836 -2.860474 -1.190293

H $-5.423167-6.3128260 .968273$

H -7.018536 -5.6633251 .269796$

H $-5.594888-4.8756221 .978594$

H $-8.385570-4.174307-0.286110$

H -6.958241-0.590216 -0.581207

H -7.835046 -0.8610210 .929573$

H $-6.123874-1.6637662 .626625$

H $0.7838232 .042741-0.125990$

H -2.0181102 .5765911 .029294$

H $0.4452984 .104960-1.399542$

H $-0.6828164 .310201-2.717885$

H $-0.0839372 .695744-2.350642$

H $-5.967100 \quad 0.3314852 .163742$

H -5.1629250 .6103070 .605595$

H -6.4008174 .7663922 .112178$

H -7.9012274 .1635791 .373052$

H $-6.9089664 .337433-0.927130$

H -5.0647736 .1949580 .667551$

H $-5.3360527 .406056-1.517806$

H $-5.8100395 .937880-2.339268$

H $-1.7796676 .801576-2.268913$ 
H -3.059458 $8.038489-1.752996$ $\mathrm{H}-2.6866144 .598615-3.127214$ H $-4.2559724 .074559-2.620392$ H -3.529425 $3.606098-0.327043$ H $-5.3193502 .741013 \quad 1.695194$ C 6.0348800 .7262493 .153479

O 5.5868291 .2424841 .883106 C $6.705531 \quad 1.2872380 .985323$ C 7.9092371 .4743521 .892760 C 7.5519690 .5324223 .030037 H $\quad \begin{array}{llll}6.770058 & 0.303583 & 0.504004\end{array}$ C $6.4886682 .356068-0.089027$ H $\quad 8.851338 \quad 1.215579 \quad 1.400947$ H 7.9816042 .5029192 .264579 C 8.3071430 .8157594 .317320 H $7.775982-0.4955582 .714161$ C $5.1079672 .289319-0.777331$ O $6.609856 \quad 3.6583070 .495881$ H $7.2874182 .289953-0.836586$ H $4.3349032 .670030-0.098155$ O $5.1284443 .180002-1.898825$ C $4.6985550 .890224-1.270098$ C $3.3405260 .900333-1.997705$ H $4.627544 \quad 0.213277-0.412737$ O $5.707225 \quad 0.371172-2.133856$ H $2.6346891 .516913-1.431835$ H $3.4695651 .346177-2.991677$ H 9.3850160 .6978804 .165333 H $\quad 8.1269591 .8361894 .671888$ H 7.9982150 .1243225 .107792 C $5.297491-0.5848933 .442159$ H 5.9559213 .7205261 .213267 H $5.3809404 .058505-1.566516$ H $5.7910350 .980034-2.887526$ H $5.666728-1.0308184 .372766$ H $5.495283-1.3056592 .638901$ H 5.7892251 .4822423 .909189 C $3.220984-2.615854-3.268227$ O $3.584124-1.246092-3.055901$ C $2.753747-0.517298-2.148343$

C $1.302959-0.489738-2.652691$

C $0.782163-1.906766-2.912539$ C $1.765541-2.731192-3.751216$ H $3.871999-2.952319-4.085623$ C $3.527657-3.480255-2.039624$ H $2.780814-0.998996-1.164467$ O $1.2295820 .278647-3.851247$ H $\quad 0.6772460 .007422-1.901940$ H -0.182966 -1.844976 -3.429702 O $0.562252-2.546305-1.659267$ H $\quad 1.470917-3.787061-3.765767$ O $1.725379-2.268002-5.104054$ C $5.013971-3.509484-1.770306$ H $3.012820-3.136534-1.137971$ H $3.208813-4.516582-2.200929$ H $\quad 0.296891 \quad 0.308380-4.123965$ H $\quad 0.199655-3.430201-1.839873$ H $\quad 0.815310-2.382743-5.426509$ O $5.301685-2.792700-0.646542$ O $5.823494-4.085950-2.485509$ C $6.686841-2.735482-0.293026$ H $6.771851-2.2275120 .670682$ H $7.100407-3.743585-0.192108$ H $7.240491-2.163123-1.043021$ SCF Energy (B3LYP/6-31G**//MMFF) $=-3245.90063576$

0700149

MM̄MF Geometry

C -1.755466 -4.066234 -2.098129

C $-1.002641-3.785361-1.025015$

C $0.475453-3.494138-1.118367$

O $0.713357-2.167912-0.588327$

C $1.259882-4.505236-0.261838$

C $2.727577-4.605101-0.630174$

C $3.749231-4.1038750 .091645$

C $3.016967-5.375959-1.894854$

C $3.692315-3.3247821 .376162$

C $3.830940-1.8182061 .149670$
C $5.166049-1.3593450 .544504$

C $6.317895-1.4409621 .548699$

C $7.617011-0.8384400 .987161$

$\begin{array}{llll}\text { O } & 4.975506 & 0.000993 & 0.135909\end{array}$

O $8.522805-0.6413422 .083877$

C $7.3161590 .556526 \quad 0.395583$

C $8.314552-1.8010050 .018226$

O $8.4597481 .084804-0.272060$

C $6.0965310 .570878-0.552172$

C $5.7251952 .022856-0.934872$

O $6.422157-0.155216-1.739460$

C $0.373941-1.109953-1.376777$

O $-0.051595-1.146119-2.520439$

C $0.5512190 .120748-0.577757$

C $-0.2530551 .167086-0.816231$

C $-0.2786542 .421062-0.060350$

C 0.8807522 .7065340 .856609

C $4.3892492 .139406-1.683845$

C $4.0369253 .577575-2.017847$

C $2.1976594 .930687-3.020956$

O $4.7934524 .522500-1.812240$

C $1.4175925 .524616-1.891150$

C $0.1131225 .827612-1.969717$

C $-0.6573116 .481382-0.860024$

C $-1.8682395 .682976-0.437630$

C $-3.1051636 .044433-0.818637$

C -1.652859 4.5210420 .504789

C $-1.3410423 .238003-0.215304$

N $2.7768843 .671984-2.594653$

H -1.285439 -4.103820 -3.078642

H $-1.463051-3.738629-0.041446$

H $0.818242-3.527914-2.159571$

H $\quad 0.828249-5.509710-0.371773$

H $1.143159-4.2484920 .794666$

H $4.760287-4.266431-0.280605$

H $4.091050-5.481297-2.082337$

H $2.596623-6.384888-1.828528$

H $2.581560-4.874069-2.763970$

H $2.757733-3.5127721 .912051$

H $4.485419-3.6862352 .040494$

H $3.019987-1.4813850 .495002$

H $3.664385-1.2948082 .100300$

H $5.383479-1.956732-0.346564$

H $6.488834-2.4745651 .871683$

H $6.047111-0.8845872 .456952$

H $8.664194-1.5019932 .514499$

H $7.108157 \quad 1.2324491 .236941$

H $8.575314-2.7359140 .529029$

H $9.261414-1.383275-0.341347$

H $7.699620-2.060269-0.846126$

H $9.197760 \quad 1.0689650 .361216$

H $5.6489712 .629402-0.022789$

H $6.5193372 .459895-1.553259$

H $5.8657510 .154890-2.470068$

H 1.2790410 .1079390 .223980

H -1.003081 $1.077782-1.601681$

H 0.9093821 .9891691 .683273

H 0.8528403 .7059541 .295252

H $1.8264462 .641587 \quad 0.309058$

H $4.4232831 .584538-2.627236$

H $3.579711 \quad 1.724455-1.073807$

H $1.5709384 .722288-3.893721$

H $2.9959695 .617595-3.320395$

H $1.9611385 .744932-0.975240$

H $-0.4247795 .629592-2.894132$

H $-0.9658477 .474630-1.211661$

H -0.0215806 .6647850 .015223$

H -3.980189 $5.491575-0.492544$

H $-3.2698836 .896402-1.469974$

H $-2.569314 \quad 4.3479801 .084808$

H $-0.8999174 .792391 \quad 1.249313$

H -2.126988 $2.929281-0.905038$

H $2.1956382 .840689-2.654328$

C $-3.990490-3.193949-2.761005$

O $-4.137960-2.079618-1.853120$

C $-5.439814-1.492432-2.056791$

C $-6.031707-2.179245-3.282373$

C $-5.411589-3.558496-3.181564$ 
H $-6.049827-1.759038-1.186903$

C $-5.3241540 .029562-2.189032$ H -7.125653 -2.195928 -3.260121 H -5.721841 -1.684508 -4.210797 C $-5.495437-4.351919-4.474044$ H $-5.911275-4.116338-2.378340$ C $-4.8243990 .744253-0.915147$ O $-4.414510 \quad 0.334864-3.254184$ H -6.290481 $0.450224-2.490180$ H $-3.7949530 .442983-0.691953$ O $-4.7892872 .150029-1.194019$ C -5.7245940 .5269010 .316554$ C $-5.375506 \quad 1.399181 \quad 1.536367$ H -5.699849 -0.5262140 .613283$ O $\quad-7.081900 \quad 0.816407-0.036336$ H -5.3983402 .4608001 .260555$ H $-6.186216 \quad 1.2667582 .265993$ H -6.540382 -4.508180 -4.761616 H -4.994605 -3.831981 -5.297631 H $-5.026845-5.334594-4.362317$ C $-3.234917-4.325531-2.063544$ H -3.558477 -0.070964 -3.033164 H $-4.2409832 .274343-1.987655$ H -7.117376 $1.744286-0.326373$ H -3.411689 -5.279718 -2.571874 H $-3.581810-4.432590-1.028674$ H -3.426929 -2.824849 -3.629063 C $-2.936634-0.8196793 .277992$ O $-4.121972-0.2988132 .663626$ C -4.0325461 .0513422 .196731$ C $-3.7179941 .999743 \quad 3.365539$ C -2.4559231 .5618124 .114871$ C -2.4790320 .0680874 .450768$ H $-3.252452-1.7844743 .693731$ C -1.860034 -1.098614 2.221304 H -3.2386351 .1228981 .446574$ O H -3.593094 3.0152832 .972178 H -2.3652752 .1342105 .046414$ O $-1.307056 \quad 1.8417853 .324158$ H $-1.492250-0.2501464 .805403$ O $-3.389763-0.1176855 .539460$ C $-0.723294-1.9331892 .760004$ H -2.292969 -1.620667 1.359330 H $-1.441160-0.1731911 .817838$ H -4.6028962 .6520574 .981766$ H -1.295277 2.7983073 .149547 H $-3.348422-1.0533425 .800992$ O $-1.179357-3.1582863 .146601$ O $0.434312-1.5401402 .827958$ C $-0.212482-4.0134103 .763242$ H $0.174268-3.5491474 .675469$ H $-0.708791-4.9502744 .030205$ H $0.602034-4.2340353 .069381$ SCF Energy (B3LYP/6-31G**//MMFF)= -3245.90649496

0700150

MM̄FF Geometry

C -2.066875 4.5616410 .561653

C $-1.1258893 .951847-0.174406$

C 0.3066273 .8008870 .270190

O $\quad 0.6875812 .410405 \quad 0.149105$

C $1.2334054 .633470-0.633705$

C $2.7090674 .498858-0.303203$

C $3.6597714 .191662-1.207687$

C 3.1000984 .7578021 .128960

C $3.4856813 .991693-2.686101$

C $3.3356992 .528490-3.125153$

C $4.5787361 .671591-2.838206$

C $4.5791660 .412728-3.708661$

C $5.781268-0.491142-3.398761$

O $4.5601961 .295293-1.456138$

O $5.547778-1.764468-4.019300$

C $5.839483-0.732517-1.877896$

C $7.0816630 .053633-4.000914$

O $7.023672-1.440504-1.517046$

C $5.7162770 .559715-1.034358$

C 5.5425760 .1801540 .454121
O $6.9094071 .333565-1.175513$

C 0.3078801 .5796911 .157826

O -0.3696891 .8699832 .131458$

C 0.8745910 .2407460 .880235

C $0.540819-0.780487 \quad 1.682874$

C $1.023387-2.1598171 .595812$

C $2.016698-2.4914460 .515036$

C $5.080310 \quad 1.328140 \quad 1.359354$

C 5.0993020 .8942222 .812952

C $3.985909-0.6725794 .408255$

O $5.9304051 .332878 \quad 3.604658$

C $3.857530-2.1504184 .205476$

C $2.892135-2.8998484 .759609$

C $2.762110-4.3818604 .542940$

C $1.373963-4.7954384 .100207$

C $0.557145-5.4683504 .928336$

C $0.958221-4.4934172 .673848$

C $0.562551-3.0527262 .496643$

N $4.121630-0.0421983 .107714$

H $-1.7958534 .994576 \quad 1.521515$

H -1.393766 $3.521580-1.136462$

H $0.4261224 .122321 \quad 1.312739$

H $0.9619065 .694583-0.558137$

H $1.0483104 .337677-1.672044$

H $4.6900044 .109074-0.863317$

H $4.1865384 .809793 \quad 1.259595$

H 2.6857415 .7128091 .468189

H 2.7303553 .9616311 .782164

H $2.6287174 .563861-3.057771$

H $4.3553004 .432458-3.190734$

H $2.4560542 .091403-2.635576$

H $3.1401772 .535568-4.204666$

H $5.4732032 .271321-3.043453$

H $4.5653160 .674271-4.774246$

H $3.656993-0.157108-3.526662$

H $5.439258-1.616663-4.974459$

H $4.996710-1.390846-1.623345$

H $6.9888590 .149265-5.089387$

H $7.914867-0.637484-3.831541$

H $7.360528 \quad 1.032767-3.606231$

H $7.053817-2.248873-2.057029$

H $4.799025-0.6207730 .531501$

H $6.485424-0.2242930 .844792$

H $7.0241711 .884482-0.385068$

H 1.5561580 .1378850 .043739

H $-0.153127-0.5877962 .500781$

H $1.641026-2.187158-0.468020$

H $2.233839-3.5584810 .435575$

H $2.965073-1.9800780 .699901$

H 5.7300442 .2036311 .259613

H 4.0639021 .6468441 .102215

H $3.101593-0.2416424 .888055$

H $4.863034-0.4537745 .024737$

H $4.600675-2.6289813 .571424$

H $2.162868-2.4281805 .414430$

H $3.020264-4.8780485 .487567$

H $3.491374-4.7417463 .806391$

H $-0.430035-5.7885964 .611541$

H $\quad 0.854379-5.7100725 .943445$

H $0.093624-5.1093312 .394049$

H $1.768812-4.8014352 .008315$

H -0.183543 -2.716380 3.218702

H $3.417121-0.2513262 .406877$

C -4.4316324 .0172771 .166709$

O -4.2008612 .5941901 .174923$

C -5.4695351 .9167071 .230311$

C -6.5075942 .9857081 .548160$

C $-5.9156254 .192474 \quad 0.846841$

H -5.6633561 .5315070 .223031$

C $-5.4120180 .755012 \quad 2.227244$

H $-7.5033002 .719277 \quad 1.180929$

H $-6.583798 \quad 3.1725702 .625625$

C -6.4995365 .5140631 .314709$

H $-6.0823114 .087537-0.233754$

C $-4.267647-0.248327 \quad 1.976794$

O

H -6.3742220 .2300942 .234975$

H $\quad-3.302014 \quad 0.2073852 .228365$ 
O $-4.442413-1.3438872 .884608$ C $-4.207382-0.808497 \quad 0.544521$ C $-3.140651-1.909827 \quad 0.392135$ H $-3.9751670 .005606-0.150142$ O $-5.486591-1.3235380 .180260$ H -2.206589-1.566678 0.848606 H $-3.476841-2.8070750 .926504$ H -7.5762725 .5487801 .119715$ H -6.3477315 .6620032 .389201$ H $-6.034328 \quad 6.3542360 .789921$ C $-3.506535 \quad 4.6831490 .147017$ H -4.4229181 .8042363 .555180$ H $-4.468775-0.9732733 .783550$ H $-5.705233-2.0369870 .804068$ H $-3.748077 \quad 5.7481100 .057157$ H -3.653582 $4.226658-0.839706$ H -4.2118954 .3810552 .179342$ C $-3.998554-3.236186-2.999670$ O $-4.049101-2.893070-1.609478$ C $-2.872649-2.276145-1.080890$ C $-1.667934-3.219022-1.223983$ C $-1.488398-3.679500-2.673647$ C $-2.806840-4.164742-3.288112$ H $-4.919387-3.805787-3.180135$ C $-4.034338-1.992815-3.895340$ H $-2.671799-1.347684-1.626745$ O $-1.848087-4.354853-0.380660$ H $-0.769691-2.692674-0.884335$ H $-0.750990-4.490965-2.704350$ O $-0.967689-2.591169-3.431197$ H $-2.696721-4.290565-4.370979$ O $-3.108687-5.459624-2.758757$ C $-5.314954-1.219077-3.683740$ H $-3.186065-1.324396-3.723261$ H $-3.995247-2.272959-4.954418$ H $-1.043978-4.897818-0.445156$ H $-0.832444-2.905429-4.341423$ H $-3.279260-5.358818-1.806348$ O $-5.048659-0.037474-3.057179$ O $-6.420074-1.624678-4.020054$ C $-6.1854910 .788326-2.787258$ H $-6.8490250 .292402-2.072682$ H $-5.829213 \quad 1.723177-2.346322$ H $-6.7186201 .020093-3.714259$ SCF Energy (B3LYP/6-31G**//MMFF) $=-3245.91382168$

07_00151

MM̄FF Geometry

C $1.408016-2.9322761 .418096$

C $1.430626-2.2762580 .247930$

C $0.193055-1.868772-0.501524$

O $0.185357-0.424260-0.618989$

C $0.172468-2.412959-1.939778$

C $0.002501-3.911099-2.050354$

C -1.188701-4.538594-1.997683

C $1.266014-4.694557-2.290696$

C $-2.536678-3.909895-1.766765$

C $-3.281790-4.622097-0.629580$

C $-4.569891-3.921952-0.167103$

C $-5.572246-3.686330-1.298903$

C $-6.824882-2.943677-0.807547$

O $-4.206975-2.678887 \quad 0.444913$

O $-7.546128-2.484295-1.961803$

C $-6.381642-1.687878-0.025054$

C $-7.778913-3.868950-0.043289$

O $-7.502929-1.0437640 .574611$

C $-5.307038-1.9761991 .045079$

C $-4.741757-0.6916251 .699195$

O $-5.895707-2.7512992 .098191$

C $-0.279340 \quad 0.2919420 .439648$

O $-0.657660-0.1425541 .515276$

C $-0.258991 \quad 1.7181810 .046398$

C -0.7857772 .6472030 .857382$

C $-0.839500 \quad 4.088336 \quad 0.598990$

C $-0.1413194 .601169-0.634134$

C -4.0296120 .2782020 .752169$

C $-4.915597 \quad 1.376626 \quad 0.190427$

C $-4.8907163 .193729-1.518972$ $\begin{array}{llll}\text { O } & -6.035133 & 1.628216 & 0.626711\end{array}$

C $-4.0516124 .409972-1.288370$

C $-4.4035805 .411218-0.468017$

C $-3.5911106 .658355-0.265170$

C $-3.0857396 .802614 \quad 1.152871$

C $-3.845698 \quad 7.3733072 .102908$

C $-1.665505 \quad 6.370942 \quad 1.442086$

C $-1.505425 \quad 4.875598 \quad 1.470193$

N $-4.2826592 .070214-0.832576$

H $\quad 0.455765-3.203823 \quad 1.866239$

H $2.386298-1.992874-0.186358$

H $-0.717758-2.186827 \quad 0.021492$

H $1.082438-2.100968-2.471001$

H $-0.629154-1.911559-2.499566$

H $-1.206610-5.619298-2.132275$

H $1.073595-5.765495-2.415321$

H $1.764540-4.342259-3.199631$

H $1.956193-4.581535-1.449829$

H $-2.453836-2.842878-1.542370$

H $-3.109737-3.999797-2.696559$

H -3.523902 $-5.645622-0.943083$

H $-2.609205-4.7012430 .235456$

H $-5.016660-4.5666600 .599240$

H $-5.846886-4.633580-1.778987$

H -5.112638 -3.071429-2.083925

H $-7.766364-3.260115-2.505628$

H $-5.970740-0.985084-0.760492$

H $-8.110779-4.692053-0.687585$

H $-8.689247-3.3377180 .255750$

H $-7.328675-4.3110540 .847698$

H $-8.144469-0.860460-0.132908$

H $-5.524371-0.1872212 .278905$

H -3.993219 -1.009556 2.439580

H $-5.354697-2.6471082 .898357$

H $0.1816371 .964055-0.912098$

H $-1.2277012 .324842 \quad 1.799265$

H $-0.5448454 .124463-1.534399$

H $0.9331204 .394485-0.579521$

H $-0.2510925 .676770-0.784541$

H -3.2526080 .7964941 .326008$

H $-3.535292-0.256564-0.065839$

H $-5.9216893 .336321-1.179662$

H -4.915304 $2.956421-2.587153$

H -3.120133 4.476395 -1.844505

H $-5.3430545 .343756 \quad 0.076377$

H $-4.2244147 .518408-0.519806$

H $-2.7488656 .703941-0.966565$

H -3.4797187 .5092443 .115127$

H -4.8536917 .7131561 .888675$

H -1.3485516 .7478192 .424026$

H $-0.992117 \quad 6.8576190 .730992$

H -1.9863354 .4064512 .329790$

H $-3.3533151 .782441-1.125774$

C $2.840274-2.424873 \quad 3.415538$

O $3.009752-1.0542472 .994905$

C $4.333676-0.6305453 .349152$

C $4.665384-1.4424744 .590089$

C $4.106559-2.7992314 .199234$

H $4.997965-0.9356012 .530247$

C 4.3949680 .8914613 .508011

H $5.737910-1.4658394 .803492$

H $4.143027-1.0624335 .475487$

C $3.838425-3.7125815 .383366$

H $4.828691-3.2947313 .536129$

C 3.7834271 .6869652 .334106

O 3.6941541 .2798544 .696076

H 5.4364401 .1940263 .666835

H 2.6890161 .6524712 .399414

$\begin{array}{lllll}\text { O } & 4.138055 & 3.064537 & 2.509031\end{array}$

$\begin{array}{llll}\text { C } & 4.212810 & 1.225209 & 0.930536\end{array}$

C $3.6830182 .157647-0.176063$

H 3.8447230 .2075370 .761606

$\begin{array}{lllll}\text { O } & 5.638134 & 1.185462 & 0.873952\end{array}$

H 2.6789902 .5096860 .084091

H $4.3452983 .029132-0.250399$

H $4.765741-3.9208435 .926981$

H $3.130480-3.2607766 .086325$

H $3.419152-4.6669095 .048620$ 
C $2.649578-3.3050782 .176149$

H 2.7774830 .9654104 .612508

H 5.1009083 .1359782 .390898

H $5.8872650 .858282-0.007211$

H $2.580980-4.3597482 .466292$

H $3.521728-3.2074351 .518448$

H $1.948563-2.4551814 .053255$

C $5.0255360 .276594-3.121552$

O $4.9289511 .040062-1.911890$

C $3.6096031 .448231-1.542464$

C $3.0071562 .346670-2.632106$

C $3.0305171 .644393-3.993461$

C $4.4076841 .044991-4.302895$

H $6.1028790 .184216-3.311188$

C $4.455910-1.140658-2.951298$

H $2.9782790 .561914-1.416005$

O $3.7452023 .564315-2.706493$

H $1.9773852 .600712-2.358362$

H $2.7620952 .365511-4.774927$

O $2.0418230 .618195-3.988290$

H $4.3456530 .384838-5.175195$

O $5.2983972 .105809-4.662935$

C $5.237578-1.956728-1.942365$

H $3.415529-1.122865-2.618363$

H $4.509033-1.669858-3.909828$

H $3.319648 \quad 4.124788-3.377564$

H $2.0509210 .197398-4.864880$

H $5.4246502 .667087-3.878730$

O $4.663797-3.192165-1.836322$

O $6.223822-1.570654-1.330610$

C $5.300509-4.093292-0.924784$

H $4.649633-4.962034-0.795427$

H $\quad 6.255677-4.425702-1.341386$

H $5.450287-3.6213090 .050846$

SCF Energy (B3LYP/6-31G**//MMFF) $=-3245.91145935$

$07 \_00152$

MM̄FF Geometry

C -0.080084 -0.710147 3.148863

C $-1.086675-0.0234583 .709024$

C $-1.572432 \quad 1.2956713 .177944$

O -2.9661841 .1079412 .835629$

C -1.4768622 .4323204 .211401$

C -0.0866912 .7285294 .733693$

C 0.8610783 .3913324 .041991

C $0.1837022 .271146 \quad 6.142851$

C 0.7563243 .9008932 .632317

C 1.9066173 .3668561 .764694

C 1.5978223 .3276270 .260055

C $1.0889544 .658257-0.293555$

C $0.7054594 .546865-1.776216$

$\begin{array}{lllll}\text { O } & 0.625658 & 2.299208 & 0.056849\end{array}$

O $-0.0499825 .717605-2.124977$

C $-0.2313523 .331565-1.969618$

C $1.9422584 .543760-2.684178$

O $-0.4817593 .132673-3.358683$

C $0.3014852 .035672-1.318707$

C $-0.7441480 .897024-1.266538$

O $1.4451631 .575525-2.038047$

C -3.4642891 .8348711 .801055$

O -2.8846292 .6803151 .139158$

C -4.8567191 .3902721 .586155$

C $-5.380538 \quad 1.4541950 .353459$

C $-6.7212221 .010541-0.027632$

C $-7.7060780 .724224 \quad 1.075282$

C $-1.2769060 .409363-2.612613$

C -1.937538 -0.955167 -2.531663

C $-3.771557-2.219809-1.420507$

O $-1.603059-1.869535-3.283134$

C -5.024065 -2.053644 -2.223391

C $-6.256026-2.236868-1.725071$

C -7.514156 -2.036075 -2.522818

C $-8.501344-1.118132-1.832490$

C $-9.570947-1.623202-1.194377$

C $-8.3010200 .379703-1.942903$

C $-7.0117310 .867780-1.337530$

N -2.950903 -1.033650 -1.590394

H $0.421703-0.3018622 .274695$
H -1.588514 -0.4247414 .586027$

H -1.0121111 .5513352 .271603$

H -2.1488672 .2134785 .053539$

H -1.901293 3.3492063 .780262

H 1.8166213 .5838094 .527820

H 1.1995532 .5142506 .472107

H -0.5130732 .7520496 .837302$

H $0.062425 \quad 1.1865386 .224260$

H -0.2080473 .6425212 .188709$

H 0.8019554 .9963722 .656696

H $2.7971503 .984587 \quad 1.936873$

H 2.1585412 .3457562 .082204

H $2.5221633 .034497-0.249856$

H $1.8322655 .450595-0.142304$

H $\quad 0.1933224 .979300 \quad 0.253912$

H $0.5022506 .496571-1.940039$

H -1.198140 $3.575312-1.507569$

H $2.5234795 .462700-2.541829$

H $1.6568314 .532393-3.741780$

H $2.6100353 .700317-2.497880$

H $-0.8348843 .967003-3.712444$

H $-0.2972060 .044422-0.735300$

H -1.582045 $1.213118-0.634520$

H $1.5898680 .643510-1.809403$

H -5.3918890 .9809292 .435324$

H $-4.7596061 .829235-0.459302$

H -7.412470 -0.1733861 .630251$

H -8.7236280 .5651690 .710495$

H -7.7611971 .5673611 .773192$

H -2.014608 $1.110706-3.017626$

H $-0.4590690 .327828-3.337672$

H -3.975165 -2.323866 -0.350158

H $-3.221587-3.101641-1.763777$

H $-4.912283-1.763255-3.265634$

H $-6.369547-2.549463-0.689461$

H $-7.967134-3.023198-2.682707$

H -7.296980 -1.642132 -3.523563

H -10.303291 -0.979082 -0.719202

H -9.737713 -2.693374 -1.129983

H $-9.142700 \quad 0.930908-1.505438$

H $-8.3088580 .652186-3.006424$

H -6.238864 $1.093744-2.072778$

H -3.235594 -0.193634 -1.096270

C $0.356328-3.1680822 .664671$

O $1.143977-2.8795641 .492739$

C $0.499661-3.4862640 .353626$

C $-0.655409-4.3192730 .904384$

C $-1.031674-3.5315772 .145171$

H $0.076794-2.670775-0.243452$

C $1.517369-4.284597-0.470678$

H - $-1.477118-4.4110790 .187239$

H $-0.330991-5.3311601 .174846$

C $-1.881633-4.3127693 .130423$

H -1.577805 -2.632998 1.830495

C $2.662002-3.436239-1.070603$

O $2.103842 \quad-5.2933510 .361332$

H $0.997545-4.822704-1.271851$

H $3.304903-3.060903-0.267319$

O $3.483153-4.306029-1.859513$

C $2.179985-2.269877-1.949814$

C $3.273610-1.557892-2.762813$

H $1.661837-1.538168-1.322425$

O $1.222249-2.777030-2.884471$

H $3.683161-2.239518-3.519515$

H $2.770292-0.767909-3.335702$

H $-2.826517-4.6152122 .667250$

H -1.370115 -5.218459 3.472943

H -2.116621 -3.704721 4.009621

C $0.432591-2.0197353 .672262$

H $2.537313-4.8426531 .106264$

H $2.942079-4.627707-2.601070$

H $0.852888-2.019038-3.369010$

H $1.481243-1.8627153 .949946$

H $-0.096933-2.3047004 .589046$

H $0.818786-4.0494983 .130074$

C $4.8025630 .495554-0.027900$

O $3.859326-0.128766-0.911424$

C $4.419667-0.949748-1.939483$ 
C $5.370115-0.127314-2.828998$

C $6.433150 \quad 0.602355-2.002911$

C $5.8143091 .346286-0.814826$

H 4.2022161 .1779340 .587103

C $5.478238-0.5178170 .909439$

H $4.976093-1.772084-1.478720$

O $4.6379600 .833995-3.589743$

H $5.852863-0.800395-3.547030$

H $6.9605201 .321321-2.641612$

O $7.392352-0.331525-1.517846$

H $6.5971161 .702585-0.135018$

O $5.1206432 .500728-1.296037$

C $4.506134-1.1477631 .882292$

H $5.950043-1.3297510 .350340$

H $6.245148-0.0116571 .507006$

H $4.128893 \quad 1.381237-2.967073$

H $7.791580-0.770239-2.288371$

H $5.7689673 .063388-1.752608$

O $5.139649-2.1692742 .532289$

O $3.352560-0.7857852 .063245$

C $4.357582-2.8694413 .503831$

H $5.002243-3.6043263 .993158$

H $3.979554-2.1770254 .261783$

H $3.535740-3.3972693 .012852$

SCF Energy (B3LYP/6-31G**//MMFF)= -3245.91797617

07_00153

MM̄FF Geometry

C -1.034559 4.645735 -1.768379

C $-1.5202343 .882882-0.777928$

C $-2.9078114 .024237-0.203804$

O $-3.5591312 .734156-0.285832$

C -2.8136794 .4347671 .275193$

C $-4.145944 \quad 4.404380 \quad 1.997339$

C -4.5225953 .4239352 .843100$

C -5.0449035 .5846531 .744056$

C $-3.745246 \quad 2.1787323 .176010$

C $-4.637765 \quad 0.9289903 .199314$

C $-3.854312-0.3965363 .186399$

C $-2.822232-0.4971164 .313969$

C $-1.990225-1.7856354 .224477$

O $-3.217997-0.5091431 .908156$

O $-0.859244-1.6442385 .098788$

C -1.434065 -1.915594 2.790105

C $-2.770380-3.0071984 .723094$

O $-0.769216-3.1648082 .614273$

C -2.511057-1.738706 1.699988

C $-1.904699-1.6955790 .280691$

O $-3.402524-2.8601961 .743543$

C $-4.064730 \quad 2.373053-1.495728$

O $-4.052302 \quad 3.024947-2.527947$

C $-4.6069221 .005429-1.363606$

C -4.545160 $0.192759-2.428516$

C $-4.977902-1.202986-2.480094$

C $-5.820411-1.716417-1.342420$

C $-0.988282-0.502724-0.007539$

C $-0.756380-0.367344-1.499670$

C $0.202167-1.577301-3.450909$

O $-1.2474220 .553988-2.147724$

C $-0.818855-2.518792-4.026513$

C $-1.519351-3.451044-3.359478$

C $-2.522415-4.370236-3.999135$

C $-3.862952-4.347812-3.296034$

C $-4.128584-5.204488-2.295228$

C $-4.922538-3.396162-3.804056$

C $-4.596805-1.954830-3.533966$

N $0.020015-1.387229-2.024332$

H - $1.6718535 .431689-2.169854$

H $-0.9132583 .087830-0.353973$

H -3.502383 $4.766310-0.751817$

H $-2.393140 \quad 5.4471151 .348950$

H -2.089072 3.7973591 .795528

H -5.4834493 .5175293 .346788$

H $-5.976300 \quad 5.5280232 .317430$

H -4.5399706 .5144272 .025394$

H -5.3133795 .6406650 .684474$

H -2.931705 2.0160462 .464132

H -3.2931832 .3203914 .164447$
H $-5.2813150 .962323 \quad 4.087458$

H -5.2967590 .9451702 .320959$

H $-4.590442-1.2043113 .276646$

H $-3.309920-0.4170325 .293334$

H $-2.1212490 .345747 \quad 4.257840$

H $-1.193855-1.4754225 .996278$

H $-0.670596-1.1365612 .666951$

H -3.054911 -2.875944 5.774077

H $-2.152198-3.9111814 .691408$

H $-3.685343-3.1938914 .157396$

H $-0.132497-3.259873 \quad 3.342514$

H $-1.380854-2.637300 \quad 0.074301$

H -2.728468 $-1.668608-0.444156$

H $-3.813009-2.9681970 .870117$

H $-4.9844140 .697179-0.396348$

H $-4.0970780 .574497-3.345783$

H $-6.744131-1.133114-1.258405$

H $-5.278664-1.647611-0.393776$

H $-6.113829-2.762210-1.457927$

H -1.4359420 .4336530 .343057$

H $-0.021578-0.606727 \quad 0.494834$

H $1.211234-1.966154-3.617139$

H $0.117191-0.606361-3.949768$

H $-0.985115-2.416692-5.097079$

H $-1.347201-3.596060-2.296243$

H $-2.109900-5.387484-3.969751$

H $-2.659421-4.139064-5.062610$

H $-5.094932-5.217705-1.802274$

H $-3.382030-5.908942-1.943313$

H $-5.908529-3.652006-3.399606$

H $-5.030616-3.529458-4.888462$

H $-3.974965-1.495888-4.303584$

H $0.355377-2.120284-1.406097$

C $1.2133773 .369668-1.990526$

O $1.7014543 .604652-0.654464$

C $3.1027263 .263281-0.599535$

C $3.3987752 .517726-1.890620$

C $2.4594193 .203874-2.860113$

H $3.640024 \quad 4.219815-0.579025$

C 3.3819202 .4626290 .679049

H $4.4484992 .589911-2.188662$

H $3.1392011 .456191-1.793698$

C $2.2263672 .408669-4.133417$

H $2.8733454 .188015-3.117783$

C $4.8186431 .918322 \quad 0.770629$

O $3.1260443 .317773 \quad 1.798433$

H $2.658396 \quad 1.6438380 .752119$

H $5.0055701 .210545-0.041550$

$\begin{array}{lllll}\text { O } & 5.720708 & 3.013359 & 0.553236\end{array}$

C $5.1817141 .279223 \quad 2.121506$

$\begin{array}{llll}\text { C } & 4.267340 & 0.140939 & 2.609341\end{array}$

H 5.2056642 .0635612 .888232

$\begin{array}{lllll} & \mathrm{H} & 6.524180 & 0.789203 & 2.021471\end{array}$

H $4.593280-0.1189393 .625347$

H 3.2397420 .5129182 .688292

H $3.1673912 .265889-4.674614$

H $1.810567 \quad 1.418812-3.917186$

H $1.5300212 .928666-4.798397$

C $0.3161014 .531781-2.430196$

H 3.7775514 .0394161 .771581

H 6.6242812 .6556280 .597105

H 6.7732050 .4384312 .893653

H $0.133804 \quad 4.453734-3.508616$

H $0.8415505 .479490-2.256882$

H $0.6497282 .430187-1.969257$

C $3.623677-1.997306-0.406008$

O $3.642777-0.904087 \quad 0.522129$

C $4.308254-1.1501801 .763231$

C $3.635762-2.3193612 .503333$

C $3.558966-3.5702251 .623552$

C $3.004467-3.2536910 .230722$

H $2.955460-1.667331-1.210999$

C $5.008132-2.256413-1.015475$

H $5.354555-1.4061631 .569176$

O $2.321190-1.9347002 .900804$

H $4.207215-2.5355953 .414308$

H $2.917305-4.3140832 .111543$

O $4.865439-4.1269671 .513638$ 
H $3.149068-4.109439-0.438115$

O $1.591831-3.059287 \quad 0.332815$

C $5.462875-1.089922-1.863387$

H $5.761995-2.449525-0.247412$

H $4.987841-3.135207-1.670428$

H $1.957346-2.6585133 .438103$

H $4.796009-4.9343340 .976325$

H $1.438933-2.3011990 .922130$

O $6.702589-0.683005-1.466210$

O $4.804968-0.606721-2.775849$

C $7.2589070 .411589-2.200954$

H $7.3748440 .140443-3.254668$

H $6.6277761 .298651-2.097246$

H $8.2450500 .633589-1.784632$

SCF Energy (B3LYP/6-31G**//MMFF) $=-3245.91182747$

0700154

MM̄FF Geometry

C 2.8065313 .5461310 .466419

C 2.1156702 .3978970 .504855

C 1.2248281 .9835151 .645168

O $\begin{array}{lllll}-0.069690 & 1.645301 & 1.092817\end{array}$

C $1.805526 \quad 0.7315702 .324267$

C $1.068194 \quad 0.3447353 .590417$

C $0.184740-0.6678443 .694135$

C 1.4126301 .1678624 .806102

C $-0.292936-1.6036352 .617270$

C $-1.786149-1.4055022 .355952$

C $-2.396096-2.4096141 .366809$

C $-1.758447-2.354594-0.022344$

C $-2.482647-3.266178-1.025950$

O $-3.784975-2.071171 \quad 1.272921$

O $-2.035617-2.915424-2.344422$

C $-3.995680-2.974482-0.970278$

C $-2.118266-4.741518-0.823479$

O $-4.724202-3.898225-1.776150$

C $-4.568927-2.957325 \quad 0.463910$

C $-6.013087-2.4043820 .446092$

$\begin{array}{lllll}\text { O } & -4.577474 & -4.284809 & 0.989032\end{array}$

C $-0.9230642 .674833 \quad 0.834242$

O $\begin{array}{llll}-0.744200 & 3.857313 & 1.078900\end{array}$

C -2.113395 $2.112843 \quad 0.157066$

C $-3.0292162 .936658-0.374181$

C $-4.2291862 .532725-1.112945$

C $-4.5718721 .067622-1.134453$

C $-6.630597-2.2449621 .841895$

C $-7.711614-1.1841401 .850354$

C -8.0289231 .2896871 .827361$

O $-8.904776-1.4759031 .871637$

C $-8.303194 \quad 1.701750 \quad 0.415520$

C $-8.0998292 .944197-0.049164$

C -8.459932 $3.371291-1.442547$

C $-7.3486024 .115732-2.144233$

C $-7.4584675 .428615-2.411463$

C $-6.1567393 .320505-2.628099$

C $-4.9487913 .485605-1.741489$

$\begin{array}{llll}\mathrm{N} & -7.194993 & 0.103520 & 1.828747\end{array}$

H $2.7421604 .239354 \quad 1.300944$

H $2.1860451 .711232-0.333557$

H 1.1145012 .7927112 .377288

H 2.8599760 .9007702 .582855

H $1.813958-0.1013171 .615152$

H $-0.262090-0.8545264 .670433$

H $0.8961180 .817838 \quad 5.706313$

H $2.488138 \quad 1.1186145 .005259$

H 1.1340232 .2151594 .655033

H $\quad 0.265378-1.4676341 .690223$

H $-0.107637-2.6324132 .948317$

H -2.341188 -1.4795573 .301715$

H $-1.971721-0.3843842 .001197$

H -2.301570 -3.4137601 .796494$

H $-0.696843-2.6188180 .017054$

H $-1.797654-1.324253-0.400370$

H $-1.066860-3.000422-2.365179$

H $-4.153746-1.984333-1.418478$

H - $-1.041053-4.892038-0.959093$

H -2.603705 $-5.375461-1.573536$

H -2.381836 -5.1155670 .168026$
H $-4.340285-3.868821-2.669287$

H $-6.012472-1.432877-0.062326$

H $-6.664531-3.066823-0.137929$

H $-5.231179-4.3372651 .704155$

H -2.187347 1.0352470 .081081

H $-2.8739224 .011368-0.287835$

H $-3.8298400 .510095-1.716032$

H $-5.5521700 .855014-1.565546$

H $-4.5946340 .663328-0.116324$

H -7.071377 -3.1909092 .176729$

H $-5.883021-1.9648232 .592806$

H -7.4973432 .0636812 .389755$

H $-8.972597 \quad 1.0700802 .337104$

H $-8.7309830 .951504-0.245542$

H $-7.706722 \quad 3.706156 \quad 0.619822$

H $-9.3515734 .007973-1.370320$

H $-8.7565862 .512712-2.058102$

H $-6.6797225 .961029-2.947684$

H $-8.3256686 .000374-2.098314$

H $-5.877558 \quad 3.673967-3.630338$

H $-6.4436222 .275754-2.773979$

H $-4.6035474 .518238-1.667929$

H $-6.190533 \quad 0.233415 \quad 1.751041$

C $5.1634123 .749983-0.471705$

O $5.4576772 .348802-0.309549$

$\begin{array}{llll}\text { C } 6.534084 & 2.216048 & 0.640608\end{array}$

C 7.0455143 .6291740 .911806

$\begin{array}{llll}\text { C } & 5.780639 & 4.448438 & 0.737192\end{array}$

H 6.0917321 .8233161 .563506

C $7.594348 \quad 1.245757 \quad 0.110423$

H 7.4858133 .7247041 .909164

H 7.8024903 .9358440 .180646

C $6.0341605 .931074 \quad 0.532642$

H $5.159284 \quad 4.3098801 .630449$

C $7.048196-0.155938-0.234620$

O $8.1561231 .801144-1.086009$

H 8.4174301 .1700310 .830767

H $6.370245-0.077708-1.091070$

O $8.155334-0.950239-0.681801$

C $6.350315-0.880044 \quad 0.932727$

C $5.990187-2.3492620 .638286$

H $5.446837-0.332061 \quad 1.221039$

O $7.230498-0.8643092 .060780$

H $6.894905-2.9087910 .370291$

H $5.635100-2.7945221 .577743$

H 6.5479696 .3566751 .400784

H $6.6569386 .114385-0.349281$

H $5.091936 \quad 6.4718970 .398946$

C $3.6649573 .950540-0.698770$

H $8.8225201 .172009-1.411967$

H $8.754351-1.0699750 .075287$

H $6.751561-1.2535002 .812253$

H $3.3663353 .373957-1.584073$

H $3.4603675 .002535-0.929213$

H $5.6853824 .063644-1.385962$

C $2.602970-1.945676-0.857043$

O $3.704381-1.9468710 .061087$

C $4.914082-2.520483-0.445086$

C $4.685643-3.999178-0.794090$

C $3.526310-4.163040-1.782542$

C $2.287262-3.369378-1.349639$

H $1.753181-1.609033-0.251730$

C $2.830869-0.908188-1.966122$

H $5.219862-1.983309-1.349070$

O $4.412109-4.7534610 .385496$

H $5.605346-4.407376-1.228781$

H $3.264018-5.225195-1.861399$

O $3.932326-3.722545-3.074508$

H $1.553816-3.325208-2.162586$

O $1.666456-4.059712-0.259382$

C $1.534214-0.302485-2.458864$

H $3.447361-0.094227-1.563740$

H $3.361370-1.326244-2.824942$

H $3.638383-4.355242 \quad 0.820139$

H $4.695940-4.261528-3.342751$

H $1.414466-4.943446-0.577310$

$\begin{array}{lllll}\text { O } & 1.800677 & 0.889991 & -3.069117\end{array}$

O $0.428666-0.812276-2.339120$ 
C $0.6571421 .599783-3.555206$

H $-0.0273511 .825548-2.731538$

H $1.0016502 .541015-3.991650$

H $\quad 0.1514481 .016509-4.330384$

SCF Energy (B3LYP/6-31G**//MMFF)= -3245.92071339

$07 \quad 00155$

MM̄FF Geometry

C 3.0193884 .0410440 .009831

C 2.0922994 .1850300 .968263

C 0.6896473 .6551880 .837267

O $0.5478092 .572401 \quad 1.788140$

C -0.3714804 .7220351 .170648$

C -0.5151455 .8290510 .147279$

C $-1.3958485 .794655-0.872267$

C 0.3307747 .0545930 .372675

C $-2.3140794 .660686-1.231536$

C $-3.7053314 .848637-0.617754$

C $-4.6880513 .750422-1.050391$

C $-6.0381453 .942789-0.359399$

C $-7.0180592 .810682-0.693871$

O $-4.1377712 .481908-0.689294$

O $-8.1165512 .891736 \quad 0.227610$

C $-6.3272701 .448935-0.455066$

C $-7.6161962 .976850-2.096974$

O $-7.1705920 .394705-0.912730$

C $-4.9245111 .347348-1.097047$

C $-4.1226430 .112842-0.621106$

O $\quad-5.046574 \quad 1.290821-2.519802$

C $-0.3239561 .577616 \quad 1.462538$

$\begin{array}{llll}\text { O } & -0.967353 & 1.473498 & 0.430283\end{array}$

C $-0.375877 \quad 0.6272432 .595895$

C -1.135826 -0.4737542 .492402$

C -1.299262 -1.520324 3.503997

C $-0.570669-1.3458204 .810534$

C $-4.735603-1.250991-0.942037$

C $-3.741148-2.388902-0.783609$

C $-3.632482-4.879313-0.793579$

O $-2.540323-2.215604-0.597875$

C $-3.591268-5.3043440 .640389$

C $-2.453802-5.5170321 .319625$

C -2.409361 -5.9813572 .746520$

C -1.682166 -5.018147 3.656988

C $-0.461690-5.3072624 .138770$

C $-2.407846-3.7623684 .084954$

C $-2.079819-2.5826323 .211982$

N $-4.356602-3.627437-0.902231$

H $2.769523 \quad 3.482714-0.889693$

H 2.3387104 .7394831 .870409

H $0.5316823 .273625-0.179896$

H $-0.166506 \quad 5.1582022 .158456$

H -1.340750 4.2266501 .313033

H -1.464102 $6.659231-1.530548$

H $0.0911917 .858396-0.331883$

H $\quad 0.169815 \quad 7.4478301 .381874$

H 1.3926626 .8236480 .253744

H -2.392527 $4.615827-2.324708$

H $-1.8836573 .703072-0.922630$

H -3.608520 4.8384020 .475822

H -4.109688 $5.829647-0.897755$

H $-4.7988013 .798629-2.140016$

H -6.472362 $4.917160-0.616547$

H $-5.891318 \quad 3.9595120 .729822$

H -8.519846 3.7719690 .136510

H $-6.217261 \quad 1.3148140 .630056$

H -8.141334 $3.936336-2.178129$

H $-8.3690072 .207343-2.300623$

H $-6.8654502 .944062-2.889155$

H $-8.0255980 .489235-0.458880$

H -3.124649 $0.171260-1.077850$

H -3.9434040 .1841700 .460120$

H $-4.2351330 .897154-2.881183$

H $\quad 0.213223 \quad 0.855471 \quad 3.476517$

H -1.691964 -0.633497 1.570080

H $0.508144-1.2620734 .642683$

H $-0.708345-2.1781655 .503552$

H $-0.916814-0.4424555 .324786$

H $-5.578825-1.444195-0.270063$
H $-5.097146-1.278277-1.976010$

H -2.630903 -4.752944 -1.215650

H $-4.170019-5.623827-1.388581$

H $-4.546016-5.4636481 .136105$

H -1.499190 -5.3756180 .818037$

H -1.915768 -6.961954 2.759965

H -3.418209 -6.1556533 .141101$

H $0.050692-4.6335074 .817392$

H $0.054933-6.2199143 .860967$

H -2.206848 -3.559388 5.141829

H $-3.491979-3.9298474 .045563$

H $-2.561497-2.6162142 .234874$

H $-5.367810-3.671928-0.989022$

C 5.5332373 .7041540 .324760

O $5.4444812 .530813-0.507723$

C $5.832106 \quad 1.3885150 .283659$

C 6.4772521 .9478691 .545148

C 5.6425993 .1966371 .761341

H 4.9082180 .8814410 .580126

C $6.7167470 .441738-0.531244$

H $\quad 6.428619 \quad 1.2465562 .383639$

H 7.5287812 .2105241 .378983

C 6.2696354 .1866802 .726677

H 4.6569602 .8987992 .141230

C $6.010118-0.150875-1.770680$

O $7.8692131 .167751-0.972583$

H $7.086528-0.3640850 .114080$

H $5.7937640 .645182-2.492276$

O $6.931278-1.034983-2.420860$

C $4.720380-0.924969-1.439670$

C $4.090116-1.602684-2.671302$

H $3.987846-0.236915-1.005179$

O $5.036802-1.908756-0.454742$

H $4.036484-0.880102-3.494025$

H $4.727839-2.439496-2.981887$

H 6.3941093 .7327913 .715260

H 7.2556454 .5152492 .381613

H 5.6391055 .0739392 .840323

C 4.3827514 .6700690 .041047

H $8.4103590 .557432-1.502321$

H $7.096436-1.780677-1.818525$

H $4.211169-2.358736-0.209296$

H $4.5399935 .111659-0.951866$

H 4.4107145 .5049750 .750993

H 6.4694134 .2062960 .044849

C $1.550715-3.760981-0.966351$

O $2.786288-3.201380-1.428312$

C $2.671378-2.132805-2.372385$

C $1.955499-2.626430-3.637084$

C $0.595573-3.246945-3.294762$

C $0.698316-4.257195-2.146049$

H $1.847439-4.637976-0.376064$

C $0.807867-2.802633-0.029010$

H $2.093773-1.314974-1.927320$

O $2.759632-3.585845-4.320206$

H $1.816269-1.780453-4.319998$

H $\quad 0.191579-3.749556-4.181972$

O $-0.319521-2.220767-2.921872$

H $-0.297763-4.533138-1.783607$

O $1.311083-5.453313-2.638140$

C $1.623585-2.5361661 .214662$

H $\quad 0.560987-1.847449-0.501315$

H $-0.141946-3.2390940 .299163$

H $2.953993-4.307846-3.698359$

H $-0.397816-1.608722-3.673297$

H $0.743099-5.803561-3.345446$

O $2.173125-1.2898571 .154819$

O $1.773177-3.3492732 .117515$

C $2.983922-0.9097662 .271531$

H 3.1500120 .1693172 .218025

H $2.484397-1.1360553 .217696$

H $3.949037-1.4205152 .216816$

SCF Energy (B3LYP/6-31G**//MMFF)= -3245.92177974

0700156

MM̄MF Geometry

C -6.434461 -1.265983 0.813294

C $-5.659473-0.9114061 .850068$ 
C $-5.312168 \quad 0.4862552 .307942$

O

C -6.0345371 .6242101 .559351$

C -5.6885993 .0085342 .077618$

C $-4.925752 \quad 3.9105101 .426851$

C -6.2940903 .3634823 .412104$

C $-4.2413883 .762513 \quad 0.094534$

C $-2.722154 \quad 3.734708 \quad 0.265714$

C $-1.9415323 .511211-1.038941$

C $-2.2090572 .138598-1.658897$

C $-1.3261381 .889119-2.888275$

O $-0.5552023 .615935-0.694133$

O $-1.422394 \quad 0.497227-3.225108$

C $0.1462882 .164702-2.522940$

C $-1.8254492 .667245-4.112603$

O $0.9546292 .089651-3.694449$

C $0.3658253 .508763-1.794693$

C $1.7640153 .604095-1.141462$

O $0.2005794 .592187-2.708751$

C -3.0352050 .2350253 .065091$

O $-3.331125-0.2539394 .143160$

C $-1.657426 \quad 0.459172 \quad 2.574178$

C -0.6028820 .0711323 .306237$

C $\quad 0.803005 \quad 0.2306392 .927571$

C 1.1065781 .0377081 .692369

C $2.9694763 .570819-2.079182$

C $4.2428203 .864627-1.309148$

C 5.7994922 .8971040 .362541

O $4.8455234 .927966-1.435671$

C 5.7492071 .8175091 .395049

C 5.4669692 .0383452 .687589

C $5.4379680 .964713 \quad 3.738436$

C 4.0351390 .7033324 .247333

C 3.5866951 .3418825 .342245

C $3.244309-0.3257193 .463043$

C $1.756836-0.329303 \quad 3.700759$

N $4.6069542 .831225-0.458703$

H $-6.926038-0.5111410 .208130$

H $-5.180043-1.7082122 .417553$

H -5.5383560 .5605393 .378695$

H -7.1215941 .4928051 .643515$

H -5.8098861 .5520520 .491202$

H -4.7611054 .8771531 .902181$

H -6.0891134 .4001713 .699720$

H $-7.3817983 .242378 \quad 3.379987$

H -5.8942472 .7195004 .201208$

H -4.581537 $2.870540-0.431360$

H $-4.5233144 .614407-0.535978$

H -2.388274 4.6810290 .713824

H -2.4384412 .9642770 .991620$

H $-2.1964574 .315775-1.738315$

H -3.262605 $2.018133-1.933934$

H $-2.0013531 .354680-0.917187$

H $-2.3583450 .293276-3.393488$

H $\quad 0.480917 \quad 1.358501-1.856856$

H -2.861136 $2.391653-4.345309$

H -1.242626 $2.416454-5.006022$

H -1.794102 $3.749944-3.973091$

H $0.8204641 .208110-4.082825$

H $1.8079374 .537099-0.560640$

H $1.8604122 .808296-0.393755$

H $0.6771545 .363218-2.358626$

H -1.543901 $0.926231 \quad 1.603239$

H $-0.780599-0.4185764 .262544$

H $0.6296862 .022790 \quad 1.750707$

H $\quad 0.7439420 .5255400 .795037$

H 2.1730221 .2280091 .550039

H $3.0830862 .591236-2.554220$

H $2.8717714 .316655-2.875338$

H 5.8690693 .8961720 .807042

H $6.6695822 .760600-0.287934$

H 5.9700920 .8068901 .061892

H 5.2501323 .0518833 .017786

H 6.0906621 .2789144 .563319

H 5.8724550 .0285153 .365260

H 2.5985331 .1689825 .752708

H 4.2098952 .0611675 .864321

H $3.627756-1.3199993 .724661$
H $3.452640-0.2135882 .393908$

H $1.452473-0.8837304 .588862$

H $4.1416611 .933093-0.562349$

C $-5.704887-3.105961-0.771788$

O $-4.327186-2.944809-0.371490$

C $-3.639233-2.198024-1.388914$

C $-4.469932-2.391356-2.649367$

C $-5.875058-2.328137-2.079513$

H -3.672884 -1.141475 -1.090862

C $-2.174070-2.632821-1.514895$

H -4.276002 -1.620304 -3.400982

H $-4.285649-3.371830-3.104365$

C $-6.936292-2.905448-2.999974$

H -6.117639-1.276801-1.883605

C $-1.337160-2.350058-0.251016$

O $-2.105375-4.021816-1.832503$

H -1.744871 -2.091749 -2.362957

H -1.402603 -1.285882 0.005458

O $-1.872987-3.0748560 .862235$

C $0.148466-2.749221-0.360406$

C $0.880994-2.040574-1.512349$

H $0.247124-3.835984-0.464655$

O $0.781489-2.3954270 .871611$

H $\quad 0.414422-2.316755-2.463580$

H $\quad 0.765377-0.959137-1.373707$

H $-6.977626-2.344135-3.939136$

H -6.730086 -3.952965 -3.244205

H $-7.924317-2.856010-2.531452$

C $-6.626354-2.6957390 .383639$

H -2.501221 -4.509078-1.089387

H $-2.770781-2.7403051 .027204$

H $0.295334-2.8395861 .587381$

H $-6.432024-3.3570671 .237938$

H -7.675132 -2.8448990 .102903$

H -5.841216 -4.179734 -0.953862

C $4.457835-1.988453-0.388029$

O $3.046002-1.758489-0.483175$

C $2.385277-2.371024-1.595061$

C $2.992559-1.868051-2.916125$

C $4.509696-2.070498-2.947187$

C $5.176126-1.544136-1.672103$

H $4.791306-1.3318090 .425275$

C $4.778491-3.4321690 .014502$

H $2.497068-3.458720-1.531012$

O $2.691690-0.483321-3.079270$

H $2.524293-2.408206-3.748080$

H $4.922993-1.550329-3.819747$

O $4.780438-3.460081-3.103351$

H $6.226231-1.855525-1.627711$

O $5.166305-0.114501-1.697803$

C $4.357069-3.6934041 .440354$

H $4.308030-4.178492-0.631741$

H $5.857883-3.619752-0.035249$

H $3.058854-0.200767-3.933978$

H $5.746030-3.568585-3.141042$

H $5.6596510 .169088-2.486190$

O $3.144103-4.3142131 .463486$

O $5.020336-3.3648972 .415582$

C $2.621212-4.5977482 .764786$

H $1.658571-5.1009152 .641373$

H $3.298277-5.2624673 .309566$

H $2.461710-3.6683913 .319287$

SCF Energy (B3LYP/6-31G**//MMFF) $=-3245.91182546$

07_00157

MM̄MF Geometry

C -1.042064 -2.780864 -0.108447

C $-0.418602-3.1819251 .011776$

C $-0.630178-2.5551522 .368937$

O $-0.777454-1.1235272 .227644$

C $-1.904694-3.1185683 .025212$

C $-2.289210-2.4094524 .309257$

C $-3.150204-1.3719404 .362815$

C -1.658168 -2.953366 5.561199

C $-3.800509-0.6932743 .187809$

C $-5.312382-0.9388953 .129622$

C $-5.900779-0.7975361 .712411$

C $-5.605316-2.0422060 .868033$ 
C $-6.130981-1.900737-0.563988$

O $-5.353070 \quad 0.3716691 .098629$

O $-5.547479-2.966965-1.330385$

C $-5.619645-0.566808-1.147892$

C $-7.647520-2.111622-0.628742$

O $-6.204358-0.349118-2.429600$

C $-5.8472590 .653426-0.217819$

C $-5.1246711 .924899-0.717612$

O $-7.232811 \quad 1.017925-0.176176$

C $0.349588-0.3657572 .211219$

O $1.503596-0.7602842 .243045$

C -0.0796321 .0507992 .171088$

C 0.8452722 .0211692 .159127

C $0.593510 \quad 3.463656 \quad 2.163788$

C $-0.836892 \quad 3.9238432 .264861$

C $-3.593899 \quad 1.859525-0.707544$

C -2.967852 $3.047364-1.419724$

C $-0.7530323 .918556-2.157738$

O $-3.6262563 .954759-1.921581$

C -0.423353 5.069819-1.261455

C $0.8257765 .511344-1.049578$

C $1.1516116 .714861-0.213348$

C 2.1646996 .4250990 .869895

C $3.450793 \quad 6.7893150 .730692$

C $1.665892 \quad 5.8023352 .153517$

C 1.6520884 .2980822 .098244

N -1.581945 $2.962408-1.448152$ H - $1.774128-1.981254-0.037952$ H $0.284961-4.009157 \quad 0.966630$ H $\quad 0.234278-2.768063 \quad 3.010554$ H $-1.773314-4.1904003 .226748$ H -2.746088 -3.0712382 .323224$ H -3.384954 -0.937005 5.332637 H -1.968577 -2.402946 6.455463 H -1.938135 -4.0022095 .703091$ H $-0.567298-2.8898415 .495353$ H -3.613061 $0.385072 \quad 3.271351$ H -3.329614 -0.992882 2.249449 H -5.574554 -1.923861 3.535501 H -5.792711 -0.1959253 .780085$ H $-6.984270-0.6684021 .818462$ H $-6.020879-2.9406241 .341551$ H $-4.522341-2.2109080 .813342$ H $-5.818151-2.845560-2.256851$ H $-4.545259-0.704775-1.314852$ H $-7.903235-3.130677-0.313929$ H -8.019293 -2.013427 -1.654434 H $-8.204681-1.4220880 .009065$ H $-5.7026510 .357205-2.869600$ H -5.492960 2.185838 -1.717892 H $-5.4243752 .769751-0.080634$ H -7.721743 $0.463970-0.805113$ H -1.144111 1.2561162 .172935 H 1.8954041 .7332012 .158795 H $-0.952904 \quad 5.0074892 .199445$ H -1.273205 3.6089923 .219240 H -1.438481 3.5037921 .452582 H -3.224385 1.8362030 .323811 H -3.240660 $0.961008-1.219502$ H -1.297941 $4.282156-3.035174$ H $0.1400843 .387236-2.499612$ H $-1.2561195 .591394-0.795498$ H $1.6573855 .012115-1.541141$ H $0.249223 \quad 7.1437200 .240108$ H $1.5377437 .490423-0.887893$ H $4.175674 \quad 6.612667 \quad 1.518591$ H $3.8083267 .265964-0.175941$ H $0.695814 \quad 6.2322742 .416969$ H $2.326648 \quad 6.0889582 .983342$ H 2.6495543 .8609242 .028322 H $-1.1177702 .213066-0.943097$ C - $0.477613-2.371732-2.558809$ O $0.709853-1.629896-2.213472$ C $0.516766-0.255321-2.599316$ C $-0.710524-0.239433-3.505385$ C $-1.552702-1.332997-2.879850$ H $0.2809780 .297238-1.682279$ C $1.7831450 .315574-3.245609$
H -1.207633 $0.734926-3.516537$

H $-0.455756-0.500903-4.539010$

C -2.662896 -1.843166 -3.781031

H $-1.998448-0.934627-1.962562$

C $3.0512010 .253053-2.369225$

O $2.061147-0.404688-4.453798$

H $1.5991911 .351671-3.552722$

H $3.399152-0.782714-2.287530$

O $4.0840820 .972464-3.055593$

C $2.892090 \quad 0.860287-0.965380$

C $4.2035131 .067474-0.185886$

H $2.2237790 .226060-0.374208$

O $2.2566822 .137916-1.065194$

H $4.8082781 .845215-0.669956$

H 3.9255641 .4849590 .790533

H $-3.360580-1.035383-4.025465$

H -2.266697 -2.241469 -4.720928

H -3.229102 -2.639730 -3.288478

C $-0.820698-3.389007-1.464422$

H $2.159639-1.343383-4.218647$

H $4.1796760 .571475-3.936513$

H $2.8198382 .704046-1.620444$

H $-0.005603-4.120630-1.404946$

H - $-1.724037-3.940206-1.750067$

H $-0.228478-2.931961-3.470158$

C $4.858356-2.4595300 .870183$

O $4.236513-1.186704 \quad 0.646874$

C $5.051544-0.1986210 .011916$

C $6.309113 \quad 0.087980 \quad 0.850950$

C $7.076858-1.199898 \quad 1.162597$

C $6.149558-2.3003101 .690794$

H $4.140973-3.0192161 .484412$

C $5.068894-3.236643-0.438951$

H $5.356759-0.561418-0.974458$

$\begin{array}{lllll}\text { O } & 5.934318 & 0.719856 & 2.074301\end{array}$

H 6.9493740 .7873750 .299892

H $7.850628-0.9860481 .910085$

O $7.729615-1.637627-0.025350$

H $6.681178-3.2579391 .724017$

O $5.789400-1.9921203 .040791$

C $3.760901-3.611059-1.101097$

H $5.645741-2.657700-1.164708$

H $5.603316-4.170027-0.228232$

H 6.7510370 .9273342 .559568

H $8.231333-2.4410340 .194599$

H $5.245779-1.1855133 .026630$

O $4.028167-4.203118-2.302873$

O $2.649956-3.417961-0.628846$

C $2.884463-4.616313-3.056985$

H $3.236057-5.176964-3.927122$

H $2.241063-5.267969-2.458290$

H $2.332037-3.739215-3.404905$

SCF Energy (B3LYP/6-31G**//MMFF)= -3245.91089938

07_00158

MM̄FF Geometry

C $0.480691-3.7522390 .729303$

C $-0.174608-2.6231190 .421911$

C $-1.293390-2.591534-0.586489$

O $-2.503223-2.2891140 .150002$

C $-1.102304-1.512778-1.668219$

C $0.233375-1.528042-2.382393$

C $1.114842-0.509093-2.336207$

C $0.516213-2.744446-3.222088$

C $0.9653180 .756159-1.538466$

C $0.240274 \quad 1.846844-2.336942$

C $-0.3438502 .975269-1.472529$

C $0.7182903 .719047-0.664363$

$\begin{array}{llll}C & 0.098953 & 4.794509 & 0.241357\end{array}$

O $-1.317245 \quad 2.395926-0.597284$

$\begin{array}{llll}0 & 1.124904 & 5.203653 & 1.158559\end{array}$

C -1.045994 4.157402 1.058135

C $-0.298628 \quad 6.043313-0.553946$

$\begin{array}{llll}\text { O } & -1.752712 & 5.145789 & 1.805111\end{array}$

C $-2.042945 \quad 3.341810 \quad 0.198337$

C -2.9962232 .5462701 .127840$

O $-2.796773 \quad 4.240242-0.613210$

C $-3.683626-2.703848-0.383654$ 
O $-3.844857-3.323835-1.422401$

C $-4.763633-2.2560070 .524426$

C $-6.043202-2.5080550 .210961$

C -7.219884 -2.115163 0.990806

C $-6.993368-1.2714122 .217882$

C $-3.947141 \quad 1.5706520 .418244$

C $-5.2426172 .196648-0.069754$

C $-7.3011461 .632913-1.360373$

O -5.5631823 .3568360 .172518$

C $-8.3729001 .219040-0.401279$

C $-9.4017560 .426019-0.737036$

C $-10.497700 \quad 0.033477 \quad 0.212080$

C -10.698999-1.463764 0.287621

C -11.710129-2.058866 -0.367565

C $-9.781334-2.2574431 .191409$

C $-8.436343-2.5150140 .564148$

N $-6.004860 \quad 1.296138-0.801875$

H $0.165923-4.6783220 .253965$

H $0.097176-1.6901610 .908590$

H -1.397126 -3.576456 -1.060591

H -1.314749 -0.532406 -1.226437

H -1.891785 -1.627243 -2.423706

H $2.033303-0.583876-2.914825$

H $1.456337-2.655168-3.777154$

H $\quad 0.589898-3.638148-2.594904$

H $-0.285105-2.896024-3.952499$

H $0.4595270 .549024-0.589511$

H $1.9662681 .107319-1.263974$

H $0.9259002 .269426-3.082053$

H $-0.5917851 .392826-2.891550$

H $-0.8532513 .667292-2.153490$

H $1.4739484 .158561-1.326932$

H $1.2565323 .011110-0.021054$

H $\quad 0.7737505 .9294621 .701578$

H -0.5870063 .4799881 .792085$

H $0.583326 \quad 6.486292-1.031919$

H -0.7045816 .8196430 .103969$

H -1.032796 $5.838929-1.336052$

H -1.122153 5.5606422 .417228

H $-2.377374 \quad 1.9382301 .801484$

H -3.5762663 .2359831 .752291$

H -3.572867 $3.772083-0.957075$

H -4.475769 -1.7241611 .424054$

H -6.254728 -3.054950 -0.707047

H -6.425408 -1.8315712 .968723$

H $-7.915960-0.9380732 .696734$

H -6.437353 -0.3621911 .963905$

H -3.448772 $1.067514-0.417056$

H $-4.236424 \quad 0.799157 \quad 1.142330$

H -7.384413 $1.117714-2.322437$

H $-7.3567712 .712153-1.534428$

H $-8.310177 \quad 1.6077280 .612556$

H $-9.4780280 .059205-1.758280$

H -11.421542 $0.518372-0.129646$

H $-10.3139290 .425140 \quad 1.220384$

H $-11.878577-3.128318-0.295990$

H -12.388862 -1.490634 -0.994987

H $-10.227421-3.2369501 .410622$

H $-9.716775-1.7578512 .161516$

H -8.491602 -3.124872 -0.339034

H $\quad-5.687221 \quad 0.335407-0.891041$

C $2.937244-4.3297841 .142278$

O $3.597392-3.2550650 .445381$

C $4.337375-3.815455-0.656960$

C $4.241043-5.335919-0.522890$

C $2.902165-5.5027320 .163528$

H $3.811375-3.506393-1.567706$

C $5.780626-3.297835-0.683737$

H $4.292369-5.838110-1.494024$

H $5.040863-5.7456820 .104485$

C $2.713439-6.8552640 .826145$

H $2.126918-5.363413-0.599004$

C $5.955095-1.767347-0.684566$

O $\quad 6.483362-3.793456 \quad 0.465815$

H $6.296768-3.726198-1.551407$

H $5.757328-1.3715040 .318137$

O $7.340470-1.504024-0.951925$

C $5.105939-0.999017-1.710635$
C $5.5475670 .458678-1.953712$

H $4.059748-1.017425-1.386524$

O $5.169082-1.676986-2.968917$

H $6.4998210 .463592-2.499930$

H $4.8142600 .906771-2.637946$

H $2.773612-7.6587710 .084821$

H $3.480459-7.0411541 .585479$

H $1.734952-6.9169001 .312881$

C $1.598292-3.8616511 .726199$

H $6.001591-3.4926831 .255485$

H $7.860524-1.978061-0.280375$

H $4.570221-1.216087-3.580881$

H $1.744021-2.8979472 .228980$

H $1.279223-4.5773662 .493608$

H $3.594685-4.5768151 .987068$

C 4.3212032 .1326301 .154153

O $4.3793221 .410215-0.083800$

C $5.6701981 .321717-0.689127$

C $6.2069692 .725542-1.006064$

C 6.2278223 .6019900 .250412

C 4.8930873 .5552240 .999121

H 3.2500992 .2225261 .375876

C 4.9685131 .3446022 .303429

H $\quad \begin{array}{lll}6.363616 & 0.833094 & 0.000917\end{array}$

O $5.4109313 .349080-2.011690$

H $7.2214382 .634658-1.411339$

H $6.4438844 .639687-0.032661$

O $7.2623383 .163531 \quad 1.126043$

H 4.9984934 .0246811 .984307

O $3.958558 \quad 4.343395 \quad 0.258428$

C 4.2408920 .0483732 .595970

H 6.0085811 .0888962 .085436

H 4.9381721 .9490473 .217420

H $4.4930933 .375260-1.691004$

H 8.1037643 .2318940 .643658

H 3.1488914 .4137250 .792150

O $4.864340-0.5766653 .639025$

O $3.262640-0.3681471 .991600$

C $4.304950-1.8332734 .032262$

H $3.232296-1.7373984 .226461$

H $4.493895-2.5800053 .256660$

H $4.799036-2.1518684 .953925$

SCF Energy $\left(B 3 L Y P / 6-31 G^{* *} / / M M F F\right)=-3245.91852836$

$07 \quad 00159$

MM̄MF Geometry

C $2.576916-3.999105-1.758694$

C $1.647659-3.385845-1.009815$

C $1.165918-3.9115920 .316098$

O $1.650846-2.9829761 .313824$

C $-0.369818-3.9860870 .387515$

C $-0.984371-4.897160-0.652844$

C $-1.694492-4.473218-1.716179$

C $-0.784461-6.371925-0.417151$

C $-2.007384-3.055306-2.108147$

C $-3.508909-2.752923-2.033248$

C $-4.059660-2.758418-0.598109$

C $-5.588634-2.720734-0.620339$

C $-6.171477-2.674444 \quad 0.796244$

O $-3.546609-1.6085790 .087980$

O $-7.566390-2.3633050 .665062$

C $-5.493578-1.5236511 .562879$

C $-6.097731-4.0407211 .487353$

O $-5.914752-1.5131322 .926060$

C $-3.944620-1.5167811 .463203$

C $-3.426995-0.1781402 .038390$

O $-3.423611-2.6000332 .232998$

C $1.775620-3.4357802 .586630$

O $1.414563-4.5251943 .011485$

C $2.445410-2.4442423 .459153$

C $3.060940-1.3420003 .002839$

C $3.813133-0.3805653 .812461$

C $3.832599-0.5805295 .305807$

C $-1.9124580 .058722 \quad 1.955251$

C -1.5688431 .4557922 .453991$

C 0.2978333 .0222892 .799119

O -2.4245622 .2787722 .775090$

C 1.7346632 .9666393 .205757 
C 2.6911603 .6893792 .600885

C 4.1495773 .8027462 .959363

C 4.6316002 .9667104 .122040

C 4.5172223 .4106555 .385328

C 5.3451951 .6718743 .813023

C 4.4550520 .6308883 .190912

N $-0.200538 \quad 1.6925172 .500177$

H $3.022436-4.926638-1.408317$

H $1.224301-2.444670-1.354031$

H $1.606164-4.8982520 .510087$

H $-0.784231-2.9724850 .346886$

H $-0.674408-4.3422431 .380371$

H $-2.098456-5.223270-2.395009$

H $-1.314077-6.986188-1.153269$

H $0.276896-6.631590-0.475191$

H $-1.158668-6.6517990 .573134$

H -1.451342 -2.326790 -1.514575

H -1.673510 -2.916082 -3.143895

H $-3.675634-1.764388-2.480647$

H -4.052029 -3.483379 -2.646226

H -3.720594 -3.663506 -0.083874

H -5.993931 -3.579061-1.170864

H -5.924158 -1.828096 -1.167223

H -7.928287 -2.248363 1.560695

H -5.871776 -0.5890531 .125760$

H $-6.634085-4.7945300 .898602$

H -6.593675 -4.018356 2.464132

H -5.074604 -4.395034 1.630032

H $-5.428781-2.2158333 .390392$

H $-3.9224940 .641771 \quad 1.500869$

H $-3.720869-0.0962403 .093053$

H -2.486700 -2.429445 2.417382

H $2.455875-2.7085334 .512039$

H $3.050932-1.1343981 .934163$

H $4.348641-1.5118825 .563068$

H $2.811633-0.6212315 .701999$

H $4.3348690 .226113 \quad 5.844888$

H -1.371767 -0.6672582 .571038$

H -1.564160 -0.0310190 .921114$

H -0.2975813 .4500373 .613277$

H 0.1381313 .6409541 .909495

H 1.9704532 .3491144 .067041

H 2.3977724 .3106581 .755468

H 4.3546914 .8606133 .172782

H 4.7485453 .5695782 .071004

H 4.8947212 .8358516 .224308

H 4.0387444 .3589425 .607257

H 5.8403011 .2713924 .705412

H 6.1690401 .8797553 .117402

H 4.3747500 .7149692 .106929

H 0.4218951 .0316032 .043632

C $4.298536-2.573700-2.883305$

O $3.873861-1.334606-2.277874$

C $4.389456-0.236420-3.049320$

C $5.543380-0.817302-3.851355$

C $4.985483-2.187050-4.194982$

H $3.5980570 .064786-3.746409$

C $4.7359610 .933887-2.125214$

H $5.784372-0.216660-4.733391$

H $6.450520-0.918326-3.244457$

C $6.041961-3.178926-4.649723$

H $4.237578-2.073233-4.991465$

C $3.5761591 .363614-1.201188$

O $5.8378410 .563200-1.286882$

H $5.0822241 .787251-2.719056$

H $3.3764140 .580760-0.459130$

O $4.0042872 .516309-0.467278$

C $2.2714701 .715560-1.939572$

C $1.1454752 .118311-0.977683$

H $1.939180 \quad 0.845521-2.512597$

O $2.5133672 .770239-2.867267$

H $0.9399681 .288199-0.289957$

H $1.4780582 .949288-0.342568$

H $6.533339-2.825954-5.562283$

H $6.813876-3.321507-3.885994$

H $5.593747-4.154709-4.862094$

C $3.072918-3.467868-3.075199$

H $5.574920-0.230976-0.790397$
H $4.8291432 .277727-0.010290$

H $2.8321263 .537609-2.361426$

H $3.314077-4.321500-3.718745$

H $2.270074-2.906570-3.570065$

H $5.015397-3.036716-2.193050$

C $-2.3682123 .327102-0.979190$

O $-1.0978322 .791482-0.588376$

C $-0.1628192 .573358-1.654460$

C $-0.7373801 .563781-2.657578$

C $-2.1134892 .000790-3.169802$

C $-3.0450072 .430849-2.031436$

H $-2.9729173 .285313-0.064263$

C $-2.2223754 .801801-1.380998$

H $0.0276323 .528850-2.156873$

O $\quad-0.845077 \quad 0.289917-2.028284$

H $-0.050321 \quad 1.460978-3.505150$

H -2.571531 $1.169522-3.719893$

O $-1.931713 \quad 3.076020-4.086338$

H -3.925895 $2.938036-2.438919$

O $-3.5286421 .255430-1.373328$

C $-3.5271235 .565480-1.323600$

H -1.524458 $5.292899-0.691533$

H $-1.8078284 .899008-2.388085$

H $-1.163877-0.337376-2.699137$

H $-2.8083273 .322088-4.427747$

H $-2.7660590 .801331-0.976059$

O $-3.3203616 .828415-1.802447$

O $-4.5885085 .124068-0.905469$

C -4.470025 $7.680649-1.806631$

H $-5.2536387 .256998-2.442039$

H $-4.8362327 .827006-0.785971$

H -4.173089 $8.650138-2.215293$

SCF Energy (B3LYP/6-31G**//MMFF)= -3245.93395701

0700160

MM̄FF Geometry

C -4.580128 -3.666080 -0.548149

C $-3.304181-3.732100-0.140206$

C $-2.156641-4.110891-1.040103$

O $-1.129757-3.099788-0.910868$

C $-1.563589-5.458289-0.594157$

C $-0.436903-5.937753-1.489352$

C $0.873455-5.886721-1.175871$

C $-0.873019-6.535695-2.802793$

C $1.506245-5.3358830 .072758$

C $2.453061-4.179186-0.254040$

C $3.147413-3.5812690 .978708$

C $2.171741-2.9133691 .951614$

C $2.904312-2.2383753 .121204$

O $4.071268-2.6013800 .488723$

O $1.971218-1.3741553 .789031$

C $4.028474-1.3395132 .564630$

C $3.359522-3.2658634 .164746$

O $4.843831-0.8303133 .616956$

C $4.912286-2.0251581 .495201$

C $5.827321-0.9811700 .815048$

O $5.783799-2.9995872 .075223$

C $-1.253713-1.981897-1.677561$

O $-2.146991-1.727866-2.469639$

C $-0.101796-1.103356-1.374331$

C $-0.0130940 .103970-1.949802$

C $1.0434471 .094679-1.726402$

C $2.2435000 .666766-0.924722$

C $6.639934-1.544354-0.358875$

C $5.914666-1.425699-1.687347$

C $5.3254980 .226659-3.449950$

O $5.462507-2.406844-2.272047$

C $5.0134081 .688128-3.483657$

C $3.8879212 .206066-3.998611$

C $3.5990193 .680935-4.000948$

C $2.2103924 .028462-3.512485$

C $1.4030504 .814268-4.246235$

C $1.7756863 .532316-2.149743$

C $0.8780022 .327765-2.248646$

N $5.836602-0.115175-2.134475$

H $-4.825150-3.911988-1.578439$

H -3.061470 -3.492771 0.892704

H $-2.479710-4.176731-2.086592$ 
H $-2.348916-6.226503-0.588516$ H -1.227461 -5.3866130 .445614$ H $1.586503-6.284662-1.897281$ H $-0.029440-6.920224-3.385867$ H -1.559862 -7.370717 -2.630192 H -1.382030 -5.786932 -3.417410 H $\quad 0.753340-5.0055910 .789007$ H $2.068308-6.1424950 .558323$ H $3.226849-4.524537-0.953887$ H $1.917516-3.389061-0.792863$ H $3.707923-4.3815331 .475980$ H $1.438512-3.6278542 .341494$ H $1.591992-2.1474161 .419813$ H $1.209558-1.9116054 .066471$ H $3.546872-0.4698282 .097288$ H $2.493686-3.7849874 .593731$ H $3.863196-2.7794685 .007143$ H $4.025241-4.0276583 .753444$ H $4.255053-0.3829344 .248888$ H $5.224071-0.1315950 .473548$ H $6.540602-0.6013521 .557811$ H $5.694409-2.9633153 .040715$ H $\quad 0.633897-1.462285-0.666611$ H $-0.8042880 .411499-2.632772$ H $2.725935-0.199847-1.390571$ H $1.949571 \quad 0.3922710 .093104$ H $3.0080491 .440738-0.835155$ H $7.579143-0.983701-0.449871$ H $6.922447-2.590644-0.200994$ H $4.453342-0.397582-3.671108$ H $\quad 6.112250-0.007097-4.174495$ H $5.7571942 .362911-3.064835$ H $3.1520961 .545849-4.451025$ H $3.7441884 .044788-5.026152$ H $4.3236484 .218486-3.375907$ H $\quad 0.4147165 .090828-3.893574$ H $1.7102645 .198139-5.213329$ H $1.2065704 .323402-1.643667$ H $2.6514273 .369653-1.515769$ H $-0.0433832 .526797-2.799125$ H $6.3960140 .581812-1.650393$ C $-6.468683-2.054685-0.207853$ O $-5.604576-0.901793-0.199830$ C $-6.372396 \quad 0.254780 \quad 0.185033$ C $-7.828702-0.1953360 .243461$ C $-7.683940-1.6548460 .626639$ H $-6.047347 \quad 0.523749 \quad 1.196683$ C $-6.1011491 .415906-0.779246$ H -8.410974 0.3836280 .966822 H $-8.323578-0.106130-0.730487$ C $-8.928120-2.4806260 .349952$ H -7.441547 -1.714034 1.696221 C $-4.6104851 .775343-0.942949$ O $-6.6027001 .072096-2.078599$ H -6.670582 2.297443 -0.463942 H $-4.094040 \quad 0.961804-1.466580$ O $-4.5325292 .911212-1.814939$ C -3.8604112 .0934500 .365763$ C -2.396709 2.4698360 .097180 H $-3.8876501 .220603 \quad 1.025058$ O -4.4901003 .1780801 .042715$ H -1.903439 $1.650946-0.436794$ H -2.352432 $3.331578-0.582792$ H $-9.779524-2.0955470 .920592$ H -9.196990 -2.457756 -0.711485 H $-8.775530-3.5258110 .636334$ C $-5.720752-3.267628 \quad 0.346852$ H $-6.1467300 .261884-2.364593$ H $-4.9313083 .666018-1.348898$ H $-5.3628922 .874847 \quad 1.343843$ H $-6.401581-4.1220180 .432097$ H -5.347257 -3.042240 1.353495 H $-6.759947-2.229131-1.252256$ C 0.6751303 .6149651 .850137 O $-0.258017 \quad 3.1494180 .868309$ C -1.5765572 .8470641 .344716$ C -1.508312 1.7446952 .410570 C -0.5488572 .1256383 .544027$
C $0.8007182 .619247 \quad 3.014125$

H $1.6393673 .637573 \quad 1.325617$

C 0.3535665 .0441042 .298668

H -2.0128583 .7542981 .776028$

O

H -2.5076601 .5791272 .826928$

H $-0.385916 \quad 1.254270 \quad 4.190059$

O -1.1311553 .1543954 .339596$

H $1.3894163 .073198 \quad 3.819877$

O 1.5456451 .4977972 .533899

C 0.4569866 .0041901 .137269

H -0.6540575 .1454762 .711705$

H 1.0434625 .3770243 .083079

$\begin{array}{llll}H & -0.233018 & 0.667561 & 1.389873\end{array}$

H -1.9702472 .8134284 .693196$

H 1.6687360 .8850743 .279001

O $1.763094 \quad 6.3227090 .908568$

O $-\begin{array}{llll}0.507779 & 6.407940 & 0.500775\end{array}$

C $1.9958477 .220785-0.181080$

H 1.5471368 .1952580 .033154

H $3.0760747 .347517-0.292226$

H $1.5945266 .807229-1.111116$

SCF Energy (B3LYP/6-31G**//MMFF) $=-3245.91149811$

$07 \_00161$

MM̄̄F Geometry

C $3.056649-0.360776-2.999122$

C $2.430323-1.383103-2.394189$

C $0.999345-1.410446-1.918878$

O $0.506822-0.055465-1.787411$

C $0.068306-2.175110-2.876628$

C $0.199809-3.683034-2.799710$

C $-0.484453-4.452164-1.929001$

C $1.111617-4.316900-3.816545$

C $-1.444642-3.977805-0.874900$

C $-2.884677-4.385437-1.207822$

C $-3.900289-3.665399-0.308342$

C $-5.308224-4.218933-0.538190$

C $-6.372911-3.4300420 .238394$

O $-3.852890-2.275038-0.646172$

O $-7.661260-3.808497-0.270550$

C $-6.191701-1.927997-0.064202$

C $-6.375343-3.7832191 .729949$

O $-7.069172-1.1349420 .731511$

C $-4.737831-1.4384930 .108612$

C $-4.5443620 .010725-0.395369$

O $-4.395641-1.4540141 .498628$

C $-0.257330 \quad 0.232503-0.699620$

O $-0.619365-0.5398590 .173590$

C $-0.5646341 .680098-0.735947$

C -1.2212132 .2320310 .295376$

C -1.5888383 .6415170 .444962$

C $-1.2163544 .579926-0.671404$

C $-4.8115950 .205494-1.888797$

C $-4.2987201 .547642-2.368607$

C $-4.8636893 .972162-2.393470$

O $-3.2652441 .641461-3.027066$

C $-4.8744824 .942852-1.251390$

C $-5.148626 \quad 4.6989290 .040194$

C -5.1154785 .7633281 .100837$

C -4.1939485 .4221082 .250491$

C -4.6770135 .2251593 .489009$

C -2.7040185 .3868611 .981654$

C $-2.2306514 .017487 \quad 1.571440$

N $-5.1034802 .604407-1.975332$

H $2.5096020 .547446-3.233751$

H $3.001059-2.284465-2.180763$

H $1.004135-1.887805-0.930864$

H $0.232299-1.838695-3.909726$

H $-0.973362-1.888854-2.678518$

H $-0.343877-5.531237-1.965406$

H $1.113147-5.410127-3.747605$

H $0.790542-4.053409-4.829604$

H $2.142596-3.979769-3.680484$

H -1.155951 -4.421018 0.085585

H $-1.376825-2.896441-0.738018$

H -3.096264 -4.137268 -2.256691

H $-2.987183-5.472163-1.098604$ 
H $-3.601820-3.7941470 .738765$ H $-5.353898-5.283881-0.278101$ H $-5.550941-4.162886-1.608895$ H -7.757877 -4.769555 -0.156599 H -6.497899-1.777252 -1.107075 H $-6.589551-4.8496231 .870126$ H -7.168654 -3.246300 2.261658 H $-5.424286-3.5722552 .223132$ H -7.970420 -1.473997 0.594597 H $-5.158646 \quad 0.7020650 .195572$ H $-3.5069310 .307776-0.195361$ H -3.645216 -0.8526461 .637988$ H $-0.2300482 .249876-1.595032$ H -1.5143471 .5888141 .124708$ H $-0.1273204 .629238-0.781546$ H $-1.5669605 .602342-0.518658$ H -1.647940 4.242381-1.620043 H -4.314194 - $0.567814-2.485105$ H $-5.882507 \quad 0.141482-2.110542$ H $-5.6536494 .234811-3.104502$ H -3.903986 4.038465 -2.915909 H -4.616644 $5.962704-1.533069$ H -5.4222663 .6987530 .364618$ H -6.142406 5.9025991 .462429 H -4.810922 6.7311130 .683121 H -4.0179565 .0055414 .322718$ H $-5.740575 \quad 5.2743723 .696867$ H -2.1597675 .6605202 .895648$ H -2.4496336 .1658801 .258420$ H -2.436012 3.2529262 .322868 H $-5.9885992 .399464-1.520337$ C $5.3355290 .729418-2.703191$ O $4.9854940 .861868-1.309155$ C $4.7200022 .251083-1.041822$ C $5.4461713 .013814-2.138840$ C $5.1651092 .115716-3.330335$ H $3.6425192 .403732-1.179368$ C 5.1115132 .6163850 .391310 H $5.0636984 .030508-2.267084$ H $6.5242623 .069468-1.947230$ C $6.0816912 .371623-4.514419$ H $4.1265562 .269391-3.649488$ C 4.3976821 .7675301 .467341 O $6.5227562 .430694 \quad 0.543311$ H 4.9211433 .6830030 .560474 H 4.7983820 .7490791 .448771 O $4.746214 \quad 2.3031872 .749280$ C 2.8656701 .7353981 .333307 C 2.1193521 .0518592 .489960 H 2.5929401 .2358710 .397596 O 2.3917503 .0831551 .244666 H $2.201217 \quad 1.6556663 .403011$ H 1.0538931 .0764842 .228700 H $5.9553493 .394496-4.884276$ H $7.1346772 .240613-4.243275$ H $5.8568691 .684394-5.336064$ C $4.519516-0.393615-3.355465$ H 6.7471742 .6553591 .462611 H 4.3558163 .1920582 .811229 H 1.4293933 .0500891 .112894 H $4.946971-1.351789-3.033796$ H $4.629094-0.355728-4.445114$ H $6.3949850 .442807-2.727140$ C $3.023936-2.4918291 .661918$ O $2.593778-1.1262051 .558509$ C $2.550435-0.3947352 .787087$ C $1.596229-1.0715923 .787640$ C $1.933850-2.5532543 .981052$ C $2.130466-3.2714412 .642754$ H $2.864460-2.9121910 .661282$ C $4.521070-2.6099621 .993698$ H $3.556337-0.3593223 .217268$ O $0.243646-0.9603713 .344060$ H $1.659900-0.5455364 .747143$ H $1.121603-3.0431484 .532311$ O $3.122835-2.6788724 .754742$ H $2.545742-4.2719342 .811378$ O $0.843834-3.4469672 .042910$
C $5.402167-2.1192060 .865602$

H $4.777106-2.0394742 .890035$

H $4.787453-3.6587852 .167556$

H $\quad 0.189447-1.3485942 .453731$

H $2.962751-2.2475035 .611396$

H $\quad 0.969717-3.9493951 .219939$

O $6.618278-1.7559221 .369819$

O $5.080646-2.092118-0.313756$

C $7.571823-1.2942790 .407841$

H $8.503664-1.0703190 .933768$

H $7.767750-2.071010-0.337528$

H $7.212188-0.380134-0.072060$

SCF Energy (B3LYP/6-31G**//MMFF)= -3245.92177302

0700162

MM̄̄FF Geometry

C $-0.5520084 .469626-0.270996$

C $-1.4022214 .543584-1.306936$

C $-2.6126313 .655786-1.511743$

O $-2.5518312 .487737-0.660329$

C $-3.9051404 .420876-1.179902$

C $-5.1641453 .637576-1.503099$

C $-6.0505503 .195577-0.589344$

C $-5.4124423 .376213-2.967169$

C -6.0176673 .3798870 .901144$

C -5.3284682 .2500951 .681198$

C -5.9843380 .8690301 .514052$

C $-5.415177-0.1210472 .534041$

C $-5.979054-1.5372962 .341391$

O

O $-5.164689-2.4429383 .102504$

C $-5.834404-1.9394910 .857938$

C $-7.402569-1.6643812 .896786$

O $-6.492588-3.1770420 .600699$

C $-6.338852-0.861924-0.123590$

C $-6.010771-1.185700-1.602837$

O $-7.765527-0.767191-0.025169$

C $-1.7269851 .479204-1.053098$

O $-1.0881801 .410918-2.091299$

C -1.6864600 .4847270 .039675$

C $-0.626208-0.3301930 .122350$

C $-0.402754-1.3768411 .118215$

C $-1.535070-1.6987492 .055743$

C $-4.522131-1.345122-1.930089$

C $-4.028299-2.783068-1.911078$

C $-1.930630-4.116048-2.121981$

O $-4.777119-3.752487-1.827482$

C $-1.404422-4.484261-0.770413$

C $-0.118795-4.793086-0.540147$

C $0.416394-5.2253390 .795718$

C $1.578298-4.3772781 .263404$

C $2.848120-4.7708021 .067212$

C $1.262054-3.1111202 .027330$

C $0.789760-2.0079031 .121798$

N -2.647071 -2.856239-2.041498

H -0.7504963 .7690960 .536385$

H -1.201827 $5.258266-2.102160$

H -2.624449 $3.333467-2.560151$

H $-3.9373215 .365286-1.739257$

H $-3.8851664 .697401-0.120272$

H $-6.9176272 .640640-0.946651$

H -6.401708 $2.942705-3.149714$

H $-5.3571374 .310855-3.534789$

H $-4.6712142 .678066-3.367452$

H $-5.543527 \quad 4.332097 \quad 1.162237$

H $-7.053793 \quad 3.4751721 .251184$

H -4.2769372 .1992121 .377108$

H -5.3427982 .5280282 .742620$

H -7.0659650 .9804041 .653882$

H -5.5985210 .2265993 .558431$

H $-4.323025-0.1657622 .425926$

H -5.184325 -2.155120 4.031230

H -4.766066 -2.109902 0.679259

H -7.416710 -1.435576 3.969260

H $-7.772229-2.6916242 .805995$

H $-8.114998-0.9961372 .408790$

H -6.129429 -3.832804 1.220411

H $-6.592913-2.052963-1.937078$ 
H $-6.376439-0.341644-2.205677$ H -8.104376 $-0.361565-0.840434$ H -2.5083850 .4634670 .744262$ H $0.168530-0.213170-0.610991$ H -2.444075 -1.9334211 .491350$ H $-1.740857-0.8490382 .715536$ H -1.342386 -2.562797 2.694401 H $-4.351731-0.979977-2.951156$ H -3.908446 -0.738722 -1.259846 H -1.129832 -3.986845 -2.856651 H -2.605954 -4.899914 -2.479556 H -2.119439 -4.5322550 .047442$ H $0.587556-4.770745-1.367247$ H $\quad 0.734685-6.272230 \quad 0.703338$ H $\quad-0.368959-5.2236391 .561777$ H $3.685323-4.1784971 .421458$ H $3.078605-5.6911610 .540865$ H $2.160319-2.7476222 .543657$ H $0.554559-3.3451302 .827190$ H $1.541638-1.6951900 .395282$ H - $2.102613-1.998851-2.063941$ C $1.9310484 .342486-0.149006$ O 1.9074083 .5038691 .023454 C 3.2460183 .3922491 .551996 C 4.1609874 .0036940 .501461 C $3.2691475 .075068-0.096420$ H 3.2532193 .9970132 .467090 C 3.5275761 .9204261 .874392 H 5.0846084 .4043490 .929266 H $4.4314723 .261028-0.259835$ C $3.7520895 .582029-1.443974$ H 3.2096375 .9166950 .606706 C 4.9682261 .6249382 .334376 O 2.6137641 .5123322 .899453 H $3.280127 \quad 1.3171170 .994476$ H 5.6897011 .8850191 .551822 O 5.2813662 .4491213 .464807 C 5.1925130 .1662042 .776932

C $4.815020-0.8983361 .739096$ H $4.622026-0.0305563 .693272$

O $6.564050-0.0087963 .137906$

H $5.054011-1.8917252 .141172$ H $3.724295-0.9045381 .622097$ H $4.7455186 .032921-1.351236$ H $3.8180804 .771385-2.177435$ H $3.0723876 .341916-1.842104$ C $0.7130955 .271040-0.143492$ H 2.8355482 .0095753 .705497 H 5.3537123 .3663953 .152126 H 6.7780210 .6686493 .802162 H $0.7905246 .001209-0.956768$ H 0.6655845 .8273030 .800355 H $1.8947213 .672696-1.019055$

C $5.175226-1.803289-1.846569$

O $4.822222-1.786008-0.458249$

C $5.444653-0.7649030 .336929$

C $6.974720-0.9131210 .288250$

C $7.492772-0.963156-1.152952$

C $6.694884-1.943098-2.020103$

H $4.707983-2.715394-2.239320$

C $4.586364-0.608296-2.605674$

H $5.1597140 .207931-0.074461$

O $7.385594-2.1065660 .951190$

H $7.446394-0.0656110 .797535$

H $8.548458-1.261447-1.139604$

O $7.4229790 .348088-1.705707$

H $6.952312-1.820905-3.078619$

O $7.045153-3.281404-1.656775$

C $3.080328-0.586385-2.518784$

H $4.9395180 .352022-2.219059$

H $4.868003-0.636866-3.665002$

H $7.263653-1.9697651 .905860$

H $7.7832490 .305530-2.607902$

H $8.001816-3.383138-1.798222$

O $2.550075-1.571145-3.297613$

O $2.455045 \quad 0.214315-1.835728$

C $1.122257-1.664839-3.291733$

H $0.668252-0.698622-3.532143$
H $\quad 0.826088-2.389028-4.055225$

H $\quad 0.779059-2.022500-2.317596$

SCF Energy (B3LYP/6-31G**//MMFF) $=-3245.90131713$

07 00163

MM̄̄FF Geometry

C -4.5086612 .0936021 .976640$

C -3.2488982 .4155191 .651003$

C -2.1181622 .5237752 .633866$

O -1.0868311 .5944942 .233203$

C - -1.5119823 .9393572 .573350$

C -0.3932064 .1485163 .575681$

C 0.9181573 .9974883 .300051

C -0.8380384 .5706094 .950961$

C 1.5312283 .5372072 .005522

C 2.4573044 .5972521 .398000

C 3.0042074 .2245150 .007030

C $1.9327154 .342587-1.078302$

C $2.4697793 .921879-2.451688$

O 3.5162312 .8918290 .058758

O $1.3500503 .774726-3.338298$

C $3.1314732 .533696-2.326582$

C $3.3719054 .998042-3.068105$

O $3.7561572 .185001-3.559443$

C $4.1341002 .423514-1.153886$

C $4.5369800 .959176-0.852834$

O $5.3100613 .177616-1.449427$

C -0.8897230 .4874442 .996222$

O -1.6163420 .0543603 .874933$

C $0.437259-0.0570992 .626302$

C $1.031155-0.9639163 .416444$

C $2.391868-1.4862723 .256315$

C $3.241847-0.8787202 .171394$

C $5.2623170 .214291-1.972437$

C $5.898679-1.074079-1.482769$

C $5.344687-3.371700-0.707084$

O $7.117429-1.233578-1.494563$

C $4.395582-3.8918110 .323844$

C $4.780883-4.5036141 .453946$

C $3.813876-5.0135222 .485839$

C $4.158799-4.5616533 .889387$

C $4.442932-5.4592574 .848366$

C $4.186408-3.0775174 .185809$

C $2.823155-2.4424024 .106043$

N $4.971063-2.012039-1.056963$

H -4.7552751 .8767493 .012847$

H -3.0162972 .6457410 .616432$

H -2.4612472 .3138503 .654363$

H -2.2935264 .6891372 .756982$

H -1.146179 4.1495521 .560397

H 1.6365694 .1841194 .097040

H 0.0046354 .7124615 .635974

H -1.384746 5.5178304 .899128

H -1.495201 3.8127915 .389137

H 2.1103392 .6307732 .219434

H 0.7677953 .2453711 .282530

H 1.9501535 .5687381 .344496

H 3.3114024 .7237272 .076971

H $3.8299534 .912604-0.209741$

H $1.5298345 .362245-1.118476$

H $1.0805153 .694762-0.838999$

H $\quad 0.8799814 .625549-3.371255$

H $2.3310691 .800193-2.162473$

H $2.8239335 .942060-3.173402$

H $3.6840304 .719915-4.080806$

H $4.2663195 .199585-2.474921$

H $3.0780062 .240134-4.254581$

H 5.1793220 .9550920 .039635

H $3.6408040 .400011-0.557213$

H $6.0409522 .827104-0.913573$

H $\quad 0.921851 \quad 0.359630 \quad 1.750518$

H $\quad 0.487882-1.3332174 .285253$

H 3.3331450 .2039262 .313941

H $2.798314-1.0661261 .189053$

H $4.258851-1.2716002 .133840$

H $4.573668-0.044582-2.784009$

H $6.0583460 .834019-2.400025$

H $6.384972-3.394626-0.366692$ 
H $5.267643-3.970436-1.620412$ H $3.333602-3.7669450 .131824$ H $5.840495-4.658788 \quad 1.642579$ H $3.817444-6.1096842 .427864$ H $2.788041-4.7021772 .253168$ H $4.694163-5.1493285 .857352$ H $4.430443-6.5255314 .648044$ H $4.920799-2.5953323 .536239$ H $4.561087-2.9034855 .203665$ H $2.132175-2.8075724 .867279$ H $3.989340-1.830299-1.244693$ C -6.2861120 .6784130 .899787$ O $-5.416410-0.232145 \quad 0.195594$ C $-6.224987-1.109533-0.615795$ C $-7.679187-0.787510-0.284172$ C $-7.593057 \quad 0.6721570 .109218$ H $-6.034649-0.842081-1.661061$ C $-5.849094-2.572478-0.357439$ H -8.344861 -0.965233 -1.134469 H $-8.046180-1.389404 \quad 0.555680$ C -8.8008321 .1562270 .893003$ H $-7.4848271 .276649-0.801352$ C $-4.398176-2.937730-0.733406$ O $-6.028113-2.8541111 .037808$ H -6.546140 -3.231761 -0.887239 H $-3.704328-2.372787-0.102185$ O $-4.204596-4.317810-0.391578$ C $-4.048380-2.738583-2.225052$ C $-2.687578-3.340121-2.631706$ H $-4.072618-1.672500-2.474301$ O $-5.057389-3.380378-3.011028$ H -2.646669 -4.390845 -2.320168 H $-2.632450-3.355427-3.728815$ H $-9.713811 \quad 1.0457180 .298627$ H $-8.9321630 .587294 \quad 1.819572$ H -8.698889 $2.212916 \quad 1.158535$ C -5.6386902 .0601090 .984556$ H -5.442011 -2.2572861 .534225$ H -4.796324 -4.844884 -0.955839 H -4.873264 -3.180286-3.944636 H -6.3771062 .8030261 .306754$ H -5.281883 2.362908 -0.007840 H $-6.444166 \quad 0.2625391 .903944$ C $-0.268807-0.504364-2.499002$ O $-1.327140-1.393647-2.881897$ C -1.486837 -2.563730 -2.070705 C $-0.202963-3.402416-2.093249$ C $0.991663-2.564986-1.632294$ C $1.078544-1.236332-2.385565$ H $-0.1880730 .204903-3.333680$ C $-0.6482120 .293604-1.247474$ H -1.697534 -2.265614 -1.040152 O $0.024634-3.897534-3.410051$ H $-0.334085-4.267557-1.432121$ H $\quad 1.917063-3.130552-1.791056$ O $0.873886-2.293036-0.239371$ H $1.811520-0.575661-1.911154$ O $1.544953-1.485299-3.714886$ C $-1.7619871 .254911-1.589093$ H $-0.998203-0.329692-0.420110$ H $\quad 0.2057770 .867759-0.868842$ H $\quad 0.834097-4.435437-3.385507$ H $\quad 0.846102-3.1457470 .227184$ H $2.426981-1.888736-3.645412$ O $-1.2418672 .470558-1.920333$ O $-2.9469800 .947480-1.602794$ C $-2.1880543 .455251-2.347634$ H $-1.6453034 .382779-2.548132$ H $-2.6790953 .129047-3.269212$ H $-2.9268613 .645279-1.564043$

SCF Energy (B3LYP/6-31G**//MMFF) $=-3245.89226420$

07 00164

MM̄FF Geometry

C -2.105117 -3.317715 -1.663718

C $-0.980428-3.911311-2.093237$

C $0.383455-3.263014-2.184895$

O $0.254935-1.847833-1.905554$
C $1.364376-3.882100-1.166906$

C $2.289796-4.922841-1.768862$

C $3.610652-4.741537-1.967788$

C $1.658890-6.250375-2.098336$

C $4.407465-3.495956-1.703930$

C $5.208754-3.612016-0.402886$

C $5.916046-2.295667-0.049724$

C $6.782041-2.4704661 .200047$

C $7.404486-1.1443701 .662252$

O $4.904122-1.3099230 .182837$

O $7.919519-1.3369462 .988808$

C $6.288933-0.0823881 .761348$

C $8.597184-0.7364410 .789986$

O 6.8301401 .2031022 .055246

C $5.402647-0.0046670 .499259$

C $4.184106 \quad 0.926706 \quad 0.692125$

O $6.1722020 .528803-0.584567$

C $1.129141-0.994065-2.498243$

O $2.071551-1.282878-3.216997$

C $0.7149550 .379267-2.131788$

C $1.4809171 .421919-2.484955$

C $1.1852642 .835847-2.237798$

C $-0.1328043 .165968-1.590231$

C $3.1999260 .481851 \quad 1.775961$

C 1.9431721 .3279491 .747271

C 1.1122603 .6196792 .283994

O $0.8805380 .887309 \quad 1.315589$

C 1.3938474 .7716261 .364795

C 2.5015354 .9950480 .639013

C $2.6869676 .205929-0.230635$

C $3.0655515 .859824-1.652542$

C $4.2856236 .151646-2.134550$

C $1.9999445 .260492-2.543013$

C $2.0904023 .759205-2.624175$

N 2.1476102 .6059452 .240380

H -2.086209 -2.275423 -1.356466

H -1.032311 -4.955930 -2.390757

H $\quad 0.740989-3.387209-3.215172$

H $0.816334-4.347681-0.335699$

H $1.945072-3.089164-0.682322$

H $4.191118-5.567836-2.376010$

H $2.402546-7.007410-2.369952$

H $1.103808-6.634941-1.236478$

H $\quad 0.971555-6.148907-2.943257$

H $5.093746-3.339349-2.545430$

H $3.763779-2.613252-1.681717$

H $4.525111-3.8833410 .412760$

H $5.946201-4.418870-0.496636$

H $6.530857-1.987707-0.903692$

H $7.562543-3.2225951 .029849$

H $6.162974-2.8636322 .018971$

H $8.569443-2.0599052 .956029$

H $5.664793-0.3599432 .619859$

H $9.383164-1.4999280 .835520$

H 9.0566350 .1881531 .156549

H $8.334149-0.599186-0.260729$

H 7.3782871 .1112692 .853401

H $4.526573 \quad 1.9517830 .881864$

H $3.6354290 .981239-0.258203$

H $5.5613310 .886157-1.250256$

H $-0.2196260 .494799-1.593875$

H $2.4117831 .225423-3.015476$

H $-0.9626202 .815126-2.213301$

H $-0.2862744 .234693-1.430090$

H $-0.2110312 .690467-0.606969$

H $2.901682-0.5628131 .632770$

H 3.6439160 .5580062 .774337

H 1.0537603 .9806253 .315608

H 0.1484383 .1749842 .021663

H 0.5878325 .5012421 .303925

H 3.3366924 .3018150 .685978

H 3.4676646 .8257630 .229240

H $1.7832156 .828074-0.245951$

H $4.5511445 .933821-3.163972$

H $5.0463336 .609822-1.511505$

H $2.1208735 .649378-3.563555$

H $1.0147315 .616167-2.229713$

H $3.0115343 .409073-3.093065$ 
H 3.0624232 .8523952 .607029 C $-3.921027-4.143074-0.102844$ O $-3.996549-2.845624 \quad 0.526027$ C $-3.366810-2.9308501 .816884$ C $-3.343405-4.4147852 .161922$ C -3.051806 -5.019755 0.802791 H $-2.333336-2.5867831 .688358$ C $-4.082391-2.0478752 .840890$ H -2.585214 -4.656920 2.912635 H -4.315732 -4.761404 2.531125 C $-3.368221-6.502147 \quad 0.710373$ H -1.986756 -4.8739140 .584643$ C $-4.173497-0.5560822 .456146$ O $-5.419304-2.5367583 .009096$ H -3.591955 -2.157153 3.815939 H $-4.898806-0.4350411 .643844$ $\begin{array}{lllll}\text { O } & -4.728674 & 0.136673 & 3.582811\end{array}$ C $-2.838240 \quad 0.1050592 .064447$ C -2.8736541 .6439371 .998426$ H $-2.505914-0.2958781 .099956$ O $-1.847425-0.2542983 .033127$ H -3.021754 2.0528943 .006060 H $-1.871914 \quad 1.9712201 .692700$ H -2.760539 -7.069976 1.422482 H $-4.421474-6.7030920 .932908$ H $-3.156588-6.882535-0.293951$ C $-3.435644-4.007908-1.549957$ H $-5.855705-1.9665963 .665161$ H -4.0800410 .0870214 .305968$ H $-0.995506 \quad 0.1008142 .725770$ H $-4.158770-3.407116-2.114272$ H -3.406973 -4.996355 -2.023036 H -4.948304 -4.529493 -0.120975 C $-4.5078772 .183117-1.315824$ O $-3.6025651 .770645-0.282628$ C -3.9155222 .2293331 .034725$ C -3.9313303 .7663781 .080223$ C $-4.869737 \quad 4.347808 \quad 0.017549$ C $-4.6350343 .717575-1.358725$ H -4.032505 $1.857972-2.250183$ C $-5.865651 \quad 1.470524-1.209343$ H -4.9031531 .8591331 .324444$ O $-2.617357 \quad 4.286571 \quad 0.886594$ H -4.255503 4.0860322 .077303 H $-4.7127215 .431168-0.053769$ O -6.2238054 .1277190 .400550$ H -5.439716 4.005896 -2.045348 O $-3.4216044 .261211-1.885798$ C $-5.752483-0.024612-1.419006$ H $-6.3300851 .624478-0.232087$ H $-6.538197 \quad 1.856973-1.984077$ H -2.300075 3.9727630 .022977 H -6.3613534 .5647841 .258344$ H -3.309761 $3.912307-2.786422$

O $-6.996622-0.577776-1.303023$ O $-4.716363-0.630569-1.651956$ C -7.064918-1.997091-1.470563 H $-8.119130-2.282584-1.519903$ H $-6.577245-2.303518-2.400913$ H $-6.607268-2.492865-0.610417$

SCF Energy (B3LYP/6-31G**//MMFF) $=-3245.92003146$

\section{$07 \quad 00165$}

MM̄FF Geometry

C -4.7726842 .4203021 .531652$

C -3.4331782 .3529261 .530911$

C -2.5850802 .4967762 .769987$

O $-1.904574 \quad 1.252203 \quad 3.074093$

C -1.622242 3.7006382 .666627

C -0.6469233 .8700023 .819165$

C 0.6430134 .2307023 .656991

C -1.190369 3.6584685 .208433

C 1.3223604 .6153242 .371351

C 2.1805763 .4822031 .809650

C 2.8487363 .8274080 .468299

C $1.8278464 .025527-0.657760$

C $2.4977914 .233425-2.024345$

O 3.7209222 .7318730 .151097
O $1.4923924 .086275-3.038904$

C $3.5265143 .108152-2.245565$

C $3.0583705 .652126-2.176421$

O $4.274743 \quad 3.325900-3.438972$

C $4.4839382 .910797-1.050752$

C $5.3952141 .674205-1.226377$

O $5.3460044 .050613-0.946523$

C -0.9468250 .7401772 .260297$

O $-0.5733351 .175821 \quad 1.185486$

C $-0.406202-0.4606092 .937502$

C $0.668368-1.0954802 .445162$

C $1.305386-2.2800663 .031119$

C $0.728815-2.8119694 .318676$

C $4.6605560 .337252-1.342258$

C $5.622143-0.835623-1.429904$

C $5.608234-3.332036-1.502270$

O $6.842064-0.708356-1.480880$

C $5.656047-3.990145-0.155642$

C $5.663524-3.3789251 .040073$

C $5.692527-4.1188922 .347174$

C $4.547208-3.7350423 .256597$

C $4.772983-3.1788784 .458889$

C $3.142670-4.0592862 .798565$

C $2.367421-2.8287882 .403190$

N $4.943194-2.044855-1.453458$

H -5.3014212 .5693172 .469978$

H -2.916254 2.2067160 .586395

H -3.246686 $2.668513 \quad 3.628259$

H -2.220028 4.6194322 .594706

H -1.074909 3.6216571 .721296

H 1.2737984 .3142584 .540885

H -0.4746803 .9544255 .983094$

H -2.094016 4.2590115 .356062

H -1.435822 2.6056655 .376342

H $0.5852094 .956114 \quad 1.639981$

H 1.9582185 .4872572 .570609

H 2.9622293 .2206332 .536109

H 1.5845492 .5696641 .691754

H $3.4533004 .731177 \quad 0.607429$

H $1.1555064 .864907-0.449673$

H $1.1866223 .136101-0.717575$

H $0.7886604 .733365-2.860059$

H $2.9526692 .186464-2.401718$

H $2.2527506 .392215-2.097585$

H $3.5017645 .797602-3.167561$

H $3.8070555 .901973-1.421906$

H $3.6369303 .441007-4.164024$

H $6.0534491 .817395-2.093458$

H $6.0687961 .615405-0.359481$

H $6.1383913 .793729-0.446577$

H $-0.906910-0.7838723 .843675$

H $1.125268-0.7173191 .531602$

H $-0.295544-3.1676584 .162106$

H $0.717518-2.0307985 .087208$

H $1.294392-3.6432494 .743748$

H $4.0116580 .185698-0.471972$

H $4.0418980 .314654-2.245340$

H $6.618552-3.229840-1.910346$

H $5.020683-3.953510-2.185746$

H $5.684782-5.077909-0.179295$

H $5.662548-2.2937811 .098194$

H $6.655116-3.9073832 .829619$

H $5.664238-5.2042752 .188396$

H $3.955274-2.9263015 .125808$

H $5.778817-2.9641954 .803854$

H $2.638652-4.6473793 .571189$

H $3.176967-4.7293331 .929643$

H $2.717314-2.3672381 .478975$

H $3.934330-2.043681-1.342939$

C $-6.420375 \quad 1.0315110 .251059$

O $-5.538238-0.071454-0.046291$

C $-6.191678-0.948487-0.984233$

C $-7.638507-0.476922-1.070820$

C $-7.4822271 .012920-0.847296$

H $-5.712211-0.787227-1.956510$

C $-6.034151-2.408165-0.549026$

H -8.097483 -0.722580 -2.033361

H $-8.257510-0.918461-0.280902$ 
C $-8.779248 \quad 1.707266-0.469169$ H $-7.0835141 .467087-1.764489$ C $-4.573137-2.892248-0.442571$ O $-6.643124-2.5792060 .738263$ H $-6.591408-3.060083-1.231758$ H $-4.068394-2.3758630 .381794$ O $-4.607142-4.281325-0.088079$ C $-3.750964-2.740463-1.738001$ C $-2.398733-3.478494-1.722846$ H -3.589291 -1.678476 -1.944018 O $-4.515945-3.266954-2.827144$ H -2.579149 -4.549529-1.568756 H -1.966824 -3.415485 -2.728727 H -9.521429 $1.595420-1.266329$ H -9.2059321 .2885990 .448502$ H -8.617780 $2.777501-0.307190$ C $-5.6166632 .330047 \quad 0.291143$ H $-6.205031-1.9657351 .353135$ H -5.126639 -4.358960 0.730485 H -4.021125 $-3.091266-3.645586$ H $-6.288283 \quad 3.1961240 .283960$ H $-4.9850212 .402114-0.602125$ H -6.8754650 .8241261 .228662$ C $-0.292090-1.279810-2.039321$ O $-1.085925-1.562404-0.879010$ C $-1.398330-2.946897-0.678466$ C $-0.108836-3.772260-0.575159$ C $0.818142-3.536705-1.763080$ C $1.039577-2.041206-1.980796$ H $-0.846327-1.557854-2.943537$ C $-0.0362610 .235133-2.085946$ H -1.886958 -3.0120080 .300532$ O $-0.413486-5.157529-0.456836$ H $0.414611-3.4860010 .342560$ H $\quad 0.424802-4.010376-2.669435$ O $2.076612-4.166232-1.510121$ H $1.661693-1.641510-1.169932$ O $1.749332-1.846373-3.201941$ C $-1.2934001 .037411-2.347575$ H $0.3833820 .564201-1.129579$ H $\quad 0.6701170 .480381-2.886984$ H $\quad 0.430419-5.638947-0.414209$ H $2.445418-3.786552-0.694409$ H $2.563415-2.376485-3.155029$ O $-1.0127642 .362409-2.172194$ O $-2.3723820 .573968-2.688184$ C -2.089776 $3.267328-2.431995$ H $-2.9695693 .001020-1.840646$ H -1.766311 $4.271194-2.144400$ H $-2.3279133 .265385-3.499556$

SCF Energy $\left(\mathrm{B} 3 \mathrm{LYP} / 6-31 \mathrm{G}^{* *} / / \mathrm{MMFF}\right)=-3245.91453353$

\section{6}

MM̄̄FF Geometry

C $-1.773515-2.714225-1.636602$

C $-0.914676-2.922103-2.644764$

C $0.037255-1.863336-3.134325$

O $1.371607-2.384321-2.937585$

C $-0.176264-1.531900-4.623209$

C $-1.569943-1.047840-4.964456$

C $-2.0414620 .182407-4.683191$

C $-2.441795-2.044875-5.682955$

C $-1.3178841 .314751-4.007729$

C $-1.9389801 .630223-2.639232$

C $-1.3636362 .878719-1.947309$

C $-1.7920244 .172718-2.645113$

C -1.169413 $5.412024-1.986846$

O $0.0612542 .771314-1.914861$

O $-1.3577856 .525104-2.874351$

C $0.3509865 .189526-1.859022$

C $-1.8713785 .780351-0.674723$

O $0.9588776 .259041-1.136668$

C $0.7282673 .833557-1.215493$

C $2.2533183 .599241-1.357847$

O 0.3726993 .8600280 .165006

C $2.375178-1.476772-2.797529$

O $2.296567-0.266717-2.935241$

C $3.591931-2.214350-2.390596$
C $4.630614-1.534171-1.882808$

C $5.882762-2.105668-1.384292$

C $6.063039-3.597434-1.486623$

C $2.7513992 .255341-0.807955$

C 3.2556572 .3077210 .625113

C 4.2883780 .8165632 .347593

O $3.2923743 .335807 \quad 1.294225$

C 5.7542840 .6091372 .131474

C $6.384531-0.5480112 .383839$

C $7.852060-0.7698682 .151366$

C $8.120974-1.9201801 .204923$

C $8.403133-3.1461071 .677742$

C $8.149028-1.635676-0.280924$

C $6.801435-1.278010-0.843262$

N 3.6694841 .0559071 .058035

H $-1.763407-1.751410-1.132166$

H $-0.906750-3.880726-3.157158$

H $-0.076239-0.957353-2.525916$

H $0.071800-2.413399-5.231209$

H $\quad 0.558247-0.783051-4.946051$

H $-3.062080 \quad 0.420521-4.980935$

H -3.435858 -1.643758 -5.907470

H $-1.980525-2.333522-6.633185$

H -2.580743 -2.945124 -5.076902

H $-0.251221 \quad 1.099816-3.892379$

H $-1.3870082 .184180-4.669802$

H -3.027785 $1.736261-2.727354$

H $-1.7609660 .768099-1.982821$

H -1.739245 $2.873527-0.916843$

H -2.885585 $4.257806-2.667770$

H $-1.4720214 .157892-3.694611$

H -2.312963 $6.629818-3.024754$

H $0.7745135 .220715-2.872872$

H $-2.9313705 .995828-0.855659$

H -1.449420 $6.695166-0.244391$

H -1.8206494 .9899080 .076857$

H $0.7073807 .087581-1.579855$

H $2.4932963 .609836-2.429761$

H $2.8090974 .421670-0.893628$

H $0.8436193 .146053 \quad 0.621519$

H $3.582927-3.294383-2.481629$

H $4.553302-0.450535-1.806042$

H $5.277595-4.119177-0.928376$

H $7.015915-3.947558-1.083437$

H $6.023759-3.918444-2.533298$

H $1.9774491 .484679-0.881769$

H $3.6033041 .933473-1.420462$

H $3.808216-0.0668962 .779124$

H 4.1211871 .6673043 .014933

H 6.3206491 .4520921 .742484

H $5.817002-1.3856832 .783244$

H $8.318244-0.9693393 .125157$

H $8.3407150 .136141 \quad 1.771905$

H $8.627388-3.9722861 .011462$

H $8.412441-3.3515562 .743174$

H $8.584760-2.474894-0.835654$

H $8.835639-0.799729-0.468387$

H $6.580469-0.210983-0.800622$

H 3.6044460 .2687830 .418765

C $-4.167474-3.064145-1.058955$

O $-4.182871-2.1590690 .063912$

C $-5.469645-2.2341410 .708162$

C $-6.341734-3.108414-0.186333$

C $-5.310551-4.040198-0.797374$

H $-5.316713-2.7577191 .658560$

C $-6.006314-0.8209540 .964267$

H -7.116326 -3.6355890 .378988$

H $-6.838162-2.521971-0.968328$

C $-5.807208-4.761822-2.037833$

H $-5.009482-4.779280-0.042948$

C $-5.0490320 .060594 \quad 1.797225$

O $-6.200816-0.179855-0.303159$

H $-6.992637-0.8766121 .439369$

H $-4.1213810 .210637 \quad 1.235497$

O $\quad-5.644551 \quad 1.3584201 .924555$

C $-4.746170-0.4876213 .205208$

C $-3.827480 \quad 0.4212844 .046986$

H $-4.298481-1.4833553 .131980$ 
O $-5.987101-0.6370283 .901185$ H -4.2326321 .4397064 .081641$ H -3.8547500 .0529295 .081207$ H -6.675619 $-5.384181-1.798382$ H $-6.105035-4.056054-2.820555$ H -5.029691 -5.412518 -2.449603 C $-2.787176-3.716116-1.158778$ H $-6.5248240 .719849-0.125673$ H -6.4532931 .2579612 .455582$ H -5.794184-1.049902 4.760297 H -2.819874 -4.575041-1.837968 H $-2.470564-4.074502-0.171941$ H $-4.375807-2.466292-1.956904$ C $-0.540339-1.0957593 .176505$ O $-1.863478-0.8946173 .688413$ C -2.372354 0.4375793 .550832 C -1.4946401 .4160054 .346142$ C $-0.026390 \quad 1.316342 \quad 3.916014$ C $0.458477-0.1371013 .845665$ H $-0.279924-2.1154123 .487243$ C $-0.554887-1.0541381 .645740$ H $-2.361070 \quad 0.7243962 .494538$ O $-1.601744 \quad 1.1259875 .738634$ H -1.8674042 .4353224 .188687$ H $\quad 0.594674 \quad 1.8733314 .628218$ $\begin{array}{lllll} & 0 & 0.117218 & 1.936000 & 2.642533\end{array}$ H $1.420990-0.1912093 .327850$ O $0.692725-0.6002655 .180185$ C $0.641134-1.7420551 .037409$ H -1.459152 -1.542732 1.263496 H $-0.595498-0.032716 \quad 1.255737$ H -1.0709651 .7857386 .216922$ H 1.0591101 .8929522 .405146 H $-0.162498-0.6156085 .643331$ O $0.548109-3.0904911 .213783$ O $1.545487-1.1499780 .463794$ C $1.631130-3.860395 \quad 0.682676$ H $1.584025-3.861519-0.409408$ H $2.593489-3.4702691 .028504$ H $1.523581-4.8881011 .039521$

SCF Energy (B3LYP/6-31G**//MMFF) $=-3245.92303926$

\section{7_00167}

MM̄FF Geometry

C $4.940519-3.647852-0.063043$

C $3.770169-3.664362-0.718504$

C $2.479563-4.154458-0.114940$

O $1.591014-3.0182500 .010110$

C $1.814614-5.195093-1.033666$

C $0.420482-5.608563-0.596657$

C $-0.660833-5.570913-1.400842$

C $0.282400-6.0994530 .821019$

C $-0.696670-5.204911-2.857435$

C $-1.000646-3.728438-3.145943$

C $-2.428285-3.309383-2.762844$

C $-2.814463-2.008789-3.472917$

C $-4.218990-1.538438-3.068152$

O $-2.484584-3.113586-1.344905$

O $-4.398862-0.198977-3.552339$

C $-4.312624-1.513587-1.529799$

C $-5.313149-2.365997-3.750949$

O $-5.644584-1.236183-1.103379$

C $-3.794980-2.805321-0.850169$

C $-3.706155-2.5711690 .674805$

O $-4.712582-3.872349-1.098731$

C $1.746438-2.2434091 .119146$

O $2.564750-2.3965642 .012725$

C $0.742471-1.158758 \quad 1.064148$

C $0.815098-0.1674141 .965027$

C -0.0972020 .9700812 .086550$

$\begin{array}{llll}\text { C }-1.248245 & 1.044752 & 1.123413\end{array}$

C -2.910353 -3.630499 1.445318

C $-3.003291-3.3662032 .936325$

C $-2.295273-1.7252994 .683221$

O $-3.689430-4.0706133 .673190$

C -2.661189 -0.2743974 .619247$

C -1.9845280 .6937035 .256121$

C -2.3491772 .1506515 .184376$
C -1.1675183 .0403564 .858620$

C $-0.6072373 .816105 \quad 5.802070$

C $-0.682088 \quad 3.087982 \quad 3.424391$

C $\quad 0.1301351 .873770 \quad 3.062672$

N -2.277493 -2.2537123 .330970$

H $4.995148-4.0361330 .950773$

H $3.717260-3.239480-1.718605$

H $2.650578-4.6026440 .872394$

H $2.444450-6.092841-1.083869$

H $1.784938-4.782989-2.048264$

H $-1.622801-5.872116-0.988226$

H $-0.709255-6.5205371 .019037$

H $1.016609-6.8863341 .022415$

H $0.438688-5.2826911 .532178$

H $0.248306-5.474443-3.342582$

H $-1.450074-5.834521-3.348270$

H $-0.269939-3.096348-2.625753$

H $-0.857223-3.575513-4.222926$

H $-3.115929-4.113888-3.048838$

H $-2.751751-2.122249-4.562331$

H $-2.090312-1.224719-3.210509$

H $-3.7110760 .363520-3.156739$

H -3.694987 -0.674359-1.181114

H $-5.222999-2.291058-4.841366$

H $-6.309972-1.981870-3.506884$

H $-5.279680-3.425105-3.487482$

H $-5.931119-0.424602-1.556138$

H -3.228675 -1.6025620 .857224$

H $-4.717369-2.5086921 .098320$

H $-4.635418-4.526838-0.386570$

H $-0.020833-1.2073990 .296138$

H $1.620510-0.1915822 .698333$

H $-0.887593 \quad 1.1104670 .091707$

H -1.8935411 .9070771 .287729$

H $-1.8842140 .160894 \quad 1.215878$

H $-3.293083-4.6384261 .254515$

H -1.854733 -3.6225391 .151411$

H $-1.297922-1.8816015 .106060$

H $-3.025889-2.2687135 .289815$

H -3.530161 -0.0076364 .021541$

H -1.1323880 .4252665 .876517$

H $-2.774542 \quad 2.433293 \quad 6.156148$

H -3.142382 2.3271714 .446993

H 0.2240424 .4744325 .572273

H $-0.963537 \quad 3.806915 \quad 6.826858$

H $-0.040198 \quad 3.965675 \quad 3.271241$

H -1.5433193 .2460802 .770557$

H $1.005648 \quad 1.7347523 .698964$

H $-1.675623-1.7947262 .653973$

C $6.377281-1.618736-0.072449$

O $5.344065-0.768211-0.610047$

C $5.9110020 .508038-0.952997$

C $7.3150180 .511727-0.361062$

C $7.692063-0.954512-0.470608$

H $5.9814790 .539332-2.047685$

C $5.0048731 .649722-0.469086$

H $7.9942841 .172938-0.907571$

H 7.3076520 .8277320 .688890

C $8.871969-1.3453670 .401686$

H $7.926464-1.181547-1.519309$

C $3.6230181 .666777-1.162794$

$\begin{array}{lllll}\text { O } & 4.844719 & 1.554858 & 0.945419\end{array}$

H $5.5080212 .602422-0.671762$

H $3.7811051 .695315-2.248170$

$\begin{array}{lllll}\text { O } & 2.898603 & 0.472265 & -0.873247\end{array}$

C $2.7458362 .876738-0.773281$

C $1.4388922 .906985-1.581772$

H $3.3120883 .795731-0.960179$

$\begin{array}{llll}H & 2.413853 & 2.844443 & 0.611206\end{array}$

H $1.6682432 .952299-2.654390$

H $0.896648 \quad 1.962125-1.443018$

H $9.766817-0.7852480 .111307$

H $8.675082-1.1391971 .459132$

H $9.094517-2.4123790 .302253$

C $6.194114-3.033783-0.623166$

$\begin{array}{llll}\text { H } & 4.388000 & 0.715829 & 1.129739\end{array}$

H $3.417604-0.279320-1.205724$

H 3.2437292 .8783071 .115932 
H $7.043817-3.670435-0.353760$

H $6.139506-3.006809-1.718675$

H $6.259762-1.6126181 .019528$

C $-1.7288964 .835191-1.925713$

O $-0.6529503 .905329-2.103117$

C $0.4614394 .040028-1.208325$

C $1.0729145 .444943-1.311710$

C $0.0107126 .531825-1.115081$

C -1.221707 6.286773 -1.987302

H $-2.3833784 .673873-2.792071$

C $-2.5471054 .532200-0.660853$

H $0.1110523 .864180-0.185589$

O $1.6909845 .599322-2.587610$

H $1.8540305 .555058-0.551112$

H $0.4366927 .510944-1.366626$

O -0.3899526 .5698010 .251593$

H -2.025652 6.975146-1.701109

O $-0.8731346 .593345-3.340282$

C $-3.3559963 .257388-0.792356$

H -1.9169874 .4236190 .225359$

H $-3.2585105 .349593-0.492623$

H $2.0806046 .489454-2.619737$

H $\quad 0.401975 \quad 6.7523970 .785332$

H -1.675973 6.496727 -3.880172

O -4.2493503 .1959120 .240386$

O $-3.2110172 .412249-1.664533$

C -5.0750722 .0279380 .275146$

H -5.8005412 .1483951 .084016$

H $-5.6179601 .913201-0.667808$

H -4.4638001 .1447830 .481455$

SCF Energy $\left(\mathrm{B} 3 \mathrm{LYP} / 6-31 \mathrm{G}^{* *} / / \mathrm{MMFF}\right)=-3245.91240883$

0700168

MM̄FF Geometry

C 4.1876381 .4050532 .999841

C 3.4147870 .4194652 .517326

C $2.007073 \quad 0.1607852 .984182$

O $1.158196 \quad 0.385227 \quad 1.834661$

C $1.849539-1.2990123 .447036$

C $0.733378-1.4897984 .455326$

C $-0.473862-2.0288234 .196020$

C $1.076299-1.0725785 .864909$

C -1.027034 -2.507453 2.881549

C $-2.364026-1.8246482 .583281$

C $-3.128403-2.393151 \quad 1.378491$

C $-2.309645-2.4011920 .087089$

C -3.148192 -2.824451-1.128225

O $-4.281288-1.5558431 .224112$

O $-2.412418-2.489437-2.314422$

C $-4.443897-1.992313-1.158417$

C $-3.369308-4.340947-1.166095$

O $-5.318260-2.449435-2.188259$

C $-5.190683-1.9578400 .193566$

C $-6.315541-0.9000910 .131811$

O $-5.759193-3.239070 \quad 0.460032$

C 0.0020491 .0765422 .012063

O -0.4535201 .5026093 .060549$

C $-0.623137 \quad 1.2299280 .678502$

C - -1.8587561 .7398350 .565093$

C $-2.5938061 .948574-0.686607$

C $-1.8857501 .604652-1.971801$

C -7.073437 -0.6974521 .451537$

C $-7.666440 \quad 0.6958251 .508638$

C $-9.2517552 .191924 \quad 0.315244$

O -7.2224171 .5535452 .268963$

C $-8.4909612 .761066-0.841726$

C -7.638546 $3.792469-0.744339$

C $-6.8430194 .321995-1.904144$

C $-5.3517404 .165965-1.695255$

C $-4.5683955 .242972-1.512037$

C $-4.7906312 .756560-1.758243$

C -3.851542 $2.435292-0.623548$

N $-8.702821 \quad 0.8804150 .606295$

H 3.8024402 .0599683 .777193

H $3.800128-0.2220741 .727866$

H 1.7565630 .8629053 .788287

H $2.776572-1.6474713 .923238$

H $1.705299-1.9527682 .579904$
H -1.163408 -2.144117 5.032413

H $\quad 0.259382-1.2662876 .568334$

H $1.952922-1.6237066 .221020$

H $1.297851-0.0019215 .908296$

H $-0.330617-2.3157542 .062441$

H -1.171994 -3.592218 2.942425

H -3.024100 -1.894771 3.459549

H -2.207904 -0.748994 2.440209

H -3.456551 -3.408613 1.629987

H -1.436540 -3.056271 0.179067

H -1.913906 -1.395219-0.099509

H -1.564777 -2.964359-2.287246

H $-4.164500-0.963350-1.425236$

H -2.408096 -4.866667-1.215495

H -3.918955 -4.638266 -2.065878

H $-3.906043-4.716929-0.292461$

H -4.814858 -2.440488 -3.020399

H $-5.8738000 .060024-0.162964$

H -7.042736 -1.172802 -0.643215

H $-6.488956-3.1333881 .090868$

H $-0.0469130 .907544-0.180193$

H -2.386088 2.0307231 .472651

H $-0.9775552 .207522-2.083635$

H $-2.4885091 .775967-2.865383$

H $-1.6075190 .544752-1.985781$

H -7.876032 -1.4354181 .561194$

H -6.412836 -0.8075122 .318368$

H $-9.1862582 .826324 \quad 1.205217$

H -10.3056842 .0663370 .050107$

H -8.636491 2.280129-1.806409

H $-7.489603 \quad 4.2738710 .219365$

H -7.107951 $5.378336-2.039202$

H -7.122033 $3.821762-2.840202$

H -3.494275 $5.151594-1.390766$

H $-4.9835776 .245163-1.485979$

H $-4.3415602 .618951-2.745071$

H -5.610144 2.030080-1.704038

H -4.2820592 .6235590 .361633$

H $-8.9486640 .120673-0.021562$

C 5.6268722 .9568731 .654657

O $4.8262402 .751603 \quad 0.472065$

C $5.6786412 .841202-0.679476$

C $6.7924803 .780380-0.249039$

C 7.0437933 .2850001 .165288

H $6.1012291 .841322-0.843927$

C $4.8751553 .255315-1.915518$

H $7.6748653 .709725-0.891536$

H $6.4586474 .824099-0.228451$

C 7.7721164 .2882772 .042979

H 7.6456472 .3676111 .111694

C $3.5814592 .437726-2.128923$

O $4.4973564 .630846-1.784230$

H $5.5183333 .197986-2.801841$

H $2.8254372 .763546-1.403278$

O $3.0767282 .786852-3.422857$

C $3.7676220 .913005-2.033586$

C $2.4672150 .130312-2.300071$

H $4.1304040 .662271-1.031097$

O $4.7709150 .513284-2.966147$

H $1.6186950 .661963-1.856669$

H $2.3080550 .059688-3.382961$

H $8.7611394 .513573 \quad 1.631079$

H 7.2180645 .2297472 .121239

H 7.9077633 .8915563 .054206

C 5.5798741 .6853592 .507481

H $3.9995264 .871560-2.584459$

H $2.1786942 .423473-3.499578$

H $4.904906-0.444216-2.862208$

H $6.2446911 .783192 \quad 3.373185$

H 5.9378240 .8326041 .918419

H 5.1858933 .8029472 .195643

C $3.834941-3.319065-1.846156$

O $3.566395-2.008840-2.361463$

C $2.529295-1.285735-1.695442$

C $1.199104-2.039668-1.819272$

C $1.329439-3.477358-1.303078$

C $2.564464-4.185630-1.874234$

H $4.553002-3.756425-2.552194$ 
C $4.512186-3.260579-0.467536$

H $2.781461-1.179932-0.634519$

O $0.762569-2.057188-3.176278$

H $0.435140-1.507177-1.242080$

H $\quad 0.426242-4.036866-1.574183$

O $1.408861-3.4429040 .118488$

H $2.753720-5.125766-1.343216$

O $2.309650-4.520708-3.242021$

C $5.833032-2.519653-0.514706$

H $3.878717-2.757090 \quad 0.267666$

H $4.716691-4.276389-0.111045$

H $1.467981-2.460620-3.710682$

H $1.448936-4.3619260 .433386$

H $1.539731-5.114561-3.259927$

O $6.234599-2.242070 \quad 0.761087$

O $6.445773-2.234644-1.534642$

C $7.478677-1.5435420 .873609$

H $7.433260-0.593200 \quad 0.332777$

H $7.657364-1.3356451 .931879$

H $8.294840-2.1656430 .494237$

SCF Energy (B3LYP/6-31G**//MMFF) $=-3245.91180954$

\section{7_00169}

MM̄FF Geometry

C $-3.0339454 .802278-0.413983$

C $-1.9036804 .378847-0.997893$

C $-0.527178 \quad 4.816887-0.567060$

O $0.204745 \quad 3.632023-0.171811$

C $0.2091945 .478875-1.744169$

C $1.5923775 .986817-1.380557$

C $2.751808 \quad 5.421015-1.773037$

C $1.6161887 .236261-0.537063$

C $2.9387764 .192476-2.619350$

C $3.4898603 .032976-1.788128$

C $3.5239981 .694842-2.542084$

C $2.1189521 .154537-2.830329$

C $2.146722-0.254296-3.439385$

O $4.2349110 .774997-1.702022$

O $0.819399-0.793914-3.351717$

C $3.059497-1.153985-2.582867$

C $2.503994-0.222967-4.929946$

O $3.228475-2.433247-3.188894$

C $4.435555-0.520919-2.278811$

C $5.206801-1.394964-1.257790$

O $5.193766-0.451039-3.489711$

C $-0.004572 \quad 3.1703591 .092994$

O $-0.707695 \quad 3.676626 \quad 1.953174$

$\begin{array}{llll}C & 0.752492 & 1.908013 & 1.245127\end{array}$

C $0.590948 \quad 1.1674512 .351482$

C $1.233109-0.1188452 .635668$

C $2.217152-0.6434201 .624973$

C $6.476754-0.738044-0.697936$

C $6.223407 \quad 0.054157 \quad 0.572168$

C $5.889121-0.2253713 .018258$

O $\quad 6.2364301 .2826250 .584594$

C $5.236167-1.2413163 .898959$

C $4.256198-0.9588454 .771217$

C $3.610479-2.0026195 .638218$

C $2.098905-1.9488175 .623855$

C $1.405470-1.8449316 .770924$

C $1.384194-2.0872224 .295909$

C $0.900559-0.7602153 .775140$

N $5.987909-0.758601 \quad 1.671138$

H $-2.979060 \quad 5.5252110 .396311$

H -1.959938 $3.643344-1.797243$

H $-0.579745 \quad 5.516750 \quad 0.276182$

H $-0.3793986 .327037-2.119513$

H $0.2606294 .772758-2.578713$

H $3.6836945 .884259-1.450542$

H $2.6330467 .609658-0.375026$

H $1.0489148 .035227-1.025815$

H $1.176167 \quad 7.047218 \quad 0.446654$

H $2.0110723 .906778-3.114735$

H $3.6446984 .430755-3.424388$

H $4.5077093 .278051-1.454534$

H $2.9107822 .912202-0.864757$

H $4.0870391 .834392-3.472345$

H $1.5560881 .827041-3.486702$
H $1.5487111 .110007-1.893075$

H $0.218673-0.194096-3.826168$

H $2.545434-1.329849-1.628940$

H $1.7634610 .361762-5.489117$

H $2.479456-1.228640-5.363697$

H $3.484357 \quad 0.215915-5.126758$

H $2.340566-2.799286-3.343092$

H $4.540666-1.658734-0.427596$

H $5.503211-2.329825-1.750418$

H $6.138947-0.520723-3.283596$

H $1.403434 \quad 1.6070310 .434536$

H $-0.0936491 .525992 \quad 3.119457$

H $1.724296-0.8077710 .661412$

H $2.674946-1.5923441 .910173$

H 3.0346220 .0707571 .478763

H $7.208335-1.519373-0.454505$

H $6.964660-0.080987-1.425130$

H $5.343643 \quad 0.7238442 .992030$

H $6.909214-0.0288173 .364065$

H $5.592273-2.2664283 .822401$

H 3.9158320 .0679874 .879713

H $3.986855-1.8629726 .659553$

H $3.919978-3.0096455 .330619$

H $0.320253-1.8317406 .773378$

H $1.904613-1.7703987 .731180$

H $0.504535-2.7326504 .425386$

H $2.019173-2.6235713 .585672$

H $0.161329-0.2906844 .426639$

H $6.168819-1.7536731 .570281$

C -4.9875813 .4698790 .364238$

$\begin{array}{lllll}\text { O } & -4.243575 & 2.242498 & 0.501274\end{array}$

C $-5.147794 \quad 1.1952330 .905681$

C -6.5124841 .8523151 .097988$

C $-6.430953 \quad 3.028995 \quad 0.143854$

H -5.2149440 .4947620 .066198$

C -4.6028090 .4831402 .150051$

H $-7.334768 \quad 1.1680540 .866073$

H -6.6540272 .2023042 .127234$

C $-7.462702 \quad 4.1071640 .424183$

H $-6.5594252 .660038-0.882610$

C $-3.200451-0.1307941 .952903$

$\begin{array}{lllll}\text { O } & -4.520709 & 1.443364 & 3.210984\end{array}$

H $-5.314748-0.2838572 .477422$

H $-2.4642380 .659645 \quad 1.766217$

O $-2.817155-0.7625803 .181024$

C $-3.121976-1.1825750 .828020$

C $-1.735498-1.8549730 .762822$

H $-3.332624-0.704650-0.134735$

O $-4.132656-2.1622801 .059550$

H $-0.967788-1.075003 \quad 0.721232$

H $-1.587334-2.4408881 .678453$

H $-8.476093 \quad 3.703067 \quad 0.330544$

H -7.3545654 .5129381 .435675$

H $-7.364606 \quad 4.936326-0.283333$

C $-4.4027804 .298449-0.779445$

H $-4.3738490 .953054 \quad 4.037426$

H -2.630004 $-0.058676 \quad 3.824969$

H $-4.102393-2.7977150 .325038$

H $-5.0399045 .163107-0.994204$

H $-4.3519103 .691565-1.691867$

H -4.8945674 .0113361 .315718$

C $-2.445056-4.858890-1.356442$

O $-2.444896-3.899414-0.291797$

C $-1.558365-2.788985-0.451868$

C $-0.107234-3.282152-0.580125$

C $0.042256-4.335853-1.681562$

C $-1.033529-5.423198-1.582349$

H $-3.078427-5.677892-0.991420$

C $-3.090327-4.301361-2.630003$

H $-1.834935-2.230177-1.352504$

O $0.344404-3.828707 \quad 0.658273$

H $\quad 0.535570-2.424206-0.802695$

H $1.036944-4.791392-1.603780$

O $-0.040667-3.689999-2.947739$

H $-1.032899-6.052920-2.479625$

O $-0.731536-6.274314-0.472837$

C $-4.553230-4.005073-2.399494$

H $-2.604102-3.393762-2.998825$ 
H $-3.034302-5.031454-3.446175$ H $-0.267127-4.5424680 .908325$ H $\quad 0.092372-4.367300-3.632524$ H $\quad 0.141397-6.670446-0.636620$ O $-4.729781-2.672000-2.173325$ O $-5.428740-4.860723-2.396300$ C $-6.077114-2.262464-1.919458$ H $-6.453242-2.742403-1.011030$ H -6.080658 -1.179468 -1.771221 $\mathrm{H}-6.714344-2.501638-2.776072$

SCF Energy (B3LYP/6-31G**//MMFF) $=-3245.90294855$

\section{7_00170}

MM̄MF Geometry

C -1.904916 -3.1574901 .477480$

C $-2.232379-2.6870190 .264280$

C $-1.504668-2.925037-1.037677$

O $-1.050109-1.656179-1.581769$

C -0.386932 -3.993540 -1.034222

C $0.247576-4.238657-2.390846$

C $1.557655-4.092272-2.674478$

C $-0.688735-4.737823-3.463661$

C $2.682042-3.662140-1.770282$

C $3.397016-2.428076-2.336215$

C $4.563508-1.904955-1.479742$

C $5.735951-2.888011-1.434793$

C $6.882941-2.366148-0.556517$

O $4.089158-1.631545-0.156865$

O $7.805754-3.445728-0.343661$

C $6.305298-1.9430420 .811192$

C $7.693354-1.281602-1.274107$

O $7.314686-1.3403601 .619159$

C $5.061704-1.0241730 .708957$

C $4.426336-0.8682662 .115670$

O $5.475548 \quad 0.250953 \quad 0.221422$

C $-0.069494-0.958316-0.951119$

O $0.507891-1.2610700 .079723$

C $0.2017900 .256419-1.751883$

C $1.3802250 .881161-1.610202$

C $1.8261302 .073753-2.332019$

C $0.7912632 .831625-3.120187$

C $3.143061-0.0288002 .169403$

C 3.3756571 .4670082 .298755

C 2.1597633 .6380082 .212371

O 4.4779791 .9575322 .527889

C 2.2883974 .1921910 .829094

C 3.4059644 .7638280 .356766

C $3.5329905 .345871-1.022145$

C $4.4553844 .537543-1.906248$

C $5.7872684 .708410-1.857070$

C $3.8239683 .589044-2.901805$

C $3.1246342 .432436-2.241065$

N 2.1986382 .1892692 .150400

H -1.021351 -3.774711 1.607399

H -3.117254 -2.059753 0.182898

H $-2.280610-3.264766-1.735062$

H $-0.808123-4.947259-0.687583$

H $\quad 0.373446-3.730432-0.296232$

H $\quad 1.882587-4.330531-3.687595$

H $-0.155723-5.065095-4.362934$

H -1.263953 -5.594804 -3.097966

H -1.385464 -3.949842 -3.765205

H $2.341687-3.455556-0.753773$

H $3.382695-4.501483-1.701647$

H $3.763226-2.638732-3.349359$

H $2.658326-1.624287-2.432426$

H $4.887666-0.961580-1.935501$

H $6.097166-3.112515-2.445996$

H $5.401634-3.845860-1.016412$

H $7.332003-4.1688480 .101122$

H $6.010627-2.8622721 .335490$

H $8.113000-1.675156-2.207906$

H $8.553132-0.966596-0.672350$

H $7.104454-0.396761-1.524104$

H $7.445826-0.4313731 .299312$

H $4.159937-1.8710072 .475954$

H $5.160819-0.4551932 .816939$

H 4.7548070 .8843780 .360116
H $-0.5606770 .584635-2.448691$

H $2.1085500 .462791-0.918171$

H $0.4488552 .238686-3.975219$

H $1.1514663 .785046-3.511125$

H $-0.0730563 .070249-2.490276$

H $2.515075-0.2258921 .298679$

H $2.576174-0.3283703 .059642$

H 2.9564743 .9979372 .872042

H 1.1982823 .9303072 .645509

H 1.4122394 .1339290 .189564

H 4.2819644 .8263480 .998656

H $3.9152386 .370576-0.925966$

H $2.5517985 .448865-1.502253$

H $6.4547544 .151265-2.505962$

H $6.2392435 .408726-1.162135$

H $4.5930573 .167038-3.562122$

H $3.1668294 .164072-3.559526$

H $3.7918701 .807895-1.645957$

H 1.3461821 .7026351 .888560

C $-2.266639-1.6469593 .499590$

O $-2.504812-0.4595552 .718861$

C -1.4041200 .4483752 .919694$

C $-0.633183-0.0871484 .120550$

C $-0.801848-1.5842893 .929318$

H -0.7645470 .3685592 .033369$

C -1.9221221 .8832193 .055304$

H 0.4119440 .2344884 .121925

H $-1.0854470 .227523 \quad 5.068113$

C -0.486586 -2.400923 5.169130

H $-0.139561-1.9018483 .114358$

C -2.8370042 .3308101 .894877$

O $-2.6783602 .000524 \quad 4.267082$

H -1.0784442 .5744353 .160122$

H -3.7862541 .7863551 .945985$

O -3.1532373 .7141832 .089190$

C -2.2108062 .1697490 .499061$

C $-3.0598372 .725001-0.658912$

H $-2.009760 \quad 1.1118870 .306949$

O -0.9478752 .8366800 .470427$

H -3.231203 $3.799756-0.521274$

H -2.456184 2.635788 -1.571856

H $0.557511-2.2596335 .466962$

H -1.119301 -2.109765 6.014199

H -0.643617 -3.4678594 .981679$

C $-2.712624-2.8822522 .715488$

H $-3.396340 \quad 1.345188 \quad 4.229960$

H -3.541929 3.8005112 .976774

H -1.1070673 .7753530 .670154$

H $-3.766224-2.7586872 .433346$

H -2.666572 -3.768208 3.359735

H $-2.914745-1.5582554 .381958$

C $-5.257353-0.233635-1.206728$

O $-4.1076800 .616974-1.107106$

C $-4.3974261 .994988-0.850136$

C $-5.2156282 .580797-2.010464$

C $-6.4925201 .767601-2.253913$

C $-6.2167150 .260014-2.306206$

H $-4.852246-1.201846-1.526922$

C $-5.898723-0.4271970 .171469$

H -4.9793822 .0787180 .073510$

O $-4.4364302 .607083-3.205180$

H -5.474606 $3.619330-1.774211$

H -6.947485 2.080405 -3.201958

O $-7.4314712 .029773-1.215517$

H $-7.159949-0.296148-2.264093$

O $-5.621537-0.023077-3.577523$

C -6.730511-1.683535 0.228087

H $-5.128961-0.4881530 .951518$

H -6.5308980 .4155750 .465558$

H $-4.1484991 .697108-3.392245$

H $-7.6250142 .982638-1.227808$

H -5.524453 -0.988047 -3.648019

O $-5.924464-2.7666790 .416961$

O $\quad-7.948189-1.700720 \quad 0.105035$

C $-6.592235-4.0303790 .478297$

H -7.112035 -4.229959 -0.463642

H -5.837455 -4.805751 0.633829

H -7.292668 -4.048369 1.318609 
C $7.365588-1.5003142 .114281$

O $4.489133-1.5898131 .689687$

O $8.343488-1.2802703 .142156$

C $6.486786-0.2370102 .004731$

C $8.172566-1.8213720 .851761$

O $7.234921 \quad 0.8553881 .474630$

C $5.195506-0.4492741 .177252$

C $4.282063 \quad 0.792418 \quad 1.325529$

O $5.543282-0.616950-0.197565$

C $1.250811-0.435568-2.976446$

O $2.069443-0.684983-3.845808$

C $0.5700720 .863840-2.780151$

C $1.0292491 .956373-3.407053$

C $0.4612183 .304022-3.317644$

C $-0.8619773 .452735-2.614884$

C 2.9286670 .6771820 .615020

C 1.9903151 .7923971 .029295

C 1.6709074 .2447730 .942829

O $1.005596 \quad 1.5719801 .731204$

C 2.0163995 .3564940 .006321

C $1.1217655 .936290-0.807322$

C $1.4329547 .074402-1.734340$

C $1.7927826 .634335-3.135846$

C $2.9439867 .032780-3.704277$

C $0.7990205 .792432-3.908727$

C $1.1457404 .327564-3.869371$

N 2.3620943 .0350320 .539941

H - $-0.334194-4.741223-2.753398$

H $0.065692-3.092023-0.200758$

H $\quad 1.286017-3.007224-3.056281$

H $2.641809-2.167535-0.435401$

H $3.357324-2.116686-1.997961$

H $3.854338-5.712820-0.057766$

H $4.097966-5.749977-2.340956$

H $2.747100-4.949598-3.147990$

H $4.337347-4.159178-3.068257$

H $2.464609-3.3032121 .301720$

H $2.481271-4.9652491 .823643$

H $3.999337-3.6987843 .192485$

H $4.923065-4.8734602 .252806$

H $5.528073-3.0301780 .626077$

H $7.065201-3.6148182 .530918$

H $6.176608-2.5327193 .582626$

H $7.874928-1.0960043 .974056$

H 6.1963350 .0531583 .024164

H $8.830904-2.6805351 .028964$

H $8.833242-0.9900680 .581980$

H $7.546906-2.057600-0.011494$

H 8.0310640 .9557132 .024215

H 4.0897770 .9685802 .392317

H 4.8023051 .6781720 .940850

H $4.759943-0.449884-0.744178$

H $-0.2865420 .886140-2.115592$

H $1.9136701 .861453-4.036007$

H -1.270459 $4.464026-2.659852$

H -0.760754 3.195299-1.555917

H -1.614591 $2.795393-3.064851$

H $3.0433890 .707843-0.473622$

H $2.437380-0.2683350 .866892$

H 1.9956374 .4932541 .958620

H 0.5946684 .0467980 .976038

H 3.0446855 .7095200 .008406

H $2.2349107 .687308-1.302118$

H $0.5558017 .731892-1.783095$

H $3.2014996 .742389-4.717696$

H $3.6545087 .655251-3.170708$

H $0.7994626 .104400-4.961997$

H -0.214655 $6.003280-3.557569$

H $2.0912094 .101150-4.365332$

H 3.2683563 .1275340 .089838

C $-2.947879-4.796323-1.543745$

O $-3.222165-3.393282-1.358910$

C $-4.614386-3.227430-1.032320$

C $-5.260582-4.598247-1.205920$

C $-4.106717-5.524037-0.867368$

H $-4.657509-2.9562710 .028524$

C $-5.224804-2.107425-1.883556$

H $-6.126390-4.732641-0.550323$

H -5.592585 -4.765300 -2.237086

C -4.310729 -6.949522 -1.348622

H $-3.965403-5.5262430 .221801$

C $-4.466797-0.765296-1.788798$

O $-5.204845-2.525122-3.254184$

H $-6.279094-1.974333-1.612833$

H $-3.476647-0.854625-2.252751$

O $-5.1887350 .206931-2.555188$

C $-4.314915-0.224408-0.354609$

C $-3.6354611 .159733-0.315992$

H $-3.711421-0.9213010 .236287$

$\begin{array}{llll}\text { O } & -5.601123 & -0.154396 & 0.257378\end{array}$

H -2.694875 $1.108650-0.877241$

H $-4.2899611 .891102-0.806255$

H -5.198874 -7.389083 -0.882780

H $-4.445350-6.991294-2.434613$

H -3.450502 -7.575495-1.091528

C $-1.575520-5.127299-0.956570$

H $-5.776033-1.917890-3.753787$

H -5.095632 $-0.031736-3.492820$

H $-6.1389310 .469903-0.259119$

H -1.387729 -6.205687 -1.000553

H -1.540863 -4.830467 0.099042

H -2.956306 -4.984073 -2.625845

C $-4.5264192 .332547 \quad 3.124503$

O $-4.5974391 .925643 \quad 1.752638$

C -3.3456121 .6413141 .121209$

C -2.4494822 .8893501 .134924$

C -2.2846773 .4456412 .554000$

C -3.6287243 .5713863 .278397$

H -5.5520512 .6278393 .381068$

C -4.1296791 .1718214 .043460$

H -2.8429920 .8283691 .656777$

$\begin{array}{lllll}\text { O } & -3.017214 & 3.893041 & 0.295169\end{array}$

H -1.473019 2.6223760 .715896

H -1.8097314 .4334622 .508776$

O -1.4350512 .5899183 .311395$

H $-3.465473 \quad 3.7738564 .343090$

O -4.3303714 .7023552 .752968$

C -5.1686690 .0758543 .996821$

H -3.1555620 .7435013 .790838$

H -4.0577411 .5055005 .085265$

H -2.402406 4.6463150 .282856

H -0.5799612 .5410342 .850968$

H -4.5458104 .5127161 .823577$

O $-4.683858-0.999147 \quad 3.311978$

O -6.2835560 .1724774 .493410$

C $-5.583288-2.1042933 .182099$

H $-6.477024-1.8024982 .628031$

H -5.073615 -2.893313 2.622842

H $-5.852582-2.4918684 .169176$

SCF Energy (B3LYP/6-31G**//MMFF $)=-3245.91445236$

0700172

MM̄FF Geometry

C $-1.616246 \quad 4.864402 \quad 0.706458$

C $-2.684212 \quad 4.055132 \quad 0.768802$

C $-3.600793 \quad 3.792224-0.398033$

O -3.358668 2.451868 -0.884451

C $-5.067016 \quad 3.872664 \quad 0.058637$

C $-6.0545153 .595468-1.057318$ 
C $-6.7197822 .432589-1.208236$

C $-6.3039294 .744162-1.998609$

C $-6.575671 \quad 1.191008-0.367076$

C $-6.698618-0.082942-1.216329$

C $-6.398721-1.391549-0.462890$

C $-7.238562-1.5659930 .805377$

C $-6.882152-2.8531141 .565265$

O $-5.001963-1.420100-0.144821$

O $-7.490781-2.7463592 .861738$

C $-5.349845-2.9050071 .756778$

C $-7.492285-4.0918220 .899820$

O -4.944628 -4.147655 2.326203

C $-4.564014-2.6514770 .451370$

C $-3.042473-2.5503540 .694768$

O $-4.767646-3.755732-0.439740$

C $-2.3273892 .286678-1.755529$

O $-1.5357743 .135440-2.134403$

C $-2.3214220 .872833-2.183463$

C $-1.1741720 .339761-2.627152$

C $-0.982864-1.033890-3.089990$

C $-2.212070-1.847574-3.397244$

C $-2.604099-1.4019341 .603380$

C $-1.124289-1.1407301 .420282$

C $1.118133-2.1099401 .887020$

O $-0.721066-0.209940 \quad 0.727279$

C $1.431657-3.0522080 .767491$

C $1.785072-2.656407-0.465041$

C $2.087789-3.607159-1.588268$

C $0.910220-3.817780-2.514463$

C $0.095845-4.874982-2.355769$

C $0.720607-2.859405-3.668984$

C $0.277812-1.495224-3.224919$

N $-0.321912-2.0699202 .063550$

H -1.385355 $5.372548-0.226654$

H -2.894109 3.5273181 .696405

H -3.430822 $4.514242-1.207061$

H -5.2744874 .8685030 .473056$

H -5.2370643 .1757350 .888566$

H -7.446699 $2.361053-2.016216$

H -7.059254 $4.505412-2.755026$

H $-6.6581105 .619642-1.444431$

H $-5.3843205 .014591-2.526510$

H $-5.617574 \quad 1.1747020 .160562$

H $-7.372611 \quad 1.2078870 .384403$

H -7.712382 -0.138848 -1.633322

H -6.004795 $-0.008466-2.064330$

H -6.607670 -2.208176 -1.164312

H -8.309562 -1.5397590 .569408$

H -7.059397 -0.7253051 .488406$

H -7.325805 -3.574990 3.342298

H -5.089467 -2.126426 2.485298

H -8.586724 $-4.021496 \quad 0.896792$

H -7.251923 -5.0018511 .460742$

H -7.167491 -4.229850 -0.133371

H -5.374955 -4.229260 3.193702

H -2.660448 -3.503295 1.083516

H -2.545338 -2.425314 -0.275886

H $-4.041935-3.769607-1.085617$

H $-3.2460040 .313298-2.104357$

H $-0.2784160 .960476-2.627441$

H $-2.834366-1.972770-2.506596$

H -1.986636 -2.851316 -3.764395

H -2.811163 -1.354018 -4.170722

H -3.135824 -0.4739691 .364785$

H -2.804225 -1.630074 2.655903

H $1.564226-2.4694202 .819131$

H $1.495428-1.1019951 .690728$

H $\quad 1.354449-4.1148540 .984887$

H $1.860214-1.593328-0.679523$

H $2.416420-4.572046-1.178761$

H $2.947790-3.231740-2.156290$

H - $0.735968-5.055182-3.028542$

H $\quad 0.240456-5.581904-1.545476$

H $0.025124-3.268039-4.411114$

H $1.670182-2.760071-4.210635$

H $1.104899-0.828559-2.979928$

H $-0.759660-2.8508972 .543658$

C 0.5927804 .2634621 .823742
O $1.302508 \quad 4.443547 \quad 0.579969$

C 1.7105113 .1436160 .097677

C 1.6200812 .2202591 .301306

C 0.3837132 .7570861 .989047

H $0.9696182 .859283-0.660438$

C $3.1036553 .231684-0.534979$

H 1.5344021 .1689551 .019057

H $2.4996042 .334641 \quad 1.945178$

C $0.2345102 .304650 \quad 3.430248$

H -0.4913682 .4196491 .422929$

C $3.6562341 .856103-0.964535$

O $3.0075274 .087624-1.675604$

H 3.8031893 .7091640 .161581

H $3.6898461 .214360-0.079046$

O $2.748827 \quad 1.244570-1.881733$

C $5.0679401 .888661-1.583296$

C $5.5888800 .508750-2.030590$

H $5.7695722 .325116-0.862815$

O $5.0938632 .716413-2.747855$

H $4.9799540 .132035-2.862600$

H $6.5899100 .673325-2.451963$

H $0.1878681 .212323 \quad 3.488992$

H 1.0753912 .6394484 .045862

H -0.6840442 .7064323 .869542$

C -0.6812815 .1143201 .856279$

H $2.6436074 .937042-1.371644$

H $2.7066741 .812903-2.670002$

H $4.8618513 .619317-2.472683$

H -0.4017586 .1747581 .815954$

H -1.2000284 .9664142 .811034$

H 1.2658434 .6312872 .609422

C $6.615054-0.8635071 .291874$

O $6.464530-0.022796 \quad 0.140334$

C $5.663079-0.552078-0.919386$

C $6.244390-1.880827-1.431495$

C $6.448910-2.876868-0.284789$

C $7.181575-2.2377850 .897893$

H $7.371532-0.3607171 .908582$

C $5.318226-0.9491462 .113903$

H $4.649193-0.734915-0.551660$

O $7.493078-1.641630-2.078164$

H $5.560481-2.298239-2.180206$

H $7.028422-3.736113-0.643722$

O $5.188477-3.3621880 .164797$

H $7.158991-2.9038641 .768354$

O $8.556842-2.0509720 .551791$

C 5.0293970 .3454192 .846163

H $4.441098-1.1769851 .503925$

H $5.432688-1.7330502 .872286$

H $7.814202-2.492692-2.421305$

H $4.772606-3.825923-0.581383$

H $8.929027-2.9254800 .346178$

O $4.1605430 .092003 \quad 3.869309$

O 5.4939711 .4387322 .554098

C 3.8284321 .2110194 .697637

H 2.9643000 .9366115 .308427

H 4.6698511 .4393695 .357977

H 3.5666152 .0863314 .096603

SCF Energy (B3LYP/6-31G**//MMFF) $=-3245.89635769$

0700173

MMFF Geometry

C $3.392703-1.8644432 .702774$

C $2.185518-2.2664823 .127852$

C $0.917488-1.5034262 .849859$

O $0.045946-2.3970802 .115047$

C $0.196993-1.0748054 .143813$

C 0.7496180 .1956634 .759013

C 0.2422461 .4280404 .554068

C 1.9221800 .0187015 .689301

C -0.9440891 .8150583 .715058$

C -0.5153722 .5588182 .443044$

C -1.685235 2.9769031 .533144

C -2.5209524 .1058542 .140991$

C -3.7277994 .4621201 .260493$

O -2.4906201 .8233621 .287272$

O -4.5815805 .3096962 .044015$

C -4.5021813 .1641690 .947862$ 
C $-3.312625 \quad 5.2848770 .035926$

O $-5.580731 \quad 3.4186520 .051032$

$\begin{array}{llll}\text { C }-3.606880 & 2.025948 & 0.413296\end{array}$

C -4.364810 $0.681010 \quad 0.323660$

O $-3.1690462 .359260-0.908000$

C $-0.774369-1.829370 \quad 1.190127$

O $-0.864062-0.6425790 .918849$

C -1.546995 -2.907256 0.535413

C $-2.153575-2.641577-0.631133$

C $-2.969045-3.559930-1.427224$

C $-3.289485-4.902799-0.828270$

C -4.8865320 .1611601 .665299$

C $-5.155804-1.3290311 .623556$

C $-6.737727-3.0216620 .710528$

$\begin{array}{llll}\text { O } & -4.445572 & -2.124781 & 2.234527\end{array}$

C $-6.817243-3.473521-0.716532$

C $-6.614500-2.746669-1.827008$

C $-6.722898-3.318091-3.212914$

C $-5.472953-3.107022-4.037435$

C $-5.484875-2.310835-5.120030$

C $-4.228890-3.880455-3.653752$

C $-3.393010-3.146282-2.640116$

N $-6.252064-1.6616210 .845921$

H $3.469160-0.9488322 .120808$

H $2.102509-3.1731453 .722054$

H $1.142346-0.6241302 .231716$

H $0.229378-1.8849614 .885554$

H $-0.874122-0.9632693 .941747$

H 0.7218072 .2686935 .054403

H $2.1811580 .946393 \quad 6.211048$

H $1.691287-0.7288906 .455343$

H $2.810441-0.3046965 .141412$

H - 1.5509630 .9452183 .452652

H -1.5846892 .4563514 .330457$

H $\quad 0.1382121 .889882 \quad 1.867947$

H 0.0837543 .4410052 .700342

H $-1.253006 \quad 3.3110500 .583133$

H -1.9016254 .9929022 .323223$

H -2.902679 3.8018743 .124152

H -5.3870625 .4768341 .524783$

H -4.9641232 .8459311 .890594$

H $-2.830663 \quad 6.2183200 .351150$

H $-4.1851865 .585196-0.554654$

H -2.616680 4.758646 -0.620737

H -5.206309 $3.520329-0.840613$

H $-5.1882160 .757805-0.397874$

H -3.683794 -0.069215 -0.098105

H -2.920803 $1.539072-1.366113$

H $-1.587811-3.8755521 .019434$

H -2.033615 -1.646705 -1.058997

H $-2.375556-5.493415-0.700811$

H $-3.973250-5.499500-1.435083$

H $-3.768188-4.783601 \quad 0.150316$

H -4.1609490 .3356012 .467992$

H $-5.809960 \quad 0.6770841 .951444$

H -7.734417 -3.0562951 .161944$

H -6.089172 -3.704455 1.268685

H -7.069150 -4.526197 -0.836269

H $-6.375795-1.689062-1.754985$

H -7.585369 -2.844325 -3.699592

H $-6.948297-4.391612-3.184354$

H $-4.597722-2.175629-5.730225$

H $-6.378719-1.773116-5.418206$

H -3.603838 $-4.036389-4.543401$

H $-4.516129-4.884091-3.329597$

H -3.087676 -2.154246 -2.978091

H $-6.820268-0.9160170 .454422$

C $5.497897-2.996571 \quad 1.815985$

O $5.736535-1.872616 \quad 0.944698$

C $5.490034-2.292686-0.410858$

C $5.546952-3.813094-0.384609$

C $4.876237-4.0922230 .949404$

H $4.463006-1.998892-0.652894$

C $6.462890-1.602946-1.370628$

H $5.024259-4.265994-1.232387$

H $6.580107-4.179444-0.378817$

C $5.112310-5.4988921 .469775$

H $3.796225-3.9312540 .838093$
C $6.409212-0.059215-1.306370$

O $7.799622-2.013077-1.062888$

H $6.271670-1.942355-2.395056$

H $\quad 6.8260780 .286058-0.353017$

$\begin{array}{lllll}\text { O } & 7.273981 & 0.458342 & -2.323969\end{array}$

C $4.9986760 .528143-1.494683$

C $4.9845272 .068544-1.504946$

H $4.3534620 .177389-0.682332$

O $4.468305 \quad 0.030895-2.722716$

H $5.6069692 .442967-0.683955$

H $5.4055472 .422866-2.453834$

H $4.701752-6.2393540 .775381$

H $6.180229-5.7086391 .591431$

H $4.627690-5.6406942 .440952$

C $4.683630-2.559143 \quad 3.036109$

H $7.975603-1.770218-0.137580$

H $6.8958930 .208183-3.184672$

H $3.5609800 .368157-2.811029$

H $5.283508-1.8414843 .610424$

H $4.508668-3.4204653 .691217$

H $6.486516-3.3206552 .166991$

C $1.4149012 .631318-2.484009$

O $2.8020042 .272257-2.506606$

C $3.5541112 .625624-1.341974$

C $3.5397284 .147491-1.146003$

C $2.1009454 .674608-1.080512$

C $1.2411714 .139024-2.230643$

H $1.0564102 .420387-3.499961$

C $0.6184121 .749901-1.516202$

H $3.1090562 .147184-0.462564$

O $4.2322964 .771561-2.224530$

H $4.0759494 .391979-0.221339$

H $2.1082625 .770638-1.120956$

$\begin{array}{lllll}\text { O } & 1.506229 & 4.287523 & 0.154671\end{array}$

H $0.1836694 .358769-2.044585$

O $1.5950534 .837111-3.428881$

C $0.6094710 .314862-1.989175$

H $1.0004521 .781939-0.492431$

H $-0.4262802 .075130-1.465938$

H $4.2295045 .729835-2.059409$

H 2.0443634 .6641030 .871586

H $2.512497 \quad 4.601420-3.650437$

O $1.485272-0.425884-1.253128$

O $-0.075805-0.087923-2.920473$

C $1.596765-1.810319-1.601746$

H $2.318242-1.921018-2.415431$

H $1.964359-2.351281-0.725777$

H $0.632306-2.237067-1.890996$

SCF Energy (B3LYP/6-31G**//MMFF) $=-3245.92852329$

0700174

MM̄FF Geometry

C $-3.714776-1.897694-3.314482$

C $-2.717484-2.269362-2.499655$

C $-1.256818-2.076027-2.804449$

O $-0.777547-1.030659-1.924722$

C $-0.480672-3.364151-2.486147$

C $0.930032-3.361243-3.038807$

C $2.034620-3.062032-2.327199$

C $1.050569-3.784694-4.480477$

C $2.103014-2.592145-0.898964$

C $3.023656-1.375505-0.736847$

C $4.531464-1.690482-0.757979$

C $4.970491-2.4444250 .502542$

C $6.494783-2.609820 \quad 0.585844$

O $5.202945-0.426608-0.839584$

O $6.821435-3.0253831 .921453$

C $7.153409-1.2326720 .370173$

C $7.001745-3.715784-0.346853$

O $8.572985-1.3498430 .311760$

C $6.632998-0.495306-0.880963$

C $7.1882840 .949489-0.986151$

O $7.073841-1.195268-2.051336$

C $-0.959580 \quad 0.255820-2.330554$

O $-1.3844650 .632711-3.411192$

C $-0.594496 \quad 1.139894-1.200637$

C $-0.7784792 .463889-1.317314$

C $-0.5059123 .474985-0.292377$ 
C -0.0469702 .9878301 .055725$

C 6.8255611 .9106550 .151824

C 5.3381742 .1824310 .253095

C $3.4348173 .151487-1.027240$

O $4.669015 \quad 1.7681411 .197430$

C $3.0228704 .369438-0.265776$

C $2.5158685 .469419-0.841804$

C $2.0152026 .654882-0.068363$

C $0.5511556 .940577-0.328645$

C $0.1704798 .039853-1.001308$

C $-0.4749195 .991007 \quad 0.256877$

C $-0.6874174 .775682-0.605879$

N $4.8467842 .900155-0.825467$

H -3.486928 -1.448845 -4.277568

H -2.964206 -2.682719 -1.526958

H -1.102309 -1.789405 -3.852115

H -1.005924 -4.233467 -2.905123

H $-0.458802-3.533028-1.402748$

H $2.996207-3.154427-2.829058$

H $2.088413-3.783790-4.829937$

H $0.658711-4.798599-4.612648$

H $0.485808-3.106504-5.127637$

H $1.112660-2.302603-0.535973$

H $2.434096-3.421129-0.266330$

H $2.795896-0.632533-1.513686$

H $2.786587-0.873350 \quad 0.210382$

H $4.771870-2.265596-1.657878$

H $4.486471-3.4253990 .568954$

H $4.637691-1.8882771 .390587$

H $6.347051-3.8550592 .101863$

H $6.927513-0.6312961 .259278$

H $6.561002-4.681189-0.070286$

H $8.085849-3.844495-0.254703$

H $6.762254-3.536052-1.396790$

H $8.858549-1.8160281 .116170$

H $8.2813610 .910078-1.078752$

H $6.8357711 .384380-1.931301$

H $7.042737-0.584514-2.806204$

H $-0.2087340 .676183-0.300203$

H -1.171472 $2.852157-2.256248$

H -0.8145452 .3496221 .504069$

H $\quad 0.1524763 .7889741 .769396$

H 0.8791152 .4098920 .968564

H 7.1848911 .5418381 .117520

H $7.3306402 .870844-0.011823$

H $2.8636982 .285116-0.677597$

H $3.2715743 .259639-2.104182$

H 3.0911944 .3206150 .818477

H $2.4364395 .516511-1.925477$

H $2.6307327 .519809-0.346807$

H 2.1630696 .5179801 .010174

H -0.877675 8.263411-1.170485

H $0.8976848 .738134-1.402309$

H -1.444248 $6.499567 \quad 0.346503$

H $-0.1875535 .748126 \quad 1.282967$

H -1.040822 5.017508 -1.609761

H $5.4940813 .260740-1.520183$

C $-5.673756-0.660022-2.401435$

O $-5.060816-0.373755-1.125590$

C $-6.0025870 .357903-0.314790$

C $-7.2509420 .553060-1.172970$

C -7.172378 -0.625679-2.123851

H $-6.257382-0.2818610 .536694$

C $-5.370856 \quad 1.668800 \quad 0.167769$

H -8.165793 $0.558522-0.571998$

H -7.215805 $1.498112-1.727907$

C -8.034773 -0.460609 -3.362627

H -7.476165 -1.533704 -1.586033

C -4.1393221 .4916331 .081523$

O $-4.9597062 .430431-0.976951$

H -6.1245632 .2787950 .678483$

H -3.3332680 .9902140 .535538$

O $-3.6582762 .804154 \quad 1.404397$

C $-4.427100 \quad 0.751362 \quad 2.405344$

C $-3.251137 \quad 0.764710 \quad 3.404388$

H $-4.718520-0.2835232 .201433$

O $-5.5441791 .370813 \quad 3.051569$

H -2.8711121 .7876093 .512972$
H $-3.6434540 .486644 \quad 4.392380$

H $-9.088840-0.354639-3.085614$

H $-7.746986 \quad 0.426530-3.936758$

H -7.946086 -1.330778 -4.020391

C $-5.164828-2.000149-2.931775$

H $-4.3055671 .898972-1.462743$

H -3.4784053 .2612890 .564862$

H $-5.3061772 .298510 \quad 3.222079$

H $-5.731628-2.310181-3.816376$

H $-5.297981-2.780156-2.173583$

H $-5.3918740 .152547-3.085272$

C -1.642209-2.581698 3.035271

O $-2.558851-1.5195613 .333346$

C -2.113056-0.193685 3.021286

C -0.8201060 .1218623 .787502$

C $0.258841-0.923543 \quad 3.492092$

C $-0.266878-2.3463553 .684621$

H $-2.083389-3.4665783 .512715$

C -1.573978 -2.850388 1.527138

H -1.921912 -0.1158881 .947029$

O

H -0.4616311 .1130453 .490723$

H $1.115906-0.7633184 .157642$

O $0.709375-0.7813862 .148319$

H $0.459269-3.0712153 .298405$

O $-0.382548-2.5836565 .091059$

C -2.765352 -3.673489 1.094866

H -1.542122 -1.946976 0.912564

H -0.682024 -3.438796 1.281158

H -1.455704 -0.671805 5.459274

H 1.0806830 .1127252 .056385

H -0.639057 -3.513862 5.212204

O $-3.896958-2.9134201 .119786$

$\begin{array}{llll}\text { O } & -2.695854 & -4.855640 & 0.783669\end{array}$

C $-5.108266-3.5981580 .786536$

H $-5.901612-2.8542650 .677562$

H $-5.003980-4.139344-0.158608$

H $-5.377755-4.2851711 .593869$

SCF Energy $\left(B 3 L Y P / 6-31 G^{* *} / / M M F F\right)=-3245.91547483$

0700175

MMFF Geometry

C $-1.473846-3.061844-2.963402$

C $-0.752509-2.083768-2.394424$

C $-0.167919-0.923682-3.156719$

$\begin{array}{lllll}\text { O } & -0.795704 & 0.286754 & -2.661773\end{array}$

C $1.342227-0.764704-2.908782$

C $2.160636-2.010437-3.163345$

C $2.703347-2.777961-2.197040$

C $2.356285-2.364031-4.614010$

C $2.619149-2.583296-0.707262$

C $3.994070-2.558160-0.025893$

C $4.954543-1.480898-0.556479$

C $6.268280-1.519010 \quad 0.229346$

C $7.237066-0.417716-0.219146$

O $4.337451-0.195885-0.422353$

O $8.277722-0.347964 \quad 0.767176$

C $6.4867240 .929639-0.217199$

C $7.923239-0.775963-1.542483$

O $7.312151 \quad 1.965329-0.746947$

C $5.1205790 .886963-0.945481$

C $4.3660872 .213666-0.689229$

O $5.3514160 .741680-2.348006$

C $-2.0390550 .587615-3.126110$

$\begin{array}{llll} & -2.657299 & 0.012138 & -4.006867\end{array}$

C $-2.5474401 .718153-2.319712$

C $-3.8428762 .056592-2.390784$

C $-4.4846203 .102839-1.599578$

C $-5.532377 \quad 3.876443-2.351745$

C $2.9284382 .249400-1.220536$

C $2.2526483 .580424-0.939391$

C $0.1319144 .807994-1.399396$

O $2.7889404 .489246-0.311560$

C $-0.772872 \quad 4.706765-0.211445$

C $-1.715745 \quad 5.6290710 .034521$

C -2.6620095 .5890381 .200277$

C $-4.109195 \quad 5.5847330 .758569$

$\begin{array}{llll}\text { C }-4.759682 & 6.737037 & 0.523202\end{array}$ 
C -4.8257614 .2581250 .659753$

C $-4.1839953 .294472-0.299679$

N $0.9764913 .630992-1.482397$

H -1.648890 -3.049603 -4.035842

H $-0.607697-2.088031-1.316112$

H $-0.349606-1.010913-4.235453$

H $1.497261-0.383300-1.895209$

H $1.7204220 .047033-3.545503$

H $3.265045-3.660582-2.501154$

H $2.988156-3.248920-4.745057$

H $1.394340-2.572478-5.092469$

H $2.837059-1.535218-5.144058$

H $2.068098-1.681669-0.429044$

H $2.046129-3.421817-0.291811$

H $3.823333-2.3959171 .046241$

H $4.465586-3.544219-0.126322$

H $5.148562-1.676572-1.616964$

H $6.743653-2.5046610 .148220$

H $6.055436-1.3777351 .298757$

H 8.8559550 .3984930 .533109

H $\quad 6.310631 \quad 1.1941660 .834541$

H $8.481235-1.714483-1.439668$

H $8.663950-0.019307-1.823444$

H $7.222010-0.896077-2.370924$

H $7.3185101 .872347-1.714870$

H 4.3260992 .4001400 .392183

H $4.9224863 .044951-1.141016$

H $4.5517931 .004601-2.829052$

H -1.856675 2.216221-1.649505

H -4.505268 $1.529407-3.074676$

H -5.113712 4.283195 -3.279489

H -5.926852 4.725282 -1.787377

H $-6.3767423 .227995-2.609977$

H $2.9132242 .092824-2.303873$

H $2.3294201 .464964-0.748654$

H $-0.4481634 .848039-2.326788$

H $0.7648245 .698241-1.325425$

H $-0.668223 \quad 3.8597970 .461319$

H -1.805961 $6.478071-0.640355$

H -2.469083 6.4721521 .823392

H -2.4670954 .7253041 .848043$

H $-5.804180 \quad 6.7485150 .230113$

H -4.2591797 .6953170 .616145$

H -5.883849 4.3960090 .407264

H -4.8354543 .7922261 .654070$

H -3.4178822 .6708810 .158954$

H $0.5947752 .803661-1.931922$

C -3.579184 -3.831387-1.865054

O $-3.606824-2.671438-1.007484$

C $-4.298691-3.0066190 .203854$

C $-5.220926-4.149821-0.184142$

C $-4.307558-4.943743-1.103346$

H $-3.548760-3.3831440 .909648$

C $-4.963834-1.759576 \quad 0.789307$

H -5.565011 -4.7243510 .680682$

H $-6.098089-3.794132-0.736867$

C -5.045349 -5.917857 -2.005131

H $-3.593928-5.506812-0.486590$

C $-4.009438-0.5538230 .939719$

O $-6.041794-1.349309-0.060750$

H $-5.419980-2.0038931 .755462$

H -3.783327 -0.132586 -0.046622

$\begin{array}{llll}\text { O } & -4.704680 & 0.468230 & 1.663471\end{array}$

C $-2.689680-0.8635531 .669353$

C $-1.817057 \quad 0.393976 \quad 1.860287$

H $-2.121359-1.6016771 .093086$

O $-3.002445-1.4448622 .933819$

H -1.7525450 .9369360 .910305$

H -2.298279 1.0443942 .601091

H -5.579648 -6.664817 -1.408992

H $-5.778607-5.404310-2.635881$

H -4.345639 -6.444607 -2.661704

C $-2.119511-4.169615-2.178437$

H $-5.671897-1.184528-0.945263$

H $-5.523904 \quad 0.661913 \quad 1.176126$

H -2.164735 -1.678590 3.367419

H -2.053564 -5.097898 -2.756608

H - $1.562927-4.328838-1.246155$
H $-4.119375-3.561741-2.781205$

C $0.751861-0.951398 \quad 4.223813$

O $-\begin{array}{llll}0.482639 & -0.520281 & 3.639811\end{array}$

C $-0.387130 \quad 0.0604332 .336966$

C 0.5163001 .3042132 .369197

C 1.8807420 .9922432 .997238

C $1.744807 \quad 0.217975 \quad 4.312407$

H $0.487591-1.2463135 .247704$

C $1.318894-2.1861233 .513010$

H $0.035371-0.6745981 .642704$

O $-0.1236632 .337760 \quad 3.115418$

H 0.6449641 .6705431 .343848

H $2.420916 \quad 1.9281223 .184567$

O 2.6622340 .2151092 .097093

H $2.720814-0.1593184 .639749$

O $1.273611 \quad 1.1050335 .330249$

C $0.361635-3.3502093 .615332$

H $1.533554-2.0039912 .456472$

H $2.264596-2.5006593 .969726$

H 0.4469783 .1240603 .077096

H 2.7838230 .7358221 .286710

H 1.9253821 .8210795 .419884

O $-0.182160-3.6155772 .392890$

O $0.113678-3.9399104 .659092$

C -1.120260 -4.694920 2.356585

H $-0.654709-5.6182622 .714322$

H -2.001163 -4.447558 2.956482

H -1.431136-4.840106 1.318835

SCF Energy (B3LYP/6-31G**//MMFF) $=-3245.90919895$

\section{6}

MM̄FF Geometry

C $3.2313342 .846613-0.892330$

C $3.1429183 .929349-0.104743$

C $2.043694 \quad 4.2186180 .885807$

$\begin{array}{llllllll}\text { O } & 0.856583 & 3.453939 & 0.581483\end{array}$

C 2.4837743 .8389092 .310715

C 1.4933444 .3092803 .359524

C 0.4772383 .5698963 .848146

C 1.7118755 .7156373 .854350

C 0.0849902 .1689163 .460552

C -0.0089901 .2581914 .690619$

C $-0.345997-0.2098564 .376686$

C $0.813494-0.9394943 .699710$

C $0.451131-2.3969093 .386995$

O $-1.508297-0.2595803 .545473$

O $1.455259-2.915838 \quad 2.503364$

C $-0.890724-2.4299452 .623120$

C $0.484150-3.2790214 .641320$

O $-1.310647-3.7788642 .442249$

C $-2.007377-1.5884013 .289206$

C $-3.229645-1.395332 \quad 2.358937$

O $-2.436659-2.2106904 .499614$

C $-0.1270794 .059808-0.134399$

O $-0.083108 \quad 5.150010-0.679554$

C $-1.3049403 .166004-0.085593$

C $-2.492198 \quad 3.589824-0.543722$

C $-3.7504662 .841882-0.477021$

C $-3.719048 \quad 1.501954 \quad 0.207325$

$\begin{array}{llll}C & -4.130226 & -2.611452 & 2.149478\end{array}$

C $-5.287216-2.2647751 .229465$

C $-5.936800-2.267484-1.187567$

O $-6.345710-1.8157811 .662990$

C $-5.577513-1.003015-1.902371$

C $-6.476056-0.074746-2.265181$

C $-6.116648 \quad 1.185058-3.002699$

C $-6.728326 \quad 2.422425-2.380631$

C -7.665108 3.130419-3.034275

C $-6.2644252 .837203-1.002186$

C $-4.8644523 .389214-1.006240$

N $-4.994923-2.489906-0.105179$

H $2.4737522 .069997-0.833852$

H $3.9239664 .684589-0.167776$

H $1.816775 \quad 5.2914350 .842680$

H 3.4667424 .2750552 .533903

H 2.6241972 .7537912 .385227

H $-0.173994 \quad 4.0274864 .592280$

H 0.9724576 .0132214 .605461 
H 2.7022825 .8064244 .312471 H $1.644926 \quad 6.4278733 .025866$ H -0.8946472 .2194452 .971031$ H $\quad 0.7783101 .7449512 .732200$ H $\quad 0.922976 \quad 1.3014885 .268227$ H -0.8016881 .6531425 .340669$ H $-0.577001-0.6897195 .335165$ H $1.718875-0.8918424 .317304$ H $1.069411-0.4474002 .752569$ H $2.316908-2.8273932 .945733$ H $-0.709100-2.0227471 .618738$ H $1.477106-3.2467015 .105803$ H $0.306994-4.3306104 .389655$ H $-0.243977-2.9787085 .397408$ H $-0.585974-4.2522621 .998747$ H -3.852542 -0.5897672 .774416$ H -2.885816 -1.008869 1.392685 H -3.306587 -1.846405 4.734373 H -1.170012 2.1905460 .368738 H -2.558249 $4.583099-0.985268$ H $-3.3755281 .607497 \quad 1.242416$ H -3.043104 $0.821820-0.321806$ H $-4.689158 \quad 1.0052570 .252309$ H $-3.581499-3.4518411 .715945$ H $-4.552348-2.9627533 .097498$ H $-6.957431-2.245008-0.792654$ H -5.841441 -3.116431-1.871604 H $-4.530475-0.850523-2.153164$ H -7.528532 $-0.239326-2.045407$ H $-6.464931 \quad 1.071463-4.037465$ H $-5.0289371 .311720-3.064423$ H -8.110848 $4.017825-2.597181$ H -8.007891 $2.839954-4.021766$ H $-6.3915732 .003801-0.306541$ H -6.918732 $3.625200-0.604863$ H -4.785212 4.366521-1.484191 H -4.071167 -2.829812 -0.356155 C $5.3302621 .544584-1.523322$ O $4.677777 \quad 0.268022-1.399337$

C $5.395253-0.513498-0.418423$

C 6.6055310 .3195390 .001172

C $6.1002011 .733175-0.219686$ H $4.731280-0.6200660 .446263$

C $5.744655-1.890185-0.999233$ H $6.8998280 .125356 \quad 1.037219$ H $7.4764160 .119705-0.634044$ C $7.2044702 .771934-0.292399$ H 5.4189951 .9857730 .602507

C $4.513928-2.702700-1.454783$ O $6.597631-1.708588-2.137318$ H $6.328994-2.465112-0.272208$ H $4.003397-2.160148-2.256883$ O $4.979100-3.925469-2.040029$ C $3.528348-3.040924-0.320157$ C $2.326431-3.892818-0.771870$ H $3.163435-2.1213290 .147002$ O $4.238082-3.7680670 .686896$ H $2.675500-4.808255-1.264920$ H $1.797803-4.2273640 .129871$ H 7.7733452 .7942900 .642965 H $7.9048062 .557667-1.106562$ H $6.7896283 .771029-0.458670$ C $4.3291832 .638223-1.896275$ H $6.108048-1.178673-2.789583$ H $5.419153-4.436420-1.339010$ H $3.619475-3.9406571 .416767$ H $3.8482422 .368407-2.844823$ H $4.8668373 .576939-2.076528$ H $6.0377571 .444892-2.358244$ C $0.005639-1.125521-1.688260$ O $0.854818-2.011321-0.945458$ C $1.352319-3.133611-1.682933$ C $0.181752-4.015818-2.140031$ C $-0.829852-3.206958-2.957841$ C $-1.199434-1.885581-2.273010$ H $-0.369266-0.418890-0.936406$ C $\quad 0.829087-0.326867-2.708667$ H $1.896102-2.776574-2.563507$
O $-0.473421-4.596182-1.013355$

H $\quad 0.572873-4.841254-2.746112$

H - $-1.734864-3.810654-3.099728$

O $-0.272911-2.951892-4.243573$

H -1.755181 -1.246166 -2.967096

O $-2.084397-2.201139-1.194708$

C $0.1828910 .988419-3.084192$

H $1.807484-0.102677-2.265333$

H $1.008789-0.893710-3.625752$

H $-0.775362-3.871734-0.439106$

H $-0.940388-2.471363-4.762348$

H $-2.393914-1.360100-0.818621$

O $1.1085871 .773938-3.709858$

O $-0.9850411 .286244-2.876913$

C $0.6446993 .062268-4.124830$

H $\quad 0.2898673 .634739-3.262989$

H $1.4845673 .596143-4.577251$

H $-0.1480622 .955556-4.871287$

SCF Energy (B3LYP/6-31G**//MMFF) $=-3245.92761594$

\section{7}

MM̄MF Geometry

C -3.348208 -0.189694 -2.268029

C $-2.5549650 .466260-3.130041$

C $-1.7124031 .664018-2.776903$

O $-0.336458 \quad 1.375222-3.147305$

C $-2.1118552 .915597-3.580962$

C $-3.3110103 .666168-3.042764$

C $-3.2409634 .826583-2.360784$

C $-4.6616493 .093123-3.389099$

C $-2.0139225 .593655-1.946178$

C $-2.104186 \quad 6.088785-0.494232$

C -1.8728844 .9774810 .541303$

C -2.2255135 .4802501 .942870$

C -1.8972904 .4448193 .026204$

O $-0.488034 \quad 4.6177380 .485504$

O -1.9910665 .1242324 .287033$

C $-0.440516 \quad 3.9767312 .836053$

C -2.9311773 .3143503 .058805$

O -0.1281382 .9041863 .722844$

C -0.1063763 .5657291 .381187$

C $1.4217763 .363511 \quad 1.248197$

O $-0.7783162 .346512 \quad 1.071263$

C $0.3407440 .466692-2.396708$

O $-0.083581-0.146474-1.431365$

C $1.7036910 .339356-2.957236$

C $2.552802-0.545268-2.413602$

C $3.927354-0.806901-2.843224$

C $4.4867800 .045252-3.951651$

C $1.8962603 .021525-0.168518$

C $3.3937593 .215920-0.300254$

C 5.5668952 .2803030 .489453

O $3.8697094 .147197-0.945347$

C $6.1543331 .405498-0.571892$

C $6.8375640 .281801-0.306579$

C $7.521800-0.555152-1.348756$

C $6.983723-1.965489-1.418378$

C $7.428539-2.920109-0.583729$

C $6.002654-2.286093-2.521386$

C $4.617197-1.788841-2.226141$

N 4.1215342 .2634420 .392146

H -3.440928 $0.162565-1.244939$

H -2.485498 $0.109636-4.155902$

H $-1.7404781 .887698-1.703010$

H $-2.2967432 .647448-4.630901$

H -1.245207 $3.584970-3.642868$

H -4.180043 $5.300350-2.075204$

H -5.474556 $3.800473-3.191164$

H $-4.7103862 .838968-4.452977$

H $-4.8641982 .194243-2.802120$

H -1.094177 $5.017466-2.079665$

H -1.940227 $6.470547-2.601021$

H - $1.3387106 .864428-0.359959$

H -3.078476 $6.565414-0.327951$

H $-2.485150 \quad 4.1063660 .283177$

H $-3.2827305 .769227 \quad 1.998285$

H -1.654789 6.3957982 .154358

H -1.8444684 .4728034 .993247$ 
H $\quad 0.212541 \quad 4.814558 \quad 3.117774$ H $-3.9266563 .713993 \quad 3.286007$ H $-2.7077652 .594976 \quad 3.854372$ H -2.998646 2.7720972 .113818 H -0.2400503 .2297934 .631500$ H 1.9303944 .2836681 .565472 H 1.7498692 .5631941 .923413 H $-0.341411 \quad 1.9292130 .312398$ H $1.9680960 .970405-3.797766$ H $2.205593-1.150592-1.577180$ H $5.545657-0.135365-4.147614$ H $4.4017841 .108675-3.700406$ H $3.947445-0.138928-4.887117$ H $1.6599601 .982361-0.411312$ H $1.4100593 .653051-0.919826$ H $5.826807 \quad 1.938231 \quad 1.496439$ H 5.9340803 .3048340 .369806 H $6.0526551 .740198-1.601175$ H $6.953896-0.0345300 .727794$ H $8.592491-0.585002-1.106324$ H $7.464722-0.083558-2.337568$ H $7.069309-3.941703-0.649366$ H $8.159159-2.6978610 .187142$ H $\quad 5.933696-3.373862-2.658961$ H $6.392078-1.914776-3.473799$ H $4.124471-2.344298-1.427877$ H 3.6310281 .4910800 .833635 C $-4.652672-2.250137-1.522746$ O $-3.559618-2.833851-0.780585$ C $-3.747231-4.258019-0.723651$ C $-5.226559-4.488776-1.008644$ C -5.502982 -3.417799-2.038364 H -3.153569 -4.679536-1.543797 C $-3.278858-4.8606990 .607318$ H -5.427331 -5.500249-1.374548 H $-5.846808-4.319217-0.121087$ C $-6.977966-3.083935-2.189193$ H -5.120248 -3.764889 -3.007769 C -1.860732 -4.487738 1.079830 O $-4.178249-4.4662441 .653883$ H $-3.370083-5.951840 \quad 0.543593$ H -1.878621 -3.481036 1.508969 O $-1.539435-5.3613892 .173133$ C $-0.743684-4.5613150 .025204$ C $0.692148-4.5232410 .590296$ H $-0.870059-3.733790-0.681909$ O $-0.869806-5.765114-0.737220$ H $\quad 0.924722-5.4847591 .066737$ H $1.368753-4.453450-0.272025$ H -7.541567 -3.966783 -2.508501 H -7.408490 -2.733815 - 1.244985 H -7.125983 -2.299615 -2.938101 C $-4.135319-1.397252-2.687747$ H -4.187077 -3.494224 1.679595 H -1.519642 -6.2717791 .832874$ H $-0.753513-6.517168-0.132348$ H $-4.990540-1.053243-3.282075$ H -3.502424 -2.020718 -3.332819 H $-5.219294-1.631434-0.815800$ C $0.893337-0.9668061 .734057$ O $0.739455-2.1382490 .919116$ C $0.984854-3.3817041 .578496$ C $2.428121-3.4369452 .106181$ C $2.731182-2.2341473 .007025$ C $2.298540-0.9156562 .358796$ H $0.814148-0.1238911 .037883$ C $-0.239388-0.8271712 .764720$ H $0.302913-3.4810962 .427636$ O $3.338176-3.4511221 .008681$ H $2.566255-4.3721822 .661863$ H $3.807248-2.1923213 .215641$ O $2.052292-2.3823334 .250318$ H $2.342607-0.1021103 .091733$ O $3.235440-0.5852811 .329801$ C -1.595812 -0.649765 2.114136 H $-0.308431-1.7015853 .416522$ H -0.0539440 .0582113 .383351$ H $4.237817-3.512278 \quad 1.372642$
H $2.362695-3.2085954 .658127$ H $3.176852-1.2706190 .642599$ O $-2.533679-0.450333 \quad 3.086923$ O $-1.815500-0.6953760 .912199$ C $-3.878419-0.2961312 .620995$ H $-4.4993150 .005026 \quad 3.468734$ H -3.9383170 .4791611 .851185$ H -4.248608 -1.248865 2.232970 SCF Energy (B3LYP/6-31G**//MMFF) $=-3245.90727073$

\section{8}

MM̄FF Geometry

C $4.046676-1.897636-3.180561$

C $2.942383-1.136212-3.211052$

C $1.547372-1.691935-3.337010$

O $0.802079-1.306654-2.156594$

C $0.839897-1.101859-4.569316$

C $-0.616608-1.512334-4.693157$

C -1.639943 -0.639790 -4.782704

C $-0.890466-2.993474-4.730180$

C $-1.5521240 .857862-4.849266$

C $-1.6164511 .570144-3.491958$

C $-2.9762481 .435676-2.789099$

C $-3.1164972 .497826-1.696683$

C $-4.4434772 .366087-0.938648$

O $-3.0650990 .132968-2.199935$

O -4.3613363 .1819390 .239856$

C $-4.5979870 .907558-0.469815$

C $-5.6282862 .896185-1.753752$

O

C $-4.328576-0.140628-1.578656$

C $-4.270198-1.545274-0.937886$

O $-5.396258-0.109493-2.527783$

C $0.944641-2.098422-1.058575$

O $1.657281-3.084706-0.953878$

C $0.078495-1.5572850 .012575$

C $0.169132-2.0870881 .241578$

C $-0.611988-1.7109592 .419657$

C - $-1.546332-0.5372812 .308307$

C $-3.727433-2.653943-1.845743$

C $-3.818992-3.985595-1.125115$

C $-2.856912-5.2154850 .824955$

O $-4.696714-4.803111-1.391991$

C $-3.227949-4.6546442 .164317$

C $-2.539681-4.8988403 .290204$

C $-2.895644-4.3137224 .628988$

C -1.712267 -3.675497 5.328509

C $-1.146287-4.2613716 .397381$

C -1.226965 -2.325700 4.834730

C $-0.458319-2.439448 \quad 3.545077$

N $-2.856288-4.128476-0.137891$

H $3.958692-2.976372-3.282409$

H $3.034445-0.060140-3.081886$

H $1.567672-2.785945-3.420768$

H $1.367488-1.413433-5.480334$

H $0.925462-0.010782-4.523159$

H -2.652391 -1.035335 -4.850751

H -1.934914 -3.215827 -4.974288

H $-0.268150-3.474825-5.491646$

H $-0.676703-3.452149-3.760028$

H $-0.6366691 .163261-5.368776$

H -2.368184 $1.218913-5.488681$

H $-0.817711 \quad 1.192129-2.841261$

H -1.410974 $2.631725-3.677575$

H $-3.7693191 .554529-3.536619$

H $-3.0180443 .506754-2.115681$

H -2.293649 $2.385578-0.977333$

H -4.187966 $4.097019-0.040363$

H $-3.8674860 .750787 \quad 0.336021$

H $-5.4752373 .951561-2.009699$

H $-6.5553072 .861403-1.170863$

H -5.786588 $2.349864-2.685803$

H $-6.002553 \quad 1.3548180 .808011$

H -3.626948 -1.504790 -0.052294

H $-5.270712-1.833293-0.589143$

H $-5.458877-0.975321-2.961037$

H $-0.604044-0.753240-0.236976$

H $\quad 0.878410-2.898708 \quad 1.402391$ 
H $-2.414877-0.798217 \quad 1.699307$ H -1.0443680 .3243241 .854427$ H -1.913514 -0.188046 3.276401 H -4.296513 -2.730530 -2.777640 H -2.682411 -2.467153 -2.116221 H $-1.852855-5.6508940 .824523$ H -3.578725 -5.9816860 .527031$ H $-4.104793-4.0123932 .208670$ H -1.678311 -5.561914 3.251930 H -3.304366 -5.124273 5.246158 H -3.698148 -3.5710174 .538298$ H -0.312991 -3.802098 6.918833 H -1.499956 -5.216047 6.772529 H $-0.563149-1.8665145 .578224$ H -2.095112 -1.665182 4.751820 H $0.282055-3.2410483 .543778$ H -2.175946 -3.386772 -0.004306 C $5.874898-1.665025-1.508993$ O $5.011314-0.973603-0.586232$ C $5.803900-0.4068770 .472026$ C $7.189043-1.0251810 .332312$ C $7.288150-1.192947-1.174542$ H 5.8793300 .6676110 .267385 C $5.098754-0.6296991 .815205$ H $7.973420-0.3849460 .746927$ H $7.252253-2.0011710 .826949$ C $8.379751-2.154304-1.610134$ H $7.472942-0.209204-1.626608$ C $3.617484-0.1930081 .817187$ O $5.128931-2.0269732 .136249$ H $5.648980-0.1258672 .617131$ H $3.043728-0.8523891 .155617$ O $3.092930-0.3926923 .135150$ C 3.3708431 .2718221 .409422 C $1.8720611 .595043 \quad 1.333839$ H $3.809143 \quad 1.4605200 .425093$ O 3.9898812 .1534192 .341811 H 1.3998680 .9553830 .579607 H 1.3855241 .3357382 .283738 H $9.361404-1.793503-1.286118$ H $8.230300-3.151578-1.182851$ H $8.399299-2.254170-2.699792$ C $5.426836-1.349745-2.936845$ H $4.695755-2.5054401 .408397$ H $3.236740-1.3265873 .365607$ H 4.9512572 .0272462 .276760 H $6.114821-1.792135-3.665452$ H $5.431581-0.264535-3.098539$ H $5.768491-2.738284-1.302022$ C $-0.4548114 .435148 \quad 0.746236$ $\begin{array}{lllll}\text { O } & 0.104961 & 3.123882 & 0.894124\end{array}$ C $1.5314183 .068371 \quad 1.045124$ C $2.2049563 .647575-0.206835$ C $1.7052115 .066830-0.497042$ C $0.1785575 .179388-0.441631$ H $-1.511944 \quad 4.2628850 .507319$ C -0.402879 5.2233952 .059799 H 1.8214443 .6541051 .923785 O $1.9759842 .814700-1.341332$ H $3.2890593 .683125-0.051866$ H $2.0621425 .376047-1.487079$ $\begin{array}{lllll}\text { O } & 2.279289 & 5.945151 & 0.469485\end{array}$ H $-0.119335 \quad 6.233510-0.408734$ O $-0.3639524 .657687-1.657178$ C $-1.296948 \quad 4.5781303 .092508$ H $0.602827 \quad 5.3154292 .479376$ H $-0.768608 \quad 6.2464361 .910197$ H $1.1016502 .401580-1.249184$ H 1.9978296 .8492680 .248320 H $-0.2996913 .689582-1.627144$ O -0.6204483 .5886773 .742899$ O $-2.464484 \quad 4.897147 \quad 3.276231$ C -1.378012 2.8559234 .710405 H -2.253945 2.3977404 .241274 H -0.7411442 .0623445 .110326$ H - 1.6783163 .5146245 .530628 SCF Energy (B3LYP/6-31G**//MMFF) $=-3245.90775287$

\section{9}

MMFF Geometry

C $1.6454004 .402796-2.771101$

C $0.9465203 .959268-1.714526$

C $-0.3399504 .569361-1.227800$

O $-1.293608 \quad 3.493383-1.076613$

C $-0.131942 \quad 5.202538 \quad 0.161070$

C $-1.402427 \quad 5.790236 \quad 0.745420$

C -2.0160495 .3330001 .855153$

C $-1.9748996 .971863 \quad 0.005363$

C $-1.582896 \quad 4.212903 \quad 2.761233$

C $-2.1906102 .872713 \quad 2.346004$

C -2.061246 1.7750943 .412967

C $-0.6067191 .423552 \quad 3.734213$

C $-0.5048810 .249740 \quad 4.722299$

O $\begin{array}{llll}-2.738702 & 0.620665 & 2.895694\end{array}$

O $0.854853-0.2129304 .718050$

C $-1.378389-0.9138124 .210012$

C $-0.8098210 .683978 \quad 6.160647$

O $-1.438335-1.971980 \quad 5.162989$

C $-2.805980-0.4853463 .808313$

C $-3.592346-1.6190053 .106571$

O $-3.540941-0.1313614 .985845$

C $-2.1881053 .271601-2.074307$

O $-2.283970 \quad 3.870072-3.132409$

C $-3.0496562 .152268-1.631462$

C $-4.087453 \quad 1.749542-2.379531$

C $-5.0121460 .663238-2.040097$

C $-4.782984-0.050943-0.733585$

C $-2.915170-2.1900931 .858728$

C $-3.842310-3.0671231 .033120$

C $-3.880581-4.302098-1.143777$

O $-4.983638-3.358307 \quad 1.378216$

C $-4.230629-3.433985-2.311664$

C $-5.443596-3.402693-2.884749$

C $-5.770957-2.547132-4.076706$

C $-7.023638-1.716595-3.888785$

C $-8.078756-1.875371-4.706007$

C $-7.065174-0.702912-2.764046$

C $-6.0058840 .360080-2.901491$

N $-3.225389-3.488498-0.136427$

H $1.2654855 .266112-3.314208$

H $1.2993053 .102663-1.147236$

H $-0.7116905 .320613-1.934570$

H 0.6234385 .9970260 .099476

H 0.2818384 .4444590 .835355

H $-2.936257 \quad 5.824043 \quad 2.169959$

H -2.806609 7.4368360 .545697

H $-1.2073957 .740160-0.134152$

H -2.350407 $6.669266-0.976830$

H -0.4915264 .1463642 .805704$

H -1.9055564 .4660553 .779010$

H -3.256052 2.9991942 .109408

H -1.726069 2.5260721 .414987

H -2.583658 2.1165454 .314284

H -0.0655292 .2902894 .131119$

H $-0.087474 \quad 1.1435142 .806741$

H 1.4269430 .5356494 .959491

H $-0.868245-1.3206673 .330418$

H -0.1058291 .4605346 .483300$

H $-0.678076-0.1483346 .860819$

H $-1.818791 \quad 1.0827726 .283890$

H $-0.522895-2.2174265 .381338$

H $-3.800939-2.4297663 .816726$

H $-4.576085-1.2218442 .818888$

H $-4.489765-0.2041764 .789787$

H $-2.8053291 .686826-0.682994$

H $-4.2810482 .258541-3.322591$

H -4.8564910 .6479050 .107370$

H -3.788162 - $0.508171-0.724763$

H $-5.496377-0.851518-0.534141$

H -2.557263 -1.3771191 .217389$

H -2.055866 -2.805495 2.147959

H $-4.758364-4.795160-0.714455$

H $-3.162759-5.067242-1.455221$

H $-3.436785-2.811875-2.719750$

H $-6.233099-4.043515-2.499148$

H $-5.887069-3.217188-4.938384$ 
H $-4.935073-1.881849-4.325917$

H $-8.982754-1.287780-4.584556$

H -8.064587 -2.596850 -5.516115

H -7.015245 -1.236948 -1.812390

H -8.035722 -0.189379-2.752080

H $-6.092136 \quad 0.945132-3.818191$

H $-2.290918-3.149938-0.344711$

C $3.4581382 .569299-2.624221$

O $3.8919082 .886210-1.289364$

C $4.8871391 .915916-0.890665$

C $5.1737471 .066940-2.127121$

C $4.6961091 .952208-3.264664$

H $5.7729282 .493001-0.600828$

C $4.3256991 .134997 \quad 0.305908$

H $6.231176 \quad 0.796741-2.208037$

H $4.5942860 .136267-2.107485$

C $4.4265681 .184298-4.546657$

H $5.4512262 .727197-3.451200$

C $5.175310-0.0690660 .740201$

O 4.1938122 .0415201 .406825

H 3.3082650 .8144180 .059645

H $5.279285-0.778076-0.084053$

O $6.501342 \quad 0.399315 \quad 1.019560$

C $4.652900-0.7989351 .990853$

C $3.173441-1.2289551 .965234$

H $4.816714-0.1592862 .867130$

O $5.472586-1.9562132 .188740$

H $2.935087-1.6420142 .954482$

H $2.546990-0.3394651 .837688$

H $5.3369650 .684381-4.893479$

H $3.6574060 .418138-4.401498$

H $4.0878891 .855221-5.342051$

C $2.9357933 .832143-3.312084$

H 5.0891942 .3197671 .665698

H $7.029758-0.3726821 .286275$

H $5.177830-2.3869013 .009339$

H $2.7748893 .624083-4.376159$

H $3.6932554 .622875-3.244085$

H $2.6604421 .816979-2.551579$

C $2.723740-2.652716-1.484368$

O $2.985182-1.761724-0.391340$

C $2.805371-2.3027740 .918558$

C $1.348472-2.766301 \quad 1.090521$

C $0.942716-3.754219-0.006169$

C $1.300551-3.230476-1.401343$

H $2.772532-2.016345-2.377574$

C $3.806235-3.731376-1.614494$

H $3.476838-3.1568431 .053778$

O $0.468636-1.6435641 .061853$

H $1.236897-3.2393862 .073290$

H $-0.137036-3.9307480 .056846$

O $1.599269-4.995308 \quad 0.232536$

H $1.182312-4.018509-2.154122$

O $0.392242-2.181281-1.750110$

C $5.133760-3.118546-1.997570$

H $3.933853-4.308700-0.694747$

H $3.550501-4.450133-2.401808$

H $0.624693-1.1624450 .231234$

H $1.309975-5.617687-0.456315$

H $-0.502882-2.560708-1.762529$

O $6.045020-3.316435-1.003069$

O $5.334593-2.529808-3.052183$

C $7.346877-2.771877-1.241726$

H $7.949596-2.926734-0.342929$

H $7.822087-3.291123-2.079220$

H $7.283906-1.697497-1.439251$

SCF Energy (B3LYP/6-31G**//MMFF)= -3245.91626227

\section{0}

MM̄FF Geometry

C 1.7100315 .7495910 .403684

C $2.2302514 .746787-0.320135$

C $3.157216 \quad 3.6979170 .239474$

$\begin{array}{lllll}\text { O } & 2.470875 & 2.423391 & 0.190653\end{array}$

C $4.4300183 .602069-0.621251$

C $5.3805432 .496061-0.200579$

C $5.7915461 .503967-1.015893$

C 5.8869022 .5605261 .216596
C $5.4405921 .314194-2.465490$

C $4.1374120 .540881-2.707787$

C $4.237150-0.963437-2.408704$

C $2.941496-1.669322-2.819561$

C $2.971528-3.166385-2.481278$

O $4.465732-1.146057-1.009669$

O $1.634423-3.674718-2.607147$

C $3.380977-3.333406-1.004044$

C $3.831986-3.960548-3.470725$

O $3.577596-4.709427-0.683407$

C $4.632686-2.512292-0.610183$

C $4.869569-2.5384780 .920272$

O $5.781577-3.097816-1.233178$

C 1.6263352 .1449511 .222154

O 1.3911722 .8550482 .187826

C 1.0139030 .8198790 .975188

C $-0.0169430 .436979 \quad 1.744064$

C $-0.745777-0.831021 \quad 1.678703$

C $-0.298708-1.8535760 .669877$

C $3.872284-1.6990661 .726294$

C $3.602604-2.2545933 .113997$

C $1.972668-2.0143684 .989792$

O $4.234306-3.1849583 .606065$

C $0.774706-2.8587794 .680661$

C $-0.465478-2.5832465 .112463$

C $-1.664780-3.4295834 .791224$

C $-2.787465-2.6417504 .150531$

C $-3.915587-2.3687584 .827742$

C $-2.637866-2.2394422 .697397$

C -1.776484 -1.017471 2.530078

N $2.560142-1.5793763 .736276$

H 1.9738185 .8490391 .453491

H $1.9328224 .636390-1.360853$

H 3.4349593 .9307831 .275186

H $4.9718954 .556317-0.581183$

H $4.1301303 .467124-1.666228$

H $6.4710760 .753722-0.614010$

H 6.6804191 .8296451 .407027

H $6.299813 \quad 3.5524491 .427310$

H 5.0784062 .3583971 .925801

H $5.3762262 .288597-2.963536$

H $6.2734380 .798744-2.960207$

H $3.3339740 .985934-2.107933$

H $3.8618480 .671666-3.761839$

H $5.087407-1.368866-2.969759$

H $2.739679-1.523499-3.888239$

H $2.093787-1.211681-2.289811$

H $1.331729-3.499632-3.514711$

H $2.530051-3.000587-0.399663$

H $3.445852-3.846257-4.490817$

H $3.793026-5.033932-3.255205$

H $4.878149-3.647555-3.477314$

H $2.765302-5.183547-0.931072$

H $4.897139-3.5810481 .259121$

H $5.872033-2.1445521 .138148$

H $6.574061-2.814323-0.748184$

H 1.4206210 .2083290 .178731

H -0.3669091 .1244612 .513982$

H $-0.213271-1.411775-0.328500$

H $-0.990909-2.6914980 .566098$

H $0.671589-2.2680430 .956696$

H $4.253845-0.6764651 .833295$

H $2.912123-1.6362361 .209109$

H $1.711486-1.1160115 .557446$

H $2.702150-2.5956355 .562049$

H $0.939421-3.7436754 .069811$

H - $0.629489-1.7092935 .738706$

H -2.009319 -3.885221 5.728695

H -1.397451 -4.266593 4.134111

H $-4.734815-1.8279884 .365276$

H -4.043793 -2.675559 5.860430

H -3.621973 -2.006664 2.272758

H -2.266776 -3.103828 2.140230

H -2.046695 -0.1979653 .198158$

H $2.076970-0.8466833 .225845$

C $-0.714840 \quad 6.293432 \quad 0.218468$

O $-1.0251085 .082630-0.498993$

C $-2.3602975 .167296-1.025106$ 
C $-2.9953316 .370187-0.339818$ C -1.798263 $7.291122-0.183478$ H $-2.2660145 .371790-2.099256$ C $-3.104286 \quad 3.838197-0.823859$ H -3.801928 $6.809600-0.934270$ H $-3.403617 \quad 6.1039280 .642384$ C $-2.016023 \quad 8.4062410 .823811$ H $-1.5610487 .730335-1.161784$ C -2.466422 $2.668064-1.608647$ O $-3.158983 \quad 3.5366520 .569332$ H -4.139608 $3.958241-1.163170$ H -2.456042 $2.944572-2.670073$ O $-1.1034752 .492335-1.224169$ C $-3.2036141 .318825-1.467087$ C $-2.5431770 .235304-2.342670$ H $-4.2511971 .441680-1.766001$ O $-3.1818670 .880279-0.112578$ H -2.426622 $0.623974-3.361543$ H $-1.544590 \quad 0.022813-1.941048$ H -2.845935 9.0487720 .512384 H -2.2523048 .0096051 .816835$ H -1.1212319 .0299820 .914501$ C $0.7064016 .729708-0.140854$ H $-2.246214 \quad 3.5275530 .904780$ H $-1.1001682 .205086-0.295903$ H -3.6636291 .5357100 .418946$ H 0.9262597 .7195290 .273920 H $0.8125486 .802255-1.230495$ H $-0.786616 \quad 6.057857 \quad 1.288700$ C -4.028348 -2.923710 -0.976021 O $-3.304168-1.693523-1.109065$ C $-3.342477-1.081838-2.401401$ C $-2.766423-2.036028-3.461613$ C $-3.473346-3.393610-3.431839$ C $-3.554981-3.957804-2.009961$ H $-3.755589-3.3020650 .017104$ C -5.544512 -2.698187 -0.989018 H $-4.379420-0.837207-2.655969$ O $-1.370159-2.217441-3.231376$ H -2.880568 -1.574695-4.450103 H $-2.929820-4.095435-4.075868$ O $-4.782830-3.236434-3.970121$ H $-4.215219-4.832306-1.978561$ O $-2.256033-4.402191-1.607628$ C $-5.984170-1.990590 \quad 0.270270$ H $-5.895866-2.123329-1.850440$ H -6.078555 -3.655505 -1.018533 H $-1.030309-2.800275-3.931222$ H -5.210237 -4.109831-3.962210 H -1.970947 -5.085444 -2.237912 O $-5.954024-0.6407290 .083169$ O $-6.291141-2.5703441 .303984$ C $-6.306814 \quad 0.1466851 .224531$ H $-6.256108 \quad 1.2007690 .938800$ H -7.328083 -0.0821241 .543452$ H -5.597686 -0.030207 2.038987

SCF Energy (B3LYP/6-31G**/MMFF) $=-3245.89617449$

0700181

MM̄FF Geometry

C $-3.132053 \quad 3.192386-0.496055$

C $-2.6926534 .452445-0.351324$

C -1.318444 4.8776510 .106543

O $-0.552573 \quad 3.7491330 .581823$

C $-0.5287305 .506532-1.055881$

C $0.770514 \quad 6.136089-0.588189$

C $1.9701855 .520713-0.584333$

C $0.6477837 .555914-0.097899$

C $2.2880294 .111468-1.009111$

C $3.4769784 .080212-1.977440$

C $3.8164112 .686244-2.532698$

C $2.7873892 .205726-3.555100$

C $3.1473990 .816738-4.096993$

O $3.9036621 .754500-1.451959$

$\begin{array}{llll}\text { O } & 2.005150 & 0.311020 & -4.805644\end{array}$

C $3.401239-0.136855-2.910921$

C $4.2968120 .876256-5.110822$

O $3.883489-1.387422-3.393919$
C $4.347853 \quad 0.435577-1.828307$

C $4.306221-0.397808-0.522908$

$\begin{array}{lllll}\text { O } & 5.687112 & 0.472882 & -2.319554\end{array}$

C -0.4386913 .5701591 .923962$

O $\begin{array}{llll}0.014259 & 4.185046 & 2.806176\end{array}$

C $0.563116 \quad 2.501096 \quad 2.130610$

C $0.956508 \quad 2.174968 \quad 3.370434$

C 1.9652871 .1682313 .709225

C 2.7164950 .5364732 .568483

C $4.944163-1.784418-0.577574$

C $4.861074-2.4762850 .770808$

C $3.334814-3.898382 \quad 2.149637$

O $5.799134-2.4614331 .564235$

C $2.443361-3.1251313 .068285$

C $2.650295-3.022264 \quad 4.390136$

C $1.733804-2.2772695 .319833$

C $2.471208-1.3084336 .220388$

C $2.517590-1.5030417 .549213$

C $3.155198-0.1160965 .587246$

C $2.174413 \quad 0.8665005 .007518$

N $3.638973-3.0946710 .979801$

H $-2.4627172 .362378-0.300226$

H -3.379075 $5.269008-0.568351$

H $-1.443421 \quad 5.6163290 .908019$

H $-1.1325786 .276814-1.553733$

H $-0.3289104 .751824-1.825530$

H $2.8260356 .077780-0.204298$

H 1.6080027 .9689180 .228675

H $0.266376 \quad 8.200586-0.896481$

H -0.0400047 .6103020 .751760$

H $2.5426513 .540244-0.108819$

H $1.4234803 .621724-1.460376$

H $3.3097704 .773783-2.811278$

H $4.3586804 .448230-1.434941$

H $4.8010662 .769450-3.008159$

H $2.6877962 .924868-4.377490$

H $1.7948952 .140548-3.090714$

H $1.7939030 .937300-5.519292$

H $2.430991-0.334340-2.436346$

H $4.0397911 .543626-5.942140$

H $4.477388-0.105844-5.561854$

H $\quad 5.233097 \quad 1.232662-4.676300$

H $3.244697-1.713412-4.050753$

$\begin{array}{llll}\text { H } & 4.819888 & 0.172881 & 0.264087\end{array}$

H $3.266336-0.474156-0.181035$

H $6.2874840 .524118-1.557235$

H 0.9668842 .0205551 .246193

H 0.5026712 .6899064 .215887

H 3.2078901 .3052481 .961429

H $2.031704-0.0320431 .930787$

H $3.500699-0.1538782 .882332$

H $4.454209-2.427146-1.314327$

H $6.001031-1.723425-0.859409$

H $4.264044-4.2090512 .637884$

H $2.810574-4.7913531 .795086$

H $1.569629-2.6487232 .631023$

H $3.505609-3.5256304 .835280$

H $1.201759-3.0236105 .924223$

H $0.958237-1.7370694 .763371$

H $3.042166-0.8151918 .204123$

H $2.031959-2.3547718 .013910$

H $3.889941-0.4674314 .858598$

H 3.7468140 .4254516 .337567

H 1.5784791 .3818765 .762191

H $2.917744-3.0108090 .270276$

C -5.2319601 .9382440 .080735$

O $-4.7730450 .573957-0.072149$

C $-5.921880-0.288562-0.169882$

$\begin{array}{lllll}\text { C }-7.086508 & 0.516762 & 0.389125\end{array}$

C $-6.7492041 .900495-0.122922$

H $-6.098928-0.459171-1.238358$

C $-5.689669-1.6257210 .542981$

H -8.0551880 .1509510 .035408$

H -7.0961210 .5084991 .485352$

$\begin{array}{llll}\text { C }-7.494995 & 3.011888 & 0.596229\end{array}$

H $-6.9859501 .946850-1.194708$

C $-4.506070-2.4646040 .013613$

O $-5.462534-1.3832071 .936619$ 
H $-6.609127-2.2211600 .492183$

H $-3.563166-2.0128600 .334612$

O $-4.564358-3.7432230 .659992$

C $-4.490633-2.670904-1.510107$

C -3.521935-3.750795-2.025095

H $-4.260310-1.716254-1.997188$

O $-5.805513-3.049943-1.933417$

H -3.889955 -4.746872 -1.745819

H -3.588104 -3.719889 -3.120864

H -8.5756242 .9000160 .460096$

H $-7.2890183 .002611 \quad 1.671846$

H $-7.205068 \quad 3.9927730 .206697$

C $-4.5269252 .842868-0.935352$

H -4.721685 -0.756933 2.005030

H $-5.372702-4.1898440 .354934$

H $-5.799166-3.096553-2.904778$

H $-5.1071173 .761153-1.083687$

H -4.458958 2.334298 -1.904624

H -4.9900302 .2401691 .108098$

C $-0.274406-1.965043-1.502146$

O $-1.623783-2.275670-1.877341$

C -2.054004 -3.607896-1.591266

C $-1.157391-4.638742-2.306036$

C $0.328796-4.402226-2.020169$

C $0.710848-2.927510-2.189653$

H $-0.099277-0.956402-1.897723$

C $-0.083242-1.9091420 .022298$

H $-2.002180-3.773664-0.510228$

O $-1.374597-4.565815-3.715434$

H -1.451280 -5.644081-1.980624

H $0.927335-5.012736-2.707828$

O $0.616039-4.841496-0.696958$

H $1.723928-2.755751-1.809801$

O $\quad 0.732966-2.640521-3.590792$

C $-0.957763-0.8701330 .686080$

H $-0.317484-2.8705650 .486222$

H $0.956236-1.6546420 .253171$

H $-0.833957-5.258172-4.132462$

H $1.570801-4.729344-0.551961$

H $\quad 0.957081-1.701073-3.700316$

O $-0.981434-1.1094182 .029430$

O -1.5392680 .0309120 .100410$

C $-1.807575-0.2659092 .841332$

H -2.087712 0.6665002 .343018

H $-2.706289-0.8226873 .117168$

$\mathrm{H}-1.255297-0.0262243 .753576$

SCF Energy (B3LYP/6-31G**//MMFF) $=-3245.90241176$

0700182

MM̄FF Geometry

C $0.3292382 .600266-1.064214$

C $1.4353853 .055136-0.458623$

C 2.1984062 .2505990 .560029

$\begin{array}{lllll}\text { O } & 3.600728 & 2.402561 & 0.231878\end{array}$

C 1.9624982 .7420212 .000432

C 0.5589012 .5217902 .519150

C 0.1011561 .3504843 .002885

C -0.3222053 .7431912 .540814$

C 0.8393430 .0419543 .114107

C $-0.007747-1.1450212 .629365$

C $-0.075285-1.2702221 .097455$

C -1.271193 -2.139297 0.701296

C -1.333162 -2.382039-0.810993

O $1.147114-1.875540 \quad 0.651216$

O $-2.281373-3.432415-1.051184$

C $0.038953-2.899896-1.275534$

C $-1.846115-1.156911-1.573712$

O $0.075717-3.026345-2.696646$

C $1.242429-2.064755-0.767147$

C $2.540163-2.850598-1.076791$

O $1.265004-0.818604-1.462077$

C 4.4595351 .4656260 .713852

$\begin{array}{lllll}\text { O } & 4.179126 & 0.488311 & 1.389017\end{array}$

C 5.8240241 .8465820 .289716

C $6.870124 \quad 1.1824300 .801627$

C 8.2812241 .4203790 .501770

C $8.6197792 .460598-0.532422$

C $3.842787-2.250382-0.530420$
C $5.032018-3.134825-0.881162$

C $7.494610-3.223434-0.872577$

O $4.911558-4.223305-1.440503$

C $8.630569-2.693385-0.059204$

C $9.743493-2.177646-0.604350$

C $10.938736-1.7126350 .180682$

C $11.228791-0.241876-0.021044$

C $11.9865500 .178105-1.048150$

C 10.6995330 .7349191 .004137

C 9.2054360 .6799151 .146959

N $6.247849-2.583893-0.491649$

H $-0.0471021 .616566-0.802339$

H $1.8038524 .050244-0.694797$

H 1.9344991 .1906180 .465768

H 2.2278343 .8059342 .074512

H 2.6717702 .2552462 .682128

H $-0.915937 \quad 1.327883 \quad 3.393344$

H -1.3238333 .5274732 .927118$

H 0.1184544 .5148883 .180602

H -0.4392514 .1553531 .535511$

H 1.7980640 .0554772 .590230

H $1.061957-0.1126564 .177200$

H $0.432502-2.0623603 .041740$

H -1.018091 -1.057707 3.048993

H $-0.170939-0.2755800 .651571$

H $-2.207665-1.6895161 .052363$

H -1.199114 -3.112355 1.207677

H $-3.128507-3.168498-0.653592$

H $\quad 0.138640-3.921330-0.882702$

H -2.828495 -0.852693 -1.196219

H $-1.993374-1.384784-2.635290$

H -1.181460 -0.296356 -1.494882

H $\quad 0.187820-2.135273-3.069453$

H $2.444259-3.861261-0.656929$

H $2.646694-2.960974-2.164023$

H $2.162910-0.454026-1.425191$

H $5.9305342 .661965-0.416279$

H 6.6776350 .3837501 .517369

H $8.1244882 .234339-1.483354$

H $9.6891872 .522683-0.748581$

H $8.3007893 .453506-0.197339$

H $4.019459-1.258290-0.957331$

H $3.793911-2.1637000 .560149$

H $7.635250-3.052738-1.945621$

H $7.399931-4.303381-0.714872$

H $8.553480-2.7785321 .021811$

H $9.822097-2.118912-1.687916$

H $11.804655-2.306155-0.141408$

H $10.823464-1.9263851 .250668$

H $12.2192801 .228462-1.188127$

H $12.388368-0.521249-1.774171$

H $11.028188 \quad 1.7582750 .789281$

H 11.1470670 .4966651 .978073

H $8.867060-0.0609481 .872390$

H $\quad 6.269278-1.606705-0.213607$

C $-1.6575834 .077838-1.457118$

O $-2.5024803 .144658-0.759741$

C $-3.8679303 .592225-0.861093$

C $-3.8449784 .894468-1.659914$

C $-2.5741014 .750987-2.474067$

H $-4.4036682 .831597-1.438928$

C -4.4880383 .7224870 .536032$

H -4.739469 $5.010042-2.280138$

H $-3.7838115 .773354-1.007969$

C $-2.0475936 .068791-3.014178$

H $-2.7716714 .068356-3.311469$

C $-4.4023302 .450374 \quad 1.403830$

O $-3.821703 \quad 4.770634 \quad 1.253589$

H -5.5323954 .0425390 .444812$

H $-3.3623452 .256676 \quad 1.693833$

O -5.1140452 .7112592 .621340$

C $-4.992928 \quad 1.1852140 .754564$

C $\begin{array}{llll}-5.060717 & 0.006491 & 1.747354\end{array}$

H $-4.3785820 .903704-0.107418$

O $\quad-6.3014821 .4911080 .275114$

H $-4.097756-0.0865592 .263879$

H $-5.833637 \quad 0.2266152 .494339$

H $-2.7880096 .539040-3.669675$ 
H -1.819761 $6.771530-2.205517$ H -1.132776 $5.915872-3.595071$ C $-0.4646993 .359563-2.086140$ H -2.876339 4.5464871 .288496 H -4.7230983 .5074353 .020357$ H $-6.6430590 .700713-0.175868$ H $\quad 0.1762154 .079775-2.607848$ H $-0.8098402 .637435-2.836498$ H -1.304428 $4.807871-0.716445$ C $-7.149466-2.402375-0.216448$ O $-6.706089-1.2502890 .511880$ C $-5.395299-1.3398431 .074393$ C $-5.327781-2.5081362 .071610$ C $-5.789084-3.8219781 .433068$ C $-7.109750-3.6578510 .670341$ H $-8.200323-2.192042-0.454871$ C $-6.396146-2.567341-1.541010$ H $-4.669860-1.5027640 .271742$ O $-6.136122-2.2320243 .214459$ H -4.296019 -2.612547 2.426886 H $-5.909627-4.5758662 .220667$ O $\quad-4.770738-4.278548 \quad 0.549079$ H -7.316706 -4.5429000 .057550$ O $-8.177426-3.5439911 .616162$ C $-6.613879-1.368087-2.433533$ H -5.321362 -2.710039-1.401926 H $-6.753742-3.448590-2.086352$ H -7.042208 -2.0630712 .903953$ H $-5.063179-5.1282380 .177507$ H -8.189411 -4.3604842 .144321$ O $-5.473838-0.622934-2.498239$ O $-7.675299-1.114264-2.987954$ C $-5.5503880 .556994-3.303551$ H $-4.5623471 .024938-3.313954$ H $-5.827636 \quad 0.301304-4.330591$ H $-6.2686251 .260559-2.872310$ SCF Energy $\left(B 3 L Y P / 6-31 G^{* *} / / M M F F\right)=-3245.92484406$

\section{3}

MM̄FF Geometry

C $0.424949-3.681219-0.126981$

C $-0.598845-2.8508600 .120339$

C $-1.469663-2.243571-0.946809$

O $-2.834309-2.490339-0.532306$

C $-1.229899-0.726609-1.064550$

C $0.177528-0.353156-1.475465$

C $1.0850410 .219251-0.661106$

C $0.520251-0.627918-2.916293$

C $0.927676 \quad 0.582475 \quad 0.789244$

C 1.0106672 .0964891 .021687

C -0.0959332 .8993460 .318730$

C 0.1257524 .3984200 .528304

C $-0.9935625 .234387-0.105297$

$\begin{array}{lllll}0 & -1.363151 & 2.509828 & 0.861663\end{array}$

O $-0.845604 \quad 6.569470 \quad 0.401268$

C -2.3528784 .6835170 .371990$

C $-0.8410985 .324501-1.628334$

O $-3.4302395 .324457-0.308947$

C -2.4878813 .1444600 .234857$

C -3.7763242 .6862120 .959846$

O $-2.5806202 .804038-1.149075$

C $-3.819584-2.339955-1.455273$

O $-3.698531-1.983382-2.615915$

C $-5.094790-2.699185-0.797372$

C $-6.257044-2.342903-1.363272$

C $-7.594330-2.669780-0.863694$

C -7.686591 -3.5987150 .319359$

C $-4.008073 \quad 1.1694350 .995787$

C $-5.174734 \quad 0.838178 \quad 1.908747$

C -7.6224541 .2332812 .169720$

O $\begin{array}{lllll}-5.006048 & 0.290900 & 2.996276\end{array}$

C $-8.4547410 .051447 \quad 1.789331$

C -9.6506950 .1577561 .190091$

C $-10.564047-1.0007380 .908871$

C $-10.807493-1.220569-0.566301$

C $-11.673944-0.453452-1.249574$

C -10.122228 -2.397607 -1.219450

C $-8.665944-2.145515-1.494732$
N $-6.395531 \quad 1.2493851 .397862$

H $\quad 0.657427-3.959767-1.151538$

H $-0.810934-2.562898 \quad 1.147985$

H -1.301871 -2.744316 -1.908780

H -1.521286 -0.243584 -0.127205

H -1.924073 -0.296165 -1.797290

H $2.0627980 .458993-1.073996$

H $1.506701-0.240405-3.190369$

H $0.521142-1.704333-3.113328$

H $-0.211133-0.152403-3.578100$

H $0.0049180 .190595 \quad 1.224671$

H 1.7435720 .1007981 .343146

H 0.9526922 .2692152 .104161

H 1.9912712 .4576970 .689309

H $-0.0765102 .669388-0.751880$

H 1.1026724 .7078090 .135629

H 0.1544524 .6147001 .605736

H -1.512120 $7.131694-0.028203$

H -2.456502 4.9447191 .434665

H $0.1157615 .793249-1.888042$

H $-1.6161235 .962796-2.067054$

H $-0.8794424 .351282-2.122134$

H $-3.3842436 .273933-0.107019$

H -3.744456 3.0426991 .998298

H -4.6458643 .1581370 .485467$

H $-2.9694461 .919359-1.229554$

H -5.039554 -3.256564 0.130942

H $-6.230346-1.764047-2.285654$

H -8.710741 -3.799895 0.639100

H -7.171557 -3.171247 1.186614

H $-7.231350-4.5670180 .083342$

H $-4.2183320 .767008-0.000641$

H $-3.1256560 .649607 \quad 1.383269$

H -8.1375642 .1791181 .972037$

H $-7.390000 \quad 1.1921213 .238970$

H -8.082660 -0.9286092 .075620$

H -10.025208 $1.145750 \quad 0.930990$

H -11.522568 -0.8043801 .407592$

H -10.186824 -1.920909 1.370953

H -11.878522 -0.628649 -2.300404

H $-12.1956160 .369019-0.771198$

H -10.598338 -2.624791 -2.183104

H $-10.289272-3.295773-0.617413$

H -8.495332 -1.474228 -2.337413

H $\quad-6.4345341 .6425830 .462169$

C $2.674088-3.5421190 .946583$

O $3.435557-3.957202-0.208852$

C $4.779547-4.2364080 .209086$

C $4.927607-3.6329481 .597379$

C $3.540775-3.8831732 .159229$

H $4.842993-5.3288170 .316403$

C $5.825024-3.817419-0.828218$

H $5.720690-4.1072112 .182794$

H $5.129269-2.5583621 .544981$

C $3.221865-3.0681823 .399678$

H $3.446587-4.9508372 .400362$

C $5.964776-2.313291-1.154297$

O $7.093839-4.285294-0.349088$

H $5.626424-4.363375-1.759109$

H $6.377230-1.785392-0.287887$

O $6.966493-2.205718-2.179393$

C $4.688296-1.604401-1.639441$

C $4.919400-0.231260-2.297689$

H $3.994572-1.493453-0.800957$

O $4.049981-2.432780-2.616810$

H $5.491814-0.358785-3.225603$

H $3.936748 \quad 0.139065-2.616687$

H $3.948719-3.2714644 .192877$

H $3.239503-1.9945803 .191189$

H $2.226705-3.3156163 .782539$

C $1.312462-4.2362410 .952131$

H $7.760261-4.050665-1.017651$

H $\quad 6.612220-2.628709-2.980585$

H $3.191802-2.027905-2.828876$

H $\quad 0.831945-4.1159541 .930031$

H $1.429549-5.3123340 .776497$

H $2.546569-2.4545190 .870355$

C 5.2561851 .9967070 .692982 
O $\quad 4.771218 \quad 1.036972-0.258307$

C $5.5982110 .820238-1.405358$

C $5.8184192 .135481-2.173580$

C $6.3574663 .238038-1.259127$

C $5.534083 \quad 3.354993 \quad 0.025704$

H 4.4235272 .1431311 .392801

C $6.456007 \quad 1.457783 \quad 1.488965$

H $6.5678310 .429426-1.082961$

O $4.6001522 .576539-2.772309$

H $\quad 6.526074 \quad 1.952367-2.990494$

H $6.3343924 .197037-1.791084$

O $7.7117322 .961514-0.916258$

H 6.0322114 .0182340 .742540

O $4.2690123 .948071-0.281658$

$\begin{array}{llll}\text { C } & 6.052143 & 0.369651 & 2.460327\end{array}$

H 7.2272361 .0423640 .835799

H 6.8924172 .2678172 .085006

H $3.9349532 .658578-2.067518$

H $8.2214942 .918467-1.743211$

H $4.4414074 .829377-0.654676$

O $7.165887-0.074178 \quad 3.115379$

O $4.910978-0.0335632 .633978$

C $6.941521-1.0909774 .097125$

H $6.200037-0.7606714 .831014$

H $6.619278-2.0157313 .611475$

H $7.886261-1.2773864 .614834$

SCF Energy (B3LYP/6-31G**/MMFF)= -3245.90578166

0700184

MM̄FF Geometry

C 2.2835123 .8915712 .910826

C 2.3592972 .5650212 .724596

C 1.5873071 .5460743 .523932

$\begin{array}{lllll}\text { O } & 0.585859 & 0.988028 & 2.636624\end{array}$

C $2.5352490 .419650 \quad 3.973740$

C $1.975193-0.4315135 .094262$

C $1.446711-1.6616554 .945481$

C $2.081728 \quad 0.177013 \quad 6.470459$

C $1.235575-2.4422213 .676758$

C $-0.260395-2.5730473 .400937$

C $-0.634403-3.4675542 .209863$

C $0.003969-3.003257 \quad 0.898978$

C $-0.554646-3.746582-0.321848$

O $-2.061188-3.3923092 .143664$

O $-0.147077-3.033867-1.499259$

C $-2.091998-3.686583-0.279689$

C $0.021172-5.161342-0.444883$

O $-2.658715-4.472596-1.327231$

C $-2.690214-4.1096021 .080447$

C $-4.205023-3.7809641 .147561$

O $-2.527378-5.520370 \quad 1.241164$

C $-0.572868 \quad 1.6951532 .502111$

O -0.9502982 .6260343 .196792$

C $-1.262952 \quad 1.2130101 .283757$

C -2.0930312 .0506730 .641991$

C $-2.7536571 .817638-0.646604$

C $-2.5521920 .480109-1.306720$

C $-4.484614-2.2968061 .410018$

C $-5.874594-1.8496861 .004835$

C $-7.1972780 .253616 \quad 0.740963$

$\begin{array}{lllll} & \text { O } & -6.745805 & -2.616418 & 0.607151\end{array}$

C $-6.973996 \quad 0.784825-0.640042$

C $-6.9042412 .091965-0.933541$

C $-6.7290032 .620405-2.327041$

C $-5.5180943 .512835-2.471933$

C $-5.6526194 .840631-2.632359$

C $-4.1550612 .856037-2.536452$

C $-3.4579132 .828760-1.199573$

N $-6.013814-0.474495 \quad 1.150741$

H $1.646454 \quad 4.2937263 .693712$

H 2.9739862 .1859071 .911523

H 1.1056282 .0092314 .393416

H 3.4841440 .8458074 .328095

H $2.801400-0.2052683 .115502$

H $1.101566-2.1774595 .841418$

H $1.700328-0.4892237 .251705$

H 3.1278350 .3963486 .708318

H 1.5097611 .1080236 .526392
H $1.734921-1.9749642 .825541$

H $1.682580-3.4355073 .800146$

H $-0.769704-2.966657 \quad 4.292327$

H $-0.693932-1.574583 \quad 3.248834$

H $-0.345404-4.5006982 .434644$

H $1.094347-3.1124450 .934314$

H $-0.181969-1.9304420 .762735$

H $0.824416-2.992139-1.503844$

H $-2.377971-2.649719-0.490801$

H $1.111188-5.124565-0.550103$

H $-0.348194-5.661975-1.346579$

H $-0.204157-5.7907350 .418699$

H -2.259859 -4.173277-2.162260

H $-4.702576-4.1335410 .236034$

H $-4.670830-4.3499241 .963656$

H -3.179352 -5.8355711 .888468$

H -1.0029910 .2332190 .905400$

H -2.268129 3.0347521 .076265

H $-3.047935-0.302628-0.728582$

H $-1.4876970 .235020-1.387120$

H $-2.9492800 .429146-2.322364$

H $-4.361734-2.0856692 .479574$

H $-3.770823-1.6708130 .868863$

H -7.3582901 .0530751 .471093$

H $-8.067700-0.4096960 .750642$

H $-6.8873410 .053401-1.440146$

H $-7.0098832 .824206-0.136335$

H -7.640728 $3.173945-2.587301$

H $-6.6622531 .804712-3.057971$

H $-4.7882555 .481478-2.772472$

H $-6.6276605 .316469-2.622435$

H $-3.5170653 .420355-3.229827$

H -4.265497 1.863149 -2.980369

H $-3.5024743 .775637-0.659260$

H $-5.206186 \quad 0.073248 \quad 1.434608$

C 2.0990135 .3814430 .905766

$\begin{array}{lllll}\text { O } & 1.761050 & 4.272444 & 0.046441\end{array}$

C $2.1164814 .600819-1.306102$

C $2.1569156 .120429-1.343169$

C $2.777595 \quad 6.421936 \quad 0.009717$

H $3.1301094 .215030-1.472153$

C $1.148753 \quad 3.928281-2.284989$

H $2.7457566 .503550-2.181681$

H $1.1516616 .553224-1.401200$

C 2.5706297 .8538990 .471340

H $3.8560596 .221892-0.048421$

C $0.9479192 .418585-2.035472$

O $-0.1393474 .549396-2.181075$

H $1.4834574 .101291-3.313978$

H $0.3892812 .270383-1.105427$

$\begin{array}{lllll} & 0 & 0.126026 & 1.902923 & -3.089341\end{array}$

C $2.2438731 .591264-1.977726$

C $1.9731650 .106700-1.686966$

H $2.8846361 .987973-1.185672$

O $2.9543221 .722159-3.205808$

H $1.4338310 .009538-0.735388$

H $1.296004-0.305223-2.447397$

H $3.0374738 .555047-0.228127$

H 1.5065278 .1042810 .538111

H 3.0167958 .0122881 .458253

C 3.0045564 .8786312 .034229

H $-0.438347 \quad 4.454275-1.260266$

H $-0.6946482 .425001-3.097947$

H $2.3753521 .393904-3.915303$

H 3.3475725 .7143502 .654288

H 3.8989964 .4042351 .610113

H $1.155105 \quad 5.7669331 .311357$

C $3.751899-3.137039-1.261114$

O $2.766139-2.098116-1.287293$

C $3.227594-0.794227-1.669601$

C $4.331166-0.318208-0.713775$

C $5.461507-1.346028-0.605546$

C $4.925585-2.756388-0.346659$

H $3.242370-3.993802-0.802655$

C $4.178587-3.548457-2.673588$

H $3.620583-0.843851-2.690766$

O $3.766677-0.0930530 .574800$

H $4.7380330 .633986-1.072637$ 
H $6.134168-1.0557000 .210804$

O $6.210508-1.324488-1.817047$

H $5.729022-3.493182-0.457025$

O $4.483746-2.8415291 .012072$

C $3.048220-4.262245-3.376500$

H $4.499450-2.710505-3.298765$

H $5.022809-4.247241-2.634612$

H $4.475241 \quad 0.239397 \quad 1.151841$

H 6.939438 -1.961002 -1.722820

H $3.720602-2.2472651 .115059$

O $2.228140-3.357053-3.982269$

O $2.900919-5.477608-3.366253$

C $1.096219-3.909454-4.661300$

H $0.481799-3.081901-5.025622$

H $1.427165-4.506369-5.516159$

H $\quad 0.495484-4.515777-3.976477$

SCF Energy (B3LYP/6-31G**//MMFF) $=-3245.92691516$

0700185

MM̄FF Geometry

C $0.727245-1.6251381 .821772$

C $0.932884-0.8131470 .772250$

C $0.015644-0.621580-0.414136$

O $-1.143304-1.476270-0.268151$

C $-0.4624130 .837768-0.539950$

C $0.6355941 .851951-0.768554$

C 1.0399062 .7559010 .145023

C $1.2547671 .829651-2.141203$

C $\quad 0.5467632 .9231531 .555999$

C -0.1585114 .2666501 .782655$

C $-1.448314 \quad 4.4486730 .964713$

C $-2.0391745 .838477 \quad 1.219312$

C $-3.373380 \quad 6.0348650 .486762$

O -2.3863553 .4377491 .353703$

O $-3.990346 \quad 7.227624 \quad 0.994332$

C -4.2975054 .8405600 .802281$

C $-3.163176 \quad 6.294465-1.008993$

O $-5.493198 \quad 4.9080690 .027626$

C -3.617709 3.4616930 .615070

C $-4.5471492 .356024 \quad 1.173737$

O $-3.4017153 .233904-0.778204$

C $-1.851267-1.789308-1.384293$

O $-1.629588-1.424030-2.527129$

C $-2.971621-2.658744-0.962269$

C $-4.019793-2.827750-1.781120$

C $-5.203996-3.646821-1.510374$

C $-5.168345-4.537597-0.296833$

C -3.9536450 .9426651 .149944$

C $-4.858494-0.0456841 .859791$

C -7.084950-1.120262 1.746605

O $-4.548179-0.5390132 .942155$

C $-8.077219-1.487556 \quad 0.691130$

C $-8.302112-2.7509810 .301951$

C $-9.311898-3.154074-0.731997$

C $-8.728604-3.318090-2.117826$

C $-9.262945-2.668490-3.166464$

C -7.573546 -4.279129-2.309830

C $-6.248646-3.563408-2.360608$

N $-6.029049-0.3129051 .165611$

H $-0.180298-2.218221 \quad 1.886817$

H $\quad 1.866330-0.2546500 .738269$

H $0.567028-0.930277-1.311214$

H -1.066100 1.0885590 .336514

H -1.176780 $0.920918-1.369631$

H $1.8285533 .452118-0.136858$

H $1.9651132 .648977-2.288937$

H $1.7924630 .891075-2.305562$

H $\quad 0.480669 \quad 1.927470-2.909324$

H -0.1027182 .1040091 .875787$

H 1.4216002 .8761842 .217194

H -0.3985684 .3345482 .851836$

H $\quad 0.5381835 .084071 \quad 1.556942$

H -1.213213 4.332235 -0.098805

H -1.327753 6.6241880 .935786

H -2.2103785 .9658902 .297494$

H -4.1210497 .1191081 .951676$

H -4.605111 4.9296741 .853584

H $-2.5624507 .200031-1.157479$
H $-4.1162156 .482218-1.515969$

H -2.656488 $5.474455-1.521923$

H -5.8887735 .7832800 .180213$

H -4.8000032 .5932702 .215764$

H -5.4867722 .3520150 .607997$

H -3.266492 $2.285357-0.926204$

H $-2.910023-3.1194890 .017226$

H -4.022095 -2.302885 -2.735743

H $-6.041276-5.186802-0.206756$

H -5.117796 -3.9388890.619199

H $-4.295146-5.199084-0.331106$

H -3.7945550 .5873330 .126492$

H $-2.9842080 .923006 \quad 1.659129$

H -7.570052 -0.5253132 .527601$

H $-6.636178-1.9978962 .225062$

H $-8.645970-0.6794700 .237542$

H -7.739893 -3.555901 0.768176

H -10.135172 -2.427767 -0.741105

H -9.763069-4.105475 -0.422857

H -8.867442 -2.796354-4.168905

H $-10.104801-1.994589-3.047540$

H -7.698380 -4.811720 -3.262555

H -7.613929 -5.061430-1.547295

H $-6.157563-2.892626-3.216837$

H $\quad-6.247046 \quad 0.247280 \quad 0.346510$

C $2.725389-2.9099492 .736796$

O $3.550470-2.6081201 .591444$

C $3.566557-3.7575660 .729022$

C $3.208787-4.9343881 .626463$

C $2.143033-4.3077192 .506729$

H $2.762142-3.616216-0.003333$

C $4.906639-3.874557-0.000575$

H $2.846157-5.797477 \quad 1.060413$

H $4.061915-5.2546502 .235774$

C $1.876016-5.0764463 .789115$

H $1.211295-4.2564521 .930546$

C $5.339975-2.608261-0.770012$

O $5.940371-4.1728690 .946397$

H $4.873898-4.731380-0.683698$

H $5.677659-1.842675-0.062463$

O $6.483258-2.956419-1.561600$

C $4.268726-2.011941-1.700420$

C $4.771363-0.924016-2.667887$

H $3.456109-1.595963-1.095918$

O $3.691909-3.051889-2.496559$

H $5.430177-1.373592-3.422360$

H $3.893590-0.566716-3.223166$

H $1.513737-6.0850113 .564712$

H $2.782513-5.1728384 .396104$

H $1.116953-4.5699044 .393588$

C $1.708373-1.7842472 .951593$

H $5.943269-3.4624581 .610670$

H $7.154581-3.317446-0.957339$

H $4.410516-3.465243-3.005368$

H $2.246247-0.8386593 .092106$

H $1.147012-1.9609853 .876532$

H $3.405104-2.9243673 .598229$

C $5.1190361 .959611-0.305831$

O $4.5883260 .859076-1.059187$

C $5.4849620 .263784-2.000462$

C $5.9451351 .302718-3.036354$

C $6.5475122 .537795-2.359044$

C $5.6506913 .066222-1.235942$

H 4.2583092 .3670460 .238736

C $6.155031 \quad 1.498848 \quad 0.732442$

H $6.363524-0.118882-1.472493$

O $4.8532181 .702512-3.862663$

H $6.6898960 .840117-3.694370$

H $6.6906623 .329413-3.104870$

O $7.8237542 .213342-1.815779$

H $6.1894093 .819797-0.649241$

O $4.5363113 .727535-1.841165$

C 5.5388900 .6350671 .811763

H $\quad \begin{array}{llll}6.959642 & 0.921173 & 0.270336\end{array}$

H $6.5906372 .372472 \quad 1.230684$

H $4.1575062 .058453-3.283999$

H $8.3875901 .914348-2.549304$

H $4.0115734 .130477-1.128666$ 
O $\quad 6.536471 \quad 0.1196582 .589495$

O $4.340786 \quad 0.446014 \quad 1.965247$

C $6.109546-0.7180513 .668263$

H $5.703629-1.6531983 .273804$

H $6.982008-0.9473344 .285779$

H $5.368068-0.2047904 .288155$

SCF Energy (B3LYP/6-31G**//MMFF) $=-3245.91378654$

0700186

MM̄FF Geometry

C 3.4431782 .4302551 .493021

C 2.6472172 .0743392 .513317

C 1.1702042 .3629852 .557437

O $\quad 0.5215821 .1295012 .957455$

C 0.8224363 .4704433 .570678

C 1.3046854 .8513283 .178247

C 0.7130415 .6273902 .248307

C 2.5075475 .3543573 .932385

C -0.4978515 .2986181 .416515$

C $-0.1432095 .257142-0.075968$

C $-1.2671404 .748905-0.995455$

C $-2.5402155 .589582-0.913501$

C $-3.6431155 .031712-1.826145$

O $-1.5572173 .392295-0.649345$

O $-4.8769445 .673386-1.468348$

C $-3.8198513 .522880-1.543597$

C $-3.3907825 .370740-3.300570$

O $-4.7302862 .953478-2.480507$

C $-2.4909672 .734571-1.528959$

C $-2.6533451 .309121-0.949957$

O $-1.9621502 .639996-2.852382$

C -0.7905970 .9858002 .630553$

O $-1.498903 \quad 1.8070862 .071810$

C -1.229515 -0.3593813 .060993$

C $-2.396450-0.8397642 .605007$

C $-2.988613-2.1420802 .918180$

C $-2.286698-2.9969153 .940094$

C $-3.458950 \quad 0.306538-1.779017$

C $-4.869408 \quad 0.077642-1.261030$

C $-6.860691-1.362774-1.674617$

O $-5.3378790 .657315-0.285573$

C $-6.811520-2.618005-0.851769$

C $-5.756149-3.423900-0.647516$

C $-5.819845-4.6721180 .187291$

C $-4.774066-4.7107531 .279590$

C $-3.782713-5.617471 \quad 1.254851$

C $-4.928552-3.7645662 .449557$

C -4.124041 -2.5026232 .282263$

N -5.540200 -0.872919-2.019840

H 3.0190582 .9782630 .655538

H 3.0759421 .5562903 .367996

H 0.8185282 .6289231 .552942

H 1.2184263 .2009264 .559907

H -0.2623233 .5054643 .733032$

H 1.1348666 .6134932 .057369

H 2.8119406 .3557683 .610259

H 2.2874255 .4049255 .003839

H 3.3626244 .6879783 .785353

H $-0.9508024 .353351 \quad 1.722380$

H -1.247835 $6.076628 \quad 1.599378$

H $\quad 0.1643806 .258029-0.404785$

H $0.7246644 .597245-0.208615$

H $-0.8678554 .769808-2.016458$

H -2.331400 $6.638896-1.155422$

H $-2.926675 \quad 5.5869160 .114130$

H $-4.7576746 .632813-1.575218$

H -4.286543 $3.424908-0.553556$

H -3.325956 $6.456791-3.437761$

H $-4.2239815 .039820-3.930515$

H -2.469467 4.932798 -3.689969

H $-5.5580553 .461151-2.425582$

H -1.644691 $0.891639-0.838632$

H -3.0388381 .3792750 .072433$

H -1.358741 $1.879104-2.886180$

H $-0.578567-0.916675 \quad 3.724227$

H $-2.980655-0.2219881 .924742$

H -1.246904 -3.176730 3.649536

H $-2.740474-3.9811774 .072610$
H $-2.293467-2.5035964 .918322$

H $-3.5033250 .590775-2.835137$

H $-2.951236-0.665625-1.724719$

H -7.401425 -1.550682 -2.607125

H -7.393552 -0.586164 -1.116083

H $-7.757326-2.885001-0.383335$

H $-4.801507-3.204417-1.118282$

H $-5.694543-5.527615-0.489000$

H $-6.810163-4.7950680 .643602$

H -3.051465 -5.673438 2.054049

H $-3.678151-6.3218230 .436338$

H $-4.682060-4.2835833 .381581$

H -5.985064 -3.490110 2.565321

H $-4.551920-1.8081541 .558657$

H -5.047679 -1.337700 -2.777108

C 5.4151841 .7489710 .069751

O $4.961610 \quad 0.419257-0.264681$

C $6.051350-0.300873-0.873727$

C $7.1402950 .728848-1.155681$

C $6.9417921 .700009-0.013405$

H $6.422114-1.003318-0.118531$

C $5.599441-1.062465-2.126411$

H $8.1389140 .281415-1.172348$

H $6.9831421 .234666-2.115652$

C $7.5970743 .050341-0.249598$

H 7.3544001 .2546860 .901977

C $4.494929-2.117097-1.910069$

O $5.117489-0.125583-3.099059$

H $6.472952-1.540647-2.585597$

H $3.538595-1.615992-1.731096$

O $4.337880-2.825008-3.148151$

C $4.768732-3.129601-0.784388$

C $3.859996-4.374261-0.787343$

H $4.687838-2.6191190 .182203$

O $6.118338-3.593101-0.901792$

H $4.113333-5.016069-1.641198$

H $4.128560-4.9582640 .103329$

H $8.6785262 .935503-0.377266$

H $7.2024233 .535878-1.148467$

H 7.4279963 .7222150 .597450

C 4.9204492 .1602651 .460170

H $4.3827570 .365210-2.693278$

H $5.160371-3.318165-3.310789$

H $6.296857-4.172722-0.141576$

H $5.4266343 .082126 \quad 1.768658$

H 5.1745321 .3784882 .186734

H $5.0125572 .423441-0.697442$

C $0.682110-2.8640930 .472185$

O $2.053781-3.2634110 .340180$

C $2.349955-4.092037-0.785489$

C $1.529301-5.393763-0.730525$

C $0.031711-5.115404-0.569765$

C $-0.238640-4.0932240 .540394$

H $\quad 0.632659-2.3547001 .442824$

C $0.263481-1.857827-0.611439$

H $2.099885-3.553760-1.704726$

O $1.968082-6.2151550 .351128$

H $1.703753-5.960423-1.652780$

H $-0.481152-6.056458-0.336143$

O $-0.482449-4.639624-1.809313$

H $-1.283954-3.7651140 .519372$

O $-0.017103-4.7205561 .806544$

C $1.063018-0.575842-0.545821$

H $0.395803-2.270591-1.614708$

H $-0.791573-1.593390-0.478359$

H $1.890487-5.6950101 .169284$

H -1.436625 -4.492148 -1.693718

H $-0.627624-5.474317 \quad 1.871979$

O $0.7898800 .173970-1.654361$

$\begin{array}{llll}\text { O } & 1.820267 & -0.264652 & 0.361787\end{array}$

C $1.4496201 .441316-1.722344$

H $1.2167701 .895161-2.689016$

H $1.0792992 .095929-0.929253$

H $2.5328871 .316368-1.646259$

SCF Energy (B3LYP/6-31G**//MMFF) $=-3245.91064110$

0700187

MM̄̄FF Geometry 
C 2.1735352 .0529091 .353240

C 2.8889963 .1649961 .119811

C $3.8795573 .379115-0.000526$

O $4.3589182 .099001-0.471851$

C $3.2373884 .156134-1.167809$

C $2.9961465 .620565-0.862729$

C $1.8182166 .145871-0.471450$

C $4.1985276 .511898-1.040942$

C $0.5262115 .418742-0.219018$

C $-0.6256406 .020186-1.034849$

C $-1.9185205 .187756-1.003762$

C -2.4784134 .9969990 .407819$

C -3.7323674 .1094130 .410178$

O $-1.6328773 .920860-1.603284$

O

C $-3.4181522 .811033-0.364042$

C $-4.9678064 .874451-0.076610$

O $-4.5888112 .009695-0.499416$

C $-2.7656283 .053608-1.741480$

C $-2.2612571 .747684-2.396500$

O $-3.7416093 .622261-2.622475$

C $5.5684732 .070100-1.090957$

O $6.2633203 .033610-1.383436$

C $5.9732690 .684659-1.419563$

C $5.526855-0.378206-0.733130$

C $5.886360-1.777919-0.967337$

C $6.766721-2.097151-2.145889$

C $-1.1970860 .995845-1.589757$

C $-0.437870-0.012439-2.436007$

C $1.615343-1.402390-2.383525$

O $-0.721163-0.261574-3.604622$

C $2.190331-2.379046-1.411323$

C $2.487779-3.647454-1.732407$

C $3.077459-4.647495-0.776734$

C $4.541733-4.910538-1.054927$

C $4.916384-5.783856-2.005059$

C $5.573237-4.208134-0.197532$

C $5.382961-2.716961-0.139937$

N $0.599633-0.601711-1.724318$

H $2.278748 \quad 1.1905250 .701112$

H 2.7424884 .0206801 .776614

H 4.7326613 .9277160 .418971

H $2.3171033 .652941-1.488843$

H $3.8793724 .091730-2.056242$

H $1.7747337 .217216-0.278218$

H $3.9747807 .561810-0.824457$

H $5.0082316 .203225-0.372503$

H $4.5602726 .458460-2.073080$

H $0.6129944 .351221-0.433972$

H $\quad 0.302826 \quad 5.5067230 .850567$

H $-0.8377767 .035856-0.677806$

H $-0.3037606 .107022-2.081432$

H -2.650150 $5.712038-1.630163$

H -2.688451 5.9657200 .877790

H -1.728875 $4.509707 \quad 1.045270$

H $-4.737736 \quad 3.130972 \quad 1.783467$

H -2.7265192 .2363520 .263935$

H -5.1527875 .7449720 .564270$

H -5.870088 $4.256923-0.006726$

H -4.874208 5.232571-1.103857

H $-5.1356472 .399603-1.202488$

H -3.104311 $1.079582-2.614732$

H -1.836887 $2.008980-3.376232$

H -3.464147 $3.456077-3.538665$

H $6.7009520 .592022-2.218878$

H $4.834143-0.2138780 .091323$

H $6.921234-3.169412-2.290158$

H $7.756229-1.644386-2.020269$

H $6.321675-1.719492-3.073296$

H $-0.4641241 .699533-1.178363$

H $-1.6626450 .459350-0.756287$

H $2.394675-0.710375-2.719682$

H $\quad 1.186405-1.893522-3.263075$

H $2.392734-2.024673-0.403948$

H $2.299209-3.995568-2.745711$

H $2.506750-5.581365-0.863694$

H $2.946182-4.3293530 .264565$

H $5.962541-5.994730-2.199684$
H $4.182562-6.306156-2.610343$

H $6.593567-4.448535-0.518821$

H $5.497753-4.6015970 .824766$

H $4.742897-2.3969040 .683305$

H $0.826513-0.218102-0.810842$

C $1.491290 \quad 0.784047 \quad 3.412293$

O $1.114943-0.4636472 .788771$

C $0.321105-1.2223263 .719880$

C $0.595663-0.6127345 .086397$

C 0.7073340 .8540564 .725324

H $-0.728422-1.0397013 .463561$

C $0.625575-2.7187753 .610803$

H $-0.203141-0.8203365 .804637$

H $1.540249-0.9730635 .510161$

C 1.3775141 .6941195 .799017

H $-0.300991 \quad 1.2490284 .540392$

C $0.437594-3.3129762 .200249$

O $1.985061-2.9520854 .001776$

H $0.008756-3.2695254 .330735$

H $1.245909-2.9637931 .548637$

O $0.602481-4.7308432 .327693$

C $-0.924958-3.0038161 .549972$

C $-1.131519-3.7480980 .217112$

H -0.994671 -1.924659 1.373562

O $-1.959775-3.3632512 .464303$

H $-0.215321-3.687555-0.377107$

H -1.337796 -4.8042110 .431407$

H 0.8009791 .6600926 .729326

H 2.3887091 .3341626 .017184

H 1.4528172 .7402545 .486187

C 1.1698801 .9448912 .469094

H $2.553823-2.4242733 .415202$

H $0.722706-5.0971861 .435545$

H -2.809561 -3.1229302 .057993$

H 1.1365292 .8850003 .032082

H $\quad 0.1809741 .7976642 .017169$

H 2.5695510 .7284823 .610863

C $-4.700849-2.898872-0.503961$

O $-3.511738-3.429108 \quad 0.093707$

C $-2.295557-3.168712-0.610764$

C $-2.353019-3.776238-2.020450$

C $-3.589765-3.288712-2.784307$

C $-4.866116-3.411150-1.945514$

H $-5.521283-3.3087720 .099029$

C $-4.760010-1.372160-0.391389$

H $-2.147137-2.086815-0.689997$

O $-2.379643-5.198872-1.923235$

H - $1.439858-3.500097-2.561407$

H -3.709040 -3.876814 -3.702631

O $-3.415514-1.926701-3.162716$

H $-5.690287-2.880015-2.436381$

O $-5.227778-4.794276-1.898554$

C $-4.779186-0.9405251 .056417$

H $-3.923020-0.879130-0.893280$

H $-5.671067-0.980720-0.858963$

H $-2.395091-5.554932-2.827754$

H -2.614730 -1.873205 -3.711755

H $-6.072344-4.861643-1.421340$

O $-3.584532-0.3741531 .389821$

O $-5.735952-1.1005491 .803016$

C -3.4798080 .1079822 .732546$

H -4.3060950 .7866682 .965197$

H -3.462611 $-0.734216 \quad 3.430259$

H -2.540999 0.6611662 .821592

SCF Energy (B3LYP/6-31G**//MMFF) $=-3245.91280439$

0700188

MM̄FF Geometry

C -3.230044 -0.609761 2.565268

C $-2.101861-1.1510263 .049133$

C $-1.303940-2.2070392 .331506$

O $0.102988-1.8448642 .385497$

C $-1.411267-3.5873203 .000476$

C $-2.781343-4.2224362 .931508$

C $-3.219305-4.9709671 .900960$

C $-3.648017-4.0429664 .151722$

C $-2.488356-5.3179510 .632561$

C $-3.351705-5.079688-0.615300$ 
C $-3.350908-3.618102-1.093737$

C $-4.430540-3.423879-2.162602$

C $-4.376933-2.030370-2.802395$

O $-2.052945-3.352951-1.638493$

O $-5.200380-2.055354-3.978456$

C $-2.931508-1.766192-3.266098$

C $-4.965693-0.953999-1.882890$

O $-2.799041-0.445762-3.788284$

C -1.870831-2.035561-2.171629

C $-0.458855-1.975430-2.802566$

O $-1.991702-1.034980-1.162957$

C $0.510361-0.720658 \quad 1.740514$

$\begin{array}{llll}\text { O } & -0.175021 & 0.029233 & 1.061037\end{array}$

C $1.926417-0.4340552 .047870$

C $2.833372-1.4090902 .206047$

C $4.248634-1.2227962 .527424$

C 4.7097590 .1607732 .905504

C $0.688951-2.339580-1.850480$

C $2.025354-2.385038-2.573223$

C $4.457990-2.527462-2.169617$

O $2.134099-2.287521-3.793110$

C $5.377234-3.036225-1.107737$

C $6.407812-2.326337-0.624147$

C $7.374158-2.8398500 .405555$

C $7.422092-1.9677511 .639894$

C $8.281160-0.9374531 .720837$

C $6.536477-2.3348172 .808733$

C $5.068603-2.2933042 .485788$

N $3.087815-2.563397-1.695525$

H -3.646737 -0.9822081 .633298$

H -1.696492 -0.7846993 .989650$

H -1.589838 -2.285649 1.276791

H -1.086611 -3.519239 4.048428

H $-0.668485-4.2591472 .549228$

H -4.209818 -5.4174711 .984410$

H $-4.607356-4.5638444 .060870$

H -3.141311 -4.442689 5.036434

H -3.867320 -2.986693 4.327345

H -1.534290 -4.7909860 .539370$

H -2.252382 -6.388296 0.682804

H - $2.948955-5.710274-1.419300$

H $-4.378420-5.417814-0.427218$

H -3.531596 -2.951100 -0.243561

H -5.427074 -3.615292 -1.745053

H $-4.293373-4.171824-2.956745$

H -6.103182 -2.295226 -3.707603

H -2.738541-2.441781 -4.110778

H $-6.010830-1.184664-1.644117$

H $-4.9825600 .022391-2.379595$

H -4.428244 $-0.853421-0.937871$

H -2.764009 $0.168397-3.036228$

H $-0.425795-2.671347-3.651477$

H -0.269357-0.970329-3.199695

H - $1.137355-0.917220-0.720722$

H 2.1858400 .6163642 .101335

H $2.514861-2.4431672 .081172$

H 4.5988990 .8496332 .062230

H 5.7590260 .1995313 .207321

H 4.1266850 .5438223 .750404

H $0.766544-1.599305-1.049545$

H $0.519187-3.323559-1.399831$

H $4.682182-1.495053-2.458291$

H $4.536635-3.151615-3.066239$

H $5.203635-4.044831-0.740587$

H $6.589419-1.326017-1.011322$

H $8.368878-2.879057-0.057931$

H $7.145181-3.8738270 .692068$

H $8.345108-0.3217962 .611840$

H $8.938279-0.6855760 .894886$

H $6.748320-1.7081863 .683620$

H $6.788024-3.3536803 .131604$

H $4.654367-3.2589592 .194426$

H $2.917195-2.447466-0.700380$

C -4.4232261 .5791112 .224068$

O $-3.3727551 .949447 \quad 1.316458$

C $-3.5501193 .325676 \quad 0.933448$

C $-4.775697 \quad 3.840417 \quad 1.689394$

C -4.8605022 .8909472 .868580$
H -2.6599993 .8596621 .283684$

C $-3.653093 \quad 3.443470-0.594261$

H -4.6575554 .8856051 .992697$

H -5.6886953 .7810051 .086695$

C -6.2341092 .8401413 .513684$

H -4.1213473 .2062063 .617117$

C -2.554045 2.702804-1.380314

O $-4.9088832 .901232-1.030057$

H -3.674055 $4.502415-0.876063$

H -2.718811 $1.623051-1.301388$

O $-2.729292 \quad 3.020648-2.768034$

C $-1.1053823 .018538-0.968125$

C $-0.0941932 .349345-1.921369$

H $-0.9360892 .669748 \quad 0.056048$

O $-0.9254994 .432764-0.983217$

H $-0.3963941 .310146-2.095082$

H $-0.1189612 .880667-2.881021$

H -6.5203423 .8285553 .887442$

H -6.9998492 .5146972 .801509$

H -6.2426712 .1447794 .358863$

C $-3.9301070 .539483 \quad 3.234040$

H $-4.9414261 .971083-0.747720$

H -2.536368 $3.967701-2.877652$

H $-0.0161784 .616716-0.692578$

H $-4.7816540 .156486 \quad 3.807779$

H -3.244496 1.0183613 .944603

H $-5.2331941 .150791 \quad 1.618284$

C $3.1046183 .894594-0.739706$

O $1.7915573 .710558-1.285665$

C $1.3512812 .355270-1.388299$

C $2.2941141 .563407-2.308460$

C $3.7509881 .669776-1.851361$

C $4.1539493 .120656-1.555962$

H $3.3065494 .967091-0.858607$

C 3.1497693 .5770720 .758246

H $1.3454321 .900314-0.392523$

O $2.1871312 .031056-3.651981$

H $1.9839530 .513671-2.310340$

H $4.3992931 .259216-2.635293$

O $3.9199590 .867554-0.687398$

H $5.1185153 .156787-1.036383$

O $4.319828 \quad 3.813728-2.796888$

C $2.1737894 .439371 \quad 1.523237$

H 2.9280212 .5282000 .965996

H 4.1484633 .7659691 .168834

H $2.3864312 .983018-3.652199$

H $4.8604870 .899694-0.442974$

H $5.0200113 .358984-3.295400$

O $1.1369193 .677944 \quad 1.976095$

O 2.3071435 .6459161 .680996

C 0.1230624 .3811602 .699912

H $-0.345786 \quad 5.1340612 .059136$

H -0.6378093 .6585223 .006792$

H 0.5484334 .8450873 .594834

SCF Energy (B3LYP/6-31G**//MMFF) $=-3245.91836112$

0700189

MM̄FF Geometry

C $0.8881693 .089181 \quad 1.491837$

C 0.0644712 .8927192 .532035

C $\quad 0.0412201 .6927543 .456734$

O $\begin{array}{llll}0.1 .083488 & 0.852315 & 3.101395\end{array}$

C $1.3099180 .818038 \quad 3.414218$

C $1.282926-0.3472104 .385175$

C $1.107990-1.6336064 .020434$

C 1.5297640 .0068115 .828011

C $0.846523-2.1515702 .631775$

C $-0.223119-3.2550062 .624601$

C $-0.765886-3.5890531 .223327$

$\begin{array}{llll}C & 0.331886 & -3.996470 & 0.236985\end{array}$

C $-0.213239-4.225047-1.180828$

O $-1.467190-2.4345770 .750366$

O $0.924004-4.297831-2.054107$

C $-1.051108-2.992909-1.586247$

C $-0.938718-5.569557-1.300082$

O $-1.694178-3.197590-2.841881$

C $-2.100054-2.597464-0.524953$

C $-2.799394-1.263028-0.861886$ 
O $-3.115258-3.606542-0.471179$

C -2.3180581 .2352383 .520376$

O -2.6114792 .2413864 .146024$

C -3.2803650 .2140423 .061160$

C -4.5044180 .6135132 .687899$

C $-5.578359-0.2448862 .190366$

C $-5.438912-1.7301712 .397300$

C $-1.868554-0.050417-0.927599$

C $-2.6602051 .237490-0.831663$

C $-4.3893892 .568082-2.029189$

O

C $-5.7787562 .091059-1.709784$

C $-6.2314400 .827786-1.770391$

C $-7.6442810 .422857-1.453967$

C $-7.713559-0.700634-0.442211$

C $-7.723481-1.984276-0.840334$

C -7.855285 -0.3404731 .018998$

C $-6.6354050 .333119 \quad 1.582769$

N -3.416868 $1.493428-1.963114$

H 1.6176702 .3317521 .227576

H -0.6642513 .6685552 .759317$

H -0.0920722 .0600634 .482533$

H $2.191321 \quad 1.4335673 .639467$

H 1.4676090 .4427292 .398242

H $1.158600-2.3983364 .794232$

H $1.570390-0.8783316 .471871$

H 2.4843010 .5331315 .932282

H $\quad 0.7320620 .652376 \quad 6.207734$

H $\quad 0.529123-1.3484301 .961633$

H $1.788246-2.5539952 .240793$

H $0.189297-4.1630803 .081944$

H -1.067645 -2.932848 3.248502

H -1.487083 -4.406107 1.343286

H $0.867158-4.8845060 .595154$

H $1.083123-3.1994990 .171198$

H $0.605356-4.500820-2.949676$

H $-0.350645-2.159553-1.725448$

H $-0.244404-6.394811-1.101547$

H -1.311814 -5.729337 -2.317731

H -1.776959-5.669732 -0.607578

H -1.001654 -3.338139 -3.508857

H -3.359156 -1.361877-1.800715

H -3.561506 -1.068264 -0.096251

H -3.932123 -3.209560 -0.126851

H -2.943572 -0.8143093 .008046$

H $-4.734770 \quad 1.6781732 .721075$

H -5.340853 -1.959061 3.464432

H $-4.556872-2.1165371 .877474$

H -6.293582 -2.301098 2.027571

H -1.145157 -0.054128 -0.104519

H -1.298227 -0.051951 - 1.861290

H -4.360336 $2.990090-3.038208$

H $-4.1039283 .350162-1.317931$

H $-6.4731182 .873140-1.408774$

H $-5.5682730 .032089-2.099674$

H $-8.1179280 .102108-2.391305$

H $-8.2381871 .274931-1.100903$

H -7.801387 -2.798108-0.127313

H -7.649209 -2.249226 -1.890069

H -8.120314 -1.218571 1.619479

H -8.7106860 .3378151 .135613$

H -6.643446 1.4177501 .470064

H $-3.3917040 .832934-2.734453$

C $0.3661004 .056077-0.774305$

O $1.0897432 .977995-1.394861$

C $1.3523483 .312259-2.769329$

C $0.5450634 .572558-3.064433$

C $0.5268725 .253449-1.710401$

H $2.4214533 .548477-2.830551$

C $1.0346972 .117751-3.678522$

H $1.0051975 .183697-3.846915$

H $-0.4780684 .341146-3.381341$

C $-0.565746 \quad 6.298976-1.569642$

H $1.5031295 .729816-1.548355$

C $1.657114 \quad 0.779136-3.227512$

O $-0.3850251 .923831-3.731932$

H $1.3385872 .347749-4.706125$

H $\quad 1.1669610 .434618-2.309757$
O $1.357695-0.198777-4.232266$

C $3.1814470 .813085-3.017549$

C $3.823930-0.571846-2.804464$

H $3.4181291 .461431-2.166943$

O $3.788826 \quad 1.400894-4.171877$

H $3.695846-1.174549-3.712676$

H $4.908210-0.427324-2.721643$

H $-0.4351997 .093881-2.311336$

H -1.559601 $5.862556-1.715424$

H $-0.541826 \quad 6.757360-0.576152$

C 0.9050754 .3216600 .632992

H $-0.7091611 .891779-2.816130$

H $\quad 0.391533-0.211897-4.344091$

H $4.742807 \quad 1.472742-3.997750$

H 0.3348285 .1308101 .103895

H 1.9481064 .6579120 .575468

H $-0.6842133 .743850-0.721709$

C $4.659789-0.3061140 .121050$

O $3.340603-0.563682-0.382432$

C $3.294878-1.344512-1.579918$

C $3.976121-2.701894-1.350984$

C $5.385388-2.547890-0.787874$

C $5.377930-1.6271550 .433170$

H $5.2240480 .260126-0.628750$

C $4.486013 \quad 0.5773381 .364741$

H $2.235307-1.552417-1.768963$

O $4.030903-3.453584-2.558827$

H $3.386239-3.287718-0.634955$

H $6.081155-2.189603-1.555605$

O $5.854716-3.839966-0.393228$

H $4.884978-2.1494461 .263456$

O $6.730797-1.3936290 .812440$

C 5.6950601 .4194061 .704259

H $3.658676 \quad 1.2758481 .200911$

H $4.222788-0.0448942 .228242$

H $3.117168-3.568701-2.871226$

H $6.739608-3.720031-0.007578$

H $6.724762-0.9950721 .698731$

O 5.5470571 .9141002 .969121

O 6.6357901 .6402410 .954601

C 6.6125872 .7500393 .431304

H 6.3779553 .0671104 .450970

H 7.5537672 .1921213 .444826

H 6.6982033 .6382632 .798230

SCF Energy (B3LYP/6-31G**//MMFF) $=-3245.90179407$

0700190

MM̄FF Geometry

C -3.985627 $3.013507-0.981404$

C $-3.1746243 .043592-2.048948$

C $-1.6872992 .830853-1.944334$

O $-1.3672691 .649502-2.716026$

C $-0.8879544 .023746-2.504936$

C $-0.9818095 .281292-1.665658$

C $-0.2518905 .503527-0.554503$

C $-1.9385526 .330442-2.167617$

C $0.7497104 .573674 \quad 0.071422$

C 2.1343515 .2252270 .172298

C 3.1859264 .2379330 .700543

C $4.5110454 .950350 \quad 0.960617$

C 5.5998583 .9665241 .406152

O $3.3737073 .227520-0.293075$

O $6.861948 \quad 4.648343 \quad 1.342436$

C 5.6681522 .7924940 .403838

C 5.4128893 .5346582 .866709

O $6.561516 \quad 1.796901 \quad 0.895951$

C 4.2888522 .1748650 .068339

C $4.3307471 .255947-1.181676$

O 3.8012131 .4465961 .191550

C $-0.2828810 .931310-2.312537$

O $0.4599871 .186409-1.377573$

C $-0.157362-0.247898-3.197369$

C $0.832001-1.123678-2.966019$

C $1.076743-2.378769-3.678041$

C $0.142060-2.738365-4.801867$

C $5.146378-0.036293-1.112082$

C $4.491902-1.158718-0.326358$

C $4.960606-3.4681470 .496669$ 
O $3.321928-1.1281290 .042756$

C $4.692948-4.472314-0.580003$

C $3.505381-5.064876-0.776760$

C $3.252313-6.070133-1.864106$

C $2.194281-5.621535-2.847921$

C $1.009966-6.251071-2.927568$

C $2.537450-4.493859-3.797747$

C $2.102109-3.154512-3.267179$

N $5.363186-2.220281-0.125821$

H -3.559152 $2.805038-0.002311$

H -3.586364 $3.257905-3.031746$

H -1.417489 2.655622 -0.894733

H -1.207899 $4.235200-3.534743$

H $0.1666693 .741243-2.620473$

H $-0.3921536 .444180-0.024013$

H -1.942128 $7.225610-1.536645$

H -1.660282 $6.642791-3.179560$

H -2.961151 5.943081-2.192961

H $\quad 0.396672 \quad 4.313352 \quad 1.076691$

H $0.8248323 .635415-0.481996$

H $2.4355935 .581704-0.821684$

H 2.0768816 .0984630 .834118

H 2.8054023 .7787971 .620352

H 4.3868795 .7481451 .703406

H 4.8474975 .4475740 .040090

H 6.8109725 .4277721 .921955

H $6.1075383 .176218-0.527321$

H $5.414740 \quad 4.409573 \quad 3.527807$

H 6.2444312 .9056723 .203241

H 4.4800892 .9928633 .037077

H 7.4172592 .2292311 .059490

H $3.2981150 .999140-1.454883$

H $4.7038451 .850914-2.026765$

H 3.1008600 .8480420 .881988

H $-0.893253-0.380018-3.981577$

H $1.523549-0.917061-2.150416$

H $-0.891745-2.789796-4.443698$

H $0.358255-3.706686-5.257543$

H $0.199066-1.990700-5.600807$

H $5.255658-0.415461-2.137154$

H $6.1572010 .141385-0.739665$

H $4.086325-3.3010541 .133227$

H $5.790485-3.8065511 .124233$

H $5.526800-4.726554-1.230665$

H $2.671305-4.822325-0.122434$

H $2.954971-7.011071-1.383534$

H $4.173247-6.297783-2.415778$

H $0.258929-5.948798-3.649925$

H $0.762356-7.077306-2.269515$

H $2.115112-4.700016-4.786322$

H $3.621823-4.468094-3.965010$

H $2.716143-2.795775-2.441254$

H $6.294744-2.175720-0.528644$

C $-6.3541552 .120432-0.685004$

$\begin{array}{lllll}\text { O } & -5.865330 & 1.411300 & 0.472143\end{array}$

C -5.937682 -0.0022070 .197806$

C $-6.887572-0.143166-0.982493$

C -6.500902 $1.080014-1.795183$

H $-4.943010-0.313212-0.139220$

C $-6.330357-0.7745001 .459108$

H $-6.744660-1.082753-1.524329$

H -7.935739 $-0.081318-0.666878$

C $-7.5186521 .448627-2.860148$

H $-5.5320340 .892521-2.276127$

C $-5.310188-0.6231592 .610563$

O $-7.598340-0.2871431 .910655$

H $-6.473206-1.8337951 .215468$

H $-5.287140 \quad 0.4183142 .951177$

O $-5.779872-1.3939603 .722912$

C $-3.888629-1.0886242 .243089$

C $-2.896935-0.9892003 .417868$

H -3.508839 -0.4779831 .417846$

O $-3.971038-2.4388581 .787829$

H -2.9818480 .0027253 .876702$

H $-3.154650-1.7459164 .168969$

H $-7.6278200 .635200-3.584872$

H -8.503992 $1.643820-2.423972$

H $-7.2064952 .345620-3.403907$
C $-5.4562173 .316968-1.007458$

H $-7.821525-0.7714282 .724048$

H $-5.757686-2.3302883 .460342$

H $-3.079651-2.7161561 .518134$

H $-5.623768 \quad 4.087527-0.243410$

H $-5.7524013 .763838-1.963501$

H $-7.3461802 .504218-0.411402$

C $-0.026564-2.9518202 .033292$

O $-1.300962-2.5811412 .573656$

C -1.441023 -1.209468 2.955494

C $-0.423166-0.8646004 .050673$

C $1.004402-1.1934733 .598012$

C $1.109161-2.6064383 .011867$

H $-0.070973-4.0451931 .944141$

C $0.181119-2.3817550 .626661$

H $-1.262187-0.5694582 .084308$

O $-0.722646-1.577306 \quad 5.248818$

H $-0.5008320 .203395 \quad 4.284745$

H $1.686546-1.1055454 .452493$

O $1.421273-0.2559802 .609675$

H $2.074810-2.7465582 .513202$

O $1.048774-3.554588 \quad 4.082106$

C $-0.850539-2.929433-0.331275$

H $0.145098-1.2889890 .599381$

H $1.162539-2.6690770 .234026$

H $-0.731072-2.5263475 .036213$

H $1.388503 \quad 0.6292323 .010783$

H $1.801383-3.3781014 .672181$

O $-1.775716-1.970567-0.620210$

O $-0.844122-4.077858-0.755238$

C $-2.812231-2.361265-1.526322$

H $-2.389350-2.793458-2.437674$

H $-3.483467-3.073855-1.039086$

H $-3.380923-1.467700-1.796910$

SCF Energy (B3LYP/6-31G**//MMFF)= -3245.93516112

$07 \quad 00191$

MM̄FF Geometry

C -3.376677 4.7694990 .197375

C $-2.3106374 .459526-0.554970$

C $-0.9091964 .933355-0.267747$

$\begin{array}{lllll}\text { O } & -0.098758 & 3.765227 & 0.002002\end{array}$

C $-0.3394025 .648799-1.505237$

C $1.0516546 .212080-1.288806$

C $2.1871995 .679380-1.783729$

C $1.1090807 .481168-0.477032$

C $2.3450594 .430345-2.607996$

C $3.1224003 .367831-1.830996$

C $3.3197932 .044799-2.586941$

C $1.9996411 .339664-2.908868$

C $2.217502-0.056612-3.511247$

O $4.1133501 .218518-1.725785$

O $0.963281-0.752890-3.467367$

C $3.201327-0.842514-2.622051$

C $2.6209440 .017781-4.988646$

O $3.541205-2.092032-3.219026$

C $4.482720-0.052347-2.276854$

C $5.292432-0.803965-1.194109$

$\begin{array}{lllll}\text { O } & 5.282059 & 0.090532 & -3.450906\end{array}$

C $-0.082467 \quad 3.302765 \quad 1.283513$

$\begin{array}{lllll}\text { O } & -0.637416 & 3.797387 & 2.251754\end{array}$

C 0.7070902 .0509561 .299909

C $0.734067 \quad 1.2920202 .405763$

C $1.403477-0.0043702 .542358$

C $2.298582-0.4473131 .417142$

C $6.542248-0.049068-0.723413$

C $6.966699-0.4883860 .662536$

C $6.408838-0.1735143 .073588$

O $7.904325-1.262700 \quad 0.837201$

C $5.572578-1.3324743 .515397$

C $4.721345-1.2823064 .551794$

C $3.955065-2.4736455 .049516$

C $2.477677-2.205148 \quad 5.213361$

C $1.921776-2.1255176 .434743$

C $1.629236-2.1360963 .964235$

C $1.163206-0.7399303 .647601$

N $6.1865910 .073704 \quad 1.662441$

H -3.252880 5.4081721 .068202 
H $-2.4410253 .803187-1.412726$ H -0.8882665 .6119710 .593909$ H -1.002903 $6.475858-1.793130$ H $-0.3493404 .964185-2.359644$ H $3.1257916 .190108-1.570075$ H $2.1253437 .882695-0.403209$ H $0.4840268 .254191-0.936127$ H 0.7518297 .3037760 .541878 H $1.3788174 .034461-2.921694$ H $2.8881934 .686378-3.525473$ H $4.1121393 .762248-1.560639$ H $2.6293233 .166379-0.872443$ H $3.8843762 .250049-3.503878$ H $1.3816891 .939443-3.586495$ H $1.4121651 .229458-1.987342$ H $\quad 0.310969-0.227533-3.961775$ H $2.678509-1.081403-1.686414$ H $1.8391310 .516445-5.574369$ H $2.725119-0.983379-5.421020$ H $3.5523640 .564116-5.151249$ H $2.709085-2.558955-3.407463$ H $4.640509-0.994070-0.333579$ H $5.612461-1.782877-1.572474$ H $\quad 6.1982830 .272998-3.189498$ H $1.207249 \quad 1.7595680 .384938$ H 0.1782651 .6264723 .280739 H $1.709935-0.6534040 .517359$ H $2.871769-1.3481431 .645153$ H $3.033480 \quad 0.3306501 .183011$ H $7.378642-0.222740-1.410071$ H $6.3793791 .034540-0.696941$ H 6.1576070 .7470813 .609610 H $7.467284-0.3978433 .240786$ H $5.707936-2.2717482 .984063$ H $4.610288-0.3532805 .106080$ H $4.392933-2.766366 \quad 6.012828$ H $4.089244-3.3389924 .388276$ H $0.854589-1.9726596 .558864$ H $2.519729-2.2093667 .336092$ H $\quad 0.733643-2.7556164 .111507$ H $2.151735-2.609178 \quad 3.128215$ H $0.490043-0.3271534 .400718$ H $5.3988980 .661142 \quad 1.405336$ C -5.1582623 .1703700 .921421$ $\begin{array}{lllll}\text { O } & -4.345971 & 1.999763 & 0.699545\end{array}$ C $-5.177608 \quad 0.8278820 .763342$ C -6.5105191 .2942121 .334859$ C $-6.603616 \quad 2.6993180 .772834$ H $-5.325717 \quad 0.489521-0.269820$ C $-4.498495-0.2861891 .573865$ H $-7.341206 \quad 0.651373 \quad 1.027920$ H -6.4910591 .3217882 .430839$ C $-7.6170703 .574476 \quad 1.489181$ H $-6.8710902 .635270-0.290575$ C -3.205579 -0.8155360 .913009$ O $-4.230560 \quad 0.1884232 .892035$ H -5.200228 -1.122806 1.673638 H -3.469724 -1.135024 -0.100274 O C -2.553041-2.001133 1.655890 C $-1.272600-2.5319580 .982099$ H -3.278746 -2.816659 1.754410 O $-2.173664-1.6143962 .978757$ H $-0.517310-1.7394110 .945949$ H $-0.858704-3.3073901 .640793$ H -8.6221083 .1492861 .401444$ H -7.383768 3.6686702 .555089 H -7.6375504 .5805851 .058768$ C $-4.7632084 .256894-0.079183$ H -3.693205 0.9949022 .810809 H $-1.967418 \quad 0.4907691 .667881$ H -2.983752 -1.373876 3.459263 H $-5.4583775 .101896-0.023952$ H -4.817006 $3.858543-1.100207$ H -4.9645913 .5034301 .949607$ C $-2.808743-4.802546-1.543855$ O $-2.417613-4.187385-0.310359$ C $-1.486667-3.107973-0.424845$
C $-0.175901-3.597273-1.059912$

C $-0.441706-4.280481-2.407175$

C $-1.577110-5.306664-2.316608$

H $-3.396708-5.680545-1.246600$

C $-3.723194-3.883673-2.368835$

H $-1.908931-2.323647-1.059263$

O $0.468459-4.515827-0.179673$

H $\quad 0.495130-2.740190-1.191969$

H $0.469668-4.786163-2.749040$

O $-0.787421-3.302039-3.382467$

H -1.881335 -5.621590 -3.321122

O $-1.083821-6.476915-1.656676$

C $-4.998559-3.538931-1.626313$

$\mathrm{H}-3.221524-2.944840-2.618281$

H -4.010612 -4.381001-3.301785

H $1.310115-4.775475-0.591692$

H -0.043674 -2.679235 -3.449673

H $-0.869962-6.233086-0.739639$

O $-5.579778-2.452723-2.216247$

O $-5.442022-4.153101-0.665539$

C $-6.804034-2.010332-1.621179$

H $-7.588504-2.754873-1.785142$

H $-6.667814-1.822731-0.551822$

H -7.098408 -1.074962 -2.104410

SCF Energy (B3LYP/6-31G**//MMFF) $=-3245.92308747$

0700192

MM̄MF Geometry

C -0.391406 $4.445315-2.972224$

C $-0.6712523 .133549-2.922667$

C $0.3154362 .053911-3.281224$

O $\quad 0.659134 \quad 1.357674-2.057343$

C $-0.3088801 .038862-4.253199$

C $0.578129-0.163974-4.522540$

C $0.215568-1.439398-4.280306$

C $1.9364150 .130752-5.104211$

C -1.113724 -1.927132 -3.780704

C $-1.211071-2.081688-2.257920$

C $-0.325483-3.201479-1.692892$

C $-0.819039-3.620929-0.307201$

C $0.070925-4.7082810 .307757$

O $1.018410-2.719753-1.593204$

O $-0.273638-4.822719 \quad 1.697307$

C $1.539354-4.2464590 .238416$

C $-0.188079-6.083880-0.317821$

O $2.398285-5.3030030 .663684$

C $1.962265-3.706804-1.153298$

C $3.350082-3.035596-1.046537$

O $2.046815-4.796297-2.074111$

C $1.6463631 .908514-1.299174$

O $2.2757102 .926653-1.542346$

C $1.8342541 .060059-0.101661$

C 2.6627891 .4895650 .862018

C 3.0135200 .7849182 .094644

C $2.304428-0.5069682 .396036$

C $3.758382-2.190718-2.259045$

C $5.167756-1.669237-2.054601$

C $6.467817-0.095108-0.615343$

O $6.132072-2.180888-2.618527$

C $6.562629-0.4920800 .826609$

C $6.8419450 .367073 \quad 1.818710$

C $6.899437-0.0244373 .269306$

C 6.0750720 .8846964 .158655

C $6.669327 \quad 1.7588024 .988634$

C 4.5649440 .7366424 .138630

C 3.9572111 .3264822 .892789

N $5.224149-0.623132-1.146990$

H $0.5764814 .777998-3.337809$

H - $-1.6248882 .810963-2.509776$

H $1.2193022 .480811-3.734069$

H $-0.5318251 .531497-5.208475$

H - $-1.2726480 .718338-3.843737$

H $0.934791-2.229868-4.490623$

H $2.462898-0.780031-5.409843$

H $1.8391980 .764517-5.991734$

H $2.5686400 .644210-4.373359$

H -1.918805 -1.267651-4.123149

H $-1.324158-2.892524-4.259139$ 
H $-0.969266-1.126969-1.774752$ H -2.257568 -2.307809 -2.028274 H $-0.358983-4.055261-2.379775$ H -1.858721 -3.966411 -0.352083 H $-0.818613-2.7482530 .361138$ H -1.222588 -5.0283971 .755064$ H $1.648496-3.4402290 .976808$ H $-1.239826-6.368879-0.193910$ H $\quad 0.393673-6.8630830 .187119$ H $0.040935-6.119816-1.384932$ H $3.280225-4.9267230 .819916$ H $3.362057-2.379887-0.168653$ H $4.119800-3.800608-0.881507$ H $2.663612-4.561042-2.785372$ H $\quad 1.3033810 .116495-0.051837$ H 3.1612552 .4488780 .725287 H $2.622135-1.2881781 .701222$ H $1.218513-0.3834662 .313872$ H $2.487190-0.8720253 .409606$ H $3.740880-2.774989-3.184502$ H $3.080744-1.340849-2.397200$ H $6.4408810 .991586-0.743020$ H $7.318121-0.499456-1.172212$ H $6.384134-1.5379851 .066468$ H 7.0450051 .4081711 .577487 H 7.9525910 .0012253 .577774 H $6.568537-1.0602703 .415606$ H 6.0895862 .3988675 .645477 H 7.7490931 .8580565 .027114 H 4.1234301 .2507955 .001636 H $4.329497-0.3249474 .259668$ H 4.3737762 .2954932 .613130 H $4.359441-0.276910-0.742955$ C $-0.9086105 .824330-0.974067$ O $-1.0983104 .644147-0.168040$ C -1.7942974 .9980121 .037505$ C -1.6615746 .5094851 .150627$ C $-1.749338 \quad 6.915152-0.312544$ H -2.8514974 .7531970 .878240$ C -1.244981 4.1692552 .203814 H -2.4480536 .9528251 .768297$ H -0.6912396 .8024541 .567826$ C $-1.2541708 .323946-0.586473$ H -2.795046 $6.833342-0.638558$ C -1.2000312 .6506231 .916636$ O 0.0947694 .5891352 .492122 H -1.826355 4.3686513 .110778 H -0.4487652 .4405201 .148208$ O -0.7600291 .9834343 .104621$ C -2.5471842 .0461121 .484518$ C $-2.4570590 .542571 \quad 1.179562$ H -2.8924442 .5568780 .581624$ O -3.5202962 .2755862 .501032$ H $-1.7350390 .369790 \quad 0.370997$ H -2.053432 0.0076152 .049717 H $-1.8598459 .056951-0.043639$ H -0.212371 8.451361 -0.273608 H -1.316421 8.557516 -1.654049 C -1.309909 $5.499971-2.415735$ H $0.6207374 .470593 \quad 1.682282$ H $\quad 0.1014232 .3643783 .347770$ H -3.204445 1.8405213 .311651 H -1.253482 $6.392788-3.047698$ H -2.346565 $5.141551-2.446509$ H $0.1591756 .075053-0.921180$ C $-4.655103-2.3253850 .256757$ O $-3.520114-1.4898370 .520821$ C $-3.807674-0.1198660 .831561$ C $-4.5485880 .538578-0.340891$ C $-5.813833-0.246214-0.703106$ C $-5.535631-1.742451-0.863402$ H $-4.230927-3.268178-0.110754$ C $-5.436553-2.6394841 .542107$ H -4.433764 -0.085924 1.729516 O $-3.6770560 .610065-1.467046$ H $-4.8271631 .562652-0.068442$ H $-6.2262750 .152003-1.638373$ O $-6.782612-0.040167 \quad 0.321552$
H $-6.479217-2.297680-0.920334$

O $-4.873025-1.927913-2.116980$

C $-4.617157-3.4526412 .524150$

H -5.753453 -1.728332 2.056152

H $-6.324863-3.2312161 .292721$

H $-4.1636491 .043563-2.188489$

H -7.590281 -0.5150110 .061628$

H $-4.753344-2.883926-2.248424$

O $-5.358183-3.6553383 .654246$

O $-3.481198-3.8630682 .330848$

C $-4.714504-4.4137574 .683093$

H -3.810396 -3.899846 5.023327

H -5.406492 -4.500593 5.524935

H $-4.475335-5.4178864 .320055$

SCF Energy $\left(B 3 L Y P / 6-31 G^{* *} / / M M F F\right)=-3245.90805962$

0700193

MM̄FF Geometry

C 5.9154031 .6669330 .838563

C 5.4593040 .4845960 .400617

C $5.157578-0.6848991 .301053$

O $3.728340-0.9075021 .255056$

C $5.869928-1.9456860 .780297$

C $5.585335-3.1890621 .602386$

C $4.820492-4.2227451 .194895$

C $6.244760-3.2298812 .956949$

C $4.095127-4.366959-0.114594$

C $2.590911-4.1548080 .064301$

C $1.842471-3.916854-1.255626$

C $2.161955-2.544993-1.861630$

C $1.320753-2.257462-3.112879$

O $0.443725-3.986068-0.950087$

O $1.427312-0.855112-3.406912$

C $-0.163624-2.534019-2.798508$

C $1.869807-2.997726-4.338009$

O $-0.957346-2.444809-3.978986$

C $-0.417339-3.886633-2.091214$

C $-1.879963-3.962951-1.594658$

O $-0.239540-4.987561-2.986495$

C $2.959923-0.1659912 .101284$

O 3.3485700 .6412752 .930665

C $1.542423-0.4877811 .825486$

C 0.5712760 .1910862 .453200

C -0.8708070 .0077692 .268297$

C $-1.318286-1.0157611 .259597$

C $-2.206626-5.247256-0.821200$

C $-1.935880-5.1287340 .667036$

C $-2.913734-4.2445042 .770662$

O $-0.971353-5.674404 \quad 1.197497$

C $-3.821233-3.1218663 .158678$

C $-3.496759-2.1635274 .040306$

C $-4.429928-1.0453504 .411230$

C $-3.7893630 .323040 \quad 4.339510$

C $-3.792378 \quad 1.1395225 .407448$

C $-3.2157420 .778128 \quad 3.014578$

C -1.7099720 .7626953 .007188$

N -2.883192 -4.355962 1.323010

H $\quad 6.109468 \quad 1.8098291 .898511$

H $5.2376960 .361965-0.657492$

H $5.472382-0.4850682 .332844$

H $6.955624-1.7787330 .770688$

H $5.594370-2.103472-0.266920$

H $4.686411-5.0635421 .874349$

H $6.077608-4.1818293 .472253$

H $7.327039-3.0981892 .856282$

H $5.854470-2.4355893 .600415$

H $4.502510-3.695633-0.870831$

H $4.273997-5.378211-0.500888$

H $2.162763-5.0333820 .566455$

H $2.393457-3.3180710 .744080$

H $2.095257-4.725350-1.951075$

H $3.226399-2.445623-2.100348$

H $1.950781-1.761506-1.122374$

H $2.370318-0.640418-3.509854$

H $-0.505589-1.732927-2.129333$

H $2.888841-2.660533-4.563974$

H $1.277539-2.778396-5.233004$

H $1.907654-4.080785-4.201369$ 
H $-0.805749-1.564080-4.363017$ H -2.102886 -3.089983 -0.970073 H -2.548381 -3.927324 -2.464402 H -0.105467 -4.641652 -3.883290 H $1.330902-1.2619701 .099655$ H $\quad 0.856023 \quad 0.961393 \quad 3.168827$ H -0.979806 -2.014559 1.554687 H $-0.905051-0.7909840 .271567$ H -2.401587 -1.066743 1.137071 H -3.273055 -5.477936 -0.942367 H -1.663407 -6.114906 -1.210506 H -1.891747 -4.1097203 .140581$ H $-3.299428-5.1913613 .162743$ H -4.805802 -3.0999962 .696197$ H -2.526899 -2.193042 4.530941 H $-4.795383-1.2427675 .427092$ H -5.314432 -1.0441563 .761494$ H -3.361703 2.134204 5.356783 H -4.2250330 .8339116 .354242$ H -3.5372971 .8101082 .819384$ H -3.6506920 .1915832 .201696$ H -1.271725 1.4824963 .700714 H -3.720701 -4.095948 0.809161 C 4.9580843 .8347450 .067723 O $3.7794193 .249352-0.524638$ C $3.0756934 .259431-1.269776$ C $3.7384325 .583420-0.907392$ C $5.1728625 .150693-0.674971$ H $3.2482524 .049759-2.333048$ C $1.5695844 .210596-0.974801$ H $3.6369016 .329066-1.701733$ H 3.3127246 .0073060 .010009 C 5.9972356 .1690100 .092677 H $5.6465154 .961378-1.647635$ C $0.8817342 .932538-1.508161$ O $1.362026 \quad 4.3512750 .429387$ H $1.0934915 .075474-1.452141$ H $1.0591582 .885066-2.589373$ O $1.4771361 .764495-0.947225$ C $-0.6410122 .901214-1.260331$ C $-1.3054691 .693099-1.950555$ H -1.079534 $3.830898-1.641751$ O -0.8835802 .8440150 .143011$ H $-0.955177 \quad 1.632856-2.987954$ H $-0.9956050 .779526-1.431853$ H $6.0537727 .112592-0.459829$ H $5.561306 \quad 6.3803141 .074797$ H 7.0184295 .8076040 .248435 C $6.1259662 .855538-0.057424$ H 1.8380803 .6273310 .870794 H 1.2684671 .7597600 .002100 H -1.8456812 .8548810 .279534$ H 7.0686983 .3361550 .226095 H $6.2285592 .525765-1.098957$ H $4.724448 \quad 4.0239581 .124074$ C $-4.7279281 .802302-0.421526$ O $-3.3104511 .676156-0.602186$ C $-2.8465151 .776666-1.951945$ C $-3.4781850 .667977-2.807884$ C $-5.0064500 .693259-2.714892$ C $-5.4859450 .761870-1.260639$ H -4.8986541 .5607030 .634520$ C $-5.2065413 .240337-0.646517$ H $-3.1268002 .753875-2.359873$ O $-3.003370-0.609831-2.392009$ H -3.169240 $0.803347-3.850901$ H -5.406699 -0.208821 -3.193684 O $-5.4877631 .821664-3.438740$ H $-6.5612700 .970014-1.215852$ O $-5.280608-0.512915-0.642848$ C $-4.650305 \quad 4.1498650 .423375$ H -4.933352 $3.643629-1.625641$ H $-6.2995373 .301111-0.583444$ H -3.188000 -0.702349 -1.441586 H $-6.458991 \quad 1.806570-3.393459$ H - $5.795516-1.167719-1.144316$ O $-3.5102944 .750534-0.021161$ O $\quad-5.1535994 .284197 \quad 1.531328$
C -2.8569935 .6069120 .920951$

H -1.9477595 .9944180 .453579$

H -3.5065026 .4482271 .180108$

H -2.5771905 .0430841 .816105$

SCF Energy $(B 3 L Y P / 6-31 G * * / / M M F F)=-3245.90709727$

0700194

MM̄FF Geometry

C -2.633465 $1.504212-3.048803$

C $-3.5075930 .678590-3.645700$

C $-3.515452-0.828321-3.544885$

O $-2.386141-1.287525-2.771730$

C $-4.791372-1.289850-2.814548$

C $-4.896868-2.798762-2.700345$

C $-4.465306-3.515048-1.642196$

C $-5.545651-3.485006-3.872556$

C $-3.754157-2.992977-0.423373$

C $-4.517374-3.3029330 .868906$

C -3.973190 -2.558949 2.102954

C $-4.396182-1.0880632 .104400$

C $-3.819386-0.3368383 .309319$

O $-2.548266-2.6681432 .119733$

O $-4.005887 \quad 1.068522 \quad 3.084474$

C -2.298595 -0.5856193 .372508$

C $-4.568276-0.6713714 .605269$

O $-1.769841-0.0094804 .564489$

C -1.902312 -2.076962 3.262404

C $-0.384771-2.2659593 .010331$

O $-2.263827-2.764736 \quad 4.460629$

C $-1.354171-1.895458-3.410047$

O $-1.205243-2.043928-4.610974$

C $-0.437258-2.382502-2.355035$

C $0.652681-3.094154-2.675650$

C $1.597516-3.682134-1.721793$

C $1.266233-3.556974-0.256788$

C $0.543341-1.7672664 .117544$

C $1.966386-2.2651103 .946390$

C $4.007334-1.9354932 .564703$

O $2.482943-3.026714 \quad 4.761289$

C $4.242231-1.9839681 .089372$

C $5.051036-2.8766760 .498100$

C $5.281786-2.930855-0.985789$

C $5.119490-4.324503-1.556241$

C $6.147734-4.940873-2.163587$

C $3.776191-5.010126-1.422191$

C $2.692203-4.311511-2.198201$

N $2.588482-1.7554442 .817571$

H -1.826595 $1.099154-2.444343$

H $-4.3070021 .112059-4.243568$

H -3.492431 -1.249799-4.556991

H $-5.680571-0.911052-3.336391$

H -4.835563 -0.838363 -1.815302

H $-4.595879-4.596007-1.662198$

H $-5.595791-4.571404-3.743375$

H $-6.569225-3.120317-4.007106$

H $-4.981983-3.287175-4.789894$

H $-2.768530-3.472796-0.381882$

H -3.564173 -1.921122 -0.499208

H $-5.586997-3.0878090 .753766$

H $-4.432227-4.3822411 .054180$

H -4.378213 -3.062689 2.988559

H -5.489495 -0.998156 2.083623

H -4.039585 -0.589101 1.194003

H -4.9581011 .2301472 .969946$

H -1.839435 -0.048637 2.531102

H $-5.632782-0.4274884 .505872$

H -4.200768 -0.0666575 .441819$

H -4.495054 -1.724785 4.883517

H $-2.006741 \quad 0.9338194 .560325$

H $-0.191848-3.3367442 .851902$

H $-0.119130-1.7874382 .058577$

H - $1.716311-3.5644584 .530424$

H $-0.704388-2.161986-1.327025$

H $0.867787-3.275882-3.727509$

H $0.286651-3.999796-0.043378$

H $1.246730-2.5027940 .039868$

H $1.978461-4.0559840 .401999$

H $\quad 0.579016-0.6726344 .137731$ 
H $0.192836-2.1026465 .100003$ H $4.364462-2.8368493 .073234$ H $4.519593-1.0700002 .996494$ H $3.727961-1.2482670 .475427$ H $\quad 5.588387-3.5962331 .111587$ H $6.297046-2.559223-1.175164$ H $4.606583-2.247115-1.514949$ H $\quad 6.045973-5.939517-2.575387$ H $7.115856-4.461663-2.264114$ H $3.537061-5.125922-0.362388$ H $3.834425-6.037113-1.807403$ H $2.837092-4.351722-3.278689$ H $2.112616-1.0202342 .302326$ C $-2.8770373 .679416-1.769782$ O $-1.7040183 .487134-0.954497$ C -2.1172453 .3600190 .421062$ C -3.6112573 .6800470 .451157$ C $-4.0550823 .208191-0.918920$ H $-1.9727792 .309147 \quad 0.697960$ C $-1.270714 \quad 4.2668631 .320656$ H -4.1281083 .1709721 .269489$ H -3.7953874 .7553490 .560310$ C $-5.397277 \quad 3.765927-1.356137$ H $-4.1122792 .112820-0.896138$ C $0.2490634 .001744 \quad 1.271860$ O -1.4824265 .6326090 .935213$ H -1.628352 4.1905532 .354061 H 0.6362584 .2761510 .284489 O $\quad 0.8701624 .9083052 .194375$ C 0.6776892 .5586391 .612624 C 2.1804902 .3906551 .915528 H $\quad 0.3928051 .8909610 .792290$ O -0.0375902 .1092942 .767712$ H 2.4494293 .0194032 .773675 H $2.335873 \quad 1.3564112 .250550$ H $-6.1862783 .457289-0.662576$ H $-5.3869184 .860533-1.387382$ H $-5.6633463 .402372-2.353726$ C $-2.7050763 .002549-3.132861$ H -1.2171795 .7181740 .003401$ H $0.511396 \quad 4.7268483 .079440$ H 0.2331052 .6597613 .521827 H - $1.7716403 .351415-3.590834$ H $-3.5230473 .311376-3.794573$ H -2.948952 4.762300-1.939755 C $3.7516871 .725313-1.406974$ O $2.9654641 .620205-0.211255$ C 3.1074912 .6912620 .727665 C 4.5717312 .8226591 .177482 C $5.5117972 .973552-0.020696$ C $5.2460181 .908712-1.087854$ H $3.6312920 .752788-1.901814$ C $3.2029422 .801813-2.349296$ H 2.7983623 .6290450 .258297 O $4.9593931 .690241 \quad 1.952059$ H 4.6626163 .7006511 .827838 H $6.5489402 .894047 \quad 0.327762$ O $5.3337894 .272764-0.577079$ H $5.7963022 .148575-2.005202$ O $5.7665330 .667624-0.605352$ C $1.8716132 .368229-2.911744$ H $3.0491763 .769603-1.864258$ H $3.8893242 .980184-3.185714$ H $4.8459380 .899657 \quad 1.397909$ H $5.9617584 .364988-1.313768$ H $5.6731760 .013718-1.318835$ O $2.0555881 .704684-4.088486$ O $0.8012512 .569206-2.353954$ C $0.864551 \quad 1.210266-4.707607$ H $1.1503550 .669383-5.613493$ H $\quad 0.348650 \quad 0.519510-4.035617$ H $\quad 0.2103542 .041879-4.985606$ SCF Energy (B3LYP/6-31G**//MMFF) $=-3245.90958382$

0700195

MM̄MF Geometry

C -5.432859 $2.353366 \quad 0.851047$

C -4.1134772 .5644070 .728080$
C -3.2045852 .8663561 .891086$

O -2.0484802 .0017361 .820123$

C -2.6986794 .3184701 .800226$

C -1.750652 4.6938992 .923969

C -0.4433734 .9858422 .770880$

C $-2.371090 \quad 4.752514 \quad 4.297611$

C 0.3693125 .0397941 .505909

C 1.3793463 .8917221 .434429

C 2.4111574 .0519970 .306034

C $1.7754064 .014752-1.086888$

C $2.8291944 .041633-2.206685$

$\begin{array}{lllll}\text { O } & 3.352867 & 2.976763 & 0.447498\end{array}$

O $2.1781263 .691038-3.438006$

C $3.9131832 .980571-1.916895$

C $3.3731565 .457391-2.427832$

O $4.9906723 .080977-2.844328$

C $4.4539743 .036470-0.472492$

C $5.4129821 .866830-0.147277$

O $5.2045994 .244660-0.296701$

C $-2.138138 \quad 0.7657512 .379421$

$\begin{array}{lllll}\text { O } & -3.125496 & 0.243000 & 2.868832\end{array}$

C $-0.791637 \quad 0.1592652 .294904$

C $-0.606307-1.1403072 .567872$

C $0.671491-1.8462082469908$

C $1.917204-1.0132262 .319316$

C $4.8268470 .469760-0.344289$

C $5.766234-0.5908920 .193587$

C $6.371548-1.7966072 .292365$

O $6.559573-1.181153-0.535733$

C $5.559369-3.0538652 .318400$

C $4.934775-3.5132943 .413618$

C $4.102679-4.7644433 .444474$

C $2.627281-4.4609963 .609567$

C $2.043333-4.5621294 .815959$

C $1.887590-4.0889432 .339888$

C $0.687851-3.1945502 .523347$

N $5.637249-0.7816531 .559512$

H -5.8920702 .4002581 .835390$

H -3.656829 $2.528309-0.258350$

H -3.723395 2.7100642 .845046

H -3.5505785 .0110771 .819864$

H -2.2200784 .4652110 .826800$

H $\quad 0.1269585 .2385003 .664862$

H -1.683161 5.1571665 .047886

H -3.256212 5.3969184 .287255

H $-2.668668 \quad 3.753770 \quad 4.631124$

H -0.2632135 .0387450 .615999$

H 0.9026615 .9985931 .496393

H 1.9203643 .8085842 .386957

H 0.8525552 .9368191 .315850

H 2.9366755 .0015930 .462276

H $1.0694124 .841950-1.224620$

H $1.1789503 .098436-1.185736$

H $1.7908412 .804989-3.335592$

H $3.4520162 .001000-2.085843$

H $2.563046 \quad 6.134617-2.724945$

H $4.0970265 .480354-3.249917$

H $3.8462685 .880100-1.539166$

H $4.6094373 .038643-3.737986$

H $\quad 6.333800 \quad 1.963262-0.737717$

H $5.733257 \quad 1.9673350 .899205$

H 5.8036114 .1283940 .459390

H $\quad 0.0209730 .802844 \quad 1.978189$

H -1.465934 -1.748246 2.844853

H $1.970641-0.2499313 .103867$

H $1.935287-0.5171631 .345650$

H $2.834486-1.5971562 .403147$

H 3.8597130 .3754290 .159163

H $4.6595220 .253654-1.404941$

H $6.557020-1.4040283 .297169$

H $7.335345-1.9835261 .808313$

H $5.478591-3.6078421 .386069$

H $5.013782-2.9556894 .344169$

H $4.462461-5.3907654 .270915$

H $4.249735-5.3643622 .537293$

H $0.985081-4.3764154 .961967$

H $2.615506-4.8432295 .694297$

H $1.559415-5.0132231 .846762$ 
H $2.575874-3.6227141 .625054$ H $-0.253393-3.7281312 .655450$ H $4.913015-0.2754572 .060813$ C $-7.0522070 .722548-0.163494$ O $-6.077659-0.336834-0.218330$ C $-6.520081-1.334463-1.155803$ C $-8.005337-1.073574-1.361803$ C $-8.045906 \quad 0.441230-1.293184$ H -5.993153 -1.135222 -2.097226 C $-6.155485-2.736601-0.654331$ H -8.370797 -1.472001 -2.312871 H -8.611545 -1.502828 -0.556043 C $-9.4350661 .003183-1.045190$ H -7.662486 $0.844358-2.240379$ C $-4.687381-2.882379-0.202369$ O $-6.980914-3.0694620 .469154$ H -6.391639-3.475085 -1.428985 H $-4.538470-2.3232640 .729513$ O $-4.455417-4.255808 \quad 0.131780$ C -3.642365 -2.421895-1.235566 C $-2.211685-2.567806-0.703132$ H -3.822125 -1.369541-1.470778 O $-3.789890-3.155933-2.446964$ H -2.123330 -2.0254780 .245062$ H -2.009095 -3.619192 -0.457678 H $-10.113808 \quad 0.723045-1.857371$ H $-9.8587840 .626597-0.108232$ H $-9.4089932 .095987-0.988179$ C $-6.3456862 .071653-0.310720$ H $-6.832970-2.3939441 .153210$ H $-4.657082-4.792423-0.653178$ H -3.528486 -4.075917 -2.272962 H $-7.0863622 .877428-0.366587$ H -5.773292 2.087155 -1.246889 H -7.5455030 .6504410 .814242$ C $1.319898-1.943999-1.649006$ O $0.120629-2.237139-0.923636$ C $-1.101756-2.109270-1.665701$ C $-1.266190-0.669231-2.178086$ C $-0.039101-0.210931-2.973363$ C $1.268201-0.526195-2.240517$ H $2.111491-1.956374-0.888629$ C $1.618080-3.059453-2.653788$ $\mathrm{H}-1.073294-2.803418-2.513512$ O $-1.4693670 .204669-1.069584$ H -2.155042 $-0.610584-2.816271$ H $-0.1071620 .870494-3.145165$ O $-0.057368-0.851921-4.244697$ H $2.124170-0.368959-2.905390$ O $1.414270 \quad 0.406780-1.166016$ C $3.057310-3.045945-3.101434$ H $1.404280-4.041482-2.212365$ H $0.981690-3.007812-3.541898$ H -1.616696 $1.098607-1.422482$ H $0.714692-0.535865-4.744484$ H $\quad 0.6826370 .260402-0.542127$ O $3.851264-3.592505-2.137048$ O $3.429899-2.598590-4.178336$ C $5.246801-3.649067-2.447175$ H $5.618569-2.663001-2.741685$ H $5.781727-3.971576-1.549876$ H $5.420920-4.378769-3.243313$ SCF Energy (B3LYP/6-31G**//MMFF) $=-3245.91529344$

07 00196

MM̄MF Geometry

C $\quad 0.397777 \quad 1.3018643 .223956$

C -0.6041802 .1594073 .470113$

C -1.251616 3.0106352 .410364

O -2.5982802 .5129262 .196748$

C -1.3903654 .4790042 .842810$

C -0.1052175 .2710672 .759904$

C 0.2653635 .9893841 .682110

C 0.7434415 .2828704 .004203

C $-0.4597396 .108546 \quad 0.368354$

C $0.4916396 .003158-0.832156$

C $1.0061564 .577271-1.092085$

C $2.1199804 .617591-2.138507$
C $2.5930583 .210686-2.515745$

O $-0.0905533 .797210-1.576918$

O $3.4514393 .323466-3.660913$

C $1.3670262 .356553-2.905827$

C $3.4661402 .603660-1.413036$

O $1.7782091 .016554-3.157041$

C $0.2100822 .419736-1.877094$

C $-1.1109151 .843216-2.446990$

O $0.577607 \quad 1.702126-0.701504$

C $-2.731395 \quad 1.386523 \quad 1.444358$

$\begin{array}{llll}\text { O } & -1.838324 & 0.750410 & 0.908485\end{array}$

C -4.1658331 .0347041 .400613$

C $-4.527154-0.1660510 .923900$

C $-5.894669-0.6758050 .809636$

C $-\begin{array}{llll}-7.017598 & 0.267628 & 1.154322\end{array}$

C $-1.2059330 .322268-2.572832$

C $-2.321233-0.071756-3.520565$

C $-4.776610-0.061875-3.806591$

O $-2.079005-0.484281-4.653724$

C $-5.958026-0.372428-2.945403$

C $-6.634882-1.528692-3.018512$

C $-7.862478-1.849039-2.213034$

C $-7.646194-3.006176-1.263185$

C -7.762621 -4.277358 -1.683113

C $-7.371117-2.6848730 .188866$

C $-6.076201-1.9436290 .382823$

N $-3.5901200 .096554-2.985848$

H 0.8034941 .2235322 .218665

H -1.025012 2.2103004 .471704

H $-0.7069772 .965726 \quad 1.459751$

H $-1.7998324 .540923 \quad 3.860692$

H -2.164387 4.9606562 .230842

H 1.1866086 .5671721 .747389

H 1.6024145 .9564793 .914203

H 0.1540595 .6194224 .863497

H 1.1338724 .2842814 .218465

H -1.260135 $5.369890 \quad 0.265445$

H -0.9323507 .0980820 .342905$

H $-0.0555776 .349907-1.718804$

H $1.3366016 .688286-0.687498$

H $1.3727314 .143070-0.155606$

H $2.9647165 .224008-1.788154$

H $1.7500585 .117576-3.044826$

H $2.9391383 .716796-4.387691$

H $0.9939972 .742426-3.863833$

H $4.3417723 .236455-1.224970$

H $3.8622301 .632359-1.721243$

H $2.9391772 .474009-0.465426$

H $1.9352630 .588631-2.297988$

H -1.935043 2.171736-1.797200

H $-1.3193832 .321412-3.413836$

H $-0.2342031 .422826-0.247214$

H $-4.878175 \quad 1.7625701 .770999$

H -3.747372 -0.8495150 .588844$

H -6.9888110 .5257252 .218595$

H $-8.010141-0.1379810 .949551$

H $-6.939671 \quad 1.1895450 .566587$

H $-0.288961-0.119510-2.966996$

H -1.386186 -0.141815 -1.597670

H $-4.597680-0.837709-4.559045$

H $-4.9392520 .882962-4.336018$

H $-6.2898910 .401344-2.257863$

H $-6.304787-2.289608-3.722943$

H $-8.672264-2.094719-2.912827$

H $-8.217140-0.971015-1.659232$

H $-7.634969-5.112994-1.003253$

H -7.986360 -4.509343 -2.719348

H -7.309295 -3.608535 0.779688

H $-8.230912-2.1461370 .596346$

H -5.195875 -2.544585 0.150103

H $-3.6816920 .589711-2.102065$

C $0.498942-1.0710824 .118003$

O $0.905399-1.6221532 .848616$

C $-0.186786-2.3941142 .315481$

C $-1.165102-2.5968683 .468542$

C $-1.011891-1.2916134 .221033$

H $-0.666670-1.7711701 .551880$

C $0.311886-3.6973451 .684770$ 
H -2.186768 -2.7748983 .118951$ H $-0.874186-3.4383134 .107897$ C -1.532753 -1.339085 5.646404 H -1.563507 -0.522012 3.669876 C $1.380836-3.5362900 .586129$ O $0.878032-4.5303252 .706387$ H $-0.543421-4.2562361 .287049$ H $2.340909-3.2645721 .038883$ O $1.569562-4.826151-0.012802$ C $1.034223-2.532629-0.528169$ C $1.937199-2.608243-1.775469$ H $1.069618-1.515331-0.122422$ O $-0.310413-2.749274-0.967887$ H $1.738151-3.541586-2.318472$ H $1.615446-1.805869-2.451619$ H -2.603515 -1.567957 5.656933 H -1.019741 -2.105857 6.236423 H $-1.388239-0.3757796 .145490$ C $\quad 0.975237 \quad 0.379524 \quad 4.259830$ H $1.594242-4.0270883 .130810$ H $1.806935-5.4442050 .699805$ H - $0.370167-3.664540-1.291411$ H 2.0663860 .4143644 .156340 H $\quad 0.742910 \quad 0.742888 \quad 5.268057$ H $1.011963-1.6702324 .881687$ C $5.045706-0.988509-0.469519$

O $3.687015-1.229811-0.864620$ C $3.443759-2.488641-1.496567$

C $4.274079-2.608818-2.783438$

C $5.764336-2.402921-2.491907$

C $6.012590-1.141420-1.660491$

H $5.0647120 .062308-0.154447$

C $5.447123-1.8427070 .743948$

H $3.731511-3.297719-0.819078$

O $3.829836-1.640837-3.731065$

H $4.107879-3.600502-3.221012$

H $6.315920-2.322654-3.436746$

O $6.275520-3.529326-1.785030$

H $7.048545-1.128361-1.301717$

O $5.859721-0.013462-2.526078$

C $4.654526-1.4886501 .984973$

H $5.298088-2.9092980 .557342$

H $6.504721-1.6695760 .975005$

H $4.364678-1.749380-4.535636$

H $6.134941-4.314000-2.342058$

H $6.107457 \quad 0.779583-2.021029$

O $5.057692-2.2995723 .008159$

O $3.795730-0.6207362 .053389$

C $4.395409-2.0968454 .260260$

H $4.929748-2.6691625 .023307$

H $4.409780-1.0401374 .543873$

H $3.369161-2.4680374 .197815$

SCF Energy (B3LYP/6-31G**//MMFF) $=-3245.91666969$

\section{7_00197}

MM̄FF Geometry

C -1.022709 -2.841712 1.292644

C $-1.213175-1.5369721 .537987$

C -1.072660 -0.904799 2.895200

O $-\begin{array}{llll}0.319732 & 0.316185 & 2.702907\end{array}$

C $-2.438187-0.5154603 .489761$

C -3.295429-1.687125 3.915797

C $-4.362910-2.1344313 .225951$

C $-2.916853-2.3198085 .228513$

C $-4.840158-1.6418071 .889028$

C $-6.005907-0.6559772 .021012$

C -6.3875690 .0338250 .699376$

C $-6.863268-0.959179-0.364685$

C $-7.177723-0.266796-1.697391$

O $-5.247313 \quad 0.768095 \quad 0.236919$

O $-7.319689-1.301422-2.683179$

C $-5.9654060 .603022-2.092481$

C $-8.5223730 .466927-1.651318$

O $-6.2505231 .354477-3.270887$

C $-5.4699101 .529199-0.955952$

C $-4.1482562 .241306-1.333772$

O $-6.4543132 .548247-0.741522$

C 0.4405130 .7656473 .732520
O $\quad 0.650474 \quad 0.191865 \quad 4.792194$

C 1.0332382 .0874963 .424701

C 0.5679392 .8917682 .455291

C 1.0944834 .2110562 .094032

C 2.2998814 .7060252 .850249

C $-2.9056791 .343546-1.344746$

C -1.721304 $2.009724-2.026935$

C $0.710206 \quad 1.682687-2.462111$

O $-1.8270163 .020520-2.717101$

C $1.3446632 .828550-1.738870$

C $1.4129714 .069420-2.245103$

C $2.0457455 .243606-1.553445$

C $1.0201296 .195836-0.977994$

C $0.3133187 .018105-1.771946$

C $0.8595396 .236790 \quad 0.528115$

C $0.4812594 .900258 \quad 1.107722$

N $-0.5359841 .317369-1.812242$

H $-0.752688-3.5048062 .110280$

H -1.514812 -0.8849010 .722008$

H $-0.524490-1.5734853 .569663$

H $-2.972617 \quad 0.1373792 .787372$

H -2.281439 0.1303324 .364829

H -4.941036 -2.959259 3.639106

H -3.596322 -3.131322 5.509989

H -1.907588 -2.739848 5.174833

H $-2.942311-1.5754016 .030884$

H -4.021836 -1.188423 1.323451

H $-5.151536-2.5183881 .308922$

H $-6.882192-1.1706922 .434319$

H -5.7232720 .1251432 .739352$

H -7.1882820 .7464490 .930326$

H -7.732948 -1.524829 -0.007859

H $-6.080448-1.703915-0.558351$

H -7.440455 -0.870467 -3.546798

H $-5.161588-0.089859-2.367952$

H $-9.332652-0.238910-1.431741$

H $-8.7676900 .907799-2.623836$

H -8.556931 $1.255292-0.896790$

H -6.815626 $2.103220-3.014750$

H $-4.2776482 .753794-2.295261$

H $-3.9545673 .046805-0.611196$

H $-6.0326183 .292707-0.281342$

H 1.8566232 .3784324 .068441

H $-0.2823762 .560547 \quad 1.861003$

H 3.1333804 .0021652 .755983

H 2.0610854 .8220363 .913506

H 2.6695805 .6736162 .507017

H $-2.6273781 .093022-0.314803$

H $-3.0971590 .410340-1.883017$

H $0.500601 \quad 1.925779-3.509533$

H $1.3706040 .811093-2.437715$

H $1.7685112 .622329-0.760604$

H $0.9820734 .262414-3.225430$

H $2.6749375 .775203-2.279177$

H $2.7348384 .912905-0.768229$

H $-0.4083527 .717887-1.364161$

H $\quad 0.4424417 .008350-2.849292$

H 0.0745526 .9504560 .809154

H $1.788574 \quad 6.630256 \quad 0.947985$

H $-0.403608 \quad 4.4611880 .644101$

H $-0.5355910 .502182-1.206172$

C $-0.102731-4.410916-0.459612$

O $1.089922-3.664929-0.787289$

C $1.629781-4.177026-2.020135$

C $0.924284-5.504557-2.271979$

C $-0.450510-5.222246-1.709767$

H $1.338013-3.470688-2.806019$

C $3.156283-4.301714-1.964397$

H $0.909552-5.772030-3.332985$

H $1.396407-6.327382-1.722494$

C $-1.259049-6.477900-1.429626$

H -0.999622 -4.595097 -2.425212

C $3.933149-3.002049-1.677452$

O $3.515467-5.244598-0.944576$

H $3.513070-4.735485-2.906594$

H $3.837204-2.735829-0.619442$

O $5.322890-3.287017-1.893409$

C $3.543121-1.795252-2.547842$ 
C $4.541850-0.621035-2.523520$

H $2.553228-1.441261-2.237973$

O $3.427739-2.221976-3.909275$

H $5.450672-0.901442-3.072171$

H $4.0884120 .191897-3.106820$

H -1.414739 -7.048206 -2.351329

H $-0.750242-7.130846-0.712520$

H -2.241835 -6.227694-1.018505

C $-1.236010-3.463828-0.057496$

H $3.142248-4.923654-0.105619$

H $5.556533-4.038777-1.322084$

H $3.112905-1.462716-4.428857$

H $-2.179723-4.019891-0.011969$

H -1.348637 -2.682035 -0.819193

H $\quad 0.159056-5.0781360 .371659$

C 3.8945250 .7605330 .887178

O $3.726354 \quad 0.304667-0.462524$

C $4.920903-0.109511-1.126587$

C $5.9239101 .052943-1.201836$

C 6.2124771 .6200130 .192116

C $4.920817 \quad 1.904757 \quad 0.964937$

H 2.9157051 .1691701 .165099

C $4.208622-0.3952321 .850144$

H $5.378568-0.932279-0.569620$

O $5.4007562 .083254-2.038129$

H $6.8504390 .691172-1.663568$

H 6.7878222 .5490520 .098123

O 7.0026240 .6964350 .934612

H 5.1415012 .1239572 .016186

O $4.304763 \quad 3.0709800 .415510$

C $3.081556-1.4016871 .927210$

H $5.107019-0.9411891 .551041$

H 4.3583840 .0061062 .859180

H $6.0659002 .790975-2.083750$

H 7.8257080 .5481110 .438533

H 4.9260583 .8110430 .523520

O $3.418799-2.3735872 .825590$

O $2.045839-1.3521021 .279647$

C $2.456317-3.4183732 .995746$

H $1.557440-3.0220103 .475788$

H $2.212585-3.8788282 .033763$

H $2.893980-4.1808353 .645675$

SCF Energy (B3LYP/6-31G**//MMFF) $=-3245.90433497$

$07 \quad 00198$

MM̄MF Geometry

C 0.2054763 .3824662 .131196

C -1.1140853 .1386452 .133636$

C - $-1.802327 \quad 1.8375032 .472252$

O $-\begin{array}{llll}0.821460 & 0.822479 & 2.796148\end{array}$

C $-2.780573 \quad 1.9740183 .657033$

C -3.884904 2.9926393 .472226

C -4.9421142 .8365252 .651767$

C $-3.749670 \quad 4.2426814 .302800$

C -5.2677541 .6564521 .777350$

C -5.1374252 .0190770 .290986$

C $-5.6241080 .931904-0.683548$

C $-7.1480540 .797280-0.675806$

C $-7.626300-0.313289-1.620422$

O $-5.009140-0.311316-0.340910$

O $-9.007554-0.574820-1.325004$

C $-6.842427-1.603709-1.304725$

C $-7.572340 \quad 0.120053-3.090222$

O $-7.183319-2.609896-2.257464$

C $-5.306760-1.398360-1.233996$

C $-4.631938-2.663906-0.647516$

O $-4.808423-1.152148-2.548073$

C -1.178304 -0.4728312 .582784$

O $-2.259688-0.8785242 .187653$

C $-0.011978-1.3286132 .896803$

C $-0.017516-2.6054202 .485274$

C $1.039079-3.5983502 .685559$

C $2.201395-3.221983 \quad 3.564247$

C $-3.107801-2.564878-0.490505$

C $-2.318092-3.191375-1.628695$

C $0.041409-3.724497-2.255621$

O $-2.839631-3.725726-2.602724$

C $0.516390-4.964413-1.564454$
C $1.770932-5.133029-1.120555$

C $2.248351-6.360697-0.398401$

C $2.808536-6.0474880 .973388$

C $4.133099-5.9188681 .160619$

C $1.850080-5.9740412 .142556$

C $0.917442-4.7965962 .075935$

N $-0.951110-3.089173-1.408942$

H 0.9108142 .6076522 .414104

H - 1.7734123 .9477461 .823240

H -2.3390971 .5249381 .569380$

H -2.2083652 .2068204 .566220$

H -3.2319000 .9986483 .880913$

H -5.6762233 .6404592 .604655$

H $-4.5639584 .952665 \quad 4.123632$

H -3.7594793 .9902765 .368370$

H -2.8097954 .7556924 .076597$

H $-4.633740 \quad 0.7957812 .010430$

H -6.2942691 .3535392 .009672$

H -5.6676482 .9560970 .077486$

H -4.0761742 .2084230 .082951$

H $-5.2848371 .226934-1.683850$

H -7.624107 $1.751794-0.932953$

H $\quad-7.4990740 .5509050 .334437$

H $-9.5035470 .251667-1.454854$

H -7.196716 -1.962367 -0.327840

H $-8.1975321 .006648-3.250949$

H -7.981419 -0.656407-3.746213

H $-6.5634250 .362725-3.429998$

H $-6.900937-3.468299-1.901063$

H $-5.045279-2.8257850 .357296$

H $-4.883853-3.545748-1.245595$

H -3.853794 -1.323732 -2.560047

H $0.817287-0.8831723 .433546$

H $-0.882662-2.9641461 .929809$

H $2.690433-2.3147303 .197706$

H $2.976413-3.9906313 .609898$

H $1.861137-3.0427254 .590020$

H -2.783381 -1.526591 -0.367701

H -2.826694 -3.1147550 .416392$

H $0.848768-3.002352-2.411794$

H $-0.393198-3.978486-3.227043$

H $-0.213912-5.755886-1.413172$

H $2.498930-4.343171-1.283729$

H $3.020233-6.834001-1.019340$

H $1.449564-7.106692-0.303219$

H $4.549733-5.7235282 .143099$

H $4.830515-5.9977420 .333073$

H $2.391675-5.986043 \quad 3.095314$

H $1.245405-6.8902292 .161287$

H $\quad 0.035640-4.9766261 .460315$

H $-0.622054-2.644788-0.556592$

C $0.9327394 .892168 \quad 0.244415$

O $1.9929724 .051721-0.259396$

C $2.8098144 .828060-1.155550$

C $2.0161606 .092164-1.462836$

C $1.3080526 .322790-0.145462$

H $3.7068905 .110933-0.592230$

C $3.2079864 .026091-2.399402$

H $2.6602886 .926019-1.757895$

H $1.2846155 .929592-2.263015$

C $0.1227297 .267311-0.252536$

H 2.0294426 .7320860 .574798

C $3.9523482 .703920-2.123970$

O $2.0207373 .705749-3.136695$

H $3.8109654 .665641-3.055924$

H $3.2393041 .955444-1.764717$

O $4.4268822 .214871-3.386058$

C $5.1339322 .806656-1.146227$

C $6.0538131 .572711-1.096330$

H $4.7484413 .009983-0.140389$

O $5.9438663 .926225-1.519695$

H $6.6583331 .522253-2.011423$

H $6.7725901 .755041-0.286294$

H $0.447488 \quad 8.253448-0.600518$

H $-0.626627 \quad 6.892374-0.957767$

H $\quad-0.364515 \quad 7.3963040 .718888$

C $0.7843274 .718473 \quad 1.758336$

H $2.2953803 .210483-3.927536$ 
H $5.1091092 .832143-3.702097$ H $6.6435154 .018335-0.850689$ H 0.1472245 .5104362 .170483 H 1.7617004 .8109322 .247827 H $0.0144754 .585146-0.272976$ C $3.751443-0.8384100 .606472$ $\begin{array}{lllll}\text { O } & 4.522358 & 0.328687 & 0.286296\end{array}$ C $5.3493880 .223628-0.873949$ C $6.362121-0.923706-0.713003$ C $5.669158-2.242499-0.362699$ C $4.658773-2.0685970 .776218$ H $3.305818-0.6151611 .583932$ C $2.604528-1.074922-0.390415$ H $4.7201030 .021944-1.746322$ O $7.312824-0.6130090 .305098$ H $6.921085-1.036480-1.649333$ H $6.430095-2.977257-0.071986$ O $5.012390-2.734671-1.525909$ H $4.035658-2.9626910 .879616$ O $5.370059-1.9098972 .007043$ C $1.5612020 .018366-0.336186$ H $2.965816-1.130849-1.420248$ H $2.096853-2.013625-0.142538$ H $\quad 6.821707-0.432542 \quad 1.124967$ H $4.658674-3.613728-1.308835$ H $5.893756-2.7169942 .148099$ O $0.613340-0.221975-1.288849$ O 1.5694730 .9604130 .442435 C $-0.4500140 .733246-1.356616$ H $-0.0525621 .741953-1.505050$ H $-1.0831660 .476403-2.209984$ H -1.053526 $0.684444-0.446528$

SCF Energy (B3LYP/6-31G**//MMFF) $=-3245.90524417$

0700199

MM̄FF Geometry

C -2.3119343 .1382751 .616774$

C -1.2007272 .5801321 .114000$

C -0.01263221886191 .952740$

$\begin{array}{lllll}\text { O } & 0.250498 & 0.786555 & 1.698044\end{array}$

C 1.2355552 .9990171 .555101

C 1.1502654 .4844801 .827288

C 1.0482875 .4330900 .875430

C 1.2455974 .8769273 .278646

C $0.9182595 .240479-0.609698$

C $2.1682345 .671521-1.389632$

C $3.3981534 .780297-1.148476$

C $4.5708825 .241456-2.014784$

C $5.7849324 .311410-1.871988$

O $3.0502653 .438786-1.494381$

O $6.7209914 .643161-2.909211$

C $5.3367112 .843371-2.072647$

C $6.5311934 .573871-0.558279$

O $6.4245801 .965043-1.795952$

C $4.0805172 .462866-1.255276$

C $3.4532171 .116402-1.687050$

O 4.4176882 .3911910 .131113

C 0.9975050 .1221272 .622420

O $1.4350850 .570746 \quad 3.670559$

C $1.216422-1.2566272 .129777$

C $2.115449-2.0364342 .749518$

C $2.451793-3.4232222 .414903$

C $1.637344-4.0985591 .345834$

C $4.313801-0.130131-1.481698$

C $3.528841-1.413058-1.706338$

C $3.867332-3.875163-1.872308$

O $2.322931-1.434672-1.935605$

C $4.393101-4.886483-0.898142$

C $5.215806-4.6834730 .143776$

C $5.670128-5.7865311 .057867$

C $5.400348-5.4951752 .517004$

C $6.410773-5.3680353 .394205$

C $3.959445-5.4359492 .976652$

C $3.453726-4.0224423 .092418$

N $4.344417-2.532403-1.604413$

H -2.3836913 .3067292 .688648$

H -1.119745 2.4275650 .040204

H -0.2418632 .3182523 .018073$
H 1.4672022 .7931380 .505498

H 2.1113792 .6095522 .090277

H 1.0280096 .4756851 .191015

H 1.2552765 .9634193 .416143

H 0.3935034 .4823863 .840577

H 2.1669984 .4822063 .719371

H $0.6434524 .214817-0.872179$

H $0.0747055 .858057-0.944301$

H $1.9093595 .638869-2.456282$

H $2.4144006 .713739-1.151279$

H $3.6658904 .824949-0.087135$

H $4.8483426 .276397-1.779112$

H $4.2614205 .248881-3.069393$

H $6.2845314 .508093-3.767367$

H $5.1038112 .713823-3.137916$

H $6.8525495 .621185-0.505047$

H $7.4499653 .979919-0.497966$

H 5.9269634 .3668620 .327450

H $6.5081731 .892089-0.829802$

H $2.5172550 .989025-1.123941$

H $3.1422591 .177485-2.738951$

H $3.779248 \quad 1.8078380 .573507$

H $0.642326-1.5857651 .272472$

H $2.667588-1.6192593 .591274$

H $\quad 0.592517-4.180817 \quad 1.661964$

H $1.975955-5.1089001 .109345$

H $1.677924-3.5312270 .410264$

H $5.149981-0.127622-2.188524$

H $4.714041-0.159425-0.462350$

H $4.197193-4.135409-2.883381$

H $2.772802-3.891033-1.861302$

H $4.039958-5.900890-1.077556$

H $5.600960-3.6898610 .355096$

H $6.744848-5.9333500 .890098$

H $5.192102-6.7394090 .797481$

H $6.222439-5.1881354 .447688$

H $7.446776-5.4374783 .079747$

H $3.878652-5.8957063 .971367$

H $3.339410-6.0650402 .332366$

H $3.973524-3.4421453 .856761$

H $5.349620-2.392279-1.553481$

C -4.7963073 .0092991 .172378$

$\begin{array}{lllll}\text { O } & -4.941847 & 1.717366 & 0.538957\end{array}$

C $-6.2125111 .676869-0.136484$

C $-7.0430142 .790156 \quad 0.486615$

C -5.9861573 .8510920 .699748$

H $-6.0202391 .929294-1.186068$

C $-6.8646950 .293817-0.042422$

H $-7.8577613 .118306-0.165734$

H -7.4746472 .4859351 .447353$

C -6.4028604 .9335321 .681361$

H $-5.7563994 .317042-0.268252$

C $-6.048152-0.874270-0.630621$

O $-7.121176-0.013978 \quad 1.334472$

H $-7.8481020 .327907-0.526673$

H $-5.219862-1.1228190 .040429$

O $-6.905089-2.024640-0.650233$

C $-5.509325-0.645771-2.053448$

C $-4.974384-1.906531-2.761593$

H $-4.7181840 .111982-2.016843$

O $-6.560575-0.115076-2.867169$

H -5.812202 -2.562557 -3.031918

H $-4.545601-1.574177-3.716552$

H -7.2883465 .4636961 .315785$

H -6.6446594 .5129732 .663261$

H $-5.6019805 .667013 \quad 1.817474$

C -3.455845 3.6376520 .779996

H $-6.2710270 .025037 \quad 1.805708$

H -7.224825 -2.1628720 .257956$

H $-6.1826630 .085460-3.740398$

H $-3.497763 \quad 4.723747 \quad 0.925497$

H -3.257823 $3.458005-0.284026$

H -4.8520712 .8391952 .255079$

C -1.809108 -2.403434 -0.836583

O $-2.846110-1.829687-1.645875$

C $-3.926848-2.705871-1.972420$

C $-3.412549-3.919352-2.763393$

C $-2.293993-4.638766-2.001322$ 
C $-1.224344-3.661110-1.503346$ H -1.018739 -1.641649 -0.812001 C $-2.277567-2.641378 \quad 0.608990$ H -4.397540 -3.062142 -1.051357 O $-2.924705-3.492139-4.033534$ H $-4.249937-4.603822-2.944540$ H $-1.823929-5.383060-2.655400$ O $-2.841864-5.330679-0.883030$ H $-0.545658-4.162072-0.804074$ O $-0.433944-3.227898-2.613782$ C $-2.574183-1.3435891 .327339$ H -3.182992 -3.250689 0.658997 H -1.483026 -3.1494971 .164964$ H -2.633044 -4.283267 -4.517701 H -3.498774 -5.962642 -1.221349 H $-0.010402-4.014702-2.997082$ O $-2.779713-1.6008612 .652231$ O $-2.636403-0.2449300 .794586$ C -3.092319 -0.4592633 .456822$ H -3.9828100 .0477543 .072828$ H $-3.298120-0.8059884 .472949$ H $-2.2396560 .225068 \quad 3.485716$

SCF Energy (B3LYP/6-31G**/MMFF) $=-3245.89517900$

07 00200

MM̄FF Geometry

C 3.1443103 .6062361 .105353

C 1.9513883 .0104931 .255203

C $\quad 0.731961 \quad 3.387773 \quad 0.454137$

O $0.2055642 .168368-0.115565$

C -0.3450973 .9820401 .382128$

C $-1.213908 \quad 5.020936 \quad 0.704657$

C $-2.476930 \quad 4.820300 \quad 0.282291$

C -0.5887656 .3885250 .581889$

C $-3.269028 \quad 3.5401670 .290509$

C $-4.0694013 .412789-1.013455$

C $-4.8514822 .096212-1.139184$

C $-5.7007552 .128711-2.414424$

C $-6.6139530 .905242-2.544152$

O -5.6869091 .9397520 .010435$

O $\quad-7.572454 \quad 1.205476-3.570562$

C $-7.3818090 .709192-1.219515$

C -5.837697 -0.324522 -3.030069

O $-8.133467-0.501147-1.290155$

C $-6.461640 \quad 0.7282580 .024789$

C $-7.303591 \quad 0.7367841 .326152$

O $-5.631655-0.429610-0.003597$

C $-0.2278982 .175627-1.400609$

O $-0.1385653 .094870-2.201711$

C $-0.8474550 .877288-1.758345$

C $-1.3164590 .028269-0.830749$

C -1.998902 -1.243875-1.071675

C $-2.029008-1.769958-2.480771$

C -6.4820320 .6597172 .622867$

C $-6.151412-0.7651443 .046761$

C $-4.675753-2.0444674 .592351$

O $-6.685438-1.7553032 .553438$

C $-3.515276-2.4788673 .753064$

C -3.472265 -3.635998 3.073921

C $-2.308339-4.0498442 .216704$

C $-2.684492-4.2603340 .762544$

C $-2.417428-5.4264340 .150208$

C $-3.368985-3.1378200 .010181$

C $-2.562546-1.868483-0.016663$

N -5.201531 -0.8005244 .058068$

H 3.2523604 .3962980 .365758

H 1.8306642 .2371082 .010158

H $1.0145704 .091029-0.338110$

H 0.1219114 .4724272 .247706

H -0.9649313 .1805241 .802588$

H $-3.0104575 .677222-0.128702$

H -1.262369 7.1149300 .114924

H $-0.322976 \quad 6.774756 \quad 1.571438$

H $0.3185086 .343987-0.028506$

H -2.624716 2.6653790 .408995

H $-3.9560293 .564371 \quad 1.143997$

H $-4.7764804 .252420-1.060361$

H $-3.3962113 .512479-1.874874$
H $-4.1349661 .268769-1.173610$

H $-5.0639212 .230609-3.302312$

H $-6.3356183 .026313-2.401758$

H $-8.197000 \quad 0.460917-3.616496$

H -8.108675 $1.529700-1.139190$

H $-5.402217-0.134171-4.018439$

H $-6.498968-1.188861-3.156473$

H -5.024635 -0.608725 -2.360087

H $-8.812402-0.473432-0.595967$

H $-7.873372 \quad 1.6749231 .355281$

H $-8.030247-0.082327 \quad 1.318773$

H $-5.111416-0.4538570 .814496$

H $-0.9543620 .688979-2.821109$

H $-1.2271970 .301903 \quad 0.219630$

H -2.654988 -1.134145 -3.115527

H -2.415232 -2.789240 -2.553103

H -1.019746 -1.797290 -2.905934

H -5.5605801 .2457152 .536649$

H -7.0763211 .0917713 .437728$

H -5.473350 -2.794414 4.613404

H $-4.347106-1.8527105 .618156$

H -2.667225 -1.799790 3.700582

H $-4.314269-4.3218973 .130743$

H -1.894885 -4.972474 2.643944

H -1.501305 -3.308936 2.270939

H -2.679097 $-5.592992-0.889406$

H -1.927119 -6.2410560 .672699$

H $-4.328618-2.9215270 .495929$

H -3.640390 -3.459184 -1.000154

H -2.484589-1.388436 0.959607

H $-4.759611 \quad 0.0652884 .353243$

C 5.6046803 .0318991 .125873

O $5.616207 \quad 1.6543210 .687140$

C 6.9075671 .0882360 .982057

C 7.8311432 .2716831 .237156

C 6.8895893 .2276601 .935397

H $6.798306 \quad 0.5242501 .915788$

C $7.3915990 .161004-0.137719$

H 8.6989091 .9997801 .845519

H 8.1949042 .7119820 .301373

C 7.3922684 .6615051 .959618

H 6.7401252 .8831182 .967809

C $6.484184-1.047898-0.441533$

O $7.5254870 .915039-1.349743$

H $8.400561-0.1955320 .101882$

H $5.581237-0.714527-0.963200$

O $7.190365-1.890424-1.362613$

C $6.093471-1.8899470 .785240$

C $5.466601-3.2611440 .466594$

H $5.401929-1.3116471 .408757$

O $7.264382-2.1314681 .572713$

H $\quad 6.232079-3.9333380 .057494$

H $5.176209-3.7040461 .428879$

H $8.342697 \quad 4.7263192 .499330$

H 7.5539015 .0470020 .947221

H 6.6750135 .3197582 .459360

C $4.348048 \quad 3.3128261 .956373$

H $6.6578361 .307666-1.546672$

H $7.419703-1.346190-2.135503$

H $6.985899-2.6045252 .375350$

H 4.5101824 .1976002 .583429

H 4.1465912 .4669272 .624999

H $5.626293 \quad 3.652188 \quad 0.220489$

C $2.098036-2.141522-0.704989$

O $3.279341-2.3412080 .084319$

C $4.253195-3.225081-0.475134$

C $3.652714-4.625302-0.687669$

C $2.369536-4.558652-1.521773$

C $1.408342-3.484300-1.002335$

H $1.430299-1.553271-0.062825$

C $2.381261-1.312396-1.968657$

H $4.585173-2.832905-1.441188$

O $3.366407-5.2205120 .576936$

H $4.397075-5.255966-1.188390$

H $1.872529-5.536122-1.494815$

O $2.720337-4.288470-2.875498$

H $0.591861-3.334957-1.716994$

O $0.821114-3.969874 \quad 0.207504$ 
C $2.8381330 .091452-1.637525$

H $3.158389-1.765205-2.588987$

H $1.463917-1.228678-2.561424$

H $3.016396-6.111948 \quad 0.409583$

H $1.898168-4.275103-3.394557$

H $\quad 0.180113-3.3044130 .509271$

O $3.0489890 .777062-2.799568$

O $3.0005220 .530792-0.508315$

C $3.5209792 .119114-2.645026$

H $4.4713252 .128501-2.103589$

H $3.6814582 .539725-3.641228$

H $2.7752432 .725880-2.124433$

SCF Energy (B3LYP/6-31G*//MMFF) $=-3245.89301786$

0700201

MMTFF Geometry

C $-3.353463-2.150259-3.271090$

C $-2.494223-2.448001-2.286343$

C $-1.006004-2.257485-2.404378$

O $-0.626712-1.221487-1.466072$

C $-0.254946-3.540978-2.022264$

C $1.240879-3.431972-2.251441$

C $2.150952-3.150725-1.296916$

C $1.690702-3.706607-3.663812$

C $1.897087-2.8399080 .152865$

C $2.520955-1.5222180 .647065$

C $4.044107-1.5876470 .847683$

C $4.526792-0.4140531 .704398$

C $6.056048-0.410538 \quad 1.860443$

O $4.672835-1.530698-0.434084$

$\begin{array}{lllll}0 & 6.440296 & 0.863609 & 2.400154\end{array}$

C $6.698715-0.5204720 .461634$

C $6.534450-1.4671982 .862786$

O $8.112398-0.6754780 .553198$

C $6.100661-1.650116-0.405291$

C $6.610997-1.624843-1.868755$

O $6.486884-2.9175530 .142630$

C $-0.8108430 .069567-1.854728$

O $-1.264415 \quad 0.462175-2.917741$

C -0.348457 $0.937149-0.750302$

C $-0.2927612 .263683-0.937965$

C $0.202633 \quad 3.251966 \quad 0.021662$

C 0.6271972 .7515001 .376583

C $6.490270-0.299713-2.625594$

C $5.0609930 .193764-2.741436$

C $3.6085932 .142586-2.234068$

O $4.179186-0.484219-3.264352$

C $3.5625173 .150702-1.130935$

C $3.4296414 .470940-1.330289$

C $3.3489235 .482527-0.220653$

C $2.0714656 .295715-0.265945$

C $2.0531517 .522368-0.814140$

C $0.834706 \quad 5.7153530 .390063$

C $0.2873004 .540255-0.371486$

N $4.8828721 .452988-2.189410$

H $-2.971637-1.809290-4.230458$

H -2.882560 -2.752521 -1.319282

H $-0.722692-1.951667-3.419620$

H $-0.636349-4.387449-2.609207$

H $-0.458265-3.800581-0.977674$

H $3.198350-3.159921-1.589369$

H $2.777916-3.635170-3.776789$

H $1.393178-4.715513-3.967414$

H $1.241451-2.987761-4.356173$

H $\quad 0.824245-2.7740590 .350615$

H $2.270996-3.673262 \quad 0.759515$

H $2.269530-0.714937-0.051857$

H $2.049989-1.2834431 .608391$

H $4.291244-2.5374651 .335969$

H $4.043612-0.4248702 .689308$

H 4.2229450 .5305721 .231765

H 5.9832520 .9787263 .250925

H $6.5253470 .442244-0.032893$

H $6.090979-1.2868753 .849481$

H $7.618970-1.4111463 .007744$

H $6.277843-2.4866732 .568082$

H $8.449257 \quad 0.054044 \quad 1.100993$

H $7.663249-1.937806-1.889309$
H $6.063954-2.391585-2.435211$

H $6.400963-3.590531-0.553013$

H -0.0395480 .4597790 .172248$

H $-0.6092562 .666758-1.899295$

H $-0.2209672 .282840 \quad 1.885626$

H 0.9952333 .5350282 .040925

H $1.4331592 .014927 \quad 1.284697$

H $7.1381480 .457461-2.172483$

H $6.860532-0.435004-3.649533$

H $2.7979151 .415717-2.117930$

H $3.5157732 .600532-3.224368$

H $3.6143422 .768659-0.114284$

H $3.3784274 .851820-2.347941$

H $4.2192626 .145703-0.309374$

H 3.4405695 .0063660 .763583

H $1.1491998 .122070-0.829503$

H $2.9418307 .952489-1.264350$

H 0.0394726 .4705970 .441005

H 1.0715995 .4797791 .430648

H $-0.0520244 .797741-1.376114$

H $5.705206 \quad 1.998601-1.947475$

C $-5.315163-0.689757-2.943640$

O $-4.925143-0.192693-1.645089$

C $-5.898804 \quad 0.781972-1.217517$

C $-6.9298030 .881218-2.340077$

C $-6.823696-0.487135-2.989842$

H $-6.3914430 .373143-0.329276$

C $-5.1937792 .103933-0.887634$

H $-7.9326311 .098254-1.959364$

H $-6.6700281 .670547-3.055778$

C $-7.420274-0.540329-4.384904$

H $-7.323636-1.225213-2.348482$

C -4.2178252 .0149810 .307298$

O $-4.449766 \quad 2.529360-2.037584$

H $-5.9361662 .888436-0.702715$

H -3.4252151 .2902980 .093176$

$\begin{array}{lllll}\text { O } & -3.579100 & 3.290447 & 0.448195\end{array}$

C -4.9030881 .6758101 .646035$

C $-3.981894 \quad 1.758798 \quad 2.878841$

H -5.3469120 .6771091 .596521$

\begin{tabular}{llll}
\hline & -5.984716 & 2.589139 & 1.861755
\end{tabular}

H -3.4820782 .7345372 .911794$

H $-4.627179 \begin{array}{lll}1.725998 & 3.767600\end{array}$

H -8.487124 $-0.295776-4.356072$

H $-6.9303920 .170917-5.058570$

H $-7.316038-1.540144-4.817589$

C $-4.846735-2.136651-3.096542$

H $-3.7933631 .838618-2.233914$

H $-3.1555213 .497699-0.402439$

H $-5.609697 \quad 3.4864491 .884892$

H $-5.300578-2.607795-3.974651$

H $-5.134129-2.724675-2.216551$

H $-4.827742-0.050730-3.693658$

C $-2.868660-1.7841293 .147342$

O $-3.671776-0.598450 \quad 3.116648$

C -2.9465610 .6263732 .961057$

C $-1.985851 \quad 0.819900 \quad 4.145877$

C $-1.047913-0.3819764 .301834$

C $-1.807913-1.7120094 .261092$

H -3.575588 -2.582597 3.407429

C $-2.314860-2.0890531 .753514$

H -2.3739790 .5930602 .028050$

$\begin{array}{lllllllll} & \text { O } & -2.737632 & 0.995666 & 5.345748\end{array}$

H -1.405806 1.7360923 .985955

H $-0.513060-0.308303 \quad 5.256890$

O $-0.075389-0.3686233 .262288$

H $-1.100825-2.5434004 .165412$

O $-2.467753-1.8707065 .521723$

C -2.044185 -3.5640101 .590704$

H $-3.027775-1.7739790 .980864$

H $-1.394231-1.5444921 .527240$

H -2.105481 1.1378136 .070561

H $0.4427480 .448662 \quad 3.355835$

H -2.887211 -2.7479765 .525474$

O $-3.136900-4.1822961 .058890$

O $-0.996736-4.105932 \quad 1.918345$

C -3.002741 -5.5905010 .845400$

H $-3.933533-5.9576310 .405026$ 
H $-2.182567-5.7918120 .149656$ H $-2.837790-6.1037061 .797601$ SCF Energy (B3LYP/6-31G**//MMFF) $=-3245.90818637$

0700202

MM̄FF Geometry

C $4.7725682 .545903-1.178815$

C $3.4944442 .818065-0.877807$

C $2.474073 \quad 3.255297-1.894172$

O $1.2769362 .464418-1.714481$

C $2.1073484 .732887-1.656887$

C $1.1092625 .289788-2.657257$

C $-0.1530315 .662757-2.361314$

C $1.6235355 .442536-4.065961$

C $-0.8346725 .609433-1.021486$

C $-1.7498484 .388431-0.925869$

C $-2.1934384 .039650 \quad 0.503045$

C -1.0200303 .5600161 .366353$

C -1.476874 3.0564252 .741880

$\begin{array}{lllll}\text { O } & -3.163435 & 2.989622 & 0.374082\end{array}$

$\begin{array}{lllll}\text { O } & -0.380739 & 2.346084 & 3.338778\end{array}$

C -2.616384 2.0411942 .542755

C -1.8148694 .2118653 .690665$

O -3.1608691 .6369253 .797065$

C -3.7411592 .5464541 .609706$

C -4.6998551 .3768351 .285399$

O -4.4668743 .5883852 .260455$

C $1.2289691 .236415-2.299779$

O $2.1259440 .664735-2.896881$

C $-0.1532240 .734288-2.133014$

C $-0.603022-0.276714-2.890890$

C $-1.985102-0.764902-2.926513$

C $-2.988807-0.067213-2.047344$

C $-5.868154 \quad 1.736447 \quad 0.354656$

C $-6.3723190 .541294-0.437700$

C $-6.986857-1.865344-0.214148$

O $-6.5118740 .596373-1.658515$

C $-5.707751-2.560691-0.568523$

C $-5.525669-3.262050-1.697846$

C $-4.215891-3.903352-2.066806$

C $-3.869138-3.733176-3.530807$

C $-3.769535-4.800901-4.340979$

C $-3.665147-2.334523-4.067743$

C $-2.301627-1.775306-3.763361$

N $-6.664979-0.5635500 .345423$

H $5.1162662 .649885-2.204898$

H 3.1555162 .7225040 .151419

H $2.8513253 .120582-2.915417$

H $3.0140245 .351395-1.702590$

H $1.7304074 .838490-0.634677$

H $-0.7794076 .045957-3.165782$

H $\quad 0.9076305 .957001-4.716120$

H $2.5483106 .028870-4.071289$

H $1.8268984 .464316-4.511813$

H $-0.1046485 .633966-0.211761$

H -1.431970 $6.521202-0.896826$

H -2.641597 $4.558355-1.544959$

H -1.265949 $3.510901-1.368480$

H -2.676679 4.9176340 .946462

H $-0.266514 \quad 4.344323 \quad 1.495808$

H -0.5053492 .7361720 .851935$

H $\quad 0.3748692 .9555163 .398776$

H -2.174511 1.1419162 .093209

H -0.9354544 .8482803 .847428$

H -2.0953403 .8399464 .682306$

H -2.6199494 .8495793 .319681$

H -2.422556 1.3177584 .343853

H -4.1199780 .5695840 .822364$

H $\quad-5.122057 \quad 0.9815492 .217666$

H -5.335552 3.6784631 .837646

H $-0.7903001 .260592-1.432530$

H $0.085104-0.756539-3.585276$

H -2.666630 -0.094483-1.001796

H -3.976774 -0.527028 -2.071665

H $-3.1098660 .978474-2.350664$

H -6.7069162 .1508310 .925026$

H $-5.5585782 .495118-0.373215$

H $-7.638258-1.731894-1.083893$
H -7.520995 -2.427470 0.557427

H $-4.881538-2.4664910 .131836$

H $-6.357220-3.382296-2.388452$

H -4.285155 -4.969377-1.815648

H -3.397908 -3.496683 -1.461939

H $-3.529281-4.691515-5.393375$

H $-3.923345-5.809264-3.971152$

H $-4.473413-1.679208-3.731572$

H $-3.768751-2.340111-5.161934$

H -1.505357 -2.225891 -4.357123

H $-6.489234-0.523273 \quad 1.344179$

C $6.3852700 .742819-0.519926$

O $5.352365-0.261318-0.559757$

C $5.840973-1.4578180 .070944$

C $7.335410-1.2467320 .292178$

C 7.4014890 .2507860 .509867

H $5.347630-1.5178391 .047789$

C $5.491945-2.694876-0.761922$

H $7.712837-1.8224421 .142825$

H $7.923603-1.526665-0.589231$

C 8.7940870 .8306190 .334046

H 7.0459840 .4713651 .525467

C $4.000497-2.841379-1.125929$

O $6.226313-2.639141-1.993049$

H $5.844528-3.591811-0.237898$

H $3.736483-2.113539-1.903357$

O $3.867566-4.138167-1.726080$

C $3.022380-2.7128790 .058553$

C $1.588894-3.143083-0.309335$

H $3.020693-1.6737960 .405259$

O $3.497830-3.5296641 .129197$

H $1.323627-2.746326-1.296739$

H $1.570165-4.238659-0.364024$

H $9.4891390 .386413 \quad 1.054192$

H $9.1842560 .639934-0.671266$

H $8.789411 \quad 1.913400 \quad 0.493711$

C $5.7832952 .101417-0.160696$

H $6.007570-3.442115-2.496567$

H $3.021868-4.158277-2.204598$

H $2.908177-3.3903711 .889384$

H $6.5739682 .858279-0.105980$

H $5.3159932 .048508 \quad 0.829941$

H $6.8335390 .777165-1.521664$

C $0.010876-2.7266853 .070186$

O $0.881823-3.1317872 .005432$

C $0.519052-2.6764610 .700726$

C $-0.878186-3.199350 \quad 0.324425$

C $-1.921472-2.8686291 .394860$

C -1.425835 -3.203945 2.805807

H $0.385706-3.2603893 .953264$

C $0.106852-1.2234293 .356044$

H $\quad 0.515533-1.5810040 .691669$

O $-0.847567-4.6134990 .132815$

H $-1.173425-2.754829-0.631974$

H $-2.836643-3.4348791 .184773$

O $-2.237465-1.4847191 .309368$

H -2.095628 -2.780428 3.563213

O $-1.442298-4.6239552 .977949$

C $1.494282-0.8589463 .826238$

H $-0.141913-0.6042822 .489595$

H $-0.591438-0.9316894 .149511$

H $-0.512426-5.0168440 .951927$

H -2.928053 -1.300368 1.968659

H -2.359842 -4.9230302 .859480$

O $2.195374-0.2852952 .807559$

O $1.911099-1.0812014 .955632$

C 3.5435140 .0792693 .116382

H $4.121120-0.8069963 .395987$

H 3.9882100 .5180812 .220386

H 3.5611240 .8232563 .918260

SCF Energy (B3LYP/6-31G**//MMFF) $=-3245.91319777$

07 00203

MM̄FF Geometry

C $1.913239-1.2891162 .420865$

C $1.371308-0.2525903 .079624$

C 1.9666451 .1315633 .219154

O 3.2546151 .1808632 .562158 
C 1.0477902 .1976152 .595711

C -0.1889712 .5081133 .409885$

C -1.444487 2.1866353 .044656

C 0.0503853 .2894344 .676455

C $-1.883891 \quad 1.4275201 .825118$

C $-2.535827 \quad 2.3422840 .782501$

C - -1.5434623 .2456770 .034630$

C $-2.2960674 .160471-0.934160$

C -1.337204 $5.039626-1.748956$

O $-0.6463022 .404069-0.696507$

O $-2.0809905 .601341-2.844123$

C $-0.2427004 .144621-2.369907$

C $-0.8317786 .232172-0.929100$

O $\quad 0.7666934 .927631-3.002418$

C $0.3831563 .106828-1.404307$

C $1.1586292 .050224-2.224085$

O $1.3326233 .695553-0.516959$

C 4.1211462 .1563762 .938898

O 3.9099953 .0525533 .744193

C 5.4048142 .0413602 .212134

C 5.8571810 .8717941 .735020

C 7.1339750 .6508901 .054704

C 7.9379021 .8591260 .654207

C $1.8649230 .982309-1.374650$

C $2.535612-0.063056-2.251580$

C $3.902153-2.128171-2.127939$

O $2.382877-0.108917-3.470190$

C $5.208517-2.440348-1.473497$

C $6.385545-2.347537-2.111428$

C $7.710388-2.731959-1.516268$

C $8.604259-1.539183-1.261256$

C $9.264225-0.937638-2.265307$

C $8.792733-1.0952520 .170603$

C $7.517399-0.6187440 .808641$

N $3.308893-0.952866-1.516820$

H $2.873480-1.1753351 .926071$

H $0.422570-0.4088213 .589626$

H 2.1227311 .3142984 .289199

H 0.7981891 .8947821 .573986

H 1.5987363 .1369042 .456567

H -2.2624342 .4920033 .696364$

H -0.8841643 .5787675 .168985$

H 0.6287942 .6984895 .392740

H $0.602274 \quad 4.2092104 .456826$

H -1.072233 0.8462451 .377719

H $-2.625526 \quad 0.6869292 .151045$

H -3.031151 1.6973540 .051282

H -3.3157732 .9507381 .257174$

H -0.9770293 .8405230 .758741$

H -3.023205 4.782918 -0.397599

H -2.883705 $3.547699-1.632482$

H -2.836049 $6.091238-2.475562$

H $-0.7320253 .587304-3.181202$

H -1.668810 $6.883197-0.647725$

H $-0.1532936 .859998-1.516429$

H $-0.3289355 .940922-0.005499$

H $1.3497035 .282983-2.311025$

H $0.4615451 .545860-2.906693$

H $1.9188642 .545295-2.842238$

H $1.0713794 .606511-0.324616$

H 5.9876432 .9543292 .152216

H $5.253417-0.0224301 .881773$

H 7.3192212 .5646960 .087972

H 8.7867101 .6179650 .009724

H 8.3318502 .3695621 .539612

H $2.6281591 .445335-0.739154$

H $1.1430250 .466699-0.731388$

H $4.009370-1.973652-3.206870$

H $3.209110-2.962666-1.980699$

H $5.178919-2.796903-0.447066$

H $6.400399-2.002188-3.143162$

H $8.210268-3.421299-2.209474$

H $7.573955-3.306426-0.591487$

H $9.926795-0.097827-2.084402$

H $\quad 9.153707-1.272255-3.291485$

H $9.581600-0.3392410 .248294$

H $9.168000-1.9458930 .754905$

H $6.862102-1.4290121 .130294$
H $3.259530-0.915428-0.502710$

C $1.258160-3.2085040 .929008$

O $0.227249-2.5533090 .157768$

C $-0.507594-3.555974-0.570835$

C $0.327842-4.827508-0.497449$

C $\quad 0.933037-4.7022140 .883569$

H - $1.441303-3.721728-0.022752$

C $-0.816851-3.102583-2.001050$

H $-0.277254-5.730528-0.622698$

H $1.116746-4.839379-1.258753$

C $2.136575-5.6045061 .098617$

H $\quad 0.163777-4.9434581 .629574$

C $-1.647796-1.809645-2.117018$

O $0.412028-2.894735-2.706026$

H -1.328108 -3.914919-2.531388

H -1.040571 -0.944121 -1.826404

O $-1.950153-1.621762-3.506751$

C $-2.959436-1.805582-1.308532$

C -3.885899-0.638134-1.700603

H $-2.715275-1.750063-0.241826$

O $-3.641836-3.039187-1.535983$

H $-3.2927250 .274431-1.830239$

H $-4.360407-0.877709-2.660444$

H $1.853871-6.6560780 .983298$

H $2.932916-5.3896740 .377639$

H $2.549157-5.4746862 .103702$

C $1.290660-2.6562402 .356207$

H $\quad 0.908680-2.211226-2.225265$

H -2.530687 -2.351914 -3.782436

H $-4.436809-3.043084-0.976363$

H $1.891178-3.3153012 .993570$

H $\quad 0.273958-2.6313682 .766900$

H $2.209435-3.0144190 .418736$

C $-6.824303-1.4732840 .469878$

O $-5.804304-1.535402-0.536581$

C $-4.984486-0.370471-0.652549$

C $-5.8480890 .844669-1.025882$

C $-6.9920411 .041155-0.025373$

C $-7.749280-0.2654730 .241534$

H -7.417846 -2.384357 0.319550

C $-6.235280-1.5287331 .884440$

H $-4.488653-0.1862580 .305993$

O $\quad-6.387730 \quad 0.686918-2.336913$

H $-5.2171491 .740207-1.047916$

H $-7.6918881 .790281-0.415237$

O $-6.471498 \begin{array}{lll}1.532880 & 1.206297\end{array}$

H -8.421030 -0.153610 1.100669

O $-8.566102-0.566155-0.894189$

C $-5.487606-2.8233542 .102287$

H $-5.561580-0.6931932 .095153$

H -7.028604 -1.481231 2.639592

H -6.888271 -0.146811-2.354421

H -6.0179232 .3726391 .019668$

H $-9.1923040 .169097-1.007492$

O $-4.148475-2.5806872 .187418$

O $-6.028328-3.9195782 .171728$

C $-3.324425-3.7335572 .383501$

H $-2.284417-3.4016362 .440927$

H $-3.585980-4.2317203 .321839$

H $-3.428155-4.4207381 .538341$

SCF Energy (B3LYP/6-31G**//MMFF) $=-3245.90431867$

0700204

MM̄MF Geometry

C $-2.932518-2.541455-2.372725$

C $-1.960823-1.945019-1.664318$

C $-0.760435-1.283263-2.287198$

O $-0.879173 \quad 0.124341-1.969901$

C $0.571793-1.814997-1.717267$

C $0.780335-3.309071-1.835704$

C $1.153828-4.108939-0.817896$

C $0.603558-3.895014-3.214031$

C $1.523181-3.7192180 .588278$

C $2.880906-4.3109321 .000654$

C $4.062326-3.8025790 .155101$

C $5.319501-4.6192480 .462812$

C $6.532961-4.103299-0.320872$

O $4.284502-2.4203740 .462971$ 
$\begin{array}{llll}\text { O } & 7.707816 & -4.736216 & 0.207811\end{array}$

C $6.657155-2.584063-0.095326$

C $6.478038-4.518860-1.794827$

O $7.684961-2.033419-0.917271$

C $5.331650-1.805358-0.305090$

C $5.526684-0.3581350 .207630$

O $5.014450-1.779553-1.696252$

C $-0.162677 \quad 1.004510-2.716897$

O $\quad 0.603507 \quad 0.748079-3.631011$

C $-0.4794462 .358675-2.216519$

C $0.290598 \quad 3.396670-2.572619$

C $0.0918634 .790950-2.172523$

C $-1.1181865 .108001-1.331383$

C $4.288792 \quad 0.5467380 .138739$

C $4.533838 \quad 1.863440 \quad 0.862793$

C 3.4365543 .9652501 .535558

$\begin{array}{lllll}\text { O } & 5.565479 & 2.097887 & 1.489425\end{array}$

C 2.4552684 .9414200 .971909

C $2.807946 \quad 6.1709290 .565182$

C 1.8452937 .2307040 .110780

C $2.0382617 .625034-1.335978$

C $3.0698838 .398149-1.715601$

C $0.9801917 .193745-2.324409$

C $0.9933265 .711422-2.574414$

N 3.4684602 .7493930 .744033

H $-2.868038-2.566779-3.457515$

H $-2.036206-1.911268-0.580059$

H $-0.784610-1.400056-3.378399$

H $0.648536-1.473847-0.680128$

H $1.406419-1.319580-2.230224$

H $1.251233-5.175118-1.021994$

H $0.933149-4.938319-3.266799$

H -0.444871 -3.867863 -3.522489

H $1.196876-3.331435-3.941916$

H $1.552304-2.6371590 .735374$

H $\quad 0.752924-4.1101991 .263343$

H $3.051726-4.0554672 .054354$

H $2.824363-5.4052810 .937969$

H $3.813180-3.897005-0.906886$

H $5.156654-5.6839330 .253586$

H $5.539724-4.5549401 .537599$

H $7.770963-4.5198651 .153629$

H $6.980415-2.4331360 .944263$

H $6.448315-5.611806-1.881746$

H $7.380608-4.201373-2.328801$

H $5.609300-4.120682-2.323233$

H $8.496937-2.540287-0.745407$

H $5.850964-0.4082671 .255872$

H $6.3305510 .131182-0.357448$

H $4.369655-1.072989-1.856602$

H - $1.3400172 .462855-1.566324$

H $1.1478973 .211240-3.218550$

H -1.205240 6.163571-1.067631

H -1.084353 $4.556232-0.385589$

H -2.037636 $4.838402-1.862877$

H $4.0294130 .768883-0.901540$

H 3.4367020 .0534200 .615161

H 4.4481494 .3826611 .592316

H 3.1384443 .6869242 .552095

H 1.4087554 .6482770 .956782

H 3.8563096 .4593450 .611533

H 1.9876178 .1150040 .746233

H $0.808508 \quad 6.9194740 .284870$

H $3.1958688 .708417-2.747527$

H $3.8122308 .737015-1.000386$

H $1.1380097 .687037-3.292923$

H $\quad 0.0057707 .557233-1.985621$

H $1.8432865 .380456-3.172823$

H 2.5968512 .4077850 .349760

C -5.420638 -2.407873 -2.052470

O $-5.326931-1.106410-1.436772$

C $-6.333376-1.012470-0.417646$

C $-7.457810-1.898901-0.925143$

C $-6.664638-3.081619-1.454891$

H $-5.911147-1.4582190 .492526$

C $-6.6922840 .450588-0.138953$

H -8.164838 -2.175086 -0.137595

H $-8.013526-1.423054-1.741344$
C $-7.431493-3.931876-2.453524$

H $-6.378354-3.717383-0.606041$

C $-5.468163 \quad 1.3688740 .073895$

$\begin{array}{llll}\text { O } & -7.430646 & 0.962433 & -1.254188\end{array}$

H -7.3655010 .4980570 .725498$

H $-4.9987701 .592003-0.892630$

O $\quad-5.9286142 .6191250 .598758$

C $-4.4022540 .808467 \quad 1.031211$

C -3.2410291 .7932001 .269632$

H $-3.994095-0.1168330 .612171$

$\begin{array}{llll}\text { O } & -5.006686 & 0.472418 & 2.279321\end{array}$

H $-2.9943192 .307360 \quad 0.333688$

H -3.551259 2.5443462 .006183

H $-8.325198-4.360517-1.988372$

H $-7.752551-3.342588-3.319170$

H $-6.809770-4.755833-2.818110$

C $-4.137692-3.190213-1.752421$

H $-7.7998311 .822799-0.993675$

H $-6.4638343 .048560-0.089370$

H $-5.407201 \quad 1.2819732 .640097$

H $-4.220151-4.212731-2.138261$

H $-3.996780-3.262375-0.667511$

H -5.531966 -2.244458 -3.131198

C $-1.231694-0.3393753 .601151$

$\begin{array}{llll}\text { O } & -2.275421 & 0.467296 & 3.038515\end{array}$

C $-1.980372 \quad 1.0657821 .774416$

C -0.7754442 .0061981 .904500$

C 0.4322891 .2649172 .487708

C $0.0679250 .472908 \quad 3.747220$

H $-1.585383-0.5928274 .608708$

C $-1.044645-1.6552902 .827911$

H $-1.743177 \quad 0.2808801 .047926$

O $\begin{array}{lllll}-1.099393 & 3.123879 & 2.727325\end{array}$

H -0.5227752 .4019880 .915291$

H 1.2191481 .9868292 .733622

$\begin{array}{lllll} & 0 & 0.949945 & 0.360605 & 1.517127\end{array}$

H $0.894016-0.1918994 .025962$

O $-0.098341 \quad 1.4079344 .817259$

C -2.279789-2.530696 2.876885

H $-0.815106-1.4747931 .774847$

H $-0.222001-2.2278893 .271311$

H -1.3875252 .7834103 .591623$

H $1.1191650 .862322 \quad 0.702121$

H -0.2593130 .8988825 .629980$

O $-2.109670-3.5834932 .022803$

O $-3.259778-2.3327973 .581637$

C -3.194192 -4.515612 1.970603

H $-4.134701-4.0024421 .747423$

H -2.988476 -5.2312691 .170227$

H -3.265770 -5.056766 2.918573

SCF Energy (B3LYP/6-31G**//MMFF) $=-3245.89981283$

07_00205

MM̄FF Geometry

C $-0.5641200 .963364 \quad 3.102870$

C $-1.521987 \quad 1.896403 \quad 3.198456$

C -1.996026 2.7211932 .034834

O -3.3800372 .3461451 .835616$

C -1.915296 4.2313552 .310295

C $-0.513827 \quad 4.7986712 .227604$

C $0.0262095 .279747 \quad 1.090168$

C $0.245751 \quad 4.878607 \quad 3.524514$

C $-0.6209795 .271715-0.267883$

C $0.3827965 .263016-1.427053$

C $1.2592114 .002098-1.507697$

C $2.3037454 .188104-2.612716$

C $3.1709072 .940499-2.802675$

O $0.4273952 .868044-1.795708$

O $3.8857943 .084326-4.039863$

C $2.2455631 .720151-2.954360$

C $4.2305932 .803837-1.705041$

O $3.005760 \quad 0.513719-2.989577$

C $1.1432801 .624697-1.867342$

C $0.1602620 .495487-2.262833$

O $1.7554941 .299670-0.619193$

C -3.8922612 .4757490 .584076$

O $-3.3618592 .995514-0.383491$

$\begin{array}{llll}\text { C } & -5.207570 & 1.804771 & 0.574148\end{array}$ 
C $-5.7178641 .415590-0.602973$

C $-6.9459190 .645985-0.796926$

C $-7.804967 \quad 0.3884120 .413418$

C $-1.0899750 .341036-1.383824$

C $-1.845071-0.931452-1.748344$

C $-3.436739-2.589130-0.811311$

O $-1.610037-1.569280-2.773086$

C $-4.925935-2.455894-0.767047$

C $-5.732368-2.941759-1.724779$

C $-7.235890-2.931334-1.668796$

C $-7.861667-2.121668-2.784745$

C -8.045132 -2.651060 -4.005952

C $-8.339434-0.722752-2.459077$

C $-7.2103430 .170296-2.031815$

N $-2.804072-1.280629-0.805716$

H $-0.079608 \quad 0.7959452 .145016$

H -2.015441 2.0573644 .154211

H -1.424977 $2.462676 \quad 1.133470$

H -2.355047 4.4638213 .290061

H $-2.566294 \quad 4.7669501 .606929$

H $1.0174705 .727017 \quad 1.139180$

H 1.2227195 .3588683 .403824

H -0.3181515 .4620294 .259660$

H $\quad 0.4219643 .880322 \quad 3.934270$

H -1.288838 $4.415256-0.379552$

H -1.231928 $6.178196-0.357345$

H $-0.1910315 .358468-2.358297$

H $\quad 1.0215416 .152489-1.350967$

H $1.7554273 .847289-0.543371$

H $2.9343735 .062882-2.409346$

H $1.7937004 .402623-3.562724$

H $4.4204523 .894893-3.984437$

H $1.7542301 .803852-3.934180$

H $4.8744413 .691199-1.680956$

H $4.8974771 .958923-1.908228$

H $3.8037562 .679741-0.708045$

H $3.6700600 .608439-3.693487$

H $-0.1858020 .667225-3.291049$

H $\quad 0.701521-0.459145-2.260791$

H $1.1175640 .833391-0.057790$

H $-5.679368 \quad 1.5916151 .526072$

H -5.157562 $1.634696-1.511424$

H $-7.262439-0.215273 \quad 1.149331$

H -8.730360 -0.1453310 .189504$

H $-8.099007 \quad 1.333970 \quad 0.882946$

H $-0.8203090 .286267-0.325203$

H -1.760862 $1.192450-1.530867$

H $-3.092010-3.1139180 .085176$

H -3.103849 -3.168780 -1.678362

H $-5.358598-1.9951570 .117068$

H - $-5.286288-3.424974-2.591782$

H -7.595912 -2.572620 -0.696850

H -7.581752 -3.971464 -1.736263

H -8.515287 -2.085911-4.803804

H -7.726042 -3.662878 -4.234128

H -9.132042 $-0.791478-1.709319$

H -8.812546 -0.260998 -3.335497

H $-6.517520 \quad 0.407214-2.840744$

H $-2.894343-0.7169140 .034720$

C $-0.363635-1.3911514 .031898$

O $0.429600-1.8825872 .932650$

C $-0.357558-2.8338332 .191046$

C $-1.578236-3.1386083 .055488$

C -1.805512 -1.801411 3.731275

H $-0.690608-2.3231591 .280395$

C $0.475983-4.0670141 .830961$

H -2.434910 -3.473348 2.463494

H -1.363743 -3.910867 3.803474

C $-2.696043-1.8764584 .958199$

H -2.263461 -1.125084 2.998818

C $1.736651-3.7879940 .985052$

O $0.903000-4.712874 \quad 3.038329$

H $-0.158597-4.795857 \quad 1.313091$

H $2.492588-3.2778361 .592929$

O $2.297649-5.0599840 .630930$

C $1.488621-2.988934-0.307608$

C $2.639175-3.033937-1.332058$

H $1.263867-1.947680-0.049748$
O $0.327804-3.520679-0.956633$

H $2.734288-4.052689-1.729880$

H $2.327285-2.415111-2.184010$

H -3.690581-2.247335 4.689184

H -2.280562 -2.548978 5.716050

H -2.815778 -0.8879765 .412860$

C $-0.1181690 .104476 \quad 4.252064$

H $1.414050-4.0638543 .552081$

H $2.456122-5.5468691 .457840$

H $0.158004-2.980306-1.747030$

H $\quad 0.955942 \quad 0.274840 \quad 4.391970$

H -0.6128940 .4176725 .179256$

H $-0.002903-1.9253024 .921178$

C $5.044619-0.6484630 .228385$

O $3.891766-1.174572-0.445365$

C $4.001009-2.547617-0.817614$

C $5.115488-2.707323-1.872881$

C $6.337048-1.830284-1.568968$

C $6.346091-1.392632-0.109816$

H $5.1361690 .391485-0.105624$

C $4.792774-0.6357861 .737158$

H $4.274999-3.1271090 .072133$

O $4.632725-2.340680-3.167589$

H $5.387715-3.767885-1.936695$

H $6.376330-0.948243-2.220526$

O $7.525204-2.577297-1.832486$

H $6.483402-2.2661030 .540170$

O $7.446831-0.519148 \quad 0.134582$

C 3.6919810 .3357802 .075478

H $4.483006-1.6226602 .100171$

H $5.691974-0.3565702 .298329$

H $5.350713-2.499800-3.803776$

H $7.504553-2.836081-2.769695$

H $8.254416-0.988953-0.135675$

O 4.2041551 .5806812 .285969

O $2.509507 \quad 0.0253092 .115212$

C 3.2419272 .5947402 .594176

H 3.7702583 .5464882 .693728

H 2.5083792 .6854221 .787628

H 2.7460192 .3667443 .542295

SCF Energy $($ B3LYP/6-31G**//MMFF $)=-3245.90233211$

07_00206

MMFF Geometry

C $-2.959586-3.787695-0.938384$

C $-1.768026-4.022245-0.368949$

C $-0.458294-3.574385-0.968612$

O $0.239557-2.7650570 .006101$

C $0.416380-4.801369-1.283096$

C $1.709911-4.459194-1.997078$

C $2.941987-4.654567-1.487858$

C $1.560052-3.890521-3.385463$

C $3.314636-5.235806-0.153392$

C $3.538221-4.1989910 .958437$

C $4.685037-3.2144870 .677622$

C $5.030303-2.4105011 .932974$

C $6.124384-1.3659731 .662063$

O $4.272269-2.317497-0.358135$

O $6.143734-0.4800102 .792142$

C $5.736089-0.5389460 .415663$

C $7.513243-2.0125081 .600706$

$\begin{array}{lllll}\text { O } & 6.796185 & 0.337017 & 0.041770\end{array}$

C $5.289996-1.401085-0.784661$

C $4.705792-0.572188-1.955177$

O $6.428194-2.103188-1.301154$

C $-0.062966-1.4394080 .048805$

O $-0.856346-0.842800-0.660768$

C $0.724358-0.8334151 .145213$

C 0.3834380 .3768951 .612128

C 1.0244551 .0826682 .722450

C 2.3695070 .5834063 .178635

C $3.5052310 .313791-1.604221$

C $3.8824951 .745399-1.259001$

C $2.9072063 .844338-0.338484$

O $4.9981412 .215686-1.464305$

C 3.0156713 .9302501 .150100

C 2.1831754 .6639841 .903717

C 2.2973414 .7959873 .394436 
C 1.0188794 .4205684 .108250

C $0.138729 \quad 5.3610104 .491269$

C $0.805994 \quad 2.964958 \quad 4.454524$

C $0.385386 \quad 2.137393 \quad 3.270934$

N $2.8113752 .450683-0.729180$

H $-3.000834-3.246809-1.881048$

H $-1.723944-4.5604120 .575026$

H - $0.628228-2.992185-1.882470$

H $-0.142037-5.504528-1.915552$

H $0.621568-5.333505-0.347996$

H $3.797149-4.373643-2.101868$

H $2.519491-3.813800-3.908428$

H $0.910276-4.530987-3.990774$

H $1.127483-2.886262-3.348017$

H $2.562851-5.9582870 .181073$

H $4.232282-5.823432-0.285927$

H $2.606017-3.6460641 .121174$

H $3.753916-4.7483291 .883279$

H $5.556545-3.788346 \quad 0.341418$

H $5.330946-3.0749432 .752717$

H $4.130698-1.8889852 .287451$

H 6.7787650 .2309262 .598118

H 4.9040920 .1046980 .723072

H $7.735721-2.5263642 .543684$

H $8.298227-1.2574821 .482063$

H $7.611281-2.7417370 .793813$

H $7.467988-0.191381-0.421951$

H $5.5025300 .003195-2.441894$

H $4.359410-1.287217-2.715501$

H $6.234101-2.373102-2.214106$

H $1.537969-1.4137931 .564263$

H $-0.468600 \quad 0.888377 \quad 1.167510$

H 3.0426130 .4622942 .322843

H $2.271104-0.3812543 .687546$

H 2.8776821 .2670093 .862261

H $2.8501420 .376601-2.482651$

H $2.919959-0.122280-0.789143$

H $2.0141874 .350930-0.714812$

H $3.7859614 .304144-0.801407$

H $3.8273503 .384317 \quad 1.625044$

H $1.3879795 .227407 \quad 1.420247$

H 2.5569255 .8390513 .618086

H 3.1265964 .1956973 .788918

H $-0.767280 \quad 5.1023985 .029314$

H $0.3023076 .410475 \quad 4.269702$

H $0.011616 \quad 2.8722525 .207566$

H 1.7022922 .5799574 .949099

H -0.5856392 .4232192 .863340$

H $1.9268591 .974672-0.577199$

C -5.110131-3.029611 0.077235

O $-4.563763-2.4938401 .302628$

C -5.645842 -2.193516 2.198098

C $-6.916976-2.2473431 .363479$

C $-6.570918-3.357146 \quad 0.386378$

H -5.685338 -3.0248342 .915992$

C $-5.400376-0.9054852 .995033$

H -7.806018 -2.466217 1.962255

H $-7.086351-1.3068840 .827374$

C $-7.470160-3.388026-0.837085$

H $-6.637259-4.3217750 .907294$

C -5.3631790 .4331162 .222853$

O $-6.418655-0.807523 \quad 3.999813$

H -4.453630 -1.012314 3.538989

H $-6.3669270 .698308 \quad 1.870666$

O -4.9922031 .4556203 .159308$

C -4.3797310 .4813121 .042911$

C $-4.214550 \quad 1.8850610 .443454$

H $-4.745498-0.1970660 .271131$

$\begin{array}{llll}\text { O } & -3.108461 & 0.013407 & 1.485779\end{array}$

H -5.1971412 .3174260 .216689$

H -3.759551 2.552968 1.187335

H -8.510927 $-3.561421-0.544607$

H -7.429305 -2.442941 -1.388999

H -7.173318 -4.189959-1.520092

C $-4.277344-4.232220-0.363898$

H -7.267945 -0.6567173 .551112$

H -5.6263281 .4180713 .895805$

H $-2.602172-0.2468170 .698032$
H $-4.804827-4.799502-1.138825$

H $-4.113659-4.9076540 .484739$

H $-5.056793-2.234391-0.677421$

C $-2.3292183 .584628-2.281327$

O $-3.2078843 .315012-1.179033$

C $-3.3202151 .931745-0.813701$

C $-3.865917 \quad 1.112114-1.995946$

C $-3.0544111 .335387-3.276635$

C $-2.7831062 .820729-3.536408$

H $-2.4573634 .656548-2.480102$

C $-0.867305 \quad 3.358119-1.878197$

H $-2.3255951 .568848-0.538252$

O $-5.229908 \quad 1.452981-2.239630$

H $-3.8407490 .046741-1.742906$

H $-3.6059940 .908684-4.123316$

O $-1.8234020 .628350-3.166154$

H $-2.0426942 .944270-4.334448$

O $-3.992313 \quad 3.436490-3.992311$

C $0.0919733 .931063-2.892397$

H $-0.6592043 .842188-0.915908$

H $-0.6432262 .299556-1.716740$

H $-5.2703742 .409423-2.411478$

H $-1.3330250 .758836-3.995534$

H $-4.2550752 .991504-4.816144$

O $0.8967512 .943289-3.378517$

O $0.125497 \quad 5.111917-3.212765$

C $1.876863 \quad 3.359526-4.333743$

H $2.4627072 .483025-4.622913$

H $1.3880563 .765905-5.224236$

H $2.5495504 .099207-3.889177$

SCF Energy (B3LYP/6-31G**//MMFF) $=-3245.91077775$

07_00207

MMFF Geometry

C -2.573164 -3.658888 -1.847085

C $-1.537936-3.430492-1.026018$

C $-0.145617-3.141553-1.529239$

O $0.334154-1.919100-0.924932$

C $0.810630-4.282764-1.138108$

C $2.249057-4.065168-1.573683$

C $3.309101-4.147910-0.745416$

C $2.467475-3.767063-3.034667$

C $3.302335-4.521108 \quad 0.709156$

C $3.231138-3.3315201 .675059$

C $4.473904-2.4295591 .639066$

C $4.539801-1.5598252 .896946$

C $5.738999-0.6015742 .863309$

$\begin{array}{llllll}\text { O } & 4.393482 & -1.589410 & 0.481967\end{array}$

$\begin{array}{lllll}\text { O } & 5.557276 & 0.369777 & 3.904656\end{array}$

C 5.7247820 .1616331 .524770

C $7.058929-1.320593 \quad 3.165288$

O $6.900323 \quad 0.9550301 .376105$

C $5.539383-0.7501010 .287122$

C $5.2982690 .130319-0.959993$

O $6.725242-1.5211620 .084740$

C $-0.078232-0.753854-1.494785$

O $-0.878971-0.626777-2.408020$

C $0.6352120 .356733-0.826345$

C $0.2973941 .619067-1.128352$

C $0.9209102 .837866-0.611062$

C 2.0208192 .6907070 .405436

C $4.792410-0.625228-2.193238$

C $4.6837160 .323239-3.371271$

C $3.3433722 .257103-4.211598$

O $5.5179640 .326160-4.273476$

C $3.4443163 .553151-3.467640$

C $2.5305964 .532291-3.553466$

C $2.6221735 .827202-2.795157$

C $1.3464106 .169016-2.053192$

C $0.5526637 .169844-2.470779$

C $1.0260255 .399481-0.786689$

C $0.4884764 .026013-1.082719$

N $3.5921341 .172298-3.278849$

H $-2.404613-3.649852-2.921912$

H $-1.690672-3.4472440 .049462$

H $-0.145969-3.029075-2.621161$

H $0.458936-5.223407-1.581827$

H $0.757752-4.412783-0.052141$ 
H $4.300663-3.963128-1.156533$

H $3.527733-3.791557-3.309213$

H $1.954025-4.509823-3.654104$

H $2.087283-2.772935-3.288941$

H $2.474509-5.2055230 .926082$

H $4.209079-5.1040450 .915857$

H $2.335823-2.739717 \quad 1.450841$

H $3.109906-3.7418692 .685279$

H $5.365408-3.0639281 .570713$

H $4.575108-2.1811023 .800492$

H $3.619122-0.9645422 .977262$

H $5.491043-0.1064534 .750096$

H 4.8806880 .8649851 .563266

H $7.019538-1.7940054 .153762$

H $7.893823-0.6121903 .206207$

H $7.301356-2.0971202 .436947$

H 6.9690361 .5215132 .163713

H $4.5545650 .896352-0.715943$

H $6.2231320 .660010-1.223507$

H $6.786865-1.765571-0.852249$

H $1.4252940 .106822-0.127665$

H $-0.5056931 .781521-1.846757$

H 1.7143382 .0292561 .223428

H 2.3033123 .6341180 .877742

H $2.9167902 .276047-0.062844$

H $5.467912-1.439699-2.473774$

H $3.808405-1.069292-2.006473$

H $2.3451542 .100375-4.632225$

H $4.0779352 .233275-5.021959$

H $4.3129643 .693372-2.828033$

H $1.6758464 .403894-4.213939$

H $2.8601936 .617953-3.518347$

H $3.4554785 .812233-2.081402$

H $-0.3508317 .436849-1.932403$

H $\quad 0.7853207 .741822-3.362810$

H $0.2670645 .934104-0.200940$

H $1.9245465 .380688-0.163928$

H $-0.3405434 .027908-1.792408$

H $2.9506181 .066862-2.498603$

C $-4.951465-2.914478-1.961401$

O $-4.891184-1.706798-1.173005$

C $-6.214431-1.131815-1.115935$

C -7.097954 -1.991790 -2.014256

C $-6.415520-3.341589-1.914378$

H $-6.564099-1.239085-0.083719$

C $-6.166396 \quad 0.345759-1.521593$

H -8.138315 -2.009428 -1.675391

H -7.090901 -1.632088 -3.050160

C $-6.823363-4.303498-3.016854$

H $-6.647114-3.786232-0.937403$

C $-5.3548091 .237420-0.557763$

O

H -7.184266 $0.739788-1.619972$

H $-4.3090420 .911753-0.536998$

O $\quad-5.3574582 .569822-1.085740$

C -5.9293341 .2906320 .872113$

C -5.2524082 .3173291 .799743$

H $-5.870794 \quad 0.3003841 .334145$

O $\quad-7.319707 \quad 1.6262010 .808699$

H $-5.294843 \quad 3.3170001 .350491$

H -5.8593862 .3771642 .713482$

H -7.900394 -4.496748 -2.978581

H -6.589242 -3.900569-4.008074

H $-6.306333-5.262216-2.912862$

C -3.977835 -3.949953-1.397871

H $-4.6848780 .065122-2.773567$

H -5.002954 2.523209 -1.990340

H -7.3918002 .4944750 .375983$

H -4.233945 -4.951331-1.760570

H -4.035452 -3.967240 -0.302822

H -4.675111 -2.640687 -2.989033

C $-2.5693640 .200558 \quad 3.277417$

O -3.8464650 .7331552 .906728$

C -3.8054201 .9591352 .168450$

C -3.1410463 .0597913 .010056$

C $-1.7468712 .629743 \quad 3.478657$

C $-1.753894 \quad 1.2243434 .089435$

H $-2.807396-0.640453 \quad 3.940989$
C -1.852903 -0.3656382 .047947$

H -3.2292511 .8113811 .249090$

O -3.9564373 .3525394 .143216$

H -3.0739943 .9733772 .407232$

H $-1.375615 \quad 3.341030 \quad 4.226607$

O $-0.845323 \quad 2.6524282 .377660$

H -0.7257870 .8695664 .222434$

O -2.3307941 .3245385 .395814$

C $-0.757598-1.3264092 .434967$

H -2.560442 -0.8915801 .394113$

H $-1.4195180 .417575 \quad 1.421077$

H -3.5208244 .0637474 .643008$

H -0.8280963 .5598202 .028511$

H -2.2661740 .4489915 .813676$

O $-1.296247-2.5303572 .779861$

O $0.431158-1.0353382 .443184$

C $-0.363464-3.532746 \quad 3.194286$

H $-0.928702-4.3706863 .611021$

H $\quad 0.204636-3.8878132 .330919$

H $\quad 0.307730-3.1459953 .967353$

SCF Energy $(B 3 L Y P / 6-31 G * * / / M M F F)=-3245.90831909$

07_00208

MMFF Geometry

C $-1.585217-0.7492673 .169080$

C $-2.1838590 .301572 \quad 3.752123$

C -1.7004241 .7339043 .728546$

O $-0.3701201 .778693 \quad 3.154162$

C -2.619348 2.6565632 .899704

C -4.0536972 .8102303 .368116$

C -5.1087342 .8528652 .528866$

C -4.2692722 .9944834 .847683$

C -5.0639422 .8398661 .027290$

C -5.5089661 .4949360 .444336$

C $-5.1411261 .396615-1.042692$

C $-5.7994310 .183450-1.696768$

C $-5.3563290 .016197-3.159292$

O $-3.7162081 .274406-1.119310$

O $-5.754307-1.294182-3.590620$

C $-3.8149280 .068749-3.227415$

C $-6.0662261 .011690-4.084356$

O $-3.3630970 .059396-4.580435$

C $-3.1907971 .253506-2.450953$

C $-1.6595061 .061186-2.356626$

O $-3.4686962 .468634-3.147263$

C 0.4073392 .8585543 .426491

O $\quad 0.1317873 .8116534 .136815$

C 1.6602052 .6987012 .660015

C 2.2799343 .7971662 .205866

C 3.4935543 .8319251 .387275

C 4.2406232 .5393191 .194959

C $-0.9409002 .070822-1.452526$

C $0.4889441 .626997-1.215429$

C $2.6983311 .447459-2.351852$

O $0.8176961 .048979-0.181982$

C $3.6955432 .562195-2.433318$

C $3.4719403 .885561-2.401524$

C $4.5747754 .900331-2.528073$

C $4.6373095 .866594-1.367185$

C $4.4047507 .178222-1.546501$

C $5.0554945 .328738-0.014692$

C 3.8710205 .0149820 .859537

N $1.3249271 .905471-2.284632$

H $-0.681621-0.6065302 .583072$

H $-3.080856 \quad 0.117428 \quad 4.339502$

$\mathrm{H}-1.6340212 .0786344 .768170$

H -2.5756252 .3014631 .863429$

H -2.1814243 .6627072 .870677$

H -6.1061132 .9647802 .950998$

H -5.3035433 .2665165 .084713$

H -4.0397292 .0769995 .396569$

H -3.628578 3.7984575 .225019

H -5.7333243 .6284020 .659453$

H $-4.071773 \quad 3.1172540 .658604$

H -5.0229830 .6796530 .994374$

H -6.5922801 .3858430 .577451$

H $-5.4563882 .318939-1.544928$

H $-6.8925240 .251764-1.632661$ 
H $-5.525473-0.724121-1.143211$ H $-6.718820-1.363760-3.487515$ H -3.445986 $-0.866051-2.784860$ H -7.154145 $0.894842-4.010005$ H $-5.8182510 .821277-5.134503$ H $-5.8289462 .052758-3.855101$ H $-3.4984560 .951166-4.943467$ H -1.454154 $0.059265-1.961004$ H -1.215838 $1.107926-3.359143$ H -2.825056 $3.141168-2.873926$ H 2.0012041 .6917212 .449978 H 1.8374294 .7678802 .428015 H 3.6110991 .7974160 .693314 H 4.5521842 .1311702 .163010 H 5.1452792 .6422000 .592612 H $-0.9377653 .073145-1.894138$ H -1.427653 2.150891-0.474486 H $2.7786050 .833117-3.254353$ H $2.9252910 .814974-1.488552$ H $4.7258922 .223706-2.535194$ H $2.4599194 .269697-2.310419$ H $4.4153855 .443634-3.468595$ H $5.552664 \quad 4.412639-2.628947$ H $4.4776967 .880707-0.722576$ H $4.1333997 .578351-2.517611$ H 5.6593516 .0836880 .506739 H $5.7284144 .478265-0.151600$ H 3.2581035 .8910311 .079176 H $\quad 0.9384882 .355907-3.109024$ C -2.429517 -2.872697 2.028414 O $-1.304068-2.9444741 .130242$ C - $1.803513-2.860153-0.220620$ C $-3.325096-2.926875-0.119482$ C $-3.563230-2.2534671 .217819$ H -1.527722 -1.869572 -0.595901 C $-1.187400-3.955735-1.096129$ H $-3.817808-2.421838-0.953979$ H $-3.683796-3.962793-0.096683$ C $-4.947682-2.4975671 .788968$ H $-3.406516-1.1746251 .094654$ C $0.344250-3.862660-1.264639$ O $-1.490107-5.234990-0.524467$ H -1.664375 -3.949096 -2.083208 H $0.842413-4.076360-0.312419$ O $0.743210-4.899327-2.170770$ C $0.836610-2.515577-1.823560$ C $2.306029-2.497545-2.280056$ H $0.680290-1.733925-1.072383$ O $0.035952-2.168901-2.959072$ H $2.441562-3.167653-3.138726$ H $2.498725-1.491876-2.671402$ H $-5.716621-2.1081931 .113908$ H -5.139376 -3.5656871 .935998$ H -5.063782 -1.9971692 .755319$ C -2.055437 -2.167075 3.331004 H -1.124794 -5.2459990 .377068$ H $0.419662-5.741606-1.807679$ H $\quad 0.309327-1.282900-3.252370$ H -1.233195 -2.717590 3.802941 H -2.902774 -2.216371 4.025197 H $-2.681238-3.9130242 .277729$ C $3.965918-2.1667321 .055722$ O $3.107835-1.965636-0.076111$ C $3.336363-2.832058-1.190299$ C $4.777411-2.677727-1.711031$ C $5.809507-2.847612-0.591545$ C $5.443788-2.0269380 .649576$ H $3.724323-1.3436091 .740271$ C $3.672197-3.4883271 .783380$ H $3.175931-3.868898-0.878604$ O $4.957933-1.397038-2.315187$ H $4.949933-3.424387-2.494721$ H $6.794643-2.527093-0.952659$ O $5.905388-4.221398-0.229594$ H $6.089872-2.3093261 .489175$ O $5.707393-0.6508660 .361013$ C $2.291667-3.5244072 .401689$ H $3.744220-4.3442751 .107662$
H $4.392683-3.6240142 .598465$

H $4.776417-0.722091-1.639125$

H $6.173223-4.716237-1.022664$

H $5.544197-0.144447 \quad 1.174935$

O $2.068451-4.7806082 .891868$

O $1.515893-2.5815212 .460016$

C $0.800594-4.9877693 .520660$

H $0.783665-6.0036223 .924468$

H $0.663221-4.2820864 .345134$

H $-0.002490-4.8905602 .785193$

SCF Energy (B3LYP/6-31G**//MMFF) $=-3245.90724972$

07_00209

MMFF Geometry

C $0.160842-3.7419101 .202126$

C $0.827285-2.5790921 .259854$

C $1.600541-2.1101722 .463731$

O $2.955347-1.8833641 .998200$

C $1.042880-0.7968303 .035146$

C $-0.145621-0.9793053 .950678$

C $-1.409068-0.6320803 .642697$

C $0.171940-1.5289055 .318029$

C $-1.923605-0.0793072 .345047$

C -2.3302341 .3970132 .445681$

C -1.1360432 .3566332 .568565$

C -1.6195013 .8022872 .696369$

C -0.4442314 .7892722 .776098$

O $-0.3379002 .227793 \quad 1.389166$

O -0.9646796 .1097942 .543583$

C 0.5456734 .4953361 .625933

C 0.1676084 .8213014 .181453

O $\quad 1.739376 \quad 5.262747 \quad 1.759792$

C 0.8716592 .9963221 .408989

C 1.5474942 .8041920 .032210

O 1.8026332 .4992952 .370391

C $3.948462-1.8982602 .923275$

O $3.814264-2.0356984 .132108$

C $5.283088-1.7241132 .304650$

C $5.508565-1.7425250 .981349$

C $6.819052-1.6419510 .331991$

C $8.012902-1.3440191 .201880$

C $1.8782801 .340940-0.294317$

C $2.3467921 .204468-1.728892$

C $4.2219041 .824180-3.248917$

O $1.6456910 .676654-2.589162$

C $5.1459680 .662595-3.429886$

C $6.4756710 .793404-3.548769$

C $7.416678-0.346888-3.811531$

C $8.355606-0.611963-2.657069$

C $9.3983400 .198022-2.407210$

C $8.139179-1.871613-1.853180$

C $6.898897-1.825437-1.002995$

N $3.6011421 .751706-1.941225$

H $0.138735-4.3846642 .078328$

H $\quad 0.871859-1.9492540 .373817$

H $1.626877-2.9009293 .223323$

H $0.818542-0.1098722 .214074$

H $\quad 1.822887-0.2714403 .601854$

H $-2.179509-0.7778904 .399117$

H $0.553647-2.5515825 .245444$

H $0.928340-0.9087605 .810495$

H - $0.707114-1.5527735 .971004$

H -1.225645 -0.231453 1.516735

H -2.811339-0.667094 2.089623

H -2.8953541 .6472071 .539826$

H $-3.0098251 .536727 \quad 3.295671$

H -0.5412932 .0751933 .444205$

H -2.276551 3.9185883 .567349

H -2.2323394 .0611801 .821483$

H -1.6617246 .2786293 .200526$

H 0.0637254 .8651690 .710700

H -0.5736355 .1680304 .912032$

H 0.9955295 .5360904 .238945

H 0.5212993 .8468224 .522057

H 2.2901834 .8462812 .443578

H $0.8907353 .190608-0.757358$

H $2.4747313 .389679-0.006572$

H 1.6820912 .9687283 .207282 
H $6.095343-1.6225373 .017860$ H $4.667646-1.8786850 .303745$ H $7.834082-0.4496381 .809599$ H $8.923582-1.1415310 .633167$ H 8.223242 -2.186970 1.868868 H $2.6551140 .950876 \quad 0.373047$ H $0.9962270 .702725-0.173630$ H $4.7477582 .782837-3.304053$ H $3.4522861 .810280-4.027571$ H $4.695040-0.323924-3.503688$ H $6.9166351 .786075-3.489909$ H $8.003265-0.108211-4.708499$ H $\quad 6.863643-1.260105-4.065014$ H $10.093240-0.006338-1.599522$ H $9.5784931 .084943-3.005839$ H $9.023244-2.106263-1.250097$ H $8.051937-2.721378-2.543189$ H $5.980914-2.021832-1.557906$ H $4.1305002 .101790-1.148085$ C -1.979098 -4.493471 -0.028852 O $-2.694450-3.247534-0.117092$ C $-4.011975-3.455810 \quad 0.438063$ C $-4.044779-4.8916310 .973044$ C $-2.574226-5.1987431 .182418$ H $-4.109659-2.767676 \quad 1.284334$ C $-5.099094-3.174715-0.611473$ H $-4.631877-4.970276 \quad 1.893760$ H -4.485305 $-5.585438 \quad 0.247330$ C -2.264944 -6.6828541 .256419$ H -2.256733 -4.711479 2.112687 C $-5.125465-1.731584-1.159191$ O $-4.897221-4.058733-1.722252$ H -6.079209-3.431474 -0.191189 H -4.241479 -1.558822 -1.781885 O $-6.252544-1.627014-2.039271$ C $-5.232244-0.643035-0.079933$ C $-5.5462740 .772023-0.593873$ H $-4.299188-0.6174290 .486824$ O $-6.269803-1.004980 \quad 0.837174$ H $-6.5664410 .807434-0.997546$ H $-5.563211 \quad 1.4241810 .289680$ H -2.795177 -7.144939 2.095548 H -2.567193 -7.202141 0.340870 H - $1.193031-6.8510731 .400832$ C $-0.465459-4.278332-0.053861$ H $-5.594659-3.869770-2.373419$ H -7.056008 -1.731577 -1.500892 H $-6.263404-0.3540911 .559711$ H -0.219916 -3.618394 -0.893616 H $\quad 0.018357-5.240542-0.264479$ H - $-2.236371-5.049930-0.941695$ C -2.177902 $1.682292-1.940828$ O $-3.2385551 .222458-1.092682$ C $-4.5592891 .333038-1.627515$ C $-4.8846512 .797056-1.976479$ C $-3.8128293 .420795-2.875849$ C $-2.4014703 .149961-2.345924$ H -1.279176 $1.638264-1.314023$ C -1.955826 $0.756943-3.146399$ H $-4.6316550 .722489-2.533131$ O $\quad-5.0032593 .580151-0.788254$ H -5.859008 $2.831697-2.477341$ H -3.970204 $4.505122-2.931480$ O $-3.9250682 .897170-4.194993$ H -1.655309 $3.449098-3.091332$ O $-2.2019253 .975455-1.195303$ C -1.487118 -0.624006 -2.743335 H $-2.8718290 .628108-3.728627$ H $-1.1828501 .182412-3.796722$ H $-4.1661603 .505488-0.298525$ H $-4.8158703 .110824-4.521161$ H -1.294131 $3.828265-0.882473$ O $-1.459858-1.414605-3.857699$ O $-1.177356-0.965289-1.610830$ C $-1.039645-2.765750-3.650987$ H $-0.028330-2.789770-3.234716$ H -1.745658 -3.282075 -2.994713 H $-1.030168-3.270773-4.620496$
SCF Energy (B3LYP/6-31G**//MMFF) $=-3245.90752519$

07_00210

MMFF Geometry

C $-1.342973 \quad 3.615181-0.018231$

C $-0.8600592 .366741 \quad 0.068643$

C $0.1757091 .816881-0.879303$

O $1.3072101 .362729-0.104499$

C $-0.4088350 .617513-1.652940$

C $0.4575790 .030062-2.755180$

C $0.314695-1.231734-3.210433$

C $1.470130 \quad 0.931017-3.414248$

C $-0.673046-2.272937-2.762352$

C $-0.038803-3.642107-2.471570$

C $0.910836-3.728395-1.264081$

C $0.213444-3.407488 \quad 0.058230$

C $1.178468-3.4621121 .251723$

O $2.022191-2.855173-1.471409$

O $0.504861-2.8361852 .354833$

C $2.429979-2.6246650 .915652$

C $1.468103-4.9050441 .679415$

O $3.427097-2.7583631 .925710$

C $3.040207-2.952184-0.467984$

C $4.151895-1.932535-0.806027$

O $3.618427-4.259773-0.427589$

$\begin{array}{llll}\text { C } 2.257852 & 2.292945 & 0.191108\end{array}$

O $2.2512943 .474514-0.116217$

C 3.3324901 .6168990 .950153

C $4.4583262 .285674 \quad 1.239605$

C 5.6244811 .7556681 .950315

C 5.6011730 .3013632 .340043

C $4.584890-1.941363-2.276163$

C $5.951014-1.312818-2.472913$

C $7.2373610 .796696-2.171448$

O $6.880406-1.950455-2.965408$

C $8.0306790 .653061-0.909378$

C $8.6466531 .681325-0.305654$

C 9.4888101 .5391550 .930981

C 9.1517522 .5599531 .995798

C 9.9315163 .6366652 .191905

C 7.9588742 .2897212 .886746

C 6.6561672 .5893602 .198773

N $6.0189850 .012941-2.073105$

H $-0.958692 \quad 4.276910-0.790389$

H $-1.232813 \quad 1.6988300 .841129$

H $0.4913862 .591570-1.588805$

H $-1.3547000 .926581-2.118021$

H $-0.639701-0.166372-0.921970$

H $0.956519-1.554053-4.029956$

H $1.9590050 .452901-4.270254$

H $\quad 0.986177 \quad 1.839356-3.787472$

H $2.259248 \quad 1.209267-2.709474$

H $-1.267978-1.948114-1.906192$

H $-1.388217-2.408441-3.584140$

H - $0.848056-4.370037-2.331096$

H $0.514277-3.959460-3.365926$

H $1.283137-4.760004-1.239918$

H $-0.629652-4.0889870 .216617$

H $-0.215350-2.3984100 .022075$

H $1.076565-2.9078413 .137775$

H $2.122591-1.5701530 .915895$

H $0.541857-5.402347 \quad 1.991680$

H $2.132394-4.9346192 .550152$

H $1.916041-5.5062620 .885468$

H $3.049809-2.4290522 .758539$

H $3.790322-0.922483-0.583769$

H $5.024699-2.116775-0.167186$

H $4.354976-4.302797-1.057922$

H 3.1746270 .5854941 .239902

H 4.5425323 .3232320 .918056

H 4.7694030 .0987893 .023755

H $6.509360-0.0340302 .843675$

H $5.488423-0.3293491 .452553$

H $4.638430-2.964177-2.664604$

H $3.860666-1.400699-2.896692$

H $6.9438561 .834310-2.358912$

H $7.8276420 .435761-3.019906$

H $8.131799-0.344991-0.489210$ 
H $8.5765872 .673214-0.746492$

H $\quad 10.538901 \quad 1.6497390 .629842$

H $9.4060110 .531807 \quad 1.357622$

H $9.712406 \quad 4.3593822 .970940$

H $10.8039873 .822523 \quad 1.574092$

H 8.0068522 .9237563 .782146

H 8.0253551 .2651533 .260984

H 6.5664913 .6292411 .880115

H $5.2090560 .451031-1.645169$

C $-3.773019 \quad 4.3167340 .235669$

O $-4.283188 \quad 3.013519-0.116319$

C $-4.804847 \quad 3.074557-1.454971$

C $-5.0271464 .554992-1.742002$

C $-3.8369435 .171178-1.033063$

H $-4.0135532 .700987-2.116401$

C $-6.0533912 .200216-1.600698$

H $-5.0499944 .772830-2.813953$

H $-5.9606904 .920635-1.298769$

C $-3.9847816 .658364-0.763120$

H -2.952883 $5.015234-1.661995$

C $-5.8872570 .733330-1.152849$

O $-7.1196152 .760832-0.822363$

H -6.397962 2.233288 -2.640915

H $-5.8632510 .682434-0.058394$

O $-7.067211 \quad 0.027074-1.560086$

C $-4.6592330 .004130-1.725675$

C $-4.666596-1.524396-1.527791$

H $-3.7519120 .424723-1.277738$

O $-4.5863930 .252674-3.132737$

H -5.457061 -1.971751-2.144397

H -3.724676 -1.905401-1.942409

H $-4.0866517 .211587-1.702467$

H $-4.8683016 .870154-0.151564$

H $-3.1078347 .045883-0.235178$

C $-2.3958594 .179785 \quad 0.893724$

H $-6.812792 \quad 2.8141080 .099139$

H $-7.8303220 .492912-1.177192$

H $-3.765840-0.154078-3.460146$

H -2.490470 3.5404291 .779863

H -2.056056 $5.159651 \quad 1.248762$

H -4.4679994 .7155720 .985773$

C $-3.756254-1.7355402 .093163$

O $-3.731628-1.4501340 .686639$

C $-4.817297-1.989040-0.071425$

C $-4.833982-3.5215790 .038970$

C $-4.877944-3.9709891 .503329$

C $-3.826681-3.2523152 .354300$

H $-2.790125-1.3740422 .468182$

C $-4.862630-0.9525262 .819134$

H -5.762487 -1.599235 0.317246

O $-3.684643-4.079696-0.595177$

H -5.711345 -3.906202 -0.493697

H $-4.704337-5.0528931 .557852$

O $-6.167997-3.7078662 .047401$

H $-4.013826-3.4389603 .418364$

O $-2.554609-3.8280722 .049424$

C -4.6360110 .5429652 .768832$

H $-5.847047-1.1471072 .386132$

H $-4.879702-1.2415133 .876314$

H -2.898231 -3.677523 -0.188751

H $-6.816617-4.2001251 .516044$

H -1.894028 -3.4117492 .628967$

O $-5.694207 \quad 1.169532 \quad 3.363697$

O -3.6610151 .0931112 .277432$

C -5.6327432 .5989093 .393122$

H $-4.6717162 .941693 \quad 3.788620$

H -5.8015312 .9952062 .388469$

H -6.4282482 .9594454 .050762$

SCF Energy (B3LYP/6-31G**//MMFF) $=-3245.91029619$

\section{1}

MM̄FF Geometry

C -0.1033740 .0998410 .273295$

C $0.627369-0.220440-0.804907$

C $0.221652-1.160255-1.917690$

O $-0.954244-1.929085-1.561418$

C $-0.149146-0.421959-3.217790$

C $0.8981610 .530695-3.749438$
C $0.8255291 .874124-3.659404$

C $2.063356-0.124855-4.442483$

C $-0.2629012 .691705-3.018308$

C $0.2757813 .534408-1.855723$

C $-0.8571774 .236893-1.091486$

C $-0.2856515 .166305-0.020464$

C -1.3926415 .8180850 .820857$

O $-1.6685303 .229654-0.476133$

O $\quad-0.7754896 .4110531 .973819$

C -2.3420524 .7138921 .330374$

C -2.0860716 .9577360 .065581$

O -3.4603855 .2797102 .011402$

C $-2.811114 \quad 3.730960 \quad 0.229437$

C -3.5375602 .5325210 .886715$

O $-3.7158984 .405409-0.645262$

C $-0.801447-3.042199-0.800266$

O $0.248989-3.489906-0.362512$

C $-2.114961-3.657435-0.490834$

C $-3.227638-3.397484-1.196353$

C $-4.565569-3.944060-0.951286$

C $-4.772177-4.7412610 .308758$

C $-3.9360181 .414357-0.083668$

C -4.3386970 .1668730 .678235$

C $-6.158869-0.8022762 .067983$

O $-3.598738-0.8110180 .751741$

C $-7.092795-1.6740871 .281453$

C -7.491883-1.519672 0.008873

C $-8.474782-2.436921-0.662223$

C $-7.951640-3.023099-1.953131$

C $-8.415409-2.601336-3.141983$

C $-6.963359-4.165422-1.864407$

C $-5.534726-3.694255-1.857135$

N -5.5813320 .2692441 .279621$

H -1.102579 -0.3055340 .380303$

H $1.6230540 .206762-0.893763$

H $1.049283-1.852849-2.116756$

H -1.117029 $0.074512-3.084687$

H $-0.364204-1.171676-3.992757$

H $1.6328032 .462216-4.094181$

H $2.8018590 .603694-4.793300$

H $2.576005-0.815006-3.765772$

H $1.715719-0.689986-5.313575$

H $-0.6875163 .356474-3.780381$

H $-1.0868032 .067785-2.665954$

H $0.8308372 .885824-1.166926$

H $0.9817354 .279351-2.243714$

H -1.463608 4.802065 -1.809062

H $0.3565285 .933266-0.471027$

H $\quad 0.3639404 .589246 \quad 0.652792$

H -0.1216907 .0607001 .663245$

H $-1.787044 \quad 4.1436342 .088235$

H -1.355665 $7.719957-0.231532$

H $-2.809745 \quad 7.4735570 .706333$

H $-2.599686 \quad 6.622924-0.837938$

H -4.0730425 .6233881 .339020$

H -2.8822802 .0961011 .650910$

H -4.4406452 .8840191 .401451$

H -4.239016 $3.744194-1.125091$

H $-2.110363-4.3550550 .340017$

H -3.161367 -2.720760 -2.046856

H $-4.453939-4.1646781 .184950$

H -4.196249 -5.6723530 .273038$

H $-5.814169-5.0138670 .486938$

H $-4.7697351 .721067-0.724562$

H $-3.1051191 .144178-0.744524$

H $-6.702074-0.3384362 .897266$

H -5.354811 -1.417783 2.484979

H -7.489270 -2.520279 1.840637

H $-7.135006-0.678003-0.578437$

H $-9.387346-1.857427-0.854051$

H $-8.779970-3.2537340 .003918$

H -8.067346 -3.040681-4.071107

H -9.148191 -1.804502 -3.213360

H -7.084233 -4.819807 -2.738531

H $-7.214260-4.795622-1.007352$

H $-5.272262-3.107538-2.739195$

H -6.1479741 .0959781 .114463$

C 0.7236410 .2618102 .667193 
O $1.842487-0.6128702 .424841$

C $1.566819-1.8900473 .029221$

C $0.450206-1.6354434 .033761$

C $-0.376374-0.6016623 .289923$

H $1.182205-2.5390482 .233357$

C $2.852380-2.4924273 .603380$

H $-0.110195-2.5439564 .273325$

H $0.831976-1.2113414 .969500$

C -1.3355270 .1719934 .176894$

H $-0.955130-1.1238672 .519210$

C $4.031401-2.5150972 .607185$

O $3.269869-1.7235404 .739167$

H $2.654070-3.5016113 .981530$

H $4.371041-1.4887072 .431541$

O $5.125626-3.1979563 .230659$

C $3.725763-3.2055751 .265243$

C $4.939370-3.3079970 .319662$

H $2.917922-2.6744150 .751153$

O $3.254046-4.528577 \quad 1.532061$

H $5.742745-3.8784730 .801675$

H $4.630122-3.905100-0.548725$

H -2.062881 -0.504002 4.638187

H $-0.806746 \quad 0.6953164 .980492$

H -1.887347 $0.916613 \quad 3.595438$

C 0.3695951 .0052921 .376459

H $3.387142-0.8039204 .444401$

H $5.298580-2.7547624 .078962$

H $2.993201-4.9237780 .682630$

H 1.2534731 .5605851 .037791

H -0.4097951 .7469041 .579853$

H $1.075044 \quad 1.0009743 .398847$

C $4.6967910 .031040-1.313233$

O $4.416013-1.312341-0.897829$

C $5.463247-1.943724-0.154004$

C $6.714970-2.072042-1.032656$

C $7.157178-0.700503-1.556139$

C $5.9898210 .098293-2.148702$

H $3.8581680 .288032-1.970410$

C $4.6513930 .981399-0.107773$

H $5.709015-1.3327280 .720314$

O $6.464429-2.945087-2.131843$

H $7.520785-2.522062-0.441588$

H $7.925665-0.836049-2.327295$

O $7.7370990 .051240-0.493769$

H $6.289098 \quad 1.141617-2.300048$

O $5.712604-0.449443-3.442441$

C $4.2670072 .394213-0.490241$

H 3.8970310 .6162960 .600538

H 5.6088741 .0162340 .418425

H $5.709991-2.586737-2.630198$

H $8.499323-0.451882-0.159936$

H $5.051023 \quad 0.123320-3.866258$

O 4.0287393 .1102540 .648625

O $4.1970742 .823551-1.633480$

C 3.6654854 .4803970 .452372

H 4.5328775 .0466400 .101092

H $2.8376694 .565652-0.257299$

H 3.3431604 .8880371 .414267

SCF Energy (B3LYP/6-31G**//MMFF) $=-3245.91773475$

0700212

MM̄FF Geometry

C $4.8905052 .684494-1.491608$

C $3.7088682 .867048-0.881544$

C $2.4454913 .244178-1.614705$

O $1.4058702 .294171-1.279282$

C $1.9675494 .635720-1.160987$

C $0.9386885 .237625-2.098336$

C $-0.3877245 .297862-1.869231$

C $1.5051435 .839743-3.361098$

C $-1.1667914 .784324-0.689421$

C $-2.1345373 .680917-1.120630$

C $-3.1895243 .307389-0.068742$

C $-2.5935942 .870447 \quad 1.269607$

C -3.6873882 .4180372 .251323$

O $-3.9403112 .225936-0.631619$

O

C $-4.5834891 .371907 \quad 1.555784$
C -4.4381413 .6141042 .849167$

$\begin{array}{lllll}\text { O } & -5.703651 & 1.034997 & 2.370337\end{array}$

C $-5.063894 \quad 1.7934820 .147235$

C $-5.6760220 .583355-0.589006$

O

C $1.3783311 .120482-1.967574$

O $2.1514350 .759742-2.840244$

C $0.2353240 .331284-1.455725$

C $0.115161-0.962786-1.787839$

C $-0.923448-1.874161-1.303516$

C $-2.160528-1.262160-0.705936$

C $-6.1575670 .922142-2.004864$

C $-6.328666-0.333601-2.833499$

C $-5.014554-2.030590-4.105843$

O $-7.431920-0.839483-3.022925$

C $-4.712056-3.183069-3.201241$

C $-3.669996-4.010783-3.373567$

C $-3.416625-5.209068-2.505514$

C $-1.996011-5.286589-1.998111$

C $-1.156529-6.239786-2.438370$

C $-1.579145-4.327329-0.906376$

C $-0.716783-3.202768-1.412576$

N $-5.122030-0.820919-3.314281$

H $4.9579052 .812938-2.569391$

H 3.6390262 .7467890 .196523

H $2.6086483 .235197-2.699467$

H $2.8175705 .329783-1.109390$

H $1.5741674 .577582-0.140100$

H $-1.0085205 .781186-2.623915$

H $\quad 0.7346056 .310135-3.981329$

H $2.2428606 .610833-3.115701$

H $1.9929035 .073387-3.971067$

H -0.5101634 .4097440 .099303$

H -1.728125 $5.624373-0.263095$

H -2.666819 $3.982674-2.033910$

H $-1.5697202 .787169-1.410199$

H -3.8532644 .1693380 .065148$

H -1.9963883 .6699631 .722745$

H -1.9039542 .0317061 .103435$

H -2.4040132 .3663473 .740589$

H -3.9882290 .4536461 .453406$

H $-3.750346 \quad 4.251174 \quad 3.418614$

H -5.2014913 .2858463 .562921$

H -4.9154634 .2430002 .094914$

H $-5.3582230 .747747 \quad 3.232960$

H $-4.933145-0.220940-0.638601$

H $-6.5325420 .189779-0.026887$

H $\quad-6.2849382 .980387 \quad 1.135264$

H $-0.4530460 .820625-0.776843$

H $0.873515-1.412042-2.427702$

H $-2.532959-0.449940-1.339918$

H -1.951646 -0.8572540 .288568$

H $-2.986550-1.970037-0.611896$

H $-7.116306 \quad 1.452092-1.970390$

H $-5.453673 \quad 1.579844-2.527738$

H $-4.225783-1.866920-4.846777$

H -5.957965 -2.209960 -4.631153

H -5.406924 -3.366736 -2.384837

H -2.992680 -3.848911-4.208979

H $-3.653050-6.101277-3.100031$

H $-4.101975-5.232107-1.648874$

H $-0.146575-6.323546-2.050252$

H -1.457879 -6.954511 -3.196898

H $-0.991591-4.876270-0.157507$

H -2.460246 -3.977211 -0.361966

H $0.220666-3.539529-1.858352$

H -4.259190 -0.355566 -3.048191

C $6.6994220 .963055-1.244517$

O $5.795790-0.072801-0.815504$

C $6.547358-1.118964-0.172299$

C $7.988924-0.910920-0.611222$

C $8.0611520 .605705-0.644120$

H $6.481965-0.9341640 .907044$

C $5.926819-2.485743-0.491907$

H $8.705733-1.3618160 .081392$

H $8.174745-1.318513-1.611389$

C $9.241706 \quad 1.142222-1.435004$

$\begin{array}{llll}\text { H } & 8.125523 & 0.976394 & 0.387834\end{array}$ 
C $4.393354-2.518992-0.318473$

O $6.209276-2.827435-1.854819$

H $6.401805-3.2627290 .116669$

H 3.940523 -1.893240 -1.097579

O $3.931114-3.849403-0.573908$

C $3.885600-2.0617391 .062642$

C $2.368339-1.8547771 .046106$

H $4.352836-1.1119141 .332163$

O $4.224181-3.0153202 .062560$

H $2.127248-1.1277110 .263066$

H $1.865422-2.7858920 .752605$

H $10.1853240 .810326-0.990012$

H $\quad 9.219748 \quad 0.795759-2.473621$

H $9.2387172 .236674-1.444821$

C $6.1642632 .319056-0.777941$

H $5.839255-2.125539-2.417640$

H $4.279121-4.4205070 .132129$

H $5.193902-3.0628432 .109094$

H $6.9030123 .103660-0.977046$

H 5.9920992 .2965980 .305536

H $6.7368910 .918788-2.340684$

C $-0.443671-0.5680903 .103990$

O $0.388352-1.0209752 .031159$

C $1.733528-1.3900282 .369796$

C $2.456731-0.2120163 .039772$

C 1.6702520 .3195024 .240087

C 0.2079710 .5866723 .880764

H -1.333045 -0.1658022 .604939$

C $-0.889542-1.7333053 .991193$

H $1.707240-2.2450403 .054341$

O $2.663607 \quad 0.8407362 .099959$

H $3.444489-0.5397913 .382171$

H $2.140406 \quad 1.2465614 .590382$

O $1.745136-0.6351985 .294398$

H $-0.381055 \quad 0.799577 \quad 4.780294$

O $0.142139 \quad 1.752726 \quad 3.054221$

C -1.870179 -2.608193 3.245712

H $-0.060761-2.3706874 .311860$

H -1.381918 -1.375385 4.903328

H 1.7999491 .0752381 .719098

H $1.261820-0.2706626 .055372$

H 0.5109282 .4945153 .563252

O $-3.116886-2.0564203 .299150$

O $-1.560306-3.6420812 .668664$

C -4.142017 -2.776562 2.608205

H -4.280631 -3.762758 3.061019

H -5.074025 -2.212453 2.700102

H -3.893713 -2.870219 1.547499

SCF Energy (B3LYP/6-31G**//MMFF) $=-3245.91069669$

\section{3}

MM̄MF Geometry

C $2.000269-2.3045752 .515731$

C $1.650229-1.3053733 .340371$

C $2.469830-0.0756233 .662748$

O $3.723155-0.0685632 .936808$

C 1.7382401 .2183903 .266152

C 0.5921061 .5968494 .178465

C -0.7114401 .4857303 .858219$

C 0.9936702 .1872205 .505930

C -1.3091040 .9244822 .598771$

C $-1.9615202 .007074 \quad 1.730810$

C -0.9507062 .9352501 .039296$

C -1.677664 4.0191520 .241763

C $-0.6945184 .918770-0.518824$

O -0.1546902 .1423390 .154353$

O $-1.4446595 .688292-1.471758$

C $0.2750734 .029053-1.326243$

C -0.0176155 .9301210 .413739$

O $1.3119384 .810508-1.915191$

C $0.8908062 .863230-0.512978$

C $1.5952221 .873181-1.464329$

O 1.8849473 .3252030 .403392

C $4.818107-0.5828573 .552752$

O $4.859862-1.0833024 .668661$

C $6.020575-0.5064422 .690905$

C 6.0721270 .1500591 .520895

C 7.2360030 .2377700 .632525
C $8.475885-0.5172821 .038535$

C $2.2234280 .669849-0.748331$

C $2.893649-0.276725-1.728144$

C $4.210031-2.388647-1.802521$

O $2.899893-0.091854-2.942537$

C $5.656075-2.008580-1.838912$

C $6.232516-1.398320-2.885296$

C $7.672646-0.973926-2.932416$

C $7.8225490 .532049-2.890389$

C $7.7041391 .268851-4.008051$

C $8.1784241 .174629-1.566745$

C $7.1318760 .957117-0.505815$

N $3.474355-1.362535-1.086266$

H $2.961394-2.2796252 .010958$

H $0.694438-1.3791743 .855553$

H $2.674185-0.0640584 .739772$

H 1.4165891 .1476062 .222170

H 2.4571922 .0499723 .259902

H -1.448373 1.8192704 .588076

H $\quad 0.1293732 .5109636 .095729$

H $\quad 1.542877 \quad 1.454627 \quad 6.104691$

H 1.6328453 .0631005 .353955

H -0.5872800 .3480652 .013014$

H -2.083260 0.2060192 .897098

H -2.5534801 .4933880 .965843$

H -2.6603312 .5981962 .335859$

H -0.3052823 .3907091 .798023$

H -2.3230284 .6196450 .894865$

H -2.345554 $3.547513-0.491397$

H -2.121525 $6.190240-0.986112$

H $-0.2988093 .597041-2.158532$

H $-0.765216 \quad 6.5924080 .866992$

H $0.6646786 .584598-0.139667$

H 0.5366055 .4586491 .227999

H $0.8851975 .509562-2.439938$

H $0.8714351 .497982-2.199979$

H $2.3866002 .392933-2.019779$

H 1.9947354 .2824830 .290890

H $6.885851-1.0269573 .089239$

H 5.1847770 .6733231 .170203

H $8.879086-0.1167881 .975217$

H $9.280420-0.4730110 .302384$

H $8.250191-1.5807091 .177824$

H $2.9791241 .008552-0.031472$

H $1.4567210 .107388-0.203743$

H $3.794108-2.503510-2.809138$

H $4.080534-3.334386-1.267859$

H $6.248682-2.241081-0.958310$

H $5.628882-1.170951-3.761466$

H $8.114768-1.365830-3.857794$

H $8.250753-1.431424-2.120211$

H $7.8329132 .345882-3.993411$

H $7.4727200 .808744-4.963136$

H $8.2927252 .260026-1.687873$

H $9.1668360 .818399-1.264705$

H $6.187399 \quad 1.460507-0.717575$

H $3.497665-1.381612-0.071313$

C $1.014106-3.8185030 .768970$

O $0.132297-2.8620130 .144176$

C $-0.692813-3.554751-0.815200$

C $-0.139816-4.972534-0.913740$

C $0.397704-5.1875040 .484992$

H $-1.700957-3.602647-0.388433$

C $-0.715713-2.809634-2.154739$

H $-0.909208-5.697764-1.195820$

H $0.670911-5.041891-1.648740$

C $1.377363-6.3445960 .581823$

H $-0.446258-5.3687661 .163899$

C $-1.285784-1.378027-2.089856$

O $0.619708-2.729881-2.666938$

H - $1.280009-3.397133-2.888421$

H -0.618113 -0.732692 -1.508393

O $-1.276468-0.850036-3.423298$

C $-2.716025-1.284792-1.521915$

C $-3.3314150 .117553-1.703857$

H $-2.694734-1.541303-0.456773$

O $-3.529128-2.252039-2.186247$

H $-2.6068090 .874200-1.382254$ 
H $-3.5414650 .272778-2.769517$ H $0.894783-7.2820850 .286430$ H $2.243795-6.192457-0.070736$ H $1.744223-6.4636481 .605899$ C $1.150385-3.5192112 .263207$ H $1.164149-2.293568-1.989894$ H -1.893978 -1.382102 -3.953899 H $-4.410040-2.229174-1.776058$ H $1.631683-4.3642012 .768302$ H $\quad 0.156098-3.3869542 .707156$ H $1.986770-3.7313440 .267918$ C $-6.886612-0.587693-0.772749$ O $-5.631297-0.570797-1.466580$ C $-4.6416310 .300726-0.910394$ C $-5.1442801 .751970-0.959097$ C $-6.4980471 .900330-0.258745$ C $-7.4959600 .823706-0.703258$ H -7.540785 -1.200665 -1.406207 C $-6.742766-1.2986800 .576745$ H -4.4520320 .0130380 .130146$ O $-5.2573972 .192816-2.310727$ H -4.409995 $2.400761-0.471197$ H $-6.9048352 .894615-0.480353$ O H $-8.3747330 .816631-0.049340$ O $\quad-7.960095 \quad 1.146425-2.018845$ C -8.075977 -1.7407721 .124982$ H $-6.098145-2.1817170 .479610$ H $-6.253382-0.6760601 .331519$ H $-5.8544851 .580589-2.773992$ H $-7.152913 \quad 1.943217 \quad 1.579957$ H -8.396527 2.014178 -1.971006 O $-8.480500-2.884686 \quad 0.503145$ O $-8.689422-1.1334191 .993011$ C $-9.746099-3.4001300 .927468$ H $-9.951001-4.3103590 .357360$ H $-9.715237-3.652057 \quad 1.991824$ H -10.538236 -2.673080 0.724514

SCF Energy (B3LYP/6-31G**//MMFF)= -3245.91777894

\section{4}

MM̄FF Geometry

C $0.973601-3.2250631 .605079$

C $0.027606-3.3174172 .550617$

C $-0.640700-2.1057613 .139405$

O $-2.075003-2.2539463 .013781$

C $-0.342262-1.9322004 .637644$

C $1.106388-1.6431724 .964360$

C $1.708683-0.4564394 .753672$

C $1.867114-2.7818175 .590392$

C 1.1023530 .7779294 .143080

C 1.8723111 .1919442 .881708

C 1.2062122 .2996972 .049365

C 0.8560603 .5420352 .866776

C 0.1614814 .6098632 .011030

O $0.024633 \quad 1.762968 \quad 1.445698$

O -0.3835055 .5754122 .923766$

C -1.0118963 .9589931 .239913$

C 1.1673775 .3619161 .129394

O

C -0.6178982 .6528290 .510926$

C $-1.8317561 .864561-0.039279$

O $0.2484932 .960101-0.582884$

C $-2.638599-1.8056731 .860204$

O $-2.058636-1.3796820 .874159$

C $-4.106852-1.8837612 .016378$

C $-4.871928-1.0800191 .263603$

C $-6.332482-0.9962611 .275300$

C $-7.081773-1.9900782 .122129$

C $-2.6763922 .583116-1.094125$

C $-3.5708681 .640499-1.885354$

C $-5.3700851 .688958-3.618552$

O $-3.5227490 .417994-1.786990$

C $-6.7204801 .707388-2.973878$

C $-7.4658280 .609133-2.775748$

C $-8.8359560 .632092-2.160256$

C $-8.954237-0.262900-0.946228$

C $-9.610686-1.433604-1.011998$
C $-8.3941920 .238580 \quad 0.365991$

C $-6.925392-0.0515850 .515034$

N -4.414192 $2.337874-2.739992$

H $1.274512-2.2399751 .255585$

H $-0.277843-4.2930812 .918987$

H $-0.333335-1.1968012 .608303$

H $-0.681719-2.8214635 .187189$

H $-0.971317-1.1254475 .038644$

H $2.752386-0.3498605 .046760$

H $2.908230-2.5189465 .805384$

H $1.398353-3.0756806 .535343$

H $1.877850-3.6494614 .923302$

H 0.0425410 .6417203 .911195

H 1.1584411 .5784724 .889832

H $2.877546 \quad 1.522626 \quad 3.173047$

H 1.9974560 .3112322 .237202

H 1.9132422 .5577891 .252264

H 1.7495063 .9544703 .351833

H 0.1677613 .2736403 .679064

H -0.7721956 .2982272 .403142$

H -1.8023793 .7219691 .965390$

H 1.9207785 .8599451 .751572

H 0.6776476 .1583530 .558262

H 1.6938654 .7116290 .427993

H -1.922249 5.6397310 .832566

H $-1.4462870 .930690-0.471320$

H -2.4766331 .5507790 .792284$

H $0.2040542 .227163-1.218538$

H -4.507914 -2.568448 2.754162

H $-4.377316-0.3978630 .575139$

H $-6.793621-3.0144901 .860315$

H -8.165557 -1.937188 1.998451

H $-6.869178-1.8264883 .184175$

H $-3.3180703 .326083-0.607721$

H -2.033065 $3.097001-1.817346$

H $-5.0323170 .672121-3.843856$

H $-5.3967012 .262652-4.550152$

H $-7.1122252 .676789-2.674427$

H -7.083760 $-0.358100-3.094458$

H $-9.5499650 .316231-2.932142$

H $-9.1329451 .651901-1.884842$

H $-9.726557-2.065745-0.138063$

H -10.042501 -1.788422 -1.941862

H -8.970148 -0.1669721 .204731$

H -8.5440871 .3237350 .436093$

H $-6.2882590 .619211-0.061896$

H -4.410304 $3.353607-2.717022$

C $1.524531-4.483236-0.525102$

O $2.001902-3.275940-1.154566$

C $1.106880-2.939960-2.232373$

C $0.237394-4.172549-2.456070$

C $0.101855-4.696560-1.039342$

H $0.466099-2.126764-1.872446$

C $1.896762-2.489319-3.464275$

H $-0.723283-3.925183-2.917786$

H $0.739506-4.911937-3.091287$

C $-0.374914-6.135893-0.962254$

H $-0.607794-4.056243-0.500398$

C $2.761171-1.227700-3.249138$

O $2.767417-3.551402-3.875552$

H $1.209347-2.324895-4.302177$

H $\quad 3.593517-1.451703-2.572367$

O $3.348825-0.887882-4.511832$

C $1.981675-0.004641-2.731639$

C $2.7492221 .328134-2.797460$

H $1.660137-0.188987-1.700316$

O $\quad 0.794050 \quad 0.147935-3.517065$

H $2.9502251 .594791-3.843135$

H $2.0665292 .105331-2.435064$

H $-1.367720-6.236561-1.412819$

H $0.303963-6.813408-1.490991$

H -0.440989 -6.468802 0.078292

C $1.683433-4.397474 \quad 0.994514$

H $3.348774-3.760249-3.124056$

H $3.833940-1.670443-4.825222$

H $0.270807 \quad 0.866862 \quad-3.123712$

H $2.747918-4.2839721 .231333$

H $1.354513-5.3370331 .453530$ 
H $2.174869-5.290261-0.888467$

C $4.892851 \quad 0.931362 \quad 0.242865$

$\begin{array}{llll}\text { O } & 3.767241 & 0.980216 & -0.645057\end{array}$

C $4.0544431 .374846-1.989505$

C $4.6839012 .779484-2.018456$

C $5.9067062 .865653-1.099239$

C $5.613677 \quad 2.2905550 .289605$

H 4.4614050 .7468001 .234208

C $5.836652-0.237591-0.081892$

H $4.7519880 .658165-2.434172$

O $3.7181843 .745988-1.605949$

H $4.9660123 .017284-3.051014$

H $6.2129273 .913609-0.992350$

O $6.9954542 .153931-1.678769$

H $6.5453192 .198123 \quad 0.860213$

$\begin{array}{lllll} & \text { O } & 4.785883 & 3.224292 & 0.989050\end{array}$

C $5.187371-1.5858590 .143240$

H $6.171594-0.209360-1.121714$

H $6.715261-0.191176 \quad 0.571810$

H $4.1355184 .622326-1.662790$

H $7.1808232 .554676-2.545143$

H 4.6482862 .8803131 .888001

O $6.034575-2.559720-0.305271$

O $4.091115-1.7669010 .653335$

C $5.569590-3.904640-0.158321$

H $6.379027-4.577759-0.452956$

H $5.307326-4.1097550 .883888$

H $4.713919-4.077098-0.816480$

SCF Energy (B3LYP/6-31G**/MMFF) $=-3245.89575694$

\section{5}

MM̄FF Geometry

C $-2.766436-0.675313 \quad 3.478804$

C $-1.639283-1.4052933 .472658$

C $-0.314072-1.0448834 .111592$

$\begin{array}{llll}\text { O } & 0.730268 & -1.224406 & 3.122418\end{array}$

C $-0.186693 \quad 0.3945884 .643849$

C $1.1550780 .673335 \quad 5.299995$

C 2.1120651 .4477234 .750321

C 1.3579970 .0616126 .660675

C 2.0227952 .1155723 .405693

C 3.3695562 .6884342 .939142

C 3.3603563 .2177951 .493119

C $2.2831014 .280076 \quad 1.255270$

C $2.2145154 .731903-0.209284$

$\begin{array}{lllll}\text { O } & 3.173872 & 2.099702 & 0.617739\end{array}$

O $\quad 0.987107 \quad 5.463459-0.357028$

C $2.136466 \quad 3.477026-1.105115$

C $3.3448905 .707063-0.554621$

O $2.209808 \quad 3.832979-2.483922$

C $3.2206882 .424888-0.777502$

C $3.0227261 .121735-1.586178$

O $4.5007052 .955762-1.143278$

C $1.352973-2.4326753 .078548$

O $1.061811-3.4358793 .710339$

C $2.475006-2.3232962 .121199$

C $3.056891-3.4427871 .668037$

C $4.157466-3.5199240 .707989$

C $4.839656-2.241200 \quad 0.304254$

C $1.8403230 .252690-1.147104$

C $1.657094-0.940116-2.071577$

C $0.599335-3.189098-2.187202$

O $2.083923-0.958976-3.224030$

C $1.684594-4.196879-1.974586$

C $2.671381-4.421416-2.856100$

C $3.760881-5.442236-2.677134$

C $5.070547-4.819781-2.241448$

C $5.825224-4.121925-3.107200$

C $5.536654-5.045701-0.818596$

C $4.492250-4.7283880 .211747$

N $0.923799-1.961788-1.483496$

H $-2.756507 \quad 0.2988313 .962126$

H -1.655291 -2.390292 3.014396

H $-0.155960-1.7403474 .945761$

H $-0.971756 \quad 0.598415 \quad 5.383199$

H $-0.355992 \quad 1.0967923 .819687$

H 3.0275691 .6174455 .313743

H 2.3051370 .3694867 .116549
H $\quad 0.5536030 .3659237 .338120$

H $1.365097-1.0306146 .595362$

H 1.6575901 .4011352 .659821

H 1.2971632 .9315053 .487769

H 3.6732473 .4963623 .616704

H $4.131170 \quad 1.9006263 .012251$

H 4.3532933 .6423061 .302337

H 2.4300445 .1393111 .921251

H 1.2987213 .8701041 .507653

H $0.940903 \quad 5.793557-1.270091$

H $1.1484603 .027498-0.948530$

H 3.2601926 .6169800 .051702

H $3.2805016 .035043-1.597992$

H $4.3397205 .289772-0.386018$

H $1.4322794 .378719-2.689838$

H $2.9574921 .360597-2.655279$

H $3.9283020 .505939-1.493833$

H $5.1255612 .218592-1.241045$

H $2.781194-1.3318591 .808964$

H $2.672464-4.4027752 .011356$

H $5.223517-1.7137011 .184444$

H $4.140696-1.584748-0.221358$

H $5.690853-2.398958-0.362834$

H $2.002350-0.104664-0.125352$

H $0.9067020 .821099-1.158533$

H $0.452220-2.970599-3.250356$

H $-0.342951-3.568787-1.782094$

H $1.640322-4.780005-1.058659$

H $2.704420-3.833724-3.771125$

H $3.903523-5.965977-3.631572$

H $3.453691-6.222538-1.970060$

H $6.773407-3.686975-2.809247$

H $5.510081-3.970889-4.134485$

H $6.456825-4.489881-0.601569$

H $5.809703-6.103779-0.711277$

H $3.937191-5.6039860 .550345$

H $0.655838-1.887228-0.506517$

C $-4.223616-2.2980002 .043790$

O $-3.769277-1.962700 \quad 0.713904$

C $-4.749419-2.424061-0.237696$

C $-5.596926-3.4423930 .509212$

C $-5.662193-2.8000231 .880174$

H $-5.384035-1.564822-0.484362$

C $-4.072086-2.955955-1.504229$

H $-6.580685-3.5835560 .051907$

H $-5.101065-4.4184170 .567279$

C $-6.111448-3.7533832 .974639$

H $-6.355884-1.9490931 .839784$

C -3.184557-1.920271-2.225901

O $-3.245971-4.074550-1.157739$

H $-4.828963-3.343243-2.195707$

H $-2.317749-1.683599-1.599377$

O $-2.672354-2.526213-3.418844$

C $-3.920446-0.628700-2.624822$

C $-3.0651690 .367574-3.429711$

H $-4.297006-0.128792-1.727531$

O $-5.061166-0.975601-3.414508$

H $-2.773938-0.081300-4.387825$

H $-3.7065291 .214108-3.705183$

H -7.122589 -4.121428 2.772301

H $-5.447058-4.6208653 .048951$

H $-6.122149-3.2534743 .948244$

C $-4.123962-1.0588412 .940729$

H -2.605642 $-3.770834-0.491349$

H $-2.269771-3.374349-3.165939$

H $-5.543912-0.154649-3.610863$

H $-4.776288-1.186442 \quad 3.813174$

H $-4.501319-0.1923192 .382245$

H -3.592909 -3.1165482 .407531$

C $-2.8881032 .427015-1.197558$

O $-2.106967 \quad 1.236636-1.327433$

C $-1.8157410 .870890-2.680327$

C $-1.0634372 .007105-3.389745$

C $-1.772572 \quad 3.354952-3.253430$

C $-2.1490293 .628512-1.794553$

H $-3.8357152 .291249-1.734106$

C -3.2834562 .5993310 .274153$

H -1.126976 $0.020652-2.625217$ 
O $-0.880991 \quad 1.709325-4.770361$

H $-0.0631452 .103161-2.952495$

H -2.651513 $3.402011-3.907311$

O $-0.877303 \quad 4.374071-3.705654$

H -1.240024 $3.849017-1.224090$

O $-2.991992 \quad 4.774912-1.724169$

C $-2.1282582 .919968 \quad 1.197289$

H $-4.029903 \quad 3.3993270 .348821$

H -3.7417601 .6782110 .652794$

H $-0.3970750 .867461-4.825509$

H $-1.3700865 .211750-3.721216$

H $-2.4689755 .543167-2.008473$

O -2.5392183 .8310802 .127360$

O $-1.017728 \quad 2.4115841 .128503$

C $-1.556508 \quad 4.2358913 .084376$

H -1.2236153 .3766273 .672166$

H $-0.712954 \quad 4.7157812 .580962$

H -2.018736 4.9617173 .758785

SCF Energy (B3LYP/6-31G*//MMFF) $=-3245.90328114$

\section{6}

MM̄FF Geometry

C 3.4363212 .7215201 .662294

C 2.3252931 .9858581 .504672

C 1.5990831 .2979672 .630354

$\begin{array}{lllll}\text { O } & 0.221084 & 1.720846 & 2.533149\end{array}$

C $1.681326-0.2250762 .439144$

C $1.224071-1.0214813 .641317$

C $0.108647-1.7751313 .685830$

C $2.160228-0.9970324 .823934$

C $-0.931709-1.9673932 .613339$

C $-2.338650-2.0154453 .226751$

C $-3.469538-2.4295042 .270147$

C $-3.286737-3.842961 \quad 1.707143$

C $-4.424401-4.2355180 .751920$

O $-3.582146-1.4737251 .207731$

$\begin{array}{llll}O & -4.004456 & -5.397596 & 0.019091\end{array}$

C -4.621098 -3.102281 -0.277689

C $-5.694407-4.6409991 .508932$

O $-5.762084-3.351914-1.095280$

C $-4.725038-1.6997620 .363598$

C $-4.802665-0.575568-0.692934$

O $-5.944631-1.6255491 .116707$

C -0.4734651 .9656033 .671997$

$\begin{array}{lllll}\text { O } & -0.079650 & 1.805497 & 4.818947\end{array}$

C -1.8224452 .5081123 .376528$

C -2.282521 2.6987312 .130458

C -3.573533 3.2772721 .750700

C $-4.479928 \quad 3.7698432 .845708$

C -3.575416 $-0.409950-1.591280$

C $-3.7931560 .699917-2.606004$

C -2.565919 $2.389926-3.945633$

O $-4.9109951 .091637-2.934522$

C -2.619440 $3.573376-3.031088$

C -3.685106 4.379131-2.903225

C $-3.7698385 .460160-1.861875$

C $-4.9069595 .251982-0.876493$

C $-5.739014 \quad 6.266308-0.584204$

C $-5.105278 \quad 3.893819-0.228355$

C $-3.865121 \quad 3.3612870 .436683$

N $-2.6002591 .193705-3.119427$

H 3.8548062 .8518002 .656576

H 1.9120361 .8566370 .507087

H 2.0289261 .6077253 .590124

H $2.719937-0.5150472 .233838$

H $1.112241-0.5124631 .548508$

H $-0.071206-2.3476764 .595624$

H $1.833743-1.6659115 .627482$

H $3.162915-1.3163144 .520747$

H 2.2281890 .0099975 .245448

H $-0.898844-1.1737391 .861387$

H - $0.701824-2.9127732 .113092$

H -2.347871 -2.704115 4.081756

H -2.568659 -1.016732 3.622189

H $-4.390684-2.3927182 .864744$

H -3.195891 -4.5752262 .519031$

H -2.349604 -3.9052521 .142044$

H $-3.794291-6.0970470 .661391$
H -3.751339 -3.124014 -0.945535

H $-5.495367-5.5057592 .153558$

H $-6.482092-4.9593450 .817160$

H $-6.091212-3.8451522 .142336$

H $-5.652866-4.236997-1.483168$

H $-5.704422-0.715840-1.303657$

H $-4.9596800 .379943-0.172546$

H $-6.168081-0.6896361 .251403$

H -2.4126022 .7655434 .250086$

H -1.6500062 .4164591 .290249$

H -3.9598584 .4964423 .480025$

H $-4.8152502 .934791 \quad 3.470327$

H $-5.373778 \quad 4.2751002 .472477$

H -2.699236 $-0.170907-0.979031$

H $-3.372319-1.324392-2.156032$

H $-3.407742 \quad 2.370764-4.645471$

H $-1.6316762 .385422-4.514463$

H -1.756231 $3.730712-2.388785$

H $-4.5577894 .221423-3.532157$

H $-3.8820636 .418292-2.385015$

H $-2.8315175 .529831-1.296698$

H -6.5599296 .1426130 .114383$

H -5.618324 $7.245803-1.035034$

H $-5.4117613 .175931-0.999569$

H $-5.946367 \quad 3.9155310 .472604$

H $-3.1319812 .971425-0.268363$

H $-1.7229770 .875125-2.718259$

C 5.4601812 .7222310 .115283

$\begin{array}{llll}\text { O } & 5.214783 & 1.353599 & -0.266048\end{array}$

C $6.244090 \quad 0.528488 \quad 0.307288$

$\begin{array}{llll}\text { C } & 7.364269 & 1.482383 & 0.703449\end{array}$

C $6.5653672 .685234 \quad 1.171017$

H 5.8253010 .0899851 .220876

C $6.650127-0.582153-0.665747$

H 8.0109851 .0660331 .481566

H $7.9906461 .751190-0.155194$

C $7.3788393 .964608 \quad 1.256496$

H 6.1527002 .4602672 .162010

C $5.492401-1.498324-1.122093$

O $7.225594 \quad 0.003927-1.839986$

H $7.444628-1.188392-0.215063$

H $4.841452-0.963056-1.821846$

O $6.065734-2.585372-1.859049$

C $4.652234-2.079784 \quad 0.027191$

C $3.676262-3.201050-0.365036$

H $4.088754-1.2721620 .503254$

O $5.523516-2.6152411 .029719$

H $4.236812-4.076564-0.717150$

H $3.186934-3.5233870 .564490$

H 8.1933303 .8545661 .979892

H 7.8217894 .2247980 .289365

H 6.7523064 .8029041 .577223

C 4.1483933 .3977310 .522859

H $6.5628470 .605075-2.221730$

H $6.587694-2.200063-2.583783$

H $6.062833-3.3075780 .610021$

H $3.4869523 .418206-0.351936$

H 4.3341374 .4427790 .795969

H $5.8313103 .223716-0.788497$

C $0.784820-1.235700-1.710179$

O $1.854889-1.716904-0.882929$

C $2.607636-2.818050-1.401758$

C $1.692149-4.017956-1.713859$

C $0.505672-3.618801-2.593371$

C $-0.194867-2.369448-2.054402$

H $0.252484-0.510358-1.081162$

C $1.296948-0.497274-2.952541$

H $3.111180-2.504120-2.321325$

O $1.201282-4.602536-0.507240$

H $2.284795-4.788619-2.220754$

H $-0.207635-4.451467-2.628997$

O $0.974957-3.387079-3.917272$

H $-0.939243-2.013295-2.775551$

O $-0.901978-2.741596-0.869932$

C $1.8399390 .860365-2.580851$

H $2.095607-1.026121-3.479579$

H $0.487482-0.354202-3.678822$

H $0.732024-3.910370-0.010598$ 
H $\quad 0.204004-3.165449-4.466884$

H $-1.346451-1.949708-0.524725$

O $0.918691 \quad 1.825454-2.857584$

O $2.9455571 .036033-2.087684$

C $1.3119323 .172492-2.573654$

H $1.1390113 .384466-1.515338$

H $2.3590403 .351422-2.836830$

H $0.6895953 .841255-3.173919$

SCF Energy (B3LYP/6-31G**//MMFF) $=-3245.91654009$

\section{$07 \quad 00217$}

MM̄FF Geometry

C $1.044748-0.5584401 .183868$

C $-0.162342-0.3917010 .624516$

C $-0.450433-0.612278-0.835268$

O $-1.485955-1.623372-0.901629$

C $-0.9959280 .656899-1.511563$

C $0.0582271 .675498-1.878081$

C $0.3020032 .810375-1.194286$

C $0.8215251 .376744-3.142902$

C $-0.322003 \quad 3.2701730 .093313$

C $-1.2423494 .485163-0.086612$

C $-2.5780814 .149404-0.769980$

C $-3.4312015 .410349-0.929832$

C $-4.8221735 .096329-1.500696$

O -3.2636953 .2009400 .053146$

O $-5.6258986 .271344-1.315451$

C $-5.4534163 .952982-0.676777$

C $-4.764544 \quad 4.850033-3.012582$

O $-6.6940653 .542647-1.247646$

C $-4.5131682 .741546-0.476927$

C -5.1270401 .7559920 .547016$

O $-4.3496732 .076531-1.731260$

C $-1.447106-2.494032-1.943334$

O $-0.603196-2.545595-2.827150$

C $-2.560731-3.464090-1.866045$

C $-3.757436-3.150872-1.345445$

C $-4.914232-4.045434-1.244702$

C $-4.689373-5.500995-1.564282$

C -4.1745580 .6318940 .980094$

C $-4.798518-0.2896762 .013012$

C $-4.318723-2.3744253 .291558$

O $-5.915609-0.1075762 .489385$

C $-5.012599-3.4757782 .554079$

C $-6.337579-3.6774472 .609848$

C $-7.065753-4.7546241 .857679$

C $-7.931616-4.1869920 .753706$

C $-9.152147-3.691370 \quad 1.019658$

C $-7.410743-4.253374-0.666216$

C $-6.099631-3.532793-0.849887$

N -3.947910 -1.336807 2.347383

H $1.879065-0.8837590 .568061$

H $-0.995574-0.0808101 .249875$

H $0.451590-0.972326-1.344865$

H -1.773597 $1.102536-0.883416$

H - $-1.5351710 .379391-2.427963$

H $1.0643993 .485198-1.581506$

H $1.5627592 .148960-3.374673$

H $1.3556670 .425914-3.055325$

H $0.1350621 .311741-3.993402$

H -0.8519902 .4643950 .608231$

H $\quad 0.4982503 .5551370 .764988$

H $-1.448775 \quad 4.8875770 .913915$

H $-0.7203205 .269551-0.648618$

H -2.375758 $3.701510-1.749049$

H -2.917411 $6.152045-1.554135$

H -3.561605 5.8870380 .052117

H $-6.5298816 .058526-1.605378$

H -5.6992584 .3692640 .309728$

H $-4.3688885 .734235-3.526987$

H $-5.7654584 .688413-3.427516$

H -4.136365 $3.999413-3.285163$

H $-6.4947362 .990700-2.023007$

H -5.4181612 .3108661 .448663$

H -6.0378791 .3062930 .131767$

H -4.145695 $1.141501-1.576207$

H -2.359592 -4.431670 -2.313395

H $-3.916774-2.141767-0.970904$
H $-5.543495-6.139583-1.332729$

H $-3.849901-5.897929-0.981903$

H $-4.469822-5.629830-2.629898$

H -3.8838210 .0230870 .119277$

H $-3.267678 \quad 1.0572331 .424182$

H -4.952387 -1.942066 4.073502

H -3.403059 -2.750400 3.757900

H $-4.394294-4.1285081 .943347$

H -6.946168 -3.017693 3.224914

H -7.689550 -5.309404 2.570604

H $-6.369155-5.4953821 .445784$

H $-9.790259-3.3025780 .233246$

H -9.539005 -3.662695 2.033156

H $-8.130098-3.794723-1.357268$

H $-7.358770-5.304816-0.959813$

H $-6.158351-2.463432-0.641276$

H -3.078225 -1.451528 1.834798

C 2.2839840 .8216642 .892990

O 3.5867320 .5019922 .361374

C 4.5361650 .5003763 .438719

C 3.9500961 .4552034 .466850

C 2.4802441 .0888204 .391501

H $4.538156-0.5160653 .853194$

C 5.9480500 .8413162 .946199

H 4.3775851 .3133795 .463724

H 4.0887342 .5027784 .176023

C 1.5546402 .1604854 .942566

H 2.3229440 .1639504 .962967

C 6.3936120 .0729331 .683878

O 6.0032372 .2397032 .638980

H $6.659430 \quad 0.6805413 .765516$

H 5.9140130 .5151810 .805054

O $7.802866 \quad 0.269019 \quad 1.516858$

C $6.123022-1.4398051 .711660$

C $6.698615-2.2295570 .521585$

H $5.043227-1.6183181 .746301$

O $6.665043-2.0024542 .909622$

H $7.790766-2.3004410 .607290$

H $6.334942-3.2598820 .631111$

H 1.7607862 .3392456 .002922

H 1.6789043 .1099354 .410791

H $0.507700 \quad 1.8552504 .847086$

C $1.322116-0.3463062 .645022$

H 6.9365762 .4769742 .508686

H 7.9472371 .2069371 .307422

H $7.618638-1.8113032 .917881$

H $0.385155-0.173013 \quad 3.188000$

H $1.756182-1.2759703 .033815$

H $1.939375 \quad 1.7226772 .370658$

C $4.416081-0.908323-2.145355$

O $4.916028-1.472397-0.923427$

C $6.329591-1.684033-0.870438$

C $6.779109-2.635144-1.993344$

C $6.304699-2.143715-3.364306$

C $4.816432-1.774221-3.351731$

H $3.323910-0.958687-2.049792$

C $4.8124940 .569159-2.311193$

H $6.839445-0.723375-0.995150$

O $6.255507-3.940434-1.754080$

H $7.873156-2.709247-1.975421$

H $6.480806-2.930539-4.107855$

O $7.087307-1.014036-3.738827$

H $4.539872-1.256536-4.277684$

O $4.036999-2.972179-3.295723$

C $4.1705551 .460133-1.270546$

H $5.8948240 .702241-2.237029$

H $4.4836190 .936218-3.290134$

H $6.589977-4.524485-2.455640$

H $6.787868-0.728179-4.618705$

H $4.255050-3.501236-4.081801$

O $4.8352002 .651943-1.227202$

O $3.1938861 .163334-0.598566$

C $4.3343853 .608238-0.286902$

H 4.1452783 .1459100 .686630

H $5.0907584 .387278-0.160083$

H $3.4210754 .063816-0.678843$

SCF Energy (B3LYP/6-31G**//MMFF) $=-3245.89865792$ 
0700218

MM̄FF Geometry

C $-2.582598-1.9843174 .043481$

C -1.844122 $-0.909678 \quad 3.729012$

C $-0.364227-0.7683533 .968536$

O $0.273456-0.6400682 .676648$

C $-0.100547 \quad 0.521896 \quad 4.767830$

C $1.366612 \quad 0.871716 \quad 4.916542$

C 1.9298291 .9949394 .427594

C $2.203041-0.1209925 .680251$

C 1.2537783 .1101943 .675869

C 1.2071272 .9184382 .151793

C 2.5750653 .0543651 .464753

C $2.4285992 .979188-0.056968$

C $3.7899403 .041979-0.767727$

O 3.4225341 .9952331 .912370

O $3.5664512 .648787-2.130646$

C $4.7470802 .012223-0.124848$

C $4.3279544 .476779-0.818631$

O $6.0645962 .147394-0.651227$

C 4.7662162 .0639761 .416815

C 5.5510760 .8946852 .058064

O $5.407408 \quad 3.276444 \quad 1.832234$

C $0.637069-1.7862392 .040437$

O $0.536796-2.9275562 .460814$

C $1.134715-1.4079390 .699262$

C $1.203189-2.341945-0.260961$

C $1.575591-2.117105-1.659047$

C $2.120509-0.763306-2.026902$

C $5.032776-0.5081501 .720701$

C $5.796385-1.1936180 .599212$

C $5.795216-3.293098-0.743453$

O $6.822499-0.7375050 .102431$

C $5.233192-2.982012-2.094183$

C $4.680335-3.907694-2.892527$

C $4.160952-3.620740-4.271803$

C $2.726605-4.060422-4.458534$

C $2.432573-5.225271-5.060588$

C $1.635332-3.107443-4.028440$

C $1.403027-3.123202-2.541890$

N $5.214021-2.4034310 .244288$

H -2.119793 -2.843549 4.520306

H -2.330792 -0.0695173 .236518$

H $0.040056-1.6318734 .510624$

H $-0.5404330 .429203 \quad 5.769803$

H $-0.635943 \quad 1.3460374 .284274$

H 2.9957122 .1472824 .592036

H $3.221306 \quad 0.242305 \quad 5.855847$

H $1.754143-0.3225146 .658354$

H $2.281750-1.0636445 .129866$

H 0.2335843 .2542254 .048130

H 1.7735294 .0477183 .910803

H $\quad 0.771814 \quad 1.9383591 .924154$

H $\quad 0.5287743 .6751691 .738219$

H $3.0085854 .017477 \quad 1.758977$

H $1.7668443 .772836-0.425819$

H $1.9385672 .033747-0.327837$

H $4.4327232 .608992-2.571473$

H $4.3964091 .025156-0.442805$

H $3.6195455 .130434-1.341739$

H $5.2604814 .531494-1.391117$

H 4.5052654 .9028440 .171062

H $6.4776382 .916158-0.222549$

H 6.6214030 .9966601 .842858

H 5.4643051 .0084413 .148518

H 5.7145203 .1641922 .747159

H $1.369707-0.364600 \quad 0.523018$

H $\quad 0.910818-3.362070-0.015117$

H $2.951714-0.497420-1.366678$

H $1.3429950 .002687-1.940101$

H $2.517355-0.713143-3.042895$

H $5.158947-1.1442902 .606461$

H $3.965318-0.4913611 .480398$

H $5.573965-4.317591-0.427841$

H $6.881753-3.159568-0.757372$

H $5.312578-1.955383-2.444020$

H $4.627980-4.939455-2.551565$

H $4.808180-4.146299-4.986287$
H $4.251293-2.556113-4.521313$

H $1.405292-5.533518-5.224604$

H $3.213152-5.899204-5.397838$

H $0.685574-3.386582-4.504616$

H $1.854366-2.109056-4.417296$

H $1.001045-4.069522-2.176666$

H $4.331281-2.6752300 .667552$

C $-4.380900-3.0729312 .641609$

O $-3.637583-2.7521911 .448549$

C $-4.557449-2.5035130 .374525$

C $-5.811654-3.2781500 .753999$

C $-5.864049-3.0375032 .250974$

H $-4.779993-1.4293900 .386529$

C -3.933807 -2.879368 -0.974282

H $-6.701014-2.9166460 .229206$

H $-5.706764-4.3503500 .553593$

C $-6.715516-4.0457503 .003577$

H $-6.277487-2.0347902 .423884$

C $-2.505463-2.351919-1.226090$

O $-3.864576-4.310488-1.075996$

H $-4.599534-2.562488-1.784808$

H -1.815101-2.890169 -0.564626

O $-2.131810-2.703090-2.565169$

C $-2.255937-0.842461-1.064053$

C $-3.1518540 .044669-1.935196$

H $-1.217374-0.653368-1.355388$

O $-2.385696-0.445500 \quad 0.294818$

H $-3.045790-0.224596-2.993437$

H -4.206155 -0.138797 -1.690339

H -7.753911 -4.006194 2.658962

H $-6.351094-5.0680852 .856232$

H -6.706256 -3.835781 4.077810

C $-4.055026-2.0589983 .744149$

H $-3.330694-4.632321-0.329180$

H -2.247082 -3.664459 -2.655933

H $-1.832989-1.0393840 .829443$

H -4.589467 -2.321944 4.664294

H -4.414292 -1.066301 3.442709

H $-4.070223-4.0797232 .945913$

C $-3.8347353 .649492-2.554890$

O $-3.8214722 .218628-2.631980$

C $-2.9044481 .556878-1.750378$

C $-1.4627632 .001287-2.046424$

C -1.328270 $3.527154-2.010662$

C $-2.4319124 .220824-2.816677$

H $-4.4865413 .966752-3.379437$

C $-4.4624074 .146312-1.247845$

H $-3.1621871 .815835-0.718024$

O $-1.0746811 .516134-3.329972$

H $-0.7865621 .561237-1.304587$

H $-0.3482793 .810497-2.414076$

O $-1.3772353 .951452-0.651386$

H $-2.4319995 .296994-2.610197$

O $-2.1413194 .076348-4.210393$

C $-5.9108673 .727668-1.161789$

H $-3.9455343 .788965-0.352555$

H -4.436421 $5.241377-1.198161$

H $-0.1454571 .766631-3.469100$

H -1.252601 $4.915670-0.640559$

H $-2.2006463 .130492-4.429883$

O $-6.0129502 .537410-0.503696$

O $-6.8348954 .368039-1.646060$

C $-7.3364602 .006939-0.387333$

H -7.9678252 .6889930 .189932$

H $-7.7627341 .830516-1.379522$

$\mathrm{H}-7.2742531 .0522370 .142005$

SCF Energy (B3LYP/6-31G**//MMFF)= -3245.92146747

\section{9}

MM̄FF Geometry

C $0.447643-2.621309-1.865633$

C $-0.610117-3.445087-1.902381$

C $-1.610156-3.591869-0.787842$

O $-2.871504-3.139715-1.336978$

C $-1.736472-5.053791-0.321466$

C $-0.501887-5.6040280 .359282$

C $-0.252157-5.4850461 .677776$

C $0.434827-6.395649-0.516820$ 
C $-1.083698-4.766100 \quad 2.706751$ C $-0.217019-4.0450903 .750708$

C $0.424447-2.7461813 .233289$

C $1.547540-2.3136424 .179453$

C $2.139282-0.9546223 .790270$

O $-0.598611-1.7433003 .192755$

O $2.950882-0.496994 \quad 4.882700$

C 0.9874660 .0539343 .629379

C $3.065275-1.0553572 .574210$

O $1.474434 \quad 1.301654 \quad 3.142294$

C $-0.165871-0.4542562 .731334$

C -1.3645750 .5246052 .845457$

$\begin{array}{lllll}\text { O } & 0.278851 & -0.506274 & 1.377664\end{array}$

C -3.854659 -2.788003 -0.467158

O $-3.862329-2.946350 \quad 0.742397$

C $-4.905155-2.097185-1.247187$

C $-5.791651-1.325574-0.600998$

C $-6.817364-0.481754-1.217957$

C $-6.962332-0.542924-2.715715$

C $-2.6286950 .076212 \quad 2.105209$

$\begin{array}{llll}C & -2.747706 & 0.701537 & 0.729985\end{array}$

C $-3.5053452 .780593-0.424583$

O $-2.3817050 .106009-0.280142$

C $-4.8849713 .356295-0.365288$

C $-5.867803 \quad 3.010174-1.210192$

C $-7.260366 \quad 3.570025-1.157861$

C $-8.2101682 .738663-0.321311$

C $-8.7595843 .241751 \quad 0.797220$

C $-8.5786151 .351432-0.809259$

C $-7.5442910 .327614-0.419258$

N $-3.287599 \quad 1.977157 \quad 0.764578$

H $0.606654-1.993560-0.993580$

H $-0.770021-4.061277-2.784575$

H $-1.340695-2.9394830 .053091$

H -2.008744 -5.693708 -1.172580

H $-2.595365-5.1564760 .353195$

H $0.638553-5.9739822 .070398$

H $1.262889-6.8343210 .050437$

H $-0.102293-7.218759-0.999311$

H $0.872705-5.763627-1.294069$

H $-1.781642-4.0569612 .252928$

H $-1.683337-5.5217903 .228820$

H $-0.858608-3.806823 \quad 4.609310$

H $0.558139-4.7331784 .111501$

H $0.817216-2.9040272 .222658$

H $2.336306-3.0755584 .219712$

H $1.152872-2.2388695 .202843$

H $3.641322-1.1631005 .042532$

H $0.5827130 .250593 \quad 4.632246$

H $3.894765-1.7425272 .779285$

H $3.526827-0.0870412 .354305$

H $2.557847-1.4100181 .674657$

H $2.187407 \quad 1.5882623 .738349$

H -1.632973 0.6074703 .906918

H -1.065691 1.5217762 .500393

H $-0.496455-0.5341290 .795240$

H - $-4.874957-2.184378-2.327051$

H $-5.731753-1.2653860 .485188$

H $-6.048630-0.188531-3.205505$

H $-7.7859000 .061320-3.100417$

H -7.158805 - $1.570949-3.040617$

H -2.686164 -1.010818 2.005566

H -3.512406 0.3732452 .684668

H -3.349330 $2.175162-1.323061$

H -2.764677 $3.586268-0.421337$

H -5.0841954 .0896900 .412592$

H $-5.6640542 .279981-1.989353$

H -7.650455 $3.644805-2.180644$

H -7.223212 4.602847 -0.786455

H -9.4576422 .6629411 .393153$

H $-8.5207674 .241073 \quad 1.145546$

H $-8.7600431 .396243-1.886062$

H $-9.533945 \quad 1.039564-0.366893$

H $-7.367498 \quad 0.2820280 .656763$

H $-3.455002 \quad 2.413724 \quad 1.666711$

C $2.877725-2.696722-2.461894$

O $3.322136-1.468698-1.845315$

C $4.682465-1.212591-2.245449$
C $5.170463-2.491436-2.914378$

C $3.895708-2.992959-3.561682$

H $4.651785-0.415024-2.996264$

C $5.519018-0.781615-1.038060$

H $5.973887-2.303385-3.633002$

H $5.537791-3.216981-2.178724$

C $3.959639-4.454872-3.969216$

H $3.682792-2.379177-4.447233$

C $5.0852130 .557654-0.403698$

O $5.434486-1.798416-0.030711$

H $6.577017-0.728408-1.319169$

H $4.072907 \quad 0.4730120 .005344$

O 5.9554950 .8047520 .708915

C $5.1665391 .768782-1.356762$

C $5.048028 \quad 3.142837-0.668177$

H $4.3965681 .682261-2.130367$

O $6.428403 \quad 1.749615-2.034334$

H 5.8105573 .2304580 .115724

H $5.3072563 .904936-1.416380$

H $4.754524-4.613911-4.705348$

H $4.163307-5.102646-3.109803$

H $3.015196-4.778525-4.417532$

C $1.450459-2.518248-2.982441$

H $4.492441-1.9277850 .174132$

H $\quad 5.8965100 .0340191 .299204$

H $7.1252391 .803545-1.357831$

H $1.232251-3.272907-3.746699$

H $1.342156-1.531887-3.448762$

H $2.916266-3.475438-1.688345$

C $1.4049613 .919286-0.883344$

O $2.7697843 .620163-1.205165$

C $3.655803 \quad 3.448645-0.093588$

C 3.6761184 .7140100 .780592

C $2.2631765 .117701 \quad 1.212365$

C 1.2981565 .1544910 .024880

H $0.9445174 .177117-1.846214$

C $0.6798972 .690255-0.328614$

H 3.3229182 .5993610 .510712

$\begin{array}{lllll}\text { O } & 4.277778 & 5.786589 & 0.057369\end{array}$

H 4.3004274 .5215161 .661464

H 2.2922526 .1079381 .682831

$\begin{array}{lllll}\text { O } & 1.770101 & 4.195040 & 2.177728\end{array}$

H $0.263801 \quad 5.260897 \quad 0.372874$

$\begin{array}{lllll}\text { O } & 1.588123 & 6.302207 & -0.778237\end{array}$

C $0.5338591 .646350-1.406899$

H 1.2058122 .2202470 .506083

H -0.3192532 .9482860 .039433$

H 4.3050046 .5595300 .646430

H $2.387507 \quad 4.1958962 .928844$

H $1.4733517 .088681-0.218016$

O $-0.565167 \quad 1.910449-2.168403$

$\begin{array}{llll}\text { O } & 1.321656 & 0.724320 & -1.567972\end{array}$

C $-0.8018811 .001150-3.247312$

H $0.0620990 .969957-3.918012$

H $-1.0253770 .004418-2.857035$

H -1.668792 $1.359274-3.808827$

SCF Energy (B3LYP/6-31G**//MMFF) $=-3245.91163116$

0700220

MM̄FF Geometry

C $1.789902-3.2289772 .297899$

C $2.093271-1.9215242 .260791$

C $1.187896-0.7812402 .656045$

O $-0.157467-1.2565852 .904274$

C $1.646557-0.0801843 .945730$

C 2.8114840 .8663243 .762665

C 2.6842882 .1318463 .317656

C $4.1585920 .335083 \quad 4.172308$

C 1.4189412 .8172842 .871656

C 1.6239633 .4843751 .506385

C 0.3635554 .0931820 .874155

C $-0.368599 \quad 5.0711291 .789954$

C -1.6241515 .6427001 .116355$

$\begin{array}{llll}0 & -0.516167 & 3.029586 & 0.500318\end{array}$

$\begin{array}{llll}O & -2.379836 & 6.296426 & 2.147841\end{array}$

C $-2.476575 \quad 4.4793690 .556121$

C $-1.267537 \quad 6.7223110 .086140$

O $-3.5526534 .997128-0.222360$ 
C $-1.660666 \quad 3.453791-0.266930$ C -2.448776 $2.157760-0.568856$ O $-1.2570404 .039891-1.506705$ C $-1.089747-1.0505211 .936617$ O $-0.921413-0.5182750 .851711$ C -2.376548-1.586053 2.434955 C $-3.500672-1.3304161 .749723$ C $-4.852905-1.7742132 .092953$ C $-5.004697-2.697858 \quad 3.272730$ C $-3.7027332 .311339-1.430515$ C $-4.2226740 .979185-1.948067$ C $-6.1370420 .021717-3.232187$ O $-3.646649-0.089603-1.766710$ C -7.202495 -0.412744 -2.275492 C $-7.300108-1.657442-1.783664$ C $-8.385966-2.098381-0.844735$ C $-7.851825-2.6412170 .462162$ C $-7.900220-3.9556780 .736716$ C $-7.347629-1.6526701 .489886$ C $-5.882214-1.3501041 .329307$ N -5.408393 1.135103 -2.653541 H $0.823411-3.5522152 .673142$ H $3.066475-1.6289051 .872775$ H $1.177943-0.0579691 .831442$ H $1.884113-0.8259594 .716929$ H 0.8017390 .4752284 .376289 H 3.5801272 .7493183 .264989 H 4.9562661 .0726104 .033627 H 4.1493360 .0533895 .230414 H $4.421682-0.5460313 .581301$ H $\quad 0.5747842 .1239412 .818241$ H 1.1701853 .5760763 .621931 H 2.3833854 .2707691 .603180 H 2.0206242 .7323580 .812542 H $0.6935864 .606248-0.036853$ H 0.2989715 .8789152 .114026 H $-0.689647 \quad 4.5570862 .705657$ H -3.151978 6.7191971 .735952 H -2.926885 3.9529591 .409172 H -0.7208037 .5420010 .567516$ H -2.168355 $7.175640-0.342319$ H $-0.6512336 .346294-0.733155$ H $-4.115575 \quad 5.5272010 .366149$ H -1.761697 $1.465286-1.072411$ H $-2.714122 \quad 1.6632260 .374411$ H -1.040312 $3.323106-2.126095$ H -2.364838 -2.163442 3.352192 H -3.428534 -0.7267620 .847304$ H -4.373434 -3.5854753 .150825$ H $-6.023608-3.0657573 .410338$ H $-4.718204-2.1875774 .198826$ H $-4.5033002 .772992-0.842593$ H -3.496365 $2.943999-2.300982$ H $-5.440490-0.788326-3.471879$ H $-6.593604 \quad 0.375913-4.161482$ H $-7.9396380 .332918-1.986561$ H -6.573185 -2.408229 -2.085318 H $-8.977710-2.865918-1.360382$ H $-9.086257-1.280511-0.632677$ H -7.548165 -4.347350 1.685219 H $-8.288658-4.669830 \quad 0.018155$ H -7.578778 -2.008922 2.499291 H -7.908372 -0.7131411 .401592$ H $-5.663401-0.6832120 .495355$ H $-5.8377442 .054739-2.700257$ C $2.352643-4.8805740 .453290$ O $2.326354-3.815329-0.519604$ C $1.083600-3.888562-1.237558$ C $0.620849-5.330096-1.083555$ C $1.000271-5.5938810 .362713$ H $\quad 0.372922-3.236504-0.715114$ C $1.264927-3.405291-2.677541$ H $-0.449479-5.449396-1.275912$ H $1.169840-6.005586-1.749871$ C $1.061994-7.0686570 .720456$ H $0.256838-5.109407 \quad 1.007014$ C $1.851410-1.981518-2.807392$ O $2.143995-4.299133-3.371433$
H $\quad 0.305554-3.461277-3.205123$

H $2.917213-1.993696-2.553044$

O $1.772753-1.607599-4.188718$

C $1.132645-0.910512-1.966743$

C $1.4992020 .544632-2.308826$

H $1.330997-1.094118-0.905006$

O $-0.277874-1.050260-2.165552$

H $1.1449130 .782126-3.320162$

H $0.916524 \quad 1.188348-1.635418$

H $0.081832-7.5380230 .587097$

H $1.779422-7.6054950 .090723$

H $1.362719-7.2022251 .764531$

C $2.722942-4.3116211 .826686$

H $2.983281-4.324072-2.880390$

H $2.234042-2.293614-4.701111$

H $-0.721586-0.483391-1.512284$

H $3.741585-3.9094191 .770730$

H $2.744118-5.1126052 .574184$

H $3.154783-5.5591530 .135758$

C $4.751346 \quad 0.990135-0.506934$

$\begin{array}{lllll}\text { O } & 3.376745 & 0.712928 & -0.814463\end{array}$

C $2.9923990 .888595-2.182166$

C $3.2743032 .325397-2.653536$

C $4.7335582 .714504-2.402277$

C $5.1619662 .400983-0.967757$

H $4.797000 \quad 0.9704470 .589439$

C $5.686162-0.101316-1.043313$

H $3.563740 \quad 0.197536-2.808764$

$\begin{array}{llll}\text { O } & 2.410453 & 3.233830 & -1.974678\end{array}$

H $3.0448942 .394176-3.723766$

H $4.8662593 .787240-2.588874$

O $5.5810732 .007011-3.302640$

H $6.2470272 .523230-0.867697$

O $4.558744 \quad 3.366128-0.103828$

C $5.620478-1.336884-0.179519$

H $5.453187-0.409426-2.066208$

H $6.7291430 .238225-1.052893$

H 2.600828 4.126953-2.307946

H $5.3090622 .240140-4.206536$

H 4.8710853 .1897630 .799997

O $6.470116-1.2077490 .879697$

O $4.895259-2.293856-0.413450$

C $6.525433-2.3252861 .771458$

H $5.592887-2.3961412 .336744$

H $6.716128-3.2519571 .221542$

H $7.348319-2.1599192 .472042$

SCF Energy (B3LYP/6-31G*//MMFF) $=-3245.90293710$

0700221

MM̄FF Geometry

C $-2.380473 \quad 3.668062-1.675287$

C $-3.6122053 .862683-1.179651$

C $-4.4922822 .816087-0.534950$

O $-3.986842 \quad 1.497468-0.845437$

C $-4.4943893 .029388 \quad 0.989801$

C $-5.5952052 .285243 \quad 1.717798$

C -5.4078241 .1798642 .465316$

C -6.9658812 .9034261 .614130$

C $-4.121796 \quad 0.427256 \quad 2.671793$

C $-4.357179-1.0900452 .639006$

C $-3.071775-1.9328602 .647696$

C $-2.190434-1.6726873 .871865$

C $-0.891830-2.4944713 .828296$

O $-2.349823-1.6519121 .444646$

O $-0.001064-1.962400 \quad 4.821759$

C $-0.218832-2.2778292 .456242$

C $-1.130610-3.9611964 .203623$

O $0.923529-3.1188642 .311806$

C $-1.177723-2.4552311 .259312$

C $-0.527769-2.035123-0.074764$

O $-1.517998-3.8424821 .141808$

C $-4.8879590 .511676-1.098756$

O $-6.1044050 .588148-1.031529$

C $-4.132881-0.697583-1.493947$

C $-4.778278-1.866696-1.604560$

C $-4.164877-3.130779-1.993543$

C $-4.516399-4.269382-1.075757$

C $-0.124357-0.559739-0.173100$ 
C $0.172060-0.207300-1.615153$

C $1.631451-0.895621-3.498050$

O $-0.5941190 .482993-2.282743$

C $1.205202-2.262242-3.936874$

C $-0.048475-2.566698-4.307507$

C $-0.490109-3.955900-4.661592$

C $-1.333927-4.584647-3.574060$

C $-0.762547-5.280660-2.576349$

C $-2.836789-4.499187-3.696976$

C $-3.413381-3.238654-3.108509$

N $1.341169-0.788621-2.081365$

H -1.929003 2.682138-1.633002

H $-4.0341804 .865454-1.217557$

H $-5.4962972 .941642-0.957963$

H -4.614833 4.0975661 .218471

H -3.5131602 .7587001 .397515$

H -6.2690860 .7654982 .987908$

H -7.714291 2.3568252 .197716

H $-6.947256 \quad 3.933268 \quad 1.985751$

H -7.306292 2.9150820 .574294

H -3.378375 0.6918001 .914915

H -3.719164 $0.715778 \quad 3.648913$

H -4.983461 -1.380499 3.492185

H $-4.920296-1.3372101 .729142$

H -3.383810 -2.984049 2.628020

H $-2.743273-1.8712054 .798229$

H -1.906601-0.612931 3.910876

H -0.445881 -2.0203395.684849

H $0.160224-1.2493582 .456416$

H $-1.566450-4.0328445 .207446$

H $-0.186911-4.5159074 .249086$

H $-1.803258-4.4768863 .515411$

H $0.606826-4.0173382 .116855$

H $0.334785-2.680259-0.284732$

H -1.239296 -2.246165 -0.884772

H $-1.809158-4.0126980 .230729$

H -3.070855 -0.604536-1.687375

H -5.839151 -1.930299-1.370249

H $-4.389682-3.971737-0.028063$

H -3.884282 -5.148628 -1.221860

H $-5.558995-4.571050-1.222229$

H -0.9290390 .0977840 .174016$

H $0.759989-0.3427720 .432556$

H $2.709311-0.769861-3.635960$

H $1.112426-0.110110-4.056577$

H $1.959341-3.044695-3.901310$

H $-0.803326-1.784630-4.326365$

H $0.382190-4.592778-4.861502$

H $-1.044523-3.927046-5.607047$

H -1.359730 -5.764403 -1.810599

H $0.315652-5.375358-2.500565$

H -3.298886 -5.391026 -3.258950

H -3.119567-4.545978-4.756595

H -3.240445 -2.344832 -3.707540

H $1.875481-1.381226-1.452732$

C $-0.2982375 .112639-1.481054$

O $0.608393 \quad 3.995321-1.421808$

C $1.2704074 .010759-0.141071$

C $0.859843 \quad 5.3149290 .539759$

C $-0.5254265 .538797-0.032651$

H 0.8662093 .1653860 .429925

C $2.790767 \quad 3.848679-0.291929$

H $0.866146 \quad 5.230952 \quad 1.630914$

H $1.526133 \quad 6.1410590 .263898$

C -1.031021 6.9614590 .123645

H -1.2162114 .8541590 .474790$

C $3.1948422 .464869-0.846530$

O $3.2753164 .895652-1.130613$

H 3.2501723 .9788210 .695704

H $2.7217541 .709810-0.210744$

O $2.673052 \quad 2.285735-2.161491$

C $4.718815 \quad 2.222147-0.865759$

C $5.1225800 .815777-1.352443$

H $\quad \begin{array}{llll}5.121712 & 2.398676 & 0.138207\end{array}$

O $5.334727 \quad 3.173452-1.737210$

H $4.7409390 .649408-2.367372$

H $6.217250 \quad 0.800352-1.439061$

H $-1.095635 \quad 7.2324501 .182614$
H $-0.3665897 .680480-0.367051$

H $-2.0283787 .070388-0.314032$

C $-1.547103 \quad 4.756262-2.291259$

H $4.2445624 .827810-1.157897$

H $3.0511632 .985619-2.721257$

H $6.2972423 .050041-1.674794$

H $-1.2406324 .406573-3.284950$

H -2.149008 $5.659991-2.444606$

H $0.2363795 .905428-2.021974$

C $4.898977-1.0235531 .885380$

O $5.282082-0.1061190 .853236$

C $4.668266-0.318135-0.421141$

C $5.040244-1.705362-0.963991$

C $4.664598-2.8008280 .040007$

C $5.163298-2.478374 \quad 1.454715$

H $5.563749-0.7921932 .727304$

C $3.456642-0.7830532 .353984$

H $3.583118-0.262525-0.312399$

O $6.439629-1.754601-1.231104$

H $4.515657-1.867532-1.913107$

H $5.096553-3.753429-0.290381$

$\begin{array}{llllll}\text { O } & 3.247347 & -2.945337 & 0.038898\end{array}$

H $4.714099-3.1675832 .179342$

O $6.575336-2.7092211 .479616$

C $3.2471870 .620006 \quad 2.884326$

H $2.750645-0.9348101 .535104$

H $3.205855-1.4796643 .161560$

H $6.639739-2.636506-1.588268$

H $3.021596-3.6459140 .674344$

H $6.880502-2.5687962 .392149$

$\begin{array}{lllll} & \text { O } & 1.907249 & 0.879439 & 2.936021\end{array}$

$\begin{array}{lllll}\text { O } & 4.138183 & 1.383446 & 3.230017\end{array}$

C 1.5435392 .1728183 .427742

H 2.0673562 .9585462 .875597

H $0.4677602 .301347 \quad 3.281169$

H 1.7653442 .2394194 .496744

SCF Energy (B3LYP/6-31G**//MMFF)= -3245.89825551

\section{2}

MM̄FF Geometry

C $4.7905113 .629247-0.574168$

C $3.5450013 .525053-0.087133$

C $2.3066703 .996213-0.806230$

O $1.3759442 .893911-0.886332$

C 1.6463555 .1182150 .018069

C $0.3375225 .620396-0.559015$

C $-0.877590 \quad 5.421425-0.007912$

C $0.4634016 .421448-1.829852$

C $-1.2220014 .671206 \quad 1.251806$

C $-1.920615 \quad 3.337054 \quad 0.970734$

C $-3.352599 \quad 3.448478 \quad 0.423302$

C $-4.337940 \quad 3.908162 \quad 1.503704$

C -5.7940963 .8854501 .014508$

O $-3.719690 \quad 2.143515-0.053743$

$\begin{array}{lllll}0 & -6.648068 & 3.992865 & 2.164356\end{array}$

$\begin{array}{llll}\text { C } & -6.082379 & 2.514531 & 0.367253\end{array}$

C $-6.1142215 .093007 \quad 0.125328$

$\begin{array}{lllll}\text { O } & -7.376575 & 2.495986 & -0.230044\end{array}$

C $-5.0166362 .091549-0.665671$

C $-5.2481730 .661441-1.202520$

O $-5.0906672 .969301-1.797103$

C $1.5279992 .005438-1.905911$

O $2.3772512 .018520-2.781279$

C $0.4795260 .976591-1.739046$

C $0.611362-0.227432-2.314103$

C $-0.289400-1.359755-2.094318$

C $-1.622144-1.082121-1.449374$

C $-5.127482-0.465973-0.173767$

C $-5.353565-1.825527-0.817149$

C $-5.271971-4.261238-0.319340$

O $-5.742123-1.961548-1.975042$

C $-3.987436-4.797154-0.868047$

C $-3.740209-4.931722-2.180400$

C $-2.472592-5.504526-2.747539$

C $-1.514054-4.427767-3.212416$

C $-1.502195-4.037741-4.498420$

C $-0.591490-3.891267-2.138386$

C $0.114047-2.598168-2.447083$ 
N $-5.097167-2.8713330 .059324$ H $4.9499264 .065742-1.556610$ H 3.3983333 .0884780 .898803 H $2.5403894 .359799-1.814381$ H 2.3366615 .9689510 .099600 H 1.5019664 .7675771 .044779 H -1.738893 $5.853167-0.516175$ H $-0.495674 \quad 6.837385-2.156737$ H $1.1509297 .260828-1.683048$ H $\quad 0.8437005 .796266-2.643360$ H -0.3322014 .4661061 .852504$ H -1.8508245 .3130391 .878331$ H -1.3132252 .7514740 .271797$ H -1.939635 2.7459761 .895510 H $-3.358831 \quad 4.138127-0.426824$ H $-4.083370 \quad 4.9078651 .874143$ H -4.2587983 .2392292 .372327$ H $-6.423321 \quad 4.8172802 .628875$ H -6.1039451 .7767391 .179070$ H $-5.978393 \quad 6.0266130 .684703$ H -7.162893 $5.084351-0.191079$ H -5.484092 $5.151039-0.764512$ H $-8.015171 \quad 2.7621230 .453562$ H $-6.2241750 .612289-1.703566$ H $-4.5130360 .466459-1.995978$ H $-4.6679372 .533016-2.555365$ H $-0.3445881 .209922-1.076261$ H $1.490340-0.427167-2.924653$ H -2.136364 -0.266534 -1.969486 H -1.495696 -0.803028 -0.399158 H -2.300911 -1.937581 -1.484561 H $-4.132968-0.456030 \quad 0.285542$ H $-5.876858-0.3513660 .615821$ H -6.086885 -4.340923 -1.046985 H -5.560013 -4.8204500 .576096$ H $-3.234524-5.105676-0.147581$ H $-4.494524-4.616547-2.897872$ H $-2.740466-6.167513-3.580519$ H $-1.971858-6.152116-2.016813$ H -0.816597 -3.285053 -4.870248 H -2.186446 -4.472091 -5.220536 H $0.172369-4.652232-1.929828$ H -1.142651 -3.782675 -1.196949 H $1.085209-2.721816-2.926078$ H $-4.711658-2.6704310 .977404$ C $6.7361872 .029982-0.466320$ O $5.894700 \quad 0.859921-0.425774$ C $6.644542-0.2386660 .122326$ C $8.1078500 .162945-0.000729$ C 8.0257891 .6522030 .267816 H $6.379935-0.3001171 .185263$

C $6.268862-1.549995-0.575249$ H $8.747318-0.3687080 .710242$ H 8.497611-0.015392-1.009331 C $9.2476462 .424228-0.199845$ H 7.9023421 .8046331 .348560 C $4.754442-1.820262-0.658535$ O $6.766511-1.531753-1.920218$ H $6.775095-2.387175-0.081211$ H $4.290064-1.124426-1.368783$ O $4.574876-3.131967-1.208701$ C $4.003674-1.7473410 .684315$ C $2.513924-2.0654980 .508994$ H $4.109532-0.7403111 .097143$ O $4.579760-2.6546511 .618950$ H $2.098815-1.406121-0.258240$ H $2.393716-3.0851440 .118105$ H 10.1455342 .0748550 .320292 H $9.4129262 .300899-1.275455$ H 9.1340683 .4938440 .003062 C 6.0115493 .2042410 .193850 H $6.383081-0.755922-2.364742$ H $5.040249-3.153873-2.062571$ H $4.519146-3.5472741 .237249$ H 6.6837704 .0671620 .265553 H 5.7284002 .9303301 .218313 H $6.9352812 .242819-1.524209$ C $-0.684748-2.3088202 .376895$
O $\quad 0.315798-2.2731321 .353750$

C $1.652960-1.9705121 .780297$

C $1.695595-0.5980992 .468076$

C $0.669513-0.5138323 .604461$

C $-0.716946-0.986873 \quad 3.161296$

H -1.634217 -2.401981 1.833357

C $-0.537585-3.5468253 .267581$

H $1.987542-2.7459732 .477561$

O 1.4350870 .4229821 .507789

H $2.701128-0.4267452 .868473$

H $0.5965600 .522638 \quad 3.956338$

O $1.101225-1.3156714 .700241$

H -1.375672 -1.086394 4.032082

$\begin{array}{llll}\text { O } & -1.280537 & 0.027069 & 2.325122\end{array}$

C $-0.882804-4.7972732 .493946$

H $0.463716-3.6703713 .689593$

H $-1.229090-3.4948734 .117212$

H 1.4850601 .2792861 .965625

H $1.967634-0.9799194 .986891$

H $-2.184663-0.2475452 .095973$

O $0.203234-5.2252761 .789342$

O $-1.991069-5.3172392 .501078$

C $-0.003062-6.3916470 .987251$

H $\quad 0.913639-6.5825780 .423079$

H $-0.210512-7.2547411 .626509$

$\mathrm{H}-0.821423-6.2296340 .279990$

SCF Energy $\left(B 3 L Y P / 6-31 G^{* *} / / M M F F\right)=-3245.90619275$

0700223

MM̄FF Geometry

C 0.6182852 .0096420 .112180

C 1.6350042 .3934000 .901099

C 2.2193451 .6402112 .073178

$\begin{array}{llll}\text { O } & 1.581890 & 0.349624 & 2.206581\end{array}$

C 3.7335401 .4147301 .884290

C 4.5827682 .6343272 .171312

C 5.2102423 .3730511 .235913

C 4.7532302 .9677373 .631771

C $5.1702683 .207473-0.257807$

C $6.5114202 .724573-0.825969$

C $6.8087831 .249117-0.514073$

C $8.2077400 .871398-1.005140$

C $8.497780-0.623089-0.807867$

O $5.8232260 .455357-1.182622$

O $9.675469-0.947608-1.562168$

C $7.335425-1.436450-1.411879$

C $8.812160-0.9584230 .654704$

O $7.489531-2.826586-1.132403$

C $5.936012-0.955876-0.956354$

C $4.850629-1.654753-1.809561$

O $5.752515-1.2884630 .419577$

C $1.609595-0.2380823 .431951$

$\begin{array}{llll}\text { O } & 2.111280 & 0.221327 & 4.449151\end{array}$

C $0.980947-1.5785333 .410319$

C $0.214413-2.0184912 .401120$

C $-0.418478-3.3358502 .302863$

C $-0.095991-4.3517823 .365931$

C $3.412615-1.191011-1.533791$

C $2.434517-1.769327-2.544736$

C $-0.008055-1.987807-2.988777$

O $2.789312-2.360670-3.561541$

C $-0.314614-3.409835-2.635413$

C $-1.456929-3.800323-2.051763$

C $-1.762180-5.219956-1.670886$

C $-1.321384-5.569626-0.265209$

C $-0.422482-6.545208-0.050079$

C $-1.960528-4.8420720 .897778$

C -1.224555 -3.577339 1.248609

N $1.114416-1.527891-2.189962$

H $\quad 0.109919 \quad 1.067053 \quad 0.285043$

H 2.0959293 .3577920 .693257

H 2.0118052 .2293732 .974724

H $3.918768 \quad 1.0193570 .879338$

H 4.0778240 .6062762 .543178

H 5.8184494 .2126751 .571090

H 5.4388623 .8071673 .789720

H 3.7935953 .2394724 .081322

H 5.1598432 .1067454 .172496 
H $4.3576442 .552589-0.584504$ H $4.9452804 .191647-0.688260$ H $6.4752072 .857590-1.915179$ H $7.3229633 .359374-0.448776$ H $6.733203 \quad 1.0858900 .566356$ H $8.9728801 .479497-0.506517$ H $8.2934401 .101953-2.076466$ H $10.401602-0.386393-1.240113$ H $7.397660-1.332039-2.504338$ H $9.687618-0.3935580 .997124$ H $9.074891-2.0158310 .769597$ H $7.987946-0.7317301 .334223$ H $8.371981-3.089056-1.445998$ H $5.070822-1.468559-2.869226$ H $4.893551-2.740378-1.654377$ H $4.803673-1.3122410 .617224$ H $1.173594-2.1851684 .289386$ H $0.027796-1.3560441 .557553$ H $-0.522492-5.3377953 .170127$ H $-0.478855-4.0234164 .338407$ H $0.987309-4.4965513 .445182$ H $3.097121-1.502761-0.532313$ H $3.341210-0.099344-1.594797$ H $\quad 0.247426-1.916341-4.050949$ H $-0.849753-1.317882-2.786012$ H $0.443292-4.154179-2.868663$ H -2.216011 -3.057707 -1.820572 H -1.301985 -5.898801-2.401101 H $-2.842645-5.386090-1.761347$ H $-0.115434-6.817760 \quad 0.954243$ H $0.029518-7.090394-0.871953$ H -2.047847 $-5.512276 \quad 1.759177$ H $-2.996950-4.5883360 .646141$ H -1.387953 $-2.765542 \quad 0.540739$ H $0.912139-1.096507-1.292606$ C $-0.9291433 .838731-0.684065$ O $-2.2233983 .197838-0.634327$ C $-3.1840144 .053526-1.285328$ C $-2.4933835 .398241-1.478574$ C $-1.0626304 .966154-1.708783$ H -3.375483 $3.617855-2.272509$ C -4.487421 4.152384 -0.484999 H $-2.9131035 .964072-2.315929$ H $-2.5638136 .022628-0.579968$ C $-0.0572556 .091271-1.528452$ H $-0.9745954 .562291-2.726440$ C -5.262597 $2.831759-0.307275$ O -4.1994244 .6675320 .821481$ H -5.144007 $4.893331-0.956850$ H $-4.734140 \quad 2.1743380 .391289$ O $-\begin{array}{llll}6.515306 & 3.154499 & 0.312221\end{array}$ C $-5.5532302 .076025-1.616178$ C $-6.6104570 .960587-1.517839$ H -4.619052 $1.648933-1.997286$ O $-6.0134642 .996300-2.612122$ H $-7.6038401 .399800-1.358316$ H $-6.658187 \quad 0.490497-2.509413$ H $-0.2508426 .897537-2.243633$ H $-0.1080526 .517023-0.520673$ H $0.9646705 .735131-1.691380$ C $0.1566712 .828554-1.062091$ H -3.557514 4.0671031 .237410 H -6.3158943 .6210631 .142099$ H $-6.8200603 .419600-2.270714$ H $1.0246353 .346131-1.488380$ H $-0.2109522 .141775-1.834488$ H $-0.7450314 .256596 \quad 0.314592$ C $-4.614579-1.6523950 .260455$ O $-5.047099-0.663003-0.683148$ C $-6.342844-0.109637-0.447079$ C $-7.414771-1.213730-0.468819$ C -7.070383 -2.356006 0.492555 C $-5.618468-2.817324 \quad 0.331797$ H -3.678404 -2.040047 -0.156248 C $-4.297661-1.0452351 .635864$ H $-6.353978 \quad 0.3778050 .532710$ O $-7.561685-1.742173-1.786417$ H -8.380332 $-0.773632-0.193752$
H $-7.737802-3.2057980 .302731$

O $-7.276795-1.9328731 .836584$

H $-5.347056-3.4950261 .149678$

O $-5.530909-3.567494-0.883309$

C $-3.159820-0.0490861 .580344$

H $-5.163781-0.5243362 .052238$

H $-4.000417-1.8398892 .329686$

H $-6.694192-2.078046-2.070312$

H -8.211657-1.680214 1.924666

H $-4.621305-3.901286-0.959419$

O $-\begin{array}{llll}-3.081913 & 0.594709 & 2.782825\end{array}$

$\begin{array}{lllll}0 & -2.429503 & 0.136665 & 0.617469\end{array}$

C -2.0585231 .5880282 .889882$

H -2.1721992 .3425512 .107028$

H -2.1586252 .0763533 .862818$

$\mathrm{H}-1.0746231 .1174512 .833473$

SCF Energy (B3LYP/6-31G**//MMFF) $=-3245.91427697$

$07 \quad 00224$

MM̄FF Geometry

C -0.1897824 .9480690 .480028$

C $0.8207784 .555866-0.310437$

$\begin{array}{llll}\text { C } & 2.028935 & 3.816699 & 0.206716\end{array}$

O $2.0874672 .529084-0.456143$

C $3.3161404 .588513-0.131497$

C $4.551876 \quad 4.0242840 .544150$

C $5.5934143 .461932-0.099417$

C 4.5913634 .1528062 .046545

C $5.7814993 .267359-1.576735$

C $5.2817001 .921957-2.125302$

C $5.9068780 .686833-1.454949$

C $5.572314-0.580756-2.245867$

C $6.083809-1.851626-1.548775$

O $5.376508 \quad 0.577113-0.130508$

O $5.449411-2.980895-2.169228$

C $5.630673-1.835105-0.072335$

C $7.592469-2.040901-1.747363$

O $6.218216-2.912602 \quad 0.651831$

C $5.923077-0.500208 \quad 0.642705$

C $5.301288-0.4162162 .059051$

$\begin{array}{llllll} & & 7.339450 & -0.350985 & 0.801158\end{array}$

C $1.2098311 .575076-0.039514$

$\begin{array}{lllll}\text { O } & 0.399151 & 1.666977 & 0.868350\end{array}$

C $1.387416 \quad 0.383555-0.896145$

C $0.441784-0.567544-0.893407$

C $0.471036-1.820792-1.649783$

C $1.745871-2.166619-2.371465$

C $3.776381-0.5547992 .119900$

C $3.290219-1.9633422 .419988$

C $1.192410-3.3045352 .555779$

O $4.034102-2.8722512 .778121$

C $1.088834-4.1196461 .305148$

C $-0.060229-4.6577390 .868335$

C $-0.167314-5.512823-0.362832$

C $-1.224579-5.021534-1.326723$

C $-2.443236-5.585865-1.360343$

C $-0.842655-3.933185-2.306833$

C $-0.627810-2.603535-1.635887$

N $1.912602-2.0751162 .278179$

H -0.1271984 .7787241 .552134$

H $0.7482284 .700532-1.385727$

H 1.9536843 .6577531 .289022

H 3.2158585 .6375750 .177818

H $3.4438664 .602857-1.219431$

H 6.4256353 .0963540 .501770

H 5.5696243 .8853212 .460501

H 4.3839345 .1854002 .345762

H 3.8499433 .4965732 .512065

H $5.3079684 .079669-2.138521$

H $6.8538643 .361291-1.792085$

H $4.1914291 .884033-2.021053$

H $5.5024801 .901480-3.199763$

H $6.9927890 .829749-1.405298$

H $5.969942-0.518343-3.266683$

H $4.482966-0.661234-2.356450$

H $5.662598-2.960610-3.117959$

H $4.547751-2.009650-0.070515$

H $7.830418-2.124359-2.814680$ 
H $7.937306-2.975257-1.290849$

H $8.185303-1.218275-1.342296$

H $5.991920-3.7342880 .183245$

H $5.799673-1.1238652 .732384$

H 5.5415870 .5802892 .457540

H 7.5044420 .2858901 .516123

H $2.2859790 .320636-1.497405$

H $-0.445222-0.416689-0.278487$

H $2.584531-2.192967-1.667606$

H $1.961384-1.426965-3.150161$

H $1.724828-3.141995-2.860479$

H 3.4036250 .0748122 .938184

H $3.314770-0.1972221 .196295$

H $\quad 0.210373-3.0242912 .950032$

H $1.729896-3.8726063 .321789$

H $2.005150-4.2987750 .747339$

H $-0.971321-4.5023961 .442186$

H $-0.404956-6.534371-0.037917$

H $0.794388-5.585481-0.885992$

H -3.197834 -5.259728 -2.068315

H $-2.715875-6.383140-0.676789$

H -1.639847 -3.795749 -3.049262

H $0.026993-4.271876-2.875350$

H -1.497872 -2.255641 -1.079252

H $1.381501-1.2822961 .930014$

C $-2.589362 \quad 4.4898250 .046574$

O $-2.3062143 .426003-0.886047$

C $-3.5145723 .090234-1.598136$

C $-4.6430613 .749809-0.822918$

C $-3.9689935 .019029-0.336181$

H $-3.4146173 .553543-2.588126$

C $-3.5989121 .565194-1.728216$

H $-5.5241273 .943449-1.441661$

H -4.9491973 .1275370 .026159$

C $-4.706165 \quad 5.698451 \quad 0.804347$

H $-3.8836255 .718828-1.178224$

C $-4.9329131 .042605-2.288037$

O $-2.5236841 .146485-2.578292$

H $-3.3970891 .114579-0.754888$

H $-5.7426901 .235872-1.576131$

O $-5.2396771 .806486-3.464066$

C $-4.917761-0.442166-2.696100$

C $-4.561748-1.468555-1.602199$

H $-4.200214-0.568972-3.516134$

O $-6.185534-0.786093-3.268022$

H $-4.432828-2.425281-2.123432$

H -3.588821 -1.215115 -1.174978

H -5.7101845 .9995550 .487981$

H -4.811563 5.0322311 .667322

H -4.1724156 .5953601 .134216$

C -1.474645 5.535104 -0.033027

H -2.692551 $1.507888-3.465542$

H $-6.0836681 .473999-3.814895$

H $-6.884811-0.437422-2.690068$

H -1.723257 6.4149860 .570215

H -1.347800 5.870406 -1.069796

H -2.619516 4.036446 1.045705

C $-4.513184-0.5723601 .384107$

O $-5.615166-0.602703 \quad 0.465133$

C $-5.619132-1.664978-0.489643$

C $-5.535451-3.0174390 .233431$

C $-4.313354-3.0909561 .136947$

C $-4.319718-1.9137162 .108837$

H $-3.595022 \quad-0.3204220 .844867$

C -4.8296050 .5697142 .358121$

H $-6.606680-1.643111-0.962881$

O $-5.522222-4.082698-0.709956$

H $-6.436782-3.1491290 .846508$

H $-3.388879-3.1276450 .550143$

O $-4.370709-4.310824 \quad 1.878827$

H $-5.119035-2.0645142 .845031$

O $-3.087538-1.9169772 .828793$

C $-3.7042680 .922210 \quad 3.294657$

H -5.1126681 .4763411 .808603$

H $-5.714795 \quad 0.3241142 .958824$

H -5.435672 $-4.912638-0.210298$

H -3.604148 -4.321019 2.477514

H $-2.366466-1.7825182 .190102$
O $-2.740148 \quad 1.6207122 .632518$

O

C -1.6332872 .0446993 .434836$

H -0.9870341 .1894133 .651685$

H -1.0646902 .7848142 .867537$

$\mathrm{H}-1.9760702 .5149104 .361837$

SCF Energy $(B 3 L Y P / 6-31 G * * / / M M F F)=-3245.90358015$

0700225

MM̄FF Geometry

C $-3.799340-0.4861292 .289724$

C $-2.881853-0.3620543 .258171$

C $-1.433857-0.6892973 .018104$

O $-\begin{array}{lllll}0.701541 & 0.549184 & 3.191481\end{array}$

C $-0.863484-1.7269353 .996980$

C $-1.401695-3.1306363 .820232$

C $-0.904427-4.0213572 .938918$

C $-2.509686-3.5277854 .758934$

C $\quad 0.180622-3.782597 \quad 1.926360$

C $1.552754-4.2338592 .437489$

C $2.652518-4.0595141 .377432$

C $4.016427-4.4408981 .953217$

C $5.153329-4.1798740 .955294$

O $2.669106-2.6912110 .967452$

O $6.395269-4.2531391 .672451$

C $5.033163-2.7398160 .406395$

C $5.213975-5.264469-0.128101$

O $5.984572-2.537260-0.635506$

C $3.610226-2.383422-0.077127$

C $3.417877-0.873145-0.346366$

O $3.310621-3.106946-1.271610$

C 0.3754860 .7505512 .385330

O $0.804122-0.008298 \quad 1.531335$

C 0.9508142 .0732502 .716789

C 2.0126032 .5168102 .026656

C 2.7065763 .7921532 .216595

C 2.1893884 .7115153 .292017

C $4.262737-0.264472-1.464766$

C $3.7681791 .115084-1.872550$

C $4.4074243 .056552-3.298079$

O $2.714674 \quad 1.603059-1.472869$

C $5.0325254 .071258-2.392524$

C $4.3561605 .090202-1.840274$

C $4.9914386 .138426-0.973007$

C $4.306503 \quad 6.2935610 .366340$

C 3.4966147 .3380060 .609263

C 4.6384435 .2996211 .456036

C 3.7594774 .0800671 .421557

N $4.6392501 .729508-2.761048$

H $-3.483817-0.8703101 .321609$

H $-3.1662840 .032788 \quad 4.229380$

H -1.308424 -1.041416 1.986645

H -1.023260 -1.392438 5.031362

H $0.230389-1.7448353 .900112$

H -1.328817 -5.023948 2.918749

H $-2.826461-4.5659054 .611825$

H -2.180045 -3.428575 5.798414

H -3.388247 -2.893229 4.611054

H $-0.075478-4.3356091 .014220$

H $\quad 0.212642-2.7288021 .636133$

H $1.806390-3.6391703 .324843$

H $1.510881-5.2842502 .751774$

H $2.403440-4.6906540 .516203$

H $4.022390-5.4883502 .279801$

H $4.210055-3.8456762 .856799$

H $6.453211-5.1332212 .082390$

H $5.303309-2.0490691 .217105$

H $5.363892-6.251267 \quad 0.326327$

H $6.069302-5.110863-0.795305$

H $4.307154-5.315373-0.734359$

H $6.861499-2.750714-0.273032$

H $2.357431-0.715781-0.584555$

H $3.586322-0.3109850 .581765$

H $2.609009-2.634018-1.749193$

H $0.4860522 .643146 \quad 3.512792$

H 2.4206411 .8829861 .241553

H 1.1385704 .9634393 .110099

H 2.7264625 .6603673 .350983 
H $2.271904 \quad 4.2364294 .275797$

H $5.301982-0.166464-1.133694$

H $4.236534-0.898392-2.357961$

H $3.3304373 .216301-3.416579$

H $4.8776263 .098155-4.285223$

H $6.0992613 .975393-2.204004$

H $3.2941625 .198646-2.048817$

H $4.9559937 .087580-1.523873$

H $6.0569455 .932563-0.811938$

H 3.0293087 .4774531 .578269

H $3.2790718 .075003-0.156724$

H 4.6069755 .7881842 .436055

H $5.6827284 .977526 \quad 1.352745$

H 4.0528353 .3512720 .665690

H $5.5206931 .276119-2.984098$

C -5.5708841 .2018051 .641268$

O -4.8173651 .2756380 .409799$

C -4.0344002 .4823440 .440990$

C -4.8414013 .4313361 .309325$

C -5.2561982 .4842462 .422535$

H -3.0986572 .2540700 .966047$

C $-3.7171072 .968491-0.972218$

H -4.2522244 .2807821 .666692$

H -5.7226633 .8121270 .780147$

C -6.4210532 .9946053 .253384$

H $-4.3973522 .324923 \quad 3.087325$

C $-2.9284841 .942448-1.818046$

O $-4.9471593 .254624-1.645806$

H $-3.1671223 .915620-0.917485$

H $-3.5873301 .108456-2.081493$

O $-2.5812212 .575639-3.055542$

C -1.647942 $1.410657-1.147044$

C $-0.7408980 .556065-2.047858$

H $-1.9168480 .823575-0.262213$

O $-0.8758602 .526569-0.691171$

H $-0.2890161 .180757-2.829068$

H $0.0975470 .228028-1.419888$

H -6.1549283 .9308923 .754732$

H -7.303766 3.1847022 .633590

H -6.6985772 .2656694 .021499$

C $-5.245633-0.0983962 .392680$

H -4.722990 $3.546592-2.546058$

H -1.950767 $3.288239-2.852890$

H $-0.0865772 .176101-0.244217$

H $-5.823165-0.916170 \quad 1.943248$

H $-5.573155-0.0305043 .436497$

H -6.6303061 .1860311 .356655$

C $-2.769977-2.592229-2.084610$

O $-2.058137-1.422670-1.652914$

C $-1.417103-0.668120-2.685693$

C $-0.399786-1.540174-3.442936$

C -1.035151 -2.834966 -3.957135

C $-1.843138-3.541816-2.863763$

H $-3.059225-3.101873-1.156534$

C -4.052461 -2.238829 -2.856084

H $-2.173592-0.307183-3.389369$

O $\quad 0.701050-1.871620-2.597915$

H $0.005230-0.963426-4.282436$

H $-0.247653-3.509600-4.314773$

O $-1.893186-2.547951-5.056630$

H -2.435515 -4.359192 -3.291383

O $-0.940784-4.126607-1.919854$

C $-5.089462-1.571111-1.978893$

H -3.842194 -1.565604 -3.691203

H -4.514067 -3.149714 -3.253835

H $\quad 0.343440-2.291715-1.796560$

H -1.354714 -2.126273 -5.747719

H $-0.385468-4.763483-2.401077$

O $-5.986356-0.912668-2.771179$

O $-5.129382-1.642940-0.759058$

C $-7.050518-0.245810-2.084952$

H $-7.7799420 .083870-2.829637$

H $-7.549011-0.923775-1.385410$

$\mathrm{H}-6.6621000 .632236-1.562634$

SCF Energy (B3LYP/6-31G**//MMFF) $=-3245.93250639$

0700226

MM̄MF Geometry
C $-4.253120-1.6334931 .962492$

C $-3.051179-2.1845372 .192541$

C $-2.060423-1.8029993 .265724$

O $-0.817532-1.5068362 .582904$

C $-2.430834-0.5628004 .106613$

C $-1.414179-0.2361945 .185672$

C $-0.4177100 .662307 \quad 5.048784$

C $-1.595817-0.9799516 .482192$

C $-0.063442 \quad 1.4387913 .810891$

C -0.2207382 .9512534 .001342$

C $-0.134167 \quad 3.7471192 .684727$

C -1.427796 3.6297561 .875483

C $-1.3271114 .360600 \quad 0.532246$

O 0.9776253 .2639961 .928244

O $-2.4459303 .955409-0.270522$

C $-0.0478713 .894324-0.195176$

C -1.4416175 .8810590 .696620$

O $0.1327914 .652822-1.388170$

C 1.2225283 .9482530 .685908

C 2.4188313 .2001220 .046720

O 1.5958115 .3062460 .920088

C $0.283298-2.2475952 .878569$

O $0.338062-3.262148 \quad 3.552824$

C $1.440767-1.5485952 .276114$

C $2.690468-1.9817602 .497241$

C $3.914396-1.3210042 .034353$

C 3.7612130 .0278561 .381858

C $2.9454883 .766493-1.271908$

C $4.3293443 .237620-1.607462$

C $5.5329951 .177701-2.323033$

O $5.3048233 .983524-1.664442$

C $5.546729-0.209465-1.764640$

C $6.608856-0.765090-1.161651$

C $6.596442-2.155324-0.588491$

C $7.156614-2.2201320 .817650$

C $8.239738-2.9679931 .089460$

C $6.479354-1.4166761 .907175$

C $5.101785-1.9291662 .237812$

N $4.3495981 .872249-1.843080$

H -4.611758 -0.833564 2.601801

H $-2.734434-3.0055711 .552421$

H -1.943375 -2.668656 3.929145

H -3.404371 -0.7154434 .590898$

H $-2.5634390 .307032 \quad 3.451633$

H 0.2463830 .8316265 .894960

H $-0.842335-0.7061697 .228216$

H -2.579704 -0.762218 6.910404

H -1.518799 -2.059231 6.316743

H 0.9831961 .2167373 .569164

H -0.6451621 .1030972 .950874$

H -1.1623393 .1873354 .512499$

H $0.588963 \quad 3.2893204 .661750$

H 0.0449234 .7962162 .948614

H -2.2819604 .0045942 .453382$

H -1.6513872 .5753561 .667657$

H $-3.259723 \quad 4.1773330 .213669$

H $-0.2023142 .851960-0.505896$

H -2.396640 6.1442091 .167188

H -1.439468 $6.384982-0.276322$

H $-0.6441936 .308138 \quad 1.307969$

H $-0.6739094 .549535-1.921754$

H 3.2433353 .1900790 .773887

H $2.1467952 .145267-0.089086$

H 2.5395045 .3282311 .150233

H $1.230531-0.6582491 .692939$

H $2.835289-2.8870713 .084914$

H 3.2364160 .7207082 .049346

H $3.192065-0.0626410 .450310$

H 4.7078930 .5068541 .128473

H $2.2782243 .512298-2.102729$

H $3.0142904 .859151-1.228057$

H $6.4311201 .743613-2.055669$

H $5.4613921 .139139-3.414708$

H $4.635489-0.793351-1.861683$

H $7.534762-0.200127-1.084501$

H $7.184404-2.793647-1.260481$

H $5.581488-2.571583-0.586336$

H 8.654061-3.021642 2.090801 
H $8.737700-3.5455420 .317647$ H $6.486470-0.3587161 .633927$ H $7.069245-1.4636502 .832779$ H $5.107014-2.9059112 .723616$ H $3.4593391 .390272-1.927864$ C $-5.561054-1.003722-0.142732$ O $-4.410647-0.598489-0.906511$ C $-4.5911530 .779518-1.302161$ C -5.972336 $1.201526-0.800995$ $\begin{array}{llll}C & -6.163621 & 0.290671 & 0.396937\end{array}$ H -3.831436 $1.359997-0.767377$ C -4.401851 $0.926125-2.818676$ H -6.007618 $2.264452-0.541976$ H $-6.7522271 .017982-1.549150$ C -7.6082500 .1645680 .845453$ H -5.5627510 .6919991 .221548$ C -3.041517 $0.415005-3.335933$ O $-5.427253 \quad 0.181634-3.490401$ H $-4.5485051 .972053-3.110744$ H -2.981390 -0.664224 -3.163313 O $-3.0067230 .584302-4.758270$ C $-1.8192321 .118987-2.718347$ C $-0.4643010 .681108-3.306193$ H $-1.8031710 .948196-1.637273$ O $-1.9409902 .531291-2.904396$ H $-0.4186140 .931315-4.373506$ H $0.3098191 .293428-2.824006$ H $-8.006723 \quad 1.1415261 .137993$ H -8.243946 -0.2320170 .046657$ H -7.691239-0.505417 1.706942 C -5.187682 -2.0859570 .874703$ H -5.340193 $-0.748459-3.219662$ H -3.175864 $1.521570-4.952446$ H -1.859886 2.717119-3.855014 H $-4.739358-2.9288950 .333439$ H $-6.100548-2.4638581 .350055$ H -6.255842 -1.458285 -0.862762 C $0.019573-2.438619-1.329419$ O $-0.133175-1.059635-1.691950$ C $-0.154738-0.809174-3.100493$ C $1.189332-1.215611-3.718103$ C $1.507965-2.684272-3.420049$ C $1.301828-3.035374-1.940651$ H $0.141134-2.415796-0.241024$ C $-1.267663-3.217089-1.637261$ H -0.949157-1.401707 -3.565435 O $2.233019-0.387920-3.211871$ H $1.147658-1.057970-4.802108$ H $2.548704-2.885898-3.702993$ O $0.670949-3.500542-4.234397$ H $1.314278-4.122845-1.807200$ O $2.423517-2.514115-1.220427$ C $-1.447851-4.427811-0.748472$ H -2.126861 -2.556034-1.467397 H $-1.304327-3.546713-2.678938$ H $2.235100-0.476620-2.243226$ H $0.913454-4.427342-4.067682$ H $2.360727-2.840425-0.306779$ O $-2.703810-4.931149-0.938913$ O $-0.603296-4.8798550 .011865$ C $-3.034573-6.075058-0.145656$ H -2.976238 -5.8278020 .918644$ H $-4.061375-6.366792-0.381571$ H -2.367999-6.908470 -0.386816 SCF Energy (B3LYP/6-31G**//MMFF)= -3245.91896607

\section{7_00227}

MM̄FF Geometry

C $0.051658 \quad 3.873309-2.021763$

C $-0.5253494 .744829-1.179254$

C $-1.8046514 .524716-0.400260$

O $-2.260146 \quad 3.167089-0.599537$

C -1.5373664 .7422221 .099253$

C -2.7967994 .8430591 .937419$

C $-3.257468 \quad 3.8536462 .727566$

C $-3.501564 \quad 6.172787 \quad 1.893124$

C -2.687315 2.4697532 .837862

C -1.7015732 .3357274 .007000$
C -0.9112841 .0143223 .989230$

C 0.1675401 .0187252 .900521

C $0.869230-0.3388582 .769593$

O $-1.846750-0.0449013 .793945$

O $1.611449-0.3282221 .542197$

C $-0.206921-1.4342462 .636820$

C $1.889532-0.5720943 .887935$

O $0.384744-2.731207 \quad 2.624101$

C $-1.291665-1.3627303 .732067$

C -2.467837 -2.327481 3.451404

O $-0.716077-1.7334394 .988549$

C -3.555035 $2.954386-0.950639$

O $-4.4462443 .783169-1.032966$

C $-3.6838061 .514054-1.277239$

C $-4.7549621 .081620-1.959526$

C $-4.980578-0.282402-2.448870$

C $-3.977216-1.339062-2.068384$

C $-3.254372-2.0058132 .175360$

C $-4.586602-2.7326922 .137427$

C -6.876806 -2.537367 1.163350

O $-4.774121-3.8064542 .703854$

C $-6.976992-3.109641-0.214144$

C $-7.841060-2.665475-1.139199$

C $-7.977310-3.269579-2.505616$

C $-7.825302-2.254277-3.614026$

C $-8.886839-1.849606-4.332160$

C $-6.429347-1.784118-3.956089$

C $-6.048390-0.512177-3.242610$

N $-5.525427-2.0561241 .372089$

H $-0.4151102 .911165-2.212694$

H $-0.0458945 .710675-1.029149$

H -2.541877 $5.245409-0.773343$

H $-0.9640065 .667044 \quad 1.251252$

H $-0.892463 \quad 3.938301 \quad 1.474755$

H -4.1462844 .0341613 .329260$

H -4.3750446 .2017682 .553167$

H -2.8239226 .9731352 .207845$

H -3.849224 6.3898230 .878579

H -3.5225861 .7713912 .975339$

H -2.222111 2.1767761 .893541

H -1.0008743 .1794544 .030532$

H -2.281330 2.3804604 .938625

H -0.4414870 .8923264 .972450$

H $\quad 0.899775 \quad 1.814433 \quad 3.087519$

H $-0.277525 \quad 1.2550561 .925773$

H 2.2171010 .4316951 .568497

H $-0.680168-1.2987931 .656016$

H $2.670590 \quad 0.1975373 .859531$

H $2.407729-1.5283623 .756170$

H $1.445546-0.5525644 .885192$

H $1.049064-2.7393371 .913501$

H -2.113887 -3.3659823 .428552$

H $-3.162810-2.2770134 .301784$

H -1.425734 -2.043256 5.575079

H -2.863777 $0.861619-0.999865$

H $-5.5240811 .805313-2.227361$

H $-3.798400-1.337728-0.988388$

H $-3.024926-1.161045-2.579184$

H $-4.299075-2.352730-2.316069$

H $-3.439130-0.9283222 .104659$

H $-2.688665-2.3109121 .288471$

H $-7.549668-1.6861671 .307797$

H -7.122433 -3.304259 1.904615

H $-6.323239-3.945544-0.451902$

H $-8.511140-1.845109-0.892052$

H $-8.966043-3.743473-2.560350$

H $-7.252950-4.079462-2.658781$

H $-8.778157-1.146047-5.151083$

H $-9.887446-2.207853-4.114323$

H $-6.372929-1.576873-5.033692$

H $-5.719215-2.599555-3.795268$

H $-6.7086630 .326348-3.470317$

H $-5.260909-1.1914060 .909553$

C $2.3644073 .033366-2.481869$

O $2.7752383 .067287-1.100778$

C $4.1926302 .793813-1.027339$

C $4.6196022 .397504-2.433980$

C $3.6505283 .186661-3.289632$ 
H $4.664253 \quad 3.739020-0.731795$

C 4.4344531 .7152790 .035632

H $5.6672092 .639001-2.637632$

H $4.4812951 .320617-2.595629$

C $3.5530502 .677667-4.717173$

H $3.9661884 .238568-3.300283$

C 5.8923321 .2256280 .103351

O $4.0725632 .251613 \quad 1.312123$

H $3.7565220 .876319-0.150658$

H $\quad 6.195416 \quad 0.798324-0.858312$

O $\quad 6.751924 \quad 2.354283 \quad 0.310071$

C $6.177976 \quad 0.204187 \quad 1.220995$

C $5.267037-1.0310931 .234836$

H 6.1057550 .6957252 .198934

O $7.534660-0.2336521 .111291$

H $5.632273-1.7407011 .989079$

H $4.276719-0.7282531 .593993$

H $4.5257432 .742613-5.215628$

H $3.2284521 .632057-4.749067$

H $2.8377263 .269933-5.296176$

C $1.3406524 .138123-2.749830$

H 3.1599342 .5808051 .240853

H 6.4767492 .7841811 .138205

H 8.0972040 .5597541 .110903

H $1.1192334 .192203-3.821561$

H $1.7540645 .107790-2.446474$

H $1.9177592 .045871-2.658457$

C $3.628114-3.487284-1.031072$

O $4.047602-2.7437460 .121365$

C $5.084461-1.775947-0.103735$

C $6.347747-2.472172-0.630466$

C $6.045973-3.318946-1.872114$

C $4.819692-4.216782-1.673107$

H $2.945470-4.251415-0.637594$

C $2.838859-2.615670-2.021509$

H $4.726359-1.063082-0.849863$

O $6.915365-3.301091 \quad 0.381129$

H $7.097344-1.718371-0.891726$

H $6.922539-3.937494-2.100630$

O $5.832996-2.443734-2.976320$

H $4.504911-4.660518-2.624772$

O $5.174698-5.297235-0.804737$

C $1.560370-2.080850-1.410159$

H $3.432533-1.762805-2.361005$

H $2.555070-3.203814-2.901344$

H $6.232592-3.9321370 .666364$

H $5.676060-2.995298-3.761629$

H $5.895176-5.791805-1.231430$

O $1.206733-0.925773-2.045741$

O $0.938582-2.625341-0.508287$

C $0.000385-0.291698-1.604136$

H $-0.4172160 .263341-2.448309$

H $-0.745910-1.016191-1.265035$

H $0.2372990 .413426-0.803311$

SCF Energy (B3LYP/6-31G**//MMFF) $=-3245.92070543$

0700228

MM̄FF Geometry

C -0.222075 -0.3174621 .831472$

C $0.090127-0.8166320 .624406$

C $-0.754596-1.747725-0.212199$

O $-1.961847-2.0789910 .511488$

C $-1.167544-1.093298-1.549097$

C $-0.033708-0.795382-2.507146$

C $0.328108 \quad 0.439180-2.908695$

C $0.694692-2.001560-3.042703$

C $-0.2717211 .758662-2.507588$

C $0.5799002 .425919-1.425309$

C $0.0370403 .790585-0.983463$

C $0.928579 \quad 4.363106 \quad 0.123341$

C 0.3811525 .6795020 .690353

O $-1.3003923 .617306-0.501932$

O 1.0742125 .9506191 .918699

C -1.1081615 .4847171 .043260$

C $0.6738206 .861456-0.240836$

O

C -1.936742 $4.833274-0.090702$

C $-3.358547 \quad 4.4976660 .427612$
O $-2.0431265 .768326-1.168720$

C $-2.628934-3.2087040 .160519$

O $-2.285266-4.058779-0.643498$

C $-3.918580-3.1712800 .878889$

C $-5.008429-3.6469250 .260507$

C $-6.383392-3.5120660 .741815$

C $-6.586214-3.0527752 .161763$

C $-4.2350943 .697073-0.547231$

C $-4.1514272 .186309-0.387374$

C $-5.2334000 .111182-1.281638$

O -3.4171331 .6260550 .420525$

C $-6.541813-0.186666-0.615570$

C $-7.614462-0.674991-1.258848$

C $-8.924806-0.994839-0.591001$

C $-9.382276-2.418315-0.843800$

C $-10.274877-2.694403-1.809496$

C -8.857961 -3.509795 0.069017

C -7.387005 -3.742814 -0.130038

N $-5.0194111 .545975-1.264433$

H -1.167021 -0.573485 2.302422

H $1.053598-0.5499980 .195271$

H $-0.179755-2.666767-0.378544$

H -1.758303 -0.198707 -1.324612

H $-1.877749-1.748770-2.070851$

H $1.1611450 .527179-3.604403$

H $1.415872-1.738403-3.823520$

H $1.246009-2.506083-2.243603$

H $-0.015234-2.710955-3.480140$

H $-0.3126822 .402144-3.394968$

H -1.306755 $1.646776-2.173685$

H $0.614641 \quad 1.762827-0.554603$

H $1.6103652 .543731-1.784151$

H $0.0232094 .456918-1.854039$

H $1.9570654 .496258-0.235508$

H 0.9942673 .6384310 .947217

H 2.0258755 .9950801 .722966

H -1.145892 4.8253331 .921412

H $1.7544266 .968283-0.395263$

H $\quad 0.342537 \quad 7.806671 \quad 0.202945$

H $0.2107176 .757666-1.224202$

H -1.858510 7.2489070 .657250

H -3.287416 3.9541941 .377638

H -3.8802485 .4415610 .633222$

H -2.885296 $5.638558-1.631147$

H -3.953953 -2.650457 1.828686

H $-4.894113-4.095014-0.725870$

H -6.274933 -2.009324 2.279139

H $-6.002847-3.6723042 .852633$

H -7.622579 -3.123789 2.498142

H $-5.2814993 .969507-0.356390$

H $-4.0160043 .946862-1.590186$

H $-4.420338-0.398545-0.756334$

H $-5.222899-0.206749-2.328892$

H $-6.597863-0.0102310 .455956$

H -7.555983 -0.843612 -2.331907

H $-9.674121-0.290068-0.974681$

H -8.877247 $-0.816216 \quad 0.490415$

H - $-10.626511-3.705943-1.983129$

H -10.670908 -1.914455 -2.451419

H $-9.373117-4.458664-0.128431$

H $-9.115167-3.247481 \quad 1.098428$

H -7.133224 -4.078348 -1.136771

H $-5.6044642 .110408-1.873846$

C $1.701479-0.0862493 .490246$

O $2.637660-0.8101752 .664918$

C $2.771077-2.1414493 .190027$

C $2.313206-2.0499704 .639687$

C $1.149048-1.0847874 .510953$

H $2.064180-2.7725692 .637800$

C $4.194589-2.6643972 .985191$

H $2.022781-3.0222285 .048590$

H $3.089575-1.6226075 .284875$

C $0.730685-0.4478935 .824530$

H $0.293524-1.6347724 .100386$

C $4.704038-2.6046811 .528671$

O $5.102303-1.8966293 .786314$

H $4.262239-3.6920253 .361059$

H $4.934180-1.5672951 .260193$ 
O $\quad 5.947312-3.316715 \quad 1.478815$

C $3.752718-3.207310 \quad 0.479548$

C $4.369586-3.409263-0.917420$

H $2.864217-2.5722450 .392378$

$\begin{array}{llll}\text { O } & 3.309331 & -4.487358 & 0.940681\end{array}$

H $5.124257-4.205303-0.875725$

H $3.570953-3.798127-1.563526$

H $0.397564-1.212506 \quad 6.533898$

H $1.5580750 .100896 \quad 6.286828$

H -0.0950920 .2538315 .669463$

$\begin{array}{llll}\text { C } & 0.669188 & 0.616372 & 2.604405\end{array}$

H $5.007292-0.9645923 .524733$

H $6.537051-2.9162862 .140491$

H $2.654286-4.8183310 .302615$

H $1.192413 \quad 1.2720351 .898717$

H $\quad 0.036021 \quad 1.268126 \quad 3.217927$

H $2.291516 \quad 0.6845834 .002496$

C $4.3674190 .114872-2.177469$

O $3.955318-1.153313-1.646466$

C $4.979332-2.146621-1.549775$

C $5.575235-2.441271-2.936132$

C $6.068959-1.159591-3.614551$

C $5.017815-0.047776-3.563389$

H $3.4358530 .677827-2.311704$

C $5.2447700 .896606-1.185824$

H $5.776450-1.783147-0.894545$

O $4.608433-3.075948-3.771396$

H $6.407869-3.144897-2.820876$

H $6.314203-1.372723-4.662329$

O $7.255150-0.702087-2.972245$

H $5.4618240 .903025-3.881422$

O $3.995095-0.368968-4.510601$

C 4.4758861 .3089640 .050645

H $6.1060080 .311812-0.853413$

H $5.6033851 .815399-1.664228$

H $3.834533-2.489795-3.828493$

H $7.919369-1.408572-3.044724$

H $3.3788450 .382419-4.542546$

O $5.312092 \quad 1.987776 \quad 0.890355$

$\begin{array}{llllll}\text { O } & 3.295048 & 1.070647 & 0.258223\end{array}$

C 4.7195062 .4410102 .111846

H 4.4647151 .5842752 .742136

H 5.4537463 .0573192 .637336

H 3.8330613 .0499581 .908554

SCF Energy (B3LYP/6-31G**/MMFF)= -3245.90371196

07_00229

MMFF Geometry

C $0.904196-3.3143490 .868727$

C $0.058279-2.2786800 .755697$

C $-0.745132-1.743182 \quad 1.911730$

O $-0.726978-0.2955881 .888163$

C $-2.224719-2.1528861 .801716$

C $-2.473572-3.6341031 .984028$

C -2.786333 -4.4868200 .989040$

C $-2.392095-4.129758 \quad 3.404744$

C $-2.909264-4.190435-0.480334$

C $-4.355371-4.314665-0.978668$

C $-5.275407-3.198217-0.460473$

C $-6.722144-3.457527-0.882928$

C $-7.651605-2.325352-0.425456$

O $-4.825725-1.960351-1.018869$

O $-8.903955-2.475910-1.112414$

C $-7.053977-0.975008-0.875023$

C -7.968335 -2.4235031 .071560$

O $-7.787676 \quad 0.116233-0.323855$

C -5.545282 -0.806522 -0.562971

C $-4.9931150 .413533-1.331019$

$\begin{array}{llll}\text { O } & -5.312768 & -0.553420 & 0.823465\end{array}$

C $0.307993 \quad 0.3334952 .497939$

O $1.311202-0.1943602 .958792$

C 0.0921251 .7981002 .574369

C $\begin{array}{llll}0 & 1.050276 & 2.414419 & 2.230201\end{array}$

C - $-1.317826 \quad 3.8540852 .325280$

C -0.1942474 .7388402 .800176$

C $-3.4907470 .655896-1.129319$

C $-3.0195691 .875005-1.905312$

C $-1.0115263 .298097-2.273016$
O $-3.7594802 .532749-2.633222$

C $-1.0615264 .420553-1.286650$

C $-2.0035095 .375139-1.302484$

C $-2.1004416 .484927-0.295918$

C -3.2873726 .3102300 .627100$

C $-4.5144496 .714957 \quad 0.257580$

C $-3.036845 \quad 5.7355882 .004453$

C $-2.537257 \quad 4.315548 \quad 1.972149$

N -1.673976 $2.142672-1.694227$

H $1.036767-3.782048 \quad 1.841639$

H $-0.118451-1.832510-0.218629$

H $-0.342030-2.0856902 .873116$

H -2.635680 -1.786607 0.854115

H $-2.803336-1.6063842 .559713$

H -2.962693 -5.5306191 .246703$

H -2.646842 $-5.191477 \quad 3.490297$

H $-1.379760-4.0023603 .800269$

H -3.087989 -3.573510 4.041238

H $-2.498771-3.212519-0.747051$

H -2.292824 -4.924283-1.014895

H $-4.332213-4.279694-2.075667$

H $-4.758341-5.294669-0.693965$

H $-5.204400-3.1499710 .631156$

H $-7.075181-4.421860-0.496851$

H -6.777010 -3.533186 -1.978047

H $-9.255131-3.359252-0.906664$

H -7.182908 $-0.914495-1.965158$

H -8.484527 $-3.365721 \quad 1.292335$

H $-8.652905-1.6277481 .385022$

H -7.077774 -2.384421 1.702476

H $-8.720523-0.008526-0.569178$

H $-5.1762370 .278679-2.405311$

H -5.531040 $1.318385-1.019499$

H $-6.161239-0.5681511 .292718$

H 0.9364992 .3501322 .973765

H -1.8793531 .8170861 .855738$

H -0.4443615 .8009812 .815493$

H 0.6725194 .6413822 .138176

H $\quad 0.1052114 .469694 \quad 3.819001$

H -3.272761 $0.819715-0.068401$

H $-2.914221-0.210301-1.472971$

H $-1.4949113 .566358-3.218329$

H $0.0270123 .027789-2.484610$

H $-0.2935224 .435849-0.518688$

H -2.769358 $5.349093-2.074845$

H $-2.188027 \quad 7.434574-0.839669$

H -1.1781396 .5730550 .291450$

H $-5.367386 \quad 6.6173800 .920894$

H $-4.6922057 .151056-0.720000$

H -3.965189 5.7347032 .591156

H -2.365772 6.4085522 .544903

H -3.284638 3.5989661 .628646

H $-1.1573601 .584691-1.020416$

C $3.078619-4.2710320 .026670$

O $3.866906-3.0644130 .022461$

C $5.158340-3.357505-0.552487$

C $5.199412-4.866293-0.773896$

C $3.736886-5.182245-1.008208$

H $5.190387-2.862094-1.529042$

C $6.280414-2.8345810 .351889$

H $5.839874-5.142306-1.617434$

H $5.568705-5.3966840 .111892$

C $3.407174-6.656127-0.845030$

H $3.463778-4.858807-2.021757$

C $6.260561-1.3050640 .547485$

O $6.135700-3.4554961 .635694$

H $7.252156-3.155242-0.042073$

H $5.320967-1.0032221 .022603$

O $7.310194-0.9642041 .462521$

C $6.488657-0.502056-0.751988$

C $6.6178251 .019017-0.534235$

H $5.678770-0.694142-1.462087$

O $7.694721-0.953078-1.376059$

H $7.359725 \quad 1.2189220 .248028$

H $7.0256421 .460386-1.453909$

H $3.977289-7.258603-1.559885$

H $3.648497-7.012123 \quad 0.162190$

H $2.343563-6.842905-1.022053$ 
C $1.616796-3.951650-0.291946$ H $6.957169-3.2964132 .130527$ H $7.044895-1.2833012 .341706$ H $8.422318-0.805973-0.747328$ H $1.081624-4.879295-0.525400$ H $1.560178-3.304857-1.174563$ H $3.168448-4.7098981 .029462$ C $3.1967872 .292806-1.282282$

O $4.5160181 .743880-1.402193$

C $5.281799 \quad 1.687233-0.192766$

C $5.472748 \quad 3.098998 \quad 0.375295$

C $4.1201593 .778344 \quad 0.600703$

C $3.2214383 .689360-0.636055$

H $2.8528282 .411630-2.318119$

C $2.2478711 .307736-0.592197$

H $4.753434 \quad 1.0774280 .544835$

O $6.2739853 .887116-0.501272$

H 6.0111793 .0283561 .327647

H $4.288544 \quad 4.8309250 .859796$

O $3.4810443 .148403 \quad 1.706749$

H $2.2021134 .003081-0.384831$

O $3.7180034 .624082-1.599409$

C $1.992513 \quad 0.130938-1.505414$

H 2.6349160 .9110880 .350000

H $1.2895121 .786441-0.362093$

H $5.8392183 .900585-1.370960$

H 2.6555743 .6310671 .878354

H $3.0998574 .629051-2.350030$

O $\quad 0.8141550 .300468-2.167679$

O $2.770079-0.803347-1.646636$

C $0.490245-0.704827-3.132900$

H $0.366058-1.675336-2.645501$

H $-0.455459-0.427690-3.606338$

H $1.266291-0.755729-3.902456$

SCF Energy (B3LYP/6-31G*//MMFF)= -3245.90593185

07_00230

MM̄FF Geometry

C -5.505780 -3.378504 1.133211

C $-5.086090-2.4578552 .014817$

C $-5.324868-0.9655491 .979526$

O $-4.054305-0.3418561 .655968$

C $-6.353981-0.4832350 .938714$

C $-6.735508 \quad 0.975154 \quad 1.113774$

C $-6.301926 \quad 1.9923450 .343128$

C $-7.710043 \quad 1.2494852 .232614$

C $-5.3482251 .950194-0.819454$

C $-4.0635632 .710845-0.488999$

C $-3.1083852 .903403-1.676500$

C $-2.6821621 .590278-2.335301$

C $-1.6207931 .810028-3.425624$

O $-1.958415 \quad 3.576282-1.147880$

O $-1.0926950 .517467-3.755415$

C $-0.4762562 .664663-2.843704$

C -2.241589 $2.366132-4.712744$

O $0.4658143 .019886-3.853413$

C $-0.9692903 .938512-2.119283$

C $0.2065794 .596080-1.359739$

O $-1.4850494 .860428-3.079694$

C -3.160399 -0.1503272 .663949$

O $-3.333798-0.359175 \quad 3.853675$

C $-1.893844 \quad 0.307246 \quad 2.050537$

C $-0.751990 \quad 0.2282462 .749640$

C $0.5826370 .562524 \quad 2.247854$

C 0.6675101 .3299650 .956362

C $-0.198550 \quad 5.809317-0.513838$

C $0.837642 \quad 6.095627 \quad 0.553095$

C 1.6115965 .3136032 .791614

$\begin{array}{lllll}\text { O } & 1.680262 & 6.979164 & 0.415944\end{array}$

C 2.7308104 .3403922 .596072

C 3.0742133 .4109113 .500818

C 4.2464912 .4888083 .331136

C 3.9140571 .0444753 .623198

C $4.397645 \quad 0.431188 \quad 4.717103$

C 3.1123580 .2797212 .596533

C $1.656792 \quad 0.1576542 .956805$

$\begin{array}{lllll}\mathrm{N} & 0.721292 & 5.253088 & 1.648958\end{array}$

H $-6.112759-3.0877190 .281676$
H $-4.464031-2.7988502 .841460$

H -5.646944 -0.6519012 .980067$

H -7.273983 -1.077827 1.015408

H $-5.967949-0.659141-0.070771$

H -6.6722712 .9923910 .569112$

H -8.0052572 .3033102 .279561$

H -8.622665 0.6605412 .093716

H -7.271671 0.9894353 .200736

H $-5.1074810 .924478-1.106530$

H $-5.8411892 .412286-1.683033$

H $-4.3097453 .705065-0.090049$

H $-3.5344582 .206030 \quad 0.327619$

H -3.600511 $3.557943-2.405377$

H $-3.5418901 .058757-2.759573$

H $-2.2635000 .922338-1.569976$

H $-0.4530720 .626134-4.479061$

H $0.0636492 .039596-2.119179$

H -2.978367 $1.660644-5.115481$

H $-1.4857102 .489702-5.496144$

H $-2.7450103 .324273-4.567840$

H $0.8481302 .197421-4.202661$

H $0.6681293 .845819-0.707255$

H $0.9803864 .919996-2.067261$

H $-1.4774705 .751768-2.697130$

H $-1.9220220 .636863 \quad 1.018881$

H $-0.787360-0.1647583 .764959$

H 0.3789990 .6989830 .110126

H 0.0010692 .1987560 .983588

H $1.6653991 .722590 \quad 0.751737$

H $-0.3030346 .702790-1.139591$

H $-1.1639505 .657029-0.018047$

H $1.0162665 .087158 \quad 3.681689$

H 2.0151776 .3266102 .886951

H $3.312390 \quad 4.4312121 .681767$

H 2.5171343 .3428384 .432431

H 5.0380272 .8354824 .008228

H 4.6669272 .5604402 .320004

H $4.193102-0.6166234 .911816$

H $5.0046980 .962018 \quad 5.442854$

H $3.513870-0.7398612 .520884$

H $3.2692000 .712177 \quad 1.605605$

H $1.483504-0.3957803 .881024$

H $0.022716 \quad 4.515764 \quad 1.635027$

C -3.909088 $-5.204776 \quad 0.364573$

$\begin{array}{llll}0 & -2.756996 & -4.456078 & 0.811964\end{array}$

C $-2.231889-3.725244-0.313481$

C $-2.641680-4.543155-1.525602$

C $-4.052903-4.946263-1.139907$

H $-2.746285-2.755198-0.310128$

C $-0.723648-3.518283-0.140234$

H $-2.604527-3.972848-2.458216$

H -2.002456 -5.428324 -1.631795

C $-4.579614-6.142780-1.913984$

H $-4.715757-4.094453-1.332048$

C $-0.061643-2.824886-1.344046$

$\begin{array}{llll}\text { O } & -0.523738 & -2.704908 & 1.019415\end{array}$

H $-0.236930-4.4791370 .060826$

H -0.081399 -3.494762 -2.211531

O $-0.854738-1.686430-1.709122$

C $1.376742-2.320589-1.120035$

C $2.352456-3.407068-0.634802$

H $\quad 1.368420-1.479080-0.418357$

O $1.823024-1.800931-2.377806$

H $1.947058-3.901208 \quad 0.253684$

H $2.461399-4.155693-1.429496$

H $-4.621654-5.920010-2.985055$

H -3.940577 -7.021994 -1.778719

H $-5.589371-6.404480-1.581582$

C $-5.126985-4.8340411 .223065$

H $-0.965646-3.1471431 .764593$

H $-0.890218-1.094108-0.938633$

H $2.699894-1.405150-2.240509$

H $-4.907297-5.0833472 .269262$

H $-5.991221-5.4411470 .930261$

H -3.669489 -6.259895 0.546687

C $5.649818-1.763750-1.321847$

O $4.372490-2.394954-1.480666$

C $3.741182-2.836404-0.276133$ 
C $4.623527-3.8747390 .432611$

C $6.032238-3.3254560 .680518$

C $6.629406-2.690670-0.581737$

H $6.025107-1.629190-2.344695$

C $5.527048-0.370976-0.683508$

H $3.586949-1.9786730 .386180$

O $4.699956-5.054858-0.364050$

H $4.153343-4.147438 \quad 1.385140$

H $6.679444-4.1421601 .022702$

O $5.965247-2.3609421 .727074$

H $7.542812-2.134686-0.340436$

O $7.001979-3.730360-1.491207$

C $4.6972170 .577885-1.523907$

H $5.062283-0.4267720 .303185$

H $6.5228410 .073583-0.575060$

H $5.238342-5.702576 \quad 0.121515$

H $6.868447-2.0385241 .887816$

H $7.659228-4.290056-1.043848$

O $4.4572891 .714781-0.804915$

O $4.315730 \quad 0.361003-2.665788$

C $3.6642702 .704365-1.468159$

H $3.4989303 .531025-0.772497$

H $4.1933183 .084231-2.347038$

H $2.6937792 .286297-1.751742$

SCF Energy (B3LYP/6-31G**//MMFF)= -3245.89087461

0700231

MM̄FF Geometry

C $-3.736268-2.283360-3.033838$

C $-2.624307-2.628999-2.369951$

C $-1.228328-2.396270-2.884554$

O $-0.665476-1.320625-2.096238$

C $-0.374165-3.660534-2.687790$

C $1.033753-3.549845-3.242510$

C $2.159923-3.717810-2.520962$

C $1.136148-3.273861-4.721603$

C $2.286202-4.056916-1.062790$

C $2.679900-2.853637-0.204598$

C $4.054550-2.232213-0.502925$

C $5.200605-3.222469-0.277469$

C $6.578210-2.559186-0.416772$

O $4.187876-1.116248 \quad 0.388177$

O $7.558250-3.4590370 .123493$

C $6.609551-1.2855280 .450293$

C $6.956561-2.337130-1.886181$

O $7.822000-0.5631420 .243549$

C $5.394203-0.356867 \quad 0.226337$

C 5.3879260 .7591591 .300654

O $5.483202 \quad 0.225638-1.072657$

C $-0.890926-0.057231-2.555045$

O $-1.3855230 .253575-3.628043$

C $-0.4933900 .900569-1.497855$

C $-0.8083862 .195192-1.659811$

C $-0.5658033 .289360-0.716941$

C 0.1027582 .9513040 .586153

C 4.2085861 .7347261 .199293

C $4.5521902 .958974 \quad 0.371249$

C $3.7789094 .304257-1.574868$

O 5.4889703 .6984150 .667195

C $2.9622115 .406386-0.976180$

C $1.9788016 .049633-1.622882$

C $1.1842207 .165495-1.006850$

C $-0.3091846 .939822-1.075093$

C -1.101148 $7.766799-1.778824$

C $-0.8980165 .804644-0.263141$

C $-0.9865164 .526812-1.054494$

N $3.7026253 .142624-0.708210$

H -3.656823 -1.830016 -4.018480

H -2.717797 -3.051092 -1.374366

H -1.249946 -2.127114 -3.947286

H $-0.861860-4.513082-3.179296$

H -0.352485 -3.896394 -1.619430

H $3.114572-3.622030-3.037392$

H $2.158685-3.391534-5.096399$

H $\quad 0.503249-3.969166-5.282864$

H $0.820552-2.250567-4.946908$

H $1.357896-4.478285-0.666437$

H $3.022098-4.861770-0.955817$
H $1.912946-2.073141-0.302219$

H $2.649182-3.1515130 .852194$

H $4.060649-1.856701-1.532038$

H $5.130653-4.074846-0.962872$

H $5.120707-3.644036 \quad 0.734311$

H $7.500459-4.296122-0.368339$

H $6.613352-1.6027631 .502609$

H $6.994683-3.294194-2.420547$

H $7.959449-1.905206-1.975599$

H $\quad 6.253158-1.692688-2.417577$

H $8.557291-1.1786630 .406130$

H 5.3239470 .2805112 .286761

H $\quad 6.331457 \quad 1.3161391 .266363$

H $4.8468020 .955069-1.131293$

H $\quad 0.0005300 .515141-0.613976$

H -1.318449 2.490136-2.576603

H 1.0608912 .4524370 .416933

H -0.5363212 .2827421 .170292$

H $\quad 0.3115993 .8207181 .211979$

H $3.308271 \quad 1.238907 \quad 0.825133$

H 3.9622412 .1103922 .200530

H $3.4131244 .003586-2.561534$

H $4.8223634 .622315-1.665382$

H 3.2048945 .7018350 .042320

H $1.7468375 .778770-2.650037$

H $1.4544318 .091635-1.530279$

H 1.4667507 .3197370 .042280

H -2.176608 $7.625909-1.810093$

H -0.693345 $8.599500-2.342130$

H -1.917633 6.0694320 .047553

H -0.3317445 .6995860 .665926$

H -1.493820 $4.649236-2.013040$

H $2.9214482 .509211-0.845237$

C $-5.629918-1.080282-1.951352$

O $-4.890890-0.683853-0.775353$

C -5.7750500 .0466250 .099393$

C $-7.1198940 .132318-0.619210$

C $-7.087103-1.098406-1.503800$

H $-5.898862-0.5555901 .005744$

C $-5.171163 \quad 1.4140010 .439528$

H -7.9608550 .1347740 .081296$

H -7.194884 $1.041654-1.227679$

C $-8.092120-1.050586-2.641121$

H -7.279744 -1.983962 -0.883539

C $-3.853096 \quad 1.3576151 .240722$

O $-4.9118232 .119827-0.782674$

H $-5.9056802 .018657 \quad 0.983671$

H $-3.0720670 .868616 \quad 0.648644$

O -3.4228912 .7112551 .443781$

C -3.9744910 .6836022 .624858$

C -2.7313630 .8504093 .523555$

H $-4.195453-0.3814542 .504263$

O $-5.084447 \quad 1.2542293 .326186$

H -2.4505961 .9096003 .568678$

H -3.016452 0.5755464 .548404

H $-9.112006-0.975544-2.249679$

H -7.918492 -0.188751 -3.294234

H $-8.033280-1.954821-3.254447$

C -5.117506 -2.427755 -2.459608

H $-4.2802421 .589472-1.298589$

H $-3.355003 \quad 3.1281380 .567792$

H -4.9108372 .2063993 .424016$

H $-5.772597-2.822242-3.243915$

H $-5.112228-3.159524-1.643971$

H $-5.472092-0.301027-2.709781$

C $-0.820512-2.3289073 .126095$

O $-1.812815-1.3540093 .474738$

C $-1.534493-0.004453 \quad 3.081024$

C -0.2244720 .4745723 .723483$

C $0.929729-0.4626923 .361726$

C $0.574214-1.9255573 .635853$

H -1.117198 -3.2329113 .674544$

C $-0.864509-2.6534531 .628080$

H $-1.439790 \quad 0.0451901 .992764$

O $-\begin{array}{llll}0.379188 & 0.532417 & 5.139582\end{array}$

H -0.0058241 .4904063 .375240$

H $1.816243-0.1834543 .944041$

O $1.236899-0.2921501 .981955$ 
H $1.329646-2.5870753 .197361$

O $0.618635-2.1511595 .048665$

C -2.009726 $-3.595276 \quad 1.337270$

H $-0.968319-1.7778250 .982040$

H $\quad 0.049860-3.1735941 .322299$

H 0.4556970 .8637465 .512335

H $2.023531-0.8306481 .790650$

H $-0.096348-1.6321775 .455607$

O $-3.199493-2.9383681 .444573$

O $-1.860409-4.7792631 .062902$

C $-4.368924-3.744481 \quad 1.271115$

H -5.233477 -3.0798881 .198347$

H $-4.304709-4.3367510 .353548$

H $-4.497916-4.3968042 .139581$

SCF Energy (B3LYP/6-31G**//MMFF)= -3245.92759622

$07 \_00232$

MM̄FF Geometry

C -2.331837 3.6853550 .922221

C -2.161438 3.0692932 .101423

C $-2.795118 \quad 1.7496242 .441909$

$\begin{array}{llll}\text { O } & -1.723295 & 0.842447 & 2.803872\end{array}$

C -3.7491561 .8236893 .647633$

C -5.1840182 .0877373 .243478$

C $-6.114706 \quad 1.123525 \quad 3.098739$

C $-5.549813 \quad 3.530523 \quad 3.018111$

C $-5.948841-0.3565493 .311080$

C $-5.154485-1.1028022 .233598$

C $-5.563601-0.8318840 .777977$

C $-7.008825-1.244160 \quad 0.492247$

C $-7.369136-1.074102-0.990972$

O $-4.666823-1.589579-0.049978$

O $-8.603096-1.769023-1.228114$

C $-6.290364-1.763927-1.849772$

C $-7.6224080 .394840-1.351516$

O $-6.505662-1.508877-3.236469$

C $-4.843210-1.379727-1.458346$

C $-3.845357-2.289979-2.217130$

O $-4.607280-0.018551-1.822263$

C -1.234249 0.0337051 .826347

$\begin{array}{lllll}0 & -1.575832 & 0.001401 & 0.655067\end{array}$

C -0.213762 -0.8420282 .440646$

C $0.080079-2.0071421 .845968$

C $1.035653-3.0067812 .321894$

C $1.955100-2.6187853 .448705$

C $-2.381880-2.138793-1.781514$

C $-1.454557-3.063920-2.553806$

C $0.968191-3.618452-2.794209$

O $-1.855261-3.895902-3.363417$

C $1.294108-4.754695-1.877613$

C $2.462462-4.879437-1.229474$

C $2.792712-6.024756-0.314605$

C $3.080993-5.5793751 .103182$

C $4.344214-5.432887 \quad 1.536547$

C $1.913857-5.4033562 .048784$

C $1.056643-4.2129071 .718054$

$\mathrm{N}-0.123001-2.849943-2.223076$

H $-2.995825 \quad 3.246304 \quad 0.181415$

H -1.493808 3.5042722 .841210

H -3.335494 1.3440131 .577558

H -3.419793 2.5876204 .364398

H -3.690543 0.8843984 .211677

H -7.118942 1.4214132 .799827

H $-6.622732 \quad 3.6633572 .841757$

H $-5.287757 \quad 4.1312453 .895188$

H -5.0239213 .9328062 .148481$

H -5.489749 -0.5397014 .290192$

H $-6.950470-0.7990323 .384933$

H $-4.087832-0.8759882 .344245$

H $-5.223257-2.1810332 .432071$

H $-5.414010 \quad 0.2317840 .563701$

H $-7.715497-0.6826221 .114119$

H -7.145220 -2.300422 0.763373

H $-9.278032-1.391243-0.638293$

H $-6.408125-2.848201-1.712941$

H -8.448364 $0.798661-0.753623$

H -7.932569 $0.497008-2.397318$

H $-6.7529901 .034020-1.184760$
H $-7.417423-1.777802-3.442192$

H $-4.130120-3.339136-2.062199$

H $-3.910215-2.090832-3.294460$

H $-3.651726 \quad 0.131085-1.890449$

H $0.237055-0.5229493 .373041$

H $-0.450255-2.2708840 .932603$

H $2.444465-1.6618323 .235815$

H $2.759577-3.3392783 .615138$

H $1.395702-2.5248584 .385685$

H -2.035544 -1.112419-1.939550

H $-2.282710-2.381222-0.718096$

H $1.814425-2.937893-2.925908$

H $0.677852-4.004041-3.776642$

H $0.528977-5.516513-1.746919$

H $3.231127-4.123944-1.370726$

H $3.667281-6.541765-0.731062$

H $1.989835-6.772497-0.303635$

H $4.562425-5.1453992 .559652$

H $5.189400-5.5940660 .875722$

H 2.256979 -5.3602103 .088689$

H $1.285239-6.3026242 .006814$

H $\quad 0.346901-4.3980550 .911639$

H $0.104605-2.176057-1.497590$

C $-0.796673 \quad 4.721948-0.744158$

O $0.1945873 .702661-0.515368$

C $1.3919784 .042988-1.235482$

C $1.0644235 .293776-2.046548$

C $-0.0096295 .946882-1.198412$

H $2.1499214 .287664-0.481846$

C $1.8773922 .858218-2.084907$

H $1.9419745 .930869-2.193955$

H $0.6678655 .039924-3.036807$

C $-0.8363036 .974886-1.950111$

H $0.4698296 .428032-0.335476$

C $2.2915811 .629746-1.244289$

O $0.8622582 .499470-3.021003$

H $2.7467693 .185623-2.667591$

H $3.0227771 .975764-0.507027$

O $1.1800641 .120585-0.511064$

C $2.9192720 .490616-2.074305$

C $3.377568-0.720806-1.240672$

H $3.7729540 .880782-2.640761$

O $1.979528-0.012641-3.026564$

H $2.509197-1.215932-0.787101$

H $3.794678-1.450459-1.947545$

H $-0.1995817 .786970-2.316048$

H $-1.3424656 .529564-2.813216$

H $-1.6005307 .412494-1.300197$

C -1.613816 $4.945577 \quad 0.528909$

H $0.0552672 .299271-2.516580$

H $0.5584680 .731199-1.149315$

H $1.7561930 .713763-3.632729$

H $-2.354613 \quad 5.7364730 .369579$

H $-0.952315 \quad 5.2701661 .341601$

H -1.446458 $4.360189-1.552621$

$\begin{array}{llll}C & 6.547521 & 0.734551 & 0.099575\end{array}$

O $5.513670 \quad 0.276540-0.781829$

C $4.416515-0.395550-0.156858$

C $4.912974-1.6548560 .567376$

C $6.010903-1.3025861 .577446$

C $7.098912-0.4232170 .951499$

H $7.3549381 .070033-0.564273$

C $6.091416 \quad 1.944906 \quad 0.929678$

H 3.9464650 .2688090 .574547

O $5.426524-2.579645-0.389072$

H $4.066593-2.1330401 .073101$

H $6.471201-2.2229171 .957322$

O $5.440189-0.6114052 .684327$

H $7.754066-0.0219341 .732859$

O $7.923665-1.2409870 .114875$

C $5.814906 \quad 3.158800 \quad 0.065009$

H 5.1798421 .7317131 .493798

H 6.8852922 .2220091 .632976

H $5.748244-3.3578250 .096033$

H $4.793428-1.2071343 .099032$

H $7.378704-1.549344-0.629359$

O $5.411715 \quad 4.189465 \quad 0.866222$

O $5.9378983 .205374-1.151101$ 
C 5.1290625 .4206390 .193004

H $6.0593555 .863716-0.173926$

H $4.4275505 .262108-0.631498$

H $4.672141 \quad 6.1047120 .912972$

SCF Energy (B3LYP/6-31G**//MMFF) $=-3245.91647287$

0700233

MM̄̄FF Geometry

C $1.610723 \quad 3.207213-2.167557$

C $2.0732872 .066711-2.703837$

C $1.355598 \quad 0.734439-2.710917$

O $\quad 0.237496 \quad 0.737645-1.791941$

C $0.8284870 .421575-4.120508$

C $0.314847-0.999323-4.253173$

C $-0.984359-1.357265-4.237796$

C $1.380046-2.047888-4.449945$

C $-2.187687-0.471336-4.068844$

C $-2.989349-0.862485-2.826259$

C $-4.292856-0.067983-2.655266$

C $-4.0621321 .434360-2.468402$

C $-5.3671512 .187892-2.166452$

O $-4.944117-0.605850-1.496300$

O $-5.0201513 .494024-1.680956$

C $-6.1129371 .467094-1.025797$

C $-6.2042442 .404760-3.433012$

O $-7.4006452 .041762-0.814242$

C $-6.241838-0.057201-1.240316$

C $-6.812216-0.7226840 .036991$

O $-7.142145-0.302574-2.324685$

C $0.5265040 .514777-0.479749$

O $1.6221460 .261855-0.004921$

C $-0.720530 \quad 0.6602630 .300329$

C $-0.6531450 .688374 \quad 1.639240$

C - -1.7818690 .7952592 .566865$

C $-3.1698650 .745867 \quad 1.985867$

C $-6.720269-2.2553160 .043159$

C $-5.427190-2.7675230 .652861$

C $-4.307054-3.1229252 .842997$

O $-4.554833-3.301830-0.027375$

C $-4.268528-2.3978314 .149131$

C $-3.137819-2.0005604 .752316$

C $-3.130187-1.2688746 .064827$

C $-2.228654-0.054596 \quad 6.075887$

C -1.280833 $0.086743 \quad 7.018791$

C -2.4628471 .0301465 .044669$

C -1.5112540 .9145573 .883559$

N -5.368391 -2.566066 2.023791

H $0.6139193 .232957-1.737055$

H $3.0684272 .062412-3.144129$

H $2.070770-0.039191-2.404888$

H $1.6260360 .566487-4.862019$

H $0.0496881 .142553-4.391333$

H -1.223849 -2.412530 -4.365426

H $0.961216-3.054396-4.553708$

H $1.957435-1.835961-5.355768$

H $2.065791-2.065554-3.598753$

H -1.903939 $0.580505-4.006921$

H -2.817405 -0.576562 -4.960301

H -3.241338 -1.931376 -2.868376

H -2.370957 -0.753490 -1.927358

H $-4.921161-0.254144-3.534407$

H -3.577019 $1.876560-3.345843$

H $-3.3708351 .594585-1.630917$

H $-4.4842483 .934719-2.362533$

H $-5.5424881 .638710-0.102490$

H -5.640756 $2.991535-4.168490$

H -7.107191 $2.986002-3.215796$

H $-6.5012301 .471358-3.915737$

H -7.272416 $2.995416-0.672637$

H $-6.291778-0.3257750 .916842$

H $-7.870768-0.4491540 .132675$

H -7.580646 -1.157461-2.190599

H -1.654192 $0.739732-0.240073$

H $\quad 0.3280930 .6226562 .108832$

H -3.343862 1.6109491 .336654

H $-3.959140 \quad 0.7504772 .740222$

H -3.308705 -0.1670241 .395469$

H $-7.544962-2.6636370 .641392$
H $-6.827767-2.683570-0.958654$

H -3.359596 -3.060527 2.299010

H -4.545664 -4.178829 3.005785

H -5.221047 -2.195203 4.634080

H -2.178194 -2.229868 4.295414

H -2.817681 -1.981664 6.838634

H $-4.143366-0.9446486 .334740$

H -0.6445430 .9652707 .050185$

H -1.118231 -0.6706707 .778184$

H -2.298921 2.0118845 .509212

H $-3.514176 \quad 1.0309364 .745012$

H -0.4627750 .9541104 .185116$

H -6.221109-2.274322 2.493714

C $2.8957474 .822838-0.690074$

O $3.7928073 .790434-0.230622$

C 3.4236273 .4292971 .113047

C 2.6009944 .5992771 .637322

C 1.8239354 .9844250 .391707

H 2.7714892 .5513701 .035245

C 4.6592463 .0683861 .941687

H 1.9574594 .3146682 .475126

H $3.2360105 .432847 \quad 1.958804$

C 1.2188256 .3759850 .451259

H 1.0149494 .2565010 .260093

C 5.5862312 .0287291 .278561

O 5.4309174 .2569612 .155870

H 4.3431852 .7249432 .934000

H 6.1083032 .4941040 .433802

O 6.6065351 .6915922 .227673

C 4.8916530 .7395730 .801543

C $5.898778-0.2721460 .224921$

H $4.161840 \quad 0.9932820 .026343$

O $4.189244 \quad 0.163442 \quad 1.899414$

H $6.5408120 .246970-0.497228$

H $6.528741-0.6490941 .040472$

H $0.5045546 .449799 \quad 1.277670$

H $1.986667 \quad 7.1422350 .602066$

H $0.6877836 .608016-0.477345$

C $2.3979804 .486766-2.101352$

H 6.2075694 .0065122 .685055

H $6.178697 \quad 1.2135192 .958933$

H $3.700325-0.6075051 .564511$

H $3.2655424 .414042-2.769435$

H $1.7725575 .302847-2.480634$

H $3.5001295 .737132-0.752951$

C $3.981608-3.4953190 .065124$

O $4.642739-2.3090270 .530490$

C $5.223801-1.470823-0.475269$

C $6.240542-2.255915-1.321576$

C $5.626879-3.533349-1.895527$

C $4.914281-4.347188-0.813081$

H $3.777424-4.0756310 .974361$

C $2.638677-3.174426-0.611468$

H $4.431624-1.087868-1.127559$

O $7.389238-2.586596-0.542901$

H $6.585493-1.614873-2.141434$

H $6.421036-4.137617-2.350418$

O $4.705436-3.175167-2.920985$

H $4.344322-5.169572-1.260838$

O $5.890900-4.9415010 .046766$

C $1.533769-2.9432740 .399863$

H $2.686105-2.286536-1.244536$

H $2.333206-4.031250-1.224257$

H $7.087172-3.0821750 .237553$

H $4.339947-3.998143-3.287987$

H $6.455459-5.512688-0.501664$

O $0.329018-3.128690-0.215815$

O $1.707159-2.628021 \quad 1.568847$

C $-0.850651-2.9970920 .586847$

H $-0.684009-2.410041 \quad 1.494110$

H -1.616914 -2.501012 -0.014678

H -1.205697 -3.9961320.853619

SCF Energy (B3LYP/6-31G**//MMFF)= -3245.91282959

0700234

MM̄MF Geometry

C $3.3606124 .193874-0.226315$

C $2.5454723 .153848-0.457646$ 
C $\quad \begin{array}{lll}1.502847 & 2.676361 & 0.518558\end{array}$

O $\quad 0.2827792 .529044-0.244773$

C $1.915306 \quad 1.314238 \quad 1.105024$

C $1.2019990 .967902 \quad 2.398054$

C 0.2403620 .0334512 .533524

C 1.6822551 .7230133 .613061

C $-0.380077-0.8272091 .468381$

C $-1.902852-0.6861951 .473877$

C -2.624622 -1.6042420 .476729$

C $-2.226664-1.337449-0.976636$

C $-3.069384-2.153912-1.966766$

O $-4.023880-1.3506280 .655111$

O $-2.857858-1.610795-3.278941$

C $-4.561278-1.958296-1.633174$

C $-2.618609-3.618431-2.024243$

O $-5.377262-2.806201-2.438950$

C $-4.892870-2.168587-0.138166$

C $-6.341488-1.7074810 .142356$

$\begin{array}{llll}\text { O } & -4.763831 & -3.551788 & 0.188512\end{array}$

C -0.8958092 .8705010 .337968$

O $-1.073513 \quad 3.2520221 .482965$

C -1.953731 2.695251 -0.684541

C $-3.2465842 .728238-0.327328$

C $-4.395398 \quad 2.559055-1.222834$

C $-4.1119402 .416730-2.695770$

C $-6.765326-1.8112311 .614655$

C $-7.861542-0.8143431 .932401$

C $-10.174708-0.1712601 .289428$

O $\begin{array}{llll}-7.660698 & 0.148363 & 2.669990\end{array}$

C $-10.0020690 .749518 \quad 0.121792$

$\begin{array}{llll}\text { C } & -9.681266 & 2.047330 & 0.235487\end{array}$

C $-9.4345332 .947900-0.942012$

C $-7.9973413 .425587-0.999811$

C $-7.6968534 .711544-0.748489$

C $-6.9579812 .389624-1.395931$

C $-5.6345212 .536578-0.687512$

N $-9.059241-1.1004231 .294740$

H 3.2867624 .7335890 .714336

H $2.6309092 .610420-1.396216$

H $1.3679253 .429702 \quad 1.303648$

H 2.9928041 .3129561 .321352

H 1.7707080 .5294640 .354609

H $-0.158400-0.1414843 .532549$

H 1.2169381 .3642804 .537438

H 2.7647641 .6111513 .728703

H 1.4514312 .7885143 .522154

H $0.009112-0.577740 \quad 0.479830$

H $-0.114321-1.8711181 .669389$

H -2.290811 -0.9026362 .479194$

H -2.186307 0.3552041 .282630

H - $2.413931-2.6446990 .750865$

H -1.163251-1.539735 -1.144368

H -2.367201 -0.272620 -1.201744

H -1.905854 -1.657470 -3.472987

H $-4.825188-0.926124-1.904161$

H - $-1.570885-3.684526-2.341851$

H -3.192663 -4.179922 -2.769434

H -2.703583 -4.131046 -1.063791

H $-5.145410-2.633574-3.367602$

H $-6.441406-0.662181-0.175437$

H $-7.044478-2.298143-0.458103$

H -5.277069 -3.734992 0.991202

H $-1.6349272 .526040-1.707008$

H -3.490662 2.8829540 .723044

H -3.589256 $3.302560-3.073479$

H $-5.0080302 .296368-3.306893$

H -3.489769 $1.534483-2.883708$

H -7.122345 -2.820391 1.849455

H -5.927832 -1.600487 2.288845

H -10.205590 0.3694512 .240997

H -11.097179 -0.7496161 .183748$

H $-10.1162140 .313214-0.867787$

H $-9.5602532 .483246 \quad 1.224531$

H - $-10.125428 \quad 3.797217-0.868578$

H -9.678313 2.445204 -1.886551

H -6.680865 5.086004 -0.810983

H -8.466861 $5.427829-0.480753$

H $-6.8698612 .410432-2.484974$
H -7.328224 $1.389412-1.143085$

H $-5.7272372 .611208 \quad 0.397278$

H $-9.098391-1.8835260 .649091$

C $5.8035054 .178009-0.950419$

O $5.8610682 .752637-1.149082$

C $6.7738052 .191512-0.184881$

C 7.4790473 .3738750 .474089

C 6.3903624 .4312190 .436475

H $6.159563 \quad 1.6896490 .571898$

C $7.7006431 .175263-0.860725$

H 7.8179213 .1407791 .488152

H $8.3497803 .703857-0.104160$

C 6.9012155 .8457300 .641922

H 5.6604504 .1960701 .220772

C $6.9560050 .071460-1.641051$

O $8.5375301 .873272-1.791721$

H $8.3707450 .734416-0.113255$

H $6.4599850 .510836-2.514427$

O $7.938289-0.832758-2.163274$

C $5.933225-0.723287-0.809525$

C $5.268724-1.845404-1.631046$

H $5.156495-0.044974-0.440087$

O $6.607735-1.274340 \quad 0.319695$

H $4.917293-1.429196-2.582666$

H $6.016515-2.619234-1.844643$

H 7.3763915 .9441861 .623411

H $7.6403966 .120642-0.118011$

H 6.0794596 .5669620 .590051

C $4.3839014 .684436-1.211371$

H $9.1136861 .213303-2.214137$

H $8.353935-1.281513-1.406965$

H $5.949904-1.7513850 .853053$

H $4.0832074 .380547-2.222509$

H $4.3731015 .780622-1.199867$

H $6.4603254 .613966-1.715279$

C $3.613464-3.8748741 .045527$

O $4.590958-3.238877 \quad 0.211382$

C $4.076779-2.491299-0.895192$

C $3.262243-3.406517-1.822413$

C $2.162632-4.143950-1.052844$

$\begin{array}{llll}\text { C } 2.702853 & -4.800810 & 0.222370\end{array}$

H $4.198779-4.5037161 .729317$

C $2.841333-2.8567901 .890170$

H $3.431856-1.687516-0.523536$

O $4.115959-4.354714-2.459927$

H $2.811472-2.797372-2.614639$

H $1.723657-4.908480-1.705351$

O $1.137323-3.212532-0.721862$

H $1.880132-5.1569680 .853192$

O $3.477618-5.947849-0.139888$

C $3.743470-2.2294452 .925585$

H $2.389536-2.0596411 .295199$

H $2.021397-3.3411902 .433452$

H $4.581999-4.850213-1.764774$

H $0.432063-3.701530-0.264641$

H $2.890652-6.558983-0.617112$

O $4.244741-1.0517702 .457285$

O $3.992214-2.7404034 .010001$

C $5.136633-0.3657883 .341110$

H $6.046042-0.9556933 .488095$

H 5.4063950 .5872312 .878432

H $4.647332-0.1643144 .298850$

SCF Energy (B3LYP/6-31G*//MMFF) $=-3245.91841043$

07_00235

MM̄FF Geometry

C -2.076030 -3.182988 -1.813738

C $-2.528233-2.412393-2.814129$

C $-3.431335-1.228112-2.584053$

O $-2.730775-0.036679-3.027401$

C $-4.743248-1.313602-3.385459$

C $-5.852611-2.014902-2.630683$

C $-6.855705-1.376029-1.996484$

C $-5.809112-3.520237-2.641166$

C $-7.0978630 .105099-1.899879$

C $-6.0667600 .919282-1.111871$

C $-5.673098 \quad 0.368858 \quad 0.265579$

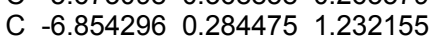


C $-6.407919-0.1694572 .630556$

$\begin{array}{lllll}\text { O } & -4.679580 & 1.263046 & 0.787391\end{array}$

\begin{tabular}{llllll}
\hline & -7.491975 & 0.073368 & 3.540002
\end{tabular}

C -5.2268150 .7098853 .089295$

C $-6.128530-1.6764682 .680375$

$\begin{array}{llll}\text { O } & -4.684533 & 0.232392 & 4.318920\end{array}$

C -4.1038790 .8491862 .033154$

C -3.1056871 .9420452 .485191$

O $-3.415965-0.3958131 .908061$

C $-1.7683660 .458376-2.199861$

O $-1.4375450 .023473-1.108067$

C $-1.1460781 .616161-2.874618$

C $0.1136351 .947863-2.555375$

C $0.8972173 .031406-3.149911$

C $0.1556014 .064514-3.955468$

C $-2.0242292 .292858 \quad 1.453582$

C -1.1898773 .4828281 .898126$

C $0.685948 \quad 4.922512 \quad 1.182264$

O $\begin{array}{llll} & -1.313343 & 4.018608 & 2.996831\end{array}$

C $1.3063055 .373357-0.099546$

C $2.6222335 .295494-0.348327$

C $3.2730385 .810211-1.600095$

C $4.0016304 .728860-2.363807$

C $5.2948824 .458369-2.119137$

C $3.2489654 .021168-3.467530$

C $2.2295223 .050900-2.937961$

N $-0.284963 \quad 3.8781320 .920447$

H -2.411198 -2.985016 -0.797973

H $-2.178849-2.587298-3.828373$

H $-3.660930-1.106278-1.518907$

H $-4.581158-1.815128-4.349026$

H -5.063532 -0.302304 -3.664930

H -7.606383 -1.981485-1.489830

H $-6.716004-3.963691-2.215708$

H -5.717145 -3.892437 -3.666747

H $-4.961586-3.887583-2.056866$

H -7.193055 $0.522143-2.910147$

H -8.083583 $0.252055-1.439763$

H $-5.155731 \quad 1.038647-1.710644$

H -6.447203 $1.943332-0.994712$

H -5.216484 -0.6170040 .134086$

H $-7.634704-0.3855390 .853081$

H -7.324413 1.2738091 .320413

H -8.266470 -0.421709 3.222185

H -5.629331 $1.711434 \quad 3.297479$

H -7.026022 -2.241626 2.401353

H $-5.876890-1.9979473 .697177$

H $-5.323040-1.9862872 .011299$

H $-5.417364 \quad 0.1754554 .955840$

H -3.666303 $2.859982 \quad 2.706874$

H -2.609193 1.6313583 .413466

H -2.554313 -0.2387381 .491188$

H - $1.7194972 .129698-3.637426$

H $0.6366021 .348620-1.810486$

H $-0.7034494 .447888-3.392697$

H $-0.205357 \quad 3.632896-4.895201$

H $0.762808 \quad 4.936491-4.207300$

H -1.343394 1.4474421 .310262

H -2.481782 2.5411360 .489444

H $1.4234514 .522521 \quad 1.886380$

H 0.1798245 .7622131 .670773

H $0.6465105 .816269-0.841652$

H 3.2798264 .8694670 .406534

H $3.9803516 .598652-1.310453$

H $2.5447366 .300953-2.257649$

H $5.8310123 .701131-2.681421$

H $5.843824 \quad 4.984919-1.345283$

H $3.9476753 .445704-4.089550$

H $2.8209754 .768510-4.140873$

H $2.6674892 .249983-2.343211$

H -0.1211613 .2529780 .135960$

C $0.270425-3.811334-1.367375$

O $0.857925-2.801279-2.212359$

C $2.297927-2.890084-2.104010$

C $2.578535-4.016031-1.115890$

C $1.343501-4.886474-1.259891$

H $2.658726-3.158494-3.104385$

C $2.819527-1.507419-1.694759$
H $3.508514-4.546784-1.340601$

H $2.649453-3.629172-0.091926$

C $1.150090-5.850334-0.102694$

H $1.411370-5.449312-2.200333$

C $4.292206-1.479704-1.259869$

O $2.655318-0.627339-2.812399$

H $2.181307-1.112504-0.897562$

H $4.439650-2.124434-0.390054$

O $5.081219-2.055787-2.309330$

C $4.847638-0.074572-0.962047$

C 4.0269420 .7977590 .005992

H $4.9616620 .467316-1.909371$

O $6.170279-0.234815-0.437922$

H $4.496412 \quad 1.790298 \quad 0.032961$

H $3.0231790 .934155-0.408742$

H $2.000584-6.535778-0.027637$

H $1.061139-5.3197230 .851361$

H $0.245234-6.450236-0.239970$

C $-1.052809-4.271873-1.977013$

H $1.719624-0.664175-3.075583$

H $6.008408-2.039452-2.015737$

H $6.5426190 .653926-0.306551$

H - $1.424172-5.170667-1.473740$

H $-0.918502-4.514219-3.038032$

H $0.099495-3.350413-0.384005$

C $3.035263-1.5614342 .753139$

O $3.202064-0.9367141 .473979$

C 3.9310820 .2914551 .460765

C $3.228006 \quad 1.3280322 .355549$

C 2.9927090 .7901923 .769957

C $2.358937-0.6044053 .748599$

H $2.341921-2.3920662 .566827$

C $4.349186-2.1537413 .276681$

H $4.9449910 .115876 \quad 1.834298$

$\begin{array}{lllll}\text { O } & 1.975778 & 1.704236 & 1.784201\end{array}$

H 3.8435272 .2340102 .400180

H 2.3333901 .4767524 .315047

$\begin{array}{lllll}\text { O } & 4.229952 & 0.725357 & 4.472159\end{array}$

H $2.372279-1.0501244 .750113$

O $0.986643-0.485898 \quad 3.359886$

C $4.807606-3.3003802 .405246$

H $5.149848-1.4110923 .334768$

H $4.222020-2.5556434 .288698$

H $1.447590 \quad 0.8965331 .663435$

H $4.597767 \quad 1.6249774 .503119$

H 0.5437890 .0791654 .015889

O $5.981688-2.9804311 .790409$

O $4.192032-4.3514632 .280951$

C $6.519994-3.9856280 .925459$

H $5.791453-4.2633160 .157741$

H $7.404308-3.5720460 .433499$

H $6.817738-4.8612641 .509606$

SCF Energy (B3LYP/6-31G**//MMFF) $=-3245.91410741$

07_00236

MM̄FF Geometry

C -4.082969 -0.496921 -3.336010

C $-2.926320-0.345070-2.671220$

C $-1.690789-1.161417-2.956010$

O $-0.581971-0.635319-2.183090$

C $-1.876249-2.630420-2.531197$

C $-0.789180-3.536170-3.076509$

C $0.246941-4.022407-2.365703$

C $-0.953664-3.928370-4.524024$

C $0.589660-3.771023-0.923426$

C $2.008222-3.212398-0.785702$

C $2.520149-3.1684220 .661280$

C $1.668717-2.2882531 .578485$

C $2.274483-2.1691552 .986266$

O $3.857528-2.6549940 .601347$

O $1.613536-1.092253 \quad 3.666665$

C $3.759871-1.7762052 .860787$

C $2.019684-3.428263 \quad 3.823731$

O $4.402255-1.7962204 .133519$

C $4.545715-2.6491161 .857015$

C $5.960135-2.0564901 .638109$

O $4.680638-3.9695192 .390553$

C $0.0450680 .475899-2.653562$ 
O $-0.249803 \quad 1.119991-3.647575$

C $1.1733390 .773043-1.742032$

C $1.8743591 .904033-1.906072$

C $3.0303692 .334761-1.115179$

C $3.6496541 .332120-0.177826$

C $6.725584-2.6704870 .457069$

C $6.461898-1.955256-0.856578$

C $7.0388450 .117709-2.102623$

O $5.824897-2.480849-1.766081$

C $7.286347 \quad 1.544493-1.732275$

C $6.6408892 .584473-2.281805$

C $6.9023154 .008266-1.878597$

C $5.6432014 .809537-1.633128$

C $5.4490705 .986339-2.253500$

C $4.6395344 .291850-0.623538$

C $3.4840253 .593322-1.290764$

N $7.019222-0.686130-0.893540$

H $-4.150990-1.238291-4.128178$

H $-2.8706990 .393807-1.875353$

H - $1.444900-1.092897-4.023105$

H -2.839488 -3.019149-2.886788

H $-1.925085-2.695366-1.438815$

H $0.939673-4.692379-2.874764$

H $-0.177630-4.625446-4.857996$

H -1.921179 $-4.417904-4.676651$

H $-0.903195-3.047928-5.171582$

H $-0.116759-3.082835-0.455029$

H $0.515406-4.723947-0.386289$

H $2.709656-3.820551-1.373869$

H $2.062870-2.210656-1.229476$

H $2.551735-4.1976031 .038243$

H $0.641831-2.6640601 .649564$

H $1.594126-1.2813901 .146646$

H $0.663295-1.2954003 .697420$

H $3.793841-0.7341232 .513034$

H $0.942379-3.6012493 .936334$

H $2.411319-3.3129574 .840424$

H $2.452773-4.3301963 .386471$

H $3.883269-1.2283334 .728590$

H $5.880721-0.9725731 .491994$

H $6.554777-2.2211512 .545740$

H $5.496021-4.3709832 .051332$

H $1.3975810 .056790-0.961412$

H $1.5695452 .591287-2.694602$

H 2.9574321 .0821720 .632153

H $4.571901 \quad 1.6842060 .288568$

H $3.9055980 .410716-0.713087$

H $7.803549-2.6025180 .652256$

H $6.504460-3.7346790 .325978$

H $6.097737-0.020234-2.645129$

H $7.857971-0.256964-2.724909$

H $8.0430451 .738171-0.974740$

H $5.9090832 .406634-3.065921$

H $7.4998584 .472556-2.673508$

H $7.5140304 .047386-0.968224$

H $4.5607596 .581182-2.067158$

H $6.1708636 .381082-2.960748$

H $4.2310165 .137975-0.054618$

H 5.1488813 .6726150 .119564

H $2.9447814 .234042-1.991123$

H $7.668254-0.435770-0.152496$

C $-6.183613-0.288247-1.907677$

O $-5.442352-0.255274-0.670061$

C $-5.505663-1.558396-0.066750$

C $-6.749512-2.207831-0.656547$

C $-6.661536-1.731241-2.095948$

H $-4.624633-2.112074-0.415023$

C -5.465837-1.456879 1.460161

H $-6.737416-3.297679-0.563252$

H -7.665773 -1.830089-0.188347

C $-7.968165-1.846643-2.861159$

H -5.901558 -2.332360 -2.610029

C -4.294583-0.613159 2.010350

O $-6.683298-0.854346 \quad 1.914914$

H -5.445888 -2.466174 1.888479

H -4.5059900 .4468791 .835274$

O $-4.273532-0.7772453 .433669$

C $-2.911605-0.9690421 .440052$
C $-1.723618-0.2699452 .122702$

H $-2.889691-0.7360010 .371284$

O $-2.715526-2.3807911 .565374$

H $-1.582408-0.6771013 .131945$

H $-0.828229-0.5625471 .560109$

H -8.288481 -2.891985 -2.920005

H $-8.768859-1.277024-2.377767$

H $-7.854086-1.468957-3.882182$

C $-5.327507 \quad 0.292320-3.039133$

H $-6.635511-0.8006782 .884956$

H $-4.033291-1.7016763 .618202$

H $-1.866983-2.5979891 .143504$

H $-5.0384251 .318290-2.781977$

H $-5.9230160 .360555-3.957178$

H $-7.0444820 .375461-1.757069$

C $-2.177694 \quad 3.191217 \quad 0.764324$

$\begin{array}{llll}\text { O } & -2.028010 & 1.766921 & 0.869555\end{array}$

C $-1.808973 \quad 1.263596 \quad 2.190113$

C -0.5321531 .8746962 .792830$

C -0.5649253 .4042822 .747762$

C $-0.9623613 .922027 \quad 1.360874$

H $-2.1858723 .392295-0.314771$

C -3.514086 3.6764891 .350061

H $-2.661997 \quad 1.5264802 .823323$

$\begin{array}{lllll}\text { O } & 0.620120 & 1.414176 & 2.090015\end{array}$

H -0.4324651 .5362623 .830703$

H 0.4270013 .7891173 .014550

O -1.4892443 .8694873 .726169$

H $-1.1652584 .998779 \begin{array}{lll}1.393926 \\ \text { H }\end{array}$

$\begin{array}{lllll}\text { O } & 0.133162 & 3.732326 & 0.461572\end{array}$

$\begin{array}{llll}\text { C } & -4.701908 & 3.172330 & 0.560270\end{array}$

H -3.639446 3.3468362 .384596

H -3.557887 $4.771272 \quad 1.327112$

H $\quad 0.505761 \quad 1.6399461 .151023$

H $-1.4783394 .841508 \quad 3.701321$

H 0.8913964 .2302260 .811912

O $\quad-5.8295893 .298261 \quad 1.320279$

O $-4.6541142 .747819-0.585268$

C -7.045562 $2.871305 \quad 0.698283$

H $-7.0301661 .787967 \quad 0.553340$

H -7.8745913 .1240231 .364564$

H -7.193298 $3.387599-0.255074$

SCF Energy (B3LYP/6-31G**//MMFF)= -3245.90449941

07_00237

MM̄FF Geometry

C -2.257868 $-3.503000 \quad 0.710505$

C $-1.257032-2.634840 \quad 0.916340$

C $-0.686638-2.2967242 .267062$

O $0.733495-2.5512542 .188496$

C $-0.909495-0.8066742 .576139$

C $-0.331207-0.369142 \quad 3.908936$

C 0.6115820 .5811924 .069115

C $-0.926307-1.0361235 .124076$

C 1.2938161 .4035573 .011247

C $2.822196 \quad 1.3907093 .175178$

C 3.5796412 .0877402 .029203

C 3.2008493 .5651281 .883699

C $3.848194 \quad 4.222677 \quad 0.656371$

$\begin{array}{lllll}\text { O } & 3.302121 & 1.362379 & 0.828005\end{array}$

O 3.1734665 .4678760 .414860

C $3.5921493 .328590-0.575098$

C $5.3203354 .569508 \quad 0.904103$

O $4.2779193 .830960-1.719386$

C $3.9700481 .852653-0.341058$

C $3.5583410 .947571-1.520700$

O $5.3920201 .746946-0.204715$

C $1.187287-3.7277352 .690989$

O $0.545248-4.5411583 .342188$

C $2.589081-3.9908262 .293208$

C $3.354286-3.1272421 .607899$

C $4.700700-3.3957411 .092152$

C $5.265344-4.7764351 .305713$

C $2.0509990 .849210-1.772559$

C $1.759523-0.362082-2.634556$

C $1.822297-2.854557-2.517764$

O $1.612183-0.271906-3.850547$

C $3.207994-3.402918-2.336805$ 
C $4.314102-2.818193-2.824659$

C $5.717033-3.298696-2.579285$

C $6.531989-2.288747-1.794862$

C $7.165753-1.281042-2.418513$

C $6.661502-2.481518-0.297161$

C $5.334113-2.4189080 .409507$

N $1.754379-1.540875-1.901967$

H -2.697804 -4.017567 1.559794

H $-0.802680-2.1386680 .062174$

H -1.155711 -2.908933 3.046334

H $-1.987337-0.5933502 .586715$

H $-0.501360-0.2087371 .755802$

H 0.9173150 .8261045 .086076

H $-0.596965-0.5688906 .058490$

H -2.019046 -0.9715675 .096186$

H $-0.638043-2.0908175 .166484$

H 1.0373451 .0721512 .003179

H 0.9211732 .4287923 .114149

H 3.0951101 .8598234 .128633

H 3.1572190 .3457393 .220587

H $4.650963 \quad 1.9985302 .246139$

H 3.4497534 .1186972 .797611

H 2.1164273 .6622421 .766045

H 3.2553206 .01310111 .216097

H $2.5199453 .389289-0.803307$

H $5.4104195 .288331 \quad 1.727602$

H $5.763858 \quad 5.0589120 .029969$

H 5.9291833 .7005111 .161348

H $4.0129154 .760054-1.831218$

H $4.0682371 .263373-2.440297$

H $3.945594-0.059220-1.322812$

H $5.6576340 .834685-0.406822$

H $2.948988-4.9785592 .564092$

H $2.953362-2.1451451 .362925$

H $4.614556-5.5325250 .852302$

H $5.360784-4.9896642 .376321$

H $\quad 6.256073-4.920270 \quad 0.871817$

H $1.4916520 .751380-0.836414$

H $1.677337 \quad 1.742373-2.283871$

H $1.551097-2.807883-3.577001$

H $1.099308-3.490780-1.997728$

H $3.300954-4.307643-1.741673$

H $4.211786-1.917388-3.426179$

H $6.190337-3.481258-3.552732$

H $5.725804-4.266503-2.063084$

H $7.767148-0.563688-1.870120$

H $7.092496-1.149748-3.493147$

H $7.298334-1.6994440 .136394$

H $7.193560-3.419377-0.120167$

H $4.840877-1.4511700 .320815$

H $1.907553-1.492339-0.898652$

C $-4.259121-3.415116-0.866748$

O $-4.426273-1.997506-0.659485$

C $-5.667664-1.7844050 .039292$

C $-6.427947-3.104702-0.037753$

C $-5.290868-4.104778 \quad 0.025034$

H $-5.410210-1.5923901 .087491$

C $-6.426833-0.589745-0.545466$

H $-7.147121-3.2179700 .779091$

H $-6.971759-3.205321-0.984451$

C $-5.676125-5.498398-0.438907$

H $-4.940810-4.1592541 .062726$

C $-5.6713660 .754846-0.498629$

O $-6.741519-0.859803-1.918199$

H -7.391118 $-0.485702-0.033956$

H $-4.8497520 .748401-1.223823$

O $-6.583722 \quad 1.772064-0.934296$

C $-5.133782 \quad 1.146027 \quad 0.889910$

C $-4.681513 \quad 2.613113 \quad 1.023290$

H -4.3021310 .4821831 .152369$

$\begin{array}{llll}H & -6.163759 & 0.930432 & 1.860699\end{array}$

H $-5.553174 \quad 3.2768540 .955468$

H -4.301595 2.7346822 .046699

H $-6.470534-5.9083210 .193472$

H $-6.039291-5.492177-1.472123$

H $-4.818908-6.177072-0.386663$

C $-2.798894-3.823875-0.655638$

H $-5.903143-1.025078-2.383119$
H $-6.9060871 .513815-1.814940$

H $-5.783574 \quad 1.1120722 .737071$

H $-2.188568-3.318304-1.414563$

H $-2.686758-4.900001-0.830548$

H $-4.507915-3.594775-1.921303$

C - $-1.3710252 .456717-0.700536$

$\begin{array}{lllll}\text { O } & -2.470046 & 2.209860 & 0.186753\end{array}$

C $-3.607116 \quad 3.059066 \quad 0.018682$

C $-3.217629 \quad 4.533914 \quad 0.219712$

C $-2.0475794 .928875-0.686431$

C $-0.8997613 .916929-0.600753$

H $-0.5629491 .819455-0.321220$

C -1.680585 2.020968 -2.142026

H $-4.0063232 .933321-0.992197$

O $-2.857532 \quad 4.753750 \quad 1.582395$

H $-4.091048 \quad 5.161013 \quad 0.003753$

H -1.684851 $5.921202-0.391633$

O $-2.5196185 .021879-2.026800$

H $-0.1521294 .112274-1.378276$

$\begin{array}{llll}\text { O } & -0.241232 & 4.067988 & 0.658552\end{array}$

C $-1.8507170 .522905-2.259981$

H $-2.5957642 .477820-2.526378$

H $-0.8437482 .308146-2.789410$

H $-2.655817 \quad 5.6994591 .683947$

H $-1.7698365 .291934-2.583989$

H $\quad 0.1102464 .9736250 .699202$

$\begin{array}{lllll}0 & -1.884086 & 0.179896 & -3.581277\end{array}$

O $-1.960537-0.248233-1.318143$

C $-2.057079-1.215321-3.848118$

H $-2.027915-1.360486-4.931143$

H $-1.246048-1.793602-3.395488$

H -3.028539-1.552446 -3.474103

SCF Energy (B3LYP/6-31G**//MMFF) $=-3245.89732555$

07_00238

MM̄FF Geometry

C $1.6273904 .580526-1.362471$

C $0.7197814 .008825-0.557183$

C $-0.6805193 .660710-0.992300$

O $-0.8912012 .251823-0.735574$

C $-1.7115864 .465849-0.181068$

C $-3.1596604 .132495-0.494765$

C $-4.100746 \quad 3.9261540 .447613$

C $-3.540040 \quad 4.067754-1.951428$

C $-3.945678 \quad 4.062061 \quad 1.935392$

C -3.6334492 .7570412 .679376$

C $-4.725372 \quad 1.685597 \quad 2.534975$

C -4.6209940 .6556843 .663094$

C -5.643424 -0.4778853 .501259$

O $-4.548899 \begin{array}{lll}1.035066 & 1.270738\end{array}$

O $-5.273105-1.5375824 .396323$

C $-5.538778-1.0383612 .069510$

C $-7.055310-0.0398353 .907565$

O $-6.567320-1.9936421 .818479$

C -5.5485980 .0521850 .972101$

C $-5.211161-0.589757-0.393582$

O $-6.852193 \quad 0.6341190 .898180$

C $-0.4109651 .383078-1.667713$

O $\quad 0.172683 \quad 1.665877-2.702464$

C $-0.7051390 .009016-1.204428$

C $-0.188336-1.027483-1.880148$

C $-0.372295-2.448320-1.579508$

C $-1.196341-2.807773-0.372418$

C $-4.8768710 .415753-1.503211$

C $-4.898858-0.249652-2.866757$

C $-3.781823-2.018678-4.217451$

O $-5.7172430 .070964-3.725906$

C $-3.320339-3.391578-3.842628$

C $-2.272892-4.008769-4.410393$

C $-1.809731-5.385331-4.023265$

C $-0.335391-5.437131-3.681590$

C $0.542492-6.040386-4.500859$

C $0.111651-4.854029-2.355657$

C $0.202393-3.351727-2.401098$

N $-3.933241-1.232597-3.006858$

H $1.3490024 .851273-2.377845$

H 1.0047183 .7287890 .454598

H $-0.8176593 .865054-2.061852$ 
H -1.561119 $5.537505-0.366263$ H -1.510591 4.2971820 .883059 H -5.1105573 .6784820 .122780$ H -4.623112 $3.986082-2.094274$ H -3.211875 $4.975216-2.468967$ H $-3.0811943 .200489-2.435768$ H -3.1739984 .8021832 .176114$ H -4.8763494 .4877122 .332680$ H -2.673387 2.3635692 .323495 H -3.509600 3.0128013 .739035 H -5.7062952 .1746212 .562137$ H $-4.738444 \quad 1.1371384 .642111$ H -3.614401 $0.216833 \quad 3.665332$ H -5.264854 -1.177652 5.299835 H -4.586852 -1.584401 2.003501 H -7.0686420 .2848064 .955029$ H -7.761439 -0.8751563 .844640$ H $-7.4399980 .783752 \quad 3.302614$ H -6.516663 -2.665081 2.520302 H -4.341471 -1.247409-0.273911 H $-6.048190-1.220843-0.719248$ H $-7.0066700 .957728-0.003320$ H - $1.333888-0.110962-0.330461$ H $0.430788-0.818427-2.752048$ H $-2.227930-2.462637-0.491574$ H $-0.775052-2.3491410 .529098$ H -1.240041 -3.880743 -0.176416 H $-5.5979651 .239490-1.525718$ H -3.886993 $0.857329-1.343550$ H -3.064443 -1.497320 -4.859261 H $-4.741926-2.074080-4.740215$ H -3.881197 -3.906985 -3.066031 H -1.725435 -3.505810 -5.204243 H -2.026502 -6.056286 -4.864559 H -2.384899 -5.774808 -3.173950 H $1.596849-6.102052-4.252353$ H $0.228308-6.481465-5.440936$ H $1.107484-5.234654-2.093726$ H $-0.559079-5.221644-1.574869$ H $0.809453-2.980406-3.228728$ H $-3.199057-1.292989-2.308028$ C $4.0118003 .997583-1.816102$ O $3.7355942 .596461-1.625126$ C $4.9768901 .886396-1.473063$ C $6.0693392 .860068-1.899687$ C $5.4825514 .183691-1.445590$ H $5.0943291 .680424-0.403119$ C $4.9295570 .569552-2.255323$ H $7.0325362 .633285-1.432914$ H $6.2127742 .863575-2.986255$ C $6.1339375 .393608-2.091841$ H $5.5897304 .258051-0.355065$ C $3.704864-0.326756-1.967472$ O $4.9075830 .864867-3.660457$ H $5.854628 \quad 0.006687-2.088030$ H $2.8057380 .167536-2.355049$ O $3.852206-1.538266-2.720013$ C $3.458865-0.727420-0.501101$ C $4.681936-1.3810470 .172019$ H $2.644257-1.462333-0.481521$ $\begin{array}{llll}\text { O } & 3.020425 & 0.393748 & 0.256511\end{array}$ H $5.024643-2.218464-0.446625$ H $5.493166-0.6462640 .235996$ H $7.1988705 .436571-1.841089$ H $6.0466925 .361478-3.183088$ H $5.6670416 .320286-1.743695$ C $3.0543784 .828333-0.958974$ H $4.128727 \quad 1.422297-3.830737$ H $3.984203-1.287015-3.650387$ H $2.2403970 .766085-0.188785$ H $3.2719465 .896043-1.072024$ H 3.1923874 .5755940 .099763 H $3.8513094 .209526-2.881432$ C $3.922839-1.0285063 .817677$ O $4.276826-0.7521982 .456645$ C $4.368595-1.8895491 .593065$ C $5.449442-2.8509022 .109062$ C $5.188107-3.2499533 .564908$
C $4.902633-2.0328944 .451376$

H $4.045710-0.0702734 .338235$

C $2.449282-1.4432123 .943947$

H $3.404914-2.4093821 .565259$

O $6.738850-2.2512051 .998971$

H $5.457610-3.7473941 .478103$

H $6.064670-3.7862013 .949229$

O $4.079547-4.1442613 .597733$

H $4.525703-2.3555295 .428865$

O $6.147431-1.3657674 .683219$

C $1.517229-0.3338283 .501144$

H $2.232537-2.3276023 .338819$

H $2.208342-1.6732384 .987784$

H $6.724125-1.4200942 .503935$

H $3.949668-4.4164504 .522182$

H $5.979228-0.6359755 .303597$

O $0.311283-0.8706993 .151781$

O $1.7990690 .856933 \quad 3.491412$

C -0.6779240 .0717832 .727399$

H -1.0035510 .6757413 .579221$

H -0.2876950 .7112701 .929871$

H -1.535454 -0.484951 2.340673

SCF Energy (B3LYP/6-31G**//MMFF)= -3245.90915720

07 00239

MM̄FF Geometry

C $2.155863-3.4525940 .044556$

C $1.591250-2.2402360 .152076$

C $0.486325-1.756797-0.750014$

O $-0.609601-1.3005680 .075297$

C $0.973281-0.572668-1.604866$

C $-0.088992-0.006516-2.529893$

C $-0.4422451 .293182-2.571775$

C $-0.749323-0.986869-3.465374$

C $0.1609452 .430031-1.799871$

C $-0.5787352 .804590-0.509593$

C $-2.0210443 .284271-0.734280$

C $-2.5036414 .094650 \quad 0.471684$

C $-3.9644374 .536330 \quad 0.313356$

O $-2.8606932 .136664-0.907609$

O -4.4224805 .0134271 .587531$

C $-4.8160173 .302874-0.041064$

C $-4.1091925 .706531-0.666477$

O $-6.157511 \quad 3.681787-0.343919$

C $-4.2315582 .447899-1.192350$

C $-5.0236821 .123525-1.287192$

O $-4.362703 \quad 3.160910-2.422751$

C $-1.559690-2.2208430 .394563$

O $-1.563038-3.4049190 .095353$

C $-2.620150-1.5334131 .166335$

C $-3.732266-2.2151431 .479059$

C $-4.896532-1.7077582 .204082$

C $-4.821249-0.3208232 .784576$

C $-4.4719650 .104947-2.292046$

C $-5.166145-1.235833-2.133769$

C $-7.429024-2.288223-2.225199$

O $-4.561145-2.235471-1.753444$

C $-8.185986-2.018396-0.960760$

C $-8.352136-2.9177800 .020903$

C $-9.140818-2.6411941 .270219$

C $-8.417729-3.0479512 .535709$

C $-8.828840-4.1069283 .254152$

C $-7.269910-2.1837903 .018184$

C $-5.969665-2.5146392 .341990$

N -6.512555-1.186740 -2.454264

H $1.802611-4.137618-0.721803$

H $1.943392-1.5520190 .916792$

H $\quad 0.151064-2.565397-1.411537$

H $1.825049-0.890677-2.220112$

H $1.3482340 .204776-0.930247$

H $-1.2274291 .590745-3.265689$

H -1.371183 -0.488523 -4.217080

H $0.007822-1.566889-4.002863$

H $-1.394763-1.676676-2.913045$

H $1.2080142 .220279-1.558544$

H $0.1962183 .305540-2.461237$

H $-0.569591 \quad 1.946806 \quad 0.174972$

H $-0.0032573 .608852-0.035758$ 
H $-2.0508903 .900527-1.640366$ H $-1.859414 \quad 4.9668240 .639407$ H -2.420782 3.4808861 .379918 H -3.845036 5.7479821 .857603 H -4.869162 2.6761050 .859878 H $-3.521586 \quad 6.566975-0.324591$ H $-5.1470366 .054079-0.715757$ H $-3.7790445 .462758-1.678318$ H -6.489359 4.1993510 .409708 H $-5.0336810 .641432-0.302520$ H $-6.0664161 .345411-1.544959$ H $-4.3018142 .530977-3.157854$ H $-2.469012-0.4938481 .432953$ H -3.812066 -3.251758 1.152368 H -4.7429440 .4291971 .992692$ H $-3.948918-0.2262093 .441146$ H -5.692511 -0.0620983 .392249$ H $-4.6025850 .444335-3.324790$ H -3.401318 -0.056255 -2.130503 H $-6.876521-3.232168-2.184718$ H -8.120271 -2.315712 -3.072958 H -8.638514 -1.034557 -0.856812 H -7.929555 -3.913457 -0.092787 H -10.088948 -3.188634 1.188102 H $-9.413278-1.5806941 .342141$ H -8.332999 -4.3902034 .176715$ H -9.666373 -4.7176802 .933953$ H $-7.134721-2.3215004 .098686$ H -7.541864 -1.133230 2.866059 H -5.909350 -3.524561 1.934771 H $-6.914020-0.306477-2.763146$ C $4.649112-3.9035490 .278719$ O $4.992675-2.546743-0.074050$ C $5.503259-2.542421-1.418345$ C $5.918875-3.980888-1.701264$ C $4.817645-4.743479-0.989412$ H $4.658241-2.290555-2.069790$ C $6.615478-1.502867-1.587779$ H $5.969316-4.197660-2.772488$ H $6.891950-4.218483-1.256172$ C $5.155350-6.199606-0.720333$ H $3.916234-4.701585-1.612320$ C $6.233782-0.070508-1.157962$ O $7.740067-1.904769-0.794695$ H $\quad 6.959890-1.508805-2.628978$ H $6.181498-0.031615-0.064803$ O $7.3180590 .791821-1.527442$ C $4.9286930 .480231-1.758817$ C $4.6679111 .971682-1.480755$ H $4.076699-0.099280-1.387986$ O $4.9500220 .346551-3.183701$ H $5.3777172 .586262-2.050221$ H $3.6831222 .198561-1.910082$ H $5.322857-6.735469-1.660215$ H $6.061348-6.296611-0.112696$ H $4.337325-6.695954-0.188505$ C $3.264908-3.9419660 .933795$ H $8.435211-1.235621-0.917030$ H $7.3494950 .822835-2.499239$ H $4.911475-0.598935-3.400994$ H $3.289743-3.3340831 .846151$ H $3.030967-4.9650791 .250631$ H $5.387773-4.2132221 .029336$ C 3.7528451 .7841442 .146710 O $3.8026531 .552551 \quad 0.730587$ C $4.7013352 .390147-0.001526$ C 4.3296643 .8737430 .174158 C 4.2397714 .2562431 .654086 C 3.4009583 .2505972 .450092 H 2.9202041 .1641942 .503216 C 5.0345841 .3183252 .856902 H 5.7196252 .2349780 .367935 O $3.0746474 .131038-0.453700$ H $5.0843344 .488666-0.331129$ H 3.7918065 .2536541 .741757 O $5.559015 \quad 4.3245432 .186321$ H 3.5074913 .4370203 .524547 O 2.0195773 .4537862 .140296
C $5.214988-0.1817112 .788342$

H 5.9243281 .7781832 .419254

H 4.9908871 .5862023 .918762

H $2.8911015 .081752-0.365263$

H $5.4860014 .591398 \quad 3.118534$

H 1.8882853 .2064341 .209092

O $6.500950-0.4917263 .127540$

O $4.337469-0.9818372 .498056$

C $6.827197-1.8852033 .116726$

H $7.818822-2.0049413 .561054$

H $6.107920-2.4586373 .709622$

H $6.858029-2.2498862 .086820$

SCF Energy (B3LYP/6-31G**//MMFF) $=-3245.90733754$

07_00240

MM̄FF Geometry

C $-2.519383-1.9120623 .076695$

C $-3.167704-3.0758042 .915205$

C $-4.195682-3.3857201 .848766$

O $-4.404260-2.2631630 .963110$

C $-3.674509-4.5595060 .997310$

C $-4.513773-4.845585-0.230988$

C $-4.163138-4.467610-1.477038$

C $-5.774148-5.6296760 .011856$

C $-2.950725-3.653352-1.847828$

C $-3.213422-2.745829-3.057375$

C $-2.118246-1.694544-3.313807$

C $-0.705886-2.284386-3.369705$

C $0.368929-1.203346-3.565120$

O $-2.202068-0.700350-2.283107$

O $1.631300-1.825169-3.281543$

C $0.139957-0.084096-2.524838$

C $0.437271-0.728424-5.020675$

O $1.0196271 .014851-2.747751$

C $-1.3207630 .416147-2.482338$

C $-1.5648591 .432336-1.345801$

O $-1.6209171 .096172-3.708295$

C $-5.362302-1.357851 \quad 1.291880$

O $-6.042186-1.3341912 .304598$

C $-5.428716-0.3756380 .187851$

C -5.9405010 .8414870 .419998$

C $-6.0584561 .925927-0.555660$

C $-5.8140471 .594479-2.004676$

C - 1.2876760 .9180620 .067078

C -1.8952681 .8511741 .096595$

C -1.7659914 .1841581 .950192$

O -2.8671011 .5222521 .772835$

C -2.5846595 .0589811 .053375$

C -3.9257735 .0950581 .069861$

C $-4.7474595 .958607 \quad 0.156177$

C $-5.2640595 .208291-1.052472$

C $-4.6360415 .307359-2.236581$

C $-6.5460504 .415922-0.918986$

C $-6.3775193 .159941-0.112312$

N $-1.2557573 .078646 \quad 1.161597$

H -2.755585 -1.068583 2.436910

H -2.913265 -3.904853 3.573059

H $-5.142628-3.6699812 .323823$

H $-3.621598-5.4683751 .612370$

H -2.636137 -4.366504 0.698545

H $-4.804283-4.762793-2.305933$

H -6.323022 -5.830122 -0.914439

H $-5.539024-6.5945510 .472639$

H $-6.443829-5.0786100 .679526$

H -2.623899-3.032281-1.008216

H $-2.139585-4.350254-2.087363$

H -3.323768 -3.371784 -3.952087

H -4.166632 -2.220912 -2.910147

H $-2.363491-1.218212-4.270621$

H $-0.634405-3.048019-4.153888$

H $-0.479853-2.798987-2.426233$

H $2.333748-1.174643-3.449371$

H $0.399984-0.500146-1.543555$

H $0.711096-1.561319-5.679671$

H $1.2180310 .028527-5.154017$

H $-0.506243-0.316518-5.384188$

H $1.9300720 .689265-2.648409$

H $-0.9815752 .342912-1.535580$ 
H $-2.613612 \quad 1.755457-1.390220$ H $-2.3884001 .673523-3.560524$ H $-5.030187-0.673587-0.775195$ H -6.2812121 .0782801 .427290$ H $-4.7868611 .249837-2.159016$ H $-5.9637122 .443660-2.674881$ H $-6.4991330 .806478-2.337076$ H -1.722970 -0.0761090 .217021$ H -0.2118340 .8373200 .257246$ H -0.9110094 .7421062 .343580$ H -2.3486473 .8009352 .794393$ H -2.042485 5.6843110 .347921 H $-4.4636894 .466136 \quad 1.774457$ H -4.154369 $6.825202-0.166251$ H $-5.587420 \quad 6.3833430 .719430$ H $-5.0093594 .800641-3.120132$ H $-3.7307085 .895098-2.347831$ H $-6.9713934 .186756-1.903079$ H -7.305437 $5.043376-0.434427$ H -6.5350693 .3036380 .957034$ H -0.4910293 .2714860 .521716$ C $-0.155451-1.1798183 .347356$ O $0.279988-2.1373502 .363638$ C $1.723174-2.1608322 .338280$ C $2.180512-1.0345643 .255328$ C $1.047129-0.9703264 .261460$ H $2.011035-3.1340722 .754631$ C $2.188940-2.0451200 .881076$ H $3.152358-1.2373163 .715178$ H $2.257024-0.0901192 .703728$ C 1.0068730 .3239655 .053809 H $1.145700-1.8164104 .954619$ C $3.719112-1.9428430 .727067$ O $1.709484-3.197486 \quad 0.183373$ H $1.718983-1.1749210 .408035$ H $4.046221-1.0419031 .254253$ O $4.341335-3.0501081 .378869$ C $4.210914-1.865423-0.734041$ C $5.738061-1.716009-0.873917$ H $3.715643-1.030295-1.242884$ O $3.866769-3.057296-1.444103$ H $6.240045-2.589719-0.439257$ H $5.965081-1.748978-1.948196$ H $1.936413 \quad 0.460618 \quad 5.615831$ H $0.878175 \quad 1.1918244 .399282$ H 0.1780330 .3175245 .768591 C -1.404594 -1.698898 4.063063 H $0.742456-3.2183180 .287115$ H $4.056819-3.8561470 .914443$ H $2.896439-3.110284-1.476048$ H - $1.734218-0.9764984 .817641$ H -1.173291 -2.639083 4.578350 H $-0.388502-0.2527492 .807748$ C $6.0212841 .978010-0.423004$ O $5.6622320 .677846-0.906594$ C $6.308922-0.425421-0.266677$ C $7.832429-0.327538-0.438617$ C 8.3530351 .0223640 .067859 C $7.5430292 .193058-0.499521$ H $5.5511812 .678484-1.125815$ C 5.4333872 .2503950 .970530 H $6.078522-0.4150050 .802224$ O $8.172506-0.485983-1.814171$ H $8.303645-1.1486010 .114866$ H $9.405174 \quad 1.140601-0.218487$ O 8.2840351 .0616941 .490344 H 7.8047813 .1257020 .014050 O $7.8801832 .367872-1.878894$ C 3.9218942 .3562560 .940151 H 5.6866541 .4710081 .693154 H 5.8212763 .2086851 .335953 H $9.141422-0.444753-1.883942$ H $8.8246650 .328803 \quad 1.831152$ H $8.8332792 .554728-1.926378$ O 3.4959082 .9167292 .111125 O 3.2040131 .9857440 .021416 C 2.0836153 .1147762 .220504 H 1.8643853 .4552193 .235947
H 1.7623013 .8855591 .514070

H 1.5462492 .1790482 .041487

SCF Energy (B3LYP/6-31G**//MMFF) $=-3245.90142548$

07_00241

MM̄FF Geometry

C $0.148093-0.4157082 .753035$

C $-0.7140040 .323708 \quad 3.466010$

C -1.4042261 .5488192 .936952$

O $-2.808817 \quad 1.1957992 .891117$

C -1.2309862 .7714913 .850887$

C 0.1208013 .4413673 .737737

C 0.3857164 .4323372 .863767

C 1.1648982 .9948824 .725278

C -0.5570294 .9714521 .822274$

C 0.1555925 .5309810 .585299

C $0.9601634 .492690-0.214354$

C $1.7734075 .213545-1.294181$

C $2.5549884 .234408-2.173823$

O $0.0557193 .552144-0.815333$

O $3.0249384 .952846-3.324824$

C $1.5844493 .153489-2.679211$

C $3.7988173 .688972-1.465659$

O $2.2887522 .139643-3.393239$

C $0.7111082 .516013-1.565485$

C $-0.3544431 .618498-2.241901$

O $1.5415181 .709798-0.728198$

C -3.5919391 .8180421 .973030$

O $-3.2807792 .735776 \quad 1.232841$

C -4.9024861 .1371691 .983595$

C -5.7217691 .2960530 .934433$

C -7.0017640 .6175340 .734110$

C $-7.520119-0.2378671 .860940$

C $-1.4229811 .006229-1.324443$

C $-2.270442-0.001420-2.093117$

C $-3.818899-1.906684-1.769381$

O $-2.185290-0.144688-3.311673$

C $-5.222083-1.955894-1.253850$

C $-6.293488-1.994791-2.062859$

C $-7.717310-2.144605-1.600792$

C $-8.587284-0.967850-1.990793$

C $-9.139834-0.890297-3.213060$

C $-8.8712980 .081401-0.937372$

C $-7.6156770 .753709-0.459811$

N -3.129838 -0.725682 -1.275203

H $\quad 0.416715-0.108699 \quad 1.746025$

H -0.991417 -0.0031104 .465890$

H $-1.057637 \quad 1.769798 \quad 1.919008$

H -1.424623 2.4960574 .896938

H -2.0185313 .5050243 .632880$

H 1.3676194 .9012782 .898814

H 2.0923713 .5707254 .636507

H 0.7978763 .1198585 .749232

H 1.4182851 .9421254 .572115

H -1.265861 4.2070761 .498428

H -1.1361915 .7845012 .276448$

H $-0.6103315 .970524-0.067041$

H 0.8173126 .3481950 .899966

H $1.632446 \quad 3.9577840 .465214$

H $2.4544565 .947367-0.844777$

H $1.0938265 .791368-1.936809$

H $3.5837165 .685373-3.013179$

H $0.9144953 .633209-3.406872$

H $4.4677704 .509242-1.178971$

H $4.3822273 .048960-2.136255$

H $3.5650093 .123302-0.561449$

H $2.8100642 .580127-4.085893$

H $-0.8830822 .206279-3.004487$

H $0.1578580 .800543-2.764817$

H $1.0086361 .003420-0.332459$

H $\quad-5.1273590 .4811592 .816354$

H -5.4051031 .9459970 .119383$

H -6.834387 -1.068882 2.060300

H -8.498702 -0.6790831 .663816$

H -7.6273690 .3565452 .775243$

H $-0.9640780 .487457-0.477484$

H -2.082479 $1.790356-0.941555$

H $-3.263472-2.776721-1.404820$ 
H $-3.785934-1.932887-2.863671$ H -5.358072 -2.016942 -0.177423 H $-6.138960-1.960352-3.139582$ H -7.770594 -2.309182 -0.517821 H -8.122066 -3.060769 -2.050948 H $-9.783977-0.063374-3.492467$ H -8.957551 -1.653780 -3.962410 H $-9.436271-0.385129-0.126051$ H $-9.5325380 .861643-1.336030$ H -7.152214 1.390719-1.214596 H -3.059071 -0.606134 -0.268645 C $0.257865-2.9309762 .462237$ O $0.771327-2.8821291 .118314$ C $-0.267721-3.2966600 .210165$ C $-1.340206-3.9529491 .073116$ C $-1.256347-3.1150342 .334802$ H $-0.676940-2.378889-0.228888$ C $0.312634-4.180070-0.900593$ H -2.327731 -3.9268210 .603763$ H -1.101202 -4.9981181 .300525$ C -1.901471 -3.760554 3.547832 H -1.756849 -2.159948 2.134654 C $1.580004-3.605438-1.565387$ O $0.669550-5.456999-0.352742$ H $-0.456462-4.385018-1.653472$ H $2.402017-3.651177-0.843426$ O $1.949512-4.470344-2.646010$ C $1.444166-2.174046-2.115591$ C $2.726525-1.641466-2.786630$ H $1.158897-1.481686-1.316527$ O $0.409329-2.130611-3.100396$ H $2.979791-2.259134-3.657371$ H $2.491660-0.647316-3.188164$ H $-2.969523-3.9266793 .372639$ H -1.444398 -4.7285513 .778721$ H $-1.798878-3.1196304 .429218$ C $0.733449-1.706628 \quad 3.252627$ H $1.303451-5.3012150 .368400$ H $2.031842-5.368924-2.283337$ H $-0.435883-2.302144-2.652959$ H $1.824196-1.6254373 .175223$ H $0.496859-1.8499224 .313666$ H $0.705826-3.8233932 .919124$ C $4.537897-0.6043390 .301423$ O $3.569712-0.686486-0.753063$ C $3.930829-1.547800-1.836473$ C $5.189388-1.007874-2.527607$ C $6.341615-0.882646-1.523153$ C $5.913850-0.165512-0.235146$ H 4.1575340 .1939020 .951870 C $4.549504-1.9098531 .110617$ H $4.149199-2.548571-1.450286$ O $4.911006 \quad 0.265729-3.102493$ H $5.467424-1.687348-3.342053$ H $7.169959-0.330851-1.983954$ O $6.825648-2.180562-1.188434$ H $6.671456-0.2982390 .545363$ O $5.8339671 .237806-0.500939$ C $4.979661-1.7099852 .546923$ H $3.532892-2.3206121 .128152$ H $5.201184-2.6627810 .659508$ H $5.7207190 .572967-3.544022$ H $7.122332-2.605064-2.011559$ H $6.7172791 .535775-0.777238$ O $4.719169-2.8535953 .248081$ O $5.486838-0.6932682 .999459$ C $5.065086-2.8164194 .636042$ H $6.141144-2.6576904 .754195$ H $4.499268-2.0309455 .146309$ H $4.801976-3.7811605 .077781$

SCF Energy (B3LYP/6-31G**//MMFF) $=-3245.89211442$

\section{7_00242}

MM̄FF Geometry

C $2.814528-2.5516292 .886429$

C $2.448675-1.2618632 .946597$

C $1.112104-0.7035543 .364960$

$\begin{array}{llll}\text { O } & 0.563808 & -0.030699 & 2.203187\end{array}$
C $0.071305-1.7357793 .849282$

C -1.251605-1.111154 4.252469

C $-2.417673-1.3271663 .612221$

C -1.216229 -0.221098 5.467910

C $-2.673140-2.2489672 .453417$

C $-2.249117-1.6672741 .099703$

C $-2.843684-2.476276-0.061034$

C $-2.122417-2.170037-1.372351$

C $-2.779008-2.894163-2.557701$

O $-4.220423-2.105156-0.170785$

O $-2.227829-2.355615-3.768828$

C $-4.296082-2.607333-2.545454$

C $-2.421877-4.384125-2.578055$

O $-4.971084-3.378624-3.536465$

C $-4.962239-2.818589-1.163282$

C $-6.411895-2.262460-1.199836$

O $-5.012699-4.215846-0.869494$

C 0.3244901 .3051922 .282949

O $0.6240322 .057346 \quad 3.196272$

C -0.4219921 .6970531 .065583$

C -1.0327972 .8906811 .026577$

C $-1.8232433 .432276-0.081617$

C $-2.0275502 .561592-1.293312$

C -7.105006-2.105194 0.164885

C $-6.506435-1.0280501 .054586$

C $-5.819806 \quad 1.360017 \quad 1.084665$

O $-6.121001-1.2769082 .195172$

C -5.5064232 .3739540 .031694$

C $-5.8014873 .677806 \quad 0.136183$

C $-5.5527654 .676397-0.957049$

C $-4.5314885 .724384-0.582042$

C $-4.9049356 .879332-0.005475$

C $-3.0939875 .479340-0.980514$

C $-2.329034 \quad 4.6781530 .038657$

N $-6.447487 \quad 0.2153190 .450281$

H $2.113727-3.3337573 .161137$

H $3.180916-0.5087962 .662178$

H 1.3099710 .0101744 .173646

H $\quad 0.467099-2.288470 \quad 4.711155$

H $-0.091210-2.4758963 .058259$

H $-3.307018-0.814993 \quad 3.977649$

H $-2.2174070 .096646 \quad 5.778336$

H $-0.767246-0.753225 \quad 6.313061$

H $-0.631903 \quad 0.682735 \quad 5.275675$

H -2.176905 -3.212993 2.618209

H $-3.745332-2.4774492 .444782$

H -2.579357 -0.6232331 .027546$

H $-1.156101-1.6803641 .033461$

H $-2.768364-3.5442680 .174748$

H -1.060111 -2.434664 -1.304027

H $-2.152267-1.087665-1.559277$

H -2.401994 -1.399159 -3.783875

H -4.429127 -1.554393 -2.830790

H -1.335201-4.514570 -2.649388

H -2.840660 -4.879047 -3.461486

H $-2.762697-4.920494-1.690138$

H $-4.527437-3.212020-4.385800$

H -6.408669 -1.287155-1.700478

H $-7.033031-2.929859-1.810978$

H $-5.734644-4.378541-0.241799$

H -0.4665690 .9858980 .248947$

H -0.9452003 .5441271 .893956$

H -2.404932 $1.574095-1.004849$

H - $1.0878222 .432724-1.837615$

H $-2.7576102 .966402-1.997925$

H $-8.158965-1.8456220 .006820$

H -7.092759 -3.052408 0.714518

H -4.9012771 .0280611 .579775$

H -6.5088601 .7406991 .845502$

H $-5.0243042 .012770-0.873954$

H -6.2851624 .0442031 .038662$

H -6.510623 $5.159655-1.191598$

H -5.249406 4.180479-1.887697

H $-4.181307 \quad 7.6521130 .232008$

H $-5.942127 \quad 7.0739150 .246709$

H -2.573041 $6.440753-1.089522$

H -3.056731 $5.039363-1.981557$

H -2.1390835 .2269250 .962264$ 
H $-6.9968660 .372953-0.389955$

C $4.238761-3.4990671 .001494$

$\begin{array}{lllll}\text { O } & 3.925660 & -2.417698 & 0.099757\end{array}$

C $3.067760-2.923777-0.939498$

C $3.172317-4.442923-0.864225$

C $3.305807-4.6524130 .632198$

H $2.044875-2.631560-0.672914$

C $3.445308-2.319776-2.295615$

H $2.297765-4.941580-1.292915$

H $4.064521-4.815269-1.380718$

C $3.838560-6.0215521 .015032$

H $2.315573-4.5179991 .083994$

C $3.446959-0.776771-2.338433$

O $4.759329-2.760463-2.661768$

H $2.774099-2.711684-3.068765$

H $4.304811-0.388819-1.777753$

O $3.646827-0.381710-3.701743$

C $2.154498-0.120969-1.825833$

C $2.0338801 .385969-2.110364$

H $2.064480-0.292795-0.747135$

O $1.035192-0.762808-2.445593$

H $1.9159941 .556621-3.188291$

H $1.0872361 .708597-1.663512$

H $3.161827-6.808650 \quad 0.666727$

H $4.824061-6.2070660 .574764$

H $3.932040-6.1121012 .101867$

C $4.179824-3.0093632 .450923$

H $5.367574-2.487048-1.954064$

H $4.468973-0.801933-4.007422$

H $0.227993-0.370999-2.071048$

H $4.894101-2.1861072 .574402$

H $4.508338-3.807533 \quad 3.126849$

H $5.277579-3.7790050 .782186$

C $4.386346 \quad 2.6954190 .488444$

O $3.3311021 .982145-0.173210$

C $3.1767352 .257357-1.567859$

C $2.8995513 .754514-1.799369$

C $3.9528264 .638030-1.126503$

C $4.205536 \quad 4.213853 \quad 0.324912$

H 4.2586872 .4599391 .552961

C 5.7789172 .2062460 .055768

H $4.0950141 .977751-2.093498$

O $1.6102124 .109590-1.300374$

H $2.8878423 .948186-2.878363$

H $3.6112235 .680093-1.151015$

O $5.1604824 .560015-1.877006$

H 5.0825494 .7306790 .731597

O 3.0842654 .5987191 .125869

C $6.044454 \quad 0.7783440 .478314$

H $5.9026572 .254154-1.028946$

H 6.5511052 .8276930 .523705

H $1.5805843 .871872-0.357738$

H $5.8088505 .146504-1.451316$

H 2.9954945 .5648901 .061097

O $7.1740530 .330971-0.145822$

$\begin{array}{lllll}\text { O } & 5.364335 & 0.139991 & 1.268226\end{array}$

C $7.559301-1.0108810 .168471$

H $6.789762-1.711864-0.166127$

H $8.489043-1.230964-0.362781$

H $7.736113-1.1151411 .243347$

SCF Energy (B3LYP/6-31G**//MMFF) $=-3245.90461988$

\section{7_00243}

MM̄FF Geometry

C $2.692752-2.382109-2.970554$

C $1.726865-1.748372-2.289583$

C $0.566837-1.038422-2.930300$

O $-0.614950-1.635060-2.337306$

C $0.5680390 .475474-2.651253$

C $1.6924451 .246285-3.310315$

C $2.5576932 .050584-2.662914$

C $1.7642451 .139649-4.813026$

C $2.6400292 .340367-1.192150$

C $2.2219703 .773960-0.843354$

C $0.7253494 .055937-1.049939$

C $0.4205165 .521428-0.740051$

C $-1.078330 \quad 5.823071-0.858769$

O $-0.0140883 .211522-0.165014$
O $-1.3117847 .128872-0.309311$

C $-1.872116 \quad 4.792862-0.023632$

C $-1.5128335 .919686-2.326248$

O $-3.268763 \quad 4.975527-0.244155$

C $-1.4458393 .324640-0.269390$

C $-1.9989532 .354450 \quad 0.801849$

O $-1.9029112 .903586-1.555185$

C $-1.797004-1.507654-2.994189$

O $-2.009083-0.889027-4.024103$

C $-2.805266-2.272416-2.228885$

C $-4.109898-2.028301-2.418146$

C $-5.218517-2.706205-1.741659$

C $-4.889433-3.926795-0.921896$

C -3.5195152 .2337440 .884819$

C $-3.918576 \quad 1.158168 \quad 1.874014$

C $-4.179476-1.3066362 .104848$

$\begin{array}{lllll}\text { O } & -4.248818 & 1.432810 & 3.025728\end{array}$

C $-5.590962-1.7639661 .881117$

C $-6.579232-1.1113011 .248083$

C $-7.958746-1.6789811 .062906$

C $-8.398099-1.697248-0.385515$

C $-9.377693-0.887001-0.821051$

C $-7.757751-2.710961-1.310418$

C $-6.464138-2.210108-1.895726$

N $-3.842971-0.1193591 .344194$

H $2.692304-2.350690-4.056999$

H $1.735777-1.787886-1.204238$

H $0.544979-1.241305-4.008053$

H $0.5303360 .634399-1.568402$

H $-0.3741780 .905923-3.013507$

H $3.3064882 .575652-3.255599$

H $2.469144 \quad 1.856610-5.247752$

H $2.087506 \quad 0.139099-5.115445$

H $0.7828621 .340539-5.255490$

H $2.0784061 .622122-0.589361$

H $3.6891272 .218253-0.900161$

H $2.474753 \quad 3.9399450 .212285$

H $2.8179004 .482328-1.432547$

H $0.4544723 .822030-2.085012$

H $0.9986786 .188553-1.391773$

H $\quad 0.743713 \quad 5.7513850 .284978$

H $-1.033777 \quad 7.1209910 .622363$

H -1.6979865 .0255621 .035308$

H $-0.9257176 .685032-2.848273$

H $-2.5573156 .240252-2.410353$

H $-1.3951984 .980813-2.871555$

H $-3.4903354 .564314-1.097016$

H $-1.577618 \quad 1.3579810 .606381$

H -1.6015612 .6365231 .786560$

H $-1.9745131 .934632-1.552344$

H $-2.443185-3.010624-1.522791$

H $-4.395672-1.247411-3.121793$

H $-4.420248-4.692189-1.550413$

H $-5.761434-4.392434-0.459113$

H -4.200646 -3.677017 -0.108269

H $-3.973712 \quad 3.167197 \quad 1.231031$

H -3.956531 $2.000723-0.092563$

H $-4.016370-1.1230233 .171643$

H -3.488747 -2.091333 1.782049

H $-5.808233-2.7527202 .281921$

H $-6.408924-0.1157340 .847405$

H -8.649839 -1.0719111 .661906$

H $-8.028577-2.6968181 .466613$

H $-9.715240-0.913528-1.851868$

H $-9.860646-0.176157-0.158884$

H $-8.433254-2.928660-2.148610$

H -7.653089 -3.662014 -0.782030

H $-6.591504-1.326470-2.523337$

H $-3.612072-0.237350 \quad 0.362047$

C $4.997380-2.506792-1.841640$

O $4.703782-1.705279-0.683274$

C $5.622442-0.592826-0.661554$

C $6.555394-0.772337-1.859432$

C $5.683844-1.561884-2.820245$

H $5.0252150 .308983-0.822866$

C $6.329574-0.5230120 .698287$

H $6.8862640 .186806-2.270186$

H $7.451628-1.345337-1.594273$ 
C $6.461412-2.262093-3.919153$

H $4.958584-0.872195-3.270616$

C $5.371616-0.4183181 .905921$

O $7.119383-1.7069630 .875463$

H 7.0372900 .3135530 .706940

H $4.789744-1.3421571 .995741$

O $6.167272-0.3251483 .093967$

C 4.4302820 .7982831 .858026

C $3.564348 \quad 0.987913 \quad 3.118045$

H $3.776860 \quad 0.7146680 .985847$

O 5.2208391 .9771251 .682464

H 4.2016551 .0470774 .008601

H 3.0761861 .9685383 .035730

H $6.997748-1.532956-4.535196$

H $7.197461-2.960953-3.507971$

H $5.788613-2.826504-4.572553$

C $3.743631-3.235147-2.319184$

H $6.518574-2.4703270 .827978$

H $6.766079-1.0913803 .102607$

H $4.6108282 .725603 \quad 1.567441$

H $3.292040-3.758220-1.465918$

H $4.029067-4.014168-3.037014$

H $5.710984-3.271341-1.503625$

C $0.595253-1.0282342 .121156$

O $1.610633-0.0176142 .171882$

C $2.493399-0.1010563 .296076$

C $1.698612 \quad 0.079845 \quad 4.597835$

C $0.565774-0.9485154 .695555$

C $-0.257470-1.0213993 .402752$

H $-0.046889-0.7182971 .286436$

C $1.213493-2.3889591 .766458$

H $2.978634-1.0828573 .304809$

O $1.153321 \quad 1.3969404 .645282$

H $2.382716-0.0259255 .448204$

H $-0.097490-0.6832875 .527977$

O $1.109615-2.2346174 .975775$

H $-0.912893-1.8995153 .413300$

O -1.1014090 .1318853 .339227$

C $0.259580-3.2738880 .993979$

H $2.096742-2.2208511 .137603$

H $1.544448-2.9370742 .651914$

H $\quad 0.6781701 .4882125 .488649$

H $\quad 1.597307-2.1715655 .814669$

H -1.700518 0.1010864 .104398

O $0.970752-4.2662310 .381466$

O $-0.953777-3.1276720 .942939$

C $0.201450-5.173989-0.413283$

H $-0.287790-4.637838-1.231966$

H $0.883617-5.914849-0.838561$

H $-0.536760-5.6908000 .207205$

SCF Energy $\left(\mathrm{B} 3 \mathrm{LYP} / 6-31 \mathrm{G}^{* *} / / \mathrm{MMFF}\right)=-3245.91096572$

0700244

MM̄̄FF Geometry

C $-1.173423-3.3039801 .462269$

C $-0.966477-3.1125070 .151651$

C -1.897879 -2.301893 -0.713540

O $-1.161743-1.168977-1.237464$

C $-2.414378-3.113120-1.918137$

C $-3.797846-3.683794-1.690905$

C $-4.919625-3.188927-2.249656$

C $-3.870858-4.891286-0.794039$

C $-5.046444-2.013779-3.179366$

C $-4.738957-0.638650-2.577103$

C $-5.400212-0.326090-1.227898$

C $-6.927506-0.349687-1.298352$

C -7.563495 0.0710840 .035322

O $-4.9500950 .983029-0.849976$

O

C -6.9323361 .4017730 .492464$

C $-7.497808-1.0510811 .078420$

O -7.3854531 .7578591 .797046$

C $-5.385031 \quad 1.4051730 .448970$

C $-4.8661202 .843350 \quad 0.686046$

O $-4.8843520 .548800 \quad 1.476721$

C $-0.962780-0.116000-0.397945$

O $-1.343985-0.002514 \quad 0.756034$

C $-0.1453380 .876499-1.121506$
C $0.7456591 .602162-0.430456$

C $1.6917632 .561673-1.000703$

C $1.4645512 .999837-2.423525$

C -3.3556873 .0227210 .479546$

C -2.9316204 .4706290 .663465$

C -0.9274045 .9220650 .673340$

O $-3.7118725 .365586 \quad 0.978464$

C $0.3634735 .981747-0.076200$

C 1.5553436 .1466740 .516985

C $2.8540506 .264729-0.227075$

C 3.8498835 .1988090 .168476

C 4.7617885 .4245561 .129203

C $3.8442103 .906510-0.614308$

C $2.7097362 .996976-0.229612$

N -1.573069 4.6462290 .431223

H $-2.061777-2.8796501 .924958$

H $-0.074982-3.522029-0.316659$

H $-2.747483-1.915098-0.136039$

H -1.729954 -3.936079-2.162446

H -2.409289-2.482779 -2.815208

H $-5.864563-3.680464-2.021494$

H $-4.872492-5.334088-0.775676$

H -3.178873 -5.665955-1.139917

H -3.612669-4.623605 0.234274

H -4.411944 -2.175210 -4.059694

H -6.072639-2.004795 -3.568685

H -3.654631 - $0.518718-2.465819$

H $-5.0225840 .133134-3.305681$

H $-5.037010-1.043340-0.484634$

H -7.300091-1.337185-1.594095

H $-7.2662550 .345068-2.079752$

H $-9.353279-0.487368-0.558439$

H $-7.2976802 .185358-0.186328$

H $-8.021247-1.9432810 .714084$

H -8.009283 -0.7612912 .003067$

H $-6.476594-1.347321 \quad 1.327075$

H -8.3578131 .7592021 .775497$

H $-5.3777243 .524662-0.006860$

H -5.1195143 .1645381 .704553$

H $-3.964150 \quad 0.788074 \quad 1.667742$

H $-0.2498220 .929986-2.198730$

H $0.826809 \quad 1.4422260 .644282$

H $1.6491782 .173160-3.117458$

H $2.1022953 .832443-2.728043$

H $0.4329343 .345129-2.558412$

H -2.7943382 .4198391 .200358$

H -3.070172 2.712165 -0.531797

H -0.7878436 .0186231 .755413$

H $-1.589911 \quad 6.7303280 .345836$

H $0.3104225 .914524-1.160123$

H 1.6010316 .2340961 .600303

H $3.2704637 .258725-0.016896$

H $2.6982626 .238326-1.312737$

H $5.4983544 .674463 \quad 1.397637$

H 4.7875926 .3633881 .672484

H $4.7740303 .351589-0.428782$

H $3.8673834 .133739-1.683790$

H 2.7789202 .6243450 .792590

H -0.9871473 .8190010 .361587$

C $0.405304-3.1082523 .375370$

O $1.501773-2.4133482 .740634$

C $2.571731-2.2755453 .697937$

C $2.014829-2.7546135 .034114$

C $1.019781-3.8059364 .585746$

H $3.365794-2.9646323 .390488$

C $3.087321-0.8331943 .720441$

H $2.795684-3.1520165 .689728$

H $1.504586-1.9458855 .571107$

C $0.018218-4.1853055 .662643$

H $1.568284-4.7021264 .266324$

C $3.756484-0.3706722 .408339$

$\begin{array}{lllll}\text { O } & 1.990058 & 0.048528 & 3.992616\end{array}$

H $3.788507-0.7037944 .553056$

H $3.018426-0.3341391 .599196$

O 4.2108190 .9742822 .608056

C $4.967405-1.2258831 .986203$

C $5.813920-0.6359700 .843360$

H $4.629687-2.2272951 .700741$ 
O $\quad 5.839502-1.3968993 .109323$ H $6.192857 \quad 0.353338 \quad 1.128885$ H $6.703170-1.2736090 .743297$ H $0.531845-4.6034816 .534618$ H $-0.559276-3.3170735 .997510$ H $-0.687112-4.9369615 .295198$ C $-0.248086-4.0596622 .372598$ H $1.327650-0.0839843 .292480$ H 3.4442991 .4993122 .895703 H $6.127887-0.512583 \quad 3.393864$ H $-0.844644-4.8185702 .890783$ H $0.520106-4.5830801 .790647$ H $-0.308123-2.3414513 .707303$

C $4.030981-2.008528-2.130676$

O $4.761177-1.892094-0.901780$

C $5.087815-0.555100-0.507419$

C $5.9645970 .117201-1.577474$

C $5.3138200 .056776-2.962733$

C $4.795597-1.346627-3.292313$

H $3.993873-3.087696-2.325833$

C $2.590765-1.521590-1.945846$

H $4.1658670 .021327-0.381353$

O $7.246541-0.507382-1.635915$

H $6.1330191 .161641-1.290745$

H $6.0478290 .352976-3.722420$

$\begin{array}{lllll} & & 4.230581 & 0.977272 & -3.025385\end{array}$

H $4.169542-1.312302-4.190765$

O $5.928312-2.163856-3.606729$

C $1.700836-1.918807-3.095977$

H $2.162855-1.921742-1.018032$

H $2.538002-0.436044-1.836644$

H $7.109075-1.450289-1.830298$

H $4.5876651 .864958-2.852550$

H $5.594179-3.031040-3.893267$

O $1.431296-3.253711-3.042342$

O $1.304079-1.134831-3.948403$

C $0.634569-3.764438-4.115589$

H $0.359232-4.794463-3.874086$

H $-0.280674-3.177211-4.236213$

H $1.216964-3.762248-5.041563$

SCF Energy (B3LYP/6-31G**//MMFF) $=-3245.91693749$

\section{7_00245}

MMFF Geometry

C -3.125798 -3.841053 -0.685963

C $-1.895824-3.501458-0.270913$

C $-0.761091-3.181180-1.208808$

O $-0.142761-1.970847-0.716930$

C $0.270741-4.321529-1.189556$

C $1.337739-4.193944-2.258757$

C $2.630150-3.903329-2.011285$

C $0.886063-4.489374-3.666117$

C $3.245643-3.613772-0.669217$

C $4.487084-2.723597-0.792812$

C $5.264742-2.5932530 .525118$

C $4.519947-1.7635861 .572454$

C $5.360167-1.5610132 .845600$

O $6.512397-1.9646840 .206588$

O $4.735496-0.525707 \quad 3.621325$

C $6.760645-1.0456242 .446298$

C $5.367217-2.8152853 .727159$

O $7.624582-0.9734873 .577872$

C $7.420790-1.8570641 .309772$

C $8.735566-1.2203850 .791599$

O $7.766087-3.1567891 .808762$

C $0.220529-1.024541-1.622590$

O $0.109129-1.079430-2.836554$

C $0.7589870 .127193-0.865175$

C $0.946094 \quad 1.296319-1.495429$

C $1.3979472 .547906-0.883537$

C 1.8196882 .5015820 .561567

$\begin{array}{lllll}\text { C } & 8.676698 & 0.246255 & 0.359147\end{array}$

C $7.6944460 .494896-0.769319$

C $5.5992761 .720891-1.298880$

O $7.774005-0.095901-1.843946$

C $5.0610643 .070769-0.952190$

C $4.8111204 .031442-1.853660$

C $4.2900255 .390729-1.488284$
C $2.8968395 .644695-2.014927$

C $2.6935436 .422753-3.091416$

C $1.7342015 .074961-1.231889$

C $1.3955963 .667557-1.637954$

N $6.7363961 .441225-0.442569$

H $-3.328582-3.898129-1.752660$

H $-1.680326-3.4775940 .794857$

H $-1.157314-3.029344-2.219445$

H $-0.235740-5.285016-1.338930$

H $0.729015-4.383248-0.195328$

H $3.318881-3.893593-2.854775$

H $1.714608-4.466280-4.381969$

H $\quad 0.435750-5.486110-3.718657$

H $0.146251-3.755818-3.999242$

H $2.521834-3.125786-0.009820$

H $3.531828-4.568590-0.212583$

H $5.165561-3.138021-1.551286$

H $4.207956-1.728816-1.164732$

H $5.467221-3.6024940 .902356$

H $3.555756-2.2173591 .829089$

H $4.283735-0.7757131 .152274$

H $3.825643-0.8066443 .819296$

H $6.617118-0.0120312 .113149$

H $4.343736-3.0899914 .009993$

H $5.896913-2.6334744 .668694$

H $5.816697-3.6814963 .237594$

H $7.915520-1.8775043 .786383$

H $9.512948-1.3218421 .560357$

H $9.088400-1.813192-0.064034$

H $8.446303-3.536712 \quad 1.227935$

H $0.943311-0.006148 \quad 0.194684$

H $\quad 0.7104761 .356601-2.557334$

H 2.6100181 .7563180 .707838

H $0.9722382 .238067 \quad 1.202641$

H 2.2192123 .4485190 .929966

H 8.4675840 .8891151 .220176

H $9.6635410 .552916-0.009266$

H $4.8448260 .952876-1.099467$

H $5.902593 \quad 1.642131-2.347975$

H 4.8560993 .2674350 .097868

H $5.0058853 .841979-2.906462$

H $4.9907046 .134863-1.889156$

H $4.2979955 .542191-0.401416$

H $1.6930236 .634445-3.453866$

H $3.5230526 .860230-3.637084$

H $0.8379375 .685591-1.407911$

H $1.9309995 .178242-0.161418$

H $1.0642953 .588316-2.674621$

H 6.6712341 .7408180 .525961

C $-5.589170-3.628899-0.169771$

O $-5.647526-2.2247350 .165870$

C $-6.925371-1.9501790 .772675$

C $-7.791360-3.1776890 .514863$

C $-6.761946-4.2838160 .563113$

H $-6.749001-1.8688801 .851230$

C $-7.542291-0.6536340 .236723$

H -8.582402 -3.2919901 .262311$

H -8.263958 $-3.140894-0.473768$

C $-7.241985-5.581483-0.065224$

H $-6.493882-4.4696611 .611963$

$\begin{array}{llll}\text { C } & -6.728856 & 0.632251 & 0.481921\end{array}$

O $-7.736985-0.775615-1.178955$

H $-8.545244-0.5313620 .663717$

H $-5.8568470 .656708-0.180246$

$\begin{array}{llll}\text { O } & -7.559031 & 1.735923 & 0.092494\end{array}$

C -6.2789140 .8525181 .936786$

C -5.8022092 .2803492 .267465$

H $-5.482800 \quad 0.1373322 .174201$

$\begin{array}{llll}\text { O } & -7.375570 & 0.563090 & 2.810809\end{array}$

H -6.6625362 .9624992 .278088$

H -5.4365492 .2579643 .303077$

H -8.125873 -5.9584320 .459825$

H $-7.510668-5.442266-1.117788$

H $-6.466800-6.352232-0.014957$

$\begin{array}{llll}\text { C }-4.240971 & -4.226732 & 0.243847\end{array}$

H $-6.869362-0.959883-1.578230$

H $-7.8206701 .589125-0.832680$

H $-7.0509280 .639343 \quad 3.724346$ 
H $-4.306481-5.320750 \quad 0.227494$

H -4.000557 $-3.921721 \quad 1.269906$

H -5.730609 -3.701302 -1.256112

C -2.4957422 .3015860 .522064$

O

C -4.7098172 .8425291 .344479$

C -4.2883334 .2616661 .762900$

C $-3.115677 \quad 4.7643410 .916277$

C -1.993910 3.7242420 .826385

H -1.6952961 .6004130 .790259$

C $-2.8368572 .085611-0.961065$

H $-5.1088682 .879910 \quad 0.326649$

O $-3.9120394 .262446 \quad 3.138978$

H -5.1490664 .9327091 .654223$

H $-2.7220195 .687679 \quad 1.358374$

O $-3.5976335 .077709-0.386854$

H -1.265117 4.0249890 .066636

O -1.2917003 .6977762 .072506$

C $-3.1049730 .631422-1.284696$

H -3.723674 2.650579-1.258685

H $-1.9934522 .408602-1.581294$

H -3.695063 5.1783523 .383107

H $-2.8467195 .416214-0.903864$

H -1.9021293 .3607772 .750705$

O $-3.464450 \quad 0.533117-2.598876$

O $-3.015419-0.297392-0.494428$

C $-3.777781-0.785075-3.058015$

H $-2.874713-1.400532-3.068642$

H $-4.549689-1.239388-2.430023$

H $-4.161061-0.706997-4.078949$

SCF Energy $\left(B 3 L Y P / 6-31 G^{* *} / / M M F F\right)=-3245.90795615$

\section{00246}

MM̄FF Geometry

C $-1.945513-2.492952-2.187838$

C $-0.724793-2.512083-1.632838$

C $0.483060-3.121550-2.289844$

O $1.540043-2.132958-2.249856$

C $0.982305-4.362233-1.518225$

C $-0.010075-5.502456-1.413801$

C $-0.701625-5.827553-0.301983$

C $-0.173697-6.317858-2.670302$

C $-0.694354-5.1200821 .026573$

C -2.095528 -4.5951321 .377571$

C $-2.060131-3.4957362 .450661$

C $-3.475783-3.0149452 .775260$

C -3.469321-1.817796 3.738393

O $-1.299644-2.4105361 .918778$

O $-4.781013-1.2325153 .725918$

C $-2.495615-0.7423443 .205890$

C $-3.208520-2.2625075 .182212$

$\begin{array}{llll}\text { O } & -2.336724 & 0.315068 & 4.148287\end{array}$

C - $1.110436-1.2998222 .802761$

C $-0.308236-0.2380442 .014482$

O $-0.330367-1.6586543 .941899$

C $2.502625-2.208641-3.204633$

O $2.496300-2.935929-4.188983$

C $3.643657-1.312507-2.910903$

C $3.773020-0.611651-1.774615$

C $4.9197280 .229840-1.448258$

C $4.5436701 .575016-0.895639$

C $1.062306-0.7362351 .530169$

C $2.186894-0.5631792 .536657$

C $4.567287-1.2299692 .898771$

O 2.0807370 .1040613 .561488

C $5.530699-0.2629682 .285616$

C $6.738771-0.6171281 .821043$

C $7.7402750 .351346 \quad 1.259749$

C $8.216720-0.031527-0.123748$

C $9.328378-0.767613-0.291656$

C $7.4603990 .520272-1.310394$

C $6.182184-0.223102-1.582989$

N $3.336939-1.2283582 .131031$

H -2.082917 -2.902771-3.185640

H $-0.594871-2.128333-0.626426$

H $\quad 0.255887-3.379477-3.331715$

H $1.345831-4.051542-0.531719$

H $1.890479-4.742278-2.006454$
H $-1.368555-6.687803-0.346332$

H $-0.870206-7.152555-2.537566$

H $-0.558282-5.694984-3.483834$

H $0.789194-6.738225-2.978423$

H $\quad-0.369089-5.8253491 .800739$

H $0.029284-4.3028861 .037899$

H $-2.562608-4.1893040 .472698$

H -2.722694 -5.429376 1.715692

H $-1.561603-3.8873003 .344854$

H -4.081252 -3.834673 3.181833

H $-3.978067-2.7055351 .847545$

H -5.419314 -1.921207 3.979346

H -2.958293 -0.296405 2.313720

H $-4.003841-2.9353285 .525354$

H -3.222192 -1.409093 5.868956

H $-2.260155-2.7908905 .301863$

H -3.2246170 .6527514 .357015$

H -0.8867160 .0356541 .122263$

H -0.1835880 .6708772 .614466$

H -0.791214 -1.360184 4.742152

H $4.416792-1.285977-3.673962$

H $2.993631-0.633127-1.018791$

H $4.0763912 .184919-1.675266$

H $5.3922022 .138050-0.501366$

H $3.8353911 .465475-0.067374$

H $1.015274-1.786107 \quad 1.222274$

H $1.347931-0.1553030 .647634$

H $4.950745-2.2552902 .892114$

H $4.362607-0.9434793 .935197$

H 5.2305250 .7815362 .252027

H $7.044292-1.6593651 .881952$

H 8.5953300 .3814321 .948239

H 7.3435601 .3739611 .242862

H $9.695730-1.020381-1.280789$

H $9.892493-1.1385880 .557811$

H $8.0767600 .446003-2.216521$

H $7.3037191 .594508-1.173494$

H $6.334326-1.237310-1.953405$

H $3.339118-1.7147451 .239137$

C $-3.992541-0.997620-2.207627$

O $-3.5712150 .321621-1.787882$

C $-4.7332981 .052560-1.354594$

C $-5.9196990 .350803-1.999706$

C $-5.488889-1.092831-1.886402$

H $-4.7988850 .926862-0.267299$

C $-4.6404782 .539036-1.706148$

H $-6.8613290 .561452-1.483727$

H $-6.0346170 .631405-3.053239$

C $-6.254378-2.030501-2.804923$

H $-5.631643-1.419104-0.846918$

C $-3.4478843 .298822-1.094427$

O $-4.5591312 .682315-3.130651$

H $-5.5748973 .032097-1.412033$

H -2.530987 $3.028895-1.629125$

O $-3.6603744 .692868-1.361954$

C $-3.2376333 .101650 \quad 0.418765$

C -2.2938154 .1241031 .082338$

H $-2.8631392 .086848 \quad 0.597101$

$\begin{array}{lllll}\text { O } & -4.502918 & 3.205421 & 1.079537\end{array}$

H -2.7924035 .1007441 .136812$

H -2.1587493 .8054202 .124778$

H -7.321677 -2.021438 -2.560796

H $-6.147422-1.739137-3.855223$

H $-5.892486-3.058459-2.702520$

C $-3.186557-2.061587-1.459183$

H $-3.7733492 .192748-3.429334$

H -4.441575 $4.973579-0.854931$

H -4.359081 3.0057492 .020491

H -3.793765 -2.967372 -1.339766

H -2.937361 -1.702050 -0.453397

H $-3.826015-1.053769-3.290561$

C $1.0043732 .964622-0.228462$

O $-0.266520 \quad 3.0108280 .435013$

C -0.9268324 .2773090 .404822$

C -0.0639705 .3462241 .092649$

C 1.3281125 .4172660 .459628

C $1.963908 \quad 4.029647 \quad 0.329262$

H $1.420279 \begin{array}{lll}1.986488 & 0.033957\end{array}$ 
C $0.8511343 .006078-1.757041$ H - $1.0886634 .578870-0.633636$ O $0.057986 \quad 5.0700822 .486229$ H -0.5613726 .3188250 .999202$ H $1.9671686 .061702 \quad 1.075485$ O $1.2125356 .017564-0.827360$ H $2.8624724 .075698-0.296055$ $\begin{array}{llllll}\text { O } & 2.380117 & 3.587028 & 1.625209\end{array}$ C $0.1634071 .765348-2.290580$ H $0.2684343 .869531-2.087706$ H $1.8426073 .052675-2.220648$ H $0.423274 \quad 4.1735952 .581197$ H $2.1073846 .082813-1.202258$ H $3.029374 \quad 4.2289701 .959781$ O $\quad 0.117590 \quad 1.835446-3.653911$ O $-0.283871 \quad 0.854819-1.607314$ C $-0.521593 \quad 0.731925-4.303540$ H $0.017132-0.196276-4.091796$ H $-1.5650080 .656350-3.983524$ H - $0.4982520 .912275-5.381508$

SCF Energy (B3LYP/6-31G**//MMFF) $=-3245.89700137$

\section{7_00247}

MM̄FF Geometry

C $3.154108-2.9427320 .749889$

C $2.746744-3.8378681 .662106$

C $1.543841-3.7171022 .567838$

O $0.882900-2.4383192 .442905$

C $0.504085-4.7981372 .221397$

C $-0.657702-4.8138963 .197050$

C $-1.868550-4.2704492 .961220$

C $-0.389764-5.5219334 .500448$

C $-2.330016-3.5257311 .736279$

C $-3.422835-4.3206771 .012684$

C $-3.995613-3.650631-0.245928$

C $-2.968014-3.527358-1.372213$

C $-3.564133-2.853344-2.617553$

O $-4.510084-2.3587610 .100311$

O $-2.464461-2.511365-3.475716$

C $-4.251745-1.541799-2.181605$

C $-4.446823-3.816886-3.417604$

O $-4.925679-0.929590-3.277536$

C $-5.204358-1.706980-0.976123$

C $-5.748172-0.357854-0.449790$

O $-6.343993-2.474608-1.386759$

C $1.347310-1.4096913 .200317$

O $2.364935-1.3882113 .873089$

C $0.363418-0.310306 \quad 3.092442$

C $0.670597 \quad 0.9081893 .560047$

C -0.2211232 .0686173 .579233$

C -1.6728091 .8378313 .246066$

C $-4.6862270 .689489-0.110191$

C -5.2856941 .9291870 .534093$

C $-4.7689084 .311171 \quad 1.007249$

O -6.4069511 .9536341 .034262$

C $-3.531722 \quad 5.132501 \quad 1.172838$

C $-3.018894 \quad 5.462252 \quad 2.367334$

C $-1.757400 \quad 6.256788 \quad 2.548294$

C $-0.539883 \quad 5.3719022 .721077$

C 0.4036885 .3369701 .763646

C -0.4508254 .5835094 .014173$

C $0.287925 \quad 3.2748353 .908073$

N $-4.398800 \quad 2.9976180 .515090$

H $2.583573-2.0316850 .594657$

H $3.333366-4.7454841 .793093$

H $1.881347-3.8499053 .603675$

H $0.973768-5.7908312 .221174$

H $0.140795-4.648786 \quad 1.197665$

H $-2.625443-4.3531263 .740647$

H -1.270796 -5.5450635 .150611$ H $-0.092031-6.5590074 .314336$ H $0.412572-5.0229095 .052467$ H -2.724071 -2.554383 2.058020 H -1.499613 -3.3102441 .060661$ H -3.043742 -5.3160070 .747068$ H $-4.252536-4.4754051 .716100$ H $-4.829104-4.282296-0.576478$ H -2.545121 -4.506473 -1.628076
H $-2.125797-2.913127-1.032382$

H $-2.822434-2.008774-4.227778$

H $-3.440701-0.859140-1.904183$

H $-3.863168-4.687666-3.740188$

H $-4.813846-3.347770-4.337030$

H $-5.305416-4.184566-2.852222$

H $-5.752035-1.419640-3.427695$

H $-6.4539330 .066670-1.176387$

H $-6.344133-0.559206 \quad 0.451370$

H $-7.069002-2.304749-0.762334$

H $-0.598405-0.5422432 .648566$

H 1.6658001 .0746903 .970440

H -2.113595 1.1127143 .939384

H $-1.783598 \quad 1.4556032 .226586$

H $-2.2809202 .741735 \quad 3.308327$

H -3.944612 0.2769150 .582881

H $-4.1731581 .002856-1.024789$

H $-5.325708 \quad 4.1963491 .943683$

H $-5.437445 \quad 4.773393 \quad 0.273618$

H $-3.028137 \quad 5.459440 \quad 0.266222$

H -3.5203805 .1260653 .271849$

H $-1.8644526 .905193 \quad 3.426657$

H -1.625950 6.9381701 .697258

H $1.299949 \quad 4.733747 \quad 1.856082$

H $0.305295 \quad 5.923948 \quad 0.856093$

H -1.4499144 .3999964 .424106$

H $0.048143 \quad 5.2081224 .766538$

H 1.3527563 .3515424 .129934

H $-3.5783212 .928130-0.079409$

C $5.372676-1.9798000 .107841$

O $4.823870-0.750119-0.406571$

C $5.882097 \quad 0.014563-1.016170$

C $7.177970-0.734735-0.722722$

C $6.692887-2.168357-0.635094$

H $5.702786-0.002518-2.097064$

C $5.8529921 .455294-0.495220$

H $7.928950-0.581790-1.503829$

H $7.621325-0.422910 \quad 0.230323$

C $7.673110-3.0963160 .060968$

H $\quad 6.502480-2.538459-1.651585$

C $4.5182552 .190773-0.736876$

$\begin{array}{lllll}\text { O } & 6.087959 & 1.442808 & 0.920155\end{array}$

H $6.6802722 .026490-0.931501$

H $3.7201531 .710475-0.160657$

O $4.6539313 .517296-0.208805$

C $4.1113752 .303236-2.220385$

C $2.8755363 .190836-2.467552$

H $3.9270281 .307244-2.635313$

O $5.1996432 .862091-2.962564$

H $3.0200234 .165998-1.986997$

H $2.8179883 .395118-3.545660$

H $8.625157-3.124432-0.479052$

H $7.877408-2.7684361 .085771$

H $7.281607-4.1172960 .106111$

C $4.377561-3.119911-0.107778$

H $5.3955750 .894127 \quad 1.327455$

H $4.9102173 .432173 \quad 0.725656$

H $5.3888003 .739940-2.588736$

H $4.855949-4.0820030 .107399$

H $4.054825-3.141014-1.155180$

H $5.553698-1.8276281 .180378$

C $0.0490120 .760720-2.657570$

O $1.2424161 .507827-2.930076$

C $1.5615272 .548167-1.997379$

C $0.4212153 .573600-1.924428$

C $-0.9012612 .886575-1.577640$

C $-1.1667041 .687044-2.490884$

H $-0.1179700 .163059-3.563761$

C $0.252232-0.210284-1.487393$

H $1.7091542 .112630-1.005392$

O $0.3046904 .252780-3.171959$

H $0.6688304 .321608-1.162885$

H -1.720851 $3.608776-1.672567$

O -0.843658 2.460359 -0.220039

H $-2.0155501 .107819-2.115658$

O $-1.5520012 .165752-3.782894$

C $1.091783-1.382125-1.945875$

H $0.7565750 .232751-0.624707$ 
H $-0.714822-0.588973-1.135663$

H $-0.4035664 .912835-3.081062$

H $-1.6632131 .974810-0.027771$

H $-0.7839462 .615672-4.174831$

O $0.352647-2.525735-1.911961$

O $2.255864-1.285548-2.311948$

C $0.989106-3.708997-2.404479$

H $1.512981-3.514196-3.345276$

H $1.678983-4.097867-1.651879$

H $\quad 0.213455-4.457331-2.588556$

SCF Energy $\left(B 3 L Y P / 6-31 G^{* *} / / M M F F\right)=-3245.91612643$

\section{00248}

MM̄FF Geometry

C $-0.110907-0.5633661 .648297$

C $-0.518176-1.6554720 .980346$

C $-0.313201-1.921269-0.490235$

O $-0.132796-0.660793-1.177594$

C $0.927590-2.789041-0.749696$

C $0.773699-4.252216-0.405376$

C $1.346984-4.8544730 .654615$

C $-0.031710-5.059585-1.390220$

C $2.146531-4.2205821 .758071$

C $3.651039-4.5134201 .671247$

C $4.380207-3.7205920 .572878$

C $5.881349-4.0242250 .601008$

C $6.674287-3.142294-0.376965$

O $4.157273-2.3300920 .821296$

O $8.067035-3.258350-0.046556$

C $6.280114-1.666143-0.150941$

C $6.531380-3.626313-1.824618$

O $6.883299-0.818549-1.125226$

C $4.754844-1.442006-0.132339$

C $4.364513-0.0053930 .282818$

O $4.231957-1.659438-1.447610$

C $-0.540935-0.578430-2.468403$

O $-1.057652-1.469796-3.128454$

C $-0.2853990 .765865-3.036219$

C $\quad 0.2598971 .780249-2.346108$

C $0.5234863 .129314-2.855140$

C $0.1831753 .410152-4.295682$

C 4.8433350 .4043831 .676989

C 4.0119631 .5442152 .227728

C 3.6145633 .9772922 .023051

O $3.216267 \quad 1.3708753 .148330$

C 3.5173645 .0217820 .952960

C $3.8942724 .933307-0.332714$

C $3.7371506 .059528-1.315540$

C $2.8993765 .679643-2.516281$

C $3.4446285 .601016-3.741862$

C $1.4140935 .475494-2.312005$

C $1.0643354 .040913-2.018935$

N 4.2388452 .7470151 .576650

H 0.4618620 .2019331 .132254

H -1.070046 -2.422913 1.518864

H -1.224654 -2.399722 -0.868503

H $1.781677-2.345976-0.229686$

H $1.208995-2.719984-1.809396$

H $1.200542-5.927410 \quad 0.774497$

H $-0.061899-6.123185-1.130784$

H - $-1.064772-4.701427-1.426815$

H $\quad 0.400751-4.977689-2.392755$

H $1.966591-3.1440551 .829547$

H $1.771650-4.6361322 .702617$

H $4.085761-4.2447022 .643281$

H $3.819152-5.5884401 .530900$

H $3.955748-3.986901-0.401530$

H $6.065654-5.0866140 .398007$

H $6.268909-3.8436651 .613755$

H $8.312213-4.197588-0.107134$

H $6.696730-1.3685390 .820042$

H $6.917058-4.647927-1.926149$

H $7.128183-3.011879-2.507565$

H $5.497510-3.630654-2.176126$

H $7.840330-0.990825-1.106187$

H $4.7224650 .714911-0.464162$

H 3.2677950 .0721570 .255303

H $3.404034-1.160029-1.541160$
H $-0.5853690 .878233-4.073379$

H $0.5382021 .621777-1.305579$

H $0.7187572 .721788-4.959072$

H $0.450014 \quad 4.417837-4.620327$

H $-0.8933403 .296503-4.464619$

H $4.763987-0.4266802 .386943$

H 5.8959670 .7081691 .655568

H 4.2142534 .3587622 .856308

H 2.6133703 .7535882 .405928

H 3.0740125 .9583401 .288276

H $4.3437424 .020717-0.713769$

H $4.7427656 .365511-1.631862$

H $3.2930906 .943558-0.841041$

H $2.8444055 .359171-4.612513$

H $4.5035415 .773833-3.902629$

H $0.8603215 .859916-3.174896$

H $1.0684986 .094928-1.474263$

H $1.2748833 .737952-0.993059$

H 5.0721502 .8181240 .999760

C $-1.1977750 .998808 \quad 3.328875$

O $-2.256175 \quad 1.1410142 .357809$

C -2.1494392 .4465101 .761259$

C -1.3823913 .2840572 .772084$

C -0.3530382 .2738343 .246547$

H -1.526272 2.3407000 .864790

C -3.5291952 .9799081 .369367$

H -0.9338044 .1756862 .324197$

H -2.0222043 .5983843 .604978$

C 0.3078562 .6552264 .560449

H 0.4221732 .1736722 .476571

C -4.3005272 .0576680 .398557$

O -4.3161023 .1317692 .555860$

H -3.423966 3.9829320 .939040

H -4.6172901 .1559290 .931901$

O $-5.5054182 .732306 \quad 0.017561$

C $-3.5150691 .675724-0.867389$

C $-4.3142190 .905945-1.931097$

H $-2.6413611 .077880-0.587280$

O $-3.0277202 .877103-1.474089$

H $-5.0610901 .564879-2.392786$

H $-3.6071350 .668649-2.736632$

H 0.8643373 .5919584 .453450

H -0.4314652 .7938035 .356479$

H 1.0082081 .8793134 .884421

C $-0.433830-0.3117793 .096451$

H -5.1909863 .4578742 .283491$

H $-5.2514043 .513828-0.502867$

H $-2.5258632 .626175-2.267666$

H -1.050511 -1.136207 3.476043

H $0.491518-0.318848 \quad 3.683170$

H $-1.6868670 .942896 \quad 4.310001$

C $-4.539664-2.385356-0.148167$

O $-4.042220-1.162217-0.712084$

C $-4.995000-0.382721-1.440731$

C $-5.575007-1.193364-2.613848$

C $-6.132145-2.543048-2.151187$

C $-5.147429-3.283004-1.241082$

H $-3.650692-2.8944080 .244268$

C $-5.503742-2.1387261 .024771$

H $-5.808336-0.092251-0.768228$

O $-4.574765-1.422213-3.606700$

H -6.367816 -0.605900 -3.091107

H $-6.346861-3.168307-3.026731$

O $-7.354444-2.343621-1.448142$

H $-5.639072-4.148972-0.782540$

O $-4.085566-3.786680-2.056787$

C -4.808680 -1.536253 2.226181

H $-6.317076-1.4674660 .737842$

H $-5.942736-3.0862141 .357587$

H -3.827810 -1.874380 -3.178040

H -7.974368 -1.905155 -2.055439

H -3.500074 -4.311625 -1.484949

O $-5.727690-0.8813902 .995408$

O $-3.618619-1.6549842 .479970$

C $-5.217460-0.2808034 .189863$

H -4.5075910 .5119413 .939406$

H -6.0572730 .1599574 .733693$

H $-4.745584-1.0359304 .825778$ 
C $4.669090-4.2838631 .899949$

O $2.499746-2.3710891 .730408$

O $5.922871-4.4675232 .575521$

C $4.773327-2.9623401 .105417$

C $4.459985-5.5287661 .028018$

O $5.672239-3.1232620 .011094$

C $3.408564-2.4290560 .616609$

C $3.479924-0.9787390 .086237$

O $2.899511-3.274227-0.416289$

C $0.496424 \quad 1.6408792 .224976$

O $\quad 0.881966 \quad 0.6294731 .661678$

C 1.1803892 .9530942 .189281

C 2.3301643 .0806231 .509442

C 3.1397114 .2958421 .390687

C 2.6577955 .5247012 .117399

C $4.334396-0.755919-1.161622$

C $4.0345330 .570868-1.841062$

C $4.8607612 .033725-3.680620$

O $3.0909551 .292772-1.532004$

C $5.6001783 .146523-3.006265$

C $5.0526064 .341635-2.736883$

C $5.8036025 .479608-2.108691$

C $5.1005386 .057257-0.900770$

C $4.4226547 .214269-0.987918$

C 5.2704445 .3551720 .426951

C 4.2652124 .2567730 .645503

N $4.9358870 .839571-2.861391$

H -3.752910 -0.0487962 .006847$

H -2.940093 1.7428974 .353889

H -1.419689 -0.0152962 .297017$ H $-0.8161020 .398618 \quad 5.296098$ H $0.266361-0.3641134 .194468$ H -1.612485 -3.617048 4.324551 H -2.878986 -2.575395 5.938139 H -2.052495 -1.207273 6.687633 H -3.341650 -0.931764 5.497783 H $-0.504801-3.5481872 .186704$ H $0.068782-1.9114882 .410927$ H $\quad 1.620027-2.6891344 .217609$ H $1.047040-4.3493174 .011984$ H $1.853053-4.3340971 .650629$ H $3.413423-5.1079013 .477185$ H $3.930410-3.4553553 .740653$ H $5.849095-5.2604383 .134080$ H $5.218699-2.2079091 .768786$ H $4.458095-6.4338181 .647396$ H $5.282882-5.6566960 .316264$ H $3.521050-5.5087880 .470823$ H $6.518969-3.4346650 .374449$ H $2.452039-0.658017-0.125084$ H $3.822141-0.3083620 .886215$ H $2.232136-2.776932-0.916633$ H $\quad 0.7238493 .7741072 .729973$ H 2.7273592 .2073860 .995334 H 1.6570475 .8076481 .772112 H 3.2936026 .3993231 .966364 H 2.6193135 .3424813 .197053 H $5.395600-0.767774-0.890319$ H $4.153223-1.544448-1.900511$ H $3.8087062 .282927-3.855384$ H $5.3302151 .802294-4.641519$ H $6.6423302 .967810-2.752456$

H $5.9265086 .256109-2.875349$

H $6.8212855 .181528-1.827450$

H $3.9465397 .657811-0.120070$

H $4.3253097 .742601-1.930581$

H $5.246414 \quad 6.082013 \quad 1.246514$

H 6.2776834 .9227840 .485730

H 4.5276893 .3222790 .149155

H $5.7251930 .216026-3.003658$

C -6.0401421 .7260691 .997811$

O

C $-5.6291921 .762008-0.301124$

C $-5.9662963 .175376 \quad 0.150224$

C $-5.5287163 .112517 \quad 1.603578$

H $-4.5422871 .700495-0.428751$

C $-6.3247951 .280375-1.575545$

H $-5.4342443 .937366-0.426760$

H -7.0417223 .3769450 .081660$

C -6.081635 4.2393742 .457985

H -4.4322093 .1448421 .640384$

C $-5.997689-0.186323-1.936182$

O $-7.7403761 .388036-1.391101$

H $-6.069321 \quad 1.945847-2.408595$

H -6.453441 -0.857980 -1.199269

O $-6.633019-0.484098-3.185193$

C $-4.491392-0.485560-2.050758$

C $-4.202161-1.935858-2.484668$

H $-4.012469-0.309651-1.081660$

O $\quad-3.9281890 .429707-2.989748$

H -4.796956-2.620898-1.869368

H $-4.500390-2.058863-3.533161$

H -5.7309575 .2088632 .089307$

H -7.1766024 .2523272 .446836$

H -5.7557594 .1349563 .497615$

C $-5.3036841 .041302 \quad 3.151385$

H $-8.1635311 .063725-2.204558$

H $-6.2019410 .056814-3.869102$

H $-2.9710450 .266177-3.026265$

H $-5.835173 \quad 0.1128393 .396743$

H $-5.344673 \quad 1.674593 \quad 4.045084$

H $-7.104407 \quad 1.7858532 .261196$

C $-0.554204-1.671879-3.258177$

O $-1.977665-1.533044-3.302412$

C $-2.707332-2.295777-2.338709$

C $-2.445371-3.795496-2.543970$

C $-0.943055-4.112257-2.531262$

C $-0.137321-3.144260-3.407359$

H $-0.196920-1.131903-4.144833$

C $0.050435-0.984241-2.030429$

H -2.390568 -2.003565 -1.331756

O $-3.004041-4.200419-3.793072$

H $-2.956867-4.358133-1.753816$

H $-0.795082-5.131666-2.907639$

O $-0.431425-4.099375-1.200911$

H $\quad 0.935999-3.242620-3.206899$

O $-0.340818-3.497010-4.780545$

C $-0.278507 \quad 0.490751-2.015528$

H $-0.285044-1.428953-1.090271$

H $1.141384-1.070831-2.046620$

H -2.862329 -5.158132 -3.881821

H $-0.896303-3.420867-0.686321$

H -0.013576 -4.404509-4.901894

O $-1.0700630 .781784-0.943758$

O $0.1094931 .285251-2.862223$

C $-1.4564742 .153517-0.816382$

H $-0.5726582 .795576-0.755687$

H -2.089171 $2.445883-1.659492$

$\mathrm{H}-2.0310242 .2603450 .107623$

SCF Energy $(\mathrm{B} 3 \mathrm{LYP} / 6-31 \mathrm{G} * * / / \mathrm{MMFF})=-3245.91015960$

0700250

MM̄FF Geometry

C $2.490579-2.384039-3.265341$

C $1.903946-1.442053-2.511587$

C $0.823287-0.526340-3.022843$

O $-0.389743-0.957853-2.358481$

C $1.0777530 .948515-2.668158$

C $2.2544251 .569869-3.389088$ 
C $3.3984291 .969656-2.800095$

C $2.0596761 .785741-4.868491$

C $3.790107 \quad 1.853040-1.354240$

C $3.9252923 .214323-0.659025$

C $2.5869353 .945472-0.477633$

C $2.801074 \quad 5.302877 \quad 0.187964$

C 1.4700006 .0290970 .418360

$\begin{array}{lllllll}\text { O } & 1.752034 & 3.144016 & 0.358732\end{array}$

$\begin{array}{llllll}\text { O } & 1.749847 & 7.123802 & 1.306208\end{array}$

C $0.4773195 .077914 \quad 1.131536$

C $0.9496006 .653736-0.883062$

$\begin{array}{llll}\text { O } & -0.823197 & 5.658276 & 1.158944\end{array}$

C 0.4138823 .6478920 .530882

C $-0.2559752 .633377 \quad 1.494190$

O $-0.3041863 .614594-0.700333$

C -1.572504-0.787287-3.006728

O $-1.757086-0.232903-4.077361$

C $-2.615941-1.422301-2.171203$

C $-3.911756-1.331311-2.502955$

C $-5.029985-1.896776-1.740706$

C $-4.693251-2.706379-0.513588$

C $-1.770546 \quad 2.737566 \quad 1.687433$

C -2.5711882 .1756530 .525939$

C $-4.8289272 .284820-0.516805$

O $-2.1144561 .354189-0.264983$

C $-5.6440211 .133919-0.018941$

C $-6.9676111 .033060-0.213435$

C $-7.794926-0.1440160 .221018$

C $-8.376635-0.899891-0.955665$

C $-9.587960-0.586552-1.445605$

C $-7.586106-2.065734-1.513427$

C $-6.289920-1.640628-2.152682$

N -3.8679072 .6702220 .498654$

H $2.185948-2.467477-4.307217$

H $2.137221-1.362400-1.455472$

H $0.700544-0.651117-4.105688$

H $1.1608121 .049678-1.580304$

H $0.1812211 .535387-2.910960$

H $4.1635692 .425186-3.428186$

H $2.8888422 .340341-5.321044$

H $1.9823200 .827860-5.391704$

H $1.1451242 .359360-5.051741$

H $3.1089761 .210699-0.790616$

H $4.7666681 .354608-1.321349$

H 4.3729103 .0357750 .327344

H $4.6230203 .847997-1.220702$

H $2.1132334 .069153-1.457201$

H $3.4816305 .927471-0.404314$

H 3.2938795 .1603521 .160224

H 0.9316287 .6353501 .422916

H 0.8059824 .9911022 .176536

H $1.6607987 .400319-1.256935$

H $0.0104587 .193244-0.719220$

H $0.7930605 .921640-1.678043$

H -0.7676206 .4869151 .663587$

$\mathrm{H}-0.0169341 .618171 \quad 1.147757$

H $\quad 0.2282722 .7182082 .475980$

H $-0.5396914 .520132-0.953858$

H $-2.282871-1.950028-1.286071$

H -4.182925 $-0.777428-3.400387$

H $-4.075654-3.570857-0.781971$

H $-5.566972-3.0975650 .010152$

H $-4.145772-2.095645 \quad 0.212628$

H -2.049206 2.1383112 .563019

H -2.077728 3.7635441 .900168

H $-4.2948411 .992131-1.426570$

H $-5.4440803 .163468-0.734735$

$\begin{array}{llll}\text { H } & -5.118907 & 0.327808 & 0.487247\end{array}$

H $-7.4901601 .834380-0.731257$

H -8.6031390 .2291720 .863426$

H $-7.216089-0.8311880 .850598$

H -10.025000 -1.137569-2.271539

H -10.167968 $0.232121-1.032317$

H -8.169318 -2.588848 -2.282868

H $-7.445432-2.801305-0.717177$

H -6.428446 -1.038908 -3.052139

H $-4.185977 \quad 3.279567 \quad 1.246435$

C $4.532789-3.094275-1.768589$
O $3.958750-2.741164-0.496941$

C $4.748086-1.6768350 .070069$

C $6.026195-1.611559-0.757428$

C $5.496657-1.968460-2.133804$

H $4.192091-0.750473-0.093382$

C $4.965664-1.8965901 .567265$

H $6.497645-0.624982-0.719283$

H $6.760239-2.354027-0.422091$

C $6.580820-2.368652-3.118215$

H $4.950008-1.105701-2.534366$

C $3.674219-1.8449152 .413744$

O $5.581679-3.1741081 .767869$

H $5.679670-1.1569551 .948354$

H $3.038022-2.7086272 .190066$

O $4.059573-1.9740073 .788534$

C $2.863130-0.5428732 .253063$

C $1.783360-0.3151533 .325369$

H $2.403207-0.5211541 .258800$

$\begin{array}{lllll}\text { O } & 3.763142 & 0.569857 & 2.319693\end{array}$

H $2.248397-0.2642814 .318062$

H 1.3691150 .6855883 .151218

H $7.283859-1.543295-3.271671$

H $7.150309-3.232741-2.760059$

H $6.148637-2.628240-4.089689$

C $3.462952-3.438882-2.808252$

H $4.991643-3.8461751 .385084$

H $4.570237-2.7976423 .869794$

H 3.2548281 .3708802 .104926

H $2.863901-4.278065-2.431325$

H $3.977468-3.810667-3.703672$

H $5.107479-4.012358-1.582427$

C -1.114836 -2.174259 1.868355

O $-0.001977-1.2886572 .052469$

C $0.641131-1.3438403 .329850$

C $-0.360140-1.0547974 .463654$

C $-1.592290-1.9586174 .371071$

C -2.179142 -1.965261 2.957352

H $-1.555611-1.8655840 .913664$

C $-0.671127-3.6356941 .737540$

H $1.069389-2.3405363 .474434$

$\begin{array}{lllll} & \text { O } & -0.771935 & 0.310120 & 4.407222\end{array}$

H $0.147750-1.2028935 .424313$

H -2.357604 -1.6112015.075591

O $-1.241003-3.2903224 .734098$

H -2.949977 -2.739576 2.866011

$\begin{array}{llll}\text { O } & -2.817147 & -0.711577 & 2.701316\end{array}$

C $-0.040740-3.8823650 .388558$

H $0.058691-3.9422422 .491806$

H $-1.527871-4.312617 \quad 1.842199$

H $-1.3726510 .467608 \quad 5.155227$

H $-0.892708-3.2656845 .641540$

H $-3.521082-0.6000053 .362673$

O $-0.947178-4.458462-0.451449$

O $1.113579-3.585034 \quad 0.113149$

C $-0.467403-4.765477-1.763874$

H $-0.235942-3.844417-2.304510$

H $\quad 0.412578-5.413464-1.708917$

H $-1.259589-5.295595-2.299337$

SCF Energy (B3LYP/6-31G**//MMFF) $=-3245.89547400$

0700251

MMFF Geometry

C $1.097843 \quad 5.6795130 .473389$

C $1.8080334 .766968-0.206300$

C 2.7797553 .8156990 .443696

$\begin{array}{lllll}\text { O } & 2.318954 & 2.464677 & 0.204174\end{array}$

C $4.178788 \quad 3.972073-0.178463$

C 5.2068262 .9868650 .347969

C $5.9754342 .209291-0.440013$

C 5.3656062 .9200301 .844989

C $6.0298362 .205157-1.941122$

C $5.1190431 .172590-2.618807$

C $5.497544-0.282614-2.302661$

C $4.892280-1.232775-3.338949$

C $5.202739-2.701059-3.014113$

O $4.993654-0.610624-1.002555$

O $4.360352-3.523040-3.836283$

C $4.812984-2.976515-1.548989$ 
C $6.648145-3.074631-3.363323$

O $5.219990-4.283180-1.148430$

C $5.352220-1.922979-0.550490$

C $4.708747-2.1590020 .834831$

O $6.769186-2.066632-0.430204$

C $1.364687 \quad 1.9897511 .051871$

O 0.8422052 .5917921 .977278

C 1.0563180 .5952390 .663742

C $0.050500-0.0363781 .287450$

C $-0.385128-1.4172151 .075874$

C $0.319477-2.2227910 .018471$

C $4.888484-1.0059851 .829290$

C $4.408428-1.4157533 .208646$

C $2.346343-2.0700674 .455916$

O $5.192693-1.5801394 .140234$

C $1.422305-3.1772994 .054789$

C $0.123613-3.2239654 .388524$

C $-0.803767-4.329716 \quad 3.968295$

C $-2.061753-3.8209283 .296427$

C $-3.254169-3.899782 \quad 3.911240$

C $-1.952255-3.2868161 .882078$

C -1.387789-1.893064 1.843238

N $3.036617-1.5988673 .269134$

H 1.2317135 .7710971 .548422

H $1.6557034 .663494-1.278197$

H 2.8418993 .9989971 .523995

H 4.5498634 .9882960 .007976

H $4.0802763 .871674-1.264997$

H $\quad 6.674824 \quad 1.5234860 .036537$

H 6.2277312 .3139432 .143820

H 5.5157523 .9240282 .255172

H 4.4790772 .4787882 .310441

H $5.7968843 .199956-2.337210$

H $7.0698132 .022105-2.241296$

H $4.077530 \quad 1.361217-2.328674$

H $5.192671 \quad 1.339845-3.700682$

H $6.590445-0.368842-2.305047$

H $5.242878-0.984881-4.348640$

H $3.800964-1.102958-3.359343$

H $4.546538-3.306811-4.766084$

H $3.714834-2.966639-1.499694$

H $6.839890-2.906726-4.429894$

H $6.832926-4.140740-3.190618$

H $7.387705-2.501279-2.800877$

H $4.847962-4.912085-1.790473$

H $3.632042-2.3154940 .704466$

H $5.112180-3.0792211 .277351$

H $7.047238-1.7446060 .441733$

H $1.6647710 .129611-0.102806$

H -0.5077050 .5080462 .048671$

H $0.309133-1.694438-0.941599$

H -0.143688 -3.194004 -0.168359

H $1.358235-2.4070630 .306164$

H $5.939032-0.7089901 .912643$

H $4.325261-0.1218331 .510524$

H $1.810041-1.2158804 .880974$

H $3.069625-2.4337705 .192026$

H $1.845173-3.9830843 .458802$

H $-0.296023-2.4316495 .004342$

H -1.061319-4.905355 4.866766

H $-0.302252-5.0350973 .293928$

H -4.163287 -3.5587603 .426833$

H -3.347961 -4.302342 4.914422

H -2.945639 -3.2495521 .421730$

H -1.372174 -4.000405 1.290991

H -1.861329 -1.2112962 .551679$

H $2.470388-1.3306122 .469841$

C $-1.341524 \quad 6.1242800 .224917$

O $-1.6166714 .851201-0.394361$

C $-2.9540364 .863494-0.922980$

C $-3.6183726 .107693-0.347329$

C $-2.4430147 .062742-0.263081$

H $-2.8619184 .974836-2.010925$

C $-3.6803883 .547926-0.608107$

H -4.429003 $6.477516-0.982453$

H -4.0295105 .9189620 .651499$

C -2.690793 8.2529210 .646821

H $-2.2094047 .423924-1.273643$
C $-3.0216742 .316682-1.270223$

$\begin{array}{lllll}\text { O } & -3.747399 & 3.373146 & 0.805957\end{array}$

H $-4.7120703 .629635-0.970416$

H -2.906846 $2.515992-2.342938$

O $-1.7173992 .093447-0.738440$

C $-3.8435881 .021350-1.107451$

C $-3.199784-0.165440-1.852306$

H $-4.8504161 .194036-1.504282$

$\begin{array}{llll}\text { O } & -3.981905 & 0.692772 & 0.272544\end{array}$

H $-2.9170180 .153509-2.862676$

H $-2.289305-0.465095-1.319813$

H $-3.533558 \quad 8.8481830 .280167$

H -2.9233557 .9352941 .668824$

H $-1.811058 \quad 8.9027690 .687970$

C $0.0686346 .575396-0.157509$

H -2.8352283 .2762631 .129836$

H -1.185929 $2.889027-0.909835$

H -3.0878930 .5922030 .642111$

H 0.2508637 .6013280 .180689

H $\quad 0.1807456 .565297-1.248919$

H -1.4176235 .9740001 .310223$

C $-5.291846-2.998499-0.568245$

O $-4.373716-1.900419-0.650424$

C $-4.141805-1.383097-1.962808$

C $-3.554884-2.478607-2.866084$

C $-4.454317-3.718903-2.884034$

C $-4.844231-4.162342-1.470078$

H $-5.230132-3.3353530 .474456$

C $-6.736301-2.553360-0.822348$

H -5.088934 -1.032797 -2.387492

O $-2.254487-2.832248-2.399538$

H -3.445312 -2.079235 -3.881695

H $-3.927591-4.536520-3.391451$

O $-5.623348-3.419774-3.641248$

H $-5.633863-4.921413-1.516623$

O $-3.701240-4.787594-0.879105$

C -7.192966 -1.604956 0.260839

H $-6.876573-2.070359-1.793540$

H -7.416936 -3.412894 -0.806548

H -1.901906 -3.512295 -2.998200

H $-6.170043-4.223540-3.666698$

H -3.967274 -5.122279-0.005736

O $-7.081807-0.316351-0.169806$

O $-7.574720-1.9676121 .366124$

C $-7.4403610 .686907 \quad 0.785383$

H -8.5005020 .6015651 .041616$

H -6.8172870 .5998691 .680758$

H -7.2649921 .6659410 .331463$

SCF Energy (B3LYP/6-31G**//MMFF $)=-3245.90307780$

0700252

MM̄FF Geometry

C $-0.2409903 .047433-1.302955$

C $0.8363653 .782582-0.992140$

C 1.6579953 .5238900 .239896

O $3.0043783 .236054-0.201728$

C 1.7001094 .7326631 .192342

C 0.3538735 .2123141 .691515

C -0.4082964 .5501082 .583440$

C -0.1027826 .5366881 .139035$

C -0.1096663 .2232953 .223751$

C -1.181804 2.191409 2.849429

C $-0.7979170 .732567 \quad 3.139276$

C $-0.3420190 .498525 \quad 4.581431$

C $0.081477-0.959940 \quad 4.818514$

$\begin{array}{lllll}\text { O } & 0.237534 & 0.352430 & 2.222458\end{array}$

O $0.796389-1.0162926 .062744$

C $1.072914-1.3776763 .710767$

C $-1.129191-1.8883594 .973256$

O $1.383424-2.7663213 .804171$

C $0.580082-1.0398622 .285564$

C $1.694715-1.3167131 .249298$

O $-0.538824-1.8738521 .975296$

C $3.7448292 .404817 \quad 0.580234$

O 3.4105981 .8928701 .636788

C $5.0462102 .202084-0.091533$

C 5.8226101 .1769840 .288096

C $7.1260930 .825973-0.274341$ 
C $7.7382741 .766240-1.278915$

C $1.382921-0.766865-0.150502$

C $2.013715-1.608135-1.241602$

C $4.180852-2.334846-2.190046$

O $1.325827-2.194447-2.075542$

C $5.522683-2.687193-1.632673$

C $6.675743-2.417858-2.263814$

C $8.032187-2.805964-1.744253$

C $8.969963-1.623819-1.634816$

C $9.770835-1.286082-2.659936$

C $9.037069-0.877791-0.320931$

C $7.713415-0.3213420 .123805$

N $3.399097-1.637350-1.186125$

H $-0.5196792 .217626-0.657152$

H $1.1294424 .611347-1.631193$

H 1.2599642 .6442210 .757885

H 2.2299795 .5604010 .700073

H 2.3380944 .4965692 .054605

H -1.353720 4.9974742 .887404

H -1.081496 6.8360051 .528895

H 0.6126047 .3230131 .401184

H $-0.183416 \quad 6.489000 \quad 0.048551$

H 0.8850392 .8585222 .955551

H -0.1017803 .3666354 .310914$

H -2.1085152 .4319843 .386137$

H -1.4035112 .2753241 .777120$

H - $-1.687907 \quad 0.1288912 .923721$

H -1.1256880 .7943885 .289610$

H $\quad 0.524625 \quad 1.1334484 .807674$

H $0.208530-0.6808326 .761338$

H $2.013137-0.8366943 .887793$

H -1.739360 -1.580841 5.831089

H $-0.814586-2.9164705 .183331$

H -1.779066 -1.898130 4.095991

H $1.695658-2.9329084 .710101$

H $2.632130-0.8548191 .583924$

H $1.870644-2.3985861 .189764$

H $-0.522199-2.1045841 .033181$

H $5.3179132 .885220-0.887988$

H 5.4639290 .5175511 .077859

H $7.1171591 .824123-2.179463$

H $8.7373821 .462681-1.600828$

H $7.8385012 .771459-0.854854$

H $0.304413-0.742801-0.343473$

H $\quad 1.7410310 .263275-0.249566$

H $4.250050-1.679388-3.064718$

H $3.650333-3.245788-2.487028$

H $5.545807-3.213199-0.681242$

H $6.645176-1.915998-3.228557$

H $8.454251-3.549364-2.433326$

H $7.962536-3.312330-0.773495$

H $10.466135-0.456698-2.585971$

H $9.745786-1.830764-3.597976$

H $9.786169-0.077070-0.350677$

H $\quad 9.396227-1.5693850 .452572$

H $7.195128-0.9518100 .847047$

H $3.874065-0.971440-0.583108$

C -2.528595 $3.701067-2.063305$

O $-3.1361492 .646094-1.290283$

C $-4.4826242 .443885-1.750661$

C $-4.8159153 .653454-2.615078$

C $-3.4684213 .949264-3.243335$

H -4.464465 $1.551284-2.387595$

C $-5.4290542 .215465-0.566782$

H $-5.595873 \quad 3.436017-3.351054$

H $-5.1470304 .506922-2.012442$

C $-3.3646185 .344996-3.832918$

H $-3.2813573 .210856-4.034691$

C -4.9982751 .0966320 .405823$

O

H $-6.4377542 .014373-0.945914$

H -4.1559681 .4408001 .018048$

O $-6.0800740 .881682 \quad 1.322108$

C $-4.623314-0.239473-0.258876$

C $-4.416310-1.3695490 .770089$

H -3.709311 -0.100299-0.847285

O $-5.668904-0.607706-1.159058$

H -3.891035 -0.9816331 .650952$
H $-5.399740-1.7366601 .088341$

H -4.104207 $5.482113-4.628539$

H -3.540323 $6.115227-3.074411$

H $-2.3720495 .514892-4.261721$

C $-1.1204703 .286513-2.494323$

H -4.6394903 .6645260 .491567$

H -6.8253010 .5161360 .815001$

H -5.394808 -1.425248 -1.608352

H $-0.6870244 .052060-3.147988$

H -1.161690 $2.352500-3.066281$

H $-2.4826764 .583799-1.411769$

C $-3.548215-4.053569-1.690069$

O $-4.261494-3.042710-0.965178$

C $-3.596145-2.5400370 .195038$

C $-3.401132-3.6757341 .209890$

C $-2.649777-4.8558800 .581676$

C $-3.234271-5.255073-0.779944$

H $-4.249013-4.388329-2.465519$

C $-2.310387-3.481663-2.399130$

H -2.620757 -2.135395 -0.095007

O $-4.664007-4.1235251 .699601$

H -2.842326 -3.296578 2.071993

H $-2.698503-5.7118101 .266087$

O $-1.279573-4.4948460 .433473$

H -2.557897 -5.946690 -1.295540

O $-4.452633-5.964852-0.534706$

C $-2.671427-2.343729-3.331370$

H -1.581300 -3.104197 -1.676997

H -1.823458 -4.260010 -2.996789

H $-5.195655-4.4067390 .936125$

H $-0.810004-5.2661110 .072886$

H $-4.786500-6.280708-1.391636$

O $-1.577012-1.548384-3.515017$

O $-3.768575-2.182476-3.848537$

C $-1.775017-0.418240-4.369860$

H $-2.055008-0.747281-5.375043$

H $-2.5401740 .242787-3.951707$

H $-0.8314640 .130462-4.430855$

SCF Energy $\left(B 3 L Y P / 6-31 G^{* *} / / M M F F\right)=-3245.92021636$

\section{3}

MM̄FF Geometry

C $-1.501757-2.520896-2.543811$

C $-0.418519-2.964990-3.200018$

C $1.000262-2.464290-3.019758$

O $1.053157-1.401187-2.043088$

C $1.892255-3.626980-2.536376$

C $3.365294-3.322504-2.316933$

C $4.143316-4.014392-1.457588$

C $3.991428-2.251849-3.173265$

C $3.756622-5.197956-0.614525$

C $4.218056-5.1268220 .848588$

C $3.661452-3.9851041 .715125$

C $2.139683-3.8566611 .651637$

C $1.623628-2.6923062 .509559$

O $4.285864-2.757407 \quad 1.325908$

O $\quad 0.263688-2.4394912 .123140$

C $2.432650-1.4209502 .169185$

C $1.589459-3.0489673 .999998$

O $2.062749-0.3668533 .054681$

C $3.963304-1.6455592 .176707$

C $4.764505-0.4229011 .673728$

O $4.388354-1.8865323 .524898$

C $0.900406-0.129753-2.509292$

$\begin{array}{lllll}\text { O } & 0.749343 & 0.215244 & -3.670546\end{array}$

$\begin{array}{llll}\text { C } & 0.951136 & 0.785454 & -1.349085\end{array}$

C $1.0867622 .102281-1.561961$

C $1.1993823 .139818-0.536965$

C 1.1352042 .7190770 .905767

$\begin{array}{lllll}\text { C } 4.479544 & 0.002573 & 0.232500\end{array}$

C $5.3172321 .201228-0.179911$

C $5.6685752 .766670-2.083606$

$\begin{array}{llllll}\text { O } & 6.143384 & 1.728719 & 0.560489\end{array}$

C $4.7670833 .952757-1.944236$

C $4.8828304 .861879-0.964034$

C $3.9996936 .069655-0.816182$

C 2.9914955 .9145170 .302191

C 3.3540826 .0716991 .586462 
C $1.5509615 .637059-0.068417$

C $1.3851994 .415684-0.932050$

N $5.0335971 .611886-1.476149$

H - $1.403533-1.720011-1.816499$

H $-0.545747-3.759821-3.932597$

H $1.364853-2.108860-3.991546$

H $1.833954-4.442960-3.269506$

H $1.456745-4.001259-1.603794$

H $5.197542-3.746814-1.392482$

H $5.079806-2.208759-3.055302$

H $3.790142-2.445810-4.231779$

H $3.601159-1.264356-2.913203$

H $2.684425-5.407069-0.647637$

H $4.236463-6.077445-1.064299$

H $3.946465-6.0787381 .323200$

H $5.314788-5.0717500 .862364$

H $3.969015-4.2097412 .743888$

H $1.656191-4.7972301 .942768$

H $1.824637-3.6618060 .620774$

H -0.247319-3.253516 2.271436

H $2.115586-1.1154271 .165302$

H $0.936974-3.9134844 .171479$

H $1.163151-2.2317014 .592178$

H $2.572782-3.2945224 .405958$

H 2.4632630 .4546392 .726392

H 4.6176990 .4257112 .354352

H $5.835738-0.6611071 .744843$

H $5.340955-1.7053813 .586059$

H $\quad 0.8996580 .351827-0.357694$

H $1.1424632 .458477-2.590082$

H 1.9684462 .0508121 .144184

H $\quad 0.195430 \quad 2.197693 \quad 1.113447$

H 1.1937313 .5565721 .605019

H $4.698550-0.820296-0.455939$

H $3.4301250 .282508 \quad 0.120233$

H $5.8332572 .542393-3.141941$

H $6.6437142 .943073-1.617058$

H $3.9912814 .070352-2.696337$

H $5.6621774 .734805-0.215704$

H $3.4871336 .300737-1.758260$

H $4.6385736 .940422-0.618750$

H $2.634407 \quad 5.9818362 .393174$

H 4.3812016 .2923421 .858496

H $1.1627896 .500748-0.624069$

H $\quad 0.913307 \quad 5.567087 \quad 0.820614$

H $1.4337104 .628475-2.000691$

H $4.2854671 .154624-1.989443$

C $-3.564748-3.492948-1.436372$

O $-3.673593-2.374316-0.530394$

C $-3.333108-2.8258010 .793603$

C $-3.386892-4.3481330 .740707$

C $-2.865611-4.609198-0.659360$

H -2.296357 -2.5220270 .974020$

C $-4.256870-2.1916461 .836609$

H -2.776683 -4.8130431 .520949$

H $-4.413108-4.7205720 .842003$

C $-3.168990-6.004029-1.176408$

H $-1.778372-4.461029-0.654419$

C $-4.204573-0.6496301 .894816$

O $-5.610143-2.5716561 .555757$

H $-4.029887-2.6042002 .826636$

H $-4.654364-0.2239050 .990812$

O $-5.028300-0.232398 \quad 2.991446$

C -2.794907 -0.0656202 .102343$

C -2.747363 1.4322612 .456001

H $-2.194045-0.2367111 .202446$

O $-2.142074-0.765748 \quad 3.167087$

H -3.190407 1.5964903 .446912

H -1.687219 1.6943642 .568083

H -2.698205 -6.760667 -0.540102

H -4.246006 -6.201604 -1.192053

H -2.787107 -6.132922 -2.194102

C $-2.890720-3.058912-2.741616$

H $-5.816293-2.2732120 .653109$

H $-5.914837-0.6047472 .845296$

H -2.683965 -0.655549 3.967309

H -3.484336 -2.256454 -3.194516

H $-2.888657-3.896257-3.448923$
H $-4.595614-3.790859-1.669584$

C $-3.3392452 .910206-0.929326$

O $-2.7999362 .147003 \quad 0.159266$

C -3.4117542 .3664781 .432892$

C $-3.281667 \quad 3.842414 \quad 1.846113$

C -3.839936 4.7725390 .764061

C $-3.2977564 .418781-0.623552$

H -2.660194 $2.717946-1.769821$

C $-4.7402282 .424791-1.335414$

H $-4.4728612 .105426 \quad 1.374614$

$\begin{array}{lllll}\text { O } & -1.909670 & 4.155777 & 2.079823\end{array}$

H -3.8189463 .9928202 .790169$

H -3.571772 5.8103330 .997474

$\begin{array}{llll}O & -5.261505 & 4.686925 & 0.743049\end{array}$

H -3.851944 $4.969359-1.392858$

O $-1.9411314 .863375-0.690768$

C $-4.727707 \quad 1.020958-1.900102$

H $-5.4308502 .425729-0.488378$

H $-5.140168 \quad 3.081701-2.116549$

H $-1.862668 \quad 5.0872382 .354310$

H -5.5844374 .9447201 .623177$

H $-1.6178164 .687905-1.590793$

O $-6.0145610 .648421-2.169950$

$\begin{array}{llll}0 & -3.728532 & 0.341667 & -2.086437\end{array}$

C $-6.181730-0.661811-2.719461$

H $-7.231148-0.779664-3.002544$

H $-5.563619-0.787623-3.613421$

H $-5.935666-1.414844-1.966438$

SCF Energy (B3LYP/6-31G**//MMFF) $=-3245.89284270$

\section{4}

MMFF Geometry

C $-2.746263-3.3526551 .508938$

C $-1.966993-2.7996072 .451475$

C $-0.493408-2.5726372 .256161$

O $-0.200239-1.2405332 .747264$

C $0.389694-3.563838 \quad 3.032556$

C $0.305693-5.0062822 .585730$

C $0.949036-5.5024471 .510576$

C $-0.499138-5.9149473 .476463$

C $1.764856-4.7378790 .507193$

C $3.259991-4.736005 \quad 0.838021$

C $4.080775-4.064089-0.273451$

C $5.563952-4.0342110 .087674$

C $6.388919-3.342931-1.006199$

O $3.614327-2.723424-0.444001$

O $7.690540-3.061140-0.466433$

C $5.747191-1.978853-1.358466$

C $6.616187-4.274185-2.204706$

O $6.379974-1.427267-2.509847$

C $4.209611-2.023324-1.552128$

C $3.564287-0.615901-1.535998$

O $3.838042-2.624445-2.794039$

C $0.855844-0.5900352 .187760$

$\begin{array}{llll}\text { O } & 1.548431 & -0.973729 & 1.259195\end{array}$

C 1.0407900 .6803532 .922970

C $1.960818 \quad 1.5588492 .497988$

C 2.2696582 .8525023 .110331

C $1.5374533 .224158 \quad 4.373498$

C $3.9298970 .287681-2.712192$

C $3.0539791 .524194-2.750305$

C $2.9891143 .907109-2.103460$

O $1.9984301 .546517-3.380180$

C $3.5839434 .796373-1.060378$

C $2.8541695 .553957-0.227680$

C $3.445996 \quad 6.4597750 .813346$

C 2.9728736 .1287632 .211721

C 2.0213906 .8649942 .810197

C 3.6618315 .0011592 .948001

C 3.1831843 .6453062 .511931

N $3.5652512 .577875-2.005761$

H $-2.291458-3.6930050 .581592$

H $-2.405923-2.4811223 .393419$

H $-0.269468-2.6074551 .185279$

H $0.163387-3.4926614 .105849$

H $1.436580-3.2373062 .971969$

H $0.863994-6.5681591 .303340$

H $-0.513991-6.9477653 .112717$ 
H $-0.076855-5.9263574 .486697$ H -1.536895 -5.573619 3.538330 H $\quad 1.603817-5.197256-0.476335$ H $1.405618-3.7106180 .413383$ H $3.411651-4.1979421 .782475$ H $3.614176-5.7637050 .985648$ H $3.917031-4.624838-1.201148$ H $5.942923-5.0468750 .273469$ H $5.701964-3.4833471 .028579$ H $8.078738-3.900528-0.164978$ H $5.959728-1.293540-0.525882$ H $7.163580-5.172048-1.892627$ H $7.240368-3.796371-2.967590$ H $5.686869-4.605356-2.672615$ H $7.332646-1.374668-2.320650$ H $2.471905-0.742382-1.529371$ H $3.795714-0.111057-0.588802$ H $4.640173-2.919937-3.251846$ H $\quad 0.4149240 .8576103 .789983$ H 2.5423701 .3136241 .609793 H 0.4544383 .2241184 .211256 H 1.7914614 .2179314 .747852 H 1.7706462 .5125465 .173365 H $4.9775330 .600027-2.656983$ H $3.802738-0.229393-3.669805$ H $1.9010263 .825805-2.018803$ H $3.2240194 .293655-3.100890$ H $4.6689124 .831615-0.995125$ H $1.7701345 .541296-0.308264$ H 3.1672417 .4897760 .554933 H 4.5425656 .4376150 .787369 H 1.6962876 .6536883 .823335 H 1.5419587 .6926272 .297954 H 3.5577095 .1264094 .031426 H 4.7439265 .0622392 .773341 H 3.6621483 .2858711 .601162 H $4.5171752 .497457-1.659298$ C $-4.967203-3.272750 \quad 0.337104$ O $-4.881602-1.876552-0.018451$ C $-6.157229-1.453798-0.537472$ C $-6.997387-2.717563-0.697813$ C $-6.460897-3.5851300 .420306$ H $-6.616592-0.8294570 .237524$ C -6.002104 -0.652885 -1.836324 H -8.069194 -2.515281 -0.609484 H $-6.826770-3.199137-1.667900$ C $-6.801347-5.0568170 .259386$ H $-6.868839-3.222178 \quad 1.373370$ C $-5.1490330 .628612-1.733492$ O $-5.393941-1.498490-2.822234$ H $-6.999403-0.405229-2.220913$ H $-4.0910940 .358464-1.659349$ O $\quad-5.285117 \quad 1.325786-2.980235$ C $-5.5263461 .576489-0.583377$ C $-4.9197962 .989770-0.668588$ H -5.2403821 .1142720 .368356$ O $\quad-6.9500001 .729630-0.563871$ H $-5.3999813 .552317-1.480245$ H -5.2155663 .5076730 .253670$ H -7.886651 -5.2008960 .246690$ H $-6.397547-5.462262-0.674436$ H $-6.393644-5.6463911 .086363$ C $-4.227944-3.5468901 .651049$ H -5.315097 $-0.979103-3.640751$ H $-6.2072901 .627613-3.048369$ H -7.1765522 .2678160 .213684$ H -4.402681 -4.583497 1.960032 H -4.616437 -2.888862 2.437981 H $-4.505459-3.836951-0.484451$ C -1.3617892 .2096820 .185446$ $\begin{array}{lllll}\text { O } & -2.794002 & 2.283387 & 0.208876\end{array}$ C $-3.3921063 .056233-0.832356$ C $-2.8844534 .509846-0.789656$ C $-1.3538304 .579335-0.791043$ C -0.7410053 .6168790 .230982$ H -1.0997491 .6926181 .115369$ C $-0.8295961 .367233-0.985433$ H $-3.1319862 .615435-1.799922$
O

H -3.283014 $5.047531-1.657814$ H -1.034696 $5.601678-0.553145$ O $-0.8603884 .260630-2.087800$ H $0.3421593 .547206 \quad 0.083323$ O -0.9520534 .1687121 .533006$ C $-1.247500-0.082503-0.896262$ H $-1.180818 \quad 1.741427-1.950230$ H $\quad 0.2662911 .390516-0.973426$ H -3.083082 4.6752751 .150537 H -1.232019 $4.907400-2.711455$ H -0.5278323 .5748292 .174521$ O $-0.687437-0.767255-1.936733$ O $-1.977400-0.553143-0.036310$ C -1.018207-2.157033-2.017875 H $-0.681960-2.529093-2.989264$ H $-0.495234-2.707847-1.231904$ H $-2.099817-2.306894-1.943100$ SCF Energy $\left(B 3 L Y P / 6-31 G^{* *} / / M M F F\right)=-3245.90438463$

\section{5}

MMFFF Geometry

C $-1.261384-3.7154092 .602174$

C $-0.492246-2.7428692 .091153$

C $-0.129257-1.4731532 .810927$

O $1.306014-1.3358362 .694715$

C $-0.769139-0.2525632 .118539$

C -0.7730191 .0003632 .976975$

C -1.8769181 .6952313 .315889$

C 0.5735131 .4935983 .444778

C $-3.301687 \quad 1.4305912 .915625$

C -4.1317142 .7126902 .731368$

C -3.8119703 .5898381 .506382$

C -4.1378652 .8977180 .181599$

C $-3.830448 \quad 3.800520-1.024493$

O $-2.4383113 .988244 \quad 1.532105$

O $-3.902793 \quad 2.971169-2.193447$

C $-2.3823764 .320344-0.885649$

C $-4.892362 \quad 4.890913-1.208280$

O $-2.075248 \quad 5.273344-1.899737$

C -2.0818374 .9261910 .503860$

C $-0.590438 \quad 5.2883310 .688471$

$\begin{array}{lllll} & \text { O } & -2.820428 & 6.146438 & 0.652607\end{array}$

C $2.103467-1.8768693 .649837$

O $1.746266-2.4278614 .680418$

C $3.531060-1.6766933 .302129$

C $3.923819-1.5134372 .029317$

C $5.282855-1.2812011 .541469$

C $6.378664-1.1361332 .561210$

C $0.402427 \quad 4.1446850 .461582$

C 1.8070914 .5186460 .908349

C 4.1003113 .5753161 .111856

O 2.0832295 .6057771 .409455

C 4.9966053 .1059820 .010401

C 5.9126192 .1384090 .168131

C $6.8658151 .693353-0.903806$

C $6.542987 \quad 0.326512-1.464948$

C $6.1712580 .177985-2.748055$

C $6.717695-0.879075-0.563070$

C $5.462174-1.1790870 .207941$

N $2.717567 \quad 3.4958720 .677151$

H -1.689234 -3.6016243 .594274$

H $-0.077729-2.8719381 .094179$

H $-0.428315-1.5113033 .866102$

H $-1.789243-0.5144631 .821775$

H $-0.224517-0.0152331 .196091$

H -1.7453772 .5596323 .966406$

H $0.5277112 .515723 \quad 3.836573$

H $0.9628130 .853188 \quad 4.241608$

H $1.287691 \quad 1.5009602 .615161$

H -3.3740350 .8213642 .012016$

H $-3.7632690 .851626 \quad 3.725445$

H -5.1915972 .4311512 .687880$

H -4.0142323 .3295343 .632703$

H -4.4340014 .4871351 .610814$

H -5.1836082 .5675570 .156999$

H -3.5302831 .9892380 .084871$

H -3.748798 $3.531145-2.972744$ 
H -1.721043 $3.460671-1.050658$ H $-5.8735174 .437697-1.394510$ H -4.675945 $5.509242-2.086569$ H $-4.9916005 .549139-0.343132$ H -2.167213 $4.829568-2.759388$ H $-0.327538 \quad 6.1323710 .037085$ H -0.4611665 .6621341 .714258$ H -2.3941516 .6819631 .342178$ H $4.229169-1.7010664 .131179$ H $3.167498-1.5462701 .247601$ H $6.474592-2.0525353 .154138$ H $6.166004-0.3012503 .238192$ H $7.359346-0.9438012 .121552$ H 0.0927923 .2557581 .019246 H $0.4476333 .889308-0.602674$ H 4.1897912 .9570562 .011174 H 4.3534824 .6061461 .378735 H $4.9079863 .605847-0.951222$ H 6.0135221 .6636711 .140538 H $7.8807871 .679190-0.487036$ H $6.8932792 .439251-1.709229$ H $5.958103-0.801610-3.163266$ H $6.063648 \quad 1.031248-3.409230$ H $7.591605-0.7254540 .075326$ H $\quad 6.952811-1.763931-1.169349$ H $4.593114-1.323065-0.435200$ H $2.3715972 .594246 \quad 0.361183$ C $-2.827985-4.990784 \quad 1.046655$ O $-2.751401-3.9726460 .029225$ C $-4.038437-3.333444-0.073614$ C $-5.013244-4.2324250 .677898$ C $-4.130090-4.7398281 .804142$ H $-3.964243-2.3814650 .465203$ C $-4.381861-3.079888-1.544313$ H $-5.893258-3.687421 \quad 1.032608$ H $-5.356945-5.0673770 .056128$ C $-4.680558-5.9666822 .508729$ H $-4.006142-3.9325032 .536598$ C -3.332159 -2.229860 -2.293763 O $-4.491256-4.336192-2.225128$ H -5.368854 -2.609733 -1.620451 H $-2.399974-2.796405-2.396797$ O $-3.816612-2.000611-3.622223$ C $-3.051536-0.865030-1.641480$ C $-2.1391560 .071081-2.453937$ H $-2.605683-1.013052-0.652928$ O $-4.291978-0.184391-1.428822$ H $-2.6122900 .327009-3.409999$ H -2.077879 $1.011445-1.891967$ H -5.647130 -5.7429192 .971607$ H -4.826680 -6.798872 1.811899 H -3.997954 -6.301454 3.296211 C -1.540999-4.999698 1.871535 H -3.645241 -4.803557 -2.115704 H -3.998397 -2.870314-4.018098 H $-4.707376-0.052262-2.298268$ H - $0.698841-5.2138121 .199666$ H -1.567496 -5.8173662 .601173$ H -2.891237 -5.9472320 .510493$ C $1.166249-1.319546-1.446965$ O $-0.139168-0.730884-1.413325$ C $-0.721756-0.468385-2.692952$ C $0.1466160 .532868-3.473449$ C $1.592398 \quad 0.039711-3.589789$ C $2.145127-0.438014-2.241384$ H $1.492377-1.315738-0.400409$ C $1.083897-2.778021-1.905255$ H $-0.791019-1.403682-3.258728$ O $\quad 0.1320691 .795987-2.809422$ H $-0.2871960 .676600-4.470285$ H $2.2204530 .853838-3.971800$ O $1.633934-1.020339-4.539857$ H $3.088708-0.971569-2.390545$ O $2.453620 \quad 0.710323-1.443400$ C $2.263885-3.577422-1.412354$ H $0.180597-3.254458-1.503031$ H $0.994469-2.880205-2.990694$ H $0.6528772 .416460-3.347138$
H $2.560101-1.305582-4.621147$

H $1.6179531 .169036-1.250040$

O $3.194474-3.695627-2.401330$

O $2.355721-4.024173-0.276181$

C $4.375918-4.423394-2.052017$

H $5.067265-4.374629-2.897445$

H $4.859560-3.975607-1.178854$

H $4.126836-5.471055-1.859712$

SCF Energy (B3LYP/6-31G**//MMFF)= -3245.91227744

\section{6}

MM̄FF Geometry

C $1.683662-2.7780521 .489701$

C $0.864512-2.957422 \quad 2.535403$

C $-0.113997-1.9052682 .985873$

$\begin{array}{llll}\mathrm{C} & -1.432842 & -2.487246 & 2.883143\end{array}$

C $0.154854-1.455588 \quad 4.435288$

C $1.539373-0.8835704 .662736$

C $1.929107 \quad 0.3486694 .282414$

C $2.502958-1.7899525 .384764$

C 1.1089841 .4055443 .595736

C 1.6343931 .6934832 .181296

C 0.9897662 .9152091 .500758

C 1.4692314 .2329442 .116784

C 0.7734645 .4484531 .488068

$\begin{array}{llll}\text { O } & -0.431071 & 2.798865 & 1.597920\end{array}$

O 1.0341236 .5875462 .322878

C -0.7502395 .2109331 .512623$

C $1.344605 \quad 5.7847310 .105527$

O $\begin{array}{lllll} & -1.435924 & 6.257319 & 0.827400\end{array}$

C $-1.171122 \quad 3.8374730 .938704$

C -2.675979 3.5918121 .221061

O $-0.9429123 .839011-0.468511$

C -2.475255 -1.623089 2.747552

O $-2.435309-0.4060592 .830385$

C $-3.680785-2.4168772 .421808$

C $-4.768491-1.7854351 .955120$

C $-6.018616-2.4110911 .521345$

C $-6.148112-3.9033591 .675613$

C -3.191206 2.2069730 .802566

C $-3.7121112 .127048-0.622831$

C $-4.567470 \quad 0.448425-2.265112$

O $-3.883946 \quad 3.111060-1.335894$

C $-6.0257970 .189372-2.051372$

C $-6.606357-1.004101-2.248172$

C $-8.066815-1.271899-2.015672$

C $-8.301639-2.389341-1.021348$

C $-8.558069-3.639615-1.442209$

C $-8.322474-2.047268 \quad 0.453047$

C $-6.978174-1.6292830 .983344$

N $-3.9774540 .814872-0.991233$

H $1.601093-1.8593210 .913892$

H $\quad 0.931539-3.868703 \quad 3.123984$

H $-0.061936-1.0421392 .309999$

H -0.017010 -2.300369 5.117071

H $-0.598463-0.7200784 .744135$

H $2.9518210 .652943 \quad 4.503097$

H $3.484388-1.3252595 .528077$

H $2.111397-2.0440806 .375281$

H $2.658228-2.7168874 .824835$

H $0.051764 \quad 1.1275383 .548076$

H 1.1618822 .3067854 .215406

H 2.7246831 .8187102 .190872

H 1.4275700 .8108791 .561395

H 1.2740812 .8790780 .442480

H 2.5589544 .3265032 .030882

H 1.2534334 .2462163 .192573

H 1.9980926 .7025172 .380321

H -1.0747895 .2624112 .561450$

H 2.4132106 .0202410 .180286

H $0.8692916 .679587-0.310921$

H $1.2382254 .970366-0.613863$

H -1.1504017 .0984861 .223462$

H -2.830038 3.6676432 .305856

H -3.2829324 .3734850 .749860$

H $-1.4527803 .117284-0.866622$

H -3.624979 -3.4938062 .531115$

H - $4.736104-0.7010871 .855453$ 
H $-5.376965-4.4191381 .092822$ H -7.110228 -4.2923121 .334782$ H $-6.045598-4.1912212 .727842$ H -2.420264 1.4435830 .944543 H -4.0383501 .9460841 .450045$ H -4.037664 -0.439188 -2.624807 H $-4.4308801 .255662-2.991050$ H $-6.6312091 .026861-1.712630$ H $-6.002103-1.837647-2.599127$ H -8.516605 -1.530492 -2.983361 H $-8.592361-0.369780-1.678936$ H $-8.756779-4.443485-0.741425$ H -8.571167 -3.888036 -2.498409 H -8.726183 -2.876209 1.046132 H $-9.030697-1.2242580 .615713$ H $-6.792597-0.5577330 .902522$ H $-3.8434800 .074985-0.308100$ C $4.115484-3.0122301 .072725$ O $4.112330-2.0020020 .048378$ C $5.459506-1.817217-0.427632$ C $6.327328-2.8203060 .330972$ C $5.317727-3.883658 \quad 0.733352$ H $5.458014-2.087739-1.488585$ C $5.855544-0.342718-0.264958$ H $7.135954-3.215249-0.292160$ H $6.788721-2.3727931 .218578$ C $5.791096-4.7662861 .874193$ H $5.095226-4.507165-0.142500$

C $4.8299960 .636664-0.876956$ O $5.941299-0.052478 \quad 1.136946$ H $6.855406-0.174346-0.680797$ H $3.8787420 .536609-0.343712$ O $5.2806321 .972405-0.611952$

C $4.6169660 .474260-2.395105$ C $3.5136321 .402151-2.942873$ H $4.369304-0.562539-2.639831$

O $5.8486990 .778027-3.052394$ H $3.6119342 .398817-2.496681$ H $3.6654351 .531881-4.022563$ H $6.706809-5.2973121 .594784$ H $6.003564-4.1794602 .774167$ H $5.033724-5.5135962 .130798$ C $2.767711-3.7342311 .076093$ H $\quad \begin{array}{llll}6.176646 & 0.887188 & 1.224064\end{array}$ H $6.1040662 .106699-1.112018$ H $5.7221010 .613953-4.002557$ H $2.800168-4.5961141 .751375$ H $2.530650-4.1030160 .072796$ H $4.272296-2.5021532 .033585$ C $0.668577-0.942193-3.539702$ O $1.917079-0.241009-3.623884$ C $2.1070460 .830491-2.688456$ C $1.010151 \quad 1.889767-2.854607$ C $-0.376898 \quad 1.252644-2.729468$ C $-0.5202440 .028570-3.636950$ H $0.645450-1.578877-4.433840$ C $0.617926-1.854256-2.306338$ H $2.0634410 .432642-1.670296$ O $1.1439252 .524166-4.123570$ H $1.1441972 .658464-2.084820$ H -1.141234 $1.993839-2.992056$ O $-0.5761480 .870269-1.371266$ H -1.448295 $-0.506087-3.406296$ O $-0.6391190 .466989-4.993412$ C $1.348384-3.152804-2.562152$ H $1.041769-1.403410-1.406462$ H -0.419874 -2.123720 -2.076356 H $\quad 0.454803 \quad 3.207814-4.182651$ H $-1.4550270 .459609-1.307220$ H $\quad 0.204127 \quad 0.879191-5.248313$ O $2.680342-2.923038-2.736277$ O $0.796222-4.245385-2.594239$ C $3.471686-4.079333-3.026505$ H $3.426080-4.789883-2.196182$ H $3.130671-4.547972-3.954483$ H $4.508292-3.758548-3.157629$

SCF Energy (B3LYP/6-31G**//MMFF) $=-3245.91969726$

\section{7}

MMFF Geometry

C $3.762913-0.8429873 .458359$

C $2.616818-1.0967822 .807548$

C $1.268351-0.6344343 .294798$

O $\quad 0.6989890 .1519652 .220908$

C $0.354858-1.8380893 .588902$

C $-0.616154-1.5878354 .726433$

C -1.947122 -1.442395 4.583488

C $-0.006469-1.5850976 .106343$

C $-2.740033-1.4296223 .308956$

C $-3.283972-2.8239682 .978195$

C $-4.374926-2.7851561 .898915$

C $-4.792094-4.2030551 .499931$

C $-5.816502-4.2033010 .354086$

O $-3.868389-2.0865800 .759606$

O $-5.883630-5.535025-0.178925$

C $-5.302526-3.289909-0.779977$

C -7.226815 -3.8660510 .852679$

O $-6.287426-3.135968-1.799378$

C $-4.830989-1.902726-0.286862$

C $-4.143875-1.129885-1.441457$

O $-5.973290-1.1675610 .159242$

C -0.0122371 .2627342 .549196$

O -0.3125441 .6430343 .669052$

C -0.3250811 .9745911 .288035$

C -0.7921633 .2310571 .337832$

C -1.0864384 .0983420 .192860$

C $-0.9335273 .513880-1.186326$

C $-3.2440700 .036414-1.007322$

C $-3.9680611 .169568-0.313367$

C $-5.6319892 .979649-0.732275$

O $-3.798726 \quad 1.4081320 .879791$

C $-5.0394784 .245622-1.264313$

C $-4.7906585 .325841-0.508421$

C $-4.2677906 .619216-1.063392$

C $-3.0674087 .145770-0.312167$

C $-3.166723 \quad 8.2281470 .478360$

C $-1.7310936 .478742-0.552162$

C -1.4479855 .3762010 .435475$

N $-4.8167271 .860078-1.160472$

H $3.736505-0.2880354 .392999$

H $2.644371-1.6468211 .869693$

H $1.405994-0.0041804 .181323$

H $0.954731-2.7136443 .873750$

H $-0.178915-2.1308672 .677298$

H -2.549185-1.310364 5.481875

H $-0.761159-1.4870456 .894207$

H $\quad 0.532389-2.5214686 .284853$

H $0.694924-0.7534996 .220841$

H $-3.569470-0.7228423 .436800$

H -2.143091-1.0440972.475330

H -2.446691 -3.445422 2.634479

H $-3.690879-3.3013183 .878668$

H $-5.231115-2.2340802 .306083$

H $-5.182328-4.7521572 .365860$

H -3.905633 -4.761275 1.167037

H $-6.130960-6.1355410 .545063$

H $-4.448638-3.799482-1.248198$

H -7.558589-4.606009 1.590865

H $-7.956076-3.9109000 .036415$

H -7.292501 -2.882516 1.322350

H $-6.550453-4.027892-2.084743$

H -3.494818 -1.822707 -1.991721

H -4.901616 -0.769783 -2.147945

H $-5.861740-0.228211-0.049002$

H -0.1228051 .4612850 .355758$

H -0.9491643 .6850142 .315730$

H $0.1153413 .267859-1.383338$

H -1.261503 $4.182916-1.984081$

H -1.530567 $2.602902-1.289855$

H -2.441714 -0.313497 -0.348107

H $-2.7492620 .452047-1.893788$

H -5.7164522 .9864560 .359140$

H $-6.6291122 .832323-1.158600$

H $-4.8409144 .288802-2.332965$

H -5.0191225 .2967860 .554516$

H $-5.0866337 .349554-1.022085$ 
H $-4.0105576 .522513-2.125620$

H $-2.301244 \quad 8.6303200 .994947$ H -4.1149298 .7329270 .631098$ H $-0.9302547 .222783-0.443705$ H -1.673623 $6.153171-1.593858$ H $-1.5064695 .702627 \quad 1.475167$ H $-4.8505861 .603620-2.142886$ C $5.952763-0.0512972 .547982$ O $5.3231820 .576307 \quad 1.412848$ C 6.2726930 .6500780 .337966 C 7.6329950 .6068561 .013906 C $7.371333-0.4203452 .101770$ H $6.147938-0.260596-0.259880$ C $5.9871361 .871259-0.539655$ H 8.4334160 .3131230 .328586 H $7.895771 \quad 1.5723751 .461051$ C $8.392101-0.3930933 .226067$ H $7.374503-1.4202191 .646858$ C $4.5132311 .994153-0.984259$ O 6.3178263 .0660970 .178023 H $6.6461491 .856252-1.415265$ H $3.8924462 .313520-0.137808$ O $4.4300223 .040037-1.959144$ C $3.9187900 .710671-1.589992$ C $2.4686640 .903825-2.072338$ H $3.927423-0.082422-0.835329$ O $4.737267 \quad 0.271967-2.672302$ H $1.9002941 .432040-1.298426$ H $2.4726391 .518141-2.981068$ H $9.392292-0.6201412 .843113$ H 8.4317440 .5892413 .708450 H $8.145584-1.1353013 .992047$ C $5.119397-1.2653042 .965697$ H 5.7952473 .0701570 .998532 H $4.8049323 .841860-1.556065$ H $4.7449230 .978534-3.340625$ H $5.625195-1.8158703 .766834$ H $5.009215-1.9508932 .116182$ H 5.9770910 .6963393 .350800

C $1.944195-2.337655-3.866650$

O $2.419341-1.037291-3.497199$

C $1.773337-0.439411-2.370965$

C $0.274618-0.261309-2.650868$

C $-0.373937-1.589360-3.057985$

C $0.431724-2.309855-4.144991$

H $2.455367-2.562540-4.811676$

C $2.358706-3.404449-2.846136$

H $1.900398-1.084339-1.494210$

O $0.0926770 .700414-3.687500$ H $-0.2050410 .133250-1.748564$ H -1.388634 -1.402765 -3.429017 O $-0.478895-2.442700-1.921830$ H $\quad 0.068087-3.335519-4.277866$

O $0.239824-1.632137-5.390293$

C $3.864219-3.498109-2.751330$

H $1.954652-3.209867-1.848720$

H $1.989656-4.393162-3.142249$

H $-0.8640430 .817937-3.814181$

H $-0.998876-1.975162-1.246692$

H $-0.709911-1.661719-5.596908$

O $4.280725-3.092311-1.517717$

O $4.583274-3.875152-3.667687$

C $5.694538-3.129340-1.301700$

H $5.887302-2.844576-0.264098$

H $\quad 6.080025-4.140647-1.463412$

H $6.194408-2.414432-1.961906$

SCF Energy $\left(B 3 L Y P / 6-31 G^{* *} / / M M F F\right)=-3245.89065928$

\section{8}

MM̄FF Geometry

C $2.622512-3.416462-0.468649$

C $1.586376-4.0834740 .063092$

C $0.199344-3.5410300 .307803$

O $0.082158-2.183786-0.169834$

C $-0.909652-4.420405-0.313996$

C -1.017101-4.384415 -1.828764

C $-1.938146-3.679240-2.516802$

C $-0.056077-5.271289-2.579730$
C $-3.006337-2.764153-1.982289$

C $-4.403645-3.289023-2.330003$

C $-5.561739-2.476919-1.727332$

C $-5.607712-1.030883-2.224787$

C $-6.769231-0.249733-1.589928$

O $-5.446318-2.504451-0.299856$

O $-6.5464321 .138955-1.879759$

C $-6.709519-0.432694-0.056861$

C $-8.112740-0.608572-2.234433$

$\begin{array}{lllll}\text { O } & -7.840795 & 0.168110 & 0.567767\end{array}$

C $-6.555368-1.9040520 .388774$

C $-6.300689-2.0593311 .909191$

O $-7.772774-2.6050440 .100870$

C $-0.824973-1.3776290 .441807$

O $-1.525871-1.6497871 .403208$

C $-0.856236-0.100239-0.302404$

C $-1.9642500 .652651-0.245782$

C $-2.1812141 .931871-0.921286$

C $-1.0261272 .531078-1.676632$

C $-5.097295-1.2844542 .457163$

C -5.4648210 .0818123 .015721$

C -4.4767872 .3086423 .541629$

O $-6.595076 \quad 0.363117 \quad 3.406680$

C -4.9117693 .1850352 .409270$

C -4.1745034 .1908131 .914558$

C -4.6155285 .0603320 .770818$

C $-3.6745064 .988374-0.412774$

C $-2.7707615 .957774-0.633617$

C $-3.8343883 .838347-1.383939$

C $-3.3880712 .523238-0.808258$

N $-4.374500 \quad 0.938827 \quad 3.070419$

H $2.502511-2.387256-0.788087$

H $1.744028-5.1147680 .374295$

H $0.067161-3.5458911 .398512$

H -1.868228 -4.1236470 .126033$

H $-0.776180-5.4624810 .006543$

H -1.925633 -3.750783 -3.604180

H $-0.327090-5.373548-3.636344$

H $\quad 0.958531-4.867292-2.542985$

H $-0.049896-6.279123-2.151643$

H $-2.925400-2.627545-0.902906$

H -2.853406 -1.778573 -2.435944

H $-4.522249-3.332366-3.420214$

H $-4.485493-4.319486-1.958024$

H $-6.484026-2.997054-2.012985$

H $-5.671313-0.995803-3.319279$

H $-4.675270-0.515320-1.960378$

H $-7.2241251 .651402-1.405975$

H -5.8367900 .1371220 .282389$

H -8.088125 $-0.392816-3.309419$

H $-8.9245240 .004240-1.826968$

H -8.381174 -1.659891-2.113197

H $-8.598559-0.4286870 .445316$

H -7.216264 -1.826521 2.466410

H $-6.107891-3.1249222 .098994$

H -7.808476 -3.3996920 .658956$

H $\quad 0.0120770 .165137-0.893842$

H $-2.804920 \quad 0.2920890 .344773$

H -1.246071 $3.512390-2.102722$

H $-0.7278341 .881552-2.506583$

H $-0.1692942 .667826-1.009826$

H -4.670070 -1.845556 3.297954

H -4.315046 -1.191131 1.697644

H -3.4963232 .5938383 .935412$

H -5.2093212 .3606584 .353722$

H $-5.8878012 .980071 \quad 1.974918$

H -3.202932 4.4060052 .353438

H $-4.6785196 .091773 \quad 1.141394$

H -5.6303734 .8046790 .440972$

H -2.110410 $5.930478-1.493965$

H -2.6682116 .7991020 .043787$

H -3.315358 $4.044576-2.326846$

H $-4.8915013 .754383-1.667502$

H $-4.1640842 .011778-0.239157$

H -3.4889720 .6431482 .670596$

C $4.984324-3.6322380 .386176$

O $5.326066-2.2326900 .278301$

C $6.757259-2.0990250 .361041$ 
C $7.275430-3.4328900 .885910$

C $6.300771-4.4007200 .252901$

H $7.117461-1.962567-0.665364$

C $7.169251-0.9009531 .224516$

H $8.314806-3.6173010 .597498$

H $7.212074-3.4929121 .978731$

C $6.289902-5.768480 \quad 0.914482$

H $6.562256-4.517978-0.807560$

C $6.632456 \quad 0.4740050 .778996$

O $6.723531-1.1137132 .571311$

H $8.264074-0.8614891 .279233$

H 5.5710520 .5607551 .033363

O 7.3128111 .4657771 .561584

C $6.8325380 .807468-0.708510$

C $6.625873 \quad 2.288313-1.083491$

H $6.1582650 .182833-1.305872$

$\begin{array}{lllll}\text { O } & 8.169197 & 0.459586 & -1.085833\end{array}$

H $7.4662632 .884907-0.704779$

H $6.6994232 .346381-2.177786$

H $7.276807-6.237460 \quad 0.843055$

H $6.026871-5.6987551 .975338$

H $5.565896-6.4316850 .431297$

C $3.975040-4.029002-0.694184$

H $5.759087-1.2363032 .543586$

H 7.1599331 .2508372 .497840

H $8.2533990 .613097-2.042340$

H $3.881132-5.120788-0.735615$

H $4.328729-3.702004-1.679884$

H $4.553978-3.775973 \quad 1.385830$

C $2.9097762 .548153-0.740993$

O $4.2251822 .126350-1.130338$

C $5.3020842 .914518-0.620465$

C $5.1716074 .374517-1.088417$

C $3.8015204 .954020-0.725635$

C $2.6611604 .014769-1.134500$

H $2.2311301 .923221-1.335842$

C $2.626403 \quad 2.267152 \quad 0.744640$

H 5.2789722 .8911420 .473141

O $5.356107 \quad 4.443722-2.501140$

H $5.9679744 .966725-0.621450$

H $3.6774165 .922123-1.225979$

$\begin{array}{lllll}\text { O } & 3.763579 & 5.186617 & 0.679235\end{array}$

H $1.7125174 .352438-0.701498$

O $2.5075744 .055784-2.556040$

C $2.636720 \quad 0.787417 \quad 1.056616$

H $3.3654132 .739798 \quad 1.396317$

H 1.6317252 .6480711 .003390

H $5.3080015 .380184-2.758225$

H $2.8970975 .574440 \quad 0.889399$

H $2.3039974 .973971-2.802729$

$\begin{array}{llll}\text { O } & 2.392312 & 0.608425 & 2.387883\end{array}$

O $2.838287-0.100653 \quad 0.241313$

C $2.395797-0.7511402 .835631$

H $1.547571-1.2893502 .404436$

H $3.337870-1.2412752 .571925$

H $2.295086-0.7506713 .924081$

SCF Energy (B3LYP/6-31G**/MMFF) $=-3245.90291997$

0700259

MM̄FF Geometry

C $0.243405-3.904664-0.509204$

C $-0.557899-2.831475-0.429599$

C $-1.598683-2.507245-1.470559$

O $-2.847846-2.358884-0.750398$

C $-1.312429-1.192396-2.217180$

C $0.030208-1.124242-2.914380$

C $1.006634-0.260278-2.572336$

C $0.207631-2.051887-4.086268$

C $0.958558 \quad 0.720813-1.435254$

C $0.3746742 .069388-1.877320$

C $-0.1309092 .945795-0.720446$

C $0.975285 \quad 3.3342990 .259600$

C $0.430606 \quad 4.1430851 .445491$

O $-1.1645172 .219701-0.046710$

O $1.470199 \quad 4.186676 \quad 2.434433$

C -0.7713153 .3848532 .049920$

C $0.1509245 .598371 \quad 1.054615$

O $\begin{array}{llll}-1.382526 & 4.153382 & 3.084246\end{array}$
C -1.8210992 .9414641 .001969$

C $-2.8494751 .987523 \quad 1.664518$

O $-2.487196 \quad 4.100397 \quad 0.504456$

C $-4.006248-2.534856-1.438889$

O $-4.127066-2.841407-2.613551$

C $-5.117960-2.265783-0.498902$

C $-6.384631-2.284508-0.938761$

C $-7.581204-2.018132-0.136222$

C $-7.381742-1.6228151 .304051$

C $-3.861937 \quad 1.3397710 .706388$

$\begin{array}{llll}C & -5.076507 & 2.199835 & 0.399809\end{array}$

C $-7.0770892 .273724-1.087889$

$\begin{array}{lllll}\text { O } & -5.322153 & 3.249637 & 0.987442\end{array}$

C $-8.2543351 .694549-0.368472$

C $-9.3256141 .184019-0.994903$

C -10.528301 $0.635438-0.281527$

C $-10.894108-0.760188-0.736142$

C $-11.900867-0.962845-1.602840$

C $-10.150770-1.924075-0.119318$

C $-8.789748-2.125431-0.727491$

N $-5.8609621 .646935-0.603478$

H $0.148994-4.546338-1.384122$

H $-0.492145-2.1587330 .420523$

H $-1.694893-3.337418-2.181939$

H $-1.455192-0.354784-1.524591$

H -2.094257 $-1.032722-2.972132$

H $1.928076-0.251568-3.151733$

H $1.162853-1.894520-4.598372$

H $0.177664-3.096053-3.760550$

H $-0.589311-1.892820-4.820016$

H $0.4035490 .298273-0.591187$

H $1.9800660 .863192-1.068650$

H $1.1241622 .619775-2.459532$

H $-0.4769661 .887914-2.546842$

H $-0.570023 \quad 3.845547-1.167589$

H $1.7760143 .883332-0.250875$

H 1.4426702 .4293100 .664005

H 1.1075384 .6235993 .224316

H $-0.3680352 .487798 \quad 2.539837$

H 1.0697616 .0792190 .697552

H $-0.1754056 .186184 \quad 1.919673$

H $-0.600753 \quad 5.6952350 .268585$

H -1.921949 4.8427322 .660712

H -2.292042 1.1622832 .127671

H -3.3847172 .5102202 .466015$

H -3.2837783 .8199500 .028489$

H $-4.861010-2.0476330 .531572$

H -6.567037 -2.514025 - 1.987971

H $-6.937206-2.4487901 .869784$

H -8.306277 -1.3449881 .813812$

H $-6.721271-0.7517101 .378305$

H $-3.3809821 .039681-0.230560$

H $-4.2463300 .429884 \quad 1.184316$

H $-7.1245442 .098808-2.167279$

H -7.029290 $3.352802-0.909916$

H -8.2349051 .7180580 .718600$

H $-9.3558621 .187259-2.082278$

H - $-11.3657111 .320406-0.469057$

H -10.3841050 .6360690 .806156$

H -12.188231 -1.962362 -1.912055

H -12.456687 - $0.133128-2.026937$

H -10.714797-2.853755 -0.274405

H -10.122065 - 1.7961150 .966088

H $-8.826806-2.414637-1.778912$

H $-5.6131440 .738507-0.983988$

C $1.322825-3.6641351 .842994$

O $1.945720-2.3693451 .702317$

C $2.946099-2.2237642 .727394$

C $2.646844-3.3019463 .758823$

C $2.168472-4.4280192 .867528$

H $3.911190-2.4482432 .259435$

C $2.964936-0.7997663 .289659$

H $3.526713-3.5662254 .352997$

H $1.849319-2.9974724 .446662$

C $1.407478-5.5093143 .616037$

H $3.039523-4.8801022 .373977$

C $3.176676 \quad 0.3105902 .242450$

O $1.720866-0.5356443 .951493$ 
H $3.736193-0.7294964 .065751$

H $2.2619280 .436540 \quad 1.652592$

O $3.355926 \quad 1.5427172 .952639$

C 4.3653900 .0973921 .287529

C 4.6576891 .3453950 .431937

H $4.145793-0.7569070 .637690$

O $5.523209-0.2269392 .056587$

H $3.717171 \quad 1.8029910 .110865$

H 5.2003282 .0737741 .047449

H $2.046463-5.9736634 .374393$

H $0.526308-5.1021224 .123184$

H $1.069702-6.2948072 .932942$

C $1.272178-4.3732830 .485207$

H $1.010237-0.6513003 .297298$

H 4.1952261 .4810643 .440409

H $6.250844-0.396476 \quad 1.434356$

H $1.074230-5.4391810 .652142$

H $2.252309-4.299118-0.002327$

H $\quad 0.312747-3.4978802 .237900$

C $7.667850 \quad 0.148515-1.442185$

O $6.7778700 .558253-0.395501$

C $5.4930641 .018178-0.820522$

C $5.6407202 .238814-1.742143$

C $6.5734291 .939652-2.919319$

C $7.8803521 .281223-2.460343$

H $8.625693-0.031326-0.936855$

C $7.223149-1.170813-2.083348$

H $4.9819120 .215487-1.363633$

O $\quad 6.143122 \quad 3.355744-1.010927$

H $4.6502742 .521037-2.118255$

H $6.7965842 .877277-3.443010$

O $5.8921351 .083661-3.831789$

H $8.4438680 .897862-3.318910$

O $8.6999682 .270776-1.830441$

C $7.253249-2.290349-1.069471$

H $6.221125-1.115893-2.518470$

H $7.897593-1.455769-2.899313$

H $6.9868253 .091087-0.606109$

H $6.485177 \quad 0.927913-4.586512$

H $8.8790532 .964721-2.487804$

O $5.982303-2.646133-0.727456$

O $8.284083-2.775230-0.620936$

C $5.875204-3.6940510 .240686$

H $6.271221-3.3565411 .203023$

H $4.816665-3.9377030 .361352$

H $6.402176-4.589476-0.102838$

SCF Energy (B3LYP/6-31G**//MMFF) $=-3245.92287499$

\section{0}

MM̄̄FF Geometry

C $-1.823520-3.496067-1.701774$

C $-0.900340-2.632140-1.252558$

C $0.210253-2.078964-2.106148$

O $0.195148-0.641764-1.923008$

C $1.583117-2.629558-1.672197$

C $1.723223-4.130652-1.802041$

C $1.797045-4.985857-0.763455$

C $1.819200-4.647482-3.214175$

C $1.731637-4.6666090 .704816$

C $3.040502-4.9964241 .435787$

C $4.240296-4.1484890 .981889$

C $5.511490-4.5973151 .707549$

C $6.715864-3.7178981 .342508$

O $3.964329-2.775376 \quad 1.281222$

O $7.780651-4.0162182 .258030$

C $6.325278-2.2359591 .520949$

C $7.267555-4.062784-0.044914$

O $7.364353-1.3780561 .053647$

C $4.982151-1.8609560 .847677$

C $4.572230-0.4344001 .286171$

O $5.145528-1.887596-0.570897$

C $0.833991 \quad 0.113623-2.856357$

O $1.412392-0.285127-3.854880$

C $0.7250221 .530317-2.442228$

C $1.5476652 .434331-2.995318$

C $1.5807993 .871133-2.711713$

C $0.479724 \quad 4.433973-1.855113$

C 3.1913900 .0155970 .795069
C $2.798165 \quad 1.334088 \quad 1.430917$

C $3.361583 \quad 3.7427861 .489107$

O $1.946108 \quad 1.3936792 .314874$

C 4.0547074 .7388420 .617621

C $3.4101475 .704383-0.053612$

C $4.0854116 .735983-0.908609$

C $4.1452036 .363647-2.373034$

C $5.3196096 .341022-3.026794$

C $2.8476046 .084177-3.102102$

C $2.5825904 .606576-3.237982$

N 3.5002122 .4157150 .921140

H -1.788556 -3.824849 -2.737479

H $-0.924139-2.319779-0.210946$

H $0.018319-2.298799-3.164033$

H $1.800219-2.287513-0.655759$

H $2.374330-2.160676-2.271611$

H $1.909767-6.047130-0.982056$

H $1.998217-5.727338-3.250606$

H $\quad 0.892645-4.449819-3.761621$

H $2.646402-4.160415-3.740775$

H $1.447701-3.6289120 .898858$

H $0.929481-5.2764221 .139512$

H $2.869099-4.8323662 .507641$

H $3.270917-6.0614201 .305486$

H $4.368419-4.263444-0.099800$

H $5.730336-5.6518711 .498111$

H $5.349117-4.5355412 .792732$

H $7.466073-3.8362323 .160256$

H $6.231070-2.0477432 .599606$

H $7.592567-5.109964-0.076085$

H $8.157683-3.467137-0.276077$

H $6.539870-3.918956-0.846320$

H 8.184146-1.642955 1.505001

H $4.567442-0.3850952 .383253$

H 5.3217940 .2856930 .935416

H $4.440314-1.362704-0.980141$

H $-0.0126921 .784564-1.689771$

H $2.2826782 .088885-3.721865$

H $0.5162895 .520091-1.752148$

H $0.5306964 .015665-0.844448$

H $-0.4995644 .198040-2.286261$

H $3.1683560 .134144-0.293352$

H $2.423418-0.719173 \quad 1.059670$

H 3.8161853 .7327172 .485449

H 2.2956353 .9624641 .611416

H $5.1376584 .671346 \quad 0.547744$

H $2.328613 \quad 5.774756 \quad 0.028145$

H $5.0923256 .932087-0.516831$

H $3.5413157 .683221-0.804846$

H $5.3712236 .098566-4.083302$

H $6.2543306 .561796-2.522250$

H $2.9030116 .503919-4.115842$

H $2.0299136 .628067-2.621839$

H $3.3216984 .094360-3.856631$

H 4.2760792 .2379840 .289393

C $-4.299053-3.822477-1.402751$

O $-4.581521-2.409452-1.357232$

C $-5.860639-2.210564-0.731017$

C $-6.566354-3.557429-0.801811$

C $-5.402182-4.504311-0.588587$

H $-5.660582-1.9756790 .320972$

C -6.612813 -1.042005-1.376025

H -7.348859-3.658863 -0.043884

H $-7.017744-3.731398-1.785161$

C $-5.691219-5.932260-1.018447$

H $-5.139897-4.5007380 .478136$

C $-5.7902260 .257387-1.495346$

O $-7.004949-1.428214-2.698896$

H $-7.540109-0.859670-0.819388$

H $-5.054648 \quad 0.162735-2.303934$

O $-6.6824021 .310500-1.881398$

C $-5.0673190 .688782-0.206287$

C $-4.3819702 .060861-0.355879$

H -4.309404 -0.0591360 .049070$

$\begin{array}{lllll}\text { O } & -5.998359 & 0.733381 & 0.873564\end{array}$

H $-3.8640322 .104983-1.321562$

H $-5.1500632 .843923-0.339903$

H $-6.522819-6.347060-0.439492$ 
H $-5.960330-5.985402-2.078739$ H $-4.817027-6.571576-0.860356$ C -2.901999-4.087335-0.838237 H -7.640868 -0.768303 -3.022188 H -6.980128 1.126287 -2.787941 H -6.6902551 .3756440 .639623$ H $-2.727014-5.167262-0.770621$ H -2.831152 -3.679612 0.177603 H -4.352553 -4.122821 -2.456998 C -3.2229632 .5796243 .169188$ O -4.0502312 .3982892 .011993$ C $-3.3588452 .335676 \quad 0.762950$ C -2.5900593 .6428820 .518441$ C $-1.6530153 .965417 \quad 1.685569$ C -2.3640143 .8499683 .038689$ H -3.9309202 .7330043 .993807$ C -2.4027051 .3240003 .487926$ H $-2.652864 \quad 1.4980500 .780614$ $\begin{array}{lllll}\text { O } & -3.496997 & 4.725886 & 0.321941\end{array}$ H $-2.0099743 .540340-0.404603$ H -1.2637564 .9833491 .560268$ O -0.5501793 .0665051 .637120$ H -1.6336193 .8916843 .855045$ O -3.2161384 .9906163 .181102$ C $-3.312830 \quad 0.165817 \quad 3.823336$ H -1.7419981 .0279082 .668318$ H $-1.757177 \quad 1.4899394 .358473$ H -4.0644454 .7850821 .109504$ H 0.0437613 .2878012 .374433 H -3.6149804 .9509134 .067164$ O $-3.293637-0.7520422 .815355$ $\begin{array}{lllll}\text { O } & -3.975357 & 0.096205 & 4.850647\end{array}$ C $-4.130121-1.8961633 .010029$ H -5.181693 -1.594581 3.018954 H $-3.967740-2.5819602 .174849$ $\mathrm{H}-3.866488-2.4101843 .939423$

SCF Energy (B3LYP/6-31G**//MMFF) $=-3245.90686634$

0700261

MM̄FF Geometry

C $2.5232423 .578349-0.279604$

C $1.7458792 .499306-0.108182$

C 0.8694462 .2612591 .092538

O $-0.455754 \quad 1.9304950 .617822$

C 1.4208521 .0661081 .888930

C 0.5671520 .6758683 .077084

C $-0.254266-0.3925143 .111808$

C 0.7118521 .5604674 .287781

C $-0.537942-1.3724702 .005355$

C $-2.027307-1.3821671 .656967$

C $-2.385717-2.3021530 .480074$

C -1.812296 -1.811788 -0.853595

C $-2.306416-2.648621-2.043975$

O $-3.818018-2.3180250 .412107$

O $-2.001111-1.927193-3.247719$

C $-3.841706-2.769089-1.969665$

C -1.568658 -3.988874 -2.144657

O $-4.333074-3.658158-2.970317$

C $-4.358396-3.198594-0.578164$

C $-5.903808-3.090981-0.533423$

O $-3.981016-4.557503-0.341231$

C -1.3007892 .9665450 .360531$

O $\begin{array}{llll}\text { O } & -1.065037 & 4.157459 & 0.488019\end{array}$

C $-2.5798932 .393740-0.116303$

C $-3.5903123 .204722-0.461420$

C $-4.9095112 .789065-0.948828$

C $-5.1859011 .311486-1.045310$

C $-6.514397-3.2511350 .866105$

C $-6.532151-1.9616131 .667765$

C $-7.7379170 .194201 \quad 1.887991$

O $-5.816306-1.8012552 .653689$

C -8.5116851 .1087020 .994568$

C -8.2711832 .4236050 .876738$

C $-9.0719203 .319195-0.025783$

C $-8.2225534 .234647-0.878408$

C $-8.4169395 .564784-0.859372$

C $-7.2008453 .612817-1.807304$

C $-5.7973313 .750256-1.279446$
N -7.433041 -1.031490 1.171294

H $2.5269304 .358746 \quad 0.475907$

H $1.7433551 .722169-0.868625$

H $\quad 0.8257503 .1453991 .740794$

H $2.427866 \quad 1.3067112 .254494$

H $\quad \begin{array}{llll}1.560897 & 0.209720 & 1.221416\end{array}$

H $-0.798737-0.5890954 .034802$

H 0.1102921 .2104325 .133395

H 1.7561871 .5871504 .615925

H $\quad 0.3926772 .5816174 .057367$

H $0.047930-1.1500831 .113259$

H $-0.234505-2.3711932 .340871$

H -2.603603 -1.700073 2.537092

H $-2.376696-0.3647701 .442957$

H $-2.028939-3.3128770 .709859$

H $-0.717374-1.796835-0.840706$

H -2.120535 -0.771698-1.019392

H -1.041609-1.769330 -3.267376

H $-4.259738-1.779818-2.202887$

H $-0.496745-3.823944-2.304887$

H - $1.912652-4.566228-3.009788$

H -1.677852 -4.606257-1.250577

H -3.995328 -3.345104 -3.826870

H $-6.212219-2.123941-0.948980$

H -6.329556 -3.873800 -1.174079

H $-4.633683-4.9767860 .240782$

H -2.657454 $1.316402-0.174633$

H $-3.4397264 .280755-0.382231$

H $-4.4971140 .838121-1.753715$

H $-6.196677 \quad 1.077541-1.385030$

H $-5.0654940 .831885-0.067424$

H -7.555610 $-3.584500 \quad 0.766571$

H $-6.004050-4.0194371 .455517$

H -6.8035490 .6413362 .243646$

H -8.342944 -0.0804702 .758474$

H -9.3267090 .6708310 .422075$

H $-7.4831302 .879321 \quad 1.471311$

H -9.7472473 .9081930 .608047$

H $-9.7125382 .727657-0.692430$

H -7.831611 $6.228487-1.487503$

H $-9.1633506 .020215-0.217308$

H -7.241452 4.124051-2.778743

H -7.477697 2.578028 -2.026096

H $-5.4809904 .790181-1.180171$

H $-8.093877-1.3453800 .465573$

C $4.9055483 .630240-1.175720$

O $5.1815322 .337455-0.595615$

C 6.0566862 .5245670 .531118

C 6.6739593 .9055190 .346962

C $5.4964774 .672147-0.224175$

H 5.4191202 .5481991 .423262

C $7.065737 \quad 1.378818 \quad 0.647951$

H 7.0459584 .3251601 .286415

H $7.5009643 .887980-0.372502$

C $5.8850125 .974396-0.902141$

H 4.8017654 .8945460 .594632

C $6.438848-0.0292040 .743022$

O $7.9171371 .398439-0.504409$

H 7.7164291 .5613031 .511820

H $6.058309-0.323206-0.240957$

O $7.496750-0.9487571 .043335$

C $5.326557-0.1756041 .795369$

C $4.892000-1.6207092 .098629$

H $4.455508 \quad 0.402023 \quad 1.470144$

O $5.7793320 .399783 \quad 3.024992$

H $5.699412-2.1489212 .622312$

H $4.069149-1.5546612 .823224$

H $6.359476 \quad 6.653894-0.186465$

H $6.5904065 .805842-1.722794$

H $5.0035106 .477014-1.312602$

C $3.4104913 .767995-1.480124$

H $8.5539900 .669886-0.406530$

H $7.818041-0.7438381 .938279$

H $5.035103 \quad 0.3775743 .650756$

H $3.146273 \quad 3.027141-2.245519$

H $3.2062264 .752413-1.916487$

H $5.4484023 .652865-2.129870$

C $2.869454-2.340895-0.964132$ 
O $3.379993-1.735033 \quad 0.231843$

C $4.440968-2.4401990 .879233$

C $3.980727-3.8519961 .285140$

C $3.387591-4.6247520 .101189$

C $2.388812-3.776952-0.693942$

H $1.987786-1.741093-1.225441$

C $3.864410-2.243700-2.131733$

H $5.289956-2.5212150 .194678$

O $2.999588-3.7716132 .320005$

H $4.835572-4.4014801 .695923$

H $2.868789-5.5110360 .486303$

O $4.412891-5.101284-0.765179$

H $2.137718-4.263614-1.643556$

O $1.176683-3.6790530 .060894$

C $4.139266-0.811119-2.536027$

H $4.824968-2.697920-1.878921$

H $3.455449-2.755374-3.010333$

H $2.266599-3.2223721 .992581$

H $5.162523-4.485511-0.741676$

H $0.822324-4.5785620 .166101$

O $5.203231-0.787489-3.392241$

O $3.5001230 .161809-2.162319$

C $5.5931110 .508368-3.858161$

H $6.028201 \quad 1.082790-3.035811$

H $6.3520630 .376060-4.633772$

H $4.7394201 .038302-4.291857$

SCF Energy (B3LYP/6-31G**//MMFF) $=-3245.90605408$

0700262

MM̄FF Geometry

C $-2.167963-1.5973912 .690418$

C $-2.229149-0.5415393 .516329$

C $-1.3294810 .669553 \quad 3.453456$

O $-\begin{array}{llll}0.034773 & 0.257424 & 2.944830\end{array}$

C $-1.898743 \quad 1.788492 \quad 2.562230$

C -3.2649252 .3110002 .949534$

C -4.3427312 .3109962 .140775$

C -3.356802 2.9301244 .320847

C $-4.453036 \quad 1.769830 \quad 0.743249$

C $-4.5838102 .866588-0.320394$

C -3.331882 $3.745131-0.474738$

C $-3.5633504 .805987-1.550667$

C $-2.3079285 .655316-1.784743$

O $-2.236332 \quad 2.908584-0.853837$

O $-2.515626 \quad 6.433902-2.973339$

C -1.095809 $4.723410-2.017753$

C $-2.113066 \quad 6.680761-0.661032$

O $0.0951465 .502684-2.090325$

C $-0.9704893 .585757-0.973867$

C $0.031890 \quad 2.484731-1.401650$

O $\begin{array}{lllll}0.555056 & 4.132375 & 0.277913\end{array}$

C 1.0357881 .0540993 .204017

O 1.0273242 .1453293 .750611

C 2.2458780 .3376892 .741392

C 3.4386330 .9493342 .789472

C 4.7359000 .3439052 .476501

C $4.755755-1.1262212 .147596$

C $1.5046572 .894439-1.454316$

C $2.4077911 .750679-1.884129$

C $4.7681051 .330259-2.579599$

O $2.0054670 .606789-2.072993$

C $5.5449240 .720874-1.458199$

C $6.8207651 .031629-1.185290$

C $7.6470360 .337369-0.141939$

C 7.8950411 .1790961 .087041

$\begin{array}{llll}\text { C } 8.707814 & 2.248746 & 1.050771\end{array}$

C $7.279881 \quad 0.707762 \quad 2.383472$

C 5.8404711 .1176932 .537129

N $3.7190642 .175943-2.047882$

H -1.446402 -1.599893 1.877320

H -2.968181 -0.5438624 .314809$

H -1.184233 1.0255854 .481392

H -1.863245 1.4532421 .520838

H -1.2107672 .6422512 .585392$

H -5.267102 2.7452912 .520164

H -4.3250753 .4111244 .496256$

H -3.2224182 .1708635 .097209$

H -2.5845553 .6966504 .443714$
H $-3.629992 \quad 1.0963870 .491251$

H $-5.358324 \quad 1.1517960 .705087$

H $-4.7912402 .368552-1.276823$

H $-5.4519243 .497758-0.091725$

H $-3.109790 \quad 4.2208350 .486544$

H $-4.4173795 .445218-1.294022$

H $-3.8328384 .312834-2.495345$

H -2.662307 $5.822972-3.715375$

H $-1.220803 \quad 4.265233-3.007841$

H $-2.9979297 .322806-0.572911$

H $-1.2789057 .356393-0.880705$

H -1.9375316 .2211740 .313868$

H $0.3525845 .735382-1.181953$

H $-0.0608321 .645171-0.696993$

H $-0.2774162 .071588-2.371477$

H -0.1138243 .4320080 .786070$

H $2.127713-0.6895212 .416921$

H 3.4775031 .9862893 .121145

H $4.381729-1.7145942 .992605$

H $5.751141-1.5066421 .909870$

H $4.130165-1.3329141 .272862$

H $1.6497003 .698683-2.180881$

H $1.8423413 .243015-0.472295$

H $5.3956161 .957638-3.220857$

H $4.330507 \quad 0.540322-3.198898$

H $5.049246-0.049156-0.874644$

H $7.3203581 .795227-1.777066$

H $8.6104110 .062600-0.591642$

H $7.185349-0.6170050 .138324$

H 8.9140922 .8323851 .941739

H 9.1839032 .5637620 .127978

H 7.8290941 .1347583 .234001

H $7.422558-0.3723192 .488339$

H 5.7196442 .1752222 .776079

H $3.9614043 .134274-1.813520$

C $-4.085670-2.9836961 .742273$

$\begin{array}{lllll}\text { O } & -3.487757 & -2.929420 & 0.432627\end{array}$

C $-4.323779-2.118260-0.416835$

C $-5.658254-1.9956280 .307865$

C $-5.199440-1.937634 \quad 1.752444$

H $-3.858778-1.127333-0.463494$

C $-4.401129-2.718790-1.822688$

H $-6.222615-1.111661-0.003129$

H $-6.290804-2.876306 \quad 0.146124$

C -6.303576 -2.217211 2.756414

H $-4.788935-0.9412101 .948726$

C -3.024839-2.948804 -2.480361

O $-5.064840-3.987773-1.754385$

H $-5.025282-2.086952-2.464552$

H $-2.491110-3.734751-1.935490$

O $-3.247482-3.456356-3.801842$

C $-2.147413-1.685946-2.586311$

C $-0.822200-1.910843-3.342158$

H $-1.929201-1.299266-1.585258$

O $-2.888082-0.674034-3.273375$

H $-1.024321-2.320567-4.339165$

H $-0.362792-0.927397-3.510266$

H -7.100846 -1.472017 2.667807

H $-6.748471-3.2056742 .599973$

H $-5.917810-2.1787743 .779956$

C $-3.018491-2.8293292 .827686$

H $-4.564019-4.548254-1.136826$

H $-3.809433-4.245793-3.718472$

H $-2.3525840 .137596-3.265512$

H $-2.337105-3.688146 \quad 2.774094$

H $-3.489774-2.8679633 .816873$

H $-4.521330-3.9873191 .834720$

C $1.391614-2.831665-0.497934$

O $0.546892-2.101826-1.394500$

C $0.161173-2.809259-2.577937$

C $1.401949-3.157759-3.411936$

C $2.404485-3.967109-2.580743$

C $2.662696-3.329201-1.210557$

H $1.699712-2.089100 \quad 0.248739$

C $0.587591-3.9199990 .219402$

H $-0.344469-3.737989-2.295594$

O $2.018955-1.959719-3.876447$

H $1.087784-3.731284-4.292096$ 
H $3.355081-4.042358-3.123067$

O $1.909822-5.289240-2.390500$

H $3.198357-4.031283-0.562614$

O $3.532719-2.209150-1.398072$

C $1.201981-4.2707431 .551156$

H $-0.444867-3.5915310 .393204$

H $0.498387-4.836583-0.370779$

H $2.785354-2.214564-4.417972$

H $1.789519-5.687585-3.269258$

H $3.046099-1.535587-1.904083$

O $0.849305-3.3408292 .483754$

O $1.920846-5.2439641 .735559$

C $1.382752-3.5458123 .795145$

H $1.018452-4.4912344 .207818$

H $2.476372-3.5311623 .766240$

H $1.037969-2.7275954 .433244$

SCF Energy (B3LYP/6-31G**//MMFF) $=-3245.91713462$

0700263

MM̄FF Geometry

C $4.214738-2.084839-2.433930$

C $4.410013-0.777693-2.664425$

C $5.089580 \quad 0.216700-1.754004$

O $4.0400381 .044733-1.196872$

C $5.873540-0.383045-0.566198$

C $6.533637 \quad 0.666857 \quad 0.311095$

C 6.2101260 .8948271 .600526

C $7.6300491 .469305-0.340600$

C 5.1905920 .1702002 .438558

C 3.7658810 .6462262 .161331

C 2.7143590 .1619883 .169624

C $2.658077-1.3604993 .283799$

C $1.509572-1.8196424 .190978$

O $1.460358 \quad 0.6578242 .686807$

O $1.371885-3.2363974 .004448$

C $0.199282-1.1468833 .728869$

C $1.849496-1.6121915 .672180$

O $-0.850892-1.4695104 .635805$

C $0.3200810 .379497 \quad 3.517039$

C $-0.879997 \quad 0.9741652 .741837$

O $0.434911 \quad 1.0355844 .779058$

C $3.7667782 .248435-1.761161$

O $4.2991432 .749990-2.736474$

C $2.6752312 .850631-0.961077$

C $2.1490134 .036195-1.300605$

C $1.0687564 .725374-0.587578$

C 0.5209514 .0612540 .648949

C $-2.229748 \quad 0.979815 \quad 3.458023$

C -3.3100481 .5959992 .588147$

C $-4.0263753 .744614 \quad 1.544897$

O -4.2163840 .9135182 .114652$

C -3.287134 $4.084240 \quad 0.288360$

C $-3.2748625 .309676-0.258313$

C $-2.5489745 .644197-1.531534$

C -1.671432 $6.871207-1.400510$

C $-1.9660858 .002149-2.063796$

C $-0.4560086 .792878-0.502185$

C $0.6202165 .903496-1.069232$

N -3.156479 2.9609452 .403600

H $4.576327-2.541698-1.517810$

H $4.007703-0.355772-3.584117$

H $5.7707780 .818785-2.367189$

H $6.651360-1.061979-0.938719$

H $5.195904-0.9933690 .040247$

H $6.742717 \quad 1.6829692 .131328$

H 8.1615992 .1040750 .376632

H $8.3695990 .802168-0.795354$

H $7.2227712 .122818-1.117839$

H $5.279847-0.9102602 .281204$

H $5.4372090 .341808 \quad 3.493821$

H 3.7348241 .7441122 .132635

H 3.4569300 .3216191 .161352

H 2.9301720 .6197114 .141802

H $3.606922-1.7692953 .650176$

H $2.509876-1.7949922 .285163$

H $0.592668-3.5259134 .509760$

H $-0.072645-1.6072852 .771354$

H $2.767275-2.1536325 .931978$
H $1.068920-2.0243526 .321152$

H $1.996312-0.5621595 .934021$

H $-0.752787-0.8992835 .417452$

H -0.6250502 .0112842 .487766$

H $-0.975561 \quad 0.4688301 .771277$

H $\quad 0.185213 \quad 1.967159 \quad 4.660651$

H $2.3226922 .287366-0.104122$

H $2.5399884 .548871-2.178104$

H 1.3113023 .9211371 .394931

H $\quad 0.096046 \quad 3.083500 \quad 0.398118$

H $-0.2722374 .626840 \quad 1.140117$

H -2.555317 -0.0371963 .693939$

H -2.1797931 .5364324 .400139$

H -4.317415 4.638195 2.105718

H -4.9256483 .1703261 .301562$

H $-2.7440793 .281217-0.203831$

H $-3.838937 \quad 6.109040 \quad 0.216549$

H -3.306385 $5.803576-2.309912$

H -1.941111 $4.798491-1.875735$

H -1.344222 $8.887085-1.977764$

H -2.835956 $8.070116-2.708722$

H $-0.7675506 .501770 \quad 0.503892$

H $-0.0139927 .790478-0.376099$

H $1.0803016 .301440-1.974905$

H -2.3738743 .4372032 .842182$

C $1.998549-3.134939-2.913302$

O $1.935195-3.708605-1.596535$

C $0.703909-4.455531-1.475541$

C $0.015549-4.377302-2.835440$

C $1.166029-4.074506-3.777037$

H $0.999678-5.484966-1.242688$

C $-0.117366-3.850055-0.330088$

H $-0.511287-5.303166-3.086299$

H $-0.718714-3.561901-2.857464$

C $0.720986-3.475077-5.099446$

H $1.720211-5.003829-3.964293$

C $-1.477330-4.535457-0.108182$

O $0.643014-3.9588460 .877640$

H $-0.251433-2.779133-0.518483$

H -2.077934 -4.510005-1.023056

O $-1.256816-5.9245720 .167647$

C $-2.294000-3.9400461 .056023$

C $-2.568498-2.4327980 .945800$

H -1.776628 -4.128005 2.004689

O $-3.540894-4.6298461 .153355$

H $-3.192339-2.1181881 .792693$

H -1.624295-1.892312 1.080006

H $0.054934-4.163998-5.629133$

H $0.182304-2.533003-4.951867$

H $1.580094-3.273463-5.746746$

C $3.453509-2.992470-3.361268$

H $1.499913-3.5263830 .720632$

H $-0.687396-5.9797810 .954446$

H $-3.343041-5.5795671 .222893$

H $3.498402-2.611431-4.387918$

H $3.952190-3.969226-3.357320$

H $1.524688-2.146320-2.856273$

C $-3.5866970 .179154-1.500382$

O $-3.121819-0.508221-0.332954$

C $-3.216358-1.940113-0.366395$

C $-4.678636-2.371494-0.559011$

C $-5.312919-1.691239-1.777754$

C $-5.051269-0.182357-1.795289$

H $-3.5516291 .241800-1.233123$

C $-2.655010-0.028910-2.704462$

H $-2.613271-2.297264-1.204942$

O $-5.427034-2.0464470 .610363$

H $-4.721411-3.457509-0.692300$

H $-6.394617-1.872883-1.766548$

O $-4.786015-2.293083-2.956946$

H $-5.3516930 .242018-2.760444$

O $\quad-5.881771 \quad 0.419478-0.798241$

C $-1.2742770 .549381-2.475858$

H -2.525528 -1.088426 -2.939641

H $-3.0773160 .476142 \quad-3.580827$

H $-6.336662-2.3617160 .474879$

H $-5.221057-1.874452-3.719083$

H $\quad-5.737914 \quad 1.380508-0.834138$ 
O $-0.496773 \quad 0.263634-3.562920$

O $-0.9207031 .173730-1.485576$

C $0.8475710 .747677-3.502686$

H $1.3392180 .397786-2.590404$

H $\quad 1.3907280 .352606-4.365232$

H $0.8526261 .840234-3.552421$

SCF Energy (B3LYP/6-31G**//MMFF) $=-3245.90615835$

0700264

MM̄FF Geometry

C $0.1009535 .275131 \quad 1.097502$

C 1.0308304 .7659780 .275646

C 2.1912263 .9262280 .746560

O 2.1113252 .6319300 .103617

C 3.5141554 .5901410 .323312

C $4.7500793 .867468 \quad 0.822713$

C $5.6741093 .291730 \quad 0.028534$

C 4.9341923 .8429972 .318728

C $5.7019453 .233143-1.472966$

C $5.0640371 .972856-2.078108$

C $5.7656850 .662606-1.685000$

C $5.238022-0.507209-2.518805$

C $5.859105-1.846149-2.088579$

O $5.5113950 .409025-0.300724$

O $5.083132-2.901225-2.677945$

C $5.731767-1.995312-0.556380$

C $7.287129-2.008759-2.623015$

O $6.446063-3.139040-0.095628$

C $6.185103-0.7436840 .223020$

C $5.864943-0.813848 \quad 1.736537$

O $7.606603-0.6046530 .104395$

C $1.2740661 .709186 \quad 0.652702$

O $0.578517 \quad 1.8447351 .646534$

C $1.3326800 .493016-0.189718$

C $0.397866-0.458068-0.044167$

C $0.292911-1.694032-0.823655$

C $1.446692-2.049574-1.722212$

C $4.384300-0.9922052 .091031$

C $3.980971-2.4367792 .338488$

C $1.973785-3.8457792 .774957$

O $4.787808-3.3589832 .417812$

C $1.510941-4.4586721 .491152$

C $0.265910-4.9225041 .306461$

C $-0.211626-5.5656540 .037202$

C -1.426653 -4.875110 -0.539077

C $-2.664453-5.345357-0.309912$

C -1.198242 -3.691969-1.451400

C $-0.824009-2.441748-0.701877$

N $2.607921-2.5702122 .497916$

H 0.1727535 .0917352 .166612

H $0.944394 \quad 4.934928-0.795269$

H 2.1663253 .7980401 .835559

H 3.5498395 .6208360 .701011

H $3.5307994 .668155-0.769180$

H 6.5253482 .8070520 .505948

H 5.9136863 .4487662 .610403

H 4.8561804 .8555362 .728244

H 4.1747353 .2131642 .791812

H $5.2213344 .117086-1.905035$

H $6.7496873 .295304-1.794397$

H $4.0094181 .931492-1.781929$

H $5.0892572 .079956-3.169677$

H $\quad 6.843270 \quad 0.787862-1.843790$

H $5.409648-0.332310-3.588190$

H $4.148318-0.575382-2.398891$

H $5.093018-2.777651-3.642612$

H $4.672512-2.181760-0.344037$

H $7.293176-1.962704-3.718731$

H $7.697163-2.990969-2.363258$

H $7.971873-1.240695-2.257708$

H $6.113706-3.907216-0.590804$

H $6.491016-1.5741272 .218988$

H $\quad 6.1809850 .1428172 .177277$

H $7.924209-0.0497330 .835966$

H $2.1262750 .426693-0.924711$

H $-0.388206-0.3043810 .693221$

H $1.520383-1.340872-2.554073$

H $1.373725-3.050999-2.151245$
H $2.386611-2.032401-1.160321$

H $4.186120-0.456773 \quad 3.028455$

H $3.738399-0.5568911 .322534$

H $1.146276-3.6537153 .465080$

H $2.690248-4.5127143 .264881$

H $2.236173-4.5424620 .685128$

H $-0.449134-4.8621132 .123736$

H $-0.448902-6.6136490 .262574$

H $0.582300-5.600136-0.719330$

H $-3.538815-4.872227-0.744748$

H $-2.831261-6.2112470 .322082$

H -2.118007 -3.465960 -2.008064

H $-0.469013-3.968607-2.217560$

H -1.602611 -2.099088 -0.019883

H $2.008994-1.7606692 .363077$

C -2.3407005 .2021120 .623021$

O $-2.2364754 .239666-0.443151$

C $-3.5417514 .015963-1.007651$

C $-4.5152494 .819923-0.150548$

C -3.6358375 .9552390 .341094$

H $-3.5265314 .430513-2.023193$

C $-3.8507102 .511666-1.068455$

H $-5.3818385 .164659-0.722976$

H -4.8876354 .2282900 .694597$

C $-4.205940 \quad 6.685770 \quad 1.543817$

H $-3.4860986 .667912-0.480927$

C $-2.9557741 .739424-2.057564$

O $-3.707196 \quad 1.9607020 .239935$

H -4.898382 2.385671 -1.362040

H -3.106612 2.146119-3.065380

O $-1.583767 \begin{array}{lll}1.982636 & -1.723427\end{array}$

C $-3.1572570 .211369-2.076731$

C $-4.592990-0.257556-2.371680$

H $-2.781515-0.231402-1.149581$

O $-2.337683-0.312285-3.134448$

H $-5.1323440 .514195-2.933844$

H -4.537133 -1.134719 -3.030426

H -5.1729457 .1362411 .296831$

H -4.3574636 .0079662 .390626$

H $-3.534677 \quad 7.4864561 .869421$

C $-1.085586 \quad 6.0724640 .628240$

H -2.7722702 .0508870 .493921$

H -1.041360 $1.698959-2.478353$

H -1.410639 -0.231650 -2.853954

H $-1.208797 \quad 6.9297651 .298878$

H $-0.897726 \quad 6.468092-0.377651$

H -2.4229874 .6380731 .562292$

C $-5.289000-2.2523020 .713176$

O $-4.711272-1.756298-0.501492$

C $-5.387588-0.651086-1.113532$

C $-6.840580-1.025012-1.449078$

C $-7.574868-1.553190-0.213469$

C $-6.763731-2.6348260 .508536$

H $-4.735019-3.1750100 .926631$

C $-5.070269-1.2896821 .886300$

H $-5.3903850 .193102-0.421485$

O $-6.857418-2.015815-2.474645$

H -7.350641 -0.136353-1.840069

H $-8.544238-1.964189-0.520672$

O $-7.826531-0.462250 \quad 0.667278$

H $-7.215416-2.8725101 .478661$

O $-6.790337-3.836243-0.267677$

C -3.611777 -1.218489 2.272359

H $-5.395826-0.2690191 .667204$

H -5.633278 -1.610983 2.770912

H $-7.787630-2.203478-2.686433$

H -8.308763 -0.8108381 .436242$

H -7.720505 -4.102942 -0.363707

O $-3.261647-2.3231782 .991211$

O $-2.872111-0.2907531 .972958$

C $-1.902252-2.3584013 .436817$

H -1.733461 -3.315049 3.938017

H -1.217368 -2.281463 2.587585

H $-1.720778-1.5502834 .151554$

SCF Energy (B3LYP/6-31G**//MMFF) = -3245.89313669

0700265

MM̄MF Geometry 
C $-0.625734-1.8435162 .345785$ C $-1.256170-1.1842583 .330109$ C -1.1628310 .2941453 .629005$ O -0.2268390 .9169212 .716123$ C -2.5219921 .0069893 .482809$ C -3.6377550 .4729294 .358536$ C $-4.754302-0.1122353 .880468$ C -3.4637830 .6858335 .838717$ C $-5.066732-0.3823432 .434805$ C $\begin{array}{llll}-5.808586 & 0.799760 & 1.797822\end{array}$ C $\begin{array}{llll}-5.801200 & 0.794497 & 0.262449\end{array}$ C $-6.531500-0.406793-0.335918$ C $-6.447463-0.410547-1.870614$ O $-4.4362660 .810138-0.164716$ O $-6.866861-1.704285-2.331217$ C $-4.971860-0.238916-2.288842$ C $-7.4051710 .606350-2.501882$ O $-4.854428-0.100067-3.702897$ C $-4.2507240 .933030-1.579317$ C $-2.7281190 .858739-1.865245$ O $-4.7715142 .166548-2.071078$ C 0.3741862 .0697683 .103518 O 0.2254702 .6475384 .171588 C 1.2353562 .6169802 .031166 C 1.6459801 .9064690 .969063 C $2.4687592 .424998-0.120936$ C $2.1368901 .863639-1.477648$ C $-1.8530831 .797844-1.021263$ C -1.698979 $3.203398-1.578180$ C $-0.6387505 .395622-1.007153$ O $-2.1377043 .548724-2.671128$ C $0.7096015 .423523-1.656907$ C $1.7965965 .988891-1.109156$ C $3.1375186 .041703-1.785802$ C $4.2475015 .433373-0.955828$ C $5.1443616 .209556-0.324180$ C $4.3720203 .924459-0.934045$ C 3.4747073 .2962250 .095108 N $-0.9921074 .020895-0.706262$ H $0.025692-1.3095131 .659989$ H -1.878209 -1.7608734 .012322$ H $-0.780254 \quad 0.3977844 .652109$ H -2.8034381 .0097602 .423152$ H -2.395530 $2.073073 \quad 3.715090$ H -5.520578 -0.4324014 .584135$ H $-4.343720 \quad 0.3700016 .409177$ H $-2.607828 \quad 0.115401 \quad 6.212132$ H -3.296825 1.7464856 .053072 H -4.149551 -0.6239071 .887784$ H -5.687120 -1.284924 2.378064 H $-6.842157 \quad 0.8278102 .164662$ H $-5.331725 \quad 1.7343072 .122949$ H $-6.2818301 .725218-0.062026$ H -7.577243 -0.430742 -0.006042 H $-6.079866-1.3386510 .028243$ H -7.772988 -1.856422 -2.012251 H -4.453980 -1.170569 -2.025771 H $-8.4404090 .386338-2.214433$ H $-7.3793280 .546591-3.595445$ H -7.193254 $1.636259-2.207565$ H $-5.305227-0.861099-4.107324$ H -2.392246 -0.159435 -1.630222 H $-2.5351731 .027301-2.931290$ H -4.132504 2.871850 -1.886296 H 1.5271043 .6549102 .163129 H 1.3458480 .8668570 .860515 H $1.6736900 .874904-1.404562$ H $3.0275891 .734089-2.099331$ H $1.4357182 .517871-2.002874$ H $-2.212074 \quad 1.8559770 .011477$ H $-0.8434741 .370328-0.987958$ H $-0.6539265 .950215-0.063572$ H -1.385136 $5.830097-1.679677$ H $0.7873674 .968617-2.641736$ H $1.7161116 .461875-0.133102$ H $3.3598097 .096400-1.995098$ H $3.1097885 .547428-2.764970$ H 5.9599045 .7790380 .247457
H $5.0797417 .292050-0.359638$

H $5.4037943 .634570-0.695276$

H $4.1916793 .542056-1.943262$

H 3.6869873 .6110261 .116627

H -0.6200753 .6201010 .148948$

C $-1.102597-3.7482320 .724267$

O $-0.049125-3.391855-0.190653$

C $-0.644816-3.065482-1.463679$

C $-2.125184-3.418573-1.346487$

C $-2.378610-3.1596830 .128267$

H $-0.556927-1.979673-1.582301$

C $0.105715-3.782248-2.592105$

H $-2.747441-2.810406-2.008314$

H $-2.312986-4.471652-1.586547$

C $-3.657711-3.7861040 .650845$

H $-2.419325-2.0742240 .285197$

C $1.620363-3.484920-2.619628$

O $-0.054375-5.198182-2.433995$

H $-0.345774-3.533513-3.559093$

H $2.089998-3.888787-1.716292$

O $2.193854-4.193005-3.724969$

C $1.961073-1.992227-2.777492$

C $3.468990-1.688395-2.859379$

H $1.533913-1.425415-1.946283$

O $1.345668-1.492151-3.967732$

H $3.932049-2.280882-3.657682$

H $3.574163-0.639372-3.166841$

H $-4.526753-3.3849960 .119575$

H -3.655948 -4.8731630 .519226$

H -3.786466 -3.575025 1.717155

C $-0.734725-3.3291042 .147651$

H $0.289814-5.436366-1.555959$

H $1.959840-5.131245-3.620597$

H $1.697187-2.004677-4.716125$

H $\quad 0.244627-3.7577992 .395393$

H -1.461048 -3.7589922 .847468$

H -1.157486 -4.845503 0.705690

C $4.157821-1.1385670 .759603$

O $3.647204-1.008273-0.572014$

C $4.207463-1.910080-1.531949$

C $5.716814-1.656990-1.660342$

C $6.413978-1.773047-0.300770$

C $5.686787-0.9743670 .789323$

H $3.728312-0.286821 \quad 1.300311$

C $3.627482-2.4201391 .408833$

H $4.042420-2.940512-1.200624$

O $5.959109-0.362238-2.208414$

H $\quad 6.142337-2.387517-2.357978$

H $7.444275-1.409966-0.398910$

O $6.473273-3.1494200 .061556$

H $6.066680-1.2374591 .782702$

O $5.961513 \quad 0.417690 \quad 0.593179$

C $3.647093-2.3313952 .914092$

H $2.595377-2.6174781 .092440$

H $4.193208-3.3067101 .107895$

H $5.5165850 .289790-1.638172$

H $6.942925-3.2094020 .910904$

H $6.9232350 .540057 \quad 0.668542$

O $2.641136-1.5183043 .346075$

O $4.463480-2.9097343 .619398$

C $2.558457-1.3368974 .762615$

H $2.310715-2.2852245 .248330$

H $3.499137-0.9361985 .152276$

H $1.761378-0.6175264 .967647$

SCF Energy (B3LYP/6-31G**//MMFF)= -3245.91832667

0700266

MM̄FF Geometry

C $4.294300-2.3218192 .094243$

C $2.980228-2.2806581 .824513$

C $1.930535-2.1232932 .894152$

O $0.999157-1.0782392 .534181$

C $1.123912-3.4287383 .037233$

C $0.012535-3.3478924 .069102$

C $-1.300140-3.5126973 .807998$

C $0.460283-3.0863035 .485564$

C -1.963294 -3.843085 2.499149

C $-2.777193-2.6642431 .958908$ 
C $-3.606823-3.0204780 .712951$ C $-2.727221-3.363284-0.494000$ C $-3.546846-3.573425-1.776053$ O $-4.428758-1.880400 \quad 0.419414$ O $-2.645701-3.541888-2.893143$ C $-4.518455-2.389926-1.950992$ C $-4.213131-4.954832-1.801119$ O $-5.397127-2.614167-3.051764$ C $-5.333030-2.073254-0.677611$ C $-6.139247-0.764411-0.876991$ O $-6.245135-3.149005-0.436156$ C 1.4023750 .2109842 .684996 O 2.5354840 .6151722 .887115 C 0.1887351 .0531862 .623885 C 0.2941102 .3812692 .472769 C -0.8269503 .3245142 .467142$ C -2.124286 2.8412643 .062742 C $-6.877618-0.2851050 .379896$ C $-6.1379180 .820358 \quad 1.105170$ C $-5.7931173 .287643 \quad 1.091437$ O C $-4.454271 \quad 3.573191 \quad 0.492614$ C -4.0644754 .7951250 .099133$ C $-2.6890555 .091032-0.425998$ C $-1.932047 \quad 6.0792950 .437074$ C $-1.5502597 .269149-0.056904$ C -1.6189505 .6977641 .870906$ C $-0.6323344 .563521 \quad 1.968196$ $\begin{array}{llll}\mathrm{N} & -6.329971 & 2.065612 & 0.524602\end{array}$ H $4.629346-2.2404083 .125531$ H $2.637281-2.3725820 .796923$ H $2.391995-1.8656073 .855775$ H $1.796222-4.2498063 .319100$ H $0.720181-3.6940942 .055246$ H -1.998349 -3.425896 4.640507 H $-0.356022-3.1990746 .207250$ H $1.246410-3.7927355 .771890$ H $\quad 0.849146-2.0684645 .586965$ H -1.238933 -4.1772501 .754161$ H -2.630605 -4.697059 2.671524 H -3.459885 -2.296090 2.737071 H -2.114043 -1.818577 1.731835 H $-4.258627-3.865074 \quad 0.966173$ H -2.112749 -4.249783 -0.300245 H -2.018436 -2.543782 -0.664971 H - $-1.975845-4.234041-2.758354$ H -3.920754 -1.504968 -2.208059 H -3.454199 -5.746127 - 1.772048 H $-4.769769-5.109193-2.732068$ H -4.890054 -5.120440 -0.960527 H $-4.844526-2.802515-3.829651$ H $-5.4668640 .027028-1.230764$ H $-6.891429-0.933243-1.658019$ H -7.064196 -2.804337 -0.048005 H $-0.767851 \quad 0.552770 \quad 2.729241$ H 1.2812622 .8183642 .329945 H -2.560704 2.0468612 .448341 H -2.878941 3.6230223 .163089 H -1.958507 2.4467084 .071948 H $-7.8622520 .106070 \quad 0.092802$ H -7.070084 -1.090746 1.095930 H -5.6920233 .1657012 .174919$ H -6.5147264 .0860160 .891905$ H -3.752114 2.7459710 .421053 H -4.756656 5.6301520 .176933 H $-2.7965805 .472860-1.449195$ H -2.100504 4.172328 -0.511458 H -1.0077797 .9864330 .549913$ H -1.769968 $7.553976-1.080538$ H $-2.554127 \quad 5.4954252 .398427$ H -1.177922 6.5522742 .401537 H 0.3546584 .8108251 .574258 H $-6.8580752 .136322-0.340243$ C $6.348873-1.3318861 .038813$ O $5.671356-0.137970 \quad 0.594084$ C $6.3414360 .359738-0.574531$

C $7.764156-0.164242-0.463534$ C $7.507825-1.5636210 .061347$
H $5.857925-0.110611-1.438490$ C $6.2021591 .878659-0.695715$ H $8.291501-0.154381-1.421766$ H 8.3531010 .4078050 .262498 C $8.725670-2.2086380 .700464$ H $7.175111-2.194134-0.774523$

C $4.7616162 .407979-0.542920$

O 6.9966872 .5024880 .320394

H $6.6287982 .199380-1.653770$

H 4.4874792 .4175470 .519232

O $4.7640453 .781727-0.953522$

C $3.6833871 .641268-1.328719$

C $2.3173182 .348835-1.277266$

H $3.5938760 .630350-0.916489$

O $4.0977891 .521170-2.689141$

H $2.1740892 .814452-0.294811$

H $2.3034643 .139740-2.037323$

H $9.534694-2.311642-0.030216$

H $9.102872-1.6117891 .537693$

H $8.482575-3.2061281 .080028$

C $5.361696-2.5024041 .053495$

H 6.9000143 .4645470 .214360

H $4.9292933 .800094-1.911907$

H $3.4193081 .004268-3.156295$

H $5.894434-3.4352721 .271172$

H $4.901170-2.6108130 .064315$

H $6.722630-1.1266002 .049651$

C $0.322328-0.236449-3.126323$

O $1.307495 \quad 0.754022-2.796064$

C $1.1445551 .379663-1.521003$

C $-0.2077602 .100655-1.455713$

C $-1.3618151 .146066-1.765523$

C $-1.1016340 .344314-3.044909$

H $0.518841-0.481701-4.178387$

C $0.504179-1.517498-2.302494$

H $1.1870800 .617097-0.735912$

O $-0.2337883 .194853-2.370253$

H $-0.3370192 .518140-0.453673$

H -2.285610 $1.727755-1.872434$

O $-1.5270020 .261645-0.661633$

H $-1.832480-0.465318-3.150442$

O $-1.2734141 .207521-4.173365$

C $1.658127-2.335780-2.828904$

H $\quad 0.661561-1.348544-1.234060$

H $-0.383317-2.152143-2.390390$

H $-0.0380632 .846547-3.257003$

H $-2.331769-0.258613-0.824317$

H $-2.1882721 .536484-4.153904$

O $2.842760-1.819358-2.396264$

O $1.518486-3.314918-3.551347$

C $4.009051-2.504584-2.862238$

H $4.041655-2.501317-3.956047$

H $4.888888-1.977779-2.486968$

H $4.023542-3.528624-2.477288$

SCF Energy (B3LYP/6-31G**//MMFF) $=-3245.91224476$

0700267

MM̄FF Geometry

C $3.426914-4.156422-0.341714$

C $2.362632-3.594307-0.934806$

C $0.946259-3.773601-0.452323$

O $0.338803-2.471787-0.284808$

C $0.124510-4.544872-1.500658$

C $-1.341631-4.694274-1.137580$

C $-2.363580-4.246106-1.893444$

C $-1.630085-5.4150790 .154507$

C $-2.293644-3.571687-3.234031$

C $-2.439157-2.043476-3.191942$

C $-3.814667-1.574799-2.690953$

C $-4.100308-0.143095-3.149035$

C $-5.4481260 .364265-2.616657$

O $-3.817124-1.624718-1.259325$

O $-5.5004941 .783038-2.829469$

C $-5.4956550 .132313-1.094157$

C $-6.633372-0.234248-3.382857$

O $-6.7841010 .445214-0.569299$

C $-5.079696-1.295973-0.664666$

C $-4.924491-1.3370190 .872272$ 
O $-6.096969-2.221835-1.051195$

C $0.565684-1.8299640 .892095$

O $1.302905-2.1910991 .795666$

C $-0.265445-0.6050550 .902535$

C $-0.127508 \quad 0.265107 \quad 1.913511$

C -0.8821701 .5036122 .109528$

C -1.9141131 .8701421 .077601$

C $-4.237529-2.5935871 .418991$

C -4.173306 -2.516738 2.931980

C $-3.044754-1.2582894 .771866$

O $-4.958408-3.1396263 .642671$

C $-3.351953 \quad 0.204160 \quad 4.877575$

C -2.5616861 .0868285 .507285$

C -2.85079225603975 .579567$

C -1.671449 3.4137985 .158504

C $-0.9868804 .136566 \quad 6.061299$

C -1.325682 3.4851633 .683057

C $-0.621504 \quad 2.2422913 .208294$

N -3.189302 -1.6502463 .381341$

H $3.281677-4.7859090 .532857$

H $2.509596-2.966530-1.810690$

H $0.923872-4.3155260 .501670$

H $0.550601-5.547286-1.639281$

H $\quad 0.231657-4.033275-2.462509$

H -3.377631 -4.404716 -1.527697

H $-2.697040-5.6278550 .281917$

H -1.101608 -6.3737570 .180460$

H -1.311730 -4.814833 1.012323

H -1.364482 $-3.822281-3.755368$

H -3.088376 -3.992346 -3.863802

H -1.643706 -1.619583 -2.565955

H -2.281194 -1.678280 -4.214452

H $-4.580654-2.254179-3.082847$

H $-4.070715-0.068027-4.243264$

H $-3.3091590 .522700-2.778815$

H $-5.399025 \quad 1.946772-3.782905$

H $-4.7950980 .846005-0.637732$

H $-6.5629390 .011109-4.449333$

H -7.581783 $0.194106-3.039629$

H -6.695530 -1.321120 -3.297354

H $-6.997551 \quad 1.352537-0.847069$

H -4.336056 -0.4704161 .194361$

H $-5.910225-1.2412121 .346122$

H $-6.052941-2.998715-0.471538$

H $-0.970470-0.461306 \quad 0.091775$

H $\quad 0.6053690 .0436502 .688942$

H -2.741023 1.1554281 .094034

H -1.4760391 .8735340 .073946$

H -2.3380482 .8662481 .222347$

H -4.781308 -3.5035341 .145734$

H $-3.219540-2.6927091 .026012$

H -2.016107 -1.486053 5.068413

H -3.732378 -1.832289 5.399810

H -4.2665920 .5551074 .404503$

H -1.659330 $0.733593 \quad 6.001319$

H -3.134092 2.791909 6.614395

H -3.718604 2.8262324 .963237

H -0.1548354 .7675545 .766714$

H -1.239411 4.110163 7.116068

H $-0.661246 \quad 4.3374853 .491806$

H -2.2468943 .6879223 .129686$

H 0.1801341 .9123413 .871201

H -2.584367 -1.198582 2.702005

C $5.697758-3.261104 \quad 0.231197$

O $5.204290-1.920248 \quad 0.418481$

C $6.306561-0.9991520 .345770$

C $7.558877-1.8428050 .536661$

C $7.162930-3.116778-0.185913$

H $6.308280-0.591992-0.672762$

C $6.1130630 .140606 \quad 1.352090$

H $8.449964-1.3677090 .115847$

H 7.751775 -2.054589 1.594562

C $8.025322-4.3143260 .172708$

H $7.230288-2.940363-1.267959$

C $4.721459 \quad 0.803777 \quad 1.295017$

O $6.284248-0.3663662 .682515$

H $\quad 6.898871 \quad 0.8921101 .216783$

H 3.9684960 .1085651 .688020
O $4.728467 \quad 1.9280552 .183037$

C $4.2816361 .283971-0.100867$

C $2.8665281 .874732-0.069034$

H $4.2924690 .439739-0.795933$

O $5.1791882 .272714-0.596552$

H 2.1767391 .1404820 .360536

H 2.8372412 .7373370 .610493

H $9.070117-4.130763-0.098426$

H $7.989381-4.5277381 .246313$

H $7.689935-5.209718-0.360150$

C $4.841510-3.973320-0.817953$

H $5.638997-1.0832922 .809043$

H 4.9950261 .6028293 .060146

H $6.0468021 .853015-0.719455$

H $5.260618-4.961416-1.038845$

H $4.848736-3.398384-1.752545$

H $5.618592-3.7672811 .201890$

C $0.2781193 .357329-2.286922$

O $0.9776222 .785254-1.175399$

C $2.3224802 .353336-1.427873$

C $2.3406781 .281349-2.529461$

C $1.5966161 .743236-3.785976$

C $0.2371112 .372696-3.468142$

H $-0.7522073 .493588-1.933020$

C $0.8296084 .741279-2.661281$

H $2.9180343 .216367-1.744031$

O $1.7715410 .061744-2.059981$

H $3.3800831 .061442-2.797892$

H $1.456406 \quad 0.884174-4.453631$

O $2.4159352 .693251-4.463960$

H $-0.1539402 .885662-4.354112$

O $-0.6927351 .326243-3.174746$

C $0.6243685 .756402-1.555145$

H $1.9002664 .707287-2.877998$

H $\quad 0.3024095 .114307-3.547039$

H $0.9496810 .272553-1.588837$

H $1.9571832 .944482-5.283711$

H $-0.6660371 .147061-2.220490$

O $1.1488556 .953750-1.953386$

O $0.0766945 .536448-0.483748$

C $1.0335068 .020637-1.006401$

H $1.5665627 .768250-0.084758$

H $1.4898818 .913170-1.442512$

H $-0.0201288 .230726-0.799059$

SCF Energy $\left(B 3 L Y P / 6-31 G^{* *} / / M M F F\right)=-3245.90724080$

07_00268

MM̄FF Geometry

C -1.916429 -3.561300 -1.715987

C -1.843024 -2.303109-2.179612

C $-0.626907-1.648232-2.798382$

O $0.519179-2.518308-2.642110$

C $-0.816594-1.338938-4.295339$

C $-1.965202-0.407906-4.616347$

C $-1.9427950 .925346-4.424990$

C $-3.181988-1.066732-5.213113$

C $-0.8188301 .761819-3.876230$

C $-1.2112502 .390513-2.532335$

C $-0.1980173 .403077-1.971311$

C $-0.1438694 .691382-2.796162$

C $0.8916575 .679941-2.241005$

O $1.0924972 .791124-1.911668$

O $1.1038226 .698870-3.230381$

C $2.2323734 .939838-2.060750$

C $0.3876936 .396427-0.983050$

O $3.1918585 .779757-1.421579$

C $2.1076533 .600398-1.295396$

C $3.4486642 .828934-1.387126$

O 1.8069943 .8717590 .072401

C $1.750557-1.947621-2.587316$

O $2.029908-0.776355-2.781028$

C $2.704316-3.004957-2.183482$

C $3.880253-2.655688-1.641760$

C $4.893786-3.567401-1.108070$

C $4.657780-5.046705-1.263953$

C $3.431503 \quad 1.431601-0.755144$

C $3.834774 \quad 1.3861920 .708954$

C $4.082973-0.2602822 .574167$ 
O $4.2630962 .357504 \quad 1.324476$

C 5.411101-0.942577 2.476894

C $5.599723-2.2466462 .728783$

C $6.928720-2.9358462 .603580$

C $6.897691-4.0842501 .617476$

C $6.754299-5.3504232 .044142$

C $7.120122-3.7791610 .151517$

C $5.969375-3.044132-0.482780$

N $3.671162 \quad 0.1119631 .234421$

H -1.040292 -4.200971 -1.799349

H -2.711472 -1.656099 -2.089660

H $-0.440037-0.726442-2.233764$

H $-0.937574-2.282635-4.845829$

H $\quad 0.110756-0.920738-4.707742$

H $-2.8275461 .496916-4.703828$

H -3.983870 -0.350251 -5.421253

H -2.921512 -1.554442 -6.158267

H -3.585694 -1.822753 -4.532873

H $\quad 0.1006391 .180725-3.760881$

H -0.603202 2.539846 -4.615962

H -2.193252 $2.874399-2.610013$

H -1.319509 $1.577972-1.801213$

H $-0.5223033 .635889-0.951136$

H -1.133771 $5.161096-2.849756$

H $0.1361124 .463165-3.832412$

H $0.2481267 .124883-3.409960$

H $2.6268384 .727751-3.064571$

H $-0.5306026 .954333-1.202651$

H $1.1131287 .139828-0.634570$

H $0.1691825 .715409-0.158526$

H $3.2491476 .600623-1.940083$

H $3.6816352 .688508-2.451207$

H $4.2596103 .424035-0.951880$

H 2.0327973 .0919850 .602214

H $2.385649-4.036314-2.283617$

H $4.113184-1.596852-1.548014$

H $3.742032-5.347293-0.742790$

H $5.465002-5.660098-0.857698$

H $4.563522-5.311259-2.322946$

H $2.4501930 .961042-0.867656$

H $4.1625490 .811877-1.289338$

H $3.308650-0.9167182 .983369$

H 4.1596940 .6279863 .208492

H $6.256960-0.3306182 .172093$

H $4.754532-2.8560203 .040884$

H $7.209141-3.3032253 .599462$

H $7.720640-2.2327772 .316520$

H $6.761768-6.1857091 .352020$

H $6.622664-5.5800623 .096464$

H $7.347274-4.692288-0.411044$

H $8.024502-3.1651000 .049488$

H $6.051556-1.959400-0.408107$

H $3.342484-0.6320810 .625632$

C $-4.395057-3.440060-0.945672$

O $-4.240462-2.370280 \quad 0.009113$

C $-5.460389-2.2394990 .764289$

C $-6.465964-3.194706 \quad 0.128552$

C $-5.561913-4.276830-0.422164$

H $-5.239726-2.5835901 .780454$

C $-5.931806-0.7812770 .778948$

H -7.193880 -3.5701630 .854568$

H -7.024664 -2.714258 -0.683443

C $-6.232462-5.142936-1.474872$

H $-5.226790-4.9114210 .409383$

C $-4.961614 \quad 0.213597 \quad 1.448469$

O $-6.134909-0.349467-0.574599$

H -6.914314 -0.718029 1.260958

H $-4.047031 \quad 0.2854650 .849726$

O -5.5758731 .5089761 .381557$

C $-4.601069-0.0917582 .919242$

C -3.9198671 .0768013 .659854$

H -3.961786 -0.980056 2.974207

O $-5.793496-0.375167 \quad 3.659412$

H -4.6009221 .9373113 .680137$

H -3.7981410 .7757974 .709797$

H -7.108566 -5.648406 -1.055388

H -6.566232 -4.547392 -2.331253

H -5.547268 $-5.911274-1.845895$
C $-3.093216-4.237631-1.061160$

H -5.284685 -0.434407 -1.039620

H $-6.378716 \quad 1.481571 \quad 1.930042$

H $-6.119402-1.2468983 .380697$

H -3.284325 -5.156584 -1.627813

H -2.768163 -4.538542 -0.056801

H -4.659148 -2.985844 -1.909575

C -0.2802090 .6664432 .929118$

O $-1.616516 \quad 0.465930 \quad 3.413913$

C -2.5586841 .4918693 .085125$

C -2.0946272 .8406663 .655211$

C -0.6914723 .1896513 .156782$

C 0.2872212 .0298703 .357819

H $0.313880-0.1051693 .436992$

C $-0.1969110 .404577 \quad 1.421151$

H $-2.639697 \quad 1.5797841 .998582$

O -2.1094802 .8151295 .080405$

H -2.7992763 .6193913 .339983$

H -0.3328814 .0766643 .692852$

O -0.7756013 .5153621 .773705$

H 1.2273072 .2171182 .826413

O $\quad 0.6100221 .9358354 .749172$

C $-0.305239-1.0821241 .178893$

H -0.9873560 .8910560 .843741$

H $\quad 0.753738 \quad 0.755628 \quad 1.006619$

H -1.550435 2.0742525 .370794

H 0.1090163 .7976251 .486029

H 1.0207612 .7772665 .011933

O $0.937527-1.6429901 .169160$

O $-1.369051-1.6781271 .076291$

C $0.972733-3.0711001 .098314$

H $\quad 0.432622-3.5065881 .944425$

H $\quad 0.547331-3.4153340 .152865$

H $2.017781-3.3879551 .151058$

SCF Energy $\left(B 3 L Y P / 6-31 G^{* *} / / M M F F\right)=-3245.91344254$

0700269

MM̄FF Geometry

C $0.271895-2.212865-0.081291$

C $0.809338-1.8995231 .109153$

C $0.512295-0.6555331 .909421$

$\begin{array}{lllll}\text { O } & 0.115587 & 0.393719 & 0.995047\end{array}$

C $-0.622233-0.8662912 .926670$

C $-0.388934-1.9609193 .945959$

C -1.034929-3.144562 3.934472

C $0.590347-1.6302065 .039329$

C -1.993161 -3.616382 2.876460

C $-3.443477-3.211883 \quad 3.167298$

C $-4.342089-3.2050461 .918280$

C $-4.437058-4.5757451 .245408$

C $-5.277208-4.521086-0.040967$

O $-3.809415-2.2375051 .005414$

O $-5.083516-5.754936-0.750028$

C $-4.760357-3.365258-0.927074$

C $-6.776653-4.4685240 .269738$

O $-5.586654-3.200077-2.077600$

C $-4.596741-2.022924-0.171804$

C $-3.847704-1.008014-1.076020$

O $-5.891684-1.5177880 .148025$

C $0.307906 \quad 1.683613 \quad 1.372946$

O 0.7965652 .0853522 .415539

C -0.2080452 .5304590 .275098$

C $-0.366328 \quad 3.8485640 .459354$

C $-0.8886574 .796327-0.528139$

C $-1.2692674 .255542-1.882405$

C $-3.4249980 .303510-0.395596$

C $-4.5121051 .362666-0.329602$

C -4.9965693 .5850560 .696703$

O $-5.5866691 .259398-0.914951$

C $-4.6554284 .682971-0.261428$

C -4.3832165 .9401280 .121223$

C $-4.0879447 .060869-0.834946$

C $-2.8164527 .806460-0.493520$

C $-2.857361 \quad 8.9727740 .172892$

C $-1.5074377 .245483-1.002279$

C $-1.0149436 .091884-0.172521$

N -4.132465 2.4444690 .453843

H $-0.474102-1.557342-0.520809$ 
H $\quad 1.535625-2.577873 \quad 1.551484$ H $1.439168-0.3512392 .411795$ H -1.561140 -1.023631 2.382199 H -0.8011220 .0729603 .467510$ H $-0.837764-3.8583064 .731889$ H $0.679525-2.4373025 .774249$ H $1.586208-1.4540194 .620820$ H $0.273109-0.7294615 .574684$ H -1.674878 -3.249776 1.896532 H -1.921823 -4.709310 2.812844 H -3.865522 -3.8820123 .926097$ H -3.458319 -2.199538 3.591943 H $-5.336171-2.8723762 .239909$ H $-4.839878-5.3236501 .939356$ H -3.432704 -4.927939 0.975930 H $-4.137881-5.841387-0.958998$ H $-3.774784-3.667598-1.306757$ H $-7.075981-5.3488330 .851485$ H -7.372766 -4.503464 -0.648950 H -7.068344 -3.5827780 .837539$ H $-6.396123-2.740170-1.796872$ H -2.921304 -1.485481-1.421581 H $-4.448907-0.783041-1.964859$ H $-5.810516-0.5809270 .383895$ H $-0.4593952 .037735-0.657391$ H -0.1002424 .2742041 .425990$ H -0.387349 $3.861074-2.398658$ H -1.719043 $5.000881-2.541177$ H -2.007295 $3.451363-1.785509$ H $-3.036427 \quad 0.1167480 .610744$ H $-2.6101010 .741226-0.985863$ H -4.8504803 .8858241 .738982$ H -6.0417743 .2888660 .561852$ H -4.660772 $4.438836-1.321055$ H $-4.408813 \quad 6.189073 \quad 1.179845$ H $-4.941247 \quad 7.751536-0.808935$ H $-4.0314206 .700584-1.869658$ H -1.9527999 .5285230 .396314$ H -3.796470 9.3949810 .515003 H $-0.7296138 .020639-0.973566$ H -1.609663 $6.998023-2.062288$ H $-0.717296 \quad 6.3833930 .835908$ H -3.196203 2.4687070 .846873 C $1.185849-3.106189-2.273220$ O $2.139239-2.023205-2.206254$ C $1.722348-1.004535-3.133203$ C $0.904242-1.742578-4.180293$ C $0.118319-2.690703-3.291738$ H $1.050767-0.330213-2.588409$ C $2.925677-0.219508-3.659449$ H $0.265875-1.073101-4.764164$ H $1.543854-2.305568-4.870085$ C $-0.501669-3.857824-4.040405$ H $-0.684792-2.126485-2.801312$ C $3.7688670 .453232-2.553061$ O $3.783281-1.101233-4.392534$ H $2.5883040 .534567-4.379704$ H $4.309070-0.314046-1.987742$ O $4.7650461 .263649-3.186681$ C $2.9638621 .340910-1.587232$ C $3.8036572 .133156-0.573215$ H $2.2463400 .729436-1.030198$ O $2.2133582 .312162-2.323461$ H $4.3821922 .911957-1.087384$ H 3.0888632 .6806220 .055238 H - $-1.217713-3.498807-4.786859$ H $\quad 0.257838-4.450268-4.561578$ H -1.034408 -4.521628 -3.351986 C $0.657276-3.434157-0.871069$ H $4.050706-1.817081-3.791357$ H $5.2636550 .688971-3.792556$ H $1.5140401 .847760-2.811643$ H $1.439193-3.981816-0.330620$ H $-0.203931-4.108229-0.941389$ H $1.737118-3.978056-2.647997$ C $4.759473-0.7050311 .687276$ $\begin{array}{lllll}\text { O } & 4.008797 & 0.195391 & 0.858272\end{array}$ C $4.742523 \quad 1.2956550 .312407$
C 5.3611462 .1490601 .434900

C 6.1844181 .2994242 .407282

C 5.4209120 .0498302 .854663

H $4.009868-1.3825672 .114415$

C $5.760693-1.547490 \quad 0.878076$

H $5.5426970 .909294-0.326637$

O 4.3416142 .8304272 .166273

H 5.9963432 .9196510 .982827

H 6.4373551 .8981553 .290998

O $7.4028340 .901516 \quad 1.786645$

H $6.091239-0.6228853 .402376$

O 4.3990890 .4585393 .768693

C $5.077539-2.565775-0.008416$

H $6.389688-0.9202840 .241530$

H $6.410948-2.1120211 .556240$

H 3.7209682 .1622792 .504682

H 7.8862151 .7096071 .543965

H $3.957420-0.3441444 .094859$

O $5.935845-2.944680-1.000827$

O $3.951505-3.0078440 .168705$

C $5.443184-3.950959-1.891183$

H $4.515365-3.622256-2.367010$

H $6.194937-4.115538-2.667628$

H $5.286623-4.887780-1.348329$

SCF Energy (B3LYP/6-31G**//MMFF) $=-3245.91585675$

0700270

MM̄FF Geometry

C -0.603663 -2.512505 0.271803

C $-1.474597-2.6128921 .288118$

C $-1.890747-1.5062242 .228822$

O $-1.216980-0.2735671 .873174$

C $-3.404937-1.2336052 .138247$

C -4.298701-2.281699 2.760354

C $-5.048189-3.1594772 .064798$

C $-4.368390-2.2511084 .265031$

C $-5.083271-3.3740800 .576399$

C $-6.368843-2.859313-0.086757$

C $-6.408192-1.331602-0.255447$

C $-7.711694-0.896297-0.930471$

C $-7.7303620 .610276-1.235220$

O $-5.294636-0.954180-1.070935$

O $-8.811504 \quad 0.863777-2.145537$

C $-6.4256800 .992273-1.968059$

C -8.0184491 .4406360 .021293$

O $-6.3301772 .404317-2.140200$

C $-5.1545340 .457586-1.274282$

C $-3.910630 \quad 0.685757-2.168734$

O $-4.9688441 .158170-0.041097$

C -0.9504570 .6124492 .869120$

O -1.1342350 .4524614 .065051$

C -0.4227801 .8439602 .241303$

C $-0.0099942 .856503 \quad 3.017087$

C 0.4894354 .1307332 .513263

C 1.8036034 .5416293 .115122

C $-2.678208-0.096139-1.696904$

C -1.424033 $0.298043-2.447407$

C $0.3437072 .039849-2.502364$

O $-0.918174-0.433639-3.294588$

C $0.3195273 .532235-2.449994$

C $0.8622704 .246008-1.452744$

C $0.8531095 .744569-1.392488$

C $-0.1806626 .283724-0.429955$

C $-1.2960656 .881593-0.882465$

C 0.1121526 .2060931 .054144

C -0.2369374 .8643021 .645829$

N $-0.9423811 .538314-2.059877$

H $-0.113592-1.5677500 .060353$

H -1.939591 -3.580570 1.466376

H -1.600445 -1.803634 3.243399

H -3.673113 -1.064909 1.091336

H -3.631456 -0.271024 2.617525

H -5.689553 -3.839796 2.624110

H $-5.071711-2.9896174 .664290$

H $-3.386863-2.4641474 .699439$

H -4.695409 -1.264861 4.610573

H $-4.200687-2.960616 \quad 0.079919$

H $-5.023990-4.4577910 .410744$ 
H $-6.427160-3.322384-1.080733$ H -7.245789 -3.1978910 .478504$ H $-6.314445-0.8624680 .730183$ H -8.578277 -1.176252 -0.318502 H -7.828437 -1.439119-1.879131 H $-9.6340320 .557504-1.726496$ H $-6.4772320 .559412-2.976907$ H -8.9966891 .1745250 .439603$ H $-8.0751942 .508834-0.215042$ H $-7.275427 \quad 1.2993130 .808954$ H $-7.1429222 .696986-2.586907$ H -4.1273620 .361147 -3.194697 H -3.679995 $1.757230-2.212824$ H -4.0213381 .2184220 .157166$ H $-0.400368 \quad 1.9029791 .158141$ H $-0.0160902 .759477 \quad 4.100534$ H 2.1509965 .5206302 .779112 H 2.5810333 .8155002 .858545 H 1.7279844 .5891514 .207376 H -2.489129 $0.061687-0.631194$ H $-2.829818-1.173078-1.835076$ H $0.5536961 .693695-3.519443$ H $1.1060711 .615089-1.840171$ H $-0.1669114 .046910-3.274913$ H $1.3418363 .724006-0.629725$ H $0.6875726 .156082-2.397126$ H $1.8494506 .097001-1.098370$ H $-2.0315847 .295256-0.200468$ H -1.501701 $6.965258-1.944510$ H $-0.478498 \quad 6.9627391 .587066$ H $\quad 1.1593546 .4786841 .213024$ H -1.210321 4.4789041 .339145 H $-1.3557611 .973446-1.239481$ C $0.983731-4.391397-0.302318$ O $2.143098-3.551190-0.489552$ C $3.143889-4.298127-1.205155$ C $2.704012-5.756856-1.150161$ C $1.199314-5.617813-1.192441$ H $3.101734-3.956715-2.245874$ C $4.546826-4.066177-0.633465$ H $3.106404-6.343173-1.981910$ H $3.011959-6.240906-0.216074$ C $0.462519-6.861249-0.723528$ H $0.898304-5.391576-2.224387$ C $5.035838-2.605726-0.592140$ O $4.598451-4.564790 \quad 0.710920$ H $5.264724-4.672071-1.199773$ H $4.544353-2.0688650 .226605$ O $6.432976-2.643583-0.264674$ C $4.867456-1.811179-1.898551$ C $5.679108-0.503044-1.984907$ H $3.805044-1.584199-2.042626$ O $5.265231-2.619771-3.010951$ H $6.742849-0.739698-2.119273$ H $5.372802-0.002908-2.913629$ H $0.709586-7.717219-1.360159$ H $0.729042-7.1208010 .306657$ H $-0.620971-6.713168-0.763692$ C $-0.297057-3.640421-0.669738$ H $3.910366-4.1031331 .220387$ H $6.519943-3.1237360 .576774$ H $6.192292-2.878393-2.870179$ H -1.144403 -4.336212 -0.694860 H $-0.208488-3.214464-1.677211$ H $0.970026-4.6797220 .756751$ C 3.8108951 .6680210 .420885 O $4.138704 \quad 0.816126-0.687937$ C $5.517350 \quad 0.456733-0.798452$ C $6.3874151 .713537-0.965638$ C 6.1420392 .7143000 .167662 C 4.6470342 .9602080 .392590 H 2.7623921 .9472950 .258786 C 3.8913750 .9199201 .762039 H $5.829756-0.0652540 .110455$ O $\quad 6.1212422 .343038-2.217749$ H $7.4423321 .415699-0.975485$ H $6.6321133 .665429-0.073940$ O 6.7152522 .2245101 .376034
H $4.4862953 .519637 \quad 1.321019$

O $4.1488683 .769661-0.676095$

C $2.878992-0.2013021 .848137$

H 4.8788180 .4795061 .921483

H 3.6792061 .6131542 .583204

H $5.1693582 .539054-2.255764$

H 7.6674572 .1024321 .221231

H $4.6299314 .614423-0.653332$

O $3.069315-0.8844343 .015172$

O $2.029444-0.4485711 .004554$

C $2.191509-1.9941033 .229970$

H $2.236410-2.6901062 .387117$

H $2.519843-2.5172244 .131862$

H $1.169619-1.6375423 .383175$

SCF Energy (B3LYP/6-31G**//MMFF)= -3245.90769076

0700271

MM̄FF Geometry

C -0.637660 $4.594326-0.402774$

C 0.4112445 .0742870 .282498

C 1.5742074 .2679060 .821077

$\begin{array}{lllll}\text { O } & 1.430128 & 2.857172 & 0.537748\end{array}$

C 2.8864304 .7513440 .174934

C 4.1198703 .9834240 .615222

C $5.0022013 .424770-0.236812$

C 4.3499263 .8825452 .100936

C $4.9930083 .501104-1.736843$

C $4.3114722 .318289-2.437880$

C $5.0447370 .982693-2.239538$

C $4.613446-0.030118-3.302993$

C $5.290287-1.392773-3.096815$

O $4.7245820 .474430-0.939177$

O $4.614374-2.351045-3.925121$

C $5.082903-1.831724-1.634495$

C $6.754764-1.382756-3.550249$

O $5.820667-3.017792-1.346906$

C $5.419239-0.732212-0.597179$

C $4.948782-1.1941050 .800750$

O $6.833598-0.530451-0.562432$

C 0.7362242 .1125451 .441720

O 0.1861482 .5146432 .455041

C 0.7499200 .7066350 .978239

C $-0.049935-0.1809801 .587713$

C $-0.160571-1.6115691 .296068$

C $0.710318-2.1819910 .210486$

C $4.911862-0.0916451 .865463$

C $4.600128-0.6858323 .226555$

C $2.788536-1.8558974 .488135$

O $5.449384-0.744814 \quad 4.112828$

C $2.111531-3.1100054 .028694$

C $0.873095-3.4696194 .399707$

C $0.191968-4.7222823 .922828$

C $-1.183976-4.4627883 .345110$

C $-2.292228-4.8406634 .004493$

C $-1.274558-3.8276111 .971795$

C $-1.031894-2.3438062 .021542$

N $3.301896-1.1600293 .321757$

H $-0.6994643 .533444-0.629513$

H 0.4431556 .1407530 .498057

H 1.6257284 .4270741 .905650

H 3.0428025 .8126990 .409236

H $2.7671134 .688659-0.912344$

H 5.8503042 .8832530 .180388

H 5.3305443 .4573362 .340699

H 4.3047704 .8757532 .559526

H 3.5941243 .2445452 .568902

H $4.5229564 .432165-2.072601$

H $6.0333503 .575231-2.079100$

H $3.2750572 .234788-2.086627$

H $4.2760582 .556043-3.508425$

H $6.1239881 .163174-2.306750$

H $4.8219950 .348657-4.311400$

H $3.524281-0.169795-3.253484$

H $4.677522-2.047384-4.846941$

H $4.020965-2.094577-1.524511$

H $6.825098-1.115260-4.611395$

H $7.201636-2.379277-3.463128$

H $7.372683-0.677821-2.990350$ 
H $5.567003-3.684282-2.008265$ H $3.936298-1.6031630 .715717$ H $5.591365-2.0105121 .155647$ H $7.082352-0.1879670 .310781$ H 1.4153960 .4443710 .164136 H -0.6907910 .1728882 .395020$ H $1.754453-2.2026980 .533317$ H $0.639457-1.585552-0.704843$ H $0.434634-3.199117-0.076490$ H 5.8719410 .4297801 .935837 H 4.1504240 .6596371 .628089 H $2.098384-1.1746274 .995786$ H $3.607947-2.1009085 .170476$ H $2.671244-3.7561833 .355864$ H $0.320875-2.8374315 .091431$ H $0.119243-5.4058214 .778650$ H $0.799154-5.244706 \quad 3.172997$ H $-3.280741-4.6769133 .588517$ H $-2.236790-5.3166604 .977930$ H -2.275393 -3.9834371 .549240$ H $-0.585093-4.3525881 .305205$ H $-1.646649-1.8279622 .761080$ H $2.652170-0.9640172 .566325$ C $-3.0300125 .262349-0.046432$ O $-3.4125053 .876695-0.000802$ C $-4.8468073 .778336-0.069268$ C $-5.3796415 .203897 \quad 0.034216$ C $-4.2507256 .002123-0.590750$ H -5.082352 3.383547-1.064855 C -5.3590712 .8070201 .002706$ H $-6.3333585 .327009-0.488047$ H -5.5280595 .5128691 .075027$ C -4.286039 $7.480181-0.244427$ H $-4.3047405 .886184-1.681678$ C -4.6057611 .4626691 .057543$ O -5.2229843 .4119852 .296373$ H -6.4326292 .6362250 .863779$ H -3.589915 1.6211951 .440940 $\begin{array}{llll}\text { O } & -5.268015 & 0.624713 & 2.013366\end{array}$ C $-4.5304500 .707949-0.281350$ C $-3.747948-0.604898-0.146442$ H $-4.0324781 .340661-1.020947$ O $-5.843586 \quad 0.440675-0.764504$ H $-2.748559-0.3886160 .246981$ H $-4.225070-1.2500020 .603823$ H -5.216228 $7.935989-0.598792$ H $-4.223743 \quad 7.6406170 .837166$ H -3.450895 $8.010876-0.712218$ C -1.777347 $5.434643-0.907640$ H $-4.283543 \quad 3.6325062 .420289$ H -5.2969751 .1123142 .854526$ H -6.298257 -0.098616 -0.094739 H - $-1.4907406 .491958-0.944727$ H -1.982235 5.121738 -1.939018 H -2.820591 5.5682230 .987205 C $-2.597098-3.531343-2.124668$ O $-2.807918-2.562556-1.091129$ C $-3.624333-1.434298-1.436730$ C $-3.004614-0.672883-2.617649$ C $-2.732807-1.602049-3.804869$ C $-1.997610-2.876844-3.379310$ H -1.843712 -4.216074 -1.713114 C $-3.864232-4.345664-2.405538$ H -4.623934 -1.789567-1.708842 O $-1.789469-0.042987-2.216420$ H -3.689287 $0.120726-2.936628$ H -2.134530 -1.061607-4.548627 O $-3.977755-1.940714-4.409406$ H -1.980829 -3.606151-4.197530 O $-0.636700-2.550870-3.083639$ C $-4.189005-5.239297-1.232104$ H $-4.742731-3.732230-2.624401$ H -3.718594 -4.998539-3.274489 H $-1.200756-0.732481-1.865716$ H -3.786979 -2.505108 -5.177928 H $-0.241305-2.180989-3.891360$ O $-4.923909-4.553012-0.311201$ O $-3.815232-6.401376-1.138418$
C $-5.273993-5.2847170 .867337$

H $-4.379979-5.6968251 .344790$

H -5.755726 -4.5946541 .565227$ H $-5.979230-6.0821520 .615632$ SCF Energy (B3LYP/6-31G**//MMFF)= -3245.91617269

0700272

MM̄FF Geometry

C $-0.289881-1.8602841 .818542$

C -1.270731 -1.3158502 .552892$

C $-1.508194 \quad 0.167967 \quad 2.613019$

$\begin{array}{lllll}\text { O } & -2.928182 & 0.369324 & 2.419433\end{array}$

C $-1.0868520 .776124 \quad 3.962514$

C $0.403390 \quad 0.743151 \quad 4.237724$

C $1.300897 \quad 1.5575303 .646580$

C $0.847793-0.2502865 .277685$

C 1.0169672 .5862862 .587119

C 1.9502812 .4224541 .379895

C $1.4289363 .023766 \quad 0.061829$

C 0.7647494 .3960140 .217870

C $0.1683554 .914570-1.100729$

O $0.5116632 .080569-0.513072$

O $-0.706276 \quad 6.001336-0.757120$

C $-0.6881773 .795320-1.739298$

C $1.2517665 .504006-2.011827$

O $-1.1427324 .176480-3.035503$

C $0.0530782 .444045-1.824000$

C $-0.8348401 .302815-2.369963$

O $1.1469942 .579446-2.741732$

C -3.3395981 .5672011 .929268$

O -2.6354932 .5356351 .677624$

C $-4.794658 \quad 1.574673 \quad 1.661013$

C -5.6195330 .5748992 .005371$

$\begin{array}{lll}\text { C }-7.041126 & 0.522326 & 1.684062\end{array}$

C $-7.9068840 .038092 \quad 2.813350$

C $-2.0211410 .896374-1.495653$

C $-2.770575-0.286004-2.092228$

C $-4.585143-1.913717-1.560329$

O $-2.557390-0.711783-3.224801$

C $-5.922560-1.377672-1.965399$

C $-7.081207-1.790260-1.428489$

C $-8.425556-1.231823-1.807294$

C $-9.198998-0.714965-0.611015$

C $-10.182587-1.447452-0.061641$

C $-8.882795 \quad 0.672088-0.090124$

$\begin{array}{llll}C & -7.480004 & 0.793520 & 0.438910\end{array}$

$\mathrm{N}-3.715923-0.801446-1.216974$

H $0.372669-1.203648 \quad 1.258931$

H $-1.923375-1.9558203 .140979$

H -0.9773010 .6479851 .783781$

H -1.6301850 .2747334 .775654$

H -1.4340351 .8159924 .025464$

H $2.345865 \quad 1.479503 \quad 3.943016$

H $1.931226-0.2301235 .436525$

H $0.368096-0.0333136 .237695$

H $0.578622-1.2674414 .976597$

$\begin{array}{llll}\text { H } & -0.023453 & 2.539381 & 2.264842\end{array}$

H 1.1650963 .5813273 .023814

H 2.9107972 .8924721 .626429

H $2.147647 \quad 1.3556351 .213552$

H $2.2951383 .100983-0.606692$

H $1.4728525 .122046 \quad 0.636317$

H $-0.060147 \quad 4.3350940 .938912$

H $-1.0482486 .386027-1.581651$

H $-1.5827023 .672130-1.115298$

H $1.7191826 .373033-1.532762$

H $0.8236455 .873456-2.950208$

H $2.0462464 .794225-2.251114$

H $-1.6941394 .970168-2.933498$

H $-1.1788991 .554087-3.381936$

H $-0.2066960 .410968-2.498518$

H $1.4071981 .695008-3.047807$

H -5.1635342 .4501111 .133941$

H $-5.249237-0.2864982 .556112$

H -7.605393 -0.9678593 .126308$

H -8.967274 -0.0086732 .553775$

H $-7.8160760 .708597 \quad 3.675298$

H -1.667958 $0.610035-0.500721$ 
H $-2.7302431 .724093-1.394631$ H $-4.647756-2.559416-0.678806$ H $-4.147958-2.485525-2.384717$ H $-5.937831-0.609055-2.734729$ H -7.067270 -2.572295 -0.672489 H -8.990618 -2.031623 -2.303467 H -8.332354 -0.429321 -2.549542 H -10.759192 -1.0799180 .780650$ H -10.431248 -2.433585 -0.439710 H -9.6020750 .9840180 .676316$ H $-9.0121541 .394209-0.906673$ H $-6.7555681 .101957-0.314998$ H $-3.849687-0.357732-0.313726$ C $0.207802-3.8420900 .315006$ O $1.309067-3.141963-0.301091$ C $0.945872-2.826006-1.657925$ C $-0.262029-3.697889-1.977537$ C $-0.977455-3.699674-0.639266$ H $0.623601-1.778905-1.665579$ C $2.146504-3.016832-2.588368$ H $-0.869197-3.287234-2.789795$ H $0.036463-4.716028-2.253923$ C $-2.017671-4.796375-0.501737$ H -1.466422 -2.727069 -0.506215 C $3.359429-2.118048-2.256889$ O $2.579547-4.381128-2.520110$ H $1.838125-2.851870-3.627178$ H $3.810111-2.427412-1.307406$ O $4.351739-2.336523-3.267374$ C $3.034979-0.614097-2.219673$ C $4.2486980 .329139-2.157496$ H $2.382546-0.401837-1.365882$ O $2.296329-0.263854-3.395529$ H $4.8177980 .271452-3.094258$ H $3.845271 \quad 1.350210-2.127122$ H $-2.798678-4.683106-1.260541$ H $-1.573137-5.789744-0.623608$ H $-2.494720-4.7581470 .482698$ C $-0.008925-3.3334061 .742533$ H $2.799055-4.572270-1.591875$ H $4.544015-3.289806-3.282609$ H $2.847292-0.485887-4.165780$ H $0.906105-3.5145882 .318525$ H -0.809873 -3.909218 2.220401 H $0.514621-4.8948950 .372330$ C $5.148285-0.0324951 .459489$ O $4.4246520 .180367 \quad 0.238958$ C $5.1940030 .109492-0.963410$ C $6.3233951 .154768-0.943614$ C 7.1812921 .0367080 .319907 C 6.3218580 .9559281 .584710 H $4.429400 \quad 0.1998682 .255553$ C $5.578187-1.4984381 .626665$ H $5.630909-0.889920-1.054074$ O $5.7844922 .474576-1.019317$ H $6.9488821 .015336-1.832861$ H $7.843688 \quad 1.9081610 .393592$ O $7.998500-0.1268040 .239450$ H 6.9455910 .6878632 .445635 O 5.7925822 .2600191 .841282 C $4.396300-2.4273271 .798352$ H $6.148393-1.8531780 .764426$ H $6.198147-1.5988882 .524994$ H $5.1654162 .585988-0.277375$ H $8.559546-0.037653-0.549753$ H 5.2986522 .2178592 .677716 O $4.847706-3.7169031 .799068$ O $3.233194-2.0732521 .927066$ C $3.846753-4.7259681 .962724$ H $3.205344-4.7607651 .078151$ H $4.351089-5.6902882 .067777$ H $3.255939-4.5414382 .865033$ SCF Energy $\left(B 3 L Y P / 6-31 G^{* *} / / M M F F\right)=-3245.92128450$

\section{7_00273}

MM̄FF Geometry

C $-2.769917-1.358044-3.133711$

C $-1.688200-1.198814-2.357126$
C $-0.353441-0.735974-2.875700$

O $-0.0358320 .486643-2.169088$

C $0.770878-1.749839-2.589797$

C $0.534392-3.138858-3.134698$

C $0.142514-4.189501-2.387119$

C $0.793279-3.303148-4.608236$

C $-0.195740-4.195062-0.921250$

C $0.685084-5.142273-0.100483$

C $2.161902-4.721842-0.011051$

C $2.939710-5.7685170 .793961$

C $4.401157-5.3607031 .012932$

O $2.241699-3.4418100 .630660$

O $4.966869-6.2366991 .999763$

C $4.437998-3.9222161 .566319$

C $5.242162-5.578824-0.249483$

O $5.782889-3.4542211 .654184$

C $3.576825-2.9226370 .754981$

C $3.506045-1.5764661 .524487$

O $4.180922-2.720607-0.520284$

C $0.782687 \quad 1.375673-2.792554$

O $1.2276311 .297690-3.926166$

C $1.0790812 .456956-1.825980$

C $1.9076673 .451286-2.177508$

C $2.3491434 .554609-1.320940$

C $1.9243594 .527947 \quad 0.123352$

C $2.763701-0.4391340 .813077$

C $3.6612500 .328548-0.141022$

C $5.5966091 .903996-0.160331$

O $3.6247200 .136596-1.354086$

C 5.5970963 .3431020 .249408

C $5.9268154 .339902-0.586759$

C $5.9470605 .789965-0.193039$

C $5.2469256 .682101-1.196086$

C $5.9496417 .471913-2.025818$

C $3.7337406 .715920-1.185526$

C $3.1353195 .510687-1.858521$

N $4.510758 \quad 1.2095280 .509734$

H -2.705440 -1.126669 -4.194149

H -1.758307 -1.424656 -1.296569

H $-0.417729-0.534328-3.952599$

H $0.966092-1.778866-1.513645$

H $1.713478-1.366910-3.003745$

H $\quad 0.012262-5.151144-2.881723$

H $\quad 0.631763-4.332127-4.946731$

H $\quad 0.126810-2.656918-5.188196$

H $1.828758-3.036894-4.843775$

H $-0.168485-3.196516-0.478849$

H -1.237089-4.530007 -0.829872

H $0.264964-5.1805430 .913242$

H $0.613965-6.156086-0.514769$

H $2.571671-4.641351-1.024051$

H $2.885253-6.7521930 .310760$

H $2.463063-5.8936661 .776501$

H $4.447036-6.1511102 .816962$

H $4.052091-3.9499392 .594857$

H $5.237805-6.638827-0.531166$

H $6.292566-5.320834-0.075035$

H $4.885225-5.008252-1.109302$

H $6.283688-4.1063002 .173559$

H $2.985229-1.7567592 .474259$

H $4.520101-1.2378191 .767146$

H $3.687615-2.025321-0.983441$

H $0.6311692 .388139-0.841910$

H $2.3168703 .453610-3.187132$

H 2.3559975 .3306540 .723954

H 2.2359363 .5906910 .597726

H $\quad 0.8357314 .6202460 .203907$

H $1.880824-0.796175 \quad 0.277721$

H $2.4001470 .272277 \quad 1.564245$

H $5.5109001 .783180-1.244861$

H $\quad \begin{array}{llll}\text { H } & 623410 & 1.425648 & 0.172548\end{array}$

H 5.3325713 .5795851 .277290

H $6.2250664 .098963-1.604855$

H $6.9980036 .091571-0.094458$

H 5.4973835 .9410040 .796206

H $5.4561838 .132716-2.730846$

H $7.0347367 .471819-2.022864$

H $3.3729477 .601767-1.725310$ 
H $3.3895516 .854839-0.157584$ H $3.3873725 .436467-2.917614$ H 4.5097151 .2206351 .525773 C -5.125697 -0.764765 -2.519830 O $-4.8532230 .017684-1.338521$ C $-6.1071610 .402121-0.737497$ C -7.201820 -0.059522 -1.694132 C $-6.555346-1.269066-2.339439$ H $-6.200280-0.1642370 .195022$ C $-6.1166931 .906920-0.448649$ H -8.133310 -0.297563 -1.171281 H -7.428971 $0.702201-2.449297$ C -7.232742 -1.697851 -3.629412 H $-6.573134-2.103108-1.625226$ C $-5.006527 \quad 2.357348 \quad 0.523875$ O $-5.9318672 .594925-1.692368$ H -7.101435 $2.205024-0.069533$ H -4.0213542 .1366430 .098103$ O $\quad-5.0862463 .7834760 .649867$ C -5.1259871 .7500461 .939262$ C -4.0871482 .2993672 .937055$ H -5.0393380 .6603651 .895425$ O $-\begin{array}{llll}6.426730 & 2.037428 & 2.461164\end{array}$ H -4.0409293 .3915922 .854697$ H -4.441941 2.0899423 .955586 H -8.277291 -1.968803 -3.443006 H -7.221547 -0.895438 -4.374564 H $-6.732269-2.568257-4.064880$ C -4.090189-1.880455 -2.641702 H $-6.1558423 .529250-1.544553$ H -4.789062 4.167148 -0.192541 H -6.5224713 .0050112 .492110$ H $-4.427127-2.642940-3.352946$ H $-3.957631-2.378821-1.675285$ H -5.066638 $-0.083693-3.379560$ C $-1.501562-0.368168 \quad 3.298374$ O $-2.715863 \quad 0.392154 \quad 3.368266$ C $-2.692891 \quad 1.6821762 .743116$ C -1.5946822 .5564713 .362735$ C -0.2343041 .8597043 .281351$ C $-0.296136 \quad 0.4317113 .823793$ H -1.664265 -1.206282 3.988647 C -1.291124 -0.9521911 .896578$ H -2.4935251 .5618701 .674184$ O -1.9041452 .8645684 .719492$ H -1.554286 3.5084482 .820534 H 0.5055682 .4323263 .853770 O $\quad 0.194437 \quad 1.812836 \quad 1.924551$ H $0.634693-0.0997713 .593191$ O $-0.387860 \quad 0.509017 \quad 5.249656$ C $-2.180964-2.1590051 .715499$ H -1.488967 -0.2456251 .085932$ H $-0.260389-1.3027711 .767068$ H -2.0151162 .0251215 .197994$ H $\quad 0.2172142 .7265521 .594400$ H $-0.346503-0.3983885 .596739$ O $-3.423234-1.7739191 .308106$ O $-1.821862-3.3057411 .951483$ C -4.379128 -2.832099 1.189795 H $-4.587678-3.2589402 .175376$ H $-5.304577-2.4139640 .786705$ H -4.016087 -3.604327 0.504698 SCF Energy (B3LYP/6-31G**//MMFF) $=-3245.91143248$

\section{$07 \quad 00274$}

MM̄FF Geometry

C $0.943297-2.391757-2.146552$

C $0.002559-3.347278-2.144126$

C $-0.908881-3.613730-0.978325$

O $-2.218444-3.154717-1.388750$

C $-0.999449-5.110176-0.630963$

C $0.252233-5.692610-0.015582$

C $0.500322-5.707337 \quad 1.308293$

C $1.210027-6.354032-0.971821$

C $-0.340154-5.1060742 .404204$

C $0.520384-4.4152503 .472054$

C $1.154021-3.0979852 .992158$

C $2.354779-2.7530613 .876608$
C $2.975174-1.4036193 .500513$

O $0.158172-2.0684363 .082223$

O $3.887133-1.0332264 .545818$

C $1.856120-0.3480863 .477048$

C $3.801757-1.4870992 .212864$

O $2.3580520 .906567 \quad 3.016975$

C $0.603435-0.7704902 .664741$

C -0.5228380 .2515942 .947695$

O $0.934085-0.7527441 .277307$

C $-3.091112-2.799760-0.409708$

O $-2.914166-2.8663640 .795654$

C $-4.312981-2.293293-1.066792$

C $-5.326089-1.846561-0.312045$

C $-6.573688-1.299752-0.832191$

C $-7.789589-1.771106-0.082104$

C $-1.873359-0.0141872 .269808$

C $-2.839741 \quad 1.1294572 .548031$

C $-4.8644822 .248272 \quad 1.701553$

O $-2.657221 \quad 1.9491443 .446615$

C -6.1367471 .8802561 .010438$

C $-6.6008062 .526097-0.070784$

C $-7.9165302 .232076-0.736091$

C $-7.7562011 .783371-2.171786$

C -7.682120 $2.677097-3.172433$

C $-7.758514 \quad 0.296368-2.449417$

C $-6.576085-0.400708-1.836860$

N $-3.9400391 .128647 \quad 1.698162$

H $1.058409-1.757674-1.272309$

H -0.133231 -3.961994 -3.030884

H $-0.582244-3.035731-0.104031$

H -1.275856 -5.685823 -1.525530

H -1.848011 -5.2784110 .044910$

H $1.404966-6.2091201 .649799$

H $2.049775-6.830922-0.454810$

H $\quad 0.696823-7.131586-1.547005$

H $1.629796-5.624362-1.669760$

H -1.078726 -4.398618 2.018915

H $-0.892481-5.9225622 .884862$

H $-0.117307-4.212917 \quad 4.342317$

H $1.299871-5.1144823 .800995$

H $1.473000-3.197467 \quad 1.948858$

H $3.112887-3.5451063 .829459$

H $2.033379-2.7087364 .927045$

H $4.562476-1.7296664 .615341$

H $1.552431-0.1842684 .520158$

H $4.593748-2.2389082 .313189$

H $4.314391-0.5394782 .016180$

H $3.207553-1.7487741 .334724$

H 2.4526500 .8506242 .050907

H $-0.6998530 .287178 \quad 4.031356$

H -0.1856651 .2505222 .647512$

H $0.115754-0.7251940 .758728$

H $-4.351108-2.284764-2.150888$

H -5.258995 -1.886274 0.773458

H -7.902751 -2.855827 -0.188651

H $-8.720066-1.314383-0.424460$

H -7.697958 -1.538843 0.985076

H -1.755036 -0.1093001 .186595$

H $-2.317748-0.9367342 .656841$

H -4.357472 3.0913661 .218945

H -5.0745222 .5350892 .737662$

H -6.7235971 .0761991 .447094$

H $-6.0183583 .345558-0.486694$

H $-8.5221103 .147478-0.702404$

H $-8.4909331 .485031-0.174491$

H $-7.5931692 .363439-4.207187$

H -7.706221 $3.744254-2.976848$

H $-7.7265270 .107570-3.530489$

H $-8.712703-0.120645-2.115374$

H $-5.622229-0.108743-2.277188$

H -3.9112230 .5340590 .874575$

C $3.304885-1.984018-2.854827$

O $3.481033-0.713822-2.190751$

C $4.738371-0.144950-2.602999$

C $5.467483-1.240201-3.373013$

C $4.309290-1.984804-4.004980$

H $4.5060030 .671913-3.295630$

C $5.5066800 .387340-1.390933$ 
H $6.169806-0.833170-4.107029$

H $6.027923-1.902697-2.702701$

C $4.681442-3.370642-4.503294$

H $3.921988-1.390503-4.843470$

C $4.7783491 .511986-0.622296$

O $5.727900-0.702429-0.487078$

H $6.4998500 .728103-1.707201$

H $3.8614311 .117731-0.171177$

$\begin{array}{lllll}\text { O } & 5.623574 & 1.889896 & 0.473278\end{array}$

C $4.4600492 .763100-1.468888$

C $4.0267523 .994382-0.650193$

H $3.6918242 .520230-2.211010$

O $5.6427003 .127552-2.189796$

H 4.7909614 .2216840 .103284

H $4.0188634 .858322-1.329095$

H $5.456997-3.306500-5.273493$

H $5.064397-3.999341-3.692403$

H $3.814379-3.876037-4.939744$

C $1.852366-2.119834-3.312040$

H $6.210332-0.348757 \quad 0.279815$

H 6.4325512 .2791760 .098896

H $5.4085003 .856895-2.788770$

H $1.762685-2.923162-4.052052$

H $1.523695-1.187264-3.786422$

H $3.557437-2.765664-2.125629$

C $0.2968623 .822674-0.629092$

O $1.6691223 .909146-1.037311$

C 2.6456633 .8617940 .010223

C $2.406116 \quad 4.9883451 .029962$

C 0.9696804 .9751381 .556336

$\begin{array}{llll}\text { C }-0.048236 & 4.897505 & 0.416587\end{array}$

H $-0.2769404 .047282-1.537766$

C $-0.0679132 .404885-0.179648$

H 2.5826762 .8975150 .522238

$\begin{array}{llllll}\text { O } & 2.687704 & 6.259504 & 0.446578\end{array}$

H 3.1047714 .8599421 .865118

H $0.796041 \quad 5.886102 \quad 2.142342$

O 0.8136063 .8573832 .424364

H $-1.052374 \quad 4.7189830 .818991$

O $-0.082198 \quad 6.171721-0.232680$

C $-0.2523161 .508853-1.380989$

H $0.688579 \quad 1.9391630 .455400$

H -1.0039532 .4120410 .390434$

H $2.1180356 .361010-0.335042$

H -0.0935653 .8804382 .773750$

H $-0.780114 \quad 6.138957-0.908996$

O $-1.579504 \quad 1.264357-1.577824$

O $0.6699231 .100995-2.074573$

C -1.899940 $0.463975-2.719081$

H -2.988614 $0.410463-2.801491$

H $-1.5017160 .919829-3.630613$

H $-1.507699-0.547500-2.590162$

SCF Energy (B3LYP/6-31G*//MMFF) $=-3245.91155638$

\section{7_00275}

MM̄FF Geometry

C $-0.318957-2.6243261 .698624$

C $0.082938-2.3492330 .448439$

C $-0.816752-1.799876-0.622764$

O $-0.323970-0.470796-0.904394$

C $-0.779215-2.652706-1.903308$

C $-1.372916-4.035718-1.748504$

C $-2.693807-4.280805-1.642649$

C $-0.381994-5.168562-1.740603$

C $-3.803796-3.264965-1.631922$

C $-4.527540-3.265247-0.279841$

C $-5.476098-2.076665-0.053089$

C $-6.491419-1.887331-1.181612$

C $-7.402991-0.675352-0.936554$

O $-4.690172-0.8869910 .110603$

O $-8.075867-0.410243-2.177255$

C $-6.5243630 .548260-0.594217$

C $-8.495722-0.988605 \quad 0.092287$

O $-7.327031 \quad 1.660539-0.203204$

C $-5.461688 \quad 0.261787 \quad 0.494975$

C -4.5154041 .4800350 .639023$

O $-6.1278550 .056617 \quad 1.745531$

C $-1.1722470 .405392-1.504288$
O $-2.273237 \quad 0.172417-1.974361$

C $-0.5367441 .734367-1.431557$

C $-1.1134132 .796302-2.010418$

C $-0.5929384 .155786-1.913983$

C $-1.6638745 .193375-1.717713$

C -3.2622241 .2126821 .485267$

C -2.5752442 .5022851 .909106$

C $-0.377153 \quad 3.3570832 .713025$

O -3.1978793 .5356522 .145027$

C $-0.0895404 .502524 \quad 1.792779$

C 1.1493534 .8950381 .457252

C 1.4518116 .1338360 .659999

C $2.2177995 .854399-0.612837$

C $3.5599125 .791274-0.615739$

C $1.4250185 .735447-1.894943$

C $0.7281804 .409586-1.998040$

N -1.203639 2.3534102 .065528

H -1.354304 -2.457979 1.983757

H $1.126100-2.5023690 .181131$

H -1.838627 -1.710709-0.237281

H $0.255381-2.726491-2.264457$

H -1.295471 -2.133958 -2.720761

H -3.022460 -5.314687 -1.547806

H $-0.867640-6.145756-1.648488$

H $0.196506-5.173227-2.670185$

H $\quad 0.311009-5.064445-0.900258$

H -3.437815 -2.262476 -1.865185

H -4.508012 -3.529297 -2.429608

H $-5.096742-4.198879-0.183476$

H -3.781241 -3.2609760 .526075$

H $-5.997648-2.2764500 .890744$

H -7.087714 -2.796831-1.326078

H $-5.965343-1.717381-2.130392$

H $-8.6994370 .321259-2.032862$

H $-6.0025160 .847110-1.514328$

H $-9.132681-1.806283-0.266397$

H $-9.162423-0.1310880 .236279$

H -8.098313 -1.281252 1.066284

H -7.902314 $1.887574-0.952804$

H $-4.1716711 .801345-0.352719$

H -5.0800042 .3169851 .068150$

H -5.5412820 .3225502 .470599$

H $\quad 0.381014 \quad 1.822237-0.860471$

H $-2.0515462 .687699-2.549646$

H $-2.2765995 .283909-2.621433$

H $-1.2695406 .184581-1.483848$

H -2.320247 $4.913920-0.885275$

H -3.5122650 .6797892 .408711$

H -2.5672620 .5798880 .926392$

H 0.5375462 .8600553 .048713

H -0.9086673 .7260093 .596930$

H -0.9398065 .0847331 .446563$

H 2.0023084 .3352561 .833196

H 2.0411246 .8048041 .299354

H 0.5360656 .6912250 .427328

H $4.1161425 .618085-1.530956$

H 4.1307795 .9048750 .300038

H $2.0901625 .826136-2.763956$

H $0.7388386 .583451-1.979210$

H $1.4112903 .576031-2.159933$

H -0.7835041 .4446281 .894508$

C $0.942823-2.0849993 .807247$

O $1.613552-0.9802003 .164190$

C $2.954931-0.9049543 .668722$

C $2.851565-1.4470235 .084904$

C $1.908927-2.6185204 .875241$

H $3.563509-1.5882823 .062026$

C 3.5108850 .5166613 .536193

H $3.821737-1.7451305 .492968$

H $2.397543-0.7188335 .766519$

C $1.213319-3.0767426 .145965$

H $2.487389-3.4617614 .474232$

C $3.317737 \quad 1.1574202 .145325$

O 2.8722591 .3744904 .490165

H $4.5730980 .513617 \quad 3.806619$

H 2.2724861 .4661692 .028881

O 4.0863642 .3661882 .109354

C 3.7074010 .2725380 .949767 
C $3.5840001 .026392-0.389149$

H $3.060875-0.6113050 .932784$

O $5.049649-0.1765011 .126021$

H $2.7025811 .676570-0.367004$

H $4.4729811 .654251-0.526678$

H $1.946213-3.4142416 .886077$

H $\quad 0.627879-2.2683296 .596732$

H $0.533931-3.9093085 .936554$

C $0.607904-3.1501302 .757570$

H 1.9140021 .3306634 .329626

H 5.0259332 .1166412 .142245

H $5.265548-0.7571970 .376527$

H $0.138380-4.0175843 .235592$

H $1.533209-3.5053872 .287452$

H $\quad 0.026376-1.6839504 .256487$

C $4.656019-1.727075-2.681997$

O $4.651829-0.704809-1.676501$

C $3.4480880 .059377-1.579789$

C $3.1825930 .794919-2.900085$

C $3.151050-0.182680-4.079857$

C $4.354857-1.132601-4.068860$

H 5.687753 -2.102184 -2.696272

C $3.738091-2.900683-2.305834$

H $2.608132-0.610611-1.367017$

O $4.1813541 .786893-3.126730$

H $2.223275 \quad 1.317773-2.829943$

H $3.1448740 .381979-5.020120$

O $1.954917-0.955554-4.029298$

H $4.211351-1.945790-4.789972$

O $5.517844-0.406922-4.479834$

C $4.168083-3.576986-1.020400$

H $2.705037-2.572355-2.170419$

H $\quad 3.763559-3.656615-3.098876$

H $5.0475661 .344719-3.116295$

H $1.203848-0.338538-4.060021$

H $5.355047-0.078487-5.380603$

O $3.213501-4.484637-0.658224$

O $5.207203-3.351912-0.415595$

C $3.476714-5.2075460 .548258$

H $3.673568-4.5190061 .375828$

H $2.589780-5.7993300 .790009$

H $4.323399-5.8841180 .399960$

SCF Energy (B3LYP/6-31G**//MMFF) $=-3245.91212037$

\section{7_00276}

MM̄FF Geometry

C -2.240617 -2.845910 -0.733196

C $-1.239778-3.132851-1.578181$

C $-0.135813-2.164892-1.907026$

O $1.090822-2.696801-1.351281$

C $0.073959-2.004459-3.423503$

C $-0.922178-1.066489-4.074933$

C $-0.7551320 .268320-4.167460$

C $-2.151912-1.716579-4.651884$

C $0.4040681 .079719-3.655297$

C $0.0198681 .837295-2.378907$

C $1.1868492 .555390-1.684574$

C $1.7043583 .745789-2.493400$

C $2.9073194 .413970-1.809841$

O $2.2271821 .601050-1.457513$

O $3.4915475 .339160-2.739631$

C $3.9549273 .327361-1.485623$

C $2.4808705 .264478-0.609092$

O $5.0409503 .869620-0.738446$

C $3.3659922 .104164-0.746942$

C $4.3865870 .946487-0.634614$

O 3.0053152 .4860570 .585020

C $1.513941-2.170631-0.168881$

O $0.981031-1.2793640 .473944$

C $2.755831-2.875504 \quad 0.223077$

C $3.403690-2.4848111 .331016$

C $4.646884-3.0466151 .864110$

C $5.255532-4.2123701 .132066$

C $4.8562190 .386185-1.978755$

C $5.579253-0.935234-1.818470$

C $7.747737-1.913621-1.081907$

O $5.070460-1.992217-2.185355$

C $8.213287-2.0929490 .331425$
C $7.946734-1.3214521 .397406$

C $8.481451-1.6134392 .771718$

C $7.398062-1.7149103 .821679$

C $7.347305-0.8488254 .848157$

C $6.420337-2.8677063 .727736$

C $5.171201-2.4904152 .976505$

N $6.830738-0.801258-1.240011$

H -2.250351 -1.882652 -0.230682

H -1.210262 -4.106454 -2.061119

H $-0.343107-1.180475-1.473101$

H $0.045371-2.983535-3.921454$

H $1.096689-1.655013-3.611441$

H $-1.5339920 .854559-4.653094$

H $-2.798203-0.998111-5.167224$

H -1.869403 -2.483066 -5.380919

H $-2.747935-2.186584-3.864602$

H $1.2796540 .450990-3.475075$

H $0.6947991 .786513-4.440736$

H $-0.7801912 .555665-2.595284$

H -0.388577 1.113063 -1.664698

H $0.8154762 .901873-0.713591$

H $0.9054124 .477283-2.666633$

H $2.0220983 .407955-3.488425$

H $3.7560094 .846404-3.535003$

H $4.3735572 .996310-2.443697$

H $1.8010226 .062800-0.930741$

H $3.3420625 .769126-0.156922$

H $1.9714274 .691406 \quad 0.168277$

H $5.3766014 .638565-1.230195$

H $5.2473161 .260532-0.030622$

H $3.9212750 .130229-0.067609$

H 2.9731401 .6867151 .136580

H $3.105177-3.682240-0.410595$

H $2.988181-1.6583721 .906846$

H $4.567648-5.0652331 .132663$

H $6.192522-4.5622331 .569373$

H $5.476534-3.9443450 .093421$

H $4.0081550 .215242-2.651483$

H $5.5306501 .086363-2.483960$

H $8.609299-1.713862-1.727024$

H $7.275043-2.838461-1.427421$

H $8.841983-2.9692130 .482504$

H $7.335524-0.4294651 .291273$

H $9.188615-0.8140383 .027848$

H $9.063929-2.5434492 .781004$

H $6.588116-0.9327365 .618891$

H $8.059526-0.0355434 .939378$

H $6.116243-3.1721834 .738331$

H $6.928732-3.7412683 .311454$

H $4.634132-1.6567763 .432789$

H $7.1859870 .131753-1.051480$

C $-4.724553-3.216973-0.814043$

O $-5.025673-2.052411-0.014952$

C $-6.252216-2.2862740 .694473$

C $-7.000448-3.307420-0.147925$

C $-5.862405-4.215831-0.570428$

H $-5.978887-2.7454651 .652858$

C $-7.003591-0.9766750 .957040$

H -7.779009 -3.8280860 .417202$

H -7.461468 -2.848358 -1.029934

C $-6.185866-5.077205-1.779428$

H -5.611399 -4.874342 0.272093

C -6.1578860 .1513351 .585591$

O $-7.531685-0.469381-0.275195$

H -7.876033 -1.183899 1.587736

H -5.5204130 .6006460 .816689$

O $\quad-7.056917 \quad 1.1830902 .011369$

C $-5.293912-0.2700672 .784001$

C -4.6219460 .8869383 .546297$

H $-4.517354-0.9632002 .440867$

O $-6.111269-0.9860943 .714665$

H -5.3698791 .4252184 .143151$

H $-3.948916 \quad 0.423807 \quad 4.280029$

H -7.029606 $-5.741033-1.564388$

H $-6.452044-4.465654-2.648114$

H $-5.327416-5.698212-2.054713$

C $-3.362413-3.787547-0.403811$

H $-6.784760-0.352182-0.886853$ 
H $-7.584011 \quad 1.4454751 .237239$ H -5.535014 -1.293772 4.435096 H $-3.203957-4.755870-0.893029$ H -3.340749 -3.959174 0.679565 H $-4.705521-2.886930-1.860254$ C $-2.1818491 .965046 \quad 0.910793$ O $\quad-2.950057 \quad 1.1676051 .824675$ C -3.8391631 .8897052 .680973$ C -3.0634022 .9007353 .542945$ C -2.1999503 .8237632 .676680$ C $-1.3682173 .035443 \quad 1.661279$ H $-1.470478 \quad 1.2616700 .461877$ C $-3.0473672 .556702-0.216404$ H -4.5649252 .4328212 .067780$ O -2.2290852 .2030864 .466194$ H -3.7808803 .4889804 .127518$ H $-1.524067 \quad 4.4036513 .317071$ O $-3.032573 \quad 4.744680 \quad 1.978217$ H $-0.906913 \quad 3.7243840 .944040$ O -0.3019852 .3909432 .363590$ C $-3.5316871 .507305-1.194204$ H -3.9302183 .0677210 .175292$ H $-2.4543503 .275012-0.794156$ H -1.772507 2.8673495 .010118 H -3.5276785 .2545512 .641745$ H 0.2586221 .9469631 .704532 O $-4.3885492 .091251-2.083752$ O $-3.1942630 .332713-1.197089$ C $-4.9157401 .221314-3.090536$ H $-5.6788881 .770233-3.648550$ H $-4.1198520 .925909-3.779925$ H -5.379799 $0.339911-2.638194$ SCF Energy (B3LYP/6-31G $\left.{ }^{* *} / / \mathrm{MMFF}\right)=-3245.90076652$

\section{7_00277}

\section{MM̄FF Geometry}

C 0.9697311 .5298022 .643352

C -0.0246760 .9354693 .322545$

C $-0.254851-0.5501233 .502075$

O $-1.548460-0.8118622 .900879$

C $0.823394-1.4436722 .857251$

C $0.657374-2.9329143 .082477$

C $0.273225-3.8105772 .133483$

C $1.030330-3.4211674 .458435$

C $-0.086905-3.5034690 .706949$

C - $1.421564-4.1384620 .291848$

C $-1.997050-3.550971-1.009823$

C -1.026964 -3.662911 -2.190777

C -1.563375 -2.971927 -3.452016

O $-2.326968-2.182161-0.747692$

O $-0.464838-2.869035-4.371574$

C -2.001256-1.540899-3.074929

C $-2.629608-3.821221-4.151427$

O $-2.599540-0.884705-4.190060$

C $-2.930524-1.486678-1.844083$

C $-3.205599-0.037720-1.387821$

O $-4.193841-2.067060-2.188986$

C $-2.389136-1.6683993 .536524$

O $-2.146743-2.3475204 .523839$

C -3.697594 -1.727811 2.854578

C $-4.308698-0.6128982 .425686$

C $-5.645870-0.5361701 .831144$

C $-6.343723-1.8349031 .520595$

C $-1.9803810 .729150-0.884347$

C $-2.4036061 .983592-0.149050$

C $-3.5492444 .160586-0.496831$

O -2.3405462 .0671681 .075282$

C $-5.0243973 .934891-0.319648$

C $-5.7714763 .021557-0.961008$

C $-7.2416702 .823863-0.735295$

C $-7.5827071 .392108-0.383805$

C $-7.9795670 .526370-1.331996$

C $-7.562000 \quad 0.9921801 .073161$

C $-6.1834180 .678795 \quad 1.588758$

N -2.882665 $2.971606-0.994328$

H 1.7334930 .9324172 .154719

H -0.7645551 .5712603 .807009$

H $-0.297639-0.7357284 .582616$
H $1.812351-1.1646983 .246196$

H $\quad 0.860487-1.2253241 .787538$

H $0.229845-4.8665352 .396145$

H $0.902769-4.5038814 .563985$

H $2.079943-3.1904914 .668498$

H $0.412672-2.9426325 .223734$

H $-0.135947-2.4278990 .526525$

H $\quad 0.715457-3.8967090 .071598$

H -1.290739 -5.2218010 .180266$

H -2.156261 -3.981432 1.092166

H $-2.924144-4.093395-1.230240$

H $-0.785460-4.713225-2.395531$

H $-0.073236-3.181169-1.939236$

H $-0.772548-2.361043-5.142027$

H -1.083596 -0.983149 -2.852788

H $-2.207487-4.787534-4.453128$

H $-2.972940-3.345068-5.076372$

H -3.499305 -4.022597 -3.522856

H -3.501603 -1.233822 -4.289866

H $-3.6965830 .518454-2.196186$

H $-3.944174-0.065793-0.577458$

H -4.862721 -1.744817 - 1.562276

H -4.169434 -2.703396 2.825023

H -3.8022280 .3407292 .562431$

H $-5.703641-2.4784960 .905992$

H $-7.270153-1.7055080 .956782$

H -6.595490 -2.367134 2.444189

H $-1.3896560 .123253-0.188922$

H -1.324226 $1.011316-1.712744$

H -3.370695 4.976839-1.203009

H -3.1081234 .4308180 .468266$

H -5.5081814 .5826480 .408586$

H $-5.3159832 .385400-1.715162$

H -7.763258 3.114001-1.656908

H $-7.624027 \quad 3.4961370 .042407$

H $-8.259708-0.492515-1.086667$

H $-8.0280450 .816842-2.376370$

H $-8.246446 \quad 0.1547601 .249339$

H -7.980165 1.8077521 .677615

H -5.5958671 .5644431 .831995$

H $-2.9402492 .782379-1.990787$

C 1.1830343 .4884071 .061077

O 2.3645922 .9603760 .419903

C 3.2433984 .0540040 .118403

C $2.3059125 .224154-0.132349$

C 1.2841145 .0191450 .970007

H 3.8307244 .2448321 .026157

C $4.2022613 .717644-1.029435$

H $2.8139606 .191016-0.071652$

H $1.8167495 .150099-1.110444$

C -0.0495145 .6948640 .696818$

H 1.6939475 .4274741 .903702

C $4.8767672 .333160-0.935691$

O $3.4830683 .753157-2.268498$

H $4.9580194 .509170-1.100620$

H $4.1550011 .570445-1.241467$

O $5.9198342 .295212-1.918286$

C $5.4604221 .965195 \quad 0.436511$

C 6.2911400 .6682330 .461589

H 4.6475431 .8756801 .165588

O $\quad 6.302556 \quad 3.0334290 .878277$

H $7.2605630 .836320-0.025596$

H 6.5291630 .4737461 .515688

H $\quad 0.077610 \quad 6.779018 \quad 0.612926$

H $-0.4968795 .335569-0.236046$

H $-0.7572175 .497302 \quad 1.508295$

C 1.1303503 .0190462 .521857

H $4.1163423 .550354-2.978277$

H $6.6087432 .921953-1.637669$

H $6.6158502 .809736 \quad 1.771233$

H 0.3160023 .5357223 .043792

H 2.0669873 .2854773 .027834

H 0.3217113 .1078340 .501053

C $3.526738-1.798890-0.117645$

O $4.309430-0.7150660 .406308$

C $5.614747-0.567609-0.158508$

C $6.446089-1.8427520 .066850$

C $5.717558-3.088687-0.447470$ 
C $4.264705-3.1389130 .036923$

H $2.642911-1.8391960 .526000$

C $3.048823-1.534721-1.554264$

H $5.521148-0.392323-1.234818$

O $6.734989-2.0123541 .454073$

H $7.407449-1.733957-0.448308$

H $6.243405-3.986916-0.101260$

O $5.730180-3.100326-1.871566$

H $3.709153-3.923372-0.490409$

O $4.247398-3.4759051 .427364$

C $2.096385-0.360506-1.637227$

H $3.892157-1.329584-2.219103$

H $2.511766-2.408121-1.941225$

H $5.888950-2.0295821 .933725$

H $6.659622-3.098448-2.157390$

H $4.659441-4.3516671 .521300$

O $2.1329170 .151116-2.902299$

O $1.3932530 .037557-0.719408$

C $1.3082391 .292257-3.164787$

H $1.7963501 .889969-3.939250$

H $\quad 0.3404450 .954358-3.542905$

H $1.1740891 .918996-2.278076$

SCF Energy (B3LYP/6-31G*//MMFF)= -3245.91342611

0700278

MM̄FF Geometry

C $0.077570-2.082681-2.970945$

C $-0.901783-2.243665-3.875118$

C $-2.349629-1.838242-3.746844$

O $-2.727681-1.550218-2.384358$

C $-2.608060-0.573991-4.582966$

C $-4.072708-0.186886-4.636873$

C $-4.622210 \quad 0.807714-3.912040$

C $-4.909493-0.964212-5.618961$

C $-3.9318901 .664774-2.884695$

C $-4.8665382 .002163-1.714858$

C $-4.1965462 .750237-0.549188$

C $-3.4934544 .040332-0.982463$

C $-2.795778 \quad 4.740175 \quad 0.195015$

O $-3.2676161 .864341 \quad 0.091624$

O $-1.9241085 .749286-0.339231$

C -1.9295443 .7060890 .948843$

C -3.795263 $5.499057 \quad 1.073737$

O $-1.373165 \quad 4.2723782 .132221$

C -2.688528 2.4054741 .290531

C $-1.774408 \quad 1.3303331 .918420$

O -3.6994472 .6982822 .265112$

C $-3.107122-2.602201-1.610435$

O $-3.006165-3.788838-1.877493$

C $-3.708270-2.050720-0.377248$

C $-3.901660-2.8527750 .679405$

C $-4.491331-2.4567461 .957596$

C $-5.244218-1.1536812 .011619$

C $-0.606477 \quad 0.860973 \quad 1.052505$

C $0.161785-0.2298191 .771525$

C $0.030073-2.6462152 .377916$

O $1.193354 \quad 0.0114892 .393300$

C $-0.834155-2.9947643 .554234$

C $-1.563528-2.1442274 .294492$

C -2.448971-2.569098 5.433567

C $-3.887784-2.1406225 .230715$

C $-4.353497-1.0136565 .795389$

C $-4.803788-3.0433434 .430497$

C $-4.334343-3.2718793 .020819$

N $-0.450458-1.4676861 .681956$

H $-0.124955-1.594271-2.022120$

H $-0.652969-2.719600-4.822596$

H -2.971055 -2.660555 -4.124437

H -2.250392 -0.720279-5.611253

H -2.013386 $0.257011-4.185886$

H $-5.675326 \quad 1.034758-4.071838$

H -5.946858 $-0.614385-5.649915$

H $-4.498823-0.866377-6.629201$

H $-4.928734-2.025407-5.352118$

H -3.038351 $1.172075-2.492088$

H $-3.6173742 .588521-3.382362$

H -5.704265 2.605819-2.087656

H $-5.2922511 .068270-1.324146$
H -4.9958492 .9844580 .164258$

H $-4.1978394 .721625-1.475281$

H $-2.7251183 .809615-1.730393$

H $-1.276215 \quad 5.316193-0.920637$

H -1.085001 3.4598020 .294319

H -4.2981596 .2799610 .490423$

H $-3.286723 \quad 6.0192901 .893067$

H $-4.5695614 .858288 \quad 1.500240$

H $-0.901536 \quad 5.082192 \quad 1.872370$

H $-1.395618 \quad 1.6850552 .886610$

H -2.3909380 .4557202 .163142$

H $-3.932571 \quad 1.8766052 .727841$

H -3.971219 $-0.999321-0.370957$

H $-3.573582-3.8895540 .615948$

H $-6.034643-1.136827 \quad 1.252711$

H $-4.574301-0.3082821 .833246$

H $-5.731890-0.9774952 .973341$

H -0.9518590 .4843380 .083157$

H $\quad 0.094308 \quad 1.6769810 .857267$

H $1.066441-2.5107062 .700710$

H $-0.006055-3.4641811 .650983$

H $-0.866723-4.0537883 .802840$

H -1.530328 -1.078612 4.083552

H -2.049951 -2.121712 6.353237

H -2.411109-3.653967 5.591790

H $-5.387119-0.7061715 .676701$

H $-3.709540-0.3707206 .386301$

H -5.834222 -2.667655 4.424654

H $-4.862288-4.0160114 .936341$

H $-3.775175-4.1990042 .890585$

H $-1.323898-1.5485621 .171084$

C $1.974353-3.504844-2.128077$

O $2.655695-2.746606-1.102945$

C $3.922775-3.378372-0.830612$

C $3.803135-4.790284-1.383727$

C $2.989731-4.532445-2.634968$

H $4.679358-2.840783-1.414284$

C $4.259800-3.3036670 .661065$

H $4.777745-5.243194-1.588288$

H $3.254436-5.449168-0.700431$

C $2.356217-5.784323-3.217575$

H $3.642526-4.075848-3.391618$

C $4.344271-1.8670801 .218336$

O $3.256374-4.0059081 .403488$

H $5.200033-3.8323740 .855660$

H $3.351404-1.4043821 .202669$

O $4.730859-1.9516762 .595090$

C $5.355736-0.9674950 .484396$

C $5.6085720 .397853 \quad 1.149429$

H $5.018483-0.808464-0.545058$

O $6.610033-1.6523930 .408900$

H $6.0848090 .249456 \quad 2.127020$

H $6.3652970 .921006 \quad 0.551494$

H $3.127041-6.503102-3.513842$

H $1.699141-6.276249-2.492356$

H $1.759501-5.543867-4.103160$

C $1.487044-2.550974-3.223188$

H $2.394335-3.6399551 .142822$

H $4.080239-2.5187333 .043739$

H $7.216126-1.099570-0.113212$

H $1.534317-3.047386-4.199739$

H $2.135621-1.667980-3.274572$

H $1.133475-4.013049-1.638141$

C $4.1775452 .068500-0.962799$

$\begin{array}{llllllllll}\text { O } & 3.576344 & 1.333607 & 0.106228\end{array}$

C 4.3545821 .2789021 .303950

C $4.6417132 .699377 \quad 1.811763$

C 5.2841753 .5755310 .736631

C $4.4727923 .519510-0.560356$

H $5.1228721 .583163-1.237625$

C $3.277643 \quad 1.935977-2.201123$

H 3.7253440 .7933692 .058334

O 5.4890402 .6647832 .955960

H 3.6992593 .1694162 .122322

H 6.3289373 .2897160 .568002

$\begin{array}{lllll}\text { O } & 5.308561 & 4.921828 & 1.218077\end{array}$

H $3.5480594 .089470-0.423169$

O $5.2059004 .147001-1.610117$ 
C $1.8655812 .455256-2.022348$

H $3.7305672 .456119-3.052129$

H $3.1873370 .870084-2.446529$

H 5.0477642 .1196573 .629658

H 5.8103295 .4538780 .578119

H $5.3012915 .087048-1.382518$

O $1.1655752 .230430-3.174676$

O $1.4211522 .982681-1.012986$

C $-0.2008732 .652842-3.147896$

H $-0.7456582 .112478-2.368119$

H $-0.2617453 .732898-2.983431$

H -0.649142 2.420297-4.117240

SCF Energy (B3LYP/6-31G**//MMFF) $=-3245.90439504$

\section{7_00279}

MMTFF Geometry

C -3.311449 $0.838146-3.044356$

C $-2.701618-0.351505-3.175860$

C $-1.247986-0.655253-2.889427$

O $-0.6512940 .485242-2.228118$

C $-1.096035-1.895538-1.981467$

C $-0.783127-3.174874-2.734851$

C $0.371860-3.864450-2.644667$

C $-1.891570-3.706449-3.608089$

C $1.590027-3.556631-1.818573$

C $2.824727-3.351682-2.707762$

C $4.105977-2.987914-1.937264$

C $4.600104-4.133407-1.049425$

C $5.838503-3.734479-0.232831$

O $3.842304-1.817772-1.158895$

O $6.040839-4.733698 \quad 0.778889$

C $5.535321-2.4096840 .496551$

C $7.112153-3.718202-1.084951$

O $6.691125-1.9236871 .175185$

C $4.969202-1.312439-0.430920$

C $4.491928-0.0729430 .359093$

O $5.999614-0.874524-1.323888$

C $0.6787460 .700161-2.400699$

O $1.467030-0.005531-3.007957$

C $1.0038131 .981234-1.732903$

C $2.2389192 .492411-1.840101$

C $2.7116323 .751760-1.257482$

C $1.7369514 .548314-0.430356$

C $3.344774-0.3430041 .334643$

C 2.7179290 .9516151 .804861

C 3.1459312 .9375303 .244325

O $1.622168 \quad 1.3210441 .390672$

C 3.9327994 .0548602 .624234

C 5.0034003 .9572491 .819290

C 5.7413255 .1494721 .280147

C $5.905395 \quad 5.112776-0.221873$

C $7.1118454 .919983-0.781913$

C $4.6877845 .391754-1.074841$

C $3.9833224 .132279-1.501818$

N $3.507727 \quad 1.6393312 .709540$

H -2.751105 $1.710155-2.719299$

H -3.297231 -1.188380 -3.533599

H $-0.745979-0.810049-3.853373$

H -2.011009 -2.058562 -1.396037

H $-0.327332-1.697752-1.230925$

H $0.460559-4.782023-3.226483$

H -1.674281 -4.708108 -3.994284

H -2.825102 $-3.774870-3.039687$

H -2.049509 $-3.051466-4.470126$

H $1.445986-2.681127-1.182528$

H $1.748901-4.406109-1.145322$

H $3.006829-4.249068-3.312596$

H $2.607937-2.535320-3.409303$

H $4.870084-2.743640-2.685076$

H $4.805854-5.028466-1.649379$

H $3.815384-4.422933-0.338866$

H $6.152435-5.5915540 .334389$

H $4.793769-2.6383401 .272363$

H $7.303927-4.712893-1.505143$

H $7.990567-3.475795-0.476702$

H $7.064104-3.014235-1.918107$

H $7.013680-2.6407571 .747652$

H $\begin{array}{llll}5.338793 & 0.385946 & 0.884951\end{array}$
H $4.1580310 .687293-0.359502$

H $5.7692210 .011335-1.649740$

H $0.2123402 .475216-1.180793$

H $2.9785211 .940235-2.418793$

H $0.8949714 .882446-1.046605$

H $2.1747145 .439826 \quad 0.022832$

H $1.3465083 .945345 \quad 0.396617$

H $2.555242-0.9377090 .862091$

H $3.690833-0.9026842 .210373$

H 3.3301392 .9120954 .322912

H 2.0776783 .1143693 .081936

H 3.5781635 .0508422 .884501

H 5.4029982 .9817801 .556110

H 6.7247055 .1761771 .767399

H 5.2450156 .0868911 .560809

H $7.2399144 .923813-1.859422$

H $7.9963824 .749488-0.177319$

H $4.9975795 .916423-1.989112$

H $4.0340286 .099830-0.558994$

H $4.6030443 .481468-2.120950$

H 4.4048721 .2484612 .982311

C $-5.5060821 .750012-2.194557$

$\begin{array}{llll}\text { O } & -5.410050 & 0.967589 & -0.986621\end{array}$

C $-6.7344840 .661876-0.526890$

C $-7.622738 \quad 1.744375-1.124811$

C $-7.0017621 .907804-2.496670$

H $-7.000361-0.306642-0.968377$

C $-6.791298 \quad 0.553828 \quad 1.000841$

H $-8.6754301 .448488-1.161498$

H -7.550182 $2.686139-0.569299$

C $-7.352552 \quad 3.221552-3.174599$

H $-7.3486681 .082459-3.133022$

C $-5.697188-0.3101651 .660951$

O -6.6855241 .8622931 .580458$

H $-7.7821150 .185337 \quad 1.292059$

H $-4.767543 \quad 0.265746 \quad 1.724104$

O $-6.107305-0.5525943 .014135$

C $-5.406612-1.6609700 .989075$

C $-4.515908-2.6166541 .808268$

H $-4.936201-1.4831170 .015269$

$\begin{array}{lllll} & \mathrm{H} & -6.644788 & -2.332273 & 0.740017\end{array}$

H $-5.102083-3.0525522 .628022$

H $-4.267971-3.4587801 .148258$

H $-8.4341613 .299990-3.325517$

H $-7.0337844 .080323-2.574479$

H $-6.8675983 .295222-4.153140$

C $-4.7698561 .046896-3.340290$

H $-5.8476582 .251103 \quad 1.274940$

H -6.2573700 .3133593 .430969$

H $-6.441289-3.1553440 .263679$

H $-4.8553311 .648258-4.252941$

H $-5.2469040 .078842-3.539796$

H $-5.0405122 .719688-1.978685$

C $-1.263968-0.7551011 .678351$

O $-2.472204-1.4330051 .299068$

C $-3.224356-2.0001272 .375225$

C $-2.383642-3.0386103 .134910$

C $-1.064495-2.4264063 .616658$

C $-0.342609-1.6820152 .491097$

H $-0.751985-0.5365340 .733791$

C $\begin{array}{lll}\text {-1.554236 } & 0.587505 & 2.369047\end{array}$

H -3.508114 -1.208490 3.074490

O $-2.110960-4.1497312 .283550$

H -2.964268 -3.4094723 .988061$

H $-0.408519-3.2171734 .000523$

O $-1.319616-1.5137844 .680521$

H $0.491176-1.1019052 .901723$

O $0.234681-2.6403811 .598891$

C $-2.031312 \quad 1.627348 \quad 1.376048$

H -2.315642 0.5112523 .148511

H -0.6303200 .9658212 .820990$

H -1.603534 -4.798050 2.800821

H $-1.752564-2.0101315 .395759$

H $-0.492262-3.104668 \quad 1.149295$

$\begin{array}{lllll}\text { O } & -1.862643 & 2.871748 & 1.912341\end{array}$

$\begin{array}{llll}0 & -2.513578 & 1.382522 & 0.278805\end{array}$

C -2.278436 $3.957801 \quad 1.078455$

H -2.0820794 .8912471 .612505$ 
H -1.7063493 .9603780 .145726$

H -3.351312 3.8884050 .873927

SCF Energy (B3LYP/6-31G**//MMFF) $=-3245.90711554$

0700280

MM̄FF Geometry

C -0.614970 -3.084410 2.665756

C $0.271922-2.1242462 .366557$

C $1.746264-2.2069672 .644551$

O $2.388146-2.0989691 .350423$

C $2.232973-1.0422583 .526515$

C $1.583188-0.9521484 .891568$

C 0.7017450 .0064325 .241376

C $2.026818-1.9863765 .890561$

C 0.1469931 .0718264 .337442

C 0.9894292 .3531254 .359920

C 0.7700883 .2573263 .135150

C -0.6757583 .7389263 .001440$

C -0.8838604 .5713551 .727812$

O $1.1592282 .513422 \quad 1.974817$

O

C $-0.3195593 .784357 \quad 0.526157$

C -0.3359505 .9935881 .885898$

O $-0.393798 \quad 4.561922-0.666769$

C 1.1151553 .2469160 .745492

C $1.4855232 .275556-0.405816$

$\begin{array}{llllll}\text { O } & 2.020377 & 4.348684 & 0.758037\end{array}$

C $3.659989-2.5573531 .222106$

O $4.343642-3.0903752 .080112$

C $4.084281-2.267752-0.166094$

C $5.364522-2.439365-0.525645$

C $5.930780-2.168523-1.849692$

C $5.009076-1.607206-2.901421$

C $2.7969911 .496597-0.223644$

C $4.0454552 .250178-0.647794$

C $6.5328742 .063153-0.599881$

O $4.0132953 .331300-1.228857$

C $7.0187841 .464370-1.882403$

C $8.1919350 .823978-2.002019$

C $8.707598 \quad 0.258046-3.294466$

C $9.118053-1.193130-3.177965$

C $10.409601-1.536716-3.037779$

C $8.041885-2.247051-3.313859$

C $7.240933-2.420405-2.053345$

N $5.202573 \quad 1.561537-0.308585$

H $-0.280271 \quad-3.997163 \quad 3.149979$

H - $0.078100-1.2220251 .869995$

H $2.001123-3.1741603 .095585$

H $2.125129-0.1031692 .970034$

H $3.318857-1.1259423 .669116$

H $\quad 0.316792 \quad 0.018780 \quad 6.259351$

H $1.567899-1.8369786 .873688$

H $1.755202-2.9901255 .549350$

H $3.112838-1.9455116 .023591$

H $0.047196 \quad 0.6859793 .318727$

H $-0.876050 \quad 1.297413 \quad 4.662868$

H 0.7797912 .9139895 .279101

H 2.0536422 .0832894 .386032

H 1.4447924 .1149133 .243006

H -0.9809504 .3060803 .889473$

H -1.3538082 .8767342 .949505$

H -2.447452 5.1517370 .692051

H $-0.987462 \quad 2.9278020 .368917$

H -0.8347416 .5028432 .719335$

H $-0.545866 \quad 6.6007320 .998401$

H 0.7390036 .0208522 .076152

H $0.3322465 .208355-0.643399$

H $\quad 0.692198 \quad 1.520772-0.473375$

H $1.5061142 .814183-1.360567$

H 2.9210264 .0124370 .632741

H $3.329230-1.902548-0.853284$

H $\quad 6.068888-2.8066270 .219569$

H $4.227515-2.332686-3.152342$

H $5.515306-1.345386-3.832544$

H $4.532099-0.686718-2.546804$

H 2.9117421 .1537620 .809952

H $2.7493540 .604713-0.861093$

H $7.171947 \quad 1.7991760 .248548$
H $6.5027823 .153688-0.689190$

H $6.3873361 .586270-2.759370$

H $8.8353670 .728625-1.130243$

H $9.5696300 .865049-3.601145$

H $7.9707860 .363399-4.100467$

H $10.715615-2.576222-2.982937$

H $11.189476-0.785516-2.969080$

H $8.496929-3.220288-3.542872$

H $7.424337-2.019599-4.186902$

H $7.825295-2.822066-1.224075$

H 5.1264970 .6397040 .111523

C $-2.488714-3.8267701 .162863$

O $-3.435213-3.0969660 .353764$

C $-4.485731-3.990645-0.053523$

C $-3.999845-5.3959350 .283796$

C $-3.181089-5.137701 \quad 1.534129$

H $-5.352817-3.7586710 .576288$

C $-4.842739-3.770177-1.527878$

H $-4.827481-6.0928110 .447059$

H -3.362026 -5.808464 -0.506421

C $-2.231637-6.269843 \quad 1.882587$

H -3.872774 -4.977668 2.372118

C $-5.173045-2.311682-1.910893$

O $-3.741820-4.181873-2.349887$

H $-5.678259-4.425370-1.800829$

H $-4.251043-1.719831-1.939884$

O $-5.684085-2.322875-3.250266$

C $-6.200528-1.616858-1.002545$

C $-6.727880-0.268668-1.529255$

H -5.759899-1.466940 -0.010281

O $-7.326897-2.483354-0.833652$

H -7.375372 -0.437833 -2.399486

H $-7.388500 \quad 0.135047-0.750103$

H -2.785674 -7.200744 2.042247

H -1.504051 -6.444347 1.083092

H $-1.678999-6.0473022 .800258$

C -2.078809 -2.959282 2.356370

H -2.963392 -3.666283 -2.076834

H $-5.017803-2.756273-3.810787$

H -7.934906 -2.055622 -0.206808

H $-2.648842-3.228178 \quad 3.253627$

H -2.328945 -1.909518 2.158193

H -1.629381 -4.019978 0.507422

C $-3.7252881 .878992-0.900356$

O $-4.8166210 .961837-0.733304$

C $-5.6473090 .767604-1.880966$

C $-6.2682152 .099220-2.336981$

C $-5.1917603 .165598-2.563724$

C $-4.2249113 .253067-1.378964$

H -3.3154512 .0116570 .108961$

C $-2.6164921 .297877-1.793672$

H $-5.0431030 .363167-2.698670$

O $-7.1919932 .560766-1.352851$

H $-6.8320791 .926110-3.261438$

H $-5.6679874 .142566-2.711072$

O $-4.4538232 .862290-3.743470$

H -3.364946 $3.884761-1.630987$

O $-4.890627 \quad 3.875222-0.276595$

C $-1.8928070 .142040-1.136934$

H $-3.0114930 .935504-2.745971$

H -1.864587 2.069568 -1.995082

H -7.589059 $3.383658-1.685349$

H $-5.0822902 .835834-4.484885$

H $-5.1598804 .765238-0.561088$

O $-1.016136-0.396669-2.036135$

O $-2.055262-0.2305880 .016003$

C -0.229797 -1.490140 -1.552953

H $0.411027-1.158471-0.731319$

H $-0.876850-2.312183-1.234660$

H $0.404874-1.840541-2.371315$

SCF Energy $\left(B 3 L Y P / 6-31 G^{* *} / / M M F F\right)=-3245.91033773$

07 00281

MM̄FF Geometry

C $1.5731055 .327098-0.099681$

C 0.7620574 .5456880 .628801

C -0.5950334 .0884970 .158765$

O -0.6670962 .6530640 .328257$ 
C -1.6938784 .7156791 .033545$ C -3.0548334 .7055010 .365293$ C $-4.016142 \quad 3.7856580 .578667$ C $-3.3144385 .866378-0.561547$ C -3.9336722 .5535851 .437803$ C $-4.403734 \quad 1.293590 \quad 0.697955$ C $-5.925786 \quad 1.1820370 .482989$ C $-6.6753300 .983591 \quad 1.805098$ C $-8.1693810 .697515 \quad 1.595239$ O O C $-8.314438-0.4436430 .569261$ C $-8.952507 \quad 1.970286 \quad 1.253392$ O $-9.684342-0.6641130 .242015$ C -7.494161 -0.216740 -0.718137 C $-7.530970-1.446754-1.663216$ O $\quad-8.0618810 .880269-1.445393$ C $-0.1210331 .867874-0.637939$ O $\quad 0.3854252 .233273-1.686210$ C $-0.2162160 .466588-0.167523$ C $0.362763-0.514429-0.875672$ C $0.407099-1.934799-0.514667$ C $-0.260239-2.3438180 .772074$ C $-6.953938-2.755746-1.112439$ C $-5.467176-2.676792-0.832207$ C $-3.279664-2.333462-1.970854$ O $-5.025961-2.6702350 .315059$ C $-2.538426-3.614608-1.764071$ C $-1.645976-4.105873-2.636583$ C $-0.823190-5.334955-2.377386$ C $0.663344-5.045301-2.372130$ C $1.461844-5.510484-3.347457$ C $1.234757-4.277116-1.196958$ C $1.047552-2.789474-1.340900$ N $-4.706167-2.583876-1.986622$ H $1.2446295 .670972-1.077473$ H 1.0844214 .2085941 .611199 H $-0.7540064 .342255-0.896887$ H -1.4462255 .7607081 .265329$ H -1.7435574 .2061642 .003770$ H -4.9697643 .9312350 .074439$ H -4.309205 $5.822210-1.017564$ H -3.243929 $6.812067-0.014274$ H -2.581304 $5.879553-1.373983$ H $-2.9061072 .375176 \quad 1.767467$ H -4.5224802 .7140642 .346002$ H -3.896188 $1.226097-0.274261$ H $-4.0639360 .409768 \quad 1.255016$ H -6.286167 $2.075903-0.035931$ H $-6.554248 \quad 1.8495982 .465348$ H -6.2358910 .1330582 .345216$ H -8.546935 0.8924623 .516231 H -7.961628 -1.3578661 .061898$ H -8.878970 2.6963552 .072163 H -10.0209291 .7578241 .137399$ H -8.596932 2.4635620 .346600 H - $10.162431-0.8053531 .077140$ H -8.566169 -1.625313 -1.981691 H $-6.987652-1.189215-2.582554$ H $-7.7768120 .816547-2.371939$ H -0.7353790 .2852780 .766984$ H $\quad 0.873527-0.253338-1.801817$ H $0.189956-1.8161931 .619400$ H $-0.179819-3.4102210 .989450$ H -1.329335 -2.107098 0.746021 H -7.470539 $-3.072687-0.201425$ H -7.115020 -3.557218 -1.844547 H -3.050192 -1.638052 -1.156611 H -3.020396 -1.851461 -2.918917 H -2.696286 -4.130020 -0.819567 H -1.474944 -3.585492 -3.576171 H -1.068858 -6.071906 -3.152664 H -1.096162 -5.803002 -1.423193 H $2.530968-5.325792-3.338109$ H $\quad 1.064527-6.081593-4.179913$ H $2.314146-4.462900-1.119451$ H $0.810978-4.679006-0.273202$ H $\quad 1.516517-2.381849-2.237912$
H $-5.162223-2.658354-2.891005$

C $4.0206245 .351584-0.631727$

O $4.1478103 .916312-0.628406$

C $5.5430203 .571184-0.729301$

C $6.2901154 .874664-0.991443$

C $5.4089055 .880597-0.277423$

H 5.8434943 .1870420 .252323

C $5.7448452 .491882-1.799384$

H $7.3138824 .848905-0.605504$

H $6.3425935 .107144-2.061319$

C $5.6565787 .315879-0.707978$

H 5.5803825 .7928740 .803957

C $4.8856861 .227238-1.600220$

O $5.4099233 .036030-3.083497$

H $6.8051582 .220219-1.855524$

H $3.8250071 .462586-1.751815$

O $5.241920 \quad 0.292821-2.627715$

C $5.0681950 .538869-0.235001$

C $4.234771-0.744562-0.120089$

H $4.763923 \quad 1.2303610 .555983$

O $6.4451800 .233534-0.027589$

H $3.178153-0.503950-0.275818$

H $4.499721-1.436672-0.930811$

H $6.6903147 .607813-0.495913$

H $5.4833627 .448567-1.781222$

H $4.9957398 .004531-0.172681$

C 2.9335345 .7772690 .355722

H $4.4865283 .339227-3.041639$

H $5.1124090 .739141-3.482101$

H $6.725090-0.359381-0.746092$

H $2.915866 \quad 6.8689420 .447888$

H 3.1538005 .3654461 .348342

H $3.7472635 .646617-1.653731$

C $3.580929-3.5809532 .186340$

O $3.530156-2.6501861 .099271$

C $4.406701-1.5188431 .196850$

C $4.076414-0.7051202 .454960$

C $4.120465-1.5878743 .708371$

C $3.319759-2.8802863 .530654$

H $2.749599-4.2724461 .997469$

C $4.876227-4.3995452 .167395$

H $5.441078-1.8752301 .247003$

O $2.781952-0.1241062 .314613$

H 4.7981120 .1128162 .558365

H $3.715210-1.0316474 .562648$

O $5.472236-1.9231034 .009968$

H $3.531760-3.5697304 .356375$

O $1.931520-2.5480423 .612727$

C $4.921457-5.2851920 .944860$

H $5.781753-3.7864392 .184817$

H $4.932511-5.0563903 .043705$

H 2.6022510 .3916013 .118930

H $5.956838-1.0918344 .150381$

H $1.426035-3.3766423 .555503$

O $5.530727-4.629631-0.083943$

O $4.447808-6.4134090 .903708$

C $5.611432-5.354020-1.314944$

H $4.612572-5.642805-1.655117$

H $6.060748-4.698866-2.065926$

H $\quad 6.247068-6.235708-1.190737$

SCF Energy (B3LYP/6-31G**//MMFF $)=-3245.90474570$

0700282

MM̄MF Geometry

C $2.047240 \quad 3.2259812 .615110$

C 1.2054672 .2961242 .138444

C 0.6524881 .1647142 .968017

O $-0.693965 \quad 0.9454792 .491547$

C $1.509402-0.0900742 .730655$

C $1.201002-1.2463323 .658916$

C $0.569856-2.3782193 .290171$

C $1.711917-1.0950435 .068784$

C $-0.007307-2.7053591 .941070$

C $-1.418974-3.2942282 .072473$

C $-2.167386-3.4394380 .738217$

C $-1.441593-4.346247-0.258761$

C $-2.178104-4.423347-1.605145$

O $-2.347168-2.129841 \quad 0.188047$ 
O $-1.285658-5.014496-2.562767$

C $-2.470010-2.987195-2.088916$ C $-3.406828-5.336920-1.538179$ O $-3.270387-2.997245-3.268349$ C $-3.128882-2.097826-1.013247$ C $-3.243681-0.623917-1.454782$ O $-4.461876-2.565201-0.771459$ C $-1.6442270 .544016 \quad 3.374873$ $\begin{array}{lllll}\text { O } & -1.501049 & 0.284521 & 4.557811\end{array}$ C -2.9205420 .4925612 .633917$ C $-4.017382-0.0434903 .185144$ C $-5.303315-0.0985522 .500016$ C $-5.907522-1.4725222 .449593$ C -1.917506 $0.075480-1.764728$ C $-2.1919971 .536660-2.049149$ C $-2.7377483 .660021-0.874137$ O $\quad-2.4497911 .933268-3.183579$ C $-4.1219943 .598471-0.300923$ C $-5.1835953 .124210-0.972029$ C $-6.5750803 .034902-0.412029$ C $-7.0111991 .607471-0.151124$ C $-7.3116920 .773540-1.161238$ C $-7.174337 \quad 1.173414 \quad 1.289382$ C -5.8576551 .0226851 .996580$ N -2.191839 2.316497 -0.903957 H 2.3356013 .1640633 .663204 H 0.9120252 .3075561 .092903 H 0.6375841 .4532834 .025966 H 2.5690180 .1647072 .868509 H $1.423785-0.4004701 .683333$ H $0.450998-3.1629694 .036529$ H $1.505139-1.9775935 .683448$ H $2.795722-0.9446105 .065520$ H $1.243563-0.2372795 .560436$ H $-0.043077-1.8259981 .293261$ H $0.653581-3.4376641 .464543$ H -1.362895 -4.273303 2.564973 H -2.012407 -2.641126 2.725513 H $-3.154300-3.8561720 .972918$ H -1.297433 $-5.349074 \quad 0.161649$ H $-0.435397-3.955279-0.456799$ H - $1.024600-5.889899-2.228896$ H -1.506017 -2.547831 -2.372125 H $-3.110281-6.357137-1.266210$ H -3.892694 -5.417146 -2.516871 H -4.149925 $-5.006275-0.809669$ H -2.819252 -3.560941 -3.920019 H $-3.915106-0.544926-2.320352$ H $-3.749199-0.064550-0.655044$ H -4.985496 -1.833660 -0.404475 H -2.9425630 .9061241 .632692$ H -3.984710 -0.492115 4.174598 H $-6.268199-1.7668273 .441046$ H -5.163332 -2.208807 2.124783 H -6.743310 -1.548432 1.749970 H -1.212150 -0.014007 -0.931179 H -1.433018 -0.350642 -2.648945 H $-2.7428504 .096827-1.877901$ H -2.090651 4.267510 -0.234047 H $-4.247314 \quad 3.9523880 .719072$ H $-5.0477142 .774323-1.993128$ H -7.266710 $3.501762-1.125370$ H $-6.662773 \quad 3.6293360 .505896$ H -7.646453 $-0.241690-0.976168$ H -7.225445 $1.088279-2.196102$ H $-7.768540 \quad 0.2559331 .346835$ H -7.769791 1.9266911 .821316 H $-5.322247 \quad 1.9626542 .132784$ H -1.968956 $1.883274-0.013754$ C $2.225903 \quad 4.5988900 .416391$ O $2.5479703 .472625-0.417661$ C $2.7866123 .937199-1.759393$ C $2.6757375 .461032-1.722760$ C $2.9228345 .777009-0.259856$ H $3.8169053 .658117-2.006717$ C $1.8129703 .257156-2.733013$ H $3.4019245 .938355-2.388644$ H $1.6823205 .809061-2.027709$
C 2.3986497 .1398890 .158290

H $4.0038985 .730653-0.072250$

C $1.7713371 .717496-2.638826$

O $0.4822373 .728206-2.476276$

H $2.0440863 .566265-3.758807$

H $1.3176911 .417932-1.687382$

O $\quad 0.893319 \quad 1.245408-3.670055$

C $3.1327571 .023165-2.815222$

C $3.039120-0.515122-2.866877$

H $3.8127741 .325126-2.011977$

O $3.711156 \quad 1.473577-4.043716$

H $2.322530-0.814642-3.641175$

H $4.001474-0.901916-3.222038$

H $2.8755727 .931772-0.428643$

H 1.3163087 .2160620 .009302

H 2.6074627 .3334691 .214793

C 2.6981934 .3525191 .852721

H $0.2572743 .490282-1.560451$

H $0.0617471 .744015-3.597619$

H $4.5992331 .083497-4.110971$

H 2.5446665 .2669922 .437754

H 3.7767924 .1497751 .847724

H 1.1358174 .7300150 .392702

C $4.888547-1.315541-0.595904$

O $3.566668-0.782227-0.468395$

C $2.667533-1.164141-1.516407$

C $2.552151-2.694593-1.569115$

C $3.916500-3.366756-1.712695$

C $4.884620-2.849542-0.645938$

H $5.331495-0.935250-1.525013$

C $5.763670-0.7311400 .523406$

H $1.680946-0.779597-1.232038$

O $1.712788-3.102342-2.644616$

H $2.085964-3.054202-0.643028$

H $4.320067-3.227682-2.722480$

O $3.736651-4.774978-1.541037$

H $4.600454-3.2760870 .321705$

O $6.204618-3.303552-0.937408$

C $5.350155-1.1058381 .931353$

H $6.802575-1.0483860 .378390$

H 5.7184650 .3637740 .466969

H $0.850778-2.669738-2.522287$

H $4.586149-5.205590-1.735230$

H $\quad 6.205074-4.273060-0.869267$

O $6.273678-0.6024182 .804644$

O $4.349576-1.7375962 .237526$

C $6.016181-0.8526664 .189863$

H $6.884819-0.5172584 .762815$

H $5.140748-0.2822564 .512679$

H $5.870791-1.9221114 .369928$

SCF Energy (B3LYP/6-31G**//MMFF $)=-3245.89350529$

0700283

MM̄MF Geometry

C $2.434710-1.0167713 .171142$

C 1.8469020 .1046483 .616868

C 2.2861481 .5261283 .343520

O $3.379859 \quad 1.5333542 .397197$

C 1.1317162 .3802992 .782701

C 0.0432842 .6786843 .792798

C -1.2179152 .2106313 .726128$

C 0.4350383 .6140804 .908874

C $-1.821030 \quad 1.3097872 .684955$

C $-2.8706702 .048748 \quad 1.844739$

C $-2.269308 \quad 3.1227360 .924425$

C -3.3786703 .9464770 .268343$

C $-2.8101775 .000447-0.691323$

O $-1.4946592 .459617-0.079829$

O $-3.8942955 .483362-1.503489$

C $-1.8099254 .317169-1.647889$

C -2.2743996 .2223590 .063635$

O $-1.1285975 .276804-2.452394$

C $-0.8071243 .346062-0.972260$

C $-0.1487162 .468678-2.060535$

O $0.2581864 .032487-0.316751$

C 4.2088712 .6089292 .403583

O 4.0868193 .6189933 .083880

C 5.3378752 .4402181 .460210 
C $5.661278 \quad 1.2690050 .890679$

C $6.806631 \quad 1.0249870 .011258$

C $7.6277032 .210008-0.424521$

C $0.8975631 .475169-1.534303$

C $1.4570420 .626355-2.665244$

C $3.063449-1.178334-3.155907$

O $1.0199050 .671608-3.813304$

C $4.386118-1.655894-2.651703$

C $5.522872-1.521714-3.351855$

C $6.854663-2.070997-2.927208$

C $7.865865-0.987098-2.631801$

C $8.477513-0.317784-3.623620$

C $8.228109-0.738365-1.187257$

C $7.071890-0.243295-0.364823$

N $2.498486-0.195650-2.250755$

H $3.323148-0.9572532 .549251$

H $0.983946-0.0022754 .271459$

H 2.6510241 .9368904 .293020

H $\quad 0.739687 \quad 1.9035531 .877634$

H 1.5214853 .3399432 .418632

H -1.9153862 .5103614 .507883$

H -0.4207183 .9032345 .528530$

H 1.1724113 .1455765 .567375

H 0.8656524 .5339964 .499789

H -1.071118 0.8475772 .037254

H -2.3125360 .4800043 .208356$

H -3.3903941 .3019941 .234342$

H -3.617612 2.5002732 .509772

H -1.616495 3.7788941 .509130

H $-4.0111124 .423747 \quad 1.027341$

H $-4.0427453 .282996-0.300061$

H $-4.5816315 .832860-0.910888$

H $-2.4178673 .726943-2.348246$

H -3.0854396 .7167410 .612661$

H -1.882590 $6.976497-0.627179$

H -1.4987635 .9766900 .790788$

H - $0.4360555 .688065-1.908840$

H $-0.9303761 .902558-2.584728$

H $0.3412523 .110628-2.804320$

H $-0.0638704 .881450 \quad 0.016151$

H 5.9320193 .3346801 .302020

H 5.0569090 .3909621 .110667

H $6.9881352 .978881-0.872611$

H $8.3762971 .962045-1.181048$

H 8.1571482 .6462670 .429323

H $1.7275242 .010966-1.060337$

H $0.4489260 .804172-0.793622$

H $3.136190-0.739759-4.157365$

H $2.363509-2.017287-3.215190$

H $4.401112-2.174281-1.696392$

H $5.491572-1.018952-4.316330$

H $7.233736-2.711432-3.734656$

H $6.748702-2.735476-2.060555$

H $9.2243180 .441840-3.418040$

H $8.242580-0.515207-4.664423$

H $9.084660-0.059835-1.105435$

H $8.582432-1.680034-0.747078$

H $\quad 6.414577-1.039345-0.013180$

H $2.657091-0.307534-1.252958$

C $1.898712-3.3182302 .304668$

O $0.902330-2.862374 \quad 1.369543$

C $0.274985-4.0120070 .766577$

C $1.003228-5.2408861 .304835$

C $1.495708-4.7502172 .650240$

H $-0.759420-4.0304821 .125922$

C $0.302490-3.897427-0.761687$

H $\quad 0.342216-6.1099661 .380283$

H $1.849826-5.5247230 .668940$

C $2.619016-5.5948373 .226045$

H $0.650463-4.7440893 .351515$

C $-0.377743-2.635045-1.327010$

O $1.666676-3.889476-1.203602$

H $-0.148878-4.793572-1.203098$

H $0.204967-1.747606-1.058410$

O $-0.323859-2.722654-2.757517$

C - $-1.841499-2.434416-0.891829$

C -2.512983 -1.272476 -1.651891

H -1.874960 -2.2361300 .185159$
O $-2.550604-3.648225-1.135947$

H -1.860464 -0.392582 -1.613959

H -2.633655 -1.568181 -2.701579

H $2.287964-6.6286203 .369234$

H $3.490324-5.6097432 .562673$

H $2.942079-5.2081304 .197538$

C $1.947334-2.3959903 .523337$

H $2.103738-3.125072-0.790314$

H $-0.875041-3.477775-3.026096$

H -3.458531 -3.530369 -0.809805

H $2.631644-2.8113564 .271648$

H $0.951134-2.3371093 .979130$

H $2.862862-3.3069771 .778577$

C $-6.097759-1.841786-0.734024$

O $-4.775951-2.004244-1.263640$

C $-3.895400-0.892011-1.086651$

C $-4.4715380 .352307-1.782095$

C $-5.9038120 .646190-1.322986$

C $-6.782914-0.610318-1.347550$

H $-6.643154-2.732779-1.071427$

C $-6.104325-1.8433870 .798861$

H -3.776369-0.694073 -0.016799

O $-4.4596770 .179049-3.198589$

H $-3.8269621 .210263-1.562242$

H $-6.3359001 .411824-1.978788$

O $-5.863131 \quad 1.179408-0.003430$

H -7.733828 $-0.428908-0.833248$

O $-7.095595-0.930036-2.706457$

C $-5.593996-3.1611681 .332649$

H $-5.505778-1.0352961 .229128$

H $-7.121804-1.7088681 .184523$

H -4.965072 -0.626173 -3.403876

H $-6.775490 \quad 1.394530 \quad 0.254815$

H -7.572814 -0.171465 -3.083808

O $-4.330941-3.0079541 .823181$

O $-6.232902-4.2047721 .295853$

C -3.723230 -4.192942 2.346597

H $-2.753422-3.9183102 .769841$

H $-4.342142-4.6222873 .140251$

H $-3.564259-4.9188531 .543853$

SCF Energy $(B 3 L Y P / 6-31 G * * / / M M F F)=-3245.90962973$

0700284

MMFF Geometry

C $3.924792-2.0974792 .396129$

C $3.192521-0.9843752 .554894$

C $1.814397-0.8684653 .160312$

O $0.958270-0.3357982 .118289$

C $1.196615-2.1833033 .671941$

C $-0.130350-1.9928594 .380931$

C $-1.331404-2.3041033 .854469$

C $-0.037204-1.4658005 .789897$

C -1.617188 -2.832520 2.475004

C $-2.816809-2.1192551 .844648$

C $-3.197066-2.6749430 .464212$

C $-2.152570-2.354538-0.607752$

C $-2.610221-2.802579-2.004518$

O $-4.444334-2.0654630 .112001$

O $-1.741527-2.197889-2.975286$

C $-4.046009-2.293536-2.266982$

C $-2.421395-4.312879-2.184813$

O $-4.566923-2.840566-3.475653$

C $-5.027321-2.559969-1.099582$

C $-6.356644-1.804301-1.332941$

O $-5.365737-3.945373-1.000145$

C 0.2650110 .8044102 .381844

O $\quad 0.275001 \quad 1.4512263 .417043$

C $-0.517100 \quad 1.146026 \quad 1.171610$

C - -1.1980022 .3011121 .136477$

C -1.999185 2.8079010 .022057

C $-2.2260151 .903004-1.158805$

C -7.382126 -1.966740 -0.200319

C $-7.076673-1.1114161 .015006$

C -7.0193791 .2469041 .794610$

O $-6.728802-1.6052522 .085081$

C -6.8749252 .5789161 .133615$

C -5.7253933 .2686911 .087348$

C -5.5761344 .5808930 .369603$ 
C $-4.7936554 .436194-0.916884$

C $-5.4085854 .056777-2.050482$

C $-3.3245374 .790766-0.917398$

C -2.5120524 .0530280 .110242$

N $\quad-7.2270680 .2431840 .768142$

H $3.537796-3.0572302 .723884$

H $3.620419-0.0414362 .220631$

H $1.901777-0.1601223 .992998$

H $1.883650-2.6689034 .377788$

H $1.084619-2.8871752 .839727$

H -2.211920 -2.169555 4.481669

H -1.016779 -1.400341 6.275522

H $0.588815-2.1240826 .401102$

H $0.400029-0.4629015 .798577$

H $-0.749800-2.7226561 .820762$

H -1.834927 -3.903933 2.555865

H -3.688250 -2.212201 2.507621

H -2.626356 -1.040895 1.769863

H -3.345384 -3.757132 0.556524

H -1.181430 -2.804300 -0.371962

H -1.980973 -1.271256-0.629301

H - $1.783468-1.232908-2.862970$

H -3.986638 -1.206610 -2.418204

H -1.359178 $-4.575743-2.107305$

H $-2.736377-4.637350-3.182623$

H $-2.959777-4.904053-1.440797$

H -3.931453 -2.637326 -4.183342

H $-6.148441-0.738882-1.488074$

H -6.825234 -2.185394 -2.249291

H -4.992364 -4.413155-1.764110

H -0.4956980 .4507130 .340915$

H -1.149684 2.9572172 .005051

H -1.276280 $1.594598-1.603996$

H -2.806865 2.372056-1.956538

H $-2.7764531 .007891-0.849819$

H $-8.373715-1.672276-0.567425$

H -7.469782 -3.0117210 .116276$

H $-6.140846 \quad 0.9733192 .388944$

H $-7.891347 \quad 1.2413992 .456598$

H -7.7597392 .9850760 .648837$

H -4.8414562 .8597721 .569833$

H -5.0891625 .3048001 .034109$

H -6.5626115 .0112420 .150179$

H -4.867730 $3.970336-2.986971$

H $-6.4679153 .822185-2.065640$

H $-3.2303505 .866555-0.718324$

H -2.877306 4.648155 -1.909222

H -2.317564 4.6361581 .010856

H -7.688265 $0.525791-0.092381$

C $5.359129-2.7072350 .382166$

O $4.624811-1.875539-0.535798$

C $3.963185-2.723806-1.495737$

C $4.531929-4.125024-1.290555$

C $4.808606-4.1209090 .201402$

H $2.900770-2.733745-1.224651$

C $4.135016-2.156469-2.909380$

H $3.829024-4.905932-1.596136$

H $5.463612-4.273891-1.848640$

C $5.758526-5.2154500 .653016$

H $3.851028-4.2410870 .722107$

C $3.710889-0.680219-3.051189$

O $5.516598-2.239980-3.285130$

H $3.590176-2.777943-3.628891$

H $4.404848-0.047664-2.487293$

O $3.854730-0.308953-4.427660$

C $2.261522-0.388451-2.623724$

C $1.8106751 .065481-2.864157$

H $2.130223-0.626110-1.563231$

O $1.373943-1.247304-3.344471$

H $1.8842601 .313490-3.930116$

H $\quad 0.742041 \quad 1.114222-2.622955$

H $5.341202-6.202950 \quad 0.430550$

H $\quad 6.728146-5.1396240 .149479$

H $5.933142-5.157527 \quad 1.732045$

C $5.301509-2.1111161 .790752$

H $6.031433-1.748028-2.622358$

H $4.772409-0.502710-4.685476$

H $1.499125-1.069416-4.292537$
H $5.693008-1.0864181 .752683$

H $5.963506-2.6753492 .457876$

H $6.404673-2.6726390 .046992$

C 3.1334712 .5658710 .276650

O $2.3752561 .773905-0.646834$

C $2.5815722 .095193-2.025953$

C $2.1241443 .535193-2.297983$

C $2.8557504 .520735-1.379367$

C 2.8431694 .0665700 .086434

H 2.7527782 .2685121 .261343

C $4.6187882 .179737 \quad 0.210793$

H $3.6472342 .012909-2.263438$

O $0.7179893 .637202-2.090059$

H $2.3236613 .778829-3.348395$

H $2.3794195 .505629-1.459998$

O $4.1977404 .653513-1.839245$

H 3.5520174 .6637450 .670605

O 1.5388884 .3427780 .607459

C 5.3496452 .4287101 .511493

H $4.6927381 .106486-0.006391$

H $5.1445812 .713512-0.584855$

H $0.4572534 .554391-2.279675$

H $4.6388465 .307844-1.271056$

H 1.5446904 .1148901 .552864

O $6.576138 \quad 1.8298361 .449534$

O 4.9128253 .0696722 .457184

C 7.3869051 .9652402 .620772

H 6.8871601 .5109843 .481691

H 8.3289191 .4392442 .444639

H 7.6041103 .0205452 .811057

SCF Energy (B3LYP/6-31G**//MMFF $)=-3245.90599214$

07_00285

MM̄MF Geometry

C $3.115057-0.324570-3.171938$

C $2.315034-1.366805-2.897208$

C $0.806571-1.360816-2.905905$

O $0.298469-0.007982-2.826222$

C $0.213945-2.015120-4.165442$

C $0.308570-3.527863-4.167208$

C $-0.522799-4.338141-3.480524$

C $1.407079-4.108984-5.016977$

C $-1.680654-3.924177-2.612750$

C $-1.375769-4.139532-1.123312$

C $-2.456919-3.594397-0.173980$

C $-3.736452-4.430652-0.210255$

C $-4.826461-3.8459970 .699929$

O $-2.726243-2.236193-0.529654$

$\begin{array}{llll}\text { O } & -6.043696 & -4.535316 & 0.375911\end{array}$

C $-5.007017-2.3548360 .346380$

C $-4.546608-4.1248392 .180840$

O $-5.913484-1.7163371 .242785$

C $-3.678545-1.5647630 .304659$

C $-3.871435-0.140864-0.272270$

O $-3.172694-1.4214271 .636433$

C $0.0088310 .460328-1.580275$

O $0.093061-0.153060-0.527432$

C $-0.4274971 .868457-1.706797$

C $-0.8187672 .519889-0.601523$

C $-1.3000943 .899177-0.515339$

C $-1.3550344 .698957-1.788704$

C $-4.395008-0.110808-1.709689$

C $-4.232376 \quad 1.251067-2.353175$

C $-5.1978393 .543318-2.378207$

O $-3.4294121 .435113-3.265619$

C $-5.0650374 .628762-1.353963$

C $-4.9375774 .505250-0.023247$

C $-4.7993725 .686390 \quad 0.896872$

C -3.5546085 .6286561 .754127$

C -3.6408565 .5617943 .093589$

C -2.2085805 .7237251 .065144$

C -1.6676544 .3679620 .695788$

N $-5.0759742 .210899-1.817495$

H $2.6769790 .618069-3.485063$

H $2.772444-2.310529-2.606792$

H $0.468581-1.916125-2.025629$

H $0.695061-1.605381-5.064209$

H $-0.836312-1.714408-4.274597$ 
H $-0.368983-5.414231-3.546036$ H $\quad 1.421360-5.203666-4.986488$ H $1.274244-3.810160-6.061901$ H $2.385878-3.757815-4.677492$ H -1.952373 -2.881071 -2.796111 H -2.551151 -4.521332 -2.907090 H $-1.207678-5.204841-0.921543$ H -0.435854 -3.626895 -0.882830 H -2.034206 -3.614097 0.836271 H -3.528128 -5.4728670 .061807$ H -4.131684 -4.462202 -1.234169 H $-6.738520-4.2219190 .979059$ H $-5.474891-2.323254-0.645338$ H -4.515652 -5.205503 2.365115 H -5.350425 -3.7350892 .815293$ H -3.601612 -3.701937 2.527802 H $-6.769655-2.1703391 .170335$ H -4.5268750 .4460250 .383662$ H -2.903976 $0.375671-0.247296$ H -2.566684 -0.6620801 .654968$ H $-0.4245342 .320428-2.691413$ H -0.7960861 .9836630 .346840$ H $-0.3512844 .808812-2.214117$ H -1.753229 $5.706575-1.654705$ H -1.994219 4.205291-2.528433 H $-3.859323-0.832717-2.336670$ H -5.456556 -0.379687 -1.746820 H $-6.1842743 .604433-2.849393$ H $-4.4439583 .689067-3.158316$ H -5.067149 5.634081-1.772854 H $-4.942287 \quad 3.5227920 .440690$ H -5.6973805 .7216821 .527002$ H $-4.790670 \quad 6.6281870 .333640$ H -2.751576 5.5445503 .715314 H -4.600096 5.5186423 .598354 H -1.481794 6.1937081 .741282 H $-2.287601 \quad 6.4047330 .213890$ H -1.5656843 .7012921 .554254$ H -5.772599 $1.921920-1.136865$ C $5.229700 \quad 0.733551-2.221939$

O $4.6016010 .810198-0.925405$

C $4.4299802 .200014-0.585641$

C $5.2919272 .983334-1.569751$

C $5.1539042 .138151-2.820335$

H $3.3786902 .445129-0.776334$

C 4.7744572 .4510060 .885771

H $4.9406334 .010962-1.703346$

H $6.3396093 .021572-1.248486$

C $6.2163172 .423643-3.867487$

H $4.1655952 .325946-3.256558$

C 3.9050101 .6785811 .900417

O $6.1411502 .084873 \quad 1.116649$

H 4.7140383 .5252901 .096382

H 4.1514560 .6116321 .868745

O 4.2631232 .1364663 .210877

C 2.3890011 .8694771 .717414

C $1.517727 \quad 1.3658292 .883231$

H $2.076553 \quad 1.3737940 .792374$

O $2.119523 \quad 3.266481 \quad 1.556749$

H 1.6723562 .0030683 .763674

H 0.4723341 .5278252 .588350

H $6.1582643 .465262-4.199849$

H $7.2242462 .252463-3.474754$

H $6.0822821 .781955-4.743959$

C $4.614114-0.389159-3.064350$

H $\quad \begin{array}{llll}6.237910 & 1.148237 & 0.872730\end{array}$

H 5.2221532 .0079433 .310321

H 1.1659883 .3669491 .397790

H $4.901492-1.347105-2.612642$

H $5.043328-0.372643-4.072348$

H $6.2805120 .474656-2.034189$

C $1.693968-2.3190822 .269049$

O $1.493102-0.9083592 .104681$

C $1.723224-0.1079643 .266279$

C $0.782762-0.5325574 .406625$

C $0.909479-2.0312534 .699013$

C $0.829557-2.8676863 .418230$

H $1.329128-2.7660741 .334868$
C $3.182501-2.6744882 .413763$

H $2.759549-0.2341623 .594298$

O $\quad-0.566394-0.2335464 .050273$

H $1.025507 \quad 0.0522995 .301917$

H $0.106715-2.3431465 .378338$

O $2.151722-2.2909395 .345562$

H $1.116858-3.9041493 .628469$

O $-0.531198-2.9073422 .980425$

C $3.974388-2.3541631 .164731$

H $3.650213-2.1337113 .240344$

H $3.282990-3.7510182 .594423$

H -1.130792 -0.4838534 .801514$

H $2.167931-1.7744306 .169165$

H $-0.781979-2.0092172 .703610$

O $5.286964-2.664181 \quad 1.382127$

O $3.509271-1.8922180 .132968$

C $6.170922-2.4096670 .286081$

H $6.245748-1.3331450 .109320$

H $7.160411-2.7908590 .551955$

H $5.827192-2.927892-0.614355$

SCF Energy $(B 3 L Y P / 6-31 G * * / / M M F F)=-3245.90719824$

\section{$07 \_00286$}

MMFF Geometry

C -1.4085753 .1426932 .458996$

C -0.1792823 .0455962 .986469$

C 0.6503971 .7917592 .928621

O 1.9316882 .1314372 .344614

C 0.9167031 .2003794 .323731

C $-0.2635370 .429446 \quad 4.881362$

C $-0.552461-0.8477024 .558239$

C $-1.1252691 .177528 \quad 5.863667$

C $0.218156-1.7440173 .627654$

C $-0.523925-1.9626272 .301621$

C $0.295842-2.7247681 .246401$

C $0.495029-4.1958201 .614361$

C $1.359122-4.9291810 .578515$

O $1.552584-2.0609981 .089482$

O $1.714169-6.1962151 .153047$

C $2.656046-4.1200000 .368609$

C $0.573722-5.242520-0.699458$

O $3.456574-4.699748-0.658848$

C $2.414081-2.6187280 .089787$

C $3.729972-1.8045900 .108942$

O $1.840890-2.475060-1.214274$

C 2.0329892 .0334150 .990330

O 1.1585431 .6863680 .211878

C 3.4081152 .4212880 .605116

C $3.7962532 .252892-0.668023$

C $5.1141052 .556525-1.230275$

C 6.132803 3.185713-0.318462

C $4.463602-1.8265641 .451404$

C $5.564717-0.7883851 .515017$

C $7.869985-0.3150630 .700889$

O 5.4656320 .2069792 .229243

C $8.2566400 .149317-0.670479$

C $7.660049-0.128670-1.840798$

C $8.1553200 .404559-3.156315$

C $7.097273 \quad 1.160036-3.928674$

C $6.6778690 .727263-5.129871$

C $6.5821342 .460193-3.347093$

C $5.3364562 .258394-2.527722$

N $6.649877-1.0990970 .711934$

H $-1.8518802 .274527 \quad 1.977117$

H 0.2629553 .9097853 .476412

H 0.1674041 .0320992 .304845

H 1.2142371 .9930255 .024122

H $\quad 1.7995360 .5496594 .282784$

H -1.427617 -1.3115585 .010855$

H $-1.947560 \quad 0.563520 \quad 6.246437$

H $-0.527212 \quad 1.4983996 .722966$

H -1.566488 2.0631825 .397939

H $1.218289-1.3446843 .439626$

H $\quad 0.367095-2.7037694 .135286$

H $-0.779695-0.983041 \quad 1.878727$

H -1.473599-2.482135 2.480730

H $-0.251165-2.6546210 .301573$

H $-0.471353-4.7009541 .735991$ 
H $\quad 0.997276-4.272708 \quad 2.587614$ H $2.327404-6.6363300 .539385$ H $3.233329-4.2258541 .294812$ H $-0.300159-5.862793-0.466337$ H $1.176700-5.828887-1.401548$ H $0.217707-4.349046-1.215531$ H $3.058385-4.462015-1.513422$ H $4.394413-2.145151-0.695312$ H $3.495058-0.760484-0.135645$ H $2.015494-1.572775-1.529554$ H 4.0613222 .8157981 .374589 H $3.0795611 .835245-1.374839$ H $5.771816 \quad 4.1534560 .047514$ H $7.0953893 .370003-0.799371$ H 6.3319592 .5404040 .543476 H $3.773760-1.6228482 .278346$ H $4.910750-2.8085071 .641053$ H $8.663229-0.9507841 .107349$ H 7.7625590 .5519421 .360366 H $9.1336430 .794840-0.687935$ H $6.790195-0.778864-1.873722$ H $8.520203-0.448906-3.742321$ H $9.0211691 .063940-3.015568$ H $5.9383961 .279435-5.700597$ H $7.060424-0.188508-5.567828$ H $6.3297003 .150975-4.162924$ H $7.3868002 .955170-2.797202$ H $4.5167951 .818049-3.098547$ H $6.660367-1.9916040 .226572$ C $-2.691434 \quad 4.8800651 .119587$ O $-3.601646 \quad 3.928688 \quad 0.534770$ C -3.332022 $3.847051-0.878629$ C $-2.4031535 .013204-1.202271$ C -1.596639 5.1140270 .079085 H -2.784209 2.911761-1.038916 C $-4.6431543 .821957-1.671442$ H -1.786582 4.819314-2.085175 H -2.959369 5.941777-1.374935 C $-0.861562 \quad 6.4319820 .241484$ H -0.8661954 .2953430 .086550$ C $-5.6290202 .718447-1.232790$ O $-5.3099945 .081644-1.518351$ H -4.422885 $3.727457-2.740913$ H $-6.0276732 .953990-0.238965$ O $-6.752403 \quad 2.757325-2.122732$ C $-5.0423201 .294502-1.224291$ C -6.104384 $0.236099-0.866487$ H -4.225435 $1.243065-0.496312$ O $-4.4968901 .024854-2.514302$ H -6.6513970 .5663140 .024730$ H $-6.8142440 .153310-1.699008$ H -0.142833 $6.573994-0.572191$ H $-1.552597 \quad 7.2814610 .231482$ H -0.3107426 .4556821 .187098$ C -2.235401 4.3967252 .498583 H $-5.4675865 .216877-0.568076$ H -6.435332 $2.499404-3.005502$ H $-4.0984970 .138775-2.487172$ H $-3.121440 \quad 4.1815573 .108720$ H -1.686605 5.2030842 .999503 H -3.2730975 .8009151 .260892$ C $-4.313669-2.932168-1.745729$ O $-4.974439-1.662645-1.823468$ C $-5.493550-1.153358-0.591375$ C $-6.531076-2.125284-0.007981$ C $-5.945924-3.5337650 .143500$ C $-5.238900-3.997358-1.134085$ H $-4.124902-3.212025-2.790340$ C -2.959548 -2.825011-1.037931 H $-4.673430-1.0266610 .123950$ O $-7.671763-2.171872-0.863130$ H -6.863464 -1.745674 0.965532 H $-6.748155-4.2422090 .383754$ O $-5.012404-3.5510321 .219609$ H -4.664747 $-4.909662-0.936485$ O $-6.228369-4.344550-2.107826$ C -2.012066 -1.960432-1.835295 H $-3.035093-2.420748-0.024500$
H $-2.490624-3.811233-0.940103$ H $-8.322609-2.765925-0.451958$ H $-5.488153-3.2872342 .025608$ H $-6.705240-3.532133-2.350144$ O $-1.923326-0.722444-1.270811$ O $-1.435478-2.336337-2.847672$ C $-1.0602680 .198929-1.943092$ H $-1.4049060 .364088-2.968275$ H $-1.0947421 .149444-1.405609$ H $-0.031329-0.172048-1.935280$ SCF Energy (B3LYP/6-31G**//MMFF) $=-3245.93044953$

07_00287

MMFF Geometry

C $-1.8429363 .312774-0.029406$

C -0.8668613 .8216110 .736346$

C 0.1061632 .9671091 .501440

O 1.4307823 .4114751 .116976

C -0.0359713 .1298543 .025197$

C -1.299399 2.5274063 .600421

C $-1.444367 \quad 1.2229353 .905981$

C -2.4176833 .4984953 .875503$

C $-0.436143 \quad 0.120530 \quad 3.721285$

C $-1.006247-0.9989762 .840510$

C $-0.013607-2.1224692 .497630$

C $0.555588-2.8215583 .731106$

C $1.556040-3.9200523 .343592$

O $1.047548-1.5691591 .716159$

O $2.261253-4.3127064 .531276$

C $2.602049-3.3303602 .370767$

C $0.849252-5.1783442 .823385$

O $3.449667-4.3675261 .883036$

C $1.980084-2.5386401 .198081$

C $3.023752-1.7110160 .413083$

O $1.334844-3.4390880 .295680$

C $2.427375 \quad 2.486519 \quad 1.134801$

$\begin{array}{llll}\text { O } & 2.325725 & 1.306763 & 1.427224\end{array}$

C 3.6845533 .1635880 .747120

C $4.7983832 .438193 \quad 0.562327$

C $6.1268842 .959704 \quad 0.230781$

C 6.2810624 .4558190 .134763

C $4.101241-2.498905-0.330784$

C $4.831426-1.645655-1.356272$

C $6.673527-1.793838-3.028625$

O $4.528501-0.483492-1.611915$

C $7.826693-1.049087-2.433755$

C $8.1278180 .218403-2.755031$

C $9.3242420 .952610-2.223513$

C $8.9804752 .313478-1.662174$

C $9.1163933 .420012-2.412982$

C $8.5918422 .406308-0.205480$

C 7.1420082 .0884030 .045096

N $5.855554-2.352604-1.970128$

H $-1.9523412 .233782-0.098130$

H -0.7529394 .9000530 .813720$

H -0.0199961 .9148191 .215199$

H $0.027671 \quad 4.193554 \quad 3.294069$

H 0.8373492 .6902843 .524672

H $-2.3879280 .900904 \quad 4.345051$

H -3.2960403 .0089944 .309737$

H -2.0860014 .2665924 .581930$

H -2.7404613 .9914762 .954349$

H 0.4967410 .4914353 .290341

H $-0.193450-0.272564 \quad 4.714722$

H $-1.885534-1.4371933 .329720$

H $-1.352678-0.5517801 .899678$

H $-0.559642-2.8429621 .878699$

H $-0.249076-3.2322914 .353204$

H $1.087668-2.0978844 .362083$

H $1.606171-4.6222295 .180132$

H $3.242314-2.6459122 .944503$

H $\quad 0.161382-5.571708 \quad 3.581574$

H $1.567278-5.9821872 .626320$

H $0.271340-5.0019441 .913789$

H $3.833628-4.8161232 .656005$

H $2.475669-1.092671-0.310614$

H $3.509196-0.9970281 .090469$

H $1.275525-3.010411-0.574219$ 
H $3.661824 \quad 4.2422160 .641758$ H $4.741017 \quad 1.3570460 .680512$ H $5.6175414 .861778-0.637063$ H $7.2909094 .774852-0.130668$ H $6.038813 \quad 4.929867 \quad 1.092444$ H $4.842706-2.8798260 .379717$ H $3.660162-3.347406-0.865419$ H $6.050177-1.153126-3.661559$ H $7.041919-2.629160-3.632144$ H $8.462005-1.588671-1.735579$ H $7.5072130 .743380-3.478063$ H $10.0368501 .065511-3.051453$ H $9.8513350 .363529-1.462878$ H $8.9126534 .406330-2.010460$ H $9.4314633 .363180-3.449672$ H 8.8543423 .3905760 .198904 H 9.2060191 .7094260 .379590 H $6.933416 \quad 1.0208480 .121174$ H $6.073240-3.291302$-1.648078 C $-4.2456693 .927364-0.349802$ O $-4.6600142 .575273-0.628733$ C $-6.0475012 .584629-1.015442$ C $-6.5361654 .019687-0.840821$ C $-5.2637624 .811944-1.064563$ H $-6.0803362 .327588-2.080368$ C $-6.8282411 .552281-0.195664$ H -7.330591 4.273480 -1.549627 H $-6.923974 \quad 4.1976540 .168925$ C $-5.3357456 .233413-0.534472$ H $-5.0500784 .836788-2.141589$ C $-6.3019530 .106578-0.314490$ O $\quad-6.7709241 .917077 \quad 1.191010$ H -7.888949 $1.587135-0.469739$ H -5.3135030 .0319320 .152129$ $\begin{array}{llll}\text { O } & -7.173910 & -0.729880 & 0.458327\end{array}$ C $-6.246980-0.443753-1.754214$ C $-5.995895-1.962333-1.838441$ H $-5.4784650 .090445-2.323735$ O $-7.502996-0.178595-2.387251$ H -6.766386 -2.490978 -1.263544 H $-6.145103-2.270782-2.882346$ H $-6.1387276 .788244-1.030645$ H $\quad-5.5311596 .2503880 .543020$ H $-4.3973516 .766752-0.714635$ C $-2.8099004 .139541-0.827428$ H -5.8330961 .9494641 .447086$ H -7.197431 -0.3686531 .361052$ H -7.429555 -0.459835 -3.315191 H -2.544454 $5.200748-0.764682$ H -2.715811 $3.838630-1.878042$ H $-4.311021 \quad 4.0664700 .737692$ C -2.290899 -2.305326 -2.163382 O $-3.670262-1.988171-2.399411$ C $-4.593451-2.389915-1.379136$ C $-4.518625-3.906239-1.143807$ C $-3.086178-4.344607-0.830183$ C -2.094532 -3.801709-1.859175 H -1.794946 -2.102676 -3.121687 C -1.679439-1.375746-1.105341 H -4.345819-1.879391-0.444605 O $-5.003501-4.615497-2.281679$ H -5.174932 -4.164872 -0.304616 H -3.031978 $-5.440231-0.821259$ O $-2.712124-3.872708 \quad 0.460563$ H -1.066949 -3.984298 -1.523604 O $-2.271218-4.540155-3.072122$ C $-1.162644-0.113963-1.761543$ H $-2.381778-1.068736-0.326868$ H $-0.835527-1.870581-0.614543$ H -4.474581 -4.342597 -3.050659 H $-3.332636-4.2549221 .104181$ H -1.586281-4.247224 -3.697113 O $0.1593110 .052410-1.470032$ O $-1.846251 \quad 0.625716-2.457579$ C $0.7995681 .168486-2.095839$ H $1.8482291 .175679-1.787060$ H $0.7557531 .066916-3.184332$ H $0.3339282 .104655-1.777721$
SCF Energy (B3LYP/6-31G**//MMFF) $=-3245.91209741$

07_00288

MMFF Geometry

C $-5.328512-2.297051-1.582218$

C $-4.051888-2.586459-1.285161$

C $-2.980257-2.761215-2.331646$

O $-1.929743-1.799033-2.074969$

C $-2.358907-4.166179-2.246261$

C $-1.415475-4.460724-3.397643$

C $-0.078013-4.592971-3.298981$

C $-2.079904-4.650992-4.739965$

C $0.801002-4.505553-2.082346$

C $1.754973-3.309696-2.161582$

C $2.983211-3.441585-1.246863$

C $2.621467-3.5606640 .234878$

C $3.873930-3.6011381 .127276$

O $3.788176-2.272478-1.460388$

O $3.462891-3.4048942 .488281$

C $4.781802-2.4106790 .758038$

C $4.565784-4.9685631 .083874$

O $6.020861-2.4720531 .459376$

C $5.040677-2.283392-0.758297$

C $5.798208-0.986176-1.125353$

O $5.858638-3.377776-1.190447$

C $-2.112407-0.535138-2.545922$

O $-3.069622-0.113076-3.174784$

C $-0.940503 \quad 0.266795-2.131705$

C $-0.9964531 .605001-2.205380$

C $0.0488042 .535123-1.774899$

C $1.3771341 .953607-1.366339$

C $5.1204380 .308744-0.672903$

C $5.7818161 .544029-1.260462$

C $5.6260204 .025175-1.212253$

O $6.731327 \quad 1.498482-2.037381$

C $4.4887484 .987155-1.094606$

C $3.8158455 .468040-2.150686$

C $2.6602626 .421803-2.047394$

C $1.3495485 .760724-2.420649$

C $0.8433165 .913792-3.656546$

C $0.6703514 .979805-1.311940$

C $-0.222853 \quad 3.857394-1.769203$

N $5.1652122 .707238-0.820501$

H -5.626253 -2.206322 -2.624119

H $-3.747697-2.661514-0.243980$

H $-3.390737-2.593612-3.334724$

H -3.150336 $-4.927521-2.252695$

H $-1.846145-4.276112-1.285118$

H $0.473404-4.816527-4.212729$

H $-1.379350-5.002413-5.505229$

H $-2.879123-5.395850-4.666076$

H $-2.509777-3.710953-5.097951$

H $0.222639-4.451758-1.156822$

H $1.378640-5.436903-2.031853$

H $2.115041-3.175455-3.190996$

H $1.212186-2.385477-1.925095$

H $3.548549-4.321861-1.574887$

H $1.999331-4.4425490 .426349$

H $2.014473-2.6926740 .525043$

H $2.803774-4.0868222 .704345$

H $4.269064-1.5069391 .110460$

H $3.884835-5.7523431 .437383$

H $5.428906-4.9958631 .758125$

H $4.900161-5.2513860 .083868$

H $5.814113-2.5449782 .406946$

H $6.821568-1.023806-0.729220$

H $5.910378-0.956483-2.218411$

H $6.295502-3.126498-2.021236$

H $-0.082749-0.262180-1.732875$

H $-1.9070092 .068341-2.583527$

H $1.7600721 .278748-2.140240$

H $1.2818881 .395484-0.428614$

H $2.1504822 .710239-1.221319$

H $4.0681700 .313621-0.978297$

H $5.1669740 .396072 \quad 0.417918$

H $6.0310793 .982519-2.229115$

H $6.4392604 .313310-0.538305$

H $4.2030275 .295444-0.092056$ 
H $4.1023465 .153181-3.151730$ H $2.8637897 .275154-2.707177$ H $2.5796816 .845714-1.038388$ H $-0.1032935 .476228-3.952756$ H $1.3682806 .493523-4.409298$ H $0.0689145 .679461-0.717028$ H $1.4216944 .595880-0.614950$ H -1.211836 4.187364 -2.089268 H $4.5042172 .640185-0.051571$ C $-6.774440-0.551175-0.547195$ O $-5.6452890 .211443-0.080091$ C $-6.0905411 .165550 \quad 0.900655$ C $-7.601734 \quad 1.2413090 .739010$ C $-7.926320-0.2033670 .395567$ H $-5.8691490 .733357 \quad 1.883989$ C $-5.314711 \quad 2.477874 \quad 0.731117$ H -8.1018041 .5912651 .646917$ H -7.891223 $1.900665-0.087223$ C $-9.302372-0.389241-0.219067$ H -7.858259 -0.8067191 .310770$ C $-3.787707 \quad 2.2752130 .621347$ O $-5.7447743 .129096-0.470853$ H $-5.553103 \quad 3.1646681 .550497$ H $-3.5560841 .749341-0.312646$ O $-3.161478 \quad 3.558993 \quad 0.525301$ C -3.1638691 .5162871 .805730$ C -1.6907051 .1767841 .554569$ H $-3.7027310 .578301 \quad 1.958593$ O -3.2967002 .2822922 .998660$ H $-1.616770 \quad 0.558090 \quad 0.653044$ H $-1.1264822 .092021 \quad 1.330387$ H -10.082123 -0.0700060 .480134$ H -9.414259 $0.196821-1.137476$ H $-9.479539-1.440590-0.466471$ C $-6.396514-2.033768-0.555194$ H $-5.5951232 .513311-1.208927$ H -3.561861 4.020011 -0.231736 H -2.8355423 .1269252 .856975$ H -7.269149 -2.651674 -0.793567 H $-6.041748-2.3339800 .438718$ H $-6.993210-0.204048-1.565648$ C $1.207978-0.5510043 .105033$ O 0.2973220 .0489762 .176786 C $-0.964515 \quad 0.4710272 .715049$ C -1.710937 -0.7375463 .299693$ C $-0.852477-1.4769124 .330465$ C $0.565442-1.7512353 .822320$ H $2.027835-0.9304942 .482579$ C 1.7979940 .4893234 .061797 H $-0.790044 \quad 1.2121413 .502584$ O $-2.107594-1.6413692 .270220$ H -2.627633 -0.393704 3.791627 H -1.340957 -2.4246304 .588158$ O $-0.790300-0.6818135 .512594$ H $1.209151-2.0635884 .652423$ O $0.515369-2.8616622 .923002$ C $2.5934191 .515648 \quad 3.289285$ H $1.039707 \quad 1.0065634 .656845$ H $2.481737 \quad 0.018198 \quad 4.777626$ H -1.449136 -1.605168 1.557097 H $-0.283245-1.1808546 .175478$ H $\quad 0.167697-2.5491872 .072158$ O 1.8314612 .6250843 .069010 O 3.7399871 .3368442 .899315 C 2.4548323 .6586052 .301546 H 1.7494434 .4889402 .211792 H 3.3574764 .0162652 .805679 H 2.6913453 .2906881 .299764

SCF Energy $\left(B 3 L Y P / 6-31 G^{* *} / / M M F F\right)=-3245.92223051$

\section{9}

MM̄MF Geometry

C $-2.966150-2.636272-3.168270$

C $-1.976216-2.821182-2.282158$

C $-0.514573-2.682784-2.622889$

O $0.073225-1.685009-1.753780$

C $0.206623-4.024802-2.397567$

C $1.711513-3.965622-2.583610$
C $2.596161-4.410262-1.668696$

C $2.203187-3.394231-3.887721$

C $2.283546-5.094707-0.368423$

C $2.032373-4.1560540 .818460$

C $3.276825-3.3775521 .271432$

C $3.028613-2.7453732 .643659$

C $4.214377-1.8881103 .105659$

O $3.552030-2.3522680 .311245$

O $3.776688-1.0920184 .217499$

C $4.591909-0.9138211 .973547$

C $5.377655-2.7463033 .615330$

O $5.782528-0.1969922 .293013$

C $4.734980-1.5914010 .589626$

C $4.882252-0.502185-0.496166$

O $5.903939-2.4127980 .584494$

C $-0.114268-0.384554-2.113676$

$\begin{array}{lllll}\text { O } & -0.781198 & 0.023870 & -3.052393\end{array}$

C $0.6528100 .473628-1.181279$

C $0.5441201 .805289-1.304939$

C $1.2561932 .833791-0.547089$

C 2.2219792 .4145270 .525797

C $4.755674-1.003357-1.937690$

C $5.0641330 .120344-2.908685$

C $4.1450802 .274166-3.776958$

O $6.136377 \quad 0.176047-3.506707$

C $4.2887263 .427802-2.832239$

C $3.5548194 .548746-2.912024$

C $3.6967575 .708902-1.966295$

C $2.3724046 .176305-1.397831$

C $1.8173617 .331776-1.800749$

C $1.7287975 .343469-0.307605$

C $1.0382494 .127357-0.865550$

N $4.0398451 .049378-3.003569$

H -2.725396 -2.384673 -4.198087

H -2.221802 -3.062187 -1.250212

H $-0.383586-2.376605-3.668959$

H $-0.196196-4.774498-3.091480$

H $-0.036366-4.379095-1.389603$

H $3.658933-4.312903-1.884890$

H $3.283656-3.522460-4.015260$

H $1.713989-3.895771-4.729249$

H $1.991830-2.322159-3.946730$

H $1.414163-5.751231-0.493645$

H $3.113927-5.771408-0.128656$

H $1.227635-3.4543890 .565222$

H $1.673716-4.7739651 .651298$

H $4.124978-4.0704861 .319935$

H $2.799870-3.5141123 .392369$

H $2.137107-2.1057912 .590227$

H $3.463560-1.6952974 .913308$

H $3.789167-0.1658261 .909284$

H $5.054431-3.3664854 .459980$

H $6.191917-2.1210683 .997949$

H $5.784048-3.4141002 .852863$

H $5.6450310 .222023 \quad 3.159888$

H $4.1012070 .249132-0.346010$

H $5.8468930 .008749-0.380588$

H $6.229189-2.497425-0.325424$

H $1.299150-0.008395-0.458089$

H $-0.1282582 .194598-2.069715$

H 1.8830611 .5211081 .057061

H 2.3436263 .1784911 .299075

H 3.2042862 .2147090 .090644

H $5.446458-1.826900-2.144436$

H $3.743508-1.372167-2.135079$

H $3.2392342 .351469-4.386382$

H $5.0145242 .224861-4.439295$

H $5.0387773 .339642-2.049461$

H $2.8248664 .648716-3.712327$

H $4.1779226 .527365-2.517444$

H $4.3742785 .466421-1.138034$

H $\quad 0.8815807 .685681-1.380881$

H $2.2830697 .947618-2.563034$

H 0.9773415 .9361130 .229809

H 2.4956815 .0969970 .432501

H $0.3029594 .356484-1.638292$

H $3.196967 \quad 0.912319-2.454567$

C $-5.108546-1.371283-2.884619$ 
O $-4.542723-0.503729-1.879908$

C $-5.6033450 .017727-1.066388$

C $-6.859557-0.114789-1.912549$

C $-6.609140-1.448660-2.588749$

H $-5.681924-0.643673-0.194732$

C $-5.292592 \quad 1.435332-0.571268$

H -7.773338 $-0.101988-1.311154$

H $-6.9286080 .682067-2.662153$

C $-7.465575-1.679215-3.821679$

H -6.809252 -2.249939-1.864602

C $-3.940302 \quad 1.5256590 .169846$

O $-5.3101622 .350369-1.665089$

H $-6.099703 \quad 1.7328250 .108558$

H $-3.831710 \quad 0.647876 \quad 0.814033$

O $-2.8609511 .505306-0.762007$

C -3.8199632 .7840811 .047754$

C -2.4536372 .9848581 .726992$

H -4.5953382 .7421161 .821927$

$\begin{array}{lllll}O & -4.087447 & 3.958023 & 0.277552\end{array}$

H $-1.710600 \quad 3.3105100 .987538$

H -2.571938 3.8388472 .407088

H -8.528197-1.677326 -3.557790

H -7.304749 $-0.899408-4.573676$

H $-7.232479-2.644512-4.282132$

C $-4.423360-2.738034-2.817432$

H $-4.5958332 .091894-2.272440$

H -2.924457 $0.677415-1.267904$

H -3.467876 $3.963561-0.472279$

H -4.900147 -3.436105 -3.514990

H -4.534252 -3.158564-1.810215

H $-4.924393-0.893674-3.855322$

C -2.591646 0.0838604 .100792

O -2.9319961 .2594583 .350789$

C -1.9047951 .7698072 .496127$

C $-0.651617 \quad 2.131797 \quad 3.311714$

C -0.1786640 .9478764 .161046$

C $-1.330893 \quad 0.3236804 .952695$

H -3.435103 -0.0646804 .786941$

C $-2.481790-1.1666353 .210446$

H -1.6394491 .0056041 .758595$

O -0.9364873 .2401834 .163821$

H 0.1388272 .4456902 .622326

H $0.594670 \quad 1.2832194 .863277$

O $0.397984-0.046285 \quad 3.320952$

H $-1.003719-0.6184745 .408104$

O $-1.663427 \quad 1.210700 \quad 6.024184$

C $-3.824986-1.6354782 .690224$

H $-1.840543-1.0016942 .341198$

H $-2.067491-1.9910703 .803248$

H -0.1193343 .4607954 .642318$

H $\begin{array}{llll}\text { H } & 1.178498 & 0.346788 & 2.895422\end{array}$

H $-2.355202 \quad 0.782773 \quad 6.556813$

O $-3.647107-2.8179482 .027588$

O $-4.887889-1.0489882 .836875$

C $-4.832782-3.4166261 .495659$

H $-4.555736-4.3700201 .038086$

H $-5.554742-3.607966 \quad 2.295307$

H -5.269109-2.773918 0.726910

SCF Energy (B3LYP/6-31G**/MMFF) $=-3245.90443284$

$07 \_00290$

MMFF Geometry

C $3.5321213 .572169-0.033199$

C $2.5349652 .676070-0.097904$

C $1.3324392 .700237 \quad 0.808232$

$\begin{array}{lllll}\text { O } & 0.168176 & 2.578576 & -0.042526\end{array}$

C 1.3727601 .5038681 .778198

C 0.3002021 .5526552 .847909

C -0.7018330 .6606572 .970150$

C $0.427756 \quad 2.671434 \quad 3.851570$

C $-0.945801-0.5826222 .163465$

C $-1.867380-0.3411240 .962568$

C -2.448752 -1.6570840 .425324$

C $-3.199051-1.430581-0.886483$

C $-3.853415-2.723599-1.401014$

O $-3.351193-2.1534341 .415486$

O $-4.798527-2.355968-2.421820$

C $-4.668409-3.368331-0.255420$
C $-2.835884-3.642191-2.086124$

O $-5.158290-4.655963-0.618055$

C $-3.945332-3.4184821 .111965$

C $-4.902950-3.7562172 .283696$

O $-2.960873-4.4581871 .161791$

C $-0.8893623 .400446 \quad 0.192418$

O $-0.979948 \quad 4.261966 \quad 1.051900$

C $-1.9410383 .069330-0.796669$

C $-3.101176 \quad 3.742851-0.778228$

C $-4.2281623 .556379-1.695482$

C $-4.1280102 .444484-2.706718$

C $-6.151203-2.8836362 .452243$

C $-5.836638-1.4072822 .605957$

C $-6.177400 \quad 0.7975391 .508536$

O $-5.155516-0.979253 \quad 3.535099$

C $-6.603602 \quad 1.2663120 .154310$

C $-7.5567702 .187354-0.049898$

C -8.032108 2.610571-1.410061

C $-7.779545 \quad 4.073711-1.690337$

C $-8.684406 \quad 5.008942-1.355527$

C $-6.5224294 .436629-2.447028$

C $-5.2891314 .384388-1.588166$

N $-6.370768-0.637953 \quad 1.585089$

H 3.4878524 .3680690 .705822

H $2.5857331 .874366-0.830946$

H 1.3142643 .6489141 .356974

H 2.3459121 .4695862 .285330

H $1.2957410 .577972 \quad 1.197386$

H -1.4231240 .8101543 .773167$

H -0.2938822 .5785094 .670548$

H 1.4275252 .6649634 .298407

H 0.2640113 .6447463 .381995

H $-0.004808-1.0292741 .826065$

H $-1.388707-1.3226172 .841926$

H -2.688672 0.3242051 .258073

$\begin{array}{llll}H & -1.297765 & 0.162143 & 0.173378\end{array}$

H $-1.634362-2.3749910 .284802$

H -2.533494 -1.008110 -1.649491

H $-3.987253-0.681759-0.729303$

H $-4.321768-1.857678-3.107648$

H $-5.559215-2.739523-0.152810$

H $-2.413578-3.150277-2.970922$

H $-3.311686-4.556711-2.456188$

H $-1.999512-3.918816-1.442337$

H $-4.423198-5.288161-0.553508$

H $-5.225382-4.8024722 .197481$

H $-4.326062-3.7043613 .218260$

H $-2.614974-4.6167070 .272904$

H $-1.7276122 .288370-1.517507$

H -3.237695 $4.520925-0.028057$

H $-3.9124431 .491011-2.211765$

H $-3.3310202 .652690-3.428619$

H $-5.0476822 .287179-3.273907$

H $-6.847214-3.0561131 .624689$

H -6.686846 -3.1905673 .359045$

H $-5.119148 \quad 1.0212881 .677082$

H -6.7572491 .2566732 .315999$

H $-6.1109860 .813087-0.702636$

H -8.0540912 .6364120 .806778$

H $-9.1090872 .403713-1.468096$

H -7.579136 $1.998412-2.199813$

H $-8.5250446 .057746-1.582731$

H $-9.6031024 .745390-0.841736$

H $-6.6006285 .461679-2.834429$

H $-6.4467813 .810727-3.340730$

H $-5.2688825 .154689-0.815828$

H $-7.048150-1.062347 \quad 0.957630$

C $5.9781812 .915366-0.315591$

$\begin{array}{lllll}\text { O } & 5.708671 & 1.539811 & 0.026715\end{array}$

C $6.182517 \quad 1.3040331 .364418$

C $7.1933772 .409715 \quad 1.636338$

C 6.5193103 .5842730 .950281

H $\quad 5.3243251 .4464582 .032721$

C $6.714326-0.1249011 .511848$

H 7.3580432 .5724932 .705555

H 8.1610602 .1999611 .166202

C $7.451548 \quad 4.751336 \quad 0.676343$

H 5.6997613 .9317671 .590857 
C $5.721658-1.2266781 .079650$

O $7.898473-0.278306 \quad 0.720083$

H $7.026780-0.2915172 .549281$

H $5.646558-1.247624-0.013273$

O $6.272104-2.4904431 .468740$

C $4.317967-1.0944681 .691896$

C $3.380082-2.2895581 .450663$

H $3.844364-0.1893871 .298884$

O $4.444049-0.9212293 .106360$

H $3.736358-3.1656852 .007613$

H $2.417865-2.0269031 .909983$

H 7.8524575 .1513781 .613384

H 8.2982294 .4511780 .050021

H $\quad 6.9210525 .5593870 .162537$

C $4.7326693 .553738-0.938771$

H $7.665130-0.067402-0.200044$

H $7.157859-2.5548931 .072211$

H $3.553884-0.7593853 .462968$

H $4.4790383 .009493-1.856138$

H $4.9545394 .583084-1.243883$

H $6.7626592 .884134-1.082770$

C $2.565531-1.647250-2.146764$

O $2.771978-1.485682-0.736218$

C $3.165655-2.663759-0.025645$

C $2.113377-3.776842-0.180965$

C $1.786766-4.041170-1.653180$

C $1.520272-2.740865-2.418773$

H $2.135924-0.693274-2.478570$

C $3.883016-1.870807-2.906930$

H $4.120734-3.020167-0.423776$

O $0.917486-3.4056350 .501956$

H $2.494073-4.6893740 .293719$

H $0.902591-4.687468-1.715971$

O $2.879277-4.740235-2.241461$

H $1.470486-2.940177-3.495042$

O $0.238154-2.232258-2.041803$

C $4.760635-0.638594-2.905774$

H $4.462799-2.690117-2.474732$

H $3.672378-2.107758-3.956074$

H $\quad 0.296268-4.150480 \quad 0.433674$

H $2.646473-4.922969-3.167623$

H $0.288875-1.961159-1.109128$

O $6.020419-0.986561-3.302005$

O $4.3862190 .490680-2.624166$

C $6.9596590 .090233-3.380134$

H $7.922135-0.320414-3.696514$

H $6.6310410 .825948-4.120260$

H $7.0835560 .558312-2.399901$

SCF Energy (B3LYP/6-31G**//MMFF) $=-3245.90626755$

\section{1}

MM̄FF Geometry

C $-3.836427 \quad 0.9350622 .529221$

C -2.498960 0.9001252 .436976

C -1.5932390 .6211693 .607656$

O $-0.783594-0.535674 \quad 3.283741$

C -0.6300081 .7913703 .878182$

C -1.3293053 .0903844 .225879$

C -1.500842 4.1245403 .377776

C -1.8518243 .1768095 .636748$

C -1.0570314 .2169701 .943619$

C -0.2653465 .5073081 .684289$

C $0.599527 \quad 5.4674560 .413612$

C $-0.1958175 .140553-0.852844$

C $0.7110915 .005970-2.086696$

O $1.623313 \quad 4.4913400 .632620$

O $-0.0774864 .402562-3.123792$

C $1.8674934 .039840-1.745598$

C $1.1409526 .376076-2.621748$

O $2.7999683 .961891-2.820591$

C $2.5845504 .386767-0.424059$

C $3.6117293 .314576-0.002878$

O $3.3032755 .614517-0.590037$

C -1.350759-1.758102 3.456334

O $-2.466040-1.9860003 .907023$

C $-0.465717-2.8528492 .997634$

C $0.781511-2.6791392 .533149$

C $1.670041-3.7386772 .044166$
C $1.152377-5.1540642 .079708$

C $3.0374121 .906867 \quad 0.184824$

C 3.9145901 .0713501 .097929

C 6.2427840 .2157421 .342885

O 3.5016750 .6476022 .175481

C $6.313640-1.2279480 .962663$

C $6.241842-2.2285261 .853645$

C $6.376270-3.6789091 .492508$

C $5.142445-4.4815881 .831612$

C $5.150110-5.3859982 .825079$

C $3.926027-4.2972910 .955617$

C $2.891304-3.3992241 .579347$

N $5.1877390 .867768 \quad 0.592094$

H $-4.313278 \quad 0.755218 \quad 3.489345$

H -2.0237341 .0746591 .474276$

H $-2.1649880 .412073 \quad 4.520950$

H $0.055606 \quad 1.9085143 .031042$

H 0.0384121 .5151324 .705654

H -2.037455 5.0005623 .740300

H -2.3141254 .1463055 .850798$

H -2.6073572 .4055745 .815240$

H -1.0350313 .0371636 .352397$

H -0.4630843 .3493621 .648382$

H $-1.9529374 .211327 \quad 1.310933$

H $-0.962455 \quad 6.3525801 .625951$

H $0.399745 \quad 5.6964262 .537735$

H 1.0736046 .4514900 .316354

H $-0.9783355 .889836-1.025039$

H $-0.7196834 .185510-0.724668$

H $0.5094024 .235883-3.881371$

H $1.423023 \quad 3.040895-1.658855$

H $0.2612446 .963371-2.911865$

H $1.7442226 .275925-3.530799$

H $1.7055906 .963863-1.895306$

H $3.3415994 .769257-2.801694$

H $4.4374473 .287136-0.725790$

H 4.0681343 .6340460 .945033

H 3.9968225 .6596380 .089049

H -0.916795 -3.838735 3.056964

H $1.194143-1.6726952 .490083$

H $1.888905-5.8990731 .772631$

H $\quad 0.287949-5.2634351 .415523$

H $0.852635-5.4289883 .097336$

H $2.039395 \quad 1.9448580 .636193$

H $2.9406571 .397115-0.779105$

H $\quad \begin{array}{llll}\text { H } & 065564 & 0.353403 & 2.414848\end{array}$

H $7.1820170 .713048 \quad 1.081242$

H $6.464893-1.458605-0.089173$

H $6.109614-1.9952332 .907874$

H $7.249381-4.0752232 .026885$

H $6.602282-3.8043100 .425981$

H $4.273926-5.9858493 .047534$

H $6.030420-5.5448553 .438942$

H $3.518586-5.2759710 .683789$

H $4.224845-3.857476-0.004818$

H $3.178973-2.3478801 .600750$

H $5.4067841 .189309-0.346137$

C $-5.539861-0.0160530 .955616$

O $-4.643819-1.021140 \quad 0.435142$

C $-5.169340-1.512586-0.809678$

C $-6.626964-1.072472-0.847961$

C $-6.5438380 .275375-0.161653$

H $-4.624766-0.993831-1.607716$

C $-4.956227-3.024539-0.933368$

H -7.017188 -1.013082 -1.868486

H $-7.272430-1.748653-0.275608$

C -7.8840690 .7913710 .332596$

H $-6.120347 \quad 1.002028-0.868289$

C $-3.498262-3.500301-0.763552$

O $-5.742904-3.6949310 .061081$

H $-5.346798-3.365356-1.899603$

H -3.211586 -3.447355 0.294314

O $-3.486879-4.890673-1.116027$

C $-2.464820-2.739840-1.617002$

C $-1.073410-3.403311-1.599302$

H $-2.388427-1.711145-1.246346$

O $-2.938847-2.682187-2.963007$

H $-0.843120-3.757638-0.587505$ 
H -1.094913 -4.269648 -2.271914 H -8.577506 $0.923704-0.504326$ H $-8.3440990 .096912 \quad 1.043625$ H -7.7695291 .7584280 .832425$ C $-4.740789 \quad 1.220716 \quad 1.365807$ H $-5.464139-3.3595140 .930524$ H $-2.656632-5.274257-0.787177$ H $-2.301445-2.158191-3.477127$ H -5.417663 2.0341251 .650775 H $-4.149471 \quad 1.5760370 .514544$ H $-6.042891-0.4584521 .825208$ C $0.663210-0.961006-3.848938$ O $-0.243289-1.953037-3.348163$ C $0.052497-2.447659-2.041077$ C $1.424259-3.136694-2.046698$ C $2.515957-2.188035-2.554660$ C $2.109417-1.490412-3.857550$ H $0.355388-0.802368-4.890267$ C $0.504106 \quad 0.375776-3.108929$ H $0.063961-1.613596-1.330454$ O $1.373596-4.295087-2.877122$ H $1.653350-3.473044-1.031961$ H $3.441667-2.751326-2.725462$ O $2.788423-1.199436-1.567348$ H $2.804094-0.671357-4.078647$ O $2.235889-2.444578-4.916750$ C $-0.912806 \quad 0.898227-3.215233$ H $\quad 0.7536990 .274665-2.049211$ H $1.1692571 .134063-3.536613$ H $2.247054-4.720692-2.841076$ H $3.098735-1.658131-0.768543$ H $2.045492-1.984120-5.751789$ O $-1.2500601 .544707-2.061779$ O $-1.6282320 .769919-4.199932$ C $-2.5703982 .094883-2.031507$ H $-2.7192362 .783591-2.868778$ H $-3.3117611 .290856-2.054717$ $\mathrm{H}-2.6838672 .653115-1.099040$

SCF Energy (B3LYP/6-31G**//MMFF) $=-3245.90836727$

0700292

MM̄FF Geometry

C $-0.8221323 .800539-0.494589$

C -1.295693 $2.549182-0.580156$

C $-1.8772611 .964980-1.839208$

O $-1.3127080 .643626-2.029113$

C -3.399761 $1.784244-1.731061$

C $-4.1845193 .073696-1.838507$

C $-4.7901153 .694649-0.807803$

C $-4.3098583 .634533-3.232066$

C $-4.780613 \quad 3.3065300 .644396$

C -6.1533152 .8216571 .131661$

C -6.5453361 .4429070 .577475$

C $-7.944631 \quad 1.047781 \quad 1.053979$

C $-8.335456-0.3551270 .566974$

O $\begin{array}{llll}-5.590942 & 0.493595 & 1.058825\end{array}$

O $-9.489850-0.7531391 .325882$

C $-7.194729-1.3427680 .901916$

C $-8.789570-0.329400-0.896786$

O $-7.436030-2.6236220 .325885$

C $-5.772638-0.8363040 .558987$

C $-4.720755-1.7216741 .264844$

O $-5.486362-0.923249-0.836603$

C $-0.0876180 .557998-2.607819$

O $0.606156 \quad 1.492389-2.986984$

C $0.362295-0.847357-2.738592$

C $-0.361752-1.910622-2.354147$

C $0.050059-3.315065-2.446741$

C $1.399633-3.602525-3.052103$

C $-3.273719-1.2630121 .031611$

C $-2.279928-2.1266451 .787754$

C $0.162891-2.4022052 .169007$

O $-2.614552-3.0816512 .484141$

C $0.626604-3.4424731 .199661$

C $0.310395-4.7423051 .300027$

C $0.734952-5.7982290 .319221$

C $-0.422605-6.294930-0.520747$

C $-1.265500-7.230193-0.051056$
C $-0.560049-5.756544-1.929418$

C $-0.778951-4.266924-1.966014$

N $-0.971273-1.7023921 .594601$

H $-0.8765694 .451555-1.363747$

H -1.258929 1.9022720 .293360

H -1.637313 2.576999 -2.717301

H -3.640075 $1.241684-0.810787$

H $-3.7381551 .106424-2.527208$

H $-5.3498084 .605669-1.017598$

H $-4.9478194 .524170-3.267180$

H $-3.3285993 .919645-3.623366$

H $-4.7499772 .889573-3.902987$

H -4.0103672 .5640840 .872199$

H -4.504963 4.2003291 .218545

H -6.1122072 .7669362 .227426$

H $-6.921383 \quad 3.5613370 .873220$

H $-6.5105241 .471392-0.516901$

H $-8.690005 \quad 1.7862720 .733207$

H -7.9700261 .0539512 .152968$

H -9.707282 -1.667266 1.073634

H $-7.238568-1.4973251 .989211$

H $-9.6974150 .277814-1.000407$

H -9.061261 -1.330353 -1.248551

H $-8.0430500 .087993-1.574757$

H -7.209944 -2.576860 -0.618093

H $-4.911046-1.7194292 .346226$

H $-4.814844-2.7589280 .918150$

H $-6.303338-0.803839-1.340356$

H $1.349600-0.958943-3.175977$

H -1.346184-1.749438-1.919548

H $1.675992-4.658021-3.035623$

H $2.186058-3.072949-2.504680$

H $1.425093-3.282568-4.099632$

H $-3.028269-1.314828-0.034780$

H $-3.143174-0.2281311 .367405$

H $-0.126016-2.840596 \quad 3.130157$

H $0.955824-1.6709012 .349042$

H $1.242128-3.1005870 .372704$

H $-0.309372-5.0718612 .131175$

H $1.171402-6.6335330 .882197$

H $1.540482-5.437899-0.331453$

H -2.083949 -7.609729 -0.653455

H $-1.155317-7.6353520 .949518$

H $-1.416953-6.221614-2.434639$

H $\quad 0.313977-6.071121-2.505412$

H $-1.733984-3.956575-1.539544$

H $-0.784329-0.9569430 .929908$

C 1.1475745 .0111330 .466261

O 2.0824884 .0108690 .017584

C 3.3826724 .3200600 .554063

C 3.2495785 .6592631 .274919

C $1.7945275 .641627 \quad 1.696869$

H 3.6061983 .5469461 .296744

C $4.4425664 .304620-0.553262$

H 3.9407945 .7426362 .119301

H 3.4416346 .5050080 .604646

C 1.2507697 .0137442 .054319

H 1.6884904 .9730852 .561907

C $4.5280062 .983414-1.345119$

O $4.1354585 .351575-1.483351$

H $5.4199494 .551394-0.120810$

H $3.6500672 .868418-1.993075$

O $5.6630323 .075776-2.216964$

C $4.6861251 .718412-0.478521$

C $5.0340370 .475021-1.322831$

H 3.7549091 .5429740 .071386

O 5.7208451 .9513120 .477218

H $4.3849890 .437789-2.205941$

H $6.0725790 .573344-1.662848$

H 1.8112727 .4430272 .891324

H 1.3242507 .7071241 .209756

H $\quad 0.198766 \quad 6.9531392 .349830$

C -0.2119334 .3729560 .753290$

H $4.9137715 .476610-2.051846$

H $5.4540253 .732345-2.902401$

H 5.7704421 .1703861 .053828

H -0.8993815 .1250021 .156280$

H -0.0982013 .5869651 .510200$ 
H $1.0576845 .750372-0.341296$

C $5.698062-1.953337 \quad 1.453340$

O $5.756601-0.8149430 .585270$

C $4.890118-0.853948-0.550643$

C $5.229115-2.066711-1.434363$

C $5.215685-3.370215-0.628791$

C $6.006586-3.2448490 .679142$

H $6.507418-1.7930292 .177349$

C $4.379545-2.0106922 .232302$

H $3.851135-0.927465-0.210543$

O $6.519664-1.886390-2.015995$

H $4.503114-2.119546-2.254123$

H $5.643366-4.173675-1.240765$

O $3.864841-3.717570-0.347351$

H $5.822380-4.1094811 .327319$

$\begin{array}{llll}\text { O } & 7.404946-3.238445 & 0.378158\end{array}$

C $4.227890-0.7968463 .118239$

H $3.504871-2.0786741 .580402$

H $4.346866-2.8935722 .881401$

H $6.686897-2.644874-2.601057$

H $3.872638-4.556108 \quad 0.144944$

H $7.612351-4.075501-0.070937$

O 3.3149510 .0614302 .580364

O $4.860681-0.6238904 .151839$

C $3.085192 \quad 1.263630 \quad 3.320999$

H 3.9990491 .8641933 .356279

H $2.307767 \quad 1.8355992 .807204$

H 2.7368761 .0283644 .331138

SCF Energy (B3LYP/6-31G**/MMFF) $=-3245.89946328$

\section{3}

MM̄FF Geometry

C $0.424691-1.8812950 .992696$

C $-0.202842-0.8573231 .593360$

C $\quad 0.468164 \quad 0.333977 \quad 2.237711$

$\begin{array}{llll}\text { O } & 1.881608 & 0.053861 & 2.403152\end{array}$

C $\quad 0.3288941 .6137691 .394168$

C -1.0691102 .1499851 .186824$

C $-1.5762372 .494328-0.012954$

C -1.8750412 .3913892 .436762$

C $-0.9405672 .359469-1.368331$

C $-0.3486793 .674222-1.895524$

C $0.9840194 .068431-1.237469$

C $1.4865355 .398646-1.804080$

C $2.8752505 .768966-1.260221$

O $1.9309713 .033204-1.516091$

O $3.3934536 .835958-2.069598$

C $3.8191014 .561173-1.442954$

C $2.797547 \quad 6.310157 \quad 0.172182$

O $5.0880834 .810709-0.841736$

C $3.2259743 .226869-0.933405$

C $4.1219242 .045982-1.378548$

$\begin{array}{llllllll}\text { O } & 3.172644 & 3.263861 & 0.493220\end{array}$

C 2.5743100 .7493093 .339716

O 2.1337621 .6181164 .079189

$\begin{array}{llll}\text { C } 4.003878 & 0.360966 \quad 3.348168\end{array}$

C $4.452989-0.7933022 .830738$

C $5.841904-1.2573852 .827347$

C $6.909665-0.2802653 .243106$

C $3.5252810 .660099-1.090539$

C $4.364184-0.456590-1.687450$

C $4.311847-2.915788-2.083908$

O $5.460776-0.270224-2.207774$

C $5.081681-3.598321-0.998815$

C $6.421839-3.647586-0.964574$

C $7.216123-4.3683390 .086774$

C $7.957437-3.4243531 .006936$

C $9.098035-2.8292150 .619501$

C $7.419600-3.2402782 .407141$

C $6.096294-2.5249552 .440783$

N $3.737825-1.689810-1.565891$

H $1.507764-1.8800470 .907752$

H $-1.288675-0.8820081 .662300$

H $\quad 0.0285810 .4650913 .234087$

H $\quad 0.843304 \quad 1.4462520 .443182$

H $0.9052522 .415242 \quad 1.874167$

H $-2.5767412 .924069-0.038867$

H $-2.7968662 .947522 \quad 2.235718$
H -2.1590401 .4422542 .901498$

H -1.2933962 .9752923 .157561$

H $-0.2026641 .552306-1.400591$

H $-1.7328212 .049574-2.060624$

H $-0.1818393 .544268-2.973169$

H $-1.0786354 .484982-1.778565$

H $0.8404224 .150259-0.155115$

H $0.7677486 .202890-1.603125$

H $1.5543965 .325823-2.898763$

H $2.7687667 .579730-2.021370$

H $4.0106724 .466888-2.520707$

H 2.1498727 .1942590 .212580

H 3.7801556 .6456430 .521853

H 2.4051825 .5820920 .885076

H 4.9867514 .7015320 .119086

H $4.2916812 .118079-2.461049$

H $5.1017582 .114146-0.889093$

H 3.2200822 .3592890 .839086

H 4.6652441 .0616903 .847189

H $3.737888-1.4837942 .386759$

H 6.8111240 .6593442 .687355

H $7.923140-0.6392403 .048171$

H $6.836936-0.064074 \quad 4.314393$

H $3.4583230 .484869-0.012640$

H $2.5175130 .585321-1.514838$

H $4.941177-2.686576-2.950561$

H $3.489473-3.555269-2.418629$

H $4.505828-4.093175-0.220666$

H $6.989064-3.154470-1.751236$

H $7.934383-5.027916-0.417868$

H $6.574771-5.0387050 .672730$

H $9.648829-2.1731391 .285233$

H $9.504023-2.984089-0.374798$

H $8.156059-2.7406863 .045669$

H $7.286818-4.2288212 .866460$

H $5.255519-3.1554492 .149636$

H $2.860828-1.753112-1.058048$

C $-0.260431-3.141398-1.077349$

O $-1.238174-2.204140-1.579490$

C $-2.212207-2.931813-2.346404$

C $-1.470398-4.158957-2.848812$

C $-0.663353-4.519937-1.617297$

H $-2.994913-3.251140-1.647883$

C $-2.835706-2.049822-3.430592$

H $-2.146347-4.957329-3.168674$

H $-0.800343-3.916287-3.681622$

C $0.520468-5.427173-1.907765$

H $-1.325467-5.026097-0.901525$

C $-3.389992-0.704402-2.920019$

O $-1.853935-1.757445-4.432512$

H $-3.625109-2.611768-3.943148$

H $-2.555465-0.026395-2.712379$

O $-4.123831-0.099479-3.991952$

C $-4.288188-0.791182-1.672837$

C $-4.9077130 .572429-1.310723$

H $-3.693442-1.156804-0.828611$

O $-5.324773-1.739583-1.919678$

H $-4.152226 \quad 1.357779-1.421930$

H $-5.7290420 .782071-2.007527$

H $0.180884-6.382842-2.320034$

H $1.205301-4.973351-2.631846$

H $1.085528-5.633269-0.992987$

C $-0.269943-3.0987240 .452367$

H -1.108638 -1.312917 -3.993112

H $-4.907789-0.650919-4.157689$

H $-5.858749-1.808394-1.110048$

H $\quad 0.247398-3.9769420 .856871$

H $-1.302365-3.1330310 .821011$

H $0.713044-2.832511-1.476407$

C $-7.129276-0.4419751 .527882$

$\begin{array}{lllll}\text { O } & -6.538854 & -0.308106 & 0.227944\end{array}$

C $-5.446408 \quad 0.609751 \quad 0.133073$

C -5.8993852 .0212570 .537550$

C $-6.5439532 .022987 \quad 1.926471$

C -7.5989920 .9198252 .065768$

H -8.021365 -1.0606041 .363989$

C $-6.208789-1.1944132 .495203$

H -4.6403090 .2846140 .800192$ 
O $-6.8236872 .539662-0.417046$ H -5.0300482 .6883610 .535698$ H -7.006863 3.0016092 .102491 O H -7.9104160 .8108153 .111027$ O $-8.761786 \quad 1.294124 \quad 1.320594$ C $-6.049510-2.6311902 .058162$ H $-5.217434-0.7424112 .589389$ H $-6.635295-1.2134703 .505174$ H -7.566156 $1.914782-0.481966$ H $-5.942676 \quad 1.8771223 .778040$ H -9.0904232 .1309841 .691033$ O $-4.851154-2.8018561 .430726$ O $-6.902784-3.4919462 .231883$ C $-4.593303-4.1253240 .952047$ H -3.563047 -4.1626010 .590771$ H $-4.704504-4.856787 \quad 1.758328$ H $-5.266476-4.3583630 .121765$

SCF Energy (B3LYP/6-31G**//MMFF) $=-3245.91008705$

\section{4}

MM̄FF Geometry

C $0.315776-2.2442012 .406081$

C $0.591634-2.2634251 .092881$

C $-0.454461-2.1698840 .013264$

O $-0.257985-0.919328-0.692616$

C $-0.312802-3.303610-1.022137$

C $-1.173630-4.507129-0.703545$

C $-2.306689-4.820414-1.362170$

C $-0.679315-5.396087 \quad 0.407060$

C $-2.943496-4.085781-2.509299$

C $-3.484883-2.685095-2.206488$

C $-4.358394-2.549829-0.952392$

C $-5.620809-3.410011-1.012612$

C $-6.531046-3.1666010 .201081$

O $-4.716946-1.163933-0.863227$

O $-7.809066-3.757114-0.080333$

C $-6.758889-1.6493920 .359705$

C $-5.999938-3.8608201 .461206$

O $-7.478371-1.3654881 .557950$

C $-5.455953-0.8156600 .313963$

C $-5.8068890 .688656 \quad 0.226190$

O $-4.700503-1.0563561 .501506$

C $-0.774623 \quad 0.202787-0.120231$

O -1.4149150 .2790650 .916143$

C $-0.377929 \quad 1.350565-0.960224$

C $-0.221168 \quad 2.551269-0.383782$

C $0.2593593 .764859-1.046356$

C $0.338198 \quad 3.751070-2.550428$

C -4.6012901 .6143770 .010757$

C $-5.0283333 .055293-0.214146$

C $-4.1063895 .318434-0.598717$

O $-6.1996213 .423846-0.182912$

C -2.881424 5.908985-1.217139

C $-2.1394086 .855048-0.622160$

C $-0.9334017 .495682-1.246369$

C $0.315077 \quad 7.312746-0.414040$

C $0.6628508 .215557 \quad 0.518583$

C $1.2032826 .130590-0.726495$

C $0.6112394 .820570-0.282586$

$\mathrm{N}-3.940873 \quad 3.884616-0.459833$

H $-0.716033-2.1684392 .739222$

H $1.628815-2.3169890 .769986$ H $-1.467987-2.1767530 .433462$ H $0.733538-3.625140-1.113106$ H $-0.556100-2.918326-2.019360$ H $-2.845600-5.715593-1.053912$ H $-1.252567-6.3266140 .479054$ H $\quad 0.366869-5.6720320 .238603$ H $-0.758241-4.8883461 .372200$ H $-2.231011-4.027311-3.341510$ H $-3.767684-4.705272-2.885691$ H $-2.652313-1.975691-2.124243$ H $-4.051684-2.334515-3.079844$ H $-3.757162-2.808590-0.074685$ H $-5.376013-4.475757-1.088080$ H $-6.184223-3.166184-1.924157$ H $-7.670373-4.704416-0.251607$
H -7.402919 -1.328464 -0.471108 H $-5.924713-4.942857 \quad 1.299071$ H -6.688896 -3.731908 2.303262 H -5.013533 -3.5041611 .764594$ H $-8.293092-1.8963701 .539366$ H $-6.4994760 .840688-0.612542$ H -6.3270821 .0040351 .139548$ H $-4.060772-0.3371411 .619766$ H $-0.1601341 .161671-2.004664$ H -0.4103982 .6442040 .685127$ H $-0.6065883 .402385-2.983133$ H $1.1438083 .089886-2.887340$ H $\quad 0.5181234 .736971-2.984374$ H -3.9437551 .5961510 .886041$ H $-4.0250381 .294509-0.864683$ H -4.3102095 .7248230 .397881$ H -4.980193 $5.517025-1.228567$ H -2.607895 $5.560061-2.209941$ H -2.4304517 .2148240 .362412$ H -1.145848 $8.566392-1.364901$ H $-0.7590757 .119413-2.262076$ H 1.5722938 .1075651 .099997 H $\quad 0.0414679 .081076 \quad 0.724012$ H $2.1676736 .237354-0.211465$ H $1.4540206 .145590-1.790856$ H 0.5163424 .7315150 .800624 H -3.008857 $3.522746-0.277453$ C $1.582853-0.9419744 .142426$ O 2.0523950 .0030953 .157360 C 3.3824780 .4078373 .512731 C 3.4101250 .3058025 .028148 C $2.657802-0.9970005 .237370$ H $4.067608-0.3361863 .088989$ C 3.7140021 .7804362 .921101 H 4.4271250 .2828625 .430120 H 2.8676681 .1327575 .500486 C $2.088700-1.1544256 .637340$ H $3.347284-1.8309045 .047840$ C 3.3763561 .9305181 .423202 O 2.9866572 .7969763 .621890 H 4.7735262 .0026753 .092079 H 2.2937612 .0537321 .301136 O $3.970990 \quad 3.146690 \quad 0.955114$ C $3.8480740 .774257 \quad 0.523894$ C $3.5662931 .044551-0.965991$ H $3.330835-0.1428850 .822632$ $\begin{array}{lllll}\text { O } & 5.243673 & 0.549276 & 0.711407\end{array}$ H $2.6027401 .552699-1.068003$ H $4.3462181 .704549-1.365403$ H $2.891467-1.1532497 .381900$ H $1.399712-0.3396536 .884128$ H $1.541775-2.0982846 .728833$ C $1.374224-2.3037523 .470670$ H 2.0398422 .5866323 .545523 H 3.6353823 .8673751 .515582 H $5.707127 \quad 1.3759540 .492853$ H $1.079233-3.0518444 .215501$ H $2.317592-2.6466603 .027840$ H $0.633579-0.5529144 .530062$ C $4.950125-2.078890-2.501291$

O $4.828229-0.828341-1.809772$

C $3.519018-0.254092-1.794084$ C $3.0225250 .003106-3.224907$ C $3.082989-1.275606-4.064851$ C $4.446150-1.964306-3.950940$ H $6.030285-2.271243-2.535949$ C $4.295163-3.225765-1.722179$ H $2.827745-0.942770-1.296323$ O $3.8176191 .020014-3.829938$ H $\quad \begin{array}{llll}1.991382 & 0.373062 & -3.180539\end{array}$ H $2.885260-1.025927-5.114391$ O $2.052716-2.155746-3.624134$ H $4.405365-2.958838-4.410415$ O $5.389689-1.197429-4.704383$ C $5.104423-3.553561-0.490700$ H $3.265262-3.019155-1.419357$ H $4.260452-4.138867-2.328636$

H $3.4675541 .173503-4.723953$ 
H $\quad 2.088214-2.951751-4.181344$

H $6.239606-1.669209-4.679344$

O $4.607136-2.8901900 .591283$

O $6.080571-4.292964-0.498687$

C $5.314571-3.1061061 .815954$

H $6.328266-2.7017581 .740054$

H $4.786413-2.5796842 .614107$

H $5.339894-4.1724042 .060617$

SCF Energy (B3LYP/6-31G**//MMFF) $=-3245.89889744$

\section{5}

MM̄FF Geometry

C $-0.029191-2.3819641 .985121$

C $-0.489918-3.2774061 .096547$

C $0.022677-3.480794-0.309900$

O $0.715718-2.302900-0.780780$

C $0.989559-4.675030-0.341566$

C $1.487475-5.003334-1.734885$

C $2.713424-4.692175-2.200497$

C $0.519324-5.766229-2.601210$

C $3.794178-3.928531-1.485139$

C $4.351790-2.799238-2.362280$

C $5.271952-1.818347-1.617258$

C $6.465059-2.500102-0.949744$

C $7.343182-1.490646-0.197333$

O $4.490421-1.144582-0.628823$

O $8.231178-2.2580050 .631878$

C $6.452573-0.627180 \quad 0.730041$

C $8.233997-0.701980-1.165478$

$\begin{array}{lllll}\text { O } & 7.211314 & 0.440748 & 1.290307\end{array}$

C $5.174733-0.0758670 .047923$

C $4.146491 \quad 0.4728401 .062915$

O $5.4615920 .996288-0.852684$

C $-0.042987-1.356725-1.401029$

O $-1.243382-1.407685-1.619627$

C $0.840037-0.224444-1.761298$

C $0.2948230 .880146-2.292819$

C $0.9971232 .100643-2.697031$

C $2.4977542 .106739-2.592215$

C 4.6015871 .6925861 .866280

C 3.4278532 .5545272 .286341

C 1.7591834 .1597401 .373403

O 3.0118822 .5699313 .441639

C $1.7093005 .096128 \quad 0.209180$

C $0.6120135 .304323-0.533767$

C $0.573176 \quad 6.244056-1.704321$

C $-0.0256895 .622047-2.946386$

C $-1.1613456 .104153-3.479991$

C $0.729040 \quad 4.493107-3.618704$

C $0.2637403 .138938-3.150961$

N $2.917873 \quad 3.2989681 .234069$

H $0.808169-1.7452941 .715973$

H -1.304762 -3.9355221 .391660$

H $-0.834155-3.690552-0.962517$

H $0.494188-5.5666290 .067115$

H $1.832460-4.4865510 .332801$

H $2.976485-5.015188-3.207102$

H $0.946750-6.023137-3.576283$

H $0.228356-6.702013-2.112895$

H $-0.382372-5.173605-2.784107$

H $3.435692-3.506106-0.543544$

H $4.595865-4.634143-1.239808$

H $4.895833-3.232353-3.211230$

H $3.510488-2.227600-2.776653$

H $5.614492-1.082527-2.354367$

H $7.058535-3.057700-1.684781$

H $6.113584-3.241201-0.219785$

H $8.838560-1.6422551 .075414$

H $6.138399-1.2643151 .568584$

H $8.919936-1.379757-1.688256$

H $8.870076 \quad 0.011667-0.630473$

H $7.667454-0.158528-1.924526$

H $7.9234330 .049947 \quad 1.823927$

H $3.2276200 .715497 \quad 0.512608$

H $3.846190-0.3251911 .755655$

H $6.421551 \quad 1.128779-0.887941$

H $1.901574-0.324780-1.571097$

H $-0.7840130 .901285-2.441949$
H $2.8082721 .966440-1.553580$

H $2.9578063 .034283-2.938055$

H $2.928470 \quad 1.299896-3.195942$

H 5.1403631 .3779742 .767122

H $5.2850882 .329047 \quad 1.292348$

H $1.844404 \quad 4.7167212 .312071$

H 0.8738683 .5180821 .428893

H $2.6228325 .634727-0.033056$

H $-0.3107844 .792309-0.274527$

H $1.5774506 .613620-1.947510$

H $-0.0085787 .124448-1.402332$

H -1.582842 $5.679674-4.385308$

H -1.697229 $6.929262-3.022791$

H $1.8017594 .651772-3.480983$

H $0.5642834 .540785-4.703428$

H $-0.8183283 .009326-3.213583$

H $3.268148 \quad 3.1147340 .298539$

C $-0.947701-0.7880053 .755275$

O $-1.820582-0.1884722 .776645$

C -1.4012861 .1746182 .571512$

C -0.4666421 .5021823 .730496$

C 0.2315660 .1707563 .925599

H $-0.8199251 .188973 \quad 1.641692$

C -2.609685 2.107729 2448298

H 0.2184602 .3206523 .496073

H $-1.0222891 .775674 \quad 4.635234$

C $0.942808 \quad 0.044741 \quad 5.261414$

H 0.9633820 .0421613 .118964

C -3.6004341 .7444541 .322555$

O -3.3396042 .1014873 .681916$

H -2.2605873 .1384122 .313626$

H $-4.1593860 .841790 \quad 1.593347$

O -4.5619552 .8037641 .234877$

C $-2.9555991 .561658-0.061276$

C $-3.9389921 .470370-1.241844$

H $-2.3326790 .661661-0.050097$

O $-2.0788642 .660105-0.327883$

H $-4.4327522 .438873-1.393987$

H $-3.3313421 .310528-2.142541$

H 1.7207230 .8088945 .357781

H 0.2484990 .1661116 .099666

H $1.419821-0.9356145 .357813$

C $-0.598002-2.2283913 .369241$

H -3.5993901 .1819963 .863570$

H -4.9650302 .9020952 .114654$

H $-2.6107043 .474056-0.303815$

H -1.510345 -2.832665 3.452840

H $0.118544-2.6370724 .090725$

H -1.523037 -0.8239084 .690369$

C $-5.168363-2.029034-0.757167$

O $-4.324223-0.882678-0.931848$

C $-4.9987010 .363186-1.124801$

C $-5.8975930 .306088-2.372330$

C $-6.859992-0.883465-2.312049$

C $-6.134821-2.182661-1.943684$

H $-4.484119-2.887220-0.773657$

C $-5.882168-2.0177620 .604207$

H $-5.6190350 .577430-0.249143$

O $\quad-5.0869630 .196663-3.541496$

H $-6.4592291 .245186-2.445880$

H -7.347235 -1.002370 -3.287572

O $-7.873265-0.599249-1.352295$

H $-6.862629-2.971043-1.721630$

O $-5.387172-2.631784-3.077923$

C $-4.913601-2.1389301 .760220$

H $-6.453249-1.0979980 .753645$

H -6.566700 -2.871618 0.665703

H $-5.6801420 .207630-4.311811$

H $-8.488618-1.352129-1.343629$

H $-4.680909-1.984030-3.243928$

O $-5.602833-2.0029382 .931607$

O $-3.711423-2.3346301 .655448$

C $-4.817249-2.0950784 .124258$

H -5.497108 -2.086176 4.980294

H $-4.247234-3.0290564 .140354$

H -4.149712 -1.232473 4.197699

SCF Energy $\left(B 3 L Y P / 6-31 G^{* *} / / M M F F\right)=-3245.91231334$ 
0700296

MM̄FF Geometry

C $1.204753-3.4160982 .039013$

C $0.445229-3.705093 \quad 3.106233$

C $-0.519040-2.7596963 .783896$

O $-0.572627-1.4958393 .084630$

C - $-1.939635-3.3568753 .741234$

C $-2.968285-2.4889204 .440240$

C $-3.750130-1.5808623 .821257$

C $-3.086256-2.7089315 .925101$

C $-3.733342-1.2002152 .364976$

C $-5.095951-1.4293231 .701208$

C $-5.104662-1.1848250 .181069$

C $-4.397127-2.304161-0.583739$

C $-4.399790-2.038052-2.093870$

O $-4.4785820 .069893-0.091020$

O $-3.484446-2.960169-2.705141$

C $-3.853786-0.618876-2.355327$

C $-5.772871-2.308561-2.721823$

O $-3.992592-0.298875-3.738009$

C $-4.4980340 .477392-1.472230$

C $-3.7052231 .808490-1.502063$

O $-5.8352740 .722270-1.910255$

C $-0.016242-0.3985243 .659049$

O $0.663725-0.3522194 .669964$

C -0.4458810 .7633152 .847140$

C -0.2021632 .0145393 .263137$

C -0.6699043 .2395202 .606433$

C -1.566907 3.0806391 .406188

C $-3.5769072 .486480-2.864488$

C $-3.0136213 .891571-2.752903$

C $-0.8941095 .132670-2.346157$

O $-3.6941034 .878789-3.024054$

C $0.0347455 .120258-1.174534$

C $0.1908286 .159965-0.340501$

C 1.1237506 .1449080 .837692

C 0.4630996 .6066262 .119550

C 0.9005707 .7036412 .760834

C -0.7046205 .8087132 .658481$

C $-0.303971 \quad 4.4329993 .119912$

N -1.689272 $3.916549-2.341847$

H $1.184739-2.4141491 .619536$

H $0.490709-4.7110043 .518851$

H -0.204070 -2.619236 4.824856

H -1.943578 -4.351914 4.206386

H -2.241844 -3.528087 2.700569

H -4.457745 -1.015084 4.426030

H -3.841283 -2.0614516 .383540$

H -3.369625 -3.7457326 .133793$

H -2.131367 -2.502561 6.418808

H -3.476421 -0.1358732 .304153$

H -2.953867 -1.733806 1.818501

H $-5.462270-2.4422141 .910799$

H -5.811940 -0.734133 2.160548

H -6.155527 -1.129911 -0.127119

H $-4.855378-3.276513-0.364266$

H -3.351983 -2.385312 -0.258111

H -3.779534 -3.861831 -2.490946

H $-2.777247-0.637369-2.146301$

H -6.086048 -3.341306 -2.527170

H $-5.735371-2.209115-3.812356$

H -6.555102 -1.647659-2.342631

H -3.538986 $-0.994670-4.244034$

H -4.186683 $2.513325-0.809024$

H -2.708261 $1.638294-1.078491$

H $-6.0926961 .614498-1.624136$

H -1.007262 $0.552973 \quad 1.943759$

H 0.3642922 .1584294 .182020

H -2.452100 2.4863281 .660050

H -1.029171 2.5815370 .593854

H -1.9379304 .0238151 .002944$

H -2.913997 $1.919381-3.526614$

H $-4.5527942 .556896-3.357466$

H -1.549758 $6.009306-2.347906$

H $-0.3173955 .132756-3.276624$

H $0.6155264 .216901-1.006581$

H $-0.3668117 .076182-0.521268$

H $1.974720 \quad 6.7936790 .593415$
H $\quad 1.5421625 .144598 \quad 0.995391$

H $0.4352198 .045807 \quad 3.679309$

H $1.734900 \quad 8.2852962 .383238$

H -1.503810 5.7936241 .913603

H -1.144858 6.3212323 .524505

H $\quad 0.344857 \quad 4.442267 \quad 3.996839$

H -1.189890 $3.033610-2.279380$

C $1.703133-4.619118-0.136910$

O $1.744374-3.359966-0.840941$

C $0.456026-3.138208-1.440681$

C $-0.107855-4.532219-1.663794$

C $0.306191-5.210322-0.368798$

H $-0.162573-2.624826-0.693352$

C $0.576812-2.251453-2.682256$

H -1.190449 -4.529268-1.817816

H $\quad 0.364721-5.028823-2.518939$

C $0.292362-6.727537-0.441104$

H $-0.387825-4.8965010 .420396$

C $1.373185-0.946555-2.459218$

O $1.231328-2.977847-3.729163$

H $-0.425008-2.019455-3.061978$

H $2.443378-1.173294-2.424461$

O $1.180094-0.110886-3.606325$

C $0.976256-0.155729-1.203205$

C $1.6554471 .215256-1.058801$

H $1.198076-0.750331-0.310989$

O $-0.435646 \quad 0.055828-1.222929$

H $1.2901861 .899327-1.835279$

H $1.3021511 .639248-0.109582$

H $-0.717743-7.093409-0.652369$

H $0.955805-7.098404-1.229625$

H $0.619596-7.1632130 .508470$

C $2.092099-4.4038201 .333012$

H $2.100396-3.257825-3.393207$

H $1.442124-0.627046-4.388032$

H $-0.6821460 .470980-0.379784$

H $3.111147-4.0012771 .375587$

H $2.102296-5.3677631 .854689$

H $2.466182-5.252379-0.606576$

C $5.0635000 .047094-0.014494$

O $3.6418480 .216607-0.119937$

C $3.1939191 .185572-1.073998$

C $3.7845312 .572575-0.766285$

C $5.3085262 .519541-0.638045$

C $5.757927 \quad 1.3869890 .289944$

H $5.197603-0.6033300 .858897$

C $5.657871-0.663663-1.238581$

H $3.5154920 .877446-2.073678$

O $3.2327513 .094621 \quad 0.441272$

H $3.5035153 .262690-1.570291$

H $5.6667373 .479004-0.244948$

O $5.8686902 .339703-1.934940$

H $\quad 6.846000 \quad 1.264622 \quad 0.235196$

O 5.4499821 .7671131 .634087

C $5.377013-2.148107-1.219494$

H $5.275329-0.275710-2.186452$

H $6.748047-0.545551-1.273146$

H 3.4065572 .4529211 .151040

H $6.8363432 .341197-1.838990$

H 5.7945891 .0738712 .222495

O $6.180713-2.762813-0.304633$

O $4.559770-2.698095-1.945657$

C $6.056448-4.186412-0.231138$

H $6.658890-4.537450 \quad 0.610837$

H $5.016211-4.476893-0.061985$

H $6.437273-4.640686-1.150618$

SCF Energy (B3LYP/6-31G**//MMFF)= -3245.91743527

\section{7}

MM̄FF Geometry

C $-1.2094404 .671216 \quad 1.288770$

C $-2.3079363 .902076 \quad 1.305475$

C $-3.2031493 .722710 \quad 0.107535$

O $-2.8593412 .427745-0.435789$

C -4.6818303 .7542430 .530471$

C $-5.6462363 .907293-0.630079$

C -6.417855 2.922565-1.131556

C $-5.7467015 .300599-1.198650$ 
C $-6.4678891 .482780-0.702807$

C $-6.2932600 .536893-1.901114$

C $-6.146390-0.944536-1.513603$

C $-7.398298-1.499483-0.828969$

C $-7.206433-2.951149-0.366122$

O $-5.003587-1.060377-0.661682$

$\begin{array}{llll}0 & -8.286965 & -3.277839 & 0.521794\end{array}$

C $-5.905545-3.0315050 .459014$

C $-7.288347-3.944059-1.530718$

O $-5.610349-4.3800110 .815801$

C $-4.689374-2.395539-0.248910$

C $-3.456471-2.3138530 .677782$

O $-4.324335-3.205887-1.371850$

C -2.806564 2.300664 -1.785492

O $-3.2176073 .099911-2.615231$

C $-2.1572341 .031782-2.183132$

C $-1.2246860 .433814-1.424782$

C $-0.445425-0.753172-1.788633$

C $-0.790036-1.447165-3.079893$

C $-3.622365-1.3932821 .890433$

C $-2.281595-1.1809672 .559489$

C $-0.567386-2.3850903 .897688$

O $-1.609288-0.1741942 .349452$

C $0.220265-3.2203462 .938850$

C $1.118060-2.7216472 .076094$

C $1.847867-3.5639051 .072111$

C $1.229847-3.488446-0.307067$

C $0.504834-4.510921-0.791526$

C $1.523868-2.276993-1.160736$

C $0.540398-1.157935-0.960050$

N $-1.908473-2.2492343 .361410$

H $-0.952413 \quad 5.2117990 .380826$

H -2.548151 3.3355052 .202107

H -2.989463 $4.511751-0.624163$

H -4.8540374 .5977141 .213628$

H -4.9161152 .8581611 .115320$

H -7.099058 3.171857-1.944468

H -6.496092 $5.372788-1.994052$

H $-6.030748 \quad 6.010657-0.415105$

H $-4.7886725 .615538-1.622971$

H -5.7073891 .2600640 .048963$

H $-7.4431251 .310946-0.234291$

H -7.137817 $0.652014-2.592155$

H $-5.3900940 .833876-2.449583$

H -5.948828 -1.501745 -2.437193

H -8.270197 -1.416194 -1.489471

H $-7.637141-0.900540 \quad 0.059259$

H -9.121047 -3.1522020 .037570$

H $-6.091317-2.496151 \quad 1.398669$

H -8.270359 -3.884426 -2.015328

H $-7.191360-4.976188-1.176565$

H $-6.532661-3.769815-2.299083$

H $-6.397925-4.7417911 .257150$

H $-3.159953-3.3205720 .999556$

H -2.605391-1.945506 0.089543

H -3.394840 $-3.029067-1.592269$

H -2.411429 $0.675044-3.175560$

H $-0.9692240 .876502-0.463176$

H $-1.854715-1.705999-3.105941$

H $-0.247740-2.383496-3.227819$

H $-0.563737-0.801795-3.935573$

H $-4.007876-0.4098031 .599383$

H $-4.323310-1.8142122 .618950$

H $-0.637581-2.8851914 .868043$

H $-0.122545-1.3964104 .044939$

H $0.011569-4.2877252 .934082$

H $1.321055-1.6538752 .073226$

H $1.878918-4.6069081 .415132$

H $2.896341-3.2489001 .029523$

H $0.083472-4.481299-1.790601$

H $0.311694-5.398252-0.197753$

H $1.592380-2.566197-2.215149$

H $2.525057-1.897699-0.923022$

H $0.702566-0.594271-0.042615$

H -2.512933 -3.0648343 .402409$

C 1.0327024 .0227532 .292236

O $1.679720 \quad 4.3404421 .042276$

C 2.0213543 .1035550 .378968
C 2.0446872 .0576451 .480970

C 0.8708712 .5009882 .328558

H $1.2049452 .903831-0.327255$

C $3.3447603 .271474-0.374512$

H 1.9390951 .0367301 .106909

H 2.9778922 .1215172 .052357

C 0.8745721 .9186093 .730720

H -0.0501762 .1913901 .821354$

C $3.8159631 .973381-1.061701$

O $3.154395 \quad 4.289307-1.360756$

H $4.122413 \quad 3.6326990 .308924$

H $3.8936541 .192815-0.298699$

O $2.8214971 .542408-1.991631$

C $5.1804062 .078944-1.774442$

C $5.5975090 .806670-2.537146$

H $5.9526212 .328922-1.037362$

O $5.171965 \quad 3.129518-2.743230$

H $4.9302420 .653275-3.395278$

H $6.5875081 .012194-2.965755$

H $\quad 0.8627340 .825122 \quad 3.693064$

H 1.7641342 .2244034 .291055

H $-0.006128 \quad 2.249303 \quad 4.290324$

C $-0.265088 \quad 4.8243792 .447843$

H $2.846710 \quad 5.087740-0.898177$

H $2.7419932 .233827-2.671305$

H $5.0156723 .964942-2.271612$

H -0.0177545 .8909852 .518720$

H $-0.7571424 .553893 \quad 3.389550$

H 1.7271064 .3421113 .080258

C $6.716650-1.3248350 .320805$

O $6.573584-0.248250-0.615906$

C $5.655146-0.472916-1.688971$

C $6.065763-1.704981-2.514016$

C $6.238444-2.934912-1.617092$

C $7.109590-2.628667-0.394910$

H $7.561199-1.0320460 .958297$

C $5.476686-1.4729001 .217556$

H $4.657626-0.653323-1.279711$

O $7.295290-1.474883-3.198077$

H $5.297479-1.905946-3.270682$

H $6.700945-3.744718-2.194584$

O $4.965541-3.392121-1.170972$

H $7.074250-3.4605570 .318394$

O $8.472046-2.494593-0.809718$

C $5.370771-0.357178 \quad 2.236508$

H $4.544219-1.4749860 .649832$

H $5.553427-2.4134701 .776098$

H $7.114616-0.872502-3.939079$

H $4.436373-3.605175-1.958468$

H $8.734203-3.330948-1.230612$

O $4.498014-0.737561 \quad 3.216498$

$\begin{array}{lllll}\text { O } & 5.963827 & 0.710933 & 2.178212\end{array}$

C 4.3213330 .1892494 .292667

H $3.435399-0.1122974 .857907$

H 5.1920110 .1518154 .953332

H 4.1681401 .2061553 .920948

SCF Energy (B3LYP/6-31G*//MMFF) $=-3245.89808330$

\section{8}

MM̄FF Geometry

C $1.259473-1.8473021 .772749$

C $0.724868-0.9137432 .575181$

C $1.453814 \quad 0.277385 \quad 3.156917$

$\begin{array}{llll}\text { O } & 2.871513 & 0.165576 & 2.880585\end{array}$

C 0.9333191 .5989372 .565155

C -0.4231142 .0298643 .078538$

C -1.5451352 .0732602 .334862$

C -0.451655 2.5015174 .509965

C $-1.722398 \quad 1.658218 \quad 0.901954$

C $-1.866505 \quad 2.859714-0.042920$

C $-0.5496603 .612955-0.285331$

C $-0.7846214 .830824-1.181148$

C $0.529776 \quad 5.543053-1.536005$

$\begin{array}{llll}\text { O } & 0.353578 & 2.706775 & -0.925342\end{array}$

O $0.258526 \quad 6.456369-2.610289$

C $1.5321834 .497916-2.070105$

C $1.0501206 .389911-0.368756$

O $2.8114135 .087426-2.294564$ 
C $1.6584023 .240535-1.176654$

C $2.4767422 .154638-1.912780$

O 2.3309503 .5982720 .031545

C 3.7281560 .8292103 .697575

O 3.4238561 .5874624 .608965

C 5.1429580 .5282333 .377276

C $5.534516-0.4384802 .531863$

C $6.920129-0.8291072 .255548$

C 8.0197030 .0109302 .852225

C $2.5199870 .796861-1.196793$

C $3.160147-0.249502-2.086771$

C $5.348414-0.904532-3.083758$

O $2.494890-1.126449-2.632756$

C $6.112841-1.880564-2.247913$

C $7.450166-1.867889-2.145513$

C $8.248764-2.874269-1.368761$

C $8.981967-2.260271-0.198270$

C $10.099532-1.536649-0.380528$

C $8.473143-2.5680351 .189272$

C $7.148737-1.9219201 .496782$

N $4.527527-0.077351-2.222076$

H $2.303526-1.7761561 .479448$

H $-0.321113-1.0119752 .858823$

H $1.3165190 .242838 \quad 4.244541$

H $\quad 0.949573 \quad 1.526845 \quad 1.473354$

H 1.6421452 .4094842 .780057

H -2.457757 2.4374532 .805726

H -1.427516 2.9104324 .793488

H $-0.2310611 .676557 \quad 5.193789$

H $0.290801 \quad 3.2913404 .664770$

H $-0.927907 \quad 0.9881240 .560497$

H -2.642562 1.0631910 .852670

H -2.230012 2.480943-1.005918

H -2.629095 3.5474640 .343196

H -0.1310213 .9274600 .676590$

H -1.486178 $5.530714-0.710170$

H -1.265738 $4.508877-2.115673$

H $-0.4284227 .076274-2.310673$

H $1.1684794 .184922-3.058486$

H $\quad 0.3025507 .136466-0.074570$

H $1.9399736 .959209-0.659271$

H 1.2907605 .7980170 .516805

H $3.2431345 .195476-1.429981$

H $2.0407441 .991274-2.907617$

H $3.5038922 .508529-2.065596$

H 2.7601892 .8117380 .402530

H 5.8620531 .1227743 .932404

H $4.778773-1.0331582 .022345$

H 7.8814341 .0671492 .594350

H $9.014742-0.2578182 .489671$

H $8.030541-0.0871073 .943075$

H $3.0831370 .851894-0.259084$

H $1.5113480 .447922-0.948465$

H $6.008891-0.232207-3.641033$

H $4.721329-1.441324-3.802472$

H $5.541624-2.648118-1.731603$

H $8.013468-1.105444-2.679269$

H $8.975115-3.335623-2.050796$

H $7.613024-3.699356-1.024239$

H $10.648160-1.1209560 .458051$

H $10.489312-1.343328-1.374581$

H $9.221128-2.3080891 .946718$

H $8.356298-3.6553921 .289498$

H $6.293720-2.4635931 .090916$

H $4.9902930 .655701-1.692612$

C $0.479451-3.048534-0.281301$

O $-0.550730-2.147811-0.746401$

C $-1.342525-2.832095-1.735259$

C $-0.510425-4.027193-2.177102$

C $0.139345-4.420390-0.867141$

H -2.235756 -3.205365 -1.222494

C -1.752604 -1.891429-2.871051

H -1.121639-4.824042 -2.611274

H $\quad 0.252187-3.740885-2.910957$

C $1.342145-5.332791-1.037629$

H $-0.607267-4.924148-0.238427$

C $-2.573684-0.662275-2.431640$

O $-0.577897-1.415549-3.539271$
H - $-2.313908-2.458665-3.622898$

H $-1.9234840 .052834-1.914635$

O $-3.0176220 .005829-3.620179$

C -3.791976 -0.977052 -1.542055

C $-4.7108440 .244300-1.347794$

H $-3.434200-1.331758-0.569051$

O $-4.539801-2.034285-2.142937$

H $-4.1044691 .143110-1.203404$

H $-5.3128320 .378409-2.255370$

H $1.047050-6.271910-1.516755$

H $2.114592-4.866857-1.658849$

H $1.790300-5.574107-0.068684$

C $0.516950-3.0437641 .247823$

H $-0.021151-0.972621-2.875803$

H -3.640182 -0.587289-4.074904

H $-5.275284-2.250688-1.544939$

H $1.038366-3.9347611 .615878$

H $-0.503353-3.0686271 .649215$

H $1.428531-2.686895-0.695347$

C $-7.445173-1.3268220 .668519$

O $-6.539561-1.000830-0.394068$

C $-5.6510670 .088781-0.136731$

C $-6.448764 \quad 1.3735330 .135722$

C -7.466495 1.1705371 .262004

C $-8.292943-0.1061511 .063021$

H $-8.119742-2.0779380 .237051$

C $-6.721918-1.9753081 .854332$

H $-5.034076-0.1458290 .737820$

O $-7.1271851 .796899-1.045431$

H -5.7503392 .1734200 .408625$

H -8.1338562 .0402841 .298860$

O -6.7656621 .1082712 .500615$

H -8.868842 -0.3400831 .966065$

O $\quad-9.2364160 .1141650 .010164$

C $-6.081798-3.2793401 .438924$

H $-5.954293-1.3278652 .287631$

H -7.425126 -2.203266 2.663905

H $-7.6939071 .064038-1.341889$

H -7.4266201 .0102813 .207080$

H $-9.808307 \quad 0.8532980 .278837$

O $-4.724939-3.147152 \quad 1.414576$

O $-6.714124-4.2878901 .152739$

C $-3.998589-4.3166611 .025260$

H -2.931266 -4.098532 1.114093

H -4.239894 -5.154967 1.685780

$\mathrm{H}-4.221676-4.567429-0.015982$

SCF Energy (B3LYP/6-31G**//MMFF) $=-3245.91797645$

0700299

MM̄FF Geometry

C $-2.838036-2.836670-2.324077$

C -1.613834 -2.822625 -1.777380

C $-0.376723-3.363131-2.442537$

O $0.582475-2.276262-2.394421$

C $0.205058-4.583620-1.695519$

C $-0.797144-5.652221-1.310418$

C $-1.228013-5.877706-0.052336$

C $-1.297768-6.499819-2.450282$

C $-0.833908-5.132228 \quad 1.192315$

C $-2.029317-4.4121681 .832839$

C $-1.556562-3.3692452 .855861$

C -2.729095 -2.806100 3.656858

C $-2.266000-1.7120994 .631615$

O $-0.930056-2.3084712 .126469$

O $-3.432190-1.0214575 .107283$

C $-1.415176-0.6768283 .862831$

C -1.586088 -2.3092395.869416

O $-0.815822 \quad 0.2604704 .753585$

C $-0.332484-1.2950482 .942692$

C $0.241622-0.2049812 .005431$

O $0.769184-1.806973 \quad 3.694273$

C $1.737484-2.384504-3.097893$

O $2.053589-3.268113-3.876513$

C $2.588905-1.242949-2.695719$

C $3.922738-1.360168-2.765674$

C $4.902095-0.350266-2.355720$

C $4.3765760 .958371-1.828339$

C $1.074805-0.7262780 .829195$ 
C $2.354139-1.4375621 .216727$

C $4.595830-1.0411352 .234914$

O $2.497060-2.6443791 .032734$

C $5.668248-0.2151821 .596296$

C $6.737120-0.7377460 .975056$

C 7.8512110 .0901230 .400305

C $8.228924-0.313467-1.007246$

C $9.323189-1.057593-1.242185$

C $7.3984780 .227663-2.148770$

C $6.214515-0.644181-2.464248$

N $3.303290-0.5884851 .757586$

H $-2.980508-3.263180-3.313484$

H $-1.488180-2.402519-0.782312$

H $-0.577903-3.611193-3.492327$

H $0.776674-4.232144-0.830598$

H $\quad 0.973578-5.053047-2.324231$

H -1.943950 -6.682008 0.110979

H -2.005256 -7.267112 -2.118340

H -1.808260 $-5.881249-3.194769$

H - $0.461203-7.010833-2.937960$

H $-0.422183-5.852131 \quad 1.910542$

H $-0.033421-4.4157930 .999145$

H -2.620389 -3.913138 1.054591

H $-2.677793-5.1529912 .316192$

H -0.822908 -3.8388593 .521509$

H $-3.253527-3.6042334 .196640$

H -3.467999 -2.370955 2.969332

H $-4.020083-1.6758705 .522338$

H $-2.108475-0.1049233 .230327$

H -2.286138 -2.9505836 .418577$

H -1.284688 -1.5255116 .572857$

H $-0.710075-2.9147695 .627305$

H -1.5306180 .6559385 .281431$

H $-0.591510 \quad 0.3562531 .562628$

H $\quad 0.8388700 .5044952 .590640$

H $0.612985-1.6371044 .636423$

H $2.093533-0.359907-2.308833$

H $4.340875-2.292703-3.143233$

H $3.8199951 .488710-2.608773$

H $5.1567151 .635094-1.474449$

H $3.7112090 .789779-0.975175$

H $0.480617-1.4063320 .211874$

H $1.344020 \quad 0.1157630 .179321$

H $4.721748-2.1102982 .037517$

H $4.608424-0.8865703 .318473$

H 5.5791390 .8654651 .680764

H $6.843512-1.8188820 .921051$

H $8.719573-0.0225351 .062935$

H 7.6044501 .1589870 .416640

H $9.619103-1.323263-2.251680$

H $9.943697-1.422573-0.430540$

H $8.0118850 .281164-3.058722$

H $7.1266541 .266383-1.942669$

H $6.493219-1.624274-2.854708$

H $3.050557 \quad 0.377809 \quad 1.938213$

C $-4.719357-1.177675-2.410455$

O $-3.831277-0.044546-2.480930$

C $-4.5888861 .158059-2.250538$

C $-6.0568780 .751657-2.308220$

C $-5.999089-0.658387-1.754969$

H $-4.3591551 .478340-1.227115$

C $-4.1703512 .254455-3.237018$

H $-6.6952361 .419084-1.721623$

H $-6.4412450 .732962-3.333479$

C -7.234775 -1.484803-2.066206

H -5.868952 -0.602495-0.665739

C $-2.6561362 .545261-3.255636$

O $-4.517186 \quad 1.859007-4.570743$

H -4.723246 $3.178965-3.030191$

H -2.113601 $1.700544-3.697811$

O $-2.4428303 .661041-4.131934$

C -2.043114 2.884823-1.884097

C $-0.5654963 .310500-2.000360$

H -2.106670 $2.008585-1.229995$

O $-2.802523 \quad 3.924674-1.271562$

H $-0.0232092 .554969-2.581608$

H $-0.5147304 .263368-2.541945$

H -8.126222 -1.022858 -1.629223
H -7.397196 -1.573421-3.145664

H -7.142879 -2.495133 -1.655451

C $-4.057463-2.307178-1.621832$

H $-5.4679732 .021361-4.689236$

H -2.817199 $3.423752-4.997667$

H -2.746011 $4.707784-1.845580$

H -4.761376 -3.136730 -1.490120

H -3.786913 -1.947156 -0.620986

H $-4.914835-1.494290-3.443377$

C $0.0299674 .895761 \quad 1.340245$

$\begin{array}{lllll}\text { O } & -0.459380 & 4.614487 & 0.022867\end{array}$

C $0.1247973 .486437-0.632875$

C $1.6391313 .693831-0.790738$

C 2.3045573 .9997860 .555083

C 1.5573205 .0896461 .329053

H -0.4292635 .8553011 .610645$

C -0.4427403 .8503242 .362075$

H $-0.0612432 .583084-0.042092$

O $1.8816114 .767326-1.698168$

H $2.0710892 .788333-1.230495$

H $3.337871 \quad 4.327617 \quad 0.387159$

O 2.3504892 .8161551 .344911

H 1.9335585 .1450262 .357281

O $1.851540 \quad 6.3446290 .708054$

C -1.9496713 .8351472 .515523$

H -0.1349352 .8426282 .075252$

H -0.017362 4.0814103 .345370

H $2.8446684 .855211-1.798989$

H 2.8696992 .1603890 .849561

H 1.4288627 .0416461 .238216

O $-2.2964712 .816612 \quad 3.357274$

O -2.7247824 .6140131 .978361$

C $-3.6991302 .667605 \quad 3.597735$

H $-3.8369281 .852494 \quad 4.312908$

H -4.1119443 .5847314 .028710$

H -4.214753 2.4099722 .667783

SCF Energy $(B 3 L Y P / 6-31 G * * / / M M F F)=-3245.88807977$

0700300

MM̄FF Geometry

C $-4.204350-0.979144-3.459372$

C $-3.131774-1.583083-2.925521$

C $-1.763925-1.568815-3.560225$

O $-0.855653-0.907964-2.643739$

C $-1.272400-3.011812-3.779475$

C $-0.252019-3.122750-4.895032$

C $1.073899-3.287601-4.727407$

C $-0.826268-3.077261-6.290511$

C $1.853821-3.381958-3.446890$

C $2.695188-2.123576-3.241618$

C $3.757647-2.241652-2.139348$

C $3.164314-2.599016-0.775558$

C $4.217958-2.5510020 .341056$

O $4.397709-0.962596-2.090544$

O $3.530541-2.599161 \quad 1.600341$

C $4.940055-1.1917600 .276436$

C $5.148290-3.7683530 .307966$

O $6.012600-1.1348081 .213847$

C $5.456060-0.832815-1.134532$

C $5.9559570 .632092-1.216093$

O $6.554158-1.687166-1.466661$

C $-0.8508300 .455032-2.659845$

O $-1.4354561 .180323-3.449772$

C $-0.0649210 .927353-1.495931$

C $-0.2347252 .191533-1.076256$

C $0.3673982 .816000 \quad 0.106037$

C 1.3880442 .0188640 .871395

C $4.8499151 .659682-0.977255$

C $5.2714833 .091573-1.238222$

C $4.2192955 .358228-1.433721$

O $6.4420343 .456015-1.274217$

C $3.5958425 .885143-0.180292$

C $2.3990406 .489548-0.138918$

C $1.7643296 .972617 \quad 1.132021$

C $0.411416 \quad 6.3463891 .386619$

C -0.6991477 .1021441 .437849$

C $\quad 0.3515624 .8578171 .672203$

C -0.0541134 .0477110 .466799$ 
N $4.159128 \quad 3.912374-1.364907$ H -4.108848 $-0.452091-4.405354$ H -3.229246 -2.094570 -1.970891 H -1.782973 -1.023100 -4.511320 H -2.114314 -3.665428 -4.046961 H $-0.875376-3.414588-2.841718$ H $1.692357-3.365454-5.621582$ H $-0.060221-3.213791-7.061363$ H -1.566771 $-3.873387-6.421215$ H -1.312718 -2.115868 -6.480052 H $1.202341-3.541223-2.584344$ H $2.505863-4.261387-3.511661$ H $3.207705-1.855222-4.176258$ H $2.037121-1.271142-3.025316$ H $4.494393-2.992879-2.447143$ H $2.692471-3.588232-0.797243$ H $2.362781-1.888462-0.529227$ H $3.008689-3.4196401 .625449$ H $4.209918-0.4390240 .596750$ H $4.572823-4.6930950 .436181$ H $5.862105-3.7423361 .138576$ H $5.707795-3.856055-0.625698$ H $5.648134-1.3648732 .085622$ H $6.7883010 .792088-0.519350$ H $6.3744980 .807289-2.216948$ H $7.094054-1.247131-2.144074$ H $\quad 0.5707670 .213655-0.985107$ H $-0.9234032 .829715-1.629528$ H 2.1783921 .6679830 .202413 H $0.923593 \quad 1.1461051 .341678$ H 1.8803102 .5858811 .663844 H $3.9867561 .434318-1.613952$ H 4.5258341 .6233440 .067952 H $3.6706925 .666232-2.329198$ H $5.2546135 .700753-1.519810$ H 4.1531725 .7395680 .742358 H $1.8429256 .639886-1.061184$ H 1.6770958 .0646781 .066461 H 2.4098806 .7745601 .997205 H -1.668807 6.6637131 .650955 H $-0.665086 \quad 8.1727451 .265622$ H -0.3937004 .6783782 .458606$ H 1.3103224 .5389462 .090868 H $-0.8337774 .513834-0.137644$ H $3.2344333 .492979-1.321172$ C $-5.8646630 .420063-2.216760$ O $-4.9470210 .679761-1.134096$ C -5.6997320 .9941530 .048688$ C $-7.0514741 .470661-0.458909$ C $-7.2711470 .514967-1.617666$ H -5.8393450 .0535370 .596242$ C $-4.925721 \quad 1.9831920 .924984$ H -7.8319921 .4076430 .304921$ H -7.008177 $2.503067-0.824257$ C $-8.3256800 .984308-2.604955$ H -7.574752 -0.460478 -1.213892 C -3.468232 1.5657731 .212464 O H -5.4657642 .1422601 .865222$ H $-2.864394 \quad 1.678827 \quad 0.304943$ O -2.9285582 .4824232 .171623$ C $-3.302214 \quad 0.1336861 .751632$ C $-1.841119-0.2022382 .079748$ H -3.668129 -0.5706790 .999780$ O $-4.101445-0.0390772 .919537$ H -1.209281 -0.0008491 .207897$ H -1.475352 0.4650152 .871879 H $-9.2992831 .077163-2.112734$ H -8.070304 $1.960257-3.031299$ H -8.429570 $0.272576-3.430056$ C $-5.558260-0.958581-2.806596$ H $-4.4624943 .136082-0.591314$ H $-3.017623 \quad 3.376727 \quad 1.799296$ H -3.801443 0.6101533 .578960 H $-6.309941-1.229118-3.556783$ H -5.600077 -1.718439 -2.015791 H $-5.7034491 .206589-2.964874$ C $0.220018-2.9841373 .439750$
O $-0.213894-1.7362622 .884242$

C -1.609468 -1.647389 2.560441

C $-1.978424-2.7168801 .520190$

C -1.570844 -4.116333 1.993118

C $-0.126040-4.1504602 .500545$

H $1.313581-2.9035073 .485846$

C -0.292161 -3.180579 4.870601

H -2.194497-1.799124 3.473536

O $-1.324812-2.4194130 .287745$

H $-3.058681-2.6939911 .339527$

H $-1.682148-4.8228871 .161372$

O $-2.460953-4.5203313 .029446$

H $\quad 0.075033-5.1000883 .009027$

O $\quad 0.755150-4.1011541 .374882$

C $0.317438-2.1538805 .795089$

H -1.380791 -3.1226304 .958376$

H $-0.006251-4.1683015 .251932$

H -1.590946 -3.096729 -0.356915

H -2.207805 -5.4192963 .299903$

H $\quad 0.639621-3.2362350 .945097$

O $-0.480204-1.0495945 .856109$

O $1.387159-2.3060896 .370586$

C 0.0065010 .0174886 .675299

H -0.7188950 .8343806 .635626$

H $\quad 0.104483-0.3155867 .712958$

H $0.965293 \quad 0.380917 \quad 6.293318$

SCF Energy $(B 3 L Y P / 6-31 G * * / / M M F F)=-3245.92579969$

0700301

MM̄FF Geometry

C $2.300578-2.2416652 .289634$

C $1.768703-1.7965203 .436408$

C $1.114497-0.4482703 .557855$

O $-0.305036-0.6574563 .751713$

C 1.6186250 .3495804 .770848

C 2.9962760 .9464494 .587044

C 3.2284942 .1102753 .948391

C 4.1293250 .1816455 .216805

C 2.2118952 .9812153 .258881

C 2.6831613 .3619581 .848720

C 1.6101854 .0225060 .967639

C 0.9383695 .2264721 .623804

C $-0.1364095 .836836 \quad 0.714072$

$\begin{array}{llllll}\text { O } & 0.627532 & 3.035047 & 0.641283\end{array}$

O $-\begin{array}{llll}0.889566 & 6.749657 & 1.527465\end{array}$

C -1.0904564 .7182810 .231932$

C $0.4873626 .669537-0.413714$

O $-1.9877615 .237992-0.746372$

C $-0.3576803 .469690-0.316730$

C $-1.2974552 .255127-0.512361$

O $0.2499523 .782572-1.571350$

C -1.082411 -0.662553 2.634825

O $-0.710005-0.5509641 .477265$

C $-2.490217-0.8115913 .064355$

C $-3.460733-0.4827392 .199659$

C $-4.904008-0.5670412 .422449$

C $-5.376486-1.1971363 .705287$

C $-2.4102512 .424543-1.548496$

C $-3.1253661 .119409-1.855776$

C $-5.0098150 .223520-3.225903$

O $-2.8249790 .047069-1.340146$

C $-6.2706650 .250162-2.419518$

C $-6.731017-0.797814-1.718766$

C $-8.010179-0.774813-0.931097$

C $-7.815022-1.1302700 .526757$

C $-8.223595-2.3156591 .009923$

C -7.230621 -0.0799811 .445415$

C $-5.726699-0.1018151 .458616$

N $-4.1425221 .299576-2.782556$

H $2.267245-1.6041791 .409204$

H $1.780803-2.4337834 .316806$

H 1.2742020 .1490062 .650826

H $1.595212-0.2752905 .674369$

H $0.903163 \quad 1.1521564 .996965$

H 4.2521802 .4797923 .907594

H 5.0947950 .6794565 .077111

H 3.9646910 .0781356 .294296

H $4.209521-0.8179984 .779384$ 
H $\quad 1.2348192 .493587 \quad 3.199692$ H 2.0846303 .8887243 .860268 H 3.5426784 .0393841 .931357 H 3.0356262 .4568001 .336799 H 2.1122324 .3318440 .044158 H 1.6811655 .9823311 .907249 H 0.4472144 .9197632 .556820 H -1.539722 7.1960670 .959311 H -1.702064 4.4065161 .089895 H 1.0982927 .4787240 .004034 H -0.283532 $7.159522-1.018856$ H $1.1224416 .081925-1.079935$ H -2.525367 $5.925658-0.319410$ H $-0.6779891 .394805-0.803547$ H $-1.736634 \quad 1.9750770 .454230$ H $0.4273552 .949588-2.040013$ H -2.685308 -1.170764 4.067803 H -3.169202 -0.1027311 .223086$ H -4.947940 -2.198700 3.823590 H -6.460373 -1.320866 3.755892 H $-5.080730-0.5852684 .564511$ H -3.164883 $3.125434-1.176893$ H -2.002184 2.810675 -2.489093 H $-4.483393-0.732653-3.139767$ H $-5.239706 \quad 0.402634-4.280683$ H $-6.8412891 .176016-2.427010$ H -6.171535 -1.730446 -1.725953 H -8.701663 -1.484722 -1.403607 H $-8.501857 \quad 0.203914-0.997532$ H -8.114515 -2.5648232 .060277$ H $-8.671379-3.0653300 .365944$ H -7.644636 -0.1867882 .453595$ H $-7.5617290 .915009 \quad 1.121242$ H -5.2770820 .3366490 .567599$ H $-4.3528172 .238903-3.107667$ C $2.111952-4.5706061 .301805$ O $1.729437-3.985817 \quad 0.041344$ C $0.351627-4.323783-0.219853$ C $0.016062-5.4616530 .735226$ C $0.817547-5.053798 \quad 1.957925$ H $-0.249287-3.4512440 .061240$ C $0.159893-4.638805-1.706495$ H - $1.057656-5.5392710 .930011$ H $\quad 0.365780-6.4281570 .353857$ C $1.014551-6.1761872 .961404$ H $0.297334-4.2230892 .450804$ C $0.582353-3.482351-2.638367$ O $0.950492-5.785553-2.044535$ H $-0.880954-4.920460-1.901088$ H $1.658185-3.308589-2.532544$ O $0.370897-3.896151-3.993505$ C $-0.198824-2.175193-2.406795$ C $0.179897-1.031111-3.367334$ H $-0.057550-1.831624-1.377264$ O $-1.592666-2.452248-2.568666$ H $-0.004104-1.333777-4.405470$ H -0.509496 -0.198646 -3.174661 H $0.049081-6.525773 \quad 3.341488$ H $1.527900-7.0324292 .511087$ H $\quad 1.610110-5.8357013 .814228$ C $2.950701-3.5836682 .118263$ H $1.875287-5.579200-1.825169$ H $0.849612-4.733342-4.118804$ H -2.080656 -1.640920 -2.347353 H $3.900097-3.4105601 .595589$ H $3.204322-4.0237823 .089557$ H $2.745570-5.4335911 .057207$ C $3.1196370 .280892-1.490781$ O $1.784366-0.092101-1.852507$ C $1.628377-0.547464-3.200443$ C $1.9831280 .585419-4.172296$ C $3.4133921 .080193-3.923737$ C $3.6797661 .358036-2.439638$ H $3.0142590 .728761-0.494931$ C $4.000788-0.964651-1.351052$ H $2.301565-1.391524-3.380805$ O $1.0634591 .662103-4.005363$ H $\quad \begin{array}{llll}1.881777 & 0.217758 & -5.200228\end{array}$
H $3.587506 \quad 1.999906-4.495769$

O $4.3434670 .103765-4.384051$

H $4.7547451 .487472-2.270336$

O $3.0582582 .605696-2.119858$

C $5.182433-0.700959-0.450685$

H $3.433403-1.791523-0.907332$

H $4.344536-1.342996-2.318280$

H $1.3085842 .358841-4.637690$

H $4.181396-0.031996-5.333189$

H $3.3158272 .833016-1.210483$

O $6.333274-0.692614-1.181121$

O $5.091257-0.5073920 .754976$

C $7.529464-0.432753-0.440124$

H 7.4819550 .5592600 .019383

H $7.679752-1.2049240 .320428$

H $8.372256-0.456917-1.135972$

SCF Energy $(B 3 L Y P / 6-31 G * * / / M M F F)=-3245.91781543$

0700302

MM̄FF Geometry

C $-2.178957-3.146826-1.924447$

C $-1.102112-3.604783-2.581033$

C $0.294190-3.023968-2.541543$

O $0.357684-1.861841-1.684449$

C $1.270085-4.067208-1.967393$

C $2.720565-3.762310-2.285829$

C $3.594806-3.196364-1.430590$

C $3.179278-4.201085-3.653388$

C $3.311942-2.676271-0.046712$

C $4.012001-1.3374810 .226614$

C $5.523750-1.4422870 .502578$

C $5.813660-2.0860521 .862302$

C $7.309148-2.0593662 .209524$

O $6.039945-0.1044510 .483075$

O $7.443771-2.3650693 .606340$

C $7.846578-0.6278792 .008540$

C $8.087272-3.1424351 .452282$

O $9.262019-0.5937362 .183321$

C 7.462931-0.009557 0.643133

C 7.8515251 .4912360 .624230

O $8.163811-0.696176-0.392762$

C $0.124101-0.640730-2.234511$

O $-0.115591-0.391057-3.404283$

C $0.1850310 .349447-1.134471$

C $-0.1243141 .633126-1.370749$

C $-0.1147092 .719623-0.386580$

C 0.2328862 .3641811 .035419

C $7.6875612 .221101-0.714782$

C $6.2875082 .175618-1.287902$

C $3.9652622 .923841-0.889019$

O $6.0282831 .529466-2.301172$

C $3.2894304 .052643-0.183041$

C $2.6978055 .076598-0.815956$

C $1.9702096 .182790-0.108253$

C $0.5204696 .286446-0.529663$

C $0.0916007 .308933-1.288867$

C $-0.4467985 .245560-0.005004$

C $-0.4072453 .969734-0.804209$

N $5.3769692 .925919-0.561939$

H $-2.098691-2.264586-1.295925$

H $-1.220288-4.483273-3.213140$

H $\quad 0.585246-2.753855-3.563940$

H $1.045004-5.064789-2.369031$

H $1.129207-4.152613-0.882631$

H $4.624608-3.088192-1.765882$

H $4.239269-3.986692-3.826354$

H $3.037631-5.280246-3.773282$

H $2.609305-3.686847-4.433248$

H $2.238474-2.5171910 .093405$

H $3.615368-3.4300000 .686008$

H $3.843181-0.653634-0.616460$

H $3.530264-0.8527021 .086499$

H $5.999925-2.007983-0.304512$

H $5.440419-3.1154161 .905648$

H $5.270004-1.5386072 .645094$

H $7.043042-3.2374603 .762227$

H 7.4205120 .0009212 .803095

H $7.703974-4.1378491 .707202$ 
H $9.143831-3.1464351 .741740$ H $8.025749-3.0356680 .367257$ H $9.455389-0.9777453 .055729$ H 7.2622052 .0180091 .385478 H 8.9073331 .5963790 .903627 H $8.022514-0.222977-1.227755$ H $0.476697-0.010267-0.153930$ H $-0.409273 \quad 1.920916-2.381853$ H $-0.465248 \quad 1.6154461 .424888$ H $\quad 0.194898 \quad 3.211581 \quad 1.722167$ H 1.2477611 .9555951 .093948 H $7.9649583 .275128-0.586002$ H $8.381618 \quad 1.821601-1.462607$ H $3.5457921 .968436-0.556947$ H $3.8502062 .979425-1.977041$ H 3.2524474 .0033810 .902613 H $2.7322595 .123507-1.902098$ H $2.4985047 .120600-0.323059$ H 2.0166716 .0582160 .980861 H $-0.9512117 .402217-1.573592$ H $0.7741248 .072398-1.647346$ H -1.472669 $5.632760-0.058192$ H -0.2573855 .0930251 .060392$ H $-0.6583124 .114236-1.856256$ H $5.661762 \quad 3.2924200 .341958$ C $-4.108704-4.160362-0.625125$ O $-4.271495-2.9794530 .187485$ C $-3.819303-3.2773081 .520724$ C $-3.742850-4.7979101 .604017$ C $-3.279227-5.143403 \quad 0.201960$ H $-2.803075-2.873821 \quad 1.605748$ C $-4.726815-2.6230262 .565416$ H $-3.052648-5.1379732 .381855$ H $-4.724763-5.2442511 .800234$ C -3.493316 -6.598608 -0.174577 H $-2.209231-4.9124760 .128415$ C -4.868565 -1.091652 2.428531 O $-6.037334-3.1922232 .450579$ H $-4.367479-2.8820073 .568799$ H -5.480601-0.860721 1.549905 O $-5.621007-0.632803 \quad 3.559998$ C -3.540385 -0.316039 2.357551 C -3.6719641 .2097962 .521979$ H -3.041470 -0.538951 1.407493 O $-2.681661-0.7845773 .402526$ H -3.9995501 .4463973 .542700$ H -2.660026 1.6264312 .440217 H -2.927558 -7.2559520 .493721$ H $-4.549545-6.880203-0.107143$ H -3.157985 -6.788198 -1.199154 C $-3.537337-3.787949-1.996412$ H $-6.597848-2.7667273 .121942$ H $-5.077322-0.7803264 .352960$ H -1.822148 -0.3422603 .295995$ H -4.209711 -3.067956-2.476995 H -3.514066 -4.679203 -2.634376 H -5.119483 -4.561485 -0.776156 C $-4.9029252 .125997-0.888178$ $\begin{array}{lllll}\text { O } & -4.119187 & 1.598481 & 0.192229\end{array}$ C -4.5965591 .8974401 .506300$ C $-4.6449563 .419321 \quad 1.724466$ C $-5.460645 \quad 4.1170120 .632751$ C $-5.0516623 .652731-0.770169$ H -4.312179 $1.918153-1.789835$ C $-6.2512141 .401913-1.030260$ H -5.6039701 .4882081 .628473$ O -3.3260393 .9626981 .744147$ H -5.0900443 .6227222 .705485$ H -5.3164325 .2009490 .719195$ O $-6.8412153 .843813 \quad 0.852529$ H $-5.7694494 .007424-1.518921$ O $-3.7866084 .230834-1.100608$ C $-6.084301-0.057302-1.393997$ H $-6.8305151 .441913-0.104387$ H $-6.8352681 .870275-1.830856$ H -2.8892093 .7078170 .913619$ H -7.346545 4.3165410 .169352 H $-3.8891725 .197351-1.074272$
O $-7.312702-0.654968-1.386694$

O $-5.021471-0.602017-1.655586$

C $-7.329535-2.049907-1.705477$

H $-6.912525-2.622525-0.872831$

H $-8.370078-2.351759-1.851968$

$\mathrm{H}-6.776713-2.247456-2.628860$

SCF Energy (B3LYP/6-31G**//MMFF)= -3245.91137855

0700303

MM̄FF Geometry

C $-5.0677452 .491433-0.456729$

C $-4.4113362 .622717 \quad 0.707317$

C -3.4505013 .7153051 .120844$

$\begin{array}{lllll}\text { O } & -2.159237 & 3.088218 & 1.335908\end{array}$

C $-3.247765 \quad 4.8487840 .096732$

C $-2.285093 \quad 5.924705 \quad 0.567690$

C $-1.087298 \quad 6.188642 \quad 0.007265$

C $-2.7594336 .751638 \quad 1.736127$

C $-0.4456635 .524673-1.179691$

C $0.7423224 .657229-0.759185$

C $1.4634543 .997417-1.945090$

C $0.6083512 .921450-2.622838$

C $1.3793342 .175717-3.723533$

O $2.6647153 .409460-1.423062$

O $0.6334730 .995714-4.061956$

C $2.7311811 .704686-3.151546$

C $1.4859583 .003681-5.009180$

O $3.545594 \quad 1.124365-4.166302$

C $3.5112252 .816082-2.419767$

C $4.777528 \quad 2.285199-1.706698$

O $3.9490943 .794053-3.370352$

C $-1.9335102 .492840 \quad 2.538890$

O -2.6861112 .4579693 .499096$

C -0.6064351 .8443812 .468699$

C $-0.277748 \quad 0.906751 \quad 3.369902$

C 0.9745260 .1498843 .398105

C $2.064940 \quad 0.572324 \quad 2.449276$

C $4.525297 \quad 1.146272-0.718386$

C $5.732450 \quad 0.841138 \quad 0.151693$

C $6.456280-0.7153651 .955155$

$\begin{array}{lllll}\text { O } & 6.783300 & 1.472894 & 0.102999\end{array}$

C $5.754449-1.5601772 .968691$

C $5.453161-1.1379034 .205838$

C $4.701546-1.9624015 .212210$

C $3.302633-1.4280855 .445863$

C $3.033760-0.6882696 .535474$

C $2.269986-1.8158544 .404584$

C $1.101608-0.8748084 .267482$

N $5.476004-0.2197771 .008182$

H $-4.9440253 .251456-1.223950$

H $-4.551548 \quad 1.8604401 .469877$

H $-3.814568 \quad 4.1437102 .062828$

H $-4.210620 \quad 5.327927-0.123984$

H $-2.902248 \quad 4.412922-0.844864$

H $-0.487433 \quad 6.9891320 .440002$

H -2.095528 7.5977631 .943453

H $-3.754143 \quad 7.161416 \quad 1.532555$

H -2.8097656 .1447042 .645056$

H $-1.1657874 .937101-1.750868$

H $-0.098607 \quad 6.312844-1.859535$

H $1.4661315 .270177-0.204624$

H $0.4172923 .883227-0.052542$

H $1.7380674 .782338-2.659485$

H $-0.3122523 .345254-3.039298$

H $0.2861982 .188566-1.869473$

H $-0.2528591 .273739-4.350173$

H $2.5028040 .904762-2.437056$

H $0.487783 \quad 3.227081-5.404491$

H $2.0014202 .444296-5.797538$

H $2.0020883 .955133-4.865669$

H $3.0220410 .424082-4.592039$

H $5.5234421 .969696-2.447949$

H $5.2395513 .123331-1.166245$

H $4.6974124 .281757-2.987774$

H 0.0496382 .1294961 .654645

H -1.0019460 .6442374 .139954$

H $2.295716 \quad 1.6363352 .574258$

H $1.762716 \quad 0.399227 \quad 1.411774$ 
H 3.0027400 .0381032 .608708 H $3.6925991 .401001-0.053505$ H $4.2689620 .230323-1.261938$ H 6.9776850 .1352132 .407433 H $7.193952-1.308758 \quad 1.405724$ H $5.459391-2.5596062 .658012$ H $5.739504-0.1336144 .509980$ H $5.277035-1.9657306 .146719$ H $4.639675-3.0131844 .901867$ H $2.040127-0.3090536 .746135$ H $3.809855-0.4469067 .255009$ H $1.885462-2.8132344 .654662$ H $2.749220-1.9397883 .427294$ H $0.270130-1.1037194 .934400$ H $4.626693-0.7586920 .866438$ C $-6.0537850 .160727 \quad 0.098048$ $\begin{array}{llll}0 & -4.763005 & -0.469472 & 0.199715\end{array}$ C $-4.949744-1.881740 \quad 0.408646$ C $-6.457657-2.1181530 .457030$ C $-6.988044-0.954931-0.361647$ H $-4.545221-2.385811-0.475637$ C $-4.187349-2.3285131 .661950$ H $-6.733666-3.0932880 .043411$ H $-6.844994-2.0741851 .481467$ C -8.461659-0.673333 -0.127766 H $-6.822663-1.170158-1.425879$ C $-2.684333-1.9768321 .645484$ O $-4.768645-1.6802662 .801348$ H $-4.328820-3.4051911 .813812$ H -2.558841 -0.8897211 .709491$ O $-2.104111-2.5073662 .844987$ C $-1.908227-2.5156370 .428858$ C $-0.393679-2.2510560 .546786$ H -2.287590 -2.042509-0.483356 O $-2.154017-3.917416 \quad 0.329909$ H $-0.235340-1.1959710 .795910$ H $\quad 0.005135-2.865087 \quad 1.363819$ H $-9.066874-1.544956-0.397568$ H $-8.663401-0.4357690 .922152$ H -8.799743 $0.171912-0.735196$ C $-5.9716411 .350256-0.862953$ H $-4.268255-1.9674403 .584474$ H -2.148562 -3.4774182 .787009$ H $-1.703604-4.240600-0.468382$ H $-6.9737801 .762844-1.025697$ H $-5.6065090 .991629-1.834154$ H $-6.3407790 .498076 \quad 1.102754$ C $0.912396-4.464765-2.175931$ O $0.289741-3.985606-0.977755$ C $0.385700-2.577647-0.744330$

C $1.861146-2.156558-0.641507$ C $2.658903-2.610373-1.868527$ C $2.401416-4.083051-2.205390$ H $0.848082-5.558298-2.105251$ C $0.146445-4.030649-3.430413$ H $-0.091330-2.038876-1.570337$ O $2.432495-2.725548 \quad 0.535473$ H $1.912536-1.066136-0.542640$ H $3.728584-2.460620-1.677355$ O $2.295885-1.790837-2.974775$ H $2.820803-4.325678-3.188255$ O $3.094635-4.899803-1.256942$ C $-1.239709-4.630967-3.440831$ H $\quad 0.059076-2.944439-3.524817$ H $\quad 0.655466-4.377046-4.337607$ H $3.363041-2.448826 \quad 0.576814$ H $2.837956-2.064908-3.734121$ H $2.687993-4.754076-0.385419$ O $-2.161223-3.684272-3.103215$ O $-1.470869-5.806024-3.694746$ C $-3.519595-4.132115-3.061575$ H $-3.811461-4.560779-4.025077$ H -3.649223 -4.862551-2.257525 H $-4.156031-3.267197-2.857287$

SCF Energy (B3LYP/6-31G**/MMFF) $=-3245.91604459$

0700304

MM̄FF Geometry
C $1.080345-1.2813711 .538122$

C $-0.136269-1.0002921 .051313$

C $-0.455757-1.057345-0.419097$

O $-1.696153-1.782599-0.598338$

C $-0.6625290 .350844-1.001666$

C $0.603731 \quad 1.178344-1.083827$

C $0.8844362 .236863-0.298996$

C $1.5693840 .773397-2.167608$

C 0.0712412 .7912210 .836834

C -0.5553294 .1516330 .502638$

C $-1.7191904 .058862-0.496495$

C $-2.2496775 .453686-0.834964$

C $-3.4862135 .390517-1.743796$

O $-2.752834 \quad 3.278492 \quad 0.111376$

O $-4.0970216 .689792-1.749595$

C $-4.5080474 .413145-1.126680$

C $-3.1124855 .080053-3.197977$

O $-5.6132884 .210097-2.004732$

C $-3.8971393 .051299-0.720925$

C $-4.9257842 .243684 \quad 0.105987$

O $-3.5595582 .320992-1.900944$

C $-1.618533-3.137287-0.657540$

O $-0.603599-3.813321-0.548924$

C $-2.944622-3.762752-0.864308$

C $-4.079424-3.082815-1.092229$

C $-5.408410-3.670532-1.294134$

C $-5.518644-5.172909-1.231909$

C -4.3729370 .9516730 .726700$

C $-5.403090 \quad 0.266574 \quad 1.608571$

C $-5.750690-1.7954022 .957852$

$\begin{array}{lllll} & 0 & -6.514367 & 0.739945 & 1.833163\end{array}$

C -6.506868 -2.7415402 .079598$

C $-7.777577-2.5394671 .700008$

C $-8.547336-3.4508320 .787381$

C $-8.799006-2.819117-0.565542$

C $-9.827834-1.974868-0.752151$

C $-7.901485-3.221337-1.716178$

C $-6.460067-2.847637-1.496577$

N $-4.930839-0.9338322 .124745$

H $1.868776-1.5762880 .850710$

H $-0.927406-0.6840041 .726480$

H $0.334285-1.568849-0.982858$

H $-1.4464550 .866530-0.437455$

H $-1.0852630 .260920-2.012008$

H $1.8205792 .766933-0.471252$

H $2.4368551 .439860-2.224383$

H $1.946376-0.237308-1.987850$

H $1.0748930 .793002-3.144174$

H $-0.693022 \quad 2.089453 \quad 1.182987$

H 0.7495642 .9261801 .688217

H -0.9269944 .5810151 .442256$

H $0.2156324 .831500 \quad 0.118910$

H $-1.3726083 .556932-1.406237$

H $-1.4653906 .067223-1.295503$

H -2.529388 5.9725870 .092799

H -3.437092 $7.329653-2.067436$

H $-4.913658 \quad 4.892148-0.224308$

H -2.440618 $5.851399-3.593416$

H $-3.9968115 .093809-3.844597$

H $-2.6127324 .116397-3.316623$

H $-5.9741465 .086399-2.223427$

H $-5.294732 \quad 2.8754940 .924972$

H $-5.788791 \quad 1.986773-0.521487$

H $-3.5673401 .371757-1.703909$

H -2.931562 $-4.847697-0.825998$

H $-4.044208-1.996313-1.134659$

H $-6.539432-5.545290-1.334277$

H $-5.154585-5.545591-0.267655$

H $-4.930681-5.633885-2.033108$

H $-4.0756330 .247062-0.055864$

H $-3.4965581 .169792 \quad 1.347314$

H $-6.422924-1.1814713 .566845$

H $-5.087827-2.3492403 .629367$

H $-5.976777-3.6257801 .736661$

H $-8.298001-1.6508492 .051093$

H -9.504150 -3.689799 1.269633

H $-8.038501-4.4144680 .663036$

H - $-10.031635-1.535175-1.722791$ 
H -10.488103 -1.7033130 .065006$ H -8.224631 -2.728658 -2.642821 H $-8.036569-4.289804-1.904420$ H -6.282602 -1.771293-1.513032 H -4.030553 -1.287673 1.814042 C $2.244436 \quad 0.101113 \quad 3.258920$

$\begin{array}{llll}\text { O } & 3.418714 & 0.161764 & 2.431825\end{array}$ C 4.4099820 .9646163 .103409 C $3.849144 \quad 1.2835404 .491278$ C $2.784518 \quad 0.217390 \quad 4.678713$ H $5.301508 \quad 0.338230 \quad 3.214244$ C $4.7364702 .202305 \quad 2.254032$ H 4.6260921 .2440665 .261733 H 3.4065552 .2850644 .534182 C 1.7396140 .5814035 .718378 H $3.277556-0.7197674 .969736$ C $5.161392 \quad 1.8721280 .807695$ O 3.5613893 .0208832 .186126 H 5.5044112 .8019412 .756821 H 4.3320091 .3925920 .276180 O $5.414000 \quad 3.1104490 .130543$ C 6.4331231 .0054750 .703200 C $6.9120950 .782354-0.745656$ H 6.2707820 .0348751 .181589 O 7.4927391 .6444801 .420986 H $6.9407361 .740959-1.277021$ H $7.9514110 .427800-0.710221$ H 2.2075360 .7374716 .695762 H 1.2086581 .5005185 .448605 H $0.998386-0.2169205 .824174$ C $1.460083-1.1813832 .986950$ H 3.8295753 .8895081 .842389 H $4.5561563 .545547-0.010757$ H 7.6367382 .5167801 .015159 H $0.567112-1.2174123 .621567$ H $2.064353-2.0604823 .235394$ H 1.6245070 .9725883 .009835 C $5.707069-2.641353-1.608366$ O $6.412590-1.534634-1.030637$ C $6.050337-0.234056-1.512203$ C $6.271151-0.143189-3.028720$ C $5.526313-1.261521-3.762343$ C $5.809638-2.632398-3.144087$ H $6.245444-3.528083-1.248858$ C $4.267369-2.723258-1.082851$ H $4.994471-0.048594-1.296581$ O $7.662056-0.206040-3.335403$ H $5.9093820 .830434-3.379826$ H $5.832621-1.262189-4.815711$ O $4.129446-0.988403-3.709449$ H $\quad 5.135728-3.385654-3.568217$ O $7.139394-3.011278-3.513589$ C $4.244549-3.3766240 .280393$ H $3.765785-1.755805-1.002592$ H $3.654092-3.351347-1.739896$ H $8.010400-1.037199-2.969641$ H $3.673832-1.690219-4.204861$ H $7.290332-3.913594-3.184034$ O $4.791341-2.5447021 .211503$ O $3.805487-4.5010870 .485836$ C $4.904033-3.0830802 .532459$ H $5.687667-3.8459012 .552940$ H $5.185061-2.2718103 .208696$ H $3.951638-3.5038072 .867615$ SCF Energy (B3LYP/6-31G**//MMFF) $=-3245.90515293$

\section{7_00305}

MM̄FF Geometry

C $-3.534123 \quad 4.1110570 .224065$

C -2.328254 $3.708679-0.200642$

C $-1.057636 \quad 3.889628 \quad 0.586708$

$\begin{array}{lllll}\text { O } & -0.547242 & 2.562852 & 0.852522\end{array}$

C $-0.030214 \quad 4.676649-0.252155$

C 1.2575594 .9902100 .483146

C 2.4635384 .4820680 .160283

C $1.1387105 .974948 \quad 1.618631$

C $2.8092833 .581776-0.993167$

C $2.6797192 .097435-0.639863$
C $3.2117471 .193070-1.759440$

C $2.944822-0.276649-1.437309$

C $3.541493-1.212159-2.497259$

O $4.6180711 .417532-1.867245$

O $3.478009-2.542535-1.960985$

C $5.028513-0.845159-2.693030$

C $2.700612-1.225298-3.778153$

O $5.604985-1.594736-3.759738$

C $5.2744420 .667105-2.894628$

C $6.7782971 .041495-2.857593$

O $4.7789591 .046818-4.185180$

C $-0.0875622 .292466 \quad 2.103922$

$\begin{array}{lllll}\text { O } & -0.059248 & 3.049476 & 3.060873\end{array}$

C $0.390160 \quad 0.8909962 .115521$

C 1.0379120 .4233803 .193316

C $1.546498-0.9379403 .379506$

C $1.356878-1.9126042 .247602$

C $7.5752510 .604467-1.623353$

C $6.9994251 .133516-0.322605$

C 5.9374760 .3926691 .801321

O $6.9191672 .337244-0.088525$

C $5.290511-0.8631732 .291749$

C $5.584040-1.4427213 .464998$

C $4.998399-2.7474053 .923505$

C $4.161320-2.6117425 .173628$

C $4.729563-2.6091236 .391368$

C $2.659229-2.5856215 .010954$

C $2.140399-1.2497664 .551063$

N $6.5592090 .123737 \quad 0.517897$

H $-3.622840 \quad 4.602764 \quad 1.189877$

H -2.245905 $3.224203-1.169766$

H $-1.2777314 .417977 \quad 1.521619$

H -0.471798 $5.626386-0.583042$

H $0.1832864 .111888-1.165657$

H 3.3202384 .7686430 .769162

H 2.1119056 .2257432 .054148

H 0.6911866 .9085031 .261642

H 0.5142365 .5782262 .423040

H $2.1984153 .814764-1.872187$

H $3.8411363 .810482-1.288195$

H 3.2302921 .8977840 .287961

H $1.6267701 .860497-0.460381$

H $2.7245281 .477093-2.699675$

H $1.872449-0.462766-1.321011$

H $3.394664-0.522522-0.465768$

H $3.922962-3.135230-2.591120$

H $5.541740-1.178580-1.784795$

H $1.677977-1.555308-3.558420$

H $3.095318-1.944634-4.504020$

H $2.638535-0.247919-4.261207$

H $5.304019-1.199107-4.595477$

H $7.2743790 .641102-3.751323$

H $6.8592862 .134582-2.940299$

H $5.2182901 .871958-4.450751$

H $\quad 0.1927580 .283852 \quad 1.240213$

H 1.2011591 .0978094 .033260

H $\quad 0.290731-2.0951292 .077833$

H $1.827722-2.8822642 .421073$

H $1.797745-1.5222121 .323951$

H $7.668627-0.486196-1.600092$

H $8.5996310 .989739-1.698325$

H $5.193174 \quad 1.1853001 .675226$

H 6.7157810 .7588712 .478936

H $4.557165-1.3296931 .638463$

H $6.322230-0.9796944 .115976$

H $5.828334-3.4411254 .112671$

H $4.408100-3.218724 \quad 3.127870$

H $4.131940-2.5470057 .294813$

H $5.806697-2.6651386 .510052$

H $2.174753-2.8037825 .972521$

H $2.351508-3.4079554 .358503$

H $2.236066-0.4689285 .306987$

H $6.818130-0.8354790 .305005$

$\begin{array}{lllll}\text { C } & -5.825050 & 3.097266 & 0.174964\end{array}$

$\begin{array}{lllll}\text { O } & -5.480701 & 1.697986 & 0.067894\end{array}$

C $-6.7002190 .934301-0.044698$

C -7.8479491 .9251590 .129084$

C $-7.2357223 .204721-0.398438$ 
H $-6.7346430 .535724-1.064430$

C $-6.729471-0.2000220 .984910$ H -8.744289 $1.618380-0.418996$ H -8.1252412 .0380691 .184229$ C $-7.992016 \quad 4.4517710 .026830$ H -7.201642 $3.155563-1.495108$ C $-5.711522-1.3343050 .743375$ O $\begin{array}{lllll}-6.484432 & 0.349926 & 2.286617\end{array}$ H -7.737690 $-0.628678 \quad 1.030311$ H $-4.688163-0.9613280 .865199$ O $-5.912319-2.3056941 .780208$ C $-5.853075-2.043132-0.621884$ C $-5.186270-3.430590-0.702836$ H -5.460164 -1.391145 -1.410116 O $-7.246013-2.237308-0.895628$ H $-5.638875-4.0944950 .044604$ H -5.450170 -3.866347 -1.676629 H $-9.0217874 .421608-0.344231$ H $-8.0304894 .545547 \quad 1.117330$ H $-7.5182795 .353994-0.371718$ C $-4.7996073 .950644-0.571188$ H -5.6116620 .7786802 .262379$ H $-5.815140-1.8450782 .631289$ H -7.321230 -2.599534-1.794903 H $-5.1985604 .955548-0.749465$ H $-4.5834703 .504025-1.549454$ H -5.8317043 .3452381 .245192$ C $-1.688740-2.726031-1.795641$ O $-3.118167-2.823486-1.735684$ C $-3.658441-3.419875-0.551302$ C $-3.108282-4.843508-0.358254$ C $-1.578354-4.853990-0.368641$ C $-1.018641-4.091708-1.571875$ H -1.476027 -2.424517 -2.829357 C -1.165181-1.632673 -0.859885 H $-3.388529-2.813430 \quad 0.317923$ O $-3.598757-5.691111-1.395650$ H $-3.481143-5.2381730 .594733$ H -1.224935 -5.891919 -0.396340 O $-1.112120-4.2645870 .840907$ H $0.063001-3.953560-1.462508$ O $-1.203930-4.883761-2.749073$ C $-1.484026-0.269723-1.424680$ H -1.594294 -1.678311 0.144756 H $-0.078568-1.700628-0.737433$ H -3.269514 -6.589167-1.220560 H $-0.140708-4.307490 \quad 0.832003$ H -2.160315 -4.959037-2.909717 O $-0.573997 \quad 0.050999-2.387927$ O $-2.435400 \quad 0.410549-1.065418$ C $-0.7570531 .307303-3.046458$ H $-1.8098891 .483723-3.286636$ H $-0.3645152 .106858-2.414549$ $\mathrm{H}-0.1866491 .286450-3.978933$ SCF Energy (B3LYP/6-31G**//MMFF) $=-3245.91725953$

0700306

MM̄MF Geometry

C $-4.9351601 .264773-0.123707$

C -5.2694262 .1867820 .791748$

C -4.3288562 .8468651 .777094$

O -2.9959222 .2919531 .696063$

C $-4.233148 \quad 4.3531821 .474946$

C -3.3486235 .1055072 .450651$

C -2.1492205 .6442822 .152663$

C -3.9132395 .2609853 .840400$

C -1.416705 5.6327050 .838926

C $-0.084423 \quad 4.8873170 .950570$

C $0.7799134 .987592-0.316167$

C $0.1519034 .281235-1.520713$

C $1.0798004 .289050-2.746496$

O $2.0422594 .381632-0.002745$

O $0.5297113 .348132-3.682081$

C $2.4751153 .784538-2.321469$

C $1.0781395 .652553-3.447172$

O $3.4206853 .917201-3.379260$

C $3.0174514 .477742-1.052166$

C $4.3259463 .836437-0.531279$
O $3.3151975 .847080-1.350307$

C -2.7227791 .1965322 .457604$

O $-3.487431 \quad 0.610423 \quad 3.206711$

C -1.3150320 .8181262 .201182$

C $-0.826935-0.3266542 .702042$

C $0.534412-0.8338102 .514516$

C 1.5301340 .0747211 .842394

C $4.2423182 .329037-0.289852$

C 5.4060261 .7880530 .520977

C $6.267703-0.3507751 .456958$

O $6.3167392 .488604 \quad 0.952078$

C $5.616064-1.5990231 .956534$

C $5.212288-1.7661543 .224756$

C $4.526500-3.0030923 .730110$

C $3.058980-2.7573594 .015109$

C $2.643068-2.5426955 .275192$

C $2.141946-2.8082422 .807611$

C $0.834915-2.0742372 .954445$

N 5.2860190 .4185190 .717097

H $-3.9015190 .942001-0.216113$

H -6.3114482 .4929210 .863053$

H -4.7304922 .6988852 .787034$

H -5.2347794 .8034141 .501954$

H $-3.882950 \quad 4.4888540 .446897$

H -1.620596 6.1739312 .945071

H $-3.2888405 .899754 \quad 4.474435$

H $-4.9078805 .716663 \quad 3.797340$

H $-3.995054 \quad 4.2885264 .335277$

H -2.018591 5.1942470 .041712

H -1.229979 6.6740710 .549306

H 0.4936095 .2853551 .796032

H $-0.260757 \quad 3.832021 \quad 1.193229$

H $0.942716 \quad 6.049994-0.532203$

H $-0.8139074 .725659-1.787011$

H $-0.0626403 .236545-1.253741$

H $1.0700163 .374321-4.489771$

H $2.3703822 .710247-2.126223$

H $0.0704865 .894726-3.805976$

H $1.7181155 .642243-4.336365$

H $1.4040606 .468590-2.798848$

H $3.1044023 .383375-4.127183$

H $5.1527264 .050902-1.221079$

H 4.5950784 .3324580 .411649

H $3.9515906 .173347-0.692467$

H -0.7203731 .4859851 .589012$

H - $-1.487924-0.9650193 .286612$

H 1.5906001 .0354222 .366429

H 1.2403560 .2610590 .803846

H $2.544584-0.3284441 .829859$

H $3.3196462 .080737 \quad 0.246414$

H $4.2410231 .804726-1.251279$

H 6.6721230 .2619352 .269935

H $7.090779-0.5952040 .778025$

H $5.455577-2.3951031 .233990$

H $5.365837-0.9632503 .942293$

H $5.047928-3.3344944 .637189$

H $4.623389-3.8321323 .018889$

H $1.599516-2.3844635 .522211$

H $3.346133-2.5257506 .102199$

H $1.922252-3.8608452 .585423$

H $2.671299-2.4419791 .921923$

H $0.055062-2.6589453 .443461$

H $4.584572-0.0863960 .181642$

C $-6.197555-0.829169-0.662945$

O $-4.973971-1.583837-0.617938$

C $-5.217337-2.909902-1.120974$

C $-6.723145-3.015855-1.342510$

C -7.094351 -1.576204-1.647010

H -4.711635 -2.970508 -2.092007

C $-4.620512-3.950051-0.163432$

H $-6.972996-3.705011-2.155129$

H -7.247558 -3.358721-0.443504

C $-8.575278-1.282545-1.484342$

H $-6.792178-1.348629-2.678108$

C $-3.160319-3.6702520 .250108$

$\begin{array}{lllll}\text { O } & -5.398445 & -3.986561 & 1.041257\end{array}$

H -4.707561 -4.948882 -0.605876

H $-3.128633-2.7917310 .906146$ 
O $-2.715359-4.7709331 .053855$

C -2.178857-3.466661 -0.919425

C $-0.752942-3.208780-0.412372$

H -2.512472 -2.614847 -1.519201

O $-2.203659-4.625685-1.747222$

H $-0.764328-2.3547750 .274318$

H $-0.412119-4.0639930 .186922$

H $-9.167273-1.902178-2.165813$

H -8.915125 -1.486374 -0.463351

H -8.792194 -0.233394 -1.708302

C $-5.9066060 .615989-1.070058$

H $-5.380756-3.0950601 .429920$

H $-2.680335-5.5560950 .480929$

H $-1.712378-4.421022-2.560215$

H -6.840286 $1.187849-1.119400$

H -5.457868 $0.643498-2.070701$

H $-6.625751-0.8559070 .348092$

C $2.703069-2.552103-1.601713$

O $1.533507-2.717147-0.792078$

C $0.310398-2.972770-1.498501$

C $-0.005131-1.807066-2.446673$

C $1.170417-1.527007-3.390747$

C $2.501447-1.435739-2.638938$

H $3.481615-2.222840-0.901587$

C $3.156391-3.879692-2.216622$

H $0.422050-3.899437-2.070888$

O $-0.293716-0.639554-1.679513$

H $-0.897992-2.047604-3.034279$

H $0.997279-0.583752-3.923385$

O $1.260731-2.565156-4.362125$

H $3.336864-1.441292-3.348040$

O $2.555893-0.179598-1.959200$

C $3.509198-4.877138-1.139670$

H $2.389960-4.343349-2.844319$

H $4.034160-3.737789-2.858259$

H $-0.5179160 .073309-2.301705$

H $0.422738-2.580189-4.855180$

H $1.835143-0.163608-1.307244$

O $4.769968-4.640998-0.677843$

O $2.747034-5.751442-0.747739$

C $5.219518-5.5207790 .357224$

H $6.179681-5.1498810 .725476$

H $4.508111-5.5348001 .188474$

H $5.360854-6.527899-0.045704$

SCF Energy (B3LYP/6-31G**/MMFF) $=-3245.91336513$

07_00307

MM̄FF Geometry

C $3.202568-1.1937302 .344144$

C $2.352156-0.8678353 .328730$

C $1.027051-0.2038783 .067995$

O $0.008250-1.2042883 .306864$

$\begin{array}{lllll}\text { C } & 0.765390 & 1.000640 & 3.990346\end{array}$

C $1.631802 \quad 2.2081243 .700842$

C $1.3934673 .087022 \quad 2.706941$

C 2.7998282 .4088104 .629103

C $0.2936303 .017761 \quad 1.686222$

C -0.5823514 .2748591 .686292$

C -1.717295 $4.154620 \quad 0.657417$

C -2.478061 5.4713510 .531623

C $-3.660793 \quad 5.342603-0.436264$

O $-2.606807 \quad 3.1287591 .104462$

O $-4.4858466 .507246-0.280006$

C $-4.518468 \quad 4.121895-0.033885$

C -3.200193 $5.329353-1.899945$

O $-5.5264273 .901964-1.016733$

C -3.697592 2.8312410 .211069

C -4.5125001 .7362520 .949103$

O $-3.2127172 .322599-1.028381$

C -1.120017-1.126641 2.549515

O $-1.397357-0.2640691 .731344$

C -1.961849 -2.302897 2.863262

C $-3.114393-2.4730312 .198192$

C $-4.044034-3.5964982 .331475$

C $-3.675032-4.6976653 .289807$

C $-5.681414 \quad 1.0797140 .213540$

C $-5.2840680 .011593-0.791655$

C $-6.311715-1.509032-2.486543$
O $-4.146516-0.440261-0.884171$

C $-6.915464-2.715852-1.839100$

C $-6.258263-3.867668-1.635222$

C $-6.876984-5.067608-0.976485$

C $-6.135264-5.507644 \quad 0.267018$

C $-5.464074-6.6714730 .295386$

C $-6.240725-4.6437371 .505619$

C $-5.158577-3.5998801 .569712$

N $-6.372916-0.406448-1.544668$

H $2.914574-0.9922601 .315111$

H $2.608825-1.0968034 .359771$

H 0.9815200 .1113732 .018778

H 0.8814330 .6978365 .040310

H $-0.292002 \quad 1.2904103 .925394$

H 2.0752683 .9273142 .585448

H 3.3754793 .3078804 .384640

H 2.4498532 .5123295 .661385

H 3.4842731 .5569294 .577434

H $\quad 0.7564762 .9096190 .699873$

H $-0.3334802 .136064 \quad 1.831659$

H -1.0055874 .4172302 .689061$

H $\quad 0.036605 \quad 5.1514391 .457975$

H $-1.2846083 .865545-0.307514$

H $-1.808958 \quad 6.281297 \quad 0.215180$

H -2.864465 5.7686331 .516680

H $-3.9395417 .289112-0.470238$

H $-5.040374 \quad 4.377503 \quad 0.898777$

H -2.630996 $6.238011-2.130392$

H $-4.0559005 .332379-2.584280$

H $-2.5683114 .472292-2.142544$

H $-6.0291994 .729410-1.107888$

H $-3.819090 \quad 0.948254 \quad 1.271694$

H -4.8941482 .1708691 .883348$

H $-2.9587451 .394197-0.894354$

H $-1.600144-3.0045123 .605619$

H $-3.403794-1.7213741 .465564$

H -2.702446 -5.1282333 .025827$

H $-4.386411-5.5261203 .297850$

H $-3.622443-4.3127194 .314098$

H -6.2977770 .5656810 .963586$

H $-6.3278221 .817493-0.265220$

H $-5.275722-1.678764-2.796828$

H $-6.898805-1.224907-3.364982$

H -7.952933 -2.634521 -1.522316

H $-5.224725-3.958104-1.961221$

H $-6.896553-5.878279-1.716332$

H $-7.925890-4.880249-0.714266$

H $-4.954789-7.0074861 .192575$

H $-5.405281-7.312683-0.577894$

H $-6.251904-5.2718662 .402476$

H $-7.214186-4.1368571 .518749$

H $-5.329495-2.751950 \quad 0.906461$

H $-7.286498-0.005706-1.352237$

C $5.675126-1.0980261 .876569$

O $5.444322-1.043566 \quad 0.452884$

C $6.454312-1.823684-0.202413$

C $7.669314-1.6774960 .697792$

C $7.026570-1.8030812 .068808$

H $6.111961-2.866797-0.184638$

C $6.633939-1.386274-1.659927$

H $8.428334-2.4421090 .508972$

H $8.135239-0.6910210 .592929$

C $7.863281-1.2091603 .189681$

H $6.869453-2.8688262 .282734$

C $5.312215-1.195819-2.435579$

O $7.329764-0.134299-1.691802$

H $7.283794-2.105598-2.172346$

H $4.854805-0.253695-2.118294$

O $5.638884-1.022260-3.819701$

C $4.294556-2.339171-2.294891$

C $3.036015-2.197579-3.171209$

H $3.980766-2.423914-1.248929$

O $4.937907-3.569111-2.638347$

H $3.288435-2.377609-4.224213$

H $2.367386-3.022083-2.890615$

H $8.826985-1.7231723 .264876$

H $8.061172-0.1451403 .021861$

H $7.348821-1.3091974 .150884$ 
C $4.528927-1.8674632 .547508$

H $7.4316560 .117602-2.625777$

H $5.995559-1.867181-4.144094$

H $4.297705-4.286124-2.490738$

H 4.741811-1.990296 3.615902

H $4.451915-2.8697292 .107509$

H $5.704073-0.0621232 .235481$

C $1.4649510 .685132-1.360883$

O $2.093865-0.571623-1.653619$

C $2.295048-0.855855-3.041191$

C $0.951349-0.866664-3.790005$

C $0.167080 \quad 0.429695-3.556630$

C $0.1042620 .799573-2.071898$

H $1.2703210 .651073-0.283349$

C $2.3935371 .879098-1.642227$

H $2.934984-0.082751-3.477657$

O $0.155988-1.975642-3.373337$

H $1.144806-0.997658-4.860916$

H $-0.8545030 .311738-3.938552$

O $0.7809021 .497625-4.271937$

H $-0.2949121 .813932-1.953373$

O $-0.817910-0.089247-1.434773$

C $3.539811 \quad 1.958718-0.655604$

H $2.8163301 .828299-2.648645$

H $1.8407232 .821172-1.554637$

H $\quad 0.026197-1.904157-2.411989$

H $0.7778871 .259224-5.214656$

H $-0.9046450 .190890-0.507332$

O $4.5979832 .592962-1.240689$

O 3.5008341 .5545900 .497933

C $5.7468822 .769668-0.405569$

H 6.0695331 .8138130 .017342

H $6.5564523 .169898-1.021604$

H 5.5214863 .4856420 .390305

SCF Energy (B3LYP/6-31G**//MMFF) $=-3245.93556864$

0700308

MM̄FF Geometry

C -0.605190 -0.374838 0.656238

C $0.136097-0.743882-0.399569$

C $-0.255706-1.724970-1.479882$

O $-1.581301-2.263186-1.254732$

C $-0.307055-1.076346-2.874908$

C $0.998743-0.486873-3.360682$

C $1.264740 \quad 0.832713-3.427805$

C $2.024602-1.491928-3.814681$

C $0.3738921 .969409-3.008968$

C $1.0402022 .826409-1.925115$

C $0.0838853 .899956-1.387361$

C $0.8180724 .855315-0.446520$

C -0.1348345 .8939310 .164598$

O $-0.9699633 .238139-0.677005$

O 0.5528506 .5358391 .249426

C -1.3500705 .1587240 .767268$

C $-0.4916826 .998845-0.836512$

O

C -1.989352 $4.114793-0.181210$

C -3.0219363 .2721440 .604628$

O $-2.6594794 .795586-1.243448$

C $-1.710827-3.370036-0.481825$

O $-0.810406-3.9712050 .087650$

C $-3.127987-3.797866-0.383403$

C $-4.138308-3.199949-1.036038$

C -5.549311 -3.599120 -1.005342

C $-5.933037-4.727097-0.083244$

C $-3.5498382 .042882-0.144330$

C $-4.423675 \quad 1.2234540 .785408$

C -6.7718650 .9995851 .586947$

O $\quad-3.9486820 .380686 \quad 1.543733$

C -7.203992 -0.3328021 .061847$

C $-8.389609-0.5499830 .473661$

C -8.862732 -1.898399 0.010160

C $-8.764383-2.084002-1.487280$

C $-9.473946-1.325503-2.340237$

C -7.893277 -3.213690 -1.988702

C $-6.424198-2.944061-1.798980$

N -5.7620901 .5709910 .716811$

H -1.605021 -0.7760520 .785880$
H $1.142085-0.337119-0.480164$

H $0.470703-2.547193-1.491185$

H $-1.121623-0.343892-2.902606$

H $-0.633291-1.835608-3.600312$

H $2.2336891 .141195-3.818105$

H $2.913506-1.015002-4.239066$

H $2.350416-2.113418-2.975116$

H $1.603724-2.142808-4.588000$

H $0.1711662 .590987-3.889476$

H $-0.5956971 .618589-2.649100$

H $1.3574922 .178278-1.099678$

H $1.9399383 .299107-2.338490$

H $-0.3382684 .447555-2.238251$

H $1.6467795 .353914-0.964682$

H 1.2775794 .2814790 .370944

H $1.359867 \quad 6.9450090 .892698$

H -0.9885274 .6343991 .662663$

H $0.4164357 .498069-1.195471$

H -1.093709 $7.781912-0.362479$

H -1.031844 $6.627519-1.709900$

H $-2.806151 \quad 6.425106 \quad 0.451532$

H -2.5608012 .9201801 .536283$

H -3.8743273 .9017030 .891040$

H -3.335388 $4.207896-1.616089$

H $-3.292226-4.6617370 .252872$

H $-3.921204-2.345118-1.674144$

H $-5.620044-4.5070800 .943918$

H $-5.460477-5.662359-0.402423$

H $-7.008537-4.909021-0.038387$

H $-4.1219602 .332207-1.032020$

H $-2.7353581 .398302-0.489711$

H -7.601958 1.7116381 .637069

H -6.3581920 .8836482 .594259$

H -6.519887 -1.1655131 .206870$

H -9.0750870 .2833450 .337435$

H $-9.906409-2.0317040 .322719$

H $-8.307573-2.6903580 .527856$

H -9.419985 -1.478852 -3.412989

H $-10.127404-0.535748-1.984563$

H -8.055780 -3.369474 -3.063684

H $-8.219009-4.146752-1.520222$

H -6.048995 -2.134294 -2.426500

H -6.0647482 .2569560 .031760$

C $0.538668-0.1177432 .911169$

O $1.731310-0.7983982 .467489$

C $1.676702-2.1566732 .933096$

C $0.746734-2.1242034 .137752$

C $-0.314642-1.1550183 .648781$

H $1.203180-2.7468742 .138836$

C $3.083588-2.6968913 .198327$

H $0.346050-3.1118264 .384511$

H $1.246458-1.7210755 .026120$

C $-1.167950-0.5644614 .757498$

H -0.971701-1.691542 2.953680

C $4.065411-2.5492912 .014969$

O $3.656147-2.0072154 .316751$

H $3.018492-3.7488763 .499567$

H $4.374806-1.5020541 .922417$

O $5.248851-3.2895172 .340365$

C $3.532454-3.0483400 .660303$

C $4.586465-3.143609-0.458714$

H $2.719225-2.3932950 .330426$

O $2.972906-4.3533780 .837097$

H $5.292565-3.953464-0.233989$

H $4.056128-3.462814-1.365971$

H $-1.714917-1.3535635 .283760$

H -0.557636 -0.030744 5.493641

H -1.8990970 .1407414 .349206$

C -0.1302290 .5758121 .720377$

H $3.658100-1.0577484 .105898$

H $5.573645-2.9558593 .194257$

H $2.566588-4.615143-0.006673$

H $0.574996 \quad 1.287971 \quad 1.276123$

H -0.9857191 .1643352 .069202$

H 0.8784760 .6561493 .611103

C $4.9682420 .481890-1.307716$

O $4.414432-0.819659-1.063508$

C $5.358261-1.844341-0.740400$ 
C $6.371008-2.022961-1.883758$

C $7.047572-0.696365-2.243946$

C $6.0290410 .433002-2.422844$

H $4.1247501 .080281-1.674782$

C $5.4850851 .140560-0.017572$

H $5.899712-1.5647750 .168296$

O $5.729529-2.554565-3.041812$

H $7.127211-2.753990-1.574876$

H $7.614937-0.817267-3.175132$

O $7.965630-0.331185-1.217920$

H $6.5463851 .397483-2.487832$

O $5.3659420 .230596-3.673765$

C $4.368014 \quad 1.4479730 .955319$

H 6.2041440 .5031910 .502932

H $5.9694452 .092884-0.261888$

H $5.016899-1.942647-3.293362$

H $8.622918-1.043909-1.144678$

H $4.7730470 .987606-3.817911$

O $4.906527 \quad 1.9295572 .114291$

O $3.176249 \quad 1.295994 \quad 0.730339$

C 3.9632042 .2707783 .134956

H 4.5089882 .7359503 .960076

H 3.2262192 .9854762 .755784

H 3.4708601 .3672713 .505110

SCF Energy (B3LYP/6-31G**//MMFF) $=-3245.91338321$

0700309

MM̄MF Geometry

C -1.908602 -3.295172 1.845290

C $-1.244098-2.4254182 .621947$

C $0.167231-1.9830662 .347369$

O $0.168931-0.5384542 .426885$

C $1.177945-2.5203763 .376991$

C $1.433791-4.0092903 .271708$

C $2.323238-4.5711812 .429256$

C $0.641005-4.8765794 .215611$

C $3.251300-3.8791461 .469933$

C $2.737816-3.9485110 .027685$

C $3.751577-3.475843-1.028364$

C $4.768716-4.569269-1.360231$

C $5.796033-4.089693-2.392973$

O $4.416815-2.303430-0.551670$

O $6.849250-5.065052-2.418346$

C $6.394276-2.752980-1.911482$

C $5.207815-4.054683-3.808524$

O $7.257736-2.226969-2.917787$

C $5.329513-1.709728-1.484098$

C $6.025578-0.518748-0.781479$

O $4.649028-1.231724-2.645836$

$\begin{array}{llll}C & 0.945124 & 0.146826 & 1.545424\end{array}$

O $1.668888-0.3152510 .678933$

C $0.7381251 .586797 \quad 1.814233$

C 1.1772162 .5027160 .938538

C 1.0164903 .9535691 .062713

C $0.406526 \quad 4.4761122 .338309$

C $5.0803590 .441660-0.046360$

C 5.8430231 .5995550 .578748

C $5.5151323 .610671 \quad 1.995342$

O $7.058277 \quad 1.7412880 .466828$

C 4.4709424 .6784312 .026865

C 4.5077935 .7673301 .244372

C 3.4750956 .8577071 .262196

C $2.6846596 .920607-0.026378$

C $3.1372747 .618976-1.081341$

C $1.3342516 .240045-0.060926$

C $1.429605 \quad 4.7420740 .047777$

N 5.0033042 .4659691 .265261

H -1.407730 -3.7228740 .980304$

H -1.728702 -2.021648 3.507705

H $0.455350-2.2917801 .338152$

H $0.844602-2.2701494 .393884$

H $2.122313-1.9710213 .276964$

H $2.420249-5.6562412 .434464$

H $0.895758-5.9373824 .118245$

H $0.841458-4.5861825 .252304$

H - $0.431994-4.7810024 .031305$

H $3.411419-2.8351841 .757052$

H $4.229890-4.3667361 .549135$
H $\quad 2.390590-4.959808-0.218848$

H $1.858203-3.298725-0.046437$

H $3.179647-3.219098-1.928213$

H $4.264809-5.476661-1.715992$

H $5.307761-4.864755-0.450197$

H $7.541027-4.735672-3.017756$

H $7.029677-2.985724-1.045262$

H $4.866103-5.054162-4.103151$

H $5.966035-3.768666-4.546138$

H $4.361349-3.371787-3.905440$

H $7.778509-1.509740-2.519812$

H $6.736919-0.905398-0.039349$

H $6.6035070 .054529-1.517440$

H $4.297357-0.346003-2.463861$

H $\quad 0.2030691 .8529792 .719101$

H 1.6860342 .1614540 .037983

H -0.6285894 .1336082 .439556$

H 0.3910275 .5655292 .403899

H 0.9768604 .1276453 .206933

H $4.3367680 .857991-0.733781$

H $4.554558-0.0882450 .755831$

H $6.444583 \quad 3.9589141 .532168$

H 5.7519833 .2824653 .012471

H 3.6549204 .5509552 .733104

H 5.3328445 .8880320 .545624

H 3.9920087 .8121801 .427679

H $2.792406 \quad 6.7480072 .113719$

H $2.5652277 .692275-2.000251$

H $4.0935018 .130850-1.048809$

H $0.8198676 .462178-1.005557$

H 0.6983356 .6806050 .711900

H $1.8695504 .269396-0.831353$

H 4.0455402 .1751931 .440184

C $-4.148474-3.9280250 .871897$

O $-4.527788-2.6348790 .352659$

C $-5.917559-2.681009-0.025349$

C $-6.320859-4.1510160 .008402$

C $-5.458266-4.6725951 .135494$

H $-6.472327-2.1499030 .756556$

C $-6.156114-2.019976-1.387555$

H $-7.392107-4.2829320 .188730$

H -6.071830 -4.662073 -0.929094

C $-5.324219-6.1860581 .141427$

H $-5.891696-4.3458232 .090398$

C $-5.784886-0.527286-1.487451$

O $-5.400698-2.717781-2.387370$

H -7.208492 -2.148597-1.668493

H $-4.696058-0.412024-1.512571$

O $-6.266963-0.057274-2.754472$

C $-6.3733130 .366408-0.382128$

C $-6.3246291 .880255-0.665992$

H $\quad-5.853816 \quad 0.1545310 .559735$

O $\quad-7.7489750 .019597-0.188187$

H -7.048798 2.131228 -1.452246

H -6.6940422 .3810900 .239094$

H $-6.305456-6.6580321 .257362$

H -4.882920 -6.5533790 .208917$

H $-4.690373-6.5209511 .968347$

C -3.300984-3.775544 2.138407

H -4.465186 -2.683401-2.123210

H $-5.873194-0.621837-3.441616$

H $-8.0811740 .543890 \quad 0.560489$

H -3.209418 -4.7483742 .635049$

H -3.797324 -3.088006 2.834200

H $-3.576587-4.4315070 .081292$

C $-2.6721952 .529220-0.202198$

O -4.0306662 .1201030 .011210$

C $-4.9438212 .439297-1.040464$

C $-4.9928283 .959268-1.273421$

C $-3.5937144 .528203-1.523315$

C $-2.5885994 .041859-0.473370$

H -2.1692232 .3322620 .752828$

C $-1.9784201 .685922-1.284241$

H $-4.6159731 .951480-1.963288$

O $-5.5691834 .600462-0.136815$

H $-5.6427644 .163423-2.132999$

H -3.643005 $5.623739-1.504834$

O $-3.1687594 .138039-2.825550$ 
H -1.567636 $4.301023-0.773620$

$\begin{array}{lllll}\text { O } & -2.844746 & 4.714711 & 0.762054\end{array}$

C $-1.8573620 .228444-0.894645$

H -2.519866 $1.720802-2.232951$

H $-0.9629602 .065165-1.446197$

H $-5.6219835 .551596-0.331555$

H -2.288945 $4.524109-2.975345$

H -2.7303375 .6675850 .606146$

O $-1.275034-0.453552-1.925313$

O $-2.224740-0.253091 \quad 0.167386$

C $-1.108611-1.860143-1.723553$

H -2.056772 -2.325963-1.439231

H $-0.772875-2.301039-2.665879$

H $-0.344908-2.037661-0.962332$

SCF Energy (B3LYP/6-31G**//MMFF) $=-3245.89455336$

07_00310

MM̄FF Geometry

C -1.769338 -3.493631 -0.896843

C $-0.551504-4.044813-1.016473$

C $0.762864-3.341583-0.768833$

O $0.515249-1.989683-0.310810$

C $1.613824-4.0322290 .313336$

C $2.396503-5.230848-0.181374$

C $3.563695-5.136649-0.849935$

C $1.823533-6.5782280 .166904$

C $4.265761-3.869828-1.254512$

C $5.622782-3.729069-0.554952$

C $6.246568-2.347426-0.805518$

C $7.665123-2.285507-0.235364$

C $8.272655-0.879594-0.353329$

O $5.412050-1.377453-0.163034$

O $9.448441-0.8366310 .469944$

C $7.274166 \quad 0.1403980 .232482$

C $8.733078-0.570790-1.782466$

O $7.727375 \quad 1.4765920 .029407$

C $5.839805-0.018138-0.316781$

C 4.8226440 .8759510 .431014

O $5.815657 \quad 0.373779-1.693687$

C $1.120541-0.963060-0.962458$

O $1.907598-1.035286-1.892088$

C $0.6319780 .292800-0.349268$

C $0.9663311 .468488-0.900855$

C $0.5657202 .794647-0.426220$

C -0.2672682 .8608220 .825008$

C 4.6762360 .5614491 .920037

C 3.4763871 .2650352 .520245

C 2.7284533 .4754393 .389041

O 2.4422690 .6558662 .785199

C 2.3821364 .7016172 .600146

C 2.8807675 .0976171 .418032

C $2.4436826 .360560 \quad 0.729450$

C $1.9556436 .130320-0.683085$

C $2.5914756 .679983-1.731858$

C $0.6847665 .330414-0.876784$

C $0.9640123 .873550-1.132062$

N 3.6881482 .6199402 .717730

H -1.866215 -2.454074 -0.595427

H -0.484664 -5.077025 -1.350923

H $1.301911-3.321966-1.723598$

H $0.980600-4.3204571 .163686$

H $2.315212-3.3064110 .747874$

H $4.066573-6.054403-1.150479$

H $2.451455-7.401259-0.190582$

H $1.733051-6.6812831 .253383$

H $0.832310-6.705203-0.277050$

H $4.415693-3.888461-2.340856$

H $3.649506-2.991594-1.048381$

H $5.485857-3.8726780 .525072$

H $6.298610-4.516841-0.910340$

H $\quad 6.257885-2.156184-1.885051$

H $8.312412-3.025313-0.722756$

H $7.642334-2.5632610 .828024$

H $10.057149-1.5287850 .159546$

H $7.260830-0.0206591 .317614$

H $9.498475-1.288645-2.101219$

H $9.2055540 .415884-1.840445$

H $7.922483-0.609586-2.512998$
H $\quad 8.629798 \quad 1.534020 \quad 0.387576$

H $5.073571 \quad 1.934606 \quad 0.286827$

H $3.8394140 .748217-0.040501$

H $4.9008750 .595150-1.934883$

H $\quad 0.004106 \quad 0.214386 \quad 0.531017$

H $1.5939131 .463054-1.791307$

H -1.2223282 .3481270 .673566$

H -0.4984203 .8784561 .144610$

H $\quad 0.2528282 .3816841 .660911$

H $4.543468-0.5137872 .085734$

H 5.5692500 .8633072 .478185

H 3.1765653 .7713344 .343215

H 1.8149052 .9133783 .606864

H 1.6242295 .3286563 .067626

H 3.6476514 .5105230 .920346

H 3.2976567 .0500900 .731046

H 1.6494456 .8650841 .294382

H $2.2304426 .540582-2.745596$

H $3.4875977 .277573-1.602011$

H $0.1389275 .717578-1.747913$

H $0.0142985 .498714-0.029759$

H $1.5573193 .705034-2.032475$

H 4.6127173 .0011232 .538486

C $-4.047401-4.187953-0.018888$

O $-4.378759-2.827766 \quad 0.331710$

C $-4.426026-2.7268021 .767489$

C $-4.463892-4.1605122 .286702$

C $-3.582416-4.8621111 .271401$

H -3.480362 -2.271043 2.083869

C $-5.603878-1.8571012 .214292$

H $-4.089130-4.241563 \quad 3.311523$

H -5.479049-4.573928 2.261495

C $-3.733491-6.3727731 .268624$

H -2.537049 -4.609404 1.489149

C $-5.569145-0.4067751 .684069$

O $-6.816663-2.4657161 .753299$

H $-5.658644-1.8556573 .309723$

H $-5.757865-0.4063550 .604947$

$\begin{array}{lllll}\mathrm{O} & -6.675378 & 0.287968 & 2.275867\end{array}$

C -4.2701420 .3624341 .990243$

C -4.3421391 .8822991 .750125$

H -3.450136 -0.0625661 .400360$

$\begin{array}{lllll}\text { O } & -3.939866 & 0.166430 & 3.369677\end{array}$

H -5.0517922 .3372882 .453051$

H -3.3608432 .2918962 .024191$

H $-3.454226-6.7887462 .242166$

H $-4.765536-6.6732871 .058774$

H $-3.088811-6.8268470 .509444$

C $-3.055558-4.215306-1.184822$

H -7.552867 -1.900179 2.043339

H -6.5096150 .3414913 .232944$

H $-3.0763180 .585324 \quad 3.527037$

H -3.513640 -3.717469 -2.047361

H -2.864458 -5.253585 -1.479597

H $-4.984688-4.646887-0.360818$

C $-3.9006841 .982014-1.959368$

O $-3.7180471 .721303-0.561350$

C $-4.6907392 .298908 \quad 0.312721$

C $-4.7226933 .830606 \quad 0.159241$

C $-4.8951944 .252949-1.303569$

C $-3.9380603 .497024-2.230990$

H -3.001532 $1.575579-2.439831$

C $-5.1178811 .239819-2.533510$

H $-5.678707 \quad 1.8971580 .068202$

O -3.5253234 .4122130 .673130$

H $-5.548996 \quad 4.225907 \quad 0.761417$

H -4.705457 $5.329382-1.396847$

O $-6.2349524 .009843-1.720031$

H -4.205853 $3.681964-3.277870$

O $-2.6242174 .027727-2.033326$

C $-4.954448-0.264157-2.495803$

H $-6.0325901 .476909-1.984396$

H $-5.2540041 .523289-3.583638$

H -2.7822664 .0759260 .144026$

H $-6.8211234 .526440-1.141160$

H $-2.0286113 .582195-2.659677$

O $-6.106163-0.838776-2.955405$

O $-3.954344-0.858811-2.120722$ 
C $-6.118919-2.268888-2.986166$

H $-7.049315-2.589897-3.461984$

H $-5.277675-2.648448-3.573787$

H -6.092415 -2.664178 -1.967172

SCF Energy (B3LYP/6-31G**//MMFF)= -3245.91830773

07 00311

MM̄FF Geometry

C -2.318270 $3.368528-0.354844$

C -2.5919123 .3436310 .958521$

C -3.3991672 .2529651 .612529$

O -2.4991871 .4930912 .460067$

C -4.5230482 .7976612 .513015$

C -5.8116603 .0632181 .764520$

C -6.8745482 .2345581 .776996$

C $-5.875707 \quad 4.3571510 .997011$

C -7.0151210 .9232112 .500340$

C $-6.137620-0.2260391 .993230$

C -6.140310 -0.459724 0.475922

C -7.522522 -0.831760 -0.061874

C $-7.477111-1.163754-1.561289$

O $-5.216857-1.5298260 .227670$

O $-8.717361-1.797349-1.910783$

C $-6.356848-2.193744-1.812543$

C $-7.3775570 .100777-2.423073$

O $-6.179324-2.421868-3.209143$

C $-5.005107-1.815755-1.160973$

C $-4.029939-3.012920-1.263927$

O $-4.448261-0.695812-1.851505$

C $-1.743580 \quad 0.540919 \quad 1.845903$

$\begin{array}{lllll}\text { O } & -1.782784 & 0.218259 & 0.669375\end{array}$

C $-0.815496-0.0239892 .846879$

C $0.316559-0.6032772 .419366$

C $1.362275-1.1927143 .258121$

C $1.086534-1.3080744 .733957$

C $-2.720714-2.840881-0.481659$

C $-1.823683-4.060668-0.605846$

C $0.407961-4.9085460 .045702$

O $-2.124342-5.051289-1.267087$

C $1.274747-4.8135731 .258961$

C $2.605229-4.6513021 .203207$

C $3.498419-4.6204772 .410255$

C $4.302145-3.3441912 .505882$

C $5.523768-3.2544421 .953270$

C $3.723200-2.2029933 .315009$

C $2.511834-1.5855492 .670001$

N - $0.645463-3.9142850 .116784$

H -2.720869 2.591969-1.000839

H -2.161809 4.1023331 .607832

H $-3.830211 \quad 1.5758710 .864911$

H $-4.201883 \quad 3.7128223 .028376$

H -4.703936 2.0867823 .328967

H -7.756295 2.5249101 .207090

H -6.8794724 .5577910 .607011$

H -5.6051295 .1982491 .643653$

H -5.1923924 .3361110 .143943$

H -6.8305871 .0759663 .570864$

H -8.067209 0.6170492 .436305

H -5.102051 -0.0699282 .319110$

H $-6.443663-1.1508202 .501205$

H $-5.7648910 .441446-0.019597$

H -8.251678 -0.0341910 .122161$

H -7.898214 -1.712232 0.477964

H -9.439548 -1.184435 -1.690470

H -6.690803 -3.148974 -1.383298

H $-8.2380810 .756065-2.241836$

H -7.408418 -0.145118 -3.490174

H $-6.4742940 .682883-2.228824$

H -7.045508 -2.671823 -3.574105

H -4.527795 -3.913593 -0.880560

H -3.785786 -3.199597 -2.317619

H -3.494125 -0.660933 -1.682015

H -1.072084 0.0802673 .894280

H $0.511052-0.6321361 .347551$

H $0.195986-1.9214954 .910585$

H $0.923812-0.3177005 .173796$

H $1.901660-1.7656905 .297842$

H $-2.160120-1.980764-0.857766$
H $-2.930658-2.6816430 .581988$

H $\quad 0.963913-4.730687-0.881103$

H $-0.040790-5.905769-0.014548$

H $\quad 0.789567-4.9177692 .226413$

H $3.089203-4.5709050 .232212$

H $4.177347-5.4811762 .346391$

H $2.928101-4.7673103 .335894$

H $6.120196-2.3524682 .039925$

H $5.951785-4.0800041 .394277$

H $4.471580-1.4088323 .433170$

H $3.517355-2.5796424 .320637$

H $2.619715-1.4207631 .597537$

H $-0.409968-2.9922300 .473751$

C $-0.0483823 .760786-1.300803$

O $0.6347713 .498398-0.057138$

C $1.9897373 .985106-0.151240$

C $2.2406714 .197501-1.634954$

C $0.8783584 .674267-2.100975$

H 1.9992574 .9441010 .381963

C 2.9233312 .9810810 .531276

H $3.0369074 .921210-1.831557$

H $2.5054033 .251801-2.124705$

C $0.6846264 .581501-3.604408$

H $\quad 0.7431155 .717520-1.785039$

C 4.4260203 .2922010 .389443

O 2.5797902 .9412641 .921712

H $2.706704 \quad 1.9814230 .138727$

H $4.7309633 .265534-0.662838$

$\begin{array}{lllll}\text { O } & 4.680893 & 4.625467 & 0.850583\end{array}$

C 5.3382932 .3626421 .214752

C 5.1088980 .8612630 .999354

H 5.2064512 .5779692 .282610

O 6.7023882 .6719820 .926618

H 5.8040410 .3008351 .637478

H $4.1142670 .602948 \quad 1.381554$

H $1.4128575 .211505-4.125542$

H $0.8116073 .554284-3.962714$

H $-0.3176524 .916353-3.889826$

C -1.415094 4.381093-1.000904

H 2.8000223 .8081192 .303827

H 4.2830845 .2417630 .213148

H 6.8249553 .6230921 .088847

H -1.891911 $4.731644-1.922912$

H -1.295406 $5.250496-0.342541$

H $-0.1659542 .794821-1.808124$

C $4.558695-1.632523-1.704762$

O $4.642326-0.968967-0.438312$

C $5.2275030 .342128-0.449313$

C $6.6668240 .273649-0.986145$

C $6.732315-0.437479-2.344002$

C $5.952974-1.755390-2.341949$

H $4.204183-2.643993-1.469102$

C $3.524852-0.978869-2.635006$

H $4.6212320 .979545-1.097991$

O $7.491361-0.418677-0.050800$

H $7.0633621 .288667-1.090376$

H $7.779066-0.645324-2.598445$

O $6.2037950 .413853-3.356616$

H $5.862489-2.139753-3.364774$

O $6.711586-2.713907-1.598927$

C $2.116221-1.056890-2.086133$

H $3.7479800 .077722-2.803756$

H $3.524000-1.494231-3.602393$

H $8.398601-0.416810-0.400209$

H $6.7388291 .225643-3.366755$

H $6.236807-3.561416-1.639925$

O $1.301665-0.279569-2.861172$

O $1.766984-1.720453-1.120236$

C $-0.074266-0.253339-2.472552$

H $-0.1690730 .114176-1.447567$

H $-0.6048000 .430826-3.140039$

H -0.511664 -1.250998 -2.571331

SCF Energy (B3LYP/6-31G*//MMFF)= -3245.89948213

$07 \quad 00312$

MM̄MF Geometry

C -3.411988 3.2878630 .910524

C $-2.8799443 .911376-0.150942$ 
C $-1.4685783 .665960-0.610330$ O $-1.5880592 .698873-1.686388$ C $-0.7786014 .923263-1.170231$ C $-0.0061325 .702232-0.122044$ C $1.3401125 .722996-0.035218$ C -0.8367546 .4953910 .851660$ C $2.3141365 .011922-0.936094$ C $2.4674183 .543044-0.545629$ C $3.3991072 .720037-1.448310$ C $2.9553522 .728779-2.914608$ C $3.7836041 .768372-3.780274$

O $3.3633841 .382434-0.926297$ O $3.128242 \quad 1.637137-5.051473$ C $3.8061520 .383406-3.104508$ C $5.167203 \quad 2.344759-4.098188$ O $4.669327-0.511082-3.802064$ C $4.1947130 .437310-1.610385$ C $4.023446-0.937718-0.917573$ O $5.5763160 .797369-1.500138$ C $-1.0262791 .474157-1.501801$ O $-0.2674821 .136405-0.607365$ C $-1.5583190 .572009-2.548196$ C $-1.154515-0.706666-2.562824$ C $-1.650821-1.773705-3.432847$ C $-2.816051-1.457599-4.331536$ C $2.578892-1.435522-0.828779$

C $2.470114-2.815149-0.201828$ C $0.720927-4.4481940 .525845$ O $3.434263-3.551879-0.017675$ C $0.283558-5.212432-0.684304$ C $-1.000655-5.414236-1.017119$ C $-1.432288-6.123952-2.268879$ C $-2.206891-5.226303-3.212142$ C $-3.539766-5.342477-3.335468$ C $-1.430785-4.253467-4.076305$ C $-1.074575-2.990589-3.341832$ N $1.150544-3.1338930 .087447$ H $-2.7878782 .623667 \quad 1.503993$ H $-3.5128954 .540672-0.771542$ H $-0.870782 \quad 3.2518590 .211392$ H -1.506660 $5.590723-1.650303$ H $-0.1149924 .619888-1.987901$ H 1.8007786 .3053880 .761081 H $-0.222695 \quad 7.1356361 .494248$ H $-1.534587 \quad 7.1464760 .315075$ H -1.408757 5.8290961 .503780 H $1.9955485 .124396-1.976406$ H $3.286858 \quad 5.513878-0.866655$ H $2.835176 \quad 3.476143 \quad 0.487638$ H $1.4859043 .058186-0.526970$ H $4.4171693 .113746-1.348418$ H $3.0046813 .736738-3.341648$ H $1.8989872 .430620-2.971315$ H $2.2289121 .300881-4.897524$ H $2.794535-0.028625-3.196781$ H $5.0696583 .279601-4.663647$ H $5.7409911 .666494-4.739331$ H $5.7595502 .560377-3.206628$ H $4.394684-0.511726-4.735081$ H $4.658374-1.683249-1.413330$ H $4.412168-0.8660490 .107776$ H $5.9036400 .504009-0.633760$ H -2.302167 $0.963973-3.232065$ H $-0.401033-1.016994-1.841013$ H -2.531060 - $0.709778-5.079643$ H $-3.658275-1.067768-3.748521$ H -3.194244 -2.326499-4.874366 H $1.977742-0.734408-0.240118$ H $2.134864-1.514522-1.825710$ H $-0.094041-4.3065121 .242410$ H $1.542146-4.9688251 .027876$ H $1.068993-5.590635-1.334466$ H $-1.786301-5.031232-0.369862$ H -2.046347 -6.983335 -1.969987 H $-0.573423-6.549015-2.803221$ H $-4.098826-4.723501-4.029247$ H -4.102592 -6.055162 -2.741725 H $-1.981632-4.038368-4.998250$
H $-0.503999-4.731140-4.418945$ H $-0.232215-3.106598-2.660990$ H $0.422915-2.468284-0.156217$ C $-5.6607642 .116247 \quad 0.920328$ $\begin{array}{llll}\text { O } & -5.025522 & 0.938600 & 1.462707\end{array}$ $\begin{array}{llll}\text { C } & -4.774025 & 0.024960 & 0.379248\end{array}$ C $-5.8271230 .361170-0.664720$ C $-5.8230991 .877106-0.587624$ H $-3.7848790 .272734-0.025776$ C $-4.766170-1.4242060 .871164$ H $-5.569756-0.018884-1.657775$ H $-6.813112-0.028962-0.387524$ C -7.065631 $2.522953-1.176403$ H $-4.9504512 .243652-1.140205$ C -3.843555 -1.682571 2.080020 O $-6.092368-1.806123 \quad 1.255829$ H $-4.492472-2.088307 \quad 0.042821$ H $-4.289914-1.2604992 .989001$ O $-3.777023-3.0990592 .285084$ C -2.410904-1.141983 1.923773 C $-1.518654-1.4935723 .122057$ H $-2.457562-0.0554651 .818522$ O $-1.823825-1.6582410 .731892$ H $-2.017451-1.2116354 .058123$ H -1.383764 -2.582537 3.175743 H $-7.1566592 .282438-2.240674$ H $-7.9752082 .175888-0.674944$ H -7.019801 3.612217-1.077560 C $-4.8610873 .372173 \quad 1.301566$ H $-6.388518-1.1859351 .944307$ H $-4.689096-3.4192052 .392697$ H $-1.850045-2.6287510 .788262$ H -4.8930873 .4992892 .390807$ H $-5.334451 \quad 4.259332 \quad 0.864568$ H -6.6457922 .1770861 .400001$ C $1.951636-0.9629194 .344276$ O $0.574443-1.349364 \quad 4.237019$ C $-0.113126-0.862526 \quad 3.074985$ C $-0.119105 \quad 0.674362 \quad 3.074389$ C 1.3005721 .2396073 .190890 $\begin{array}{llll}\text { C } 2.097247 & 0.569200 & 4.314946\end{array}$ H $2.253788-1.3104045 .340721$ C $2.797842-1.7154593 .308159$ H $0.400845-1.2320882 .181518$ O $\quad-0.913438 \quad 1.145594 \quad 4.160875$ H $-0.579249 \quad 1.0369152 .149624$ H 1.2494432 .3187643 .379455 \begin{tabular}{llll}
\hline & 1.993378 & 1.053428 & 1.961258
\end{tabular} H $3.156900 \quad 0.838781 \quad 4.244087$ O $1.6237821 .062690 \quad 5.572292$ C $4.271889-1.7452953 .644378$ H $2.452860-2.7550383 .247562$ H $2.681902-1.2756752 .315379$ H -0.9265182 .1167394 .115334$ H 1.4593601 .4566661 .255994 H 1.7687032 .0240695 .583099 O $4.950486-2.2976622 .595272$ O $4.768451-1.3496624 .689668$ C $6.368738-2.3922992 .758085$ H $6.785364-2.8396091 .851761$

H $6.802189-1.3962812 .888935$ H $6.610081-3.0348213 .610255$ SCF Energy (B3LYP/6-31G**//MMFF) $=-3245.91251342$

0700313

MM̄FF Geometry

C $0.356032-1.0876410 .551519$

C $-0.185599-1.659284-0.535879$

C $-0.437703-0.989414-1.863087$

O $-0.5295920 .442159-1.671169$

C $0.684219-1.278562-2.875193$

C $0.673042-2.683448-3.441844$

C $1.539036-3.653922-3.088195$

C $-0.356019-2.936895-4.512235$

C $2.593199-3.572545-2.020663$

C $3.955271-3.154032-2.584994$

C $5.000383-2.824645-1.505787$

C $5.406590-4.046079-0.680044$ 
C $6.399678-3.674073 \quad 0.432224$

O $4.451205-1.812151-0.657838$

O $6.484142-4.7854301 .337678$

C $5.827023-2.4840051 .230516$

C $7.815428-3.450257-0.111511$

O $6.770802-2.0122942 .190107$

C $5.344505-1.3115680 .342903$

C $4.563579-0.2901761 .212344$

O $6.480352-0.676267-0.241662$

C $-1.3701371 .127539-2.487590$

O $-2.0366740 .662753-3.403081$

C $-1.4403032 .562723-2.129689$

C $-0.6483573 .161686-1.226157$

C $-0.7186534 .571060-0.826608$

C $-1.8169545 .413802-1.423077$

C $3.7639790 .763602 \quad 0.431845$

C $4.5754291 .953874-0.043008$

C $4.3614083 .994260-1.459717$

$\begin{array}{lllll}\text { O } & 5.718621 & 2.185017 & 0.340310\end{array}$

C $3.8761395 .110568-0.590701$

C $3.1191096 .124344-1.035653$

C $2.6199657 .232875-0.155303$

C $1.1154947 .384336-0.205249$

C $0.552928 \quad 8.388983-0.898264$

C $0.270495 \quad 6.4396790 .621782$

C 0.1882515 .0487250 .051948

N $3.8534222 .744046-0.927309$

H $0.687055-0.0544670 .507522$

H $-0.490138-2.702334-0.478760$

H - $1.404322-1.357462-2.228596$

H $1.655647-1.036379-2.425058$

H $0.608796-0.573459-3.714408$

H $1.474240-4.617672-3.590446$

H $-0.287460-3.949868-4.923171$

H $-1.366393-2.813016-4.111169$

H $-0.222410-2.235139-5.341858$

H $2.278209-2.890129-1.226826$

H $2.669720-4.558910-1.548325$

H $4.341620-3.934401-3.252039$

H $3.815301-2.252718-3.196977$

H $5.876167-2.414619-2.022696$

H $5.823503-4.829934-1.324207$

H $4.521666-4.487633-0.203488$

H $6.773385-5.563494 \quad 0.830817$

H $4.967150-2.8607641 .802277$

H $8.183446-4.357744-0.605032$

H $8.521755-3.2436480 .700126$

H $7.873815-2.635252-0.835656$

H $7.039215-2.7744112 .731299$

H $3.828138-0.8454481 .808605$

H 5.2449240 .2039831 .915272

H $6.2334690 .220942-0.512970$

H -2.206398 $3.114141-2.666142$

H $0.1181622 .575094-0.722760$

H -1.702377 $5.479636-2.510780$

H -1.832143 $6.438549-1.046948$

H -2.799823 $4.985678-1.197692$

H $3.2421110 .313286-0.418975$

H $2.996671 \quad 1.1718381 .102312$

H $4.0003024 .081378-2.489252$

H $5.4556253 .977479-1.470616$

H 4.1615525 .0806380 .458354

H $2.8465796 .165184-2.087762$

H $3.1047148 .161470-0.483776$

H 2.9320897 .0896510 .886664

H $-0.5216688 .535114-0.913994$

H $1.1550649 .089245-1.467919$

H $-0.735593 \quad 6.8475530 .776563$

H 0.6932286 .3756191 .632899

H $0.953238 \quad 4.3709900 .431716$

H $2.8826252 .511962-1.117440$

C - $0.157521-1.0900503 .037834$

O $-1.486367-0.6683222 .664818$

C -1.630017 0.7217223 .008968

C -0.5959600 .9696014 .095939$

$\begin{array}{llll}\text { C } & 0.567280 & 0.156004 & 3.555859\end{array}$

H -1.3385621 .3038442 .126020$

C -3.0793141 .0420473 .378571$
H -0.3590402 .0308484 .216185$

H $-0.9236590 .573655 \quad 5.064296$

C $1.638319-0.1411914 .590897$

H 1.0256190 .7084562 .726322

$\begin{array}{llll}\text { C } & -4.093180 & 0.703545 & 2.261746\end{array}$

$\begin{array}{llll}\text { O } & -3.433202 & 0.289323 & 4.543630\end{array}$

H -3.1598822 .0992263 .658200$

H $-4.187245-0.3836192 .175875$

$\begin{array}{lllll}\text { O } & -5.378708 & 1.177288 & 2.679440\end{array}$

C $-3.750311 \quad 1.3144130 .892090$

C $-4.827473 \quad 1.150342-0.191679$

H -2.8153350 .8795660 .522754$

$\begin{array}{llll}\text { O } & -3.522068 & 2.716230 & 1.069386\end{array}$

H $-5.707391 \quad 1.7586550 .054582$

H $-4.4171591 .599583-1.105457$

H 2.0832440 .7882344 .960761

H $1.227912-0.6824145 .449901$

H $2.438328-0.7507364 .158938$

C $0.519917-1.8061061 .863645$

H -4.3606430 .4958724 .751335$

H -5.3363082 .1484812 .713079$

H -3.2762413 .0862060 .205047$

H $0.084667-2.8099331 .781577$

H $1.586231-1.9462392 .072109$

H $-0.282385-1.8111403 .855993$

C $-4.333502-2.500426-0.884527$

O $-4.094886-1.103989-0.651589$

C $-5.262477-0.296411-0.474240$

C $-6.171668-0.378185-1.713896$

C $-6.494090-1.827281-2.088229$

C $-5.235765-2.701453-2.114521$

H $-3.347080-2.916758-1.123834$

C $-4.863367-3.2199640 .367252$

H $-5.814099-0.6535920 .401011$

O $-5.553367 \quad 0.261659-2.830550$

H $-7.1001760 .166974-1.507625$

H $-6.969785-1.842119-3.076725$

O $-7.429963-2.342899-1.146773$

H $-5.512608-3.758237-2.205178$

O $-4.488450-2.363352-3.286624$

C $-3.834422-3.2836341 .474539$

H $-5.752333-2.7245600 .765681$

H $-5.128444-4.2550610 .123275$

H $-4.690746-0.163284-2.977020$

H -7.646714 -3.251673 -1.416422

H $-3.724219-2.962957-3.331190$

O $-4.471617-3.4441972 .671646$

$\begin{array}{llllll}0 & -2.623907 & -3.235947 & 1.309827\end{array}$

C $-3.618462-3.5482413 .815914$

H -2.924414 -4.3862593 .700599$

H -3.073046 -2.6125293 .965139$

H $-4.246137-3.7314544 .692009$

SCF Energy (B3LYP/6-31G**//MMFF) = -3245.91544929

07_00314

MM̄FF Geometry

C $3.200559-2.916516-1.881609$

C $2.389006-2.476948-2.857080$

C $0.933676-2.105793-2.730948$

O $0.557900-1.970602-1.345247$

C $0.577376-0.823192-3.517928$

C $1.1029420 .481815-2.949921$

C $0.3570341 .376344-2.271685$

C $2.5476470 .799655-3.241923$

C $-1.0886921 .277457-1.869052$

C $-1.9010292 .448032-2.433068$

C $-3.4046042 .389178-2.117309$

C $-3.7082152 .480352-0.620203$

C $-5.2171342 .395309-0.340931$

O $-3.935796 \quad 1.174430-2.657667$

\begin{tabular}{llll}
\hline & -5.369423 & 2.188551 & 1.071923
\end{tabular}

C $-5.7849571 .156135-1.068501$

C $-5.927434 \quad 3.716003-0.656837$

O $-7.203457 \quad 1.102552-0.942416$

C $-5.3632861 .058512-2.551744$

C $-5.770983-0.280050-3.216383$

O $-6.014628 \quad 2.101721-3.288606$

C $-0.750312-2.155575-1.027908$ 
O $-1.658726-2.464371-1.782262$

C $-0.899691-1.8736310 .415240$

C $-2.092984-1.4733290 .877361$

C -2.417494-1.146244 2.265703

C $-1.359238-1.3857463 .309236$

C $-5.262697-1.543649-2.513001$

C $-6.266328-2.127443-1.530944$

C $-6.408733-3.574348 \quad 0.491622$

O $-7.476493-1.932112-1.616365$

C $-6.572097-2.6128081 .626639$

C $-6.106082-2.8317032 .865404$

C $-6.268567-1.8581263 .998343$

C $-4.943131-1.4109364 .576204$

C -4.471384-1.951839 5.712166

C $-4.205938-0.2829453 .887319$

C $-3.649953-0.6752772 .546356$

N -5.661695 -2.929348 -0.573301

H $2.822416-3.062710-0.874661$

H $2.800389-2.395812-3.862335$

H $\quad 0.371610-2.940010-3.173131$

H $-0.512725-0.771322-3.609873$

H $0.927697-0.919701-4.554906$

H $0.8463712 .289983-1.935793$

H $2.8033751 .835355-2.992389$

H $3.212157 \quad 0.154067-2.662017$

H $2.7631130 .662747-4.306544$

H -1.540032 $0.335246-2.183743$

H -1.126418 $1.290354-0.774779$

H -1.492211 3.398126 -2.066359

H -1.784068 $2.449696-3.525221$

H $-3.8647573 .235695-2.641160$

H -3.286681 $3.397652-0.190927$

H $-3.224791 \quad 1.650162-0.089136$

H -6.3146782 .0401041 .247965$

H $-5.3986860 .284501-0.527481$

H $-5.5040534 .528705-0.054329$

H $-6.9887713 .669404-0.389011$

H -5.849521 4.006335-1.706439

H $-7.5808561 .743331-1.568941$

H -6.857836 $-0.309115-3.360870$

H $-5.342950-0.283824-4.229138$

H $-6.0271531 .852156-4.227628$

H $-0.024770-1.978887 \quad 1.045127$

H -2.911854 -1.360193 0.168284

H -1.702733 -1.191977 4.327855

H $-0.496213-0.7366863 .133894$

H -1.024595 -2.428875 3.287894

H $-5.093913-2.323289-3.266486$

H $-4.304180-1.356953-2.018492$

H $-5.855062-4.4723490 .783122$

H $-7.392105-3.8748080 .115735$

H -7.105555 -1.688094 1.417227

H $-5.583999-3.7607803 .082476$

H $-6.872628-2.3470704 .773862$

H -6.844289 -0.9761583 .690538$

H $-3.537140-1.6195396 .152115$

H -5.007746 -2.742667 6.226315

H -3.4140590 .1207294 .529073$

H -4.896605 0.5582463 .743997

H -4.353144 -0.5392611 .724830$

H -4.648142 -2.985810 -0.539107

C $5.573714-3.010938-0.958001$

O $5.551476-1.626308-0.553775$

C $6.897571-1.124341-0.540873$

C $7.796708-2.354065-0.477280$

C $7.025839-3.336243-1.328640$

H $7.044932-0.618971-1.503087$

C $7.138172-0.1267800 .598960$

H $8.803384-2.152892-0.856259$

H $7.890355-2.743300 \quad 0.542663$

C $7.410953-4.786457-1.087697$

H $7.211038-3.094644-2.384014$

C 6.1052681 .0016910 .773143

O $7.180759-0.8296581 .850916$

H 8.1383880 .3063610 .476785

H 5.2262860 .6118351 .297420

O $\quad 6.6958191 .970777 \quad 1.653366$

C $5.6459451 .721989-0.503973$
C $4.8767843 .039898-0.261400$

H $5.0211081 .039645-1.090578$

O $6.7910482 .033249-1.301764$

H 5.5866773 .8230720 .035744

H $4.4744773 .360600-1.231742$

H $8.469244-4.946835-1.318143$

H $7.246206-5.077175-0.044718$

H $6.821644-5.456637-1.721340$

C $4.639265-3.256610-2.148189$

H $6.334256-1.2982021 .952096$

H $6.941206 \quad 1.5069612 .472392$

H $6.4720452 .437753-2.126491$

H $4.692459-4.315630-2.427794$

H $4.991340-2.668294-3.005692$

H $5.246334-3.596731-0.090074$

C $1.697540 \quad 1.747708 \quad 1.230977$

$\begin{array}{llll}\text { O } & 2.776550 & 2.008839 & 0.319972\end{array}$

C 3.7423702 .9589820 .773410

C 3.0750784 .3210891 .011817

C 1.9204994 .1873002 .010465

C $0.9707483 .045797 \quad 1.631651$

H 0.9884431 .1345960 .661358

C 2.1644250 .9116612 .433358

H 4.1693992 .6203051 .720824

O $2.5919684 .839737-0.224631$

H 3.8255535 .0216861 .397436

H 1.3665075 .1331142 .046233

O $2.464475 \quad 3.9537713 .306572$

H 0.2752672 .8325872 .451712

O $0.1778303 .461616 \quad 0.516884$

C $2.582029-0.4828462 .014703$

H 3.0140881 .3639522 .950138

H 1.3365050 .8095103 .143453

H $2.1930035 .707578-0.042845$

H 1.7212253 .8971603 .931022

H -0.3314554 .2425190 .792549$

O $2.808679-1.2361223 .130854$

O $2.708938-0.8637980 .859392$

C $3.222642-2.5833832 .882726$

H $2.449444-3.1205592 .324962$

H $4.170901-2.5939652 .336862$

H $3.367087-3.077746 \quad 3.847001$

SCF Energy (B3LYP/6-31G**//MMFF) $=-3245.89864191$

07_00315

MM̄FF Geometry

C -3.849258 -2.039734 -3.126101

C $-2.616522-2.117007-2.604691$

C -1.391681 -1.603659 -3.314814

O $-0.792939-0.622664-2.434970$

C $-0.388233-2.744050-3.568195$

C $0.827569-2.315971-4.367540$

C $2.090518-2.326428-3.897279$

C $0.564969-1.901364-5.793234$

C $2.571582-2.813133-2.559556$

C $2.368066-1.795710-1.432955$

C $3.081742-2.233630-0.146442$

C $2.652428-1.3629781 .033316$

C $3.421150-1.7146962 .314800$

O $4.488899-2.103731-0.366036$

O $3.155695-0.6667223 .259683$

C $4.932703-1.7050652 .000075$

C $2.902323-3.0082822 .951509$

O $5.691728-2.1466293 .122742$

C $5.308670-2.510330 \quad 0.735713$

C $6.790679-2.3232690 .322000$

O $5.127623-3.9067131 .006649$

C $-0.4937320 .600444-2.948328$

O $-\begin{array}{llll}0.654982 & 0.978082 & -4.097786\end{array}$

C $0.0651691 .427032-1.853372$

C $0.5174792 .659544-2.131392$

C $1.0704663 .625152-1.178023$

C 1.1310923 .2156390 .269026

C $7.280875-0.8865520 .120166$

C $6.523176-0.146197-0.965537$

C $4.9703851 .754640-1.324061$

O $6.498679-0.544813-2.128051$

C $4.6957813 .067944-0.667354$ 
C $4.8440374 .253357-1.276753$

C $4.5727545 .569548-0.607463$

C $3.4500696 .339471-1.264756$

C $3.7075397 .355466-2.105745$

C $2.0309665 .992586-0.870306$

C $1.4826684 .824246-1.642031$

N $5.883840 \quad 0.990733-0.495111$

H -3.979597 -1.613938-4.118691

H -2.481860 -2.535252 -1.610673

H -1.689246 -1.145286 -4.265079

H $-0.882801-3.560056-4.111665$

H -0.080941 -3.164244 -2.605646

H $2.886986-1.987403-4.558712$

H $1.491160-1.732422-6.353171$

H $0.007303-2.684608-6.317220$

H $-0.014286-0.975663-5.835090$

H $2.085747-3.762122-2.304100$

H $3.638274-3.050137-2.660973$

H $2.749125-0.818582-1.753528$

H $\quad 1.297423-1.685982-1.233375$

H $2.841271-3.2850360 .048327$

H $1.572162-1.4446021 .201354$

H $2.839095-0.3064650 .793284$

H $3.694847-0.8398894 .050675$

H $5.201817-0.6537961 .852918$

H $1.839678-2.9104883 .200505$

H $3.410835-3.2157783 .899507$

H $3.012092-3.8804822 .304018$

H $5.617443-3.1151503 .167572$

H $7.437148-2.8085091 .064970$

H $6.955831-2.872685-0.615607$

H $5.657781-4.4131460 .368951$

H $\quad 0.077981 \quad 1.005087-0.854838$

H $0.471773 \quad 3.006957-3.163110$

H $\quad 0.120278 \quad 3.0659720 .663962$

H 1.6217393 .9491290 .911729

H $1.6963452 .283947 \quad 0.383027$

H $7.259965-0.3366901 .066518$

H $8.333592-0.906019-0.188471$

H $4.0430641 .180065-1.408983$

H $5.3992651 .861401-2.326013$

H 4.3399473 .0423660 .360283

H $5.1944294 .284879-2.305776$

H $5.5009266 .155250-0.636357$

H 4.3460825 .4373180 .457996

H $2.9067557 .932586-2.556190$

H $4.7245737 .628351-2.366865$

H $1.3717676 .848467-1.069920$

H 1.9809385 .8535690 .212865

H $1.3917815 .021760-2.711351$

H $5.832774 \quad 1.1305480 .509825$

C $-5.981245-1.264743-2.121422$

O $-5.436511-0.541127-0.998209$

C $-6.5278150 .073224-0.279949$

C $-7.802751-0.289848-1.037467$

C -7.410401-1.593703 - 1.707187

H $-6.565444-0.3980470 .707511$

C $-6.2791871 .581569-0.156193$

H -8.663445 $-0.392641-0.369207$

H $-8.0553360 .469692-1.787314$

C $-8.324762-1.977440-2.857201$

H -7.410704 -2.394315 -0.955480

C $-5.045676 \quad 1.9424550 .701988$

O $-6.0867232 .124745-1.469090$

H -7.1696782 .0771090 .246329$

H -4.1423791 .4936190 .274275$

$\begin{array}{lllll}\text { O } & -4.865261 & 3.363132 & 0.636137\end{array}$

C -5.1907161 .5463092 .185263$

C -4.030827 2.0248073 .079901

H $-5.300530 \quad 0.4612192 .274772$

O

H -3.812168 3.0812982 .884691

H $-4.376278 \quad 1.9809274 .121851$

H -9.352752 -2.108016 -2.503348

H -8.332893 -1.209080 -3.637598

H $-8.005150-2.918644-3.315125$

C $-5.103025-2.478159-2.421571$

H $\quad-5.3128131 .682326-1.858287$
H $-4.7925153 .602654-0.303594$

H $-6.530577 \quad 1.7998393 .592236$

H -5.625689 -3.182630 -3.077485

H $-4.856623-3.007172-1.493033$

H $-5.989518-0.575445-2.977471$

C $-2.009849-1.0886723 .376282$

O $-3.068232-0.1265303 .440780$

C -2.7542221 .1780542 .941272$

C -1.5895441 .7872963 .740084$

C $-0.3719450 .860778 \quad 3.755057$

C $-0.750754-0.5827014 .103084$

H -2.399154 -1.944820 3.941931

C $-1.776054-1.5475851 .928567$

H $-2.477792 \quad 1.1028561 .883789$

O $-2.0081872 .032090 \quad 5.082575$

H -1.3247152 .7541253 .294777$

H 0.3531661 .2341914 .488818

O 0.2469190 .9059122 .475583

H $0.090323-1.2519273 .896019$

O $-0.989927-0.6611465 .512585$

C $-1.116366-2.9087011 .858013$

H -2.739563 -1.621866 1.409605

H -1.162971 -0.8284201 .378608$

H -1.270312 2.4638995 .545870

H 1.0798330 .4088852 .540360

H $-1.773447-0.1203065 .711427$

O $-0.760726-3.1725180 .566092$

O $-0.948250-3.6596982 .809423$

C $-0.150614-4.4500250 .355479$

H $0.754214-4.5460760 .962848$

H $-0.860245-5.2488060 .590910$

H $0.127081-4.529565-0.697622$

SCF Energy (B3LYP/6-31G**//MMFF) $=-3245.92887749$

0700316

MM̄FF Geometry

C $3.531758-3.070345-1.917269$

C $2.502966-2.526836-1.250988$

C $1.217269-2.093372-1.900843$

O $0.147896-2.662754-1.111255$

C $1.126151-0.557135-1.872396$

C $-0.048677-0.000348-2.648064$

C $-1.1430340 .541785-2.079598$

C $0.091115-0.028053-4.147908$

C $-1.4371200 .673127-0.611104$

C $-1.0391372 .044422-0.046102$

C $-1.7953173 .221976-0.678018$

C $-1.3124294 .556270-0.108257$

C $-2.1219165 .735900-0.671655$

O $-3.1848053 .051835-0.390939$

O $-1.833100 \quad 6.8917310 .129694$

C -3.627450 $5.436101-0.507930$

C $-1.7026986 .086051-2.104366$

O $-4.4217756 .427462-1.155133$

C $-4.0394494 .027596-0.995286$

C $-5.5061053 .732260-0.582862$

O $-3.9555163 .975857-2.422584$

C $-0.958514-3.109483-1.763442$

O $-1.172386-3.074974-2.964561$

C $-1.883406-3.663002-0.748226$

C $-3.118638-4.035515-1.116094$

C $-4.144256-4.625673-0.251506$

C $-3.769821-4.9042441 .180672$

C $-5.8836562 .242814-0.623893$

C -5.4528491 .4816950 .622712$

C $-5.093348-0.8245681 .470760$

O -5.1038322 .0391381 .660569$

C $-5.974486-2.0300161 .597229$

C -7.016361 -2.382870 0.827970

C $-7.834783-3.6205831 .066004$

C $-7.749671-4.606378-0.077285$

C -8.745962 -4.715828 -0.972395

C $-6.551512-5.530011-0.125273$

C $-5.355726-4.895389-0.783139$

N -5.5409110 .1064540 .451933$

H $3.448488-3.235287-2.988146$

H $2.596160-2.355061-0.182106$

H $1.178728-2.472195-2.929424$ 
H $2.043208-0.120963-2.292064$ H $1.090868-0.214174-0.832879$ H $-1.9212780 .935096-2.732117$ H $-0.7607030 .439802-4.652863$ H $\quad 0.9920350 .513723-4.453896$ H $\quad 0.163941-1.056547-4.513005$ H -2.508245 $0.497224-0.456691$ H $-0.931884-0.111105-0.036981$ H -1.249571 2.0254261 .030460 H $0.0413402 .189856-0.166967$ H -1.640811 3.204141 -1.762118 H $-0.2413994 .697960-0.299743$ H -1.423552 4.5494010 .985189 H -0.8737827 .0480940 .094891$ H -3.856045 5.5083820 .564806 H $-0.6373526 .344089-2.139079$ H -2.236505 $6.971578-2.466625$ H $-1.8679775 .270505-2.811495$ H $-4.1426757 .292764-0.809726$ H -5.6938834 .1145260 .427763$ H -6.180179 4.274669 -1.258408 H $-4.5656893 .294870-2.748474$ H -1.519530 $-3.755116 \quad 0.268713$ H $-3.411871-3.900613-2.156684$ H -3.437205 -3.987287 1.679566 H -2.959905 -5.640559 1.227742 H -4.593744 -5.296776 1.779554 H $-6.9763272 .159915-0.674935$ H $-5.4704661 .758540-1.514958$ H -5.043166 -0.3137552 .437898$ H $-4.078286-1.1359911 .202602$ H -5.711509-2.685654 2.425894 H -7.319237 -1.758665 -0.007902 H -8.875591 -3.306604 1.219368 H -7.542598 -4.1216211 .997366$ H -8.705165 -5.442747 -1.776851 H -9.621610 -4.077155 -0.923956 H $-6.799569-6.428415-0.706685$ H $-6.342495 \quad-5.9017990 .881291$ H -5.520815 -4.657919-1.835313 H -5.684242 -0.253950 -0.487121 C $5.952550-2.438485-1.481863$ O $5.605941-1.183217-0.863033$ C $6.030147-0.111470-1.728339$ C $6.952700-0.746869-2.763866$ C $6.315289-2.113646-2.930684$ H $5.1315620 .256029-2.238260$ C $6.6629921 .017126-0.906786$ H $6.989583-0.172431-3.694304$ H $7.977526-0.849875-2.388665$ C $7.225095-3.139649-3.582455$ H $5.415010-1.994946-3.545451$ C $5.766800 \quad 1.555626 \quad 0.228937$ O $7.8739790 .534175-0.308407$ H $6.9631821 .835779-1.570960$ H $\begin{array}{llll}5.655606 & 0.775272 & 0.989873\end{array}$ O $\quad 6.4849672 .630218 \quad 0.847788$ C $4.3862592 .063190-0.233933$ C 3.5368762 .7208520 .873155 H $3.8199841 .233453-0.670455$ O $4.5816583 .027651-1.271633$ H 4.0555353 .5998411 .274280 H 2.6246683 .1084630 .399049 H $7.506145-2.818845-4.590870$ H $8.145674-3.283792-3.007128$ H $6.722386-4.108719-3.663423$ C $4.829289-3.456147-1.264368$ H $7.642967-0.2316300 .244880$ H 6.0735662 .8020831 .711385 H $3.7044133 .284259-1.603530$ H $4.669245-3.575445-0.185072$ H $5.133257-4.437352-1.646829$ H $\quad 6.842387-2.799336-0.948928$ C $1.988357-0.3328752 .322854$ O $2.340456 \quad 0.731131 \quad 1.430038$ C 3.1557101 .7565482 .004179 C 2.3922232 .4536143 .138414 C 1.9293721 .4406494 .193175
C 1.2596530 .2100313 .566545

H $1.275747-0.9378771 .748010$

C $3.217483-1.2048922 .620760$

H 4.0663891 .3108502 .416956

O 1.2634343 .1543482 .622384

H 3.0481033 .2000813 .601063

H 1.2193331 .9249184 .875052

O 3.0479471 .0134314 .965399

H $1.152937-0.5812514 .317052$

O $\quad-0.061952 \quad 0.597071 \quad 3.174974$

C $2.855106-2.6441132 .913312$

H $3.871526-1.2029141 .739561$

H $3.799487-0.8210553 .462408$

H 0.6830152 .5029012 .194451

H 3.4270451 .8000905 .393428

H $-0.516553-0.1977582 .847346$

O $3.985831-3.4076252 .842508$

O $1.732685-3.0497593 .181341$

C $3.800328-4.8057023 .083664$

H $3.424757-4.9682124 .098351$

H $3.114639-5.2299002 .343960$

H $4.770910-5.2990352 .985277$

SCF Energy (B3LYP/6-31G**//MMFF) $=-3245.90609050$

$07 \_00317$

MM̄FF Geometry

C -1.725183 -3.672001-1.860364

C $-0.835288-2.862505-1.267840$

C $0.228306-2.107169-2.018548$

O $-0.075055-0.702279-1.840955$

C $1.639073-2.416529-1.479795$

C $2.033186-3.877500-1.547285$

C $2.403140-4.625240-0.489218$

C $2.051704-4.482396-2.928852$

C $2.531426-4.2087840 .949851$

C $3.986272-4.2833711 .439355$

C $4.936097-3.3193470 .708226$

C $6.378162-3.5265911 .176211$

C $7.336224-2.5237260 .516154$

O $4.519328-1.9792360 .988836$

O $8.597486-2.596611 \quad 1.197745$

C $6.770455-1.0994540 .694741$

C $7.640044-2.902742-0.937324$

O $7.548711-0.148017-0.028341$

C $5.276958-0.968750 \quad 0.307348$

C 4.7505480 .4123340 .762498

O $5.145225-1.076637-1.110464$

C $0.4931680 .175731-2.709395$

O $1.319016-0.071480-3.573810$

C $-0.0920861 .509261-2.450720$

C $0.5300562 .598375-2.925117$

C $0.0561053 .979848-2.827064$

C $-1.3594914 .200094-2.362073$

C 3.2371410 .6017970 .610248

C $2.813811 \quad 1.8525081 .350384$

C 2.6959874 .3145401 .154771

O 2.5051431 .8162492 .539955

C 2.2468445 .2848840 .108768

C 1.1417816 .0365500 .236861

C $0.6115437 .088932-0.701050$

C $1.2725127 .193146-2.057262$

C $2.3320697 .996501-2.251577$

C $0.6273946 .455611-3.209049$

C $0.8960664 .975067-3.181218$

N 2.8610982 .9969230 .568887

H -1.692547 $-3.803230-2.939223$

H -0.872969-2.726632 -0.190730

H $\quad 0.169709-2.336813-3.090460$

H $1.720727-2.010462-0.467127$

H $2.379679-1.845668-2.053405$

H $2.672216-5.665568-0.670819$

H $2.486315-5.487856-2.936782$

H $1.038467-4.560600-3.333641$

H $2.651641-3.863840-3.604654$

H $2.127050-3.2120611 .141677$

H $1.924655-4.8962611 .552389$

H $3.987360-4.0485632 .511646$

H $4.351907-5.3126301 .333411$ 
H $4.868294-3.500281-0.369591$ H $6.708754-4.5548180 .983635$ H $6.427852-3.3948782 .266158$ H $8.449045-2.3888932 .135927$ H $6.872416-0.8377641 .757146$ H 8.099741-3.897371-0.983607 H $8.369602-2.215839-1.380817$ H $\quad 6.752571-2.918464-1.573413$ H $8.474145-0.2595880 .248934$ H $5.000511 \quad 0.552924 \quad 1.822798$ H $5.258730 \quad 1.2094840 .204995$ H $4.333623-0.624000-1.388182$ H $-1.0242261 .554112-1.899410$ H $1.4799342 .469464-3.443135$ H -1.668480 $5.246980-2.381184$ H - $1.4857123 .858061-1.331139$ H -2.062961 $3.653794-3.000229$ H $2.9455860 .676720-0.442665$ H $2.678408-0.2363321 .040411$ H 3.6715764 .6185461 .548534 H 1.9979324 .2550271 .996839 H $2.8870855 .392941-0.761756$ H 0.5471355 .9129011 .140998 H $0.6835288 .056410-0.185989$ H $-0.4634526 .918795-0.839135$ H $2.7868308 .106814-3.230368$ H $2.7704888 .557415-1.432672$ H $1.0132036 .834907-4.165076$ H $-0.4411896 .688828-3.237245$ H $1.9055064 .713226-3.501427$ H $3.3038082 .936241-0.343906$ C $-4.174118-3.846069-1.419404$ O $-4.309774-2.567242-0.767272$ C $-5.690852-2.388291-0.390080$ C $-6.435278-3.635866-0.861606$ C $-5.332814-4.677044-0.878757$ H $-5.720713-2.3570800 .703967$ C $-6.227886-1.078702-0.980160$ $\mathrm{H}-7.261514-3.898127-0.193247$ H -6.854846 -3.502534 -1.865846 C $-5.671153-5.899215-1.714022$ H $-5.123787-4.9871950 .153898$ C $-5.4823470 .177788-0.481440$ O $-6.090188-1.147918-2.405351$ H -7.301474 -0.992966 -0.774322 H $-4.4233340 .124731-0.757945$ O $-6.0283011 .312551-1.166817$ C -5.6260240 .4270611 .035211$ C -5.0168021 .7605651 .510837$ H $-5.176872-0.3961141 .598861$ $\begin{array}{llll}\text { O } & -7.016617 & 0.443320 & 1.374171\end{array}$ H -5.3375782 .5738320 .849177$ H $-5.441941 \quad 1.9922712 .497321$ H $-6.565380-6.395984-1.323378$ H -5.864727 -5.630834 -2.758035 H -4.851136 -6.623674 -1.699091 C -2.794295 -4.429525 -1.124862 H $-6.621686-0.427635-2.784402$ H $-5.7375831 .260499-2.093071$ H $-7.432977 \quad 1.161890 \quad 0.867574$ H $-2.742792-5.476620-1.443103$ H -2.598593 -4.407393 -0.047719 H -4.296691 -3.676031 -2.497924 C -1.772634 0.7811853 .071064 $\begin{array}{llll}\text { O } & -3.165778 & 0.933031 & 2.767482\end{array}$ C -3.4833821 .7254421 .615654$ C -2.8938913 .1391841 .746520$ C -1.3945903 .0934162 .049035$ C -1.083510 2.1483693 .209787 H -1.756847 0.3046194 .059902 C -1.077947 -0.1642502 .080292$ H -3.0703621 .2471330 .722642$ O -3.5670813 .8701032 .769703$ H -3.0635273 .6781070 .807021$ H -1.048288 4.1048512 .293627 O $-\begin{array}{llll}0.708110 & 2.658991 & 0.880603\end{array}$ H -0.0021032 .0114713 .307410$ O -1.5310812 .7683654 .418523$
C $-1.313436-1.6028822 .477790$

H $-1.401066-0.0428041 .043021$

H $\quad 0.007787-0.0115282 .100052$

H -3.4826973 .3683773 .598501$

H $\quad 0.2438432 .6847491 .073293$

H -1.2677322 .1947065 .158202$

O $-2.615442-1.9445512 .261849$

O $-0.442040-2.3296752 .938318$

C $-2.985073-3.2625042 .678456$

H $-3.030980-3.3027493 .770551$

H $-3.977365-3.4817132 .276292$

H -2.280693 -4.008047 2.297576

SCF Energy (B3LYP/6-31G**//MMFF) $=-3245.91239716$

0700318

MM̄MF Geometry

C $2.918137-1.9839261 .992920$

C $2.074186-2.0726133 .030491$

C $0.904465-1.1439693 .205955$

O $-0.284120-1.9668383 .111706$

C $0.890048-0.4458974 .575321$

C 1.8801720 .6920214 .698511

C 1.6017571 .9709304 .376049

C 3.2221320 .3328845 .280580

C $0.3205802 .507683 \quad 3.794227$

C 0.6005143 .4365272 .603611

C -0.6524343 .9276671 .858240$

C -1.6407194 .6702752 .757035$

C -2.890923 5.1105731 .980284

O

O -3.8849985 .5115432 .936316$

C -3.4650573 .8973141 .213758$

C $-2.6147406 .338119 \quad 1.102956$

O $-4.532963 \quad 4.3160930 .367778$

C -2.4036413 .1194010 .404852$

C $-2.9202411 .755711-0.105841$

O $-1.9789473 .900607-0.712655$

C $-1.341490-1.4587162 .424274$

O $-1.405188-0.371421 \quad 1.874830$

C $-2.424068-2.4673112 .448193$

C $-3.541806-2.2539451 .736973$

C $-4.707203-3.1383681 .664281$

C $-4.667584-4.4073002 .475877$

C $-4.0252901 .791416-1.160534$

C $-4.2160400 .440005-1.832363$

C $-5.636797-0.683295-3.544293$

O $-3.519304-0.541910-1.590224$

C $-6.679688-1.465478-2.810003$

C $-6.554292-2.767057-2.509457$

C $-7.622779-3.571356-1.827739$

C $-7.128285-4.280286-0.587159$

C $-6.849696-5.594689-0.610203$

C $-7.059525-3.4942820 .701950$

C $-5.748345-2.7778380 .883151$

N $-5.2545420 .469634-2.752183$

H $2.756086-1.2036271 .253262$

H $2.207876-2.8604013 .767435$

H $0.903001-0.3931762 .405035$

H $1.061488-1.1779585 .376606$

H $-0.122927-0.0766014 .782942$

H 2.3714082 .7218114 .550371

H 3.8654561 .2095965 .412533

H $3.097481-0.1322916 .263992$

H $3.753793-0.3665254 .629499$

H -0.3466151 .7018353 .477480$

H -0.1923863 .0656884 .585325$

H 1.1734834 .3067762 .948402

H 1.2342832 .8982501 .885617

H $-0.3004274 .589781 \quad 1.058097$

H - 1.1598335 .5308153 .238085

H -1.9739994 .0144653 .571764$

H -3.510384 6.2299613 .474359

H -3.901832 3.2124111 .953836

H -2.262869 7.1758101 .717131

H -3.5305496 .6900790 .615352$

H $-1.862664 \quad 6.1548720 .332719$

H -5.1826564 .7758320 .926611$

H $-2.0594031 .213065-0.518270$ 
H $-3.256222 \quad 1.1508030 .745915$ H -1.581115 3.302494 -1.367018 H -2.271224 -3.355904 3.049937 H -3.618515 -1.338559 1.152635 H -3.809790 -5.023668 2.183852 H -5.552357 -5.0345792 .350481$ H -4.588714 -4.1777633 .544255$ H $-4.9769072 .073149-0.697615$ H -3.787955 $2.518242-1.945083$ H -4.747031 -1.283454 -3.762443 H $-6.046310-0.311271-4.488428$ H -7.593446 -0.941170 -2.540182 H -5.649809-3.295639-2.802405 H -7.996288 -4.305446 -2.553829 H -8.488826 -2.949470 -1.569189 H $-6.530918-6.1210590 .283043$ H $-6.930500-6.173614-1.524402$ H -7.279089 -4.146029 1.554802 H -7.867412 $-2.751400 \quad 0.721691$ H $-5.679787-1.8436570 .325156$ H $\quad-5.819586 \quad 1.309840-2.834834$ C $5.358181-2.1600081 .462645$ O $5.205489-1.382240 \quad 0.259694$ C $6.423790-1.445552-0.502738$ C $7.445093-2.1522050 .382596$ C $6.558840-3.0676821 .205481$ H $6.215653-2.073984-1.376470$ C $6.825673-0.037525-0.953943$ H $8.195392-2.692442-0.202987$ H $7.975241-1.4500891 .036364$ C $7.225877-3.5860102 .467441$ H $\quad 6.259215-3.9193260 .580029$ C $5.7159560 .732633-1.701200$ $\begin{array}{lllll}\text { O } & 7.177791 & 0.740867 & 0.199030\end{array}$ H $7.731377-0.088127-1.568691$ H $4.8959320 .961220-1.011527$ O $6.2649831 .996929-2.099307$ C $5.1674670 .022397-2.955681$ C $4.1741610 .884012-3.761691$ H $4.689535-0.922060-2.674144$ O $6.271563-0.297675-3.806393$ H $4.6041291 .879632-3.923501$ H $4.0638860 .442536-4.761621$ H $8.119517-4.1677502 .218861$ H $7.530917-2.7658273 .125845$ H $6.547175-4.2346853 .030036$ C $4.063541-2.9231391 .742250$ H 6.4072450 .7510900 .792743 H $6.6067812 .429712-1.298168$ H $5.924751-0.808646-4.557391$ H $4.200428-3.6001412 .592831$ H $3.802429-3.5338480 .869298$ H $5.570153-1.4548412 .277146$ C $0.752906-0.328182-2.877498$ O $2.086521-0.221301-3.396136$ C $2.7895770 .992428-3.098886$ C $2.0098302 .209273-3.618171$ C $0.5788522 .219150-3.075087$ C $-0.1142540 .873650-3.291350$ H $0.337321-1.218137-3.368063$ C $0.756630-0.589068-1.365749$ H $2.9071431 .082113-2.015328$ O $1.9871152 .218577-5.043860$ H $2.5300763 .121157-3.302096$ H $0.0015113 .005791-3.576148$ O $0.5990452 .504816-1.679569$ H -1.067392 $0.851411-2.751967$ O $-0.4247860 .755195-4.682808$ C $1.116793-2.033127-1.094562$ H $1.4642300 .032429-0.811049$ H $-0.239618-0.399431-0.950389$ H $1.5937141 .380426-5.341011$ H $1.0086273 .379142-1.563991$ H -0.935582 $-0.063771-4.800892$ O $0.095395-2.652754-0.436925$ O $2.173727-2.549665-1.433660$ C $0.295016-4.039918-0.148626$ H $-0.594076-4.4109940 .368001$
H $\quad 0.422317-4.604601-1.077055$

H $1.161146-4.1724620 .505419$

SCF Energy (B3LYP/6-31G**//MMFF) $=-3245.91521115$

07_00319

MM̄FF Geometry

C $0.482966-0.8423903 .212247$

C $-0.536310-0.3224203 .912483$

C $-1.233848 \quad 0.957674 \quad 3.537747$

$\begin{array}{llll}\text { O } & -2.631197 & 0.668602 & 3.280157\end{array}$

C $-1.206564 \quad 1.9863334 .680258$

C 0.0705032 .7935564 .740561

C $0.235807 \quad 3.9769214 .118035$

C 1.1654012 .2318735 .607879

C $-0.7518194 .682323 \quad 3.227061$

C -0.0913095 .2742121 .974216$

C $0.344716 \quad 4.220753 \quad 0.942110$

C $1.1776944 .891318-0.150465$

C $1.5622173 .905925-1.255648$

O $-0.8338893 .643744 \quad 0.371400$

O $2.0946464 .681175-2.340292$

C $0.2881643 .194966-1.756281$

C $2.6871892 .968094-0.803895$

O $0.6500632 .183380-2.692872$

C $-0.6022842 .627515-0.623358$

C $-2.0144822 .232265-1.130857$

O $0.0340011 .484771-0.051738$

C $-2.944107 \quad 0.0738532 .097458$

O $-2.170202-0.3014871 .231963$

C $-4.416425-0.0385382 .033176$

C $-4.980483-0.7732181 .063453$

C -6.416224 $-0.973800 \quad 0.861377$

C $-7.356583-0.1590051 .711779$

C $-2.0679591 .084802-2.141031$

C $-3.478105 \quad 0.637522-2.492675$

C $-5.5142041 .419181-3.701127$

O $-3.822271-0.538540-2.392189$

C $-6.608707 \quad 1.005337-2.767422$

C $-7.4517080 .001933-3.058223$

C $-8.624530-0.408988-2.213747$

C $-8.594404-1.877515-1.851691$

C $-8.952514-2.818552-2.742090$

C $-8.226789-2.248735-0.434097$

C $-6.819067-1.857872-0.075402$

$\mathrm{N}-4.269148 \quad 1.661225-2.993561$

H $0.857086-0.3140142 .338830$

H $-0.916794-0.8584484 .778707$

H $-0.805713 \quad 1.4063242 .633344$

H -1.3822301 .4950235 .647386$

H -2.065641 2.6628024 .580359

H 1.1766564 .5051614 .266771

H 2.0262922 .9056215 .677383

H 0.7971642 .0659006 .625553

H 1.5272821 .2804925 .208580

H - 1.5793134 .0324682 .927065

H $-1.1863565 .507123 \quad 3.805240$

H -0.8188465 .9504481 .506008$

H 0.7695865 .8854842 .273367

H $0.9253493 .437328 \quad 1.441340$

H 2.0748705 .3597330 .273740

H $0.5988285 .710288-0.600891$

H $2.2788004 .071159-3.075481$

H $-0.2970443 .935638-2.317902$

H $3.5635943 .546919-0.489302$

H $3.0286142 .339220-1.631676$

H 2.4000272 .3192850 .026326

H $0.9431111 .406406-2.186911$

H -2.633339 $1.957840-0.266131$

H $-2.498813 \quad 3.126758-1.542941$

H -0.6496590 .9166940 .340072$

H -4.9948150 .4828622 .787274$

H $-4.332176-1.2888830 .356244$

H -7.283269 -0.4608762 .762312$

H -8.404471 -0.2528491 .421096$

H -7.1183880 .9081351 .634907$

H - $1.5915321 .368492-3.084560$

H $-1.5333000 .211470-1.750611$

H $-5.3240830 .667911-4.475445$ 
H $-5.7909812 .359886-4.188234$ H $-6.7415531 .574027-1.850983$ H -7.321699 $-0.532323-3.997522$ H $-9.541140-0.198802-2.780732$ H $-8.6962270 .203464-1.306822$ H -8.959818 $-3.871829-2.482161$ H $-9.246100-2.554139-3.752808$ H $-8.305135-3.334698-0.291373$ H -8.972127 -1.8200260 .241169$ H $-6.058691-2.394488-0.644747$ H -3.822782 2.554666 -3.176189 C $0.915079-3.2380772 .495038$ O $1.507857-2.8586421 .236923$ C $0.619531-3.2535870 .174616$ C $-0.415690-4.1799690 .807096$ C $-0.543200-3.5872712 .195394$ H $\quad 0.114963-2.341890-0.167075$ C $1.399668-3.887082-0.981712$ H -1.359449 -4.1853460 .253216$ H $-0.055208-5.2133770 .868537$ C -1.174951 -4.5250263 .208677$ H -1.157469 -2.683244 2.121035 C $2.518628-3.012303-1.581578$ O $2.010853-5.102889-0.527144$ H $0.700013-4.183961-1.771926$ H $3.358957-2.948705-0.881008$ O $3.014588-3.693157-2.742597$ C $2.087365-1.595891-2.000738$ C $3.107077-0.841669-2.876138$ H $1.874821-1.001929-1.104985$ O $0.864308-1.663428-2.740749$ H $3.188281-1.327668-3.857146$ H $2.678808 \quad 0.147737-3.079878$ H -2.191660 -4.792924 2.902802 H $-0.600200-5.4514173 .312132$ H -1.232369 -4.0514454 .193854$ C $1.142293-2.1496913 .549082$ H $2.583238-4.8784780 .226749$ H $3.296091-4.580322-2.459691$ H $1.021952-2.226023-3.518425$ H $2.217478-1.9575393 .643571$ H $0.798733-2.5137324 .524660$ H $1.452361-4.1391532 .819068$ C $5.5735290 .180714-0.260632$ O $4.363708-0.018519-1.003691$ C $4.505736-0.685340-2.259793$

C $5.4520210 .095980-3.187397$ C $6.798430 \quad 0.366811-2.507631$ C $6.6189100 .939366-1.098681$ H $5.283806 \quad 0.8256020 .578693$ C $6.110382-1.1356570 .323335$ H $4.920266-1.684234-2.093514$ O $4.849309 \quad 1.334699-3.557435$ H $5.600596-0.483552-4.106419$ H $7.3776891 .077870-3.109514$ O $7.547459-0.841858-2.425741$ H $7.5810660 .948683-0.573075$ O $6.2009512 .301213-1.228013$ C $5.173816-1.7348211 .350532$ H $6.263393-1.889978-0.452704$ H $7.065839-0.9481340 .826912$ H $5.4609411 .791511-4.159595$ H $7.676182-1.169782-3.332085$ H $6.1421432 .677756-0.333516$ O $5.685994-2.9320851 .764395$ O $4.133979-1.2247651 .742423$ C $4.910458-3.6360672 .739000$ H $5.497309-4.4900483 .087437$ H $4.687219-2.9936213 .596019$ H $3.989886-4.0070612 .280983$ SCF Energy (B3LYP/6-31G**//MMFF) $=-3245.92461504$

\section{00320}

MM̄FF Geometry

C $1.375295-3.5884491 .453730$

C $1.624251-2.9036172 .581978$

C $0.592963-2.0874583 .323275$

O $-0.131048-1.2621332 .381156$
C $-0.394857-3.0069924 .064037$

C $-1.506880-2.2582914 .770210$

C $-2.772852-2.1625604 .315674$

C $-1.119511-1.6179476 .077323$

C $-3.333595-2.7166943 .033086$

C $-3.864471-1.5939202 .140554$

C $-4.316938-2.0664980 .749980$

C $-3.147132-2.540920-0.120711$

C $-3.574949-2.843206-1.564945$

O $-4.954224-0.9376730 .137512$

O $-2.387315-2.934061-2.367585$

C $-4.392410-1.653351-2.106948$

C $-4.277129-4.201946-1.674870$

O $-4.932001-1.951172-3.392880$

C $-5.517757-1.196387-1.152273$

C $-6.1538730 .117307-1.673872$

O $-6.521301-2.213667-1.100751$

C $0.414820-0.0505402 .080364$

O 1.4490930 .4207592 .526457

C $-0.4702840 .615913 \quad 1.099879$

C -0.1972321 .8662120 .700290$

C $-0.9628852 .656152-0.268692$

C $-2.1902882 .026113-0.871738$

C $-7.1500490 .776324-0.709260$

C -6.4898901 .6299960 .357908$

C -5.4499753 .8466930 .730563$

O $-6.464461 \quad 1.2872861 .537885$

C $-4.7550684 .888468-0.084387$

C -3.5751065 .4348890 .246179$

C $-2.9030776 .487413-0.589151$

C $-1.4429936 .202189-0.858118$

C $-0.4991087 .110938-0.557007$

C $-1.0776494 .900347-1.539462$

C $-0.5181363 .893993-0.570071$

N $-5.9535742 .805009-0.146716$

H $0.364854-3.5836351 .054941$

H $2.621779-2.9275243 .013434$

H $1.103090-1.4481274 .054776$

H $\quad 0.149735-3.6079854 .805380$

H $-0.815322-3.7410633 .369416$

H -3.494599 -1.6151734 .920853$

H -1.966719 -1.125961 6.566866

H $-0.731980-2.3724906 .769631$

H $-0.346020-0.8601075 .918890$

H -2.590994 -3.301696 2.490886

H $-4.151292-3.4044763 .279339$

H -4.712976 -1.105723 2.640058

H $-3.111220-0.8046542 .027144$

H $-5.056487-2.8655880 .877174$

H $-2.656363-3.4203680 .311139$

H $-2.378300-1.758925-0.152489$

H -1.832605 -3.644193 -2.002111

H $-3.697380-0.813767-2.249328$

H $-3.603005-5.007684-1.359728$

H $-4.545357-4.425982-2.713023$

H $-5.177699-4.269849-1.061186$

H -4.191622 -2.224276 -3.961391

H $-5.3592390 .835497-1.910406$

H $-6.690929-0.098256-2.606283$

H $-7.387255-1.809380-0.938227$

$\mathrm{H}-1.3210880 .0615580 .728061$

H 0.6794482 .3616691 .116442

H -1.922185 $1.124058-1.433161$

H -2.721852 $2.682082-1.563802$

H $-2.9062901 .750812-0.089365$

H $-7.8186321 .432718-1.281561$

H -7.796807 $0.044915-0.214835$

H -4.7961643 .3914041 .482179$

H $-6.3120554 .285741 \quad 1.243689$

H $-5.2555885 .226472-0.989240$

H -3.0828785 .1305631 .166678$

H -3.014041 $7.445799-0.065787$

H -3.412644 6.602551-1.554340

H $\quad 0.5488806 .925950-0.770027$

H $-0.7508778 .058004-0.091512$

H $-0.3051085 .095858-2.295543$

H -1.933760 $4.523742-2.105438$

$\mathrm{H} \quad 0.3930354 .239284-0.078222$ 
H $-6.1897173 .059785-1.101910$

C $2.463173-4.139273-0.787763$

O $2.723720-2.749333-1.064125$

C $1.992145-2.377635-2.249267$

C $1.438116-3.673006-2.837901$

C $1.213375-4.500483-1.588417$

H $1.148714-1.761168-1.916901$

C $2.879818-1.585005-3.214363$

H $0.523373-3.506830-3.415259$

H $2.164895-4.164417-3.495140$

C $1.054893-5.985543-1.860647$

H $0.308305-4.130661-1.093364$

C $3.463953-0.280411-2.633800$

O $3.978607-2.417531-3.607775$

H $2.315407-1.369682-4.129932$

H $4.226254-0.521824-1.885269$

$\begin{array}{llllll}\text { O } & 4.157826 & 0.385082 & -3.697898\end{array}$

C $2.4238110 .680615-2.032541$

C $2.9492102 .094924-1.729392$

H $2.0125790 .239070-1.118185$

O $1.3386640 .817673-2.956202$

H $3.2011632 .610244-2.665293$

H $2.1084712 .659592-1.310960$

H $0.189482-6.167367-2.506425$

H $1.938303-6.399661-2.358197$

H $0.901753-6.538631-0.928675$

C $2.406618-4.3985930 .720964$

H $4.529107-1.900103-4.220445$

H $3.4949010 .655516-4.356337$

H $0.6562291 .362616-2.528556$

H $3.396853-4.1876981 .143038$

H $2.203987-5.4609710 .898471$

H $3.323360-4.693435-1.187644$

C 4.8107591 .3839301 .435004

$\begin{array}{lllll}\text { O } & 3.787683 & 1.459135 & 0.431854\end{array}$

C $4.1421632 .157580-0.763967$

C $4.5323773 .611524-0.443161$

C $5.620808 \quad 3.6808190 .631305$

C 5.2893562 .7899041 .834520

H 4.3162190 .9404812 .308794

C $5.9604560 .451761 \quad 1.021095$

H $4.9907531 .654494-1.237793$

O $3.3940344 .349597 \quad 0.000247$

H $4.8874354 .093185-1.361872$

H 5.7280394 .7212170 .961855

$\begin{array}{lllll}\text { O } & 6.858583 & 3.280063 & 0.052195\end{array}$

H 6.1545462 .7039952 .502143

$\begin{array}{lllllllll}\text { O } & 4.237713 & 3.399411 & 2.589138\end{array}$

C $5.511244-0.984858 \quad 0.865826$

H 6.4039260 .7571090 .070029

H 6.7393710 .4659311 .792149

H $3.0165003 .882410 \quad 0.765201$

H 7.5420223 .3539960 .739950

H 4.5553734 .2697062 .884502

O $6.545686-1.715213 \quad 0.352711$

O $4.405781-1.4153671 .160985$

C $6.277558-3.1040940 .138188$

H $5.541731-3.221815-0.661816$

H $7.209271-3.586363-0.169078$

H $5.928227-3.5763701 .061333$

SCF Energy (B3LYP/6-31G**//MMFF) $=-3245.90172172$

07_00321

MM̄FF Geometry

C -1.5608991 .9906601 .868971$

C $-2.6053832 .735296 \quad 1.479038$

C -2.848643 $3.112104 \quad 0.043260$

O $-4.0209872 .363505-0.353692$

C $-3.0832494 .619750-0.156408$

C -1.992586 5.5294090 .371900

C $-0.7437635 .612550-0.128193$

C $-2.3978596 .397451 \quad 1.534829$

C $-0.1567974 .833667-1.273764$

C $0.9409383 .888560-0.765245$

C $1.4326802 .853545-1.787498$

C $2.0915173 .487993-3.011755$

C $2.5472802 .422437-4.021633$

O $0.3195612 .048729-2.188743$
O $2.855326 \quad 3.088119-5.256049$

C $1.3693771 .466399-4.305023$

C $3.8351041 .721053-3.576174$

$\begin{array}{llllll}\text { O } & 1.786671 & 0.371647 & -5.118658\end{array}$

C $0.6654630 .939053-3.029775$

C $-0.6413760 .206929-3.428494$

O $1.5314740 .026093-2.359589$

C $-4.2002122 .108758-1.673126$

O $-3.6166192 .642905-2.606169$

C $-5.1812691 .016986-1.883497$

C $-5.5578700 .178393-0.904424$

C $-6.413741-1.002406-1.046903$

C $-6.919403-1.335839-2.425512$

C $-1.562866-0.170670-2.262085$

C $-1.285702-1.514713-1.615776$

C $-2.128729-2.948754 \quad 0.250458$

O $-0.474638-2.321927-2.057786$

C $-3.403001-3.660182-0.085112$

C $-4.321114-4.009786 \quad 0.828821$

C $-5.592209-4.7426800 .502946$

C $-6.824110-4.0410491 .032868$

C $-7.355877-4.3847482 .218263$

C $-7.488962-3.0015440 .153016$

C $-6.689661-1.732050 \quad 0.055154$

N -2.086619 $-1.706586-0.497030$

H $-0.853957 \quad 1.6351891 .122963$

H -3.3307723 .0718812 .215601$

H -2.007010 $2.775658-0.571136$

H $-4.046093 \quad 4.8915860 .298869$

H -3.232249 $4.833929-1.222547$

H -0.0512206 .3144310 .334991$

H -1.5787847 .0333601 .887247$

H $-3.225767 \quad 7.053607 \quad 1.246639$

H -2.720851 5.7783102 .377864

H $-0.9273344 .282916-1.819886$

H $0.2703515 .548438-1.986716$

H $1.7944824 .477771-0.406054$

H $0.5542613 .338703 \quad 0.101673$

H $2.1549482 .216092-1.263139$

H $2.9344874 .124249-2.715495$

H $1.3772454 .149366-3.519697$

H $3.5494573 .745542-5.076818$

H $0.6322212 .027620-4.896524$

H $4.6444142 .449969-3.448773$

H $4.1848941 .014929-4.337341$

H $3.7236091 .183775-2.633557$

H $2.2054630 .744605-5.913306$

H $-1.2226100 .883896-4.068921$

H $-0.408591-0.684858-4.022313$

H $1.001686-0.527573-1.765532$

H $-5.5023340 .882402-2.911024$

H $-5.1736040 .339246 \quad 0.101642$

H -7.473383 -0.489763-2.847717

H $-7.604537-2.185792-2.445921$

H $-6.084380-1.577445-3.092208$

H $-1.5603450 .603272-1.489590$

H $-2.582913-0.242143-2.659056$

H -2.065668 -2.6912131 .312356$

H $-1.271594-3.576329-0.010475$

H $-3.570022-3.910183-1.130328$

H $-4.139955-3.7789641 .876300$

H $-5.519910-5.746067 \quad 0.942935$

H $-5.698457-4.901938-0.577321$

H $-8.254744-3.9083872 .595257$

H $-6.900267-5.1479612 .840652$

H $-8.475546-2.7414490 .557651$

H -7.672204 $-3.458819-0.823346$

H $-6.303975-1.3817721 .013939$

H $-2.746900-0.980408-0.234059$

C -0.0057942 .1663013 .827119$

$\begin{array}{lllll}\text { O } & 1.115604 & 1.695272 & 3.052546\end{array}$

C 2.1643521 .2885673 .947762

C 1.7894291 .8454535 .315708

C $0.278053 \quad 1.752246 \quad 5.271232$

H 2.1313340 .1940453 .991672

C 3.5307951 .7420823 .425894

H 2.2326221 .2698956 .133991

H 2.0995572 .8903515 .430702 
C $-0.415572 \quad 2.6166796 .309835$ H -0.0119730 .7041325 .426299$ C 3.8516291 .3282951 .975831 O 3.6091353 .1733893 .480789 H 4.3148571 .3759734 .099010 H 3.2734551 .9390741 .271195 O 5.2299101 .6462951 .736466 C $3.626070-0.1594391 .648157$ C $4.206792-0.5375290 .271416$ H $2.550928-0.3685321 .669863$ O $4.245574-0.9542922 .658339$ H $3.9826270 .256562-0.449201$ H $5.296849-0.6176760 .367328$ H -0.1230112 .3129757 .320378$ H $-0.157506 \quad 3.6741376 .188810$ H -1.5033542 .5244206 .231425$ C -1.3057441 .5692353 .285974$ H 2.8752633 .5276002 .949464 H 5.3474872 .5918291 .932136 H $4.058874-1.8863142 .454253$ H $-2.145194 \quad 1.8563143 .929670$ H -1.2486170 .4743693 .295833$ H -0.0196083 .2600123 .731155$ C $3.463002-4.2119580 .289532$ O $3.974110-2.9161630 .632279$ C $3.653056-1.865567-0.281933$ C $4.225422-2.181276-1.673894$ C $3.764509-3.554678-2.172857$ C $3.945956-4.640969-1.107641$ H $3.911638-4.8898431 .026895$ C $1.940671-4.2899170 .452378$ H $2.564303-1.767494-0.347072$ O $5.651671-2.145463-1.644563$ H $3.904787-1.405255-2.377063$ H $4.339819-3.829546-3.065535$ O $2.390567-3.495235-2.541965$ H $3.431289-5.558926-1.415157$ O $5.341304-4.947767-1.029736$ C $1.548032-4.0831651 .895494$ H $1.406556-3.561934-0.164118$ H $\quad \begin{array}{llll}1.566325 & -5.276357 & 0.154227\end{array}$ H $5.951475-2.786074-0.977112$ H $2.297686-2.810885-3.226509$ H $5.445248-5.687376-0.407052$ O $1.040013-2.8298372 .066164$ O $1.696626-4.9277492 .769283$ C $0.644546-2.5037803 .401366$ H $0.228909-1.4935663 .394125$ H -0.126601-3.197055 3.750615 H $1.512661-2.5214994 .066873$

SCF Energy $($ B3LYP/6-31G**//MMFF $)=-3245.92562889$

\section{$07 \quad 00322$}

MM̄FF Geometry

C $-0.216252-4.453877-1.740811$

C $0.936404-4.214165-1.097945$

C $2.044200-3.369730-1.672806$

O $2.163671-2.189199-0.841135$

C $3.384038-4.124041-1.646779$

C $4.566202-3.291686-2.111669$

C $5.706056-3.143152-1.408321$

C $4.428021-2.622011-3.454834$

C $6.068561-3.803995-0.109599$

C $5.767568-2.9744551 .145772$

C $6.550501-1.6537301 .212522$

C $6.645229-1.1582902 .657724$

C 7.3698370 .1918142 .749319

O $5.863186-0.6798950 .416538$

$\begin{array}{lllll}\text { O } & 7.152111 & 0.721293 & 4.065827\end{array}$

C 6.7167371 .1696151 .754233

C 8.8870070 .0444422 .585393

O 7.4443902 .3943721 .689001

C 6.5325420 .5853830 .332251

C $5.6585141 .551815-0.500292$

O $7.8097640 .460363-0.296784$

C $1.276758-1.185299-1.087334$

O $0.420572-1.161371-1.958356$

C $1.508277-0.111032-0.097640$
C $0.6176660 .889320-0.027455$

C 0.6801992 .0637040 .842428

C 1.8329802 .1709551 .802590

C $5.1209170 .966791-1.812033$

C $4.4871172 .049452-2.667594$

C $2.6299933 .714779-2.685153$

O $4.9501462 .356033-3.764088$

C $2.1759444 .649339-1.607589$

C $0.9075995 .061422-1.458648$

C $0.4573495 .991305-0.365941$

C -0.7015695 .4400260 .438432$

C $-1.9327735 .967357 \quad 0.329545$

C -0.4203434 .3171561 .418150$

C $-0.2925282 .990466 \quad 0.722244$

N $3.3798252 .632730-2.073203$

H $-0.354842-4.080475-2.752427$

H $1.056238-4.556337-0.072551$

H $1.814951-3.073558-2.704422$

H $3.315437-5.014204-2.285409$

H $3.552136-4.484780-0.626234$

H $6.488919-2.509343-1.823033$

H $5.370477-2.180873-3.797317$

H $4.116254-3.350094-4.210811$

H $3.687141-1.817704-3.413854$

H $5.574800-4.778005-0.019797$

H $7.141113-4.036789-0.138324$

H $4.689612-2.7760041 .199755$

H $6.028739-3.5979832 .010104$

H $7.554769-1.8176060 .804791$

H $7.144214-1.9005343 .293283$

H $5.633194-1.0408473 .070318$

H 7.4968270 .0776674 .708374

H $5.724121 \quad 1.4214682 .154188$

H $9.292311-0.6212793 .356805$

H 9.3938841 .0058492 .723634

H $9.176620-0.361147 \quad 1.613752$

H 7.5236582 .7329942 .597298

H $4.794819 \quad 1.854745 \quad 0.102964$

H $6.2276392 .463986-0.722611$

H $7.6958370 .516169-1.258755$

H $2.394250-0.1645610 .523776$

H $-0.2455510 .858703-0.692299$

H 1.9002491 .2734792 .427722

H 1.7455173 .0136582 .491867

H 2.7751722 .2912271 .259576

H $5.9224040 .504692-2.397787$

H $4.3717630 .191261-1.618534$

H $1.792043 \quad 3.265772-3.227923$

H $3.2660424 .245471-3.400358$

H $2.9336505 .008239-0.914493$

H $0.1518674 .722096-2.163656$

H $0.1730036 .941621-0.836039$

H 1.2815626 .2304750 .317666

H -2.7632435 .5890520 .916611$

H -2.139926 $6.784277-0.353636$

H -1.2408194 .2347692 .142550$

H $\quad 0.4683114 .581599 \quad 1.997897$

H -1.100316 2.7946090 .016024

H $2.9857142 .194622-1.246296$

C $-2.460041-4.065541-0.759981$

O $-1.942532-3.1755200 .250963$

C $-2.997680-2.8557101 .182205$

C $-4.273289-3.4246930 .578342$

C $-3.748031-4.633665-0.172511$

H $-2.744790-3.3813122 .111289$

C $-3.002896-1.3393531 .407421$

H -5.019288 -3.680898 1.336274

H $-4.725975-2.709275-0.119427$

C $-4.719411-5.170695-1.208638$

H $-3.514954-5.4256110 .551741$

C $-4.183326-0.8373332 .255046$

O $-1.783838-0.9860352 .070512$

H -2.985554 -0.8321170 .436919$

H $-5.127832-1.0555591 .751003$

O $-4.225146-1.585993 \quad 3.478677$

C -4.1293130 .6570992 .622535$

C -3.9389611 .6432011 .454941$

H -3.3196920 .8150243 .346014$ 
O $\quad \begin{array}{llll}-5.341624 & 0.979499 & 3.312760\end{array}$ H -3.804675 2.6426931 .889714 H $-3.009729 \quad 1.3960670 .931662$ H -5.650130 -5.494533 -0.731461 H -4.972044 -4.409508 -1.954596 H $-4.292908-6.030077-1.735201$ C -1.401400 -5.118211-1.096920 H -1.050712 -1.312196 1.520525 H -3.375280 -1.452936 3.932522 H -5.2792351 .9076223 .596056$ H -1.802897 $-5.867725-1.787204$ H $-1.090125-5.642343-0.184887$ H $-2.681288-3.450051-1.642547$ C $-6.2505010 .381046-1.215729$ O C -5.1098831 .7260810 .453564$ C $-4.9211242 .896280-0.528030$ C $-6.0125082 .904615-1.603270$ C $-6.1904191 .523744-2.242701$ H -6.048322 $-0.556814-1.749309$ C $-7.6186250 .249840-0.533973$ H -6.0365551 .8764181 .016826$ O $-3.6419712 .795092-1.151044$ H -4.9387213 .8363280 .036551$ H $-5.7529923 .630739-2.383248$ O $-7.2504953 .312270-1.028709$ H -7.092769 $1.501145-2.864897$ O $-5.080056 \quad 1.263014-3.106257$ C -7.715658 -1.042594 0.244707 H $-7.825881 \quad 1.0850570 .140549$ H $-8.4257160 .235136-1.275621$ H -3.532949 $3.571041-1.726705$ H -7.122807 $4.200588-0.654605$ H $-5.0694841 .956447-3.787730$ O $-7.969541-0.777846 \quad 1.558149$ O $-7.584618-2.153272-0.253424$ C -8.086331 -1.9234792 .407815$ H -8.955039 -2.520600 2.115564 H -7.173555 -2.525039 2.368024 H -8.229035 -1.572270 3.433180

SCF Energy (B3LYP/6-31G**//MMFF) $=-3245.89942826$

\section{7 _00323}

MM̄FF Geometry

C $1.751904-2.912091 \quad 1.230197$

C $0.672390-2.1471921 .008382$

C $0.216683-1.0248281 .898629$

O -0.1105050 .0644440 .999382$

C -1.047451-1.400658 2.694781

C $-0.893670-2.5927203 .616877$

C $-1.481099-3.7872353 .403089$

C $-0.069038-2.3551784 .853234$

C $-2.306012-4.1658272 .204716$

C $-3.798166-3.8909312 .428647$

C $-4.616988-3.806444 \quad 1.130545$

C $-4.587909-5.103106 \quad 0.320247$

C -5.355594 -4.961003-1.004396

O $-4.090014-2.7230460 .356855$

O $\quad-5.049129-6.103055-1.819367$

C $-4.859148-3.693582-1.736349$

C $-6.870801-5.020642-0.784789$

O $-5.628166-3.453576-2.913112$

C $-4.817114-2.429531-0.841632$

C $-4.069883-1.290808-1.585090$

O $-6.155028-2.025067-0.558014$

C -0.2296021 .3161191 .510113$

O -0.0360891 .6654792 .662575$

C -0.6715122 .1920480 .401355$

C -1.030566 3.4593520 .651350

C $-1.4920794 .435937-0.338057$

C $-1.6968443 .954403-1.750870$

C -3.787753 -0.030192 -0.752252

C $-4.9384480 .959900-0.697682$

C $-5.6300793 .068870 \quad 0.440265$

O $-5.952451 \quad 0.850628-1.381492$

C $-5.2680284 .239748-0.418151$

C $-5.0771565 .478020 \quad 0.062138$

C $-4.7492436 .665942-0.796535$
C $-3.5182167 .408013-0.325192$

C -3.6255348 .5429690 .386065$

C $-2.1665616 .881431-0.753441$

C -1.7193145 .7032210 .066633$

N $-4.690517 \quad 1.9892990 .200539$

H $2.354399-2.7544302 .120004$

H $\quad 0.066335-2.3374240 .124448$

H $1.027743-0.7058762 .565023$

H $-1.882420-1.5365871 .996541$

H -1.364487 -0.5420673 .301341$

H $-1.347592-4.5771234 .139884$

H $-0.043141-3.2318855 .508946$

H $0.964267-2.1137094 .585493$

H $-0.482703-1.5225865 .431574$

H -1.937194 -3.648782 1.313085

H $-2.152338-5.2335802 .006028$

H -4.219298 -4.662978 3.084392

H -3.910854 -2.931946 2.952222

H $-5.647269-3.5638001 .416829$

H $-4.983101-5.9400950 .908671$

H $-3.549755-5.3687740 .081416$

H -4.089739 -6.117270 -1.976025

H -3.838018 -3.902825 -2.083068

H -7.149771 $-5.969778-0.311198$

H -7.410295 -4.995080 -1.738126

H $-7.246895-4.213247-0.153194$

H -6.477422 -3.068180 -2.637801

H -3.090142 -1.678398 -1.895159

H $-4.615114-1.015868-2.495828$

H $-6.139847-1.117426-0.217875$

H $-0.6951341 .764909-0.594989$

H -0.9759763 .8219671 .676966$

H $-0.7407923 .667015-2.201484$

H -2.150761 $4.700826-2.405562$

H -2.367363 $3.088043-1.770463$

H $-3.482293-0.2907780 .266588$

H $-2.949900 \quad 0.502573-1.219405$

H $-5.584414 \quad 3.307847 \quad 1.507483$

H -6.6440052 .7335060 .199739$

H $-5.1810744 .064864-1.487969$

H -5.1916585 .6569301 .128997$

H -5.618382 $7.336715-0.780254$

H $-4.6184316 .377412-1.846900$

H $-2.7481009 .096694 \quad 0.703437$

H -4.593662 8.9410650 .671879

H -1.404916 $7.664297-0.635376$

H -2.186073 $6.674665-1.826833$

H -1.5428045 .9505691 .114565$

H -3.7908532 .0322670 .670227$

C $3.632549-3.924019-0.131884$

O $3.911188-2.634114-0.713895$

C $4.301933-2.819956-2.080745$

C $4.896875-4.220870-2.126568$

C $3.960617-4.973541-1.203785$

H $3.378334-2.793351-2.672811$

C $5.237984-1.706028-2.564779$

H $4.923087-4.631478-3.140357$

H $5.913876-4.247310-1.719398$

C $4.555677-6.252568-0.638557$

H $3.056994-5.234285-1.770814$

C $4.848079-0.266788-2.173509$

O $6.559448-1.922726-2.048192$

H $5.340055-1.781858-3.653982$

H $5.128033-0.088674-1.129258$

O $5.6605520 .617342-2.959822$

C $3.3739540 .123220-2.369588$

C $3.0882191 .635925-2.247348$

H $2.757780-0.418583-1.643256$

O $2.952640-0.293957-3.670659$

H $3.4677322 .150727-3.139723$

H $1.9972841 .760005-2.278098$

H $4.806280-6.949680-1.444671$

H $5.470319-6.052403-0.070186$

H $3.844535-6.7481260 .030073$

C $2.162601-4.0129160 .295470$

H $6.498689-1.923922-1.077693$

H $6.5894340 .379215-2.796669$

H $2.006775-0.084175-3.753682$ 
H $\quad 1.985712-4.975688 \quad 0.789305$ H $1.524085-3.986588-0.597149$

H $\quad 4.287402-4.0232320 .742104$

C 3.5564902 .1477631 .430905

$\begin{array}{lllll}\text { O } & 3.070309 & 1.685151 & 0.161824\end{array}$

C $3.6537672 .308336-0.984774$

C $3.3858263 .820387-0.967939$

C 3.8971594 .4480210 .333515

C $3.4175943 .676877 \quad 1.565996$

H 2.8919781 .6849832 .171247

C 4.9843771 .6491441 .710117

H $4.7361652 .154253-0.970749$

O $1.9884624 .060108-1.106176$

H $3.8864274 .278130-1.829438$

H $3.5486845 .485901 \quad 0.402644$

$\begin{array}{lllll}\text { O } & 5.321919 & 4.467506 & 0.329185\end{array}$

H $3.957774 \quad 4.0211502 .455813$

$\begin{array}{lllll} & & 2.040428 & 3.998841 & 1.769728\end{array}$

C $5.045153 \quad 0.1434801 .857710$

H $5.672966 \quad 1.9290380 .908772$

H 5.3467832 .0782602 .651253

H $1.8507455 .022412-1.092862$

H $5.6062014 .986895-0.442165$

H 1.7501093 .5537962 .584106

O $6.353193-0.2442561 .925883$

O $4.075900-0.5990101 .921006$

C $6.569510-1.6499532 .083952$

H $6.135487-2.1990591 .243804$

H $7.647939-1.8284222 .098983$

H $6.142744-1.9933523 .031054$

SCF Energy (B3LYP/6-31G**//MMFF) $=-3245.91029445$

\section{7_00324}

MMFF Geometry

C $3.8506973 .826758-0.897979$

C $2.6359123 .756421-0.334629$

C $1.3644924 .156886-1.036312$

$\begin{array}{lllll}\text { O } & 0.447543 & 3.039475 & -0.974453\end{array}$

C $0.7251825 .354225-0.312624$

C $-0.5427295 .851277-0.980019$

C $-1.7916475 .642058-0.517337$

C $-0.3316206 .660557-2.234233$

C $-2.208153 \quad 4.870607 \quad 0.704834$

C -3.057167 $3.657248 \quad 0.322332$

C -3.4733452 .7902171 .520360$

$\begin{array}{llll}\text { C }-2.281218 & 2.105947 & 2.197976\end{array}$

C $-2.719144 \quad 1.123410 \quad 3.294397$

O -4.3732221 .7998031 .004938$

$\begin{array}{llll}0 & -1.589031 & 0.303342 & 3.628411\end{array}$

C -3.804898 0.1887492 .724577

C -3.1197931 .8509304 .583393$

O $-4.342477-0.6493663 .744966$

C -4.9425890 .9399031 .998138$

C $-5.875977-0.0724031 .287306$

O -5.7048681 .6730402 .960901$

C $0.6147342 .051493-1.896792$

O $1.4545252 .006308-2.781838$

C $-0.407643 \quad 1.014411-1.635776$

C $-0.419986-0.105412-2.372879$

C $-1.359242-1.222080-2.238892$

C $-2.469781-1.082636-1.231273$

C -6.8977350 .5680550 .336735$

C $-6.3654790 .757215-1.072468$

C $-5.910640-0.482132-3.174953$

O $-6.0987791 .869108-1.521939$

C $-5.490728-1.869174-3.539671$

C $-4.427408-2.153900-4.306770$

C $-4.024660-3.560203-4.649717$

C $-2.552039-3.834665-4.442090$

C $-1.802872-4.343187-5.435853$

C $-1.958116-3.590751-3.070533$

C -1.171757-2.306953 -3.019309

$\mathrm{N}-6.232694-0.440048-1.759595$

H $3.9508164 .208401-1.910843$

H 2.5393703 .3762050 .679869

H $1.5546104 .417597-2.084889$

H $1.4404346 .187222-0.271742$

H $\quad 0.535274 \quad 5.090638 \quad 0.732863$
H -2.622645 $6.068151-1.078689$ H $-1.2705037 .052898-2.639510$

H $0.3195067 .516524-2.028896$

H $0.1318646 .049255-3.014468$

H -1.345101 4.5527621 .290012

H -2.792215 5.5364551 .351401

H $-3.9649363 .995606-0.196367$

H $-2.5273603 .036203-0.410182$

H $-4.013573 \quad 3.4240512 .233299$

H -1.5842502 .8389892 .618956$

H -1.7082341 .5470891 .446331$

H -0.8681930 .8886743 .917773$

H -3.317499 -0.4782332 .000657$

H -2.272639 $2.424616 \quad 4.978317$

H -3.3914601 .1394395 .370994$

H -3.9496712 .5466094 .443463$

H -3.596004 -1.114754 4.159866

H $-5.269474-0.7959240 .729057$

H $-6.433852-0.6306452 .049820$

H -6.6243521 .7428932 .660596$

H $-1.1199341 .194829-0.841130$

H $0.336846-0.223585-3.147638$

H $-2.060139-0.974607-0.221113$

H $-3.146952-1.938449-1.206520$

H -3.082762 $-0.202276-1.453896$

H $-7.779203-0.0823670 .266652$

$\begin{array}{lllll}H & -7.260959 & 1.533496 & 0.702988\end{array}$

H $-5.1381890 .263685-3.390175$

H $-6.818396-0.208067-3.722177$

H -6.098654 -2.687754 -3.160071

H $-3.832433-1.343002-4.720155$

H $-4.301443-3.737698-5.696805$

H $-4.594280-4.284002-4.052747$

H $-0.751304-4.567822-5.289552$

H $-2.221729-4.545882-6.415784$

H $-1.270039-4.411247-2.825054$

H $-2.741447-3.647823-2.310124$

H $-0.320818-2.300516-3.703014$

H $-6.647940-1.269907-1.344898$

C $5.8346022 .295383-0.972552$

O $5.0309591 .097262-0.997554$

C $5.885338-0.038790-0.772348$

C $7.3178170 .479059-0.852197$

C $7.1593831 .890495-0.327270$

H $5.693810-0.3752650 .252670$

C $5.573778-1.169412-1.757448$

H $8.010083-0.126010-0.258773$

H $7.6882210 .497596-1.883755$

C $8.3243042 .801347-0.674204$

H 7.0487001 .8474230 .764690

C $4.111197-1.657900-1.756901$

O $5.874522-0.714920-3.083851$

H $6.251499-2.009685-1.562393$

H $3.468432-0.911171-2.238564$

O $4.056413-2.816466-2.602366$

C $3.530115-2.010327-0.374188$

C $2.199333-2.781509-0.481166$

H $3.386612-1.0857110 .195560$

$\begin{array}{llllll}\text { O } & 4.479447 & -2.814119 & 0.327148\end{array}$

H $1.580937-2.345368-1.273446$

H $2.427992-3.817856-0.758850$

H $9.2546292 .416041-0.244018$

H $8.4625392 .880899-1.757729$

H $8.1632663 .809511-0.280293$

C $5.1111153 .400408-0.201578$

H $5.680896-1.448427-3.692479$

H $4.569446-3.520132-2.169010$

H $4.122596-2.9812021 .215918$

H $5.760048 \quad 4.278174-0.105712$

H $4.8802193 .052430 \quad 0.812714$

H $5.9900052 .595898-2.017235$

C $1.599150-3.2949053 .184792$

O $2.193326-3.3042321 .880156$

C $1.381742-2.7806350 .827505$

C $0.092275-3.6095770 .695520$

C $-0.659202-3.7249152 .026710$

C $0.273822-4.073942 \quad 3.191929$

H $2.311133-3.8446083 .814483$ 
C $1.481362-1.8767983 .753699$

H $1.131999-1.7382951 .051516$

O $0.397965-4.9228740 .225121$

H $-0.556443-3.147019-0.053444$

H -1.416389-4.512913 1.933445

O $-1.364173-2.5224142 .314443$

H $-0.233420-3.9264554 .152568$

O $0.609120-5.4639653 .103881$

C $2.850878-1.2726203 .956948$

H $0.896430-1.2052753 .120874$

H $0.984829-1.8874934 .731161$

H $1.025084-5.3237560 .851193$

H $-0.829865-1.7637472 .031198$

H $-0.217547-5.9700923 .182705$

O $3.117672-0.3773942 .963846$

O $3.601286-1.5786654 .874493$

C 4.4019890 .2495703 .027584

H $5.192592-0.4989732 .919485$

H 4.4742430 .9584092 .199008

H 4.5124500 .7974803 .968142

SCF Energy (B3LYP/6-31G**//MMFF) $=-3245.91327262$

\section{7_00325}

MM̄FF Geometry

C -1.044422 $3.315751-0.499143$

$\begin{array}{llll}C & -0.085630 & 3.906532 & 0.229221\end{array}$

C $0.779196 \quad 3.154133 \quad 1.205337$

$\begin{array}{llll}\text { O } & 2.153130 & 3.453240 & 0.869775\end{array}$

C 0.4887953 .5816082 .659540

C $-0.956536 \quad 3.4195373 .086979$

C -1.540298 2.2401603 .375493

C -1.7452244 .6990383 .196048$

C -0.9067590 .8769943 .371613$

C $-1.501965-0.0183042 .274866$

C $-1.007859-1.4760022 .311524$

C $-1.587322-2.248093 \quad 3.500083$

C $-1.041812-3.6807323 .576774$

O $0.420737-1.4723472 .359297$

O $-1.373381-4.2099474 .870200$

C $0.496447-3.628803 \quad 3.491419$

C $-1.703855-4.6052922 .550172$

O $1.043760-4.9444293 .422400$

C $1.030261-2.7710462 .318560$

C $2.558976-2.5736032 .483376$

O $\quad 0.760765-3.438748 \quad 1.087814$

C $3.099398 \quad 2.5750851 .299487$

O $2.928066 \quad 1.6042562 .019040$

C 4.3899492 .9719770 .693390

C $5.411798 \quad 2.1029110 .706488$

C $6.734202 \quad 2.306948 \quad 0.112671$

C $7.0223283 .644242-0.517143$

C $3.214743-1.6784231 .422932$

C $3.798357-2.4311020 .238079$

C $5.132157-2.005057-1.835316$

O $3.720792-3.6485810 .103935$

C $6.594382-1.776398-1.615027$

C $7.330439-0.921560-2.341225$

C $8.795215-0.676305-2.114319$

C $9.1028910 .778856-1.833020$

C $9.5113911 .596636-2.818125$

C $9.0212891 .266372-0.402830$

C 7.6192821 .2883450 .140433

N $4.421094-1.565313-0.649951$

H $-1.1717482 .238654-0.434855$

H $\quad 0.030813 \quad 4.9868390 .189243$

H $0.6190992 .075321 \quad 1.086338$

H $\quad 0.802818 \quad 4.625624 \quad 2.799761$

H 1.1358003 .0251043 .349290

H -2.588497 2.2450633 .672222

H -2.786532 4.5229733 .486500

H -1.297298 5.3538073 .950810

H -1.757482 5.2311502 .240254

H 0.1789200 .9399253 .251636

H -1.081196 0.4380294 .359604

H $-2.598420-0.0045552 .318261$

H -1.2218560 .4087241 .303428$

H -1.327635 -1.9474851 .374753$

H $-2.683561-2.2552183 .459856$
H $-1.326897-1.7436214 .439218$

H $-2.340585-4.1785744 .967588$

H $0.861254-3.1912474 .431590$

H $-2.788475-4.6399942 .708051$

H -1.351792 -5.6367532 .661888$

H $-1.531080-4.2916681 .520240$

H $0.696608-5.4428494 .182014$

H $2.729097-2.0870963 .453352$

H $3.065834-3.5449342 .515740$

H $1.329634-3.0634560 .397705$

H 4.4505353 .9462000 .222388

H 5.2630551 .1343461 .182462

H $6.3253263 .840718-1.339289$

H $8.0280933 .718256-0.936758$

H $6.928166 \quad 4.4461210 .223588$

H $2.514134-0.9246341 .049971$

H $4.052503-1.1505361 .896912$

H $4.746127-1.424446-2.679000$

H $4.941371-3.065603-2.025360$

H $7.066875-2.340659-0.814393$

H $6.857732-0.367615-3.149310$

H $9.332401-1.005375-3.013624$

H $9.184894-1.295671-1.296906$

H $9.7637962 .633840-2.625017$

H $9.5988051 .247182-3.841688$

H $9.4875612 .252728-0.294273$

H 9.6281330 .6068610 .231340

H 7.3197860 .3505870 .609352

H $4.434065-0.571495-0.439688$

C $-3.4281503 .926007-0.679436$

O $-3.924467 \quad 2.586494-0.870306$

C $-5.3652972 .636094-0.918842$

C $-5.7535234 .103197-0.747878$

C $-4.5159084 .819956-1.259962$

H $-5.6602512 .315788-1.923547$

$\begin{array}{llll}\text { C }-5.945032 & 1.689223 & 0.140998\end{array}$

H $-6.6583354 .357231-1.308871$

H -5.9370684 .3524420 .304130$

C $-4.435332 \quad 6.270727-0.819154$

H $-4.5019114 .767608-2.356724$

C $-5.5283600 .214239-0.055086$

$\begin{array}{llll}\text { O } & -5.489286 & 2.108626 & 1.434792\end{array}$

H $-7.036598 \quad 1.7749180 .165552$

H $-4.440426 \quad 0.1350110 .040555$

O $-6.074663-0.550717 \quad 1.026394$

C $-5.994157-0.408278-1.386149$

C $-5.604313-1.888470-1.558128$

H $-5.5998870 .157465-2.235843$

O $-7.421253-0.345887-1.467955$

H -5.979551-2.482136-0.715544

H $-6.138203-2.259864-2.443414$

H $-5.2972726 .833762-1.191495$

$\begin{array}{llll}\text { H } & -4.422468 & 6.358446 & 0.272552\end{array}$

H -3.529677 $6.748028-1.205478$

C -2.051292 $4.061849-1.329844$

H $-4.5181352 .052072 \quad 1.437144$

H $-7.043563-0.5207910 .945883$

H $-7.6694030 .583948-1.603668$

H $-1.7683715 .116752-1.412415$

H -2.054235 $3.633838-2.338810$

H -3.3519544 .0902360 .404651$

C $-2.279738-1.389208-3.165840$

O $-3.685736-1.367413-2.890343$

C $-4.094378-2.113298-1.739381$

C $-3.748878-3.598892-1.930757$

C $-2.263180-3.792582-2.251893$

C $-1.775961-2.831957-3.344548$

H $-2.189646-0.880022-4.133776$

C $-1.515167-0.562221-2.122446$

H $-3.571767-1.732698-0.855612$

O $-4.531705-4.161020-2.983011$

H $-4.004129-4.143235-1.014018$

H $-2.102450-4.828919-2.573392$

O $-1.512946-3.589033-1.060701$

H $-0.681752-2.835833-3.403439$

O $-2.264115-3.293734-4.608761$

C $-0.153911-0.121613-2.611001$

H $-2.0859260 .342611-1.882893$ 
H $-1.392237-1.120032-1.190223$

H $-4.365500-3.640444-3.787456$

H $-0.578148-3.759698-1.267071$

H -1.900429 -4.183479 -4.755891

O $0.6095260 .244287-1.539868$

O $0.189410-0.072318-3.784206$

C $1.9180920 .726190-1.857511$

H $2.444714 \quad 0.024316-2.511038$

H $1.8468521 .711922-2.326185$

H $2.478840 \quad 0.819310-0.926057$

SCF Energy (B3LYP/6-31G**//MMFF $)=-3245.91707573$

\section{00326}

MM̄MF Geometry

C -3.074505 -0.402819 -3.666520

C $-2.258626-1.088608-2.852199$

C $-0.775409-1.217502-3.079117$

O $-0.060745-0.675116-1.948932$

C $-0.308675-2.677460-3.287883$

C $-0.658092-3.661120-2.184810$

C $0.068158-3.851808-1.065421$

C $-1.882059-4.507398-2.433600$

C $1.345302-3.165349-0.664301$

C $2.428588-4.194527-0.320367$

C $3.806498-3.591941-0.007543$

C $3.812090-2.7140171 .244647$

C $5.206979-2.1259251 .513032$

O $4.236064-2.832212-1.141078$

O $5.044392-1.1051552 .510016$

C $5.720907-1.4530810 .220317$

C $6.154244-3.1676152 .118877$

O $7.067503-1.0137890 .379006$

C $5.578302-2.334079-1.040772$

C $5.879525-1.570176-2.352912$

O $6.516570-3.414707-0.959957$

C $0.3215740 .627815-1.999357$

O $0.061947 \quad 1.443539-2.868742$

C $1.1217670 .894700-0.784124$

C $1.3288022 .160656-0.394636$

C 2.0448252 .5810680 .810397

C 2.8454641 .5370761 .542647

C $5.048882-0.299817-2.577864$

C $5.7785550 .976300-2.186519$

C $5.4183733 .425255-1.925729$

O $6.9838271 .017733-1.952921$

C $5.3909103 .710986-0.457221$

C 4.9217064 .8579750 .057260

C 4.9240695 .1807251 .524172

C 3.5644485 .6102202 .028472

C 3.2728576 .9093352 .209589

C 2.5736904 .5294622 .398347

C 1.9595873 .8725161 .191853

N $4.9352822 .079349-2.176150$

H -2.654296 $0.130851-4.515800$

H -2.684532 -1.605919-1.996555

H $-0.481413-0.667710-3.983549$

H $0.779468-2.679974-3.431630$

H $-0.721309-3.049722-4.235320$

H $-0.289400-4.587303-0.345401$

H -2.082284 -5.201774 -1.610403

H $-2.773619-3.888047-2.560772$

H -1.746328 -5.105318 -3.340966

H $1.711957-2.502576-1.451609$

H $1.133319-2.5410370 .209579$

H $2.102514-4.8107740 .527231$

H $2.544025-4.869074-1.179903$

H $4.490823-4.4379860 .128244$

H $3.455018-3.2744942 .117318$

H $3.113438-1.8765361 .117705$

H $5.904754-0.6659722 .625062$

H $5.122029-0.5451870 .091719$

H $5.740631-3.5579653 .056587$

H $7.118985-2.7202962 .382959$

H $6.338740-4.0166391 .457542$

H $7.644452-1.7921300 .297259$

H $6.955095-1.371284-2.431169$

H $5.651448-2.252443-3.184544$

H $\quad 6.673775-3.752180-1.857509$
H $1.4818250 .042223-0.219591$

H $0.9020912 .965845-0.991064$

H 3.4918941 .9484742 .320545

H 3.5086501 .0098890 .848901

H 2.1835200 .8056592 .018611

H $4.838587-0.208266-3.651233$

H $4.084924-0.354748-2.060709$

H $4.7808734 .110646-2.492971$

H $6.4448783 .511237-2.297104$

H 5.7995802 .9566510 .211078

H $4.5427265 .624397-0.615421$

H 5.6536115 .9850391 .686131

H 5.2814464 .3325082 .121197

H 2.3093167 .2245132 .595931

H 3.9900687 .6876121 .970140

H 1.7502234 .9581392 .985390

H 3.0571433 .8187333 .074114

H 1.3522734 .5507050 .590414

H $3.9391461 .950044-2.327290$

C $-5.1390110 .889167-2.947747$

O $-5.1531490 .765493-1.505208$

C $-6.5210350 .801140-1.064399$

C $-7.2306951 .651548-2.104624$

C $-6.5971601 .121696-3.374350$

H $-6.901702-0.226353-1.122861$

C $\quad-6.649714 \quad 1.3073250 .375399$

H -8.317053 $1.525315-2.082044$

H $-7.0011972 .716581-1.984328$

C $-6.7386362 .057442-4.563150$

H $-7.0740270 .164492-3.626344$

C $-5.786804 \quad 0.567287 \quad 1.417713$

$\begin{array}{lllll}\text { O } & -6.287620 & 2.692706 & 0.431055\end{array}$

H $-7.703703 \quad 1.2663580 .674965$

H -4.7487430 .9026121 .336448$

O

C $-5.837930-0.9666411 .340905$

C $-5.169039-1.7037852 .516898$

H $-5.369622-1.2938420 .405340$

O $-7.205951-1.3826381 .292244$

H -5.793139-1.615768 3.415984

H $-5.180571-2.7715512 .261269$

H -7.794543 2.216018 -4.805019

H $-6.2897893 .035473-4.359548$

H $-6.2470971 .638165-5.446995$

C $-4.573304-0.398671-3.555647$

H -5.3837492 .7713010 .081195$

H -6.1803661 .9441672 .752130$

H -7.215074 -2.348781 1.182488

H $-4.958213-0.534737-4.574175$

H $-4.906599-1.266501-2.972302$

H $-4.5120331 .755966-3.189218$

C -1.601750 -0.848053 1.765523

O $-2.958736-1.2988561 .641224$

C $-3.733493-1.2605802 .842313$

C $-3.094152-2.1427313 .928750$

C $-1.627067-1.7662914 .159579$

C $-0.854771-1.6581962 .839396$

H -1.140907 -1.074769 0.795837

C -1.512169 0.6714061 .990263

H -3.779452 -0.2301463 .207807$

O $-3.174753-3.5133523 .541339$

H -3.665399-2.027402 4.857796

H - $1.158256-2.5253574 .797549$

O $-1.578835-0.5262644 .858605$

H $\quad 0.134679-1.2159483 .004563$

O $-0.642452-2.9724142 .317507$

C $-1.982522 \quad 1.4653540 .791018$

H -2.1113120 .9972542 .843858$

H -0.4670290 .9459732 .172124$

H -2.799987 -4.045326 4.263915

H $-0.642869-0.3117995 .012008$

H $-0.120328-3.4671882 .971688$

O -1.8035322 .7959581 .043380$

O $-2.449097 \quad 0.989213-0.233608$

C $-2.1910263 .682073-0.011463$

H $-1.714783 \quad 3.396624-0.954323$

H $-3.2791443 .683065-0.115697$

H -1.8612254 .6900590 .254058$ 
H -6.7215124 .3993900 .672200$

H -5.6852965 .8619094 .450595$

H -7.464350 5.7079093 .957985

H $-3.968756 \quad 5.1198782 .993829$

H $-4.395024 \quad 4.7723581 .348784$

H -4.2654902 .8026023 .736890$

H $-6.150079-0.6588891 .507950$

C $3.129549-0.8426853 .405902$

O $3.655318-1.8515342 .520626$

C $5.086453-1.6784002 .412763$

C $5.416694-0.4071293 .184094$

C $4.324260-0.3840974 .235389$

H $5.531593-2.5515822 .905079$

C $5.458533-1.6459850 .925750$

H $6.423984-0.4264303 .610766$

H 5.3392210 .4750292 .535787

C 4.1438520 .9747714 .888668

H $4.558139-1.1310765 .005407$

C $6.919788-1.2550740 .642669$

$\begin{array}{llll}\text { O } & 5.200320 & -2.942947 & 0.376089\end{array}$

H $4.773366-0.9636920 .412534$

H $7.129423-0.2541471 .035783$

O $7.769409-2.1529211 .367979$

C $7.313701-1.319996-0.845202$

C $6.403207-0.532274-1.799352$

H $7.338744-2.369160-1.164634$

O $8.657442-0.847381-0.965468$

H $6.820244-0.567649-2.814130$

H $5.441948-1.057674-1.878088$

H 5.0637531 .2820535 .396984

H 3.8942291 .7450264 .151051

H 3.3410890 .9481005 .632020

C $1.983620-1.4326004 .227680$

H $5.821101-3.5662850 .791090$

H $8.688615-1.8974441 .178407$

H $8.939274-0.999842-1.883558$

H $1.678861-0.7361225 .016173$

H $2.308666-2.3616274 .711874$

H $2.766447-0.0196932 .775043$

C $4.5634922 .683598-2.093857$

O $5.0998801 .377722-2.340915$

C $6.1141120 .936046-1.426084$

C $7.3209531 .885073-1.481764$

C $6.8926193 .337310-1.238538$

C $5.6840893 .736506-2.094662$

H $3.9114302 .886488-2.953529$

C $3.6908812 .722093-0.828317$

H $5.6925610 .953384-0.418339$

O $7.9611521 .813664-2.752854$

H $8.0536821 .603140-0.717877$

H $7.7379523 .998634-1.465896$

$\begin{array}{lllll} & \text { O } & 6.582426 & 3.492796 & 0.143572\end{array}$

H $5.277854 \quad 4.699712-1.764718$

O $6.1092833 .905573-3.450112$

C $2.4845281 .810241-0.925125$

H 4.2516562 .4211000 .060268

H $3.3182023 .741927-0.676918$

H $8.4189140 .958461-2.806652$

H 6.3444874 .4245600 .287663

H $6.7915714 .598359-3.459656$

$\begin{array}{llllll}\text { O } & 1.803174 & 1.857811 & 0.258667\end{array}$

O $2.1735321 .145334-1.903342$

$\begin{array}{llll}\text { C } & 0.630202 & 1.041429 & 0.328277\end{array}$

H $\quad 0.1366511 .2390801 .283259$

H $-0.0621101 .292476-0.480880$

H $0.908422-0.0156840 .286771$

SCF Energy (B3LYP/6-31G**//MMFF) $=-3245.89144588$

\section{8}

MM̄FF Geometry

C $4.676679-1.7566292 .064422$

C $3.341986-1.7669361 .933900$

C $2.397503-1.515783 \quad 3.079985$

O $1.581679-0.3601792 .774850$

C $1.468227-2.729488 \quad 3.275838$

C $0.518155-2.6044124 .453400$ 
C $-0.805236-2.8518104 .392358$

C $1.133646-2.2113255 .772648$

C -1.606324 -3.350403 3.222841

C $-2.378132-2.2197622 .543833$

C $-3.492357-2.7156191 .609077$

C $-2.966492-3.5546390 .443236$

C $-4.081566-3.935934-0.544251$

O $-4.155715-1.5464141 .111531$

O $-3.459498-4.422905-1.743771$

C $-4.867423-2.665081-0.928430$

C $-4.949819-5.081324-0.011046$

O $-6.004825-2.983551-1.725804$

C $-5.299355-1.818306 \quad 0.287624$

C $-5.904419-0.453091-0.116473$

O $-6.305304-2.5224821 .024585$

C 2.1432220 .8601453 .002033

O 3.2870531 .0837623 .367360

C $1.113071 \quad 1.8943172 .760176$

C 1.4352293 .1841472 .939547

C 0.5235134 .3137982 .793452

C 1.1196545 .4746352 .045931

C $-5.0066320 .388292-1.024496$

C $-5.3854411 .858201-1.052838$

C $-4.5356474 .019985-1.965558$

O $-6.3004482 .337829-0.390651$

C $-3.4985834 .505527-1.002872$

C -3.7667655 .2809660 .057814$

C -2.7232265 .7105091 .049643$

C -2.8852745 .1547112 .453016$

C $-3.967514 \quad 4.4980712 .906964$

C -1.7182475 .4130153 .382926$

C -0.7041614 .3006343 .349760$

N $-4.5488732 .571212-1.902663$

H $5.116689-1.5614323 .039475$

H $2.901812-1.9404490 .957051$

H $2.959523-1.3307984 .004096$

H $2.074161-3.6323693 .428328$

H $0.904411-2.8791282 .348090$

H -1.392621 -2.717231 5.300395

H $\quad 0.437670-2.3359676 .609428$

H $2.008049-2.8355445 .984296$

H $1.443507-1.1619095 .758334$

H $-0.974241-3.8730802 .498580$

H -2.308725 -4.103224 3.602550

H -2.834210 -1.566438 3.300630

H $-1.684931-1.5745381 .987181$

H -4.203605 -3.2958582 .208879$

H -2.459226 -4.4589060 .798789$

H -2.206984 -2.978174 -0.099612

H -2.894818 -5.177396 -1.503695

H -4.201952 -2.067293 - 1.563415

H -4.338704 -5.974837 0.164806

H $-5.707602-5.377240-0.744748$

H $-5.452988-4.8375890 .926856$

H -5.691218 -3.510178 -2.480834

H $-6.880670-0.596603-0.597620$

H -6.111548 0.1091830 .804563

H $-6.808569-1.878888 \quad 1.550434$

H 0.1169511 .5784032 .469829

H 2.4519393 .4596553 .213825

H 1.4980715 .1455431 .071173

H 1.9530535 .9074262 .610076

H $\quad 0.403223 \quad 6.275237 \quad 1.848190$

H $-3.964547 \quad 0.328987-0.688558$

H $\quad-5.0625690 .005868-2.049874$

H $-5.5326574 .409190-1.736123$

H -4.267356 4.307757 -2.986256

H -2.476034 4.177820-1.176966

H -4.7863425 .6187410 .227551$

H -2.732932 $6.806141 \quad 1.103540$

H -1.7315985 .4264250 .675429$

H -4.0126764 .1366813 .929861$

H -4.833785 4.2978152 .287385

H -2.077443 5.5020574 .417427

H $-1.275421 \quad 6.3902453 .164949$

H -1.0207653 .4062243 .887534$

H $-3.7727672 .082837-2.338766$

C $6.204363-0.6577630 .427134$
O $5.1871190 .026577-0.335775$

C $5.7717000 .510050-1.558222$

C $7.2785680 .433752-1.355922$

C $7.393098-0.824958-0.518611$

H $5.491115-0.197600-2.347131$

C $5.2352751 .905111-1.886658$

H $7.8236040 .372855-2.302644$

H $7.6575921 .298482-0.798736$

C $8.729686-0.9644770 .188861$

H $7.237809-1.696937-1.168243$

C $3.7004381 .976208-2.036787$

O $5.6122292 .811990-0.842436$

H $5.7156322 .280907-2.797410$

H $3.2206881 .823450-1.063165$

O $3.3682993 .308743-2.446784$

C $3.1165610 .991694-3.067787$

C $1.6376061 .223906-3.421535$

H $3.239392-0.032482-2.705460$

O $3.8617491 .082936-4.286741$

H $1.5311132 .216029-3.877342$

H $1.3758710 .520175-4.221190$

H $9.546038-1.017525-0.538849$

H $8.924473-0.1139090 .850553$

H $8.757375-1.8756250 .794644$

C $5.636700-1.9830600 .931552$

H $5.2526112 .463879-0.008270$

H $3.7379193 .916195-1.783062$

H $3.7999082 .001678-4.600475$

H $6.438303-2.6360821 .294556$

H $5.133798-2.5093990 .111704$

H $6.470699-0.0005521 .265431$

C $0.306536-1.319946-2.320116$

O $0.789799-0.189383-1.581342$

C $0.6598191 .076927-2.240062$

C $-0.8103511 .332676-2.609500$

C $-1.3874920 .187166-3.435990$

C $-1.167957-1.134678-2.707034$

H $0.912411-1.452828-3.224676$

C $0.472703-2.562201-1.427832$

H $0.9347151 .837711-1.499823$

O $-0.9626692 .569152-3.297972$

H -1.394984 $1.420856-1.684754$

H $-0.956794 \quad 0.153342-4.443358$

O $-2.7899950 .382886-3.608814$

H -1.800710 -1.166936 -1.811127

O $-1.570468-2.206970-3.556702$

C $1.885005-3.106895-1.426446$

H $\quad 0.211006-2.323706-0.390389$

H -0.173072 -3.371592-1.787409

H $-0.6490142 .451450-4.210415$

H $-2.9050871 .208382-4.110267$

H -2.504716 -2.061131-3.784434

O $1.881131-4.323272-0.802666$

O $2.864662-2.542266-1.891174$

C $3.142196-4.995744-0.726318$

H $2.953022-6.040853-0.466960$

H $3.665701-4.966138-1.686931$

H $3.751420-4.5444450 .060554$

SCF Energy $\left(B 3 L Y P / 6-31 G^{* *} / / M M F F\right)=-3245.91885322$

0700329

MM̄FF Geometry

C $3.291233-3.806655-0.508393$

C $2.001823-3.688849-0.154397$

C $0.830276-3.405450-1.067916$

O $0.001754-2.409516-0.412417$

C $1.210754-2.865442-2.462139$

C $0.034019-2.468763-3.333476$

C $-0.069440-1.275218-3.950982$

C $-1.039989-3.508160-3.522423$

C $0.946091-0.168068-3.961962$

C $0.773450 \quad 0.880126-2.855049$

C $-0.5319791 .682845-2.968815$

C $-0.4384042 .967390-2.143670$

C $-1.7507103 .762377-2.172772$

O $-1.6076050 .874473-2.478141$

O $-1.6847564 .769317-1.152223$

C $-2.9089232 .815875-1.801740$ 
C -1.941072 $4.506265-3.499718$ O $-4.1674843 .469042-1.954856$ C $-2.9010661 .483424-2.589914$ C $-3.9464590 .523360-1.975835$ O $-3.2457231 .740539-3.951845$ C -1.012636 -2.874309 0.369495 O $-1.294354-4.0438370 .582550$ C $-1.734267-1.7132140 .937815$ C $-2.821215-1.9401131 .691490$ C $-3.668413-0.9385502 .338722$ C -3.2134580 .4949202 .310460$ C $-3.947104-0.892504-2.564601$ C $-4.856744-1.813812-1.772857$ C $-7.245361-2.119555-1.113663$ O $-4.413319-2.762696-1.130251$ C -7.581253-1.272995 0.075168 C -7.701933 $-1.751307 \quad 1.322891$ C $-8.082323-0.9004292 .501499$ C -7.183745 -1.107137 3.700829 C $-7.626729-1.7572954 .790506$ C $-5.800882-0.4883213 .676606$ C $-4.791231-1.3502322 .964412$ N -6.193199-1.464405-1.867303 H $3.570412-3.731883-1.553464$ H $\quad 1.750400-3.8110670 .897902$ H $0.268999-4.339965-1.192405$ H $1.783502-3.626360-3.007547$ H $1.878143-2.008619-2.318725$ H $-0.958028-1.077802-4.548959$ H -1.773220 -3.212807 -4.280737 H $-0.597192-4.454876-3.848714$ H -1.585261 -3.679126 -2.589649 H $1.960793-0.578879-3.908757$ H $\quad 0.897034 \quad 0.327326-4.940401$ H $\quad 0.8275930 .388254-1.875215$ H $\quad 1.6279161 .565203-2.923064$ H $-0.706572 \quad 1.922867-4.024069$ H $\quad 0.3945303 .593798-2.486719$ H $-0.2102922 .708462-1.101620$ H $-0.8950255 .312291-1.317919$ H $-2.8103612 .583327-0.732260$ H -1.113549 $5.205827-3.668095$ H -2.850320 $5.117445-3.482792$ H - $1.9898713 .839846-4.363286$ H -4.132643 4.290208-1.435020 H $-3.7588790 .430433-0.899809$ H -4.947265 $0.956859-2.090802$ H $-3.5432520 .913752-4.362226$ H -1.364134 -0.7195820 .716416$ H -3.137967 -2.971638 1.844096 H -3.2170470 .8848491 .288959$ H -2.199412 0.5800892 .716142 H $-3.838501 \quad 1.1586612 .913302$ H -4.276757 -0.896437 -3.608688 H -2.940204 -1.320368 -2.536365 H $-6.927638-3.127517-0.828684$ H -8.115790 -2.194773 -1.772593 H -7.759378 $-0.214990-0.104895$ H -7.558047 -2.8145271 .501031$ H -9.119354 -1.147746 2.764188 H $-8.087307 \quad 0.1648782 .238928$ H -6.998290 -1.886140 5.665542 H -8.624975 -2.1802794 .830481$ H $-5.448133-0.3356264 .704808$ H -5.8776990 .5050243 .221342$ H -5.007791 -2.418955 2.982870 H -6.454406 -0.657073 -2.425361 C $5.586306-3.0985880 .347621$

O $5.161656-1.7247760 .468299$

C $5.943575-0.929151-0.445770$

C $7.063783-1.834072-0.954292$

C $6.383829-3.187018-0.953511$

H $5.288489-0.690867-1.291654$

C $6.438474 \quad 0.3607750 .218268$

H $7.421179-1.532574-1.943775$

H $7.924264-1.845394-0.276704$

C $7.352321-4.355714-1.010323$

H $5.718793-3.233969-1.823418$
C $5.329396 \quad 1.344136 \quad 0.646624$

O $7.176004 \quad 0.0335831 .403743$

H $7.1373700 .877051-0.451695$

H 4.7785680 .9294721 .498340

O $5.9853702 .521891 \quad 1.141572$

C $4.3401601 .752572-0.464047$

C $3.5162853 .020984-0.162812$

H $3.6675230 .912646-0.672658$

O $5.0768042 .008513-1.664838$

H $4.1840193 .891669-0.131464$

H $2.8568443 .189395-1.025318$

H $7.951478-4.314237-1.925919$

H $8.040559-4.349383-0.158413$

H $6.814431-5.308776-1.002595$

C $4.400379-4.0610220 .474412$

H $8.043302-0.3086981 .131241$

H 6.4558902 .9314240 .395351

H $4.4324392 .182786-2.372071$

H $4.005260-3.9864431 .494203$

H $4.751254-5.0913720 .346028$

H $6.256579-3.2821361 .198484$

C $0.843727 \quad 1.6883712 .068451$

O 1.7045491 .9255810 .948156

C 2.6787942 .9561981 .121773

C 1.9990494 .3019531 .425637

C 1.0476854 .1863872 .620739

C 0.1150572 .9778852 .490138

H $0.093746 \quad 0.9805661 .696704$

C 1.5846131 .0133153 .232491

H 3.3346722 .6982011 .958099

O $1.278111 \quad 4.7462320 .278815$

H 2.7755665 .0478911 .634368

H 0.4495115 .1031002 .693093

O 1.8233544 .0804233 .810588

H -0.4199672 .8102463 .432105$

O -0.8699253 .2961421 .505256$

C $2.090354-0.3682452 .876392$

H 2.4500551 .5942093 .561130

H 0.8941580 .9016894 .076828

H $0.878743 \quad 5.605393 \quad 0.497251$

H 1.2059024 .0342924 .560379

H -1.473529 2.5385351 .432371

O $2.583256-0.9555954 .007372$

O $2.075421-0.8628901 .758314$

C $3.129628-2.2661593 .836822$

H $3.374916-2.6637224 .825186$

H $2.399679-2.9307033 .364772$

H $4.046680-2.2100863 .243852$

SCF Energy (B3LYP/6-31G**//MMFF) $=-3245.87817108$

0700330

MMFF Geometry

C $-1.707241-4.0168660 .699369$

C $-0.758749-3.077815 \quad 0.571725$

C $0.376214-2.8841781 .540634$

O $1.582032-2.955107 \quad 0.738467$

C $0.314125-1.5186722 .246258$

C $-0.747343-1.4300153 .320778$

C $-1.845018-0.6531923 .256136$

C $-0.480525-2.2446834 .561296$

C -2.2820830 .2337262 .127618$

C $-2.257503 \quad 1.7233812 .493805$

C -0.8510522 .2772772 .772681$

C -0.9239803 .7674413 .102643$

C 0.4717864 .3685233 .314586

O -0.0530642 .0850191 .604651$

O $\quad 0.337306 \quad 5.7983113 .316638$

C 1.3718063 .9971922 .114445

C 1.0494413 .9856194 .683333

O 2.7084404 .4233712 .368218

C 1.3195362 .4989431 .730868

C 1.9461722 .2106760 .347158

O 2.0015391 .7259052 .719032

C $2.755351-3.2077381 .375307$

O $2.931321-3.3567722 .573519$

C $3.811776-3.2595590 .342948$

C $5.076044-2.9734610 .684586$

C $6.227978-2.959471-0.218984$ 
C $6.056863-3.601167-1.571200$

C 3.4433632 .4751150 .202831

C $3.9180462 .068061-1.177868$

C $4.5306090 .078661-2.542847$

O $4.0805692 .895192-2.072504$

C $6.013705-0.137244-2.617763$

C $6.9550140 .268670-1.750265$

C $8.419129-0.028044-1.922725$

C $9.020347-0.744030-0.732013$

C $9.892858-0.1229800 .079499$

C $8.671614-2.204319-0.529122$

C $7.369992-2.3813910 .208542$

N $4.1082790 .701950-1.304564$

H $-1.706022-4.6609821 .574973$

H - $0.758696-2.445646-0.311929$

H $0.401251-3.7083142 .263921$

H $0.214599-0.7271451 .495751$

H $1.280846-1.3004662 .718025$

H $-2.525942-0.656478 \quad 4.106503$

H $-1.209347-2.0452945 .354506$

H $-0.523930-3.3149604 .338901$

H $0.510632-2.0111604 .963641$

H -1.7138600 .0589801 .210310$

H $-3.314035-0.0420911 .889476$

H -2.700689 2.2738601 .653993

H -2.901215 1.8981773 .365096

H -0.4097581 .7239813 .608888$

H -1.5557513 .9421263 .982529$

H -1.4091314 .3030272 .274249$

H -0.2799016 .0405974 .028053$

H 1.0226414 .5842121 .254100

H 0.3693314 .2948915 .486173

H 1.9922054 .5095694 .875719

H 1.2214162 .9124934 .789591

H 3.1165753 .7767792 .968973

H 1.7548641 .1554740 .105473

H $1.4026762 .774518-0.422024$

H 2.2744400 .8855662 .314851

H $3.513600-3.501522-0.670651$

H $5.284640-2.6920591 .716346$

H $5.748952-4.647060-1.459331$

H $6.968910-3.608453-2.170774$

H $5.296511-3.078146-2.159811$

H 3.6746343 .5380230 .318409

H $4.021544 \quad 1.9298170 .956805$

H $4.2064070 .690690-3.390726$

H $4.018359-0.887034-2.601176$

H $6.335996-0.706888-3.488014$

H $6.6790470 .849625-0.874586$

H $8.932596 \quad 0.927519-2.090646$

H $8.601466-0.626193-2.824411$

H $10.347345-0.637798 \quad 0.919516$

H $10.1685570 .914976-0.074272$

H $9.453404-2.6963140 .065024$

H $8.691231-2.712596-1.496551$

H $7.387257-1.959861 \quad 1.215105$

H $4.0017980 .106916-0.488202$

C $-4.163504-3.907770-0.065133$

O $-4.335121-2.487855-0.218050$

C $-5.500831-2.0989990 .542742$

C $-5.975993-3.3486991 .290734$

C $-4.728637-4.2154241 .314296$

H -5.168611 -1.357836 1.275792

C $-6.556862-1.484754-0.390092$

H $-6.344237-3.1077452 .293193$

H $-6.790344-3.8556670 .760195$

C $-5.011194-5.6862491 .559130$

H -4.065109 -3.833805 2.100670

C $-6.057014-0.274077-1.207808$

O $-6.995477-2.482307-1.323141$

H $-7.445026-1.2061630 .188309$

H -5.267952 -0.596331-1.895714

$\begin{array}{llll}\text { O } & -7.141617 & 0.186107 & -2.024028\end{array}$

C $-5.5665680 .906275-0.351708$

C -5.183102 2.160167-1.160509

H -4.7099630 .5914550 .247941$

O

H $-6.0294652 .482012-1.779422$
H $-5.0136142 .974083-0.442361$

H $-5.495484-5.8284042 .530758$

H -5.671309-6.101921 0.790410

H $-4.083547-6.2671021 .556041$

C $-2.723436-4.319560-0.364740$

H $-6.224447-2.748836-1.852510$

H $-7.451320-0.571828-2.548788$

H $-6.2553731 .960407 \quad 1.147118$

H $-2.414967-3.852146-1.309184$

H $-2.695009-5.403384-0.534457$

H $-4.794935-4.373405-0.835159$

C $-1.5763621 .446657-1.773411$

O $-2.8316761 .719854-1.138333$

C $-3.9247901 .975264-2.026141$

C $-3.6299923 .217345-2.880862$

C $-2.3074353 .061229-3.639167$

C $-1.1729572 .590440-2.721050$

H $-0.8567501 .428230-0.946421$

C $-1.5901190 .047711-2.406035$

H $-4.0653861 .111979-2.684759$

O $-3.5669014 .370313-2.043595$

H $-4.4569453 .365770-3.585595$

H -2.028656 $4.021593-4.089912$

O $-2.4691892 .122203-4.697797$

H $-0.3013842 .290863-3.313464$

O $-0.7636093 .690276-1.900952$

C $-0.203042-0.533354-2.579155$

H -2.151518 $-0.630077-1.750336$

H $-2.0887620 .049687-3.378773$

H $-3.4051755 .140143-2.615062$

H $-3.1693692 .457041-5.283624$

H $-0.4379924 .390902-2.491261$

O $-0.313445-1.806267-3.063461$

$\begin{array}{llll}0 & 0.844430 & 0.043918 & -2.323640\end{array}$

C $0.924605-2.498227-3.255180$

H $\quad 0.698429-3.510220-3.601303$

H $1.525868-1.991569-4.015991$

H $1.473472-2.565565-2.310995$

SCF Energy (B3LYP/6-31G**//MMFF) $=-3245.90529678$

0700331

MMFF Geometry

C $-3.557106-0.8541812 .585446$

C $-2.229143-0.6739742 .524928$

C $-1.275817-1.184153 \quad 3.574659$

O $-0.263408-2.0083892 .951044$

C $-0.533458-0.020328 \quad 4.267625$

C $-1.4303770 .944898 \quad 5.017177$

C $-1.533818 \quad 2.261422 \quad 4.747180$

C $-2.228875 \quad 0.350675 \quad 6.149767$

C $-0.768675 \quad 3.062556 \quad 3.731281$

C -1.455649 3.0876692 .362283

C $-0.8594194 .147863 \quad 1.424964$

C $-1.4828804 .048393 \quad 0.029981$

C $-0.845250 \quad 5.033486-0.962687$

\begin{tabular}{lllll}
\hline & 0.554813 & 3.945255 & 1.343359
\end{tabular}

O $-1.268647 \quad 4.627895-2.273427$

C $0.6927144 .894820-0.888173$

C $-1.371656 \quad 6.458751-0.757964$

O $1.3421085 .893716-1.669732$

C 1.2384794 .9310710 .555600

C $2.751468 \quad 4.6288150 .641951$

$\begin{array}{lllll}\text { O } & 1.050228 & 6.244458 & 1.098542\end{array}$

C $-0.506818-3.3407312 .860757$

O $-1.540773-3.915653 \quad 3.175115$

C $0.653487-4.0791812 .309021$

C $1.863459-3.5371362 .096897$

C $3.034461-4.2274331 .545231$

C $2.869472-5.6756581 .161336$

C 3.1872743 .3034270 .014490

C 4.6036042 .9175240 .406491

C $6.3864901 .280526-0.092385$

O $5.256967 \quad 3.520471 \quad 1.254158$

C $6.521381-0.058951-0.740644$

C $6.692562-1.200492-0.055943$

C $6.943045-2.533884-0.700402$

C $5.797401-3.505203-0.539308$

C $5.121902-3.952421-1.611578$ 
C $5.498777-4.0318470 .847612$

C $4.183506-3.5353141 .386081$

N $5.045258 \quad 1.794703-0.283462$

H -3.975624 -1.452688 3.391231

H -1.808998 -0.0785411 .719467$ H $-1.798920-1.7744134 .337276$ H 0.0826950 .4961253 .521568 H $0.193683-0.4402294 .976209$ H -2.228490 2.8526875 .342871 H -2.7404451 .1160246 .743247$ H -2.992620 -0.3347625 .770969$ H -1.569890 -0.2002546 .828914$ H -0.6778954 .0860884 .117114$ H $\quad 0.2579222 .6910903 .639348$ H -1.336138 2.1016401 .902269 H -2.532049 3.2673582 .474991 H -1.0562975 .1332001 .863896$ H -2.5688534 .1978400 .076618$ H -1.339341 $3.029385-0.357214$ H $-0.9215275 .269775-2.915518$ H $0.9445343 .927863-1.340932$ H -2.454205 $6.492561-0.930287$ H $-0.9311717 .152071-1.482809$ H -1.184552 6.8460090 .245664 H $1.0532665 .782224-2.590892$ H 3.3265705 .4504990 .194831 H 3.0304324 .6241141 .705152 H $1.664726 \quad 6.3613341 .842137$ H $\quad 0.452949-5.1241952 .093947$ H $2.020933-2.4881082 .341420$ H $2.060159-5.7901340 .431032$ H $2.636910-6.2823422 .043486$ H $3.757905-6.1118420 .700880$ H 2.5234802 .4923900 .333763 H $3.1459553 .381758-1.077224$ H $6.599947 \quad 1.2462680 .981775$ H $7.0874931 .989155-0.546391$ H $6.526428-0.081481-1.827699$ H $\quad 6.702371-1.1705701 .031201$ H $7.846175-2.971932-0.257074$ H $7.179946-2.395496-1.763675$ H $4.321366-4.677891-1.513460$ H $5.352238-3.602423-2.612306$ H $6.286844-3.7193691 .544773$ H $5.563893-5.1242860 .840698$ H $4.201600-2.4846821 .678440$ H $4.5209971 .494948-1.100998$ C $-5.147760-1.0831170 .616917$ O $-4.294048-1.057769-0.551134$ C $-5.027363-0.454571-1.629730$ C $-6.475132-0.814287-1.344025$ C $-6.525861-0.5839630 .152809$ H $-4.9023980 .631584-1.534036$ C $-4.505244-0.904383-2.996628$ H -7.182024 -0.190122 -1.898605 H $-6.685445-1.865716-1.571402$ C -7.685682 -1.286489 0.838708 H $-6.617456 \quad 0.4957030 .334069$ C -2.988633 -0.728141 -3.211047 O $-4.802421-2.292982-3.191116$ H $-5.054108-0.371859-3.782287$ H -2.452755 -1.524430 -2.685242 O $-2.722477-0.920365-4.606238$ C $-2.4197240 .637589-2.792682$ C $-0.952797 \quad 0.889942-3.191200$ H $-2.5038410 .744634-1.705473$ O $-3.2065821 .685547-3.365741$ H $-0.8819411 .083071-4.269350$ H $-0.6619381 .830341-2.706537$ H -8.641666 -0.9198020 .450840$ H $-7.650481-2.3692740 .678081$ H -7.664800 -1.104584 1.918088 C $-4.547530-0.167328 \quad 1.688005$ H -4.394226 -2.784892 -2.458073 H -3.070686 -1.795441-4.849424 H $-3.187844 \quad 1.571779-4.331583$ H $-5.340772 \quad 0.2137132 .343643$ H $-4.0799880 .707001 \quad 1.216711$
H $-5.195478-2.1240110 .958347$ C $0.651971-1.594807-0.902791$ O $-0.135151-0.510177-1.412496$ C $0.028722-0.227566-2.803961$ C $1.4909210 .133922-3.117461$ C $2.443761-0.958208-2.624817$ C $2.145604-1.367779-1.179109$ H $0.506457-1.5525820 .183191$ C $0.155349-2.964670-1.397196$ H $-0.238524-1.115711-3.384523$ O $1.8578451 .359990-2.491903$ H $1.6088090 .262401-4.200414$ H $3.475713-0.593408-2.698327$ O $2.317930-2.087335-3.484805$ H $2.700107-2.273899-0.919010$ O $2.591398-0.339969-0.293315$ C -1.167947 -3.351812 -0.774886 H $0.023971-2.993164-2.481293$ H $0.880403-3.735160-1.109940$ H $1.4548912 .084251-2.999318$ H $2.939701-2.766027-3.172455$ H $3.559109-0.291769-0.369485$ O $-1.463875-4.639593-1.120113$ O $-1.861257-2.622734-0.080956$ C $-2.694352-5.145476-0.593073$ H $-2.659930-5.1581330 .500512$ H -3.536064 -4.542186 -0.946083 $\mathrm{H}-2.822256-6.170105-0.952047$ SCF Energy $(B 3 L Y P / 6-31 G * * / / M M F F)=-3245.90164561$

\section{2}

MM̄FF Geometry

C $-0.471042-1.927477-0.349523$

C $-1.287715-2.332277 \quad 0.636118$

C $-1.613994-1.5713301 .900328$

O $-0.970174-0.275508 \quad 1.873365$

C $-3.132683-1.3513032 .045539$

C $-3.878629-2.5304992 .631835$

C $-4.668718-3.3694281 .934048$

C $-3.739005-2.6953714 .124076$

C $-4.929163-3.385604 \quad 0.453773$

C $-6.364652-2.974957 \quad 0.097881$

C $-6.632901-1.472066 \quad 0.274274$

C $-8.087165-1.141176-0.069321$

C $-8.3589290 .370074-0.022642$

O $-5.753480-0.767433-0.606885$

$\begin{array}{lllll}0 & -9.622006 & 0.611735 & -0.661202\end{array}$

$\begin{array}{llll}C & -7.284258 & 1.097580 & -0.858047\end{array}$

C $-8.495747 \quad 0.8811351 .416598$

O $-7.4093932 .512067-0.727487$

C $-5.8399410 .660336-0.522010$

C $-4.8543861 .251807-1.558455$

\begin{tabular}{lllllll}
\hline & -5.498217 & 1.138087 & 0.780028
\end{tabular}

C $-0.657646 \quad 0.2998853 .062049$

O $-0.866010-0.1679394 .173651$

C -0.0130011 .6224872 .882966$

C $0.2649042 .170178 \quad 1.689186$

C 0.9102663 .4677391 .461300

C $1.220307 \quad 4.3152462 .667097$

C $-3.4105190 .753528-1.406170$

C $-2.551343 \quad 1.162346-2.585885$

C $-1.560583 \quad 3.138217-3.714010$

O $-2.1281620 .336186-3.391683$

C $-1.0386714 .459616-3.252869$

C $0.2532634 .683584-2.972233$

C $0.7870625 .994342-2.474367$

C $0.9097586 .044957-0.967099$

C $0.2289396 .955320-0.250493$

C $1.8696025 .091058-0.291502$

C 1.1899883 .8350700 .192602

N $-2.3025292 .524227-2.629164$

H $0.035095-0.969063-0.286186$

H $-1.762801-3.3061450 .535844$

H $-1.214018-2.1501082 .741237$

H $-3.553837-1.0537361 .080016$

H $-3.324128-0.4812852 .688283$

H $-5.178959-4.1625862 .479943$

H $-4.343843-3.5227214 .510243$ 
H -2.698077 -2.896049 4.395132 H -4.063019 -1.784561 4.638337 H $-4.209518-2.777608-0.101577$ H -4.769420 -4.4152140 .108646$ H $-6.529082-3.243076-0.954107$ H $-7.078549-3.5560270 .694821$ H $-6.414283-1.1883711 .309573$ H $-8.775585-1.6766090 .596439$ H -8.312667 -1.499986 -1.083543 H -10.299745 $0.098512-0.188532$ H -7.478241 $0.865621-1.914837$ H -9.3246610 .3742691 .925263$ H -8.741612 1.9486301 .435144 H -7.596654 0.7228662 .015621 H -8.325526 2.744136 -0.957259 H $-5.1946510 .988269-2.568698$ H -4.862118 2.346326 -1.489749 $\begin{array}{llll}\text { H } & -4.533777 & 1.204768 & 0.853097\end{array}$ H 0.2331592 .1282373 .811369 H 0.0107841 .6196750 .785074 H 1.6149695 .3023412 .417840 H 1.9656103 .8217103 .300740 H 0.3145054 .4902373 .258619 H -2.945972 $1.140840-0.492766$ H $-3.379515-0.339960-1.345644$ H $-2.244577 \quad 3.272882-4.558169$ H $-0.7610042 .461293-4.033929$ H -1.760202 $5.263943-3.131735$ H $0.971093 \quad 3.874666-3.087324$ H $\quad 0.1518716 .812658-2.838364$ H $1.7728426 .168209-2.923161$ H $\quad 0.3296787 .0135630 .828249$ H $-0.4458027 .660222-0.724442$ H 2.3965765 .6073170 .517290 H $2.6646804 .808306-0.993532$ H $0.9281663 .144450-0.609152$ H $-2.8069873 .131875-1.989832$ C $0.914212-3.721046-1.470679$ O $2.177924-3.040263-1.314428$ C $3.145221-3.655868-2.185284$ C $2.521510-4.965523-2.655182$ C $1.056751-4.595806-2.717897$ H $3.254786-2.989352-3.048526$ C $4.499839-3.842400-1.491803$ H $2.924611-5.294751-3.617902$ H $2.676145-5.773943-1.931165$ C $0.128753-5.798654-2.741482$ H $\quad 0.886387-3.994134-3.620919$ C $5.160609-2.567657-0.932253$ O $4.348550-4.754524-0.394890$ H $5.190891-4.332262-2.188705$ H $4.654136-2.256256-0.012483$ O $6.498770-2.920652-0.552853$ C $5.232994-1.379230-1.905354$ C $6.189138-0.242900-1.491744$ H $4.226479-0.967391-2.039862$ O $5.660858-1.834622-3.193387$ H $7.229522-0.569507-1.621178$ H $6.0466290 .565886-2.221003$ H $\quad 0.327574-6.420289-3.620599$ H $0.259404-6.423747-1.851611$ H $-0.918776-5.484626-2.781000$ C $-0.233461-2.717817-1.603285$ H $3.675135-4.3847770 .201557$ H $\quad 6.435519-3.6559360 .080788$ H $6.531394-2.254232-3.082752$ H -1.157991 -3.237944 -1.882002 H $-0.018978-2.004459-2.409142$ H $0.778690-4.343905-0.576898$ C 4.2918131 .2302831 .375785 O $4.6501520 .745703 \quad 0.073369$ C $5.999710 \quad 0.299094-0.066496$ C $6.978646 \quad 1.4452590 .239173$ C 6.7083042 .0593191 .616665 C 5.2232102 .3728271 .819373 H 3.2880971 .6486431 .247124 C 4.1947210 .0967322 .409525 H $6.184851-0.5177410 .637308$
O $6.8833932 .462605-0.756520$

H $8.002568 \quad 1.0559950 .200558$

H 7.2872822 .9850791 .723350

O 7.1329471 .1593452 .635802

H 5.0321852 .6241642 .869341

O 4.9131803 .5364891 .047668

C $3.095024-0.8883322 .077725$

H $5.128016-0.4678082 .477983$

H 3.9647640 .5184873 .394755

H $5.9611082 .770508-0.779711$

H $8.084021 \quad 1.0006922 .509908$

H 3.9993643 .7913541 .259716

O $3.156570-1.9330702 .954992$

O $2.282531-0.7589511 .173501$

C $2.174960-2.9574822 .768488$

H $2.138841-3.2811031 .724179$

H $2.455570-3.8133413 .388045$

H $1.197725-2.5925513 .094473$

SCF Energy $(B 3 L Y P / 6-31 G * * / / M M F F)=-3245.91016359$

\section{3}

MM̄FF Geometry

C $1.594839-3.0864390 .991779$

C $1.222168-2.3019432 .014180$

C $1.907667-1.0015662 .335480$

$\begin{array}{lllll}\text { O } & 0.886455 & 0.019758 & 2.439048\end{array}$

C $2.669853-1.0521423 .672453$

C $3.831699-2.0204503 .713206$

C $5.002769-1.8253023 .075813$

C $3.620237-3.246664 \quad 4.560325$

C $5.369253-0.6654342 .190933$

C $5.676438-1.1483450 .767701$

C $5.691370-0.044964-0.301916$

C 6.5749541 .1495940 .063903

C $6.5317952 .242647-1.017215$

$\begin{array}{lllll}\text { O } & 4.340766 & 0.386558 & -0.523008\end{array}$

O $7.1311173 .429089-0.473305$

C $5.0565652 .555772-1.357005$

C $7.4023961 .872196-2.222649$

O $4.970005 \quad 3.468512-2.449200$

C $4.1995131 .295429-1.624995$

C $2.7084311 .686158-1.760829$

O $4.623366 \quad 0.705500-2.858727$

C $1.261121 \quad 1.2935192 .139921$

O 2.3583931 .6700991 .758783

$\begin{array}{lllll}\text { C } & 0.096737 & 2.176779 & 2.352744\end{array}$

C $0.028495 \quad 3.325799 \quad 1.665499$

C $-1.0401694 .320612 \quad 1.745065$

C -1.9928604 .2403202 .908796$

C $1.7445720 .490504-1.752482$

C $0.5017840 .780341-2.570481$

C - $-1.5308212 .204877-2.656981$

$\begin{array}{llllllll}\text { O } & 0.266947 & 0.172850 & -3.613067\end{array}$

C $-1.7232653 .670108-2.425073$

C $-2.8482844 .200750-1.922706$

C $-3.0575725 .668913-1.679593$

C $-3.3035675 .980702-0.218715$

$\begin{array}{lllll}C & -4.548402 & 5.974114 & 0.287123\end{array}$

C -2.1180916 .3678240 .637996$

C -1.112417 5.2593730 .778985

N $-0.2875691 .784988-2.034814$

H $2.430661-2.7767330 .368072$

H $0.409814-2.6162092 .661800$

H $2.582781-0.7426631 .512092$

H $1.963780-1.2798314 .483810$

H $3.036177-0.0484443 .926737$

H $5.783966-2.5755483 .188869$

H $4.488758-3.9136284 .551667$

H $3.432646-2.9603765 .600352$

H $2.760952-3.8195134 .197224$

H 4.5850360 .0947462 .175108

H $6.257767-0.1824802 .614737$

H $6.650203-1.6550160 .771247$

H $4.928283-1.8955510 .471069$

H $6.056845-0.514339-1.223255$

H 7.6074530 .8291490 .249511

H 6.2246261 .5961501 .003596

H 6.6195523 .6980360 .308575 
H $4.6303883 .081523-0.491753$

H $8.4430171 .723390-1.909354$

H $7.4277742 .684578-2.957461$

H $7.0760110 .960220-2.726435$

H $5.1498452 .971225-3.265242$

H $2.4227122 .344039-0.930656$

H $2.5721822 .261645-2.685834$

H $3.8738580 .247019-3.270450$

H -0.6692221 .8537543 .046587$

H 0.8205493 .5517440 .951872

H -2.5286293 .2865402 .918462$

H -2.7546265 .0233672 .898469$

H -1.446435 4.3386193 .853536

H $2.208537-0.408787-2.172162$

H $1.4463360 .239112-0.728882$

H -2.333531 $1.603165-2.219089$

H -1.492299 $1.998749-3.731409$

H $-0.8978124 .327348-2.688360$

H -3.682085 $3.545480-1.684484$

H $-3.9196845 .990767-2.278390$

H -2.210194 $6.261525-2.046485$

H $-4.7385246 .218357 \quad 1.326934$

H $-5.4049505 .718953-0.328055$

H -2.439283 6.7265501 .622449

H -1.6183857 .2312830 .179612$

H $-0.3745925 .237447-0.023985$

H -0.090755 2.109262 -1.092174

C $0.609953-4.488601-0.828644$

O $-0.567379-3.701377-1.096066$

C -1.325132 -4.350365 -2.140120

C $-0.510995-5.565060-2.576088$

C $0.259055-5.892791-1.311899$

H $-2.255791-4.703040-1.683241$

C -1.620810 -3.354752 -3.268508

H -1.147906 -6.387435 -2.916638

H $\quad 0.177639-5.319308-3.393218$

C $1.459881-6.790236-1.555544$

H $-0.420477-6.377761-0.598398$

C -2.455771 -2.136549-2.820629

O $-0.377059-2.872305-3.793425$

H -2.120049 -3.866198 -4.099015

H -1.911600 -1.577560 -2.051947

O $-2.593001-1.254799-3.942123$

C $-3.868475-2.501808-2.322942$

C $-4.731274-1.280571-1.951158$

H -3.801390 -3.173221 -1.461434

O $-4.539586-3.220914-3.361883$

H $-4.721902-0.550053-2.768818$

H -5.772670 -1.617965 -1.862157

H $\quad 1.143853-7.746017-1.986395$

H $2.172082-6.328646-2.247764$

H $1.988040-7.001889-0.620801$

C $0.964401-4.4093050 .656393$

H $0.093824-2.424802-3.069305$

H -1.696185 -1.031166-4.245517

H -5.399137 -3.510276 -3.011443

H $1.689109-5.1904380 .910852$

H $0.067586-4.570647 \quad 1.266673$

H $1.421586-4.072584-1.441460$

C $-4.162559-1.1388791 .726128$

O $-4.575676-1.5525930 .417932$

C $-4.297766-0.620845-0.634050$

C $-5.048036 \quad 0.693507-0.377537$

C $-4.683488 \quad 1.270878 \quad 0.992870$

C -4.7813660 .2204492 .104572$

H $-4.586872-1.8980012 .395768$

C $-2.638015-1.2095511 .852449$

H -3.221644 $-0.421921-0.665324$

O $-6.457180 \quad 0.489716-0.452949$

H $-4.7868751 .409238-1.164434$

H -5.3530862 .1071821 .229220$

O -3.3521331 .7710240 .951557$

H -4.3230830 .6038023 .023036$

O $\quad-6.1706390 .0186862 .385090$

C -2.218096 -1.357000 3.293593

H -2.241805 -2.0617991 .285125$

H -2.133002 -0.334586 1.433689

H $-6.694687-0.1915240 .199170$
H $-3.3336082 .507260 \quad 0.318129$

H -6.231975 -0.581685 3.147556

O $-2.232090-2.6733303 .650005$

O $-1.931663-0.4155204 .021264$

C -1.847012 -2.947153 5.000638

H -1.877170 -4.029714 5.149769

H -2.548231 -2.476506 5.696223

H $-0.826470-2.5966065 .183182$

SCF Energy (B3LYP/6-31G**//MMFF) $=-3245.92298527$

\section{4}

MM̄FF Geometry

C $-1.241640 \quad 4.8740470 .669470$

C $-0.508258 \quad 4.205914-0.233360$

C $0.9807894 .000584-0.122768$

O $1.2518792 .583709-0.237116$

C $1.6908964 .714705-1.286777$

C $3.2027924 .606752-1.238941$

C $3.9563113 .990812-2.171209$

C $3.8696715 .273931-0.063058$

C $3.5007563 .303905-3.428045$

C $3.2633001 .792265-3.285513$

C $4.5222790 .990527-2.916605$

C $4.267527-0.512666-3.052816$

C $5.485284-1.342479-2.611750$

O $4.869703 \quad 1.290891-1.562360$

O $5.071791-2.713308-2.502446$

C $5.948369-0.860597-1.217115$

C $6.580516-1.339934-3.683958$

O $7.163496-1.500403-0.836294$

C $6.0817790 .673509-1.108729$

C 6.3353681 .1651390 .337164

O $7.2012051 .099466-1.895816$

C $1.058577 \quad 1.8152180 .868784$

$\begin{array}{lllll}\text { O } & 0.710344 & 2.190682 & 1.976546\end{array}$

C 1.3123950 .4102100 .480526

C $0.893032-0.581428 \quad 1.279944$

C $1.024042-2.0154271 .015785$

C $1.907670-2.433185-0.129032$

C $5.247543 \quad 0.8063101 .355369$

C $5.541213-0.4510802 .156752$

C $4.503073-1.9573823 .849495$

O $6.617481-1.0409202 .118345$

C $3.920457-3.1511123 .161539$

C $2.947583-3.9048873 .695611$

C $2.379634-5.1298423 .038327$

C $0.873915-5.0758102 .910517$

C $0.081610-5.6929993 .803187$

C $0.291892-4.3791701 .701849$

C $0.358079-2.8811461 .808359$

N $4.464176-0.8170992 .953488$

H -0.7550765 .3224561 .531756$

H -1.002101 $3.756603-1.092292$

H 1.3595784 .3706930 .837590

H $1.4262935 .780550-1.285255$

H $1.3075884 .309806-2.229546$

H $5.0367073 .982850-2.029340$

H $4.9603775 .296242-0.161866$

H 3.5299506 .3107340 .028787

H $3.636598 \quad 4.7442390 .865467$

H $2.5899323 .771297-3.817156$

H $4.2597093 .477018-4.201832$

H $2.483147 \quad 1.624282-2.533691$

H $2.8731421 .426853-4.243687$

H $5.3361051 .301437-3.582109$

H $3.982091-0.770084-4.080381$

H $3.408793-0.790407-2.426782$

H $4.359391-2.765719-1.843410$

H $5.190471-1.200973-0.502766$

H $6.192166-1.745776-4.626118$

H $7.413189-1.993356-3.401054$

H $6.977376-0.344328-3.892042$

H $7.889499-1.070116-1.319341$

H 7.3287400 .8461630 .675124

H 6.3814042 .2632650 .300546

H $7.4850231 .973373-1.580000$

H $1.8064480 .229908-0.467116$

H $\quad 0.373566-0.3228382 .201705$ 
H $2.889550-1.954695-0.047548$ H $1.456832-2.151010-1.086418$ H $2.100954-3.507452-0.161526$ H 5.1757421 .6223512 .086182 H 4.2700790 .7127020 .872815 H $3.942293-1.6824464 .748552$ H $5.539586-2.1648064 .134075$ H $4.334754-3.4222492 .193294$ H $2.555138-3.6463524 .676770$ H $2.671382-5.9979143 .643900$ H $2.823887-5.2978792 .049373$ H $-0.998744-5.6825083 .703443$ H $\quad 0.496047-6.2180434 .657468$ H $-0.767388-4.6467021 .589527$ H $\quad 0.772809-4.7661140 .799343$ H -0.254206 -2.477452 2.616084 H $3.593695-0.2982352 .883540$ C $-3.443373 \quad 4.354474 \quad 1.748571$ O -3.1454792 .9446051 .751724$ C $-4.3677902 .204476 \quad 1.908482$ C -5.3967273 .2105282 .409899$ C $-4.967544 \quad 4.4635941 .670977$ H $-4.666409 \quad 1.873040 \quad 0.907167$ C $-4.146728 \quad 0.9871132 .812589$ H -6.4222862 .9055452 .180796$ H -5.3225263 .3682093 .491855$ C -5.5246625 .7446742 .266458$ H $-5.298491 \quad 4.3825920 .626757$ C $-2.953076 \quad 0.0886212 .421335$ O $-3.902336 \quad 1.4626114 .144279$ H $-5.070613 \quad 0.398772 \quad 2.863615$ H $-2.020740 \quad 0.6340612 .615627$ O $-2.944882-1.051282 \quad 3.290845$ C -2.919854 -0.450062 0.978684 C $-4.204280-1.1749170 .556429$ H -2.094725 -1.166283 0.914500 $\begin{array}{lllll}\text { O } & -2.637209 & 0.592637 & 0.052652\end{array}$ H $-4.437719-1.9822261 .261685$ H $-5.055243-0.4840230 .611513$ H -6.6190775 .7394982 .234282$ H $-5.2197295 .867492 \quad 3.311157$ H -5.1739406 .6184901 .708256$ C $-2.734215 \quad 5.0254570 .570441$ H -3.996306 $0.706315 \quad 4.747577$ H -2.715319 -0.7388264 .182059$ H $-1.827103 \quad 1.0410420 .349346$ H -2.976321 6.0938440 .543751 H $-3.0901864 .586896-0.370428$ H -3.0715344 .7584512 .699089$ C $-5.707844-2.805831-2.426354$ O $-5.467224-2.311111-1.103376$ C $-4.175339-1.728123-0.882132$ C $-3.073131-2.767948-1.135351$ C $-3.210885-3.396597-2.527494$ C $-4.646473-3.846770-2.816908$ H $-6.675385-3.320424-2.359702$ C -5.855605 -1.668584 -3.443215 H -4.048615 -0.883032 -1.566812 O $-3.148875-3.787046-0.140920$ H -2.092123 -2.288362 -1.044914 H $-2.543370-4.263376-2.606910$ O $-2.815823-2.452062-3.518369$ H -4.755655 -4.098921 -3.877802 O $-4.906626-5.048471-2.084790$ C -7.050179 -0.807823 -3.106479 H $-4.973656-1.024938-3.508667$ H -6.016040 -2.069480 -4.451111 H $-2.423025-4.412581-0.306480$ H $-1.892451-2.205966-3.338418$ H $-4.881294-4.830521-1.137161$ O $-6.6642010 .235131-2.316865$ O $-8.191236-1.050898-3.477054$ C $-7.7134401 .108380-1.888905$ H -8.183474 $1.586766-2.753353$ H -8.453004 $0.553852-1.303400$ $\mathrm{H}-7.2733001 .882488-1.254431$

SCF Energy (B3LYP/6-31G**//MMFF) $=-3245.90411653$

\section{5}

MMFF Geometry

C $1.012118-3.3081940 .940285$

C $0.203381-2.3512660 .460853$

C $-0.600700-1.4302501 .339840$

O $-0.360085-0.0864820 .859748$

C -2.110663 -1.723512 1.248286

C $-2.500880-3.1049781 .725300$

C $-2.880145-4.1181540 .922273$

C $-2.462634-3.3010783 .219198$

C $-2.989368-4.125197-0.577940$

C $-4.386789-4.541824-1.058658$

C $-5.495802-3.540987-0.694897$

C $-6.863293-4.106867-1.087345$

C $-7.992990-3.102067-0.824953$

O $-5.249430-2.318498-1.399994$

O $-9.181880-3.589418-1.465023$

C $-7.617071-1.750804-1.466647$

C $-8.341629-3.0193110 .665067$

O $-8.571115-0.747010-1.125286$

C $-6.187296-1.269633-1.113464$

C $-5.834139-0.046279-1.998363$

O $-6.147652-0.8932960 .261616$

C $-0.5325190 .938142 \quad 1.737097$

O

C $-0.3170872 .197000 \quad 0.988860$

C -0.6631823 .3690321 .542197$

C -0.5591484 .6861720 .907922$

C $0.0313084 .742809-0.476887$

C $-4.4156430 .509274-1.826250$

C $-4.2677521 .447855-0.645086$

C -4.9412823 .6864910 .209597$

O

C $-3.9866334 .752354-0.227321$

C -4.1427496 .0466190 .089107$

C $-3.1910767 .135557-0.319107$

C $-2.437827 \quad 7.715714 \quad 0.858610$

C -2.9668248 .7065881 .596261$

C $-1.0393077 .201767 \quad 1.127868$

C -1.020122 5.7661101 .574665

N $-4.9699502 .633365-0.789365$

H $1.095409-3.4407472 .016357$

H $0.091851-2.234560-0.614498$

H $-0.255847-1.5058272 .378480$

H -2.457033 -1.530150 0.227345

H $-2.659522-0.9861161 .848791$

H -3.131421 -5.0699161 .389665$

H -2.815062 -4.294082 3.517866

H -1.442259 -3.187156 3.597525

H -3.102730 -2.563698 3.714752

H $-2.715586-3.167944-1.028164$

H $-2.263940-4.855818-0.957433$

H $-4.342438-4.648151-2.150296$

H $-4.630337-5.530086-0.648134$

H $-5.465830-3.3480830 .382866$

H $-7.061595-5.050178-0.563237$

H -6.857604 -4.355670 -2.157899

H $-8.999404-3.683213-2.415429$

H -7.676727 -1.875361-2.556835

H -8.669865 -3.998580 1.034206

H $-9.182565-2.3383810 .837462$

H -7.504178 -2.695737 1.286534

H -9.449441 -1.086758 -1.367919

H $-5.924763-0.351375-3.049328$

H $-6.5636680 .753528-1.826514$

H $-5.282176-0.4945810 .442938$

H $0.0908622 .119374-0.012659$

H -1.084808 3.3660282 .546586

H $0.0602715 .746518-0.904281$

H $-0.5522064 .128619-1.171754$

H $1.0610674 .374241-0.464548$

H -3.678065 -0.295340 -1.735378

H $-4.1411221 .078901-2.723406$

H $-4.6197583 .270114 \quad 1.169494$

H -5.9620374 .0691310 .307458$

H $-3.1202434 .450643-0.809965$

H -5.005146 6.3463590 .680676

H $-3.7711637 .925273-0.814538$ 
H -2.481220 $6.781722-1.077087$ H -2.4238909 .1480342 .425200$ H $-3.9580869 .095813 \quad 1.387545$ H $-0.5612637 .794674 \quad 1.918917$ H -0.4252627 .3746800 .239850$ H -1.442312 5.6200012 .569889 H $-5.4571742 .820183-1.660893$ C $3.221895-4.4505680 .539974$ O $4.020618-3.308494 \quad 0.159363$ C $5.278379-3.780642-0.361507$ C $5.352421-5.264382-0.017484$ C $3.896226-5.663359-0.103305$ H $5.222166-3.682746-1.451578$ C $6.460888-2.9713670 .183884$ H $5.992997-5.819074-0.710081$ H $5.732864-5.4275490 .997766$ C $3.591608-6.9833310 .584999$ H $3.611858-5.734683-1.161857$

C $6.453226-1.468880-0.163315$ O $6.466132-3.0950501 .612028$ H $7.395247-3.426252-0.168734$ H $5.694059-0.9490660 .430572$ O $7.721200-0.9305830 .236302$ C $6.251749-1.150045-1.655901$ C $6.5928690 .291552-2.078847$ H $5.213006-1.365141-1.930317$ O $7.069822-2.017831-2.448933$ H $7.6792730 .444110-2.035988$ H $6.330927 \quad 0.374317-3.142340$ H $4.162188-7.7968250 .125139$ H $3.851040-6.9504851 .648522$ H $2.528766-7.2314630 .505696$ C $1.773772-4.2716350 .075781$ H $7.321837-2.7646221 .932934$ H $7.747532-0.9321181 .207844$ H $7.994346-1.874314-2.182843$ H $\quad 1.248957-5.2325180 .127821$ H $\quad 1.757753-3.938707-0.969251$ H $3.273704-4.523177 \quad 1.634244$ C $3.6677572 .121207-0.638119$

O $4.4787431 .197589-1.378698$

C $5.8896481 .399492-1.279885$ C $6.2700432 .796898-1.798758$

C $5.4682433 .895470-1.094446$

C $3.9713933 .572632-1.054102$

H $2.6375201 .890866-0.938222$

C 3.7714191 .8974290 .879168

H $6.1946151 .314838-0.232458$

O $6.0485892 .884929-3.205719$

H $7.3421912 .955192-1.634815$

H $5.6140474 .847392-1.620007$

O $5.943127 \quad 4.057520 \quad 0.238434$

H $3.4597114 .269425-0.380397$

O $3.4423753 .788608-2.365741$

C 3.2980530 .5215721 .293666

H 4.8005372 .0034261 .231772

H 3.1445082 .6306561 .399548

H $5.1125192 .680405-3.372733$

H $\quad 6.883726 \quad 4.298514 \quad 0.187804$

H $2.4827013 .638352-2.325480$

O $3.522470 \quad 0.3634342 .631691$

O $2.800289-0.3084940 .546690$

C $3.154789-0.9077583 .175289$

H $3.637250-1.7186032 .621380$

H $3.495762-0.9445144 .213303$

H $2.067648-1.0186933 .159548$

SCF Energy (B3LYP/6-31G**//MMFF) $=-3245.90383820$

\section{6}

MM̄FF Geometry

C $4.099618-0.0848323 .157791$

C $3.090434-0.7194742 .541123$

C $1.674353-0.7123073 .054548$

O $0.833191-0.1114932 .041702$

C $1.179436-2.148323 \quad 3.290324$

C $0.025757-2.2052074 .271718$

C -1.265630 -2.395862 3.941107

C $0.417988-2.0980325 .725319$
C $-1.862576-2.5495512 .569447$

C $-3.182632-1.7768922 .450206$

C $-4.041687-2.232581 \quad 1.262650$

C $-3.440473-1.857740-0.090151$

C $-4.365303-2.261256-1.251418$

O $-5.322097-1.6055111 .407942$

O $-3.890164-1.602268-2.436116$

C $-5.790992-1.729898-0.977222$

C $-4.298132-3.766695-1.537758$

O $-6.694789-2.231242-1.959419$

C $-6.300621-2.0362450 .450616$

C $-7.640523-1.339356 \quad 0.801854$

O $-6.534229-3.4466870 .570805$

C $0.716928 \quad 1.244408 \quad 2.060016$

$\begin{array}{lllll}\text { O } & 1.202504 & 2.012664 & 2.875094\end{array}$

C $-0.083383 \quad 1.644422 \quad 0.880829$

C $-0.2464262 .949105 \quad 0.615170$

C $-0.952482 \quad 3.528188-0.530419$

C $-1.5055322 .580516-1.562112$

C -7.7561880 .1537310 .490915$

C $-6.711696 \quad 1.0051161 .187672$

$\begin{array}{llll}C & -4.931818 & 2.672873 & 0.720347\end{array}$

O $\begin{array}{llll}-6.498549 & 0.926540 & 2.394986\end{array}$

C $-4.654193 \quad 3.682142-0.345923$

C $-4.4650904 .989743-0.117529$

C $-4.2086395 .985397-1.211654$

C $-2.8154646 .568397-1.163743$

C $-2.6064077 .831570-0.756045$

C $-1.6763935 .716325-1.682677$

C -1.051409 $4.872710-0.604678$

$\begin{array}{llll}\mathrm{N} & -6.070603 & 1.870593 & 0.314874\end{array}$

H $3.9083180 .460037 \quad 4.078828$

H $3.279706-1.2526411 .612262$

H $1.605328-0.1324653 .982981$

H $1.988558-2.7700563 .697377$

H $0.900772-2.6128712 .337100$

H -1.984669 -2.473189 4.756378

H -0.440421 -2.200561 6.397692

H $1.132670-2.8863625 .984180$

H $0.880637-1.1283565 .932052$

H $-1.176955-2.210517 \quad 1.788100$

H -2.047819 -3.617834 2.406506

H -3.778915 -1.905406 3.364269

H $-2.985708-0.6984602 .383363$

H $-4.172016-3.3182941 .340852$

H $-2.451823-2.309103-0.222998$

H $-3.282968-0.770677-0.131710$

H -2.971376 -1.882327 -2.585693

H $-5.741625-0.644890-1.123973$

H -3.271951 -4.065743 -1.781475

H $-4.901033-4.029338-2.414039$

H $-4.626947-4.379196-0.695885$

H -7.548736 -1.784355 -1.836646

H $-8.462554-1.8571840 .290050$

H -7.828769 -1.480984 1.875411

H -7.132392 -3.5946201 .322279$

H -0.4877760 .8601280 .251911$

H 0.1943503 .6740621 .298982

H $-2.1882391 .857896-1.101839$

H $-0.6950422 .030027-2.050636$

H $-2.0717763 .078418-2.351724$

H $-7.7397270 .312105-0.592345$

H -8.7342180 .5159610 .831641$

H $-4.077809 \begin{array}{lll}1.996903 & 0.833302\end{array}$

H $-5.142644 \quad 3.128194 \quad 1.693620$

H $-4.5933213 .315004-1.368403$

H -4.5126095 .3697040 .900005$

H -4.960380 $6.780252-1.120790$

H $-4.3762665 .540736-2.200939$

H - $-1.611778 \quad 8.265058-0.750829$

H $-3.4234088 .457897-0.413654$

H $-0.8869846 .366215-2.084422$

H $-2.0194135 .133805-2.541928$

H -0.6035635 .4649470 .195360$

H $-6.212856 \quad 1.733450-0.681726$

C 5.8685331 .3252412 .080217

$\begin{array}{lllll}\text { O } & 5.029140 & 1.594838 & 0.937984\end{array}$

C $5.8638711 .705771-0.224220$ 
C $7.184223 \quad 2.2287340 .313671$

C $7.317734 \quad 1.3976291 .578708$

H $6.0140880 .689509-0.611275$

C $5.1786122 .557249-1.296304$

H $8.0121812 .081399-0.385742$

H 7.1261693 .2935990 .566469

C 8.2829141 .9838332 .594807

H 7.6709240 .3948841 .301796

C $3.7229582 .139184-1.599244$

O $5.1509973 .927428-0.879629$

H $5.7758472 .536976-2.215062$

H $3.0628452 .468779-0.787169$

O $3.2939952 .839341-2.772321$

C $3.523236 \quad 0.631052-1.826634$

C $2.0743650 .276288-2.209809$

H $3.7838290 .093346-0.909154$

O $4.4119350 .177061-2.846092$

H $1.3822500 .881123-1.616340$

H $1.9153230 .506892-3.270129$

H 9.2925052 .0454692 .175862

H 7.9825872 .9922522 .898349

H $8.325367 \quad 1.3595703 .493183$

C $5.508892-0.0554862 .637319$

H $4.6846583 .965830-0.026642$

H $3.4175563 .789195-2.603619$

H $4.2123560 .678147-3.655432$

H $6.189332-0.3258543 .452733$

H $5.624773-0.8121551 .852249$

H 5.6622482 .1082962 .820124

C $2.521530-3.413517-2.617550$

O $2.602101-1.995387-2.807034$

C $1.766040-1.211066-1.953202$

C $0.290178-1.554591-2.197623$

C $0.045097-3.057612-2.023447$

C $1.072923-3.901777-2.786716$

H $3.115315-3.841442-3.435624$

C $3.166755-3.847725-1.292068$

H $2.013261-1.420980-0.906600$

O $-0.076136-1.156176-3.516600$

H -0.330522 -0.987053 -1.494413

H $-0.961974-3.300658-2.381459$

O $\quad 0.100962-3.366457-0.633747$

H $1.003955-4.951727-2.480729$

O $0.748095-3.864523-4.180172$

C $4.635388-3.480569-1.230072$

H $2.672126-3.380692-0.436190$

H $3.095317-4.935436-1.180989$

H $-1.019808-1.357936-3.633878$

H $-0.097715-4.313113-0.535077$

H $0.873809-2.951205-4.490319$

O $5.061245-3.558160 \quad 0.065634$

O $5.329032-3.185254-2.193583$

C $6.441607-3.2449660 .276717$

H $6.641579-3.2970841 .350208$

H $7.075965-3.974701-0.235057$

H $6.660289-2.230827-0.071726$

SCF Energy (B3LYP/6-31G**//MMFF) $=-3245.89710970$

0700337

MM̄FF Geometry

C $-4.195866-2.800064-2.312294$

C $-3.021491-2.866753-1.666674$

C $-1.697371-2.979317-2.372903$

O $-0.872119-1.857952-1.982126$

C $-0.953449-4.263133-1.962469$

C $0.425912-4.379845-2.590035$

C $1.580204-4.490513-1.902085$

C $0.460319-4.386294-4.098129$

C $1.772356-4.600594-0.416399$

C $2.300058-3.3303050 .255804$

C $3.646958-2.774570-0.240957$

C $4.748723-3.834782-0.282687$

C $6.111738-3.230523-0.645662$

O $4.006838-1.7351910 .682695$

O $7.114137-4.234234-0.422378$

C $6.397523-2.0405880 .291348$

C $6.200853-2.895458-2.138837$

O $7.602069-1.393001-0.114716$
C $5.224113-1.0331510 .387412$

C $5.477927-0.0496011 .560698$

O $5.128643-0.305367-0.834930$

C $-1.019577-0.700467-2.680367$

O $-1.867472-0.446727-3.520353$

C $0.0842890 .195177-2.273113$

C $0.2447251 .376825-2.886723$

C $1.3324962 .327625-2.645724$

C $2.3698041 .940877-1.625752$

C 4.3221500 .9168431 .862356

C 4.5245312 .3233561 .319107

C 3.5886694 .6344861 .580624

O 5.3627232 .6060550 .468499

C 2.4661144 .8934230 .626403

C $2.5629265 .721910-0.424499$

C $1.4322925 .979119-1.380803$

C $1.8551865 .878378-2.831256$

C $1.7548096 .938835-3.650660$

C $2.4027754 .560481-3.334613$

C $1.3633883 .472578-3.359551$

N 3.6305473 .2174461 .892493

H -4.215869 -2.861007-3.397600

H -3.001010 -2.786475 -0.582335

H -1.837105 -2.970607 -3.461335

H -1.543915 -5.142156 -2.251248

H $-0.882532-4.285712-0.870302$

H $2.505640-4.554186-2.473509$

H $1.448523-4.650406-4.490045$

H $-0.250682-5.120839-4.490488$

H $\quad 0.202828-3.400194-4.496347$

H $0.844124-4.8945900 .083265$

H $2.460052-5.433116-0.224169$

H $1.547227-2.5346090 .170629$

H $2.380598-3.5281691 .333535$

H $3.496822-2.328681-1.230724$

H $4.508194-4.640829-0.984822$

H $4.826960-4.3116760 .704355$

H $7.081292-4.4948530 .513662$

H $6.587359-2.4568031 .290502$

H $6.052806-3.799740-2.741603$

H $7.196713-2.521385-2.401723$

H $5.461154-2.159248-2.459907$

H $7.885932-0.8072360 .606339$

H $5.627657-0.6493292 .468657$

H $6.400146 \quad 0.517048 \quad 1.392407$

H $4.7741630 .578187-0.649503$

H $0.759511-0.161986-1.503417$

H $-0.4697821 .667136-3.655849$

H $2.8497260 .998481-1.910404$

H $1.9110331 .818056-0.638441$

H $3.1688982 .674114-1.511713$

H 3.3688310 .5399851 .479688

H 4.2310481 .0021772 .953136

H 4.5558754 .9528511 .178722

H 3.4027615 .1616922 .521360

H 1.5224554 .3860160 .812693

H $3.4940806 .256849-0.596739$

H $1.0429376 .983157-1.168588$

H $0.5998515 .287444-1.202427$

H $2.0568106 .880845-4.691168$

H $1.3676057 .890142-3.301236$

H $3.2881304 .291722-2.753125$

H $2.7719584 .673396-4.363038$

H $0.5646173 .655038-4.080088$

H 2.9344902 .8646682 .543427

C $-6.051310-1.180223-1.926948$

O $-5.134679-0.201816-1.394948$

C $-5.8594150 .704517-0.549636$

C $-7.3079650 .594331-0.998505$

C $-7.405902-0.896028-1.272512$

H -5.7783380 .3176320 .472813$

C $-5.2367922 .101811-0.611851$

H $-8.0107760 .938506-0.234164$

H -7.492851 $1.160712-1.918422$

C $-8.595256-1.282380-2.134089$

H -7.478314 -1.424570 -0.312364

C $-3.7098832 .126511-0.380726$

O $-5.4821382 .678849-1.900164$ 
H -5.7375332 .7579480 .109383$ H $-3.1893821 .738910-1.265425$ O $-3.3067753 .494767-0.247603$ C -3.2355221 .3526220 .861845$ C -1.7223931 .5043001 .110457$ H -3.4632780 .2896040 .730902$ O H -1.1853651 .3815580 .164232$ H $-1.5268572 .514003 \quad 1.492128$ H -9.533324 -1.014274 -1.637364 H $-8.570928-0.773267-3.103457$ H -8.606274 -2.361022 -2.319992 C $-5.514023-2.581231-1.623902$ H $-5.1079172 .077688-2.567320$ H -3.605414 $3.963330-1.046103$ H -3.7789892 .7505302 .115679$ H -6.221919 -3.344702 -1.964979 H $-5.392850-2.707167-0.540710$ H -6.098599 -1.012293 -3.010493 C -1.448182 -0.1583944 .449634$ $\begin{array}{lllll}\text { O } & -1.767888 & 0.760779 & 3.398051\end{array}$ C -1.1927330 .4686002 .122059$ C 0.3413540 .4710162 .216608 C $0.828328-0.4819743 .312685$ C $0.074050-0.2746174 .631658$ H $-1.860696 \quad 0.302306 \quad 5.356691$ C -2.144169-1.510586 4.254216 H -1.527554 -0.520906 1.791074 O $\quad 0.823302 \quad 1.7795282 .510603$ H $\quad 0.7655680 .166881 \quad 1.251976$ H $1.900845-0.3211623 .474421$ O $0.654881-1.8209172 .860631$ H $\quad 0.294730-1.0858945 .335068$ O $\quad 0.525394 \quad 0.9391665 .239636$ C $-3.646346-1.3463404 .268781$ H $-1.852460-2.001714 \quad 3.321660$ H -1.885821 -2.2038885 .063068$ H $\quad 0.6961282 .325914 \quad 1.717076$ H $1.004534-2.4113203 .549637$ H $1.4815800 .852253 \quad 5.393640$ O $-4.161186-1.6445183 .041708$ O $-4.283460-0.9857355 .250087$ C $-5.581908-1.5233712 .924346$ H $-5.868253-1.8429281 .919091$ H $-6.081895-2.1697153 .651882$ $\mathrm{H}-5.882944-0.4806163 .061733$

SCF Energy (B3LYP/6-31G**//MMFF) $=-3245.90607833$

\section{8}

MM̄FF Geometry

C $4.036523 \quad 1.2144143 .580452$

C $2.8957640 .592213 \quad 3.245045$

C 1.5300811 .1846693 .472958

O 0.8736001 .2054122 .183500

C 0.7230550 .3091684 .444850

C $-0.532950 \quad 0.9823854 .962043$

C $-1.787516 \quad 0.6080174 .644035$

C -0.3105862 .0948335 .955713$

C $-2.197058-0.4944013 .706021$

C $-3.548534-0.1983603 .045980$

C $-4.137893-1.4097022 .309257$

C $-3.354344-1.7782191 .048579$

C $-4.027937-2.9239840 .273873$

O $-5.484125-1.0627491 .960909$

O $-3.430030-2.983823-1.029919$

C $-5.519567-2.5862310 .066394$

C $-3.770335-4.285604 \quad 0.929638$

O $-6.221828-3.684389-0.510111$

C $-6.232515-2.1244311 .354793$

C $-7.673213-1.6120941 .100407$

O $-6.346496-3.2374772 .251888$

C 0.1855122 .3262971 .838237

O $-0.014143 \quad 3.3122702 .529689$

C -0.2570722 .1650780 .433824$

C $-0.7416623 .229601-0.223740$

C $-1.1690943 .271089-1.624489$

C $-1.1738811 .976268-2.392960$

C $-7.848858-0.5238980 .038901$
C $-7.077991 \quad 0.7438840 .352858$

C $-5.2324292 .172615-0.499702$

O $\quad \begin{array}{llll}-7.259598 & 1.375024 & 1.391547\end{array}$

C $-4.7930832 .609618-1.859238$

C $-4.8966063 .868650-2.308832$

C $-4.4765964 .294925-3.685597$

C $-3.3493715 .301127-3.667082$

C $-3.5906816 .614106-3.819120$

C $-1.9355934 .772197-3.568457$

C -1.527964 $4.461104-2.151859$

N $-6.1809061 .085003-0.646940$

H 3.9940972 .1904854 .057638

H $2.940038-0.3816882 .762709$

H 1.6416202 .2030143 .863350

H 1.3410390 .0512785 .316027

H $0.479984-0.645188 \quad 3.963419$

H $-2.609119 \quad 1.1342905 .128369$

H -1.249630 2.4704996 .376395

H $0.302549 \quad 1.740883 \quad 6.791063$

H $\quad 0.1975772 .941403 \quad 5.485087$

H -1.444865 -0.6443132 .925913$

H -2.274160 -1.421501 4.286222

H -4.2731100 .1205413 .808130$

H -3.4574280 .6531972 .358685$

H -4.163930 -2.253197 3.009051

H -2.318168 -2.045069 1.286875

H $-3.294221-0.9015880 .388333$

H -2.473680 -3.110074 -0.910591

H -5.553057-1.777570 -0.672040

H -2.693898 -4.4912270 .974056$

H $-4.205293-5.0979850 .337026$

H -4.160502 -4.352665 1.947242

H $-5.745753-3.941136-1.318432$

H $-8.316303-2.461070 \quad 0.833425$

H -8.071885 -1.228041 2.049925

H -7.056962 -3.047762 2.887063

H $-0.1351871 .192193-0.028213$

H $-0.8090184 .178780 \quad 0.307062$

H -1.730407 $1.203754-1.850082$

H $-0.1498951 .624504-2.553100$

H -1.648350 $2.054172-3.373406$

H -7.593246 $-0.911548-0.952441$

H $-8.907838-0.242516-0.015990$

H $-4.377650 \quad 1.789477 \quad 0.066994$

H -5.6912452 .9819340 .077617$

H $-4.3614281 .849854-2.506777$

H -5.326354 $4.632249-1.664713$

H $-5.3561554 .722159-4.185047$

H $-4.1849003 .433590-4.299668$

H $-2.783357 \quad 7.338730-3.838397$

H $-4.6018716 .994060-3.921940$

H -1.230781 $5.525493-3.946225$

H -1.818983 $3.922277-4.246527$

H -1.490705 $5.342552-1.509693$

H $-5.9999320 .407083-1.381462$

C $6.1446301 .493198 \quad 2.271378$

O 5.4489901 .3955021 .012280

C $6.3893151 .039591-0.013846$

C 7.7563841 .3967160 .549203

C 7.5727431 .0112342 .005808

H $6.332578-0.048768-0.130854$

C $6.0119801 .712690-1.335929$

H 8.5667900 .8502440 .058052

H $7.9638282 .469607 \quad 0.465019$

C 8.6088331 .6207182 .933813

H $7.621912-0.0830052 .087701$

C $4.5412121 .502066-1.755947$

O $6.2274553 .125458-1.231709$

H $6.6840021 .365483-2.129193$

H $3.8781202 .103142-1.121403$

O $4.3883332 .008742-3.087314$

C $4.0711950 .036560-1.733271$

C $2.633688-0.128498-2.263957$

H $4.107346-0.338704-0.705111$

O $4.968048-0.759697-2.505624$

H $1.9893900 .625396-1.797330$

H $2.6354950 .039960-3.347944$

H 9.6140151 .2805242 .664212 
H 8.5994362 .7146822 .882667 H $8.420021 \quad 1.3301463 .972113$ C $5.405893 \quad 0.653536 \quad 3.315023$ H $5.6939363 .449808-0.485724$ H $4.6816422 .936132-3.079505$ H $4.951687-0.421046-3.417216$ H $5.965076 \quad 0.639047 \quad 4.257182$ H $5.323200-0.3836452 .966791$ H 6.1393412 .5535592 .554809 C $2.476202-3.862105-2.468114$ O $2.830433-2.491674-2.687681$ C $2.053423-1.527578-1.972966$ C $0.574091-1.632434-2.376638$ C $0.049881-3.062161-2.207164$ C $0.998703-4.100815-2.817035$ H $3.086639-4.426735-3.184860$ C $2.853688-4.331738-1.058708$ H $2.148349-1.710455-0.896916$ O $0.423446-1.229830-3.736723$ H $-0.010993-0.941040-1.758785$ H $-0.931661-3.143459-2.689572$ O $-0.125646-3.316399-0.816364$ H $0.708247-5.109943-2.504217$ O $0.862944-4.063499-4.241193$ C $4.345262-4.225854-0.840947$ H $2.341728-3.765187-0.275704$ H $2.577156-5.382098-0.909889$ H $-0.525531-1.255866-3.946801$ H $-0.454126-4.226800-0.722387$ H $1.182333-3.197380-4.547744$ O $4.614677-3.263520 \quad 0.086957$

O $5.171184-4.907372-1.434492$ C $6.000261-3.059451 \quad 0.379217$ H $6.074059-2.2984351 .160419$ H $6.452353-3.9849890 .748360$ H $6.524460-2.701359-0.511873$ SCF Energy (B3LYP/6-31G*//MMFF) $=-3245.91437721$

0700339

MM̄FF Geometry

C $0.462438-1.9904710 .297125$

C $1.278697-2.353563-0.704566$

C $1.526545-1.564605-1.971684$

O $0.894398-0.265669-1.858733$

C $3.037975-1.335030-2.180786$

C $3.775616-2.476131-2.845416$

C $4.685464-3.261994-2.237514$

C $3.495323-2.649787-4.316619$

C $5.096924-3.264358-0.791726$

C $6.475504-2.629060-0.554799$

C $6.453133-1.091929-0.572808$

C $7.855926-0.524048-0.345194$

C $7.8434491 .008517-0.222739$

$\begin{array}{llll}\text { O } & 5.584208 & -0.664101 & 0.479117\end{array}$

$\begin{array}{lllll}\text { O } & 9.113807 & 1.419641 & 0.305172\end{array}$

C $6.7711401 .422302 \quad 0.808816$

C $7.7017391 .687623-1.590124$

$\begin{array}{llll}\text { O } & 6.621584 & 2.839502 & 0.851202\end{array}$

C 5.3997710 .7536230 .570688

C 4.4534241 .0227171 .766200

O $4.8165811 .294657-0.616414$

C $0.627354 \quad 0.421260-2.997552$

$\begin{array}{llll}\text { O } & 0.831750 & 0.042628 & -4.142575\end{array}$

C $0.0890101 .769325-2.698835$

C $-0.4211752 .112008-1.505387$

C $-0.9800193 .417137-1.143658$

C $-0.808858 \quad 4.547997-2.123247$

C $3.1724310 .179571 \quad 1.734420$

C $2.346090 \quad 0.3968812 .984152$

C $0.595397 \quad 1.8477363 .957195$

O $2.409274-0.3709483 .941822$

C $0.123083 \quad 3.2522793 .774909$

C -1.1138793 .5663483 .362476$

C $-1.588244 \quad 4.9716673 .138076$

C $-1.4215725 .427711 \quad 1.704468$

C $-0.5981516 .444787 \quad 1.398967$

C -2.259399 $4.763095 \quad 0.634894$

C $-1.595627 \quad 3.5437770 .050553$
N 1.5608291 .5364552 .920356 H $-0.121807-1.0774080 .221793$

H $1.838474-3.280532-0.601188$

H $1.073072-2.109222-2.807859$

H $3.493525-1.073017-1.221049$

H $3.201561-0.439177-2.794928$

H $5.185760-4.021500-2.837765$

H $4.124597-3.422001-4.772012$

H $2.452863-2.938754-4.480415$

H $3.685819-1.714273-4.853059$

H $4.339606-2.806405-0.148620$

H $5.144827-4.314848-0.476194$

H $6.820372-2.9623430 .433033$

H $7.195868-3.004449-1.291921$

H $6.057581-0.751012-1.535728$

H $8.538142-0.841245-1.143690$

H $8.270546-0.9392820 .584274$

H $9.806711 \quad 1.100226-0.298155$

H $7.136498 \quad 1.119246 \quad 1.800014$

H $8.5380821 .410842-2.243327$

H $7.7444042 .778332-1.495920$

H $6.7782271 .421102-2.108138$

H 7.5027513 .2188291 .011221

H 4.9778420 .7917972 .702825

H 4.1843802 .0858771 .800162

H $3.8500921 .252155-0.548554$

H $\quad 0.1056902 .461333-3.534472$

H $-0.456124 \quad 1.364213-0.714914$

H $-1.148913 \quad 5.511712-1.738437$

H -1.371095 4.352399 -3.042298

H $0.2486644 .676898-2.380207$

H 2.5604530 .4164350 .857709

H $3.402495-0.8904941 .679393$

H 1.0651391 .7230014 .938311

H $-0.219475 \quad 1.119156 \quad 3.880164$

H $0.8376404 .046343 \quad 3.977084$

H -1.825669 2.7701703 .156203

H -1.061550 5.6498313 .822553

H -2.646232 5.0393503 .420405

H $-0.4908836 .797616 \quad 0.378772$

H -0.0103576 .9458572 .160830$

H $-2.523408 \quad 5.486780-0.143874$

H $-3.224942 \quad 4.463711 \quad 1.062472$

H -1.665975 2.6604460 .685279

H $1.457797 \quad 1.9965382 .019811$

C $-0.926810-3.5560891 .735847$

O $-2.097597-2.7321441 .559269$

C $-2.905710-2.8159852 .742247$

C $-2.519098-4.1353993 .398316$

C $-1.029540-4.1741203 .136433$

H $-2.596150-1.9837163 .386723$

C $-4.400198-2.6822182 .428082$

H $-2.769799-4.1612514 .462992$

H $-3.000943-4.9918482 .913447$

C $-0.426603-5.5655293 .234584$

H $-0.534857-3.5273663 .873644$

C $-4.805261-1.5372961 .480826$

O -4.877535 -3.894277 1.824751

H $-4.950453-2.5942683 .372664$

H $-4.586273-1.8253970 .446611$

O $-6.232878-1.4114651 .566335$

C $-4.181581-0.1598241 .760316$

C -4.8439861 .0134901 .005924$

H -3.116031 -0.1882711 .507072$

$\begin{array}{llll}\text { O } & -4.260319 & 0.133564 & 3.157679\end{array}$

H -5.8227221 .2300301 .453775$

H -4.2313301 .9041901 .198044$

H $-0.566228-5.9754584 .240311$

H $-0.891864-6.2555402 .522628$

H $0.647566-5.5391093 .025747$

C $0.350207-2.7233861 .600252$

H $-4.355816-4.0453051 .017936$

H $-6.613865-2.2792391 .347331$

H -5.1987830 .1058013 .411582$

H $1.226340-3.3654941 .752824$

H $0.379179-1.9626032 .390743$

H $-0.962583-4.3261880 .955836$

C $-3.7043980 .342971-2.495440$ 
O $-3.7156390 .628784-1.088420$

C $-5.009757 \quad 0.805290-0.508393$

C $-5.741793 \quad 1.979972-1.174843$

C $-5.8195451 .788547-2.693351$

C $-4.458744 \quad 1.423736-3.292595$

H $-2.6485060 .395676-2.784832$

C $-4.191462-1.084457-2.793463$

H -5.604049 -0.098536 -0.667207

O $-5.086453 \quad 3.210754-0.880541$

H -6.753406 $2.049062-0.758284$

H -6.180843 $2.713408-3.159725$

O $-6.7477780 .752106-2.999853$

H $-4.5808851 .102123-4.333517$

O $-3.6623062 .611459-3.314353$

C $-3.251764-2.132232-2.233866$

H $-5.181787-1.271201-2.370601$

H $-4.236257-1.238399-3.877674$

H $-4.1770773 .150789-1.219374$

H -7.612245 $1.017257-2.642145$

H -2.829752 2.397897-3.768898

O $-3.807710-3.367519-2.409007$

O $-2.166120-1.904207-1.719217$

C -3.023647 -4.465542 -1.931893

H $-2.874933-4.382811-0.851716$

H -3.569514 -5.389409-2.140664

H $-2.062040-4.500255-2.452836$

SCF Energy (B3LYP/6-31G**/MMFF) $=-3245.91287489$

0700340

MM̄FF Geometry

C $-0.357544-2.262478 \quad 3.895974$

C $-0.034787-1.244398 \quad 3.084704$

C $1.013476-0.2165703 .415522$

O $2.090021-0.3944272 .465596$

C $0.4563351 .213586 \quad 3.280198$

C $-0.713648 \quad 1.525647 \quad 4.186183$

C $-1.976716 \quad 1.7237243 .760529$

C -0.3776591 .6581535 .648645$

C -2.5053381 .6304902 .355365$

C -2.8863153 .0005841 .780166$

C -1.6811323 .9111721 .491200$

C -2.1589395 .2660790 .963024$

C $-0.983502 \quad 6.1875770 .612368$

O $-0.860511 \quad 3.273040 \quad 0.507918$

O $-1.5211127 .270939-0.165174$

C $0.008347 \quad 5.417115-0.286062$

C $-0.387705 \quad 6.832922 \quad 1.868443$

$\begin{array}{lllll}\text { O } & 1.194636 & 6.174998 & -0.508603\end{array}$

C $0.344414 \quad 3.982548 \quad 0.190391$

C $1.0359813 .211959-0.958612$

$\begin{array}{llll}\text { O } & 1.266703 & 3.973436 & 1.279520\end{array}$

C 3.3005870 .1311042 .797037

O $3.5877730 .736093 \quad 3.817875$

C $4.229207-0.142948 \quad 1.681517$

C $5.376548 \quad 0.5449851 .597680$

$\begin{array}{llll}\text { C } & 6.364082 & 0.384350 & 0.537694\end{array}$

C $6.8664951 .685628-0.023887$

C $1.3995361 .762859-0.606679$

C $2.0114951 .030136-1.789097$

C $2.754348-1.243909-2.487985$

O $2.234889 \begin{array}{lll}1.568435 & -2.870323\end{array}$

C $4.247125-1.331697-2.417853$

C $4.905808-2.498970-2.331090$

C $6.394543-2.734718-2.324274$

C $7.276275-1.509883-2.227432$

C $7.620968-0.816732-3.326097$

C $7.842716-1.150561-0.872823$

C $6.786867-0.8348090 .149893$

N $2.266784-0.302668-1.497065$

H $0.176499-2.3919694 .833844$

H $-0.565701-1.1280492 .142419$

H $1.402277-0.3851384 .427907$

H $\quad 0.213840 \quad 1.4000552 .229419$

H 1.2530691 .9392433 .490012

H -2.734699 1.9684854 .503874

H -1.242402 1.9620986 .248244

H $-0.018944 \quad 0.705057 \quad 6.049176$

H 0.4026002 .4127525 .792104
H -1.817779 1.1121791 .680980

H $-3.406806 \quad 1.0049282 .386569$

H -3.432995 2.8245020 .845529

H $-3.578403 \quad 3.5071212 .464798$

H -1.103966 4.0451342 .412285

H -2.825471 5.7541041 .685113

H $-2.761650 \quad 5.111157 \quad 0.056823$

H $-0.7755247 .827092-0.450013$

H $-0.4759025 .334602-1.269391$

H $-1.1307407 .481376 \quad 2.349105$

H $0.457297 \quad 7.484111 \quad 1.620683$

H $-0.061517 \quad 6.107262 \quad 2.615407$

H $1.7520426 .101280 \quad 0.284147$

H $0.3750263 .199024-1.834920$

H $1.9562783 .733006-1.252736$

H 1.1389834 .7679631 .815893

H $3.946256-0.8888120 .947273$

H 5.6118381 .3112522 .333503

H $7.4836441 .558290-0.916827$

H 7.4635052 .2201940 .722773

H $6.0256492 .325888-0.315294$

H $2.1216751 .743966 \quad 0.216232$

H $0.503251 \quad 1.213287-0.297066$

H $2.452055-0.909296-3.485822$

H $2.274042-2.205811-2.285231$

H $4.793506-0.395801-2.486738$

H $4.314319-3.412865-2.292224$

H $6.647133-3.290494-3.237277$

H $6.633547-3.416264-1.498041$

H $8.2811230 .042258-3.270220$

H $7.245216-1.092718-4.305936$

H $8.564086-0.329223-0.951854$

H $8.431296-2.000383-0.502715$

H $6.360748-1.7140050 .633376$

H $2.071135-0.653799-0.564178$

C $-0.992927-4.4457992 .808631$

O $-0.775882-4.0443891 .437367$

C $-1.578653-4.8754940 .582920$

C $-1.824278-6.1443931 .384226$

C -2.040011 -5.5642592 .767900$

H $-2.535609-4.3590930 .442716$

C $-0.906622-5.081480-0.776291$

H -2.684197 -6.712255 1.016924

H $-0.948114-6.8030041 .382365$

C $-1.890782-6.581828 \quad 3.885911$

H $-3.050509-5.1356622 .813923$

C $-0.530580-3.774586-1.504464$

O $0.295380-5.834929-0.577389$

H $-1.556861-5.696671-1.410263$

H $0.335104-3.307373-1.018736$

O $-0.112902-4.119540-2.831670$

C -1.666941 - $2.738049-1.611338$

C $-1.295468-1.557032-2.529219$

H $-1.898445-2.356103-0.611126$

O $-2.846221-3.370343-2.107153$

H $-0.275782-1.230717-2.303316$

H -1.323431 -1.900595 -3.570762

H -2.634081 -7.3790513 .782229$

H $-0.898302-7.0448353 .877419$

H -2.033999-6.109782 4.863055

C $-1.467046-3.2342243 .612397$

H $0.606297-6.128566-1.450031$

H $\quad 0.732092-4.594105-2.759779$

H $-2.639376-3.732755-2.985688$

H $-1.884161-3.5534644 .574786$

H $-2.270400-2.7163433 .073835$

H $-0.033787-4.8192323 .188689$

C $-4.5743690 .254297-2.613777$

O $-3.557931-0.746872-2.754221$

C $-2.238923-0.347733-2.375381$

C $-1.7757140 .842612-3.231558$

C $-2.7779601 .998467-3.170167$

C $-4.214403 \quad 1.519982-3.409246$

H $-5.466218-0.188758-3.075809$

C $-4.8986370 .533118-1.141845$

H $-2.236907-0.058043-1.318671$

$\begin{array}{llll}\text { O } & -1.614825 & 0.423185 & -4.585266\end{array}$

H -0.796127 1.174948 -2.871801 
H $-2.508568 \quad 2.746855-3.925358$

O $-2.6775462 .619391-1.893393$

H $-4.9311882 .314454-3.171154$

O $-4.3774461 .213087-4.796996$

C $-5.505047-0.688333-0.493676$

H $-4.0249190 .840688-0.561616$

H -5.630788 $1.343922-1.048865$

H -1.278763 $1.183198-5.090053$

H $-3.2973213 .368440-1.880294$

H -4.194772 2.024745 -5.300388

O $-4.581193-1.3082890 .294364$

O $-6.656188-1.058813-0.685442$

C $-5.032359-2.4983360 .947484$

H -5.918222 -2.2906351 .555252$

H $-5.243770-3.2750070 .206589$

H $-4.233840-2.8488131 .605414$

SCF Energy (B3LYP/6-31G**//MMFF) $=-3245.88611650$

0700341

MM̄FF Geometry

C $2.6482161 .906863 \quad 3.912445$

C 2.0010310 .8283163 .446066

C 0.5280350 .5827213 .653732

O -0.1035650 .4890102 .354450$

C $0.314721-0.7464234 .400477$

C $-1.142116-1.1093714 .624571$

C -1.693464 -2.281052 4.250027

C $-1.983463-0.0823955 .336607$

C -1.002606 -3.443658 3.594687

C $-0.959774-3.3754132 .062604$

C $-2.323840-3.5898911 .387099$

C $-2.147371-3.684620-0.130845$

C $-3.488301-3.824262-0.862037$

O $-3.177757-2.4892211 .708647$

O $-3.227756-3.601538-2.256426$

C $-4.436699-2.711219-0.370195$

C $-4.044815-5.247959-0.751391$

O $-5.741202-2.880842-0.921663$

C $-4.502297-2.5894031 .172351$

C $-5.297449-1.3361791 .611496$

O $-5.188389-3.7346941 .691069$

C $-0.4018701 .665742 \quad 1.736686$

O -0.1914672 .7906372 .163486$

C $-1.029218 \quad 1.3564580 .432280$

C -1.241295 2.359286-0.433777

C $-1.8677232 .266088-1.754033$

C $-2.3397680 .914554-2.217987$

C $-4.562456-0.0111161 .388358$

C -5.5029701 .1709541 .229601$

C $-5.468522 \quad 3.5553810 .491137$

O -6.7200931 .0919051 .367866$

C $-5.4992643 .591845-1.006103$

C $-4.9630894 .577938-1.740969$

C $-4.9775094 .596551-3.243385$

C $-3.5972334 .764578-3.843143$

C $-3.2389545 .914943-4.438202$

C $-2.6578683 .574228-3.822386$

C $-1.9930763 .395924-2.482035$

N -4.809868 2.3284160 .899973

H 2.1065812 .6483074 .494406

H 2.5432170 .0979832 .849627

H $\quad 0.0724991 .4008754 .225919$

H $0.807551-0.6979475 .380575$

H $0.822001-1.5393393 .839860$

H -2.751229 -2.442002 4.453955

H $-2.967527-0.4739945 .616194$

H -1.488410 $0.241318 \quad 6.257985$

H -2.1482790 .7918194 .699364$

H $\quad 0.020330-3.5359193 .977474$

H -1.505920 -4.367119 3.908025

H $-0.540926-2.4087891 .754854$

H $-0.267918-4.1528141 .715312$

H -2.762331 $-4.514981 \quad 1.779561$

H -1.479843 -4.513829 -0.396263

H -1.652235 -2.773079 -0.492583

H $-4.082612-3.609437-2.720853$

H -4.057481 -1.773219-0.791580

H $-3.335760-5.967675-1.178006$
H $-4.967174-5.360184-1.331906$

H $-4.246424-5.5510980 .278135$

H $-6.181803-3.591640-0.425478$

H $-6.277988-1.3447781 .119908$

H -5.519805 -1.404798 2.685475

H $-5.509586-3.5236772 .583323$

$\begin{array}{llll}H & -1.298356 & 0.327254 & 0.225618\end{array}$

H $-0.932130 \quad 3.363452-0.144414$

H $-3.1973080 .584454-1.624642$

H -1.541779 $0.170041-2.127662$

H $-2.6434470 .898319-3.266994$

H $-3.894790 \quad 0.1881452 .235015$

H -3.947347 -0.0573560 .486539$

H -4.8995104 .3886920 .914588$

H -6.4880343 .5834550 .887471$

H $-5.9850652 .759144-1.510002$

H $-4.4929175 .420582-1.239208$

H $-5.6299545 .421222-3.558311$

H $-5.4326713 .684270-3.649141$

H -2.260966 $6.035399-4.892356$

H $-3.9131976 .763960-4.480523$

H -1.864564 $3.710368-4.568617$

H -3.220192 2.691448 -4.138982

H -1.580004 4.319386-2.073004

H -3.803892 2.2770010 .774286

C 4.2615823 .3648672 .680870

O 3.7016423 .0119961 .398832

C 4.6723123 .2835790 .373637

C 5.6510684 .2664870 .999092

C 5.7243003 .7321762 .418061

H 5.2006902 .3405050 .185236

C $3.9792733 .736565-0.916434$

H $6.619526 \quad 4.2737890 .490513$

H 5.2579455 .2888741 .008024

C 6.2967114 .7239263 .415824

H 6.3462852 .8268512 .420052

C $2.8380752 .803114-1.373301$

O $3.3883405 .027222-0.722446$

H $4.7160773 .847984-1.721217$

H $1.9810842 .905578-0.696191$

O $2.3870583 .253258-2.657163$

C $3.2222481 .316438-1.482651$

C $2.0682840 .463454-2.042942$

H $3.4955590 .942125-0.490023$

O $4.3684111 .196844-2.322582$

H $1.1391050 .746900-1.539613$

H $1.9602620 .670014-3.114993$

H 7.3246094 .9892033 .147759

H 5.7085295 .6471413 .446826

H 6.3088944 .2982754 .424092

C 4.1005602 .1851433 .642504

H $4.1028655 .685422-0.751040$

H $2.1258834 .185188-2.561012$

H $4.6112940 .256146-2.358206$

H 4.5976012 .3983724 .595417

H 4.5779591 .2931583 .217627

H 3.7003394 .2360243 .042254

C $3.766311-2.827228-2.556441$

O $3.368878-1.455335-2.694939$

C $2.297697-1.046240-1.840305$

C $1.033850-1.858814-2.157777$

C $1.298160-3.363452-2.058487$

C $2.575941-3.774731-2.800144$

H $4.492884-2.978951-3.364747$

C $4.499550-3.040358-1.223573$

H $2.583030-1.213088-0.794916$

O $0.556390-1.543536-3.464067$

H $0.246184-1.575321-1.450677$

H $0.439948-3.902964-2.478305$

O $1.400315-3.713979-0.681882$

H $2.848257-4.802407-2.535859$

O $2.279399-3.769399-4.200786$

C $5.370309-4.277648-1.215765$

H $5.151271-2.178501-1.033022$

H $3.796386-3.107967-0.388999$

H $1.270844-1.734050-4.095743$

H $1.514270-4.678327-0.632737$

H $3.062599-4.106932-4.667937$ 
O $5.910100-4.428920 \quad 0.030345$

O $5.563722-5.013549-2.173375$

C $6.768491-5.5620590 .195291$

H $6.214153-6.4873160 .011222$

H $7.628983-5.486211-0.476167$

H $7.128446-5.5682921 .227512$

SCF Energy (B3LYP/6-31G*//MMFF) $=-3245.92096715$

07_00342

MM̄FF Geometry

C $-1.873797 \quad 4.705116-0.670619$

C $-0.7202454 .883598-0.008685$

C $0.6811304 .635113-0.515558$

O 1.2245613 .5878860 .327696

C $0.7925114 .183248-1.988274$

C $2.2190624 .109193-2.501586$

C $2.8374412 .975067-2.885851$

C $2.9423265 .427697-2.617360$

C $2.2660641 .587729-2.963489$

C $2.4602250 .797269-1.666197$

C $2.079315-0.679105-1.840926$

C $2.091259-1.406499-0.497336$

C $1.794782-2.904520-0.658898$

O $3.036680-1.271033-2.720845$

O $2.106003-3.5501100 .585586$

C $2.753228-3.482438-1.722102$

C $0.309976-3.174646-0.927533$

O $2.431589-4.837594-2.025197$

C $2.807511-2.651781-3.024042$

C $3.935599-3.110515-3.983907$

O $1.568781-2.808077-3.728547$

C 2.4410803 .7782760 .903483

O $3.1612204 .758248 \quad 0.805011$

C 2.7554012 .5638291 .691433

C $3.9599362 .443282 \quad 2.269289$

C 4.4166391 .3262843 .100105

C $3.475230 \quad 0.165143 \quad 3.287302$

C $5.356596-3.171900-3.412527$

C $5.829370-1.846722-2.843225$

C $6.499985-0.751056-0.712979$

O $5.939303-0.840745-3.540447$

C $6.359121-1.0666150 .742046$

C $7.373129-1.0110111 .617818$

C $7.253911-1.4068753 .061488$

C $7.501546-0.2579324 .010445$

C $8.748740 \quad 0.0772054 .381913$

C $6.295853 \quad 0.430434 \quad 4.606747$

C $5.643967 \quad 1.3985983 .657964$

N $6.076105-1.906977-1.481203$

H -1.868484 4.341488-1.693919

H $-0.772390 \quad 5.2434541 .018135$

H $1.2303025 .572699-0.369150$

H $0.2468494 .881001-2.636610$

H $\quad 0.3000593 .210390-2.092869$

H $3.8704203 .045071-3.225384$

H $3.9195185 .323844-3.101314$

H $2.3545786 .128114-3.220030$

H $3.1119165 .874261-1.634490$

H $1.2045031 .612333-3.233345$

H $2.7650021 .075443-3.795769$

H $3.5074690 .871946-1.347868$

H $1.8404631 .241499-0.881031$

H $1.085209-0.732515-2.299311$

H $1.384531-0.9479550 .202788$

H $3.081099-1.297730-0.033796$

H $1.563140-3.1386741 .279967$

H $3.748079-3.499561-1.263499$

H $-0.304023-2.795318-0.102180$

H $\quad 0.108118-4.250217-0.979877$

H $-0.052086-2.710768-1.847302$

H $2.408192-5.325252-1.184011$

H $3.684107-4.096833-4.395118$

H $3.949185-2.427181-4.844657$

H $1.718586-2.591424-4.663761$

H 1.9843351 .8063441 .778592

H 4.6815823 .2486782 .136716

H $3.161101-0.2367882 .317820$

H 2.5840340 .4782333 .841959
H $3.918594-0.6718873 .830693$

H $5.430704-3.971342-2.667930$

H $6.056982-3.443249-4.211914$

H $5.8722090 .103527-0.984116$

H $7.532211-0.521490-0.997293$

H $5.375547-1.3754501 .087942$

H $8.358646-0.7081881 .270885$

H $7.984323-2.2042563 .253126$

H $6.276301-1.8590053 .270022$

H $8.9323050 .878748 \quad 5.089766$

H $9.613831-0.4411193 .981412$

H 6.5939881 .0048055 .494517

H $5.601784-0.3229344 .990574$

H 6.2542882 .2796143 .454003

H $6.111390-2.817625-1.031832$

C $-3.983984 \quad 3.663046 \quad 0.173004$

O $-4.3130643 .043353-1.084489$

C $-5.5796242 .358200-0.949680$

C $-6.008387 \quad 2.539273 \quad 0.501719$

C $-5.3234493 .837848 \quad 0.880435$

H $-6.2732442 .873832-1.624621$

C $-5.3863120 .898679-1.377979$

H -7.0962232 .5778940 .613050$

H $-5.634746 \quad 1.717328 \quad 1.125838$

C $-5.2151214 .045346 \quad 2.381089$

H -5.8791194 .6737090 .434778$

C $-6.601678-0.009016-1.122362$

O $-5.0675530 .880680-2.773627$

H $-4.5038910 .505152-0.865912$

H $-6.867693-0.004026-0.059765$

O $-7.7257590 .546037-1.815896$

C $-6.407601-1.461219-1.598689$

C $-5.153500-2.163798-1.052952$

H $-6.375341-1.480022-2.694954$

O $-7.573873-2.202707-1.234129$

H $-5.152235-3.210539-1.384520$

H $-4.267620-1.722020-1.528443$

H -6.2098864 .0730992 .837683$

H $-4.648728 \quad 3.2381792 .857914$

H -4.7132734 .9902342 .610934$

C $-3.2293264 .969124-0.071143$

H $-5.8506801 .195493-3.256903$

H -8.483664 - $0.042047-1.655016$

H -7.460678 $-3.109798-1.565986$

H $-3.126643 \quad 5.5264150 .866759$

H $-3.7867945 .605278-0.769361$

H $-3.3502382 .957478 \quad 0.727099$

C -3.169252 -2.587683 2.065970

O $-3.626792-2.6331630 .708316$

C $-4.950376-2.1243810 .477909$

C $-5.976209-2.9337321 .285701$

C $-5.602312-2.9877542 .771836$

C $-4.130605-3.3593202 .982963$

H -2.211095 -3.1233892 .055061$

C $-2.897716-1.1541162 .538612$

H $-4.973844-1.0815420 .803377$

O $-6.049781-4.2590340 .765060$

H $-6.965467-2.4761751 .179612$

H $-6.239834-3.7244943 .275790$

O $-5.870831-1.713528 \quad 3.350271$

H $-3.845728-3.1980934 .028730$

O $-3.968115-4.758206 \quad 2.730172$

C $-1.766328-0.5372341 .752284$

H $-3.768088-0.4959882 .464457$

H -2.599526 -1.146110 3.593964

H $-6.733912-4.7338991 .267086$

H $-5.659110-1.7710514 .297495$

H -4.150095 -4.9130761 .787384$

$\begin{array}{lllll}\text { O } & -2.263176 & 0.142544 & 0.680457\end{array}$

O $-0.584733-0.6698762 .042828$

C $-1.293636 \quad 0.740952-0.184215$

H $-0.577217 \quad 1.3383420 .386601$

H $-0.783307-0.038212-0.755007$

H $-1.817857 \quad 1.396673-0.883655$

SCF Energy (B3LYP/6-31G**//MMFF)= -3245.91432233

0700343

MM̄FF Geometry 
C 0.9724382 .2214842 .965670

C $1.045674 \quad 1.1710483 .797647$

C -0.0472870 .1524574 .051910$

O -1.2281060 .5116253 .297378$

C $0.423847-1.2538303 .636084$

C $-0.387980-2.3783184 .252918$

C $-1.273673-3.1523743 .594723$

C $-0.103812-2.644605 \quad 5.710696$

C -1.681215 -3.066817 2.150875

C $-3.209586-3.0445431 .991688$

C $-3.687331-2.8346570 .543108$

C $-3.317393-4.005164-0.372449$

C $-3.713116-3.745686-1.833320$

O $-3.118642-1.612798 \quad 0.067735$

O $-3.055533-4.744798-2.627962$

C $-3.163511-2.366468-2.254955$

C $-5.216718-3.943390-2.053886$

O $-3.599486-2.027181-3.569706$

C -3.504870 -1.237455-1.259130

C $-2.7677310 .076861-1.595489$

O $-4.910176-0.970374-1.329699$

C -2.4440220 .3360813 .872550$

O $-2.679736-0.2144854 .938988$

C $-3.5246800 .895423 \quad 3.028173$

C $-3.324370 \quad 1.9088562 .171243$

C -4.3469292 .5756321 .360756$

C -5.7238811 .9662341 .334805$

C $-1.2431150 .006682-1.472232$

C $-0.6716521 .405605-1.459171$

C $-0.2506513 .346376-2.944454$

O $-0.447188 \quad 1.995469-0.404834$

C - $1.5750344 .024611-3.106063$

C $-2.1912744 .695843-2.121523$

C $-3.5570175 .300637-2.262478$

C $-4.6355094 .442189-1.636526$

C $-5.4022703 .640151-2.394734$

C $-4.8899824 .578773-0.152875$

C $-4.005904 \quad 3.6914740 .680919$

N $-0.5092861 .934760-2.729960$

H 0.0690562 .3891332 .386900

H 1.9546561 .0353234 .380321

H $-0.2653420 .196248 \quad 5.125923$

H $1.467670-1.405783 \quad 3.942398$

H $0.428625-1.3275702 .543480$

H -1.761248 -3.9530834 .149831$

H $-0.658039-3.5089036 .092146$

H $0.961856-2.8484805 .858382$

H $-0.381661-1.7823836 .323877$

H -1.256069 -2.184300 1.668073

H -1.271101-3.946481 1.643902

H $-3.641308-3.9720472 .387897$

H -3.606903 -2.223409 2.601741

H $-4.777496-2.7206900 .576044$

H -3.768703 -4.937196 -0.009842

H -2.234031-4.173350 -0.348533

H $-3.238367-4.545205-3.562347$

H -2.074791 -2.471414 -2.321683

H -5.506913 -4.969494 -1.797237

H -5.484110 -3.814052 -3.108453

H -5.831085 -3.266477 -1.456553

H -4.526661 -1.740379 -3.509271

H -3.052331 $0.426701-2.596378$

H -3.128232 $0.857265-0.911757$

H -5.069173 -0.062139-1.024008

H $-4.5111160 .482502 \quad 3.211063$

H -2.3227852 .3228942 .075618$

H -5.6703380 .8982191 .095526$

H -6.3796372 .4100790 .582406$

H -6.213243 2.0825832 .307774

H $-0.932640-0.480756-0.541924$

H $-0.804972-0.559572-2.299939$

H $0.3459023 .450748-3.855295$

H $\quad 0.3200713 .753119-2.102978$

H -2.066594 $3.918085-4.070148$

H -1.702106 $4.790465-1.155545$

H -3.782796 $5.469480-3.324080$

H -3.559769 $6.298963-1.808508$

H $-6.1978443 .043814-1.960873$
H $-5.2465383 .557192-3.465290$

H $-5.949594 \quad 4.4059620 .065020$

H -4.720785 5.6198550 .151209

H -2.979892 4.0504830 .762954

H $-0.8106491 .387248-3.531001$

C 2.5228573 .3240141 .334978

O 3.5072212 .2962911 .097319

C 4.5498062 .8461320 .273063

C $4.0627904 .209237-0.201494$

C 3.2078564 .6410800 .973605

H 5.4077123 .0215060 .937007

C $5.0021241 .857494-0.812538$

H $4.8867314 .900111-0.405269$

H $3.4557204 .132617-1.109097$

C 2.2448195 .7665210 .634753

H 3.8658244 .9601441 .793079

C $3.8681271 .033634-1.465020$

O $5.7699082 .571631-1.776165$

H $5.6789261 .138241-0.332501$

H $3.3563250 .496250-0.661617$

O $2.9023681 .889936-2.066545$

C $4.3656800 .013752-2.510253$

C $3.270130-0.943133-3.022923$

H $5.189611-0.567327-2.079973$

O $4.8946170 .717475-3.636643$

H $2.462877-0.371832-3.497883$

H $3.716075-1.549697-3.822183$

H 2.7936216 .6575360 .312365

H $1.5626365 .481245-0.173421$

H 1.6394766 .0400691 .504364

C 2.0508093 .2550352 .788174

H $6.1385771 .925384-2.401339$

H $3.3456192 .362636-2.791961$

H $5.2637490 .058102-4.248632$

H 1.6234974 .2170093 .093505

H 2.8982763 .0445143 .451673

H 1.6936593 .1359200 .642300

C $3.463223-3.418598-0.236107$

O $3.792108-2.576515-1.348218$

C $2.698000-1.865777-1.935557$

C $1.662955-2.858828-2.481863$

C $1.200139-3.819588-1.380019$

C $2.380289-4.440337-0.624520$

H $4.384281-3.977260-0.024647$

C $3.119901-2.5936301 .015201$

H $2.214445-1.242278-1.178336$

O $2.206696-3.604525-3.568425$

H $0.805843-2.301011-2.874420$

H $0.591189-4.617250-1.822360$

O $0.391576-3.116793-0.444019$

H $2.032055-4.9610330 .275192$

O $3.000649-5.420045-1.462682$

C $4.299855-1.7711291 .494218$

H $2.295032-1.9015280 .828659$

H $2.836708-3.2661461 .832833$

H $3.014029-4.045156-3.252491$

H $-0.370764-2.756718-0.925910$

H $2.333087-6.097918-1.664185$

O $3.892473-0.9620472 .516658$

O $5.435529-1.8350221 .043809$

C $4.910929-0.1379353 .092535$

H $5.631884-0.7602333 .630668$

H 5.4137050 .4533412 .321390

H 4.4369900 .5451643 .801893

SCF Energy (B3LYP/6-31G**//MMFF)= -3245.91660166

0700344

MM̄MF Geometry

C -3.131588 -2.448325 -2.557557

C $-1.988144-2.143064-1.927827$

C $-0.767933-1.599185-2.617387$

O $-0.650085-0.228765-2.159785$

C $0.505568-2.372638-2.236466$

C $0.476752-3.853251-2.549418$

C $0.434767-4.830885-1.621996$

C $0.546898-4.201055-4.013232$

C $0.354191-4.672569-0.129833$

C $1.601603-5.1656410 .615715$ 
C $2.912019-4.4814010 .193549$

C $4.070870-4.9456721 .078437$

C $5.379687-4.2207660 .729985$

O $2.757946-3.063676 \quad 0.312208$

O $6.324955-4.4802441 .779245$

C $5.125056-2.6964840 .674502$

C $6.018006-4.797719-0.539021$

O $6.290006-2.0191820 .215382$

C $3.887231-2.309971-0.171219$

C $3.512383-0.816545-0.002190$

O $4.128417-2.575367-1.553353$

C $0.1173970 .627893-2.882717$

O $0.7986660 .364938-3.859899$

C $-0.0428701 .971517-2.281992$

C $0.7329392 .986341-2.691387$

C $0.6425894 .377429-2.238954$

C $-0.4541474 .721634-1.264871$

C $4.3849730 .174133-0.778289$

C 4.8350011 .3208380 .099836

C 4.0635833 .4385141 .122329

O 5.9468101 .3328590 .623456

C 3.1604114 .5549300 .708653

C 3.6091755 .7074620 .189648

C $2.7324616 .871191-0.173133$

C $2.7589507 .184125-1.651851$

C $3.7654617 .891724-2.192753$

C $1.5691816 .759823-2.480665$

C $1.5225385 .277156-2.727504$

N 3.8681822 .3035340 .242273

H -3.203489 -2.320771 -3.634650

H -1.937309 -2.249119-0.847610

H $-0.909654-1.596600-3.705594$

H $0.727642-2.181701-1.182293$

H $1.363334-1.933127-2.760790$

H $\quad 0.440230-5.865129-1.962925$

H $0.594433-5.282118-4.182071$

H $-0.334886-3.824253-4.540669$

H $1.440091-3.758246-4.466168$

H $\quad 0.143753-3.641190 \quad 0.159620$

H $-0.509386-5.2523250 .220343$

H $1.429414-4.9880401 .685384$

H $1.701345-6.2507240 .485591$

H $3.112043-4.738896-0.852618$

H $4.203397-6.0327081 .011958$

H $3.827512-4.7380002 .129841$

H $5.952838-4.1462302 .613110$

H $4.952718-2.3513151 .703345$

H $6.243583-5.862256-0.401737$

H $6.977994-4.315327-0.754658$

H $5.381103-4.706068-1.421178$

H $7.027384-2.2834700 .791734$

H $2.480424-0.680993-0.354704$

H $3.471336-0.5732001 .067820$

H $3.525180-2.025844-2.079818$

H $-0.8166612 .092652-1.531849$

H $1.4954342 .787304-3.443424$

H $-0.4439485 .764276-0.942123$

H $-0.3597014 .121132-0.354943$

H -1.437554 $4.533041-1.709487$

H $5.280381-0.284431-1.207917$

H $3.8220310 .579075-1.628951$

H $5.1192243 .730881 \quad 1.106117$

H 3.8241493 .1170942 .141126

H $2.094847 \quad 4.4199870 .874481$

H 4.6792175 .8391890 .043353

H 3.0802537 .7468470 .390862

H 1.6997826 .7080920 .158444

H $3.7754028 .148154-3.246762$

H $4.6038858 .227479-1.591291$

H $1.6005297 .248177-3.464144$

H $0.6548847 .140743-2.016597$

H $2.2875204 .930091-3.423501$

H $2.9279532 .107603-0.089754$

C -5.358891-1.770962 -1.674155

O $-4.846533-0.833630-0.705070$

C $-5.957219-0.2469230 .003107$

C -7.222832 -0.852630 -0.599954

C $-6.719281-2.184743-1.124378$
H $-5.878127-0.5709321 .045867$

C $-5.880567 \quad 1.281913-0.085182$

H -8.020954 -0.9586920.141452

H -7.614899-0.239527 -1.420004

C -7.642621 -2.817385-2.150560

H $-6.590141-2.871736-0.277380$

C -4.5881091 .8729250 .519580$

O $-5.9347821 .646271-1.470543$

H $-6.763901 \quad 1.7251870 .389499$

H $-3.7112461 .489070-0.013847$

O $-4.609784 \quad 3.2899470 .300644$

C -4.4369671 .6303252 .037707$

C -3.2168792 .3373442 .661377$

H -4.3777380 .5581902247643$

O $\quad-5.6071002 .1142122 .704127$

H -3.1674933 .3720132 .302356$

H $-3.3758882 .403738 \quad 3.746481$

H -8.628506 -3.008315 -1.714230

H -7.780222 -2.167605 -3.021247

H -7.240566 -3.772080 -2.503264

C $-4.368195-2.921358-1.844918$

H -6.107137 $2.601994-1.513739$

H $-4.4759453 .439393-0.650632$

H -5.6801763 .0652732 .513632$

H -4.810809 -3.732976 -2.432515

H $-4.100741-3.337366-0.866736$

H -5.479291 -1.226091-2.620718

C $-0.666924-0.3350493 .196261$

$\begin{array}{llll}\text { O } & -1.834139 & 0.493476 & 3.286170\end{array}$

C -1.8934391 .6060002 .383643$

C -0.6832522 .5259882 .593564$

C 0.6267721 .7471532 .453262

C 0.6292490 .4854903 .315758

H $-0.725117-0.9862714 .078253$

C $-0.714890-1.2270871 .951922$

H $-1.890903 \quad 1.2381331 .353244$

O $-0.7431563 .149702 \quad 3.874194$

H $-0.717306 \quad 3.328737 \quad 1.849321$

H 1.4648602 .3875102 .751936

O $\quad 0.817690 \quad 1.370424 \quad 1.093335$

H $1.493752-0.1403073 .064552$

O $0.789531 \quad 0.887642 \quad 4.679387$

C $-1.646792-2.3899202 .199491$

H -1.034953 -0.706884 1.045261

H $\quad 0.271357-1.655700 \quad 1.738144$

H $-0.7862482 .447776 \quad 4.545907$

H 0.8200932 .1847100 .562749

H $\quad 0.8716600 .0809615 .216121$

O $-2.929564-2.0415411 .898000$

O $-1.278014-3.4698812 .644265$

C $-3.908033-3.0539832 .152453$

H -3.948157 -3.277590 3.222708

H $-4.883374-2.6753061 .838363$

$\mathrm{H}-3.681545-3.9570971 .577681$

SCF Energy (B3LYP/6-31G**//MMFF) $=-3245.89923939$

0700345

MM̄FF Geometry

C $1.7333332 .795640 \quad 0.290455$

C 2.7751043 .0525171 .095157

C 3.2985382 .1326872 .175846

O 2.3783611 .0423722 .435786

C $4.629617 \quad 1.4822681 .763889$

C 5.8087992 .4276621 .710866

C 6.3761032 .8817220 .576431

C 6.3828782 .8141813 .049441

C $5.9432542 .627741-0.841155$

C $6.9181151 .719071-1.601848$

C $6.8464730 .246802-1.166348$

C $7.902007-0.580107-1.903835$

C $7.815675-2.068367-1.538813$

O $5.540922-0.241545-1.489215$

O $8.604258-2.781341-2.506839$

C $6.352641-2.539855-1.691551$

C $8.471134-2.347993-0.181848$

O $6.188982-3.871618-1.211117$

C $5.293189-1.591459-1.077784$

C $3.892943-1.969934-1.612167$ 
O $5.218224-1.709066 \quad 0.342485$

C $1.3470591 .273607 \quad 3.288653$

$\begin{array}{lllll}\text { O } & 1.114575 & 2.317797 & 3.883048\end{array}$

C 0.4628080 .0916653 .418433

C $0.712777-1.1044682 .862235$

C $-0.133544-2.2981332 .964252$

C $-1.442620-2.1607463 .698689$

C $2.760075-1.076928-1.084196$

C $1.413224-1.490840-1.654079$

C $-1.026841-1.080970-1.387211$

O $1.292095-2.337596-2.536282$

C $-1.544700-2.081322-0.403221$

C -1.506403 -3.407078 -0.603192

C $-1.999769-4.423605 \quad 0.385476$

C $-0.868214-5.2089871 .010551$

C $-0.353557-6.287453 \quad 0.396085$

C $-0.391022-4.788726 \quad 2.383555$

C $0.286711-3.4438272 .385554$

N $0.361254-0.794780-1.072546$

H $1.204303 \quad 1.8520950 .381989$

H 3.2968094 .0007570 .984748

H 3.4347762 .7048013 .101284

H 4.4939230 .9568650 .812343

H 4.8669690 .6751642 .471340

H 7.2433283 .5353600 .664110

H 7.2722893 .4462522 .953963

H 5.6469433 .3701813 .638115

H 6.6745081 .9200343 .610223

H $4.9252982 .232408-0.901625$

H $5.909483 \quad 3.600394-1.348415$

H $6.6659831 .786286-2.668289$

H $7.9422042 .095434-1.486860$

H $7.0012880 .182460-0.084056$

H $8.909816-0.194881-1.704412$

H $7.751488-0.478397-2.987919$

H $8.501617-3.732030-2.327425$

H $6.163572-2.593029-2.773062$

H $9.543806-2.122114-0.226363$

H $8.402730-3.4083100 .084104$

H $8.050379-1.7561660 .632928$

H $6.147406-3.838516-0.240671$

H $3.895758-1.909428-2.708596$

H $3.662945-3.009067-1.342971$

H $6.090972-1.9353100 .692627$

H -0.4245540 .2644514 .019663$

H $1.623404-1.2353622 .281212$

H $-1.267003-1.9510294 .759490$

H -2.071095 -3.051741 3.646360

H -2.039439-1.346230 3.273074

H $2.708030-1.1414940 .007960$

H $2.935763-0.031567-1.361526$

H -1.106807 -1.448332 -2.415858

H $-1.591965-0.146945-1.311544$

H $-1.965775-1.6929950 .519317$

H -1.085410 -3.790667 -1.529862

H -2.682844 -5.105910 -0.137050

H -2.605244 -3.9550351 .170571$

H $0.439989-6.8726790 .848399$

H $-0.713920-6.605984-0.576602$

H $0.335119-5.5156312 .771616$

H $-1.233585-4.8385143 .078789$

H $1.244323-3.4406341 .862822$

H $0.550025-0.186768-0.281647$

C $-0.155034 \quad 4.280425-0.385565$

O $-1.093273 \quad 3.199674-0.233029$

C -2.379286 $3.620929-0.717921$

C $-2.2385415 .095445-1.085310$

C $-0.7684245 .192344-1.443827$

H -2.573237 $3.037617-1.625910$

C -3.480106 3.3359580 .316473

H $-2.9011345 .379900-1.908708$

H -2.467581 $5.747636-0.234263$

C $-0.2321856 .612933-1.429074$

H $-0.6226784 .760293-2.442892$

C -3.6426241 .8325660 .637250$

O $-3.2059734 .069291 \quad 1.509299$

H $-4.4293483 .714080-0.081642$

H $-3.7537671 .307944-0.317446$
O $-2.4536691 .347781 \quad 1.258986$

C $-4.862087 \quad 1.5346201 .535836$

C $-5.1171830 .037702 \quad 1.804599$

H $-5.753812 \quad 1.9765071 .075299$

$\begin{array}{llll}\text { O } & -4.686806 & 2.174026 & 2.802290\end{array}$

H $-4.288114-0.3891372 .381836$

H $-5.993354-0.0229292 .464243$

H $-0.767147 \quad 7.233279-2.155718$

H $-0.3472597 .075149-0.442991$

H $0.8310766 .632008-1.687997$

C $1.2190313 .729584-0.767063$

H $-2.380203 \quad 3.716252 \quad 1.882947$

H $-2.5169650 .380373 \quad 1.311543$

H $-5.502752 \quad 2.0424213 .314421$

H $1.9233884 .552416-0.933213$

H $1.1522053 .162600-1.703681$

H -0.0993484 .7949370 .583279$

C $-6.815994-0.821485-1.405682$

O $-6.476470-0.215897-0.152097$

C $-5.368718-0.7982640 .539944$

C $-5.654241-2.2732030 .860792$

C $-6.022563-3.049838-0.408323$

C $-7.093366-2.324174-1.231797$

H $-7.758642-0.343545-1.702588$

C $-5.770966-0.509062-2.488868$

H $-4.476143-0.744637-0.089133$

O $-6.722112-2.3614851 .801561$

H $-4.764661-2.7106131 .328740$

H $-6.386277-4.045147-0.125796$

O $-4.848227-3.225432-1.193831$

H $-7.203794-2.789324-2.218364$

O $-8.354403-2.451196-0.567839$

C $-5.7007430 .971770-2.803903$

H $-4.769007-0.826212-2.188264$

H $-6.039909-1.026580-3.416802$

H $-6.855605-3.3024462 .006843$

H $-5.092645-3.748094-1.976424$

H -8.555273 -3.399980 -0.498359

O $-4.676888 \quad 1.189112-3.682117$

O $-6.449386 \quad 1.827542-2.352897$

C $-4.4957422 .547100-4.097145$

H $-3.544427 \quad 2.615122-4.631429$

H $-5.3037682 .835892-4.775285$

H $-4.4599903 .219470-3.234641$

SCF Energy (B3LYP/6-31G**//MMFF) = -3245.88456745

07_00346

MM̄FF Geometry

C $3.1908754 .012295-1.509923$

C $4.3084153 .406392-1.079866$

C $5.0244092 .237196-1.718134$

O $4.900876 \quad 1.155699-0.759763$

C $4.4652881 .787983-3.084085$

C $5.3261810 .771477-3.810360$

C $5.018862-0.532410-3.961382$

C $6.5773021 .326151-4.444157$

C $3.817040-1.260031-3.425374$

C $4.210218-2.586149-2.755386$

C $3.047735-3.293539-2.036104$

C $1.920058-3.695865-2.990435$

C $0.725581-4.314886-2.250095$

O $2.564086-2.410813-1.021184$

O $-0.357950-4.369255-3.191198$

C $0.317609-3.367068-1.101961$

C $1.006729-5.760146-1.824964$

O $-0.699087-3.951354-0.290710$

C $1.505801-2.938921-0.213337$

C $1.108165-1.8385500 .794137$

O $1.953845-4.069487 \quad 0.543340$

C $5.9749520 .356954-0.539010$

O $7.0376910 .365415-1.143584$

C $5.705365-0.6014080 .554613$

C $4.956226-0.2716251 .618349$

C $4.705703-1.1142362 .790676$

C $5.130934-2.5572642 .714594$

C $0.705586-0.5036160 .160596$

C $\quad 0.6684440 .5695891 .225303$

C $-\begin{array}{llll}0.654534 & 1.310521 & 3.191148\end{array}$ 
O $1.6224451 .320347 \quad 1.415547$ C -0.2204880 .4262024 .317424$ C 0.9838200 .5092944 .902081 C $1.456238-0.4412575 .961786$ C $2.356156-1.5230145 .403356$ C $1.881757-2.7587215 .170889$ C $3.819898-1.2050005 .200230$ C $4.107242-0.5641663 .868905$ N -0.5040640 .5521651 .964166$ H $2.7026453 .679661-2.421176$ H $4.7568683 .762031-0.153052$ H $6.0762002 .532619-1.818577$ H $4.3693032 .658063-3.747749$ H $3.4478391 .404355-2.953354$ H $5.698238-1.152525-4.545262$ H $7.1347650 .564814-5.000290$ H $6.3232682 .126109-5.147405$ H $7.2502361 .733257-3.683866$ H $3.263610-0.647629-2.709668$ H $3.148882-1.457872-4.270552$ H $4.644060-3.264277-3.500888$ H $4.993939-2.381227-2.014324$ H $3.462995-4.185234-1.551592$ H $2.291984-4.379111-3.764046$ H $1.550136-2.811679-3.525521$ H -1.110312 -4.809650 -2.761556 H -0.124152 -2.474963 -1.563549 H $1.189866-6.386800-2.706165$ H $\quad 0.140014-6.199878-1.319001$ H $1.872504-5.854275-1.166314$ H $-1.469207-4.119845-0.858800$ H $\quad 0.312136-2.2012481 .457260$ H $1.963951-1.6538041 .457614$ H $2.415616-3.7492631 .335827$ H $6.229893-1.5481320 .484324$ H 4.5180190 .7245751 .659558 H $4.745501-3.0261911 .802035$ H $4.757855-3.1624713 .543845$ H $6.223276-2.6363282 .715163$ H $1.421568-0.184601-0.604871$ H $-0.274871-0.571180-0.321822$ H -1.709162 $1.579746 \quad 3.300210$ H -0.0613532 .2292273 .135104$ H -0.915271 -0.3499384 .629389$ H $1.680344 \quad 1.2779894 .577894$ H $0.593038-0.8946816 .467528$ H $1.982971 \quad 0.119183 \quad 6.743445$ H $2.523947-3.5502784 .799356$ H $0.838815-3.0044995 .340811$ H $4.426048-2.1058295 .346615$ H $4.157144-0.5247025 .992949$ H 3.8327750 .4897093 .822920 H -1.185288 -0.1793051 .781007$ C $1.2162134 .652312-0.130920$ O $0.2661734 .286012-1.148046$ C -1.066702 $4.555923-0.656612$ C -0.8991535 .0983450 .757809$ C 0.4944655 .6968950 .712338 H -1.479058 $5.325127-1.320554$ C $-1.8823793 .260702-0.754674$ H -1.6722095 .8276871 .018456$ H -0.9403074 .2872621 .495724$ C 1.0996915 .9162432 .087742 H 0.4518616 .6526690 .173556 C $-3.2811203 .336236-0.120857$ O $-2.0246632 .929926-2.140251$ H -1.306573 $2.448906-0.298340$ H -3.1912513 .5058420 .958610$ O $-3.9538064 .482971-0.656166$ C $-4.1738752 .102456-0.372800$ C -3.5217840 .7824160 .073577$ H $-4.4566042 .057172-1.431047$ $\begin{array}{lllll}\text { O } & -5.379197 & 2.314626 & 0.369747\end{array}$ H -2.539114 $0.678390-0.395163$ H $-3.388150 \quad 0.821716 \quad 1.161597$ H 0.4833346 .6062142 .673276 H 1.1769264 .9772802 .646566 H 2.1034296 .3451562 .010060
C $2.5126105 .139038-0.776958$

H -1.130545 $2.885644-2.520695$

H $-4.8351804 .518628-0.246223$

H -5.9762161 .5714330 .180476$

H $3.1818135 .555776-0.016001$

H $2.3033085 .935333-1.501278$

H 1.4109973 .7539500 .467757

C $-6.443928-1.5553800 .251592$

O $-5.540132-0.4688090 .493638$

C $-4.343113-0.470747-0.287413$

C $-3.538757-1.756558-0.029176$

C $-4.390773-3.009503-0.246520$

C $-5.745060-2.9084460 .464880$

H -7.215412 -1.451439 1.025797

C -7.131933-1.435889-1.112791

H $-4.602506-0.410430-1.349947$

O $-3.020467-1.761017 \quad 1.300260$

H -2.677958 -1.774792 -0.707503

H $-3.842212-3.8823170 .127683$

O $-4.587153-3.187051-1.645999$

H $-6.412206-3.7173700 .145172$

O $-5.541556-3.065770 \quad 1.872470$

C $-8.026481-0.219790-1.152656$

H -6.429292 -1.380065 -1.948937

H -7.767779 -2.308316 -1.305411

H $-3.771222-1.6711931 .912018$

H -5.108391 -3.998620 -1.768611

H $-5.146979-3.9429672 .015287$

O $-7.3825150 .813129-1.766859$

O $-9.152160-0.186817-0.672630$

C $-8.1184762 .037551-1.848276$

H -7.483412 $2.783489-2.333412$

H $-9.0203271 .896629-2.451303$

H -8.374473 $2.394214-0.845904$

SCF Energy $\left(B 3 L Y P / 6-31 G^{* *} / / M M F F\right)=-3245.91095392$

$07 \quad 00347$

MM̄FF Geometry

C $-0.2648691 .213356-2.167313$

C $-0.7607860 .279081-2.993242$

C $-0.206154-1.118330-3.179113$

O $1.130919-1.252697-2.640847$

C $-1.106143-2.135194-2.460839$

C $-0.625967-3.566351-2.586690$

C $0.034332-4.226254-1.613558$

C $-0.976416-4.254325-3.878433$

C $0.474290-3.667547-0.284899$

C $1.857748-4.1953780 .121169$

C $2.479825-3.4904571 .339875$

C $1.564024-3.4807722 .566980$

C $2.192323-2.7286223 .750342$

O $2.821221-2.1503460 .960904$

O $1.145483-2.5074294 .708248$

C $2.683546-1.3495663 .257075$

C $3.248678-3.5811544 .462011$

O $3.385789-0.6627254 .290383$

C $3.535318-1.4227841 .972248$

C $3.875047-0.0240201 .413422$

O $4.783197-2.0576442 .281796$

C $2.159891-0.751813-3.375276$

O $2.086783-0.121683-4.417971$

C $3.415570-1.103200-2.679797$

C $4.487534-0.311290-2.826487$

C $5.805901-0.525030-2.228599$

C $6.087236-1.878661-1.629449$

C 2.6751470 .8296600 .998984

C 3.1275122 .0004710 .147902

C 4.5096554 .0632510 .217728

O $2.9053122 .046928-1.059850$

C $5.9123883 .695431-0.177314$

C 6.6730142 .7374690 .377369

C $8.0756702 .418523-0.055917$

C $8.2726990 .945585-0.341763$

C 8.6514260 .1019970 .633563

C $8.1238470 .467016-1.767329$

C $6.7029780 .482978-2.257982$

N 3.8231732 .9604410 .864926

H $0.5861930 .966554-1.541728$ 
H -1.613709 $0.532753-3.619102$ H - $0.174822-1.346209-4.252339$ H $-2.132369-2.069051-2.845949$ H -1.178270 -1.870591-1.401670 H $\quad 0.289777-5.271039-1.784125$ H $-0.636122-5.295084-3.900405$ H -2.061505 -4.258419 -4.024619 H $-0.512833-3.738051-4.724886$ H $\quad 0.506265-2.574857-0.302503$ H $-0.266286-3.967306 \quad 0.465081$ H $1.781886-5.2699250 .331168$ H $2.544339-4.080668-0.728099$ H $3.407277-4.0287101 .569801$ H $1.292639-4.5031402 .857708$ H $0.619786-2.9772282 .323330$ H $1.513524-1.9643105 .426514$ H $1.787201-0.7516503 .055292$ H $2.797327-4.5054654 .842671$ H $3.650972-3.0639215 .340059$ H $4.081941-3.8621213 .814896$ H $4.271878-1.0573754 .358648$ H 4.4923520 .5217842 .138514 H $4.520089-0.1519480 .534502$ H $5.437012-1.7999401 .610885$ H $3.415470-1.992151-2.060332$ H $4.3888010 .589795-3.431074$ H $5.407128-2.085441-0.796945$ H $7.100584-1.976174-1.233826$ H $5.963378-2.663982-2.383576$ H 1.9619090 .2441570 .409305 H 2.1391091 .2148221 .873052 H 4.5199134 .9131060 .906710 H $3.9461374 .348153-0.677113$ H $6.3306264 .280963-0.993519$ H 6.2881972 .1640541 .216821 H 8.7539152 .7286710 .750070 H $8.3705113 .008715-0.932234$ H $8.827774-0.950060 \quad 0.436413$ H $8.789680 \quad 0.445643 \quad 1.653482$ H $8.560333-0.530661-1.894660$ H $8.725690 \quad 1.109767-2.423104$ H $6.4053121 .433460-2.702020$ H 4.0022262 .8076381 .852974 C $-0.9567123 .111013-0.645442$ O $-2.2605812 .711854-0.168205$ C -2.9393253 .8706190 .347961$ C $-1.8453174 .892453 \quad 0.626013$ C $-0.9107974 .638619-0.536879$ H -3.574257 $4.247820-0.462934$ C $-3.8077503 .534388 \quad 1.565318$ H -2.2281845 .9170700 .655105$ H -1.331706 4.6918601 .573326 C $0.4884795 .193564-0.324579$ H -1.341897 $5.093540-1.439044$ C $-4.8456772 .413566 \quad 1.352826$ O $-2.952106 \quad 3.1269362 .641387$ H -4.3080224 .4493781 .905803$ H -4.3342401 .4471551 .355343$ O $\quad \begin{array}{llll}-5.698840 & 2.401173 & 2.506223\end{array}$ C -5.7104902 .5404390 .088073$ C $-6.912267 \quad 1.5772380 .020948$ H -5.078941 2.392205 -0.795199 O -6.2254053 .8738510 .017908$ H $-7.6721401 .879696 \quad 0.753257$ H $-7.3798571 .728086-0.961329$ H $0.4566196 .281330-0.204847$ H 0.9562794 .7706050 .570939 H $1.1333014 .965782-1.179254$ C $-0.7546332 .631579-2.088067$ H -3.5205142 .9336453 .406695$ H -6.2122613 .2273082 .499561$ H $-6.7122063 .956509-0.819916$ H $0.0097593 .249421-2.574614$ H -1.685982 $2.747270-2.655672$ H -0.2207172 .6572290 .030500$ C $-5.090522-1.619721-0.649892$ O $-5.575999-0.272213-0.742738$ C -6.5753120 .0891790 .212224$
C $-7.815380-0.8057360 .048506$

C $-7.447381-2.2926300 .112677$

C $-6.247435-2.626250-0.780801$

H $-4.435963-1.740724-1.522610$

C $-4.233233-1.8449490 .607242$

H -6.175671 -0.0414581 .222636$

O -8.463689 $-0.537288-1.193941$

H $-8.533108-0.5645820 .841202$

H -8.307511 -2.894618 -0.205781

O $-7.137966-2.6529331 .455569$

H $-5.887420-3.638977-0.564316$

O $-6.699159-2.622352-2.138521$

C $-3.008591-0.958730 \quad 0.627425$

H $-4.793203-1.6404151 .523228$

H $-3.888798-2.8852930 .629244$

H $-7.817190-0.687178-1.904943$

H -7.923415 - 2.4677131 .997906

H $-5.954200-2.903453-2.696714$

O $-2.297131-1.2008931 .766902$

O $-2.714422-0.148719-0.239669$

C $-1.123946-0.3993621 .936190$

H -1.3771490 .6644991 .905804$

H $-0.697838-0.6272122 .916358$

H $-0.388108-0.6430841 .164619$

SCF Energy (B3LYP/6-31G*//MMFF) $=-3245.90564483$

0700348

MM̄FF Geometry

C -0.2769942 .6390400 .510356$

C $-0.1677291 .469867-0.139306$

C $-1.0689090 .980032-1.248564$

O $-2.302372 \quad 1.739376-1.237747$

C $-0.4145731 .092050-2.636025$

C $0.7978450 .213529-2.841627$

C $0.742989-1.100507-3.134061$

C $2.1246650 .919100-2.773537$

C $-0.486136-1.959160-3.265554$

C $-0.323638-3.326396-2.585609$

C $-0.264599-3.267016-1.049189$

C $0.201100-4.620821-0.504444$

C $0.199244-4.6582251 .028398$

O $-1.576863-2.962498-0.554762$

O $0.348431-6.0270201 .435420$

C $-1.178119-4.1829271 .525722$

C $1.391000-3.8983351 .618904$

O $-1.194966-4.0810532 .948781$

C -1.661782 -2.862282 0.875217

C $-3.140723-2.6246641 .272512$

O $-0.863082-1.7869001 .374605$

C $-3.3980661 .164740-1.801450$

O $-3.4416130 .115982-2.424210$

C $-4.5663382 .012464-1.481349$

C $-5.7949701 .511568-1.675898$

C $-7.0630772 .177616-1.382603$

C $-7.0132463 .565021-0.799975$

C $-3.833255-1.4776780 .529444$

C $-5.177009-1.1620681 .159939$

C -7.443284-2.130662 1.535270

O $-5.345145-0.156131 \quad 1.846055$

C -8.457410 -1.5103270 .626455$

C $-9.266576-0.5092361 .007031$

C -10.3637140 .0667590 .155720$

C -10.196164 $1.549190-0.092636$

C -10.614204 2.4530770 .809600

C $-9.6186911 .984885-1.419103$

C $-8.208592 \quad 1.508882-1.627799$

$\mathrm{N}-6.136373-2.1282640 .905799$

H $-1.055817 \quad 3.3442410 .232400$

H 0.6242330 .7843610 .157588

H $-1.314196-0.063274-1.018819$

H $-0.1556402 .141921-2.832860$

H $-1.1501680 .862721-3.417572$

H $1.682833-1.622070-3.312651$

H $2.9554660 .250595-3.017439$

H $2.1540441 .748387-3.487838$

H $2.2999561 .317707-1.770151$

H $-1.380929-1.465124-2.881754$

H $-0.654547-2.130802-4.335875$ 
H -1.175257 -3.950638 -2.885997 H $0.581893-3.809119-2.975400$ H $0.429394-2.477383-0.740655$ H $1.196974-4.873553-0.889708$ H $-0.469756-5.410773-0.871337$ H $1.181622-6.362077 \quad 1.062210$ H -1.896306 -4.9754861 .273894$ H $2.332475-4.3194331 .246596$ H $1.428390-4.0038052 .708674$ H $1.383755-2.834527 \quad 1.373817$ H $-0.726575-3.2645403 .192262$ H -3.715502 -3.539267 1.076261 H -3.202832 -2.432505 2.351679 H -1.386419 -0.9705501 .371632$ H -4.386103 2.998373 -1.069416 H $-5.8861220 .503456-2.079172$ H -6.4400353 .5702530 .133930$ H -7.999177 $3.968929-0.557955$ H -6.544574 4.260707 -1.504439 H -3.235004 -0.5630740 .562887$ H -3.984865 -1.734360 -0.524402 H -7.382737 -1.614810 2.499477 H $-7.708808-3.1761981 .721012$ H -8.564599 -1.936398 -0.368019 H $-9.170399-0.1078262 .013690$ H $-11.317345-0.1101290 .670619$ H -10.448206 -0.465212 -0.800050 H $-10.522457 \quad 3.5187730 .628267$ H -11.055083 2.1452751 .752110 H -9.684515 $3.071625-1.544243$ H - $10.2418521 .576720-2.225611$ H -8.146295 $0.499360-2.035712$ H $-5.912673-2.8967180 .280271$ C 1.5122524 .2292601 .284340 O $2.583197 \quad 3.7655730 .432058$ C 3.8377204 .1626861 .014213 C 3.5026145 .3263101 .934607 C 2.1825664 .8566252 .511409 H 4.1807113 .3206281 .628333 C $4.8834454 .467636-0.062763$ H 4.2703365 .4928712 .695985 H 3.3613666 .2590931 .376575 C 1.3689905 .9670973 .153994 H 2.3844804 .0837053 .265562 C $5.1005173 .333996-1.085864$ O $4.4719605 .632096-0.788947$ H 5.8318154 .7288300 .422358 H $4.2510243 .298823-1.778436$ O $6.2391413 .680818-1.884003$ C $5.3150011 .940060-0.473869$ C $5.6844230 .880426-1.531008$ H 4.3980861 .6386150 .044741 O $\quad 6.3564152 .0191190 .499252$ H $5.1327501 .070162-2.457895$ H $6.757440 \quad 0.951039-1.747936$ H 1.9167776 .4091563 .992766 H 1.1466496 .7666412 .439322 H 0.4181585 .5824143 .535921 C 0.6094333 .0499681 .652457 H $5.1528195 .810489-1.460327$ H $7.0214943 .650598-1.306805$ H 6.4476411 .1393220 .903228 H -0.0411253 .3197442 .492748$ H $1.2227052 .200317 \quad 1.975755$ H $0.957920 \quad 4.9864230 .715387$ C $5.894011-2.0616420 .761027$ O $6.167831-0.820807 \quad 0.098794$ C $5.350330-0.540174-1.039176$ C $5.570934-1.609139-2.116904$ C $5.309649-3.012838-1.557731$ C $6.022813-3.243367-0.218264$ H $6.684827-2.1567161 .516061$ C $4.548886-2.0260381 .501333$ H $4.295816-0.544861-0.741624$ O $6.901072-1.533263-2.624163$ H $4.894412-1.415662-2.957202$ H $5.653073-3.755583-2.288346$ O $3.903447-3.179832-1.395022$
H $5.654136-4.1611290 .254436$

O $7.411628-3.445455-0.499286$

C $4.480988-0.9020832 .514014$

H $3.719235-1.8906310 .802612$

H $4.396372-2.9667292 .041464$

H $7.511854-1.644863-1.875667$

H $3.750531-4.084835-1.074157$

H $7.852602-3.6642780 .339180$

O $3.173955-0.6696732 .835466$

O $5.448036-0.3142862 .979058$

C 2.9517950 .3776363 .784665

H $1.8737940 .473173 \quad 3.939174$

H 3.4230420 .1285004 .740130

H 3.3365581 .3268743 .399157

SCF Energy (B3LYP/6-31G**//MMFF)= -3245.91496882

0700349

MM̄FF Geometry

C -1.984237 $-5.092446 \quad 0.732115$

C $-2.598178-4.4237301 .720341$

C $-3.922747-3.7011491 .661235$

O $-3.628674-2.2819421 .606147$

C $-4.806574-4.0382960 .443599$

C $-6.166555-3.3609760 .473132$

C $-6.562838-2.385251-0.369912$

C $-7.119647-3.8716171 .523999$

C $-5.783062-1.763327-1.495868$

C $-5.283087-0.369542-1.113577$

C $-4.3087680 .243863-2.131059$

C $-2.973616-0.501700-2.173395$

C $-1.9720890 .188172-3.106071$

O $-4.0821991 .595763-1.713646$

O $-0.687446-0.414582-2.897961$

C $-1.8710791 .684384-2.739377$

C $-2.306768-0.077063-4.579225$

O $-1.0753502 .355413-3.711133$

C $-3.2431312 .378715-2.586092$

C $-3.1491513 .762556-1.893127$

O $-3.8497922 .540614-3.868754$

C $-3.353084-1.6357182 .771224$

O $-3.355492-2.1080653 .896180$

C -3.030232 -0.2329072 .428587$

C $-2.6688830 .632373 \quad 3.387230$

C -2.3486222 .0482493 .185461$

C $-2.5386362 .607523 \quad 1.800347$

C $-2.3510544 .859438-2.603814$

C $-0.8990024 .966137-2.165196$

C $0.5463085 .373083-0.173837$

O $0.0240994 .880061-2.972904$

C 0.6010134 .4979731 .037160

C 0.8443934 .9473272 .277510

C 0.9171394 .0552403 .484696

C -0.0748074 .4467844 .558386$

C 0.3409194 .9681535 .724837

C -1.5477614 .2323964 .282090$

C $-1.9167132 .772898 \quad 4.239040$

N $-0.7481975 .204567-0.808825$

H -2.448477 -5.186534 -0.244361

H $-2.076144-4.3385842 .672490$

H $-4.475069-3.9304422 .580776$

H $-4.968573-5.1229710 .390309$

H -4.268942 -3.768076 -0.469578

H -7.565122 -1.976521 -0.246792

H $-8.121050-3.4395121 .422129$

H $-7.224725-4.9586341 .445518$

H $-6.758185-3.6265282 .527050$

H -4.960378 -2.407250 -1.807814

H $-6.440739-1.686091-2.370582$

H $-6.1432390 .303954-0.995661$

H $-4.804701-0.392189-0.128086$

H $-4.7919790 .251346-3.114648$

H -3.098739 -1.546379 -2.477817

H -2.544450 -0.536030 -1.162021

H $-0.434827-0.277382-1.968921$

H $-1.3387671 .764960-1.782082$

H -2.311235 -1.155127 -4.781041

H -1.543325 $0.345505-5.242074$

H $-3.2790520 .320771-4.877312$ 
H $-0.2136431 .906006-3.743626$ H -4.177205 4.138565 -1.785879 H -2.795599 $3.617270-0.867116$ H -4.492057 $3.267726-3.815738$ H $-3.0969210 .054078 \quad 1.385915$ H -2.6033190 .2766524 .414509$ H -3.5732922 .4674071 .467075$ H -1.871253 2.108831 1.088992 H -2.3344073 .6765781 .726350$ H -2.389182 4.741790 -3.691908 H $-2.8085415 .833012-2.383673$ H 0.6499036 .4339940 .075371 H $1.3479785 .099595-0.866832$ H 0.4425103 .4339250 .880748 H 1.0204136 .0087012 .435440 H 1.9420584 .1082443 .874155 H 0.7595583 .0049243 .212891 H -0.3625705 .2499226 .501325$ H 1.3944395 .1253105 .930760 H -1.825737 4.771182 3.373007 H -2.152769 4.6934865 .074455 H -1.816972 2.2733915 .203946 H - $1.5737095 .401734-0.250336$ C $0.512681-4.734148 \quad 0.451089$ O $0.509911-3.562463 \quad 1.294714$ C $0.645997-2.3920700 .459625$ C $1.191844-2.907299-0.863147$ C $0.459804-4.228856-0.991467$ H $-0.368064-1.9918330 .331420$ C $1.537749-1.3709911 .172348$ H $0.997547-2.229672-1.697920$ H $2.274581-3.073084-0.799553$ C $1.085076-5.178233-1.998074$ H $-0.571997-4.018471-1.298214$ C $1.852487-0.128990 \quad 0.322840$ O $0.867727-0.9448092 .363531$ H $2.461431-1.8650481 .491322$ H $2.353580-0.419097-0.603369$ O $\quad 0.6167320 .473748-0.089111$ C 2.6809630 .9546491 .038298 C 3.9933300 .4948391 .698384 H 2.0621681 .4291831 .808729 O 2.9968951 .9854830 .096314 H 4.3941021 .3491552 .260529 H $3.768064-0.2873512 .431266$ H $1.073417-4.737827-3.000428$ H $2.125806-5.405280-1.743959$ H $0.532644-6.122443-2.035950$ C $-0.609626-5.686148 \quad 0.880723$ H $0.659064-1.7423662 .879964$ H $\quad 0.1142050 .6888640 .715582$ H $2.1692392 .242955-0.344430$ H $-0.452175-5.9669641 .930150$ H $-0.561844-6.6129610 .297670$ H $1.472281-5.2340210 .636724$ C $5.538365-1.670676-0.946035$ O $4.650433-1.1469640 .049350$ C 5.0996420 .0258210 .728614 C $6.413207-0.2725841 .472320$ C $7.472729-0.8488170 .526359$ C $6.914705-1.987346-0.335830$ H $5.079044-2.617415-1.257477$ C $5.617793-0.762880-2.181029$ H $5.2732750 .823681-0.000821$ O $6.160975-1.2001132 .526617$ H $\quad 6.7800630 .6548341 .928460$ H $8.317324-1.2213601 .118863$ O $7.9581520 .201961-0.303960$ H $7.624609-2.242426-1.131345$ O $6.787712-3.1433860 .497618$ C $4.261621-0.603614-2.830782$ H $6.0060280 .228759-1.932699$ H $6.291526-1.187312-2.933982$ H $6.999799-1.3470432 .995615$ H $8.648854-0.172262-0.877065$ H $6.491409-3.879959-0.063659$ O $3.9625020 .721020-2.959776$ O $3.554164-1.539071-3.181628$
C $2.6897111 .014547-3.543756$

H $2.6283050 .598125-4.553537$

H $1.8879980 .621091-2.913599$

H $2.5851472 .101160-3.604114$

SCF Energy (B3LYP/6-31G**//MMFF)= -3245.91368337

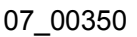

MM̄FF Geometry

C -2.508237 3.7817130 .005486

C -1.8870014 .8435830 .541642$

C -0.5939354 .8257341 .320457$

O -0.0634123 .4862941 .421242$

C 0.4735885 .6785210 .608916

C 1.7295085 .8410561 .445011

C 2.8310895 .0703671 .342692

C 1.6834806 .9638322 .449305

C 3.0578003 .8982660 .425461

C $4.3802754 .040238-0.338778$

C $4.6521932 .923620-1.361771$

C $3.7652573 .046199-2.600080$

C $4.0375861 .915311-3.600736$

O $4.450728 \quad 1.654097-0.736316$

O $2.9769471 .918920-4.568790$

C $3.9738990 .559442-2.864450$

C $5.3377322 .145254-4.381894$

O $4.376370-0.486569-3.744832$

C $4.7961420 .518854-1.554047$

C $4.463945-0.722885-0.690578$

O $6.1919270 .517372-1.854231$

C -0.2006972 .8188202 .596235$

O $-\begin{array}{llll}0.845820 & 3.159093 & 3.573459\end{array}$

C 0.6423471 .6067192 .493819

C 0.8838860 .8569113 .579051

C $1.780896-0.3008443 .633464$

C $2.565673-0.6204932 .388842$

C $4.902105-2.074385-1.252122$

C $4.707741-3.182321-0.233542$

C $2.955133-4.7054660 .692955$

O $5.648728-3.6360880 .413252$

C $2.060870-4.1942791 .776333$

C $2.168447-4.5521273 .064949$

C $1.241014-4.0594204 .140597$

C $1.975391-3.4490815 .314974$

C $1.929858-4.0231226 .529141$

C $2.763922-2.1778025 .087287$

C $1.879581-0.9960894 .785872$

N $3.388942-3.593148-0.133847$

H $-2.0764582 .792020 \quad 0.109724$

H -2.3470365 .8235290 .428044$

H -0.7908395 .2378992 .317723$

H $0.073922 \quad 6.675607 \quad 0.380598$

H $\quad 0.7190945 .235617-0.363419$

H 3.6626205 .2803522 .014771

H 2.6113617 .0467383 .025175

H 1.5247517 .9204821 .940727

H $0.8669596 .808604 \quad 3.161434$

H 3.0937262 .9930361 .042827

H $2.2317383 .769035-0.275397$

H $4.4300505 .014897-0.840404$

H 5.1947554 .0243970 .398605

H $5.7062013 .012068-1.651589$

H $3.8983224 .023838-3.079813$

H $2.7083562 .996577-2.311930$

H $2.9630412 .793290-4.994735$

H $2.9219250 .367875-2.612743$

H $5.2999293 .103104-4.914665$

H $5.4759641 .380357-5.154010$

H $6.2245712 .155842-3.744815$

H $3.812621-0.434167-4.535649$

H $4.942397-0.593896 \quad 0.290924$

H $3.386827-0.736612-0.479973$

H $6.6682110 .173713-1.079992$

H 1.0884631 .3938491 .528405

H $0.406310 \quad 1.127970 \quad 4.519459$

H $3.171907 \quad 0.2409852 .086631$

H $1.889409-0.8806851 .567338$

H $3.256732-1.4565622 .504040$

H $4.332233-2.337160-2.148974$ 
H $5.960620-2.064038-1.534187$ H $3.822518-5.2425611 .089315$ H $2.392516-5.3829090 .042814$ H $1.268153-3.5098281 .484934$ H $2.942375-5.256783 \quad 3.360162$ H $0.638967-4.9150924 .472727$ H $0.525430-3.3294363 .743063$ H $2.452019-3.5943057 .377948$ H $1.369077-4.9354536 .703498$ H $3.518774-2.3544634 .317091$ H $3.341675-1.9243205 .986416$ H $1.271549-0.6806035 .634888$ H $2.691275-3.147159-0.721892$ C $-4.9089953 .078748-0.059372$ O $-4.7055581 .660602-0.248549$ C $-5.9838101 .040538-0.489533$ C -7.036256 2.110779-0.219020 C $-6.2990973 .369668-0.621726$ H $-6.0156910 .782699-1.553987$ C $-6.158535-0.2125120 .374212$ H $-7.9524191 .944928-0.794026$ H $-7.308713 \quad 2.1495850 .842485$ C $-6.9366414 .639286-0.083604$ H $-6.258792 \quad 3.420853-1.718092$ C $-5.191253-1.3739280 .064346$ $\begin{array}{lllll}\text { O } & -5.988679 & 0.144169 & 1.752823\end{array}$ H $-7.190710-0.5726750 .286750$ H $-4.171513-1.1146300 .369687$ O $-5.587429-2.4799600 .887991$ C $-5.192901-1.839825-1.405796$ C $-4.597093-3.237932-1.653994$ H $-4.663086-1.102934-2.019138$ O $-6.540156-1.879783-1.892707$ H $-5.204435-3.990117-1.133880$ H $-4.722160-3.452662-2.724532$ H $-7.9583534 .746381-0.462630$ H $-6.983078 \quad 4.632497 \quad 1.010590$ H $-6.369845 \quad 5.523640-0.390447$ C $-3.7999643 .866332-0.758050$ H -5.0986490 .5251091 .848064$ H $-5.569848-2.1740251 .811160$ H $-7.035020-2.506809-1.337645$ H $-4.096354 \quad 4.915027-0.872803$ H -3.625563 $3.462003-1.762467$ H $-4.890312 \quad 3.262607 \quad 1.023268$ C $-0.923984-2.611531-1.947755$ O $-2.344701-2.581319-2.147446$ C $-3.119282-3.402522-1.268822$ C $-2.685753-4.875265-1.379365$ C $-1.180805-5.036807-1.156586$ C $-0.375492-4.048241-2.002046$ H $-0.515926-2.068670-2.809647$ C $-0.511778-1.857758-0.677868$ H $-2.977457-3.063548-0.238165$ O $-3.034427-5.406682-2.656777$ H $-3.235225-5.460092-0.632271$ H $-0.879687-6.058968-1.417206$ O $-0.877704-4.827697 \quad 0.218255$ H $0.675262-4.058922-1.692332$ O $-0.413840-4.498521-3.359499$ C $-0.791338-0.381910-0.817840$ H $-1.040029-2.2058860 .214285$ H $0.558936-1.975752-0.476260$ H $-2.608943-4.854639-3.334987$ H $-1.366973-5.4926020 .732141$ H $0.160936-3.912065-3.880329$ O $\quad 0.123630 \quad 0.186508-1.653660$ O $-1.717710 \quad 0.189197-0.258711$ C $-0.0615351 .577043-1.932737$ H $0.5442531 .830293-2.806166$ H $-1.1078601 .796855-2.165932$ H $\quad 0.2806242 .170115-1.081287$

SCF Energy (B3LYP/6-31G**//MMFF) $=-3245.91770290$

0700351

MM̄FF Geometry

C 1.8185901 .3319241 .676813

C 2.5147992 .4742401 .779617
C 4.0000072 .6171861 .540563

$\begin{array}{lllll}\text { O } & 4.557511 & 1.338007 & 1.170993\end{array}$

C 4.3166363 .6830570 .470600

C $3.6602203 .491991-0.886976$

C $2.7097094 .307853-1.387405$

C $4.1920532 .360407-1.728237$

C $2.0862245 .486106-0.690553$

C $0.8431256 .066244-1.389271$

C $-0.4341125 .205042-1.410367$

C $-0.9718004 .888791-0.014074$

C $-2.2452324 .029635-0.078317$

O $-0.1814583 .998595-2.129975$

O -2.4983293 .5542101 .253067$

C $-1.9739122 .805414-0.978866$

C -3.472832 $4.857446-0.475192$

O $-3.1763762 .064075-1.164743$

C $-1.3338793 .168479-2.336449$

C $-0.858211 \quad 1.926863-3.125470$

O $-2.307736 \quad 3.837920-3.145905$

C 5.8729271 .0882741 .377869

O $6.7115361 .867881 \quad 1.803895$

C $6.201349-0.2973940 .961066$

C $5.281321-1.2754680 .978485$

C $5.464703-2.671590 \quad 0.576390$

C $6.793736-3.057967-0.013955$

C $0.188908 \quad 1.072869-2.404761$

C $0.8254120 .048797-3.327954$

C $2.754880-1.527190-3.427776$

O $0.413665-0.180681-4.461881$

C $2.465551-2.910289-2.938645$

C $3.403018-3.705047-2.400681$

C $3.163466-5.118620-1.957030$

C $3.090077-5.275420-0.454672$

C $1.972949-5.7374700 .132126$

C $4.330452-4.978447 \quad 0.365170$

C $4.427349-3.5207960 .737813$

N $1.908268-0.579402-2.728974$

H 2.3217160 .4078651 .407790

H 1.9855793 .3843442 .053379

H 4.4529602 .9269562 .491373

H 5.4011543 .7194060 .305600

H 4.0562194 .6662030 .881448

H $2.3655894 .119307-2.402399$

H $3.8138762 .393966-2.755844$

H $5.2846082 .404205-1.782950$

H $3.8993431 .394946-1.306659$

H $1.839110 \quad 5.236283 \quad 0.345477$

H $2.8382676 .285020-0.657183$

H $0.6024437 .023058-0.908331$

H $1.115596 \quad 6.299428-2.427475$

H - $1.1805815 .785209-1.966588$

H -1.1573945 .8100170 .551689$

H -0.2186004 .3311770 .556543$

H -3.256538 $2.946123 \quad 1.209648$

H $-1.2929072 .158162-0.417805$

H -3.642342 5.6593280 .253506

H $-4.3826694 .246823-0.464435$

H $-3.3811255 .318408-1.460652$

H $-2.9397501 .204297-1.551489$

H $-1.7154511 .305850-3.414521$

H $-0.4230902 .275057-4.073273$

H -2.046688 $3.751572-4.077787$

H $7.233017-0.4713430 .676650$

H $4.277503-1.0366261 .325404$

H $7.599257-2.8875270 .708986$

H $6.999792-2.468180-0.914216$

H $6.853561-4.108656-0.304269$

H $0.9855791 .708348-2.003855$

H $-0.2696560 .531688-1.571625$

H $3.791673-1.230290-3.238211$

H $2.573277-1.470506-4.505717$

H $1.450930-3.282220-3.058773$

H $4.419577-3.331236-2.305318$

H $3.975709-5.747944-2.342103$

H $2.248986-5.503251-2.427784$

H $1.918304-5.8811551 .206181$

H $1.086594-5.978270-0.445281$

H $5.205787-5.344398-0.177523$ 
H $4.302205-5.5546421 .299528$

H $3.517197-3.133377 \quad 1.198991$

H $2.148225-0.348058-1.769099$

C $-0.060015 \quad 0.385598 \quad 3.070677$

O $-1.341015-0.2118002 .784659$

C -2.3029790 .3065793 .712315$

C -1.491527 0.6013114 .962617

C $-0.233324 \quad 1.2047294 .357429$

H -2.677037 1.2489693 .292210

C $-3.470953-0.6715363 .871160$

H $-2.006141 \quad 1.2816335 .647257$

H -1.240864 -0.315535 5.508307

C $0.968861 \quad 1.1454365 .285509$

H -0.4348162 .2570104 .117662$

C $-4.027736-1.2149272 .536224$

O $-3.055824-1.8018984 .647419$

H $-4.270123-0.1942234 .449706$

H -3.328243 -1.950096 2.122158

O $-5.237148-1.9274132 .820624$

C $-4.331682-0.1404831 .479152$

C $-5.021969-0.6611490 .206478$

H $-3.4035280 .360772 \quad 1.186880$

O -5.1723840 .8662552 .049391$

H $-6.034059-1.0126230 .444942$

H $\quad-5.1662140 .207095-0.449655$

H $\quad 0.769699 \quad 1.6995386 .208607$

H $1.213831 \quad 0.1136245 .559037$

H 1.8505381 .5866184 .810875

C $\quad 0.338451 \quad 1.221875 \quad 1.852614$

H -2.310182 -2.219385 4.183641

H $-5.024384-2.6056153 .484642$

H $-5.987497 \quad 0.4304202 .352348$

H -0.1327342 .2100291 .893312$

H $-0.0603350 .751150 \quad 0.946137$

H $0.637545-0.4474953 .216416$

C $-2.060597-2.235127-1.477867$

O $-2.924651-1.304341-0.807683$

C $-4.254593-1.765908-0.544231$

C $-4.966734-2.154842-1.851588$

C $-4.142460-3.164213-2.654764$

C $-2.684975-2.716076-2.798675$

H -1.167894 -1.655332 -1.736359

C -1.627945 -3.389054 -0.559371

H -4.206983 -2.644462 0.106606

O $-5.184664-0.987725-2.642855$

H -5.948714 -2.577251 - 1.606564

H -4.587828 $-3.282061-3.650228$

O $-4.203206-4.426119-1.997092$

H -2.079041 -3.529321 -3.213710

O $-2.619840-1.644536-3.744435$

C $-0.669139-2.9343940 .521644$

H -2.488428 -3.854794 -0.072456

H $-1.101255-4.156242-1.138556$

H -5.673339 -1.259642 -3.438355

H $-3.696219-5.060336-2.532157$

H $-3.088025-0.882106-3.363308$

O $-0.706006-3.8277801 .553677$

O $0.048882-1.946606 \quad 0.453132$

C $0.187144-3.5739352 .643452$

H $\quad 0.305371-4.5031173 .207321$

H $1.172488-3.2564122 .289431$

H -0.245845 -2.817539 3.301531

SCF Energy (B3LYP/6-31G**//MMFF) $=-3245.91903544$

0700352

MM̄MF Geometry

C $3.284801-2.6314492 .855507$

C $2.615991-1.5542503 .293024$

C $1.161887-1.4657973 .679168$

O $0.552968-0.5796332 .707812$

C $0.377532-2.7952523 .672946$

C -1.074414 -2.637200 4.084869

C $-2.123950-2.846303 \quad 3.265367$

C -1.310582 -2.250145 5.522250

C $-2.101430-3.3286651 .842051$

C $-1.722224-2.2436860 .827599$

C $-2.048230-2.683431-0.605619$

C $-1.315181-1.816853-1.627991$
C $-1.715598-2.183799-3.065297$

O $-3.459977-2.540665-0.779811$

O $-1.209063-1.166080-3.942006$

C $-3.255820-2.192918-3.174280$

C $-1.046739-3.483826-3.523765$

O $-3.677439-2.679272-4.446299$

C $-3.960276-2.982830-2.043798$

C $-5.489038-2.714651-2.102081$

O $-3.735722-4.381979-2.228186$

C $-0.0570750 .552337 \quad 3.144764$

O $-\begin{array}{llll}0.119432 & 0.967124 & 4.290286\end{array}$

C -0.6709571 .1998741 .963732$

C -1.5063822 .2350462 .133334$

C -2.194066 2.9718741 .070545

C $-2.0205582 .476088-0.340989$

C $-6.281917-3.105422-0.842377$

C $-5.998279-2.230278 \quad 0.368617$

C -5.8649590 .1439851 .101919$

O $-5.628745-2.7112351 .437871$

C -5.5889891 .4169450 .366914$

C -6.1366762 .5997630 .681970$

C $-5.9119183 .855975-0.109370$

C -5.1758434 .9220240 .667770$

C $-5.8433415 .831472 \quad 1.397756$

C -3.6745154 .9975000 .506965$

C -2.9395444 .0448641 .408739$

N $-6.190796-0.8813920 .127642$

H $2.781686-3.5890382 .768569$

H $3.160079-0.6150893 .376669$

H $1.128226-1.0286994 .684217$

H $0.851951-3.5111244 .356611$

H $0.435164-3.2413792 .674171$

H -3.125394 -2.691383 3.665244

H -2.373461 -2.265705 5.786723

H $-0.798839-2.9503046 .190772$

H $-0.938943-1.2421415 .723946$

H -1.423563 -4.185527 1.744722

H $-3.098602-3.7232801 .612533$

H -2.262459-1.317438 1.060106

H $-0.649977-2.0400200 .909667$

H $-1.766483-3.735944-0.726902$

H $-0.229101-1.898860-1.498854$

H - $1.558986-0.760242-1.452394$

H -1.583352 -0.312197 -3.665685

H -3.589601 -1.147354 -3.117821

H $\quad 0.044773-3.388597-3.475969$

H -1.281865 -3.701208 -4.571734

H -1.331087 -4.349147 -2.921411

H -3.219720 -2.153432 -5.124486

H -5.660064 -1.650879 -2.303515

H $-5.914970-3.267380-2.949570$

H -4.451614 -4.872678 -1.793478

H -0.4264920 .7977850 .986754$

H -1.702769 2.5840833 .146232

H -2.348012 $1.433790-0.423007$

H $-0.9704192 .532504-0.642422$

H -2.598516 $3.038582-1.076918$

H -7.355980 -3.030515 -1.052060

H $-6.083159-4.147608-0.570703$

H $-4.981094-0.1675501 .668078$

H $-6.7112280 .229777 \quad 1.790844$

H -4.913048 $1.358520-0.483124$

H -6.8151712 .6643781 .529393$

H $-6.8945484 .235431-0.420007$

H $-5.3789343 .646762-1.045440$

H -5.3234536 .6219261 .928909$

H -6.9244865 .8013101 .484013$

H -3.3253696 .0091850 .755864$

H -3.412102 $4.873170-0.547587$

H -3.0079234 .3143832 .463813$

H $-6.716212-0.606089-0.697346$

C $4.993150-2.8034830 .971265$

O $4.400883-1.7442870 .190884$

C $3.986544-2.294606-1.077076$

C $4.491683-3.736963-1.109629$

C $4.478282-4.1037390 .360345$

H $2.890944-2.308831-1.072880$

C $4.509185-1.443800-2.241211$ 
H $3.853261-4.383168-1.720175$

H $5.510210-3.800307-1.510754$

C $5.318657-5.3231710 .693631$

H $3.438161-4.2916420 .652907$

C $3.9687620 .000952-2.301575$

O $5.939354-1.373402-2.160037$

H $4.290194-1.953295-3.187250$

H $4.3773540 .583539-1.469308$

O $4.494404 \quad 0.599547-3.495025$

C $2.4327790 .118703-2.320051$

C $1.8856311 .498732-2.736339$

H $2.037727-0.150186-1.333831$

O $1.918812-0.836993-3.254452$

H $2.0914801 .670224-3.800840$

H $0.7913441 .441285-2.656484$

H $4.949618-6.2028890 .156109$

H $6.367974-5.1761690 .416919$

H $5.279795-5.5419811 .765331$

C $4.736563-2.5968922 .466788$

H $6.163782-0.964776-1.306337$

H $4.118050 \quad 0.121180-4.253753$

H $0.949513-0.763349-3.250792$

H $5.170203-1.6344362 .763347$

H $5.264568-3.3668423 .041282$

H $\quad 6.077067-2.7255200 .808769$

C $2.487106 \quad 3.4598210 .384479$

O $2.0427932 .462346-0.545337$

C $2.4005362 .686772-1.910404$

C $1.8236004 .022488-2.413464$

C $2.2145705 .184396-1.495362$

C $1.9702364 .851878-0.018318$

H $2.0137493 .187576 \quad 1.336719$

C 4.0099553 .4242740 .589503

H $3.4906832 .719887-1.997225$

O $0.4023493 .937169-2.488779$

H $2.1951834 .203573-3.429389$

H $1.6328896 .071976-1.772526$

O $3.5896165 .488548-1.708370$

H 2.4289825 .6094650 .627646

O $\quad 0.564604 \quad 4.8803350 .240256$

C 4.4863272 .1194231 .188416

H $4.5512373 .559104-0.350319$

H 4.2985614 .2258191 .279508

H $0.0750934 .780151-2.846062$

H $3.8161416 .240965-1.135555$

H $0.243845 \quad 5.773200 \quad 0.027938$

O $5.8378372 .192077 \quad 1.375281$

O $3.7760561 .159370 \quad 1.449328$

C 6.4498821 .0209611 .922680

H 7.5109311 .2330682 .078480

H 5.9980690 .7692692 .886709

H $6.358750 \quad 0.187893 \quad 1.220075$

SCF Energy (B3LYP/6-31G**//MMFF) $=-3245.89668220$

0700353

MM̄FF Geometry

C $\quad \begin{array}{llll}0.546227 & 0.119767 & 0.326007\end{array}$

C $-0.068020-0.728985-0.514533$

C $-0.437134-0.443806-1.951314$

O $-0.4320990 .987081-2.155570$

C $0.538054-1.113182-2.935146$

C $0.319032-2.606095-3.072142$

C $1.011242-3.553353-2.408528$

C $-0.757378-3.000183-4.050603$

C $2.091271-3.348219-1.382433$

C $3.396137-4.047947-1.784274$

C $4.600210-3.681748-0.898829$

C $4.395463-4.0470520 .573577$

C $5.576686-3.5956291 .447776$

O $4.831239-2.276826-1.041295$

O $5.168318-3.6870262 .821867$

C $5.859853-2.1053241 .160661$

C $6.791400-4.5175761 .295990$

O $7.027582-1.6635071 .847976$

C $5.983321-1.784261-0.343635$

C $6.069131-0.266537-0.624628$

O $7.182707-2.376573-0.855336$

C $-1.2682621 .506524-3.089480$
O $\quad-1.922640 \quad 0.885312-3.914338$

C -1.298075 $2.985535-3.001922$

C $-1.0635183 .626401-1.845933$

C -1.103740 $5.071050-1.617821$

C $-1.3148905 .970307-2.805243$

C $4.8778030 .548581-0.112354$

C $4.7996691 .925559-0.750089$

C $3.2668073 .862342-0.945536$

O $5.6175712 .339497-1.567052$

C $2.4230524 .661711-0.007331$

C 2.4471336 .0021990 .038869

C 1.5686516 .8320630 .933524

C 0.4858827 .5528180 .157744

C $0.7364618 .729176-0.441582$

C $-0.901107 \quad 6.946993 \quad 0.124127$

C $-0.9199735 .524603-0.360499$

N $3.6932482 .635439-0.298729$

H $0.8550451 .099326-0.025593$

H $-0.363830-1.706871-0.141560$

H -1.461682 -0.806879-2.102922

H $1.573657-0.881323-2.657337$

H $0.433545-0.655423-3.928019$

H $\quad 0.759431-4.596959-2.593649$

H $-0.891978-4.085582-4.107658$

H -1.718128 -2.563583 -3.760686

H $-0.504236-2.645114-5.055028$

H $2.281925-2.287729-1.203791$

H $1.727360-3.763854-0.435612$

H $3.250466-5.135362-1.771754$

H $3.638150-3.766695-2.818106$

H $5.467576-4.219282-1.300406$

H $4.220064-5.1240420 .685461$

H $3.494225-3.5539390 .960582$

H $4.908819-4.6079602 .996488$

H $5.019161-1.5374531 .579309$

H $6.534485-5.5408271 .595492$

H $7.607165-4.2076391 .958249$

H $7.173561-4.5616490 .274208$

H $6.910827-1.8827202 .788330$

H $7.0016630 .144521-0.216753$

H $6.144101-0.133030-1.713011$

H $7.440261-1.903741-1.664102$

H $-1.5880943 .506323-3.907668$

H $-0.8326313 .034709-0.961335$

H $-0.5622965 .773386-3.576854$

H -1.235305 $7.032389-2.559691$

H -2.309554 $5.811149-3.235607$

H $3.9382290 .029789-0.335315$

H 4.9508240 .6801180 .972968

H $2.6739543 .568566-1.818407$

H $4.1439634 .417606-1.293825$

H 1.7311014 .1245050 .634886

H $3.1248386 .542986-0.618239$

H 2.2029807 .5610391 .454682

H $1.1183926 .223115 \quad 1.727713$

H $-0.0359859 .265104-0.982784$

H $1.7224849 .180921-0.404928$

H -1.591270 $7.561610-0.465427$

H -1.309979 6.9627281 .143038

H $-0.764808 \quad 4.7963240 .436334$

H 3.0070362 .1358220 .260165

C $-0.086516 \quad 0.5941802 .732280$

O $-1.467095 \quad 0.4406492 .335306$

C -2.0291361 .7525812 .158206$

C -1.210509 2.6474093 .073968

C 0.1822262 .1015242 .807171

H -1.8315472 .0510011 .121550$

C $-\begin{array}{llll}3.539123 & 1.737987 & 2.395577\end{array}$

H -1.3103873 .7081432 .826888$

H -1.481009 2.505555 4.126651

C 1.2066162 .4913713 .857827

H 0.5213852 .4800301 .836356

C -4.2950020 .7069611 .526596$

O

H -3.942582 2.7444532 .231933

H -4.095032 -0.2989921 .909841$

$\begin{array}{llll}\text { O } & -5.698328 & 0.925368 & 1.712228\end{array}$

C $-\begin{array}{llll}-3.966805 & 0.770175 & 0.024225\end{array}$ 
C $-4.835666-0.123167-0.876632$

H $-2.9166660 .502735-0.128734$

O $-4.1237432 .121277-0.420038$

H $-5.8611410 .266526-0.915172$

H -4.444479 -0.008979-1.896424

H 1.3242503 .5793213 .894335

H 0.9085982 .1514874 .855278

H 2.1829222 .0525263 .628458

C $0.816088-0.1924691 .772902$

H -4.7515941 .4093793 .894280$

H $-5.913324 \quad 1.7924291 .327357$

H -3.858768 2.156017 -1.354995

H $0.656986-1.263851 \quad 1.945521$

H $1.871100 \quad 0.0046301 .994980$

H $-0.003136 \quad 0.1479243 .731264$

C $-3.360748-3.4481830 .027567$

O $-3.513780-2.077194-0.369099$

C $-4.860865-1.612592-0.494944$

C $-5.626117-2.449271-1.535262$

C $-5.540137-3.946991-1.229141$

C $-4.101098-4.387539-0.941531$

H -2.286556 -3.649006 -0.070564

C $-3.755708-3.6753911 .496208$

H $-5.363257-1.7010440 .473296$

O $-5.111454-2.210439-2.844944$

H $-6.675528-2.132141-1.541499$

H -5.929942 -4.508544 -2.087115

O $-6.371377-4.230417-0.107956$

H $-4.091398-5.410931-0.548810$

O $-3.392416-4.411521-2.183573$

C $-2.809331-2.9921282 .459484$

H $-4.763793-3.3051801 .699570$

H $-3.727647-4.745268 \quad 1.732411$

H -4.161697 -2.419711 -2.834080

H $-6.330438-5.1885540 .052852$

H -2.496708 -4.745858 -2.005579

O $-3.438432-2.8208373 .659610$

O $-1.654406-2.6803512 .206142$

C -2.653543 -2.209023 4.687907

H -1.733533 -2.776403 4.858009

H -2.425642 -1.174061 4.420395

H -3.241014 -2.211058 5.609958

SCF Energy (B3LYP/6-31G**//MMFF)= -3245.90943509

07 00354

MM̄FF Geometry

C $0.9551460 .608153-2.473186$

C $1.331591-0.678904-2.550378$

C $0.780963-1.833597-1.746442$

O $0.017379-1.319578-0.634500$

C $-0.114347-2.719502-2.626806$

C $-0.366815-4.091478-2.034879$

C $-1.524457-4.495260-1.476272$

C $0.786535-5.057745-2.147080$

C $-2.788488-3.705963-1.272423$

C $-3.208321-3.727747 \quad 0.203367$

C $-4.513644-2.9778620 .511495$

C $-5.737710-3.661571-0.100463$

C $-7.028250-2.8956380 .218322$

O $-4.403460-1.6359610 .030362$

O $-8.054666-3.433908-0.631041$

C $-6.832284-1.410539-0.162004$

C $-7.493058-3.1514841 .656686$

$\begin{array}{llll}\text { O } & -7.960526 & -0.651033 & 0.264263\end{array}$

C $-5.514398-0.793214 \quad 0.372584$

C $-5.2246510 .605640-0.209273$

O $-5.579334-0.5822391 .789493$

C $0.216672-1.8601230 .593582$

O $0.872621-2.8588950 .856054$

C $-0.441905-1.0583341 .653578$

C $-1.037143 \quad 0.122373 \quad 1.423676$

C -1.622396 1.0177052 .426180

C -1.5966040 .5723003 .863215$

C $-5.0147140 .665764-1.723811$

C $-4.8404412 .107118-2.159444$

C $-3.2400544 .009173-2.004410$

O $-5.7689122 .749770-2.644859$

C $-3.4462954 .625325-0.655436$
C $-2.445597 \quad 5.0486620 .130825$

C -2.6432415 .5637251 .526980$

C -2.0560304 .6346422 .569675$

C -0.9919834 .9970943 .305491$

C $-2.748773 \quad 3.3063352 .807141$

C -2.1320752 .1933802 .003217$

N $-3.5710732 .599096-1.901473$

H $0.1390850 .895943-1.817634$

H $2.140325-0.942449-3.229587$

H $1.649284-2.392432-1.377317$

H $\quad 0.351633-2.866474-3.610992$

H -1.057847 -2.200850 -2.828031

H -1.582665 -5.523520 -1.119961

H $0.534825-6.053476-1.766784$

H $1.084700-5.173157-3.194364$

H $1.649069-4.699287-1.577537$

H -2.683104 -2.670059-1.603439

H -3.565756 -4.163919-1.893208

H -3.297411 -4.7633110 .556066$

H -2.406192 -3.264483 0.791062

H $-4.608792-2.9510381 .603630$

H $-5.814517-4.7024290 .237683$

H $-5.635648-3.706510-1.192484$

H $-8.861865-2.911377-0.484289$

H -6.830054 -1.378711-1.258240

H $-7.725129-4.2141901 .797290$

H -8.420431 -2.611625 1.876764

H -6.747236 -2.876812 2.405676

H $-7.9096300 .221429-0.160269$

H -6.0158451 .3065040 .088057$

H $-4.3180790 .992690 \quad 0.275290$

H -6.467983 -0.8164012 .100553$

H $-0.357012-1.4709222 .653689$

$\begin{array}{llll}H & -1.077300 & 0.498643 & 0.403281\end{array}$

H $-0.564556 \quad 0.446907 \quad 4.208893$

H $-2.123865-0.3815013 .978352$

H $-2.076147 \quad 1.2747154 .547498$

H $-4.1356870 .085122-2.025381$

H $-5.8731100 .263623-2.269956$

H $-3.8824964 .496122-2.744438$

H $-2.2013294 .078168-2.339446$

H -4.470016 $4.683644-0.292551$

H -1.422118 $4.982756-0.227218$

H -3.7073225 .7165131 .746607$

H -2.1810376 .5571571 .588197$

H -0.5849914 .3401264 .067195$

H -0.5018315 .9537243 .159205$

H -3.8074083 .3903952 .531723$

H -2.748342 3.0931183 .879958

H -2.1091532 .3902370 .932098$

H $-2.8975971 .995991-1.437625$

C $2.1697462 .838719-2.336369$

O $2.8540702 .293431-1.188052$

C $2.3283852 .929035-0.008296$

C $1.7434914 .245668-0.493774$

C $1.1062413 .800390-1.797368$

H 1.5051652 .3018510 .355215

C 3.4037223 .0477921 .073859

H 1.0276174 .6718910 .214618

H $2.5257774 .989521-0.685618$

C $0.7848684 .947763-2.740146$

H $0.1789013 .260562-1.568774$

C 4.0306971 .7000241 .492898

O 4.4586983 .8954460 .606049

H 2.9842503 .5501911 .953230

H 4.6755801 .3229790 .691597

O 4.8828181 .9426792 .618029

C 3.0020660 .6268181 .886179

C $3.581410-0.6541402 .507121$

H $2.417440 \quad 0.347393 \quad 1.003727$

O $2.077876 \quad 1.1765722 .830777$

H $4.004623-0.4378423 .496703$

H $2.727466-1.3165142 .700575$

H $0.0709325 .637700-2.279004$

H $1.6827725 .519063-2.998694$

H $0.3428774 .574859-3.669402$

C $1.6408041 .711843-3.234223$

H $4.8048333 .506254-0.214979$ 
H 5.5249702 .6235322 .353028

H 2.5886351 .4690703 .605190

H $2.491550 \quad 1.293400-3.786874$

H $\quad 0.9521412 .119678-3.982681$

H $2.9231203 .402506-2.901626$

C $4.970885-2.194317-0.613252$

O $4.096994-1.5590240 .331327$

C $4.627244-1.3836031 .648725$

C $5.015796-2.7390212 .267228$

C $5.950018-3.5291571 .347302$

C $5.426805-3.568206-0.092586$

H $4.348345-2.368728-1.499994$

C $6.144844-1.288387-1.020988$

H $5.515651-0.7466191 .594731$

O $3.838976-3.5095142 .509341$

H $5.498426-2.5594763 .235517$

H $6.047616-4.5537031 .726736$

O $7.240115-2.9272501 .384993$

H $6.193528-3.975579-0.761138$

O $4.315328-4.466593-0.159088$

C $5.697199-0.089471-1.828072$

H $6.685196-0.914906-0.147511$

H $6.846089-1.848273-1.650446$

H $4.112747-4.3359092 .942340$

H $7.826404-3.4541760 .815801$

H $3.588148-4.0799330 .358828$

O $6.7071690 .829529-1.860949$

O $4.615478 \quad 0.018791-2.386799$

C $6.4390372 .010628-2.623285$

H $7.3056182 .671996-2.541332$

H $\quad 6.290811 \quad 1.753719-3.676311$

H $5.5629352 .530621-2.226326$

SCF Energy (B3LYP/6-31G**//MMFF) $=-3245.91467933$

\section{7 _00355}

\section{MM̄FF Geometry}

C -4.075827 -2.982754 -1.964248

C $-3.048156-3.035177-1.104662$

C $-1.625242-3.302772-1.523781$

O $-0.805448-2.162897-1.169766$

C $-1.089411-4.528344-0.760188$

C $0.261998-5.011815-1.250421$

C $1.426391-4.882119-0.583128$

C $0.240253-5.728203-2.578237$

C $1.678808-4.2276610 .747959$

C $2.411107-2.8891850 .614874$

C $3.843216-2.9599790 .062989$

C $4.813719-3.5938461 .064056$

C $6.271335-3.5336740 .579896$

O $4.241463-1.608678-0.220482$

O $7.121011-3.8245291 .700922$

C $6.593145-2.0908050 .134704$

C $6.565349-4.608010-0.473989$

O $7.887436-2.015377-0.457412$

C $5.538544-1.498378-0.823623$

C $5.795143-0.010102-1.150004$

O $5.591016-2.204317-2.070375$

C $-0.919534-1.037478-1.929256$

O $-1.666741-0.854806-2.876592$

C $0.034107-0.043488-1.393775$

C $-0.0982931 .258473-1.685057$

C $0.7714172 .324505-1.185461$

C $2.0746601 .924598-0.541419$

C 5.7561480 .9493590 .041907

C $5.9511592 .392085-0.398493$

C 6.0443364 .7090330 .505772

O $6.1763872 .713814-1.562633$

C 4.7163725 .3601630 .279596

C $4.2998655 .793718-0.920202$

C $2.9920656 .492633-1.159958$

C $1.9599115 .577644-1.784420$

C $1.8441975 .498674-3.121200$

C $1.0869804 .828705-0.798364$

C $0.3868653 .609285-1.332196$

N 5.8623203 .2788230 .666148

H -3.896696 -3.151512 -3.023330

H -3.227870 -2.851163 -0.049664

H - $-1.555743-3.467408-2.605925$
H $-1.796905-5.363859-0.853612$

H -1.051955 -4.296143 0.309356

H $2.319825-5.302562-1.043578$

H $1.219152-6.140509-2.845525$

H $-0.467789-6.562940-2.547985$

H $-0.057592-5.047104-3.380876$

H $0.745684-4.0518901 .290293$

H $2.253832-4.9188811 .373979$

H $1.821860-2.226784-0.027296$

H $2.431432-2.3947941 .594726$

H $3.837265-3.518433-0.878532$

H $4.534460-4.6305981 .284497$

H $4.747863-3.0566982 .020809$

H $6.877343-4.7021932 .042209$

H $6.630780-1.4790721 .044767$

H $6.405948-5.608884-0.054689$

H $7.614816-4.578852-0.786512$

H $5.936554-4.522168-1.362524$

H $8.519367-2.3928870 .178123$

H $6.7513530 .091149-1.680525$

H $5.0320230 .319403-1.869862$

H $5.184109-1.650228-2.756832$

H $\quad 0.818117-0.406864-0.742614$

H $-0.9312711 .576101-2.309979$

H $1.902377 \quad 1.4731110 .440066$

H $2.7593402 .764831-0.402797$

H $2.6111901 .204687-1.169798$

H 4.7951050 .8720940 .561836

H $\quad 6.5563860 .7130340 .750678$

H $6.7360854 .897952-0.322295$

H 6.4991655 .0946931 .423437

H 4.0796605 .4994441 .149645

H $4.9397055 .649156-1.787967$

H $3.1804257 .356392-1.810808$

H $2.5944786 .917090-0.229224$

H $1.1024614 .872255-3.603363$

H $2.4959296 .071697-3.773274$

H $0.3230235 .520979-0.420568$

H 1.6776364 .5550710 .083127

H $-0.5546903 .822695-1.837112$

H 5.6069312 .9298901 .585427

C $-5.848550-1.237557-1.984067$

O $-5.147699-0.295175-1.146838$

C $-5.9745210 .874670-0.977623$

C $-7.2377190 .633929-1.800351$

C $-7.321092-0.881118-1.813933$

H -6.2509880 .9203620 .080399$

C $-5.1855332 .126394-1.377822$

H -8.116725 $1.107598-1.352359$

H $-7.1366521 .023253-2.820412$

C $-8.226519-1.425688-2.904291$

H -7.681243 -1.224719 -0.835019

C $-3.9103672 .356605-0.539532$

O $-4.7904132 .001736-2.751187$

H $-5.8317183 .009862-1.327961$

H $-3.2232161 .512503-0.661993$

O $-3.2418003 .507632-1.072658$

C -4.1784952 .6167160 .956534$

C -2.9025472 .9096731 .771332$

H $-4.7025101 .767101 \quad 1.403459$

O $-5.0496833 .744707 \quad 1.082460$

H -2.260225 3.6084801 .222530

H -3.198696 3.4292372 .692773

H $-9.250218-1.060343-2.771666$

H -7.886176 -1.118864 -3.899132

H $-8.254219-2.519481-2.880634$

C $-5.487876-2.660636-1.562918$

H $-4.2351991 .206401-2.825485$

H $-3.0820673 .337623-2.016869$

H -4.6028154 .5060430 .674020$

H $-6.151314-3.388251-2.042842$

H -5.605946 -2.775277 -0.479849

H -5.529375 -1.051805 -3.018951

C $-2.332260-0.2697963 .638646$

O $-2.879980 \quad 0.961795 \quad 3.150388$

C -2.1234411 .6343622 .134399$

C -0.7081691 .9519102 .638007$

C -0.0107450 .6870103 .146442$ 
C $-0.888445-0.0820284 .135175$

H -2.949990 -0.519493 4.511253

C -2.490002 -1.3982902 .610579$

H -2.054129 $0.994333 \quad 1.249868$

O $-0.7766292 .922374 \quad 3.680048$

H -0.1335392 .3940381 .816495$

H 0.9323510 .9664683 .631668

O $0.297503-0.1450512 .033206$

H -0.441405 -1.058037 4.357021

O

C $-3.879473-1.9881542 .688443$

H -2.309709 -1.088979 1.577984

H - $-1.793276-2.2170652 .827217$

H $\quad 0.133720 \quad 3.113942 \quad 3.962770$

H $0.748454-0.9357752 .374064$

H -1.424382 0.1270096 .006850

O $-4.796621-1.1075012 .196633$

O $-4.119121-3.0982153 .146410$

C $-6.161654-1.5241452 .298038$

H $-6.785356-0.7776181 .800356$

H $-6.309779-2.4895081 .805564$

H -6.456252 -1.581167 3.350186

SCF Energy (B3LYP/6-31G**//MMFF)= -3245.90260975

$07 \quad 00356$

MM̄FF Geometry

C 1.0751723 .9230260 .622288

C $1.6449872 .891601-0.017203$

C $21351042.964552-1.438502$

O $3.5256602 .557139-1.454638$

C $1.3920491 .982755-2.358955$

C $-0.0865352 .259067-2.530232$

C $-1.0603351 .470343-2.035140$

C $-0.4334043 .466658-3.358925$

C $-0.8729180 .258916-1.167891$

C $-0.760260-1.030271-1.989715$

C $-0.290369-2.247382-1.174535$

C $-1.273487-2.626249-0.066483$

C $-0.757753-3.791146 \quad 0.789643$

O $0.996154-1.941265-0.626499$

O $-1.596168-3.8950521 .951203$

C $0.676737-3.461557 \quad 1.255398$

C $-0.903239-5.1341560 .067869$

O $1.256249-4.5646591 .947154$

C $1.607271-3.0111590 .106939$

C $2.972372-2.4985970 .623647$

O $1.872174-4.129753-0.747496$

C $4.4728923 .493724-1.197357$

O $4.2808694 .678081-0.957821$

C $5.8359252 .909993-1.217140$

C $6.0938871 .629021-1.525152$

C $7.4101810 .984005-1.538920$

C $8.6007101 .819511-1.148269$

C $2.890413-1.2786841 .543594$

C $4.263582-0.6913561 .797912$

C $6.395157-1.1323933 .011966$

O 4.6215320 .3606631 .273432

C $7.322534-1.9346722 .155206$

C $8.156409-1.3936881 .254460$

C $9.103110-2.1920340 .406284$

C $8.610188-2.384188-1.010458$

C $8.180204-3.585472-1.432473$

C $8.692806-1.209986-1.962306$

C $7.480925-0.320199-1.879912$

N $5.028656-1.4636322 .657061$

H 0.9613104 .8697510 .100448

H 1.7777551 .9472580 .504695

H $2.0530563 .979541-1.846514$

H $1.5735420 .956909-2.014012$

H $1.8649792 .005308-3.350958$

H $-2.0964241 .726688-2.244136$

H -1.512267 $3.563911-3.518990$

H $-0.0907104 .381815-2.866441$

H $0.0402103 .401348-4.343816$

H $-0.0033210 .387897-0.516066$

H $-1.7363040 .194890-0.497941$

H -1.724293 -1.252066 -2.464138

H $-0.035484-0.871141-2.799025$
H $-0.179874-3.081137-1.878066$

H $-2.253298-2.866310-0.489519$

H - $1.434443-1.7685310 .597554$

H -1.565407 -3.047526 2.426725

H $\quad 0.593607-2.647028 \quad 1.985195$

H $-1.958742-5.334850-0.153095$

H $-0.567705-5.962106 \quad 0.702277$

H $-0.355369-5.179243-0.875440$

H $0.639891-4.8197422 .654958$

H $3.513479-3.3147191 .119504$

H $3.593256-2.228635-0.242680$

H $2.693614-3.956647-1.236458$

H $6.6275953 .604598-0.955108$

H $5.2641710 .974405-1.786525$

H $8.7317732 .651591-1.848843$

H $9.5407371 .264703-1.136983$

H $8.4705642 .226513-0.139107$

H $2.268454-0.4931361 .100895$

H $2.443719-1.5380582 .509560$

H $6.538131-1.3953564 .064535$

H $6.555695-0.0551372 .898956$

H $7.310457-3.0136912 .290218$

H $8.172684-0.3143561 .130052$

H $9.290580-3.1660970 .877556$

H $\quad 10.078137-1.689557 \quad 0.390538$

H $7.849899-3.739551-2.454424$

H $8.142599-4.439551-0.764534$

H $8.757651-1.575484-2.996187$

H $9.632515-0.677272-1.795537$

H $6.555561-0.832539-2.148511$

H $4.652409-2.3423633 .001042$

C -0.8967353 .9842672 .247079$

O

C -2.8880893 .1472101 .358364$

C -3.0451474 .6463991 .617470$

C -1.6118265 .1338481 .544446$

H $-2.9229312 .963647 \quad 0.277697$

C -3.9593572 .2959052 .058387$

H -3.699030 5.1237370 .880766

H -3.4672704 .8431652 .610258$

C -1.3914756 .4899532 .189300$

H -1.330126 5.1854230 .485798

C $-3.8807090 .798546 \quad 1.690478$

O -3.8337012 .4742773 .468305$

H -4.9442792 .6795811 .764159$

H -3.8833020 .7306770 .598388$

$\begin{array}{llll}\text { O } & -2.651524 & 0.233790 & 2.139518\end{array}$

C $-5.057104-0.0327042 .240825$

C $-5.062178-1.5087971 .796935$

H -5.9975480 .4367611 .930723$

O $-5.052728-0.0117013 .670064$

H -4.172374 -2.022940 2.181894

H $-5.916607-1.9856332 .295334$

H -1.9970927 .2551391 .692568$

H -1.665897 6.4802093 .249517

H $-0.341476 \quad 6.7898892 .115336$

C 0.6172533 .8739942 .051942

H -4.5769732 .0122453 .891220$

H $-2.5454830 .467473 \quad 3.077342$

H $-4.248547-0.467614 \quad 3.971401$

H 0.9629582 .9419022 .517148

H 1.1103924 .6946512 .586813

H -1.084864 4.0380293 .328522

C $-6.507121-0.986886-1.608786$

O $-6.302264-0.981757-0.190514$

C $-5.165897-1.7134200 .276339$

C $-5.295729-3.195477-0.108894$

C $-5.506592-3.351518-1.619589$

C $-6.614287-2.426930-2.139867$

H -7.483235 -0.505126 -1.752704

C $-5.457087-0.137922-2.344245$

H $-4.258963-1.307344-0.181274$

O $-6.395633-3.777140 \quad 0.588191$

H -4.389855 -3.726347 0.205407

H $-5.764934-4.394662-1.838359$

O $-4.281821-3.060916-2.286224$

H -6.620917 -2.410634 -3.235826

O $-7.882686-2.941633-1.723758$ 
C $-5.5683241 .333647-1.998620$

H $-4.434397-0.445918-2.112569$

H $-5.617150-0.235233-3.424626$

H -6.430172 -4.7187900 .348464$

H $-4.427435-3.191141-3.238697$

H $-7.972971-3.835467-2.095875$

O $-4.8133082 .054080-2.880341$

O $-6.2134721 .795313-1.067563$

C $-4.8056353 .469723-2.670037$

H $-4.1815773 .924770-3.443569$

H $-5.8196293 .871566-2.756028$

H -4.377933 $3.704726-1.691138$

SCF Energy (B3LYP/6-31G**/MMFF) $=-3245.89870243$

\section{7}

\section{MMFF Geometry}

C -1.914813 3.1030421 .162777

C -1.4888312 .3780082 .208254$

C -2.122264 1.0776072 .620975

O

C -2.8887991 .1791423 .951975$

C -4.0145702 .1893513 .987855$

C $-5.1970092 .040378 \quad 3.359475$

C -3.7562813 .4061474 .837345$

C $-5.624646 \quad 0.9081432 .465612$

C -6.1072561 .4490141 .112325$

C $-6.2486580 .398710-0.000747$

C $-7.048131-0.8372010 .417436$

C -7.124204 -1.879000 -0.711009

O $-4.9328640 .022694-0.429966$

O $-7.585845-3.113892-0.140768$

C $-5.698271-2.136376-1.246910$

C $-8.148841-1.490383-1.782867$

O $-5.728167-2.991230-2.387253$

C $-4.922518-0.841888-1.577604$

C $-3.450519-1.113648-1.967020$

O $-5.534114-0.206974-2.708236$

C $-1.341430-1.1933902 .693128$

O $-2.446160-1.6941092 .557216$

C $-0.071030-1.9436092 .764398$

C $0.008125-3.1403162 .164922$

C $1.188532-4.0021502 .114983$

C $2.367561-3.6233862 .971465$

C $-2.602427-1.758918-0.872382$

C $-1.140779-1.825688-1.269096$

C $0.411389-2.971693-2.854328$

O $-0.294757-1.114128-0.732781$

C $0.950373-4.280813-2.370577$

C $2.169092-4.426623-1.828213$

C $2.740542-5.750189-1.403211$

C $3.232243-5.7486040 .027133$

C $4.526232-5.5204560 .308326$

C $2.252391-6.1070281 .121329$

C $1.166723-5.0847251 .310230$

N $-0.898605-2.748181-2.273103$

H -2.7752562 .7601990 .593097$

H -0.640146 2.7261912 .792157

H $-2.775736 \quad 0.723143 \quad 1.815924$

H $-2.1753501 .389894 \quad 4.761317$

H -3.2945360 .1946354 .219796$

H -5.9475842 .8192373 .490788$

H -4.597672 4.107032 4.829201

H -3.580609 3.1114045 .877196

H -2.875230 3.9452644 .475060

H $-4.825711 \quad 0.1776162 .320927$

H -6.4476690 .3788602 .960612$

H -7.077706 1.9409291 .259402

H -5.4077112 .2204120 .763261$

H -6.749410 $0.902113-0.836691$

H -8.053793 -0.5546040 .752060$

H $-6.567390-1.3197381 .278627$

H -8.449299 -2.947639 0.274873

H -5.159368 -2.682880 -0.462700

H -9.150289-1.409456 -1.342956

H -8.223696 -2.262839 -2.556202

H -7.925975 $-0.537381-2.266751$

H -6.219182 -3.792153 -2.135891

H -3.415503 -1.714730 -2.884615
H $-2.979161-0.157041-2.235056$

H $-4.8882900 .398420-3.108757$

H $0.764704-1.4762313 .271642$

H $-0.868638-3.5175511 .639367$

H $2.752930-2.6414582 .679884$

H $3.203095-4.3226862 .895547$

H $2.078800-3.5869504 .027698$

H -2.670505-1.172685 0.047707

H -2.942074 -2.774083 -0.642409

H $1.074445-2.136226-2.611943$

H $\quad 0.279408-3.007006-3.940166$

H $0.320488-5.157139-2.505377$

H $2.809260-3.554165-1.721246$

H $3.573145-5.984012-2.079910$

H $2.015895-6.562644-1.538982$

H $4.898930-5.549257 \quad 1.326686$

H $5.242767-5.298081-0.475342$

H $2.768519-6.2926792 .070896$

H $1.781993-7.0678500 .874487$

H $\quad 0.273493-5.276153 \quad 0.715042$

H $-1.668094-3.313992-2.619222$

C $-0.9943664 .382242-0.785031$

O $-0.0266613 .363770-1.105567$

C $0.8947223 .888301-2.079114$

C $0.3151825 .219663-2.544541$

C $-0.3947975 .692948-1.292196$

H $1.8314914 .085613-1.546523$

C $1.1432352 .871682-3.197795$

H $1.0906105 .911475-2.887471$

H $-0.4037635 .089311-3.361586$

C $-1.4172736 .785753-1.550596$

H $0.3566606 .062955-0.581639$

C $1.5930491 .477243-2.715920$

O $-0.0744282 .697242-3.935221$

H $1.8732243 .283468-3.905268$

H $\quad 0.7422600 .956052-2.261376$

O $1.9375050 .737477-3.895216$

C $2.7846901 .485376-1.739196$

C $3.3092100 .069381-1.429758$

H $2.4752701 .967926-0.805640$

O $3.8313372 .270899-2.308991$

H $2.463006-0.600925-1.242760$

H $3.856389-0.297115-2.306950$

H -0.934955 $7.669313-1.981290$

H $-2.1930586 .452753-2.248257$

H $-1.9082967 .089636-0.621057$

C $-1.273887 \quad 4.3874350 .719207$

H $0.1079832 .060406-4.647342$

H $2.002426-0.200268-3.649396$

H $4.5542372 .313652-1.660480$

H -1.9521815 .2113290 .967991$

H -0.3379244 .5483631 .268483$

H $-1.9089034 .138078-1.342049$

C 6.3364880 .9613170 .586602

O $5.3889050 .837193-0.481185$

C $4.2448670 .025969-0.204484$

C $4.681233-1.4095630 .128982$

C $5.702399-1.4284281 .271263$

C $6.835353-0.4204531 .041171$

H 7.1865551 .4959420 .143064

C 5.7918431 .8207601 .733387

H 3.6981810 .4485230 .645894

O $5.253141-2.011259-1.031099$

H $3.795182-1.9946260 .402712$

H $6.120593-2.4384201 .360706$

O $5.029477-1.1307952 .490547$

H $7.442311-0.3068461 .946902$

O $7.701382-0.9201530 .018124$

C 5.4993203 .2242911 .256575

H 4.8840991 .4049442 .179792

H 6.5251371 .8997202 .544645

H $5.504391-2.920505-0.797561$

H $5.685289-1.1770963 .207017$

H $8.054392-1.7731920 .323209$

O $4.152114 \quad 3.4297051 .209188$

$\begin{array}{lllll}\text { O } & 6.364437 & 4.033161 & 0.946394\end{array}$

C 3.7402424 .7245010 .760797

H 4.1585905 .5027571 .406223 
H $4.0464174 .877306-0.278310$ H 2.6494604 .7724560 .817368

SCF Energy (B3LYP/6-31G**//MMFF) $=-3245.90145471$

0700358

MM̄FF Geometry

C -3.608723 -1.262906 -3.601466

C $-2.621377-1.679704-2.794091$

C -1.158382 -1.591159-3.144595

O $-0.516734-0.734360-2.170648$

C $-0.514302-2.984853-3.064747$

C $0.945646-2.995689-3.473765$

C $1.983541-3.216967-2.642715$

C $1.202343-2.783826-4.945397$

C $1.960462-3.484079-1.163109$

C $2.714481-2.438975-0.332683$

C $4.199239-2.232233-0.675579$

C $5.015967-3.523601-0.587507$

C $6.514046-3.272234-0.815934$

O $4.703406-1.276087 \quad 0.268713$

O $7.226398-4.449989-0.406612$

C $6.974396-2.1243420 .103863$

C $6.837643-3.063438-2.300289$

O $8.323902-1.757756-0.176816$

C $6.062403-0.8757410 .044318$

C 6.4678960 .1097621 .171380

O $6.220233-0.245110-1.225333$

C $-0.5853130 .606940-2.397043$

O $-1.0905391 .165284-3.358463$

C $0.0282601 .295618-1.240121$

C $-0.2237842 .600935-1.058328$

C 0.2345583 .4346590 .056223

C 1.1890442 .8171091 .041367

C 5.5167151 .2946721 .387127

C 5.7721572 .4897640 .485151

C $4.8145304 .710399-0.085343$

O $6.6954162 .547622-0.321240$

C 3.9420965 .7127930 .596935

C 2.8989536 .3210710 .012350

C 2.0369247 .3266420 .720261

C 0.5582407 .0337430 .603879

C -0.2788287 .9309460 .054801$

C 0.0331435 .7420041 .195231

C -0.2339174 .6976490 .141933$

N $4.843063 \quad 3.4964500 .707315$

$\mathrm{H}-3.368152-0.846501-4.576300$

H -2.869356 -2.075877 -1.811484

H - $1.021508-1.177593-4.151391$

H -1.056001 -3.681218 -3.719392

H $-0.638644-3.381771-2.052361$

H $2.980164-3.232806-3.082108$

H $2.256333-2.923675-5.208925$

H $\quad 0.621225-3.496890-5.539238$

H $\quad 0.922811-1.769290-5.244785$

H $\quad 0.939435-3.535549-0.777584$

H $2.388249-4.477629-0.986214$

H $2.200678-1.471437-0.415141$

H $2.631977-2.7116300 .728135$

H $4.267405-1.797038-1.678723$

H $4.656248-4.274859-1.299694$

H $4.885407-3.9676490 .409386$

H $6.894884-5.198551-0.931502$

H $6.966346-2.508998 \quad 1.133531$

H $6.549395-3.946762-2.882872$

H $7.915133-2.941002-2.456376$

H $6.325496-2.202376-2.734481$

H $8.861892-2.565862-0.117802$

H $6.479142-0.4478892 .117510$

H 7.4853960 .4806540 .999147

H $5.8862180 .663101-1.166039$

H $\quad 0.6222320 .706292-0.551586$

H $-0.8593143 .109332-1.782952$

H 0.6950592 .0217121 .609124

H 1.5880363 .5300111 .765662

H 2.0522872 .3872250 .521836

H 4.4708490 .9852981 .286455

H 5.6526931 .6533692 .415938

H $4.4509014 .447537-1.084413$
H $5.8356565 .093279-0.181731$

H 4.1869465 .9549821 .628885

H $2.6648576 .102319-1.026672$

H 2.2649748 .3135520 .297760

H 2.2949957 .3843391 .785427

H $-1.3456807 .741815-0.006149$

H $\quad 0.0816808 .872168-0.346627$

H $-0.9177115 .944406 \quad 1.706711$

H 0.7077765 .3962621 .982899

H $-0.9401985 .030112-0.621104$

H 4.0498403 .2960651 .309557

C $-5.5881560 .096423-2.892889$

O $-4.885540 \quad 0.605343-1.740065$

C $-5.8425520 .937602-0.722591$

C $-7.1377551 .195778-1.475051$

C $-7.0761520 .101587-2.525998$

H $-5.9667730 .044243-0.098885$

C -5.3249342 .0885660 .144369$

H $-8.0188801 .123941-0.830787$

H -7.139249 2.180584 -1.956061

C $-7.9960850 .339686-3.710941$

H -7.350714 -0.851434 -2.053931

C $-3.888887 \quad 1.887123 \quad 0.673125$

O $-5.3303213 .302396-0.617072$

H -6.0144922 .2567920 .979489$

H $-3.1699382 .027555-0.142781$

O -3.6188972 .9222931 .625646$

C -3.6297100 .5249651 .341323$

C -2.2106190 .4254821 .931399$

H $-3.756430-0.2693200 .598303$

O $-4.594654 \quad 0.3016832 .367101$

H -1.494667 0.8320731 .211941

H -2.160706 1.0277222 .846945

H $-9.0409640 .375235-3.385719$

H $-7.767691 \quad 1.285959-4.212585$

H $-7.897049-0.464610-4.446773$

C $-5.061799-1.303971-3.218719$

H $-4.7772123 .157980-1.404066$

H -3.7632153 .7732111 .177030$

H -4.504166 1.0189523 .017557

H $-5.630260-1.742304-4.046797$

H -5.195164 -1.961841-2.350683

H $-5.3833870 .788153-3.719585$

C -2.436245 -2.850732 3.720497

O $-2.631548-1.486178 \quad 3.330013$

C $-1.808935-1.0263472 .255415$

C $-0.323194-1.1386432 .631870$

C $0.030208-2.5627733 .073350$

C $-0.967693-3.1086304 .100827$

H $-3.046593-2.9706014 .624717$

C $-2.971868-3.8259952 .665665$

H -2.000349-1.634736 1.364470

O $-0.028498-0.2218313 .683441$

H $0.283748-0.8524251 .764178$

H $1.038470-2.5644593 .505316$

O $0.050011-3.4020091 .922856$

H -0.806052 -4.182504 4.250963

O $-0.700947-2.4674695 .351463$

C $-4.462253-3.6514582 .487583$

H $-2.484858-3.7077681 .693542$

H $-2.801489-4.8648362 .971695$

H $0.921140-0.2948373 .878951$

H $0.314333-4.2917292 .212845$

H -1.295938 -2.856741 6.014800

O $-4.726844-3.101881 \quad 1.268009$

O $-5.289415-3.952222 \quad 3.338759$

C $-6.110193-2.8745460 .982408$

H $-6.678747-3.8052411 .072101$

H $-6.510602-2.1097611 .654430$

H -6.190450 -2.515673 -0.046660

SCF Energy (B3LYP/6-31G**//MMFF) $=-3245.90740021$

07_00359

MM̄MF Geometry

C $3.301519-2.6035992 .670716$

C $2.674556-1.4492662 .941365$

C $1.300800-1.2660803 .538542$

O $0.458094-0.7125112 .498144$ 
C $0.615949-2.5503204 .048227$

C $-0.763131-2.3081944 .638475$

C $-1.927574-2.6732494 .062553$

C $-0.773774-1.6293805 .984677$

C $-2.133861-3.3661752 .742695$

C $-2.874425-2.4654601 .750607$

C $-2.938702-3.0383560 .324954$

C $-1.560097-3.075592-0.345632$

C $-1.645353-3.530741-1.807860$

O $-3.815711-2.183381-0.421621$

O $-0.388870-3.243669-2.439466$

C $-2.714561-2.685872-2.529493$

C $-1.837827-5.048524-1.913774$

O $-2.928844-3.172424-3.852896$

C $-4.062911-2.604884-1.770756$

C $-4.979378-1.548311-2.434349$

O $-4.758705-3.851942-1.817503$

C 0.4107740 .6394152 .357942

$\begin{array}{lllll}\text { O } & 1.042440 & 1.474147 & 2.984147\end{array}$

C -0.5937390 .9242391 .312130$

C $-1.0575642 .169177 \quad 1.136449$

C -2.1198992 .5570750 .204267$

C $-2.7637951 .460853-0.607128$

C $-6.419185-1.524269-1.910884$

C -6.569851-0.925747 -0.527960

C $-6.849634 \quad 1.2296440 .649242$

O $-6.770111-1.6299300 .459563$

C -6.2930112 .6139200 .546914$

C -5.6159813 .2001731 .546096$

C $-5.027538 \quad 4.5852161 .566470$

C $-4.637445 \quad 5.179343 \quad 0.227404$

C $-5.1703276 .344604-0.178561$

C -3.611765 $4.470172-0.640717$

C $-2.487404 \quad 3.854808 \quad 0.151449$

N $-6.4731960 .458868-0.521567$

H $2.829868-3.5526652 .905513$

H $3.182626-0.5223172 .684153$

H $1.384837-0.5553374 .370185$

H $1.240694-3.0233294 .817357$

H $0.554584-3.2752423 .231719$

H $-2.852198-2.4521814 .594933$

H $-1.781377-1.5614026 .408703$

H $-0.156971-2.1876616 .696493$

H $-0.381781-0.6106935 .906520$

H -1.188483 -3.7031272 .319259$

H $-2.725304-4.2732532 .917610$

H -3.898407 -2.294691 2.110691

H -2.415691 -1.470460 1.719431

H $-3.380217-4.0403320 .373163$

H -0.861616 -3.7204580 .198384$

H $-1.116262-2.071318-0.323543$

H $0.309858-3.695303-1.936108$

H - $2.316973-1.666154-2.632252$

H $-0.980055-5.574261-1.476970$

H - $1.884123-5.370116-2.959984$

H $-2.733496-5.404427-1.400166$

H -2.064538 -3.187968 -4.298854

H $-4.532078-0.553188-2.322972$

H -5.046521 -1.760350 -3.508769

H -4.316958 $-4.430964-2.459411$

H $-0.9597920 .083950 \quad 0.735422$

H -0.6511842 .9700101 .752260$

H -2.014767 $0.937457-1.212116$

H -3.521731 $1.815737-1.307035$

H -3.2534670 .7341560 .050665$

H -7.036089 $-0.930122-2.597763$

H $-6.864349-2.525346-1.907261$

H $-6.5103070 .705124 \quad 1.549041$

H $-7.943857 \quad 1.2698820 .672075$

H $-6.4810653 .158722-0.374123$

H -5.4841382 .6413442 .471433$

H -4.1344004 .5654972 .203981$

H -5.7380815 .2370882 .091214$

H $-4.8962586 .790752-1.128972$

H $-5.895420 \quad 6.8780290 .427001$

H -3.160716 $5.179489-1.346981$

H $-4.141243 \quad 3.736228-1.251994$

H -1.9424944 .5725390 .766620$
H $-6.4700220 .937304-1.418203$

C $4.588846-2.9807770 .511246$

O $4.004419-1.868848-0.198900$

C $3.168237-2.385668-1.251456$

C $3.485856-3.875564-1.353662$

C $3.794942-4.2173310 .090293$

H $2.132071-2.268824-0.912959$

C $3.385869-1.609861-2.555034$

H $2.650022-4.450972-1.763004$

H $4.363448-4.062641-1.983336$

C $4.549083-5.5231010 .265113$

H $2.842952-4.2799310 .631169$

C $3.149889-0.087970-2.466857$

O $4.735887-1.805325-2.998598$

H $2.750509-2.035719-3.340564$

H $3.9556540 .383757-1.893660$

$\begin{array}{llll}\text { O } & 3.243931 & 0.436306 & -3.798480\end{array}$

C $1.7883160 .324687-1.882404$

C $1.4189661 .807483-2.072635$

H $1.7658470 .082587-0.814431$

O $0.748467-0.444116-2.494182$

H $1.2567792 .019849-3.137417$

H $0.4386551 .947615-1.601354$

H $3.959957-6.361894-0.119809$

H $5.504376-5.510453-0.270143$

H $4.757088-5.7130251 .322852$

C $4.651364-2.6814762 .012941$

H $5.324656-1.499223-2.287403$

H $4.1136480 .179328-4.149915$

H $0.784770-0.279432-3.452051$

H $5.187459-1.7366112 .165391$

H $5.235429-3.4575082 .521171$

H $5.620258-3.059345 \quad 0.142384$

C $3.562796 \quad 3.331874 \quad 0.584215$

O $2.6147332 .509130-0.109884$

C $2.4254622 .813862-1.493848$

C $1.9424184 .264694-1.673936$

C $2.8638305 .258189-0.960813$

C $3.1745114 .815845 \quad 0.473831$

H 3.4699563 .0402451 .638387

C 5.0086863 .0548100 .140189

H $3.3747962 .687505-2.023273$

O $0.6216504 .397832-1.152778$

H $1.8979634 .490327-2.746253$

H $2.3837746 .244262-0.940907$

O $4.0688625 .378189-1.710185$

H 3.9751505 .4344740 .894346

O $2.019522 \quad 5.0406151 .288051$

C $5.455967 \quad 1.649438 \quad 0.475184$

H $5.1319363 .184184-0.938005$

H 5.6878753 .7428190 .656697

H $\quad 0.3258125 .307242-1.327782$

H $4.6321916 .028907-1.257649$

H 1.3162504 .4448710 .977177

O $6.6957591 .439524-0.058234$

$\begin{array}{llll}\text { O } & 4.809727 & 0.839822 & 1.123774\end{array}$

C $7.2535150 .141862 \quad 0.170802$

H $6.667956-0.614645-0.358671$

H $8.2730220 .135726-0.223728$

H $7.291600-0.0772291 .242371$

SCF Energy (B3LYP/6-31G**//MMFF) $=-3245.90679526$

07_00360

MM̄FF Geometry

C $2.246879 \quad 3.0245192 .359048$

C 1.5090072 .8438193 .465450

C 1.1978851 .5198344 .129718

$\begin{array}{llllll}\text { O } & -0.228325 & 1.244814 & 4.104664\end{array}$

$\begin{array}{llll}\text { C } 2.004388 & 0.296174 & 3.649199\end{array}$

C $1.821891-0.9381294 .511505$

C $1.298290-2.1042324 .085440$

C $2.352466-0.8331045 .919813$

C $0.750485-2.4371992 .723467$

C $-0.495170-3.3256412 .840539$

C -1.132368 -3.7352511 .503396$

C $-0.169299-4.481148 \quad 0.576147$

C $-0.832249-4.863424-0.756678$

O $-1.642174-2.5654200 .851854$ 
O $0.226956-5.255547-1.644290$

C -1.515397 -3.609660 -1.346417

C $-1.747117-6.083799-0.606470$

O $-2.279706-3.934723-2.504589$

C $-2.405084-2.859076-0.329847$

C $-2.954974-1.531225-0.893266$

O $-3.545901-3.669747-0.014986$

C -0.8824021 .1308762 .918995$

O -0.4211751 .2658911 .797204$

C -2.2957620 .8138203 .219850$

C $-3.232757 \quad 1.1587732 .325580$

C -4.6727350 .9204852 .424363$

C $-5.1621850 .078216 \quad 3.571947$

C -1.899822 -0.538410 -1.382267

C $-2.5177160 .815024-1.669596$

C $-4.225781 \quad 1.937990-3.089485$

O $\quad-2.2341841 .807282-1.003079$

C $-5.5607661 .907550-2.399632$

C $-6.1480530 .848045-1.819118$

C $-7.4850210 .899789-1.135712$

C -7.4295370 .4002770 .292330$

C $-7.850600-0.8360310 .608204$

C $-6.973096 \quad 1.3613631 .367193$

C -5.475996 1.4449011 .474347

N -3.401634 $0.797372-2.736621$

H 2.6590652 .1680531 .832692

H 1.0986453 .7206813 .962993

H 1.4323301 .6806055 .189141

H 3.0740090 .5441203 .655218

H 1.7604090 .0760282 .606350

H $1.279090-2.9337584 .792123$

H $2.365927-1.8008996 .432740$

H $3.380248-0.4557025 .913670$

H $1.734051-0.1556286 .516171$

H $0.498035-1.5411102 .151378$

H $1.536560-2.9711742 .179827$

H $-0.242382-4.2362713 .399122$

H -1.248978 -2.786469 3.430260

H $-1.977933-4.3859471 .757965$

H $\quad 0.240927-5.369350 \quad 1.072133$

H $\quad 0.691274-3.841670 \quad 0.342121$

H $-0.172328-5.544793-2.481961$

H $-0.713427-2.939044-1.677551$

H $-1.168233-6.954367-0.275177$

H -2.190621 -6.367154 -1.567383

H $-2.554942-5.9306270 .112002$

H -1.670355 -4.300297 -3.167493

H -3.667676 -1.748855-1.698995

H -3.549747 -1.041829-0.109341

H -4.242166 -3.094196 0.343005

H $-2.520507 \quad 0.3224604 .159013$

H -2.913999 1.6871351 .430221

H $-4.995956 \quad 0.5932764 .524303$

H -4.636072 -0.8830593 .596206$

H -6.226641 -0.1592183 .513121$

H -1.119000 -0.399132 -0.626306

H -1.413457 -0.890905 -2.298170

H $-4.363647 \quad 1.933677-4.174755$

H -3.696512 $2.855029-2.809916$

H $-6.0847332 .861195-2.379208$

H $-5.662776-0.123705-1.845423$

H -8.180906 $0.284614-1.721109$

H -7.904704 $1.913234-1.150004$

H -7.842099 -1.190834 1.633518

H -8.207585 -1.522915 -0.152045

H $-7.427364 \quad 1.1002272 .329030$

H -7.363946 2.3633141 .147975

H -5.0172372 .0369430 .682022$

H -3.584769 -0.079668 -3.214854

C $1.705646 \quad 4.7247940 .577307$

O $1.8370943 .732911-0.456774$

C $1.8282384 .388075-1.739519$

C $1.5438955 .864365-1.472796$

C $2.1042836 .046450-0.077185$

H $2.8430034 .290415-2.142742$

C $0.8272033 .710789-2.685471$

H $2.0187026 .514781-2.214191$

H $\quad 0.4707106 .085724-1.479965$
C $1.572966 \quad 7.2788520 .634114$

H $3.1980846 .115378-0.148673$

C $0.9828192 .179102-2.789751$

O $-0.4959883 .978649-2.203456$

H $0.8941264 .174302-3.677151$

H $\quad 0.669330 \quad 1.713224-1.848762$

O $\quad 0.0771451 .712792-3.798615$

C $2.3966831 .700044-3.165166$

C $24898950.184636-3.433489$

H $3.1043021 .976299-2.376507$

O $2.8059012 .383993-4.353298$

H $1.800460-0.087702-4.242142$

H $3.486085-0.023805-3.841496$

H 1.8264138 .1850860 .074373

H $\quad 0.483491 \quad 7.242398 \quad 0.739841$

H 2.0055057 .3697791 .635181

C 2.5821424 .3688541 .778819

H $-1.1203373 .753477-2.913164$

H $-0.8266941 .876961-3.483412$

H $3.7237532 .123363-4.540865$

H 2.4951055 .1457632 .547539

H 3.6370154 .3379871 .478224

H 0.6480084 .7396780 .872643

C $4.455114-0.669634-1.271306$

O $3.073907-0.339938-1.091102$

C $2.227009-0.692044-2.191224$

C $2.316205-2.200809-2.457060$

C $3.758221-2.655053-2.675725$

C $4.653124-2.169266-1.534307$

H $4.840689-0.102343-2.127671$

C $5.242985-0.152457-0.057791$

H $1.200605-0.489711-1.866143$

O $1.515983-2.548923-3.581319$

H $1.904657-2.734563-1.591276$

H $4.137622-2.311847-3.644541$

O $3.799612-4.083457-2.729084$

H $4.430180-2.757427-0.637255$

O $6.018334-2.409451-1.867246$

C $4.931945-0.8693421 .239296$

H $6.315311-0.253096-0.262395$

H 5.0189710 .9082590 .106560

H $1.609403-3.507236-3.718651$

H $3.449844-4.426352-1.888824$

H $6.106814-3.357593-2.065111$

O $6.006160-0.7671252 .076078$

O $3.873496-1.4244531 .498655$

C $5.866023-1.4101783 .346975$

H $6.840678-1.3990413 .842179$

H $5.152224-0.8594293 .964888$

H $5.549619-2.4505673 .223717$

SCF Energy (B3LYP/6-31G**//MMFF) $=-3245.89084862$

07 00361

MM̄FF Geometry

C $1.270922-3.994792-0.927314$

C $0.732350-3.289000-1.933541$

C $-0.640259-2.674544-1.863417$

O $-0.515938-1.300037-2.299916$

C -1.647060 -3.408559-2.770541

C $-2.143820-4.711776-2.174782$

C $-3.057759-4.781982-1.185177$

C $-1.546943-5.965161-2.755718$

C $-3.746920-3.610447-0.539199$

C $-5.144731-3.970485-0.019620$

C $-5.992449-2.7530090 .393570$

C $-6.542275-2.007247-0.827247$

C $-7.350727-0.764602-0.430717$

O $-5.201117-1.8802991 .208395$

O $-7.543705-0.000531-1.631514$

C -6.5035870 .0784780 .545360$

C $-8.748386-1.1331020 .079411$

O -7.2553801 .1756251 .059985$

C $-5.912423-0.7427561 .716164$

C -4.9412510 .0980812 .581202$

O $-6.984046-1.1533602 .576205$

C $-1.412758-0.412069-1.789656$

O $-2.339469-0.657367-1.033915$

C $-1.0606090 .935959-2.285594$ 
C $-1.7516411 .990830-1.827761$

C $-1.5084943 .399551-2.141623$

C $-0.4393223 .711186-3.153491$

C $-3.651094 \quad 0.541746 \quad 1.884734$

C -2.8682081 .5482962 .714189$

C -0.8567793 .0219172 .572407$

O -3.2413651 .9485513 .813634$

C -1.1193394 .2612931 .771901$

C $-0.1648224 .934411 \quad 1.109593$

C $-0.326130 \quad 6.1669720 .256404$

C $-1.7223136 .453689-0.255332$

C $-2.5370607 .300144 \quad 0.396765$

C $-2.1348585 .829748-1.574642$

C $-2.2359104 .332093-1.491090$

N -1.7107371 .9602822 .067089$

H $0.693097-4.143455-0.018097$

H $1.289003-3.163892-2.859008$

H $-0.983910-2.680039-0.821964$

H -1.201598 -3.590091 -3.758342

H -2.502276 -2.757616 -2.990782

H $-3.325969-5.763000-0.796955$

H -1.941354 -6.870893 -2.282904

H -1.769938 -6.028007 -3.825825

H $-0.460662-5.973030-2.627076$

H -3.133337 -3.266005 0.301616

H $-3.830546-2.787535-1.251222$

H -5.695496 -4.564498 -0.759805

H $-5.014530-4.6116550 .862667$

H $-6.826220-3.1361880 .994013$

H -7.150440 -2.676234-1.448890

H $-5.714427-1.678335-1.468676$

H $-8.1019370 .765441-1.415998$

H -5.684572 $0.510192-0.041761$

H $-9.318456-1.641497-0.707549$

H $-9.325083-0.2375670 .336308$

H $-8.729246-1.7904120 .950964$

H $-7.519608 \quad 1.7314850 .308051$

H -5.4783090 .9661042 .985539$

H $-4.650747-0.4914873 .462382$

H -6.615370 -1.363314 3.450167

H $-0.2298621 .025415-2.975399$

H -2.563641 $1.811972-1.124183$

H $-0.7084273 .295091-4.130735$

H $0.5190373 .282625-2.847149$

H $-0.2688794 .779215-3.299086$

H $-3.010077 \quad-0.3269021 .697369$

H -3.871450 1.0228660 .927806

H 0.1785322 .6807652 .480943

H -1.072333 3.2084923 .628816

H -2.1483594 .6093561 .746054$

H 0.8590154 .5744821 .188150

H $0.3464586 .078889-0.606876$

H 0.0504207 .0240580 .829988

H -3.526962 7.5362920 .020817

H -2.232213 7.7719631 .325149

H -1.439300 $6.165935-2.347975$

H -3.117600 $6.208246-1.885031$

H $-2.9985353 .993314-0.787835$

H -1.4963191 .5801441 .149716$

C $3.415319-4.4941750 .295190$

O $3.970073-3.1625560 .372068$

C $5.334736-3.2598900 .824190$

C $5.520091-4.6909881 .315201$

C $4.608247-5.4479530 .375574$

H $5.969033-3.111289-0.057048$

C $5.651532-2.2071051 .891536$

H $6.563272-5.0168411 .259649$

H $5.183773-4.8106652 .351965$

C $4.259207-6.8423470 .867927$

H $5.096619-5.523661-0.605366$

C $5.523010-0.7378991 .442707$

O $4.776227-2.3947203 .011675$

H $6.664548-2.3783622 .275231$

H $4.467389-0.4657821 .337445$

O $6.043861 \quad 0.0751012 .503620$

C $6.280481-0.3880930 .149734$

C $6.4735341 .115371-0.120453$

H $5.759944-0.839651-0.702144$
O $7.587361-0.9730450 .182799$

H $7.194557 \quad 1.5317660 .595330$

H $6.9615001 .194424-1.101444$

H 5.164051 -7.449628 0.975128

H $3.758831-6.8104731 .841729$

H $3.593717-7.3520450 .164489$

C $2.612182-4.666790-0.996905$

H $3.863204-2.3217792 .684238$

H $5.545649-0.1466803 .309066$

H $8.047799-0.6200030 .963630$

H $2.427643-5.732442-1.175139$

H $3.187272-4.279014-1.846664$

H $2.770006-4.619751 \quad 1.174749$

C $3.0050902 .064166-1.142684$

O $4.2819091 .415325-1.061884$

C $5.1926781 .960761-0.105606$

C $5.4919743 .436011-0.422995$

C $4.2017604 .253833-0.528293$

C $3.1684263 .569746-1.427807$

H $2.5127771 .605520-2.009609$

C $2.132667 \quad 1.7825230 .089982$

H $4.749387 \quad 1.8947290 .892815$

O $6.2116173 .525972-1.651494$

H 6.1362713 .8426270 .365861

H $4.4274215 .246547-0.936957$

O 3.6436294 .4372430 .768758

H $2.2013024 .075590-1.328863$

O $3.5912213 .733959-2.784392$

C 1.8039960 .3150490 .254480

H 2.6205202 .1094021 .011828

H $1.1808132 .314633-0.013620$

H $6.4074034 .465137-1.809620$

H 4.3037454 .9028441 .309896

H $2.9078923 .345117-3.356263$

O $1.1163540 .151183 \quad 1.423232$

O $2.102868-0.569907-0.534056$

C $0.730913-1.1884201 .743185$

H $0.382826-1.2022142 .779395$

H -0.091099-1.499155 1.094111

H $1.579570-1.8726351 .652363$

SCF Energy $\left(B 3 L Y P / 6-31 G^{* *} / / M M F F\right)=-3245.89881024$

07_00362

MMFF Geometry

C $2.256863 \quad 3.6495281 .298252$

C 2.0456473 .0172892 .464377

C 0.7495022 .3921032 .933238

O -0.2204912 .4021301 .862105$

C 0.9841610 .9388313 .380016

C $-0.1767380 .339995 \quad 4.148817$

C $-0.988476-0.6235143 .670531$

C -0.3465390 .8390225 .560834$

C $-0.939273-1.2472042 .302060$

C $-2.312358-1.7769251 .873682$

C $-2.264217-2.5990470 .577467$

C $-2.010111-1.738486-0.659693$

C $-2.063313-2.568337-1.953390$

O $-3.534889-3.2486660 .455515$

O $-2.123042-1.650870-3.057250$

C $-3.372642-3.388104-1.972218$

C $-0.793412-3.403961-2.155147$

O $-3.376419-4.278080-3.086017$

C $-3.640203-4.151214-0.654100$

C $-5.044654-4.805164-0.591517$

O $-2.685889-5.214400-0.530507$

C -1.4883992 .7982082 .152009$

O -1.9371293 .0994923 .246196$

C -2.2430572 .8404330 .878637$

C -3.4445513 .4366240 .849336$

C $-4.2923043 .628743-0.330286$

C $-3.8273913 .021463-1.627747$

C $-6.246137-3.904759-0.888916$

C $-6.354234-2.7231270 .056095$

C $-6.254762-0.2404690 .097840$

O $-6.450269-2.869006 \quad 1.272621$

C $-6.7011400 .846352-0.825145$

C $-7.6569601 .737633-0.525526$

C $-8.1198632 .804049-1.474988$ 
C $-7.8074854 .199001-0.986376$ C $-8.7549914 .961369-0.414926$ C $-6.4210684 .743048-1.252323$ C $-5.4280604 .346031-0.193918$ N $-6.331767-1.506380-0.606826$ H 1.4491053 .7464800 .578258 H 2.8652312 .9759393 .179959 H 0.4015283 .0013593 .776004 H 1.8735380 .8790344 .021993 H 1.2124140 .3242752 .504573 H -1.754388 -1.0176314 .337006$ H -1.1449690 .3125316 .094483$ H 0.5781850 .6931916 .128658 H -0.5953371 .9042215 .569095$ H $-0.587277-0.5246841 .559833$ H $-0.226540-2.0795332 .331960$ H -2.725347 -2.413859 2.668110 H -3.024423 -0.9478951 .766463$ H -1.487099 -3.3643630 .687167$ H - $1.053278-1.212877-0.588689$ H $-2.776889-0.953265-0.720681$ H -1.336276 -1.080422 -3.015621 H -4.177655 -2.666632 -2.149848 H $0.085286-2.753521-2.231396$ H - $0.831461-3.959739-3.098430$ H $-0.611284-4.112135-1.344109$ H -4.265241 -4.664114 -3.156592 H -5.077766 -5.663134 -1.275429 H $-5.178999-5.2283580 .414018$ H -3.039351 -5.8727390 .090821$ H -1.773249 2.419591-0.002723 H -3.837783 3.8549851 .775269 H -3.638634 $1.948574-1.506442$ H -2.903861 $3.502636-1.967549$ H $-4.5558483 .111526-2.436131$ H $-6.228571-3.579007-1.933983$ H -7.169426 -4.486085 -0.773915 H $-5.208856-0.0863180 .382663$ H $-6.855700-0.2952301 .011569$ H $-6.2082880 .906379-1.793143$ H $-8.151701 \quad 1.6872600 .441496$ H -9.202784 2.682781-1.609817 H -7.687475 2.664678 -2.473940 H -8.544826 $5.975600-0.091827$ H $-9.7598694 .585940-0.252143$ H $-6.4532725 .841174-1.267333$ H -6.109162 $4.463008-2.262182$ H $-5.669403 \quad 4.7451490 .792690$ H -6.090826 -1.503364 -1.593633

C $4.0011094 .045969-0.498605$

O $4.4858202 .692817-0.640165$

C $5.7428542 .721218-1.341857$

C $5.8414304 .106482-1.968988$

C $5.1588014 .955366-0.919958$

H $6.5261082 .625418-0.580511$

C $5.8633001 .577828-2.355880$

H $6.8772704 .404731-2.157602$

H $5.294040 \quad 4.165271-2.916913$

C $4.7213716 .316578-1.434680$

H $5.8512035 .096739-0.078949$

C $5.6896220 .156129-1.786483$

O $4.8634521 .757712-3.368382$

H $\quad 6.830858 \quad 1.659706-2.866737$

H $4.629311-0.023289-1.586610$

O $6.047670-0.761841-2.829727$

C $6.516205-0.163157-0.530011$

C $6.565157-1.655254-0.143591$

H 6.1240520 .4173030 .313030

O $7.864026 \quad 0.266863-0.745673$

H $7.189142-2.203730-0.861366$

H $7.102329-1.7144890 .812433$

H $5.5861086 .892786-1.779992$

H $4.023536 \quad 6.222821-2.273548$

H $4.2255036 .892455-0.647157$

C 3.5593834 .3137850 .944524

H $4.9677801 .034867-4.010692$

H $7.002650-0.667045-2.988386$

H 8.3544050 .1191510 .080929
H $3.4178385 .391594 \quad 1.086534$

H 4.3476213 .9883101 .634724

H $3.1557604 .154021-1.190295$

C $3.065372-2.0783191 .098480$

O $4.407460-1.6062930 .916254$

C $5.198107-2.345963-0.015768$

C $5.331654-3.809807 \quad 0.436748$

C $3.956942-4.4455380 .670345$

C $3.055661-3.5537111 .533055$

H $2.671378-1.4843471 .932109$

C $2.184702-1.806209-0.131582$

H $4.715509-2.323991-0.997435$

O $6.093470-3.8707241 .641133$

H $5.880969-4.367888-0.331216$

H $4.090233-5.4171991 .161119$

O $3.344280-4.681859-0.594000$

H $2.024621-3.9262831 .528658$

O $3.510751-3.6027562 .888348$

C $2.037848-0.328427-0.418902$

H $2.590019-2.270644-1.033808$

H $1.182102-2.2074760 .050139$

H $6.189829-4.8081401 .880574$

H $2.482006-5.100097-0.429311$

H $3.460273-4.5285133 .181251$

O $1.260984-0.162881-1.529238$

O 2.5430300 .5695540 .238699

C $1.0302451 .194368-1.919251$

H $1.9749031 .675314-2.189708$

H $\quad 0.374627 \quad 1.189604-2.793900$

H $0.5341161 .742238-1.112831$

SCF Energy (B3LYP/6-31G**//MMFF) $=-3245.88407709$

07 00363

MMFF Geometry

C $2.314565-3.657485-0.989629$

C $1.498909-2.981943-1.813181$

C $0.053261-2.695907-1.504771$

O $-0.127519-1.266865-1.658390$

C $-0.903537-3.402883-2.484263$

C $-1.262146-4.814683-2.066706$

C $-2.363149-5.144772-1.362287$

C $-0.316977-5.895290-2.524958$

C $-3.448546-4.234235-0.857756$

C $-3.440732-4.1410020 .674190$

C $-4.551604-3.2465791 .255463$

C $-5.938232-3.8808721 .116889$

C -7.052129 -2.953648 1.625598

O $-4.503474-1.9826410 .590708$

O $-8.293913-3.5201721 .178729$

C $-6.883765-1.5734720 .955173$

C -7.111842 -2.924239 3.156651

O $-7.806799-0.6251951 .485376$

C $-5.447924-1.0120531 .059175$

C $-5.255704 \quad 0.2621520 .202495$

O $-5.180108-0.6561812 .420150$

C $-0.942077-0.639395-0.768846$

O $-1.540115-1.1433050 .167935$

C $-0.9745380 .795816-1.123760$

C $-1.4257111 .675421-0.217510$

C $-1.5364643 .124990-0.388369$

C $-1.2681023 .687353-1.758096$

C $-5.4853310 .054554-1.296169$

C $-4.9189601 .192788-2.119186$

C $-5.3117663 .579468-2.714622$

O $-3.9377751 .033859-2.842169$

C $-4.9135984 .725882-1.834908$

C $-4.9252744 .785329-0.493475$

C $-4.511747 \quad 6.010186 \quad 0.273945$

C -3.4304065 .7361671 .294463$

C -3.6642745 .8942282 .608283$

C -2.0539275 .3580970 .789320$

C - 1.8788733 .8673970 .685162

N $-5.6237432 .375686-1.969064$

H $1.922533-4.033218-0.047517$

H $1.879148-2.631668-2.770051$

H $-0.172654-2.988484-0.471501$

H -0.476236 -3.418369 -3.496456

H - $-1.812286-2.801980-2.603883$ 
H $-2.522463-6.196158-1.124272$ H $-0.689564-6.898501-2.291187$ H $-0.178914-5.845653-3.609983$ H $0.658241-5.791176-2.043640$ H $-3.367625-3.233230-1.288123$ H $-4.403472-4.640559-1.208354$ H -3.505899 -5.1415661 .119445$ H -2.472907 -3.721897 0.980666 H -4.319535 -3.093459 2.316232 H -5.976161-4.847315 1.634959 H $-6.144157-4.1010830 .061508$ H $-9.018726-2.9799211 .535883$ H $-7.142030-1.703277-0.103268$ H -7.326308 -3.9264333 .547552$ H -7.927688 -2.2834683 .509309$ H $-6.182791-2.5812083 .616713$ H -8.703638 -0.9561431 .310041$ H $-5.898608 \quad 1.0685980 .577764$ $\begin{array}{llll}H & -4.229130 & 0.623742 & 0.346658\end{array}$ H -4.460735 -0.0033622 .432069$ H $-0.6203821 .086521-2.105613$ H $-1.735362 \quad 1.2992520 .757200$ H $-0.2345283 .489533-2.059328$ H -1.416030 4.766915 -1.824967 H -1.937132 $3.234003-2.497538$ H $-5.005559-0.867668-1.643359$ H -6.553963 -0.035209-1.520950 H $-6.2082943 .848973-3.282048$ H $-4.5072213 .376182-3.428437$ H $-4.5796865 .607535-2.379988$ H -5.2666603 .9353840 .090931$ H $-5.4082586 .409490 \quad 0.765645$ H $-4.1627956 .801737-0.401145$ H -2.884397 5.7262983 .343684 H -4.639769 6.1919492 .978120 H -1.292319 5.7290551 .488481 H $-1.8558585 .883329-0.148756$ H -2.0357093 .3514071 .634239$ H $-6.4556682 .383937-1.386416$ C $4.685557-3.841372-0.137710$ O $5.004492-2.4468970 .065190$ C $6.423682-2.3277750 .285371$ C $6.933628-3.7411490 .538939$ C $6.024689-4.548129-0.360203$ H $6.857638-1.965139-0.653571$ C $6.741960-1.3552141 .426151$ H $7.993743-3.8512890 .291352$ H $6.797582-4.0404361 .584993$ C $6.008544-6.030572-0.027977$ H $6.351006-4.416406-1.400812$ C $6.2714260 .098042 \quad 1.219104$ O $6.138885-1.8351282 .635542$ H $7.822920-1.3609121 .611792$ H $5.183711 \quad 0.1583231 .329853$ O $\quad 6.827442 \quad 0.8792642 .286029$ C $6.6914250 .733310-0.117920$ C $6.5366122 .264593-0.194639$ H $6.1164750 .269109-0.927479$ O $8.070637 \quad 0.435616-0.360054$ H 7.2981742 .7447870 .433709 H $6.7850732 .554107-1.224761$ H $7.011227-6.458363-0.131166$ H $5.671667-6.2081630 .998988$ H $5.339349-6.576066-0.700367$ C $3.735703-4.009353-1.327360$ H $5.181272-1.9008012 .478033$ H $6.542827 \quad 0.472070 \quad 3.122290$ H $8.2980220 .788536-1.237155$ H $3.734748-5.057253-1.649166$ H $4.086777-3.400904-2.169813$ H $4.217366-4.1975820 .789391$ C $2.8136142 .523227-0.412278$ O $4.1815972 .173313-0.668030$ C 5.1474302 .8039380 .176164 C 5.0737824 .3336310 .035615 C 3.6514134 .8452680 .289717 C $2.6116744 .047625-0.502680$ H $2.2475892 .059799-1.230420$
C 2.3055811 .9215950 .908736

H 4.9465512 .5372851 .218085

O $5.4904154 .715134-1.274261$

H 5.7734104 .7876150 .747465

H 3.5841065 .9016750 .002204

O 3.3467254 .7534361 .678012

H $1.6024424 .310213-0.165462$

O $2.7118564 .442853-1.873948$

C 2.2641090 .4085030 .872254

H 2.9363682 .2047261 .754997

H $1.2864962 .272987 \quad 1.101125$

H $5.4619465 .685913-1.320569$

H 3.9992455 .2907232 .158742

H $2.0303483 .961272-2.372163$

O $1.820692-0.0496222 .080222$

O $2.583741-0.284182-0.083138$

C $1.758688-1.4725062 .217140$

H $1.518030-1.7033813 .258334$

H $\quad 0.969657-1.8749341 .576725$

H $2.723916-1.9266071 .974319$

SCF Energy (B3LYP/6-31G**//MMFF) $=-3245.89076828$

0700364

MMFF Geometry

C $-0.735583 \quad 3.0013490 .984325$

C 0.0900712 .7873362 .021051

C 0.0341091 .6338832 .996578

O -1.0516450 .7345472 .685771$

C 1.3496160 .8411532 .891309

C $1.381861-0.3971133 .761857$

C $1.218684-1.6503033 .293401$

C $1.663974-0.1552235 .220153$

C $0.920816-2.0466561 .871885$

C $-0.071089-3.2162951 .806344$

C $-0.567406-3.5512300 .388705$

C $0.571724-3.804277-0.602720$

C $0.053901-4.084460-2.022158$

O $-1.395621-2.472280-0.066424$

O $1.170287-4.014429-2.923599$

C $-0.953065-2.977803-2.409728$

C $-0.475985-5.515669-2.153591$

O $-1.557985-3.258889-3.669421$

C $-2.029275-2.719398-1.330915$

C $-2.925554-1.508510-1.670464$

O $-2.893496-3.861683-1.260554$

C $-2.2412750 .942693 \quad 3.309374$

O $-2.5135401 .823937 \quad 4.108229$

C $-3.181180-0.0859472 .813470$

C $-4.499747 \quad 0.1193312 .942771$

C $-5.557965-0.7584022 .441768$

C $-5.168077-2.1461132 .007632$

C $-2.213503-0.159154-1.735702$

C $-3.2061120 .938313-2.064708$

C $-5.0146532 .325094-1.051025$

O $-3.3128271 .387108-3.204062$

C $-6.3875331 .719804-0.979417$

C $-6.7065630 .434695-0.755595$

C $-8.116603-0.079141-0.669740$

C $-8.684180-0.0501930 .734842$

C $-9.7675570 .691887 \quad 1.021105$

C $-8.040610-0.9223901 .796295$

C -6.815521 -0.2727812 .381722$

N $-3.958600 \quad 1.333252-0.970832$

H -1.5748412 .3366810 .803151$

H 0.9216703 .4745112 .167687

H -0.0749862 .0279484 .014419$

H $2.193867 \quad 1.4867193 .169529$

H $1.5365590 .570811 \quad 1.844707$

H $1.311933-2.4771483 .995781$

H $1.713865-1.0868875 .793717$

H 2.6232590 .3585195 .341175

H $0.8783980 .463676 \quad 5.664614$

H $0.514744-1.2085461 .298616$

H $1.867444-2.3423031 .406944$

H $0.397846-4.1088542 .239869$

H $-0.943360-2.9751822 .428479$

H -1.190673 -4.448809 0.480485

H $1.218035-4.620233-0.256761$ 
H $1.211168-2.915876-0.658127$ H $1.543128-3.117318-2.879570$ H $-0.368960-2.061287-2.554337$ H $\quad 0.318400-6.237465-1.927423$ H $-0.787162-5.730063-3.181960$ H $-1.313650-5.729120-1.486834$ H -2.225062 -3.952301-3.529585 H $-3.461639-1.693882-2.610833$ H $-3.712722-1.438673-0.909898$ H -3.729752 -3.591896 -0.846184 H -2.766031 -0.9536292 .313635$ H -4.839616 1.0411423 .413226 H -4.589163 -2.645811 2.793007 H $-4.561623-2.1121121 .098828$ H -6.023865 -2.793952 1.806643 H - $1.7157310 .076230-0.788402$ H -1.447446 - $0.150614-2.515835$ H -4.915764 2.892759-1.981802 H -4.875352 $3.013709-0.211647$ H -7.201734 2.431400 -1.106671 H $-5.924354-0.305267-0.616495$ H -8.139378 -1.110002 -1.044780 H $-8.7538170 .493459-1.356697$ H -10.194380 0.7063562 .018520 H -10.249593 1.3048840 .266860 H -7.840159-1.904742 1.360281 H -8.746996 -1.100758 2.617495 H -6.9954890 .7370952 .754437$ H -3.798155 $0.886990-0.072636$ C $-0.3286913 .502406-1.428120$ O $0.7246032 .520008-1.405588$ C $1.6513702 .798484-2.463168$ C $0.9006503 .698282-3.433918$ C $0.077556 \quad 4.540264-2.475526$ H $2.4744603 .362797-2.007425$ C $2.2289611 .505976-3.057787$ H $1.5734694 .291926-4.059632$ H $\quad 0.238502 \quad 3.122845-4.091084$ C -1.100055 5.240069-3.131235 H $0.7344105 .295659-2.023901$ C $2.7499180 .529068-1.977867$ $\begin{array}{lllll}\text { O } & 1.250737 & 0.854571 & -3.865391\end{array}$ H $3.0521351 .789372-3.724081$ H $3.2684751 .104368-1.204525$ O $1.658420-0.142624-1.348524$ C $3.731982-0.518596-2.532020$ C $4.173785-1.598454-1.527182$ H $4.6176270 .006947-2.909681$ O $3.141798-1.188111-3.648668$ H $3.365966-2.325784-1.383967$ H $4.981664-2.160688-2.014510$ H $-0.7539735 .930427-3.907405$ H -1.783325 $4.523194-3.598884$ H $-1.6688395 .816070-2.394447$ C $-0.5171754 .092586-0.027365$ H $0.5255700 .581454-3.278766$ H $1.0864170 .534949-0.949683$ H $3.808271-1.794020-4.015069$ H -1.376458 $4.772041-0.017729$ H $\quad 0.367156 \quad 4.680960 \quad 0.246834$ $\mathrm{H}-1.2389252 .976970-1.737876$ C $6.171342 \quad 0.5266390 .824672$ O $5.651902-0.090020-0.362883$ C $4.648102-1.087742-0.156262$ C $5.185857-2.2339380 .719061$ C $5.777106-1.7058112 .028820$ C $6.736038-0.5351131 .785744$ H 7.0115591 .1421250 .478352 C $5.146711 \quad 1.4543621 .498225$ H $3.788971-0.6342070 .347852$ O $6.192690-2.9498930 .005100$ H $4.367486-2.9338310 .926832$ H $6.314158-2.5173952 .534880$ O $4.708705-1.2959482 .876181$ H $7.000740-0.0618922 .737989$ O $7.955812-1.0401211 .234881$ C $4.8641452 .704751 \quad 0.692318$ H 4.1892840 .9559351 .662335
H $\quad 5.543798 \quad 1.7831552 .466083$

H $6.476664-3.6929560 .564366$

H $5.097581-0.9859783 .711681$

H $7.757557-1.4008280 .353558$

O $4.0386043 .514874 \quad 1.421115$

O $5.2992962 .943070-0.425308$

C $3.690674 \quad 4.760442 \quad 0.809225$

H $3.0751294 .584788-0.076350$

H 3.1088515 .3415791 .529688

H 4.5910725 .3252300 .549281

SCF Energy (B3LYP/6-31G**//MMFF) $=-3245.91793430$

07_00365

MM̄FF Geometry

C $0.919858-3.902610-1.587685$

C $-0.071355-3.016696-1.410290$

C $-1.106821-2.700883-2.458536$

O $-2.381644-2.808414-1.779046$

C $-0.978298-1.276404-3.025933$

C $0.405334-0.914577-3.512922$

C $1.261847-0.131288-2.829363$

C $0.788352-1.481260-4.853180$

C $1.0454250 .480762-1.473795$

C $1.0525722 .013279-1.507337$

C $-0.1212332 .622925-2.288436$

C $-0.0722324 .150618-2.213802$

C $-1.2833854 .798245-2.901764$

O $-1.338272 \quad 2.142990-1.709564$

O $-1.322914 \quad 6.179784-2.510937$

C -2.571956 4.137384-2.364515

C $-1.1436734 .785821-4.428534$

O $-3.7163804 .611853-3.071475$

C $-2.5266312 .589697-2.370314$

C $-3.752706 \quad 1.969992-1.652320$

O $-2.563783 \quad 2.138999-3.730297$

C $-3.507976-2.982871-2.510715$

O $-3.577330-3.109279-3.725085$

C $-4.713613-2.983113-1.645100$

C $-4.662085-2.894406-0.306124$

C $-5.801349-2.8771540 .613952$

C $-7.184754-2.8809740 .019555$

C $-3.7809662 .139909-0.129733$

C $-3.015210 \quad 1.0642970 .626336$

C $-2.323342 \quad 0.4658102 .946932$

$\begin{array}{llll}\text { O } & -2.614077 & 0.027476 & 0.105669\end{array}$

C $-3.388619-0.2713253 .708064$

C $-4.714175-0.0553543 .676737$

C $-5.708948-0.8363484 .489747$

C $-6.793728-1.4623823 .640209$

C $-7.973749-0.8440473 .464113$

C $-6.547541-2.8409003 .070190$

C -5.554706 -2.840155 1.940612

N $-2.876331 \quad 1.384743 \quad 1.970093$

H $1.003258-4.419477-2.540521$

H - $0.162439-2.503963-0.455355$

H $-1.068083-3.441703-3.267357$

H $-1.345600-0.556977-2.287095$

H - $1.684339-1.155559-3.858631$

$\begin{array}{llll}\text { H } & 2.236376 & 0.075573 & -3.268914\end{array}$

H $0.772193-2.575292-4.825555$

H $0.087637-1.141752-5.622803$

H $1.793183-1.174216-5.162214$

H $0.1325780 .125951-0.988544$

H $1.8559310 .134887-0.824994$

H $1.0015952 .361924-0.469942$

H $2.0030322 .373358-1.920637$

H $-0.061943 \quad 2.293315-3.331701$

H $0.8631214 .531325-2.642782$

H $-0.0666524 .463822-1.160079$

H $-0.4837956 .591846-2.779329$

H $-2.6932474 .488849-1.333188$

H $-0.2326545 .314750-4.733541$

H $-1.971716 \quad 5.321598-4.905637$

H $-1.0945683 .778693-4.847235$

H -3.738485 $4.162696-3.933641$

H -4.667176 $2.402836-2.078158$

H -3.810410 $0.900202-1.893695$

H -2.831251 $1.205076-3.738176$ 
H $-5.655807-3.061420-2.177827$ H $-3.689861-2.8295800 .179739$ H -7.306551 -2.045096 -0.678635 H $-7.977101-2.7744970 .763993$ H -7.369347 -3.817685 -0.517472 H -3.4194013 .1265970 .172692$ H -4.8235512 .0585200 .203938$ H -1.713281 1.0512643 .641441 H -1.675367 -0.2559892 .439055$ H -3.014158 -1.0634384 .354118$ H $-5.1218680 .739906 \quad 3.058284$ H $-6.158566-0.1470785 .216777$ H -5.218005 -1.613912 5.088076 H -8.769110 -1.297492 2.882050 H $-8.168523 \quad 0.1327193 .894733$ H -7.489138 -3.3104232 .764052$ H $-6.166222-3.4939473 .866124$ H $-4.516830-2.8319332 .275182$ H -3.290518 2.2450222 .317110 C $3.349364-3.856023-0.741720$ O $3.531290-2.458570-0.443757$ C $4.704705-2.003422-1.153660$ C $5.186616-3.180287-2.009183$ C $3.938769-4.033774-2.134587$ H $4.378084-1.197421-1.819187$ C $5.773086-1.490909-0.173653$ H $5.573617-2.847633-2.977882$ H $5.989788-3.739663-1.514890$ C $4.220826-5.475995-2.513471$ H $3.292569-3.574990-2.893540$ C $5.346647-0.2860470 .692065$ O $6.123729-2.5619400 .713406$ H $6.685094-1.244757-0.731184$ H $4.572994-0.6007731 .399566$ O 6.4769860 .0942651 .488520 C $4.8697080 .936918-0.105928$ C 4.6792882 .2274390 .710661 H $3.9337390 .691767-0.610703$ O $5.8272321 .216802-1.132994$ H 5.6568312 .6239611 .014904 H 4.2723762 .9725030 .013995 H $4.722398-5.527193-3.485494$ H $4.865495-5.966167-1.776181$ H $3.291618-6.050197-2.582726$ C $1.899426-4.293390-0.518929$ H $6.801872-2.2231221 .322888$ H 7.1656920 .4201320 .883678 H $5.4722791 .941343-1.675953$ H $1.557984-3.9090010 .449109$ H $1.870887-5.387828-0.445052$ H $3.963019-4.399633-0.009193$ C 1.5667391 .3103492 .590884 O $2.526142 \quad 1.5270931 .546132$ C 3.7650582 .1157651 .944728 C 3.5247803 .4925832 .592156 C 2.4915643 .4221403 .720562 C 1.2449712 .6246293 .324709 H 0.6559860 .9864572 .071779 C $1.992407 \quad 0.188013 \quad 3.549729$ H 4.2560731 .4596902 .670262 O 3.0884974 .4461541 .624478 H 4.4777873 .8635202 .987196 H 2.1904894 .4375164 .006525 O 3.0816462 .8057224 .862564 H 0.6495392 .4038944 .218113 O 0.4152853 .4391202 .492166 C $2.044035-1.1649652 .875606$ H $2.9807170 .379563 \quad 3.975662$ H $1.268421 \quad 0.1079704 .368931$ H 2.4224264 .0227161 .058889 H 3.8395843 .3516825 .132424 H 0.6889463 .3161961 .569093 O $2.658263-2.0483813 .717834$ O $1.593067-1.420871 \quad 1.768517$ C $2.791154-3.3855703 .228499$ H $1.811735-3.7977672 .968788$ H $3.463003-3.4020002 .366272$ H $3.227184-3.9963634 .023418$
SCF Energy (B3LYP/6-31G**//MMFF) $=-3245.90844598$

\section{7_00366}

MM̄FF Geometry

C $0.670901-2.9498741 .360532$

C $0.549649-2.0172872 .318595$

C $1.555241-0.9169782 .536187$

O 0.8644520 .3549192 .566586

C $2.268185-1.0383813 .895922$

C $3.157301-2.2513404 .051962$

C $4.457836-2.2881443 .703932$

C $2.527233-3.4442794 .725067$

C $5.280471-1.1954353 .075534$

C $6.143489-1.7234491 .919279$

C $5.374532-1.8512600 .593917$

C $6.170843-2.715847-0.386915$

C $5.525387-2.756945-1.776913$

O $5.211564-0.5274840 .071315$

O $6.475405-3.328988-2.688604$

C $5.271407-1.309397-2.238023$

C $4.288808-3.662889-1.810799$

O $4.560398-1.290257-3.474782$

C $4.532654-0.445246-1.189225$

C $4.5624151 .037557-1.639337$

O $3.183755-0.896564-1.089352$

C 0.6599140 .9820621 .380137

$\begin{array}{lllll}\text { O } & 0.925899 & 0.552801 & 0.270326\end{array}$

C 0.0629342 .3042551 .666962

C $-0.200436 \quad 3.143114 \quad 0.654027$

C $-0.8024894 .473122 \quad 0.767105$

$\begin{array}{llll}\text { C } & -1.066677 & 4.999628 & 2.153444\end{array}$

C $3.9064012 .018875-0.660105$

C $4.1751173 .470609-1.019889$

C $3.6528595 .778521-0.257015$

O $4.9212723 .811744-1.933464$

$\begin{array}{llll}\text { C } 2.382237 & 6.454329 & 0.149219\end{array}$

C $1.6726567 .241655-0.673442$

C $0.4165517 .965777-0.279291$

C $-0.7796747 .538484-1.100710$

C -1.040921 $8.108263-2.289365$

C $-1.7148576 .510283-0.504905$

C -1.079594 $5.154009-0.365077$

N $3.4752744 .340251-0.191278$

H $1.512603-2.8991060 .674106$

H - $0.269556-2.0853613 .030277$

H $2.311722-0.9005981 .741495$

H $1.527224-1.0179194 .707857$

H $2.854848-0.1257564 .068096$

H $5.011954-3.2018053 .917532$

H $3.241766-4.2600424 .879576$

H $2.137825-3.1616785 .708805$

H $1.703353-3.8435904 .128239$

H $4.670685-0.3529982 .735978$

H $5.951959-0.8105583 .852954$

H $6.975925-1.0214931 .777395$

H $6.583324-2.6887532 .199993$

H $4.388793-2.2925750 .778632$

H $6.296615-3.7325330 .006276$

H $7.186824-2.308534-0.489671$

H $6.703113-4.217632-2.365478$

H $6.250541-0.851467-2.437068$

H $4.560385-4.691197-1.543257$

H $3.867155-3.717723-2.820522$

H $3.501728-3.342586-1.124960$

H $5.062759-1.833377-4.105979$

H $5.6096661 .337909-1.775934$

H $4.0633441 .145204-2.610742$

H $2.642980-0.193855-0.697066$

H -0.1499922 .5476162 .701609$

H $0.0343012 .823350-0.360587$

H -1.4226856 .0314032 .170485$

H -0.1484104 .9864892 .751632$

H $-1.824153 \quad 4.3891082 .657263$

H $2.8227581 .880330-0.669016$

H $4.274018 \quad 1.8531350 .358477$

H $3.9727036 .065479-1.264397$

H $4.4558526 .042860 \quad 0.438781$

H 2.0448746 .3063121 .172098 
H $2.0266477 .397580-1.690359$ H $0.5903519 .041471-0.414490$ H 0.1996457 .8391660 .788571 H -1.911350 7.828094 -2.873202 H $-0.3859528 .866403-2.706115$ H -2.600301 $6.386381-1.142793$ H -2.102862 6.8963420 .441517 H -0.846799 $4.685408-1.322303$ H 3.0057543 .9562970 .624445 C $-0.719669-4.449403-0.154816$ O $-1.952925-3.732473-0.394513$ C $-2.970844-4.681862-0.759508$ C $-2.215896-5.888390-1.297381$ C -1.044076 -5.937609 -0.342990 H $-3.481310-4.9668960 .168592$ C $-3.989011-4.095266-1.743898$ H -2.820084 -6.800451-1.284891 H $-1.860909-5.723811-2.321369$ C $0.127647-6.756672-0.858735$ H $-1.387060-6.3714440 .606374$ C $-4.678009-2.790521-1.298681$ O $-3.343042-3.829399-2.995910$ H $-4.748053-4.856321-1.960608$ H $-3.977104-1.958800-1.414317$ O $-5.741369-2.525174-2.223923$ C $-5.238185-2.788582 \quad 0.134426$ C $-6.151437-1.5954730 .480719$ H $-4.401241-2.8059520 .841532$ $\begin{array}{lllll}\text { O } & -5.985758 & -3.985583 & 0.367594\end{array}$ H -7.118377 -1.704488 -0.027521 H $-6.376299-1.6735431 .552710$ H $-0.170479-7.798539-1.015066$ H $0.500593-6.367077-1.811935$ H $\quad 0.956047-6.745285-0.143352$ C $-0.210610-4.1640891 .263402$ H -2.612519 -3.211444 -2.822616 H -6.372267 -3.262984 -2.175061 H -6.753424 -3.980827 -0.228602 H $0.403184-5.0072581 .604886$ H - $1.059257-4.0769421 .953208$ H $-0.002788-4.109333-0.912485$ C -3.5639871 .1039530 .545670$ O $-4.290391-0.1049790 .809248$ C $-5.561692-0.2113140 .166075$ C $-6.4869250 .929726 \quad 0.617433$ $\begin{array}{llll}\text { C } & -5.830703 & 2.295056 & 0.380248\end{array}$ $\begin{array}{llll}\text { C } & -4.397538 & 2.340806 & 0.921367\end{array}$ H -2.704792 1.0637221 .226372 C $-3.0247871 .148138-0.894250$ H -5.427101 $-0.143698-0.917608$ $\begin{array}{lllll} & -6.789935 & 0.781188 & 2.003031\end{array}$ H -7.4306440 .8600290 .063493$ H -6.424395 3.0773450 .868412 O $-5.804502 \quad 2.579894-1.015544$ H $-3.885168 \quad 3.2448290 .572578$ $\begin{array}{llll}\text { O } & -4.439193 & 2.409936 & 2.349589\end{array}$ C $-1.9758760 .085382-1.141878$ H $-3.8144291 .000090-1.634738$ H -2.554098 2.120651-1.075328 H -7.395591 1.5004382 .250079 H $-6.7228492 .570560-1.334791$ H -4.9171253 .2218642 .589841$ O $-1.4725550 .241505-2.401811$ O $-1.644086-0.776722-0.340671$ C $-0.472187-0.705344-2.791964$ H $-0.301041-0.594916-3.866022$ H $0.461928-0.495981-2.264267$ H $-0.803634-1.729335-2.595950$

SCF Energy (B3LYP/6-31G**/MMFF) $=-3245.89801247$

\section{7}

MM̄FF Geometry

C $0.196999-1.8376901 .385343$

C $-0.318655-2.7472750 .542328$

C $0.068460-2.918174-0.909563$

O $0.653196-1.703964-1.435052$

C $1.093403-4.057789-1.034456$

C $1.550097-4.302781-2.458481$
C $2.712085-3.841876-2.962915$

C $0.630745-5.147777-3.298062$

C $3.700488-2.956125-2.257143$

C $4.943650-3.732209-1.804612$

C $5.804978-2.971680-0.779728$

C $5.190071-3.0276060 .622555$

C $5.988038-2.1957301 .634220$

O $5.941922-1.621925-1.223758$

O $5.176845-2.0837062 .814209$

C $6.187009-0.7786951 .057993$

C $7.276538-2.9051092 .064812$

O $7.023184-0.0207931 .929044$

C $6.740187-0.775553-0.385266$

C $6.7221690 .629186-1.038737$

O $8.100939-1.220492-0.364407$

C $-0.208099-0.724786-1.824894$

O $-1.428016-0.767208-1.800380$

C $0.5958830 .438110-2.262372$

C $0.0108991 .644406-2.300625$

C $0.6374072 .909178-2.689763$

C $1.9902782 .847169-3.346209$

C $5.3329801 .269704-1.148854$

C 4.9867532 .1885120 .011194

C 3.0296363 .3298961 .054251

O $5.8377952 .736320 \quad 0.706973$

C 2.7737664 .5999550 .304657

C 1.5734215 .1941170 .225272

C $1.3323686 .468431-0.533163$

C $0.1946006 .363318-1.525083$

C $-0.9242397 .091970-1.372331$

C $0.3811235 .471455-2.736461$

C $-0.0430304 .052861-2.465231$

N 3.6166492 .3686540 .140157

H $0.986333-1.1787261 .035895$

H -1.078688 -3.433924 0.908029

H $-0.828124-3.165473-1.492030$

H $\quad 0.666537-4.988729-0.636616$

H $1.960247-3.849913-0.395099$

H $2.973448-4.094422-3.989054$

H $1.024263-5.312877-4.306590$

H $0.487439-6.129168-2.834463$

H $-0.346004-4.664826-3.400186$

H $3.997382-2.160180-2.952163$

H $3.234683-2.440889-1.413608$

H $4.671441-4.713402-1.395942$

H $5.556312-3.923685-2.695939$

H $6.794745-3.443405-0.767516$

H $5.100932-4.0660380 .965425$

H $4.164459-2.6365230 .598906$

H $5.641156-1.4965523 .435502$

H $5.199367-0.3019931 .074991$

H $7.041128-3.8630462 .544073$

H $7.819352-2.3204722 .815740$

H $7.953840-3.1089101 .232832$

H 7.0089810 .9030841 .628616

H $7.4400031 .291300-0.540769$

H $7.0995290 .514942-2.065328$

H $8.546571-0.889145-1.161670$

H $1.6378690 .275650-2.512361$

H - $-1.0314781 .721329-1.993858$

H $1.9677302 .167234-4.205637$

H $2.7492442 .491646-2.644779$

H $2.3315403 .810704-3.730119$

H $5.3127681 .904114-2.044310$

H $4.5549540 .509261-1.276539$

H 3.7138523 .5177041 .887228

H $2.1091832 .888317 \quad 1.444387$

H $3.6249565 .057475-0.194803$

H 0.7239434 .7536630 .742035

H $2.2358176 .787185-1.068384$

H $\quad 1.1243507 .256295 \quad 0.202177$

H -1.733849 $7.039301-2.092887$

H -1.058638 $7.752880-0.522669$

H $1.4181385 .551952-3.073574$

H $-0.2303085 .845613-3.568051$

H -1.037408 3.977411 -2.022492

H $2.9911901 .900244-0.508092$

C $-0.707153-0.2823993 .192572$ 
O $\begin{array}{llll}-1.827770 & 0.113840 & 2.376132\end{array}$

C -1.7016361 .5197392 .083793$

C -0.6012882 .0463572 .999961$

C 0.3189810 .8438963 .075801

H -1.3540231 .5955711 .046726$

C -3.0476452 .2345372 .242746$

H -0.1165652 .9391602 .594567$

H -0.9860402 .2946873 .996149$

C 1.3120940 .9041994 .222280

H 0.8702220 .7765402 .130681

C $-4.183591 \quad 1.6905481 .351370$

O -3.4802622 .1275573 .605320$

H -2.914973 3.3066702 .055606

H $-4.5024490 .705987 \quad 1.710354$

O -5.3108872 .5619871 .506764$

C $-3.8364671 .613910-0.144676$

C $-5.0212351 .336187-1.087189$

H $-3.0774480 .840251-0.297227$

O $-3.2483952 .850397-0.561680$

H -5.693803 2.203446 -1.108651

H $-4.6024891 .268494-2.100377$

H $1.964238 \quad 1.7778664 .120113$

H $0.804900 \quad 0.9750405 .190258$

H $1.944790 \quad 0.0113394 .236696$

C $-0.228392-1.6892532 .819225$

H $-3.539803 \quad 1.1807693 .819792$

H -5.5219502 .5954972 .455698$

H $-3.8950243 .556315-0.389360$

H -1.039585 -2.3964563 .032433$

H $0.613781-1.9707533 .461826$

H $-1.085759-0.3235254 .223052$

C $-5.510067-2.312138-0.416673$

O $-4.926484-1.043619-0.746736$

C $-5.8274620 .066479-0.769886$

C $-6.947532-0.164284-1.799944$

C $-7.660156-1.498307-1.560300$

C $-6.664314-2.648926-1.376314$

H $-4.709850-3.043453-0.589871$

C $-5.915850-2.3950511 .063785$

H -6.2757860 .1882650 .221015$

O $\quad-6.396087-0.155907-3.115954$

H -7.662689 $0.664781-1.735351$

H $-8.314141-1.715709-2.413664$

O $-8.482384-1.372042-0.404296$

H -7.184952 -3.544967 -1.020188

O $-6.107110-2.986150-2.650168$

C -4.722486 -2.327835 1.991271

H $-6.590978-1.5827781 .344576$

H -6.417468 -3.3512291 .252303$

H -7.132219 $-0.257333-3.743157$

H -8.946496 -2.217510 -0.280417

H -5.574856 -2.229719 -2.951596

O $-5.166731-2.302187 \quad 3.282760$

O $-3.553130-2.3068691 .635701$

C $-4.147353-2.2382734 .284886$

H -4.627480 -2.319356 5.263656

H -3.443036 -3.068024 4.171121

H -3.627536 -1.278151 4.227783

SCF Energy (B3LYP/6-31G**//MMFF) $=-3245.92007815$

\section{8}

MM̄FF Geometry

C $0.395356-2.8144121 .221189$

C $1.671402-2.7341001 .630205$

C $2.407236-1.4839432 .049437$

O $1.498664-0.3579382 .092628$

C $3.061696-1.6104493 .439366$

C $4.193089-2.6111683 .544292$

C $5.414395-2.4381943 .000603$

C $3.890550-3.8419724 .357326$

C $5.879936-1.2724752 .171756$

C $6.312586-1.7320800 .773089$

C $6.374171-0.618089-0.285385$

C $7.158794 \quad 0.616346 \quad 0.163433$

C $7.1464531 .721802-0.905368$

O $5.029007-0.254496-0.625310$

O $7.5966572 .937928-0.287862$

C $5.6906841 .961758-1.362811$
C $8.1298841 .426411-2.043924$

O $5.6450082 .875159-2.456796$

C $4.9348360 .662681-1.725572$

C $3.4391220 .967683-1.985698$

O $5.500850 \quad 0.115847-2.920832$

C 2.0105310 .8717421 .818387

O $3.163996 \quad 1.149058 \quad 1.533331$

C 0.9040441 .8451211 .904324

C 0.9828842 .9812201 .197215

C -0.0414464 .0210461 .131454$

C -1.1586573 .9757722 .138984$

C $2.556677-0.284582-2.056785$

C $1.2027380 .030319-2.659457$

C $-0.9892441 .099689-2.146894$

O $0.927611-0.287306-3.814705$

C $-1.0282752 .578166-2.372504$

C $-1.9986033 .364188-1.880981$

C $-2.0785904 .849253-2.101654$

C $-2.1882165 .620546-0.803585$

C $-3.3909385 .945302-0.299276$

C $-0.9150626 .074534-0.121329$

C 0.0506834 .9580810 .165391

N $0.3587510 .698644-1.788425$

H $-0.224200-1.9234811 .181567$

H $2.265633-3.6456891 .621572$

H $3.163570-1.3013621 .278667$

H $2.285261-1.8447124 .181329$

H $3.435989-0.6291143 .761559$

H $6.165355-3.2109133 .159727$

H $4.738944-4.5333524 .398361$

H $3.640232-3.5633315 .386270$

H $3.042594-4.3857023 .929239$

H $5.111346-0.5013782 .099797$

H $6.732496-0.8088932 .682622$

H $7.299628-2.2058270 .850793$

H $5.615580-2.5009950 .413013$

H $6.844343-1.059688-1.172458$

H $\quad 8.187877 \quad 0.3466290 .430289$

H $6.710797 \quad 1.0349301 .074306$

H 8.4846002 .7782560 .075740

H $5.1610232 .447558-0.531128$

H $9.1525181 .348566-1.655451$

H $8.1486872 .244624-2.772287$

H $7.9084370 .497479-2.573145$

H $6.1189743 .678988-2.182633$

H $3.0482881 .605576-1.184177$

H $3.3374101 .533679-2.920827$

H $4.819570-0.376562-3.404668$

H $0.045725 \quad 1.5841182 .509213$

H 1.8608623 .1482870 .574289

H -1.7966293 .1044301 .963458$

H -1.800579 4.8596542 .108953

H -0.7587973 .9192313 .157656$

H $3.012852-1.068375-2.670639$

H $2.410395-0.713821-1.059660$

H $-1.6425200 .794774-1.323505$

H -1.300610 $0.581546-3.058473$

H $-0.239306 \quad 3.018457-2.977216$

H -2.800137 $2.910389-1.302260$

H -2.960221 $5.047425-2.725399$

H -1.220912 $5.218572-2.677329$

H -3.484853 6.5171890 .617740

H $-4.3092835 .641263-0.790849$

H -1.126186 6.6241050 .804428

H $-0.4152046 .800134-0.776334$

H $0.8893994 .913519-0.529980$

H $0.6885540 .926478-0.855930$

C $-0.578177-4.065741-0.713859$

O $-1.737938-3.237754-0.937908$

C $-2.550403-3.846818-1.962902$

C $-1.741716-5.016387-2.512662$

C $-0.940881-5.430669-1.294684$

H -3.442663 -4.243599-1.465916

C $-2.944841-2.803933-3.014557$

H $-2.381282-5.815583-2.899900$

H $-1.072226-4.702140-3.321924$

C $0.256512-6.303404-1.629719$

H $-1.601629-5.970280-0.602950$ 
C $-3.747965-1.614447-2.447477$ O $-1.754195-2.284755-3.620499$ H -3.507551 -3.285086 -3.822410 H -3.124854 -1.065874 -1.735298 O $-4.011442-0.707892-3.525543$ C $-5.083333-2.005242-1.786722$ C $-5.955549-0.805220-1.370115$ H $-4.894305-2.638558-0.913930$ O $-5.833899-2.789102-2.719294$ H -6.168833 -0.174865 -2.242097 H $-6.927295-1.204873-1.050007$ H $-0.067362-7.229501-2.116005$ H $0.948394-5.792275-2.307590$ H $0.810134-6.574194-0.725468$ C $-0.248238-4.1007390 .778611$ H -1.207078 -1.902683 -2.912943 H -4.583352 -1.169209 -4.162921 H $-6.640414-3.090923-2.267630$ H $0.415994-4.9443380 .998502$ H -1.162544 -4.242167 1.367503 H $0.249433-3.619586-1.282129$ C $-4.627568-0.2043882 .057546$ O $-5.245419-0.807708 \quad 0.913724$ C $-5.3532150 .038514-0.235832$ C -6.2254341 .2610250 .091853$ C -5.6931812 .0170151 .312410$ C $-5.374047 \quad 1.0739842 .479984$ H $-4.757117-0.9448052 .857923$ C -3.124176 -0.029523 1.823611 H $-4.354957 \quad 0.379057 \quad-0.530374$ $\begin{array}{lllll}\text { O } & -7.572507 & 0.860493 & 0.337697\end{array}$ H $-6.2421481 .927940-0.778226$ H $-6.4431512 .752921 \quad 1.627335$ $\begin{array}{llll}\text { O } & -4.523494 & 2.731454 & 0.928756\end{array}$ H -4.8039091 .5983873 .254056$ O $\quad-6.606457 \quad 0.6598203 .080439$ C -2.369443 -0.027509 3.130307 H -2.738630 $-0.842348 \quad 1.194457$ H -2.8764180 .8878991 .282598$ H $-7.5622020 .213757 \quad 1.064042$ H $-4.2314483 .249976 \quad 1.697659$ H -7.057885 1.4585063 .403034 O $-1.851736-1.2648173 .372831$ $\begin{array}{llll}0 & -2.270866 & 0.950532 & 3.859955\end{array}$ C $-1.095257-1.3911064 .580831$ H $-0.269432-0.6732034 .591831$ H -1.745827 -1.245508 5.448113 $\mathrm{H}-0.679781-2.4018334 .617358$

SCF Energy (B3LYP/6-31G**//MMFF) $=-3245.91290229$

\section{9}

MM̄FF Geometry

C -1.801022 -3.094162 2.475395

C -1.573226 -1.812894 2.154669

C $-0.992927-0.8028143 .106936$

O $0.137771-0.2256332 .407961$

C -1.986965 $0.318013 \quad 3.453130$

C $-3.189213-0.1358604 .257025$

C $-4.444362-0.1992753 .769220$

C $-2.916747-0.4694115 .699709$

C -4.8605630 .0840742 .354231$

C -5.2917801 .5433652 .165732$

C -5.2759352 .0065280 .700474$

C $-6.2600741 .236219-0.179878$

C $-6.1655641 .676717-1.649890$

O $-3.940927 \quad 1.8403320 .214493$

O $\quad-6.906791 \quad 0.736085-2.442285$

C $-4.6863961 .626777-2.092583$

C $-6.8532353 .026511-1.879006$

O $-4.5320262 .145068-3.412168$

C $-3.7122822 .325779-1.111982$

C $-2.2528651 .969173-1.493747$

O $-3.9065273 .735851-1.194867$

C $1.1505240 .292196 \quad 3.149267$

O $1.208648 \quad 0.372144 \quad 4.365256$

C 2.1987340 .7362512 .204598

C 3.3210461 .2997012 .673225

C 4.4473421 .7320451 .851127
C $5.784651 \quad 1.4376372 .475591$

C -1.177158 $2.422999-0.495037$

C $-0.6738153 .838433-0.715784$

C 0.9345495 .4882130 .239164

O $-1.0400114 .547361-1.648827$

C $2.2214055 .330324-0.507219$

C 3.4292325 .5410590 .036931

C $4.7182985 .365752-0.712587$

C $5.6375134 .352537-0.065893$

C 6.7413564 .7462920 .591593

C $5.3302022 .883504-0.258178$

C 4.2693802 .3782070 .680975

N $0.246404 \quad 4.210577 \quad 0.255398$

H -1.580920 -3.404096 3.496214

H -1.765879 -1.454323 1.150212

H $-0.633125-1.2995304 .016455$

H -2.285271 0.8352582 .533346

H -1.4679141 .0985014 .025953$

H -5.251673 -0.498435 4.435241

H -3.832309 -0.7123896 .249411$

H $-2.249089-1.3329665 .775734$

H $-2.446180 \quad 0.380797 \quad 6.204208$

H -4.051798 -0.1771331 .666341$

H -5.690595 -0.5853792 .097621$

H -6.2896931 .6935922 .595352$

H -4.6033562 .1926602 .723379$

H -5.5215183 .0750650 .697069$

H $-7.286114 \quad 1.3466780 .191916$

H $-6.0383620 .161724-0.133765$

H -6.513128 -0.144319 -2.317868

H $-4.4082500 .566911-2.154421$

H -7.908076 $2.971674-1.583955$

H $-6.8557293 .293813-2.941597$

H -6.394399 $3.843792-1.318857$

H $-4.5709803 .114920-3.355271$

H $-2.1822610 .875297-1.539359$

H -2.018867 $2.353760-2.493462$

H $-3.1279264 .179829-0.825018$

H 2.0351540 .5712611 .144663

H 3.4579381 .4274863 .745191

H $5.882287 \quad 1.9576203 .435321$

H 6.6310681 .7445201 .857815

H 5.8918570 .3618592 .655660

H -1.524597 2.3220040 .538246

H $-0.3057311 .765265-0.611669$

H 1.0916915 .7878951 .280043

H $0.3083416 .239681-0.252067$

H $2.1537485 .025639-1.549075$

H 3.4993885 .8587791 .074720

H $5.2089396 .346492-0.763439$

H $4.5383105 .071617-1.754295$

H 7.4247804 .0276421 .030965

H 6.9853045 .7971290 .708521

H $6.2445052 .285084-0.173570$

H $4.9929372 .715526-1.289081$

H 3.2518992 .5623640 .338668

H 0.5473603 .5222980 .939766

C $-2.615916-3.9664380 .115860$

O $-1.492306-3.409978-0.591866$

C $-1.994300-2.509752-1.601025$

C $-3.508525-2.706745-1.636659$

C $-3.808825-3.066739-0.193914$

H -1.782548 -1.493374 -1.253571

C -1.296855 -2.763594-2.940090

H -4.035480 -1.809919-1.974271

H $-3.792300-3.530630-2.302239$

C $-5.160992-3.729620-0.000353$

H -3.779617 -2.152015 0.404924

C $0.232362-2.548016-2.926244$

O $-1.543013-4.118017-3.342426$

H - $1.749565-2.135488-3.716241$

H $\quad 0.712326-3.308944-2.300448$

O $0.704108-2.769622-4.262709$

C $0.684470-1.142180-2.479296$

C $2.152481-0.803083-2.806092$

H $0.506182-1.024698-1.404397$

O $-0.127209-0.172488-3.151233$

H $2.292401-0.819208-3.894618$ 
H $2.3241250 .239650-2.504353$

H -5.965829 -3.059098 -0.319540

H -5.243210 -4.654054 -0.581462

H $-5.326590-3.9764571 .052909$

C $-2.285347-4.2128931 .591823$

H -1.199050 -4.698265 -2.641607

H $\quad 0.415355-3.659615-4.528197$

H $\quad 0.139576 \quad 0.706924-2.833700$

H -1.492850 -4.973156 1.621326

H -3.161001-4.661930 2.075916

H -2.790374 -4.951460 -0.340185

C $4.089072-2.1603300 .086469$

O $3.176170-1.406942-0.725382$

C $3.191420-1.705043-2.125416$

C $4.595317-1.477323-2.709999$

C $5.653065-2.270681-1.940557$

C $5.533117-2.048606-0.430810$

H $4.053845-1.6727611 .069313$

C $3.619125-3.6078070 .262492$

H $2.911802-2.751039-2.277828$

O $4.927417-0.090306-2.687363$

H $4.595158-1.789637-3.760976$

H $6.648435-1.962307-2.282777$

O $5.495260-3.653963-2.241464$

H $6.171645-2.7519530 .116018$

O $6.000995-0.734473-0.117918$

C $2.420881-3.6505551 .179703$

H $3.328164-4.092961-0.673067$

H $4.410471-4.2275430 .701183$

H $4.8504670 .218351-1.768361$

H $6.191696-4.138740-1.766769$

H $6.929851-0.677744-0.399655$

O $2.823479-3.9076192 .456795$

O $1.272576-3.4473210 .808846$

C $1.782822-3.9509953 .437201$

H $2.224264-4.2617474 .387806$

H $1.347535-2.9561023 .562024$

H $1.016779-4.6787383 .153260$

SCF Energy (B3LYP/6-31G**//MMFF) $=-3245.90491062$

0700370

MM̄MF Geometry

C $1.447812-2.6578282 .126717$

C $0.343475-2.6022892 .884185$

C $-0.528605-1.3765942 .942317$

O $-1.874040-1.7527962 .562317$

C $-0.578448-0.7541814 .347426$

C $0.736699-0.1367604 .786282$

C $1.214076 \quad 1.0403804 .332428$

C $1.516846-0.9317025 .799778$

C $0.551551 \quad 1.964198 \quad 3.347727$

C 1.2478431 .9319291 .977983

C 0.4567852 .6319810 .858204

C $0.447066 \quad 4.1526551 .022927$

C $-0.4183264 .840470-0.043661$

O $-\begin{array}{llll}0.872942 & 2.105412 & 0.862768\end{array}$

O $-0.614547 \quad 6.204341 \quad 0.361692$

C $-1.8054514 .168614-0.053601$

C $0.2710964 .890865-1.410393$

O $-2.6190234 .685369-1.104165$

C $-1.7470532 .627418-0.144666$

C -3.1383891 .9825520 .077333$

O $-1.2988562 .245871-1.449115$

C $-2.126489-1.7986751 .222769$

O $-1.335599-1.5715490 .319471$

C $-3.548255-2.1600241 .031372$

C $-4.068019-2.085272-0.203192$

C $-5.453638-2.355016-0.590881$

C $-6.392498-2.8496730 .476162$

C -3.7586652 .2914961 .441637$

C -4.9213311 .3794521 .774354$

C -7.3236520 .9965691 .275823$

$\begin{array}{lllll}\text { O } & -4.838892 & 0.540733 & 2.669577\end{array}$

$\begin{array}{llll}\text { C } & -8.015389 & 0.476528 & 0.053341\end{array}$

C $-7.6230390 .564833-1.227401$

C $-8.409718-0.028024-2.363919$

C -7.610602 -1.011356-3.190561

C $-7.369209-0.782959-4.492725$
C $-7.152261-2.295493-2.528317$

C $-5.806803-2.138152-1.875484$

N -6.0422341 .6154200 .993506$

H $1.736627-1.7780251 .556083$

H $\quad 0.037597-3.4699183 .462234$

H $-0.180538-0.6146692 .237867$

H -0.905444 -1.507262 5.077703

H -1.3724040 .0034584 .382362$

H $2.1754501 .389823 \quad 4.705726$

H $2.456419-0.4427756 .077984$

H $0.927874-1.0590466 .713945$

H $1.765193-1.9218455 .406086$

H $-0.509545 \quad 1.719753 \quad 3.247412$

H 0.5881202 .9799993 .758174

H 2.2506252 .3687112 .057990

H 1.3905210 .8862591 .678239

H $0.9336082 .366928-0.092031$

H 1.4690404 .5509231 .003926

H 0.0414754 .4180012 .008164

H $\quad 0.2604876 .6203950 .446340$

H -2.2953524 .4543010 .885552$

H $1.2133145 .447375-1.342462$

H $-0.3414885 .429468-2.141970$

H $0.4981313 .902051-1.813249$

H -2.630928 $5.653529-1.012108$

H $-3.8237072 .277856-0.727429$

H -3.035568 $0.893985-0.017303$

H -1.582534 $1.331470-1.615832$

H -4.126622 -2.4449781 .902031$

H -3.417168 -1.770069-1.018486

H $-6.034051-3.7993360 .888998$

H -7.407520 -3.0279900 .116268$

H -6.469634 -2.123606 1.291922

H -3.018378 2.1774812 .242030

H -4.118195 $3.325777 \quad 1.483817$

H -7.9485951 .7590041 .752441$

H -7.191455 0.1790151 .991559

H $-8.946938-0.0471220 .263454$

H $-6.7055461 .085398-1.487175$

H $-8.7630930 .801388-2.989960$

H $-9.312116-0.534312-1.998654$

H -6.817081 -1.495134 -5.097032

H $-7.7138890 .121465-4.982847$

H $-7.062843-3.088546-3.282714$

H -7.929184 -2.639792 -1.840773

H $-5.034085-1.798413-2.567637$

H -6.0366672 .4182260 .370665$

C $2.372170-4.4097890 .556377$

O $2.733849-3.362961-0.370630$

C $1.794680-3.379892-1.460369$

C $1.185393-4.775182-1.444697$

C $1.058008-5.0104050 .048430$

H $1.007102-2.657147-1.215640$

C $2.472350-2.985181-2.774091$

H $0.226694-4.818911-1.969882$

H $1.858326-5.516391-1.891513$

C $0.863858-6.4689840 .423928$

H $0.198749-4.4360930 .416487$

C $3.116756-1.581509-2.758781$

O $3.497505-3.944847-3.055684$

H $1.745714-3.057342-3.592616$

H $4.008621-1.584979-2.121600$

O $3.566587-1.295822-4.089339$

C $2.169920-0.446195-2.321287$

C $2.6876710 .979076-2.591015$

H $1.948791-0.547832-1.253059$

O $0.920219-0.573895-3.008512$

H $2.770621 \quad 1.147628-3.672419$

H $1.9036131 .667863-2.250442$

H $-0.060557-6.859183-0.014510$

H $1.692595-7.0897050 .067355$

H $\quad 0.798469-6.5848991 .510407$

C $2.332875-3.8621981 .987220$

H $3.781543-3.809917-3.975307$

H $4.316123-1.885153-4.278202$

H $1.103283-0.523988-3.962424$

H $3.344209-3.5525102 .276371$

H $2.033238-4.6558682 .681138$ 
H $3.172946-5.1580340 .496264$

C $5.0197981 .400110 \quad 0.302516$

O $3.8500861 .166933-0.495374$

C $4.0197181 .326279-1.906409$

C $4.4723332 .759350-2.238488$

C $5.7048373 .170240-1.427377$

C 5.5736232 .8151580 .056911

H $4.6668711 .356306 \quad 1.340904$

C $6.0791720 .301707 \quad 0.117529$

H $4.7804450 .624674-2.261203$

O $3.4249193 .696442-2.001289$

H $4.7013982 .813459-3.309224$

H $5.8641324 .251311-1.523912$

O $\quad 6.8558802 .512222-1.951525$

H 6.5484582 .9134840 .548300

O $4.7148383 .769606 \quad 0.687208$

C $5.604045-1.0470180 .611862$

H $6.3615150 .182185-0.931484$

H 6.9745590 .5591820 .695147

H $2.9995083 .470514-1.158301$

H $6.9558192 .788671-2.878429$

H 3.7979623 .4625440 .599472

O $6.568236-1.977076 \quad 0.344359$

O $4.534567-1.2595541 .164628$

C $6.274003-3.3153850 .756463$

H $5.968803-3.3417911 .806906$

H $5.495588-3.7366950 .115030$

H $7.182571-3.9127090 .643353$

SCF Energy (B3LYP/6-31G**//MMFF) $=-3245.90619262$

\section{1}

MM̄FF Geometry

C - $-1.732262-3.481983-2.586253$

C $-0.488826-3.050994-2.849595$

C $0.776243-3.336745-2.069836$

O $1.223520-2.090417-1.477754$

C $0.625652-4.358728-0.924736$

C $1.933382-4.709083-0.238580$

C $2.208845-4.4653901 .057965$

C $2.956820-5.408094-1.097136$

C $1.327583-3.8228552 .092003$

C $1.528355-2.3084342 .253653$

C $2.929771-1.9032862 .738290$

C $2.964782-0.4172163 .100603$

C 4.3738490 .0434223 .501117

O $3.863527-2.1549371 .686102$

O 4.3549011 .4793383 .514186

C $5.384803-0.4089572 .423205$

C $4.722238-0.3942384 .929163$

O $6.710838-0.1288392 .865035$

C $5.232504-1.8933642 .026884$

C $6.084913-2.3021700 .800076$

O $5.654957-2.714793 \quad 3.122194$

C $1.917161-1.219726-2.258137$

O $2.251925-1.375085-3.421396$

C $2.172371-0.007658-1.449563$

C $2.4494241 .149915-2.067475$

C $2.7070062 .434644-1.414020$

C 2.9276192 .4252230 .075502

C $5.799438-1.523601-0.488917$

C $6.721352-0.336302-0.710212$

C $7.0519231 .606280-2.235717$

O $7.715891-0.117609-0.023882$

C $6.4637642 .828491-1.604146$

C $6.0961463 .912753-2.303520$

C $5.5427825 .163182-1.682538$

C $4.2233715 .580719-2.292350$

C $4.1721736 .520466-3.251403$

C $2.9596784 .970571-1.729373$

C $2.7525933 .548757-2.174344$

N $6.3197960 .434398-1.793129$

H -1.926847 -4.114967 -1.725771

H $-0.351485-2.410985-3.720034$

H $\quad 1.530337-3.697278-2.779996$

H $0.192917-5.290811-1.311189$

H $-0.088668-3.962871-0.195906$

H $3.183075-4.7724411 .437491$

H $3.804370-5.781598-0.512096$
H $2.506124-6.268829-1.601927$

H $3.357312-4.726861-1.853829$

H $\quad 0.271615-4.0194631 .878648$

H $1.518794-4.3158693 .053710$

H $1.314544-1.8191351 .296643$

H $0.783008-1.9502612 .974623$

H $3.185037-2.5165483 .610427$

H $2.246975-0.1928343 .899648$

H 2.6387160 .1739792 .234201

H $5.260021 \quad 1.7837763 .699697$

H 5.2049000 .2233081 .545851

H 4.0076320 .0331285 .642918

H $5.706090-0.0184955 .231144$

H $4.713177-1.4783805 .059617$

H $7.315091-0.2965622 .122907$

H $7.151127-2.2884491 .055207$

H $5.855295-3.3563790 .586306$

H $5.874676-3.5969502 .779371$

H $2.099087-0.095149-0.371889$

H $2.466647 \quad 1.167423-3.156599$

H 3.6841861 .6806260 .345028

H 1.9973742 .1878950 .601512

H $3.292713 \quad 3.376141 \quad 0.468831$

H $5.959753-2.195736-1.341712$

H $4.757928-1.191179-0.525933$

H $6.9905381 .633849-3.328247$

H $8.1034911 .511483-1.946603$

H $6.3591812 .831676-0.521691$

H $6.2282573 .916710-3.383261$

H $6.2853705 .959973-1.820636$

H $5.4253265 .054880-0.597134$

H $3.2279046 .846664-3.674439$

H $5.0750536 .981282-3.638569$

H $2.0842785 .542612-2.065419$

H $2.9631155 .082626-0.641736$

H $2.5877643 .449769-3.248268$

H $5.4524110 .208194-2.271387$

C $-3.816901-2.108751-2.693560$

O $-4.486578-2.752594-1.592503$

C $-5.800437-2.164796-1.439126$

C $-5.888391-1.049382-2.473869$

C $-4.940233-1.535839-3.551269$

H $-6.514029-2.964347-1.671579$

C $-5.956465-1.7001240 .013819$

H $-6.908900-0.895453-2.837081$

H $-5.531348-0.098925-2.056883$

C -4.501224 -0.437933 -4.504648

H $-5.429661-2.338908-4.118158$

C -7.200435 -0.8356120 .290319$

O $-5.988159-2.8583490 .854134$

H $-5.049920-1.1574490 .293692$

H -7.216545 $0.049460-0.355056$

O $-8.378353-1.586694-0.027506$

C -7.333198 -0.3854171 .757889$

C $-6.105102 \quad 0.328146 \quad 2.342877$

H $-7.562448-1.2520962 .390124$

O

H $\quad-6.3511890 .7047543 .344590$

H $-5.314480-0.4156182 .510195$

H $-5.366428-0.008846-5.020722$

H $-3.9896980 .372878-3.974923$

H -3.816730 -0.828021 -5.263972

C $-2.927178-3.113259-3.424194$

H $-6.809739-3.3406160 .658236$

H -8.385136 -1.735456 -0.987930

H $-9.2316960 .011078 \quad 1.515291$

H -2.598248 -2.699110 -4.384028

H -3.485583 -4.032066 -3.640361

H -3.208919-1.296492 -2.272234

C -3.4767402 .8183921 .492679$

O -4.2773451 .8366872 .163228$

C -5.5064421 .4820321 .510600$

C -6.4053772 .7189291 .375594$

C -5.6692353 .8641690 .669801$

C -4.2783144 .1101661 .266319$

H -2.6664683 .0441772 .198273$

C -2.8357882 .2635520 .214568$

H $\quad-5.2650021 .1019230 .515111$ 
O -6.8263523 .1439362 .669062$ H -7.299941 2.456846 0.800486 H $-6.270078 \quad 4.7782340 .750070$ O $-5.555617 \quad 3.543153-0.714119$ H -3.6989634 .7900110 .630681$ O -4.4252484 .7498842 .537455$ C -1.8971351 .1253580 .538693$ H -3.565444 $1.911351-0.520397$ H $-2.2456253 .037968-0.289932$ H -7.4225573 .9026222 .549466$ H -5.111924 $4.288968-1.152821$ H -4.8916265 .5903482 .390898$ O $-2.551512-0.0641190 .414105$ O $-0.733893 \quad 1.277620 \quad 0.887777$ C $-1.786160-1.2279160 .737261$ H $-0.904776-1.2907970 .094454$ H $-1.496885-1.2066501 .791693$ H $-2.411179-2.1075530 .562741$

SCF Energy (B3LYP/6-31G*//MMFF) $=-3245.90459326$

\section{2}

MM̄FF Geometry

C $3.403208-2.1135821 .640232$

C $2.844256-2.8598142 .604213$

C $1.495412-2.6506653 .245497$

O $0.776216-1.5490582 .649250$

C $0.615172-3.9028763 .061989$

C $-0.697343-3.8031163 .817077$

C $-1.885550-3.4880813 .263399$

C $-0.610253-4.1060135 .291161$

C $-2.185066-3.1467691 .827281$

C $-3.140570-4.1806051 .220418$

C $-3.553015-3.915449-0.236485$

C $-2.385040-4.040357-1.215296$

C $-2.823048-3.774514-2.663320$

O $-4.141171-2.612227-0.332275$

O $-1.623963-3.614140-3.437737$

C $-3.598228-2.439602-2.704055$

C $-3.562922-4.974680-3.263174$

O $-4.137271-2.201207-4.001447$

C $-4.700502-2.324141-1.625333$

C $-5.339696-0.915527-1.565845$

O $-5.757364-3.241123-1.937875$

C $0.609471-0.4261053 .397553$

O $1.234029-0.1026654 .394282$

C $-0.563871 \quad 0.2890252 .844820$

C -1.144385 1.2617303 .564039

C -2.4000991 .9437643 .237121$

C $-3.187027 \quad 1.4104412 .069584$

C $-4.3440570 .238324-1.451682$

C $-5.0092971 .574425-1.162014$

C $-4.4389634 .015675-1.066664$

O $-6.1837371 .686608-0.824551$

C -3.6185754 .5017010 .086054$

C -4.1210865 .1582191 .142378$

C $-3.2796615 .616765 \quad 2.300804$

C -3.857588 $5.221993 \quad 3.643885$

C $-4.169035 \quad 6.158179 \quad 4.556514$

C -4.0888223 .7540623 .933554$

C -2.808631 2.9650464 .019800

N $-4.1105132 .621686-1.305496$

H $2.871708-1.2546161 .241926$

H $3.406099-3.7118932 .983682$

H $1.659478-2.4742904 .315620$

H $1.149734-4.7972283 .409006$

H $0.428980-4.0718291 .995217$

H -2.756842 -3.4505583 .916933$

H $-1.587630-4.0635775 .783722$ H $-0.207112-5.1117775 .448390$ H $0.042427-3.3862465 .794587$ H - $2.650378-2.154573 \quad 1.805655$ H -1.272936 -3.0725821 .231847$ H -2.697986 -5.182595 1.291701 H $-4.055657-4.1951381 .828321$ H -4.317736 -4.663418 -0.478793 H - $1.904184-5.022658-1.131325$ H -1.614951 -3.302808 -0.961487 H $-1.887518-3.365090-4.340428$
H $-2.849390-1.655476-2.537990$

H $-2.920263-5.863354-3.248398$

H $-3.808691-4.802969-4.316954$

H $-4.483664-5.222075-2.731142$

H - $-4.923255-2.764603-4.103307$

H $-5.975899-0.756029-2.446668$

H $-6.019979-0.885691-0.703472$

H -6.562851 -2.947096 -1.480352

H $-0.969764-0.0657561 .904029$

H $-0.683793 \quad 1.5610904 .504684$

H -3.4301940 .3526412 .219538$

H -2.6103831 .5082121 .145289$

H $-4.134503 \quad 1.9248691 .903761$

H $-3.6241630 .041872-0.649772$

H $-3.7955430 .340326-2.394586$

H $-5.5133134 .127442-0.891237$

H $-4.1693164 .568739-1.971597$

H -2.549831 4.3032440 .050347

H $-5.184161 \quad 5.384648 \quad 1.174637$

H $-3.1900606 .708500 \quad 2.231444$

H -2.2584245 .2233162 .225433$

H $-4.5868595 .891298 \quad 5.521617$

H $-4.0125997 .213943 \quad 4.361280$

H -4.7906873 .3508213 .199542$

H $-4.600403 \quad 3.6370354 .898475$

H -2.1713573 .2646004 .853124$

H $-3.1631262 .412137-1.603445$

C $5.716809-1.248857 \quad 1.174743$

$\begin{array}{llllll}\text { O } & 5.280303 & -0.159989 & 0.337554\end{array}$

C $6.4233380 .402753-0.333560$

C $7.649974-0.2085640 .332487$

C $7.133736-1.5827300 .708390$

H $6.3788750 .059563-1.373809$

C $6.3633341 .932527-0.282540$

H $8.513047-0.241449-0.339300$

H 7.9448270 .3448601 .231619

C $7.982164-2.2831341 .755751$

H $7.095089-2.202905-0.197354$

C $5.0550112 .531086-0.839542$

$\begin{array}{lllll}\text { O } & 6.486285 & 2.360136 & 1.080898\end{array}$

H $7.2274172 .354055-0.808536$

H $4.2229862 .247990-0.186383$

O $5.150822 \quad 3.958522-0.741555$

C $4.7349652 .141489-2.298475$

C $3.4978612 .859487-2.871828$

H $4.5861731 .059501-2.372846$

O $5.8565692 .457269-3.127097$

H $3.5807613 .936128-2.681345$

H $3.5108902 .746587-3.964534$

H $9.002663-2.4307501 .387516$

H $8.039644-1.7000652 .680938$

H $7.567432-3.2654302 .002331$

C $4.746232-2.4206341 .033656$

H 5.7576701 .9554501 .582391

H $5.9375804 .240396-1.237885$

H $5.938183 \quad 3.425218-3.164417$

H $5.174248-3.317748 \quad 1.495397$

H $4.588495-2.643290-0.028453$

H $5.726779-0.8770292 .207714$

C $0.6938480 .404018-2.637998$

O $1.895261 \quad 1.090993-3.014299$

C $2.1744002 .310440-2.313309$

C $1.0265753 .312623-2.492758$

C $-0.3012432 .696281-2.051671$

C $-0.5317711 .330456-2.701735$

H $0.559137-0.363859-3.411614$

C $0.855567-0.312008-1.289213$

H $2.2903542 .092721-1.248097$

O $0.9488803 .713656-3.858372$

H $1.2421614 .207098-1.895576$

H $-1.1186443 .375672-2.320699$

O $-0.286705 \quad 2.560216-0.633758$

H -1.387255 $0.830201-2.236933$

O $-0.882377 \quad 1.525662-4.075006$

C $1.640143-1.593051-1.480199$

H $1.3770330 .281035-0.533842$

H $-0.131555-0.571417-0.887634$

H $0.2340364 .368704-3.931020$ 
H -1.137058 $2.172032-0.368767$ H $-0.1056391 .892849-4.531072$ O $0.957727-2.640172-0.935742$ O $2.722401-1.658241-2.048645$ C $1.540802-3.932608-1.128374$ H $1.547466-4.183975-2.193084$ H $2.553785-3.966182-0.718977$ H $0.925154-4.664660-0.599069$ SCF Energy (B3LYP/6-31G**//MMFF) $=-3245.91044484$

\section{3}

MM̄FF Geometry

C $-0.885534-3.1550590 .992169$

C $-0.624385-2.898562-0.297905$

C -1.532488 -2.078138-1.176252

O $-0.819914-0.890685-1.608031$

C $-1.946718-2.840694-2.451005$

C $-3.205780-3.663544-2.279036$

C $-4.413050-3.305710-2.759400$

C $-3.047342-4.973140-1.552268$

C $-4.772712-2.068447-3.534795$

C $-4.649199-0.736174-2.789004$

C $-5.276490-0.682408-1.389829$

C $-6.786042-0.923142-1.404783$

C $-7.398616-0.756610-0.005221$

O $-4.9976750 .626964-0.875023$

O $-8.824985-0.685640-0.153397$

C -6.9378990 .5913850 .586349$

C $-7.114754-1.9688150 .890274$

$\begin{array}{lllll}\text { O } & -7.360846 & 0.724043 & 1.941811\end{array}$

C -5.4122890 .8298240 .481496$

C -5.0899002 .2934390 .865038$

O $-4.734683-0.052041 \quad 1.377554$

C $-0.6895050 .124303-0.709990$

O

C $0.0011581 .237614-1.392802$

C $0.7119692 .113421-0.667551$

C $1.4697423 .247192-1.198478$

C $1.1719123 .687626-2.607717$

C -3.6340022 .7148490 .618380$

C -3.4212894 .1908230 .911197$

C -1.6802025 .9477110 .898280$

O $-4.2980614 .918206 \quad 1.370976$

C $-0.481678 \quad 6.2754580 .068908$

C 0.6937296 .6557220 .591823

C $1.8908747 .046448-0.225905$

C 3.1045956 .2002120 .081981

C 3.9957676 .5810341 .012887

C $3.3276584 .959758-0.751286$

C $2.3805833 .845276-0.403031$

N -2.1321614 .6029390 .596522$

H -1.802201 -2.772068 1.435037

H $\quad 0.293271-3.272724-0.741853$

H $-2.428958-1.753979-0.633415$

H -1.132290 -3.491762 -2.797038

H - $-2.075582-2.125984-3.273058$

H -5.251268 -3.979850 -2.587006

H -3.944413 -5.597741 -1.624780

H -2.217997 -5.548429-1.976575

H -2.852162 -4.805175 -0.490034

H -4.171954 -2.028515 -4.451923

H -5.808801 -2.180540 -3.879615

H -3.591958 $-0.456384-2.704451$

H $-5.0883000 .055080-3.411924$

H -4.777507 -1.418226 -0.751458

H -7.029740 -1.914418-1.804055

H -7.263441 -0.200993 -2.081829

H -9.121714 -1.502015 -0.590930

H $-7.449857 \quad 1.3849470 .023854$

H $-7.523124-2.8814450 .439618$

H -7.611307 -1.8672121 .861589$

H -6.049488 -2.135219 1.062796

H $-8.323046 \quad 0.5833801 .960152$

H -5.7328622 .9631420 .278216$

H -5.3266352 .4601751 .923755$

H $-3.8498750 .300751 \quad 1.559666$

H $-0.0685301 .286290-2.473364$

H $0.776397 \quad 1.9681560 .410196$
H $1.5806892 .975848-3.331283$

H $1.5791074 .672898-2.845983$

H $\quad 0.090388 \quad 3.768027-2.767392$

H $-2.9543982 .147641 \quad 1.262586$

H $-3.3559472 .533526-0.425931$

H $-1.4705905 .990176 \quad 1.972497$

H $-2.488617 \quad 6.6547580 .683773$

H $-0.5960136 .227736-1.011310$

H 0.7951056 .7240641 .672731

H $2.1111078 .101096-0.014203$

H $1.6744877 .004029-1.300617$

H 4.8844235 .9939091 .219774

H 3.8528287 .4869941 .592567

H $4.3451374 .577414-0.591690$

H $3.2958905 .230167-1.810658$

H $2.513513 \quad 3.4642260 .609075$

H -1.432164 3.8919710 .403673

C $0.620958-3.0212932 .968458$

O $1.623534-2.1745532 .371006$

C $2.658821-1.9283813 .345746$

C $2.220421-2.6297864 .628369$

C $1.350947-3.7510974 .090615$

H $3.566231-2.4157812 .976531$

C $2.884851-0.4187453 .490383$

H $3.072788-2.9904725 .212396$

H $1.634749-1.9645695 .273959$

C $0.436829-4.3604545 .138907$

H $1.998944-4.5328163 .673004$

C 3.3142580 .2732082 .179787

O $1.661394 \quad 0.189446 \quad 3.926814$

H $3.620292-0.2211594 .278192$

H 2.5407900 .1387351 .416017

O 3.3968841 .6817662 .433454

C $4.684387-0.1902191 .646390$

C 5.0953270 .4969670 .328563

H $4.692088-1.2735061 .500454$

O 5.6851770 .0932972 .628267

H $4.872149 \quad 1.569168 \quad 0.378071$

H $\quad \begin{array}{llll}6.185971 & 0.415652 & 0.226749\end{array}$

H $1.023813-4.7884885 .958213$

H $-0.239950-3.6122625 .565101$

H $-0.173753-5.1606174 .709209$

C $0.021780-3.9351581 .901198$

H $0.983436-0.0012223 .255582$

H 2.5322391 .9651482 .777015

H $5.680717 \quad 1.0540472 .780843$

H -0.570601 -4.732162 2.363551

H $0.819055-4.4117891 .320050$

H $-0.152040-2.3618173 .386700$

C $4.518345-2.214219-2.168222$

O $5.021859-1.427436-1.080987$

C $4.419536-0.138450-0.898708$

C $4.5900550 .715722-2.162160$

C $4.045012-0.016240-3.393429$

C $4.594039-1.439610-3.495546$

H $5.212458-3.061656-2.241991$

C $3.123069-2.774250-1.854766$

H $3.352293-0.261032-0.691820$

O $5.9692921 .023652-2.349823$

H $4.0597351 .663987-2.021894$

H $4.3150460 .538114-4.300481$

O $2.623978-0.082511-3.325484$

H $4.063042-1.991542-4.280088$

O $5.963240-1.356442-3.901030$

C $3.221081-4.030717-1.017959$

H $2.470720-2.072939-1.328841$

H $2.613625-3.061187-2.782486$

H $6.041758 \quad 1.572127-3.149380$

H $2.2910710 .830011-3.308459$

H $6.288314-2.263988-4.027742$

O $3.777453-3.7543450 .195122$

O $2.839449-5.129692-1.400716$

C $3.990903-4.8852141 .045446$

H $4.449240-4.5331121 .973131$

H $3.039957-5.3690701 .284419$

H $4.673374-5.5938560 .566332$

SCF Energy (B3LYP/6-31G**//MMFF) $=-3245.90253842$ 


\section{4}

MMFF Geometry

C -1.427692 3.3375450 .253281

C $-0.508608 \quad 3.9797610 .987981$

C 0.4619623 .2688951 .892575

O $1.782403 \quad 3.6782841 .464527$

C 0.2561623 .6339423 .373942

C $-1.1212543 .307368 \quad 3.912257$

C -1.546532 2.0673994 .223868

C -2.0332024 .4905164 .111162$

C $-0.780842 \quad 0.7762604 .124366$

C -1.405130 -0.1574803 .076805$

C $-0.766832-1.5560633 .001459$

C -1.081192 -2.404990 4.235778

C $-0.396223-3.7780244 .175261$

O $0.645346-1.4091272 .838707$

O $-0.477726-4.3695995 .481206$

C $1.100619-3.5690723 .867362$

C -1.114603 -4.737699 3.219567

O $1.756286-4.8207953 .672662$

C $1.362374-2.6400972 .657927$

C $2.871051-2.2973742 .581535$

O $0.967941-3.312106 \quad 1.464245$

C 2.8172292 .8405861 .739056

O $2.785973 \quad 1.8323612 .425280$

C 3.9993193 .3394511 .000956

C $5.0295692 .509610 \quad 0.778917$

C 6.2407422 .8128470 .014453

C $6.4175034 .220010-0.491889$

C $3.245793-1.2977331 .479137$

C $3.624877-1.9269670 .149352$

C $4.356162-1.259476-2.144701$

O $3.727609-3.137635-0.023125$

C $5.834640-1.026032-2.148864$

C $6.436387-0.070956-2.873861$

C $7.9184440 .176452-2.863830$

C $8.2685121 .590390-2.451002$

C $8.5116252 .534436-3.375919$

C $8.4219391 .891202-0.975653$

C $7.1244041 .819313-0.217327$

N $3.853063-0.956950-0.818141$

H -1.474885 2.2517420 .294247

H -0.4537615 .0650230 .950318$

H 0.3717052 .1844791 .752068

H 0.4708234 .7019893 .519598

H 1.0148433 .1335203 .988977

H -2.559339 1.9555294 .609787

H -3.014924 4.199217 4.499531

H -1.590606 5.1918964 .825991

H -2.197183 5.0161193 .165696

H $\quad 0.2731150 .9498553 .888449$

H -0.8033880 .3088465 .114446$

H -2.483434 $-0.262953 \quad 3.252482$

H -1.293166 0.3183542 .093510

H -1.174096 -2.042023 2.107359

H -2.164774 -2.520652 4.361796

H -0.727449 -1.896741 5.141777

H -1.417735 -4.436990 5.721606

H $1.558572-3.1146214 .757302$

H -2.151325 -4.893555 3.541542

H $-0.646443-5.7281923 .228998$

H -1.141259-4.378763 2.189117

H $1.576419-5.3670274 .457151$

H $3.158041-1.8347623 .535417$

H $3.463906-3.2127942 .472153$

H $1.356132-2.8524830 .703608$

H 3.9671724 .3537850 .620257

H 4.9727991 .4959581 .172328

H $5.6075554 .486315-1.179932$

H $7.3526734 .370645-1.036043$

H 6.4192884 .9311450 .341683

H $2.442043-0.5737081 .311379$

H $4.130818-0.7429091 .814917$

H $3.831204-0.606679-2.849121$

H $4.139162-2.300590-2.402972$

H $6.437413-1.674018-1.516684$

H $5.8335510 .569208-3.514073$

H $8.297012-0.023026-3.874908$
H $8.440321-0.533166-2.209830$

H $8.7930723 .543734-3.094648$

H $8.4308872 .319561-4.436360$

H $8.9016082 .864476-0.818338$

H $9.1225451 .168065-0.537919$

H $\quad \begin{array}{llll}6.908462 & 0.826713 & 0.179289\end{array}$

H $3.7900690 .022526-0.555627$

C $-3.8556613 .817381-0.166041$

O $-4.1637392 .409744-0.134522$

C $-5.3259842 .167684-0.941882$

C $-6.0547233 .501547-0.992678$

C $-4.8847614 .462366-1.101000$

H $-4.9642821 .921016-1.947405$

C $-6.1293530 .982904-0.398030$

H $-6.7466213 .569302-1.837381$

H $-6.6147733 .695705-0.070917$

C $-5.2330915 .893791-0.731211$

H $-4.5158814 .446020-2.135584$

C $-5.279283-0.261251-0.065437$

O

H $-6.9253850 .730335-1.108714$

H $-4.725618-0.0822580 .864523$

O $-6.179714-1.3387290 .221491$

C $-4.292660-0.700223-1.161977$

C $-3.559823-2.001349-0.783610$

H $-3.557740 \quad 0.095097-1.325079$

O $-5.010991-0.885305-2.379853$

H -3.275502 -1.964052 0.275040

H -4.242922 -2.847010 -0.930727

H $-6.0030036 .288218-1.402382$

H -5.6133625 .9622490 .293567$

H -4.352888 $6.539919-0.808089$

C $-2.4161444 .017497-0.649564$

H $-7.2960920 .617818 \quad 1.130458$

H -6.635188 -1.567488 -0.607212

H $-4.367910-1.134826-3.065093$

H -2.197032 $5.088568-0.729891$

H $-2.2926883 .584229-1.649458$

H -3.967986 4.1881580 .860485

C $-1.582051-2.574556-3.914132$

O $-2.665028-2.417858-2.986711$

C $-2.285836-2.224130-1.621895$

C $-1.479328-3.428489-1.116328$

C $-0.266929-3.695324-2.012005$

C $-0.655417-3.728862-3.494378$

H $-2.064100-2.860266-4.858147$

C $-0.838996-1.254345-4.147346$

H -1.672644 -1.319717 -1.540708

O $-2.314979-4.583217-1.081772$

H -1.155707 -3.225296 -0.091021

H $0.185604-4.653642-1.729500$

O $\quad 0.698746-2.672767-1.784182$

H $\quad 0.243310-3.721268-4.121109$

O $-1.325462-4.963948-3.765762$

C $-1.743915-0.253735-4.826247$

H $-0.449649-0.811358-3.226286$

H $\quad 0.025075-1.403046-4.805815$

H -1.789102 $-5.313827-0.714044$

H $1.479126-2.880584-2.325649$

H -2.163047 -4.962871 -3.271393

O $-2.1750010 .675766-3.926926$

O $-2.049870-0.308804-6.010629$

C $-3.0576721 .672871-4.450246$

H $-2.6086412 .171125-5.314762$

H $-4.0155201 .218096-4.719181$

$\mathrm{H}-3.2288552 .419095-3.670746$

SCF Energy $\left(B 3 L Y P / 6-31 G^{* *} / / M M F F\right)=-3245.92744206$

0700375

MM̄FF Geometry

C -0.2263003 .0400490 .697693$

C $-1.2713693 .556895 \quad 0.035337$

C $-1.9749372 .818382-1.069926$

O $-3.3900532 .869664-0.771255$

C $-1.7184743 .450015-2.451934$

C $-0.263127 \quad 3.602547-2.843879$

C $0.5452002 .585663-3.200953$

C $0.2540985 .017447-2.852845$ 
C $0.208127 \quad 1.120751-3.243744$

C $1.2257580 .309107-2.429803$

C $0.811861-1.140528-2.130954$

C $0.368896-1.917161-3.373151$

C $-0.092030-3.342520-3.028357$

O $-0.238593-1.112032-1.155056$

O $-0.794375-3.866407-4.166683$

C $-1.108008-3.268837-1.867258$

C $1.090679-4.285499-2.782915$

O $-1.460331-4.574062-1.414939$

C $-0.622155-2.411059-0.678080$

C $-1.710341-2.2321760 .405704$

O $0.479382-3.073448-0.044561$

C $-4.1829971 .925894-1.345833$

O $-3.849717 \quad 1.071100-2.151041$

C $-5.5437622 .088514-0.791803$

C $-6.3916051 .050366-0.839001$

C $-7.7605221 .027726-0.323891$

C -8.3340752 .3167100 .203264$

C $-2.955252-1.468509-0.043509$

C $-3.916758-1.2690461 .111863$

C $-5.505223-2.5243992 .575377$

O $-4.045353-0.1789451 .663992$

C $-6.904123-2.6414802 .057417$

C $-7.922457-1.8914602 .505928$

C $-9.344527-2.0457132 .044483$

C $-9.954125-0.7419641 .580069$

C -10.6368810 .0395462 .433975$

C $-9.850085-0.3856540 .114855$

C $-8.438024-0.139044-0.339678$

N $-4.584124-2.4323021 .458214$

H 0.1218462 .0404180 .447882

H -1.611115 4.5634960 .265799

H -1.660869 $1.768503-1.059260$

H -2.217959 $4.428250-2.497008$

H $-2.2319462 .864641-3.225787$

H $1.5672162 .822754-3.495061$

H $1.3145885 .072448-3.120796$

H $-0.3034785 .619064-3.578235$

H $\quad 0.1415905 .473299-1.864285$

H $-0.8061020 .924664-2.889075$

H $\quad 0.2415350 .799174-4.291600$

H $2.179490 \quad 0.300391-2.972983$

H $1.4070240 .815078-1.471994$

H $\quad 1.685267-1.625517-1.678761$

H $1.169498-1.939462-4.122637$

H -0.477506 -1.406356 -3.850942

H $-0.189848-3.843739-4.928122$

H -2.024030 -2.823896 -2.275645

H $1.725438-4.344528-3.675175$

H $\quad 0.748019-5.308265-2.590893$

H $1.722629-3.971507-1.951376$

H -1.763490 -5.077215 -2.189979

H -1.985795 -3.2114540 .817853$

H -1.272663 -1.6828401 .251746$

H $\quad 0.582107-2.7144650 .852426$

H -5.796467 $3.039749-0.338265$

H $-6.0461020 .117542-1.283877$

H -7.754200 2.6729611 .061849

H -9.3690072 .2249360 .540652$

H $-8.3230623 .089116-0.573940$

H -2.675410 -0.481685-0.421890

H -3.485720 -1.990098 -0.846576

H -5.378279 -1.6614113 .236985$

H -5.243133 -3.430219 3.131207

H $-7.095794-3.4033321 .305420$

H -7.733280 -1.151672 3.280816

H -9.926289 -2.443956 2.886504

H -9.430023 -2.795830 1.248466

H -11.105204 0.9619362 .107386

H -10.739225 -0.2248893 .481372$

H -10.484688 $0.474007-0.131711$

H -10.264188-1.210830 -0.479272

H -7.939511-1.028703 -0.725919

H -4.419752 -3.275072 0.915283

C 1.8319924 .3672011 .174268

O $2.6616203 .326284 \quad 0.624112$

C 4.0366773 .6164060 .930307
C 4.0569325 .0151621 .538013

C 2.7001345 .0748102 .211658

H 4.3433212 .8977991 .698222

C $4.9102963 .444930-0.316821$

H 4.8897495 .1512342 .234871

H 4.1334515 .7932990 .769701

C 2.2473566 .4858832 .542880

H 2.7411044 .4821523 .135423

C $4.7749522 .081841-1.026464$

O $4.5700624 .454557-1.277385$

H $5.9590083 .625646-0.053347$

H $3.8223162 .030257-1.567365$

O $5.8016862 .013445-2.025211$

C $4.8981250 .852073-0.108890$

C $4.991369-0.463327-0.907557$

H 4.0298980 .8183170 .558315

$\begin{array}{lllll}\text { O } & 6.064549 & 1.000738 & 0.700322\end{array}$

H $4.272227-0.445686-1.734443$

H $6.000593-0.538310-1.331198$

H 2.9514416 .9638903 .231777

H $2.182128 \quad 7.107770 \quad 1.643734$

H 1.2620736 .4786213 .019426

C 0.5489873 .7708031 .754162

H $3.6223124 .362528-1.476485$

H $5.6982122 .790600-2.600775$

H $\quad 6.1004910 .241803 \quad 1.306709$

H -0.0651874 .5595702 .203685$

H 0.7862863 .0535662 .549154

H 1.5904065 .0488080 .347642

C $5.517852-2.8314641 .947153$

O $5.677046-1.7599121 .008348$

C $4.713982-1.712165-0.046429$

C $4.775534-3.004116-0.877682$

C $4.629701-4.2492420 .003069$

C $5.544966-4.1922801 .232410$

H $6.406323-2.7718142 .589372$

C $4.284905-2.6343042 .836569$

H $3.712131-1.6022220 .383158$

O $6.008756-3.077501-1.591901$

H $3.975338-2.982420-1.625716$

H $4.869696-5.136713-0.595068$

O $3.272190-4.3592530 .417092$

H $5.286210-4.9831511 .946009$

O $6.893575-4.4279340 .816587$

C $4.416016-1.3731643 .657878$

H $3.352587-2.5874132 .266688$

H $4.173240-3.4686743 .539030$

H $6.731574-3.018230-0.943698$

H $3.189290-5.1748000 .939949$

H $6.924564-5.3104360 .409412$

O $3.567841-0.4167203 .183306$

O $5.193453-1.2482354 .595242$

C 3.6072340 .8379213 .869765

H 3.3402770 .7038694 .922399

H $4.6001561 .288145 \quad 3.776617$

H 2.8748171 .5027303 .404511

SCF Energy $\left(B 3 L Y P / 6-31 G^{* *} / / M M F F\right)=-3245.91227306$

0700376

MM̄̄FF Geometry

C $0.596263-3.654255-0.301602$

C $0.535361-2.5998200 .526786$

C $-0.101532-2.5357651 .897269$

O $-0.988402-1.3872741 .908244$

C $-0.931576-3.7724382 .289943$

C $-1.681947-3.6068823 .597812$

C $-3.003073-3.3488313 .680016$

C $-0.858358-3.7909814 .844061$

C $-3.943164-3.1173982 .527369$

C $-4.931955-1.9760292 .807106$

C $-5.679220-1.4761871 .556889$

C $-6.393377-2.5966280 .793849$

C $-7.057050-2.090192-0.495594$

O $-4.725170-0.8186530 .713045$

O $-7.407716-3.248540-1.268855$

C $-6.015199-1.281064-1.299502$

C $-8.371877-1.359531-0.202471$

O $-6.611511-0.695667-2.454640$ 
C $-5.288963-0.210798-0.457000$

C $-4.145120 \quad 0.475148-1.234644$

O $-6.2282050 .813870-0.106569$

C $-0.438850-0.1776232 .198186$

$\begin{array}{llllll}\text { O } & 0.730344 & 0.061660 & 2.457138\end{array}$

C - $1.509072 \quad 0.8357172 .104871$

C $-1.1726302 .062888 \quad 1.684483$

C -2.072375 3.1946181 .474328

C $-3.481308 \quad 3.0784551 .990213$

C $-3.000438-0.443162-1.670957$

C $-1.8047870 .386442-2.098932$

C -1.127611 2.094054 -3.781067

O $-0.7879210 .455957-1.412565$

C -1.745331 $3.419086-3.457152$

C $-1.301214 \quad 4.232344-2.487104$

C -1.938022 $5.549506-2.144077$

C $-2.8900465 .456068-0.969926$

C $-4.2169735 .381612-1.170065$

C -2.321984 5.5258130 .431513

C $-1.593457 \quad 4.272100 \quad 0.818575$

N $-2.016621 \quad 1.055745-3.294798$

H $0.175118-4.612042-0.012480$

H $0.994083-1.6709380 .189501$

H $0.691238-2.3922042 .642529$

H $-0.275198-4.6488692 .372756$

H $-1.636355-4.0182051 .487384$

H -3.457954 -3.294443 4.667856

H -1.458122 -3.697664 5.755674

H $-0.397728-4.7842394 .852116$

H -0.065133 -3.038452 4.893287

H -3.395356 -2.889209 1.609142

H $-4.499969-4.0462042 .357893$

H -5.661345 -2.308660 3.556382

H $-4.385416-1.1284373 .241257$

H -6.407269 -0.7305401 .898191$

H $-7.126801-3.0979421 .437419$

H $-5.670896-3.3706420 .503873$

H -7.759727 -2.938070 -2.120933

H $-5.277743-2.002618-1.673114$

H -9.078836 -2.0348900 .294589$

H -8.865013 -1.042077 -1.127878

H $-8.244696-0.4850320 .438873$

H -7.126973 $0.075566-2.162989$

H $-4.5559841 .007311-2.102491$

H $-3.7193081 .263022-0.599566$

H -5.7401751 .6313820 .085589$

H $-2.524147 \quad 0.535856 \quad 2.332787$

H -0.1322052 .2470931 .424167$

H -3.997541 2.2277821 .536299

H -4.091929 3.9603581 .784034

H -3.477375 2.9400653 .077131

H -2.678605 -1.094623 -0.850846

H -3.302220 -1.087668 -2.503425

H $-1.0317281 .977460-4.864602$

H $-0.1379351 .982422-3.328632$

H -2.612595 $3.713308-4.043804$

H $-0.4349813 .932891-1.903437$

H -2.464904 $5.944562-3.023297$

H $-1.1525776 .284454-1.929331$

H -4.913370 $5.339853-0.339355$

H -4.636397 5.355095 -2.170476

H -3.1046945 .7610521 .161449$

H $-1.625168 \quad 6.3714950 .494732$

H $-0.5526654 .260506 \quad 0.493631$

H -2.907096 $0.942709-3.770539$

C $2.596720-4.244014-1.706441$

O $3.508600-3.472964-0.895664$

C $4.623071-3.071982-1.707791$

C $4.671096-4.085715-2.840162$

C $3.191894-4.270511-3.118700$

H $4.376629-2.087125-2.122079$

C $5.897268-2.963547-0.867449$

H $5.229635-3.720009-3.706714$

H $5.115194-5.034761-2.518754$

C $2.869534-5.536577-3.893634$

H $2.835064-3.405600-3.694290$

C $5.760801-2.1018210 .404722$

O $6.308569-4.272078-0.450914$
H $\quad 6.712737-2.581169-1.492487$

H $5.187970-2.6435571 .167621$

O $7.075913-1.9222310 .946950$

C $5.125682-0.7148830 .192019$

C $5.1850730 .152408 \quad 1.465009$

H $4.079329-0.840003-0.106230$

O $5.793011-0.043923-0.875535$

H $4.893779-0.4530612 .331788$

H $\quad \begin{array}{lll}6.219948 & 0.486007 & 1.611208\end{array}$

H $3.354463-5.520822-4.875164$

H $3.214011-6.430510-3.362895$

H $1.790373-5.632019-4.050008$

C $1.215769-3.587578-1.670025$

H $5.571810-4.666498 \quad 0.047041$

H $7.453637-2.8071651 .089651$

H $6.7282850 .049971-0.625740$

H $0.540066-4.084339-2.375934$

H $1.293633-2.540134-1.986926$

H $2.560091-5.251719-1.274218$

C $3.899973 \quad 3.3929680 .085231$

$\begin{array}{lllll}\text { O } & 4.691072 & 2.220106 & 0.317720\end{array}$

C 4.2651201 .3893881 .400019

C 4.2866832 .1778612 .720355

C $3.474646 \quad 3.4728792 .613274$

C 3.8261994 .2647001 .349644

H $4.4556353 .955822-0.676229$

C $2.5246663 .049310-0.498833$

H 3.2470741 .0356971 .204095

O 5.6348522 .4928293 .065311

H 3.8833851 .5416233 .517276

H 3.6651904 .0987773 .493567

$\begin{array}{lllll}\text { O } & 2.084396 & 3.169923 & 2.594559\end{array}$

H 3.1051905 .0752541 .190769

$\begin{array}{lllll}\text { O } & 5.109050 & 4.873367 & 1.523277\end{array}$

C $2.6679242 .464955-1.883463$

H 1.9555082 .3489800 .117985

H $1.9058703 .949444-0.593411$

H $5.6187572 .957593 \quad 3.919073$

H 1.8776452 .6762503 .406291

H 5.0511495 .4649532 .292718

O $2.576404 \quad 1.105876-1.830153$

$\begin{array}{llll}\text { O } & 2.864659 & 3.137553 & -2.887487\end{array}$

C $2.7330240 .428133-3.080139$

H $2.0550050 .838267-3.834549$

H $3.7720800 .502047-3.414081$

H $2.485795-0.624912-2.928893$

SCF Energy (B3LYP/6-31G*//MMFF) $=-3245.90157157$

\section{7}

MM̄FF Geometry

C $2.516128 \quad 0.3994772 .377966$

C $1.8164941 .214006 \quad 3.181461$

C 0.9353022 .3200112 .663203

O $-0.4416592 .030052 \quad 3.017594$

C 1.2748773 .6795613 .298500

C 2.5951664 .2717042 .855808

C 2.7317575 .1437631 .838328

C 3.7977343 .8986763 .685532

C 1.6614275 .6799740 .927235

C $2.0882015 .644758-0.547761$

C $1.9326454 .260467-1.198962$

C $2.6033114 .260430-2.575694$

C $2.3817582 .942335-3.325834$

$\begin{array}{llll}\text { O } & 0.530137 & 4.013796 & -1.336892\end{array}$

$\begin{array}{lllll}\text { O } & 2.767797 & 3.169477 & -4.691877\end{array}$

C $0.8734232 .616267-3.317285$

C $3.3137151 .841306-2.807210$

O $0.6249241 .333207-3.883280$

C $0.185867 \quad 2.759542-1.938401$

C $-1.3508152 .771693-2.124849$

O $0.4708181 .660368-1.075626$

C - $-1.074431 \quad 1.0501042 .315660$

$\begin{array}{llll}\text { O } & -0.593561 & 0.343017 & 1.444371\end{array}$

C -2.4776970 .9899672 .781530$

C -3.296646 0.0611792 .265750

C $-4.707017-0.1404452 .600466$

C $-5.350355 \quad 0.856073 \quad 3.528423$

C $-2.1223782 .903526-0.806512$ 
C $-3.5994023 .130075-1.054284$

C $-5.6822921 .944893-1.742730$

O $-4.1065744 .244162-0.944616$

C $-6.4442701 .445314-0.555707$

C $-7.1882300 .328963-0.576718$

C $-8.022402-0.1536350 .575480$

C $-7.626440-1.5348911 .045465$

C $-8.086214-2.6355620 .426435$

C $-6.772297-1.6366242 .287848$

C $-5.352178-1.1947282 .057802$

N $-4.267871 \quad 1.975955-1.427254$

H 2.4388950 .5286121 .300926

H 1.8609331 .0739884 .258691

H 0.9966402 .4124941 .571529

H 1.2570443 .5975394 .394490

H 0.4581694 .3819743 .086916

H 3.7267485 .5437011 .645268

H 4.6889824 .4702573 .404298

H 3.6064314 .0989784 .744818

H 4.0424612 .8399003 .569202

H 0.7058875 .1625151 .052040

H 1.4944516 .7273051 .208030

H $1.4563106 .360772-1.090256$

H $3.1235955 .995201-0.642620$

H $2.3746623 .494380-0.552378$

H $3.6760824 .473587-2.486183$

H $2.1871435 .078048-3.181669$

H $2.5696252 .359075-5.192388$

H $\quad 0.4016343 .335599-4.001388$

H $4.3595322 .106273-3.006427$

H $3.1435940 .894767-3.331010$

H $3.2252591 .667565-1.733263$

H $0.8766220 .664647-3.225030$

H -1.632559 $3.609519-2.776210$

H -1.673336 $1.850423-2.625939$

H $1.3536841 .316456-1.268915$

H $-2.7966901 .708063 \quad 3.527991$

H -2.894981 -0.6324651 .529449$

H -6.4265990 .7137573 .644492$

H -5.2198121 .8757403 .148063$

H -4.9051330 .7938804 .527328$

H -2.003890 $2.000699-0.201392$

H -1.752071 $3.743284-0.207982$

H -5.803706 $1.294671-2.615116$

H $-6.0278212 .948660-2.009583$

H -6.4098492 .0492330 .347676$

H -7.234693 -0.257193-1.492145

H $-9.071438-0.1666380 .250831$

H $-7.9883090 .551421 \quad 1.415208$

H -7.831151 -3.6303390 .775938$

H $-8.726138-2.563966-0.446957$

H $-6.732043-2.6778842 .634864$

H -7.261006 -1.092054 3.100601

H -4.797465 -1.854093 1.388289

H -3.769873 $1.091756-1.424349$

C $4.714424-0.8046842 .135432$

O $4.460897-1.1437770 .754479$

C $4.946796-2.4740700 .523914$

C $6.154131-2.5803881 .438726$

C $5.614533-1.9178402 .695327$

H $4.169472-3.1675700 .870942$

C $5.202252-2.714353-0.965453$

H $6.469998-3.6147341 .602298$

H $7.007467-2.0121281 .050997$

C $6.700316-1.4045293 .626333$

H $5.009097-2.6520863 .243991$

C $4.028913-2.306987-1.882320$

O $6.347100-1.954965-1.370943$

H $5.464707-3.767827-1.119861$

H $4.005311-1.214529-1.974573$

O $4.312189-2.800749-3.197402$

C $2.641484-2.803602-1.438543$

C $1.546300-2.466376-2.469597$

H $2.388772-2.347518-0.475088$

O $2.698408-4.216459-1.247876$

H $1.729365-1.470958-2.889345$

H $1.592333-3.201977-3.281842$

H $7.332032-2.2288243 .973143$
H $7.344856-0.6736253 .126522$

H $6.259630-0.9214444 .504406$

C $3.373786-0.7283292 .875539$

H $6.492455-2.132324-2.316330$

H $4.271375-3.771970-3.159942$

H $1.824570-4.505601-0.933934$

H $3.550314-0.6301493 .953171$

H $2.814490-1.6564862 .708790$

H 5.2192490 .1686662 .142759

C $-1.377666-3.965661-0.674292$

O $-0.142039-3.803583-1.384748$

C $0.138662-2.478802-1.842293$

C $-0.950942-2.016726-2.820717$

C $-2.336534-2.112904-2.172603$

C $-2.569445-3.480485-1.520015$

H $-1.483663-5.049915-0.538932$

C -1.320220 -3.3233050 .720529$

H $0.160698-1.795698-0.985753$

O $-0.945407-2.814220-4.001769$

H $-0.759057-0.980560-3.119786$

H $-3.106074-1.938510-2.934277$

O $-2.474416-1.101663-1.179118$

H -3.472614 -3.461193 -0.898545

O $-2.788164-4.454578-2.544671$

C $-0.271850-3.9620301 .607298$

H $-1.087540-2.2581250 .659570$

H -2.288027 -3.442985 1.220991

H $-0.149933-2.585634-4.511048$

H $-2.208809-0.256733-1.578560$

H $-3.566896-4.173720-3.054825$

O $-0.165005-3.2342012 .758775$

O $\quad 0.361731-4.973171 \quad 1.338252$

C $0.781673-3.7251373 .713148$

H $1.761291-3.8732303 .248488$

H $\quad 0.881307-2.9800514 .506878$

H $\quad 0.417576-4.6598874 .149376$

SCF Energy $(B 3 L Y P / 6-31 G * * / / M M F F)=-3245.91334378$

0700378

MM̄FF Geometry

C $3.625523-2.8291081 .608674$

C $2.331325-2.4914531 .506723$

C $1.470557-2.1390432 .690155$

O $0.620973-1.0201022 .346698$

C $0.564620-3.3374613 .035099$

C $-0.422481-3.0745884 .157571$

C $-1.751698-3.2726674 .049916$

C $0.160943-2.5869855 .457878$

C $-2.490455-3.8311792 .865638$

C $-2.986981-2.7257721 .934546$

C $-3.751210-3.2608990 .713716$

C $-2.834922-3.986665-0.277362$

C $-3.576067-4.394898-1.561209$

O $-4.350578-2.1235480 .079819$

O $-2.592954-4.761143-2.541744$

C $-4.328138-3.167419-2.115833$

C $-4.452159-5.634202-1.344450$

O $-5.146974-3.524587-3.226299$

C $-5.174865-2.439914-1.051668$

C $-5.771337-1.111467-1.568479$

O $-6.275485-3.272479-0.668695$

C 1.0692270 .2248862 .663409

O 2.1877720 .5294263 .045476

C $-0.077428 \quad 1.1546682 .543637$

C $-0.0253542 .338158 \quad 3.173556$

C -1.1012713 .3263363 .231434$

C -0.6142254 .7499733 .198699$

C $-4.739475-0.112203-2.097416$

C $-5.2759151 .307353-2.145671$

C $-4.4667243 .641372-2.428956$

O $-6.4270171 .607613-1.842266$

C $-3.9453774 .041814-1.085244$

C $-4.7045974 .518978-0.088561$

C -4.1543424 .8433501 .272068$

C -4.5032913 .8458632 .361411$

C -5.5547773 .0084232 .321950$

C -3.5901833 .8551333 .569281$

C -2.3913672 .9629223 .382577$ 
N $-4.2966422 .205633-2.551663$ H $4.097476-2.8363292 .588178$ H $1.847932-2.5139700 .534498$ H $2.090898-1.8879553 .560005$ H $1.186146-4.1963533 .321549$ H $\quad 0.033762-3.6335832 .123474$ H -2.382256 -3.038593 4.906250 H $-0.579936-2.5721186 .264587$ H $\quad 0.976526-3.2441615 .777207$ H $0.550443-1.5700105 .351460$ H - $1.867531-4.5538612 .330962$ H -3.346620 -4.4067223 .239534$ H -3.644767 -2.046361 2.494082 H -2.149390 -2.101611 1.595242 H -4.546821 -3.9265561 .068057$ H $-2.370389-4.8679940 .178979$ H -2.004772 $-3.322000-0.554621$ H - $2.058690-5.486048-2.174288$ H -3.566089-2.477435 -2.500450 H -3.837212 -6.488359-1.036219 H $-4.942371-5.939517-2.275479$ H -5.218935 -5.490200 -0.580680 H -4.578026 -3.977089 -3.872284 H $-6.524890-1.306681-2.342426$ H $-6.320860-0.648757-0.736779$ H -6.970588 -2.709948 -0.288700 H $-0.953178 \quad 0.8236791 .996235$ H 0.8756552 .6211893 .715168 H -0.0408734 .9792594 .103584$ H -1.420753 5.4827673 .123572 H 0.0339114 .9153642 .331883 H -3.852308 -0.104782 -1.453063 H $-4.428018-0.394548-3.109479$ H $-5.5217903 .902450-2.559505$ H -3.882412 4.119797 -3.220351 H $-2.8836043 .893559-0.910636$ H $-5.7699734 .665242-0.247828$ H -4.5107095 .8362461 .571919$ H -3.0620184 .9219771 .200292$ H $-5.7545202 .322318 \quad 3.139197$ H -6.2459572 .9801181 .487499$ H -4.133438 3.4941994 .453318 H -3.3188674 .8858203 .817842$ H -2.631184 1.9002123 .426028 H -3.354722 $1.859061-2.710837$ C $5.744791-2.4340700 .323862$

O $5.405019-1.094731-0.081153$ C $6.352440-0.652924-1.071652$

C $7.470064-1.688484-1.085568$

C $6.708868-2.953511-0.742592$

H $5.834200-0.683833-2.037346$

C $6.8039520 .778357-0.765584$ H $7.976099-1.739260-2.054508$ H $8.227763-1.482301-0.320836$

C $7.601177-4.089374-0.273173$

H $6.148998-3.278301-1.630027$

C $5.6445231 .782805-0.603985$

O 7.5497110 .7866370 .459332

H $7.4977201 .122867-1.540681$

H 5.0900161 .5578540 .313881

O $6.2166863 .083872-0.419822$

C $4.6775421 .838333-1.800487$

C $3.6278372 .959527-1.707522$

H $4.1699720 .875808-1.909479$

O $5.4409742 .047231-2.992068$

H $4.1391273 .927229-1.634561$

H $3.0935173 .000110-2.664166$

H $8.314877-4.365932-1.056086$

H $8.172336-3.8089990 .618370$

H $7.009033-4.976549-0.028227$

C $4.471059-3.2682180 .445590$

H 6.9749070 .4238261 .155395

H 6.8226703 .0291150 .338950

H $4.8270262 .006548-3.745175$

H $4.728539-4.3223240 .599087$

H $3.895633-3.201033-0.485486$

H $6.251467-2.3569861 .294801$

C $0.9689451 .451564-1.685153$
O $1.9113511 .553609-0.610468$

C $2.6294702 .791123-0.544529$

C $1.6440723 .954949-0.402052$

C $0.6186163 .965173-1.529129$

C $-0.0547682 .597575-1.642478$

H $1.5035681 .464756-2.642741$

C $0.2532160 .095267-1.551201$

H 3.2116582 .7600490 .383419

O $2.3466165 .192325-0.364639$

H 1.1179243 .8553070 .554220

H $1.0762654 .269620-2.477445$

O $-0.3630194 .956341-1.218337$

H $-0.7397692 .457634-0.796360$

O $-0.8329462 .553930-2.837949$

C $1.116686-1.070965-1.983430$

H $-0.040291-0.080121-0.510215$

H $-0.6408500 .081153-2.185316$

H $1.6841725 .900333-0.289477$

H -0.937252 $5.056258-1.996191$

H -1.493463 $3.264381-2.788961$

O $0.334904-2.189576-2.063978$

O $2.317062-1.019724-2.210292$

C $1.004536-3.382064-2.484739$

H $1.777713-3.658204-1.762763$

H $\quad 0.267553-4.187582-2.531739$

H $1.436849-3.243528-3.480213$

SCF Energy $\left(B 3 L Y P / 6-31 G^{* *} / / M M F F\right)=-3245.90530431$

\section{9}

MM̄FF Geometry

C - $-1.744257-2.4744502 .351803$

C $-0.603457-1.8603852 .695566$

C $-0.415395-0.4230153 .113269$

$\begin{array}{llll}\text { O } & 0.486335 & 0.155361 & 2.126922\end{array}$

C -1.6572120 .4964013 .173331$

C $-2.6771960 .213108 \quad 4.258938$

C -4.0011470 .0787294 .034784$

C -2.1461900 .1776555 .667665$

C -4.6933000 .1018852 .700618$

C -5.1891001 .5012552 .316471$

C -5.3049871 .7098910 .796291$

C -6.2388370 .7016470 .122194$

C $-6.2529670 .867874-1.406229$

$\begin{array}{llll}\text { O } & -3.982904 & 1.607568 & 0.254365\end{array}$

O $-6.904695-0.280876-1.970657$

C $-4.7959100 .900056-1.920846$

C $-7.1096602 .064864-1.831226$

O $-4.7577851 .171280-3.320193$

C $-3.8819871 .880817-1.146981$

C $-2.4053291 .664188-1.572726$

O $-4.2828663 .214674-1.453383$

C $1.323511 \quad 1.1446682 .540890$

O 1.4710561 .5518153 .682191

C 2.0036311 .6882231 .342954

C 2.7875382 .7697271 .466208

C 3.4896573 .4665430 .386418

C $3.2304693 .021086-1.028121$

C $-1.3542312 .406190-0.731348$

C -1.161272 $3.865296-1.105817$

C $-0.1066785 .963436-0.266664$

O $-1.6240234 .361643-2.129284$

C $1.2129856 .154118-0.945004$

C $2.2119556 .884593-0.426197$

C $3.5234367 .119536-1.119826$

C $4.7149216 .768965-0.256623$

C 5.4059887 .7242680 .388064

C $5.1577115 .323785-0.213733$

C $4.3122234 .487680 \quad 0.706767$

N $-0.4001634 .545954-0.165182$

H $-2.678619-1.9332672 .373898$

H $0.315335-2.4439612 .664547$

H $\quad 0.089753-0.4367804 .086945$

H -2.1105080 .5296772 .177008$

H -1.3142541 .5289743 .330986$

H $-4.661557-0.0730644 .886891$

H $-2.947978 \quad 0.137601 \quad 6.412792$

H -1.514143 -0.702990 5.818301

H -1.5533551 .0748395 .873609$ 
H $-4.040153-0.3016901 .923249$

H -5.545027 -0.5885742 .744149$

H -6.1589381 .6884542 .793044$

H -4.4894682 .2541202 .703449$

H -5.6705442 .7311760 .636109$

H -7.2539920 .7789410 .530453$

H $-5.906501-0.3211740 .343044$

H -6.400587 -1.072794-1.717462

H -4.391050 -0.113689-1.800629

H -8.141892 $1.937104-1.482853$

H -7.172274 2.139947 -2.922456

H $-6.7448133 .016417-1.439207$

H -4.913344 2.123488 -3.441289

H -2.179603 $0.595214-1.466484$

H -2.277797 $1.922246-2.630769$

H -3.579112 $3.822483-1.179814$

H 1.8177091 .2030030 .392068

H 2.9271703 .1975852 .458512

H $3.7222352 .063975-1.219834$

H $3.5897373 .730113-1.777484$

H $2.1570992 .910730-1.217379$

H -1.583392 2.3291180 .336626

H $-0.384344 \quad 1.919236-0.892727$

H $-0.112332 \quad 6.369413 \quad 0.749849$

H $-0.893636 \quad 6.458483-0.844777$

H $1.3425805 .692101-1.920803$

H 2.0696427 .3697190 .536866

H $3.5648728 .180626-1.399067$

H $3.5829326 .563891-2.064054$

H 6.2792637 .4846690 .985549

H 5.1102048 .7669270 .337071

H $6.1917595 .259658 \quad 0.151417$

H $5.2018374 .927128-1.232122$

H 4.4283924 .7502331 .759209

H 0.0043894 .0332230 .612955

C $-2.087501-4.0764070 .411710$

O $-0.949190-3.588849-0.329283$

C -1.429482 -2.836609-1.458204

C $-2.895415-3.221778-1.620941$

C $-3.315330-3.371098-0.171304$

H -1.376874 -1.780431 -1.170980

C $-0.560152-3.087599-2.694074$

H -3.468551 -2.462238 -2.160600

H -3.009894 -4.175491 -2.149127

C $-4.614361-4.1327380 .018001$

H $-3.432753-2.3650180 .248611$

C $0.943870-2.802358-2.499875$

O $-0.687959-4.460165-3.088785$

H - $0.943416-2.500485-3.536595$

H $1.382163-3.572486-1.855220$

O $1.579354-2.939502-3.777592$

C $1.281831-1.409628-1.939801$

C $2.778986-1.054600-1.959500$

H $0.912523-1.320275-0.911772$

O $0.633658-0.394341-2.713827$

H $3.114392-0.907335-2.994594$

H $2.863698-0.069084-1.487484$

H -5.440744 -3.611335 -0.476221

H $-4.553958-5.141916-0.402898$

H $-4.859446-4.2237151 .081027$

C -1.846882 -3.9087541 .915515$

H -0.418498 -5.008930 -2.332476

H $1.366249-3.827005-4.113561$

H $-0.324737-0.477903-2.584381$

H $-0.930499-4.4427472 .192153$

H -2.663595 -4.3804062 .474440$

H -2.143629 -5.1518930 .197737$

C $3.999921-3.1744750 .875808$

O $3.247929-2.2418690 .084251$

C $3.716235-2.046169-1.252324$

C $5.170091-1.538643-1.248971$

C $6.086105-2.446963-0.423522$

C $5.482282-2.7663890 .946856$

H $3.580749-3.0848441 .886029$

C $3.801647-4.6262480 .407724$

H $3.673121-2.996951-1.792368$

O $5.237793-0.213386-0.724149$

H $5.528581-1.489172-2.283608$
H $7.055564-1.953848-0.279939$

O $6.316253-3.664477-1.125079$

H $6.063698-3.5553871 .438092$

O $5.592648-1.5952991 .761093$

C $2.386253-5.1107490 .627648$

H $4.024659-4.742108-0.655786$

H $4.459703-5.2908500 .979494$

H $4.847839-0.2250860 .166668$

H $6.730321-3.439559-1.975679$

H $5.269678-1.8231962 .649527$

O $2.229673-6.3219510 .016412$

O $1.530881-4.5150821 .266034$

C $0.934402-6.9159440 .148678$

H $\quad 0.191073-6.313803-0.380655$

H $\quad 0.968390-7.909849-0.305285$

H $0.665883-7.0221351 .204194$

SCF Energy $(B 3 L Y P / 6-31 G * * / / M M F F)=-3245.91516707$

0700380

MM̄FF Geometry

C $2.5493763 .895816-0.652962$

C $3.111551 \quad 4.3977690 .456220$

C $3.652323 \quad 3.5928581 .617231$

O 3.1963082 .2178841 .602059

C 5.1883673 .5859311 .572891

C 5.8150672 .9660452 .805786

C $6.3512371 .730552 \quad 2.848625$

C 5.8637003 .8557714 .020887

C $6.3839510 .712802 \quad 1.739595$

C $6.153675-0.705542 \quad 2.281206$

C $6.044542-1.7973771 .203367$

C $7.259305-1.850664 \quad 0.272400$

C $7.101501-2.920423-0.819742$

O $4.845865-1.573791 \quad 0.450782$

O $8.105674-2.682729-1.818933$

C $5.731243-2.726638-1.504188$

C $7.354680-4.333346-0.282131$

O $5.472871-3.775655-2.434166$

C $4.559242-2.605930-0.505882$

C $3.227561-2.256687-1.203548$

$\begin{array}{lllll}\text { O } & 4.365210 & -3.869508 & 0.143131\end{array}$

C 1.9048051 .9815311 .963146

$\begin{array}{llll}\text { O } & 1.049766 & 2.811195 & 2.230825\end{array}$

C 1.6983920 .5178131 .980236

$\begin{array}{lllll}\text { C } & 0.465228 & 0.028613 & 1.781204\end{array}$

C $0.081415-1.3839871 .818935$

C $1.057433-2.3496712 .439410$

C $3.206692-0.921751-1.950202$

C $1.779956-0.559660-2.308727$

C $-0.139875-1.350532-3.685080$

$\begin{array}{lllll}\text { O } & 1.166077 & 0.311642 & -1.698028\end{array}$

C - $0.773602-2.513022-2.984785$

C -1.609613 -2.394592 -1.942602

C $-2.201502-3.560886-1.208554$

C -1.438362 -3.8991590 .053332$

C $-0.573820-4.9280490 .070369$

C $-1.740534-3.1176101 .312630$

C $-1.124700-1.7453811 .331924$

N $1.269528-1.337163-3.337399$

H $2.5053282 .819089-0.794633$

H 3.1485515 .4783350 .579456

H 3.3068494 .0582882 .549062

H 5.5678114 .6120601 .473973

H 5.5285513 .0637480 .670477

H 6.8234261 .4122913 .777238

H 6.3749523 .3802114 .864644

H 6.3996624 .7826353 .791813

H 4.8523814 .1108774 .352018

H 5.6339930 .9284060 .973268

H $7.3711940 .771291 \quad 1.268606$

H $6.969437-0.9664482 .967796$

H $5.225371-0.7089862 .868149$

H $5.943014-2.7500511 .737085$

H $8.180830-2.0144810 .844365$

H $7.385072-0.884977-0.233917$

H $8.975155-2.720048-1.384474$

H $5.795074-1.800507-2.089979$

H $8.375430-4.4156160 .110364$ 
H $7.280501-5.078948-1.081316$ H $6.671589-4.6178470 .520523$ H $6.229418-3.814941-3.044120$ H $2.939372-3.072506-1.879397$ H $2.435333-2.229648-0.443490$ H $\quad 3.458090-3.9038670 .489200$ H $2.563850-0.1099612 .157727$ H -0.3431360 .7258291 .562383$ H $1.985720-2.3934581 .861604$ H $0.677036-3.3711882 .501282$ H $1.300171-2.0426443 .463036$ H $3.613689-0.113540-1.332377$ H $3.803151-0.966803-2.867508$ H $-0.218730-1.475690-4.769032$ H $-0.601724-0.397829-3.408808$ H $-0.506829-3.505035-3.342019$ H $-1.875207-1.406705-1.582793$ H $-2.240869-4.435350-1.872064$ H $-3.247496-3.342309-0.963616$ H $-0.043300-5.2084140 .973863$ H $-0.373885-5.512262-0.821911$ H - $-1.441085-3.6821192 .203558$ H -2.828139-3.013162 1.413980 H $-1.764687-0.9731340 .904622$ H $1.855780-2.062619-3.739984$ C $0.3751874 .626906-1.585687$ O $-0.028395 \quad 3.259779-1.765112$ C -1.356999 $3.236379-2.327707$ C $-1.7667914 .690964-2.524759$ C $-0.4236795 .395104-2.630802$ H $-1.2595452 .724328-3.292393$ C $-2.2487572 .435776-1.373335$ H $-2.3951204 .829248-3.409832$ H $-2.3265875 .061125-1.656740$ C $-0.5020256 .890459-2.381606$ H $-0.0085535 .212364-3.630852$ C -3.724438 2.339249-1.791061 O $-1.7136321 .112489-1.292127$ H -2.172004 2.861670 -0.366281 H -4.205987 $3.318954-1.689174$ O $-3.7755002 .006032-3.185183$ C $-4.5314561 .265949-1.030859$ C $-4.503620 \quad 1.4275530 .499883$ H $-4.1730730 .271426-1.320930$ O $-5.884591 \quad 1.363851-1.489809$ H -3.4837301 .6111590 .849898$ H $-5.120145 \quad 2.2952130 .765762$ H - $1.1577767 .368726-3.116492$ H $-0.897257 \quad 7.111026-1.384307$ H $0.4869887 .352136-2.461727$ C $1.8941864 .730508-1.719373$ H $-0.7786501 .189798-1.034889$ H $-4.713993 \quad 1.947644-3.434113$ H $-6.3902460 .649037-1.067286$ H $2.2129675 .776162-1.646385$ H $2.2224774 .352321-2.694828$ H $0.0668994 .925124-0.573736$ C $-7.019283-1.1819641 .452919$ O $-6.401506-0.0117540 .900625$ C $-5.026294 \quad 0.1777841 .238342$ $\begin{array}{llll}C & -4.866998 & 0.308422 & 2.761271\end{array}$ C $-5.494511-0.8816543 .495308$ C $-6.907914-1.1875052 .986548$ H $-8.081471-1.083328 \quad 1.193279$ C $-6.498453-2.4675780 .800217$ H $-4.445519-0.6820660 .886947$ $\begin{array}{lllll}\text { O } & -5.470360 & 1.516884 & 3.220671\end{array}$ H $-3.799440 \quad 0.3725553 .002267$ H $-5.537097-0.6646024 .569573$ O $-4.682348-2.0382023 .318865$ H -7.257072 -2.151968 3.373555 O $-7.803958-0.1871313 .480631$ C $-6.842414-2.494664-0.670042$ H $-5.418285-2.6003780 .907749$ H $-6.961065-3.3503431 .257413$ H -6.4033291 .5064772 .946061$ H -3.798339 -1.8337163 .668821$ H $-7.779643-0.2263374 .451936$
O $-5.768136-2.092259-1.406274$

O $-7.941447-2.809855-1.107310$

C $-5.969308-2.044320-2.821746$

H $-5.041652-1.696825-3.284355$

H -6.201333 -3.042421 -3.204883

H $-6.769004-1.339091-3.067094$

SCF Energy (B3LYP/6-31G**//MMFF)= -3245.92217804

0700381

MM̄FF Geometry

C $3.164670-2.9066800 .460986$

C $2.652148-3.8178881 .301767$

C $1.468919-3.6297022 .220489$

O $0.775174-2.3961401 .928821$

C $0.453727-4.772864 \quad 2.017374$

C $-0.676451-4.7379753 .028545$

C $-1.901898-4.2241102 .801399$

C $-0.350881-5.346267 \quad 4.368672$

C $-2.413873-3.5589281 .551742$

C $-3.700560-4.231206 \quad 1.056434$

C $-4.282289-3.630446-0.234945$

C $-3.448351-3.981196-1.465832$

C $-4.041019-3.362868-2.739058$

O $-4.370283-2.211347-0.091923$

O $-3.062423-3.480688-3.783085$

C $-4.275716-1.854556-2.505680$

C $-5.281856-4.124961-3.221442$

O $-4.966006-1.295008-3.620079$

C $-5.017496-1.533379-1.186040$

C $-4.950671-0.029253-0.826259$

O $-6.385772-1.928201-1.287352$

C $0.682536-1.4551642 .906081$

O $1.292241-1.4165053 .961517$

C $-0.377370-0.5103582 .487124$

C -0.8005770 .4607493 .309393$

C $-1.928494 \quad 1.3622363 .050705$

C -2.7340741 .1159341 .802100$

C $-5.7235390 .922430-1.737262$

C $-5.7479842 .324930-1.158557$

C $-4.3442604 .381348-0.939011$

$\begin{array}{llll}\text { O } & -6.737451 & 2.766025 & -0.578821\end{array}$

C $-3.313068 \quad 4.407718 \quad 0.143104$

C $-3.446313 \quad 5.086946 \quad 1.292475$

C $-2.386136 \quad 5.1291142 .358008$

C $-2.906923 \quad 4.734498 \quad 3.722642$

C -2.9716005 .6318824 .720799$

C $-3.3580513 .306125 \quad 3.933584$

C -2.2125952 .3299293 .947671$

N $-4.5578713 .006398-1.353516$

H $2.728042-1.9146720 .404830$

H $3.129822-4.7950491 .351447$

H $1.841207-3.641762 \quad 3.251784$

H $0.957333-5.7457242 .096032$

H $0.054334-4.7368860 .997264$

H -2.623923 -4.2532753 .617177$

H $-1.203241-5.3218675 .055789$

H $-0.056053-6.3938804 .247804$

H $0.472034-4.8057744 .846572$

H -2.625516 -2.512154 1.796750

H $-1.662944-3.5482470 .759816$

H $-3.539935-5.3080410 .918912$

H $-4.458509-4.1244311 .844770$

H $-5.294237-4.038675-0.343769$

H $-3.350256-5.068284-1.575498$

H -2.427794 -3.597631 -1.348048

H $-2.853293-4.424205-3.892583$

H $-3.291291-1.367844-2.471594$

H $-5.036586-5.177765-3.405773$

H $-5.640729-3.730546-4.178628$

H $-6.107029-4.097420-2.506895$

H -4.446162 -1.499117 -4.416353

H -5.3353680 .0944390 .196506$

H $-3.8989020 .279961-0.770449$

H $-6.892539-1.441170-0.616246$

H $-0.836798-0.686767 \quad 1.521949$

H $-0.304950 \quad 0.586148 \quad 4.270815$

H -3.143913 $0.099776 \quad 1.805812$

H -2.1109401 .2406880 .909888$ 
H $-3.583173 \quad 1.7901911 .684546$ H $-5.2743650 .974310-2.733997$ H $-6.7613460 .596504-1.866221$ H -5.289844 $4.833035-0.623132$ H -3.972172 $4.923563-1.813931$ H $-2.3991373 .848993-0.036256$ H $-4.3496315 .666667 \quad 1.467391$ H -1.987475 6.1516162 .384945 H -1.536785 4.4870862 .098814 H -3.343741 5.3599885 .703047 H -2.6533816 .6595214 .579663$ H -4.124009 3.0538923 .195941 H -3.8703003 .2120924 .900776$ H -1.571969 2.4265724 .825226 H $-3.8006902 .543006-1.847888$ C $5.563017-2.367087-0.044347$

O $5.345375-0.970650-0.325556$ C $6.581756-0.391361-0.789710$ C $7.645188-1.477190-0.656114$ C $6.821966-2.738615-0.824809$ H $6.443777-0.167081-1.853401$ C $6.8894980 .894976-0.014649$ H $8.434796-1.375543-1.407046$ H $8.122152-1.4621470 .330767$ C $7.523503-3.987906-0.320351$ H $6.581248-2.862254-1.889145$ C $5.7663871 .950017-0.081798$ O $7.0819650 .552791 \quad 1.364666$ H $7.8382811 .319487-0.363179$ H $4.877868 \quad 1.5584360 .423736$ O 6.1895413 .0812880 .691753 C $5.4126442 .422893-1.506018$ C $4.3670413 .555504-1.538477$ H $5.0541511 .578442-2.103489$ O $6.6085232 .902833-2.126504$ H $4.6819834 .371551-0.877273$ H $4.3555273 .978874-2.551940$ H $8.458737-4.149985-0.866128$ H $7.765146-3.9098560 .744900$ H $6.895642-4.873516-0.458909$ C $4.332380-3.178142-0.448345$ H 7.2672981 .3781681 .844619 H $6.955206 \quad 3.4751470 .239015$ H $6.3905763 .131732-3.046106$ H $4.569500-4.248176-0.444101$ H $4.027524-2.917196-1.469315$ H $\quad 5.742098-2.4544401 .035830$ C $1.2122201 .644160-2.040094$ O $2.4875072 .263721-2.253566$ C $2.9555333 .076124-1.170511$ C $1.9879974 .245383-0.947902$ C $0.5725803 .731633-0.673323$ C $0.1272752 .684613-1.704593$ H $0.9614751 .203333-3.013840$ C $1.3395950 .506766-1.022900$ H $3.0008382 .472076-0.258676$ O $1.9768535 .107765-2.082901$ H $2.3364824 .838809-0.094381$ H $-0.1187584 .582512-0.685546$ O $0.549026 \quad 3.169700 \quad 0.635664$ H $-0.7814832 .171521-1.370419$ O $-0.2010523 .359985-2.923543$ C $0.423287-0.639027-1.379321$ H $2.3707650 .132951-0.991006$ H 1.1110410 .8173990 .000016 H $1.737144 \quad 4.574145-2.859783$ H $-0.3536672 .851508 \quad 0.803874$ H $-0.9294293 .974882-2.730365$ O $1.135358-1.626364-1.993084$ O $-0.780755-0.646469-1.159314$ C $0.388642-2.788185-2.363878$ H $0.024652-3.298557-1.467535$ H $-0.441633-2.515642-3.021725$ H $1.056967-3.463672-2.904585$ SCF Energy (B3LYP/6-31G**//MMFF $)=-3245.91751090$

\section{2}

MM̄FF Geometry
C $-2.6408553 .392759-0.023222$

C -2.0281954 .5146820 .388584$

C -0.5814754 .6699160 .799004$

O $\quad 0.108725 \quad 3.401647 \quad 0.783102$

C $0.1252205 .617649-0.188371$

C 1.5781145 .8823430 .155874

C $2.6330185 .370316-0.509648$

C 1.8017926 .8186051 .315630

C $2.6150864 .421085-1.676634$

C $3.3253973 .112826-1.325511$

C $3.2149802 .036198-2.416018$

C $1.7830061 .513614-2.579585$

C $1.7006450 .321679-3.544088$

O $4.0809560 .968222-2.009808$

O $0.416328-0.296628-3.372442$

C $2.762949-0.720443-3.140803$

C $1.7701310 .769800-5.008691$

O $2.816413-1.784470-4.088376$

C $4.170142-0.115203-2.941167$

C $5.127170-1.181852-2.350787$

O $4.6737410 .312578-4.209238$

C 0.2411982 .7473801 .969264

O -0.1823963 .1021293 .057763$

C 0.9978121 .5013631 .713000

C 1.2025340 .6297022 .710885

C $1.907683-0.6511862 .613478$

C $2.599803-0.9718661 .314720$

C $6.499604-0.642926-1.921776$

C $6.507173-0.044638-0.526889$

C $6.578353-0.6672501 .870769$

O $6.6139521 .165427-0.341052$

C $6.143497-1.8210952 .714319$

C $5.394145-1.7000573 .820656$

C $4.970904-2.8793344 .649738$

C $3.497624-2.8796564 .989725$

C $3.088713-2.9808116 .266322$

C $2.495667-2.8361003 .855137$

C $1.890302-1.4674563 .687853$

N $6.396417-1.0023820 .469665$

H -2.092330 $2.459011-0.083167$

H $-2.6117035 .433700 \quad 0.424385$

H -0.5554505 .1092041 .803653$

H $-0.4002276 .582229-0.217546$

H $0.0331655 .216762-1.202966$

H $3.6321215 .653697-0.180255$

H 2.8635927 .0309541 .480466

H 1.3004607 .7748871 .133980

H 1.4060686 .3872842 .240333

H $1.5978754 .216202-2.009428$

H $3.1268344 .898052-2.520985$

H $4.3888153 .316994-1.137739$

H $2.9435002 .710104-0.379498$

H $3.5867352 .455483-3.358171$

H $1.1023302 .304675-2.912787$

H $1.4026651 .186520-1.603330$

H $-0.2662070 .376551-3.536536$

H $2.441995-1.166965-2.189274$

H $0.929761 \quad 1.433240-5.246129$

H $1.677756-0.084365-5.688389$

H $2.6893851 .307295-5.250180$

H $1.915699-2.142153-4.171576$

H $4.647061-1.662891-1.489934$

H $5.298772-1.957342-3.108200$

H $5.639867 \quad 0.232534-4.220359$

H $1.350881 \quad 1.322677 \quad 0.706064$

H $\quad 0.7974490 .8650813 .694397$

H $3.326287-0.1909521 .063377$

H $1.873661-1.0464920 .498257$

H $3.149833-1.9146541 .335373$

H $7.223305-1.468405-1.924557$

H $6.8914920 .103727-2.619375$

H 6.0247280 .2513242 .092548

H $7.645322-0.4726512 .022273$

H $6.471800-2.8120972 .407928$

H $5.089416-0.7116854 .156040$

H $5.573863-2.8727015 .566626$

H $5.202881-3.8202984 .134685$

H $2.033610-3.0083276 .519062$ 
H $3.796735-3.0383247 .086325$ H $1.676634-3.5352034 .072768$ H $2.952919-3.2197252 .939256$ H $1.340979-1.1340034 .570261$ H $6.492653-1.9773440 .199005$ C -4.9979932 .5976270 .460868$ $\begin{array}{lllll}\text { O } & -4.876741 & 1.172815 & 0.234936\end{array}$ C $-6.1830490 .645137-0.058042$ C -7.1648111 .6501630 .529314$ C -6.4687012 .9525690 .208542$ H $-6.2812280 .654372-1.149597$ C $-6.379608-0.7798010 .471188$ H $-8.160180 \quad 1.5727200 .081766$ H -7.2662741 .5346431 .614577$ C -6.9654324 .1275291 .034688$ H $-6.6248453 .178081-0.855369$ C $-5.361467-1.830215-0.011358$ O $-6.310529-0.7453751 .903910$ H -7.397157 -1.109289 0.226482 H $-4.412127-1.6639070 .506051$ O $\quad-5.833172-3.108060 \quad 0.442023$ C $-5.114505-1.901465-1.528539$ C $-4.299530-3.125006-1.996507$ H $-4.608140-0.988490-1.862465$ O $-6.360841-1.981860-2.228298$ H -4.936079 -4.019756-1.974346 H -4.070495 -2.959825 -3.058091 H -8.0299724 .3050470 .849580$ H -6.8364593 .9467032 .107232$ H -6.4206205 .0419230 .779749$ C $-4.0716073 .369060-0.484956$ H -6.455316 -1.652592 2.223502 H -6.645228 -3.312968 -0.052671 H $-6.834783-1.143595-2.108441$ H $-4.4313864 .399538-0.594881$ H -4.093316 2.915215 -1.483656 H -4.7281382 .7770971 .509112$ C $-0.957476-2.332283-0.508640$ O $-2.178822-2.243723-1.259833$ C $-3.004458-3.409217-1.222168$ C $-2.249188-4.619380-1.796806$ C $-0.913347-4.835325-1.077466$ C $-0.111668-3.534304-0.966590$ H $-0.403629-1.419660-0.764215$ C -1.215313 -2.320126 1.007646 H $-3.274931-3.629694-0.185628$ O $-2.010061-4.441967-3.191885$ H -2.876398 -5.512149-1.690850 H $-0.321068-5.578428-1.625367$ O $-1.147244-5.345576 \quad 0.231567$ H $\quad 0.738411-3.661163-0.285720$ O $0.425686-3.215621-2.252788$ C $-1.813122-1.0105991 .473405$ H -1.901936 -3.115048 1.308715 H $-0.270283-2.4588681 .541856$ H $-1.523594-3.607452-3.306257$ H $-1.631697-6.1835110 .138123$ H $1.015857-3.943485-2.512543$ O $-2.143998-1.1163932 .794084$ O $-1.971863-0.020594 \quad 0.773804$ C $-2.755366 \quad 0.0430593 .368245$ H -3.692404 0.2723292 .852481 H $-2.976352-0.1732924 .416684$ H -2.072180 $0.895393 \quad 3.322542$

SCF Energy (B3LYP/6-31G**//MMFF) $=-3245.90634456$

0700383

MM̄FF Geometry

C -0.499769-4.007203-1.935968

C $-0.104607-3.618438-0.715456$

C $1.290957-3.125217-0.423377$

O $1.203574-1.7703340 .078822$

C $1.921061-3.9838730 .686460$

C $3.428064-4.0974510 .587709$

C $4.296553-3.352621 \quad 1.298598$

C $3.928896-5.183068-0.328885$

C $3.948757-2.2018882 .201573$

C $5.132209-1.2737012 .525445$
C $5.905328-0.7479991 .305237$

C 7.0136710 .2138051 .743068

C 7.7880690 .7747540 .541138

O $5.000495-0.0835420 .414745$

O 8.5705491 .8768091 .025913

C $6.7739241 .324590-0.484931$

C $8.787002-0.245770-0.015764$

O $7.4324291 .741195-1.678248$

C $5.6208530 .346179-0.808029$

C $4.5399020 .979171-1.713256$

O $6.152782-0.771471-1.533476$

C $0.907119-0.779292-0.802250$

O $0.773363-0.871790-2.011262$

C $0.7004980 .451063-0.012950$

C $-0.2702221 .295792-0.388908$

C -0.6766272 .5100300 .321262$

C 0.2277733 .0124941 .414798

C $3.8747142 .242135-1.164714$

C $2.8460002 .806052-2.132170$

C $1.5018304 .865355-2.523418$

O $2.5090742 .234613-3.165455$

C $0.5158065 .571384-1.650371$

C $-0.8034145 .584757-1.892372$

C -1.804882 $6.271809-1.010145$

C $-2.8781905 .330225-0.510756$

C $-4.1454895 .429162-0.946297$

C -2.4954464 .3176010 .547684$

C $-1.8374243 .098588-0.034393$

N $2.3516114 .027982-1.696143$

H $\quad 0.211128-3.976257-2.758621$

H $-0.812335-3.6371560 .109634$

H $1.916844-3.131728-1.324648$

H $1.516964-5.0053970 .662866$

H $1.645102-3.5831401 .668425$

H $5.355251-3.5874521 .209302$

H $5.022661-5.226894-0.363833$

H $3.568451-6.1610810 .006667$

H $3.573637-5.013285-1.350429$

H $3.152304-1.5983021 .755659$

H $3.565189-2.6008113 .148171$

H $4.732877-0.4247993 .095590$

H $5.828637-1.8018943 .189318$

H $6.353358-1.6026040 .787816$

H $7.700128-0.2741572 .446442$

H $6.568144 \quad 1.0545702 .293395$

H 9.0052482 .2889940 .259441

H $6.3579392 .233957-0.035863$

H $9.507802-0.5340240 .759044$

H $\quad 9.3817150 .184156-0.829287$

H $8.311749-1.157984-0.381863$

H $7.6496740 .943389-2.189983$

H $4.9685001 .187620-2.702759$

H $3.7552620 .230329-1.891011$

H $5.421046-1.211958-1.997080$

H 1.2934510 .5836820 .884152

H $-0.8565691 .050116-1.274066$

H -0.0784003 .9764191 .825524$

H 1.2479863 .1479861 .039396

H 0.2553082 .3025612 .248548

H $3.3783302 .030336-0.212059$

H $4.6223933 .024456-1.000095$

H $1.0176084 .259310-3.295963$

H $2.1523055 .594716-3.016656$

H $0.9024786 .095549-0.779513$

H -1.188553 $5.076033-2.773381$

H $-2.2532557 .089902-1.588428$

H -1.318480 $6.745924-0.148476$

H $-4.9230554 .769448-0.575338$

H $-4.4328836 .167156-1.687766$

H -3.3961243 .9780931 .075585$

H -1.8857324 .8143961 .306548$

H -2.420656 $2.633442-0.829250$

H $2.7869724 .460136-0.885854$

C $-2.622872-3.450228-3.117604$

O $-3.163412-2.421798-2.257593$

C $-4.481286-2.075810-2.728759$

C $-4.642522-2.760451-4.080738$

C $-3.821013-4.016773-3.876533$ 
H $-5.197633-2.522269-2.030289$ C $-4.654546-0.555002-2.776825$ H -5.690493 -2.964653 -4.320180 H -4.222504 -2.154374 -4.892394 C $-3.460072-4.718835-5.174297$ H $-4.382014-4.708051-3.233357$ C $-4.5908180 .144869-1.402706$ O $-3.6267040 .004751-3.604902$ H -5.602504 -0.307320 -3.268662 H $-3.582170 \quad 0.063849-0.982701$ O $-4.8318031 .541274-1.623213$ C $-5.632129-0.363640-0.386641$ C -5.7702020 .4932370 .885119$ H -5.396926 -1.393887 -0.100917 O $-6.920585-0.399343-1.011213$ H -6.0255881 .5256140 .615261$ H -6.6401040 .1096851 .436045$ H -4.364577 $-5.032389-5.705674$ H -2.889705 -4.062755 -5.840408 H $-2.855898-5.610886-4.982697$ C - $-1.883262-4.486321-2.271228$ H -2.770118 $-0.247457-3.218908$ H $-4.1881791 .845763-2.285919$ H -7.133802 $0.506245-1.295447$ H -1.778281 -5.429947 -2.818135 H $-2.446976-4.698958-1.354722$ H -1.936705 -2.952132 -3.816015 C $-3.241750-1.089743 \quad 3.125982$ O $-4.368910-0.8688342 .267290$ C $-4.537568 \quad 0.474564 \quad 1.801886$ C -4.7101961 .4382532 .988683$ C -3.5581061 .3080483 .988838$ C $-3.281562-0.1550394 .348436$ H $-3.375082-2.1160763 .491180$ C -1.937265 -1.048892 2.321972 H -3.6572320 .7685161 .221962$ O H -4.7651202 .4635182 .604097$ H -3.8028451 .8614834 .903596$ O $-2.376676 \quad 1.883472 \quad 3.442648$ H $-2.345824-0.2393074 .911830$ O $-4.324656-0.626667 \quad 5.207921$ C $-0.752237-1.532174 \quad 3.121112$ H -2.022183 -1.663547 1.417562 H $-1.715323-0.0414081 .962427$ H -6.0412701 .8126334 .370188$ H -2.564073 2.8183893 .253464 H $-4.320476-0.071347 \quad 6.006025$ O $-0.881549-2.8601313 .398171$ O $0.171454-0.8070593 .467397$ C $0.133388-3.4307934 .231072$ H $1.130306-3.0780443 .951570$ H $-0.076861-3.1863565 .275959$ H $0.102070-4.5166384 .108408$ SCF Energy (B3LYP/6-31G**//MMFF)= -3245.90414655

0700384

MM̄FF Geometry

C $2.009770-1.984517-3.189674$

C $2.861001-3.017842-3.084671$

C $4.125632-3.063566-2.253701$

O $4.274561-1.868147-1.456416$

C $4.071237-4.266662-1.287505$

C $5.299465-4.396417-0.404239$

C $5.289241-4.2753470 .939178$

C $6.588971-4.701201-1.121684$

C $4.102350-4.0146751 .828262$

C $3.665119-2.5531191 .783880$

C $2.585084-2.1518672 .797243$

C $1.347437-3.0497112 .734584$

C $0.203111-2.5198773 .609984$

O $2.248505-0.8027862 .452223$

O $-0.989061-3.243104 \quad 3.268497$

C $-0.045976-1.0439973 .247159$

C $0.452678-2.7767225 .100258$

O $-1.018191-0.463984 \quad 4.113929$

C $1.235347-0.1848963 .248674$

C 0.9873751 .2221182 .651712
O $1.681159-0.0141374 .597694$

C $4.931569-0.807045-1.993257$

O $5.427185-0.730688-3.105323$

C $4.9137890 .272089-0.979100$

C $5.2404751 .522629-1.334958$

C $5.2811762 .689717-0.452040$

C 4.9922982 .4811911 .011107

C $0.662108 \quad 1.2272101 .152601$

C 0.5431712 .6343910 .596354

C $0.4408783 .859760-1.571610$

O 0.4335043 .6299511 .307204

C $1.8037714 .458379-1.728420$

C $2.1799585 .590162-1.114276$

C $3.5396596 .216841-1.240741$

C $4.4229355 .937480-0.043380$

C $4.0893386 .365577 \quad 1.186821$

C $5.7281185 .209385-0.273214$

C $5.5769743 .892957-0.985695$

N $0.5417242 .639895-0.793335$

H $2.205794-1.059391-2.655512$

H $2.645314-3.921752-3.651852$

H $4.981787-3.173724-2.930336$

H $3.960725-5.197350-1.860105$

H $3.166812-4.188043-0.673104$

H $6.232718-4.3812351 .472824$

H $7.410543-4.910049-0.428082$

H $6.468921-5.582707-1.760089$

H $\quad 6.893516-3.855642-1.745828$

H $3.280512-4.6819441 .546388$

H $4.375887-4.2858862 .855302$

H $4.534422-1.8968021 .928523$

H $3.289254-2.3128620 .783647$

H $3.022287-2.1646163 .802293$

H $1.590483-4.0784063 .025801$

H $0.992305-3.1072121 .695924$

H $-0.817113-4.1897943 .409805$

H -0.481029 -1.036242 2.240799

H $\quad 0.540657-3.8526845 .293432$

H $-0.391510-2.4315165 .707316$

H $1.362812-2.3011645 .471696$

H $-1.804123-1.0363734 .092992$

H $\quad 0.203073 \quad 1.7395423 .218466$

H 1.8931161 .8277452 .797689

H 2.2345350 .7828684 .642226

H 4.6223400 .0089990 .031402

H $5.505406 \quad 1.713675-2.374239$

H 5.7051251 .7732901 .448250

H 3.9782322 .0907861 .150300

H 5.0586193 .4003391 .598457

H 1.4480110 .7038200 .595955

H -0.2902410 .7228220 .959703$

H -0.252403 $4.545434-1.072716$

H $\quad 0.0265903 .601577-2.550997$

H $2.5063103 .936578-2.373193$

H $1.4685466 .101581-0.469059$

H $3.4193727 .301687-1.356355$

H $4.0262995 .884071-2.165628$

H 4.7300706 .1828522 .043023

H 3.1697916 .9130501 .365520

H 6.2768395 .0618350 .665158

H $6.3778515 .852655-0.881388$

H $5.7581443 .956682-2.059151$

H $0.7019891 .770559-1.293958$

C $-0.445091-1.479221-3.251968$

O $-0.734838-2.344544-2.138157$

C $-2.166879-2.472294-2.003720$

C $-2.776006-1.486040-2.990464$

C $-1.726820-1.439963-4.080866$

H $-2.401648-3.504186-2.291532$

C $-2.555016-2.215577-0.542533$

H $-3.756748-1.807650-3.352860$

H -2.890988 -0.496137 -2.531661

C $-1.847664-0.223329-4.982287$

H $-1.811619-2.350858-4.688241$

C $-4.073174-2.237087-0.298481$

O $-1.946327-3.2248680 .268121$

H $-2.126958-1.259240-0.223364$

H $-4.566358-1.482882-0.916138$ 
O - $-4.579564-3.500752-0.751343$

C $-4.488788-2.0593121 .172217$

C $-3.957489-0.7995061 .880884$

H $-4.172224-2.9434911 .739160$

O $-5.920066-2.0510431 .220009$

H $-4.246050-0.8727572 .937856$

H -2.864240 -0.8094011 .847601$

H $-1.7569850 .708014-4.413218$

H -2.818975 $-0.214644-5.487761$

H $-1.067861-0.226459-5.750148$

C $0.761645-2.010709-4.028072$

H $-0.989595-3.1970110 .095379$

H $-5.539734-3.499236-0.595730$

H $-6.181097-1.9955332 .155072$

H $0.932938-1.390450-4.915042$

H $0.560595-3.033775-4.369137$

H $-0.225297-0.486631-2.836109$

C $-4.417247 \quad 1.944975-0.637436$

$\begin{array}{llll}\text { O } & -3.975462 & 0.755128 & 0.029765\end{array}$

C $-4.4951720 .544246 \quad 1.344098$

C $-4.092906 \quad 1.712882 \quad 2.260650$

C -4.5260103 .0578741 .670482$

C $-4.108146 \quad 3.1950920 .202242$

H -3.805662 $1.995641-1.547569$

C $-5.888508 \quad 1.852273-1.062007$

H $-5.587100 \quad 0.483777 \quad 1.297344$

O $-2.679027 \quad 1.7062322 .445291$

H $-4.556374 \quad 1.567077 \quad 3.243965$

H -4.0769293 .8679962 .257848$

O $-5.940922 \quad 3.171867 \quad 1.788574$

H $-4.586164 \quad 4.068526-0.256067$

$\begin{array}{lllll}\text { O } & -2.696723 & 3.411868 & 0.138397\end{array}$

C $-6.085707 \quad 0.777716-2.106986$

H $-6.5511391 .649570-0.216220$

H -6.226874 2.794636 -1.508229

H -2.457219 2.4318743 .053088

H $-6.196074 \quad 4.0397121 .432047$

H -2.499338 4.2221020 .638323

O $-6.972671-0.154731-1.655861$

O $-5.521906 \quad 0.768638-3.193817$

C $-7.249013-1.231466-2.556993$

H $-6.326739-1.756530-2.822206$

H $-7.917532-1.933578-2.051813$

H $-7.748320-0.851868-3.453338$

SCF Energy (B3LYP/6-31G**/MMFF) $=-3245.89911308$

07_00385

MM̄FF Geometry

C -1.855479 -0.492681 3.916115

C $-0.961600-0.3412962 .928014$

$\begin{array}{llll}\text { C } & 0.097325 & 0.727007 & 2.923196\end{array}$

$\begin{array}{lllll}\text { O } & 1.354297 & 0.056237 & 2.669841\end{array}$

C $-0.148251 \quad 1.7525241 .797446$

C $-0.447578 \quad 3.1404212 .325139$

C $0.438156 \quad 4.1558302 .356535$

C -1.8634393 .3571812 .791609$

C 1.8870504 .1293281 .960796

C $2.251408 \quad 5.1003790 .829191$

C $1.9354084 .640742-0.605494$

C $0.4479744 .705365-0.954953$

C $0.1766294 .226844-2.388961$

O $2.444727 \quad 3.315291-0.790962$

O $-1.2382334 .016729-2.522514$

C $0.8564032 .855699-2.584947$

C $0.555819 \quad 5.283172-3.432632$

$\begin{array}{lllll}\text { O } & 0.755999 & 2.431694 & -3.941905\end{array}$

C $2.334005 \quad 2.823572-2.133356$

C $2.9268871 .394235-2.175691$

O $3.1088713 .623209-3.036494$

C 2.4790440 .5997693 .206273

$\begin{array}{llll}\text { O } & 2.555128 & 1.565453 & 3.947937\end{array}$

C $3.625728-0.1800002 .697739$

C 4.8709640 .2980512 .828082

C $6.065787-0.3703822 .324983$

C 7.0230570 .5511451 .621039

C $2.3175250 .397200-1.185386$

C $2.894665-0.998519-1.357375$

C $2.625358-3.325685-0.511880$
O $3.749791-1.274468-2.194977$

C $3.785885-3.6234460 .383793$

C $5.001907-3.959719-0.072685$

C $6.167463-4.3259190 .802235$

C $7.245403-3.2646390 .819383$

C $8.029130-3.050361-0.251220$

C $7.464213-2.5021472 .105991$

C $6.267693-1.6889432 .517148$

N 2.323625 -1.907191-0.475902

H $-1.8235270 .178303 \quad 4.771089$

H $-0.998918-1.0058762 .068268$

H $\quad 0.138780 \quad 1.2101463 .908093$

H -0.9891711 .4479681 .159980$

H $0.707000 \quad 1.781713 \quad 1.114919$

H 0.0998355 .1174842 .740659

H -2.0339464 .3801523 .143634$

H $-2.564370 \quad 3.171111 \quad 1.971717$

H -2.1035442 .6817103 .618001$

H 2.4553694 .4289232 .851470

H 2.2396383 .1243401 .719213

H 1.8117956 .0888051 .011296

H 3.3409155 .2337680 .883796

H $2.4869295 .318307-1.269090$

H $0.0550615 .718357-0.805165$

H $-0.1260604 .056628-0.286939$

$\mathrm{H}-1.6861004 .856614-2.323107$

H $0.2872212 .133139-1.988446$

H $-0.0181206 .203565-3.270703$

H $\quad 0.3026154 .946915-4.444145$

H $1.6145675 .548581-3.412162$

H $-0.1881302 .429160-4.175909$

H $2.8633831 .001650-3.199140$

H $4.0032521 .455289-1.959792$

H $4.0384343 .348031-2.973229$

H $3.420577-1.1208462 .200621$

H 5.0420191 .2636423 .298915

H 7.4685841 .2558622 .331518

H 6.5009541 .1259510 .847307

H $7.8380040 .022999 \quad 1.119687$

H $2.5132110 .723861-0.158836$

H $1.2349930 .319888-1.327086$

H $2.821685-3.625401-1.547132$

H $1.738657-3.865005-0.164593$

H $3.605289-3.592247 \quad 1.455028$

H $5.169779-3.997583-1.146989$

H $6.592699-5.2691250 .434921$

H $5.832967-4.5428431 .824333$

H $8.819314-2.307301-0.234993$

H $7.896771-3.614919-1.168389$

H $8.359557-1.8729382 .045401$

H $7.683729-3.2199872 .907180$

H $5.509823-2.2597803 .053894$

H $1.656247-1.5785600 .216098$

C -4.284004-1.048166 3.402690

O $-4.195413-0.7686611 .989895$

C $-4.8723800 .472375 \quad 1.726476$

C -5.7372180 .7434942 .952800$

C -4.8506750 .2107814 .061320$

H -4.0962061 .2435941 .665061$

C $-5.6419970 .409176 \quad 0.404255$

H -5.9771491 .8050623 .065849$

H -6.6786150 .1830292 .919856$

C $-5.585621-0.0558665 .362970$

H $-4.0625890 .950128 \quad 4.246738$

C $-4.808147-0.006863-0.825313$

O $-6.715496-0.534251 \quad 0.525759$

H -6.1190841 .3782700 .216855$

H -4.594262 -1.080956 -0.780449

O $\quad-5.6303200 .189459-1.983817$

C $-3.4935620 .768487-1.022573$

C $-2.8308330 .574528-2.400534$

H $-2.788070 \quad 0.480981-0.235197$

O $-3.7503782 .167368-0.864657$

H $-3.4499461 .042346-3.176789$

H -1.896385 $1.149014-2.387038$

H -6.0223610 .8687445 .754329$

H $-6.397097-0.7784725 .225822$

H $-4.902507-0.4546996 .119565$ 
C $-2.931100-1.5418673 .923537$ H $-6.325697-1.3921370 .767074$ H -6.448093 -0.319509-1.848376 H -2.897534 $2.631080-0.926091$ H -2.605711 $-2.397347 \quad 3.319182$ H -3.042306 -1.909988 4.950286 H $-5.003304-1.8722833 .496612$ C -1.297344 -2.805694 -1.965555 O $-1.650017-1.425634-1.792361$ C $-2.530891-0.883569-2.779556$ C $-1.902225-0.990646-4.179002$ C $-1.480251-2.427616-4.496352$ C $-0.672414-3.047002-3.350387$ H $-0.521105-2.992320-1.213058$ C $-2.477458-3.743763-1.664987$ H $-3.470927-1.442953-2.773394$ O $-0.766963-0.134971-4.284121$ H -2.632008 $-0.647452-4.921785$ H $-0.878838-2.426941-5.413561$ O $-2.649216-3.202379-4.743988$ H $-0.540273-4.123738-3.507277$ O $0.632882-2.463325-3.338710$ C $-2.917006-3.668861-0.218761$ H -3.348869 -3.507608 -2.280863 H -2.178683 -4.780271 -1.860026 H $-0.145541-0.373788-3.575070$ H -2.361560 -4.102766 -4.971860 H $1.050999-2.659838-4.194238$ O $-4.022319-4.454131-0.052656$ O $-2.365466-3.0133220 .653490$ C $-4.568909-4.4842031 .269500$ H -5.048586 -3.527302 1.491617 H -5.325310 -5.272752 1.306291 H -3.793561-4.710313 2.008026 SCF Energy (B3LYP/6-31G**//MMFF) $=-3245.90795703$

07 00386

MM̄FF Geometry

C -0.610840 -3.759466 0.401359

C $0.099785-3.8154121 .539574$

C $-0.229049-3.0302532 .784951$

O $-0.407740-1.6392462 .437237$

C $-1.527549-3.5375813 .445585$

C -1.985215 -2.687832 4.616988

C $-3.119327-1.9570634 .627413$

C $-1.081318-2.6975915 .821651$

C $-4.156083-1.8629513 .539704$

C $-3.853042-0.7273222 .561770$

C -4.874173 -0.589969 1.422055

C $-4.854343-1.7943090 .478456$

C $-5.787448-1.595401-0.723647$

$\begin{array}{lllll}\text { O } & -4.506344 & 0.588277 & 0.694291\end{array}$

O $-5.469930-2.602737-1.697885$

C $-5.490678-0.229419-1.379124$

C $-7.254149-1.820796-0.335668$

O $-6.4599920 .039804-2.389639$

C $-5.3978020 .945433-0.372264$

C $-4.8007052 .195769-1.056434$

$\begin{array}{lllll}0 & -6.683477 & 1.326990 & 0.119156\end{array}$

C $0.685650-0.8314892 .460432$

O $1.840804-1.1570042 .677980$

C 0.2113920 .5460802 .201791

C 1.0748721 .5724342 .211132

C 0.7302302 .9835812 .010598

C -0.7279223 .3154451 .828388$

C $-4.6179623 .382539-0.101151$

C $-3.9741834 .565652-0.799716$

C -1.812073 5.325205-1.776159

O $-4.6090335 .579842-1.077836$

C $-0.3744545 .015002-1.509975$

C $0.5114925 .889317-1.010485$

C $1.9412985 .527822-0.717074$

C 2.3808425 .9272030 .676561

C $3.431248 \quad 6.747340 \quad 0.851560$

C 1.6117435 .3942981 .868337

C 1.7201493 .9014792 .015726

$\mathrm{N}$-2.629302 4.362843 -1.060169

H - $1.496660-3.1317310 .371616$
H $\quad 0.972832-4.460767 \quad 1.593180$

H $0.600766-3.1301553 .495778$

H $-1.386761-4.5703413 .790505$

H -2.316529 -3.585661 2.688387

H -3.344050 -1.365263 5.513524

H -1.512878 -2.153434 6.668605

H $-0.900703-3.7258546 .151590$

H $-0.119444-2.2312485 .587524$

H -4.251089-2.821544 3.022830

H -5.131426 -1.688178 4.010881

H $-3.8039320 .224324 \quad 3.108670$

H $-2.856787-0.8583872 .124397$

H $-5.868858-0.4436351 .858407$

H $-5.119477-2.7197561 .001725$

H -3.832692 -1.944719 0.102998

H -5.568752 -3.472835 -1.274666

H $-4.522479-0.328921-1.890582$

H -7.403588 -2.849102 0.015678

H -7.916621 -1.702171-1.200063

H -7.593969-1.152930 0.458788

H $-6.1277710 .771255-2.935917$

H -3.829691 $1.934776-1.495094$

H -5.448424 2.525417 -1.878006

H -7.358702 $0.774691-0.305262$

H $-0.850436 \quad 0.6814802 .030151$

H 2.1296061 .3657842 .388350

H -1.299996 3.0321112 .719022

H -1.1361072 .7778770 .966290$

H $-0.922300 \quad 4.374826 \quad 1.654574$

H $-5.584227 \quad 3.7102780 .298744$

H -3.9922003 .1093350 .756424$

H -2.092359 $6.337448-1.466596$

H $-2.0318405 .217358-2.843135$

H $-0.0453584 .003787-1.733711$

H $0.1984326 .909627-0.803209$

H $2.5675006 .022629-1.470429$

H $2.1080334 .451263-0.844316$

H 3.7582887 .0453901 .842235

H $3.990576 \quad 7.1393110 .008637$

H 0.5784555 .7437371 .808790

H 2.0031725 .8339422 .795569

H 2.7433253 .5557712 .168827

H $-2.2536543 .427863-0.930732$

C $-0.248166-3.704014-2.122412$

O $0.685612-2.612273-2.009977$

C $0.129410-1.467802-2.686222$

C $-1.082070-1.975445-3.463334$

C $-1.576601-3.084882-2.557397$

H $-0.221217-0.781678-1.906652$

C $1.187111-0.784385-3.558884$

H $-1.826633-1.190547-3.628473$

H $-0.796773-2.380487-4.441413$

C $-2.519777-4.057354-3.243150$

H $-2.095600-2.624216-1.709208$

C $2.431550-0.276349-2.801992$

O $1.638179-1.706386-4.560000$

H $\quad 0.7260790 .046679-4.105579$

H $3.046367-1.122566-2.476021$

O $3.227804 \quad 0.464016-3.736669$

C $2.1224490 .635471-1.602110$

C $3.3298731 .401727-1.032798$

H $1.6689820 .038797-0.803479$

O $1.1471561 .610622-1.981866$

H $3.6912732 .133973-1.766387$

H $2.9543251 .999230-0.193592$

H $-3.406843-3.534927-3.615667$

H -2.039266 -4.550741-4.094703

H $-2.852557-4.833371-2.546741$

C $-0.272069-4.538329-0.837920$

H $1.991551-2.487426-4.100380$

H $3.423233-0.128292-4.483076$

H $1.5215902 .131281-2.713206$

H $\quad 0.713653-5.003409-0.712694$

H $-0.997682-5.352442-0.947102$

H $0.134193-4.345554-2.928111$

C $4.940723-1.3608320 .899640$

$\begin{array}{lllll}\text { O } & 3.988527 & -0.419611 & 0.386322\end{array}$

C $4.4956490 .520350-0.563671$ 
C $5.627392 \quad 1.3591640 .054819$

C $6.722198 \quad 0.467302 \quad 0.648187$

C $6.138053-0.6349741 .538309$

H $4.408061-1.8946551 .696742$

C $5.356526-2.396330-0.157346$

H $4.885242-0.018878-1.432671$

O $5.0962572 .200618 \quad 1.077090$

H $6.0484572 .009378-0.721504$

H $7.408803 \quad 1.08648111 .238611$

O $7.468111-0.108313-0.419947$

H $6.916851-1.3609211 .799325$

O $5.705756-0.0294712 .760235$

C $4.201227-3.273255-0.590444$

H $5.755309-1.919666-1.056343$

H $6.124910-3.056526 \quad 0.261440$

H 5.8268502 .7414051 .422273

H $8.174006-0.649145-0.026532$

H $5.375971-0.7371983 .339826$

O $4.628242-4.100328-1.590637$

O $3.074633-3.244378-0.116158$

C $3.643633-4.996732-2.114272$

H $4.133910-5.656103-2.835495$

H $3.217270-5.609321-1.314270$

H $2.862054-4.432537-2.630355$

SCF Energy (B3LYP/6-31G*//MMFF) $=-3245.90101617$

$07 \quad 00387$

MM̄FF Geometry

C -2.962183 -4.201479-1.052323

C $-1.944395-3.872461-0.243536$

C $-0.494649-3.886125-0.654387$

O $0.074642-2.583745-0.386993$

C $0.262806-4.9183790 .201006$

C $1.738388-5.028483-0.127444$

C $2.735528-4.7331550 .729755$

C $2.065180-5.532784-1.509558$

C $2.626232-4.250746 \quad 2.149772$

C $2.649352-2.7226462 .311415$

C $3.968844-2.0663411 .873108$

C $4.000757-0.5903322 .275084$

C 5.2729800 .1140311 .776163

O $4.078910-2.171513 \quad 0.451838$

O $5.071561 \quad 1.5301261 .904565$

C $5.458296-0.1801820 .271312$

C $6.490890-0.2303942 .641654$

$\begin{array}{lllll}\text { O } & 6.713565 & 0.309148 & -0.193502\end{array}$

C $5.310813-1.672890-0.086813$

C $5.281479-1.934274-1.612913$

O $6.430778-2.3949520 .440247$

C $-0.163645-1.594202-1.289456$

O $-0.810343-1.676762-2.321244$

C $0.487730-0.365886-0.782742$

C $0.1370250 .825650-1.289036$

C $0.6579312 .127143-0.866359$

C $1.8489392 .134258 \quad 0.053518$

C $4.144662-1.245451-2.376827$

C $4.5401190 .075091-3.017281$

C $3.556107 \quad 1.993312-4.268960$

O $5.698906 \quad 0.476353-3.078068$

C $3.3514443 .131632-3.320248$

C $2.4639714 .115675-3.531148$

C $2.280708 \quad 5.289214-2.612114$

C $0.8445895 .469535-2.174860$

C $0.0450446 .365965-2.777303$

C $0.3642874 .674096-0.982965$

C $0.0559443 .244224-1.324705$

N $3.4424250 .737281-3.551711$

H $-2.760481-4.505791-2.075966$

H -2.159647 -3.5756590 .779977$

H $-0.385766-4.123505-1.719641$

H $-0.189905-5.9106600 .070956$

H $\quad 0.125462-4.661737 \quad 1.257024$

H $3.760435-4.8586630 .381845$

H $3.135497-5.726941-1.638504$

H $1.537558-6.471825-1.706083$

H $1.772147-4.800160-2.267481$

H $1.721320-4.6442652 .624431$

H $3.456625-4.6861532 .720381$
H $1.814256-2.2917691 .746375$

H $2.472553-2.4976533 .370542$

H $4.796304-2.6080742 .346087$

H $3.902770-0.4794753 .362162$

H $3.130221-0.0791921 .843219$

H 4.8753451 .7215812 .837729

H $4.687306 \quad 0.388963-0.259507$

H $\quad \begin{array}{llll}\text { H } & 416780 & 0.066876 & 3.682839\end{array}$

H 7.3768020 .3261162 .316728

H $6.731436-1.2954662 .639183$

H $6.755102 \quad 1.2554860 .027113$

H 6.260722 -1.710764 -2.052978

H $5.142440-3.016355-1.751942$

H $6.521007-3.227321-0.052732$

H $1.213329-0.4728930 .015043$

H $-0.6291180 .857445-2.062721$

H $1.581491 \quad 1.7337751 .035822$

H $2.275153 \quad 3.1270480 .210801$

H $2.6549621 .523636-0.365547$

H $3.836073-1.901161-3.201304$

H $3.271078-1.094874-1.735211$

H $2.8078341 .979796-5.067743$

H $4.5496932 .065868-4.722713$

H $3.9810083 .162587-2.434049$

H $1.8553514 .096773-4.432554$

H $2.6232356 .185650-3.145670$

H $2.9239605 .210034-1.726786$

H $-0.9776606 .521235-2.449899$

H $0.3941006 .960425-3.615030$

H $-0.5604915 .115104-0.587289$

H $1.0865194 .773558-0.167961$

H $-0.7952883 .134273-1.998200$

H $2.5115160 .358177-3.404262$

C $-5.233767-3.195397-1.428974$

O $-4.748622-1.853772-1.222578$

C $-5.864497-0.982905-0.974818$

C $-7.106271-1.767878-1.386982$

C $-6.704914-3.179355-1.010729$

H $-5.914629-0.8098490 .105460$

C $-5.6795750 .350013-1.698005$

H $-8.005973-1.423120-0.868164$

H -7.293802 -1.706264 -2.464856

C $-7.548820-4.251380-1.678067$

H $-6.788085-3.2865150 .079263$

C $-4.3471101 .101591-1.491418$

$\begin{array}{llll}\text { O } & -5.802819 & 0.120574 & -3.112325\end{array}$

H $-6.5144001 .010472-1.434579$

H -3.527484 $0.576398-1.995659$

O $-4.4912242 .344076-2.213944$

C $-3.9311411 .456314-0.047011$

$\begin{array}{llll}\text { C } & -3.286619 & 0.292924 & 0.733098\end{array}$

H -4.7870151 .8564350 .508309$

O $-2.959157 \quad 2.506119-0.123078$

H $-4.015378-0.5010180 .895726$

H -2.473838 -0.1178600 .123654$

H $-8.599687-4.149081-1.388207$

H $-7.493298-4.184171-2.769692$

H $-7.213360-5.251031-1.384648$

C $-4.398626-4.181240-0.610814$

H $-5.102092-0.503585-3.369114$

H $-5.2089612 .848465-1.793949$

H $-3.3590643 .242941-0.614963$

H $-4.804234-5.194009-0.715680$

H $-4.457771-3.9161710 .452551$

H -5.136069 -3.407013 -2.501643

C -0.9257552 .0348993 .075799$

O $-1.553762 \quad 1.5156321 .897168$

C -2.7133340 .7044402 .110257$

C $-2.335891-0.5236152 .955389$

C $-1.666587-0.1091654 .269302$

$\begin{array}{llll}C & -0.536830 & 0.900841 & 4.037732\end{array}$

H 0.0014602 .4989832 .716988

C $-1.7695643 .133142 \quad 3.731852$

H $-3.479228 \quad 1.2886412 .631809$

O $-1.447655-1.3542442 .211272$

H -3.242378 -1.1053813 .161210$

H $-1.265236-1.0020944 .763839$

O $-2.656093 \quad 0.4517095 .126582$ 
H $-0.1995601 .329623 \quad 4.988432$ O $0.584873 \quad 0.221296 \quad 3.465227$ C -1.8922644 .3274112 .813209$ H -2.771428 2.7889484 .002633 H -1.3011193 .4872764 .657607$ H -1.243877 -2.132458 2.757194 H -2.2196630 .6898745 .962150$ H $0.860522-0.4699674 .090959$ O -3.1860344 .4763162 .409669$ O -0.9486345 .0356102 .485836$ C -3.4325205 .5719381 .522916$ H $-4.4898605 .553147 \quad 1.245850$ H -3.2169536 .5194212 .025769$ H -2.831225 5.4710010 .614569 SCF Energy (B3LYP/6-31G**//MMFF) $=-3245.88361710$

0700388

MM̄FF Geometry

C -2.896959 $1.634581-1.801970$

C $-1.8621362 .165452-2.471108$

C $-0.7185112 .901991-1.827403$

O $\quad 0.4867342 .312679-2.374849$

C $-0.7585854 .408828-2.143226$

C -1.914252 5.145070 -1.501470

C $-1.9146545 .600329-0.233205$

C $-3.0903285 .422802-2.402394$

C -0.8253825 .4752120 .799495$

C -1.3693695 .0328542 .167592$

C -1.561643 3.5115322 .292459

C -2.4556093 .1972753 .494713$

C -2.5749611 .6901623 .748595$

O -0.2654262 .9277132 .478892$

O -3.1651171 .5065715 .044563$

C -1.1602891 .0864303 .806445$

C -3.5092341 .0065832 .746384$

O $-1.217949-0.3362353 .901471$

C -0.2559871 .5004652 .621638$

C 1.1965601 .0513582 .923153

O -0.7133220 .8544551 .433668$

C $1.6583532 .538438-1.724711$

O $1.8389083 .278684-0.772035$

C $2.6938621 .690681-2.356949$

C $3.9315581 .666553-1.840086$

C $5.0549840 .855305-2.315497$

C $4.786129-0.118912-3.432574$

C 2.2352661 .5423201 .911978

C 3.6490101 .2378212 .367465

C $5.248681-0.6513232 .678454$

O $\quad 4.4207142 .1280692 .716932$

C $6.140086-0.5985891 .476321$

C $6.447822-1.6713750 .730354$

C $7.356523-1.744488-0.470009$

C $8.084736-0.475125-0.853792$

C $9.220628-0.116536-0.231082$

C $7.5544710 .317877-2.028544$

C $6.2573291 .014802-1.723369$

N $3.947730-0.1131982 .332161$

H $-2.9431501 .737347-0.721730$

H -1.841137 $2.083372-3.556004$

H $-0.7219552 .730751-0.744463$

H $-0.7756674 .559065-3.231876$

H $0.1804994 .885865-1.838122$

H -2.787982 6.1590850 .101933

H -3.881109 $5.984396-1.893513$

H -2.771826 $6.014794-3.266759$

H $-3.5341774 .492053-2.765723$

H -0.0196594 .8092190 .480943$

H $-0.382912 \quad 6.4722220 .917982$

H -0.6521355 .3596852 .932171$

H -2.313195 5.5540822 .370968

H -2.0098043 .1200381 .372912$

H -3.4503913 .6422363 .365462$

H -2.033454 3.6671144 .394556

H -4.0324441 .9466805 .044032$

H -0.6936411 .4394254 .736968$

H $-4.513407 \quad 1.4442772 .795069$

H -3.631878 -0.0535682 .992354$

H $\quad-3.1643481 .0848251 .713764$
H -1.779955 -0.552861 4.665001

H $1.492117 \quad 1.430533 \quad 3.910642$

H $1.239672-0.0437872 .972433$

H 0.0114450 .8329410 .789648

H $2.4035821 .092549-3.213122$

H $4.1466752 .297351-0.978211$

H $3.964956-0.794483-3.168723$

H $5.638802-0.757036-3.672021$

H $4.5175410 .415995-4.350101$

H 2.0842191 .0779110 .932580

H 2.1622422 .6268981 .779298

H $5.097684-1.6747133 .035949$

H $5.685707-0.0630993 .491969$

H 6.5632010 .3735681 .242595

H $6.000390-2.6252111 .006683$

H $6.772941-2.106965-1.325515$

H $8.099622-2.529488-0.273793$

H $9.7699890 .772572-0.521739$

H $9.622848-0.7044960 .587564$

H $7.489136-0.344461-2.895796$

H $8.2763041 .093461-2.317454$

H $6.3450131 .755822-0.927180$

H $3.250313-0.7637251 .984815$

C $-4.8877850 .092618-1.560429$

O $-4.128131-1.019707-1.051818$

C $-5.005193-2.158508-0.918118$

C $-6.402650-1.680807-1.304673$

C $-6.097156-0.536903-2.249380$

H -4.669999 -2.902101-1.649592

C $-4.918398-2.7304880 .503147$

H $-6.993987-2.475500-1.770433$

H -6.965043 -1.318842 -0.436139

C $-7.2684580 .409626-2.448120$

H $-5.800272-0.955118-3.220575$

C $-3.499523-3.1585430 .929695$

O $-5.368887-1.7352941 .431510$

H $-5.611629-3.5726010 .609319$

H -2.838048 -2.285707 0.946708

O $-3.572300-3.6376712 .278898$

C $-2.893652-4.2762220 .058837$

C $-1.546002-4.8228790 .570242$

H -2.769134 -3.921328 -0.968801

O $-3.808751-5.3751190 .000921$

H -1.645757 -5.1626631 .608195$

H -1.315041 -5.720764 -0.018963

H $-8.128504-0.125443-2.864109$

H -7.581006 $0.865015-1.502395$

H $-7.0082601 .216172-3.140415$

C $-4.0328910 .941749-2.503368$

H $-4.851137-0.9293941 .265281$

H $-3.958397-2.9257982 .817705$

H $-3.950943-5.686158 \quad 0.911728$

H $-4.662826 \quad 1.710367-2.965562$

H -3.628859 $0.306752-3.301320$

H $-5.2061240 .689497-0.695051$

C $0.699146-2.469944-1.213134$

O $-0.168802-3.577670-0.944897$

C $-0.395530-3.8174980 .445880$

C $0.915674-4.3075491 .085983$

C $2.135826-3.5402250 .554325$

C $1.721273-2.221221-0.091705$

H $1.238429-2.727575-2.134103$

C $-0.150755-1.227139-1.484607$

H $-0.688205-2.8755000 .924496$

O $1.115650-5.694136 \quad 0.803647$

H $0.829595-4.2240922 .175908$

H $2.710225-4.138852-0.163699$

O $3.018858-3.2615741 .640772$

H $1.315067-1.5456920 .671228$

O $2.856237-1.570611-0.658436$

C $-0.801284-1.323851-2.843079$

H $-0.951704-1.108487-0.746245$

H $0.454364-0.315946-1.448976$

H $1.930450-5.9704941 .256948$

H $3.284704-4.1137372 .026647$

H $3.540809-1.5191180 .029249$

O $-0.057367-0.647333-3.763824$

O $-1.830417-1.948852-3.063712$ 
C $-0.565907-0.667388-5.100622$

H $0.095054-0.059833-5.724396$

H $-0.571393-1.691181-5.486461$

H -1.571265 -0.236680 -5.132551

SCF Energy (B3LYP/6-31G**//MMFF) $=-3245.91147732$

0700389

MM̄MF Geometry

C 2.775924 -3.557162 -1.090021

C $2.121920-2.396036-0.933518$

C $0.901149-2.006813-1.722216$

O $-0.068247-1.453048-0.800874$

C $1.247707-0.926632-2.761638$

C $0.056720-0.469420-3.585810$

C $-0.3004120 .820233-3.743954$

C $-0.736435-1.549860-4.275380$

C $0.4205702 .033036-3.233862$

C $-0.0538002 .550492-1.871033$

C -1.520907 $3.005098-1.847928$

C $-1.7641103 .939465-0.659619$

C $-3.2337444 .366887-0.561011$

O $-2.3562841 .847700-1.726964$

O $-3.431058 \quad 4.9771350 .723361$

C $-4.1155663 .105180-0.603159$

C $-3.5968915 .427153-1.606833$

O $-5.4976753 .453429-0.661481$

C -3.762069 2.131911-1.754937

C $-4.5334620 .808716-1.546121$

O $-4.1551052 .711621-2.999382$

C $-0.901792-2.332949-0.183523$

O -0.916268 -3.546486 -0.318442

C $-1.811112-1.5602720 .693333$

C $-2.814471-2.2064801 .305519$

C $-3.827480-1.6219062 .183414$

C $-3.675322-0.1772642 .578109$

C $-4.186272-0.313150-2.532118$

C $-4.823615-1.621791-2.100277$

C -7.052604 -2.647304-1.628649

O $-4.146808-2.584966-1.748239$

C $-7.573010-2.225269-0.288512$

C $-7.569224-3.0108910 .799244$

C $-8.127014-2.5843612 .128361$

C -7.205168 -2.888706 3.289375

C -7.496502 -3.869142 4.161272

C $-5.988400-2.0079203 .492704$

C $-4.830848-2.4117862 .620179$

$\mathrm{N}-6.207315-1.587680-2.146725$

H $2.433682-4.266119-1.839567$

H $2.467414-1.684875-0.187998$

H $\quad 0.478949-2.883396-2.229145$

H $2.012933-1.309435-3.449428$

H $1.696364-0.077017-2.235556$

H -1.183634 $1.041541-4.341698$

H -1.463897 -1.141242 -4.985120

H $-0.068145-2.209227-4.838983$

H - $-1.291602-2.149528-3.547740$

H $1.4995001 .846554-3.187707$

H $\quad 0.3069022 .830624-3.979729$

H $0.1125921 .777323-1.110093$

H $0.5881593 .402979-1.619544$

H -1.745048 $3.521997-2.788263$

H -1.115250 4.822381-0.716964

H -1.488775 3.4239010 .271071

H -2.823703 5.7336910 .791877

H -3.9708532 .5771710 .349650$

H $-2.9685286 .317343-1.483332$

H -4.629634 $5.770209-1.479113$

H -3.474824 5.076833 -2.633887

H -5.6782334 .0481810 .086595$

H $-4.3300300 .432024-0.536976$

H -5.610610 $1.007760-1.601842$

H -4.231202 $2.007772-3.662485$

H -1.644983 -0.4940250 .794041$

H $-2.922983-3.2764801 .130106$

H -3.7894910 .4780331 .710435$

H -2.688043 -0.0019383 .020056$

H -4.4056850 .1423993 .326087$

H -4.525493 -0.079566 -3.546708
H $-3.103651-0.469317-2.573269$

H -6.490085 -3.584986-1.578890

H -7.885009 -2.767278 -2.328635

H -7.995978 -1.225538 -0.215169

H -7.179132 -4.0232340 .722678$

H $-9.084080-3.1044992 .264913$

H -8.365360 -1.513279 2.134808

H -6.855557 -4.077685 5.011649

H -8.378776 -4.489296 4.041995

H -5.660146 -2.067277 4.538387

H $-6.283944-0.9680693 .314650$

H -4.822804 -3.462556 2.327869

H $-6.668714-0.740675-2.464745$

C $5.277113-3.276680-0.595960$

O $5.188070-1.865648-0.313389$

C $5.812800-1.145352-1.391034$

C $6.649752-2.172507-2.144054$

C $5.759022-3.398031-2.041415$

H $5.006422-0.815003-2.057064$

C $6.5701350 .075377-0.857923$

H $6.850513-1.870955-3.176331$

H $7.608469-2.363534-1.649816$

C $6.473531-4.702561-2.345733$

H $4.924087-3.277721-2.743026$

C $5.6918851 .060395-0.052991$

O $7.616828-0.3529900 .021276$

H $7.0547160 .605297-1.687113$

H 5.4354230 .6143730 .914289

$\begin{array}{lllll}\text { O } & 6.495228 & 2.209606 & 0.246567\end{array}$

C $4.4104991 .517334-0.772132$

C $3.6706972 .690395-0.106098$

H $3.7260410 .667559-0.866271$

O $4.7544251 .927450-2.099911$

H $4.2801053 .601284-0.166353$

H $2.7899732 .892711-0.727855$

H $\quad 6.854157-4.702082-3.372237$

H $7.322832-4.862417-1.673143$

H $5.792714-5.552828-2.237637$

C $3.956980-3.972058-0.257086$

H $8.333328-0.715701-0.525779$

H $6.7002762 .651648-0.595276$

H $3.9268422 .134214-2.566727$

H $3.715740-3.7911180 .797445$

H $4.080939-5.057055-0.357917$

H $6.046003-3.6672200 .083988$

C 1.9930040 .8551532 .691095

O 2.4685861 .2439671 .394668

C 3.2317792 .4528731 .348547

C 2.4156963 .6415471 .891802

C 1.8271953 .3442853 .273785

C 1.1416001 .9744603 .316063

H $1.333789-0.0014622 .502113$

C 3.1312000 .3874023 .613680

H 4.1327392 .3308821 .957908

O 1.3539263 .9479920 .990416

H 3.0687084 .5214651 .939667

H 1.0977504 .1230003 .529013

O 2.8745893 .4026924 .236617

H 0.8857021 .7136074 .349565

O -0.0888832 .0718932 .593048$

C $3.780376-0.8890433 .127565$

H 3.9146021 .1450113 .695784

H 2.7411290 .1835984 .617326

H 0.8840634 .7238801 .340272

H 2.4806263 .2350815 .109692

H -0.5371131 .2115162 .656403$

O $5.014352-1.0090493 .699834$

O $3.260666-1.6938702 .368505$

C $5.753666-2.1830563 .348469$

H $6.608106-2.2609764 .026088$

H $5.139475-3.0820633 .458232$

H $6.125552-2.0907892 .324830$

SCF Energy (B3LYP/6-31G*//MMFF)= -3245.88943144

0700390

MM̄FF Geometry

C $4.5775852 .701400-1.759794$

C $3.5231282 .947499-0.969883$ 
C $2.1752513 .376894-1.482102$

O $1.2916482 .242869-1.322118$

C $1.6263984 .543309-0.643428$

C $0.2960035 .068513-1.147996$

C $-0.8685894 .994003-0.473853$

C $0.3324225 .741817-2.497071$

C $-1.1213394 .419737 \quad 0.891374$

C -1.7299183 .0153790 .863803$

C -3.0459592 .8585620 .085068$

C $-4.158553 \quad 3.7639070 .620561$

C $-5.5018633 .504051-0.078378$

$\begin{array}{lllll}\text { O } & -3.428945 & 1.480612 & 0.211941\end{array}$

$\begin{array}{llll}\text { O } & -6.529760 & 4.152134 & 0.686997\end{array}$

C $-5.7960611 .991216-0.038161$

C $-5.5483084 .125895-1.478931$

O $-6.9644741 .676190-0.791379$

C $-4.612751 \quad 1.122806-0.514357$

C $-4.863848-0.386555-0.293229$

O $-4.4255091 .313008-1.922180$

C $1.1746891 .394624-2.380647$

O $1.6443581 .540089-3.497947$

C $0.4067370 .215026-1.925535$

C $0.149959-0.783043-2.784808$

C $-0.541289-2.034408-2.468351$

C $-0.893743-2.280769-1.028076$

C $-5.083460-0.8030691 .162724$

C $-5.185100-2.3104551 .286858$

C $-3.802089-4.3850991 .196534$

O $-6.265722-2.873746 \quad 1.445460$

C $-3.699408-4.970414-0.182693$

C $-4.022018-4.383050-1.346671$

C -3.853724 -5.038206 -2.689078

C $-2.983154-4.219129-3.619096$

C $-3.530040-3.546001-4.646468$

C $-1.489842-4.248093-3.361169$

C $-0.816592-2.902382-3.464332$

N $-3.957422-2.9430491 .181338$

H $4.4895902 .842715-2.834072$

H 3.6120842 .7706480 .097718

H $2.2386193 .673823-2.535625$

H $2.3451165 .373661-0.645106$

H 1.5443554 .2191320 .399052

H -1.756526 $5.410955-0.948139$

H $-0.6152156 .233862-2.741566$

H $1.1118676 .510637-2.517226$

H $0.5370145 .014280-3.288201$

H -0.2056634 .3927501 .489956$

H -1.7855805 .1039941 .432770$

H -0.9958802 .3124260 .448292$

H $-1.8857542 .681303 \quad 1.898497$

H $-2.8526753 .067137-0.973089$

H -3.892692 4.8229470 .525869

H -4.2863753 .5817841 .696904$

H -6.3159685 .0997590 .734435$

H -6.0237141 .7444571 .006096$

H $-5.4136555 .212775-1.419514$

H -6.526395 $3.970937-1.947623$

H $-4.7784003 .736856-2.148198$

H -7.684903 $2.237929-0.457639$

H -5.712587 -0.719872 -0.905083

H $-3.998892-0.935904-0.687502$

H -3.936711 $0.550770-2.274426$

H $0.0986560 .187946-0.886902$

H $\quad 0.483717-0.683579-3.816935$

H -1.329858 -3.262939-0.846336

H -1.616961 -1.537370 -0.679069

H $0.001219-2.223343-0.402387$

H $-4.269139-0.4514861 .805679$

H $-6.012254-0.3795381 .558388$

H -4.634461 $-4.847608 \quad 1.736140$

H $-2.876446-4.5906221 .744229$

H -3.303634 -5.984035 -0.214058

H $-4.437317-3.379517-1.354434$

H -4.853046 -5.175907 -3.121651

H -3.427683 -6.044911 -2.594839

H $-2.925062-2.977291-5.344270$

H -4.601497 -3.553773 -4.819085

H $-1.024690-4.927927-4.087310$
H -1.277699-4.703171-2.388069

H $-0.525153-2.636283-4.480917$

H $-3.126888-2.3832751 .013084$

C $5.9838710 .662444-1.538001$

O $5.052661-0.062005-0.704746$

C $5.617866-1.354136-0.401374$

C $6.966841-1.414440-1.115651$

C $7.3421330 .051432-1.214872$

H $5.791192-1.3830210 .679536$

C $4.647697-2.463932-0.823435$

H $7.699748-2.007812-0.559859$

H $6.874380-1.857022-2.114812$

C $8.4171790 .329701-2.250576$

H $7.6853390 .395950-0.230209$

C $3.316321-2.487198-0.042075$

O $4.339435-2.301658-2.215199$

H $5.139963-3.439476-0.738554$

H $2.759392-1.560061-0.215708$

O $2.521025-3.548466-0.589152$

C $3.472904-2.7400201 .473627$

C $2.150792-3.0329912 .212830$

H $3.971187-1.8869251 .944428$

O $4.326975-3.8719231 .672865$

H $1.610735-3.8361591 .696855$

H $2.403617-3.4350963 .203664$

H $9.336521-0.212167-2.005185$

H $8.1003940 .018157-3.251601$

H $8.6564441 .396796-2.289967$

C $5.8834772 .159914-1.250040$

H $3.922278-1.429456-2.322825$

H $2.415043-3.370356-1.539617$

H $3.905863-4.6361281 .242529$

H $6.6948442 .706929-1.742563$

H $5.9747752 .343363-0.173570$

H $5.7114720 .456316-2.582353$

C 1.1644470 .2535493 .672191

O $1.840650-0.9781893 .389995$

C $1.248763-1.8006422 .378416$

C $-0.180694-2.1928922 .782161$

C -1.034365 -0.956538 3.078572

C -0.3206670 .0253754 .010563$

H 1.6509990 .6280054 .582615

C 1.4069721 .2874502 .564665

H $1.221674-1.2510381 .434501$

O $-0.131527-3.0297603 .936714$

H $-0.633196-2.7768551 .972565$

H -1.969214 -1.278109 3.553798

O $-1.397553-0.3081821 .865275$

H $-0.8539470 .982944 \quad 4.028679$

O $-0.396971-0.5159805 .335177$

C $2.760514 \quad 1.9343842 .747494$

H 1.3666380 .8801751 .551396

H 0.6664292 .0928012 .622495

H -1.044267 -3.285675 4.153005

H $-0.591259-0.0503011 .392710$

H $-0.0290580 .145823 \quad 5.945274$

O 3.7503331 .0493872 .437380

O 2.9153203 .0854453 .134797

C 5.0864371 .5167272 .646891

H 5.7741380 .7953672 .198162

H 5.2406472 .4897902 .171513

H 5.2906951 .5779373 .719604

SCF Energy (B3LYP/6-31G**//MMFF) $=-3245.90028803$

07 00391

MM̄MF Geometry

C $0.187750-1.7425090 .816486$

C $0.706669-2.205946-0.330029$

C $1.460849-1.329327-1.294646$

O $2.766163-1.903965-1.558472$

C $0.752826-1.217672-2.657220$

C $-0.592025-0.524420-2.628021$

C $-0.763810 \quad 0.806152-2.746089$

C $-1.795394-1.429247-2.544630$

C $0.2894791 .869213-2.896092$

C $0.0525713 .052965-1.945114$

C $0.5719472 .805609-0.519723$

C 0.0712023 .9071440 .417946 
C $0.6777413 .795807 \quad 1.822536$

O $2.0021412 .824416-0.580300$

O $\quad 0.370675 \quad 5.0230272 .501414$

C 2.2109213 .6906101 .687572

C 0.0241822 .6709802 .633910

O 2.8216993 .4457482 .953007

C 2.6664082 .6160630 .672228

C 4.1898342 .7469440 .423725

$\begin{array}{lllll}\text { O } & 2.383834 & 1.325751 & 1.212469\end{array}$

C $3.696015-1.849469-0.566961$

O $3.534134-1.4459500 .573579$

C $4.975256-2.342916-1.123879$

C $6.059868-2.399738-0.336472$

C $7.397429-2.840671-0.738422$

C $7.628612-3.108935-2.202707$

C $4.7223151 .860337-0.708942$

C $6.1739532 .173410-1.009880$

C $8.4770191 .901234-0.097809$

O $6.4998392 .816230-2.005627$

C $9.1537780 .677031-0.630195$

C $10.126451 \quad 0.031784 \quad 0.031614$

C $10.874063-1.155648-0.505589$

C $10.778275-2.3618970 .401349$

C $11.614666-2.5125311 .442419$

C $9.770015-3.4315260 .048873$

C $8.347717-2.9672510 .211925$

N $7.0440861 .682040-0.049885$

H $\quad 0.325046-0.695411 \quad 1.074827$

H $\quad 0.570909-3.249676-0.601229$

H $1.611443-0.320765-0.891612$

H $\quad 0.642822-2.217075-3.101688$

H $1.420170-0.700960-3.360174$

H $-1.7852151 .183769-2.768314$

H -2.738470 -0.872497 -2.567869

H -1.804376 -2.123985 -3.391094

H $-1.786209-2.012028-1.620597$

H $1.3025791 .476874-2.767511$

H $0.2237822 .242338-3.925805$

H $\quad 0.5772903 .922195-2.363364$

H -1.015555 $3.303224-1.921759$

H $\quad 0.236170 \quad 1.822523-0.172307$

H -1.024515 3.9041430 .472933

H 0.3437524 .8877990 .001832

H 0.6916724 .9510583 .415947

H $2.579140 \quad 4.668627 \quad 1.347102$

H -1.044476 2.8738752 .772959

H 0.4506322 .6092523 .641109

H 0.1168001 .6907852 .161802

H 2.6061644 .1938813 .534422

H 4.4231103 .7886050 .165717

H 4.7369062 .5128251 .345423

H 3.0107300 .6817110 .848686

H $4.991702-2.634983-2.167765$

H $5.962856-2.1008210 .706614$

H $8.672967-3.305742-2.452506$

H $7.333716-2.241279-2.804076$

H $7.047442-3.977726-2.530342$

H $4.6457820 .801462-0.453226$

H $4.1522462 .010044-1.632612$

H 8.8000342 .1424320 .919959

H $8.7005452 .756483-0.743528$

H $8.8563580 .330139-1.616710$

H $\quad \begin{array}{llll}10.435825 & 0.402613 & 1.006559\end{array}$

H $11.927682-0.867409-0.618947$

H $10.534661-1.419436-1.514861$

H $11.572373-3.3882452 .081437$

H $12.358971-1.7594261 .679663$

H $9.899869-4.3053270 .701294$

H $9.986255-3.798401-0.958185$

H $8.080703-2.7420901 .245505$

H $6.677464 \quad 1.1169510 .710585$

C -2.066376 -2.116084 1.803392

O $-2.155211-0.7714262 .321621$

C $-3.268486-0.7000533 .222348$

C $-4.078968-1.9682993 .002316$

C $-2.973080-2.9624662 .695920$

H -2.842855 -0.751491 4.234491

C -4.0264610 .6253123 .107677$
H $-4.675319-2.2443153 .876719$

H $-4.749747-1.8691482 .143029$

C $-3.461539-4.2443722 .045958$

H $-2.454125-3.2105733 .631661$

C $-4.7843890 .920799 \quad 1.793481$

O $\quad-4.979171 \quad 0.651640 \quad 4.179797$

H -3.3215601 .4440183 .300345$

H -5.6544990 .2598491 .717746$

O -5.32255122475941 .918152$

C $-3.959250 \quad 0.8455240 .497392$

C $-4.6305361 .472965-0.738196$

H $-3.716170-0.1989320 .282953$

O $-2.725479 \begin{array}{lll}1.540457 & 0.698265\end{array}$

H -4.722296 $2.557755-0.596855$

H -3.932170 $1.348272-1.575861$

H $-4.192857-4.7477232 .686583$

H -3.935883 -4.049491 1.079484

H -2.629746 -4.935179 1.875498

C $-0.608144-2.5717751 .783687$

H $-5.441387 \quad 1.5062424 .135495$

H $-4.5722532 .866527 \quad 1.940721$

H -2.172903 $1.387917-0.087199$

H $-0.547187-3.6332881 .518636$

H $-0.159587-2.4532792 .777310$

H -2.463463 -2.091303 0.780760

C $-6.988642-1.238995-1.779793$

O $-5.809166-0.512199-1.403943$

C $-5.9948990 .877582-1.118985$

C $-6.6010451 .610584-2.328741$

C $-7.8886370 .931690-2.802616$

C $-7.696114-0.576945-2.974931$

H -6.619325 -2.214850 -2.120756

C $-7.922245-1.473607-0.580683$

H $-6.6712780 .983947-0.265607$

O $-5.6565841 .637658-3.397595$

H $-6.8035962 .650065-2.043782$

H $-8.2026571 .366010-3.759553$

O $-8.9308051 .161680-1.859570$

H -8.660836 -1.069294 -3.144573

O $-6.895586-0.819515-4.135258$

C $-7.358272-2.4827360 .396652$

H -8.111289-0.551917 -0.024841

H -8.877818 -1.878202 -0.934318

H $-6.0573412 .133728-4.131540$

H $-9.0503372 .123600-1.782219$

H $-7.364045-0.441632-4.898879$

O $-8.210964-2.5930991 .458495$

O $-6.317173-3.1062650 .247254$

C -7.829493 -3.540878 2.460472

H -7.712238 -4.536149 2.020951

H $-6.903903-3.2218742 .946893$

H -8.624061 -3.581122 3.210224

SCF Energy (B3LYP/6-31G**//MMFF) $=-3245.90267686$

$07 \quad 00392$

MM̄FF Geometry

C -3.871921 -2.516435 -2.466128

C $-2.730191-2.776905-1.812350$

C -1.387665 -2.780696-2.498695

O $-0.522884-1.819211-1.845900$

C $-0.728192-4.163963-2.389669$

C $0.447030-4.317352-3.335765$

C $1.743593-4.239475-2.979016$

C $0.080129-4.626708-4.765994$

C $2.317013-3.961632-1.617435$

C $3.384151-2.862276-1.672470$

C $4.212394-2.767361-0.382869$

C $3.392056-2.2997210 .819839$

C $4.263689-2.1289052 .074823$

O $5.277248-1.838364-0.632421$

O $3.499853-1.3917113 .042323$

C $5.494093-1.2660991 .718237$

C $4.597019-3.4766682 .726719$

O $6.386962-1.2127242 .827929$

C $6.224828-1.7366990 .439213$

C $7.372321-0.795405-0.006999$

O $6.835677-3.0105440 .694980$

C $-0.735886-0.505294-2.131667$ 
O $-1.599773-0.042422-2.860547$

C $0.2713730 .290721-1.396740$

C $0.1046541 .618248-1.307910$

C $0.9987592 .573899-0.650591$

C $2.2939562 .050856-0.090040$

C $7.0612050 .696140-0.131188$

C $6.2516251 .101377-1.351036$

C $5.2828093 .141893-2.401400$

O $5.988285 \quad 0.344944-2.279887$

C $4.7384154 .445278-1.911961$

C $3.517627 \quad 4.914812-2.210464$

C $2.9947506 .215799-1.668575$

C $1.6057896 .115211-1.076848$

C $0.6460626 .985296-1.436788$

C $1.335215 \quad 5.057162-0.025961$

C $0.6168703 .867356-0.605763$

N $5.895616 \quad 2.443585-1.284784$

H -3.837188 -2.335173 -3.538360

H $-2.748394-2.951810-0.740580$

H -1.491012 -2.500071-3.554563

H $-1.457027-4.951197-2.626279$

H $-0.419540-4.351752-1.355036$

H $2.491741-4.410788-3.752562$

H $0.961131-4.776706-5.399254$

H $-0.517893-5.542596-4.814206$

H $-0.500910-3.808747-5.202581$

H $1.542502-3.674599-0.902229$

H $2.768708-4.891147-1.250687$

H $4.073667-3.050037-2.507141$

H $2.919928-1.892344-1.893589$

H $4.645829-3.756190-0.191969$

H $2.564313-2.9860751 .031915$

H $2.929641-1.3313270 .585131$

H $2.704910-1.9099603 .255019$

H $5.113608-0.2481941 .568207$

H $3.677731-3.9962163 .022914$

H $5.171830-3.3378573 .649033$

H $5.154030-4.1472152 .069517$

H $7.060253-0.5387582 .637503$

H $8.205773-0.9055380 .700215$

H $7.761290-1.152865-0.970590$

H $7.524484-3.1598710 .026065$

H $1.112402-0.230490-0.955911$

H $-0.7813232 .057147-1.766251$

H $2.875853 \quad 1.547745-0.870037$

H $2.105145 \quad 1.3357210 .716856$

H 2.9345932 .8311880 .325261

H 6.5804361 .0798900 .773364

H $8.0198961 .225238-0.220006$

H $4.5152672 .501190-2.847948$

H $6.0659893 .316080-3.146299$

H $5.3854795 .040360-1.270754$

H $2.8724394 .347296-2.876380$

H $3.0078256 .944955-2.488604$

H $3.6650756 .608452-0.893196$

H $-0.3478776 .938216-1.003493$

H $0.8309037 .762398-2.170775$

H 0.6927115 .4816940 .757265

H $2.265022 \quad 4.794480 \quad 0.485666$

H $-0.3525574 .125764-1.035912$

H $6.328155 \quad 3.007657-0.558004$

C $-5.710640-0.954206-1.902611$

O $-4.990662-0.149937-0.946803$

C $-5.8573510 .913103-0.496815$

C $-7.173426 \quad 0.746059-1.251962$

C $-7.178214-0.742921-1.550393$

H $-6.045456 \quad 0.741466 \quad 0.567137$

C $-5.1630842 .265055-0.707278$

H -8.033485 $1.066250-0.655550$

H -7.181880 $1.326367-2.182275$

C -8.149068 -1.133309-2.650656

H -7.428515 -1.288905 -0.631592

C -3.8377002 .4014320 .072713$

O $-4.873107 \quad 2.420321-2.102838$

H $-5.8413383 .084052-0.443959$

H $-3.1455001 .608901-0.230958$

O $-3.2229343 .641579-0.299756$

C -4.0183882 .4043631 .604188$
C -2.692714 2.5162292 .381847

H -4.5449581 .5033811 .929829$

$\begin{array}{lllll} & \text { O } & -4.845205 & 3.511762 & 1.973474\end{array}$

H -2.061812 3.2961271 .939090

H -2.923761 2.8517943 .401787

H $-9.174067-0.870531-2.369355$

H -7.916759 -0.622108 -3.590958

H $-8.117470-2.211200-2.836792$

C $-5.224233-2.400821-1.820688$

H $-4.2869761 .689088-2.363777$

H $-3.1175513 .633067-1.266593$

H $-4.398426 \quad 4.322513 \quad 1.674454$

H $-5.909521-3.071511-2.349649$

H $-5.175911-2.728737-0.776639$

H -5.502134 - $0.536856-2.897892$

C $-2.168517-1.0160253 .468449$

O $-2.6883850 .309572 \quad 3.299337$

C $-1.935384 \quad 1.1794752 .442402$

C -0.5055851 .3486152 .972630$

C $0.168307-0.0140913 .153918$

C $-0.710208-0.9780183 .954465$

H -2.769184-1.450055 4.278783

C $-2.382913-1.8676752 .207672$

H -1.8933200 .7513681 .436959$

$\begin{array}{lllll}\text { O } & -0.533660 & 2.045549 & 4.215829\end{array}$

H $0.063814 \quad 1.9610962 .264978$

H 1.1263810 .1241203 .668568

O $0.429841-0.5654801 .867759$

H $-0.288158-1.9888263 .924158$

O $-0.700397-0.5826825 .329321$

C $-3.771835-2.4668592 .186703$

H -2.243807 -1.320669 1.272030

H $-1.683924-2.7126012 .200937$

H $\quad 0.3866852 .1617804 .507075$

H $0.839087-1.4372521 .997861$

H -1.1434150 .2808485 .394088$

O $-4.716749-1.4847252 .179782$

O $-3.987591-3.6722552 .167763$

C $-6.073446-1.936017 \quad 2.236185$

H $-6.725794-1.0594302 .209997$

H $-6.302940-2.5706841 .375794$

H $-6.251235-2.4763273 .170803$

SCF Energy (B3LYP/6-31G**//MMFF) = -3245.90420815

07_00393

MM̄FF Geometry

C -1.836057 -3.165056 0.632490

C $-0.522591-3.410704 \quad 0.523217$

C $0.355303-2.753529-0.510599$

O $1.272362-1.8693440 .175293$

C $1.171535-3.810867-1.275412$

C $2.150818-3.227706-2.278186$

C $3.467530-3.515201-2.299412$

C $1.573270-2.292858-3.309324$

C $4.200631-4.485591-1.418327$

C $4.796874-3.876210-0.142459$

C $5.921706-2.864093-0.409875$

C $6.745266-2.6340810 .859966$

C $7.841451-1.5809250 .645301$

O $5.334261-1.627213-0.830018$

O $8.351300-1.2051091 .933619$

C $7.202848-0.3226230 .026490$

C $9.029646-2.140275-0.145721$

O $8.1996720 .631930-0.332581$

C $6.288812-0.618531-1.187440$

C $5.5238570 .667868-1.572515$

O $7.093908-1.026751-2.295239$

C $0.817956-0.6122530 .436604$

O $-0.286015-0.162151 \quad 0.170676$

C $1.903183 \quad 0.142187 \quad 1.102119$

C $1.644522 \quad 1.3832531 .541446$

C 2.5916522 .3087052 .164597

C 3.9931901 .8220422 .414334

C $4.3743450 .461281-2.564734$

C $3.8010911 .805353-2.968599$

C $2.4883123 .723156-2.055826$

O $4.071147 \quad 2.320522-4.051126$

C $3.1973404 .554606-1.031145$ 
C $2.5668385 .363813-0.166230$

C 3.2732036 .1753040 .883885

C 2.6777755 .9960152 .265715

C 1.9650386 .9789722 .841563

C 2.9641674 .7054423 .009819

C 2.1617253 .5506022 .472079

N $3.0076362 .369351-1.982163$

H -2.307765 -2.476599-0.064930

H $-0.044349-4.0802151 .234033$

H $-0.249302-2.182275-1.226733$

H $0.488966-4.483399-1.811307$

H $1.701490-4.428805-0.542322$

H $4.089588-3.032430-3.051962$

H $2.297469-2.039652-4.091335$

H $0.711849-2.756530-3.801023$

H $1.251247-1.355063-2.846362$

H $3.546117-5.320304-1.142497$

H $5.002679-4.944552-2.010796$

H $3.998149-3.4032130 .442860$

H $5.193068-4.7057230 .456346$

H $6.561470-3.255740-1.209368$

H $7.185781-3.5738811 .215733$

H $6.082801-2.2887151 .666392$

H $8.693693-2.0063802 .365687$

H $6.592648 \quad 0.1483950 .809815$

H $9.470762-2.9957890 .379887$

H $9.830566-1.397790-0.232799$

H $8.758455-2.474080-1.149352$

H $8.739050 \quad 0.7995790 .459199$

H $5.0981521 .115348-0.667355$

H $6.224736 \quad 1.402473-1.990258$

H $6.628330-0.817153-3.120102$

H $2.876672-0.3264931 .186162$

H $\quad 0.638278 \quad 1.779787 \quad 1.406846$

H 3.9828460 .8740042 .963783

H 4.5888462 .5104343 .018377

H 4.5215651 .6734991 .469018

H $4.707126-0.051535-3.472713$

H $3.578019-0.146904-2.121906$

H $1.4113183 .668381-1.868703$

H $2.6560104 .137198-3.054467$

H $4.2822564 .483617-0.996105$

H $1.4841545 .454679-0.217526$

H $3.215588 \quad 7.2292670 .582574$

H 4.3423535 .9319380 .923124

H 1.5531626 .8688523 .839180

H 1.7728257 .9144852 .326690

H 2.7126704 .8181484 .072049

H 4.0423664 .5253922 .968746

H 1.1118583 .7902952 .295266

H $2.8479611 .852169-1.123056$

C -3.122054 -2.764104 2.781977

O $-3.710254-1.5809822 .201648$

C -3.114675 -0.4276262 .823682$

C $-2.506164-0.9331254 .124641$

C $-1.981453-2.2859483 .684855$

H -2.301909-0.096342 2.166935

C -4.1393470 .6966582 .987795$

H -1.723077 -0.2703824 .504617$

H -3.264363 -1.054645 4.907100

C -1.671967 -3.2262904 .836428$

H -1.064334 -2.125869 3.104994

C -4.8041051 .1615761 .675124$

O

H -3.665862 1.5517913 .484436

H -5.5060330 .3979381 .321666$

O

C $-3.819778 \quad 1.5233470 .547919$

C $-4.4420992 .251519-0.658190$

H -3.3256320 .6121530 .193425$

O $-2.787942 \quad 2.370087 \quad 1.065437$

H -4.772959 $3.254398-0.358671$

H -3.629362 2.415901-1.378550

H $-0.894414-2.8025065 .480341$

H -2.556934 -3.409443 5.454919

H -1.313884 -4.191589 4.464778

C $-2.723128-3.7609251 .688402$

H $-5.571999-0.5430343 .487207$
H -6.2099652 .0750302 .684899$

H -3.214985 3.1655921 .427042

H -3.628994 -4.116873 1.182974

H -2.253162 -4.641034 2.142731

H -3.909158 -3.224902 3.392958

C $-6.092234-0.634616-2.370247$

O $-5.1219370 .223034-1.752732$

C $-5.6000191 .506282-1.340198$

C $-6.1494612 .291775-2.543183$

C $-7.2131151 .484126-3.294169$

C $-6.7422720 .055282-3.582480$

H -5.513951 -1.488569-2.746143

C $-7.117068-1.167061-1.356054$

H $-6.4018001 .375965-0.607096$

O $-5.0823892 .610006-3.434965$

H $-6.5723303 .237610-2.183867$

H -7.449516 $1.979229-4.244000$

O $-8.4088101 .430949-2.522084$

H -7.581771 $-0.551290-3.940683$

O $-5.7857350 .089988-4.646108$

C $-6.483206-2.071988-0.321204$

H -7.612861 -0.355256 -0.817916

H -7.876873 -1.758648 -1.879864

H $-5.4549363 .137153-4.162210$

H $-8.7070482 .345434-2.378809$

H $-4.9993650 .562723-4.323472$

O $-7.429381-2.415350 \quad 0.602422$

O $-5.316392-2.437084-0.322454$

C $-6.986079-3.2765961 .656022$

H -7.865781 -3.616593 2.208845

H $-6.467733-4.1521411 .253244$

H -6.336568 -2.721375 2.337575

SCF Energy $\left(B 3 L Y P / 6-31 G^{* *} / / M M F F\right)=-3245.91735413$

0700394

MM̄FF Geometry

C 4.0902091 .7660553 .044400

C 3.3292220 .7362842 .641612

C 1.9077920 .5255973 .091702

O 1.0934630 .6195341 .899651

C $1.736722-0.8752353 .707147$

C $0.590547-0.9585104 .696346$

C $-0.606326-1.5315724 .462720$

C $0.888074-0.3836696 .060027$

C -1.115453 -2.158649 3.194027

C $-2.445944-1.5265352 .779985$

C $-3.166070-2.2323201 .621684$

C $-2.308417-2.3714850 .364278$

C $-3.107310-2.938029-0.819237$

O $-4.318294-1.4294741 .340079$

O $-2.336486-2.728555-2.012331$

C $-4.411533-2.132961-0.982881$

C $-3.310427-4.452741-0.692693$

O $-5.225182-2.735993-1.987343$

C $-5.197410-1.9534020 .337727$

C $-6.329178-0.9189440 .142309$

O $\quad-5.763398-3.202648 \quad 0.731175$

C -0.0727951 .3134961 .967468$

O -0.5625591 .8458592 .949749$

C -0.6597961 .3133720 .607983$

C -1.8949551 .7950630 .402442$

C $-2.5924231 .855820-0.886150$

C $-1.840507 \quad 1.382821-2.103749$

C $-7.095919-0.5633101 .424977$

C -7.6828120 .8296041 .317829$

C $-9.2532572 .184911-0.050043$

O $\quad-7.239031 \quad 1.7671321 .977213$

C $-8.4781872 .617158-1.255753$

C $-7.6242673 .651977-1.267191$

C $-6.8144784 .044171-2.470867$

C $-5.3258563 .917165-2.227013$

C $-4.5439285 .009160-2.169787$

C $-4.7627512 .512313-2.108975$

C $-3.8560162 .330690-0.915622$

N -8.7127820 .9133890 .393425$

H 3.6837822 .4956193 .740347

H 3.7353430 .0187001 .931918

H 1.6328291 .3113053 .805404 
H $2.648818-1.1655014 .246969$ H $1.619735-1.6207522 .912605$ H -1.321267 -1.557032 5.285101 H $0.050636-0.5039686 .755663$ H $1.755713-0.8846906 .501847$ H 1.1033600 .6868635 .990116 H $-0.395316-2.0509382 .380633$ H -1.254861 -3.231330 3.370481 H -3.133807 -1.5066693 .637255$ H -2.292441 -0.4715862 .524267$ H -3.494642 -3.2175661 .973002$ H $-1.431174-3.0003600 .552165$ H $-1.917754-1.3871240 .078227$ H $-1.484652-3.184186-1.904807$ H $-4.127388-1.141775-1.363740$ H -2.342474 -4.966301-0.647152 H $-3.822193-4.858404-1.572518$ H $-3.876004-4.7352240 .197817$ H $-5.907677-2.093958-2.244246$ H $-5.8922380 .000714-0.266274$ H $-7.054527-1.288466-0.592587$ H $-6.513265-3.034727 \quad 1.323778$ H $-0.0565990 .903468-0.192790$ H $-2.4518352 .179082 \quad 1.256250$ H $-0.9324611 .978435-2.250290$ H -2.413936 $1.452348-3.029525$ H $-1.555900 \quad 0.330146-1.994803$ H -7.902571-1.281111 1.611665 H $-6.444120-0.5756132 .305142$ H -9.1939922 .9157240 .763103$ H $-10.3049012 .033407-0.310252$ H $-8.6142682 .029441-2.160708$ H -7.484996 4.240505 -0.363370 H $-7.0785995 .076808-2.731575$ H $-7.0813143 .437062-3.345226$ H $-3.4712374 .933931-2.025967$ H $-4.9585036 .006607-2.273083$ H $-4.285026 \quad 2.261543-3.059408$ H $-5.5828471 .793356-1.994630$ H $-4.3192302 .621100 \quad 0.028852$ H -8.958285 $0.087540-0.144675$ C 5.5583813 .1678501 .576248 $\begin{array}{lllll}\text { O } & 4.797946 & 2.818782 & 0.401339\end{array}$ C $5.6790112 .813795-0.731830$

C $6.7658263 .810968-0.368733$

$\begin{array}{llll}\text { C } & 6.984685 & 3.463137 & 1.094451\end{array}$

H $6.1226661 .812221-0.788476$

C $4.8986973 .084851-2.020267$

H $7.6667803 .692057-0.977454$

H $6.415594 \quad 4.845306-0.461995$

C $7.6786324 .558846 \quad 1.885352$

H 7.5966922 .5526821 .149243

C $3.6254132 .223274-2.172414$

O $4.493570 \quad 4.458774-2.036497$

H $5.5619822 .950737-2.883041$

H $2.8531572 .600039-1.489644$

O $3.1320932 .439692-3.499439$

C $3.8396070 .716873-1.932828$

C $2.550125-0.103593-2.122508$

H $4.2014890 .566239-0.910391$

$\begin{array}{llll}\text { O } & 4.850946 & 0.248575 & -2.822415\end{array}$

H $1.7094620 .435627-1.673807$

H $2.356962-0.221540-3.195805$

H 8.6759214 .7547711 .478395

H $7.1127915 .496038 \quad 1.854411$

H 7.7912724 .2672082 .934460

C 5.4961621 .9998932 .565412

H $4.0079754 .607207-2.866141$

H $2.2366902 .064880-3.548005$

H $4.998345-0.693347-2.631561$

H $6.133178 \quad 2.202569 \quad 3.433944$

H 5.8778061 .0881722 .089237

H 5.0903524 .0629072 .004302

C $3.889995-3.568910-1.683508$

O $3.619553-2.256831-2.193356$

C $2.650758-1.495630-1.467669$

C $1.299545-2.224454-1.454046$

C $1.443861-3.652774-0.916877$
C $2.599746-4.400900-1.588866$

H $4.531852-4.034410-2.442780$

C $4.680631-3.515047-0.371110$

H $2.993797-1.359987-0.435930$

O $0.742686-2.262046-2.766067$

H $0.600480-1.660956-0.825910$

H $\quad 0.511085-4.203671-1.087092$

O $1.678602-3.6178910 .487512$

H $2.809727-5.339584-1.062887$

O $2.215515-4.744291-2.923539$

C $6.047937-2.915666-0.602262$

H $4.172056-2.9429790 .410227$

H $4.835646-4.5219380 .034148$

H $1.388449-2.693441-3.351592$

H $\quad 0.928619-3.1579100 .899526$

H $1.432645-5.318212-2.864499$

O $6.112227-1.667819-0.056232$

O $6.944415-3.482828-1.213862$

C $7.361607-0.989724-0.219675$

H $7.508478-0.728992-1.271847$

H $7.330123-0.0705720 .370192$

H $8.187927-1.6075620 .145267$

SCF Energy (B3LYP/6-31G**//MMFF) $=-3245.90343802$

07_00395

MMFF Geometry

C $0.010323-2.9824720 .541945$

C $-0.175923-2.122987 \quad 1.555367$

C $0.915767-1.2307682 .086689$

$\begin{array}{lllll}\text { O } & 0.509861 & 0.153050 & 1.966741\end{array}$

C $1.180197-1.4643783 .585826$

C $1.741256-2.8236363 .937644$

C $3.055838-3.1122423 .984358$

C $0.730188-3.8681624 .337853$

C $4.215656-2.2053933 .672153$

C $5.259394-2.8999522 .784224$

C $4.882675-2.9055641 .293497$

C $5.757360-3.9108170 .539807$

C $5.525253-3.855708-0.974720$

O $5.106253-1.5810330 .796105$

O $6.576173-4.599103-1.610233$

C $5.664198-2.392533-1.435188$

C $4.208662-4.528517-1.380074$

O $5.337419-2.263874-2.817781$

C $4.827634-1.395892-0.598227$

C $5.2399520 .050718-0.969231$

O $3.446101-1.595587-0.889713$

C $0.7134350 .762918 \quad 0.769679$

$\begin{array}{lllll}\text { O } & 1.108846 & 0.244453 & -0.261340\end{array}$

C $\quad 0.377375 \quad 2.193938 \quad 0.936621$

C $0.5312163 .036464-0.095644$

C $0.2192054 .467136-0.094954$

C $-0.239300 \quad 5.077677 \quad 1.204068$

C $4.5477491 .148344-0.150417$

C $5.1506962 .520785-0.401937$

$\begin{array}{llll}\text { C } 4.899956 & 4.898839 & 0.274974\end{array}$

O $6.1433532 .701149-1.102347$

C 3.7174855 .8132880 .304446

C $3.4371276 .683254-0.677755$

C $2.2863297 .648302-0.652272$

C $1.3132687 .420616-1.787333$

C $1.5116667 .983074-2.991636$

C $0.0688816 .612284-1.498132$

C $0.3627275 .159892-1.244498$

$\begin{array}{lllll}\text { N } & 4.445117 & 3.521341 & 0.255568\end{array}$

H $0.974982-3.010074 \quad 0.040618$

H -1.133976 -2.099019 2.068963

H $1.855673-1.3747651 .538849$

H $0.258236-1.2872504 .158113$

H $1.855988-0.6788143 .950076$

H $3.342044-4.1131984 .306596$

H $1.203349-4.7975934 .672783$

H $\quad 0.112977-3.5013095 .164590$

H $0.074613-4.1216683 .500962$

H $3.900263-1.2629653 .214300$

H $4.694022-1.9524444 .626403$

H $6.210287-2.3645202 .906450$

H $5.421061-3.9250333 .140790$ 
H $3.823331-3.1615461 .180268$ H $5.590376-4.9283700 .915292$ H $\quad 6.816608-3.6910820 .735744$ H $6.547163-5.509798-1.270021$ H $6.724638-2.118850-1.341352$ H $4.211646-5.584311-1.083727$ H $4.081346-4.523322-2.468239$ H $3.330752-4.062963-0.927550$ H $5.891143-2.896628-3.306420$ H $6.3238250 .150617-0.824103$ H $5.034851 \quad 0.238308-2.030847$ H $2.954063-0.791174-0.664010$ H 0.0002922 .5120421 .902078 H $0.9076932 .641491-1.038309$ H -0.3601696 .1617811 .160262$ H $0.4913124 .886821 \quad 1.998283$ H -1.204687 $4.657191 \quad 1.505929$ H $3.4892961 .202015-0.416479$ H 4.6292800 .9394120 .921880 H $5.5489285 .088484-0.586487$ H 5.4983705 .0337351 .181897 H 3.0809705 .7758561 .184796 H $4.0900006 .726424-1.547154$ H $2.6970288 .664561-0.718357$ H 1.7552627 .6127090 .306951 H $0.8044077 .849036-3.803318$ H $2.3899338 .588105-3.192245$ H $-0.6210476 .658131-2.351455$ H $-0.4756097 .083755-0.675206$ H $0.708994 \quad 4.627891-2.131705$ H 3.7253723 .2496060 .919794 C - $1.513496-3.791416-1.316240$ O $-2.671846-2.924863-1.271680$ C $-3.799105-3.649576-1.793143$ C $-3.194400-4.661519-2.752676$ C $-1.982586-5.104379-1.959904$ H $-4.249598-4.185682-0.948327$ C $-4.843851-2.719368-2.418571$ H $-3.879222-5.482229-2.985204$ H -2.880251 -4.193736 -3.692849 C $-0.915025-5.781704-2.802951$ H $-2.313871-5.807888-1.183717$ C $-5.339829-1.576141-1.509536$ O $-4.303073-2.120139-3.602620$ H -5.696427 -3.321009-2.755402 H $-4.576017-0.794556-1.471335$ O $-6.472874-0.970756-2.145198$ C $-5.717324-1.988473-0.076626$ C $-6.418876-0.902007 \quad 0.759815$ H -4.810485 -2.300022 0.453598 O $-6.576181-3.131871-0.109039$ H -7.450263 -0.7644590 .409524$ H $-6.511870-1.3065521 .776544$ H -1.310324 -6.691607 -3.266223 H - $0.558966-5.124405-3.603353$ H $-0.053612-6.060688-2.187724$ C $-0.991556-4.014406 \quad 0.107367$ H -3.493837 -1.645719 -3.346067 H -7.164600 -1.647952 -2.232972 H -7.407095 -2.871055 -0.540712 H $-0.477541-4.9819090 .168954$ H -1.830302 -4.050775 0.813788 H $-0.765842-3.291672-1.944300$ C $-3.517623 \quad 1.4330881 .166089$ O C $-5.707676 \quad 0.4619960 .797329$ C -6.3820961 .4340861 .780469$ C -5.5972842 .7449221 .892255$ C -4.1007722 .4943802 .114298$ H -2.5602181 .1010581 .587561$ C $-3.2521831 .973833-0.249471$ H $-5.7566620 .888246-0.209350$ O $-6.4731120 .827003 \quad 3.068129$ H -7.4039001 .6325911 .435121$ H -5.9985493 .3334302 .726159$ O $-5.795123 \quad 3.4969160 .698823$ H -3.534354 3.4268202 .008645 O -3.8955452 .0330323 .452398$
C $-2.3845081 .044088-1.069709$

H $-4.1750332 .130524-0.812811$

H -2.722095 2.930151-0.174116

H -6.9368201 .4490453 .654136$

H $-5.307174 \quad 4.3325340 .793766$

H -4.2272772 .7209304 .054160$

O $\quad-2.0871291 .649445-2.257707$

O $-2.017558-0.069373-0.722861$

C $-1.2660190 .887864-3.148351$

H -1.739603 -0.070225 -3.381124

H $-1.1520861 .456396-4.075107$

H $-0.2768050 .732230-2.708812$

SCF Energy (B3LYP/6-31G**//MMFF) $=-3245.89473810$

07_00396

MM̄MF Geometry

C $5.459721-0.3679972 .701101$

C $4.371117-1.0347172 .286462$

C $3.078727-1.1261483 .053798$

O $2.080450-0.3841282 .312480$

C $2.618520-2.5928303 .130489$

C $1.300705-2.7752853 .859851$

C $0.100005-2.9010493 .257486$

C $1.407939-2.8458135 .360673$

C $-0.196323-2.8205531 .783430$

C $-1.216704-1.7258001 .457175$

C -2.690168 -2.079877 1.702256

C $-3.192531-3.1656090 .745900$

C $-4.707068-3.3938840 .865430$

O $-3.419146-0.8618701 .485788$

O $-5.126238-4.170502-0.267632$

C $-5.424324-2.0319640 .766871$

C $-5.064049-4.2152252 .109515$

O $-6.822329-2.1701441 .008224$

C $-4.831304-0.9600991 .704719$

C $-5.4382960 .442026 \quad 1.464769$

O $-5.117056-1.3139183 .063106$

C 2.0172860 .9625902 .499441

O $2.7077721 .639553 \quad 3.243152$

C 0.9522591 .4776041 .611126

C 0.7610772 .7959251 .462290

C -0.2434203 .4138510 .593502$

C $-1.2973742 .516999-0.006335$

C -5.2152921 .0162240 .062826$

C $-5.7073992 .448772-0.053822$

C $-5.9180424 .297327-1.710641$

O -6.1983723 .0699530 .885090$

C $-4.7063865 .174054-1.691749$

C $-4.4238346 .024212-0.693241$

C $-3.234906 \quad 6.941438-0.677477$

C $-2.122307 \quad 6.4191580 .206914$

C -2.0919376 .7406951 .511841$

C $-1.0826285 .573597-0.503349$

C -0.1917854 .7464380 .386868$

N $-5.5359042 .948689-1.338343$

H $5.456676 \quad 0.129083 \quad 3.667107$

H $4.381864-1.4899701 .298126$

H $3.176009-0.7113944 .064918$

H $3.386182-3.1953703 .635031$

H $2.546309-3.0166472 .121788$

H $-0.772292-3.0654553 .888179$

H $\quad 0.434891-2.9930335 .841263$

H $2.052128-3.6802035 .656681$

H $1.834053-1.9189365 .757227$

H $0.709950-2.5934911 .215612$

H $-0.537159-3.7993311 .433825$

H -0.979395 -0.821874 2.032024

H -1.102078 -1.431759 0.405821

H $-2.818437-2.3860932 .746028$

H -2.660716 -4.111344 0.899318

H $-2.973696-2.864126-0.287609$

H $-4.619455-5.000635-0.266473$

H $-5.320240-1.696196-0.272174$

H $-4.589745-5.2030402 .064707$

H -6.142052 -4.403314 2.163088

H $-4.748213-3.7423803 .041669$

H -7.155939 -2.850173 0.398288

H $\quad-6.5108550 .4308801 .698559$ 
H $-4.991415 \quad 1.1364422 .190555$ H -5.060301 -0.5128453 .609797$ H $\quad 0.3654830 .749688 \quad 1.064968$ H 1.4086733 .4839692 .003507 H $-1.756971 \quad 1.8887660 .765654$ H $-0.8597941 .867981-0.772482$ H -2.120944 $3.065899-0.469407$ H $-4.1487531 .006125-0.186758$ H $-5.7538150 .420738-0.681911$ H $-6.6960114 .663605-1.032357$ H $-6.3375374 .260717-2.720679$ H $-4.0425375 .117656-2.550766$ H -5.0889286 .0735920 .166131$ H -3.567542 $7.927995-0.329305$ H -2.853320 $7.110598-1.692614$ H $-1.303995 \quad 6.4050372 .176161$ H -2.8666677 .3609571 .952082$ H $-0.4417806 .243700-1.091465$ H -1.577215 4.934495-1.241793 H $\quad 0.599411 \quad 5.3191890 .872019$ H $-5.0661612 .377636-2.034973$ C 6.6131361 .1319991 .070564 O 5.4445121 .1186780 .228949 C $5.8069871 .511755-1.104438$ C $7.1520922 .208297-0.962384$ C 7.7967531 .3713090 .132108 H $5.9478100 .588283-1.679380$ C $4.6641332 .328484-1.720876$ H $7.7225052 .209549-1.895789$ H $7.0410313 .245544-0.626554$ C $8.981821 \quad 2.0458470 .799827$

H $8.1283510 .419754-0.304753$ C $3.2763361 .676767-1.530333$ O $4.6159663 .617641-1.095606$ H $4.8612202 .513114-2.782361$ H $3.0281841 .662381-0.463817$ O $2.2923042 .504654-2.160340$ C $3.1519910 .252669-2.101235$ C $1.822730-0.407524-1.707184$ H $3.962735-0.369904-1.713982$ O $3.285490 \quad 0.290818-3.518752$ H $1.782843-0.511623-0.615762$ H $\quad 0.9850630 .254348-1.965063$ H 9.7762252 .2374340 .071114 H 8.6982263 .0040441 .247795 H 9.3941891 .4124551 .591746 C $6.680827-0.1927151 .836394$ H $4.4892443 .477355-0.141352$ H $2.3714753 .393451-1.773147$ H $2.573060 \quad 0.855891-3.864404$ H $7.575206-0.2302402 .467899$ H $6.749674-1.0292781 .129182$ H $6.491775 \quad 1.970751 \quad 1.768054$ C $-0.163899-3.508918-2.203122$ O $0.362996-2.273859-1.706069$ C $1.535356-1.770799-2.362910$ C $2.677481-2.789280-2.251890$ C $2.247547-4.155104-2.797441$ C $0.909302-4.609502-2.208988$ H -0.928569 -3.800184 -1.471719 C $-0.861827-3.314006-3.553592$ H $1.304002-1.590345-3.418283$ O $3.066617-2.913087-0.886132$ H $3.544869-2.428880-2.816272$ H $3.021431-4.895710-2.561696$ O $2.145557-4.063464-4.215729$ H $0.532884-5.483324-2.752609$ O $1.122276-5.038549-0.860431$ C -2.158789 -2.560168 -3.366902 H $-0.257810-2.779325-4.291700$ H - $-1.117116-4.283724-3.997447$ H $3.812153-3.536261-0.848833$ H $1.901633-4.943500-4.549795$ H $1.420198-4.268459-0.346851$ O $-1.908677-1.230013-3.200139$ O $-3.259626-3.095654-3.348924$ C -3.055476 -0.415042 -2.940181 H $-2.7114330 .602899-2.738348$
H -3.711406 -0.399522 -3.815471

H $-3.592636-0.782581-2.060857$

SCF Energy (B3LYP/6-31G**//MMFF) $=-3245.91664246$

07 00397

MM̄FF Geometry

C $2.8534031 .830012-3.037269$

C $3.1321683 .057523-2.569413$

C $2.1948873 .952865-1.789393$

O $0.880838 \quad 3.345867-1.754156$

C $2.6502974 .184883-0.336682$

C $4.0561084 .722150-0.165350$

C 5.0597524 .0243200 .403785

C $4.2731626 .138860-0.623873$

C 4.9795022 .6062970 .895802

C 4.5829412 .5422682 .375962

C 3.9900451 .1915512 .806284

C 4.9576310 .0241022 .615926

C $4.311189-1.3168382 .995676$

O 2.7985720 .9771302 .042341

O $5.180900-2.3459012 .499828$

C $2.954907-1.4396042 .268952$

C $4.237200-1.5015394 .515810$

O $2.244563-2.5978202 .699896$

C $2.053324-0.1892962 .410884$

C $0.854040-0.3101511 .436085$

O $1.581768-0.1079293 .754091$

C $-0.2046304 .148030-1.607040$

O $-0.2185855 .361282-1.485535$

C -1.391205 $3.263371-1.617641$

C $-2.6158903 .766283-1.405575$

C $-3.8628322 .997421-1.383141$

C $-3.7620851 .504499-1.556274$

C $-0.049026 \quad 0.926810 \quad 1.327217$

C -1.1643720 .9889552 .355948$

C -3.1544532 .3598232 .971447$

O $-1.312178 \quad 0.152793 \quad 3.242370$

C -4.3689851 .9096932 .221311$

C -5.4350212 .6954802 .006841$

C -6.6686442 .2526691 .273495$

C $-7.0792783 .221000 \quad 0.185612$

C -8.0541794 .1216850 .395690$

C $-6.4214233 .087785-1.169351$

C $-5.0276073 .653689-1.198668$

N -1.978008 2.0972782 .163615

H $1.8826761 .381441-2.845036$

H $4.1075193 .483908-2.796350$

H $2.1287884 .906822-2.328005$

H $2.499193 \quad 3.258973 \quad 0.230147$

H 1.9586674 .8896800 .145035

H $6.030790 \quad 4.5012330 .522258$

H $5.2850576 .495357-0.403775$

H $4.1230696 .220415-1.704758$

H $3.5704086 .812297-0.122448$

H 4.2863242 .0320410 .274406

H 5.9588812 .1335280 .753411

H 5.4541422 .7809282 .998601

H 3.8264423 .3121762 .578971

H $3.715793 \quad 1.2859913 .863827$

H 5.8794640 .1845943 .188529

H $5.265156-0.0382601 .563508$

H $4.818866-3.2055552 .773158$

H $3.167110-1.5870671 .203234$

H $5.243614-1.4830344 .950919$

H $3.816104-2.4790624 .775974$

H $3.645222-0.7305865 .013464$

H $2.784629-3.3752522 .480071$

H $1.257483-0.4776870 .429818$

H $0.250796-1.1868691 .693936$

H 0.8110700 .4800823 .778283

H $-1.2203212 .207726-1.798665$

H -2.718147 $4.836807-1.233354$

H $-4.7128050 .987551-1.419381$

H $-3.0752791 .074660-0.818497$

H $-3.3995911 .255916-2.559621$

H 0.5335221 .8526431 .374931

H $-0.5378670 .894496 \quad 0.345129$

H -3.1712593 .4341323 .180032$ 
H $-3.082727 \quad 1.818813 \quad 3.920180$ H $-4.378006 \quad 0.886674 \quad 1.852520$ H -5.4337743 .7086472 .403095$ H -7.477095 2.1571172 .010150 H -6.5412831 .2522940 .841203$ H -8.375155 4.801173 -0.386932 H -8.5498004 .2034061 .357523$ H -7.004047 $3.632182-1.925065$ H $-6.4670412 .042854-1.488038$ H -4.997554 4.737189-1.074546 H -1.8001182 .7116831 .374494$ C $4.126754-0.362096-3.326042$ O $2.935080-1.167117-3.218769$ C $3.059279-2.003811-2.048447$ C $4.504438-1.857074-1.577582$ C $4.783848-0.413112-1.949733$ H $2.401562-1.574189-1.286369$ C $2.625082-3.438632-2.360925$ H $4.622992-2.060508-0.510553$ H $5.179606-2.527716-2.122082$ C $6.256843-0.050424-1.944002$ H $4.2504980 .232625-1.242163$ C $1.184989-3.550181-2.904491$ O $3.516775-3.964227-3.351945$ H $2.746907-4.062668-1.467466$ H $1.107408-3.042051-3.873498$ O $0.913123-4.936471-3.149587$ C $0.095229-3.011714-1.952597$ C -1.318579 -3.212879-2.538584 H $0.254096-1.941725-1.780009$ O $0.217913-3.675896-0.697605$ H -1.356370 -2.718176 -3.516751 H -1.489756 -4.286315 -2.688590 H $6.682471-0.189380-0.944723$ H $\quad 6.826967-0.672403-2.642102$ H $6.400605 \quad 0.996607-2.228584$

C $3.7990451 .023731-3.883012$ H $3.380786-4.926170-3.385879$ H $1.449203-5.208719-3.913444$ H $-0.427435-3.280569-0.088621$ H $3.3187940 .898599-4.861893$ H $4.7310571 .575064-4.054703$ H $4.767563-0.870979-4.059366$ C -3.535904-3.098543 0.465984 O $-2.632526-3.562689-0.546923$ C $-2.448748-2.666060-1.644861$ C -3.780581-2.524659-2.409255 C $-4.993434-2.453904-1.471717$ C $-4.567038-2.082810-0.055887$ H $-4.068777-3.987716 \quad 0.827041$ C $-2.731003-2.5137481 .633459$ H -2.148944 -1.685694 -1.251666 O $-3.970338-3.643930-3.279138$ H -3.720736 -1.641302 -3.056611 H -5.543571 -3.403413 -1.463342 O $-5.893735-1.473674-1.987267$ H -4.155706 -1.067028 -0.041412 O $-5.691562-2.0865720 .820336$ C -1.958699 -3.5707702 .395210$ H $-2.009745-1.7843501 .252618$ H $-3.404510-2.0246712 .346178$ H $-4.796023-3.493661-3.770879$ H $-6.704713-1.509390-1.452643$ H -6.275038 -1.3568500 .552299$ O $-1.288600-2.9714323 .423664$ O $-1.942363-4.7647252 .130301$ C $-0.510613-3.8452474 .247471$ H $0.062793-3.2311244 .947016$ H -1.170969 -4.505839 4.816826 H $\quad 0.187988-4.4287853 .640254$

SCF Energy (B3LYP/6-31G**//MMFF) $=-3245.89325180$

07 00398

MM̄MF Geometry

C $2.941345-1.8577332 .391209$

C $1.999157-2.7587192 .707639$

C $\quad 0.552397-2.6752702 .285132$

O $0.174118-1.2775342 .205646$
C $\quad 0.314099-3.3266340 .908483$

C $0.122194-4.8295370 .957530$

C $-1.082756-5.4340770 .941076$

C $1.379239-5.6587160 .951564$

C $-2.427277-4.7636990 .922037$

C $-3.080964-4.879804-0.459849$

C $-4.312066-3.971288-0.588070$

C $-5.034392-4.233597-1.911652$

C $-6.191722-3.251130-2.143427$

O $-3.849484-2.616867-0.535552$

O $-6.597019-3.362594-3.516528$

C $-5.665843-1.814457-1.943523$

C $-7.421410-3.603292-1.298841$

O $-6.727515-0.865821-2.016187$

C $-4.879735-1.625748-0.628342$

C $-4.202505-0.238061-0.544450$

O $-5.784309-1.7216050 .477892$

C $-1.096262-0.9423912 .554619$

O $-1.990950-1.6994112 .894956$

C -1.209232 0.5319712 .470047

C -2.3646951 .1222802 .810727$

C -2.6483442 .5596032 .797899$

C -1.5389163 .4833012 .372840$

C $-3.1353990 .022121-1.609717$

C $-2.3980191 .319477-1.343659$

C $-2.6768033 .794883-1.474716$

O $-1.2424971 .329088-0.925481$

C $-3.5082334 .619299-0.538413$

C -4.6424924 .2680700 .088436$

C -5.3890465 .1902411 .010752$

C -5.6203674 .5952772 .381391$

C -6.8633504 .3379052 .822774$

C $-4.4170864 .375798 \quad 3.273489$

C -3.8780272 .9722933 .171070$

N -3.168071 2.438401 -1.616864

H $2.670814-0.9923221 .790945$

H $2.282739-3.6322603 .289456$

H $-0.050205-3.1622093 .062337$

H $1.140843-3.0892420 .224261$

H $-0.550789-2.8539170 .426679$

H $-1.118906-6.5223670 .945798$

H $1.169564-6.7274130 .833994$

H $2.027864-5.3626070 .120523$

H $1.930405-5.5363921 .887460$

H -3.068139 -5.243917 1.671767

H $-2.351331-3.7174281 .226452$

H -2.348935 -4.599143 -1.229021

H $-3.363086-5.924680-0.639486$

H $-4.981112-4.1589960 .259878$

H $-5.394489-5.268939-1.959034$

H $-4.321695-4.127789-2.741910$

H -6.856641 -4.285946 -3.678277

H $-5.001788-1.601738-2.790394$

H -7.787062 -4.605055 -1.554811

H -8.251937 -2.919606 -1.506538

H $-7.223452-3.590936-0.225208$

H -7.193580 -1.016406 -2.856460

H $-4.9650030 .550973-0.562010$

H $-3.722147-0.1473550 .438613$

H $-5.380622-1.2829861 .245132$

H $-0.339979 \quad 1.088297 \quad 2.138123$

H -3.191690 0.4910023 .134448

H -0.6924583 .4100653 .064567$

H -1.832126 4.5342462 .339164

H -1.1901453 .2261131 .367455$

H $-2.393824-0.784562-1.627737$

H -3.575451 $0.078306-2.611047$

H $-2.6866494 .248660-2.470778$

H -1.643784 $3.779875-1.117319$

H -3.116305 $5.619409-0.359876$

H $-5.0765263 .284798-0.070542$

H -6.3469075 .4321570 .532522$

H -4.8628116 .1459621 .127978$

H $-7.036573 \quad 3.9368863 .816062$

H -7.7341974 .5179722 .201315$

H -4.7057544 .5399394 .320595$

H -3.665741 5.1428433 .068040

H -4.6030942 .2136143 .471162$ 
H $-4.0869842 .312176-2.031254$

C $5.308675-2.3226231 .612193$

O $5.054716-1.4565490 .485132$

C $4.856443-2.278771-0.678573$

C $5.548636-3.595729-0.358624$

C $5.172477-3.7605331 .101106$

H $3.778477-2.465321-0.758429$

C $5.352748-1.575372-1.942079$

H $5.196835-4.417844-0.988688$

H $6.636970-3.518249-0.465397$

C $6.036951-4.7621511 .846691$

H $4.129302-4.0932841 .153227$

C $4.689400-0.207838-2.213087$

O $6.764525-1.362654-1.822644$

H $5.214606-2.239912-2.803632$

H $5.0666430 .526609-1.493090$

O $5.1413610 .236541-3.499182$

C $3.148950-0.215163-2.192805$

C $2.4859271 .060019-2.745898$

H $2.801897-0.389362-1.167903$

O $2.686864-1.313821-2.985659$

H $2.6753811 .138314-3.824287$

H $1.4005890 .920434-2.649672$

H $5.939273-5.7587691 .403953$

H $7.095334-4.4824781 .813945$

H $5.737223-4.8278022 .897406$

C $4.388284-1.9606452 .783489$

H $7.059600-0.914334-2.633749$

H $4.775805-0.371694-4.164437$

H $1.719349-1.353496-2.895672$

H $4.682463-0.9788603 .174876$

H $4.525754-2.6762413 .602305$

H $6.345660-2.1313321 .916457$

C $2.944613 \quad 3.362500 \quad 0.162595$

O $2.5770792 .245471-0.659311$

C $2.9030962 .362542-2.046618$

C $2.2023723 .585262-2.661562$

C $2.5130334 .863001-1.875693$

C $2.3236364 .667825-0.368534$

H 2.4939143 .1502261 .140622

C $4.466602 \quad 3.4571190 .358030$

H $3.9848532 .482594-2.157187$

O $\quad 0.7904653 .386161-2.696365$

H $2.5338593 .697722-3.700265$

H $1.8531995 .670385-2.217156$

O $3.857167 \quad 5.264147-2.123285$

H $2.736568 \quad 5.5256230 .175306$

O $0.9185084 .642930-0.103615$

C $5.0244642 .269481 \quad 1.112746$

H $4.9933303 .511506-0.598134$

H 4.7056064 .3529090 .942729

H $0.4997893 .177095-1.792178$

H $3.9470425 .416266-3.079498$

H $\quad 0.803513 \quad 4.6043450 .860988$

$\begin{array}{lllll}\text { O } & 6.387302 & 2.360467 & 1.131477\end{array}$

$\begin{array}{lllll}\text { O } & 4.357237 & 1.382384 & 1.625207\end{array}$

C 7.0749201 .3041661 .809732

H $8.112648 \quad 1.614923 \quad 1.956825$

H $6.628788 \quad 1.1078722 .789602$

H 7.0630820 .4028681 .191179

SCF Energy (B3LYP/6-31G**//MMFF) $=-3245.91733805$

07_00399

MM̄FF Geometry

C -2.913151 -3.499510-1.988416

C $-2.174763-2.410354-2.250485$

C $-0.667671-2.305782-2.266438$

O $-0.283677-1.337114-1.258970$

C $0.093022-3.609477-1.956439$

C $1.600193-3.465676-2.059221$

C $2.441349-3.386652-1.008192$

C $2.148978-3.469465-3.463493$

C $2.091661-3.367938 \quad 0.453752$

C $2.748245-2.2328451 .261255$

C $4.279273-2.3456501 .360774$

C $4.791657-1.6977012 .649324$

C $6.324227-1.7519892 .739837$

O $4.855580-1.6702820 .235564$
O $6.734743-0.8752843 .800073$

C $6.918928-1.1916431 .432833$

C $6.830139-3.1505843 .112244$

O $8.334092-1.3628841 .398503$

C $6.281860-1.7814430 .151040$

C $6.785398-0.988665-1.083660$

O $\quad 6.686852-3.145576 \quad 0.003594$

C $-0.217932-0.029630-1.627431$

O $\quad-0.5253820 .449624-2.706746$

C $0.3367500 .722392-0.480773$

C $0.6413552 .019943-0.625876$

C 1.2631982 .8801010 .383618

C 1.6945882 .2337021 .674438

C $5.962580-1.200806-2.361894$

C $4.809591-0.218896-2.465792$

C $4.3211762 .205666-2.757409$

O $3.642649-0.566239-2.302049$

C $4.3614112 .865664-1.414578$

C $4.5308854 .185065-1.240363$

C 4.5417954 .8606850 .102518

C 3.3670535 .7985380 .283931

C $3.4597907 .094891-0.056848$

C 2.1106945 .2469750 .926244

C 1.4524704 .1839230 .088212

N $5.2277491 .073958-2.744573$

H $-2.429377-4.445621-1.767214$

H -2.698537 -1.484279 -2.479486

H $-0.366003-1.948042-3.258952$

H $-0.218416-4.398622-2.653791$

H $-0.184115-3.971915-0.960360$

H $3.507274-3.350476-1.222721$

H $3.243442-3.431722-3.483630$

H $1.842730-4.379905-3.989170$

H $1.781085-2.603551-4.022173$

H $1.011291-3.2787510 .601001$

H $2.384550-4.3315030 .888435$

H $2.469547-1.2668630 .824129$

H $2.314320-2.2744062 .268255$

H $4.559127-3.4051621 .337295$

H $4.342970-2.1723513 .530934$

H $4.475516-0.6453802 .679571$

H $6.308798-1.1776694 .620490$

H $6.739139-0.1071521 .436715$

H $6.409061-3.4663674 .074390$

H $7.917565-3.1538653 .246604$

H $6.572164-3.9116952 .373052$

H $8.693766-0.9655862 .210131$

H $6.8019780 .081280-0.843923$

H $7.820683-1.286845-1.293943$

H $6.644939-3.384516-0.936407$

H 0.5090380 .1799830 .441460

H $0.4369132 .497945-1.583107$

H 2.3850481 .4054661 .478208

H 0.8266861 .8457072 .218596

H 2.2150852 .9114902 .353129

H $6.605061-1.045021-3.237689$

H $5.572996-2.220747-2.439958$

H $3.3043191 .861822-2.971701$

H $4.6452182 .876271-3.559280$

H $4.2182572 .229731-0.543964$

H $4.6659004 .822774-2.111104$

H 5.4834485 .4179520 .192666

H 4.5579134 .1287210 .919853

H 2.6321537 .7803480 .091330

H $4.3665437 .498290-0.495610$

H 1.3733376 .0472671 .072669

H 2.3613584 .9008251 .932116

H $1.1020304 .551337-0.877664$

H $6.2224491 .268269-2.814351$

C $-5.036553-3.593351-0.588091$

O $-4.748615-2.3935140 .159290$

C -4.441303 -2.764023 1.516026

C $-4.895277-4.2129291 .669518$

C $-4.593108-4.7644920 .289904$

H $-3.348558-2.7339791 .604101$

C $-5.062444-1.7803372 .514035$

H -4.359581-4.733584 2.469031

H $-5.968531-4.2905411 .873394$ 
C $-5.303678-6.069950-0.020104$ H $-3.510588-4.924526 \quad 0.221836$ C $-4.686368-0.300468 \quad 2.289694$ O $-6.492225-1.8471392 .428403$ H -4.797302 -2.076371 3.536626 H -5.2180390 .0785131 .410024$ $\begin{array}{llll}\text { O } & -5.202766 & 0.440043 & 3.405518\end{array}$ C $-3.181075-0.0094122 .148073$ C $-2.799910 \quad 1.4807102 .254827$ H -2.823855 -0.412568 1.193456 O $-2.479857-0.7022383 .185813$ H $-2.967683 \quad 1.8314253 .281266$ H -1.7148711 .5424662 .099067$ H $-4.999017-6.8503840 .684815$ H $-6.391299-5.9620070 .048242$ H $-5.061389-6.413469-1.030659$ C $-4.417108-3.510569-1.986144$ H -6.780886 -2.639469 2.911195 H $-4.713778 \quad 0.1564444 .197103$ H $-1.528268-0.5683373 .035812$ H $-4.790843-2.607683-2.484450$ H $-4.753269-4.362419-2.588823$ H $-6.128172-3.614309-0.706289$ C $-3.8215282 .699615-1.124418$ O $-3.194796 \quad 1.972343-0.059224$ C $-3.517528 \quad 2.409692 \quad 1.262910$ C $-3.114483 \quad 3.8807261 .465155$ C $-3.7114424 .780065 \quad 0.378447$ C $-3.4969924 .200202-1.024576$ H $-3.3539232 .315919-2.040425$ C $-5.3302492 .416991-1.209130$ H $-4.5960052 .317948 \quad 1.421302$ O $-1.694104 \quad 3.994704 \quad 1.444128$ H -3.456156 4.2059892 .455213 H -3.247062 5.7722020 .436948 \begin{tabular}{lllll}
\hline & -5.103437 & 4.937728 & 0.635592
\end{tabular} H $-4.0970314 .751917-1.756779$ O $-2.1295244 .391346-1.397746$ C $-5.6312070 .966586-1.516729$ H -5.839052 2.660016-0.272804 H $-5.7694683 .018412-2.013378$ H $-1.4699914 .925347 \quad 1.614552$ H $-5.463040 \quad 5.528687-0.047650$ H $-1.581303 \quad 3.870728-0.786532$ O $-6.983307 \quad 0.777640-1.468213$ O $-4.795996 \quad 0.110152-1.768137$ C -7.433524 $-0.556848-1.721042$ H $-7.003414-0.945229-2.649180$ H -7.175119-1.199127 -0.874869 H -8.521675 -0.533945 -1.824526

SCF Energy (B3LYP/6-31G**//MMFF) $=-3245.89015004$

\section{7_00400}

MM̄FF Geometry

C -1.514474 -3.2176262 .553579$

C $-0.607431-2.3220242 .138396$

C $-0.228386-1.0790922 .895270$

O $1.210851-0.9514242 .843934$

C -0.8169290 .1579592 .197694$

C -0.7395891 .4070193 .052982$

C -1.7693311 .9141833 .758031$

C $0.591203 \quad 2.115076 \quad 3.067277$

C -3.1499601 .3457693 .919813$

C $-4.268214 \quad 2.3178603 .521537$

C -4.4532912 .6065272 .021532$

C $-4.7788391 .352921 \quad 1.206294$

C $-4.960277 \quad 1.671720-0.284749$

O $-3.291713 \quad 3.261757 \quad 1.498316$

O $-4.9494050 .417350-0.980200$

C $-3.7499982 .505511-0.767685$

C $-6.3328272 .293292-0.569474$

O $-3.9590162 .918191-2.115722$

C -3.450553 3.7173630 .142725

C $-2.1794254 .496834-0.260704$

O $-4.534897 \quad 4.652910 \quad 0.041812$

C $1.976944-1.692298 \quad 3.682675$

O $1.584754-2.4727834 .537402$

C $3.415660-1.4928673 .378403$
C $3.856950-0.4180472 .706847$

C $5.227161-0.1443242 .272619$

C $6.256958-1.2194252 .485668$

C $-0.8742443 .699115-0.345382$

C $0.3150264 .630394-0.546189$

C $2.7785994 .714262-0.546768$

O $0.1890615 .846562-0.681144$

C $3.9017153 .918172-1.131036$

C $5.0549313 .696337-0.480506$

C $6.2603233 .021657-1.074603$

C $6.5654861 .658981-0.491452$

C $6.7435510 .594559-1.292977$

C $6.731906 \quad 1.523367 \quad 1.008564$

C 5.4663081 .0317411 .655505

N $1.5355013 .962317-0.565870$

H -2.046493 -3.0549193 .486834$

H $-0.096827-2.4945411 .195341$

H $-0.558900-1.1265513 .940744$

H $-1.853900-0.0363971 .907538$

H -0.2860720 .3524651 .255272$

H -1.5945112 .8249474 .330214$

H 0.5140783 .1316253 .468004

H 1.3068311 .5683623 .687713

H $0.999626 \quad 2.197798 \quad 2.055536$

H -3.277464 0.3854393 .414988

$\mathrm{H}-3.2718401 .124845 \quad 4.988635$

H $-5.213971 \quad 1.9194123 .911057$

H -4.0972093 .2722594 .037429$

H $-5.296075 \quad 3.306141 \quad 1.959215$

H -5.6685660 .8490851 .603716$

H -3.9620240 .6271631 .291760$

H $-4.9538080 .608334-1.934059$

H -2.883772 $1.831286-0.771932$

H $-7.130927 \quad 1.591715-0.297414$

H $-6.4630162 .493409-1.638747$

H $-6.5103993 .220701-0.021777$

H $-3.1182323 .270163-2.452759$

H -2.350223 $5.014015-1.214556$

H $-2.038104 \quad 5.300122 \quad 0.477224$

H $-4.2241135 .519867 \quad 0.351337$

H $4.081862-2.2732643 .729229$

H 3.1358540 .3437402 .414609

H $5.943529-2.1529202 .006282$

H $6.399417-1.4060913 .555706$

H $7.237637-0.9721702 .074641$

H $-0.719048 \quad 3.1340440 .576781$

H $-0.9041622 .998948-1.186618$

H 2.9677134 .9963660 .494967

H $2.6515215 .636849-1.123660$

H $3.7881163 .568272-2.153573$

H 5.1674314 .0797510 .531463

H $7.1288833 .671425-0.906877$

H $6.1457672 .955050-2.164476$

H $6.982693-0.382705-0.886253$

H $6.6501430 .673924-2.370930$

H 7.0008032 .4931181 .445241

H 7.5866820 .8749231 .222374

H 4.6382381 .7364511 .570003

H $1.5578562 .991434-0.267303$

C -2.856202 -4.3267330 .681597$

O $-2.343431-3.480320-0.366119$

C $-3.438858-2.717270-0.912827$

C $-4.710514-3.294066-0.296804$

C $-4.210290-3.7372041 .066181$

H $-3.314451-1.691619-0.550984$

C $-3.394091-2.756954-2.445560$

H $-5.515425-2.554878-0.240135$

H $-5.082313-4.153596-0.866951$

C $-5.134123-4.7142631 .769956$

H $-4.084310-2.8451051 .692739$

C $-2.097407-2.170470-3.045922$

O $-3.500577-4.120569-2.875006$

H $-4.266487-2.239827-2.860365$

H -1.236702 -2.755879 -2.705412

O $-2.152631-2.315850-4.469854$

C $-1.892270-0.678507-2.731852$

C $-0.646966-0.041205-3.370998$

H $-1.840560-0.526668-1.649573$ 
O $-3.028365 \quad 0.057992-3.194195$ H $-0.733815-0.047200-4.464940$ H $-0.658623 \quad 1.019012-3.084144$ H $-6.113042-4.2573411 .948125$ H -5.289642 -5.6203561 .174932$ H $-4.719473-5.0132312 .737745$ C -1.820292 -4.4789761 .796031$ H $-2.762481-4.612180-2.475166$ H -2.304415 -3.257781 -4.658745 H -3.113114 $-0.101700-4.150048$ H $-0.887068-4.8603431 .360490$ H $-2.156499-5.2366622 .513645$ H $-3.000004-5.3151050 .223794$ C $1.876993-1.370207-0.964955$ O $0.727678-0.715769-1.519159$ C $0.683853-0.681928-2.949588$

C $1.8921140 .091964-3.504726$ C $3.212200-0.472431-2.969210$ C $3.166268-0.692008-1.452409$ H $1.800786-1.1936250 .114445$ C $1.805480-2.882092-1.197929$ H $0.708003-1.706916-3.334659$ O $1.787471 \quad 1.466409-3.134793$ H $1.8724460 .041008-4.600065$ H $4.0237020 .224631-3.211158$ O $3.485380-1.700535-3.635584$ H $4.037872-1.267686-1.124888$ O $3.2562480 .581769-0.807643$ C $2.703684-3.635732-0.249605$ H $0.782863-3.244636-1.034641$ H $2.036995-3.165874-2.228592$ H $2.5304501 .938020-3.548233$ H $4.334184-2.034742-3.298546$ H $2.4598391 .089237-1.039529$ O $3.845461-4.014843-0.890860$ $\begin{array}{lllll}\text { O } & 2.431374 & -3.846975 & 0.924978\end{array}$ C $4.789387-4.733952-0.091618$ H $4.364291-5.6924940 .220355$ H $5.676396-4.924088-0.701658$ H $5.083609-4.1407490 .779107$

SCF Energy (B3LYP/6-31G**//MMFF) $=-3245.91542281$

07_00401

MM̄FF Geometry

C -2.200435 3.3655561 .597401

C - $-1.407722 \quad 3.8165552 .581456$

C -0.4292832 .9761443 .367186$

$\begin{array}{lllll}\text { O } & -0.721643 & 1.569931 & 3.219079\end{array}$

C $0.983112 \quad 3.209802 \quad 2.794740$

C 2.0896022 .5641773 .603113

$\begin{array}{llll}\text { C } 2.579474 & 1.326368 & 3.389179\end{array}$

C $2.663298 \quad 3.428425 \quad 4.695031$

C 2.0903820 .2985482 .406739

C $3.165667-0.226687 \quad 1.458579$

C $3.643611 \quad 0.759212 \quad 0.386214$

C $2.5438621 .144288-0.605798$

C $3.0848912 .030155-1.737703$

O $4.6934560 .084719-0.319138$

O $2.0661092 .087373-2.747390$

C $4.3297281 .358399-2.355874$

C $3.3028613 .472558-1.264855$

O $4.9313852 .236446-3.304531$

C $5.3597380 .879340-1.305244$

C $6.452865 \quad 0.005459-1.975684$

O $5.9930962 .017384-0.713356$

C -1.574276 0.9628334 .081476

O -2.1908961 .4886414 .995995$

C -1.613352 $-0.499143 \quad 3.821074$

C $-1.213418-1.0224812 .651370$

C -1.047212 -2.439354 2.327001

C $-1.552305-3.4522443 .316519$

C $7.244190-0.866782-0.987766$

C $6.556781-2.193171-0.690101$

C $6.269457-3.8955521 .110420$

O $5.916068-2.811455-1.537535$

C $4.833100-3.7398421 .503535$

C $3.850490-4.5287931 .041436$

C $2.401038-4.3830641 .418629$
C $1.438802-4.152998 \quad 0.264691$

C $1.797341-4.007017-1.023394$

C $-0.032224-4.1052720 .644831$

C $-0.420897-2.7500241 .172603$

N $6.779041-2.6317640 .606821$

H -2.188412 2.3107041 .336099

H -1.4117714 .8794572 .813295$

H $-0.469323 \quad 3.2561084 .426690$

H $1.187656 \quad 4.287107 \quad 2.726069$

H 1.0283982 .8451841 .761631

H 3.3886840 .9793774 .030715

H 3.4512942 .9191065 .259743

H 3.0969014 .3396554 .269974

H 1.8802553 .7134595 .404938

H $1.728808-0.5515932 .998662$

H 1.2349830 .6504441 .829302

H $4.033660-0.5650912 .039866$

H $2.785409-1.1290130 .961774$

H $4.056537 \quad 1.6446160 .881868$

H $1.7065621 .646954-0.111646$

H $2.1215700 .231839-1.050036$

H $2.4213432 .595811-3.496816$

H $3.9702630 .486665-2.920768$

H $2.3596643 .901786-0.905228$

H $3.6283034 .115726-2.090072$

H $4.0337893 .553133-0.457430$

H $5.6082501 .734344-3.788414$

H $5.997070-0.639504-2.736045$

H $7.1636410 .660766-2.494968$

H $6.8693231 .755164-0.387905$

H $-1.940971-1.1138224 .652194$

H $-0.919399-0.3438491 .852174$

H -2.604603 -3.262311 3.556957

H $-0.968081-3.4114124 .241980$

H $-1.506532-4.4784802 .945513$

H $8.210950-1.124300-1.437730$

H $7.453880-0.319427-0.063093$

H $6.869465-4.1665001 .984532$

H $6.404892-4.6614220 .339341$

H $4.594019-2.9461462 .206092$

H $4.101659-5.3410710 .362568$

H $2.282416-3.5651592 .140451$

H $2.105880-5.3051441 .935288$

H $1.052253-3.847217-1.796733$

H $2.830519-4.030225-1.349600$

H $-0.239583-4.9162831 .348087$

H $-0.652881-4.310515-0.234622$

H $-0.119528-1.9306760 .516956$

H $7.273846-2.0310761 .259344$

C -2.671926 $4.289752-0.706568$

O $-2.6975332 .968123-1.284767$

C $-1.532338 \quad 2.809748-2.116627$

C $-0.973314 \quad 4.213709-2.323074$

C $-1.2752134 .849938-0.981019$

H $-0.8071792 .223288-1.540170$

C $-1.8933352 .079482-3.412085$

H $0.093164 \quad 4.206737-2.565920$

H -1.499741 $4.746329-3.123739$

C $-1.209503 \quad 6.366573-0.992292$

H $-0.5480294 .469067-0.253467$

C $-2.4842550 .666641-3.218708$

O $-2.858592 \quad 2.857589-4.133027$

H $-1.0111632 .024364-4.060627$

H $-3.4792640 .732370-2.764139$

O $-2.6752640 .105585-4.525047$

C $-1.600484-0.297560-2.402367$

C $-2.004091-1.781271-2.499623$

H $-1.5941460 .010822-1.351395$

O $-0.247363-0.205117-2.861557$

H -1.951973 - $2.108796-3.545815$

H $-1.231461-2.359238-1.975181$

H $-0.2041116 .705565-1.263027$

H $-1.9137436 .794178-1.713710$

H $-1.4487616 .773356-0.004652$

C $-3.0949094 .238703 \quad 0.765155$

H -3.629592 $2.977758-3.552283$

H $-3.2407790 .719894-5.024009$

H $-0.239882-0.442205-3.804891$ 
H -4.1084093 .8253260 .831998$

H -3.1368575 .2556591 .171942$

H -3.422251 $4.873422-1.256264$

C $-4.494439-2.191717 \quad 0.253680$

O $-3.305281-1.907659-0.495508$

C $-3.383182-2.117061-1.909566$

C $-3.790045-3.566182-2.236731$

C $-5.060382-3.978701-1.490631$

C $-4.978821-3.627205-0.002761$

H $-4.183541-2.1308471 .304671$

C $-5.580813-1.1379960 .017946$

H -4.127785 -1.436887 -2.333297

O $-2.730555-4.454904-1.889513$

H -3.944869 -3.648777 -3.319402

H -5.209768 -5.059639-1.602196

O $-6.172332-3.320338-2.089357$

H $-5.955216-3.7732070 .472753$

O $-4.077329-4.5310230 .643028$

C $-5.2048380 .159036 \quad 0.689525$

H $-5.752064-0.915644-1.038924$

H $-6.544501-1.4661370 .425843$

H -2.999358 -5.350999 -2.154325

H $-6.972775-3.610313-1.619499$

H -3.184644 -4.358572 0.297595

O -5.6944450 .1831391 .961859$

O $-4.536801 \quad 1.032406 \quad 0.153186$

C $-5.399578 \quad 1.3632422 .714301$

H -4.3198371 .4634842 .848043$

H -5.8121142 .2454242 .215898$

H -5.8665241 .2654953 .697913$

SCF Energy $\left(\mathrm{B} 3 \mathrm{LYP} / 6-31 \mathrm{G}^{* *} / / \mathrm{MMFF}\right)=-3245.91388165$

\section{00402}

MM̄FF Geometry

C $0.3548851 .856519-2.510960$

C $0.7508820 .712673-3.091570$

C $0.376250-0.693661-2.677630$

O $-0.380306-0.664664-1.446385$

C $-0.478365-1.336453-3.789138$

C $-0.756536-2.815324-3.596383$

C $-1.956284-3.346248-3.283357$

C $0.424087-3.723722-3.833475$

C $-3.255425-2.627933-3.038865$

C -3.729503 -2.819825 -1.592422

C $-4.849597-1.859922-1.161104$

C $-6.156722-2.105330-1.914031$

C -7.240604-1.096881-1.504729

O $-4.391766-0.521685-1.374547$

O $-8.314812-1.195299-2.452464$

C $-6.6599980 .327069-1.625430$

C $-7.841256-1.421095-0.131848$

O $-7.5846841 .295321-1.133142$

C $-5.2880450 .504346-0.930212$

C $-4.6784231 .871529-1.339938$

O $\begin{array}{llll}-5.472638 & 0.458425 & 0.483246\end{array}$

C $-0.079725-1.590311-0.496566$

O $0.757118-2.476033-0.569357$

C $-0.961902-1.3519070 .667110$

C $-0.931686-2.2121021 .695034$

C -1.743712 -2.120203 2.904052

C $-0.985116-2.4831864 .152533$

C $-3.2489182 .123832-0.839318$

C -3.1789482 .6934910 .565567$

C -1.624259 2.9395402 .491331

O $-4.132528 \quad 3.242028 \quad 1.111930$

C -1.9587581 .7999563 .401645$

C -3.1126601 .7178174 .080504$

C $-3.4866490 .577478 \quad 4.981704$

C $-4.489836-0.3585004 .343713$

C -5.7650800 .0154194 .142362$

C $-4.025561-1.7571064 .008935$

C -3.051793 -1.794828 2.861904

N $-1.9176972 .544514 \quad 1.127296$

H $-0.3049201 .823905-1.650882$

H $1.403084 \quad 0.775789-3.961255$

H $\quad 1.315335-1.242908-2.539867$

H $\quad 0.026851-1.219798-4.758132$

H - $1.412298-0.773840-3.884872$
H -2.027319 -4.429753 -3.192808

H $\quad 0.160344-4.782633-3.739505$

H $0.820238-3.573172-4.843140$

H $1.222919-3.521349-3.114384$

H -3.177269 -1.564600 -3.272034

H $-4.003740-3.036746-3.728060$

H $-4.051375-3.857625-1.440046$

H -2.877840 -2.655232 -0.924301

H $-5.004837-2.010782-0.086490$

H -6.508144 -3.132995-1.759705

H $-5.991474-2.003043-2.994695$

H -8.643333 -2.110632 -2.438696

H $-6.5335550 .537367-2.696788$

H -8.290010 -2.421763 -0.137577

H $-8.654353-0.7299140 .116108$

H $-7.107450-1.3940540 .676398$

H -8.425470 $1.157718-1.602285$

H $-4.6316391 .904133-2.436757$

H -5.334936 2.688090 -1.017646

H -4.6651090 .7799050 .912981$

H $-1.622825-0.4932580 .649539$

H $-0.243648-3.0554811 .675959$

H $-0.716250-3.5452344 .142351$

H -1.542002 -2.291151 5.071905

H -0.062263 -1.896075 4.222246

H $-2.6482841 .211985-0.905587$

H -2.780458 $2.872316-1.491102$

H $-2.194743 \quad 3.8407342 .740047$

H -0.5580413 .1770362 .554964$

H -1.221422 1.0076783 .495965

H -3.8460202 .5143413 .974401$

H -3.9074300 .9876565 .908873$

H -2.5932990 .0233715 .293829$

H $-6.491628-0.6613713 .705220$

H -6.1094191 .0093494 .408365$

H $-4.881615-2.3841673 .725304$

H -3.626553 -2.224860 4.914072

H -3.502376 -1.567263 1.896173

H -1.215689 2.0018410 .633197

C $1.5547964 .064392-2.045553$

O $2.7853933 .393640-1.711874$

C $3.0681113 .634504-0.318526$

C 2.1188784 .7437010 .121537

C $0.8944924 .419258-0.714541$

H 2.7910002 .7207140 .218427

C $4.5572413 .926333-0.113834$

H 1.9239654 .7210791 .197999

H $2.5057845 .736731-0.135076$

C $-0.1120565 .551431-0.797859$

H $0.4081053 .540791-0.276541$

C $5.4867432 .814308-0.645280$

O $4.8822275 .138569-0.804430$

H 4.7532724 .1155070 .948079

H $5.4480082 .801248-1.741018$

O $6.8330723 .167691-0.305103$

C $5.183276 \quad 1.405344-0.102759$

C $6.1691690 .347437-0.633784$

H $4.1697651 .106896-0.391179$

O 5.2523891 .4160521 .322326

H $\quad 6.284707 \quad 0.470425-1.717019$

H $7.1462520 .501218-0.159137$

H -0.4894165 .8038800 .198581$

H $0.3335826 .454806-1.227469$

H $-0.9668355 .267105-1.419679$

C $0.7346193 .219831-3.022435$

H $5.8319215 .301591-0.672308$

H 6.9131453 .1173830 .662990

H $4.515871 \quad 1.9553461 .654180$

H $1.3143273 .110331-3.948121$

H $-0.1836243 .755779-3.289352$

H $1.8456334 .987361-2.565614$

C $5.204325-2.5542591 .528740$

O $5.704451-1.2910201 .073432$

C $5.684732-1.088387-0.342304$

C $6.552961-2.144887-1.040475$

C $6.111221-3.560165-0.652646$

C $5.962794-3.716641 \quad 0.865915$

H $5.433232-2.5736392 .602337$ 
C $3.682275-2.6494221 .374521$

H $4.654880-1.172178-0.706917$

O $7.919327-1.950140-0.682673$

H $6.471648-2.009254-2.125612$

H $6.848764-4.280607-1.026472$

O $4.870079-3.839496-1.294426$

H $5.464216-4.6616811 .110809$

O $7.265229-3.7678121 .455785$

C $2.992037-1.6383022 .260656$

H $3.349292-2.5010490 .344211$

H $3.319879-3.6396861 .674753$

H $8.445122-2.614486-1.159493$

H $4.613319-4.746263-1.055228$

H $7.730615-4.5312751 .073552$

O $2.546333-0.5868591 .515890$

O $2.883588-1.7606313 .474093$

C $1.873053 \quad 0.4480272 .240192$

H 2.6002731 .0339292 .808870

H 1.3776021 .1017481 .517515

H $\quad 1.1136070 .0306232 .907258$

SCF Energy $\left(B 3 L Y P / 6-31 G^{* *} / / M M F F\right)=-3245.88634037$

\section{00403}

MM̄̄FF Geometry

C $-3.806154-4.611165-0.278134$

C $-2.686661-4.2432330 .362736$

C -1.303192 -4.392892 -0.216287

O $-0.622666-3.121574-0.103563$

C $-0.508340-5.4283770 .599261$

C $0.840028-5.765939-0.008644$

C $2.028833-5.3060390 .431147$

C $0.791301-6.726210-1.170614$

C $2.295691-4.3487881 .560739$

C $2.890118-3.0223891 .077914$

C $4.321411-3.0801650 .522961$

C $5.349634-3.4332781 .601220$

C $6.793667-3.3285061 .085314$

O $4.592557-1.773501-0.008409$

O $7.670762-3.3444692 .222594$

C $6.979631-1.9616240 .393341$

C $7.180155-4.5375740 .225024$

O $8.260965-1.871129-0.224295$

C $5.874866-1.642875-0.635280$

C $5.982276-0.208498-1.201155$

O $5.998479-2.537593-1.747924$

C $-0.866204-2.184033-1.059612$

O $-1.610846-2.284857-2.020854$

C $-0.057369-0.992677-0.719506$

C $-0.1472990 .126167-1.452521$

C $0.578366 \quad 1.368082-1.183627$

C $1.7938601 .286506-0.296515$

C $5.8349370 .917560-0.174092$

C $5.8524922 .286233-0.835021$

C $5.5997124 .705688-0.305917$

O $6.0642582 .450208-2.033970$

C $4.1964705 .104548-0.637786$

C $3.7472265 .245382-1.894171$

C $2.3536155 .675103-2.251512$

C $1.4794274 .502389-2.643099$

C $1.4361484 .087569-3.920748$

C $0.6712563 .902399-1.511069$

C $0.1386432 .514412-1.743014$

N $5.623811 \quad 3.3070490 .078437$

H -3.734194 -5.053939 -1.268366

H -2.759428 -3.8080121 .356909$

H -1.344543 -4.694301 -1.270360

H -1.085233 -6.3598570 .682325$

H $-0.388044-5.0728081 .628339$

H $2.925366-5.656339-0.078419$

H $1.789076-6.979038-1.544760$

H $0.307070-7.661249-0.870202$

H $\quad 0.228599-6.294583-2.003883$

H $1.379628-4.1179672 .111260$

H $2.961141-4.8322332 .283417$

H $2.240719-2.600600 \quad 0.302573$

H $2.858607-2.2980151 .902870$

H $4.357901-3.801363-0.300184$

H $5.170995-4.4356382 .006902$
H $5.236378-2.7428582 .448872$

H $7.509514-4.1681962 .713943$

H $6.960962-1.2016481 .184363$

H $7.113951-5.4615260 .812120$

H $8.221607-4.469144-0.108192$

H $\quad 6.542947-4.661900-0.652902$

H $8.925719-2.0746730 .455726$

H $6.927026-0.095653-1.749368$

H $5.193951-0.077783-1.956666$

H $5.547091-2.143836-2.512787$

H $0.576949-1.0527380 .157100$

H $-0.8407190 .153648-2.291508$

H $2.4388140 .454038-0.600958$

H $1.501770 \quad 1.1380330 .747264$

H $2.4214812 .179927-0.344706$

H 4.8927310 .8108390 .374323

H 6.6609930 .8918540 .543932

H $6.2780834 .863419-1.151396$

H 5.9670375 .2941230 .540433

H 3.5266685 .2999190 .195870

H $4.4207185 .042688-2.724141$

H $2.4171496 .395801-3.077310$

H $1.8869976 .227346-1.425667$

H $0.8075563 .265902-4.244128$

H $2.0355464 .571668-4.685534$

H $-0.1847894 .560947-1.317241$

H $1.2607693 .928869-0.588801$

H $-0.7431452 .478364-2.383593$

H 5.3889003 .0723201 .038526

C $-6.077476-3.590434-0.587636$

O $-5.569939-2.242167-0.615514$

C $-6.671738-1.323583-0.490803$

C $-7.937189-2.152966-0.673382$

C -7.513912 -3.480437 -0.075961

H $-6.646740-0.9426590 .537207$

C $-6.511204-0.161747-1.477947$

H -8.800474 -1.708868 -0.168615

H $-8.194034-2.279681-1.731299$

C -8.406646 -4.640692 -0.480419

H -7.517389 -3.388844 1.018508

C $-5.1451510 .549725-1.410513$

O $-6.665150-0.654417-2.816045$

H -7.320497 $0.562419-1.330153$

H -4.357237 -0.112779-1.790155

O $\quad-5.1917791 .670803-2.302664$

C $-4.7528021 .057682-0.011301$

C $-3.4043291 .789464-0.025197$

H -4.6868920 .2045920 .669606$

$\begin{array}{llll}\text { O } & -5.763336 & 1.927552 & 0.491746\end{array}$

H -2.637843 $1.125854-0.439425$

H $-3.4507882 .646389-0.710925$

H $-9.434580-4.470084-0.144187$

H $-8.425993-4.772283-1.567513$

H $-8.056826-5.576801-0.034039$

C $-5.185623-4.4591240 .300714$

H $-5.996043-1.347284-2.952324$

H -5.429286 $1.330655-3.182456$

H $-5.8458832 .671291-0.129692$

H $-5.622087-5.4585160 .406888$

H $-5.124623-4.0190101 .303922$

H $-6.063720-3.957849-1.622248$

C -1.031464 3.5718732 .210321

O -1.6506132 .9239301 .094197$

C -2.9356792 .3340131 .335845$

C -2.8316741 .2705992 .438054$

C -2.1986211 .8498743 .708760$

C -0.9084822 .6163393 .408941$

H -0.0175903 .8115721 .864246$

C -1.7282104 .8929172 .550614$

H -3.6340783 .1196541 .643913$

O -2.0523690 .1740841 .965265$

H -3.8327450 .8903012 .669980$

H -1.9752941 .0366334 .410309$

O -3.1206112 .7307864 .344358$

H -0.5876303 .1732594 .297314$

O 0.1138651 .6569353 .126412

C -1.5566585 .8835241 .423148$

H -2.7965354 .7796112 .756145$ 
H $-1.2884445 .345823 \quad 3.447159$ H $-2.017651-0.4899492 .674508$ H -3.9195462 .2181054 .554662$ H 0.9466232 .1430533 .001627 O -2.6454865 .8500940 .602940$ O -0.5621516 .5823341 .276416$ C $-2.6014036 .738998-0.517371$ H $-1.7346646 .518207-1.146985$ H -3.509764 $6.587877-1.106546$ H $-2.5739687 .777194-0.173204$

SCF Energy (B3LYP/6-31G**//MMFF) $=-3245.90636712$

\section{00404}

MM̄FF Geometry

C $0.605879 \quad 5.390250 \quad 0.260005$

C 1.4436334 .3438630 .295419

C 2.1145613 .8522591 .551734

O $1.7371502 .468861 \quad 1.752914$

C 3.6418823 .9202431 .377765

C 4.4001733 .5856262 .646770

C 4.9593572 .3881412 .909635

C 4.5353284 .7178403 .631641

C 4.8954861 .1496612 .063296

C $6.282472 \quad 0.7018521 .586474$

C $6.165613-0.5143200 .657891$

C $7.527793-1.1532610 .394883$

C $7.414815-2.337916-0.577686$

O $5.599987-0.070087-0.582012$

O $8.752503-2.666415-0.982195$

C $6.628289-1.886950-1.828328$

C $6.848229-3.5839870 .113180$

O $6.343858-2.990161-2.686274$

C $5.316665-1.128482-1.503584$

C $4.726169-0.533631-2.809551$

O $4.374660-2.050315-0.956612$

C 0.5418612 .2220002 .350144

O -0.2485003 .0589422 .766706$

C 0.2460110 .7709772 .427587

C $1.112884-0.1948832 .086106$

C $0.866595-1.6399582 .115130$

C $-0.501216-2.1028622 .542735$

C $3.783170 \quad 0.662030-2.641704$

C $2.4694000 .369509-1.951051$

C $0.280055-0.777400-2.249344$

O $2.206700 \quad 0.855503-0.853552$

C $0.211666-2.072166-1.497475$

C $1.101572-3.076165-1.519397$

C $0.955603-4.337819-0.715885$

C $2.070219-4.5161180 .291292$

C $3.180505-5.203856-0.025061$

C $1.857303-3.9755971 .689723$

C $1.863794-2.4716661 .743678$

N $1.624577-0.445074-2.685605$

H 0.3887435 .9342961 .175904

H $1.6510743 .796373-0.621268$

H 1.8132804 .4458922 .424029

H 3.9370344 .9293541 .058828

H 3.9556013 .2590470 .561105

H 5.4958472 .2646873 .849356

H 5.1143944 .4327914 .516453

H 5.0438905 .5679953 .165137

H 3.5502395 .0475903 .975671

H 4.4514930 .3517152 .671934

H 4.2321601 .2773251 .204532

H $6.769614 \quad 1.5293761 .055058$

H 6.9005020 .4521712 .457489

H $5.491824-1.2403581 .128200$

H $7.998764-1.4725961 .332880$

H $8.203637-0.404529-0.041759$

H $8.710877-3.447964-1.558590$

H $7.285072-1.212172-2.395190$

H $7.502613-3.8934010 .936938$

H $6.808643-4.436045-0.574402$

H $5.848621-3.4283610 .523512$

H $7.191825-3.379194-2.958304$

H $5.552877-0.167305-3.432419$

H $4.225069-1.325072$-3.380261

H $3.492373-1.651105-1.004832$
H $-0.750954 \quad 0.5372632 .788397$

H 2.1041240 .0898751 .743339

H $-0.687611-1.8311043 .587601$

H $-0.645232-3.1817622 .462809$

H -1.275358 -1.644671 1.918579

H $4.2833081 .473864-2.100519$

H $3.5450241 .068345-3.633349$

H $-0.361276-0.823806-3.134801$

H $-0.0858880 .028770-1.604644$

H -0.665752 -2.181792 -0.864235

H $1.976292-3.010307-2.160667$

H $0.946321-5.183206-1.416484$

H $-0.011855-4.377214-0.201099$

H $3.972518-5.3644980 .698460$

H $3.324201-5.616424-1.018219$

H $2.657988-4.3248302 .355293$

H $\quad 0.941675-4.4131302 .095994$

H $2.819985-2.0334621 .454676$

H $1.940302-0.818984-3.575522$

C $-1.5810475 .592013-0.933372$

O $-1.8100854 .169654-0.999531$

C $-2.9077573 .911971-1.892354$

C $-3.5531025 .265126-2.165242$

C $-2.3520586 .187553-2.109925$

H -2.473316 $3.524431-2.822602$

C $-3.8651142 .866286-1.304718$

H -4.072268 $5.290912-3.128089$

H $-4.2753635 .531982-1.384716$

C -2.717899 $7.651029-1.934289$

H -1.774918 $6.069332-3.036971$

C $-3.2166901 .477544-1.111462$

O $-4.3831483 .344488-0.064381$

H $-4.7168762 .760288-1.987051$

H -2.757432 $1.173128-2.060167$

O $-2.178051 \quad 1.533019-0.134926$

C $-4.2277620 .388156-0.693617$

C $-3.568719-1.003219-0.634502$

H $-5.0510750 .364476-1.417280$

$\begin{array}{lllll}0 & -4.779539 & 0.683079 & 0.586913\end{array}$

H -2.998046 -1.169041-1.555637

H $-2.873010-1.0238990 .212513$

H -3.326518 $7.998674-2.775439$

H -3.290751 $7.814063-1.015201$

H -1.820227 8.275231-1.886670

C $-0.0785805 .867129-0.990119$

H -3.6329503 .4294140 .549394$

H -1.511131 $2.168485-0.445687$

H -5.2506201 .5303110 .518535$

H $0.1102246 .941514-1.093109$

H $0.3566875 .373483-1.868142$

H -2.0026405 .9395540 .019150$

C $-6.236515-2.9790871 .103836$

O $-5.206110-2.0259690 .813205$

C $-4.591091-2.146847-0.471890$

C $-3.929096-3.526706-0.612645$

C $-4.934535-4.651494-0.342256$

C $-5.713456-4.4181570 .956858$

H $-6.475023-2.8156882 .162986$

C -7.508606 -2.707776 0.292880

H $-5.351685-2.028473-1.251521$

O $-2.842073-3.6285890 .304365$

H -3.517562 -3.620832 -1.624620

H $-4.404185-5.609250-0.275896$

O $-5.856440-4.742444-1.424261$

H $-6.552475-5.1197581 .033640$

O $-4.850745-4.6742952 .069075$

C -8.113218 -1.380032 0.683451

H -7.341193 -2.711223 -0.787824

H $-8.266053-3.4762310 .487979$

H -2.434195 -4.502391 0.181557

H $-5.344983-4.918669-2.232295$

H -4.559083 -5.599743 2.007063

O $-7.760580-0.422585-0.220721$

O $-8.795849-1.2113891 .685691$

C $-8.2441120 .895378 \quad 0.056130$

H -7.851975 $1.568716-0.710779$

H -9.3371990 .9120160 .014673$

H -7.8884251 .2344151 .033900$ 
C $-4.853352-3.828865-1.835334$

C $-5.586445-2.621691-1.226188$

C $-6.971278-2.461626-1.857890$

C $-7.695887-1.215868-1.327125$

O $-4.799937-1.449990-1.468797$

O $-8.847498-0.983987-2.152334$

C $-6.7560910 .000925-1.454103$

C $-8.248861-1.4406230 .084350$

O $-7.3292671 .153006-0.839565$

C $-5.334349-0.248690-0.893401$

C $-4.4141130 .929137-1.293046$

O $-5.397872-0.330280 \quad 0.531151$

C -1.132342 -0.3696112 .982713$

O $-2.007881-0.5293093 .817760$

C -0.4035050 .8936842 .734109$

C -0.8584422 .0340693 .273649$

C $-0.251943 \quad 3.3599943 .131150$

C 1.0904633 .4357502 .454517

C $-2.9304260 .713608-0.978014$

C $-2.1093301 .832147-1.586456$

C $-1.2633234 .116684-1.176529$

O $-1.589814 \quad 1.720324-2.695180$

C -1.633846 $5.290752-0.330530$

C -0.7709695 .8943170 .499629$

C $-1.1020867 .081308 \quad 1.354857$

C -1.490961 6.7158602 .770302

C -2.6390477 .1687873 .302872$

C -0.5310195 .8829173 .595071$

C -0.9208104 .4277583 .614523$

N $-2.0619402 .968369-0.793626$

H $1.251578-2.2727920 .878374$

H $-0.500116-4.6611951 .639764$

H -1.334646 -2.9069623 .220441$

H -2.404888 $-2.207970 \quad 0.429679$

H $-3.227421-1.9037151 .915096$

H $-4.202480-5.5308710 .365747$

H -4.268534 -5.430597 2.645022

H -2.710421 -4.8680533 .253619$

H -4.143220 -3.819407 3.352310

H -2.837632 -3.278312 -1.264028

H -3.022515 -4.954499-1.712084

H -4.657039 -3.632957 -2.897420

H $-5.495536-4.717363-1.783600$

H -5.687049-2.771703 -0.146087

H -7.582470 -3.358033 -1.694347

H $-6.863406-2.367628-2.947536$

H -8.545862 -0.861062 -3.068452

H $-6.665748 \quad 0.234736-2.524033$

H -8.960384 -2.275309 0.086221

H -8.812161 -0.5675230 .432125$

H -7.474090 -1.664895 0.820338

H -8.214311 $1.276646-1.223441$

H $-4.5013171 .098296-2.374833$

H $-4.7489901 .848656-0.796208$

H -4.532174 -0.0972030 .900954$

H $\quad 0.480810 \quad 0.8503852 .108328$

H -1.769595 1.9992823 .869606

H $1.516374 \quad 4.4407082 .438682$

H 1.0107633 .1044921 .413662

H 1.8183572 .7988952 .969651

H -2.748402 $0.674850 \quad 0.101055$

H -2.566465 -0.230133 -1.397429

H -1.440257 4.338516 -2.234229

H $-0.2104613 .837484-1.063513$

H $-2.6521015 .663300-0.408963$

H -1.894853 7.6692740 .874023

H $-0.225058 \quad 7.7401411 .387232$

H -2.918132 6.9315094 .324376

H -3.3256347 .7843082 .731259$

H $-0.5383576 .241743 \quad 4.633286$

H $\quad 0.4931086 .0504843 .250994$

H -1.8832034 .2506604 .098059$

H -2.3758832 .8990390 .170690$

C $3.263247-4.2554701 .022071$

O $3.929595-3.0509690 .589830$

C $5.323370-3.3430460 .368811$

C $5.545739-4.7684440 .864737$

C $4.190434-5.3966470 .610485$

H $5.484034-3.321679-0.714936$

C $6.204676-2.2967051 .059261$

H $6.359130-5.269340 \quad 0.330686$

H $5.783972-4.7934641 .934649$

C $3.969767-6.6874781 .379779$

H $4.089492-5.593778-0.465355$

C $5.987023-0.8542510 .554014$

O $5.911093-2.3277392 .462134$

H $7.259248-2.5796720 .956505$

H $4.970815-0.5218350 .793551$

O $6.879048-0.000481 \quad 1.282618$

C $6.266625-0.659266-0.951682$

C $6.3135060 .813042-1.408290$

H $5.516722-1.196474-1.541292$

O $7.534011-1.242029-1.273552$

H $7.0487311 .362319-0.807517$

H $6.7014060 .828379-2.436230$

H $4.715580-7.4368601 .094795$

H $4.048819-6.5289372 .460658$

H $2.978999-7.1032841 .172474$

C $1.875040-4.3375190 .386628$

H $6.614192-1.8354862 .918551$

H 6.5516510 .0514722 .196557

H $8.209206-0.788288-0.740283$

H $1.459092-5.3424160 .520599$

H $1.945938-4.151852-0.691812$

H $3.182366-4.2032382 .116243$

C $2.8236101 .492217-2.539862$

O $4.1552970 .972628-2.422170$

C $4.9515871 .521040-1.365724$

C $5.0956593 .040863-1.538657$

C $3.7273483 .719076-1.645454$

C $2.8269453 .025158-2.670089$

H $2.4509181 .081498-3.487498$

C $1.9196870 .967816-1.418386$

H $4.4723971 .317996-0.404124$

O $5.8720703 .340606-2.696716$

H $5.6392063 .444122-0.676085$

H $3.8710954 .767944-1.932514$

O $3.1072373 .694961-0.363903$

H $1.8030193 .408798-2.596663$

O $3.3056673 .368964-3.974186$

C $1.596062-0.485355-1.678359$

H $2.364998 \quad 1.028939-0.421977$

H $0.9828071 .534056-1.379261$

H $5.4348602 .930488-3.462488$

H $2.2631024 .171746-0.439221$

H $2.6891542 .989126-4.623333$

O $0.315286-0.605933-2.126010$

O $2.401510-1.396013-1.537333$

C $-0.107152-1.923819-2.490385$

H $-0.327366-2.500912-1.589624$

H -1.023110 -1.834116 -3.080536

H $\quad 0.649653-2.428778-3.098460$

SCF Energy (B3LYP/6-31G**//MMFF) $=-3245.90546008$

0700406

MM̄FF Geometry

C $1.435526-4.040161-1.176643$

C $0.485874-3.102424-1.053302$

C $-0.218055-2.464741-2.223299$

O $-1.638270-2.613135-2.004582$

C $0.108058-0.960979-2.239306$

C $-0.171784-0.187335-3.514798$ 
C $0.3683821 .030966-3.727196$ C $-1.084154-0.788844-4.547843$ C $1.2182011 .807608-2.756904$ C $0.8165983 .284020-2.638925$ C $-0.6609443 .531699-2.290677$ C $-0.9060605 .032360-2.103701$ C $-2.3511605 .332988-1.687849$ O $-0.9928882 .821745-1.091819$ O $-2.3825036 .695549-1.236540$ C $-2.7222954 .427520-0.495760$ C $-3.3069905 .255320-2.883990$ O $-4.0994234 .583969-0.157783$ C $-2.3731772 .933946-0.711937$ C -2.5922482 .1628570 .611881$ O $-3.2429432 .401611-1.713163$ C $-2.233942-3.744538-2.458165$ O $-1.711696-4.625407-3.127886$ C $-3.637701-3.845686-1.993566$ C $-4.268491-2.890745-1.291593$ C $-5.621975-2.978343-0.732841$ C $-6.384590-4.257236-0.964350$ C $-2.1776120 .687654 \quad 0.575405$ C $-2.443886-0.0114641 .896949$ C $-2.555980-2.2885562 .893657$ O -2.8167890 .5811242 .906036$ C $-3.956703-2.7719082 .677891$ C $-5.029883-2.2098063 .254504$ C $-6.449386-2.6354833 .003869$ C $-7.219950-1.5995872 .210786$ C $-7.774270-0.5373512 .819433$ C $-7.402000-1.8277790 .723258$ C $-6.092442-1.936543-0.013325$ N $-2.214026-1.3799071 .813843$ H $1.700501-4.398018-2.167488$ H $\quad 0.210372-2.750252-0.062085$ H $\quad 0.084574-2.933054-3.167445$ H $1.176826-0.848128-2.010958$ H $-0.458406-0.474570-1.438885$ H $\quad 0.1651641 .534375-4.670886$ H -1.247407 -0.115672 -5.396683 H $-0.663617-1.715423-4.949361$ H -2.066380 -0.999399-4.113802 H $1.1935421 .366745-1.755752$ H $2.2599551 .758235-3.095787$ H $1.4509613 .729719-1.862251$ H $1.0561813 .800272-3.577323$ H -1.279312 $3.153277-3.112513$ H -0.645968 5.586135-3.014609 H $-0.2350175 .415629-1.322147$ H -3.277600 $6.873485-0.899671$ H $-2.157158 \quad 4.7938560 .372066$ H -3.009284 $5.975066-3.656057$ H $-4.3269575 .533596-2.596642$ H -3.336498 4.266612 -3.346407 H -4.621662 $4.082949-0.807250$ H -2.019376 2.6527611 .410402 H -3.651758 2.2134980 .894406 H $-3.198557 \quad 1.433498-1.686377$ H $-4.121751-4.785763-2.239489$ H -3.741981 -1.964238 -1.072238 H $-7.389738-4.255557-0.539881$ H -5.855251 -5.106196 -0.517311 H -6.505073 -4.442890 -2.037669 H $-2.7294170 .151663-0.202452$ H $-1.108891 \quad 0.6025470 .364774$ H -2.444924 -1.777698 3.855912 H -1.857082 -3.129588 2.864964 H -4.088962 -3.6082121 .996701$ H $-4.885727-1.3702673 .931364$ H -6.935321 -2.797406 3.974855 H -6.491598 -3.605559 2.493399 H -8.346652 0.1989412 .265122 H -7.665534 $-0.380923 \quad 3.887679$ H -7.962471 $-0.997628 \quad 0.274430$ H -8.032651 -2.7110790 .597419$ H $-5.463767-1.0502520 .086553$ H -1.977407 -1.7856970 .912841$ C $3.660506-4.3019620 .031024$
O $3.870182-2.8783180 .148638$

C $5.034511-2.527321-0.626283$

C $5.687546-3.844603-1.043801$

C $4.484904-4.754865-1.172899$

H $4.670291-2.018769-1.526125$

C $5.977468-1.6106200 .160902$

H $6.255598-3.746883-1.974113$

H $6.369899-4.222447-0.273126$

C $4.832783-6.232861-1.166020$

H $3.977406-4.511598-2.113718$

C $5.387640-0.2458960 .571548$

O $6.371821-2.2998731 .354839$

H $6.893931-1.455534-0.422288$

H $4.635281-0.3801431 .357555$

O $\quad 6.454856 \quad 0.515078 \quad 1.155911$

C $4.7853730 .581592-0.582374$

C $4.6020172 .083244-0.279121$

H $3.8250040 .140875-0.874493$

O $5.6509120 .490281-1.719658$

H $5.5870582 .557406-0.180085$

H $4.1473142 .538048-1.169931$

H $5.490423-6.477011-2.006678$

H $5.347237-6.520573-0.242970$

H $3.930389-6.845877-1.254489$

C $2.166069-4.636180-0.007038$

H $7.132925-1.8256491 .729570$

H 6.6659960 .1118972 .014663

H $5.2146330 .949870-2.457485$

H $1.707994-4.2850780 .925392$

H $2.031155-5.723541-0.032754$

H $4.076867-4.7417740 .947611$

C 1.4835702 .0857221 .768106

O 2.4236651 .9169250 .700230

C 3.7435042 .3986530 .955466

C 3.7166773 .9057671 .259964

C 2.7536524 .2198742 .408620

C 1.3871243 .5589542 .198780

H $\quad 0.518308 \quad 1.8071091 .334107$

C 1.7620751 .1259942 .934051

H $4.161373 \quad 1.8728261 .818768$

O 3.3217414 .6243950 .093435

H 4.7315774 .2304571 .520239

H 2.6290825 .3068662 .485446

O $3.3340483 .763963 \quad 3.626898$

H 0.7779153 .6325923 .107129

O $0.6856974 .261544 \quad 1.169851$

C $1.608295-0.3258322 .531608$

H $2.774041 \quad 1.2435523 .329696$

H $1.043057 \quad 1.322228 \quad 3.738164$

H 3.3422745 .5719040 .310626

H $2.720156 \quad 3.988828 \quad 4.346714$

H 0.5765775 .1819441 .463329

O $1.709576-1.1040293 .649690$

O $1.441906-0.7304071 .389629$

C $1.618279-2.5147563 .430957$

H $2.516535-2.8668692 .915853$

H $1.555655-3.0067034 .405201$

H $\quad 0.720447-2.7621112 .857083$

SCF Energy (B3LYP/6-31G**//MMFF) $=-3245.89516068$

0700407

MM̄FF Geometry

C $4.481524-2.463148-0.234774$

C $3.875136-2.755623-1.394182$

C $2.390393-2.624919-1.582221$

O $2.200880-1.393329-2.324077$

C $1.769178-3.760408-2.410039$

C $1.623692-5.076172-1.678428$

C $0.540754-5.405312-0.946481$

C $2.742961-6.063047-1.871914$

C $-0.631677-4.518660-0.634449$

C $-1.818259-4.760788-1.571286$

C $-3.050574-3.934370-1.166469$

C $-4.181662-4.132952-2.174736$

C $-5.407064-3.278835-1.823656$

O $-2.687689-2.552797-1.130038$

O $-6.278425-3.264908-2.965866$

C $-4.966302-1.815372-1.581247$ 
C $-6.212392-3.906426-0.678115$

O $-6.058222-1.056849-1.067877$

C $-3.726684-1.669788-0.665160$

C $-3.098018-0.258392-0.715623$

O $-4.040319-1.9186810 .707017$

C $1.171132-0.583281-1.962697$

O $0.358002-0.774410-1.073628$

C $1.2079460 .599433-2.852314$

C $\quad 0.2484521 .531787-2.757949$

C $0.1409172 .750494-3.563630$

C $1.2324503 .019283-4.566850$

C $-3.9885750 .891303-0.241185$

C -3.2011142 .1587080 .052275$

C -3.5447064 .5769400 .551921$

O

C $-3.3561915 .326505-0.728949$

C $-2.1877975 .865614-1.108919$

C $-2.0060176 .648125-2.377199$

C $-0.9247886 .081558-3.269777$

C $0.2579446 .705496-3.402614$

C $-1.2476314 .848436-4.083182$

C $-0.9134113 .569610-3.362427$

N $-4.039368 \quad 3.2460370 .255529$

H $3.876298-2.1675850 .619295$

H $4.475549-3.015407-2.262151$

H $1.895597-2.555059-0.605643$

H $2.341935-3.910853-3.335726$

H $0.784385-3.435925-2.772799$

H $0.497549-6.394766-0.494394$

H $2.548522-7.014966-1.366438$

H $2.880449-6.278371-2.936592$

H $3.680950-5.665877-1.473104$

H $-0.932294-4.7040760 .404030$

H $-0.334242-3.468091-0.679711$

H -1.517033 -4.491001-2.591787

H -2.080275 -5.826203 -1.580601

H -3.359944 -4.251367 -0.163634

H -4.458661 -5.191971 -2.248073

H $-3.834219-3.843496-3.176494$

H $-6.502585-4.185860-3.183926$

H $-4.715092-1.381337-2.559157$

H $-6.579047-4.898858-0.967655$

H -7.105420 -3.315100 -0.448474

H $-5.630388-4.0286600 .237742$

H -6.794263 -1.144683 -1.697543

H $-2.192128-0.278357-0.096728$

H -2.742299 -0.046940 -1.732872

H $-4.983761-2.1316800 .779511$

H $2.0226380 .667868-3.564259$

H $-0.5419731 .388303-2.024053$

H $2.2104243 .047876-4.073143$

H $1.1243863 .974006-5.085361$

H $1.2487752 .238210-5.334821$

H $-4.7334381 .121157-1.010904$

H -4.5127330 .6194760 .681933$

H -2.6160924 .4992881 .127151$

H -4.297069 5.0774561 .169113

H -4.230349 $5.446930-1.364561$

H -1.318308 $5.761500-0.463753$

H -1.761741 $7.680857-2.096205$

H -2.942695 $6.711762-2.945030$

H $1.0260476 .324741-4.066994$

H $\quad 0.4863167 .609067-2.847068$

H $-0.7518264 .900595-5.058446$

H -2.317624 $4.839475-4.328093$

H -1.648674 $3.289959-2.607918$

H -5.0396093 .1334310 .118090$

C $6.556546-1.014850-0.061439$

O $5.798707-0.142363 \quad 0.805845$

C 5.3124470 .9580590 .015412

C $6.3299491 .103389-1.104666$

C $6.576337-0.354585-1.447188$

H $4.3543760 .644749-0.419193$

C 5.0876532 .2047450 .872507

H $5.9459481 .683314-1.949099$

H $7.2565721 .571472-0.752949$

C $7.865485-0.591736-2.215584$

H $5.740462-0.709106-2.061421$
C 4.2032161 .9696862 .114458

O $6.3571342 .686623 \quad 1.328244$

H $4.664483 \quad 3.0001550 .247207$

H 4.7754761 .4153672 .868261

O 3.9297153 .2500712 .697690

C 2.8751811 .2352401 .845738

C $2.0067391 .142608 \quad 3.109274$

H 3.1043510 .2330681 .474550

$\begin{array}{llll}\text { O } & 2.159601 & 1.926683 & 0.824304\end{array}$

H 2.6157930 .8062323 .958303

H 1.6474212 .1443273 .381987

H $7.844158-0.064346-3.174746$

H $8.737756-0.236772-1.656470$

H $8.005121-1.658471-2.417934$

C $5.970504-2.435519-0.031833$

H $\quad 6.189873 \quad 3.4780191 .868485$

H 3.3592543 .7375152 .078568

H $1.407981 \quad 1.3703370 .559471$

H $6.165827-2.8794950 .952332$

H $6.481261-3.067084-0.767977$

H $7.571633-1.0509730 .352930$

C $-1.129079-0.4256284 .363260$

O $\quad 0.090970 \quad 0.317818 \quad 4.245034$

C 0.7680310 .2322792 .982242

C $1.109413-1.2310902 .656324$

C $-0.120204-2.1391342 .755870$

C $-0.893467-1.9137224 .056581$

H $-1.403710-0.3464685 .423408$

C -2.2656570 .1966693 .536079$

H $0.114146 \quad 0.6439252 .206915$

O $2.123888-1.7130123 .535809$

H $1.514504-1.2900561 .641152$

H $0.196911-3.1873522 .697328$

O $-0.996128-1.8876831 .664129$

H -1.856657 -2.436717 4.027705

O $-0.148760-2.4650565 .145775$

C -2.7087551 .5347744 .090293$

H -1.9808140 .3600832 .494521$

H -3.134227 -0.4719693 .564735$

H $1.802271-1.6055684 .447384$

H $-0.472369-1.9192030 .846395$

H $-0.038986-3.4158094 .973786$

O -3.8818121 .8958873 .490033$

O -2.1035862 .1887224 .928350$

C -4.4295443 .1476593 .914921$

H -4.6936283 .1024624 .975709$

H -3.7185973 .9581593 .728055$

$\mathrm{H}-5.3366083 .3363663 .334653$

SCF Energy (B3LYP/6-31G**//MMFF) $=-3245.92116878$

0700408

MM̄FF Geometry

C $-2.2097233 .605839-0.125482$

C -2.6225263 .4532571 .141984$

C -3.4742272 .2963161 .597774$

O -2.7030251 .5146312 .548397$

C -4.7515202 .7551762 .327181$

C -5.9570612 .8292971 .415450$

C -6.9215941 .8891931 .366008$

C -6.0487844 .0542990 .545285$

C -7.0052580 .6142212 .160910$

C $-5.965746-0.4573901 .812394$

C $-5.809377-0.7753820 .318959$

C $-7.077940-1.376646-0.288165$

C $-6.869718-1.782020-1.755421$

O $-4.725539-1.7099200 .205468$

O $-7.970819-2.617978-2.143072$

C $-5.591555-2.639265-1.863417$

C $-6.897503-0.567086-2.691213$

O $-5.275930-2.909999-3.227754$

C $-4.366519-2.022701-1.146957$

C -3.207482 -3.047071-1.124398

O $-3.944079-0.860311-1.861605$

C -1.7277880 .7100622 .044510$

$\begin{array}{llll}\mathrm{O} & -1.422198 & 0.565123 & 0.871497\end{array}$

C -1.0576820 .0462743 .180726$

C $0.227213-0.3114613 .043563$

C $1.060753-0.9558734 .058895$ 
C $0.364206-1.5497755 .253646$ C $-1.998153-2.632100-0.274777$ C $-0.887414-3.668497-0.330875$ C $1.428507-4.0779120 .463606$ O $-0.956095-4.687644-1.013395$ C $2.013076-4.0940491 .839159$ C $3.273190-3.7225172 .111622$ C $3.894143-3.8038833 .477383$ C $4.412436-2.4696213 .963543$ C $5.693868-2.113398 \quad 3.772317$ C $3.461481-1.5831334 .738856$ C $2.395104-0.9870313 .862411$ N $0.192953-3.3168340 .469205$ H -2.537382 $2.897458-0.882775$ H -2.2719304 .1434341 .905502$ H -3.7402921 .6394590 .759506$ H -4.601643 3.7305112 .809387 H -4.9574802 .0748653 .162613$ H -7.750178 2.0401210 .675336 H $-6.9959574 .103690-0.002533$ H -5.9771934 .9610041 .154848$ H $-5.2427204 .063936-0.193572$ H -6.9451290 .8450013 .231570$ H -8.0089130 .1940982 .017872$ H $-4.987323-0.1638372 .210373$ H $-6.213875-1.3792452 .355421$ H $-5.5273440 .142552-0.207443$ H -7.925703 $-0.686384-0.207594$ H -7.364400 -2.2715810 .281969$ H -8.791529 -2.113102 -2.010837 H -5.809152 -3.610634 -1.397461 H -7.859382 -0.046051-2.613355 H $-6.807743-0.873430-3.739171$ H $-6.1118810 .159886-2.474943$ H -6.063868 -3.310915 -3.633180 H -3.578180 -4.000967 -0.726016 H -2.865851 -3.234040 -2.150691 H -3.054366 -0.618145 -1.562110 H -1.621318 -0.0941284 .095134$ H $0.729682-0.0892152 .102396$ H $-0.417252-2.2486804 .934161$ H $-0.096677-0.7626735 .860423$ H $1.029157-2.1107215 .913016$ H - $1.580575-1.688180-0.636775$ H $-2.295877-2.5037880 .771863$ H $2.095116-3.607122-0.266772$ H $1.231641-5.103200 \quad 0.134368$ H $1.381264-4.4656072 .642535$ H $3.910869-3.3758831 .301095$ H $4.719399-4.5263203 .425984$ H $3.192064-4.2146684 .213717$ H $6.079452-1.1677834 .138281$ H $6.384001-2.7574403 .237182$ H $4.011881-0.7505965 .196140$ H $3.055039-2.1627485 .571754$ H $2.791108-0.4991792 .972115$ H $\quad 0.212916-2.3849020 .873634$ C $0.1222604 .023947-0.869672$ $\begin{array}{lllll}\text { O } & 0.704628 & 3.543732 & 0.358284\end{array}$ C 2.1300243 .7770660 .327312 C $2.4432694 .271492-1.077753$ C $1.1557344 .982173-1.452314$ H 2.3174694 .5672751 .064323 C $2.8377812 .479178 \quad 0.729677$ H $3.3194584 .926113-1.106395$ H $2.6264083 .428568-1.756314$ C $1.0082115 .219181-2.945033$ H $1.1169475 .944663-0.925122$ C 4.3721832 .5045540 .605835 O 2.4852572 .1941022 .088120 H 2.4217861 .6614960 .131325 H $4.6812832 .724430-0.421790$ O $4.8936153 .563270 \quad 1.418680$ C 5.0491671 .2010841 .076964 C $4.550922-0.0788470 .389118$ H 4.9122161 .0863312 .159316 O $\quad 6.4599751 .3101020 .878966$ H $5.132161-0.9333410 .758885$
H $3.522621-0.2801950 .714546$

H $1.8216085 .851264-3.316060$

H $1.0315934 .278116-3.504970$

H $0.0617125 .720772-3.169215$

C -1.235463 $4.661723-0.569036$

H 2.8800042 .8857812 .646425

H 4.6181974 .4057901 .020064

H 6.7553902 .1172391 .334172

H -1.639033 $5.154075-1.460329$

H -1.1264845 .4258690 .210318$

H $-0.0006403 .153155-1.526773$

C $3.626346-1.413889-2.956371$

O $3.828517-1.250051-1.547197$

C $4.575481-0.088596-1.153655$

C $5.981313-0.131316-1.772733$

C $5.922579-0.326848-3.293288$

C $4.976444-1.462796-3.692450$

H $3.149106-2.396882-3.058711$

C $2.661529-0.363854-3.531497$

H $4.0421100 .795419-1.512074$

O $6.725888-1.196233-1.185338$

H $6.5047270 .803892-1.549103$

H $6.927060-0.551739-3.672856$

O $5.487236 \quad 0.879338-3.914293$

H $4.810568-1.449821-4.776151$

O $5.621333-2.700944-3.381216$

C $1.271078-0.468036-2.942362$

H $3.0131400 .654484-3.347681$

H $2.567705-0.511449-4.613655$

H $7.617810-1.180057-1.572096$

H $6.124021 \quad 1.576291-3.681220$

H $5.042276-3.419573-3.687530$

O $0.509478 \quad 0.555522-3.432427$

O $0.893736-1.333287-2.165221$

C $-0.8470880 .582865-2.979946$

H $-0.8828360 .658107-1.889523$

H $-1.3313851 .464984-3.407038$

$\mathrm{H}-1.375031-0.310926-3.324956$

SCF Energy $\left(B 3 L Y P / 6-31 G^{* *} / / M M F F\right)=-3245.89610793$

\section{9}

MM̄FF Geometry

C $-0.097423-1.705860-0.779627$

C $-0.571640-0.713428-0.009187$

C $0.014072-0.224897 \quad 1.296001$

O $1.149349-1.0516951 .642284$

C $0.502846 \quad 1.232271 \quad 1.207558$

C -0.5425092 .2427760 .794713$

C $-0.6229802 .791399-0.433962$

C $-1.505642 \quad 2.6447841 .878773$

C $0.2320052 .475721-1.631364$

C $1.0997313 .653667-2.091506$

C $2.1071384 .149994-1.041852$

C $2.9586755 .283888-1.619821$

C $4.0282445 .762210-0.627508$

O $2.9468863 .057803-0.649793$

O $4.968566 \quad 6.573325-1.349003$

C $4.8024464 .535409-0.099700$

C $3.4343396 .659126 \quad 0.465256$

$\begin{array}{lllll}\text { O } & 5.702638 & 4.914258 & 0.939752\end{array}$

C 3.8846363 .3864850 .384662

C $4.6909592 .115036 \quad 0.754377$

O 3.2115943 .8116701 .577506

C $1.531570-1.1400612 .938938$

O $0.992939-0.6046043 .897399$

C $2.751904-1.9723313 .079562$

C $3.291433-2.6651432 .063672$

C $4.497012-3.4943602 .111355$

C $5.286684-3.5137943 .392866$

C $5.3438021 .374586-0.417053$

C $4.4779430 .280084-1.021862$

C $4.547501-1.446587-2.823190$

O $3.399221-0.069930-0.553222$

C $5.268652-2.713163-2.457357$

C $6.367664-2.841171-1.695268$

C $7.030821-4.158155-1.399483$

C $7.173064-4.4217060 .084193$

$\begin{array}{llll}\text { C } & 8.318832 & -4.140035 & 0.727369\end{array}$ 
C $6.023733-5.0913350 .803487$

C $4.849719-4.1750691 .000656$

N $5.082633-0.286249-2.136484$ H $0.804121-2.239817-0.494899$ H $-1.488356-0.219471-0.325814$ H $-0.756797-0.3310832 .069805$ H $1.377024 \quad 1.274716 \quad 0.551335$ H $0.910014 \quad 1.5362872 .181463$ H -1.404999 $3.526174-0.620212$ H -2.192570 3.4310031 .551930 H -2.1070351 .7864172 .193804$ H -0.9617843 .0275252 .748563$ H $0.8632371 .596900-1.472680$ H $-0.4417592 .202114-2.453500$ H $1.6445323 .325063-2.986144$ H $0.4533484 .485568-2.398779$ H $1.5506974 .508386-0.168646$ H $2.3266706 .122958-1.936646$ H $3.4654674 .930913-2.529233$ H $4.4802717 .311595-1.752087$ H $5.4247364 .172441-0.926466$ H 2.9692937 .5470310 .020162 H 4.2150217 .0353151 .135433 H 2.6740356 .1577091 .067374 H $6.262907 \quad 5.6290320 .591968$ H $5.4697702 .394052 \quad 1.476171$ H $4.040214 \quad 1.4216781 .303167$ H $2.891757 \quad 3.0251792 .049269$ H $3.181991-1.9873594 .075783$ H $2.808261-2.6306941 .088895$ H $5.561803-2.4959573 .691719$ H $6.222100-4.0731113 .317252$ H $4.700849-3.9703754 .197994$ H $5.6583052 .059978-1.208791$ H $6.2457160 .873901-0.040928$ H $4.647211-1.265212-3.897840$ H $3.483767-1.553311-2.586787$ H $4.829425-3.617072-2.876301$ H $6.844492-1.960070-1.274043$ H $8.022323-4.144210-1.871239$ H $6.495248-4.992788-1.868715$ H $8.441251-4.3523101 .784130$ H $\quad 9.158545-3.685966 \quad 0.211687$ H $6.354891-5.5156461 .757873$ H $5.694291-5.9609520 .220050$ H $4.214276-4.0920760 .118444$ H $5.9957030 .051786-2.426813$ C $-1.803438-3.252881-1.861389$ O $-2.886407-2.753996-1.047588$ C -3.122054 -3.6901180 .016891$ C $-2.564956-5.014137-0.488315$ C $-1.308366-4.541303-1.196860$ H $-2.517051-3.3585260 .870042$ C $-4.598459-3.7063720 .418349$ H -2.361471 -5.7196450 .322665$ H $-3.243637-5.494486-1.202676$ C $-0.737510-5.552650-2.175662$ H $-0.548086-4.325120-0.436602$ C $-5.188500-2.318858 \quad 0.753216$ O $-5.363773-4.250403-0.664287$ H $-4.733732-4.3928161 .262889$ H $-5.341490-1.760966-0.176738$ O $-6.496575-2.532188 \quad 1.299694$ C $-4.361641-1.4706051 .735866$ C -5.070973 -0.2095142 .264090$ H $-3.421025-1.1786971 .257039$ O $-4.023860-2.2796432 .866722$ H $-5.891880-0.4997302 .932544$ H -4.3475860 .3162282 .901718$ H $-0.449950-6.471925-1.655039$ H $-1.465705-5.818949-2.949147$ H $0.151808-5.151787-2.672444$ C $-0.756047-2.151604-2.056516$ H $-6.295908-4.255457-0.386359$ H $-6.386570-2.9786202 .156979$ H $-3.437580-1.7546443 .438044$ H - $-1.230923-1.285102-2.532351$ H $\quad 0.022301-2.495348-2.747809$
H -2.245363 -3.480757 -2.839684

C $-4.831021 \quad 1.988590-0.755235$

$\begin{array}{llll}\text { O } & -4.500328 & 1.133243 & 0.349028\end{array}$

C $-5.596700 \quad 0.746168 \quad 1.181176$

C $-6.2673351 .985907 \quad 1.795595$

C $-6.6833952 .989128 \quad 0.714960$

C $-5.5482803 .264379-0.275772$

H $-3.8631202 .287689-1.176867$

C $-5.6090501 .239975-1.849836$

H -6.3357700 .2076840 .580456$

O -5.3868322 .6247402 .718521$

H $-7.148097 \quad 1.6660632 .364658$

H -6.9812773 .9327451 .188755$

O $-7.8078202 .486062-0.000238$

H $-5.9307143 .823071-1.138152$

$\begin{array}{llll}\text { O } & -4.594329 & 4.106640 & 0.376512\end{array}$

C $-4.783717 \quad 0.146646-2.492901$

H $-6.5150630 .776399-1.451229$

H $-5.8940901 .937521-2.645424$

H $-4.5751792 .862424 \quad 2.238639$

H $-8.5271162 .351500 \quad 0.640279$

H $-3.9100874 .335900-0.275265$

O $-5.604071-0.641448-3.248407$

$\begin{array}{llll}0 & -3.575466 & 0.009806 & -2.364837\end{array}$

C $-4.970706-1.730206-3.927958$

H $-4.076277-1.394611-4.461685$

H $-4.721118-2.515676-3.209899$

H $-5.679274-2.133275-4.656349$

SCF Energy (B3LYP/6-31G**//MMFF) $=-3245.91361399$

\section{$07 \_00410$}

MMFF Geometry

C $-0.981566 \quad 3.031913-1.498970$

C $-1.4217492 .082817-2.340575$

C $-0.6766730 .837568-2.768457$

$\begin{array}{llll}\text { O } & 0.623904 & 0.770285 & -2.142821\end{array}$

C $-1.488858-0.395683-2.329961$

C $-0.851950-1.718096-2.706801$

C $-0.271590-2.568204-1.836088$

C $-0.924987-2.071310-4.169899$

C $-0.100813-2.391474-0.352202$

C $1.375980-2.2298520 .008618$

C $1.613660-1.8166251 .468877$

C $1.154260-0.3811731 .748541$

C $1.545813 \quad 0.0950853 .153729$

O $3.025724-1.9255391 .689245$

$\begin{array}{lllll}\text { O } & 1.360421 & 1.518958 & 3.178169\end{array}$

C $3.044085-0.1936083 .385274$

C $0.606505-0.4782354 .221586$

O 3.3888830 .1227834 .732303

C $3.446727-1.6440753 .028814$

C $4.986564-1.8119893 .088117$

O $2.846806-2.5340943 .973878$

C $1.6779791 .292486-2.828127$

$\begin{array}{lllll}\text { O } & 1.659013 & 1.785681 & -3.944598\end{array}$

C $2.871857 \quad 1.189692-1.958152$

C $4.0309971 .738924-2.348989$

C $5.2880821 .742831-1.594167$

C $5.3078501 .031918-0.266670$

C $5.503951-3.1475372 .533491$

C $5.726007-3.1345831 .031977$

C $7.332809-2.396187-0.704521$

O $4.990340-3.7485050 .262504$

C $8.332798-1.298881-0.873563$

C $8.373128-0.479921-1.935686$

C $9.3917320 .614827-2.082242$

C $8.7927341 .946994-2.473366$

C $9.2331902 .604882-3.559918$

C $7.7384142 .555076-1.572226$

C $6.3493272 .382616-2.128819$

N $6.829068-2.3818010 .657349$

H $0.0168522 .972711-1.075823$

H -2.429076 2.178484 -2.743142

H $-0.5780690 .842706-3.860603$

H $-2.490079-0.358597-2.781362$

H $-1.663790-0.341212-1.250905$

H $\quad 0.141662-3.498224-2.224778$

H $-0.527854-3.070628-4.377003$ 
H $-1.964122-2.054076-4.514121$ H $-0.348228-1.357983-4.766745$ H $-0.678820-1.5463020 .019799$ H - $0.504781-3.2771710 .152872$ H $1.899465-3.176831-0.183149$ H $1.857540-1.500953-0.654538$ H $\quad 1.095988-2.5283072 .122298$ H $\quad 0.073811-0.2703281 .607802$ H 1.6172180 .2954191 .018282 H 1.6579661 .8396204 .047067 H 3.6075660 .5000912 .745686 H $-0.419907-0.1346114 .049491$ H $0.875769-0.1210195 .221703$ H $0.590798-1.5700024 .237831$ H $4.3582310 .135084 \quad 4.797254$ H $5.462811-0.9878062 .543651$ H $5.308330-1.7470544 .134959$ H $3.408650-3.3162764 .086335$ H $2.7622440 .681523-1.008877$ H $4.0643172 .245104-3.313076$ H $4.603467 \quad 1.5002980 .429767$ H 6.2854181 .0412670 .219214 H $5.029154-0.020655-0.389054$ H $6.469360-3.3785043 .002091$ H $4.842053-3.9845282 .777390$ H $6.489351-2.309707-1.397748$ H $7.812021-3.367900-0.863130$ H $9.074499-1.176739-0.087123$ H $7.658627-0.615512-2.743971$ H $\quad 10.121206 \quad 0.289310-2.834992$ H $9.9528680 .752285-1.149108$ H $8.8282963 .573688-3.834026$ H $10.0060532 .191583-4.199180$ H $7.9247373 .633570-1.478227$ H $7.8472242 .164222-0.556992$ H $6.2130082 .875199-3.093376$ H $7.457876-2.0688171 .391998$ C $-1.968857 \quad 4.1919140 .487904$ O -2.4553992 .9164230 .947416$ C -3.5235173 .1271321 .884111$ C -3.4362184 .5933002 .289563$ C -2.9681025 .2324240 .995739$ H -4.4563842 .9678811 .330297$ C -3.4445212 .1172463 .033687$ H -4.3952084 .9853232 .641289$ H -2.6926904 .7567463 .077883$ C -2.375282 6.6186221 .177018 H -3.8249605 .2979660 .311807$ C -3.2354360 .6536752 .593396$ O $-2.3382912 .462468 \quad 3.878507$ H -4.3426702 .2097503 .655848$ H -2.1945390 .5165552 .277791$ O $-3.399064-0.1712773 .754753$ C $-4.165306 \quad 0.149368 \quad 1.476395$ C $-3.977236-1.3587301 .217343$ H -3.9594820 .7083000 .557442$ $\begin{array}{lllll}\text { O } & -5.516177 & 0.409705 & 1.852076\end{array}$ H -2.908730 -1.602955 1.240173 H -4.476522 -1.9161572 .019600$ H -3.1197347 .3036451 .595754$ H - 1.5151446 .6018461 .854767 H -2.041007 7.0272520 .218148 C $-1.8338884 .181624-1.037966$ H -2.310043 1.8113124 .600548 H $-4.334914-0.1253274 .016312$ H -6.0862820 .1108891 .123340$ H -1.384487 $5.120574-1.379977$ H -2.828017 4.111938-1.495789 H -0.9845754 .3369890 .951916$ C $-6.642118-1.889705-1.359060$ O $-5.962741-1.587829-0.133050$ C $-4.550413-1.805971-0.141390$ C $-4.238898-3.281933-0.434021$ C $-4.905859-3.741865-1.733681$ C $-6.386129-3.344469-1.786671$ H $-7.708444-1.794077-1.116227$ C $-6.319167-0.868786-2.456110$ H $-4.095578-1.179290-0.915047$
O $-4.695805-4.0886280 .649735$

H -3.152240 -3.407468 -0.503661

H $-4.814218-4.831524-1.817330$

O $-4.205944-3.164613-2.831646$

H $-6.793590-3.504368-2.791690$

O $-7.125416-4.186844-0.897584$

C $-6.7901520 .508774-2.054608$

H $-5.252581-0.820688-2.693300$

H -6.830125 -1.125738 -3.391402

H $-4.452797-5.0093510 .453310$

H $-4.623109-3.487165-3.648531$

H -7.003822 -5.104878 -1.194215

O $\quad-5.721728 \quad 1.293651-1.735909$

$\begin{array}{llll}\text { O } & -7.968131 & 0.839257 & -2.009235\end{array}$

C $-6.0401072 .624314-1.318756$

H $-5.1032543 .160913-1.152305$

H $-6.6069503 .144200-2.097010$

H -6.602605 2.599244 -0.380661

SCF Energy (B3LYP/6-31G**//MMFF)= -3245.89492368

\section{1}

MM̄FF Geometry

C $1.4879390 .266374 \quad 3.037710$

C $2.000826 \quad 1.413616 \quad 3.509748$

C 2.6411182 .4989422 .679401

O 3.4126081 .9340101 .590174

C 1.6083013 .4549892 .060642

C 0.9020554 .3406733 .063461

C -0.3801814 .1946913 .449428$

C 1.7227515 .4850353 .601186

C $-1.358356 \quad 3.1314863 .034969$

C -2.513934 3.6915692 .195495

C $-2.084037 \quad 4.166098 \quad 0.798879$

C $-3.271723 \quad 4.781853 \quad 0.055661$

C $-2.898430 \quad 5.182804-1.378790$

O $-1.589827 \quad 3.033338 \quad 0.076322$

O $-4.109775 \quad 5.485624-2.087028$

C $-2.2189893 .983712-2.072391$

C $-2.0780426 .476750-1.409069$

O $-1.7235994 .353468-3.357438$

C $-1.0898613 .337566-1.232813$

C $-0.6539512 .011968-1.899239$

O $0.025135 \quad 4.227976-1.179401$

C 4.6548151 .4750931 .897236

O $5.158109 \quad 1.431092 \quad 3.012874$

C 5.3920121 .0032710 .704530

C $4.9720161 .150283-0.561549$

C $5.6950950 .706652-1.758271$

C $7.022738 \quad 0.020967-1.555135$

C $0.3345081 .169387-1.080611$

C $0.586961-0.179282-1.734411$

C $2.176263-2.091763-1.719822$

O $-0.125320-0.636107-2.625431$

C $3.030482-1.825510-2.919626$

C $4.280468-2.289242-3.073632$

C $5.188266-2.019989-4.246390$

C $4.998916-0.678171-4.925819$

C $4.308072-0.576476-6.073826$

C $5.6892250 .526742-4.319861$

C $5.1357390 .907289-2.970779$

$\mathrm{N} 1.690901-0.826841-1.196488$

H 1.4800050 .0833451 .966564

H 1.9730561 .5989544 .581487

H 3.3272543 .0721423 .315409

H 0.8979712 .8843271 .453172

H 2.1137724 .0987541 .326947

H -0.7838994 .9126994 .162511$

H 1.1422146 .1406254 .259108

H 2.5740635 .1123604 .178625

H 2.1015436 .1000352 .778194

H -0.8785272 .3007212 .510662$

H $-1.7756452 .700668 \quad 3.953367$

H -3.261138 2.8951582 .088337

H -2.9961044 .5151872 .737006$

H -1.2831294 .9058050 .904057$

H -3.671382 5.6446630 .602891

H -4.0901974 .0495510 .012604$

H -4.676834 $4.695825-2.072066$ 
H $-2.9935243 .223521-2.246877$ H -2.649986 7.300821 -0.965496 H -1.861705 $6.783950-2.438439$ H -1.133148 $6.400800-0.867172$ H -2.463378 $4.745910-3.851882$ H $-1.5486421 .404977-2.081400$ H $-0.2009762 .217423-2.877782$ H $0.8292113 .719008-0.993039$ H $6.343646 \quad 0.533358 \quad 0.934212$ H $4.0184421 .640203-0.748822$ H $7.486602-0.323546-2.481155$ H $6.904373-0.867439-0.924310$ H $7.7368320 .700889-1.077520$ H $1.2902501 .694376-0.981868$ H $-0.059657 \quad 0.977245-0.077137$ H $1.321873-2.709986-2.009647$ H $2.726914-2.591349-0.917713$ H $2.584231-1.219716-3.704128$ H $4.698089-2.926553-2.296851$ H $5.067385-2.840248-4.965618$ H $\quad 6.228831-2.086867-3.904281$ H $4.1930760 .374404-6.583561$ H $3.837483-1.442949-6.526590$ H $5.5743811 .401386-4.974133$ H $6.7671770 .342531-4.293362$ H $4.1630121 .398095-3.021149$ H $2.267515-0.343203-0.514242$ C $1.356825-2.1966563 .491974$ O $0.567585-2.6256372 .359606$ C $0.201828-4.0078562 .544510$ C $1.045219-4.5114403 .709701$ C $1.146470-3.2646214 .563812$ H $-0.851418-4.0233172 .846162$ C $0.402239-4.798175 \quad 1.248083$ H $\quad 0.575799-5.3526884 .228777$ H $2.040532-4.8312143 .378599$ C $2.255535-3.3310775 .599630$ H $0.185753-3.1039985 .071676$ C $-0.540446-4.3765570 .098907$ O $1.757989-4.6098350 .825221$ H $\quad 0.288000-5.869821 \quad 1.451247$ H $-0.377967-3.325717-0.164635$ O $-0.189067-5.152399-1.054641$ C -2.036897 -4.6245300 .393504$ C -2.966202 -4.445232 -0.824635 H $-2.372555-3.973317 \quad 1.206502$ O $-2.202609-5.9704540 .855112$ H -2.556568 -4.980727-1.689238 H -3.918003 -4.943191 -0.591971 H $2.083198-4.1597016 .294401$ H $3.233771-3.4835135 .131122$ H $2.302935-2.4065536 .183342$ C $0.908847-0.7996583 .927850$ H $1.949692-5.2822920 .150210$ H $\quad 0.687039-4.855187-1.352122$ H - $1.894569-6.562473 \quad 0.147324$ H $1.212357-0.6160094 .964956$ H $-0.183041-0.7290303 .882732$ H $2.405403-2.1864393 .165747$ C $-4.490023-1.085014-0.305040$ O $-4.104046-2.457973-0.166443$ C -3.242276 -2.977238 -1.185433 C $-3.915108-2.853098-2.562422$ C $-4.364383-1.416458-2.846372$ C $-5.144586-0.820156-1.670606$ H $-5.258345-0.9354720 .464261$ C -3.324610 $-0.141680 \quad 0.005617$ H $-2.299932-2.420338-1.186260$ O $-5.043568-3.723048-2.645785$ H -3.205367 -3.178983 -3.331656 H -4.997743 -1.404589-3.741740 O $-3.230231-0.597482-3.108052$ H $-5.2810050 .259352-1.805310$ O $-6.449230-1.407085-1.636274$ C $-2.800424-0.3655131 .401535$ H $-2.483914-0.294571-0.675286$ H -3.624335 $0.908224-0.087362$ H $-5.649037-3.494780-1.919664$
H $-2.753371-0.983964-3.862109$

H -6.888561 -1.193496 -2.477181

O

O $-1.699773-0.8447631 .638355$

C $-3.361725-0.2017953 .689542$

H -3.233525 -1.273498 3.869848

H $-2.4474850 .342186 \quad 3.940339$

$\mathrm{H}-4.1735530 .1666824 .322192$

SCF Energy (B3LYP/6-31G**//MMFF) $=-3245.91579490$

\section{2}

MM̄FF Geometry

C 0.3138514 .5905620 .053563

C $1.187765 \quad 4.611424 \quad 1.072326$

C $2.392126 \quad 3.7071961 .227509$

$\begin{array}{llll}\text { O } & 2.340919 & 2.594025 & 0.305549\end{array}$

C 3.6953654 .4824690 .969480

C 4.9374223 .7097821 .375155

C $5.8765023 .254513 \quad 0.523053$

C 5.1081263 .4803672 .856304

C $5.9320413 .405546-0.969799$

C $5.3069212 .250152-1.765780$

C $5.9164780 .867866-1.475259$

C $5.451335-0.150005-2.519002$

C $5.959738-1.566600-2.211778$

$\begin{array}{llll}\text { O } & 5.488213 & 0.445539 & -0.177023\end{array}$

O $5.222893-2.484150-3.035781$

C $5.634236-1.915966-0.742629$

C $7.436286-1.734521-2.591453$

O $6.242075-3.159249-0.399992$

$\begin{array}{llll}\text { C } & 6.038857 & -0.808128 & 0.255318\end{array}$

C $5.528912-1.0569821 .697937$

O $7.468605-0.7417690 .325367$

C 1.4912601 .5761220 .613915

O 0.7999411 .4705351 .614679

C $1.5105690 .618328-0.511187$

C $0.463916-0.202030-0.675079$

C $0.317237-1.228747-1.706217$

C $1.522290-1.546523-2.549775$

C $4.008721-1.1750501 .850672$

C $3.482716-2.6005271 .812885$

C $1.339750-3.8777211 .781231$

O $4.211150-3.5883351 .849754$

C $1.006839-4.2753490 .377780$

C $-0.235739-4.584324-0.024425$

C $-0.579374-5.038519-1.415119$

C $-1.676990-4.211004-2.046562$

C $-2.954977-4.625195-2.025187$

C $-1.278428-2.942802-2.768834$

C $-0.873303-1.852421-1.818634$

N $2.094649-2.6380181 .771155$

H $0.4836593 .931236-0.793543$

H 0.9991875 .2812371 .908966

H 2.3837733 .3159912 .252243

H 3.6896445 .4280541 .527745

H $3.7379094 .755679-0.090345$

H 6.7233342 .7111090 .941683

H 6.0919573 .0659263 .101695

H 5.0090354 .4247373 .401420

H 4.3564082 .7788313 .229940

H $5.4651874 .345890-1.282371$

H $6.9871143 .504046-1.256649$

H $4.2301462 .227273-1.565399$

H $5.4299942 .482247-2.831065$

H $7.0089060 .958682-1.490324$

H $5.7604650 .156347-3.526302$

H $4.353668-0.173091-2.539440$

H $5.363928-2.232969-3.964741$

H $4.549451-2.070482-0.694292$

H $7.581339-1.537076-3.660437$

H $7.771434-2.765129-2.430636$

H $8.099691-1.064830-2.040406$

H $5.902214-3.4288040 .469287$

H $6.047706-1.9142452 .142961$

H $5.837291-0.1902652 .300832$

H $7.716627-0.3060411 .157729$

H $2.3716020 .618560-1.167423$

H $-0.374888-0.1098810 .012488$ 
H $2.378391-1.796662-1.914006$ H $1.788834-0.689535-3.177810$ H $1.378248-2.399760-3.215392$ H $3.726766-0.7735722 .832529$ H $3.496493-0.5778141 .093588$ H $0.442969-3.7075802 .384828$ H $1.935401-4.6642622 .255490$ H $1.828802-4.342843-0.331077$ H $-1.051056-4.5429690 .694805$ H $-0.894861-6.088408-1.353433$ H $0.302276-5.033855-2.068034$ H $-3.744449-4.049661-2.496659$ H $-3.241360-5.546168-1.527958$ H $-2.122076-2.563073-3.360348$ H $-0.503143-3.182456-3.501204$ H $-1.679164-1.542010-1.154045$ H $1.574709-1.7679431 .702711$ C $-2.158747 \quad 4.4238750 .097513$ O $-2.3096383 .740669-1.164398$ C $-3.7204893 .583389-1.443672$ C $-4.4471284 .045791-0.187846$ C -3.5003825 .1048650 .343580$ H -3.934670 $4.263180-2.277853$ C $-3.980128 \quad 2.128110-1.856420$ H $-5.447902 \quad 4.434742-0.396576$ H -4.5443123 .2233370 .532161$ C $-3.758137 \quad 5.469252 \quad 1.795211$ H $-3.5867036 .004968-0.279643$ C $-5.4659131 .728252-1.935889$ O $-3.364568 \quad 1.919515-3.132989$ H $-3.4491961 .470016-1.161471$ H $-5.9426411 .815173-0.952948$ O $-6.1550272 .638378-2.804390$ C $-5.719622 \quad 0.318239-2.502136$ C $-4.942578-0.826068-1.841420$ H $-5.4895320 .309477-3.574903$ O $-7.117497 \quad 0.027722-2.411797$ H -5.279113 -1.779734 -2.269822 H $-3.888435-0.747400-2.136550$ H $-4.767785 \quad 5.875602 \quad 1.915691$ H -3.6667604 .5965802 .450617$ H $-3.047427 \quad 6.2258282 .141586$ C $-0.965570 \quad 5.380454 \quad 0.029715$ H -3.847731 2.457526 -3.783560 H $-6.1826293 .506131-2.368437$ H $-7.5933380 .751554-2.854056$ H $-0.992233 \quad 6.0831310 .869747$ H $-0.9931725 .965476-0.896914$ H $-1.985567 \quad 3.650607 \quad 0.859147$ C -3.899176 -2.217678 1.431374 O $-4.145520-2.038097 \quad 0.030767$ C $-4.995232-0.929471-0.305027$ C $-6.387752-1.1519790 .301530$ C $-6.299416-1.3738531 .816220$ C -5.220469-2.3973392.197664 H $-3.344545-3.1633421 .486598$ C -2.987696 -1.100965 1.961316 H $-4.558074-0.0198430 .118529$ O $-7.015111-2.288888-0.285639$ H $-7.022945-0.2798440 .112287$ H $-7.275755-1.7171862 .180242$ O $-6.026696-0.1212402 .438710$ H $-5.027366-2.3704793 .275777$ O $-5.706272-3.7092851 .893431$ C $-2.114783-1.5492403 .112548$ H $-2.324441-0.7791161 .150857$ H $-3.553336-0.2240512 .286129$ H $-7.237125-2.061024-1.204206$ H $-6.009998-0.2691923 .399644$ H $-6.515807-3.8503442 .413164$ O $-1.076530-0.6720803 .248847$ O $-2.326418-2.5243883 .820018$ C $-0.166864-0.946520 \quad 4.319005$ H $0.685220-0.268276 \quad 4.222867$ H $-0.658221-0.7632925 .278898$ H $0.199111-1.9759334 .264346$

SCF Energy (B3LYP/6-31G**//MMFF) $=-3245.90249815$
0700413

MMFF Geometry

C $-0.347938-0.8516180 .161829$

C $0.264624-0.9854541 .349250$

C $\quad 0.383214 \quad 0.0618852 .431764$

$\begin{array}{lllll}\text { O } & 0.030664 & 1.371909 & 1.925552\end{array}$

C $-0.561750-0.205792 \quad 3.617801$

C $-0.344895-1.5222764 .333158$

C $-1.188139-2.5730724 .286885$

C $0.909817-1.599738 \quad 5.164995$

C $-2.477522-2.6906043 .521972$

C $-2.382655-3.7805622 .446871$

C $-3.632922-3.8211151 .555705$

C $-3.529007-4.9661310 .546381$

C $-4.701073-4.968369-0.446060$

O $-3.717374-2.569466 \quad 0.869098$

O $-4.361200-5.852670-1.525092$

C $-4.844082-3.554209-1.046258$

C $-5.980290-5.5275790 .186925$

O $-5.994579-3.472247-1.885118$

C $-4.857861-2.4265910 .012981$

C $-4.731712-1.051330-0.682344$

O $-6.084404-2.4834800 .741070$

C 1.0295102 .2197001 .571606

O 2.2311961 .9989051 .618466

C $0.484118 \quad 3.492022 \quad 1.044916$

C $-0.7220013 .962201 \quad 1.399473$

C $-1.348035 \quad 5.2021490 .931931$

C $-0.623823 \quad 5.988342-0.128943$

C $-4.7365740 .145182 \quad 0.281271$

C $-3.776261 \quad 1.235084-0.150762$

C $-3.1720792 .702557-2.069508$

O -2.8861401 .6323790 .597594$

C $-3.5798194 .119174-1.797029$

C $-4.5797534 .555506-1.014862$

C $-4.8875526 .010601-0.799534$

C $-4.753266 \quad 6.423467 \quad 0.649628$

C -5.8358706 .5472411 .435872$

C -3.3722056 .7761861 .160866$

C -2.538598 5.5595181 .459338

N $-4.022567 \quad 1.720725-1.423791$

H $-0.8419720 .082025-0.082570$

H $0.740948-1.937296 \quad 1.577164$

H 1.4194600 .0729802 .790375

H $-1.597373-0.0869643 .280925$

H -0.4412600 .6066984 .348720$

H $-0.927976-3.4630314 .858985$

H $0.983189-2.5406725 .720819$

H $1.798005-1.5234174 .530165$

H $0.931789-0.7847085 .895781$

H $-3.276678-2.9453694 .228666$

H $-2.767479-1.7423193 .065128$

H $-1.501140-3.5883831 .821792$

H $-2.236519-4.7565362 .926419$

H - $-4.516540-3.9447612 .192732$

H $-3.458425-5.9327381 .060711$

H -2.595146 $-4.863751-0.024672$

H $-4.196216-6.734430-1.149545$

H -3.979662 -3.399885 -1.706141

H $-5.813642-6.550056 \quad 0.547120$

H $-6.787362-5.600106-0.550444$

H $-6.334670-4.9342821 .032429$

H $-6.775876-3.407046-1.310060$

H $-3.798495-1.041769-1.257573$

H $-5.556435-0.914194-1.392735$

H $-6.268445-1.6106401 .122603$

H $1.146204 \quad 4.0386820 .382223$

H -1.314881 3.3894832 .111095

H $-0.4140545 .357407-1.000226$

H $0.324401 \quad 6.374698 \quad 0.260570$

H - $1.1893486 .844215-0.502110$

H $-5.741726 \quad 0.5754740 .356990$

H $-4.440396-0.1595311 .291649$

H -3.216912 $2.507956-3.145636$

H $-2.1394542 .553971-1.735580$

H $-2.9629504 .864037-2.297384$

H $-5.222433 \quad 3.846294-0.500602$

H $-5.9120676 .190351-1.150568$ 
H $-4.2494516 .652803-1.419414$ H -5.7512006 .8698462 .468231$ H -6.8308306 .3226601 .065949$ H -3.454914 7.3489202 .094235 H $-2.897077 \quad 7.4600320 .452717$ H -2.9761674 .9166142 .224937$ H -4.838968 1.394312 -1.932108 C $0.075916-1.513493-2.266968$ O $1.362148-0.867316-2.184215$ C $1.3114490 .348904-2.951277$ C $0.1277230 .190454-3.894927$ C $-0.854941-0.554804-3.012984$ H $1.0882011 .159790-2.247448$ C $2.6518770 .612147-3.644804$ H $-0.2562841 .152165-4.248065$ H $0.387504-0.415963-4.770511$ C -1.953646 -1.252131 -3.797692 H -1.314255 $0.158182-2.319269$ C $3.8675280 .682969-2.696348$ O $2.907454-0.430420-4.594585$ H $2.5842541 .537351-4.229198$ H $4.109017-0.319613-2.326115$ O $4.9971841 .109967-3.467549$ C $3.6932371 .645678-1.510555$ C $4.9727281 .927028-0.702543$ H $2.9250571 .245446-0.841166$ O $3.2096322 .900231-2.000730$ H $5.6833452 .496907-1.314904$ H 4.6868712 .6035260 .113961 H -2.574808 -0.518913 -4.322436 H - $-1.539965-1.937674-4.545075$ H -2.600646 -1.833333 -3.135361 C $-0.398592-1.944965-0.873337$ H $2.901806-1.274410-4.111265$ H $5.091657 \quad 0.489333-4.210539$ H $3.0400303 .470217-1.231498$ H $0.229805-2.782116-0.544485$ H - $-1.423250-2.327555-0.936891$ H $0.228790-2.418423-2.869376$ C $5.180792-1.2621171 .243897$ O $4.710542-0.039690 \quad 0.658074$

C $5.6652150 .682367-0.124132$ C $6.887551 \quad 1.0609900 .729989$ C $7.491992-0.1643551 .423178$ C $6.421841-1.0106162 .119180$ H $4.366534-1.5867681 .904270$ C $5.400077-2.3609750 .190686$ H $5.9951330 .056955-0.959300$ O $\quad 6.5285362 .0266041 .717712$ H 7.6377591 .5312350 .083853 H $8.227640 \quad 0.1639722 .167928$

O $8.170899-0.972057 \quad 0.466597$ H $6.850661-1.9664502 .442241$ O $6.011155-0.3150403 .300154$ C $4.106350-2.795608-0.462408$ H $\quad 6.071597-2.029179-0.605180$ H $5.832144-3.2475180 .669011$ H 5.8090091 .6505082 .253234 H $8.866728-0.4262340 .062295$ H $5.379887-0.8843303 .772351$

O $4.392273-3.644329-1.493587$

O $2.988249-2.449343-0.109967$ C $3.260392-4.149155-2.209158$ H $3.614835-4.902635-2.917483$ H $2.548540-4.620639-1.524555$ H $2.782089-3.340123-2.767530$

SCF Energy (B3LYP/6-31G**//MMFF)= -3245.90420352

0700414

MM̄FF Geometry

C $1.359494-1.993364-2.449401$

C $0.425659-2.868979-2.846795$

C $-0.642587-3.397848-1.929317$

O $-1.890397-2.822232-2.382896$

C $-0.757846-4.931505-1.982465$

C $0.382981-5.669113-1.318923$

C $0.402836-5.995751-0.011968$

C $1.499877-6.109247-2.228077$
C $-0.624403-5.6382061 .030089$

C $0.013379-5.3040052 .385732$

C $0.809819-3.9868922 .391596$

C $1.788156-3.9926013 .569474$

C $2.557275-2.6740743 .682082$

O $-0.116325-2.8958002 .517621$

O $3.216652-2.6558884 .957391$

C $1.539252-1.5214873 .681951$

C $3.658071-2.5520582 .622732$

O $2.207293-0.2616263 .659179$

C $0.497138-1.5973232 .534687$

C $-0.592347-0.5276122 .796912$

O $1.147861-1.3102881 .296862$

C $-2.885067-2.708306-1.462728$

O $-2.852564-3.066842-0.296322$

C $-4.036659-2.052857-2.116808$

C $-5.171081-1.902865-1.418192$

C $-6.389288-1.231957-1.869404$

C $-6.383580-0.620826-3.245164$

C $-1.808631-0.5404751 .857962$

C -2.7452710 .6190312 .179790$

C -4.7593821 .8095061 .399560$

O -2.5281951 .4154613 .091779$

C -6.0900081 .4647190 .808907$

C $-6.6464752 .164112-0.194047$

C $-8.0033271 .992494-0.829314$

C $-8.8314400 .809641-0.378036$

C -9.6803970 .9132580 .658336$

C $-8.744351-0.460581-1.196717$

C -7.427192 -1.157856-1.010722

N -3.8545020 .6735841 .342062$

H $1.334988-1.628917-1.426459$

H $\quad 0.422524-3.218713-3.876097$

H $-0.448155-3.065400-0.900756$

H $-0.861671-5.264754-3.024562$

H $-1.704947-5.245038-1.524117$

H $1.243420-6.5841900 .353428$

H $2.254757-6.702575-1.701010$

H $1.106474-6.727911-3.041412$

H $2.009921-5.245295-2.663280$

H $-1.253904-4.8036000 .715146$

H -1.279356 -6.507229 1.165614

H $-0.789004-5.2439073 .132254$

H $0.662283-6.1395442 .678551$

H $1.358972-3.8815651 .449663$

H $2.488409-4.8337903 .489896$

H $1.232423-4.1537004 .504294$

H $3.811948-3.4239524 .998020$

H $0.999393-1.5656764 .638654$

H $4.363606-3.3875652 .705801$

H $4.252243-1.6448702 .777231$

H $3.274358-2.5453491 .600389$

H $2.835778-0.2520704 .401251$

H $-0.973089-0.6541683 .819518$

H $-0.130748 \quad 0.4666772 .745903$

H $\quad 0.497389-0.9522370 .673316$

H -3.914943 -1.697305 -3.133152

H -5.208713 -2.291055 -0.400676

H $-5.5825730 .121612-3.333097$

H -7.312494 -0.106414 -3.498755

H $-6.231870-1.393442-4.006995$

H -1.498411 -0.4434200 .812948$

H -2.365276 -1.476288 1.974779

H -4.2747572 .6381600 .872573$

H -4.8903302 .1106612 .444681$

H $-6.6284330 .644473 \quad 1.273941$

H $-6.0755732 .995987-0.605223$

H -7.873036 $1.944828-1.917707$

H $-8.5683052 .917178-0.649142$

H $-10.302064 \quad 0.0793830 .966512$

H $-9.767317 \quad 1.8349081 .224418$

H -8.958087 -0.229431-2.244126

H $-9.532917-1.164218-0.898586$

H -7.325518 $-1.627622-0.031080$

H -3.8577330 .0961350 .505878$

C $3.816555-1.560767-2.658504$

O $3.835890-0.711738-1.491751$

C $4.9389100 .201259-1.599422$ 
C $5.918771-0.476335-2.543584$

C $4.962302-1.081379-3.555373$

H $4.5553721 .109300-2.079705$

C $5.4785170 .552519-0.210944$

H $6.6271390 .228161-2.989136$

H $\quad 6.487014-1.267440-2.041121$

C $5.581041-2.183648-4.397525$

H $4.608846-0.283731-4.222851$

C 4.3995901 .0205080 .788396

O $6.116545-0.5973340 .358484$

H $6.2615291 .313931-0.303566$

H $3.7877680 .167747 \quad 1.104477$

O 5.0660571 .4937581 .964794

C 3.4720652 .1331380 .268519

C 2.4936622 .6297281 .350416

H $2.8933431 .751916-0.579287$

O $4.2560093 .223699-0.210930$

H 2.0609521 .7670191 .868063

H 3.0474643 .2313112 .081723

H $\quad 6.420373-1.795203-4.983375$

H $5.956371-3.002138-3.774096$

H $4.845727-2.599891-5.093374$

C $2.446188-1.450284-3.333179$

H $5.459233-1.3139180 .386581$

H 5.6286070 .7697692 .289337

H 4.7867883 .5545040 .533783

H $2.459071-1.974472-4.295498$

H $2.215257-0.395813-3.527989$

H $3.993229-2.585867-2.308875$

C $0.9638455 .541725-0.438150$

O $1.897626 \quad 4.6721990 .215446$

C 1.3450853 .4747740 .766339

C 0.2920453 .8179081 .830750

C $-0.7899894 .738740 \quad 1.256977$

C -0.1844225 .9317980 .508652$

H $1.538706 \quad 6.450161-0.660011$

C $0.4828514 .957075-1.771391$

H $0.8745832 .890336-0.032171$

O 0.9235854 .4520302 .940683

H -0.1580192 .8878472 .195424$

H -1.425729 5.1084322 .070558

O $-1.619023 \quad 4.003768 \quad 0.361590$

H $-0.957823 \quad 6.463209-0.058398$

O $\quad 0.346057 \quad 6.857252 \quad 1.462082$

C $1.6404314 .789725-2.728169$

H $-0.0193273 .992773-1.653489$

H $-0.2409515 .625113-2.252753$

H 0.2381454 .6268523 .607603

H -1.987929 3.2516350 .854923

H -0.3879627 .1455752 .030811$

O $1.9058843 .465241-2.914394$

O $2.2458615 .725340-3.235353$

C $2.9938563 .173179-3.796391$

H $3.0462542 .088899-3.923826$

H $2.827738 \quad 3.631717-4.775892$

H $3.9341273 .522583-3.359765$

SCF Energy (B3LYP/6-31G**//MMFF) $=-3245.91372214$

\section{5}

MM̄FF Geometry

C $-1.122577 \quad 3.401787 \quad 1.173011$

C $-1.634843 \quad 4.2951350 .312657$

C $-1.5200204 .260452-1.193274$

O $-0.6978613 .161725-1.646229$

C $-2.9238094 .101536-1.812989$

C $-2.9182703 .885138-3.315374$

C $-3.4898042 .824985-3.921901$

C $-2.2369764 .947982-4.136860$

C $-4.2901341 .734229-3.265060$

C $-3.4148960 .610352-2.708629$

C $-4.219569-0.549372-2.097652$

C $-5.060973-0.112439-0.894514$

C $-5.752914-1.299684-0.209590$

O $-3.263110-1.536947-1.689577$

O $-6.242077-0.8516201 .063783$

C $-4.705826-2.3949230 .067699$

C $-6.977284-1.778883-0.998836$

O $-5.326800-3.5737180 .576503$
C $-3.833743-2.742222-1.160646$

C $-2.671405-3.662859-0.719856$

O -4.634028 -3.418477 -2.129877

C $0.6291823 .412738-1.817572$

O $1.2293564 .430572-1.511119$

C $1.2248562 .242203-2.498053$

C $2.5122762 .286763-2.871391$

C $3.1921371 .255166-3.647833$

C $4.0616021 .814734-4.742524$

C $-1.661531-3.987785-1.828442$

C $-0.429383-4.687220-1.280477$

C $1.893263-5.295030-1.938503$

O $-0.331845-5.066754-0.116679$

C $2.746880-4.090107-1.695587$

C $3.872109-3.822268-2.374118$

C $4.680730-2.574658-2.161976$

C $4.880925-1.793281-3.441559$

C $6.107296-1.633186-3.966322$

C $3.663166-1.208426-4.121753$

C $3.064592-0.056182-3.363349$

N $0.551920-4.835869-2.251211$

H -0.5471172 .5575160 .804386$

H -2.2028745 .1326910 .713767$

H -1.089544 $5.212423-1.529351$

H -3.524707 $4.993723-1.592199$

H $-3.4284953 .268741-1.310473$

H $-3.4163942 .744139-5.005111$

H $-2.3872544 .800286-5.211702$

H -2.637248 $5.935299-3.884287$

H -1.158413 $4.950455-3.953743$

H $-4.9238662 .167532-2.484815$

H $-4.9823091 .323271-4.010764$

H $-2.7757440 .210122-3.507518$

H -2.718822 $1.001966-1.955301$

H -4.856505 -0.975910 -2.881394

H $-5.8114290 .633075-1.179760$

H $-4.4109150 .381849-0.159651$

H $-6.844068-0.1036200 .908070$

H -4.045959 -2.024956 0.864468

H -7.713029 -0.970933 -1.091829

H $-7.491558-2.592324-0.475220$

H $-6.731806-2.117425-2.007487$

H $-5.849006-3.3131231 .354540$

H $-2.129353-3.1720330 .099347$

H -3.067922 -4.608200 -0.328484

H $-4.053669-3.905454-2.735149$

H $0.5843041 .396688-2.725661$

H $3.1124463 .167981-2.653604$

H $4.7637642 .553282-4.338580$

H $3.4447332 .306900-5.502514$

H $4.665973 \quad 1.058206-5.247798$

H -2.111677 -4.644533 -2.580302

H -1.333574 -3.066912 -2.323854

H $2.243381-5.889271-2.787955$

H $1.873781-5.927056-1.045387$

H $2.409086-3.390790-0.933905$

H $4.223691-4.523029-3.127475$

H $5.646772-2.867715-1.731921$

H $4.203717-1.921803-1.422667$

H $6.260912-1.077513-4.885481$

H $6.986584-2.052724-3.488759$

H $2.893984-1.983010-4.232325$

H $3.898077-0.920090-5.152508$

H $2.441859-0.351109-2.518764$

H $0.397456-4.432945-3.171131$

C $-1.9330032 .240548 \quad 3.282728$

O -1.0394571 .1178673 .166069$

C $-1.829675-0.0836173 .037419$

C $-3.2876860 .338719 \quad 3.210649$

C -3.2591591 .7656532 .695041$

H -1.688732 -0.444335 2.012122

C -1.343693 -1.137335 4.041047

H -3.972396 -0.3065822 .654209$

H $-3.596423 \quad 0.319873 \quad 4.262201$

C -4.4596022 .5935633 .114208$

H -3.2105771 .7315111 .599650$

C $0.164160-1.4510893 .936410$

O $-1.594609-0.6626585 .371169$ 
H -1.932595 -2.055025 3.932337 H $0.733914-0.5518694 .190800$ O $0.485326-2.4155934 .947673$ C $0.608037-2.0006392 .566754$ C $2.110336-2.3432212 .497947$ H $\quad 0.362127-1.2866131 .774527$ O $-0.137680-3.1921712 .307802$ H $2.385087-2.9909063 .338975$ H $2.280872-2.9392311 .591815$ H -5.3839232 .1535362 .725580$ H -4.547011 2.651798 4.204259 H $-4.382393 \quad 3.6142882 .726793$ C -1.3033893 .4891052 .662134$ H $-1.1091390 .172858 \quad 5.481547$ H $0.011451-3.2365494 .729455$ H $0.107135-3.5060491 .420610$ H -0.3109613 .6399013 .103585$ H -1.908646 4.3648362 .924951 H -2.047423 2.4246424 .359835 C $3.4501580 .812344 \quad 1.046046$ O $2.775749-0.4427231 .209208$ C $3.004915-1.0944152 .463905$ C $4.493751-1.4377992 .605840$ C $5.363606-0.1839402 .457877$ C 4.9708410 .6553541 .234510 H $3.2671671 .080497-0.001817$ C 2.7941321 .8847461 .927891 H $2.717214-0.4234313 .279075$ O $4.877923-2.4036901 .630675$ H $4.661039-1.8917383 .589399$ H $6.415772-0.4808732 .368622$ O $5.240674 \quad 0.622797 \quad 3.625954$ H 5.4409251 .6439501 .282015 $\begin{array}{lllll}\text { O } & 5.474818 & 0.010690 & 0.060064\end{array}$ C $3.022187 \quad 3.291817 \quad 1.421229$ H 1.7106821 .7137041 .944868 H 3.1510741 .8288532 .959769 H $4.683977-2.0309470 .754408$ H $5.5294650 .087487 \quad 4.384769$ H $6.443262-0.0277720 .138795$ $\begin{array}{lllll}\text { O } & 2.351033 & 4.163583 & 2.231046\end{array}$ O 3.6980273 .5944060 .448005 C 2.4617545 .5458371 .878480 H 3.5089425 .8618391 .902897 H 2.0277195 .7200910 .889830 H 1.9018046 .1295272 .614053 SCF Energy (B3LYP/6-31G**//MMFF)= -3245.92172728

\section{6}

MM̄FF Geometry

C $2.138411-3.7416870 .412974$

C $1.397244-3.010152-0.432692$

C $-0.059112-2.700696-0.205533$

O $-0.164051-1.267093-0.024449$

C $-0.930695-3.106697-1.410281$

C $-1.250712-4.587888-1.466250$

C $-2.367004-5.135882-0.945187$

C $-0.265586-5.448872-2.211883$

C $-3.453898-4.420694-0.193540$

C $-4.739199-4.337591-1.025653$

C $-5.744057-3.342591-0.426555$

C $-7.074530-3.406405-1.180141$

C $-8.058597-2.325192-0.709744$

O $-5.167733-2.037654-0.546840$

O $-9.129897-2.258294-1.663649$

C $-7.341450-0.959429-0.740303$

C -8.699022 -2.678827 0.637092

O $-8.1660100 .064804-0.189760$

C $-5.971464-0.970663-0.029814$

C $-5.1882000 .344559-0.247586$

O $-6.172089-1.116871 \quad 1.380200$

C $-1.126491-0.8194360 .828671$

O $-1.933692-1.4964441 .446061$

C $-1.0244940 .655506 \quad 0.904345$

C $-1.8034231 .318951 \quad 1.772256$

C -1.8358892 .7662891 .995410$

C $-0.8829293 .617448 \quad 1.200540$

C $-4.8161980 .631739-1.703307$
C $-3.8251221 .773470-1.798915$

C $-3.6760884 .258453-1.688384$

O $-2.6343481 .573155-2.027223$

C $-3.8204725 .113970-0.465623$

C $-4.589584 \quad 4.8920690 .612494$

C -4.6478495 .8280001 .786822$

C -4.3404445 .1451903 .100488$

C -5.2803025 .0232954 .053313$

C -2.921666 $4.675963 \quad 3.342955$

C $-2.707207 \quad 3.2534982 .904070$

N $-4.4102703 .011509-1.591336$

H $1.681720-4.1250571 .322433$

H $1.849270-2.632216-1.346626$

H $-0.408688-3.2093280 .702594$

H $-0.451202-2.797274-2.349507$

H $-1.860753-2.524101-1.401331$

H -2.522958 -6.207358 -1.059654

H $-0.614300-6.482221-2.315364$

H $-0.105802-5.057132-3.221742$

H $0.695980-5.480168-1.693173$

H $-3.655355-4.9676270 .735690$

H -3.129923 -3.4241930 .114561$

H $-4.487939-4.018529-2.046217$

H -5.190113 $-5.335276-1.094280$

H $-5.889529-3.5783640 .634060$

H $-7.525290-4.402363-1.087888$

H -6.891467 -3.260849 -2.254274

H -9.543192 -3.137378 -1.712127

H $-7.193952-0.703956-1.796999$

H $-9.258622-3.6187090 .559619$

H $-9.429232-1.9196730 .938452$

H -7.970404 -2.796397 1.441790

H $-9.0153590 .038573-0.662907$

H $-5.743188 \quad 1.189454 \quad 0.179787$

H $-4.258410 \quad 0.2909520 .333414$

H $-5.379996-0.7920241 .839623$

H $-0.314118 \quad 1.1497720 .253225$

H -2.492740 0.7439672 .389883

H 0.1507553 .3908191 .482286

H $-1.026767 \quad 4.6894551 .348598$

H $-1.001972 \quad 3.4376930 .126567$

H $-4.356248-0.243935-2.175151$

H $-5.7011270 .887893-2.295916$

H $-4.066103 \quad 4.795791-2.558868$

H -2.615478 $4.053137-1.864115$

H $-3.2111076 .016291-0.479586$

H $-5.223846 \quad 4.0113580 .662926$

H -5.6538516 .2662991 .810898$

H -3.9569796 .6707721 .658362$

H -5.0579074 .5580165 .008037$

H -6.2928995 .3809843 .899318$

H -2.699270 $4.723022 \quad 4.417677$

H $-2.222800 \quad 5.3791432 .882528$

H -3.3457292 .5441283 .433651$

H $-5.4206223 .065392-1.497827$

C $4.519215-3.6500651 .251752$

O $5.004331-2.3282640 .917681$

C $6.440800-2.3265001 .019873$

C $6.792007-3.5398911 .869422$

C $5.762145-4.5357561 .383107$

H $6.828925-2.4885990 .007255$

C $6.976785-1.0042011 .577240$

H $7.821984-3.8745941 .713934$

H $6.653496-3.3408432 .938668$

C $5.577208-5.7211052 .315644$

H $6.068385-4.9035400 .394232$

C $6.632819 \quad 0.250624 \quad 0.748743$

O $6.446271-0.8165862 .895460$

H $8.064627-1.0836291 .695224$

H 5.5763560 .4975850 .887652

$\begin{array}{llll}\text { O } & 7.365755 & 1.345800 & 1.315980\end{array}$

C $6.9543730 .154424-0.753008$

C $6.9024451 .489047-1.522021$

H $6.267764-0.560396-1.220786$

O $8.276835-0.372201-0.905451$

H $7.7463412 .123912-1.222102$

H $7.0815371 .249249-2.578754$

H $6.511430-6.2839592 .411720$ 
H $5.274174-5.399718 \quad 3.317813$

H $4.809949-6.4023421 .934644$

C $3.563842-4.1466950 .161130$

H $6.803303 \quad 0.0219883 .234977$

H 8.3108691 .1797581 .156806

H $8.435866-0.495015-1.856967$

H $3.571008-5.2427530 .127394$

H $3.896646-3.789031-0.820963$

H $4.008349-3.5629852 .219191$

C $3.1996691 .981486-1.635454$

O $4.5130891 .421621-1.775297$

C $5.5897702 .275481-1.384315$

C $5.5940913 .553255-2.240484$

C $4.2348124 .261393-2.200427$

C $3.0744113 .288868-2.438177$

H $2.5293501 .240018-2.089153$

C $2.7911612 .138567-0.161877$

H $5.4695172 .553829-0.333070$

O $5.9212043 .245355-3.594824$

H $6.3758784 .225580-1.868699$

H $4.2087565 .043113-2.969754$

O $4.0584274 .890830-0.934878$

H $2.1207563 .778391-2.207956$

O $3.0546002 .964252-3.831423$

C 2.7438790 .8124170 .563480

H 3.4849012 .7811640 .385851

H $1.7903342 .579221-0.111500$

H $5.2689072 .600822-3.918921$

H $4.7835915 .528314-0.820338$

H $2.2752572 .405006-3.991503$

O 2.4492591 .0326391 .878402

O $2.946011-0.2775310 .048294$

C $2.408667-0.1361162 .703445$

H $1.675777-0.8522332 .321333$

H $3.401486-0.5920612 .756598$

H 2.1077900 .1686473 .709158

SCF Energy (B3LYP/6-31G**//MMFF) $=-3245.91139304$

0700417

MM̄FF Geometry

C $1.8781323 .078904-2.657085$

C $3.1273993 .051327-3.146782$

C $4.1899822 .045389-2.766990$

O $3.8263411 .351695-1.551001$

C $4.3627350 .988593-3.871254$

C $5.5245180 .054973-3.586445$

C $5.425130-1.119282-2.930725$

C $6.8637170 .536544-4.080894$

C $4.183033-1.743921-2.351363$

C $4.143740-3.254288-2.615538$

C $2.833938-3.944152-2.197362$

C $1.693991-3.647643-3.171015$

C $0.399859-4.351125-2.743730$

O $2.471252-3.524186-0.879522$

O $-0.676416-3.798651-3.517359$

C $0.110671-4.022386-1.263318$

C $0.432853-5.852432-3.057989$

O $-0.996712-4.795744-0.809176$

C $1.329190-4.207898-0.325169$

C $1.097260-3.5644101 .066521$

O $1.607242-5.598093-0.156724$

C $4.1760901 .915691-0.364482$

O $4.7721772 .965824-0.191825$

C 3.6847081 .0155720 .702216

C 3.8445791 .3494511 .991005

C 3.4040540 .5575703 .142257

C $2.769218-0.7785242 .859640$

C $0.083905-4.2630571 .971436$

C $-0.019635-3.5864523 .326132$

C -1.227335 -1.733539 4.492184

O $0.562533-4.0260964 .314816$

C $-0.483924-0.4386874 .559472$

C 0.0545950 .0419835 .690806

C 0.7654561 .3624545 .779904

C 2.1338841 .2500826 .419034

C 2.3960541 .8490437 .593163

C 3.2002710 .4493055 .702043

C 3.5815991 .0627914 .380740
N $-0.830592-2.464192 \quad 3.302629$

H $1.5558972 .301275-1.968999$

H $3.4352363 .822635-3.849214$

H $5.1363992 .578913-2.612995$

H $4.5288821 .476504-4.840873$

H $3.4364100 .414845-3.990294$

H $6.340153-1.685306-2.760013$

H $7.671834-0.164017-3.845045$

H $\quad 6.8428460 .661912-5.168393$

H $7.117373 \quad 1.498543-3.624424$

H $4.192980-1.572794-1.268749$

H $3.278668-1.274990-2.743279$

H $4.346865-3.467120-3.672766$

H $4.961885-3.711767-2.042201$

H $3.038158-5.021578-2.183249$

H $1.967625-3.936522-4.193307$

H $1.498479-2.567961-3.206022$

H $-0.473282-3.941174-4.457874$

H $-0.199756-2.969381-1.214337$

H $0.610048-6.015774-4.127780$

H $-0.532455-6.323120-2.840756$

H $1.208682-6.388763-2.507624$

H -1.740972 -4.617159-1.409289

H $2.059334-3.5439831 .598195$

H $0.826808-2.5084930 .935899$

H $2.144020-5.703470 \quad 0.646494$

H 3.1946290 .0985130 .394403

H 4.3364022 .2916642 .228673

H $3.457283-1.4191242 .296633$

H $1.852003-0.6480552 .275367$

H $2.493968-1.3365083 .755839$

H $-0.915620-4.2698611 .527250$

H $\quad 0.362758-5.3087162 .142251$

H -1.062788 -2.353073 5.379702

H -2.299655 -1.535148 4.397743

H $-0.4074180 .144176 \quad 3.644911$

H -0.048690 -0.526897 6.612109

H $0.1290932 .039266 \quad 6.364824$

H $\quad 0.8629201 .8275254 .791379$

H 3.3729251 .7769118 .059794

H 1.6401542 .4255068 .115957

H $2.866734-0.5872815 .612509$

H $4.113143 \quad 0.399648 \quad 6.310337$

H 4.0566072 .0401094 .478204

H -1.236442 -2.169259 2.420173

C $0.4312864 .861196-1.692371$

O $-0.2597353 .937462-0.826672$

C $-1.4265504 .582902-0.288615$

C $-1.2602796 .066072-0.591578$

C $-0.5448546 .012421-1.929229$

H $-2.2834504 .208471-0.860035$

C -1.602286 4.2232541 .189959

H -2.218885 $6.591522-0.636648$

H $-0.632654 \quad 6.5664990 .154810$

C $0.1228777 .320703-2.314424$

H $-1.2703535 .736224-2.706165$

C -1.598207 2.7052591 .474831

O $-0.5248454 .804401 \quad 1.934862$

H -2.5222634 .6834291 .569687$

H -0.5824282 .3106811 .351332$

O -1.9206422 .5237852 .859552$

C -2.5755341 .8841120 .614244$

C $-2.609907 \quad 0.398178 \quad 1.022951$

H $-2.2796821 .959421-0.437650$

O

H -1.5833680 .0325331 .144745$

H $-3.1274130 .309647 \quad 1.986267$

H $-0.6205578 .119415-2.404814$

H $0.8599027 .630959-1.566093$

H $0.6375717 .228102-3.275791$

C $0.8593154 .138455-2.972166$

H -0.6522274 .5594922 .867568$

H -2.843152 2.8056362 .984775

H -4.4746481 .9636510 .156229$

H $1.2603114 .856447-3.696065$

H $-0.0053663 .646268-3.433498$

H $1.3130225 .213538-1.140611$

C $-5.508544-0.765258-1.043522$ 
O $-4.703027-0.090046-0.068763$

C $-3.329387-0.483302-0.018719$

C $-3.221494-1.9777870 .318260$

C $-4.042291-2.833084-0.651563$

C $-5.463719-2.287696-0.833504$

H $-6.532997-0.428552-0.837877$

C $-5.154193-0.336463-2.471931$

H -2.863351-0.297161 -0.992630

O $-3.663895-2.2192801 .652323$

H $-2.168323-2.2743120 .269602$

H $-4.086360-3.860169-0.269014$

O $-3.371313-2.865637-1.907380$

H $-5.966589-2.785701-1.670623$

O $-6.223604-2.577097 \quad 0.343785$

C $-5.4074541 .140631-2.663952$

H $-4.114271-0.552289-2.732586$

H -5.771822 $-0.869270-3.204409$

H $-4.575429-1.8889591 .728153$

H -3.885412 -3.439551-2.500415

H -6.240842 -3.5433830 .451284$

O $-4.2231991 .805696-2.784919$

O $-6.5233041 .643725-2.684969$

C -4.325560 $3.221611-2.964306$

H $-4.9418853 .454179-3.838118$

H -4.740469 $3.684013-2.063822$

H -3.319896 3.616124 -3.131893

SCF Energy (B3LYP/6-31G**//MMFF) $=-3245.92456062$

0700418

MM̄FF Geometry

C $1.591724-3.103111-0.013537$

C $0.536750-3.5764920 .665399$

C $-0.438581-2.6812631 .381165$

O $-1.772341-2.989490 \quad 0.911319$

C $-0.410962-2.8850412 .905960$

C $0.858726-2.3790703 .565041$

C $1.120550-1.0789353 .811271$

C $1.852669-3.4404453 .956568$

C 0.2295220 .0950313 .509460

C $0.7455890 .903066 \quad 2.309951$

C $-0.249741 \quad 1.9527061 .786556$

C -0.3963393 .1388102 .738953$

C -1.430724 4.153056 2.229318

O -1.5096221 .3113491 .574152$

O $-1.682167 \quad 5.060515 \quad 3.313207$

C -2.7399873 .3976091 .917526$

C -0.8848734 .9956411 .070923$

O -3.7039414 .2585441 .315928$

C -2.5364622 .1487221 .028878$

C $-3.822224 \quad 1.290440 \quad 0.932661$

O $-2.1967902 .564522-0.298337$

C -2.198433 -2.304561-0.186984

O $-1.570141-1.469796-0.819625$

C $-3.581777-2.733248-0.490460$

C $-4.255890-2.093375-1.458083$

C $-5.636910-2.339950-1.877096$

C $-6.377580-3.466795-1.209000$

C $-4.316843 \quad 0.7528192 .276989$

C $-5.311460-0.3782002 .116210$

C -7.690204-0.841566 1.572489

O $-5.018147-1.5340232 .415317$

C -8.448443 -0.7498310 .283938$

C $-8.2221140 .063540-0.759857$

C $-9.0547510 .035316-2.011909$

C -8.238647 -0.209481-3.262075

C $-8.1789480 .711292-4.239015$

C $-7.550506-1.551213-3.414589$

C $-6.163777-1.541266-2.829186$

N $-6.5392540 .039742 \quad 1.626927$

H $1.751974-2.028813-0.055635$

H $0.365888-4.6485430 .718921$

H $-0.230597-1.6287001 .166901$

H -0.563395 -3.9467433 .144989$

H -1.283071 -2.391546 3.354596

H $2.067131-0.8198454 .282802$

H $2.749844-3.0186974 .422047$

H $1.401797-4.1323804 .675419$

H $2.174893-4.0118383 .081058$
H $-0.797873-0.2367043 .336440$

H $\quad 0.1938080 .7332854 .399972$

H 1.6992531 .3844712 .560632

H $\quad 0.9590290 .210957 \quad 1.486301$

H 0.1276402 .3016740 .819916

H 0.5715813 .6284872 .903862

H $-0.7242202 .785120 \quad 3.725584$

H -2.294110 5.7465622 .997534

H -3.1587943 .0867222 .882596$

H -0.0026895 .5610101 .394406$

H $-1.617648 \quad 5.742545 \quad 0.745754$

H $-0.5968854 .399443 \quad 0.202548$

H -3.888326 4.9788551 .941667

H $-4.6189191 .857514 \quad 0.434454$

$\begin{array}{llll}\text { H } & -3.620358 & 0.437564 & 0.272085\end{array}$

H -2.407565 $1.839813-0.910359$

H $-4.010217-3.5354870 .098458$

H -3.752198 -1.287021 -1.990766

H $-5.870195-4.420049-1.395132$

H $-7.404505-3.583875-1.560462$

H $-6.431437-3.307507-0.126987$

H -3.4837920 .3679432 .876432$

H -4.7932401 .5456902 .864509$

H $-8.346743-0.5618172 .403116$

H -7.373044 -1.876982 1.732085

H $-9.275757-1.4539140 .208164$

H $-7.4139600 .788993-0.726882$

H $-9.5825240 .995279-2.080112$

H $-9.835789-0.733126-1.950566$

H $-7.614540 \quad 0.532386-5.148344$

H $-8.6881541 .665197-4.150795$

H -7.454832 -1.797519-4.480526

H $-8.191690-2.331776-2.996889$

H $-5.524763-0.767069-3.257988$

H $-6.7075001 .037171 \quad 1.531611$

C $3.980397-3.825587-0.109842$

O $4.480227-2.488737-0.309271$

C $5.905231-2.550664-0.513524$

C $6.303918-4.014296-0.343136$

C $5.030686-4.735949-0.738935$

H $6.089776-2.252855-1.551701$

C $6.612632-1.591578 \quad 0.450434$

H $7.161314-4.282440-0.968356$

H $6.567667-4.2456270 .695620$

C $4.977838-6.175573-0.258165$

H $4.939899-4.714588-1.833268$

C $6.180439-0.115962 \quad 0.315680$

O $6.335909-2.0004621 .798038$

H $7.698555-1.6756700 .329199$

H $5.129014-0.0095850 .604315$

$\begin{array}{lllll} & \text { O } & 6.937281 & 0.641776 & 1.269894\end{array}$

C $6.4115420 .495408-1.081946$

C $6.1893232 .020772-1.141105$

H $5.7663620 .000105-1.815200$

$\begin{array}{lllll} & & 7.765005 & 0.236726 & -1.467557\end{array}$

H $6.7896702 .509317-0.364151$

H $6.5898412 .385774-2.096877$

H $5.807065-6.752366-0.680656$

H $5.047039-6.2369240 .833124$

H $4.043583-6.656700-0.563770$

C $2.591905-3.961993-0.733443$

H $5.370817-1.9811641 .917915$

H 6.7848420 .2424902 .143666

H $7.8769210 .556423-2.379056$

H $2.272910-5.010129-0.718944$

H $2.617234-3.640590-1.781475$

H $3.928152-3.9918290 .974748$

C $2.6822652 .394014-2.359232$

$\begin{array}{llll}\text { O } & 4.084502 & 2.109137-2.267807\end{array}$

C $4.7129432 .429774-1.020513$

C $4.5616933 .926544-0.713000$

C $3.0867674 .337579-0.727783$

C $2.379443 \quad 3.858205-1.997556$

H $2.4407112 .261427-3.422198$

C $1.8545461 .379864-1.563172$

H $4.2429611 .854645-0.218236$

O $5.2866254 .689264-1.674951$

H 5.0013014 .1308720 .270741 
H $3.0066215 .429593-0.664118$

$\begin{array}{lllll}\text { O } & 2.426544 & 3.781722 & 0.404871\end{array}$

H $1.2965503 .994181-1.898316$

O $2.7893344 .684433-3.092068$

C $1.8536660 .047468-2.276649$

H $2.2278421 .204978-0.551106$

H $0.8177741 .723166-1.467665$

H $5.1932775 .627157-1.436183$

H 2.8647114 .1279221 .200812

H $3.7359594 .524068-3.247423$

O $0.626170-0.190441-2.820007$

O $2.834378-0.680306-2.363022$

C $0.502221-1.408356-3.560814$

H $1.231415-1.435566-4.376173$

H $0.631249-2.267956-2.897682$

H $-0.503234-1.444190-3.988694$

SCF Energy (B3LYP/6-31G*//MMFF) $=-3245.90990701$

0700419

MM̄FF Geometry

C $-1.371083-3.3541022 .775020$

C -1.100954 -2.090459 2.410956

C $-0.193071-1.1736273 .188973$

O $1.069782-1.1520302 .485152$

C $-0.789782 \quad 0.246297 \quad 3.235082$

C $-0.195424 \quad 1.1239854 .318899$

C $0.656513 \quad 2.1447894 .101851$

C -0.6718340 .8286595 .718640$

C 1.2436712 .5900482 .793036

C $0.557288 \quad 3.863758 \quad 2.285544$

C 1.2088464 .4033191 .004456

C $0.465614 \quad 5.640493 \quad 0.495706$

C $1.0353056 .146658-0.838605$

$\begin{array}{llllll}\text { O } & 1.168570 & 3.374299 & 0.013731\end{array}$

O $0.0847127 .081240-1.371676$

C $1.1409094 .960826-1.823168$

C $2.3359676 .930641-0.629464$

O $1.7933095 .357058-3.027444$

C $1.8200443 .712479-1.216071$

C $1.6860592 .505255-2.174286$

O $3.2086273 .994636-1.020444$

C $2.206159-1.1200843 .232721$

O $2.288293-1.0128364 .446024$

C $3.355608-1.2759432 .314033$

C $4.603541-1.2194992 .801361$

C $5.817014-1.3950612 .010574$

C $6.856752-0.3392282 .265476$

C $2.050916 \quad 1.167656-1.517182$

C $2.0839130 .016068-2.505963$

C $2.606801-2.428470-2.559065$

$\begin{array}{lllll}\text { O } & 1.920947 & 0.153068 & -3.714351\end{array}$

C $4.088399-2.563900-2.709887$

C $4.848915-3.316743-1.901228$

C $6.341718-3.409044-2.015330$

C $7.057020-2.448938-1.089466$

C $7.637693-1.337060-1.572044$

C $7.182527-2.8146970 .372147$

C $5.968127-2.4488231 .182643$

N $2.344105-1.184681-1.861248$

H $-0.904794-3.7657953 .666453$

H $-1.554618-1.6908231 .507240$

H -0.060989 -1.5779224 .200056$

H $-1.8722150 .192403 \quad 3.416783$

H $-0.6826650 .721742 \quad 2.252956$

H 0.9901762 .7272714 .959858

H $-0.268242 \quad 1.5340826 .452924$

H -1.763802 0.8924305 .769273

H $-0.366975-0.175575 \quad 6.027883$

H $2.312605 \quad 2.7821102 .947637$

H 1.1880111 .7971372 .040914

H -0.498012 3.6356122 .091948

H $\quad 0.583774 \quad 4.6398123 .060855$

H $2.253707 \quad 4.647242 \quad 1.229976$

H $0.476862 \quad 6.4394121 .247524$

H $-0.593619 \quad 5.3896330 .343670$

H $0.3985467 .350582-2.252189$

H $\quad 0.1157314 .694544-2.111731$

H 2.1596517 .7924680 .025622
H $2.7059217 .346073-1.573217$

H $3.1306606 .330708-0.181125$

H $2.7476075 .401844-2.846137$

H $0.6496022 .424414-2.526349$

H $2.3181632 .664470-3.056987$

H $3.7152543 .169228-1.072754$

H $3.156255-1.4565701 .264253$

H $4.772059-1.0145743 .856619$

H $7.230568-0.4092753 .292839$

H $6.428590 \quad 0.659747 \quad 2.122715$

H $7.716578-0.4086431 .595403$

H $3.0403121 .223013-1.050116$

H $1.3223190 .925317-0.734551$

H $2.124694-2.424996-3.541102$

H $2.177925-3.238049-1.960252$

H $4.557422-1.991184-3.506412$

H $4.377901-3.881243-1.100705$

H $6.643580-3.228535-3.055896$

H $6.657477-4.437379-1.801337$

H $8.180951-0.656449-0.925182$

H $7.578807-1.079783-2.624350$

H $8.087375-2.3674600 .799540$

H $7.348643-3.8959470 .462969$

H $5.149991-3.1641501 .103973$

H $2.515675-1.171315-0.860740$

C $-1.645302-4.954737 \quad 0.836045$

O $-1.395279-3.969549-0.188795$

C $-2.242097-4.261305-1.311236$

C $-2.357403-5.774197-1.292087$

C $-2.555313-6.0155920 .196128$

H $-3.226973-3.827577-1.097862$

C $-1.687193-3.626348-2.588235$

H $-3.188832-6.142590-1.899878$

H $-1.433713-6.255541-1.633144$

C $-2.227336-7.4342800 .630449$

H $-3.605143-5.8110610 .446580$

C $-1.328259-2.133244-2.423983$

O $-0.496860-4.325668-2.966720$

H $-2.403729-3.765405-3.406496$

H $-0.411688-2.042169-1.829813$

O $-1.033739-1.591033-3.715339$

C $-2.427610-1.271788-1.776625$

C $-2.0068850 .205059-1.659050$

H -2.645334 -1.655549-0.775108

O $-3.619762-1.383402-2.550535$

H $-0.9658120 .256782-1.321987$

H $-2.0788240 .677146-2.646546$

H $-2.880387-8.1527830 .124403$

H $-1.190778-7.6968580 .393673$

H -2.366764 -7.550074 1.710143

C $-2.312250-4.2582912 .027583$

H $-0.251183-4.027677-3.858591$

H $-0.219712-2.013983-4.035161$

H $-4.304932-0.848897-2.114845$

H -2.697485 -5.004182 2.733112

H $-3.172471-3.6682311 .686795$

H $-0.674797-5.3771181 .122829$

C $-5.1710791 .714639-0.345746$

O $-4.2054711 .070973-1.188153$

C -2.882481 $0.979220-0.654535$

C $-2.3227772 .379980-0.370773$

C $-3.259586 \quad 3.179772 \quad 0.538111$

$\begin{array}{lllll}C & -4.710223 & 3.128771 & 0.045328\end{array}$

H $-6.0637861 .817835-0.976468$

$\begin{array}{llll}\text { C } & -5.546028 & 0.841460 & 0.857192\end{array}$

$\begin{array}{llll}\text { H } & -2.907385 & 0.407941 & 0.280002\end{array}$

O $-2.118763 \quad 3.090088-1.589745$

H $-1.3438332 .275208 \quad 0.108971$

H $-2.917413 \quad 4.2210010 .574029$

$\begin{array}{llll}\text { O } & -3.177179 & 2.647988 & 1.857056\end{array}$

H -5.3909703 .5374860 .801099$

O $-4.8329833 .953506-1.117398$

C $-6.257455-0.4095260 .398373$

H $-4.6839120 .554631 \quad 1.465835$

H -6.2325051 .3728091 .526902$

H -2.967330 $3.118538-2.063960$

H -3.747179 3.1903132 .428327

H - $4.5870534 .859045-0.861909$ 
O $-5.422059-1.483930 \quad 0.483073$

O $-7.411919-0.423287-0.009578$

C $-5.981759-2.7307310 .058827$

H $-6.918581-2.9338500 .586860$

H $-6.142077-2.717524-1.023079$

H $-5.270253-3.5241660 .299649$

SCF Energy (B3LYP/6-31G*//MMFF) $=-3245.90661264$

0700420

MM̄FF Geometry

C $1.6690594 .666094-0.250804$

C $2.9142084 .629021-0.751608$

C $4.092867 \quad 3.922126-0.120404$

O $3.6052452 .805361 \quad 0.660452$

C $5.053153 \quad 3.388592-1.204505$

C $6.4684513 .156993-0.707197$

C $7.0478571 .952930-0.533191$

C $7.2730074 .410254-0.460998$

C $6.4471710 .591151-0.742339$

C $6.671878-0.3070290 .484382$

C $6.090485-1.724398 \quad 0.346156$

C $6.845628-2.563815-0.687964$

C $6.214589-3.950592-0.880360$

O $4.709602-1.608084-0.001843$

O $6.811990-4.513536-2.058622$

C $4.705521-3.766198-1.146432$

C $6.554943-4.896955 \quad 0.275976$

O $4.034498-5.022877-1.202176$

C $4.009819-2.853186-0.113053$

C $2.555866-2.526177-0.514896$

$\begin{array}{lllll}\text { O } & 3.960332 & -3.526167 & 1.150441\end{array}$

C 4.2286562 .5127901 .827292

O 5.2104373 .0749572 .293416

C 3.5858871 .3714522 .519845

C $2.365737 \quad 0.893917 \quad 2.224697$

C $1.681527-0.1947462 .931962$

C $2.485595-0.9864923 .931547$

C $2.409036-1.748962-1.827035$

C $1.004625-1.196594-1.924869$

C -1.344067-1.949749 -2.188087

O $0.742749-0.045874-1.583222$

C $-1.720717-2.578615-0.882954$

C $-1.815676-1.8969470 .268305$

C $-2.101059-2.5423651 .590913$

C $-0.868408-2.6201752 .464977$

C $-0.143189-3.7496632 .531179$

C $-0.531297-1.4309353 .333817$

C $0.380616-0.4374542 .661568$

N $0.084533-2.140232-2.357653$

H 1.4522324 .1754970 .694528

H $3.1116295 .174112-1.672521$

H $4.592408 \quad 4.6559890 .523192$

H $5.1256594 .105265-2.034121$

H $4.6353562 .477180-1.645229$

H $8.088906 \quad 1.934022-0.211441$

H $8.3143504 .192478-0.200916$

H $7.2857115 .035963-1.359526$

H 6.8461634 .9909110 .361960

H $5.3770770 .649988-0.955482$

H $6.9284530 .151808-1.622198$

H $7.742596-0.3753220 .714524$

H $6.194156 \quad 0.172566 \quad 1.348128$

H $6.163760-2.1989451 .332040$

H $7.903816-2.657233-0.413848$

H $6.833969-2.056855-1.661402$

H $6.470935-5.417084-2.168474$

H $4.610471-3.316392-2.142779$

H $7.639255-5.0503330 .335077$

H $6.119580-5.8895860 .116344$

H $6.219517-4.5278591 .247317$

H $4.426002-5.534862-1.929494$

H $1.957741-3.445928-0.550276$

H $2.104966-1.9264090 .287805$

H $3.237610-3.1446981 .676136$

H 4.1692980 .9675923 .341727

H $1.795148 \quad 1.357575 \quad 1.421889$

H $3.421955-1.3375203 .482721$

H $1.973705-1.8823634 .289410$
H $2.723244-0.3725044 .806764$

H $3.104108-0.903688-1.877096$

H $2.609604-2.387686-2.693792$

H $-1.858472-2.448484-3.014556$

H - $1.588075-0.883057-2.215960$

H $-1.876601-3.654682-0.883385$

H $-1.646248-0.8239470 .264586$

H -2.511646 -3.549361 1.437869

H $-2.894563-1.9870802 .104395$

H $\quad 0.723672-3.827795 \quad 3.178665$

H $-0.396544-4.6190151 .933464$

H $-0.117012-1.7730344 .288520$

H -1.455253 -0.9129603 .619920$

H -0.1154720 .1800561 .912930$

H $0.407986-3.085691-2.541892$

C $-0.7514524 .525980-1.022468$

$\begin{array}{llllll}\text { O } & -1.344473 & 4.380387 & 0.282646\end{array}$

C $-1.845207 \quad 3.0325300 .415629$

C $-1.8173562 .438189-0.986447$

C $-0.5969043 .112376-1.579830$

H -1.1329822 .5137821 .069283$

C -3.2364693 .0698751 .060491$

H -1.744281 $1.347748-0.981109$

H $-2.7193422 .718237-1.543301$

C $-0.5280323 .051050-3.094425$

H $0.2916232 .622213-1.164677$

C $-3.8476551 .667914 \quad 1.250086$

$\begin{array}{lllll}\text { O } & -3.110782 & 3.705897 & 2.335417\end{array}$

H $-3.910165 \quad 3.6921250 .459268$

H -3.843728 1.1557290 .283057

$\begin{array}{lllll} & \text { O } & -3.009776 & 0.911212 & 2.123488\end{array}$

C $-5.293161 \quad 1.6572081 .789472$

C $\begin{array}{llll}-5.841257 & 0.249102 & 2.090957\end{array}$

H -5.9507802 .1577051 .068963$

O $\begin{array}{llll}-5.386561 & 2.384830 & 3.015919\end{array}$

H $-5.288800-0.1898312 .932095$

H -6.8701150 .3806132 .452699$

H $-0.5125202 .011727-3.438478$

H $-1.3884053 .546371-3.555948$

H $0.3787163 .542627-3.460907$

C $0.5242625 .369161-0.927824$

H $-2.708146 \quad 4.5785852 .185601$

H $-3.006273 \quad 1.3621072 .985362$

H $-5.144092 \quad 3.3078582 .830971$

H $0.3072386 .276219-0.350282$

H $0.8168395 .684435-1.936552$

H $-1.4839385 .086707-1.618364$

C $-6.682384-0.900525-1.365306$

O $-6.611296-0.143535-0.149111$

C $-5.831391-0.7224440 .900419$

C $-6.378541-2.1056611 .288528$

C $-6.483797-3.0204440 .064269$

C $-7.197078-2.326403-1.099656$

H $-7.438198-0.385157-1.972369$

C $-5.354280-0.867755-2.139040$

H $-4.799084-0.8408180 .561011$

O $-7.664178-1.9629191 .888965$

H $-5.712307-2.5499262 .038015$

H $-7.033975-3.9304890 .331952$

O $-5.181483-3.410591-0.360461$

H $-7.106115-2.922887-2.015094$

O $-8.593032-2.229404-0.803100$

C $-5.0883400 .490961-2.753435$

H $-4.494204-1.110045-1.511844$

H $-5.406260-1.591332-2.961527$

H $-7.967210-2.8494022 .149133$

H $-4.756456-3.8723810 .382149$

H -8.930292 -3.133016 -0.680177

O $-4.1421020 .356660-3.729563$

O $-5.6286251 .535326-2.416733$

C $-3.810511 \quad 1.549552-4.447469$

H -2.882691 $1.368412-4.996690$

H $-4.6059651 .777995-5.162332$

H $-3.6546212 .392330-3.768378$

SCF Energy (B3LYP/6-31G*//MMFF) $=-3245.89683819$

0700421

MM̄FF Geometry 
C $-3.9940883 .166073 \quad 1.175155$ C -2.6873803 .1935000 .871969$ C $-1.5871213 .189248 \quad 1.901756$ O $-0.6361872 .171528 \quad 1.515180$ C -0.8764894 .5559331 .904323$ C 0.2890464 .6399332 .870572 C 1.5862924 .7232112 .511491 C -0.0883444 .6723654 .330123$ C 2.1715664 .7495901 .125424 C 3.1600053 .6012540 .906409 C $3.8624873 .654790-0.460290$ C $2.8977353 .429444-1.628704$ C $3.6287833 .360630-2.979306$ O $4.8641142 .627245-0.449362$ O $2.7123162 .831570-3.950313$ C $4.7929832 .355936-2.865329$ C $4.0349724 .751336-3.481260$ O $5.5912632 .357780-4.045982$ C $5.6842772 .588673-1.627349$ C $6.7337991 .469785-1.430965$ O $6.4079973 .814473-1.788553$ C -0.3054091 .2290422 .438610$ O -0.7545531 .1077713 .567072$ C $\quad 0.720698 \quad 0.3504461 .835454$ C $1.078514-0.7755702 .470388$ C $2.063964-1.7516322 .003773$ C $2.952577-1.3480530 .856808$ C $6.1531850 .059198-1.315129$ C $7.171662-0.953226-0.820398$ C $7.347350-3.354311-0.183203$ O $8.343509-0.674406-0.583678$ C $6.410414-4.2543580 .555240$ C $6.306392-4.2713851 .892493$ C $5.346998-5.1437412 .649698$ C $4.197905-4.3425973 .227267$ C $4.248747-3.9144714 .500556$ C $3.028693-4.1133052 .287493$ C $2.139088-2.9455912 .629329$ N $6.599344-2.207578-0.661282$ H -4.2975203 .1277462 .218450$ H $-2.3853583 .263117-0.170741$ H -2.007428 2.9845092 .893480 H -1.596848 5.3431712 .166014 H -0.5528464 .7937970 .886292$ H 2.3286804 .8010913 .305368 H 0.7806084 .8126414 .981987 H $-0.7794185 .499306 \quad 4.524057$ H $-0.572537 \quad 3.7379134 .627674$ H $1.396466 \quad 4.714607 \quad 0.359144$ H $2.693148 \quad 5.706156 \quad 0.998444$ H 3.9269713 .6181461 .692933 H 2.6518392 .6358181 .019941 H $4.3584354 .628294-0.550834$ H $2.1270584 .207332-1.667491$ H $2.3606382 .482582-1.476320$ H $1.9338633 .414042-3.976532$ H $4.3400461 .358794-2.797338$ H $3.1484625 .381946-3.619760$ H $4.5159284 .691850-4.463738$ H $4.7063815 .275557-2.798305$ H $4.9951762 .205019-4.799172$ H $7.4737351 .498801-2.241544$ H $7.2974221 .692190-0.513847$ H $7.1896113 .789134-1.211973$ H 1.1399610 .6432780 .879669 H $0.585878-1.0245053 .409492$ H $3.406604-0.3687111 .047025$ H $2.380210-1.292954-0.074946$ H $3.784396-2.0367080 .693308$ H $5.3112320 .054868-0.614573$ H $\quad 5.793787-0.281459-2.292435$ H $8.179070-3.0185330 .445428$ H $7.767967-3.871444-1.051421$ H $5.778713-4.908396-0.040557$ H $6.933679-3.6068492 .482588$ H $5.903566-5.6515593 .447870$ H $4.950595-5.9468162 .015315$ H $3.440213-3.3568814 .959037$
H $5.112446-4.1194725 .125477$ H $2.411356-5.0211922 .282963$

H $3.395699-4.0175081 .260819$

H $1.456633-3.1419043 .457315$

H $5.666081-2.365777-1.031776$

C $-6.2102542 .257670 \quad 0.348383$

O $\quad-5.8136780 .966919-0.167524$

C $-6.8629210 .469584-1.019535$

C $-8.0975791 .296716-0.685912$

C $-7.4788522 .649221-0.416026$

H $-6.5612230 .689832-2.050342$

C -7.076197 -1.037833 -0.846776

H $-8.8234481 .310256-1.504572$

H -8.6048420 .9261720 .212562$

C $-8.394816 \quad 3.5915840 .347159$

H -7.211776 $3.110796-1.376630$

C $-5.854137-1.929766-1.137414$

O $-7.488386-1.3049820 .500689$

H -7.913639-1.354838-1.479983

H $-5.130827-1.848896-0.319569$

O $-6.312695-3.289313-1.134653$

C $-5.149893-1.661797-2.478877$

C $-4.147266-2.744625-2.925388$

H $-4.630841-0.698452-2.423096$

O $-6.124667-1.538706-3.519686$

H $-4.689279-3.647655-3.235458$

H -3.663015 -2.368564 -3.836619

H $-9.309303 \quad 3.784212-0.223291$

H -8.6854533 .1713111 .315851$

H -7.9035204 .5520570 .531095$

C -5.0876743 .2814770 .150431$

H $-6.796280-0.9613231 .091313$

H $-6.735672-3.450167-0.273630$

H $-6.631014-2.368752-3.547646$

H $-5.497277 \quad 4.294456 \quad 0.242144$

H $-4.6727783 .183110-0.860417$

H -6.4319732 .1187241 .414235$

C $-1.389373-2.093669-0.488037$

O $-2.376308-1.931670-1.517085$

C $-3.083113-3.118409-1.882920$

C $-2.108062-4.183029-2.410975$

C $-0.997189-4.463284-1.394202$

C $-0.359973-3.168710-0.876692$

H $-0.865280-1.130111-0.450767$

C $-2.033096-2.3346880 .887594$

H $-3.597132-3.520665-1.004962$

O $-1.532225-3.738934-3.637508$

H $-2.668337-5.102327-2.621148$

H $-0.229194-5.088777-1.865045$

O $-1.549895-5.201298-0.308098$

H $\quad 0.289094-3.377707-0.019536$

O $0.469695-2.615601-1.902388$

C $-2.826576-1.1354251 .360294$

H $-2.714960-3.1886040 .877524$

H -1.249449 -2.523653 1.628831

H $-0.948413-4.445926-3.960771$

H $-0.829038-5.3876850 .317350$

H $1.155581-3.272568-2.110526$

O $-3.324219-1.3934892 .605532$

O $-3.000339-0.1084030 .719961$

C $-4.125816-0.3531213 .173973$

H -5.003745 -0.166696 2.548766

H $-4.461979-0.6838244 .160237$

$\mathrm{H}-3.5360120 .5601253 .292610$

SCF Energy $(B 3 L Y P / 6-31 G * * / / M M F F)=-3245.90062527$

0700422

MM̄FF Geometry

C 2.4773870 .5341852 .202362

C 1.7356181 .4040712 .901991

C 0.8348602 .4211222 .257044

O -0.5186102 .0053042 .556866$

C 1.0662443 .8400002 .806658

C 2.4031454 .4423692 .437885

C 2.6443025 .0967001 .285451

C 3.4794384 .3380343 .487243

C 1.6980075 .3200800 .134856

C $2.3713995 .053018-1.219587$ 
C $2.5403173 .556328-1.537125$

C $3.6538773 .371474-2.569499$

C $3.7974671 .908753-3.000698$

O $1.2951933 .078500-2.064373$

O $4.652706 \quad 1.874438-4.153855$

C $2.4155671 .392841-3.442826$

C $4.4821111 .056349-1.926243$

O $2.497545-0.001845-3.731970$

C $1.2805661 .689670-2.426741$

C $-0.0856811 .379494-3.086530$

O $1.4501730 .848353-1.286926$

C -1.502559 $2.465137 \quad 1.739662$

O -1.3817293 .2425950 .806483$

C -2.7719081 .8426052 .166446$

C -3.9175502 .2725921 .620599$

C -5.2371541 .7238801 .903648$

C -6.2905522 .7748632 .121603$

C $-1.3210141 .812038-2.284221$

C $-2.6057821 .447933-3.015108$

C $-5.0730551 .542909-2.810060$

O $-2.6172360 .929175-4.129200$

C $-6.0493341 .352973-1.693491$

C $-6.6448950 .180602-1.423511$

C $-7.688061-0.018820-0.359016$

C $-7.234603-0.9530620 .741869$

C $-7.319158-2.2862860 .598251$

C $-6.756390-0.3336602 .037375$

C $-5.4550860 .394567 \quad 1.865466$

N $-3.7407691 .755840-2.275224$

H $2.417726 \quad 0.547674 \quad 1.118887$

H 1.7614341 .3750623 .988698

H 0.9732602 .4073451 .168494

H 0.9442323 .8406383 .898864

H 0.2643674 .5066062 .464406

H 3.6318195 .5369411 .150449

H 4.4036034 .8430843 .186370

H 3.1435024 .7991804 .421865

H 3.7294063 .2921093 .686036

H 0.7877494 .7212840 .219027

H $1.392538 \quad 6.3732120 .163005$

H $1.7559925 .518067-2.000719$

H $3.3451795 .559183-1.236181$

H $2.7790553 .005643-0.620920$

H $4.6092333 .748858-2.183000$

H $3.4315063 .977011-3.459632$

H $5.5111722 .255681-3.901969$

H $2.1796211 .895162-4.391754$

H $5.4706451 .465038-1.684780$

H $4.6617000 .037972-2.286659$

H $3.9130231 .000698-0.996621$

H $1.700505-0.252907-4.227457$

H $-0.1328261 .886417-4.059592$

H $-0.1629300 .302085-3.277348$

H $\quad 0.5948390 .736597-0.843945$

H -2.7400631 .0422272 .897704$

H -3.9100703 .0967450 .910039$

H -6.0537253 .3676843 .012250$

H -7.292795 2.3658052 .263757

H -6.3434233 .4533011 .262579$

H -1.337129 $1.319924-1.307064$

H -1.313963 2.896528 -2.130168

H $-5.0595840 .685585-3.492083$

H $-5.3413152 .428346-3.395588$

H $-6.3070412 .232261-1.109234$

H -6.392878 $-0.689717-2.025821$

H $-8.586246-0.428934-0.839530$

H $-8.005124 \quad 0.9393470 .070608$

H -7.019040 -2.961895 1.392148

H -7.686541 -2.730954 -0.320847

H -6.601882 -1.1083002 .799900$

H -7.5482340 .3053542 .438948$

H -4.610254 -0.2645871 .669081$

H -3.638588 2.334240 -1.446084

C $4.622955-0.7658301 .984811$

O $4.211715-1.3485410 .728317$

C $4.530928-2.7453640 .768600$

C $5.844761-2.7786231 .527846$

C $5.550283-1.7965752 .651911$
H $\quad 3.754847-3.2422341 .365427$

C $4.539226-3.349469-0.635648$

H $6.098163-3.7788621 .890762$

H $6.674784-2.4097100 .914331$

C $6.800655-1.1762693 .253986$

H $5.013866-2.3274643 .449831$

C $3.287896-3.022357-1.479286$

O $5.679132-2.857725-1.350191$

H $4.675986-4.434682-0.555889$

H $3.369366-2.001382-1.867993$

O $3.311475-3.875898-2.630734$

C $1.935236-3.170129-0.758979$

C $0.743262-3.006196-1.721150$

H $1.869363-2.4240030 .040444$

O $1.876932-4.462403-0.155336$

H $\quad 0.963771-2.231568-2.465073$

H $0.594976-3.955544-2.250036$

H $7.443153-1.9485263 .689190$

H $7.384292-0.6375932 .499891$

H $6.536615-0.4676614 .045796$

C $3.367403-0.5008482 .827727$

H $5.660585-3.257493-2.236640$

H $3.165935-4.786874-2.322372$

H $1.037515-4.5194430 .332195$

H $3.657539-0.1943853 .839545$

H $2.784293-1.4265772 .905043$

H 5.1441010 .1710761 .756190

C $-1.975530-3.2931670 .851393$

O $-0.834189-3.5715790 .027695$

C $-0.559760-2.611424-0.995315$

C $-1.747982-2.506929-1.966218$

C $-3.063466-2.253290-1.225598$

C $-3.244033-3.170652-0.012612$

H -2.088174 -4.181033 1.486964

C $-1.755564-2.0806661 .772165$

H $-0.385543-1.634972-0.530696$

O $-1.872029-3.690084-2.752314$

H -1.558438 -1.685519-2.666316

H -3.899781 -2.390492 -1.922133

O $-3.071351-0.898140-0.789545$

H $-4.070934-2.8081640 .608189$

O $-3.631815-4.469385-0.467542$

C $-0.724815-2.3512482 .847871$

H -1.429367 -1.189768 1.230654

H $-2.700038-1.8536182 .281036$

H $-1.754003-4.457083-2.168685$

H -3.962188 -0.707315 -0.451615

H $-2.828794-4.990509-0.631891$

O $-0.847612-1.4009613 .822279$

O $0.088498-3.2645252 .834607$

C $0.030986-1.5416984 .942619$

H -0.094114 -0.665419 5.584328

H $-0.234009-2.4370125 .512537$

H $1.072992-1.5890194 .616073$

SCF Energy (B3LYP/6-31G**//MMFF) $=-3245.90860457$

0700423

MM̄FF Geometry

C $-3.498851 \quad 3.234008 \quad 0.625174$

C -3.6474992 .6808501 .838577$

C -4.1865481 .2951172 .053926$

O -3.1307990 .5603532 .720662$

C -5.4347291 .2567692 .949997$

C -6.6549501 .9490122 .385543$

C -7.4070761 .4591181 .380791$

C -7.0266423 .2464063 .052284$

C -7.1662980 .1945090 .602710$

C $-6.9764900 .512357-0.885948$

C $-6.336855-0.613113-1.711933$

C $-7.032459-1.965922-1.552508$

C $-6.330753-3.065866-2.366675$

O $-4.958856-0.718313-1.329677$

O $-6.821504-4.335424-1.908670$

C $-4.817414-3.030210-2.059051$

C $-6.676891-2.982054-3.858041$

O $-4.104482-3.931815-2.902868$

C $-4.199883-1.615706-2.153181$

C $-2.738030-1.633609-1.646426$ 
O $-4.193484-1.200015-3.523190$ C -3.026791-0.770665 2.468394 O $-3.756465-1.4507881 .765965$ C -1.829951-1.258254 3.187200 C -1.253954 -2.406897 2.804680 C $-0.056339-3.0055953 .394544$ C $0.502149-2.3791144 .645365$ C $-2.154542-0.235643-1.393005$ C $-0.647796-0.206565-1.551136$ C $1.463534-1.143907-0.669419$ O $-0.1055560 .534643-2.369207$ C $1.898960-2.482424-0.169501$ C $2.774718-2.6436250 .833969$ C $3.266689-3.9802471 .313047$ C $3.021128-4.1924072 .790221$ C $3.964695-3.8781023 .694080$ C $1.721518-4.8385673 .217964$ C $0.496389-4.0785652 .791802$ N $0.015993-1.074330-0.697792$ H -3.818954 2.686402 -0.257936 H -3.3142503 .2250382 .719056$ H $-4.389460 \quad 0.828745 \quad 1.083645$ H -5.1887741 .6773693 .935310$ H $-5.689657 \quad 0.2121513 .175181$ H -8.2835362 .0253161 .068392$ H -7.9154573 .7047092 .605954$ H $-7.237823 \quad 3.0797394 .113722$ H -6.2070853 .9672562 .969520$ H $-6.307553-0.3607250 .987478$ H -8.038674 -0.4573640 .730508$ H $-7.9527430 .764713-1.319759$ H $-6.3420081 .403689-0.985357$ H $-6.380734-0.290015-2.759144$ H -8.091199 -1.894239-1.830211 H -7.016412 -2.273331 -0.498541 H $-7.787028-4.343053-2.025528$ H -4.683346 -3.395877 -1.031163 H -7.758116 -3.089309-4.006298 H -6.215772 -3.803593 -4.417506 H -6.374444 -2.038916-4.317435 H $-4.515338-4.807742-2.803372$ H -2.682986 -2.188605 -0.701663 H -2.111262 -2.169925 -2.370631 H -3.451941 -0.591660 -3.670478 H -1.439704 -0.643354 3.990243 H - $-1.685426-2.9515731 .965531$ H $-0.261552-2.3374505 .429956$ H $\quad 0.854200-1.3619334 .441590$ H $1.347331-2.9305065 .064184$ H -2.566695 $0.499927-2.092737$ H $-2.4054380 .112025-0.385650$ H $1.820319-0.326850-0.035763$ H $1.846496-0.984433-1.681607$ H $\quad 1.501453-3.357988-0.677040$ H $3.190555-1.7636731 .320289$ H $4.343371-4.0336301 .103930$ H $2.816769-4.803340 \quad 0.743893$ H $3.813278-4.0500064 .754519$ H $4.910128-3.4394443 .392842$ H $1.694833-5.0078494 .301495$ H $1.671798-5.8429042 .777011$ H $0.035654-4.4630921 .881399$ H $-0.500983-1.4890430 .072144$ C -1.547099 $4.407372-0.397759$ O C 0.6871524 .1751470 .294044 C $0.6168375 .251378-0.778582$ C $-0.8164295 .726786-0.641095$ H $\quad 0.8463204 .6262331 .281371$ C 1.7210113 .0739360 .028714 H $1.3498616 .048371-0.623359$ H $0.7840914 .821491-1.774218$ C -1.320666 $6.498431-1.847806$ H $-0.888023 \quad 6.3662030 .249007$ C $3.1666853 .589657-0.092676$ O $1.647721 \quad 2.1349551 .107932$ H $1.4277982 .526111-0.871824$ H $3.2518454 .294524-0.927749$
O $3.4624414 .328321 \quad 1.100569$

C $4.2239272 .477181-0.242346$

C $4.1087721 .705911-1.571120$

H 4.1763871 .7984350 .617130

O $5.5075493 .113904-0.196454$

H $3.0649531 .471957-1.798910$

H $4.4921982 .350284-2.372129$

H $-0.7196637 .399787-2.006426$

H -1.268667 $5.894996-2.760288$

H -2.361312 $6.806685-1.706504$

C -2.8463704 .5684110 .396386$

H 1.9285872 .5955391 .917207

H $4.3790684 .644678 \quad 1.024035$

H $6.1826922 .414966-0.225457$

H -3.545806 $5.214550-0.145291$

H -2.6338975 .0495681 .359180$

H -1.741712 $3.890580-1.346903$

C $7.100143-0.503325-1.100143$

O $6.2659480 .651165-1.265496$

C $4.8951350 .378041-1.561232$

C $4.790870-0.369733-2.899782$

C $5.643975-1.643385-2.889187$

C $7.061548-1.382708-2.361794$

H $8.117136-0.102932-0.998384$

C $6.768036-1.2706620 .188462$

H $4.471063-0.239327-0.762454$

O $5.2289920 .485645-3.954225$

H $3.741255-0.619000-3.091443$

H $5.703103-2.044271-3.908493$

O $4.991616-2.616467-2.078968$

H $7.572101-2.331093-2.160644$

O $7.815231-0.726210-3.386290$

C $6.855429-0.3835871 .412002$

H $5.758511-1.6873280 .147474$

H $7.468628-2.1013260 .328128$

H $5.1129470 .003076-4.790420$

H $5.528195-3.426931-2.103456$

H $7.4158340 .148195-3.534761$

O $6.018572-0.8651822 .377289$

O $7.580410 \quad 0.5966901 .515234$

C $5.981360-0.1114993 .592923$

H $5.260754-0.5856724 .264631$

H $6.965104-0.1173844 .071514$

H 5.6533340 .9133013 .393732

SCF Energy $\left(B 3 L Y P / 6-31 G^{* *} / / M M F F\right)=-3245.92089340$

0700424

MM̄FF Geometry

C $-3.382740 \quad 3.325398 \quad 0.457440$

C -2.5040632 .3165660 .564282$

C $-1.4187402 .022456-0.438873$

$\begin{array}{lllll}\text { O } & -0.185347 & 1.965577 & 0.313545\end{array}$

C $-1.6904460 .653568-1.088547$

C $-0.9751140 .421921-2.402868$

C $0.048095-0.434469-2.590283$

C $-1.5380081 .183305-3.577441$

C $0.745651-1.289516-1.568507$

C $2.251244-1.021800-1.585152$

C $3.068342-1.907659-0.633483$

C $2.654985-1.7509470 .830948$

C $3.577846-2.5350651 .774520$

O $4.429640-1.496531-0.798769$

O $3.329788-2.0804713 .114424$

C $5.045688-2.1881971 .450889$

C $3.250646-4.0329761 .760014$

O $5.940278-3.0156772 .190701$

C 5.392271-2.253511-0.054969

C $6.763203-1.592396-0.307362$

O $5.491218-3.597097-0.526623$

C $0.9558382 .408696-0.274947$

O $1.0873442 .831716-1.411572$

C 2.0390542 .2851300 .728599

C 3.3191862 .4471130 .360928

C 4.4900492 .3474791 .238297

C 4.2409002 .0950632 .702659

C $7.206229-1.636605-1.776479$

C $8.224234-0.551876-2.063803$

C $10.4755190 .254506-1.387646$ 
O $7.952070 \quad 0.411476-2.777458$

C $10.2263021 .121692-0.193121$

C $9.7918712 .388911-0.267849$

C 9.4709243 .2290750 .936238

C 7.9965623 .5718511 .012419

C 7.5783134 .8309460 .795260

C 7.0591032 .4353821 .387005

C 5.7187062 .4803090 .694920

N $9.438093-0.760310-1.426808$

H $-3.3183304 .002070-0.389917$

H $-2.572371 \quad 1.642703 \quad 1.415616$

H -1.374273 2.826553 -1.182730

H -2.761871 $0.555336-1.296865$

H -1.461071 $-0.142043-0.372322$

H $0.441510-0.539979-3.601103$

H $-1.0426300 .922778-4.518933$

H -2.604129 $0.962934-3.696960$

H -1.419243 2.261209-3.433029

H $0.354645-1.109458-0.566023$

H $0.555611-2.342369-1.807335$

H $2.641788-1.164771-2.602637$

H $2.4468820 .032806-1.356497$

H $2.969068-2.950114-0.957654$

H $1.616468-2.0635500 .988993$

H $2.695333-0.6901361 .109835$

H $2.384868-2.2094813 .305304$

H $5.218692-1.1588641 .796175$

H $2.226210-4.2045472 .112555$

H $3.899529-4.5877192 .446231$

H $3.331699-4.4786140 .766164$

H $5.707075-2.9259453 .130760$

H $6.717672-0.5453950 .016868$

H $7.534356-2.0859370 .297096$

H $5.324799-4.2025290 .212740$

H $1.7501392 .049098 \quad 1.746697$

H $3.5348232 .667062-0.684019$

H 3.6536392 .9108443 .138509

H 5.1530772 .0103063 .295874

H 3.6957661 .1548232 .843279

H $7.637703-2.612729-2.025498$

H $6.359602-1.476338-2.453469$

H $\quad 10.4652820 .825700-2.321807$

H $11.441317-0.250803-1.295378$

H $10.381608 \quad 0.6679370 .783025$

H $9.6292902 .841460-1.243369$

H $10.081306 \quad 4.139636 \quad 0.885261$

H 9.7650442 .7238931 .864867

H 6.5329335 .1093020 .871672

H 8.2779495 .6210260 .542390

H 6.9796022 .4213542 .476919

H 7.5164141 .4801801 .104357

H $5.7905762 .620382-0.384964$

H $\quad 9.536797-1.556085-0.803393$

C $-5.890610 \quad 3.362322 \quad 0.921441$

O -6.0142202 .0520600 .329922$

C $-6.7211542 .183061-0.917864$

C -7.415789 $3.536900-0.856023$

C $-6.3650634 .361555-0.134955$

H $-5.9608212 .227241-1.707112$

C -7.643333 $0.983525-1.158760$

$\mathrm{H}-7.6618313 .925937-1.848470$

H $-8.3379403 .493089-0.264945$

C -6.8994735 .6613220 .440772$

H $-5.5593274 .592416-0.842388$

C $-6.926845-0.384914-1.121959$

O $-8.6583570 .974229-0.148538$

H -8.163581 $1.114003-2.115352$

H $-6.664714-0.626147-0.086739$

O $-7.868496-1.383547-1.530889$

C $-5.678739-0.471869-2.014040$

C $-5.049173-1.868604-2.140811$

H $-4.9242160 .221783-1.634028$

O $-6.024331-0.030788-3.330878$

H -5.703492 -2.523426 -2.730929

H -4.143582 -1.744033 -2.749621

H $-7.284172 \quad 6.305370-0.356871$

H -7.714678 $5.481917 \quad 1.149798$

H $-6.109905 \quad 6.2082910 .965499$
C -4.4734473 .5760461 .463850$

H $-9.2277170 .203852-0.316764$

H $-8.078500-1.222429-2.466883$

H -5.207719 -0.014947 -3.858751

H -4.3321332 .9070002 .321904$

H -4.370962 4.5969551 .849151

H -6.5830923 .3748921 .773705$

C $-3.556746-2.1274031 .289219$

O $-3.938929-1.647756-0.008567$

C $-4.691634-2.558475-0.813417$

C $-3.893441-3.852885-1.049874$

C $-3.425284-4.4759390 .268506$

C $-2.760901-3.4399151 .180796$

H $-2.874963-1.3615221 .680786$

C $-4.758373-2.2289862 .242990$

H $-5.627520-2.801638-0.300235$

O $-2.753610-3.595227-1.870420$

H -4.525142 -4.563684 -1.594992

H -2.710113 -5.2803990 .057405$

O $-4.535268-5.0500200 .951462$

H $-2.603961-3.8546402 .183416$

O $-1.470218-3.1140810 .656241$

C $-5.339725-0.8721752 .576411$

H $-5.558047-2.8373121 .812747$

H -4.449713 -2.683703 3.191124

H -2.231462 -2.894584 -1.443095

H $-4.923693-5.7235380 .367457$

H $-0.947575-3.9337380 .632409$

O $-6.618730-1.0305173 .028006$

O -4.7406550 .1896232 .483155$

C -7.2942550 .1749913 .399729$

H -7.4135570 .8245802 .528336$

H $-8.285396-0.0928223 .775230$

H $-6.7456920 .690216 \quad 4.194017$

SCF Energy $\left(B 3 L Y P / 6-31 G^{* *} / / M M F F\right)=-3245.91533171$

0700425

MM̄FF Geometry

C -3.018796 -2.122280 -2.386711

C $-1.730143-2.039008-2.748265$

C $-0.747858-1.078826-2.129143$

O $0.363747-1.889834-1.676326$

C $-0.246852-0.032033-3.146186$

C $-0.9286491 .316559-2.999963$

C $-0.3251912 .453466-2.598809$

C $-2.3888531 .353762-3.375580$

C $1.1100932 .669390-2.206191$

C $1.2177243 .149330-0.752128$

C $2.6584973 .349272-0.253105$

C $3.3743874 .496796-0.970498$

C $4.8301574 .645172-0.504898$

O $3.3673672 .119767-0.429194$

O $5.4780455 .529455-1.432466$

C $5.5159433 .266035-0.603707$

C 4.9211795 .3226130 .866611

O $6.8420533 .320008-0.082995$

C 4.7129862 .1273740 .063784

C $5.3231260 .738084-0.226062$

O $4.7370682 .311774 \quad 1.483960$

C $1.117300-1.405429-0.653175$

O $1.006176-0.319494-0.108160$

C $2.096707-2.453299-0.287089$

C $2.859734-2.2919350 .804166$

C $3.859342-3.2345231 .314357$

C $4.100602-4.4923670 .522487$

C $5.3513180 .348558-1.706101$

C $5.620280-1.132446-1.877010$

C $7.391042-2.857362-1.552796$

O $4.746658-1.903129-2.268195$

C $7.628165-3.390554-0.169872$

C $7.629427-2.7056760 .985372$

C $7.909459-3.3375212 .319262$

C $6.844331-3.0374443 .349162$

C $7.099204-2.2306714 .393428$

C $5.513662-3.7446153 .219226$

C $4.500069-2.9306312 .462751$

N $6.916379-1.487200-1.544079$

$\mathrm{H}-3.394320-1.450153-1.619097$ 
H -1.353196 -2.697480 -3.527634 H -1.199407 -0.592839-1.255707 H $-0.402958-0.384256-4.175123$ H $\quad 0.838966 \quad 0.067601-3.061035$ H $-0.9312573 .358315-2.551354$ H $-2.7711382 .377202-3.456498$ H $-2.5476280 .872849-4.346312$ H $-2.9922870 .839744-2.622469$ H $1.7136091 .769914-2.343871$ H $1.5222313 .426465-2.882283$ H $\quad 0.7413252 .397533-0.110152$ H $0.6545734 .081977-0.620536$ H 2.5964473 .5650060 .820375 H $2.8287865 .439291-0.837643$ H $3.3898184 .309996-2.051975$ H $6.4202325 .568059-1.193443$ H $5.6214703 .050873-1.674109$ H 4.4693496 .3212720 .827877 H 5.9640885 .4793641 .163442 H 4.4184094 .7623261 .657526 H $\quad \begin{array}{llll}\text { H } & 778916 & 3.323244 & 0.887448\end{array}$ H 6.3315590 .6768250 .202421 H $4.737518-0.0158100 .315835$ H $4.5406401 .459857 \quad 1.908052$ H $2.152256-3.334066-0.916548$ H $2.735579-1.3830251 .392125$ H $3.199045-5.1147070 .507435$ H $4.909371-5.1096440 .918304$ H $4.377094-4.251868-0.510011$ H $4.3926690 .567078-2.190082$ H $6.1226890 .906916-2.247712$ H $8.326610-2.877589-2.120259$ H $6.663774-3.494691-2.065885$ H $7.829109-4.459920-0.130313$ H $7.457233-1.6330100 .985888$ H $8.883097-2.9645592 .662908$ H $8.022132-4.4252832 .230234$ H $6.347870-2.0364185 .151851$ H $8.061234-1.7431794 .511150$ H 5.100653 -3.928895 4.220529 H $5.668182-4.7415722 .798172$ H $4.246180-1.9924772 .959080$ H $7.569686-0.763320-1.259053$ C -4.843290 -3.850972 -1.976194 O $-5.598971-2.924268-1.170082$ C $-5.388177-3.2532280 .214863$ C $-4.972806-4.716100 \quad 0.214984$ C $-4.066604-4.745541-1.005362$ H -4.536975 -2.654253 0.559505 C $-6.623751-2.8928401 .043428$ H $-4.461499-5.0072911 .137150$ H $-5.831283-5.3815400 .067872$ C $-3.802417-6.142350-1.539189$ H -3.107177 -4.286622 -0.735253 C -7.103805 -1.437597 0.840169 O $-7.704671-3.7626670 .689785$ H -6.421538 -3.080434 2.104049 H -7.565155 -1.340393 -0.149901 O $-8.148013-1.1767961 .784751$ C $-6.001339-0.3742070 .994543$ C -6.5349761 .0591870 .814206$ H $-5.220801-0.5540280 .248064$ O $-5.412206-0.5172112 .285591$ H -7.209518 $1.088130-0.049524$ H $-7.1001961 .344803 \quad 1.709951$ H $-3.293299-6.751993-0.785717$ H -4.733171 -6.651898 -1.810336 H -3.167028 -6.103065 -2.429753 C -4.006458 -3.076503 -3.000878 H -7.865969-3.658666 -0.263793 H -7.751935 -1.200561 2.672840 H $-4.696060 \quad 0.1361302 .356752$ H -4.686517 -2.475604 -3.617730 H -3.500981 -3.784286 -3.667941 H $-5.585980-4.446671-2.522346$ C -3.4813723 .0203951 .738972$ O -4.6323322 .1677621 .793224$ C -5.3929602 .0714360 .585068$
C $-5.921263 \quad 3.452697 \quad 0.172191$

C -4.7762054 .4639290 .055656$

C -3.8685334 .4395421 .290198$

H -3.1327603 .0836532 .778131$

C -2.3550082 .4035860 .902667$

H $-4.7542251 .679825-0.214579$

O -6.8690893 .9061901 .135826$

H $-6.4420643 .360600-0.788556$

H $-5.1953005 .469603-0.070635$

O $-4.0177834 .156161-1.110956$

H $-2.9604665 .023807 \quad 1.103579$

O -4.5463465 .0846322 .372874$

C $-1.7756891 .197763 \quad 1.603873$

H -2.667062 2.107168 -0.102353

H -1.5355883 .1197710 .772033$

H -7.2094224 .7645860 .830727$

H -3.306769 4.815698 -1.181888

H -5.3199454 .5425062 .604802$

O -2.3724960 .0600231 .149649$

O $\quad-0.916879 \quad 1.2675112 .473779$

C $-1.931425-1.1638731 .747872$

H -2.437447 -1.301573 2.707187

H -2.205343 -1.985472 1.080930

H $-0.845889-1.1782391 .882928$

SCF Energy $\left(B 3 L Y P / 6-31 G^{* *} / / M M F F\right)=-3245.92375351$

0700426

MM̄MF Geometry

C -0.0431660 .4657902 .583423$

C $-1.120441-0.1666043 .074944$

C $-1.760076-1.4404142 .571444$

O $-3.160870-1.1592302 .317096$

C $-1.166119-2.0149751 .272064$

C $-1.813759-3.3158610 .836346$

C $-2.639109-3.444240-0.222063$

C $-1.423684-4.5302661 .638535$

C $-3.153569-2.365985-1.133219$

C $-2.722896-2.513177-2.599926$

C -1.297190 -2.060752 -2.965097

C $-0.206604-3.017642-2.483529$

C $1.197237-2.516352-2.846870$

O $-1.079372-0.744340-2.449398$

O $2.129574-3.302644-2.089044$

C $1.334106-1.048127-2.391505$

C $1.526900-2.768339-4.322438$

O $2.588636-0.529978-2.829042$

C $0.161181-0.147026-2.850864$

C $0.273504 \quad 1.252325-2.191704$

O $0.2323030 .011530-4.271273$

C $-4.037354-1.2929483 .346903$

O $-3.764709-1.5848184 .503587$

C $-5.428462-1.0086562 .923658$

C $-5.812564-0.9686801 .639049$

C -7.164752 -0.7092611 .141469$

C $-8.220938-0.3090652 .136426$

C $-0.9655502 .146042-2.353669$

C $-2.0406101 .909904-1.306118$

C $-4.3883802 .554545-0.766718$

O $-1.8868651 .178445-0.332799$

C $-5.4105031 .748218-1.503886$

C $-6.6786632 .148625-1.681660$

C $-7.7257171 .345171-2.401835$

C $-8.8005690 .834560-1.466122$

C $-9.8684211 .592354-1.164510$

C $-8.673185-0.575536-0.931536$

C $-7.389617-0.809236-0.184794$

N -3.189540 $2.639321-1.578985$

H 0.4844240 .0663521 .722953

H $-1.6033090 .267641 \quad 3.949373$

H -1.674944 -2.193353 3.364969

H $-0.093497-2.2017351 .399563$

H -1.238344 -1.271136 0.473386

H -3.019713 -4.438268 -0.454568

H -1.859682 -5.4514641 .237583$

H $-0.335859-4.6544631 .634389$

H -1.760101 -4.433033 2.675080

H -4.248792 -2.440742 -1.106361

H $-2.930047-1.361266-0.767574$ 
H $-2.885254-3.542071-2.944376$ H -3.411005 -1.882909-3.180693 H -1.267759 -2.010503-4.060650 H - $0.371127-4.028393-2.876597$ H $-0.239276-3.109675-1.394700$ H $3.017564-2.941662-2.254301$ H $1.361363-1.054625-1.293063$ H $1.495032-3.842611-4.540783$ H $2.546026-2.445078-4.562110$ H $\quad 0.838505-2.270779-5.008580$ H $2.7392730 .312955-2.370782$ H $0.497607 \quad 1.142404-1.123582$ H $1.118208 \quad 1.782221-2.649213$ H $-0.1625540 .861765-4.520057$ H -6.133894 -0.8685643 .736307$ H $-5.069385-1.1507870 .866135$ H -7.894742 0.5615232 .716659 H -8.430488 -1.133321 2.826624 H $-9.168918-0.0296511 .669850$ H $-0.6537443 .192081-2.243173$ H -1.406556 $2.037937-3.349649$ H $-4.7261373 .578232-0.577780$ H -4.1596542 .0767640 .191060$ H $-5.0986090 .779525-1.887876$ H $-6.9905403 .112652-1.285815$ H $-7.2794600 .505620-2.949427$ H -8.175072 $1.985664-3.172194$ H $-10.655437 \quad 1.228376-0.512487$ H $-9.9767802 .596252-1.561861$ H -8.707581 -1.273377 -1.778683 H $-9.535208-0.846741-0.311390$ H -6.561056 -1.099193 -0.831789 H $-3.2270783 .210180-2.418608$ C 0.6063452 .8446832 .121270 O 1.7768462 .6199991 .306938 C 2.4713043 .8717591 .134859 C 1.5032084 .9532601 .595366 C $0.781944 \quad 4.2397742 .721314$ H 3.3258353 .8560811 .821128 C $2.9590844 .021056-0.309507$ H 2.0188145 .8618101 .920881 H 0.7954285 .2294800 .805035 C -0.5219354 .9077253 .123064$ H 1.4474234 .1929283 .593911 C $3.8842352 .874979-0.772360$ O $1.8130934 .045626-1.167500$ H $3.4565644 .989753-0.435175$ H $3.3304091 .930642-0.741260$

O $4.1998953 .123477-2.147374$

C 5.1927912 .7738820 .036908

C $6.1974721 .722410-0.469672$

H 4.9635622 .5611991 .085476

O 5.8545794 .0430800 .017843

H $6.4628361 .922251-1.514639$

H 7.1254831 .8647820 .101001 H -0.3377825 .9257243 .481328$ H -1.217809 4.9692842 .279469 H -1.015600 4.351497 3.926080 C 0.5006451 .7396943 .174332 H $2.1370944 .136422-2.080041$ H $4.5459202 .296754-2.523905$ H $6.0390054 .263406-0.911451$ H -0.1395302 .0697024 .000460$ H 1.4921891 .5221803 .589925 H -0.2603542 .8272301 .447703$ C $5.018221-1.2489761 .440020$ O $5.544694 \quad 0.0383081 .096824$ C $5.713610 \quad 0.273561-0.305482$ C $6.713892-0.734575-0.893506$ C $6.287213-2.176411-0.600118$ C $5.900516-2.3745370 .871165$ H $5.091249-1.2912932 .534370$ C $3.537291-1.3374951 .061903$ H $4.7492220 .160480-0.810989$ O $8.006776-0.501789-0.336967$ H $6.784522-0.572840-1.976014$ H $7.112843-2.853021-0.852990$ O $5.188269-2.504426-1.443879$
H $5.400713-3.3398711 .003715$

O $7.100711-2.4337531 .650226$

C $2.825965-2.4099611 .847006$

H $3.031703-0.3807901 .244012$

H $3.390290-1.537595-0.002213$

H $8.624045-1.123865-0.758578$

H $4.956998-3.433324-1.271793$

H $7.532678-1.5637501 .596944$

O $2.536835-1.9549593 .099517$

O $2.571845-3.5224981 .404447$

C $1.882666-2.8908703 .961512$

H $\quad 0.939013-3.2240193 .520747$

H $2.539338-3.7436694 .157347$

H $1.667357-2.3878794 .907942$

SCF Energy (B3LYP/6-31G**//MMFF) $=-3245.91042402$

$07 \quad 00427$

MM̄FF Geometry

C $3.155796-4.355890-1.148019$

C $2.314810-3.428760-1.630231$

C $0.834662-3.418443-1.357504$

O $0.511383-2.176322-0.692384$

C $0.050543-3.454819-2.678827$

C -1.447896-3.564064 -2.471888

C $-2.314394-2.532704-2.534581$

C $-1.956553-4.960912-2.221606$

C $-2.003058-1.077281-2.757825$

C $-2.582579-0.134263-1.687091$

C $-4.0907790 .128431-1.835669$

C $-4.5139371 .327941-0.985907$

C $-6.0263971 .581579-1.078960$

O $-4.799026-1.028184-1.393805$

O $-6.3810642 .502772-0.034238$

C $-6.7802640 .263120-0.789518$

C $-6.4036472 .265771-2.398384$

O $-8.1726700 .427086-1.045479$

C $-6.222104-0.962975-1.553554$

C $-6.798107-2.313399-1.061498$

O $-6.570893-0.913922-2.943727$

C $0.669722-2.1205700 .656510$

O $1.090224-3.0010241 .388890$

C $0.216400-0.7816461 .090825$

C $0.157145-0.4853152 .396715$

C -0.3214990 .7749872 .969837$

C -0.7264751 .8663942 .012351$

C $-6.725558-2.5987400 .440722$

C $-5.307900-2.6129580 .978503$

C $-3.835001-1.5423142 .665543$

O $-4.454708-3.3821610 .541380$

C $-3.738616-0.1705023 .251691$

C -3.5966970 .0746384 .563149$

C $-3.468977 \quad 1.454188 \quad 5.147137$

C -2.1777321 .6448015 .915489$

C -2.1512251 .5484897 .255471$

C $-0.9347072 .021775 \quad 5.134967$

C $-0.408614 \quad 0.874598 \quad 4.313034$

N -5.103072 -1.671872 1.975614

H $2.769316-5.167729-0.537184$

H $2.711250-2.610355-2.227005$

H $0.539332-4.264807-0.724640$

H $0.379730-4.308782-3.286169$

H $\quad 0.285621-2.567614-3.278757$

H $-3.371037-2.764868-2.423848$

H $-3.044967-4.993788-2.104401$

H -1.695341 -5.615870 -3.059225

H $-1.516125-5.371569-1.307771$

H $-0.921925-0.910071-2.779432$

H $-2.377953-0.786714-3.746317$

H $-2.370510-0.539943-0.690165$

H $-2.0510530 .821224-1.772389$

H $-4.3114970 .313094-2.893213$

H -3.958078 2.228523 -1.275426

H -4.2551801 .1406020 .065325$

H $-5.8529383 .310659-0.154042$

H -6.6805240 .0955030 .288987$

H $-5.9096273 .241749-2.478269$

H -7.478793 $2.471300-2.444849$

H $-6.121648 \quad 1.685252-3.279133$ 
H $-8.631559-0.362683-0.713694$ H -7.846494 -2.398040 -1.376308 H -6.270430 -3.120507 -1.589318 H -7.124996 -0.133091 -3.099827 H $-0.078291-0.0786730 .319942$ H $\quad 0.459433-1.2446503 .116708$ H $-1.538667 \quad 1.5276191 .359394$ H $\quad 0.1238582 .162247 \quad 1.388230$ H -1.081661 2.7732622 .504662 H -7.354735 -1.8940380 .993805$ H -7.146667 $-3.592266 \quad 0.638716$ H -3.018087 -1.710087 1.955946 H -3.791610 -2.327797 3.427225 H -3.7608930 .6640422 .555084$ H -3.574224 -0.7597355 .260578$ H -4.3286821 .6166425 .810222$ H -3.5456092 .2263574 .371424$ H -1.2364291 .7080017 .816462$ H $-3.043873 \quad 1.3028137 .821200$ H -0.1326602 .3260615 .820338$ H -1.1534762 .9121494 .540151$ H $-0.086146 \quad 0.0243204 .916159$ H $-5.911957-1.2138412 .385946$

C $5.388651-3.975541-0.086326$

O $5.017427-2.647118 \quad 0.335922$

C $6.209328-1.8810580 .575102$

C $7.320314-2.9059680 .752439$

C $6.909023-3.956115-0.262414$

H $6.402397-1.304699-0.338102$

C $6.000361-0.9213751 .750842$

H $8.311465-2.4851000 .559239$

H $7.321150-3.3364041 .760487$

C $7.569303-5.306637-0.045906$

H $7.167225-3.591765-1.265940$

C $4.728916-0.0525641 .650535$

O $5.903355-1.6731162 .967676$

H $6.884376-0.2832701 .863866$

H $3.837768-0.6687191 .824530$

O $4.768897 \quad 0.9034592 .717398$

C 4.5668040 .7038880 .319650

C 3.3354681 .6200830 .317038

H $4.472944-0.026497-0.488454$

O $5.732797 \quad 1.4812220 .056745$

H 2.4466991 .0492240 .607056

H 3.4485042 .3960831 .086434

H $8.657342-5.221392-0.133715$

H $7.342900-5.7095560 .946999$

H $7.225408-6.031654-0.790376$

C $4.641125-4.321717-1.375922$

H $5.164300-2.2978692 .868577$

H 4.8543690 .4058093 .548656

H 5.8421582 .1048220 .795063

H $4.960009-5.300742-1.750597$

H $4.880609-3.583073-2.151524$

H $5.097671-4.657741 \quad 0.722648$

C $1.4877233 .975021-1.889337$

O $1.9024443 .146818-0.796438$

C $3.0713072 .343970-1.017783$

C $2.8529841 .398751-2.210467$

C $2.4036992 .164312-3.458977$

C $1.2568143 .132495-3.154396$

H $0.5161204 .382378-1.580768$

C $2.4452525 .156269-2.108446$

H $3.9208733 .005076-1.218831$

O $1.8671300 .427464-1.867956$

H $3.7854450 .865586-2.426824$

H $2.081077 \quad 1.446512-4.223233$

O $3.5211222 .879158-3.977897$

H $1.0847893 .796318-4.008975$

O $\quad 0.0530382 .379096-2.979747$

C $2.4449016 .119871-0.939258$

H $3.4761124 .822920-2.252856$

H $2.1285315 .719739-2.993672$

H $1.777902-0.180871-2.621301$

H $3.228143 \quad 3.336599-4.784295$

H $0.160271 \quad 1.828498-2.185085$

O $3.3114867 .141269-1.210635$

O $1.776768 \quad 6.0001960 .078214$
C $3.4241778 .139454-0.191193$

H $4.1459298 .888544-0.527382$

H $2.4585758 .628697-0.032106$

H 3.7895017 .6924340 .738315

SCF Energy $(B 3 L Y P / 6-31 G * * / / M M F F)=-3245.91249497$

0700428

MM̄MF Geometry

C -4.362009 2.416624 1.972347

C -3.1286752 .7131041 .536067$

C -1.9503922 .9390662 .447514$

O -0.9032142 .0181642 .073461$

C -1.422646 4.3745842 .260864

C -0.1646064 .6680003 .055038$

C 1.0597944 .8508322 .520117

C -0.3590844 .7823704 .545357$

C 1.4608414 .7914581 .070544

C 2.5319393 .7244210 .831965

C $2.9864503 .628695-0.633381$

C $1.8891023 .090534-1.556259$

C $2.3937912 .881035-2.993162$

O $4.1126712 .741248-0.653836$

O $1.4154132 .094338-3.691726$

C $3.6965212 .054566-2.952929$

C $2.5051814 .206206-3.756796$

O $4.2575951 .967006-4.260547$

C $4.7322202 .594410-1.941186$

C $5.9465251 .654527-1.754981$

O $5.2417673 .847835-2.411048$

C -0.6292900 .9993622 .932986$

$\begin{array}{llll}\text { O } & -1.229982 & 0.712324 & 3.956042\end{array}$

C 0.5604800 .2881552 .416590

C $0.978755-0.8359343 .016829$

C $2.132066-1.6416872 .614483$

C $3.085365-1.0447291 .611831$

C $5.5899860 .226404-1.337555$

C $6.793690-0.555926-0.840846$

C $7.383248-2.7614740 .149915$

O $7.935634-0.105916-0.826291$

C $6.650781-3.7226791 .028840$

C $6.591194-3.6054582 .363879$

C $5.817457-4.5368783 .252751$

C $4.565727-3.8798973 .800017$

C $4.554966-3.3836305 .049207$

C $3.375866-3.8665462 .858607$

C $2.303091-2.8587523 .172571$

N $6.426668-1.819099-0.398492$

H -4.5348022 .2925513 .038635$

H -2.961784 2.8530380 .470394

H -2.243568 2.7997403 .495076

H -2.1985085 .0922332 .560761$

H -1.259209 4.5643941 .195616

H 1.8822295 .0770923 .198241

H 0.5589675 .0798605 .063396

H -1.121406 $5.534860 \quad 4.772137$

H $-0.677766 \begin{array}{lll}3.824277 & 4.967246\end{array}$

H 0.6045414 .6031750 .422597

H $1.8569465 .773720 \quad 0.785916$

H 3.4093213 .9402381 .457253

H 2.1744152 .7435141 .167420

H $3.3145134 .623419-0.956730$

H $1.0139983 .749819-1.565985$

H $1.5320342 .125369-1.169711$

H $0.5676802 .569860-3.657404$

H $3.4052121 .036072-2.668327$

H $1.5274644 .700076-3.810776$

H $2.8129114 .039523-4.794996$

H $3.2029934 .909249-3.297649$

H $4.9729271 .309874-4.234511$

H $6.557170 \quad 1.632882-2.666776$

H $6.5955182 .092820-0.983652$

H $6.0994594 .011337-1.984631$

H 1.0544830 .7008961 .545052

H $0.414572-1.2169593 .866921$

H $3.403685-0.0449581 .929299$

H $2.614315-0.9635620 .627556$

H $4.001623-1.6269561 .490994$

H $4.8488590 .243469-0.530710$ 
H $\quad 5.164235-0.318714-2.187343$ H $8.163167-2.2156710 .691679$ H $7.858370-3.289562-0.682871$ H $6.127020-4.5396260 .538660$ H $7.106201-2.7793302 .849255$ H $6.477825-4.8562844 .069085$ H $5.541616-5.4572182 .722494$ H $3.674456-2.9242285 .483613$ H $5.438563-3.4303115 .678006$ H $2.919882-4.8652322 .868086$ H 3.717448 -3.724120 1.827724 H $1.577613-3.2041783 .909683$ H $5.488242-2.151128-0.604865$ C -6.3006741 .0044311 .143674$ O C $-6.665961-0.409669-0.686522$ C -8.000293 -0.1963220 .012984$ C -7.7584901 .1370420 .683893$ H $-6.6161100 .250199-1.561449$ C $-6.418028-1.856558-1.122320$ H $-8.841743-0.180178-0.685971$ H -8.193396 -0.9680690 .767008$ C -8.7309611 .4355531 .812909$ H $-7.843977 \quad 1.928374-0.073489$ C $-5.030160-2.145942-1.725764$ O $-6.576073-2.7132340 .016625$ H -7.202316 -2.148793-1.831785 H $-4.295208-2.202320-0.917368$ O $-5.086287-3.463253-2.291873$ C $-4.542675-1.156372-2.797900$ C $-3.304311-1.616131-3.594576$ H $-4.329769-0.191880-2.322422$ O $-5.598912-0.938775-3.738221$ H $-3.591877-2.411022-4.295332$ H -2.998713 $-0.769283-4.223639$ H $-9.757945 \quad 1.475326 \quad 1.435380$ H -8.6888300 .6671562 .592174$ H -8.5037122 .3997552 .278315$ C -5.5708592 .3503001 .081846$ H -6.434462 -3.626895 -0.285559 H -5.685864 -3.428033 -3.056996 H -5.295461 $-0.262842-4.368131$ H -6.2430353 .1499981 .416945$ H -5.2930242 .5742890 .044414$ H -6.2468580 .5675412 .148373$ C $-0.709350-1.348577-0.917102$ O $-1.747126-1.028916-1.855677$ C $-2.117375-2.086827-2.742282$ C $-0.915160-2.503355-3.603151$ C $0.273287-2.898480-2.721176$ C $0.554006-1.851346-1.638003$ H $-0.460545-0.392281-0.439962$ C -1.205461 -2.3089340 .176707$ H $-2.444623-2.953248-2.160558$ O $-0.540715-1.422850-4.454909$ H -1.208993 -3.345415 -4.241218 H $1.163349-3.022421-3.350158$ O $-0.011499-4.156669-2.115798$ H $1.257928-2.254543-0.902212$ O $1.203389-0.729480-2.241841$ C $-2.273285-1.6765111 .043531$ H -1.628867 -3.224570 -0.243470 H $-0.369339-2.5804340 .829725$ H $0.201429-1.726499-5.004983$ H $0.761196-4.406953-1.581137$ H $0.570215-0.308030-2.848153$ O $-2.648345-2.5653552 .009904$ O $-2.730657-0.552788 \quad 0.890899$ C -3.681484 -2.110886 2.889912 H -3.364053 -1.205643 3.415849 H -4.602131 -1.9282172 .327688$ H -3.870296 -2.896103 3.626710 SCF Energy (B3LYP/6-31G**//MMFF) $=-3245.90760252$

\section{9}

MM̄FF Geometry

C -2.305861 -3.546962 2.750932

C $-1.428790-2.5577102 .517621$
C $-0.425402-2.1122873 .555710$

O $0.938023-2.3084953 .088340$

C $-0.633412-0.6760294 .078690$

C -1.994951-0.421681 4.692485

C -2.9171010 .4108604 .169915$

C $-2.264107-1.1233605 .998143$

C -2.8118801 .2040322 .895580$

C -2.3899672 .6472113 .193916$

C -2.1534253 .5238081 .954748$

C -3.4208943 .7472051 .130542$

C $-3.1415364 .608139-0.111558$

O $-1.1421612 .912693 \quad 1.148744$

O $-4.2780834 .506046-0.983135$

C -1.935719 $4.015239-0.870059$

C $-2.997150 \quad 6.093496 \quad 0.239534$

O $-1.5455194 .863143-1.948546$

$\begin{array}{llll}\text { C } & -0.715724 & 3.707848 & 0.032129\end{array}$

C $0.3258702 .886640-0.770061$

O -0.1303874 .9379800 .456626$

C $1.443015-1.5830612 .057109$

O $0.859968-0.7586771 .372919$

C $2.866088-1.9665391 .907591$

C $3.655510-1.2798931 .068616$

C $5.066837-1.5490760 .787557$

C $5.721881-2.6912201 .518420$

C 1.5033062 .3456640 .051936

C 2.6769223 .3004260 .181933

C 4.8992643 .4760971 .306140

O $2.7606624 .356121-0.438753$

C 5.9354382 .8487430 .428196

C 6.9815642 .1505410 .894555

C 8.0117111 .5030450 .014242

C 8.0876950 .0052460 .217225

C $9.027486-0.5331001 .012698$

C $7.136889-0.872585-0.566708$

C $5.710128-0.765498-0.103438$

N 3.6315752 .8134751 .064325

H -2.258748 -4.049138 3.716537

H -1.445756 -2.024366 1.572909

H $-0.494098-2.7834614 .421085$

H -0.4195080 .0522883 .291580$

H $0.135309-0.4676594 .835956$

H $-3.8537990 .548303 \quad 4.708084$

H $-3.216329-0.8178836 .444952$

H -2.302301 -2.207352 5.854767

H $-1.474920-0.8944386 .721735$

H -2.120376 0.7330622 .191017

H $-3.793831 \quad 1.1871162 .409511$

H -3.1375363 .1265643 .838794$

H -1.451206 2.6180543 .764020

H -1.7748674 .4855282 .321843$

H -4.2100864 .1995681 .743513$

H $-3.8198112 .783615 \quad 0.789650$

H -5.060679 $4.809333-0.491697$

H -2.269265 $3.074522-1.325742$

H -3.9100806 .4631850 .721633$

H -2.868434 $6.701740-0.662702$

H -2.1626396 .2962310 .913874$

H -2.332931 $5.011285-2.499897$

H $-0.1868042 .011077-1.184778$

H $0.7004093 .473600-1.616733$

H $\quad 0.7691834 .762524 \quad 0.772727$

H $3.223393-2.8094362 .488406$

H $3.227257-0.4410930 .523063$

H $5.654092-2.5444332 .602226$

H $6.783762-2.8017851 .288020$

H $5.238600-3.6400361 .260492$

H 1.1793872 .0321401 .049726

H $1.8930191 .457429-0.460696$

H 5.1307503 .3587412 .369527

H 4.8144954 .5439141 .082368

H $5.8124242 .971805-0.645496$

H 7.1085632 .0359571 .968721

H $8.982254 \begin{array}{lll}1.963313 & 0.241361\end{array}$

H $7.8266621 .717509-1.045763$

H $9.112616-1.606871 \quad 1.142217$

H 9.7313260 .0890981 .555564

H $7.467698-1.917723-0.555528$ 
H $7.179841-0.586324-1.625657$ H $5.1541310 .039942-0.584173$ H 3.4975691 .8946321 .476668 C -3.614372 -3.3919290 .498544$ O $-4.022664-2.0222960 .686490$ C $-5.247096-1.797464-0.025453$ C $-5.353576-2.912548-1.054380$ C $-4.738561-4.071963-0.295985$ H $-6.053651-1.9359920 .708386$ C $-5.331002-0.363345-0.559136$ H $-6.387131-3.101332-1.359989$ H $-4.769548-2.705409-1.955715$ C $-4.253673-5.196985-1.195429$ H $-5.495299-4.4733530 .391559$ C $-4.491435-0.021354-1.807619$ O $-6.706876-0.122325-0.885739$ H -5.0759640 .3320990 .250148$ H $-4.931571-0.510499-2.684484$ O $-4.6337111 .384993-2.052033$ C -2.996131 -0.366787-1.730933 C $-2.2262940 .115727-2.975730$ H -2.879607 -1.449155 -1.632095 $\begin{array}{lllll} & \text { O } & -2.440528 & 0.236404 & -0.564461\end{array}$ H $-2.8563600 .014702-3.867550$ H $-1.9759451 .175489-2.850568$ H $-5.085772-5.615016-1.771524$ H $-3.496744-4.844932-1.904513$ H $-3.811351-6.005177-0.604390$ C $-3.399627-4.0660331 .858263$ H $-6.7736390 .796956-1.196562$ H $-4.2268061 .853939-1.304168$ H $-1.506147-0.027922-0.513217$ H -3.209979 -5.134834 1.700503 H $-4.329765-3.9877832 .437040$ H -2.680984 -3.378084 -0.076893 C $1.072107-1.401839-2.083818$ O $-0.096278-0.568709-2.059915$ C $-0.938631-0.697162-3.208199$ C $-0.181854-0.238031-4.462154$ C $1.117243-1.031637-4.634970$ C $1.924281-1.109651-3.332286$ H $1.649452-1.085683-1.209127$ C $0.676409-2.871297-1.880691$ H $-1.230494-1.748143-3.325095$ O $0.117962 \quad 1.151796-4.350185$ H $-0.826970-0.371745-5.338783$ H $1.727039-0.557773-5.413938$ O $0.786584-2.343175-5.082524$ H $2.716891-1.858519-3.426609$ O $2.581198 \quad 0.147781-3.132402$ C $1.776137-3.691200-1.244209$ H $-0.197087-2.903102-1.216164$ H $0.385725-3.361072-2.813692$ H $0.565602 \quad 1.422328-5.169936$ H $1.620000-2.825069-5.219485$ H $1.8948520 .822053-2.989464$ O $1.219331-4.673987-0.476950$ O $2.973496-3.515174-1.422560$ C $2.146261-5.549495 \quad 0.172702$ H $1.575846-6.2624100 .773923$ H $2.727234-6.102447-0.571307$ H $2.807042-4.9829470 .835279$ SCF Energy (B3LYP/6-31G**//MMFF) $=-3245.91981636$

\section{7_00430}

MM̄FF Geometry

C 3.5621112 .3057991 .524969

C $2.741266 \quad 2.015196 \quad 2.546241$

C 1.2779202 .3668122 .563834

$\begin{array}{lllll}\text { O } & 0.574731 & 1.178016 & 3.005512\end{array}$

C $0.962993 \quad 3.5237923 .530973$

C 1.4920164 .8717223 .088229

C 0.9199815 .6382452 .138039

C 2.7179575 .3561143 .816768

C -0.3084445 .3270201 .325239$

C $0.0290115 .251473-0.169788$

C $-1.118608 \quad 4.764983-1.071608$

C $-2.3649375 .645120-0.990982$
C $-3.4890715 .111082-1.893602$

O $-1.4483593 .423342-0.703768$

O $-4.6943825 .818802-1.563844$

C $-3.7099393 .608157-1.598319$

C $-3.2238385 .436126-3.368185$

O $-4.6508473 .061651-2.518454$

C -2.404152 2.780130-1.570008

C $-2.6098871 .370058-0.967862$

O $-1.8829582 .648036-2.893350$

C -0.7319961 .0651882 .647776$

O $\begin{array}{llll}\text { O } & -1.389950 & 1.876777 & 2.017646\end{array}$

C $-1.239688-0.2301413 .148995$

C $-2.402197-0.697782 \quad 2.669367$

C $-3.064805-1.9490593 .041593$

C $-2.472290-2.7406624 .177121$

C $-3.4381620 .374540-1.783543$

C $-4.852070 \quad 0.179877-1.260697$

C $-6.864196-1.242715-1.639025$

O $-5.314576 \quad 0.797624-0.306112$

C $-6.842828-2.478727-0.786428$

C $-5.804082-3.297967-0.552816$

C $-5.896947-4.522124 \quad 0.314955$

C $-4.876410-4.538073 \quad 1.431699$

C $-3.905487-5.4662031 .469268$

C -5.031159 -3.534789 2.553457

C $-4.168671-2.3170642 .356492$

N $-5.533929-0.785691-1.990508$

H 3.1710732 .8480190 .667849

H 3.1386781 .5041953 .420082

H $\quad 0.9474422 .609812 \quad 1.546414$

H $1.344773 \quad 3.2809064 .532497$

H $-0.121047 \quad 3.599554 \quad 3.684674$

H 1.3750156 .6020621 .913152

H 3.0547646 .3352503 .459953

H 2.5086485 .4493414 .887527

H 3.5481504 .6558333 .685536

H $-0.7818384 .398705 \quad 1.651055$

H $-1.035813 \quad 6.127303 \quad 1.502657$

H $0.3641526 .237216-0.517231$

H $0.8748794 .563268-0.299882$

H $-0.7275994 .759124-2.096112$

H $-2.1272316 .685649-1.243502$

H -2.741656 5.6630350 .039895

H -4.899076 $5.651754-0.628025$

H $-4.1786163 .538252-0.607724$

H -3.106995 $6.518163-3.505055$

H $-4.0744465 .148927-3.996485$

H $-2.3266364 .952927-3.760350$

H $-4.1954492 .935189-3.368037$

H $-1.6133860 .927694-0.844783$

H $-2.997117 \quad 1.4684360 .051755$

H $-1.2933461 .875773-2.912366$

H $-0.643466-0.760848 \quad 3.881542$

H -2.924952 $-0.109458 \quad 1.916545$

H $-1.419316-2.9707903 .987968$

H $-2.967274-3.6989604 .346480$

H -2.538980 -2.171972 5.111190

H $-3.4799180 .646175-2.842978$

H $-2.949883-0.606945-1.719555$

H $-7.404875-1.442694-2.569168$

H $-7.384715-0.443149-1.101643$

H $-7.797042-2.717268-0.319460$

H $-4.841169-3.106892-1.019336$

H $-5.765059-5.397553-0.334024$

H $-6.898020-4.6227040 .753160$

H $-3.190944-5.5005872 .285033$

H -3.799972 -6.2089180 .685528$

H $-4.838443-4.023063 \quad 3.513862$

H $-6.078029-3.2114582 .618622$

H $-4.516952-1.6645771 .555148$

H $-5.046493-1.279761-2.732503$

C 5.5214701 .5266640 .135183

O $4.9974920 .225881-0.209544$

C $6.052011-0.552083-0.809635$

C $7.2055620 .412738-1.063945$

C $7.043546 \quad 1.3879880 .080472$

H $6.367959-1.283164-0.056553$

C $5.575072-1.271100-2.077617$ 
H $8.175798-0.093358-1.064889$

H $7.0973220 .931688-2.023554$

C $7.7821112 .698241-0.134781$

H 7.4114940 .9139441 .000460

C $4.409221-2.262843-1.892190$

O $5.159275-0.296274-3.043929$

H $6.424296-1.794220-2.533593$

H $3.476353-1.716957-1.716089$

O $4.239640-2.954100-3.137891$

C $4.615008-3.307332-0.782173$

C $3.647135-4.506207-0.821220$

H $4.542019-2.8100560 .192015$

O $5.942523-3.834714-0.883149$

H $3.883835-5.145261-1.681887$

H $3.871963-5.1171230 .063492$

H $8.8568942 .519985-0.243741$

H $7.4335643 .211491-1.037307$

H 7.6379783 .3742410 .713596

C $5.025404 \quad 1.967127 \quad 1.516141$

H $4.4564540 .237762-2.636233$

H $4.098357-2.280851-3.825583$

H $6.076891-4.437457-0.131882$

H 5.5703052 .8652961 .828888

H 5.2304791 .1771282 .249268

H $5.1735592 .223731-0.638548$

C $0.526599-2.8639820 .415924$

O $1.878152-3.3281820 .298055$

C $2.151590-4.150890-0.837913$

C $1.267519-5.411521-0.823354$

C $-0.217153-5.057224-0.686306$

C $-0.456694-4.0477210 .441151$

H $0.485448-2.367975 \quad 1.393632$

C $0.176509-1.819868-0.655711$

H $1.944871-3.584096-1.750877$

O $1.653556-6.2532960 .262465$

H $1.438453-5.971628-1.750733$

H $-0.786845-5.972290-0.482017$

O $-0.678906-4.530967-1.926082$

H $-1.486302-3.674040 \quad 0.402604$

O $-0.306810-4.7425881 .681725$

C $1.032421-0.577181-0.554738$

H $0.306866-2.220749-1.663934$

H $-0.866981-1.509156-0.535351$

H $1.102825-7.0535510 .224230$

H -1.626236 -4.335011-1.826472

H $-0.466695-4.1059332 .397366$

O $\quad 0.8256120 .192287-1.664412$

O $1.775234-0.3071220 .377632$

C $1.5422421 .429279-1.702874$

H $1.3430781 .908623-2.664867$

H $1.1909812 .087678-0.904403$

H $2.6175151 .253509-1.614770$

SCF Energy (B3LYP/6-31G**//MMFF) $=-3245.90002176$

\section{7_00431}

MM̄FF Geometry

C -1.325397 -4.336459-1.881583

C $-0.075020-4.195796-1.417557$

C $0.892731-3.163071-1.935520$

O $1.232804-2.270740-0.848898$

C $2.181946-3.843004-2.431486$

C $3.254546-2.874264-2.895594$

C $4.521536-2.877781-2.435640$

C $2.845211-1.880468-3.951510$

C $5.131903-3.843026-1.460059$

C $5.059503-3.4078940 .009776$

C $5.899165-2.1605520 .325907$

C $6.120277-2.0340321 .835462$

C $6.904100-0.7629762 .192092$

O $5.201628-1.005425-0.153413$

O $6.798445-0.5619913 .609722$

C $6.226593 \quad 0.442578 \quad 1.512389$

C $8.399267-0.903704 \quad 1.883266$

O $6.999442 \quad 1.628268 \quad 1.686513$

C $5.9225990 .223103 \quad 0.010864$

C $5.0406161 .382535-0.504671$

O $7.1490420 .214395-0.722682$

C $0.378370-1.233837-0.628149$
O $-0.661088-1.003022-1.226333$

C $0.929938-0.407068 \quad 0.468629$

C $0.223524 \quad 0.6534850 .887628$

C 0.6087591 .6175051 .918750

C 1.9208611 .4095042 .624045

C $4.4194341 .152270-1.886357$

C $3.6868612 .402129-2.331931$

C $1.6646183 .771014-1.814531$

O $4.1755303 .175959-3.152044$

C $1.7105704 .508700-0.511339$

$\begin{array}{llll}\text { C } & 0.618776 & 4.985426 & 0.105378\end{array}$

C $0.6448215 .701561 \quad 1.425862$

C -0.3492495 .1356482 .419235$

C -1.4654095 .8123392 .738731$

C -0.0190903 .8168453 .092319$

C -0.2276572 .6456272 .172236$

N $2.4712942 .570649-1.687731$

H $-1.676269-3.687027-2.680060$

H $\quad 0.268261-4.835728-0.608049$

H $\quad 0.447691-2.591645-2.760225$

H $1.942928-4.516363-3.265190$

H $2.565660-4.475638-1.623748$

H $5.213838-2.131549-2.823212$

H $3.700509-1.326321-4.353118$

H $2.367226-2.395233-4.791493$

H $2.143012-1.147099-3.543102$

H $4.670180-4.831665-1.562051$

H $6.181566-3.995047-1.742978$

H $4.011878-3.2348650 .286959$

H $5.422327-4.2490250 .613685$

H $6.861991-2.247598-0.191068$

H $6.632376-2.9200692 .231117$

H $5.146666-1.9976992 .344601$

H $7.158400-1.3500644 .051678$

H $5.2749340 .615123 \quad 2.034704$

H $8.828438-1.7429322 .443854$

H $8.953913-0.0150062 .204423$

H $8.604876-1.0747420 .824626$

H 7.1540541 .7359912 .640684

H $4.2187061 .547340 \quad 0.200766$

H $5.6276902 .309944-0.529962$

H $6.9735860 .487942-1.636835$

H $1.895434-0.6768920 .879972$

H -0.7337590 .8556780 .408773$

H 1.9808880 .3980103 .041242

H 2.0735982 .0901063 .464623

H 2.7546761 .5529841 .931922

H $5.1778690 .916966-2.639720$

H $3.7129670 .315410-1.864623$

H $0.6493343 .453887-2.072945$

H $2.0552984 .404346-2.616464$

H $2.6859364 .654231-0.052455$

H $-0.3503174 .860661-0.369552$

H 0.4240456 .7594821 .233490

H 1.6469645 .6793281 .871781

H -2.173913 5.4248103 .463346

H $-1.700367 \quad 6.765952 \quad 2.277365$

H -0.6625193 .6689563 .969037$

H 1.0040913 .8781853 .473498

H -1.1786842 .6755561 .639673$

H $2.1631041 .866186-1.024412$

C $-3.413838-4.661122-0.547226$

O $-2.870815-4.1843910 .705049$

C $-3.723721-4.6230671 .772178$

C $-5.046598-5.0013761 .123153$

C $-4.560406-5.606353-0.180269$

H $-3.269063-5.5421932 .168075$

C $-3.788494-3.6054352 .916870$

H $-5.629864-5.7013851 .728467$

H $-5.665680-4.120690 \quad 0.921137$

C $-5.640003-5.704324-1.244475$

H $-4.167864-6.6117980 .023383$

C $-4.531445-2.2779812 .656746$

O $-4.418116-4.2476094 .034704$

H $-2.766164-3.3733743 .239638$

H $-5.605338-2.4614882 .535369$

O $-4.400249-1.4753913 .840150$

C $-4.012071-1.4545361 .466360$ 
C $-4.739045-0.1097851 .316533$

H -4.156975 -2.0291690 .549870$

O $-2.613361-1.2237841 .613188$

H -5.816648 -0.2824151 .198565$

H -4.6369520 .4750062 .240697$

H $-6.457612-6.348391-0.904625$

H -6.062046 -4.721327 -1.479589

H -5.237912 -6.128154-2.169895

C $-2.307045-5.343612-1.352606$

H $-5.343435-4.4256393 .795003$

H $-4.739827-2.0002184 .585264$

H -2.482042 -0.7283852 .439798$

H $-2.731813-5.882663-2.206874$

H -1.789512 -6.080568 -0.725785

H -3.796703 -3.783795 -1.084291

C $-4.6769672 .972615-0.755832$

$\begin{array}{lllll}\text { O } & -5.056280 & 1.947612 & 0.171597\end{array}$

C -4.2207920 .7802990 .169941$

C $-4.2550160 .109992-1.211324$

C $-3.8775991 .098643-2.321166$

C $-4.6325352 .425410-2.194006$

H -5.491084 $3.706659-0.699983$

C $-3.3947073 .666302-0.287662$

H -3.196062 1.0845890 .409642

O $-5.562513-0.406982-1.450564$

H -3.558303 -0.735331 -1.223131

H $-4.1021630 .653849-3.298478$

O $-2.4772131 .356655-2.278325$

H $-4.1965543 .169803-2.869386$

O $-5.9766872 .202098-2.632846$

C $-3.2655925 .050135-0.871780$

H $-3.383007 \quad 3.7477230 .806851$

H -2.489552 $3.105694-0.536533$

H $-5.550564-0.844745-2.318658$

H $-2.0166750 .505503-2.374243$

H $-6.4352053 .059549-2.622805$

O $-3.8611625 .960171-0.049759$

O $-2.7149035 .291092-1.938129$

C -3.797377 $7.319771-0.490577$

H $-4.294837 \quad 7.9431180 .257125$

H $-2.7548837 .641245-0.577605$

H $-4.3185737 .432784-1.445898$

SCF Energy (B3LYP/6-31G**//MMFF) $=-3245.90191091$

07 00432

MM̄FF Geometry

C $4.572031-3.7330180 .685342$

C $3.263334-3.8412610 .414033$

C $2.204149-4.0846971 .456001$

O $1.209643-3.042508 \quad 1.321718$

C $1.521852-5.4407061 .214366$

C $0.378132-5.7066732 .174337$

C $-0.927894-5.6167601 .851652$

C $0.788215-6.1263353 .562091$

C -1.517962 -5.2177350 .526921$

C $-2.564928-4.1092040 .677452$

C $-3.185240-3.701580-0.668097$

C $-2.212645-2.908425-1.546553$

C $-2.874684-2.450079-2.855044$

O $-4.334165-2.893448-0.380562$

O $-2.023279-1.464129-3.460236$

C $-4.210233-1.748588-2.521512$

C $-2.982374-3.598155-3.865806$

O $-4.940914-1.448293-3.707253$

C $-5.104388-2.541438-1.537429$

C $-6.353511-1.752940-1.080257$

O $-5.645662-3.712574-2.167267$

C $1.410637-1.8984012 .030134$

O $2.347044-1.6478502 .772074$

C $0.285588-0.9838951 .732412$

C $0.277705 \quad 0.243248 \quad 2.273095$

C -0.7234881 .2895672 .048327$

C $-1.916621 \quad 0.9401001 .201791$

C $-6.121155-0.431320-0.347128$

C $-5.780684-0.5615181 .128517$

C -5.3218890 .8634293 .116897$

O $-5.756737-1.627361 \quad 1.735108$

C -4.8450202 .2586483 .358956$
C -3.7416592 .5730524 .054587$

C -3.2781363 .9905134 .239948$

C -1.8258144 .2066973 .875406$

C -0.9977334 .8435424 .721804$

C -1.3424693 .7537852 .512659$

C -0.5090952 .5027382 .599146$

N $-5.5312200 .684017 \quad 1.691405$

H $4.918542-3.8459111 .709535$

H $2.922545-3.727987-0.612884$

H $2.630610-4.0683412 .467015$

H $2.259359-6.2487921 .314383$

H $1.175644-5.5034440 .177222$

H -1.662290 -5.862534 2.617844

H $-0.072732-6.3588504 .198006$

H $1.415467-7.0226663 .517907$

H $1.353473-5.3287634 .053878$

H $-0.746922-4.889982-0.171748$

H -1.991136 -6.103386 0.085923

H -3.366068 -4.450665 1.347154

H $-2.129833-3.2295741 .168379$

H $-3.516854-4.613574-1.177953$

H -1.308077 -3.484322 -1.771138

H -1.873131 -2.017437 -1.000446

H -1.147239 -1.865192 -3.593837

H -3.948069 -0.787145 -2.065799

H $-1.984746-3.966230-4.134562$

H -3.437985 -3.259443 -4.802723

H -3.552813 -4.449840 -3.489671

H $-4.352389-0.937265-4.289008$

H -6.975290 -1.549416-1.962805

H $-6.965497-2.407464-0.444134$

H -5.498763 -3.643922 -3.124430

H $-0.488596-1.3336551 .060216$

H 1.1021930 .5249382 .927388

H -2.4866770 .1263691 .663072$

H -1.5993790 .6176980 .205124$

H $-2.606638 \quad 1.772877 \quad 1.055122$

H $-5.3592900 .177944-0.840530$

H -7.058996 $0.138778-0.390999$

H -4.6159220 .1045303 .470348$

H -6.2824430 .6994813 .616161$

H -5.4418113 .0609342 .929587$

H $-3.149867 \quad 1.7833414 .510337$

H -3.4541684 .2623935 .288566$

H -3.884360 4.6777523 .635860

H 0.0396115 .0288644 .462090

H -1.336612 5.1950005 .690641

H -0.7141234 .5413362 .075637$

H -2.187655 3.6609561 .825383

H 0.4061162 .6422453 .177709

H $\quad-5.7452901 .5069081 .134179$

C $6.369251-2.153804-0.056348$

O $5.466783-1.032174-0.112154$

C $6.1573030 .094941-0.685225$

C $7.608423-0.337987-0.876058$

C $7.465486-1.834763-1.069670$

H $5.709130 \quad 0.270252-1.669920$

C 5.9764391 .3319420 .202538

H $8.0787290 .162429-1.728177$

H $8.218067-0.1259440 .009959$

C $8.758416-2.600614-0.850699$

H $7.101793-2.022456-2.088867$

C $4.509597 \quad 1.6961750 .506248$

O $6.625706 \quad 1.1035141 .461590$

H $6.4898252 .188931-0.248262$

H 4.0497690 .9246551 .136107

O 4.5164062 .9003981 .285545

C $3.6326481 .930958-0.738331$

C $2.2111462 .372113-0.361688$

H $3.5771331 .004599-1.317464$

O $4.2329952 .919311-1.572043$

H 1.7527851 .6063780 .271251

H 2.2536843 .2775920 .258620

H $9.526078-2.268908-1.557588$

H $9.146976-2.4509310 .162257$

H $8.606884-3.674434-0.997875$

C $5.613140-3.447195-0.361468$

H $\quad 6.2223890 .313127 \quad 1.860294$ 
H 5.0619102 .7320202 .073085

H $4.3068823 .737009-1.050452$

H $6.309172-4.292715-0.392713$

H $5.141602-3.373626-1.349439$

H $6.781254-2.1839540 .961300$

C $-0.9693453 .540718-1.885990$

O $0.0346513 .096131-0.966306$

C $1.2834282 .692207-1.546256$

C $1.0593781 .518862-2.509296$

C $0.0130661 .873914-3.571673$

C $-1.2554142 .473423-2.954404$

H $-1.8743903 .654172-1.274560$

C $-0.6294534 .915114-2.471775$

H $1.7119693 .541353-2.088979$

$\begin{array}{llll}\text { O } & 0.633478 & 0.374988 & -1.772558\end{array}$

H $2.0064051 .266252-2.999526$

H $-0.2437420 .969742-4.136910$

O $0.592656 \quad 2.803601-4.483239$

H $-1.8982732 .901308-3.732415$

O $-2.0055031 .431338-2.326451$

C $-0.6576135 .969705-1.390871$

H $0.3452344 .951506-2.966588$

H $-1.368703 \quad 5.207763-3.227022$

H $0.512498-0.353955-2.404210$

H $-0.0712912 .997652-5.166671$

H $-2.2139380 .768152-3.006378$

O $0.575776 \quad 6.088240-0.821806$

O $-1.6626326 .593384-1.074846$

C $0.675528 \quad 7.0408320 .240983$

H $1.691503 \quad 6.9969340 .642175$

H $0.4906888 .049575-0.139946$

H -0.0280146 .7955541 .042184$

SCF Energy (B3LYP/6-31G**/MMFF) $=-3245.90776854$

\section{7_00433}

MM̄FF Geometry

C -3.708668 -1.729227 -2.940427

C $-2.558040-1.040883-2.867279$

C $-1.183849-1.609272-2.598466$

O $-0.484568-0.701804-1.707686$

C $-1.154660-3.001621-1.934581$

C $0.247700-3.502325-1.633499$

C $0.726930-3.761175-0.400329$

C $1.125974-3.737543-2.836817$

C $0.014767-3.6511180 .917288$

C $0.435085-2.4457941 .771046$

C $1.888885-2.5102092 .265391$

C $2.082571-1.5968023 .478075$

C $3.537985-1.6048783 .966171$

O $2.753689-2.0824131 .207354$

O $3.699570-0.5180294 .890074$

C $4.464756-1.3227382 .768152$

C $3.879196-2.8855174 .737040$

O $5.834372-1.4766173 .136083$

C $4.151274-2.1791891 .516798$

C $4.955502-1.6293140 .316522$

O $4.541269-3.5313301 .760465$

C $0.3863880 .172034-2.282522$

$\begin{array}{lllll}\text { O } & 0.522714 & 0.393948 & -3.475729\end{array}$

C $1.2076580 .774207-1.208602$

C $2.3607741 .367226-1.551730$

C $3.3469261 .969489-0.655732$

C 3.0369162 .0318110 .814958

C $4.625914-2.275028-1.035473$

C $5.261182-1.489474-2.167256$

C $7.478843-0.724454-3.006184$

O $4.584001-0.847765-2.966996$

C $7.8402780 .495907-2.216760$

C $7.5345391 .748671-2.586528$

C $7.8638742 .959470-1.759538$

C $6.6362823 .745659-1.349624$

C $6.4673185 .021125-1.737140$

C $5.6356493 .073111-0.429906$

C $4.5036472 .422980-1.183788$

N $6.645156-1.567968-2.169452$

H $-3.700685-2.805824-2.807511$

H $-2.5917090 .032669-3.045100$

H $-0.662655-1.667605-3.562210$
H $-1.637352-3.739914-2.587436$

H $-1.756902-2.959851-1.022224$

H $1.750709-4.125135-0.320202$

H $2.064476-4.238412-2.575158$

H $\quad 0.610675-4.372484-3.564911$

H $1.384663-2.789838-3.318697$

H $-1.070076-3.6168240 .783489$

H $0.200971-4.574907 \quad 1.480019$

H $\quad 0.270274-1.5229661 .201471$

H $-0.235199-2.4207802 .638964$

H $2.122097-3.5473962 .533215$

H $1.407324-1.8815874 .294646$

H $1.809169-0.5670233 .207398$

H $3.069505-0.6488095 .619365$

H $4.337335-0.2646332 .502744$

H $3.214032-3.0007985 .601342$

H $4.894918-2.8416135 .145624$

H $3.791185-3.7885274 .129346$

H $5.991741-0.9092223 .910194$

H $4.768184-0.5525820 .225356$

H $6.028118-1.7498850 .512954$

H $4.661505-3.980827 \quad 0.909123$

H $0.8696610 .674683-0.184310$

H $2.6299811 .395185-2.607593$

H 2.7319301 .0518011 .195657

H 2.2325382 .7482781 .003084

H 3.8955492 .3406851 .418252

H $4.979142-3.310320-1.084719$

H $3.544989-2.287577-1.210026$

H $6.945866-0.478490-3.930082$

H $8.380439-1.291167-3.256337$

H $8.3699620 .334244-1.280370$

H $7.0127531 .916284-3.525837$

H $8.5463733 .588120-2.345440$

H $8.4159402 .678832-0.853463$

H $5.5995985 .593572-1.426001$

H $7.1888215 .519679-2.375799$

H $5.235173 \quad 3.8224580 .264950$

H $6.1526982 .321768 \quad 0.179465$

H $4.6538152 .312749-2.257752$

H $7.108544-2.100586-1.439222$

C $-6.080190-1.272131-2.125039$

O $-5.570750-0.730633-0.889476$

C $-5.773663-1.7048660 .148709$

C $-6.887717-2.608993-0.361246$

C $-6.520278-2.709504-1.830154$

H $-4.850355-2.2948550 .207732$

C $-6.024455-1.0285621 .499825$

H $-6.905949-3.5780320 .145735$

H -7.874246 - $2.144397-0.256207$

C $-7.656563-3.195668-2.713425$

H $-5.680641-3.407486-1.926414$

C $-4.9583640 .015274 \quad 1.895886$

O $-7.277955-0.3353291 .471625$

H $-6.108293-1.7907332 .284347$

H -5.1084170 .9232541 .302152$

O $-5.2110830 .392514 \quad 3.256099$

C -3.498031 -0.4526961 .765686$

C -2.4569750 .4894332 .398053$

H -3.255856 -0.5965540 .706501$

O $-3.370215-1.7266092 .403986$

H -2.5447260 .4579653 .491788$

H -1.4692340 .0668942 .172723$

H $-7.968227-4.203202-2.419237$

H $-8.530715-2.539785-2.641744$

H $-7.345400-3.230206-3.762298$

C $-5.044427-1.102742-3.241587$

H $-7.983989-0.9988461 .548125$

H $-5.034317-0.3847703 .813355$

H -2.472136 - 2.0528942 .226821

H $-4.908629-0.030370-3.427458$

H $-5.431732-1.535520-4.171174$

H $-6.957218-0.663755-2.381682$

C $-2.471518 \quad 3.242598-0.135313$

$\begin{array}{llll}\text { O } & -2.372697 & 1.955013 & 0.490914\end{array}$

C $-2.519722 \quad 1.946806 \quad 1.913514$

C -1.434086 2.8120332 .573401

C -1.432617 4.2317621 .998532 
C $-1.454346 \quad 4.2285360 .465990$ H -2.186291 $3.068852-1.181077$ C $-3.9104533 .782863-0.119408$ H -3.5005742 .3519522 .179263$ O $-\begin{array}{llll}0.156802 & 2.212867 & 2.367367\end{array}$ H -1.615146 2.8421423 .654633 H -0.5384004 .7609822 .349635$ O $-2.572844 \quad 4.9232262 .500059$ H -1.657250 5.2371910 .088866 O $-0.1535293 .876314-0.011053$ C $-4.8513922 .928436-0.940788$ H -4.309852 3.8289900 .897066 H $-3.9326584 .790502-0.549720$ H 0.5001042 .7548782 .836687 H -2.544415 5.8294882 .148992 H 0.0168382 .9568340 .254846 O $\quad-6.1367163 .311665-0.682937$ O $-4.5069822 .050406-1.718888$ C -7.153910 2.592570 -1.387602 H $-8.1009553 .120784-1.248322$ H $-6.9335462 .549543-2.458723$ H -7.249087 $1.586415-0.971116$ SCF Energy (B3LYP/6-31G**//MMFF)= -3245.89917626

\section{7_00434}

\section{MM̄FF Geometry}

C $0.7053435 .284524-0.039745$

C $-0.574304 \quad 4.9045170 .090318$

C $-1.5194184 .746771-1.071798$

O $-1.8480863 .339386-1.173116$

C $-2.8167885 .532553-0.815991$

C $-3.8181755 .424920-1.951355$

C $-4.9504184 .692623-1.919267$

C $-3.4863176 .239493-3.175896$

C $-5.4635563 .824641-0.802119$

C $-5.4479932 .347947-1.202924$

C $-5.8837181 .388219-0.083561$

C -4.9267381 .4035381 .108830$

C $\begin{array}{llll}-5.316141 & 0.357667 & 2.160125\end{array}$

O $-5.8999350 .076311-0.659364$

\begin{tabular}{lllll}
\hline & -4.239152 & 0.254858 & 3.104228
\end{tabular}

C -5.496733 -1.017747 1.482443

C -6.5295230 .8172362 .978825$

O $-6.034373-1.9385492 .426136$

C $-6.369336-0.9749020 .208457$

C $-6.256607-2.261470-0.650762$

O $-7.736579-0.7685240 .564762$

C $-0.9325962 .529830-1.776885$

O $\quad 0.1280082 .863923-2.280724$

C $-1.4434341 .143851-1.708628$

C $-0.6919510 .128107-2.158764$

C $-1.083343-1.283738-2.183474$

C $-2.476142-1.622774-1.722063$

C $-6.771162-3.574735-0.052516$

C $-5.782272-4.3478190 .805420$

C $-3.483239-5.301463 \quad 0.838954$

O $-6.099021-4.7690161 .917352$

C $-2.190275-4.6041330 .559850$

C -1.153599-5.173570 -0.073133

C $0.140201-4.462666-0.354390$

C $0.499126-4.472496-1.825197$

C $1.560210-5.165247-2.272405$

C $-0.371241-3.682855-2.778456$

C $-0.192057-2.195290-2.626308$

N $-4.555345-4.5584560 .200886$

H $1.0927115 .547464-1.020754$

H -0.9430544 .6059111 .069178$

H -1.059855 $5.082993-2.009554$

H -2.581838 $6.594542-0.662107$

H -3.2639305 .1987560 .125706$

H $-5.5937584 .707538-2.798539$

H -4.272248 $6.186518-3.936940$

H -3.360857 $7.293701-2.908107$

H -2.559651 $5.884022-3.636553$

H -4.8891953 .9760410 .111703$

H $-6.4913004 .129760-0.571044$

H -6.108417 2.197425 -2.068236

H $-4.4506092 .062474-1.556711$
H -6.9014381 .6554660 .223099$

H -4.8781742 .3920271 .578333$

H -3.9078341 .1880220 .759418$

H -3.437264 -0.010587 2.622687

H -4.501986 -1.391132 1.203974

H $-6.317477 \quad 1.7744343 .470536$

H -6.7538630 .1121663 .786975$

H -7.4301850 .9462622 .374803$

H -5.430409-1.961934 3.188256

H $-6.844763-2.086779-1.563875$

H $-5.224612-2.361565-1.002890$

H -8.295074 -1.090448 -0.162187

H $-2.4306670 .993368-1.290600$

H $0.299303 \quad 0.346904-2.553883$

H -3.221928 -1.085878 -2.319176

H $-2.608279-1.351565-0.668714$

H -2.719372 -2.682692 -1.805969

H -7.681017 $-3.401780 \quad 0.532610$

H -7.051409-4.249909-0.871519

H $-3.505297-6.3180140 .433360$

H $-3.652904-5.356575 \quad 1.918717$

H -2.103574 $-3.575600 \quad 0.902915$

H $-1.231502-6.207983-0.399925$

H $\quad 0.924546-4.9574080 .232773$

H $\quad 0.110231-3.4264450 .003823$

H $1.826617-5.176339-3.324028$

H $2.189808-5.734425-1.596485$

H -1.411242 -4.000993 -2.670676

H $-0.113914-3.930813-3.817206$

H $\quad 0.792105-1.844931-2.940185$

H $-4.458662-4.357823-0.790096$

C 2.5843694 .0270660 .987551

O 1.7828542 .8396181 .162641

C 2.5046341 .8997211 .984746

C 3.9167022 .4528012 .103985

C 3.6670263 .9477362 .059285

H 2.0037771 .9101412 .960790

C 2.4034900 .5102891 .344711

H 4.4196152 .1294083 .019896

H $4.5290212 .142573 \quad 1.248151$

C 4.9109064 .7599601 .745290

H 3.2616704 .2667073 .028815

C $3.266002-0.5554482 .040103$

O 1.0343590 .0941391 .396051

H 2.6646050 .5828610 .283675

H $4.321811-0.2798651 .986470$

O $2.949068-0.5689263 .439602$

C $3.083102-1.9885991 .509581$

C $3.242956-2.185203-0.007402$

H $2.091004-2.3582451 .797590$

O $4.025312-2.8428612 .167468$

H $3.001175-3.233430-0.222789$

H $2.498770-1.570162-0.525333$

H 5.6745534 .6065622 .514925

H 5.3443054 .4738660 .780926

H 4.6797715 .8289891 .707015

C 1.6886235 .2628781 .097050

H $\quad 0.5079290 .7769180 .945685$

H $1.998012-0.7553803 .522510$

H $3.910984-2.7177923 .125294$

H $2.2853146 .180773 \quad 1.063726$

H 1.1539305 .2536372 .055103

H $3.0299923 .964457-0.014438$

C $6.234784-0.107836-0.910807$

O $4.940699-0.524879-0.457188$

C $4.652655-1.918641-0.576408$

C $4.746436-2.355989-2.049602$

C $6.096511-1.974062-2.663043$

C $6.457852-0.511905-2.377349$

H $6.2035490 .988590-0.868168$

C $7.352927-0.5846780 .025057$

H $5.376478-2.4890210 .014636$

O $3.695383-1.744361-2.795514$

H $4.601992-3.441738-2.104693$

H $6.056570-2.137580-3.746748$

O $7.097287-2.838120-2.133170$

H $7.498437-0.309359-2.655902$

O $5.6370020 .337616-3.184101$ 
C $7.2362330 .062411 \quad 1.386268$

H $7.353880-1.6710860 .149389$

H $8.337755-0.313221-0.372535$

H $3.749858-2.077699-3.707263$

H $7.943093-2.596593-2.547706$

H $5.8075420 .112985-4.114598$

O $7.079652-0.8941252 .345538$

O 7.2810751 .2712151 .575191

C $6.960411-0.4101943 .687044$

H 7.8892890 .0799413 .993297

H $\quad 6.1144640 .278014 \quad 3.772839$

H $6.781531-1.2669104 .342121$

SCF Energy (B3LYP/6-31G**//MMFF)= -3245.90484185

\section{7_00435}

MM̄MF Geometry

C -0.634324 -3.1341621 .458357$

C $-0.333239-2.8861250 .174724$

C -1.312212 -2.314436 -0.817712

O $-0.799796-1.032768-1.261847$

C - $-1.452012-3.207145-2.067229$

C $-2.565905-4.226289-1.951517$

C $-3.748538-4.128626-2.589950$

C -2.279917 -5.424183 -1.084569

C $-4.212239-3.041916-3.519765$

C $-4.411423-1.654484-2.902385$

C -5.212092 -1.599819-1.594709

C $-6.638159-2.129186-1.747460$

C $-7.448922-1.953479-0.453923$

O $-5.243754-0.219923-1.205442$

O $-8.832407-2.176619-0.766493$

C -7.322883 $-0.491514 \quad 0.021230$

C $-7.068861-2.9962770 .604258$

O $-7.939400-0.3153901 .295174$

C $-5.865060 \quad 0.0267770 .061852$

C $-5.865587 \quad 1.5556430 .298680$

O $-5.164721-0.6166611 .126805$

C $-1.0225960 .040297-0.453690$

O

C $-0.3402881 .202835-1.060595$

C $0.0067842 .236546-0.279354$

C $0.7556913 .419902-0.705748$

C $0.8847133 .664786-2.185991$

C $-4.4845532 .218373 \quad 0.195334$

C $-4.579040 \quad 3.7329390 .273059$

C $-3.180827 \quad 5.7726160 .251868$

$\begin{array}{llll}\text { O } & -5.635631 & 4.334242 & 0.449177\end{array}$

C $-1.8576516 .188705-0.302671$

C $-0.925628 \quad 6.826910 \quad 0.420595$

C $0.3921477 .293540-0.127060$

C 1.5681586 .6894460 .605539

C 2.1047047 .3005871 .675316

C 2.1736305 .4237800 .044096

C $1.294658 \quad 4.218117 \quad 0.239941$

N -3.338014 4.3375830 .116811 H -1.639010 -2.933232 1.821704 H $\quad 0.677274-3.074618-0.180842$ H -2.298981 -2.156021 -0.365141 H $-0.509135-3.727327-2.284245$ H -1.610340 -2.574215 -2.948502 H -4.476247 -4.925399 -2.441071 H -3.043813 -6.202363-1.188486 H -1.320342 -5.874383 -1.358653 H -2.246859 -5.138768 -0.029802 H -3.516084 -2.966852 -4.364373 H $-5.162117-3.365979-3.964108$ H -3.436364 -1.182822 -2.728592 H -4.894178 -1.007782 -3.647949 H -4.672259 -2.163646 -0.827071 H -6.644010 -3.182026 -2.051940 H -7.145777 -1.580805 -2.553315 H -8.915779 -3.073094 -1.134368 H $-7.8906670 .130214-0.685393$ H -7.244076 -4.0095830 .223127$ H -7.696574 -2.899980 1.496908 H $-6.022292-2.9360780 .910093$ H -8.854051 -0.637725 1.221255 H $-6.5195942 .027046-0.447040$
H $-6.284574 \quad 1.779896 \quad 1.287844$

H $-4.378864-0.0934571 .349049$

H $-0.0917321 .150532-2.114314$

H -0.2319382 .1916880 .782519$

H $1.5605932 .932385-2.639642$

H $1.2643234 .659627-2.429070$

H $-0.0931373 .591250-2.675945$

H -3.8370071 .8829711 .012032$

H $-4.0064921 .960635-0.756290$

H -3.285894 6.0175351 .314354

H $-3.9909656 .271594-0.290499$

H -1.668358 $5.976097-1.351942$

H -1.1297007 .0543031 .464812$

H $0.4237848 .387620-0.039920$

H $0.4778507 .084178-1.200582$

H 2.9668106 .8890122 .189503

H 1.6893768 .2251632 .062748

H 3.1282335 .2092390 .543503

H $2.4388075 .592332-1.003289$

H $1.1438883 .954972 \quad 1.287837$

H -2.5075073 .7539060 .166262$

C $0.710394-2.6183513 .505595$

O $1.419480-1.5342812 .869797$

C $2.640773-1.2929033 .587935$

C $2.432114-1.9137904 .961003$

C $1.642003-3.1558454 .595345$

H $3.428574-1.8493513 .066774$

C 2.9844160 .1992283 .582670

H $3.376142-2.1370955 .466752$

H $1.836467-1.2662365 .614696$

C $0.913516-3.7839765 .770722$

H $2.332253-3.8963804 .168888$

C 2.9827450 .8508412 .184562

$\begin{array}{lllll}\text { O } & 2.032834 & 0.911212 & 4.383869\end{array}$

H 3.9558540 .3521214 .066784

H $1.952376 \quad 0.968511 \quad 1.828467$

O 3.5140502 .1749712 .318284

C 3.8011140 .1008321 .118597

C $3.8670850 .870088-0.214573$

H $3.344675-0.8782080 .938631$

O $5.121355-0.1310951 .605943$

H $2.8679121 .236350-0.468228$

H $4.5323021 .734075-0.093577$

H $1.625647-4.1024126 .539015$

H $\quad 0.213815-3.0791686 .232380$

H $0.344702-4.6629715 .451398$

C $\quad 0.352708-3.6720782 .455464$

H $\quad \begin{array}{llll}1.149217 & 0.733142 & 4.017747\end{array}$

H 2.9702672 .6399912 .977077

H $\begin{array}{llll}5.524211 & 0.735130 & 1.788458\end{array}$

H $-0.087630-4.5533132 .935592$

H $1.261779-4.0033171 .938050$

H $-0.197602-2.1876653 .946434$

C $6.344659-1.231963-2.069790$

O $5.735419-0.356407-1.113158$

C $4.376924-0.001299-1.378551$

C $4.2703330 .729089-2.726485$

C $4.891907-0.097824-3.856885$

C $6.281302-0.627759-3.483450$

H $7.398787-1.281731-1.768389$

C $5.771475-2.651815-1.989237$

H $3.763601-0.908715-1.409990$

O $4.9283961 .991200-2.636616$

H $3.2121850 .922635-2.940057$

H $4.9688070 .525421-4.756363$

O $4.018641-1.182899-4.156058$

H $6.613360-1.369102$-4.219673

O $7.1982640 .468329-3.551806$

C $6.019844-3.252021-0.624873$

H $4.698575-2.685800-2.198781$

H $6.249877-3.308464-2.725320$

H $4.8173122 .441630-3.491099$

H $4.411225-1.680488-4.893648$

H $8.0878340 .120084-3.370279$

O $4.841324-3.3859940 .048046$

O $7.129893-3.551001-0.203499$

C $4.942131-3.9372341 .364433$

H $3.931353-4.0411211 .767425$ 
H $5.407936-4.9268361 .329708$ H $5.511689-3.2637642 .011640$

SCF Energy (B3LYP/6-31G**//MMFF) $=-3245.90255958$

\section{6}

MM̄MF Geometry

C $3.184831-2.878425-2.214191$

C $4.370911-3.176652-1.661950$

C $5.189937-2.305616-0.737057$

O $4.671282-0.961513-0.630967$

C $5.188759-2.9158540 .675025$

C $6.139837-2.215573 \quad 1.625293$

C $5.758766-1.3530262 .588149$

C $7.592000-2.5879091 .473517$

C $4.363439-0.8655392 .875467$

C $4.3639100 .619668 \quad 3.267596$

C $2.967767 \quad 1.2381933 .454376$

C 2.1130580 .4969784 .486043

C $\quad 0.707713 \quad 1.1036864 .616926$

O 2.3094021 .2623082 .182696

O $-0.079104 \quad 0.1605925 .360382$

C $\quad 0.096617 \quad 1.2361643 .204224$

C 0.7215542 .3972795 .439058

O -1.1499841 .9258113 .247277$

C 1.0380891 .9296812 .196288

C $0.473837 \quad 1.9091240 .759876$

O 1.1834143 .3074812 .565128

C $4.997093-0.085549-1.618548$

O $5.602355-0.326739-2.650706$

C $4.4858291 .242885-1.221725$

C $4.2007382 .141973-2.174249$

C $3.7055443 .500962-1.955139$

C $3.8254224 .073003-0.566961$

C $\quad 0.2728210 .5190630 .153062$

C $0.1320410 .634945-1.350824$

C $-1.3497161 .601233-3.100488$

O $1.0326850 .289612-2.111935$

C $-1.1261553 .077540-3.197711$

C $-0.0425413 .631679-3.761922$

C $0.1923675 .113506-3.832720$

C $1.1306955 .607870-2.752986$

C $0.6458756 .136565-1.616426$

C $2.6197065 .569081-3.016811$

C $3.1844464 .173444-3.002438$

N -1.074965 $1.196497-1.735479$

H $2.711226-1.924280-2.005351$

H $4.815582-4.144206-1.889570$

H $6.216465-2.263073-1.122694$

H $5.469687-3.9769300 .628982$

H $4.169662-2.9039451 .080328$

H $6.528632-0.9540613 .247473$

H $8.227391-2.0974862 .218652$

H $7.721534-3.6687151 .591356$

H $7.962278-2.2983580 .485313$

H $3.702852-1.0081252 .015540$

H $3.969817-1.4646573 .703730$

H 4.9350570 .7478654 .196080

H $4.888107 \quad 1.1868612 .486717$

H 3.1278392 .2736853 .778235

H 2.6153510 .4703635 .460929

H $1.988244-0.5505914 .182422$

H -0.9632030 .5420985 .493068$

H $-0.116156 \quad 0.2204892 .847573$

H 1.0716812 .1957506 .458640

H $-0.2878332 .809973 \quad 5.544705$

H 1.3657633 .1704915 .015778

H -1.754323 1.4066323 .803714

H -0.4655712 .4760460 .721181$

H 1.1629702 .4652560 .110540

H 1.4584293 .8114791 .781565

H $4.3485501 .435591-0.164271$

H $4.3203201 .850503-3.217309$

H 3.2485393 .4845950 .151874

H $3.4654895 .101251-0.489146$

H $4.8734804 .078593-0.247083$

H $\quad \begin{array}{llll}\text { H } & 127028 & -0.135677 & 0.357964\end{array}$

H $-0.619707 \quad 0.0318450 .560925$

H $-2.393622 \quad 1.362335-3.322187$
H $-0.7119271 .042302-3.793039$

H -1.890541 $3.719639-2.766595$

H $0.7211672 .985990-4.187838$

H $-0.7662975 .645662-3.765819$

H $0.5910465 .369601-4.821992$

H $1.3041676 .521308-0.844654$

H $-0.4217206 .191817-1.430336$

H $3.1613586 .210114-2.311586$

H $2.8203606 .015866-3.999301$

H $3.1433243 .675250-3.971614$

H $-1.7418431 .467347-1.018989$

C $1.069840-4.236714-2.633956$

O $0.168533-3.114357-2.613758$

C $-0.706171-3.248663-1.470560$

C $-0.453076-4.640431-0.904088$

C $1.012110-4.845727-1.234752$

H $-0.378248-2.482936-0.756894$

C $-2.152827-2.983102-1.911498$

H $-0.662634-4.7013590 .168128$

H -1.069971 -5.389174 -1.416604

C $1.458764-6.294951-1.171763$

H $1.605367-4.256472-0.524503$

C $-3.180440-3.191582-0.782647$

O $-2.212855-1.634491-2.373147$

H -2.403300 -3.620132 -2.767844

H $-3.093476-4.237431-0.461148$

O $-2.821026-2.3952390 .340051$

C $-4.661163-2.968342-1.154347$

C $-5.090081-1.595722-1.706971$

H $-5.265407-3.176458-0.262358$

O $-5.006853-3.960853-2.127584$

H -4.621812 -1.419226 -2.682055

H $-6.163983-1.677196-1.927828$

H $1.322820-6.695478-0.161861$

H $\quad 0.885522-6.922721-1.862083$

H $2.518008-6.387076-1.431889$

C $2.449072-3.807988-3.139874$

H $-1.545563-1.535584-3.073921$

H $-3.420048-2.6358841 .066718$

H $-5.964230-3.898148-2.285883$

H $2.330052-3.285046-4.096950$

H $3.050100-4.703643-3.338161$

H $\quad 0.650179-4.942069-3.364218$

C -5.5375110 .4034531 .422885$

O $-5.623640-0.6171600 .418249$

C $-4.871355-0.390265-0.777722$

C $-5.3081300 .923918-1.449271$

C $-5.2390172 .103731-0.475230$

C -5.9207911 .7820110 .856268$

H $-6.299640 \quad 0.1291752 .163301$

C -4.1729350 .3970982 .129306$

H -3.809042 -0.317572 -0.532365

O $-6.6364360 .810652-1.957451$

H -4.655685 $1.114038-2.308765$

H $-5.7291002 .976506-0.924352$

O $-3.8788032 .445079-0.228670$

H -5.6962942 .5638021 .591453$

O -7.3351541 .8060420 .640022$

C $-3.968383-0.8567302 .954715$

H $-3.351780 \quad 0.4608621 .410014$

H -4.1042081 .2467052 .817784$

H -7.220088 $0.578135-1.215129$

H $-3.4996862 .746689-1.071682$

H -7.7666101 .6792001 .502181$

O $-2.645999-0.9676013 .276156$

O $-4.851513-1.6355153 .286436$

C -2.288134 -2.129010 4.031874

$\mathrm{H}-1.197217-2.1793904 .081354$

H $-2.685311-2.0476065 .047651$

H $-2.654821-3.0375373 .544196$

SCF Energy $\left(B 3 L Y P / 6-31 G^{* *} / / M M F F\right)=-3245.90638626$

$07 \_00437$

MM̄MF Geometry

C $-0.535093-3.1328840 .358567$

C $-1.077346-2.0075620 .846310$

C $-0.976906-1.5428162 .272647$

O $-0.567332-0.1558452 .182308$ 
C $-2.334734-1.6201582 .995940$

C -2.909004 -3.017188 3.117000

C $-3.969242-3.4618762 .412683$

C $-2.244349-3.9029364 .136762$

C $-4.706707-2.7053041 .344249$

C $-5.985473-2.0471441 .879581$

C $-6.457374-0.8489321 .037809$

C $-6.740397-1.220563-0.419661$

C $-7.0977170 .008849-1.267563$

O $\quad \begin{array}{lllll}-5.434764 & 0.148809 & 1.122152\end{array}$

O $-7.042611-0.401732-2.642616$

C $-6.0130811 .086969-1.050029$

C $-8.5375690 .469348-1.016533$

O $-6.3537402 .288597-1.737119$

C $-5.717218 \quad 1.3740740 .436900$

C -4.4961512 .3061340 .642934$

O

C $-0.060078 \quad 0.457896 \quad 3.278684$

O $0.093284-0.0279234 .389424$

C $0.251271 \quad 1.8742972 .976333$

C $0.6282302 .265714 \quad 1.748843$

C 0.9846773 .6245011 .338742

C 0.6448934 .7505672 .277008

C $-3.149003 \quad 1.7412920 .176608$

C $-2.7799802 .099962-1.251715$

C $-1.1284331 .530827-3.027821$

O $-3.3425632 .985830-1.889272$

C $0.0880732 .388039-2.881120$

C $0.0873663 .715359-3.075654$

C $1.2991264 .591283-2.930736$

C $1.2332225 .470721-1.700355$

C $0.5234436 .611543-1.700162$

C $2.0587375 .066870-0.498944$

C 1.5762833 .7944400 .138469

N -1.719764 1.332519-1.718280

H $\quad 0.011120-3.801277 \quad 1.017415$

H -1.642665 -1.367616 0.172211

H $-0.206351-2.1068432 .812376$

H $-3.042068-0.9333412 .515159$

H -2.233751-1.205834 4.007632

H -4.334099 -4.470763 2.596094

H -2.734222 -4.878832 4.221110

H -1.198953 -4.081793 3.866415

H -2.273470 -3.432814 5.125055

H $-4.050458-1.9654430 .876979$

H $-4.964318-3.4094400 .543338$

H $-6.784135-2.7965401 .940682$

H -5.804604 -1.685952 2.900898

H -7.365336 -0.455499 1.509940

H -7.532581 -1.977175 -0.479758

H $-5.851785-1.683969-0.868000$

H $-7.1881790 .388043-3.191443$

H $-5.103000 \quad 0.708158-1.530204$

H $-9.242184-0.332458-1.268714$

H -8.803365 $1.312808-1.663268$

H -8.7223160 .7578440 .020128$

H -7.034297 2.746008 -1.214454

H -4.6949793 .2930680 .207987$

H -4.4009142 .4805621 .724426$

H -6.5637362 .5072751 .808736$

H $\quad 0.2134642 .5561503 .818564$

H $\quad 0.7139281 .5137630 .964972$

H 0.7620595 .7384351 .825406

H 1.2845234 .7156393 .165330

H -0.4018144 .6860142 .595526$

H $-2.360532 \quad 2.1719270 .806114$

H -3.111663 0.6551750 .305712

H -1.863043 $1.980031-3.704435$

H $-0.8514360 .549432-3.425123$

H $1.0109591 .886789-2.601540$

H $-0.8417254 .208585-3.353221$

H $1.3790345 .217726-3.828827$

H $2.2188063 .992752-2.915394$

H $0.4934467 .259527-0.830676$

H $-0.0468596 .919526-2.570542$

H 2.1130075 .8842910 .227022

H $3.0960224 .917326-0.824464$

H $1.7871762 .905930-0.456289$
H $-1.2582020 .686783-1.084215$

C $0.618480-4.014132-1.722311$

O $1.632364-2.988284-1.681859$

C $2.241253-2.883560-2.980702$

C $1.803044-4.121238-3.758195$

C $0.419242-4.360260-3.200177$

H $1.808569-1.993589-3.452517$

C $3.764926-2.735156-2.891137$

H $1.808639-3.953668-4.839575$

H $2.443943-4.985587-3.549951$

C $-0.100077-5.767595-3.441619$

H $-0.268964-3.642655-3.666972$

C $4.303076-1.603093-1.996471$

O $4.331707-3.956280-2.391139$

H $4.167120-2.620381-3.905167$

H $4.227424-1.896287-0.943753$

O $5.706949-1.488195-2.277870$

C $3.656303-0.220583-2.183210$

C $4.4505820 .953009-1.569480$

H $2.644833-0.241281-1.760609$

O $3.5207950 .045120-3.582577$

H $5.3376651 .151233-2.185609$

H $3.8228621 .848866-1.665556$

H $-0.158826-5.976972-4.514808$

H $\quad 0.553898-6.519759-2.987716$

H $-1.101585-5.892519-3.018433$

C $-0.685226-3.529034-1.081824$

H $3.928201-4.131984-1.523584$

H $\quad 6.106337-2.361094-2.120765$

H $3.0668510 .899865-3.676954$

H $-1.434645-4.327439-1.136714$

H -1.072873 -2.678139-1.657039

H $1.017376-4.873607-1.168714$

C 3.9262790 .3595352 .083785

O 3.6979310 .6184200 .690718

C $4.8747810 .768764-0.105095$

C $5.7196571 .948496 \quad 0.401039$

C 6.0502091 .7885471 .889098

C 4.8059641 .4538312 .716193

H 2.9352680 .4183572 .547462

C $4.457978-1.0629522 .323421$

H $5.478471-0.140052-0.033432$

O $5.0122093 .167695 \quad 0.194747$

H $6.6431821 .999492-0.188057$

H 6.4927962 .7174402 .269472

O 7.0101620 .7495842 .058849

H 5.0989361 .1557283 .729798

O 4.0310782 .6494232 .841036

C $3.454809-2.1191461 .911478$

H $5.378375-1.2550001 .766467$

H $4.656639-1.1995423 .392782$

H 5.5760703 .8933660 .511977

H $7.8022350 .995757 \quad 1.551325$

H 3.2816102 .4541573 .428694

O $3.979043-3.3541272 .167233$

O $2.356827-1.8960221 .421942$

C $3.141463-4.4599731 .816056$

H $2.917736-4.4423220 .745424$

H $3.681866-5.3821302 .045573$

H $2.219536-4.4370582 .404766$

SCF Energy (B3LYP/6-31G**//MMFF) $=-3245.89768514$

07 00438

MM̄FF Geometry

C $1.1205061 .013574-2.195130$

C $1.523675-0.253092-2.003658$

C $0.832526-1.298267-1.159777$

O $-0.308602-0.706235-0.502598$

C $0.361330-2.480865-2.020166$

C $0.217585-3.762645-1.222577$

C $-0.948183-4.288563-0.799723$

C $1.511402-4.487643-0.946373$

C $-2.332452-3.723791-0.961725$

C $-3.073719-3.7082050 .383709$

C $-4.480246-3.0903580 .332494$

C $-5.449564-3.905140-0.528214$

C $-6.834592-3.245828-0.613211$

O $-4.368493-1.751504-0.159033$ 
O $-7.564845-3.888042-1.670211$

C $-6.648724-1.770749-1.025684$

C $-7.653074-3.4544820 .665622$

O $-7.890584-1.071451-0.997109$

C $-5.602701-1.020894-0.171434$

C $-5.3003470 .391880-0.714896$

O $-6.116201-0.856801 \quad 1.156193$

C $-0.456315-0.8963100 .833490$

O $0.196434-1.630121 \quad 1.556752$

C $-1.547020-0.0023521 .276185$

C $-1.928145 \quad 0.0089172 .560584$

C -2.9629550 .8857823 .095139$

C -3.9855780 .1750213 .935715$

C $-4.7298680 .436803-2.134499$

C $-4.356073 \quad 1.863247-2.474962$

C $-2.6171183 .574216-1.999219$

O $-5.1517722 .623411-3.021960$

C -2.682583 $4.043828-0.578556$

C $-3.8090434 .490323-0.001992$

C -3.9082994 .9424721 .426788$

C $-4.650677 \quad 3.955522 \quad 2.303784$

C -5.9718173 .7509602 .166624$

C -3.8679473 .2641913 .397744$

C -2.931476 2.2142102 .866077

N -3.083383 2.201754 -2.042267

H $0.1817611 .354680-1.769516$

H $2.471848-0.566560-2.435707$

H $1.569905-1.624457-0.416391$

H $1.079970-2.677264-2.827710$

H $-0.578549-2.226613-2.522920$

H $-0.912676-5.240563-0.270684$

H $1.356533-5.427906-0.406574$

H $2.020970-4.727869-1.885223$

H $2.176457-3.867430-0.338740$

H $-2.311283-2.713265-1.377702$

H -2.866862 -4.358830 -1.676131

H $-3.143332-4.7280180 .783536$

H -2.475484 -3.1293231 .099065$

H $-4.845825-3.0520001 .365757$

H -5.533733 -4.932237 -0.152189

H -5.061158 -3.990672 -1.551117

H -7.620143 -4.836645 -1.462738

H $-6.318805-1.775906-2.071885$

H $-7.811506-4.5243740 .847838$

H -8.652447 -3.0160040 .570178$

H -7.176523 -3.0378261 .555063$

H $-8.517482-1.571340-1.547618$

H $-6.202147 \quad 1.015982-0.657464$

H $-4.5807830 .874103-0.038192$

H -5.686342 -0.0864531 .562651$

H $-2.003806 \quad 0.6508910 .540673$

H -1.475486 -0.6704373 .279230$

H -3.571468 -0.0587644 .922285$

H $-4.289807-0.7660973 .463067$

H -4.8999430 .7570284 .076621$

H $-3.848508-0.206805-2.232123$

H $-5.4626330 .100256-2.874877$

H $-3.2209804 .207024-2.657785$

H $-1.5851723 .589908-2.359978$

H -1.7641783 .9974290 .000679$

H $-4.7231484 .527343-0.590878$

H -4.4241125 .9109831 .452277$

H -2.909764 5.1363791 .838364

H $-6.507217 \quad 3.0617162 .811228$

H -6.5461734 .2673381 .404734$

H -4.5475472 .8522564 .150765$

H -3.275777 4.0141893 .938117

H -2.113457 2.6134552 .265908

H -2.520012 $1.500038-1.571471$

C $2.5182053 .120058-2.001706$

O $3.2148182 .496833-0.899470$

C $2.660026 \quad 3.007753 \quad 0.325341$

C $2.1220304 .382046-0.039194$

C $1.4926334 .079529-1.387032$

H 1.8136182 .3617490 .590557

C 3.6939292 .9769961 .451912

H 1.4043014 .7608420 .694289

H $2.9280545 .116336-0.151348$
C $1.2258365 .314169-2.230021$

H $0.5406123 .566676-1.212278$

C 4.3181191 .5871551 .705306

$\begin{array}{llllll}\text { O } & 4.759849 & 3.883488 & 1.146484\end{array}$

H 3.2382143 .3531512 .375213

H 5.0190001 .3456320 .898602

$\begin{array}{lllll}\text { O } & 5.098029 & 1.671418 & 2.904048\end{array}$

C 3.2988710 .4449881 .863761

C $3.886792-0.8936392 .344333$

H 2.7885930 .2850530 .908333

$\begin{array}{lllll} & & 2.300664 & 0.843491 & 2.808807\end{array}$

H $4.211612-0.8048423 .389107$

H $3.057865-1.6138702 .358147$

H $0.5201785 .980359-1.723027$

H $2.1451835 .878620-2.418273$

H $0.7950305 .037104-3.197497$

C $1.9398022 .041409-2.929393$

H 5.1408603 .6115620 .294050

H 5.7373452 .3943972 .783517

H 1.6320390 .1382122 .844558

H $2.7729361 .542318-3.439712$

H $1.3254072 .503164-3.710468$

H $3.2743263 .686406-2.559720$

C $5.562669-1.993613-0.817790$

O $4.590253-1.530516 \quad 0.130910$

C $5.036850-1.4458321 .487164$

C $5.507789-2.8215781 .990291$

C $6.557909-3.4317651 .058480$

C $6.120309-3.367990-0.409142$

H $5.000214-2.130486-1.750450$

C $6.663992-0.951043-1.073273$

H $5.872676-0.7421311 .547932$

O $4.404205-3.7189162 .102738$

H $5.928675-2.7047162 .995812$

H $6.726308-4.4762901 .347959$

O $7.784719-2.7294191 .232485$

H $6.951825-3.634551-1.071849$

O $5.086789-4.332147-0.629530$

C $6.140274 \quad 0.283572-1.775006$

H $7.132079-0.625398-0.140921$

H $7.437276-1.379757-1.721093$

H $3.974128-3.7709001 .232279$

H $8.446508-3.1472490 .655578$

H $5.452055-5.207727-0.416218$

O $7.0894681 .264320-1.716736$

O $5.053340 \quad 0.373608-2.327251$

C $6.7474712 .492095-2.367403$

H $6.5599112 .319042-3.431428$

H $5.8734622 .942860-1.889674$

H $7.5935803 .177061-2.267373$

SCF Energy (B3LYP/6-31G**//MMFF) $=-3245.90357257$

07_00439

MM̄FF Geometry

C $-0.1947252 .823500 \quad 0.337463$

C $-0.145546 \quad 1.552310-0.089564$

C $-1.0548340 .916156-1.113135$

O $-2.317826 \quad 1.618092-1.238820$

C $-0.4514520 .882789-2.526789$

C $0.8643640 .155546-2.663314$

C $0.987702-1.171051-2.858220$

C $2.0901441 .029584-2.656083$

C $-0.106441-2.199658-2.944461$

C $0.194357-3.426406-2.070269$

C $-0.128552-3.210360-0.582460$

C $0.508808-4.322466 \quad 0.254952$

C $0.095601-4.2472941 .730443$

O $-1.554737-3.242876-0.453200$

$\begin{array}{lllll}\text { O } & 0.485369 & -5.477758 & 2.359381\end{array}$

C -1.441577 -4.168601 1.800826

C $0.831535-3.1271282 .474153$

O $-1.878489-3.9924173 .147504$

C $-2.053183-3.0844930 .880950$

C -3.590896 -3.2664010 .832292$

O $-1.733967-1.8011951 .415878$

C $-3.2252791 .476762-0.235340$

O $-3.059587 \quad 0.9074630 .831233$

C $-4.4796572 .125901-0.675541$ 
C $-5.5800832 .026182 \quad 0.085033$ C -6.895716 $2.592867-0.220939$ C -7.056631 3.303993-1.539709 C $-4.322346-2.316841-0.125835$ C $-5.794129-2.669119-0.270974$ C $-7.865574-1.903616-1.408679$ O $-6.293286-3.681800 \quad 0.212296$ C -8.548563 $-0.575566-1.486340$ C $-9.604529-0.248038-0.726196$ C $-10.3478111 .053953-0.822404$ C -10.319479 1.8361500 .471822 C -11.2183981 .5999701 .442370$ C $-9.303034 \quad 2.947780 \quad 0.602056$ C -7.8911212 .4367110 .677400$ N -6.481701-1.716960 -1.014439 H $-0.9184693 .511986-0.090184$ H $\quad 0.590447 \quad 0.883670 \quad 0.353721$ H - $1.275958-0.110277-0.796796$ H -0.344072 $1.910741-2.901576$ H -1.187852 $0.442181-3.213040$ H $1.992140-1.571154-2.994115$ H $2.9933020 .465034-2.903292$ H $1.9966501 .827997-3.399602$ H $2.2389911 .484380-1.673294$ H -1.089334 -1.789190 -2.694476 H $-0.157494-2.529715-3.989385$ H - $0.414618-4.259397-2.445903$ H $1.244680-3.717231-2.194491$ H $0.243061-2.231105-0.260370$ H $\quad 1.602010-4.2987710 .163215$ H $0.198180-5.299738-0.141166$ H $1.447198-5.5767722 .255284$ H -1.825912 -5.148847 1.486904 H $1.913979-3.2939312 .438545$ H $\quad 0.568897-3.118567 \quad 3.537567$ H $0.636964-2.1353702 .060339$ H -1.736296 -3.060409 3.385222 H -3.809488 -4.2965870 .521230$ H $-4.015532-3.1324281 .835299$ H -2.392290 -1.156664 1.114017 H $-4.4662122 .656145-1.621022$ H -5.5165571 .4751821 .022666$ H -6.426556 4.199583 - 1.572040 H -8.079951 3.624788 -1.743418 H -6.774955 $2.645304-2.369036$ H $-4.264243-1.290967 \quad 0.244742$ H $-3.871781-2.353538-1.123825$ H $-8.362008-2.584523-0.709277$ H -7.863060 -2.379079-2.394953 H -8.176629 $0.133977-2.221187$ H $-9.982187-0.972530-0.007553$ H -11.388247 $0.829071-1.092116$ H $-9.9641871 .674223-1.641726$ H -11.223865 2.175261 2.362162 H -11.967555 $0.821906 \quad 1.338347$ H -9.488436 3.5274611 .516309 H $-9.4525663 .659914-0.214184$ H -7.677420 1.8956951 .600392 H $-5.946022-0.995393-1.489262$ C 1.6345004 .4478230 .976462 $\begin{array}{lllll}\text { O } & 2.695738 & 3.858474 & 0.192766\end{array}$ C $3.958600 \quad 4.249157 \quad 0.760065$ C 3.6663445 .4994011 .576980 C 2.3119695 .1527802 .157074 H 4.2588313 .4444121 .441650 C $5.0343204 .429191-0.314354$ H $4.426858 \quad 5.686817 \quad 2.340883$ H 3.5879106 .3911630 .944663 C 1.5447356 .3579292 .674321 H 2.4579574 .4451692 .984658 C $5.2194343 .229897-1.263174$ O $4.6937285 .563683-1.121901$ H $5.9851504 .677500 \quad 0.172941$ H $4.3897083 .199564-1.979530$ O $6.394713 \quad 3.484427-2.045486$ C $5.3496151 .854310-0.584751$ C $5.7447590 .755638-1.590772$ H $4.3951401 .602653-0.109603$ $\begin{array}{llll}\text { O } & 6.338157 & 1.932370 & 0.440860\end{array}$

H $5.2325830 .927778-2.543748$

H $6.8259480 .815757-1.768825$

H 2.0982906 .8486953 .481361

H 1.3777547 .0969031 .883437

H $0.5679536 .058761 \quad 3.067441$

C $0.6684113 .357628 \quad 1.445259$

H $5.3972675 .669102-1.785129$

H $7.1594833 .447139-1.445423$

H $6.379110 \quad 1.0629840 .874511$

H $-0.001418 \quad 3.758748 \quad 2.215300$

H 1.2330882 .5379981 .905801

H 1.1239685 .1714630 .328904

C $5.948042-2.2094220 .686967$

O $6.185156-0.9398320 .063851$

C $5.391930-0.659201-1.091915$

C $5.631347-1.718400-2.179923$

C $5.409553-3.134860-1.642600$

C $6.139183-3.361716-0.314629$

H $6.731828-2.2930001 .450786$

C $4.593229-2.2520291 .401966$

H $4.332743-0.663110-0.812224$

O $6.956231-1.608589-2.697152$

H $4.945848-1.529697-3.014403$

H $5.767136-3.857876-2.385952$

O $4.010827-3.340149-1.470069$

H $5.815804-4.306514 \quad 0.137517$

O $7.535277-3.490537-0.598630$

C $4.584881-1.3023572 .575270$

H $3.749407-2.0112650 .749144$

H $4.396626-3.2543121 .800825$

H $7.575262-1.712242-1.954311$

H $3.883736-4.254889-1.165988$

H $7.986901-3.702827 \quad 0.235954$

O $3.937477-0.1501142 .241327$

O $5.114682-1.5480153 .651429$

C 3.8858250 .8423993 .269922

H 3.2820721 .6772412 .907397

H 3.4150300 .4374234 .170943

H 4.8936821 .2073303 .489248

SCF Energy (B3LYP/6-31G**//MMFF) = -3245.92356139

07_00440

MMFF Geometry

C -3.421340 $3.742638-1.527870$

C $-2.544703 \quad 3.028918-0.805559$

C $-1.3550122 .307697-1.381676$

$\begin{array}{llll}\text { O } & -0.222547 & 2.716935 & -0.575310\end{array}$

C $-1.531798 \quad 0.776377-1.293687$

C $-1.7983120 .125990-2.638933$

C $-1.005963-0.788333-3.233097$

C $-3.091640 \quad 0.521091-3.306890$

C $0.285384-1.376912-2.738725$

C $1.425104-1.139773-3.739239$

C $2.793676-1.661239-3.268981$

C $2.827482-3.186413-3.135239$

C $4.175792-3.686728-2.595472$

O $3.102193-1.034617-2.020928$

O $4.014779-5.061095-2.210947$

C $4.511354-2.896835-1.313643$

C $5.271620-3.663078-3.666526$

O $5.811793-3.230051-0.833595$

C $4.389344-1.366776-1.484959$

C $4.539397-0.617155-0.142703$

O $5.436407-0.904867-2.346227$

C $1.0173342 .606633-1.122156$

O $1.3018152 .139075-2.212505$

C $1.977798 \quad 3.186928-0.155807$

C $3.2834463 .236685-0.458506$

C $4.346198 \quad 3.806524 \quad 0.375686$

C $3.9531604 .382577 \quad 1.710723$

C $3.471558-0.952748 \quad 0.901687$

C $3.479705 \quad 0.0574402 .030301$

C 4.8959330 .8915163 .904246

$\begin{array}{lllll}\text { O } & 2.567774 & 0.868218 & 2.174266\end{array}$

C 6.0008481 .8424173 .544034

C $6.8291291 .773278 \quad 2.488877$

$\begin{array}{lll}\text { C } 7.929970 & 2.762398 & 2.232960\end{array}$ 
C $7.8945193 .333380 \quad 0.833688$

C $8.8153172 .982798-0.080254$

C $6.850338 \quad 4.3818960 .523136$

C $5.6098193 .799156-0.098548$

N $4.602421-0.0420842 .834318$

H -3.295324 $3.820199-2.604348$

H $-2.688527 \quad 2.9526920 .270499$

H -1.177906 $2.638514-2.413247$

H $-2.3692730 .518718-0.631155$

H $-0.6562920 .336640-0.808254$

H $-1.331778-1.193960-4.190913$

H $-3.325769-0.112747-4.169059$

H -3.925985 $0.429588-2.605071$

H $-3.042464 \quad 1.553256-3.664297$

H $0.566899-0.986670-1.758792$

H $0.122551-2.452457-2.611637$

H $1.176193-1.594136-4.706537$

H $1.513378-0.058691-3.909107$

H $3.531818-1.337995-4.012897$

H $2.593661-3.665206-4.094142$

H $2.049643-3.519652-2.436209$

H $3.722526-5.558918-2.993705$

H $3.802639-3.227031-0.543591$

H $4.996596-4.308909-4.509150$

H $6.213219-4.064951-3.276361$

H $5.461391-2.665247-4.066688$

H $5.847083-4.197475-0.739810$

H $5.541919-0.7892650 .269140$

H $4.4953050 .461555-0.342820$

H $5.5752350 .042677-2.182234$

H 1.5797323 .5731420 .776022

H $3.6090232 .831380-1.415856$

H 3.4397773 .6310642 .320286

H 3.2848175 .2402511 .576360

H 4.8016354 .7276182 .304731

H $2.469682-0.9438330 .457906$

H $3.630449-1.9501001 .325885$

H 5.1845280 .3070644 .783235

H 3.9940091 .4616454 .149347

H $6.1291162 .667916 \quad 4.241995$

H 6.7497620 .9496551 .784880

H 8.8835172 .2461182 .404969

H 7.9039693 .5865162 .956839

H $8.8134553 .413713-1.076121$

H 9.5872172 .2542720 .144310

H $7.2635725 .104026-0.194588$

H $6.6458204 .975114 \quad 1.418441$

H $5.7875313 .357609-1.080471$

H $5.290276-0.7627862 .636089$

C -5.879042 $3.645837-0.852297$

\begin{tabular}{llllll}
\hline & -5.706294 & 2.548043 & 0.064558
\end{tabular}

C $-6.394146 \quad 1.396393-0.461792$

C $-7.2537621 .907286-1.613569$

C $-6.3882783 .031966-2.154386$

H $-5.6249430 .733020-0.873202$

C $-7.152903 \quad 0.6741360 .656636$

H $-7.459732 \quad 1.128591-2.354166$

H -8.213809 2.302144 -1.261673

C -7.135815 4.007745-3.045097

H -5.565133 $2.586066-2.725855$

C -6.2722640 .3114911 .871278$

O $-8.200410 \quad 1.5343131 .122655$

H -7.644462 $-0.218757 \quad 0.253737$

H $-5.971842 \quad 1.2321692 .385701$

O $-7.086710-0.4091982 .804892$

C $-5.021720-0.5268301 .544469$

C $-4.189681-0.8287492 .805057$

H -4.3853110 .0099080 .833496$

O $-5.414756-1.7578630 .943645$

H $-4.021782 \quad 0.108414 \quad 3.349177$

H $-4.756485-1.5095123 .452639$

H $-7.5364053 .495328-3.925872$

H $-7.9750204 .472753-2.516718$

H $-6.4711304 .805722-3.390893$

C $-4.5916004 .469616-0.926784$

H -8.664363 1.0649771 .837230

H -7.311567 -1.263605 2.397986

H $-5.791915-1.5562530 .071340$
H $-4.322938 \quad 4.796803 \quad 0.086317$

H $-4.7669345 .381022-1.510471$

H $-6.6592634 .281984-0.412917$

C -1.891134 -3.5589371 .653859$

O $-3.055339-2.8136212 .036413$

C $-2.824962-1.4706152 .475761$

C $-1.888599-1.4566693 .694782$

C $-0.587599-2.207903 \quad 3.400851$

C $-0.855616-3.5864012 .790284$

H $-2.252848-4.5855941 .510601$

C $-1.314943-3.0717660 .319638$

H $-2.365449-0.8972231 .663510$

O $-2.548524-2.0553934 .808241$

H $-1.669667-0.4152293 .960191$

H $-0.019854-2.3240274 .332060$

O $0.194781-1.4243332 .505011$

H $0.078901-4.0246332 .422307$

O $-1.333444-4.4622223 .815721$

C $-2.198057-3.503969-0.826140$

H $-1.182503-1.9882580 .265784$

H $-0.327334-3.5130650 .138382$

H $-1.947378-1.9985315 .570443$

H $1.027146-1.9020202 .349588$

H $-2.202144-4.134748 \quad 4.105671$

O $-3.185254-2.587436-1.030628$

O $-2.038102-4.544709-1.450787$

C $-4.104674-2.899535-2.081555$

H $-4.814566-2.072741-2.169706$

H $-3.574969-3.009004-3.032714$

H $-4.657145-3.812120-1.839064$

SCF Energy (B3LYP/6-31G**//MMFF) $=-3245.89687011$

07_00441

MMFF Geometry

C -1.135986 -2.888121 1.006153

C $-1.090188-1.9618410 .035613$

C $-0.433354-2.064401-1.320616$

O $0.522295-0.975766-1.345732$

C $0.281951-3.398227-1.610850$

C $0.770414-3.543365-3.040024$

C $2.058204-3.454413-3.428340$

C $-0.299917-3.861379-4.053741$

C $3.255420-3.158942-2.568875$

C $4.105753-2.020134-3.152989$

C $5.181452-1.491105-2.187995$

C $6.195523-2.567002-1.788881$

C $7.201499-2.056153-0.747783$

O $4.507179-0.970820-1.038652$

O $7.894288-3.205313-0.236344$

C $6.413927-1.4187850 .416641$

C $8.269422-1.160481-1.384140$

O $7.301265-0.8376611 .369593$

C $5.359042-0.390885-0.045074$

C $4.4582590 .078828 \quad 1.117504$

O $6.0330540 .764763-0.556329$

C $0.608772-0.216000-2.466407$

O $0.102857-0.443345-3.555731$

C $1.4325770 .985942-2.217898$

C $1.3427171 .648601-1.054381$

C $2.0136162 .904414-0.714472$

C $3.0519913 .423011-1.672837$

C $3.572694-1.0114951 .728302$

C $2.529385-0.3802342 .625300$

C 2.3111110 .8565324 .770983

O $1.375246-0.2001992 .243533$

C 2.7579952 .2633504 .524670

C 2.0428983 .1620723 .832028

C $2.5276134 .549893 \quad 3.530691$

C 3.1176804 .6702072 .141830

C 4.4488734 .7072991 .961231

$\begin{array}{llll}\text { C } 2.174149 & 4.835564 & 0.972367\end{array}$

C 1.6730663 .5233570 .435700

N $3.041515-0.0012243 .856970$

H $-0.670643-3.8591880 .870647$

H $-1.579298-1.006300 \quad 0.224854$

H -1.222186 -1.888522 -2.062827

H $-0.403075-4.235139-1.419014$

H $1.108768-3.530274-0.905185$ 
H $2.288076-3.616290-4.480835$ H $\quad 0.108145-3.997552-5.060918$ H $-0.817226-4.787003-3.780945$ H -1.036972 -3.054478 -4.105862 H $2.963421-2.904304-1.547808$ H $3.856418-4.073472-2.512183$ H $4.580279-2.354224-4.084148$ H $3.442947-1.184584-3.412779$ H $5.692767-0.663165-2.693568$ H $\quad 6.716027-2.956395-2.672542$ H $5.675055-3.426358-1.346653$ H $8.478536-2.9020610 .480053$ H $5.909181-2.2413630 .937973$ H $8.827077-1.717508-2.146697$ H $9.012887-0.842568-0.645012$ H $7.855431-0.270274-1.862038$ H 7.6210180 .0036341 .001432 H 5.0670250 .5570391 .895748 H 3.8022580 .8768590 .745168 H $5.4298241 .524276-0.502741$ H $2.0108701 .350491-3.058911$ H $0.6791461 .257396-0.284040$ H $3.7875722 .644524-1.904947$ H $3.6207834 .268224-1.278871$ H $2.582433 \quad 3.750154-2.606740$ H $3.044547-1.5816110 .955912$ H $4.163916-1.7252362 .311634$ H 2.5576460 .5536295 .792896 H 1.2340310 .7336274 .619511 H 3.7366222 .5415964 .909042 H 1.0689402 .8798593 .441286 H 3.2681714 .8556514 .281922 H 1.6964065 .2560883 .646248 H 4.8817654 .8221520 .973090 H $5.135046 \quad 4.6172122 .796731$ H 2.6470395 .4342410 .186325 H 1.3095285 .4356831 .284189 H 0.9084553 .0613441 .059687 H $4.030756-0.1429914 .040163$ C $-3.144576-3.3892172 .433450$ O $-4.040833-2.8695371 .429151$ C $-5.147263-2.2371282 .088334$ C $-5.288387-2.998988 \quad 3.395327$ C $-3.830116-3.1595263 .787911$ H -4.845322 -1.204088 2.304212 C $-6.379356-2.2242561 .179798$ H $-5.875428-2.4528034 .139426$ H $-5.747293-3.9827843 .244445$ C $-3.586883-4.2771834 .787736$ H -3.483580 -2.216135 4.231341 C $-6.114687-1.700000-0.248044$ O $-6.898626-3.5538851 .053577$ H -7.175073 -1.638522 1.654565 H -5.577642 -2.458168 -0.831664 O $-7.382299-1.524075-0.892569$ C $-5.340716-0.371922-0.319349$ C $-5.2089590 .154314-1.762668$ H $-4.337655-0.5163380 .095426$ $\begin{array}{lllll}\text { O } & -5.994057 & 0.604119 & 0.490567\end{array}$ H -4.957710 -0.675653 -2.433743 H $-6.1731860 .573031-2.076278$ H -4.124606 -4.0826285 .721326$ H $-3.925735-5.2435804 .399511$ H -2.520608 -4.3622445 .020990$ C -1.807110 -2.650912 2.331272 H $-6.187531-4.1143060 .697751$ H -7.851315 -2.374968 -0.846183 H $-6.894725 \quad 0.7206570 .142475$ H -1.130699 -2.979012 3.129065 H -1.968783 -1.575054 2.471208 H $-3.014780-4.4578542 .224409$ C $-3.5360583 .419679-1.020819$ O $-4.4935882 .355285-1.086135$ C $-4.1183441 .236706-1.892369$ C $-3.9136881 .680561-3.348529$ C $-2.9033362 .830566-3.439634$ C -3.205082 $3.942907-2.428672$ H $-4.0474584 .224883-0.477824$
C $-2.2964783 .018502-0.205990$

H $-3.1844650 .809105-1.510813$

O $-5.1619372 .098181-3.897892$

H $-3.5654380 .821598-3.934562$

H $-2.9183383 .251738-4.452234$

O $-1.5900102 .336633-3.199131$

H $-2.3654064 .645148-2.366261$

O $-4.3402144 .685906-2.883852$

C -2.652184 2.6529721 .220044

H -1.791673 2.158978 -0.654118

H $-1.5860533 .851251-0.164429$

H -5.006946 2.344631 -4.825582

H -1.406249 $1.651270-3.864015$

H -4.114263 $5.065445-3.750030$

O

O -3.6489123 .0394871 .815306$

C $-1.878567 \quad 1.4328763 .089959$

H $-1.0590990 .765613 \quad 3.367462$

H -1.8681212 .2983743 .759182$

$\mathrm{H}-2.8213700 .884467 \quad 3.179851$

SCF Energy $\left(B 3 L Y P / 6-31 G^{* *} / / M M F F\right)=-3245.90058560$

07 00442

MM̄FF Geometry

C $-3.243992-2.934029-1.714364$

C $-2.513517-2.361404-0.745377$

C $-1.010467-2.315666-0.732612$

O $-0.663221-0.918271-0.900950$

C $-0.420461-2.8086520 .605418$

C $-0.216496-4.3092460 .683416$

C $0.986222-4.9171350 .723211$

C -1.468071 -5.1456410 .754498$

C $2.346592-4.2776020 .724578$

C $2.971685-4.292703-0.674779$

C $4.309989-3.541540-0.776987$

C $5.426263-4.2343410 .008117$

C $6.743348-3.447336-0.054959$

O $4.114132-2.203287-0.308649$

O $7.629295-3.9901610 .936560$

C $6.457984-1.9855150 .346613$

C $7.451744-3.612537-1.403939$

O $7.621031-1.1766280 .182080$

C $5.266218-1.356720-0.411872$

C 4.8874690 .0316820 .157027

O $5.640041-1.165856-1.781814$

C $0.506221-0.633305-1.533177$

O $1.326656-1.429480-1.959366$

C $0.6179330 .838986-1.623780$

C $1.7139901 .386546-2.168618$

C $1.9877792 .818001-2.316632$

C $0.9291743 .781802-1.848572$

C $4.2711210 .008117 \quad 1.558065$

C 4.1030571 .4095462 .110970

C 2.7875123 .5043091 .805407

O $4.805066 \quad 1.826787 \quad 3.029813$

C 3.3794714 .4833780 .835815

C 4.4247564 .2897380 .015616

C $4.9261085 .332185-0.943580$

C $4.9994814 .831156-2.369539$

C $6.1822484 .680541-2.989357$

C $3.6986794 .576937-3.102754$

C $3.1689383 .190418-2.853660$

N 3.1071922 .1296051 .473529

H -2.747534 -3.409151 -2.556081

H -3.024295 -1.874105 0.081717

H $-0.604470-2.895117-1.571998$

H -1.056526 -2.495894 1.443993

H $0.521656-2.2804990 .782618$

H $1.009688-6.0053420 .770801$

H - $1.257436-6.1815701 .041908$

H $-2.158538-4.7395991 .501097$

H -1.970649 -5.172304 -0.215961

H $2.308173-3.2604261 .123281$

H $2.975144-4.8429591 .422551$

H $3.104671-5.327327-1.015269$

H $2.266609-3.821963-1.371127$

H $4.573633-3.503250-1.840734$

H $5.573643-5.260767-0.350153$ 
H $5.141900-4.3234911 .064590$

H $7.755672-4.9344030 .740635$

H $6.237068-1.9913651 .420612$

H $7.686957-4.668225-1.585451$

H $8.412606-3.086210-1.411911$

H $6.857440-3.258889-2.248784$

H $8.342103-1.6015390 .677516$

H 5.7604310 .6969280 .133563

H $4.1616070 .498834-0.520925$

H $5.055251-0.495061-2.171515$

H $-0.2015461 .431490-1.236957$

H $2.4972330 .723237-2.534080$

H $\quad 0.7174403 .636884-0.783748$

H $0.0008513 .633981-2.411727$

H $\quad 1.2070554 .830367-1.969677$

H $3.291621-0.4845951 .546852$

H $4.899002-0.5432922 .264302$

H 3.1142893 .7384102 .823309

H 1.6977163 .5829711 .767189

H 2.8792345 .4498520 .808615

H 4.9637513 .3464970 .031864

H $5.9188995 .649346-0.599341$

H $4.2980256 .231588-0.918012$

H $6.2406564 .344863-4.019470$

H $7.1184224 .884167-2.480315$

H $3.8577774 .676861-4.184866$

H $2.9851805 .365009-2.848335$

H $3.8589732 .405925-3.169216$

H $2.599273 \quad 1.7012710 .705553$

C $-5.340254-2.016178-2.758622$

O $-4.948637-0.661206-2.453293$

C $-6.125504 \quad 0.087447-2.116487$

C $-7.228916-0.570817-2.927505$

C $-6.876055-2.037251-2.749998$

H -6.314973 -0.076749-1.047700

C $-5.909467 \quad 1.583630-2.364442$

H -8.228168 $-0.322361-2.558206$

H -7.172258 -0.297464 -3.987252

C -7.475316 -2.941632 -3.813602

H -7.243594 -2.366733 -1.768754

C $-4.6019502 .150756-1.770033$

O $-5.881818 \quad 1.839645-3.774116$

H $-6.7722832 .139915-1.980059$

H -3.754894 $1.847217-2.397544$

O $-4.6607083 .579363-1.864014$

C $-4.3125071 .747939-0.313793$

C -3.052756 2.4404050 .240809

H $-4.1826930 .661938-0.263231$

O -5.4394952 .0883520 .491762$

H -2.287937 $2.496443-0.542077$

H -3.3142123 .4619010 .543454$

H -8.568811 -2.895126 -3.787275

H -7.150070 -2.650080 -4.817821

H -7.175595 -3.981893 -3.650471

C $-4.746885-2.956828-1.704286$

H $-5.1628551 .306839-4.155627$

H $-5.3633013 .883161-1.263836$

H $-5.249525 \quad 1.795996 \quad 1.399400$

H -5.081467 -3.984927 -1.885181

H -5.110651 -2.671272 -0.708757

H -4.940071 -2.248107 -3.752902

C -3.059464 0.9520723 .676024

O -3.4210961 .6966482 .505048$

C -2.4619941 .6817661 .444751$

C -1.1382912 .2943791 .921090$

C -0.6176381 .5668693 .164497$

C -1.704305 1.4210104 .235788

H $-3.834741 \quad 1.2009134 .412061$

C -3.123581 -0.5589313 .427169$

H $-2.286910 \quad 0.647727 \quad 1.128163$

O -1.3339843 .6765242 .210426$

H $-0.4022962 .225610 \quad 1.112572$

H 0.2312022 .1248643 .578383

$\begin{array}{lllll}\text { O } & -0.142247 & 0.282668 & 2.770218\end{array}$

H -1.3693820 .7349665 .022441$

O -1.8845532 .7011094 .849013$

C -4.535408 -0.9855163 .102400$

H $-2.490627-0.8848602 .598103$
H $-2.786403-1.1176774 .308318$

H -0.4872054 .0365072 .523423$

H $\quad 0.215159-0.153843 \quad 3.562106$

H -2.524686 2.5935245 .572907

O $-5.231219-1.2103514 .253120$

O $-4.972900-1.0831361 .963218$

C $-6.596794-1.5999674 .077397$

H $-7.037723-1.7494685 .066607$

H $-7.152072-0.8111033 .561030$

H $-6.654070-2.5407323 .521561$

SCF Energy $(B 3 L Y P / 6-31 G * * / / M M F F)=-3245.90398187$

07 00443

MM̄FF Geometry

C $2.047519-3.074797-0.655245$

C $2.130608-2.8207630 .660891$

C $1.176493-1.9921691 .488361$

O $0.275924-1.2694440 .621309$

C $0.372117-2.9020992 .430204$

C $-0.460948-2.1407023 .440897$

C $-1.805220-2.0497723 .420324$

C $0.317111-1.5004784 .563263$

C $-2.761934-2.6361092 .418715$

C $-3.747203-1.5839601 .902777$

C $-4.903944-2.1884631 .092949$

C $-4.448884-2.806728-0.230057$

C $-5.640131-3.298077-1.069670$

O $-5.821775-1.1210170 .831259$

O $-5.166841-3.548978-2.401906$

C $-6.690609-2.171386-1.168487$

C $-6.192224-4.630520-0.548110$

O $-7.878190-2.634623-1.807211$

C $-7.037778-1.5249140 .192983$

C -7.923304 -0.270884 -0.030138

O $-7.765008-2.4650530 .986997$

C 0.1219510 .0645220 .839915

$\begin{array}{llll}\text { O } & 0.574077 & 0.719618 & 1.765277\end{array}$

C $-0.6574820 .613990-0.292668$

C $-0.7481211 .944714-0.437983$

C -1.383452 $2.656302-1.549707$

C $-2.1145811 .833107-2.577245$

C -8.1308680 .6198381 .206877$

C -6.8868731 .3853221 .620568$

C -5.2408283 .0559530 .805814$

O -6.3464221 .2024452 .709216$

C $-4.8419653 .589653-0.531611$

C $-4.5608794 .879464-0.766396$

C $-4.2099625 .420414-2.121841$

C $-2.7844735 .913016-2.203276$

C $-2.4792937 .196154-1.946467$

C $-1.7326144 .938855-2.683010$

C -1.263126 $4.000040-1.606277$

N -6.4475292 .2670810 .648348$

H $1.205211-2.699838-1.228540$

H $2.979512-3.2212761 .212254$

H $1.794660-1.2937502 .064896$

H $1.054659-3.5600702 .985697$

H $-0.254485-3.5750981 .835488$

H -2.287284 -1.504023 4.231014

H $-0.335341-1.0361015 .310341$

H $0.924245-2.2507885 .080416$

H $0.981006-0.7205294 .180009$

H -2.240157 -3.081932 1.569459

H $-3.315277-3.4411182 .917016$

H -4.175452 -1.026804 2.747491

H $-3.220229-0.8332481 .298995$

H -5.402205 -2.937317 1.719709

H -3.744237 -3.629964 -0.065163

H -3.900199-2.053548 -0.812882

H -4.453559-4.208062 -2.347305

H $-6.271463-1.392765-1.821577$

H $-5.414241-5.403109-0.572624$

H -7.000858 -5.000806 -1.187734

H $-6.562708-4.5691820 .477183$

H -7.616350 -3.020011 -2.660834

H $-7.4858520 .338466-0.830051$

H -8.914390 -0.591924 -0.375055

H $-8.302403-1.9847811 .635887$ 
H $-1.082017-0.086523-1.002521$ H -0.2696882 .5815140 .305534$ H - $1.4111551 .213163-3.143605$ H -2.673760 2.434611-3.297253 H -2.850024 $1.178252-2.095868$ H $-8.912645 \quad 1.3590180 .991743$ H -8.482851 0.0373402 .064171 H -4.4520872 .4135381 .211321$ H $-5.4502983 .848247 \quad 1.531898$ H -4.787978 $2.878050-1.352198$ H -4.612019 5.5941450 .051694 H -4.903035 $6.242484-2.344720$ H $-4.3885024 .675298-2.907190$ H -1.462803 $7.564577-2.036020$ H -3.239860 $7.904754-1.635234$ H $-0.8497305 .489619-3.035596$ H -2.100417 4.416104-3.570496 H $-0.7136004 .504003-0.809697$ H -7.070282 $2.485659-0.124614$ C $3.738978-3.060217-2.554623$ O $4.086024-1.729574-2.116700$ C $3.584348-0.791103-3.085961$ C $3.414020-1.592422-4.367371$ C $2.873997-2.896278-3.807398$ H $2.588922-0.480438-2.745660$ C $4.4986720 .431630-3.185124$ H $2.731210-1.112742-5.074686$ H $4.373595-1.760085-4.870341$

C $2.969390-4.061100-4.777526$

H $1.821371-2.751632-3.533315$

C $4.6945781 .172659-1.843431$

O $5.7844020 .006568-3.649560$

H $4.1080641 .118509-3.945488$

H $5.3164000 .562400-1.180971$

O $5.4501852 .360838-2.106768$

C $3.3839641 .555087-1.134842$

C 3.5427252 .4519720 .103454

H $2.8492970 .645432-0.840632$

O $2.5524542 .248141-2.071265$

H $3.8651613 .457293-0.197065$

H 2.5351792 .5883650 .517474

H $2.376971-3.861794-5.676604$

H $4.003505-4.240341-5.090133$

H $2.591103-4.980622-4.320022$

C $3.097560-3.849584-1.407213$

H $6.3523990 .794955-3.691708$

H $4.8963332 .945344-2.652428$

H $1.7186992 .465758-1.622157$

H $3.894563-4.143763-0.712567$

H $2.659390-4.779024-1.787906$

H $4.683780-3.550675-2.822476$

C $4.964949-0.1143482 .440037$

O $4.143080 \quad 0.5501501 .468185$

C 4.4858381 .9113511 .192544

C 4.4095972 .7594972 .475079

C 5.2412592 .1516973 .607315

C 4.9639500 .6531813 .773557

H $4.469297-1.0778002 .614327$

C $6.382392-0.3935511 .912455$

H $5.505780 \quad 1.949496 \quad 0.796777$

O 3.0563292 .8856772 .912198

H 4.7693323 .7708672 .252379

H 5.0060072 .6752334 .541948

O $\quad 6.6206442 .365963 \quad 3.325449$

H $5.6920570 .198994 \quad 4.455634$

O $3.673121 \quad 0.4793014 .365044$

C $6.390067-1.4039210 .786283$

H 6.8574990 .5194321 .544805

H $7.007649-0.8074732 .711792$

H 2.6929051 .9895483 .018107

H 7.1321921 .9968864 .065360

H 3.6831450 .9271655 .228108

O $7.527814-1.2450130 .047761$

O $5.527741-2.249706 \quad 0.597094$

C $7.685769-2.155133-1.045299$

H $8.645952-1.946858-1.524628$

H $7.690911-3.187938-0.683968$

H $6.888773-2.006313-1.778603$
SCF Energy (B3LYP/6-31G**//MMFF)= -3245.91297511

\section{7_00444}

MM̄FF Geometry

C -0.2964512 .9900351 .955563$

C -0.0228422 .1456812 .963459$

C $-0.897737 \quad 1.021321 \quad 3.471723$

O -2.0628560 .8792332 .630283$

C $-0.125006-0.3110953 .480638$

C $-0.795387-1.3916674 .311307$

C $-1.508041-2.4187073 .806375$

C $-0.578239-1.2842375 .798705$

C $-1.832370-2.6679552 .361560$

C $-0.982718-3.8087791 .788105$

C $-1.064462-3.8623110 .256018$

C $-0.253499-5.038250-0.291795$

C $-0.173635-5.021756-1.825829$

O $-0.527377-2.630113-0.226963$

O $0.850651-5.954739-2.201387$

C $0.268349-3.616744-2.291327$

C $-1.473505-5.532867-2.457646$

O $0.238377-3.522941-3.713822$

C $-0.544427-2.470675-1.648749$

C $0.121083-1.105980-1.943681$

O $-1.870194-2.487264-2.175300$

C $-3.2633330 .668337 \quad 3.232912$

O $-3.490947 \quad 0.606515 \quad 4.430304$

C -4.2701840 .5022332 .163595$

C $-5.517614 \quad 0.1272732 .481506$

C $-6.578726-0.0957131 .506783$

C $-7.304249-1.3987851 .696865$

C $-0.6072210 .090632-1.306089$

C $-1.4866200 .858452-2.279646$

C $-3.1882612 .680848-2.327919$

O $-1.4308510 .715457-3.497916$

C $-4.426314 \quad 1.966692-2.770097$

C $-5.650812 \quad 2.223050-2.286671$

C $-6.8965541 .535509-2.766166$

C $-7.339796 \quad 0.405995-1.864013$

C $-7.292385-0.869172-2.285637$

C $-7.9137020 .751332-0.508016$

C -6.8561680 .8208630 .558375$

N -2.314791 $1.759898-1.625161$

H $-1.223917 \quad 2.889911 \quad 1.399134$

H 0.8927492 .3049013 .529411

H $-1.192971 \quad 1.2993434 .491012$

H $0.881355-0.1575113 .891510$

H $0.029244-0.6567312 .451321$

H $-1.920669-3.1534764 .495980$

H $-1.047047-2.107700 \quad 6.348077$

H $0.492287-1.3031786 .028142$

H $-1.000147-0.3509286 .183674$

H -2.895022 -2.928110 2.280955

H -1.710171 -1.7547261 .772644$

H $\quad 0.063961-3.6571522 .083933$

H $-1.309889-4.7635512 .217708$

H $-2.116327-3.942547-0.042340$

H $-0.663815-5.9917010 .063454$

H $0.769996-4.9916900 .105537$

H $0.960234-5.898815-3.166376$

H $1.322221-3.501134-2.005298$

H $-1.675935-6.560539-2.132585$

H $-1.394613-5.576935-3.549413$

H $-2.343167-4.925689-2.197867$

H $-0.688861-3.408414-3.983406$

H $1.131993-1.129892-1.517842$

H $\quad 0.230831-0.963995-3.023977$

H -2.281133 -1.621125 -2.028201

H $-3.963767 \quad 0.6724311 .136560$

H -5.788359 $-0.072595 \quad 3.515837$

H $-8.029076-1.6109280 .908002$

H -7.844883 -1.4000002 .649668$

H -6.593509-2.233323 1.703922

H $-1.204077-0.216979-0.440492$

H $0.1399880 .805068-0.941516$

H -2.660108 $3.080310-3.200117$

H -3.407853 $3.508976-1.646486$

H -4.311020 $1.214768-3.547395$ 
H $-5.7718492 .982189-1.517865$ H $-6.7430811 .168324-3.789828$ H -7.702423 $2.275833-2.845816$ H -7.634217 -1.684844 -1.657274 H $-6.906957-1.127326-3.266467$ H $-8.7046100 .041569-0.241895$ H -8.424825 $1.721172-0.559706$ H $-6.271111 \quad 1.7398670 .546113$ H -2.321254 $1.783335-0.609752$ C 0.7729564 .3265510 .087888 O $1.6448103 .301906-0.428304$ C $2.5008343 .882038-1.436892$ C $2.0166995 .313832-1.642119$ C $1.4460585 .648278-0.280574$ H $3.5126313 .906049-1.017335$ C $2.4717413 .036535-2.716036$ H $2.8276415 .983451-1.944931$ H $1.2346625 .373345-2.408258$ C $0.5079816 .843233-0.299691$ H $2.2773505 .851240 \quad 0.408079$ C $2.9409321 .581391-2.524231$ O $1.1267413 .001876-3.213016$ H $3.0673233 .520550-3.498205$ H $2.2936521 .079088-1.801393$ O $2.7638010 .895996-3.771198$ C $4.4162911 .435607-2.099079$ C $4.889172-0.031704-2.031710$ H $4.5787041 .919574-1.130696$ O $5.2292352 .121934-3.054877$ H $4.600346-0.554728-2.951013$ H $5.987415-0.040480-2.015283$ H $1.0324327 .737594-0.652274$ H $-0.3467406 .671534-0.962590$ H 0.1199557 .0543770 .701481 C 0.5828424 .1587621 .597297 H $0.5601512 .667064-2.497576$ H $1.8297140 .992771-4.024695$ H $6.1484122 .083298-2.740254$ H 0.1030815 .0560342 .005203 H 1.5612504 .0583652 .082014 H $-0.1871664 .226243-0.435701$ C $4.762574-0.8708491 .598207$ O $5.122349-0.3180680 .325786$ C $4.355685-0.773962-0.795893$ C $4.472198-2.297572-0.944830$ C $4.074126-3.0058170 .353054$ C $4.791293-2.4076821 .566890$ H $5.556523-0.5387042 .280065$ C $3.437788-0.2933052 .105036$ H $3.306101-0.511475-0.642701$ O $5.811197-2.641895-1.292887$ H $3.821752-2.622294-1.766077$ H $4.317337-4.0720790 .271303$ O $2.664628-2.8880950 .519800$ H $4.357102-2.7996382 .493511$ O $6.157317-2.8330701 .552383$ C 3.6138711 .1662812 .450009 H $2.622271-0.3579791 .379438$ H $3.101181-0.8266463 .001639$ H 5.843163 -3.606044 -1.415179 H $2.424325-3.3640821 .332854$ H $6.584896-2.4346230 .774972$ O 3.8378731 .3069773 .787502 O $3.5906492 .068511 \quad 1.623345$ C 4.0728112 .6458784 .234615 H 4.9758783 .0475473 .764909 H 3.2087743 .2794444 .014048 H 4.2218352 .6213535 .317333 SCF Energy (B3LYP/6-31G**//MMFF)= -3245.91227533

\section{5}

MM̄̄F Geometry

C $0.207598-4.0952290 .688132$

C $0.861629-4.429977-0.435595$

C $2.174114-3.831432-0.894305$

O $2.405680-2.551299-0.261850$

C $3.337658-4.769341-0.529388$

C $4.682865-4.298837-1.048448$
C $5.720448-3.936195-0.268571$

C $4.832861-4.274172-2.548164$

C $5.791373-3.9226691 .232493$

C $5.390166-2.5910391 .885659$

C $6.293596-1.4050761 .509465$

C $5.977738-0.1893222 .382657$

C 6.8047241 .0406831 .976913

O $6.063303-1.0735010 .138258$

O 6.2222162 .1888502 .614263

C 6.6813001 .2577550 .451921

C 8.2479360 .9482082 .488221

O 7.5741762 .2902230 .040337

C $6.906497-0.030062-0.371702$

C $6.5697990 .125272-1.875769$

O $8.288098-0.401544-0.290040$

C $1.743942-1.477422-0.774534$

O $1.031618-1.453722-1.765564$

C $1.995844-0.330248 \quad 0.123685$

C 1.1673950 .7236720 .088950

C 1.2616401 .9229790 .923085

C $2.5011562 .089671 \quad 1.762223$

C $5.1300420 .547213-2.188702$

C $4.9501782 .044918-2.373955$

C $3.1724773 .761674-2.697356$

O $5.8897292 .831233-2.457239$

C $2.8727524 .412787-1.383914$

C $1.7251495 .059675-1.129021$

C 1.4252925 .7570110 .167170

C 0.1097385 .3212580 .773802

C -1.0074456 .0452550 .591428$

C $0.1107864 .096873 \quad 1.660947$

C 0.2523782 .8179430 .883341

N $3.6104742 .396405-2.473261$

H $\quad 0.659128-3.3841261 .374064$

H $0.417101-5.161806-1.106350$

H $2.128241-3.691469-1.981069$

H $3.148906-5.773385-0.932268$

H $3.365017-4.8816990 .559891$

H $6.639780-3.616573-0.758366$

H $5.863376-4.071185-2.859188$

H $4.548809-5.241760-2.974653$

H $4.199936-3.497284-2.987623$

H $5.177289-4.7254411 .654560$

H $6.820102-4.1703041 .524623$

H $4.351288-2.3637231 .620151$

H $5.420722-2.7336942 .973116$

H $7.337999-1.7107331 .643210$

H $6.132320-0.4207183 .443981$

H 4.9127650 .0615112 .284592

H 6.2334952 .0368173 .574739

H $5.665450 \quad 1.629978 \quad 0.274184$

H 8.2619110 .8602533 .581299

H 8.8091051 .8599742 .255604

H 8.7927140 .0934702 .082161

H $7.3775552 .503899-0.886752$

H $7.2998530 .781943-2.363518$

H $\quad 6.715637-0.860633-2.341067$

H $8.496363-0.981775-1.040886$

H $2.835614-0.397400 \quad 0.804884$

H $\quad 0.3262630 .700928-0.602512$

H $3.3990581 .986043 \quad 1.143406$

H 2.5323411 .3358942 .556282

H 2.5781913 .0684682 .239767

H $4.8331720 .086185-3.139631$

H $4.4398760 .186725-1.421079$

H $2.2932393 .717518-3.347848$

H $3.9615964 .318318-3.212920$

H $3.6441544 .374216-0.618479$

H $0.9680095 .121463-1.907768$

H $1.4021276 .836279-0.033445$

H 2.2303495 .6102640 .897908

H -1.948945 5.7585911 .047470

H -1.005826 $6.940339-0.021800$

H -0.8341094 .0329592 .217141$

H 0.8782544 .2158192 .430601

H $-0.6002562 .609707 \quad 0.235654$

H $2.897471 \quad 1.684560-2.343048$

C $-2.255186-3.5811521 .014916$ 
O - $-2.401924-3.099594-0.336676$

C $-2.530719-1.662035-0.303420$

C $-2.881353-1.3161731 .135845$

C $-2.074396-2.3460151 .899697$

H $-1.541058-1.268897-0.563550$

C $-3.573602-1.221237-1.335761$

H $-2.616070-0.2885401 .399581$

H -3.953599 -1.455363 1.320521

C -2.537381 -2.543697 3.332532

H -1.030322 -2.013169 1.910630

C $-3.8873410 .285745-1.310542$

O $-3.095964-1.580000-2.636331$

H $-4.485439-1.805347-1.176018$

H $-4.244417 \quad 0.591270-0.324726$

O $-2.677398 \quad 1.021943-1.538225$

C $-4.891943 \quad 0.745104-2.382409$

C $-6.2501660 .020415-2.403023$

H $-4.4338090 .644743-3.374210$

O $-5.142272 \quad 2.144228-2.205571$

H $-6.8062970 .395204-3.272675$

H $-6.077740-1.048165-2.569305$

H -2.442918 -1.6115173 .899033$

H -3.585634 -2.857858 3.376484

H -1.934165 -3.3074443 .833502$

C $-1.141108-4.6339141 .075972$

H -2.294387 -1.056862 -2.809380

H -2.117186 $0.917787-0.751132$

H -4.280385 2.594895 -2.204593

H $-1.407733-5.463666 \quad 0.409107$

H -1.074984 -5.047199 2.088908

H $-3.203486-4.0770901 .260183$

C -7.187091-0.242724 1.221504

O $-6.519524-0.397980-0.037646$

C $-7.1391670 .235414-1.158760$

C -8.558304 -0.326628 -1.365357

C $-9.392414-0.216918-0.085624$

C $-8.636366-0.7486651 .137751$

H -6.641839 -0.9033471 .907961$

C $-7.077500 \quad 1.189697 \quad 1.760526$

H -7.208226 $1.311916-0.971781$

O $-8.507728-1.699371-1.749220$

H -9.053821 $0.227045-2.172400$

H -10.321987 -0.783952-0.218122

$\begin{array}{llll}\text { O } & -9.743533 & 1.149227 & 0.113277\end{array}$

H -9.166161 -0.4898732 .061990$

O -8.586266 -2.176907 1.072706

C -5.6432851 .5431052 .083440$

H -7.4740611 .9288681 .059022$

H -7.6488201 .3022252 .689516$

H -8.191492 -1.739506 -2.667250

H $-10.296003 \quad 1.1968450 .912071$

H -9.502287 -2.502271 1.052442

O -5.2423942 .6032671 .325319$

$\begin{array}{llll}O & -4.962176 & 0.949391 & 2.909579\end{array}$

C -3.893904 3.0383521 .526837

H -3.6552093 .7697610 .750491$

H -3.800730 3.5172332 .505699

H -3.196037 2.2004611 .440015

SCF Energy (B3LYP/6-31G**//MMFF)= -3245.90079038

\section{$07 \_00446$}

MM̄FF Geometry

C $2.034075-3.1996832 .565434$

C $0.776794-3.3827992 .994379$

C $-0.247708-2.2815363 .028107$

O $-1.359436-2.6797542 .191179$

C $-0.790374-2.0295184 .445493$

C $0.167682-1.2437805 .319338$

C $0.291456 \quad 0.098762 \quad 5.287983$

C $1.000157-2.0609276 .271540$

C -0.4810201 .0608054 .427184$

C $\quad 0.3862291 .646906 \quad 3.303194$

C $-0.398248 \quad 2.497884 \quad 2.288517$

C $-0.883855 \quad 3.816768 \quad 2.893444$

C -1.7322914 .6243251 .900430$

O -1.4974541 .7170551 .811222$

O -2.3853995 .6744762 .630314$

C $-2.8389953 .704626 \quad 1.345685$
C $-0.875521 \quad 5.3090890 .830963$

$\begin{array}{lllll}\text { O } & -3.589397 & 4.362632 & 0.327576\end{array}$

C -2.3133402 .3508140 .818740$

C $-3.463397 \quad 1.372953 \quad 0.476013$

O $-1.5705652 .571356-0.384104$

C -1.305574 -2.294723 0.885638

O $-0.421106-1.647050 \quad 0.347071$

C $-2.522812-2.795716 \quad 0.209511$

C $-2.770023-2.402496-1.048908$

C $-3.923348-2.767495-1.873653$

C $-4.922487-3.728881-1.288690$

C -4.3520491 .0037121 .665492$

C $-5.256113-0.1723411 .358101$

C $-7.320137-0.7923280 .110895$

O $-5.081026-1.2692341 .884507$

C $-7.452077-1.002077-1.367178$

C $-6.776712-0.394703-2.355890$

C $-7.006794-0.700167-3.810045$

C $-5.746599-1.111314-4.537792$

C $-5.261383-0.372969-5.550452$

C $-5.091274-2.419237-4.145106$

C $-4.021546-2.223007-3.104743$

N -6.2645250 .1387500 .460669$

H $2.342647-2.2120252 .230811$

H $0.462763-4.367543 \quad 3.331202$

H $0.171939-1.3467052 .641236$

H -1.049547 -2.981056 4.930021

H -1.751842 -1.5045054 .380477$

H 1.0145380 .5682655 .953295

H $1.654849-1.4381556 .890584$

H $0.352588-2.6293086 .947279$

H $1.636059-2.7642785 .726465$

H -1.3700660 .5822864 .008015$

H -0.8482951 .8654725 .074360$

H $1.2067102 .238363 \quad 3.728445$

$\begin{array}{llll}\mathrm{H} & 0.850516 & 0.818816 & 2.753147\end{array}$

H $\quad 0.2751542 .7019261 .448212$

H $-0.0358794 .414638 \quad 3.250284$

H $-1.5029403 .617723 \quad 3.777785$

H $-1.6982096 .217127 \quad 3.053924$

H $-3.535302 \quad 3.5202572 .173207$

H $-0.1644516 .002771 \quad 1.295652$

H -1.492488 5.9166910 .159762

H $-0.300376 \quad 4.604897 \quad 0.228331$

H -3.903715 5.2051750 .698085

H $-4.070862 \quad 1.779286-0.342624$

H -3.0248110 .4490340 .076766$

H $-1.5428601 .739477-0.885513$

H -3.182235 -3.4526990 .764239$

H -2.060477 -1.727674 -1.525667

H $-5.344087-3.331290-0.359605$

H $-4.444513-4.690358-1.070371$

H $-5.765880-3.938535-1.949403$

H -3.7463000 .7317112 .537443$

H -4.9831371 .8481121 .963914$

H $-8.254779-0.3803400 .505249$

H $-7.144563-1.7559460 .599306$

H -8.193718 $-1.750338-1.643246$

H $-6.0365780 .367562-2.128654$

H $-7.4397450 .196639-4.271583$

H $-7.755410-1.492867-3.934496$

H $-4.372075-0.676292-6.093194$

H $-5.7384380 .552087-5.856909$

H $-4.613703-2.868521-5.026328$

H $-5.861677-3.134624-3.845828$

H $-3.226769-1.549401-3.430588$

H $-6.367288 \quad 1.102056 \quad 0.154835$

C $3.585162-4.599758 \quad 1.123238$

$\begin{array}{lllll}\text { O } & 4.160015 & -3.425318 & 0.518144\end{array}$

C $3.803421-3.410762-0.879097$

C $3.234336-4.792090-1.184703$

C $2.549204-5.1230140 .128729$

H $2.996829-2.676777-0.991123$

C $5.008751-2.999297-1.730148$

H $2.548206-4.779024-2.036952$

H $4.025767-5.520504-1.396655$

C $2.220395-6.595687 \quad 0.294390$

H $1.621017-4.5406800 .189072$ 
C $5.610150-1.634062-1.334111$

O $6.031661-3.990898-1.573802$

H $4.733397-3.006239-2.791179$

H $6.056953-1.709387-0.335829$

O $6.695196-1.357573-2.229022$

C $4.618493-0.455333-1.369913$

C $5.2862860 .875118-0.971623$

H $3.789333-0.640339-0.678575$

O $4.076880-0.328152-2.682582$

H $5.7970180 .739790-0.010815$

H $6.0349211 .140727-1.728488$

H $1.533115-6.926702-0.490888$

H $3.120084-7.2174210 .237363$

H $1.742562-6.7812361 .261549$

C $3.076789-4.2797932 .530626$

H $6.793105-3.708016-2.108715$

H $6.316363-1.218010-3.113981$

H $3.504706-1.096702-2.844287$

H $3.920128-3.9284803 .138431$

H $2.704649-5.1962853 .003307$

H $4.406077-5.3234311 .217466$

C $2.8485153 .409496-2.253030$

O $3.8439182 .382987-2.169753$

C $4.2684712 .028756-0.850107$

C $4.8716203 .251353-0.142245$

C $3.8890274 .427127-0.139253$

C $3.3086414 .689232-1.533062$

H $2.770800 \quad 3.633775-3.324555$

C $1.4789092 .910339-1.782789$

H $3.4086451 .666747-0.275470$

O $6.079738 \quad 3.631134-0.797319$

H 5.1268532 .9730950 .887512

H $4.4075345 .326400 \quad 0.215525$

O $2.839948 \quad 4.139713 \quad 0.781009$

H $2.4759045 .399431-1.467800$

O $4.3277375 .313553-2.319553$

C $0.9618191 .823568-2.695398$

H $1.4927322 .532996-0.756765$

H $\quad 0.742513 \quad 3.721609-1.806404$

H $6.447525 \quad 4.392440-0.317201$

H 2.2416784 .9059470 .792431

H $3.939376 \quad 5.534323-3.183265$

O $1.0322380 .613130-2.071908$

O $0.557982 \quad 2.026836-3.833378$

C $0.586122-0.502784-2.848378$

H $0.757592-1.412779-2.267538$

H $-0.483981-0.411155-3.055983$

H $1.155105-0.572566-3.780516$

SCF Energy (B3LYP/6-31G**//MMFF) $=-3245.90118498$

\section{7}

MM̄FF Geometry

C $3.372373 \quad 0.7171224 .135963$

C 2.1755790 .3962823 .621535

C 0.9620561 .2816993 .701007

$\begin{array}{lllll}\text { O } & 0.636148 & 1.610047 & 2.330061\end{array}$

C $-0.211706 \quad 0.5312294 .352088$

C -1.4453691 .3891384 .556640$

C -2.619306 1.2081603 .919708

C -1.3238402 .4612115 .610471$

C -2.962782 0.1645212 .891766

C -3.780124 -0.9590373 .540521$

C $-4.255019-2.0638682 .585269$

C $-3.102535-2.8646001 .976759$

C -3.605732 -3.942485 1.002190

O $-5.056374-1.4724221 .557199$

$\begin{array}{llll}0 & -2.480975 & -4.405121 & 0.237526\end{array}$

C $-4.591369-3.2898630 .006612$

C $-4.152649-5.1687321 .741771$

O $-5.184537-4.271300-0.840473$

C $-5.679228-2.4237080 .684749$

C $-6.569841-1.666691-0.333511$

O $-6.558277-3.289568 \quad 1.418581$

C 0.3929592 .9122102 .026117

$\begin{array}{lllll}\text { O } & 0.289525 & 3.846676 & 2.804381\end{array}$

C $0.309407 \quad 3.0192010 .551954$

C $-0.0775214 .177453-0.002624$

C $-0.1776644 .465262-1.435478$
C $0.3127753 .410393-2.392537$

C $-5.870003-0.686490-1.276760$

C $-5.4998770 .647448-0.649780$

C $-4.1011232 .656591-1.079943$

$\begin{array}{lllll}\text { O } & -5.924857 & 1.029173 & 0.436473\end{array}$

C $-3.5108573 .326993-2.278265$

C $-3.7999944 .581159-2.655118$

C $-3.2105525 .240448-3.869656$

C $-2.3514356 .436989-3.527224$

C $-2.8482377 .684174-3.584191$

C $-0.8925296 .189428-3.207065$

C $-0.6925615 .654158-1.815540$

N $-4.6709321 .385001-1.485355$

H 3.4950831 .6500314 .679799

H $2.070791-0.5292083 .058997$

H 1.2061752 .1856584 .271408

H $\quad 0.0973130 .147098 \quad 5.333858$

H $-0.451618-0.355193 \quad 3.754746$

H $-3.446922 \quad 1.8706894 .170940$

H -2.2715262 .9823795 .784187$

H -1.0143392 .0205646 .563920$

H -0.5861353 .2143355 .321195$

H -3.5423170 .6466992 .096103$

H -2.064558 -0.2313162 .412802$

H -3.200509 -1.415651 4.353365

H $-4.670983-0.5098104 .000875$

H $-4.889393-2.732253 \quad 3.180450$

H -2.483666 -3.314192 2.763065

H $-2.436898-2.1956351 .420204$

H $-1.808724-4.7317260 .859907$

H $-3.983560-2.655439-0.648089$

H $-3.376514-5.6068012 .381052$

H -4.439152 -5.9588591 .039108$

H $-5.010805-4.9411112 .377040$

H $-5.865220-4.735434-0.323936$

H $-7.107996-2.406260-0.941533$

H $-7.350388-1.1242550 .217335$

H -7.388981 -2.813997 1.585221

H $\quad 0.5860382 .150289-0.034154$

H $-0.350875 \quad 5.0012360 .656077$

H $0.2151823 .691724-3.442632$

H $-0.2512562 .480483-2.267141$

H $1.3741353 .200189-2.219018$

H $-4.986955-1.138392-1.736450$

H $-6.565246-0.451364-2.093569$

H $-3.3216032 .450459-0.339224$

H $-4.8790283 .257906-0.597733$

H $-2.7964252 .752248-2.863368$

H $-4.510253 \quad 5.163169-2.072494$

H $-4.0410025 .546665-4.519417$

H -2.623673 $4.529877-4.465431$

H -2.230246 $8.549572-3.368787$

H $-3.8861517 .866585-3.842427$

H $-0.3280047 .128968-3.276639$

H -0.464548 $5.547412-3.981208$

H $-1.0096196 .350796-1.037654$

H $-4.2592780 .912577-2.285622$

C 5.5898810 .5586382 .996645

$\begin{array}{llll}\text { O } & 4.956920 & 0.774879 & 1.718597\end{array}$

C $5.791500 \quad 0.234383 \quad 0.683083$

$\begin{array}{llll}\text { C } 7.179719 & 0.134797 & 1.300778\end{array}$

C $6.836245-0.2843672 .718323$

H $5.426459-0.7782840 .477006$

C $5.6935581 .091071-0.582854$

H $7.817243-0.5865640 .781127$

H 7.6931181 .1031951 .308719

C $7.962376-0.0606543 .712422$

H $6.575918-1.3514552 .712449$

C $4.2610691 .366713-1.094218$

O $6.2980942 .368130-0.327554$

H $6.2930330 .637078-1.379910$

H $3.7482362 .029371-0.385111$

O $4.3652452 .096652-2.324484$

C $3.3639970 .145468-1.366029$

C $4.031113-0.921810-2.258450$

H $2.4582930 .502075-1.873668$

O $2.952332-0.409386-0.121661$

H $4.483923-0.437046-3.131034$ 
H $\quad 4.826470-1.413427-1.686859$

H $8.842426-0.6504543 .435920$

H 8.2615550 .9922283 .749778

H $7.656729-0.3602984 .719974$

C $4.600252-0.1281803 .941830$

H 5.8279132 .7696410 .423614

H $4.9088302 .883764-2.148640$

H $2.344469-1.144078-0.308561$

H $5.065882-0.3091324 .916911$

H $4.315532-1.1054883 .531574$

H 5.8643301 .5483553 .383484

C $1.600446-3.739790-1.871006$

O $2.600849-2.746932-1.610667$

C $3.040373-1.998239-2.746966$

C $3.678050-2.940610-3.779356$

C $2.715977-4.070944-4.165357$

C $2.088288-4.737579-2.935224$

H $1.492230-4.284766-0.924425$

C $0.242433-3.104373-2.202377$

H $2.185232-1.485077-3.200548$

O $4.873681-3.498524-3.236782$

H $3.956992-2.359131-4.666141$

H $3.254917-4.830572-4.744752$

O $1.677095-3.552852-4.990890$

H $1.256174-5.382913-3.239106$

O $3.065297-5.591463-2.330871$

C $-0.229631-2.185512-1.095422$

H $\quad 0.298016-2.519600-3.124707$

H $-0.519745-3.879476-2.338905$

H $5.272418-4.061022-3.922470$

H $2.093551-3.170202-5.781904$

H $3.783870-5.028986-1.994383$

O $-1.020135-1.210619-1.629703$

O $0.047988-2.3205890 .088588$

C $-1.518347-0.221637-0.723675$

H $-0.828627-0.0349590 .104478$

H - $-1.6453370 .708574-1.281192$

H $-2.491616-0.540329-0.344381$

SCF Energy (B3LYP/6-31G**//MMFF) $=-3245.91773717$

\section{8}

MM̄FF Geometry

C -4.456469-2.846498 -1.677515

C $-3.163391-2.954696-1.335160$

C $-2.066665-3.170037-2.346184$

O $-1.053333-2.152427-2.166447$

C -1.391311-4.538648 -2.140267

C $-0.374258-4.867737-3.219885$

C $0.952464-5.001084-3.022071$

C $-0.941005-5.078675-4.602304$

C $1.721959-4.902508-1.734643$

C $2.407865-3.543553-1.587903$

C $3.420245-3.477463-0.432643$

C $2.776661-3.761718 \quad 0.926334$

C $3.765385-3.5696302 .084011$

O $3.972294-2.153325-0.448910$

O $3.013341-3.5412283 .306890$

C $4.443600-2.1954331 .939978$

C $4.742487-4.7457762 .200512$

O $5.475119-2.0368182 .913042$

C $5.000360-1.9172740 .524164$

C $5.399482-0.4244780 .425150$

O $6.142292-2.7423310 .299528$

C -1.295377 -0.921521 -2.693247

O $-2.321413-0.537873-3.230790$

C $-0.059841-0.123154-2.531485$

C $-0.0166871 .141523-2.975644$

C $1.1539632 .021297-2.950580$

C $2.4427471 .449173-2.422522$

C $6.073085-0.017121-0.892514$

C $5.9949561 .467979-1.221461$

C $5.8917833 .753446-0.226462$

O $5.9224631 .847112-2.390904$

C $4.4262344 .060511-0.284614$

C $3.8985434 .997424-1.087211$

C $2.4227255 .268078-1.187828$

C $1.9634875 .472020-2.616540$

C $1.4639686 .654109-3.015893$
C $2.1022794 .322185-3.588994$

C $1.0298473 .279607-3.422744$

N $6.0674492 .314162-0.128678$

H $-4.739728-2.948887-2.722681$

H $-2.870675-2.849051-0.293658$

H $-2.465237-3.099460-3.365669$

H $-2.149071-5.333223-2.132930$

H $-0.924210-4.554810-1.150025$

H $1.575967-5.228271-3.886533$

H $-0.196545-5.477611-5.299978$

H $-1.768844-5.794603-4.568328$

H - $1.308629-4.136723-5.019935$

H $1.078015-5.102813-0.874363$

H $2.476801-5.698788-1.734948$

H $2.929884-3.281243-2.518403$

H $1.651318-2.761169-1.451154$

H $4.222265-4.196168-0.638365$

H $2.357965-4.7739410 .965480$

H $1.927674-3.0804321 .074633$

H $2.523491-4.3785463 .376857$

H $3.691267-1.4302162 .166229$

H $4.196496-5.6823082 .365844$

H $5.402053-4.6276153 .067316$

H $5.361420-4.8777191 .310459$

H $5.079315-2.2059973 .785317$

H 4.4969940 .1855220 .565431

H $6.093787-0.1811411 .239002$

H $6.642473-2.388572-0.452519$

H $\quad 0.795573-0.609306-2.077490$

H $-0.914737 \quad 1.569202-3.419440$

H $2.3261921 .119657-1.384699$

H $3.2619842 .167556-2.424687$

H $2.7653170 .595471-3.028544$

H $7.132349-0.297583-0.883694$

H $5.594197-0.544937-1.725813$

H $6.4242734 .118652-1.110705$

H $6.3340654 .195140 \quad 0.671385$

H 3.7653383 .4750330 .350621

H $4.5613175 .602790-1.701502$

H $2.2075866 .163031-0.590100$

H $1.8410444 .452770-0.741227$

H $1.1377286 .813558-4.038326$

H $1.3676437 .489305-2.330040$

H $3.1109903 .904000-3.534864$

H $2.0242564 .694961-4.619786$

H $0.0511713 .605943-3.777680$

H $6.118467 \quad 1.9260290 .806708$

C $-6.225480-1.209092-1.041471$

O $-5.277235-0.147635-0.811412$

C $-5.9121120 .886643-0.040206$

C $-7.4070090 .631227-0.163947$

C -7.437834 -0.886658 -0.166243

H $\quad-5.6198540 .723845 \quad 1.003594$

C $-5.4262312 .267105-0.491577$

H $-7.975648 \quad 1.0724340 .659959$

H $-7.8102321 .019596-1.106293$

C $-8.742283-1.468698-0.681790$

H -7.267462 -1.2437210 .858434$

C $-3.8890472 .402566-0.553937$

O $-5.9358762 .526852-1.805240$

H -5.8543603 .0365880 .161976$

H -3.509855 $1.838252-1.415360$

O $-3.6035653 .781525-0.816119$

C -3.1610641 .9537210 .726157$

C -1.6371452 .1634600 .651403$

H -3.3611160 .8899750 .893149$

O -3.6969662 .6747291 .834373$

H -1.278081 $1.853139-0.335077$

H -1.415593 3.2292850 .786017

H -9.579049 -1.157661 -0.047846

H $-8.953549-1.137079-1.703973$

H -8.707228 -2.562736 -0.682931

C $-5.568074-2.550737-0.709645$

H $-5.6194083 .408076-2.069119$

H -2.665435 $3.848464-1.061504$

H -3.2572852 .3500542 .638268$

H -6.304144 -3.360034 -0.769457

H $\quad-5.178699-2.5313990 .315851$ 
H -6.497159-1.165569-2.104335

C $-0.676392 \quad 1.2051854 .137320$

O -1.2275651 .8931193 .006887$

C $-0.891892 \quad 1.3502731 .727445$

C $0.629178 \quad 1.376247 \quad 1.522006$

C 1.3518820 .6581072 .665049

C 0.8565001 .1261994 .038993

H $-0.925374 \quad 1.8402444 .997479$

C $-1.344721-0.1579664 .353603$

H -1.243833 0.3142721 .669158

$\begin{array}{llll}\text { O } & 1.096630 & 2.719056 & 1.427265\end{array}$

H 0.8657830 .8842350 .572237

H 2.4295720 .8419782 .577043

O $1.132391-0.7422772 .523808$

H 1.2382950 .4733414 .832368

$\begin{array}{lllll}\text { O } & 1.369206 & 2.437538 & 4.292819\end{array}$

C -2.824672 0.0050684 .613398

H $-1.209528-0.8291043 .500937$

H $-0.916081-0.6701255 .222865$

H $0.821938 \quad 3.187927 \quad 2.233777$

H $1.590568-1.1884203 .256466$

H $2.3399212 .380124 \quad 4.279744$

O $-3.541297-0.5700413 .605603$

$\begin{array}{llll}\text { O } & -3.287136 & 0.583329 & 5.588286\end{array}$

C $-4.963672-0.4816933 .731881$

H -5.2811260 .5638553 .678121$

H -5.412912 -1.029745 2.899339

H -5.293333 -0.9389824 .669572$

SCF Energy (B3LYP/6-31G**/MMFF) $=-3245.92006772$

\section{9}

MM̄FF Geometry

C $-3.211580 \quad 0.230293 \quad 2.887255$

C $-1.9893380 .039323 \quad 2.366271$

C $-0.869961-0.633863 \quad 3.116041$

O $-0.363182-1.6929882 .268614$

C 0.2865310 .3431653 .416598

C -0.1024691 .5389814 .256870$

C $-0.1001122 .816113 \quad 3.828378$

C $-0.487767 \quad 1.2257615 .680774$

C $0.2759013 .350407 \quad 2.472032$

C $1.370175 \quad 4.4170762 .605966$

C $1.9155884 .974078 \quad 1.281338$

C $0.8229995 .474533 \quad 0.335045$

C $1.409686 \quad 6.028919-0.973057$

O $2.704987 \quad 3.9635750 .637167$

O $\quad 0.331913 \quad 6.161802-1.914029$

C $2.3917724 .986829-1.554010$

C $1.9938737 .433930-0.788267$

O $3.0663365 .505988-2.697963$

C $3.4077894 .441585-0.520524$

C $4.2871423 .300436-1.092107$

O $4.3101075 .502708-0.170509$

C $0.343373-2.6814182 .881667$

O $0.506128-2.835904 \quad 4.081870$

C $0.956503-3.5157791 .826824$

C $2.090735-4.1711352 .114648$

C $2.895224-4.9671101 .187350$

C $2.308838-5.279210-0.163232$

C $3.5590222 .034035-1.537540$

C $3.3343181 .052195-0.405849$

C $4.468319-0.8084740 .821528$

$\begin{array}{llllllllll}\text { O } & 2.261304 & 0.986050 & 0.187863\end{array}$

C $4.864610-2.0677770 .114058$

C $5.951386-2.7917990 .422937$

C $6.358271-4.045315-0.302156$

C $6.430865-5.2534950 .607531$

C $7.589906-5.6183521 .180909$

C $5.174999-6.0824440 .785796$

C $4.126021-5.3551971 .582594$

N $4.4500400 .271503-0.148697$

H -3.423453 -0.1228033 .893563$

H $-1.7687700 .404424 \quad 1.365918$

H -1.253789 -1.0759934 .044321$

H $\quad 0.7532420 .6377602 .471555$

H $1.088661-0.1933083 .941045$

H -0.4133773 .5856934 .533864$

H $-0.662157 \quad 2.130304 \quad 6.273287$
H -1.405552 0.6310175 .712502

H 0.3107560 .6615116 .173732

H $0.6042072 .567437 \quad 1.786387$

H -0.6255363 .7956692 .036473$

H $0.9780815 .253734 \quad 3.199338$

H 2.2103213 .9900183 .170111

H 2.5785095 .8039031 .554612

H 0.1946316 .2271250 .826929

H $\quad 0.1539414 .6463870 .069333$

H $-0.3347286 .753496-1.524877$

H $1.7726664 .160593-1.920891$

H $1.2231798 .123117-0.422176$

H $2.3339457 .848978-1.743361$

H $2.8249837 .465110-0.081094$

H $3.7523156 .119323-2.383615$

H $4.8567553 .694636-1.944147$

H $5.0409783 .030286-0.340142$

H 5.1066635 .1111730 .224719

H $\quad 0.502195-3.513657 \quad 0.843723$

H $2.502177-4.075867 \quad 3.119289$

H $1.283928-5.654827-0.065927$

H $2.857508-6.052220-0.705341$

H $2.297128-4.384136-0.791630$

H $2.6032322 .238370-2.022749$

H $4.1671101 .526782-2.298439$

H $3.481018-0.9289211 .277841$

H $5.182958-0.5309901 .602697$

H $4.217277-2.402929-0.692832$

H $\quad 6.602273-2.455607 \quad 1.226934$

H $7.340758-3.863263-0.757328$

H $5.683926-4.259536-1.140736$

H $7.656931-6.4978391 .812589$

H $8.497348-5.0417821 .034084$

H $5.403617-7.0157601 .316456$

H $4.824545-6.388087-0.203573$

H $4.453920-5.0914262 .589384$

H $5.3093990 .459339-0.657301$

C -5.5502130 .0613891 .976210$

O $-5.225326-0.9703181 .021682$

C $-6.090746-0.827347-0.115921$

C $-7.347288-0.1781410 .439245$

C $-6.739080 \quad 0.835738 \quad 1.390686$

H $-5.600428-0.123497-0.801171$

C $-6.286731-2.167391-0.829948$

H $-7.9639270 .279605-0.339756$

H $-7.963353-0.8939510 .995388$

C $-7.710246 \quad 1.345593 \quad 2.441806$

H $-6.378398 \quad 1.6918050 .804187$

C $-4.974527-2.924092-1.125414$

O $-7.097699-3.018208-0.011459$

H $-6.853749-2.002478-1.753975$

H $-4.602396-3.379896-0.199816$

O $-5.286424-4.016270-1.998623$

C $-3.858618-2.072032-1.751539$

C $-2.620154-2.908774-2.123409$

H $-3.567610-1.287401-1.044736$

O $-4.370689-1.433218-2.920799$

H $-2.453580-3.687066-1.369204$

H $-2.797668-3.394636-3.090568$

H $-8.5556081 .856351 \quad 1.969263$

H -8.1094480 .5270463 .050150$

H -7.2170862 .0554983 .113354$

C -4.3236210 .9541092 .182941$

H $-7.212992-3.856905-0.490256$

H -5.549951 -3.639221 -2.855786

H $-3.663707-0.870944-3.280779$

H -4.5958991 .8271672 .787514$

H $-3.969302 \quad 1.323512 \quad 1.213244$

H -5.827859 -0.4429462 .909767$

C $-0.499296-0.070257-3.329377$

O $-1.550966-1.036113-3.205035$

C -1.351963 -2.037331-2.205033

C $-0.097771-2.860704-2.523298$

C $1.125369-1.948274-2.668845$

C $0.850225-0.762770-3.599877$

H $-0.7616100 .516983-4.218796$

C $-0.463140 \quad 0.893155-2.131569$

H $-1.227541-1.555597-1.228586$ 
O $-0.300021-3.594439-3.728374$

H $0.061723-3.586753-1.718194$

H $1.971085-2.525132-3.062784$

O $1.497184-1.446005-1.390517$

H $1.664392-0.032003-3.527736$

O $0.848049-1.255066-4.943103$

C $-1.7204111 .730653-2.022566$

H $-0.3432880 .362802-1.183523$

H $\quad 0.373818 \quad 1.587969-2.255354$

H $0.505093-4.113546-3.894768$

H $1.700685-2.204967-0.819564$

H $0.739823-0.491922-5.535944$

O $-1.6127962 .544384-0.930054$

O $-2.6686441 .688891-2.793983$

C $-2.717720 \quad 3.425237-0.703582$

H -2.5934613 .8801910 .282544$

H $-2.7194974 .216041-1.459243$

H -3.664483 2.876861-0.717569

SCF Energy (B3LYP/6-31G**//MMFF) $=-3245.90186215$

\section{0}

MM̄FF Geometry

C $2.199328-2.3309472 .656003$

C $2.118732-0.9942632 .559291$

C $0.854338-0.1761572 .460834$

$\begin{array}{llll}0 & -0.286044 & -1.044168 & 2.256087\end{array}$

C $0.589236 \quad 0.653492 \quad 3.729298$

$\begin{array}{llll}\text { C } & 1.326093 & 1.977460 & 3.746174\end{array}$

C 0.8718673 .1002163 .153939

C 2.6285221 .9943484 .501505

$\begin{array}{llll}\text { C } & -0.410127 & 3.273613 & 2.385274\end{array}$

C $-0.138583 \quad 3.4392150 .885017$

C $-1.401507 \quad 3.5924730 .020916$

C -2.094114 4.9393590 .240732

C -3.387467 $5.057951-0.579310$

$\begin{array}{llll}0 & -2.286613 & 2.510372 & 0.319915\end{array}$

O $-4.117076 \quad 6.194257-0.090576$

C $-4.2551133 .812625-0.304806$

C $-3.1093875 .327324-2.062204$

O $-5.4158673 .804638-1.132579$

C $-3.4889652 .480323-0.460616$

C $-4.323073 \quad 1.270538 \quad 0.021729$

O $-3.191412 \quad 2.266080-1.844777$

C $-0.885740-1.0226001 .035303$

O $-0.622805-0.2894290 .095390$

C $-1.922098-2.0795401 .023959$

C $-2.573095-2.339250-0.119884$

C $-3.633706-3.329422-0.319170$

C $-4.043020-4.1610650 .866645$

C $-4.712907 \quad 1.3187051 .500344$

C $-5.179214-0.0318802 .004336$

C $-7.086526-1.6291091 .879423$

O $-4.508368-0.6843072 .801200$

C -7.406204 -2.526375 0.721831

C $-7.228137-2.287174-0.587336$

C $-7.598744-3.276038-1.657247$

C $-6.452823-3.601605-2.588546$

C $-6.513834-3.290583-3.894536$

C $-5.269406-4.359680-2.024405$

C $-4.181981-3.436361-1.547738$

N $-6.403592-0.4132081 .481160$

H $1.292872-2.9259172 .713541$

H $3.043215-0.4256582 .501229$

H $\quad 0.9690240 .4933531 .600961$

H $\quad 0.8431350 .072827 \quad 4.626674$

H -0.4883210 .8320173 .834496$

H 1.4780494 .0024983 .224700

H 3.0851782 .9897534 .519278

H $2.467528 \quad 1.6891815 .540725$

H 3.3513121 .3118124 .046506

H - 1.0919372 .4349462 .549830

H $-0.915772 \quad 4.1614812 .780410$

H $0.526904 \quad 4.2942820 .713562$

H $0.396206 \quad 2.5458190 .537786$

H - $1.0848693 .506312-1.025538$

H -1.413922 5.7681340 .008140

H -2.357612 5.0567591 .299746

H -3.548844 $6.978084-0.184775$
H $-4.612708 \quad 3.8999960 .728905$

H $-2.5543376 .265476-2.182792$

H $-4.0427205 .457417-2.621045$

H $-2.5273294 .537728-2.541552$

H $-5.8682714 .656088-1.005614$

H $-5.217917 \quad 1.157200-0.603502$

H $-3.7376380 .358824-0.149889$

H $-3.0341151 .317244-1.982527$

H $-2.119749-2.6092171 .948620$

H $-2.309681-1.761968-1.005776$

H -3.200272 -4.7668121 .217936$

H $-4.861965-4.8510710 .654959$

H -4.379504 -3.521393 1.689615

H $-3.862753 \quad 1.6229922 .121522$

H -5.5140092 .0461461 .672460$

H -8.014686 -1.3293662 .376454$

H $-6.476832-2.1777562 .604274$

H -7.833969 -3.4855431 .009817$

H $-6.819264-1.337670-0.921622$

H -8.440119 -2.852618 -2.220940

H $-7.970628-4.211597-1.220786$

H $-5.705970-3.545588-4.572600$

H $-7.368810-2.771413-4.314380$

H $-4.838738-4.999934-2.806263$

H $-5.617010-5.051982-1.253343$

H $-3.796098-2.789590-2.337911$

H $-6.911500 \quad 0.2414280 .893339$

C $3.774327-3.853711 \quad 1.372415$

O $3.706990-2.953432 \quad 0.245685$

C $2.813437-3.518272-0.729328$

C $2.813823-5.013161-0.446008$

C $2.816227-5.011261 \quad 1.071889$

H $1.813235-3.122308-0.513836$

C $3.228371-3.117780-2.146809$

H $1.944392-5.521240-0.873538$

H $3.720544-5.497588-0.826590$

C $3.245033-6.333710 \quad 1.684194$

H $1.801553-4.7805051 .417894$

C $3.355417-1.595140-2.371062$

O $4.496862-3.707235-2.456814$

H $2.519301-3.541500-2.867610$

H $4.258105-1.222597-1.873926$

O $3.549750-1.376602-3.773700$

C $2.137172-0.775328-1.911967$

C $2.1382490 .699989-2.351851$

H $2.064835-0.822022-0.819755$

O $0.951109-1.379606-2.437739$

H $2.0053210 .764528-3.439459$

H $1.2371151 .156508-1.920539$

H $2.555090-7.1331091 .394804$

H $4.249292-6.6220031 .355844$

H $3.252067-6.2709402 .776970$

C $3.502612-3.0840492 .670696$

H $5.133032-3.402504-1.787494$

H $4.323743-1.899914-4.043942$

H $0.189694-0.903612-2.063811$

H $\quad 4.326322-2.3782852 .834433$

H $3.507623-3.7715803 .524214$

H $4.807355-4.2230721 .402963$

C $4.6586842 .062177 \quad 0.054842$

O $3.5162301 .401842-0.509311$

C $3.3783841 .504886-1.929622$

C $3.2772932 .977209-2.369675$

C $4.4136953 .827147-1.792688$

C $4.6427293 .559243-0.301578$

H 4.5253751 .9783411 .141288

C $5.9803491 .369366-0.317115$

H $4.2539401 .056397-2.408490$

O $2.0282353 .542393-1.978834$

H $3.3058293 .015474-3.464935$

H $4.1842494 .890488-1.933580$

O $5.620298 \quad 3.545932-2.497320$

H $5.586376 \quad 4.0173920 .015984$

$\begin{array}{llllll} & 0 & 3.611618 & 4.211212 & 0.446042\end{array}$

C $6.102884-0.0093730 .293564$

H $6.086672 \quad 1.259338-1.399150$

H 6.8247541 .9590870 .057912

H $1.9112503 .377406-1.029848$ 
H $5.473043 \quad 3.767976-3.432507$

H 2.9244893 .5586370 .660533

O $7.153714-0.661262-0.286743$

O $5.389028-0.4528231 .181133$

C $7.410585-1.9787710 .208899$

H $6.565762-2.636154-0.013425$

H $8.298503-2.365481-0.298195$

H $7.605824-1.9506321 .285273$

SCF Energy (B3LYP/6-31G**//MMFF)= -3245.91686164

\section{1}

MM̄FF Geometry

C $-2.292117-1.2434113 .155088$

C $-3.458192-1.907872 \quad 3.187751$

C $-4.404085-2.0975902 .025467$

O $-4.045801-1.2494220 .912702$

C $-4.345274-3.5586131 .542834$

C $-5.196954-3.8091880 .313475$

C $-4.718649-3.815954-0.947438$

C $-6.653730-4.0745290 .582031$

C $-3.303812-3.535026-1.378886$

C $-2.640327-4.789564-1.955900$

C $-1.110954-4.692128-2.088503$

C $-0.412625-4.833967-0.731752$

C $1.111993-4.710736-0.858107$

O $-0.768432-3.446383-2.704007$

O $1.629628-4.5546810 .472230$

C $1.436497-3.426615-1.649674$

C $1.740664-5.993684-1.412520$

O $2.836120-3.334632-1.903371$

C $0.633530-3.284094-2.963604$

C $0.840163-1.905133-3.634355$

O $1.097222-4.272424-3.893261$

C $-4.597530-0.0089320 .852208$

O

C $-4.1955600 .577295-0.445700$

C $-4.5342531 .838951-0.748672$

C $-4.2254752 .532205-2.002482$

C $-3.5661501 .725119-3.091452$

C $0.396562-0.698879-2.802227$

C $0.5850620 .613626-3.547370$

C $0.3804743 .069721-3.212850$

O $1.0556180 .686375-4.679193$

C $-0.5516433 .970548-2.468473$

C $-1.2600314 .951087-3.049357$

C $-2.1943665 .851021-2.290117$

C -3.554806 $5.994028-2.942647$

C $-4.0580927 .212592-3.204198$

C $-4.3398584 .747176-3.299027$

C $-4.5583743 .835089-2.120382$

N $0.166666 \quad 1.697684-2.785847$

H -1.950031 -0.7921992 .227563$

H $-3.770426-2.3687914 .122760$

H -5.424478 -1.8624742 .353469$

H -4.669615 -4.231824 2.347863

H $-3.305893-3.8446671 .340226$

H -5.410478 -4.018317 -1.763602

H -7.218940 -4.258982 -0.337621

H $-6.767281-4.9553401 .222409$

H -7.111882 -3.217091 1.084850

H $-3.330766-2.750082-2.145178$

H -2.709570 -3.124172 -0.560289

H $-2.893930-5.675903-1.360421$

H -3.061495 -4.955323 -2.956822

H $-0.798292-5.510542-2.748360$

H $-0.681226-5.784134-0.253105$

H - $0.759570-4.048823-0.047396$

H $2.583753-4.3791450 .398977$

H $1.193399-2.589682-0.984602$

H $1.510979-6.843147-0.757992$

H $2.833849-5.922754-1.434278$

H $1.391781-6.244696-2.416164$

H $3.044619-3.939031-2.636031$

H $1.891273-1.791402-3.929986$

H $\quad 0.273558-1.890882-4.576277$

H $0.858748-3.986322-4.790636$

H -3.636543 -0.056356 -1.125499

H $-5.0869222 .421785-0.012998$
H $-4.2105460 .890616-3.390375$

H -2.611149 $1.318462-2.745221$

H $-3.3516722 .293541-3.997520$

H $-0.661979-0.792019-2.534249$

H $0.984538-0.633280-1.881049$

H $\quad 0.2507873 .134990-4.298182$

H $1.4168193 .327568-2.974109$

H $-0.6411963 .819006-1.394807$

H -1.150588 $5.129200-4.116575$

H -1.709264 $6.832167-2.206600$

H $-2.3335805 .491481-1.262782$

H -5.029163 $7.336223-3.672131$

H $-3.5115188 .116496-2.956328$

H $-3.8344944 .253136-4.132147$

H -5.332352 $5.020471-3.680666$

H $-5.0554664 .320111-1.278994$

H $-0.0453031 .534545-1.805171$

C $-1.1779530 .367140 \quad 4.722678$

O -0.4216321 .0456473 .696645$

C 0.6015901 .8397514 .323422

C 0.2373741 .9043585 .801438

C $-0.3769170 .536688 \quad 6.014116$

H 1.5398691 .2824614 .218857

C 0.7262523 .2089883 .646513

H 1.1090982 .0887126 .436562

H -0.5017412 .6881506 .004546$

C $-1.2124710 .437190 \quad 7.278755$

H $0.429640-0.2078356 .057140$

C 1.0262013 .1690012 .133783

O $-0.4962533 .936463 \quad 3.823913$

H 1.4979043 .7943064 .160369

H 0.1349442 .8433321 .584316

O 1.2674494 .5160891 .704733

C 2.2171702 .2801721 .726040

C 2.6602232 .5109050 .266983

H 1.9337351 .2302341 .860476

O 3.3225552 .5282012 .596059

H $1.7792512 .622246-0.374587$

H 3.2347023 .4440380 .219989

H $-0.5965300 .633281 \quad 8.162605$

H $-2.034437 \quad 1.1609427 .274746$

H -1.644828 -0.5627217 .384477$

C -1.378945 -1.099977 4.339465

H -1.212461 3.4055623 .434644

H 2.0419124 .8500612 .188306

H 3.6136903 .4454182 .459885

H -1.776334 -1.659321 5.194118

H $-0.416134-1.5510384 .071823$

H $-2.145100 \quad 0.881553 \quad 4.797573$

C 5.4663310 .0300730 .266242

O $4.6388611 .157426 \quad 0.581344$

C $3.5206031 .356846-0.285539$

C $4.0145701 .628329-1.714811$

C $4.9367430 .507486-2.206296$

C $6.0072980 .143246-1.169173$

H $\quad \begin{array}{llll}6.318213 & 0.105021 & 0.954287\end{array}$

C $4.750352-1.2991310 .548345$

H $2.8925730 .459136-0.279673$

O $4.7184812 .868660-1.746291$

H $3.1502921 .721118-2.379900$

H $5.4240010 .824767-3.136405$

O $4.136769-0.631189-2.504554$

H $6.502444-0.793999-1.446987$

O $7.0185031 .156355-1.185509$

C $4.284411-1.3889731 .985849$

H $3.877104-1.418868-0.097820$

H $5.422029-2.1441490 .362078$

H $4.9793023 .034277-2.668303$

H $4.727428-1.327961-2.837719$

H $6.613206 \quad 1.985362-0.877578$

O $3.143015-2.1353962 .045551$

O $4.861097-0.8834722 .939445$

C $2.578277-2.294733 \quad 3.350383$

H $3.287982-2.7994134 .012706$

H $2.291623-1.3210383 .759332$

H $1.682624-2.9148493 .259011$

SCF Energy (B3LYP/6-31G**//MMFF) $=-3245.91201187$ 


\section{2}

MMFF Geometry

C $0.112359-2.6701322 .328940$

C $0.795411-1.5498782 .047576$

C $1.440816-0.6618183 .077201$

O $2.862881-0.7042912 .795089$

C $0.990473 \quad 0.8030142 .953246$

C -0.3601831 .0969673 .562038$

C -1.495105 1.2731182 .859581

C $-0.366596 \quad 1.2512135 .061009$

C $-1.696070 \quad 1.1215381 .379433$

C -1.9798682 .4502850 .666500$

C $-0.718316 \quad 3.2925270 .426095$

C -1.069733 $4.591124-0.302429$

C $0.1852115 .403998-0.655373$

O $0.1689842 .518864-0.383957$

O $\quad-0.1933196 .413280-1.604595$

C $1.2040954 .482924-1.364377$

C $\quad 0.733107 \quad 6.147890 \quad 0.568453$

O $2.4486535 .150595-1.556879$

C $1.4367133 .135111-0.643669$

C $2.2325382 .165498-1.544089$

O $2.203074 \quad 3.300800 \quad 0.551139$

C $3.721919-0.6182613 .842167$

O $3.428982-0.5229025 .025731$

C $5.126296-0.7162853 .385206$

C $5.523448-0.3253892 .163822$

C $6.889350-0.3936401 .635799$

C $7.909355-1.1369952 .459245$

C $2.4309330 .786780-0.900061$

C $3.186842-0.175168-1.797196$

C $4.087068-2.499347-1.815729$

O $3.5864180 .123660-2.919040$

C $5.522173-2.410486-1.402701$

C $6.495203-1.962860-2.210289$

C $7.937619-1.833749-1.810322$

C $8.365187-0.386674-1.691394$

C $8.7076650 .323515-2.779823$

C $8.4586570 .217738-0.305876$

C 7.1528330 .1820780 .442801

N $3.355112-1.414315-1.190776$ H $-0.019990-2.9417603 .374766$

H $0.948967-1.2568411 .014559$

H $1.252986-1.0511204 .085018$

H 1.0300841 .1139191 .903745

H $1.731031 \quad 1.4543113 .438207$

H -2.404203 1.5175473 .407839

H -1.3586081 .5054325 .449273$

H -0.0532800 .3209025 .544813$

H 0.3195952 .0490315 .363753

H -0.8685380 .5943410 .897642$

H -2.564105 0.4679351 .248934

H -2.429646 $2.210265-0.305403$

H -2.7203533 .0325961 .228058$

H -0.2415473 .5056991 .389209$

H -1.7658795 .1953330 .292315$

H -1.600375 4.354208 -1.235569

H $-0.8977236 .951884-1.205248$

H $0.8073984 .269474-2.366919$

H $-0.007879 \quad 6.863408 \quad 0.944917$

H 1.6164836 .7416960 .309297

H 0.9913455 .4814991 .394368

H $2.2625375 .978112-2.033030$

H $1.7027352 .029557-2.495927$

H $3.2185362 .589132-1.773020$

H $2.491425 \quad 4.2252500 .612194$

H 5.824321-1.088412 4.127549

H $4.7851560 .093966 \quad 1.483201$

H $8.876039-1.2512901 .966116$

H $7.558781-2.1514502 .681540$

H $8.093874-0.6139723 .403978$

H 2.9889060 .8866620 .036920

H $\quad \begin{array}{llll}1.458136 & 0.336969 & -0.675647\end{array}$

H $3.967884-2.441012-2.902861$

H $3.653004-3.442330-1.470783$

H $5.764961-2.720857-0.389640$

H $\quad 6.240685-1.653486-3.221956$

H $8.550354-2.340810-2.567032$
H $8.140746-2.363747-0.871456$

H $9.037018 \quad 1.354317-2.703898$

H $8.663973-0.112447-3.772531$

H $8.765072 \quad 1.270070-0.369841$

H $9.265998-0.2902220 .227404$

H $6.3430830 .704386-0.069270$

H $3.064211-1.535115-0.224941$

C $-0.221808-3.376810-0.141537$

O $-1.149178-2.370145-0.596332$

C $-1.528363-2.675162-1.956287$

C $-0.663578-3.854101-2.386201$

C $-0.455379-4.570580-1.064909$

H -2.569805 -3.007977 -1.927784

C $-1.390600-1.426550-2.834443$

H -1.155921 -4.475629 -3.140385

H $\quad 0.295848-3.519907-2.799730$

C $0.687843-5.569908-1.098700$

H $-1.383261-5.087443-0.787722$

C $-2.370986-0.305636-2.426605$

O $-0.048863-0.946745-2.705143$

H $-1.530363-1.692344-3.888604$

H -2.266353 -0.088714 -1.359238

O $-2.015446 \quad 0.891717-3.127209$

C -3.842492 -0.634754 -2.764233

C $-4.8355580 .472686-2.362410$

H $-4.145024-1.572882-2.291037$

O $-3.960159-0.838066-4.176125$

H $-4.4326831 .453226-2.642276$

H $-5.7528860 .339728-2.951744$

H $\quad 0.492961-6.350836-1.841127$

H $1.635537-5.087659-1.360114$

H $0.814580-6.055123-0.126189$

C $-0.463819-3.6612731 .344060$

H $0.084700-0.266658-3.386916$

H $-1.1784201 .210784-2.748350$

H $-3.677727-0.016587-4.614011$

H $-0.032461-4.6360701 .599707$

H -1.542669 -3.722074 1.533257

H $\quad 0.792626-2.994114-0.309156$

C $-6.460505-0.9193950 .701017$

O $-6.023560-0.700922-0.647950$

C $-5.1850620 .441815-0.865066$

C $-5.8939541 .728810-0.418766$

C -6.3859221 .6208941 .026591$

C $-7.1682160 .326777 \quad 1.263265$

H $-7.210297-1.7173820 .622324$

C -5.321131-1.430639 1.593931

H $-4.2647030 .322215-0.290603$

O $-6.9931292 .017231-1.280385$

H $-5.1919592 .566421-0.506545$

H -7.0256672 .4834021 .250680$

O $\quad-5.2591191 .6700081 .896381$

H -7.3641820 .1943502 .333749$

$\begin{array}{lllll}\text { O } & -8.440421 & 0.458058 & 0.622493\end{array}$

C $-5.130852-2.9213121 .435466$

H $-4.358877-0.9524641 .403843$

H -5.561562 -1.262351 2.650635

H $-7.5931001 .252037-1.263996$

H -5.5907041 .6316732 .809483$

H $-8.959406-0.3368890 .832910$

O $-4.643797-3.2098310 .195696$

O $-5.383258-3.7329522 .317042$

C $-4.459561-4.601991-0.078940$

H $-5.395960-5.1488200 .068121$

H $-4.157496-4.707646-1.123940$

$\mathrm{H}-3.670397-5.0089910 .559784$

SCF Energy (B3LYP/6-31G**//MMFF) $=-3245.91222113$

0700453

MM̄FF Geometry

C $3.0117280 .445963 \quad 3.868992$

C $2.075594-0.3322443 .304655$

C $0.596335-0.0803473 .443900$

O $-0.012787-0.0462732 .132236$

C $-0.054697-1.2239344 .242722$

C $-1.556147-1.0805914 .407953$

C $-2.454338-2.0018504 .006918$

C -2.0288740 .1775355 .089859$ 
C $-2.177270-3.3405553 .384496$ C $-2.223867-3.3574181 .850196$ C $-3.619799-3.0693631 .276142$ C $-3.709408-3.533986-0.177481$ C $-5.092578-3.241295-0.773765$ O $-3.850743-1.659398 \quad 1.325315$ O $-5.000889-3.414275-2.197769$ C $-5.449302-1.760120-0.524395$ C $-6.142457-4.247688-0.287084$ O $-6.805039-1.519338-0.894021$ C $-5.168484-1.2630790 .920352$ C $-5.214654 \quad 0.2784340 .955260$ O $-6.158865-1.7116331 .846164$ C 0.1213991 .1074141 .423552 O $0.7989972 .077482 \quad 1.725072$ C $-0.739174 \quad 1.0071320 .222543$ C $-0.7118812 .006854-0.671117$ C $-1.5380682 .146928-1.869742$ C $-2.5344721 .069336-2.200185$ C -4.7771050 .8975592 .286292$ C -4.8952062 .4072812 .209102$ C -3.8714844 .3897181 .086187$ O -5.8282313 .0082552 .737057$ C $-4.2426234 .497158-0.361851$ C $-3.5631655 .238046-1.251190$ C $-3.9255565 .333356-2.707660$ C $-2.7444055 .107769-3.630340$ C $-2.2134786 .123971-4.331614$ C $-2.2206833 .694270-3.795278$ C $-1.3925473 .262173-2.615533$ N $-3.8821532 .986566 \quad 1.460228$ H 2.7078631 .3035324 .464177 H $2.379330-1.1911332 .710761$ H $0.410644 \quad 0.874776 \quad 3.951605$ H $\quad 0.396151-1.2795735 .242454$ H $0.187712-2.168482 \quad 3.744413$ H -3.511512 -1.792647 4.166321 H $-3.0980490 .144755 \quad 5.326314$ H -1.4913210 .3232116 .032410$ H $-1.861571 \quad 1.0502174 .451337$ H -1.208054 -3.729248 3.714318 H -2.912172 -4.0553083 .776913$ H -1.497724 -2.6350981 .455615$ H -1.899281 -4.3554191 .529375$ H -4.366429 -3.586324 1.890105 H -3.476082 $-4.602546-0.264581$ H -2.953092 -3.009013 -0.776541 H -4.697067 -4.321719-2.371516 H $-4.828876-1.173910-1.217454$ H -5.864240 -5.265405 -0.587146 H $-7.117830-4.057687-0.748242$ H $-6.265254-4.2498510 .797887$ H -6.937691 -0.557909-0.937263 H -4.5625990 .6718120 .169822$ H $-6.230517 \quad 0.6287380 .733544$ H $-6.738579-2.3504031 .403632$ H -1.3923650 .1474190 .133149$ H $-0.0271412 .835821-0.492107$ H -2.148256 $0.068789-1.983351$ H -2.793708 $1.052954-3.262694$ H $-3.4538091 .220743-1.630467$ H -5.4013080 .5422583 .113488$ H $-3.739977 \quad 0.6379012 .522869$ H -2.864599 $4.770747 \quad 1.282382$ H $-4.5890014 .944497 \quad 1.697927$ H $-5.1144573 .938490-0.695174$ H -2.707635 $5.819007-0.913755$ H -4.348836 $6.331723-2.877434$ H -4.719293 $4.621817-2.967124$ H -1.382495 $5.967850-5.011395$ H $-2.5944367 .135403-4.238513$ H -1.588167 $3.624921-4.689248$ H -3.074783 $3.035255-3.978269$ H $-0.6222973 .980768-2.331219$ H -3.168274 2.3894661 .053918 C 5.1861801 .3476063 .000442 O 4.6997811 .4183751 .644691 C 5.8218631 .4315720 .747228
C $7.017623 \quad 1.8437631 .593834$

C 6.7007251 .1469372 .902022

H 5.9657640 .3984350 .409570

C $5.5403922 .326628-0.462949$

H $7.968626 \quad 1.5268451 .155415$

H 7.0556772 .9283921 .745196

C 7.4709401 .6989564 .089206

H 6.9334610 .0788702 .793539

C $4.2032162 .050132-1.178303$

O $5.5184773 .699026-0.048430$

H $6.3699552 .244967-1.175011$

H $3.3692222 .441045-0.581889$

O $4.2035742 .798843-2.400975$

C $3.9295230 .572265-1.509706$

C $2.6667440 .400505-2.374993$

H $3.8049750 .013277-0.576056$

O $5.0524390 .014057-2.189891$

H $1.8803211 .068595-2.006546$

H $2.9047850 .687418-3.406810$

H 8.5485371 .5754943 .939712

H 7.2722612 .7659984 .235530

H 7.1947431 .1751785 .009696

C 4.4880080 .2025893 .737200

H 4.8365333 .7865720 .639635

H $4.3558283 .731076-2.169218$

H $5.1929310 .531667-3.001243$

H $4.909123 \quad 0.091057 \quad 4.742794$

H $4.661883-0.7407563 .205787$

H 4.9499372 .3100703 .471757

C $2.778703-3.302317-2.943614$

O $3.109391-1.906881-2.936740$

C $2.125950-1.042508-2.364001$

C $0.803833-1.153689-3.137517$

C $0.323273-2.606137-3.210016$

C $1.439816-3.551643-3.662272$

H $3.573459-3.772242-3.537129$

C $2.829663-3.906831-1.535650$

H $1.959454-1.325793-1.318733$

O $0.974797-0.637164-4.455533$

H $0.053940-0.533171-2.637846$

H $-0.515376-2.681198-3.913026$

O $-0.146014-3.019467-1.929899$

H $1.129264-4.593353-3.520665$

O $1.633948-3.360131-5.066641$

C $4.237002-3.861617-0.989980$

H $2.161647-3.405376-0.829466$

H $2.524856-4.960093-1.551846$

H $0.115533-0.693659-4.906806$

H $-0.854729-2.408191-1.666401$

H $2.295665-4.008009-5.363200$

O $4.341670-2.884570-0.044844$

O $5.139388-4.594373-1.374588$

C $5.638493-2.7337470 .539332$

H $5.572287-1.9759771 .323386$

H $5.967531-3.6746140 .990991$

H $6.353223-2.395285-0.216460$

SCF Energy (B3LYP/6-31G**//MMFF) $=-3245.89457995$

\section{4}

MM̄FF Geometry

C $3.011027-1.315233-2.157081$

C $2.990935-2.656124-2.126065$

C $2.589420-3.416579-0.887414$

O $1.323550-4.078752-1.129701$

C $3.620443-4.483816-0.470758$

C $4.924217-3.9179220 .058691$

C $5.236924-3.7940021 .364256$

C $5.951048-3.537723-0.979793$

C $4.431533-4.1740622 .574631$

C $3.092177-3.4676392 .788042$

C $3.085401-1.9406422 .641788$

C $3.985404-1.2208143 .643877$

C $3.8524200 .307673 \quad 3.515776$

O $1.723987-1.5383232 .841535$

O 4.4742850 .9013794 .665448

C 2.3569670 .6901973 .562235

C 4.6000840 .8485382 .289494

O 2.1767622 .0796073 .295566 
C $1.471451-0.1474132 .611142$

C -0.0241740 .1133892 .917016$

O 1.7524340 .2174651 .260603

C $0.211142-3.301039-0.990788$

O $0.181281-2.112577-0.710199$

C $-0.984818-4.131001-1.245801$

C $-2.159851-3.514002-1.446748$

C $-3.450446-4.154408-1.706631$

C -3.529517 -5.647391-1.528921

C $-0.986739-0.7840912 .128105$

C $-2.418829-0.6751112 .619289$

C $-4.602532-1.8409372 .488003$

O $-2.808921 \quad 0.216552 \quad 3.367793$

C $-5.278124-2.8086511 .570814$

C $-6.304648-2.4787200 .773018$

C $-7.027186-3.451509-0.114803$

C $-6.933660-3.082498-1.577786$

C $-7.794737-2.209383-2.127345$

C $-5.894998-3.791286-2.419016$

C $-4.489449-3.376373-2.077985$

N $-3.218146-1.6815642 .089908$

H $2.689301-0.774645-1.269632$

H $3.282115-3.225630-3.004088$

H $2.454256-2.731673-0.045243$

H $3.825261-5.158732-1.313051$

H $3.163184-5.139306 \quad 0.281262$

H $6.219086-3.3901221 .609895$

H $6.941186-3.372561-0.540830$

H $6.060096-4.336439-1.720865$

H $5.668066-2.616663-1.494696$

H $4.269082-5.2591292 .565260$

H $5.058137-3.9894793 .457185$

H $2.345176-3.8861882 .101999$

H $2.715684-3.7328023 .785801$

H $3.382739-1.6809841 .622028$

H $5.033690-1.5186473 .525356$

H $3.702418-1.5115394 .665033$

H 5.4029160 .6129924 .686160

H $2.010450 \quad 0.524667 \quad 4.591957$

H 5.6649980 .5933702 .347622

H 4.5564491 .9427052 .251382

H 4.2185890 .4566111 .344339

H 2.7359052 .5699223 .922228

H -0.201975 -0.060409 3.986495

H $-0.275422 \quad 1.1611152 .711796$

H $1.019612-0.0737860 .695590$

H - 0.866205 -5.207617 -1.269972

H -2.182632 -2.424702 -1.435131

H -2.916230 -6.156361 -2.280406

H -4.541981-6.045840 -1.616930

H -3.174141 $-5.935105-0.532746$

H $-0.988161-0.5021231 .071481$

H $-0.677998-1.8323212 .207048$

H -5.084236 -0.8569202 .491322$

H -4.614794 -2.223306 3.513999

H $-4.920452-3.8354661 .583472$

H -6.669024 -1.453555 0.776212

H -8.081097 -3.4709730 .192600$

H $-6.662803-4.4756370 .034314$

H -7.752872 -1.959670 -3.182182

H $-8.559089-1.721830-1.530925$

H -6.049858 -3.561462 -3.481571

H $-6.055948-4.868831-2.331613$

H -4.317474 -2.304470 -2.187286

H -2.766786 -2.448382 1.598444

C $4.3470590 .669580-2.870206$

O $3.5876811 .541067-2.006599$

C $3.6375102 .878255-2.526867$

C $4.9023962 .918782-3.368782$

C $4.8494021 .546627-4.021235$

H $2.7704062 .997322-3.188698$

C $3.5425763 .888981-1.379369$

H $4.8990853 .739181-4.092199$

H $5.8012663 .007156-2.747946$

C $6.1787951 .092923-4.598571$

H $4.1040761 .570515-4.827709$

C $2.3768923 .617154-0.401688$

O $4.7547783 .857104-0.615799$
H $3.4690004 .904248-1.785074$

H 2.5946122 .7224100 .193765

O 2.3070094 .7073940 .523964

C $1.0018363 .456834-1.075627$

C $-0.1129873 .143792-0.068719$

H $1.0570592 .636325-1.795146$

O $0.6760804 .638453-1.802263$

H 0.1557632 .2533810 .510505

H -0.1929643 .9561350 .666104$

H $6.5099941 .777769-5.386175$

H $6.959451 \quad 1.060445-3.831261$

H $6.0918340 .092193-5.033456$

C $3.441555-0.479543-3.328777$

H $4.8729912 .947941-0.290250$

H 3.1778384 .7801260 .950950

H $0.6567935 .375685-1.168022$

H $3.958860-1.093747-4.074235$

H $2.536025-0.070092-3.793058$

H $5.1849060 .284744-2.275059$

C $-3.7433172 .343355 \quad 0.089727$

O -2.3575102 .5329630 .399920$

C $-1.5141212 .950073-0.683829$

C $-1.5671291 .924695-1.827040$

C $-3.0092391 .645114-2.264443$

C $-3.9211321 .350960-1.072775$

H -4.1718051 .8837100 .989644$

C $-4.4621813 .682173-0.138178$

H -1.858756 $3.924285-1.047201$

O $-0.9471770 .713413-1.402853$

H $-0.9974772 .306251-2.681715$

H $-3.0253190 .785398-2.945808$

O $-3.5225132 .769501-2.972814$

H $-4.9690181 .339098-1.395431$

O $-3.6141780 .036052-0.604975$

C -4.5195574 .5243301 .120119$

H $-3.9690334 .282815-0.906738$

H -5.496252 $3.490171-0.447186$

H $-0.9772690 .088916-2.147347$

H -2.947543 $2.919579-3.742520$

H $-4.239919-0.1757670 .108021$

O -5.1884565 .6836180 .843547$

O -4.0438804 .2143922 .203468$

C -5.3238046 .5920831 .941008$

H -5.8733147 .4697151 .590141$

H -5.8898926 .1245992 .752216$

H -4.3379186 .9123172 .291367$

SCF Energy $\left(B 3 L Y P / 6-31 G^{* *} / / M M F F\right)=-3245.91678587$

\section{5}

MM̄FF Geometry

C -0.1169114 .7826180 .173719$

C $0.870128 \quad 4.471139-0.679247$

C $2.1710063 .849905-0.235336$

O $2.3043842 .565751-0.896260$

C $3.3591134 .732800-0.665234$

C 4.5824794 .5589690 .215481

C $5.6993703 .884027-0.120326$

C $4.5050525 .250617 \quad 1.554965$

C $5.9966513 .143352-1.393799$

C $5.6899881 .647963-1.293522$

C $6.4534480 .881574-0.203921$

C $7.927320 \quad 0.677791-0.559534$

C $8.647902-0.2173720 .463115$

O $5.804601-0.390428-0.077701$

O $9.894338-0.634205-0.115703$

C $7.811781-1.4950810 .699767$

C $9.001240 \quad 0.5544251 .740502$

O $8.365036-2.279162 \quad 1.754162$

C $6.319952-1.2086680 .980243$

C $5.506828-2.5247280 .998505$

$\begin{array}{lllll} & 0 & 6.200470 & -0.573229 & 2.255608\end{array}$

C $1.5312011 .537608-0.447658$

$\begin{array}{llllll}\text { O } & 0.771277 & 1.545673 & 0.507621\end{array}$

C $1.7272870 .395061-1.366767$

C $0.731763-0.488359-1.533664$

C $0.740715-1.632304-2.449313$

C $2.069595-2.061143-3.012348$

C $3.986132-2.3063931 .004011$ 
C $3.213213-3.6099961 .087274$

C $0.870935-4.466751 \quad 1.114465$

O $3.752137-4.7085351 .188590$

C $0.572402-4.946270-0.270151$

C $-0.656135-4.947354-0.808712$

C $-0.956309-5.467729-2.184469$

C -1.594365 -4.429062 -3.077316

C $-2.901093-4.489117-3.384973$

C $-0.697828-3.371609-3.685517$

C $-0.433987-2.226948-2.745784$

N $1.841903-3.3919591 .047658$

H 0.0286844 .6201611 .239216

H $0.7214854 .609746-1.747383$

H $2.179713 \quad 3.6880390 .849318$

H $3.0803965 .794682-0.622059$

H $3.6037164 .533739-1.714255$

H 6.5099343 .8495830 .607317

H 5.4272905 .1399182 .135399

H 4.3317096 .3231831 .417858

H 3.6887464 .8412332 .157365

H $5.4341883 .564712-2.232859$

H $7.0522403 .296065-1.646909$

H $4.6179441 .517787-1.116691$

H $5.8742421 .176903-2.268077$

H $\quad 6.352579 \quad 1.411756 \quad 0.748498$

H $8.447120 \quad 1.638323-0.655856$

H $7.9997070 .198122-1.545678$

H $10.402650 \quad 0.164252-0.339389$

H $7.881145-2.106618-0.210855$

H 9.6656201 .3955551 .507974

H $9.554390-0.0772482 .444160$

H 8.1263310 .9599312 .252698

H $9.297418-2.4450651 .533034$

H $5.751999-3.1179050 .107765$

H $5.787743-3.1214141 .875626$

H $5.328085-0.7649042 .632360$

H $2.6573770 .343263-1.919944$

H $-0.195261-0.338295-0.981092$

H $2.794246-2.224683-2.207205$

H $2.465454-1.296816-3.689843$

H $2.024037-2.995441-3.575021$

H $3.683986-1.6902501 .857171$

H $3.680245-1.7914730 .086846$

H -0.017247 -4.074309 1.619274

H $1.277360-5.2894541 .711595$

H $1.407005-5.333910-0.849846$

H -1.496143 -4.578925 -0.224314

H -1.622566 -6.333133 -2.073373

H $-0.052209-5.853168-2.672205$

H -3.359365 -3.762228 -4.047426

H -3.543834 -5.260845 -2.974696

H -1.178068 -2.952792 -4.580070

H $0.215934-3.852095-4.044431$

H -1.339875 -1.815751 -2.301362

H $1.494368-2.4512940 .883868$

C $-2.5032174 .166248-0.084380$

O $-2.2863563 .168079-1.102785$

C $-3.5665022 .643316-1.521499$

C $-4.6009333 .276217-0.601455$

C $-3.9512954 .605964-0.268242$

H $-3.7089632 .988957-2.552831$

C -3.490598 $1.113171-1.472817$

H -5.578733 $3.382889-1.080376$

H -4.7306352 .6783320 .309105$

C -4.5566635 .2827810 .948841$

H $-4.0367655 .271522-1.137467$

C $-4.8418490 .402995-1.651236$

O $-2.6075450 .685783-2.515839$

H $-3.0234690 .811045-0.529665$

H $-5.5250830 .690761-0.848233$

O $-5.4354940 .881391-2.865293$

C $-4.749148-1.132904-1.733650$

C $-3.981573-1.834159-0.597845$

H -4.281496 -1.415476 -2.685240

O $-6.072553-1.677157-1.765398$

H -3.923973 -2.900395 -0.853200

H -2.954362 -1.456042 -0.576593

H $\quad-5.617308 \quad 5.4971450 .781194$
H $-4.4789534 .650767 \quad 1.840021$

H $-4.050828 \quad 6.2295301 .162170$

C -1.470985 $5.281777-0.246918$

H -1.763531 $1.152939-2.391130$

H $-6.3648480 .597009-2.869364$

H -6.485929 -1.397319-2.599072

H $-1.727406 \quad 6.1446920 .376852$

H -1.443329 $5.622286-1.289261$

H -2.3801613 .6661100 .886341$

C $-5.139046-0.0941292 .519739$

$\begin{array}{llll}\text { O } & -4.561337 & -0.372378 & 1.237762\end{array}$

C $-4.611749-1.7332660 .807084$

C $-3.865661-2.6305841 .811347$

C $-4.390259-2.4391313 .237832$

C $-4.488750-0.957268 \quad 3.614278$

H -4.8756970 .9524802 .720791$

C $-6.669005-0.1981052 .494240$

H -5.656165 -2.054793 0.742411

O $-2.466125-2.3513011 .783564$

H -3.987141 -3.677354 1.509041

H $-3.720365-2.9461773 .942896$

$\begin{array}{llll}\text { O } & -5.679260 & -3.032621 & 3.359807\end{array}$

H -5.041142 -0.833495 4.553117

O $-3.170659-0.4448913 .832406$

C $-7.2686790 .870534 \quad 1.609177$

H $-7.014647-1.1771162 .151000$

H -7.088051 -0.051954 3.496694

H -2.348256 -1.403357 1.966482

H $-5.589346-3.977223 \quad 3.147193$

H -2.775737 -0.953497 4.561181

$\begin{array}{llll}\text { O } & -7.934766 & 0.295152 & 0.567702\end{array}$

O -7.1564002 .0718691 .816773$

C $-8.5493471 .204712-0.350468$

H -7.809203 $1.900385-0.757015$

H $-8.9700220 .622372-1.174615$

$\mathrm{H}-9.3579651 .748617 \quad 0.146444$

SCF Energy $(B 3 L Y P / 6-31 G * * / / M M F F)=-3245.90964616$

\section{6}

MM̄FF Geometry

C $-2.135245-3.000924-3.171780$

C $-1.208454-3.897421-3.544411$

C $0.290871-3.736059-3.491826$

O $0.666785-2.450039-2.954404$

C $0.906257-4.809586-2.571212$

C $2.422548-4.762819-2.547320$

C $3.165970-4.232224-1.555364$

C $3.098008-5.380954-3.745036$

C $2.697705-3.567478-0.287144$

C $3.268866-4.2872200 .940355$

C $2.863179-3.6922662 .299245$

C $1.378752-3.8918202 .615962$

C $0.996728-3.2722963 .971293$

O $3.201872-2.2993172 .331658$

O $-0.436698-3.2292494 .053030$

C $1.527400-1.8219224 .031964$

C $1.437415-4.1583535 .141444$

O $1.329448-1.2566595 .324991$

C $3.010714-1.6899593 .620731$

C $3.488273-0.2192263 .538648$

O $3.831894-2.3257044 .610172$

C $1.425482-1.629833-3.730855$

O $1.631052-1.721931-4.929574$

C $2.052311-0.641975-2.824423$

C $2.9919810 .201018-3.278300$

C $3.7823881 .117847-2.450823$

C $3.6115731 .009475-0.958774$

C 2.6299110 .7069692 .676548

C 3.2664422 .0747932 .474867

C 2.7350904 .3249841 .509034

O 4.4249002 .3324982 .789208

C $2.8506184 .403790 \quad 0.019383$

C $3.9086474 .904086-0.636490$

C $3.9869664 .962244-2.137624$

C $5.2715904 .381724-2.690652$

C $6.1083245 .142050-3.417369$

C $5.6004292 .932670-2.401349$

C $4.6412881 .969537-3.050109$ 
N $2.3845342 .963724 \quad 1.876513$ H -1.834676 -2.030809 -2.786599 H -1.550596-4.854353 -3.936021 H $0.670591-3.862003-4.512739$ H $\quad 0.593635-5.810614-2.897512$ H $\quad 0.503727-4.698263-1.557905$ H $4.249906-4.255495-1.666820$ H $4.189951-5.368160-3.660317$ H $2.790162-6.425844-3.856666$ H $2.833484-4.840159-4.658853$ H $3.047733-2.528959-0.300367$ H $1.609061-3.529294-0.230231$ H $2.990316-5.3486820 .914745$ H $4.364948-4.2473410 .874218$ H $3.467103-4.2157793 .050509$ H $1.115325-4.9564842 .593447$ H $\quad 0.766213-3.4189761 .839940$ H $-0.768804-2.6942333 .312199$ H $\quad 0.914813-1.2337013 .338074$ H $\quad 0.950985-5.1394705 .080070$ H $1.125800-3.7306576 .100887$ H $2.515630-4.3269885 .173732$ H $0.387497-1.3574625 .544816$ H 3.5668020 .2028334 .549569 H $4.513432-0.2183533 .142329$ H $4.733542-1.9710834 .534871$ H $1.774388-0.687860-1.778386$ H $3.2327130 .196770-4.340247$ H $3.830774-0.008541-0.617641$ H $2.5845041 .262466-0.674506$ H $4.2682961 .669617-0.391414$ H 2.4704270 .2632091 .689059 H 1.6547220 .8591603 .151906 H 3.6588014 .6248702 .013551 H 1.9205454 .9709191 .850762 H $2.0089824 .031844-0.558924$ H $4.7486575 .303000-0.073237$ H $3.8892806 .015634-2.430492$ H $3.1370644 .442216-2.596824$ H $7.0337064 .741789-3.818224$ H $5.8879196 .182938-3.629969$ H $5.6809442 .793010-1.320707$ H $6.6010022 .689604-2.784356$ H $4.6989541 .965366-4.139376$ H 1.4510462 .6418831 .640611 C $-4.355450-3.185295-1.925390$ O $-4.377710-1.826415-1.448510$ C $-4.134182-1.835397-0.031106$ C $-4.328505-3.2781080 .419892$ C $-3.796307-4.034070-0.782971$ H $-3.081785-1.5596520 .097523$ C -5.002743 -0.8043980 .704016$ H $-3.786551-3.5022331 .343531$ H -5.387231 -3.5135320 .579044$ C $-4.221507-5.490433-0.833322$ H -2.700746 -3.985332 -0.755050 C -4.8030290 .6286090 .166145$ O $-6.376301-1.1768970 .619146$ H $-4.728750-0.829247 \quad 1.765338$ H -3.7278300 .7886610 .037766$ O $-5.397848 \quad 0.757311-1.124463$ C -5.3454231 .7292981 .099655$ C -5.2395293 .1581470 .529078$ H -4.8062241 .6745972 .053082$ O $\quad-6.722521 \quad 1.479031 \quad 1.385277$ H $-5.9654093 .286320-0.284321$ H -5.5631813 .8445881 .322721$ H $-3.847082-6.030811 \quad 0.042237$ H -5.311906 -5.590633 -0.848621 H -3.824723 -5.979927 -1.728367 C $-3.614883-3.265940-3.263831$ H -6.595712 -1.276150 -0.323066 H $-6.362757 \quad 0.726421-1.006365$ H -7.018280 2.1519542 .021880 H -4.036344 -2.519594 -3.948642 H $-3.798201-4.250442-3.711180$ H -5.405691 -3.450436 -2.106322 C -1.5406113 .6236270 .805073$
O $-2.919784 \quad 3.382526 \quad 1.120593$

C -3.8386383 .5509260 .037228$

C $-3.7859774 .992407-0.489485$

C $-2.3567875 .379064-0.884268$

C -1.3461665 .0322410 .213772$

H -1.023388 3.5989721 .772626

C $-0.9574992 .502403-0.071092$

H $-3.5682072 .877580-0.780962$

O -4.2743765 .9021130 .493634$

H -4.445262 $5.076402-1.361358$

H $-2.3230216 .456517-1.088187$

O $-2.0162414 .694025-2.086387$

H $-0.3244075 .139917-0.166981$

O -1.5004545 .9900581 .265696$

C $-0.834749 \quad 1.195256 \quad 0.685701$

H $-1.5710902 .315311-0.955680$

H $0.0503592 .773799-0.399817$

H $-3.7360345 .790846 \quad 1.295858$

H -1.122418 $4.978325-2.342936$

H -0.8058605 .8182901 .924176$

O $-\begin{array}{llll}0.474255 & 0.211838 & -0.189604\end{array}$

O $-1.006397 \quad 1.0579701 .888992$

C $-0.268401-1.0906060 .366810$

H $-0.479233-1.830038-0.409581$

H $\quad 0.776883-1.1850210 .669083$

$\mathrm{H}-0.927691-1.2878451 .217263$

SCF Energy $(B 3 L Y P / 6-31 G * * / / M M F F)=-3245.90250509$

\section{7}

MM̄FF Geometry

C $1.538800 \quad 0.312578-2.671978$

C $0.425919-0.382143-2.393754$

C $0.372990-1.474411-1.356109$

O $-0.681708-1.180121-0.410163$

C $0.050597-2.817225-2.031083$

C $-0.034509-3.972406-1.052574$

C -1.189791-4.520364 -0.625444

C $1.287443-4.533601-0.599545$

C $-2.583635-4.072248-0.974322$

C $-3.519544-4.1202020 .242545$

C $-4.879445-3.4345880 .018834$

C $-5.638281-3.983666-1.193354$

C $-6.954395-3.231596-1.442032$

O $-4.642261-2.029791-0.128724$

O $-7.411640-3.609543-2.749990$

C $-6.657061-1.716130-1.462865$

C $-8.049151-3.671181-0.463856$

O $-7.863074-0.964915-1.580476$

C $-5.831317-1.237270-0.249958$

C $-5.400600 \quad 0.239420-0.378681$

O $-6.641085-1.3362050 .928410$

C $-0.443180-0.2081550 .510255$

$\begin{array}{lllll}\text { O } & 0.546766 & 0.500337 & 0.600567\end{array}$

C - $-1.612405-0.1486281 .410918$

C -1.8919151 .0065602 .031314$

C -2.9987811 .2422782 .957543$

C -3.7324580 .0399213 .491111$

C $-4.502150 \quad 0.555801-1.577285$

C $-3.7867391 .877642-1.375552$

C $-4.2246424 .305875-1.024151$

O $-2.5701881 .932322-1.209855$

C -4.5559554 .6384980 .403435$

C $-5.4725314 .038121 \quad 1.180367$

C -5.7626244 .4366452 .600556$

C $-5.6849903 .267353 \quad 3.558055$

C $-6.7793542 .543583 \quad 3.848905$

C -4.3652682 .9879484 .240332$

C -3.2991282 .5142113 .293487$

N $-4.6459242 .964671-1.383073$

H $2.4596800 .071157-2.146286$

H $-0.498969-0.144425-2.913860$

H $1.322380-1.555661-0.813147$

H $\quad 0.819379-3.050758-2.780322$

H $-0.883373-2.732935-2.599332$

H $-1.135042-5.3803890 .040349$

H $1.167571-5.3879030 .075024$

H $1.871955-4.872352-1.461044$

H $1.863951-3.772911-0.065943$ 
H $-2.585564-3.057957-1.382653$ H $-2.963312-4.743787-1.752562$ H $-3.023329-3.6278111 .089648$ H -3.685878 -5.1662850 .529094$ H $-5.467319-3.5901330 .931261$ H -5.820709 -5.059714-1.082680 H $-5.026065-3.874670-2.097928$ H $-8.202922-3.080023-2.949541$ H -6.089483 -1.521026 -2.381363 H $-8.247314-4.744378-0.573173$ H $-8.999228-3.170315-0.679744$ H $-7.791660-3.4877150 .581164$ H $-8.297507-0.964460-0.710516$ H -6.289336 $0.883356-0.393528$ H -4.8621710 .5214300 .535501$ H $-6.297495-0.7187361 .594965$ H -2.212740 -1.043561 1.521510 H $-1.262147 \quad 1.8719041 .826332$ H -4.204942 -0.521415 2.679094 H -4.5263510 .2945344 .196820$ H -3.040571 -0.626777 4.017766 H -3.736223 $-0.216206-1.711960$ H $-5.0833000 .606160-2.504448$ H -4.722722 $5.009476-1.698047$ H -3.143884 4.391195-1.170847 H -3.9833565 .4622640 .825518$ H $-6.080923 \quad 3.2337890 .775613$ H -6.7722404 .8676912 .626960$ H -5.092602 5.2362022 .939950 H -6.7434901 .7210714 .555290$ H -7.7359222 .7558553 .382688$ H -4.488934 2.2769215 .065572 H -4.0146263 .9088914 .724376$ H -2.717062 3.3225222 .849616 H $-5.6384962 .808567-1.531664$ C $1.9744512 .785243-3.053688$ O $3.2681822 .703513-2.421383$ C $3.1610703 .250842-1.094854$ C $1.9255284 .140369-1.122994$ C $0.9991633 .320464-2.000826$ H $2.9730422 .407610-0.418501$ C $4.4607623 .952147-0.689313$ H $1.5199034 .323722-0.123651$ H $2.1319135 .108654-1.593381$ C $-0.1530914 .118800-2.584876$ H $0.5870132 .504799-1.395633$ C $5.7313053 .090898-0.847833$ O $4.6369725 .127503-1.490461$ H 4.3735294 .3069690 .344092 H $5.9756822 .987845-1.911878$ O $6.8227953 .817155-0.267579$ C $5.6567401 .691745-0.209243$ C $7.0069950 .951696-0.282444$ H $4.8889681 .102680-0.722924$ O $5.2592811 .834606 \quad 1.153403$ H $7.4427591 .087486-1.279431$ H $7.6877771 .391800 \quad 0.456795$ H $-0.7713114 .538871-1.785602$ H $0.2049844 .949322-3.202515$ H $-0.7890563 .483226-3.209169$ C $1.6055381 .432366-3.673043$ H $4.6522484 .848070-2.422139$ H 6.6620163 .8706130 .690250 H 5.1613960 .9417061 .525579 H $2.3717451 .159915-4.409620$ H $0.6574761 .526278-4.215678$ H $2.0850513 .514218-3.866762$ C $6.180814-2.0932231 .737965$ O $6.420687-0.7376301 .336842$ C $6.873218-0.559954-0.007768$ C $8.202578-1.299850-0.226233$ C $8.085059-2.777000 \quad 0.162267$ C $7.440349-2.9535621 .542189$ H $5.978679-2.0328622 .815408$ C $4.935329-2.6770021 .061846$ H $6.120570-0.956589-0.697928$ O $9.225455-0.6805370 .551083$ H $8.488052-1.209279-1.281350$
H $\quad 9.084657-3.2281710 .162802$

O $7.309123-3.446300-0.827402$

H $7.192551-4.0063311 .720868$

O $8.381177-2.5700382 .549064$

C $3.695618-1.9395151 .506954$

H $4.963255-2.621979-0.029912$

H $4.820881-3.7388691 .311467$

H $\quad 10.058303-1.146588 \quad 0.365269$

H $7.264878-4.385603-0.579783$

H $9.162028-3.1415432 .454091$

O $3.085570-2.6194672 .518525$

O $3.325826-0.8784771 .021285$

C $1.882811-2.0310163 .023360$

H $1.596927-2.5684383 .931398$

H $2.038632-0.9776203 .275922$

H $1.083382-2.1389682 .285265$

SCF Energy (B3LYP/6-31G**//MMFF) $=-3245.90490939$

\section{8}

MM̄FF Geometry

C - $-1.743620-0.2675672 .445059$

C $-2.058281-1.0245731 .381554$

C $-1.176754-1.3567320 .200412$

O $0.129541-0.7513020 .323183$

C $-0.994933-2.8809780 .100505$

C $-0.297314-3.310838-1.175314$

C $0.988707-3.706387-1.256091$

C $-1.164496-3.311316-2.409031$

C $2.007190-3.776205-0.151086$

C $3.231342-2.918550-0.478326$

C $4.337588-2.9866810 .585244$

C $3.902793-2.4138271 .937384$

C $5.062459-2.3563072 .944171$

O $5.438008-2.2265330 .068492$

O $4.658788-1.5136644 .034760$

C $6.275148-1.6775872 .276665$

C $5.359473-3.7341973 .548177$

O $7.421575-1.7351063 .122420$

C $6.613858-2.2550020 .884607$

C $7.701866-1.3866250 .204313$

O $7.117788-3.5844591 .040675$

C $0.2907060 .494928-0.201376$

O $-0.5549531 .170945-0.765264$

C 1.6897160 .9112950 .043187

C $2.0635572 .168663-0.234325$

C $3.4030782 .737890-0.059605$

C 4.5166651 .8000920 .326137

C $7.928270-1.703416-1.280997$

C $6.991966-0.943335-2.203740$

C $6.6159761 .303720-3.190488$

O $6.102684-1.508704-2.835593$

C $6.8474592 .719081-2.770131$

C $5.9032863 .671899-2.793897$

C $6.1653955 .086954-2.361778$

C $5.1056945 .643670-1.437738$

C $4.4887736 .803648-1.722820$

C $4.8061444 .902788-0.150888$

C $3.5608534 .062473-0.263898$

N $7.2680770 .414848-2.245724$

H $-0.752431 \quad 0.1650692 .538223$

H $-3.067639-1.4277151 .322353$

H -1.674552 -0.990576 -0.706095

H -1.973333 -3.3798250 .135825$

H $-0.455909-3.2450260 .981633$

H $1.365130-4.019613-2.229560$

H $-0.630848-3.676285-3.293045$

H -2.034398 -3.959103 -2.260675

H - $1.517445-2.300401-2.634313$

H $1.583668-3.4604550 .803260$

H $2.314231-4.821972-0.031964$

H $3.657150-3.236602-1.440267$

H $2.933694-1.874044-0.631960$

H $4.647153-4.0332140 .690280$

H $3.071399-2.9850272 .365512$

H $3.522942-1.3938831 .793889$

H $3.848311-1.8892654 .419723$

H $6.030496-0.6126532 .158927$

H $4.479033-4.1180244 .077358$ 
H $\quad 6.155827-3.6741014 .298209$ H $5.643110-4.4780942 .800760$ H $7.167764-1.3540453 .980513$ H $7.439143-0.3271960 .309755$ H $8.653793-1.5414580 .728114$ H $7.736050-3.7813860 .319654$ H $2.372003 \quad 0.177757 \quad 0.452532$ H $1.3134602 .857023-0.622301$ H 4.3364831 .3790481 .321314 H 5.4980932 .2777750 .355528 H $4.593797 \quad 0.976397-0.392746$ H $8.952941-1.421869-1.556181$ H $7.837156-2.772779-1.496197$ H $5.5524141 .048928-3.248611$ H $7.0702821 .123536-4.170145$ H $7.8495002 .981926-2.437611$ H $4.9081153 .430749-3.159551$ H $6.2403655 .700940-3.268369$ H $7.1357215 .164726-1.854684$ H $3.7448167 .224750-1.054344$ H $4.7115227 .357377-2.628786$ H 4.6416765 .6317410 .654270 H 5.6861074 .3334700 .159962 H $2.6753184 .644743-0.525811$ H $8.1360490 .731380-1.822287$ C -3.3949901 .4166003 .393930$ O -4.0235761 .5137752 .097783$ C $-3.5881312 .735447 \quad 1.476233$ C -3.1732343 .6372252 .628533$ C $-2.4726112 .632173 \quad 3.526845$ H -2.6925862 .4948320 .890321$ C $-4.668778 \quad 3.290906 \quad 0.546083$ H -2.5209364 .4548472 .308192$ H -4.0425284 .0655963 .141076$ C -2.2938753 .1111694 .957010$ H -1.483465 2.4182493 .103222 C $-5.1511882 .286962-0.525628$ O $\begin{array}{llll}-5.802729 & 3.672270 & 1.332884\end{array}$ H -4.3016254 .2087090 .071353$ H $-5.7754161 .524368-0.048557$ O $-6.0134882 .991525-1.426628$ C $-4.0227671 .614914-1.325532$ C $-4.4774320 .759205-2.518858$ H -3.428912 $0.988644-0.652277$ O $-3.1536482 .633476-1.830430$ H $-4.8796001 .402970-3.311809$ H $-3.568320 \quad 0.312567-2.943467$ H -1.669981 4.0103314 .986196 H -3.254946 3.3542285 .422549 H -1.8085882 .3419245 .566031$ C -2.7239270 .0466473 .543740$ H $-6.477606 \quad 4.0139930 .721560$ H $-5.4693083 .640138-1.905497$ H -2.390849 2.190285 -2.239904 H -3.504618 -0.7240703 .555811$ H $-2.211117-0.0206704 .510026$ H $-4.207018 \quad 1.4824854 .129453$ C $-5.844756-2.169700-0.621885$ O $-4.989746-1.114277-1.086939$ C $-5.491851-0.346758-2.184856$ C $-5.759526-1.252229-3.400426$ C $-6.663418-2.433119-3.037770$ C $-6.187499-3.141343-1.764068$ H $-5.237517-2.7202350 .107963$ C $-7.091575-1.632430 \quad 0.100342$ H $-6.4271750 .136956-1.886327$ O $-4.532613-1.752823-3.931318$ H $-6.229551-0.654390-4.190070$ H -6.671701 -3.144080 -3.873028 O $-7.992631-1.952878-2.862780$ H $-6.939498-3.859651-1.417390$ O $-5.002971-3.886944-2.059538$ C $-6.749722-0.9376751 .400428$ H -7.638170 $-0.921479-0.524503$ H $-7.766962-2.4581680 .351787$ H -4.066797 -2.217200 -3.214991 H -8.556588 -2.718011 -2.658105 H $-5.228438-4.534447-2.749078$
O $-7.760636-0.0808291 .730586$

O $-5.742896-1.1448192 .062722$

C -7.5889710 .6328382 .959104$

H -6.7262871 .3006622 .889291$

H -8.485093 1.2350603 .129871

$\mathrm{H}-7.471338-0.0651763 .793498$

SCF Energy $(B 3 L Y P / 6-31 G * * / / M M F F)=-3245.91615121$

\section{9}

MM̄FF Geometry

C $-0.035066-3.7960971 .447078$

C $-1.074039-3.2682252 .114563$

C $-0.998574-2.2098913 .191912$

O $0.352031-1.7036473 .270420$

C -1.936148 -1.0443202 .823337$

C -2.0020420 .0397653 .879290$

C -1.4623341 .2656613 .729431$

C $-2.774749-0.3065545 .124329$

C $-0.720507 \quad 1.7569082 .515101$

C 0.0330883 .0674142 .784229

C $0.5514073 .778266 \quad 1.520862$

C -0.5887714 .3580750 .679932$

C $-0.0724995 .034073-0.595081$

$\begin{array}{lllll}\text { O } & 1.325312 & 2.859523 & 0.746342\end{array}$

O $-1.1962125 .253449-1.462484$

C $0.8801274 .065579-1.327827$

C $0.5276556 .415509-0.305268$

O $1.4790874 .731589-2.436630$

C $1.9591993 .441903-0.410169$

C $2.7249002 .281657-1.091451$

$\begin{array}{lllll}\text { O } & 2.902474 & 4.443688 & -0.023441\end{array}$

C $0.933704-1.5520494 .485016$

O $0.423046-1.7682505 .575180$

C $2.334416-1.0820734 .356884$

C $2.948175-0.8941743 .177888$

C $4.337097-0.4770442 .975226$

C $5.184361-0.2245674 .194050$

C $3.5169642 .657055-2.345611$

C $4.5889071 .633572-2.657267$

C $4.914002-0.734135-3.346426$

O $5.7833151 .892078-2.523149$

C $5.155378-1.440150-2.049893$

C $6.356281-1.508078-1.455663$

C $6.584798-2.151826-0.118550$

C $7.049448-1.1599580 .929714$

C $8.221154-1.3331871 .564031$

C $6.1686850 .031416 \quad 1.250396$

C $4.794394-0.3688901 .710610$

N $4.0747300 .421006-3.087609$

H $0.981426-3.4992021 .689694$

H $-2.077270-3.6019021 .855263$

H $-1.303674-2.6733174 .137325$

H -2.954934 -1.419187 2.655254

H -1.619154 -0.6195361 .862649$

H $-1.5960861 .985077 \quad 4.535274$

H -2.831993 0.5339015 .824358

H $-3.800590-0.5890194 .865702$

H -2.308132 -1.142418 5.653271

H $-0.003128 \quad 1.0014412 .175051$

H -1.4538991 .9033631 .719805$

H $-0.597843 \quad 3.7670533 .347005$

H 0.8924112 .8306003 .425904

H 1.2064044 .5888211 .862521

H $-1.191174 \quad 5.058628 \quad 1.271509$

H $-1.275273 \quad 3.5614010 .372385$

H $-1.8410005 .803018-0.984810$

H $\quad 0.2725323 .249860-1.742716$

H $-0.220848 \quad 7.067800 \quad 0.160577$

H $0.8271206 .919301-1.230932$

H $1.3914836 .376577 \quad 0.361464$

H $0.7588615 .071194-2.994930$

H $3.4176351 .851275-0.354328$

H $2.0239771 .469871-1.324766$

$\begin{array}{llll}\text { H } 3.727069 & 4.002059 & 0.240004\end{array}$

H $2.846460-0.9201575 .299886$

H $2.391493-1.0754502 .259885$

H $5.215782-1.1139594 .833402$

H 4.7807850 .6122004 .774814 
H $\quad 6.2225600 .019626 \quad 3.957722$

H $2.8561612 .737320-3.216056$

H $4.0176613 .623969-2.223388$

H $5.846058-0.403123-3.816475$

H $4.382954-1.389349-4.043216$

H $4.291918-1.890613-1.565975$

H $7.218728-1.054554-1.938751$

H $7.327782-2.948021-0.254769$

H $5.676122-2.6516860 .238716$

H $8.566193-0.6321962 .316883$

H $8.863192-2.1803541 .346502$

H $\quad 6.0630280 .6549160 .353406$

H $6.6480450 .690571 \quad 1.982563$

H $4.108768-0.5860110 .890742$

H $3.0678200 .287081-3.103451$

C $0.452765-4.220865-0.968309$

O $-0.097472-2.926787-1.281247$

C $-0.416834-2.875103-2.681048$

C $0.279776-4.074585-3.312841$

C $0.191539-5.095376-2.194684$ H -1.502195 -3.014432 -2.756999 C $-0.032698-1.511462-3.268288$ H $-0.211051-4.401835-4.234400$ H 1.329562 -3.862614 -3.545719 C $1.163507-6.252359-2.344722$ H $-0.832884-5.490547-2.165042$ C $-0.536552-0.291082-2.468405$ O $1.395403-1.407548-3.334367$ H $-0.382343-1.454257-4.305549$ H $0.066596-0.172204-1.560203$ O $-0.2834620 .878678-3.257228$ C -2.023261 -0.330172 -2.078042 C $-2.4915230 .987406-1.427107$ H $-2.188589-1.162832-1.385135$ O $-2.796097-0.581830-3.251441$ H -1.703775 $1.378793-0.773452$ H -2.681981 $1.723493-2.217734$ H $0.964863-6.803988-3.269412$ H $2.201087-5.903046-2.377739$ H $1.069460-6.951046-1.507381$ C $-0.190893-4.7659820 .309383$ H $1.740697-1.536391-2.434083$ H $-0.8600220 .834782-4.039403$ H -3.728757 $-0.638257-2.982140$ H $\quad 0.272916-5.7192830 .585858$ H -1.254844 -4.9608160 .131333$ H $1.530216-4.074941-0.816026$ C $-6.016375-0.078953-0.770487$ O $-4.7980760 .281837-1.433975$ C $-3.7681920 .793504-0.585865$ C -4.2432532 .0910640 .081936$ C $-5.550621 \quad 1.8629510 .848898$ C $-6.592931 \quad 1.1202150 .001456$ H $-6.715219-0.323842-1.580927$ C $-5.841747-1.339808 \quad 0.090620$ H -3.5333490 .0533150 .186708$ O $-4.4342123 .095472-0.911417$ H -3.4679702 .4480090 .767104$ H -5.9549502 .8329451 .162904$ O H -7.4296880 .7826460 .623983$ O $-7.1306832 .027855-0.965380$ C $-5.349052-2.516858-0.725728$ H -5.123769 -1.1708860 .897638$ H $-6.799689-1.6245090 .539653$ H $-4.7047313 .910874-0.456322$ H -6.0898661 .0034572 .517606$ H -7.543569 2.762629 -0.480412 O $-4.785364-3.4380830 .110176$ O $-5.459624-2.625699-1.939452$ C $-4.265433-4.608025-0.528872$ H -3.858236 -5.2648860 .244169$ H -5.063342 -5.139974 -1.055791 H -3.460864 -4.335206 -1.218604 SCF Energy $\left(B 3 L Y P / 6-31 G^{* *} / / M M F F\right)=-3245.90999762$

0700460

MM̄MF Geometry
C $4.061200-1.7710322 .891734$

C $3.049028-0.9042323 .051944$

C $1.611498-1.2184733 .393518$

O $0.765541-0.6738792 .349635$

C $1.260117-2.7149263 .514421$

C $-0.143084-2.9579714 .041485$

C -1.199658 -3.333837 3.293140

C $-0.298958-2.8043595 .533824$

C -1.260744 -3.534054 1.802061

C $-2.484032-2.8288981 .205573$

C $-2.691009-3.084678-0.296204$

C $-1.541399-2.551089-1.154530$

C $-1.828310-2.700143-2.655298$

O $-3.911469-2.420762-0.652447$

O $-0.886279-1.889339-3.373314$

C $-3.224834-2.126077-2.963776$

C $-1.601997-4.141439-3.126953$

O $-3.593283-2.391238-4.315699$

C $-4.334105-2.622445-2.006664$

C $-5.623183-1.796727-2.231623$

O $-4.669791-3.988482-2.258260$

C 0.3522220 .6165922 .470227

O $0.728330 \quad 1.4402993 .288112$

C -0.7065510 .8292451 .459087$

C $-1.451370 \quad 1.943229 \quad 1.494115$

C -2.6111632 .2346290 .647142$

C $-3.0865331 .147839-0.282027$

C $-6.821361-2.241731-1.386470$

C $-6.772503-1.7722380 .053855$

C -7.1888540 .2104891 .477587$

O $-6.611087-2.5610890 .982481$

C -6.8318151 .6620741 .424288$

C -6.0919072 .2606302 .370313$

C -5.6982993 .7111542 .437933$

C -5.5131204 .4247051 .113356$

C -6.2296695 .5257880 .829612$

C -4.4855283 .9083820 .119748$

C $-3.215363 \quad 3.4348350 .775234$

N $-6.949583-0.4017310 .183844$

H $3.888570-2.8363603 .003539$

H 3.2700310 .1567012 .949914

H $1.382381-0.7211624 .344556$

H $1.959075-3.2138184 .198329$

H $1.391094-3.2057902 .543168$

H -2.141170 -3.523235 3.808258

H $-1.311667-3.045125 \quad 5.874285$

H $\quad 0.390969-3.4733276 .058919$

H $-0.087063-1.7754225 .839869$

H $-0.354887-3.1641321 .316798$

H -1.327147 -4.609491 1.600912

H $-3.391684-3.1541341 .733218$

H -2.422449 -1.749439 1.390413

H $-2.819442-4.162933-0.446290$

H $-0.594801-3.044949-0.907448$

H -1.388190 -1.486601 -0.939209

H $\quad 0.007178-2.172823-3.115355$

H -3.157224 -1.032909 -2.869578

H $-0.554669-4.432198-2.979463$

H -1.791304 -4.242380 -4.201138

H -2.220057 -4.867531-2.594325

H $-2.884144-2.042628-4.882576$

H -5.415147 -0.736102 -2.045170

H -5.925945 -1.893489 -3.282089

H -4.202539-4.283346 -3.056011

H -0.8833410 .0322540 .748660$

H -1.2164502 .7014972 .240048$

H -2.283803 $0.842794-0.962081$

H -3.919881 $1.445816-0.920694$

H -3.4171220 .2747180 .289775$

H -7.740133 -1.834429 -1.828202

H $-6.940346-3.330604-1.396422$

H -6.627601 -0.331705 2.246016

H -8.2568020 .0984151 .692967$

H $-7.2233102 .243870 \quad 0.594262$

H -5.7535301 .6598203 .213066$

H -4.7576043 .7824582 .998750$

H -6.440396 4.2268143 .061273

H $-6.1041996 .055642-0.108980$ 
H -6.9608205 .9216041 .526729$ H -4.214069 $4.700929-0.589410$ H -4.964932 $3.122250-0.468050$ H -2.772642 4.1667561 .452413 H $-7.2399110 .117667-0.640050$ C $5.947236-1.7235301 .178712$

O $5.131075-1.0591870 .193012$

C $4.814198-2.003113-0.844918$

C $5.808767-3.147162-0.684357$

C $5.934925-3.211808 \quad 0.825201$

H $3.808009-2.381399-0.628093$

C $4.830916-1.328885-2.218695$

H $5.445805-4.079637-1.126854$

H $6.779027-2.909715-1.135874$

C $7.160555-3.9682511 .306628$

H $5.038671-3.7046421 .219813$

C $3.918329-0.091772-2.350131$

O $6.166584-0.907420-2.525053$

H $4.568895-2.065819-2.987048$

H $4.3610790 .756414-1.816378$

O $3.8935460 .273590-3.736584$

C $2.468062-0.299390-1.877509$

C $1.4779660 .808161-2.287800$

H $2.453607-0.401781-0.786699$

O $1.968210-1.529902-2.409382$

H $1.3098590 .771189-3.372022$

H $\quad 0.5109300 .552894-1.833265$

H $7.127459-5.0090730 .968397$

H $8.084650-3.5208920 .925340$

H $7.211719-3.9690902 .400031$

C $5.475207-1.3538922 .587092$

H $6.454240-0.307291-1.815655$

H $4.8144520 .418549-4.014190$

H $2.062820-1.488270-3.376776$

H $5.569597-0.2677452 .711353$

H $6.137911-1.8119313 .330516$

H $6.960842-1.3225511 .046717$

C 2.4623153 .5090670 .102294

O $2.0207292 .262219-0.452832$

C $1.8903412 .230114-1.875910$

C $0.8729463 .281249-2.348147$

C $1.2394994 .675816-1.831202$

C $1.5430804 .666786-0.329405$

H 2.3599373 .3837981 .187905

C $3.9455123 .780000-0.200279$

H $2.8587582 .451593-2.333878$

O $-0.4288502 .926324-1.890222$

H $0.8486823 .280715-3.444678$

H $0.4081225 .362893-2.031569$

O $2.3730255 .144513-2.555722$

H $1.9865335 .623535-0.029647$

O $\quad 0.300006 \quad 4.5520250 .366446$

C 4.8607342 .7633420 .447142

H $4.1496423 .762278-1.273800$

H 4.2236574 .7639020 .194699

H -1.050440 $3.599849-2.214509$

H $2.5742726 .041024-2.237707$

H $0.489346 \quad 4.596140 \quad 1.319112$

O $\quad 6.1484543 .029766 \quad 0.077341$

O 4.5001321 .8552691 .181738

C 7.1400622 .1445590 .607595

H $7.0424991 .159403 \quad 0.143877$

H 8.1246292 .5518740 .362589

H 7.0537972 .0710971 .696013

SCF Energy (B3LYP/6-31G**//MMFF) $=-3245.90567517$

0700461

MM̄FF Geometry

C -2.596487 -4.269176 -0.462455

C $-1.680252-3.734700 \quad 0.356845$

C $-0.255094-3.467988-0.046659$

O $0.023791-2.0651370 .157496$

C $0.706803-4.2677420 .846895$

C $2.150562-4.1718570 .391872$

C $3.082479-3.365848 \quad 0.939407$

C $2.528052-5.103800-0.731714$

C $2.900641-2.3668692 .049856$

C $3.408088-0.9503051 .720194$
C $4.937730-0.8020721 .792325$

C $5.3372840 .675707 \quad 1.814384$

C 6.8639780 .8559661 .812690

O $5.511429-1.4320210 .645495$

O 7.1439432 .2344931 .522942

C 7.4625100 .0220880 .660088

C 7.4783120 .5694023 .187557

$\begin{array}{lllll}\text { O } & 8.886939 & 0.028227 & 0.703388\end{array}$

C $6.944245-1.4317910 .616950$

C $7.396979-2.199926-0.651289$

O $7.468314-2.1512971 .741108$

C $-0.278252-1.210727-0.855778$

O $-0.838972-1.478197-1.905872$

C $0.2339930 .121526-0.467909$

C $0.1790161 .137507-1.340663$

C $0.7012192 .488598-1.123396$

C 1.3724922 .7732640 .195698

C $7.127711-1.534291-2.003354$

C $5.656116-1.268798-2.255733$

C $4.0518530 .551965-2.784348$

O $4.819811-2.169292-2.235928$

C $3.9685092 .000176-2.423566$

C $3.6558352 .972055-3.294108$

C $3.5466074 .424425-2.921412$

C $2.1750904 .998865-3.208408$

C $1.9467085 .717588-4.320406$

C $1.0912654 .804474-2.168142$

C $0.590313 \quad 3.386834-2.124676$

N $5.3864750 .070959-2.486689$

H -2.310112 -4.539678-1.475992

H $-1.964743-3.4760431 .372287$

H -0.086504 -3.729632 -1.098992

H $\quad 0.418237-5.3276370 .854351$

H $0.614094-3.9365521 .887957$

H $4.095310-3.444500 \quad 0.551560$

H $3.585350-5.020828-1.005428$

H $2.343798-6.143144-0.440821$

H $1.939687-4.883331-1.627778$

H $1.843655-2.2727652 .316690$

H $3.411836-2.7416082 .944597$

H $3.051300-0.6572620 .725198$

H $2.954257-0.2650372 .447334$

H $5.292578-1.3018652 .701019$

H 4.8941641 .1871712 .677911

H $4.926776 \quad 1.176680 \quad 0.926391$

H 6.7086652 .7777172 .202057

H $7.1797770 .532191-0.267970$

H 7.0569661 .2437133 .943033

H 8.5573060 .7599923 .185140

H $7.309371-0.4537623 .529374$

H 9.1723950 .9576180 .725831

H $8.472241-2.410725-0.580731$

H $6.903230-3.181767-0.650913$

H $7.421630-3.1018951 .544691$

H $0.6677650 .223306 \quad 0.520291$

H $-0.2681300 .959629-2.317901$

H $0.6575582 .674527 \quad 1.019194$

H $1.7968213 .776548 \quad 0.264538$

H 2.2008452 .0769530 .367571

H $7.720350-0.619160-2.100634$

H $7.470291-2.198766-2.806448$

H $3.321510-0.025192-2.207707$

H $3.8658280 .373058-3.848496$

H $4.1529402 .258258-1.383461$

H $3.4716482 .714829-4.334677$

H $4.3100794 .972540-3.488726$

H $3.7929374 .586533-1.864581$

H $0.9743666 .154500-4.522066$

H $2.7265215 .880792-5.056940$

H $0.2290105 .446121-2.393152$

H $1.4615415 .159334-1.202825$

H $0.0886233 .078305-3.043162$

H $6.1642740 .701257-2.659923$

C $-5.004863-3.832461-0.985441$

O $-5.057068-2.425799-0.663395$

C $-6.408294-1.960573-0.866263$

C -7.195498 -3.144349-1.422584

C $-6.443027-4.322350-0.840952$ 
H $-6.810504-1.7060520 .120321$

C $-6.423550-0.739954-1.792438$

H -8.247981 -3.116241-1.123554

H -7.162873 -3.169816 -2.518603

C $-6.734627-5.631738-1.553575$

H $-6.703259-4.4192950 .221557$

C $-5.7894550 .535627-1.199425$

O $-5.721906-1.066524-2.999787$

H -7.453561 -0.521969-2.098106

H $-4.7178010 .384441-1.027097$

O $\quad-5.8985291 .563408-2.194191$

C -6.4674251 .0419910 .092640$

C $-6.1229062 .493200 \quad 0.480311$

H -6.2194410 .3727410 .923345$

O

H $-6.3931303 .166387-0.342881$

H -6.7797122 .7738401 .315432$

H -7.800105 $-5.875627-1.488013$

H -6.466353 -5.579925 -2.614145

H $-6.173697-6.456969-1.104548$

C $-4.018491-4.548061-0.063159$

H -4.811834 -1.305624 -2.752927

H $-5.4641221 .233678-2.999619$

H -8.126371 $1.559152-0.813185$

H -4.165904 -5.632264 -0.118941

H -4.191095 $-4.243340 \quad 0.976438$

H -4.681792 -3.911444 -2.032488

C -3.1612212 .1683442 .724201$

$\begin{array}{llll}\text { O } & -4.475298 & 2.077080 & 2.158444\end{array}$

C -4.6587692 .7111360 .887756$

C -4.3193124 .2080680 .974729$

C $-2.909656 \quad 4.4233631 .531934$

C -2.6853093 .6284012 .819564$

H -3.2742961 .7872423 .747285$

C $-2.174476 \quad 1.257723 \quad 1.988837$

H -4.0062182 .2399660 .146950$

O $\quad-5.2720694 .8681581 .805741$

H $-4.3995704 .644866-0.027916$

H -2.753966 5.4894851 .738223

O

H -1.624916 3.6569913 .097163

O -3.4077794 .2773863 .869890$

C -2.474831 -0.1905972 .290079$

H -2.206149 1.3770820 .903037

H -1.142391 1.4601022 .297862

H -5.0409135 .8122801 .825056$

H -2.086071 $4.556259-0.233785$

H -3.208886 3.8079714 .697815

O $-1.853039-0.5555113 .447026$

O $-3.192667-0.8947091 .592845$

C - $-2.069922-1.8934213 .908867$

H -1.277734 -2.537215 3.518485

H -2.009797 -1.887340 5.000596

H -3.053020 -2.277092 3.619749

SCF Energy (B3LYP/6-31G**//MMFF) $=-3245.89914220$

0700462

MM̄MF Geometry

C -1.141375 4.900113-0.450730

C $-2.037624 \quad 4.4154390 .421729$

C -3.0997573 .4148300 .047703$

O $-2.879417 \quad 2.212966 \quad 0.836164$

C -4.5156703 .9344180 .370528$

C $-5.3112434 .255020-0.881789$

C $-6.2723083 .458856-1.392601$

C $-4.9606675 .554319-1.557328$

C $-6.7374662 .143459-0.830752$

C $-5.8062481 .000478-1.233024$

C $-6.159657-0.364018-0.622656$

C $-6.152931-0.3407850 .909353$

C $-6.319958-1.7395101 .518428$

O $-5.161482-1.270140-1.112942$

O $-5.986028-1.6557672 .912858$

C $-5.294608-2.6855630 .867031$

C -7.771967 -2.225554 1.448371

O $-5.489868-4.0284431 .304910$

C -5.298502 -2.626163 -0.676508

C $-4.129077-3.436069-1.286829$
O $-6.516841-3.201485-1.160974$

C $-1.874767 \quad 1.3853240 .432881$

O $-1.158924 \quad 1.524433-0.546309$

C -1.7580020 .2866051 .416051$

C $-0.588271-0.3635411 .514715$

C $-0.263874-1.4473562 .444070$

C $-1.386681-2.0340303 .257674$

C $-2.740303-2.845048-1.027038$

C $-1.623757-3.754315-1.507222$

C $0.863624-3.913758-1.467628$

O $-1.818759-4.861565-2.000146$

C $1.266194-4.470805-0.138399$

C $2.379913-4.1105180 .515693$

C $2.755978-4.6361831 .869744$

C $2.855650-3.5396382 .907445$

C $4.046879-3.1597903 .399875$

C $1.566965-2.9370663 .433352$

C $1.020466-1.8530522 .541342$

N $-0.376801-3.183104-1.289085$

H -1.196315 $4.603586-1.495267$

H $-1.9667674 .693047 \quad 1.470810$

H -3.032252 3.139778 -1.013246

H -4.4783914 .8367250 .995600$

H -5.0459613 .1970010 .983184$

H $-6.7764693 .769938-2.305772$

H $-5.6019075 .758961-2.421270$

H -5.075682 $6.388817-0.857906$

H $-3.9259535 .536427-1.912436$

H -6.8345092 .2265240 .255161$

H -7.748845 $1.940666-1.203817$

H $-5.7999860 .902768-2.327553$

H $-4.7692281 .240145-0.969469$

H -7.139933 -0.675098 -1.001767

H -6.9337290 .3209291 .300846$

H $-5.200346 \quad 0.079293 \quad 1.260747$

H $-6.576511-1.0012823 .323868$

H $-4.308877-2.3813761 .237195$

H -8.429379 -1.549580 2.008387

H -7.882929 -3.2085731 .919222$

H $-8.155868-2.2848000 .427903$

H $-5.474782-4.0213392 .277367$

H -4.180674 -4.476108 -0.940183

H $-4.263911-3.491267-2.376330$

H -6.382898 -3.479915 -2.081821

H -2.613152 0.0715312 .045337

H $0.229802-0.0565340 .863936$

H -2.145749 -2.474254 2.602659

H -1.860167 -1.263940 3.876456

H -1.058898 -2.820197 3.942459

H -2.641919-1.878931-1.534911

H $-2.580922-2.6879440 .043419$

H $1.602486-3.213575-1.865443$

H $\quad 0.724087-4.724990-2.188686$

H $0.594832-5.196807 \quad 0.314684$

H $3.057091-3.3911550 .064036$

H $3.716401-5.1581351 .770348$

H $2.041611-5.3928922 .217163$

H $4.123660-2.3906054 .161244$

H $4.972234-3.6024673 .046657$

H $1.744815-2.4997934 .424365$

H $0.843638-3.7473473 .573731$

H $1.769042-1.3316691 .944409$

H $-0.325110-2.282329-0.821544$

C $1.3270135 .015449-0.177871$

O $1.340528 \quad 3.9356190 .777415$

C 2.6610273 .8294511 .350491

C 3.5458444 .7640070 .538159

C 2.5642695 .8497610 .139869

H 2.5679204 .1919442 .381780

C 3.0847392 .3561791 .332213

H 4.3955585 .1431111 .113765

H $3.9364354 .255744-0.352567$

C $3.0459736 .709863-1.015001$

H 2.3735826 .4890391 .012225

C $4.5323222 .106217 \quad 1.783969$

O 2.1943381 .6340232 .189791

H $2.929078 \quad 1.9577060 .323803$

H 5.2293082 .6163031 .110719 
O $4.709140 \quad 2.716940 \quad 3.069760$

C $4.9121780 .617047 \quad 1.914667$

C $4.620228-0.2520060 .676042$

H 4.3849580 .1892092 .776295

$\begin{array}{lllll}\text { O } & 6.302982 & 0.556499 & 2.235442\end{array}$

H $4.869389-1.2893600 .928845$

H $3.539722-0.2406080 .497603$

H $3.9738327 .226474-0.748551$

H $3.2409276 .108480-1.909313$

H $2.3005227 .467863-1.274713$

C $0.0094105 .783652-0.058223$

H 2.3407751 .9487253 .098345

H 5.6283832 .5538493 .342778

H $6.542976-0.3816342 .325245$

H $0.0140886 .662724-0.711593$

H $-0.122918 \quad 6.1391330 .971219$

H $1.4156894 .560777-1.173809$

C $3.975170-1.054102-2.166958$

O $4.485050 \quad 0.212082-1.731395$

C $5.3818520 .147895-0.611314$

C $6.588125-0.735091-0.973244$

C $6.167492-2.108246-1.493599$

C $5.126339-1.968051-2.602611$

H $3.415995-1.526421-1.351876$

C $3.005303-0.791912-3.328286$

H $5.759646 \quad 1.167507-0.486441$

O $7.451703-0.8892380 .145933$

H $7.168337-0.228569-1.755940$

H $5.801222-2.742738-0.678038$

O $7.324602-2.762814-2.019328$

H $5.610641-1.565251-3.500849$

O $4.630120-3.261574-2.941152$

C $1.691472-0.205951-2.854781$

H $3.445638-0.085789-4.041526$

H $2.772001-1.731422-3.841560$

H $8.190526-1.457419-0.131673$

H $7.033483-3.615869-2.384967$

H $4.191105-3.629364-2.155427$

O $0.809314-0.203916-3.898126$

O $1.4663630 .203526-1.724567$

C $-0.4922880 .313074-3.605311$

H $-1.1046250 .222740-4.506404$

H $-0.960463-0.267523-2.804964$

H $-0.424903 \quad 1.369982-3.331409$

SCF Energy (B3LYP/6-31G**/MMFF) $=-3245.90669234$

07_00463

MM̄FF Geometry

C -0.567479 4.417068 -0.121804

C $-1.4238944 .557839-1.145901$

C $-2.6424953 .691158-1.389632$

O $-2.5656092 .471636-0.615727$

C $-3.9252164 .434765-0.980079$

C $-5.1923823 .665706-1.306044$

C -6.052853 $3.178466-0.390210$

C $-5.4796613 .473090-2.773534$

C $-5.978957 \quad 3.293047 \quad 1.105890$

C $-5.2692092 .127988 \quad 1.811935$

$\begin{array}{llll}C & -5.946451 & 0.760450 & 1.620558\end{array}$

C $-5.339155-0.2741872 .571060$

C $-5.928798-1.6741272 .351039$

$\begin{array}{lllll}\text { O } & -5.748928 & 0.336334 & 0.269047\end{array}$

O $-5.5 .087145-2.5970103 .059965$

C $-5.858877-2.0246450 .848259$

C $-7.324275-1.8005872 .974401$

O $-6.545886-3.2512790 .612805$

C $-6.394306-0.901698-0.067753$

C $-6.133084-1.154544-1.573898$

O $-7.813935-0.800698 \quad 0.098536$

C -1.821665 $1.456264-1.131199$

O $-1.2935551 .412438-2.230431$

C $-1.7109510 .413907-0.089503$

C $-0.669581-0.429549-0.122212$

C $-0.407469-1.5148250 .823684$

C $-1.498564-1.8633151 .801027$

C $-4.661670-1.317155-1.969815$

C $-4.186626-2.759710-2.035976$

C $-2.118745-4.103372-2.416059$
O $-4.943109-3.723034-1.949500$

C $-1.528486-4.537741-1.111286$

C $-0.235429-4.864417-0.961228$

C $0.364113-5.3598970 .324406$

C $1.550625-4.5358720 .773219$

C $2.808921-4.9285170 .511324$

C $1.275086-3.2967371 .594845$

C $0.776559-2.1571140 .750593$

N -2.815673 -2.842080 -2.242730

H $-0.7653393 .666900 \quad 0.639984$

H $-1.2298565 .323147-1.893916$

H -2.676491 $3.443659-2.457396$

H $-3.9738055 .407258-1.487525$

H -3.8740274 .6551210 .091707$

H $-6.9288932 .639332-0.749538$

H $-6.4728663 .045750-2.949732$

H -5.441294 $4.433575-3.297751$

H $-4.7482162 .796573-3.225848$

H $-5.4956664 .231773 \quad 1.397274$

H $-7.004523 \quad 3.3727661 .489040$

H -4.2319122 .0793901 .462033$

H -5.2364152 .3643882 .882966$

H -7.0190350 .8754011 .815504$

H -5.4662400 .0338653 .616432$

H -4.254092 -0.324739 2.406068

H $-5.409990-3.4948672 .870324$

H -4.801615 -2.206694 0.622097

H -7.276155 -1.618145 4.054850

H -7.719854 -2.815977 2.861090

H -8.047980 -1.100551 2.551970

H $-6.370865-3.521094-0.303917$

H -6.743516 -1.992415 -1.931190

H $-6.508414-0.274551-2.116595$

H -8.184842 $-0.355543-0.681507$

H -2.4717850 .3846190 .680808$

H $0.071872-0.308989-0.910926$

H -2.440789 -2.047199 1.273691

H -1.649209-1.047065 2.515676

H -1.295831 -2.766002 2.380377

H -4.528287 -0.907215 -2.979213

H $-4.012564-0.750285-1.298180$

H -1.354391 -3.947269 -3.183877

H -2.820474 -4.863680 -2.773656

H -2.201567 -4.618931 -0.260977

H $0.428336-4.807871-1.821312$

H $0.673011-6.4016650 .167284$

H $-0.382307-5.3928351 .127911$

H $3.663566-4.3536210 .852355$

H $3.011628-5.830695-0.056311$

H $2.195293-2.9605052 .089490$

H $0.597656-3.5558102 .412670$

H $1.500015-1.8237400 .005396$

H $-2.263093-1.989806-2.261090$

C $1.915534 \quad 4.2916700 .034715$

O $1.8590263 .378661 \quad 1.148005$

C $3.1788103 .238717 \quad 1.714429$

C 4.1259353 .9121320 .732995

C 3.2509395 .0177110 .171850

H 3.1521183 .7887282 .662978

C 3.4524321 .7507071 .958383

H 5.0345054 .2861831 .213830

H $4.4221743 .216464-0.062490$

C $3.7733195 .601268-1.129314$

H 3.1694185 .8171450 .920383

C 4.8662111 .4279182 .479352

O 2.4852581 .2772062 .903521

H 3.2588831 .2037331 .028911

H $5.629051 \quad 1.7280611 .752340$

O 5.1173922 .1901543 .667254

C $5.065005-0.0538752 .857298$

C $4.730866-1.0607501 .748031$

H $4.448250-0.2923923 .732713$

O $6.415652-0.253174 \quad 3.275888$

H $4.895547-2.0767972 .129440$

H $3.654485-1.0145571 .542502$

H $4.7628856 .045775-0.981059$

H $3.8625674 .834051-1.905906$

H $3.104616 \quad 6.382792-1.503169$ 
C $\quad 0.693944 \quad 5.214694 \quad 0.061822$

H $2.657647 \quad 1.7249203 .749562$

H 5.2146403 .1214173 .406713

H $6.597693 \quad 0.3871043 .985011$

H $0.7825245 .982454-0.714732$

H 0.6291895 .7254551 .029935

H $1.9058773 .681506-0.876645$

C $5.274427-1.687145-1.878854$

O $4.808369-1.750566-0.524610$

C $5.487554-0.8998940 .412725$

C $6.979867-1.2696820 .472315$

C $7.623020-1.313161-0.918346$

C $6.764970-2.057420-1.946975$

H $4.710087-2.465615-2.408427$

C $4.957833-0.339848-2.546461$

H $5.3762980 .134377 \quad 0.076582$

O $7.165659-2.5303591 .112133$

H $7.510743-0.5265441 .076055$

H $8.604712-1.796794-0.842266$

O $7.8344920 .029145-1.351407$

H $7.140991-1.863527-2.958029$

O $6.907052-3.464620-1.734004$

C $3.471648-0.066099-2.622533$

H $5.4154830 .492471-2.005472$

H $5.342008-0.338271-3.572922$

H $6.515810-3.154098 \quad 0.749522$

H $8.288417-0.008008-2.210553$

H $6.237249-3.747385-1.089779$

O $3.283900 \quad 1.238392-2.983610$

O $2.589504-0.886166-2.411462$

C $1.9202791 .654922-3.088882$

H $1.392513 \quad 1.052152-3.833989$

H $1.4331101 .579692-2.113017$

H $1.9062692 .700228-3.408611$

SCF Energy (B3LYP/6-31G*//MMFF) $=-3245.91387922$

$07 \quad 00464$

MM̄FF Geometry

C $1.145392-3.2734210 .574950$

C $0.179744-3.6598821 .421385$

C $-0.712186-2.6976082 .159028$

O $-2.069205-3.067843 \quad 1.823424$

C $-0.499637-2.7772953 .683015$

C $0.900589-2.4151024 .132678$

C $1.377572-1.1568824 .200410$

C $1.770742-3.5763464 .538626$

C $0.655748 \quad 0.122722 \quad 3.876212$

C $1.260510 \quad 0.8027602 .639950$

$\begin{array}{llll}C & 0.707930 & 2.208734 & 2.345992\end{array}$

C $1.187148 \quad 3.241224 \quad 3.369547$

C $0.6075444 .635523 \quad 3.089613$

O $-\begin{array}{llll}0.720591 & 2.153331 & 2.321830\end{array}$

$\begin{array}{lllllllllllll} & 0 & 0.840708 & 5.448811 & 4.250283\end{array}$

C $-0.920407 \quad 4.5085022 .923445$

C 1.3225275 .3302721 .925706

O \begin{tabular}{llll}
\hline & -1.492175 & 5.758385 & 2.540147
\end{tabular}

C -1.3582613 .3850441 .950640$

C -2.8909893 .1836882 .064708$

O $-1.020548 \quad 3.7708730 .619373$

C -3.035485 -2.127894 1.999602

O $-2.918469-1.0449872 .549166$

C $-4.258822-2.6214111 .331441$

C $-5.284164-1.7798071 .136882$

C $-6.509825-2.0994140 .411597$

C $-7.744494-1.5175941 .043097$

C -3.4674192 .0339301 .228498$

C $-3.9154172 .430758-0.168614$

C $-4.9693131 .447504-2.202592$

O $-3.7698503 .558089-0.631434$

C $-6.2202270 .638690-2.338428$

C $-6.344824-0.411226-3.164286$

C $-7.616120-1.194276-3.334716$

C $-7.437012-2.670813-3.059549$

C $-7.190697-3.534570-4.059075$

C $-7.631175-3.155771-1.638524$

C $-6.478364-2.792126-0.745071$

$\mathrm{N}-4.5056911 .362643-0.831540$

H $1.289714-2.2140340 .381683$
H $\quad 0.039042-4.7191071 .624164$

H $-0.536490-1.6764671 .798732$

H $-0.757557-3.7857414 .035911$

H $-1.222563-2.1338784 .199331$

H $2.402226-1.0152844 .542846$

H $2.770319-3.2578204 .853027$

H $1.316223-4.112668 \quad 5.378017$

H $1.896514-4.2765813 .707417$

H $-0.414092-0.0453003 .721143$

H 0.7413670 .7733814 .752760

H 2.3532210 .8567982 .726952

H 1.0483230 .1667201 .770700

H 1.0656832 .4885821 .348098

H 2.2830813 .2795243 .399073

H $0.8711042 .945276 \quad 4.377921$

H 1.7999515 .4776304 .408313

H -1.3305874 .2837313 .917724$

H 2.3968515 .4121322 .129809

H $\quad 0.964543 \quad 6.357921 \quad 1.798722$

H 1.2058494 .8036170 .977506

H -1.316324 5.8854721 .592261

H -3.1184782 .9497203 .113617$

H -3.414348 $4.115268 \quad 1.819055$

H $-1.507170 \quad 3.2035910 .000763$

H $-4.268540-3.6419470 .963743$

H $-5.237177-0.7654231 .526916$

H -7.822612 -1.8354062 .089303$

H -8.671215 -1.8227350 .553537$

H $-7.706162-0.4228301 .018961$

H $-2.755373 \quad 1.2074101 .148038$

H -4.3592261 .6559281 .744754$

H $-4.1617391 .077617-2.842588$

H $-5.1658972 .490987-2.467975$

H $-7.0739480 .949923-1.740793$

H $-5.496399-0.708610-3.776520$

H $-7.960827-1.048539-4.366966$

H $-8.418961-0.798943-2.700062$

H $-7.089515-4.599029-3.875509$

H $-7.079520-3.194497-5.083387$

H -7.721796 -4.249810-1.622665

H -8.590468 -2.779589-1.273379

H $-5.513694-3.145089-1.111455$

H $-4.4323560 .438139-0.417586$

C $3.528939-3.949162 \quad 0.329614$

O $3.993875-2.727309-0.279598$

C $5.379009-2.888981-0.644941$

C $5.821354-4.227515-0.064106$

C $4.527612-5.018227-0.105179$

H $5.412507-2.957502-1.738083$

C $6.191576-1.681926-0.163040$

H $6.625107-4.686578-0.647832$

H $6.174260-4.1250300 .968767$

C $4.545289-6.2507840 .782089$

H $4.328974-5.319341-1.142549$

C $5.699066-0.329327-0.721474$

O $6.119327-1.6133561 .267697$

H $7.251027-1.824084-0.404143$

H $4.695952-0.116091-0.335944$

$\begin{array}{lllll}\text { O } & 6.556284 & 0.697675 & -0.207731\end{array}$

C $5.706957-0.240267-2.259377$

C $5.3675351 .156233-2.816321$

H $5.008055-0.973532-2.675169$

O $7.012072-0.594734-2.726819$

H $6.079758 \quad 1.897115-2.432887$

H $5.5310571 .123104-3.901976$

H $5.335521-6.9398790 .466673$

H $4.726647-5.9885501 .829852$

H $3.591813-6.7854090 .729453$

C $2.089692-4.223181-0.107593$

H $5.180110-1.5415561 .510711$

H 6.5509060 .6216030 .761874

H $6.984565-0.601795-3.698805$

H $1.814466-5.2585710 .122616$

H $1.988080-4.084782-1.190668$

H $3.579379-3.8042861 .417552$

C $1.6538000 .883280-2.942827$

O $3.0518130 .670105-3.176819$

C $3.9254451 .603220-2.530640$ 
C $3.6502733 .026374-3.041796$

C $2.1758963 .401935-2.858996$

C $1.2365622 .301896-3.369003$

H $1.1506630 .173845-3.612692$

C $1.2926720 .504432-1.504390$

H $3.7531051 .573815-1.449991$

O $3.994843 \quad 3.111789-4.423211$

H $4.290875 \quad 3.729147-2.495454$

H $1.9726354 .334130-3.400187$

O $1.9397343 .644143-1.475864$

H $0.2106382 .501836-3.043285$

O $1.2171602 .348090-4.800126$

C $-0.1864740 .262531-1.350261$

H $1.832094-0.401246-1.200778$

H $1.5924321 .264063-0.777472$

H $3.8488294 .030920-4.705408$

H $1.0055453 .895012-1.376278$

H $2.1065802 .114200-5.116942$

O $-0.489165-1.024202-1.681392$

O $-0.981176 \quad 1.126077-1.003947$

C $-1.873532-1.376662-1.595668$

H -2.410023 $-0.967604-2.456371$

H $-1.949418-2.466929-1.616311$

H $-2.314039-1.014764-0.662775$

SCF Energy (B3LYP/6-31G*//MMFF) $=-3245.92147978$

$07 \quad 00465$

MM̄FF Geometry

C -1.351544 -3.821865 -0.326349

C $-1.177188-3.388007-1.585003$

C $-0.018924-2.555865-2.082573$

O $0.616154-1.855638-0.988769$

C $1.025530-3.446599-2.777333$

C $2.229712-2.685415-3.301613$

C $3.505795-2.991181-2.994785$

C $1.939808-1.539964-4.237160$

C $3.988667-4.147032-2.167110$

C $4.147719-3.852035-0.670168$

C $5.219084-2.795188-0.358218$

C $5.662235-2.9055391 .102978$

C $6.684731-1.8226371 .471629$

O $4.660803-1.497219-0.594132$

O $6.813073-1.8046902 .901468$

C $6.122929-0.4519081 .050088$

C $8.078820-2.1326610 .914139$

$\begin{array}{llll}\text { O } & 7.095071 & 0.574822 & 1.237430\end{array}$

C $5.582581-0.415466-0.400867$

C $4.8275420 .915501-0.621932$

O $6.677772-0.490130-1.314037$

C $0.136183-0.611994-0.711404$

O $-0.762739-0.028360-1.297178$

C $0.889470-0.0683880 .440361$

C $\quad 0.636577 \quad 1.191570 \quad 0.824594$

C $1.261016 \quad 1.9206851 .926880$

C 2.2218011 .1774072 .815054

C $4.115397 \quad 1.045667-1.973566$

C $3.2170592 .269644-1.993764$

C $3.2919094 .755670-1.751741$

O $1.9954952 .172731-2.082346$

C $3.3977345 .173999-0.317131$

C 2.3760225 .6730820 .395222

C 2.5060006 .1236281 .823411

C $1.402143 \quad 5.6012952 .717136$

C 0.4354526 .4190923 .167748

C 1.4585014 .1522293 .157676

C $0.919373 \quad 3.212806 \quad 2.113577$

N $3.9228803 .457421-1.900627$

H $-0.590021-3.6115390 .418214$

H -1.929201 -3.632852 -2.332470

H $-0.414432-1.834716-2.808771$

H $0.558949-3.973592-3.619797$

H $1.340928-4.218063-2.066511$

H $4.299852-2.371976-3.410014$

H $2.849700-1.141709-4.699102$

H $1.281216-1.870071-5.047155$

H $1.456038-0.715529-3.704874$

H $3.324102-5.009695-2.292012$

H $4.952926-4.476578-2.575216$
H $3.180824-3.539018-0.256138$

H $4.419113-4.798061-0.184938$

H $6.074649-2.952307-1.025448$

H $6.071794-3.9015151 .313073$

H $4.786671-2.7964971 .758524$

H $7.099264-2.6890673 .187689$

H $5.293182-0.2188071 .731503$

H $8.439216-3.0946651 .298086$

H $8.810541-1.3864401 .243225$

H $8.103962-2.182339-0.176340$

H 7.3933250 .5262032 .161893

H $4.068244 \quad 1.0292300 .160352$

H $5.5296991 .750694-0.510576$

H $6.388195-0.158257-2.178274$

H $1.620284-0.7030000 .927119$

H $-0.103091 \quad 1.7621410 .263342$

H $3.1236650 .898002 \quad 2.264199$

H $1.7566500 .266502 \quad 3.208604$

H 2.5382201 .7581993 .685482

H $4.8291651 .125242-2.800009$

H $3.4861780 .170635-2.164175$

H $2.2536894 .709560-2.095111$

H $3.8452055 .457058-2.383557$

H 4.3740985 .0826850 .153934

H $1.4066435 .798499-0.081694$

H 2.4983967 .2215021 .823514

H 3.4752955 .8316882 .246598

H $-0.345526 \quad 6.059494 \quad 3.829555$

H 0.3992117 .4646972 .880688

H 0.8635634 .0196104 .070467

H 2.4947743 .9142023 .421082

H $\quad 0.165344 \quad 3.645797 \quad 1.454775$

H $4.9364393 .425512-1.844734$

C $-3.181560-4.1144891 .427380$

O $-3.469391-2.702721 \quad 1.368817$

C $-3.150405-2.1207682 .647902$

C $-2.954462-3.2896973 .607981$

C $-2.346354-4.3302402 .689169$

H -2.190165 -1.605307 2.530027

C $-4.234130-1.1292843 .084300$

H $-2.308078-3.0296504 .451815$

H $-3.909391-3.6475774 .010629$

C -2.412584 -5.7432093 .242513$

H -1.296570 -4.0657502 .515700$

C $-4.448530 \quad 0.063720 \quad 2.128541$

O $-5.482973-1.8228123 .204020$

H $-4.001948-0.7581874 .089522$

H $-4.922485-0.2865701 .205434$

$\begin{array}{llll}\text { O } & -5.396828 & 0.943913 & 2.746652\end{array}$

C $-3.1725530 .856502 \quad 1.793109$

C -3.4093372 .2039501 .086279$

H $-2.5148850 .233876 \quad 1.176902$

$\begin{array}{llll} & -2.472382 & 1.130542 & 3.011245\end{array}$

H -3.8943372 .9066601 .775988$

H -2.4189302 .6333830 .886666$

H $-1.860654-5.8129114 .185668$

H -3.445679 -6.051684 3.434922

H -1.972382 -6.458082 2.540452

C $-2.557262-4.6044980 .116083$

H -5.686553 - $2.210722 \quad 2.335558$

H -4.9829431 .3033783 .550163$

H $-1.642117 \quad 1.5795512 .778804$

H $-3.325625-4.551206-0.665482$

H $-2.276747-5.6591560 .216106$

H -4.151712 -4.6163421 .544217$

C $-4.1912910 .960399-2.353684$

O $-3.554611 \quad 1.194508-1.090280$

C $-4.210448 \quad 2.122443-0.223760$

C $-4.3355103 .499149-0.898825$

C $-5.014833 \quad 3.387982-2.266909$

C $-4.3912292 .278183-3.121228$

H $-3.4749770 .351524-2.920739$

C $-5.4865720 .146452-2.202951$

H -5.2118861 .7496290 .012551$

O $-3.039915 \quad 4.074717-1.058910$

H $-4.9124994 .161925-0.242484$

H $-4.9262234 .347697-2.790213$

O $-6.401757 \quad 3.132183-2.067095$ 
H $-5.0002522 .089920-4.013025$

O $-3.1051402 .706600-3.578134$

C $-5.228674-1.256060-1.695871$

H $-6.1828590 .619749-1.506254$

H $-5.9745920 .052089-3.179981$

H $-3.1562914 .955636-1.453632$

H $-6.8205023 .083549-2.943365$

H $-3.2339063 .517103-4.099547$

O $-6.427549-1.882240-1.503262$

O $-4.125543-1.744404-1.498061$

C $-6.352132-3.226818-1.019633$

H -7.363042 -3.642886 -1.013878

H $-5.726221-3.838573-1.676341$

H -5.964573 -3.233785 0.002753

SCF Energy (B3LYP/6-31G**//MMFF) $=-3245.88056980$

0700466

MM̄MF Geometry

C $3.753093-0.6944652 .889430$

C $2.531308-0.5487382 .352983$

C 1.4258750 .2392263 .004568

O $1.157993 \quad 1.3514832 .115179$

C $0.129134-0.5813753 .169380$

C $0.267949-1.8800813 .937445$

C $-0.145721-3.0806503 .483612$

C $0.881104-1.7711225 .310517$

C $-0.884206-3.3719232 .203951$

C $-1.962579-4.4467882 .423371$

C $-3.017071-4.5320631 .307443$

C $-2.412085-4.763026-0.077542$

C $-3.486274-4.778348-1.177586$

O $-3.769678-3.3146651 .328206$

O $-2.816519-4.682004-2.444740$

C $-4.360628-3.513539-1.028687$

C $-4.263703-6.098817-1.200392$

O $-5.447584-3.530241-1.950648$

C $-4.871690-3.278110 \quad 0.410432$

C $-5.566282-1.9056810 .579031$

O $-5.840506-4.283273 \quad 0.730628$

C 0.5508332 .4528782 .631704

O 0.1993042 .6310283 .786088

C $0.3704563 .410147 \quad 1.517877$

C -0.4030594 .4944551 .673545$

C -0.6998735 .4912090 .639760$

C $-0.0449735 .309669-0.706313$

C $-4.674563-0.7045520 .259486$

$\begin{array}{llll}C & -5.250915 & 0.614016 & 0.743981\end{array}$

C -4.6110783 .0051630 .976822$

$\begin{array}{lllll}\text { O } & -6.320407 & 0.707424 & 1.338937\end{array}$

C $-4.011776 \quad 4.0186750 .055434$

C $-4.5757515 .212472-0.182620$

C $-3.9834796 .266889-1.075049$

C $-3.5187567 .480914-0.298337$

C $-4.378770 \quad 8.458518 \quad 0.034026$

C -2.0440147 .5944240 .027178$

C $-1.559883 \quad 6.4906890 .930846$

N $-4.4078761 .675350 \quad 0.433584$

H $3.975051-0.2362433 .850040$

H $2.316102-0.9965531 .385164$

H 1.7573320 .6314963 .974612

H $-0.288493-0.7442012 .170220$

H -0.6205750 .0359373 .681694$

H $\quad 0.038863-3.9523144 .110795$

H $\quad 0.816728-2.7110825 .869294$

H $1.938342-1.4991545 .248838$

H $0.358257-1.0085325 .897316$

H -1.358307 -2.470209 1.808260

H $-0.162573-3.7253051 .458724$

H -1.476921 -5.4240252 .539271$

H -2.487966 -4.235249 3.364721

H -3.689031 -5.3588601 .567076$

H -1.823699 -5.688569 -0.096566

H -1.709848 $-3.953957-0.313970$

H -2.201467 -5.431997 -2.516661

H -3.728485 -2.668424 -1.324678

H -3.580609 -6.941903 -1.359582

H -4.970296 -6.126475 -2.037101

H $-4.812673-6.290535-0.276323$
H $-6.116567-4.147508-1.608588$

H -6.480488 -1.863768 -0.026937

H $-5.903598-1.8223761 .621768$

H $-6.396958-3.9548711 .456378$

H 0.8664703 .1800040 .582475

H -0.8834914 .6581522 .637146$

H $1.0462775 .325597-0.607834$

H $-0.3032716 .083346-1.431174$

H $-0.3442344 .355368-1.153385$

H $-3.700480-0.8267750 .744962$

H -4.520511 $-0.627286-0.822271$

H -4.0966063 .0328401 .943266$

H $-5.679086 \quad 3.175278 \quad 1.147777$

H -3.061762 $3.777488-0.414998$

H -5.5206625 .4559420 .298637$

H $-4.7476866 .563924-1.805064$

H -3.155138 $5.865849-1.672065$

H -4.0515299 .3441040 .568292$

H $-5.4316118 .392029-0.220035$

H -1.8368548 .5444420 .537232$

H $-1.4872147 .649649-0.911859$

H -1.987070 6.5317721 .933785

H -3.4838881 .4561350 .071846$

C $5.974980-0.5093761 .745099$

O $5.434647 \quad 0.344144 \quad 0.715506$

C $6.1574080 .108038-0.502439$

C $7.533672-0.341280-0.043017$

C $7.156019-1.2556181 .110477$

H $5.662832-0.729993-1.009772$

C $6.1051531 .345962-1.403969$

H $8.094399-0.852931-0.830542$

H 8.1342930 .4992840 .323238

C $8.296767-1.5219762 .077486$

H $6.816440-2.2156590 .698253$

C $4.6846731 .925963-1.589453$

O $6.9222522 .381155-0.844958$

H $6.5503731 .109076-2.376709$

H $4.3851932 .439398-0.667561$

O $4.7416502 .939955-2.598297$

C $3.6031360 .897416-1.963987$

C $2.2099741 .541008-2.106060$

H $3.5492970 .121030-1.193694$

O $3.9376840 .265077-3.198484$

H $2.0725802 .302720-1.331076$

H $2.1352742 .025207-3.087468$

H $9.126375-2.0211081 .566191$

H $8.679972-0.5924242 .511710$

H $7.966340-2.1668682 .898036$

C $4.870917-1.4533432 .230733$

H $6.5866612 .571506 \quad 0.047954$

H $4.9429342 .501631-3.442988$

H $4.743794-0.258891-3.059698$

H $5.276584-2.1728982 .950921$

H $4.476019-2.0270441 .383836$

H $\quad 6.3105580 .1457112 .558387$

C $0.325509-1.566432-2.983409$

O $1.213430-0.443033-3.038740$

C $1.0948470 .487882-1.961200$

C $-0.3086091 .105632-1.957545$

C $-1.3825910 .013178-1.891036$

C $-1.140213-1.098371-2.918856$

H $0.474019-2.087945-3.937482$

C $0.708374-2.541507-1.858818$

H $1.256873-0.032060-1.010314$

O $-0.5042471 .905550-3.120384$

H $-0.4048631 .769432-1.092022$

H -2.368509 $0.459450-2.069300$

O $-1.389961-0.564112-0.588776$

H -1.794032 -1.952696 -2.712050

O $-1.509412-0.588072-4.203454$

C $2.129273-3.046383-1.998658$

H $0.620197-2.069357-0.876675$

H $0.047165-3.414004-1.882808$

H $-0.3595851 .340120-3.898402$

H $-1.5379440 .152240 \quad 0.051625$

H -1.429843 -1.316435 -4.842765

O $2.543181-3.553139-0.799447$

O $2.786741-3.021469-3.030076$ 
C $3.876727-4.072115-0.780266$

H $3.967412-4.909163-1.478965$

H $4.594067-3.282966-1.025985$

H $4.086102-4.4339060 .229969$

SCF Energy (B3LYP/6-31G ${ }^{* *} / /$ MMFF $)=-3245.90569305$

0700467

MM̄MF Geometry

C $1.672304-3.5795061 .911115$

C $0.852770-3.7912892 .952239$

C $-0.175057-2.8141993 .479531$

O $-0.322860-1.7058012 .562731$

C $-1.542192-3.5055363 .617571$

C -2.603414 -2.615095 4.234329

C $-3.687903-2.1423363 .589031$

C $-2.409318-2.2995855 .696536$

C -4.094498 -2.359309 2.157697

C $-4.320550-1.0300451 .432495$

C -4.981091-1.202142 0.056276

C $-4.075752-1.907732-0.955055$

C $-4.716244-1.964513-2.351861$

O $\quad-5.294437 \quad 0.112819-0.417196$

O $-3.693015-2.328103-3.291336$

C $-5.206866-0.554275-2.742112$

C $-5.792521-3.053455-2.439961$

O $-5.943950-0.590137-3.962316$

C $-6.039740 \quad 0.141647-1.639501$

C $-6.296376 \quad 1.620113-2.032565$

O $-7.296719-0.525576-1.509662$

C $0.102044-0.4779402 .962797$

O $0.533318-0.1653974 .060728$

C $-0.014406 \quad 0.4294201 .797728$

C 0.4358301 .6898941 .890750

C 0.4323902 .6944680 .822917

C $-0.0885232 .268436-0.524035$

C $-6.9148242 .505516-0.937424$

C -5.9859312 .7864980 .229850$

C $-3.746342 \quad 3.6898440 .780973$

O $-6.2426952 .397148 \quad 1.367241$

C $-2.739848 \quad 4.5960110 .151390$

C $-2.138568 \quad 5.6089410 .791634$

C -1.136395 $6.514598 \quad 0.137543$

C $0.2642316 .320508 \quad 0.669324$

C 0.8329607 .2331281 .475054

C 1.0416455 .1154520 .182937

C 0.9059213 .9285391 .098377

N $-4.8587083 .505764-0.131817$

H $1.635791-2.6313091 .381897$

H $0.931384-4.7341703 .489802$

H $\quad 0.171013-2.4752034 .462878$

H -1.445034 -4.401325 4.245923

H -1.858844 -3.872912 2.636401

H -4.390482 -1.535422 4.159925

H -3.261579 -1.7591786 .122577$

H -2.289354 -3.223505 6.271868

H -1.521327 -1.677883 5.844168

H -3.359302 -2.948906 1.607227

H $-5.026870-2.9367392 .160752$

H -4.960500 -0.376535 2.041387

H $-3.371137-0.4883261 .326668$

H $-5.915184-1.7591400 .195260$

H -3.816772 -2.919526 -0.622763

H -3.123849 -1.363796-1.031315

H -3.323768 -3.184658 -3.015800

H $-4.3149090 .057735-2.937295$

H $-5.360346-4.037425-2.221378$

H -6.200334 -3.125295 -3.454291

H $-6.619828-2.895811-1.745009$

H -5.381314 -1.021768 -4.628110

H $-5.3508462 .071165-2.355335$

H $-6.9751281 .645907-2.894618$

H -7.941520 $0.084759-1.119490$

H $-0.446017 \quad 0.0266110 .888242$

H 0.8601102 .0203152 .838168

H $0.4884801 .420225-0.905814$

H $-0.0354643 .050917-1.283022$

H -1.139085 $1.965947-0.453741$

H $-7.1927153 .473419-1.373574$
H $-7.8393732 .067768-0.547331$

H -3.3042212 .7041620 .962248$

H $-4.1300794 .074847 \quad 1.731637$

H $-2.4734254 .398922-0.884910$

H -2.3833485 .8075791 .832233$

H -1.4683867 .5488710 .296370$

H -1.128595 $6.377806-0.951371$

H 1.8508427 .1134281 .831468

H $\quad 0.2925468 .1159321 .800043$

H 2.1078345 .3716550 .134702

H $0.7628114 .895682-0.850795$

H 1.2737614 .1217752 .107480

H $-4.7076303 .703280-1.116606$

C $2.652554-4.775668-0.099014$

O $2.962758-3.539523-0.775106$

C $2.101496-3.416286-1.922431$

C $1.466834-4.789680-2.120059$

C $1.328172-5.261649-0.685347$

H $1.312791-2.704284-1.652483$

C $2.886977-2.901082-3.130615$

H $0.510683-4.732600-2.649012$

H $2.125187-5.463367-2.681334$

C $1.105579-6.757224-0.549939$

H $0.480874-4.733060-0.230655$

C $3.531367-1.511034-2.931736$

O $3.934805-3.836136-3.418609$

H $2.234717-2.891419-4.012212$

H $4.331841-1.579220-2.186945$

O $4.175077-1.166772-4.166841$

C $2.546676-0.387808-2.545360$

C $3.1144871 .038285-2.684843$

H $2.190920-0.549638-1.521735$

O $1.405807-0.474568-3.406805$

H $3.430640 \quad 1.206245-3.722102$

H $2.2873871 .740948-2.518187$

H $\quad 0.177742-7.054657-1.049397$

H $1.925884-7.328038-0.997971$

H $1.029786-7.0449430 .503447$

C $2.687570-4.5720091 .418284$

H $4.427756-3.492158-4.183093$

H $3.480730-1.062071-4.840087$

H $\quad 0.7548320 .179264-3.099409$

H $3.677086-4.1884391 .695234$

H $2.561476-5.538477 \quad 1.919975$

H $3.457437-5.473489-0.365945$

C 4.6568621 .6952210 .657593

O $3.7267311 .392150-0.392962$

C $4.2630241 .371422-1.720369$

C $4.8989252 .729093-2.064933$

C $5.9419543 .138657-1.023621$

C 5.3927573 .0203530 .400580

H 4.0301761 .8333851 .548659

C 5.6085460 .5247480 .924638

H $5.0254750 .590475-1.792411$

O $3.8975343 .741371-2.157422$

H $5.3727702 .656829-3.051007$

H $6.2509594 .173195-1.216539$

O $7.0868022 .304457-1.173114$

H 6.1971223 .1390681 .135807

O 4.4621524 .0799810 .632471

C $4.865342-0.5965391 .605906$

H 6.0596010 .1117520 .018267

H $6.4381910 .828475 \quad 1.574230$

H $3.4076883 .751919-1.317365$

H $7.7485332 .594786-0.522434$

H 4.9369214 .9200420 .512249

O $4.946748-0.4546022 .959452$

O $4.259730-1.472351 \quad 1.003702$

C $4.251739-1.4411843 .727534$

H $4.612307-2.4438273 .479475$

H $4.449841-1.2500524 .785509$

H $3.175726-1.3605943 .552696$

SCF Energy (B3LYP/6-31G*//MMFF)= -3245.90590504

0700468

MM̄MF Geometry

C 0.0798123 .2597522 .597372

C 0.4726612 .8372443 .808478 
C $0.325811 \quad 1.4285924 .340742$

O -0.5381140 .6384623 .492240$

C 1.7178650 .7661454 .385913

C $1.743958-0.6726754 .867181$

C $2.509268-1.6290624 .303199$

C $0.893070-1.0076996 .064130$

C $3.513621-1.4574983 .196510$

C $2.942603-1.8589091 .838035$

C $3.993260-1.8716200 .716010$

C $4.550949-0.4802720 .406877$

C $5.488533-0.497897-0.811410$

O $3.336555-2.395225-0.442583$

O $5.7320720 .860169-1.207287$

C $4.792541-1.224991-1.982513$

C $6.866193-1.063297-0.447875$

O $5.689173-1.391175-3.079090$

C $4.179294-2.588929-1.584654$

C $3.299329-3.125357-2.743885$

O $5.230942-3.515695-1.318385$

C -1.8647240 .6331723 .802859$

O $-2.402149 \quad 1.2114504 .734708$

C -2.567982 -0.2025162 .804133$

C $-3.860597-0.5011043 .004510$

C $-4.708339-1.2913622 .115426$

C $-6.128152-0.7940032 .055470$

C $2.661072-4.507365-2.535283$

C $1.829510-4.640172-1.275160$

C $-0.154643-3.676310-0.114917$

O $2.145211-5.420945-0.379669$

C -1.521841-4.140590 -0.497871

C $-2.257078-4.9726910 .254716$

C $-3.652970-5.396906-0.097317$

C $-4.692765-4.763270 \quad 0.801411$

C $-5.353769-5.4856241 .721195$

C $-5.027131-3.3035440 .566786$

C $-4.246524-2.3764141 .461742$

N 0.725472 -3.805870 -1.260354

H -0.4132222 .5692981 .918163$

H $0.9792633 .539503 \quad 4.467378$

H -0.0859181 .4830525 .356085$

H $2.374421 \quad 1.3518885 .043092$

H 2.1444820 .8246253 .377069

H $2.453982-2.6399524 .704945$

H $1.093830-2.0143276 .446617$

H $1.094552-0.3054196 .879749$

H $-0.170430-0.9600545 .812941$

H $3.893315-0.4315763 .172220$

H $4.381081-2.0870983 .431891$

H $2.495069-2.8601641 .905576$

H $2.115147-1.1916761 .561267$

H $4.797511-2.5574561 .007015$

H $5.073631-0.0561341 .271821$

H 3.7175190 .2035910 .197662

H $4.8797961 .267041-1.436363$

H $3.983391-0.575638-2.343206$

H $7.337500-0.4483730 .328609$

H $7.546541-1.032166-1.306197$

H $6.825982-2.089498-0.077011$

H $6.046277-0.513284-3.297572$

H $2.498269-2.402554-2.943155$

H $3.904356-3.194639-3.656568$

H $4.853850-4.407611-1.263679$

H -2.015954 -0.5441061 .935387$

H -4.370110 -0.1216853 .888898$

H $-6.643415-0.9945303 .001088$

H $-6.712706-1.2478561 .251945$

H $-6.147777 \quad 0.287527 \quad 1.875970$

H $2.013883-4.739121-3.390435$

H $3.431942-5.286058-2.522139$

H $-0.186470-2.6142410 .147204$

H $\quad 0.248882-4.2291410 .739304$

H -1.944977 -3.743702 -1.417231

H -1.846369 -5.3562531 .185767$

H -3.698850 -6.491419-0.030665

H -3.888163 -5.157985 -1.142357

H $-6.120198-5.0368202 .344576$

H -5.140075 -6.5381101 .874163$

H $-6.106695-3.1734430 .695789$
H $-4.819757-3.047365-0.478272$

H -3.195422 -2.632405 1.581189

H $0.550384-3.201979-2.058042$

C 1.2971274 .6796720 .894185

O $0.8581923 .789047-0.154547$

C $1.9657802 .950098-0.527735$

C $3.2097883 .706627-0.082293$

C 2.7352984 .2791081 .238950

H 1.8840562 .0323340 .067246

C $1.9190512 .605457-2.016962$

H 4.0818443 .0549580 .022715

H $3.4642894 .514816-0.777787$

C 3.5865085 .4287951 .749557

H 2.7501213 .4757041 .985471

C $0.6178851 .912871-2.475212$

O $2.0589583 .816093-2.770612$

H $2.7862281 .984911-2.271950$

H $-0.1998522 .642154-2.478439$

O $\quad 0.804467 \quad 1.526942-3.843852$

C $0.2109290 .675554-1.653045$

C $-0.892732-0.189055-2.289970$

H $-0.1031260 .992611-0.652015$

O $1.361039-0.157916-1.489022$

H $-0.516359-0.649570-3.212345$

H -1.080255 -1.023380 -1.602043

H 4.6152855 .0974071 .924500

H 3.6176176 .2554231 .031949

H 3.1902145 .8157072 .693696

C 0.3103534 .6471322 .067527

H $2.0263593 .576497-3.712675$

H $1.4934220 .840440-3.863737$

H $1.116203-0.887239-0.894617$

H -0.6602755 .0286791 .729300$

H 0.6561495 .3200232 .860671

H $1.2831705 .687910 \quad 0.460864$

C $-3.9381361 .776475-1.388620$

O $-2.7179131 .025406-1.319767$

C $-2.2106360 .548069-2.568655$

C $-3.238409-0.369335-3.252167$

C $-4.5980750 .319344-3.394921$

C $-5.0477670 .965152-2.079440$

H $-4.2360271 .923581-0.342195$

C $-3.7254293 .162281-2.017947$

H $-2.0029871 .397533-3.225853$

O $-3.399811-1.576700-2.510945$

H $-2.859871-0.649233-4.242238$

H $-5.340251-0.422420-3.714189$

O $-4.5038021 .307038-4.416505$

H $-5.9209491 .607578-2.242217$

O $-5.450580-0.064725-1.173274$

C $-2.8273974 .044865-1.178147$

H -3.272934 $3.093025-3.010360$

H -4.691653 $3.672755-2.105709$

H -3.637275 -1.334398 -1.599435

H $-5.3811831 .715264-4.511985$

H $-6.185649-0.549287-1.585875$

O $-2.6780795 .246489-1.810845$

O $-2.3223253 .727569-0.110804$

C -1.847166 $6.201809-1.144194$

H $-0.8025945 .883928-1.198102$

H $-1.9493407 .159607-1.661176$

H -2.160543 $6.332330-0.103881$

SCF Energy (B3LYP/6-31G**//MMFF) $=-3245.91131463$

0700469

MM̄FF Geometry

C $0.5109076 .014886-0.110466$

C $1.1841275 .032016-0.726550$

C $2.2479874 .205685-0.050508$

O $1.7880112 .833898-0.003962$

C $3.5599094 .253648-0.853677$

C $4.6639143 .371937-0.297533$

C $5.3726872 .496586-1.037781$

C 4.9759893 .5304791 .168251

C $5.2827922 .269167-2.519741$

C $4.3951371 .090113-2.941425$

C $4.895646-0.269505-2.429198$

C $4.317791-1.409988-3.271787$ 
C $4.749312-2.784021-2.738108$

O $4.475993-0.420289-1.067555$

O $3.968311-3.788086-3.402922$

C $4.441146-2.849997-1.228554$

C $6.204237-3.102038-3.099087$

O $4.956906-4.048058-0.653249$

C $4.947234-1.618724-0.437333$

C $4.384402-1.6695061 .001895$

O $6.374770-1.651527-0.374388$

C $0.9430412 .509598 \quad 1.013696$

O $0.521363 \quad 3.2565151 .883248$

C $0.608981 \quad 1.073647 \quad 0.892130$

C -0.3271520 .5582001 .703214$

C $-0.770088-0.8361161 .752215$

C $-0.122019-1.8057720 .803697$

C $4.533006-0.3628991 .791932$

C $4.197075-0.5789013 .255726$

C $2.316829-1.1924134 .774382$

O $5.053835-0.4909534 .132463$

C $1.314368-2.2948114 .634368$

C $0.069789-2.2417455 .132732$

C $-0.934696-3.3495624 .978905$

C $-2.253140-2.8758694 .404079$

C -3.359743 -2.823272 5.164822

C $-2.306757-2.5324652 .928972$

C $-1.724288-1.1732852 .644946$

N $2.865266-0.8979563 .463065$

H $0.752065 \quad 6.2656420 .919672$

H $0.9198574 .763017-1.746684$

H 2.4340214 .5673490 .968960

H $3.9294445 .286959-0.886800$

H $3.3341503 .968523-1.887155$

H $6.1363051 .900778-0.539090$

H 5.8839882 .9914051 .459895

H 5.1331964 .5867291 .409970

H $4.156674 \quad 3.146557 \quad 1.783734$

H $4.9383353 .174369-3.031892$

H $6.3007512 .105662-2.897115$

H $3.3687461 .267792-2.595482$

H $4.3738051 .081127-4.038381$

H $5.990645-0.282355-2.478873$

H $4.605674-1.304705-4.325298$

H $3.220229-1.352983-3.253148$

H $3.029014-3.602204-3.236897$

H $3.348793-2.900901-1.120132$

H $6.334784-3.103974-4.188089$

H $6.484425-4.106597-2.763059$

H $6.918443-2.389198-2.681935$

H $4.609712-4.792672-1.173596$

H $3.315143-1.9078780 .957652$

H $4.869412-2.4820771 .558561$

H $6.668580-1.1860150 .424526$

H $\begin{array}{llll}1.141318 & 0.486311 & 0.153024\end{array}$

H -0.8173301 .2215942 .415157$

H $-0.274430-1.484163-0.232487$

H $-0.518616-2.8192370 .877480$

H $\quad 0.953025-1.8738440 .994129$

H $5.5565640 .022351 \quad 1.737740$

H $3.872744 \quad 0.4144741 .391277$

H $1.870420-0.2689075 .156775$

H $3.120635-1.4991965 .450924$

H $1.631861-3.1857124 .096965$

H $-0.242709-1.3623345 .691170$

H -1.092195 -3.793352 5.970464

H $-0.542834-4.1571324 .347935$

H -4.313111 -2.507455 4.754243

H -3.336683 -3.089901 6.216195

H -3.348260 -2.522625 2.582074

H -1.818181 -3.335794 2.372044

H -2.150149 -0.3815863 .263824$

H $2.213329-0.7848652 .692722$

C -1.961183 $6.229699-0.199446$

O $-2.1831654 .904817-0.725011$

C $-3.5763344 .756277-1.052368$

C $-4.2725625 .991173-0.492742$

C $-3.1827947 .038848-0.624215$

H $-3.6485004 .768630-2.147223$

C $-4.1179793 .422514-0.519237$
H $-5.180926 \quad 6.242870-1.048436$

H -4.548146 5.8537690 .559837

C $-3.429050 \quad 8.279088 \quad 0.216260$

H -3.094967 $7.327574-1.680135$

C $-3.4953832 .188874-1.212282$

$\begin{array}{lllll}\text { O } & -3.905658 & 3.357983 & 0.889998\end{array}$

H $-5.2020963 .398497-0.681120$

H $-3.6566192 .284702-2.293331$

O $-2.0859622 .141088-0.996786$

C $-4.1069490 .849646-0.748099$

C $-3.608863-0.324954-1.605004$

H $-5.1979420 .914099-0.826443$

O $-3.783106 \quad 0.586797 \quad 0.613239$

H -3.902775 -0.166328 -2.650976

H -2.510274 -0.338314 -1.613041

H $-4.3574758 .772451-0.089755$

H $-3.5141748 .032901 \quad 1.280031$

H $-2.6114818 .997650 \quad 0.101448$

C $-0.6329606 .763711-0.735799$

H $-2.9442513 .376417 \quad 1.039262$

H $-1.7004482 .955181-1.362046$

H -4.1485451 .3101391 .149728$

H $-0.5172367 .827601-0.502504$

H $-0.5987116 .660592-1.827505$

H $-1.926567 \quad 6.1380300 .894776$

C $-3.866821-4.029460-1.906563$

O $-3.607099-2.639799-2.146266$

C $-4.065260-1.722611-1.140805$

C $-5.581377-1.855706-0.942593$

C $-5.973896-3.307019-0.645649$

C $-5.364536-4.277599-1.658124$

H -3.598094 -4.528339-2.846813

C $-2.962405-4.595611-0.802083$

H -3.552036 -1.946979 -0.199793

O $-6.253083-1.399458-2.114459$

H $-5.895770-1.215783-0.110467$

H -7.066207 -3.404307 -0.669764

O $-5.531120-3.6663080 .660294$

H $-5.522019-5.311168-1.327932$

O $-6.066155-4.125161-2.895158$

C $-1.571117-4.892922-1.315777$

H -2.860954 -3.938030 0.064433

H $-3.367800-5.549357-0.442924$

H -7.208709 -1.488545 -1.958863

H $-5.942964-3.0508961 .290389$

H $-5.717283-4.788315-3.514898$

O $-0.983569-3.757891-1.787611$

O $-1.056791-6.003525-1.271962$

C $0.353869-3.908258-2.272653$

H $\quad 0.709313-2.926256-2.596128$

H $1.008591-4.271736-1.474846$

H $\quad 0.371832-4.588942-3.128913$

SCF Energy (B3LYP/6-31G**//MMFF) $=-3245.90260993$

$07 \quad 00470$

MM̄FF Geometry

C -3.408725 -2.502142 -3.061465

C $-2.494918-2.724950-2.107188$

C $-1.013625-2.561473-2.306084$

O $-0.599111-1.421234-1.515314$

C $-0.264900-3.800226-1.791785$

C $1.234009-3.713989-2.003921$

C $2.131306-3.392359-1.050195$

C $1.703643-4.060733-3.393694$

C $1.859986-3.0114040 .379552$

C $2.435588-1.6459080 .794294$

C $3.951510-1.6564321 .050758$

C $4.383404-0.3828481 .782686$

C $5.905121-0.3346251 .995625$

O $4.629321-1.736091-0.204395$

O $6.251367 \quad 1.0006582 .395382$

C $6.604927-0.5973590 .645204$

C $6.356269-1.2574913 .133415$

O $8.015514-0.7172120 .808882$

C $6.056248-1.830559-0.105004$

C $6.627741-1.972244-1.539033$

O $6.431974-3.0188490 .604330$

C $-0.779748-0.189222-2.066196$ 
O $-1.2375310 .064567-3.169203$

C $-0.3154480 .814074-1.084471$

C $-0.264926 \quad 2.105572-1.442281$

C $0.2255943 .212563-0.619147$

C 0.6198132 .9003600 .799460

C $6.546079-0.742157-2.446033$

C $5.126672-0.262465-2.679802$

C $3.6873191 .754544-2.528991$

O $4.252046-1.005136-3.120156$

C $3.6231042 .922606-1.598114$

C $3.5059724 .196144-2.004412$

C $3.4056955 .368685-1.068514$

C $2.1372996 .171018-1.274316$

C $2.1462867 .302038-1.999742$

C $0.8764955 .700750-0.577423$

C $0.3299284 .434082-1.183441$

N $4.9498941 .069254-2.338008$

H -3.085548 -2.233744 -4.064005

H -2.830915 -2.956925 -1.102590

H $-0.766971-2.389998-3.361464$

H $-0.637722-4.698798-2.301954$

H -0.491083 -3.960412 -0.731409

H $3.183021-3.419744-1.325910$

H $2.794051-4.018514-3.488085$

H $1.389294-5.075546-3.658542$

H $1.284465-3.363803-4.125863$

H $0.784768-2.9750980 .575333$

H $2.262032-3.7953451 .032322$

H $2.188483-0.8972710 .031620$

H $1.928795-1.3516601 .720822$

H $4.193610-2.5386931 .654627$

H $3.861160-0.2876812 .742921$

H $4.0863530 .495078 \quad 1.191528$

H $5.757396 \quad 1.2077503 .207324$

H $6.4397140 .297697 \quad 0.034454$

H $5.871243-0.9703154 .074175$

H $7.433363-1.1658103 .312361$

H $6.124920-2.3089262 .951789$

H $8.321296 \quad 0.077290 \quad 1.279009$

H $7.678896-2.283791-1.478908$

H $6.102734-2.799647-2.036821$

H $6.382186-3.770300-0.009708$

H $-0.0032610 .460712-0.108947$

H $-0.5816172 .379430-2.447907$

H -0.2420362 .5086641 .348552$

H 0.9788913 .7656721 .358743

H 1.4225042 .1553120 .826117

H $7.1785940 .060471-2.053501$

H $6.959008-0.993572-3.430872$

H $2.8639961 .061841-2.326239$

H $3.6297112 .053195-3.581005$

H $3.6471652 .703658-0.533155$

H $3.4827174 .413558-3.069908$

H $4.2841956 .005335-1.236082$

H $3.4671965 .051342-0.020011$

H $1.2484917 .896442-2.132615$

H $3.0518157 .656201-2.481435$

H $0.0908856 .463865-0.653942$

H 1.0828215 .6108970 .492057

H $0.0124994 .553645-2.220582$

H $5.7722251 .638504-2.158605$

C $-5.377370-1.046257-2.703711$

O $-4.905242-0.451456-1.474936$

C $-5.8735010 .524163-1.037058$

C $-6.990745 \quad 0.519264-2.078984$

C $-6.891002-0.884026-2.647607$

H $-6.2811800 .166989-0.085496$

C $-5.194343 \quad 1.888055-0.860751$

H $-7.9680100 .733401-1.634859$

H -6.812919 1.266911-2.861526

C $-7.588407-1.042666-3.986896$

H -7.319808 -1.591307-1.925166

C $-4.157115 \quad 1.939762 \quad 0.283015$

O $-4.5268942 .231415-2.083092$

H $-5.9518852 .664457-0.705485$

H -3.3474901 .2272010 .092106$

O $-3.562970 \quad 3.244813 \quad 0.269270$

C -4.7563331 .7021581 .686129$
C -3.8006612 .0126822 .855626$

H $-5.1085700 .669301 \quad 1.770183$

$\begin{array}{llll}\text { O } & -5.907232 & 2.538458 & 1.852009\end{array}$

H $-3.403173 \quad 3.0296272 .750673$

H -4.4001222 .0230153 .776441$

H -8.656724 $-0.822356-3.892195$

H $-7.170964-0.365565-4.739718$

H -7.488131-2.065728 -4.362105

C $-4.888814-2.491748-2.796809$

H $-3.8604561 .544103-2.255724$

H $-3.1842633 .381090-0.616255$

H -5.6145393 .4615111 .757995$

H $-5.387550-3.024064-3.613600$

H $-5.113705-3.027293-1.866323$

H $-4.964028-0.450980-3.530170$

C $-2.323725-1.3217623 .627083$

O $-3.231403-0.226788 \quad 3.455340$

C -2.6529721 .0033183 .006829$

C $-1.598196 \quad 1.490267 \quad 4.012547$

C $-0.5338030 .419075 \quad 4.271284$

C $-1.152292-0.9521294 .554677$

H -2.917597 -2.089650 4.140608

C $-1.896753-1.9050702 .275900$

H -2.1839020 .8535992 .030794$

$\begin{array}{llll}\text { O } & -2.240366 & 1.828818 & 5.241443\end{array}$

H -1.1294382 .4021083 .624929$

H $\quad 0.0655690 .719521 \quad 5.139429$

$\begin{array}{lllll}\text { O } & 0.363784 & 0.336397 & 3.169921\end{array}$

H $-0.380346-1.7295084 .513983$

O $-1.643056-0.9253515 .900861$

C $-3.049914-2.6810961 .681560$

H $-1.605811-1.1545601 .537075$

H - $-1.043583-2.5821472 .398795$

H $-1.5575832 .163776 \quad 5.847230$

H $-0.143856 \quad 0.1622332 .362399$

H $-1.953905-1.8206066 .118699$

O $-2.806045-4.0198931 .765281$

O $-4.058524-2.1582861 .224640$

C $-3.851915-4.8690101 .282075$

H -3.536557 -5.9068861 .417902$

H $-4.769151-4.7032611 .855016$

H $-4.025101-4.6923140 .216727$

SCF Energy (B3LYP/6-31G**//MMFF) $=-3245.90155756$

07_00471

MM̄FF Geometry

C 0.7823543 .9828690 .415884

C $-0.104094 \quad 3.0033790 .650908$

C -0.9989672 .9603891 .860197$

O $-2.357720 \quad 3.001512 \quad 1.352183$

C $-0.838041 \quad 1.6627642 .667489$

C $0.395003 \quad 1.5994853 .538013$

$\begin{array}{llll}\text { C } & 1.414016 & 0.738062 & 3.358139\end{array}$

C 0.3992882 .5307284 .723350

C $1.602829-0.251600 \quad 2.245521$

C $1.356224-1.7031212 .676492$

C $-0.130249-2.0653762 .825338$

C $-0.283434-3.540000 \quad 3.210207$

C $-1.754404-3.9861013 .239190$

O $-0.770170-1.8176621 .569705$

O $-1.777216-5.421803 \quad 3.273793$

C -2.431653 -3.5546171 .919273$

C $-2.464715-3.5129194 .512629$

O $-3.829246-3.8341591 .944580$

C -2.180126 -2.076091 1.556999

C $-2.705674-1.706610 \quad 0.152008$

O $-2.871986-1.243166 \quad 2.495034$

C $-3.315236 \quad 3.5246312 .159339$

$\begin{array}{lllll} & -3.148522 & 3.990198 & 3.279105\end{array}$

C -4.653540 $3.487102 \quad 1.525476$

C $-4.9254962 .861563 \quad 0.369053$

C $-6.2363782 .793598-0.286421$

C $-7.388615 \quad 3.494658 \quad 0.386721$

C $-2.088208-2.509257-0.994097$

C $-2.317561-1.819644-2.322948$

C $-4.064901-1.348663-4.018496$

O $-1.405047-1.253968-2.921086$

C $-5.503093-0.924787-4.030049$ 
C $-6.390841-0.975291-3.023837$

C -7.815341 $-0.516066-3.156949$

C $-8.1874350 .543299-2.143864$

C $-9.0925260 .292335-1.182810$

C $-7.5766891 .918762-2.296681$

C $-6.3497962 .106030-1.443009$

N -3.637521 -1.884881 -2.740947

H $\quad 0.894937 \quad 4.780517 \quad 1.145714$

H $-0.2444072 .222424-0.093077$

H -0.8280143 .8439412 .486633$

H $-0.896990 \quad 0.8111521 .982979$

H -1.7107791 .5289343 .321370$

H 2.2312490 .7544094 .078467

H $0.4539773 .573042 \quad 4.395590$

H -0.5123302 .3976885 .315193$

H 1.2501352 .3516085 .389591

H 1.0144560 .0020951 .358790

H $2.647123-0.1676561 .928422$

H $1.798413-2.3481671 .907924$

H $1.888061-1.9140163 .612601$

H $-0.577472-1.4239303 .592721$

H $0.198689-3.7405134 .175194$

H $0.248062-4.1639002 .478195$

H -1.284963 -5.7108084 .061143$

H -2.010548 -4.192083 1.131773

H -1.962587 -3.9133305 .401734$

H -3.491961 -3.890736 4.556464

H $-2.489127-2.4258294 .612050$

H $-4.256511-3.1586372 .498189$

H $-3.799610-1.7933730 .125170$

H -2.497684 -0.640802 -0.022218

H -3.034174 -0.378519 2.082855

H -5.4256644 .0027472 .088026$

H $-4.1220982 .344512-0.152733$

H $-7.5435653 .097407 \quad 1.396265$

H $-8.3395253 .378303-0.136768$

H $-7.195217 \quad 4.5707580 .457047$

H -1.007742 -2.618424 -0.864272

H -2.510363 -3.519190 -1.040568

H -3.905549 -2.132291-4.766679

H -3.430366 $-0.496254-4.283498$

H -5.840256 -0.525530 -4.985474

H -6.102531 -1.371385 -2.054171

H -8.459456 -1.397414 -3.042633

H -8.016643 -0.128016-4.163410

H $-9.3876891 .056641-0.471820$

H -9.557297 -0.682817 -1.083525

H -8.331894 $2.686022-2.096675$

H -7.298621 $2.085351-3.345533$

H -5.458571 $1.626478-1.848598$

H $-4.244220-2.558846-2.282529$

C $3.0766104 .092206-0.755027$

O $3.5627592 .763606-0.491965$

C 4.8537482 .8830510 .144949

C $5.122214 \quad 4.3806590 .324174$

C 3.7231794 .9660120 .312386

H 4.7573802 .4265311 .136121

C $5.9362982 .163190-0.674025$

H 5.6648514 .5900851 .251565

H $5.7180634 .788724-0.500774$

C 3.6851826 .4528000 .010012

H 3.2723744 .7809831 .295212

C $5.7055090 .650569-0.876015$

O $6.0027422 .769451-1.971865$

H $6.9138872 .333470-0.206570$

H $4.859404 \quad 0.497227-1.554128$

O $\quad 6.854085 \quad 0.126662-1.555566$

C $5.489872-0.1396490 .423705$

C $5.525335-1.6713120 .279974$

$\begin{array}{llll}\text { H } & 4.535544 & 0.160471 & 0.863227\end{array}$

O $6.511331 \quad 0.2255411 .358062$

H $6.546732-2.0008640 .049086$

H $5.308320-2.0802301 .275942$

H 4.2436787 .0135710 .766455

H $4.1262106 .675383-0.967441$

H 2.6552986 .8235800 .007100

C $1.5517894 .120656-0.867272$

H $6.6944852 .304949-2.473541$
H $7.6136840 .208027-0.953296$

H $6.311572-0.2185742 .199855$

H $1.2371563 .337979-1.566858$

H $1.2544765 .076552-1.316887$

H $3.4747084 .365185-1.742904$

C $2.188267-2.228190-1.320450$

O $3.233455-1.789586-0.441573$

C $4.545973-2.265161-0.744921$

C $4.582054-3.804271-0.730500$

C $3.501358-4.407222-1.633760$

C $2.132502-3.765389-1.388018$

H $1.263528-1.876753-0.847521$

C $2.285354-1.577008-2.708526$

H $4.836488-1.909361-1.738478$

O $4.403817-4.2965880 .597618$

H $5.572712-4.136412-1.061732$

H $3.428697-5.485491-1.444859$

O $3.860489-4.228107-2.999934$

H $1.425643-4.079191-2.165174$

O $1.632795-4.262057-0.143510$

C $2.053674-0.082714-2.667658$

H $3.266763-1.739669-3.161083$

H $1.519461-2.001874-3.367565$

H $3.555081-3.9566940 .929093$

H $4.714290-4.671878-3.140122$

H $\quad 0.741697-3.895383-0.015165$

O $2.315002 \quad 0.431119-3.906721$

O $1.6844720 .550409-1.689089$

C $2.1481851 .844941-4.041546$

H $1.1268252 .136069-3.779048$

H $2.8767862 .368562-3.416658$

H $2.3290502 .110355-5.086605$

SCF Energy (B3LYP/6-31G**//MMFF) $=-3245.91766025$

$07 \quad 00472$

MM̄FF Geometry

C $0.8215155 .400048 \quad 0.081425$

C $1.6214274 .581276-0.617937$

C 2.7159963 .7607210 .017341

O $2.6452482 .401457-0.472309$

C $4.0820024 .343385-0.393261$

C 5.2636253 .7279040 .329860

C $6.2640183 .054507-0.270946$

C 5.3049843 .9446121 .821432

C $6.4372622 .752188-1.732896$

C $5.8604491 .399246-2.179218$

C $6.4879160 .183709-1.476443$

C $6.032178-1.120079-2.135425$

C $6.558605-2.358169-1.392204$

O $6.071500 \quad 0.193835-0.109185$

O $5.823523-3.486998-1.889695$

C $6.247849-2.2148780 .115345$

C $8.031023-2.625539-1.725668$

O $6.849555-3.2715540 .858511$

C $6.644090-0.8427540 .697020$

C $6.147548-0.6213772 .146306$

$\begin{array}{lllll}\text { O } & 8.073447 & -0.744674 & 0.725481\end{array}$

C 1.6834681 .5889560 .045339

O 0.8355621 .8825690 .872369

C $1.8429280 .258532-0.584284$

C $0.871112-0.660573-0.478237$

C $0.903554-2.010715-1.049031$

C $2.212037-2.487487-1.623577$

C $4.628028-0.6849022 .337354$

C $4.120885-2.0491692 .772381$

C $2.005335-3.3207743 .100356$

O $4.858792-2.9510633 .159761$

C $1.812454-4.1926831 .900235$

C $0.627482-4.7176021 .554323$

C $0.431091-5.6225060 .372595$

C $-0.708621-5.181582-0.518299$

C $-1.915679-5.764229-0.424522$

C $-0.421860-4.142972-1.579481$

C $-0.217107-2.762780-1.017125$

N $2.735696-2.1280882 .715527$

H $0.9550125 .481214 \quad 1.157745$

H $1.5060234 .508698-1.696556$

H $2.6146153 .754048 \quad 1.109224$ 
H $4.1006695 .423611-0.193499$ H $4.1947664 .233742-1.477311$ H 7.0678472 .6710430 .357130 H 6.2592933 .6334092 .260006 H 5.1703515 .0055602 .056102 H 4.5157353 .3719702 .317640 H $6.0019143 .546147-2.348807$ H $7.5114552 .775258-1.957177$ H $4.7771721 .405077-2.008901$ H $6.0172631 .310464-3.261417$ H $7.5788900 .277823-1.529277$ H $6.332209-1.149681-3.190391$ H $4.934716-1.157396-2.138290$ H $6.092980-4.265317-1.371865$ H $5.165380-2.3488180 .220699$ H $8.154529-2.782253-2.804064$ H $8.385615-3.546466-1.249751$ H $8.692270-1.807854-1.431377$ H $7.795389-3.0668100 .952557$ H $6.672547-1.2971092 .832086$ H 6.4589150 .3907872 .443179 H $8.322240-0.0589981 .367470$ H $2.7616220 .074380-1.129791$ H $-0.039319-0.399296 \quad 0.059744$ H $2.454377-1.936030-2.538251$ H $2.221853-3.550870-1.870859$ H $3.021219-2.344346-0.899562$ H 4.3499520 .0107343 .139532 H $4.101812-0.3661631 .432129$ H $1.054625-2.9951103 .533972$ H $2.572633-3.8634053 .863450$ H $2.690906-4.4227011 .301665$ H $-0.244410-4.5122462 .171529$ H $0.233742-6.6319020 .757013$ H $1.347149-5.707953-0.225038$ H $-2.730380-5.489541-1.084723$ H $-2.116328-6.5324750 .315059$ H - $1.261933-4.081794-2.283852$ H $0.421082-4.482793-2.187522$ H -1.115906 -2.336745 -0.569109 H $2.207338-1.3532862 .324922$ C -1.619641 5.9603260 .062231 O $-2.074705 \quad 4.674434-0.404563$ C $-3.4669824 .772148-0.754490$ C $-3.9442146 .116029-0.217932$ C $-2.6942256 .958300-0.365643$ H -3.516540 $4.784052-1.850706$ C $-4.2599453 .571647-0.222070$ H $-4.7973606 .508141-0.779770$ H $-4.236738 \quad 6.0481520 .836646$ C $-2.721891 \quad 8.2331350 .459637$ H -2.567239 $7.217539-1.425431$ C -3.838033 2.228842 -0.857710 $\begin{array}{llll}\text { O } & -4.132620 & 3.512827 & 1.197217\end{array}$ H $-5.3211173 .740940-0.441170$ H -3.857362 2.339426-1.949152 O $-2.5033341 .885647-0.488951$ C $-4.775074 \quad 1.065404-0.475039$ C $-4.410085-0.231629-1.223228$ H $-5.8045881 .353084-0.719280$ O $-4.7063690 .843193 \quad 0.931256$ H $-4.242949-0.003094-2.282544$ H $-3.478037-0.629772-0.802968$ H -3.556209 8.8720360 .151833 H -2.839560 8.0178681 .526904 H $-1.796143 \quad 8.8025430 .331275$ C $-0.244317 \quad 6.263712-0.531432$ H -3.198390 3.3295871 .397159 H $-1.9197472 .605831-0.781432$ H $-5.3340150 .134173 \quad 1.150210$ H $0.0249057 .309016-0.342073$ H $-0.2684696 .120938-1.618934$ H -1.5657835 .9020361 .157459$ C -6.608472 -2.6967090 .540705$ O $-5.586463-1.7365170 .244751$ C $-5.513719-1.304430-1.116781$ C $-5.238920-2.497979-2.043890$ C $-6.265075-3.615342-1.832674$
C $-6.458339-3.942639-0.347543$

H $-6.414027-2.9990371 .578093$

C $-8.009085-2.0771440 .489007$

H $-6.463949-0.839108-1.400399$

O $-3.928872-2.999532-1.792639$

H $-5.266817-2.151346-3.083995$

H $-5.931585-4.515019-2.364026$

O $-7.503389-3.205105-2.405434$

H $-7.329601-4.593597-0.213624$

O $-5.325033-4.6854760 .110869$

C $-8.169650-1.0390321 .574016$

H $-8.243943-1.614973-0.474020$

H $-8.778137-2.8403390 .657545$

H $-3.761438-3.711846-2.432906$

H $-8.136115-3.934179-2.289349$

H $-4.551064-4.0972870 .076947$

$\begin{array}{lllll}0 & -7.941249 & 0.205463 & 1.066422\end{array}$

$\begin{array}{lllll}0 & -8.438845 & -1.306886 & 2.737932\end{array}$

C $-8.026203 \quad 1.2809882 .006372$

H -9.0416551 .3524142 .407111$

H $-7.300327 \quad 1.1392612 .812914$

H -7.788040 2.2099781 .481434

SCF Energy (B3LYP/6-31G**//MMFF) $=-3245.91887960$

0700473

MM̄FF Geometry

C -0.438629 -2.690123 -0.517010

$\begin{array}{lllll}C & 0.315742 & -3.263706 & 0.435737\end{array}$

C $0.116223-3.0483131 .919161$

O $-0.387121-1.7130362 .149700$

C $-0.904510-4.0670172 .466850$

C $-1.423525-3.732003 \quad 3.852265$

C $-2.701276-3.3849414 .113128$

C $-0.409877-3.8040574 .962921$

C -3.837309 $-3.307776 \quad 3.126254$

C $-3.829168-2.000592 \quad 2.332744$

C $-4.944712-1.8747761 .283813$

C $-4.862295-2.9610690 .206830$

C $-5.901473-2.754960-0.903683$

O $-4.764015-0.5866480 .679420$

O $-5.519523-3.609980-1.994835$

C $-5.822023-1.298911-1.405977$

C $-7.287783-3.237120-0.462283$

O $-6.869402-1.014200-2.329319$

C $-5.754738-0.229306-0.290837$

C $-5.309514 \quad 1.119853-0.902251$

$\begin{array}{llll}\text { O } & -7.024505 & 0.007872 & 0.315132\end{array}$

C $0.512033-0.7051292 .300691$

$\begin{array}{llll}\text { O } & 1.728280 & -0.794103 & 2.316486\end{array}$

C -0.2663350 .5486982 .414951$

$\begin{array}{llll}\text { C } & 0.364529 & 1.729453 & 2.494814\end{array}$

C $-0.280301 \quad 3.0444942 .555825$

C $-1.785774 \quad 3.0712082 .610431$

C $-5.1938382 .247741 \quad 0.129086$

C $-4.7152113 .530350-0.521203$

C $-2.6887414 .611267-1.477332$

O $-5.489476 \quad 4.448269-0.782226$

C $-1.2876974 .733310-0.969589$

C $-0.7848305 .857600-0.436112$

C $0.6320465 .982302 \quad 0.047124$

$\begin{array}{llll}\text { C } & 0.737290 & 6.420687 & 1.493173\end{array}$

C 1.4263797 .5268111 .821780

C 0.0579045 .5908932 .565956

C 0.4956924 .1493032 .556654

N $-3.3529243 .528807-0.776478$

H $-1.269222-2.058785-0.214756$

H $1.122309-3.9335590 .149592$

H $1.074601-3.1730312 .437954$

H $-0.450752-5.0668102 .485323$

H $-1.741307-4.1458321 .763916$

H $-2.974202-3.1435275 .139041$

H $-0.858127-3.6228495 .945620$

H $0.051583-4.7965854 .990299$

H $0.376838-3.0575164 .817153$

H $-3.803128-4.1801622 .466838$

H $-4.781644-3.3925843 .678222$

H -3.900020 -1.150579 3.025434

H $-2.869101-1.8707481 .823272$ 
H $-5.913487-1.8981791 .795814$ H -4.980737 -3.9621820 .635879$ H $-3.860902-2.945496-0.246171$ H -6.133684 -3.440886 -2.730191 H $-4.892735-1.230489-1.987360$ H $-7.273300-4.319838-0.285200$ H -8.036004 -3.075428 -1.245535 H -7.636979 -2.7645520 .457562$ H -7.685050 -0.863704 -1.822896 H $-4.3375390 .990573-1.395705$ H $-6.023470 \quad 1.435511-1.673984$ H $-7.549099-0.8043540 .291360$ H -1.347641 0.4653362 .401366 H 1.4534801 .7395112 .492378 H -2.146516 2.5237873 .488731 H -2.2079862 .6080721 .713727$ H -2.209631 4.0742572 .679263 H -6.1626202 .4493220 .599834$ H -4.4986221 .9803420 .932659$ H -3.259467 $5.537800-1.355536$ H $-2.6792384 .353607-2.541235$ H -0.650343 $3.856562-1.061254$ H -1.413266 $6.741862-0.361350$ H $1.1394456 .702485-0.607725$ H $1.1718235 .037230-0.076892$ H 1.5130897 .8549042 .852449 H 1.9204978 .1314201 .068441 H -1.021664 5.7040832 .440602 H 0.2890865 .9944973 .560242 H 1.5788784 .0193412 .546701 H $-2.8389382 .660829-0.652788$ C $-0.072683-1.570497-2.771320$ O $1.057193-0.828566-2.271346$ C $0.7124130 .569588-2.249690$ C $-0.5651930 .703739-3.071859$ C $-1.260436-0.606364-2.755092$ H $0.4819890 .820624-1.207482$ C $1.8831231 .423192-2.748738$ $\mathrm{H}-1.1522621 .582167-2.786889$ H $-0.3527330 .771747-4.145120$ C $-2.361401-0.966540-3.736768$ H -1.693859 -0.525832 -1.751333 C $3.2037031 .232461-1.972201$ O $2.144778 \quad 1.109448-4.122896$ H $1.5946192 .480750-2.734237$ H $3.6405610 .256011-2.208927$ O $4.1312242 .216098-2.447832$ C $3.0725181 .389146-0.448645$ $\begin{array}{llll}C & 4.398021 & 1.477127 & 0.328019\end{array}$ H $2.4856560 .555139-0.050933$ O $2.3374172 .581467-0.158748$ H 4.9077832 .4204180 .092879 H 4.1308391 .5488341 .391116 H -3.145151 -0.201998 -3.732483 H -1.977842 -1.047846 -4.759273 H $-2.821170-1.923868-3.471658$ C -0.242667 -2.880805 -1.993812 H $2.3352530 .156976-4.174480$ H $4.2071342 .100195-3.410614$ H $2.8291303 .329107-0.539804$ H $0.643236-3.504333-2.165154$ H -1.102288 -3.432256 -2.391241 H $\quad 0.172740-1.828323-3.810337$ C $5.421872-2.1198340 .243273$ O $4.674062-0.9097550 .430725$ C 5.3631410 .3012670 .110800 C $6.632751 \quad 0.446248 \quad 0.968108$ C $7.528879-0.7898490 .851527$ C $6.734217-2.0863841 .045434$ H $4.790617-2.9088780 .672331$ C $5.634313-2.441378-1.245144$ H $5.647526 \quad 0.286231-0.945898$ O $\quad 6.269707 \quad 0.6396152 .334122$ H 7.1776481 .3403180 .641464 H $8.322927-0.7296741 .605740$ O $8.150287-0.782689-0.430047$ H $7.345345-2.9565230 .778501$ O $6.394713-2.2246532 .428063$
C $4.333693-2.735814-1.959679$

H $6.114179-1.613482-1.773109$

H $6.264477-3.333056-1.341268$

H $7.089490 \quad 0.7671082 .840950$

H $8.730603-1.561390-0.479245$

H $7.225027-2.2450322 .933756$

O $4.585489-2.886270-3.293815$

O $3.239711-2.830195-1.422277$

C $3.444413-3.169783-4.109316$

H $3.795059-3.377203-5.123767$

H $2.912172-4.050404-3.736573$

H $2.782263-2.300402-4.137961$

SCF Energy (B3LYP/6-31G**//MMFF)= -3245.89564653

$07 \quad 00474$

MM̄̄FF Geometry

C -3.396406 -3.561760 -1.106294

C $-2.185515-3.379221-0.558040$

C $-0.917004-3.261272-1.361723$

O $-0.219254-2.087582-0.886701$

C $-0.027228-4.491020-1.103470$

C $1.127773-4.608287-2.076994$

C $2.383392-4.187049-1.829616$

C $0.797333-5.282314-3.383148$

C $2.879271-3.452751-0.615318$

C $3.860357-4.2964930 .206294$

C $4.532046-3.5262701 .357128$

C $3.542813-3.0830042 .438140$

C $4.229747-2.2484803 .531293$

O $5.194217-2.391308 \quad 0.794477$

O $3.208035-1.6435844 .338945$

C $5.045842-1.1210512 .861686$

C $5.035117-3.1285374 .492650$

O $5.809405-0.4024473 .827563$

C $5.965713-1.6188331 .722682$

C $6.544727-0.4119890 .946931$

O $7.036322-2.3803012 .281511$

C $0.265985-1.205610-1.799541$

O $0.122254-1.233963-3.010612$

C $1.027778-0.180029-1.051979$

C $1.3215610 .990470-1.636475$

C $2.0244012 .106527-1.000387$

C $2.815298 \quad 1.8086150 .245389$

C $7.353716-0.805423-0.294990$

C $7.6080230 .389448-1.189817$

C $6.5088791 .841887-2.889634$

O $8.6992300 .953985-1.217427$

C $6.0433893 .092411-2.213894$

C $5.1346113 .923427-2.746776$

C $4.7258995 .219346-2.109635$

C $3.2282585 .410950-2.065252$

C $2.6215116 .305095-2.864775$

C $2.4441854 .639967-1.028215$

C $1.9132183 .333960-1.549867$

N $6.4968490 .745604-1.940180$

H $-3.485917-3.620770-2.188295$

H $-2.089828-3.3495880 .525038$

H -1.156056 -3.172093 -2.428228

H $-0.624402-5.410403-1.175999$

H $0.349520-4.471925-0.073050$

H $3.139683-4.345135-2.597475$

H $1.665237-5.351053-4.047589$

H $\quad 0.436809-6.300585-3.204391$

H $0.019095-4.725799-3.914718$

H $3.370941-2.537544-0.966300$

H $2.051698-3.1293420 .018811$

H $3.349780-5.1821540 .604472$

H $4.651448-4.657156-0.464958$

H $5.285659-4.1937161 .791748$

H $3.030078-3.9480582 .876288$

H $2.755498-2.4623191 .992098$

H $2.654980-1.0871593 .764754$

H $4.330221-0.4034882 .436750$

H $4.376271-3.8503264 .990559$

H $5.483709-2.5310685 .294120$

H $5.828918-3.6926143 .998744$

H $5.195783-0.1083024 .522331$

H $\begin{array}{llll}5.718057 & 0.237873 & 0.635759\end{array}$ 
H $7.1910830 .185053 \quad 1.602547$ H $7.772805-2.4025641 .650948$ H $1.298971-0.404697-0.026251$ H $0.9727401 .170445-2.652492$ H 3.4977872 .6132170 .527358 H 3.4416940 .9214850 .099085 H $2.146618 \quad 1.6263841 .092883$ H $8.324113-1.228163-0.012216$ H $6.837632-1.568111-0.889280$ H $5.8616601 .556744-3.725069$ H $7.527473 \quad 1.983410-3.265460$ H $6.5054033 .349215-1.263499$ H $4.701623 \quad 3.687221-3.716219$ H $5.1897536 .030140-2.686556$ H $5.1290885 .308934-1.093141$ H $1.5502716 .470465-2.814385$ H $3.1802316 .887112-3.590100$ H $1.5767605 .237177-0.714376$ H $3.0485984 .528801-0.124106$ H $1.3020533 .439700-2.447649$ H $5.6206180 .251493-1.798317$ C $-5.822850-2.921528-0.790339$ O $-5.703140-1.574699-0.279269$ C $-6.968954-1.1828950 .285493$ C $-7.990449-2.180876-0.245200$ C $-7.167742-3.448774-0.283041$ H $-6.883337-1.3181741 .370040$ C $-7.3164110 .274910-0.035828$ H $-8.871249-2.2574720 .399638$ H -8.329782 -1.918795 -1.254180 C $-7.774215-4.536886-1.153494$ H $-7.060421-3.8286610 .742186$ C $-6.305479 \begin{array}{lll}1.325280 & 0.465133\end{array}$ O $-7.417622 \quad 0.407719-1.459671$ H -8.3126600 .4986800 .366291$ H $-5.4101161 .310555-0.165051$ $\begin{array}{llll}\text { O } & -6.905549 & 2.616032 & 0.288804\end{array}$ C -5.8996821 .1892801 .944156$ C $-5.1584702 .402898 \quad 2.540135$ H -5.2728170 .2985172 .062146$ $\begin{array}{llll}\text { O } & -7.065679 & 0.976321 & 2.746835\end{array}$ H $-5.857842 \quad 3.2395022 .668027$ H $-4.856178 \quad 2.1186903 .557154$ H $-8.760653-4.825369-0.775971$ H -7.895731-4.201061-2.188723 H $-7.142013-5.430175-1.161728$ C $-4.650549-3.781486-0.308013$ H $-7.8375781 .263884-1.646793$ H $-6.9563262 .788318-0.666355$ H -7.657852 1.7361502 .612164 H $-4.906851-4.842391-0.412333$ H $-4.463372-3.5894060 .755800$ H $-5.830557-2.845567-1.885280$ C $-1.857742 \quad 2.0342380 .825147$ O -3.0274001 .7791421 .617107$ C $-3.9344222 .876179 \quad 1.743049$ C $-3.237707 \quad 4.0708252 .413932$ C -1.9557194 .4573951 .668116$ C $-1.074600 \quad 3.238990 \quad 1.377239$ H $-1.233126 \quad 1.1409930 .953497$ C $-2.1958302 .158664-0.670065$ H $-4.275145 \quad 3.1833630 .750102$ O $-2.9213993 .766599 \quad 3.771245$ H -3.929260 4.9212052 .430133 H -1.385781 5.1757792 .270199 $\begin{array}{llll}\text { O } & -2.288432 & 5.088228 & 0.434778\end{array}$ H $-0.274197 \quad 3.5152950 .682636$ O $-0.449017 \quad 2.8495442 .603451$ C -2.720780 $0.858662-1.240509$ H -2.954002 $2.922695-0.857696$ H -1.291765 2.422178 -1.228468 H -2.3615332 .9714313 .774988$ H -2.8156895 .8784750 .642316$ H $\quad 0.1546662 .1136972 .405289$ O $-2.9683761 .024219-2.573130$ O $-2.903129-0.167904-0.601641$ C -3.505366 -0.120765 -3.242981 H $-2.802545-0.957022-3.189168$
H $-4.469854-0.397045-2.806280$

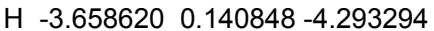

SCF Energy (B3LYP/6-31G**//MMFF) $=-3245.89882941$

07_00475

MMFF Geometry

C 5.7785792 .9186880 .846484

C $5.1784062 .460011 \quad 1.956068$

C 5.2541941 .0667852 .536243

$\begin{array}{lllll}\text { O } & 3.946256 & 0.459561 & 2.367155\end{array}$

C $6.2739720 .126789 \quad 1.860454$

C $6.311806-1.2647572 .465339$

C $5.948999-2.3900401 .816831$

C $6.811715-1.3451853 .884836$

C $5.473269-2.5288240 .395548$

C $3.946921-2.5320380 .314538$

C $3.398303-2.840365-1.085745$

C $3.724418-1.743906-2.102081$

C $3.058593-2.000180-3.463692$

O $1.975504-2.958144-0.946910$

O $3.163879-0.776338-4.207287$

C $1.560450-2.308241-3.251042$

C $3.817971-3.064690-4.265000$

O $0.943381-2.727538-4.466786$

C $1.296892-3.356452-2.145022$

C $-0.219743-3.431641-1.838627$

O $1.741398-4.636201-2.602484$

C 2.9711890 .7398103 .271660

$\begin{array}{lllll}\text { O } & 3.056997 & 1.457249 & 4.254426\end{array}$

C $1.7512570 .029107 \quad 2.827736$

$\begin{array}{lllll}\text { C } & 0.550465 & 0.386447 & 3.305949\end{array}$

C $-0.732599-0.2003262 .913260$

C $-0.703078-1.4209052 .030901$

C $-0.561255-4.237042-0.576943$

C $-2.060213-4.333065-0.343914$

C $-3.707320-4.9249461 .441084$

O $-2.890878-4.118895-1.222194$

C $-4.285140-3.6101231 .858796$

C $-4.377271-3.1993263 .132312$

C $-4.993093-1.8931383 .548299$

C $-3.963024-0.9162434 .073870$

C $-3.725412-0.827285 \quad 5.394010$

C $-3.287500-0.044888 \quad 3.033795$

C $-1.8739450 .363233 \quad 3.361760$

$\begin{array}{lllll}\text { N }-2.354981 & -4.730159 & 0.953208\end{array}$

H 6.4389512 .2789870 .269985

H 4.5116093 .1392212 .485607

H 5.4873391 .1514393 .604629

H $7.2809840 .558427 \quad 1.926333$

H 6.0354600 .0612720 .794269

H $6.004354-3.3337672 .358425$

H $6.945969-2.3794374 .219472$

H $7.781888-0.845668 \quad 3.974582$

H $6.106608-0.8672684 .571635$

H $5.897391-1.747201-0.240276$

H $5.865087-3.4746970 .000743$

H $\quad 3.539409-3.2697451 .019579$

H $3.553211-1.5647920 .646509$

H $3.805154-3.805727-1.408551$

H $4.806763-1.631176-2.233445$

H $3.369758-0.777298-1.717780$

H $2.789343-0.926698-5.091537$

H $1.069361-1.369684-2.963904$

H $4.847679-2.737472-4.453098$

H $3.367217-3.215219-5.252168$

H $3.863868-4.030464-3.757204$

H $\quad 1.022967-1.998697-5.104636$

H $-0.614004-2.416887-1.694147$

H $-0.746607-3.865978-2.697600$

H $1.235152-5.330575-2.153177$

H $1.869922-0.7507112 .084921$

H $\quad 0.496275 \quad 1.2020974 .025613$

H $-0.322930-1.1710831 .035408$

H $-0.064529-2.1976732 .466908$

H - $1.684131-1.8791181 .895649$

H $-0.181828-5.261474-0.651762$

H $-0.097707-3.7687040 .297840$

H $-3.656866-5.6331872 .274281$ 
H $-4.321544-5.3664860 .649518$

H $-4.668982-2.9728511 .067765$ H -3.999166 -3.8392273 .926311$ H $-5.746814-2.1043704 .318007$ H -5.546873 -1.430473 2.721259 H -3.015914 -0.118634 5.806219 H -4.246606 -1.464401 6.101493 H -3.8895040 .8633762 .900331$ H -3.312618 -0.5402322 .057451$ H $-1.806026 \quad 1.2384674 .008372$ H -1.598796 $-4.817157 \quad 1.626014$ C $4.3481074 .322159-0.712208$ O $3.1314103 .955414-0.025175$ C $2.5344832 .844742-0.723544$ C $3.0252912 .982819-2.153569$ C $4.4694393 .391860-1.925415$ H $2.9446631 .937198-0.261482$ C $1.0159332 .887143-0.536608$ H $2.9326962 .056173-2.727478$ H $2.477743 \quad 3.775157-2.678527$ C $5.1164594 .048950-3.132586$ H $5.0427622 .491035-1.676162$ C $0.2652421 .810169-1.337495$ $\begin{array}{lllll}\text { O } & 0.741794 & 2.693183 & 0.854431\end{array}$ H $0.6399893 .883116-0.796379$ H $0.3580942 .019196-2.409670$ O $0.9086590 .545607-1.126777$ C $-1.2227761 .626548-0.980156$ C $-2.0315272 .935535-0.988556$ H -1.310516 $1.135296-0.004242$ O $-1.7611830 .718358-1.946704$ H $-1.5638753 .658526-0.312734$ H -2.014045 $3.348881-2.004876$ H $5.1389213 .357342-3.981102$ H $4.5690274 .944780-3.444115$ H $6.1465324 .343317-2.907011$ C 5.5205094 .2895380 .279650 H 1.2384583 .3698871 .345900 H $\quad 0.9129210 .374518-0.169658$ H -2.673310 $0.512137-1.681966$ H 5.3086494 .9831911 .103475 H $6.4348014 .647631-0.207458$ H $4.2052995 .359073-1.041241$ C $-5.5735291 .762076-1.335969$ O $-4.1949152 .062501-1.592508$ C $-3.4966932 .740373-0.543757$ C $-4.1756534 .083330-0.230948$ C -5.6610653 .8916910 .087001$ C $-6.3601723 .031391-0.970719$ H $-5.9600221 .395610-2.296026$ C $-5.7262430 .635747-0.308199$ H -3.497716 2.1161920 .356530 O $-4.0292934 .961169-1.345281$ H $-3.6662264 .545605 \quad 0.623310$ H $-6.145707 \quad 4.8740510 .142321$ O H $-7.3666412 .752088-0.638057$ O $-6.5159413 .798455-2.167963$ C $-5.205905-0.663583-0.872979$ H -5.1858010 .8221330 .623259$ H $-6.7777490 .494879-0.031064$ H -4.440555 $5.809313-1.106781$ H -6.7236053 .1825931 .563732$ H -7.050951 $4.580035-1.948271$ O $-6.201519-1.311621-1.541849$ O $-4.047464-1.040935-0.754493$ C $-5.831798-2.550985-2.153791$ H -6.668716 -2.887632 -2.771305 H $-4.955227-2.420111-2.795823$ H $-5.639753-3.302649-1.383166$

SCF Energy (B3LYP/6-31G**//MMFF) $=-3245.89977747$

\section{7 _00476}

MM̄FF Geometry

C $-2.478494-2.7488341 .560561$

C $-1.686908-1.6758011 .408164$

C $-0.662096-1.436548 \quad 0.323059$

O $0.606449-1.1321710 .954880$
C $-0.433660-2.607599-0.652994$

C $0.591151-2.320584-1.736571$

C $1.663212-3.098831-1.985269$

C $0.340703-1.099741-2.584553$

C $2.023398-4.394382-1.316079$

C $2.989006-4.258968-0.131063$

C $4.397443-3.796978-0.535284$

C $5.398075-4.0836560 .587032$

C $6.806001-3.5817180 .235952$

O $4.361716-2.389265-0.794909$

O $7.594808-3.6050321 .435196$

C $6.709178-2.110945-0.213642$

C $7.511906-4.502732-0.766113$

O $7.965645-1.640490-0.696796$

C $5.604225-1.850440-1.265982$

C $5.434897-0.326858-1.460856$

O $5.992237-2.435790-2.510626$

C $0.8479540 .174917 \quad 1.251477$

O $0.084015 \quad 1.1152301 .095687$

C $2.2216700 .277306 \quad 1.793426$

C 2.6342371 .4577912 .279362

C 3.9644491 .7772362 .799570

C 4.9919320 .6786442 .829487

C $4.1933510 .085634-2.259920$

C $4.2069221 .585470-2.476822$

C $3.9526933 .735491-1.236136$

O $4.5698982 .078325-3.542323$

C $5.0948124 .030348-0.312596$

C 5.0167214 .8899350 .714488

C 6.1473555 .1523761 .669685

C 5.7319875 .0234593 .121230

C 5.6281416 .1091863 .906564

C 5.5010273 .6329923 .683698

C 4.2015843 .0391003 .215690

N $3.8241052 .293726-1.348506$

H $-2.408569-3.5869380 .873949$

H -1.803898 -0.8556702 .115156$

H -1.001763 -0.569318 -0.252755

H -1.377034 -2.867338-1.149411

H $-0.131757-3.481398-0.066550$

H $2.343928-2.799675-2.781189$

H $1.013151-1.049938-3.447823$

H $-0.683679-1.109951-2.971002$

H $0.488054-0.184794-2.002803$

H $1.121009-4.917797-0.981311$

H $2.464814-5.056735-2.071835$

H $2.564016-3.5676830 .607865$

H $3.056636-5.2451760 .345032$

H $4.690568-4.329967-1.447476$

H $5.424512-5.154908 \quad 0.823226$

H $5.067781-3.5810751 .507118$

H $7.605086-4.5183871 .769304$

H $6.480428-1.5148840 .680644$

H $7.595836-5.517876-0.359679$

H $8.538931-4.169579-0.952626$

H $6.992820-4.573060-1.724272$

H $8.624720-1.807937-0.001432$

H $5.3689130 .155556-0.478838$

H $6.3254630 .082581-1.955464$

H $5.556633-1.959634-3.234798$

H $2.854134-0.6019881 .755304$

H 1.9322502 .2913732 .274917

H $4.610804-0.1927533 .373918$

H 5.9184760 .9659043 .332036

H 5.2527380 .3699231 .813840

H $4.159686-0.398417-3.241098$

H $3.274945-0.190359-1.730317$

H $3.0019964 .118181-0.852162$

H $4.1436514 .174149-2.219981$

H $6.0289493 .502217-0.491463$

H 4.0907705 .4363080 .880019

H 6.5200966 .1657711 .472098

H 6.9926224 .4786951 .481200

H 5.3522546 .0261344 .952610

H 5.8120477 .1063723 .520529

H 5.4753843 .6697704 .780081

H 6.3633093 .0150443 .415588

H 3.3721853 .7482433 .203492 
H $3.5548871 .781389-0.513954$

C -4.923105 -2.991207 2.098942

O $-5.316506-1.7441181 .484572$

C -6.570620-1.326063 2.048207

C -7.183541-2.582850 2.650804

C $-5.951595-3.2718253 .198914$

H -6.331697-0.626679 2.858606

C $-7.452955-0.6260641 .008366$

H -7.928454 -2.356895 3.419637

H -7.657988 -3.2108271 .887928$

C $-6.156524-4.7496013 .485539$

H -5.655168 -2.768500 4.129353

C -6.8049410 .5582990 .261643$

O $-7.862234-1.574980 \quad 0.013892$

H -8.377353 -0.2913551 .494380$

H $-6.124700 \quad 0.183210-0.509260$

O $-7.8518411 .248856-0.434309$

$\begin{array}{llll}\text { C } & -6.063958 & 1.570488 & 1.148912\end{array}$

C $-5.6595952 .886073 \quad 0.457133$

H -5.1636081 .0976461 .557040$

$\begin{array}{lllll} & \mathrm{O} & -6.883981 & 1.918731 & 2.269140\end{array}$

H -6.549336 3.5070590 .288135

H -5.0540983 .4430631 .184730$

H $-6.937947-4.8904974 .239417$

H $-6.456650-5.2951892 .584583$

H $-5.235761-5.2048073 .863579$

C -3.500608 -2.874541 2.655590

H -7.056333 -1.953692 -0.377822

H -8.296240 $0.599561-1.006210$

H -7.714305 2.2876171 .921822

H $-3.254630-3.7652363 .245160$

H $-3.439070-2.0101243 .328690$

H $-4.971220-3.7580001 .315221$

C $-2.9521091 .612870-1.797960$

O $-3.7537221 .893938-0.640651$

C $-4.8853692 .738327-0.863926$

C -4.441776 4.106189-1.413128

C -3.566291 $3.950167-2.660731$

C -2.456634 $2.915710-2.449387$

H -2.073468 $1.085414-1.409378$

C -3.661938 $0.676150-2.790154$

H -5.549301 2.263018-1.592614

O $-3.7081184 .809222-0.411220$

H -5.334392 $4.699523-1.645385$

H -3.109560 $4.914648-2.913995$

O $-4.3718203 .547144-3.764057$

H -1.971598 2.685307-3.404715

O $-1.4462013 .482762-1.609945$

C -3.878651-0.708895-2.221870

H $-4.6417461 .055700-3.090006$

H -3.036910 $0.566744-3.684282$

H -3.478458 $5.681850-0.773626$

H $-5.0477594 .232775-3.900358$

H $-1.8337303 .619201-0.728270$

O $-4.306806-1.539319-3.217833$

O $-3.715971-1.024317-1.052332$

C $-4.572645-2.887286-2.817025$

H $-4.930975-3.437659-3.690968$

H -3.655521 -3.361401-2.454872

H -5.349201-2.909016-2.046182

SCF Energy (B3LYP/6-31G**//MMFF) $=-3245.90783446$

\section{7_00477}

\section{MM̄FF Geometry}

C $-0.375804-4.071425-0.654730$

C $0.615349-4.527017-1.436577$

C $2.032881-3.994376-1.437924$

O $2.073292-2.652502-0.899849$

C $2.924788-4.893152-0.563329$

C $4.375642-4.454951-0.516772$

C $5.010176-4.0344460 .595481$

C $5.123987-4.531074-1.822503$

C $4.455711-3.9187941 .988322$

C $3.836158-2.552847 \quad 2.322213$

C $4.843107-1.3912762 .332048$

C $4.192535-0.1174282 .875440$

C $5.150276 \quad 1.0820272 .823270$

$\begin{array}{llll}\text { O } & 5.289136 & -1.159852 & 0.994097\end{array}$
O $4.3586812 .257824 \quad 3.054675$

C 5.7586121 .1893651 .406592

C 6.1796921 .0275813 .958864

$\begin{array}{lllll}\text { O } & 6.768414 & 2.195463 & 1.395795\end{array}$

C $6.304085-0.1524560 .871136$

C $6.713773-0.108171-0.621839$

O $7.475407-0.5104221 .615013$

C $1.788712-1.630897-1.752239$

O $1.588840-1.705557-2.953945$

C $1.703772-0.392834-0.948529$

C $1.0208040 .654511-1.432920$

C $0.8084611 .934949-0.756306$

C 1.5649332 .1812410 .522061

C $5.6013840 .288343-1.598844$

C $5.5875881 .766315-1.952677$

C $4.2485823 .448066-3.213565$

O $6.4830102 .544121-1.634522$

C $3.4025254 .218831-2.249861$

C $2.2938084 .881883-2.612538$

C $1.4586935 .699621-1.669357$

C $-0.0014515 .306613-1.693307$

C $-0.890085 \quad 5.990802-2.433587$

C $-0.4437494 .176445-0.792685$

C $-0.0376812 .825832-1.314688$

N $4.4698772 .108048-2.702444$

H $-0.166081-3.2843550 .064462$

H $0.409681-5.337365-2.132213$

H $2.402908-3.986745-2.470287$

H $2.889126-5.926346-0.933845$

H $2.502798-4.9222380 .447106$

H $6.056908-3.7450800 .508127$

H $6.194335-4.333027-1.699893$

H $5.025157-5.530113-2.259492$

H $4.734988-3.796811-2.534409$

H $3.711316-4.7013362 .171091$

H $5.267136-4.1303002 .696390$

H $3.032651-2.3410011 .607569$

H $3.372756-2.6311463 .313664$

H $5.695531-1.6818712 .957189$

H $3.826589-0.2709523 .898358$

$\begin{array}{llll}\text { H } & 3.304140 & 0.120834 & 2.274684\end{array}$

H 4.9426413 .0296142 .956332

H 4.9547591 .5431940 .750802

H $5.673487 \quad 1.0349694 .931722$

H 6.8257531 .9123993 .952408

H 6.8132080 .1389243 .921313

H 7.0413112 .3356970 .473912

H $7.6087910 .511615-0.752678$

H $7.027007-1.125270-0.899246$

H $7.992462-1.1435431 .090013$

H $2.171936-0.3913400 .028384$

H $0.5486710 .559364-2.410074$

H 2.6361552 .0117230 .370429

H 1.2122381 .5127741 .314388

H 1.4732563 .2034050 .894622

H $5.763034-0.248155-2.542764$

H $4.619600-0.007586-1.218730$

H $3.7723293 .346745-4.193904$

H $5.2114923 .952573-3.343425$

H $3.7317864 .257062-1.214109$

H $1.9840014 .865194-3.655260$

H $1.5648606 .753299-1.959348$

H $1.8368255 .637544-0.641246$

H $-1.9439495 .733164-2.434173$

H $-0.5816936 .821252-3.060063$

H $-1.5382254 .171530-0.708765$

H $-0.089145 \quad 4.367786 \quad 0.223764$

H $-0.5346992 .554596-2.247258$

H $3.7525501 .409512-2.874910$

C $-2.807545-3.449132-0.783439$

O $-4.092777-3.936038-0.351663$

C $-5.121606-3.171312-1.022436$

C $-4.399799-2.226129-1.978466$

C $-3.061665-2.909631-2.187792$

H $-5.725397-3.901042-1.573931$

C $-5.961541-2.4544740 .043233$

H $-4.955141-2.076592-2.909682$

H -4.249753 -1.240502 -1.519630 
C $-2.001608-1.964796-2.724345$

H -3.201572 -3.740375 -2.891989

C $-6.995039-1.457831-0.514538$

O $-6.636641-3.4441550 .826426$

H $-5.274698-1.9490860 .729671$

H -6.520122 -0.714066 -1.163175

O $-7.934775-2.161982-1.335154$

C $-7.799523-0.7263930 .576626$

C -6.9553000 .0131611 .626289$

H -8.452063 -1.439185 1.095576

O $\quad-8.676615 \quad 0.215180-0.046425$

H -7.620724 0.5920502 .280446

H $-6.489141-0.7284372 .288854$

H -2.346013 -1.481810 -3.644863

H -1.760343 -1.178404 -2.001419

H - $-1.082088-2.504687-2.962624$

C -1.784858 $-4.581369-0.672760$

H $-7.280623-3.8870810 .247587$

H -7.459247 -2.484520 -2.119135

H $-9.210534-0.272281-0.697012$

H -1.942243 -5.313594 - 1.474038

H $-1.957637-5.1253850 .264864$

H $-2.541330-2.632950-0.097147$

C -3.9319582 .1566491 .985891$

O

C $\begin{array}{llll}-5.844319 & 0.945611 & 1.094098\end{array}$

C $-6.3502542 .177407 \quad 0.328189$

C $-5.2030373 .136159-0.010596$

C -4.3193673 .4243881 .207723$

H -3.579324 2.469179 2.977536

C -2.8011571 .3551671 .319055$

H $\quad-5.1841590 .3624600 .447647$

O $-7.3251042 .858178 \quad 1.114490$

H $-6.8347041 .853488-0.599210$

H $-5.6225174 .075780-0.390228$

O $-4.4228272 .559411-1.054409$

H -3.4112103 .9595450 .909067$

O -5.0287614 .2806492 .107512$

C -2.2373350 .2818502 .226692$

H -3.1266200 .8606690 .400731$

H -1.9794692 .0393781 .079799$

H -7.657691 3.6050360 .588224

H $-3.7193003 .191931-1.278765$

H -5.2397115 .0999661 .628157$

O $-1.125083-0.2527981 .638932$

O $-2.708930-0.0541213 .303821$

C $-0.460228-1.2716012 .392246$

H $-0.189475-0.899733 \quad 3.385170$

H -1.099160 -2.156208 2.470168

H $\quad 0.456126-1.5439201 .862559$

SCF Energy (B3LYP/6-31G**//MMFF) $=-3245.89625776$

\section{7 _00478}

MM̄̄FF Geometry

C $2.5462952 .662879-2.194509$

C $2.3178401 .341871-2.271037$

C $0.9902960 .672264-2.546172$

O $-0.0441691 .669306-2.703281$

C $1.030096-0.220584-3.800668$

C $1.792779-1.515320-3.594867$

C $1.315536-2.563327-2.892598$

C $3.155562-1.580190-4.231375$

C $-0.040359-2.650824-2.244197$

C $-0.531797-4.101214-2.144924$

C -1.999702 -4.247762 -1.707107

C $-2.970394-3.863467-2.827764$

C $-4.432844-4.003126-2.383183$

O $-2.230554-3.442722-0.547511$

O $-5.256015-3.337921-3.354927$

C $-4.616135-3.247376-1.049786$

C $-4.886778-5.467085-2.367597$

O $-5.917340-3.468741-0.511881$

C $-3.543784-3.5806210 .016852$

C -3.632705 -2.650794 1.247141

O $-3.734702-4.8983760 .548422$

C - $-1.2856351 .358144-2.242756$

O $-1.669470 \quad 0.276256-1.828848$

C $-2.0913452 .598721-2.263325$
C $-3.2580792 .626047-1.602617$

C $-4.1289153 .788779-1.429215$

C $-3.7410355 .068212-2.121842$

C $-3.351412-1.1720760 .971861$

C $-3.450821-0.3267052 .231173$

C -3.1345002 .0228063 .012360$

O $-3.861090-0.762807 \quad 3.303424$

C -4.4102742 .7773762 .803945$

C -4.4657574 .0885282 .524250$

C -5.7489264 .8389012 .298570$

C -5.7892205 .5363300 .955040$

C $-5.530070 \quad 6.8512750 .857270$

C $-6.2050804 .734549-0.260532$

C $-5.2091503 .669814-0.629327$

N -3.0454850 .9812282 .005333$

H $1.7201713 .348447-2.370075$

H $3.1347640 .654749-2.072938$

H $0.7608510 .070855-1.659481$

H $1.457500 \quad 0.340841-4.642719$

H $0.008958-0.448879-4.131163$

H $1.955968-3.435181-2.769304$

H $3.657809-2.534827-4.041379$

H $3.069746-1.462961-5.316694$

H $3.803453-0.786673-3.848012$

H $0.025326-2.225943-1.236368$

H $-0.764102-2.060418-2.809955$

H $-0.383485-4.626121-3.097165$

H $0.096395-4.613197-1.403338$

H -2.145157 $-5.300952-1.438471$

H -2.784531 -4.464490 -3.726572

H $-2.807995-2.819909-3.126293$

H $-5.084616-3.744127-4.221858$

H $-4.557351-2.178872-1.289319$

H $-4.826496-5.896980-3.374834$

H -5.937155 -5.552435 -2.068360

H $-4.285595-6.096667-1.708467$

H $-6.560433-3.232397-1.202187$

H $-4.614569-2.7647231 .725458$

H -2.908053 -2.999734 1.996969

H $-4.574001-5.2509810 .212094$

H $-1.6761803 .466087-2.763479$

H -3.595251 $1.717195-1.105751$

H -2.768905 $5.422144-1.760935$

H $-4.4525185 .881158-1.959825$

H -3.680236 $4.915555-3.205050$

H -2.347551 -1.056916 0.551750

H $-4.074466-0.7650220 .258117$

H -2.2525512 .6611182 .902828$

H -3.1226611 .5775944 .012038$

H -5.3359482 .2121272 .885875$

H -3.5413794 .6577552 .456448$

H -5.8505195 .5746163 .107140$

H -6.6209964 .1793942 .388970$

H $-5.5828157 .369723-0.094275$

H $-5.255857 \quad 7.4390091 .727223$

H $-6.4022705 .387273-1.119112$

H -7.169480 $4.252568-0.053670$

H -5.407862 2.705216 -0.161081

H -2.7618271 .2556941 .069463$

C $5.0872172 .471684-1.704462$

O $4.9523721 .607070-0.561978$

C $6.2621191 .343658-0.023529$

C $7.2496932 .167838-0.849018$

C $6.3672453 .256109-1.429819$

H 6.2601191 .7150651 .007195

C $6.541179-0.165117-0.043869$

H $8.0643422 .563893-0.234318$

H $7.707597 \quad 1.574884-1.649257$

C $6.9673763 .927637-2.652910$

H $6.1857054 .010387-0.652548$

C $5.501440-1.0225930 .706455$

O $6.564314-0.619943-1.405108$

H $7.543945-0.3607140 .352615$

H $4.538699-0.9784550 .185379$

O $5.937393-2.3870080 .630167$

C $5.315281-0.6581602 .192954$

C $4.468293-1.6707592 .990028$

H 4.8658960 .3367462 .278040 
O $6.598141-0.5847322 .822747$

H $4.904460-2.6722592 .890302$

H $4.555594-1.4145774 .054872$

H $7.9284404 .390153-2.404562$

H $7.1387373 .209074-3.461333$

H $6.3063684 .711900-3.034058$

C $3.8443653 .352054-1.853142$

H $5.689805-0.438694-1.790499$

H $6.043628-2.605000-0.311820$

H $7.019843-1.4566612 .731929$

H $4.0264074 .097790-2.635838$

H $3.6769063 .899838-0.916864$

H $5.2114341 .836945-2.592122$

C $0.995853-0.3113042 .823185$

O $2.391175-0.492126 \quad 3.103287$

C $2.986097-1.6890682 .586677$

C $2.263932-2.930843 \quad 3.131370$

C $0.762147-2.8646732 .844339$

C $0.166827-1.5238303 .279365$

H 0.6955720 .5407193 .447806

$\begin{array}{llll}\text { C } & 0.771939 & 0.074922 & 1.356944\end{array}$

H $2.909576-1.6915941 .496262$

O $2.482406-3.0620424 .534109$

H $2.688942-3.8227562 .656028$

H $\quad 0.260823-3.6831603 .374908$

O $0.555521-3.0543061 .447515$

H $-0.859116-1.4222662 .912761$

O $0.100388-1.4844584 .707879$

C $1.067401 \quad 1.5452751 .166412$

H $1.400609-0.4759920 .653506$

H $-0.266933-0.106296 \quad 1.063594$

H $2.184058-2.2401854 .960125$

H -0.403446 -3.039097 1.286558

H $-0.470139-2.2179164 .994747$

$\begin{array}{lllll}\text { O } & -0.090981 & 2.225009 & 0.931367\end{array}$

$\begin{array}{llll}\text { O } & 2.187744 & 2.032152 & 1.245177\end{array}$

C $0.035163 \quad 3.6438590 .799899$

H -0.9681674 .0650970 .693075$

H $0.499603 \quad 4.0702831 .694054$

H $0.6163503 .890362-0.092226$

SCF Energy (B3LYP/6-31G**//MMFF) $=-3245.91966518$

\section{7_00479}

MM̄FF Geometry

C 1.0475873 .4715812 .425706

C $-0.1894213 .055622 \quad 2.116935$

C $-0.957658 \quad 3.512047 \quad 0.904323$

O -1.1465132 .3317220 .090994$

C -2.312650 4.0936401 .349599

C $-3.081026 \quad 4.804030 \quad 0.252556$

C $-4.192164 \quad 4.328164-0.344350$

C -2.549106 $6.163791-0.125303$

C $-4.878240 \quad 3.014377-0.093601$

C $-5.0999252 .232512-1.397350$

C $-5.5616130 .780749-1.180245$

C $-6.9045540 .691765-0.450483$

C -7.307239 $-0.758923-0.148637$

O $-4.5362010 .106903-0.444666$

O $-8.400205-0.696906 \quad 0.780859$

C $-6.121540-1.4598150 .549076$

C $-7.836581-1.473364-1.396929$

O $-6.384158-2.8485340 .737219$

C $-4.782775-1.282255-0.199585$

C $-3.589147-1.8288230 .613286$

O $-4.830698-2.017458-1.427979$

C $-1.1443292 .481356-1.257326$

O $-1.147696 \quad 3.535470-1.878442$

C $-1.1320331 .177078-1.954994$

C $-0.6765990 .046502-1.393442$

C $-0.526646-1.247045-2.067233$

C $-1.046220-1.365472-3.476118$

C $-3.305835-1.0943691 .927101$

C -1.921519-1.444897 2.434585

C $-0.593257-3.3577293 .311186$

O $-0.979261-0.6622952 .333958$

C $-0.010403-4.1054822 .145058$

C $-0.687898-4.5907601 .092021$

C $-0.055101-5.325211-0.053383$
C $-0.419061-4.725603-1.393970$

C $-1.432247-5.225276-2.121691$

C $0.450425-3.616475-1.936120$

C $0.079432-2.256536-1.406857$

N -1.851867 -2.723443 2.965638

H 1.5566704 .1787551 .776355

H -0.6847172 .3394232 .769561$

H -0.3694654 .2576500 .356619$

H $-2.151817 \quad 4.8203412 .158474$

H -2.9187493 .2993231 .797872$

H $-4.6755454 .950763-1.096351$

H -3.168124 $6.657151-0.882188$

H -2.521566 6.8174020 .752798

H $-1.5363686 .085611-0.531611$

H -4.3173682 .3939260 .608331$

H -5.8452813 .2309850 .374042$

H -5.829722 $2.759622-2.024564$

H -4.155774 $2.207657-1.956338$

H -5.641388 $0.315363-2.169984$

H $-7.690866 \quad 1.200542-1.021874$

H $-6.843676 \quad 1.2205790 .509515$

H -8.712377 -1.6034160 .941380$

H -6.031006 -1.019902 1.550468

H $-8.737617-0.970978-1.769077$

H -8.139082 - $2.500913-1.166763$

H $-7.113721-1.503537-2.214736$

H -7.172804 -2.9263731 .299690$

H -3.724918 -2.901564 0.799403

H -2.689528 $-1.764044-0.010958$

H -3.920282 -2.190398 -1.720271

H $-1.4339111 .218252-2.996548$

H $-0.3303080 .074928-0.361887$

H -2.094240 -1.048283 -3.528070

H $-1.023998-2.386692-3.862790$

H $-0.455101-0.744163-4.157628$

H $-3.342463-0.0072531 .795211$

H -4.044284 -1.354129 2.693339

H -0.771011 -4.040133 4.147716

H $0.110166-2.5847233 .636464$

H $1.066821-4.2538522 .181363$

H $-1.768697-4.4844691 .050660$

H $-0.394555-6.368386-0.009329$

H $1.036262-5.3665050 .048654$

H -1.678661 -4.822962 -3.098530

H -2.041514 -6.044149-1.753338

H $0.446315-3.632707-3.031392$

H $1.497392-3.816213-1.674030$

H $0.405748-2.079288-0.382170$

H $-2.682784-3.3075952 .951697$

C $2.9321102 .065113 \quad 3.310054$

$\begin{array}{llll}\text { O } & 2.385349 & 0.819245 & 2.826318\end{array}$

C $2.968793-0.2603363 .573643$

C $4.2389920 .302043 \quad 4.189932$

C 3.7873031 .7098494 .530899

H $2.269827-0.4873844 .391308$

C $3.091264-1.5157412 .703818$

H $4.574112-0.2669415 .061913$

H 5.0608880 .3507373 .470611

C 4.9339162 .6752184 .777892

H 3.1593501 .6673585 .431217

C $3.993996-1.3733511 .459088$

O $3.526245-2.5890413 .533631$

H $2.079497-1.7521902 .352236$

H $3.795382-0.4006700 .998960$

O $5.368916-1.3912911 .835394$

C $3.747570-2.4709870 .404172$

C $4.669135-2.393487-0.827089$

H $2.703640-2.4138050 .083048$

O $3.926148-3.7569881 .001124$

H $5.700569-2.627603-0.534104$

H $4.365234-3.201566-1.505836$

H 5.5397672 .3433515 .627371

H 5.5910612 .7494043 .904897

H 4.5571613 .6781145 .001912

C 1.7885293 .0208993 .653265

H $3.524253-3.3974462 .993944$

H $5.541531-2.2455892 .267053$

H $3.684376-4.4248630 .336714$ 
H 2.1767593 .9113524 .161010

H 1.0938192 .5307214 .347404

H 3.5531482 .4746192 .502996

C $3.0548820 .487322-2.570040$

O $3.276401-0.792330-1.958186$

C $4.629767-1.049374-1.570405$

C $5.541760-1.045728-2.805974$

C $5.420570 \quad 0.278583-3.568000$

C $3.9590730 .674089-3.804330$

H $2.0164150 .446919-2.919394$

C $3.1450731 .603161-1.517759$

H $4.967314-0.265806-0.884351$

O $5.187340-2.127803-3.664447$

H $6.577620-1.204775-2.483126$

H $5.9272590 .191759-4.537090$

O $\quad 6.067320 \quad 1.314768-2.834648$

H $3.9087221 .708902-4.160346$

O $3.453462-0.152951-4.858227$

C $2.5117612 .901574-1.965750$

H $2.6231741 .279549-0.608625$

H $4.1842061 .804042-1.242422$

H $5.789051-2.106481-4.427770$

H $6.9993751 .060133-2.726016$

H $2.551504 \quad 0.148787-5.061161$

O $2.7426803 .854923-1.014801$

O $1.8929603 .065043-3.007908$

C $2.2305405 .158260-1.309397$

H $1.1524785 .117874-1.487990$

H $2.4173465 .800031-0.444469$

H $2.7492885 .576398-2.177093$

SCF Energy (B3LYP/6-31G**//MMFF) $=-3245.90145772$

\section{7_00480}

MM̄FF Geometry

C -0.281533 -0.3285361 .039778$

C $0.416386-0.203034-0.100281$

C $0.080210-0.817218-1.436897$

O $-1.053222-1.705380-1.296427$

C $-0.266132 \quad 0.255075-2.484818$

C $0.9362820 .962219-3.078198$

C $1.3042052 .222898-2.775072$

C $1.6944410 .179099-4.117842$

C $0.680438 \quad 3.112521-1.738159$

C $-0.421156 \quad 4.002842-2.324334$

C -1.298128 $4.681100-1.258635$

C $-0.5241545 .685656-0.404247$

C $-1.405378 \quad 6.288947 \quad 0.700711$

O $-1.853418 \quad 3.651110-0.435945$

O $-0.545088 \quad 6.964386 \quad 1.631183$

C -2.089952 5.140296 1.471053

C $-2.368850 \quad 7.348547 \quad 0.154130$

O -3.0227465 .6490922 .422253$

C -2.781709 $4.100844 \quad 0.556054$

C -3.209212 2.8696921 .399530

O $-3.9307924 .701556-0.038395$

C $-1.104194-2.789777-2.111134$

O $-0.303903-3.077826-2.991326$

C $-2.261979-3.659512-1.799134$

C $-3.251629-3.329459-0.954170$

C $-4.403051-4.169646-0.609723$

C $-4.457157-5.557688-1.194069$

C $-3.631472 \quad 1.6309510 .594133$

C -5.0628151 .6597180 .091512$

C $-6.6185190 .396199-1.391845$

$\begin{array}{lllll}\text { O } & -5.891382 & 2.483547 & 0.468704\end{array}$

C $-7.336086-0.612723-0.552624$

C $-7.776370-1.790572-1.019431$

C $-8.483743-2.807019-0.170513$

C $-7.799076-4.156271-0.185398$

C $-8.299856-5.169947-0.911676$

C $-6.595379-4.359891 \quad 0.708879$

C $-5.354904-3.669861 \quad 0.206623$

N $-5.3102020 .635291-0.812218$

H -1.204759 -0.8997951 .056468$

H $1.3430050 .366756-0.074254$

H $0.953422-1.404417-1.746911$

H $-0.9807870 .969669-2.057394$

H $-0.826132-0.205230-3.310312$
H $2.1547252 .657950-3.296152$

H $2.5056160 .761330-4.567074$

H $2.138217-0.717855-3.675336$

H $1.023915-0.127481-4.927292$

H $0.2951422 .512354-0.909515$

H $1.4730493 .734587-1.305592$

H $0.0204524 .760492-2.983059$

H -1.078877 $3.382736-2.948141$

H $-2.1140135 .188060-1.787440$

H $-0.0922136 .476562-1.029614$

H $\quad 0.3260425 .188768 \quad 0.081194$

H -0.0505817 .6445781 .142561$

H -1.3086304 .6262592 .048585$

H -1.810540 8.166091 -0.317741

H $-2.948387 \quad 7.8091810 .961640$

H $-3.0652446 .954191-0.588679$

H -2.5475776 .2877152 .980941$

H -2.346351 2.5569032 .002041

H -4.0081713 .1506922 .095961$

H -4.534514 $4.002400-0.332066$

H $-2.257217-4.609649-2.324455$

H -3.226633 -2.357331 -0.465115

H -4.499222 -5.513081-2.287952

H $-5.327664-6.132365-0.871253$

H -3.574001 -6.134817 -0.897870

H $-2.9512931 .455825-0.245934$

H $-3.560306 \quad 0.7564721 .254139$

H $-6.4622010 .048282-2.417628$

H $-7.1879211 .330484-1.420553$

H -7.500925 -0.3598360 .492348$

H $-7.627080-2.038083-2.067917$

H -9.510086 -2.899662 -0.548933

H -8.581983 -2.465012 0.867382

H -7.840988 -6.152786 -0.905250

H -9.180604 -5.039479-1.531921

H $-6.401030-5.4272300 .867337$

H $-6.826538-3.9763381 .711294$

H -5.237304 -2.653106 0.582011

H -4.581302 $-0.048123-0.996905$

C $0.882324-0.712636 \quad 3.263384$

O $2.023836-1.2670342 .574169$

C $1.931494-2.7000812 .632413$

C $1.096484-2.9864063 .871294$

C $0.044203-1.8974193 .756124$

H $1.364756-3.0202951 .749045$

C $3.319328-3.3424842 .602230$

H $0.672539-3.9948103 .868457$

H $1.678221-2.8560124 .791203$

C $-0.696875-1.6221515 .053194$

H $-0.686496-2.2026742 .997121$

C $4.199641-2.8979711 .413040$

O $4.012216-3.0002483 .808278$

H $3.211638-4.4338542 .610228$

H $4.557931-1.8789601 .594501$

O $5.367914-3.7279711 .409014$

C $3.520295-2.9718970 .034075$

C $4.455726-2.755569-1.168965$

H $2.712080-2.235179-0.007727$

O $2.919774-4.262372-0.112639$

H $5.138286-3.609662-1.266858$

H $3.824202-2.782418-2.067263$

H -1.237595 -2.514889 5.384022

H $-0.009981-1.3295475 .854457$

H -1.424131 -0.8150044 .919916$

C 0.1580650 .2784462 .344903

H $4.889283-3.4188223 .767099$

H $5.083581-4.6326781 .193121$

H $2.429061-4.263380-0.952371$

H $\quad 0.828137 \quad 1.1223552 .139404$

H $-0.717094 \quad 0.6956272 .855837$

H $1.286291-0.1564714 .118696$

C $4.9447100 .948499-0.932194$

O $4.347302-0.353684-1.022095$

C $5.261524-1.447095-1.142134$

C $6.127550-1.289468-2.403444$

C $6.8326600 .069170-2.433161$

C $5.8653561 .219716-2.136172$

H $4.1012951 .647711-0.992215$ 
C 5.6474001 .1701150 .417372

H $5.915613-1.469543-0.265243$

O $5.329961-1.431784-3.577904$

H $6.872781-2.093273-2.423486$

H $7.2824450 .215118-3.423011$

O $7.8850910 .056424-1.473548$

H $6.4236092 .149471-1.976284$

O $5.0522721 .416624-3.295799$

C 4.6761371 .1766251 .577969

H $6.390646 \quad 0.3927850 .612849$

H 6.1530192 .1425150 .419051

H $4.630508-0.756910-3.545250$

H $8.3431040 .912406-1.529049$

H $4.5017392 .202054-3.137102$

O 5.3743721 .0281542 .742372

O $3.4655611 .318606 \quad 1.481317$

C 4.5924011 .0270743 .940942

H 3.9220661 .8915003 .971134

H 4.0256150 .0950694 .011573

H 5.2760511 .0869664 .791895

SCF Energy (B3LYP/6-31G**//MMFF) $=-3245.91774787$

\section{00481}

MM̄MF Geometry

C $-0.053675-4.4660300 .110370$

C $-0.413331-3.663813-0.903299$

C $0.405137-3.313384-2.122893$

O $0.822305-1.933739-1.967397$

C $1.668762-4.164450-2.343551$

C $2.389838-3.825874-3.634325$

C $3.525128-3.103302-3.707134$

C $1.776285-4.398743-4.885774$

C $4.273025-2.457259-2.572630$

C $4.637471-1.002764-2.900101$

C $5.271286-0.228825-1.731747$

C $6.532139-0.898158-1.185906$

C $7.130423-0.107659-0.015560$

O $4.300428-0.109177-0.688940$

O $8.099767-0.9669440 .606740$

C $6.0195690 .186670 \quad 1.021739$

C $7.9022641 .120889-0.513027$

O 6.5068351 .0742642 .023711

C 4.7050590 .7338610 .405991

C 3.5220260 .7001411 .401305

O $4.8299402 .093850-0.016606$

C $0.060111-0.968993-2.547541$

O $-0.946746-1.130337-3.218413$

C $0.6537210 .341544-2.195300$

C $0.002413 \quad 1.463482-2.535305$

C $0.3922372 .836773-2.205720$

C $1.7268733 .045878-1.545087$

C 3.6840591 .5829792 .639736

C 2.3602812 .1546383 .104865

C 0.8254064 .0666722 .741216

O $1.734297 \quad 1.6648504 .041334$

C 0.5602285 .0937611 .687181

C $-0.607516 \quad 5.228307 \quad 1.039697$

C -0.8867096 .3334220 .061088$

C -1.369603 $5.836994-1.281854$

C $-2.6625985 .949767-1.632748$

C $-0.3355965 .308786-2.253773$

C $-0.4730223 .830430-2.496552$

N $1.967426 \quad 3.2586382 .360028$

H $0.918981-4.9486290 .110216$

H -1.388581 -3.182306 -0.853058

H $-0.245607-3.421341-2.999538$

H $1.402436-5.229471-2.368555$

H $2.349520-4.051974-1.493161$

H $3.976855-2.959293-4.687837$

H $2.369300-4.176183-5.779230$

H $1.698588-5.487999-4.806457$

H $\quad 0.774703-3.988677-5.046868$

H $3.700898-2.478878-1.641934$

H $5.186459-3.039580-2.408681$

H $5.323486-0.983193-3.756422$

H $3.725221-0.473723-3.206270$

H $5.5040430 .774343-2.108005$

H $7.276040-1.037569-1.979999$
H $\quad 6.292662-1.903381-0.815013$

H $8.538482-0.4656101 .314400$

H $5.783981-0.7601251 .527801$

H $8.7482670 .810193-1.138276$

H $8.337897 \quad 1.682470 \quad 0.320622$

H $7.2888491 .802659-1.105796$

H 7.2407330 .6307452 .481177

H 2.6121860 .9882050 .859146

H $3.335526-0.3350761 .717923$

H 5.7407372 .3883840 .138369

H $1.5796090 .347146-1.632564$

H $-0.9364191 .369200-3.080421$

H $1.7364612 .576469-0.557844$

H $1.9836434 .096492-1.397205$

H $2.5303512 .610757-2.149778$

H $4.121313 \quad 1.0074703 .463771$

H 4.3575952 .4280082 .457332

H 1.0723574 .5623203 .686566

H -0.0304003 .4102722 .926811$

H 1.3633425 .7973731 .478700

H -1.4259664 .5495891 .267083$

H $-0.0045926 .968987-0.087215$

H -1.644294 6.9872650 .513171

H -3.011528 $5.632815-2.610107$

H -3.400743 $6.358693-0.950890$

H $0.6664905 .589381-1.919498$

H -0.461343 $5.820866-3.217331$

H -1.412762 $3.561649-2.982144$

H 2.6358363 .6545521 .704316

C $-0.761935-3.7077742 .437759$

O $-1.360931-2.4549182 .049276$

C $-0.519209-1.3820122 .508103$

C $0.466176-2.0070743 .491460$

C $0.664810-3.3811872 .881683$

H $\quad 0.037804-1.0258171 .633361$

C $-1.361988-0.2432103 .090830$

H $1.394129-1.4324393 .570137$

H $0.038056-2.0972904 .496452$

C $1.259631-4.3988693 .838154$

H $1.332838-3.2722252 .018518$

C -2.4508730 .3199782 .154308$

O $-2.021668-0.7008934 .279102$

H $-0.7005550 .567952 \quad 3.413977$

H $-3.287327-0.3846002 .084231$

O $-2.973901 \quad 1.5025582 .774925$

C -1.9681500 .6867710 .741060$

C $-2.9487201 .546576-0.078162$

H $-1.744134-0.2311180 .187003$

$\begin{array}{lllll}\text { O } & -0.740914 & 1.414361 & 0.830365\end{array}$

H -3.0534892 .5378850 .381246$

H $-2.4752111 .728086-1.049451$

H $2.251578-4.0781374 .172787$

H $0.632225-4.5308524 .726076$

H $1.365237-5.3735943 .351423$

C $-0.916022-4.7349161 .311897$

H $-2.560105-1.4735674 .035778$

H -3.285058 1.2507223 .661438

H $-0.9258702 .235171 \quad 1.315433$

H - $1.966161-4.7760180 .998418$

H $-0.666047-5.7332791 .690468$

H -1.347255 -4.060672 3.297228

C -5.354309-1.092557-1.173532

O $-4.156263-0.341767-0.934042$

C $-4.3354460 .924504-0.294458$

C $-5.237148 \quad 1.837069-1.144021$

C $-6.5660171 .152429-1.476530$

C $-6.356432-0.271852-2.003686$

H $-5.034208-1.945240-1.786241$

C $-5.956996-1.6455800 .127738$

H $-4.800750 \quad 0.774250 \quad 0.684414$

O $-4.5659892 .179054-2.356011$

H -5.417233 $2.767651-0.592155$

H -7.097455 $1.745452-2.230909$

O $\quad-7.3687691 .125764-0.300304$

H -7.314020 -0.802698 -2.048475

O $-5.872287-0.198577-3.348196$

C $-5.036913-2.6345020 .810499$

H $-6.167130-0.8485550 .845391$ 
H $-6.891626-2.172295-0.096432$

H $-5.1386542 .794157-2.844959$

H $-8.2169450 .711449-0.533344$

H $-4.9857220 .200853-3.323639$

O $-5.578370-2.9825882 .015330$

O $-3.984824-3.053610 \quad 0.349838$

C -4.815877 -3.9128922 .790507$

H $-5.417694-4.2093643 .653644$

H -4.581838 -4.806886 2.204424

H $-3.900888-3.4337833 .148746$

SCF Energy (B3LYP/6-31G**//MMFF)= -3245.90869105

\section{00482}

MM̄MF Geometry

C $-0.789110 \quad 5.8133850 .827061$

C $-0.0487345 .200578-0.109282$

C $1.3248624 .631460 \quad 0.135842$

O 1.2229883 .1881850 .096523

C $2.2785155 .075900-0.988437$

C $3.6888694 .537778-0.847426$

C $4.2755633 .694229-1.719809$

C 4.4578555 .0270320 .352841

C $3.7002193 .117328-2.984239$

C $3.0086671 .754996-2.813649$

C $3.9540860 .617566-2.393721$

C $3.233553-0.732344-2.437636$

C $4.129051-1.878690-1.938838$

O $4.4049390 .869997-1.061518$

O $3.292224-3.021326-1.701767$

C $4.749514-1.485257-0.580039$

C $5.150789-2.304128-3.000000$

O $5.719599-2.443292-0.165293$

C $5.368400-0.072411-0.572239$

C 5.7924390 .4010320 .839376

O $6.547499-0.071448-1.386617$

C $0.8112482 .562997 \quad 1.233754$

O $\quad 0.5415473 .0838432 .304537$

C 0.7180631 .1153160 .945186

C $0.0259380 .324463 \quad 1.778775$

C $-0.173763-1.1189861 .635749$

C $0.626758-1.8282260 .577323$

C 4.6646870 .4697681 .875790

C $4.572949-0.7551632 .771071$

C $3.167433-1.7280964 .584628$

O $5.418533-1.6453672 .787329$

C $2.297867-2.7915623 .992755$

C $1.149904-3.1996254 .554499$

C $0.293688-4.3030524 .003279$

C -1.136609-3.871631 3.772276

C $-2.101889-4.1662334 .659391$

C $-1.460934-3.1807282 .468210$

C -1.048737 -1.733868 2.458683

N $3.448153-0.7147873 .584856$

H -0.3942755 .9371631 .832008$

H $-0.4722235 .052565-1.100600$

H $1.718374 \quad 4.9516981 .108357$

H $2.3304636 .172588-1.017411$

H $1.8506224 .770430-1.949424$

H $5.2987733 .380477-1.515290$

H 5.5079354 .7161620 .328485

H $4.442306 \quad 6.1209460 .394048$

H 4.0222214 .6348291 .276785

H $2.9943083 .818607-3.441319$

H $4.5141823 .019945-3.714107$

H $2.2007141 .855544-2.079649$

H $2.5425101 .495005-3.772154$

H $4.8117930 .614467-3.076672$

H $2.865922-0.946060-3.449080$

H $2.340628-0.687679-1.799879$

H $2.826381-3.228767-2.529729$

H $3.942060-1.5212250 .159450$

H $4.639759-2.636819-3.911546$

H $5.741393-3.161595-2.659007$

H $5.836295-1.501860-3.280910$

H $5.283211-3.312342-0.156658$

H $6.640007-0.1949251 .198536$

H $\quad 6.183417 \quad 1.423416 \quad 0.732476$

H $7.1055480 .677973-1.120239$
H $\quad \begin{array}{llll}1.203723 & 0.745351 & 0.050191\end{array}$

H -0.4617990 .7780172 .640862$

H $\quad 0.325497-1.496358-0.421084$

H $0.524469-2.9146250 .602746$

H $1.693515-1.6219480 .708436$

H $4.861247 \quad 1.3186492 .543570$

H 3.6965260 .6482561 .397984

H $2.689335-1.2249685 .431051$

H $4.108045-2.1707114 .928035$

H $2.643265-3.2686363 .078439$

H $0.821023-2.7394935 .483706$

H $0.321661-5.1347484 .719438$

H $0.708218-4.7045193 .070093$

H -3.135390 -3.886521 4.483717

H -1.878671 -4.689550 5.583263

H -2.543725 -3.2076052 .289921$

H -1.028537 -3.7562191 .646649$

H -1.578396 -1.128116 3.195438

H 2.7709540 .0325983 .462918

C -3.2110065 .1856750 .847193$

O $-3.0623964 .163506-0.161448$

C $-4.3532293 .858642-0.708151$

C -5.3655224 .3869160 .294381$

C -4.6655275 .6543910 .757548$

H $-4.4567494 .461170-1.622133$

C $-4.4148512 .385074-1.128543$

H $-6.3442874 .578762-0.155119$

H $-5.502743 \quad 3.7105321 .141921$

C -5.2119186 .2112952 .060647$

H $-4.7662126 .417661-0.026023$

C $-4.0334311 .349669-0.050406$

O $-5.6969602 .089822-1.674380$

H -3.695195 2.288459-1.947901

H -3.074309 1.6202190 .406017

O -4.9978801 .3755831 .005630$

C $-3.943969-0.105819-0.563892$

C $-2.874308-0.299936-1.654364$

H $-4.920442-0.443149-0.930448$

O $-3.610302-0.9374430 .550030$

H -3.092595 $0.353232-2.504528$

H $-1.902874-0.007425-1.235987$

H -6.2677146 .4799001 .951321$

H -5.1332435 .4814922 .873322$

H -4.663215 7.1097452 .360415

C -2.1921036 .2963290 .579488$

H $-6.3589862 .225015-0.976064$

H -5.8395431 .0427730 .650921$

H $-4.283153-0.7909891 .236604$

H -2.380949 7.1592311 .227959

H -2.279481 $6.645969-0.457047$

H $-3.0169794 .707053 \quad 1.815924$

C $-2.124406-3.971387-1.462033$

O $-2.247262-2.578538-1.147487$

C $-2.776762-1.748208-2.183797$

C $-1.882857-1.825038-3.434095$

C $-1.681656-3.273424-3.887590$

C -1.271579-4.180872 -2.723242

H -1.574377 -4.401802 -0.615960

C $-3.490496-4.662019-1.528821$

H $-3.788547-2.083526-2.436729$

O $-0.616849-1.230423-3.152287$

H -2.349459 -1.242176 -4.237404

H $-0.909182-3.301465-4.665708$

O $-2.896085-3.741030-4.466743$

H -1.315553 -5.235094-3.020455

O $0.087415-3.903076-2.373124$

C -4.147589-4.670032 -0.168867

H -4.173332 -4.197491 -2.245848

H $-3.384301-5.708284-1.839245$

H $-0.087189-1.265844-3.966886$

H $-2.745398-4.653152-4.768221$

H $\quad 0.634267-4.082412-3.156807$

O $-5.096426-3.693498-0.102504$

$\begin{array}{lllll} & -3.837708 & -5.438912 & 0.732217\end{array}$

C $-5.782871-3.5846461 .148148$

H $-6.487096-2.7513871 .077063$

H -6.342424 -4.502359 1.352494

H $\quad-5.074488-3.3758201 .955255$ 
H $-5.729685-2.875013-4.371321$

H $-4.772822-1.614683-3.616853$

H $-8.808960-0.934730-3.953360$

H -7.890574 -2.198858 -4.945189

H $-6.820682-1.083208-1.138910$

H -7.918175 -0.038849-1.985611

H $-6.0810691 .262790-3.032581$

H -2.228932 -3.2298270 .017261$

C $2.7366922 .987871-2.885199$

O $3.4587092 .917655-1.641269$

C $4.5122771 .934991-1.760125$

C $4.3377361 .291234-3.128410$

C $3.6954432 .410363-3.924571$

H $5.4513492 .500192-1.716747$

C $4.4148500 .959183-0.580653$

H $5.2862480 .950252-3.553983$

H $3.6627190 .427674-3.070617$

C $3.0212781 .929248-5.198028$

H $4.4661493 .150623-4.177692$

C $5.420935-0.206379-0.650400$

O $4.645110 \quad 1.720913 \quad 0.609521$

H $3.3882180 .583507-0.521660$

H $5.262341-0.791829-1.562179$

O $6.7448530 .331895-0.763142$

C $5.398322-1.1515780 .567228$

C $4.026689-1.7597680 .890241$

H $5.755601-0.6158991 .455007$

O $6.335423-2.2089170 .357662$

H $4.120160-2.4084011 .770981$

H $3.352550-0.9570611 .211973$

H $3.749647 \quad 1.450536-5.860855$

H $2.232277 \quad 1.200565-4.983642$

H $2.5694822 .763967-5.742654$

C $2.3136954 .434895-3.169853$

H 4.3646841 .1815431 .367700

H $\begin{array}{llll}6.896346 & 0.902391 & 0.009987\end{array}$

H $7.194628-1.8005720 .154767$

H $1.9750254 .503291-4.210495$

H $3.1794945 .100341-3.065053$

H $1.8536562 .341988-2.789668$

C $1.103389-3.440474-0.671645$

O $1.998198-2.8080120 .251206$

C $3.324466-2.548117-0.236799$

C $4.005310-3.865804-0.643735$

C $3.136448-4.676645-1.613669$

C $1.682890-4.783437-1.140045$

H $\quad 0.199224-3.647147-0.085080$

C $0.706568-2.517177-1.830218$

H $3.244757-1.894699-1.110098$

O $4.263788-4.6737830 .502118$

H $4.964949-3.654090-1.127196$

H $3.553549-5.686034-1.717535$

O $3.158933-4.066967-2.901242$

H $1.049372-5.196438-1.933882$

O $1.613551-5.687499-0.034258$

C $-0.078660-1.324278-1.344441$

H $1.557090-2.141766-2.406100$

H $0.068121-3.056990-2.540272$

H $5.002552-4.2689780 .987235$

H $4.083299-4.050004-3.202608$

H $1.946775-6.548911-0.338066$

O $0.766049-0.400466-0.806947$

O $-1.294359-1.223302-1.438421$

C $0.1479920 .793414-0.325449$

H $\quad 0.900461 \quad 1.367213 \quad 0.220538$

H $-0.2079161 .386725-1.171464$

$\mathrm{H}-0.6743360 .5609700 .355856$

SCF Energy (B3LYP/6-31G**//MMFF) $=-3245.90766573$

\section{4}

MM̄FF Geometry

C $0.972250-3.3413190 .101814$

C $0.617546-2.6794691 .212738$

C $1.477933-1.6140201 .835209$

O $0.706852-0.388752 \quad 1.818568$

C $1.864353-1.935858 \quad 3.288986$

C $2.793073-3.1196503 .451765$ 
C $4.107735-3.096107 \quad 3.156120$

C $2.161082-4.3631164 .017986$

C $4.882166-1.9437242 .577167$

C $5.529138-2.3353681 .241977$

C $5.944186-1.151750 \quad 0.352770$

C $6.772697-0.0924951 .083298$

C $7.1184291 .094270 \quad 0.166970$

O $4.752006-0.563638-0.185524$

O $7.6203512 .158996 \quad 0.990007$

C $5.8286141 .586594-0.530143$

C $8.2692370 .748621-0.784200$

O $6.1234652 .599707-1.489235$

C $4.9979130 .450169-1.170892$

C $3.637316 \quad 0.995512-1.667944$

O $5.717062-0.065133-2.296019$

C $1.401044 \quad 0.779614 \quad 1.753379$

O $2.613637 \quad 0.9191631 .750411$

C 0.4222501 .8818311 .652945

C 0.8234473 .0669051 .170882

C $-0.0224294 .241747 \quad 0.971053$

C $-1.395068 \quad 4.235687 \quad 1.588426$

C $2.633239-0.101823-2.042413$

C $1.5057740 .451727-2.889007$

C $-0.552181 \quad 1.852956-2.764787$

O $1.4503240 .239855-4.098539$

C $-0.3164803 .330353-2.804202$

C $-1.2374804 .228613-2.422651$

C -1.031991 $5.717528-2.464115$

C $-1.3537626 .381263-1.142295$

C -2.583376 $6.869258-0.904004$

C $-0.2465116 .547290-0.123314$

C $0.457032 \quad 5.267970 \quad 0.238029$

N $0.5966311 .201872-2.162469$

H $1.926494-3.114529-0.368835$

H $-0.333664-2.9013681 .688597$

H $2.374604-1.4724971 .221425$

H $0.954230-2.0910713 .884390$

H $2.326035-1.0542383 .753356$

H $4.695015-3.9952783 .336435$

H $2.877069-5.1857734 .117194$

H $1.750384-4.1599265 .012394$

H $1.349261-4.7089663 .370479$

H $4.251623-1.0620842 .448716$

H $5.664220-1.6624163 .292715$

H $6.411580-2.9541361 .449172$

H $4.828543-2.9579630 .668858$

H $6.522895-1.575445-0.476754$

H $7.683440-0.5335281 .506650$

H $\quad 6.2046620 .300658 \quad 1.936487$

H 6.9266332 .4066001 .624684

H 5.2110052 .0710710 .238182

H $9.1638730 .470315-0.213795$

H $8.560268 \quad 1.616637-1.386203$

H $8.037865-0.078214-1.458616$

H $6.4941582 .163986-2.275553$

H $3.1791731 .611967-0.885244$

H $3.7993601 .649215-2.534755$

H $5.089023-0.414186-2.947655$

H -0.6030501 .6816561 .937043$

H 1.8612673 .1805140 .859729

H -2.041133 3.5195841 .070157

H $-1.8864815 .210947 \quad 1.548520$

H -1.3445443 .9626362 .648562$

H $3.104969-0.908609-2.613211$

H $2.209733-0.560781-1.142589$

H -1.424071 $1.592086-2.156770$

H $-0.7041371 .476937-3.780836$

H $0.644616 \quad 3.677262-3.176208$

H -2.207428 $3.875477-2.078473$

H -1.683875 $6.122940-3.249158$

H $-0.009628 \quad 5.976163-2.765897$

H -2.823075 $7.369710 \quad 0.028252$

H -3.378860 $6.775819-1.635919$

H $-0.609366 \quad 7.0309430 .792254$

H $0.4961707 .241177-0.538971$

H $1.4616995 .189438-0.178182$

H $\quad 0.7400891 .323402-1.165136$

C $-0.333437-3.919329-1.939305$
O $-1.457722-3.027697-1.777711$

C $-2.403342-3.283488-2.836432$

C $-1.720754-4.253439-3.794386$

C $-0.836022-5.038791-2.847173$

H $-3.260390-3.792736-2.382279$

C $-2.842023-1.966853-3.485085$

H $-2.439559-4.879518-4.331821$

H - $1.112804-3.725632-4.538931$

C $0.265471-5.810898-3.552595$

H $-1.460505-5.737258-2.274087$

C $-3.585405-1.005805-2.531864$

O $-1.682341-1.287634-3.983230$

H $-3.466690-2.173777-4.361549$

H $-2.913646-0.677468-1.731068$

O $-3.9301750 .167702-3.279678$

C $-4.879631-1.593447-1.934276$

C $-5.750476-0.577116-1.171088$

H $-4.633743-2.431895-1.274299$

O $-5.671114-2.121626-3.004152$

H $-5.9933050 .271892-1.821787$

H $-6.710145-1.066959-0.956075$

H $-0.161985-6.541464-4.247275$

H $0.918846-5.144588-4.126018$

H $0.886506-6.353624-2.833542$

C $0.139854-4.397988-0.566895$

H $-1.080613-1.146565-3.232120$

H $-3.1134670 .503735-3.686655$

H $-6.441326-2.564937-2.609424$

H $0.763052-5.293740-0.664838$

H $-0.720901-4.6609790 .059753$

H $0.456075-3.348682-2.447415$

C $-4.422674-0.9576702 .290185$

O $-5.026038-1.2154421 .015880$

C $-5.130452-0.0827230 .145025$

C -5.9895891 .0153210 .796010$

C $-5.460487 \quad 1.391827 \quad 2.182906$

C $-5.1768810 .153791 \quad 3.040991$

H $-4.559306-1.8901982 .853309$

C $-2.917726-0.726795 \quad 2.132486$

H $-4.1295950 .313061-0.056086$

$\begin{array}{lllll}\text { O } & -7.336875 & 0.559980 & 0.913543\end{array}$

H -5.9936331 .8947550 .140609$

H -6.1993972 .0243112 .690019$

$\begin{array}{llll}\text { O } & -4.272567 & 2.159233 & 2.021653\end{array}$

H $-4.6229690 .438452 \quad 3.941279$

$\begin{array}{lllll} & -4.622964 & 0.438452 & 3.941279\end{array}$

C $-2.194946-0.893692 \quad 3.445880$

H $-2.494034-1.4540001 .428564$

H $-2.6820160 .248963 \quad 1.698300$

H -7.8622941 .2907251 .281659$

H -3.9602212 .4085652 .908127$

H $-6.911664-0.7021892 .715730$

$\begin{array}{lllll}0 & -1.873858 & 0.327330 & 3.959472\end{array}$

O $-1.964325-1.9786823 .963836$

C -1.1930250 .3098845 .217443$

H $-1.027543 \quad 1.3449505 .527830$

H $-0.222807-0.1844335 .114938$

H $-1.804643-0.1898125 .974597$

SCF Energy (B3LYP/6-31G**//MMFF) $=-3245.91988010$

0700485

MMFF Geometry

C $1.104601-2.7672682 .533430$

C $1.627140-3.1101471 .345169$

C $0.886785-3.214646 \quad 0.033740$

O $-0.482630-2.7850640 .191883$

C $1.547988-2.280844-1.000433$

C $0.888813-2.334187-2.364025$

C $-0.029738-1.451680-2.806630$

C $1.328007-3.476187-3.242251$

C $-0.637099-0.297383-2.054405$

C $-0.4826831 .024956-2.815456$

C $-1.1077212 .240920-2.105816$

C $-0.306713 \quad 2.671399-0.877494$

C $-0.9620023 .864677-0.169199$

O $-2.448519 \quad 1.918537-1.729253$

O $-0.317305 \quad 4.0405351 .101500$

C -2.4523313 .5503910 .087571$ 
C $-0.7181815 .173281-0.929793$ O $-3.106722 \quad 4.7071010 .602064$ C $-3.1991613 .005110-1.153481$ C -4.573555 $2.393015-0.785839$ O $-3.3906564 .049910-2.106698$ C -1.478435 -3.642941-0.153516 O $-1.376220-4.821795-0.448533$ C $-2.730017-2.852638-0.176964$ C $-3.871617-3.400092-0.619526$ C $-5.142317-2.691993-0.798132$ C $-5.124591-1.195971-0.627725$ C $-5.6498573 .367010-0.310913$ C $-6.9770442 .657437-0.112403$ C -8.2140791 .2362721 .531839$ O $-7.8807442 .736834-0.941240$ C -7.943455 -0.2349341 .537531$ C -8.765082 -1.145475 0.992731 C -8.500261 -2.625325 1.019706 C -8.623915 -3.269309 -0.345248 C $-9.596989-4.160374-0.600062$ C $-7.628815-2.880711-1.416524$ C $-6.242765-3.397302-1.133442$ N -7.0289151 .9375701 .070502$ H $0.041520-2.5659162 .629069$ H $2.696398-3.3092801 .290821$ H $0.942841-4.254510-0.309237$ H $2.608714-2.542074-1.114893$ H $1.548655-1.251033-0.623578$ H $-0.436450-1.597126-3.806850$ H $0.850759-3.451452-4.227466$ H $2.410065-3.443055-3.400017$ H $1.074948-4.434086-2.776914$ H - $1.703265-0.517597-1.927865$ H $-0.219745-0.202934-1.050280$ H $0.575972 \quad 1.224039-3.023933$ H $-0.9808320 .913157-3.788220$ H - $-1.1330223 .056440-2.838346$ H $0.7279542 .910668-1.150892$ H $-0.246043 \quad 1.841812-0.162139$ H $-0.410458 \quad 3.215628 \quad 1.607680$ H -2.5009012 .7868080 .876066$ H $0.3578365 .359312-1.032513$ H $-1.1172476 .032465-0.379241$ H -1.153269 $5.175779-1.931370$ H -2.612422 4.9957221 .388395 H $-4.9621311 .867858-1.670463$ H $-4.4242181 .606037-0.035447$ H -4.119531 $3.793364-2.695873$ H -2.663558 -1.8121740 .122718$ H -3.874466 -4.452081 -0.900619 H $-4.381351-0.743994-1.294289$ H $-4.878115-0.9304970 .406148$ H $-6.075151-0.713677-0.859398$ H $-5.375796 \quad 3.8368690 .638506$ H $-5.8046584 .172149-1.037336$ H $-9.074137 \quad 1.4979990 .907557$ H -8.408926 1.5774762 .553380 H -7.034442 -0.5703122 .031808$ H $-9.689279-0.8152860 .523999$ H -9.219263 -3.075373 1.716439 H $-7.507728-2.8426321 .432775$ H $-9.695598-4.627565-1.574348$ H - $10.317876-4.4443200 .159491$ H -7.658001-1.798257 -1.564416 H -7.934386 -3.297056 -2.386053 H $-6.152757-4.479448-1.237957$ H -6.2194531 .9418341 .684513$ C $1.854897-1.1337314 .272140$ O $2.239384-0.2336873 .215060$ C $3.2001120 .706397 \quad 3.720371$ C 3.0844430 .6345155 .236969 C $2.799785-0.8425075 .439681$ H 4.1870330 .3327323 .422588 C 2.9786042 .0879153 .096626 H 3.9954550 .9707715 .740995 H $2.246210 \quad 1.2327375 .611793$ C $2.214473-1.1722566 .801488$ H $3.738707-1.3979245 .312404$
C 2.8032122 .0731331 .563597

$\begin{array}{llll}\text { O } & 1.787100 & 2.661243 & 3.650802\end{array}$

H 3.8009132 .7523633 .387536

H 1.8100061 .6794611 .317755

$\begin{array}{lllll}\text { O } & 2.803578 & 3.435310 & 1.116737\end{array}$

C 3.8657851 .2825930 .780478

C $3.6686841 .418813-0.742390$

H 3.8108770 .2250031 .059282

$\begin{array}{lllll}\text { O } & 5.154832 & 1.766221 & 1.152260\end{array}$

H $2.6073901 .288458-0.983423$

H $3.9728412 .428444-1.045312$

H $2.912124-0.8928737 .597751$

H $1.274823-0.6372526 .974902$

H $2.012927-2.2445896 .888832$

C $1.937662-2.5788613 .771990$

H 1.6710993 .5373663 .244239

H $3.6981983 .789751 \quad 1.259447$

H 5.8166491 .2374180 .675454

H $1.587010-3.2673004 .548659$

H $2.982465-2.8354213 .558089$

H $0.819130-0.8836584 .535951$

C $6.769944-0.293308-1.947687$

O $5.8700620 .630588-1.322864$

C $4.4802560 .385365-1.547247$

C $4.1607460 .452834-3.049156$

C $5.063381-0.487896-3.853707$

C $6.539890-0.335735-3.467298$

H $7.7681220 .127516-1.769606$

C $6.720729-1.674932-1.284588$

H $4.224401-0.608461-1.165890$

O $4.333709 \begin{array}{llll}1.790687 & -3.512365\end{array}$

H $3.107787 \quad 0.185017-3.198500$

H $4.939199-0.275857-4.922626$

O $4.637766-1.827717-3.628013$

H $7.138976-1.144200-3.902195$

O $7.0374960 .891230-4.008846$

C $7.118470-1.5841450 .170086$

H $5.733321-2.140975-1.349615$

H $7.418771-2.366208-1.770931$

H $4.0853451 .810306-4.452205$

H $5.199059-2.409598-4.168135$

H $6.936196 \quad 0.847931-4.974985$

O $6.028980-1.786157 \quad 0.964994$

O $8.256173-1.343108 \quad 0.552125$

C $6.270666-1.7100422 .372873$

H $7.022658-2.4465252 .671462$

H $6.588322-0.6994022 .645890$

H $5.335021-1.9363382 .890568$

SCF Energy (B3LYP/6-31G**//MMFF) $=-3245.90819327$

\section{$07 \quad 00486$}

MMFF Geometry

C $3.162151 \quad 0.3428832 .743695$

C $1.994039-0.3088762 .635580$

C $1.104662-0.571078 \quad 3.821026$

$\begin{array}{lllll}\text { O } & -0.241394 & -0.126248 & 3.538147\end{array}$

C $1.059790-2.0528904 .239417$

C $0.572851-3.051917 \quad 3.206590$

C $1.353683-3.9910692 .638757$

C $-0.899971-3.0300552 .891064$

C $2.836078-4.1645112 .816895$

C $3.496354-4.9637141 .681594$

C $3.592668-4.2801710 .305305$

C $4.582777-3.1160300 .292690$

C $4.682932-2.484419-1.104073$

O $2.303914-3.823546-0.109514$

O $5.397115-1.247867-0.955361$

C $3.262691-2.159088-1.613754$

C $5.517861-3.347553-2.058242$

O $3.331239-1.726045-2.970786$

C $2.256389-3.325981-1.452945$

C $0.817517-2.827567-1.740155$

O $2.576889-4.351702-2.397445$

C $-0.512016 \quad 1.201309 \quad 3.624370$

$\begin{array}{lllll}\text { O } & 0.287207 & 2.093345 & 3.875278\end{array}$

C $-1.947477 \quad 1.484573 \quad 3.383109$

C $-2.8667510 .534862 \quad 3.149118$

C -4.2998790 .7405402 .926020$ 
C -4.8179202 .1549172 .966268$

C $-0.285611-3.766245-1.231401$

C $-1.642185-3.429330-1.828048$

C -4.071994 -3.776020 -1.460395

O $-1.769188-2.921051-2.939566$

C $-4.982596-3.426988-0.325541$

C $-5.918894-2.468597-0.411606$

C $-6.925025-2.1686390 .664051$

C -6.954043 -0.714191 1.080962

C -7.4039820 .2448760 .253708$

C $-6.565276-0.3857832 .502831$

C $-5.076016-0.3406722 .697605$

N $-2.694429-3.808053-1.003726$

H $3.502593 \quad 0.6620253 .726212$

H $1.644945-0.6327621 .658441$

H $1.462500-0.0113794 .696394$

H $0.395156-2.1426315 .110054$

H $2.052933-2.3384624 .607257$

H $\quad 0.876053-4.7107991 .975597$

H -1.232066 -3.950202 2.397727

H -1.491850 -2.925660 3.806023

H -1.131460 -2.198528 2.219545

H $3.338661-3.1989712 .926349$

H $2.994337-4.7221903 .748333$

H $4.506250-5.2516322 .000528$

H $2.936858-5.9009341 .556195$

H $3.930586-5.055831-0.392644$

H $5.572487-3.4377340 .639346$

H $4.253895-2.3412810 .996415$

H $5.392729-0.799080-1.818443$

H $2.903344-1.301381-1.029210$

H $6.527698-3.491925-1.655853$

H $5.650957-2.852839-3.026798$

H $5.084886-4.334432-2.235114$

H $2.455114-1.390253-3.222700$

H $0.657784-1.857261-1.251916$

H $\quad 0.702087-2.666668-2.818829$

H $1.770393-4.848394-2.609402$

H -2.2118152 .5365243 .423340$

H -2.552582 $-0.505398 \quad 3.126574$

H -4.2700902 .7908052 .262245$

H -4.7088252 .5732003 .972767$

H -5.8709902 .2420562 .690743$

H - $0.071562-4.806837-1.497924$

H $-0.341070-3.703306-0.139519$

H -4.308694 -4.780862 -1.825785

H -4.171895 -3.080113 -2.299894

H -4.906153 -4.020875 0.581905

H -6.009658 -1.907470 -1.339314

H -6.754411 -2.807472 1.539375

H $-7.919522 \quad-2.4451770 .289451$

H -7.456915 1.2823070 .567016

H $-7.7274040 .013311-0.755748$

H $-6.980630-1.1466503 .176587$

H $-7.045070 \quad 0.5441962 .823899$

H -4.605245 -1.324426 2.676483

H -2.486218 -4.359340 -0.175841

C 4.2409522 .2163461 .497964

O $2.9830432 .840014 \quad 1.169228$

C 3.1933773 .7719720 .094758

C 4.6929994 .0246740 .058381

C 5.2211572 .6444320 .404354

H $2.9077073 .256605-0.828654$

C 2.3096845 .0090220 .279096

H $5.0325964 .383780-0.917607$

H $5.001748 \quad 4.7512190 .818804$

C 6.6748232 .6389460 .842946

H $5.1110831 .994770-0.474455$

C $0.814377 \quad 4.6907750 .497355$

O $2.760618 \quad 5.752306 \quad 1.417749$

H $2.4296995 .677068-0.581570$

H 0.6600494 .2793581 .502506

O 0.0880035 .9241680 .452261

C $0.2112503 .730101-0.541441$

C $-1.3003403 .508741-0.343368$

H $0.7157012 .761106-0.466589$

O $0.4556734 .233278-1.853790$

H $-1.505863 \quad 3.3443690 .720670$
H -1.841142 $4.406381-0.667353$

H 7.3200783 .0090160 .039516

H $6.831953 \quad 3.2753021 .720218$

H 7.0005971 .6258011 .098558

C 4.0457780 .7016311 .583305

H 2.7307445 .1576262 .187037

H $\quad 0.4809576 .5158891 .116642$

H $0.0261735 .103403-1.919851$

H 5.0114710 .2018301 .716560

H 3.6037890 .3346490 .649931

H 4.5501562 .6210042 .470604

C -2.042805 $1.516543-3.425036$

O $-1.6984562 .588273-2.537327$

C -1.807386 2.292529-1.143208

C $-3.2574311 .935470-0.795080$

C $-3.7521010 .770598-1.656669$

C $-3.4640220 .997565-3.144638$

H $-2.0443341 .972943-4.423576$

C $-0.9762830 .415093-3.418671$

H -1.160502 $1.442153-0.899488$

O $-4.0935223 .072748-0.986788$

H -3.3054721 .6645140 .263332$

H -4.831006 $0.640315-1.515292$

O $-3.110979-0.429988-1.238963$

H $-3.6338940 .076482-3.714589$

O $-4.379924 \quad 1.975574-3.647015$

C $0.3492130 .956328-3.902252$

H $-0.838588-0.030707-2.429930$

H -1.255445 -0.402118 -4.093541

H $-4.9963532 .819247-0.729505$

H $-3.339626-0.576470-0.305143$

H $-5.2788471 .625879-3.523677$

O $1.2529840 .968216-2.881509$

O $0.5500131 .340260-5.047536$

C $2.5452501 .489560-3.208417$

H $3.2125891 .295672-2.364396$

H $2.9529830 .993179-4.094336$

H $2.4802202 .569547-3.368354$

SCF Energy $\left(B 3 L Y P / 6-31 G^{* *} / / M M F F\right)=-3245.89947299$

\section{$07 \quad 00487$}

MM̄FF Geometry

C $-1.549440 \quad 4.719049-0.500073$

C $-0.6475295 .700267-0.344527$

C $0.8540825 .507025-0.348747$

O $1.196467 \quad 4.129344-0.063824$

C $1.4162075 .872564-1.733108$

C $2.9305455 .853521-1.789298$

C $3.6714484 .869233-2.336423$

C $3.6061617 .066758-1.202395$

C $3.1985293 .583518-2.960534$

C $3.7184302 .381700-2.170924$

C $3.3671091 .016222-2.780553$

C $1.861068 \quad 0.766137-2.883050$

C $1.542870-0.662667-3.353995$

O $3.9588730 .040640-1.913967$

O $0.150223-0.907151-3.104425$

C $2.337197-1.669400-2.497846$

C $1.748424-0.822673-4.864803$

O $2.196606-2.995777-3.002824$

C $3.834850-1.316065-2.358007$

C $4.487141-2.212425-1.279713$

O $4.491351-1.525853-3.608108$

C 1.1992533 .7450391 .243873

O 1.0147494 .4546802 .219696

C 1.4199212 .2818231 .279889

C $1.2772361 .607896 \quad 2.430897$

C 1.4129000 .1578532 .601202

C $1.911546-0.6352541 .423595$

C $5.954127-1.865907-0.992369$

C $6.354310-2.2772720 .409289$

C $6.103417-1.5972042 .796251$

O $7.035732-3.277188 \quad 0.620946$

C $4.990646-2.4050783 .385906$

C $4.283623-2.0246334 .461116$

C $3.230577-2.8830235 .099992$

C $1.938244-2.1463855 .361812$

C $1.551467-1.8556216 .616006$ 
C $1.044023-1.8411334 .181417$

C $1.072190-0.3867583 .787763$

N $5.864520-1.4131521 .378224$ H -1.204594 $3.703590-0.680579$ H $-0.9959816 .719039-0.190332$ H 1.2951826 .1540270 .419374 H $1.0750696 .875995-2.022370$ H $0.9961765 .199471-2.488516$ H $4.7548434 .987180-2.333770$ H $4.6956067 .028859-1.307928$ H $3.2590437 .975083-1.705645$ H $3.3829657 .152926-0.134570$ H $2.1096633 .542749-3.020899$ H $3.5745463 .540331-3.989426$ H $4.8123282 .443725-2.082721$ H $3.3522022 .425246-1.138310$ H $3.8384110 .949669-3.768246$ H $1.3814471 .490360-3.551392$ H $1.3989960 .915461-1.898045$ H - $0.363133-0.231962-3.580383$ H $1.886977-1.672508-1.496400$ H $1.104811-0.125561-5.414847$ H $1.457705-1.824548-5.199569$ H $2.778199-0.638110-5.178146$ H $1.245103-3.184600-3.064252$ H $3.907014-2.127705-0.353113$ H $4.442987-3.265244-1.585801$ H $5.440249-1.650984-3.449294$ H 1.6565351 .7880290 .346087 H $1.0059072 .161908 \quad 3.328443$ H $1.182060-0.6062020 .607355$ H $2.101188-1.6858471 .651849$ H $2.860036-0.2258811 .059038$ H $6.615049-2.373805-1.704167$ H $\quad 6.150669-0.792467-1.093932$ H $6.177827-0.6026363 .247059$ H $7.054510-2.1200132 .939296$ H $4.786280-3.3719612 .931524$ H $4.510346-1.0748274 .939783$ H $3.644486-3.2620276 .043728$ H $3.017075-3.770826 \quad 4.491478$ H $0.605791-1.3611666 .813160$ H $2.170661-2.1025227 .471954$ H $0.008614-2.0879824 .452078$ H $1.277998-2.5141593 .352335$ H $\quad 0.7120300 .2814394 .571673$ H $5.260570-0.6460911 .098031$ C $-3.643383 \quad 4.1012240 .705427$ O $\quad-3.3689772 .701100 \quad 0.512841$ C $-4.526777 \quad 1.9336690 .879010$ C -5.4919872 .9144521 .535163$ C -5.1630714 .2036570 .805180$ H $-4.9645331 .566672-0.057288$ C $-4.1311320 .736222 \quad 1.757620$ H -6.5360662 .6081901 .418568$ H $-5.291348 \quad 3.0223252 .607637$ C -5.6548585 .4500291 .519492$ H -5.609392 4.162374 -0.197494 C $-3.156670-0.2347411 .052529$ O H $-5.041707 \quad 0.1869092 .024046$ H -3.600426 -0.4933680 .085576$ O C -2.878268 -1.537095 1.833549 C -1.863980 -2.473609 1.146356 H $-3.820331-2.0741241 .994768$ O $-2.337988-1.242618 \quad 3.122843$ H $-0.886830-1.9803501 .083378$ H -1.716982 -3.3349221 .811948$ H -6.7448285 .4300261 .621334$ H -5.2251265 .5332252 .523420$ H -5.3845306 .3519850 .961718$ C $-3.0398674 .904468-0.448574$ H -2.811135 1.7864632 .745157 H -1.473222 0.5749191 .625071 H $-3.005735-0.7356773 .614686$ H -3.297489 $5.964502-0.346012$ H -3.449994 $4.555135-1.403944$
H $-3.1730104 .390447 \quad 1.654828$

C $-4.120672-4.169454-1.288687$

O $-3.512416-3.697180-0.080306$

C $-2.298105-2.960125-0.244043$

C $-1.230586-3.832305-0.919496$

C $-1.748208-4.391869-2.250933$

C $-3.135076-5.027135-2.104001$

H $-4.936796-4.822844-0.954725$

C -4.746427 -3.017681-2.091320

H -2.479919-2.087102 -0.876907

O $-0.869401-4.905015-0.052958$

H $-0.332566-3.225734-1.084681$

H -1.046940 -5.145964 -2.628763

O $-1.825612-3.348678-3.218015$

H $-3.557953-5.238966-3.093030$

O $-2.970145-6.284557-1.441307$

C $-5.838911-2.312375-1.312818$

H $-3.997604-2.268147-2.361076$

H $-5.197589-3.405746-3.011329$

H $-0.182618-5.426687-0.501691$

H $-0.942025-2.951225-3.297280$

H -3.840521 -6.716998-1.409657

O $-6.115602-1.117640-1.914261$

O $-6.394212-2.756660-0.317226$

C $-7.138514-0.336276-1.288369$

H $-7.1816880 .633153-1.792003$

H -8.106627 -0.833932 -1.396599

H $-6.905625-0.171011-0.231883$

SCF Energy $(B 3 L Y P / 6-31 G * * / / M M F F)=-3245.90891806$

\section{8}

MM̄FF Geometry

C 3.1212722 .7737051 .647813

C $2.165127 \quad 1.859149 \quad 1.421665$

C 1.4324351 .1253332 .516260

$\begin{array}{lllll}\text { O } & 0.008958 & 1.266625 & 2.292938\end{array}$

C $1.769937-0.3730072 .463110$

C $1.373228-1.1184933 .720564$

C $0.357856-1.9995743 .803363$

C $2.260701-0.8819934 .917365$

C $-0.618228-2.3863762 .723138$

C $-1.979210-2.7543113 .334528$

C -3.049694 -3.227685 2.338361

C $-2.629784-4.4707071 .550129$

C $-3.715222-4.9122370 .556293$

O $-3.370339-2.153681 \quad 1.448075$

O $-3.122749-5.851357-0.355058$

C $-4.154212-3.690680-0.279229$

C $-4.863005-5.6523971 .253042$

O $-5.270342-4.016355-1.105723$

C $-4.472789-2.4406750 .572663$

C $-4.739677-1.195714-0.303358$

O $-5.673119-2.689123 \quad 1.317251$

C -0.5495322 .4637782 .618321$

$\begin{array}{lllll}\text { O } & 0.008559 & 3.421322 & 3.130891\end{array}$

$\begin{array}{llll}\text { C }-1.967427 & 2.430280 & 2.199982\end{array}$

C -2.592433 3.5953521 .974614

C $-3.978600 \quad 3.771273 \quad 1.545135$

C -4.8723572 .5623481 .530540$

C $-3.518819-0.634756-1.037591$

C $-3.895007 \quad 0.537173-1.928457$

C $-2.9006562 .450550-3.168381$

$\begin{array}{lllll}\text { O } & -5.045020 & 0.742561 & -2.309877\end{array}$

C $-3.359482 \quad 3.643982-2.388443$

C $-4.5654954 .206618-2.560692$

C $-5.0721615 .399982-1.799520$

C $-6.0660395 .026502-0.718946$

C $-7.2200224 .402252-1.014110$

C $-5.756416 \quad 5.4202090 .708956$

C $-4.3896525 .003682 \quad 1.182554$

N $-2.793941 \quad 1.304153-2.284897$

H 3.4100623 .0040242 .669913

H 1.8782101 .6276650 .399301

H 1.6847821 .5402783 .499567

H $2.850719-0.5098572 .328473$

H $1.298054-0.8281931 .584846$

H $\quad 0.219645-2.5174264 .752022$

H $1.993847-1.5207325 .766306$ 
H $3.304617-1.0947724 .664402$ H 2.1886650 .1561435 .254885 H $-0.754463-1.5775411 .998461$ H $-0.199224-3.2497082 .196393$ H -1.840254 -3.539470 4.089393 H -2.366822 $-1.871418 \quad 3.860798$ H -3.940180 -3.456402 2.936619 H $-2.371811-5.2926172 .229336$ H -1.720729 -4.2611270 .973929$ H -2.772384 -6.594055 0.166089 H -3.330637 -3.454539 -0.961289 H $-4.486641-6.5467201 .764204$ H $-5.600066-6.0116920 .526566$ H -5.379757 -5.0421961 .996445$ H $-5.025426-4.798848-1.628673$ H -5.550894 -1.412783 -1.010135 H $-5.133755-0.3949220 .336719$ H -6.050012 -1.836577 1.590631 H -2.440012 1.4646322 .063630 H -2.027187 4.5188402 .098435 H -4.871104 2.066219 2.507369 H $-4.530717 \quad 1.8506150 .773784$ H -5.9146462 .8004191 .303858$ H $-2.763665-0.308467-0.313524$ H -3.072017 -1.393473 -1.686525 H $-3.5908312 .203760-3.982687$ H -1.914273 $2.636987-3.601755$ H -2.670652 4.058154 -1.656782 H $-5.2434913 .780412-3.297326$ H $-5.5506186 .091631-2.504805$ H $-4.2314885 .962080-1.375436$ H -7.940268 4.150007 -0.242990 H -7.474619 $4.133979-2.034021$ H $-6.5144305 .042174 \quad 1.406345$ H $-5.820280 \quad 6.5141940 .780800$ H $-3.672015 \quad 5.824157 \quad 1.217564$ H -1.885820 $1.097013-1.879359$ C 5.2051133 .0262480 .201000 O $5.1234321 .650069-0.218453$ C $6.207976 \quad 0.924651 \quad 0.387199$ C 7.2025381 .9808320 .850726 C 6.2579473 .0786001 .307672 H 5.7994930 .4261951 .274450 C $6.758099-0.118339-0.588803$ H $7.8573451 .616567 \quad 1.647967$ H 7.8306402 .3364340 .025687 C 6.9236024 .4345961 .460685 H 5.8257492 .7840772 .271970 C $5.698956-1.112525-1.116128$ O $7.3304430 .546661-1.721295$ H $7.581146-0.666399-0.115876$ H $5.026763-0.607576-1.819109$ O $6.382751-2.122956-1.866228$ C $4.877062-1.798059-0.012159$ C $3.977901-2.953389-0.477264$ H $4.253754-1.0522600 .489726$ O $5.761908-2.3248920 .982456$ H $4.593076-3.778182-0.859365$ H $3.492163-3.3474370 .425901$ H 7.7115124 .3924942 .219784 H 7.3794124 .7671910 .522026 H 6.1961035 .1918981 .769531 C 3.8107623 .5432570 .555472 H $6.634911 \quad 1.097779-2.119849$ H $6.895725-1.671783-2.558648$ H $6.351328-2.9616350 .542590$ H $3.1918603 .508767-0.348401$ H 3.8654014 .5975070 .850442 H $5.5612183 .586742-0.673688$ C $1.032448-1.047062-1.801811$ O $2.117318-1.508576-0.982308$ C $2.905956-2.578055-1.515251$ C $2.031981-3.798340-1.864183$ C $0.844918-3.413797-2.747494$ C $0.096826-2.205442-2.177842$ H $0.467192-0.366116-1.152055$ C $1.522639-0.257364-3.021529$ H $3.409752-2.229453-2.422102$
O $1.544041-4.426189-0.678294$

H $2.653540-4.538479-2.381825$

H $0.162563-4.269541-2.820176$

O $1.322414-3.123674-4.056978$

H $-0.660173-1.851051-2.886767$

O $-0.593352-2.595384-0.988876$

C $1.8837191 .157167-2.639292$

H $2.402292-0.697574-3.498971$

H $0.746828-0.196721-3.794878$

H $1.069482-3.754252-0.159218$

H $0.551945-2.904786-4.608187$

H -1.148674 -3.360859-1.212617

O $0.7603231 .929784-2.618066$

O $3.0228011 .521278-2.381652$

C $0.9318243 .313294-2.290348$

H $0.8288073 .440274-1.209750$

H $1.8959553 .700962-2.632721$

H $\quad 0.1394263 .880385-2.785707$

SCF Energy (B3LYP/6-31G**//MMFF) $=-3245.91159481$

\section{9}

MMFF Geometry

C $0.946167-3.511019-1.363317$

C $0.058099-2.536416-1.114500$

C $-0.933025-2.058584-2.144550$

O $-2.238058-2.458995-1.657691$

C $-0.942237-0.530474-2.339321$

C $0.4112930 .135083-2.427688$

C $0.9125490 .953602-1.481895$

C $1.188023-0.140021-3.687544$

C $0.2916311 .318315-0.162063$

C $-0.0579882 .806977-0.049540$

C $-1.1160013 .281241-1.058677$

C $-1.4448624 .758608-0.830700$

C $-2.5628075 .249679-1.763210$

O $-2.2925942 .485101-0.889292$

O $-3.0211996 .517892-1.268481$

C $-3.7528664 .269284-1.670024$

C $-2.0475225 .503054-3.184823$

O $-4.7651234 .608332-2.615765$

C $-3.3464262 .782611-1.812242$

C $-4.5312921 .819824-1.540004$

O $-2.9290012 .554960-3.165018$

C $-3.248858-2.578511-2.553949$

O $-3.172884-2.414568-3.764033$

C $-4.524405-2.946121-1.893689$

C $-4.672569-3.064968-0.564491$

C $-5.904845-3.4218020 .143306$

C $-7.139685-3.674924-0.681338$

C $-5.0472751 .784092-0.098379$

C -4.2222110 .9081300 .830581$

C -3.9227510 .4052983 .254427$

$\begin{array}{lllll}\text { O } & -3.450787 & 0.041494 & 0.429215\end{array}$

C $-4.868305-0.6414163 .769619$

C $-6.169170-0.7864243 .468555$

C $-7.044401-1.8621004 .048123$

C -7.734803 -2.687523 2.984548

C $-8.998075-2.4155432 .615807$

C $-6.999346-3.8802382 .416768$

C $-5.878096-3.4981031 .490782$

N $-4.489690 \quad 1.1774162 .165471$

H $0.930111-3.986550-2.340651$

H $0.026109-2.074180-0.131087$

H $-0.740536-2.551853-3.106557$

H -1.560236 -0.078381-1.558240

H $-1.497320-0.298846-3.258643$

H $1.8878491 .404175-1.656843$

H $1.410440-1.207930-3.777160$

H $0.6104320 .170249-4.564430$

H $2.142147 \quad 0.397267-3.713038$

H $-0.586851 \quad 0.711857 \quad 0.072730$

H 1.0169891 .0741290 .620063

H -0.4304992 .9766930 .969193$

H $0.8521893 .409058-0.162255$

H $-0.7248553 .136470-2.071892$

H $-0.5476055 .379448-0.946179$

H -1.775422 4.9017430 .207906

H $-2.2618737 .125416-1.251539$ 
H $-4.2023674 .426104-0.682820$

H -1.241680 $6.246987-3.172465$ H -2.833022 $5.926723-3.820130$ H -1.657535 $4.604712-3.667214$ H -4.466053 4.305783 -3.490169 H $-5.3610142 .083554-2.208549$ H $-4.2408770 .802860-1.836528$ H $-2.9870971 .603341-3.350675$ H $-5.352937-3.108611-2.575921$ H -3.812934 -2.886510 0.079286 H -7.384624 -2.795480 -1.287441 H $-8.026342-3.890900-0.081259$ H $-6.988292-4.531243-1.347610$ H -5.1375262 .7900590 .319918$ H $-6.054540 \quad 1.347842-0.105461$ H -3.6711111 .1034704 .058619$ H -3.000408 -0.0762672 .913248$ H -4.422374 -1.347389 4.468200 H $-6.652977-0.0873952 .791325$ H -7.793391 -1.373583 4.685108 H $-6.479657-2.5253264 .715075$ H -9.515037 -3.019746 1.877771 H -9.542436 -1.578942 3.041241 H -7.693999 -4.5683901 .921229$ H $-6.581193-4.4691243 .243657$ H -4.939839 -3.288077 2.005112 H -5.1897731 .8755652 .398972$ C $3.401035-3.973328-0.784512$ O $3.888673-2.620177-0.702016$ C $4.850312-2.418566-1.756506$ C $5.095516-3.788022-2.390299$ C $3.754798-4.463186-2.187948$ H $4.360961-1.772962-2.495453$

C $6.124658-1.739043-1.239649$ H $5.383539-3.709217-3.443173$ H $5.881627-4.347420-1.870376$ C $3.799869-5.974502-2.322914$ H $3.066961-4.055635-2.937613$

C $5.912088-0.431970-0.451189$ O $6.809917-2.648835-0.367260$ H $6.800125-1.564640-2.086253$ H $5.548795-0.6744370 .552711$ O $7.203967 \quad 0.162826-0.263960$ C $4.9735430 .593986-1.104228$ C $4.9945401 .994630-0.461538$ H $3.9520590 .199680-1.085816$ O $5.3402680 .747190-2.478304$ H $5.9395712 .498248-0.703758$ H $4.2147312 .582951-0.963574$ H $4.134010-6.261414-3.325330$ H $4.488588-6.422347-1.598646$ H $2.808681-6.409250-2.159542$ C $1.928094-4.056157-0.364792$ H $7.623579-2.207434-0.068813$ H $7.5261860 .444626-1.137518$ H $4.687267 \quad 1.337901-2.891343$ H $\quad 1.803850-3.5406180 .595287$ H $1.670300-5.108970-0.196511$ H $3.982772-4.539108-0.043973$ C 3.1865971 .2608422 .731374 O $3.529601 \quad 1.3733891 .342627$ C 4.7691752 .0241121 .059687 C 4.7590313 .4606971 .607433 C 4.4098763 .4864873 .099508 C 3.1702392 .6421953 .409783 H 2.1589650 .8764222 .737768 C $4.0682680 .232923 \quad 3.457444$ H 5.5843901 .4724151 .537066 O $3.821808 \quad 4.2621330 .889947$ H 5.7475903 .9068041 .448769 H 4.2252054 .5214893 .412681 O 5.5077062 .9897463 .858880 H 3.0569842 .5244174 .493737 O 2.0242453 .3617032 .943894 C $3.865389-1.1687642 .920667$ H $5.1300750 .471783 \quad 3.356645$ H 3.8115210 .2128784 .522655 H $2.948003 \quad 3.8440200 .974845$
H $\quad 6.274322 \quad 3.558517 \quad 3.673511$

H 1.2346282 .8538893 .196872

O $4.808605-1.9922593 .466702$

O $2.992285-1.5034042 .132357$

C $4.758816-3.3567573 .038752$

H $3.759726-3.7756603 .193126$

H $5.049499-3.4256931 .987164$

H $5.473146-3.9272683 .638381$

SCF Energy (B3LYP/6-31G**//MMFF) $=-3245.91497768$

\section{$07 \quad 00490$}

MM̄FF Geometry

C $-0.667895-3.883871-0.518074$

C $-0.526773-3.036799-1.548302$

C $-1.583668-2.041284-1.945229$

O $-0.975841-0.727127-2.010801$

C $-2.166218-2.326869-3.340461$

C $-2.964775-3.608840-3.444149$

C $-4.215866-3.763103-2.967419$

C $-2.277565-4.743698-4.156168$

C $-5.042468-2.735603-2.244240$

C $-5.240068-3.133627-0.775492$

C $-5.860563-2.0355360 .102012$

C $-7.283856-1.669212-0.321562$

C $-7.862972-0.5415370 .547542$

$\begin{array}{llll}0 & -5.008810 & -0.886845 & 0.046602\end{array}$

O $-9.053347-0.057650-0.093741$

C -6.8565090 .6281500 .572842$

C $-8.290732-1.0415201 .932035$

O $\begin{array}{llll}-7.275988 & 1.643516 & 1.482458\end{array}$

$\begin{array}{llll}\text { C } & -5.409261 & 0.190757 & 0.902617\end{array}$

C $-4.398709 \quad 1.344480 \quad 0.703423$

O $-5.344536-0.1989752 .279562$

C $-0.789639-0.061461-0.838510$

O $-1.065367-0.4534150 .283755$

C $-0.183408 \quad 1.248404-1.166734$

C $0.1389612 .098870-0.181171$

C $0.7636333 .412887-0.349094$

C $0.9261843 .933420-1.753753$

C $-4.2453791 .819559-0.745807$

C $-4.4343093 .317780-0.848883$

C $-3.3691775 .470360-0.237442$

O $-5.4743353 .808587-1.281499$

C $-1.9824906 .013866-0.354647$

C $-1.313915 \quad 6.571827 \quad 0.665667$

C 0.0544437 .1773090 .541204

C $1.076568 \quad 6.5016891 .426149$

C 1.3033846 .9362882 .677340

C 1.9038295 .3896550 .824117

C $1.1634194 .083370 \quad 0.751834$

N $-3.3346994 .029232-0.391397$

H $-1.594266-3.8800760 .051366$

H $\quad 0.395490-3.039718-2.124187$

H $-2.396459-2.007809-1.210447$

H -1.357011 -2.329206 -4.084531

H -2.793851 -1.479904 -3.649377

H $-4.706315-4.726195-3.101781$

H -2.900071 -5.643453 -4.202767

H $-2.037694-4.454551-5.184701$

H $-1.348064-5.012801-3.645399$

H $-4.591690-1.741493-2.312977$

H $-6.013747-2.667907-2.747696$

H $-5.854954-4.040590-0.717169$

H $-4.260782-3.387119-0.348217$

H $-5.864425-2.417461 \quad 1.129947$

H $-7.936631-2.550322-0.294711$

H $-7.287098-1.323323-1.363548$

H -9.669342 $-0.804878-0.185092$

H $-6.8769331 .085788-0.423713$

H $-9.052261-1.8248631 .837276$

H $-8.757560-0.2393302 .514187$

H -7.464878 -1.4544912 .514656$

H $-8.181798 \quad 1.897347 \quad 1.235417$

H $-4.6539172 .171638 \quad 1.378422$

H -3.410708 0.9988101 .037681

H $-4.421356-0.1350282 .575299$

H $-0.0097391 .473052-2.212987$

H $-0.048568 \quad 1.7984860 .848857$ 
H $-0.0335203 .918935-2.282879$

H $1.6422963 .321547-2.312750$

H $1.2774804 .966483-1.796619$

H -3.247336 $1.569870-1.121926$

H -4.949926 $1.339301-1.432186$

H -3.8178325 .6823510 .739006$

H -4.013506 $5.910812-1.005327$

H -1.520280 $5.972820-1.337847$

H -1.788095 $6.627270 \quad 1.643106$

H $-0.023799 \quad 8.2388670 .811012$

H $0.4027037 .169108-0.499121$

H 2.0586116 .4781553 .307395

H 0.7339647 .7573613 .100474

H 2.8001345 .2163081 .435452

H $2.2954785 .711347-0.145286$

H 0.9925473 .6269821 .727946

H -2.604045 3.5200200 .098416

C $0.788463-4.607698 \quad 1.378528$

O $1.448811-3.3308821 .495244$

C $2.581353-3.4723592 .371794$

C $2.443757-4.841073 \quad 3.027508$

C $1.780804-5.6359331 .920106$

H $3.472181-3.4808961 .734413$

C $2.656166-2.2959983 .350365$

H $3.409772-5.2530023 .334402$

H $1.795583-4.8061713 .910858$

C $1.138329-6.9257762 .399353$

H $2.534185-5.8706701 .156039$

C $2.684562-0.9038862 .685020$

O $1.518532-2.3331334 .221926$

H $3.532139-2.4185293 .997908$

H $1.696003-0.6677242 .273304$

O $2.9152820 .062912 \quad 3.718667$

C $3.746476-0.7310011 .582901$

C 3.8605680 .7314691 .110185

H $3.487281-1.3729350 .733738$

O $5.005416-1.1683902 .093450$

H $2.857146 \quad 1.1511500 .980136$

H 4.3791021 .3087491 .885749

H $1.890050-7.5887912 .839958$

H $0.372432-6.734776 \quad 3.158553$

H $\quad 0.664217-7.458377 \quad 1.569133$

C $0.391917-4.853513-0.077849$

H $0.721643-2.2852753 .666032$

H $3.811846-0.0869074 .065176$

H $5.655414-1.0981851 .373904$

H $-0.003924-5.868323-0.195374$

H $\quad 1.275098-4.765442-0.722723$

H -0.103637 -4.563555 2.017302

C $6.8006100 .398623-1.185207$

O $5.9661130 .405767-0.020157$

C $4.6298010 .872337-0.218855$

C $4.6453332 .324013-0.721460$

C $5.5082352 .469136-1.978739$

C $6.8777441 .800788-1.813712$

H $7.7986350 .139330-0.809417$

C $6.381965-0.690408-2.179763$

H $4.1305920 .236805-0.958604$

O $\quad 5.1431493 .1798950 .304619$

H $3.6173682 .631565-0.936894$

H $5.6461523 .535129-2.197933$

O $4.8093171 .888860-3.076135$

H $7.3911401 .747038-2.780878$

O $7.6710212 .636885-0.965867$

C $6.505126-2.060542-1.554957$

H $5.358974-0.560297-2.543799$

H $7.027359-0.678575-3.065878$

H $5.0976504 .092889-0.026943$

H $5.3545452 .015611-3.871259$

H $8.5583592 .243422-0.911180$

O $5.266443-2.585817-1.333244$

O $7.573794-2.591229-1.280671$

C $5.244963-3.883525-0.731253$

H $5.783826-4.602584-1.355637$

H $5.679473-3.8402770 .271935$

H $4.202555-4.203022-0.649938$

SCF Energy (B3LYP/6-31G**//MMFF) $=-3245.90444230$
0700491

MMFF Geometry

C $0.113193 \quad 3.825475-0.169887$

C $-1.0156734 .447247-0.544086$

C $-2.2126673 .839350-1.237910$

O $-1.9869722 .432363-1.482426$

C $-3.4778164 .022318-0.375359$

C $-4.7625834 .085575-1.181774$

C $-5.7140173 .131842-1.212145$

C $-4.9736675 .368924-1.948161$

C $-5.7204301 .804125-0.509272$

C $-6.0102620 .655942-1.489089$

C $-5.972156-0.741327-0.846786$

C -7.121276 -0.9588970 .141216$

C -7.026426 -2.316646 0.851399

O $-4.706332-0.892008-0.202119$

O $\quad-7.963015-2.2771841 .939345$

C $-5.607719-2.4578521 .442250$

C -7.464334 -3.468439-0.059735

O $-5.411059-3.7525402 .006176$

C $-4.488437-2.1628540 .419475$

C $-3.095709-2.1105451 .084402$

O $-4.457973-3.211197-0.555843$

C $-2.4702141 .908000-2.636734$

O $-3.1452442 .488431-3.476887$

C $-2.0954120 .485442-2.802369$

C -1.116402 -0.126427 -2.117392

C $-0.672589-1.510817-2.316041$

C -1.475419-2.370384 -3.258838

C $-2.909946-0.9767212 .098094$

C $-1.447469-0.8581942 .466440$

C $0.351208-2.1022093 .641593$

O $-0.719886-0.0104531 .954192$

C $0.774633-3.2207102 .741053$

C $1.338970-3.0255131 .540232$

C $1.720483-4.1307940 .603053$

C $0.803928-4.204714-0.597830$

C $-0.182379-5.115698-0.653575$

C $1.089582-3.293971-1.769700$

C $0.420545-1.949532-1.656022$

N $-1.046962-1.829143 \quad 3.372934$

H $0.2353652 .760345-0.340473$

H -1.087473 $5.520800-0.374077$

H $-2.3020304 .369204-2.193954$

H -3.418416 4.9606010 .193760

H -3.5215453 .2304860 .379700$

H -6.608459 $3.325333-1.803921$

H $-5.9536825 .406547-2.436071$

H $-4.9127506 .228739-1.273021$

H -4.216503 $5.485104-2.729072$

H $-4.773717 \quad 1.6148430 .001635$

H $-6.498627 \quad 1.843860 \quad 0.260192$

H $\quad-6.9825080 .808539-1.974392$

H $-5.2526980 .684291-2.282688$

H -6.038846 -1.473753 -1.660331

H $-8.090487-0.854235-0.362295$

H -7.102170 -0.1801070 .914423$

H -7.964678 -3.149167 2.368794

H $-5.534687-1.7416782 .270133$

H -8.513273 -3.342753 -0.353781

H $-7.412457-4.4292660 .464153$

H -6.871519 -3.543694 -0.973602

H $-6.063958-3.8674092 .716756$

H -2.862281 -3.0788291 .545555$

H $-2.340640-1.9847130 .296533$

H $-3.569713-3.244855-0.947606$

H -2.644840 -0.027269 -3.585951

H $-0.5617670 .437846-1.370002$

H $-2.535522-2.362275-2.981148$

H -1.172785 -3.419402 -3.257320

H $-1.378381-2.006480-4.287457$

H $-3.229380-0.0126771 .687363$

H -3.494550 -1.152745 3.007093

H $0.447144-2.4005264 .689649$

H $0.946805-1.1982573 .480143$

H $0.579647-4.2331713 .085568$

H $1.517258-2.0089131 .203037$

H $1.720586-5.0919331 .134330$ 
H $2.756175-3.9849970 .273484$ H $-0.825145-5.201412-1.523103$ H $\quad-0.372456-5.7928980 .172558$ H $\quad 0.823894-3.792675-2.708339$ H $2.172251-3.137643-1.852152$ H $\quad 0.935293-1.256337-0.990564$ H -1.724255 -2.522391 3.678123 C 1.7863153 .9690151 .761718 O 2.4898162 .7356601 .533564 C 2.4597641 .9750912 .760191 C 1.5921892 .7606733 .748259 C 0.7550443 .6345032 .831449 H 1.9518291 .0322652 .533365 C 3.8935651 .7067323 .242605 H 0.9840872 .0961324 .370852 H 2.1918413 .3765604 .426424 C 0.1554044 .8469653 .519326 H $-0.050833 \quad 3.0157672 .416967$ C 4.7391810 .8620632 .264210 O $4.585422 \quad 2.9524173 .405485$ H $3.879831 \quad 1.2243374 .227307$ H 4.9007941 .4144351 .331599 O 6.0320260 .6790062 .856997

C $4.151044-0.5279881 .962566$ C $5.092688-1.4864381 .212304$ H $3.225278-0.4144071 .391202$ O $3.801349-1.1654843 .196118$ H $\quad 6.018607-1.6218541 .785369$ H $4.608773-2.4722141 .201541$ H $-0.509048 \quad 4.5380424 .332884$ H 0.9314985 .4918353 .945099 H -0.4302305 .4450812 .814032$ C 1.2751034 .5547060 .446231 H 4.2489803 .3794154 .210409 H $6.393697 \quad 1.5634913 .038725$ H $4.614032-1.2488423 .724079$ H $2.0927204 .541001-0.285317$ H 1.0122135 .6068470 .612110 H 2.5295944 .6711782 .165133

C $4.305059-0.750998-2.365643$

O $4.202164-1.110309-0.980171$

C $5.418493-1.061117-0.225637$

C $6.481045-1.982613-0.847949$

C $6.694582-1.668814-2.330534$

C $5.365927-1.599820-3.086461$

H $3.329782-1.014122-2.795231$

C $4.5059570 .758985-2.548293$

H $5.797860-0.035157-0.215413$

O $6.104854-3.351435-0.703557$

H $7.423104-1.852538-0.302533$

H $7.326694-2.443886-2.780722$

O $7.366971-0.420521-2.466815$

H $5.519672-1.209550-4.099341$

O $4.835818-2.921055-3.225635$

C $3.1966681 .487804-2.344989$

H $5.2291601 .197220-1.856094$

H $4.8616530 .967256-3.564480$

H $5.225652-3.462357-1.104370$

H $8.218138-0.493148-2.002271$

H $5.485474-3.446199-3.723362$

O $2.8684472 .174184-3.476727$

O $2.5453661 .447414-1.309682$

C $1.6369282 .901591-3.435320$

H $1.3901953 .209638-4.454789$

H $\quad 0.8245442 .275758-3.055996$

H $1.7599333 .795630-2.818706$

SCF Energy (B3LYP/6-31G**//MMFF)= -3245.90294178

\section{7 _00492}

MM̄FF Geometry

C $-2.4397340 .056694 \quad 3.318674$

C -1.2389680 .3387172 .792711$

C 0.0579630 .2086453 .547289

O $0.874142-0.7399952 .823222$

C 0.8076741 .5564003 .607052

C 0.0668762 .6513014 .341326

C -0.5604883 .6840973 .745537$

C 0.0895402 .5426745 .843834
C -0.7163683 .9491342 .271975$

C $-0.0095385 .247162 \quad 1.867674$

C -0.1035475 .6017030 .373331$

C -1.546576 $5.723777-0.123692$

C $-1.6098786 .078098-1.616411$

O $0.6063674 .617424-0.391902$

O $-2.9659535 .865611-2.039337$

C $-0.7096505 .092375-2.393212$

C $-1.3166927 .562931-1.858874$

O $-0.6359795 .457897-3.769911$

C $0.7069334 .936418-1.788715$

C $1.5317073 .837477-2.505405$

O $1.4168936 .168727-1.984469$

C $1.910939-1.3105193 .494020$

O $2.195822-1.1797924 .673274$

C $2.696147-2.0776932 .507824$

C $4.028788-2.1309752 .643609$

C $4.956199-2.7230091 .679209$

C $4.382195-3.6444570 .635423$

C $1.0327762 .399643-2.344697$

C $1.5825841 .718321-1.107296$

C $3.6855620 .819599-0.128300$

O $0.8719491 .475876-0.135342$

C $4.734991-0.087052-0.688211$

C $6.0498340 .052724-0.458833$

C $7.095867-0.886441-0.994279$

C $7.931165-1.5223870 .098833$

C $9.160772-1.0616630 .384018$

C $7.370652-2.7403350 .807707$

C $6.258505-2.3778771 .751492$

N $2.927953 \quad 1.399320-1.220527$

H -2.500675 -0.3165424 .338087$

H -1.1828040 .7201681 .775962$

H $-0.127213-0.1784654 .557310$

H $1.085301 \quad 1.8675652 .593378$

H 1.7801381 .4131234 .096776

H -1.050629 4.4194454 .382746

H -0.4204393 .3812836 .329754$

H -0.4062401 .6230976 .169857$

H $1.1220362 .530068 \quad 6.208277$

H $-0.3513773 .121673 \quad 1.660681$

H -1.7914004 .0273262 .075125$

H -0.4182596 .0807492 .453546$

H 1.0526915 .1594562 .132701

H $0.410895 \quad 6.563370 \quad 0.257078$

H $-2.1042586 .457003 \quad 0.471757$

H $-2.068551 \quad 4.767017 \quad 0.007907$

H -3.000515 $6.009435-3.000926$

H -1.220892 $4.123230-2.368601$

H -2.043933 8.185921-1.324109

H -1.426242 $7.821551-2.917861$

H $-0.3196057 .864524-1.531938$

H $-0.0287576 .213746-3.842996$

H $1.5789904 .077195-3.575772$

H $2.5700353 .899174-2.154046$

H $2.3676785 .995401-1.886568$

H $2.173755-2.4871791 .653335$

H $4.487259-1.6303853 .495669$

H $5.141988-4.1817720 .064239$

H $3.770521-3.085451-0.079629$

H $3.756616-4.4132241 .103486$

H $-0.0579082 .328524-2.329939$

H $1.3614561 .809553-3.210230$

H 3.0127500 .2585490 .526807

H 4.1129861 .6464430 .448088

H $4.389808-0.924131-1.290001$

H 6.3987110 .8875530 .144673

H $7.740005-0.313675-1.674126$

H $6.646793-1.676279-1.609547$

H $9.776035-1.526247 \quad 1.147315$

H $9.577715-0.205868-0.136555$

H $8.154564-3.2355121 .395453$

H $7.070787-3.4716610 .052651$

H $6.572689-1.7084682 .553923$

H $3.4466891 .755475-2.018481$

C $-4.548700-0.9692902 .371852$

O $-4.114746-1.5931161 .139987$

C $-5.264873-1.7914720 .297871$ 
C $-6.461334-1.768148 \quad 1.237984$

C $-6.043937-0.6791652 .200922$

H $-5.323840-0.921591-0.367231$

C -5.155772 -3.074699-0.531120

H $-7.398021-1.5471140 .717584$

H -6.579048 -2.719794 1.769384

C $-6.823385-0.6892353 .505238$

H $-6.184940 \quad 0.294074 \quad 1.710958$

C $-3.938400-3.165764-1.472853$

O $-5.100314-4.203806 \quad 0.350986$

H $-6.074987-3.201251-1.115928$

H $-3.034023-3.370229-0.891280$

O $-4.138213-4.310218-2.314673$

C $-3.707759-1.936788-2.369868$

C $-2.709588-2.146947-3.526263$

H $-3.370262-1.100342-1.746884$

O $-4.955985-1.548464-2.953001$

H -3.159792 -2.796426 -4.288416

H -2.581149 -1.171325 -4.014042

H $-7.889711-0.5260203 .317264$

H $-6.717105-1.6452164 .028733$

H -6.4729550 .1022364 .175253$

C $-3.7441390 .310558 \quad 2.617327$

H $-4.335262-4.0779780 .938360$

H -4.267269 -5.078634 -1.732580

H $-4.802159-0.731934-3.458345$

H -4.3133790 .9929923 .260319$

H $-3.5715820 .831091 \quad 1.666887$

H -4.386105 -1.7018383 .172342$

C $0.489811-2.250354-1.595871$

O $-0.765639-1.826169-2.146711$

C -1.339291 -2.707203 -3.114861

C $-0.397367-2.864365-4.319462$

C $0.996243-3.322017-3.872559$

C $1.527844-2.477137-2.710800$

H $0.829396-1.404446-0.984799$

C $0.325629-3.461550-0.661839$

H -1.497428 -3.691129-2.663750

O $-0.293164-1.620832-5.010184$

H $-0.831314-3.592726-5.014957$

H $1.694581-3.248459-4.715365$

O $0.947636-4.686880-3.467957$

H $2.429299-2.939560-2.292496$

O $1.916255-1.206365-3.239438$

C $-0.504825-3.1256920 .557594$

H $-0.159590-4.305627-1.157473$

H $1.313102-3.782276-0.312649$

H $\quad 0.291646-1.757539-5.774824$

H $0.635564-5.207485-4.227656$

H $2.297332-0.688693-2.510476$

O $-0.476659-4.1805791 .423887$

O $-1.114819-2.079557 \quad 0.725700$

C -1.240281 -4.0088362 .622222$

H $-0.834729-3.1830583 .213845$

H -2.292733 -3.830988 2.381415

$\mathrm{H}-1.165772-4.9290813 .207427$

SCF Energy (B3LYP/6-31G**//MMFF) $=-3245.89687368$

\section{3}

MM̄FF Geometry

C $-1.8715793 .839610-0.529238$

C $-0.6768023 .248456-0.375156$

C 0.2352063 .5259430 .793952

O $\quad 0.722108 \quad 2.246374 \quad 1.264244$

C 1.4240664 .3867740 .316812

C 2.2439625 .0218841 .426662

C 3.5563614 .7921201 .636613

C 1.5143296 .0165792 .293655

C 4.4596353 .8937620 .837952

C 4.4812412 .4853421 .433218

C 5.0544281 .4049530 .511421

C $6.546591 \quad 1.5817060 .240072$

C $7.0757360 .474835-0.684457$

O $4.3235411 .425837-0.720300$

O $8.3845810 .877874-1.114410$

C $6.1673960 .391938-1.930118$

C $7.258906-0.8486000 .066838$

O $6.537633-0.713715-2.752094$
C $4.6500340 .354230-1.611027$

C $3.8511250 .530607-2.927867$

O $4.332882-0.907908-1.031700$

C 0.7759422 .0251492 .602921

O 0.4358262 .8008913 .486089

C 1.3602560 .6993152 .922925

C $1.574057-0.2694952 .018787$

C $2.173680-1.5833342 .278677$

C $2.809207-1.7970093 .627412$

C $2.3471670 .799195-2.784128$

C $1.472519-0.397405-2.461442$

C $0.785518-2.650929-3.269063$

O $0.710970-0.384480-1.495894$

C $1.413209-3.685770-2.382153$

C $2.689979-3.738141-1.971851$

C $3.229200-4.787397-1.042977$

C $3.695004-4.2135360 .278694$

C $5.001981-4.0460810 .540406$

C $2.651647-3.9339011 .339260$

C $2.140380-2.5173311 .303797$

N $1.576491-1.436927-3.370276$

H -2.208452 4.5500230 .222230

H $-0.3150492 .564878-1.139548$

H -0.3320314 .0359181 .580265$

H $1.0563495 .207477-0.314530$

H $2.0564213 .768622-0.329314$

H 4.0468625 .3032752 .463556

H 2.1910106 .5555042 .965478

H 1.0114096 .7643541 .671619

H 0.7650415 .5194022 .915498

H $4.1607093 .883599-0.214367$

H 5.4707204 .3183930 .850062

H 5.0242902 .4901342 .386477

H 3.4521402 .1948661 .660988

H 4.8766220 .4451521 .011339

H $7.115927 \quad 1.6053751 .177348$

H $6.7254062 .549988-0.246464$

H $8.6974560 .221619-1.760768$

H $6.372178 \quad 1.291222-2.527190$

H $7.956084-0.7185560 .903236$

H $7.706598-1.613326-0.577448$

H $6.324665-1.2424600 .470845$

H $6.181266-1.519522-2.340234$

H $4.258187 \quad 1.404993-3.453445$

H $4.017971-0.337997-3.574249$

H $3.367434-0.997135-1.010219$

H 1.6055200 .5604773 .971304

H $1.278093-0.0942790 .988162$

H $3.523078-0.9951303 .848352$

H $3.372790-2.7289213 .703078$

H $2.045538-1.8142264 .412532$

H $2.1651221 .572500-2.029386$

H $1.9717161 .205338-3.732647$

H $0.650463-3.052299-4.277991$

H $-0.199210-2.387131-2.867740$

H $0.730862-4.458739-2.034156$

H $3.402213-2.999591-2.326830$

H $4.061898-5.288515-1.553740$

H $2.487886-5.574162-0.855088$

H $5.339844-3.6679001 .499600$

H $5.761051-4.274327-0.200353$

H $3.054309-4.2016152 .320489$

H $1.803030-4.6140101 .199214$

H $1.644360-2.2578900 .369330$

H $2.218500-1.359144-4.152320$

C $-4.2258373 .449982-1.346031$

O $-4.4637492 .070994-0.985733$

C $-5.6899421 .638917-1.608144$

C $-6.3755382 .901208-2.115717$

C $-5.183133 \quad 3.748467-2.501185$

H $-5.4056911 .028603-2.472976$

C $-6.5538150 .821171-0.642088$

H -7.050413 2.699508 -2.953054

H $-6.9542743 .393806-1.325321$

C $-5.5193015 .221165-2.665602$

H $-4.7671883 .365910-3.443056$

C $-5.918478-0.487885-0.131714$

O -6.8723051 .6252200 .501150$ 
H $-7.5138700 .593550-1.120643$

H $-5.111715-0.2641830 .574370$

O $-6.923721-1.187017 \quad 0.615034$

C - $-5.397603-1.421812-1.238089$

C $-5.082686-2.860387-0.788297$

H $-4.502581-0.977216-1.688316$

O $-6.384933-1.510514-2.271499$

H $-6.015086-3.386761-0.545962$

H -4.677143 -3.379934 -1.667107

H $-6.2618235 .359375-3.458436$

H $-5.9305995 .644253-1.742947$

H $-4.6296225 .799587-2.933203$

C $-2.757133 \quad 3.654677-1.729489$

H -6.0329861 .9030220 .906035$

H -7.236773 -0.585675 1.312412

H $-6.007443-2.041227-2.993585$

H -2.657890 $4.563159-2.334853$

H $-2.4076882 .810248-2.335952$

H $-4.4916404 .056159-0.469958$

C -1.874412 $-2.267277 \quad 1.035564$

O $-2.897601-2.2911310 .030568$

C $-4.097471-2.983147 \quad 0.384377$

C $-3.800120-4.4559940 .718663$

C $-2.705465-4.5791241 .783510$

C $-1.496140-3.697473 \quad 1.455554$

H $-1.004239-1.8217190 .537663$

C -2.249363 -1.371000 2.225914

H $-4.545405-2.503098 \quad 1.259946$

O $-3.381810-5.139840-0.462492$

H -4.725008 -4.9327891 .064439$

H -2.374799 -5.6228321 .852366$

O $-3.225646-4.2054353 .055002$

H $-0.815516-3.6593812 .313504$

O $-0.769510-4.3019520 .382602$

C $-2.4359990 .076388 \quad 1.827417$

H -3.181786 -1.697930 2.693430

H -1.451909 -1.403229 2.976785

H -3.244072 -6.073285 -0.227809

H -3.970275 -4.7981583 .253935$

H -1.326291-4.262335 -0.413876

O $-2.953034 \quad 0.7597772 .891445$

O $-2.163811 \quad 0.548493 \quad 0.733320$

C -3.2292762 .1454712 .669411$

H -3.7685182 .5278613 .540090$

H -2.2943272 .7003562 .565130$

$\mathrm{H}-3.8585312 .2779651 .785347$

SCF Energy (B3LYP/6-31G**//MMFF)= -3245.91648985

\section{4}

MM̄FF Geometry

C -1.622639 $5.422255-0.703730$

C -2.3186714 .5555010 .046666$

C $-3.3016473 .565515-0.523716$

O $-2.8462622 .233406-0.188306$

C $-4.695154 \quad 3.7747830 .095047$

C $-5.7346022 .764525-0.356795$

C -6.5184982 .0635380 .486070$

C $-5.8887242 .579660-1.844451$

C -6.5837632 .1798631 .982136$

C $-5.723147 \quad 1.1660702 .748208$

C $-6.141381-0.2942752 .516004$

C $-5.621158-1.1896923 .643094$

C $-5.971441-2.6654653 .404025$

O $-5.589179-0.729017 \quad 1.267639$

O $-5.201413-3.4562844 .321962$

C $-5.522694-3.0560201 .982486$

C -7.445103 -2.963479 3.704376

O $-5.959267-4.3726871 .651931$

C $-5.972674-2.0573380 .888554$

C $-5.270276-2.410487-0.442741$

O $-7.386326-2.1620650 .705469$

C $-1.8928281 .695766-0.998326$

O $-1.3724162 .224440-1.968632$

C $-1.5795300 .338275-0.500053$

C $-0.552513-0.325730-1.051131$

C $-0.105441-1.679845-0.723329$

C $-0.841623-2.4192540 .360844$

C $-5.358347-1.323016-1.520508$
C $-4.857682-1.845763-2.854253$

C $-2.811546-2.714576-3.985635$

O $-5.611011-1.982100-3.815683$

C $-1.847296-3.749080-3.495904$

C $-0.549490-3.781825-3.834704$

C $0.415294-4.818446-3.330504$

C $1.653831-4.215316-2.701241$

C $2.848309-4.298777-3.310983$

C $1.520050-3.576166-1.333087$

C $0.932583-2.192041-1.416901$

N $-3.507521-2.155532-2.841362$

H -1.781749 $5.447581-1.778926$

H -2.1442734 .5183951 .119450$

H $-3.3702573 .668327-1.614234$

H $-5.0597494 .778941-0.158175$

H $-4.5892623 .746527 \quad 1.185152$

H $-7.225167 \quad 1.3519200 .060872$

H $-6.760267 \quad 1.966582-2.098318$

H $-6.0195183 .549667-2.335149$

H $-5.0083972 .086823-2.268020$

H -6.3134913 .1916642 .304273$

H -7.632871 2.0620312 .283584

H -4.6697641 .3037712 .472656$

H -5.8166431 .4066093 .814523$

H -7.235528 -0.3435112 .470475$

H $-6.009265-0.8600054 .614963$

H -4.527876 -1.096275 3.708270

H $-5.424150-3.1696475 .224281$

H $-4.423858-3.0874401 .988190$

H -7.681565 -2.714058 4.745830

H -7.661527 -4.0321693 .596579$

H -8.134583 -2.406266 3.066727

H $-5.644269-4.9673132 .354036$

H $-4.207525-2.592876-0.245386$

H $-5.685098-3.345213-0.841958$

H $-7.608993-1.897749-0.201088$

H $-2.199359-0.0745110 .287237$

H $\quad 0.0180070 .167297-1.837819$

H $-0.860528-1.8306591 .285070$

H $-0.384746-3.3759650 .622440$

H $-1.871471-2.6245680 .054451$

H $-6.389886-0.983214-1.660102$

H $-4.762568-0.446421-1.242681$

H -2.310563 -1.887699-4.499111

H $-3.532270-3.168437-4.672860$

H $-2.239956-4.514131-2.829539$

H -0.159450 -3.032336 -4.519546

H $0.694203-5.452886-4.181763$

H $-0.062657-5.486731-2.603162$

H $3.743842-3.887607-2.858103$

H $2.956832-4.774447-4.280099$

H $2.505197-3.489471-0.859041$

H $0.941415-4.250350-0.695935$

H $1.422424-1.554125-2.154797$

H -2.948475 -1.861775 -2.046118

C $0.8186455 .905310-0.594772$

O 1.1442524 .6548040 .045436

C 2.5192944 .6848350 .466566

C $3.1241295 .931696-0.168259$

C $1.9318886 .868594-0.191843$

H 2.5141664 .8016161 .557622

C $3.239203 \quad 3.3788980 .099221$

H 3.9680256 .3230490 .407898

H $3.4748995 .732800-1.188030$

C $2.1041548 .046994-1.134097$

H 1.7567597 .2422080 .826000

C 2.6996082 .1347730 .835529

O $3.1561373 .168323-1.310520$

H 4.2995753 .5049740 .342939

H 2.8004372 .2740421 .918971

O 1.3015651 .9734940 .575152

C 3.3710040 .8066110 .428400

C 4.8978950 .8300910 .558468

H $3.0793530 .540246-0.593845$

O $2.864435-0.2244881 .282794$

H $5.3300701 .554963-0.141758$

H 5.1672351 .1947751 .559598

H $2.9604238 .658683-0.831697$ 
H $2.2746497 .716033-2.163971$

H $1.2149288 .685095-1.128210$

C $-0.5763116 .351244-0.154377$

H $2.2144513 .068395-1.533622$

H 0.8422902 .7576970 .919654

H $1.895965-0.2238021 .193257$

H $-0.7896437 .363084-0.516329$

H $-0.631984 \quad 6.3756710 .941037$

H $0.8409365 .724764-1.677800$

C $7.862678-1.4137740 .432912$

O $6.998390-0.273700 \quad 0.513165$

C $5.594492-0.5320580 .367354$

C $5.306360-1.194463-0.988189$

C $6.172967-2.442050-1.196082$

C $7.648254-2.171524-0.887499$

H $8.877681-0.9957200 .413357$

C $7.748011-2.3072971 .677991$

H $5.275210-1.1948541 .178529$

O $5.555009-0.258782-2.035297$

H $4.246891-1.469283-1.036558$

H $6.081115-2.782948-2.234399$

O $5.713937-3.494358-0.352960$

H $8.214044-3.110233-0.863407$

O $8.206146-1.373579-1.935258$

C $8.237216-1.6121162 .932157$

H $6.716517-2.6173931 .863168$

H $8.367538-3.2010461 .539961$

H $5.331308-0.692722-2.876143$

H $4.787788-3.675286-0.584724$

H $8.114713-1.872800-2.764586$

O $8.127181-2.476075 \quad 3.985537$

O $8.659451-0.4656252 .989150$

C $8.550617-1.9596695 .251268$

H $8.410688-2.7423786 .001662$

H $9.611034-1.6926115 .215926$

H $7.942369-1.0937585 .529669$

SCF Energy (B3LYP/6-31G**//MMFF) $=-3245.90236065$

0700495

MM̄FF Geometry

C $3.445280-3.452612-1.330487$

C $2.265633-2.847588-1.536732$

C $0.964576-3.341201-0.957724$

O $0.396740-2.268060-0.171593$

C $-0.010453-3.690483-2.102825$

C $-1.348411-4.221837-1.627972$

C $-2.523369-3.584267-1.799852$

C $-1.318369-5.574379-0.963154$

C $-2.778272-2.300230-2.538895$

C $-2.447104-1.042979-1.726275$

C $-3.050867 \quad 0.214033-2.369283$

C -2.465535 $1.482619-1.752506$

C $-3.1435612 .746216-2.303427$

O $-4.459274 \quad 0.171088-2.131404$

O $-2.7642673 .848911-1.465225$

C $-4.6747082 .590794-2.181968$

C $-2.6548653 .086486-3.716151$

O $-5.3247773 .687810-2.820725$

C $-5.2087091 .238566-2.715986$

C $-6.6977281 .056679-2.317544$

O $\quad-5.113100 \quad 1.220269-4.141967$

C $-0.081679-2.5726001 .065839$

O $0.012699-3.6386791 .652930$

C $-0.790697-1.3816141 .585583$

C -1.584706 -1.509639 2.659760

C $-2.375556-0.4507693 .292112$

C -2.2752180 .9351962 .712832$

C $-7.250577-0.367308-2.500846$

C -7.389006 -1.088813 -1.170296

C $-6.016112-2.1365470 .633428$

O $-8.469964-1.175821-0.592021$

C -5.717860 -1.014569 1.579798

C $-6.367554-0.8302672 .738568$

C -6.1170220 .3205353 .671034$

C -5.498184 -0.1103084 .981590$

C $-6.252330-0.6232345 .968489$

C -4.0200410 .1419915 .177003$

C $-3.155664-0.7694354 .347191$
N $-6.186168-1.581720-0.696113$

H $3.485441-4.336331-0.697964$

H $2.221306-1.971991-2.180347$

H $1.151363-4.225946-0.337700$

H $0.444546-4.444077-2.759586$

H $-0.157422-2.800866-2.726056$

H $-3.423770-4.055058-1.406880$

H -2.322072 -5.941243 -0.723002

H $-0.853131-6.309958-1.627775$

H $-0.750524-5.544521-0.030183$

H $-2.224782-2.289585-3.485619$

H -3.837455 -2.295169 -2.825554

H -2.835692 -1.153958 -0.706211

H -1.359266 -0.934455 -1.662442

H $-2.8591860 .188789-3.448401$

H $-1.3811041 .527099-1.911312$

H -2.608609 $1.460055-0.664202$

H -1.793514 3.910110-1.471405

H $-4.9147822 .665388-1.111800$

H -1.568212 $3.234555-3.719828$

H $-3.0860584 .030812-4.066694$

H -2.885206 $2.309093-4.447633$

H -6.262362 $3.668627-2.566611$

H $-6.8350601 .356850-1.270861$

H -7.308306 $1.731179-2.931344$

H $-5.7893520 .619204-4.494176$

H $-0.661494-0.4449471 .056291$

H -1.674954 -2.491731 3.122987

H -2.5300970 .9268841 .648352$

H -1.2597251 .3255012 .829916$

H -2.9500031 .6557273 .178996$

H $-8.255525-0.313270-2.937387$

H $-6.645833-0.971153-3.185928$

H -5.169475 -2.828411 0.593602

H $-6.918924-2.6884530 .913569$

H $-4.935968-0.3159401 .291322$

H -7.149848 -1.529950 3.024523

H -7.0776470 .8159103 .865014$

H -5.4928331 .0865073 .194731$

H -5.820907 -0.9058526 .922843$

H $-7.319291-0.7739105 .840685$

H $-3.743517-0.0206296 .227513$

H $-3.811915 \quad 1.2008374 .998319$

H $-3.171709-1.8060304 .686713$

H $-5.341587-1.379477-1.220896$

C $5.842050-2.722811-0.980824$

O $5.714095-1.357205-0.521985$

C $6.975867-0.689750-0.707206$

C $8.010129-1.800555-0.816680$

C $7.236414-2.832433-1.607540$

H $6.918824-0.167981-1.669918$

C 7.2601820 .3160150 .413032

H $8.925702-1.471586-1.317242$

H $8.281447-2.2016250 .166886$

C $7.834854-4.227300-1.534058$

H $7.202195-2.515114-2.658758$

C 6.2072281 .4270020 .596134

O $7.367093-0.382247 \quad 1.660514$

H 8.2430710 .7718890 .243801

H 5.3127431 .0162851 .074590

O 6.7502752 .3830611 .517148

C $5.8116012 .169049-0.691851$

C $4.9917913 .457862-0.487248$

H $5.2440941 .489087-1.336776$

O $6.9897502 .531146-1.419695$

H $5.6298174 .242806-0.060693$

H $4.7206063 .817385-1.488979$

H $8.847813-4.233307-1.949610$

H $7.893655-4.584484-0.500518$

H $7.231710-4.941608-2.103190$

C $4.732734-3.041615-1.988860$

H $6.533526-0.8644401 .797628$

H 6.9801121 .9024522 .330988

H $7.5322923 .092654-0.839606$

H $5.038796-3.881719-2.623921$

H $4.567130-2.178953-2.646140$

H $5.767638-3.363455-0.092948$

C $1.728725 \quad 1.9385250 .531915$ 
O $2.9211872 .264630-0.197316$

C 3.7271413 .2998370 .371116

C 2.9307904 .6124190 .466051

C $1.613636 \quad 4.4091121 .221966$

$\begin{array}{lllll}\text { C } & 0.845862 & 3.185736 & 0.708491\end{array}$

H $1.1867361 .235353-0.114152$

C 2.0410721 .2199341 .855478

H 4.0399503 .0046031 .377119

O $2.6511665 .097435-0.846215$

H 3.5470965 .3644350 .973172

H 0.9907505 .3046251 .106819

O $1.904610 \quad 4.2567842 .607882$

H 0.0134022 .9524631 .380680

O $0.2637913 .506551-0.558238$

C $2.677945-0.1328591 .627447$

H 2.7228491 .7935032 .487740

H $1.109022 \quad 1.0559952 .406124$

H $2.1885595 .947565-0.752215$

H $1.058415 \quad 4.1534693 .075688$

H $0.9881863 .642950-1.192898$

O $2.804279-0.7779922 .823834$

O $3.035132-0.5659710 .541586$

C $3.421098-2.0681312 .760740$

H $3.432791-2.4888623 .769559$

H $2.848648-2.7350072 .109438$

H $4.451889-1.9760832 .406011$

SCF Energy (B3LYP/6-31G**/MMFF) $=-3245.90899248$

0700496

MM̄FF Geometry

C $0.458168-2.623309-1.861054$

C $-0.602549-3.442191-1.916993$

C -1.615371 -3.595658 -0.814967

O $-2.869236-3.134638-1.373783$

C $-1.750853-5.061376-0.363654$

C $-0.525014-5.6207340 .325414$

C $-0.287713-5.5113261 .646825$

C $0.417806-6.409185-0.546989$

C $-1.126727-4.7969692 .672925$

C $-0.266989-4.078644 \quad 3.724444$

C $0.368239-2.772252 \quad 3.217986$

C $1.487953-2.3431204 .169047$

C $2.088124-0.9912583 .770706$

O $-0.656985-1.773346 \quad 3.193859$

O $2.899287-0.527986 \quad 4.861871$

C $0.9444070 .027137 \quad 3.599959$

C $3.023365-1.1185092 .564247$

O $1.435928 \quad 1.2586713 .079175$

C $-0.238008-0.477817 \quad 2.736042$

C $-1.442457 \quad 0.4816202 .909374$

$\begin{array}{lllll}\text { O } & 0.084283 & -0.493149 & 1.347128\end{array}$

C -3.860924 -2.789108 -0.511360

O $-3.883970-2.9625980 .696022$

C $-4.897790-2.081841-1.294877$

C $-5.783056-1.308247-0.649168$

C $-6.793582-0.446987-1.266856$

C $-6.921767-0.487228-2.766851$

C -2.693378 0.0631582 .130201

C -2.771706 0.7050290 .759026

C $-3.4580362 .818393-0.380778$

O $-2.4310180 .103722-0.256275$

C $-4.8393363 .391936-0.336360$

C $-5.8121743 .047178-1.193459$

C $-7.2041773 .609483-1.158173$

C -8.167946 $2.774607-0.341327$

$\begin{array}{llll}C & -8.724465 & 3.267297 & 0.778320\end{array}$

C $-8.542373 \quad 1.396976-0.851418$

C $-7.5221010 .358886-0.465908$

$\begin{array}{llll}N & -3.257940 & 2.002305 & 0.802924\end{array}$

H $0.610219-2.003951-0.981665$

H $-0.755549-4.049392-2.806858$

H $-1.352993-2.9517110 .034666$

H -2.015302 $-5.692623-1.223663$

H $-2.617287-5.1683870 .300778$

H $0.597735-6.0058712 .044842$

H $1.239559-6.8532440 .025230$

H $-0.116858-7.228098-1.039474$

H $0.864234-5.773440-1.316297$
H $-1.823088-4.0873782 .217620$

H -1.728458 -5.5553683 .188619$

H $-0.912264-3.8503594 .583123$

H $0.510825-4.7650664 .082380$

H $0.758021-2.9166742 .204303$

H $2.272405-3.1088604 .217483$

H $1.088551-2.2588885 .189754$

H $3.577110-1.2025925 .039655$

H $\quad 0.5593020 .2492384 .605358$

H $3.865889-1.7800132 .799906$

H $3.466710-0.1506862 .308609$

H $2.529705-1.5222231 .677683$

H 2.1566931 .5540113 .661436

H -1.7149920 .5006023 .972638$

H -1.1530691 .4999642 .622667$

H $1.001368-0.2003391 .230784$

H $-4.857083-2.157711-2.375151$

H $-5.733384-1.2605050 .438252$

H $-5.998400-0.137245-3.241451$

H $-7.7336000 .131736-3.153376$

H $-7.126072-1.508668-3.107318$

H -2.765148 -1.0220452 .018454$

H -3.5865680 .3683342 .690777$

H $-3.2882902 .223773-1.283731$

H -2.719366 $3.625429-0.357240$

H -5.0476164 .1242890 .439988$

H $-5.5993182 .317988-1.971123$

H -7.578874 $3.692556-2.185951$

H $-7.1707994 .639603-0.778987$

H -9.4323952 .6859691 .359800$

H -8.481339 4.2602281 .141918

H $-8.7119971 .457220-1.929392$

H $-9.5048561 .087459-0.423387$

H -7.3577140 .2975570 .611297$

H $-3.423726 \quad 2.433734 \quad 1.707824$

C $2.894600-2.698957-2.434284$

O $3.339554-1.470820-1.818089$

C $4.701398-1.217633-2.214915$

C $5.191209-2.501271-2.873023$

C $3.919504-3.003462-3.525321$

H $4.673633-0.425081-2.971279$

C $5.533935-0.778355-1.007743$

H $5.999700-2.319402-3.587544$

H $5.551922-3.223169-2.130540$

C $3.982400-4.468120-3.923807$

H $3.713696-2.394389-4.415915$

C $5.0965800 .564629-0.383757$

O $5.447082-1.7885050 .005938$

H $6.592789-0.726039-1.285786$

H 4.0824730 .4818200 .021180

$\begin{array}{lllll}\text { O } & 5.961550 & 0.819698 & 0.731068\end{array}$

C $5.1809871 .769593-1.344496$

C $5.0626393 .148203-0.665274$

H $4.4124071 .678736-2.118846$

O $6.443957 \quad 1.744841-2.019821$

H 5.8270533 .2419580 .116077

H $5.3191503 .905482-1.419284$

H $4.781139-4.633568-4.654332$

H $4.179377-5.111076-3.059233$

H $3.039819-4.792049-4.375827$

C $1.472488-2.515150-2.967073$

H $4.504232-1.922330 \quad 0.203613$

H $5.9000130 .053077 \quad 1.326430$

H $7.1398311 .802840-1.342665$

H $1.260578-3.265297-3.737662$

H $1.369911-1.526254-3.429303$

H $2.923593-3.475342-1.657980$

C $1.4184153 .921716-0.874823$

O $2.7825613 .621267-1.198716$

C $3.6718903 .456361-0.088783$

C 3.6936814 .7261690 .778894

C 2.2816205 .1300211 .213035

C 1.3136125 .1612260 .027695

H $0.9549754 .174013-1.837493$

C $0.6957952 .695776-0.310423$

H 3.3412382 .6103440 .521118

$\begin{array}{llllll}\text { O } & 4.292089 & 5.795540 & 0.048454\end{array}$

H 4.3208844 .5385501 .658762 
H 2.3109306 .1222901 .679419

O $1.792825 \quad 4.2105692 .183769$

H $\quad 0.2802595 .269102 \quad 0.377889$

O $1.6011146 .305331-0.781439$

C $\quad 0.547320 \quad 1.642707-1.379142$

H $1.2258142 .233107 \quad 0.525254$

H -0.3020252 .9548630 .059889$

H $4.319804 \quad 6.5717490 .633346$

H 2.4100054 .2191742 .935023

H $1.4873427 .094265-0.224657$

O $-0.5501241 .903376-2.143925$

O $1.3319170 .716059-1.529749$

C $-0.7920910 .982845-3.211956$

H $0.0735450 .934566-3.879501$

H $-1.026605-0.006410-2.809315$

H $-1.6536791 .342298-3.780768$

SCF Energy (B3LYP/6-31G*//MMFF) $=-3245.90331266$

0700497

MM̄FF Geometry

C -2.039177 -2.610642 -2.101593

C $-0.863585-2.147431-1.651289$

C $0.116776-1.397048-2.512361$

O $0.471805-0.198994-1.780504$

C $1.406870-2.199608-2.765501$

C $1.206686-3.548265-3.424636$

C $1.406140-4.725431-2.798425$

C $0.813554-3.510615-4.876895$

C $1.782670-4.907743-1.354195$

C $3.303089-4.991479-1.171025$

C $3.775940-4.7112530 .264226$

C $3.207552-5.6964531 .285169$

C $3.644060-5.3418642 .715479$

O $3.387101-3.374168 \quad 0.595757$

O $2.858119-6.1275743 .625535$

C $3.346102-3.8481292 .980808$

C $5.096245-5.7545632 .980651$

O $3.869667-3.4743394 .253794$

C $3.863451-2.9043461 .863542$

C $3.304111-1.4711022 .074370$

O $5.288681-2.8748711 .910276$

C $0.9354110 .862133-2.492003$

O $1.0150730 .957891-3.705728$

C $1.3752271 .885041-1.516853$

C $2.0358702 .970344-1.945747$

C $2.5777764 .041183-1.105763$

C $2.454672 \quad 3.887150 \quad 0.387071$

C $3.513273-0.4949850 .904899$

C $4.875696 \quad 0.1754350 .871813$

C $6.2738711 .657168-0.568187$

O $5.6965290 .075319 \quad 1.779529$

C $6.1059173 .071090-0.107613$

C $6.3136144 .137041-0.895842$

C $6.1876555 .558812-0.427218$

C $5.2831336 .391222-1.308805$

C $5.793813 \quad 7.237985-2.218748$

C $3.7912876 .307729-1.073470$

C $3.1746535 .091365-1.707656$

N $5.0592320 .912915-0.290480$

H -2.308565 -2.449652 -3.142723

H $-0.578107-2.329636-0.617867$

H $-0.352357-1.115872-3.463288$

H $1.961851-2.289895-1.824142$

H $2.080216-1.605720-3.398607$

H $1.275706-5.647855-3.361024$

H $0.746780-4.512426-5.314363$

H $-0.163116-3.032594-4.998895$

H $1.552111-2.946734-5.455825$

H $1.353139-4.106224-0.744917$

H $1.314921-5.831352-0.991775$

H $3.656736-5.979098-1.491886$

H $3.783645-4.252466-1.826317$

H $4.871051-4.765738 \quad 0.255600$

H $3.494543-6.7256041 .036825$

H $2.110359-5.6748201 .252228$

H $1.921034-5.9100013 .485150$

H $2.254122-3.7457373 .049204$

H $5.215380-6.8365182 .845116$
H $5.382403-5.5510694 .018564$

H $5.812591-5.2587422 .322596$

H $3.497805-2.6078614 .487774$

H $2.217577-1.5511212 .212520$

H $3.719758-1.0344412 .989793$

H $5.601712-2.0916341 .432684$

H $1.1674401 .702615-0.468815$

H $2.2084693 .084242-3.015238$

H 1.4026873 .9035120 .685435

H $2.961567 \quad 4.6711860 .952699$

H 2.8959862 .9407250 .717200

H $3.324784-0.983282-0.056675$

H 2.7792360 .3140331 .008564

H $6.4561681 .591252-1.645383$

H 7.114741 1.194309 -0.041606

H 5.8242483 .2251370 .931524

H $6.6232153 .982409-1.927125$

H $7.1961765 .992710-0.412338$

H 5.8295215 .6088490 .608573

H $5.1528137 .860093-2.834642$

H $6.8644847 .323779-2.372137$

H $3.2939177 .185358-1.508316$

H $3.5914096 .381369-0.001122$

H $3.2195085 .105432-2.797642$

H $4.292410 \quad 0.996401-0.951436$

C $-4.444959-2.927444-1.414329$

O $-4.653613-1.711510-0.661428$

C $-5.887974-1.8309930 .071455$

C $-6.613390-3.036159-0.515514$

C $-5.452205-3.939429-0.864652$

H $-5.617056-2.0614931 .108127$

C $-6.713545-0.540591 \quad 0.013278$

H -7.312104 -3.4871630 .195743$

H $-7.174591-2.771475-1.419565$

C $-5.819642-5.044066-1.841201$

H $-5.066411-4.3903330 .059767$

C -6.0558710 .7185050 .616278$

O $-7.031301-0.249652-1.354892$

H $-7.676391-0.714240 \quad 0.509446$

H $-5.2848351 .093860-0.065274$

\begin{tabular}{lllll}
\hline & -7.088499 & 1.714336 & 0.655172
\end{tabular}

C $-5.472767 \quad 0.5254252 .028369$

C -5.0932881 .8149142 .780319$

H $-4.592366-0.1243231 .962206$

O $-6.439354-0.160498 \quad 2.832725$

H -6.0024832 .3701273 .045401$

H $-4.658121 \quad 1.5006163 .738729$

H $-6.594413-5.690531-1.415950$

H $-6.202116-4.636217-2.782921$

H -4.950839 $-5.667908-2.072963$

C $-2.998802-3.409368-1.266812$

H $-6.191310-0.161250-1.837126$

H -6.6712762 .5772440 .809962$

H -6.024806 -0.3514533 .691356$

H -2.921947 -4.450536 -1.600724

H $-2.703212-3.375630-0.211029$

H -4.673264 -2.693568 -2.462452

C $-1.9278162 .712061 \quad 1.003788$

O $-2.930926 \quad 2.000036 \quad 1.742522$

C -4.111434 2.7436282 .051113

C -3.7640723 .9759202 .904393$

C $-2.691355 \quad 4.8359642 .228653$

C $-1.506440 \quad 3.993403 \quad 1.746491$

H -1.0652502 .0340760 .979225$

C -2.354592 $2.975581-0.449702$

H $-4.5780253 .077174 \quad 1.120102$

O $-3.2959353 .555471 \quad 4.184840$

H -4.6765664 .5625413 .064210$

H -2.326318 5.5912512 .935546

O -3.2567515 .5229881 .116467$

H -0.8583204 .5999891 .104018$

O $-\begin{array}{llll}-0.735486 & 3.623557 & 2.893052\end{array}$

C $-2.5844331 .697157-1.224791$

H $-3.2813203 .552533-0.501972$

H $-1.563403 \quad 3.533874-0.963496$

H -3.111191 4.3543454 .707370

H -3.982276 6.0769891 .451227

H 0.0423413 .1314462 .580024 
O $-2.9763822 .015658-2.493676$

O $-2.4463630 .567409-0.778571$

C $-3.2623510 .907275-3.352104$

H $-3.9587920 .212592-2.873332$

H $-3.7283701 .294334-4.262103$

H $-2.3330770 .399798-3.622526$

SCF Energy (B3LYP/6-31G*//MMFF) $=-3245.88368834$

\section{8}

MM̄FF Geometry

C $-1.710154 \quad 3.173734 \quad 0.083358$

C $-0.746637 \quad 3.833646 \quad 0.742880$

C $0.404338 \quad 3.1635581 .441846$

O $1.598274 \quad 3.599340 \quad 0.745785$

C 0.5023603 .5743572 .922076

C $-0.5578752 .966262 \quad 3.817086$

C -0.4154781 .7773294 .436389$

C $-1.780532 \quad 3.8094654 .062128$

C $0.743798 \quad 0.832573 \quad 4.277555$

C $0.328296-0.6311134 .479036$

C $1.387582-1.6588234 .044633$

C $2.766229-1.4033264 .659711$

C $3.813844-2.4098834 .164160$

O $1.467201-1.6449652 .612111$

O $5.100263-1.8948534 .543225$

C $3.760288-2.4624562 .620884$

C $3.674052-3.7654524 .866285$

O $4.639209-3.4803792 .147649$

C $2.329200-2.6567422 .067321$

C $2.262871-2.5717360 .525687$

O $1.872285-3.9694122 .421362$

C 2.6999632 .8062700 .827323

O $2.841241 \quad 1.8068431 .512943$

C $3.7026283 .337052-0.122025$

C $4.7423492 .561834-0.464227$

C $5.7563302 .868546-1.473546$

C $5.7317314 .240309-2.094106$

C $2.612647-1.213397-0.084184$

C $2.617785-1.265372-1.604687$

C $2.8732990 .213954-3.599009$

O $2.533144-2.313104-2.240151$

C $4.3202430 .396309-3.943832$

C $4.7888091 .446222-4.637934$

C $6.2192211 .721049-5.026067$

C $7.2850121 .135156-4.123218$

C $7.919813-0.003030-4.449640$

C $7.6796631 .928158-2.893158$

C $6.6180121 .892249-1.828945$

N $2.755188-0.004946-2.167755$

H -1.674521 2.0899930 .024922

H $-0.779997 \quad 4.9202830 .783313$

H $0.3195402 .073628 \quad 1.346194$

H $0.480617 \quad 4.6695213 .011211$

H 1.4949093 .3112453 .310668

H -1.200542 1.4516705 .116588

H -2.469749 3.3429044 .774002

H -1.494693 4.7834424 .472313

H -2.333298 3.9718943 .132715

H 1.1905340 .9398803 .286691

H 1.5018161 .0992955 .022569

H $0.090223-0.7889135 .538718$

H $-0.589531-0.8259593 .910672$

H $1.013509-2.6411134 .357215$

H $2.710826-1.4084955 .755299$

H $3.119595-0.4020094 .380637$

H $5.775568-2.4897364 .173878$

H $4.164658-1.5070082 .263572$

H $3.828824-3.6514245 .946105$

H $4.442138-4.4692304 .527050$

H $2.695672-4.2276004 .720612$

H $4.762688-3.3467251 .193138$

H $2.882716-3.3636960 .086506$

H $1.239157-2.8123540 .210952$

H $1.135717-4.2093761 .835106$

H $3.5223464 .311475-0.560664$

H $4.8325041 .579896-0.000891$

H $5.8380915 .011538-1.322957$

H $4.7891994 .404835-2.628075$
H $6.5338094 .408573-2.815333$

H $1.881796-0.463266 \quad 0.237189$

H $3.609549-0.8895850 .229832$

H $2.2748841 .097506-3.842250$

H $2.467844-0.646057-4.140948$

H $5.001872-0.382504-3.612877$

H $4.0737782 .188948-4.987729$

H $6.3632132 .808753-5.062536$

H $6.3599251 .377398-6.059155$

H $8.707356-0.411112-3.824937$

H $7.660932-0.554792-5.347405$

H $7.9450892 .946079-3.192433$

H $8.5984021 .518492-2.452801$

H $6.5428070 .925415-1.328868$

H $2.8482440 .801027-1.557859$

C $-4.2056973 .432551-0.025754$

O $-4.502616 \quad 2.070974-0.408947$

C -5.803116 2.039287-1.020821

C $-6.506463 \quad 3.301350-0.542614$

C $-5.359596 \quad 4.289805-0.554957$

H $-5.6429052 .114927-2.103116$

C $-6.5441680 .738401-0.697336$

H -7.333393 $3.592744-1.197084$

H $-6.8970793 .188616 \quad 0.475405$

C $-5.623576 \quad 5.5388300 .268768$

H $-5.1628544 .585301-1.594619$

C -5.792973 -0.560506 -1.056192

$\begin{array}{llll}\text { O } & -6.832948 & 0.689600 & 0.705518\end{array}$

H $-7.5194430 .748103-1.198610$

H $-4.993981-0.745653-0.330234$

O $-6.722522-1.642987-0.914930$

C $-5.210649-0.602455-2.477990$

C $-4.752603-1.987258-2.967373$

H $-4.3654850 .092145-2.539524$

O $-6.189587-0.134813-3.412818$

H $-5.628840-2.620344-3.160337$

H $-4.290812-1.827336-3.951068$

H $-6.4884246 .083092-0.124453$

H $-5.829315 \quad 5.2927691 .315949$

H $-4.760743 \quad 6.2117740 .244384$

C $-2.8550923 .853210-0.611936$

H $-5.9892880 .782704 \quad 1.179588$

H -7.064383 -1.613781 -0.004752

H $-6.970180-0.709512-3.333154$

H $-2.7425534 .941700-0.543983$

H $-2.8081103 .583792-1.674196$

H $-4.1777943 .454575 \quad 1.071170$

C -1.621560 -2.462560 -0.958093

O $-2.638635-1.903837-1.804634$

C $-3.774392-2.738028-2.050044$

C $-3.353458-4.079362-2.679457$

C $-2.272190-4.775469-1.849746$

C $-1.131374-3.818379-1.499306$

H $-0.786382-1.754797-1.026942$

C -2.066782 -2.539886 0.510768

H $-4.282035-2.936399-1.101014$

O $-2.863351-3.857467-4.001264$

H $-4.239042-4.720730-2.762636$

H $-1.864707-5.623826-2.413161$

O $-2.840134-5.286001-0.647724$

H $-0.462022-4.291277-0.771303$

O $-0.364769-3.589471-2.684194$

C $-2.132849-1.1730721 .154355$

H -3.043439 -3.020497 0.611967

H -1.352371 -3.1228291 .103970$

H $-2.634016-4.723387-4.379357$

H $-3.541186-5.911946-0.896893$

H $0.397696-3.037657-2.439839$

O $-3.283755-1.0614651 .878046$

$\begin{array}{llll}0 & -1.254327 & -0.326283 & 1.063007\end{array}$

C -3.4656410 .1669992 .588867$

H -3.232302 1.0294311 .958907

H -4.5131360 .2310762 .893955$

H -2.842639 0.1644083 .485953

SCF Energy (B3LYP/6-31G**//MMFF) $=-3245.91667618$

0700499

MM̄FF Geometry 
C $3.283450-2.635850-1.324709$

C $2.586032-2.814325-2.456096$

C $1.252044-2.166021-2.746277$

O $0.519977-1.922963-1.522254$

C $1.471126-0.817442-3.452521$

C $0.178064-0.108518-3.802900$

C $-0.3305550 .944103-3.130578$

C $-0.528972-0.636147-5.025054$

C $0.2211551 .609976-1.900275$

C $-0.7704271 .732307-0.729405$

C $-1.9830202 .629318-1.027045$

C $-2.5248053 .257100 \quad 0.258984$

C $-3.7672964 .119476-0.010726$

O $-3.0070761 .820482-1.619387$

O $-4.3775204 .417111 \quad 1.254521$

C $-4.7809303 .286761-0.821217$

C $-3.4020885 .467424-0.643224$

O $-5.8934974 .083415-1.222835$

C $-4.1646852 .552839-2.038152$

C $-5.2092781 .568403-2.625521$

O $-3.8343933 .511510-3.047630$

C $-0.193794-2.962988-1.010377$

O $-0.258905-4.098886-1.452648$

C $-0.905036-2.4692930 .189340$

C $-1.776485-3.2712440 .818623$

C $-2.611017-2.9114041 .967920$

C -2.465129-1.519832 2.525759

C $-4.6295510 .515018-3.579089$

C $-4.210574-0.744923-2.843337$

C $-5.148426-2.691542-1.601095$

O $-3.029670-1.022012-2.648657$

C $-5.375690-2.315589-0.170214$

C $-6.199345-2.9841710 .651380$

C $-6.407161-2.6372392 .099781$

C $-5.929812-3.7336953 .029425$

C $-6.766384-4.7009713 .441802$

C $-4.502925-3.6665193 .535610$

C $-3.493147-3.8226272 .431124$

N -5.292096 -1.505021 -2.422761

H $2.884317-1.977637-0.556668$

H $2.995251-3.449532-3.237796$

H $0.665163-2.832350-3.390811$

H $2.047082-0.970161-4.375491$

H $2.101433-0.172049-2.830285$

H -1.241623 $1.394047-3.520325$

H - $-1.428565-0.060362-5.267270$

H $0.133473-0.592454-5.895596$

H $-0.836130-1.675387-4.872268$

H $1.0990541 .080137-1.520110$

H $0.5670882 .612453-2.180951$

H $-1.1055230 .732383-0.430579$

H -0.2083942 .1538160 .111694$

H -1.679602 $3.414400-1.729354$

H -1.7522333 .8536770 .758964$

H -2.7977332 .4618150 .966835$

H $-3.7216334 .882707 \quad 1.801314$

H $-5.1861122 .531557-0.133412$

H -2.699311 $6.013222-0.002163$

H $-4.2834236 .111994-0.734532$

H -2.942621 $5.368130-1.628758$

H $-5.6082484 .632589-1.972904$

H -5.728248 $1.059056-1.804554$

H $-5.9644392 .144771-3.175300$

H $-3.8321503 .067241-3.910918$

H $-0.714242-1.4505530 .503436$

H -1.920996 -4.281305 0.437219

H -2.629240 -0.7673141 .746157$

H -1.461980 -1.384483 2.942583

H -3.171762 -1.289159 3.324756

H $\quad-5.393967 \quad 0.228369-4.312279$

H $-3.7779130 .895557-4.151847$

H -4.141837 -3.104285 -1.720902

H -5.877065 -3.426262 -1.957135

H $-4.813504-1.4685360 .216209$

H $-6.756014-3.835767 \quad 0.266679$

H -7.479242 $-2.457258 \quad 2.252792$

H -5.913392 -1.693056 2.361197

H $-6.441491-5.4773064 .126460$
H $-7.795354-4.7392203 .099777$

H -4.316478 -4.468192 4.262206

H -4.378479 -2.737351 4.097774

H -3.523636 -4.803294 1.954002

H $-6.233260-1.163414-2.594125$

C $5.786695-2.309560-1.073389$

O $5.481655-1.090427-0.362800$

C $5.8285270 .021672-1.208421$

C $6.825160-0.534142-2.214930$

C $6.217041-1.897346-2.483714$

H $4.9198590 .311612-1.749317$

C $6.3416851 .196624-0.374989$

H $6.9042420 .086366-3.112455$

H $7.824934-0.639289-1.777335$

C $7.174700-2.872690-3.145562$

H $5.341843-1.766966-3.132634$

C 5.3071691 .7707970 .618710

O $7.491114 \quad 0.7772540 .369801$

H $6.6921191 .992387-1.042531$

H 5.1280861 .0499941 .424136

O 5.9024232 .9162861 .241966

C $3.9666532 .182812-0.021919$

C 3.0557813 .0391800 .874703

H $3.4266701 .285082-0.342603$

O $4.2422352 .950165-1.199261$

H 3.5570953 .9844901 .118342

H 2.1846963 .3202300 .267132

H $7.493324-2.494986-4.122723$

H $8.072142-3.029777-2.537791$

H $\quad 6.696024-3.845038-3.298335$

C $4.603175-3.279671-1.011624$

H 7.2183830 .0336320 .934588

H $6.0404383 .587456 \quad 0.551584$

H $3.3943173 .117692-1.645048$

H $4.525866-3.6728710 .009887$

H $4.787607-4.142487-1.661816$

H $6.634422-2.766577-0.546456$

C 1.2498190 .4261342 .843796

O $1.821314 \quad 1.194373 \quad 1.777101$

C 2.5773992 .3472342 .161261

C 1.7363933 .3063533 .024404

C 1.0946682 .5816534 .208749

C $0.383611 \quad 1.3015423 .762240$

H $0.582420-0.2822062 .339545$

C $2.312380-0.3740443 .605677$

H 3.4518182 .0268222 .735720

O $\quad 0.7164723 .9064042 .227783$

H 2.3871284 .1128453 .383995

H 0.3749743 .2519364 .694044

O $2.1096962 .272720 \quad 5.158815$

H 0.0666450 .7137064 .631640

O -0.8015461 .6447063 .039964$

C $2.730830-1.5927262 .819930$

H 3.2178140 .2012543 .817724

H $1.931410-0.7235284 .572989$

H 0.2330894 .5335962 .791825

H 1.6822351 .8295225 .911420

H -1.366378 2.1681623 .633535

O $1.821931-2.5938683 .000646$

O $3.737662-1.6433712 .126799$

C $2.106128-3.8225462 .325220$

H $1.385217-4.5710482 .664834$

H $1.991140-3.6901741 .246883$

H $3.114184-4.1730712 .566979$

SCF Energy (B3LYP/6-31G**//MMFF) $=-3245.88887037$

0700500

MM̄MF Geometry

C 1.6990824 .1875951 .203056

C 0.4663473 .6585221 .173489

C $-0.485833 \quad 3.8374470 .018415$

O $-0.7500652 .520998-0.521522$

C -1.7904564 .4786770 .530050$

C $-2.6941435 .012766-0.564146$

C $-3.8672974 .462599-0.935080$

C $-2.2276416 .291406-1.214319$

C $-4.5095803 .213970-0.398138$

C $-4.9659982 .284886-1.533214$ 
C $-5.5106820 .928473-1.053168$ C $-6.7772021 .070902-0.203792$ C $-7.265856-0.2811750 .334960$ O $-4.473855 \quad 0.273572-0.316479$ $\begin{array}{lllll}0 & -8.253053 & 0.000318 & 1.338958\end{array}$ C $-6.078168-0.9887431 .021130$ C $-7.978026-1.098605-0.747890$ O $-6.448486-2.296878 \quad 1.451059$ C $-4.805140-1.0398030 .148810$ C $-3.589268-1.579750 \quad 0.930449$ O $-5.031574-1.932021-0.949053$ C $-0.9431712 .424207-1.862637$ O $-0.9747593 .347525-2.666003$ C -1.151382 $1.026568-2.298987$ C $-0.702302-0.045454-1.628522$ C $-0.811462-1.437626-2.080276$ C $-1.688989-1.718091-3.272262$ C $-3.123081-0.7047172 .098359$ C $-1.777747-1.1987412 .587359$ C $-0.739724-3.1888913 .649944$ O $-0.728956-0.656066 \quad 2.247882$ C $-0.733833-4.2641512 .607836$ C $0.060972-4.2473591 .527765$ C $-0.009449-5.265198 \quad 0.429018$ C $-0.755682-4.747794-0.782437$ C $-2.019292-5.132151-1.029603$ C $-0.013425-3.858321-1.752807$ C $-0.127800-2.394568-1.417836$ N $-1.881650-2.3360793 .375635$ H 2.0541214 .7598780 .350841 H $0.113314 \quad 3.0826162 .026010$ H - $0.0124424 .469117-0.742786$ H -1.552144 5.3228011 .192713 H -2.324772 $3.760774 \quad 1.161560$ H -4.445922 4.968799-1.707208 H -2.947767 $6.672958-1.946111$ H -2.084557 $7.070853-0.458810$ H - $1.2799546 .137725-1.738617$ H $-3.835761 \quad 2.6663370 .264737$ H -5.374332 3.5213510 .199876 H -5.727544 2.787599-2.142455 H $-4.1075322 .093344-2.189777$ H $-5.7279980 .334773-1.949173$ H $-7.5714151 .571291-0.771459$ H -6.5767341 .7154670 .661699$ H -8.512633 -0.8460731 .742171$ H $-5.863779-0.4210831 .934908$ H -8.848901 $-0.549839-1.126576$ H $-8.371348-2.037255-0.342486$ H -7.338221 -1.332775 -1.601027 H $-6.458407-2.8736720 .668138$ H -3.790516 -2.602145 1.275951 H -2.748805 -1.6819540 .231792$ H $-4.171157-2.233899-1.284551$ H $-1.6364450 .929456-3.265326$ H $-0.1661290 .105391-0.693440$ H -2.679320 -1.267958 -3.137873 H $-1.866254-2.782930-3.439038$ H -1.238669-1.312937 -4.184810 H $-3.006014 \quad 0.3417261 .795720$ H -3.838450 -0.7278532 .926922$ H $-0.867310-3.6236244 .645388$ H $0.181383-2.5978293 .638208$ H $-1.459173-5.0658462 .725409$ H $\quad 0.774787-3.4386541 .406218$ H $-0.483863-6.1837740 .799711$ H $1.007240-5.5619190 .144324$ H -2.551652 -4.788832 -1.910360 H -2.551550 -5.792536 -0.352981 H $-0.341961-4.065008-2.776920$ H $1.049576-4.129717-1.754296$ H $\quad 0.474330-2.094224-0.562991$ H $-2.803625-2.7300563 .540571$ C $3.528972 \quad 2.8406432 .290290$ O $2.733442 \quad 1.6837122 .626745$ C 3.3185751 .0422253 .765614 C 4.7865991 .4292903 .735175 C $4.6814212 .880383 \quad 3.302407$
H 2.8621351 .5049564 .652554

C $2.993333-0.4510903 .799542$

H 5.2774461 .3083434 .705257

H 5.3449680 .8518412 .990996

C 5.9724843 .4397932 .729269

H 4.3944283 .4842664 .174245

C $3.675436-1.3658282 .763545$

O $3.330508-0.9439195 .104291$

H $1.907304-0.5729363 .706452$

H $4.737855-1.4880993 .006581$

O $3.091348-2.6702942 .911500$

C $3.543830-0.9460321 .286366$

C $3.997470-2.0660460 .328964$

H $4.147896-0.0509691 .107771$

O $2.190639-0.6052040 .997690$

H $4.926115-2.5127130 .704565$

H $3.222684-2.8432910 .318828$

H 6.7702273 .4122743 .478705

H 6.3076832 .8641861 .859912

H $5.838625 \quad 4.4795262 .413981$

$\begin{array}{lll}\text { C } 2.638253 & 4.082226 & 2.370857\end{array}$

H $4.294408-0.8729955 .210286$

H $3.194113-2.9308863 .842991$

H $1.629895-1.3438441 .287825$

H 3.2519134 .9907652 .387941

H 2.0587974 .0705373 .302962

H 3.9036672 .6885861 .270539

C $3.077954-0.537471-2.973568$

O $3.021697-1.046090-1.634514$

C $4.238251-1.589115-1.118468$

C $4.741761-2.743412-2.005199$

C $4.835699-2.328465-3.475377$

C $3.557960-1.626239-3.947413$

H $2.038283-0.297175-3.228890$

C $3.8961670 .758630-3.073294$

H $4.995754-0.798563-1.085024$

O $3.859671-3.858699-1.886466$

H $5.725329-3.063008-1.639628$

H $5.012772-3.219528-4.089708$

O $5.957508-1.465227-3.633479$

H $3.705294-1.187012-4.940905$

O $2.507225-2.588456-4.069868$

C $3.1911041 .929493-2.422947$

H $4.8815410 .683494-2.607515$

H $4.0357821 .002051-4.133795$

H $4.226968-4.582838-2.421151$

H $6.013332-1.225755-4.574276$

H $2.796908-3.259892-4.710517$

O $3.541778 \quad 3.076408-3.076418$

O $2.4595051 .853065-1.445886$

C $2.9743264 .286188-2.566612$

H $1.8928654 .187283-2.437695$

H $3.4578184 .547171-1.621515$

H $3.1662825 .082640-3.290574$

SCF Energy (B3LYP/6-31G**//MMFF) $=-3245.88618721$

0700501

MM̄FF Geometry

C 2.6300513 .6651390 .207104

C $2.2094064 .303289-0.895733$

C $2.0986673 .711039-2.284571$

O $2.5910212 .353387-2.339086$

C $0.611298 \quad 3.682721-2.682346$

C $0.353713 \quad 3.065467-4.040931$

C $-0.1376681 .823456-4.224223$

C $0.6368293 .957864-5.219786$

C $-0.4444260 .804391-3.157097$

C $-0.063759-0.610838-3.614997$

C $-0.138446-1.684936-2.516596$

C $-1.506711-1.761203-1.832389$

C $-1.529412-2.814758-0.713516$

O $0.890113-1.417304-1.554192$

O $-2.723712-2.5946380 .049079$

C $-0.320760-2.5701350 .216763$

C -1.646138 $-4.234913-1.278055$

O $-0.232380-3.5886201 .210349$

C $1.017047-2.427285-0.541739$

$\begin{array}{lll}\text { C } 2.184099 & -2.035565 & 0.388692\end{array}$ 
O $1.359308-3.695458-1.117021$

C $3.9291612 .171161-2.492712$

O $4.791923 \quad 3.032025-2.553432$

C $4.1706510 .714155-2.544743$

C $5.337950 \quad 0.233747-2.093268$

C $5.733284-1.174876-2.053059$

C $4.892913-2.158626-2.824585$

C $2.038167-0.6899731 .100193$

C $3.362393-0.3054801 .727740$

C $4.945078-0.9894153 .514533$

O $4.1097110 .518671 \quad 1.205973$

C $5.744425-2.1278812 .960323$

C $6.688273-1.9860542 .017067$

C $7.463249-3.1336341 .435798$

C $6.923385-3.5828280 .094880$

C $6.081732-4.6274180 .012496$

C $7.419220-2.886730-1.153261$

C $6.824222-1.517562-1.336231$

N $3.636761-1.0140642 .887845$

H 2.9652162 .6354900 .140059

H $1.8684605 .332553-0.799804$

H $2.6608904 .334657-2.990556$

H $0.205013 \quad 4.703537-2.679405$

H $0.0363723 .148958-1.915986$ H $-0.3374491 .498001-5.244177$

H $0.394348 \quad 3.476627-6.173242$

H $0.0436664 .875640-5.153206$

H $1.6967514 .229308-5.248313$

H $\quad 0.082675 \quad 1.030093-2.225635$

H -1.519880 $0.846711-2.951756$

H $-0.716006-0.906173-4.446909$

H $0.963573-0.591034-4.002889$

H $0.088024-2.641102-3.003638$

H -2.299274 -1.957115 -2.565104

H $-1.750243-0.792064-1.378793$

H -2.710030 -3.206559 0.805062

H $-0.525645-1.6378810 .757645$

H -2.571597-4.337520 -1.857871

H - $-1.715288-4.977005-0.475026$

H $-0.815496-4.509432-1.931111$

H $0.130360-4.3849310 .786237$

H $2.355702-2.8334531 .123220$

H $3.105604-2.002311-0.207769$

H $2.314886-3.708339-1.291716$

H $3.3726260 .083309-2.918077$

H $6.0655760 .935800-1.687177$

H $3.864759-2.174421-2.450519$

H $5.261342-3.184980-2.765088$

H $4.871240-1.890295-3.886803$

H 1.7502170 .1045730 .402969

H $1.273115-0.7351951 .881869$

H $4.808349-1.1116144 .592953$

H $5.426475-0.0229773 .331506$

H $5.516537-3.1198903 .343260$

H $6.907790-0.9933241 .632812$

H $7.462494-3.9773202 .139442$

H $8.516988-2.8455881 .338769$

H $5.709058-4.980635-0.943089$

H $5.742326-5.1499500 .900869$

H $7.246963-3.506393-2.040708$

H $8.511249-2.788778-1.100135$

H $7.367748-0.731754-0.810612$

H $2.982939-1.7289343 .193672$

C 1.6840413 .4329582 .488449

$\begin{array}{lllll}\text { O } & 0.343348 & 3.481276 & 1.970710\end{array}$

C -0.5867293 .4358723 .065836$

C $0.251502 \quad 3.307669 \quad 4.336104$

C 1.5663203 .9431383 .919112

H -1.1096344 .4001033 .070169$

C -1.607863 2.3056532 .854365

H $-0.215214 \quad 3.8101265 .188995$

H 0.4054812 .2588724 .615333

C 2.7346603 .5620064 .810886

H $1.445708 \quad 5.0345903 .921865$

C -2.422898 2.4540261 .548350

$\begin{array}{lllll}\text { O } & -0.927355 & 1.050825 & 2.862737\end{array}$

H -2.298338 2.3018603 .705767

H -2.8571463 .4608101 .513360$ $\begin{array}{llll}\text { O } & -1.572559 & 2.315319 & 0.408546\end{array}$

C $-3.567153 \quad 1.427363 \quad 1.418454$

C $-4.383816 \quad 1.6426840 .127716$

H $-4.231822 \quad 1.524767 \quad 2.284574$

$\begin{array}{lllll}\text { O } & -3.042177 & 0.104786 & 1.438542\end{array}$

H -4.7242182 .6846730 .097405$

H $-3.7310581 .469795-0.737456$

H 2.5526593 .8833385 .841733

H $2.8963602 .478743 \quad 4.819568$

H 3.6588014 .0373114 .467723

C 2.6033284 .2589901 .588750

H $-0.345281 \quad 1.0337422 .083733$

H -0.9047693 .0203940 .449884$

$\begin{array}{llll}\text { H } & -2.420556 & 0.024177 & 0.695661\end{array}$

H 3.6228024 .2680061 .988819

H 2.2518565 .2970161 .547263

H 1.9936272 .3794182 .482723

C $-6.116572-1.644910-0.328954$

O $-5.120224-0.619518-0.235274$

C $-5.602408 \quad 0.7028870 .019822$

C $-6.555315 \quad 1.143998-1.102841$

C $-7.6894880 .132918-1.298004$

C $-7.162297-1.301533-1.403538$

H $-5.568783-2.534545-0.665084$

C $-6.731607-1.967101 \quad 1.037463$

H -6.1340190 .7192780 .977435$

O $-5.822964 \quad 1.287154-2.318600$

H $-6.9685802 .127627-0.848566$

H $-8.2429350 .386626-2.210308$

$\begin{array}{llll}\text { O } & -8.589480 & 0.243078 & -0.199333\end{array}$

H $-7.993680-2.014328-1.354746$

O $-6.559383-1.452722-2.692157$

C $-5.696719-2.5814291 .950436$

H $-7.163396-1.0927751 .532512$

H -7.543841 -2.6969250 .937746$

H $-6.4452171 .587011-3.002892$

H $-9.315578-0.385590-0.350748$

H $-6.273785-2.378228-2.777254$

O $-5.240598-1.6497142 .834689$

O $-5.321875-3.7442741 .868686$

C $-4.222249-2.0997973 .733661$

H $-4.611385-2.8941634 .377564$

H -3.347702 -2.447690 3.175422

H $-3.923609-1.2543154 .359010$

SCF Energy (B3LYP/6-31G**//MMFF) $=-3245.90501566$

0700502

MM̄FF Geometry

C $-3.080538-4.6994910 .176029$

C $-2.110882-3.8802930 .610586$

C $-0.657772-4.0256690 .241021$

O $-0.295381-2.831219-0.488567$

C $0.185922-4.138269 \quad 1.527055$

C $1.647154-4.4553541 .288184$

C $2.675802-3.6165961 .524280$

C $1.927414-5.8588520 .806782$

C $2.654446-2.2241402 .095188$

C $3.216876-1.1326951 .175136$

C $4.601277-1.3866260 .558370$

C $5.674644-1.7224851 .594927$

C $7.039625-1.9442970 .927783$

O $4.978444-0.182234-0.126880$

O $8.038580-1.9636641 .960169$

C $7.356063-0.7369250 .022650$

C $7.116100-3.3090440 .232407$

O $8.521822-0.974920-0.762651$

C $6.186068-0.288114-0.890653$

C $6.5204591 .100567-1.489944$

O $6.012262-1.164264-2.007752$

C $0.585698-2.959820-1.514439$

O $1.155440-3.980022-1.875423$

C $0.848308-1.658911-2.161528$

C $-0.100028-0.720811-2.304771$

C $0.0710460 .572153-2.974198$

C $1.4710340 .962916-3.369516$

C $5.3580771 .786128-2.220609$

C $4.5590572 .724869-1.327281$

C $2.9025924 .586860-1.461942$ 
O $4.6377732 .722541-0.101709$

C $1.5233434 .041084-1.279375$

C $0.4256544 .597277-1.814559$

C $-0.9558724 .048097-1.609889$

C $-1.6759893 .748259-2.906423$

C $-2.8151284 .387407-3.221671$

C $-1.0917642 .700465-3.832136$

C $-1.0172701 .341212-3.187595$

N $3.7410983 .568302-2.067210$

H -2.834457 -5.516265 -0.497687

H $-2.370812-3.0599861 .276213$

H $-0.530231-4.912613-0.391114$

H $-0.224967-4.9314612 .167054$

H $\quad 0.081042-3.2161642 .105761$

H $3.676955-3.9983601 .328562$

H $2.999608-6.0599080 .708503$

H $1.522394-6.5877341 .516665$

H $1.471943-6.039761-0.170410$

H $1.646602-1.9212912 .391562$

H $3.235069-2.2388693 .025935$

H $2.505455-0.9484770 .359325$

H $3.247001-0.1892921 .736693$

H $4.501230-2.190686-0.179218$

H $5.411131-2.6033592 .189794$

H $5.762921-0.8933262 .310820$

H $7.807040-2.6687722 .588788$

H 7.6012460 .1010210 .690792

H $6.975234-4.1172620 .960357$

H $8.105080-3.473874-0.209034$

H $\quad 6.362422-3.438074-0.547058$

H $9.238527-1.216905-0.151317$

H $6.8924051 .768886-0.703550$

H $7.3367880 .975620-2.213848$

H $6.619219-1.914788-1.915876$

H $1.843562-1.546559-2.578226$

H $-1.098326-0.922653-1.919027$

H $\quad 1.857517 \quad 0.284180-4.137675$

H $2.1365490 .924489-2.500187$

H $1.5492511 .973034-3.774269$

H $5.7800002 .393351-3.031773$

H $4.677974 \quad 1.055845-2.670624$

H $2.9193045 .457680-2.124922$

H $3.317646 \quad 4.879237-0.492203$

H $1.4224253 .150189-0.663042$

H $0.5190685 .499903-2.413344$

H -1.520451 4.778364 -1.016939

H $-0.9212123 .135138-1.007019$

H -3.345461 4.177394 -4.144519

H -3.245811 $5.134549-2.563087$

H $-0.1245043 .054452-4.196657$

H -1.716363 $2.596639-4.729065$

H -1.988957 $0.956271-2.877466$

H $3.6867913 .448571-3.074568$

C $-5.383840-3.974270-0.524010$

O $-5.001731-2.597033-0.726964$

C $-6.147958-1.762911-0.495344$

C -7.342913 -2.658942 -0.778575

C $-6.873142-3.961019-0.158421$

H $-6.141903-1.5008160 .570281$

C $-6.066944-0.484938-1.333770$

H $-8.267069-2.279302-0.333124$

H -7.506089 -2.791884 -1.854175

C $-7.632177-5.181102-0.651608$

H $-6.992887-3.8910310 .931389$

C $-4.7358550 .283067-1.205538$

O $-6.236869-0.810162-2.719407$

H -6.905607 $0.172752-1.077366$

H $-3.940990-0.252899-1.738773$

O $\quad-4.892906 \quad 1.538790-1.878474$

C -4.2750300 .5531010 .239085$

C $-3.022677 \quad 1.4378630 .289866$

H $-4.066276-0.4031140 .726526$

O

H $-2.2547421 .037283-0.381066$

H -3.265006 $2.436972-0.096839$

H $-8.694577-5.101477-0.399509$

H -7.551874 -5.291377-1.738279

H -7.241493 -6.093686 -0.190408
C $-4.519796-4.5707560 .589153$

H -5.543607 -1.449550 -2.957967

H $-5.154214 \quad 1.345521-2.795267$

H -5.5407172 .0153110 .518904$

H $-4.886967-5.5670200 .862131$

H $-4.590278-3.9447551 .488219$

H $-5.213323-4.496473-1.473440$

C -0.6846742 .9753842 .736013$

O -1.3573272 .5750651 .537482$

C -2.4246911 .6291571 .696286$

C $-1.887641 \quad 0.3336492 .322811$

C $-1.1427050 .615595 \quad 3.631943$

C $-0.1270291 .752588 \quad 3.480357$

H 0.1680073 .5743942 .389999

C -1.565275 3.8770053 .607190

H -3.1945492 .0668282 .340157$

O $-1.005333-0.3011711 .399612$

H -2.719881 -0.3522152 .515394$

H $-0.624047-0.2959603 .953269$

O $\quad-2.100028 \quad 0.9487294 .632963$

H 0.2453052 .0618244 .463362

O 1.0054531 .2636832 .755540

C -1.817091 5.1957762 .915061

H -2.531178 3.4330623 .864341

H -1.0659364 .1013004 .557393$

H $-0.694330-1.1247821 .810409$

H -1.6164401 .0980375 .463271$

H $\quad 0.712712 \quad 1.050537 \quad 1.852757$

O -2.8922335 .0883092 .083076$

O -1.1257326 .1928123 .078175$

C -3.2141776 .2651051 .335620$

H $-2.3561606 .584704 \quad 0.736324$

H $-4.040344 \quad 6.0237100 .661630$

H -3.532230 7.0638742 .011954

SCF Energy $\left(B 3 L Y P / 6-31 G^{* *} / / M M F F\right)=-3245.90962823$

0700503

MM̄FF Geometry

C $1.680299-3.5692341 .493445$

C $1.474190-2.9918880 .301558$

C $0.123464-2.619480-0.238771$

O $0.075573-1.171636-0.299305$

C $-0.106770-3.150594-1.663352$

C $-0.490081-4.615763-1.699065$

C $-1.750849-5.069569-1.546599$

C $0.642742-5.579339-1.935006$

C $-2.990914-4.244680-1.331611$

C $-3.477632-4.3180760 .122435$

C $-4.587885-3.3085460 .463609$

C $-5.901016-3.620818-0.256859$

C $-6.979417-2.5686980 .039548$

O $-4.117010-2.001143 \quad 0.121947$

O $-8.049524-2.793820-0.891042$

C $-6.386807-1.172660-0.244601$

C $-7.581374-2.7485741 .437129$

O $-7.310752-0.1461480 .108859$

C $-5.017425-0.9300290 .429515$

C $-4.3565970 .383482-0.053251$

O $\quad-5.209177-0.8163281 .843854$

C $-0.356362-0.5271300 .820109$

O $-0.765372-1.0381891 .851297$

C $-0.237330 \quad 0.928130 \quad 0.584546$

C $-0.786717 \quad 1.774793 \quad 1.467810$

C -0.7864883 .2368311 .394009$

C $0.0121423 .877740 \quad 0.292036$

C $-4.0568810 .424734-1.553142$

C $-3.1429881 .575786-1.921940$

C $-3.1134174 .046681-2.219307$

O $-1.989101 \quad 1.381606-2.298465$

C $-3.1391185 .111267-1.164604$

C -3.7236695 .0705810 .043489$

C -3.6705616 .2157981 .016510$

C -3.1255875 .8212922 .371141$

C $-3.8904725 .900823 \quad 3.473272$

C -1.6707305 .4097772 .462587$

C -1.4957553 .9215632 .315548$

N $-3.7519262 .814120-1.799243$

H $0.833526-3.8284552 .123283$ 
H $2.334512-2.705404-0.293348$ H $-0.678204-2.9783200 .415633$ H $\quad 0.782061-2.980508-2.286050$ H $-0.879210-2.546868-2.155775$ H -1.923002 -6.144198 -1.581802 H $0.299672-6.618080-1.987753$ H $1.143388-5.350411-2.881563$ H $1.379497-5.517734-1.129043$ H $-2.825906-3.204883-1.625284$ H $-3.767883-4.626077-2.004300$ H $-3.817338-5.335616 \quad 0.352784$ H $-2.628810-4.1130980 .786764$ H $-4.739811-3.3514121 .548686$ H -6.259416 -4.624084 0.004706 H -5.736624 -3.636127 -1.342375 H $-8.702526-2.083630-0.766310$ H $-6.268486-1.108497-1.332808$ H $-8.033560-3.7433021 .530910$ H -8.393321 -2.034639 1.614273 H -6.847759 -2.641282 2.238717 H $-7.297346-0.0568811 .077060$ H $-4.974585 \quad 1.2430320 .236179$ H $-3.411130 \quad 0.5146410 .488814$ H $-4.450380-0.3391862 .219037$ H $\quad 0.2829551 .258885-0.305965$ H -1.3095541 .3567042 .327502$ H 1.0703493 .6134440 .388843 H $-0.040744 \quad 4.9679540 .289112$ H $-0.3414323 .544226-0.688842$ H -3.566323 $-0.499689-1.879072$ H $-4.9785340 .523008-2.137110$ H $-3.6495074 .399300-3.106478$ H -2.076712 $3.849056-2.510090$ H -2.602544 $6.018442-1.438941$ H $-4.280827 \quad 4.1913640 .354993$ H -4.689289 $6.612507 \quad 1.115308$ H -3.063985 7.0416220 .623614 H -3.497457 5.6440314 .451555 H $-4.926158 \quad 6.2199223 .422878$ H -1.270162 5.6922313 .445454 H -1.085206 5.9881581 .743208 H -2.025072 3.3515123 .081067 H -4.743287 $2.850206-1.580255$ C $3.440985-2.6494292 .981153$ O $3.637183-1.4539312 .197412$ C $4.645591-0.6444632 .833529$ C $5.076724-1.4003764 .089152$ C $4.758801-2.8387173 .722099$ H $5.495327-0.5926452 .144366$ C 4.0823220 .7564023 .101149 H $6.134574-1.2417414 .320924$ H 4.497719-1.089715 4.966965 C $4.673932-3.7644484 .922412$ H $5.529806-3.2056703 .031491$ C 3.6555061 .5210621 .828541 O $2.928722 \quad 0.635283 \quad 3.945994$ H 4.8085181 .3497543 .668025 H 2.8530250 .9804191 .315518 O 3.0963942 .7735922 .247460 C 4.8099951 .8118410 .846671 C $4.4091822 .725916-0.329653$ H 5.2203590 .8725140 .461871 O 5.8582042 .4632891 .571383 H 3.8426683 .5864590 .045050 H $5.3268773 .142181-0.767489$ H $5.628597-3.7872805 .458245$ H $3.900733-3.4391495 .626547$ H $4.437769-4.7869934 .612023$ C $3.055005-3.8030932 .057192$ H 2.2739670 .0941823 .472021 H 2.3618252 .5746612 .853249 H 6.6142962 .5627630 .968067 H $3.050448-4.7555292 .598049$ H $3.784201-3.8934381 .242894$ H $2.628984-2.4425053 .692081$ C $4.0313290 .406535-3.227465$ O $4.5555441 .179043-2.139778$ C $3.6167391 .995230-1.426850$
C $2.9294022 .985104-2.380351$

C $2.2866812 .255943-3.562968$

C $3.2703031 .291952-4.228923$

H $4.9178380 .014896-3.743744$

C $3.213987-0.790098-2.726435$

H $2.8602451 .355772-0.962237$

O $3.8845383 .929957-2.859499$

H $2.1688243 .543079-1.823498$

H $1.9441862 .986826-4.305583$

O $1.152547 \quad 1.520017-3.115197$

H $2.7434790 .658393-4.951306$

O $4.2253402 .047669-4.980294$

C $4.129334-1.866239-2.182417$

H $2.506750-0.535109-1.933474$

H $2.642140-1.215345-3.559783$

H $3.4131564 .558090-3.432919$

H $0.5211562 .154275-2.734646$

H $4.7601742 .561027-4.350718$

O $3.783386-3.076523-2.709358$

O $5.025459-1.665298-1.372944$

C $4.561510-4.190779-2.261979$

H $5.609652-4.058426-2.546085$

H $4.466487-4.310236-1.178983$

H $4.176248-5.090995-2.748280$

SCF Energy $\left(B 3 L Y P / 6-31 G^{* *} / / M M F F\right)=-3245.92371713$

0700504

MM̄FF Geometry

C -3.274310 -3.220175 -0.713334

C $-3.158153-2.632782-1.913681$

C $-3.750922-1.285447-2.230611$

O $-2.682614-0.380881-2.616711$

C $-4.727619-1.336076-3.417471$

C $-6.027406-2.056379-3.135197$

C $-7.140134-1.458134-2.667632$

C $-6.056552-3.521386-3.489404$

C $-7.331380-0.010548-2.303015$

C $-8.0306420 .154684-0.944868$

C -7.082777 -0.0124690 .253969$

C $-7.891719-0.1215771 .549217$

C $-6.992513-0.1462842 .791784$

$\begin{array}{lllll}\text { O } & -6.239977 & 1.144807 & 0.293918\end{array}$

O $-7.8246290 .057024 \quad 3.943949$

C -6.0228311 .0492622 .718460$

C $-6.312123-1.5070772 .980747$

O -5.0779061 .0033003 .785858$

C -5.2861251 .1657221 .363668$

C -4.5334992 .5180881 .299944$

$\begin{array}{llll}\text { O } & -4.348512 & 0.095324 & 1.255295\end{array}$

C $-1.8343880 .048215-1.642832$

O $-1.835908-0.280551-0.467135$

C $-0.8967401 .015377-2.254659$

C $0.0853441 .539756-1.506219$

C $1.0898792 .506926-1.950759$

C $0.9329113 .086039-3.332399$

C $-3.9182392 .836218-0.068107$

C $-3.3503574 .240802-0.096138$

C -1.4684355 .6201990 .780609$

O $-3.9245785 .154846-0.683915$

C $-0.3121725 .683126-0.167341$

C 0.9541115 .8738340 .233392

C $2.1253776 .016919-0.696590$

C $3.2009904 .985068-0.441462$

C 4.1353205 .1795610 .504439

C $3.2387223 .771956-1.341828$

C $2.0861412 .836072-1.102069$

$\begin{array}{llll}\mathrm{N} & -2.165917 & 4.359761 & 0.613362\end{array}$

H $-3.841528-2.7251440 .071297$

H -2.577918 -3.117833 -2.694882

H $-4.264287-0.852653-1.363618$

H $-4.235191-1.788362-4.289808$

H -4.935077 -0.310226 -3.749762

H $-8.033500-2.071256-2.551768$

H -7.050848 $-3.961225-3.355922$

H $-5.776580-3.663501-4.538424$

H $-5.363572-4.090372-2.864534$

H $-6.393147 \quad 0.551817-2.312043$

H $-7.9744930 .433218-3.073006$ 
H $-8.4610851 .164562-0.914714$ H -8.866064 -0.552889-0.870852 H $-6.465950-0.9069270 .112451$ H -8.539891 -1.0067401 .528517$ H -8.5683430 .7410591 .629837$ H -8.491887 -0.6504003 .960378$ H -6.6162831 .9619062 .869842$ H $-7.063417-2.2968053 .101738$ H -5.710078 -1.523750 3.896073 H -5.672651 -1.788538 2.141509 H -5.5812610 .9393364 .615613$ H -5.2319713 .3295111 .544432$ H -3.7410212 .5356132 .058574$ H -3.615536 0.3685430 .682389 H - $-1.039851 \quad 1.268106-3.298922$ H $0.173606 \quad 1.223416-0.467398$ H $1.0762152 .310066-4.092157$ H $1.6387163 .889020-3.553464$ H $-0.0658503 .519969-3.456484$ H -3.115909 2.137845 -0.313327 H $-4.6649742 .762661-0.866566$ H -1.148940 5.6746401 .826336 H -2.1518116 .4525990 .585016$ H $-0.5323755 .606523-1.229350$ H 1.1608955 .9753351 .296727 H $2.5445317 .022529-0.558382$ H $1.8098075 .975861-1.746418$ H 4.9268804 .4588760 .676928 H $4.1251186 .065151 \quad 1.131392$ H $4.1585693 .198832-1.169499$ H $3.3094594 .105385-2.380743$ H $2.1077352 .363733-0.118854$ H -1.746736 3.5298491 .022982 C -1.457883 -4.2903560 .609514$ O $-0.389330-3.621420-0.091368$ C $0.866595-4.2041480 .298598$ C $0.558166-5.0988071 .493055$ C $-0.842529-5.574244 \quad 1.159621$ H $1.202284-4.826338-0.540866$ C $1.917187-3.1199290 .578753$ H $1.277533-5.9168411 .596036$ H $0.556463-4.5297612 .430454$ C - $-1.585093-6.1573792 .349032$ H $-0.778904-6.3315320 .366558$

C $2.321498-2.301151-0.665025$

O $1.434489-2.2374031 .591765$ H $2.806312-3.6162010 .982303$ H $2.729190-2.971337-1.431724$

O $1.170694-1.676680-1.242474$

C $3.334006-1.170772-0.387547$

C $4.605218-1.6513130 .321379$

H $2.853533-0.3742900 .192089$

O $3.709238-0.596316-1.644002$

H $4.366269-2.0121881 .329061$

H $5.007068-2.523405-0.213198$

H -1.056657 -7.0327912 .740459$

H -1.676666 -5.428934 3.161710

H -2.593863 -6.471925 2.063772

C $-2.622976-4.524979-0.352145$

H $0.636224-1.804371 \quad 1.242777$

H $0.557319-2.378713-1.517618$

H $2.893104-0.292377-2.077461$

H $-3.379482-5.1704640 .107144$

H -2.265096 -5.030861-1.257482

H -1.763150 -3.629090 1.431720

C $8.006119-0.5001461 .297616$

O $6.800733-1.2572141 .133884$

C $5.740473-0.6119240 .413893$

C 5.3344910 .6949761 .110717

C $6.5469731 .605701 \quad 1.338274$

C 7.7175420 .8517551 .973457

H $8.619045-1.0946551 .987259$

C $8.783327-0.369484-0.022062$

H $6.088034-0.396947-0.602095$

O $4.717790 \quad 0.3930562 .360517$

H 4.5930831 .2159820 .495454

H 6.2621192 .4384751 .992900

O 6.9766542 .1559560 .096584
H $\quad 8.618848 \quad 1.475822 \quad 1.956704$

O $7.396320 \quad 0.617774 \quad 3.347544$

C $9.303139-1.703435-0.518249$

H $\quad 8.1658370 .052077-0.819196$

H 9.6529040 .2795650 .133587

H $4.449273 \quad 1.2347792 .766433$

H $6.2289132 .646236-0.283824$

H 8.1665390 .1924583 .761586

O $10.007794-1.499674-1.671424$

O $9.118972-2.7833180 .025726$

C $10.563921-2.675520-2.268213$

H $11.093254-2.377626-3.177262$

H $11.277259-3.145794-1.584646$

H $9.767887-3.375685-2.539085$

SCF Energy (B3LYP/6-31G**//MMFF)= -3245.91387517

0700505

MM̄̄F Geometry

C -1.121410 $5.695587-0.576422$

C $-2.1928704 .934369-0.307829$

C $-3.0510404 .301453-1.372021$

O $-2.8005892 .875195-1.310198$

C $-4.5412504 .548520-1.088121$

C $-5.4524353 .968662-2.153389$

C $-6.2925912 .930242-1.972676$

C $-5.3982034 .657642-3.494149$

C $-6.5409082 .131250-0.723491$

C $-5.9661940 .718572-0.841926$

C $-6.383427-0.2103690 .308914$

C $-5.836444 \quad 0.242804 \quad 1.665273$

C $-6.159512-0.7619702 .783429$

O $-5.877199-1.513982-0.012434$

O $-5.347998-0.4313743 .921331$

C $-5.737340-2.1726882 .323799$

C $-7.617093-0.6535273 .246462$

O $-6.143353-3.1632693 .264596$

C $-6.254575-2.5404600 .917400$

C $-5.675137-3.8770750 .397494$

O $-7.678303-2.693408 \quad 0.961525$

C $-1.7112642 .415695-1.988030$

O $-1.0047803 .047909-2.757938$

C $-1.4779871 .013198-1.576954$

C $-0.3147300 .427584-1.900764$

C $0.117451-0.920583-1.525640$

C $-0.846309-1.765151-0.737548$

C $-4.147209-3.9434390 .361178$

C $-3.629732-5.137073-0.423312$

C $-1.503953-6.258352-1.052233$

O $-4.358130-5.920861-1.025071$

C $-0.069313-5.854039-1.161176$

C $0.508706-5.468126-2.308486$

C $1.951950-5.069128-2.427447$

C $2.112646-3.601780-2.762187$

C $2.363783-3.217903-4.025904$

C $2.035672-2.636102-1.595955$

C $1.348609-1.330302-1.898309$

$\mathrm{N}-2.243344-5.202759-0.387717$

H $-0.8736145 .933160-1.607792$

H $-2.4056094 .662558 \quad 0.723757$

H -2.792233 $4.686407-2.365791$

H $-4.7333625 .627844-1.021667$

H $-4.7881634 .142820-0.102143$

H $-6.8953012 .610668-2.822545$

H $-6.1660034 .288511-4.182718$

H $-5.5594875 .734013-3.374910$

H -4.4268794 .499948 -3.972393

H -6.1403632 .6281530 .162083$

H -7.626400 $2.075442-0.573687$

H $-6.2904300 .262488-1.787674$

H $-4.8708910 .760563-0.902865$

H $-7.478761-0.253787 \quad 0.330133$

H -6.2168021 .2337961 .938182$

H $-4.744490 \quad 0.348358 \quad 1.598084$

H $-5.547996 \quad 0.485728 \quad 4.176040$

H -4.640219 -2.182719 2.315206

H $-7.8207000 .350724 \quad 3.637325$

H $-7.821167-1.3428204 .073245$

H $-8.336958-0.8470472 .448734$ 
H $-5.795738-2.8978214 .133191$ H $-6.065410-4.7144600 .990669$ H -6.057738 -4.037788 -0.620253 H -7.953139 $-3.239150 \quad 0.206119$ H $-2.2421480 .521844-0.986346$ H $0.4099850 .999534-2.479273$ H -1.063434 -1.3023760 .231377$ H $-0.473000-2.769524-0.533828$ H -1.785687 -1.889242 -1.287558 H -3.740097 -3.039692 -0.105557 H $-3.752353-4.0158951 .380687$ H -1.959272 -6.451717 -2.029798 H -1.598325 -7.169702 -0.452781 H $\quad 0.521414-5.884054-0.248837$ H $-0.084636-5.439807-3.219658$ H $2.411094-5.693038-3.205180$ H $2.507439-5.294041-1.508017$ H $2.517985-2.178151-4.291661$ H $2.429640-3.943131-4.830765$ H $3.059023-2.418740-1.262290$ H $1.563186-3.124031-0.738331$ H $1.968404-0.638493-2.468707$ H $-1.750851-4.6167080 .280760$ C $1.071376 \quad 5.209558 \quad 0.471637$ O $0.6602903 .890436 \quad 0.886041$ C 1.6848183 .3164581 .724920 C 2.8686214 .2695251 .644581 C 2.1766485 .6057791 .444016 H 1.2712413 .3104032 .741062 C 1.9500381 .8840511 .246190 H 3.4926904 .2395152 .542741 H 3.5021234 .0339190 .780408 C 3.0987106 .6940990 .924118 H 1.7408925 .9250922400153 C 3.1525361 .2005931 .926936 O $\quad 0.7531821 .133387 \quad 1.473784$ H 2.1003891 .8892670 .161372 H 4.0389991 .8158871 .742047 O 2.9295531 .1837223 .336002 C $3.417878-0.2284611 .408119$ C $4.642285-0.9258692 .029449$ H $3.536418-0.1859710 .319478$ O $2.282078-1.0553541 .671544$ H $4.446104-1.1321443 .089491$ H $4.724104-1.9176891 .566973$ H 3.9105276 .8818771 .634553 H $3.5490716 .414638-0.034401$ H $2.553151 \quad 7.6318270 .779336$ C $-0.144418 \quad 6.139448 \quad 0.477519$ H 0.6513831 .0284882 .435236 H $3.757615 \quad 0.9113373 .765554$ H $2.452239-1.9270181 .276291$ H $\quad 0.151998 \quad 7.1733520 .271017$ H -0.6243726 .1251311 .463960$ H $1.4737285 .112788-0.546042$ C $6.458287-0.725680-0.415642$ $\begin{array}{lllll}\text { O } & 6.193055 & 0.300166 & 0.547493\end{array}$ C $5.973809-0.1649171 .881322$

C $7.201616-0.9421842 .375552$

C $7.592807-2.0617281 .413933$

C $7.702868-1.528155-0.014605$

H $5.588988-1.390070-0.495115$

C $6.664254-0.040587-1.770582$

H $5.896745 \quad 0.7307622 .509470$

O $6.953899-1.4745103 .672434$

H $8.046180-0.2470112 .469526$

H $\quad 6.887875-2.8988431 .476890$

O $8.862575-2.577851 \quad 1.819507$

H $8.598162-0.898302-0.090777$

O $7.889011-2.624030-0.908328$

C $5.3555720 .463484-2.329832$

H $7.3609460 .803077-1.694277$

H $7.081212-0.731305-2.512533$

H $7.746345-1.9718273 .938610$

H $9.118133-3.2584701 .173293$

H $7.095766-3.184816-0.863193$

O $5.0890301 .711896-1.850712$

O $4.650262-0.184459-3.092310$
C $3.8726972 .304803-2.315978$

H $3.9401962 .502766-3.389697$

H $3.0204881 .655564-2.097456$

H $3.7331323 .253175-1.790432$

SCF Energy $(B 3 L Y P / 6-31 G * * / / M M F F)=-3245.89451889$

0700506

MM̄FF Geometry

C -2.816608 $4.160118-0.972559$

C $-2.0863803 .245156-0.318014$

C $-0.9970122 .433666-0.966618$

O $\quad 0.1625482 .473427-0.103625$

C $-1.4393400 .966898-1.090343$

C $-0.5407910 .160164-2.007048$

C $0.425324-0.680849-1.588626$

C $-0.8253880 .310329-3.479497$

C $0.866235-0.923488-0.170478$

C $2.390011-1.047265-0.045431$

C $2.961395-2.403673-0.497486$

C $2.597080-3.5297730 .475870$

C $3.286811-4.8530480 .114812$

O $4.385953-2.252215-0.548666$

O $3.162515-5.736921 \quad 1.239846$

C $4.793548-4.595005-0.091251$

C $2.593825-5.550425-1.062204$

O $5.443930-5.772198-0.567077$

C $5.094337-3.403041-1.031086$

C $6.610496-3.081244-0.997347$

O $4.708969-3.749077-2.360455$

C $1.0479833 .491058-0.269713$

O $0.9949074 .387936-1.095205$

C 2.1009493 .3261450 .759032

C 3.1033074 .2140660 .837946

C 4.2034044 .1944211 .806738

C 4.2051163 .0926222 .834591

C $7.107668-2.032551-2.000452$

C $6.415490-0.689820-1.902710$

C $6.0999711 .267668-0.425570$

O $5.650082-0.296217-2.780717$

C 6.8835221 .9660290 .636638

C 7.4761013 .1565830 .461180

C 8.2210183 .8825761 .544017

C 7.6569435 .2604951 .815754

C 8.3213476 .3660321 .439078

C 6.3626055 .3569302 .596202

C 5.1491715 .1545521 .726468

N $6.7431210 .010476-0.752991$

H -2.625505 4.341909-2.027074

H $-2.2861793 .056614 \quad 0.734320$

H $-0.7420532 .837145-1.954348$

H $-2.4624680 .913077-1.486872$

H -1.486035 $0.501618-0.097890$

H $0.963673-1.245894-2.347256$

H $-0.192977-0.338671-4.094573$

H $-1.8666330 .053824-3.695437$

H $-0.649173 \quad 1.341311-3.801239$

H $\quad 0.550813-0.1010450 .478484$

H $0.376244-1.8256660 .202781$

H $2.875784-0.239891-0.610119$

H $2.677303-0.8758691 .000681$

H $2.600100-2.629482-1.505873$

H $1.512803-3.6789340 .529467$

H $2.909049-3.2459701 .490942$

H $2.215043-5.8517511 .427611$

H $5.229998-4.3757990 .893483$

H $1.549398-5.774679-0.813063$

H $3.062411-6.516080-1.281426$

H $2.591612-4.951181-1.975092$

H $5.238808-6.4889770 .057349$

H $6.879125-2.7527200 .014679$

H $7.180748-3.996000-1.202001$

H $5.019890-3.055672-2.963296$

H 2.0157572 .4796071 .431209

H 3.1229195 .0387010 .126339

H 4.1989602 .1099212 .350235

H 3.3220323 .1697363 .478621

H 5.0780853 .1056753 .489835

H $8.182320-1.870234-1.847866$ 
H $7.006676-2.400068-3.027939$ H $5.0923261 .042238-0.061375$ H $\quad 6.0000861 .867604-1.336614$ H 6.9332001 .4838581 .610137 H $7.4241583 .639255-0.512064$ H 9.2714933 .9553511 .234288 H 8.2262683 .3083772 .479076 H 7.9357807 .3572131 .653843 H 9.2627326 .3051200 .903066 H $6.2740196 .353723 \quad 3.048773$ H 6.4051674 .6681223 .443904 H 5.0490015 .9114790 .946888 H $7.256530-0.472858-0.021376$ C $-5.3151364 .431394-0.569042$ O -5.4737443 .1610620 .091803$ C $-6.3041232 .316437-0.729783$ C $-6.8691813 .212636-1.828476$ C $-5.7366424 .204744-2.019503$ H $-5.6397021 .576338-1.190946$ C -7.3563331 .6095240 .132496$ H -7.108407 $2.652237-2.737441$ H -7.778472 $3.731866-1.504061$ C $-6.1465805 .471702-2.748056$ H -4.941256 $3.706305-2.587048$ C $-6.772358 \quad 0.814862 \quad 1.319878$ O -8.2563962 .5878940 .669582$ H -7.967558 $0.953007-0.497270$ H -6.3434841 .5086602 .052547$ $\begin{array}{lllll}\text { O } & -7.860240 & 0.163541 & 1.989049\end{array}$ C $-5.719109-0.2391880 .932163$ C $-5.233436-1.0439022 .153956$ H $-4.861080 \quad 0.2567530 .465549$ O $-6.297375-1.115914-0.032429$ H $-4.978010-0.3467592 .960840$ H -6.049859 -1.690717 2.498820 H $-6.5072295 .236764-3.754804$ H $-6.9477055 .998459-2.218934$ H -5.298109 6.156576 -2.843931 C $-3.9042024 .973075-0.329561$ H -7.729485 3.2148251 .194432 H $-8.229482-0.4943701 .375080$ H -5.618845 -1.763596 -0.287161 H -3.7250225 .0222420 .752300$ H -3.831978 $6.000520-0.705080$ H $-6.0234385 .113698-0.079683$ C $-3.376418-3.859475 \quad 0.522157$ O $-4.412392-2.9692270 .960014$ C $-4.002173-1.9186191 .840430$ C $-3.384740-2.5075583 .118104$ C $-2.242755-3.4748262 .791029$ C $-2.647103-4.4919601 .719416$ H -3.906475 -4.662672 -0.006536 C $-2.433793-3.182743-0.478714$ H -3.259756 -1.290014 1.336868 O $-4.378561-3.1865423 .883556$ H -3.008362 $-1.687183 \quad 3.740005$ H -1.945742 -4.0089203 .701917$ O $-1.112361-2.7440742 .326285$ H -1.770246 -5.044626 1.361962 O $-3.536767-5.4496302 .301270$ C -3.141101-2.944039-1.791167 H -2.035319 -2.229968 -0.122658 H -1.568304 -3.820657 -0.694412 H -4.777961 -3.867679 3.315509 H $-0.851976-2.1250623 .029553$ H -3.059662 -5.8913973 .024302$ O $-3.725695-1.712883-1.790264$ O $-3.192771-3.768137-2.695104$ C $-4.470219-1.380519-2.966387$ H -4.770379 -0.331939-2.892434 H -3.856095 -1.506499-3.863067 H -5.368980 -2.001388 -3.024428 SCF Energy (B3LYP/6-31G**//MMFF) $=-3245.91514862$

\section{7}

MM̄MF Geometry

C -1.668071 -3.593683 1.068256

C $-0.931164-2.5591181 .500153$
C $0.418121-2.2050260 .935023$

O $0.343215-0.829966 \quad 0.489415$

C $1.528467-2.2762952 .000478$

C $2.040894-3.6750682 .263945$

C $3.069830-4.2317241 .595027$

C $1.370308-4.4235923 .384773$

C $3.850221-3.6225280 .463621$

C $5.293392-3.3230220 .886188$

C $6.096177-2.679633-0.253028$

C $7.574412-2.5634560 .124506$

C $8.387040-1.842279-0.960810$

O $5.549428-1.378794-0.500464$

O $9.662712-1.522498-0.384183$

C $7.675786-0.516479-1.308998$

C $8.675688-2.759301-2.154511$

O $8.323114 \quad 0.138082-2.396852$

C $6.163521-0.676357-1.586655$

C $5.4391310 .681317-1.777282$

O $5.995924-1.399518-2.814616$

C $0.955273-0.500305-0.676916$

O $1.485879-1.265534-1.470228$

C $0.9110160 .960201-0.916578$

C 0.6853151 .8505130 .061462

C $0.6755333 .306789-0.076194$

C $0.9013653 .889454-1.445767$

C $5.5543211 .704103-0.643475$

C 4.7059691 .4052200 .580091

C 4.2959662 .2587522 .892640

$\begin{array}{lllll}\text { O } & 3.818857 & 0.557831 & 0.595818\end{array}$

C 3.4623593 .4988343 .050121

C 3.4897784 .6056872 .289653

C 2.6168105 .8080582 .527040

C 1.8043346 .1826981 .305926

C 2.2583527 .0987880 .433891

C 0.4393905 .5546521 .129717

C 0.4829764 .0553291 .029433

N $5.0296362 .240528 \quad 1.640562$

H -1.285555 -4.218356 0.264575

H -1.302504 -1.945198 2.317516

H $0.657499-2.8614640 .089201$

H $1.185567-1.8228202 .941000$

H $2.363630-1.6301921 .701214$

H $3.384304-5.2367751 .872335$

H $1.841074-5.3941273 .574836$

H $1.425689-3.8462444 .313432$

H $0.318368-4.610733 \quad 3.153502$

H $3.852664-4.335105-0.370559$

H $3.371925-2.7155650 .083629$

H $5.279025-2.6463911 .750808$

H $5.780113-4.254207 \quad 1.202518$

H $5.980213-3.298725-1.150453$

H $8.003861-3.5517190 .331284$

H $7.665998-1.9961701 .061544$

H $10.157656-0.997714-1.036849$

H $7.8148540 .134177-0.438191$

H $9.242258-3.640120-1.828740$

H $9.306497-2.258109-2.896809$

H $7.771310-3.113477-2.653415$

H $8.063600-0.320238-3.214380$

H $5.8147841 .148719-2.697555$

H $4.3741080 .486236-1.964450$

H $5.095751-1.238529-3.143502$

H $\quad 1.132857 \quad 1.265734-1.933654$

H $\quad 0.5076491 .4859331 .071908$

H $1.8853793 .598437-1.829649$

H $0.8682154 .981340-1.461311$

H $\quad 0.1334543 .540732-2.144772$

H $6.5953331 .855930-0.346273$

H $5.1953132 .668691-1.027097$

H 5.0279822 .2018693 .704200

H 3.6480621 .3781052 .949823

H 2.7657833 .4701503 .886319

H 4.1864214 .6774431 .458455

H 3.2697936 .6443772 .809509

H 1.9454705 .6577593 .381512

H $1.6751787 .394547 \quad-0.431812$

H 3.2264607 .5710200 .565064

H $-0.0876845 .975845 \quad 0.265113$ 
H -0.1834915 .8235591 .992904$ H 0.3381253 .5500111 .984871 H 5.7831872 .9124131 .527490 C $-4.083225-4.2016680 .641521$ O $-4.663637-2.927057 \quad 0.282196$ C -6.098665 -3.045930 0.325699 C $-6.393909-4.5403540 .349772$ C $-5.237098-5.053398 \quad 1.177431$ H -6.420298 -2.613832 1.280538 C $-6.772842-2.309541-0.836563$ H $-7.370957-4.7644730 .788680$ H $-6.364923-4.976462-0.655744$ C $-5.015722-6.5506881 .043283$ H $-5.424581-4.8116142 .232463$ C $-6.511377-0.791617-0.905049$ O $-6.315029-2.886080-2.066810$ H -7.853005 -2.497232 -0.795974 H -5.488331 -0.614996-1.250674 O $-7.365191-0.264682-1.930996$ C $-6.761263-0.019648 \quad 0.402440$ C $-6.823931 \quad 1.5136720 .256752$ H $-5.983532-0.2852961 .127698$ O $-8.012487-0.4433490 .953900$ H -7.749183 $1.798914-0.260656$ H $-6.9211021 .922307 \quad 1.271561$ H -5.899187 -7.101373 1.382462 H -4.820831 -6.8357690 .003847$ H $-4.163193-6.8767041 .646940$ C -2.975808 -4.0078841 .681989$ H $-6.760635-2.414563-2.791418$ H $-8.283779-0.358784-1.624925$ H -8.118301 -0.0017761 .813885$ H $-2.790329-4.9537732 .204468$ H -3.295234 -3.2739402 .432124$ H -3.683247 -4.639244 -0.282205 C $-3.2029402 .234750-0.318916$ $\begin{array}{lllll}\text { O } & -4.435456 & 1.775389 & 0.254271\end{array}$ C $-5.6197182 .155875-0.448332$ C $-5.7390793 .687618-0.506840$ C $-4.4785254 .317435-1.110008$ C $-3.2020883 .766396-0.465322$ H $-2.437047 \quad 1.9686860 .420466$ C -2.869402 $1.499462-1.627589$ H -5.577386 $1.766418-1.469892$ O $\quad-5.9445014 .2030320 .807225$ H $-6.6193783 .950512-1.105582$ H -4.509616 $5.404973-0.972004$ O $-4.4358614 .061563-2.510689$ H -2.320834 $4.076745-1.038098$ O $-3.066338 \quad 4.320854 \quad 0.846348$ C $-2.6606550 .016430-1.411217$ H $-3.6655101 .607217-2.368555$ H -1.942997 $1.905140-2.048421$ H -6.0446715 .1671530 .732328$ H -5.240216 $4.441165-2.903723$ H $-2.993219 \quad 5.2856200 .750180$ O $-2.390320-0.574232-2.612539$ O $-2.734095-0.555377-0.332960$ C -2.208435 -1.993084 -2.573325 H -2.009579 -2.338268 -3.591357 H -1.352184 -2.249404 -1.943518 H -3.117032 -2.481538 -2.209343 SCF Energy (B3LYP/6-31G**//MMFF) $=-3245.89805842$

\section{8}

MM̄FF Geometry

C 1.7877656 .0612850 .685191

C 2.5371294 .9923650 .377490

C 3.2542224 .1521781 .401601

O 2.7178582 .8099731 .331620

C 4.7569264 .0907171 .078384

C 5.5497683 .2633722 .072156

C 6.1235732 .0729691 .805579

C 5.7054963 .8775713 .440907

C 6.1218421 .3002900 .515121

C $5.378002-0.0287840 .663717$

C $5.470320-0.923428-0.582539$

C $4.748698-0.325447-1.793807$
C $4.739845-1.285715-2.994648$

O $4.874046-2.179919-0.229923$

O $3.783489-0.792757-3.946139$

C $4.237487-2.666683-2.525743$

C $6.092508-1.312036-3.715762$

O $4.343723-3.633635-3.567334$

C $4.950833-3.179985-1.257015$

C $4.315247-4.473577-0.696104$

O $6.314056-3.488363-1.571448$

C 1.5813382 .5625912 .040260

O 0.9646273 .3508202 .739516

C $1.201373 \quad 1.1514801 .806821$

C 0.0509360 .6859452 .316657

C $-0.477051-0.6706142 .163713$

C $0.387707-1.6798911 .455695$

C $2.822540-4.368373-0.379187$

C $2.316809-5.5302520 .458449$

C $0.231803-6.382281 \quad 1.511293$

O $3.036765-6.4358560 .868395$

C $-1.055188-5.7672671 .955309$

C $-1.253150-5.2643923 .182805$

C $-2.532542-4.6137953 .626407$

C $-2.360894-3.1245253 .845125$

C $-2.184433-2.6407485 .086991$

C $-2.441740-2.2619962 .599571$

C $-1.695503-0.9540722 .671450$

N $0.957444-5.4136300 .712446$

H $1.6927106 .369551 \quad 1.723325$

H $2.6123784 .676469-0.660604$

H 3.1118304 .5564442 .411513

H 5.1747125 .1065831 .065207

H 4.8898693 .7090420 .061564

H 6.6776381 .5856122 .607551

H 6.3786043 .2988904 .082573

H 6.1214834 .8870663 .359176

H 4.7391353 .9377213 .950185

H $5.6901211 .877792-0.303519$

H $7.165280 \quad 1.106477 \quad 0.237877$

H $5.782242-0.5855091 .520461$

H $\quad 4.323770 \quad 0.152538 \quad 0.909119$

H $6.530799-1.087790-0.806691$

H $5.1916760 .631977-2.090636$

H $3.707641-0.102382-1.520527$

H $4.0444080 .111217-4.192520$

H $3.166728-2.554213-2.313347$

H $6.343827-0.313794-4.094342$

H $\quad 6.059077-1.965289-4.594672$

H $\quad 6.914981-1.636174-3.074889$

H $3.882006-3.274563-4.344402$

H $4.486551-5.309908-1.386458$

H $4.852267-4.7411600 .224662$

H $6.651020-4.109601-0.904575$

H 1.8678980 .5404001 .209551

H $\quad-0.5769291 .3658502 .891191$

H $0.513680-1.4083550 .401988$

H $-0.020911-2.6913081 .483716$

H $1.376553-1.7393891 .924353$

H $2.617943-3.4469560 .177506$

H $2.243136-4.347794-1.308932$

H $0.857900-6.6933952 .354567$

H $0.041522-7.2640530 .891100$

H -1.856319 -5.7232461 .222985$

H $-0.446044-5.3059373 .910927$

H -2.859668 -5.1049154 .551888$

H -3.340782 -4.783806 2.904125

H -2.082313 -1.579909 5.285651

H -2.144149 -3.302989 5.946006

H -3.498974 -2.044967 2.400974

H $-2.103510-2.8322631 .729003$

H -2.247473 -0.161074 3.177307

H $0.425580-4.7207900 .192283$

C $-0.4799536 .588412-0.215149$

O $-0.7511745 .227355-0.608556$

C $-1.9035125 .205538-1.467819$

C $-2.547416 \quad 6.579457-1.331868$

C $-1.3299077 .461578-1.136694$

H -1.531257 $5.081366-2.492765$

C $-2.8327784 .033700-1.120378$ 
H $-3.139023 \quad 6.850398-2.211564$ H -3.201530 $6.632357-0.453432$ C $-1.6554748 .830100-0.564593$ H $-0.8277797 .587211-2.105490$ C $-2.1811022 .651074-1.345526$ O -3.2741224 .1608160 .230162$ H -3.725403 4.102005 -1.753519 H $-1.8056662 .619899-2.375491$ O $-1.0515082 .483011-0.492903$ C $-3.1520541 .467593-1.151151$ C $-2.4858240 .119478-1.489276$ H $-4.0245511 .614301-1.798530$ O $-3.610581 \quad 1.4127840 .197689$ H $-1.9132460 .218846-2.418993$ H $-1.790793-0.143478-0.682990$ H -2.318397 $9.381094-1.239682$ H -2.155680 8.7505730 .406454 H $-0.7453049 .422238-0.427147$ C $1.0207666 .859338-0.331585$ H -2.483227 4.2119820 .793916 H -1.380787 2.425116 0.420109 H -4.0937542 .2362240 .379567$ H $1.2340347 .921402-0.167649$ H $1.3666036 .609224-1.342479$ H -0.8110346 .6905740 .826750$ C $-5.216425-2.224419-0.428523$ O $-4.163089-1.251816-0.410927$ C $-3.514136-1.020820-1.663398$ C $-2.858826-2.317372-2.161598$ C $-3.889803-3.446622-2.257567$ C $-4.720487-3.573698-0.975299$ H $-5.479988-2.3671950 .627665$ C $-6.462309-1.699883-1.151095$ H $-4.253663-0.685110-2.398747$ O $-1.809847-2.693632-1.272069$ H -2.410595 -2.133150 -3.145530 H -3.368718 -4.391251-2.454791 O $-4.746383-3.184819-3.365879$ H -5.578211 -4.237654 -1.134504 O $-3.914044-4.1763830 .039254$ C $-7.052736-0.528135-0.402957$ H -6.268102 -1.395602 -2.183508 H $-7.238014-2.473256-1.200996$ H $-1.401197-3.502296-1.624546$ H -5.377937 -3.921496 -3.429202 H $-3.651462-5.056296-0.280085$ O $-6.6469640 .641981-0.972360$ O $-7.766666-0.6417490 .585125$ C $-7.106674 \quad 1.834002-0.328201$ H -6.676548 $2.691100-0.853252$ H -8.197404 $1.896602-0.384979$ H -6.7709711 .8577670 .713063$

SCF Energy (B3LYP/6-31G*//MMFF) $=-3245.90245660$

07_00509

MM̄FF Geometry

C $2.014504-2.6176280 .972635$

C $1.438636-1.9273091 .967412$

C $1.878398-0.5423252 .359145$

$\begin{array}{lllll}\text { O } & 0.737245 & 0.350498 & 2.343712\end{array}$

C $2.440862-0.4883003 .790230$

C $3.708541-1.2821274 .010158$

C $4.948637-0.8106923 .778252$

C $3.524498-2.6571724 .600018$

C 5.3397410 .5393293 .240193

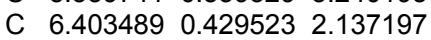

C $5.823904 \quad 0.0630830 .760517$

C $6.953474-0.362584-0.180716$

C $6.460831-0.597670-1.613241$

$\begin{array}{lllll}\text { O } & 5.167796 & 1.230250 & 0.252427\end{array}$

O $7.631261-0.704398-2.437650$

C $5.6640230 .642031-2.064047$

C $5.709091-1.927190-1.744976$

O $5.0651110 .427434-3.340934$

C $4.5832021 .086160-1.048720$

C $4.0319082 .471157-1.469004$

O $3.5280250 .126805-1.049922$

$\begin{array}{llll}\text { C } & 0.264028 & 0.756876 & 1.135754\end{array}$ $\begin{array}{llll}\text { O } & 0.623675 & 0.373191 & 0.034807\end{array}$

C $-0.762664 \quad 1.790526 \quad 1.392268$

C -1.2980202 .4666230 .365216$

C $-2.310105 \quad 3.5205260 .463866$

C $-2.7373093 .947174 \quad 1.844961$

C $3.0121863 .082809-0.498770$

C $2.6872724 .526060-0.849588$

C $1.3201386 .449250-0.072075$

O $3.2447305 .138212-1.756910$

C -0.1444486 .5782050 .197161$

C $-1.0176267 .050760-0.705137$

C $-2.4839687 .244509-0.444362$

C $-3.3516416 .407862-1.357551$

C $-3.7076376 .854498-2.573932$

C $-3.876305 \quad 5.095385-0.822613$

C $-2.8011764 .054583-0.674493$

N $1.7008015 .049912-0.023051$

H $2.833959-2.1637230 .421638$

H $0.628652-2.3731822 .536506$

H $2.630637-0.1464931 .665861$

H $1.671302-0.8146254 .504198$

H $2.6104760 .562043 \quad 4.063969$

H $5.789884-1.4579874 .024302$

H $2.945197-3.2975923 .929512$

H $4.479118-3.1605884 .786983$

H $2.997074-2.5902595 .557291$

H 4.4808021 .1129192 .879562

H 5.7718981 .1056754 .074531

H 6.9043901 .4038242 .060516

H $7.166872-0.3002362 .435132$

H $5.093291-0.7456600 .870249$

H $7.459424-1.2586390 .200581$

H $7.7211980 .424152-0.204096$

H $7.345447-0.911410-3.343321$

H $6.3810891 .465384-2.190250$

H $6.370027-2.764914-1.491732$

H $5.387694-2.100147-2.777995$

H $4.831830-1.990003-1.098570$

H $5.7799380 .267491-3.979403$

H $4.8764753 .167519-1.557482$

H $3.5612652 .403025-2.458125$

H $2.7159340 .545787-0.725451$

H -1.0420681 .9742512 .423611$

H $-0.969740 \quad 2.225999-0.645184$

H -3.425994 4.7941051 .848210

H -1.8691634 .2606572 .435949$

H -3.2368343 .1233262 .366162$

H $2.0775442 .516563-0.528721$

H 3.3978203 .0669700 .526489

H $1.6018056 .879689-1.038838$

H 1.8887796 .9705640 .704897

H $-0.494856 \quad 6.298376 \quad 1.187462$

H $-0.6543237 .345299-1.687482$

H $-2.7145658 .308007-0.590651$

H -2.735203 7.0361340 .602849

H $-4.3538276 .274091-3.223989$

H -3.356006 $7.810675-2.947460$

H $-4.6326094 .682765-1.504120$

H -4.4176955 .2814050 .109189$

H -2.419874 $3.686187-1.627885$

H 1.3988174 .5032800 .778737

C $1.142794-4.054604-0.876614$

O $-0.202935-3.537856-0.955709$

C $-0.911303-4.263774-1.983660$

C $0.093665-5.232797-2.601350$

C $1.055038-5.463804-1.454264$

H $-1.695170-4.842938-1.484076$

C $-1.518525-3.292151-3.001998$

H $-0.384235-6.152232-2.953742$

H $0.614421-4.782773-3.455318$

C $2.387624-6.044283-1.895419$

H $0.584259-6.140098-0.728139$

C $-2.648505-2.400813-2.444829$

O $-0.479148-2.439947-3.501799$

H $-1.888596-3.851153-3.869304$

H $-2.254445-1.760440-1.648386$

O $-3.055091-1.512045-3.494589$

C $-3.884241-3.177869-1.935354$ 
C $-5.135039-2.308824-1.695102$ H -3.627337 -3.708825 -1.012915 O $-4.243972-4.181854-2.890386$ H -5.385990 -1.764128 -2.613654 H -5.980833 -2.986066 -1.513117 H $2.241423-7.012841-2.384854$ H $2.898584-5.383398-2.603667$ H $3.052234-6.197878-1.039735$ C $1.637987-4.0132270 .568234$ H $-0.116450-1.946706-2.745820$ H $-3.345938-2.052667-4.248322$ H $-4.532193-3.732321-3.702803$ H $2.532182-4.6362850 .681248$ H $\quad 0.873526-4.4148261 .241379$ H $1.770175-3.420446-1.518175$ C $-4.896500-1.3899461 .910412$ O $\quad-5.045060-2.1162520 .682674$ C $-4.995097-1.335712-0.516861$ C $-6.110059-0.280546-0.513096$ C $-6.0202680 .596144 \quad 0.738950$ C $-5.917764-0.2445892 .012901$ H -5.147475 -2.117766 2.693708 C $-3.438549-0.9548262 .123899$ H $-4.028846-0.828338-0.585228$ O $-7.381035-0.923337-0.567131$ H $-6.0138260 .337268-1.414216$ H $-6.904627 \quad 1.2415890 .800934$ $\begin{array}{lllll}\text { O } & -4.873085 & 1.433581 & 0.653427\end{array}$ H -5.6702630 .3881272 .873301$ O $-7.193535-0.833406 \quad 2.284473$ C -2.625203 -2.109037 2.666881 H -2.944178 -0.5917481 .219717$ H -3.384862 -0.1545612 .871419$ H -8.061312 -0.228755 -0.579256 H $-4.9658421 .978189-0.146677$ H $-7.831735-0.1093652 .402084$ O $-2.555617-3.1107051 .744854$ O $-2.130090-2.1214263 .786533$ C -1.948471-4.329192 2.184818 H -1.709912 -4.928552 1.302365 H -1.026487 -4.1406112 .741118$ H -2.658234 -4.884001 2.804982

SCF Energy (B3LYP/6-31G**//MMFF) $=-3245.89601430$

\section{$07 \_00510$}

MM̄FF Geometry

C -2.871949 -2.280343 -2.407847

C $-2.123129-3.188360-3.052208$

C $-0.616121-3.301178-3.010519$

O $-0.043044-2.194883-2.275437$

C $-0.223124-4.600415-2.282889$

C $1.191747-5.043253-2.593781$

C $2.271454-4.747059-1.845562$

C $1.335735-5.899498-3.825512$

C $2.329477-3.872664-0.625819$

C $2.769586-4.6491910 .623732$

C $3.139089-3.7431871 .812684$

C $1.953691-2.9148162 .314966$

C $2.365864-1.9290743 .415964$

O $4.208021-2.9008871 .379145$

O $1.264163-1.0263913 .601366$

C $3.571570-1.1083812 .911473$

C $2.576887-2.6315744 .761292$

O $4.038599-0.2533893 .952520$

C $4.718011-1.9847392 .354003$

C $5.814177-1.1536341 .642774$

O $5.338096-2.6825763 .438567$

C $0.478543-1.149520-2.970318$

O $0.585496-1.046971-4.181186$

C $0.867355-0.115859-1.980392$

C $1.3185811 .076888-2.398381$

C $1.6713182 .216466-1.546270$

C $1.5499502 .039176-0.055978$

C $5.334310-0.4542740 .368542$

C $6.4477060 .272920-0.362448$

C $6.8041211 .568602-2.468790$

O 7.5453990 .5051090 .134722

C $6.4021962 .966029-2.119254$
C $5.3476163 .587909-2.668147$

C $4.8692784 .941325-2.231846$

C $3.8555304 .850429-1.111561$

C 4.2400464 .9912410 .168749

C $2.3959164 .678595-1.463309$

C $2.0725593 .366959-2.128002$

N $6.0363180 .667951-1.628951$

H $-2.397162-1.516329-1.798291$

H $-2.628723-3.942507-3.652780$

H $-0.243816-3.313420-4.041911$

H $-0.898964-5.419185-2.566116$

H $-0.353059-4.480638-1.199913$

H $3.237838-5.137325-2.162716$

H $2.374762-6.191875-4.010988$

H $\quad 0.748013-6.817301-3.720513$

H $0.982780-5.359532-4.709824$

H $3.039278-3.065570-0.842329$

H $1.369045-3.390384-0.432890$

H $1.979986-5.3499180 .921931$

H $3.653673-5.2494410 .370053$

H $3.511017-4.3903212 .615790$

H $1.143272-3.5678752 .661902$

H $1.529507-2.3255941 .491794$

H $1.541548-0.3514124 .244790$

H $3.187295-0.4543172 .119322$

H $1.646090-3.1093655 .090386$

H $2.839011-1.9138155 .546436$

H $3.350087-3.4018314 .727926$

H $4.6528760 .387964 \quad 3.558147$

H $6.261109-0.4324502 .337681$

H $6.637095-1.8270061 .362996$

H $6.236982-2.9356433 .170659$

H $0.735596-0.354563-0.930518$

H $1.4146851 .249561-3.469606$

H $0.510928 \quad 1.8344350 .221299$

H 1.8681212 .9166710 .511375

H $2.1745301 .206400 \quad 0.285029$

H $4.895110-1.185250-0.319930$

H 4.5713680 .2937750 .607139

H $6.5779591 .327568-3.512190$

H $7.8754241 .425014-2.297954$

H $6.9727033 .463061-1.338559$

H $4.7671073 .077606-3.432741$

H $4.4383295 .471904-3.089470$

H $5.7260075 .552650-1.917555$

H $3.523954 \quad 4.9561330 .982990$

H 5.2820875 .1407370 .431891

H $2.1002765 .489514-2.141832$

H $1.7635574 .818911-0.578829$

H $2.1430663 .402888-3.215527$

H $5.0707830 .500187-1.898517$

C -5.019264 -2.484369-1.075877

O $-4.607515-1.457315-0.149820$

C $-4.263704-2.0822811 .100068$

C $-4.892525-3.4698611 .054376$

C $-4.704341-3.824767-0.407640$

H -3.172611 -2.192721 1.108416

C $-4.707761-1.2180912 .283133$

H $-4.401828-4.1722021 .734808$

H $-5.959666-3.4410181 .303441$

C $-5.583627-4.969110-0.879736$

H -3.653904 -4.102059 -0.558907

C $-4.1497090 .220458 \quad 2.285291$

O $-6.138363-1.1224752 .287402$

H -4.440329 -1.719604 3.220597

H -4.6424660 .8120801 .505579$

O $\quad-4.514097 \quad 0.8195793 .535914$

C $-2.622046 \quad 0.3240612 .126345$

C -2.0274941 .7157602 .416801$

H $-2.3424940 .023866 \quad 1.110435$

O $-1.984508-0.6010003 .012830$

H -2.1320731 .9504243 .484013$

H $-0.946140 \quad 1.6395532 .241619$

H $-5.356698-5.883693-0.322200$

H $-6.646184-4.744594-0.738209$

H $-5.419948-5.171799-1.942880$

C $-4.375046-2.256703-2.447265$

H $-6.409079-0.7457551 .432338$ 
H -5.4815710 .7578013 .615430$ H -2.253866 $-0.373013 \quad 3.919259$ H $-4.674512-1.272180-2.825006$ H $-4.758333-2.998908-3.157417$ H $-6.105468-2.370345-1.186890$ C -2.965052 $3.484748-0.742196$ O -2.4217492 .5433410 .194253$ C -2.6152132 .8597281 .575329$ C -1.966109 4.2117861 .916892 C $-2.471100 \quad 5.3191490 .987255$ C $-2.4129194 .896957-0.484897$ H -2.591342 $3.155423-1.720486$ C -4.500526 $3.437843-0.781787$ H -3.6871982 .9210861 .785941$ O $-0.5486624 .106167 \quad 1.800928$ H -2.195355 4.4597172 .960313 H -1.859851 6.2182051 .133066 O -3.8104365 .6401491 .350646$ H -2.953824 $5.619378-1.106433$ O $-1.0515364 .931568-0.920190$ C -5.020412 2.114705-1.300244 H -4.9366303 .5930270 .208244$ H -4.872073 4.217649 -1.456701 H -0.1678854 .9633612 .057044$ H $-4.106996 \quad 6.3617300 .770184$ H $-0.5698894 .237620-0.438107$ O $-6.3848732 .122062-1.233753$ O $-4.3263371 .196734-1.712966$ C -7.036818 $0.932379-1.689145$ H $-6.6699890 .638572-2.677379$ H $-6.8846030 .128858-0.964000$ H -8.107715 $1.139710-1.763303$

SCF Energy (B3LYP/6-31G**//MMFF)= -3245.91208611

\section{7_00511}

MM̄FF Geometry

C -1.642082 4.7344291 .413039

C -2.6907743 .8989031 .378025$

C $-3.642232 \quad 3.806407 \quad 0.212639$

O $-3.3970722 .563909-0.486259$

C -5.0937323 .7882370 .719320$

C -6.113095 $3.696890-0.398805$

C $-6.7806542 .572478-0.726991$

C $-6.3939484 .987070-1.123302$

C $-6.6125891 .206240-0.115364$

C $-6.7344430 .097853-1.171884$

C $-6.415765-1.318006-0.658823$

C -7.255251-1.723691 0.555526

C $-6.879083-3.1174361 .081940$

O $-5.019452-1.380032-0.344949$

$\begin{array}{llll}0 & -7.493608 & -3.248117 & 2.373544\end{array}$

C $-5.346886-3.1758221 .270832$

C $-7.465151-4.2314510 .207140$

O $-4.921534-4.491213 \quad 1.619319$

C $-4.561779-2.6875490 .034003$

C $-3.043535-2.6010810 .302352$

O $-4.741670-3.625355-1.035013$

C $-2.3855422 .557323-1.395604$

O $-1.6244913 .474048-1.662089$

C $-2.351677 \quad 1.221442-2.024732$

C $-1.1909320 .782933-2.532527$

C $-0.965615-0.503520-3.189578$

C $-2.173683-1.288275-3.627944$

C $-2.632377-1.6121161 .393001$

C $-1.157014-1.2970921 .269104$

C $1.100715-2.285308 \quad 1.597397$

$\begin{array}{lllll}\text { O } & -0.766853 & -0.255898 & 0.746469\end{array}$

C $1.452458-3.0300030 .348150$

C $1.816102-2.436406-0.799065$

C $2.160722-3.191430-2.051535$

C $0.996482-3.300022-3.011861$

C $0.215567-4.393721-3.019649$

C $0.782700-2.188057-4.014300$

C $0.306218-0.912931-3.376383$

N $-0.341514-2.3063801 .755377$

H -1.4571515 .3936000 .568212$

H -2.856239 3.2214202 .212599

H -3.507196 4.649482 -0.476924
H -5.2958524 .6955251 .304707$

H -5.2322312 .9569001 .421223$

H -7.530379 $2.635353-1.514592$

H -7.173396 $4.875282-1.884503$

H $-6.7305045 .753670-0.417711$

H -5.492376 $5.348859-1.627056$

H -5.6474901 .1101630 .390355$

H -7.3992951 .0832980 .637031$

H $-7.7514190 .104927-1.584722$

H $-6.0489320 .325142-1.999204$

H $-6.610966-2.002450-1.493068$

H $-8.326030-1.6740050 .322345$

H -7.091976 -1.011946 1.375237

H $-7.317484-4.1456662 .702730$

H -5.102705 -2.530869 2.124856

H $-8.560645-4.1800970 .209890$

H $-7.210410-5.2210180 .602642$

H $-7.134467-4.181819-0.832193$

H $-5.354358-4.7295382 .456134$

H -2.644546 -3.598432 0.529248

H $-2.544484-2.305912-0.629726$

H $-4.011461-3.518164-1.666770$

H $-3.2645990 .638121-2.032882$

H $-0.3093601 .416015-2.435137$

H -2.804838 $-1.549781-2.774001$

H $-1.922690-2.225848-4.128804$

H $-2.772798-0.701230-4.333079$

H $-3.180530-0.6672311 .307679$

H -2.833951 -2.015936 2.391099

H $1.542219-2.7807312 .467148$

H $1.455432-1.2503191 .571231$

H $1.397808-4.1151120 .392775$

H $1.869060-1.351347-0.842028$

H $2.523972-4.194993-1.791089$

H $3.008937-2.702024-2.545705$

H $-0.605825-4.498123-3.720511$

H $0.378032-5.207725-2.320916$

H $\quad 0.099059-2.501219-4.811658$

H $1.730217-1.985963-4.530569$

H $1.115431-0.272623-3.025271$

H $-0.766710-3.1639972 .095305$

C $0.627793 \quad 4.0281412 .326553$

$\begin{array}{lllll}\text { O } & 1.265823 & 4.428956 & 1.096014\end{array}$

C 1.6906373 .2398210 .392890

$\begin{array}{llll}\text { C } & 1.673768 & 2.126971 & 1.429724\end{array}$

$\begin{array}{llll}\text { C } & 0.466498 & 2.509062 & 2.257780\end{array}$

H $0.9310143 .057550-0.377563$

C $3.0568633 .482625-0.259400$

H $1.5882751 .134600 \quad 0.979681$

H 2.5827852 .1502462 .043723

C $0.417063 \quad 1.826398 \quad 3.613275$

H -0.4324422 .2389591 .693498$

C $3.6486232 .211979-0.901363$

O $2.8966324 .492276-1.257600$

H 3.7580843 .8803860 .484122

H $3.7024891 .443683-0.126805$

O $2.761944 \quad 1.721472-1.905550$

C $5.0588842 .387904-1.496316$

C $5.6577351 .092899-2.081477$

H $5.7323022 .774525-0.722284$

O $5.0466363 .344003-2.557202$

H $5.1011630 .795119-2.979652$

H $6.6673101 .343764-2.433115$

H 0.3737190 .7386383 .495265

H 1.3013012 .0626304 .214756

H -0.4678662 .1408924 .175153$

C $-0.666364 \quad 4.8175052 .553250$

H $2.5031495 .269251-0.824441$

H $2.6998862 .403870-2.595933$

H $4.7447154 .190760-2.187564$

H -0.4172925 .8783192 .683340$

H -1.1389314 .4929463 .487945$

H 1.3299134 .2893473 .129026

C $6.428430-0.6038881 .153019$

$\begin{array}{llllllll}\text { O } & 6.400137 & 0.352124 & 0.082686\end{array}$

C $5.725044-0.088435-1.099287$

C $6.449123-1.305866-1.693831$

C $6.575370-2.430273-0.658454$ 
C $7.086720-1.9181640 .694447$

H $7.079802-0.1456681 .908462$

C $5.029278-0.7541221 .769477$

H $4.700394-0.381482-0.846506$

O $7.749497-0.919211-2.133797$

H $5.892124-1.656283-2.571037$

H $7.263894-3.197172-1.033940$

O $5.308526-3.051493-0.466921$

H $6.957629-2.6855331 .465550$

O $8.492265-1.6670780 .586112$

C $5.048828-1.2795533 .187324$

H 4.5510850 .2326601 .797444

H $4.392846-1.4099031 .170030$

H $8.169091-1.700383-2.532643$

H $5.033939-3.424757-1.321584$

H $8.923955-2.5087330 .360674$

O $3.771736-1.2723113 .673719$

O $6.043979-1.6507223 .793452$

C $3.618318-1.7498125 .013628$

H $4.184644-1.1208355 .706966$

H $2.558101-1.6935965 .274559$

H $3.942292-2.7925225 .083289$

SCF Energy (B3LYP/6-31G**//MMFF) $=-3245.90203030$

07_00512

MM̄FF Geometry

C -1.123888 -2.7507130 .783465$

C $-0.508677-2.564550-0.394708$

C $-1.150335-1.842851-1.546026$

O $-0.306225-0.697470-1.821799$

C $-1.263780-2.691067-2.823353$

C $-1.990962-4.010640-2.685132$

C $-3.323055-4.142609-2.531518$

C $-1.116853-5.233788-2.783547$

C $-4.343328-3.046120-2.392330$

C $-5.217812-3.282081-1.151684$

C $-5.975672-2.046803-0.641224$

C $-6.749280-1.301343-1.730731$

C $-7.437750-0.039603-1.183218$

O $-5.022657-1.176565-0.017687$

O $-7.845554 \quad 0.761678-2.303564$

C $-6.4022810 .789468-0.390698$

C $-8.716020-0.375070-0.406315$

$\begin{array}{lllll}\text { O } & -7.021465 & 1.899581 & 0.254303\end{array}$

C -5.609301 -0.0428510 .640501$

C $-4.4686720 .760376 \quad 1.307229$

O $-6.492085-0.455247 \quad 1.691131$

C $-0.8939980 .398681-2.369141$

O $-2.0475210 .511126-2.751392$

C $0.1109831 .480500-2.374439$

C $-0.3008832 .740092-2.171283$

C $0.554210 \quad 3.924214-2.098584$

C $1.989043 \quad 3.782855-2.532638$

C $-3.413296 \quad 1.2923010 .339818$

C -2.1680241 .7434291 .074937$

C $-1.318217 \quad 3.554528 \quad 2.567842$

O -1.1310421 .0849931 .046045$

C -0.9685614 .8819501 .973361$

C $0.284861 \quad 5.255698 \quad 1.674352$

C 0.6410306 .6055551 .117811

C $1.4576496 .520274-0.152789$

C $2.7966986 .628132-0.115601$

C $0.7231406 .399293-1.469879$

C $0.0246405 .078961-1.643913$

N -2.349697 2.9330761 .758857

H $-2.143736-2.397717 \quad 0.917477$

H $0.515862-2.904005-0.526274$

H $-2.136469-1.481569-1.236827$

H $-0.256113-2.865771-3.226796$

H - $1.753965-2.099164-3.608115$

H $-3.735379-5.150278-2.488839$

H - $1.685620-6.163375-2.675765$

H $-0.614718-5.263513-3.756173$

H $-0.354053-5.221619-1.998463$

H $-3.875394-2.060871-2.348605$

H -4.977446 -3.051985 -3.286976

H -5.942351 -4.073831-1.382166

H $-4.588766-3.653494-0.331151$
H $-6.667850-2.4041730 .130739$

H -7.479817 -1.964260 -2.210363

H $-6.063423-0.982953-2.526729$

H $-8.4361880 .223149-2.857895$

H $-5.701503 \quad 1.207413-1.124686$

H $-9.435805-0.886506-1.056771$

H $-9.2189750 .535138-0.061501$

H -8.538562 -1.0200660 .456538$

H $-7.5050652 .397485-0.427000$

H $-4.8868791 .583332 \quad 1.901006$

H -3.9664590 .1069082 .035159$

H $-5.959241-0.6767572 .472806$

H $1.1516701 .204902-2.489577$

H $-1.3634552 .919438-2.009352$

H $2.5424773 .159412-1.824213$

H $2.5166914 .736903-2.599708$

H $2.0512343 .327215-3.527275$

H -3.114894 $0.513617-0.365988$

H $-3.7984782 .132880-0.247518$

H -0.4496112 .8940402 .646039$

H -1.7368503 .6977363 .568925$

H $-1.7854155 .579777 \quad 1.803421$

H 1.1048784 .5681391 .868539

H $1.208717 \quad 7.142761 \quad 1.889008$

H $-0.253613 \quad 7.213598 \quad 0.934708$

H $3.3927556 .600828-1.021560$

H 3.3277866 .7429690 .823654

H $1.3931676 .586089-2.317642$

H $-0.0263897 .198961-1.532343$

H -1.022886 $5.091599-1.341781$

H $-3.257307 \quad 3.388071 \quad 1.723137$

C $-0.274909-2.3620603 .097165$

O $0.629361-1.3328112 .646740$

C $1.674475-1.1704143 .618650$

C $1.126346-1.7719674 .904206$

C $0.354416-2.9590694 .357737$

H $2.518126-1.7823613 .278258$

C 2.1159870 .2935993 .698060

H $1.918135-2.0577745 .602934$

H $0.445304-1.0841105 .418259$

C $-0.656318-3.5328315 .335162$

H $1.070151-3.7461844 .084503$

C $2.426100 \quad 0.9382672 .329901$

$\begin{array}{lllll}\text { O } & 1.067882 & 1.058985 & 4.305636\end{array}$

H 2.9814020 .3756414 .366699

H $1.486697 \quad 1.1307051 .798317$

O 3.0039452 .2211092 .601744

C 3.3708040 .1184781 .430837

C $3.719614 \quad 0.849107 \quad 0.120245$

H $2.893009-0.8368201 .187900$

$\begin{array}{lllllll} & & 4.563005 & -0.165447 & 2.160868\end{array}$

H $2.8118321 .305577-0.290051$

H $4.441744 \quad 1.6442750 .342567$

H $-0.154669-3.897808 \quad 6.237351$

H -1.391278 -2.780439 5.640453

H $-1.198706-4.3711624 .886647$

C $-0.470173-3.3869631 .976967$

H $1.371967 \quad 1.9817854 .349490$

H 2.9982362 .7301581 .773722

H $5.130124-0.7171441 .596081$

H $-1.100448-4.2139202 .322586$

H $\quad 0.498949-3.8118131 .688012$

H $-1.224872-1.8657843 .334282$

C $6.219974-1.556233-1.288363$

O $5.557289-0.604019-0.446477$

C $4.318643-0.092566-0.944401$

C $4.5400610 .623856-2.285385$

C $5.239506-0.292740-3.294781$

C $6.471734-0.973043-2.688681$

H $7.195918-1.716130-0.811781$

C $5.485632-2.901705-1.311519$

H $3.616150-0.921058-1.088148$

O $5.3259951 .795291-2.076763$

H $3.5696480 .944435-2.679628$

H $5.5461110 .292409-4.170317$

O $4.331202-1.294919-3.742115$

H $6.839078-1.765373-3.351362$

O $7.521765-0.009038-2.566071$ 
C $5.473510-3.524148 \quad 0.065047$

H $4.455598-2.816667-1.669257$

H $5.987361-3.609818-1.981383$

H $5.4182062 .243628-2.934471$

H $3.570968-0.843444-4.146807$

H $7.7210130 .322569-3.458203$

$\begin{array}{lllll}\text { O } & 4.213040 & -3.487743 & 0.583983\end{array}$

O $6.469558-3.971496 \quad 0.618833$

C $4.071813-4.0420691 .895240$

H $4.432441-5.0750041 .917281$

H $4.612374-3.4274652 .621113$

H $3.009863-4.0392582 .152624$

SCF Energy (B3LYP/6-31G**/MMFF) $=-3245.89414743$

07_00513

MMFF Geometry

C $1.695670-0.0022131 .958329$

C 0.3791590 .1826791 .782560

C $-0.411381-0.4036660 .643756$

O $-1.464731-1.192681 \quad 1.247588$

C $-1.0552320 .695609-0.221668$

C $-0.0728861 .444095-1.094835$

C $0.3985192 .680605-0.841246$

C $0.336390 \quad 0.722335-2.353211$

C $0.103653 \quad 3.5593020 .342459$

C $-0.6224444 .855780-0.046415$

C $-2.0198094 .628320-0.644235$

C $-2.6865565 .959265-0.988936$

C $-4.1117425 .751815-1.523463$

O $-2.813975 \quad 3.9369230 .322141$

O $-4.7615847 .032020-1.557580$

C $-4.8963094 .832286-0.556232$

C -4.094751 5.271661-2.979755

O $-6.1687364 .517528-1.113193$

C $-4.1233593 .554884-0.140798$

C -4.7810352 .8281941 .062180$

O $-4.0320462 .667173-1.252026$

C -2.009975 -2.191917 0.502657

O $-1.703685-2.515407-0.633528$

C -3.072534-2.820626 1.319709

C $-3.874724-3.7483410 .775902$

C $-4.985564-4.4291621 .447751$

C -5.225874 -4.098020 2.898354

C -6.0628382 .0332830 .795341$

C -5.8207240 .6665940 .176203$

C $-7.027992-1.213881-0.923753$

$\begin{array}{lllll}\text { O } & -4.717491 & 0.126515 & 0.156058\end{array}$

C -7.468737 -2.2065090 .105058$

C $-8.317780-3.211458-0.157799$

C $-8.747909-4.2365660 .853562$

C -8.228521 -5.6206270 .526748$

C -8.922061 -6.446726 -0.274558

C -6.945262 -6.071992 1.192500

C $-5.745974-5.286413 \quad 0.733463$

N $-6.9887160 .107078-0.323633$

H $2.247648-0.6202141 .255256$

H -0.1702590 .7913112 .497567$

H 0.225222 -1.062022 0.039297

H $-1.631325 \quad 1.3756800 .416169$

H $-1.8203400 .250892-0.871960$

H $1.0884653 .121388-1.559302$

H $1.0350191 .307246-2.960698$

H $0.823909-0.227288-2.113219$

H $-0.5424980 .513324-2.971819$

H -0.4630893 .0373321 .118124$

H $1.062642 \quad 3.8247600 .804995$

H $-0.713728 \quad 5.4646910 .862463$

H $-0.0065335 .424438-0.754475$

H -1.920801 4.012111-1.544570

H -2.083735 $6.525275-1.709802$

H $-2.7386916 .584067-0.086448$

H $-4.7799867 .387993-0.652974$

H -5.0999765 .4168690 .350865$

H $-3.5515105 .985806-3.610514$

H $-5.1082555 .223772-3.393631$

H -3.624342 $4.294090-3.104390$

H $-6.0331023 .837927-1.795469$

H -4.0368342 .1537501 .508378$
H $-4.983793 \quad 3.571993 \quad 1.844570$

H $-3.7498811 .799629-0.918308$

H -3.177892 -2.485345 2.345474

H $-3.718159-4.025145-0.265838$

H $-4.352618-4.3682523 .502471$

H $-6.081702-4.6174583 .332723$

H $-5.421029-3.0268613 .022091$

H -6.5531611 .8443441 .759100$

H -6.7700312 .5977980 .184596$

H $-6.030470-1.478253-1.289028$

H -7.716697 -1.166238 -1.772945

H -7.051472 -2.1131491 .104538$

H -8.726821 -3.311335-1.160804

H $-9.845440-4.2467000 .880133$

H $-8.436924-3.9538591 .866969$

H $-8.571709-7.449660-0.494026$

H $-9.855535-6.134953-0.731814$

H $-6.747381-7.1273700 .962623$

H $-7.086090-6.0415442 .276164$

H $-5.493423-5.453382-0.314688$

H $-7.8699680 .595823-0.194099$

C 3.5243111 .5943612 .646487

O 4.5622550 .9164641 .907600

C $5.810600 \quad 1.100426 \quad 2.593504$

C 5.6443782 .4084443 .350320

C $4.2136892 .277243 \quad 3.835173$

H $\begin{array}{llll}5.897771 & 0.276334 & 3.313227\end{array}$

C $6.996013 \quad 1.0457231 .622992$

H $6.3662102 .516964 \quad 4.165058$

H $\quad 5.7365523 .2767592 .688104$

C 3.5778613 .6009784 .225853

H 4.2025481 .6124924 .709635

C $6.981075-0.1433760 .639799$

$\begin{array}{lllll}\text { O } & 7.030565 & 2.241486 & 0.833494\end{array}$

H 7.9301701 .0460042 .196773

H $6.2552810 .055156-0.155020$

$\begin{array}{llll}\text { O } & 8.262820 & -0.202326 & 0.001945\end{array}$

C $6.686117-1.5110121 .273244$

C $6.835086-2.7132910 .322813$

H $5.667760-1.5111761 .677604$

O $7.572978-1.7103162 .377301$

H $7.897536-2.906795 \quad 0.125526$

H $6.482044-3.5920970 .878391$

H 4.1288304 .0641495 .050905

H 3.5718234 .3060673 .387703

H 2.5425133 .4537864 .549515

C $2.470077 \quad 0.580676 \quad 3.106388$

H 6.1790682 .3095240 .369070

H $8.4262320 .667704-0.400914$

H $7.335060-2.5550162 .796155$

H 1.7870551 .0585403 .819198

H $2.956616-0.2517283 .630269$

H 3.0806752 .3328311 .967488

C $3.899013-2.000962-1.900892$

O $4.726956-2.207187-0.746118$

C $6.076973-2.598093-1.012537$

C $6.115958-3.916412-1.804879$

C $5.262595-3.829308-3.074575$

C $3.869193-3.261318-2.783579$

H $2.888232-1.854789-1.498251$

C $4.288770-0.732883-2.680815$

H $6.565341-1.816450-1.603028$

O $5.633995-4.982070-0.987662$

H $7.157851-4.143007-2.060664$

H $5.157320-4.828144-3.515271$

O $5.910709-3.000778-4.034695$

H $3.343995-3.037084-3.719517$

O $3.094412-4.247600-2.096032$

C $4.0091950 .538495-1.908729$

H $5.350549-0.729808-2.939324$

H $3.702333-0.676053-3.605334$

H $5.705570-5.803465-1.502982$

H $6.780130-3.393293-4.222863$

H $3.032068-5.026840-2.674190$

O $4.564287 \quad 1.592310-2.576104$

O $3.3685190 .605324-0.870291$

C $4.3789572 .889713-1.997535$

H $4.3115022 .852589-0.906248$ 
H $\quad 5.239368 \quad 3.507080-2.269702$

H $3.4756293 .339917-2.416754$

SCF Energy (B3LYP/6-31G**//MMFF) $=-3245.90571470$

0700514

MM̄FF Geometry

C $4.181636-2.6560640 .185220$

C $3.277933-2.225367-0.706274$

C $2.933558-2.957778-1.975252$

O $1.544262-3.356104-1.860736$

C $3.067022-2.059840-3.217006$

C $4.502639-1.699852-3.547895$

C $5.112222-0.552027-3.188603$

C $5.252352-2.736550-4.343716$

C $4.5252240 .571810-2.382420$

C $4.6543731 .923666-3.097983$

C $3.6542942 .978910-2.596243$

C $3.7482923 .231953-1.088924$

C $2.6623244 .201329-0.597176$

O $2.3489842 .511348-2.949836$

O 2.6485854 .1192510 .836110

C $1.2908443 .712343-1.114155$

C $3.0075825 .654734-0.938729$

O $\quad 0.2639484 .643805-0.783053$

C $1.2796633 .405184-2.626551$

C $-0.0304472 .729418-3.087453$

O $1.4006554 .633947-3.352292$

C $1.298113-4.550189-1.259454$

O $2.130741-5.390351-0.942045$

C $-0.138233-4.747487-0.962662$

C $-1.088880-3.814594-1.128441$

C $-2.501855-3.960680-0.761240$

C $-2.930752-5.270012-0.148986$

C $-0.3414281 .391943-2.406105$

C $-1.3100180 .544689-3.212229$

C $-3.5650010 .590586-4.279453$

O $-1.029977-0.605153-3.545929$

C $-4.6091840 .045929-3.358391$

C $-5.068265-1.212733-3.424510$

C $-6.146020-1.752524-2.530715$

C $-5.720164-2.995643-1.783766$

C $-6.215645-4.201385-2.109258$

C $-4.798038-2.827903-0.598833$

C -3.341825 -2.924698 -0.970265

N $-2.5024981 .188400-3.494427$

H $4.726749-3.577499-0.002286$

H $2.714265-1.319836-0.492221$

H $3.561953-3.846942-2.109920$

H $2.438233-1.167780-3.109283$

H $2.623924-2.577781-4.078971$

H $6.152412-0.407904-3.477344$

H $6.278344-2.426893-4.569365$

H $5.307325-3.679475-3.790920$

H $4.746777-2.920934-5.297352$

H $3.478200 \quad 0.380607-2.139092$

H $5.0617410 .618708-1.426722$

H $5.6782632 .300044-2.982233$

H $4.4822491 .779341-4.172966$

H $3.8536393 .905206-3.148104$

H $4.7468013 .597503-0.819174$

H $3.6184532 .289299-0.541065$

H 1.9080544 .6629151 .156083

H $1.0569862 .795373-0.558887$

H $3.9586565 .938977-0.472451$

H $2.2602206 .346200-0.534174$

H $3.0987555 .833639-2.012007$

H $\quad 0.3340995 .393512-1.398341$

H $-0.8688813 .425071-2.956444$

H $0.0362042 .557052-4.171311$

H $1.0626384 .494695-4.252492$

H $-0.368314-5.719839-0.537488$

H -0.813030 -2.845604 -1.539979

H $-4.001060-5.3285340 .057427$

H -2.413412 -5.4366770 .802288$

H $-2.705490-6.102790-0.824807$

H $\quad 0.5739730 .801822-2.281794$

H $-0.7642401 .547427-1.408616$

H $-3.148831-0.179082-4.937647$
H $-3.9954391 .383845-4.898465$

H $-5.0242640 .726577-2.618741$

H $-4.669662-1.886260-4.179869$

H -7.019619-1.968910-3.159339

H $-6.481533-0.997563-1.808695$

H $-5.941938-5.092952-1.555393$

H $-6.903779-4.325098-2.938951$

H -5.062646 -3.542499 0.187944

H $-4.972360-1.849492-0.134601$

H -2.942239-2.017046-1.422831

H $-2.6718512 .113111-3.113060$

C $3.635756-2.5849462 .612170$

O $2.245390-2.2389132 .436570$

C $1.666731-1.9641063 .726011$

C $2.713691-2.3725934 .756115$

C $4.001174-2.0846574 .007067$

H $1.525752-0.8790143 .786809$

C $0.320098-2.6832753 .857619$

H $2.621420-1.8057875 .687624$

H $2.645192-3.4386485 .002948$

C $5.218319-2.7624244 .611245$

H $4.163643-0.9986273 .986927$

C $-0.721397-2.2863332 .788123$

O $0.537128-4.0970993 .753077$

H $-0.092134-2.5164484 .859452$

H $-0.391045-2.6349361 .802397$

O $-1.919938-3.0105193 .095613$

C $-1.031128-0.7751372 .735710$

C -2.201365 -0.439515 1.789312

H $-0.136566-0.2365792 .403967$

O $-1.337885-0.3353574 .058464$

H -2.040176 -0.9433100 .830431$

H $-3.128550-0.8209362 .234324$

H $5.381879-2.4152765 .636693$

H $5.098817-3.8507054 .639984$

H $6.118912-2.5379354 .031376$

C 4.455001-1.954565 1.486405

H $0.942474-4.2679312 .885520$

H -2.488037 -2.9901292 .307177$

H -1.459462 0.6285654 .029191

H $5.527943-2.0267861 .695449$

H $4.212140-0.8884951 .393783$

H $3.700785-3.6807282 .570530$

C -2.8093853 .1349302 .728136$

O -2.6815871 .7085582 .770094$

C -2.3669641 .0729281 .529172$

C -3.4681191 .3582860 .494202$

C -3.7281822 .8626580 .349794$

C -3.8832873 .5522931 .708298$

H -3.1662043 .4142743 .727712$

C -1.4551583 .8277872 .506778$

H -1.4090601 .4533351 .158802$

$\begin{array}{llll}\text { O } & -4.674377 & 0.708762 & 0.891646\end{array}$

H $-3.1662050 .928848-0.467790$

H $-4.6419173 .021947-0.236162$

O $-2.6520823 .473787-0.352470$

H -3.871872 4.6411461 .580379

O -5.1684913 .2053462 .231676$

C -0.4855303 .5744423 .642540$

H -0.9702723 .4863611 .589005$

$\mathrm{H}-1.6083444 .9112252 .442500$

H -5.3360280 .8665930 .196758$

H -2.619298 $3.076973-1.238299$

H -5.2879403 .6913013 .065311$

O 0.6953054 .2012553 .360565

O -0.7238292 .9150574 .644737$

C 1.7274474 .0374914 .338126

H 2.6095224 .5854433 .996076

H 1.4084094 .4508045 .299605

H 1.9886252 .9795704 .438531

SCF Energy $($ B3LYP/6-31G**//MMFF $)=-3245.90355905$

07 00515

MM̄MF Geometry

C $3.440419-2.1577131 .369874$

C $3.177841-2.7150852 .560728$

C $1.943983-2.4979073 .404552$

O $1.063452-1.4894122 .858713$ 
C $1.134297-3.8044023 .510540$ C $-0.058645-3.6903604 .442155$ C $-1.348453-3.7884514 .063913$ C $0.272968-3.4688995 .897099$ C -1.909921-4.067493 2.697714 C -2.475664 -2.804182 2.044947 C $-3.391936-3.0965550 .846443$ C $-2.663512-3.792914-0.304411$ C $-3.580646-3.992767-1.521243$ O $-3.910626-1.8368850 .397017$ O $-2.754362-4.340783-2.642698$ C $-4.266417-2.655321-1.864008$ C $-4.551748-5.161874-1.316858$ O $-5.246061-2.831360-2.885455$ C $-4.894648-1.942927-0.642750$ C $-5.322857-0.512288-1.047778$ O $-6.043359-2.673373-0.211362$ C $1.302530-0.1935953 .200128$ $\begin{array}{lllll}\text { O } & 2.283282 & 0.246748 & 3.777497\end{array}$ C 0.1155410 .5946012 .797290 C 0.0658491 .9119013 .046571 C - 1.0851962 .7905472 .812949 C -2.3486492 .1507262 .298985$ C -5.8188350 .3567360 .114564$ C $-5.9828661 .800518-0.322571$ C $-4.6556543 .815794-0.941240$ O $-7.0862742 .291332-0.546882$ C $-3.3185284 .353479-0.547888$ C -3.1278865 .4839660 .147189$ C -1.7678135 .9569460 .580323$ C -1.701449 6.2711342 .060286 C -1.378788 7.5050602 .484139 C -2.0252655 .1728423 .051093$ C -0.9688984 .1030513 .108561$ N $-4.7699682 .459756-0.435647$ H $2.710953-1.4977960 .909674$ H $3.926646-3.3723722 .999515$ H $2.274610-2.1870304 .403496$ H $1.776825-4.6175363 .873576$ H $0.814649-4.0979282 .505426$ H -2.112663 -3.675765 4.832929 H $-0.608610-3.5529676 .541811$ H $0.997146-4.2156256 .239459$ H $0.697327-2.4722566 .050972$ H -1.168123 -4.532092 2.043091 H $-2.708578-4.8104032 .818209$ H -3.048281 -2.221596 2.779688 H -1.654634 -2.147483 1.730745 H -4.225255 -3.712846 1.204322 H -2.249831 -4.757955 0.010228 H $-1.802621-3.183826-0.612664$ H -2.264161 -5.149374 -2.414600 H -3.499407 -1.994641 -2.287405 H -3.999201-6.092229-1.138301 H -5.151885 -5.339338 -2.216124 H $-5.229120-5.013264-0.473326$ H -4.807034 -3.265635 -3.636862 H -4.468869 -0.011484 -1.519172 H $-6.119019-0.558274-1.801779$ H $-6.617373-2.0845590 .302945$ H -0.7138600 .0549932 .355336$ H 0.9350562 .3894703 .497107 H -2.178351 1.7087121 .311853 H -3.181108 2.8472842 .190982 H -2.6895351 .3640382 .981404$ H -6.7865290 .0040860 .486948$ H $-5.121191 \quad 0.3318300 .959483$ H $-5.4858484 .416057-0.554603$ H -4.740434 $3.770684-2.031619$ H -2.452412 $3.765111-0.836848$ H $-3.984685 \quad 6.0918860 .427457$ H -1.523024 $6.848237-0.011441$ H $-0.997077 \quad 5.2131730 .342382$ H -1.332637 7.7467873 .540862 H -1.151303 8.3027681 .784755 H $-3.022354 \quad 4.7778732 .842314$ H -2.104993 5.5944594 .062540 H $-0.010544 \quad 4.4644263 .484679$
H $-3.9198761 .907280-0.368024$

C $5.516880-1.0703360 .517843$

O $4.785686-0.111245-0.264768$

C $5.7249440 .762747-0.923240$

C $7.1198130 .260740-0.552127$

C $6.857374-1.193066-0.197377$

H $5.5789460 .624503-1.999826$

C $5.4279882 .218605-0.530751$

H $7.8290120 .376184-1.378006$

H 7.5333090 .7980460 .309000

C $7.958843-1.8188600 .639511$

H $6.735379-1.764947-1.127071$

C $3.9511952 .613390-0.745056$

O 5.7218942 .3721890 .863865

H $6.0987402 .896177-1.071513$

H $3.3088331 .981071-0.121902$

O $3.7633023 .955808-0.278961$

C $3.4951852 .551380-2.215838$

C $1.9852192 .817997-2.376329$

H $3.7351121 .578156-2.652889$

O $4.2246443 .532019-2.955910$

H $1.6781063 .637996-1.716499$

H $1.7946163 .159741-3.402236$

H $8.911847-1.7944610 .100796$

H $8.094432-1.2865461 .586993$

H $7.729758-2.8637520 .870188$

C $4.722145-2.3715420 .610809$

H 5.7399773 .3252961 .054227

H 3.8443273 .9421010 .689660

H $3.9725103 .441344-3.890666$

H $5.327165-3.1494481 .089737$

H $4.462177-2.729464-0.390310$

H $5.653847-0.6391751 .519193$

C $0.643599-0.572882-3.160483$

O $1.323500 \quad 0.686592-3.222151$

C $1.1517751 .554311-2.094355$

C $-0.3329411 .875689-1.886899$

C $-1.1711810 .598989-1.781803$

C $-0.861652-0.379579-2.917964$

H $\quad 0.763897-1.003448-4.163570$

C $1.306990-1.529239-2.160031$

H $1.5385391 .060642-1.197585$

O $-0.8221052 .682053-2.956466$

H -0.437012 2.461716 -0.966477

H -2.232272 $0.871506-1.806264$

O $-0.906399-0.022650-0.528883$

H -1.321739 -1.354863 -2.727790

O $-1.4324860 .121155-4.130058$

C $2.629509-2.026611-2.703313$

H $1.506436-1.074854-1.187180$

H $\quad 0.653359-2.393660-1.992478$

H -0.661792 2.203148 -3.787670

H $-1.500286-0.788549-0.451012$

H $-2.3930250 .190257-3.994611$

O $2.667025-3.389478-2.662316$

O $3.523160-1.297118-3.113062$

C $3.856256-3.990132-3.185623$

H $4.742233-3.607621-2.670079$

H $3.793393-5.068789-3.019388$

H $3.926410-3.803221-4.261253$

SCF Energy (B3LYP/6-31G**//MMFF) $=-3245.90626258$

$07 \_00516$

MM̄FF Geometry

C $-3.261275-1.916440-3.151058$

C $-2.234257-2.392986-2.433742$

C $-0.791463-2.173982-2.807404$

O $-0.178375-1.353823-1.782614$

C $-0.037325-3.512758-2.866350$

C $1.445425-3.358434-3.151574$

C $2.424596-3.794237-2.334839$

C $1.802247-2.681549-4.450095$

C $2.273358-4.551635-1.047097$

C $2.307934-3.6829430 .217730$

C $3.653197-2.9727040 .435861$

C $3.820055-2.5762381 .903525$

C $5.153036-1.8555222 .140738$

O $3.681023-1.791252-0.371438$ 
O $5.105207-1.2574843 .446176$

C $5.281411-0.7022461 .125239$

C $6.333521-2.8333772 .159260$

O $6.580282-0.1169921 .174855$

C $4.929169-1.085501-0.335890$

C $4.7643600 .204224-1.165057$

O $5.971643-1.834705-0.961975$

C $-0.402484-0.013362-1.860565$

O $-1.092663 \quad 0.567782-2.683816$

C $0.3485620 .643923-0.768670$

C $0.1398401 .950397-0.548716$

C $0.827320 \quad 2.801247 \quad 0.421419$

C 1.8448372 .1710121 .331681

C $4.278560-0.017575-2.600857$

C $4.250070 \quad 1.309461-3.335126$

C $3.0718483 .513382-3.329857$

O $5.1088301 .606641-4.162268$

C $3.3453274 .372067-2.132121$

C $2.5543905 .385157-1.745381$

C $2.8164606 .231384-0.529842$

C 1.5995906 .3816250 .361396

C 0.9311567 .5458050 .424220

C 1.1963625 .2069701 .232360

C $0.532742 \quad 4.117937 \quad 0.434138$

N $3.2022022 .122845-2.932032$

H $-3.061566-1.384402-4.078262$

H $-2.432216-2.910790-1.500584$

H $-0.710683-1.666930-3.777280$

H $-0.476548-4.151194-3.643941$

H $-0.192996-4.036107-1.917194$

H $3.457862-3.617340-2.631254$

H $1.274263-3.156863-5.283231$

H $1.532481-1.621502-4.421822$

H $2.874275-2.740630-4.667781$

H $1.351207-5.142296-1.046844$

H $3.079450-5.295153-0.996495$

H $1.494538-2.9476910 .177227$

H $2.105846-4.3481291 .066445$

H $4.461827-3.6457320 .128668$

H $3.738512-3.4510062 .560442$

H $3.004378-1.9002932 .193032$

H $4.939869-1.9639494 .094031$

H $4.5793920 .080306 \quad 1.446893$

H $6.213556-3.5619182 .970313$

H $7.275915-2.3129712 .362756$

H $6.441344-3.3954451 .229368$

H 6.7466830 .1415302 .097584

H $4.0462530 .861732-0.664245$

H $5.7195570 .744642-1.202092$

H $6.654158-2.035456-0.303485$

H $1.0626490 .051922-0.208682$

H $-0.5942812 .465583-1.168014$

H 1.4747181 .2295851 .749078

H 2.0902702 .7964292 .193809

H $2.771137 \quad 1.9718790 .787742$

H $4.943086-0.697964-3.144142$

H $3.274844-0.456197-2.617691$

H $2.0545013 .648839-3.709815$

H $3.7847733 .745022-4.126673$

H $4.2382804 .146927-1.552921$

H $1.6762395 .628448-2.339310$

H $3.149697 \quad 7.217237-0.879117$

H 3.6449795 .8272240 .065187

H 0.0724707 .6703711 .075654

H $1.2242698 .399526-0.177835$

H 0.4884505 .5321202 .005293

H $2.087162 \quad 4.858526 \quad 1.763299$

H $-0.2501784 .479495-0.234302$

H $2.5452321 .771093-2.242618$

C $-5.210272-0.588188-2.392660$

O $-4.619326-0.124125-1.160369$

C $-5.5624330 .747105-0.500891$

C $-6.7871820 .824475-1.410598$

C $-6.712332-0.495814-2.154733$

H $-5.8555880 .252627 \quad 0.430966$

C $-4.908478 \quad 2.104813-0.211116$

H -7.714680 $0.944287-0.841984$

H $-6.7173251 .666509-2.109776$
C $-7.550877-0.522558-3.420303$

H $-7.037519-1.301221-1.482709$

C $-3.7256242 .022808 \quad 0.779573$

O $-4.4272642 .635529-1.452304$

H -5.6641772 .8085380 .157073$

H -2.9500621 .3583900 .383674$

$\begin{array}{lllll}\text { O } & -3.139391 & 3.325850 & 0.884650\end{array}$

C $-4.138268 \quad 1.5780772 .197865$

C $-3.011328 \quad 1.644342 \quad 3.247697$

H -4.5389340 .5608032 .169553$

O -5.2014152 .4178042 .661762$

H -2.573672 2.6497673 .271087

H -3.479838 1.4987224 .230659

H $-8.607545-0.359255-3.184527$

H $-7.2394440 .255915-4.124897$

H -7.465198 - $1.488829-3.926732$

C $-4.698307-1.992143-2.716526$

H $-4.2064193 .570285-1.303163$

H -2.6922643 .5122750 .041855$

H -4.8688203 .3319272 .671784$

H $-5.277504-2.439089-3.531531$

H $-4.800177-2.642408-1.839423$

H $-4.9100310 .117798-3.179858$

C $-1.667056-1.7865982 .794129$

$\begin{array}{llllll} & \text { O } & -2.506921 & -0.699973 & 3.203226\end{array}$

C -1.9150020 .5914203 .044732$

C $-0.7482490 .731538 \quad 4.040977$

C $0.058075-0.5683894 .174363$

C $-0.168905-1.4869212 .976838$

H -1.945849-2.634979 3.432118

C $-1.986414-2.1555391 .343817$

H $-1.521630 \quad 0.6761432 .025273$

\begin{tabular}{llll}
\hline & -1.241081 & 1.069663 & 5.339867
\end{tabular}

H $-0.116197 \quad 1.5708733 .728435$

H -0.190939 -1.093245 5.105033

O $1.441941-0.2284694 .266844$

H $0.252245-1.0213292 .078100$

O $0.537284-2.7133343 .157616$

C $-3.342672-2.813148 \quad 1.248314$

H -2.008271 -1.273633 0.694292

H $-1.233286-2.838267 \quad 0.932729$

H $-0.470468 \quad 1.187431 \quad 5.921615$

H $1.943071-1.0609664 .308908$

H $0.217797-3.1259123 .978146$

O $-3.199394-4.1655371 .151484$

O $-4.401863-2.2000891 .274642$

C $-4.419677-4.905498 \quad 1.048746$

H $-4.168121-5.9661230 .964489$

H $-5.027894-4.7589871 .946332$

H -4.971871 -4.6036290 .153509$

SCF Energy (B3LYP/6-31G**//MMFF) $=-3245.89846851$

07_00517

MM̄FF Geometry

C $0.526416 \quad 3.767929-2.205851$

C $0.142152 \quad 4.747752-1.373717$

C $-1.072726 \quad 4.731838-0.471388$

O $-1.8399673 .517566-0.638123$

C $-0.6123214 .808901 \quad 0.998256$

C -1.743874 $4.781622 \quad 2.008122$

C -1.8385643 .8862213 .011180$

C -2.7912835 .8541641 .859359$

C $-0.860783 \quad 2.7990393 .359878$

C -1.1026861 .4853632 .614207$

C -2.496966 0.8646772 .785712

C $-2.8062050 .502463 \quad 4.240854$

C $-4.144527-0.2411794 .380457$

O $-2.514501-0.3173771 .972107$

O $-4.193610-0.8187175 .694392$

C $-4.173374-1.4097653 .373731$

C -5.3404170 .7134524 .282816$

O $-5.449098-2.045017 \quad 3.360385$

C $-3.779578-0.9919441 .942161$

C $-3.641400-2.194742 \quad 0.986234$

O $-4.799540-0.144756 \quad 1.398353$

C $-2.860677 \quad 3.543445-1.538164$

O $-3.205410 \quad 4.487737-2.230858$

C $-3.4874082 .202658-1.552056$ 
C $-4.4764931 .952384-2.422626$

C $-5.1900510 .685275-2.543181$

C $-6.6814680 .847844-2.661375$

C $-2.657822-3.280817 \quad 1.426594$

C $-2.418232-4.2328270 .272294$

C $-1.138109-4.396758-1.858782$

O $-3.131239-5.216140 \quad 0.086762$

C $-1.766007-3.486418-2.866868$

C $-2.853245-3.798908-3.587527$

C $-3.523388-2.834089-4.524779$

C $-4.931775-2.485959-4.085574$

C $-5.981491-2.733013-4.887024$

C $-5.133724-1.867015-2.717490$

C $-4.534471-0.491093-2.604400$

N -1.378975 -3.822451 -0.547955

H $-0.0717652 .866595-2.298160$

H $0.7634595 .638795-1.302521$

H -1.688272 $5.608386-0.707938$

H -0.0377385 .7313341 .156414$

H $0.082446 \quad 3.981737 \quad 1.181715$

H -2.699258 3.9457023 .676029

H -3.486801 5.8748772 .705365

H $-2.3187476 .840306 \quad 1.802279$

H -3.3827795 .6952800 .952627$

H $\quad 0.1661113 .134146 \quad 3.173789$

H -0.9041532 .6296644 .442450$

H -0.9210511 .6383751 .542507$

H -0.3431940 .7562362 .926951$

H $-3.242806 \quad 1.5653602 .394147$

H -2.808077 1.3920564 .881211

H $-2.008098-0.1454974 .629689$

H $-4.097638-0.0993986 .341941$

H $-3.456737-2.157348 \quad 3.737129$

H -5.2975511 .4632575 .082144$

H -6.2857050 .1784154 .425251$

H -5.3860641 .2499993 .332933$

H -5.656944 -2.296772 4.276450

H $-4.626352-2.6458680 .806238$

H -3.318585 -1.8111810 .008577$

H $-4.740355-0.1725940 .429158$

H $-3.1262441 .455069-0.854027$

H $-4.8239982 .736433-3.092467$

H $-6.932141 \quad 1.571351-3.445821$

H -7.098205 $1.209455-1.714967$

H -7.200370 -0.078016-2.919953

H - $-1.704335-2.8491631 .750443$

H -3.053647 -3.8613692 .266549$

H -1.545881 -5.411427 -1.904591

H $-0.056771-4.443859-2.014450$

H -1.319417 -2.501178 -2.979710

H $-3.308001-4.780071-3.474668$

H -3.529378 $-3.288147-5.523710$

H -2.941664 -1.908999-4.620772

H -6.994519 -2.491503 -4.582604

H -5.852964 -3.180621 -5.866889

H $-4.673209-2.508413-1.956815$

H -6.195899-1.861103 -2.451043

H $-3.446047-0.482379-2.544599$

H $-0.904766-2.951841-0.329800$

C $2.7632952 .732936-2.525809$

O $3.2657773 .108160-1.227872$

C $4.6498252 .702788-1.127212$

C $4.9533251 .922912-2.399933$

C $4.0044422 .562825-3.394720$

H $5.2328843 .631458-1.098881$

C 4.8263621 .9119250 .174236

H $6.0036441 .998974-2.696750$

H $4.7120030 .859689-2.274122$

C $3.7813171 .724905-4.641295$

H $4.4020293 .545411-3.681934$

C 6.1936651 .2219450 .322584

O 4.6292922 .8127321 .269964

H $4.017613 \quad 1.1773180 .243977$

H $6.3503510 .509021-0.494575$

O 7.2140362 .2181350 .181385

C 6.4007430 .5122361 .674433

C $5.290690-0.4720332 .074219$

H 6.5059941 .2657072 .464618
O $7.655703-0.1702931 .622487$

H $5.574756-0.9809433 .004532$

H 4.3911110 .1015682 .334994

H $4.7248621 .571678-5.175402$

H $3.3709020 .739703-4.394644$

H $3.0838072 .218473-5.325094$

C $1.7890533 .801441-3.021963$

H 5.3577573 .4571231 .254357

H $8.071439 \quad 1.769570 \quad 0.280347$

H $7.796808-0.5925232 .487107$

H $1.5233783 .624076-4.069646$

H $2.2555544 .792236-2.961357$

H $2.2492381 .769559-2.405101$

C $3.077969-3.0944770 .642634$

O $3.701716-2.1495531 .521664$

C $4.898233-1.5315801 .024555$

C $5.965721-2.6015880 .753792$

C $5.433521-3.705932-0.167517$

C $4.059827-4.2204460 .279544$

H $2.265700-3.5358911 .235067$

C $2.445575-2.416179-0.583700$

H $4.654331-1.0201550 .090706$

O $6.409440-3.1801601 .979057$

H $6.836255-2.1363990 .280360$

H $6.151736-4.534932-0.178291$

O $5.349191-3.188160-1.492442$

H $3.613810-4.857754-0.492830$

O $4.227477-5.0312421 .446336$

C $1.329973-1.462660-0.208396$

H $3.177474-1.843644-1.158800$

H $2.014232-3.184611-1.235200$

H $5.631203-3.5456132 .433961$

H $5.044483-3.908603-2.069922$

H $4.807637-5.7737861 .206325$

O $0.800691-0.935137-1.352588$

O $0.962489-1.2122810 .930792$

C $-0.275871-0.012351-1.163354$

H $0.0774020 .869281-0.621243$

H $-0.6331440 .299004-2.148383$

H $-1.100893-0.490880-0.628275$

SCF Energy $(B 3 L Y P / 6-31 G * * / / M M F F)=-3245.90361864$

07_00518

MM̄FF Geometry

C - $0.129275-1.4058792 .354117$

C $0.322316-1.7987841 .153011$

C $-0.549969-1.896709-0.065198$

O $-0.095338-0.854554-0.960848$

C $-0.428686-3.240838-0.798199$

C $-0.948499-4.440552-0.037646$

$\begin{array}{llll}C & -2.251170 & -4.780774 & 0.026623\end{array}$

C $0.101168-5.3077490 .604536$

C $-3.404870-4.000410-0.542173$

C $-4.607929-3.9946670 .412445$

C -5.574531-2.813627 0.221194

C $-5.972355-2.566901-1.237219$

C $-6.868685-1.326622-1.390306$

O $-4.949274-1.6479070 .777669$

O $-6.899415-0.973431-2.782577$

C $-6.216352-0.141925-0.641672$

C $-8.319502-1.617703-0.988930$

O $\begin{array}{llll}\text { C } 7.077210 & 0.994031 & -0.638817\end{array}$

$\begin{array}{llll}\text { C } & -5.796330 & -0.489598 & 0.802497\end{array}$

C $-5.0297870 .658541 \quad 1.498861$

O $-6.974559-0.7248481 .585489$

C $-1.027152-0.225484-1.723955$

O $-2.211549-0.495499-1.832210$

C $-0.3520670 .924063-2.356567$

C $-1.0236822 .077920-2.472390$

C $-0.4623503 .346931-2.931191$

C $0.898123 \quad 3.326551-3.576742$

C -3.6847311 .0303810 .873796$

C -2.9764712 .1204011 .662558$

C $-0.8431293 .413394 \quad 1.694948$

$\begin{array}{llll}\text { O } & -3.491116 & 2.707203 & 2.610861\end{array}$

C - 1.0474314 .6648970 .899330

C $-0.061784 \quad 5.309418 \quad 0.255648$

C $-0.2662346 .572098-0.536222$ 
C $0.214306 \quad 6.449697-1.967133$

C $1.4350436 .887293-2.319423$

C $-0.7387495 .890019-3.003461$

C -1.166102 4.477296 -2.714840

N -1.7046332 .3713021 .167955$

H -1.180742 -1.162109 2.482808

H $1.379949-2.0162961 .025255$

H $-1.591085-1.7053080 .217646$

H $\quad 0.616952-3.410179-1.088039$

H $-0.951096-3.172761-1.762354$

H $-2.520300-5.6995190 .545450$

H $-0.330440-6.1821181 .102970$

H $0.806866-5.672299-0.149345$

H $\quad 0.658505-4.742148 \quad 1.357215$

H -3.107589 -2.973611 -0.764288

H -3.703621 -4.462582 -1.490570

H $-5.159999-4.9339090 .279146$

H -4.252352 -3.976825 1.451439

H -6.465792 -3.040426 0.818662

H -6.460822 -3.453452 -1.659850

H $-5.077003-2.398035-1.849228$

H -7.230142 -1.741469 -3.279254

H $-5.3240610 .144730-1.212578$

H $-8.743349-2.400708-1.629248$

H -8.954221 -0.736750 -1.134449

H $-8.421456-1.9510060 .045634$

H -7.312954 $1.181209-1.563663$

H -5.6731661 .5456191 .566256$

H $-4.840186 \quad 0.3633812 .540999$

H $-6.746847-0.6269862 .524738$

H $0.6944050 .819175-2.611621$

H -2.058774 2.114496 -2.134372

H $1.6687293 .068741-2.842613$

H $1.1783594 .286313-4.017309$

H $0.9285322 .594345-4.391571$

H -3.0317880 .1526490 .842782$

H -3.824244 $1.401468-0.146488$

H $\quad 0.1838473 .046144 \quad 1.629966$

H -1.085353 3.5960362 .746566

H -2.0575085 .0665830 .860791$

H $\quad 0.952257 \quad 4.9195130 .310447$

H $0.2808417 .376243-0.026650$

H -1.318179 $6.883586-0.532169$

H $1.7828606 .829765-3.345446$

H $2.1158657 .310106-1.587882$

H $-0.3171625 .951910-4.014106$

H -1.633865 $6.524904-3.032677$

H $-2.1394914 .404759-2.228810$

H $-1.383626 \quad 1.8639680 .349671$

C $0.987760 \quad 0.242572 \quad 3.876741$

O 1.6682150 .8654652 .766221

C 2.9592651 .3065983 .210664

C 2.7637741 .6056104 .687983

C $1.892066 \quad 0.433327 \quad 5.102375$

H $3.6391990 .451628 \quad 3.104723$

C 3.4711242 .4667732 .350647

H 3.7089831 .6524695 .236618

H $2.2239702 .546676 \quad 4.844319$

C $1.123441 \quad 0.669812 \quad 6.391392$

H $2.534913-0.4470245 .238141$

C 3.3460342 .2391170 .829162

O 2.7216723 .6462982 .669069

H 4.5101432 .6871942 .622982

H 2.2930832 .3426190 .538597

O $4.045923 \quad 3.315018 \quad 0.191917$

C $3.8867530 .890050 \quad 0.317287$

C $3.760130 \quad 0.754112-1.212574$

H 3.3358950 .0699930 .790534

$\begin{array}{lllll}\text { O } & 5.263677 & 0.748423 & 0.663381\end{array}$

H $2.8280101 .219687-1.549350$

H $4.5992161 .279584-1.684805$

H 1.8126390 .8346207 .225917

H $\quad 0.471896 \quad 1.546794 \quad 6.314883$

H $0.497734-0.1951496 .633224$

C $0.757793-1.2375773 .553945$

H 3.0677064 .3678982 .116168

H $3.8102653 .306683-0.750840$

H 5.3282700 .6945981 .630935
H $\quad 0.295483-1.7457124 .407926$

H $1.721050-1.7276413 .368191$

H 0.0312950 .7657343 .997850

C $5.095246-2.730084-1.533301$

O $4.982860-1.326090-1.265598$

C $3.754375-0.718219-1.669064$

C $3.569438-0.853465-3.187475$

C $3.657251-2.320724-3.622825$

C $4.886044-3.020483-3.030282$

H $6.135294-2.980568-1.286880$

C $4.184209-3.561667-0.615987$

H $2.920823-1.213517-1.159384$

O $4.571230-0.092837-3.859077$

H $2.596120-0.434564-3.466671$

H $3.705112-2.375858-4.717092$

O $2.485250-3.015637-3.207241$

H $4.823484-4.104179-3.184499$

O $6.055026-2.566558-3.719210$

C $4.542103-3.4017440 .847317$

H $3.135093-3.277462-0.727159$

H $4.286077-4.624616-0.862925$

H $4.415785-0.180548-4.814753$

H $1.719958-2.569495-3.608298$

H $5.950850-2.798054-4.657682$

O $3.593546-4.0288651 .604590$

O $5.528120-2.8181521 .275578$

C $3.797248-3.9710963 .019839$

H $4.662083-4.5812603 .295809$

H $3.934061-2.9366283 .349791$

H $2.907573-4.3781693 .507768$

SCF Energy (B3LYP/6-31G**//MMFF $)=-3245.88496157$

07_00519

MMFF Geometry

C $0.978664 \quad 4.6637270 .939935$

C 1.2892983 .4156540 .559268

C 1.8848722 .3859781 .481594

O $0.976421 \quad 1.2584031 .439968$

C 3.2857291 .9380181 .027647

C 4.3006233 .0557770 .922840

C $4.8969653 .442996-0.221796$

C 4.6720363 .7169612 .225353

C $4.6980612 .885746-1.604411$

C $5.9508242 .168655-2.127102$

C $6.3475990 .927921-1.309239$

C $7.6540860 .336911-1.843957$

C $8.066888-0.918262-1.064865$

O $5.299702-0.041118-1.415115$

O $9.097504-1.564225-1.831192$

C $6.863243-1.882010-0.995432$

C $8.712139-0.5567820 .277806$

O $7.142372-2.989977-0.143054$

C $5.509986-1.221006-0.626257$

C $4.364073-2.202685-0.961490$

O $5.400648-0.9492080 .770346$

C 1.0440260 .3432942 .441187

O $1.837614 \quad 0.3177023 .367582$

C $-0.050953-0.6271482 .220331$

C $-0.085752-1.7650892 .928586$

C $-1.133308-2.7868592 .865675$

C $-2.333480-2.4996992 .001575$

C $2.962582-1.680002-0.620324$

C $1.912082-2.629906-1.161054$

C $1.051229-4.952557-0.878615$

O $1.263689-2.360555-2.169633$

C $-0.178838-5.096108-0.042218$

C $-0.399651-6.1369340 .774980$

C $-1.678031-6.3597761 .530751$

C -1.495781 -6.309398 3.029874

C $-1.036333-7.3737983 .709404$

C $-1.945573-5.0581123 .746729$

C $-0.980870-3.914513 \quad 3.591332$

N $1.822097-3.807286-0.435903$

H 1.1828684 .9786961 .960278

H $1.0827993 .102140-0.461594$

H 1.9158902 .7680122 .510095

H $3.190478 \quad 1.379687 \quad 0.092033$

H 3.6754591 .1935191 .733733 
H $5.6246224 .252152-0.171051$ H 5.4858034 .4403462 .107193 H 3.8160434 .2524202 .646795 H 5.0049192 .9660982 .949520 H $3.8326162 .221704-1.672981$ H $4.4748363 .727113-2.272497$ H $5.7519971 .869025-3.164125$ H $6.7886142 .877213-2.150771$ H $6.4697751 .214360-0.259454$ H $8.4594201 .081740-1.821294$ H $7.5253190 .065905-2.901518$ H $9.319491-2.398347-1.382309$ H $\quad 6.759032-2.307372-2.003622$ H 9.6376310 .0086900 .112478 H $9.008493-1.4535500 .832480$ H 8.0709130 .0519700 .917482 H 7.045382 -2.693206 0.777226 H $4.391406-2.437947-2.034051$ H $4.520131-3.145626-0.422033$ H $6.276191-0.7422821 .125970$ H $-0.810624-0.3667181 .492230$ H $\quad 0.721303-1.9647203 .632421$ H -3.060068 -3.314026 1.974191 H $-2.025909-2.3294070 .964653$ H -2.862386 -1.6102232 .361035$ H $2.829946-1.5723260 .461773$ H $2.787513-0.697581-1.071616$ H $1.704799-5.827542-0.799418$ H $\quad 0.771123-4.834645-1.930387$ H $-0.947475-4.337828-0.158864$ H $0.363785-6.9060640 .870535$ H -2.071150 -7.344804 1.245374 H $-2.450313-5.6453711 .220982$ H $-0.935672-7.3554544 .789468$ H $-0.747651-8.2862073 .197923$ H -2.041356 $-5.254046 \quad 4.823463$ H -2.959997 -4.800797 3.427343 H $-0.069552-4.0349574 .178561$ H $2.387019-3.9172570 .401248$ C -1.050721 6.0686120 .569246 O C -3.2259565 .3248160 .107182$ C $-3.208443 \quad 6.8490430 .069234$ C -1.757481 $7.125548-0.276955$ H $-3.3350484 .951788-0.918236$ C -4.3074034 .7064861 .000105$ H -3.910837 $7.253104-0.666056$ H -3.4549477 .2850931 .044073$ C -1.322348 8.5501420 .019091 H -1.605533 $6.916722-1.344460$ C -4.1914203 .1763351 .168018$ O -4.2084595 .2887912 .306484$ H $-5.297674 \quad 4.9786180 .616178$ H -3.3091292 .9384401 .774236$ O $\quad-5.3210792 .741516 \quad 1.936514$ C $-4.1373302 .382431-0.150813$ C $-4.1166640 .864757 \quad 0.090641$ H -3.240765 2.678455 -0.703620 O $\quad-5.2744642 .731255-0.937585$ H -3.2821920 .6029590 .753353$ H -5.0239320 .5665100 .633426$ H -1.914123 9.262253 -0.565294 H -1.449852 8.7968381 .078500 H $-0.2684178 .697616-0.236664$ C 0.3273765 .6738050 .036731 H -4.9013274 .8850882 .856856$ H -6.115961 2.8838461 .394071 H -5.123679 $2.401932-1.839472$ H $0.9774206 .553493-0.025973$ H $0.2297885 .263839-0.976310$ H $-0.9766826 .398799 \quad 1.614013$ C $-4.127286-2.356333-1.770140$ O $-4.065219-1.361007-0.740941$ C $-4.0619020 .003251-1.183991$ C $-2.8418760 .264202-2.080975$ C $-2.797459-0.727660-3.248099$ C -2.983993 -2.175481-2.782304 H $-3.962935-3.309129-1.249782$
C $-5.515045-2.413718-2.426918$

H $-4.9810140 .186612-1.749659$

O $-1.6336760 .137117-1.334455$

H $-2.8834671 .282952-2.483013$

H -1.832482 -0.630830 -3.761218

O $-3.822503-0.380861-4.175113$

H $-3.152710-2.831293-3.643700$

O $-1.771204-2.621827-2.171950$

C $-6.593756-2.841604-1.452842$

H $-5.810786-1.444034-2.835451$

H $-5.501835-3.149015-3.239825$

H $-1.5792100 .891076-0.723739$

H -3.758209-0.995108 -4.926198

H -1.603147 -2.061791-1.394983

O $-7.796774-2.843333-2.101179$

O $-6.412901-3.138792-0.280158$

C $-8.923156-3.227536-1.306384$

H $-9.814983-3.184750-1.937351$

H $-8.798724-4.252274-0.943475$

H $-9.051079-2.533447-0.470233$

SCF Energy (B3LYP/6-31G**//MMFF) $=-3245.90967252$

0700520

MMFF Geometry

C 3.5763803 .1218850 .798450

C 3.1392021 .9263631 .219575

C 2.8695591 .6061642 .666643

O 1.4904161 .1652072 .783130

C 3.7528560 .4562563 .177618

C 5.2149920 .8131593 .362679

C 6.2101500 .3725442 .566624

C 5.5273531 .6634774 .565894

C $6.052940-0.4547701 .322903$

C $6.049267-1.9599511 .621408$

C $5.435000-2.8083080 .496127$

C $6.248969-2.760418-0.796965$

C $5.565761-3.548427-1.925146$

O $4.110936-2.3186660 .270602$

O $6.239782-3.201783-3.144477$

C $4.099943-3.077151-2.040097$

C $5.746627-5.060108-1.749164$

O $3.390399-3.858092-2.999641$

C $3.343124-3.047680-0.690003$

C $1.992757-2.306066-0.867485$

O $3.108612-4.388126-0.263761$

C 0.5311202 .1321762 .789323

O $0.706968 \quad 3.3386742 .716822$

C $-0.789247 \quad 1.4698362 .885377$

C -1.8964922 .2153752 .758123$

C -3.2733441 .7355392 .829186$

C -4.2056822 .7163563 .489851$

C $1.243987-1.9849870 .435355$

C $0.336359-3.0952230 .930816$

C $-1.230665-3.6102062 .801048$

O $0.179355-4.1568390 .335345$

C $-2.615463-3.2377592 .375213$

C $-3.558926-2.8064153 .225451$

C $-4.965175-2.4798582 .816764$

C $-5.364792-1.0676393 .177281$

C $-6.058808-0.8124094 .299212$

C -5.0607910 .0296992 .185549$

C -3.6578780 .5605352 .290963$

N $-0.281673-2.7490792 .124452$

H 3.7808523 .9017991 .528232

H $2.920703 \quad 1.1491970 .490928$

H 3.0068872 .4845633 .309437

H $3.631867-0.4153152 .523372$

H 3.3593140 .1090344 .143321

H 7.2351950 .6475912 .808416

H $6.603491 \quad 1.8223674 .693813$

H 5.0590962 .6480964 .473518

H 5.1555511 .1842745 .477450

H $5.145679-0.1514510 .791932$

H $6.880065-0.2160230 .643158$

H $5.458295-2.1421092 .528940$

H $7.069770-2.3002641 .835258$

H $5.371971-3.8394400 .863696$

H $7.268476-3.129208-0.629611$ 
H $6.358399-1.720071-1.131252$

H $5.766753-3.635011-3.875843$

H $4.127753-2.056374-2.445537$

H $\quad 6.812772-5.316292-1.733797$

H $5.320338-5.611941-2.594337$

H $5.299952-5.441423-0.828661$

H $3.171734-4.709622-2.583902$

H $2.201996-1.338238-1.342658$

H $1.339853-2.868787-1.545094$

H $2.388938-4.3873340 .386004$

H $-0.822777 \quad 0.399843 \quad 3.054528$

H -1.806174 3.2887782 .598215

H -5.1922422 .2991653 .703281$

H -4.3441773 .5987732 .855596$

H -3.7956053 .0450704 .452076$

H $1.936911-1.7091381 .236413$

H $0.599263-1.1156230 .254785$

H -1.078282 -3.480499 3.877099

H -1.031745 -4.655512 2.544310

H -2.858849 -3.3535361 .321744$

H $-3.318865-2.7120984 .282231$

H $-5.631538-3.194823 \quad 3.317372$

H $-5.119428-2.6424291 .742899$

H -6.3846930 .1922864 .546554$

H $-6.313153-1.6027254 .997735$

H -5.7972650 .8363562 .273230$

H -5.206866 -0.3516971 .168163$

H -2.906859 -0.0695291 .816028$

H $-0.133847-1.8194442 .505992$

C $2.7168704 .515504-1.071916$

O $1.4202013 .886779-1.111496$

C $0.6600684 .455348-2.196456$

C $1.5152395 .577400-2.777618$

C $2.9187305 .085782-2.471911$

H $\quad 0.5552173 .670922-2.954097$

C $-0.7199714 .889540-1.687033$

H $1.3342595 .722211-3.847191$

H $\quad 1.326814 \quad 6.533691-2.275963$

C $3.9729426 .175394-2.555099$

H $3.1718154 .279995-3.173837$

C -1.524511 $3.754314-1.019387$

O $-0.5487745 .927627-0.711768$

H -1.298991 $5.336588-2.502243$

$\mathrm{H}-1.0038443 .414208-0.116189$

O $-2.7777064 .295355-0.582534$

C $-1.8119042 .544596-1.930376$

C $-2.5674581 .436928-1.182476$

H $-0.8701142 .128527-2.300179$

O $-2.5849482 .949647-3.056985$

H -1.948013 $1.074869-0.351990$

H -3.470356 $1.851111-0.714212$

H $4.0100746 .597988-3.564476$

H $3.7622126 .992031-1.856258$

H $4.9652895 .778564-2.319550$

C $3.7652013 .487110-0.647949$

H -0.0003525 .5679370 .006836$

H -2.580291 5.0468830 .002431

H -2.026291 $3.520122-3.610755$

H $4.7756273 .891969-0.769655$

H $3.6889362 .592311-1.277797$

H $2.6580795 .328724-0.335317$

C $-4.146343-1.889110-1.700267$

O $-3.588300-0.702410-1.125478$

C $-3.0268630 .245605-2.045026$

C -1.906593 -0.401771 -2.872049

C $-2.377424-1.694215-3.546080$

C -3.107517 -2.617560 -2.566497

H $-4.373326-2.533537-0.841373$

C $-5.459396-1.601496-2.435246$

H $-3.8199340 .598658-2.713078$

O $-0.799211-0.684754-2.022026$

H -1.566543 $0.306168-3.636513$

H -1.508911 -2.219019-3.962561

O $-3.238654-1.351988-4.627914$

H -3.594228 -3.435839 -3.108798

O $-2.144535-3.224219-1.699917$

C -6.524194 -1.130759-1.473627

H $-5.358291-0.830836-3.204687$
H $-5.832329-2.498676-2.943695$

H $-0.087844-1.043519-2.579352$

H -3.506981 -2.179416 -5.062568

H -1.750082 -2.521763 -1.154841

O $-7.062062-2.196331-0.814134$

O $-6.8311950 .044397-1.320573$

C $-8.089029-1.8824410 .131684$

H $-7.728746-1.1565370 .866448$

H $-8.971033-1.498083-0.388849$

H $-8.361498-2.8027020 .655101$

SCF Energy $(B 3 L Y P / 6-31 G * * / / M M F F)=-3245.92045581$

07_00521

MMFF Geometry

C $3.073141-1.6991782 .464356$

C $2.588394-1.7179793 .715715$

C $1.319037-1.0357734 .177670$

O $0.852087-0.1312653 .150383$

C $0.219159-2.0722924 .466095$

C $-1.026766-1.4820355 .100905$

C $-2.275702-1.5972504 .607771$

C $-0.818393-0.761774 \quad 6.410557$

C $-2.744164-2.3444053 .391174$

C $-3.217760-1.3945442 .289882$

C $-4.037071-2.0993371 .197294$

C $-3.228982-3.1442420 .428019$

C $-4.031711-3.745971-0.734439$

O $-4.487951-1.0807490 .297559$

O $-3.098392-4.454920-1.563967$

C $-4.649095-2.607702-1.575892$

C $-5.039323-4.789632-0.235486$

O $-5.511309-3.164447-2.566323$

C $-5.380813-1.538412-0.726568$

C $-5.762657-0.323441-1.611453$

O $-6.567351-2.115370-0.181325$

C $0.950643 \quad 1.2042313 .386590$

$\begin{array}{lllll}\text { O } & 1.232228 & 1.750392 & 4.441237\end{array}$

C 0.7116871 .9031992 .103306

C 0.6645813 .2439002 .081879

C $0.5112094 .087356 \quad 0.894151$

C $0.5874093 .410150-0.447493$

C $-6.4419020 .851899-0.892989$

C $-5.568283 \quad 1.5510260 .129142$

C -3.4871112 .8842780 .358224$

O $\begin{array}{lllll}-5.809827 & 1.485574 & 1.332700\end{array}$

C $-2.9192804 .035130-0.407114$

C $-2.898553 \quad 5.295855 \quad 0.050594$

C -2.310406 $6.447979-0.711769$

C -1.1876407 .1323910 .036456$

C $-1.378405 \quad 8.3201870 .635246$

$\begin{array}{llll}\text { C } & 0.178915 & 6.481833 & 0.012154\end{array}$

C $0.3301895 .414287 \quad 1.062007$

N $-4.5162832 .248270-0.441514$

H $2.511371-1.2003811 .679022$

H $3.153661-2.2343824 .488727$

H $1.572043-0.4886465 .093015$

H $\quad 0.606083-2.8440445 .144866$

H $-0.025600-2.5810103 .528306$

H $-3.085612-1.1262635 .165028$

H $-1.763447-0.5444306 .920434$

H $-0.219185-1.3761487 .090645$

H $-0.305530 \quad 0.1912936 .253731$

H -1.975761 -3.0173473 .003539$

H $-3.576480-2.9873913 .704393$

H $-3.838034-0.5961802 .720271$

H $-2.357574-0.8804801 .840103$

H $-4.909865-2.5604501 .674472$

H -2.875938 -3.944016 1.089363

H $-2.324530-2.6728100 .022232$

H $-3.585955-4.789913-2.336237$

H $-3.826158-2.122853-2.116856$

H $-4.517815-5.6086050 .274478$

H $-5.578157-5.253037-1.069304$

H $-5.772496-4.3787420 .461716$

H $-5.690442-2.476649-3.228636$

H $-4.8592450 .047689-2.110971$

H $-6.457744-0.651872-2.393590$

H -7.140448 -1.4058710 .147463$ 
H $\quad 0.616273 \quad 1.296773 \quad 1.209644$ H 0.7542903 .7826553 .024476 H $1.5549662 .912589-0.570259$ H $0.4722724 .091012-1.292670$ H $-0.2035672 .660246-0.542650$ H $-6.7388191 .600541-1.639004$ H -7.368922 $0.538303-0.401970$ H -2.7090772 .1365450 .544188$ H $-3.9040373 .187899 \quad 1.324149$ H -2.480409 $3.815138-1.377613$ H -3.3351965 .5178631 .021639$ H -3.120473 $7.160818-0.914347$ H -1.945092 $6.130867-1.696681$ H $-0.569775 \quad 8.830248 \quad 1.148257$ H -2.3484158 .8060570 .630930$ H $0.953722 \quad 7.2363970 .204534$ H $0.3893716 .123072-0.998828$ H 0.2967325 .8050662 .080285 H -4.337206 2.128215-1.434273 C $5.294511-1.2603501 .427276$ O $4.719038-0.7715480 .201220$ C $5.784901-0.368836-0.682592$ C $7.092630-0.6865360 .041919$ C $6.670268-1.771721 \quad 1.016977$ H $5.713483-1.002796-1.572423$ C $5.6042241 .106071-1.069193$ H $7.873525-1.016800-0.650381$ H 7.4827040 .1848470 .580610 C $7.639605-1.9568622 .170816$ H $6.571482-2.7178610 .468449$ C $4.2218011 .420451-1.680503$ O $5.742551 \quad 1.8991090 .118013$ H $6.4080201 .416262-1.746621$ H $3.4456961 .206634-0.938673$ O $4.1575972 .834045-1.916525$ C $3.9240060 .684552-3.001630$ C $2.5248571 .002994-3.569742$ H $4.024983-0.396099-2.865998$ O $4.9072021 .080262-3.961353$ H $2.3365162 .081940-3.519794$ H $2.5166840 .740957-4.636191$ H $8.630926-2.2362121 .798968$ H $7.746692-1.0375192 .756311$ H $7.298655-2.7479752 .845638$ C $4.378942-2.3170732 .046980$ H $5.6108662 .828223-0.137636$ H $4.8110963 .044892-2.605598$ H $4.7542270 .560639-4.768912$ H $4.871904-2.7892782 .903667$ H $4.158451-3.1027621 .317292$ H $5.391155-0.4019172 .106655$ C $0.627123-2.065005-2.629546$ O $1.547168-1.147273-3.233274$ C $1.4118540 .226410-2.846112$ C $0.0103670 .741968-3.200143$ C $-1.067226-0.137786-2.557195$ C $-0.827687-1.621127-2.850415$ H $0.767034-3.003173-3.183198$ C $0.985592-2.329051-1.158546$ H $1.560700 \quad 0.317208-1.766399$ O $-0.1508040 .756071-4.616231$ H $-0.0877201 .774989-2.848906$ H $-2.0505110 .161894-2.939703$ O $-1.0620040 .080801-1.149373$ H -1.486877 -2.247570 -2.243322 O $-1.155070-1.883611-4.218196$ C $2.080391-3.368429-1.047207$ H $1.313600-1.440062-0.614595$ H $\quad 0.117930-2.738889-0.628801$ H -1.038865 $1.102878-4.807122$ H $-1.789295-0.440435-0.768687$ H $-2.092861-1.659310-4.343111$ O $3.200559-2.955785-1.705019$ O $1.953161-4.422209-0.436264$ C $4.277214-3.897212-1.749772$ H $5.113057-3.432580-2.279255$ H $4.604088-4.158930-0.740057$ H $3.969490-4.792613-2.297859$
SCF Energy (B3LYP/6-31G**//MMFF) $=-3245.89715750$

\section{7_00522}

MM̄FF Geometry

C $0.448898-0.9290550 .117087$

C $-0.220947-1.564761-0.857607$

C $-0.580482-0.993583-2.206145$

O $-0.4196140 .437915-2.182621$

C $0.203273-1.641476-3.362675$

C $1.697055-1.375608-3.406841$

C $2.640434-2.290130-3.105528$

C $2.114561-0.023649-3.927471$

C $2.408361-3.687927-2.601223$

C $3.689943-4.435121-2.186028$

C $4.417046-3.950812-0.915750$

C $3.588946-4.1459700 .354804$

C $4.335357-3.6435161 .600910$

O $4.779478-2.579454-1.068091$

O $3.382240-3.6070322 .674377$

C $4.822124-2.2007481 .342738$

C $5.432231-4.6209182 .039727$

O $5.625122-1.7583892 .434456$

C $5.567672-2.0354480 .000300$

C $5.841603-0.555637-0.357931$

O $6.836012-2.6945250 .085558$

C -1.189066 1.205985 -2.992351

O $-1.9666350 .818634-3.851787$

C $-0.9460612 .643848-2.722013$

C $-0.5099793 .070442-1.526233$

C $-0.2498204 .452331-1.120248$

C $-0.3058965 .512885-2.185753$

C $4.5898860 .323383-0.454840$

C $4.8701991 .644036-1.151670$

C $3.6755353 .577312-2.181596$

O $6.0039582 .047559-1.396094$

C $3.4034334 .757705-1.298799$

C 3.3023194 .7798510 .039805

C $2.986936 \quad 6.0242190 .822106$

C $1.6998385 .904907 \quad 1.609203$

C 1.7149575 .7892782 .947733

C 0.3920955 .9885840 .846928

C 0.0451914 .6876910 .175928

N $3.6983972 .321435-1.457359$

H $0.8461880 .065103-0.063941$

H $-0.595437-2.568185-0.665618$

H $-1.647962-1.209203-2.351523$

H $-0.216788-1.287389-4.313577$

H $0.010416-2.721758-3.353550$

H $3.679894-2.005914-3.256928$

H $3.1925360 .035401-4.113490$

H $1.6104340 .195176-4.874338$

H $1.8677820 .761840-3.209526$

H $1.697627-3.683291-1.769950$

H $1.949981-4.263786-3.415336$

H $3.440058-5.495935-2.056655$

H $4.397911-4.383515-3.024372$

H $5.337157-4.543874-0.846008$

H $3.302348-5.1978230 .476775$

H $2.648645-3.586638 \quad 0.274860$

H $3.823633-3.2130913 .446596$

H $3.929127-1.5668791 .339696$

H $4.996603-5.5983382 .279786$

H $5.923283-4.2790472 .957633$

H $6.199406-4.7776671 .278872$

H $5.776061-0.8046162 .326125$

H $6.553829-0.1148880 .350971$

H $6.354015-0.535516-1.330520$

H $7.428358-2.311433-0.582408$

H $-1.193063 \quad 3.324252-3.529187$

H $-0.3350452 .332748-0.745044$

H $-1.3186825 .590923-2.596257$

H $0.3854575 .274963-3.002100$

H $-0.0334246 .507208-1.826742$

H $3.804058-0.195432-1.014098$

H 4.2067140 .5505820 .546218

H $2.8787523 .494398-2.927966$

H $4.6242723 .722281-2.707934$

H $3.2570625 .692980-1.836887$ 
H 3.4464923 .8698250 .615986 H $2.923651 \quad 6.9029190 .167928$ H 3.8313756 .2171501 .496217 H 0.7950215 .7241553 .519501 H 2.6470375 .7506663 .501578 H 0.4455016 .8300930 .151471 H $-0.4275806 .230211 \quad 1.536387$ H $0.038103 \quad 3.8414540 .864488$ H $2.8071921 .925726-1.171655$ C $0.109786-0.6084012 .614656$ O $-1.241285-0.1971622 .317961$ C -1.3147591 .2323932 .460279$ C $-0.174780 \quad 1.597004 \quad 3.398543$ C $0.911464 \quad 0.6703602 .881877$ H -1.0988231 .6618691 .476102$ C $-2.711091 \quad 1.6648572 .909712$ H 0.0977192 .6545903 .338084 H -0.4170781 .3587684 .440746$ C $2.0669210 .479557 \quad 3.849127$ H 1.3067291 .0852301 .946197 C -3.8467841 .2042831 .968751$ O $-2.977171 \quad 1.1336894 .212445$ H -2.739523 2.7551103 .019142 H -3.9961300 .1234092 .066356$ O -5.0608121 .8219902 .411534$ C -3.6281161 .5703820 .490321$ C $-4.8251091 .321587-0.441667$ H $-2.768731 \quad 1.013767 \quad 0.101323$ $\begin{array}{llll}\text { O } & -3.295408 & 2.958803 & 0.389273\end{array}$ H $-5.6359942 .024374-0.210362$ H -4.489378 $1.590668-1.452292$ H $2.5677861 .433878 \quad 4.042029$ H $1.7253950 .079227 \quad 4.809512$ H $2.807352-0.2141863 .438215$ C $0.650769-1.5023441 .492697$ H $-2.891960 \quad 0.1664834 .157608$ H -5.1839821 .5809223 .345804$ H $-4.031563 \quad 3.4659310 .772480$ H $0.150531-2.476468 \quad 1.557909$ H $1.715175-1.6845241 .652882$ H $0.052475-1.2068293 .533103$ C $-4.620436-2.413562-0.662628$ O $-4.286115-1.017884-0.673213$ C $-5.372128-0.116744-0.437182$ C $-6.475742-0.301512-1.494372$ C $-6.924829-1.762098-1.585790$ C $-5.730064-2.716605-1.684285$ H -3.710337 -2.923238 -1.004205 C $-4.952522-2.920463 \quad 0.750846$ H $-5.791723-0.3123970 .554579$ O $-5.992203 \quad 0.119939-2.769199$ H -7.325143 $0.341465-1.233891$ H -7.564694 -1.887849 -2.467804 O $-7.705238-2.069989-0.435040$ H $-6.064552-3.752993-1.562651$ O $-5.169954-2.625550-2.997744$ C $-3.745105-2.918307 \quad 1.662607$ H $-5.724486-2.309576 \quad 1.225523$ H $-5.308655-3.9558490 .700034$ H $-6.724706 \quad 0.036407-3.403252$ H $-8.002677-2.991950-0.520328$ H $-4.794681-1.734021-3.100174$ O $-4.159929-3.0882362 .952956$ O $-2.584597-2.810741 \quad 1.293943$ C $-3.117834-3.1367413 .932615$ H $-3.579194-3.2859714 .912393$ H $-2.445123-3.9756883 .729832$ H $-2.565290-2.1934303 .945394$ SCF Energy (B3LYP/6-31G**//MMFF) $=-3245.91012869$

\section{3}

MMFF Geometry

C $4.682389-1.2850362 .408778$

C $3.360242-1.1692462 .212106$

C $2.407266-0.6701393 .264318$

$\begin{array}{lllll}\text { O } & 1.679196 & 0.466752 & 2.744938\end{array}$

C $1.388742-1.7654813 .628864$

C $0.296829-1.2803754 .564388$
C $-1.011106-1.2104284 .244926$

C $0.752671-0.8935665 .948057$

C $-1.665203-1.5794042 .940802$

C $-2.468943-2.8748973 .106096$

C $-3.001413-3.4806031 .798850$

C $-1.889248-4.116308 \quad 0.961537$

C $-2.434380-4.701566-0.347806$

O $-3.671839-2.4655821 .049553$

O $-1.300054-5.000029-1.175730$

C $-3.276494-3.622500-1.059414$

C $-3.159123-6.033021-0.118961$

O $-3.892496-4.183025-2.216924$

C $-4.321361-2.940221-0.139716$

C $-4.942159-1.719076-0.858696$

O $-5.355655-3.878134 \quad 0.163639$

C 2.2694251 .6842792 .861951

$\begin{array}{lllll}\text { O } & 3.429669 & 1.901926 & 3.185808\end{array}$

C 1.3138012 .7895672 .618896

C 0.0265972 .6179742 .279538

C -0.9700653 .6816112 .123031$

C -0.5501095 .0935012 .442183$

C $-5.813451-0.827564 \quad 0.036665$

C $-6.3096590 .401285-0.706699$

C $-7.7205892 .426082-0.378706$

$\begin{array}{llll}\text { O } & -5.968212 & 0.676535 & -1.853924\end{array}$

C $-6.868107 \quad 3.534766 \quad 0.151591$

C $-5.9396834 .169911-0.579328$

C $-5.0837425 .288638-0.060275$

C $-3.6606814 .840547 \quad 0.201311$

C $-2.718695 \quad 5.009487-0.742044$

C $-3.405473 \quad 4.268568 \quad 1.581878$

C $-2.214733 \quad 3.356327 \quad 1.713774$

$\begin{array}{llll}\mathrm{N} & -7.172418 & 1.161130 & 0.072434\end{array}$

H $5.108092-1.0166933 .372281$

H $2.937882-1.4296951 .244186$

H $2.947914-0.3702124 .171582$

H $1.910230-2.6060654 .106308$

H $0.957860-2.1791692 .711492$

H $-1.706376-0.8565135 .005421$

H $-0.087092-0.6738836 .616053$

H $1.325176-1.7095016 .401151$

H $1.384390-0.0007835 .911145$

H -2.335261 -0.7611082 .649555$

H $-0.937313-1.6659392 .132333$

H -1.874498 -3.6323583 .633404$

H $-3.329967-2.6500093 .750459$

H $-3.730957-4.2481412 .084547$

H $-1.359855-4.8871571 .535265$

H $-1.133342-3.3624560 .705652$

H $-1.635724-5.297524-2.039071$

H $-2.572581-2.860523-1.417443$

H $-2.473901-6.7682580 .319956$

H $-3.497352-6.468130-1.065977$

H $-4.021364-5.9452100 .545009$

H $-4.225643-3.450793-2.761948$

H $-4.135521-1.097245-1.264864$

H $-5.551769-2.054245-1.707156$

H $-6.164218-3.3960070 .396643$

H 1.7304303 .7790022 .780708

H -0.3487361 .6085542 .122021$

H 0.2907045 .3974371 .808595

H -0.2498725 .1780723 .492233$

H $-1.340483 \quad 5.8292702 .273708$

H $-6.685216-1.381850 \quad 0.399730$

H $-5.239227-0.4785450 .902499$

H $-8.738268 \quad 2.5127700 .014113$

H $-7.7727442 .439038-1.472587$

H $-7.027476 \begin{array}{lll}3.827507 & 1.186333\end{array}$

H $-5.7789483 .869463-1.612383$

H $-5.506136 \quad 5.7210530 .855753$

H $-5.1015216 .101925-0.797440$

H $-1.6827094 .733378-0.585615$

H -2.964413 $5.441475-1.707206$

H $-4.2756353 .669877 \quad 1.882959$

H $-3.364148 \quad 5.0888802 .307245$

H -2.434068 2.3142091 .480498

H $-7.3383330 .888926 \quad 1.037232$

C $6.542749-0.6253250 .866257$ 
O $5.729444 \quad 0.3825790 .230157$

C $6.1880720 .562274-1.118187$

C $7.6417770 .116982-1.098762$

C $7.555305-1.091872-0.185858$

H $5.611954-0.130685-1.744607$

C $5.9388021 .998823-1.585583$

H $8.020329-0.123742-2.096443$

H $8.2945890 .877574-0.655173$

C $8.892335-1.5134580 .398928$

H $7.142217-1.932854-0.759278$

C $4.4982962 .506901-1.363100$

O $6.8151552 .890624-0.885247$

H $6.2087122 .087931-2.644395$

H $4.3380292 .733284-0.301435$

O $4.3612253 .750650-2.061518$

C $3.3928501 .551098-1.843834$

C $1.9871022 .175316-1.744693$

H $3.4204220 .638316-1.238880$

O $3.6479151 .168913-3.195153$

H $1.8955212 .733433-0.805315$

H $1.8518872 .877142-2.576766$

H $9.588740-1.795822-0.397237$

H $9.351382-0.7033740 .975558$

H $8.770736-2.3741541 .064348$

C $5.640214-1.7607091 .354116$

H 6.6485982 .7782090 .066574

H $5.060148 \quad 4.343804-1.736217$

H $3.6578691 .978690-3.733774$

H $6.245088-2.5731231 .772574$

H $5.079775-2.1759520 .508070$

H $7.046305-0.1400621 .711584$

C $0.054602-0.695124-3.179452$

O $0.9644370 .402271-3.027358$

C $0.8734851 .110334-1.789566$

C $-0.523697 \quad 1.727971-1.646445$

C $-1.6092340 .652058-1.758597$

C $-1.402002-0.233216-2.993866$

H $0.173446-1.015619-4.222575$

C $0.440161-1.880063-2.280340$

H $1.052430 \quad 0.417487-0.959411$

O $-0.7130742 .712295-2.660562$

H $-0.5904152 .233956-0.678899$

H -2.595090 $1.129312-1.817812$

O $-1.594685-0.166305-0.592353$

H $-2.058861-1.109043-2.956936$

O $-1.7661460 .508760-4.161918$

C $1.833286-2.393196-2.578670$

H $0.407794-1.604360-1.222869$

H $-0.254921-2.710227-2.443112$

H $-1.6167553 .058634-2.571116$

H -1.7581700 .4090840 .173923$

H $-2.701702 \quad 0.757831-4.071039$

O $2.263956-3.151481-1.527380$

O $2.456948-2.178933-3.609129$

C $3.572567-3.712047-1.671529$

H $3.821980-4.235521-0.744785$

H $3.584277-4.431002-2.496030$

H $4.311020-2.921554-1.837415$

SCF Energy (B3LYP/6-31G**//MMFF)= -3245.89845780

$07 \_00524$

MMFF Geometry

C $-2.538785-1.8154834 .143373$

C $-1.780182-0.767406 \quad 3.789240$

C $-0.293071-0.6600563 .999264$

O $0.322765-0.5587592 .694553$

C $0.0192990 .629374 \quad 4.781574$

C 1.4983730 .9357414 .907372

C 2.0886812 .0382744 .403516

C $2.315586-0.0767545 .666091$

C 1.4367723 .1702053 .656206

C 1.3770682 .9811932 .132242

C $2.7459253 .072738 \quad 1.439930$

C $2.5900123 .029557-0.082398$

C $3.9530033 .053176-0.796049$

O 3.5510201 .9717901 .865273

O $3.7353802 .726058-2.177151$

C $4.8712031 .972548-0.176947$
C $4.5506064 .464509-0.809480$

O $6.1922882 .064357-0.703190$

C 4.8952851 .9945501 .366484

C $5.6337020 .783137 \quad 1.984275$

O $5.586593 \quad 3.171704 \quad 1.803234$

C $0.643065-1.7194492 .061392$

O $0.520064-2.8542852 .492845$

C $1.126072-1.3650140 .708508$

C $1.155056-2.308498-0.244427$

C $1.509811-2.103660-1.650155$

C $2.093330-0.770806-2.034116$

C $5.054701-0.5914741 .630308$

C $5.787617-1.2972670 .501109$

C $5.716485-3.399036-0.836264$

O $6.815739-0.865470-0.012708$

C $5.131657-3.083803-2.176087$

C $4.531885-3.999562-2.951683$

C $3.987621-3.709305-4.320415$

C $2.534412-4.099499-4.464872$

C $2.183552-5.258195-5.048299$

C $1.489223-3.104899-4.015007$

C $1.287059-3.107187-2.524338$

N $5.171902-2.4939720 .158240$

H -2.087866 -2.673819 4.633026

H -2.2544040 .0733703 .285776$

H $0.100031-1.5291344 .540894$

H $-0.4092310 .559937 \quad 5.790218$

H -0.497694 1.4640534 .295847

H 3.1609752 .1586024 .552050

H $3.345946 \quad 0.258067 \quad 5.827135$

H $1.873424-0.2602696 .650896$

H $2.360505-1.0240275 .120060$

H $0.4227453 .341104 \quad 4.033913$

H 1.9819814 .0939003 .888672

H $0.9082362 .016202 \quad 1.905744$

H $\quad 0.7226223 .7611931 .723673$

H $3.2199104 .012141 \quad 1.748076$

H $1.9589363 .854534-0.435646$

H $2.0618712 .108503-0.365393$

H $3.3377111 .840373-2.221990$

H $4.4819871 .004630-0.509618$

H $3.8678115 .162539-1.309214$

H $5.4808224 .495236-1.387677$

H $4.7513584 .856674 \quad 0.189486$

H $6.6347112 .811678-0.265773$

H 6.7062700 .8429431 .763932

H $\begin{array}{llll}5.558527 & 0.883558 & 3.076898\end{array}$

H 5.8909803 .0298582 .715061

H $1.384134-0.3294670 .519074$

H $\quad 0.840789-3.3189780 .014337$

H $2.940049-0.525532-1.385059$

H $1.3413300 .020112-1.944844$

H $2.480564-0.741160-3.054721$

H $5.150849-1.2422332 .509065$

H $3.989651-0.5251411 .387904$

H $5.482919-4.417692-0.510786$

H $6.804929-3.286330-0.873595$

H $5.233114-2.063254-2.537507$

H $4.456640-5.025927-2.599540$

H $4.597225-4.265097-5.045040$

H $4.108804-2.651435-4.584714$

H $1.142190-5.531279-5.182459$

H $2.930635-5.962158-5.399766$

H $0.519757-3.349952-4.469987$

H $1.736633-2.116602-4.412248$

H $0.860422-4.037702-2.146685$

H $4.290275-2.744500 \quad 0.596445$

C -4.389032 -2.909394 2.817441

O $-3.666698-2.6481411 .597291$

C $-4.605547-2.432268 \quad 0.532505$

C $-5.864694-3.1664020 .972854$

C $-5.879358-2.8587002 .458247$

H $-4.811490-1.3551790 .502904$

C $-4.020138-2.874800-0.813662$

H $-6.758919-2.8121740 .451587$

H $-5.783089-4.2483530 .818840$

C $-6.735028-3.8136803 .272993$

H $-6.267373-1.8402482 .594927$ 
C $-2.589031-2.378285-1.109968$ O $-3.977730-4.309462-0.831500$ H -4.704255 -2.581627 -1.618862 H -1.894451 -2.898382 -0.437982 O $-2.240564-2.779415-2.441462$ C $-2.320398-0.865308-1.012959$ C $-3.227421-0.003822-1.898752$ H -1.287259 -0.699772 -1.336170 O $-2.412057-0.4120920 .331234$ H -3.151041-0.318539-2.946964 H $-4.277329-0.163127-1.620795$ H -7.779927 -3.7660862 .949377$ H $-6.395837-4.8493613 .163465$ H -6.697369 -3.557055 4.336522 C $-4.017837-1.8625583 .873546$ H -3.838992 -4.587316 - 1.752550 H -2.143911 -3.746497 -2.441055 H -1.859873 -0.9958230 .877811$ H -4.541011 -2.076942 4.812569 H -4.357865 -0.8733903 .539913$ H -4.091616 -3.9107883 .152022$ C $-3.8858223 .579719-2.653628$ O $-3.8906082 .146817-2.669732$ C $-2.9579111 .511622-1.784882$ C $-1.5202211 .924135-2.138787$ C $-1.3675263 .449411-2.169121$ C $-2.4836274 .122439-2.973959$ H $-4.5539683 .869677-3.475144$ C $-4.4766654 .138463-1.354444$ H -3.184362 $1.817363-0.757999$ O $-1.174404 \quad 1.382751-3.411890$ H $-0.8284981 .504004-1.400014$ H $-0.3993773 .713030-2.612519$ O $-1.3872953 .956886-0.837906$ H -2.465458 $5.205802-2.809665$ O $-2.2312873 .918436-4.367683$ C $-5.9281003 .743754-1.219373$ H -3.944057 $3.810611-0.457092$ H $-4.4356335 .234187-1.350408$ H $-0.2495591 .623415-3.591624$ H $-0.6550573 .539277-0.353346$ H $-2.3093272 .965691-4.547493$ O $-6.0312202 .587365-0.503583$ O $\quad-6.8541954 .372658-1.714846$ C $-7.3587852 .081705-0.334773$ H -7.9709552 .8019650 .216160$ H -7.805685 $1.858186-1.308104$ $\mathrm{H}-7.2984751 .1560850 .244116$

SCF Energy (B3LYP/6-31G**//MMFF) $=-3245.90345392$

\section{5}

MM̄MF Geometry

C $-4.578673 \quad 3.746876 \quad 0.194051$

C $-3.3688563 .624281-0.372934$

C $-2.105350 \quad 4.146368 \quad 0.261835$

O -1.1799923 .0422860 .408195$

C -1.444862 $5.199243-0.640835$

C $-0.341615 \quad 5.9569750 .070953$

C $0.9765635 .723553-0.079371$

C -0.8097587 .0753670 .968008$

C $1.6423084 .672967-0.925167$

C $2.7118363 .914296-0.130859$

C $3.6105803 .041556-1.018573$

C $2.8775821 .838178-1.612751$

C $3.8251980 .934878-2.419537$

O $4.6958042 .586385-0.197570$

O $3.152211-0.312661-2.647500$

C $5.0739110 .618112-1.568110$

C $4.1299251 .520757-3.803557$

O $6.029860-0.089600-2.353830$

C $5.7081541 .867080-0.914980$

C 6.8624121 .5421450 .067164

O $6.2793362 .691680-1.941563$

C -1.351085 2.2284831 .485081

O -2.1936902 .3377302 .361963$

C -0.3417091 .1491981 .399667$

C -0.4641440 .0835842 .204502$

C $0.406187-1.0940632 .240691$
C $1.643188-1.0790441 .383411$

C 6.6258320 .4405621 .100348

C 5.6550590 .7801282 .217684

C $4.542216-0.2981664 .166904$

O $5.284271 \quad 1.9203582 .476410$

C $4.059371-1.6715184 .507186$

C $2.837328-1.9427594 .989538$

C $2.379261-3.3408325 .297559$

C $1.007968-3.6676274 .748353$

C $0.064127-4.2041485 .541273$

C $0.739484-3.4369303 .274980$

C $0.044841-2.1206933 .038401$

N $5.297075-0.3599692 .927701$

H -4.6721404 .2688831 .143487$

H -3.269191 $3.091719-1.315740$

H -2.3136124 .5786411 .248665$

H -2.192487 $5.927992-0.982427$

H -1.060374 $4.725692-1.551764$

H 1.6687906 .3706860 .458670

H $\quad 0.0235797 .619878 \quad 1.424887$

H -1.4000337 .7987530 .395896$

H -1.430295 6.6851301 .780365

H $0.9206873 .959412-1.329985$

H $2.1113335 .179210-1.777379$

H $3.352148 \quad 4.6272960 .406721$

H 2.2434793 .3002900 .649540

H $4.0147753 .678211-1.814293$

H $2.0341802 .151270-2.238915$

H $2.4430151 .241687-0.799490$

H $2.338931-0.126784-3.146504$

H $4.735212-0.070600-0.784153$

H $3.2038981 .648120-4.377335$

H $4.7540440 .839722-4.392631$

H $4.6249412 .492870-3.759032$

H $6.735164-0.398799-1.761584$

H $7.7497341 .263286-0.517488$

H 7.1395032 .4644810 .595992

H $6.9208543 .293454-1.528147$

H 0.4472631 .2449560 .663828

H -1.3040180 .0569802 .898454$

H $2.270497-0.214171 \quad 1.626835$

H $1.373987-1.0276480 .322436$

H $2.269902-1.9643501 .507871$

H $6.322375-0.4924040 .615373$

H 7.5863640 .2331591 .591108

H 3.7254710 .4224854 .053618

H $5.2234680 .065004 \quad 4.943170$

H $4.753993-2.4947854 .352561$

H $2.145550-1.1272235 .185365$

H $2.390392-3.4597076 .388444$

H $3.088629-4.0766124 .897371$

H -0.916369 -4.462457 5.154258

H $0.248310-4.3962186 .593031$

H $0.081187-4.2309862 .897644$

H $1.669795-3.5457272 .711269$

H $-0.890174-2.0265783 .594204$

H $5.818923-1.2116492 .738514$

C -6.3357922 .0109250 .499228$

O $\begin{array}{lllll}-5.400206 & 0.918549 & 0.412137\end{array}$

C $-6.129232-0.3193440 .322600$

C $-7.5802740 .023950 \quad 0.635623$

C -7.6809841 .4294380 .069401$

H $-6.068097-0.645882-0.722153$

C $-5.481229-1.3681561 .234076$

H $-8.281709-0.6775300 .174136$

H $-7.7731910 .034395 \quad 1.714460$

C -8.8823632 .2055430 .579192$

H $-7.7306151 .365670-1.025923$

C $-3.964062-1.5385751 .006343$

O $-5.665921-0.9809222 .602080$

H $-5.993148-2.3301981 .120184$

H -3.436845 -0.627129 1.311961

O $-3.498514-2.5829611 .869398$

C $-3.577105-1.894909-0.439861$

C $-2.058614-2.013178-0.623330$

H $-3.944831-1.115328-1.112583$

O $-4.206877-3.116108-0.818730$

H $-1.586223-1.055787-0.374085$ 
H -1.649632 -2.7382940 .092898$ H $-9.812848 \quad 1.7023350 .297117$ H -8.867385 2.2972281 .670445 H $-8.902833 \quad 3.2148760 .156652$ C $-5.8413363 .162332-0.376919$ H $-5.267367-0.1006602 .714479$ H -3.737884 -2.334674 2.778796 H -3.897043 -3.806842 -0.207805 H $-6.5953103 .954987-0.432788$ H $-5.6620602 .804670-1.398363$ H -6.3711192 .3174411 .553402$ C $0.495559-2.866466-3.182648$ O $-0.168901-2.404852-2.000747$ C -1.602821-2.460401-2.024937 C $-2.148729-1.600306-3.173975$ C $-1.520360-2.000730-4.513257$ C $0.005377-2.099826-4.421549$ H $1.551325-2.610851-3.026747$ C $0.404509-4.389478-3.324382$ H $-1.915653-3.501020-2.162524$ O $-1.875128-0.226729-2.904229$ H -3.236582 -1.719280 -3.232431 H $-1.792873-1.258519-5.273552$ O $-2.066148-3.254578-4.912234$ H $0.410673-2.569194-5.324991$ O $0.546603-0.775908-4.375975$

C $1.166509-5.065849-2.209129$ H $-0.620539-4.770575-3.323017$ H $0.854257-4.717338-4.269245$ H -2.266796 $0.296826-3.624120$ H - $1.679568-3.481785-5.775075$ H $0.211039-0.346639-3.570202$ O $0.354903-5.236919-1.126548$ O $2.348521-5.376189-2.282929$ C $0.973947-5.824074 \quad 0.021777$ H $0.214153-5.929248 \quad 0.800711$ H $1.366016-6.815976-0.222082$ H $1.770750-5.1731620 .394190$

SCF Energy (B3LYP/6-31G**//MMFF)= -3245.91063714

\section{6}

MM̄FF Geometry

C $2.630296-0.052290-4.193314$

C $1.964800-0.594748-3.162548$

C $2.235772-1.975432-2.626717$

O $2.734050-1.810575-1.277504$

C $0.949646-2.822911-2.581633$

C $0.313253-3.080943-3.929141$

C $-0.836459-2.517164-4.349069$

C $1.030904-4.079379-4.798704$

C $-1.672789-1.493535-3.632444$

C $-3.017654-2.050386-3.149871$

C -2.895031 -3.068486 -2.003894

C $-4.276394-3.620903-1.642887$

C $-4.209119-4.601758-0.465628$

O $-2.327889-2.404477-0.869594$

O $-5.557502-4.792864-0.004842$

C -3.411821 -3.9514020 .685583$

C $-3.725605-5.983665-0.919525$

O $-3.184904-4.8823991 .740682$

C $-2.093263-3.2587450 .258791$

C -1.594070 -2.359302 1.413819

O $-1.045395-4.1941440 .006003$

C $3.409168-2.860193-0.735014$

O $3.662561-3.928796-1.268148$

C $3.773797-2.4937000 .648660$

C $4.107522-3.4600581 .515679$

C $4.462141-3.2384582 .912008$

C $3.794309-4.1922383 .863701$

C $-0.270000-1.6411871 .121360$

C $0.121372-0.7028352 .250743$

C 1.8298841 .0114632 .844655

O $\begin{array}{llll}1.0 .531090 & -0.587171 & 3.284975\end{array}$

C 2.9128540 .4187123 .691314

C $4.124017 \quad 0.983496 \quad 3.824546$

C 5.2753950 .5340524 .686915

C $5.153898-0.8312695 .325338$

C $4.523347-0.9918876 .501096$
C $5.855154-1.9902634 .654813$

C $5.339588-2.2814203 .272455$

N $1.285043-0.0033621 .962862$

H $3.433364-0.611680-4.666549$

H $1.171996-0.023580-2.684719$

H $3.006097-2.471710-3.230716$

H $0.243896-2.360748-1.884158$

H $1.163670-3.793522-2.115273$

H $-1.218343-2.798035-5.329809$

H $0.494399-4.278929-5.732409$

H $2.027209-3.712285-5.063902$

H $1.139482-5.033074-4.271938$

H -1.136067 -1.031136-2.800906

H $-1.869692-0.678771-4.340889$

H $-3.620863-1.199440-2.807535$

H $-3.550196-2.503914-3.995188$

H -2.231348 -3.882029 -2.315560

H $-4.744940-4.099086-2.512171$

H $-4.940178-2.790034-1.365673$

H $-5.521124-5.3602860 .784738$

H $-4.068791-3.1797621 .111263$

H $-4.445385-6.424735-1.620397$

H $-3.665547-6.682397-0.078318$

H $-2.757837-5.960117-1.423318$

H -2.452337 -5.4638731 .476809$

H $-2.356575-1.6026601 .639218$

H -1.457472 -2.9630312 .320470$

H $-1.424338-5.020808-0.323104$

H $3.743706-1.4473280 .930182$

H $4.091802-4.5030391 .205994$

H $3.937827-3.9227904 .913097$

H $4.179819-5.207633 \quad 3.722149$

H $2.711753-4.2077503 .691985$

H $0.535824-2.3720250 .992342$

H $-0.352035-1.0506800 .202646$

H $1.034271 \quad 1.4052983 .485343$

H $2.200593 \quad 1.8230132 .212433$

H $2.663497-0.4844334 .240447$

H $4.316722 \quad 1.9048343 .276299$

H 5.4196391 .2899305 .470564

H 6.1920030 .5671824 .084222

H $4.462336-1.9616756 .983586$

H $4.049852-0.1551127 .004353$

H $5.815997-2.8895575 .280075$

H $6.924989-1.7552394 .577091$

H $5.772851-1.6502472 .496426$

H $1.763343-0.175098 \quad 1.083556$

C $3.0924742 .420188-4.098258$

O $2.4970432 .686063-2.809659$

C $2.1998564 .090418-2.716118$

C $3.0937144 .757720-3.750124$

C $3.032963 \quad 3.739978-4.872882$

H $1.1551764 .217328-3.025515$

C $2.3743464 .584109-1.277231$

H $2.7280195 .745919-4.044345$

H $4.1237804 .864196-3.390567$

C $4.141530 \quad 3.898466-5.899345$

H $2.0632913 .834473-5.380335$

C $1.5686683 .775003-0.239258$

O $3.7612754 .484193-0.932992$

H $2.1142065 .648043-1.221347$

H $1.9831392 .763499-0.147165$

$\begin{array}{lllll}\text { O } & 1.736129 & 4.404398 & 1.037185\end{array}$

C $0.0585503 .682967-0.533671$

$\begin{array}{llll}\text { C } & -0.702396 & 2.909462 & 0.552937\end{array}$

H $-0.0877783 .175576-1.491440$

O $-0.4883514 .993724-0.657914$

H -0.2870001 .8994630 .639361$

H $-0.538018 \quad 3.379621 \quad 1.531810$

H $4.0779784 .877250-6.385913$

H $5.1310263 .816600-5.437114$

H $4.0672193 .129365-6.674659$

C $2.3125821 .295186-4.779467$

H $3.8958594 .983495-0.110086$

H $2.6566894 .262677 \quad 1.315156$

H -0.3390385 .4544780 .185624$

H $2.5505821 .256746-5.848930$

H $1.2339471 .482189-4.698711$ 
H $4.1328752 .123042-3.915074$

C -4.1504011 .8949771 .511968$

O $\begin{array}{llll}-2.727449 & 2.055130 & 1.449459\end{array}$

C $-2.2267792 .824906 \quad 0.347255$

C $-2.642842 \quad 2.179346-0.982370$

C $-4.1554731 .949898-1.051587$

C $-4.691846 \quad 1.273030 \quad 0.214032$

H $-4.315414 \quad 1.1708922 .320716$

C -4.8575923 .2023191 .902078$

H -2.634151 3.8391790 .411316

O $-1.9656090 .937621-1.155868$

H -2.342422 $2.829581-1.811262$

H $-4.3798231 .327292-1.926354$

O $-4.7967193 .208350-1.237564$

H $-5.787646 \quad 1.2935170 .229422$

$\begin{array}{lllll} & -4.301255 & -0.102563 & 0.204935\end{array}$

C -4.504990 3.6499163 .305950

H -4.599848 4.0221601 .226906

H -5.9426523 .0484321 .873128$

H -2.204816 $0.361102-0.410236$

H -5.752142 $3.042969-1.310900$

H -4.681312 -0.509928 -0.591675

O -5.1938484 .7960243 .588402$

O $-3.729560 \quad 3.077804 \quad 4.059190$

C $-4.962321 \quad 5.347888 \quad 4.888359$

H -5.2629204 .6350285 .662004$

H -3.908698 5.6205695 .001478

H -5.570877 6.2505804 .987823

SCF Energy (B3LYP/6-31G**/MMFF) $=-3245.90577327$

\section{7}

MM̄FF Geometry

C - $-1.457003 \quad 4.6952370 .019057$

C $-2.390208 \quad 3.733605-0.017450$

C $-3.2267593 .425375-1.232946$

O $-3.1446962 .005943-1.498014$

C $-4.6953953 .785281-0.954040$

C -5.637826 3.380199-2.070415

C $-6.4554442 .309262-2.017711$

C $-5.647158 \quad 4.289859-3.269857$

C $-6.546576 \quad 1.303284-0.902307$

C $-6.639239-0.136551-1.429171$

C $-6.394555-1.210221-0.353049$

C $-7.318663-1.067327 \quad 0.860218$

C $-6.991312-2.0810221 .967094$

O $-5.021395-1.117710 \quad 0.043995$

O $-7.692631-1.6465393 .142942$

C $-5.475827-2.0172052 .261319$

C $-7.527690-3.4773731 .632461$

O $-5.088047-3.0332393 .183076$

C $-4.607424-2.1124700 .989512$

C -3.108684 -1.882910 1.281088

O $-4.730118-3.4301970 .439158$

C -2.018739 $1.552598-2.111668$

O $-1.049108 \quad 2.207951-2.458354$

C -2.156777 $0.089882-2.265059$

C -1.047997 -0.663408 -2.275040

C $-0.996582-2.121039-2.374130$

C $-2.256153-2.839456-2.780818$

C $-2.752244-0.5056851 .848809$

C $-1.270573-0.2268751 .679068$

C $0.967225-1.1341262 .287960$

O -0.8602050 .6411040 .912153$

C $1.374030-2.3174241 .455299$

C $0.643909-3.4130901 .188536$

C $1.129135-4.5704500 .360716$

C $0.201492-4.894612-0.789747$

C $-0.770694-5.811966-0.651492$

C $0.450650-4.220191-2.120209$

C $0.166371-2.744163-2.092479$

N $-0.472427-1.0567582 .449157$

H $-1.2954855 .313184-0.860872$

H -2.560920 3.1221780 .865663

H $-2.8759453 .976923-2.114678$

H $-4.7850654 .868325-0.793110$

H -5.015816 $3.336303-0.006495$

H -7.127313 2.131602 -2.856002

H $-6.3750493 .974300-4.024880$
H -5.904246 $5.311124-2.970308$

H $-4.662594 \quad 4.304188-3.747725$

H -5.687983 $1.377171-0.229829$

H $-7.443717 \quad 1.534158-0.316603$

H -7.626871 $-0.292621-1.880914$

H $-5.893899-0.273325-2.224171$

H $-6.549916-2.184475-0.831592$

H -8.370126 -1.1488840 .557888$

H $-7.209880-0.0658061 .296437$

H -7.548597 -2.306399 3.841958

H $-5.281588-1.0557092 .754009$

H -8.621177 -3.4545761 .550377$

H -7.301591 -4.190975 2.432318

H $-7.134793-3.8762010 .695011$

H $-5.575811-2.8834124 .010139$

H -2.737996 -2.6721621 .947390$

H $-2.552678-2.0190250 .344224$

H $-3.939397-3.622304-0.091015$

H $-3.157087-0.322745-2.318674$

H - $0.083573-0.169702-2.165208$

H $-3.053138-2.673660-2.049206$

H $-2.130054-3.921068-2.866457$

H -2.600877 -2.482356 -3.757764

H $-3.298950 \quad 0.2899001 .330219$

H -3.006086 -0.4386092 .912175$

H $1.415894-1.2058383 .283143$

H $1.326360-0.2163891 .810669$

H $2.377020-2.2562731 .041550$

H $-0.351499-3.5162501 .611624$

H $1.215039-5.4417681 .023309$

H $2.139501-4.395524-0.024594$

H $-1.424010-6.073696-1.477133$

H $-0.937707-6.3185210 .293354$

H $-0.117433-4.705192-2.922388$

H $1.501269-4.367023-2.402703$

H $1.023783-2.139468-1.795558$

H $-0.913540-1.7372973 .060657$

C 0.8795934 .9654950 .870400

$\begin{array}{llllll}\text { O } & 1.305335 & 3.617198 & 0.590269\end{array}$

C 2.6854793 .4804350 .987976

C 3.1100504 .8347021 .553549

C 1.7900925 .4270362 .002742

H 2.7165802 .7279361 .785176

C $3.5511963 .024061-0.196544$

H 3.8319604 .7318792 .369494

H 3.5727645 .4635350 .783059

C 1.8407896 .9326142 .194602

H 1.4915634 .9485932 .945087

C $3.3079401 .559630-0.626473$

O $3.3407183 .911116-1.294083$

H 4.6062423 .1171270 .088003

H 3.5152930 .9237780 .240799

$\begin{array}{lllll}\text { O } & 1.946168 & 1.348005 & -0.989377\end{array}$

C $4.2042981 .123918-1.805986$

C $4.141831-0.382430-2.122460$

H $5.243807 \quad 1.405866-1.603154$

O $3.8150451 .802386-3.003690$

H $3.106172-0.683682-2.321531$

H $4.682068-0.533347-3.066838$

H 2.5830107 .1982932 .954568

H $2.111487 \quad 7.4466731 .266201$

H 0.8712457 .3193072 .523152

C $-0.606840 \quad 4.988176 \quad 1.223889$

H $2.4066993 .831181-1.555124$

H $1.3935361 .559078-0.217872$

H $3.9544072 .753592-2.860948$

H $-0.889090 \quad 5.978427 \quad 1.598157$

H $-0.810722 \quad 4.2608192 .019235$

H $1.0657835 .559844-0.034832$

C $6.817825-1.517416 \quad 0.219633$

O $6.103562-0.846402-0.825311$

C $4.755607-1.278711-1.033436$

C $4.728415-2.767351-1.416192$

C $5.478187-3.623528-0.387621$

C $6.849322-3.033047-0.037425$

H $7.847298-1.1443500 .142482$

C $6.284223-1.1408051 .610522$

H $4.184127-1.137239-0.112780$ 
O $5.330010-2.940771-2.698176$

H $3.684676-3.092080-1.494652$

H $5.609627-4.634998-0.791157$

O $4.684664-3.7273420 .789620$

H $7.269162-3.5419280 .837534$

O $7.747667-3.285614-1.122165$

C $6.413436 \quad 0.342419 \quad 1.890295$

H $5.228518-1.4036311 .717739$

H $6.853610-1.6709862 .382198$

H $5.250438-3.880605-2.934696$

H $5.157607-4.3043811 .413248$

H $7.429747-2.785984-1.893814$

O 5.6669430 .6523692 .991740

O $7.093974 \quad 1.126453 \quad 1.243284$

C $5.6967542 .026346 \quad 3.390565$

H 4.9505652 .1688304 .176686

H $6.6839912 .275303 \quad 3.790566$

H 5.4463672 .6792632 .549623

SCF Energy (B3LYP/6-31G**/MMFF) $=-3245.90793581$

\section{8}

MM̄FF Geometry

C -6.431051 -0.7225450 .611209$

C $-5.777009 \quad 0.368048 \quad 0.185315$

C $-5.323386 \quad 1.4755431 .100315$

O $-3.878425 \quad 1.4157451 .154173$

C $-5.7310072 .845640 \quad 0.530862$

C $-5.3359214 .008892 \quad 1.422874$

C $-4.349893 \quad 4.889812 \quad 1.156945$

C $-6.154675 \quad 4.1602132 .680176$

C $-3.4373384 .928878-0.038814$

C $-2.032006 \quad 4.452718 \quad 0.330177$

C $-1.0455914 .412104-0.847050$

C $-1.4654073 .420605-1.935323$

C $-0.4130813 .317034-3.048165$

O $0.2156224 .007050-0.298628$

O $-0.719677 \quad 2.158948-3.841042$

C $0.9692953 .068295-2.413521$

C $-0.4793004 .519011-3.997836$

O $2.0003503 .139163-3.395042$

C $1.3070074 .003916-1.229882$

C $2.5480153 .467235-0.480018$

O $1.6346845 .324127-1.664456$

C -3.324752 0.5521462 .050556

O $-3.910908-0.1373202 .869655$

C $-1.861424 \quad 0.566742 \quad 1.838402$

C $-1.074945-0.2735282 .527033$

$\begin{array}{lllll}C & 0.377963 & -0.384901 & 2.392144\end{array}$

C $1.074818 \quad 0.616277 \quad 1.509952$

C $2.957328 \quad 4.3358490 .714997$

C 3.7707553 .5879281 .755948

C $5.884077 \quad 2.3239292 .102147$

O $3.441576 \quad 3.5911102 .941596$

C $5.483708 \quad 0.9043232 .357362$

C 5.1166570 .4504953 .565670

C $4.791254-0.9828743 .875788$

C $3.320310-1.1905014 .166999$

C $2.837462-0.9776435 .403401$

C $2.492114-1.6874473 .001224$

C $1.023978-1.3626693 .060908$

$\begin{array}{llll}\mathrm{N} & 4.906057 & 2.975740 & 1.251976\end{array}$

H -6.690976 -0.8161741 .662618$

H $-5.4860670 .438157-0.860508$

H $-5.741071 \quad 1.3533952 .107087$

H -6.8190202 .8780980 .384452$

H -5.299052 2.960928 -0.468274

H -4.163586 5.6832621 .880102

H $-5.8934635 .064306 \quad 3.240592$

H -7.219572 4.2252352 .434063

H -6.001653 3.3060673 .346730

H -3.843302 4.339854 -0.861877

H -3.390469 $5.962118-0.404065$

H $-1.616647 \quad 5.106151 \quad 1.109974$

H -2.080540 3.4603640 .791220

H - $0.9489765 .425634-1.253077$

H -2.433403 $3.687686-2.372990$

H -1.600868 $2.425418-1.488923$

H - $-1.6265632 .257386-4.178409$
H $\quad 0.9651182 .036869-2.034703$

H $-1.4567704 .561926-4.493498$

H $0.2592834 .430820-4.801962$

H $-0.3291705 .474336-3.490178$

H $1.7805872 .496691-4.091659$

H $2.3487372 .448532-0.131681$

H $3.4002063 .410180-1.168918$

H $1.5622345 .364001-2.630824$

H $-1.468728 \quad 1.2495551 .094698$

H $-1.534412-0.9561653 .240196$

H 0.8047551 .6385841 .798725

$\begin{array}{llll}\text { H } & 0.799142 & 0.461899 & 0.461214\end{array}$

H 2.1643490 .5675701 .572298

H $3.538638 \quad 5.202270 \quad 0.379422$

H 2.0705334 .7242191 .229426

H 5.9823312 .8947673 .032109

H 6.8470932 .3506791 .582710

H $5.539480 \quad 0.2134941 .520756$

H 5.0747201 .1471734 .400441

H $5.390145-1.2885304 .743836$

H $5.108336-1.6469613 .061568$

H $1.795860-1.1421305 .654964$

H $3.484744-0.6353726 .204767$

H $2.599483-2.7776182 .944756$

H $2.917543-1.3130212 .065696$

H $0.444029-2.0398073 .688520$

H $5.1040123 .025170 \quad 0.257593$

C $-5.744611-3.012246-0.075621$

O $-4.483404-2.617706-0.657960$

C $-3.919943-3.739319-1.361676$

C $-4.750286-4.948022-0.947879$

C $-6.119208-4.321592-0.765093$

H $-4.069835-3.552036-2.432538$

C $-2.418314-3.876689-1.071317$

H $-4.736263-5.740119-1.702637$

H $-4.393707-5.373915-0.002248$

C $-7.081467-5.1872720 .029137$

H $-6.549580-4.116516-1.754530$

C $-1.575823-2.729165-1.673212$

O $-2.215895-3.9712790 .336969$

H $-2.065760-4.819599-1.506241$

H $-1.770917-2.704725-2.752244$

O $-1.995176-1.468318-1.155086$

C $-0.056815-2.894000-1.456628$

C $0.742147-1.825173-2.229107$

H $0.241927-3.892900-1.795812$

O $\quad 0.231113-2.799494-0.064939$

H $0.379564-1.789504-3.263415$

H $0.561990-0.846871-1.766163$

H $-7.254418-6.139196-0.483439$

H $-6.690827-5.409668 \quad 1.027837$

H $-8.048065-4.6882650 .149111$

C $-6.765350-1.890110-0.274254$

H -2.589117 -3.1697820 .742017$

H $-1.749740-1.445007-0.214769$

H $1.188196-2.9223020 .050416$

H $-7.775350-2.232431-0.023581$

H $-6.777567-1.574096-1.324784$

H $-5.561452-3.1832730 .993811$

C $4.168336-2.132205-0.743599$

O $2.766729-1.886581-0.922753$

C $2.260075-2.103210-2.243301$

C $2.990347-1.201954-3.252280$

C $4.509661-1.364475-3.162781$

C $5.003427-1.282156-1.714554$

H $4.386145-1.7826770 .272836$

C $4.500720-3.626874-0.802173$

H $2.411358-3.151966-2.521014$

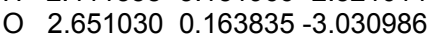

H $2.651641-1.456254-4.263459$

H $4.987090-0.578306-3.760435$

O $4.867971-2.620620-3.730032$

H $6.056365-1.579296-1.646117$

$\begin{array}{llll}\text { O } & 4.921413 & 0.076328 & -1.272659\end{array}$

C $3.892240-4.3553540 .372079$

H $4.165152-4.112140-1.723091$

H $5.584530-3.785468-0.749889$

H $2.8749210 .381532-2.109777$ 
H $5.836667-2.695159-3.689614$

H $5.4863670 .609664-1.857417$

O $2.686954-4.8847430 .018034$

O $4.416799-4.4244961 .475926$

C $1.994323-5.5925531 .051005$

H $1.751933-4.9162981 .875822$

H $1.062022-5.9790360 .630588$

H $2.596751-6.4345281 .404551$

SCF Energy (B3LYP/6-31G**//MMFF) $=-3245.91333865$

\section{9}

MM̄FF Geometry

C -1.486873 -2.878961 2.825618

C $-0.244496-2.9525932 .325453$

C $0.043279-3.3107900 .888843$

O $0.634372-2.1733890 .219631$

C $1.048623-4.4722860 .829659$

C $1.349832-4.924800-0.585637$

C $2.473869-4.610876-1.260645$

C $0.312303-5.820800-1.210103$

C $3.595521-3.722907-0.790707$

C $4.123902-2.832966-1.926065$

C $5.119676-1.748877-1.476264$

C $6.324224-2.313273-0.717107$

C $7.268345-1.205095-0.226084$

O $4.411120-0.805552-0.664301$

O $8.168905-1.8093380 .715333$

C $6.433224-0.1466480 .527609$

C $8.140761-0.661816-1.362875$

O 7.2442380 .9625440 .907459

C $5.1915150 .328204-0.257984$

C 4.2807681 .2475530 .583649

O $5.6240481 .090866-1.391452$

C $-0.211436-1.181160-0.165509$

O $-1.406989-1.1003010 .065242$

C $0.566888-0.193211-0.937618$

C $0.1432371 .078376-0.965798$

C $0.7628272 .176382-1.705431$

C $1.7700381 .821260-2.767388$

C 3.7083220 .6076661 .850042

C 2.4696491 .3502202 .306459

C 1.7012303 .5609863 .153611

O 1.3506520 .8544832 .200143

C 1.4474624 .5490332 .050629

C 2.2625304 .8442971 .024893

C $1.9444195 .863836-0.033056$

C $2.0759105 .306727-1.433595$

C $3.2336285 .411686-2.108138$

C $0.8500214 .706977-2.083542$

C $0.3845953 .440262-1.422783$

N 2.7430642 .6106372 .810156

H -2.333385 -3.085647 2.174627

H $0.604664-2.7299572 .966999$

H $-0.874948-3.597521 \quad 0.360913$

H $0.661395-5.3308541 .394813$

H $1.976640-4.1874411 .339950$

H $2.608625-5.034574-2.254858$

H $0.595762-6.146949-2.216453$

H $\quad 0.169867-6.718923-0.600272$

H $-0.646841-5.300605-1.292039$

H $3.280617-3.0880480 .041948$

H $4.401848-4.370685-0.429074$

H $3.271894-2.335492-2.408627$

H $4.601532-3.464230-2.686250$

H $5.457040-1.235208-2.384603$

H $6.866847-3.040233-1.333775$

H $5.981940-2.8655960 .167859$

H $8.721095-1.1015591 .090392$

H $6.106220-0.6156571 .464063$

H $8.752469-1.465935-1.789439$

H $8.8499690 .088081-0.995724$

H $7.561833-0.220141-2.176382$

H 7.3923341 .5060790 .114870

H 4.8164452 .1707990 .839798

H $3.4431411 .571424-0.047914$

H $4.9002491 .677998-1.665330$

H $1.457181-0.536588-1.450252$

H $-0.7382801 .346516-0.383824$
H $2.6383041 .318956-2.330265$

H $2.1509902 .689082-3.310215$

H $1.3207151 .154100-3.511702$

H $3.419538-0.4348671 .674756$

H 4.4458040 .6092012 .660262

H 2.0100864 .0863374 .062394

H $\quad 0.7759173 .015923 \quad 3.365911$

H 0.4966325 .0744272 .119300

H $3.231551 \quad 4.358327 \quad 0.946263$

H $2.6379816 .705450 \quad 0.095319$

H $\quad 0.941267 \quad 6.286337 \quad 0.102469$

H $3.3331615 .043375-3.123741$

H $4.1098255 .865649-1.657115$

H $1.0110844 .539159-3.155087$

H $\quad 0.0331375 .439335-2.041884$

H $-0.3511993 .601322-0.634204$

H 3.7052872 .9351582 .835911

C $-2.369196-1.1129044 .431724$

O $-3.490924-0.8864993 .554981$

C -3.3556560 .4253942 .971388$

C -2.3330211 .1573353 .831702$

C -1.3824140 .0240544 .166960$

H -2.9225670 .2916621 .974496$

C -4.7211121 .1079942 .860371$

H -1.8502901 .9802573 .295938$

H -2.7840431 .5603854 .745915$

C $-0.4556090 .324677 \quad 5.331128$

H $-0.779896-0.193176 \quad 3.278072$

C -5.7805180 .3239832 .052415$

O $-5.245286 \quad 1.3071574 .180846$

H -4.5970132 .1112752 .437778$

H -6.019638 -0.5984692 .596045$

O -6.9931251 .0884852 .034291$

C -5.419796 -0.0439330 .597879$

C $-4.962258 \quad 1.157610-0.255597$

H $-6.317439-0.4672560 .129092$

O $-4.437809-1.0721640 .613371$

H $-5.734581 \quad 1.933628-0.212324$

H -4.0380051 .5682840 .167717$

H $\quad 0.175563 \quad 1.190676 \quad 5.107931$

H -1.0171540 .5458056 .244918$

H $0.200754-0.5269285 .535794$

C -1.813631 -2.528904 4.249554

H -5.3168210 .4331184 .602120$

H $-6.782796 \quad 1.9829151 .717513$

H $-4.242889-1.316268-0.306327$

H -2.569898 -3.249615 4.585922

H $-0.939702-2.6715784 .895903$

H -2.761266 -1.030481 5.454021

C $-3.006768-0.307760-3.092865$

O $-3.4253770 .137958-1.796321$

C $-4.6994040 .787616-1.730932$

C $-4.7075412 .030346-2.633964$

C $-4.3256091 .666455-4.070996$

C $-3.0411960 .834959-4.121523$

H $-1.956768-0.598361-2.962024$

C $-3.785021-1.551319-3.534694$

H $-5.4807760 .094209-2.060394$

O $-3.7934292 .996756-2.119393$

H -5.709302 2.476469 -2.613520

H $-4.1888772 .587179-4.651307$

O $-5.4003930 .936226-4.655024$

H $-2.8945720 .424976-5.126995$

O $-1.9272881 .698396-3.875963$

C $-3.390274-2.740378-2.688970$

H $-4.868846-1.416283-3.479481$

H -3.554298 -1.812900 -4.574028

H $-3.840228 \quad 3.779689-2.694017$

H $-5.1533380 .735032-5.573744$

H $-2.0029212 .028166-2.964213$

O $-4.406792-3.074218-1.844731$

O $-2.298771-3.291395-2.757125$

C $-4.141305-4.174006-0.968194$

H $-3.926074-5.077762-1.546083$

H $-3.307717-3.932696-0.302388$

$\mathrm{H}-5.034278-4.348575-0.362214$

SCF Energy (B3LYP/6-31G**//MMFF $)=-3245.91143128$ 


\section{0}

MMFF Geometry

C $0.1863904 .172832-0.449329$

C $-0.6822653 .154263-0.537910$

C -1.3648872 .5210330 .645000$

$\begin{array}{lllll}\text { O } & -2.786730 & 2.704344 & 0.441371\end{array}$

C -1.057223 1.0141660 .726262

C 0.3757780 .7051151 .095765

C $\quad 1.309421 \quad 0.2541190 .236553$

C 0.7162670 .8940862 .551273

C $1.1647650 .005279-1.238731$

C $1.332519-1.473793-1.610179$

C $0.255633-2.394343-1.015938$

C $0.513154-3.845333-1.417894$

C $-0.580001-4.775667-0.879198$

O $-1.016906-1.981146-1.516749$

O $-0.433999-6.048102-1.527909$

C $-1.963650-4.217990-1.279577$

C $-0.418228-5.033166 \quad 0.624765$

O $-2.988439-4.979787-0.646368$

C $-2.141154-2.708393-0.984694$

C $-3.374231-2.102616-1.697822$

O $-2.266271-2.499017 \quad 0.421915$

C -3.5935862 .5734491 .528191$

O -3.2548342 .3221942 .673822$

C -4.9875632 .7656631 .075142$

C -5.9815702 .2133301 .785889$

C -7.4105252 .2817931 .474459$

C -7.845682 3.2655260 .420589

C $-4.744376-2.602108-1.240088$

C $-5.846791-1.910408-2.017381$

C -7.063385 $0.252131-2.212192$

O $-6.421656-2.466680-2.950800$

C $-8.3978670 .246707-1.527482$

C $-8.794343-0.503430-0.486837$

C $-10.166971-0.4070880 .119815$

C -10.139763 -0.0780691 .596664$

C -10.526287 -0.981224 2.513566

C -9.7415251 .3252462 .005734$

C -8.2508721 .4703492 .150525$

N $-6.091763-0.618795-1.581231$

H $0.408224 \quad 4.592906 \quad 0.527370$

H $-0.9286112 .750942-1.517794$

H -1.0747773 .0338281 .570713$

H -1.352730 $0.529355-0.211208$

H $-1.7109480 .539199 \quad 1.469521$

H 2.3034630 .0505910 .629512

H 0.6453221 .9499932 .829304

H 0.0280020 .3200843 .180430

H $1.730000 \quad 0.5566822 .788367$

H $\quad 0.2176870 .380496-1.635394$

H $1.9468150 .581277-1.749312$

H $1.303031-1.542792-2.705312$

H $2.322401-1.818568-1.292805$

H $\quad 0.266770-2.2962000 .074999$

H $1.501910-4.177601-1.080177$

H $\quad 0.529219-3.925852-2.513923$

H $\quad 0.457599-6.383890-1.332334$

H -2.082165 -4.370432 -2.361379

H $\quad 0.571624-5.4550440 .836809$

H - $-1.141130-5.7765740 .978765$

H -0.529764 -4.130004 1.228539

H -2.852547 -5.910886 -0.892015

H -3.340987 -1.011843 -1.561991

H -3.272821 -2.248395 -2.782076

H $-2.711166-1.6471960 .565011$

H -5.1514453 .3248220 .161419$

H -5.7244031 .6284902 .668530$

H -7.536980 4.2800990 .696887

H $-8.926586 \quad 3.2984610 .272491$

H -7.401466 $3.021078-0.549497$

H -4.859922 -3.675231-1.416204

H -4.896424 -2.427543 -0.169225

H -7.190360 -0.040349 -3.259636

H $-6.6449851 .263098-2.187748$

H $-9.1113070 .956934-1.942816$

H -8.118878 -1.231918 -0.046911

H $-10.670529-1.368041-0.047352$
H $-10.7790840 .342907-0.397013$

H $-10.534030-0.7425533 .572064$

H -10.838846 -1.980149 2.227768

H -10.190115 1.5704412 .977685

H -10.179921 2.0393651 .303956

H -7.8267470 .8086582 .907782$

H $-5.604701-0.269215-0.761099$

C $2.3610274 .904954-1.595928$

O $2.9742323 .601725-1.571131$

C $4.2482723 .722138-0.899192$

C $4.4117665 .196724-0.526159$

C $2.9720385 .658701-0.419345$

H 4.1753633 .1363650 .022602

C $5.3813743 .192285-1.787680$

H 4.9737095 .3242010 .404496

H $4.9386505 .756118-1.308466$

C $2.8090017 .166313-0.488983$

H 2.5699915 .2936750 .533244

C $5.2949291 .689748-2.121967$

O $5.3804743 .917599-3.024723$

H $6.3481403 .406152-1.317044$

H $4.4114201 .483572-2.737771$

O $6.4355421 .357401-2.925026$

C $5.2949000 .768291-0.887689$

C $5.400989-0.725195-1.258570$

H $4.3733100 .928657-0.321195$

O $6.3800951 .125861-0.032306$

H $4.663701-0.955832-2.036444$

H $6.401710-0.918234-1.664674$

H $3.3505737 .649900 \quad 0.330560$

H $3.1954527 .569247-1.431078$

H $1.7546317 .448386-0.408677$

C $0.8341954 .808549-1.647336$

H $4.5160453 .774839-3.447018$

H $6.4264541 .946578-3.698808$

H $7.2042360 .993512-0.531337$

H $0.5591114 .247739-2.549980$

H $0.4162005 .815863-1.760964$

H $2.6862665 .373397-2.535737$

C $6.123037-2.1860662 .114524$

O $6.231081-1.4550920 .886491$

C $5.169581-1.656906-0.050656$

C $5.108557-3.135021-0.466098$

C $4.979687-4.0459460 .760530$

C $6.006507-3.6952311 .842749$

H $7.077168-2.0104072 .628279$

C $5.003838-1.6384603 .007099$

H $4.219882-1.3731050 .412812$

O $6.289295-3.473479-1.191104$

H $4.255936-3.283046-1.138662$

H $5.121996-5.0911960 .459879$

O $3.671427-3.924868 \quad 1.311240$

H $5.763310-4.2162922 .775673$

O $7.289624-4.1775631 .431984$

C $5.301177-0.2163173 .420999$

H $4.020734-1.6669422 .528460$

H $4.913887-2.2295983 .926216$

H $6.209646-4.403335-1.464209$

H $3.038337-4.1991480 .626869$

H $7.557728-3.6747180 .643686$

O 4.5190230 .6485242 .715271

$\begin{array}{lllll}\text { O } & 6.142516 & 0.088031 & 4.256722\end{array}$

C 4.7004272 .0354693 .016381

H 3.9381052 .6024082 .475519

H 4.5735142 .2167814 .088067

H 5.6887492 .3641092 .682294

SCF Energy (B3LYP/6-31G**//MMFF) $=-3245.91165057$

0700531

MM̄FF Geometry

C -0.5417910 .9960083 .086176$

C -1.5016701 .9272423 .180580$

C -1.9906112 .7352602 .011241$

O -3.3754022 .3533521 .832227$

C -1.9124814 .2491872 .265400$

C -0.5138524 .8195822 .162055$

C 0.0166125 .2808871 .012111

C 0.2538344 .9266163 .452221 
C $-0.6406785 .246708-0.340783$

C $0.3548135 .218196-1.506628$

C $1.2275763 .953866-1.574322$

C $2.270714 \quad 4.124606-2.682841$

C $3.1335502 .873401-2.866287$

O $0.3919452 .819589-1.849634$

O $3.8427073 .042189-4.103386$

C $2.2034181 .653727-3.004701$

C $4.1955322 .749738-1.769191$

O $2.955974 \quad 0.442246-3.022104$

C $1.1045801 .574097-1.911762$

C $0.1167720 .443436-2.292053$

O $1.7218701 .260743-0.663013$

C $-3.901798 \quad 2.4635390 .584714$

O $-3.3822082 .967620-0.396915$

C $-5.2181791 .794793 \quad 0.601057$

C $-5.7489151 .397599-0.564289$

C $-6.9824470 .630289-0.731486$

$\begin{array}{llll}C & -7.818707 & 0.379784 & 0.496177\end{array}$

C $-1.1271260 .295236-1.403032$

C $-1.888989-0.975869-1.759462$

C $-3.481203-2.625752-0.809425$

O $-1.666096-1.614671-2.786308$

C $-4.968264-2.485089-0.732073$

C $-5.797878-2.969592-1.670644$

C $-7.299780-2.951675-1.582375$

C $-7.945205-2.145154-2.689375$

C $-8.154636-2.679087-3.904412$

C $-8.411989-0.743283-2.360500$

C $-7.271903 \quad 0.150236-1.958941$

N $-2.840992-1.320794-0.808214$

H $-0.066503 \quad 0.8201562 .125443$

H -1.987802 2.0963884 .138444

H $-1.4285782 .466098 \quad 1.107330$

H -2.344335 4.4935703 .245778

H -2.571170 4.7730841 .560385

H $1.0067965 .731962 \quad 1.045527$

H $1.2311655 .401638 \quad 3.314893$

H -0.3046645 .5278504 .177219$

H $0.429740 \quad 3.937435 \quad 3.883364$

H $-1.3083334 .387795-0.431958$

H $-1.2531216 .150922-0.442179$

H $-0.225298 \quad 5.300949-2.435072$

H $0.9959786 .107268-1.448288$

H $1.7243013 .808007-0.608977$

H $2.9052124 .999059-2.489835$

H $1.7578294 .333684-3.632649$

H $4.4344432 .279848-4.219446$

H $1.709657 \quad 1.728189-3.983934$

H $4.8521783 .627993-1.773090$

H $4.8486351 .889172-1.950257$

H $3.7706142 .657221-0.767735$

H $3.5490670 .471844-3.791111$

H $-0.2365370 .609320-3.318765$

H $0.656013-0.512332-2.288459$

H $1.0827470 .809184-0.091204$

H -5.6753191 .5908161 .562101$

H $-5.2035741 .608631-1.483689$

H $-7.262127-0.219167 \quad 1.225434$

H -8.747871 -0.1556890 .292965$

H $-8.104215 \quad 1.3281720 .965265$

H $-0.849797 \quad 0.242027-0.346209$

H - $-1.7964231 .148463-1.546783$

H $-3.119981-3.1586670 .075702$

H -3.170059-3.201006 -1.687536

H $-5.379160-2.0197280 .159846$

H $-5.372850-3.457446-2.545713$

H $-7.637101-2.585830-0.604959$

H -7.652023 $-3.990320-1.636819$

H -8.638860 -2.115870 -4.695049

H -7.843352 -3.692736 -4.134757

H $-9.190327-0.806210-1.595583$

H $-8.900316-0.283571-3.229617$

H $-6.5942210 .381655-2.782161$

H -2.920618 -0.7569140 .033307$

C $-0.330745-1.3519224 .027806$

O $0.472661-1.8520372 .938990$

C $-0.319919-2.7812012 .176243$
C -1.544969 -3.091548 3.032478

C $-1.771933-1.7608273 .719711$

H $-0.644950-2.2459901 .277463$

C $0.498451-4.018844 \quad 1.794754$

H -2.399789 -3.418835 2.433858

H $-1.335196-3.8706193 .774864$

C $-2.665499-1.8451184 .943949$

H -2.229427 -1.078957 2.992278

C $1.763994-3.7480280 .954754$

O $0.915720-4.6868712 .994275$

H $-0.144712-4.7329141 .266986$

H $2.513980-3.2355151 .567998$

O $2.326122-5.0242590 .615996$

C $1.541643-2.959886-0.350175$

C $2.726174-2.996368-1.335963$

H $1.296271-1.917385-0.117839$

O $\quad 0.433471-3.510738-1.071287$

H $2.840653-4.014834-1.730227$

H $2.433552-2.381956-2.198142$

H $-3.660158-2.2112194 .669305$

H $-2.253232-2.5255695 .696602$

H $-2.783787-0.8606265 .407322$

C $-0.0871430 .145847 \quad 4.238837$

H $1.436539-4.0523333 .516242$

H $2.477490-5.5044311 .448146$

H $-0.381048-3.294319-0.589643$

H $0.987114 \quad 0.3199814 .373680$

H -0.5796870 .4629525 .165744$

H $0.022894-1.8798594 .923498$

C $5.091961-0.600348 \quad 0.276448$

O $3.955342-1.129722-0.422494$

C $4.074843-2.503588-0.786951$

C $5.216420-2.665974-1.813023$

C $6.432365-1.793296-1.477225$

C $6.400681-1.349371-0.020599$

H $5.1947780 .436925-0.063532$

C $4.800776-0.5749341 .777760$

H $4.326822-3.0800660 .111219$

O $4.768625-2.296208-3.119357$

H $5.486646-3.727285-1.870889$

H $6.495068-0.914348-2.131126$

O $7.624471-2.546742-1.702328$

H $6.516435-2.2202810 .636976$

$\begin{array}{llll}\text { O } & 7.496453 & -0.477792 & 0.251364\end{array}$

C 3.6912860 .3994092 .075462

H $4.480857-1.5584382 .141116$

H $5.684410-0.2902012 .360471$

H $5.501339-2.460358-3.737371$

H $7.630262-2.810030-2.638492$

H $8.310340-0.9516950 .008557$

$\begin{array}{lllll} & \text { O } & 4.197572 & 1.645651 & 2.292171\end{array}$

$\begin{array}{lllll}\text { O } & 2.508075 & 0.089630 & 2.080072\end{array}$

C 3.2263622 .6625852 .558500

H 2.5144992 .7395601 .731359

H 2.7058662 .4479623 .496624

H 3.7505633 .6167712 .657637

SCF Energy (B3LYP/6-31G**//MMFF) = -3245.88867996

\section{2}

MM̄FF Geometry

C $-5.026907 \quad 3.453133 \quad 0.266978$

C $-3.7697973 .384370-0.195658$

C $-2.566366 \quad 3.909786 \quad 0.541925$

$\begin{array}{llll}0 & -1.609430 & 2.836076 & 0.673917\end{array}$

C $-1.898853 \quad 5.027972-0.280076$

$\begin{array}{llll}\text { C }-0.604343 & 5.532889 & 0.328437\end{array}$

C $0.623775 \quad 5.343628-0.195871$

C $-0.760073 \quad 6.318016 \quad 1.606241$

C $1.0049704 .629614-1.464570$

C $1.7536563 .310999-1.236448$

C $3.0650513 .398462-0.436906$

C $4.0897924 .321195-1.104360$

C $5.444517 \quad 4.303504-0.381323$

O $3.5853582 .060726-0.349806$

O $6.4073654 .958522-1.221715$

C $5.8890752 .839070-0.189496$

C $5.4062305 .132822 \quad 0.906824$

O 7.0743102 .7663300 .599160 
C 4.7904771 .9409490 .420909

C 5.2072200 .4504110 .445751

O 4.5714442 .3351021 .781219

C -1.7320111 .9968881 .736578$

O -2.5983122 .0044142 .594716$

C -0.6077671 .0418281 .651505$

C $-0.624935-0.1019512 .350372$

C $0.385246-1.1556092 .250378$

C $1.647597-0.8397991 .491646$

C $5.366319-0.206816-0.926261$

C $5.853795-1.637644-0.797067$

C $5.129168-3.919959-0.138868$

O $6.992247-1.961205-1.129353$

C $4.318597-4.5040650 .976480$

C $4.159505-3.9556932 .191836$

C $3.321535-4.5645473 .282166$

C $2.177500-3.6595563 .695366$

C $2.220042-3.0081814 .870430$

C $1.019360-3.5815282 .719925$

C $0.149298-2.3553512 .821428$

N $4.900212-2.492593-0.268268$

H -5.2199733 .9123211 .233012$

H -3.586002 2.921471 -1.162968

H -2.8399264 .2891381 .534339$

H $-2.5910815 .874426-0.383024$

H -1.728186 $4.667982-1.299385$

H 1.4706065 .7721310 .339581

H $\quad 0.187690 \quad 6.745803 \quad 1.950372$

H -1.458543 7.1482061 .458828

H -1.141324 5.6786322 .408182

H $\quad 0.1316094 .415938-2.086397$

H $1.6201425 .308424-2.066595$

H $1.0853042 .598829-0.737361$

H $1.9655782 .857215-2.214248$

H $2.834213 \quad 3.7430970 .577027$

H $3.7204215 .351170-1.167081$

H $4.2424143 .994764-2.142498$

H $6.4451264 .483746-2.069377$

H $6.1536142 .453949-1.181360$

H 5.1793196 .1812770 .677819

H 6.3834045 .1402621 .402217

H 4.6591294 .7841381 .622719

H 7.7359723 .3453080 .183301

H $\quad \begin{array}{lll}6.129313 & 0.330738 & 1.029915\end{array}$

H $4.449680-0.1122861 .005529$

H 4.1498521 .5980952 .253334

H $0.191798 \quad 1.283727 \quad 0.961466$

H -1.474474 -0.3126382 .997632$

H 2.1317020 .0487861 .911904

H $1.434019-0.6552300 .435001$

H $2.386377-1.6415241 .533143$

H $4.418691-0.207452-1.475916$

H $6.0958850 .328035-1.542022$

H $6.194211-4.1305880 .002217$

H $4.822765-4.368051-1.089959$

H $3.831793-5.4525040 .761676$

H $4.659570-3.0184872 .425652$

H $3.978936-4.7712614 .136204$

H $2.913819-5.5366392 .977056$

H $1.411290-2.3694305 .207510$

H $3.072350-3.1049505 .535485$

H $\quad 0.388499-4.4689012 .859672$

H $1.387201-3.6563701 .689361$

H - $0.780586-2.5077053 .368741$

H $3.942404-2.160579-0.200258$

C -6.8907821 .7669620 .228436$

$\begin{array}{lllll}\text { O } & -5.986836 & 0.645473 & 0.281133\end{array}$

C $-6.683070-0.540069-0.142668$

C -8.164780 -0.193254 -0.083695

C $-8.1420571 .265659-0.495168$

H $-6.400936-0.712148-1.188708$

C $-6.260265-1.7434630 .707544$

H -8.765699 $-0.819653-0.749710$

H $-8.567961-0.2916510 .930692$

C $-9.4086322 .021178-0.131381$

H -7.995085 $1.319277-1.582370$

C $-4.734825-1.9380570 .823940$

O $-6.769905-1.5847192 .038493$
H $-6.729942-2.6522220 .314408$

H -4.311761 -1.139055 1.445070

O $-4.504717-3.154786 \quad 1.546714$

C $-3.977332-1.992728-0.517031$

C $-2.476751-2.223861-0.295878$

H $-4.130798-1.048596-1.047767$

O $-4.519735-3.040763-1.314382$

H -2.101174 -1.472299 0.404944

H -2.319177 $-3.192267 \quad 0.198593$

H -10.276478 $1.581072-0.633335$

H $-9.595471 \quad 1.9951490 .947450$

H $-9.3380583 .070078-0.435943$

C $-6.2160512 .934417-0.493472$

H $-6.411152-0.7529772 .393234$

H -4.820312 -3.888559 0.991802

H $-4.131017-2.969489-2.202305$

H $-6.9262953 .759289-0.621359$

H $-5.9055192 .615382-1.496614$

H -7.122732 2.0376081 .266547

C $0.767089-2.466897-2.088927$

O $-0.251104-2.375161-1.086470$

C -1.597625 -2.218292 -1.559539

C $-1.718313-0.944146-2.408279$

C $-0.682208-0.928909-3.537218$

C $0.725491-1.248973-3.027412$

H $1.711536-2.430566-1.530478$

C $0.716663-3.810485-2.824932$

H -1.863613 -3.092813 -2.162258

O $-1.5429910 .199006-1.574733$

H -2.724866 -0.890499-2.838361

H $-0.6830130 .060807-4.010368$

O $-1.071775-1.882467-4.521607$

H $1.406645-1.406782-3.871675$

O $1.202945-0.103491-2.316511$

C $1.141322-4.936281-1.911112$

H $-0.268571-4.055938-3.230899$

H $1.412163-3.807586-3.672885$

H $-1.6443360 .989233-2.131908$

H $-0.422380-1.839914-5.244263$

H $2.115922-0.287653-2.038408$

O $0.112404-5.284790-1.087262$

O $2.258476-5.436773-1.922921$

C $0.397657-6.328161-0.150826$

H $0.608340-7.261752-0.680580$

H $1.239899-6.0481160 .488565$

$\mathrm{H}-0.485455-6.4710260 .477669$

SCF Energy $\left(B 3 L Y P / 6-31 G^{* *} / / M M F F\right)=-3245.89546082$

0700533

MM̄MF Geometry

C $1.065308-2.9128681 .739889$

C $\quad 0.060767-2.8504222 .625595$

C $-0.611413-1.5514892 .984315$

O $-2.050695-1.7028352 .882425$

C $-0.310760-1.1243284 .432700$

C $1.146283-0.8236394 .721409$

C $1.776302 \quad 0.309144 \quad 4.352027$

C $1.881969-1.8787715 .505054$

C 1.1882601 .4594323 .584075

C 1.9357211 .6897562 .261429

C 1.1658852 .5569971 .250832

C 0.7870083 .9289961 .809051

C $-0.084962 \quad 4.722667 \quad 0.826365$

O $-\begin{array}{llll}0.001154 & 1.824342 & 0.877631\end{array}$

O $-0.615010 \quad 5.8617891 .521740$

C $-1.270878 \quad 3.838470 \quad 0.373927$

C $0.7493645 .302798-0.321432$

O $-2.0218004 .514788-0.630446$

C $-0.8371132 .441119-0.116799$

C $-2.0057641 .443266-0.286999$

O $-0.1544972 .565501-1.364439$

C -2.594433 -1.753426 1.635130

O $-1.995008-1.7590410 .572100$

C $-4.066938-1.7976351 .786260$

C $-4.850133-1.8358500 .696804$

C $-6.314104-1.9196610 .687146$

C -7.023312 -1.821817 2.013116

C $-3.0900451 .787614-1.306169$ 
C $-4.3287712 .409212-0.682497$

C -6.687067 2.983321-1.244115

$\begin{array}{llll}\text { O } & -4.392499 & 2.770457 & 0.489226\end{array}$

C $-7.4812541 .836932-0.699992$

C $-8.4811161 .244524-1.369029$

C $-9.3093100 .118561-0.819752$

C $-9.062509-1.193311-1.529627$

C $-9.476491-1.392181-2.792827$

C $-8.419003-2.307119-0.736306$

C $-6.948222-2.090577-0.492910$

N $-5.3640122 .511587-1.601528$

H $1.417300-1.9915221 .280815$

H $-0.297062-3.756702 \quad 3.106547$

H $-0.311034-0.7486252 .299938$

H -0.676312 -1.894924 5.126092

H $-0.921831-0.2462024 .682519$

H $2.822636 \quad 0.433537 \quad 4.627438$

H $2.927648-1.6086495 .686857$

H $1.405033-2.0267806 .479554$

H $1.877634-2.8321944 .968379$

H $\quad 0.121769 \quad 1.3085533 .396039$

H $1.267593 \quad 2.356623 \quad 4.209430$

H 2.9092172 .1486992 .471685

H $2.1312420 .717011 \quad 1.790239$

H 1.8009632 .6697040 .365466

H 1.6837824 .4987602 .081730

H 0.2143003 .8106732 .737766

H -1.145281 5.5411172 .270973

H $-1.938694 \quad 3.707992 \quad 1.235925$

H 1.5171905 .9805720 .071041

H $0.1314615 .908817-0.993203$

H $1.2566034 .539435-0.914923$

H -2.285393 $5.376826-0.265261$

H $-1.5580800 .495865-0.619040$

H -2.442770 1.2205330 .694797

H $-0.2023041 .714752-1.831078$

H $-4.466069-1.8119132 .794326$

H -4.377412 -1.831208 -0.284678

H -6.790851 -2.690189 2.638874

H -8.110200 - 1.7673071 .923955

H $-6.720000-0.9127442 .545116$

H -2.713755 $2.441253-2.099323$

H -3.414692 $0.852020-1.780839$

H -7.138485 $3.409261-2.145750$

H -6.606741 $3.772786-0.489729$

H $-7.228756 \quad 1.4913830 .299647$

H -8.734309 $1.599716-2.365435$

H -10.369116 $0.388176-0.916109$

H -9.1393520 .0022240 .257638$

H -9.328891-2.342715 -3.294316

H $-9.973271-0.607079-3.353228$

H -8.515286 -3.258087 -1.277888

H -8.984915 -2.458633 0.187375

H -6.347176 - $2.140745-1.401795$

H -5.243842 2.107393-2.525888

C $1.706291-4.437027-0.177029$

O $2.247559-3.312024-0.902854$

C $1.340198-2.981616-1.969606$

C $0.486039-4.225932-2.176577$

C $0.308186-4.692733-0.744818$

H $0.696329-2.175229-1.600000$

C $2.106428-2.520444-3.212130$

H $-0.460986-4.003438-2.677272$

H $1.014711-4.986744-2.762702$

C $-0.150501-6.135275-0.623061$

H $-0.433759-4.046378-0.260573$

C $2.982542-1.266177-3.002864$

O $2.960842-3.583759-3.653203$

H $1.401082-2.345717-4.032869$

H $\quad 3.828132-1.518267-2.353597$

O $3.552757-0.926113-4.274139$

C $2.246841-0.033405-2.441125$

C $3.0567261 .275538-2.489648$

H $1.934318-0.220601-1.407441$

O $1.0618240 .214991-3.205755$

H $3.2160291 .576465-3.533415$

H $2.4163702 .056841-2.061446$

H -1.127216 -6.269507 -1.099312
H $\quad 0.554121-6.823045-1.102357$

H $-0.243751-6.4257140 .428176$

C $1.767831-4.1752711 .331231$

H $3.563789-3.797954-2.920590$

H $2.824260-0.656960-4.859795$

H $0.422340-0.488984-3.008516$

H $\quad 2.817745-4.0684701 .628494$

H $1.369115-5.0406011 .873140$

H $2.362873-5.286769-0.404587$

$\begin{array}{llll}\text { C } & 5.318064 & 0.774768 & 0.447671\end{array}$

$\begin{array}{lllll}\text { O } & 4.156729 & 0.876699 & -0.389240\end{array}$

C $4.4002351 .250281-1.747194$

C $5.1023332 .618486-1.814408$

C $6.3700962 .645193-0.955775$

C 6.1136282 .0916510 .449515

H $4.922617 \quad 0.625778 \quad 1.460661$

C $6.178635-0.4482750 .093205$

H $5.0390640 .498197-2.220228$

O $4.223558 \quad 3.655769-1.379685$

H $5.3556582 .833775-2.858875$

H $6.7339773 .676844-0.875304$

O $7.3910451 .872156-1.578781$

H $7.059767 \quad 1.9448920 .983515$

O $5.3552013 .047688 \quad 1.195915$

C $5.467343-1.7536840 .375599$

H $6.459220-0.454503-0.962942$

H $7.090850-0.4408120 .701038$

H $3.9361133 .442572-0.475536$

H $7.5570392 .256908-2.456263$

H 5.8795093 .8652861 .242775

O $6.264022-2.784848-0.034895$

$\begin{array}{llll}\text { O } & 4.364396 & -1.859651 & 0.892112\end{array}$

C $5.734698-4.0985990 .167040$

H $4.896724-4.269119-0.513995$

H $6.523296-4.820751-0.060327$

H $5.424826-4.2370021 .207279$

SCF Energy (B3LYP/6-31G**//MMFF)= -3245.92069203

0700534

MM̄FF Geometry

C $0.269260-4.055156-0.814976$

C $0.002590-3.403011-1.955620$

C $0.935543-2.509131-2.734177$

O $0.346686-1.180156-2.870822$

C $2.407602-2.353396-2.287150$

C $3.294308-3.571137-2.445819$

C $4.060438-4.092181-1.466431$

C $3.363588-4.158481-3.832464$

C $4.173300-3.656165-0.031032$

C $5.487764-2.921011 \quad 0.262945$

C $5.531320-1.489542-0.298357$

C $6.897613-0.855620-0.026665$

C $6.9490440 .608132-0.486357$

$\begin{array}{lllll}0 & 4.504645 & -0.732938 & 0.349799\end{array}$

$\begin{array}{lllll}\text { O } & 8.127302 & 1.207274 & 0.075766\end{array}$

C $5.7378301 .358578 \quad 0.109172$

C $7.0945210 .718941-2.008310$

O $5.6494542 .683184-0.409290$

C $4.3887320 .627860-0.088388$

$\begin{array}{llll}\text { C } & 3.244264 & 1.278019 & 0.719292\end{array}$

O $3.9546090 .695885-1.451827$

C $0.127753-0.419254-1.764451$

O $0.271741-0.749185-0.598455$

C $-0.2819180 .925566-2.227082$

C $-0.4170101 .912392-1.329491$

C $-0.7561283 .307420-1.615672$

C $-0.9018143 .712283-3.058062$

C $3.410744 \quad 1.218616 \quad 2.238141$

C $2.165561 \quad 1.7238562 .938635$

C 0.9958883 .8158283 .599096

$\begin{array}{lllll}\text { O } & 1.358068 & 0.952062 & 3.450966\end{array}$

C 0.5221675 .0334732 .862870

C 1.0172025 .5644361 .732973

C $0.423305 \quad 6.768979 \quad 1.056826$

C $0.0028296 .491353-0.371296$

C $0.6524307 .054199-1.404133$

C $-1.2172875 .625666-0.602584$

C $-0.8912864 .157970-0.577097$ 
N 2.0541913 .1059822 .905582 H $1.253574-3.981861-0.371231$ H $-0.992859-3.503512-2.384099$ H $0.935955-2.896141-3.760002$ H $2.436421-1.965731-1.265294$ H $2.856653-1.549719-2.888279$ H $4.689095-4.947575-1.712916$ H $4.159499-4.904849-3.929211$ H $2.420696-4.650202-4.090221$ H $3.564146-3.373259-4.568826$ H $3.320293-3.0554380 .295119$ H $4.139100-4.5640890 .584964$ H $5.600000-2.8758011 .354157$ H $6.333430-3.502501-0.125084$ H $5.334115-1.518010-1.375178$ H $7.697460-1.435652-0.503799$ H $7.107188-0.8872071 .051909$ H $8.8967150 .690483-0.218991$ H 5.9316101 .4589941 .184140 H $8.0341490 .258448-2.337302$ H $7.1472601 .765929-2.326013$ H $6.2841990 .230348-2.553597$ H $6.507936 \quad 3.113125-0.254018$ H 3.0967762 .3152200 .391228 H $2.307440 \quad 0.765066 \quad 0.462497$ H $4.5550911 .279728-1.941955$ H $-0.4290501 .074136-3.290520$ H $-0.2484631 .682929-0.277642$ H $\quad 0.0126633 .483247-3.616678$ H -1.085796 $4.780133-3.194182$ H $-1.7395563 .181586-3.523067$ H 3.5896760 .1915942 .576084 H 4.2632181 .8193022 .572043 H 1.3865804 .1051934 .580133 H $0.149936 \quad 3.139677 \quad 3.760311$ H $-0.344908 \quad 5.5147883 .312799$ H 1.8848655 .1221931 .251467 H 1.1737487 .5694361 .085638 H $-0.4423207 .151813 \quad 1.611983$ H $0.3406836 .880939-2.428732$ H $1.5125947 .697008-1.249466$ H -1.714150 $5.908293-1.536775$ H -1.964194 5.8327970 .174701 H -0.7691413 .7576380 .429123$ H 2.8664593 .6411982 .612287 C $-1.052642-4.4334801 .332569$ O $-1.697053-3.1442401 .309826$ C -1.319028 -2.428299 2.503819 C $-0.510215-3.404126 \quad 3.358337$ C $0.118525-4.2904212 .301829$ H $-0.656359-1.6153332 .185741$ C -2.551426-1.860255 3.217203 H $0.226942-2.8906763 .983556$ H -1.153642 -3.995693 4.020096 C $0.645240-5.6080182 .840557$ H $\quad 0.944755-3.7328391 .844928$ C -3.391666 -0.870894 2.382931 O $-3.404444-2.9524673 .586089$ H -2.237768 -1.386575 4.155636 H -3.908407 -1.412335 1.583516 O $-4.423058-0.3619563 .240254$ C $-2.602410 \quad 0.309936 \quad 1.792360$ C $-3.466246 \quad 1.4537051 .231089$ H -1.933620 -0.0622001 .009392$ O H -4.0085611 .9488042 .047004$ H -2.772318 2.2115530 .848985 H $1.421286-5.4331333 .592869$ H $-0.150019-6.1976053 .308973$ H $1.083424-6.2093812 .037881$ C $-0.726478-4.911693-0.085759$ H -4.177019 -2.575426 4.040972 H $-3.9925180 .164765 \quad 3.935485$ H -1.204234 1.5358872 .402635 H -1.659283 -4.957950 -0.660446 H $-0.330866-5.933231-0.043024$ H -1.791345 -5.1302991 .752238$ C $-4.489313-0.094808-2.013727$
O $\quad-3.713247 \quad 0.393543-0.910929$

C -4.4504111 .0487540 .123430$

C $-5.2052322 .267274-0.438003$

C $-6.0668611 .894170-1.649052$

C $-5.2902411 .047423-2.663018$

H $-3.748549-0.446427-2.743357$

C $-5.368565-1.293323-1.621986$

H $\quad-5.173380 \quad 0.347020 \quad 0.550744$

O $-4.2891063 .292153-0.820599$

H $-5.8362112 .686146 \quad 0.354364$

H $-6.4165742 .809324-2.142838$

O $\quad-7.213651 \quad 1.167942-1.218323$

H $-5.9757930 .641605-3.416148$

O $-4.3770811 .910051-3.347373$

C $-4.555569-2.493425-1.189838$

H $-6.041404-1.046704-0.796821$

H -5.970059-1.602575 -2.484543

H $-3.6896172 .918811-1.489023$

H $-7.7085541 .737173-0.604638$

H $-3.9258041 .381336-4.027209$

O $-5.407459-3.456621-0.727979$

O $-3.337956-2.581448-1.252528$

C $-4.789447-4.661798-0.267611$

H $-5.579842-5.3662200 .004699$

H $-4.182103-5.107855-1.060708$

H $-4.183235-4.4562060 .618821$

SCF Energy $(B 3 L Y P / 6-31 G * * / / M M F F)=-3245.90534195$

0700535

MM̄FF Geometry

C $1.3872074 .636799-0.322701$

C 0.8877974 .8189090 .909202

C -0.4896604 .4190421 .387283$

$\begin{array}{llll}\text { O } & -1.246047 & 3.829090 & 0.305404\end{array}$

C -0.3658243 .3737912 .515540$

C -1.6876153 .0177063 .169267$

C -2.2810931 .8086993 .110442$

C $-2.3289664 .121633 \quad 3.973502$

C -1.7834250 .5452702 .465708$

C $-2.541548 \quad 0.215801 \quad 1.174073$

C $-2.172306-1.1781240 .644120$

C $-2.795233-1.426575-0.729396$

C $-2.521993-2.851865-1.234094$

O $-2.662373-2.1401121 .581228$

O $-3.384365-3.091472-2.357293$

C $-2.923867-3.854943-0.131809$

C $-1.087566-3.017738-1.747378$

O $-2.551295-5.184992-0.483837$

C $-2.357828-3.5025361 .261465$

C $-2.937975-4.3914012 .391686$

O $-0.939204-3.7098671 .257616$

C $-2.5120354 .275198 \quad 0.090392$

O -3.0915395 .1747810 .677165$

C $-3.1009393 .442431-0.981616$

C $-4.4332643 .425575-1.135604$

C $-5.1860382 .636125-2.112625$

C $-4.4008251 .789022-3.078850$

C $-4.463820-4.4776692 .498355$

C $-5.127560-3.1256302 .682596$

C $-6.734165-1.5639491 .606177$

O $-4.868583-2.3973673 .638108$

C $-7.353373-1.4226770 .252793$

C $-6.978637-0.507416-0.654162$

C -7.585186 -0.407305 -2.025469

C $-8.2866890 .910228-2.264708$

C $-9.5761311 .075951-1.924993$

C -7.519456 $1.997515-2.982976$

C $-6.5341992 .696842-2.085518$

N $-6.009033-2.8188831 .658893$

H $\quad 0.7789434 .173077-1.093797$

H 1.5235025 .2865011 .659205

H -0.9752935 .3302891 .755011$

H 0.3080053 .7466483 .298468

H 0.1166932 .4795942 .107355

H -3.2359251 .6868653 .621652$

H -3.2229953 .7818934 .507328$

H -1.6260114 .4985004 .723881$

H -2.6295514 .9536673 .331616$ 
H $-0.709677 \quad 0.5818742 .260468$ H -1.916170 -0.2633943 .195290$ H $-3.621590 \quad 0.260013 \quad 1.365123$ H -2.3063600 .9720850 .417934$ H -1.080579 -1.252194 0.587520 H -2.439245 -0.689519-1.458556 H $-3.882674-1.284059-0.667238$ H -3.193638 -2.415831-3.030552 H -4.018677 -3.843758 -0.089102 H $-0.883439-2.303302-2.553879$ H $-0.936567-4.011943-2.182230$ H $-0.335675-2.864351-0.972010$ H -2.928537 -5.369111-1.361185 H -2.540543 -5.4099712 .291944$ H -2.553031 -4.0176303 .350922$ H $-0.642320-3.8140692 .176860$ H -2.428413 $2.844758-1.585866$ H $-5.0391904 .039600-0.469982$ H -3.773811 $1.072028-2.538869$ H -3.756936 $2.417025-3.704437$ H $-5.025778 \quad 1.201539-3.754207$ H -4.873471 -5.0056701 .631057$ H $-4.731738-5.0849943 .371731$ H $-6.043299-0.7471671 .841237$ H -7.508314 -1.585186 2.379877 H $-8.153503-2.1174900 .007606$ H $-6.1723430 .179347-0.410479$ H -8.292527 -1.230825 -2.192701 H -6.795529 -0.552120 -2.772905 H -10.097805 2.006806 -2.120882 H $-10.1364170 .284049-1.438770$ H -8.212237 $2.762159-3.359906$ H $-7.0565491 .580931-3.882062$ H $-7.0094303 .332516-1.336804$ H $-6.266095-3.5486211 .000212$ C $3.657273 \quad 3.767557-0.868406$ O 3.9599803 .2491250 .443566 C 5.3357862 .8059770 .466755

C $5.8122192 .857312-0.977315$

C $5.0118254 .024070-1.521928$

H 5.8779583 .5426931 .072862

C 5.3893791 .4228101 .124892

H $6.8934263 .003109-1.058378$

H $5.5501931 .933390-1.508556$

C $4.9693344 .071661-3.039287$

H $5.4436194 .959181-1.140922$

C $6.743899 \quad 0.7031310 .996544$

O 5.0664451 .5823542 .511161

H $4.5893490 .806637 \quad 0.701961$

H $\quad 6.9812190 .530760-0.059271$

O 7.7641331 .5775451 .495127

C $6.835852-0.6256191 .771560$

C $5.689459-1.6154131 .517061$

H $6.883774-0.4116652 .846426$

O $8.084881-1.2379881 .443186$

H $5.887333-2.5472952 .062595$

H $4.774867-1.2153471 .974785$

H $5.9790254 .181019-3.448664$

H $4.5343813 .157680-3.457520$

H $4.3706254 .919048-3.387417$

C $2.7854965 .015033-0.728096$

H 5.7848162 .0925592 .922969

H 8.6132171 .1113901 .406234

H $8.154922-2.0580851 .961154$

H $2.7265325 .555747-1.679259$

H 3.2226575 .6979530 .010767

H $3.1194562 .977332-1.409092$

C $3.556339-2.912372-1.230595$

O $4.118683-2.6268330 .055376$

C $5.384914-1.9515350 .043370$

C $6.435472-2.826865-0.655119$

C $5.966987-3.248199-2.054513$

C $4.526955-3.777991-2.050901$

H $2.661272-3.512176-1.019702$

C $3.101619-1.640617-1.965288$

H $5.268285-1.016560-0.509005$

O $6.684060-3.9888710 .132821$

H $7.375326-2.271073-0.738868$
H $\quad 6.639001-4.025463-2.438501$

O $6.069077-2.121487-2.920956$

H $4.154590-3.871227-3.077162$

O $4.527332-5.097861-1.497996$

C $2.054044-0.863589-1.195138$

H $3.932475-0.955607-2.152024$

H $2.662149-1.925320-2.928565$

H $7.378014-4.503328-0.313637$

H $5.800401-2.409727-3.809940$

H $4.790777-5.028820-0.564199$

O $1.6308510 .183873-1.963760$

O $1.661660-1.126888-0.067223$

C $0.636321 \quad 1.021700-1.367941$

H $-0.2944370 .464079-1.243721$

H $0.9852801 .410390-0.406587$

H $0.4521151 .861561-2.042575$

SCF Energy (B3LYP/6-31G**//MMFF) $=-3245.90311969$

\section{6}

MM̄FF Geometry

C $-4.696993-1.890494-2.192388$

C $-3.493128-2.163689-1.666299$

C $-2.247957-2.303551-2.503038$

O $-1.177181-1.531442-1.912950$

C $-1.802620-3.777394-2.530510$

C $-0.706423-4.052066-3.542984$

C $0.591274-4.266117-3.248028$

C $-1.167376-4.123625-4.977864$

C $1.259046-4.252087-1.900140$

C $2.341604-3.172189-1.841820$

C $3.166255-3.163731-0.544923$

C $2.308965-3.010572 \quad 0.712319$

C $3.163883-2.8676421 .980140$

O $4.070063-2.055969-0.654764$

O $2.308671-2.4123943 .039395$

C $4.224125-1.7727091 .753465$

C $3.737421-4.2153902 .433949$

O $5.133769-1.7101662 .850133$

C $5.002098-1.926347 \quad 0.427433$

C $5.843474-0.6520910 .172538$

O $5.871515-3.055450 \quad 0.512774$

C $-1.189447-0.183427-2.100101$

O $-2.0664680 .483066-2.624526$

C $0.094251 \quad 0.338832-1.584430$

C $0.3621631 .652262-1.608996$

C $1.6255002 .258198-1.186668$

C $2.823926 \quad 1.352625-1.065171$

C $6.590003-0.650551-1.167784$

C $6.8065310 .748950-1.712774$

C $7.8844792 .933627-1.206459$

O $6.387511 \quad 1.074486-2.822325$

C $6.8026813 .864942-0.754494$

C $6.0560904 .591988-1.600053$

C $5.0151835 .588158-1.173480$

C $3.6144475 .155944-1.553660$

C $3.1066315 .494926-2.751530$

C $2.8651624 .381534-0.487871$

C $1.6796643 .588561-0.969871$

N $7.5306351 .569507-0.863737$

H $-4.791349-1.766345-3.268651$

H $-3.393448-2.309144-0.593696$

H $-2.421720-1.942711-3.524424$

H $-2.656764-4.422571-2.778595$

H $-1.489627-4.084662-1.526865$

H $1.270292-4.480160-4.073256$

H $-0.356972-4.394186-5.663229$

H $-1.953071-4.878488-5.086903$

H $-1.564052-3.158142-5.305806$

H $0.535049-4.091041-1.099486$

H $1.710707-5.236303-1.728635$

H $3.036428-3.294948-2.684406$

H $1.889986-2.185441-1.994681$

H $3.745832-4.093528-0.504161$

H $1.623295-3.8567210 .828814$

H $1.674244-2.1198940 .612996$

H $1.597388-3.0659253 .153087$

H $3.701226-0.8073001 .733772$

H $2.928004-4.9255202 .642060$ 
H $4.293120-4.1126243 .372553$

H $4.394504-4.6726911 .691263$

H $4.605259-1.5998173 .659235$

H $\begin{array}{lllll}5.182387 & 0.221607 & 0.208153\end{array}$

H $6.582143-0.5296120 .974545$

H $6.585025-2.952570-0.136837$

H $0.806809-0.382490-1.203846$

H $-0.4024792 .342752-1.960557$

H $2.7453590 .727261-0.171221$

H 3.769008 1.898466-1.009599

H $2.9114780 .700394-1.941861$

H $7.566557-1.137991-1.066889$

H $6.032178-1.206029-1.930052$

H $8.0586432 .999745-2.285800$

H $8.8205723 .172819-0.692427$

H 6.6526203 .9686300 .317195

H $6.2200224 .490468-2.670830$

H $5.2529776 .550417-1.645831$

H $5.0633265 .777614-0.093630$

H $2.1013395 .226013-3.054344$

H $3.6937826 .062992-3.466583$

H 2.5121195 .0934020 .269894

H 3.5628913 .7255390 .044279

H $0.7728514 .178708-1.104665$

H $7.809046 \quad 1.2238980 .049542$

C $-6.687671-0.454897-1.644227$

O $\quad-5.9102290 .639554-1.113434$

C $-6.7175881 .354813-0.164845$

C $-8.1550431 .060964-0.566999$

C -8.049799 $-0.403407-0.941257$

H $-6.525537 \quad 0.8996130 .814828$

C $-6.3482202 .840109-0.113381$

H $-8.864299 \quad 1.2475200 .244782$

H $-8.4632021 .650510-1.438153$

C $-9.200614-0.898244-1.800750$

H $-8.019946-0.998023-0.018087$

C -4.8454313 .1370170 .067665$

O $-6.7618793 .465892-1.334587$

H -6.9292083 .3199710 .683861$

H $-4.3274493 .009170-0.890625$

O $\quad \begin{array}{llll}-4.722038 & 4.528936 & 0.390122\end{array}$

C $-4.1266552 .298778 \quad 1.137104$

C -2.7091572 .8170291 .444238$

H $-4.082772 \quad 1.2576920 .797676$

O

H $-2.258036 \quad 3.2604750 .548238$

H -2.782992 3.6018352 .206720

H -10.150974 -0.797635-1.266223

H -9.277962 -0.330065 -2.733721

H $-9.067246-1.953606-2.058767$

C $-5.959168-1.777367-1.387020$

H -6.533553 $4.409137-1.269000$

H -5.1054864 .6595931 .274563$

H -4.4373341 .7710702 .993300$

H -6.609643 -2.616043-1.661170

H $-5.730408-1.871386-0.317956$

H -6.795997 -0.273480 -2.720889

C $-1.703796-0.2195643 .395659$

O -2.4193530 .9514922 .969544$

C -1.7710661 .6979771 .935709$

C -0.4302302 .2421092 .450260$

C 0.4565881 .1165812 .991977

C $-0.311360 \quad 0.169027 \quad 3.924142$

H -2.290155 -0.6063174 .238934$

C -1.709102 -1.285542 2.287425

H -1.5949101 .0376351 .079278$

O -0.6493313 .2019353 .482969$

H 0.0832642 .7608771 .633764

H 1.3028841 .5588583 .531757

O $0.982268 \quad 0.387250 \quad 1.889726$

H $0.273645-0.734661 \quad 4.123947$

O $\quad-0.506393 \quad 0.824608 \quad 5.181894$

C -1.396837 -2.6785262 .784439$

H -2.705233 -1.316333 1.828726

H $-0.995760-1.0415451 .495508$

H -1.170514 2.771966 4.182573

H $\quad 1.534537-0.3267192 .250054$

H $\quad 0.3723641 .0214565 .548770$
O $-1.296163-3.515001 \quad 1.708187$

O $-1.268110-3.000726 \quad 3.956981$

C -1.010626 -4.883196 2.014291

H -1.838367 -5.322646 2.578706

H $-0.899710-5.4256051 .071532$

H $-0.074619-4.9622812 .575655$

SCF Energy (B3LYP/6-31G**//MMFF) $=-3245.92111506$

$07 \quad 00537$

MM̄FF Geometry

C $1.689372-0.8057472 .376294$

C $0.409571-0.4448232 .205852$

C $-0.404376-0.8467451 .005446$

O $-1.669724-1.3274441 .518638$

C $-0.695007 \quad 0.3381180 .066133$

C $0.5271421 .053966-0.461069$

C $0.9470352 .257672-0.024872$

C $1.2686770 .340169-1.559224$

C 0.3612003 .0879521 .084339

C -0.1307654 .4609150 .615005$

C $-1.3354084 .419013-0.341381$

C $-1.6924875 .847514-0.767241$

C $-2.9528675 .894236-1.638119$

O $-2.4436643 .809466 \quad 0.334325$

O $-3.3702347 .263552-1.747719$

C $-4.0728305 .103285-0.933195$

C $-2.6626885 .444869-3.074126$

O $-5.2217585 .006668-1.773663$

C $-3.6355533 .693688-0.462176$

C $-4.756013 \quad 3.087257 \quad 0.425867$

O $-3.4228652 .870160-1.606248$

C $-2.359044-2.2296150 .772866$

O $-1.999279-2.757001-0.266648$

C $-3.665789-2.4338411 .438362$

C $-4.563203-3.2742750 .903513$

C $-5.913592-3.5301721 .410654$

C $-6.388455-2.7010692 .575531$

C -4.5345351 .6400770 .883205$

C $-5.0302820 .635062-0.141648$

C $-7.136005-0.249283-1.148069$

O $-4.2573550 .051405-0.897712$

C $-8.162383-1.115689-0.489127$

C $-8.474059-2.344259-0.930687$

C $-9.507141-3.226154-0.288418$

C $-9.014092-4.639116-0.059652$

C $-9.449327-5.654546-0.824856$

C $-8.075633-4.8872791 .101942$

C $-6.665067-4.4650990 .792530$

N $-6.4092430 .494758-0.134256$

H $2.172504-1.4182151 .619878$

H -0.0714890 .1854192 .950040$

H $0.100741-1.6542160 .459942$

H $-1.373350 \quad 1.0354970 .565825$

H -1.286146 $-0.018023-0.788514$

H $1.8324242 .689765-0.489115$

H $2.1307950 .910855-1.920214$

H $1.639946-0.624929-1.202344$

H $0.6067660 .164675-2.413427$

H $-0.4384892 .573078 \quad 1.622701$

H $1.1559663 .247241 \quad 1.824258$

H -0.4088105 .0296641 .512123$

H 0.6986054 .9998570 .139176

H -1.074747 $3.816222-1.218329$

H $-0.850596 \quad 6.321581-1.287382$

H -1.8679956 .4571810 .130391$

H $-3.5448157 .601548-0.852930$

H $-4.3830345 .678153-0.049520$

H -1.921970 $6.107497-3.538055$

H -3.559195 $5.515229-3.700133$

H $-2.2773614 .425175-3.137311$

H $-5.4629895 .910157-2.040633$

H -4.8411173 .7038231 .330294$

H $-5.7155863 .147604-0.101079$

H $-3.2462301 .965956-1.301783$

H -3.865354 -1.861230 2.337248

H -4.291631 -3.8187420 .000097$

H -7.432727 -2.874988 2.841274

H $-6.304889-1.6321592 .348234$ 
H $-5.791219-2.917548 \quad 3.468159$ H -3.4860571 .4394361 .114630$ H -5.0909551 .4652341 .813019$ H -6.440286 -0.827261 -1.764563 H $-7.6319450 .492103-1.782875$ H $-8.683578-0.7164550 .377835$ H -7.976332 -2.729446 -1.818179 H $-10.386679-3.238174-0.945253$ H $-9.850750-2.8081580 .665962$ H $-9.119711-6.673810-0.652575$ H - $-10.140804-5.489155-1.644315$ H -8.048878 -5.9587651 .341657$ H -8.484717 -4.4090881 .995482$ H -6.223956 -5.011391-0.042888 H $-6.952828 \quad 1.0956130 .478724$ C 3.4228100 .7966853 .205397 O 4.2584950 .5005232 .073756 C 5.4711401 .2732092 .182445 C 5.4023222 .0112333 .519995 C 4.4033321 .1825314 .306754 H $6.299746 \quad 0.557778 \quad 2.210971$ C 5.6103482 .1918120 .959802 H 6.3822432 .0671784 .004981 H 5.0419843 .0395823 .402221 C 3.7766441 .9323135 .468657 H 4.9143710 .2867054 .684376 C $5.5263421 .458349-0.394783$ O $4.556957 \quad 3.1655780 .982447$ H 6.5445832 .7605591 .025295 H $4.5324181 .013949-0.515303$ O $5.6534922 .442350-1.430817$ C $6.6181150 .391612-0.610727$ C $6.567629-0.265313-2.004771$ H $6.546768-0.3858970 .155722$ O $7.9022561 .002236-0.457135$ H $6.4684790 .509260-2.774380$ H $7.534852-0.752034-2.189929$ H 4.5461492 .2567936 .176739 H 3.2365422 .8218405 .127343 H 3.0692911 .2958626 .009096 C $2.534967-0.3982263 .548087$ H 3.7128242 .6834140 .971043 H $4.9599903 .108383-1.283853$ H $7.9722741 .707915-1.122913$ H $1.902302-0.1602794 .410962$ H $3.147611-1.2609713 .832123$ H 2.7958261 .6552542 .930892 C $4.957545-3.580728-1.427039$ O $5.877664-2.481011-1.446001$ C $5.439087-1.301796-2.133041$ C $5.120363-1.623186-3.599475$ C $4.105291-2.765443-3.704281$ C $4.513362-3.966609-2.849494$ H $5.540557-4.420018-1.025698$ C $3.792431-3.318591-0.463780$ H $4.536429-0.916283-1.650807$ O $6.310291-1.966925-4.305544$ H $4.714503-0.723771-4.077182$ H $4.018570-3.082163-4.750979$ O $2.825323-2.313070-3.272724$ H $3.691740-4.690852-2.801717$ O $5.605442-4.619158-3.504923$ C $4.220263-3.5879880 .960762$ H $3.394816-2.301935-0.511805$ H $2.959064-3.999781-0.673045$ H $6.718196-2.723855-3.851201$ H $2.568267-1.569661-3.844411$ H $5.810263-5.426011-3.002331$ O $5.059369-2.6056231 .395392$ O $3.862222-4.5674821 .602476$ C $5.598437-2.7910272 .707943$ H $6.311749-3.6203312 .700259$ H $6.125361-1.8769582 .993004$ H $4.802415-2.9770833 .434820$ SCF Energy (B3LYP/6-31G**//MMFF) $=-3245.91681708$

0700538

MM̄FF Geometry
C $-0.237081-1.9882011 .439343$

C $-0.124458-2.3930080 .164609$

C $-1.218948-2.390806-0.879878$

O $-2.503116-2.234993-0.228343$

C $-1.063155-1.252024-1.906470$

C $0.295502-1.135497-2.563094$

C $1.182568-0.165655-2.265073$

C $0.599014-2.156720-3.624272$

C $1.0216010 .868118-1.187247$

C $0.4695792 .190463-1.728621$

C $-0.0745613 .122777-0.633530$

C 1.0036073 .5734970 .352030

C 0.4220234 .4367121 .481186

$\begin{array}{llll}\text { O } & -1.114751 & 2.420320 & 0.054350\end{array}$

O 1.4445254 .5414382 .484136

C -0.7857163 .6977992 .098569$

C 0.1258395 .8644531 .008635

O -1.4546344 .5177593 .054739$

C -1.8038273 .1847051 .049378$

C $-2.8326122 .249217 \quad 1.739828$

O $-2.477167 \quad 4.3032590 .474614$

C $-3.612152-2.669398-0.882100$

O $-3.659435-3.226418-1.966373$

C $-4.778557-2.317668-0.041222$

C $-6.020237-2.469127-0.524516$

C $-7.265359-2.1415550 .175107$

C -7.154917 -1.542542 1.553196

C $-3.758826 \quad 1.4659620 .796059$

C -4.9771652 .2360090 .317704$

C $-6.8881542 .045723-1.274759$

O $\quad \begin{array}{llll}-5.292849 & 3.337060 & 0.759886\end{array}$

C $-8.0812251 .499342-0.555341$

C $-9.0859880 .856643-1.170011$

C $-10.3064350 .342823-0.460927$

C $-10.582652-1.116213-0.748808$

C $-11.522224-1.479085-1.638374$

C $-9.832961-2.1521980 .058878$

C $-8.436971-2.380286-0.450794$

N $-5.6789411 .536537-0.655157$

H -1.189768 -1.578951 1.770278

H $\quad 0.831099-2.769108-0.185964$

H -1.194815 -3.366867 -1.381325

H -1.357608 -0.306119-1.434634

H -1.815187 -1.376997 -2.697515

H $2.122945-0.125329-2.810791$

H $1.582464-2.000686-4.080278$

H $0.590392-3.164630-3.197433$

H $-0.148574-2.109576-4.422693$

H $0.393106 \quad 0.483914-0.378816$

H $1.9996421 .033811-0.729018$

H $1.2447812 .706875-2.308095$

H $-0.3560261 .972776-2.419455$

H $-0.5115123 .992442-1.138357$

H $1.8078684 .108104-0.168114$

H 1.4758472 .6968910 .814561

H 1.1198195 .1295443 .186618

H -0.3914962 .8326902 .650309$

H $1.047356 \quad 6.350716 \quad 0.666748$

H $-0.252721 \quad 6.4831331 .829906$

H $-0.5945355 .904800 \quad 0.189075$

H $-0.8192644 .728657 \quad 3.759032$

H -2.272244 1.4943812 .307888

H -3.4342932 .8181292 .458599$

H -3.2948083 .9943850 .055794$

H $-4.581228-1.9262920 .950321$

H $-6.140366-2.865360-1.532059$

H -6.702187 -2.259229 2.247109

H -8.114803 -1.245358 1.979941

H $-6.538471-0.6370401 .533218$

H $-3.2079231 .081568-0.068679$

H $-4.1453160 .599499 \quad 1.347560$

H $-6.8653051 .744739-2.326916$

H $-6.8942923 .139108-1.223558$

H $-8.132267 \quad 1.6624730 .518642$

H $-9.0470530 .719560-2.248335$

H $-11.1589750 .954435-0.784178$

H -10.2317180 .4903020 .623746$

H - $-11.746838-2.523156-1.829350$ 
H -12.083909 $-0.739862-2.199874$

H -10.356107 -3.1167910 .008082$

H -9.865758 -1.8758161 .116055$

H -8.409041 -2.814460 -1.451509

H $-5.3780760 .599414-0.906415$

C $2.002694-2.9805552 .423603$

O $2.917510-2.6236091 .372422$

C $3.419149-3.8358490 .776581$

C $2.974460-4.9809971 .685078$

C $1.658983-4.4560982 .225967$

H $2.911794-3.938792-0.189446$

C $4.933585-3.7625150 .550318$

H $2.869506-5.9239701 .139645$

H $3.679666-5.1474222 .507394$

C $1.193943-5.1571353 .490738$

H $0.895984-4.5885321 .451527$

C $5.439570-2.521845-0.212887$

O $5.606346-3.774386 \quad 1.817447$

H $5.261984-4.6727680 .034445$

H $5.435710-1.6523840 .453882$

O $6.813648-2.761451-0.547983$

C $4.675488-2.176707-1.501433$

C $5.370340-1.142897-2.410208$

H $3.676490-1.814053-1.235304$

O $4.496362-3.369679-2.270425$

H $6.247091-1.602174-2.885345$

H $4.674619-0.925724-3.231836$

H $1.039298-6.2248973 .303672$

H $1.928178-5.0606784 .297484$

H $0.247490-4.7353963 .843290$

C $0.823830-2.0018192 .510670$

H $5.273886-3.0194442 .332510$

H $7.284224-2.9564030 .280689$

H $3.949433-3.141742-3.041574$

H $1.216627-0.9797962 .583115$

H $0.305733-2.2032643 .457444$

H $2.578635-2.8694193 .352862$

C $4.8379371 .977199-0.417007$

O $4.619400 \quad 0.765502-1.154261$

C $5.7855600 .166258-1.721350$

C $6.4624681 .136983-2.702250$

C $6.7662792 .481460-2.032447$

C $5.5507203 .031925-1.281666$

H $3.8346372 .359582-0.197035$

C $5.532358 \quad 1.7115190 .927507$

H $6.494125-0.074377-0.923448$

O $5.6339451 .352738-3.843384$

H $7.3925770 .683790-3.064117$

H $7.0750353 .207343-2.794938$

O $7.8431932 .327561-1.112789$

H $5.8465363 .886288-0.661465$

O $4.6211343 .521746-2.253554$

C 4.6624850 .9011061 .867343

H $\quad 6.4706341 .1660560 .798909$

H 5.7429762 .6657821 .423952

H $4.7773401 .689946-3.529587$

H $8.6138622 .010160-1.613676$

H $3.8826033 .933339-1.773216$

O 5.3658970 .6463643 .010506

O 3.5169920 .5390501 .637703

C $4.671633-0.1185154 .000819$

H $3.744250 \quad 0.3840814 .292109$

H $4.464575-1.1227423 .621399$

H $\quad 5.316067-0.2016374 .879955$

SCF Energy (B3LYP/6-31G $\left.{ }^{\star *} / / M M F F\right)=-3245.90850098$

\section{9}

MM̄FF Geometry

C -1.671424 $3.239127-1.826780$

C $-2.2180824 .316251-1.241802$

C $-3.2399044 .313025-0.127888$

O -3.8265143 .0085290 .074720$

C -2.5510764 .7309561 .182890$

C -3.4904414 .7690192 .371411$

C -3.5396683 .8160123 .323776$

C -4.3636345 .9915722 .466943$

C -2.7491292 .5346243 .352525$

C -3.5638341 .3790973 .951502$
C $-2.915204-0.0075803 .794587$

C $-1.479343-0.0701884 .323329$

C $-0.835655-1.4472124 .096319$

O $-2.952682-0.3633042 .406534$

O $0.572160-1.2862014 .331642$

C $-1.032540-1.8461962 .616767$

C $-1.330891-2.4794235 .115213$

O $-0.582957-3.1774842 .377962$

C $-2.492514-1.6956082 .134215$

C $-2.640302-1.9527100 .618961$

O $-3.309304-2.6684802 .798300$

C $-4.8612732 .657770-0.734617$

O $-5.2999593 .285017-1.685395$

C $-5.3797261 .357977-0.257433$

C $-6.0638030 .579116-1.107663$

C $-6.640502-0.731125-0.805907$

C $-6.755722-1.1292080 .642158$

C $-1.845179-1.006779-0.280144$

C $-2.285233-1.153385-1.722393$

C $-2.370433-2.807087-3.577839$

O $-2.954537-0.289506-2.284261$

C $-3.536429-3.713493-3.331896$

C $-4.814652-3.350638-3.518016$

C $-5.985261-4.249023-3.237689$

C $-6.617414-3.983394-1.887867$

C $-6.321516-4.758756-0.830704$

C -7.648236 -2.881562 -1.775880

C $-7.043132-1.507057-1.833866$

N $-1.879752-2.351108-2.291098$

H -1.964953 2.242464 -1.511743

H -1.892703 $5.303130-1.566223$

H $-4.0333715 .030721-0.372488$

H -2.0941195 .7232581 .065976$

H -1.7119914 .0549361 .389726$

H $-4.2133863 .966348 \quad 4.165920$

H -4.9886415 .9864043 .366277$

H -3.7484686 .8966692 .497899$

H -5.0317396 .0536631 .602366$

H -2.417386 2.2512212 .349297

H -1.8571192 .7067273 .965285$

H -3.7318161 .5760555 .018037$

H -4.5498491 .3529903 .468662$

H -3.548545 -0.7120354 .347192$

H $-1.4438200 .203774 \quad 5.384975$

H -0.8580260 .6688863 .800721$

H $0.995172-2.156704 \quad 4.241817$

H -0.390101 -1.185927 2.020259

H -1.058470 -2.171055 6.131799

H $-0.851898-3.4523254 .958415$

H -2.413206 -2.621993 5.092525

H $0.366099-3.2104472 .584832$

H -2.381454 -2.995621 0.394725

H $-3.700687-1.863170 \quad 0.350090$

H $-4.115673-2.8047532 .273699$

H $-5.168041 \quad 1.0734290 .766593$

H $-6.1918510 .919352-2.134829$

H -5.771497 -1.181115 1.116737

H $-7.223798-2.1052230 .787011$

H $-7.365209-0.4010741 .189079$

H -1.9924170 .0385970 .012483$

H $-0.774315-1.218494-0.215570$

H -1.563621 -3.354639-4.073902

H -2.641814 -1.947464 -4.199294

H $-3.313188-4.712007-2.963399$

H $-5.032781-2.350020-3.881770$

H $-5.670025-5.299286-3.303085$

H $-6.732743-4.126520-4.030932$

H $-6.786601-4.5996650 .136342$

H $-5.602364-5.567659-0.907764$

H $-8.252265-3.000185-0.868955$

H $-8.369653-2.980522-2.597288$

H -6.922152 -1.127153 -2.848868

H -1.364910 -3.017175 -1.722642

C $0.7238622 .721204-2.507401$

O $0.612768 \quad 1.295170-2.338434$

C $1.4393360 .898613-1.221754$

C $2.2865352 .114312-0.868955$

C $1.3498933 .252849-1.218623$ 
H $\quad 0.7447550 .672946-0.403865$

C $2.242177-0.352162-1.603057$

H 2.5954142 .1212980 .180721

H $3.1886102 .153912-1.490346$

C $2.0430334 .594000-1.376398$

H $0.6023073 .329949-0.419649$

C $3.212348-0.809037-0.495553$

O $1.315665-1.400254-1.890862$

H $2.802838-0.168194-2.527485$

H $3.909520 \quad 0.012582-0.308277$

O $2.488536-1.0204970 .717587$

C $4.024979-2.076359-0.833750$

C $5.027979-2.4839020 .263856$

H $4.560663-1.926532-1.778340$

O $3.157390-3.197844-1.014701$

H $4.490112-2.7218861 .190446$

H $5.492005-3.425697-0.059264$

H $2.5411924 .881924-0.444870$

H $2.7996364 .565049-2.167019$

H $1.3208295 .376575-1.629670$

C $-0.6279303 .320931-2.906523$

H $0.713495-1.074758-2.582081$

H $1.860473-1.7457850 .556861$

H $2.574795-3.000317-1.767264$

H -1.016873 $2.779447-3.777814$

H $-0.4801604 .362400-3.216425$

H $1.4176262 .867775-3.346133$

C $7.851639-0.212823-0.633042$

O $6.829351-1.216278-0.678503$

C $6.120390-1.4398820 .543084$

C $7.086958-1.8888011 .648793$

C $8.223813-0.8746531 .820439$

C $8.871663-0.5142840 .479178$

H $8.374418-0.301870-1.594601$

C $7.2567891 .202736-0.555933$

H $5.638677-0.5116210 .862158$

O $7.627713-3.167471 \quad 1.325320$

H $6.527428-1.9940342 .586015$

H $8.987463-1.2894222 .489435$

O 7.7204090 .3150672 .421442

H 9.5461940 .3421130 .596164

O $9.670273-1.6164200 .037599$

C $6.5360071 .584377-1.833853$

H 6.5421821 .3148480 .263067

H $8.0726931 .921199-0.413021$

H $8.216256-3.4304042 .053095$

H 7.3373690 .0704593 .281098

H $\quad 10.349127-1.7766590 .715127$

O $6.3428132 .936670-1.848385$

O $6.175054 \quad 0.801661-2.702055$

C $5.6999063 .455969-3.017227$

H $6.3606013 .349513-3.882666$

H $4.7479252 .947599-3.195861$

H $5.5032954 .518710-2.852875$

SCF Energy (B3LYP/6-31G**//MMFF)= -3245.89905991

$07 \quad 00540$

MM̄MF Geometry

C -3.977046 -2.117884 -3.047587

C $-2.809063-2.306101-2.417033$

C $-1.489964-1.811422-2.941038$

O $-1.096581-0.705387-2.092800$

C $-0.415239-2.906046-2.848741$

C $0.928057-2.439209-3.375709$

C $1.963579-2.056896-2.601994$

C $1.066246-2.441659-4.876257$

C $2.007417-1.983148-1.099541$

C $2.986326-3.000565-0.494904$

C $4.450477-2.546333-0.595661$

C $5.405121-3.678458-0.215551$

C $6.869037-3.210944-0.227201$

O $4.622640-1.4473720 .306946$

O $7.658351-4.2163580 .426746$

C $6.987178-1.9239600 .615199$

C $7.421429-3.084899-1.651869$

O $8.294463-1.3632580 .516143$

C $5.924279-0.8511320 .273092$

C $5.9782440 .287346 \quad 1.326643$
O $6.215983-0.304703-1.015571$

C -1.407607 $0.547240-2.525299$

O $-1.9429120 .849948-3.580240$

C $-0.9959281 .506419-1.476345$

C $-1.0224452 .820351-1.746599$

C $-0.6740643 .906244-0.828258$

C -0.3831833 .5417110 .602661$

C 4.6910421 .1040671 .492149

C 4.2386401 .8579360 .261061

C $4.8333113 .769661-1.211146$

O $3.1677921 .605855-0.287500$

C $4.1892754 .986777-0.623392$

C $2.8610185 .173299-0.590165$

C 2.2013476 .3671370 .037360

C $1.0889946 .941657-0.811496$

C $1.3289217 .930160-1.689158$

C $-0.3160276 .437127-0.561797$

C $-0.6224675 .167067-1.308010$

N $5.1313112 .840958-0.136879$

H -3.993586 -1.616752 -4.012036

H $-2.807902-2.773963-1.437093$

H -1.583257 -1.484272 -3.984061

H $-0.730699-3.788644-3.421253$

H $-0.321686-3.254389-1.814004$

H $2.879581-1.739550-3.097913$

H $2.068627-2.144354-5.202303$

H $0.876275-3.444366-5.272863$

H $\quad 0.353436-1.746573-5.330405$

H $2.285436-0.963969-0.810297$

H $1.019358-2.152511-0.666506$

H $2.727258-3.1239260 .564940$

H $2.852435-3.975389-0.979654$

H $4.654287-2.211983-1.618469$

H $5.273343-4.540274-0.881536$

H $5.162291-4.038460 \quad 0.794019$

H $7.535111-5.053189-0.053334$

H $6.855257-2.2161451 .666610$

H $7.361723-4.048530-2.172062$

H $8.483552-2.816355-1.640939$

H $\quad 6.886281-2.350020-2.256695$

H $8.928127-2.0629150 .750452$

H $6.175260-0.1507952 .313973$

H 6.8187980 .9563611 .105110

H $5.8977830 .610524-1.053347$

H $-0.6897801 .107596-0.516561$

H $-1.3249903 .139014-2.743560$

H -1.241348 3.0300161 .049974

H -0.1645774 .4027541 .237533$

H 0.4892462 .8819930 .660309

H 3.8693920 .4557361 .818724

H 4.8317111 .8381532 .295652

H $4.1872663 .283638-1.950125$

H $5.7748394 .037711-1.699745$

H $4.8493745 .738061-0.197470$

H $2.2209734 .396336-0.997677$

H $2.945144 \quad 7.147770 \quad 0.245209$

H 1.7998886 .0764621 .015669

H $\quad 0.5299878 .370524-2.276348$

H $2.3314348 .315885-1.842861$

H $-1.046327 \quad 7.189692-0.889926$

H $-0.492766 \quad 6.3448820 .514291$

H -0.832162 $5.328510-2.366323$

H 5.9391873 .0393070 .446082

C $-5.980217-1.234842-1.879303$

O $-5.249025-0.770578-0.724776$

C $-6.175601-0.1417800 .183916$

C $-7.554120-0.241301-0.467437$

C $-7.397866-1.454207-1.366415$

H $-6.177979-0.7367591 .102928$

C $-5.720084 \quad 1.2929740 .475450$

H $\quad-8.350872 \quad-0.3581440 .273969$

H -7.785688 $0.651367-1.060591$

C $-8.452225-1.538229-2.455543$

H $-7.438368-2.360529-0.747558$

C -4.3428261 .3941461 .166896$

O $\quad-5.643210 \quad 2.012442-0.763545$

H $-6.476706 \quad 1.8101211 .076349$

H -3.5645620 .9692850 .523702$ 
O $-\begin{array}{llll}-4.036510 & 2.788151 & 1.309803\end{array}$

C -4.288897 0.7483112 .567746

C $-2.971277 \quad 1.002594 \quad 3.328557$

H $-4.461525-0.3295202 .493345$

O $\begin{array}{lllll}-5.353841 & 1.278176 & 3.364194\end{array}$

H -2.7116572 .0664613 .274923$

H -3.149518 $0.791748 \quad 4.392105$

H $-9.453250-1.604112-2.016892$

H $-8.427579-0.658067-3.106826$

H -8.299972 -2.423761-3.080333

C $-5.306386-2.482952-2.448012$

H $-4.9894491 .560527-1.324534$

H -4.056862 3.1814410 .420390

H -5.2235612 .2403353 .424349$

H -5.923904 -2.938951-3.229305

H $-5.171596-3.232023-1.659797$

H $-5.964290-0.423895-2.620865$

C $-1.056493-2.1766632 .946080$

O $-2.035976-1.1936183 .305864$

C -1.8021240 .1379402 .829521$

$\begin{array}{llll}\text { C } & -0.451070 & 0.658355 & 3.342471\end{array}$

C $0.682951-0.2928532 .952683$

C $0.363169-1.7370193 .342302$

H $-1.307773-3.0529553 .558644$

C -1.189265 -2.580800 1.473074

H $-1.796457 \quad 0.1375421 .735536$

$\begin{array}{lllll}\text { O } & -0.498977 & 0.803347 & 4.759970\end{array}$

H -0.2701601 .6521012 .918628$

H 1.6112360 .0175713 .447551

O $0.896020-0.2339371 .546757$

H $1.094391-2.4248962 .901582$

O $0.471882-1.8718044 .762685$

C $-2.320850-3.5669301 .301088$

H $-1.359353-1.7418580 .793283$

H -0.281429 -3.0930111 .134093$

H $0.362770 \quad 1.1483475 .049338$

H 1.1655750 .6741331 .327400

H $1.384303-1.6437165 .009718$

O $-3.522525-2.9341641 .418973$

O $-2.151778-4.762875 \quad 1.099155$

C $-4.671490-3.783214 \quad 1.340340$

H -5.563187-3.151892 1.322954

H $-4.649257-4.3829580 .425655$

H $-4.715866-4.4295962 .221849$

SCF Energy (B3LYP/6-31G**//MMFF) $=-3245.90374317$

07_00541

MM̄FF Geometry

C $-0.260396 \quad 4.932569-0.395824$

C $-1.133794 \quad 4.5848310 .560910$

C $-2.367262 \quad 3.764640 \quad 0.275573$

O -2.2828692 .5362791 .042546$

C -3.636659 4.5166550 .723755

C $-4.855094 \quad 4.172484-0.112948$

C $-5.871975 \quad 3.377962 \quad 0.274229$

C $-4.8981244 .827761-1.472231$

C -6.0450472 .6476321 .575947$

C -5.502469 1.2173991 .540593

C $-6.070768 \quad 0.3124690 .438473$

C $-7.516685-0.101427 \quad 0.712779$

C $-8.030870-1.097951-0.339396$

O $-5.239827-0.8540930 .402416$

O $-9.237652-1.6916680 .165007$

C $-7.005283-2.243569-0.506760$

C $-8.415188-0.384444-1.641606$

O $-7.358533-3.089329-1.598266$

C $-5.545869-1.757704-0.668584$

C $-4.557732-2.938824-0.571680$

O $-5.328556-1.159637-1.949317$

C -1.3807401 .5984860 .640362$

O $-0.6506551 .644604-0.336733$

C $-1.393826 \quad 0.506337 \quad 1.635966$

C $-0.258310-0.1654921 .875263$

C $-0.085408-1.2441472 .850194$

C -1.327956-1.909702 3.378435

C $-3.090826-2.489203-0.630657$

C $-2.121923-3.657042-0.616963$

C $0.326070-4.136308-0.591268$
O $-2.481291-4.830973-0.638826$

C $0.664378-4.5004730 .819211$

C $1.868116-4.2933641 .373714$

C $2.216625-4.7031622 .775538$

C $2.674314-3.5434813 .629579$

C $3.972896-3.3764243 .932636$

C $1.615289-2.6256504 .202245$

C $1.168632-1.5812523 .215634$

$\mathrm{N}-0.802230-3.225032-0.593650$

H $-0.4647254 .655587-1.427735$

H $-0.926667 \quad 4.8361091 .597986$

H $-2.4261793 .502219-0.787740$

H -3.488587 5.6022220 .642887

H $-3.826151 \quad 4.3212381 .785002$

H $-6.6903823 .221513-0.428194$

H $-5.8127274 .584395-2.023554$

H $-4.8584365 .917209-1.368443$

H $-4.0514514 .506656-2.086309$

H $-5.563255 \quad 3.1915192 .395280$

H -7.112601 2.6360051 .825959

H -4.4154861 .2553361 .425659$

H $-5.6642170 .747272 \quad 2.519583$

H $-5.9845480 .822017-0.526564$

H -8.1767390 .7726370 .762117$

H -7.577681 -0.5804051 .700028$

H $-9.867808-0.9757290 .355495$

H $-7.059425-2.8647930 .398321$

H $-9.2266700 .331101-1.460823$

H $-8.801204-1.092064-2.383190$

H -7.585906 $0.169537-2.086912$

H $-8.270145-3.392678-1.446322$

H $-4.713772-3.4781750 .371442$

H -4.743414 -3.645992 -1.390045

H $-6.118468-1.298651-2.495923$

H $-2.3152810 .317298 \quad 2.173095$

H $0.6407020 .122714 \quad 1.331458$

H -1.969263 -2.239210 2.553650

H $-1.896874-1.2177134 .008819$

H $-1.124055-2.7985773 .978729$

H -2.906496 -1.916348 -1.546189

H $-2.863509-1.8469700 .226473$

H $1.154250-3.634497-1.101184$

H $0.065060-5.038664-1.153598$

H $-0.114369-4.9827601 .405765$

H $2.655303-3.8306150 .783074$

H $3.008059-5.4614282 .711907$

H $1.372112-5.2008623 .268427$

H $4.304583-2.5604834 .566237$

H $4.734122-4.0461073 .546543$

H $2.018994-2.0952835 .075043$

H $0.793425-3.2336374 .588948$

H $1.993411-1.0119352 .788540$

H $-0.606768-2.231521-0.507670$

C $2.1972154 .627697-0.330900$

$\begin{array}{lllll}\text { O } & 2.223795 & 3.710998 & 0.783582\end{array}$

C 3.6009023 .4290761 .122291

C $4.438597 \quad 4.064556 \quad 0.022566$

C $3.5847845 .259048-0.359650$

H 3.7824233 .9365212 .078239

C $3.7560971 .912597 \quad 1.286328$

H 5.4394834 .3440260 .364561

H $4.5462323 .382925-0.830710$

C $3.9633915 .868699-1.697866$

H $3.668723 \quad 6.0223280 .425462$

C $5.212416 \quad 1.4267781 .375469$

$\begin{array}{lllll}\text { O } & 3.072546 & 1.534917 & 2.486874\end{array}$

H $3.236966 \quad 1.4060150 .465755$

H 5.7391921 .6461780 .439975

$\begin{array}{lllll}\text { O } & 5.876210 & 2.187326 & 2.393807\end{array}$

C $5.370924-0.0655161 .722095$

C $4.594934-1.0434340 .828932$

H $5.067032-0.2257332 .764021$

O 6.765668 -0.378980 1.678507

H $4.855061-2.0723181 .110986$

H $3.525764-0.9535341 .055483$

H $4.9963076 .231219-1.676190$

H $3.8807975 .138157-2.509758$

H $3.3138226 .715517-1.939636$ 
C $1.0503625 .621340-0.140258$

H 2.1563281 .8524772 .411693

H $6.793973 \quad 1.8685292 .440962$

H $6.865273-1.3111721 .937064$

H $1.1385146 .458108-0.841493$

H 1.0724256 .0345490 .875489

H $2.0390154 .024794-1.235799$

C $3.730921-1.821385-2.696323$

O $3.849470-1.849881-1.269523$

C $4.762826-0.901654-0.694910$

C $6.179906-1.148914-1.227735$

C $6.201595-1.140799-2.762061$

C $5.104325-2.026795-3.358113$

H $3.103218-2.688274-2.940573$

C $2.994321-0.567022-3.187383$

H $4.4397880 .103680-0.977043$

O $6.656246-2.402474-0.744861$

H $6.850789-0.369402-0.851835$

H $7.177310-1.494922-3.116697$

O $6.0245590 .191691-3.235387$

H $5.027484-1.854038-4.438086$

O $5.497101-3.390777-3.179516$

C $1.547251-0.585190-2.744280$

H $3.426600 \quad 0.368758-2.823930$

H $3.027067-0.533748-4.283089$

H $7.558278-2.525374-1.086045$

H $6.749536 \quad 0.730194-2.874854$

H $4.829459-3.948479-3.614009$

O $0.727460-0.460099-3.826983$

O $1.187349-0.691127-1.578880$

C $-0.675397-0.478340-3.545165$

H $-0.9358840 .313031-2.836296$

H $-1.212431-0.299976-4.480509$

H $-0.964764-1.458604-3.156179$

SCF Energy (B3LYP/6-31G**//MMFF) $=-3245.89988147$

0700542

MM̄FF Geometry

C $-0.730948 \quad 4216713 \quad 1.644258$

C $-0.2941805 .085850 \quad 0.719849$

C 1.0636755 .0869010 .061588

O 1.9286724 .0988300 .668340

C $1.0190844 .889009-1.471450$

C $0.1668313 .741831-1.982391$

C $0.5927292 .474710-2.147179$

C $-1.2357714 .110887-2.399031$

C $1.9509741 .906738-1.854639$

C $2.5504601 .239012-3.099654$

C $3.8879360 .554940-2.785794$

C $4.5298900 .004594-4.061267$

C $5.817937-0.779757-3.764458$

O $3.627764-0.513237-1.868069$

O $6.167134-1.518224-4.945606$

C $5.525987-1.813516-2.655803$

C $7.0005410 .150485-3.470882$

O $6.719656-2.470432-2.238039$

C $4.801236-1.212800-1.433246$

C $4.351132-2.285111-0.418535$

O $5.693135-0.331589-0.739157$

C 3.2512384 .3989640 .754058

O 3.7987085 .4030210 .316396

C 4.0180703 .3632911 .483727

C 3.5173552 .1918031 .906738

C 4.2621161 .1555452 .632357

C 5.6652431 .4860693 .073432

C $3.457130-3.392863-0.978561$

C $2.882875-4.1975550 .170373$

C $0.973718-4.2176891 .771243$

O $3.527878-5.0907790 .715401$

C $1.139023-3.1346962 .789094$

C $1.912932-3.2269843 .880345$

C $2.137624-2.0734784 .818455$

C $3.581443-1.6110014 .837544$

C $4.261163-1.5378405 .994216$

C $4.251999-1.2548693 .525725$

C $3.663844-0.0304702 .872497$

N $1.621326-3.7685950 .551068$

H $-0.082163 \quad 3.4158991 .987511$
H $-0.965170 \quad 5.880650 \quad 0.398735$

H $1.486576 \quad 6.081376 \quad 0.258759$

H $2.0405514 .775631-1.854777$

H $0.6486225 .816939-1.928446$

H $-0.1195611 .744805-2.531294$

H -1.809742 $4.501714-1.555475$

H -1.207170 $4.876807-3.181342$

H $-1.7874373 .255208-2.802148$

H $2.6460182 .669548-1.496654$

H $1.8431251 .171825-1.049547$

H $1.8425390 .492151-3.482891$

H $2.6925111 .992699-3.884273$

H $4.5498461 .288626-2.310513$

H $4.7288840 .812333-4.776674$

H $3.822060-0.670934-4.562146$

H $6.279227-0.883416-5.673761$

H $4.887251-2.584513-3.105099$

H $7.1995230 .799608-4.332157$

H $7.921253-0.420558-3.307975$

H $\quad 6.8360470 .795253-2.605167$

H $7.141349-2.838779-3.033304$

H $5.228071-2.7325370 .068117$

H $3.802596-1.7779910 .386301$

H $5.380677-0.234240 \quad 0.175560$

H 5.0586973 .6248251 .650683

H 2.4762181 .9562081 .696323

H 5.6830682 .4297703 .630697

H 6.3284151 .5760472 .206248

H $6.100793 \quad 0.7405343 .741802$

H $2.645438-2.978865-1.586740$

H $4.023309-4.079314-1.617067$

H $1.407689-5.1665652 .101746$

H $-0.086338-4.3732561 .552061$

H $0.620391-2.2007262 .592194$

H $2.443772-4.1525814 .087825$

H $1.816785-2.3890335 .819232$

H $1.499584-1.2212624 .552560$

H $5.296490-1.2145306 .022381$

H $3.798066-1.7965976 .940688$

H $4.153181-2.0959522 .828900$

H $5.332622-1.1575563 .667352$

H $2.644446-0.1737572 .513563$

H $1.201718-2.9816780 .063879$

C -2.9328803 .0483241 .869803$

O $-3.285568 \quad 3.1321120 .474525$

C -4.6613192 .7206850 .310420$

C -5.0737352 .1167541 .644640$

C -4.2584752 .9417462 .619922$

H -5.2275113 .6396990 .113732$

C $-4.7512341 .767933-0.887317$

H -6.1511042 .1848221 .822062$

H -4.7827311 .0599631 .701530$

C -4.1425362 .3096923 .995972$

H -4.7191633 .9341382 .714621$

C $-6.1299411 .100023-1.049191$

O $-4.4485422 .537243-2.055840$

H -3.958520 $1.017778-0.792491$

H $-6.3862040 .538873-0.143533$

O $-7.1165792 .133237-1.164783$

C $-6.2586420 .173468-2.273526$

C $-5.177731-0.913018-2.385890$

H $-6.2524670 .778494-3.188216$

O $-7.550594-0.437451-2.226081$

H $-5.409006-1.569005-3.235347$

H -4.226004 -0.435803 -2.654378

H -5.1319182 .1943524 .450534$

H -3.678894 1.3185853 .944555

H -3.5359062 .9310204 .661887$

C -2.102009 4.270382 2.261616

H $-4.3030391 .918985-2.790958$

H $-7.980476 \quad 1.698444-1.267684$

H -7.641457 -0.993594 -3.018675

H -1.979195 4.3146253 .349512

H -2.620194 5.1867091 .952654

H -2.3492932 .1282491 .998314$

C $-3.254295-3.312869-0.299567$

O $-3.745186-2.527908-1.393739$

C $-4.942567-1.781035-1.132478$ 
C $-6.089620-2.739110-0.781477$

C $-5.703839-3.6691960 .377116$

C $-4.326012-4.3093980 .171861$

H -2.419970 -3.890279 -0.718951

C $-2.693055-2.4358270 .830998$

H $-4.752105-1.117628-0.285747$

O $-6.425184-3.517174-1.927803$

H $-6.974861-2.159214-0.501021$

H -6.455886 -4.461465 0.475626

O $\quad-5.693024-2.933251 \quad 1.597319$

H $-3.987215-4.7984381 .092812$

O $-4.431824-5.325858-0.829253$

C $-1.549127-1.5590130 .365878$

H -3.460439 -1.7765221 .244890$

H -2.313876 -3.0718431 .638492$

H -7.169387 -4.093644 -1.684470

H $-6.583758-2.5654081 .727106$

H $-5.067352-5.988274-0.508517$

O $-1.286629-0.629748 \quad 1.332065$

O $-0.950676-1.679400-0.693997$

C $-0.2099890 .269322 \quad 1.051262$

H $0.676045-0.2727480 .706884$

H -0.5309581 .0013380 .306048$

H $\quad 0.042941 \quad 0.793556 \quad 1.976267$

SCF Energy (B3LYP/6-31G**//MMFF) $=-3245.89208547$

0700543

MM̄MF Geometry

C -1.729995 5.0144260 .225139

C $-0.8775924 .362304-0.579201$

C $0.5067323 .940220-0.165171$

O $0.6064902 .510890-0.358565$

C $1.5635624 .607135-1.059476$

C $2.9826874 .304316-0.616440$

C $3.7878723 .379621-1.177992$

C 3.4939865 .1602670 .514524

C $3.4603502 .430725-2.298906$

C $3.7382190 .949138-1.982344$

C $5.227120 \quad 0.567119-2.042353$

C $5.393254-0.953920-2.098084$

C $6.873545-1.368553-2.087632$

O $5.8749191 .073039-0.873526$

O $6.932950-2.780455-1.831362$

C $7.578629-0.665587-0.908450$

C $7.542764-1.147455-3.449011$

O $8.985580-0.890851-0.939082$

C $7.2900750 .849675-0.831194$

C 7.8388121 .5058050 .461551

O $7.9342461 .507552-1.930556$

C $0.198441 \quad 1.7074250 .659231$

O $-\begin{array}{llll}0.266886 & 2.053085 & 1.732839\end{array}$

C $0.436364 \quad 0.311176 \quad 0.232372$

C $0.225429-0.6930811 .095033$

C $0.472726-2.1134360 .839463$

C $0.992829-2.495132-0.522355$

C 7.4555930 .8520591 .791629

C 5.9579670 .8060922 .025671

C $4.093849-0.7607492 .512347$

O 5.2673881 .8228342 .020804

C $3.784223-2.1642682 .100533$

C $3.347173-3.1120762 .943785$

C $3.001241-4.5133252 .521946$

C $1.565943-4.8796682 .839653$

C $1.263048-5.5967883 .934880$

C $0.492603-4.4775021 .848677$

C $0.256244-2.9914671 .841405$

N $5.486842-0.4824722 .224349$

H -1.416524 5.2886181 .229301

H -1.197333 $4.084140-1.580940$

H 0.6997294 .1885890 .886081

H $1.4241415 .696742-1.054787$

H $1.4184074 .303033-2.102704$

H $4.8033203 .307091-0.795837$

H 4.5303794 .9254310 .779899

H $3.456841 \quad 6.218622 \quad 0.236475$

H 2.8851575 .0143631 .412039

H $2.4039542 .504774-2.573462$

H $4.0295522 .729953-3.187041$
H $3.3280410 .703007-0.995134$

H $3.1921430 .351551-2.723422$

H $5.6682821 .026860-2.934233$

H $4.887843-1.369931-2.978495$

H $4.898855-1.406241-1.226880$

H $6.428393-3.233028-2.528894$

H $7.208473-1.1486510 .002939$

H $7.031235-1.728766-4.225508$

H $8.578581-1.504346-3.441925$

H $7.540072-0.102364-3.764930$

H $9.124298-1.852528-0.982503$

H $8.934276 \quad 1.5523740 .405209$

H 7.4997592 .5510330 .485283

H $8.0330532 .448361-1.708483$

H $\quad 0.8053340 .149993-0.774184$

H $-0.139632-0.4532772 .092930$

H $1.944371-1.991959-0.728221$

H $0.272758-2.213803-1.299110$

H $1.176028-3.564606-0.638973$

H $7.901658-0.1448811 .867287$

H $7.885267 \quad 1.4346692 .615991$

H $3.460982-0.0562251 .962864$

H $3.939051-0.5946043 .583440$

H $3.899909-2.4032001 .046034$

H $3.231136-2.8724593 .998399$

H $3.686842-5.1966523 .039807$

H $3.185268-4.6668021 .451233$

H $\quad 0.241192-5.884840 \quad 4.158140$

H $2.030739-5.9078374 .635682$

H -0.461393 -4.957823 2.103213

H $0.757451-4.8758880 .866040$

H -0.125545 -2.610054 2.789813

H $6.159075-1.2266782 .387806$

C $-4.1574104 .670605 \quad 0.722575$

O $-4.059241 \quad 3.2442350 .544430$

C -5.3818852 .6793280 .522388$

C -6.3297473 .8043070 .923629$

C -5.6049015 .0229320 .387433$

H $-5.5799872 .393432-0.517694$

C $-5.471551 \quad 1.433758 \quad 1.418770$

H -7.3289653 .6754090 .496498$

H -6.4364183 .8730682 .012659$

C $-6.082374 \quad 6.330310 \quad 0.994577$

H $-5.7409795 .060162-0.701869$

C $-4.548677 \quad 0.283591 \quad 0.957182$

$\begin{array}{llll}\text { O } & -5.169400 & 1.802977 & 2.763547\end{array}$

H -6.5080821 .0762561 .408431$

H $-4.751900 \quad 0.106547-0.104541$

$\begin{array}{lllll}\text { O } & -3.188276 & 0.694761 & 1.071878\end{array}$

C $-4.760400-1.0281141 .744244$

C $-3.850858-2.1980461 .319366$

H -5.807879 -1.334319 1.643040

O $-4.535903-0.8124573 .139437$

H -2.805774 -1.962126 1.552248

H -4.107938 -3.051255 1.961741

H $-7.144044 \quad 6.4892780 .778680$

H -5.9559496 .3375002 .082331$

H -5.5244847 .1781120 .585063$

C $-3.1349835 .374137-0.171633$

H -4.2452872 .1061322 .779600$

H -2.6453780 .0672140 .566455$

H -3.639150 -0.449094 3.239164

H -3.248569 $6.461203-0.094544$

H -3.310252 $5.099503-1.219347$

H $-3.9451864 .875497 \quad 1.780310$

C $-5.682659-3.300145-1.738817$

O $-5.347771-2.965371-0.385890$

C $-3.984309-2.601406-0.156044$

C $-3.052788-3.763261-0.529964$

C $-3.290132-4.208342-1.977286$

C $-4.778820-4.426695-2.269874$

H -6.705721 -3.694636 -1.685100

C $-5.709117-2.054601-2.638807$

H $-3.726207-1.736247-0.773524$

O $-3.271143-4.8600350 .353553$

H $-2.014937-3.438473-0.401894$

H $-2.744939-5.140608-2.168064$

O $-2.784301-3.221195-2.871722$ 
H $-4.945579-4.548588-3.346641$

O $-5.200192-5.641367-1.641560$

C $-6.774597-1.065332-2.210503$

H -4.750196 -1.529818 -2.626134

H -5.934190 -2.349353-3.670049

H $-2.643338-5.5618280 .111244$

H -1.835058 -3.117501 -2.688549

H $-4.669483-6.364913-2.016248$

O $-6.6183160 .090854-2.921355$

O $-7.641745-1.278004-1.374268$

C $-7.5672831 .123541-2.635128$

H $-7.2426712 .034428-3.145027$

H $-8.5521280 .837370-3.015659$

H -7.613051 $1.322892-1.560113$

SCF Energy $\left(B 3 L Y P / 6-31 G^{* *} / / M M F F\right)=-3245.88550632$

07 00544

MM̄MF Geometry

C $\quad 0.707812-2.5394972 .135424$

C $-0.052520-1.7496891 .360913$

C $-0.943496-2.2704110 .263803$

O $-2.299047-2.0647320 .736919$

C $-0.783613-1.496832-1.057121$

C $0.602426-1.509472-1.663424$

C $1.370364-0.409404-1.794670$

C $1.066127-2.836813-2.199116$

C $1.0384030 .967149-1.293462$

C $0.2840001 .798693-2.337693$

C $-0.4690852 .999763-1.743063$

C $0.4516393 .983779-1.019423$

C $-0.3389805 .126991-0.362940$

O $-1.4550152 .483802-0.841917$

O 0.5486635 .8281810 .521986

C -1.487152 4.5238960 .478176

C $-0.7812006 .168502-1.396058$

O -2.3222135 .5516891 .007127$

C $-2.3300093 .468481-0.280297$

C -3.2830912 .7502810 .711311$

O $-3.0920674 .130581-1.287880$

C $-3.286351-2.8319490 .204992$

O $-3.166245-3.719241-0.623527$

C $-4.561156-2.3774370 .804888$

C $-5.724960-2.8722290 .358768$

C $-7.059451-2.5026150 .837651$

C -7.150195 -1.433230 1.895303

C -4.0418051 .5404450 .142152$

C $-5.3092191 .890041-0.618838$

C -7.049465 $0.849751-2.072067$

O $-5.7931073 .018559-0.635691$

C $-8.2240960 .445917-1.237578$

C $-9.095673-0.507878-1.599968$

C -10.300432 -0.891105 -0.788798

C $-10.381383-2.378219-0.523845$

C -11.195399-3.165574 -1.247204

C $-9.586511-2.9354700 .636028$

C $-8.130621-3.1245240 .301700$

N $-5.8428840 .784953-1.268388$

H $0.693622-3.6108571 .941851$

H $-0.091071-0.6798941 .540810$

H $-0.773771-3.3430720 .106773$

H -1.152342 -0.474087 -0.913719

H -1.479259 -1.911330 -1.799543

H $2.334414-0.497586-2.290796$

H $2.006679-2.754239-2.753508$

H $1.222636-3.546441-1.380798$

H $\quad 0.320399-3.252984-2.884475$

H $\quad 0.4800380 .901967-0.353895$

H $1.9763881 .472874-1.040268$

H $0.9860782 .144579-3.106367$

H $-0.4534571 .160483-2.842340$

H $-0.981073 \quad 3.501495-2.572789$

H $1.2131794 .380366-1.702039$

H $1.0033443 .461527-0.228041$

H 0.8728925 .2001101 .189646

H -1.025802 4.0351411 .347071

H $0.0918496 .590174-1.909056$

H -1.280555 $7.016687-0.914617$

H -1.448036 $5.762212-2.159135$
H $-2.896226 \quad 5.8657110 .287779$

H -2.6767382 .3669831 .542985$

H -3.9977073 .4679121 .131600$

H -3.790835 $3.532014-1.593130$

H -4.506804 -1.633757 1.591956

H $-5.702458-3.616760-0.436054$

H $-6.684903-1.7749962 .826335$

H -8.174251 -1.141387 2.136022

H $-6.644357-0.518867 \quad 1.565719$

H $\quad-3.3904740 .931076-0.493207$

H -4.3572930 .9124910 .985021$

H $-6.9036260 .186175-2.930163$

H $-7.1910021 .871238-2.439653$

H $-8.3787620 .980619-0.303326$

H -8.955745 -1.021771 -2.548463

H $-11.190287-0.562214-1.341603$

H -10.329546 -0.3506580 .165622$

H -11.281269-4.228071-1.044837

H -11.790204-2.766072 -2.061961

H -9.980942 -3.919999 0.922085

H $-9.747813-2.3075801 .516391$

H -7.958638 -3.883436 -0.463136

H $-5.409113-0.124451-1.139246$

C $1.821253-0.6358153 .503761$

O $2.745064-0.1744652 .496234$

C 3.6741890 .7460903 .105550

C 3.1367681 .0263704 .503289

C $2.481585-0.2979034 .840598$

H 4.6308020 .2205893 .199681

C 3.8273321 .9931132 .230480

H 3.9293921 .3061945 .203958

H 2.3931751 .8323124 .495586

C $1.514993-0.2118346 .009080$

H $3.264787-1.0325725 .071073$

C 4.4552621 .7126180 .848663

O 2.5305022 .5694822 .024598

H 4.4145182 .7533672 .757742

H $3.829581 \quad 1.0079580 .292559$

O 4.4480452 .9400360 .108372

C 5.9073171 .1928300 .907320

C $6.5899631 .087668-0.471450$

H $5.935848 \quad 0.2186661 .405877$

O $6.6758552 .096548 \quad 1.707751$

H $6.4819202 .035368-1.012489$

H $7.6686340 .962076-0.305265$

H $2.0370050 .109296 \quad 6.916376$

H $\quad 0.710603 \quad 0.505048 \quad 5.812865$

H $1.056657-1.1847646 .211137$

C $1.561295-2.1315923 .310479$

H $1.972963 \quad 1.8917091 .604938$

H 3.5306493 .2621780 .089275

H 7.5662451 .7180581 .805280

H $\quad 1.081837-2.5413454 .207315$

H $2.525148-2.6430883 .201433$

H $0.898141-0.0534343 .396765$

C $6.177049-2.512588-1.359156$

O $6.554531-1.287134-0.718080$

C $6.071301-0.081658-1.323963$

C $6.5499760 .020883-2.780498$

C $6.164198-1.228344-3.576903$

C $6.570748-2.511216-2.845425$

H $6.775881-3.284832-0.858076$

C $4.699718-2.836937-1.120690$

H $4.977833-0.080294-1.307062$

O $7.9651210 .193322-2.809135$

H $\quad 6.0995350 .911123-3.236070$

H $6.652451-1.193243-4.558294$

O $4.755897-1.215285-3.791381$

H $6.137003-3.389705-3.337356$

O $7.992104-2.658145-2.912434$

C $4.480400-3.2390720 .317949$

H $4.032925-1.996181-1.320956$

H $4.373233-3.659939-1.767349$

H $8.230790 \quad 0.286894-3.739744$

H $4.530103-2.005636-4.310735$

H $8.239020-2.698648-3.851987$

O $4.346627-4.592551 \quad 0.408727$

O $4.459260-2.4456051 .249557$ 
C $4.167196-5.111778 \quad 1.729862$

H $3.174206-4.8452562 .101376$

H $4.242036-6.2012401 .679281$

H $4.945629-4.7400552 .403341$

SCF Energy (B3LYP/6-31G**//MMFF) $=-3245.90607715$

0700545

MM̄̄FF Geometry

C $-0.7550505 .479330-0.835435$

C $-1.368916 \quad 4.6987390 .066194$

C $-2.6265793 .917192-0.213471$

O -2.3660172 .5228350 .072336$

C $-3.750728 \quad 4.3936810 .723915$

C -5.0783653 .6989750 .491688$

C -5.7041422 .9212061 .396952$

C $-5.7207923 .946565-0.849410$

C -5.2601972 .5773962 .791340$

C -4.4597541 .2705362 .907391$

C -5.2466220 .0140792 .499885$

C $-4.480781-1.2548882 .882716$

C $-5.207810-2.5265102 .412523$

$\begin{array}{lllll}\text { O } & -5.439040 & 0.040881 & 1.083446\end{array}$

O $-4.303705-3.6330082 .555175$

C $-5.567118-2.3809520 .915195$

C $-6.394171-2.8604953 .323660$

O $-6.373225-3.471571 \quad 0.477739$

C $-6.237893-1.0339490 .571518$

C $-6.399572-0.801269-0.950229$

O $-7.553976-1.0117371 .138777$

C $-1.6949391 .805348-0.869282$

O $-1.3067862 .198216-1.957681$

C $-1.4847440 .445309-0.326115$

C $-0.596230-0.368992-0.914110$

C $-0.249926-1.724154-0.482461$

C $-1.119527-2.3618790 .567647$

C $-5.096567-0.793927-1.757256$

C $-4.765932-2.127035-2.407634$

C $-2.980064-3.243047-3.739833$

O $-5.537749-3.081800-2.422270$

C $-2.118430-4.063105-2.832744$

C $-0.860411-4.415921-3.136932$

C $0.006409-5.273701-2.260952$

C $1.330117-4.618636-1.938115$

C $2.435477-4.908594-2.644963$

C $1.383327-3.688466-0.747784$

C $0.813158-2.330238-1.051139$

N -3.513190 -2.110767 -3.006450

H -1.178053 5.588670 -1.830547

H -0.9329934 .5865421 .056712$

H -2.934841 4.025928 -1.260324

H -3.9062635 .4735530 .597623$

H -3.422566 4.2553091 .759697

H -6.6597772 .4798051 .115605$

H $-6.7400233 .548649-0.901661$

H -5.780298 5.021309-1.049883

H $-5.1435123 .471680-1.648339$

H -4.672085 3.3936863 .223820

H $-6.1548672 .508272 \quad 3.423411$

H -3.5526101 .3541102 .297310$

H -4.1392431 .1701143 .951813$

H -6.2200660 .0387963 .003322$

H -4.303650 -1.294114 3.964610

H -3.485527 -1.228328 2.418533

H -3.518709 -3.458275 2.009250

H -4.625531 -2.459273 0.360365

H $-6.053067-3.0029504 .356290$

H -6.862501-3.807416 3.033235

H -7.161736 -2.083971 3.333643

H -7.278713 -3.3180590 .796945$

H -7.130169 -1.506780 -1.364033

H $-6.8535040 .192313-1.077903$

H -8.081403 -0.3461120 .666793$

H $-2.045596 \quad 0.1617690 .556876$

H - $0.047236-0.001817-1.780220$

H -2.174501 $-2.298776 \quad 0.279638$

H $-0.987992-1.8608531 .532713$

H -0.913944 -3.423437 0.719550

H -5.203134 -0.073369-2.578331
H $-4.251962-0.467215-1.143110$

H -2.421774 -2.843232 -4.592247

H -3.804305 -3.855614 -4.118985

H $-2.557003-4.403332-1.897567$

H -0.435342 -4.095794-4.085805

H $\quad 0.178243-6.221685-2.787435$

H $-0.505793-5.543411-1.329000$

H $3.393009-4.457824-2.408152$

H $2.404728-5.596147-3.483871$

H $2.424794-3.537770-0.436106$

H $0.907130-4.1739160 .108300$

H $1.376833-1.782445-1.807760$

H $-2.920383-1.292547-2.899910$

C $1.6815965 .653267-1.395378$

O $1.8878964 .263494-1.071269$

C $3.2741924 .054898-0.756523$

C $4.0083405 .242829-1.363785$

C $3.0111506 .358734-1.117319$

H 3.3601034 .1084020 .334392

C $3.7361012 .680195-1.251271$

H $4.9785125 .419957-0.890257$

H 4.171374 5.115669-2.440106

C $3.2448577 .588584-1.977321$

H $3.0693916 .649933-0.059825$

C $2.8617651 .488283-0.805380$

O $3.7383202 .681657-2.687268$

H $4.7778532 .513229-0.956692$

H $1.8801351 .579202-1.286799$

O $3.4400970 .286306-1.324146$

C 2.6459601 .2977910 .706460

C 3.8940631 .4068691 .603690

H 2.1896630 .3131920 .866980

O $1.6873712 .262638 \quad 1.147487$

H $4.3372782 .404715 \quad 1.513124$

H 3.5415301 .3420362 .642381

H $4.2296358 .020553-1.771354$

H $3.2007297 .346418-3.044500$

H $2.4900178 .354620-1.774006$

C $0.5293946 .207423-0.553667$

H $2.8351042 .891619-2.981301$

H $3.5452000 .404365-2.283816$

H 1.4783532 .0679772 .076887

H $\quad 0.3828037 .272361-0.766776$

H $\quad 0.777706 \quad 6.121114 \quad 0.511781$

H $1.4314945 .694849-2.463089$

C $5.230190-2.0748551 .399814$

O $4.374321-0.9450901 .620965$

C $4.9694540 .333244 \quad 1.374824$

C 6.1838220 .5461522 .295798

C $7.193317-0.5972052 .158547$

C $6.514586-1.9691772 .241017$

H $4.656020-2.9297251 .779041$

C $5.460022-2.293347-0.099518$

H $\begin{array}{llll}5.304513 & 0.384457 & 0.334761\end{array}$

O $5.7473160 .630785 \quad 3.651548$

H 6.6574161 .5016992 .040084

H $7.942143-0.5132052 .955663$

O $7.869636-0.4576890 .913080$

H $7.217873-2.7552311 .946852$

O $6.174739-2.2291723 .607383$

C $6.060764-3.644143-0.398565$

H $4.515051-2.211039-0.649378$

H $\quad 6.108075-1.527463-0.535735$

H 6.5296560 .8083354 .200901

H $8.518119-1.1795150 .848318$

H $5.499285-1.5821143 .873894$

O $5.151489-4.628683-0.149686$

O $7.203581-3.803174-0.807940$

C $5.603654-5.963065-0.399008$

H $5.872743-6.081209-1.452900$

H $6.453903-6.2013250 .247030$

H $4.783701-6.648102-0.167738$

SCF Energy (B3LYP/6-31G**//MMFF) $=-3245.90987579$

0700546

MM̄FF Geometry

C 3.0575444 .0256880 .014370

C 2.1379214 .1648540 .980535 
C 0.7315993 .6444560 .853389 O $\quad 0.588186 \quad 2.5555501 .797010$ C $-0.321642 \quad 4.714727 \quad 1.200520$ C $-0.459848 \quad 5.8332530 .188983$ C $-1.3393275 .813188-0.831869$ C $0.390614 \quad 7.052798 \quad 0.428693$ C $-2.2627334 .687574-1.203515$ C $-3.6548524 .880629-0.593543$ C $-4.643446 \quad 3.792552-1.037896$ C $-5.995906 \quad 3.992453-0.353477$ C $-6.9832022 .870828-0.700847$ O $-4.1058012 .517729-0.680398$ O -8.0869662 .9557850 .213802$ C $-6.3054711 .502056-0.465469$ C $-7.5704173 .049975-2.107072$ O $-7.154847 \quad 0.457954-0.935002$ C $-4.8997251 .391902-1.098307$ C $-4.111850 \quad 0.148100-0.622875$ O $-5.0131371 .343118-2.522064$ C -0.2892091 .5670131 .467937$ O -0.9350241 .4714770 .436243$ C $-0.345490 \quad 0.6123812 .597630$ C $-1.124714-0.4753322 .497040$ C $-1.297655-1.5218023 .507012$ C $-0.558208-1.3617574 .809295$ C $-4.737272-1.208359-0.950473$ C $-3.756997-2.357917-0.787815$ C $-3.680660-4.849836-0.795417$ O $-2.554335-2.199014-0.600439$ C $-3.644899-5.2739730 .638858$ C $-2.510143-5.4994691 .318692$ C $-2.472147-5.9654592 .745230$ C $-1.730962-5.0139003 .656668$ C $-0.514643-5.3214124 .137846$ C $-2.438424-3.7486984 .087026$ C $-2.096925-2.5709293 .217978$ N $-4.387758-3.588799-0.905673$ H $2.7981903 .478797-0.889366$ H 2.3936884 .7079291 .887108 H $0.565705 \quad 3.271686-0.165664$ H -0.1114425 .1391382 .192343$ H -1.2938984 .2245421 .339739$ H $-1.403126 \quad 6.685023-1.480961$ H $0.1562897 .864560-0.268469$ H $\quad 0.228697 \quad 7.4366591 .441234$ H $1.451820 \quad 6.8185120 .310211$ H -2.336955 $4.651523-2.297408$ $\mathrm{H}-1.840103 \quad 3.724673-0.899976$ H -3.562194 4.8618390 .500178 H $-4.0510865 .866373-0.868087$ H -4.747563 $3.847424-2.127860$ H $-6.4203124 .971833-0.607098$ H -5.8553004 .0010570 .736659$ H -8.4824443 .8399050 .125029$ H -6.2039831 .3606450 .619559$ $\mathrm{H}-8.0863464 .014605-2.186293$ H -8.328824 2.288484 -2.319888 H $-6.814705 \quad 3.014560-2.894404$ H -8.012211 $0.557382-0.486682$ H -3.111183 $0.197742-1.075029$ $\begin{array}{llll}\text { H } & -3.936739 & 0.214395 & 0.459347\end{array}$ H -4.203677 $0.942366-2.880355$ H $0.2544530 .827933 \quad 3.474115$ H -1.691688 -0.6222361 .579310$ H $\quad 0.520790-1.2956254 .634894$ H $-0.705127-2.1931085 .501608$ H $-0.886247-0.4537905 .327296$ H $-5.586977-1.393327-0.284373$ H $-5.092215-1.229617-1.986871$ H -2.677838 -4.737285 -1.217931 H $-4.228102-5.587950-1.389545$ H $-4.601521-5.4226531 .134044$ H $-1.553668-5.368900 \quad 0.817712$ H $-1.992666-6.9530432 .757858$ H $-3.483423-6.1255803 .139881$ H $0.007614-4.6563774 .817541$ H $-0.011305-6.2408643 .858473$ H -2.231927 -3.548689 5.143511
H $-3.524722-3.901508 \quad 4.050527$ H $-2.585419-2.5951832 .244094$ H -5.399168 -3.620460 -0.995676 C $5.569678 \quad 3.667075 \quad 0.313747$

O $5.4635222 .498637-0.523580$

C $5.842091 \quad 1.348274 \quad 0.260469$

C $6.503887 \quad 1.894727 \quad 1.519104$

C 5.6851493 .1517341 .747368

H $4.9138100 .852090 \quad 0.561972$

C $6.7084280 .393777-0.564653$

H $6.454271 \quad 1.1904132 .355019$

H 7.5568662 .1463301 .345196

C 6.3309534 .1303942 .711939

H 4.6993092 .8632962 .134214

C $5.984177-0.186485-1.799628$

O $7.865592 \quad 1.107520-1.013646$

H $7.074166-0.4182510 .074829$

H $5.7687050 .614488-2.516029$

O $6.890652-1.076866-2.461992$

C $4.690066-0.949422-1.459437$

C $4.043680-1.619119-2.687469$

H $3.967307-0.256080-1.017054$

O $5.006606-1.938110-0.479322$

H $3.988830-0.894256-3.508102$

H $4.673088-2.459617-3.004642$

H 6.4578563 .6708413 .697704

H 7.3180504 .4489902 .360684

H 5.7115495 .0243192 .834202

C 4.4255994 .6445480 .043358

H $8.3946220 .492519-1.550165$

H $7.052635-1.827416-1.864847$

H $4.180137-2.384695-0.230907$

H $4.5811855 .092681-0.946992$

H 4.4636855 .4737100 .759537

H 6.5083994 .1618380 .029257

C $1.504545-3.754845-0.957592$

O $2.738175-3.205899-1.432994$

C $2.622732-2.140223-2.379215$

C $1.897059-2.631468-3.640224$

C $0.540374-3.262206-3.299246$

C $0.632533-4.244558-2.124923$

H $1.802955-4.636389-0.374604$

C $0.786517-2.7985990 .000357$

H $2.057941-1.316678-1.929497$

O $2.713775-3.595977-4.302814$

H $1.759551-1.785986-4.324742$

H $\quad 0.172383-3.801799-4.180129$

O $-0.425312-2.253545-3.018234$

H $-0.365727-4.498072-1.752835$

O $1.221499-5.461495-2.599368$

C $1.616758-2.5687731 .242214$

H $\quad 0.556333-1.829284-0.448462$

H $-0.168885-3.2221120 .328934$

H $2.254278-3.859371-5.118318$

H - $0.028692-1.586969-2.435933$

H $0.635085-5.822696-3.285960$

O $2.203351-1.3389991 .190295$

O $1.746307-3.3941762 .136973$

C $3.030736-0.9934302 .305799$

H $2.525472-1.2049413 .252336$

H $3.976472-1.5391302 .246318$

H 3.2363630 .0790022 .256069

SCF Energy (B3LYP/6-31G**//MMFF) $=-3245.90880296$

0700547

MM̄FF Geometry

C $0.0249722 .172013-2.206810$

C $-0.846297 \quad 1.399646-2.872913$

C $-0.642897-0.047298-3.267838$

O $0.615610-0.575360-2.789802$

C $-1.765379-0.886135-2.626933$

C $-1.623474-2.375693-2.862936$

C $-1.157833-3.241214-1.939770$

C $-2.084783-2.863558-4.209303$

C $-0.643615-2.894197-0.567694$

C $0.550164-3.767991-0.160556$

C $1.286709-3.2861031 .102392$

C $0.356507-3.0220822 .289009$ 
C $1.126414-2.5090883 .515757$

O $2.007781-2.0929280 .771783$

O $0.169220-1.9826464 .449203$

C $2.028198-1.3285343 .085225$

C $1.849172-3.6482544 .244197$

O $2.863572-0.9360864 .171457$

C $2.870760-1.6229901 .820578$

C $3.622406-0.3879551 .288155$

O $3.908722-2.5729292 .098390$

C $1.726355-0.375632-3.546206$

$\begin{array}{llll}\text { O } & 1.818840 & 0.285516 & -4.567467\end{array}$

C $2.834390-1.108394-2.897372$

C $4.091194-0.662615-3.035935$

C $5.290599-1.282697-2.470053$

C $5.157131-2.678041-1.916382$

C $2.748351 \quad 0.799842 \quad 0.885551$

C 3.5598791 .7970990 .081391

C $5.487003 \quad 3.3545420 .239049$

O $3.396987 \quad 1.938875-1.128284$

C $6.705712 \quad 2.597453-0.208134$

$\begin{array}{llll}\text { C } & 7.132492 & 1.416388 & 0.267604\end{array}$

C $8.3672790 .711302-0.216916$

C $8.103295-0.731966-0.585909$

C $8.217302-1.705902 \quad 0.333360$

C $7.805304-1.059791-2.030608$

C $6.450291-0.592007-2.487162$

N $4.494706 \quad 2.4826250 .840182$

H $\quad 0.998447 \quad 1.778750-1.939517$

H $-1.8162331 .821290-3.131194$

H -0.690836 $-0.138049-4.360026$

H $-2.741711-0.559411-3.010559$

H $-1.805676-0.682223-1.549703$

H -1.142173 -4.301082 -2.189041

H $-1.993183-3.950058-4.311138$

H -3.136716 -2.604497 -4.367825

H $-1.489478-2.406358-5.005864$

H $-0.348593-1.842767-0.507392$

H -1.460942 -3.054813 0.143238

H $\quad 0.197782-4.794507 \quad 0.002532$

H $1.269827-3.801339-0.989022$

H $2.012778-4.0673061 .357065$

H $-0.219294-3.9213442 .539951$

H $-0.381232-2.2540322 .023777$

H $-0.468200-2.6873724 .656117$

H $1.357541-0.4834052 .882392$

H $1.125644-4.3838434 .616493$

H $2.381139-3.2793235 .127828$

H $2.558212-4.1828933 .608848$

H $3.260777-0.0783253 .945915$

H $4.374824-0.0685302 .020657$

H $4.205695-0.6974640 .410396$

H $3.969838-2.6922113 .059893$

H $2.579434-1.982561-2.309805$

H $4.2588740 .251337-3.604781$

H $4.772014-3.359859-2.682923$

H $4.474102-2.693141-1.060655$

H $6.102691-3.098785-1.567708$

H 1.9027250 .4780690 .267717

H 2.3368511 .3074901 .764553

H 5.7626524 .1182360 .972428

H $5.0341793 .850847-0.625875$

H $7.2841453 .078173-0.994583$

H $6.5903060 .934851 \quad 1.077239$

H 9.1136950 .7526610 .587295

H $8.8219231 .233928-1.067416$

H 8.064703 -2.748679 0.076643

H $8.462440-1.4800681 .366002$

H $7.922219-2.132976-2.222175$

H $8.566817-0.589069-2.666314$

H $6.4495340 .419307-2.894858$

H 4.6003872 .2534891 .824039

C $-0.0212883 .678167-0.219125$

$\begin{array}{llllll}\text { O } & -0.774411 & 2.671794 & 0.483659\end{array}$

C -1.446310 $3.278766 \quad 1.597956$

C $-0.7656864 .625306 \quad 1.805584$

C $-0.451351 \quad 5.0197930 .373838$

H -2.480862 $3.452844 \quad 1.279377$

C -1.4461372 .3356172 .804969$
H -1.4118215 .3439852 .318482$

H $\quad 0.1623374 .5297002 .380780$

$\begin{array}{lllllllll}\text { C } & 0.600550 & 6.108731 & 0.256098\end{array}$

H $-1.3779455 .364651-0.104450$

C -1.8775440 .8884352 .486165$

$\begin{array}{lllll}\text { O } & -0.118896 & 2.270857 & 3.343239\end{array}$

H -2.0751442 .7622063 .595339$

H -1.0581900 .3704631 .972363$

$\begin{array}{lllll}0 & -2.044933 & 0.204832 & 3.734886\end{array}$

C $-3.1631250 .745397 \quad 1.652293$

C $-3.588226-0.728148 \quad 1.503911$

H $-2.997963 \quad 1.1794990 .660457$

O -4.2055321 .4858462 .284092$

H -2.701661 -1.3454011 .323734$

H $-4.053947-1.0557872 .441550$

H $0.2617347 .025745 \quad 0.748923$

H 1.5450145 .8064170 .720919

H $0.8018416 .343064-0.794007$

C $-0.280103 \quad 3.562062-1.724315$

H $-0.143927 \quad 1.6662874 .104825$

H -2.8127930 .5988064 .183575$

H -4.9981231 .4116091 .725657$

H $0.3473664 .277185-2.267456$

H $-1.3261343 .808720-1.941778$

H $1.0366323 .484023-0.003432$

C $-6.789316-0.278345-0.386337$

O $-5.784248-0.2373240 .635816$

C $-4.577763-0.9451830 .342172$

C $-4.876902-2.4351940 .116544$

C $-5.946823-2.633387-0.961034$

C $-7.163699-1.729890-0.732833$

H -7.6666520 .1981300 .069709$

C $-6.3925440 .553624-1.611407$

H $-4.126050-0.526137-0.562783$

O $-5.306532-3.044558 \quad 1.332566$

H $-3.953757-2.941796-0.185578$

H $-6.264085-3.683505-0.958387$

O $-5.365743-2.355990-2.231702$

H $-7.817654-1.746382-1.612449$

O $-7.913594-2.2765870 .356188$

C $-6.2604392 .011794-1.240876$

H $-5.4573540 .219616-2.069705$

H $-7.1586120 .487231-2.392961$

H $-6.093018-2.5645511 .643989$

H -6.044306 -2.523486 -2.907619

H $-8.721113-1.7437800 .454121$

O $-4.946210 \quad 2.373963-1.225941$

O $-7.211862 \quad 2.735197-0.975096$

C $-4.6889013 .735277-0.869644$

H -3.615986 $3.915442-0.969394$

H $-5.2205364 .415066-1.542343$

H -4.9802643 .9120140 .169927$

SCF Energy (B3LYP/6-31G**/MMFF)= -3245.90747699

07_00548

MM̄FF Geometry

C $5.777481-1.1422930 .549754$

C $4.867253-1.880573-0.100707$

C $4.330854-3.2006330 .387820$

O $2.918891-3.022630 \quad 0.638218$

C $4.502638-4.265271-0.712819$

C $3.790554-5.573425-0.424793$

C $2.694362-6.009129-1.080496$

C $4.396823-6.4072990 .673264$

C $1.960582-5.317519-2.198293$

C $0.678152-4.650050-1.693879$

C $0.221074-3.455435-2.547219$

C $1.152793-2.247693-2.384695$

C $0.631526-1.004946-3.117930$

O $-1.097379-3.108356-2.097590$

$\begin{array}{lllll}0 & 1.371601 & 0.131602 & -2.644559\end{array}$

C $-0.840566-0.776002-2.726315$

C $0.883466-1.088784-4.627658$

O $-1.408561 \quad 0.276402-3.501021$

C $-1.716404-2.045679-2.836821$

C $-3.108601-1.772618-2.216275$

O $-1.874881-2.382869-4.215879$

C $2.542601-2.5734161 .866937$ 
O $3.263527-2.3318542 .820784$

C $1.075391-2.3923831 .829890$

C $0.423152-1.8201892 .852104$

C $-1.010276-1.5222252 .871721$

C $-1.831232-1.9674471 .688989$

C $-4.002605-3.012812-2.099290$

C $-5.329667-2.674786-1.445724$

C $-6.371471-2.2482430 .769661$

O $-6.371665-2.613379-2.094120$

C $-5.971722-1.4314151 .956638$

C $-5.746583-1.9493103 .173927$

C $-5.367549-1.1372124 .380260$

C $-3.908860-1.3083154 .750345$

C $-3.541619-2.2256315 .661536$

C $-2.947473-0.3601104 .063640$

C $-1.532171-0.8536833 .921401$

N -5.215063 -2.443873 -0.083909

H $6.185778-1.4961671 .492613$

H $4.450486-1.499094-1.031089$

H $4.837659-3.5316421 .302813$

H $5.571652-4.470924-0.859097$

H $4.162179-3.849824-1.665996$

H $2.260901-6.963041-0.783340$

H $3.887331-7.3693280 .794099$

H $5.448304-6.6180660 .452920$

H $4.339332-5.8821731 .631726$

H $2.605969-4.609462-2.719597$

H $1.699768-6.067270-2.956011$

H $-0.124711-5.399556-1.665721$

H $0.788818-4.321792-0.654485$

H $0.167072-3.774454-3.594580$

H $2.171645-2.474155-2.716868$

H $1.237988-1.997404-1.319518$

H $2.316317-0.038803-2.800842$

H -0.853737 $-0.435263-1.681239$

H $1.957683-1.172602-4.832069$

H $0.553275-0.175801-5.135374$

H $0.391886-1.943361-5.097244$

H $-0.8350441 .055082-3.395784$

H $-2.973817-1.354736-1.211308$

H $-3.640023-1.016571-2.808482$

H -2.674995 $-2.919586-4.323961$

H $0.563483-2.6998460 .926753$

H $0.987390-1.5078643 .729110$

H -1.491162 -1.473460 0.772946

H $-2.892919-1.7435531 .796995$

H -1.756952 -3.0521981 .552690$

H $-4.219048-3.443021-3.082590$

H $-3.515512-3.796916-1.508226$

H $-6.736794-3.2404111 .056091$

H -7.169024 -1.7465810 .212138$

H $-5.872216-0.3593371 .805081$

H -5.842971 -3.0229133 .320192$

H $-6.012405-1.4448665 .213731$

H -5.584353 -0.0723964 .226417$

H $-2.509478-2.3546875 .967699$

H $-4.275478-2.871864 \quad 6.132857$

H -2.9295250 .5819904 .627121$

H -3.335273 -0.0839083 .076120$

H $-0.877558-0.5768834 .747817$

H $-4.343804-2.7076720 .366381$

C 5.6313411 .3366620 .846279

$\begin{array}{lllll}\text { O } & 4.220022 & 1.407843 & 0.558591\end{array}$

C $3.8160922 .790050 \quad 0.525487$

C $5.040215 \quad 3.6014140 .940448$

C 6.1783392 .7130110 .479846

H $3.5617833 .023666-0.515638$

C $2.5923573 .022106 \quad 1.424330$

H $5.054194 \quad 4.592857 \quad 0.477008$

H $5.079544 \quad 3.7418462 .027408$

C 7.5091003 .0481061 .130179

H $6.2745992 .801929-0.610608$

C $1.2989052 .356407 \quad 0.898871$

$\begin{array}{lllll}\text { O } & 2.894487 & 2.569695 & 2.743561\end{array}$

H 2.4089434 .1008811 .495991

H $1.0979782 .777957-0.091557$

$\begin{array}{lllll}\text { O } & 1.490216 & 0.956033 & 0.712619\end{array}$

C $0.072238 \quad 2.587007 \quad 1.806724$
C $-1.2447792 .025740 \quad 1.239232$

H -0.0435023 .6586922 .004996$

\begin{tabular}{lllll}
\hline & 0.264486 & 1.946886 & 3.070917
\end{tabular}

H -1.1413570 .9534701 .041183$

H -1.996807 2.1117182 .035048

H 7.8054064 .0749400 .892232

H 7.4572142 .9574032 .220383

H 8.2984202 .3789220 .773980

$\begin{array}{lllll}\text { C } & 6.259001 & 0.192467 & 0.050884\end{array}$

H 3.1443861 .6317372 .682430

H 1.6381800 .5627371 .589205

H 1.0214862 .3747593 .505268

H 7.3509310 .2149340 .137611

H $6.0164830 .303425-1.013616$

H $5.732241 \quad 1.1559681 .925005$

C $-2.2713094 .977693-0.793423$

$\begin{array}{lllll}\text { O } & -1.878182 & 4.128584 & 0.290857\end{array}$

C $-1.7510912 .739350-0.023431$

C $-3.0982212 .176242-0.507064$

C $-3.6658543 .003422-1.667680$

C $-3.6112024 .508880-1.384691$

H $-2.4350145 .961916-0.335765$

C $-1.1522315 .125039-1.835913$

H $-1.0065122 .608611-0.814358$

$\begin{array}{lllll}\text { O } & -4.033105 & 2.178676 & 0.571044\end{array}$

H -2.954336 $1.134396-0.814328$

H $-4.7081002 .713946-1.849662$

O $-2.9293782 .736318-2.855734$

H $-3.8222275 .072684-2.300437$

O $-4.6491874 .837708-0.455825$

C $0.106813 \quad 5.719647-1.238352$

H $-0.885724 \quad 4.158338-2.271537$

H $-1.4791035 .789829-2.643370$

H $-4.8528491 .766506 \quad 0.249022$

H $-3.0194441 .787364-3.048933$

H -4.4440954 .3973750 .386903$

O $1.1535345 .490558-2.085773$

O $\quad 0.1654906 .324365-0.176591$

C $2.4191056 .001203-1.654604$

H $2.4037707 .094901-1.668959$

H $2.6636985 .632120-0.654429$

H $3.1834875 .649507-2.352542$

SCF Energy (B3LYP/6-31G**//MMFF)= -3245.92083779

07_00549

MM̄FF Geometry

C 1.2203431 .8276973 .034894

C 0.3609042 .8488463 .167951

C -0.2779793 .5519182 .000413$

O $-1.674853 \quad 3.167541 \quad 1.938891$

C $-0.252405 \quad 5.081546 \quad 2.159585$

C 1.0697705 .7159071 .791368

C $1.343684 \quad 6.2256570 .574674$

C 2.0833245 .8194032 .900300

C $0.4544536 .219915-0.639889$

C $1.2146565 .836894-1.917114$

C $1.6060194 .351163-1.984740$

C $2.5289104 .119184-3.181272$

C $2.8749732 .636565-3.348248$

O $0.407592 \quad 3.585399-2.137780$

O $3.5342992 .475348-4.613483$

C $1.5670181 .816011-3.370551$

C $3.8968352 .182787-2.299598$

$\begin{array}{lllll}\text { O } & 1.877860 & 0.425637 & -3.394617\end{array}$

C $0.5910712 .158900-2.218255$

C $-0.8320931 .589775-2.461346$

O $1.109071 \quad 1.636299-0.995363$

C $-1.965967 \quad 1.9502821 .403730$

O $-1.1750101 .111317 \quad 1.003550$

C -3.4366571 .8093651 .380991$

C -3.9766630 .6267091 .051544$

C $-5.406420 \quad 0.325456 \quad 0.968634$

C $-6.367511 \quad 1.4745091 .131203$

C $-0.9440450 .066130-2.520570$

C $-2.371921-0.452485-2.591580$

C $-4.348699-0.499007-4.109551$

O $-2.778367-1.317291-1.817899$

C $-5.465630-0.278576-3.136884$ 
C $-6.317274-1.258474-2.795504$

C $-7.508620-1.089617-1.895511$

C $-7.497698-2.052487-0.728362$

C $-7.829426-3.344657-0.892249$

C $-7.180783-1.4994610 .641398$

C $-5.784257-0.9496830 .738527$

N $-3.1035440 .084219-3.640733$

H 1.4964861 .4858752 .040565

H $0.0659563 .167524 \quad 4.164891$

H 0.1990553 .2815721 .050968

H -0.5263935 .3665893 .184896$

H -1.057311 5.5189791 .554653

H 2.3095256 .7079590 .429377

H 2.9704016 .3874422 .599754

H 1.6491086 .3281553 .767278

H 2.4216164 .8270783 .210741

H $-0.4090645 .558996-0.520967$

H $0.0636037 .236890-0.767114$

H $0.5655846 .072761-2.770740$

H $2.1091856 .465790-2.009821$

H $2.1038654 .061035-1.053141$

H $3.4441654 .717890-3.092244$

H $2.0322554 .468727-4.097457$

H $2.9264942 .769194-5.313194$

H $1.0618052 .031257-4.321473$

H $4.7985802 .804820-2.351377$

H $4.2341661 .159929-2.493712$

H $3.5170342 .232751-1.276994$

H $2.1062710 .155688-2.488686$

H -1.494967 $1.952358-1.663954$

H $-1.2322902 .037303-3.380077$

H $0.3658181 .465871-0.393900$

H -4.031952 2.6788111 .635015

H $-3.311149-0.2066160 .829162$

H $-6.319614 \quad 1.8734802 .150344$

H $-7.408277 \quad 1.2075370 .938534$

H -6.1276872 .2793130 .426782$

H $-0.427928-0.332828-3.399426$

H $-0.478919-0.375147-1.632777$

H -4.171453 -1.562612 -4.303063

H $-4.592871-0.008398-5.057438$

H $-5.6049940 .722143-2.737087$

H $-6.178592-2.245347-3.233320$

H -8.413501 -1.262244 -2.493184

H -7.590724 -0.057190 -1.534507

H -7.850236 -4.035214 - 0.055765

H -8.087220 -3.740100 -1.869290

H -7.271355 $-2.286911 \quad 1.401504$

H $-7.945490-0.7637230 .904073$

H -5.009141 -1.7096210 .628132$

H $-2.6052090 .657049-4.314496$

C $1.098526-0.2244604 .530589$

O $1.063192-1.1090743 .391849$

C $-0.224016-1.7564983 .360325$

C $-0.878115-1.4714274 .708785$

C $-0.348356-0.0844675 .006779$

H $-0.807838-1.2586112 .577996$

C $-0.084065-3.2479083 .043986$

H -1.970357 -1.513702 4.656694

H $-0.548323-2.1785925 .478868$

C $-0.4852120 .323388 \quad 6.462856$

H $-0.901907 \quad 0.627996 \quad 4.384178$

C $0.595199-3.5754921 .699663$

O $0.684520-3.877796 \quad 4.077987$

H -1.072890 -3.720483 3.078805

H $1.667414-3.3577061 .758676$

O $0.481929-4.9916461 .501720$

C $-0.011063-2.8660550 .474865$

C $0.423901-3.434338-0.889884$

H $\quad 0.239417-1.8001430 .515500$

O $-1.438706-2.9505450 .529908$

H $0.003621-4.439861-1.022822$

H $-0.054408-2.817070-1.661056$

H $-1.5381940 .329825 \quad 6.762845$

H $\quad 0.048419-0.3664157 .125331$

H -0.0819291 .3277776 .625630$

C 1.8210601 .0833894 .191806

H $1.545664-3.4270494 .117966$
H $\quad 0.881131-5.4249092 .275713$

H -1.678186 -3.8932010 .530869$

H 2.8608940 .8598823 .923924

H 1.8609241 .7245265 .080438

H $1.688371-0.7447165 .297192$

C $3.869287-2.008112-1.147077$

O $2.446500-2.141466-1.019576$

C $1.941199-3.474880-1.120329$

C $2.292287-4.078164-2.489243$

C $3.802832-4.022612-2.740463$

C $4.373580-2.628712-2.464477$

H $4.046103-0.926774-1.193944$

C $4.607822-2.5463610 .088856$

H $2.389491-4.094184-0.337951$

O $1.607154-3.363775-3.516028$

H $1.941394-5.116580-2.518864$

H $4.012258-4.294350-3.782331$

O $4.464424-4.967446-1.904528$

H $5.469288-2.667557-2.461606$

O $3.990766-1.781183-3.549988$

C $4.297860-1.7542151 .342029$

H $4.350233-3.5884870 .293921$

H $5.689189-2.477308-0.077731$

H $1.838760-3.775900-4.365663$

H $4.108609-5.847074-2.116702$

H $4.431120-0.924026-3.423655$

O $4.980808-2.3090382 .387663$

O $3.558777-0.7818121 .402631$

C $4.795118-1.6766913 .657303$

H $5.492750-2.1288504 .367195$

H $5.009798-0.6057773 .591507$

H $3.775175-1.8481504 .011119$

SCF Energy (B3LYP/6-31G**//MMFF) $=-3245.91338435$

0700550

MM̄MF Geometry

C -1.604948 -1.633532 3.158572

C $-2.465377-0.7873113 .746230$

C -2.3173410 .7109793 .885355$

O $-1.062621 \quad 1.185003 \quad 3.334991$

C -3.4379891 .4784043 .158268$

C -4.8284641 .2667223 .726652$

C -5.8306820 .6381423 .080262$

C -5.0722451 .8677365 .086836$

C $-5.768893-0.0027551 .722603$

C -6.3666820 .9224550 .654132$

C $-5.9801360 .552130-0.785142$

C $-6.492120-0.822347-1.212403$

C $-5.999098-1.207068-2.617195$

O $-4.5545820 .599530-0.863642$

O $-6.260480-2.610442-2.773134$

C $-4.470290-0.990822-2.693296$

C $-6.799482-0.489977-3.710613$

O $-3.986687-1.185649-4.020459$

C $-4.0143560 .391904-2.171182$

C $-2.4677700 .431360-2.049075$

O $-4.4520521 .395092-3.086805$

C $0.020800 \quad 1.179348 \quad 4.155385$

O $0.0556850 .773729 \quad 5.310119$

C 1.2285751 .7255523 .494404

C 1.2447802 .2921652 .277444

C 2.4202472 .8732681 .617207

C 3.7392182 .7911262 .342344

C $-1.9099981 .617706-1.246102$

C $-1.8025432 .914869-2.026054$

C $-1.3472415 .343705-1.680399$

O $-1.8880202 .973621-3.249365$

C $0.1129485 .512037-1.953417$

C $0.9410026 .216795-1.168267$

C $2.4128606 .348716-1.426782$

C $3.2481375 .637703-0.385569$

C 3.9763126 .3273700 .508362

C $3.3119814 .126667-0.441009$

C 2.2643513 .4638560 .413408

N -1.570643 $3.996930-1.189081$

H $-0.693611-1.2581892 .702286$

H -3.354798 -1.2085194 .211828$

H -2.3498520 .9649214 .951960$ 
H $-3.394393 \quad 1.2518972 .086611$ H -3.212539 2.5532963 .203206 H -6.8018710 .5703893 .568241$ H $-6.120467 \quad 1.7881685 .394377$ H $-4.468823 \quad 1.3619605 .846525$ H -4.8123312 .9314405 .084876$ H $-4.743066-0.2902151 .473843$ H $-6.335580-0.9409551 .759989$ H -7.4590650 .9442590 .752284$ H -6.0096901 .9456150 .835963$ H $-6.395793 \quad 1.327049-1.440155$ H -7.587427 -0.860937-1.162996 H $-6.137300-1.585643-0.509230$ H $-6.008328-2.866568-3.676349$ H -4.000340 -1.766075 -2.074543 H -7.858867 -0.766748 -3.650341 H $-6.466973-0.793610-4.709404$ H $-6.7367350 .598188-3.644102$ H -4.190298 -2.100365 -4.277813 H -2.144755 -0.472226 -1.515618 H -2.009442 $0.404222-3.044821$ H -3.912776 $2.190943-2.965300$ H 2.1290401 .6550664 .097209 H $\quad 0.3163202 .3562931 .712732$ H 3.6819023 .3112643 .305126 H 4.5701033 .2427111 .796901 H 4.0131371 .7456722 .522161 H $-2.4929161 .784762-0.334333$ H $-0.8899251 .368973-0.927619$ H -1.707890 $6.036792-0.913918$ H -1.922584 5.506713 -2.597099 H $0.5088545 .013252-2.834965$ H $0.5481856 .711777-0.283323$ H $2.6544207 .419224-1.451427$ H $2.6782785 .965613-2.420297$ H 4.6021245 .8220091 .236209 H 3.9601887 .4118270 .534835 H $4.3200853 .789277-0.180579$ H $3.1758153 .792619-1.477319$ H $1.2691923 .469485-0.029786$ H -1.416722 $3.828708-0.198895$ C -1.664066 -3.7610181 .748748$ O $-0.306469-3.661007 \quad 1.285932$ C $-0.319710-3.605495-0.155338$ C $-1.782984-3.722627-0.585559$ C $-2.521107-3.1853800 .627107$ H $0.050657-2.611967-0.432732$ C $0.600160-4.689623-0.732836$ H -1.986253 -3.154943 -1.498626 H -2.071909-4.762451 -0.777193 C $-3.976958-3.6097620 .693573$ H -2.461115 -2.090109 0.606843 C $2.037262-4.665559-0.173507$ O $0.041695-5.972651-0.417012$ H $0.609939-4.618641-1.826892$ H $2.021835-4.9358850 .888830$ O $2.774887-5.706166-0.829889$ C $2.782562-3.331428-0.358075$ C $4.229237-3.4122430 .169529$ H $2.249760-2.5363800 .174564$ O $2.780600-3.006372-1.746428$ H $4.211047-3.8143051 .189708$ H $4.797711-4.103757-0.464894$ H -4.514749 -3.285191-0.201145 H $-4.075535-4.6983910 .763229$ H -4.470772 -3.170512 1.566005 C -1.808049 -3.123503 3.131181 H $0.636078-6.646734-0.789125$ H $2.853994-5.461167-1.768002$ H $3.234272-2.153214-1.850800$ H $-1.053903-3.5622243 .796819$ H -2.790841 $-3.383123 \quad 3.542581$ H -1.865816 -4.835802 1.857001 C $5.828775-0.397623-1.367633$

O $5.192450-1.667638-1.177330$

C $4.947629-2.0478270 .180028$

C $6.269682-2.1115040 .962351$

C $7.055308-0.8020680 .846785$
C $7.163834-0.328321-0.605972$

H $6.050688-0.357671-2.441823$

C $4.8875410 .766044-1.042904$

H $4.282461-1.3145230 .648768$

O $7.073854-3.1916310 .491373$

H $6.047072-2.3152532 .016298$

H $8.060531-0.9525471 .259450$

O $6.402971 \quad 0.189348 \quad 1.634481$

H $7.5615100 .692670-0.642112$

O $8.111085-1.169306-1.270860$

C $3.7522300 .822595-2.036113$

H $4.4681890 .716176-0.034567$

H $5.4185361 .722873-1.109213$

H $7.227070-3.056076-0.459367$

H 6.9291591 .0049251 .575136

H $8.228883-0.824315-2.172448$

O $2.6465420 .217034-1.517926$

O $3.8468631 .324832-3.148638$

C $1.5072800 .176420-2.382245$

H $1.1459321 .190772-2.574954$

H $1.756183-0.330357-3.319644$

H $0.720526-0.391211-1.878796$

SCF Energy (B3LYP/6-31G**//MMFF) $=-3245.91719215$

0700551

MM̄FF Geometry

C -2.254563 -4.365410 -1.578874

C $-1.422851-3.955467-0.610812$

C $0.033530-3.663997-0.865262$

O $0.285165-2.281171-0.519664$

C $0.926666-4.5415420 .028549$

C $2.372308-4.543842-0.430575$

C $3.304203-3.645438-0.053633$

C $2.728957-5.646808-1.393391$

C $3.105591-2.4654110 .856477$

C $4.293626-2.1581131 .781124$

C $5.588880-1.7564631 .053024$

C $6.606270-1.1748772 .039941$

C $7.870779-0.6589731 .335015$

O $5.264863-0.7873560 .052365$

O $8.583764 \quad 0.1430352 .289233$

C $7.4516600 .257406 \quad 0.162668$

C $8.810574-1.8100560 .958343$

O $8.587491 \quad 0.660494-0.598178$

C $6.375987-0.369751-0.749370$

C $5.8439520 .612443-1.816587$

O $6.953648-1.475810-1.454151$

C $-0.109881-1.320736-1.397634$

O $-0.661515-1.480056-2.474071$

C $0.218682-0.014798-0.788489$

C $-0.4912081 .070053-1.130392$

C $-0.3380762 .406818-0.551892$

C 0.8737352 .6586140 .305922

C $5.1251201 .846791-1.266954$

C $4.4899152 .670977-2.375214$

C $3.1528194 .724323-2.743230$

O $4.6237652 .413210-3.568577$

C $2.1312565 .509552-1.986225$

C $0.8589895 .656683-2.386705$

C $-0.1446516 .503213-1.658285$

C $-1.4320045 .772135-1.356515$

C $-2.5774476 .101053-1.978108$

C $-1.4137374 .726125-0.265432$

C $-1.2905393 .327897-0.805326$

N $3.7545573 .735200-1.869249$

H $-1.860814-4.528341-2.579953$

H -1.806143 -3.7754760 .390317$

H $0.292781-3.821356-1.920549$

H $0.562874-5.5778420 .027363$

H $0.864203-4.2109231 .070783$

H $4.296962-3.741358-0.488094$

H $3.772635-5.594345-1.720850$

H $2.579014-6.623757-0.922103$

H $2.099088-5.590963-2.287109$

H $2.900418-1.5865600 .235122$

H $2.228034-2.6062171 .493338$

H $3.980183-1.3272562 .427245$

H $4.491445-3.0127382 .439830$ 
H $\quad 6.005243-2.6467200 .568852$ H $6.866263-1.9118752 .809918$ H $6.147901-0.3324812 .577274$ H $\quad 9.3483670 .5340091 .832220$ H $7.049631 \quad 1.1730160 .613468$ H $9.122121-2.3556901 .857338$ H $9.734220-1.4364250 .502932$ H $8.355259-2.5306540 .275942$ H $8.848882-0.089052-1.159798$ H $6.6598320 .917908-2.484730$ H $5.1354190 .068489-2.457675$ H $6.405767-1.665186-2.233875$ H $0.9924290 .005712-0.029860$ H -1.290331 $0.960466-1.862639$ H $\quad 0.9990093 .706517 \quad 0.585627$ H $1.7862542 .373198-0.228017$ H $\quad 0.8215172 .0766401 .231755$ H $4.3339181 .547496-0.570548$ H $5.8311812 .493504-0.735463$ H $2.7300914 .219308-3.618536$ H $3.9533985 .387118-3.088223$ H $2.4640626 .011274-1.080472$ H $0.5316615 .180985-3.308356$ H $-0.3480257 .383045-2.282720$ H $\quad 0.270213 \quad 6.893921-0.720573$ H $-3.5106355 .600637-1.740613$ H -2.607532 $6.872692-2.739987$ H $-2.356974 \quad 4.7753570 .296228$ H $-0.643137 \quad 4.9714240 .470454$ H -2.129392 $3.035782-1.438445$ H $3.8561263 .962301-0.883766$ C $-4.504662-3.514719-2.180781$ O $-4.534139-2.289218-1.416107$ C $-5.804727-1.643024-1.639712$ C $-6.525195-2.468171-2.701125$ C $-5.969017-3.854016-2.437944$ H $-6.370738-1.719642-0.705247$ C $-5.591779-0.175533-2.026993$ H -7.613466 -2.414371 -2.598737 H $-6.267127-2.134311-3.713503$ C $-6.194688-4.820888-3.587153$ H $-6.429331-4.256297-1.525626$ C $-4.9931740 .698495-0.902647$ O $-4.709964-0.116914-3.155660$ H $-6.5387370 .261146-2.364333$ H $-3.9825540 .355337-0.654317$ O $-4.8569962 .030378-1.414400$ C -5.8623530 .7576730 .369186$ C $-5.435441 \quad 1.8229211 .395670$ H -5.875712 -0.2260420 .849968$ O $-7.210352 \quad 1.051552-0.015172$ H -5.4663492 .8197590 .938278$ H -6.2046051 .8373022 .179954$ H -7.265615 -4.951449 -3.774490 H -5.733492 -4.459507 -4.512444 H $-5.770443-5.804096-3.361738$ C -3.731116 -4.582602 -1.407048 H -3.872121 -0.536552 -2.894389 H $-4.3199301 .975858-2.223435$ H -7.7620070 .9999920 .783940$ H $-3.964657-5.583646-1.785272$ H $-4.003146-4.555637-0.345067$ H -4.003560 -3.285149 -3.131567 C $-2.905364-0.1085673 .337261$ O $-4.124036 \quad 0.3138332 .711918$ C -4.0619821 .5748732 .037504$ C -3.695010 2.6935173 .027933 C -2.4018682 .3723853 .783016$ C $-2.404463 \quad 0.945128 \quad 4.341401$ H -3.187141 -1.001989 3.909017 C -1.880743 -0.5351532 .280650$ H -3.3051981 .5262371 .247746$ O $-4.7550582 .868806 \quad 3.967028$ H -3.588612 3.6333952 .473100 H -2.2761973 .0804614 .611150$ O -1.2868772 .5290492 .914000$ H -1.405180 0.6722424 .698200 O $\quad-3.2770640 .8948875 .475209$
C $-0.740465-1.3188602 .880345$

H -2.356042 -1.149319 1.505435

H $-1.4618150 .321346 \quad 1.747298$

H -4.5115183 .6071894 .550849$

H -1.2883003 .4472312 .594145$

H $-2.933579 \quad 1.518827 \quad 6.137234$

O $-1.162633-2.567953 \quad 3.225758$

O $0.385360-0.8649883 .039043$

C $-0.195328-3.3939093 .881626$

H $\quad 0.761026-3.3827573 .351528$

H $-0.064045-3.0546364 .913052$

H -0.575482 -4.418827 3.892261

SCF Energy (B3LYP/6-31G**//MMFF) $=-3245.90840055$

0800001

MM̄MF Geometry

C $-0.0531870 .246186-2.919156$

C $-1.042215-0.604563-3.232963$

C -1.359654 -1.933872 -2.590645

O $-2.705465-1.835511-2.061607$

C $-0.428243-2.391530-1.450801$

C $-0.500949-3.885056-1.192151$

C $-1.149870-4.465307-0.164362$

C $0.276650-4.739509-2.162421$

C $-1.980140-3.8113230 .903845$

C $-1.304937-3.8771892 .281908$

C $-0.149969-2.8753612 .428122$

C $0.575296-3.0623563 .761686$

C $1.660350-1.9944743 .973729$

O $-0.716974-1.5636522 .365121$

O $2.065900-2.0433525 .349883$

C $1.042802-0.6009913 .733547$

C $2.915050-2.2798793 .140630$

$\begin{array}{llll}\text { O } & 2.041038 & 0.415617 & 3.775950\end{array}$

C $0.237482-0.4979402 .417578$

C -0.5463250 .8349172 .384264$

O $1.136754-0.5428911 .309857$

C $-3.589091-2.816800-2.375952$

O $-3.386803-3.775645-3.109349$

C $-4.901713-2.612197-1.720884$

C $-5.142609-1.691583-0.774202$

C $-6.423928-1.473554-0.094141$

C $-7.595181-2.321526-0.518945$

C -1.5777860 .9242041 .250976$

C -2.3607382 .2246461 .298790$

C -4.3022573 .3502820 .209306$

O -2.1207103 .1277282 .095233$

C -5.4911683 .0477751 .064234$

C -6.7131272 .7875910 .576445$

C -7.8916162 .4403251 .438577$

C -8.4403701 .0609191 .144490$

C -9.6021050 .9100740 .486350$

C $-7.695174-0.1376671 .692738$

C $-6.500276-0.5237940 .861938$

N $-3.358487 \quad 2.2552690 .334515$

H $0.6047150 .036152-2.081942$

H -1.668113 -0.361327 -4.090798

H -1.324709 -2.664583 -3.408110

H $-0.661086-1.830801-0.539663$

H $0.618305-2.164567-1.688317$

H - $-1.100385-5.550486-0.078431$

H $0.223974-5.804972-1.914476$

H $-0.109079-4.621428-3.179331$

H $1.333287-4.453814-2.155740$

H $-2.245178-2.7806860 .650978$

H $-2.930853-4.3571400 .956122$

H -2.069734 -3.650765 3.036217

H $-0.948741-4.8966012 .475692$

H $0.551140-3.0103921 .597391$

H $1.007501-4.0682673 .832202$

H $-0.148990-2.9885164 .585080$

H $2.391524-2.9411315 .534568$

H $0.360938-0.3993824 .571792$

H $3.346287-3.2496563 .416095$

H $3.696420-1.5379753 .337411$

H $2.719446-2.3003292 .067096$

H $2.518698 \quad 0.318378 \quad 4.617577$

H -1.0877030 .9570053 .331918$ 
H $\quad 0.151863 \quad 1.6765982 .298846$ H $0.753088-0.0571850 .563393$ H -5.674349 -3.298638 -2.053741 H -4.332229 -1.038632 -0.455185 H -7.793036 -2.196546 -1.589464 H -8.524492 -2.071573 -0.003259 H -7.396103 -3.380000 -0.318096 H $-1.087326 \quad 0.8610110 .274673$ H -2.295716 0.0993521 .327456 H -4.564418 $3.437002-0.849582$ H $-3.833811 \quad 4.2849360 .532869$ H -5.3313903 .0265072 .139924$ H $-6.8761952 .812416-0.498574$ H $-8.6635153 .202637 \quad 1.270599$ H -7.640478 2.5059152 .504633 H $-10.023430-0.0717980 .299304$ H -10.157745 1.7656490 .116916 H -8.373721 -0.9889411 .818843$ H $-7.353130 \quad 0.0850732 .711834$ H -5.5985680 .0383311 .104016$ H $-3.5198041 .428966-0.234217$ C $0.3562312 .772394-2.944998$ O $1.3626352 .685911-1.919972$ C $0.9350923 .481305-0.794191$ C $-0.3371304 .202772-1.234863$ C $-0.9161183 .221559-2.237412$ H 0.6765542 .7793810 .005214 C $2.0723244 .402866-0.338313$ H $-1.0073574 .410177-0.395841$ H $-0.1135325 .158415-1.723354$ C - $-1.9576373 .831698-3.157732$ H -1.364376 $2.384095-1.691665$ C 3.3756663 .6535140 .012967 O $2.3665125 .313425-1.406075$ H 1.7386415 .0142400 .508040 H $3.7654193 .170537-0.888926$ $\begin{array}{lllll}\text { O } & 4.354841 & 4.631111 & 0.388490\end{array}$ C 3.2317632 .6293711 .154299 C 4.5529921 .9321561 .534388 H $2.487467 \quad 1.8745060 .885668$ O 2.7379383 .3132112 .309029 H 5.3338692 .6792041 .720034 H $4.397674 \quad 1.4182762 .492201$ H -2.802737 4.219161-2.579954 H -1.542521 $4.660424-3.740943$ H $-2.3424543 .084897-3.859255$ C $0.2778381 .466943-3.734938$ H $3.0949045 .884230-1.106781$ H 4.0516915 .0456871 .214666 H 2.5662712 .6442602 .993996 H $1.2609981 .283360-4.186822$ H - $0.4317851 .585461-4.562140$ H $0.6925183 .559337-3.634196$ C $4.262302-1.135007-0.555525$ O $4.045558-0.1310680 .443353$ C 5.0219190 .9144320 .480918 C $6.3990120 .324998 \quad 0.817105$ C $6.785176-0.778231-0.175096$ C $5.649112-1.785597-0.393567$ H $3.501801-1.895552-0.337589$ C $3.969818-0.570710-1.953303$ H $5.0689701 .402413-0.497983$ O $6.404372-0.2043852 .141406$ H 7.1448001 .1278140 .790261 H $7.667514-1.3114820 .200074$

O $7.132264-0.193959-1.426924$ H $5.873946-2.420470-1.257826$ O $5.608732-2.640430 \quad 0.754586$ C $3.518343-1.638269-2.925907$ H $3.1613010 .166216-1.870427$ H $4.836622-0.060208-2.380468$ H $5.716961-0.8904812 .189360$ H $7.8807310 .407447-1.273076$ H $4.943035-3.3286280 .584898$ O $2.981740-1.037320-4.028407$ O $3.628587-2.844036-2.752149$ C $2.472279-1.926359-5.027172$ H $3.283778-2.529994-5.444222$
H $\quad 1.691348-2.565905-4.604278$

H $2.034947-1.323217-5.827338$

SCF Energy (B3LYP/6-31G**//MMFF) $=-3245.92870194$

08_00002

MMFF Geometry

C -0.975835 -4.399529 1.769479

C $0.211890-3.8412721 .490039$

C $1.119343-3.2581642 .543879$

O $1.525320-1.9320382 .129704$

C $2.380051-4.1298832 .679232$

C $3.326945-3.6559393 .765117$

C $4.495813-3.0208003 .546050$

C $2.899882-3.9798995 .174097$

C $5.089447-2.6111672 .226613$

C $5.347031-1.0993332 .180966$

C $5.805782-0.6385590 .789263$

C $6.130800 \quad 0.8561810 .799164$

C $6.4636791 .388214-0.602554$

O $4.742725-0.906394-0.131263$

O $6.4193772 .822911-0.551163$

C $5.360240 \quad 0.939414-1.584867$

C $7.8876121 .014133-1.030190$

O $5.7057631 .280691-2.924769$

C $5.032387-0.565196-1.493838$

C $3.804457-0.974214-2.346167$

O $6.149338-1.317563-1.984172$

C $0.645180-0.9152662 .344243$

O $-0.486496-1.0042912 .793121$

C 1.3152360 .3469481 .957302

C 0.6762561 .5164412 .110968

C 1.2246972 .8461461 .830800

C 2.6220182 .9251921 .276294

C $2.476557-0.318541-1.953214$

C $2.1589320 .957413-2.714150$

C $0.5385712 .854767-2.713852$

O $2.7717331 .316325-3.715601$

C $1.0009484 .011515-1.886770$

C $0.1614874 .871995-1.291594$

C $0.6044086 .072555-0.508218$

C 0.0483196 .0933430 .897117

C -1.0579696 .7962071 .194060$

C 0.8323665 .3817401 .975084

C $0.464768 \quad 3.9285312 .099317$

N $1.0772101 .627765-2.160031$

H -1.290507 -4.437301 2.811236

H $\quad 0.569290-3.7907330 .465780$

H $0.609669-3.1923623 .513399$

H $2.894334-4.1834741 .713890$

H $2.091618-5.1656262 .905958$

H $5.103758-2.7563994 .410634$

H $3.647086-3.6773045 .915586$

H $1.965992-3.4663125 .421790$

H $2.745380-5.0580735 .285789$

H $6.038017-3.1447272 .092225$

H $4.450342-2.8974711 .388866$

H $4.422994-0.5732702 .451229$

H $6.104804-0.8336212 .928646$

H $6.688643-1.2216810 .501829$

H $\quad 6.9501541 .0708531 .496604$

H 5.2644121 .4117891 .180185

H 7.0573663 .1186200 .120758

H $4.4608771 .515305-1.335375$

H $8.6203651 .438336-0.333142$

H $8.1298091 .436510-2.011546$

H $8.055714-0.064150-1.066159$

H $5.8815812 .237071-2.944167$

H $4.022249-0.838272-3.412393$

H $3.667059-2.057347-2.214700$

H $\quad 5.837817-2.200276-2.244143$

H $2.329677 \quad 0.282077 \quad 1.582529$

H -0.3401751 .5049182 .502793$

H 2.9124053 .9316360 .967379

H 2.7242732 .2962860 .385763

H 3.3458742 .5921102 .027369

H $1.664659-1.018785-2.188174$

H $2.437542-0.124611-0.877450$

H $\quad-0.5514682 .759447-2.715589$ 
H $0.8761542 .979296-3.747516$ H $2.0741034 .163425-1.799022$ H $-0.9099284 .730744-1.408584$ H $0.2703846 .965880-1.052480$ H $1.6984216 .146102-0.471415$ H -1.443563 6.8408732 .207247 H $-1.606415 \quad 7.3355640 .428864$ H $0.628501 \quad 5.8487642 .948577$ H 1.9033795 .5404651 .819721 H -0.5354403 .7710332 .504784$ H $0.650887 \quad 1.265662-1.312104$ C $-1.654845-4.852479-0.693287$ O $-1.728235-3.459501-1.060364$ C $-2.579872-3.325340-2.210260$ C $-2.654884-4.714181-2.826823$ C $-2.670958-5.578184-1.580988$ H $-3.574184-3.055775-1.835651$ C $-2.072648-2.220664-3.139572$ H $-3.541685-4.845746-3.453983$ H -1.769406 -4.939979 -3.432116 C $-2.328810-7.034230-1.845419$ H $-3.671245-5.524391-1.130174$ C $-1.825314-0.866288-2.444460$ O $-0.829738-2.638563-3.718017$ H -2.774116 -2.101034-3.974067 H $-0.911776-0.926038-1.840979$ O $-1.5526460 .095723-3.471645$ C $-2.980360-0.356942-1.563619$ C $-2.7169881 .065092-1.032385$ H -3.117087 -1.041922 -0.719813 O $-4.180144-0.368668-2.335350$ H -1.679632 $1.138439-0.686050$ H $-2.8609411 .777584-1.853994$ H $-3.058167-7.482808-2.527783$ H $-1.337075-7.137634-2.298443$ H $-2.336181-7.611402-0.915501$ C $-1.960440-5.0012720 .800663$ H $-0.526948-1.922930-4.303110$ H $-2.3729470 .223613-3.978495$ H $-4.906590-0.087459-1.753654$ H -2.056551 -6.064894 1.048785 H -2.930286 -4.5315421 .009179$ H $-0.638656-5.196766-0.921838$ C $-5.998828 \quad 1.7225410 .652081$ O $-4.9963591 .454087-0.337392$ C $-3.647395 \quad 1.447554 \quad 0.135905$ C $-3.285703 \quad 2.8198890 .722634$ C $-4.274703 \quad 3.2319751 .816263$ C $-5.728858 \quad 3.060691 \quad 1.361174$ H $-6.931114 \quad 1.8277920 .082054$ C $-6.171750 \quad 0.5466241 .619803$ H -3.5381380 .6803410 .910506$ O $-3.290675 \quad 3.797450-0.315137$ H -2.2702292 .7713521 .128889$ H -4.097072 4.280352 2.085600 $\begin{array}{llll}\text { O } & -4.026961 & 2.437689 & 2.972275\end{array}$ H $-6.407658 \quad 3.1641562 .215166$ $\begin{array}{lllll}\text { O } & -6.054266 & 4.120989 & 0.456782\end{array}$ C $-6.658886-0.6789550 .883006$ H $-5.250903 \quad 0.2954942 .153800$ H -6.9186850 .7792792 .387952$ H -3.016392 4.6434460 .078083 H -4.6439552 .7278093 .665467$ H $-5.5099634 .008488-0.341481$ O $-5.652349-1.592507 \quad 0.777349$ $\begin{array}{llll}0 & -7.792556 & -0.798798 & 0.436529\end{array}$ C $-5.986120-2.7990310 .084674$ H $-6.178604-2.583305-0.970431$ H $-5.133027-3.4783430 .155235$ H $-6.853610-3.2804880 .546124$

SCF Energy (B3LYP/6-31G**//MMFF) $=-3245.92397586$

08_00003

MMFFF Geometry

C -2.785089 1.2794862 .390022

$\begin{array}{llll}C & -1.815625 & 0.351745 & 2.378562\end{array}$

C $-0.951965 \quad 0.061903 \quad 3.575894$

$\begin{array}{llll}\text { O } & 0.442908 & 0.159312 & 3.207050\end{array}$
C -1.201852 -1.324210 4.202614

C $-1.070296-2.5341393 .294535$

C $-2.095149-3.3374752 .944905$

C $0.327909-2.8845522 .856958$

C $-3.546541-3.1583703 .287962$

C $-4.520559-3.705177 \quad 2.229569$

C $-4.460507-2.9694360 .880530$

C $-5.701931-3.2961260 .044368$

C $-5.646403-2.649080-1.347037$

O $-3.285931-3.3942520 .177908$

O $-6.665354-3.254604-2.157345$

C $-4.290881-2.988328-1.998429$

C $-5.967983-1.150495-1.294510$

O $-4.127346-2.284678-3.228368$

C $-3.078638-2.728338-1.074150$

C $-1.794017-3.286164-1.731491$

O $-2.930031-1.322727-0.895423$

C $0.999446 \quad 1.3960403 .140789$

$\begin{array}{lllll}\text { O } & 0.425858 & 2.463553 & 3.312088\end{array}$

C $2.453307 \quad 1.3305402 .859870$

C 3.1201430 .1902752 .621905

C $4.561083 \quad 0.055138 \quad 2.392062$

C 5.3921181 .3117212 .407043

C $-0.565147-3.313919-0.812404$

C $0.671891-3.813911-1.541140$

C $2.983394-4.590676-1.141233$

O $0.690480-4.053589-2.745991$

C $4.085270-4.351756-0.159493$

C $5.266985-3.815549-0.500755$

C $6.417531-3.6254100 .445391$

C $6.778373-2.1718240 .659273$

C $7.399105-1.456082-0.293865$

C $6.506414-1.5779232 .021675$

C $5.068063-1.1819512 .201191$

N $1.760720-3.954733-0.687682$

H $-2.986276 \begin{array}{lll}1.814895 & 3.314141\end{array}$

H $-1.606693-0.1983501 .465798$

H -1.1400660 .7982184 .369506$

H $-2.187188-1.3109874 .682358$

H $-0.486725-1.4617255 .025708$

H $-1.868055-4.2260442 .357713$

H $0.391541-3.8987222 .448458$

H $0.667839-2.1945932 .079548$

H $1.021815-2.8358373 .702362$

H $-3.737687-3.6921634 .226960$

H $-3.787057-2.1036963 .458428$

H $-4.324175-4.7745992 .078859$

H $-5.532184-3.6163622 .645518$

H $-4.403882-1.8913631 .071959$

H $-6.616324-2.9919020 .569154$

H $-5.777993-4.385431-0.082473$

H -7.521431 -3.112499-1.718152

H $-4.307556-4.057423-2.252942$

H $-6.974711-0.989137-0.890784$

H $-5.974522-0.711984-2.298504$

H $-5.271153-0.582965-0.674890$

H $-4.901150-2.485884-3.782167$

H $-1.976389-4.316624-2.064231$

H $-1.555221-2.694162-2.624494$

H $-2.007410-1.124224-0.672957$

H 2.9564542 .2920792 .879323

H $2.569502-0.7474582 .611606$

H 4.9787862 .0636311 .726901

H 6.4241111 .1565352 .085870

H 5.4219371 .7362993 .416481

H $-0.343856-2.316641-0.421146$

H $-0.752830-3.9840930 .033365$

H $3.226345-4.218404-2.142487$

H $2.783712-5.664734-1.221403$

H $3.917315-4.6650850 .867940$

H $5.435376-3.527510-1.536228$

H $7.288546-4.1556550 .038233$

H $6.210654-4.1081341 .408619$

H $7.684106-0.422379-0.128157$

H $7.631158-1.888113-1.261710$

H $7.188705-0.7438072 .210845$

H $6.761524-2.3156602 .793573$

H $4.381720-2.0296532 .214578$ 
H $\quad 1.594295-3.8967690 .312917$ C $-3.441382 \quad 3.0692490 .697704$ O -2.0922513 .2675400 .229703$ C -1.673448 $4.597596 \quad 0.588684$ C $-2.929185 \quad 5.3257041 .062722$ C -3.712355 4.192837 1.696850 H -0.9916654 .4899191 .440418$ C $-0.9490395 .283870-0.574818$ H -2.699239 6.1348161 .762690 H -3.492448 5.7544520 .225725 C -5.1855394 .5014581 .895379$ H -3.259139 3.9742412 .670855 C $0.2735124 .521535-1.128816$ O $-1.8774895 .449955-1.654981$ H $-0.6552016 .294913-0.267180$ H $-0.0697113 .650567-1.696443$ O $\quad 0.912217 \quad 5.380397-2.083609$ C $1.3057984 .083849-0.075930$ C $2.6492743 .590796-0.648003$ H $0.866836 \quad 3.300576 \quad 0.551349$ O $1.588592 \quad 5.1967350 .778380$ H $3.1833784 .425999-1.119062$ H 3.2677613 .2992840 .211123 H -5.311891 5.3619342 .560362 H -5.6788204 .7355950 .946002$ H -5.7044823 .6485922 .344231$ C -3.633743 1.6354391 .202846 H -1.403356 $5.894162-2.378876$ H $1.2731936 .141561-1.597326$ H $2.170754 \quad 4.879274 \quad 1.489623$ H -3.4123840 .9487420 .378886$ H -4.6848901 .4779871 .471087$ H $-4.0846003 .199468-0.183338$ C $1.6361630 .174499-1.762426$ O $1.8629621 .349602-0.972276$ C $2.5489602 .415767-1.630757$ C $3.9361251 .952564-2.112629$ C $3.8514410 .673756-2.952888$ C $2.967579-0.387100-2.285349$ H $1.216118-0.554846-1.058844$ C $0.6010660 .405955-2.874383$ H $1.9633042 .740752-2.496391$ O $4.7992921 .714584-1.002128$ H $4.3910722 .757413-2.701234$ H $4.8593830 .264031-3.092840$ O $3.3269580 .975354-4.241528$ H $2.770768-1.215898-2.975287$ O $3.663140-0.928147-1.159895$ C $-0.7433230 .831356-2.331175$ H $0.9249881 .186128-3.567807$ H $0.452524-0.524137-3.434811$ H $4.3824521 .035622-0.444445$ H $3.9204731 .624358-4.656372$ H $4.512235-1.275449-1.480846$ O $-1.567634 \quad 1.136222-3.376369$ O $-1.0329120 .894912-1.145274$ C $-2.8694201 .613814-3.023190$ H -3.439795 $1.756589-3.944804$ H $-3.3931600 .885046-2.399122$ H -2.787685 2.575774-2.509678

SCF Energy (B3LYP/6-31G**//MMFF) $=-3245.91467096$

\section{8_00004}

MM̄FF Geometry

C $0.561014-2.7543581 .423761$

C $1.204948-2.0156802 .341958$

C $0.725828-0.7344772 .986482$

O $-0.580561-0.352056 \quad 2.505771$

C $1.701564 \quad 0.4038032 .647010$

C 1.4111351 .6894433 .391774

C 0.8329952 .7744572 .841159

C $1.876357 \quad 1.7228104 .824464$

C 0.3092502 .9167901 .437965

C $-1.0025813 .713871 \quad 1.413482$

C $-1.5195843 .911809-0.018429$

C $-2.6593034 .931176-0.038036$

C -3.220623 $5.117892-1.453385$

O $-1.9939192 .652270-0.503385$
O $-4.460608 \quad 5.834854-1.346787$

C $-3.5539373 .732408-2.043843$

C $-2.297166 \quad 5.982564-2.319745$

O $-3.9127493 .836777-3.419803$

C -2.434921 $2.673570-1.869195$

C $-3.0015641 .276566-2.196635$

O $-1.3465312 .890793-2.768071$

C $-1.658810-0.777677 \quad 3.216794$

O $-1.653572-1.469506 \quad 4.222261$

C -2.868672 $-0.270108 \quad 2.533034$

C $-4.063833-0.788746 \quad 2.851079$

C $-5.348749-0.4401812 .242154$

C -5.3803730 .7371391 .305650$

C $-1.997766 \quad 0.135960-2.011028$

C $-2.695777-1.188540-2.242115$

C $-4.051580-2.925921-1.074921$

O $-2.784412-1.681120-3.364700$

C $-5.494853-2.533451-0.999188$

C $-6.369194-3.079110-0.139528$

C $-7.817070-2.682722-0.061149$

C $-8.275938-2.3874821 .351751$

C $-9.115564-3.2184451 .992528$

C $-7.823013-1.0921391 .995173$

C $-6.426024-1.1952362 .542573$

N $-3.241755-1.721044-1.084935$

H $-0.422380-2.4530151 .077031$

H $2.184778-2.3537522 .676292$

H $0.701583-0.8846074 .072789$

H 1.6944640 .5833291 .566749

H 2.7283790 .0996452 .887561

H 0.7279123 .6634773 .461959

H 1.6948682 .6932385 .298589

H 1.3535720 .9655715 .416543

H 2.9522631 .5266484 .878996

H 1.0623543 .4494910 .845751

H $\quad 0.144593 \quad 1.9441660 .964967$

H -1.756014 3.1840922 .010840

H -0.836492 4.6928381 .880735

H $-0.6886994 .252787-0.646934$

H $-2.3304315 .894468 \quad 0.371550$

H -3.470853 4.5862720 .618050

H $-4.281597 \quad 6.679672-0.899581$

H -4.448469 $3.368079-1.518506$

H $-2.1912906 .983666-1.884365$

H -2.719285 $6.134932-3.318995$

H $-1.2936225 .565398-2.426214$

H $-4.6366614 .483297-3.482362$

H -3.874215 $1.081585-1.563372$

H $-3.3534601 .252092-3.236431$

H $-1.5228873 .690288-3.288083$

H -2.738881 0.4859921 .766902

H $-4.105323-1.5707303 .608569$

H $-4.938157 \quad 1.6211881 .779061$

H $-6.387927 \quad 1.0289921 .003217$

H -4.8205890 .5112930 .394930$

H $-1.1723200 .215122-2.726608$

H $-1.5564900 .147833-1.008077$

H -3.738909 $-3.520253-0.210666$

H $-3.869072-3.502863-1.986809$

H $-5.839544-1.767310-1.690063$

H $-6.032813-3.8661900 .531785$

H -8.408066 $-3.505329-0.484215$

H -8.024448 $-1.808748-0.691412$

H $-9.468196-3.0021262 .995565$

H $-9.465322-4.1359771 .531211$

H $-8.486947-0.8344092 .830728$

H $-7.943145-0.2846331 .268433$

H $-6.299497-2.0126453 .254449$

H $-3.155099-1.206138-0.214306$

C $1.323190-3.961187-0.690656$

O $2.057283-2.778040-1.072152$

C $1.361738-2.151650-2.165513$

C $0.498806-3.248613-2.772310$

C $0.034699-3.962258-1.516803$

H $0.701226-1.395294-1.725103$

C $2.334972-1.476340-3.134005$

H $-0.321685-2.852904-3.375802$

H $1.088441-3.928420-3.398638$ 
C $-0.534927-5.345780-1.775516$

H $-0.735538-3.351765-1.032231$

C $3.287562-0.457357-2.473220$

O $3.139342-2.484980-3.756424$

H $1.766102-0.997289-3.940012$

H $4.049268-0.997321-1.901338$

O $3.992097 \quad 0.218946-3.521902$

C $2.5987650 .582647-1.573897$

C $3.5055861 .725891-1.087351$

H $2.1662890 .074868-0.705605$

O $1.5144761 .171352-2.298025$

H $3.7620262 .384272-1.927552$

H $2.8953662 .342090-0.414881$

H -1.418369 -5.282703 -2.419393

H $\quad 0.195227-5.995491-2.269473$

H $-0.833795-5.824812-0.837894$

C $1.139770-4.0120610 .830074$

H $3.747821-2.034430-4.366923$

H $3.3436270 .751107-4.014279$

H $\quad 1.0350211 .756097-1.686350$

H $2.119572-4.2079281 .283606$

H $0.497798-4.8587161 .098893$

H $1.948671-4.813799-0.985971$

C $5.527211-0.1706881 .432129$

$\begin{array}{lllll}\text { O } & 4.434400 & 0.374739 & 0.678612\end{array}$

C $4.7890881 .284980-0.364590$

C 5.5554592 .4887240 .209366

C 6.7647462 .0407751 .035848

C 6.3905090 .9473102 .041484

H $5.054057-0.7086392 .263670$

C $6.337675-1.1874090 .612687$

H $5.425607 \quad 0.770199-1.090921$

O $4.6984293 .283173 \quad 1.029013$

H $5.8857193 .126138-0.618925$

H 7.1754342 .9020991 .576614

O $7.782049 \quad 1.544393 \quad 0.171531$

H 7.2912210 .5100072 .487979

O 5.6363501 .5332043 .107326

C $5.528724-2.4231140 .279777$

H $6.685064-0.755066-0.329146$

H $7.209656-1.5209391 .186624$

H 4.3217932 .7046731 .714307

H $8.0248442 .261309-0.438914$

H 6.1983902 .2056413 .528634

O $6.157544-3.112876-0.717331$

O $4.491779-2.7560890 .835973$

C $5.510346-4.318272-1.137095$

H $\quad 6.158091-4.816387-1.863314$

H $5.357536-4.989551-0.286491$

H $4.558271-4.082610-1.620129$

SCF Energy (B3LYP/6-31G**//MMFF) $=-3245.92432520$

08_00005

MM̄FF Geometry

C $0.369914-3.373781 \quad 1.240239$

C $1.241989-3.6665722 .217613$

C 2.132551-2.697728 2.962792

O $2.062319-1.3730712 .388994$

C $3.593765-3.1781122 .875986$

C $4.571712-2.2934863 .625681$

C $5.450229-1.4525163 .043539$

C $4.542456-2.4327385 .126049$

C $5.617819-1.1743561 .575529$

C 5.4791890 .3236441 .272075

C $5.3682700 .587161-0.236775$

C $5.3523072 .088920-0.524201$

C $5.1142252 .389091-2.011652$

O $4.149841-0.013027-0.691090$

O $4.7897053 .782995-2.132803$

C $3.8794641 .596008-2.491568$

C $6.3789652 .164657-2.848587$

O $3.7110201 .724897-3.900796$

C $3.9153920 .103512-2.100986$

C $2.592515-0.642708-2.413258$

O $4.949558-0.550347-2.846947$

C $1.273384-0.4572663 .013586$

O $0.575844-0.6364393 .998585$

C 1.4171190 .8282662 .294661
C $0.600613 \quad 1.8524312 .584530$

C 0.6724663 .2037952 .020525

C 1.7712833 .4916741 .031858

C $1.349813-0.115756-1.688241$

C $0.5740030 .931767-2.467707$

C $-1.2606522 .595036-2.177671$

O $\quad 0.7587111 .158019-3.660158$

C $-0.8752983 .899619-1.557759$

C $-1.7079684 .631622-0.803184$

C $-1.3539625 .962139-0.209126$

C -1.4800635 .9797851 .296700$

C -2.6026996 .4172621 .891990$

C -0.2663325 .5939822 .109244$

C -0.2182354 .1274402 .439442$

N $-0.3776151 .559832-1.676714$

H $\quad 0.226163-2.3433980 .927676$

H $1.338399-4.7078552 .521514$

H $1.819525-2.6845644 .013892$

H $3.882730-3.2732041 .824092$

H $3.674156-4.1955863 .282981$

H $6.125776-0.8905993 .687493$

H $5.312861-1.8272425 .615246$

H $3.573759-2.1142095 .523112$

H $4.712526-3.4752165 .414356$

H $6.613745-1.5141551 .266984$

H $4.899276-1.7353240 .974664$

H $4.5842320 .711424 \quad 1.773721$

H $\quad 6.3430990 .8597001 .684304$

H $6.2175610 .108421-0.738598$

H $6.2813332 .560450-0.180722$

H 4.5475812 .5607240 .054954

H $5.5288804 .296039-1.763538$

H $3.0044972 .064947-2.025201$

H $7.1885322 .817913-2.501299$

H $6.2107112 .430095-3.898145$

H $6.745300 \quad 1.137032-2.806012$

H $3.6684252 .675525-4.101900$

H $2.435184-0.695163-3.497346$

H $2.724501-1.685136-2.088813$

H $4.759232-1.502750-2.869713$

H $\quad 2.2129910 .905927 \quad 1.564288$

H $-0.180996 \quad 1.698361 \quad 3.327739$

H 1.7412732 .7816900 .198632

H 2.7493023 .4200581 .519294

H 1.7040324 .4847450 .582447

H $0.657660-0.952153-1.531545$

H $1.6105520 .278190-0.702276$

H $-2.2820672 .301736-1.921210$

H -1.186937 $2.665290-3.267223$

H $0.1282734 .267035-1.757940$

H $-2.7189764 .272380-0.627652$

H -2.025979 $6.712105-0.647039$

H $-0.343166 \quad 6.274714-0.498950$

H -2.6861606 .4661382 .972670$

H $-3.464530 \quad 6.729293 \quad 1.311378$

H -0.2794446 .1375893 .064294$

H 0.6415545 .9491671 .613275

H -0.9798613 .8222313 .158507$

H $-0.4679191 .292873-0.700373$

C $-0.355134-4.227797-0.995933$

O $-1.250910-3.165791-1.387255$

C -1.932341-3.553315 -2.597085

C $-1.211136-4.794170-3.106665$

C $-0.795887-5.448005-1.802653$

H $-2.949827-3.840678-2.309122$

C $-1.973941-2.379147-3.579631$

H -1.858203 -5.428395 -3.719950

H $-0.328062-4.534690-3.702435$

C $0.284920-6.501764-1.972388$

H -1.679309-5.906851-1.338425

C $-2.666333-1.125164-3.005267$

O $-0.625058-2.029159-3.914425$

H -2.451904 -2.689848 -4.515469

H -2.089626 -0.762974 -2.149204

O $-2.594530-0.092764-3.996760$

C $-4.141361-1.326613-2.603813$

C $-4.813670-0.051001-2.061068$

H $-4.235621-2.119468-1.855490$ 
O $-4.892032-1.736887-3.750204$ H $-4.6490600 .785410-2.751047$ H -5.898115 $-0.226708-2.055718$ H $-0.068371-7.313948-2.616086$ H $1.187910-6.080926-2.427323$ H $\quad 0.565072-6.934265-1.006899$ C $-0.439671-4.4198150 .519597$ H $-0.662977-1.258736-4.507010$ H -3.139739 $-0.373474-4.751805$ H $-4.625420-2.645826-3.968223$ H $-0.083123-5.419308 \quad 0.792845$ H $-1.481633-4.3327390 .849817$ H $0.656136-3.920926-1.295344$ C $-4.373024-0.5775961 .600710$ $\begin{array}{lllll}\text { O } & -4.785639 & -0.720847 & 0.235477\end{array}$ C $-4.3525340 .323864-0.643433$ C $-4.9399781 .668000-0.185553$ C $-4.579642 \quad 1.964311 \quad 1.273267$ C $-4.853923 \quad 0.7622982 .186173$ H -4.902671-1.380795 2.129813 C -2.870206 -0.8419981 .736161$ H $-3.2595990 .380219-0.621860$ O $-6.3590051 .667415-0.332357$ H $-4.5530242 .464184-0.831073$ H -5.1629282 .8282881 .614280$ $\begin{array}{llll}\text { O } & -3.202421 & 2.319128 & 1.338763\end{array}$ H -4.4066390 .9185493 .173476$ O -6.2677030 .6517322 .384513$ C $-2.532192-1.3241933 .124868$ H -2.550395 -1.600236 1.010006 H -2.2545090 .0352311 .519069$ H -6.7102620 .9178360 .178231$ H -2.998866 2.5379972 .264087 H $-6.569626 \quad 1.4745872 .805600$ O - $-2.417357-2.6823273 .132176$ O $-2.411886-0.5842414 .092555$ C $-2.100003-3.2684034 .398155$ H $-2.897511-3.0654765 .119166$ H -2.017459 -4.3498284 .260273$ H -1.141643 -2.888262 4.764044

SCF Energy (B3LYP/6-31G**//MMFF) $=-3245.91917322$

08_00006

MM̄FF Geometry

C -1.183614 -2.288822 2.551658

C $-0.552967-1.270714 \quad 3.157662$

C -1.029656 0.1494453 .329943

$\begin{array}{lllll}\text { O } & -2.368620 & 0.314705 & 2.812084\end{array}$

C $-0.101947 \quad 1.1428822 .605188$

C $0.0351552 .446335 \quad 3.367472$

C -0.6424473 .5790023 .100038$

C 1.0482462 .4226054 .484770

C -1.676906 3.8140672 .035450

C -1.2233824 .8716581 .018650$

C $-0.061624 \quad 4.3957360 .134060$

C $0.429246 \quad 5.523737-0.774465$

C $1.5325965 .042077-1.728684$

O $-0.538418 \quad 3.304918-0.659107$

O $1.7143836 .047724-2.736795$

C $1.0478983 .764897-2.444623$

C $2.8816554 .892197-1.016394$

O $2.0906193 .193148-3.231406$

C $0.4587522 .698649-1.489340$

C $-0.2261151 .587967-2.318284$

O $1.5130332 .127786-0.713773$

C $-3.2818170 .933968 \quad 3.603587$

O -3.1082791 .3373184 .746114$

C -4.593655 1.0718492 .931535

C $-4.784106 \quad 0.9187941 .611862$

C $-6.060884 \quad 1.0708450 .906431$

$\begin{array}{llll}C & -7.291236 & 1.338278 & 1.734538\end{array}$

C $-1.0539540 .590954-1.495560$

C $-1.729773-0.438036-2.386932$

C $-3.490438-2.199960-2.329892$

O $-1.469411-0.567641-3.580407$

C $-4.727869-1.527667-2.832594$

C $-5.955886-1.768123-2.349645$

C $-7.188203-1.061670-2.834744$
C $-7.863294-0.256747-1.745512$

C $-9.012923-0.678189-1.192096$

C $-7.258827 \quad 1.078060-1.364582$

C $-6.0797880 .949791-0.437696$

N $-2.649860-1.206080-1.687264$

H $-2.150770-2.1313682 .084614$

H $0.400869-1.4777503 .640851$

H -1.0186350 .3302254 .411888$

H -0.4635001 .3246761 .587283$

H 0.9063230 .7239482 .485315

H -0.4432514 .4508353 .722672$

H 1.1223243 .3855295 .001251

H $0.781813 \quad 1.6685715 .231869$

H 2.0412862 .1839204 .089341

H -1.9620472 .8934461 .519042$

H -2.586538 4.1754052 .531286

H -2.0853125 .1122860 .382946$

H $-0.9411085 .792077 \quad 1.544957$

H 0.7576594 .0491770 .773258

H $0.7809056 .377194-0.181526$

H $-0.4099095 .900852-1.375634$

H $1.9519546 .879010-2.291291$

H $0.2608254 .068131-3.149312$

H $3.1915625 .848220-0.577635$

H $3.6727334 .617343-1.722632$

H $2.8616184 .152507-0.214004$

H $2.4246953 .890543-3.820978$

H $-0.9051142 .052046-3.046345$

H $0.530864 \quad 1.038567-2.888987$

H $1.264127 \quad 1.229586-0.445468$

H -5.4065001 .3509333 .594801$

H -3.9313140 .6730920 .981617$

H -7.4302530 .5506302 .483726$

H -8.2123301 .3701401 .149033$

H -7.2067752 .3023992 .247820$

H $-0.4239410 .051080-0.781733$

H $-1.8318621 .121701-0.934666$

H -3.713933 -2.972838-1.588578

H $-2.949192-2.659023-3.163105$

H $-4.602731-0.796470-3.627934$

H $-6.085095-2.502256-1.557806$

H $-7.874483-1.821818-3.229890$

H $-6.963255-0.400536-3.681129$

H $-9.524164-0.093610-0.434815$

H $-9.468181-1.619410-1.482041$

H $-8.021278 \quad 1.738753-0.936623$

H $-6.932498 \quad 1.599301-2.273967$

H $-5.1377550 .759737-0.951407$

H -2.846331 $-0.979291-0.716687$

C $-0.352582-4.310202 \quad 1.195142$

$\begin{array}{llllll} & 0 & 0.613103 & -3.535333 & 0.459671\end{array}$

C $0.330986-3.686484-0.947284$

C $-0.807316-4.699889-1.055723$

C $-1.538988-4.4815950 .254767$

H - $0.039028-2.718331-1.298684$

C $1.595403-4.083854-1.718029$

H $-1.434004-4.524808-1.935180$

H $-0.428874-5.727263-1.117489$

C $-2.475808-5.6169320 .624621$

H $-2.111049-3.5495490 .183830$

C $2.736130-3.044490-1.660347$

O $2.089584-5.313901-1.173469$

H $1.333001-4.293864-2.762065$

H $3.146622-3.006567-0.646450$

O $3.797506-3.523145-2.496756$

C $2.331982-1.634436-2.123127$

C $3.494242-0.647305-2.328850$

H $1.625044-1.207554-1.404229$

O $1.643661-1.754343-3.372997$

H $4.091892-0.947980-3.199111$

H $3.0400760 .311492-2.610995$

H $-3.249632-5.740663-0.140175$

H $-1.939192-6.5670510 .717152$

H $-2.974074-5.4165801 .578308$

C $-0.636964-3.6921612 .563871$

H $2.892397-5.548323-1.669945$

H $3.473042-3.518264-3.413732$

H $1.362744-0.865122-3.645719$ 
H $\quad 0.296187-3.711583 \quad 3.141243$ H -1.347073 -4.326913 3.107146 H $0.115770-5.2891631 .370476$ C $4.313148 \quad 0.044861 \quad 1.258242$ O $3.606854-0.1311580 .021788$ C $4.415217-0.447026-1.114102$

C $5.4510120 .663439-1.367803$

C $6.2767320 .960616-0.112668$

C $5.3889641 .136773 \quad 1.124142$

H 3.5546360 .4041281 .965414

C $4.873249-1.2804541 .801349$

H $4.938682-1.390786-0.930612$

O $4.7835301 .854101-1.782853$

H $6.108098 \quad 0.350826-2.188328$

H $6.859861 \quad 1.875517-0.274917$

O $7.196706-0.1074780 .089529$

H 6.0063221 .1631472 .029707

O 4.7413162 .4078391 .024926

C $3.781207-2.2651162 .153527$

H $5.527571-1.7688891 .074903$

H $5.445069-1.0898752 .716847$

H $5.4631922 .524190-1.967295$

H 7.7302620 .1092500 .873053

H 4.2105952 .5330131 .829931

O $4.350623-3.4685212 .458916$

O $2.585019-2.0141822 .167138$

C $3.436209-4.5137202 .803292$

H $4.018115-5.3972153 .078807$

H $2.822241-4.2183073 .659346$

H $2.808996-4.7618641 .942484$

SCF Energy (B3LYP/6-31G**//MMFF) $=-3245.89979947$

08_00007

MM̄FF Geometry

C $-1.207305-4.3396981 .651158$

C $0.009185-3.8380971 .389040$

C $0.936483-3.3188242 .459153$

O $1.391703-1.9977352 .081704$

C $2.163602-4.2403212 .571495$

C $3.117398-3.8411663 .681283$

C $4.310825-3.2424243 .495233$

C $2.665666-4.1998525 .074280$

C $4.933304-2.8083912 .197293$

C $5.236403-1.3044512 .205219$

C $5.738859-0.8140780 .839068$

C 6.1009030 .6706190 .902068

C $6.4869641 .234687-0.473908$

O $4.691105-1.025412-0.112711$

O $6.4771762 .667954-0.378096$

C $5.3992690 .842777-1.498472$

C $7.9144720 .837739-0.867673$

O $5.7829101 .227827-2.816276$

C $5.021788-0.653272-1.457095$

C $3.803113-0.999395-2.348578$

O $6.127795-1.422719-1.946022$

C $0.545642-0.9571552 .318653$

O $-0.589432-1.0189532 .763782$

C $1.2566780 .291198 \quad 1.960989$

C 0.6501951 .4751852 .133345

C 1.2371142 .7941951 .882661

C 2.6399452 .8460991 .339247

C $2.488394-0.311119-1.967633$

C $2.2258130 .988795-2.708949$

C $0.6649132 .936103-2.698924$

O $2.864890 \quad 1.344390-3.694793$

C $1.1231944 .061897-1.828012$

C $0.2820024 .913062-1.221764$

C $0.7240706 .089261-0.401621$

C 0.1468906 .0821990 .995103

C -0.9564096 .7896221 .292346$

C 0.9071355 .3367922 .067258

C 0.5039473 .8909882 .166197

N $1.158019 \quad 1.684026-2.158735$

H -1.531498 -4.378472 2.689955

H $\quad 0.376008-3.7873150 .368130$

H $0.427732-3.2604813 .429748$

H $2.683898-4.2770521 .608590$

H $1.836130-5.2724142 .758326$
H $4.919342-3.0320944 .374141$

H $3.417251-3.9539455 .832128$

H $1.750060-3.6601365 .333948$

H $2.468974-5.2746235 .144888$

H $5.867254-3.3658272 .056750$

H $4.297073-3.0481121 .342899$

H $4.322377-0.7596532 .472801$

H $5.985058-1.0848552 .976673$

H $6.613140-1.4111600 .553692$

H 6.9048930 .8438381 .628321

H $5.237991 \quad 1.2372621 .274928$

H 7.1051522 .9264420 .318166

H $4.5142681 .441119-1.251786$

H $8.6319511 .200689-0.121710$

H $8.2084111 .304860-1.813991$

H $8.050379-0.242088-0.955702$

H $6.4368480 .582898-3.135336$

H $4.050975-0.842116-3.405261$

H $3.628350-2.080575-2.250458$

H $5.797612-2.290214-2.232805$

H $2.2713860 .203672 \quad 1.591580$

H -0.3693151 .4850772 .517419$

H 2.9597073 .8501641 .052388

H 2.7321202 .2318630 .437448

H 3.3488482 .4792952 .088871

H $1.660098-0.980714-2.233175$

H $2.433298-0.137992-0.888926$

H $-0.4262222 .865042-2.739515$

H $1.0388433 .079365-3.717542$

H $2.1961384 .201255-1.719345$

H $-0.7888884 .784806-1.358693$

H $0.4070456 .998881-0.928793$

H $1.8180306 .151319-0.346592$

H -1.356993 6.8140362 .300501

H -1.487574 7.3530750 .532196

H $0.7075445 .794578 \quad 3.046011$

H 1.9827205 .4718421 .921399

H -0.5023343 .7520462 .563338$

H $0.7079041 .321053-1.323572$

C $-1.885796-4.728364-0.823129$

O $-1.878658-3.330700-1.179097$

C $-2.703056-3.141522-2.341664$

C $-2.850548-4.521258-2.966768$

C $-2.931583-5.389337-1.726432$

H -3.686433 -2.816548 -1.980347

C $-2.113183-2.066426-3.259945$

H -3.735594 -4.598008 -3.605235

H -1.972585 -4.795107 -3.563109

C $-2.671153-6.861411-1.995754$

H -3.932292 -5.279712 -1.286812

C $-1.807080-0.726805-2.557675$

O $-0.884395-2.560743-3.806693$

H $-2.788832-1.907398-4.109297$

H $-0.910194-0.825991-1.933668$

O $\quad-1.4940590 .237927-3.569433$

C $-2.955601-0.170864-1.697597$

C $-2.6518931 .241030-1.158581$

H $-3.133931-0.850979-0.857240$

O $-4.144235-0.134437-2.487087$

H $-1.6049761 .297356-0.840224$

H $-2.8117791 .968350-1.963821$

H -3.417540 -7.263261 -2.688740

H -1.681996 -7.019605 -2.438478

H -2.722499 -7.442293 -1.069541

C $-2.214688-4.8732590 .666253$

H $-0.615605-1.957087-4.519393$

H -0.640906 -0.012860 -3.961638

H $-4.8663020 .180768-1.917493$

H $-2.375020-5.9316760 .902730$

H $-3.157351-4.3495340 .869687$

H $-0.887888-5.126368-1.044895$

C $-5.867088 \quad 1.7857370 .675086$

O $-4.9062441 .587114-0.370296$

C -3.5395031 .6069050 .045409$

C $-3.1934752 .984156 \quad 0.625317$

C -4.1359373 .3425701 .778849$

C -5.6081393 .1139771 .408854$

$\begin{array}{llll}\mathrm{H} & -6.831714 & 1.870437 & 0.158106\end{array}$ 
C -5.9441520 .5697121 .611130$ H -3.3786720 .8395330 .810806$ O $-3.2953823 .970692-0.398742$ H -2.155341 2.9704000 .972071 H -3.986489 4.394703 2.051045 O -3.7874342 .5482082 .908902$ H -6.2372523 .1638652 .304681$ O $-6.032790 \quad 4.183397 \quad 0.556928$ C $-6.271892-0.7014840 .854707$ H $-4.996477 \quad 0.4145262 .133962$ H -6.7263140 .7200662 .363286$ H -3.030899 $4.822682-0.011801$ H $-4.3728842 .806713 \quad 3.641012$ H -5.531387 $4.117933-0.274124$ O $-5.827898-1.7778331 .568102$ O $-6.859700-0.747167-0.217597$ C $-6.059155-3.0537630 .962415$ H $-7.131431-3.2187810 .820822$ H -5.527606 -3.1205880 .008223$ H -5.671922 -3.824271 1.634320

SCF Energy $\left(\mathrm{B} 3 L Y P / 6-31 G^{* *} / / \mathrm{MMFF}\right)=-3245.91602849$

08_00008

MMFF Geometry

C $3.268868 \quad 3.2944870 .050611$

C 2.9664374 .2168540 .976423

C 1.7935574 .1735491 .931701

O 1.0238572 .9589341 .780379

C 0.8559625 .3604521 .637506

C -0.2932895 .4841762 .618717$

C -1.5929825 .2944982 .316416$

C 0.0959625 .8798334 .021075

C $-2.206247 \quad 4.931950 \quad 0.991394$

C -2.692062 3.4829410 .999087

C $-3.3284623 .029536-0.323419$

C $-4.6545443 .742396-0.602546$

C $-5.3740853 .174598-1.836335$

O $-3.5392371 .617864-0.195962$

O $-6.7213523 .672317-1.826047$

C $-5.4574941 .640352-1.697188$

C $-4.7398293 .670242-3.141116$

O $-6.0163561 .050035-2.868435$

C $-4.1062410 .981518-1.348316$

C $-4.254248-0.521875-1.017308$

O $\quad-3.2325701 .086154-2.477577$

C 1.4227891 .8606702 .479202

O 2.3734411 .7744323 .239275

C 0.4984050 .7584812 .129098

C $0.749838-0.4937472 .539375$

C $-0.071250-1.6722832 .245576$

C $-1.379665-1.4430531 .537032$

C -5.132020 -0.8192650 .201438$

C $-4.950215-2.2379440 .713853$

C $-5.301580-3.5950612 .781407$

O $-4.430049-3.1322490 .052875$

C $-4.022488-3.5041923 .555404$

C -3.135134 -4.506059 3.648972

C -1.848555 -4.400742 4.419578

C $-0.658905-4.9336003 .650110$

C $0.015360-6.0109354 .087204$

C $-0.253848-4.2336592 .371722$

C $0.377983-2.8887992 .619339$

N $-5.457755-2.3833971 .997840$

H $2.6408612 .416455-0.063304$

H 3.6129385 .0875761 .069763

H 2.1790434 .2434732 .955928

H $\quad 0.482414 \quad 5.2661160 .612737$

H $\quad 1.4234186 .3007551 .660417$

H -2.323432 5.4161743 .116252

H -0.7754476 .0807024 .653687$

H 0.6730945 .0842164 .501664

H $\quad 0.7026256 .7912604 .004104$

H -3.045881 5.6151510 .821019

H -1.516871 5.0859140 .157304

H -1.8465112 .8203121 .221291$

H -3.4044413 .3231941 .820013$

H -2.615039 3.205681-1.135627

H $-4.5069664 .821922-0.722043$
H -5.3177043 .6262350 .266342$

H -6.682506 4.644081-1.836892

H $-6.1726021 .441400-0.889446$

H $-4.7769264 .765213-3.192418$

H $-5.2986273 .313079-4.013067$

H $-3.6951083 .372440-3.251198$

H $-5.3269211 .042247-3.554132$

H -4.624128 -1.071783-1.892120

H $-3.251780-0.925333-0.825508$

H $-2.5555750 .393267-2.411047$

H -0.3630081 .0065051 .520117$

H $1.647014-0.6801563 .127989$

H -2.025200 -0.782263 2.126639

H -1.948138 -2.356651 1.361159

H -1.211973 -0.9838090.558689

H $-4.888241-0.1293601 .017415$

H $-6.190194-0.691260-0.051118$

H -5.321609-4.464172 2.115951

H -6.151466 -3.648482 3.468193

H -3.816985 -2.566921 4.068061

H $-3.350663-5.4546003 .162685$

H $-1.976670-4.9626285 .353942$

H -1.648332 -3.363408 4.714603

H $0.867893-6.4040713 .543277$

H $-0.268140-6.5249734 .999564$

H $-1.109306-4.1871361 .693474$

H $\quad 0.487502-4.8405531 .834748$

H $1.338579-2.9497153 .132767$

H $-5.813812-1.5628062 .479617$

C $5.4808362 .296421-0.686529$

O $4.8957220 .990959-0.865161$

C 5.4988400 .0888860 .083601

C 6.6752950 .8410160 .700164

C 6.1639602 .2674940 .679216

H $4.753781-0.0943170 .866251$

C $5.890762-1.223043-0.601382$

H $\quad 6.913630 \quad 0.483141 \quad 1.706451$

H 7.5800380 .7496860 .087278

C $7.253323 \quad 3.3108410 .850517$

H 5.4295972 .3801561 .486462

C $4.707934-2.025567-1.185435$

O $6.800825-0.935345-1.671334$

H $6.448944-1.8506090 .103222$

H $4.268523-1.480698-2.028142$

O $5.249461-3.232662-1.739926$

C $3.608827-2.387124-0.165060$

C $2.629167-3.475445-0.640657$

H $3.057131-1.4826650 .114087$

O $4.238774-2.8787791 .023485$

H $3.183904-4.391221-0.880383$

H $1.995334-3.738310 \quad 0.214859$

H 7.7566293 .1862271 .814862

H 8.0110003 .2337040 .063612

H 6.8334074 .3211350 .816968

C $4.4337673 .392215-0.892025$

H $6.345191-0.339370-2.290336$

H $5.625821-3.749213-1.006663$

H $3.543671-3.0106271 .690721$

H $4.0304913 .309540-1.909178$

H $4.9117804 .376185-0.820053$

H $6.2364992 .399680-1.476967$

C $-0.070185-1.523303-2.329285$

O $0.862784-2.032231-1.366222$

C $1.737205-3.069575-1.823775$

C $0.931982-4.273022-2.345172$

C $-0.082066-3.845832-3.409393$

C $-0.915188-2.651188-2.941724$

H $-0.746668-0.885715-1.747954$

C $0.619656-0.644059-3.376444$

H $2.367147-2.683091-2.630533$

O $0.246689-4.897528-1.260417$

H $1.630237-5.009727-2.760449$

H $-0.754164-4.682515-3.636186$

O $0.599607-3.493916-4.609200$

H $-1.504927-2.254376-3.775837$

O $-1.854895-3.097013-1.960060$

C $1.0459370 .663038-2.754705$

H $1.511721-1.103808-3.810718$ 
H $-0.053435-0.418036-4.212143$ H $-0.223419-5.670884-1.616059$ H $1.099794-4.274431-4.902749$ H -1.356485 -3.369674 -1.170510 O $0.0134451 .552993-2.791348$ O $2.152973 \quad 0.854409-2.270395$ C $0.2684262 .834295-2.207631$ H $-0.5226783 .516755-2.529326$ H $\quad 0.2395772 .751337-1.117865$ H $\quad 1.2290973 .237018-2.542267$

SCF Energy $($ B3LYP/6-31G**//MMFF $)=-3245.91660288$

08_00009

MM̄FF Geometry

C -1.7799614 .6813871 .851965$

C -2.4495193 .5590191 .546656$

C -2.844349 2.5266452 .573098

O -2.4179851 .2221032 .111170$

C -4.3747642 .5076282 .728484$

C -4.8546751 .5730573 .822534$

C $-5.4360090 .374853 \quad 3.614059$

C -4.6823812 .0902635 .228334$

C $-5.696201-0.3083872 .300403$

C $-5.040604-1.6950262 .257870$

C $-5.205209-2.3627160 .884522$

C $-4.608244-3.7705380 .895703$

C $-4.646160-4.428204-0.492303$

O $-4.530529-1.550581-0.081843$

O $-3.781415-5.574735-0.457434$

C $-4.054148-3.446638-1.528047$

C $-6.046059-4.953430-0.831601$

O $-4.196536-3.959710-2.850368$

C $-4.636760-2.020801-1.432173$

C $-3.905658-1.000125-2.339428$

O $-6.004937-2.051343-1.858883$

C $-1.115286 \quad 0.8841072 .320405$

O $-0.244987 \quad 1.5872332 .808317$

C $-0.933666-0.5169511 .875429$

C $0.272745-1.0968721 .968652$

C $0.593298-2.490898 \quad 1.647114$

C $-0.521051-3.3660531 .138627$

C $-2.420468-0.784062-2.031437$

C $-1.481716-1.662110-2.841593$

C $0.925277-2.300225-2.985555$

O $-1.842679-2.315989-3.815962$

C $1.305461-3.462907-2.126974$

C $2.509436-3.596956-1.550229$

C $2.922739-4.807063-0.764978$

C $3.414425-4.4744830 .624104$

C $4.729352-4.4165950 .895055$

C $2.385346-4.3304791 .720953$

C $1.852885-2.9293781 .854154$

N $-0.179322-1.593430-2.367500$

H - 1.5217484 .8541412 .895606

H $-2.734954 \quad 3.3500950 .519877$

H $-2.3769102 .736713 \quad 3.543376$

H -4.838596 2.2523051 .769770

H $-4.737073 \quad 3.5184352 .961637$

H -5.766428 -0.1908614 .484702$

H $-5.104576 \quad 1.4108665 .976567$

H -3.6219082 .2196485 .464829$

H $-5.185653 \quad 3.0560975 .341942$

H $-6.780335-0.4156892 .175221$

H $-5.341640 \quad 0.2875321 .456691$

H -3.972646 -1.589784 2.486426

H $-5.479762-2.3335603 .034570$

H -6.273948 -2.4011020 .642377$

H -5.116430 -4.402595 1.634688

H -3.561595 $-3.716807 \quad 1.221838$

H -4.099280 -6.1690720 .243623$

H $-2.976406-3.405498-1.331447$

H $-6.372383-5.684708-0.082271$

H $-6.047253-5.487883-1.787885$

H $-6.801232-4.165843-0.872405$

H -5.119831 -3.824456 -3.123499

H -4.067172 -1.249956-3.395111

H -4.398609 -0.027949 -2.193782

H $-6.268266-1.152059-2.115696$
H -1.808188 -1.045371 1.515538

H $1.105545-0.5048482 .346466$

H -1.263872 -3.528422 1.926585

H $-0.184124-4.3480770 .799497$

H -1.016139 -2.905617 0.277538

H $-2.162910 \quad 0.250952-2.290544$

H $-2.216773-0.912816-0.964409$

H $1.746848-1.587761-3.102160$

H $0.638531-2.653684-3.980931$

H $\quad 0.565642-4.251144-2.009551$

H $3.256705-2.818862-1.689754$

H $3.718296-5.310433-1.330443$

H $2.107439-5.538181-0.697156$

H $5.090690-4.2174501 .898549$

H $5.473859-4.5570770 .118255$

H $2.840752-4.5920762 .686455$

H $1.596615-5.0762421 .586271$

H $2.591701-2.2195212 .227867$

H $0.020807-1.032194-1.544567$

C $-1.5159795 .520106-0.588571$

O $-0.7098734 .398342-0.997432$

C $0.0303494 .754633-2.177187$

C $-0.6538115 .995275-2.732986$

C $-1.0607176 .699609-1.452506$

H $1.0395595 .027855-1.845142$

C $0.1111553 .559785-3.132129$

H $0.0131596 .593422-3.360846$

H -1.542315 $5.742535-3.322816$

C -2.126295 $7.762953-1.654743$

H $-0.1696637 .167069-1.012067$

C $0.5894052 .250433-2.472157$

O $-1.1873213 .303544-3.683824$

H $\quad 0.754116 \quad 3.811284-3.983214$

H $-0.1893231 .860112-1.805506$

O $0.757812 \quad 1.277668-3.510561$

C $1.9115132 .365788-1.692701$

C $2.3325721 .027613-1.070857$

H $1.7902973 .101158-0.892663$

O $2.9417772 .840337-2.555411$

H $1.5337690 .656947-0.416192$

H $2.4396760 .272218-1.858815$

H -1.763043 8.548716 -2.325139

H $-3.0364547 .340731-2.094086$

H -2.397062 8.229686 -0.702495

C -1.3191745 .7650310 .910878$

H $-1.7948993 .140552-2.941785$

H $-0.0937631 .197784-3.973583$

H $3.0313692 .201819-3.283572$

H -1.8289056 .6918961 .199303$

H -0.2483045 .9111301 .104522$

H $-2.5608475 .268674-0.809163$

C $5.057604-0.5073040 .924466$

O $3.819125-0.2643820 .246391$

C $3.6673481 .052313-0.303874$

C 3.7320222 .1015460 .814950

C 5.0153011 .9608901 .639649

C 5.2783980 .5106532 .057153

H $4.923252-1.4930941 .383515$

C $6.206392-0.618235-0.087413$

H $4.4742411 .227194-1.025132$

$\begin{array}{llll}\text { O } & 2.596097 & 1.953581 & 1.662369\end{array}$

H $3.691643 \quad 3.1045270 .375183$

H 4.9344832 .5888242 .535638

O $6.1051242 .454170 \quad 0.866242$

H $6.292571 \quad 0.4146702 .460092$

O $4.3756390 .199476 \quad 3.124039$

C $7.393140-1.3899640 .446657$

H $5.844563-1.148747-0.977314$

H $6.5514230 .367080-0.411414$

H 2.6430062 .6464912 .342699

H 6.9060262 .3912941 .414293

H $4.585499-0.6977353 .434737$

O $8.357289-1.440219-0.520266$

O $7.470825-1.8895631 .560472$

C $9.548630-2.148744-0.164249$

H $10.231564-2.115772-1.017244$

H $10.032624-1.6692890 .691990$

H $9.315210-3.1943240 .058355$ 
C $2.910952-2.147599-2.213332$

O $4.917618-3.422908-1.892764$

C $0.466501-0.4720882 .121889$

O $-0.614844-0.0835852 .534037$

C $1.6553590 .381576 \quad 1.905568$

C 1.5578871 .7105532 .053210

C 2.6278842 .6841991 .825442

C 3.9541932 .1665371 .335478

C $1.989682-0.989569-1.815241$

C $2.2206890 .295300-2.592232$

C $1.5525042 .700193-2.598133$

O $2.896694 \quad 0.350614-3.615683$

C $2.5613023 .544548-1.885388$

C $2.2692484 .726476-1.320365$

C $3.2887345 .619771-0.671457$

C 2.8940316 .0537750 .722749

C 2.2809437 .2310280 .932504

C 3.2921065 .1652281 .879278

C 2.3680343 .9900422 .041736

N $1.5427591 .367049-2.026323$ H $-0.880990-4.701666-0.162785$ H -1.316210 -1.692781 0.165310 H $-0.974570-2.4959722 .567163$ H $1.043027-4.3981351 .275901$ H $-0.371659-4.8754582 .176501$ H $2.985358-4.1695344 .485521$ H $0.961751-4.1620225 .570559$ H $-0.206603-3.1389304 .731187$ H -0.358396 -4.909245 4.667843 H $4.216439-5.0274602 .530925$ H $3.040247-4.4064141 .407740$ H $3.657396-2.0418682 .522241$ H $5.110320-2.8993443 .043334$ H $5.488156-3.5577200 .602839$ H $6.666493-1.5634201 .581168$ H $5.261597-0.5694351 .270670$ H 7.5983580 .2464310 .193291 H $4.567519-0.140681-1.240813$ H $8.320633-1.933031-0.254387$ H $7.871651-1.723628-1.931110$ H $7.183616-3.063684-0.991524$ H $4.847576-0.681210-3.393631$ H $3.135745-2.134562-3.286361$ H $2.335884-3.071291-2.051803$ H $4.265624-4.098889-2.141288$ H $2.574341-0.1038241 .599959$ H 0.6002642 .1305982 .357967 H 3.8237661 .5573320 .434744 H 4.4318861 .5563502 .109109 H 4.6594532 .9549991 .064958 H $0.953789-1.284897-2.025509$ H $2.059885-0.787950-0.743701$ H $0.5393553 .101875-2.504332$ H $1.8049172 .643432-3.661822$ H $3.5874693 .184873-1.868909$

H $3.4084206 .503962-1.311539$

H $4.2769625 .144256-0.641138$

H 2.0210387 .5629591 .932143

H 2.0198167 .8857910 .107687

H 3.2612435 .7335472 .818833

H 4.3406454 .8737461 .770723

H 1.3758434 .2692252 .396911

H $1.0411881 .230861-1.153552$

C $-3.412350-3.523212-1.664221$

O $-3.882578-2.328184-1.007697$

C $-4.933712-2.692412-0.096017$

C $-5.375580-4.093023-0.507448$

C -4.055601-4.704072 -0.935748

H $-4.480168-2.7448150 .901131$

C $-6.048773-1.643798-0.105557$

H $-5.855212-4.6355040 .312748$

H $-6.072860-4.068371-1.352804$

C -4.202689-5.947846-1.794017

H $-3.493253-4.963860-0.030627$

C $-5.593097-0.1980350 .181963$

O $-6.683669-1.640354-1.391680$

H $-6.827584-1.9356960 .608825$

H $-5.0544840 .202205-0.684846$

$\begin{array}{lllll}\text { O } & -6.776914 & 0.599330 & 0.323392\end{array}$

C $-4.734258-0.0196781 .447616$

C -4.5611561 .4378881 .917872$

H $-3.746053-0.4617721 .279262$

O $-5.324745-0.7425122 .532986$

H -5.5249791 .8261532 .272078$

H -3.9122181 .4116902 .803939$

H -4.725284 -6.736221-1.242506

H $-4.773015-5.741913-2.706039$

H $-3.221827-6.333918-2.088989$

C $-1.883465-3.516201-1.730660$

H $-5.997218-1.458206-2.056470$

H -7.298318 $0.490481-0.490509$

H $-6.222161-0.3916942 .666088$

H -1.545096 -2.575430 -2.181689

H -1.542064 -4.317547 -2.397024

H $-3.795805-3.465151-2.691341$

C $-1.9601642 .698215-0.453483$

O $-2.654808 \quad 1.9385910 .545775$

C -3.9709842 .3940880 .869971$

C -3.9320293 .8424601 .385269$

C -3.2347984 .7652560 .382135$

C -1.892657 4.188482 -0.077069

H $-0.9351462 .308905-0.435965$

C $-2.5379212 .459670-1.857533$

H $-4.5984072 .358738-0.025166$

O -3.2445523 .8874002 .634029$

H -4.9597074 .1831741 .560032$

H -3.0718275 .7451440 .847140$

O $-4.0945934 .951094-0.738320$

H -1.505446 $4.762545-0.926361$

O -0.9419324 .3453350 .978320$

C -2.273605 $1.054384-2.352895$

H -3.618935 2.616681-1.888644

H -2.060213 $3.145321-2.566991$

H -3.2702074 .8065982 .949918$

H $-3.6487545 .559150-1.352370$

H -1.224705 3.7834461 .719992

O $-2.7477330 .939957-3.628851$

O $-1.7264850 .169847-1.710620$

C $-2.568503-0.340799-4.241003$

$\mathrm{H}-1.518013-0.645116-4.201334$

H -3.205529 -1.080042 -3.747807

$\mathrm{H}-2.869192-0.261576-5.289047$

SCF Energy (B3LYP/6-31G**//MMFF) $=-3245.91963257$

0800011

MMFFF Geometry

C -0.431952 -3.8461200 .609899$

C $0.263283-2.701845 \quad 0.688002$

C $0.863580-2.1827161 .966388$

$\begin{array}{lllll} & 0 & 0.598902 & -0.764264 & 2.063516\end{array}$

C $2.388670-2.3650881 .929443$

C $3.059566-2.0877943 .258850$ 
C $3.772370-0.9796923 .540346$

C $2.940852-3.1887624 .281164$

C 3.9925070 .2267482 .670371

C 5.4199030 .3006362 .110088

C $5.670720-0.6758050 .949111$

C $7.130638-0.6105670 .496030$

C $7.385737-1.483860-0.741539$

O $4.809861-0.294567-0.128279$

O $8.668727-1.125991-1.277624$

C $6.334890-1.141907-1.818117$

C $7.463618-2.972942-0.384786$

O $6.443351-2.023507-2.933685$

C $4.882715-1.137571-1.286528$

C $3.935006-0.533619-2.349683$

O $4.481363-2.476845-0.998403$

C $-0.586459-0.3702402 .592436$

O $-1.506139-1.0968992 .942955$

C $-0.636748 \quad 1.1008292 .770005$

C $\quad 0.261158 \quad 1.9498432 .244343$

C 0.2972123 .4025662 .438620

C -0.7285674 .0097273 .360183$

C $2.472002-0.422999-1.894389$

C $1.7594150 .699302-2.620319$

C $1.6437773 .173938-2.745556$

O $0.9968310 .488592-3.559494$

C $2.0414254 .335614-1.894076$

C $1.1760365 .244443-1.421981$

C $1.5739796 .408621-0.562660$

C $\quad 0.8834096 .4028090 .783201$

C -0.2022627 .1641141 .001219$

C 1.5079665 .6006731 .902960

C 1.2441694 .1217551 .798814

N $2.0893521 .947381-2.112888$

H $-0.561923-4.4422791 .508648$

H $0.428133-2.113526-0.210192$

H $0.439851-2.6968282 .837910$

H $2.809765-1.7198651 .153063$

H $2.642297-3.3911091 .630016$

H $4.240831-0.9083574 .521399$

H $3.497292-2.9669515 .198146$

H $1.894711-3.3436464 .562010$

H $3.335521-4.1265763 .876753$

H 3.2565770 .2949341 .864184

H 3.8203111 .1115153 .296854

H $5.576981 \quad 1.325426 \quad 1.748814$

H $\quad 6.146160 \quad 0.1254462 .913511$

H $5.421327-1.6921311 .272477$

H $7.804637-0.8960321 .313394$

H $7.388647 \quad 0.4279180 .244591$

H $9.334095-1.268421-0.582678$

H $6.567951-0.136000-2.194753$

H $8.274907-3.1530350 .330845$

H $7.702431-3.578269-1.266198$

H $6.542549-3.3568930 .058699$

H $7.365146-1.988328-3.242235$

H $4.3071340 .462017-2.623062$

H $3.955767-1.142381-3.262516$

H $3.513820-2.534865-1.040609$

H -1.465139 1.4506913 .377837

H 1.0583041 .5519391 .618992

H -1.7423373 .7891943 .009642$

H -0.6599335 .0970583 .432618$

H -0.6143363 .6136684 .375283$

H $1.936197-1.358304-2.091609$

H $2.390263-0.231545-0.818646$

H $0.5618153 .112085-2.904350$

H $2.1280963 .240546-3.725364$

H $3.0977714 .435243-1.654704$

H $0.1240045 .160236-1.680090$

H $1.3236157 .327159-1.109459$

H $2.6604506 .444929-0.413965$

H $-0.6852797 .196576 \quad 1.971897$

H $-0.635961 \quad 7.7682640 .211198$

H 1.1893375 .9857792 .878350

H 2.5942275 .7599671 .896176

H 1.9427153 .5968151 .146888

H $2.8386412 .003233-1.428109$

C $-2.519455-4.609060-0.647327$
O $-3.215642-3.368695-0.412131$

C $-4.329129-3.6335310 .462210$

C $-4.483553-5.1515790 .509018$

C $-3.042302-5.602148 \quad 0.390285$

H $-4.029650-3.2837271 .456448$

C $-5.590011-2.898000-0.002097$

H $-4.966523-5.4907241 .430545$

H $-5.069725-5.527836-0.337567$

C $-2.887366-7.057828-0.013617$

H $-2.559668-5.4501631 .362602$

C $-5.455223-1.365834-0.104679$

O $-5.944943-3.388771-1.302465$

H $-6.424738-3.1587310 .659885$

H $-4.804516-1.118677-0.950748$

O $-6.751303-0.851692-0.444308$

C $-4.952378-0.6512131 .163658$

C $\quad \begin{array}{llll}-5.108673 & 0.880777 & 1.141579\end{array}$

H $-3.900570-0.902211 \quad 1.339840$

O $-5.696901-1.0959402 .302935$

H -6.1741551 .1414861 .192591$

H -4.6722901 .2550772 .077854$

H -3.347994 -7.714721 0.731446

H $-3.363273-7.259886-0.978982$

H -1.829954 -7.328739-0.095080

C $-1.004579-4.387324-0.670143$

H $-6.749077-2.917386-1.580514$

H -7.337865 -1.011209 0.315191

H $-5.441790-2.0134182 .492653$

H $-0.768369-3.699863-1.490933$

H $-0.501565-5.334298-0.897896$

H $-2.823488-4.935933-1.651030$

C $-2.2919561 .900075-1.093033$

O $-3.0574671 .325834-0.023435$

C $-4.4608641 .595884-0.050507$

C $-4.7248863 .110873-0.004875$

C $-3.9645723 .845706-1.111804$

C $-2.4936233 .423416-1.159196$

H $-1.2446131 .713808-0.821037$

C -2.560924 $1.202869-2.435679$

H $-4.8893431 .193262-0.972959$

O -4.3452333 .6469961 .261580$

H $-5.8015153 .285484-0.114585$

H $-4.0237964 .927256-0.938159$

O $-4.5657913 .574903-2.373823$

H -2.010604 $3.816137-2.061237$

O $-1.8166373 .990560-0.036281$

C -2.080872 -0.231491-2.446689

H $-3.6245381 .186436-2.685573$

H $-2.0190271 .732249-3.228269$

H -3.4105013 .4229631 .408711$

H $-5.4902743 .873055-2.329120$

H -1.903315 $4.956925-0.097908$

O $-2.226801-0.732826-3.709283$

O $-1.643595-0.838202-1.479760$

C -1.810411 -2.089521 -3.887483

H $-0.763977-2.211085-3.591354$

H -2.457978 -2.757637 -3.313011

H -1.903815 -2.337314 -4.948125

SCF Energy (B3LYP/6-31G**//MMFF $)=-3245.90832657$

0800012

MM̄FF Geometry

C $0.6189372 .772283-1.827842$

C $-0.2918553 .748443-1.697749$

C $-1.1694253 .888070-0.485691$

O $-2.5175283 .589620-0.917866$

C -1.125467 $5.299747 \quad 0.125414$

C 0.2470835 .7961940 .526203

C 0.9515565 .3344351 .577604

C $0.7996636 .910152-0.323910$

C 0.5720204 .2252292 .518788

C 1.6721153 .1553332 .550912

C 1.2170651 .8773713 .270502

C 2.4328871 .0492163 .689202

C $2.027196-0.2926784 .310531$

O $0.407940 \quad 1.130332 \quad 2.352960$

O $3.201537-1.1146044 .389957$

C $1.044195-0.9969233 .358548$ 
C $1.509870-0.1308365 .744073$ O $0.531379-2.1907023 .946488$ C $-0.118044-0.0974562 .876368$ C $-0.870279-0.8279011 .737283$ $\begin{array}{lllll}\text { O } & -1.015170 & 0.139097 & 3.960853\end{array}$ C $-3.391781 \quad 3.1525240 .028489$ O $-3.209324 \quad 3.1012401 .234285$ C $-4.6062972 .682335-0.672661$ C $-5.4624091 .874848-0.028511$ C $-6.6796801 .281339-0.586493$ C -7.030667 $1.627904-2.010166$ C -1.9409130 .0107801 .036168$ C $-2.453460-0.690501-0.206068$ C $-3.807132-2.654737-0.932972$ O $-2.159495-0.302107-1.334419$ C $-5.160162-2.150810-1.325376$ C $-6.305718-2.751853-0.969930$ C $-7.671105-2.298198-1.403198$ C $-8.501801-1.766476-0.256387$ C $-9.118911-2.6004220 .597724$ C $-8.682930-0.268216-0.154565$ C -7.4055320 .4480190 .189063$ N -3.244836 -1.7888810 .084451$ H $0.7216852 .031108-1.038554$ H - $0.4171514 .477601-2.494252$ H $-0.871926 \quad 3.1421210 .258232$ H $-1.785003 \quad 5.342436 \quad 1.002223$ H - $1.5863676 .008646-0.576920$ H 1.9168965 .7936041 .788061 H 1.7940797 .2313100 .003564 H $0.8852786 .588377-1.366533$ H $0.1388547 .782135-0.281670$ H $0.4542024 .648163 \quad 3.523883$ H $-0.390742 \quad 3.7774832 .262405$ H 1.9728272 .9048571 .524600 H 2.5490993 .5799423 .055999 H $\quad 0.618452 \quad 2.149470 \quad 4.147587$ H 3.0689181 .6136284 .382723 H 3.0563620 .8517812806592 H $3.858131-0.6471384 .934300$ H $1.615862-1.3101682 .476107$ H $2.279697 \quad 0.3224906 .380165$ H $1.284433-1.103822 \quad 6.194423$ H $\quad 0.6171730 .4946825 .809529$ H $1.293858-2.7386664 .200084$ H $-0.143608-1.1329740 .974635$ H -1.334562 -1.7424282 .126501$ H $-1.8813390 .388973 \quad 3.602513$ H $-4.7392992 .979822-1.706312$ H $-5.243863 \quad 1.6041341 .003943$ H -7.202770 $2.705140-2.111567$ H -7.929756 $1.128107-2.375451$ H $-6.2205291 .335545-2.687807$ H -2.7877050 .2195831 .697931$ H -1.5254390 .9705620 .719290$ H $-3.849667-3.664923-0.512829$ H -3.148525 -2.674932 -1.807532 H $-5.192284-1.267100-1.957510$ H $-6.263262-3.642957-0.347135$ H -8.185313 $-3.153633-1.860828$ H $-7.603522-1.546339-2.199156$ H $-9.739398-2.2272351 .405644$ H $-9.014464-3.6766860 .507232$ H -9.412164 -0.0244690 .629558$ H $-9.1369500 .094879-1.080724$ H $-7.059997 \quad 0.2519841 .205055$ H -3.491055 -1.978849 1.051477 C $2.9917322 .442260-2.668035$ O $3.1998171 .203541-1.960583$ C $4.2932141 .385347-1.038355$ C $4.8553582 .781876-1.304114$ C $3.6247843 .521238-1.794339$ H $3.8617481 .373907-0.031031$ C $5.3177910 .255020-1.193010$ H $5.2946203 .226708-0.405846$ H $5.6286872 .766486-2.081312$ C $3.9346254 .813276-2.527444$ H $2.9913653 .743794-0.926401$
C $4.762110-1.158830-0.910910$

O $5.809966 \quad 0.273733-2.539346$

H $6.1814570 .455635-0.547574$

H $4.044159-1.434638-1.690474$

O $5.852343-2.081725-1.037456$

C $4.125171-1.326690 \quad 0.479344$

C $3.810534-2.7784550 .882095$

H $3.209439-0.7279360 .534557$

O $5.029185-0.799958 \quad 1.457357$

H $4.742193-3.3481030 .995665$

H $3.384168-2.7371601 .892004$

H $4.4599945 .512546-1.868631$

H $4.5674634 .636616-3.403669$

H $3.0140445 .297825-2.867876$

C $1.5142002 .620957-3.022456$

H $6.453026-0.451190-2.622487$

H $6.486276-1.885605-0.326252$

H $4.602580-0.8807762 .327437$

H $1.1733501 .736987-3.572890$

H $1.4063573 .480545-3.694447$

H $3.5512492 .349473-3.609031$

C $0.711860-3.273470-1.167514$

O $1.697016-2.725380-0.279495$

C $2.852470-3.536613-0.053955$

C $2.450112-4.899704 \quad 0.540119$

C $1.385426-5.595930-0.313542$

C $0.235652-4.648294-0.670384$

H $-0.140378-2.585990-1.104022$

C $1.193296-3.296190-2.626148$

H $3.370538-3.694505-1.005293$

O $1.935753-4.7135331 .859086$

H $3.345832-5.5268830 .621633$

H $0.979311-6.4560270 .232802$

O $1.976270-6.084264-1.513630$

H $-0.408111-5.108327-1.428629$

$\begin{array}{llll} & -0.581254 & -4.449153 & 0.487408\end{array}$

C $1.401408-1.906276-3.185694$

H $2.139491-3.833748-2.728110$

H $\quad 0.442011-3.786454-3.255773$

H $1.734321-5.5925662 .222532$

H $2.679977-6.706622-1.262963$

H $-0.051607-3.9677391 .146236$

O $2.046972-2.009181-4.385457$

O $1.034516-0.868787-2.652777$

C $2.321528-0.773836-5.052445$

H $1.398039-0.208555-5.208848$

H $3.043218-0.190136-4.474723$

H $2.758743-1.004394-6.027520$

SCF Energy (B3LYP/6-31G**//MMFF) $=-3245.91963619$

0800013

MMFF Geometry

C $-\begin{array}{llll}2.728636 & 1.261501 & 2.383728\end{array}$

C $-1.754403 \quad 0.3390832 .357096$

C $-\begin{array}{llll}0.870866 & 0.052139 & 3.540034\end{array}$

$\begin{array}{llll}\text { O } & 0.517995 & 0.128070 & 3.145892\end{array}$

C $-1.121633-1.3265404 .181880$

C $-1.034076-2.5412313 .274517$

C $-2.084440-3.3215442 .949725$

C $0.346172-2.9198162 .803950$

C $-3.522993-3.1026183 .322073$

C $-4.533628-3.6360312 .292440$

C $-4.484528-2.9233140 .929979$

C $-5.751868-3.2388960 .128604$

C $-5.713126-2.628509-1.278958$

$\begin{array}{llll}\text { O } & -3.334215 & -3.385830 & 0.212202\end{array}$

O $-6.765586-3.227895-2.050052$

C $-4.382312-3.019481-1.951259$

C $-5.995561-1.121393-1.257626$

O $-4.233181-2.355220-3.204821$

C $-3.142324-2.761275-1.063741$

C $-1.891412-3.379109-1.732106$

O $-2.956222-1.355310-0.933460$

C $1.098114 \quad 1.3534383 .077033$

$\begin{array}{lllll}\text { O } & 0.544619 & 2.432123 & 3.242935\end{array}$

C 2.5536531 .2546942 .811794

C $3.193790 \quad 0.0965792 .587081$

C $4.634220-0.0818352 .387464$ 
C 5.5057961 .1460482 .434372

C $-0.615312-3.335986-0.880089$

C $0.557190-3.988304-1.593889$

C $2.988071-4.382704-1.417174$

O $0.445878-4.578116-2.666235$

C $4.072327-4.301926-0.391215$

C $5.272980-3.758329-0.642935$

C $6.409309-3.7115420 .337560$

C $6.847576-2.3016310 .668533$

C $7.566042-1.571295-0.201227$

C $6.536610-1.7765462 .050270$

C $5.105532-1.3329862 .196841$

N $1.744585-3.860581-0.883027$

H -2.915346 1.7992443 .309528

H $-1.562580-0.2154141 .443240$

H $-1.036478 \quad 0.7974294 .330412$

H -2.092565 -1.297472 4.689700

H $-0.385245-1.4710134 .984925$

H -1.891416 -4.216890 2.360827

H $0.379188-3.9312932 .385016$

H $0.684400-2.2313212 .024504$

H $1.058830-2.8930833 .634475$

H -3.706682 -3.620237 4.271441

H -3.733293 -2.040259 3.484585

H -4.369264 -4.712565 2.153792

H $-5.533198-3.5141812 .728423$

H -4.400291 -1.8435811 .100138$

H $-6.646644-2.9000350 .665834$

H $-5.857128-4.3287440 .030561$

H -7.607240 -3.053665 -1.594846

H $-4.430017-4.094827-2.173963$

H $-6.989324-0.924233-0.837729$

H -6.012214 -0.707570 -2.271930

H $-5.271893-0.557162-0.666593$

H -5.024683 -2.553970 -3.733763

H -2.098804 -4.431455 -1.967902

H -1.689450 -2.866184 -2.681466

H -2.035742 -1.175641 -0.688952

H 3.0806982 .2030472 .838761

H $2.618114-0.8258562 .567917$

H $5.1298361 .921601 \quad 1.759274$

H $\quad 6.5370320 .9590612 .126979$

H 5.5328891 .5547503 .450418

H $-0.339890-2.304054-0.643706$

H $-0.772639-3.8779320 .057713$

H $3.226110-3.814161-2.322953$

H $2.824934-5.426267-1.707831$

H $3.874332-4.7360490 .585576$

H $5.468640-3.352891-1.633430$

H $7.257240-4.258874-0.094926$

H $6.154063-4.2524891 .257145$

H $7.907174-0.5717280 .046771$

H $7.824586-1.956879-1.181892$

H $7.237673-0.9806762 .320514$

H $6.730311-2.5670642 .786970$

H $4.392584-2.1582712 .181501$

H $1.778520-3.217836-0.096263$

C $-3.449184 \quad 3.0392880 .703765$

O -2.1081353 .2679230 .225864$

C -1.7115184 .6017650 .595049$

C -2.9802645 .3054181 .071054$

C -3.7405814 .1568331 .704482$

H $-1.0323704 .496162 \quad 1.448374$

C $-0.9980845 .310546-0.562251$

H $-2.765008 \quad 6.117918 \quad 1.771612$

H $-3.552557 \quad 5.7238310 .234821$

C $-5.2192914 .437071 \quad 1.904780$

H -3.2828163 .9453842 .677823$

C $0.2484464 .584077-1.109857$

O $-1.9262985 .450186-1.646881$

H $-0.7349406 .329641-0.253754$

H $-0.0694203 .688527-1.653290$

O $0.8445595 .443735-2.091547$

C $1.3139414 .208001-0.064960$

C $2.6492853 .713097-0.652908$

H $0.917313 \quad 3.4415540 .609199$

O $1.6409035 .351208 \quad 0.732138$

H $3.1659124 .541858-1.154542$
H $3.285840 \quad 3.452913 \quad 0.203844$

H -5.3614205 .2945422 .570790$

H $-5.717845 \quad 4.6627270 .956220$

H $-5.721573 \quad 3.5740722 .353215$

C $-3.605587 \quad 1.6022601 .212512$

H $-1.4611205 .905183-2.369749$

H $1.1871226 .226317-1.626191$

H $0.8761415 .556731 \quad 1.294390$

H $-3.387114 \quad 0.9195170 .384638$

H $-4.648487 \quad 1.4254201 .499779$

H $-4.1015113 .153892-0.172612$

C $1.6651380 .257526-1.689026$

O $1.8744491 .455692-0.928137$

C $2.5523902 .512278-1.610197$

C $3.9438612 .052136-2.084767$

C $3.8747490 .750707-2.890082$

C $3.005245-0.301509-2.191646$

H $1.250675-0.459227-0.968919$

C $0.6344050 .447550-2.813236$

H $1.9623312 .813745-2.481458$

O $4.8126871 .853321-0.970853$

H $4.3880792 .846744-2.695261$

H $4.8875740 .349758-3.019828$

O $3.3460031 .010264-4.185783$

H $2.821597-1.152773-2.857272$

O $3.704861-0.799207-1.048835$

C $-0.7232460 .852429-2.288766$

H $\quad 0.9480791 .222796-3.516977$

H $0.509266-0.494404-3.359385$

H $4.4020311 .188110-0.392229$

H $3.9301471 .656248-4.618267$

H $4.569224-1.123788-1.352015$

O $-1.5310601 .165248-3.344179$

O $-1.0353320 .891751-1.107737$

C $-2.845170 \quad 1.622453-3.009753$

H $-3.3651900 .889353-2.387651$

H $-2.7849412 .588719-2.501593$

$\mathrm{H}-3.4064361 .750340-3.939114$

SCF Energy (B3LYP/6-31G**//MMFF) $=-3245.91667856$

0800014

MMFF Geometry

C 3.0841375 .0439810 .665596

C 3.7252633 .8657240 .682684

C $4.4166273 .277464-0.520235$

O $3.8421581 .972347-0.775376$

C $5.9175983 .105158-0.233485$

C $6.7055112 .636322-1.442194$

C $7.1789571 .386829-1.619537$

C $6.9836923 .697509-2.476873$

C $7.0168880 .200454-0.711431$

C $6.267016-0.937150-1.415986$

C $5.985190-2.108508-0.464012$

C $5.299323-3.255777-1.206855$

C $4.899376-4.398609-0.262374$

O $5.136532-1.6274650 .583638$

O $4.000148-5.247648-0.992180$

C $4.127067-3.8104270 .939800$

C $6.106784-5.2688270 .105435$

O $3.861355-4.8211531 .909127$

C $4.827339-2.5996911 .591089$

C $3.960214-1.8954132 .663731$

O $6.017418-3.0533882 .248958$

C $2.6787461 .941793-1.483857$

O $2.0726372 .899921-1.937678$

C $2.2598970 .528317-1.622136$

C $1.0644010 .246435-2.162532$

C $0.522884-1.086841-2.439175$

C $1.380280-2.275351-2.095750$

C $2.630136-1.3170822 .167230$

C $1.442421-2.2466862 .352575$

C $-0.979858-2.4291851 .795958$

O $1.500706-3.3002472 .980352$

C $-1.189831-3.1422400 .498389$

C $-2.261707-2.945234-0.282817$

C $-2.518072-3.677660-1.565326$

C $-2.645767-2.747486-2.749087$

C $-3.848332-2.308990-3.158178$ 
C $-1.391161-2.415617-3.521442$ C $-0.692858-1.183208-3.018127$ N $0.295106-1.7387581 .758389$ H $3.0780505 .632211-0.248232$ H 3.7285413 .2722831 .594106 H $4.2816473 .915562-1.402414$ H 6.0538042 .4200310 .609638 H 6.3444804 .0606030 .100908 H $7.7516651 .180488-2.523107$ H $7.6132173 .327982-3.293336$ H $6.0510724 .058929-2.920327$ H $7.5044184 .545626-2.020184$ H $8.014228-0.148679-0.417885$ H $6.4994790 .464917 \quad 0.213129$ H $5.318199-0.548762-1.806538$ H $6.853920-1.289105-2.273738$ H $6.937062-2.442956-0.034597$ H $5.936019-3.631518-2.017586$ H $4.391285-2.877505-1.694464$ H $\quad 3.679167-5.932047-0.379881$ H $3.151010-3.4945280 .553853$ H $6.552693-5.701741-0.798170$ H $5.808522-6.1206650 .726347$ H $6.889524-4.7164140 .629269$ H $4.680759-4.9750632 .409383$ H $3.816395-2.5571573 .526379$ H $4.546629-1.0469123 .045469$ H $6.271589-2.3937532 .915505$ H $2.952849-0.238563-1.298379$ H $\quad 0.424718 \quad 1.075485-2.462627$ H $0.869771-3.232315-2.224210$ H $1.690619-2.238627-1.046394$ H $2.274535-2.294098-2.727400$ H $2.405111-0.4168322 .753812$ H $2.697325-1.0146141 .117710$ H -1.749851 -1.675780 1.983082 H $-0.991598-3.1485822 .620605$ H $-0.437605-3.8686460 .200543$ H $-3.025879-2.2408820 .034061$ H -3.446436 -4.251226 -1.442019 H -1.739853 -4.424938 -1.763168 H -3.951112 -1.668325 -4.027657 H -4.754875 -2.576321 -2.626339 H -1.649623 -2.230719-4.573493 H $-0.734833-3.289952-3.558694$ H - $-1.242836-0.263637-3.223541$ H $\quad 0.351417-0.877314 \quad 1.222288$ C 0.8185675 .4920271 .724892 $\begin{array}{lllll}\text { O } & 0.440725 & 4.103271 & 1.627947\end{array}$ C $-0.506373 \quad 3.9601560 .548433$ C $-1.060758 \quad 5.3553870 .311615$ C $0.176666 \quad 6.2044290 .532740$ H $0.0747413 .629226-0.320283$ C -1.5393472 .8944200 .923292$ H -1.487402 $5.478142-0.688147$ H -1.8330475 .5992561 .051552$ C $-0.1243367 .671420 \quad 0.785298$ H $\quad 0.807330 \quad 6.124540-0.360553$ C -2.661045 $2.734767-0.122244$ O $-0.8400841 .653746 \quad 1.067040$ H $-1.977762 \quad 3.123577 \quad 1.901970$ H -3.177521 $3.694859-0.233918$ O $-2.0855542 .426696-1.392430$ C $-3.715724 \quad 1.667766 \quad 0.237470$ C $-4.8328681 .589184-0.822519$ H $-4.1556391 .911027 \quad 1.211949$ $\begin{array}{lllll}\text { O } & -3.108848 & 0.384783 & 0.341725\end{array}$ H -5.206049 2.599094 -1.029669 H -4.410609 $1.179854-1.749036$ H $-0.6334448 .113541-0.077340$ H -0.7695967 .8046891 .660136$ H 0.7992998 .2328590 .958026 C 2.3422275 .6071321 .846208 H -0.1342601 .7918441 .721935$ H -1.662430 $1.554512-1.312395$ H -2.4561220 .4229981 .060537$ H 2.6595435 .0809422 .755691 H 2.6296786 .6571221 .973123
H $\quad 0.3745425 .8585122 .659609$

C -6.503446 -1.600958 0.199544

O $-5.551984-0.650357-0.294412$

C $-6.0113490 .700860-0.374306$

C $-7.1974510 .785691-1.346236$

C $-8.316600-0.182304-0.943604$

C $-7.783812-1.591218-0.653869$

H $-6.017321-2.5762400 .066609$

C -6.765738 -1.412127 1.698702

H $-6.328773 \quad 1.0392730 .618194$

O $\quad-6.7709930 .490781-2.675170$

H -7.579592 $1.812999-1.352681$

H $-9.057693-0.236024-1.750503$

O

H $-8.551553-2.201790-0.164330$

O $-7.471052-2.227902-1.896449$

C $-5.482765-1.5278512 .491511$

H -7.225516 -0.443215 1.913163

H -7.454889-2.177015 2.074221

H $-6.358503-0.389861-2.666315$

H $-9.338287 \quad 1.1842950 .005545$

H -8.292283 -2.278889 -2.414659

O $-5.287460-0.394288 \quad 3.224579$

O $-4.743456-2.5031402 .461536$

C $-4.100058-0.3660144 .022367$

H $-4.1436960 .517998 \quad 4.664180$

H -4.037004 -1.255287 4.656961

H -3.221076 -0.287340 3.377334

SCF Energy $\left(B 3 L Y P / 6-31 G^{* *} / / M M F F\right)=-3245.92884196$

\section{8_00015}

MM̄MF Geometry

C $-0.860576-4.189406-0.233806$

C $-2.105824-3.693511-0.259379$

C $-2.895628-3.445628-1.517952$

O $-3.094658-2.014744-1.613249$

C $-4.268440-4.132871-1.419169$

C $-5.174360-3.859924-2.605216$

C $-6.268982-3.072412-2.571192$

C $-4.788438-4.558227-3.883569$

C $-6.808504-2.305637-1.395698$

C $-6.553905-0.800582-1.547830$

C $-6.617241-0.071079-0.197831$

C $-6.4995881 .440522-0.396241$

C -6.4114632 .1987470 .936199$

$\begin{array}{lllll}\text { O } & -5.523517 & -0.547774 & 0.595184\end{array}$

$\begin{array}{lllll}\text { O } & -5.989328 & 3.534525 & 0.620158\end{array}$

C $-5.321977 \quad 1.5458331 .816005$

C -7.7858802 .3221641 .604138$

O $-5.3162432 .108846 \quad 3.125380$

C -5.4516020 .0111351 .913277$

C $-4.251378-0.6550832 .633294$

O $-6.625531-0.3114172 .669338$

C $-2.084655-1.289298-2.170296$

O $-1.065929-1.722599-2.685357$

C $-2.4093180 .144428-1.999624$

C -1.487664 $1.070484-2.303480$

C $-1.6315162 .519914-2.150141$

C $-2.9062293 .034913-1.536862$

C $-2.885189-0.455876 \quad 1.967240$

C -2.0906420 .7218392 .504585$

C -0.0133032 .0243562 .053851$

O -2.3940571 .3300903 .527064$

C -0.3831643 .2888541 .344300$

C 0.4875274 .0044810 .616519

C $0.1535465 .304528-0.057303$

C $0.5069415 .311876-1.528126$

C $1.6749665 .820873-1.955567$

C $-0.5318684 .813805-2.507384$

C $-0.613953 \quad 3.313406-2.544534$

N -0.9712360 .9855151 .725301$

H -0.375372 -4.477700 -1.162567

H $-2.571237-3.3874290 .674972$

H $-2.363498-3.810794-2.405026$

H -4.752652 -3.830892 -0.485562

H $-4.130438-5.219190-1.330920$

H $-6.844885-2.949976-3.487544$

H $-5.516265-4.390952-4.684798$ 
H $-3.818406-4.199529-4.241152$ H $-4.723009-5.639011-3.720997$ H -7.889069 -2.483521 -1.329639 H -6.399309 -2.674222 -0.452857 H - $5.563591-0.640502-1.991251$ H -7.291976 -0.378849 -2.241229 H $-7.562809-0.3239470 .295963$ H $-7.3340801 .819529-0.999421$ H -5.593475 $1.657550-0.976966$ H -5.9719564 .0486991 .444962$ H -4.3555891 .7930351 .361429$ H -8.4733742 .8826920 .959227$ H -7.7231842 .8893562 .539276$ H -8.246725 1.3555061 .818329 H -5.1059003 .0537253 .039616$ H -4.233071 -0.3594993 .689148$ H -4.440434 -1.738395 2.639054 H $-6.530433-1.2134283 .017396$ H -3.383175 $0.397980-1.599101$ H $-0.5268160 .738238-2.695135$ H $-3.7566262 .830321-2.195976$ H -2.897768 4.109594-1.345035 H -3.087018 2.557421 -0.568068 H -2.271964 -1.343981 2.167114 H $-2.987319-0.3663030 .882413$ H $\quad 0.975719 \quad 1.658102 \quad 1.761237$ H -0.017362 2.1937123 .135364 H - 1.4031163 .6481621 .459412 H 1.5142123 .6575850 .534569 H $\quad 0.708830 \quad 6.0982580 .459666$ H $\quad-0.906240 \quad 5.5595130 .066178$ H $1.9243155 .856674-3.010920$ H $2.4115156 .205640-1.257947$ H $-0.2830945 .149586-3.523289$ H $-1.4916155 .289880-2.288339$ H $\quad 0.2714302 .843198-2.973962$ H $-0.823547 \quad 0.4583550 .869413$ C $1.090627-3.2937221 .027604$ O $2.136583-3.750581 \quad 0.145199$ C $3.415174-3.5112950 .774690$ C $3.126552-2.6244831 .975219$ C $1.753700-3.1110722 .390579$ H $3.774572-4.4946281 .103589$ C $4.373111-2.912413-0.261991$ H $3.874460-2.7288762 .766368$ H $3.083958-1.5694891 .677592$ C $1.026378-2.1439213 .309215$ H $1.857030-4.0821232 .892999$ C $5.692427-2.3911660 .332272$ O $4.674631-3.930730-1.222138$ H $\quad 3.860957-2.113987-0.809019$ H $5.494761-1.5647991 .019800$ O $6.279055-3.4236981 .138038$ C $6.748717-1.955890-0.699493$ C $6.292220-0.955623-1.774766$ H $7.143861-2.843932-1.208503$ O $7.858559-1.380114-0.001179$ H $7.128344-0.832008-2.476107$ H $5.463475-1.396045-2.340247$ H $0.028573-2.5161483 .560860$ H $1.582540-2.0097464 .242749$ H $0.911521-1.1594442 .844005$ C $-0.055513-4.3070691 .030766$ H $3.829161-4.242291-1.588813$ H $6.415772-4.1989140 .566643$ H $8.146465-2.0247280 .667947$ H -0.700954 -4.144505 1.901762 H $0.334381-5.3297021 .096326$ H $\quad 0.749226-2.3232890 .641797$ C 4.2020131 .5676860 .057189 O $4.6980820 .347859-0.510267$ C $5.9043090 .450687-1.269156$ C $5.7097001 .426151-2.444431$ C $5.1882072 .785820-1.971556$ C $3.9917392 .636013-1.028329$ H 3.2151451 .3041270 .458604 C 5.0719152 .0543001 .222696 H $6.7070470 .821090-0.623570$
O $4.799720 \quad 0.880017-3.398821$

H $6.6691841 .556686-2.958247$

H $4.8886843 .383803-2.840977$

O $6.2242623 .490855-1.294901$

H $3.7552413 .595805-0.554952$

O $2.8453502 .246763-1.789741$

C 4.9949811 .1006112 .393079

H 6.1193582 .1764050 .933773

H 4.7282873 .0294971 .587092

H $3.9672310 .682384-2.936379$

H $6.9617493 .600198-1.919175$

H $2.6806822 .943970-2.447124$

O 6.2394140 .6316982 .694596

O $3.955942 \quad 0.8110262 .972470$

C $6.306464-0.2781823 .796911$

H $5.9944410 .222784 \quad 4.718177$

H $5.684061-1.1569023 .605486$

H $7.344634-0.6025043 .906930$

SCF Energy (B3LYP/6-31G**//MMFF)= -3245.91982536

\section{6}

MM̄FF Geometry

C $-4.294433-2.101898 \quad 2.259286$

C $-3.020455-2.2563531 .869836$

C $-1.839161-1.9438352 .746530$

O $-0.982303-1.0116652 .048333$

C -1.014010 -3.215899 3.007222

C $0.093784-2.9760454 .013809$

C $1.370957-2.6815283 .700148$

C $-0.323241-3.0866315 .458148$

C $1.978110-2.5175312 .332302$

C $3.263011-3.3460412 .206781$

C $3.903516-3.2526910 .812388$

C $5.215219-4.044622 \quad 0.789335$

C $5.972664-3.889556-0.537543$

O $4.146923-1.8745150 .515740$

O $7.310183-4.374576-0.339223$

C $6.081596-2.385852-0.870161$

C $5.360074-4.755345-1.644446$

O $6.673167-2.189901-2.152562$

C $4.735164-1.636703-0.770475$

C $4.896228-0.108797-0.946300$

O $3.880119-2.092393-1.825063$

C -1.258624 $0.314476 \quad 2.154795$

O -2.2119640 .8292752 .715186$

$\begin{array}{llll}\text { C } & -0.164208 & 1.037567 & 1.469596\end{array}$

$\begin{array}{llll}\text { C } & -0.210318 & 2.371830 & 1.344587\end{array}$

$\begin{array}{lllllll}\text { C } & 0.800386 & 3.203914 & 0.686838\end{array}$

C $2.0633392 .524422 \quad 0.228831$

C $5.696368 \quad 0.5858370 .157957$

C $5.7023012 .097183-0.005767$

C 6.1485894 .1809851 .300218

O $5.4059682 .660764-1.055790$

C $4.8295704 .660278 \quad 1.822688$

C 4.1995895 .7516301 .360545

$\begin{array}{llll}\text { C } 2.882379 & 6.236524 & 1.899385\end{array}$

C 1.9034126 .6242570 .810670

C 1.4769057 .8932590 .692098

C $1.4190715 .554507-0.143860$

C $0.556410 \quad 4.5219450 .529705$

N $6.1071752 .736785 \quad 1.157288$

H $-4.500744-1.7508673 .266823$

H $-2.808690-2.6185120 .868250$

H $-2.158930-1.5045793 .700107$

H $-0.598099-3.5944192 .066151$

H $-1.662592-4.0180303 .384011$

H $2.067033-2.5110034 .521029$

H $0.510220-2.9135716 .147245$

H $-1.100977-2.3528685 .691453$

H $-0.716696-4.0877835 .662877$

H $1.286670-2.7980191 .533487$

H $2.210455-1.4549512 .194340$

H $3.980029-2.9855622 .956729$

H $3.052483-4.3977632 .439297$

H $3.196126-3.6563610 .078248$

H $5.031812-5.1045531 .006071$

H $5.868107-3.6856701 .597772$

H $7.253698-5.301887-0.051717$ 
H $\quad 6.784454-1.957193-0.145445$

H $5.383759-5.814011-1.358730$

H $5.941937-4.684621-2.569948$

H $4.321892-4.499476-1.865209$

H $5.996795-2.384242-2.823473$

H $5.3313830 .112141-1.929433$

H $3.8951810 .343452-0.969059$

H $3.187402-1.426864-1.970740$

H $\quad 0.654207 \quad 0.444058 \quad 1.076785$

H -1.071613 2.9046301 .744858

H $\quad 1.842324 \quad 1.776102-0.538127$

H 2.5603892 .0284261 .070214

H $2.7953293 .207904-0.201959$

H 5.2681220 .3434281 .137273

H 6.7399610 .2548090 .146134

H 6.3998864 .6345980 .336023

H 6.9395484 .4096582 .020888

H 4.3758834 .0906902 .630800

H 4.6666726 .3426250 .576051

H 3.0896437 .1005582 .543924

H 2.4189905 .4815732 .546308

H $0.7765118 .185302-0.083392$

H 1.8174758 .6709891 .367813

H $2.2766985 .121794-0.664656$

H $0.8117736 .005789-0.940250$

H -0.3894634 .9210910 .898583$

H 6.2745152 .1809881 .991039

C $-6.297742-1.1779540 .985979$

O $-5.498147-0.2765290 .197147$

C $-5.880097 \quad 1.0764230 .520767$

C -7.1282620 .9729341 .393800$

C $-6.881715-0.3366672 .119096$

H $-5.066511 \quad 1.4942951 .125378$

C $-6.0578861 .902896-0.757950$

H -7.2314411 .8268982 .070255$

H -8.0429710 .9083110 .793327$

C $-8.127499-0.9302392 .751808$

H $-6.134421-0.1548462 .900636$

C $-4.8313791 .879481-1.693064$

O $-7.1690161 .369575-1.490381$

H $-6.3256422 .933518-0.497186$

H $-4.7124630 .867956-2.094640$

O $-5.1235482 .716722-2.819252$

C -3.519296 $2.354990-1.044098$

C $-2.3273282 .426678-2.018613$

H $-3.2614501 .698939-0.206554$

O $-3.7290633 .661826-0.502468$

H $-2.5493333 .120438-2.838735$

H -1.490822 $2.876086-1.470542$

H -8.542187 -0.2462713 .499636$

H -8.905065 -1.122593 2.005058

H -7.897152 -1.876752 3.250791

C $-5.477482-2.4075161 .384242$

H -7.264636 $1.901420-2.299051$

H -5.194111 $3.630677-2.493604$

H $-2.9162093 .919320-0.034582$

H -5.133142 -2.905301 0.468499

H $-6.119007-3.1240151 .910085$

H -7.107566 -1.5176310 .325883$

C $-1.266177-1.135371-1.789066$

O $-1.5604010 .228601-1.462283$

C $-1.9047281 .058347-2.576289$

C $-0.7188121 .149767-3.549744$

C $-0.269451-0.246406-3.994308$

C $-0.119002-1.212995-2.810871$

H $-0.906306-1.567849-0.846865$

C $-2.548937-1.876778-2.191545$

H $-2.7574230 .619563-3.104669$

O $\quad 0.391293 \quad 1.800652-2.936270$

H -1.010698 $1.739617-4.427155$

H $0.690947-0.161091-4.517955$

O $-1.224141-0.754040-4.921556$

H $-0.014030-2.240499-3.174521$

O $1.097809-0.900208-2.124776$

C $-2.471241-3.366722-1.942568$

H $-3.381059-1.486734-1.591774$

H $-2.799347-1.717743-3.243416$

H $0.1825302 .747669-2.871728$
H $-0.907935-1.623124-5.222004$

H $1.005104-0.007632-1.750503$

O $-3.716196-3.905900-2.104269$

O $-1.456807-3.983667-1.648423$

C $-3.809748-5.315770-1.878462$

H -3.519455 -5.554658 -0.850768

H $-4.850614-5.615430-2.027147$

H -3.182829 -5.856292 -2.593908

SCF Energy $\left(B 3 L Y P / 6-31 G^{* *} / / M M F F\right)=-3245.93316834$

\section{7}

MM̄FF Geometry

C $0.891726 \quad 3.637309-1.080529$

C $-0.252399 \quad 4.198359-0.659300$

C $-1.262133 \quad 3.460743 \quad 0.176928$

O $-2.5071843 .458596-0.564122$

C $-1.5027804 .117983 \quad 1.549474$

C $-0.2865194 .203227 \quad 2.442655$

C $0.2651353 .157062 \quad 3.087763$

C $0.2781475 .588427 \quad 2.621306$

C $-0.172563 \quad 1.7166443 .066653$

C $0.9599210 .811562 \quad 2.563054$

C $0.621376-0.6832752 .689491$

C $1.861453-1.5258692 .378337$

C $1.558227-3.0291222 .401104$

O $-0.431453-0.9964541 .771213$

O $2.666786-3.7161321 .801053$

C $0.326911-3.2980031 .515618$

C $1.439450-3.5671213 .831677$

O $-0.077038-4.6625391 .613008$

C $-0.871371-2.3656911 .813732$

C -1.946778 -2.5519830 .713143$

O $-1.425546-2.703902 \quad 3.084716$

C $-3.377828 \quad 2.457961-0.265424$

O -3.2249521 .5867420 .576558$

C $-4.5646502 .580178-1.136930$

C $-5.3292151 .490325-1.297990$

C $-6.5832341 .386639-2.041603$

C $-7.1794092 .652707-2.592933$

C $-3.180759-1.6529960 .868415$

C $-4.400996-2.3469121 .455068$

C $-6.818224-1.8995611 .928246$

$\begin{array}{llll}\text { O } & -4.408108 & -3.521028 & 1.812372\end{array}$

C $-7.642203-2.1264270 .698208$

C $-8.710867-1.3852410 .365985$

C $-9.531658-1.605981-0.875060$

C $-9.575959-0.387323-1.774529$

C $-10.6115310 .467495-1.725481$

C $-8.464180-0.200903-2.785918$

C $-7.1586780 .170384-2.142592$

N $-5.487911-1.4838491 .521772$

H $1.1016242 .602102-0.823476$

H $-0.4701695 .232855-0.912209$

H -0.9288662 .4245150 .309125$

H -2.296698 3.5814512 .084986

H $-1.928092 \quad 5.120567 \quad 1.399820$

H 1.1274853 .3510203 .725256

H 1.1939675 .5919003 .221728

H $\quad 0.5187556 .033667 \quad 1.651407$

H $-0.4526906 .231072 \quad 3.123212$

H -0.4375351 .4327794 .092519$

H -1.0704951 .5668052 .461383$

H 1.1669771 .0564681 .513247

H $1.873087 \quad 1.0229273 .134365$

H $\quad 0.276415-0.877873 \quad 3.711747$

H $2.677146-1.290548 \quad 3.073561$

H $2.236736-1.2622861 .380914$

H $3.468313-3.4859892 .301593$

H $0.630846-3.1411850 .472346$

H $2.374748-3.4038214 .380587$

H $1.276651-4.6505553 .834318$

H $\quad 0.636476-3.095847 \quad 4.402288$

H $0.698191-5.2104291 .401837$

H $-1.485793-2.297327-0.249857$

H -2.249791 -3.6031620 .653607$

H -2.340873 $-2.385403 \quad 3.125163$

H $-4.7800703 .538549-1.592774$

H $-5.0091540 .565420-0.820690$ 
H -7.302635 $3.398955-1.799884$ H -8.167378 2.508017 -3.036261 H -6.533420 $3.072745-3.371500$ H -2.954813 -0.7767081 .483332$ H -3.472177 -1.300356 - 0.128975 H -7.228735-1.104927 2.559095 H -6.762166 -2.820236 2.517065 H $-7.338379-2.9432970 .047603$ H $-9.020400-0.5754721 .022913$ H - $-10.548257-1.873805-0.558545$ H -9.166894 -2.467209-1.448525 H -10.665968 $1.328646-2.383265$ H -11.425817 $0.327742-1.022180$ H $-8.7493470 .530910-3.549339$ H -8.325289-1.137708 -3.340744 H $-6.636734-0.683253-1.709176$ H $-5.381959-0.5338451 .178388$ C $3.3048374 .410321-1.201781$ O $3.8724303 .089928-1.079639$ C 4.5591923 .0043780 .184405 C $4.606838 \quad 4.4240150 .747290$ C $3.322526 \quad 5.0112180 .202569$ H 3.9324202 .3875020 .839000 C $5.9410992 .364303 \quad 0.019013$ H 4.6542094 .4328201 .840729 H 5.4697374 .9851960 .370572 C 3.2811186 .5285590 .226867 H 2.4958854 .6201420 .805946 C $5.9402340 .954294-0.604209$ O $6.728276 \quad 3.213711-0.827199$ H 6.4467492 .3451720 .992563 H $5.6827951 .013614-1.668305$ O $7.2865980 .462829-0.537747$ C $5.022040-0.0710560 .091018$ C $5.309246-1.541171-0.273405$ H $3.9772530 .164533-0.134963$ O $5.166160 \quad 0.0411831 .511219$ H $6.315204-1.8017900 .079316$ H $4.639237-2.1677520 .326528$ H 3.3649286 .8987841 .253967 H $4.1013616 .962347-0.354746$ H $2.3392686 .898924-0.189879$ C $1.9373094 .349533-1.889786$ H $7.6545822 .931093-0.746706$ H $7.8155550 .969864-1.176421$ H $6.100433-0.1236181 .725973$ H $2.0394713 .810221-2.839468$ H $1.6077505 .367332-2.129721$ H $3.9876514 .968895-1.856015$ C $2.850928-2.559723-1.783569$ O $3.847471-1.649799-2.265085$ C $5.170921-1.879096-1.771171$ C $5.627930-3.300051-2.132710$ C $4.650882-4.350211-1.613982$ C $3.234214-4.018564-2.077436$ H $2.720967-2.415701-0.706500$ C $1.526450-2.214012-2.482136$ H $5.826552-1.195048-2.321827$ O $6.932554-3.536602-1.614957$ H $5.694377-3.384471-3.225442$ H $4.699316-4.443451-0.522728$ O $5.001369-5.629662-2.145481$ H $3.159438-4.214175-3.154670$ O $2.314818-4.900734-1.433596$ C $0.892121-0.947778-1.950877$ H $1.691679-2.074743-3.556826$ H $0.800383-3.020426-2.327353$ H $7.240918-4.387754-1.968843$ H $5.852872-5.886779-1.753540$ H $2.374344-4.745587-0.475398$ O $-0.346082-0.814620-2.513895$ O $1.406060-0.175880-1.154446$ C -1.103285 $0.318411-2.082207$ H $-1.151940 \quad 0.351905-0.990304$ H $-0.6609001 .235060-2.481126$ $\mathrm{H}-2.1192820 .218064-2.472781$

SCF Energy (B3LYP/6-31G**//MMFF) $=-3245.91594347$
0800018

MMFF Geometry

C -0.4861114 .5776510 .090925$

C $0.777976 \quad 4.137466 \quad 0.175418$

C $1.6892374 .000414-1.017510$

O $2.1446642 .627209-1.090548$

C $2.9140504 .915309-0.846724$

C $3.8523254 .894358-2.038694$

C $5.050968 \quad 4.277863-2.067573$

C $3.375915 \quad 5.666095-3.243108$

C $5.6980363 .468173-0.978714$

C $5.9314452 .020739-1.429945$

C $6.4043701 .131175-0.270765$

C $6.706824-0.283719-0.765793$

C $7.053011-1.242050 \quad 0.383177$

$\begin{array}{llllll}\text { O } & 5.356687 & 1.095531 & 0.703618\end{array}$

O $6.994976-2.570383-0.159543$

C $5.972279-1.1240381 .481377$

C $8.490655-1.0339870 .873991$

O $6.338540-1.8677442 .640788$

C $5.6611520 .333953 \quad 1.879298$

C $4.448668 \quad 0.4587842 .835985$

O 6.7939050 .8819652 .565520

C $1.2864231 .719192-1.635498$

O $0.1580941 .932877-2.050584$

C $1.9633410 .402349-1.648837$

C $1.303671-0.680106-2.089091$

C $1.842594-2.038033-2.205627$

C $3.258650-2.270034-1.750776$

C $3.109136-0.0339972 .276602$

C $2.774598-1.4727872 .632330$

C $1.083098-3.2412432 .157519$

O $3.428134-2.1420293 .427474$

C $1.568287-4.1289051 .055537$

C $0.749710-4.8265140 .253896$

C $1.223916-5.761385-0.819717$

C $0.658404-5.424893-2.180453$

C $-0.429055-6.057285-2.653296$

C $1.414648-4.431982-3.031947$

C $1.056664-3.003960-2.726452$

N $1.631158-1.9106261 .976673$

H $-0.8837014 .877153-0.875740$

H $1.1727643 .825068 \quad 1.139371$

H $1.1652314 .256315-1.947026$

H 3.4460464 .6455550 .071198

H $2.5833365 .951445-0.691185$

H $5.6441364 .354147-2.978256$

H $4.1178785 .680232-4.048697$

H $2.4609415 .223340-3.648157$

H $3.1691826 .706098-2.970234$

H $6.6608463 .929855-0.728082$

H $5.106573 \quad 3.477669-0.061168$

H $4.9948961 .618718-1.836005$

H $6.6719842 .003944-2.239234$

H $7.298576 \quad 1.5832200 .174122$

H $7.511418-0.271096-1.511659$

H $5.825388-0.677523-1.288434$

H $7.258485-3.1933850 .538466$

H $5.061833-1.5870501 .082881$

H $9.201831-1.2323680 .063050$

H $8.743512-1.7396751 .672996$

H $8.677203-0.0214321 .237851$

H $6.404810-2.8025102 .383464$

H $4.679558-0.0061673 .802040$

H $4.319877 \quad 1.5288993 .054176$

H 6.4966931 .6381433 .098128

H $2.9920380 .362073-1.311644$

H $0.271358-0.561070-2.416461$

H $3.959514-1.746423-2.409128$

H $3.546163-3.323647-1.739078$

H $3.399862-1.909911-0.726376$

H 2.3084130 .5791602 .710246

H 3.0648260 .0921451 .191004

H $-0.006596-3.1457372 .165418$

H $1.402840-3.6441533 .123851$

H $2.644553-4.2230390 .930380$

H $-0.325136-4.7542510 .400480$

H $0.921428-6.777315-0.532991$ 
H $2.319263-5.787843-0.873791$ H $-0.820745-5.844672-3.642428$ H $-0.955878-6.795726-2.057992$ H $1.179931-4.600414-4.092192$ H $2.488162-4.628653-2.959233$ H $0.040861-2.740492-3.022964$ H $1.182458-1.3021091 .297825$ C -2.4943173 .5608101 .144660$ O $-3.3822393 .859747 \quad 0.051119$ C -4.6775853 .2839710 .343572$ C -4.5669502 .6703831 .736172$ C -3.4162073 .4432452 .351437$ H -5.3870604 .1197450 .342137$ C $-5.0102482 .286553-0.773685$ H -5.4976352 .7588602 .304547$ H -4.3120111 .6057251 .676145$ C $-2.7975182 .741993 \quad 3.548192$ H $-3.775640 \quad 4.4364182 .651469$ C $-6.2414641 .409871-0.496779$ O $-5.2386063 .024184-1.979185$ H $-4.1303151 .661962-0.958595$ H -6.1009630 .8346010 .420474$ O $-7.3689122 .257595-0.239304$ C $-6.6221690 .448584-1.638899$ C $-5.491975-0.432659-2.201976$ H $-7.0494251 .024501-2.469068$ O $-7.668304-0.413984-1.179489$ H -5.904463 $-0.991667-3.052768$ H $-4.7079530 .218814-2.603512$ H -1.9736633 .3295803 .964458$ H $-3.5426222 .600724 \quad 4.337919$ H -2.4048361 .7560813 .276753$ C $-1.4274684 .647557 \quad 1.261298$ H $-4.4469523 .565870-2.141048$ H -7.489690 2.828154-1.017778 H $-8.3800630 .151507-0.833700$ H -0.8724504 .5361522 .199553$ H -1.889995 5.6415981 .267979 H -2.0251182 .5906430 .927659$ C -3.665895-1.583915 0.872836 O $-4.257872-0.771012-0.147224$ C $-4.883181-1.465471-1.226728$ C $-3.857147-2.364659-1.941307$ C -3.144295 -3.298304 -0.957858 C $-2.628769-2.544040 \quad 0.271292$ H $-3.129077-0.8747131 .516283$ C -4.722391 -2.2907131 .730489$ H -5.696068 -2.085215 -0.834377 O $-2.889122-1.550617-2.602490$ H -4.376281 -2.948770 -2.710622 H $-2.299210-3.782635-1.460760$ O $-4.037654-4.322386-0.532151$ H $-2.297543-3.2482301 .042916$ O $-1.491180-1.760994-0.100849$ C $-5.516078-1.2905282 .538644$ H $-5.413868-2.8930411 .134653$ H -4.251732 -2.974155 2.446912 H $-2.290429-2.143294-3.087747$ H $-4.326990-4.807811-1.323484$ H $-0.815359-2.369862-0.443100$ O $-6.811847-1.2699552 .115385$ O $-5.038705-0.6039663 .433016$ C -7.669629 -0.3500452 .798044$ H -8.649013 -0.3753342 .312789$ H -7.785557 -0.6514243 .843199$ H -7.2728190 .6673642 .731144$ SCF Energy (B3LYP/6-31G**//MMFF)= -3245.91049566

0800019

MM̄FF Geometry

C $-0.455516-0.364661-2.299342$

C $-0.807749-1.633592-2.037959$

C $-0.194450-2.527741-0.982290$

O $0.823022-1.805129-0.253466$

C $0.456041-3.751056-1.647209$

C $1.045277-4.728365-0.649857$

C $2.361406-4.835591-0.379409$

C $0.052220-5.6398780 .022169$
C $3.471126-3.991502-0.943903$

C $4.479337-3.5808860 .139135$

C $5.534861-2.617439-0.422195$

C $6.668360-2.4029330 .580084$

C $7.692891-1.3791570 .069601$

O $4.888466-1.368807-0.693174$

O $8.527595-1.0417951 .187687$

C $6.944358-0.105583-0.377035$

C $8.613249-1.981932-0.998394$

O $7.8289330 .817355-1.008847$

C $5.734086-0.385497-1.302461$

C $4.9142980 .914229-1.480108$

O $6.203912-0.817440-2.580133$

C $0.657431-1.6336271 .083750$

O $-0.220633-2.0971021 .792860$

C $1.721765-0.7161961 .540713$

C $1.464376 \quad 0.1055102 .568214$

C $2.365408 \quad 1.1288283 .099721$

C 3.8080891 .0786642 .673713

C $3.5520330 .727312-2.160597$

C $2.7720342 .028843-2.133587$

C $1.5409973 .536724-0.564243$

O $2.6776642 .745399-3.126992$

C 2.2150834 .1662860 .615488

C 1.5673544 .5657081 .720751

C 2.2450965 .2046352 .899709

C 1.8889954 .5471034 .216274

C 1.0598435 .1485985 .086049

C 2.5593293 .2334844 .562654

C 1.8579372 .0574123 .936868

N $2.2391062 .305638-0.884440$

H $0.3375850 .105327-1.726079$

H -1.605152 -2.083036 -2.626826

H -1.000626 -2.852661 -0.315650

H $1.223042-3.421637-2.357889$

H $-0.286095-4.283768-2.257434$

H $2.680093-5.6053990 .321999$

H $0.534259-6.3507880 .701661$

H $-0.665921-5.0592980 .609460$

H $-0.497907-6.220034-0.725885$

H $3.990432-4.573704-1.714406$

H $3.083195-3.090213-1.426080$

H $3.944974-3.0995060 .968131$

H $4.967600-4.4796650 .535901$

H $5.925853-3.035827-1.357165$

H $7.163455-3.352200 \quad 0.820127$

H $6.250417-2.0349641 .528030$

H $9.219275-0.4337830 .876434$

H $\quad 6.5755750 .3890300 .532689$

H $9.165602-2.835460-0.587387$

H $9.372642-1.260513-1.320008$

H $8.074376-2.328578-1.882778$

H $8.5145671 .058450-0.363881$

H $4.7443791 .360842-0.493983$

H $5.4932201 .644581-2.060092$

H $5.521584-0.633314-3.244970$

H $2.650550-0.6958800 .983695$

H 0.4811850 .0589953 .035779

H $4.459617 \quad 1.7191263 .271863$

H 3.9086741 .3905941 .631429

H 4.2096480 .0637952 .775909

H $3.6678340 .419377-3.205007$

H $2.956849-0.045375-1.661987$

H $0.4998583 .274911-0.353202$

H $1.5664104 .218724-1.419267$

H $3.291843 \quad 4.3074270 .552436$

H 0.4874234 .4533711 .772707

H 1.9498026 .2619372 .916980

H 3.3359615 .2023842 .781991

H 0.8212954 .6969726 .043426

H 0.5965576 .1029714 .858531

H 2.5477963 .0825245 .650165

H 3.6162833 .2998534 .291489

H $0.8014201 .989467 \quad 4.201618$

H $2.3113491 .604010-0.154004$

C $-1.6705281 .774443-2.832044$

O $-2.8987121 .483350-2.130435$

C $-3.9568772 .262393-2.712608$ 
C $-3.2620473 .437943-3.384299$

C $-2.0324902 .757919-3.950314$

H $-4.4173621 .634244-3.485556$

C $-5.0182962 .646920-1.676228$

H $-3.8839203 .910098-4.150575$

H $-2.9661864 .205097-2.659470$

C $-0.9095483 .719904-4.300699$

H $-2.3227732 .208657-4.856267$

C $-5.5634581 .478162-0.829631$

O $-4.4556133 .603083-0.768449$

H $-5.8407493 .162827-2.186720$

H $-4.822767 \quad 1.215725-0.068632$

O $-6.7027621 .966547-0.109041$

C $-5.9635450 .220427-1.616115$

C $-6.710533-0.849828-0.798137$

H $-5.066582-0.230102-2.055977$

O $-6.8130210 .608136-2.700078$

H -7.733492 -0.511599-0.587087

H -6.825518 -1.719782 -1.458275

H -1.236238 $4.429941-5.067462$

H $-0.5855854 .294451-3.426145$

H $-0.0403803 .179029-4.688193$

C $-1.0672600 .477920-3.381714$

H -5.150095 $3.841201-0.130526$

H -7.403578 2.149234 -0.758520

H -7.004359 $-0.189512-3.222481$

H $-0.273600 \quad 0.713917-4.100546$

H -1.837370 -0.092453 -3.915581

H $-0.9952632 .242742-2.105928$

C $-3.880454-1.9934851 .373427$

O $-4.673817-1.626636 \quad 0.235222$

C $-6.030775-1.2751510 .515567$

C $-6.767322-2.4495411 .183559$

C $-6.017179-2.9461452 .423597$

C $-4.526580-3.1612532 .139513$

H $-2.936898-2.3544430 .949196$

C $-3.558759-0.7898182 .275682$

H $-6.045653-0.4163301 .193900$

O $-6.902466-3.5218910 .252284$

H -7.777144 -2.121614 1.458286

H $-6.463931-3.8901472 .759277$

O $-6.184899-1.9925663 .468196$

H -3.985690 -3.335564 3.077024

O $-4.393833-4.3503751 .355486$

C $-2.692447 \quad 0.238749 \quad 1.582559$

H -4.467029 -0.2791652 .605061$

H -3.007458 -1.132162 3.159029

H $-7.397315-4.2333200 .693148$

H $-5.724246-2.3335474 .253625$

H $-3.443843-4.512578 \quad 1.225234$

O $-2.565644 \quad 1.3315712 .391755$

$\begin{array}{llll}\text { O } & -2.186418 & 0.106721 & 0.477787\end{array}$

C -1.7841322 .4022601 .854254$

H -1.7106393 .1845372 .614194$

H -0.7766382 .0549221 .609641$

H -2.2724392 .8166970 .967577$

SCF Energy (B3LYP/6-31G**//MMFF) $=-3245.89999352$

0800020

MM̄FF Geometry

C $3.096568-3.6779680 .119559$

C $1.798112-3.343580 \quad 0.184637$

C $0.859967-3.505135-0.984011$

O $-0.143778-2.463266-0.950828$

C $0.133021-4.858275-0.888705$

C $-0.696598-5.176771-2.117799$

C $-2.041380-5.112606-2.178947$

C $0.088485-5.641600-3.318358$

C $-2.995138-4.680641-1.100076$

C $-3.958177-3.600689-1.611508$

C $-4.932204-3.139619-0.517405$

C -5.929051-2.125449-1.078640

C $-6.850627-1.5587390 .010429$

O $-4.167950-2.5453540 .536138$

O $-7.508091-0.401706-0.531243$

C $-5.990236-1.0750091 .199024$

C -7.959525 -2.548606 0.388245

$\begin{array}{llll}\text { O } & -6.840722 & -0.689208 & 2.276267\end{array}$
C $-4.944365-2.1133641 .664006$

C $-3.949776-1.5685482 .720930$

O $-5.627181-3.2191592 .269141$

C $0.189076-1.245492-1.457703$

O $1.290265-0.881612-1.837318$

C $-1.053338-0.440369-1.517857$

C $-1.0337860 .782150-2.069599$

C $-2.190798 \quad 1.658873-2.278392$

C $-3.5447851 .140523-1.873472$

C $-3.082555-0.3869122 .274841$

C -3.6829040 .9761112 .574372$

C -3.3302403 .3955762 .073040$

O $-4.668781 \quad 1.1368693 .287958$

C -3.8882673 .8453510 .759563$

C -3.3274554 .8044040 .007501$

C $-3.9032615 .303829-1.284405$

C $-2.9498845 .157447-2.447879$

C $-2.139986 \quad 6.167271-2.809715$

C $-3.0182903 .885311-3.260538$

C $-1.9923842 .866114-2.848386$

N -2.9624751 .9962881 .970595$

H $3.476092-4.093576-0.809569$

H $1.383501-2.9653881 .115570$

H $1.411298-3.439852-1.930614$

H $-0.487007-4.8806040 .014293$

H $0.863275-5.668471-0.756591$

H -2.522991 -5.406187-3.111313

H $-0.559010-5.926888-4.154445$

H $0.753698-4.849578-3.675076$

H $\quad 0.694572-6.516349-3.060483$

H $-3.570680-5.556979-0.778990$

H -2.468586 -4.305166 -0.219554

H $-3.372553-2.743565-1.967108$

H $-4.525022-3.987544-2.467847$

H -5.458782 -4.019900 -0.129936

H $-6.520156-2.568792-1.889744$

H -5.380864 -1.290385 -1.533484

H -7.999996 -0.679440 -1.322856

H $-5.475832-0.1680850 .858017$

H $-8.573474-2.788355-0.488429$

H $-8.645996-2.1126671 .122448$

H -7.576816 -3.4898460 .788053$

H $-6.293774-0.2433932 .944032$

H $-4.472567-1.3517473 .660195$

H -3.256475 -2.387721 2.961904

H $-5.001101-3.6943282 .840320$

H -1.962857 -0.895770 -1.144261

H $-0.0821541 .173734-2.427769$

H $-3.5483040 .841358-0.820553$

H $-3.8166600 .274509-2.486128$

H $-4.3442741 .876706-1.979842$

H -2.134153 -0.427381 2.826257

H $-2.842821-0.4572891 .209549$

H -2.428195 3.9493162 .350850

H $-4.076303 \quad 3.5363222 .861261$

H -4.8097583 .3742760 .425822$

H -2.417668 5.2878850 .356874

H $-4.1583686 .363109-1.146968$

H -4.852676 $4.806662-1.518600$

H $-1.4811286 .081968-3.667534$

H $-2.1142797 .097749-2.252244$

H -2.830340 4.122006 -4.317369

H $-4.0379683 .489835-3.254523$

H $-0.9719753 .152534-3.106827$

H $-2.161411 \quad 1.761971 \quad 1.390543$

C $5.382245-2.8866420 .946694$

O $5.163510-1.543207 \quad 0.474819$

C $6.090081-1.281044-0.596256$

C $7.116830-2.409146-0.556961$

C $6.262526-3.573789-0.098148$

H $5.519283-1.359765-1.529040$

C $6.6931640 .121180-0.469120$

H $7.586508-2.576542-1.531062$

H $7.911535-2.2120570 .171911$

C $7.064727-4.744520 \quad 0.442512$

H $5.669454-3.916034-0.953795$

C $5.6694111 .271897-0.405879$

O 7.4831710 .1895820 .726048 
H $7.3912030 .292650-1.297029$

H 5.1688051 .2701990 .568887

O $6.4025222 .501977-0.480180$

C $4.6167911 .267826-1.528049$

C $3.7807452 .556563-1.643476$

H $3.9407660 .417771-1.384808$

O $5.260567 \quad 1.067516-2.790379$

H $4.4135603 .379912-1.999265$

H $3.0464432 .385536-2.442383$

H $7.729625-5.144288-0.330082$

H $7.682631-4.4484151 .296772$

H $6.402445-5.5524540 .768941$

C $4.052221-3.5728401 .275234$

H $6.902250-0.0324671 .473959$

H 7.0523462 .4955940 .243583

H $5.9005611 .790054-2.911564$

H $3.571546-3.0206142 .091915$

H $4.252456-4.5837561 .648876$

H $5.944958-2.7868221 .884592$

C 1.4807172 .1065521 .266332

O 2.2022031 .9049160 .043332

C $3.0616592 .976522-0.354389$

C $2.2616994 .275910-0.547797$

C 1.4471254 .6139980 .704264

C $0.6562903 .402188 \quad 1.207071$

H $\quad 0.7757041 .2664341 .318888$

C 2.3973672 .0334582 .496785

H $3.815222 \quad 3.1425190 .421126$

O $1.3835174 .135657-1.663029$

H $2.9596465 .090329-0.776582$

H $0.753016 \quad 5.4311700 .472207$

O $2.3369905 .072241 \quad 1.717006$

H 0.2298663 .6105932 .194416

O $-0.4454863 .181594 \quad 0.326300$

C 2.9842940 .6527682 .695005

H 3.2332472 .7337132 .421968

H 1.8181342 .2699423 .396906

H $0.9144394 .979844-1.776505$

H 1.8017595 .3045032 .494916

H $-0.0845202 .976415-0.552667$

O $3.8313210 .686562 \quad 3.766408$

O $2.734208-0.3272582 .008160$

C $4.475992-0.5495324 .088373$

H $3.743267-1.3557944 .190005$

H $5.215472-0.7909593 .320564$

H $4.991325-0.4246775 .044369$

SCF Energy (B3LYP/6-31G**//MMFF) $=-3245.92288432$

$08 \quad 00021$

MM̄̄FF Geometry

C 1.0691823 .3621390 .539098

C $1.5961474 .160316-0.402561$

C $2.6023293 .746506-1.453714$

O $2.7782782 .312078-1.472456$

C $3.9624784 .391280-1.140889$

C $5.0340624 .042053-2.153748$

C $5.9964123 .119715-1.952383$

C $4.9955834 .836666-3.432240$

C $6.1640702 .232381-0.748908$

C $6.4901360 .787358-1.153073$

C $6.595107-0.1256950 .076999$

C $7.166375-1.491097-0.305435$

C $7.216353-2.4403890 .900682$

O $5.281086-0.2879370 .624667$

O $7.465811-3.765780 \quad 0.408688$

C $5.829648-2.4607321 .574086$

C $8.372296-2.1041231 .849954$

O $5.861783-3.2108412 .786666$

C $5.238706-1.0536021 .836527$

C $3.761849-1.1954022 .273642$

O $5.973316-0.4177182 .883447$

C $1.9246181 .583767-2.241911$

O $1.0261482 .005814-2.951500$

C $2.2541840 .157440-2.030454$

C $1.399352-0.788158-2.447176$

C $1.549083-2.230801-2.244388$

C $2.846923-2.723480-1.661025$

C 2.9769200 .1210402 .332044
C $1.509584-0.1697752 .586710$

C $-0.503179-1.1285151 .454191$

O $1.016353-0.0724003 .707516$

C $-0.413634-2.5911171 .142612$

C $-1.123151-3.1916960 .175393$

C $-0.996575-4.649570-0.168398$

C $-0.890613-4.900196-1.659778$

C $-1.886732-5.504378-2.329485$

C $0.398395-4.522826-2.364141$

C $0.519350-3.037549-2.575985$

N $0.845094-0.5894961 .444853$

H 1.3702602 .3208050 .596427

H $1.2849435 .202507-0.436761$

H $2.2495314 .087806-2.434874$

H $4.2864094 .103613-0.133492$

H $3.8585945 .484317-1.103456$

H $6.7447542 .982333-2.731644$

H $5.8127174 .571605-4.111415$

H $4.0554074 .661015-3.964355$

H $5.0797665 .906933-3.217186$

H $6.9860762 .629456-0.141314$

H $5.2690172 .230714-0.120895$

H $5.7066810 .414216-1.825151$

H $7.4370620 .773921-1.707148$

H 7.2396760 .3624670 .817486

H $8.163448-1.386865-0.751337$

H $6.535678-1.946674-1.081910$

H $8.306740-3.750649-0.079938$

H $5.150331-2.9980100 .897254$

H $9.331013-2.1601591 .320564$

H $8.438069-2.8329562 .665316$

H $8.293586-1.1058492 .285521$

H $6.222957-4.0886902 .574803$

H $3.248979-1.8672061 .575890$

H $3.709916-1.6714373 .261609$

H 5.4122160 .2584903 .295018

H $3.167433-0.073684-1.494360$

H $\quad 0.482988-0.477932-2.948005$

H $2.949948-3.810407-1.681981$

H $2.942537-2.405290-0.619746$

H $3.699738-2.331171-2.226918$

H 3.3428390 .7703463 .134111

H 3.0674410 .6823871 .395894

H $-1.078513-0.5811670 .701268$

H $-0.964974-0.9787732 .434578$

H $0.279959-3.1829521 .735882$

H -1.831950 -2.603783 -0.401940

H -1.875935 -5.1649120 .238340$

H $-0.126735-5.098560 \quad 0.327217$

H -1.810747 -5.709761 -3.392054

H $-2.803289-5.803798-1.832212$

H $\quad 0.444517-5.001219-3.350923$

H $1.229652-4.940613-1.789884$

H $-0.365744-2.585664-3.026922$

H $1.357393-0.6331720 .569367$

C -1.2749663 .0956151 .347473$

O -1.8765373 .5722550 .125045$

C $-3.225883 \quad 3.9662100 .401463$

C -3.6020613 .2850721 .706731$

C $-2.287953 \quad 3.3738722 .460416$

H -3.1962275 .0498010 .584852$

C $-4.1480123 .720824-0.793747$

H $-4.426543 \quad 3.7874312 .220585$

H -3.8775402 .2376701 .550741$

C -2.1847722 .4125003 .631814$

H -2.1665834 .4004382 .831783$

C $-4.4980102 .259899-1.149007$

O $-5.3770644 .406836-0.514898$

H -3.713605 4.208660 -1.675560

H $-5.1740351 .858856-0.385938$

O $-5.2713652 .303927-2.359478$

C $-3.3153691 .295542-1.353323$

C $-3.688948-0.044463-2.018134$

H $-2.8325291 .101809-0.390378$

O $-2.3485921 .927361-2.197745$

H $-3.9914950 .139407-3.057279$

H $-2.768305-0.640836-2.082287$

H -2.9803402 .6034884 .359263$ 
H $-2.268573 \quad 1.3712123 .304606$ H $-1.2235192 .525146 \quad 4.143140$ C 0.0614943 .8067291 .560326 H -5.957590 4.285525 -1.285884 H -4.683225 2.610841 -3.070980 H -1.567943 $1.348448-2.235132$ H 0.4601533 .5770672 .554974 H -0.0794594 .8931661 .503796$ H -1.123124 2.0158471 .226338 C $-5.146225-2.0083270 .815610$ O $-4.257065-1.249759-0.017145$ C $-4.776756-0.851316-1.289064$ C $-5.206706-2.075570-2.113092$ C $-6.200385-2.941034-1.333647$ C $-5.696858-3.2431710 .078961$ H $-4.516936-2.3746471 .637042$ C $-6.250292-1.1239201 .417144$ H $-5.650798-0.211683-1.139253$ O $-4.059531-2.849320-2.454825$ H -5.660952 -1.728654 -3.049073 H $-6.362636-3.886366-1.865412$ O $-7.453128-2.269152-1.241939$ H $-6.498615-3.6952540 .673775$ O $-4.658409-4.222786-0.000441$ C $-5.716824-0.1948292 .487618$ H $-6.737816-0.505006 \quad 0.659861$ H -7.003802 -1.758675 1.897958 H -4.360616 -3.595704 -3.000554 H -7.769414 -2.112172 -2.147965 H -3.921698 -3.832448 -0.500313 O $-6.754852 \quad 0.5334642 .996403$ O $-4.548858-0.1201402 .842246$ C -6.4146351 .4372394 .052299$ H -7.3321991 .9264214 .389736$ H -5.9781820 .8915974 .894355$ H -5.7234762 .2017203 .687968$ SCF Energy (B3LYP/6-31G**//MMFF) $=-3245.91460819$

08_00022

MM̄FF Geometry

C $1.348513 \quad 3.424625-1.657601$

C $2.5049214 .106533-1.674855$

C $3.6538523 .888102-0.717792$

O $3.7517622 .491231-0.351949$

C 3.4465234 .7323790 .551414

C 4.5518614 .5683001 .575896

C 4.4487473 .8378132 .704704

C 5.8222905 .3195731 .273998

C 3.2746893 .0150243 .159593

C 3.6606901 .5383533 .316185

C 2.4269750 .6417883 .498986

C $2.847691-0.8047343 .763452$

C $1.644130-1.7568403 .823595$

O $1.653106 \quad 0.7156032 .295768$

O $2.141313-3.1016503 .738431$

C $0.757812-1.5256852 .580886$

C $0.902517-1.6532925 .161500$

O $-0.450597-2.2761902 .674822$

C $0.434994-0.0401822 .323605$

C $-0.294281 \quad 0.199058 \quad 0.979044$

O -0.4378490 .4322063 .358250$

C $4.3912641 .672838-1.235662$

O $4.8752261 .990783-2.310846$

C $4.4021780 .305213-0.670342$

C $4.937291-0.701963-1.377032$

C $5.040571-2.100679-0.952730$

C $4.417881-2.479170 \quad 0.364551$

C $0.472514-0.186233-0.287635$

C $0.207242-1.593501-0.790268$

C $0.917494-3.164502-2.590976$

O $-0.626180-2.345122-0.294527$

C $1.922996-4.113463-2.020137$

C $2.874836-4.710785-2.753006$

C $3.849127-5.711234-2.201862$

C $5.288513-5.344216-2.481867$

C $5.951636-5.887270-3.516834$

C $5.985720-4.425576-1.505122$

C $5.687260-2.973028-1.754454$
N $0.999400-1.898165-1.889428$

H $1.1849492 .669808-0.894268$

H $2.6514764 .880871-2.424530$

H $4.5864894 .194011-1.208826$

H 2.4741254 .4959940 .996172

H 3.3784275 .7950510 .280391

H 5.3033483 .8126083 .379844

H 6.5713035 .2055312 .064843

H 6.2700304 .9583020 .343018

H 5.6150856 .3895681 .169200

H 2.9287243 .4033674 .125010

H 2.4296243 .1024892 .473852

H 4.2125851 .2152742 .425240

H 4.3346401 .4280454 .174946

H 1.8340811 .0294264 .335960

H $3.439810-0.8749804 .684607$

H $3.512607-1.1410542 .957262$

H $2.762167-3.2389014 .474534$

H $1.308505-1.9230031 .720091$

H $1.572293-1.9097255 .991062$

H $0.076207-2.3709135 .212221$

H $0.506161-0.6546815 .356238$

H $-0.203397-3.2066742 .812112$

H -1.279152 -0.2748241 .002515$

H $-0.490656 \quad 1.2783230 .906208$

H $-0.904101 \quad 1.2206863 .034344$

H 3.9738860 .1630920 .314166

H $5.355736-0.482687-2.358559$

H $3.372140-2.1564870 .403875$

H $4.963015-2.0119661 .191203$

H $4.404996-3.5554670 .548624$

H $\quad 0.1483810 .481223-1.096304$

H $1.547596-0.044989-0.153383$

H $1.094634-2.960549-3.651615$

H $-0.089115-3.580950-2.479915$

H $1.843340-4.343272-0.960185$

H $2.934832-4.499867-3.818577$

H $3.618698-6.683717-2.656837$

H $3.711539-5.853033-1.122700$

H $6.994306-5.653719-3.705300$

H $5.464346-6.572974-4.202253$

H $7.073986-4.549119-1.590775$

H $5.755959-4.739708-0.483011$

H $6.099838-2.604175-2.694671$

H $1.706923-1.234508-2.191464$

C $-0.9594874 .349134-2.035939$

O $-1.6563873 .477577-1.119187$

C $-3.0746663 .652817-1.305827$

C $-3.2422834 .857538-2.225539$

C -1.997362 $4.761747-3.079682$

H $-3.4339892 .762569-1.832902$

C -3.804102 3.8004630 .033029

H $-4.1682834 .808599-2.806779$

H -3.247979 $5.799616-1.664692$

C $-1.667826 \quad 6.051714-3.811364$

H $-2.1344193 .955214-3.812773$

C -3.6726152 .6003950 .990749$

O -3.2855434 .9549520 .706850$

H $-4.8640724 .008915-0.158383$

H -2.6558272 .5571851 .397557$

O -4.5269182 .8640712 .113698$

C -4.0414831 .2320260 .385619$

C $-4.1882360 .137512 \quad 1.462277$

H -3.271576 $0.946578-0.339560$

O

H -3.3845480 .2353622 .201456$

H -5.1461680 .2858611 .976287$

H -2.485652 $6.331619-4.483554$

H $-1.5087586 .879921-3.112626$

H $-0.7608605 .941124-4.413588$

C $0.2418713 .630949-2.651521$

H -3.767162 5.0402251 .547518

H -5.4453672 .8480081 .793491$

H $-5.4670680 .506721-0.738993$

H $0.6194324 .199396-3.509536$

H $-0.0589782 .644807-3.025960$

H -0.630187 $5.222514-1.457309$

C $-5.259406-2.692335-0.747172$ 
O $-5.219672-1.438842-0.051808$

C $-4.148572-1.290460 \quad 0.881801$

C $-4.256882-2.3572601 .982552$

C $-4.327124-3.7699011 .391282$

C $-5.345076-3.864095 \quad 0.246774$

H $-6.196206-2.663083-1.318570$

C $-4.107662-2.831562-1.750336$

H $-3.198145-1.4080410 .353348$

O $-5.415323-2.1271162 .783413$

H -3.388569 -2.273144 2.645842

H $-4.605831-4.4796942 .179766$

O $-3.045159-4.1509990 .901716$

H -5.230289-4.810157 -0.295132

O $-6.664467-3.8532240 .800716$

C $-4.108210-1.693668-2.744174$

H $-3.132679-2.864814-1.257876$

H $-4.196243-3.765037-2.318168$

H -6.188689-2.125011 2.193624

H -2.415937 -4.088184 1.640428

H -6.742369 -4.6258151 .386200$

O $-2.972286-0.952155-2.601586$

O $-5.011939-1.484293-3.542986$

C $-2.8507490 .172179-3.477781$

H -2.708902 $-0.171164-4.506564$

H -3.732278 $0.815997-3.403892$

H $-1.9720000 .746568-3.172526$

SCF Energy (B3LYP/6-31G**/MMFF) $=-3245.92564891$

0800023

MMFF Geometry

C $2.124944 \quad 3.466528 \quad 1.244510$

C $2.718693 \quad 4.292925 \quad 0.369124$

C $3.7822843 .884368-0.625162$

O $3.6425342 .483937-0.968665$

C $5.1818834 .119943-0.040480$

C $6.2865863 .905660-1.055891$

C $7.0701652 .810906-1.113100$

C $6.5120345 .047694-2.012960$

C $6.9877931 .585033-0.247213$

C $7.081753 \quad 0.299620-1.081680$

C $6.996618-0.946572-0.190362$

C $7.306414-2.214018-0.986745$

C $7.156879-3.474893-0.121845$

O $5.668433-1.0210110 .339198$

O $7.154916-4.612739-0.997202$

C $5.782680-3.438794 \quad 0.577414$

C $8.343387-3.662061 \quad 0.831139$

O $5.654868-4.5097321 .510123$

C $5.468359-2.0906941 .271680$

C $3.988830-2.0751641 .719175$

O $6.300768-1.9449342 .423649$

C $2.6498122 .175608-1.850566$

O $1.9245712 .961936-2.440499$

C $2.5499260 .701976-1.938017$

C $1.4359450 .170385-2.464469$

C $1.124623-1.252394-2.612053$

C $2.207611-2.242228-2.278155$

C $3.460177-0.6954152 .134790$

C $2.004128-0.7954262 .548195$

C $-0.262537-1.2673651 .617858$

O $1.658449-0.708702 \quad 3.724073$

C $-0.635819-2.4154740 .734216$

C $-1.605381-2.356471-0.190310$

C $-1.991927-3.506856-1.073597$

C $-2.012714-3.138498-2.541131$

C $-3.173292-3.030713-3.210091$

C $-0.684869-2.967745-3.251136$

C $-0.106866-1.593505-3.047461$

N $1.159980-1.0272191 .474665$

H 2.4567062 .4339061 .318071

H 2.3895455 .3280040 .311124

H $3.6556624 .485226-1.534560$

H 5.3331713 .4755790 .833089

H $5.261135 \quad 5.146723 \quad 0.342094$

H $7.8637982 .789295-1.858925$

H $7.3526724 .863147-2.690419$

H $5.6241815 .214235-2.630419$

H $6.7305365 .967970-1.461258$
H 7.8207811 .6140100 .465403

H $6.063728 \quad 1.5624380 .336841$

H $6.2650720 .287301-1.815345$

H $8.0282850 .293830-1.636363$

H $7.711010-0.8316860 .633373$

H $8.312026-2.165991-1.422459$

H $6.613572-2.290086-1.836700$

H $7.989576-4.608061-1.496472$

H $5.022098-3.611611-0.197396$

H $9.280186-3.7357770 .265892$

H $8.256777-4.6005261 .389818$

H 8.452599-2.844131 1.546198

H $5.844820-5.3349801 .031756$

H $3.366236-2.4405360 .894628$

H $3.845270-2.7728332 .554659$

H $5.863948-1.3523263 .055698$

H $3.3647740 .106432-1.543908$

H $0.6539060 .847163-2.807736$

H $3.120728-2.019555-2.841937$

H $1.945056-3.274316-2.518751$

H $2.441365-2.209980-1.210931$

H $4.026978-0.2892872 .978949$

H 3.5398430 .0258511 .313690

H $-0.780897-0.3450491 .340699$

H $-0.500422-1.5024882 .659579$

H $-0.085662-3.3440380 .869073$

H $-2.170874-1.436002-0.304340$

H $-2.985892-3.847930-0.756000$

H $-1.321121-4.362973-0.929685$

H -3.195331 - 2.788719-4.267428

H $-4.127760-3.172329-2.713727$

H $-0.819243-3.111747-4.331225$

H $-0.013684-3.768493-2.929917$

H $-0.804901-0.790979-3.292112$

H $1.531408-0.9385510 .533482$

C $-0.2280952 .929279 \quad 1.823640$

$\begin{array}{lllll}\text { O } & -0.618662 & 3.042856 & 0.442280\end{array}$

C -2.056709 2.9572740 .350689

C -2.5486202 .6139591 .750577$

C $-1.485141 \quad 3.2547892 .622672$

H -2.3963903 .9585570 .058731$

C $-2.415551 \quad 1.932631-0.733052$

H $-3.5541292 .997776 \quad 1.947277$

H -2.5647041 .5279501 .901038$

C $-1.4644672 .731354 \quad 4.047528$

H -1.6522494 .3400952 .637750$

C $-3.9318321 .700839-0.884971$

O $-1.8817402 .411561-1.971370$

H $-1.9148540 .981032-0.524093$

H -4.3334391 .4002410 .087800$

O $-4.5624332 .935379-1.227246$

C $-4.3166870 .617658-1.916216$

C $-5.8314610 .476796-2.152829$

H $-3.910984-0.346729-1.593123$

O $-3.7468830 .901194-3.195658$

H $-6.220317 \quad 1.394507-2.612767$

H $-5.961599-0.308512-2.910125$

H -2.4312582 .8967894 .533877$

H $-1.249812 \quad 1.6581264 .077696$

H -0.6973213 .2418964 .638277$

C $0.956522 \quad 3.8548862 .106297$

H $-0.9295752 .561243-1.839042$

H $-4.2114493 .209060-2.092372$

H -2.779812 $0.878119-3.099640$

H 1.2522473 .7834073 .158495

H 0.6713594 .8953251 .909017

H $\quad 0.0674661 .8848451 .985837$

C $-6.804838-1.5826950 .812411$

O $-6.168079-1.110529-0.383279$

C $-6.656767 \quad 0.129432-0.903731$

C $-8.157154 \quad 0.032537-1.227128$

C $-8.954306-0.445661-0.009888$

C $-8.327755-1.6921490 .620473$

H $-6.418451-2.6006780 .953119$

C $-6.405851-0.7498902 .041950$

H -6.509799 $0.918703-0.160717$

O $-8.357444-0.872897-2.310959$

H $-8.5124801 .018175-1.551185$ 
H $-9.983621-0.676924-0.310252$ $\begin{array}{llll}\text { O } & -9.005850 & 0.589508 & 0.967191\end{array}$ H -8.803488 -1.9037361 .584790$ O $-8.596411-2.820584-0.217171$ C $-4.971283-1.001612 \quad 2.456019$ H -6.5100210 .3243881 .872740$ H $-7.039776-1.0389122 .888790$ H $-9.308544-0.882676-2.513134$ H -9.4335381 .3601140 .556561$ H -8.120095 -2.689444 -1.055068 O $-4.763997-0.4582373 .692033$ O $-4.136359-1.5918201 .785350$ C $-3.455384-0.650543 \quad 4.238212$ H $-3.311251-1.7043134 .493542$ H $-2.688289-0.3188183 .533352$ H $-3.375209-0.0521515 .149337$

SCF Energy (B3LYP/6-31G*//MMFF) $=-3245.92131252$

0800024

MM̄FF Geometry

C - -1.0417352 .1863312 .260329$

C $-1.540101 \quad 1.0600622 .795362$

C $-0.836673-0.2710232 .943573$

O $0.491565-0.1925302 .382798$

C $-1.633599-1.3480132 .187073$

C $-1.252318-2.7692342 .537379$

C $-0.503286-3.5757021 .761041$

C $-1.831093-3.287618 \quad 3.828637$

C $0.179347-3.233200 \quad 0.464342$

C $1.625648-3.7442880 .476876$

C $2.387023-3.401982-0.811048$

C $3.772429-4.053437-0.777330$

C $4.625788-3.663911-1.989570$

O $2.510523-1.977729-0.903747$

O $5.983536-4.040988-1.714150$

C $4.603964-2.130723-2.134735$

C $4.209752-4.428132-3.251577$

O $5.270349-1.727165-3.329594$

C $3.181759-1.521099-2.086786$

C $3.296924 \quad 0.018818-2.019104$

O $2.475078-1.891210-3.270825$

C $1.517512-0.7611943 .069640$

O $1.455646-1.3743454 .122550$

C $2.756057-0.4892302 .307626$

C $3.947907-0.8531862 .802373$

C $5.231275-0.6158972 .144618$

C $6.186135-1.7719562 .251305$

C $1.9698550 .771198-1.867771$

C $2.2512642 .259208-1.807110$

C $3.1811423 .993226-0.277383$

$\begin{array}{lllll}\text { O } & 2.219891 & 2.962748 & -2.814084\end{array}$

C $4.6692223 .838594-0.332296$

C 5.4648323 .8853640 .746671

C $6.937463 \quad 3.5939870 .700180$

C 7.2973602 .3215641 .440758

C 8.0981972 .3481832 .519157

C 6.7839051 .0049070 .887962

C $5.5061450 .565104 \quad 1.555045$

N $2.6014572 .687265-0.537106$ H -0.0197402 .2076251 .894816$ H -2.563559 1.0800633 .167506 H - $0.793036-0.4943354 .016205$ H $-1.535807-1.1853311 .108751$ H $-2.704227-1.2385742 .403230$ H $-0.347813-4.6004482 .097123$ H $-1.563224-4.3331304 .015273$ H -1.466392 -2.699469 4.676319 H -2.924006 -3.226951 3.807452 H $-0.368739-3.711538-0.355688$ H $0.176182-2.1564140 .275063$ H $2.149815-3.3087831 .336779$ H $1.622987-4.8330700 .617270$ H $1.809336-3.770389-1.666996$ H $3.687463-5.145078-0.703934$ H $4.300323-3.7348340 .132165$ H $6.000185-4.998557-1.544635$ H $5.185826-1.713855-1.300134$ H $4.322939-5.508328-3.099539$
H $4.857986-4.176040-4.098089$

H $3.173580-4.244084-3.542643$

H $6.160887-2.117721-3.310694$

H $3.9382960 .288562-1.170348$

H $3.7945540 .390168-2.924559$

H $1.728034-1.286305-3.396333$

H $2.662086 \quad 0.0134101 .351700$

H $4.007809-1.3748203 .755292$

H $6.547575-1.8746773 .280135$

H $7.055838-1.6756761 .597663$

H $5.689770-2.7071571 .968044$

H $1.3055190 .597712-2.719344$

H $1.4324220 .459532-0.965346$

H 2.8325894 .3177720 .707845

H $2.8450194 .714466-1.028309$

H $5.1039053 .622144-1.305697$

H 5.0318184 .0898061 .723072

H 7.4655844 .4558391 .126967

H $7.2905153 .508303-0.335232$

H 8.3753781 .4354543 .036610

H 8.4911143 .2807832 .910157

H $7.573348 \quad 0.252796 \quad 0.981883$

H $6.6025711 .104232-0.189378$

H $4.7216211 .319944 \quad 1.552855$

H 2.6556702 .0022890 .210223

C $-2.031607 \quad 3.928906 \quad 0.682787$

O $-2.541312 \quad 2.855879-0.137453$

C -1.741235 2.782274-1.331624

C $-1.0927424 .153148-1.462977$

C $-0.7717194 .451369-0.010948$

H $-0.9549332 .042217-1.138664$

C $-2.5751712 .336345-2.535515$

H $-0.2099674 .142162-2.107366$

H $-1.794953 \quad 4.897794-1.855754$

C $-0.478155 \quad 5.916174 \quad 0.261099$

H 0.1022003 .8597800 .282259

C $-3.3463191 .015593-2.325860$

O $-3.535773 \quad 3.356518-2.833562$

H $-1.9247082 .262032-3.415360$

H $-4.1964661 .199218-1.660776$

O $-3.9114910 .639911-3.587974$

C $-2.502213-0.149092-1.782813$

C $-3.204868-1.517848-1.774207$

H $-2.1740190 .091424-0.766433$

O $-1.324718-0.276804-2.584656$

H $-3.330012-1.880513-2.802787$

H $-2.507308-2.222759-1.302451$

H $0.3988386 .243801-0.306851$

H $-1.3208156 .554815-0.024044$

H $-0.2738356 .080792 \quad 1.323683$

C $-1.843392 \quad 3.455502 \quad 2.128804$

H $-4.0504023 .054609-3.601776$

H $-3.1755750 .416880-4.183814$

H $-0.768028-0.964501-2.180532$

H $-2.837448 \quad 3.301957 \quad 2.567374$

H $-1.361107 \quad 4.2430282 .719077$

H $-2.804763 \quad 4.7082430 .677357$

C $-5.557451-0.9529001 .082328$

O $-4.377221-1.0277740 .270039$

C $-4.556842-1.557259-1.044928$

C $-5.121512-2.986509-0.978203$

C $-6.400006-3.045825-0.137845$

C $-6.230788-2.331052 \quad 1.206697$

H $-5.194188-0.6708372 .078595$

C $-6.515546 \quad 0.1487120 .602474$

H $-5.257831-0.922592-1.596057$

O $-4.155070-3.880280-0.426526$

H $-5.331990-3.331973-1.997245$

H $-6.662668-4.0965020 .036789$

O $-7.459566-2.447944-0.878261$

H $-7.201792-2.2281741 .704916$

O $-5.417864-3.1596992 .043341$

C $-5.920314 \quad 1.531123 \quad 0.767208$

H $-6.7733020 .022255-0.452144$

H $-7.438506 \quad 0.1251851 .192702$

H -3.901006 -3.5408290 .448900$

H -8.268937 $-2.522767-0.344700$

H $-5.363682-2.7342912 .915958$ 
O $-6.6420352 .418953 \quad 0.021545$

O $-4.9615841 .804261 \quad 1.475528$

C $-6.199743 \quad 3.7786690 .081524$

H $-6.168916 \quad 4.1287251 .117865$

H $-5.2169303 .873062-0.388021$

H -6.913642 4.392839-0.473661

SCF Energy (B3LYP/6-31G**//MMFF) $=-3245.91116734$

08_00025

MM̄FF Geometry

C $0.302808-1.6949142 .261296$

C $0.998222-2.6269541 .592073$

C $0.561100-3.2405270 .278584$

O $-0.361949-2.356048-0.405017$

C $-0.129864-4.5907750 .525254$

C $-0.510598-5.309337-0.755134$

C $-1.762123-5.395306-1.249579$

C $0.630825-5.979745-1.476427$

C $-3.021283-4.800114-0.682678$

C $-3.582742-3.712806-1.607376$

C $-4.757397-2.959953-0.964963$

C $-5.324378-1.921229-1.934434$

C $-6.418384-1.058810-1.286905$

O $-4.270437-2.312455 \quad 0.215210$

O $-6.644417 \quad 0.077216-2.136205$

C $-5.899869-0.5153530 .062184$

C $-7.754119-1.806561-1.199208$

O $-6.937370 \quad 0.1575940 .771056$

C $-5.268962-1.6001470 .958703$

C $-4.580892-1.0201242 .218979$

O $-6.297950-2.4870371 .414882$

C $0.187073-1.294445-1.061091$

O $1.376256-1.072578-1.226061$

C $-0.909698-0.405503-1.503912$

C $-0.606100 \quad 0.807710-1.989537$

C $-1.549248 \quad 1.817252-2.474492$

C $-3.0178081 .499904-2.394221$

C -3.413395 -0.0629731 .955600$

C $-3.786477 \quad 1.409087 \quad 1.990417$

C -2.774611 3.6634031 .656162

$\begin{array}{llll}\text { O } & -4.891860 & 1.819796 & 2.331301\end{array}$

C -3.1270814 .1718890 .294755$

C -2.358761 $5.026941-0.397148$

C $-2.7482955 .614573-1.722272$

C -1.711908 $5.385278-2.797233$

C $-0.7977326 .325975-3.089529$

C $-1.8030224 .110446-3.602662$

C $-1.0559072 .970038-2.973338$

N -2.713628 2.2152361 .632032

H $-0.674765-1.3982251 .890834$

H $1.966108-2.9429781 .971295$

H $1.447592-3.386643-0.351201$

H - $1.008182-4.4386451 .160692$

H $\quad 0.532526-5.247681 \quad 1.105458$

H - $-1.910355-5.950958-2.174938$

H $0.296596-6.534848-2.359509$

H $1.363311-5.239277-1.812081$

H $1.133089-6.691352-0.812988$

H $-3.762824-5.600443-0.569636$

H -2.861561 -4.3936120 .317958$

H $-2.783490-3.000022-1.843777$

H -3.904257 -4.165830 -2.553550

H $-5.527633-3.688953-0.686776$

H $-5.703235-2.404451-2.843725$

H $-4.514870-1.257723-2.266188$

H $-6.899996-0.251721-3.015086$

H $-5.1398080 .239979-0.169449$

H $-8.099880-2.090949-2.200369$

H $-8.539459-1.166120-0.783040$

H -7.697001-2.717894 -0.600497

H $-7.291506 \quad 0.845804 \quad 0.182234$

H $-5.328210-0.5744272 .886527$

H $-4.165641-1.8710172 .778539$

H $-5.985142-2.9369692 .217203$

H $-1.927391-0.762686-1.405870$

H $0.4436201 .093272-2.049140$

H $-3.2933451 .208034-1.375654$

H $-3.2685900 .680968-3.076320$
H -3.661035 $2.344988-2.648729$

H $-2.675001-0.2011632 .755990$

H $-2.916899-0.2909871 .007217$

H -1.7994714 .0255971 .995881$

H -3.531742 3.9874102 .377521

H $-4.080518 \quad 3.854731-0.121208$

H $-1.419418 \quad 5.365122 \quad 0.034545$

H -2.896151 $6.692783-1.575150$

H $-3.7191565 .231948-2.060555$

H $-0.0728456 .181178-3.883756$

H $-0.7504317 .258275-2.536533$

H $-1.3655764 .271883-4.597730$

H -2.851784 $3.874675-3.804382$

H $0.0260653 .109071-2.981573$

H $-1.8544601 .784101 \quad 1.302773$

C $0.7854840 .541194 \quad 3.193886$

$\begin{array}{lllll}\text { O } & 1.808474 & 0.860774 & 2.231389\end{array}$

C 2.2322252 .2219272 .454226

C 1.4654082 .7212553 .679052

C 1.1050811 .4278354 .389183

H 3.2984172 .1879552 .699198

C 2.0060713 .0488091 .181990

H 2.0723673 .3910974 .296531

H $\quad 0.5608553 .271675 \quad 3.394211$

C $-0.0388621 .581985 \quad 5.375678$

H 1.9940761 .0504544 .911767

C $2.7528722 .505181-0.053099$

$\begin{array}{lllll}\text { O } & 0.603601 & 3.032718 & 0.882405\end{array}$

H 2.2709014 .0965851 .363723

H $2.3646521 .511011-0.295670$

O $2.4079243 .341321-1.167019$

C 4.2900182 .4606910 .077201

C $4.9880501 .944884-1.196737$

H 4.5933631 .8391530 .925366

$\begin{array}{llllll}\text { O } & 4.781614 & 3.781124 & 0.320347\end{array}$

H $4.5579022 .433453-2.078987$

H $6.0411342 .255916-1.162173$

H 0.2205642 .3060456 .154935

H $-0.951044 \quad 1.932242 \quad 4.880777$

H -0.2658810 .6293925 .864065$

C $0.787329-0.9610183 .478563$

H $0.476633 \quad 3.5310200 .056742$

H $2.7860924 .221832-1.000180$

H 4.5167854 .0335301 .220762

H $0.115930-1.1943194 .311521$

H $1.793867-1.2957533 .751136$

H -0.1760510 .8389892 .754283$

C $5.928452-1.567160-0.354263$

O $5.851041-0.135731-0.401560$

C $4.9119460 .415196-1.334646$

C $5.235726-0.049891-2.761211$

C $5.304891-1.577165-2.840603$

C $6.203641-2.159606-1.746261$

H $6.809352-1.7752870 .267622$

C $4.704419-2.1717800 .345261$

H $3.9052930 .080836-1.072263$

$\begin{array}{llll}\text { O } & 6.469670 & 0.512610 & -3.201853\end{array}$

H $4.4556490 .318013-3.437969$

H $5.687878-1.865353-3.827021$

O $3.986736-2.101404-2.710830$

H $6.105973-3.250540-1.704315$

O $7.569358-1.877993-2.068068$

C $4.854860-2.0765801 .845937$

H $3.757650-1.7018940 .070057$

H $4.612928-3.2386530 .108739$

H $7.1567480 .261913-2.560892$

H $4.046104-3.068978-2.787237$

H $7.758854-2.290428-2.928152$

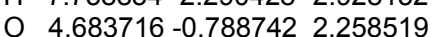

O $5.107884-3.0359832 .563691$

C $4.873331-0.5644913 .658857$

H $5.908081-0.7859103 .937316$

H $4.6737390 .489623 \quad 3.866747$

H $4.179078-1.1751094 .243098$

SCF Energy (B3LYP/6-31G*//MMFF)= -3245.91502234

0800026

MM̄FF Geometry 
C $-1.103883-2.4347102 .366248$ C $-0.473167-1.4665773 .048998$ C $-0.941207-0.0543713 .298368$ $\begin{array}{llll}\text { O } & -2.280219 & 0.161451 & 2.798719\end{array}$ C $-0.016805 \quad 0.9694302 .614272$ C $0.120404 \quad 2.232297 \quad 3.442592$ C $-0.594492 \quad 3.3597893 .265711$ C 1.1747882 .1700754 .519333 C -1.671606 3.6270632 .251957 C $-1.277850 \quad 4.7516181 .281820$ C $-0.125772 \quad 4.3596460 .345098$ C $0.3085945 .543370-0.519574$ C $1.4061575 .139703-1.516806$ O $-0.5901053 .296401-0.490677$ O $1.5353586 .194608-2.482060$ C $0.9481713 .879744-2.280744$ C $2.7738235 .002386-0.838268$ O $1.9941653 .374237-3.107686$ C $0.4062692 .755193-1.365622$ C $-0.266496 \quad 1.663474-2.229849$ $\begin{array}{llll}\text { O } & 1.488947 & 2.178249 & -0.634781\end{array}$ C -3.3064940 .0845373 .683500$ O $-3.225034-0.1822984 .875280$ C $-4.612040 \quad 0.373861 \quad 3.046248$ C $-4.783282 \quad 0.624817 \quad 1.738782$ C $-6.0632240 .904061 \quad 1.079475$ C $-7.296892 \quad 0.968798 \quad 1.942563$ C $-1.0707480 .626632-1.432802$ C $-1.779646-0.352445-2.353041$ C $-3.638710-2.010436-2.358222$ O $-1.511073-0.467787-3.546159$ C $-4.830101-1.238428-2.829399$ C $-6.076654-1.434761-2.375111$ C $-7.255656-0.620403-2.823417$ C -7.929856 $0.107652-1.680185$ C $-9.122124-0.303140-1.215916$ C $-7.272396 \quad 1.359844-1.138176$ C $-6.0832271 .075530-0.259241$ N $-2.738594-1.095798-1.679163$ H -2.062575 -2.232902 1.898815 H $0.472961-1.7122913 .528529$ H $-0.9210850 .096847 \quad 4.385012$ H $-0.385462 \quad 1.205272 \quad 1.610095$ H 0.9902580 .5566942 .467588 H -0.3926584 .2004613 .929022$ H $1.240232 \quad 3.1002445 .093568$ H $\quad 0.957745 \quad 1.362185 \quad 5.225121$ H 2.1587801 .9875524 .074463 H $-1.9520982 .729197 \quad 1.694369$ H -2.571913 3.9362442 .797378 H -2.163332 4.9982790 .681799 H -1.0092045 .6510391 .849710$ H 0.7199164 .0078740 .945723 H $\quad 0.6462996 .3798790 .104794$ H $-0.5547415 .919858-1.085887$ H $1.7547437 .012603-2.004031$ H $\quad 0.140854 \quad 4.189515-2.959361$ H $3.0618875 .947210-0.362203$ H $3.5585204 .786202-1.571504$ H $2.7940644 .226817-0.070649$ H $2.2949554 .105307-3.674266$ H $-0.960800 \quad 2.142615-2.933148$ H $0.4920041 .145928-2.828762$ H $1.2612451 .266681-0.394152$ H $-5.451607 \quad 0.3600943 .734651$ H -3.9155370 .6200631 .081811$ H $-7.469945 \quad 0.0077142 .439483$ H -8.206685 $1.201092 \quad 1.385477$ H -7.191587 1.7474242 .706245 H -0.420216 $0.049973-0.767984$ H $-1.8277901 .129498-0.820063$ H -3.909021 -2.796415-1.646727 H -3.127510 -2.467393 -3.211459 H $-4.652387-0.465905-3.574192$ H $-6.261044-2.210871-1.635983$ H $-7.963846-1.301731-3.312727$ H $-6.9695310 .109194-3.591458$ H $-9.6344640 .228182-0.421072$
H -9.613715 - $1.182185-1.619457$

H $-8.000183 \quad 1.984515-0.607153$

H $-6.943503 \quad 1.984473-1.978910$

H $-5.141146 \quad 1.014245-0.804059$

H $-2.933468-0.882255-0.705162$

C $-0.293943-4.356062 \quad 0.863302$

$\begin{array}{llllll}\text { O } & 0.706701 & -3.557408 & 0.203292\end{array}$

C $0.411223-3.542121-1.208130$

C $-0.745439-4.516799-1.419747$

C $-1.477793-4.410541-0.095949$

H $0.044808-2.536312-1.434280$

C $1.666520-3.846294-2.037547$

H $-1.364663-4.242067-2.278770$

H $-0.395388-5.542671-1.577016$

C $-2.441777-5.554177 \quad 0.161434$

H $-2.030289-3.464686-0.080952$

C $2.797166-2.803938-1.893973$

O $2.214178-5.106009-1.628236$

H $1.400007-3.950032-3.096508$

H $3.222259-2.866769-0.887030$

O $3.851933-3.183912-2.788730$

C $2.388131-1.352418-2.201058$

C $3.551029-0.350212-2.297562$

H $1.684638-0.996681-1.441652$

O $1.719233-1.295993-3.465635$

H $4.156920-0.561829-3.188471$

H $3.095900 \quad 0.629919-2.490865$

H $-3.215568-5.585546-0.612703$

H $-1.927642-6.5211970 .162374$

H -2.938355 -5.4351601 .129399$

C $-0.572320-3.8409122 .276387$

H $1.639366-5.808554-1.974127$

H $3.513671-3.092731-3.696149$

H $0.840915-1.697332-3.360266$

H $0.358627-3.9142362 .852605$

H $-1.292940-4.5058552 .767396$

H $0.140685-5.3608330 .961056$

C $4.351867-0.0249451 .345982$

O $3.649896-0.078601 \quad 0.095434$

C $4.464793-0.266731-1.064131$

C $5.4880870 .875910-1.200265$

C $6.3057391 .056607 \quad 0.082749$

C 5.4113951 .0890791 .326425

H 3.5896950 .2538392 .085116

C $4.927474-1.392274 \quad 1.749710$

H $4.998550-1.218522-0.977151$

O $4.8064622 .095636-1.491049$

H $\quad 6.1490150 .658294-2.047690$

H 6.8727801 .9940540 .029390

$\begin{array}{llll}\text { O } & 7.241615 & -0.009011 & 0.209579\end{array}$

H 6.0254721 .0255562 .231892

$\begin{array}{lllll}\text { O } & 4.736996 & 2.349693 & 1.380646\end{array}$

C $3.846713-2.4204361 .994813$

H $5.588626-1.7949320 .978234$

H $5.495726-1.2911612 .681419$

H $5.4793532 .786152-1.617410$

H $7.8165280 .009437-0.574570$

H 4.1235912 .3891880 .626906

O $4.429260-3.6416772 .180138$

O $2.647624-2.1860982 .027109$

C $3.526100-4.7267372 .413422$

H $2.911804-4.9005851 .525392$

H $4.117686-5.6245082 .611207$

H $2.898441-4.5220093 .285881$

SCF Energy (B3LYP/6-31G**//MMFF)= -3245.90727234

0800027

MM̄FF Geometry

C $1.2185653 .218539-2.467243$

C $2.0379714 .225318-2.124788$

C $3.3416024 .121170-1.362762$

O $3.6000822 .768214-0.926373$

C $3.2698815 .017275-0.110780$

C 4.5440485 .0226800 .713032

C 4.6899324 .4250681 .913157

C 5.6895725 .7990710 .115584

C 3.6755093 .6075332 .664155

C 4.1933112 .1873832 .929988 
C 3.0737891 .2519153 .411432

C $3.636438-0.1267823 .759389$

C $2.532487-1.1381464 .101612$

$\begin{array}{llll}\text { O } & 2.125236 & 1.141506 & 2.346357\end{array}$

O $3.148850-2.4352424 .096742$

C $1.463722-1.1098712 .986557$

C $1.987955-0.9192505 .517825$

O $0.338667-1.9127753 .334928$

C $\quad 0.990177 \quad 0.3146032 .630588$

C $0.075974 \quad 0.353103 \quad 1.380975$

O $0.2322690 .841878 \quad 3.726118$

C $4.3234291 .966680-1.755663$

O $4.7490132 .246642-2.864661$

C $4.5058790 .667042-1.071086$

C $5.063347-0.363120-1.724190$

C $5.327499-1.694845-1.172180$

C $4.898010-1.9616960 .246611$

C $0.732355-0.1003020 .074477$

C $0.506386-1.559101-0.278452$

C $1.128553-3.239916-2.013761$

O $-0.215597 \quad-2.3101200 .369996$

C $2.282150-4.054267-1.520340$

C $3.174146-4.641160-2.332615$

C $4.298227-5.511004-1.848863$

C $5.648253-5.067883-2.364915$

C $6.213199-5.666827-3.426942$

C $6.384603-3.999971-1.589623$

C $5.945233-2.606259-1.952833$

N $1.194334-1.913447-1.431920$

H $1.4792822 .194377-2.218082$

H $1.7526145 .236697-2.410453$

H $4.1531254 .470785-2.012678$

H 2.4130904 .7147300 .499928

H $3.0530856 .052637-0.408445$

H 5.6490024 .5227682 .420749

H 6.5591725 .8361460 .780658

H $6.0150115 .343035-0.824423$

H $5.3855856 .831816-0.084005$

H 3.4679614 .1008943 .621260

H 2.7236103 .5590262 .132264

H 4.6250261 .7847022 .005273

H 4.9971002 .2260093 .675681

H 2.5879071 .7030964 .284489

H $4.360430-0.0551984 .580705$

H $4.201320-0.5129602 .900170$

H $2.478194-3.088924 \quad 4.357317$

H $1.918061-1.5675012 .099606$

H $2.786208-1.0547086 .257550$

H $1.220196-1.6603245 .766652$

H $1.565036 \quad 0.0766515 .665424$

H $0.647012-2.8299323 .425160$

H $-0.856478-0.1877121 .572389$

H $-0.2242081 .400578 \quad 1.231818$

H $-0.349467 \quad 1.5441383 .391114$

H $4.1805660 .594612-0.040327$

H $5.368418-0.217530-2.759714$

H $5.484594-1.3526230 .942452$

H $5.013837-3.0042870 .549652$

H $3.836792-1.7264610 .381382$

H $\quad 0.2940340 .480238-0.743847$

H 1.8058210 .1053620 .079088

H $1.141136-3.118266-3.101507$

H $\quad 0.187600-3.721030-1.728269$

H $2.368126-4.192823-0.445134$

H $3.069735-4.523625-3.408994$

H $4.091050-6.536259-2.182831$

H $4.324786-5.559818-0.753171$

H $7.195998-5.376194-3.783121$

H $5.702638-6.457125-3.967295$

H $7.460536-4.061477-1.803465$

H $6.308318-4.209122-0.518923$

H $6.212809-2.319202-2.970705$

H $1.810164-1.234968-1.870866$

C -1.237111 $2.729146-2.454448$

O $-1.190877 \quad 1.311384-2.724539$

C $-2.5300210 .839266-2.989826$

C $-3.4536441 .969690-2.567276$

C $-2.6203433 .186643-2.911731$
H $-2.5830820 .690240-4.075866$

C $-2.752003-0.491415-2.258591$

H $-4.4141021 .948431-3.089588$

H -3.644835 $1.930759-1.487869$

C $-3.1064634 .458500-2.238362$

H $-2.6301413 .326673-4.001024$

C $-4.212049-0.979723-2.300158$

O $-1.904378-1.455441-2.891909$

H -2.401466 -0.392272 -1.225858

H $-4.862886-0.256742-1.802020$

O $-4.650332-1.005330-3.666979$

C $-4.466821-2.378039-1.709446$

C $-3.967767-2.623925-0.275487$

H $-4.015404-3.135083-2.362527$

O $-5.876216-2.633596-1.734710$

H $-4.143341-3.684298-0.050039$

H -2.885344 -2.464092 -0.244481

H $-2.4673005 .307958-2.497948$

H $-4.1275014 .694684-2.555388$

H $-3.1084674 .359440-1.147553$

C $-0.0872673 .423143-3.188820$

H -1.872622 -2.242722 -2.323413

H -4.051858 -1.596758 -4.155221

H -6.181866 -2.485548 -2.646089

H $-0.2958734 .494645-3.285587$

H $0.0152643 .009461-4.199317$

H -1.135433 $2.851275-1.367807$

C $-4.8334160 .493793 \quad 1.621962$

$\begin{array}{llll}\text { O } & -4.264172 & -0.428986 & 0.684066\end{array}$

C $-4.658587-1.7946470 .828548$

C $-4.283523-2.3147602 .229354$

C $-4.818373-1.4070333 .340039$

C -4.5158950 .0703973 .065283$

H -4.3130441 .4409171 .429878$

C $-6.331426 \quad 0.717772 \quad 1.376641$

H $-5.742300-1.8727670 .696660$

O $-2.867301-2.4271042 .357067$

H $-4.693646-3.3243202 .350520$

H $-4.363475-1.7052724 .292567$

O $-6.224522-1.6048293 .450731$

H -5.0644790 .7140093 .762603$

$\begin{array}{llll}\text { O } & -3.122614 & 0.309625 & 3.283416\end{array}$

C $-6.576440 \quad 1.3918330 .045910$

H $-6.898783-0.2165281 .407178$

H $-6.759423 \quad 1.3708362 .146044$

H -2.485379 -1.5479562 .196978$

H -6.543387 -1.044385 4.178597

H -2.9251330 .0766944 .206620$

O $-7.3851720 .613035-0.728284$

O $-6.1129362 .481275-0.265451$

C $-7.7107471 .146996-2.015170$

H $-8.3476050 .423877-2.531500$

H $-8.2612162 .086143-1.906429$

H $-6.8028711 .297790-2.605919$

SCF Energy (B3LYP/6-31G**//MMFF $)=-3245.92118293$

08_00028

MM̄FF Geometry

C -3.119114 $4.835498-0.128057$

C $-3.8248613 .698899-0.029640$

C -4.2355703 .0759621 .279794$

O -3.7346001 .7170561 .296181$

C $-5.7695243 .035173 \quad 1.385023$

C -6.2692542 .4747542 .703967$

C -6.8417071 .2641862 .862948$

C -6.1181843 .3934203 .890007$

C $-7.089371 \quad 0.217247 \quad 1.813668$

C $-6.222650-1.0249862 .055538$

C $-6.236747-1.9731670 .847848$

C $-5.442680-3.2447611 .149951$

C $-5.319097-4.157076-0.079063$

O $-5.642547-1.274390-0.251035$

O $-4.310289-5.1326650 .225287$

C $-4.823175-3.319276-1.278591$

C $-6.612968-4.941101-0.326020$

O $-4.823987-4.097829-2.472465$

C -5.609525 -2.007994 -1.481340

C $-4.981389-1.081669-2.551635$ 
O - $-6.934183-2.326150-1.925480$ C $-2.442077 \quad 1.540667 \quad 1.688779$ O $-1.667362 \quad 2.4030782 .071743$ C -2.117289 0.1025841 .557906 C $-0.849548-0.3068391 .717167$ C $-0.366221-1.6879671 .643696$ C $-1.372898-2.7651911 .339198$ C $-3.558543-0.597416-2.249306$ C $-2.466573-1.438107-2.889399$ C $0.013515-1.700527-2.907705$ O $-2.686119-2.297619-3.738241$ C $0.420684-2.787057-1.963891$ C $1.648955-2.877194-1.432308$ C $2.088584-3.980874-0.515894$ C $2.625959-3.4672900 .800311$ C $3.944663-3.2903470 .985873$ C $1.645773-3.2582221 .930816$ C $0.943743-1.9281091 .862826$ N $-1.206807-1.080570-2.427183$ H $-2.846390 \quad 5.3718630 .776607$ H -4.094705 $3.159231-0.935006$ H -3.821284 3.6333792 .129078 H -6.173916 2.4674660 .541288 H $-6.175561 \quad 4.049616 \quad 1.270217$ H -7.1706870 .9792873 .861755$ H -6.5701402 .9789784 .797682$ H -5.0611523 .5766244 .104892$ H -6.6058654 .3534633 .691235$ H -8.148187 -0.0670891 .849058$ H -6.9200630 .6058330 .807614$ H $-5.190635-0.7092272 .252133$ H $-6.579258-1.5502072 .950180$ H -7.277655 $-2.216328 \quad 0.603973$ H $-5.886820-3.7904781 .991973$ H $-4.431733-2.9684511 .477093$ H $-4.168264-5.668607-0.574047$ H -3.773616 -3.079377 -1.073968 H $-6.853611-5.5620040 .545369$ H $-6.502221-5.637042-1.164912$ H -7.473098 -4.297525 -0.522109 H $-5.741892-4.155810-2.788139$ H $-5.050708-1.547226-3.542081$ H $-5.611936-0.182595-2.610088$ H -7.307911 -1.544826 -2.365563 H -2.928910 -0.5793871 .335402$ H $-0.0872870 .439777 \quad 1.935984$ H $-0.925163-3.7475381 .174244$ H $-1.924540-2.5298940 .423037$ H -2.084962 -2.8648872 .165062$ H -3.442500 $0.413081-2.662336$ H $-3.384947-0.529712-1.171291$ H $0.769551-0.912382-2.982575$ H $-0.154635-2.120106-3.904615$ H $-0.320690-3.546048-1.725239$ H $2.397539-2.135314-1.700030$ H $2.867034-4.554893-1.035838$ H $1.276621-4.694217-0.328099$ H $4.341434-2.9525811 .937449$ H $4.655761-3.471510 \quad 0.186794$ H $2.176545-3.2900942 .892391$ H $0.954508-4.1047801 .976731$ H $1.599110-1.0803842 .068102$ H $-1.128923-0.408267-1.669960$ C -1.139671 $5.291435-1.655356$ O $-0.7346393 .910492-1.546855$ C $0.3950643 .837060-0.651593$ C $0.9948275 .232745-0.655130$ C $-0.2593806 .085875-0.687205$ H $-0.017798 \quad 3.6057820 .337754$ C $1.3260092 .709522-1.104409$ H 1.6198605 .4294820 .220795 H $1.5967465 .396380-1.557530$ C $-0.0115377 .520141-1.121737$ H $-0.693568 \quad 6.0960310 .319508$ C $2.6410072 .637697-0.304219$ $\begin{array}{lllll}\text { O } & 0.609247 & 1.480390 & -0.960633\end{array}$ H $1.5528822 .813812-2.172203$ H $3.1991073 .565100-0.477088$
O 2.3518472 .5852611 .093419

C $3.5489571 .449597-0.684864$

C $4.917742 \quad 1.5445610 .004462$

H $3.6834391 .441270-1.771744$

$\begin{array}{llllll}\text { O } & 2.951459 & 0.213767 & -0.304179\end{array}$

H $5.4012922 .496007-0.251774$

H 4.7791261 .5722821 .094131

H $0.6658298 .022149-0.423190$

H $\quad 0.4402587 .565990-2.118352$

H $-0.9489598 .084600-1.149579$

C $-2.6527855 .409110-1.438754$

H $-0.2212261 .571552-1.458779$

H $1.8761981 .753324 \quad 1.259516$

H $2.0939570 .147309-0.756583$

H -3.158914 4.886232 -2.260701

H $-2.9607976 .459213-1.497156$

H $-0.9187575 .589956-2.688326$

C $8.101675-0.3490440 .363107$

$\begin{array}{lllll}\text { O } & 7.076419 & 0.650062 & 0.435725\end{array}$

C $5.874857 \quad 0.374588-0.297277$

C $6.1909150 .197381-1.790029$

C $7.285191-0.851814-2.015677$

C $8.491615-0.628988-1.097951$

H 8.9702210 .1041650 .858703

C $7.725658-1.615877 \quad 1.146077$

H $5.426208-0.5424500 .097849$

O $6.601807 \quad 1.439807-2.357873$

H $5.282773-0.111974-2.318480$

H $7.615964-0.812178-3.060659$

O $6.758333-2.153930-1.776992$

H $9.168827-1.490297-1.135423$

O $9.227630 \quad 0.504638-1.567077$

C $7.547830-1.3499822 .626513$

H $6.792184-2.0483970 .779698$

H $8.522479-2.3611701 .042002$

H $7.3708241 .756940-1.853706$

H $6.011570-2.285901-2.385511$

H $9.5243030 .307065-2.471793$

O $7.084181-2.4874913 .225200$

O $7.788939-0.291776 \quad 3.190462$

C $6.854817-2.3918304 .634455$

H $6.480500-3.3575894 .984145$

H $7.790315-2.1677075 .155882$

H $6.101275-1.6267974 .844628$

SCF Energy (B3LYP/6-31G**//MMFF) $=-3245.93245728$

$08 \_00029$

MM̄FF Geometry

C 1.0211185 .0346780 .424809

C $2.128105 \quad 4.280916 \quad 0.495331$

C $2.9897053 .979259-0.705091$

O $3.014607 \quad 2.542564-0.891097$

C $4.4275664 .471012-0.470023$

C $5.2898424 .402284-1.717452$

C $6.2569953 .491300-1.943921$

C $5.0287245 .483015-2.737115$

C $6.6842672 .356220-1.057898$

C $6.3825211 .001543-1.710611$

C $6.707447-0.168454-0.771102$

C $6.432669-1.506850-1.457867$

C $6.627250-2.696632-0.505297$

O $5.882091-0.036198 \quad 0.389771$

O $6.035483-3.852887-1.118124$

C $5.846770-2.430438 \quad 0.800522$

C $8.110886-3.025548-0.301970$

$\begin{array}{lllll}\text { O } & 6.128651 & -3.432449 & 1.774028\end{array}$

C $6.105467-1.0320071 .396586$

C $5.174219-0.6940362 .586454$

O $7.450778-0.973366 \quad 1.886674$

C $1.9230461 .987572-1.489862$

O $0.9407322 .577684-1.913102$

C $2.1146660 .520768-1.536417$

C $1.086548-0.257074-1.908388$

C $1.100442-1.713599-2.060380$

C $2.386072-2.437159-1.764174$

C $3.675561-0.6718982 .265027$

C $2.953225-1.9691902 .586323$

C $0.703175-3.0228112 .405388$ 
O $3.477782-2.9067903 .180248$

C $0.629795-3.9025561 .198869$

C $-0.509545-4.1522590 .536165$

C $-0.615827-5.103337-0.619281$

C $-1.193178-4.461164-1.858591$

C $-2.499410-4.574364-2.153565$

C $-0.235329-3.778642-2.806377$

C $-0.027217-2.326437-2.478120$

N $1.632304-1.9379832 .160210$

H $0.7484945 .486561-0.525936$

H 2.4029663 .8156231 .438707

H $2.5813404 .451241-1.607366$

H 4.8780883 .9030860 .350123

H $4.4139485 .514971-0.127817$

H $6.8167113 .560230-2.876228$

H $5.7273765 .438214-3.579469$

H $4.0178565 .394573-3.145966$

H $5.1340186 .471319-2.277795$

H $7.7634412 .443362-0.881890$

H $6.2116312 .410570-0.074982$

H $5.3193390 .967466-1.979148$

H $6.9573340 .902084-2.639765$

H $7.761145-0.095472-0.477014$

H $7.059803-1.622005-2.350655$

H $5.395969-1.521112-1.819324$

H $6.467233-3.990033-1.978670$

H $4.781593-2.5212850 .557583$

H $8.581083-3.280167-1.259486$

H $\quad 8.236657-3.9071290 .336087$

H $8.677625-2.1997260 .132850$

H $5.930809-4.2939981 .368630$

H $5.397915-1.3466733 .438966$

H 5.4365690 .3195012 .922987

H $7.509482-0.2613112 .544963$

H $3.0898490 .126029-1.278497$

H $\quad 0.136561 \quad 0.220847-2.146214$

H $2.287748-3.524694-1.780946$

H $2.750918-2.182967-0.763853$

H $3.152481-2.166825-2.497893$

H 3.2021560 .1000972 .885751

H $3.496278-0.4040081 .219072$

H $-0.262043-2.5722662 .649918$

H $\quad 1.036537-3.6082993 .268325$

H $1.549261-4.3899570 .883493$

H -1.432008 -3.6859210 .872558$

H - $-1.254730-5.938658-0.302921$

H $0.355256-5.554944-0.857350$

H -2.913137 -4.143517 -3.059339

H -3.185080 -5.092179-1.491033

H $-0.636107-3.824317-3.828675$

H $\quad 0.700315-4.342715-2.859209$

H - $0.910797-1.713532-2.662870$

H $\quad 1.295770-1.1351761 .636087$

C -1.179017 4.4152091 .531253

O -0.8454993 .0361621 .787170$

C - -1.6370392 .1973040 .917812$

C $-2.710003 \quad 3.1030620 .327483$

C -1.9763204 .4250710 .226814$

H -0.9526961 .8478910 .135573$

C -2.1843071 .0083791 .717689$

H $-3.0797542 .745438-0.638262$

H -3.563192 3.1886771 .010895

C -2.8893765 .6281760 .074526$

H -1.314069 4.370258 -0.645134

C $-3.050821 \quad 0.0588830 .866723$

O $\begin{array}{llll}-1.069379 & 0.283129 & 2.243719\end{array}$

H -2.762059 1.3723542 .575742

H -3.8257220 .6495870 .368637$

O $-2.243122-0.511902-0.163885$

C $-3.754872-1.0622931 .661017$

C $-4.480001-2.0968330 .778986$

H -4.470858 -0.611984 2.358642

O $-2.820805-1.8003112 .448078$

H -3.746746 -2.6465930 .175887$

H -4.921515 -2.841165 1.455549

H $-3.4969235 .538868-0.831960$

H -3.569385 5.7272110 .926926

H -2.3052596 .5511240 .001434$
C $0.087576 \quad 5.278137 \quad 1.575605$

H -0.5321560 .9096712 .758482$

H -1.550771 -1.040668 0.269232

H -2.423791-1.187221 3.089393

H 0.6139185 .0887242 .519730

H $-0.187877 \quad 6.3391621 .573281$

H -1.824765 4.7228322 .364572

C $-7.636890-0.248540 \quad 0.031237$

O $-6.552080-0.8962880 .708598$

C $-5.571193-1.512103-0.130169$

C $-6.215243-2.587840-1.018154$

C -7.382092 -2.005327 -1.822379

C $-8.347020-1.217892-0.930600$

H $-8.3497740 .008770 \quad 0.825851$

C $-7.1900221 .061266-0.638328$

H $-5.116832-0.755650-0.776322$

O $-6.680016-3.665574-0.208951$

H $-5.451883-2.989421-1.695152$

H -7.929407 -2.817498 -2.316136

O $-6.882455-1.139160-2.837073$

H $-9.067133-0.661612-1.541864$

O $-9.100109-2.136044-0.132629$

C -6.8645332 .1345600 .381054$

H $-6.3053350 .934533-1.266679$

H $-8.0119281 .438906-1.258320$

H $-7.055658-4.338001-0.802334$

H -6.290574 -1.661743 -3.404479

H $-9.588645-2.718782-0.738532$

O $-6.8650313 .351220-0.240209$

O -6.6270601 .9349121 .564359$

C -6.6115054 .4796790 .602785$

H -7.4850284 .6716631 .232291$

H -5.7231404 .3184381 .219851$

H $-6.4387565 .349530-0.036607$

SCF Energy $\left(B 3 L Y P / 6-31 G^{* *} / / M M F F\right)=-3245.91124597$

08_00030

MM̄FF Geometry

C $3.364941-1.5893712 .408522$

C $3.130917-2.2420993 .558314$

C $1.766667-2.4723294 .170674$

O $0.728400-2.3136843 .177400$

C $1.517682-1.4561045 .299035$

C $0.190440-1.6545856 .006157$

C $-0.856489-0.8079305 .935249$

C $0.091387-2.8969176 .854837$

C -0.9569050 .4905835 .182455$

C -1.8008760 .3308173 .917533$

C -1.8523731 .5961243 .047726$

C -2.6113132 .7379123 .729565$

C -2.7858803 .9542932 .806024$

O -2.5095331 .2241691 .828062$

O -3.7672104 .8189743 .399555$

C -3.3622073 .4745101 .457504$

C $-1.494848 \quad 4.7748242 .699829$

O $-3.430605 \quad 4.5477940 .521249$

C -2.6013702 .2738590 .855695$

C $-3.308636 \quad 1.707125-0.396784$

O -1.3000172 .7040070 .446430$

C $0.481606-3.3729182 .357214$

O $0.986730-4.4822672 .410489$

C $-0.483866-2.9176831 .334041$

C $-0.720096-3.6707300 .249513$

C $-1.596911-3.306678-0.866232$

C $-2.352474-2.010141-0.750302$

C $-4.6866231 .095820-0.131264$

C $-5.1765370 .263860-1.305242$

C $-6.762701-1.595158-1.821134$

O $-4.715150 \quad 0.363677-2.438884$

C $-5.985607-2.865791-1.661067$

C $-5.537752-3.602845-2.689111$

C $-4.752107-4.873102-2.514110$

C $-3.533770-4.941056-3.411018$

C $-3.435864-5.882404-4.365279$

C $-2.434280-3.920038-3.216571$

C $-1.668695-4.126443-1.935803$

N $-6.203318-0.592567-0.932314$

H $2.538016-1.1361941 .867787$ 
H $3.970093-2.6724864 .100201$ H $1.732150-3.4931524 .570299$ H $1.586784-0.4457384 .883664$ H $2.319879-1.5235876 .046155$ H -1.760101-1.066552 6.486654 H $-0.839228-2.9334827 .431482$ H $0.126133-3.7953686 .231432$ H $0.919395-2.9350547 .570207$ H -1.4160941 .2258165 .853285$ H 0.0262290 .8936914 .925479 H - $1.397286-0.4882973 .310327$ H -2.821839 0.0196714 .176965 H -0.8245071 .8976272 .813886$ H -2.117096 3.0443224 .658703 H -3.6088672 .3832894 .024773$ H -3.449671 5.0709144 .283662 H -4.400516 3.1773341 .650454 H -1.186156 5.1317293 .689929 H -1.646367 5.6747522 .093962 H -0.6599674 .2095142 .280732$ H -2.530866 4.7111810 .191178 H -3.382951 2.478779 -1.173800 H -2.665219 $0.931597-0.830856$ H $-0.9528952 .069082-0.201940$ H $-0.945017-1.9482781 .480897$ H $-0.204807-4.6252920 .152415$ H $-3.012630-2.0252670 .124194$ H -2.982638 -1.792871-1.612733 H $-1.656197-1.170794-0.650613$ H -4.6471490 .4503820 .753399$ H $-5.4264101 .882746 \quad 0.050194$ H -6.740364 -1.223361 -2.850627 H $-7.802346-1.752335-1.519028$ H $-5.784162-3.199135-0.645171$ H -5.761822 -3.289462 -3.706086 H $-5.427886-5.710446-2.731188$ H $-4.436359-5.001446-1.471507$ H -2.569641 -5.940640 -5.016041 H $-4.218800-6.617684-4.518197$ H -2.851964 -2.914462 -3.309144 H $-1.706787-3.989631-4.036835$ H -1.079896 $-5.044733-1.924679$ H $-6.471316-0.6395880 .046574$ C $4.788911-2.0107460 .357970$ O $3.936589-1.255367-0.527655$ C $3.305848-2.168661-1.445702$ C $4.026170-3.504291-1.280080$ C $4.381386-3.4748010 .193011$ H $2.268747-2.285705-1.110147$ C $3.337645-1.613690-2.873938$ H $3.391555-4.352957-1.553067$ H $4.936283-3.554304-1.889233$ C $5.463599-4.4647380 .584371$ H $3.472215-3.6975620 .764446$ C $2.666176-0.235996-3.058597$ O $4.702189-1.486983-3.296897$ H $2.881727-2.342039-3.554761$ H $3.2834490 .536683-2.588294$ O $2.674765 \quad 0.056905-4.462952$ C $1.221413-0.137108-2.536453$ C $0.4514561 .120998-2.984980$ H $1.226714-0.189648-1.441726$ O $0.492405-1.274496-3.010135$ H $\quad 0.232897 \quad 1.054164-4.058829$ H $-0.526783 \quad 1.088157-2.486530$ H $5.140501-5.4899320 .376070$ H $6.391389-4.2856980 .030644$ H $5.689003-4.3918851 .653031$ C $4.721777-1.4456081 .781042$ H $5.148570-0.890695-2.671178$ H $2.099531-0.593287-4.901701$ H - $0.419421-1.199000-2.682913$ H $4.958018-0.3756261 .753397$ H $5.486509-1.9296422 .399727$ H $5.811371-1.865170-0.015135$ C $2.0495593 .717777-0.817462$ O $1.3574422 .545143-1.271009$ C $1.1435302 .459148-2.681896$
C $0.3083673 .652838-3.173650$

C $0.9554394 .981918-2.770943$

C $1.3404185 .001313-1.288643$

H 1.9780533 .6808570 .277285

C $3.5390433 .686606-1.195802$

H $2.1088372 .470156-3.196579$

O $-1.005043 \quad 3.577990-2.622835$

H $0.213103 \quad 3.592047-4.264311$

H $0.2591485 .805974-2.969186$

O $2.1245945 .202019-3.554418$

H $1.9781025 .867540-1.078865$

O $0.155245 \quad 5.178747-0.508764$

C $4.2891742 .567231-0.505954$

H $3.6813643 .556577-2.271566$

H $4.0110234 .627335-0.889343$

H - $-1.5163474 .324569-2.978857$

H $1.8538965 .218874-4.488228$

H $-0.3921104 .382183-0.616676$

O $5.5901132 .592024-0.923404$

$\begin{array}{llllllll}\text { O } & 3.805572 & 1.776471 & 0.291433\end{array}$

C $6.4423621 .588055-0.364536$

H $7.4699391 .809123-0.665402$

H 6.3893261 .5982230 .728330

H $6.1654500 .606672-0.758332$

SCF Energy (B3LYP/6-31G*//MMFF) $=-3245.92576090$

0800031

MM̄FF Geometry

C $3.557691-3.603860-1.274485$

C $2.301373-3.193808-1.045706$

C $1.279074-2.997404-2.130391$

O $0.642404-1.708960-1.979131$

C $0.170657-4.073998-2.129857$

C $-0.716965-4.117744-0.897096$

C $-1.838267-3.385377-0.732988$

C $-0.311628-5.1136710 .159924$

C $-2.429608-2.379976-1.684995$

C $-3.843964-2.752728-2.152998$

C $-4.928845-2.561671-1.081130$

C $-6.292910-2.999070-1.622040$

C $-7.424585-2.721243-0.621945$

O $-4.964861-1.173371-0.731098$

O $-8.672619-2.853067-1.319275$

C $-7.323395-1.256071-0.150418$

C -7.451128 $-3.752267 \quad 0.512510$

$\begin{array}{lllll}\text { O } & -8.263167 & -0.989456 & 0.888576\end{array}$

$\begin{array}{llll}\text { C }-5.902562 & -0.847518 & 0.303187\end{array}$

C -5.8492890 .6823200 .538192$

O $-5.593432-1.5201111 .525987$

C $1.289724-0.598876-2.416734$

O $2.423264-0.528768-2.860702$

C $0.3382560 .527953-2.279182$

C $0.7262351 .781816-2.550666$

C $-0.1248692 .974115-2.496731$

C -1.584781 $2.777020-2.181050$

C $-4.434248 \quad 1.237362 \quad 0.750071$

C $-4.4407612 .721871 \quad 1.077947$

C -2.8804304 .5620451 .721867$

$\begin{array}{llll}\text { O } & -5.465440 & 3.396793 & 1.127919\end{array}$

C -2.5590025 .3888460 .517003$

$\begin{array}{llll}C & -1.376929 & 5.995969 & 0.330452\end{array}$

C $-1.0730976 .912441-0.818402$

C $0.0811156 .431584-1.667105$

C $1.3287356 .883920-1.452049$

C $-0.2410915 .519251-2.829119$

C $0.4331024 .176774-2.746968$

N $-3.159430 \quad 3.197381 \quad 1.319495$

H $3.866803-3.824497-2.292769$

H $1.992332-2.980666-0.027297$

H $1.759392-3.027685-3.117741$

H $0.623866-5.062678-2.282028$

H $-0.462567-3.917358-3.012466$

H $-2.391643-3.5078600 .196667$

H $-0.999902-5.1198221 .011936$

H $-0.301515-6.124840-0.260148$

H $0.685140-4.8928490 .549109$

H $-2.436486-1.399041-1.195960$

H - $-1.809275-2.268252-2.578553$ 
H $-4.082537-2.107190-3.008677$ H -3.852333 -3.787760 -2.516984 H $-4.667771-3.151750-0.196319$ H -6.278756 -4.060239-1.900547 H $-6.510048-2.449817-2.549086$ H -8.717298 -3.752554-1.686795 H $-7.610994-0.619270-0.998823$ H $-7.599233-4.7612850 .109152$ H -8.294567 -3.574121 1.188687 H $-6.532185-3.7656611 .102279$ H -9.141076 -1.245936 0.557752 H $-6.2771321 .199039-0.331035$ H $-6.4673850 .941198 \quad 1.407356$ H -4.952546 -0.994765 2.029184 H $-0.6724570 .289670-1.967810$ H $\quad 1.759876 \quad 1.953077-2.848343$ H -2.064700 $2.163734-2.951556$ H $-2.1483493 .709791-2.118444$ H -1.706467 2.279982 -1.213200 H $-3.939350 \quad 0.719570 \quad 1.577656$ H -3.833962 $1.092967-0.155359$ H -2.048938 4.5269952 .433036 H -3.7531254 .9802252 .233841$ H -3.353225 $5.531098-0.211978$ H -0.5937855 .8714961 .075100$ H -0.839962 $7.900916-0.400541$ H -1.956213 $7.070387-1.450072$ H $2.1570076 .581277-2.084014$ H $1.5484927 .568051-0.639094$ H $0.0917706 .005189-3.756594$ H -1.324006 5.423687 -2.950694 H $1.5021384 .213417-2.961169$ H -2.3685542 .5708401 .200255$ C $5.737955-2.824308-0.207626$ O $5.256902-1.4886180 .030451$ C $6.054957-0.570236-0.744647$

C $7.212991-1.384322-1.316249$ C $6.567610-2.747279-1.487256$ H $5.424414-0.234057-1.576075$ C 6.4695760 .6301630 .114149 H $7.589277-0.965556-2.254502$ H $8.053715-1.447214-0.615776$ C $7.564078-3.881018-1.648536$ H $5.922008-2.708014-2.372800$ C 5.2854021 .3397700 .802708 O $7.3530690 .167171 \quad 1.143851$ H $7.0461041 .337890-0.492804$ H 4.8551930 .6648021 .549799 O 5.8062552 .4553271 .536207 C $4.1933981 .839696-0.158524$ C 3.0535682 .6088570 .534190 H $3.7706340 .995584-0.711912$ O $4.7980722 .707918-1.120384$ H 3.4481753 .4965751 .043751 H $2.3976152 .994897-0.255954$ H $8.180275-3.727420-2.540575$ H $8.234471-3.952891-0.785604$ H $7.047783-4.840240-1.755688$ C $4.574743-3.818295-0.188837$ H 7.6000820 .9388851 .681803 H 6.1573663 .0917510 .889809 H $4.1101052 .962819-1.758728$ H $4.080524-3.7539890 .789089$ H $4.961922-4.839951-0.278088$ H $6.393352-3.0636160 .640966$ C $1.045571-0.3489971 .596302$ O 1.7482280 .6136720 .799590 C 2.2361051 .7533291 .513975 C 1.0583662 .5329212 .115645 C 0.1883111 .6318042 .998739 C $-0.150087 \quad 0.3023822 .313134$ H $0.649394-1.0647810 .865777$ C $2.022824-1.1051062 .507409$ H 2.8946951 .4196702 .322921 O 0.2548613 .0884451 .078013 H 1.4479643 .3709842 .704593 H $-0.7448732 .153948 \quad 3.243571$ O 0.8666551 .3623874 .221689
H $-0.584736-0.392083 \quad 3.040251$

O

C $1.522943-2.4810922 .888652$

H $2.969603-1.2357381 .968324$

H $2.241918-0.5526943 .424497$

H -0.0392272 .3576170 .508298$

H 1.0339792 .2146154 .659035

H $-1.433920-0.2859450 .955737$

O $2.559578-3.1987713 .414274$

O $0.373304-2.8771852 .755760$

C $2.242282-4.5361693 .811973$

H $3.149357-4.9954394 .213956$

H $1.476708-4.5280464 .593564$

H $1.907935-5.1184932 .948163$

SCF Energy (B3LYP/6-31G**//MMFF) $=-3245.92397170$

$08 \_00032$

MM̄FF Geometry

C 0.9749424 .6816450 .575497

C 2.1560074 .0456900 .574309

C $2.9971963 .862970-0.663866$

O $3.0910222 .440266-0.923270$

C $4.4135784 .416783-0.435876$

C $5.2730334 .390004-1.686732$

C $6.2812193 .525021-1.916384$

C $4.9535955 .454268-2.706520$

C $6.7630582 .411457-1.030036$

C $6.4863341 .042047-1.662787$

C $6.808066-0.109255-0.698910$

C $6.565017-1.460806-1.372413$

C $6.748275-2.635319-0.398879$

O 5.9553320 .0292630 .441511

O $6.183849-3.806187-1.009985$

C $5.932677-2.3599660 .883125$

C $8.229752-2.945360-0.154338$

O $6.198829-3.3465121 .876791$

C $6.163778-0.9513291 .466303$

C $5.199816-0.6090572 .628568$

O $7.495900-0.8731901 .988089$

C $2.0305231 .865965-1.558535$

O $1.0366792 .434373-1.984555$

C $2.2635520 .405293-1.621892$

C $1.265148-0.391728-2.031573$

C $1.297345-1.850967-2.151338$

C $2.579237-2.555401-1.799989$

C $3.709900-0.6011252 .267769$

C $2.981566-1.8931182 .599090$

C $0.729722-2.9453602 .389288$

O $3.492401-2.8183303 .223615$

C $0.709928-3.8736871 .216839$

C $-0.402801-4.1679550 .527543$

C $-0.455148-5.164408-0.593235$

C $-1.003518-4.581425-1.874734$

C $-2.304325-4.703239-2.189581$

C $-0.023611-3.948080-2.835299$

C $0.181369-2.483512-2.570748$

N $1.670469-1.8696592 .142576$

H $0.6064745 .127242-0.345197$

H 2.5116413 .5770041 .488921

H $2.5403344 .363063-1.526892$

H 4.8929093 .8650250 .378958

H $4.3545785 .456662-0.086459$

H $6.8320693 .617213-2.851838$

H $5.6501965 .443420-3.551819$

H $3.9467035 .313674-3.110547$

H $5.0105716 .447521-2.249105$

H $7.8429802 .531233-0.879663$

H $6.3140612 .463773-0.036099$

H $5.4281240 .989938-1.947191$

H $7.0764680 .934668-2.581394$

H $7.853975-0.017927-0.382957$

H $7.215121-1.581314-2.247896$

H $5.537919-1.491615-1.759074$

H $6.637125-3.949534-1.858480$

H $4.874691-2.4643370 .615458$

H $8.725721-3.210320-1.095913$

H $8.349012-3.8153140 .500870$

H $8.777225-2.1066240 .280452$ 
H $\quad 6.021170-4.2153071 .477556$

H $5.406433-1.2514403 .493094$

H 5.4458690 .4097742 .961407

H $7.531807-0.1518932 .638050$

H $3.2331170 .028455-1.320158$

H $\quad 0.3168280 .069233-2.307014$

H $3.368341-2.285431-2.509226$

H $2.494795-3.644206-1.807422$

H $2.904198-2.283849-0.790411$

H 3.2181390 .1832402 .857961

H $3.556732-0.3575411 .211665$

H $-0.247408-2.4873492 .571970$

H $1.026730-3.4944203 .288681$

H $1.646539-4.3578300 .950750$

H -1.343314 -3.704621 0.816354

H $-1.090166-5.998393-0.265720$

H $0.530502-5.607616-0.782149$

H -2.697785 -4.314681 -3.123089

H $-3.005671-5.185387-1.516463$

H $-0.403415-4.041226-3.862049$

H $0.911001-4.516104-2.840694$

H $-0.700417-1.880931-2.794017$

H $1.354426-1.0820421 .584524$

C -1.099386 3.7879481 .610249

O

C -3.3358173 .8966690 .907429$

C -3.2508083 .0960732 .200117$

C -1.9956403 .6623882 .836854$

H -3.8463944 .8510431 .087843$

C $-3.9723463 .172934-0.286738$

H $-4.140763 \quad 3.2152352 .825285$

H -3.123133 2.0269961 .992543

C -1.430057 2.7861013 .940520

H -2.2213674 .6579493 .241761$

C $-5.326598 \quad 2.5051130 .021995$

O $-4.1365104 .136305-1.331253$

H -3.282022 2.414276 -0.674581

H $\quad-5.1464291 .7278450 .769602$

O $\quad-6.210430 \quad 3.452156 \quad 0.621037$

C $-6.0098401 .869270-1.208475$

C $-7.2524271 .027351-0.857673$

H $-5.2898831 .246626-1.751791$

O $-6.4527942 .886773-2.110033$

H -7.958296 $1.629197-0.272062$

H $-7.7661050 .793110-1.799994$

H $-0.523953 \quad 3.2279294 .366005$

H -2.158972 2.6690454 .749226

H -1.1758791 .7872923 .571249$

C 0.0701054 .7591351 .773862

H -3.261388 $4.520703-1.512193$

H $-6.3802464 .147014-0.038114$

H -5.668888 $3.381125-2.403106$

H 0.6317344 .5282722 .686031

H -0.2932715 .7896581 .862483$

H $-0.7265122 .798714 \quad 1.312519$

C $-5.618318-2.299616-0.430087$

O $-6.100707-1.068218-0.981856$

C $-6.924053-0.279345-0.117250$

C $-8.184545-1.0664390 .268877$

C $-7.819719-2.4198110 .891881$

C $-6.784509-3.1795210 .054646$

H -5.137448 -2.813903 -1.272108

C $-4.543196-2.0578030 .640503$

H $-6.370066-0.0360430 .793118$

O $-9.012310-1.275750-0.873257$

H $-8.768007-0.4734610 .982590$

H -8.724386 -3.033292 0.984770

O $-7.292764-2.2185032 .200258$

H $-6.396406-4.034756 \quad 0.620370$

O $-7.458473-3.706646-1.092755$

C $-3.335501-1.3370760 .077253$

H -4.931835 -1.456025 1.466280

H -4.194851 -3.016009 1.041821

H -8.484484 -1.746202 -1.540962

H -7.976970 -1.770615 2.726396

H $-6.820325-4.251942-1.583652$

O $-2.573948-0.8702521 .110331$

O $-3.077618-1.215886-1.112502$
C $-1.378964-0.1855970 .725787$

H $-0.703660-0.8761230 .215844$

H -1.614236 0.6683690 .084081

H -0.8884580 .1792461 .631363$

SCF Energy $\left(B 3 L Y P / 6-31 G^{* *} / / M M F F\right)=-3245.91974719$

0800033

MM̄FF Geometry

C 0.9718714 .6704780 .107466

C 2.1161703 .9704240 .118695

C $2.9319123 .682556-1.115790$

O $3.0801272 .245286-1.215077$

C $4.3288374 .313317-0.988577$

C 5.176468 4.154123-2.238022

C $6.2386503 .331791-2.353996$

C $4.7769375 .027789-3.400670$

C $6.8017182 .402905-1.316050$

C $6.5638830 .936501-1.697272$

C $6.865507-0.011183-0.527761$

C $6.703764-1.469883-0.956925$

C $6.861944-2.4417270 .222622$

O 5.9402630 .2960110 .520041

O $6.373137-3.725784-0.195974$

C $5.959314-1.9742241 .385057$

C $8.333730-2.6408120 .602490$

O $6.190788-2.7548622 .554765$

C $6.110969-0.4756291 .715655$

C 5.0640530 .0314722 .738351

O $7.403867-0.2505602 .290699$

C $2.0507561 .559642-1.786172$

O $1.0400462 .032759-2.281619$

C $2.3516040 .114075-1.685053$

C $1.396199-0.779009-1.984655$

C $1.527853-2.237711-1.956572$

C $2.857208-2.812053-1.545625$

C $3.600616-0.0931772 .298445$

C $2.908510-1.3511922 .795707$

C $0.770026-2.6091232 .552708$

O $3.394148-2.1015713 .637479$

C $0.946250-3.7149091 .560942$

C $-0.077918-4.2859850 .909760$

C $0.067689-5.425936-0.054869$

C $-0.520205-5.119280-1.413391$

C $-1.777374-5.481316-1.720835$

C $0.382606-4.483531-2.444688$

C $0.463423-2.989386-2.308229$

N $1.657614-1.5126222 .214600$

H $0.6197045 .095882-0.828212$

H $2.4692803 .539614 \quad 1.053053$

H $2.4331824 .065395-2.014618$

H $4.8355853 .891517-0.115202$

H $4.2334395 .387364-0.778234$

H $6.7724573 .313059-3.303466$

H $5.4728994 .944412-4.242315$

H $3.7840394 .752373-3.768574$

H $4.7575046 .079455-3.096293$

H $7.8803972 .585738-1.234494$

H $6.3918612 .610010-0.325629$

H $5.5173200 .810101-2.000811$

H $7.1878370 .677371-2.561530$

H $7.8872030 .177376-0.177508$

H $7.409660-1.719695-1.758894$

H $5.703650-1.611044-1.387307$

H $6.883166-4.000541-0.977210$

H $4.924244-2.1680551 .080210$

H $8.896020-3.049180-0.245930$

H $8.438045-3.3734991 .410344$

H $8.826538-1.7156020 .908415$

H $6.063974-3.6876102 .310519$

H $5.235834-0.4328573 .716830$

H 5.2546151 .1038622 .890027

H 7.3759700 .5760392 .800374

H $3.345484-0.174472-1.364924$

H $0.418887-0.408578-2.291954$

H $2.847703-3.898434-1.433442$

H $3.166140-2.412513-0.574165$

H $3.624004-2.567424-2.288056$

H 3.0390480 .7484212 .724752 
H $3.506908-0.028308 \quad 1.210096$ H $-0.249905-2.2117702 .553386$ H $1.003989-2.9739083 .557975$ H $1.956579-4.0788251 .389949$ H -1.089484 -3.941878 1.111120 H $-0.439816-6.2972220 .380015$ H $1.117008-5.725767-0.166403$ H -2.195806 -5.292471 -2.703931 H -2.418710 $-5.966831-0.992694$ H $0.000197-4.694901-3.452987$ H $1.363366-4.967712-2.421981$ H $-0.462186-2.481005-2.582230$ H $1.368653-0.8799101 .474586$ C -1.205945 4.1055751 .287786 O -0.9437342 .6954651 .130321$ C -1.8086232 .1818290 .095165$ C -2.933359 $3.195994-0.031043$ C -2.1835194 .4971790 .177566$ $\mathrm{H}-1.2074042 .169713-0.821852$ C $-2.2373890 .756377 \quad 0.457477$ H $-3.4405413 .148779-0.999111$ H -3.6798033 .0496210 .757598$ C -3.0818115 .6680590 .536968$ H $-1.6501084 .736411-0.749857$ C $-3.3075030 .179784-0.490153$ O $-1.067850-0.064570 \quad 0.423987$ H -2.610240 0.7264691 .488418 H $-4.1950810 .812189-0.400653$ O $-2.8616160 .282814-1.842626$ C $-3.705171-1.280845-0.195008$ C $-4.858773-1.802224-1.074197$ H -3.978448 -1.3819210 .861552$ O $-2.600827-2.155511-0.432247$ H $-4.562059-1.781711-2.130524$ H -4.997092 -2.863475 -0.826131 H $-3.7979705 .864753-0.267404$ H -3.650780 5.4724861 .451992 H $-2.491347 \quad 6.5763180 .694407$ C $0.1158694 .881973 \quad 1.326379$ H $-0.4149420 .339127 \quad 1.021059$ H -2.073902 $-0.281115-1.931117$ H - $1.872406-1.8748570 .146572$ H $\quad 0.673849 \quad 4.5675982 .217879$ H -0.0812635 .9540351 .439602$ H $-1.693373 \quad 4.2138082 .265470$ C $-7.730021-0.437014 \quad 0.881421$ O $-6.548721-1.1497070 .494009$ C $-6.185942-1.050704-0.885558$ C -7.306956-1.609238-1.773887 C $-8.635743-0.903912-1.476974$ C $-8.936267-0.862914 \quad 0.025415$ H -7.925820 -0.7586281 .912843$ C -7.4955391 .0824230 .904734$ H -6.028679 -0.001249-1.148648 O $-7.445487-3.010160-1.549663$ H -7.028672 $-1.468331-2.825084$ H -9.450937 -1.425414 -1.992964 O $-8.5929360 .431151-1.972786$ H $-9.781612-0.195416 \quad 0.230185$ O $-9.323758-2.1710120 .456928$ C -6.5156241 .4908501 .987239$ H -7.103868 $1.458083-0.043668$ H $-8.446217 \quad 1.5850601 .118419$ H -8.144992 -3.334859 -2.141640 H $-8.426140 \quad 0.384078-2.929515$ H -10.117646 -2.422396 -0.045178 O -6.5401372 .8508052 .117509$ O $\begin{array}{lllll}-5.812028 & 0.724361 & 2.630597\end{array}$ C -5.6832313 .3896203 .129726$ H -6.0505853 .1016674 .119045$ H -4.6528503 .0497392 .988833$ H $-5.7056204 .479608 \quad 3.049037$ SCF Energy (B3LYP/6-31G**//MMFF) $=-3245.90756181$

0800034

MM̄MF Geometry

C 2.9597103 .3994451 .458156

C 3.6866984 .0907840 .566656
C $4.228173 \quad 3.562553-0.745514$

O $3.7799392 .209750-0.997305$

C $5.7665613 .569717-0.720987$

C $6.3893163 .249315-2.067777$

C $7.0014942 .090303-2.382901$

C $6.3203794 .359087-3.086610$

C $7.1848290 .878225-1.514651$

C $6.396721-0.318110-2.062566$

C $6.399089-1.498065-1.080614$

C $5.669033-2.701743-1.677543$

C $5.540342-3.856587-0.672253$

O $5.736641-1.0673010 .112435$

O $4.574570-4.785132-1.189709$

C $4.971589-3.3087870 .655118$

C $6.854019-4.633645-0.526872$

O $4.985041-4.3129831 .666358$

C $5.698581-2.0452261 .159878$

C $5.002856-1.3848132 .375856$

O $7.022554-2.4012561 .576804$

C $2.5419692 .061352-1.548665$

O $1.7836942 .951723-1.899867$

C $2.2445640 .612629-1.616727$

C $0.9968050 .209897-1.901445$

C $0.536956-1.174920-2.033124$

C $1.544481-2.271111-1.811339$

C $3.566520-0.9065052 .135142$

C $2.502447-1.9046432 .559459$

C $0.034281-2.2418822 .504716$

O $2.749100-2.9281643 .191172$

C $-0.364688-3.0765891 .329723$

C $-1.550909-2.9610590 .714402$

C $-2.007890-3.851790-0.402901$

C $-2.466675-3.083353-1.620209$

C $-3.769704-2.825167-1.823982$

C -1.427088 -2.712049-2.651082

C $-0.752793-1.398941-2.362245$

N $1.232199-1.4925032 .180002$

H 2.7497902 .3470161 .288421

H $3.8789295 .144368 \quad 0.760621$

H $3.8708574 .219347-1.548232$

H 6.1169652 .8756450 .049344

H $6.1316754 .557260-0.407344$

H $7.4219841 .988151-3.382783$

H $6.8586974 .112363-4.008035$

H $5.2821054 .569004-3.360425$

H $6.7667475 .273626-2.682655$

H $8.2525970 .628472-1.488475$

H $6.8969831 .075059-0.480036$

H $5.361770-0.007128-2.251182$

H $\quad 6.824128-0.630487-3.023489$

H $7.438885-1.755036-0.846341$

H $6.166307-3.042836-2.594059$

H $4.659453-2.397067-1.983001$

H $4.883270-5.083479-2.062431$

H $\quad 3.918737-3.0638340 .471239$

H $7.155718-5.059072-1.491621$

H $6.736597-5.4858560 .151412$

H $7.679927-4.016433-0.167467$

H $4.498536-5.080347 \quad 1.319348$

H $5.070871-2.0408693 .252049$

H $5.589888-0.4924042 .637275$

H $7.346750-1.7215452 .190564$

H $3.056335-0.079674-1.428891$

H $\quad 0.234457 \quad 0.969137-2.070962$

H $2.303261-2.255989-2.600535$

H $1.105280-3.270937-1.799843$

H $2.040537-2.150234-0.842546$

H $3.396674-0.0075922 .741997$

H $3.410627-0.627247 \quad 1.088413$

H $-0.739339-1.5197172 .782677$

H $0.222646-2.8884043 .367917$

H $0.336754-3.8394321 .000680$

H $-2.257288-2.2142331 .067125$

H $-2.835417-4.463849-0.019697$

H $-1.226654-4.565715-0.691675$

H -4.111104 -2.303252 -2.711709

H $-4.522714-3.121468-1.101054$

H - $-1.903949-2.616901-3.636431$ 
H $-0.718389-3.536098-2.774796$ H -1.410741 - $0.539275-2.496120$ H $1.130585-0.6588871 .608328$ C $0.836000 \quad 4.1339262 .629287$ O 0.2253952 .8665142 .305987 C -0.6700553 .0644081 .190446$ C - $-1.0104014 .544471 \quad 1.215639$ C $0.3294175 .143751 \quad 1.594724$ H -0.0899872 .8202420 .291329$ C $-1.8617192 .110608 \quad 1.316938$ H -1.3821564 .9118140 .254663$ H -1.761383 $4.759177 \quad 1.985922$ C $0.236336 \quad 6.5653352 .121788$ H 0.9671365 .1443950 .702745 C $-2.9457492 .338794 \quad 0.244721$ O H -2.307883 2.1944852 .314933 H -3.3313413 .3591850 .350160$ O $-2.3558522 .254956-1.053367$ C -4.1420301 .3674420 .329352$ C $-5.2114061 .699409-0.730689$ H -4.5771861 .4323591 .333602$ $\begin{array}{lllll}\text { O } & -3.684300 & 0.032917 & 0.134743\end{array}$ H $-5.4137302 .777053-0.713935$ H -4.813134 $1.438375-1.719389$ H -0.1800117 .2318031 .359370$ H -0.4065596 .6247133 .006448$ H 1.2262346 .9425282 .397425 C 2.3616473 .9862202 .706446 H $-0.6670990 .677488 \quad 1.890848$ H -2.029482 $1.345215-1.161114$ H -4.444104 -0.5615920 .251586$ H 2.6126093 .3080123 .531612 H 2.8164114 .9553442 .942333 H $0.4662034 .401623 \quad 3.627222$ C -7.419877 -1.318557-0.486485 O $-6.297391-0.454259-0.704779$ C $-6.5382300 .942508-0.511475$ C $-7.6343741 .433067-1.471222$ C $-8.9081680 .592001-1.335922$ C $-8.605832-0.909146-1.375436$ H -7.079447 -2.307681 -0.819181 C $-7.781002-1.4231530 .998975$ H -6.8559091 .1159950 .522393$ O $\quad-7.157471 \quad 1.360684-2.813799$ H $-7.8496382 .486166-1.254023$ H $-9.6001730 .837217-2.150900$ O $-9.5567420 .900302-0.105793$ H -9.495227 -1.479549-1.084174 O $-8.307455-1.285050-2.723344$ C $-6.689602-2.1323941 .764431$ H -7.964752 -0.455006 1.473200 H $-8.697888-2.0093751 .134676$ H -7.856452 $1.709854-3.392649$ H $-9.7721641 .848374-0.116747$ H $-7.477770-0.845554-2.977917$ O $-5.823024-1.2223792 .292595$ O $-6.609979-3.350021 \quad 1.862973$ C -4.729183-1.764499 3.039555 H $-4.253033-2.5877592 .499255$ H $-3.991035-0.9711353 .185281$ H -5.084576 -2.102185 4.017115 SCF Energy (B3LYP/6-31G**//MMFF) $=-3245.91580097$

08_00035

MM̄FF Geometry

C $-0.981092 \quad 4.4016270 .745359$

C $0.270826 \quad 4.0078180 .464559$

C $0.7921533 .817155-0.936804$

O $1.2205932 .438599-1.068566$

C $2.0026254 .732776-1.182366$

C $2.5007624 .689564-2.614702$

C $3.6125314 .049234-3.028284$

C $1.6705265 .466552-3.605188$

C $4.5694323 .233215-2.205202$

C $4.5884941 .771118-2.668954$

C $5.4703260 .896149-1.766252$

C $5.509130-0.542362-2.281510$
C $6.287249-1.472527-1.338843$

O $4.9144020 .925334-0.448625$

O $5.993753-2.826236-1.720208$

C $5.766282-1.2861360 .104002$

C $7.803081-1.297979-1.493833$

O $6.585507-2.023807 \quad 1.008383$

C 5.6639340 .1942130 .532498

C 4.9465240 .4000911 .889940

O 6.9853920 .7306450 .672058

C $0.237658 \quad 1.513672-1.254995$

O $-0.9601291 .731482-1.348310$

C $0.8612630 .173481-1.317640$

C $0.069081-0.908183-1.366032$

C $0.496118-2.304332-1.475551$

C $1.973355-2.584673-1.524548$

C $3.497865-0.0960201 .954176$

C $3.343810-1.4968072 .522458$

C $1.589001-3.2116252 .975855$

O $4.272728-2.136273 \quad 3.007177$

C $1.503001-4.2013691 .858165$

C $0.376156-4.8546891 .537206$

C $0.288523-5.8993510 .464079$

C $-0.735005-5.565187-0.595753$

C $-1.981327-6.064919-0.538446$

C $-0.281075-4.736821-1.774595$

C $-0.453242-3.260334-1.550764$

N $2.028402-1.9334332 .451412$

H -1.652635 $4.643819-0.074627$

H $\quad 0.9546843 .767179 \quad 1.274497$

H $0.0126704 .026635-1.680170$

H $2.8053174 .475105-0.483727$

H $1.7371705 .772237-0.945310$

H $3.8746904 .109855-4.084137$

H $2.1065805 .460035-4.609925$

H $0.6643975 .043655-3.682112$

H $1.5862976 .512322-3.292016$

H $5.5725613 .663076-2.314387$

H $4.3307963 .280189-1.140734$

H $3.5621811 .383792-2.660587$

H $4.949047 \quad 1.717143-3.703814$

H $6.478787 \quad 1.325567-1.744348$

H $5.929397-0.581695-3.294158$

H $4.482950-0.922372-2.370564$

H $\quad 6.255416-2.939289-2.650101$

H $4.770457-1.7440100 .135554$

H $8.109749-1.514121-2.524361$

H $8.349744-2.009094-0.864869$

H $8.145235-0.289184-1.254024$

H $6.150736-2.0186391 .877264$

H $5.552325-0.0079452 .707756$

H 4.9126141 .4841602 .073362

H 6.9426721 .5128991 .246572

H $1.9423640 .113521-1.334884$

H -1.009777 -0.759369-1.343812

H $2.482072-2.126155-0.670408$

H $2.405931-2.185983-2.447863$

H $2.216140-3.648741-1.483123$

H 2.9366520 .5674032 .625379

H $3.017843-0.0471740 .972104$

H $\quad 0.620937-3.0479873 .460048$

H $2.297479-3.5668813 .730984$

H $2.418175-4.4129681 .310331$

H $-0.530470-4.6599912 .105534$

H $0.026766-6.8503100 .946762$

H $1.263906-6.072496-0.007384$

H $-2.709929-5.854661-1.314574$

H $-2.301958-6.688340 \quad 0.289533$

H $-0.878424-5.000637-2.658426$

H $\quad 0.741668-5.010701-2.049161$

H -1.498314 -2.953312 -1.500435

H $1.335133-1.3457821 .997434$

C -1.3519863 .2310632 .968258$

O -2.0575602 .1244342 .372053$

C -2.4713381 .2257153 .421216$

C -1.9818331 .8382654 .732684$

C -1.9020873 .3135414 .387140$

H -3.5664001 .2170113 .423979$

C $-1.916714-0.1768373 .146750$ 
H -2.663455 1.6290155 .563199 H -0.9939321 .4529145 .012907$ C -1.039802 4.113267 5.347420 H -2.9183503 .7293274 .374665$ C -2.487234 -0.8518541 .880735$ O $-0.493473-0.0850872 .996383$ H -2.084762 -0.8222894 .016192$ H $-2.223415-0.2705160 .990597$ O $-1.839263-2.1223161 .742992$ C $-4.010890-1.0943861 .922383$ C $-4.542357-2.0402000 .827021$ H $-4.539536-0.1373991 .864961$ O $-4.360940-1.6776683 .182718$ H -4.011161 -2.998923 0.877306 H -5.588746 $-2.270421 \quad 1.071253$ H -1.442019 4.0557226 .364246 H -0.0110693 .7382915 .371153$ H -1.0068015 .1680775 .057974$ C -1.564949 4.4936482 .130523 H $-0.316320 \quad 0.5025382 .241500$ H $-0.884612-1.9527301 .670527$ H -3.880448 -2.520165 3.259268 H -1.148818 5.3749272 .630826 H -2.643213 4.6657272 .017909 H -0.2891882 .9591883 .000668$ C $-5.5064560 .281567-1.929568$ O $-5.431106-0.407307-0.674241$ C $-4.465688-1.462096-0.593099$ C $-4.751995-2.536106-1.654943$ C $-4.818010-1.921155-3.055019$ C $-5.739838-0.697963-3.091965$ H $-6.3982450 .916534-1.845117$ C $-4.2972941 .196510-2.140421$ H $-3.465324-1.053007-0.758100$ O $-5.986145-3.186295-1.357522$ H -3.962031 -3.294963-1.613117 H -5.183001-2.675339 -3.762803 O $-3.500108-1.551593-3.450044$ H $-5.626200-0.167866-4.044143$ O $-7.098866-1.142703-3.039784$ C $-4.3213552 .326622-1.139690$ H $-3.3416010 .679491-2.025891$ H $-4.2981481 .625614-3.149580$ H -6.118312 $-3.882760-2.023128$ H -3.555374 -1.182961 -4.348162 H -7.244936 -1.551333 -2.169361 O $-4.9769703 .401192-1.663453$ O $-3.8444942 .249605-0.015362$ C $-5.1048264 .528235-0.791155$ H $-5.614276 \quad 4.2409350 .133729$ H $-4.1193554 .949800-0.574654$ H $-5.7062455 .285641-1.300781$

SCF Energy (B3LYP/6-31G**//MMFF) $=-3245.92123223$

08_00036

MM̄FF Geometry

C $1.789497-3.674865-0.174794$

C $0.842999-2.727202-0.258693$

C $0.023074-2.512328-1.503518$

O $0.048424-1.105787-1.845328$

C $-1.445635-2.910485-1.277597$

C -2.142682 -3.227266 -2.586970

C $-2.955269-2.389376-3.258516$

C $-1.894851-4.616948-3.120020$

C $-3.351696-0.984452-2.899846$

C $-4.868792-0.863390-2.688664$

C $-5.351580-1.544056-1.398413$

C $-6.879438-1.576377-1.340408$

C $-7.383296-2.163887-0.013893$

O $-4.838743-0.789007-0.297466$

O $-8.787298-1.8823430 .088368$

C $-6.687749-1.4290451 .149583$

C -7.241264 -3.689854 0.030320

O $-7.012304-2.0323712 .400466$

C $-5.151210-1.3270660 .994070$

C $-4.591057-0.3463902 .049533$

O $-4.566115-2.6151091 .183268$

C $1.094582-0.659339-2.585570$
O $2.057994-1.317266-2.955904$

C $0.9425030 .767301-2.953301$

C $-0.0304871 .571899-2.496401$

C $-0.2193612 .981715-2.856965$

C $0.7187363 .565529-3.882926$

C $-3.084757-0.0830931 .927499$

C -2.7137421 .2124202 .619975$

C -2.8489953 .6756882 .361333$

O $-2.210687 \quad 1.2319803 .740255$

C -2.9426174 .6416661 .225088$

C -1.9217345 .4094940 .816692$

C $-2.0185916 .401222-0.304243$

C $-1.0997486 .069996-1.457781$

C $0.0889106 .682952-1.588838$

C $-1.6050155 .114383-2.515115$

C $-1.2072503 .684187-2.261145$

N -3.020630 2.3282821 .854945

H $1.942977-4.332284-1.026485$

H $\quad 0.651955-2.0761620 .589145$

H $0.441972-3.089972-2.337399$

H $-1.975560-2.118795-0.735931$

H -1.510930 -3.802067 -0.639568

H -3.394802 -2.746201-4.189725

H $-2.437425-4.809075-4.051891$

H $-0.830759-4.771237-3.322942$

H $-2.221450-5.365230-2.390339$

H -2.815566 $-0.605487-2.025504$

H $-3.063750-0.336004-3.736643$

H $-5.1155450 .205357-2.645791$

H $-5.394437-1.283434-3.555329$

H $-4.954497-2.563822-1.355208$

H -7.290105 -2.138236 -2.188650

H -7.271325 -0.554291-1.439325

H $-9.229718-2.282920-0.679586$

H -7.099316 $-0.410218 \quad 1.182217$

H $-7.804447-4.150500-0.790277$

H -7.667987 -4.0993110 .952595$

H $-6.205930-4.027298-0.050060$

H -7.982320 -2.064315 2.463696

H -5.1286760 .6063051 .959738$

H $-4.785774-0.727293 \quad 3.060227$

H -3.630494 -2.504304 1.415253

H $1.7018871 .128625-3.639820$

H $-0.7601251 .173011-1.794168$

H $1.7557203 .506350-3.533729$

H $0.5295154 .617736-4.102991$

H $0.6347513 .024749-4.832169$

H -2.509150 -0.896959 2.381832

H $-2.764001-0.0138640 .881774$

H -1.892119 3.7378322 .890888

H -3.6471393 .8665873 .086280$

H $-3.903755 \quad 4.7211220 .722537$

H -0.9649215 .3399881 .328665$

H -1.7687817 .3894930 .103823$

H -3.048593 $6.493223-0.670357$

H $0.7394696 .481515-2.432967$

H $0.4407767 .395989-0.850601$

H -1.283444 $5.450889-3.506598$

H -2.700542 $5.163093-2.564029$

H - $1.8291313 .181178-1.520200$

H $-3.5797352 .197951 \quad 1.016084$

C $4.152781-3.7591890 .777420$

O $4.457274-2.4033470 .400591$

C $5.479280-2.425851-0.616586$

C $6.014241-3.854529-0.649217$

C $4.768942-4.648763-0.302346$

H $4.973252-2.218850-1.567290$

C $6.534269-1.345237-0.349751$

H $6.435007-4.117059-1.624679$

H $6.789626-4.0198370 .107676$

C $5.054599-6.0677580 .156339$

H $4.135572-4.684877-1.195954$

C $5.9448210 .056289-0.095787$

O $7.275422-1.7126110 .821854$

H $7.256550-1.321824-1.173753$

H 5.4263340 .0373910 .867927

$\begin{array}{llll}\text { O } & 7.035991 & 0.970957 & 0.067017\end{array}$

C $5.0066660 .589172-1.194511$ 
C $4.4555182 .000855-0.909642$

H $4.161593-0.091648-1.337637$

O $5.708475 \quad 0.657114-2.437676$

H $5.2789962 .722460-0.841965$

H $3.8675872 .302181-1.787151$

H $5.560837-6.632705-0.633196$

H $5.696559-6.0820091 .043552$

H $4.125388-6.5908550 .403492$

C $2.649416-3.9177151 .033374$

H $7.936299-1.0161110 .977029$

H $7.4865661 .044346-0.791902$

H $5.902810-0.252710-2.718480$

H $2.357884-3.2245331 .832071$

H $2.447545-4.9305461 .400902$

H $4.671793-3.9277221 .730680$

C 1.6533600 .9866921 .318436

$\begin{array}{lllll}\text { O } & 2.498725 & 1.151132 & 0.173595\end{array}$

C 3.5794542 .0715030 .349624

C 3.0295423 .4796310 .614345

C 2.0785673 .4798151 .818518

C $1.0571892 .336744 \quad 1.753305$

H $\quad 0.832977 \quad 0.356516 \quad 0.954593$

C 2.3929620 .2155382 .421052

H $4.180493 \quad 1.7674691 .212447$

O $2.342800 \quad 3.971124-0.533455$

H 3.8686504 .1595920 .800802

H 1.5419784 .4360991 .856469

O $2.831174 \quad 3.3584083 .021746$

H $0.545396 \quad 2.2328962 .716664$

$\begin{array}{llllll}\text { O } & 0.071013 & 2.708363 & 0.786736\end{array}$

C $1.465173-0.5578773 .330048$

H $3.066775-0.5124311 .953774$

H 3.0029330 .8806823 .037629

H $1.610747 \quad 3.360143-0.722607$

H 3.4414804 .1142453 .064150

H -0.6239592 .0285930 .796674$

O $2.215025-1.2553094 .234681$

O $0.244671-0.5623523 .258610$

C $1.473073-2.0597795 .156533$

H $2.184011-2.5610065 .818834$

H $0.812198-1.4317025 .761362$

H $0.899005-2.8199454 .618139$

SCF Energy (B3LYP/6-31G**//MMFF) $=-3245.91667500$

08_00037

MM̄FF Geometry

C -3.884088 -3.424470 1.227041

C $-2.597241-3.0480351 .202498$

C $-1.803241-2.6610322 .419547$

O $-1.203568-1.3635962 .210668$

C $-0.649749-3.6620122 .611744$

C $0.245107-3.3177973 .784893$

C $1.439364-2.7028833 .669684$

C $-0.267956-3.744157 \quad 5.134305$

C $2.086100-2.1856802 .412041$

C $3.184795-3.1354601 .928797$

C $3.904954-2.6156270 .674154$

C $4.905461-3.6668630 .186772$

C $5.743460-3.161744-0.992706$

O $4.585734-1.4065891 .020083$

O $6.843617-4.066450-1.175073$

C $6.346753-1.791994-0.619707$

C $4.944179-3.182200-2.301159$

O $7.018269-1.215964-1.738322$

C $5.314199-0.787638-0.052994$

C 6.0413730 .4383860 .550393

O $4.444166-0.309996-1.076618$

C $-1.946738-0.2544152 .452166$

O $-3.143198-0.1988172 .680390$

C -1.010484 0.8929302 .432432

$\begin{array}{llll}C & -1.475977 & 2.149351 & 2.465985\end{array}$

C -0.6589813 .3660562 .505956$

C $0.828693 \quad 3.2007642 .673715$

C $5.103674 \quad 1.5669621 .008003$

C $4.7634122 .562321-0.092258$

C $3.3925834 .572122-0.549569$

O $5.2470522 .516530-1.220514$

C 2.6491565 .6130890 .223819
C $1.407756 \quad 6.019288-0.084227$

C $0.6798027 .115187 \quad 0.638537$

C $-0.661801 \quad 6.6732501 .177080$

C $-1.8000957 .004962 \quad 0.543521$

C -0.6750605 .9389522 .499766$

C -1.277694 $4.561762 \quad 2.416006$

$\begin{array}{lllll}\text { N } 3.871898 & 3.534278 & 0.343748\end{array}$

H -4.418469 -3.4484212 .172740$

H $-2.071262-3.0350650 .252230$

H -2.429792 -2.632366 3.319802

H $-0.049018-3.7175371 .695291$

H $-1.050655-4.6744762 .754760$

H $2.017613-2.5267124 .575626$

H $\quad 0.431091-3.4992885 .941049$

H $-1.216688-3.2466405 .358124$

H $-0.429023-4.8268525 .154680$

H $1.354734-2.0302021 .613438$

H $2.506115-1.1963892 .630812$

H $3.916123-3.2696752 .737134$

H $2.745988-4.1188361 .717358$

H $3.159766-2.399669-0.100256$

H $4.391087-4.599050-0.079186$

H $5.588769-3.9278141 .007549$

H $6.479308-4.955221-1.327769$

H $7.113674-1.9666460 .148127$

H $4.646194-4.207562-2.551773$

H $5.552590-2.833557-3.142696$

H $4.033850-2.580151-2.256919$

H $7.668733-1.867191-2.052567$

H 6.6071380 .1024791 .429540

H $6.7681430 .840031-0.165401$

H $4.785057-0.599599-1.938118$

H 0.0498360 .6665022 .408118

H -2.5540752 .3044432 .468951$

H 1.0532332 .6796373 .611034

H 1.3734354 .1460132 .700115

H 1.2501122 .6231061 .844809

H 4.1788451 .1636291 .434519

H 5.6121302 .1380121 .795328

H $2.7747754 .095220-1.317193$

H $4.2571975 .023128-1.048504$

H $3.170696 \quad 6.079768 \quad 1.056073$

H $0.8997905 .575417-0.936971$

H $0.5454147 .944537-0.068530$

H 1.2830767 .5259041 .457817

H -2.7707906 .7303150 .942680$

H $-1.7867307 .560203-0.388664$

H -1.2673256 .5231593 .217293$

H 0.3292545 .9185962 .932339

H -2.362289 4.5702422 .298263

H 3.4296783 .4229101 .251655

C $-5.657367-2.861860-0.515326$

O $-5.006363-1.628879-0.876059$

C $-5.892995-0.537376-0.556888$

C $-7.234017-1.167185-0.190852$

C $-6.787631-2.4758600 .434865$

H $-5.486379-0.0541420 .338738$

C $-5.9431770 .458966-1.719429$

H $-7.813827-0.5388430 .492156$

H -7.849711 $-1.357730-1.077693$

C $-7.891942-3.5122250 .538630$

H $-6.399539-2.2622641 .438445$

C $-4.5624041 .025056-2.114420$

O $-6.485066-0.212786-2.864368$

H $-6.6392281 .272365-1.483274$

H $-3.9539430 .216327-2.535593$

O $-4.788623 \quad 1.961616-3.174890$

C $-3.8114421 .725216-0.966090$

C $-2.4815602 .382135-1.370026$

H $-3.6109851 .015069-0.157328$

O $-4.6259712 .766052-0.417488$

H -2.654416 $3.175305-2.108016$

H $-2.1085772 .901533-0.477684$

H -8.707423 -3.1431351 .169335$

H $-8.310712-3.753633-0.444130$

H $-7.515375-4.4394460 .981788$

C $-4.630454-3.8691260 .000081$

H $-6.5068490 .430804-3.593250$ 
H $-3.9323702 .141214-3.598169$ H -5.3631232 .3477110 .057899$ H -3.911487 $-4.069475-0.805381$ H $-5.122536-4.8234480 .220444$ H $-6.081360-3.263654-1.445881$ C $-0.269325-0.627094-1.213286$ O $-1.2272870 .389928-0.892510$ C $-1.4106851 .404217-1.884579$ C $-0.0830792 .129570-2.163865$ C $1.0410211 .150700-2.513836$ C $1.104800-0.014420-1.522526$ H $-0.165951-1.204176-0.287388$ C $-0.814778-1.574507-2.287984$ H -1.771511 $0.940207-2.808443$ O $0.3091402 .890836-1.023890$ H $-0.2320192 .838437-2.986519$ H $2.0014151 .680783-2.505856$ O $0.8408720 .634812-3.825382$ H $1.785472-0.791281-1.886717$ O $1.6366160 .461195-0.281611$ C $0.054935-2.793603-2.484740$ H -1.825094 -1.910481-2.024633 H $-0.923015-1.074420-3.254956$ H $0.3687852 .282098-0.268209$ H $\quad 0.841837 \quad 1.387802-4.440660$ H $2.5378760 .781102-0.456428$ O $0.141356-3.509102-1.328100$ O $0.586486-3.080714-3.549832$ C $0.962581-4.679221-1.383121$ H $\quad 0.532590-5.408286-2.076235$ H $1.981786-4.416125-1.681178$ H $0.994030-5.119632-0.383053$

SCF Energy (B3LYP/6-31G**//MMFF) $=-3245.91655704$

08_00038

\section{MM̄FF Geometry}

C -2.1992433 .1690931 .676408$

C -1.5204352 .0301251 .469842$

C -0.8127971 .2696922 .560957$

O $0.579476 \quad 1.1386282 .193500$

C - $-1.412338-0.1412532 .681837$

C $-0.907463-0.8885993 .899752$

C $0.036479-1.8497763 .875278$

C -1.579091-0.523909 5.199165

C $0.807592-2.3489022 .685628$

C $2.311645-2.4253862 .981055$

C $3.093628-3.0004741 .790776$

C $4.558955-3.2220242 .165818$

C $5.381757-3.7259450 .971451$

O $3.007277-2.0717850 .706020$

O $6.770865-3.6107411 .316708$

C $5.134294-2.796091-0.234392$

C $5.126590-5.2108290 .686274$

O $5.775115-3.327448-1.392554$

C $3.634920-2.515960-0.505826$

C $3.489753-1.384198-1.550011$

O $3.018687-3.699929-1.016232$

C 1.3955592 .1867152 .488504

O 1.0900483 .2177973 .066544

C 2.7360891 .8800621 .943820

C 3.6076232 .8848231 .769929

C 4.9466552 .7802261 .188904

C 5.5084361 .4021500 .969301

C $2.065805-0.832401-1.694026$

C $2.0707490 .382908-2.599587$

C $2.7664642 .783047-2.635871$

O $1.742354 \quad 0.308069-3.781014$

C $4.2460253 .015395-2.666148$

C $4.8271724 .166354-2.293958$

C $6.3132504 .392923-2.315180$

C $6.8438324 .976178-1.021650$

C $7.287656 \quad 6.243534-0.967837$

C 6.9309104 .0707730 .191288

C 5.6004103 .9185240 .876231

N $2.5159701 .526387-1.953698$

H -2.2452643 .5947892 .675525$

H -1.481011 1.6059630 .468813

H -0.8947771 .7924863 .522109$
H -1.208431 -0.7131091 .769238$

H -2.507439 -0.081609 2.747696

H $0.282862-2.3451934 .813823$

H -1.214498 -1.124557 6.039389

H -1.3989610 .5269925 .444999$

H -2.659814 -0.6840815 .125632$

H $0.436903-3.3508912 .438808$

H $0.650679-1.7178061 .807262$

H $2.679784-1.4177333 .214024$

H $2.479518-3.0520603 .866022$

H $2.627218-3.9477091 .494940$

H $4.645108-3.9170913 .010393$

H $4.994267-2.2738152 .512032$

H $6.926453-4.1430562 .115576$

H $5.636204-1.844561-0.008259$

H $5.374643-5.8176351 .565482$

H $5.773135-5.574994-0.119903$

H $4.089308-5.4246320 .420193$

H $5.786001-2.633889-2.072895$

H $4.152600-0.557759-1.274327$

H $3.820922-1.736181-2.535486$

H $2.342716-3.454605-1.667215$

H 2.9499830 .8559591 .660447

H 3.2977083 .8933652 .041603

H $5.4102230 .792576 \quad 1.874834$

H $6.5721841 .400783 \quad 0.722073$

H 4.9800150 .9041270 .153699

H $1.397061-1.581569-2.128036$

H $1.641068-0.542689-0.726326$

H $2.2377623 .564502-2.081332$

H $2.3731092 .742015-3.655991$

H $4.8702892 .195373-3.014551$

H $4.2026614 .996753-1.972219$

H $6.5317765 .070396-3.150777$

H $6.8536643 .463120-2.532585$

H $7.6914766 .662324-0.051994$

H $7.2528146 .892908-1.836356$

H 7.6338264 .4886270 .923581

H $7.3628733 .117023-0.123472$

H 5.1184814 .8694921 .108869

H $2.7641791 .470905-0.970460$

C $-4.4534453 .843470 \quad 0.821832$

$\begin{array}{lllll}\text { O } & -4.877978 & 2.466536 & 0.846237\end{array}$

C $-5.9167992 .279529-0.126370$

C $-6.5074233 .663838-0.344042$

C $-5.2537944 .519385-0.296131$

H $-5.4282261 .951589-1.051845$

C $-6.890904 \quad 1.194198 \quad 0.340265$

H $-7.0475013 .744423-1.291902$

H -7.187942 3.9486490 .466509

C $-5.5273985 .992478-0.047350$

H $-4.7253214 .415997-1.253591$

C $-6.209512-0.1020130 .830890$

O $\quad-7.6812121 .693344 \quad 1.426695$

H $-7.6000380 .968333-0.464303$

H $-5.7536250 .066094 \quad 1.814747$

O $-7.230348-1.0878471 .028957$

C $-5.146709-0.677457-0.121913$

C $-4.579477-2.0166820 .388916$

H $-4.3267140 .041345-0.224938$

O $-5.734618-0.853347-1.409231$

H -4.365644 -1.934856 1.461348

H $-5.336817-2.796996 \quad 0.243404$

H $-6.1386936 .411782-0.853012$

H -6.0619236 .1477540 .895855$

H $-4.5915956 .558670-0.001880$

C -2.941092 3.8975710 .590641

H -7.0706831 .9672742 .132780$

H -7.886748 -0.7072251 .637411$

H $-5.043016-1.184547-2.006703$

H $-2.599013 \quad 4.9382670 .563961$

H -2.699154 $3.452402-0.382344$

H $-4.7068864 .269521 \quad 1.800768$

C $-2.496915-3.005886-2.559422$

O $-3.604682-2.676904-1.712175$

C $-3.284334-2.427222-0.341172$

C $-2.639958-3.6703290 .292004$

C $-1.429370-4.142447-0.518974$ 
C $-1.740429-4.229949-2.017327$ H -2.952500 -3.289707 -3.516821 C -1.593058 -1.793895-2.810996 H -2.584865 -1.586179 -0.280110 O $-3.603118-4.719944 \quad 0.362448$ H -2.336841 -3.428469 1.317964 H $-1.113100-5.126836-0.152719$ O $-0.357341-3.231884-0.302541$ H $-0.815536-4.369071-2.588287$ O $-2.540372-5.392615-2.252881$ C $-2.350842-0.700516-3.525832$ H -1.170808 -1.383917 -1.889149 H $-0.743047-2.066468-3.447266$ H -3.181060 -5.476231 0.804556 H $\quad 0.423798-3.588236-0.757553$ H $-3.396343-5.259440-1.810475$ O $-2.5441620 .358623-2.689019$ O $-2.739566-0.786159-4.683619$ C $-3.2657941 .459561-3.248942$ H $-2.7564451 .836773-4.140877$ H $-4.289594 \quad 1.155912-3.486566$ H -3.300373 $2.257798-2.503546$

SCF Energy (B3LYP/6-31G**//MMFF)= -3245.91343107

08_00039

MM̄FF Geometry

C $0.685074 \quad 1.3180862 .885715$

C 1.5696530 .3493853 .169211

C $1.461957-1.1308372 .878707$

O $2.521477-1.4672291 .949578$

C $0.150756-1.6092602 .225132$

C $-0.103076-3.0927412 .420752$

C $0.225027-4.065706 \quad 1.548454$

C $-0.821035-3.4458823 .699461$

C $1.003491-3.9553320 .268213$

C $0.147619-4.173746-0.986932$

C $-0.564361-2.894353-1.448866$

C $-1.427788-3.163348-2.682483$

C $-2.052853-1.873336-3.237870$

O $0.449740-1.936499-1.773829$

O $-2.557565-2.157586-4.551420$

C $-0.950314-0.804351-3.396951$

C $-3.252780-1.417310-2.402948$

O $-1.5078810 .458045-3.755253$

C $-0.049920-0.649950-2.149180$

C $1.1557880 .262643-2.477646$

O $-0.803730-0.053301-1.093667$

C $3.760506-1.7432582 .428663$

O $4.126339-1.7448763 .591682$

C $4.613476-2.0107121 .250882$

C $5.942017-1.8611591 .341512$

C $6.895732-2.0126990 .240502$

C $6.358304-2.474922-1.089601$

C $2.2795060 .227658-1.430626$

C $3.3930561 .209040-1.761418$

C $5.5884412 .024444-0.900357$

O $3.3730021 .938108-2.749863$

C $6.7033031 .272500-1.558362$

C $7.9562661 .239493-1.077705$

C $9.0923000 .520277-1.750288$

C $9.878827-0.360099-0.802232$

C $11.0570820 .046495-0.300043$

C $9.340200-1.742097-0.502670$

C $8.190135-1.7055150 .465826$

N $4.4112301 .177874-0.817723$

H $-0.241657 \quad 1.0823112 .371800$

H 2.4794640 .6386943 .693937

H $1.616751-1.6684953 .822883$

H $\quad 0.160021-1.3734171 .155769$

H $-0.712532-1.0801702 .647017$

H $-0.051511-5.0888941 .802413$

H - $0.988798-4.5232783 .801036$

H $-0.237315-3.1210324 .566578$

H -1.798353 -2.955653 3.736369

H $1.551011-3.0127120 .202097$

H $1.772774-4.7380330 .299340$

H $\quad 0.821515-4.505582-1.788052$

H $-0.579647-4.977760-0.821410$
H $-1.180738-2.514334-0.626538$

H $-2.206370-3.903250-2.458421$

H $-0.807008-3.611791-3.471007$

H -3.207839-2.876734 -4.473214

H $-0.317923-1.113844-4.240940$

H $-4.015252-2.204298-2.362979$

H $-3.743745-0.548885-2.855378$

H $-2.981390-1.166245-1.376713$

H $-2.0571270 .318678-4.545731$

H $1.595604-0.046735-3.434961$

H $\quad 0.808948 \quad 1.297029-2.598858$

H $-0.1909780 .350374-0.459560$

H $4.115449-2.2715510 .323949$

H $6.367993-1.5657102 .299546$

H $5.602650-1.777078-1.466589$

H $5.901799-3.466450-0.993406$

H $7.119454-2.553890-1.867784$

H $1.8917680 .475109-0.438021$

H $2.718316-0.775582-1.386445$

H 5.8417152 .3276830 .120450

H $5.3524862 .919153-1.485327$

H $6.4772410 .752757-2.486489$

H $8.1886411 .788442-0.167779$

H $9.7540881 .281203-2.184607$

H $8.738955-0.083657-2.595296$

H $11.640673-0.588210 \quad 0.358424$

H $11.4621801 .025916-0.532114$

H $10.124756-2.365163-0.052401$

H $9.095610-2.240943-1.444167$

H $8.477143-1.3751831 .465329$

H $4.3594770 .511430-0.053386$

C 0.7763983 .7561622 .120408

O $-0.537448 \quad 3.689643 \quad 1.534268$

C -0.3972353 .6347730 .102966$

C $1.0031474 .154923-0.190423$

C 1.7771073 .5720160 .978915

H $-0.4357752 .575849-0.173907$

C -1.548832 $4.377059-0.581379$

H $1.3770973 .821742-1.162930$

H $1.0452255 .249857-0.162431$

C 3.1155134 .2461701 .223298

H $1.9497182 .507966 \quad 0.784223$

C $-2.9482803 .979213-0.064466$

O $-1.3889135 .782298-0.348831$

H -1.482288 $4.236650-1.666713$

H $-3.0814674 .382760 \quad 0.947314$

O $-3.9039414 .646849-0.896434$

C $-3.2292922 .463955-0.060657$

C $-4.6175042 .122546 \quad 0.497014$

H -2.4742451 .9608610 .548451$

O $-3.1441541 .945345-1.385202$

H -4.7815272 .6409211 .450026$

H $-5.3927942 .498000-0.184324$

H 3.7572754 .1594230 .341194

H 2.9943195 .3119101 .444105

H 3.6344143 .7830222 .068381

C $0.8824382 .759773 \quad 3.279216$

H -2.124733 $6.234746-0.796049$

H $-4.7672654 .599860-0.452528$

H -2.218044 2.007424-1.673059

H 0.1006812 .9964814 .012096

H 1.8462642 .8958793 .783802

H 0.8660964 .7686782 .535055

C -6.713932 -0.8439991 .309489$

O $\quad-6.227071 \quad 0.491824 \quad 1.128126$

C $-4.869750 \quad 0.6106860 .680375$

C $-3.914755-0.0656241 .679930$

C $-4.336936-1.5024932 .002720$

C $-5.833822-1.6097542 .309705$

H $-7.705352-0.7217271 .764791$

C $-6.898737-1.579423-0.026976$

H $-4.7836470 .131713-0.299201$

O $-3.855867 \quad 0.6872582 .890609$

H $-2.902175-0.0804981 .262363$

H $-3.761072-1.8525822 .867286$

O $-4.005313-2.3366710 .897560$

H -6.148644 -2.659241 2.343147

O $-6.081230-1.0563383 .605740$ 
C $-7.946206-0.926380-0.904850$ H $-5.969606-1.612344-0.601296$ H $-7.229623-2.6066020 .164992$ H -4.7603050 .7547043 .242161$ H $-4.239412-3.2493671 .137460$ H $-5.564682-1.5721604 .248133$ O $-7.986172-1.581431-2.103560$ O $-8.6443390 .026080-0.586411$ C $-8.933698-1.076044-3.049511$ H $-8.704191-0.035153-3.296788$ H $-8.859406-1.677271-3.959507$ H $-9.949289-1.164778-2.652050$

SCF Energy (B3LYP/6-31G**/MMFF) $=-3245.91339150$

08_00040

MM̄FF Geometry

C -2.192122 -3.8193360 .645300$

C -1.117841 -3.0172470 .678809$

C $-0.287127-2.7854031 .912906$

O $-0.234334-1.3627522 .173584$

C $1.151752-3.2642291 .654008$

C $2.002496-3.3476982 .905894$

C $2.863815-2.3973193 .320640$

C $1.865586-4.6326393 .681285$

C $3.096593-1.0429642 .715876$

C $4.513735-0.8308002 .170817$

C $4.870470-1.7134420 .962747$

C $6.186089-1.2419770 .336360$

C $6.520656-1.998857-0.956831$

O $3.811210-1.6159840 .010787$

O $7.560132-1.275766-1.634973$

C $5.285012-1.983725-1.884015$

C $7.084514-3.394985-0.666647$

O $5.504963-2.807789-3.027824$

C $3.979009-2.402841-1.171656$

C $2.729296-2.215812-2.075659$

O $4.070438-3.798158-0.855524$

C $-1.292204-0.7828572 .795158$

O $-2.334819-1.3321793 .124023$

C -1.0310380 .6457203 .097542$

C 0.0448801 .3186462 .659505

C 0.4034352 .7001342 .991660

C -0.5986003 .5111413 .771235$

C $1.996299-0.876572-1.975403$

C $2.8160590 .304730-2.443017$

C $4.1771312 .202870-1.622564$

O $3.019173 \quad 0.518532-3.636009$

C $4.2548863 .032349-0.381939$

C $3.7122114 .254366-0.275806$

C 3.8159275 .1209120 .944908

C 2.4889225 .3168731 .643487

C 1.6149786 .2492931 .228344

C 2.2241834 .5064192 .891690

C 1.6094893 .1657402 .599774

N $3.2922641 .078050-1.394697$

H $-2.485983-4.3476041 .548227$

H $-0.809014-2.496977-0.224008$

H $-0.708191-3.3008192 .785327$

H $1.626725-2.6045190 .921875$

H $1.141609-4.2580551 .185663$

H $3.441347-2.5867834 .224474$

H $2.505258-4.6535834 .569894$

H $0.832538-4.7692484 .016069$

H $2.144045-5.4860373 .054231$

H $2.370978-0.8173521 .931349$

H $2.918743-0.2992583 .503136$

H $4.580070 \quad 0.224002 \quad 1.872427$

H $5.253381-0.9799962 .967242$

H $4.954952-2.7528871 .300167$

H $7.009727-1.3239031 .056454$

H $6.109539-0.1724990 .092931$

H $8.321673-1.210682-1.033501$

H $5.174399-0.961019-2.261658$

H $8.008926-3.320045-0.081329$

H $7.358975-3.911572-1.592892$

H $6.393632-4.029284-0.107353$

H $\quad 6.326305-2.503417-3.450561$

H $2.986708-2.425319-3.121247$
H $1.985970-2.982524-1.816852$

H $3.186194-4.116741-0.610490$

H $-1.774007 \quad 1.1124263 .736508$

H 0.7556350 .8092932 .013784

H -1.586835 3.4655023 .301140

H -0.3484114 .5723143 .830918$

H -0.6821623 .1334034 .796044$

H $1.099851-0.909762-2.607168$

H $1.629978-0.711948-0.955314$

H $3.8124522 .772146-2.484570$

H $5.1667371 .808922-1.876983$

H 4.7989542 .6127850 .460653

H $3.1755654 .671117-1.125427$

H 4.2145736 .0960510 .635055

H 4.5565674 .7212961 .648746

H $0.6776326 .415306 \quad 1.748967$

H $1.815761 \quad 6.8605980 .354721$

H 1.6201825 .0933963 .590043

H 3.1684764 .3419163 .426455

H 2.2663142 .4947192 .045245

H $3.2381550 .696389-0.454015$

C $-4.434732-3.488342-0.520485$

O $-4.397884-2.052285-0.399684$

C $-5.469376-1.6413270 .471147$

C $-6.348259-2.8723070 .678670$

C $-5.322128-3.9866410 .618914$

H -5.008526 -1.379202 1.430835

C $-6.210183-0.429138-0.102819$

H $-6.891812-2.8376781 .627797$

H -7.083348 -2.988627 -0.126443

C $-5.922731-5.3604030 .380199$

H $-4.775821-3.9952801 .569591$

C $-5.3237950 .803322-0.378877$

O $-6.816814-0.812279-1.344067$

H -7.033518 -0.1591220 .569626$

H $-4.6895650 .606858-1.249863$

O $-6.1948321 .875695-0.763900$

C $-4.456106 \quad 1.2599940 .806552$

C -3.8022962 .6441480 .636645$

H -3.679295 0.5100690 .992336

O $-5.274556 \quad 1.315807 \quad 1.979309$

H -4.5733313 .4254250 .644120$

H -3.2033982 .8195831 .539567$

H $-6.607648-5.6267431 .191838$

H $-6.485231-5.395685-0.558850$

H $-5.140464-6.1245110 .334065$

C -3.016030 -4.059999 -0.588380

H -7.286574 -0.033906-1.689345

H -6.7370442 .1051940 .010487$

H -4.6938561 .5207392 .732131$

H $-2.506545-3.624842-1.456984$

H $-3.063564-5.141137-0.763750$

H $-4.923125-3.695286-1.482436$

C $-1.0253331 .734094-1.686602$

O $-1.9277941 .767446-0.573202$

C $-2.9161512 .798852-0.606478$

C $-2.2558454 .188531-0.667888$

C $-1.2333074 .285283-1.805528$

C $-0.2917423 .077137-1.829101$

H $\quad-0.2840670 .972850-1.416995$

C $-1.7126121 .282834-2.985091$

H -3.544068 2.666411-1.492983

O -1.6054664 .4895250 .565295$

H -3.038848 $4.943329-0.804347$

H $-0.6373685 .198317-1.682753$

O $-1.9095354 .372355-3.055350$

H $0.3067013 .082186-2.747520$

O $0.6149713 .209128-0.732650$

C $-2.225402-0.137159-2.900760$

H $-2.5656181 .920100-3.231472$

H $-0.9942091 .319833-3.812112$

H $-0.9232513 .812601 \quad 0.712027$

H -2.472071 5.165016 -3.030658

H $1.2373932 .463228-0.772540$

O $-2.962799-0.409700-4.017498$

O $-2.005185-0.911347-1.981026$

C $-3.532315-1.721152-4.076711$

H $-3.986103-1.850153-5.062876$ 
H $-2.760064-2.485070-3.945062$

$\mathrm{H}-4.311368-1.821904-3.316153$

SCF Energy (B3LYP/6-31G**//MMFF)= -3245.91359912

$08 \_00041$

MM̄FF Geometry

C $-1.871697-2.736292-2.875201$

C $-2.794902-3.683631-2.646554$

C - $4.052485-3.549728-1.814601$

O $-4.140414-2.255496-1.177550$

C $-4.047187-4.622360-0.708065$

C $-5.311616-4.6420510 .130765$

C $-5.398936-4.220331 \quad 1.408541$

C $-6.523877-5.222713-0.551960$

C $-4.315298-3.6145262 .256622$

C $-4.698841-2.2016362 .716324$

C $-3.510970-1.4611283 .348949$

C $-3.946986-0.0873403 .861671$

C $-2.761653 \quad 0.751981 \quad 4.361187$

O $-2.510909-1.3160782 .336161$

O -3.2104132 .1091954 .499945$

C - -1.6572820 .7518943 .282302$

C $-2.293806 \quad 0.3058025 .751309$

O $-0.478770 \quad 1.394632 \quad 3.761042$

C -1.311359-0.657236 2.761348

C $-0.348627-0.6355391 .548822$

O $-0.661032-1.3958873 .802273$

C $-4.769232-1.259766-1.860971$

O $-5.240848-1.322518-2.985028$

C $-4.779150-0.059941-0.994017$

C $-5.2118911 .112981-1.479893$

C $-5.3091712 .373732-0.739127$

C -4.8632802 .3764340 .699139$

C -0.8931930 .0489190 .291659$

C -0.4962491 .5069610 .145097$

C $-0.9313923 .486860-1.309782$

$\begin{array}{lllll}\text { O } & 0.336473 & 2.056927 & 0.859675\end{array}$

C $-1.9862874 .356642-0.703816$

C $-2.7844075 .159369-1.424088$

C $-3.8040046 .082854-0.822839$

C $-5.1847355 .894663-1.408844$

C $-5.6429626 .709039-2.374565$

C $-6.068034 \quad 4.827137-0.805856$

C $-5.8010383 .459183-1.372597$

N -1.159438 $2.109790-0.916342$

H -1.996736 -1.741258 -2.458206

H -2.644859-4.663411-3.097595

H -4.918338 -3.706466 -2.469522

H -3.162942 -4.484046 -0.077536

H -3.923802 -5.618107 -1.156222

H $-6.360579-4.310747 \quad 1.912603$

H -7.386959 -5.290633 0.118979

H -6.819432 -4.603767-1.404501

H $-6.309101-6.234148-0.912370$

H -4.162396 -4.251803 3.135969

H -3.359698 -3.585485 1.729769

H -5.058315 -1.630880 1.850955

H -5.527020 -2.262395 3.433454

H -3.110978 -2.070121 4.168234

H -4.705356 -0.1894264 .647992$

H -4.4371300 .4628403 .047899$

H -3.958976 2.1154045 .121041

H $-2.028391 \quad 1.3589442 .447724$

H $-3.104840 \quad 0.415227 \quad 6.481378$

H -1.476751 0.9382556 .115678

H -1.965404 -0.7351555 .779710$

H -0.7346212 .2842704 .059430$

H $\quad 0.626598-0.230793 \quad 1.843763$

H -0.157094 -1.682208 1.272671

H $-0.142883-2.1113363 .397964$

H $-4.436338-0.1766540 .026961$

H -5.541023 $1.155189-2.517421$

H -5.5325811 .7561831 .304407$

H -4.8450483 .3710691 .149510$

H -3.8434831 .9876390 .790326$

H $-0.479166-0.465915-0.583087$

H -1.981555 -0.0393040 .233128$

H $-0.9472403 .517693-2.403933$
H $\quad 0.0567793 .810297-0.967647$

H $-2.074954 \quad 4.3487010 .380035$

H -2.673231 $5.185704-2.505872$

H $-3.4633407 .111899-0.998448$

H -3.8518545 .9722820 .267625$

H $-6.6446506 .600541-2.777150$

H $-5.0241467 .498483-2.788172$

H -7.122463 $5.058483-1.010844$

H -5.9906304 .8619620 .284545$

H $-6.0930463 .360050-2.419108$

H $-1.8651921 .584343-1.424350$

C $0.619473-2.523492-2.927983$

O $0.699068-1.083133-2.948288$

C $2.075657-0.691717-3.149286$

C $2.894971-1.965106-3.010989$

C $1.930403-3.006833-3.542657$

H $2.131985-0.316708-4.179189$

C $2.4114630 .429821-2.159446$

H $3.833378-1.924240-3.571844$

H $3.133736-2.165415-1.959818$

C $2.308046-4.428001-3.163032$

H $1.886464-2.923041-4.636874$

C $3.9108530 .768409-2.074321$

O $1.6812291 .589213-2.567278$

H $2.0478130 .165209-1.159201$

H $4.428103-0.128793-1.724538$

O $4.4186051 .054789-3.376820$

C $4.2411921 .938254-1.122499$

C $5.7529712 .164152-0.921662$

H $3.7677501 .766102-0.149025$

O $3.7137903 .163837-1.635312$

H $6.2330342 .365129-1.887579$

H $5.8683303 .084384-0.333149$

H $1.579596-5.144726-3.554667$

H $3.289780-4.687097-3.572757$

H $2.353819-4.554780-2.076561$

C - $-0.628285-2.970390-3.692184$

H $0.741093 \quad 1.342373-2.604153$

H $3.9658431 .855218-3.694090$

H $2.7468173 .072290-1.677202$

H $-0.547818-4.030437-3.957485$

H $-0.721859-2.397697-4.622785$

H $\quad 0.557688-2.827527-1.874414$

C $6.306887-0.2677661 .851219$

O $5.840210 \quad 0.837887 \quad 1.069043$

C $6.4636231 .003980-0.207150$

C $7.9682501 .249742-0.031949$

C 8.6100400 .1131530 .774110

C $7.829648-0.1892762 .058961$

H $5.830172-0.1385562 .831857$

C $5.827775-1.6109721 .277161$

H $6.3293240 .096855-0.802092$

O 8.1777612 .4919650 .634949

H $8.4329661 .322852-1.022406$

H 9.6402080 .3849321 .034190

O $8.661444-1.068996-0.019934$

H $8.182860-1.1224782 .513108$

O $8.075626 \quad 0.855278 \quad 3.005770$

C $4.317831-1.7438591 .320515$

H $6.134160-1.7475230 .237011$

H $6.246666-2.4262331 .878438$

H 9.1376092 .6256130 .712423

H $9.181864-0.865847-0.815711$

H $9.0315620 .871514 \quad 3.182659$

O $3.973078-3.0110630 .942810$

O $3.536934-0.8561721 .634253$

C $2.569360-3.2896680 .944471$

H $2.168988-3.2043881 .959214$

H $2.045776-2.6106510 .265198$

H $2.425175-4.3152950 .594946$

SCF Energy $\left(B 3 L Y P / 6-31 G^{* *} / / M M F F\right)=-3245.90688652$

08_00042

MM̄FF Geometry

C -1.2158573 .2472500 .185016$

C $-0.1391573 .763039-0.425468$

C $0.8244592 .929934-1.223962$

O $2.1446383 .095121-0.652776$ 
C $0.9265823 .380963-2.691840$

C $-0.366687 \quad 3.334727-3.474380$

C $-0.9293492 .206849-3.949187$

C -1.001498 $4.672969-3.748908$

C $-0.4265230 .798695-3.792725$

C $-1.485662-0.089035-3.125753$

C $-0.992387-1.533105-2.956060$

C $-2.143048-2.444356-2.527403$

C $-1.667670-3.877835-2.256225$

O $0.031096-1.535464-1.955226$

O $-2.721528-4.572881-1.573095$

C $-0.463964-3.824407-1.295378$

C $-1.408771-4.652079-3.553646$

O $0.102733-5.121149-1.119097$

C $0.632221-2.818158-1.722832$

C $1.660077-2.662134-0.574003$

O $1.289280-3.308391-2.890243$

C 2.5444762 .1937240 .282803

O $1.895605 \quad 1.2683350 .742175$

C 3.9433722 .5192580 .637824

C $4.7143391 .582492 \quad 1.209104$

C $6.1189151 .736646 \quad 1.590584$

C 6.7200943 .1155981 .514995

C $2.733087-1.588277-0.795929$

C $3.996773-2.087612-1.474095$

C $6.258096-1.281051-2.156293$

O $4.166307-3.254535-1.814771$

C $7.193642-1.444640-0.999141$

C $8.227367-0.624480-0.757536$

C $9.168183-0.7832230 .403271$

$\begin{array}{llll}\text { C } & 9.227506 & 0.453658 & 1.272972\end{array}$

C $10.175764 \quad 1.3852541 .074798$

C 8.2480090 .5749392 .419858

C $6.808416 \quad 0.645392 \quad 1.983817$

N $4.919804-1.063178-1.640203$

H $-1.398336 \quad 2.176996 \quad 0.122441$

H $0.0562664 .830499-0.361237$

H $0.5437181 .870042-1.195962$

H $1.6837882 .768157-3.200239$

H $1.3488454 .395249-2.734472$

H -1.849733 2.296639 -4.524866

H $-1.9528464 .581369-4.283706$

H -1.200809 5.204968 -2.813864

H $-0.334025 \quad 5.288413-4.360883$

H $-0.2012520 .405852-4.791783$

H $0.5079840 .757963-3.226158$

H $-1.7398930 .331388-2.143473$

H -2.398384 - $0.080387-3.734975$

H $-0.570153-1.874138-3.908576$

H -2.945104 -2.441210 -3.275804

H $-2.590897-2.049490-1.606643$

H $-3.515181-4.540617-2.134192$

H $-0.847460-3.518628-0.312905$

H -2.323836 $-4.706428-4.155687$

H -1.126453 -5.689805 -3.344459

H $-0.632251-4.201690-4.175266$

H $-0.613192-5.712625-0.830034$

H $1.110913-2.3657540 .328087$

H $2.137549-3.625473-0.359822$

H $2.137662-2.848523-2.986578$

H 4.3080773 .5094790 .389380

H 4.2854870 .5992451 .397384

H 6.7418953 .4693380 .478302

H 7.7460983 .1608601 .887293

H 6.1383083 .8229522 .116620

H $2.337212-0.735685-1.357334$

H $3.044419-1.2217680 .190383$

H $6.512769-0.416133-2.776591$

H $6.276329-2.179101-2.781503$

H $7.011157-2.282738-0.330438$

H $8.4178430 .203476-1.436726$

H $10.165018-1.002405-0.001555$

H $8.904597-1.651407 \quad 1.020042$

H 10.2492002 .2625851 .708587

H $\quad 10.898467 \quad \begin{array}{llll}H & 1.292289 & 0.270881\end{array}$

H $8.486423 \quad 1.433707 \quad 3.059284$

H $8.366543-0.2983583 .074768$

H $6.298539-0.3184641 .997820$
H $4.705134-0.142614-1.268279$

C -3.647084 $3.914713 \quad 0.472300$

$\begin{array}{llll}\text { O } & -4.082317 & 2.546853 & 0.593019\end{array}$

C $-4.9278392 .234431-0.533294$

C $-5.2083163 .559739-1.235619$

C $-3.9147444 .310448-0.978292$

H $-4.3343411 .606445-1.207866$

C $-6.1713371 .473163-0.062969$

H $-5.424596 \quad 3.426762-2.299956$

H $-6.0538954 .090327-0.782709$

C $-4.0176925 .807397-1.204902$

H $-3.1448663 .898813-1.641735$

C $-5.8450920 .189050 \quad 0.729651$

$\begin{array}{lllll}\text { O } & -6.929204 & 2.336457 & 0.794351\end{array}$

H $-6.8146251 .244932-0.920725$

H -5.3376600 .4618841 .660597$

O $-7.086648-0.4113691 .121117$

C $-5.014792-0.845645-0.053191$

C $-4.747482-2.147151 \quad 0.727391$

H $-4.061121-0.405253-0.359882$

O $-5.726778-1.178164-1.248410$

H $-5.689873-2.5589211 .108304$

H $-4.365012-2.8904740 .016424$

H $-4.280877 \quad 6.021128-2.245987$

H $-4.782836 \quad 6.259250-0.564881$

H $-3.0640316 .299893-0.990129$

C -2.2068524 .0588160 .968655$

H $-7.706767 \quad 1.835607 \quad 1.095175$

H $-7.538990-0.7020960 .310556$

H -5.162676 -1.774107 -1.770139

H -2.159866 3.7120812 .007722

H $-1.925765 \quad 5.1183640 .970666$

H -4.2940624 .4977191 .141632$

C $-1.439048-1.3014752 .217556$

O $-2.484388-1.6027181 .284201$

C $-3.737260-1.9705621 .871613$

C $-3.567472-3.2554622 .694066$

C $-2.484573-3.077729 \quad 3.763927$

C $-1.197685-2.4750153 .185898$

H $-0.545761-1.1915951 .590361$

C -1.7016000 .0515852 .894353$

H $-4.079490-1.1645182 .529029$

O $-3.215349-4.3330101 .828978$

H -4.526699-3.506056 3.162466

H -2.254159 -4.050127 4.215946

O $-2.969852-2.228028 \quad 4.799163$

H $-0.530401-2.1558203 .994098$

O $-0.506741-3.4923192 .453763$

C $-0.4284880 .729914 \quad 3.352984$

H $-2.1893470 .715878 \quad 2.170167$

H $-2.370070-0.0430033 .753790$

H $-3.133420-5.1334992 .374577$

H $-3.765586-2.6440315 .172405$

H $-0.289430-4.206553 \quad 3.076826$

O $-\begin{array}{llll}0.707118 & 2.028672 & 3.669934\end{array}$

\begin{tabular}{lllll}
\hline & 0.667621 & 0.193617 & 3.437543
\end{tabular}

C 0.4112672 .8118314 .098129

H 0.8343082 .3965045 .017691

H 1.1679802 .8559333 .309006

H 0.0576973 .8262784 .300542

SCF Energy (B3LYP/6-31G**//MMFF)= -3245.91105817

08_00043

MM̄FF Geometry

C $-0.921519-4.3940951 .787678$

C $0.260950-3.8250691 .507672$

C $1.163763-3.2327372 .560494$

O $1.557916-1.9036852 .144285$

C $2.431953-4.0934702 .696261$

C $3.376912-3.6089643 .779308$

C $4.541059-2.9665883 .556243$

C $2.953552-3.9302605 .190014$

C $5.130325-2.5579422 .234658$

C $5.370146-1.0434752 .180324$

C $5.815415-0.5844320 .783789$

$\begin{array}{llll}\text { C } & 6.123967 & 0.913519 & 0.784351\end{array}$

C $6.4491601 .441246-0.621342$

O $4.748984-0.867133-0.128036$ 
O $6.3898872 .875779-0.575128$

C $5.3475200 .974533-1.598569$

C $7.8783171 .081822-1.044097$

O $5.6787611 .325614-2.939863$

C $5.026004-0.531397-1.493961$

C $3.795262-0.952373-2.335431$

O $6.144676-1.277999-1.989448$

C $0.668080-0.8946382 .355919$

O $-0.463042-0.9936542 .804374$

C 1.3259250 .3731051 .966115

C 0.6739731 .5362072 .112770

C 1.2085342 .8707481 .828796

C 2.6064792 .9629731 .277639

C $2.467231-0.300082-1.937710$

C $2.1360370 .965130-2.710507$

C $0.5120272 .859360-2.712522$

O $2.7339871 .311586-3.725113$

C $0.9649824 .023755-1.891340$

C $0.117864 \quad 4.879422-1.300088$

C $0.5498276 .086092-0.520181$

C $-0.005202 \quad 6.103546 \quad 0.885510$

C -1.1193876 .7939061 .181812$

C $0.787293 \quad 5.4019151 .963836$

C $0.436128 \quad 3.9457732 .091211$

N $1.0595391 .639550-2.151174$

H $-1.235567-4.4346732 .829518$

H $0.617601-3.7717090 .483294$

H $0.653845-3.1699793 .530088$

H $2.944941-4.145448 \quad 1.730143$

H $2.152577-5.1309662 .926287$

H $5.148090-2.6942904 .419016$

H $3.699841-3.6204375 .929434$

H $2.016898-3.4213805 .436984$

H $2.805708-5.0090475 .305714$

H $6.085076-3.0811382 .103325$

H $4.494913-2.8570961 .398462$

H $4.441710-0.5266382 .453296$

H $6.129007-0.7656042 .922459$

H $6.702598-1.1594530 .492981$

H 6.9416581 .1413521 .479564

H $5.251898 \quad 1.4617361 .163402$

H $7.028223 \quad 3.1812990 .092057$

H $4.4486291 .549338-1.346207$

H $8.6032481 .496730-0.333255$

H $8.1273511 .525107-2.014458$

H $8.0513090 .005193-1.098611$

H $6.3462260 .693315-3.255871$

H $4.005854-0.822655-3.403877$

H $3.664169-2.035279-2.195900$

H $5.838358-2.166583-2.235213$

H $2.342096 \quad 0.317751 \quad 1.594806$

H -0.3433321 .5152562 .501928$

H 2.8868853 .9716190 .966690

H $2.7174442 .332778 \quad 0.389064$

H 3.3321922 .6396742 .031338

H $1.657225-1.007388-2.157559$

H $2.436724-0.094167-0.864003$

H $-0.5772482 .755664-2.713882$

H $0.8487382 .980985-3.746816$

H $2.0367204 .184880-1.803940$

H - $0.9524114 .728306-1.416109$

H $0.206166 \quad 6.974874-1.065881$

H $1.6429906 .170636-0.484742$

H -1.505185 6.8357992 .195040

H -1.6744907 .3254960 .415980$

H $\quad 0.579184 \quad 5.8685262 .936629$

H 1.8563825 .5719171 .807166

H $-0.563688 \quad 3.7790532 .493679$

H $0.6444891 .287293-1.293562$

C $-1.598290-4.849502-0.674829$

O $-1.686688-3.456591-1.038443$

C -2.534499 -3.328716 -2.192001

C $-2.596673-4.718125-2.809207$

C $-2.607185-5.583145-1.563897$

H -3.532803 -3.067868 -1.821865

C $-2.030614-2.218979-3.118578$

H -3.481529 -4.856972 -3.437543

H $-1.708859-4.936227-3.413718$
C $-2.252156-7.036019-1.828795$

H $-3.608460-5.538226-1.114304$

C $-1.800613-0.863107-2.419112$

O $-0.781103-2.633251-3.684533$

H -2.728179 $-2.104296-3.957160$

H $-0.891754-0.906598-1.806516$

O $-1.5600860 .116659-3.436521$

C $-2.970494-0.374862-1.544701$

C $-2.7297371 .046486-0.998782$

H $-3.104049-1.066107-0.705508$

O $-4.165524-0.397474-2.323352$

H $-1.6997651 .124915-0.631521$

H -2.862573 $1.762881-1.818601$

H $-2.976912-7.490308-2.512283$

H $-1.259133-7.130749-2.280895$

H $-2.255391-7.613968-0.899218$

C $-1.900898-5.0049130 .819089$

H $-0.563697-2.015118-4.402197$

H $-0.695675-0.079030-3.835381$

H $-4.899532-0.131309-1.744243$

H $-1.985282-6.0700011 .065180$

H -2.875582 -4.5460061 .029660$

H $-0.578896-5.182536-0.905297$

C -6.0498001 .6755050 .620953$

O $-5.024381 \quad 1.437744-0.353465$

C $-3.687381 \quad 1.4135290 .152105$

C $-3.3378262 .773904 \quad 0.774280$

C -4.3592073 .1845871 .837697$

C $-5.806163 \quad 3.0006671 .364938$

H -6.9715181 .7842710 .034821$

C -6.2332040 .4790961 .561891$

H -3.6012970 .6330520 .916020$

O $-3.2593093 .788654-0.223087$

H $-2.3453192 .703272 \quad 1.230451$

H $-4.190877 \quad 4.2339012 .109751$

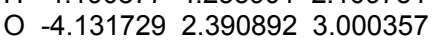

H -6.4898653 .0674252 .218807$

O -6.1488624 .0880800 .500982$

C $-6.674843-0.7420690 .789929$

H $-5.3250890 .237252 \quad 2.121371$

H -7.0082570 .6855232 .309404$

H $-3.9680863 .638134-0.869477$

H -4.7527632 .6904863 .686012$

H $-5.8373653 .878381-0.394580$

O $-5.671706-1.6647450 .758758$

\begin{tabular}{lllll}
\hline & -7.774259 & -0.853296 & 0.262608
\end{tabular}

C $-5.965963-2.8696410 .045636$

H $-6.856339-3.3523230 .459788$

H $-6.102508-2.652053-1.017777$

H -5.116802 -3.548081 0.159981

SCF Energy (B3LYP/6-31G**//MMFF) $=-3245.90710418$

08_00044

MM̄FF Geometry

C $-0.617525-3.337037-0.184643$

C $0.678797-3.4646010 .132775$

C $1.223201-3.2761911 .525426$

O $1.829030-1.9639391 .572006$

C $2.298587-4.3379511 .817569$

C $2.990561-4.154703 \quad 3.154719$

C $4.251138-3.7006783 .310725$

C $2.171696-4.539547 \quad 4.359377$

C $5.207948-3.2677382 .234112$

C $5.430255-1.7500482 .265203$

C $5.961790-1.2222600 .924116$

C $6.3060350 .263097 \quad 1.030028$

C $6.7113270 .861479-0.324756$

O $4.930180-1.415580-0.050655$

$\begin{array}{lllll}\text { O } & 6.695637 & 2.291957 & -0.193212\end{array}$

C $5.6457850 .492993-1.380955$

C $8.1454750 .477584-0.708766$

O $6.085696 \quad 0.910943-2.671188$

C $5.274650-1.006570-1.381976$

C $4.061978-1.343890-2.287686$

O $6.388290-1.761175-1.875515$

C $1.021990-0.9316191 .947565$

O $-0.139374-1.0026752 .318393$

$\begin{array}{llll}\text { C } & 1.785380 & 0.325506 & 1.789755\end{array}$ 
C 1.1480611 .4990601 .911719

C 1.7367382 .8284961 .742100

C 3.1830222 .9093441 .333748

C $2.745797-0.648476-1.921413$

C $2.4916740 .650405-2.667063$

C $0.8978942 .566917-2.733307$

O $3.1704101 .023924-3.619583$

C $1.4355443 .725356-1.954610$

C $0.6571134 .668590-1.402054$

C $1.1875435 .880764-0.690587$

C $0.608796 \quad 6.0519150 .695740$

C $-0.446364 \quad 6.855758 \quad 0.910854$

C $1.3108005 .361407 \quad 1.842747$

C 0.9506033 .9062131 .940872

N $1.387145 \quad 1.325947-2.163541$

H -1.330666 -3.079443 0.593951

H $1.396300-3.669143-0.658981$

H $0.426117-3.3580612 .274961$

H $3.030454-4.3445051 .004180$

H $1.843038-5.337378 \quad 1.794277$

H $4.646327-3.6146264 .322157$

H $2.737032-4.4484515 .292951$

H $1.289194-3.8981114 .445756$

H $1.840341-5.5798194 .276656$

H $6.166892-3.7761352 .394075$

H $4.873552-3.5847881 .244653$

H $4.483033-1.2472122 .494714$

H $6.131235-1.5058603 .072921$

H $6.845678-1.8053980 .640271$

H $7.0953540 .426901 \quad 1.774333$

H 5.4306830 .8119161 .400841

H 7.3129512 .5344800 .518028

H $4.7557761 .087422-1.142980$

H $\quad 8.853044 \quad 0.833754 \quad 0.049680$

H $8.4463390 .958118-1.646267$

H $8.288145-0.600243-0.810734$

H $5.3397940 .815862-3.286490$

H $4.319661-1.191774-3.342462$

H $3.880070-2.424146-2.190137$

H $6.063414-2.620226-2.192523$

H $2.838466 \quad 0.2536541 .547847$

H $\quad 0.0848611 .4896712 .149932$

H 3.3606642 .3237030 .425732

H 3.8261242 .5259902 .132800

H 3.5178543 .9238481 .108108

H $1.916664-1.316290-2.188825$

H $2.686426-0.468376-0.845497$

H $-0.1943782 .527760-2.711058$

H $1.2186252 .644481-3.777091$

H $2.5166483 .814038-1.878529$

H $-0.4229014 .592728-1.506482$

H $0.9423796 .758217-1.303607$

H $2.2827495 .862962-0.630461$

H -0.8494307 .0050351 .906977$

H -0.9355657 .3756600 .093780$

H 1.0259465 .8305772 .794178

H 2.3882865 .5313221 .764567

H -0.0858903 .7399952 .233649$

H $0.9081490 .958654-1.346156$

C $-2.589913-3.894149-1.701982$

O $-3.444858-2.891758-1.114296$

C $-4.477510-3.558050-0.364732$

C $-4.443608-5.016870-0.808840$

C -2.958471 -5.220702 -1.039495

H -4.183699 -3.5083380 .690293$

C $-5.824960-2.860381-0.567933$

H -4.858778 -5.690954 -0.053514

H $-4.997826-5.169776-1.742294$

C -2.629370 -6.441412 -1.880205

H $-2.468969-5.320046-0.062691$

C $-5.848436-1.366621-0.178769$

O $-6.198359-2.951269-1.949271$

H -6.602526 -3.398675 -0.013204

H -5.273576 -0.780183 -0.904910

O $-7.206559-0.919162-0.283953$

C $-5.347423-1.0629521 .245440$

C $-5.661670 \quad 0.353391 \quad 1.762880$

H -4.265591 -1.229064 1.291999
O $-5.940915-1.9850062 .166421$

H -6.7472140 .4749331 .871349$

H -5.2574830 .4138392 .782636$

H -2.977622 -7.354108 -1.385722

H $-3.104821-6.391118-2.865538$

H $-1.548819-6.529611-2.031134$

C -1.125192 -3.464211-1.593262

H -5.487240 -2.542002 -2.472095

H -7.504313 -1.108653-1.190257

H $-6.905990-1.8786672 .107532$

H -1.021217 -2.478774 -2.060639

H $-0.500269-4.165703-2.158676$

H -2.855367 -3.923565 -2.767211

C -2.9583432 .3557630 .129744$

O

C -5.0918331 .4995060 .912290$

C -5.5163522 .8719031 .464684$

C -4.8300264 .0137830 .710751$

C -3.3212063 .7802890 .585614$

H -1.8990212 .1909250 .365564$

C $-3.1471952 .144147-1.379944$

H -5.472286 $1.396141-0.108203$

O -5.1849722 .9530052 .850200$

H -6.6058812 .9639741 .379826$

H -5.0100364 .9559331 .242859$

O $-5.4180164 .124817-0.581424$

H -2.882591 $4.507897-0.106312$

O $-2.7148964 .017794 \quad 1.858527$

C $-2.4962700 .869437-1.872590$

H -4.202565 2.094276-1.659084

H -2.678486 2.975189-1.920104

H -5.504933 3.8104583 .178477

H -4.986932 $4.867880-1.037041$

H -3.020759 3.3247012 .468622

O $-2.633580 \quad 0.810190-3.231184$

O $-1.9419360 .037306-1.169013$

C $-2.056215-0.333544-3.866791$

H -2.618414 -1.231496 -3.595947

H $-2.123565-0.190868-4.948445$

H -1.001467 -0.435619-3.594097

SCF Energy $(B 3 L Y P / 6-31 G * * / / M M F F)=-3245.92223498$

08_00045

MMFF Geometry

C $-2.5864223 .931378-0.994151$

C $-3.2179532 .809224-0.619332$

C -3.7949402 .5998090 .756230$

O -3.1947291 .4046181 .306465$

C -5.3062452 .3397670 .667120$

C -6.0269542 .5982841 .974281$

C -6.3283981 .6585422 .891175$

C -6.4554514 .0274632 .188976$

C -5.9529100 .2028922 .891876$

C -7.145984 -0.723607 2.623084

C $-7.497620-0.8305111 .131290$

C $-8.742285-1.6971970 .931410$

C $-9.050782-1.920016-0.556701$

O $-6.376672-1.4249540 .466547$

O $-10.017130-2.977365-0.653768$

C $-7.769777-2.412076-1.261778$

C $-9.692402-0.684733-1.199431$

O $-7.965348-2.498909-2.671733$

C $-6.521681-1.552469-0.952475$

C $-5.255314-2.254275-1.499708$

O $-6.663515-0.280674-1.587999$

C -1.9478951 .4934431 .835663$

O -1.2720102 .4979131 .980268$

C -1.5300840 .1134172 .161751$

C $-0.225851-0.1750472 .272055$

C $0.340285-1.5027502 .518179$

C $-0.622775-2.6410442 .738985$

C $-3.926167-1.631803-1.042001$

C $-2.734763-2.333996-1.675186$

C $-0.253766-2.195510-1.851408$

O $-2.847648-3.311462-2.411233$

C $0.386543-3.137167-0.879814$

C $1.679798-3.063145-0.528372$

C $2.357296-4.0345910 .396504$ 
C $3.188049-3.3541751 .463071$

C $4.519631-3.2351141 .328001$

C $2.488825-2.8936952 .722354$

C $1.682317-1.6423962 .510550$

$\mathrm{N}-1.529058-1.738733-1.328162$ H $-2.5097974 .756479-0.291119$ H $-3.2951511 .980615-1.319407$ H -3.5851133 .4529371 .413491$ H -5.4855641 .3097160 .341416$ H $-5.7628892 .978301-0.101146$ H $-6.882731 \quad 1.9696953 .776243$ H -6.9713894 .1694163 .144550$ H -5.5859044 .6922922 .182739$ H $-7.139618 \quad 4.342012 \quad 1.394130$ H -5.135538 -0.0130582 .199273$ H $-5.557947-0.0239173 .890629$ H $-6.875668-1.7218502 .991927$ H -8.019709 -0.3913343 .197009$ H -7.6686610 .1746030 .730799$ H -9.608539 -1.257965 1.441828 H $-8.583659-2.6779391 .401754$ H -10.805093 -2.710176 -0.150301 H -7.578594 -3.436451-0.912313 H -10.632495 -0.434755 -0.693202 H -9.957485 -0.876493 -2.245145 H $-9.0532950 .199906-1.161731$ H -8.748348 -3.055856 -2.821661 H $-5.254392-3.302277-1.171480$ H $-5.285455-2.257993-2.596988$ H $-5.7850770 .077375-1.789158$ H $-2.307826-0.6368362 .249660$ H $\quad 0.496897 \quad 0.6292612 .142196$ H -1.225985 -2.462647 3.636130 H $-0.135543-3.6089522 .870707$ H -1.295330 -2.751838 1.880980 H -3.878712 $-0.573634-1.317602$ H -3.825679 -1.7099000 .046585$ H $\quad 0.359330-1.308208-2.034234$ H -0.415984 -2.708253 -2.805016 H $-0.229173-3.936859-0.475327$ H $2.299791-2.284274-0.964630$ H $3.000935-4.679364-0.216358$ H $1.632127-4.7055830 .873273$ H $5.128177-2.7733502 .098360$ H $5.030573-3.5893830 .438811$ H $3.226378-2.6719963 .505659$ H $1.897991-3.7201613 .126572$ H $2.295217-0.7581632 .331346$ H -1.525283 -0.963561 -0.671545 C $-0.4189124 .110908-2.310378$ O $0.0595912 .846958-1.806123$ C $1.1285753 .100553-0.868130$ C $1.5698224 .532419-1.131594$ C $0.2473655 .195551-1.464054$ H $0.677803 \quad 3.0204090 .128651$

C $2.2187592 .037244-1.038781$

H $2.0683544 .986760-0.270272$

H $2.2506904 .579052-1.990717$

C $0.3928786 .528616-2.176518$ H $-0.2973515 .354569-0.526039$

C $3.4641942 .296648-0.166481$

O $1.6196650 .786078-0.692784$

H $2.5046581 .971629-2.095422$

H $3.8683163 .284350-0.415295$

O 3.0779122 .3458831 .207607

C $4.6005301 .268827-0.350302$

C 5.8163921 .6113060 .533220

H $4.8940971 .258635-1.406516$

O $4.124739-0.031594-0.017323$

H 6.0366732 .6820180 .446139

H 5.5583161 .3959021 .577760

H $0.9316377 .244370-1.546869$ H $0.9469886 .424361-3.115431$ H $-0.5885006 .954068-2.408976$ C -1.951128 4.118185-2.344195 H $2.2769710 .089742-0.858498$ H $2.729698 \quad 1.4684331 .441238$ H $4.842827-0.662731-0.193375$
H $-2.2863813 .317249-3.015635$

H -2.310655 $5.060482-2.772929$

H $-0.0580924 .176000-3.345354$

C $7.916905-1.4616160 .086558$

O $6.846871-0.5682600 .424857$

C 7.0827530 .8164450 .156272

C 8.3106731 .3097470 .939070

C 9.5393920 .4369310 .664918

C $9.218271-1.0550040 .798065$

H $7.606017-2.4337090 .490699$

C $8.077524-1.616146-1.429850$

H $7.2573350 .952357-0.916653$

O $8.038612 \quad 1.3152672 .339579$

H 8.5208102 .3464490 .651413

H $10.337018 \quad 0.698433 \quad 1.370723$

O $10.0227010 .687577-0.651436$

H $10.047434-1.6630950 .417818$

O $9.063887-1.3763892 .183626$

C $6.896449-2.348060-2.020237$

H $8.194147-0.666005-1.958614$

H $8.969371-2.209553-1.665321$

H $7.772570 \quad 0.4150612 .594521$

H $10.252899 \quad 1.630798-0.705325$

H $9.904236-1.1699822 .627435$

O $5.929897-1.458247-2.383629$

O $6.836049-3.566079-2.127226$

C $4.744750-2.027399-2.948400$

H $3.982793-1.244959-2.998542$

H $4.953487-2.384195-3.960866$

H $4.367365-2.842564-2.324137$

SCF Energy $($ B3LYP/6-31G**//MMFF $)=-3245.92566263$

08_00046

MMFF Geometry

C $-1.2225693 .708255-1.025331$

C $-2.0372242 .730759-0.601472$

C -2.8604042 .8123890 .654125$

O $-2.3500341 .781106 \quad 1.531334$

$\begin{array}{llll}\text { C } & -4.335270 & 2.501449 & 0.363758\end{array}$

C -5.2364362 .8363631 .535024$

C -5.7152501 .9393712 .418817$

C -5.6277214 .2877951 .646821$

C -5.4363630 .4634682 .485484$

C $-6.676529-0.3803872 .158307$

C $-7.060060-0.3462710 .670629$

C $-8.355276-1.1239150 .428588$

C $-8.706384-1.196626-1.065181$

O $-5.989817-0.943006-0.068069$

O $-9.731830-2.187861-1.229954$

C $-7.469811-1.691385-1.843854$

C $-9.2917290 .123235-1.580973$

O $-7.698622-1.638745-3.250494$

C $-6.168649-0.933476-1.489936$

C $-4.955902-1.656077-2.123851$

O $-6.2467430 .396660-2.004684$

C -1.5527192 .1478912 .564620$

O -1.3112533 .2888872 .934120$

C -0.9412700 .9694593 .223893$

C $-0.998227-0.2744852 .721962$

C $-0.337120-1.4620013 .270615$

C $0.460323-1.2981644 .537849$

C $-3.589816-1.118991-1.672497$

C $-2.445611-1.947236-2.232572$

C $0.010547-2.282810-1.987092$

O $-2.601897-2.820822-3.081773$

C $0.174158-3.420527-1.028322$

C $1.215460-3.550193-0.192880$

C $1.374051-4.6904380 .771067$

C $1.453446-4.2327692 .211314$

C $2.636938-4.1507572 .842307$

C $0.159050-3.9608302 .947642$

C $-0.443720-2.6276662 .598036$

N -1.224510 -1.584669-1.678947

H -1.169298 $4.640486-0.469431$

H $-2.0656191 .791653-1.148931$

H -2.7651203 .8033521 .113479$

H -4.4454901 .4467810 .089913$

H $-4.6804883 .071417-0.509170$ 
H -6.3743922 .3009573 .207358$ H -6.3076244 .4724402 .485332$ H $-4.7438274 .915101 \quad 1.796791$ H -6.1357794 .6147840 .733596$ H $-4.5956480 .168214 \quad 1.851290$ H -5.1240280 .2369853 .512936$ H -6.455013 -1.416635 2.445547 H -7.523196 -0.0478752 .771852$ H -7.182984 0.6953580 .355062 H -9.184935 -0.6851930 .996953$ H $-8.241715-2.1493190 .807628$ H - $10.493355-1.924507-0.685275$ H -7.329935 -2.752330-1.592715 H -10.203786 $0.379093-1.028505$ H $-9.5923460 .038120-2.631080$ H $-8.602526 \quad 0.964811-1.486133$ H -8.514595 -2.136615 -3.430116 H $-4.997275-2.720751-1.857767$ H -5.014586 -1.591365 -3.217869 H $-5.3497880 .737835-2.144623$ H $-0.381465 \quad 1.1964904 .125532$ H -1.549969 -0.4454611 .799014$ H $-0.185598-0.9638845 .357094$ H $1.260321-0.5628734 .397532$ H $0.942812-2.2191324 .871419$ H $-3.449611-0.088667-2.013479$ H -3.518061-1.135309 -0.579070 H $0.825168-1.556737-1.913668$ H -0.027971 -2.663306 -3.012602 H $-0.605926-4.178514-1.024277$ H $2.005613-2.804156-0.207777$ H $2.288661-5.2323870 .496908$ H $0.560511-5.4191450 .667304$ H $2.703828-3.8539403 .883564$ H $3.567185-4.3714122 .329088$ H $0.302845-4.0537614 .029883$ H $-0.566984-4.7474922 .704778$ H -1.036929 -2.645320 1.683646 H -1.206063 -0.901890 -0.926663 C $1.1258093 .192934-1.831774$

O $1.1269351 .943974-1.108041$

C 1.8841782 .1206790 .107527

C $2.7951973 .305047-0.165188$

C $1.8702464 .207852-0.959544$

H 1.1528462 .3673910 .887639

C 2.5980960 .8107290 .444299

H $3.165933 \quad 3.7754190 .750193$

H $3.6560673 .002271-0.774349$

C $2.5965085 .278682-1.755108$

H $1.1865104 .699302-0.257194$

C 3.5387750 .9060561 .657218

O $1.600600-0.175807 \quad 0.720638$

H $3.1430980 .459481-0.437229$

H 4.3394711 .6272841 .459041

O 2.7933891 .4334912 .762527

C $4.142934-0.4418392 .092845$

C $4.875593-1.2164980 .987358$

H $3.347522-1.0759642 .502403$

O $5.034009-0.1938193 .182379$

H $5.327326-2.1199501 .418096$

H $4.135067-1.5917370 .269344$

H $3.1513225 .944971-1.086329$

H $3.3105984 .840496-2.460312$

H $1.8867255 .885642-2.326225$

C $-0.3130173 .562901-2.214787$

H $0.974419-0.174714-0.023048$

H 3.3963991 .4755613 .524471

H $5.386280-1.051783 \quad 3.474883$

H $-0.7098292 .781920-2.876431$

H $-0.3244924 .496844-2.788037$

H $1.6894053 .006215-2.754814$

C $7.278190-0.764954-1.813921$

O $6.346869-1.315346-0.873676$

C $5.956434-0.4463490 .200534$

C $7.189674-0.0438191 .022960$

C 8.2905040 .5525380 .138128

C $8.560798-0.304485-1.103740$

H $7.540568-1.605913-2.468986$
C $6.6387600 .322384-2.685605$

H $5.4989180 .446968-0.232765$

O $7.706902-1.1728011 .723923$

H $6.9008130 .699783 \quad 1.772615$

H 9.2096940 .6440620 .729653

O $7.8952101 .864352-0.253315$

H $9.1980850 .232968-1.815545$

O $9.273507-1.480113-0.707339$

C $5.493827-0.244611-3.491931$

H $6.2712591 .171918-2.102825$

H $7.3664760 .730412-3.396814$

H $7.911819-1.8611351 .068208$

H $8.6172132 .242281-0.783827$

H $10.110524-1.193330-0.303953$

O $4.307254 \quad 0.227095-3.013450$

O $5.637233-1.027917-4.421865$

C $3.137338-0.255446-3.680584$

H $3.049050-1.338056-3.549376$

H $2.265907 \quad 0.225309-3.229017$

H $3.1693250 .004873-4.742782$

SCF Energy (B3LYP/6-31G**//MMFF) $=-3245.91415792$

0800047

MMFF Geometry

C $-4.5422002 .891860-1.049421$

C $-3.2407143 .026479-0.752488$

C $-2.1712053 .196695-1.798004$

O $-1.1483822 .200062-1.574336$

C $-1.5062554 .580268-1.678660$

C $-0.4995884 .850261-2.783414$

C $0.8340254 .953725-2.614052$

C $-1.088538 \quad 5.036546-4.160082$

C $1.6303794 .858505-1.342689$

C $2.4185503 .548368-1.271538$

C 3.2464873 .4117580 .015706

C $4.453298 \quad 4.355577 \quad 0.027738$

C $5.347274 \quad 4.142228 \quad 1.260294$

O 3.6925002 .0480140 .090999

O $\quad 6.596316 \quad 4.809063 \quad 1.017303$

C 5.6578622 .6361841 .395852

C 4.7491294 .7928662 .513374

$\begin{array}{lllll}\text { O } & 6.375988 & 2.368605 & 2.597828\end{array}$

C 4.4059621 .7381961 .297262

C $4.7530610 .233331 \quad 1.288546$

O 3.5852471 .9593642 .452442

C $-1.3219750 .980733-2.151139$

O $-2.297642 \quad 0.586822-2.769036$

C $-0.0718870 .219081-1.939236$

C $0.136456-0.921460-2.612064$

C $1.356491-1.728102-2.573284$

C $2.520442-1.206492-1.770482$

C $5.658663-0.2412050 .150436$

C $5.847337-1.7500680 .188931$

C $6.985630-3.604510-1.017706$

O $5.343640-2.4677621 .049834$

C $5.990441-4.238310-1.937115$

C $4.980555-5.014931-1.515267$

C $3.990061-5.678745-2.428818$

C $2.658378-4.956798-2.447090$

C $1.655467-5.366071-1.651138$

C $2.571435-3.806744-3.430997$

C $1.393586-2.884941-3.267185$

N $6.654838-2.202230-0.845763$

H $-4.8502272 .935486-2.090638$

H -2.9199493 .0009490 .285689$

H $-2.591714 \quad 3.072727-2.803486$

H $-1.0348754 .664824-0.694272$

H -2.271115 5.367415-1.713463

H $1.4443145 .146709-3.496201$

H $-0.342603 \quad 5.368809-4.890207$

H $-1.5139754 .099348-4.530891$

H -1.878374 $5.794572-4.135995$

H $2.3145145 .714241-1.321408$

H $0.9998474 .957101-0.455111$

H $1.7206752 .705389-1.329447$

H $3.0740663 .443013-2.146193$

H 2.5878493 .6120770 .868901

H $4.1399415 .404381-0.027333$ 
H $5.0591954 .177600-0.871836$ H 6.4080325 .7491970 .854659 H 6.3406282 .3880190 .574004 H 4.6193375 .8709252 .359453 H 5.4241674 .6947453 .370716 H 3.7757234 .3817282 .788098 H 5.7418302 .3978263 .334409 H $5.193632-0.0485642 .254307$ H $3.814564-0.3332571 .225115$ H 2.9862981 .2007022 .553839 H $0.6644660 .640694-1.265762$ H - $0.647747-1.288812-3.272532$ H $2.893369-0.270101-2.199688$ H $3.361319-1.902271-1.726467$ H $2.227006-1.021872-0.732339$ H $5.2275420 .030026-0.819116$ H $6.649808 \quad 0.2165920 .233258$ H $7.007002-4.097566-0.039779$ H $7.988648-3.663585-1.451247$ H $6.117502-4.057334-3.001590$ H $4.853095-5.189101-0.449093$ H $3.861562-6.716984-2.096414$ H $4.381948-5.750499-3.451561$ H $0.682858-4.887456-1.647468$ H $1.785836-6.204468-0.974083$ H $2.532245-4.225438-4.445385$ H $3.498829-3.224100-3.402627$ H $\quad 0.503329-3.201811-3.810849$ H $6.970015-1.546360-1.554671$ C $-6.3616041 .361981-0.130955$ O C $-5.816057-0.797766-0.842979$ C $-7.187247-0.425844-1.400748$ C $-7.0905971 .086304-1.445702$ H -5.088807 -0.771454 -1.663179 C $-5.768095-2.162137-0.147403$ H -7.371496 -0.875267 -2.381230 H -7.997483 -0.734780 -0.729965 C $-8.4348331 .782202-1.565247$ H $-6.4699151 .360099-2.306901$ C $-4.410522-2.5194790 .492787$ O $-6.753658-2.1776160 .893989$ H -6.065526 $-2.939947-0.861208$ H -4.262251 -1.910418 1.390487 O $-4.500205-3.8753960 .951768$ C $-3.193319-2.382635-0.437669$ C -1.890773 -2.998009 0.106372 H $-3.029495-1.322688-0.660321$ O $-3.487766-3.031317-1.678632$ H -1.981556 -4.0913020 .146787$ H -1.113451 -2.803817 -0.642501 H -8.940419 $1.485701-2.490059$ H $-9.0936701 .531173-0.727295$ H -8.309887 2.869513 -1.582339 C $-5.6241732 .700565-0.023872$ H $-6.712963-3.0517761 .318416$ H -4.581334 -4.445211 0.167628 H -2.742882 -2.862801 -2.280878 H -5.1916122 .7791940 .981174$ H $-6.3416593 .523532-0.121818$ H $-7.076855 \quad 1.3085200 .701067$ C -1.011513 -0.367817 2.615971 O $-1.366828-1.0380101 .398322$ C -1.439004 -2.464226 1.475022 C $-0.082245-3.055376 \quad 1.896511$ C $0.426929-2.4160543 .192641$ C $0.333780-0.8874023 .149332$ H -0.8713920 .6822422 .328036$ C -2.136639 -0.4269403 .661567$ H -2.194353 -2.747826 2.214286 O $0.875514-2.841254 \quad 0.861946$ H $-0.193565-4.1390012 .022590$ H $1.472351-2.7033033 .359284$ O $-0.336203-2.8955344 .295399$ H $0.517839-0.471374 \quad 4.146348$ O $1.369450-0.3844782 .302197$ C $-3.376891 \quad 0.3120913 .210382$ H -2.430961 -1.457164 3.877581
H -1.8008130 .0441414 .592437$

H $1.706713-3.2648311 .136269$

H $-0.235218-3.8622334 .324690$

H $1.181004-0.6806121 .395145$

O -4.3971790 .0356354 .075248$

O -3.4425451 .0455892 .234712$

C -5.6504970 .6581693 .775764$

H -6.3181610 .5036564 .627489$

H -5.5256521 .7343113 .621407$

H -6.0933130 .1888702 .893291$

SCF Energy $(B 3 L Y P / 6-31 G * * / / M M F F)=-3245.92918016$

08_00048

MMFF Geometry

C $-2.721266 \quad 1.2422542 .380961$

C -1.7395380 .3279632 .349681$

C -0.8504390 .0466533 .529929$

O $0.536748 \quad 0.128870 \quad 3.131904$

C -1.092138 -1.332827 4.173769

C $-1.000945-2.5481023 .267713$

C $-2.047317-3.3360232 .948871$

C $0.379508-2.9183302 .791135$

C $-3.485632-3.1268673 .327695$

C $-4.496912-3.6681992 .302688$

C $-4.459345-2.9547880 .940224$

C $-5.727520-3.2803380 .144176$

C $-5.699896-2.668874-1.263289$

O $-3.308303-3.4077120 .217518$

O $-6.750817-3.276258-2.030024$

C $-4.368798-3.048929-1.941492$

C $-5.994087-1.164142-1.239838$

O $-4.230542-2.382817-3.195319$

C $-3.126880-2.781356-1.058961$

C $-1.873832-3.389002-1.732872$

O $-2.951294-1.374105-0.929058$

C 1.1126821 .3562313 .065429

O 0.5555322 .4328603 .232450

C 2.5691001 .2623132 .802717

C 3.2125010 .1061832577110

C $4.653758-0.0689302 .380556$

C 5.5233501 .1598942 .435705

C $-0.594826-3.336176-0.885953$

C $0.580436-3.977065-1.605547$

C $3.015208-4.350764-1.439111$

O $0.470290-4.564350-2.679399$

C $4.099652-4.275166-0.412958$

C $5.297703-3.723315-0.659599$

C $6.434856-3.6829390 .320532$

C $6.872614-2.2752940 .661227$

C $7.594319-1.540376-0.201968$

C $6.557139-1.7578422 .044929$

C $5.127192-1.3185522 .185223$

N $1.769156-3.842299-0.898014$

H $-2.908472 \quad 1.7766573 .308476$

H -1.547210 -0.2251611 .435099$

H $-1.017397 \quad 0.791676 \quad 4.319996$

H -2.061773 -1.308146 4.684508

H $-0.352691-1.4727104 .974813$

H -1.850862 -4.230794 2.360050

H $0.417038-3.9300502 .372906$

H $0.709928-2.2285022 .009521$

H $1.095855-2.8863073 .618424$

H -3.661361 -3.645159 4.278317

H $-3.702740-2.066028 \quad 3.490517$

H -4.325034 -4.743455 2.163234

H $-5.495468-3.5540042 .743293$

H -4.383042 -1.874385 1.110521

H $-6.622898-2.9491490 .685378$

H $-5.824259-4.3709610 .045979$

H -7.591849 -3.108910-1.571100

H $-4.408940-4.124385-2.164661$

H -6.987266 -0.975014 -0.814894

H $-6.018858-0.750163-2.253913$

H $-5.271979-0.594259-0.652144$

H $-5.022817-2.587680-3.720944$

H $-2.073888-4.442725-1.968391$

H -1.680001 -2.873927 -2.682647

H $-2.030545-1.186755-0.691423$ 
H 3.0936762 .2119382 .832874 H $2.638973-0.8175022 .554107$ H 5.1485781 .9378081 .762665 H 6.5559250 .9758202 .131420 H 5.5462541 .5643273 .453453 H $-0.327228-2.302425-0.648999$ H $-0.743658-3.8810290 .051551$ H $\quad 3.248890-3.770067-2.338168$ H $2.858467-5.391869-1.741852$ H $3.904544-4.7206670 .559281$ H $5.490913-3.306611-1.645891$ H $7.282746-4.226550-0.116954$ H $6.181016-4.2307811 .236415$ H $7.935423-0.5425530 .052993$ H $7.855935-1.920703-1.183871$ H $7.256395-0.9625532 .321491$ H $6.749418-2.5521902 .778022$ H $4.415387-2.1447032 .164358$ H $1.800879-3.199095-0.111531$ C -3.4779523 .0152540 .709725$ O C -1.7607504 .5979840 .575678$ C -3.0302085 .2856451 .071769$ C $-3.763078 \quad 4.126522 \quad 1.719153$ H -1.075794 4.4936061 .424630 C -1.049805 $5.311670-0.582259$ H -2.814303 6.1019261 .767718 H $-3.628335 \quad 5.6920980 .249139$ C -5.2409854 .3871091 .950290$ H -3.282672 3.9198062 .682550 C $0.2055794 .591633-1.119732$ O $-1.9502065 .432672-1.691890$ H $-0.7858416 .333380-0.282526$ H $-0.1024183 .691871-1.662292$ O $0.8047475 .450906-2.100629$ C $1.2687584 .224877-0.068740$ C $2.6107053 .738857-0.649281$ H $\quad 0.874574 \quad 3.4563620 .604659$ $\begin{array}{lllll}\text { O } & 1.583850 & 5.371630 & 0.728047\end{array}$ H $3.1242074 .571238-1.148121$ H $3.244413 \quad 3.4830330 .210888$ H -5.3805505 .2414172 .620727$ H -5.7618714 .6077331 .012491$ H -5.7225163 .5167742 .406989$ C -3.6091971 .5747221 .215650$ H $-2.5602956 .164418-1.500738$ H $1.1325016 .240779-1.637138$ H 0.8139255 .5751561 .283594 H -3.3871450 .8971990 .384446$ H -4.6475841 .3830151 .509651$ H $-4.147715 \quad 3.124857-0.154177$ C $1.6610370 .275177-1.686271$ O $1.8569341 .475089-0.924375$ C $2.5280052 .537537-1.603909$ C $3.9253842 .089806-2.072960$ C $3.8706970 .788304-2.879074$ C $3.007714-0.271932-2.184690$ H $1.250685-0.445459-0.967686$ C $0.6322060 .456305-2.813688$ H $1.9389562 .833580-2.477585$ O $4.7913321 .898269-0.955664$ H $4.3650752 .888498-2.681400$ H $4.8875710 .396284-3.005239$ O $3.3447261 .043668-4.176845$ H $2.833564-1.124038-2.851790$ O $3.707566-0.764866-1.039960$ C $-0.7309190 .847889-2.293336$ H $0.940887 \quad 1.234918-3.515918$ H $0.517773-0.486497-3.360722$ H $4.3841731 .229777-0.378687$ H $3.9243421 .695594-4.606496$ H $4.575445-1.082276-1.340357$ O $-1.5400531 .148770-3.351335$ O $-1.0459200 .887499-1.113091$ C $-2.8596221 .592266-3.020391$ H $-3.4170841 .722600-3.951700$ H -3.376115 $0.849499-2.406876$ H $-2.8109682 .554886-2.504137$
SCF Energy (B3LYP/6-31G**//MMFF) $=-3245.90776529$

08_00049

MMFF Geometry

C 3.4548263 .1822540 .084928

C 3.1476504 .1082721 .005664

C 1.9314294 .1021891 .905967

O 1.1034052 .9386141 .681845

C 1.0739565 .3475501 .616352

C -0.1318175 .4695972 .527272$

C -1.4053845 .2279582 .157485$

C 0.1695065 .9342863 .929458

C $-1.9125254 .764100 \quad 0.818917$

C -2.7451893 .4868210 .957446$

C $-3.1922922 .897264-0.389733$

C $-4.1749953 .811235-1.127391$

C $-4.7315573 .161167-2.404475$

O $-3.812270 \quad 1.638017-0.097465$

O $-5.8616893 .936189-2.834708$

C $-5.2550721 .751979-2.056666$

C $-3.7156753 .205975-3.552207$

O $-5.6706251 .056532-3.229942$

C $-4.2467810 .905361-1.251429$

C $-4.856870-0.424690-0.755666$

O $\quad-3.1397390 .571690-2.095925$

C 1.4129371 .7988492 .358395

O $2.338438 \quad 1.6300303 .135307$

C 0.4189950 .7743891 .967672

C $0.568261-0.4966172 .369018$

C $-0.341650-1.6046882 .065335$

C $-1.631243-1.2664231 .365794$

C -6.076131-0.267469 0.154981

C $-6.306906-1.5134850 .987112$

C $-5.484995-2.686543 \quad 3.020719$

O $-7.202973-2.3122120 .726214$

C $-4.150850-2.8460283 .676193$

C $-3.486483-4.0094203 .747085$

C $-2.140983-4.1540024 .400871$

C -1.122032 -4.825030 3.504233

C $-0.577584-6.0048413 .847293$

C $-0.733509-4.1411652 .210449$

C $0.011995-2.8536012 .434892$

N $-5.397521-1.6322662 .027630$

H $2.7974482 .332156-0.066936$

H 3.8263494 .9484551 .140909

H 2.2693594 .1196122 .949474

H 0.7639695 .3400240 .565756

H $\quad 1.6828376 .2551891 .728588$

H -2.1881275 .3817272 .900003$

H -0.7382846 .0726794 .526488$

H 0.7964715 .2046614 .450838

H 0.6960376 .8939963 .906482

H -2.5266615 .5688370 .400162$

H $-1.100874 \quad 4.5802740 .110803$

H -2.1665772 .7259301 .496870$

H -3.6266903 .6742941 .585548$

H -2.300876 $2.714253-0.999833$

H $-3.7136304 .774687-1.373025$

H $-5.0192214 .044615-0.463270$

H -5.559976 $4.848388-2.986396$

H $-6.1613341 .896463-1.455466$

H -3.438425 4.242999-3.777342

H $-4.1457582 .806219-4.477055$

H $-2.7953982 .661125-3.333034$

H $-4.8716130 .750219-3.691591$

H $-5.117213-1.064223-1.609249$

H -4.075283 -0.978099-0.220214

H $-2.700041-0.211113-1.727213$

H $-0.410911 \quad 1.0957201 .349280$

H $1.439999-0.7556722 .968452$

H $-2.218060-0.5614671 .965528$

H -2.272235 -2.130104 1.183454

H -1.435714 -0.8114740 .390506$

H -5.9625450 .5808010 .840116$

H $-6.977949-0.078130-0.438023$

H $-5.833078-3.6078432 .541650$

H -6.233078 -2.380815 3.759292

H $-3.708675-1.9566794 .119624$ 
H -3.934085 -4.906357 3.325408 H -2.282079 -4.7377125 .319621$ H -1.750077 -3.180422 4.721064 H $0.152833-6.4968843 .213566$ H $-0.848696-6.5067684 .770101$ H -1.622708 -4.0186001 .587490$ H -0.072022 -4.794692 1.626736 H $0.970591-2.9913292 .937510$ H -4.811410 -0.829591 2.240842

C $5.6701862 .108823-0.521851$

O $5.0560640 .816928-0.705702$

C $5.580909-0.0817150 .291889$

C $\begin{array}{llll}6.747320 & 0.648326 & 0.953667\end{array}$

C 6.2836932 .0887090 .877014

H $4.789988-0.2209881 .037278$

C $5.970985-1.422274-0.336365$

H $6.921907 \quad 0.3057981 .978209$

H 7.6792720 .5157880 .391145

C 7.3963973 .1011611 .081370

H $5.5145842 .241803 \quad 1.644114$

C $4.795952-2.213815-0.947601$

O $6.924736-1.167200-1.376528$

H $6.489608-2.0385710 .407771$

H $4.396049-1.669695-1.810231$

O $5.341864-3.432900-1.472860$

C $3.654243-2.5585830 .032210$

C $2.669519-3.621392-0.489078$

H $3.102371-1.6521750 .304606$

O $4.203297-3.1019581 .238465$

H $3.210817-4.551555-0.704251$

H $1.994468-3.8665910 .341193$

H 7.8472662 .9820232 .071990

H 8.1892562 .9833080 .335226

H 7.0115814 .1231441 .006434

C $4.6685743 .231680-0.797781$

H $7.157800-2.024615-1.772338$

H $5.668594-3.955775-0.720404$

H $4.612812-2.374177 \quad 1.735093$

H $4.3155663 .145256-1.833147$

H $5.1711054 .202352-0.714631$

H $6.4668742 .174661-1.275134$

C $0.099304-1.608581-2.302907$

O $0.982488-2.129180-1.300537$

C $1.839565-3.199394-1.710894$

C $1.020967-4.383158-2.257675$

C $0.053761-3.936553-3.357088$

C $-0.763829-2.719293-2.922472$

H $-0.568975-0.935676-1.755124$

C $0.849039-0.771655-3.344288$

H $2.514573-2.841213-2.493777$

O $0.286184-4.991076-1.196811$

H $1.715062-5.137297-2.648158$

H $-0.631273-4.757639-3.601049$

O $0.781944-3.610910-4.536820$

H -1.327083 -2.321639-3.774944

O $-1.722874-3.151733-1.953312$

C $1.2717550 .546152-2.743309$

H $1.750718-1.258102-3.726499$

H $\quad 0.216225-0.557711-4.213946$

H $-0.196915-5.748164-1.569110$

H $1.272616-4.405492-4.807822$

H $-2.280357-2.388599-1.726962$

O $0.253567 \quad 1.448545-2.839234$

O $2.364596 \quad 0.736470-2.227376$

C $0.5092522 .740423-2.279890$

H $-0.2826983 .417314-2.611462$

H $\quad 0.4831302 .678526-1.188857$

H $1.4700703 .134804-2.624540$

SCF Energy $\left(B 3 L Y P / 6-31 G^{* *} / / M M F F\right)=-3245.89814581$

0800050

MM̄FF Geometry

C $-0.9358651 .467246 \quad 3.228244$

C -1.7217392 .4980533 .578888$

C -2.5318513 .3611602 .640283$

O -2.8655962 .6482541 .428148$

C - 1.7347314 .6275142 .270755

C -2.4871045 .6032721 .380853$
C -2.0862325 .9683790 .145074$

C $-3.741386 \quad 6.195312 \quad 1.968651$

C $-0.8439945 .510203-0.568112$

C $-1.0766444 .183331-1.288423$

C $0.2236583 .440592-1.607993$

C $0.9978294 .063949-2.768409$

C $2.2358023 .226910-3.142066$

O $-0.1517402 .101227-1.944905$

O $2.695576 \quad 3.672418-4.427567$

C $1.8229351 .744114-3.297204$

C $3.3916263 .467410-2.163836$

O $2.9633550 .911893-3.495879$

C $0.9578911 .216285-2.130653$

C $0.396140-0.185827-2.456477$

O $1.7670811 .126839-0.956846$

C $-3.925948 \quad 1.7951871 .487471$

O -4.5887201 .5187542 .475220$

C $-4.161182 \quad 1.2627540 .125438$

C $-5.2076520 .449898-0.085733$

C $-5.624511-0.129506-1.365888$

C $-4.7922360 .186192-2.579923$

C $-0.707637-0.635131-1.487882$

C $-1.207442-2.034999-1.788945$

C $-2.896869-3.693764-1.009272$

O $-0.727831-2.746977-2.666757$

C $-4.003809-3.641626-2.014547$

C $-5.280443-3.943238-1.734208$

C $-6.377303-3.939343-2.759578$

C -7.542685 -3.057783 -2.372705

C $-8.701574-3.589337-1.948174$

C $-7.394952-1.564284-2.566123$

C $-6.736682-0.894368-1.389620$

N $-2.247288-2.398985-0.943367$

H -0.8087541 .2140482 .179021$

H -1.8072272 .7551114 .632829$

H -3.4597333 .6466483 .151751$

H -0.7997644 .3162011 .791942$

H -1.442340 5.1601743 .185656

H -2.701876 $6.668562-0.417059$

H $-4.153758 \quad 6.9956291 .344677$

H -4.5166115 .4304752 .076087$

H -3.532904 6.6248252 .953862

H $-0.5481616 .279465-1.291472$

H -0.0095435 .4369780 .138053$

H -1.696998 $3.522903-0.672343$

H $-1.6592074 .345283-2.204936$

H $0.8367393 .414729-0.699753$

H $1.2892605 .095994-2.539796$

H $\quad 0.3445874 .124667-3.650105$

H $2.8932024 .622525-4.362830$

H $1.2348321 .673388-4.222435$

H $3.6674654 .528659-2.149878$

H $4.2935712 .930323-2.477131$

H $3.1510193 .177227-1.139495$

H $3.3861970 .778298-2.630927$

H $-0.032981-0.182942-3.466928$

H $1.208925-0.923125-2.448762$

H $1.4137930 .440171-0.370337$

H $-3.4884151 .573668-0.665209$

H -5.8406610 .1926290 .763123$

H $-4.777076 \quad 1.265885-2.765913$

H -5.154274 -0.285707 -3.495015

H -3.763526 $-0.159258-2.441156$

H $-0.347193-0.623858-0.454604$

H - $-1.5605330 .048195-1.554518$

H $-3.258400-3.929993-0.003468$

H -2.163726 -4.451060 -1.305231

H $-3.735061-3.368509-3.032522$

H $-5.548299-4.241283-0.723122$

H $-6.715085-4.976292-2.886168$

H $-6.003736-3.629100-3.743471$

H -9.551685 -2.963248 -1.698076

H -8.824518 -4.661381-1.835859

H -8.386903 -1.107951 -2.685606

H $-6.883315-1.376898-3.513511$

H -7.276139-1.045163 -0.452923

H -2.624099-1.712619 -0.295932

C $-0.323891-0.8824674 .021528$ 
O $0.501520-1.3171552 .925215$

C $-0.065376-2.5288642 .387195$

C $-1.340832-2.8116793 .186010$

C $-1.709900-1.4370413 .711996$

H $-0.338471-2.3139541 .349963$

C $0.961086-3.6688582 .439226$

H $-2.123863-3.2536192 .561399$

H $-1.156510-3.5042214 .015508$

C $-2.648776-1.4662284 .903702$

H -2.181671 -0.8806632 .892408$

C $2.273822-3.4085881 .667812$

O $1.308154-3.9240283 .807623$

H $0.494899-4.5919332 .074952$

H $2.848610-2.6248072 .172558$

O $3.067072-4.5999541 .768070$

C $2.094704-3.0494500 .180507$

C $3.380945-3.132557-0.663916$

H $1.661319-2.0474110 .098752$

O $1.155984-3.967201-0.390722$

H $3.744367-4.168335-0.669791$

H $3.099816-2.915380-1.703811$

H $-3.594090-1.9501074 .636741$

H -2.214151-2.017641 5.744112

H -2.874259 -0.4515255 .246751$

C -0.1956350 .6285894 .231799$

H $1.708156-3.1125154 .164822$

H $2.591587-5.3090141 .301923$

H $1.015294-3.707370-1.317143$

H 0.8635390 .9061914 .181566

H $-0.5448890 .868057 \quad 5.243878$

H $0.084011-1.3730624 .916365$

C $4.9963230 .199211-0.152037$

O $4.070700-0.843244-0.490546$

C $4.509343-2.180387-0.231479$

C $5.810508-2.482152-0.995153$

C $6.891462-1.443634-0.683735$

C $6.359206-0.016549-0.828759$

H $4.560891 \quad 1.108070-0.582839$

C 5.0939270 .4000611 .363297

H $4.690583-2.3014940 .840483$

O $5.550770-2.492160-2.397975$

H $6.157195-3.484851-0.717389$

H $7.740437-1.578792-1.364869$

O $7.364583-1.627346 \quad 0.647068$

H $7.0848210 .698194-0.424255$

O $6.2241800 .290421-2.219806$

C 3.7984080 .9515091 .906360

H $5.305087-0.526221 \quad 1.905139$

H 5.8947211 .1029621 .621662

H $6.381465-2.721327-2.848518$

H $7.723335-2.5288780 .709549$

H $5.536264-0.290358-2.588061$

$\begin{array}{llll}\text { O } & 3.721295 & 2.291880 & 1.667899\end{array}$

$\begin{array}{lllll}\text { O } & 2.949692 & 0.266069 & 2.460041\end{array}$

C 2.5156652 .9277912 .103928

H 1.6449572 .4551091 .640185

H 2.4427122 .8876043 .194552

H 2.5507853 .9748261 .791217

SCF Energy (B3LYP/6-31G**//MMFF) $=-3245.92348328$

0800051

MMFF Geometry

C $-0.708995-1.062267-2.312965$

C $0.469525-1.078825-1.673775$

C $0.795172-1.984114-0.515268$

O $0.795848-1.142612 \quad 0.658685$

C $2.199598-2.584913-0.701924$

C $2.499520-3.7430670 .225771$

C $3.240055-3.657667 \quad 1.348180$

C $1.941819-5.072585-0.212519$

C $3.840786-2.4280391 .971036$

C $5.374090-2.4151861 .911847$

C $5.930819-2.103242 \quad 0.513704$

C $7.460844-2.150926 \quad 0.523557$

C $8.059191-1.722043-0.824871$

O $5.487815-0.7914710 .151694$

O $9.461635-1.483831-0.630120$

C $7.427729-0.378614-1.247042$
C $7.949941-2.832564-1.876449$

O $7.843973-0.010171-2.560231$

C $5.884278-0.367041-1.158550$

C $5.3527191 .072266-1.370517$

O $5.354550-1.219682-2.175480$

C $0.285192-1.637253 \quad 1.814074$

O $-0.149754-2.7649702 .000637$

C $0.257430-0.6027472 .874760$

C 0.8640870 .5888452 .760569

C $0.8126811 .685564 \quad 3.729455$

C -0.0588691 .5077584 .944452$

C $3.8526201 .230932-1.086347$

C $3.3725582 .659709-1.272316$

C $1.3573904 .086934-0.909485$

O $4.079433 \quad 3.553238-1.730216$

C 1.6488844 .8307730 .356155

C $0.7143365 .183784 \quad 1.251491$

C 1.0194965 .9215262 .524213

C $0.6245215 .144204 \quad 3.761692$

C $-0.498785 \quad 5.445715 \quad 4.434401$

C 1.5721454 .0780234 .269399

C 1.5053342 .8119123 .460287

N $2.0488472 .810890-0.877093$

H $-1.506230-1.727525-1.991381$

H $1.243283-0.387841-1.995415$

H $0.033274-2.768650-0.431696$

H $2.952226-1.799493-0.574056$

H $2.322655-2.941948-1.733576$

H $3.410802-4.5704121 .918242$

H $2.207660-5.8827990 .474596$

H $0.849457-5.031922-0.265463$

H $2.328632-5.339146-1.201522$

H $3.434183-1.5058711 .546231$

H $3.537633-2.4218873 .026027$

H $5.720597-1.6418052 .610002$

H $5.770327-3.3736822 .269187$

H $5.532470-2.831714-0.201128$

H $7.816783-3.1507630 .801518$

H $7.841209-1.4721481 .299974$

H $9.860751-2.303637-0.291630$

H $7.8184660 .394093-0.570085$

H $8.486747-3.729044-1.543412$

H $8.423467-2.533340-2.818031$

H $6.919065-3.125846-2.085601$

H $8.816501-0.018434-2.568711$

H $5.8905041 .757860-0.702421$

H $5.5543541 .394377-2.400020$

H $4.457728-0.930654-2.403318$

H $-0.313218-0.8735033 .757206$

H $1.4399290 .801894 \quad 1.861523$

H $-1.090418 \quad 1.2841714 .649645$

H $-0.105527 \quad 2.394907 \quad 5.579646$

H $0.314700 \quad 0.6879245 .567701$

H $3.2710410 .604132-1.767021$

H $3.6280030 .927487-0.057423$

H $0.2899873 .877998-1.032557$

H $1.7038424 .667150-1.770571$

H 2.6875995 .0929220 .544410

H $-0.3270184 .936831 \quad 1.060751$

H $0.481704 \quad 6.8781132 .490978$

H 2.0824546 .1857192 .588796

H $-0.7763334 .915067 \quad 5.338929$

H $-1.169304 \quad 6.2272364 .092564$

H 1.3954873 .8758355 .331694

H 2.5998584 .4618054 .234152

H 2.1059262 .8440262 .551245

H $1.5703842 .027322-0.441716$

C -1.692368 $1.143459-3.059300$

O $-2.987166 \quad 0.858189-2.494344$

C $-3.9883301 .571035-3.238214$

C $-3.2457402 .736746-3.874088$

C $-1.9368712 .071860-4.254298$

H $-4.3259870 .889088-4.029479$

C $-5.192008 \quad 1.935284-2.357691$

H $-3.7801663 .158571-4.730283$

H $-3.0520173 .540744-3.155480$

C $-0.803638 \quad 3.052622-4.505441$

H $-2.0986181 .479852-5.165257$ 
C $-5.6966550 .794684-1.448770$

O $-4.8513013 .016991-1.481559$

H $-6.0102072 .300410-2.990654$

H $-5.0142900 .695903-0.599126$

O $-6.9522831 .203566-0.890717$

C $-5.871412-0.570013-2.132123$

C $-6.517789-1.655657-1.251859$

H $-4.897604-0.934490-2.476413$

O $-6.687700-0.399832-3.293974$

H -7.588543 -1.448764 -1.125110

H $-6.472406-2.588707-1.828888$

H $-1.047598 \quad 3.716517-5.341093$

H $-0.6103253 .676327-3.626189$

H $0.1219842 .522788-4.752159$

C -1.000683 $-0.158204-3.477050$

H $-4.8581153 .834707-2.007174$

H $-7.5956231 .247407-1.618959$

H -6.742274 -1.260705 -3.742840

H $-0.070906 \quad 0.072069-4.011743$

H -1.637418 $-0.716951-4.174500$

H -1.112377 1.652405 -2.279139

C $-3.688827-2.1292801 .165445$

O $-4.453089-1.988731-0.041657$

C $-5.867562-1.8609190 .129398$

C $-6.441748-3.0920960 .852185$

C $-5.704988-3.3606822 .168848$

C $-4.183995-3.3336391 .985021$

H -2.671237 -2.356295 0.824614

C $-3.639205-0.8278301 .985565$

H $-6.075752-0.9695250 .729584$

O $-6.326050-4.2383750 .010172$

H -7.509505 -2.926063 1.038304

H -5.997698 -4.342253 2.561312

O $-6.073157-2.379638 \quad 3.133543$

H $-3.687782-3.3394582 .962238$

O $-3.775563-4.5312991 .317415$

C $-2.860798 \quad 0.2682331 .291595$

H -4.640398 -0.4382282 .185104$

H $-3.137879-1.0166662 .941947$

H $-6.732714-4.9879140 .477482$

H $-7.035993-2.4359043 .257408$

H $-4.142828-4.5098230 .416970$

O -2.9825361 .4249022 .007249$

$\begin{array}{llll}0 & -2.218603 & 0.132317 & 0.260987\end{array}$

C -2.304748 2.5607561 .460386

H -2.3412833 .3661952 .198640$

H $-1.2570192 .327393 \quad 1.249621$

H -2.811906 2.8923620 .550247

SCF Energy (B3LYP/6-31G**//MMFF) $=-3245.90425173$

0800052

MMFF Geometry

C $-0.087364-1.6336721 .915972$

C $-0.696689-2.4784801 .068385$

C $-0.289266-2.776893-0.356251$

O $1.023679-2.254110-0.651942$

C $-1.303663-2.117515-1.312921$

C $-1.006544-2.323800-2.786682$

C $-0.726988-1.325245-3.650016$

C $-1.062292-3.748451-3.271138$

C $-0.674500 \quad 0.146742-3.341919$

C $0.696100 \quad 0.554294-2.806115$

C $0.717414 \quad 1.949142-2.171685$

C $0.6120513 .069480-3.205971$

C $0.7903424 .460467-2.571053$

O $1.9648652 .046104-1.474997$

O $1.0263275 .398999-3.632385$

C $2.0536564 .455625-1.680086$

C $-0.4864814 .925777-1.860970$

O $2.176703 \quad 5.681396-0.962079$

C $2.1255103 .249572-0.717054$

C $3.5052123 .189691-0.021217$

$\begin{array}{llllll}\text { O } & 1.111600 & 3.392707 & 0.278850\end{array}$

C $2.094581-3.042935-0.365314$

$\begin{array}{llll}\text { O } & 2.075750 & -4.143746 & 0.161683\end{array}$

C $3.311036-2.329766-0.820680$

C $4.509198-2.918762-0.688836$

C $5.799767-2.379373-1.127083$
C $5.809078-1.010647-1.754486$

C $3.7454151 .886671 \quad 0.756974$

C $5.120598 \quad 1.845757 \quad 1.398474$

C 6.6823840 .2662112 .531347

O 5.8435292 .8331501 .501694

C $7.716763-0.0772961 .506030$

C $8.404171-1.2293821 .498254$

C $9.493471-1.5380280 .512168$

C $9.278937-2.844148-0.216837$

C $9.986788-3.9404700 .104660$

C $8.316222-2.853284-1.382832$

C $6.901430-3.142154-0.963574$

N 5.4293210 .5760531 .870087

H $0.803716-1.0998001 .600217$

H $-1.589613-3.0000631 .406590$

H - $0.295962-3.863909-0.503019$

H $-1.346470-1.048260-1.080786$

H -2.308814 - $2.509618-1.110755$

H $-0.517366-1.579190-4.687829$

H $-0.960709-3.820739-4.359218$

H $-0.256515-4.339908-2.825725$

H -2.021393 -4.203048 -3.001488

H $-0.8989070 .702597-4.260058$

H $-1.4658910 .415011-2.635051$

H $1.025935-0.160449-2.045091$

H $1.4500060 .489434-3.602018$

H $-0.0976702 .012015-1.441533$

H $-0.3397683 .017701-3.747885$

H $1.3956452 .936301-3.964947$

H $\quad 0.266574 \quad 5.363722-4.238588$

H $2.9200084 .413104-2.354287$

H $-1.3317894 .937137-2.559751$

H $-0.3854125 .955369-1.500324$

H $-0.7630914 .289356-1.018587$

H $1.5436795 .657277-0.224338$

H $4.2968693 .274332-0.777194$

H 3.6139614 .0407530 .662840

H 1.3706872 .8976601 .071023

H $3.185261-1.352344-1.271973$

H $4.551913-3.906945-0.231783$

H $5.237151-1.012807-2.688839$

H $6.809884-0.644466-1.991358$

H $5.368266-0.272957-1.076998$

H $3.0100631 .769977 \quad 1.559884$

H $3.652976 \quad 1.0273230 .084175$

H $6.491780-0.5606963 .222596$

H $7.010581 \quad 1.1368263 .108325$

H 7.9374740 .6767380 .753972

H $8.206107-1.9733222 .266497$

H $10.440709-1.5696151 .066557$

H $9.609825-0.730609-0.221578$

H $9.860656-4.872408-0.436456$

H $\quad 10.703197-3.932346 \quad 0.919339$

H $8.608242-3.638748-2.093404$

H $8.418582-1.921453-1.945042$

H $6.782537-4.125190-0.504836$

H $4.798027-0.1944801 .670595$

C - -1.0748070 .0717713 .529706$

$\begin{array}{llll}\text { O } & -2.108427 & 0.405745 & 2.578879\end{array}$

C $-1.872186 \quad 1.746027 \quad 2.105566$

C -0.9027832 .3687003 .102814$

C -0.0219251 .1757303 .423449$

H $-1.362716 \quad 1.653797 \quad 1.138548$

C -3.1903132 .5060891 .927039$

H $-0.352953 \quad 3.2149242 .682223$

H - -1.4176612 .7141484 .007059$

C $0.818412 \quad 1.354727 \quad 4.674896$

H 0.6449650 .9994272 .570849

C $-4.204027 \quad 1.824557 \quad 0.981525$

O -3.8225762 .6629153 .203191$

H -2.976931 3.5216201 .573740

H $-4.6189460 .937549 \quad 1.471285$

$\begin{array}{llllll}\text { O } & -5.304767 & 2.724498 & 0.801932\end{array}$

C $-3.637267 \quad 1.443188-0.396327$

C $-4.6773960 .978443-1.430163$

H $-2.8836090 .658805-0.270750$

O $-2.9704072 .584538-0.943874$

H -5.295768 1.828485 -1.746724 
H $-4.1137630 .683054-2.325331$ H 1.5012782 .2030254 .560397 H $\quad 0.1943591 .5420905 .555065$ H 1.4196890 .4613444 .871067 C $-0.582569-1.3626263 .310434$ H $-3.969273 \quad 1.7738113 .568617$ H -4.9701063 .5068640 .330710$ H $-2.5828452 .321388-1.795653$ H -1.407984 -2.047513 3.541665 H $0.220602-1.5933244 .019596$ H $-1.550691 \quad 0.1152404 .518598$ C $-5.449478-2.402232-0.011541$ O $-4.756881-1.248169-0.509496$ C -5.584484 -0.181396-0.980632 C $-6.492852-0.661293-2.126666$ C -7.296903 -1.900417-1.721097 C $-6.406349-2.966614-1.075928$ H $-4.664547-3.1512210 .155328$ C $-6.139964-2.1268931 .334770$ H $-6.2095060 .178984-0.157586$ O $-5.696765-0.974066-3.269422$ H $-7.1671040 .156320-2.408488$ H -7.782432 -2.330748 -2.605431 O $-8.318645-1.529700-0.800950$ H -7.026022 -3.752938 -0.630174 O $-5.623593-3.596862-2.094673$ C $-5.146623-1.8801302 .449019$ H $-6.794973-1.2537901 .281225$ H -6.737635 -2.997732 1.627794 H -6.300840 -1.233654 -3.985849 H -8.888692 $-0.876877-1.241893$ H -5.005680 -2.933985 -2.448119 O $-5.817655-1.4482573 .557570$ O $-3.939991-2.0588252 .369214$ C $-5.003307-1.1882624 .705301$ H $-5.655823-0.8555435 .516847$ H -4.489019-2.101386 5.019920 H $-4.282625-0.3953134 .487146$

SCF Energy (B3LYP/6-31G**//MMFF) $=-3245.91391536$

0800053

MM̄FF Geometry

C $-2.6778673 .907141-1.016115$

C $-3.3181532 .790886-0.638548$

C -3.9651992 .6103020 .708542$

O $-3.347167 \quad 1.4633791 .335622$

C -5.4541672 .2745810 .538403$

C -6.2586482 .4802371 .804984$

C -6.5573011 .5158462 .697006$

C -6.7786453 .8809162 .004123$

C $-6.096490 \quad 0.0846932 .713317$

C -7.206271 -0.9098752 .349478$

C $-7.410534-1.0493580 .833017$

C $-8.588202-1.9778910 .532017$

C $-8.748043-2.226920-0.974700$

O $-6.204334-1.593727 \quad 0.282741$

O $-9.651809-3.329143-1.146594$

C -7.386401 -2.664172 -1.552401

C $-9.383420-1.028653-1.689651$

O $-7.447631-2.773353-2.972996$

C $-6.211194-1.744257-1.141510$

C $-4.871746-2.397198-1.561593$

O $-6.349715-0.487578-1.806693$

C $-2.1365291 .622368 \quad 1.927675$

O -1.5124582 .6590922 .076632$

C $-1.680517 \quad 0.2706742 .315422$

C -0.3788620 .0488422 .544229$

C $0.225491-1.2455292 .864174$

C $-0.696736-2.4292903 .007215$

C -3.610618 -1.706721-1.016605

C $-2.346050-2.382698-1.527205$

C $0.133880-2.148281-1.535999$

O $-2.364137-3.401774-2.213935$

C $0.740767-2.993331-0.460457$

C $1.987588-2.8201600 .005900$

C $2.621447-3.715346 \quad 1.033749$

C $3.273513-2.9546042 .167171$

C $4.601759-2.7564612 .187680$
C $2.406245-2.516376 \quad 3.325072$

C $1.566685-1.3155292 .993171$

N $-1.193387-1.716359-1.131725$

H -2.641209 $4.756596-0.339841$

H $-3.3459361 .939296-1.314810$

H $-3.8341273 .495506 \quad 1.343257$

H $-5.560698 \quad 1.2394140 .197020$

H $-5.9000852 .894968-0.250748$

H -7.1781241 .7867113 .550493$

H -7.3537873 .9848302 .930340$

H -5.9488394 .5934062 .050449$

H -7.4344874 .1639931 .174338$

H $-5.218422-0.0732702 .082762$

H $-5.763224-0.1295293 .737245$

H -6.916254 -1.888371 2.754496

H $-8.146490-0.6229682 .836162$

H -7.592908 -0.058302 0.404053

H -9.517730 -1.577582 0.955785

H -8.426172 -2.9456331 .027237$

H -10.494478-3.095063 -0.720846

H -7.181587 -3.675333 -1.173131

H -10.375736 -0.817062 -1.273636

H -9.543915 -1.242360 -2.752153

H $-8.790480-0.115389-1.606975$

H -8.186797 -3.368485 -3.186071

H $-4.853765-3.436296-1.206326$

H -4.809671 -2.427863 -2.657108

H $-5.474411-0.084663-1.915419$

H -2.425219 -0.5166022 .351770$

H $\quad 0.313170 \quad 0.8861412 .465733$

H $-1.388314-2.2764903 .843104$

H -0.177184 -3.371344 3.191534

H -1.280573 -2.578052 2.091911

H $-3.579947-0.656787-1.323864$

H -3.598243 -1.747386 0.078370

H $0.723747-1.251000-1.742076$

H $0.060447-2.731246-2.459886$

H $0.139622-3.813319-0.074018$

H $2.603488-2.024111-0.406488$

H $3.374141-4.3262940 .517766$

H $1.895908-4.4274611 .446464$

H $5.085092-2.2433823 .012127$

H $5.234488-3.1034621 .377988$

H $3.033196-2.2457954 .185549$

H $1.816409-3.3686963 .673166$

H $2.149761-0.4030912 .859089$

H -1.267794 -0.912797 -0.514796

C $-0.4418904 .036681-2.210091$

O $0.0011542 .830118-1.554661$

C $1.0557833 .172795-0.626784$

C $1.4970694 .577838-1.007795$

C $0.1806355 .197263-1.435681$

H $0.591480 \quad 3.177270 \quad 0.366993$

C $2.1452882 .097597-0.696568$

H $1.9706385 .111693-0.178466$

H $2.2012024 .551616-1.848840$

C $0.3403336 .467941-2.251594$

H $-0.4007735 .424463-0.533962$

C 3.4242442 .4464410 .080623

O $1.6051150 .888287-0.156358$

H $2.3842961 .895655-1.746746$

H $3.8968363 .327730-0.368423$

O 3.0674202 .8293301 .415915

C 4.4704201 .3169190 .183300

C $4.8257580 .686353-1.175330$

H 4.1216730 .5442980 .878975

$\begin{array}{lllll}\text { O } & 5.636450 & 1.904177 & 0.768454\end{array}$

H $3.9231790 .248280-1.614292$

H $5.1823941 .482417-1.841362$

H $0.8531527 .238408-1.666612$

H $0.9258496 .291481-3.160162$

H $-0.6355146 .863661-2.550389$

C $-1.9686584 .041413-2.334791$

H $\quad 0.7532550 .727987-0.597040$

H 2.6041232 .0767381 .822249

H $6.286436 \quad 1.1954920 .906641$

H -2.262802 $3.210435-2.988933$

H $-2.3046784 .961353-2.826519$ 
H $-0.0202704 .002187-3.223546$

C $8.293880-0.655375-0.704930$

$\begin{array}{lllll}\text { O } & 7.163738 & 0.217877 & -0.834746\end{array}$

C $5.904654-0.413450-1.087519$

C $5.970134-1.234418-2.385837$

C $7.136049-2.226815-2.366731$

C $8.445765-1.554646-1.942997$

H $9.1608690 .017292-0.673077$

C $8.263074-1.4405480 .610204$

H $5.655808-1.073844-0.250796$

O $6.101133-0.370609-3.513857$

H $5.025133-1.776680-2.508389$

H $7.260833-2.661561-3.365928$

O $6.847927-3.289172-1.462528$

H $9.221173-2.306485-1.754388$

O $8.913667-0.729942-3.014367$

C $8.429802-0.5155151 .792244$

H $7.343465-2.0159060 .739969$

H $9.086765-2.1629900 .654054$

H $6.8954080 .174566-3.380206$

H $6.028690-3.717886-1.763555$

H $9.062503-1.305923-3.783685$

O $7.230761-0.3312642 .413631$

O $9.495226-0.0046492 .112484$

C $7.251646 \quad 0.5330963 .553897$

H 7.5933051 .5321433 .267142

H 6.2316560 .6090973 .940096

H 7.8934540 .1133224 .334235

SCF Energy (B3LYP/6-31G**//MMFF)= -3245.91655445

\section{$08 \_00054$}

MM̄FF Geometry

C -2.095077 4.5605221 .699640

C $-2584524 \quad 3.3142301 .612314$

C $-2.7315492 .386102 \quad 2.790069$

O $-2.0887151 .131992 \quad 2.460888$

C -4.2242502 .1050403 .049335$

C -4.4787481 .1185234 .175327$

C $-5.095284-0.0706354 .017059$

C $-4.009876 \quad 1.546567 \quad 5.541518$

C $-5.670823-0.6323442 .746133$

C $-4.631387-1.4333451 .963542$

C $-5.078617-1.7805580 .537962$

C $-6.240802-2.7743310 .509553$

C $-6.588974-3.207142-0.923992$

O $-3.936948-2.345960-0.118087$

O $-7.437562-4.362582-0.842351$

C $-5.297754-3.645795-1.646776$

C $-7.392813-2.130716-1.663434$

O $-5.550800-3.912953-3.024378$

C $-4.136857-2.634323-1.507878$

C $-2.829310-3.252300-2.057372$

O $-4.448694-1.457481-2.255340$

C $-0.750535 \quad 1.0295342 .682684$

$\begin{array}{lllll}\text { O } & -0.002347 & 1.909232 & 3.076991\end{array}$

$\begin{array}{llll}\text { C }-0.357323 & -0.370147 & 2.394251\end{array}$

C $0.898014-0.7732922 .642770$

C $1.416966-2.1371522 .502310$

C $0.474898-3.1954661 .992786$

C -1.572080 -2.415354-1.779399

C $-0.310057-3.115429-2.254229$

C $2.171275-2.899956-2.179188$

O $-0.323944-4.143293-2.926373$

C $2.675813-3.784077-1.083336$

C $3.778898-3.519643-0.367352$

C $4.352680-4.4546640 .655785$

C $4.579390-3.8003141 .997848$

C $5.807963-3.4115462 .379691$

C $3.405337-3.7063242 .943243$

C $2.692246-2.3826752 .869827$

N $0.835333-2.440822-1.854118$

H -1.8005114 .9288512 .680769$

H -2.895064 2.9137100 .651708

H -2.272272 2.8122923 .690533

H $-4.674971 \quad 1.745138 \quad 2.118087$

H -4.740103 3.0438993 .290715

H -5.214421 -0.7127204 .888526$

H -4.3461630 .8625326 .328225$
H -2.9168911 .5815705 .582172$

H $-4.4021582 .539737 \quad 5.783453$

H $-6.517300-1.2765733 .012137$

H -6.0937500 .1653392 .126392$

H -3.696560 -0.8649111 .892307$

H $-4.368537-2.3499032 .508607$

H $-5.351106-0.8487820 .028202$

H -7.128386 -2.362171 1.003489

H $-5.966477-3.6700301 .084245$

H $-8.225227-4.120081-0.326082$

H $-4.980493-4.598451-1.199772$

H $-8.331384-1.923469-1.135339$

H -7.681902 -2.470769-2.663999

H $-6.855341-1.185315-1.761348$

H $-6.274026-4.561964-3.065371$

H $-2.679698-4.238636-1.598241$

H -2.917161 -3.406284 -3.140358

H $-3.626765-0.995682-2.481830$

H $-1.124802-1.0427532 .028100$

H $1.610168-0.0431483 .024694$

H $-0.341769-3.3584242 .704405$

H $\quad 0.046456-2.897517 \quad 1.031049$

H $0.955043-4.1610321 .821359$

H $-1.626647-1.450321-2.293946$

H $-1.475477-2.225746-0.705275$

H $2.800464-2.016581-2.319654$

H $\quad 2.148370-3.457983-3.120935$

H $2.134202-4.710159-0.905946$

H $4.334266-2.607488-0.567949$

H $5.305466-4.8305750 .259915$

H $3.721059-5.3423680 .785725$

H $5.982829-2.9763833 .358209$

H $6.663479-3.5121301 .720158$

H $3.763651-3.8212143 .975925$

H $2.739229-4.5607142 .794719$

H $3.295538-1.5478693 .228968$

H $0.747644-1.631816-1.245946$

C -2.139401 $5.026814-0.848976$

O $-1.196784 \quad 3.980514-1.152859$

C $-0.6472804 .205505-2.461883$

C $-1.5521415 .238161-3.120030$

C $-1.9348646 .093785-1.926617$

H $\quad 0.3436664 .651769-2.314078$

C $-0.506242 \quad 2.875827-3.210630$

H $-1.0395165 .800061-3.906502$

H $-2.4455754 .778166-3.557519$

C $-3.1531246 .967364-2.170452$

H $-1.0813946 .733935-1.665733$

C $0.2428561 .778960-2.422850$

O $-1.808895 \quad 2.363776-3.519717$

H $-0.0165733 .051026-4.175381$

H $-0.3888661 .418764-1.601646$

$\begin{array}{lllll}\text { O } & 0.418860 & 0.654003 & -3.292734\end{array}$

C $1.6077612 .209999-1.852765$

C $2.3445121 .054670-1.162259$

H $1.4446063 .011664-1.127042$

$\begin{array}{llll}\text { O } & 2.423891 & 2.744168 & -2.892717\end{array}$

H $1.7035270 .606952-0.392211$

H $2.5322630 .256279-1.890470$

H -3.399189 7.551565 -1.278089

H -2.966141 $7.667155-2.991510$

H $-4.0309486 .367841-2.433590$

C -1.8848845 .5402690 .572038$

H $-2.2895462 .257704-2.680748$

H $0.9315610 .949215-4.063948$

H $2.6513772 .018240-3.498069$

H -2.521122 6.4116310 .765902

H $-0.842573 \quad 5.877757 \quad 0.641717$

H $-3.1446894 .594827-0.931752$

$\begin{array}{llll}\text { C } 5.535927 & 0.305566 & 0.593217\end{array}$

$\begin{array}{lllll}\text { O } & 4.226826 & 0.219857 & 0.018169\end{array}$

C $3.7117221 .429688-0.556462$

C 3.6415732 .5408740 .502249

$\begin{array}{llll}\text { C } 4.987958 & 2.733041 & 1.207474\end{array}$

C 5.5886961 .4015351 .668243

H $5.683239-0.6587391 .093944$

C $6.6223050 .449804-0.477440$

H $4.3717821 .739002-1.373707$ 
O 2.6431102 .2100541 .463779

H 3.3440503 .4801210 .022589

H $4.848583 \quad 3.3893872 .075215$

O $\quad 5.879867 \quad 3.384910 \quad 0.308002$

H 6.6257281 .5455741 .991318

O 4.8680820 .9426002 .815599

C $6.689235-0.784889-1.343782$

H $6.460158 \quad 1.300854-1.144812$

H $7.6071250 .602613-0.019933$

H 2.5818242 .9496142 .092001

H $\quad 6.7192893 .5261730 .778086$

H 3.9558120 .7534212 .536446

O $7.481479-1.725533-0.754761$

O $6.095093-0.902488-2.407772$

C $7.634753-2.947647-1.483256$

H $6.659651-3.390681-1.705778$

H $8.193480-2.764343-2.405666$

H $8.201220-3.645325-0.860804$

SCF Energy (B3LYP/6-31G**//MMFF) $=-3245.91497547$

\section{5}

MM̄FF Geometry

C $-2.4025984 .904714-1.002448$

C -3.261189 3.877196-1.078910

C $-4.2743873 .566350-0.006406$

O -4.0084912 .2239070 .467638$

C $-5.6997673 .612469-0.581461$

C $-6.776127 \quad 3.4920740 .483010$

C -7.545726 2.4046850 .690249

C $-6.9839004 .724578 \quad 1.327602$

C $-7.5146001 .097750-0.049315$

C $-6.969762-0.028678 \quad 0.837733$

C $-6.735283-1.3188650 .039437$

C $-6.236189-2.4388350 .952900$

C $-5.868207-3.707416 \quad 0.166595$

O $-5.753820-1.031802-0.960604$

O $-5.112592-4.5607931 .040852$

C $-4.937080-3.321313-1.004545$

C $-7.114807-4.501416-0.241150$

O $-4.684553-4.444436-1.845304$

C $-5.451116-2.126289-1.833831$

C $-4.418502-1.612261-2.865902$

O $-6.611965-2.535446-2.567271$

C -2.998917 2.0869961 .372606

O $-2.3464872 .983804 \quad 1.884117$

C -2.7943220 .6424271 .618736$

C - $1.695601 \quad 0.2438632 .277408$

C $-1.341131-1.1319602 .631861$

C -2.283295 -2.2285132 .213623$

C $-3.102250-1.094554-2.274417$

C $-1.978074-2.116408-2.277576$

C $0.373576-2.403212-1.505530$

O $-2.048882-3.198345-2.853463$

C $0.389324-3.199555-0.239666$

C $1.351272-3.0807030 .686910$

C $1.443147-3.9228251 .924443$

C $1.589143-3.0975773 .181851$

C $2.799625-2.8492583 .710442$

C $0.326247-2.6423403 .873628$

C $-0.201411-1.3451073 .322338$

$\mathrm{N}-0.863691-1.652702-1.591643$

H -2.464444 $5.582833-0.155416$

H $-3.2023103 .190528-1.920145$

H -4.189146 4.2724690 .828650

H -5.808786 2.831255-1.340242

H $-5.8567814 .560528-1.113657$

H -8.290705 2.4471971 .484106

H -7.830010 4.620062 2.015246

H $-6.0961594 .935641 \quad 1.931278$

H -7.186973 5.5916830 .690486

H $-8.5371110 .851862-0.361435$

H $-6.9331011 .171366-0.970556$

H $-6.021727 \quad 0.296590 \quad 1.283894$

H -7.668973 -0.2203061 .661147$

H $-7.675679-1.605948-0.445673$

H $-6.977958-2.6677361 .728149$

H -5.342035 -2.095486 1.489746

H $-5.661093-4.7529831 .820574$
H $-3.972443-3.056080-0.557192$

H $-7.694950-4.7848180 .645467$

H $-6.841764-5.442837-0.730327$

H -7.779306 $-3.944508-0.904881$

H $-5.469554-4.580322-2.402686$

H $-4.248586-2.369817-3.640209$

H $-4.881420-0.763430-3.389862$

H $-6.731613-1.930627-3.318194$

H -3.545284 -0.0473421 .252930$

H $-0.983867 \quad 1.0018942 .603063$

H -3.234702 -2.138162 2.747914

H -1.896518 -3.231912 2.404662

H -2.477235 -2.180497 1.137107

H $-2.752116-0.258091-2.893207$

H -3.244874 -0.710460 -1.259390

H $1.195138-1.681748-1.552826$

H $0.456826-3.076185-2.364917$

H $-0.400056-3.934178-0.101057$

H $2.149585-2.3625570 .525226$

H $2.314247-4.5820741 .813092$

H $0.579331-4.5920762 .019907$

H $2.909295-2.2824894 .629002$

H $3.707629-3.1998123 .230771$

H $0.527171-2.4811934 .941815$

H $-0.415078-3.4465783 .857665$

H $0.421808-0.4853083 .572151$

H $-0.917869-0.769785-1.092067$

C $0.0893954 .851739-1.495223$

O $0.2062023 .443700-1.203390$

C 0.9258453 .2883840 .039882

C 1.5819234 .6353750 .307013

C $0.5387055 .592101-0.234944$

H $\quad 0.1635703 .0731710 .797402$

C $1.9039122 .114224-0.080603$

H 1.7991624 .7969301 .366883

H $2.5194354 .731007-0.254925$

C $1.0680216 .990536-0.499606$

H -0.2732105 .6544120 .498777$

C 2.8065051 .9181661 .151416

O $1.1431730 .919232-0.280321$

H $2.5145912 .252828-0.979481$

H $3.4183212 .811177 \quad 1.326131$

O 1.9896961 .7449472 .316392

C 3.7349060 .6892071 .075250

C $4.6722170 .714370-0.138443$

H $3.141759-0.2305791 .085338$

O $4.540314 \quad 0.6716872 .259157$

H $4.0977260 .665056-1.070742$

H $5.1939421 .681210-0.167160$

H $1.434461 \quad 7.4448750 .426734$

H $1.8951336 .978243-1.217226$

H $0.2799947 .634083-0.903350$

C $-1.3196945 .169908-2.010416$

H $0.5785751 .062201-1.059255$

H 1.5584162 .5946202 .506796

H 3.9340450 .6495783 .019610

H -1.505899 $4.569793-2.910369$

H -1.376396 $6.221753-2.313473$

H $0.7978935 .046484-2.311133$

C $7.675039-1.027876-1.489555$

O $6.552156-0.153330-1.313636$

C $5.751351-0.386938-0.146618$

C $5.179310-1.811530-0.174179$

C $6.282695-2.858202-0.354484$

C $7.228424-2.499288-1.505715$

H $8.062799-0.788113-2.488325$

C $8.791695-0.742215-0.472925$

H $6.380616-0.2552110 .739408$

O $4.229696-1.939862-1.230768$

H $4.649841-2.0072520 .764457$

H $5.818349-3.832842-0.548521$

O $7.014866-2.9602520 .863182$

H $8.111667-3.148334-1.498246$

O $6.554277-2.726404-2.747369$

C $9.4012670 .632335-0.658833$

H $8.429925-0.7994500 .556773$

H $9.596486-1.474641-0.605282$

H $4.674326-1.699770-2.061798$ 
H $7.690445-3.6490010 .742416$

H $6.312286-3.667591-2.781568$

O 10.3960710 .8008310 .263036

O $9.0567341 .453676-1.497087$

C $11.0710632 .061866 \quad 0.215878$

H 11.8360062 .0673030 .996990

H $11.5593772 .194239-0.754442$

H $\quad 10.3661832 .8761840 .409022$

SCF Energy (B3LYP/6-31G**//MMFF)= -3245.91615819

\section{8_00056}

MM̄FF Geometry

C $0.9028013 .647452-1.075309$

C $-0.2337974 .213754-0.640936$

C -1.2406973 .4781710 .200484$

O $-2.4912933 .486436-0.530730$

C -1.465623 4.1305321 .577679

C -0.2399244 .2079052 .458592$

C 0.3102603 .1584793 .099857

C 0.3349775 .5895402 .632131

C -0.1390841 .7215613 .086157$

C 0.9940030 .7986592 .615833

C $0.636162-0.6899632 .761062$

C $1.877252-1.5542222 .518453$

C $1.549873-3.0521352 .574626$

O $-0.384019-1.013191 \quad 1.809248$

O $2.686226-3.7809512 .088347$

C $0.355911-3.3307471 .639758$

C $1.353174-3.5354774 .015439$

O $-0.068358-4.6875631 .754176$

C $-0.842193-2.3764191 .863848$

C $-1.872942-2.574350 \quad 0.723016$

O $-1.452857-2.6785493 .117924$

C $-3.3660852 .490187-0.229954$

O -3.2167901 .6179420 .611609$

C $-4.5516892 .616331-1.102050$

C $-5.2998451 .519474-1.290325$

C $-6.5519771 .416234-2.036781$

C $-7.1616642 .685855-2.564949$

C -3.112730 -1.675006 0.820020

C $-4.338992-2.3511621 .415814$

C $-6.750311-1.8785501 .892456$

O $-4.357565-3.5229631 .780233$

C -7.581143 -2.108423 0.667719

C $-8.651850-1.3681070 .339760$

C $-9.478300-1.590864-0.897162$

C $-9.530369-0.371939-1.796022$

C $-10.571610 \quad 0.475789-1.746674$

C -8.418895 -0.176307 -2.806004

C $-7.1151970 .196291-2.159800$

N -5.417859 -1.478155 1.477301

H $1.1081052 .609429-0.826019$

H $-0.4470275 .251130-0.885784$

H -0.9122652 .4393780 .325277$

H -2.2560183 .5940892 .118872$

H $-1.889078 \quad 5.1350361 .436622$

H $1.1786263 .347833 \quad 3.730357$

H 1.2576565 .5869353 .221963

H 0.5671216 .0345961 .659975

H -0.3863956 .2358623 .142929$

H -0.4256881 .4527854 .110163$

H -1.026443 1.5713892 .465394

H $1.222711 \quad 1.0249381 .566340$

H 1.8979431 .0104063 .201497

H $0.249027-0.857346 \quad 3.772874$

H $2.671351-1.3103693 .235244$

H $2.289415-1.3220721 .526914$

H $2.872538-3.4850511 .182492$

H $\quad 0.705225-3.2032440 .606536$

H $2.268756-3.3766724 .598260$

H $1.161682-4.6138214 .048020$

H $\quad 0.539243-3.0261934 .535221$

H $\quad 0.710439-5.2494521 .600203$

H -1.373764 -2.330277 -0.223544

H -2.174953 -3.6257920 .663028$

H $-2.354189-2.3204093 .124487$

H -4.779323 $3.582063-1.535641$

H $-4.9662370 .588272-0.834814$
H $-7.2981693 .413877-1.757404$

H $-8.1452882 .537932-3.016787$

H $-6.5174673 .129936-3.331567$

H -2.897052 $-0.772814 \quad 1.399617$

H -3.391766 -1.365575 -0.195203

H $-7.150922-1.0750692 .518443$

H $-6.700078-2.7949262 .488463$

H -7.280734 -2.926440 0.016969

H $-8.958410-0.5573010 .996804$

H $-10.492540-1.861560-0.575563$

H $-9.113849-2.450750-1.472836$

H $-10.6314601 .337246-2.403588$

H $-11.3853320 .329907-1.043811$

H $-8.7051970 .559339-3.565305$

H -8.276896 -1.109519 -3.366074

H $-6.584993-0.659918-1.742015$

H $-5.303681-0.5309941 .129030$

C $3.3194234 .405436-1.210281$

O $3.8773013 .080045-1.097901$

C 4.5647522 .9801480 .164602

C 4.6304614 .3965410 .733537

C 3.3505025 .0000800 .196760

H 3.9313612 .3676220 .817074

C $5.9381502 .323905-0.006362$

H 4.6824804 .3999191 .826795

H 5.4977454 .9501930 .355751

C 3.3257396 .5175710 .227283

H 2.5224434 .6157610 .802530

C $5.9189260 .919607-0.641572$

O $6.7360333 .170118-0.845720$

H 6.4435892 .2902720 .966792

H $5.6613740 .991124-1.704890$

O $7.2594500 .411381-0.581241$

C $4.988838-0.1011380 .045461$

C $5.261522-1.571208-0.332177$

H $3.9465110 .147627-0.177744$

O $5.134813-0.0028021 .466402$

H $6.267237-1.8388590 .014049$

H $4.590099-2.1982210 .264985$

H 3.4173886 .8828531 .255376

H $4.1484936 .944746-0.355676$

H $2.3864266 .899894-0.184627$

C $1.9465744 .357544-1.888765$

H $7.6591222 .876711-0.765665$

H $7.7938340 .918083-1.215646$

H $6.067412-0.1788891 .679470$

H $2.0381843 .822628-2.842117$

H $1.6216995 .378790-2.120770$

H $4.0020344 .961551-1.866651$

C $2.783256-2.551061-1.850681$

O $3.789252-1.641288-2.314276$

C $5.113430-1.895014-1.831191$

C $5.547950-3.317089-2.217341$

C $4.556790-4.366755-1.724745$

C $3.146893-4.006927-2.184186$

H $2.659369-2.431463-0.769710$

C $1.460616-2.172762-2.535265$

H $5.774743-1.213085-2.377665$

O $6.857386-3.604238-1.737623$

H $5.602073-3.381406-3.311818$

H $4.599826-4.485388-0.635934$

O $4.900775-5.636624-2.282551$

H $3.075260-4.170415-3.267074$

O $2.213363-4.895952-1.571534$

C $0.832817-0.923819-1.958413$

H $1.626769-1.996286-3.604332$

H $\quad 0.728837-2.978952-2.408808$

H $\quad 6.799386-3.800031-0.787418$

H $5.818968-5.827505-2.024800$

H $2.276187-4.779952-0.608326$

O $-0.392610-0.750743-2.538276$

O $1.339621-0.197458-1.115910$

C $-1.1478110 .368606-2.069631$

H $-1.1920740 .371428-0.977131$

H $-0.706606 \quad 1.295717-2.444400$

H $-2.1655250 .279745-2.458796$

SCF Energy (B3LYP/6-31G**//MMFF) $=-3245.90938640$ 
0800057

MM̄FF Geometry

C $0.729943 \quad 4.2142550 .390065$

C $1.9665263 .711745 \quad 0.265350$

C $2.7308983 .659768-1.032256$

O $2.9280422 .260254-1.347213$

C $4.1067044 .326694-0.862746$

C $4.9603204 .288539-2.117300$

C $6.0654853 .531225-2.274198$

C $4.5085015 .197541-3.231403$

C $6.6731332 .581792-1.279889$

C $6.3988371 .122128-1.663683$

C $6.5570280 .176623-0.464073$

C $6.396748-1.280106-0.900768$

C $6.395457-2.250000 \quad 0.290025$

O $5.538373 \quad 0.519606 \quad 0.481993$

O $5.925327-3.511653-0.209567$

C $5.388237-1.7465201 .348164$

C $7.814014-2.4913310 .819034$

O $5.468887-2.5211652 .541737$

C 5.556591-0.251666 1.689593

C $4.427618 \quad 0.2936982 .600230$

O $6.790386-0.0687662 .394930$

C $1.8986681 .619513-1.968918$

O $0.8665122 .120611-2.386524$

C $2.2200000 .175375-2.005207$

C $1.274648-0.699089-2.380673$

C $1.407359-2.157034-2.423557$

C $2.710340-2.754403-1.963861$

C 3.0140760 .2289882 .010743

C $2.228983-1.0109652 .400209$

C $0.089064-2.1843661 .899264$

O $2.582622-1.7822923 .287752$

C $\quad 0.387647-3.3497961 .010254$

C $-0.550185-3.9749110 .282706$

C $-0.286307-5.176294-0.578774$

C $-0.738652-4.982120-2.009033$

C $-1.948645-5.403281-2.414419$

C $0.246526-4.380402-2.986129$

C $0.354729-2.886323-2.848982$

N $1.055311-1.1282851 .666122$

H $0.2409534 .659224-0.472756$

H $2.4402203 .251176 \quad 1.129148$

H 2.178026 4.151531-1.842004

H $4.6284333 .855996-0.023988$

H $3.9740785 .376620-0.568179$

H $6.5981023 .586077-3.222821$

H $5.2079435 .201558-4.074283$

H $3.5335924 .882669-3.615695$

H $4.4258306 .228054-2.870526$

H $7.7570082 .750329-1.254224$

H $6.3278102 .789968-0.265060$

H $5.3771051 .033090-2.052408$

H $7.0802410 .826647-2.471153$

H $7.5429920 .339460-0.013262$

H $7.175773-1.557526-1.621882$

H $5.445187-1.392393-1.436985$

H $5.962848-4.1569180 .516521$

H $4.385583-1.9063680 .934900$

H $8.439399-2.9419340 .039045$

H $7.811571-3.2050631 .650250$

H $8.308416-1.5766171 .152695$

H $5.237040-3.4372992 .315580$

H $4.476825-0.1796913 .588164$

H 4.6425671 .3574282 .778038

H $\quad \begin{array}{llll}\text { H } & \text {.740217 } & 0.762657 & 2.894962\end{array}$

H $3.209612-0.131105-1.688883$

H $0.298993-0.313950-2.675618$

H $3.522248-2.464913-2.639482$

H $2.701335-3.845387-1.920041$

H $2.954071-2.412990-0.952507$

H 2.4393521 .0783882 .401814

H 3.0368620 .3236960 .921490

H $-0.902501-1.7624731 .708171$

H $0.143780-2.4973092 .946852$

H $1.411801-3.7143850 .985323$

H -1.579169 -3.629130 0.341136

H $-0.819291-6.027505-0.134711$
H $\quad 0.774517-5.455298-0.564038$

H $-2.270562-5.292486-3.444517$

H $-2.647910-5.860179-1.721805$

H $-0.072626-4.586313-4.016772$

H $1.208789-4.890714-2.889310$

H $-0.547795-2.358611-3.159432$

H $0.865688-0.4675490 .918109$

C $-1.2636683 .199593 \quad 1.482349$

O -2.2732693 .8638700 .695889$

C -3.5730713 .5604141 .249210$

C -3.3415522 .4737692 .289150$

C -1.9519732 .8134682 .788959$

H $-3.9170894 .484640 \quad 1.729925$

C -4.5133323 .1694150 .103029$

H -4.0962982 .4818943 .081203$

H -3.3463831 .4800971 .823917$

C -1.2822661 .6648173 .525087$

H -2.016069 3.6852533 .453570

C -5.8727562 .6234420 .571019$

O $-4.7420394 .331190-0.701969$

H $-4.0073962 .445334-0.542734$

H -5.7371641 .7269781 .183588$

O $-6.4818873 .588521 \quad 1.440965$

C $-6.8709202 .318236-0.560744$

C $-6.3824101 .371273-1.672044$

H $-7.1951003 .256674-1.027274$

O -8.0487641 .7411130 .016156$

H $-7.2007861 .274681-2.397823$

H $-5.5397161 .840308-2.192916$

H $-1.202991 \quad 0.7748872 .891854$

H -0.2728851 .9396803 .846508$

H -1.8579401 .3915114 .415553$

C -0.0644504 .1326241 .664660$

$\mathrm{H}-3.8726644 .660712-0.987883$

H -6.5721434 .4177490 .940390$

H -8.3619932 .3510230 .705974$

H $0.572048 \quad 3.7743982 .481826$

H -0.4001305 .1432931 .925353$

H $-0.9705712 .296590 \quad 0.929338$

C $-4.286588-1.1940030 .067949$

O $-4.7529040 .033390-0.504860$

C $-5.996125-0.048916-1.206254$

C $-5.851479-1.011410-2.397493$

C $-5.349399-2.387629-1.944784$

C $-4.137747-2.278648-1.013137$

H $-3.284284-0.9552140 .446226$

C $-5.154036-1.5876191 .266772$

H $-6.771445-0.424327-0.529124$

O $-4.940311-0.457139-3.345136$

H -6.824354 -1.109104 -2.893982

H $-5.069570-2.982589-2.822923$

O $-6.394484-3.081483-1.270673$

H $-3.932446-3.250148-0.550600$

O $-3.004168-1.950119-1.822706$

C $-4.423568-2.5132182 .206209$

H $-5.459756-0.6970541 .830436$

H -6.092078 -2.067741 0.973959

H $-4.884093-1.074244-4.094395$

H -7.137316 -3.169797 -1.891776

H $-2.223996-1.923476-1.243397$

O $-3.654400-1.7887343 .068288$

O $-4.518334-3.7330372 .171041$

C $-2.921159-2.5516764 .031487$

H $-2.235197-1.8757264 .549032$

H $-3.609763-2.9848244 .762803$

H $-2.336029-3.3365123 .542683$

SCF Energy (B3LYP/6-31G**//MMFF) $=-3245.90332846$

0800058

MM̄FF Geometry

C $1.790055-4.042388-0.003769$

C $0.474005-3.787433-0.025834$

C $-0.322728-3.688066-1.301843$

O $-1.058321-2.442809-1.297105$

C $-1.330221-4.849215-1.374049$

C $-2.134023-4.875974-2.660785$

C $-3.436890-4.545175-2.765772$

C $-1.381049-5.352988-3.876905$ 
C $-4.354324-4.054469-1.680676$

C $-4.890304-2.653049-2.001338$

C $-5.627882-2.035481-0.803760$

C $-6.219141-0.676205-1.179976$

C -6.8422380 .0429110 .026446$

O $-4.675249-1.8827850 .252685$

O

C $\begin{array}{llll}-5.824970 & 0.044209 & 1.189017\end{array}$

C -8.219375 -0.5320670 .378006$

O -6.4086640 .5817142 .373385$

C $-5.205874-1.3412411 .468214$

C $-4.047003-1.2929032 .494447$

O $-6.215915-2.1979892 .015025$

C $-0.382051-1.318637-1.664570$

O $0.802190-1.230293-1.947624$

C -1.351595 -0.200267-1.679577

C $-0.9445751 .038973-1.992157$

C $-1.7955302 .228772-2.081283$

C $-3.2512162 .076717-1.728171$

C $-2.825240-0.4722472 .067393$

C -2.8226430 .9591372 .575518$

C -1.5466403 .0899772 .346678$

O -3.6239661 .3840083 .402783$

C $-2.1923463 .958847 \quad 1.314570$

C $-1.5315904 .899750 \quad 0.623393$

C $-2.1772645 .809972-0.380699$

C $-1.4930655 .777508-1.728647$

C $-0.596724 \quad 6.718425-2.071158$

C $-1.9214924 .714725-2.714025$

C $-1.2425703 .392957-2.480682$

N $-1.787839 \quad 1.6982932 .017063$

H $2.315806-4.193342-0.943924$

H $-0.060732-3.6396960 .909404$

H $0.334351-3.715641-2.180100$

H -1.992035 -4.810614 -0.502912

H $-0.797958-5.806477-1.286261$

H -3.908989 -4.628914 -3.744098

H -2.023852 -5.431576-4.760257

H $-0.568802-4.662654-4.123379$

H $-0.953466-6.344268-3.694309$

H $-5.195643-4.752728-1.593579$

H -3.861034 -4.047901-0.706914

H -4.049643 -2.005125 -2.279817

H -5.561869 -2.707068 -2.867197

H -6.418060 -2.724569 -0.483027

H $-6.954961-0.782849-1.986849$

H -5.424874 -0.037510 -1.587057

H -7.639439 $1.425666-1.124069$

H -5.0278780 .7397380 .901340$

H -8.900415 $-0.443539-0.477037$

H -8.6885010 .0330881 .190804$

H -8.184066 -1.585704 0.661962

H $-6.979549-0.1036872 .760275$

H -4.422961 -0.9831853 .476924$

H -3.695403 -2.325675 2.632646

H -5.780374 -2.923823 2.491767

H -2.384685 -0.431795 -1.449629

H $\quad 0.108419 \quad 1.198688-2.221254$

H $-3.7585301 .456488-2.474478$

H $-3.7887743 .025598-1.672313$

H $-3.365773 \quad 1.610162-0.744297$

H -1.929127 -0.9468312 .486177$

H -2.712014 -0.4710540 .980073$

H -0.4624743 .2319502 .387940$

H -1.9650373 .3134303 .333168$

H -3.2582353 .8241381 .146722$

H -0.4726295 .0524850 .816465$

H -2.145672 6.8289440 .027373

H -3.242094 $5.577959-0.506060$

H $-0.1249566 .720477-3.048112$

H $-0.3089417 .502591-1.378706$

H -1.666755 $5.030308-3.735132$

H -3.012707 4.639720 -2.718676

H $-0.1782523 .405357-2.717421$

H $-1.205858 \quad 1.274637 \quad 1.299935$

C $3.660479-3.0404171 .317051$

O $4.602146-3.1933230 .232136$

C $5.922041-2.9564640 .735670$
C $5.754982-2.3530642 .123366$

C $4.500081-3.0623442 .594448$

H $6.383443-3.9462290 .861022$

C $6.791345-2.161898-0.241622$

H $6.619151-2.5348912 .769280$

H $5.583695-1.2719862 .075722$

C $3.846706-2.3956213 .790220$

H $4.755952-4.0979162 .856373$

C $6.331763-0.731797-0.603889$

O $8.097183-2.0790590 .346596$

H $6.903015-2.743474-1.165044$

H $6.341457-0.1038810 .291914$

O $7.346004-0.181787-1.463009$

C $4.970331-0.618153-1.323926$

C $4.6646260 .804425-1.834999$

H $4.161157-0.941051-0.664737$

O $4.984823-1.499244-2.449510$

H $5.5444291 .198904-2.356525$

H $3.8741200 .742989-2.595570$

H $4.517279-2.4172024 .655605$

H $3.608729-1.3486043 .580557$

H $2.920703-2.9078534 .068448$

C $2.610120-4.1483181 .251093$

H $8.664816-1.593343-0.276158$

H $7.330029-0.687342-2.293972$

H $4.092923-1.493215-2.837109$

H $1.964794-4.1073922 .135897$

H $3.099199-5.1301361 .246232$

H $3.183045-2.0602211 .192317$

C $2.1669842 .397710 \quad 0.436971$

O $2.8052941 .583108-0.555527$

C $4.2208941 .759276-0.712228$

C $4.5559703 .219458-1.048452$

C $3.9728994 .168598-0.000344$

C $2.488793 \quad 3.8893510 .239654$

H $1.0920672 .274707 \quad 0.251584$

C 2.4659421 .8883801 .855001

H $4.719987 \quad 1.4852190 .221804$

O $4.0410823 .542597-2.338393$

H $5.6464413 .329726-1.089486$

H $4.1014415 .203478-0.340154$

O $4.700068 \quad 4.010160 \quad 1.214050$

H 2.1326974 .4584251 .105500

O $1.7467714 .368348-0.884627$

C 1.6212970 .6766062 .197997

H 3.5122301 .6058601 .994490

H 2.2112662 .6732402 .577114

H $4.2963654 .460126-2.534195$

H $4.3317914 .636427 \quad 1.860365$

H $1.9853223 .823012-1.653691$

O $1.5160530 .580918 \quad 3.555667$

O $1.128637-0.0895921 .381486$

C $0.734611-0.5031204 .072232$

H $0.750249-1.3777543 .414931$

H $1.154532-0.7905045 .039900$

$\mathrm{H}-0.291626-0.1600644 .224366$

SCF Energy (B3LYP/6-31G**//MMFF) $=-3245.90913115$

0800059

MM̄FF Geometry

C $-0.702128-2.012778-2.600223$

C $0.103253-3.082320-2.677620$

C $0.871142-3.610675-1.498426$

O $2.269495-3.374004-1.775233$

C $0.648133-5.116727-1.273492$

C $-0.775593-5.511664-0.943917$

C $-1.337942-5.3633930 .271511$

C $-1.545246-6.160822-2.063588$

C $-0.708943-4.7370691 .484518$

C $-1.678410-3.7687612 .177092$

C $-0.950312-2.8898933 .204782$

C $-1.930363-2.3520774 .248046$

C $-1.247704-1.3879715 .227103$

O $-0.346028-1.8033712 .488367$

O $-2.274841-0.7030865 .960012$

C $-0.481895-0.3229344 .419216$

C $-0.394234-2.1284856 .263113$

$\begin{array}{llll}\text { O } & 0.286291 & 0.514716 & 5.280937\end{array}$ 
C $0.420143-0.9077713 .304160$

C $0.939994 \quad 0.2506932 .417139$

O $1.534507-1.5726123 .901720$

C $3.094087-3.231879-0.702856$

O $2.817919-3.404766 \quad 0.473246$

C $4.391451-2.742502-1.211085$

C $5.131596-1.956900-0.415454$

C $6.368543-1.274156-0.793249$

C $7.017993-1.678466-2.090586$

C $1.642111-0.1842351 .124101$

C 2.1470351 .0151620 .337704

C $2.9656281 .671752-1.920590$

O 2.2566342 .1352170 .830577

C $4.4471161 .535279-2.079280$

C $5.3348562 .398966-1.561851$

C $6.8225942 .312778-1.767830$

C $7.5792452 .040083-0.484897$

C 7.9205493 .0414310 .343400

C $8.0053570 .613678-0.205951$

C $6.827503-0.2947010 .013873$

N $2.485050 \quad 0.688115-0.967754$

H $-0.783033-1.475688-1.657843$

H $\quad 0.201555-3.614560-3.620289$

H $0.588999-3.044263-0.602823$

H $1.310938-5.469802-0.472199$

H $0.995454-5.674140-2.154786$

H -2.353972 -5.7279160 .416238$

H -2.567913 -6.420133 -1.770070

H -1.612229 $-5.490107-2.925267$

H -1.047145 -7.083113 -2.379945

H $-0.442242-5.5349712 .188374$

H $\quad 0.218873-4.2164661 .237032$

H -2.166189 -3.130283 1.428600

H -2.461011 -4.360570 2.668381

H $-0.168245-3.4826513 .693763$

H -2.406077 -3.176004 4.794971

H -2.747669 -1.819909 3.743872

H -2.810751 $-1.371496 \quad 6.420424$

H $-1.232940 \quad 0.326242 \quad 3.948893$

H -1.012980 -2.825813 6.840918

H $\quad 0.032607-1.4320836 .993449$

H $0.420431-2.7023485 .816743$

H -0.3199940 .8750785 .950644$

H 0.0959940 .8888262 .124915

H 1.6280110 .8739293 .002745

H $2.264493-1.6011923 .263595$

H $4.652840-2.963921-2.239006$

H $4.765398-1.7385130 .587042$

H $7.198217-2.759504-2.108264$

H $7.986127-1.204296-2.261615$

H $6.379075-1.416222-2.940829$

H $2.507357-0.8142991 .347466$

H $0.948334-0.7516420 .494321$

H $2.6855042 .677844-1.592113$

H $2.4689391 .478070-2.876590$

H $4.7994640 .702788-2.682377$

H $4.9721613 .238303-0.972367$

H $7.1590643 .265712-2.197142$

H $7.0769831 .554223-2.518502$

H 8.4852062 .8580551 .251324

H 7.6388414 .0676650 .131442

H $8.629050 \quad 0.566866 \quad 0.696257$

H $8.6546840 .279829-1.019588$

H $6.276549-0.0752470 .929726$

H $2.400208-0.275117-1.278939$

C $-3.023550-1.390267-3.391681$

O $-3.215056-0.407938-2.351882$

C $-4.145900-0.937055-1.390784$

C $-4.838808-2.106067-2.083041$

C -3.699374 -2.674394 -2.905627

H $-3.548972-1.328652-0.558006$

C $-5.0908040 .154651-0.883078$

H $-5.255074-2.824145-1.370048$

H -5.648852 -1.768488 -2.739946

C $-4.147428-3.596333-4.025405$

H -3.041446 -3.234308 -2.230233

C $-4.3904721 .388308-0.274788$

O $\quad-5.8921440 .610930-1.980745$
H $-5.787585-0.281859-0.157182$

H -3.934195 $1.978949-1.076844$

O

C -3.3388421 .0822060 .807363$

C -2.8859722 .3025691 .631609$

H -2.4666300 .6095580 .341461$

$\begin{array}{llll}\text { O } & -3.890591 & 0.132684 & 1.724672\end{array}$

H -3.7200102 .6607102 .248910$

H $-2.131877 \quad 1.9454172 .343937$

H -4.675455 -4.465435 -3.619627

H $-4.823493-3.086431-4.719797$

H -3.287428 -3.959752 -4.596786

C $-1.536302-1.504988-3.741278$

H $-6.4885131 .298604-1.638108$

H $-5.804921 \quad 1.7379341 .037308$

H $-3.192901-0.0932352 .362617$

H -1.154505 -0.513491-4.010313

H -1.419359-2.147312 -4.621970

H -3.551437-1.000681-4.271864

C $-0.5419403 .930125-0.785531$

O $\quad-1.1745292 .9800550 .084029$

C -2.3068023 .4653190 .810039$

C $-1.9017684 .645123 \quad 1.708539$

C -1.228933 5.7527180 .891502

C $-0.1278815 .197951-0.017019$

H $0.3751153 .433684-1.124777$

C -1.398293 $4.229732-2.025335$

H $-3.073430 \quad 3.8034190 .106613$

O -1.0059964 .1890562 .720714$

H -2.797033 5.0327732 .209298

H -0.7915896 .4965541 .568723$

O $-2.201516 \quad 6.4138750 .087842$

H $\quad 0.1937975 .966884-0.728540$

O $1.0152174 .889601 \quad 0.784946$

C $-1.5645043 .015499-2.913931$

H $-2.3996654 .573627-1.754374$

H $-0.9089665 .005466-2.625710$

H -0.7897304 .9498413 .286225$

H $-2.873278 \quad 6.7847860 .685105$

H $\quad 0.785324 \quad 4.134981 \quad 1.354189$

O $-2.3519763 .356112-3.977122$

O $-1.0720501 .915503-2.707792$

C $-2.6137542 .310551-4.918113$

H -1.683484 $1.831629-5.238616$

H $-3.2951201 .579110-4.475844$

$\mathrm{H}-3.0958382 .754343-5.793298$

SCF Energy $\left(B 3 L Y P / 6-31 G^{* *} / / M M F F\right)=-3245.91365812$

0800060

MM̄FF Geometry

C $-2.4166242 .376801-1.504942$

C $-3.2867003 .124549-0.810594$

C -3.6115022 .9395910 .655305$

O -2.6356312 .0725031 .282065$

C -4.9928152 .2901370 .820623$

C -5.5081532 .3796312 .242893$

C $-5.493367 \quad 1.3671273 .131316$

C -6.1134963 .7067002 .623690$

C $-4.935884-0.0178942 .951473$

C $-6.040193-1.0833802 .899560$

C $-6.823383-1.0775141 .577314$

C $-8.001568-2.0508331 .642158$

C $-8.739538-2.1402540 .298251$

O $-5.921571-1.4733910 .539016$

O $-9.614323-3.2775030 .349733$

C $-7.709312-2.411742-0.816786$

C $-9.630617-0.9174470 .050411$

O $-8.324688-2.362262-2.102331$

C $-6.485623-1.465278-0.778498$

C $-5.403922-1.980655-1.757658$

O $-6.892589-0.154093-1.171017$

C -1.552638 2.6485421 .863072

O -1.3616833 .8460402 .026569$

C -0.5594881 .6370262 .293917$

C $-0.549950 \quad 0.3668341 .858139$

C $0.442958-0.6560192 .202844$

C $1.555743-0.2612733 .138809$

C $-4.061612-1.238850-1.676339$ 
C $-2.997686-1.917728-2.523623$ C $-0.533066-1.950680-2.885754$ O $-3.248908-2.816470-3.322598$ C $0.035764-2.965279-1.943401$ C $1.283454-2.912180-1.454526$ C $1.843448-3.912532-0.485968$ C $2.260379-3.281000 \quad 0.824418$ C $3.549223-2.9872021 .064882$ C $1.205845-3.0872701 .891936$ C $0.328948-1.8858511 .656863$ N -1.733618 -1.393097 -2.289474 H -1.931595 $1.536955-1.015603$ H -3.783095 $3.954333-1.308295$ H -3.5971183 .9266081 .132926$ H $-4.947097 \quad 1.245076 \quad 0.495910$ H -5.7257432 .7730720 .160597$ H -5.9169181 .5479524 .118805$ H $-6.515923 \quad 3.7037543 .642379$ H -5.3648214 .5026882 .569439$ H -6.9370073 .9559321 .946389$ H $-4.283907-0.0956112 .076543$ H $-4.293515-0.2248343 .816820$ H -5.561289-2.063155 3.025483 H $-6.726322-0.9464123 .744732$ H -7.185965 -0.063692 1.376909 H -8.696395 -1.768705 2.443075 H -7.634056 -3.053157 1.903507 H $-10.224990-3.152176 \quad 1.096278$ H -7.352062 -3.442843 -0.684714 H -10.377814 -0.8206560 .847329$ H $-10.198582-1.024970-0.880402$ H -9.0714490 .0195240 .007061$ H $-9.076370-2.979275-2.087466$ H $-5.211247-3.040491-1.543447$ H -5.774467 -1.919161 -2.788881 H $-6.1240380 .327350-1.514607$ H 0.2118042 .0193712 .954822 H $-1.3270890 .040214 \quad 1.169197$ H $1.152570 \quad 0.0302254 .114822$ H 2.1239250 .5797502 .726004 H $2.278738-1.0594863 .319361$ H $-4.167740-0.210143-2.035016$ H -3.704778 -1.209815 -0.640567 H $0.156232-1.121384-3.071252$ H $-0.779242-2.425209-3.840819$ H $-0.617626-3.780610-1.640703$ H $1.946859-2.111823-1.771474$ H $2.706912-4.393124-0.964576$ H $1.130774-4.722984-0.288759$ H $3.868215-2.5663032 .012447$ H $4.315353-3.1583420 .316039$ H $1.666506-3.0444042 .885500$ H $0.567391-3.9794841 .933361$ H $-0.502914-2.0772460 .979018$ H -1.614316 -0.706205 -1.550969 C $-0.5268062 .918234-3.093874$ O $\quad 0.2584851 .887316-2.455825$ C $1.1165322 .516146-1.479961$ C $1.3263833 .922207-2.009055$ C $-0.0743964 .254193-2.491274$ H $0.5462942 .541432-0.542808$ C $2.3761901 .668292-1.299537$ H $1.6874244 .617053-1.245500$ H $2.0344583 .922294-2.846670$ C $-0.1231565 .412217-3.473245$ H $-0.6869274 .514758-1.619310$ C $3.4524152 .314615-0.411893$ O $1.9790570 .431796-0.697887$ H $2.7919481 .420892-2.282420$ H $3.8687743 .194289-0.916996$ O 2.8184932 .7951660 .782203 C 4.5913141 .3686000 .019428 C $5.3174520 .693692-1.157748$ H 4.1996090 .6164120 .714634 O $5.5244622 .165570 \quad 0.757659$ H $4.5916990 .235701-1.836570$ H $5.869277 \quad 1.464515-1.710170$ H $\quad 0.249415 \quad 6.329222-3.005399$
H $\quad 0.489878 \quad 5.214234-4.358939$ H -1.149797 $5.594229-3.806782$ C $-2.0221862 .605763-2.936550$ H $1.2365890 .080102-1.217906$ H 3.5089603 .2037251 .331831 H $6.217222 \quad 1.5722601 .093591$ H -2.248588 $1.681245-3.482312$ H $-2.6198743 .403814-3.391390$ H $-0.2743752 .877527-4.160659$ C $8.322616-0.7125830 .588789$ O 7.3586710 .1940160 .035780 C $6.296113-0.409752-0.705197$ C $6.861300-1.193164-1.900302$ C $7.916364-2.210633-1.453603$ C $8.950664-1.584390-0.511521$ H $9.111005-0.0651450 .994290$ C $7.743677-1.5266231 .751918$ H $5.745615-1.094032-0.052353$ O $7.437224-0.299260-2.851169$ H $6.038114-1.710826-2.406095$ H $8.429246-2.614192-2.335114$ O $7.282349-3.296696-0.784079$ H $9.572637-2.360724-0.050728$ O $9.823226-0.744495-1.273359$ C $7.386862-0.6233752 .908044$ H $6.858772-2.1072691 .477080$ H $8.479485-2.2495232 .124119$ H $8.119350 \quad 0.221856-2.394114$ H $6.651988-3.698146-1.406209$

H $10.265221-1.304569-1.934055$

O $6.053754-0.3381582 .883404$

O $8.201360-0.2024013 .719240$

C 5.5925720 .5398573 .914570

H 6.1329121 .4905763 .876650

H 4.5310950 .7355923 .744429

H 5.7114220 .0656034 .893128

SCF Energy $(B 3 L Y P / 6-31 G * * / / M M F F)=-3245.92302437$

0800061

MM̄FF Geometry

C $-3.352487-3.7311440 .603152$

C $-2.037716-3.4635300 .589411$

C $-1.202372-3.3597081 .837349$

O $-0.341217-2.2028491 .729722$

C $-0.301072-4.6025521 .974872$

C $0.579926-4.5783153 .211193$

C $1.927877-4.5766033 .185312$

C $-0.143203-4.5742764 .533356$

C $2.821857-4.6411091 .977813$

C $3.098127-3.2539001 .400057$

C $3.992381-3.2779060 .152553$

C $5.444550-3.6299910 .486024$

C $6.368518-3.508616-0.737242$

O $3.922174-1.963826-0.416447$

O $7.725233-3.529100-0.265914$

C $6.140038-2.132516-1.399120$

C $6.221509-4.708870-1.679673$

O $6.883015-2.017744-2.610379$

C $4.650233-1.805886-1.640685$

C $4.432026-0.357225-2.132333$

O $4.142834-2.674789-2.660438$

C $-0.826969-1.0028502 .144141$

O $-1.968660-0.7507552 .492553$

C $0.310149-0.0521812 .154545$

C 0.1467451 .1789922 .661363

C 1.1917882 .1951722 .826755

C 2.5908241 .8338872 .401895

C $4.8758850 .729499-1.150736$

C $4.4384842 .111674-1.602528$

C $4.2853644 .454017-0.801980$

O $4.0229662 .349706-2.733420$

C 4.0158805 .1078030 .514406

C 2.8434145 .6757660 .834349

C 2.5908816 .3943652 .127502

C 1.4509245 .7972212 .920122

C 0.2583706 .4153952 .978058

C 1.7411494 .5475973 .724304

C 0.8529663 .3821873 .373037 
N $4.5780983 .050676-0.589533$ H -3.841722 -3.899961 1.558242 H -1.527975 -3.324331 -0.360628 H -1.840483 -3.262318 2.724332 H $\quad 0.311860-4.691117 \quad 1.070854$ H -0.920468 -5.508378 2.012717 H $2.461186-4.5411434 .134354$ H $0.538142-4.7165075 .379194$ H $-0.663599-3.6242024 .687695$ H $-0.877095-5.3862364 .567249$ H $3.759331-5.1190472 .284655$ H $2.393673-5.296703 \quad 1.211526$ H $2.149116-2.7764081 .128597$ H $3.542037-2.6004702 .163101$ H $3.572160-3.993254-0.564082$ H $5.521145-4.6361510 .914053$ H $5.812986-2.9463121 .263751$ H $7.861812-4.3606110 .219767$ H $6.563138-1.387437-0.714260$ H $6.460570-5.640553-1.152619$ H $6.931613-4.647985-2.511506$ H $5.214549-4.812925-2.088611$ H $\quad 6.415956-2.527702-3.293873$ H $4.922397-0.211332-3.103630$ H $3.358502-0.219371-2.326965$ H $3.345578-2.270717-3.041377$ H $1.266059-0.4097211 .788633$ H $-0.842273 \quad 1.4651623 .017374$ H 2.9786501 .0152513 .018146 H 3.2989412 .6605572 .482878 H 2.6089121 .5181111 .354093 H $4.4505520 .539413-0.159011$ H $5.9673150 .744762-1.062467$ H $3.4427384 .548008-1.495556$ H $5.1626854 .907969-1.274486$ H $4.8364445 .140561 \quad 1.227328$ H 2.0323905 .6617130 .110739 H 2.3757757 .4436171 .885835 H 3.4909506 .4185962 .754683 H -0.5547236 .0232123 .579608$ H 0.0683827 .3297802 .425763 H 1.5895694 .7782774 .787559 H 2.8014024 .2907163 .649354 H -0.1903263 .5341723 .654002$ H 5.0954412 .7879830 .244984 C $-5.414576-2.937590-0.676857$ O $-5.016773-1.554982-0.591980$ C $-5.975671-0.856557 \quad 0.225007$ C -7.159231 -1.8044180 .399178$ C $-6.462915-3.1505050 .414630$ H $-5.503013-0.7089281 .203217$ C $-6.3419710 .497829-0.388958$ H -7.722449 -1.600066 1.314765 H -7.855463 -1.746459-0.445675 C -7.393607 -4.322961 0.159084 H $-5.991708-3.2776421 .395964$ C $-5.1487731 .443858-0.635158$ O $-6.9796430 .268390-1.652378$ H -7.089163 $0.989910 \quad 0.245668$ H $-4.5607881 .073670-1.482126$ O $-5.6883722 .704787-1.055150$ C -4.2305391 .6668990 .579340$ C -3.2377532 .8351040 .435621$ H -3.6802720 .7426660 .786998$ O H -3.7849103 .7865470 .425539$ H -2.636683 2.8539461 .354894 H $-8.167843-4.374620 \quad 0.931687$ H -7.893489 -4.235642 -0.811348 H $-6.841600-5.267993 \quad 0.170622$ C -4.191485 -3.858593 -0.637835 H -7.210357 $1.137693-2.022558$ H $-6.1752663 .080761-0.301547$ H $-4.454073 \quad 1.9910432 .496873$ H -3.571520 -3.644600 -1.517062 H -4.518484 -4.900927 -0.727536 H -5.886824 -3.053766 -1.661726 C $-0.669908 \quad 1.270694-1.781358$
O

C $-2.3103982 .753752-0.786779$

C $-1.3661103 .965553-0.859586$

C $-0.3377543 .809609-1.984529$

C $0.3258512 .429240-1.959611$

H $-0.1005650 .386193-1.466889$

C -1.418104 $0.916386-3.076007$

H -2.928072 2.734085-1.689513

O $-0.683540 \quad 4.1112830 .383129$

H -1.966029 4.870458 -1.013778

H $0.4382524 .578370-1.883390$

O $\quad-0.9730833 .996926-3.244990$

H $0.9073452 .274364-2.875393$

O $1.2552332 .387528-0.875185$

C $-2.216103-0.363095-2.951103$

H $-2.1170321 .704475-3.367666$

H $-0.6942750 .770146-3.886020$

H $-0.1579124 .927157 \quad 0.333539$

H -1.356819 $4.890279-3.250924$

H $\quad 0.7515812 .486424-0.049400$

O $-2.903981-0.566168-4.114014$

O $-2.236340-1.090621-1.968860$

C $-3.723160-1.738480-4.153162$

H -4.114432 -1.845659 -5.168300

H $-3.135858-2.629022-3.909680$

H $-4.564029-1.626562-3.463438$

SCF Energy $(B 3 L Y P / 6-31 G * * / / M M F F)=-3245.91469733$

0800062

MM̄FF Geometry

C -1.297444 3.0867991 .460198

C -0.6350082 .2399382 .263465$

C $-1.187476 \quad 1.0520173 .010249$

O $-0.534314-0.1195512 .461889$

C -2.7089850 .8305582 .908579$

C $-3.234626-0.1383943 .950606$

C $-3.546738-1.4284893 .720567$

C $-3.4483880 .452090 \quad 5.321737$

C $-3.395609-2.2096852 .445576$

C $-4.749419-2.6049791 .839889$

C $-5.476627-1.4303311 .167198$

C $-6.860581-1.8623760 .678730$

C $-7.564907-0.745037-0.105148$

O $-4.677474-1.0101290 .057771$

O $-8.690331-1.323434-0.783463$

C $-6.604626-0.217556-1.191218$

C -8.1265640 .3385380 .822646$

O $-7.1591390 .916640-1.854235$

C $-5.1917600 .118838-0.659469$

C $-4.2367790 .386514-1.846592$

O -5.2650051 .2817930 .165281$

C $0.103713-0.9629503 .310934$

O $0.270174-0.8041694 .512714$

C $0.576825-2.1849652 .618714$

C $0.418904-2.4212911 .306750$

C $0.823400-3.6375780 .594049$

C $1.517802-4.7152281 .386215$

C $-2.7750750 .606740-1.438871$

C $-1.8713080 .594081-2.654089$

C $-0.778532-0.937296-4.271675$

O $-1.481311 \quad 1.632465-3.182407$

C $-0.320494-2.359131-4.266858$

C $0.953140-2.725395-4.061293$

C $1.425219-4.149094-4.046371$

C $1.879673-4.592621-2.674183$

C $3.180272-4.786905-2.398577$

C $0.812681-4.895375-1.646118$

C $0.546940-3.735855-0.723783$

N $-1.566995-0.689218-3.080158$

H $-2.3548432 .945867 \quad 1.259315$

H 0.4268282 .4205652 .428765

H $-0.907360 \quad 1.196944 \quad 4.060726$

H $-2.9652670 .483189 \quad 1.901933$

H -3.2452051 .7781353 .046681$

H $-3.938868-2.0140794 .551689$

H $-3.869065-0.2727686 .026772$

H -2.5019220 .8060545 .741480$

H -4.1427301 .2970185 .266868$ 
H -2.782731-1.686589 1.706323 H -2.845254 -3.127082 2.690967 H -4.558962 -3.381955 1.087799 H -5.387727 -3.051345 2.612513 H $-5.568437-0.6054571 .882232$ H $-7.483750-2.1939991 .518560$ H $-6.758507-2.7356390 .018841$ H $-9.269872-1.725050-0.113547$ H -6.508690 -1.004599-1.952388 H -8.852239 -0.0946651 .521561$ H -8.6749561 .0988330 .255393$ H $-7.3569380 .837749 \quad 1.414853$ H $-8.0382970 .659653-2.181328$ H -4.281638 $-0.467490-2.534785$ H -4.571097 $1.268412-2.407640$ H $-4.388231 \quad 1.6929500 .213672$ H $1.052242-2.9093833 .272721$ H $-0.069342-1.6682230 .690493$ H $1.871811-5.5485950 .776309$ H $0.841327-5.1314532 .140851$ H $2.403636-4.3168791 .891609$ H -2.647987 $1.570218-0.936262$ H -2.428691 -0.164456-0.741382 H $0.056303-0.229521-4.301858$ H -1.412096 -0.744983 -5.143570 H - $-1.075330-3.122555-4.438979$ H $1.704404-1.960376-3.888828$ H $2.244940-4.237975-4.770708$ H $0.643570-4.831046-4.404187$ H $3.503580-5.132298-1.422155$ H $3.949864-4.597069-3.139320$ H $1.077556-5.806232-1.100523$ H $-0.126592-5.150573-2.153647$ H $0.038260-2.900523-1.204995$ H -2.073136 -1.468121 -2.667358 C $-0.4888834 .098735-0.699210$ O $0.4466953 .027651-0.944768$ C $1.6000883 .574375-1.597003$ C $1.0652974 .764175-2.374903$ C $0.0972285 .351500-1.365509$ H $2.2728163 .923139-0.803499$ C $2.3437832 .523178-2.427941$ H $1.8531575 .462950-2.670520$ H $0.5246924 .448097-3.274501$ C $-0.9546116 .256312-1.984805$ H $0.6704955 .935975-0.633078$ C $2.6752931 .246581-1.625235$ O $1.5894952 .183182-3.588147$ H $3.2756602 .981659-2.779671$ H $3.0236561 .542487-0.630193$ O $1.5039040 .450530-1.445813$ C $3.7708900 .383828-2.280042$ C $4.052248-0.945135-1.556491$ H $4.699590 \quad 0.965151-2.330537$ O $3.4234680 .047924-3.624212$ H $3.204838-1.629549-1.688104$ H $4.890507-1.416895-2.085989$ H $-0.4838857 .113973-2.476375$ H -1.550384 $5.724298-2.734124$ H -1.637572 $6.636785-1.218390$ C -0.6528394 .2805330 .811895$ H $\quad 0.7449901 .808257-3.284833$ H $0.8452130 .995733-0.983155$ H $3.3390080 .875399-4.126947$ H -1.273937 5.1599091 .019328 H $0.3273384 .464623 \quad 1.270251$ H -1.439811 3.808528 -1.161149 C 5.8137770 .4352491 .444349 O $5.417540 \quad 0.152913 \quad 0.094877$ C $4.380010-0.819367-0.058987$ C $4.801033-2.1665110 .551060$ C $5.250708-2.0013072 .006652$ C $6.251536-0.8523192 .164411$ H 6.6982801 .0784341 .348780 C 4.7385221 .2219402 .213315 H $3.478458-0.4683880 .453038$ O $5.868220-2.727892-0.211273$ H $3.955035-2.8595880 .489155$
H $\quad 5.714674-2.9319302 .355744$

O $4.121946-1.7429712 .835296$

H $6.422078-0.6358423 .225611$

O $7.510334-1.2463481 .611305$

C $4.5834172 .642021 \quad 1.708798$

H 3.7544740 .7507462 .151373

H 5.0324721 .2879993 .267716

H $6.078804-3.5958110 .173094$

H $3.526287-2.5074002 .764313$

H $7.809622-2.0344622 .095733$

O 3.7522573 .3189812 .557083

O 5.1092403 .0999550 .704163

C 3.5237484 .6941792 .234902

H 2.8512015 .1124002 .988611

H 4.4660275 .2497352 .257767

H 3.0478184 .7818801 .254989

SCF Energy (B3LYP/6-31G**//MMFF) $=-3245.91274460$

08_00063

MM̄FF Geometry

C $2.297856-1.118492-2.588647$

C $3.053518-2.224557-2.664503$

C $3.947270-2.741726-1.560428$

O $3.427173-2.365799-0.263179$

C $5.359080-2.142690-1.669305$

C $6.421476-3.038931-1.066569$

C $6.870173-2.9693260 .201699$

C $7.010943-4.046851-2.020237$

C $6.387354-2.0857951 .318020$

C $7.425146-1.0358041 .735852$

C 7.4142440 .2038300 .828943

C 8.5491951 .1575851 .206874

C 8.4939942 .4616560 .397532

O 6.1501630 .8545481 .007458

O 9.3767023 .4066001 .021573

C 7.0688363 .0446270 .490716

C $9.0067502 .268975-1.034480$

O $6.9258574 .180221-0.359848$

C 5.9555042 .0139930 .189714

C 4.5738732 .6209180 .537868

O $5.9857891 .688482-1.201696$

C $2.345971-3.035370 \quad 0.214808$

O $1.768831-3.976900-0.302974$

C $1.953689-2.3667151 .474002$

C $0.712321-2.5350631 .950520$

C $0.154247-1.8971013 .144193$

C $1.072015-1.0295533 .966087$

C 3.4197831 .6071900 .543544

C 2.0699912 .2814580 .731549

C -0.3732551 .7835390 .718687$

O $1.945566 \quad 3.4853680 .941513$

C $-0.867298 \quad 1.6038272 .119594$

C $-2.0152130 .976523 \quad 2.419166$

C -2.5608320 .8390053 .811787$

C $-3.002213-0.5718124 .135137$

C $-4.300883-0.9154004 .096791$

C -1.947557-1.558936 4.582729

C -1.147687 -2.103785 3.432048

N $1.018502 \quad 1.379467 \quad 0.632410$

H $2.353884-0.497826-1.699980$

H $3.008840-2.834996-3.563405$

H $3.981190-3.836947-1.620659$

H $5.387257-1.157885-1.187520$

H $5.624894-1.965574-2.720132$

H $7.660019-3.6586530 .499418$

H $7.764949-4.681987-1.543455$

H $6.227807-4.703245-2.412935$

H $7.492077-3.537161-2.861517$

H $5.432871-1.6077941 .086038$

H $6.191033-2.7387842 .178130$

H $7.183676-0.7228462 .760231$

H $8.426734-1.4823511 .760459$

H $7.516842-0.114151-0.214361$

H 9.5243980 .6701621 .083406

H 8.4739641 .4095572 .274053

H $10.268668 \quad 3.019119 \quad 1.035902$

H 6.9326223 .4088421 .518838

H $\quad 10.046640 \quad 1.920710-1.024945$ 
H $9.0140923 .217717-1.582362$ H $8.4226231 .544675-1.606012$ H $7.6336444 .806000-0.128787$ H 4.6150903 .0721231 .538028 H $4.3410023 .425058-0.171961$ H $5.0981321 .424115-1.489251$ H $2.682978-1.7188931 .947010$ H $0.029694-3.1834981 .403279$ H $1.898355-1.6241044 .371070$ H $0.578999-0.5504444 .814014$ H $1.489468-0.2199453 .357312$ H $3.3917051 .058556-0.401138$ H 3.5547100 .8852201 .357037 H $-0.933132 \quad 1.1749940 .002109$ H -0.4650292 .8353610 .429146$ H -0.274272 2.0394862 .920194 H -2.621017 0.5733241 .611834 H -3.4148171 .5234083 .900696$ H -1.8327671 .1725814 .561492$ H -4.628606 -1.917851 4.351149 H -5.064851-0.204612 3.799177 H -2.420249 -2.415834 5.081499 H $-1.327523-1.0975135 .356144$ H $-1.729223-2.7451492 .768158$ H 1.2196360 .3910390 .513243 C $-0.034147-0.359886-3.000903$ O $-0.567039-1.525546-2.345159$ C $-2.005474-1.520705-2.481603$ C $-2.361435-0.209065-3.170260$ C $-1.1142100 .063643-3.989989$ H -2.244893 -2.371672 -3.131006 C $-2.626635-1.716923-1.092565$ H -3.267310 -0.288441-3.778835 H -2.516945 $0.588136-2.433199$ C $-0.995403 \quad 1.500396-4.464372$ H -1.112951 -0.606812 -4.859777 C $-4.164910-1.622487-1.083174$ O $-2.224645-3.006712-0.621660$ H $-2.209911-0.984660-0.391759$ H $-4.448085-0.644465-1.483872$ O $-4.704164-2.602979-1.969512$ C $-4.806998-1.7761420 .312330$ C $-6.346244-1.7351460 .307165$ H -4.426562 -0.9895920 .972355$ O $-4.450294-3.0276670 .902901$ H $-6.737650-2.585839-0.265416$ H $-6.672223-1.9026981 .342928$ H -0.093462 $1.637249-5.069287$ H $-1.858523 \quad 1.776605-5.078637$ H $-0.9423442 .198107-3.622889$ C $1.315999-0.687592-3.641305$ H -1.253474 -3.041609-0.663528 H -4.462776 -3.476444 -1.615773 H -3.485363 -3.033852 1.019746 H $1.7126650 .196469-4.151965$ H $1.191865-1.481636-4.387519$ H $\quad 0.096070 \quad 0.409471-2.228131$ C $-6.918713 \quad 1.9573830 .248654$ $\begin{array}{lllll}\text { O } & -6.481444 & 0.640523 & 0.610638\end{array}$ C $-6.954890-0.425307-0.217724$ C $-8.491617-0.470787-0.218484$ C $-9.0818730 .884934-0.617371$ C $-8.455133 \quad 2.032147 \quad 0.180304$ H $-6.5915082 .598077 \quad 1.077746$ C $-6.2282642 .458213-1.030788$ H $-6.610055-0.269506-1.243879$ O $-8.961904-0.8366121 .077188$ H -8.820656 -1.246608 -0.920485 H - $-10.1652710 .869409-0.445860$ O $-8.8640591 .083791-2.011004$ H $-8.7624092 .995982-0.241902$ O -8.9758141 .9778661 .511572$ C $-4.7594422 .750562-0.804725$ H $-6.2923561 .738828-1.850504$ H -6.699622 $3.397180-1.344945$ H $-9.932351-0.8802761 .036030$ H $-9.269253 \quad 1.934057-2.252718$ H -8.6221612 .7420741 .997862$
O $-4.2720853 .437752-1.880124$

O -4.1165392 .4169320 .180593$

C $-2.894826 \quad 3.816495-1.793677$

H $-2.7597184 .554461-0.997443$

H -2.264774 $2.940550-1.616204$

H -2.605714 4.268529-2.746130

SCF Energy (B3LYP/6-31G**//MMFF)= -3245.90689255

0800064

MM̄FF Geometry

C $-0.303295-4.1486890 .339931$

C $-1.526830-3.6626730 .087556$

C $-2.102050-3.504377-1.295775$

O $-2.353518-2.090926-1.485280$

C $-3.435749-4.262206-1.405680$

C $-4.098263-4.130425-2.765103$

C $-5.219864-3.422254-3.010127$

C $-3.425732-4.882574-3.884757$

C $-6.033071-2.627371-2.027342$

C $-5.816506-1.120098-2.212766$

C $-6.234596-0.328069-0.965632$

C $-6.1309701 .176080-1.219266$

C -6.4123631 .9979920 .048544$

$\begin{array}{lllll}0 & -5.348168 & -0.706853 & 0.093461\end{array}$

O $-5.975942 \quad 3.344356-0.196578$

C $-5.550509 \quad 1.446613 \quad 1.206087$

C $-7.913518 \quad 2.078097 \quad 0.349395$

$\begin{array}{lllll}\text { O } & -5.881809 & 2.087543 & 2.435517\end{array}$

C $-5.623076-0.0871821 .355627$

C $-4.600765-0.6472542 .375877$

O $-6.927467-0.445322 \quad 1.828690$

C $-1.285896-1.317860-1.830974$

O $-0.159899-1.700648-2.107458$

C $-1.7119280 .097118-1.758483$

C $-0.7893621 .066435-1.848567$

C $-1.0326732 .507332-1.754815$

C $-2.4361952 .963781-1.459677$

C $-3.126526-0.3902202 .042330$

C -2.5400510 .8393842 .714255$

C $-0.4632832 .215372 \quad 2.761787$

\begin{tabular}{llll}
\hline & -3.117266 & 1.464677 & 3.599312
\end{tabular}

C -0.6691863 .4441181 .934197$

C $0.337168 \quad 4.145416 \quad 1.390021$

C $0.144908 \quad 5.4239250 .625328$

C $0.8085985 .405121-0.732759$

C $2.0257345 .945197-0.913582$

C $0.0207114 .847693-1.896523$

C $0.0112693 .345492-1.919756$

N $-1.268978 \quad 1.1304592 .235746$

H $0.312836-4.507679-0.480482$

H $-2.129325-3.288320 \quad 0.912016$

H $-1.405225-3.863726-2.062909$

H $-4.105734-3.918905-0.611753$

H $-3.271763-5.329526-1.204638$

H $-5.603305-3.399572-4.029350$

H $-3.993110-4.830666-4.820092$

H $-2.430252-4.473378-4.081863$

H $-3.323125-5.940839-3.623314$

H $-7.093697-2.861091-2.183216$

H $-5.822718-2.927308-0.998628$

H $-4.756589-0.926094-2.418018$

H $-6.384654-0.778262-3.086702$

H -7.261275 $-0.605502-0.699069$

H $-6.8023991 .480222-2.031859$

H $-5.1175561 .415519-1.567182$

H $-6.4581323 .679820-0.971634$

$\begin{array}{llll}H & -4.516560 & 1.733568 & 0.984358\end{array}$

H -8.449693 $2.527187-0.495475$

H -8.110894 2.7307691 .206823

H $-8.364296 \quad 1.1027710 .543775$

H -6.7131651 .6998912 .757613$

H $-4.853015-0.3082063 .387780$

H $-4.730751-1.7390632 .395790$

H $-6.876456-1.3255682 .236822$

H -2.763850 $0.302325-1.601408$

H $0.2512830 .780291-1.998982$

H $-3.1019152 .715710-2.293187$

H -2.519946 $4.038704-1.287763$ 
H -2.813058 $2.482584-0.551135$

H -2.537237 -1.240422 2.409236

H -2.971295 -0.3270510 .961547$

H $\quad 0.577114 \quad 1.8804672 .753891$

H -0.7543582 .4168363 .797776$

H -1.690876 3.7958191 .811262

H 1.3606723 .8098151 .541363

H $0.565194 \quad 6.2378891 .230840$

H -0.9190265 .6653310 .508636$

H $2.4959165 .965522-1.891091$

H $2.5819906 .373511-0.086327$

H $0.4639405 .182343-2.844289$

H $-0.9830825 .281690-1.893694$

H $0.9897082 .911754-2.124099$

H -0.8874820 .5856491 .467697$

C $1.531266-3.2284931 .740948$

O $2.646635-3.8963341 .109875$

C $3.796602-3.7554821 .953557$

C $3.477766-2.6190632 .912106$

C $1.999534-2.8683613 .151502$

H $3.855598-4.6796052 .546732$

C $5.100350-3.6389621 .161947$

H $4.073361-2.6562023 .828896$

H $3.630002-1.6444302 .437466$

C $1.265456-1.6870213 .761681$

H $1.891259-3.7367193 .815695$

C $5.285283-2.417636 \quad 0.234279$

O $6.167132-3.6327732 .121271$

H $5.225836-4.5533560 .568109$

H $5.380357-1.5095690 .839306$

O $6.561249-2.584120-0.407398$

C $4.211848-2.210404-0.852040$

C $4.626136-1.265685-1.998718$

H $3.290744-1.843984-0.387165$

O $3.916143-3.477324-1.449734$

H $5.460859-1.714348-2.552889$

H $3.788968-1.231981-2.709771$

H $1.693258-1.4291334 .735769$

H $1.326319-0.8015393 .122109$

H $0.205986-1.919513 \quad 3.908713$

C $0.309410-4.1494701 .713031$

H $7.002671-3.5917031 .624951$

H $6.498560-3.363920-0.985604$

H $3.164496-3.348065-2.053120$

H $-0.428239-3.8205782 .454071$

H $0.596291-5.1775751 .962738$

H $1.341168-2.3132651 .164144$

C $3.9332372 .141702-0.636915$

O $3.7905920 .792816-1.103766$

C $4.9879740 .163444-1.569057$

C $5.6013210 .958729-2.734259$

C $5.8271802 .422476-2.348038$

C $4.5769333 .036419-1.711530$

H $2.9060382 .495062-0.481073$

C 4.6600982 .2049860 .714965

H $5.7146840 .116617-0.753043$

O $4.736051 \quad 0.891457-3.866500$

H $6.5528420 .492468-3.017029$

H $6.0986752 .993478-3.244263$

O $6.9220302 .489864-1.439840$

H $4.8157504 .013675-1.277371$

O $3.6134193 .278105-2.740323$

C 3.8469811 .5746371 .824730

H 5.6253251 .6932980 .692514

H 4.8217703 .2556820 .983689

H $5.1694121 .367209-4.595353$

H $7.0667053 .426785-1.223484$

H $3.3251242 .416237-3.086762$

O 4.4194211 .8861873 .025004

O 2.8506450 .8845411 .662483

C 3.7640251 .3549314 .180852

H 3.8630250 .2662654 .198447

H $4.253727 \quad 1.7658585 .067654$

H 2.7102681 .6488904 .196370

SCF Energy (B3LYP/6-31G**//MMFF) $=-3245.91195602$

0800065

MM̄FF Geometry
C $-3.939465-1.2711813 .258382$

C $-2.637105-1.3304872 .940941$

C $-1.544726-0.7691103 .809377$

O $-0.897694 \quad 0.3244263 .118291$

C $-0.464062-1.8009944 .203110$

C $0.333546-2.4147443 .064551$

C $1.432661-1.8630842 .509368$

C $-0.136802-3.7701272 .601334$

C $2.080791-0.5522202 .863039$

C $3.570783-0.6779963 .216502$

C $4.483795-0.9842742 .017830$

C $5.950886-1.0130452 .457407$

C $6.908760-1.1895421 .268805$

O 4.2894320 .0423851 .040686

O $8.234630-0.8705301 .718691$

C $6.545052-0.1630670 .174414$

C $6.954073-2.6440630 .785769$

O $7.310581-0.384808-1.007830$

C $5.038280-0.138122-0.167555$

C $4.7204531 .048809-1.109581$

O $4.694929-1.358087-0.827734$

C $-1.503100 \quad 1.5414103 .129730$

O -2.5843931 .8253093 .618454$

C -0.6001952 .4869522 .436533$

C -1.0519693 .6935612 .065402$

C -0.2723364 .7391991 .396534$

C 1.1730464 .4463401 .091347

C $3.2181591 .322242-1.265359$

C $2.9185992 .400418-2.291977$

C $0.9813863 .630411-3.279310$

O $3.7791862 .920682-2.996050$

C $1.0286045 .013032-2.709973$

C $-0.0582015 .775191-2.512659$

C $0.0042487 .205598-2.060731$

C $-0.8522157 .488304-0.849303$

C $-2.0367888 .111940-0.970704$

C -0.2834197 .1542730 .510337$

C -0.8802485 .9082441 .106502$

N $1.5613392 .691081-2.340984$

H $-4.241367-0.8158404 .198342$

H $-2.349943-1.7574391 .984570$

H $-1.965215-0.3806114 .747023$

H $-0.931765-2.6051244 .786781$

H $0.235731-1.3199424 .898527$

H $1.913462-2.4031291 .695592$

H $0.503786-4.1841391 .816228$

H -0.126881 -4.478491 3.436287

H -1.153067 -3.7213272 .202542$

H 1.9493310 .1368252 .020928

H $1.585711-0.0853353 .719121$

H $3.877591 \quad 0.2834213 .650125$

H $3.707194-1.4392223 .994677$

H $4.198476-1.9512111 .589685$

H $6.116578-1.7981083 .205638$

H $6.198616-0.0666192 .958554$

H $8.449309-1.4607592 .461186$

H 6.8311090 .8302470 .547641

H $7.301914-3.3035021 .590069$

H $7.671981-2.766792-0.032696$

H $5.982936-3.0166540 .453307$

H $8.248393-0.382349-0.750044$

H $5.1807451 .964491-0.716107$

H $5.1593730 .861770-2.097727$

H $3.914537-1.215090-1.385454$

H 0.4168092 .1619402 .250276

H -2.0899483 .9466022 .278899$

H 1.2618453 .5339650 .492787

H 1.7416864 .3131462 .018185

H 1.6673615 .2341630 .519495

H $2.6988400 .416076-1.589598$

H $2.7933781 .635780-0.304905$

H $-0.0424593 .299869-3.480884$

H $1.5473873 .599208-4.216163$

H $2.0125905 .427557-2.503053$

H -1.040632 $5.373510-2.750049$

H $-0.3246087 .829419-2.902470$

H $1.0363667 .516024-1.855272$

H -2.637203 8.354974 -0.100142 
H $-2.4333348 .389437-1.941659$ H $-0.499337 \quad 7.982061 \quad 1.200067$ H 0.8085967 .1214880 .463726 H -1.933645 $6.018746 \quad 1.368915$ H $0.9357602 .246387-1.676049$ C $-5.414684-0.8167921 .222511$ $\begin{array}{llll}\text { O } & -4.332188 & -0.743794 & 0.271436\end{array}$ C $-4.0724770 .639149-0.021563$ C $-5.3444551 .380606 \quad 0.368890$ C $-5.7477330 .623737 \quad 1.621182$ H $-3.263240 \quad 0.9552450 .647414$ C $-3.6250290 .821474-1.474533$ H -5.1684342 .4454980 .547986$ H $-6.1228811 .284489-0.396636$ C -7.1984010 .8270252 .022038$ H -5.1077150 .9631282 .444154$ C -2.461182 -0.094261-1.912076 O $-4.7352960 .543324-2.337088$ H -3.366595 $1.874397-1.640466$ H -2.841124 -1.111371-2.055583 O $-2.0322490 .348763-3.206498$ C $-1.252197-0.122488-0.962782$ C $-0.007595-0.838755-1.514968$ H $-1.543829-0.590085-0.017027$ O $-0.873871 \quad 1.224469-0.667020$ H $\quad 0.427153-0.245427-2.329364$ H $\quad 0.740847-0.830492-0.711345$ H -7.3923451 .8816822 .242653$ H -7.8828850 .5196621 .224245$ H -7.4386230 .2435932 .916616$ C $-5.043494-1.7678492 .366950$ H $-4.4313120 .672957-3.251823$ H $-1.6383491 .231498-3.098020$ H $-0.143451 \quad 1.186637-0.026378$ H $-4.748513-2.7393011 .952729$ H -5.927365 -1.952382 2.989245 H $-6.257919-1.2583850 .676199$ C $-1.096855-4.406893-1.218156$ O $-0.777027-3.040430-0.914824$ C $-0.232492-2.280606-1.996050$ C $1.075821-2.918225-2.491695$ C $0.874413-4.390651-2.862917$ C $0.131282-5.157780-1.765580$ H $-1.358020-4.854740-0.251050$ C -2.326662 -4.519419-2.133857 H $-0.949942-2.257227-2.821879$ O $2.087321-2.819695-1.490807$ H $1.434251-2.359826-3.364261$ H $1.851196-4.861512-3.029691$ O $0.132481-4.483753-4.075028$ H $-0.169633-6.144974-2.135703$ O $1.047885-5.376803-0.689383$ C $-3.581227-3.994023-1.470911$ H -2.186749 -3.960555 -3.062697 H -2.509313 -5.571067-2.382636 H $1.755602-3.256866-0.687937$ H $\quad 0.637399-4.020620-4.764907$ H $0.598901-5.933305-0.030235$ O $-4.562685-3.870867-2.412563$ O $-3.693193-3.738823-0.280394$ C $-5.819796-3.376997-1.939172$ H $-5.733521-2.312135-1.707413$ H $-6.556304-3.503781-2.737025$ $\mathrm{H}-6.155810-3.938584-1.062128$ SCF Energy (B3LYP/6-31G**//MMFF) $=-3245.92284564$

08_00066

MM̄FF Geometry

C $-0.467139-0.334795-2.267293$

C $-0.822487-1.601297-1.998953$

C $-0.215425-2.490470-0.935788$

O $0.807846-1.769876-0.213309$

C $0.426722-3.723100-1.592018$

C $1.022291-4.691112-0.589375$

C $2.340961-4.800361-0.331932$

C $0.033085-5.5911050 .103316$

C $3.446577-3.966686-0.919226$

C $4.480726-3.5635370 .141941$
C $5.533772-2.613003-0.444931$

C $6.687391-2.4024610 .532797$

C $7.724013-1.421462-0.033338$

O $4.897930-1.357884-0.698749$

O $8.597857-1.0383321 .041175$

C $7.005400-0.137736-0.506524$

C $8.613009-2.088079-1.090622$

O $7.9228610 .701977-1.203700$

C $5.728710-0.389349-1.353043$

C $4.9044120 .911885-1.464820$

O $6.038908-0.782000-2.690498$

C $0.647583-1.5918231 .123801$

O $-0.237331-2.0378421 .835415$

C $1.730682-0.6935571 .576016$

C 1.5029380 .1128402 .622469

C 2.4216041 .1242183 .145475

C $3.856721 \quad 1.0662202 .696252$

C $3.5426510 .733429-2.148357$

C $2.7601022 .032532-2.105411$

C $1.5421513 .527166-0.512719$

O $2.6495262 .752895-3.094257$

C 2.2361254 .1599210 .653954

C 1.6100724 .5523321 .773998

C 2.3097965 .1958112 .938184

C 1.9798064 .5436234 .264291

C 1.1711325 .1505525 .149588

C 2.6528653 .2286484 .600978

C 1.9349332 .0538943 .993529

N $2.2424212 .301182-0.848100$

H $0.3212520 .139606-1.691364$

H -1.615408 -2.054347 -2.591283

H -1.022921 -2.805414 -0.266293

H $1.188604-3.403543-2.312790$

H $-0.321553-4.260295-2.190524$

H $2.664544-5.5635920 .374250$

H $\quad 0.519515-6.295137 \quad 0.786827$

H $-0.677527-5.0010490 .690509$

H $-0.525920-6.178578-0.632344$

H $3.944723-4.554057-1.699852$

H $3.056593-3.061948-1.393679$

H $3.968393-3.0742990 .980325$

H $4.967704-4.4664110 .530912$

H $5.898734-3.034950-1.388666$

H $7.163878-3.3570730 .788773$

H $6.299241-1.9958481 .477268$

H $9.001727-1.8460011 .402437$

H $\quad 6.7141650 .4064330 .403521$

H $9.164167-2.928695-0.651806$

H $9.374957-1.394683-1.462829$

H $8.050438-2.476340-1.942098$

H $7.5128841 .577351-1.301908$

H $4.7425011 .319413-0.461133$

H $5.4666391 .667692-2.027603$

H $6.996257-0.915053-2.766062$

H $2.647764-0.6706490 .999655$

H $0.5301930 .066812 \quad 3.111219$

H 4.2459090 .0435562 .762172

H 4.5252641 .6784503 .305273

H 3.9459571 .4085111 .662603

H $3.6671270 .437824-3.195955$

H $2.947853-0.047533-1.661886$

H $0.5071203 .257910-0.281451$

H $1.5457284 .210107-1.367362$

H 3.3100874 .3103560 .567353

H 0.5324454 .4316331 .849636

H 2.0139816 .2529382 .957197

H 3.3981005 .1936242 .799552

H 0.9514274 .7026686 .113100

H 0.7060406 .1055224 .928844

H 2.6638293 .0805475 .688788

H 3.7038643 .2903704 .306197

H $0.883016 \quad 1.9924154 .277092$

H $2.3292591 .597309-0.121563$

C $-1.6836411 .795071-2.839255$

O $-2.9160291 .502886-2.145933$

C $-3.9774932 .253882-2.757708$

C $-3.2906693 .428938-3.437990$

C $-2.0427722 .759352-3.975321$ 
H $-4.4179401 .605660-3.525460$

C $-5.0556042 .639437-1.738640$ H -3.908356 $3.879226-4.220640$ H -3.017367 4.212060 -2.721428 C $-0.927896 \quad 3.732659-4.321034$ H -2.310956 2.194696-4.878617 C $-5.6002911 .480371-0.879059$ O $-4.5321403 .621413-0.834805$ H -5.882699 3.133046 -2.262642 H $-4.8733561 .226901-0.101081$ O $-6.7654291 .959086-0.195011$ C $-5.9821310 .212420-1.657584$ C $-6.733110-0.858533-0.844945$ H -5.076365 -0.241130 -2.074718 O $\quad-6.8104600 .562323-2.770521$ H $-7.760990-0.527653-0.646537$ H $-6.834749-1.730291-1.505058$ H -1.252505 $4.428072-5.101814$ H -0.625591 4.322769 -3.449343 H $-0.0455753 .198859-4.688070$ C $-1.0660610 .496077-3.365904$ H $-3.7364583 .243189-0.423225$ H -6.5023432 .7498120 .306475$ H -7.596996 $1.015006-2.420125$ H $-0.262781 \quad 0.728937-4.075009$ H - $-1.825125-0.082941-3.906449$ H -1.018772 $2.278223-2.113126$ C $-3.926301-1.984707 \quad 1.370436$ $\begin{array}{llll}\text { O } & -4.703753 & -1.617443 & 0.220938\end{array}$ C $-6.067565-1.2758820 .479437$ C $-6.805589-2.4554031 .137840$ C $-6.070608-2.9573592 .383678$ C $-4.574951-3.1629562 .116967$ H -2.973582 -2.338583 0.960443 C -3.628544 -0.7858572 .286702$ H $-6.098823-0.4160971 .155989$ O $-6.947250-3.5349050 .215222$ H -7.817342 -2.132139 1.409772 H $-6.520991-3.9045612 .704852$ O -6.254148 -2.011420 3.432554 H -4.035962 -3.341501 3.054776 O $-4.400577-4.3293431 .307182$ C $-2.729356 \quad 0.240073 \quad 1.631948$ H -4.541712 -0.2667272 .588460$ H -3.110079 -1.139416 3.185748 H $-6.058752-3.776101-0.098382$ H -5.802728 -2.356889 4.221498 H $-4.767454-5.0842801 .798133$ O $-2.502640 \quad 1.2563032 .516161$ $\begin{array}{lllll}\text { O } & -2.286588 & 0.169834 & 0.495044\end{array}$ C - -1.6776782 .3218822 .035957$ H -1.411310 2.9541212 .886951 H -0.7584591 .9347201 .587270$ H $-2.2335272 .922170 \quad 1.311157$

SCF Energy (B3LYP/6-31G**/MMFF) $=-3245.91157093$

0800067

MM̄MF Geometry

C $1.008233-2.1165192 .385512$

C $-0.114441-2.6172731 .850670$

C $-0.180338-3.2141100 .468573$

O $-0.901814-2.266606-0.355437$

C $-0.948170-4.5455940 .492391$

C - $1.105638-5.176970-0.878955$

C $-2.260432-5.229747-1.573884$

C $0.144696-5.792231-1.452949$

C $-3.600196-4.684490-1.165921$

C $-3.964412-3.434191-1.976634$

C $-5.068213-2.616424-1.291273$

C $-5.507862-1.449936-2.174727$

C $-6.520265-0.539174-1.463982$

O $-4.528087-2.117134-0.062849$

O $-6.6228490 .676208-2.223023$

C $-5.971177-0.157613-0.070722$

C $-7.923778-1.157240-1.444502$

O $\begin{array}{llll}-6.968090 & 0.554416 & 0.658720\end{array}$

C $-5.451916-1.3672800 .737893$

C $-4.702198-0.9728722 .035917$
O $-6.566082-2.179552 \quad 1.127827$

C $-0.176209-1.237809-0.879343$

O $1.034705-1.088628-0.824522$

C $-1.122625-0.279685-1.491409$

C $-0.6747000 .921119-1.886695$

C $-1.4758251 .996965-2.473501$

C $-2.958510 \quad 1.772792-2.606248$

C $-3.466316-0.0858141 .844261$

C $-3.743241 \quad 1.401767 \quad 1.982064$

C -2.6327273 .6157771 .705510$

O -4.8089851 .8534572 .392125$

C -2.9439854 .1914200 .360234$

C $-2.1456505 .065280-0.272006$

C $-2.4889585 .716411-1.581155$

C $-1.4005985 .565132-2.619484$

C $-0.4789306 .526198-2.800256$

C $-1.4410184 .346257-3.511747$

C $-0.850548 \quad 3.131106-2.852133$

N -2.640971 2.1671351 .626659

H $1.941881-2.1820401 .835085$

H $-1.054230-2.4999102 .385768$

H $0.821466-3.3777410 .055259$

H -1.924117 -4.388351 0.961280

H $-0.427881-5.2595881 .145242$

H -2.252484 -5.710574 -2.551287

H $-0.038444-6.289515-2.411402$

H $\quad 0.909330-5.027934-1.621643$

H $0.546681-6.544171-0.766232$

H $-4.358290-5.458960-1.337205$

H -3.639555 -4.475020 -0.095024

H -3.074772 -2.803631 -2.094552

H -4.284702 -3.735803 -2.981549

H $-5.914431-3.279293-1.075881$

H $-5.918883-1.814475-3.124359$

H -4.630414 -0.846916 -2.442750

H $-6.9039180 .443896-3.124571$

H $-5.1467240 .543816-0.244984$

H $-8.276340-1.338364-2.467070$

H $-8.651459-0.472461-0.995171$

H -7.966073 -2.107785 -0.908852

H -6.5540740 .9085691 .463191$

H -5.400672 -0.5420452 .763127$

H -4.347601 -1.905916 2.497709

H $-6.301628-2.7216101 .889437$

H -2.161800 -0.574082 -1.572354

H $\quad 0.384470 \quad 1.145697-1.765308$

H $-3.3932731 .493886-1.640620$

H -3.160952 $0.974711-3.328279$

H $-3.5066592 .656890-2.938134$

H -2.740120 -0.3317702 .629811$

H $-2.985739-0.2821380 .881504$

H -1.645862 3.9188312 .067273

H -3.381815 3.9496352 .430613

H $-3.8905803 .908283-0.094131$

H $-1.2141695 .369627 \quad 0.200478$

H $-2.6643976 .782537-1.384585$

H -3.435571 $5.334126-1.982913$

H $0.2860496 .439419-3.564783$

H $-0.4657977 .418419-2.182833$

H $-0.8604764 .532429-4.425658$

H $-2.4633484 .187396-3.866410$

H $\quad 0.2282773 .201126-2.707700$

H -1.8140641 .7082091 .255628$

C 0.8282040 .1533293 .359388

O 1.9293150 .6543702 .578476

C 2.0915072 .0574062 .866258

C 1.0586262 .4059173 .938281

C 0.7848381 .0589164 .582449

H 3.0943082 .1803193 .289300

C 1.9543982 .8703171 .571659

H 1.4397343 .1477234 .647398

H 0.1421982 .8168893 .500059

C $-0.526766 \quad 1.012623 \quad 5.346650$

H 1.6139340 .8163685 .260221

C 2.9555902 .4813730 .464014

O 0.6370052 .6811721 .037485

H 2.0374143 .9402211 .793946

H $2.749873 \quad 1.4627290 .117050$ 
O $2.718643 \quad 3.344830-0.655972$

C $4.4350862 .615601 \quad 0.869436$

C $5.4313942 .414002-0.288490$

H 4.6677421 .9066251 .670295

O $4.657256 \quad 3.924025 \quad 1.405161$

H $5.1953043 .103053-1.108649$

H 6.4221722 .7168950 .077236

H -0.5264431 .7507296 .155355$

H $-1.379316 \quad 1.2289284 .693923$

H -0.6858540 .0256725 .791747$

C $1.032760-1.3306713 .665648$

H $\quad 0.5298561 .7327190 .850651$

H $1.776483 \quad 3.269362-0.884875$

H $4.431624 \quad 4.5670130 .711041$

H $0.255764-1.6937914 .347701$

H $2.001133-1.4970324 .151911$

H -0.0817450 .2895222 .758167$

C $6.147019-1.234562-0.039663$

$\begin{array}{lllll}\text { O } & 6.054507 & 0.168277 & 0.240346\end{array}$

C $5.4973380 .968949-0.807219$

C $6.3623130 .861306-2.072915$

C $6.530512-0.598099-2.503969$

C $6.942072-1.497637-1.332973$

H $6.727018-1.6350300 .801607$

C $4.756179-1.8846570 .004254$

H $4.4852210 .616711-1.031615$

O $7.642598 \quad 1.439851-1.826971$

H $5.8870541 .438592-2.875312$

H $7.292475-0.653375-3.291221$

O $5.300705-1.049617-3.062839$

H $6.856067-2.550538-1.623392$

O $8.327438-1.253106-1.067560$

C $4.816653-3.3734420 .273254$

H $4.176919-1.4250640 .814723$

H $4.205297-1.720841-0.925564$

H $8.155742 \quad 1.378523-2.650671$

H $5.431749-1.966704-3.358374$

H $8.608343-1.875996-0.375564$

O $3.544492-3.864237 \quad 0.342316$

O $5.841535-4.027168 \quad 0.409184$

$\begin{array}{llll}\text { C } 3.435429 & -5.262098 & 0.629907\end{array}$

H $3.768550-5.845374-0.233226$

H $4.015723-5.521848 \quad 1.520693$

H $2.384048-5.4877520 .825121$

SCF Energy (B3LYP/6-31G**/MMFF) $=-3245.91898206$

08_00068

MM̄FF Geometry

C -3.404228 $5.058610-0.289178$

C $-4.0552423 .908155-0.515415$

C $-4.870634 \quad 3.207160 \quad 0.541706$

O $-4.286306 \quad 1.900077 \quad 0.763984$

C $-6.321545 \quad 3.0241210 .067091$

C $-7.2443172 .524546 \quad 1.163600$

C -7.7186551 .2670371 .266117$

C $-7.661905 \quad 3.5652642 .172758$

C $-7.441512 \quad 0.097290 \quad 0.365290$

C $-6.687850-1.010008 \quad 1.112570$

C $-6.320238-2.1760730 .183804$

C $-5.599084-3.2794630 .958599$

C $-5.106982-4.4083770 .040286$

O $-5.466739-1.664644-0.843687$

O $-4.188638-5.195407 \quad 0.814447$

C $-4.330987-3.793061-1.146347$

C $-6.252736-5.344876-0.361245$

O $-4.005184-4.786322-2.115098$

C $-5.080697-2.630623-1.830128$

C $-4.223471-1.888688-2.884755$

O $-6.227459-3.151951-2.513869$

C -3.1697041 .8587611 .544712$

O $-2.632722 \quad 2.8020882 .105150$

C -2.6900210 .4596641 .595066$

C $-1.491677 \quad 0.205872 \quad 2.142533$

C $-0.870919-1.1079512 .323372$

C $-1.630422-2.315178 \quad 1.844856$

C $-2.947002-1.228132-2.351769$

C $-1.694852-2.072205-2.520712$

C $0.742638-2.036045-2.005055$
O $-1.672336-3.134526-3.135503$

C $1.038402-2.797026-0.752674$

C $2.061053-2.5049250 .064769$

C $2.425851-3.3126481 .274689$

C $2.428110-2.4962542 .544963$

C $3.563098-1.9594713 .023425$

C $1.130681-2.3844113 .309932$

C $0.334285-1.1688642 .927238$

N $-0.590967-1.473545-1.928749$

H -3.4968345 .5377110 .682474$

H -3.957220 $3.415701-1.479833$

H $-4.8589803 .771377 \quad 1.482648$

H $-6.3422102 .353157-0.797760$

H $-6.7188843 .981046-0.298066$

H -8.3891701 .0395292 .094294$

H -8.383925 3.1756562 .898413

H -6.7968183 .9290062 .735031$

H -8.132408 4.4156151 .668223

H $-8.400472-0.2942540 .004349$

H $-6.8803250 .394541-0.523188$

H $-5.772917-0.5854001 .543864$

H $-7.300402-1.3788701 .944777$

H -7.241353 - $2.561820-0.268545$

H $-6.241092-3.6816751 .752261$

H $-4.728888-2.8485141 .471519$

H $-3.898076-5.9449870 .267929$

H $-3.381470-3.418919-0.746235$

H $-6.689276-5.8150960 .528180$

H $-5.891829-6.167840-0.987995$

H $-7.057021-4.833923-0.894645$

H $-3.430146-5.441117-1.685015$

H $-4.014806-2.549016-3.735153$

H $-4.846120-1.080028-3.294288$

H $-6.492396-2.518043-3.200733$

H $-3.332535-0.3151611 .195157$

H $-0.905768 \quad 1.0456542 .515549$

H $-1.056960-3.2427651 .905065$

H -1.912661 -2.202294 0.793311

H $-2.538694-2.4536322 .440406$

H -2.771789 $-0.307800-2.924197$

H $-3.054528-0.942511-1.300679$

H $1.435173-1.204843-2.159038$

H $0.814910-2.705481-2.868316$

H $\quad 0.406530-3.654188-0.532560$

H $2.707892-1.664235-0.171818$

H $3.425046-3.7336061 .102098$

H $1.763522-4.1789941 .393742$

H $3.575348-1.3986853 .952068$

H $4.501597-2.0591192 .489776$

H $1.346262-2.3041264 .384483$

H $\quad 0.561314-3.3137143 .218301$

H $0.805066-0.234143 \quad 3.236074$

H $-0.712869-0.613100-1.402027$

C -1.012721 5.488771-1.024122

O $-0.6846894 .107521-1.273476$

C $0.2801273 .673400-0.288961$

C $0.747586 \quad 4.9350650 .425698$

C $-0.498243 \quad 5.7973790 .381267$

H $-0.268636 \quad 3.0199330 .399786$

C $1.3966492 .891358-0.991392$

H 1.0931124 .7363191 .444709

H $1.5658815 .415617-0.125038$

C $-0.234518 \quad 7.271517 \quad 0.630254$

H $-1.1883545 .424752 \quad 1.147275$

C $2.5084552 .419540-0.033692$

O $0.804765 \quad 1.751569-1.621607$

H $1.8308333 .504783-1.790107$

H 2.8921043 .2814710 .523485

$\begin{array}{lllll} & 1.951074 & 1.523820 & 0.928699\end{array}$

C $3.7102221 .755376-0.740666$

$\begin{array}{llll}\text { C } & 4.787618 & 1.309001 & 0.259427\end{array}$

H $4.1375942 .478379-1.444044$

O $3.3040060 .616667-1.490223$

$\begin{array}{llll}\text { H } & 5.117246 & 2.164986 & 0.862220\end{array}$

H 4.3539330 .5973050 .975044

H 0.1963637 .4225131 .625416

H $0.4647467 .684388-0.104464$

H -1.1637317 .8472000 .572787$ 
C $-2.5040075 .729522-1.286466$ H $0.0980792 .076897-2.205475$ H 1.6227020 .7456990 .445756 H $2.6815630 .918584-2.173008$ H $-2.7488295 .374579-2.295715$ H -2.714424 6.805193-1.268858 H $-0.4436946 .067800-1.763849$

C $8.084004-0.4280510 .359415$

$\begin{array}{lllll}\text { O } & 6.886486 & 0.279496 & 0.709067\end{array}$

C $6.0086490 .613137-0.376237$

C $6.7415141 .475750-1.415442$

C $8.0450870 .819153-1.877876$

C $8.8937510 .342644-0.695809$

H $8.680096-0.4422261 .281174$

C $7.793149-1.883829-0.039070$

H $5.663908-0.315219-0.843733$

O $7.0236532 .765628-0.875786$

H $6.092108 \quad 1.625681-2.284875$

H $8.6150101 .542599-2.473699$

O $7.724354-0.283121-2.721276$

H $9.726037-0.280635-1.042909$

O $9.4678601 .481157-0.046896$

C $7.325406-2.7182451 .135874$

H $7.022971-1.945226-0.812184$

H $8.709999-2.351979-0.415736$

H $7.5452192 .640697-0.064422$

H $8.561371-0.670802-3.028641$

H $\quad 10.020054 \quad 1.943490-0.700415$

O $\quad 6.955312-3.9526140 .681246$

O $7.303933-2.3499822 .302124$

C $6.516805-4.8736271 .684985$

H $6.203849-5.7952951 .187096$

H $7.339714-5.1045532 .367869$

H $5.663354-4.4652052 .233811$

SCF Energy (B3LYP/6-31G**//MMFF)= -3245.92610186

0800069

MM̄FF Geometry

C 22404873.2730161 .739295

C 2.8928554 .1994491 .020012

C $3.9343743 .890934-0.032324$

O $3.6907512 .585681-0.613464$

C 5.3398363 .9095060 .584319

C $6.4371163 .749195-0.448353$

C $7.1145182 .605649-0.670130$

C $6.7838674 .997018-1.218514$

C $6.9000961 .275412-0.003042$

C $6.8880140 .129475-1.024872$

C $6.679916-1.226228-0.336345$

C $6.895261-2.377630-1.318444$

C $6.634022-3.740080-0.657668$

O $5.340819-1.2674710 .169363$

O $6.551968-4.724462-1.699676$

C $5.257889-3.6976580 .038306$

C $7.792918-4.1662070 .251052$

O $5.025015-4.8911730 .783517$

C $5.036994-2.4465630 .924957$

C $3.554859-2.3829901 .359387$

O $5.855501-2.5481822 .091780$

C $2.6712702 .504942-1.514977$

O $2.0040213 .430571-1.951208$

C $2.4596641 .078121-1.844103$

C $1.3000120 .718652-2.415901$

C $0.893280-0.635347-2.798403$

C $1.908833-1.735747-2.644462$

C $3.124904-1.0472031 .980522$

C $1.658586-1.0988852 .366705$

C $-0.621054-1.250051 \quad 1.359779$

O $1.300637-1.1728203 .539573$

C -1.052303 -2.217942 0.304303

C $-1.985441-1.944383-0.618969$

C -2.405254 -2.910282 -1.689295

C $-2.367244-2.304374-3.075371$

C $-3.502362-2.029088-3.740045$

C - $-1.014236-2.094713-3.724170$

C $-0.355342-0.817139-3.278960$

N $0.817770-1.0940421 .266020$

H 2.5099072 .2252711 .632380
H $2.6275845 .247451 \quad 1.136393$

H $3.8664424 .647154-0.824526$

H 5.4198723 .1305911 .351339

H 5.5026214 .8556471 .118193

H $7.9156182 .621893-1.408066$

H $7.6142894 .838906-1.914992$

H $5.9251915 .339071-1.804132$

H $7.0787685 .797513-0.532025$

H 7.7188001 .1153240 .708734

H $5.967387 \quad 1.2520610 .566882$

H $6.0837720 .304308-1.751516$

H $7.8376520 .122870-1.574411$

H $7.386832-1.3021840 .498408$

H $7.906574-2.346083-1.742565$

H $\quad 6.208527-2.265259-2.169332$

H $7.391831-4.712615-2.190024$

H $4.502928-3.691375-0.760177$

H $8.730807-4.202516-0.316220$

H $7.639506-5.1801410 .637007$

H $7.944175-3.4928391 .097315$

H $5.541579-4.8321671 .605248$

H $2.921469-2.5674870 .484176$

H $3.342921-3.1877812 .075473$

H $5.460857-2.0153842 .800340$

H $3.2394410 .371630-1.585242$

H $0.5596661 .493696-2.613581$

H $1.569976-2.701118-3.024734$

H $2.162888-1.878330-1.590795$

H $2.825474-1.493214-3.194156$

H $3.706363-0.8197022 .879819$

H $3.272074-0.2175221 .280144$

H -1.067660 -0.261939 1.220201

H $-0.895747-1.6229062 .350809$

H $-0.569141-3.1924260 .299116$

H -2.483110 -0.978596 -0.596663

H $-3.421879-3.249273-1.454375$

H -1.780755 -3.812348 -1.678088

H $-3.483506-1.618187-4.744052$

H -4.476064 -2.201130 -3.293356

H -1.129897 -2.040481-4.814713

H $-0.400929-2.980355-3.538804$

H $-0.9995390 .058403-3.378005$

H $1.211370-0.8901360 .351933$

C -0.1569592 .8274312 .141197$

$\begin{array}{lllll}\text { O } & -0.531943 & 3.337758 & 0.847257\end{array}$

C -1.9708773 .3093560 .731016$

C -2.4852662 .6557362 .008983$

C -1.3972062 .9968393 .009060$

H $-2.287768 \quad 4.3584870 .685252$

C $-2.3406512 .581678-0.569802$

H -3.4702283 .0315072 .302477$

H -2.5623911 .5683271 .889220$

C -1.4153452 .1121084 .243555$

H -1.5085004 .0471583 .308772$

C $-3.8527182 .323280-0.717695$

O $-1.8611383 .388923-1.647713$

H -1.793009 $1.632584-0.619782$

H $-4.175251 \quad 1.7543680 .156570$

O $-4.5614893 .560828-0.685740$

C $-4.2416171 .532985-1.982404$

C $-5.741411 \quad 1.182131-2.066875$

H -3.648529 $0.613506-2.019721$

O $-3.9033012 .292482-3.143760$

H $-6.3348792 .100293-2.160899$

H $-5.8925700 .634036-3.006325$

H $-2.3650782 .217320 \quad 4.778105$

H -1.292555 1.0553443 .982224

H -0.6088612 .3844614 .931391$

C 1.0815383 .5676382 .648162

H $-2.0039432 .894007-2.472118$

H $-4.2708554 .080414-1.455091$

H -4.095039 $1.741167-3.921607$

H 1.3368013 .2410883 .661902

H 0.8876574 .6465692 .680864

H 0.0710631 .7621842 .009584

C $-5.708541-1.6535550 .346350$

O $-5.456320-0.837856-0.806265$

C $-6.2581060 .344160-0.889209$ 
C $-7.734701-0.045221-1.037954$ C $-8.177899-0.9285210 .135949$ C $-7.189381-2.070856 \quad 0.408775$ H $-5.114249-2.5589280 .172888$ C $-5.168877-0.9654131 .608850$ H -6.1462350 .9293320 .029531$ O $-7.921158-0.748796-2.263856$ H $-8.3422050 .866624-1.080277$ H -9.165248 -1.355774 - 0.078025 O $-8.297673-0.131958 \quad 1.311192$ H $-7.403000-2.5292831 .380430$ O $-7.399654-3.089926-0.575919$ C $-4.685324-1.9479292 .651725$ H $-4.310094-0.3439851 .326878$ H -5.910885 -0.3112702 .073789$ H -8.867626 $-0.958293-2.340481$ H -8.9574160 .5593651 .131809$ H $-7.155570-2.720313-1.442031$ O $-3.825497-1.3069303 .498404$ O $-5.029200-3.1190722 .730132$ C $-3.271640-2.1131934 .542612$ H -2.583539-1.491989 5.122204 H $-4.065628-2.4715105 .204448$ H -2.712519-2.952636 4.118099 SCF Energy (B3LYP/6-31G*//MMFF) $=-3245.91409258$

0800070

MM̄FF Geometry

C -1.031754 -1.469119-2.418756

C $0.138476-2.045282-2.109231$

C $0.356490-2.928237-0.907853$

O $1.209701-2.2015230 .009475$

C $1.069933-4.221664-1.339786$

C $1.418464-5.144824-0.188102$

C $2.665353-5.3422390 .287677$

C $0.254428-5.8891940 .412546$

C $3.940077-4.707037-0.195658$

C $4.454403-3.6576300 .797699$

C $5.416201-2.6617070 .131662$

C $6.009610-1.7117401 .171877$

C $6.861684-0.6067770 .530473$

O $4.661210-1.920886-0.833783$

$\begin{array}{llll}\text { O } & 7.092641 & 0.401798 & 1.527239\end{array}$

C $6.0566010 .058670-0.607967$

C $8.242852-1.1264230 .113013$

O $6.8884370 .981506-1.307488$

C $5.416689-0.956323-1.580071$

C $4.443836-0.308849-2.598931$

O $6.452949-1.593208-2.337152$

C $0.602543-1.2712320 .799559$

O $-0.593385-1.0348260 .866375$

C $1.649578-0.5397241 .544853$

C 1.3002110 .5215532 .287320

C 2.2038761 .3923373 .041258

C 3.6808691 .1209362 .938982

C $3.2321790 .408723-1.994339$

C $3.4266671 .898100-1.771919$

C $2.2945023 .864937-0.737613$

O $4.3742952 .529657-2.230594$

C 2.9622924 .1504600 .570954

C 2.3830214 .8524301 .557182

C 3.0638175 .1979902 .851714

C 2.2427614 .8231654 .065897

C 1.4708645 .7322554 .685194

C 2.3841903 .4194474 .610529

C 1.6711672 .4014353 .761925

N $2.3858262 .447480-1.033498$

H $-1.907016-1.657186-1.802573$

H $1.012514-1.821957-2.717268$

H $-0.590598-3.177885-0.414445$

H $1.967054-3.962023-1.909903$

H $0.434314-4.773199-2.046142$

H $2.796689-6.0326651 .119794$

H $0.563528-6.5691421 .213540$

H $-0.472717-5.1915570 .836912$

H $-0.246881-6.489490-0.353705$

H $4.697095-5.492205-0.314244$

H $3.820636-4.272336-1.190150$
H $3.605069-3.1048371 .216727$

H $4.953556-4.1656521 .632198$

H $6.208811-3.222060-0.377893$

H $6.595344-2.2670861 .915083$

H $5.195250-1.2357511 .733301$

H $7.537601-0.0191932 .282576$

H $5.2670450 .647747-0.126633$

H $8.785560-1.5129980 .984092$

H $8.863927-0.319334-0.290982$

H $8.193677-1.928982-0.625721$

H $6.3229331 .502237-1.901669$

H $4.9928170 .338236-3.293189$

H $4.043502-1.122501-3.221413$

H $6.063227-1.958877-3.148456$

H $2.675715-0.867628 \quad 1.435414$

H $0.2463470 .792922 \quad 2.341710$

H 3.9984181 .1211751 .891038

H 3.9221130 .1487203 .381770

H 4.3006591 .8653073 .442116

H $2.3945720 .320500-2.697999$

H $2.924597-0.064569-1.058604$

H $1.2315704 .126531-0.727026$

H $2.7869464 .432577-1.533773$

H 3.9812663 .7930290 .700602

H $1.3755865 .236591 \quad 1.412192$

H 3.2488546 .2803872 .850522

H $4.053644 \quad 4.7300662 .921843$

H 0.9007595 .4798415 .573164

H 1.3879626 .7485954 .314219

H 1.9522623 .3598265 .618679

H 3.4452983 .1959664 .749857

H 0.5865552 .5192853 .765054

H $1.6674611 .837475-0.654159$

C $-1.1830090 .941028-3.037114$

O $-2.4052661 .202043-2.318263$

C $-2.8975252 .500935-2.697187$

C $-1.7638663 .176895-3.458273$

C $-1.1207771 .989260-4.146686$

H $-3.7298372 .331478-3.390465$

C $-3.3916673 .266911-1.465627$

H -2.127435 $3.935712-4.157764$

H $-1.047757 \quad 3.656675-2.780871$

C $0.2831442 .271516-4.653938$

H -1.754756 $1.682410-4.989276$

C $-4.4551272 .520679-0.632581$

O $-2.2806543 .535898-0.599470$

H $-3.7766674 .246532-1.771488$

H -3.989421 $1.675332-0.113655$

$\begin{array}{llll}\text { O } & -4.918604 & 3.415255 & 0.386443\end{array}$

C $-5.6727932 .033184-1.437090$

C $-6.8297271 .469046-0.591053$

H $-5.3549301 .264583-2.149606$

O $-6.196523 \quad 3.114684-2.213599$

H $-7.283677 \quad 2.2693740 .007202$

H -7.610389 $1.152465-1.295756$

H $0.2673973 .070482-5.402410$

H $0.9475942 .585779-3.842427$

H $0.7181581 .381584-5.119229$

C - $-1.185455-0.498371-3.555608$

H $-1.8745532 .682332-0.369585$

H -4.1395623 .7107360 .888370$

H $-6.4459503 .824416-1.596985$

H $-0.376065-0.640317-4.281138$

H $-2.127511-0.721623-4.070391$

H $-0.3592621 .092999-2.326602$

C $-5.356269-1.8649500 .222198$

O $-5.861630-0.729182-0.494847$

$\begin{array}{lllll}\text { C } & -6.440593 & 0.294595 & 0.320637\end{array}$

C $-7.649325-0.2595971 .092680$

C $-7.263408-1.4951761 .912467$

C $-6.460247-2.5057331 .083511$

H $-5.082688-2.583247-0.561058$

C $-4.079298-1.4933140 .987053$

H $-5.6948650 .651362 \quad 1.038560$

$\begin{array}{llll}\text { O } & -8.683501 & -0.606638 & 0.173316\end{array}$

H -8.039950 0.5255621 .751065

H -8.174955 -1.9759532 .288133$

O $-6.503206-1.0713153 .039883$ 
H $-6.024237-3.2675621 .738472$

O $-7.361361-3.1952530 .210236$

C $-3.305583-2.7074271 .448062$

H -3.416113 -0.9026820 .344236$

H $-4.307841-0.8626241 .850869$

H -9.446964 -0.9117490 .692351$

H $-6.287238-1.8622743 .562667$

H $-7.714086-2.547683-0.424100$

O $-2.740494-2.4500012 .663528$

O $-3.186611-3.741303 \quad 0.804522$

C $-1.944421-3.5028633 .216000$

H -1.703235 -3.2392004 .249255$

H $-2.494388-4.4489223 .216610$

H -1.012705 -3.596186 2.651252

SCF Energy (B3LYP/6-31G**//MMFF) $=-3245.92805096$

08_00071

MM̄MF Geometry

C -0.5315733 .0307180 .674928$

C 0.1962203 .4797491 .710206

C 0.6530722 .6485352 .886681

O 1.6769101 .7348242 .424206

C -0.4857311 .8453993 .541961$

C -0.0326390 .9722004 .696348$

C $0.100527-0.3688264 .635384$

C $0.248244 \quad 1.7028825 .983379$

C $-0.126286-1.2590863 .444076$

C $1.170173-1.9554623 .013299$

C $1.053526-2.5795471 .613226$

C $2.332007-3.3438741 .265861$

C $2.327863-3.865459-0.178650$

O $0.844635-1.5138650 .679939$

O $3.669347-4.262449-0.504767$

C $1.957084-2.704606-1.127231$

C $1.458201-5.119523-0.324962$

O $1.813395-3.171959-2.466468$

C $0.700997-1.925561-0.685952$

C $0.445417-0.651096-1.529011$

O $-0.442195-2.771113-0.853901$

C 2.9407082 .2291472 .303397

O 3.3130653 .3637262 .558196

C 3.8053411 .1447301 .786537

C 5.0897991 .3994541 .495150

C $6.0681390 .438574 \quad 0.979607$

C $5.589662-0.9593460 .690279$

C $1.5463150 .412114-1.465603$

C $2.582316 \quad 0.312570-2.572018$

C $4.7647211 .297831-3.271589$

O $2.449542-0.405943-3.558483$

C $5.8916640 .416189-2.834530$

C $7.1411990 .862245-2.634225$

C $8.294775-0.020334-2.252885$

C $9.0314580 .473463-1.028177$

C $10.1534011 .204098-1.144292$

C 8.5197920 .0405300 .326481

C 7.3391750 .8501760 .787926

N $3.657547 \quad 1.162057-2.344313$

H $-0.845625 \quad 1.9917060 .646879$

H $\quad 0.5386614 .5123181 .702260$

H 1.0791293 .3206323 .642794

H -0.9901921 .2345972 .792104$

H -1.255847 2.5375533 .907869

H $\quad 0.417549-0.8954805 .534544$

H 0.5133431 .0217826 .799031

H 1.0806302 .4015695 .855073

H -0.6351172 .2667606 .300431$

H -0.873764 -2.016188 3.710204

H $-0.543484-0.7055152 .601848$

H $1.986239-1.2226593 .011042$

H $1.428838-2.7275663 .748537$

H $\quad 0.186921-3.2498301 .596689$

H $2.495247-4.1678571 .971886$

H $3.194961-2.6763141 .386767$

H $3.944928-4.9419240 .134232$

H $2.815922-2.023900-1.133037$

H $1.822071-5.9164490 .334945$

H $1.516786-5.525508-1.340769$

H $0.408733-4.944768-0.080243$
H $\quad 0.950208-3.614159-2.535270$

H $0.204574-0.923481-2.563686$

H $-0.466828-0.180687-1.133936$

H -1.231122 -2.210793 -0.941310

H $3.3605610 .166070 \quad 1.653427$

H $5.4685512 .408287 \quad 1.654456$

H $6.332520-1.5808360 .186355$

H $4.717185-0.9395570 .028407$

H $5.316016-1.4676161 .620765$

H $1.0795851 .397569-1.583974$

H $2.0453800 .402602-0.492902$

H $5.0477302 .355121-3.285493$

H $4.4402881 .010957-4.276982$

H $5.676149-0.642126-2.708191$

H $7.3589681 .916453-2.790852$

H $8.981678-0.056578-3.108720$

H $7.971480-1.056289-2.091930$

H $\quad \begin{array}{lllll}10.704924 & 1.536077 & -0.270887\end{array}$

H $10.5418421 .491213-2.115839$

H $9.3084310 .164118 \quad 1.081263$

H $8.322366-1.0351150 .312663$

H 7.5888131 .8868531 .018238

H $3.7025251 .685600-1.474753$

C $-2.3743714 .128567-0.674138$

O $-3.0812752 .903863-0.974379$

C $-3.8096643 .081289-2.200440$

C $-3.9799284 .586972-2.354585$

C $-2.6498105 .087752-1.838982$

H -3.161754 $2.709679-3.003615$

C $-5.1329442 .307383-2.209863$

H $-4.1784354 .880667-3.389666$

H -4.792169 4.972963 -1.728093

C $-2.6650876 .552883-1.435690$

H -1.898321 $4.948436-2.628144$

C $-5.0607220 .806017-1.871118$

O $-6.0391242 .899580-1.267405$

H $-5.6070972 .437088-3.190335$

H $-4.9685120 .677621-0.787892$

O $-6.328403 \quad 0.235966-2.231046$

C $-3.9512740 .004574-2.570755$

C $-4.106720-1.529196-2.488875$

H $-2.9823190 .296131-2.149121$

O $-3.9281650 .355637-3.957522$

H -4.914684 -1.849718 -3.159906

H -3.188996 -1.963396 -2.908083

H $-2.9126627 .185650-2.294246$

H -3.405218 $6.745339-0.651706$

H $-1.6858076 .863384-1.057870$

C $-0.8731123 .862286-0.526840$

H $-5.6085862 .878525-0.395542$

H $-7.0153180 .733486-1.754845$

H -3.181303 -0.114112 -4.366531

H $-0.3327494 .815879-0.486340$

H -0.506989 3.324318 -1.410114

H -2.7927194 .5144020 .263809$

C $-3.368460-2.1106281 .130555$

O $-3.256847-1.724019-0.246559$

C $-4.354036-2.095630-1.081413$

C $-4.515780-3.623590-1.099222$

C $-4.677933-4.1718580 .322845$

C $-3.604668-3.6260361 .269891$

H -2.385930 -1.886716 1.561501

C $-4.411222-1.2659601 .880537$

H -5.274461 -1.653452 -0.689375

O $-3.373904-4.217932-1.712930$

H $-5.391212-3.880834-1.707298$

H $-4.615109-5.266816 \quad 0.305215$

O $-5.961622-3.8170870 .829142$

H $-3.866325-3.8595392 .308163$

O $-2.373825-4.3046391 .005865$

C -4.0340250 .1988531 .923189$

H -5.400095-1.331275 1.419546

H -4.480116 -1.618184 2.916320

H -3.519856 -5.179053 -1.735433

H $-6.628187-4.199090 \quad 0.233213$

H -2.087618 -4.064405 0.108018

$\begin{array}{llll}\text { O } & -4.922874 & 0.866902 & 2.715637\end{array}$

$\begin{array}{llll}\text { O } & -3.093579 & 0.701228 & 1.324916\end{array}$ 
C -4.7013292 .2760902 .831572$

H $-3.7585152 .465443 \quad 3.352350$

H -4.6978972 .7472521 .844016$

H -5.5191272 .7016273 .419119$

SCF Energy (B3LYP/6-31G**//MMFF) $=-3245.92075457$

08_00072

MM̄MF Geometry

C -0.574941 -2.045469 1.257487

C $0.597816-1.8769780 .629795$

C $0.718945-1.817131-0.871036$

O $2.100865-2.108355-1.181520$

C $0.387484-0.403649-1.379836$

C $0.115106-0.336504-2.867924$

C $0.946916 \quad 0.208237-3.777082$

C $-1.226122-0.873424-3.299182$

C $2.316070 \quad 0.788064-3.552067$

C $2.3370542 .319203-3.674881$

C $1.6715313 .036334-2.489482$

C $1.6580804 .551157-2.704739$

C $1.0944205 .296521-1.484079$

O $2.4248112 .723304-1.314117$

O $1.4090346 .689389-1.632980$

C $1.8217204 .802175-0.215353$

C $-0.4345105 .201985-1.412953$

O $1.232400 \quad 5.3541960 .959877$

C $1.8852013 .261955-0.101026$

C 2.8232692 .8519031 .060357

$\begin{array}{lllll}\text { O } & 0.573341 & 2.761230 & 0.163111\end{array}$

C $2.387281-2.863844-2.269532$

O $1.586616-3.365250-3.046070$

C $3.850488-3.058749-2.413133$

C $4.759624-2.357031-1.717499$

C $6.214814-2.513319-1.780178$

C $6.766771-3.600426-2.664077$

C 3.1848951 .3601261 .067646

C 4.0763280 .9854142 .237930

C $5.343439-0.9625893 .148277$

O 4.4389151 .7872403 .093998

C $6.728674-0.8614872 .590323$

C $7.478428-1.9210482 .250677$

C $8.859732-1.8093021 .670144$

C $8.976713-2.4578450 .306984$

C $9.576198-3.6517410 .162898$

C $8.480840-1.687593-0.898553$

C $6.981469-1.702353-1.021057$

N $4.419779-0.3599412 .204698$

H -1.479571 -2.1524300 .662735$

H $1.501144-1.7446941 .220320$

H $0.061868-2.577264-1.310081$

H $1.2027420 .273409-1.109821$

H -0.502197 -0.011004-0.869601

H $\quad 0.6238360 .229550-4.817463$

H -1.407984 -0.728030 -4.369346

H -1.296840 -1.946307 -3.097301

H $-2.030127-0.364287-2.758344$

H $2.7490990 .470972-2.598541$

H $2.9756800 .370232-4.323467$

H $3.3885522 .629358-3.732510$

H $1.8577302 .622955-4.613823$

H $0.6473992 .664490-2.376401$

H $1.0926564 .811080-3.608269$

H $2.6840454 .903547-2.881480$

H $1.0169326 .997501-2.468126$

H $2.8498845 .188089-0.257183$

H $-0.8871865 .636617-2.312358$

H -0.827642 $5.781619-0.570449$

H $-0.7975184 .176020-1.325501$

H $1.222403 \quad 6.3207610 .852166$

H 3.7621543 .4162940 .985774

H 2.3607823 .1142582 .020451

H 0.6435801 .9238520 .646634

H $4.139906-3.814041-3.136619$

H $4.414215-1.592211-1.023950$

H $6.355391-4.575224-2.378921$

H $7.853269-3.695938-2.608564$

H $6.516518-3.406009-3.712845$

H 2.2837130 .7448851 .125473
H $3.717611 \quad 1.098718 \quad 0.145707$

H $5.029673-2.0006533 .295535$

H $5.287618-0.4390074 .107796$

H 7.1316400 .1396122 .454268

H $7.082106-2.9234932 .395267$

H $9.555628-2.2821662 .375334$

H $9.180150-0.7622881 .598300$

H $9.693180-4.112186-0.812455$

H $9.960189-4.1981711 .017995$

H $8.953076-2.056168-1.816117$

H $8.815149-0.644824-0.822296$

H $6.487213-0.943915-0.413946$

H $4.126776-0.9226881 .411149$

C -1.239515 -0.744133 3.338032

O $-2.350641-0.2296292 .572642$

C -2.0631281 .1379122 .229293$

C -1.0588991 .6065503 .271530$

C -0.1886520 .3700703 .386661$

H -1.5653831 .1220511 .252853$

C -3.3426941 .9687422 .135789$

H -0.5052892 .4945512 .953146$

H -1.542029 1.8237724 .231246

C 0.6843650 .3467004 .628525

H $\quad 0.4601980 .3228952 .505994$

C $-4.373125 \quad 1.433822 \quad 1.118095$

O -3.9674831 .9944653 .424156$

H -3.082191 3.0075951 .899374

H -4.8371720 .5253301 .516825$

O -5.4253552 .4027641 .025369$

C $-3.8123831 .159297-0.289889$

C $-4.8743090 .886973-1.370585$

H $-3.1201390 .311112-0.243428$

O $-3.0542052 .297653-0.711031$

H -5.456683 $1.797835-1.559469$

H $-4.3313680 .691228-2.304781$

H $1.3762821 .195390 \quad 4.627331$

H $0.0842160 .403005 \quad 5.542798$

H $1.277787-0.5721804 .669256$

C $-0.748839-2.0726442 .749844$

H -4.7787062 .5247013 .342203$

H -5.0500583 .2056170 .624464$

H -2.629933 2.071220-1.556413

H -1.491423 -2.850609 2.967576

H $\quad 0.176788-2.3848713 .247440$

H $-1.625037-0.9350574 .347698$

C $-5.757838-2.638538-0.487837$

O $-5.029673-1.451951-0.835702$

C $-5.821777-0.284202-1.067725$

C $-6.811941-0.525428-2.219739$

C $-7.662156-1.776773-1.977032$

C $-6.805136-2.977366-1.564480$

H $-5.006069-3.438130-0.489453$

C $-6.353170-2.5563390 .927233$

H $-6.384513-0.042236-0.161053$

O $-6.114988-0.664745-3.457146$

H $-7.4611100 .352454-2.317501$

H -8.211051 -2.027943 -2.893141

O $-8.617908-1.517584-0.953318$

H -7.447161 -3.795240 -1.216660

O $-6.121056-3.448128-2.729766$

C $-5.283840-2.4836131 .996125$

H -6.992259-1.677134 1.043124

H $-6.950251-3.4527291 .129660$

H -5.486033 -1.400603 -3.362921

H $-9.172684-0.776173-1.250125$

H $-5.634625-4.252278-2.480185$

O $-5.871743-2.1769213 .190275$

O $-4.091364-2.6832751 .814105$

C $-4.988596-2.0706774 .311606$

H $-5.595808-2.0505055 .220557$

H $-4.312860-2.9299524 .363936$

H -4.423469 -1.137242 4.246810

SCF Energy (B3LYP/6-31G*//MMFF)= -3245.91288224

08 00073

MM̄FF Geometry

C $-0.388082-3.776816 \quad 0.408525$

C $0.276981-2.6195060 .539456$ 
C $0.862649-2.143731 \quad 1.841167$ O $0.627118-0.7212921 .956335$ C $2.383976-2.3597751 .835826$ C $3.020704-2.1456033 .193629$ C $3.712476-1.0461453 .550828$ C $2.887101-3.2991434 .154121$ C 3.9370510 .2066622 .750670 C 5.3855890 .3469922 .263168 C $5.728188-0.5817701 .086630$ C $7.199023-0.4283720 .695354$ C $7.548123-1.268792-0.541161$ O $4.897266-0.208662-0.014589$ O $8.828548-0.833840-1.025192$ C $6.523368-0.970817-1.657660$ C $7.702811-2.752627-0.187600$ O $6.707316-1.848224-2.766107$ C $5.049179-1.016303-1.190600$ C $4.130073-0.399442-2.266582$ O $4.597646-2.354590-0.981514$ C $-0.522074-0.3043302 .543052$ O $-1.430149-1.0127812 .955018$ C -0.5458531 .1706432 .694319$ C 0.3218761 .9981082 .089566 C $0.377140 \quad 3.4557022 .237268$ C -0.5785034 .0963823 .209954$ C $2.640403-0.464370-1.903303$ C $1.8428650 .520798-2.732028$ C $1.3018072 .940857-2.898709$ O $1.216150 \quad 0.178429-3.731026$ C $1.7426514 .200477-2.226216$ C $0.8961765 .088502-1.685506$ C $1.3346026 .329592-0.964895$ C $0.806136 \quad 6.3954230 .452137$ C $-0.239537 \quad 7.1823510 .757718$ C 1.5439645 .6318001 .530501 C 1.2761694 .1497851 .506804 N $1.9456531 .815632-2.249611$ H $-0.501726-4.415178 \quad 1.279966$ H $0.428735-1.988487-0.331904$ H $\quad 0.407215-2.6674912 .690675$ H $2.843315-1.6978821 .095730$ H $2.623218-3.3801301 .506673$ H 4.154516-1.020739 4.546300 H $3.406130-3.1146475 .100828$ H $1.834584-3.4861334 .388078$ H $3.313749-4.2080883 .717549$ H $3.2396050 .293743 \quad 1.912977$ H $3.709621 \quad 1.0551653 .408897$ H 5.5222641 .3879951 .942206 H $6.078546 \quad 0.1713323 .095383$ H $5.513717-1.6180021 .369309$ H $7.854915-0.6896171 .535238$ H 7.4092530 .6260980 .467502 H $9.472873-0.936544-0.303909$ H $6.7324970 .043185-2.026854$ H $8.522507-2.8921490 .527664$ H $7.971854-3.344338-1.069300$ H $\quad 6.803869-3.1828140 .259085$ H $7.636197-1.772684-3.044360$ H $4.4233600 .645804-2.429033$ H $4.261975-0.920941-3.223215$ H $5.319477-2.966955-1.193334$ H -1.3317761 .5426373 .343797$ H 1.0729051 .5773681 .423245 H -1.616050 3.8786852 .934262 H -0.4926855 .1841273 .251105$ H -0.3998243 .7240284 .224835$ H $2.243000-1.470659-2.077172$ H $2.472301-0.234556-0.845142$ H $\quad 0.2188882 .787974-2.844496$ H $1.5937862 .945630-3.953845$ H $2.8133714 .381283-2.165043$ H $-0.1734674 .917153-1.765087$ H $0.9780047 .193150-1.541325$ H $2.4279486 .418488-0.947004$ H -0.6073847 .2656911 .774731$ H $-0.7552927 .757446-0.004179$ H 1.3238676 .0482912 .520323
H 2.6231885 .7913891 .407244 H 1.9255603 .5997790 .825554 H $2.4169001 .969120-1.361891$ C $-2.443015-4.585426-0.873966$ O $-3.203424-3.405860-0.543527$ C $-4.253575-3.7857350 .365899$ C $-4.347406-5.306426 \quad 0.289454$ C $-2.891681-5.6802150 .095592$ H $-3.906291-3.5134911 .369796$ C $-5.556783-3.0427790 .053965$ H $-4.788123-5.7415791 .191377$ H $-4.935106-5.641123-0.572097$ C $-2.686139-7.091012-0.428010$ H $-2.385926-5.5855121 .063300$ C $-5.440922-1.5045400 .050600$ O $-6.023260-3.414626-1.249622$ H $-6.335257-3.3510210 .763212$ H $-4.906414-1.183171-0.850522$ O $-6.771428-0.981883-0.077396$ C $-4.774535-0.8863301 .293966$ C $-4.9749190 .633718 \quad 1.445814$ H -3.703662 -1.117201 1.277561 O $-5.322472-1.5032542 .463368$ H -6.0311560 .8420751 .660560$ H -4.4258860 .9426982 .345945$ H $-3.093415-7.8249420 .274883$ H -3.183395 -7.238539-1.392601 H -1.621096 -7.305240 -0.561131 C $-0.943624-4.275146-0.896677$ H $-6.440540-4.289238-1.176635$ H $-7.256877-1.2142850 .732835$ H -4.830159-1.164280 3.230619 H $-0.757704-3.527696-1.677525$ H $-0.390667-5.176927-1.184225$ H -2.743361 -4.856916 -1.895028 C $-2.4904991 .986380-0.996601$ $\begin{array}{lllll}\text { O } & -3.096933 & 1.302140 & 0.108769\end{array}$ C $-4.509974 \quad 1.4716470 .244188$ C -4.8625382 .9566550 .421923$ C $-4.2969193 .793621-0.728788$ C $-2.8156723 .490533-0.968360$ H -1.409957 $1.875603-0.836782$ C $-2.8360151 .322766-2.338754$ H $-5.0083071 .100353-0.655803$ O $-4.3415513 .428243 \quad 1.662635$ H -5.9537903 .0580660 .462373$ H -4.413996 $4.859634-0.499309$ O $-5.0234663 .526263-1.924193$ H -2.477235 $3.953208-1.902729$ O -2.0520874 .0727530 .088859$ C $-2.249190-0.067106-2.460728$ H $-3.915017 \quad 1.230041-2.485677$ H $-2.417728 \quad 1.924481-3.154271$ H -4.5943544 .3627921 .751413$ H $-5.9564363 .742734-1.756056$ H -2.2121985 .0314690 .074477$ O $-2.476957-0.530258-3.725685$ O $-1.678336-0.674952-1.566500$ C -1.991846-1.848296-3.998351 H -2.134748 -2.049432 -5.063344 H $-0.924491-1.922317-3.769120$ H -2.564304 -2.581071-3.422960 SCF Energy (B3LYP/6-31G**//MMFF) $=-3245.89437984$

0800074

MM̄FF Geometry

C $2.260322-0.5167553 .164212$

C $2.897799-1.6836773 .345542$

C $3.971156-2.2827762 .466847$

O $4.368036-1.3815601 .412074$

C $3.414426-3.5393611 .774562$

C $4.489934-4.4924081 .296659$

C $4.969967-4.5516790 .039387$

C $4.983455-5.4636352 .338779$

C $4.660469-3.650026-1.122741$

C $3.753835-4.305400-2.173800$

C $2.259015-4.136916-1.859839$

C $1.401178-4.875166-2.888512$ 
C $-0.097789-4.621368-2.668769$

O $1.970520-2.734234-1.899801$

O $-0.798345-5.079791-3.835090$

C $-0.334852-3.100470-2.567348$

C $-0.648934-5.427062-1.486613$

O $-1.690141-2.817395-2.228911$

C $0.618544-2.386947-1.580150$

C $0.472989-0.852553-1.731547$

O $0.278329-2.761352-0.244549$

C $5.345901-0.4741771 .658650$

O $5.930789-0.2786352 .710372$

C $5.562244 \quad 0.260007 \quad 0.392752$

C $6.222396 \quad 1.4261100 .393494$

C $6.4780302 .263424-0.780898$

C $6.043651 \quad 1.734417-2.124037$

C $1.534155-0.033173-0.980758$

C $1.3494481 .461195-1.192822$

C $2.2729873 .660642-0.458980$

O $0.4820811 .932074-1.924200$

C $3.3004354 .166079-1.422357$

C $4.1848735 .128694-1.117543$

C $5.1828385 .688920-2.091824$

C $6.5891345 .736607-1.534655$

C $7.0957016 .876440-1.034685$

C $7.4352744 .487528-1.637595$

C $7.0719563 .461324-0.600263$

N $2.2652862 .208114-0.462977$

H 2.5117950 .1204352 .321898

H $2.614593-2.2958444 .200635$

H $4.838969-2.5335613 .089098$

H $2.782613-3.2378470 .932323$

H $2.753698-4.0965572 .452554$

H $5.714118-5.315514-0.185246$

H $5.752897-6.1385691 .949336$

H $5.417036-4.9257163 .187778$

H $4.156565-6.0815862 .704040$

H $4.250278-2.690624-0.797928$

H $5.620507-3.409431-1.598184$

H $3.962759-3.818878-3.135928$

H $4.003832-5.367347-2.286673$

H $2.058211-4.524910-0.855875$

H $1.615287-5.951068-2.873613$

H $1.665499-4.531527-3.898713$

H $-0.597692-6.024108-3.953222$

H $-0.172263-2.678633-3.569275$

H $-0.504485-6.500984-1.655010$

H -1.730046 -5.283880 -1.381563

H $-0.173199-5.176096-0.536383$

H -2.252753 -3.269231 -2.880735

H $0.546775-0.589846-2.795203$

H $-0.521479-0.540187-1.387444$

H $0.551899-2.057900 \quad 0.364406$

H $5.145444-0.173398-0.509668$

H 6.5946591 .8131401 .341115

H $6.5796750 .808870-2.361483$

H $6.2245292 .427327-2.947900$

H $4.9679231 .526800-2.130632$

H $1.478732-0.2302510 .093713$

H $2.537973-0.298826-1.330524$

H 2.4765873 .9798110 .568068

H $1.2866484 .032724-0.752924$

H $3.2940653 .747895-2.426110$

H $4.1643495 .570297-0.123625$

H $4.8547426 .703287-2.354537$

H $5.1836165 .125178-3.033005$

H $8.1123656 .926954-0.659384$

H $6.5026067 .783717-0.984396$

H $8.4950454 .735862-1.490291$

H $7.3838534 .100474-2.658795$

H 7.3424203 .7583380 .414090

H $2.977171 \quad 1.7285750 .080265$

C $-0.1765490 .003344 \quad 3.325981$

O $-0.202649 \quad 1.1291202 .424133$

C -1.5068611 .7502552 .492823$

C -2.3837270 .7899853 .280606$

C -1.3817330 .1896274 .244917$

H -1.364721 2.6803613 .058194

C -1.9767772 .0658271 .067723$
H $-3.213816 \quad 1.294104 \quad 3.784246$

H -2.799407 0.0134852 .627650

C -1.867756-1.090570 4.901903

H -1.1511960 .9301565 .022704$

C -3.4600532 .4688710 .970996$

O $-1.160173 \quad 3.1315950 .578207$

H -1.7923841 .2041450 .414339$

H $-4.051763 \quad 1.6217151 .327472$

O -3.7240733 .5575201 .855162$

C $-3.9287102 .835891-0.452992$

C $-5.4433623 .105770-0.556716$

H -3.655434 $2.033380-1.147939$

O $-3.2762174 .024331-0.905182$

H -5.7189933 .9464660 .092380$

H -5.641185 $3.444472-1.582693$

H -1.110604 -1.494282 5.581204

H -2.776464 -0.903033 5.483181

H -2.097593 -1.860999 4.158947

C $1.159977-0.0317244 .068373$

H -0.2334442 .8579150 .686094$

H $-3.2040904 .317463 \quad 1.541402$

H -2.321691 $3.842111-0.934321$

H $1.085287-0.6801884 .948789$

H 1.4267250 .9716704 .421328

H $-0.302442-0.8995412 .713255$

C $-6.600303-0.419219-0.944439$

O $-5.9522240 .841863-1.147995$

C $-6.3186191 .883222-0.239182$

C -7.819385 $2.183966-0.355290$

C $-8.6447250 .910951-0.133355$

C $-8.131780-0.260351-0.979492$

H -6.304975 -1.028058 -1.808882

C $-6.082797-1.1294710 .317284$

H $-6.109399 \quad 1.5662790 .785826$

O $-8.1017852 .724960-1.643334$

H -8.0872482 .9426180 .389993$

H $-9.6923451 .114399-0.387347$

O $-8.590627 \quad 0.570622 \quad 1.249350$

H -8.609966 -1.194074 -0.661445

O $-8.534137-0.027207-2.332826$

C $-4.619603-1.5084990 .196591$

H -6.183334 -0.512928 1.213932

H $-6.652427-2.0552190 .460828$

H -9.053736 $2.919829-1.677738$

H $-9.143483-0.2187681 .378871$

H $-8.262806-0.799249-2.858080$

O $-4.296374-2.3634371 .212024$

O $-3.852655-1.100564-0.664571$

C $-2.942226-2.8275771 .219054$

H $-2.242785-1.9867521 .189872$

H $-2.778964-3.3863142 .144271$

H -2.776259 -3.497416 0.370942

SCF Energy $\left(B 3 L Y P / 6-31 G^{* *} / / M M F F\right)=-3245.90907671$

08_00075

MM̄FF Geometry

C -2.426138 $4.165448-0.903269$

C $-3.1070953 .026008-0.710065$

C -3.9798072 .7776010 .491517$

O -3.4745421 .6070371 .173723$

C -5.4159722 .4502040 .054398$

C -6.4339772 .7068151 .146662$

C $-6.896711 \quad 1.7816612 .009435$

C -6.9658404 .1166341 .195526$

C -6.4680300 .3488222 .162462$

C $-7.527308-0.6447781 .667726$

C $-7.471540-0.8609420 .148137$

C $-8.632785-1.741260-0.315877$

C $-8.540002-2.059696-1.815127$

O $-6.223462-1.499699-0.149105$

O $-9.467861-3.117454-2.100039$

C -7.128796 -2.601354 -2.119869

C $-8.960223-0.867390-2.682608$

O $-6.944429-2.780898-3.522761$

C $-5.986732-1.729815-1.542528$

C $-4.642873-2.485069-1.683734$

O $-5.916799-0.505580-2.275115$

C $-2.3975141 .748451 \quad 1.987360$ 
O $\begin{array}{llll}-1.799733 & 2.776800 & 2.255860\end{array}$

C -2.053821 0.3944532 .471946

C -0.8370120 .1609632 .982298$

C $-0.343460-1.1290293 .467709$

C $-1.302813-2.2912123 .463166$

C $-3.453745-1.848292-0.946110$

C $-2.173725-2.637479-1.183025$

C $0.281023-2.553970-0.770924$

O $-2.147148-3.694100-1.809954$

C $0.689145-3.2775350 .473586$

C $1.826787-3.0183211 .136971$

C $2.298791-3.7961022 .332452$

C $2.731418-2.9135973 .482620$

C $4.031044-2.6550233 .706021$

C $1.664441-2.4091754 .426946$

C $0.937964-1.2122673 .881345$

N $-1.063069-2.025645-0.618046$

H -2.521409 $4.971037-0.179873$

H $-3.0083082214797-1.427735$

H -3.969108 3.6341421 .177454

H $-5.4760171 .406364-0.272912$

H $-5.7009093 .052392-0.818783$

H -7.655652 2.0910852 .727717

H $-7.692870 \quad 4.2610502 .001637$

H -6.149276 4.8275021 .357569

H $-7.463416 \quad 4.3672610 .252855$

H -5.5046420 .1524421 .685919$

H -6.3066470 .1777613 .234674$

H -7.338411 -1.6050262 .165567$

H -8.527950 -0.312822 1.970565

H $-7.5132590 .111411-0.354111$

H $-9.595917-1.270517-0.082628$

H -8.619199 -2.6885350 .241552$

H -10.355834 -2.813987 -1.844380

H -7.060785 -3.601552 -1.669071

H $-9.993140-0.575593-2.457469$

H -8.948594 -1.129762 -3.746265

H -8.330215 $0.012636-2.537322$

H $-7.671301-3.343523-3.840663$

H $-4.762720-3.503089-1.289354$

H -4.387421 -2.577530 -2.747285

H $-5.013812-0.155380-2.225241$

H -2.804350 $-0.380642 \quad 2.365065$

H -0.1292630 .9870103 .035928$

H -2.144148 -2.094548 4.136826

H $-0.851376-3.2328023 .781976$

H -1.695744 -2.467360 2.455844

H $-3.288519-0.821802-1.289367$

H $-3.643028-1.8264950 .132897$

H $0.940144-1.711061-0.994863$

H $0.312301-3.244122-1.620346$

H $\quad 0.044020-4.0829630 .815788$

H $2.482557-2.232940 \quad 0.770535$

H $3.143652-4.4178232 .007312$

H $1.531489-4.4983202 .681088$

H $4.350366-2.0470864 .546063$

H $4.804614-3.0416803 .051133$

H $2.118738-2.1050765 .379752$

H $\quad 0.996437-3.2327744 .693775$

H $1.555469-0.3142123 .841437$

H $-1.182286-1.172470-0.079590$

C $-0.0200194 .307215-1.705845$

O $0.2895392 .978358-1.240215$

C $1.1789833 .082858-0.106453$

C $1.7000784 .511740-0.120506$

C $0.4870145 .266896-0.629623$

H 0.5549102 .9116860 .778976

C $2.2524261 .996584-0.217369$

H $2.031776 \quad 4.8493110 .865829$

H $2.5388924 .614838-0.820228$

C $0.8042516 .658861-1.146373$

H $\quad-0.231467 \quad 5.3495420 .194686$

C 3.3388162 .0735460 .868152

$\begin{array}{lllll}\text { O } & 1.599427 & 0.727768 & -0.110328\end{array}$

H $2.7061592 .035766-1.213828$

H 3.9016103 .0089510 .771833

$\begin{array}{lllll}\text { O } & 2.706718 & 2.122441 & 2.153613\end{array}$

C 4.3242510 .8868930 .877035
C $5.0712640 .713896-0.451112$

H $3.798599-0.0343671 .148651$

$\begin{array}{lllll} & & 5.294529 & 1.123458 & 1.902603\end{array}$

H $4.3691100 .533215-1.272896$

H $5.5796661 .655862-0.700168$

H $1.2243767 .279143-0.347906$

H $1.5312836 .627909-1.964865$

H $-0.1004127 .151289-1.516844$

C $-1.505824 \quad 4.400671-2.067916$

H $0.9028770 .702780-0.788811$

H 2.1626991 .3211122 .240092

H 4.8096201 .2431872 .737273

H $-1.7207963 .663661-2.852484$

H $-1.7258175 .386399-2.493544$

H $0.5610144 .444845-2.627409$

C $7.887283-1.241220-1.905668$

O $6.792506-0.333909-1.719644$

C $6.138649-0.398525-0.444238$

C $5.561840-1.803955-0.220350$

C $6.635071-2.884852-0.387024$

C $7.442483-2.694954-1.675064$

H $8.155980-1.137431-2.965108$

C $9.110691-0.839314-1.074455$

H $6.870693-0.1678900 .336330$

O $4.495694-2.051838-1.135111$

H $5.141217-1.8656580 .787888$

H $6.156867-3.871893-0.403135$

O $7.526263-2.8514660 .724026$

H $8.321543-3.350183-1.679661$

O $6.631218-3.074013-2.791017$

C $9.6644940 .481840-1.551902$

H $8.905629-0.762047-0.002933$

H $9.911152-1.581367-1.180052$

H $4.842552-1.932577-2.035791$

H $7.000267-3.0039391 .527545$

H $6.394110-4.010130-2.676030$

O $9.103821 \quad 1.504989-0.846057$

$\begin{array}{llllll}\text { O } & 10.477288 & 0.590603 & -2.460858\end{array}$

C $9.5244102 .818486-1.226118$

H $10.6019612 .930532-1.073204$

H $9.2590653 .013830-2.269529$

H $9.0027493 .539232-0.590915$

SCF Energy (B3LYP/6-31G**//MMFF)= -3245.92231084

08_00076

MMFF Geometry

C -1.258892 2.9454652 .651172

C -1.8460591 .8567942 .131376$

$\begin{array}{lll}\text { C }-2.337870 & 0.702692 & 2.966914\end{array}$

O $-1.813001-0.531116 \quad 2.428428$

C -3.8669920 .6181012 .847615$

C $-4.515854-0.277386 \quad 3.881234$

C $-4.925168-1.5418353 .658998$

$\begin{array}{llll}C & -4.754641 & 0.369239 & 5.221572\end{array}$

C $-4.736976-2.3823742 .425834$

C $-6.006344-2.4988601 .568128$

C $-6.139780-1.3562430 .550478$

C $-7.459697-1.443973-0.214113$

C $-7.558972-0.350823-1.290716$

O $-5.043025-1.469133-0.364093$

O $-8.664098-0.678024-2.146795$

C $-6.282713-0.390213-2.158076$

C $-7.871481 \quad 1.022368-0.684339$

O $-6.2659930 .687881-3.091608$

C $-4.965937-0.428301-1.343117$

C $-3.781780-0.741408-2.289875$

O $-4.751915 \quad 0.849010-0.740624$

C $-0.526455-0.8653392 .700461$

O $0.295009-0.2113293 .320267$

C $-0.302297-2.200298 \quad 2.102593$

C $0.935031-2.7112662 .038824$

C $1.292038-4.0236021 .495881$

C $0.171593-4.9212721 .037470$

C $-2.458792-1.088294-1.586443$

C $-1.389762-1.462863-2.602835$

C $0.775507-2.647979-2.839516$

O $-1.440328-1.106485-3.778257$

C $1.286040-3.961419-2.339662$ 
C $2.573910-4.188466-2.037433$

C $3.115673-5.530042-1.632250$

C $3.932370-5.484655-0.360126$

C $5.272365-5.392281-0.401098$

C $3.203602-5.6491910 .953476$

C $2.596385-4.3624661 .437726$

N $-0.368523-2.225775-2.050898$

H -1.114857 2.9833953 .729736

H $-2.0123981 .779077 \quad 1.061008$

H -2.0333540 .8012004 .016187$

H $-4.122487 \quad 0.270320 \quad 1.842761$

H -4.3151261 .6170052 .938662$

H -5.423947 -2.065591 4.474072

H $-5.248007-0.3062855 .928481$

H -3.8065710 .6800865 .671292$

H $-5.392690 \quad 1.2520155 .109208$

H -3.887260 -2.041169 1.827719

H -4.461989 -3.387869 2.770750

H -5.946781 -3.447643 1.018703

H -6.896234 -2.548873 2.207146

H $-6.084265-0.4005721 .080257$

H -8.313274 -1.3854660 .472468$

H -7.535494 -2.424430 -0.705010

H $-9.465707-0.722813-1.597825$

H -6.341695 -1.306898 -2.760861

H $-8.8025990 .982642-0.106246$

H -8.038884 $1.770427-1.467245$

H $-7.0850321 .386837-0.020166$

H $-6.0159071 .493693-2.608198$

H -4.046889 -1.601337 -2.919734

H $-3.614897 \quad 0.111026-2.961033$

H -3.801105 $0.970053-0.589018$

H -1.172215 -2.7312101 .731468$

H $1.764722-2.1157792 .417747$

H $\quad 0.515377-5.8558320 .589525$

H $-0.438945-4.425147 \quad 0.275377$

H $-0.473649-5.187677 \quad 1.881882$

H -2.086073 -0.236256-1.008588

H -2.605078 -1.931562 -0.902295

H $1.529889-1.859606-2.774370$

H $\quad 0.477745-2.745810-3.888598$

H $0.571318-4.778135-2.271936$

H $3.291276-3.378915-2.145042$

H $3.741646-5.895099-2.457437$

H $2.312859-6.270125-1.525506$

H $5.867119-5.3914630 .506367$

H $5.803464-5.307356-1.343408$

H $3.902392-5.9892301 .729999$

H $2.475769-6.4612940 .870242$

H $3.334780-3.6447311 .796945$

H $-0.312721-2.309458-1.039789$

C -1.010391 4.2110040 .402005

O $-0.1638793 .237883-0.242163$

C $0.4924343 .858811-1.360502$

C $-0.2767675 .143176-1.633099$

C $-0.6467515 .560477-0.223301$

H $1.5015264 .124168-1.024714$

C $0.5872282 .890318-2.541023$

H $0.3282515 .888213-2.158595$

H -1.181481 $4.959072-2.223889$

C -1.763472 $6.588714-0.168312$

H 0.2450755 .9744550 .266569

C $1.2271801 .529860-2.198418$

O $-0.7345122 .631165-3.031702$

H $1.1303423 .373688-3.362078$

H $\quad 0.5159800 .923978-1.624281$

O $1.426520 \quad 0.831343-3.434358$

C $2.561621 \quad 1.604012-1.433928$

C $3.2146610 .218015-1.278520$

H $2.3847662 .037315-0.443538$

O $3.4429762 .474484-2.141717$

H $2.450270-0.514424-0.994103$

H $3.637908-0.078431-2.246192$

H -1.465755 $7.507175-0.684646$

H -2.676187 6.215931 -0.645227

H $-2.006204 \quad 6.8470020 .867100$

C $-0.777598 \quad 4.1697151 .915495$

H $-0.6513312 .009829-3.775586$
H $2.110807 \quad 1.309080-3.934022$

H $4.2641042 .544295-1.625920$

H -1.269117 5.0344442 .377214

H 0.2971454 .2612442 .117034

H -2.0486993 .9516440 .159236$

C 6.4360191 .2446950 .341887

O $5.3577341 .102975-0.593072$

C $4.3259730 .191213-0.210713$

C $4.907518-1.219407-0.036549$

C $6.063888-1.2185100 .966524$

C $7.083225-0.1156500 .654765$

H $7.1803051 .856700-0.184300$

C 6.0026132 .0129181 .595592

H 3.8814420 .5229340 .734227

O $5.368770-1.701534-1.296818$

H $4.110651-1.8888980 .303759$

H $6.559835-2.1964840 .942538$

O $5.529987-1.0365972 .274489$

H 7.7919580 .0010201 .482859

O $7.844895-0.499492-0.494023$

C 5.5987673 .4243391 .239203

H $5.175611 \quad 1.5319692 .125626$

H 6.8274102 .0854312 .314303

H $5.710745-2.601110-1.160435$

H $6.273188-1.0618862 .901046$

H $8.293351-1.336045-0.282771$

O $4.2436903 .555841 \quad 1.312234$

O $6.392646 \quad 4.2977770 .913976$

C 3.7320714 .8505790 .982386

H 4.1907105 .6174171 .614132

H $3.9086635 .063998-0.075964$

H 2.6547164 .8468361 .166023

SCF Energy $\left(B 3 L Y P / 6-31 G^{* *} / / M M F F\right)=-3245.91485922$

08_00077

MM̄̄FF Geometry

C $0.389398-0.8305932 .508842$

C $0.925519-1.9709632 .048095$

C $0.385615-2.788298 \quad 0.895127$

O $-0.538247-2.0067430 .099921$

C $-0.341310-4.0326141 .422916$

C $-0.774811-4.971130 \quad 0.314625$

C $-2.036050-5.071787-0.148948$

C $0.310457-5.857199-0.240193$

C $-3.230348-4.2664300 .281123$

C $-4.029127-3.753866-0.925681$

C $-5.232264-2.914305-0.475575$

C $-6.155346-2.601459-1.653012$

C $-7.328760-1.706015-1.227347$

O $-4.735196-1.6941130 .086153$

O $-7.959414-1.209970-2.417793$

C $-6.772551-0.485165-0.466933$

C $-8.401319-2.489114-0.461340$

$\begin{array}{lllll}0 & -7.828507 & 0.300413 & 0.081974\end{array}$

C $-5.751029-0.8476880 .639323$

C $-5.080840 \quad 0.444244 \quad 1.161037$

O $-6.430260-1.4841561 .723308$

C $0.013833-1.147406-0.802132$

O $1.197559-1.038421-1.081415$

C $-1.067811-0.302489-1.353403$

C $-0.7348160 .859703-1.935743$

C $-1.6516531 .848026-2.507410$

C $-3.110127 \quad 1.484182-2.581558$

C -3.8314010 .2170332 .022769$

C -3.2603461 .5491972 .471583$

C -2.1541663 .6002081 .574516$

O -3.3784761 .9460413 .628204$

C $-2.607106 \quad 4.3874490 .385143$

C $-1.7823225 .094088-0.402096$

C $-2.2409145 .874495-1.600203$

C $-1.4982985 .499555-2.864211$

C $-0.595576 \quad 6.333409-3.408128$

C $-1.8611934 .194608-3.544037$

C -1.139566 $3.017899-2.944894$

N $-2.656623 \quad 2.2458801 .437558$

H $-0.543025-0.4655012 .086691$

H $1.849217-2.3336352 .491740$

H $1.228748-3.0967380 .264556$ 
H -1.201950 -3.730654 2.030465 H $\quad 0.313329-4.5875702 .108769$ H $-2.239883-5.814033-0.919585$ H $-0.065969-6.548495-1.001688$ H $1.099198-5.256672-0.703601$ H $\quad 0.754982-6.4589960 .559209$ H $-3.874193-4.9100510 .892473$ H -2.942969 -3.414631 0.903166 H -3.371224 -3.147597 -1.562068 H -4.374868 -4.607858 -1.521322 H $-5.775892-3.4745330 .294442$ H $-6.526734-3.524650-2.114983$ H $-5.585644-2.081170-2.435802$ H -8.253750 -1.974663 -2.941779 H $-6.2662740 .151455-1.206432$ H -8.791280 -3.307693 -1.078097 H $-9.263093-1.853712-0.228599$ H -8.032337 -2.9240930 .469848$ H $-8.435757 \quad 0.518189-0.645747$ H $-4.791070 \quad 1.0636190 .304775$ H -5.8057341 .0305781 .740801$ H $-5.917897-1.3524842 .536832$ H -2.091485 -0.636229-1.233133 H $0.3197231 .130771-1.981927$ H -3.531312 $1.385216-1.577727$ H $-3.2442920 .534133-3.111296$ H $-3.7175482 .218421-3.114028$ H $-4.063976-0.3728782 .915238$ H -3.058217 -0.3256101 .467734$ H -1.064303 3.5425581 .642469 H -2.5389454 .0518772 .493871$ H -3.671476 4.3745010 .160958 H $-0.7221785 .130646-0.165677$ H -2.094881 $6.939545-1.377701$ H -3.318005 $5.750888-1.768858$ H $-0.078486 \quad 6.083014-4.328651$ H $-0.3489567 .281941-2.942860$ H -1.588632 4.241690-4.606627 H -2.948599 $4.084789-3.525170$ H $-0.0602403 .163519-2.874151$ H $-2.496215 \quad 1.7695010 .554846$ C 1.2884961 .4277112 .956052 O 2.3033711 .3382071 .938116 C 2.9767542 .6110471 .854751 C 2.3962773 .4848452 .968082 C 1.8320292 .4586733 .935216 H 4.0349092 .4269692 .066392 C 2.8117793 .1908470 .443941 H 3.1589444 .1239863 .424378 H 1.6026024 .1431752 .595556 C 0.7916303 .0304344 .881893 H $2.6590132 .027746 \quad 4.515063$ C $3.3431652 .272611-0.675643$ $\begin{array}{lllll}\text { O } & 1.412460 & 3.394764 & 0.205867\end{array}$ H $3.280740 \quad 4.1797850 .388511$ H $2.7566241 .348646-0.689379$ O $3.0733382 .922615-1.926437$ C $4.8497821 .947267-0.597983$ C $5.3402051 .079010-1.773761$ H $5.093267 \quad 1.4446780 .343435$ O $5.5981433 .165080-0.636728$ H $4.9446531 .475649-2.716262$ H $6.4326081 .175169-1.841233$ H 1.2242693 .8312515 .490433 H -0.0622993 .4462694 .336194$ H 0.4136612 .2594555 .560291 C 1.0227280 .0459773 .552069 H $1.3189153 .737841-0.699394$ H $3.6245163 .723174-1.964559$ H 5.4582583 .6243080 .208335 H $0.338555 \quad 0.1202844 .403909$ H $1.957927-0.3989573 .909526$ H 0.3783091 .8072722 .473477 C $5.694007-2.347657-0.339060$ O $5.874026-0.959222-0.649680$ C $4.967401-0.403961-1.611857$ C $5.059179-1.166244-2.941386$ C $4.839061-2.666738-2.733760$
C $5.720230-3.215214-1.608292$

H $6.577182-2.6141790 .256767$

C $4.452466-2.5689270 .532198$

H $3.946343-0.478880-1.229420$

O $6.325951-0.944990-3.557443$

H $4.297156-0.774596-3.625511$

H $5.063167-3.192495-3.669882$

O $3.466381-2.888648-2.423477$

H $5.430546-4.241545-1.354555$

O $7.076143-3.267720-2.062402$

C $4.755867-2.2299051 .972658$

H $3.583261-1.9851110 .220781$

H $4.149605-3.6227720 .517440$

H $7.015180-1.210031-2.924613$

H $3.340089-3.846699-2.315976$

H $7.102437-3.851968-2.839146$

O $4.759770-0.8783222 .149433$

O $4.979165-3.0710742 .834037$

C $5.089527-0.4364213 .469725$

H 5.0208800 .6537683 .493180

H $4.384879-0.8477504 .198352$

H $\quad 6.114481-0.7278103 .717951$

SCF Energy (B3LYP/6-31G*//MMFF)= -3245.91490780

08_00078

MM̄FF Geometry

C $-4.2494540 .299086 \quad 3.053974$

C $-3.123177-0.3158922 .660523$

C $-1.761761-0.0157363 .227778$

O -0.9378890 .5445492 .180503$

C $-1.092941-1.3195663 .699434$

C $0.157235-1.0685684 .517534$

C $1.417652-1.1704514 .053447$

C $-0.085522-0.7140745 .963033$

C $1.878049-1.4978852 .659805$

C $2.668109-2.8142632 .639597$

C $3.315925-3.0851741 .273494$

C $4.050883-4.4273001 .280298$

C $4.804845-4.671879-0.036551$

O $4.246951-2.0330151 .016215$

O $5.691907-5.784576 \quad 0.155373$

C $5.661651-3.429010-0.376107$

C $3.844181-5.112281-1.146797$

O $6.241770-3.547293-1.672292$

C $4.887732-2.095585-0.265129$

C $5.847029-0.888346-0.353466$

O $3.953599-1.942485-1.335774$

C -0.9373921 .8976292 .036698$

O -1.6000182 .7068812 .665518$

C 0.0298762 .2279210 .965634

C 0.1945293 .5022460 .578399

C $1.0940213 .974674-0.479536$

C $1.9217042 .937827-1.193702$

C $5.1205190 .442187-0.121527$

C $6.0314831 .652002-0.222564$

C 6.0093854 .1028050 .236911

O $7.1825301 .604214-0.643988$

C 4.9516735 .1347350 .461623

C $4.6855836 .142146-0.383009$

C $3.5869507 .139461-0.144097$

C $2.6208977 .250565-1.305568$

C $2.3776888 .443146-1.876071$

C $1.9367755 .998264-1.820359$

C $1.1180345 .293092-0.771630$

N 5.3717122 .8011650 .190898

H -4.2004871 .0778013 .810505$

H -3.178378 -1.084949 1.893891

H $-1.835850 \quad 0.6886494 .065621$

H $-0.862610-1.9555102 .836141$

H - $1.791883-1.9022914 .314750$

H $2.232144-0.9731644 .750270$

H $\quad 0.847025-0.5820046 .522136$

H $-0.6500390 .219901 \quad 6.042303$

H -0.654978 -1.507867 6.457641

H $1.049334-1.5565021 .948332$

H $2.512872-0.6700042 .321953$

H $3.454252-2.7685073 .405200$

H $2.000660-3.6437932 .904623$ 
H $2.535033-3.0726910 .505980$ H $3.356866-5.2519581 .485546$ H $4.777954-4.4370812 .104491$ H $6.303267-5.5651230 .878870$ H $\quad 6.496983-3.398220 \quad 0.337079$ H $3.364660-6.062489-0.881144$ H $4.379072-5.299512-2.084207$ H $3.050136-4.387979-1.338908$ H $6.729371-4.388498-1.696159$ H $6.637880-0.9842980 .401420$ H $6.331081-0.863529-1.337806$ H $4.105010-2.649240-1.983717$ H $\quad 0.5729591 .4048170 .514543$ H -0.3908384 .2760561 .073512$ H $2.5114432 .356513-0.478471$ H $2.6306603 .354016-1.911105$ H $1.2746802 .250910-1.749945$ H $4.3237090 .570365-0.863213$ H 4.6622250 .4455630 .874489 H $6.5663814 .257863-0.693002$ H 6.7223004 .0998351 .067640 H $4.3617665 .038527 \quad 1.370862$ H $5.2791136 .256217-1.286731$ H 4.0601458 .1097220 .054416 H $3.019696 \quad 6.8892060 .761256$ H $1.6912318 .542845-2.710375$ H $2.8586929 .349166-1.522535$ H $2.7001085 .354115-2.263269$ H $1.2528116 .254827-2.639964$ H $\quad 0.4523475 .955924-0.216837$ H 4.4858522 .6905350 .676779 C $-6.0514290 .770149 \quad 1.308771$ O C $-4.8662181 .845589-0.394317$ C $-5.9795782 .769618 \quad 0.082175$ C -6.1619832 .2816301 .508726$ H -3.920956 2.2089580 .026886 C $-4.7321081 .696784-1.913567$ H -5.7007393 .8257000 .020735$ H $-6.9021312 .623560-0.491600$ C -7.4669702 .7253382 .145410$ H -5.3260842 .6566522 .112629$ C $-3.6675090 .663678-2.348099$ O $-5.9964431 .280769-2.441242$ H $-4.5171112 .676415-2.357245$ H $-4.026208-0.350134-2.140773$ O $\quad-3.5048290 .757099-3.767355$ C $-2.2923620 .875673-1.694873$ C -1.156042 $-0.010878-2.226582$ H $-2.373720 \quad 0.711645-0.615593$ O $-1.8901542 .237742-1.872679$ H $-0.8962590 .277943-3.253354$ H $-0.2701050 .238781-1.629209$ H -7.515283 3.8177262 .201623 H -8.3327482 .3802201 .570271$ H $-7.5576972 .329847 \quad 3.161917$ C -5.612425 -0.0491502 .523640$ H $-5.9535331 .364723-3.408357$ H $-4.3254030 .437277-4.178404$ H -1.843591 $2.407999-2.829128$ H $-5.623369-1.1121942 .254663$ H -6.3412340 .0655143 .334678$ H -7.0149010 .3722020 .963860$ C $-2.222419-3.250176-0.664751$ O $-1.898114-1.866425-0.866547$ C - $-1.414128-1.526459-2.169159$ C $-0.137920-2.321737-2.497268$ C $-0.347856-3.827114-2.314457$ C $-1.010707-4.148644-0.971111$ H -2.436210 -3.3332130 .408210$ C $-3.483802-3.671100-1.437725$ H -2.186350 -1.758705 -2.909498 O $0.920217-1.890261-1.643407$ H $0.159154-2.102265-3.529901$ H $0.622837-4.334679-2.372100$ O $-1.151363-4.307550-3.387592$ $\mathrm{H}-1.312520-5.202215-0.943336$ O $-0.035209-3.9687210 .058905$
C $-4.731772-3.004628-0.903180$

H $-3.400381-3.427651-2.499997$

H $-3.641306-4.752006-1.347999$

H $1.725209-2.366577-1.908151$

H $-1.253446-5.267489-3.270724$

H $-0.447481-4.2163990 .903923$

O $-5.656551-2.907408-1.902868$

O $-4.891107-2.649518 \quad 0.255917$

C $-6.907083-2.319660-1.531119$

H $-7.384083-2.908357-0.741701$

H $-6.757846-1.286698-1.205843$

H -7.557041-2.319453 -2.409995

SCF Energy (B3LYP/6-31G**//MMFF)= -3245.91102495

0800079

MM̄FF Geometry

C $-2.269726 \quad 3.7222020 .034221$

C $-2.3564004 .008527 \quad 1.342658$

C -2.8406513 .0532812 .411384$

O $-2.705091 \quad 1.6822861 .965627$

C -4.3161263 .3267532 .736405$

C -4.8236322 .5041183 .903776$

C -5.5938631 .4050023 .784717$

C -4.4495513 .0226895 .268178$

C -6.0543760 .7497172 .512420$

C $-5.873151-0.7740162 .565784$

C -6.336371 -1.432551 1.259356

C $-6.372137-2.9547541 .394400$

C $-6.770468-3.6292680 .072933$

O $-5.417776-1.0603540 .225642$

O $-6.482484-5.0309200 .188627$

C -5.879041-3.075235-1.056879

C $-8.274980-3.515945-0.199656$

O $-6.307980-3.555333-2.329163$

C $-5.782692-1.529839-1.078493$

C $-4.684541-1.098987-2.077766$

O $-7.032168-0.981689-1.502195$

C -1.4668591 .1266392 .083519$

O -0.4870091 .6256282 .614775$

C -1.484011 -0.1842411 .398440$

C $-0.314350-0.7183441 .014963$

C $-0.117398-1.9883970 .313287$

C $-1.326366-2.8576240 .095195$

C $-4.2610730 .372474-1.971006$

C $-3.1824890 .679319-2.992964$

C $-0.8129710 .128556-3.554710$

O $-3.4153201 .346034-3.998319$

C $-0.203499-1.238075-3.574106$

C $1.089971-1.484897-3.318962$

C $1.692409-2.860190-3.330753$

C $2.420257-3.193060-2.046655$

C $3.761900-3.253081-2.009457$

C $1.592467-3.539094-0.825649$

C 1.131511-2.314104-0.082152

N -1.967042 $0.095282-2.676151$

H $-2.6135882 .756824-0.327633$

H -2.026255 4.9838191 .693231

H -2.2311223 .2012783 .311738$

H -4.9314413 .1499321 .846710$

H -4.4570064 .3895602 .975769$

H -5.9377940 .9206114 .697762$

H -4.8818812 .4211006 .074939$

H -3.363086 3.0140025 .398607

H -4.8078194 .0494295 .395675$

H -7.1173200 .9816712 .374223$

H -5.5230331 .1394561 .640019$

H -4.814412 -1.004432 2.742267

H $-6.447458-1.1789263 .408309$

H -7.334274 -1.049835 1.014346

H -7.051581 -3.260154 2.199692

H $-5.376952-3.3192871 .685885$

H $-6.986728-5.3790130 .943955$

H $-4.869407-3.480253-0.898575$

H $-8.847159-3.9765030 .614745$

H $-8.553822-4.062564-1.107287$

H $-8.616250-2.483650-0.300390$

H $-6.339328-4.525937-2.277177$

H $-3.795101-1.718125-1.914787$ 
H $-5.019179-1.294703-3.105052$ H -6.876345 -0.107978 -1.894407 H $-2.443254-0.6500011 .206364$ H $0.599181-0.1607731 .220485$ H -2.039455 -2.362553 -0.569256 H -1.826068 -3.0688641 .047504$ H -1.094067 -3.826817 -0.350107 H $\quad-5.1056451 .044940-2.153469$ H -3.872180 $0.603131-0.972949$ H $-0.1256020 .884745-3.166396$ H -1.119085 $0.419092-4.564243$ H -0.864062 -2.069197-3.811636 H $1.759392-0.655626-3.107100$ H $2.384049-2.911532-4.181730$ H $\quad 0.931612-3.627907-3.518972$ H $4.288453-3.519410-1.099181$ H $4.363624-3.032549-2.884719$ H $2.192649-4.138069-0.128071$ H $\quad 0.770271-4.188951-1.136367$ H $1.941904-1.6238420 .157680$ H -1.847673 -0.314797 -1.754502 C $-0.5009963 .959590-1.688315$ O $0.5738623 .784448-0.746187$ C $1.8289753 .940484-1.432948$ C $1.4868444 .135576-2.908283$ C $0.1053644 .757623-2.835816$ H $2.2921684 .855152-1.043329$ C $2.7543682 .743180-1.164298$ H $2.216814 \quad 4.770273-3.420158$ H $1.4486503 .177999-3.441517$ C $-0.6709824 .659372-4.137386$ H $0.2073685 .812825-2.549518$ C 3.2040682 .5956700 .303592 O $2.1028621 .541395-1.574710$ H $3.6400062 .861511-1.797454$ H 3.7764223 .4803560 .608078 O $2.0610402 .533611 \quad 1.164142$ C $4.025144 \quad 1.3230140 .599642$ C $5.2774541 .165227-0.279320$ H 3.3889020 .4352670 .506067 O 4.4385851 .3881841 .969215 H $5.0025631 .223170-1.336945$ H $5.9615941 .992744-0.053454$ H -0.141245 $5.180994-4.941186$ H $-0.8076723 .617253-4.445585$ H -1.661879 $5.112968-4.036964$ C -1.683244 $4.641287-0.999765$ H $1.3125791 .433736-1.017752$ H 1.5813083 .3750901 .083180 H 3.6349421 .4748022 .510326 H -2.463156 $4.882771-1.729905$ H -1.356488 $5.579118-0.534519$ H $-0.7905202 .958786-2.033385$ C 7.206271-1.400256 1.662190 O $6.542200-0.1914151 .271137$ C $6.009676-0.175799-0.055163$ C $7.138644-0.380628-1.079031$ C $7.951469-1.644923-0.779466$ C $8.356529-1.7289630 .696038$ H $7.646264-1.1704762 .641471$ C $6.222178-2.5598681 .853615$ H $5.273612-0.980076-0.160193$ O $8.0110460 .748616-1.086218$ H $6.699622-0.450864-2.081019$ H $8.854798-1.652165-1.401666$ O $7.186808-2.799225-1.112116$ H $8.757077-2.7223240 .930320$ O $9.402814-0.7851560 .943864$ C $5.280055-2.2752332 .998817$ H $5.630195-2.7765300 .960475$ H $6.755576-3.4861352 .098658$ H $8.3486830 .868591-0.182136$ H $6.970411-2.745632-2.058616$ H $10.153812-1.0241390 .374259$ O $4.086577-1.8208732 .521010$ O $5.579790-2.4179294 .177251$ C $3.115150-1.4813513 .515114$ H 2.249841-1.045336 3.010231
H $2.795893-2.3810614 .049169$

H $3.520845-0.7409064 .211277$

SCF Energy (B3LYP/6-31G**//MMFF) $=-3245.91927493$

08_00080

MM̄FF Geometry

C -0.656585 -4.047314 -0.198180

C $-1.926514-3.617228-0.205313$

C $-2.757937-3.421510-1.446026$

O $-3.077927-2.012946-1.521049$

C $-4.066033-4.223113-1.324205$

C $-5.027662-4.003697-2.476511$

C $-6.178299-3.304619-2.393706$

C $-4.629147-4.649627-3.778089$

C $-6.733859-2.596097-1.189230$

C $-6.623940-1.073649-1.340618$

C $-6.668879-0.3584400 .017740$

C $-6.7031121 .157349-0.175185$

C $-6.607278 \quad 1.913377 \quad 1.157740$

$\begin{array}{lllll}\text { O } & -5.488062 & -0.733057 & 0.737037\end{array}$

$\begin{array}{llll}\text { O } & -6.318402 & 3.289076 & 0.860423\end{array}$

C -5.4106831 .3650961 .966797$

C -7.9424581 .9046391 .912158$

O $-5.402897 \quad 1.9514373 .266432$

C $-5.384031-0.1766992 .055481$

C $-4.083269-0.7399892 .684531$

O $-6.470672-0.6139312 .881028$

C $-2.222888-1.220470-2.224888$

O $-1.254202-1.579367-2.875232$

C $-2.6461100 .184653-2.029700$

C $-1.8489151 .181047-2.442935$

C $-2.0983452 .615357-2.283885$

C $-3.369523 \quad 3.031510-1.592905$

C $-2.785115-0.4009611 .943570$

C -2.0847340 .8500542 .443535$

C -0.1991302 .3769201 .873239$

O

C -0.7374573 .5701461 .147616$

C $0.018094 \quad 4.3635710 .373336$

C $-0.4895825 .593589-0.322669$

C $-0.1918575 .590867-1.805575$

C $0.9164396 .177523-2.288880$

C $-1.2231384 .988643-2.732246$

C -1.177174 $3.485265-2.748778$

N $-1.042653 \quad 1.2271641 .605674$

H $-0.165725-4.294214-1.135853$

H -2.401344 -3.365032 0.740447

H $-2.215477-3.741739-2.344012$

H $-4.545754-3.983721-0.370469$

H $-3.835128-5.295585-1.263968$

H $-6.792680-3.214669-3.288703$

H -5.390138 -4.522601-4.555405

H $-3.698753-4.214319-4.155114$

H $-4.480007-5.725231-3.637948$

H -7.789841 -2.872206 -1.078348

H $-6.251742-2.928023-0.267593$

H -5.681435 -0.823104 -1.842279

H -7.438038 -0.716968 -1.983604

H $-7.554247-0.6988980 .567365$

H $-7.603778 \quad 1.458877-0.724484$

H $-5.8569801 .461003-0.804932$

H -7.0305003 .6291260 .291886$

H -4.5055351 .7152751 .456293$

H $-8.7267732 .375614 \quad 1.307358$

H -7.8810182 .4953592 .832699$

H -8.2838950 .8994032 .167439$

H -4.5626311 .7181183 .694599$

H -4.024911 -0.4692343 .745473$

H $-4.170332-1.8363762 .672863$

H $-6.269793-1.5059773 .209523$

H $-3.5852970 .362151-1.520260$

H $-0.9134450 .925432-2.938832$

H $-4.2406242 .736439-2.187530$

H $-3.4446874 .107451-1.423409$

H -3.441133 $2.564779-0.604837$

H -2.074385 -1.222714 2.099494

H -2.959947 -0.3182620 .867817$

H $0.8141032 .117673 \quad 1.551985$ 
H -0.1843152 .5760292 .949577$ H -1.7888713 .8091531 .288478$ H $1.076574 \quad 4.1419660 .263426$ H -0.0113546 .4631920 .147345$ H -1.566244 $5.729818-0.163204$ H $1.1231016 .205623-3.353646$ H $1.6448596 .636819-1.628823$ H -1.046945 5.328024 -3.762041 H -2.211293 $5.383891-2.480237$ H $-0.2812883 .085886-3.226215$ H -0.8933370 .7219010 .736957$ C $1.315033-3.2132821 .107643$ O $2.337029-3.6350020 .182345$ C $3.621977-3.5722820 .837953$ C $3.401018-2.7455512 .094659$ C $1.990711-3.1446542 .476448$ H $3.875884-4.6080251 .095203$ C $4.652510-2.986850-0.133081$ H $4.133739-2.9628642 .877430$ H $3.447710-1.6725731 .868727$ C $1.326471-2.1738803 .438828$ H $2.018545-4.1430752 .933224$ C $6.045216-2.7439490 .479394$ O $4.776361-3.880292-1.244663$ H $4.250747-2.054658-0.540515$ H $5.984228-2.0727741 .343063$ O $6.567503-3.9852670 .969709$ C $7.074177-2.188585-0.524344$ C $6.659211-0.890535-1.232981$ H $7.289095-2.945938-1.288304$ O $8.312714-1.9653670 .153013$ H $7.488827-0.546892-1.865017$ H $5.848025-1.116151-1.937892$ H $1.886236-2.1204604 .378188$ H $\quad 1.277147-1.1632893 .019098$ H $\quad 0.305019-2.4909073 .671338$ C $0.146773-4.2000431 .064321$ H $5.167580-4.707365-0.914446$ H $6.034653-4.247272 \quad 1.739133$ H $8.562526-2.8007840 .583852$ H $-0.498431-4.0533321 .938552$ H $\quad 0.520120-5.2307131 .103316$ H $0.988750-2.2137160 .792440$ C $5.0881032 .420680-0.593569$ O $5.6699571 .270615-1.219896$ C $6.2042300 .277376-0.330845$ C 7.3146090 .8897530 .537553 C 6.8326252 .1514561 .262944 C 6.1091773 .1180080 .318390 H $4.8561533 .101448-1.422978$ C 3.7718302 .0863550 .116509 H $5.391684-0.0766410 .309097$ O $8.4350591 .212630-0.282964$ H 7.6427010 .1534451 .278801 H 7.6959472 .6564971 .713277 O $5.963527 \quad 1.7603612 .322349$ H 5.6078863 .9108130 .885877 O $7.0732393 .753440-0.525916$ C $2.7437301 .579696-0.866397$ H 3.8889361 .3434800 .910751 H 3.3522672 .9785360 .595940 H 9.1361051 .5538430 .297796 H 5.6887272 .5675952 .789495 H 7.6961234 .2314360 .047754 O $2.6310360 .225680-0.760738$ O $2.1284492 .300295-1.640899$ C $1.684117-0.396234-1.633687$ H $1.929348-1.459049-1.702365$ H $0.684204-0.292615-1.205655$ H $1.7217210 .031380-2.640278$ SCF Energy $\left(B 3 L Y P / 6-31 G^{* *} / / M M F F\right)=-3245.90166405$

08_00081

MM̄FF Geometry

C 3.0869245 .0359420 .662685

C 3.7302433 .8588100 .673852

C $4.4146883 .272996-0.534206$

O $3.8401501 .967598-0.787178$
C $5.9176273 .102003-0.257142$

C $6.6975602 .629020-1.469406$

C $7.1699951 .378793-1.645025$

C $6.9683123 .686427-2.509764$

C $7.0134240 .196134-0.731126$

C $6.260959-0.945354-1.427018$

C $5.981420-2.110801-0.467308$

C $5.294676-3.263202-1.201552$

C $4.897649-4.400171-0.248960$

O $5.134259-1.6234440 .578633$

O $3.997943-5.254856-0.971551$

C $4.126924-3.8051740 .950876$

C $6.106454-5.2669130 .122448$

O $3.863939-4.8099651 .926947$

C $4.827546-2.5892031 .593038$

C $3.961586-1.8785312 .662398$

O $6.019090-3.0375142 .251949$

C $2.6734431 .936136-1.490180$

O $2.0640172 .893475-1.940940$

C $2.2556520 .522257-1.626470$

C $1.0586670 .238669-2.162660$

C $0.517763-1.095606-2.436201$

C $1.376928-2.282818-2.092706$

C $2.630203-1.3046332 .164253$

C $1.443703-2.2342202 .356521$

C $-0.978742-2.4232331 .802361$

O $1.503406-3.2836232 .990900$

C $-1.190661-3.1410430 .507849$

C $-2.264377-2.947506-0.271869$

C $-2.522544-3.684081-1.551520$

C $-2.651352-2.757619-2.738068$

C $-3.854307-2.320465-3.147449$

C $-1.397363-2.428093-3.512230$

C $-0.699090-1.193476-3.012326$

N $0.295471-1.7315371 .759654$

H $3.0736455 .625397-0.250278$

H 3.7406023 .2642751 .584508

H $4.2735773 .912305-1.414672$

H 6.0597462 .4199720 .587384

H 6.3462024 .0588380 .071052

H $7.7368921 .169030-2.551468$

H $7.5924353 .314019-3.329327$

H $6.0325774 .045859-2.948244$

H $7.4920814 .536351-2.059963$

H $8.012496-0.150822-0.440906$

H $\quad \begin{array}{lll}6.500264 & 0.464014 & 0.194700\end{array}$

H $5.311479-0.558831-1.817246$

H $6.845373-1.302417-2.284232$

H $6.934204-2.442021-0.037415$

H $5.930086-3.643565-2.011136$

H $4.385341-2.888570-1.689553$

H $3.678500-5.935443-0.354063$

H $3.149785-3.4926120 .564666$

H $6.551630-5.705017-0.779014$

H $5.810145-6.1151720 .749167$

H $6.889464-4.7104630 .641796$

H $4.684469-4.9599602 .426741$

H $3.819597-2.5347093 .529590$

H $4.547925-1.0272023 .037612$

H $6.273748-2.3734912 .913937$

H $2.950511-0.243827-1.304588$

H $0.4171621 .066794-2.461416$

H $0.866585-3.240374-2.217356$

H $1.690470-2.243738-1.044388$

H $2.269136-2.302505-2.727043$

H $2.404930-0.4011932 .745814$

H $2.695899-1.0082051 .113021$

H -1.748992 -1.670046 1.988131

H $-0.988156-3.1396872 .629729$

H $-0.438201-3.8677410 .210946$

H $-3.028399-2.2427760 .044186$

H -3.450848 -4.257065 -1.425091

H -1.744650 -4.432221 -1.747928

H $-3.958080-1.682582-4.018728$

H $-4.760205-2.586517-2.614074$

H - $1.656623-2.245818-4.564542$

H $-0.741223-3.302647-3.547620$

H - $-1.250160-0.274609-3.217962$ 
H $\quad 0.350358-0.873223 \quad 1.218408$

C 0.8276685 .4831951 .736423

O $0.4478664 .095497 \quad 1.630886$

C $-0.5033363 .961196 \quad 0.553851$

C -1.0591345 .3583800 .331673$

C $\quad 0.179676 \quad 6.2055180 .553027$

H $0.0745073 .638679-0.320193$

C -1.5341702 .8915750 .923088$

H -1.491009 $5.489484-0.664777$

H -1.827516 5.5959851 .077838

C $-0.119727 \quad 7.6704670 .818981$

H $0.8056796 .132855-0.344154$

C $-2.6596292 .739957-0.119745$

O -0.8335751 .6501671 .053393$

H -1.9691553 .1118381 .905333$

H $-3.1773243 .700444-0.220909$

O $-2.0887322 .443918-1.394856$

C -3.7122181 .6690720 .234067$

C $-4.8325921 .597798-0.823172$

H $-4.1490551 .904122 \quad 1.212053$

$\begin{array}{lllll}0 & -3.104147 & 0.385556 & 0.325365\end{array}$

H -5.204067 2.609220 -1.025425

H $-4.4129591 .190958-1.752094$

H $-0.6322068 .119852-0.037760$

H -0.7612367 .7966871 .697490$

H $\quad 0.804911 \quad 8.2301940 .992527$

C 2.3520555 .5957831 .849386

H $-0.124573 \quad 1.783370 \quad 1.706013$

H -1.664806 $1.571168-1.324609$

H $-2.4475090 .418547 \quad 1.040728$

H 2.6734865 .0657292 .755295

H 2.6415726 .6448671 .979070

H $\quad 0.3898745 .8428492 .676599$

C $-6.506932-1.5882630 .199908$

O $-5.555885-0.639930-0.297456$

C $-6.0134370 .711246-0.377700$

C $-7.1998040 .798730-1.350651$

C $-8.319870-0.180201-0.972819$

C $-7.787217-1.583165-0.652412$

H $-6.020100-2.5633810 .066707$

C $-6.764841-1.4041251 .699797$

H -6.3248721 .0497490 .615895$

O $-6.7624620 .515867-2.679848$

H -7.588769 $1.823494-1.351028$

H -9.018796 -0.252337 -1.815001

$\begin{array}{llll}\text { O } & -9.071555 & 0.312874 & 0.132707\end{array}$

H -8.557486 -2.187355 -0.159162

O $-7.468866-2.237037-1.886343$

C $-5.479589-1.5232872 .488779$

H -7.222802 -0.437052 1.921235

H -7.452929 -2.1701632 .074921$

H -6.355823 -0.367640 -2.676671

H $-8.478914 \quad 0.7940880 .731454$

H $-8.290433-2.307327-2.401565$

O $-5.275553-0.387253 \quad 3.215616$

O $-4.745727-2.5024832 .460516$

C -4.086222 -0.3607804 .010768$

H $-3.209840-0.2713363 .363604$

H -4.1325350 .5171154 .660738$

H -4.016278 -1.255346 4.637148

SCF Energy (B3LYP/6-31G**//MMFF) $=-3245.92497563$

08_00082

MM̄FF Geometry

C $3.424065-3.344372-2.110947$

C $2.173497-3.209391-1.645747$

C $1.000703-2.835899-2.512341$

O $0.378840-1.658232-1.948274$

C $-0.040668-3.968358-2.504238$

C $-1.136386-3.762096-3.530981$

C $-2.365742-3.282612-3.258955$

C $-0.781518-4.173737-4.937102$

C $-2.901835-2.804667-1.938431$

C $-4.042653-3.704010-1.443126$

C $-4.638384-3.198552-0.120662$

C $-5.747467-4.1303560 .372171$

C -6.437555 -3.586249 1.634136

O $-5.173403-1.892903-0.351666$
O $-7.652067-4.327413 \quad 1.827644$

C $-6.840375-2.1145751 .394568$

C $-5.587170-3.8128062 .889856$

O $-7.344962-1.5207272 .588331$

C $-5.697883-1.2503570 .817267$

C $-6.2313390 .141696 \quad 0.395065$

O $-4.692042-1.0737021 .818827$

C $0.914499-0.447264-2.261096$

O $1.883276-0.228292-2.969905$

C $0.0989890 .579863-1.575272$

C $0.3935951 .881432-1.715100$

C $-0.3235612 .995443-1.086380$

C $-1.5268692 .648056-0.250614$

C $-5.2472210 .911737-0.494023$

C $-5.6901292 .331784-0.799708$

C $-4.9974024 .286276-2.193836$

O $-6.6674852 .866178-0.285908$

C $-3.6821804 .761329-2.724246$

C $-3.0474095 .863571-2.297924$

C $-1.7065036 .290211-2.827227$

C $-0.6988736 .588345-1.735748$

C $-0.0747927 .778140-1.693506$

C $-0.4095095 .531774-0.688096$

C $0.1318034 .253075-1.270888$

N $-4.8230792 .932718-1.701885$

H $3.610088-3.202680-3.172087$

H $1.975077-3.364305-0.588400$

H $1.318897-2.625096-3.541011$

H $-0.473265-4.072676-1.501986$

H $\quad 0.446508-4.930465-2.713311$

H $-3.073785-3.195260-4.082528$

H -1.615333 -4.037094 -5.634070

H $\quad 0.059334-3.582029-5.311432$

H $-0.501410-5.231985-4.963124$

H -2.122843 -2.745017 -1.172917

H $-3.265660-1.779920-2.081587$

H $-4.829182-3.731662-2.208914$

H $-3.673930-4.729191-1.313142$

H $-3.832108-3.1354150 .619773$

H $-5.356855-5.1396960 .551845$

H $-6.506951-4.239538-0.414915$

H -7.419760 -5.2687301 .904490$

H $-7.670262-2.1160180 .673939$

H $-5.405109-4.8837313 .040597$

H $-6.110604-3.4694423 .788999$

H $-4.615090-3.3175452 .844889$

H -8.066877 -2.087120 2.910500

H $-7.1633140 .025494-0.172954$

H -6.4651340 .7362451 .286962$

H $-4.237323-0.2293981 .672942$

H $-0.7269820 .230608-0.965232$

H $1.2427552 .161037-2.336927$

H $-2.2469262 .079008-0.845678$

H -2.063702 3.5134660 .140182

H -1.232971 2.0395140 .610588

H $-4.2657200 .973831-0.012769$

H -5.124165 $0.385171-1.448139$

H $-5.3837824 .917476-1.386927$

H $-5.7418214 .255283-2.995591$

H -3.219684 $4.154471-3.499992$

H $-3.5100316 .488441-1.537832$

H -1.866821 $7.179303-3.450557$

H -1.286346 $5.524961-3.492003$

H $0.6476768 .011970-0.918529$

H $-0.2704098 .547312-2.433326$

H $-1.3126885 .380219-0.092583$

H $\quad 0.3433435 .9017470 .020845$

H $1.0260344 .394134-1.879410$

H $-4.1595552 .336913-2.189762$

C $5.666361-2.613114-1.142531$

O $5.135833-1.443138-0.488605$

C $5.754653-0.278120-1.069986$

C $6.854437-0.790271-1.999435$

C $6.280434-2.118244-2.450483$

H $4.9867160 .212427-1.678963$

C 6.2596810 .6775110 .017546

H $7.048165-0.101561-2.827602$

H $7.799698-0.945199-1.466188$ 
C $7.310501-3.059986-3.047736$

H $5.505240-1.913322-3.198262$

C $5.176150 \quad 1.191554 \quad 0.988407$

$\begin{array}{lllll}\text { O } & 7.251317 & -0.010816 & 0.791268\end{array}$

H $6.7705101 .524622-0.456884$

H $4.853156 \quad 0.381877 \quad 1.652226$

$\begin{array}{lllll}\text { O } & 5.783251 & 2.184855 & 1.825748\end{array}$

C 3.9484891 .8240890 .308107

C 3.0310892 .6289831 .248467

H $3.3670291 .037755-0.186445$

O $4.3957722 .719251-0.715522$

H 3.5557713 .5252601 .604220

H 2.2044883 .0065070 .635570

H $7.771591-2.613068-3.934566$

H $8.108540-3.288156-2.333219$

H $6.846302-4.004504-3.348294$

C $4.600733-3.707496-1.250426$

H 7.7187710 .6525901 .326029

H 6.4081191 .7297072 .414789

H $3.6083323 .046781-1.183083$

H $4.251092-3.952890-0.240118$

H $5.053059-4.619621-1.656999$

H $6.461382-2.989298-0.484072$

C $1.236231-0.1825202 .933431$

O $1.782872 \quad 0.6972301 .941530$

$\begin{array}{llll}\text { C } & 2.469921 & 1.845228 & 2.444484\end{array}$

C 1.5264442 .7103073 .299070

C 0.8563061 .8864374 .402956

C 0.2723340 .5752213 .864361

H $0.651623-0.9169012 .364727$

C $2.329582-0.9449323 .699932$

H 3.3119591 .5200993 .063156

O 0.5237893 .2919282 .468310

H 2.1053843 .5314173 .739045

H $0.0552572 .481466 \quad 4.858905$

O $1.821313 \quad 1.6147425 .414815$

H -0.026242 -0.0730684 .696495$

O -0.9168690 .8899103 .135205$

C $3.140401-1.8548762 .803584$

H $3.031975-0.2628914 .185624$

H $1.864924-1.5745434 .467615$

H -0.0316603 .8591283 .029499$

H $1.374181 \quad 1.1173576 .120679$

H -1.312057 0.0521382 .839686

O $4.154049-2.4039853 .536728$

O $2.917306-2.0622381 .619738$

C $5.026112-3.2861972 .823909$

H $5.593776-2.7254252 .076223$

H $5.726653-3.7233463 .540308$

H $4.456646-4.0941722 .354931$

SCF Energy (B3LYP/6-31G**//MMFF) $=-3245.89581993$

0800083

MM̄FF Geometry

C $-0.620474 \quad 4.609475 \quad 0.305819$

C $0.642455 \quad 4.159404 \quad 0.341392$

C $1.5383274 .101707-0.869408$

O $1.987800 \quad 2.735179-1.039749$

C $2.7683354 .999334-0.651681$

C $3.6966365 .050058-1.850419$

C $4.8921144 .431620-1.927952$

C $3.2136725 .898000-2.999722$

C $5.5437283 .552210-0.897589$

C $5.7612572 .133278-1.438678$

C $6.2331901 .170095-0.339479$

C $6.524422-0.212876-0.922668$

C $6.871239-1.242228 \quad 0.163073$

O 5.1893541 .0800260 .635816

O $6.802286-2.534109-0.460362$

C $5.796992-1.1863071 .272774$

C $8.312703-1.0726680 .657171$

O $6.165916-2.0012942 .382356$

C $5.495523 \quad 0.2461351 .761026$

C 4.2888840 .3187032 .730569

$\begin{array}{lllll}\text { O } & 6.634746 & 0.745406 & 2.473441\end{array}$

C $1.123287 \quad 1.870373-1.641826$

O $-0.0041212 .118293-2.040038$

C $1.7906380 .552149-1.740294$
C $1.122315-0.494848-2.247804$

C $1.647284-1.848907-2.445815$

C $3.061483-2.121690-2.008486$

C 2.944547-0.138354 2.152686

C $2.608834-1.5942092 .429016$

C $0.914043-3.3323601 .863713$

O $3.267643-2.3088903 .178819$

C $1.381930-4.1458940 .698569$

C $0.551150-4.792460-0.132762$

C $1.007978-5.651151-1.275329$

C $0.426681-5.218147-2.601803$

C $-0.680966-5.798492-3.093857$

C $1.188818-4.188809-3.403696$

C $0.849589-2.774942-3.018618$

N $1.458717-1.992071 \quad 1.759495$

H $-1.0306714 .977261-0.631451$

H 1.0486083 .7794781 .276042

H $1.0041764 .421972-1.772713$

H 3.3069334 .6699770 .242759

H $2.4431656 .024625-0.427662$

H $5.4782414 .562370-2.837120$

H $3.9494115 .960971-3.808540$

H $2.2941955 .484285-3.424781$

H $3.0124656 .919177-2.659838$

H $6.5125813 .990514-0.629328$

H 4.9619903 .5099970 .025237

H $4.8188961 .765186-1.862792$

H $6.4970242 .160499-2.252160$

H $7.132151 \quad 1.5881670 .128451$

H $7.325109-0.159057-1.671047$

H $5.637765-0.568622-1.463656$

H $7.066053-3.2005420 .196204$

H $4.881951-1.6195310 .851930$

H $9.018063-1.223869-0.168745$

H $8.566153-1.8280531 .409165$

H $8.506758-0.0857331 .082337$

H $6.226428-2.9187082 .067887$

H $4.524497-0.2015243 .666839$

H 4.1643281 .3748113 .010681

H 6.3440841 .4687983 .053123

H $2.8182750 .481665-1.404834$

H $0.091491-0.346304-2.567721$

H $3.766342-1.565287-2.635076$

H $3.339195-3.176424-2.062129$

H $3.207551-1.826119-0.964299$

H 2.1482430 .4512792 .625337

H 2.8939730 .0489311 .076125

H $-0.175392-3.2389351 .895660$

H $1.249563-3.7966352 .796581$

H $2.455926-4.2270680 .548239$

H $-0.521253-4.7345580 .037640$

H $\quad 0.704610-6.683712-1.057134$

H $2.102473-5.677948-1.344415$

H $-1.084705-5.516724-4.060722$

H -1.212792 -6.562572 -2.536376

H $0.944942-4.297266-4.469690$

H $2.260570-4.400650-3.350123$

H $-0.164366-2.483620-3.296270$

H $1.006050-1.3454591 .119617$

C -2.6233543 .5364851 .312983$

$\begin{array}{lllll}\text { O } & -3.540503 & 3.938238 & 0.278076\end{array}$

C -4.8303133 .3410340 .554034$

C -4.6855012 .6093461 .885669$

C -3.5106183 .3184512 .531814$

H -5.5361134 .1751130 .644872$

C $-5.1962562 .447989-0.639351$

H -5.599186 2.6567812 .486022

H $-4.4439401 .551238 \quad 1.730978$

C -2.8620322 .5112113 .643011$

H -3.8530704 .2832562 .928375$

C $-6.4113441 .536508-0.403247$

O $-5.4727663 .292355-1.762643$

H $-4.3182681 .857219-0.916381$

H -6.2494090 .8880850 .462493$

O $-7.5393962 .351955-0.057596$

C $-6.8060710 .666169-1.611486$

C $-5.692434-0.199796-2.231149$

H $-7.224391 \quad 1.302807-2.400719$ 
O $-7.866228-0.210445-1.211180$ H $-6.134730-0.745759-3.075022$ H $-4.9213140 .461293-2.642916$ H -2.026779 3.0591254 .089818 H -3.586113 2.2970014 .435870 H -2.4768131 .5552903 .272198$ C -1.543088 4.6008321 .493305 H -4.692092 $3.856424-1.899444$ $\mathrm{H}-7.6837632 .976717-0.789017$ H $-8.5734980 .342559-0.836879$ H -0.9740214 .4134642 .410700$ H -1.994462 5.5960111 .582595 H -2.1706732 .5856850 .996820$ C $-3.657781-1.351588 \quad 0.693258$ O $-4.266706-0.540295-0.315803$ C -5.050406-1.239289-1.284222 C $-4.151882-2.222395-2.054976$ C $-3.420515-3.174288-1.101835$ C $-2.760581-2.4277970 .063209$ H $-3.007291-0.6584471 .242623$ C $-4.712315-1.8769981 .671097$ H -5.846454 -1.794300 -0.775253 O $-3.195375-1.491094-2.820888$ H $-4.771569-2.792136-2.757611$ H -2.651106 -3.725344 -1.655207 O $-4.339176-4.129061-0.579515$ H -2.433427 -3.133231 0.834296 O $-1.590519-1.760089-0.419072$ C $-4.096491-2.2589832 .993334$ H -5.482081 -1.117293 1.857099 H $-5.257190-2.7415651 .281463$ H -2.666931 -2.135039 -3.322334 H $-4.719149-4.613813-1.331847$ H $-0.995885-2.436709-0.783485$ O $-3.884269-1.142453 \quad 3.747173$ O $-3.820329-3.408802 \quad 3.309673$ C $-3.272373-1.3671425 .020863$ H -3.159116 -0.400405 5.518756 H -3.907940 -2.008360 5.638882 H -2.281708 -1.813248 4.891072

SCF Energy $\left(B 3 L Y P / 6-31 G^{* *} / / M M F F\right)=-3245.90820872$

0800084

MM̄FF Geometry

C $0.259143 \quad 4.1894620 .063487$

C 1.5354103 .7787450 .077634

C $2.4064333 .682788-1.147047$

O $2.7932232 .295216-1.284330$

C $3.6729614 .532086-0.960457$

C $4.6328434 .429095-2.128946$

C $5.7532983 .679639-2.127317$

C $4.2802005 .268332-3.328680$

C $6.2396282 .779819-1.024946$

C $6.6452861 .397411-1.555545$

C $7.0225890 .455693-0.403309$

C $7.656683-0.831529-0.927861$

C $7.972435-1.8125250 .211240$

O $5.824925 \quad 0.1421950 .316973$

O $8.278533-3.071957-0.405845$

C $6.708837-1.9932791 .079019$

C $9.222814-1.3915790 .992303$

O $6.989763-2.7645762 .245063$

C $6.034263-0.6594261 .485823$

C $4.657502-0.9558442 .124586$

$\begin{array}{lllll}\text { O } & 6.853110 & 0.014334 & 2.442979\end{array}$

C $1.9463781 .486004-1.977600$

O $\quad 0.9338491 .817830-2.573648$

C $2.4350190 .097236-1.833089$

C $1.602207-0.913631-2.122789$

C $1.872921-2.340459-1.940321$

C $3.268625-2.747918-1.553270$

C 3.7611550 .2729732 .326194

C $2.394981-0.1663872 .818562$

C $0.326108-1.3311802 .034648$

O $2.083999-0.0982714 .005096$

C $0.474065-2.7796921 .681342$

C $-0.378888-3.4412950 .884360$

C $-0.217719-4.8865800 .504248$
C $-0.314728-5.122395-0.989850$

C -1.376238 $-5.753205-1.520268$

C $0.850205-4.695752-1.862887$

C $0.855359-3.210292-2.109348$

N $1.601313-0.6764801 .802824$

H $-0.1949994 .504011-0.872551$

H 1.9756053 .4501981 .017030

H $1.8730104 .016896-2.045757$

H $4.1776404 .253143-0.028295$

H $3.3951405 .586296-0.825708$

H $6.3932043 .710531-3.008057$

H $5.0261865 .187420-4.126441$

H $3.3177304 .956313-3.745820$

H $4.2124086 .324200-3.046983$

H $7.1093573 .253036-0.553365$

H $5.4844462 .651423-0.245290$

H $5.8105730 .968212-2.124745$

H $7.4942441 .508705-2.241484$

H 7.7198890 .9814220 .259620

H $8.562608-0.614119-1.507225$

H $6.963250-1.320054-1.627113$

H $8.532872-3.6977160 .293098$

H $5.990514-2.576240 \quad 0.485749$

H $10.092970-1.3530800 .326062$

H $9.471291-2.1248881 .767452$

H $9.122828-0.4130111 .466740$

H $7.307189-3.6356461 .953734$

H $4.117940-1.6671611 .489746$

H $4.795920-1.4471003 .096529$

H 6.2971100 .6145722 .964759

H $3.430722-0.054494-1.433412$

H $0.601618-0.674333-2.481959$

H $4.006768-2.292437-2.223268$

H $3.436992-3.825637-1.612526$

H $3.486253-2.435872-0.529044$

H $4.1869670 .961705 \quad 3.063174$

H 3.6329860 .8357551 .394729

H $-0.416209-0.8280631 .406963$

H $0.034653-1.2291263 .084056$

H $1.328657-3.3084602 .097738$

H $-1.246611-2.9185790 .490988$

H -0.998861 -5.4542661 .026259$

H $\quad 0.737665-5.287898 \quad 0.864849$

H -1.444415 -5.946980 -2.585644

H -2.204598 -6.084101-0.902744

H $\quad 0.792123-5.193172-2.839466$

H $1.772370-5.054304-1.397040$

H $-0.108957-2.816081-2.431994$

H $1.961330-0.6786180 .853606$

C -1.7122673 .1496661 .186600$

O -2.7178703 .5875090 .249196$

C -4.0229573 .2679310 .781924$

C -3.7725512 .3720731 .984694$

C -2.4540292 .9105472 .499134$

H $-4.457894 \quad 4.2236901 .100391$

C $-4.8711332 .638774-0.329865$

H -4.5753192 .4267902 .725471$

H -3.6612011 .3263511 .673187$

C - -1.7464741 .9594933 .449149$

H -2.6352203 .8659853 .009597$

C -6.1978162 .0355140 .161808$

O $-5.1608933 .656217-1.294399$

H $-4.276876 \quad 1.880615-0.850307$

H -6.0041781 .2194530 .862352$

$\begin{array}{lllll}\text { O } & -6.905787 & 3.026784 & 0.920349\end{array}$

C $-7.1428281 .539813-0.947551$

C $-6.5436290 .573500-1.983534$

H -7.552964 2.403251-1.486171

O $\quad-8.2639240 .892069-0.335602$

H -7.316962 $0.391844-2.741967$

H -5.709688 1.071809 -2.490275

H -0.7862032 .3709083 .774894$

H $-2.357708 \quad 1.7816364 .340096$

H -1.5551090 .9908312 .976455$

C -0.6110234 .2054361 .289606$

H $-4.3106614 .021683-1.593874$

H -7.044997 3.7951970 .340260

H $-8.643011 \quad 1.5148300 .308297$ 
H $-0.001581 \quad 4.0283172 .183275$ H -1.048295 $5.206513 \quad 1.384210$ H -1.3068302 .2037690 .802125$ C $-4.416523-1.7916170 .010635$ O $-4.961492-0.611717-0.593914$ C $-6.094825-0.801879-1.442615$ C $-5.740633-1.757869-2.596972$ C $-5.145519-3.072914-2.084825$ C $-4.036269-2.829178-1.057675$ H -3.489279 -1.453304 0.490956 C $-5.335224-2.3566461 .100891$ H $-6.915145-1.231399-0.858514$ O $-4.812670-1.139389-3.487948$ H -6.648331 -1.963206 -3.176262 H -4.735369 -3.640133-2.929354 O $-6.166409-3.863978-1.485248$ H $-3.750713-3.767225-0.568040$ O $-2.877594-2.336453-1.736242$ C -5.430105 -1.411626 2.276846 H $-6.343251-2.5599720 .729026$ H -4.945021 -3.304955 1.488608 H -4.032606 $-0.876798-2.969594$ H -6.845207 -4.029869-2.161325 H -2.604240 -3.014071 -2.377860 O $-6.725098-1.0269902 .462467$ O $-4.470556-1.0608102 .951460$ C $-6.953685-0.1347903 .557547$ H $-6.689197-0.6207894 .501425$ H -6.3811230 .7875143 .424475$ H -8.017961 0.1143183 .576216

SCF Energy (B3LYP/6-31G $\left.{ }^{* *} / / \mathrm{MMFF}\right)=-3245.92271260$

08_00085

MM̄FF Geometry

C $1.6735245 .043863-0.673345$

C $2.8142594 .369458-0.464720$

C 3.3573663 .8969520 .864910

O $3.4585602 .451950 \quad 0.797691$

C 2.4877374 .2265882 .093134

C 3.1053883 .7723463 .401315

C 2.6980862 .6936184 .099180

C 4.2044974 .6524693 .936652

C 1.6306041 .7064983 .707378

C $1.984728 \quad 0.2875584 .176955$

C $0.891758-0.7274093 .810555$

C $1.142242-2.0597334 .520262$

C $0.114713-3.1249244 .114237$

O $0.914046-0.9184842 .391535$

O $0.594088-4.3983874 .571566$

C $0.054026-3.1903362 .575922$

C - $-1.243085-2.9028174 .791040$

O $-0.988795-4.0637292 .146591$

C $-0.100061-1.8064511 .898978$

C $0.096330-1.9637910 .374103$

O $-1.410215-1.3011612 .155757$

C 4.6411791 .9102030 .405625

O $5.6490362 .505580 \quad 0.061538$

C 4.4872090 .4392570 .454796

C $5.404072-0.338229-0.139475$

C $5.373754-1.799946-0.222739$

C $4.332917-2.5239570 .588675$

C $0.091162-0.648951-0.408246$

C $0.471589-0.911764-1.851593$

C $2.452266-1.176820-3.343303$

O $-0.372423-1.201054-2.696522$

C $3.069325-2.536314-3.223221$

C $4.320278-2.821590-3.616195$

C $4.944616-4.182486-3.482876$

C $6.311961-4.145735-2.832004$

C $7.422012-4.405583-3.543575$

C $6.388638-3.880602-1.341543$

C $6.268245-2.414344-1.024787$

N $1.836750-0.834873-2.073409$

H 1.0466435 .3249320 .167865

H $3.4177424 .106195-1.331892$

H 4.3515814 .3402171 .000782

H 1.4944633 .7770221 .975546

H 2.3196955 .3097532 .155585
H 3.1792542 .4973745 .056541

H 4.5730324 .3120014 .910165

H 5.0563004 .6648893 .249797

H 3.8428945 .6782374 .062553

H 0.6883802 .0144714 .176078

H 1.4743891 .6945832 .624351

H $2.937009-0.0134113 .720927$

H 2.1260950 .2952375 .265285

H $-0.080785-0.3161394 .105699$

H $1.144486-1.9272365 .609430$

H $2.145212-2.4275874 .261042$

H $0.707299-4.3472315 .536199$

H $0.995968-3.6398822 .230792$

H -1.135793 -2.931334 5.882107

H -1.946011 -3.704397 4.538643

H -1.702237 -1.947037 4.530026

H $-0.843963-4.9224442 .579699$

H $1.049754-2.4717870 .186981$

H $-0.690312-2.607471-0.039344$

H -1.592048 -0.5766721 .536769$

H 3.6089700 .0393030 .949205

H $6.2420520 .140525-0.644823$

H $3.332925-2.3123940 .201778$

H $4.375010-2.2121641 .638651$

H $4.456922-3.608690 \quad 0.590744$

H $-0.903399-0.193758-0.401504$

H $0.783340 \quad 0.0818980 .024290$

H $3.195554-0.404062-3.562575$

H $1.696918-1.178249-4.135098$

H $2.455884-3.325381-2.793835$

H $4.924987-2.040479-4.071242$

H $5.018209-4.612561-4.490023$

H $4.298871-4.861472-2.912052$

H $8.403102-4.404391-3.080233$

H $7.375803-4.620290-4.606115$

H $7.353232-4.225319-0.946711$

H $5.633250-4.493109-0.842671$

H $\quad 6.999393-1.796374-1.548687$

H $2.453267-0.629257-1.293629$

C $-0.1221284 .680552-2.430941$

O $-1.1283224 .813067-1.400085$

C $-1.4561763 .489148-0.931988$

C $-1.2293852 .608888-2.146983$

C $0.0747943 .177091-2.669190$

H $-0.7332263 .254639-0.139553$

C $-2.8779873 .453884-0.363651$

H -1.162707 $1.547274-1.909473$

H -2.032652 2.750894 -2.879873

C $0.3596862 .817481-4.117355$

H $0.8928662 .785918-2.052172$

C -3.2465802 .0609350 .189166$

O -2.9470834 .4162970 .691486$

H $-3.6007713 .761216-1.128364$

H $-3.0923031 .325041-0.605363$

O -2.3439351 .7219051 .244651$

C -4.6979591 .9193490 .688839$

C $-4.9992460 .566522 \quad 1.365886$

H $-5.3765602 .072235-0.158510$

O -4.9859952 .9422131 .643510$

H -4.4508990 .4992852 .314548$

H -6.0625160 .5768551 .641264$

H $0.4410251 .731879-4.235163$

H - $0.436046 \quad 3.168732-4.782695$

H $1.3010503 .266639-4.449378$

C $1.1502505 .439890-2.027957$

H -2.6954915 .2772610 .315462$

H -2.469856 2.3764481 .953348

H -5.9260552 .8678661 .880420$

H $0.9222126 .512086-1.980619$

H $1.9173595 .311464-2.800566$

H $-0.5403805 .156247-3.326714$

C $-5.294974-1.623690-1.646415$

O $-5.485423-0.573932-0.688243$

C $-4.700961-0.6698810 .503646$

C $-5.005230-1.9774441 .252023$

C $-4.820330-3.1897490 .333623$

C -5.540543 -3.001214-1.005092

H $-6.078939-1.463047-2.398211$ 
C $-3.935599-1.516367-2.355823$

H -3.639725 -0.6612850 .240950$

O $-6.344639-1.9500721 .740603$

H -4.338793 -2.051965 2.119687

H $-5.210988-4.0885360 .826104$

O $-3.433407-3.395827 \quad 0.083976$

H -5.241894 -3.789285 -1.705628

O $-6.948008-3.157137-0.799655$

C $-3.886257-0.332126-3.299734$

H -3.098067 -1.410385 -1.662695

H $-3.778984-2.422711-2.953120$

H $-6.493259-2.7740362 .235131$

H $-2.995366-3.5287040 .942122$

H -7.249488 -2.423181 -0.237189

O $-2.997566-0.595115-4.302694$

O $-4.5258820 .701943-3.162697$

C $-2.8496130 .433700-5.285827$

$\mathrm{H}-3.7927250 .581202-5.820261$

H -2.521711 $1.365822-4.818392$

$\mathrm{H}-2.0865810 .113753-6.000322$

SCF Energy (B3LYP/6-31G**//MMFF) $=-3245.92881943$

08_00086

MM̄FF Geometry

C $-4.0535764 .948782-0.147336$

C $-4.4102083 .701730-0.489432$

C $-5.1363462 .767068 \quad 0.443402$

O $-4.325940 \quad 1.5853460 .652614$

C $-6.4707442 .333270-0.186639$

C -7.3227831 .4885410 .741013$

C -7.4769750 .1530470 .643457$

C $-8.0638382 .255711 \quad 1.806445$

C $-6.827319-0.780956-0.339751$

C $-6.099662-1.9244810 .380090$

C $-5.370111-2.849444-0.605398$

C $-4.717651-4.0179200 .136128$

C $-3.882880-4.903847-0.799519$

O $-4.372088-2.076985-1.281008$

O $-3.078633-5.7510790 .035635$

C $-2.931948-4.006302-1.622045$

C $-4.772391-5.838484-1.627170$

O $-2.234457-4.775404-2.598502$

C $-3.635135-2.799437-2.277378$

C $-2.649524-1.805856-2.941283$

O $-4.504057-3.277575-3.312267$

C -3.3132561 .6822941 .558969$

O -2.9570852 .6858202 .156600$

C $-2.7284190 .333274 \quad 1.735534$

C - $1.712483 \quad 0.1597172 .594708$

C -1.093861-1.116456 2.965983

C $-1.615921-2.3654902 .307504$

C -1.632586 -1.154344 -1.998737

C $-0.303582-1.886687-1.913804$

C $1.805548-1.912235-0.580035$

O $0.036801-2.759651-2.706947$

C $1.775268-2.6842440 .698450$

C $2.461088-2.3195071 .791932$

C $2.504928-3.1226923 .057424$

C $2.043373-2.3393374 .263128$

C $2.928806-1.7847695 .108426$

C $0.560020-2.2918334 .544768$

C $-0.124133-1.1131213 .905193$

N $0.474453-1.414238-0.866613$

H $-4.303158 \quad 5.3217270 .843039$

H -4.149307 $3.321494-1.474100$

H -5.3267973 .2471001 .411805$

H $-6.2807301 .801730-1.125511$

H $-7.0532573 .220123-0.472176$

H -8.146249 -0.3360981 .350590$

H $-8.709140 \quad 1.6101942 .411719$

H -7.361494 2.747151 2.486660

H -8.700458 3.0208191 .350233

H -7.607655 -1.198501-0.987257

H $-6.123303-0.257490-0.990660$

H $-5.375487-1.4959891 .084075$

H $-6.820482-2.5072580 .967334$

H -6.098105 -3.218562 -1.337579

H $-5.472679-4.6168770 .660562$
H $-4.056001-3.6242450 .918971$

H -2.489648 -6.263196 -0.545032

H $-2.172020-3.640789-0.921486$

H -5.358704 -6.488852 -0.966695

H $-4.171890-6.511405-2.249019$

H $-5.472604-5.304286-2.272600$

H $-2.845070-4.942018-3.336580$

H -2.158719 -2.278715 -3.800507

H $-3.251276-0.988922-3.365115$

H $-4.683361-2.546448-3.926522$

H $-3.180633-0.4869191 .191376$

H -1.3161911 .0333283 .111414$

H -1.031504 -3.257991 2.541131

H -1.593257 -2.269156 1.217186

H -2.647341 -2.557560 2.621393

H $-1.399332-0.152289-2.381104$

H -2.051253-1.029982 -0.995807

H $2.469571-1.047021-0.513169$

H $2.158725-2.552353-1.394287$

H $1.191298-3.6012010 .711568$

H $3.061995-1.4131401 .765114$

H $3.540973-3.4563143 .202786$

H $1.913474-4.0425192 .969500$

H $2.603999-1.2510905 .995384$

H $3.996996-1.8466714 .927921$

H $0.398242-2.2058075 .628386$

H $\quad 0.102397-3.2491504 .280028$

H $0.184645-0.1545434 .325056$

H $0.093544-0.701871-0.250911$

C $-1.7768855 .829643-0.857657$

O $-1.2792464 .561625-1.328648$

C $-0.3244764 .054776-0.373491$

C 0.0591455 .2443900 .493693

C -1.2550415 .9974840 .570109$

H -0.8624893 .3040450 .218415$

C $0.8377203 .402264-1.128029$

H $\quad 0.4369644 .9460891 .476091$

H $0.824767 \quad 5.8561310 .000517$

C -1.109330 7.4457661 .002298

H -1.8978475 .4783271 .290782$

C $1.9375542 .857528-0.197522$

O $0.2983602 .325030-1.900866$

H $\quad 1.2714674 .115607-1.838732$

H 2.3071513 .6718050 .435825

O 1.3651181 .8859590 .679882

C $3.1530842 .250373-0.933175$

C 4.1858921 .6848540 .053750

H $3.6159553 .031837-1.545699$

O $2.7559941 .199907-1.808741$

H 4.5175542 .4717630 .742998

H 3.7102840 .9250740 .688583

H -0.6777027 .5059232 .006730$

H $-0.456994 \quad 8.004416 \quad 0.322877$

H -2.0836497 .9444611 .021610$

C $-3.2961905 .884454-1.046441$

H $-0.4023762 .694896-2.465231$

H $1.054238 \quad 1.1485190 .128054$

H $2.1616051 .579114-2.477545$

H $-3.5350385 .650238-2.091926$

H $-3.656116 \quad 6.903578-0.862234$

H $-1.3258996 .586669-1.512568$

C $7.366362-0.2470260 .089329$

O $6.199661 \quad 0.493378 \quad 0.470815$

C $5.4042061 .010247-0.604971$

C $6.2390551 .955793-1.481479$

C $7.5327321 .287749-1.957750$

C $8.2807510 .602067-0.809198$

H $7.902186-0.4301721 .029933$

C $7.010497-1.612458-0.520288$

H $5.0435340 .169300-1.206244$

O $6.5566323 .145372-0.761296$

H $5.6487472 .256249-2.354177$

H $8.1770862 .048304-2.415280$

O $7.2063180 .332545-2.963140$

H $9.094290-0.022972-1.195376$

O 8.8802721 .6042950 .017424

C $6.368000-2.5448790 .486649$

H $6.318765-1.521298-1.361234$ 
H $7.929531-2.097051-0.870488$

H 7.0248812 .8850500 .050447

H $8.038809-0.060576-3.275843$

H $9.4969522 .111485-0.537752$

O $6.218588-3.775592-0.088582$

O $6.032487-2.240057 \quad 1.622538$

C $5.629985-4.7814960 .741931$

H $\quad 5.704620-5.7389840 .219485$

H $6.164602-4.8605801 .693354$

H $4.573821-4.5546260 .909007$

SCF Energy (B3LYP/6-31G**//MMFF) $=-3245.92922808$

08_00087

MMFF Geometry

C $-2.7914263 .730188-0.290145$

C $-3.0153364 .152706 \quad 0.963879$

C -3.6475453 .3158042 .053494$

O -3.4064801 .9070381 .815629$

C -5.1628763 .5578772 .101626$

C -5.8377462 .8371553 .251345$

C -6.5423351 .6952543 .126278$

C -5.7176313 .5111884 .593393$

C $-6.7558460 .889838 \quad 1.874307$

C $-6.567701-0.6124562 .131382$

C $-6.781306-1.4272570 .848145$

C $-6.829254-2.9248101 .151391$

C $-6.975872-3.756479-0.131863$

O $-5.693332-1.149160-0.040610$

O $-6.703309-5.1269560 .197014$

C $-5.898013-3.309639-1.140142$

C $-8.406573-3.717709-0.681677$

O $-6.084610-3.943734-2.403588$

C $-5.810996-1.774810-1.325216$

C $-4.552625-1.428325-2.154928$

O $-6.966940-1.314935-2.027706$

C $-2.165447 \quad 1.4385352 .127544$

O -1.2537982 .0645852 .645508$

C -2.0837880 .0303661 .680768$

C $-0.875978-0.5459321 .583290$

C $-0.593227-1.9085461 .127756$

C $-1.764507-2.8200400 .877544$

C $-4.1718320 .057734-2.145408$

C $-2.9060640 .285483-2.950115$

C $-0.454230-0.190907-2.917391$

O $-2.9286340 .837178-4.047537$

C $0.162088-1.542560-2.745912$

C $1.358871-1.751162-2.176862$

C $1.956909-3.116108-1.988241$

C $2.342890-3.399380-0.552463$

C $3.631157-3.549741-0.202490$

C $1.235121-3.5887550 .465899$

C $0.695259-2.2768530 .967843$

$\mathrm{N}-1.777467-0.216617-2.322820$

H $-3.1387272 .745541-0.592145$

H -2.676455 5.1428351 .259250

H -3.1968663 .5931043 .014774$

H -5.6171643 .2632551 .148486$

H -5.3680574 .6328312 .196900$

H -7.023474 1.2919134 .016444

H -6.2593452 .9736985 .378959$

H -4.6691503 .5722064 .900735$

H -6.1267774 .5256974 .546718$

H $-7.7790641 .069613 \quad 1.523255$

H $-6.079577 \quad 1.199777 \quad 1.072845$

H -5.554994 -0.7871332 .517490$

H $-7.278537-0.9402502 .900220$

H -7.721075 -1.103470 0.385446

H -7.641264 -3.156101 1.851787

H $-5.900810-3.2251511 .657382$

H -7.333984 -5.401135 0.884782

H $-4.930959-3.664528-0.756319$

H $-9.113760-4.0988730 .064735$

H -8.511755 -4.371629-1.554536

H -8.731675 -2.713641 -0.962071

H -6.117894 -4.902638 -2.244383

H $-3.702367-1.998505-1.764397$

H -4.692742 -1.748041 -3.195857

H -6.750622 $-0.484639-2.481018$
H $-3.004741-0.4756641 .415842$

H $\quad 0.0024410 .0464191 .838989$

H $-2.423098-2.8525861 .752995$

H -1.475234 -3.8544940 .679188$

H -2.344111 -2.472635 0.018591

H $-4.9665170 .674533-2.577660$

H $-4.0018340 .417927-1.124771$

H $0.1172710 .587450-2.404913$

H $-0.5216640 .063372-3.979394$

H $-0.409848-2.394717-3.106506$

H $1.942783-0.900457-1.833346$

H $2.837315-3.184670-2.640092$

H $1.269605-3.900788-2.328767$

H $3.914124-3.7805510 .819215$

H $4.432011-3.442005-0.926392$

H $1.616203-4.1448611 .332068$

H $0.463418-4.2204580 .016893$

H $1.470529-1.5507831 .216085$

H $-1.855987-0.541544-1.363704$

C $-0.7787923 .697256-1.724379$

O $0.1049513 .570258-0.593932$

C $1.4705043 .632477-1.058015$

C $1.3994933 .650025-2.578757$

C $0.065414 \quad 4.331737-2.822733$

H $1.8667134 .585702-0.686224$

C $2.2435832 .459897-0.441119$

H $2.2419024 .182577-3.030673$

H $1.3879822 .630439-2.982567$

C $-0.4773774 .114543-4.224178$

H $0.1785595 .408090-2.637624$

C $3.6700212 .288192-0.998318$

$\begin{array}{llll}\text { O } & 2.296750 & 2.681434 & 0.971224\end{array}$

H $1.6862761 .526253-0.584192$

H $3.5948442 .042252-2.063718$

O $4.3739493 .527641-0.912083$

C $4.4911651 .182109-0.301896$

C $5.8299780 .937711-1.026249$

H $3.9034280 .256388-0.290252$

O $4.7419251 .563306 \quad 1.048291$

H $5.6351500 .797703-2.096282$

H $6.4612251 .826851-0.903671$

H $0.210746 \quad 4.524482-4.970664$

H $-0.6133953 .049556-4.441445$

H -1.445205 $4.609836-4.349500$

C $-2.0151004 .501019-1.320312$

H $1.3801902 .761298 \quad 1.287569$

H 4.4572583 .7472200 .032046

H 5.2236550 .8362871 .476979

H -2.660021 $4.676800-2.187734$

H -1.715202 $5.476831-0.920108$

H $-1.0721782 .681132-2.014497$

C $7.750579-1.0479061 .505883$

O 7.0482980 .0042510 .831365

C $6.591646-0.293179-0.492017$

C $7.777842-0.658893-1.398926$

C $8.611329-1.792108-0.794198$

C $8.953826-1.5240160 .675285$

H $8.138812-0.5814672 .420692$

C $6.814427-2.1874591 .921058$

H $5.891336-1.134303-0.452793$

O $8.6012810 .490161-1.590043$

H $7.392482-0.957155-2.381528$

H $9.537906-1.904402-1.370232$

O $7.875328-3.006347-0.910008$

H $9.381140-2.4229981 .133555$

O $9.970086-0.5188660 .736866$

C $5.863563-1.7335083 .002531$

H $6.230963-2.5932171 .091439$

H $7.385859-3.0280012 .333011$

H $9.3180730 .239489-2.197603$

H $8.426909-3.719454-0.545592$

H 9.5886230 .3108110 .401803

O $4.705292-1.2767752 .447694$

O $6.130493-1.7625064 .197128$

C $3.728495-0.7863053 .371775$

H $3.303608-1.6192313 .939123$

H $4.169217-0.0436214 .044008$

H $2.931411-0.3041322 .800098$ 
$08 \_00088$

MM̄FF Geometry

C $3.099399-3.6868590 .119080$

C $1.801045-3.3527750 .187664$

C $0.859026-3.517719-0.977387$

O $-0.140712-2.471727-0.948892$

C $0.127311-4.867555-0.870784$

C $-0.703373-5.193987-2.097072$

C $-2.048004-5.127297-2.158858$

C $0.080534-5.670769-3.293831$

C $-3.001285-4.684778-1.083850$

C $-3.956438-3.601674-1.603049$

C $-4.932343-3.130628-0.515027$

C $-5.915806-2.108137-1.083369$

C $-6.849644-1.550423-0.000685$

O $-4.170256-2.5361870 .538358$

O $-7.495818-0.384104-0.537065$

C -6.004801-1.079715 1.206103

C $-7.968703-2.542398 \quad 0.339346$

O $-6.866061-0.7204982 .283449$

C $-4.945425-2.1091541 .669094$

C $-3.947425-1.5573072 .715143$

O $-5.562179-3.2277572 .320343$

C $0.198120-1.256401-1.457947$

O $1.301659-0.897987-1.835931$

C $-1.040588-0.445769-1.522828$

C $-1.0134990 .776673-2.074178$

C $-2.1662331 .658187-2.288502$

C $-3.5240791 .145335-1.889925$

C $-3.086527-0.3742742 .261941$

C -3.6934240 .9870482 .557508$

C $-3.344727 \quad 3.4074272 .056317$

O $-4.684716 \quad 1.1449763 .264190$

C $-3.8878413 .857000 \quad 0.736422$

C $-3.3167194 .814015-0.010280$

C $-3.8767635 .313325-1.309141$

C $-2.9116515 .160321-2.462171$

C -2.091747 6.164847 -2.815754

C $-2.9794953 .888392-3.275201$

C -1.960126 $2.864351-2.857806$ N -2.9729952 .0090531 .957060$

H $3.476017-4.104443-0.810345$

H $1.389324-2.9729571 .119102$

H $1.407702-3.460789-1.926071$

H $-0.492734-4.8801640 .032270$

H $0.854731-5.679163-0.731877$

H -2.529991 -5.427324 -3.088859

H $-0.567844-5.960577-4.127677$

H $0.748226-4.883731-3.656893$

H $0.683828-6.545347-3.028925$

H -3.582671 -5.556386 -0.760092

H $-2.474569-4.308281-0.203864$

H -3.364495 -2.749375 -1.959780

H $-4.522213-3.988778-2.459854$

H -5.466787 -4.005243 - 0.126065

H -6.497497-2.542121-1.906256

H $-5.358159-1.270899-1.522444$

H -7.968091 $-0.647212-1.345451$

H $-5.498265-0.1621770 .882476$

H -8.588165 -2.738108 -0.544359

H -8.647995 -2.131217 1.094073

H -7.594667 $-3.505150 \quad 0.694154$

H -6.328079 -0.2759032 .959550$

H -4.465162 -1.337067 3.656638

H -3.254288 -2.376625 2.957011

H -6.505993 -3.036062 2.440895

H - $-1.953742-0.897475-1.153347$

H $-0.0587551 .164317-2.428022$

H $-3.5340050 .845853-0.837138$

H -3.796687 $0.280813-2.504360$

H $-4.3198691 .885136-1.999907$

H -2.136694 -0.4084872 .811313$

H -2.849133 -0.4473371 .196408$

H -2.446844 3.9627002 .345122

H -4.1000253 .5461942 .835908$

H $-4.806566 \quad 3.3872970 .393256$
H $-4.1284926 .374028-1.176539$

H $-4.8259254 .819950-1.552211$

H $-1.424205 \quad 6.074765-3.666341$

H -2.066272 $7.095457-2.258493$

H $-2.7841374 .124529-4.330708$

H $-4.0005253 .496375-3.275624$

H $-0.9373593 .146733-3.111324$

H $-2.166532 \quad 1.7768901 .383662$

C $5.386608-2.8911570 .938989$

O $5.164352-1.5470850 .470894$

C $6.089971-1.279923-0.599904$

C $7.116557-2.408251-0.567321$

C $6.263537-3.574452-0.110410$

H $5.518217-1.353993-1.532483$

C $6.6935870 .121570-0.466211$

H $7.583752-2.572161-1.543209$

H $7.913330-2.2135420 .159931$

C $7.067403-4.7469730 .423858$

H $5.667953-3.913658-0.965522$

C $5.6704391 .272516-0.397552$

$\begin{array}{llllll}\text { O } & 7.483769 & 0.184030 & 0.729046\end{array}$

H $7.3915730 .296475-1.293313$

H $5.169224 \quad 1.265972 \quad 0.576880$

O $6.4044812 .502426-0.465178$

C $4.6186971 .274721-1.520414$

C $3.7834402 .564602-1.629955$

H $3.9421300 .424434-1.381955$

O $5.263337 \quad 1.080610-2.783384$

H $4.417383 \quad 3.389762-1.979804$

H $3.0505152 .398519-2.431030$

H $7.730421-5.143799-0.352003$

H $7.687804-4.4537491 .277431$

H $6.406300-5.5562780 .749278$

C $4.058753-3.5798831 .271515$

H $6.903110-0.0416641 .476070$

H 7.0543222 .4917970 .258520

H $5.9041431 .803033-2.900080$

H $3.579523-3.0288022 .089818$

H $4.262300-4.5905041 .644164$

H $5.952906-2.7926521 .874866$

C 1.4754902 .1002921 .273894

$\begin{array}{llllll}\text { O } & 2.200115 & 1.906184 & 0.051648\end{array}$

C $3.0599252 .980381-0.337874$

C $2.2600704 .280687-0.525458$

C 1.4414914 .6110410 .726056

C $0.649994 \quad 3.395928 \quad 1.219458$

H $\quad 0.7709891 .259503 \quad 1.320115$

C 2.3890122 .0213732 .506187

H 3.8117643 .1418680 .440263

O $1.3852154 .147402-1.644026$

H $2.9583945 .096771-0.747044$

H 0.7475445 .4290860 .496602

$\begin{array}{llll}\text { O } & 2.327960 & 5.064247 & 1.744029\end{array}$

H 0.2199283 .5983942 .206458

$\begin{array}{lllll} & 0 & -0.448639 & 3.179252 & 0.333643\end{array}$

C $2.9770120 .640203 \quad 2.698554$

H 3.2242512 .7228742 .437611

H 1.8072542 .2521283 .406046

H $0.9165204 .992282-1.753863$

H 1.7901415 .2910292 .521771

H $-0.0845102 .978108-0.544842$

O $3.819340 \quad 0.668837 \quad 3.773933$

O $2.731397-0.3359092 .004559$

C $4.463876-0.5682624 .091388$

H $3.730741-1.3743924 .191092$

H $5.202741-0.8078283 .322262$

H $4.980035-0.4467775 .047373$

SCF Energy (B3LYP/6-31G**//MMFF) $=-3245.92612322$

0800089

MMFF Geometry

C - $1.358337-4.122732-0.633787$

C $-2.593669-3.681458-0.356821$

C $-3.637638-3.371249-1.398139$

O $-3.750419-1.931863-1.470304$

C $-4.994266-3.958256-0.967258$

C $-6.141809-3.593635-1.889524$ 
C $-7.122714-2.718624-1.585792$

C -6.147335 -4.302042 -3.219289

C $-7.279699-1.921507-0.319744$

C $-6.969390-0.438076-0.557016$

C -6.6005830 .2907100 .744401$

C -6.4184581 .7869540 .484092$

C $-5.8914762 .538044 \quad 1.714942$

O $\quad-5.375765-0.2800691 .220189$

O $\quad-5.4636553 .8295201 .255112$

C -4.6569681 .7921832 .268196$

C -7.0045402 .7866252 .739337$

O $\quad-4.2269712 .3598553 .502719$

C $-4.889616 \quad 0.2775112 .448068$

C $-3.598607-0.4913102 .829695$

O $-5.828717 \quad 0.071671 \quad 3.510711$

C $-2.919550-1.295445-2.342677$

O $-2.141970-1.813082-3.128974$

C $-3.084540 \quad 0.161312-2.145281$

C $-2.2489531 .008161-2.764304$

C $-2.2413412 .466594-2.636982$

C $-3.2469373 .092259-1.707546$

C $-2.472951-0.4521861 .790943$

C -1.4529590 .6529262 .001872$

C $\quad 0.4957961 .7262290 .873863$

O

C 0.0384553 .0381470 .316172

C $0.6720863 .676110-0.680195$

C $0.2724775 .026425-1.204092$

C $0.1875575 .067819-2.714240$

C $1.2150185 .523039-3.451238$

C $-1.1156754 .657382-3.361519$

C -1.322892 $3.168116-3.333544$

$\mathrm{N}-0.6134420 .791866 \quad 0.903411$

H - $1.063800-4.288910-1.666631$

H -2.863656 -3.494369 0.680306

H $-3.359115-3.783237-2.375943$

H $-5.211697-3.6441430 .058087$

H -4.921081 -5.053319 -0.918255

H -7.899026 -2.536709 -2.327818

H -7.031242 -4.056543 -3.817721

H $-5.266149-4.025769-3.806380$

H -6.142478 $-5.386919-3.071561$

H -8.314402 -2.0222350 .030474$

H $-6.662799-2.3209510 .487393$

H -6.132961 -0.349280 -1.260758

H $-7.8381630 .040892-1.025472$

H -7.3924280 .1195881 .483029$

H -7.3553132 .2363140 .131323$

H $-5.7002581 .923542-0.334869$

H -5.1712234 .3431172 .026663$

H -3.8430651 .9444051 .549376$

H -7.792404 3.4111212 .301129

H -6.6294833 .3420273 .606055$

H -7.472175 1.8665023 .096230

H -3.9674243 .2802143 .330165$

H $-3.247530-0.1739323 .818911$

H -3.876447 -1.548886 2.946645

H $-5.722193-0.8338053 .846237$

H -3.863139 $0.495853-1.470926$

H -1.482527 $0.595474-3.419508$

H -4.263682 2.926171 -2.079174

H -3.122203 4.169827-1.585564

H -3.162195 $2.662688-0.703678$

H -1.909395 -1.3911611 .861775$

H $-2.878075-0.3898850 .777925$

H 1.2870391 .2710330 .269268

H $\quad 0.872924 \quad 1.8706291 .891463$

H -0.8282753 .5036970 .779527$

H $1.5572133 .221976-1.118734$

H $1.0212615 .750963-0.857406$

H $-0.6810965 .357613-0.774647$

H $1.1536945 .580940-4.532855$

H $2.1432025 .842224-2.988831$

H -1.129565 $4.965987-4.415480$

H $-1.9359695 .213931-2.899878$

H $-0.6286342 .622073-3.973953$

H $-0.787657 \quad 0.2382820 .069596$

C $0.714217-3.2681280 .504579$
O $1.519439-3.259271-0.690659$

C $2.912194-3.265012-0.318623$

C $2.948203-2.8263111 .135725$

C $1.682016-3.4564661 .674292$

H $3.243307-4.306068-0.419595$

C $3.702378-2.353480-1.264890$

H $3.847145-3.1674691 .656758$

H $2.897287-1.7331691 .215067$

C $1.199840-2.8305442 .972568$

H $1.867630-4.5260461 .841052$

C $5.204973-2.299325-0.937038$

O $3.548906-2.847308-2.598970$

H $3.266406-1.348706-1.248836$

H $5.360088-1.9369090 .081215$

O $5.726012-3.636997-0.946957$

C $6.049114-1.464926-1.916850$

C $5.6446550 .012224-2.074367$

H $6.026729-1.935240-2.907554$

O $7.417270-1.505756-1.494991$

H $6.2574030 .428406-2.885290$

H $4.6004670 .069081-2.400465$

H $0.274173-3.3043163 .314054$

H $1.951805-2.9497233 .759430$

H $1.005261-1.7591582 .853527$

C $-0.326711-4.3886560 .425712$

H $2.594723-2.891147-2.783234$

H $5.558588-4.009575-1.829737$

H $7.663622-2.441756-1.399254$

H $-0.814372-4.5124371 .400081$

H $\quad 0.159482-5.342286 \quad 0.185677$

H $0.222836-2.2887480 .561210$

C 5.0430731 .2106081 .432091

$\begin{array}{llll}\text { O } & 4.923450 & 0.522646 & 0.178332\end{array}$

C $5.8697480 .893357-0.826652$

C $5.7153122 .386818-1.159146$

C 5.8420953 .2538210 .097660

C 4.9637172 .7352201 .240560

H 4.1585640 .9004092 .003376

C 6.2882110 .7684212 .212735

H $6.8838690 .714909-0.455645$

O $4.4532202 .628509-1.778278$

H $6.4872342 .671754-1.883289$

H $5.5529004 .284644-0.141003$

O $7.1982863 .272448 \quad 0.533417$

H 5.2211353 .2345502 .181984

O 3.6007023 .0576080 .957193

C $6.193039-0.6861992 .613436$

H 7.2070070 .9187701 .639383

H 6.3945551 .3459773 .138356

H $3.7622652 .292255-1.182231$

H $7.7355153 .632526-0.192732$

H 3.5342464 .0256640 .893839

O $7.282353-1.3674472 .156298$

O $5.267642-1.1595313 .260358$

C $7.318068-2.7619012 .475643$

H $8.205185-3.1949902 .006256$

H $7.390764-2.8972493 .558802$

H $6.431925-3.2682512 .081970$

SCF Energy $\left(B 3 L Y P / 6-31 G^{* *} / / M M F F\right)=-3245.92241652$

0800090

MM̄FF Geometry

C $-0.893795-3.8281161 .357645$

C $-0.721341-3.5789302 .665495$

C $-1.281917-2.4173203 .454521$

O $-2.068596-1.5356852 .623770$

C $-0.132579-1.5863974 .048233$

C $-0.609246-0.5665165 .064007$

C $-0.724430 \quad 0.7547354 .826846$

C $-0.911049-1.1166196 .434501$

C -0.4654481 .4757473 .533524$

C -1.527224 2.5509173 .264155

C -1.2146173 .3359881 .982805$

C -2.1426704 .5436101 .846745$

C -1.8885185 .3075950 .539504$

O -1.3914322 .4599300 .864058$

$\begin{array}{lllll}\text { O } & -2.980591 & 6.217817 & 0.340678\end{array}$

C $-1.9137394 .306638-0.632665$ 
C $-0.617480 \quad 6.1622140 .610906$ O $-1.5357214 .941130-1.852616$ C $-1.0454583 .046119-0.398326$ C -1.336402 $2.014563-1.511519$ O $0.3355503 .405553-0.448546$ C $-3.404343-1.7739162 .538537$ O $-4.030549-2.6721153 .077501$ C $-3.977113-0.7513231 .636003$ C $-5.194128-0.9512551 .109523$ C $-5.882026-0.0696270 .164629$ C $-5.2798151 .285920-0.090048$ C $-0.7101040 .633094-1.292768$ C $-1.243308-0.326420-2.337644$ C $-3.217700-1.767257-2.845364$ O $-0.667915-0.494845-3.410146$ C $-4.416939-0.999648-3.309160$ C $-5.669356-1.479989-3.268047$ C $-6.872681-0.706766-3.730373$ C -7.999692 -0.702019-2.718674 C $-9.133618-1.386634-2.945789$ C $-7.845148 \quad 0.156249-1.479103$ C $-7.007224-0.519755-0.428603$ N -2.439821 $-0.917191-1.962648$ H - $-1.496572-3.1572520 .753745$ H $-0.127467-4.2820043 .247665$ H $-1.900576-2.8239664 .263897$ H $0.419047-1.0936993 .239559$ H $0.597732-2.2418274 .541953$ H $-1.028903 \quad 1.3951785 .653710$ H -1.193180 -0.3321657 .144934$ H $-1.738363-1.8311916 .389852$ H - $0.031474-1.6265416 .841138$ H 0.5183501 .9544803 .603027 H -0.4366740 .7860932 .685543$ H -2.510197 2.0702493 .176595 H -1.569577 3.2417214 .115661 H -0.1691863 .6640362 .024903$ H -2.039342 5.2151772 .708110 H -3.187908 $4.203521 \quad 1.854429$ H -3.019191 6.8119621 .109737 H -2.957198 $3.985372-0.761747$ H $-0.690166 \quad 6.8880811 .429798$ H $-0.487754 \quad 6.753592-0.302331$ H $\quad 0.2877795 .572995 \quad 0.771507$ H -2.115012 $5.713678-1.968988$ H $-2.4210601 .886263-1.602463$ H $-0.9845042 .400813-2.477222$ H $0.8566892 .607665-0.628811$ H -3.3688810 .1147341 .400902$ H $-5.726637-1.8665991 .365630$ H $-5.919068 \quad 1.943995-0.681741$ H $-4.332104 \quad 1.186126-0.624519$ H $-5.095458 \begin{array}{lll}1.811719 & 0.853871\end{array}$ H $\quad 0.3800600 .665611-1.385692$ H $-0.9360450 .239975-0.295288$ H $-3.493300-2.660557-2.276354$ H -2.611686 -2.067180 -3.705508 H $-4.245266-0.000610-3.704102$ H -5.839095 -2.489393 -2.900105 H -7.213670 -1.158179-4.671063 H $-6.6093350 .330336-3.973096$ H -9.955567 -1.369003 -2.237600 H $-9.264316-1.984038-3.841911$ H $-8.8293930 .358625-1.036734$ H -7.454554 1.131641-1.780620 H -7.374554-1.509264 -0.151335 H -2.842610 -0.686408 -1.059689 C $0.671165-4.702699-0.461258$ O $1.778141-3.9308340 .045165$ C $2.110941-2.920549-0.925191$ C $1.385981-3.317260-2.206147$ C $0.112881-3.921220-1.648847$ H $1.693685-1.975762-0.552527$ C $3.628615-2.769022-1.092785$ H $1.209174-2.463212-2.865589$ H $1.949539-4.069816-2.770783$ C $-0.650726-4.770445-2.648717$ H $-0.533114-3.104901-1.308908$
C $4.345845-2.2795770 .179800$

O $4.199015-4.008112-1.512208$

H $3.794360-2.054604-1.904690$

H $3.874079-1.3660380 .550063$

O $4.199020-3.249671 \quad 1.225335$

C $5.858687-2.0479410 .009668$

C $6.296276-1.122649-1.140695$

H $6.358075-3.015967-0.122183$

O $6.375722-1.5018811 .229263$

H $7.394392-1.124569-1.160720$

H $5.960692-1.553690-2.090278$

H $-0.949851-4.170007-3.513925$

H $-0.043487-5.605356-3.013996$

H $-1.556909-5.184327-2.195437$

C $-0.313270-5.0314810 .664009$

H $4.034576-4.656541-0.806061$

H $3.249444-3.3362021 .415049$

H $6.109482-2.0988351 .949616$

H $\quad 0.207359-5.6580491 .399743$

$\begin{array}{llll}H & -1.138558 & -5.633422 & 0.266237\end{array}$

H $1.102083-5.648800-0.816089$

C $3.8174291 .689699-1.032830$

$\begin{array}{llll}\text { O } & 4.417998 & 0.395941 & -1.166434\end{array}$

C $5.838458 \quad 0.346897-1.028327$

C $6.4987221 .223625-2.107179$

C $5.9517452 .655519-2.077354$

C $4.4194942 .681618-2.041182$

H $2.7665431 .533871-1.304651$

C 3.8588532 .1955550 .415301

H $6.1198050 .720100-0.038080$

O $6.2633210 .652333-3.393246$

H $7.5829091 .229186-1.942739$

H $6.2985593 .198485-2.964979$

O $6.4538893 .337831-0.932270$

H $4.0560253 .691867-1.818970$

O $3.9174782 .330568-3.334104$

C 3.1162311 .2613641 .344199

H 4.8857762 .3026740 .775436

H 3.3859373 .1806470 .498607

H $6.7176291 .207939-4.049198$

H $7.4245983 .333513-0.989427$

H $4.2522022 .988331-3.967092$

$\begin{array}{llll}\text { O } & 3.916370 & 0.928341 & 2.397595\end{array}$

$\begin{array}{lllll}\text { O } & 1.969325 & 0.876094 & 1.159530\end{array}$

C $3.3549010 .020043 \quad 3.350076$

H 2.5992090 .5343053 .949337

H $2.925829-0.8542922 .852027$

H $4.157970-0.3172524 .010682$

SCF Energy (B3LYP/6-31G**//MMFF) $=-3245.91905889$

0800091

MMFF Geometry

C $0.737780 \quad 0.885069-2.758143$

C $1.357430-0.289462-2.957194$

C $0.879880-1.657108-2.521244$

O $-0.424061-1.601820-1.902862$

C $1.875534-2.229604-1.496370$

C $1.513650-3.607990-0.981956$

C $0.968123-3.850737 \quad 0.227145$

C $1.854871-4.746933-1.905648$

C $0.549460-2.832928 \quad 1.252378$

C $-0.860259-3.1165671 .791751$

C $-1.412489-1.9139842 .571775$

C $-2.723246-2.2710413 .266831$

C $-3.341795-1.0500513 .959903$

O $-1.642958-0.8712571 .624031$

O $-4.685011-1.390877 \quad 4.335194$

C -3.4353470 .1169192 .952322$

C $-2.602492-0.6979525 .257413$

O $-3.8459481 .302503 \quad 3.627912$

$\begin{array}{llll}\text { C }-2.130671 & 0.367779 & 2.164302\end{array}$

C -2.3359681 .2610450 .915819$

$\begin{array}{lllll} & \text { O } & -1.157879 & 0.968599 & 3.019132\end{array}$

C $-1.506631-1.701576-2.722645$

O $-1.503677-1.755349-3.942056$

C $-2.712370-1.754393-1.866021$

C $-3.927725-1.837468-2.427593$

C $-5.202121-1.962892-1.715557$ 
C $-5.166575-1.949901-0.210833$

C -2.8080702 .6951721 .150733$

C -4.3030092 .8953920 .975782$

$\begin{array}{llll}C & -6.123570 & 2.812827 & -0.723427\end{array}$

O

C $-6.9418941 .586469-0.464965$

C $-7.7677971 .051111-1.377531$

C $-8.693778-0.096800-1.094752$

C $-8.652449-1.171434-2.156440$

C $-9.484422-1.132972-3.211402$

C $-7.730844-2.346435-1.935973$

C $-6.334568-2.099394-2.436162$

N $-4.7532722 .595399-0.301556$

H $-0.2221170 .911271-2.252388$

H $2.315870-0.284684-3.473775$

H $0.846003-2.315451-3.398458$

H $\quad 1.979977-1.531528-0.660014$

H $2.875712-2.283791-1.946404$

H $0.792833-4.8867040 .513446$

H $1.609824-5.722240-1.471837$

H $1.301530-4.656420-2.845506$

H $2.926687-4.745661-2.130262$

H $1.260385-2.8632082 .086239$

H $0.581225-1.8201870 .843306$

H -1.531505 -3.343115 0.953008

H $-0.827056-4.0031192 .436989$

H - $0.661529-1.5863353 .300033$

H -2.574499 -3.086916 3.985211

H -3.441124 -2.651116 2.526713

H -4.644614 -2.162956 4.925290

H -4.230899 -0.1252482 .236571$

H -2.600423 -1.554768 5.941807

H -3.1094640 .1111995 .794825$

H -1.563960 -0.403446 5.091222

H -4.6907391 .1084974 .069093$

H -1.3656351 .3235140 .403186$

H $-2.990058 \quad 0.736000 \quad 0.210490$

H $-0.515240 \quad 1.4447102 .467285$

H -2.565322 -1.755022 -0.793151

H -3.998688 -1.841456 -3.514572

H -4.675765 -1.040212 0.147202

H -4.620254 -2.819419 0.170158

H $-6.155984-1.9595230 .251165$

H -2.505892 3.0673662 .134983

H -2.329412 3.3548200 .416160

H -6.099011 3.078119-1.785211

H $-6.5411003 .655710-0.162856$

H $-6.901711 \quad 1.1578580 .533701$

H -7.837092 $1.510701-2.361148$

H $-9.7126890 .308811-1.031491$

H -8.494627 $-0.538603-0.110822$

H -9.487429 -1.920285 -3.957963

H $-10.176538-0.309391-3.354061$

H -8.114422 -3.223327 -2.475889

H -7.759495 -2.647725 -0.884669

H $-6.261782-2.086013-3.524549$

H $-4.0981752 .244536-0.993643$

C $1.5448883 .197882-2.051085$

O $2.3203502 .585234-0.999486$

C 1.6954402 .8913370 .260538

C $0.8047754 .096387-0.008296$

C $0.2835093 .761013-1.392888$

H 1.0547582 .0371190 .509838

C 2.7378303 .0893071 .364117

H $\quad 0.013220 \quad 4.2055120 .738358$

H $1.3809215 .028688-0.035581$

C $-0.3169294 .949777-2.122795$

H $-0.4840772 .984581-1.298223$

C 3.7031471 .8993951 .550305

O 3.5248574 .2444551 .050623

H 2.2255563 .3139712 .307553

H 4.4094041 .8747120 .714137

O 4.4898162 .1597042 .719441

C $3.0174570 .531078 \quad 1.696745$

C $3.948008-0.6247842 .101477$

H 2.5199720 .2773210 .755212

O $1.996926 \quad 0.6337952 .694319$

H $4.280122-0.4930823 .139408$
H $3.331221-1.5321662 .110377$

H -1.177367 5.343769-1.571939

H $0.4098665 .761245-2.235721$

H $-0.6583354 .660953-3.121770$

C $1.3136472 .205718-3.196663$

H 4.1792254 .3512821 .762336

H 3.8924452 .1326863 .486747

H $1.521628-0.2143352 .717169$

H $2.2752562 .032887-3.696168$

H $0.6487142 .651032-3.945365$

H $2.1570304 .023970-2.436397$

C $5.740508-1.089122-1.153459$

O $4.716124-0.930765-0.161240$

C $5.168403-0.8374401 .191399$

C $5.953083-2.1007421 .585517$

C $7.088484-2.3893150 .598447$

C $6.609219-2.323858-0.854657$

H $5.198395-1.283199-2.087426$

C $6.5606650 .196743-1.345497$

H 5.8214730 .0348081 .293353

O $5.084217-3.2320141 .640763$

H $6.361080-1.9634662 .593550$

H $7.497218-3.3882020 .794844$

O $8.137848-1.444197 \quad 0.782657$

H $7.468245-2.349978-1.535293$

O $5.832757-3.497577-1.113989$

C $5.7269821 .323288-1.917737$

H $6.9843100 .545670-0.400402$

H $7.3806260 .015594-2.049661$

H $4.653316-3.3190570 .773069$

H $8.449475-1.5264321 .700092$

H $5.580480-3.482679-2.052975$

O $6.3888542 .504287-1.738003$

O $4.6455881 .185935-2.471777$

C $5.7216913 .667888-2.236977$

H $4.8158253 .857253-1.654680$

H $6.3960014 .520844-2.123585$

H $5.4822383 .549925-3.298118$

SCF Energy $\left(B 3 L Y P / 6-31 G^{* *} / / M M F F\right)=-3245.90900401$

08_00092

MM̄FF Geometry

C $4.290011-0.2187142 .803524$

C $3.155697-0.6646013 .366869$

C 2.0561810 .1596653 .996619

O $0.800611-0.1776573 .356128$

C 2.2084011 .6890983 .885512

C 1.1097912 .4390954 .616871

C 0.0377212 .9993344 .022201

C 1.2990512 .5641806 .106495

C -0.3290612 .9695242 .563502$

C $-0.1023364 .340823 \quad 1.920125$

C -0.4631574 .3540870 .427886$

C $-0.0968315 .705815-0.187185$

C $-0.5047455 .788656-1.662906$

O

O $-0.4230157 .163819-2.068653$

C $-1.9835385 .369128-1.794813$

C $0.4673785 .015317-2.561756$

O $-2.3616225 .275014-3.166762$

C $-2.3357014 .050940-1.061101$

C $-3.8725063 .882510-1.002964$

O $-1.8048082 .915720-1.741473$

C $0.071603-1.1996713 .875841$

O $0.418683-1.9923394 .736132$

C $-1.260848-1.1572943 .233975$

C $-2.108892-2.1835113 .392645$

C $-3.471179-2.2651282 .860524$

C $-4.055077-1.0164392 .255489$

C $-4.3524772 .564348-0.377368$

C $-4.5637991 .448566-1.391159$

C $-5.688711-0.739024-1.664374$

O $-4.1469871 .491011-2.545371$

C $-6.308114-1.791895-0.804930$

C $-5.817117-3.033478-0.674487$

C $-6.476772-4.1090090 .139695$

C $-5.599816-4.6103791 .264200$

C $-4.972825-5.7956181 .166720$ 
C $-5.536586-3.7654772 .519771$

C $-4.130847-3.4383452 .956853$ N $-5.2683310 .381843-0.845670$ H 4.4933780 .8467082 .753725 H $3.006580-1.7418753 .421176$ H $2.007892-0.1126865 .058899$ H 2.2249861 .9849322 .830739 H 3.1732702 .0071084 .300992 H -0.6763923 .5322394 .648976$ H $\quad 0.5035103 .149883 \quad 6.579515$ H 1.3044621 .5768616 .578137 H 2.2490243 .0615046 .327915 H 0.2310262 .2049302 .017319 H -1.385220 2.6844432 .484594 H -0.7114255 .0859092 .448973$ H 0.9495534 .6286572 .043360 H $0.0803793 .545751-0.069889$ H $0.9758925 .907570-0.074817$ H $-0.6114416 .507898 \quad 0.360611$ H $0.4873007 .469058-1.913386$ H -2.591628 $6.174918-1.359624$ H $1.4781375 .433591-2.481863$ H $0.1887015 .106394-3.617296$ H $0.5324533 .955020-2.310071$ H -2.149014 $6.127080-3.585004$ H $-4.2799504 .699930-0.392982$ H -4.303914 $3.982900-2.006129$ H -1.426043 $3.205188-2.586708$ H $-1.511252-0.2744532 .656397$ H -1.777076 -3.048940 3.964926 H $-4.038864-0.1943512 .980144$ H -5.092877 -1.130255 1.939480 H $-3.485237-0.7125801 .371937$ H -3.6635242 .2106440 .397318$ H -5.3222932 .7536410 .100264$ H -4.823306 -1.107197 -2.224243 H $-6.421223-0.369377-2.390106$ H -7.225698 -1.525524 -0.285353 H -4.910451 -3.306978 -1.208489 H $-6.730633-4.932122-0.541057$ H -7.433864 -3.768002 0.554130 H $-4.372792-6.1899441 .979766$ H $-5.049385-6.4027180 .270733$ H $-6.030544-4.3134723 .333386$ H $-6.137823-2.8598802 .397194$ H -3.614666 -4.2799593 .421099$ H $-5.700190 \quad 0.5043200 .066233$ C $5.878259-0.7188640 .895018$

O $4.938694-1.123067-0.125840$

C $5.658069-1.803151-1.171999$

C $7.125736-1.453425-0.964213$

C $7.203152-1.4026910 .545352$

H $5.525516-2.877061-0.995250$

C $5.128457-1.434640-2.562610$

H $7.797162-2.196161-1.405579$

H $7.375009-0.474194-1.389210$

C $8.431300-0.6730341 .063461$

H $7.208997-2.4311810 .931199$

C $3.631485-1.710855-2.802964$

O $5.349063-0.037982-2.800230$

H $5.721180-1.959585-3.321467$

H $3.025124-0.972283-2.268918$

O $3.377949-1.503948-4.199330$

C $3.165538-3.128487-2.433086$

C $1.765661-3.517949-2.944754$

H $3.187721-3.239868-1.343288$

O $4.086433-4.090472-2.958187$

H $1.789638-3.653402-4.034002$

H $1.551984-4.514182-2.534456$

H $9.345493-1.1739570 .728597$

H $\quad 8.464515 \quad 0.3612630 .704867$

H $8.440785-0.6502162 .157582$

C $5.370530-1.1268102 .282024$

H $4.8998070 .453457-2.091831$

H $3.661440-0.598555-4.413907$

H $4.117441-3.971067-3.923032$

H $6.196727-1.0808233 .001465$

H $5.013987-2.1639562 .253136$
H $5.978108 \quad 0.371494 \quad 0.819818$

C $-0.326050-1.443025-0.642618$

O $0.639423-2.370697-1.156079$

C $0.634130-2.546040-2.574170$

C $-0.735399-3.060534-3.051236$

C -1.870642 -2.154380 -2.566799

C -1.748075 -1.840914-1.071409

H $-0.262187-1.5503880 .446542$

C $0.0193410 .012182-0.993754$

H $0.840081-1.585243-3.056459$

O $-0.947732-4.382739-2.558268$

H $-0.731782-3.111980-4.146723$

H $-2.830441-2.648758-2.758341$

O $-1.844354-0.949324-3.325601$

H $-2.446303-1.043649-0.796757$

O $-2.136756-2.996465-0.323678$

C $1.3401560 .450367-0.403265$

H $\quad 0.0789560 .160606-2.074987$

H $-0.7513040 .677239-0.588606$

H -1.798770 -4.694055 -2.910665

H $-2.573972-0.387489-3.013253$

H -1.483312 -3.696163 -0.495096

O $1.6992381 .645897-0.954537$

$\begin{array}{llll}\text { O } & 1.968228 & -0.165150 & 0.445089\end{array}$

C $2.9132492 .225288-0.465849$

H $3.2212303 .006328-1.166000$

H 2.7324972 .6807480 .511377

H $3.7117941 .481538-0.400668$

SCF Energy (B3LYP/6-31G**//MMFF) $=-3245.91503863$

0800093

MMFF Geometry

C $3.541560-3.613255-1.263039$

C $2.288282-3.214789-0.999722$

C $1.235759-3.036669-2.058202$

O $0.608721-1.743402-1.910795$

C $0.126043-4.110356-2.012018$

C $-0.761269-4.103446-0.778400$

C $-1.884348-3.366703-0.647811$

C $-0.354403-5.0522000 .320181$

C $-2.469762-2.398340-1.641137$

C $-3.873827-2.795174-2.119237$

C $-4.978223-2.576565-1.072867$

C $-6.332599-3.025052-1.629658$

C $-7.484516-2.718601-0.661544$

O $-5.018588-1.180203-0.757503$

O $-8.717814-2.864152-1.382178$

C $-7.388620-1.242186-0.224593$

C $-7.539067-3.7211960 .497263$

O $-8.349661-0.9470360 .786724$

C $-5.976165-0.8279530 .249417$

C $-5.919627 \quad 0.706677 \quad 0.452947$

O $-5.696184-1.4748881 .493253$

C $1.246725-0.644954-2.389511$

O $2.364162-0.589228-2.874701$

C $0.3076840 .489730-2.236337$

C $0.6923811 .736043-2.544309$

C $-0.1486982 .934423-2.475397$

C $-1.6007802 .747868-2.121003$

C $-4.503730 \quad 1.2570530 .672584$

C -4.5042802 .7374801 .018481$

C -2.9370354 .5601741 .698578$

O $-5.525362 \quad 3.4173791 .069700$

C -2.5824185 .3949300 .508602$

C -1.3821645 .9706520 .339397$

C $-1.0434516 .892815-0.795324$

C $0.0957846 .384163-1.648050$

C $1.355316 \quad 6.802608-1.433821$

C $-0.2542605 .481179-2.809705$

C $0.4106714 .132126-2.744995$

N -3.2219093 .2024401 .276771$

H $3.819038-3.845074-2.287929$

H $2.008399-2.9876530 .024079$

H $1.689225-3.085345-3.057553$

H $0.575600-5.105601-2.127899$

H $-0.508369-3.986264-2.898940$

H -2.441674 -3.451249 0.283706

H -1.040895 -5.019916 1.173168 
H $-0.345634-6.080930-0.054870$ H $0.643495-4.8148610 .696828$ H -2.490470 -1.402228 -1.184227 H -1.836156 -2.309616 -2.528060 H -4.101412 -2.178619 -2.999071 H -3.870663 -3.841097 -2.450391 H -4.734303 -3.145556-0.169299 H -6.315336 -4.093161 -1.880042 H -6.529229 -2.499961 -2.575099 H -8.757527 -3.772328 -1.727994 H -7.655072 $-0.625523-1.094507$ H -7.684520 -4.7391730 .115771$ H $-8.394802-3.5223191 .151866$ H -6.632042 -3.724698 1.105160 H -9.221009 -1.2096920 .443628$ H $-6.332097 \quad 1.208691-0.432183$ H -6.5489510 .9850021 .307928$ H $-5.073387-0.9358882 .004656$ H $-0.6912750 .263293-1.881564$ H $\quad 1.7150511 .896463-2.882763$ H -2.101398 2.123545 -2.869297 H -2.160289 $3.683024-2.061124$ H -1.698601 2.266427 -1.142732 H $-4.013448 \quad 0.7275621 .494703$ H -3.901777 $1.119222-0.232884$ H -2.1198354 .5085242 .425286$ H -3.814612 4.9832892 .197996 H $-3.3663175 .569255-0.224430$ H -0.6079385 .8128591 .087050$ H $-0.7781937 .866646-0.362503$ H -1.918344 $7.089546-1.427548$ H $2.1747316 .479593-2.067247$ H $1.5943417 .478951-0.619785$ H $\quad 0.0638615 .970840-3.740271$ H -1.340008 $5.392458-2.910279$ H $1.4739134 .160131-2.987471$ H -2.4321172 .5792321 .138644$ C $5.747313-2.779472-0.297497$ O $5.256751-1.446571-0.060777$ C $5.998562-0.531119-0.892752$

C $7.145017-1.335892-1.499784$

C $6.519155-2.713396-1.613712$ H $5.322323-0.226467-1.699934$

C $6.4313320 .696920-0.083416$ H $7.470712-0.930104-2.462463$ H $8.017530-1.368944-0.837165$

C $7.528995-3.832241-1.795473$ H $5.835873-2.704272-2.471369$

C $5.270557 \quad 1.404596 \quad 0.644717$

O 7.3704330 .2733940 .913973

H $6.9680381 .398535-0.732352$

H 4.8986330 .7420651 .433089

O 5.8078772 .5500261 .318021

C $4.1096931 .857187-0.259844$

C 2.9936732 .6077170 .489537

H $3.6683700 .997647-0.773786$

O $4.6006272 .744204-1.268068$ H 3.3951583 .5131750 .961557 H $2.2825682 .965607-0.265217$ H $8.102810-3.686435-2.716496$ H $8.237638-3.873718-0.961466$ H $7.026813-4.802575-1.860247$ C $4.601051-3.790464-0.212238$ H 7.6291101 .0617491 .421451 H 6.1007413 .1793140 .636430 H $5.1539442 .225256-1.875622$ H $4.143217-3.7125570 .781938$ H $5.000560-4.807853-0.295703$ H $6.442256-2.9942450 .525685$

C $1.127452-0.3752141 .727380$ O 1.7442980 .5923340 .867484 C 2.2588261 .7504461 .531732 C 1.1071842 .5131622 .200068 C 0.3397691 .6095333 .170878 C $-0.014741 \quad 0.2553112 .542800$ H $0.682277-1.1035481 .037875$ C $2.192677-1.1035652 .559768$ H 2.9759351 .4415162 .299561
O $\quad 0.2088113 .0136691 .213060$

H 1.5132863 .3784572 .736111

H $-0.5829552 .113823 \quad 3.483768$

O $1.1261101 .384310 \quad 4.337008$

H $-0.351406-0.446653 \quad 3.313530$

O $-1.111977 \quad 0.446224 \quad 1.644094$

C $1.760770-2.4914242 .979134$

H $3.098302-1.2100621 .949564$

H $2465890-0.5431323 .457497$

H -0.0836072 .2620240 .669664$

H $\quad 1.309687 \quad 2.250124 \quad 4.739781$

H -1.869629 0.7379092 .178755

O $2.855187-3.1837013 .413830$

O $0.614044-2.9156812 .943876$

C $2.605576-4.5301783 .828761$

H $3.556214-4.9714594 .139990$

H $1.917052-4.5429734 .678953$

H $2.203650-5.1148362 .995752$

SCF Energy $\left(B 3 L Y P / 6-31 G^{* *} / / M M F F\right)=-3245.91747441$

\section{4}

MM̄MF Geometry

C $-0.293074-3.9085400 .196549$

C -1.582852 -3.542524 0.173805

C $-2.421157-3.467855-1.075318$

O $-2.862579-2.096057-1.193412$

C -3.652720 -4.376557 -0.942169

C $-4.566555-4.314941-2.150336$

C $-5.722468-3.622953-2.196417$

C $-4.120088-5.127802-3.337292$

C $-6.304357-2.755166-1.115417$

C $-6.697982-1.370150-1.648561$

C $-7.130959-0.444013-0.503561$

C -7.733567 $0.853756-1.039700$

C $-8.093724 \quad 1.8208220 .098811$

$\begin{array}{lllll}0 & -5.967800 & -0.148073 & 0.277347\end{array}$

O $-8.3558893 .107365-0.481727$

C -6.8691861 .9819541 .023017$

C -9.3777681 .4014990 .824352$

O -7.1972502 .7508582 .178035$

C -6.2229680 .6402231 .445785$

C -4.8710070 .9159832 .143562$

O $-7.087131-0.0382332 .358139$

C $-2.167921-1.290185-2.040697$

O $-1.286714-1.619640-2.818385$

C $-2.6120510 .099026-1.793974$

C $-1.7893611 .101178-2.135861$

C $-1.9782532 .519465-1.834031$

C $-3.3163032 .957711-1.305848$

C $-4.003756-0.3276752 .378649$

C -2.6345510 .0864562 .883086$

C -0.5103891 .1501002 .108105$

O $-2.344346 \quad 0.0372704 .075906$

C -0.6077642 .6162701 .814734$

C 0.2713083 .2790341 .047660

C 0.1819574 .7497140 .749283

C $0.3473445 .068540-0.722835$

C $1.4794805 .626296-1.184617$

C $-0.8244164 .806378-1.648803$

C $-0.9311113 .350972-2.012608$

N -1.8087020 .5470671 .868951$

H $0.201322-4.189121-0.729958$

H $-2.068213-3.2575441 .105026$

H -1.840245 -3.770116 -1.955300

H $-4.206696-4.125813-0.030568$

H $-3.331341-5.417210-0.799133$

H -6.319151 -3.679218 -3.105900

H $-4.834849-5.081348-4.165798$

H $-3.158527-4.763876-3.712350$

H -4.009084 -6.180326 -3.056799

H $-7.195732-3.253107-0.715371$

H -5.610443 -2.630844 -0.280539

H $-5.842417-0.927709-2.175267$

H -7.514518 -1.479678 -2.372759

H $-7.861300-0.9763940 .117314$

H $-8.6140180 .648565-1.661080$

H -7.007736 $1.348600-1.700245$

H $-9.0828593 .005572-1.119926$ 
H -6.1206412 .5641010 .467313$ H -10.218291 $1.359207 \quad 0.121314$ H -9.6603092 .1385571 .584269$ H $-9.2993760 .423923 \quad 1.304626$ H -7.584757 $3.587606 \quad 1.868774$ H -4.2955861 .6197561 .532957$ H -5.0425821 .4067333 .110460$ H $-6.558859-0.6484542 .896694$ H -3.541810 $0.253251-1.259635$ H $-0.8489380 .854943-2.627335$ H -4.131050 2.539556-1.907787 H -3.454593 4.041136-1.330772 H -3.440772 $2.630870-0.270841$ H -4.456033 -0.994496 3.120147 H -3.874196 -0.9095391 .459417$ H $\quad 0.207793 \quad 0.645511 \quad 1.454076$ H $-0.212000 \quad 0.9975323 .149509$ H -1.437497 3.1597952 .261070 H 1.1157462 .7387470 .628995 H 0.9662395 .2521291 .330355 H -0.7682415 .1702211 .101316$ H $1.5985515 .877593-2.233377$ H $2.3158235 .836954-0.526302$ H $-0.7049405 .373199-2.581093$ H $-1.7300105 .198701-1.177927$ H $-0.0115292 .938913-2.429984$ H -2.1582140 .5533010 .916046$ C $1.687943-2.9588701 .409723$ O $2.677936-3.4122830 .464064$ C $3.986570-3.3047491 .064216$ C $3.804929-2.4249892 .290113$ C $2.417504-2.8264602 .746391$ H $4.266229-4.3243211 .358036$ C $4.969853-2.7579090 .024435$ H $4.572286-2.5969943 .050648$ H $3.825811-1.3629432 .014200$ C $1.782582-1.8277823 .699012$ H $2.478340-3.8057673 .239868$ C $6.376559-2.4785730 .584087$ O $5.082617-3.718522-1.030749$ H $4.551294-1.851777-0.424635$ H $6.326348-1.7309601 .382846$ O $6.873712-3.6723711 .203336$ C $7.403367-2.027282-0.472060$ C $6.982122-0.811435-1.311763$ H $7.618633-2.863726-1.148235$ O $8.634620-1.7483340 .197878$ H $7.806011-0.534019-1.982473$ H $6.167443-1.112455-1.983871$ H $\quad 0.769320-2.1370303 .973959$ H $2.372234-1.7452764 .617875$ H $1.721068-0.8309233 .250353$ C $0.528778-3.9564911 .455468$ H $4.185874-3.882226-1.370157$ H $\quad 6.894665-4.3681480 .523925$ H $9.286357-1.495370-0.478000$ H -0.104808 -3.752231 2.326639 H $0.915189-4.9773581 .563667$ H $1.339171-1.9772561 .064326$ C $5.4309382 .554934-1.034751$ O $6.0177971 .346486-1.532339$ C $6.5278750 .444471-0.538214$ C $7.630501 \quad 1.1337790 .279699$ C 7.1464702 .4648730 .868032 C $6.4388523 .335613-0.175535$ H $5.2162113 .149396-1.931976$ C $4.0989152 .293748-0.321871$ H $5.703417 \quad 0.1634200 .122532$ O $\quad 8.764317 \quad 1.363642-0.553516$ H 7.9431690 .4741051 .095979 H 8.0073173 .0104721 .273833 O 6.2640502 .1853181 .951325 H 5.9362874 .1786920 .313073 O $7.4406493 .886856-1.035274$ C $3.1040011 .647839-1.258825$ H 4.2149481 .6575500 .560029 H 3.6556633 .2322550 .030504 H $9.455417 \quad 1.770616-0.003861$
H 5.9924703 .0361972 .335534

H $6.9960074 .485213-1.659477$

O $2.783705 \quad 0.403627-0.805224$

O $2.6722762 .188510-2.268389$

C $1.851087-0.363054-1.576434$

H $\quad 0.885897-0.351990-1.064303$

H $1.7364730 .007462-2.598952$

H $2.212522-1.393718-1.620754$

SCF Energy (B3LYP/6-31G**//MMFF) $=-3245.90681962$

\section{5}

MMFF Geometry

C $-3.375875 \quad 4.645757-0.526490$

C $-3.8518563 .391364-0.507346$

C $-4.406442 \quad 2.724788 \quad 0.724218$

$\begin{array}{llll}\text { O } & -3.685956 & 1.484175 & 0.921855\end{array}$

C -5.8954832 .3940790 .520250$

C $-6.551907 \quad 1.7850151 .746499$

C -6.9197890 .4931401 .866679$

C -6.8158862 .7466912 .877542$

C $-6.756446-0.607362 \quad 0.856420$

C $-5.691710-1.6172781 .303143$

C $-5.250394-2.5290250 .149541$

C $-4.245692-3.576242 \quad 0.635061$

C $-3.693177-4.423763-0.521341$

O $-4.646036-1.698526-0.848854$

O $-2.557000-5.137814-0.011263$

C $-3.198279-3.479483-1.639984$

C $-4.700579-5.489229-0.967883$

O $-2.790063-4.222499-2.785247$

C $-4.225160-2.395500-2.028690$

C $-3.664217-1.340228-3.014156$

O $-5.337845-3.021256-2.679949$

C $-2.544379 \quad 1.536578 \quad 1.662132$

O -2.0389792 .5275552 .164770$

C -2.0207810 .1581131 .786581$

C $-0.989952-0.0881022 .608196$

C $-0.453195-1.4141662 .901822$

C $-0.146521-1.6159044 .361885$

C $-2.435841-0.568435-2.521453$

C $-1.119106-1.199926-2.942068$

C $1.273976-1.341856-2.260836$

O $-1.006434-1.918001-3.932216$

C $1.385528-2.619426-1.492075$

C $2.122378-2.756046-0.380260$

$\begin{array}{llll}\text { C } 2.215650 & -4.031845 & 0.402132\end{array}$

C $1.765271-3.8697381 .837103$

C $2.650007-3.9395722 .845744$

C $0.283583-3.7316402 .109863$

C $-0.230265-2.328642 \quad 1.936587$

N $-0.074521-0.829382-2.108548$

H -3.404762 5.2402930 .382571

H $-3.8181992 .794920-1.416428$

H -4.2893083 .3686921 .604477$

H -6.000953 $1.735079-0.346980$

H $-6.445832 \quad 3.308006 \quad 0.258001$

H $-7.3891910 .176514 \quad 2.797379$

H -7.370653 2.2806023 .698929

H $-5.876144 \quad 3.125583 \quad 3.290312$

H -7.4093193 .5958632 .523212$

H $-7.718564-1.1222560 .743104$

H $-6.518597-0.211360-0.132731$

H $-4.819506-1.0744091 .684942$

H $-6.085440-2.2208082 .130268$

H -6.139786 -3.013176 - 0.270783

H $-4.693305-4.2186951 .403660$

H -3.402153 -3.070644 1.125361

H $-2.148351-5.612359-0.755677$

H $-2.294111-2.995339-1.251890$

H $-4.938390-6.160592-0.133803$

H $-4.281494-6.128194-1.753014$

H $-5.639935-5.067066-1.330699$

H $-3.591899-4.499729-3.260190$

H -3.486312 -1.799892 -3.993855

H $-4.457695-0.597653-3.181120$

H $-5.806327-2.349439-3.202813$

H $-2.518862-0.6320731 .236511$

H -0.5332260 .7266343 .166573$ 
H $\quad 0.298430-2.5879254 .583744$ H -1.063356 -1.535546 4.956640 H $0.560116-0.8565934 .715590$ H -2.444387 $0.432324-2.971913$ H -2.468135 -0.433219-1.435616 H $1.960428-0.573830-1.892089$ H $1.486735-1.521307-3.319354$ H $\quad 0.825642-3.472152-1.869247$ H $2.681691-1.904463-0.003400$ H $3.259716-4.368646 \quad 0.364242$ H $1.628377-4.831329-0.066912$ H $2.335862-3.8614193 .880907$ H $3.710698-4.0721632 .659786$ H $0.041094-4.1136593 .108210$ H $-0.270767-4.3904601 .430112$ H -0.467694 -2.0640680 .907032$ H $-0.282509-0.316801-1.256817$ C $-1.2442955 .389960-1.692996$ O $-0.6801654 .076876-1.492733$ C $0.2026134 .134535-0.353734$ C $0.6334595 .587450-0.269313$ C $-0.6728476 .290253-0.592676$ H -0.4085413 .8679130 .516861$ C $1.3243573 .112253-0.538568$ H 1.0297855 .8585190 .713351 H $1.3930025 .814119-1.027537$ C - $0.4981957 .737772-1.018759$ H $-1.306993 \quad 6.2663410 .301528$ C 2.4059873 .1649410 .552434 O $\quad 0.7256471 .812099-0.520160$ H $1.7754413 .238821-1.528832$ H 2.9584094 .1089440 .483987 O $1.768622 \quad 3.1721431 .838324$ C 3.4005181 .9870750 .544039 C $4.1344351 .798363-0.794661$ H $2.886627 \quad 1.0598680 .824245$ O 4.3767322 .2402781 .560379 H $3.4141441 .759740-1.617438$ H $4.7946442 .660159-0.952965$ H $-0.0445128 .323929-0.212907$ H $\quad 0.1470837 .822593-1.899478$ H -1.465070 8.189003 -1.263525 C $-2.7730015 .285550-1.748183$ H $\quad 0.0334001 .806435-1.203609$ H 1.2315962 .3636751 .904065 H 3.8982722 .3607022 .398546 H -3.046879 $4.696394-2.633085$ H $-3.2138476 .279632-1.884025$ H $-0.8873485 .726061-2.674786$ C $6.853292-0.5615290 .255804$ O $6.033794 \quad 0.6063820 .112710$ C $4.9690630 .501259-0.834419$ C $5.5356580 .228099-2.236050$ C $6.442442-1.006464-2.238781$

C $7.464547-0.969504-1.095232$ H $7.672668-0.2458840 .914876$ C $6.109072-1.7047230 .955546$ H $4.311485-0.323753-0.546483$ O $6.268709 \quad 1.358987-2.702714$ H $4.7029790 .074667-2.932611$ H $6.966539-1.060198-3.200761$

O $5.625176-2.167983-2.123500$ H $7.965151-1.939111-0.990767$ O $8.476201-0.008104-1.411504$ C $5.708770-1.3057722 .356010$ H $5.215669-2.0303650 .416421$ H $\quad 6.750285-2.5900301 .041515$ H $6.963826 \quad 1.552302-2.050493$ H $6.211018-2.943345-2.154619$ H $8.899986-0.290371-2.239932$ O $4.397673-0.9331172 .377628$ O $6.476612-1.3034583 .309338$ C $3.897788-0.4982693 .645667$ H $2.861325-0.1785663 .509204$ H $3.921381-1.3232364 .363644$ H 4.4802300 .3513704 .014649

SCF Energy (B3LYP/6-31G**//MMFF) $=-3245.91818644$

\section{6}

MMFF Geometry

C $-3.2802064 .601563-0.971451$

C $-3.8815943 .409457-0.846960$

C -4.5928032 .9644050 .405263$

O -4.0015121 .7117150 .826296$

C -6.0844652 .7304090 .112629$

C -6.8910422 .3628241 .345206$

C -7.3976221 .1399511 .602907$

C -7.143494 3.4945982 .309270

C $-7.273169-0.1026080 .767584$

C $-6.373301-1.1411551 .448908$

C $-5.978160-2.2696790 .485870$

C $-5.159951-3.3386521 .211324$

C $-4.630109-4.413506 \quad 0.250944$

O $-5.197831-1.681987-0.559956$

$\begin{array}{llll}\text { O } & -3.637628 & -5.162364 & 0.969476\end{array}$

C $-3.932526-3.722057-0.941529$

C $-5.727988-5.412691-0.131253$

O $-3.544993-4.679348-1.923818$

C $-4.771942-2.595592-1.577796$

C $-3.988919-1.773913-2.631011$

O $-5.896051-3.178235-2.248485$

C -2.8164371 .7864081 .494650$

O -2.2191122 .8004221 .819948$

C $-2.3507960 .405406 \quad 1.748301$

C -1.121589 0.2037272 .246296

C $-0.520734-1.0920622 .569362$

C -1.321726 -2.329867 2.263778

C $-2.752477-1.037136-2.102738$

C $-1.450590-1.797456-2.289751$

C $0.964254-1.678238-1.687353$

O $-1.351978-2.807772-2.980424$

C $1.227681-2.572253-0.517157$

C $2.240001-2.3725870 .339517$

C $2.582922-3.2906241 .475524$

C $2.734325-2.5622242 .792057$

C $3.947540-2.2284613 .263770$

C $1.487646-2.3082963 .606256$

C $0.709220-1.1139133 .124237$

N $-0.397512-1.184408-1.623862$

H -3.340090 $5.312277-0.151562$

H -3.825616 2.693579 -1.663952

H -4.483472 3.7059261 .206264

H $-6.1817731 .963682-0.661926$

H $-6.5239653 .638775-0.321690$

H -7.967772 1.0032382 .521074

H -7.808342 3.2023663 .129185

H -6.2058973 .8395302 .755352$

H -7.6146454 .3365501 .791430$

H $-8.274302-0.5281890 .626081$

H $-6.905100 \quad 0.121777-0.235514$

H $-5.464551-0.6456251 .812571$

H -6.890981 -1.555638 2.322761

H $-6.890065-2.7039600 .059347$

H $-5.745924-3.7973642 .017591$

H $-4.301612-2.8626371 .703530$

H -3.239115 -5.7953520 .347501$

H -3.001911 -3.296706 -0.549619

H $-6.119420-5.9089610 .765110$

H $-5.332938-6.213848-0.765621$

H -6.570458 -4.947049 -0.646895

H $-4.339239-4.925217-2.427890$

H $-3.749759-2.400947-3.498284$

H -4.673496-1.002369 -3.012344

H $-6.221992-2.546024-2.910359$

H -3.030092 -0.406610 1.518206

H -0.4944591 .0709382 .450411$

H - $0.762395-3.2578292 .399792$

H -1.648682 -2.328960 1.218626

H $-2.204094-2.3820812 .910094$

H -2.639883 -0.102520 -2.667327

H -2.872231 -0.767960 -1.049051

H $1.625990-0.806547-1.701151$

H $1.110075-2.237211-2.617338$

H $0.585950-3.441289-0.394845$

H $2.890020-1.5165440 .187933$

H $3.524918-3.7939331 .220284$ 
H $1.840016-4.0904631 .582932$ H $4.065942-1.7328994 .221470$ H $4.849616-2.4407652 .700646$ H $1.758180-2.1131594 .653097$ H $0.888285-3.2222273 .647194$ H $1.217291-0.1643243 .298668$ H $-0.583299-0.382651-1.028106$ C $-0.9922075 .149068-1.929795$ O $-0.4419663 .888350-1.495812$ C $0.5314424 .143053-0.456738$ C $0.7941705 .642529-0.495035$ C $-0.5670456 .181285-0.886923$ H 0.0434843 .8659640 .485880

C $1.7642283 .259628-0.694080$ H 1.1462796 .0330540 .464425 H $1.5393045 .888831-1.261962$ C $-0.5336487 .607319-1.407356$ H $-1.2144406 .140385-0.003135$ C 2.9249013 .5599790 .275975 O $1.3519781 .900752-0.532472$ H $2.0997443 .370485-1.731662$ H 3.1529014 .6293920 .194224 O 2.5142013 .3372941 .623168 C 4.2550762 .8248260 .003154 C $4.251357 \quad 1.312628 \quad 0.257839$ H 5.0064093 .2715200 .665012 O $4.6690673 .068977-1.339091$ H 3.9300601 .0933631 .283238 H $3.5163070 .830236-0.396208$ H $-0.1535388 .288302-0.638860$ H $\quad 0.1129417 .699480-2.286499$ H -1.536892 $7.942144-1.689386$ C $-2.4973145 .015578-2.185567$ H $0.6213081 .742484-1.154741$ H 2.1311432 .4447181 .671455 H $4.7237744 .032225-1.459623$ H -2.648001 4.277712 -2.984049 H $-2.8987675 .966538-2.553977$ H $-0.5072025 .373841-2.889227$ C $6.490317-1.649149-0.002878$ O $5.391808-0.773626 \quad 0.276292$ C $5.5976290 .612968-0.023570$ C $6.778031 \quad 1.1532990 .795712$ C $8.039737 \quad 0.307428 \quad 0.584694$ C $7.759497-1.1910750 .733219$ H $6.189177-2.6159290 .421170$ C $6.687413-1.844222-1.509470$ H $5.812244 \quad 0.714394-1.092634$ O $\quad 6.4450401 .1760922 .182307$ H 6.9868802 .1860450 .497282 H 8.8027380 .6056961 .313965 O $8.5655530 .547490-0.717700$ H $8.615419-1.7810220 .385033$ O $7.579499-1.4917122 .120448$ C $5.472509-2.500728-2.121529$ H $6.851550-0.906371-2.047481$ H $7.561959-2.473142-1.714268$ H $\quad 6.1883330 .2742922 .441159$ H $8.7674911 .496350-0.782922$ H $8.402637-1.2547162 .580706$ O $5.549541-3.854061-1.974266$ O $4.556093-1.881297-2.646546$ C $4.445858-4.594338-2.504688$ H $4.648677-5.658619-2.358142$ H $3.526773-4.335922-1.971539$ H $4.338118-4.402277-3.576499$ SCF Energy (B3LYP/6-31G**//MMFF)= -3245.92398859

0800097

MMFF Geometry

C $-3.187795-0.6363463 .252614$ C $-2.649438-1.5774304 .043157$ C $-1.195326-1.9847884 .108353$ O $-0.362257-1.1499153 .274176$ C $-1.040898-3.4313783 .602947$ C $0.322705-4.020023 \quad 3.906825$ C $1.337027-4.1059743 .024074$ C $0.485543-4.5697825 .300596$
C $1.377597-3.5932331 .611927$

C $1.389397-4.7421870 .594997$

C $1.500238-4.223197-0.846092$

C $1.429631-5.371851-1.853553$

C $1.642244-4.882580-3.296069$

O $2.754087-3.547718-0.974510$

O $1.888089-6.032021-4.120436$

C $2.914018-4.007519-3.350458$

C $0.385651-4.206909-3.858539$

O $3.067283-3.404512-4.633011$

C $2.962774-2.926189-2.249294$

C $4.350057-2.235761-2.224109$

O $1.967145-1.935148-2.520862$

C $0.172075-0.0242903 .818957$

O -0.0199340 .4189814 .938808$

C 1.0520900 .5767212 .791366

C 1.6080821 .7818942 .989171

C 2.4991172 .4748302 .052475

C 2.9405321 .7054250 .836419

C $4.592281-1.450175-0.929581$

C $5.805613-0.538230-0.980868$

C $7.073208 \quad 1.0225130 .512721$

O $6.473809-0.348316-1.991441$

C 6.6185681 .9176651 .622458

C 6.5331533 .2535551 .533054

C 6.0217214 .1158072 .654069

C 4.9873015 .1295392 .207905

C 5.1752466 .4396832 .442581

C 3.7450034 .6499041 .485289

C 2.8815383 .7409242 .323142

N $6.013957 \quad 0.0656420 .251396$

H -2.559520 -0.0683622 .577025$

H $-3.309550-2.1233534 .715248$

H $-0.863232-1.9146105 .151626$

H -1.237901 -3.471633 2.524736

H $-1.800758-4.0787454 .061697$

H $2.264237-4.5737513 .353101$

H $1.475879-5.0088495 .462266$

H $\quad 0.352149-3.7780286 .044265$

H $-0.256293-5.3527715 .488783$

H $0.542508-2.9209011 .396662$

H $2.285848-2.9870941 .506643$

H $2.240268-5.4011360 .812228$

H $\quad 0.474235-5.3373600 .703975$

H $\quad 0.685645-3.511484-1.019994$

H $\quad 0.476738-5.908488-1.766114$

H $2.210915-6.108946-1.620516$

H $1.123972-6.628218-4.039042$

H $3.776541-4.675464-3.216572$

H $-0.458769-4.906538-3.856159$

H $0.528802-3.914752-4.904719$

H $\quad 0.082616-3.324366-3.291694$

H $3.041510-4.118072-5.293403$

H $5.144998-2.988425-2.302033$

H $4.446360-1.569616-3.090357$

H $2.263741-1.079663-2.172904$

H $1.2165930 .001427 \quad 1.886508$

H 1.3858162 .3160423 .911778

H 2.0883421 .4741590 .191753

H 3.4070970 .7634621 .141302

H 3.6719812 .2282880 .218986

H $3.725810-0.821173-0.699736$

H $4.728979-2.151701-0.097364$

H $7.3073721 .570705-0.405441$

H 7.9604690 .4595760 .818275

H 6.3260021 .4332672 .552061

H 6.8428213 .7498840 .616548

H 6.8868904 .6259963 .096690

H 5.5881943 .5019833 .453520

H 4.4489307 .1819652 .128402

H 6.0598886 .8024042 .955692

H 4.0417154 .1999400 .534769

H 3.1191315 .5078691 .204429

H 2.5058894 .2115043 .232576

H $5.505204-0.2999571 .051757$

C $-5.345796-0.7635031 .967669$

$\begin{array}{llll}\text { O } & -4.947384 & 0.053782 & 0.843418\end{array}$

$\begin{array}{llll}\text { C } & -6.128769 & 0.459427 & 0.127918\end{array}$ 
C $-7.243901-0.4617370 .607083$

C $-6.869056-0.6442962 .061085$

H -6.3562561 .4806740 .455967$

C $-5.9197240 .434976-1.390162$

H $-8.235883-0.0208360 .469779$

H $-7.225918-1.4269230 .087501$

C -7.544063 -1.838036 2.715246

H -7.1418600 .2665582 .610999$

C $-4.7671631 .310710-1.922209$

O $-5.656760-0.915539-1.793929$

H $-6.8593450 .720211-1.879653$

H -3.811085 $0.826025-1.701510$

O $-4.8768281 .322129-3.352766$

C $-4.7437642 .759210-1.406333$

C $-3.8186503 .719284-2.178857$

H $-4.4607252 .754634-0.347313$

O $-6.068443 \quad 3.296712-1.484331$

H $-4.2609573 .951280-3.156684$

H -3.825644 $4.668557-1.626703$

H -8.632753 -1.722365 2.695298

H -7.296070 -2.771693 2.199253

H -7.234839 -1.938529 3.760231

C $-4.649657-0.2910393 .247197$

H -5.541225 -0.912269 -2.759737

H $-5.6905741 .803718-3.580879$

H $-6.0487584 .183688-1.086031$

H $-5.137644-0.7338884 .123465$

H $-4.737514 \quad 0.798112 \quad 3.344352$

H -5.054979 -1.794569 1.727437

C $-0.4818362 .404610-1.105436$

O $-1.8210892 .920133-1.093199$

C $-2.3694003 .240681-2.372849$

C $-1.5108674 .305452-3.078549$

C $-0.0431663 .874929-3.159835$

C $0.4799413 .379428-1.807624$

H $-0.1967862 .355940-0.047444$

C $-0.4143890 .978497-1.679507$

H $-2.3913262 .338131-2.991070$

O $-1.6035515 .540891-2.371036$

H -1.912248 $4.470905-4.085658$

H $0.5626974 .725497-3.495473$

O $0.0750982 .846438-4.138169$

H $1.4611482 .907650-1.934481$

O $0.6723384 .519191-0.965198$

C -1.165796 -0.013537-0.821315

H $-0.8385410 .930271-2.685588$

H $\quad 0.6278340 .644542-1.723408$

H -1.076331 $6.197856-2.856632$

H $1.0147382 .602925-4.195962$

H $1.0528544 .205224-0.127343$

O $-1.323638-1.180943-1.511061$

$\begin{array}{lllll}\text { O } & -1.559132 & 0.202757 & 0.315576\end{array}$

C -2.052363 -2.203258 -0.823712

H -2.070810 -3.093129 -1.458437

H -1.559813 -2.4556430 .119504$

$\mathrm{H}-3.081007-1.876754-0.645094$

SCF Energy (B3LYP/6-31G**//MMFF)= -3245.89905395

\section{8}

MM̄FF Geometry

C $2.2570474 .162603-0.446570$

C 2.7904184 .6864960 .668369

C 3.4608743 .9351211 .797436

O 3.6054922 .5281111 .503214

C 2.6029594 .0714263 .070140

C 3.2038923 .3985254 .289595

C 2.7570452 .2483074 .833699

C 4.3564954 .1340614 .923268

C 1.6384781 .3705154 .342263

C $2.120673-0.0703994 .123666$

C $1.073706-0.9259143 .392901$

C $1.565744-2.3684683 .254844$

C $0.626257-3.2265372 .396000$

O $0.873748-0.3444222 .101640$

O $1.335907-4.4343542 .080294$

C $0.342847-2.4829381 .072752$

C $-0.618965-3.653623 \quad 3.181173$

O $-0.620481-3.1896350 .295723$
C $-0.084812-1.0152401 .274547$

C $-0.171519-0.224268-0.052357$

O $-1.388310-0.990698 \quad 1.868620$

C 4.7370242 .1403000 .852263

O 5.6168772 .8629090 .412310

C 4.7272780 .6618610 .778704

C 5.6985290 .0167070 .115528

C $5.842596-1.436593-0.009901$

C $4.777079-2.3010070 .611227$

C $1.150376-0.065553-0.809256$

C $1.367360-1.079959-1.918056$

C $3.084019-1.756395-3.598394$

O $0.525340-1.906050-2.256576$

C $3.918703-2.869606-3.049320$

C $5.191793-3.087025-3.412078$

C $6.020351-4.232398-2.906890$

C $7.329829-3.785156-2.298470$

C $8.474888-3.846353-2.999299$

C $7.326031-3.361165-0.847660$

C $6.920550-1.924760-0.659260$

N $2.617438-0.927172-2.504197$

H $2.2967423 .091083-0.618810$

H 2.7273315 .7641840 .810890

H 4.4481724 .3797501 .974003

H 1.5973663 .6845912 .873103

H 2.4549745 .1346853 .305007

H $3.251575 \quad 1.8821975 .732817$

H 4.7124853 .6435685 .835611

H 5.2022054 .1930474 .231323

H 4.0553655 .1513215 .194023

H 0.8364501 .3734665 .090109

H 1.2042381 .7542883 .416963

H $3.044458-0.0495703 .531657$

H $2.364432-0.5255105 .091810$

H $0.132845-0.8905323 .954508$

H $1.710772-2.8255164 .241830$

H $2.556962-2.3677202 .781823$

H $0.780142-4.9513931 .471906$

H $1.275483-2.5090270 .496903$

H $-0.330106-4.2382684 .063058$

H -1.258069 -4.313232 2.584212

H -1.219356-2.809396 3.526220

H -1.499414 -2.996451 0.664652

H -0.956546 -0.642729 -0.691001

H -0.5107490 .7910600 .198506$

H $-1.809095-0.139748 \quad 1.662138$

H 3.9272170 .1408281 .290530

H $6.4758060 .605951-0.369229$

H $4.797025-2.2047681 .702008$

H $4.882757-3.3620720 .376240$

H $3.784305-2.0116230 .250811$

H $1.1569020 .919868-1.290561$

H $2.002499-0.096882-0.125797$

H $3.649698-1.110161-4.277023$

H $2.228011-2.168205-4.142278$

H $3.443223-3.543554-2.340540$

H $5.656634-2.426265-4.140520$

H $\quad 6.213096-4.901758-3.755661$

H $5.466856-4.836609-2.177220$

H $9.423242-3.557413-2.558432$

H $8.488251-4.180993-4.031317$

H $8.336297-3.465981-0.428711$

H $6.717011-4.059086-0.266503$

H $7.636602-1.215869-1.077619$

H $3.266822-0.248469-2.117005$

C $0.0718034 .612485-1.562967$

O $-0.0797913 .356281-2.252288$

C $-1.3387523 .370028-2.970333$

C -1.993293 4.707538-2.650208

C $-0.7930175 .587191-2.354498$

H $-1.0764353 .313178-4.033784$

C $-2.1338172 .131615-2.541752$

H -2.606185 $5.082057-3.475396$

H $-2.6342224 .628129-1.763218$

C $-1.1510216 .858480-1.604760$

H $-0.3002315 .846247-3.301120$

C $-3.6047442 .091818-2.989572$

O $-1.4734420 .978940-3.077222$ 
H -2.076757 $2.048321-1.451015$

H $-4.1633232 .931037-2.559043$

O $-3.675786 \quad 2.236205-4.412703$

C $-4.3299650 .774067-2.637445$

C $-4.2637030 .425156-1.145636$

H $-3.925371-0.043744-3.243753$

O $-5.708057 \quad 0.911597-2.997993$

H -3.224578 $0.314652-0.818478$

H $-4.6661521 .265293-0.562535$

H -1.846942 7.467317 -2.191492

H $-1.6266206 .637086-0.643379$

H $-0.2600987 .462566-1.408264$

C $1.5523144 .986737-1.492658$

H $-0.5324781 .045884-2.844183$

H $-3.372797 \quad 3.131645-4.637184$

H -5.737735 1.139557 -3.943035

H $1.6629256 .052704-1.265020$

H $2.0393094 .802776-2.457586$

H $-0.3383134 .479118-0.552116$

C $-5.514880-2.0568021 .321209$

$\begin{array}{lllll} & -5 & -4.838410 & -0.967297 & 0.683585\end{array}$

C $-5.042651-0.838450-0.731457$

C $-4.587611-2.113934-1.457980$

C $-5.244332-3.363958-0.863947$

C $-5.139701-3.393607 \quad 0.664288$

H $-5.121439-2.0658062 .346259$

C -7.026441-1.818694 1.403282

H $-6.107192-0.663218-0.918732$

O $-3.169805-2.230320-1.366723$

H -4.846491-2.034778 -2.519918

H $-4.760965-4.255118-1.282207$

O $-6.612712-3.388901-1.259783$

H -5.766845 -4.191166 1.079314

O $-3.789971-3.6880951 .035165$

C -7.329711 -0.6585102 .320943$

H $-7.491929-1.6225060 .433225$

H $-7.535425-2.6976821 .816620$

H -2.905229 -3.019722 -1.868929

H $-7.008568-4.199339-0.896664$

H $-3.562257-4.5528460 .653179$

O -7.3746170 .5021041 .606546$

O $-7.481676-0.7693753 .530706$

C -7.624922 1.6858222 .370130

H -7.6343312 .5368891 .684066$

H -8.600047 1.6203862 .862190

H -6.8293161 .8369143 .105967$

SCF Energy (B3LYP/6-31G*//MMFF) $=-3245.91149575$

0800099

MM̄FF Geometry

C -1.961927 3.8585801 .100549

C -2.037408 3.6604802 .426408

C -2.390622 2.3679593 .126096

O $-2.266997 \quad 1.2406632 .229775$

C -3.8365342 .4259413 .642335$

C $-4.175431 \quad 1.275248 \quad 4.569325$

C $-4.903375 \quad 0.197432 \quad 4.217719$

C -3.680969 1.4231225 .985621

C $-5.472691-0.1171772 .862761$

C $-5.229514-1.5842112 .479693$

C $-5.826362-1.9061061 .103381$

C $-5.766220-3.4065820 .817234$

C $-6.295624-3.735686-0.586808$

O $-5.071442-1.1952290 .116641$

O $-5.922226-5.087521-0.893507$

C $-5.587132-2.826494-1.611399$

C -7.826573 -3.682604 -0.650675

O $-6.150559-2.985536-2.911680$

C $-5.576775-1.329276-1.217800$

C $-4.638462-0.554361-2.170505$

O $-6.897508-0.797749-1.335087$

C $-1.047230 \quad 0.6389432 .166096$

O -0.0548870 .9175822 .820848$

C $-1.108906-0.4030581 .117456$

C $0.040612-0.8984890 .635263$

C $0.182841-1.876396-0.443822$

C $-1.057400-2.582526-0.919324$

C $-4.3129430 .877614-1.725682$
C $-3.310348 \quad 1.497945-2.679339$

C $-0.9094721 .351921-3.352375$

O $-3.6512552 .310616-3.535380$

C $-0.2769350 .082749-3.833317$

C $1.030117-0.197133-3.713965$

C $1.660238-1.461008-4.229211$

C $2.513433-2.168476-3.198126$

C $3.854517-2.157208-3.285894$

C $1.812591-2.959299-2.112472$

C $1.413543-2.085000-0.955609$

N -2.026892 $1.010460-2.493037$

H -2.1981783 .0493710 .414777$

H $-1.8143004 .494593 \quad 3.089213$

H -1.7040772 .2438713 .972657$

H -4.531962 2.4560932 .795631

H -4.0054533 .3641054 .188646$

H -5.122364 -0.5426044 .986461$

H -3.998221 0.5931596 .625914

H -2.587584 $1.456976 \quad 6.010870$

H -4.068473 2.3463786 .428616

H -6.5522180 .0731162 .893447$

H -5.0520450 .5279222 .086599$

H -4.148862 -1.777809 2.469899

H -5.677836 -2.238219 3.238066

H $-6.865870-1.5575741 .087222$

H $-6.319675-3.9720681 .577196$

H $-4.724453-3.7500910 .886108$

H $-6.306047-5.666099-0.212277$

H $-4.545424-3.170780-1.682054$

H $-8.264067-4.3917380 .062249$

H -8.191187 $-3.987918-1.637791$

H $-8.231660-2.694278-0.424046$

H -6.124099 -3.934120 -3.124845

H $-3.695229-1.104404-2.261734$

H $-5.077425-0.515615-3.176087$

H $-6.8403840 .160816-1.473570$

H $-2.082889-0.6953940 .742597$

H $0.978591-0.5218941 .041935$

H $-1.709085-1.890501-1.457400$

H $-1.614083-3.001206-0.073010$

H $-0.850216-3.421655-1.586709$

H $-5.2095441 .505941-1.714809$

H $-3.8914310 .898964-0.714205$

H $-0.2219191 .961113-2.761195$

H $-1.2553181 .943423-4.205481$

H $-0.929718-0.637244-4.322205$

H $1.6895690 .534042-3.253922$

H $2.272327-1.191605-5.099931$

H $\quad 0.901710-2.159643-4.603901$

H $4.471109-2.685252-2.566479$

H $4.365760-1.615030-4.074224$

H $2.482729-3.737716-1.725836$

H $0.971294-3.496726-2.557735$

H $2.252844-1.549804-0.510023$

H $-1.8397060 .414390-1.692474$

C $-0.372248 \quad 4.993287-0.472959$

$\begin{array}{lllll}\text { O } & 0.753144 & 4.445700 & 0.232350\end{array}$

C $1.9682574 .948856-0.352634$

C $1.5480365 .896245-1.476511$

C $0.1453856 .300697-1.062764$

H 2.4798305 .5149070 .435028

C $2.870873 \quad 3.789739-0.813177$

H $2.2280876 .749293-1.567115$

H $1.5288975 .392022-2.449562$

C $-0.6929176 .842166-2.207273$

H $0.2217287 .063564-0.276336$

C $3.237898 \quad 2.7964770 .313114$

O $2.2196323 .091666-1.874331$

H $3.7929124 .213111-1.228478$

H 3.6810253 .3494861 .150246

$\begin{array}{llll}\text { O } & 2.060892 & 2.151273 & 0.801387\end{array}$

C $4.2323331 .699827-0.129368$

C 4.5644550 .7293311 .015272

H $5.1508422 .181662-0.480992$

O $3.7038370 .955310-1.223578$

H 5.0415161 .2738501 .840146

H 3.6353540 .3229121 .437785

H $-0.2251297 .733246-2.638500$ 
H $-0.8069656 .101644-3.006212$

H -1.693215 7.120791-1.861292

C -1.5514485 .1695280 .484795$

H $1.4409272 .653657-1.490498$

H 1.4894042 .8327161 .193339

H $2.8240800 .635660-0.960482$

H -2.410362 $5.585472-0.053550$

H -1.2809545 .8830651 .273038$

H -0.634454 $4.283103-1.268943$

C $6.277859-2.512701 \quad 1.628065$

O $5.585617-1.2687281 .794438$

C $5.422835-0.4818140 .607103$

C $6.793505-0.1128490 .024953$

C $7.655351-1.359995-0.206283$

C $7.670138-2.2848701 .015954$

H $6.421297-2.8921222 .648285$

C $5.430064-3.5415740 .863191$

H $4.855715-1.066194-0.124499$

$\begin{array}{lllll}\text { O } & 7.476243 & 0.782024 & 0.900643\end{array}$

H $6.6547870 .412144-0.926383$

H $8.682470-1.053144-0.438315$

O $7.156683-2.087708-1.325003$

H $8.119105-3.2523440 .762387$

O $8.488785-1.6949902 .030797$

C $4.152630-3.8939241 .598137$

H $5.144368-3.172766-0.124914$

H $6.002784-4.4678660 .739938$

H 7.5466740 .3513041 .769821

H 7.176933 -1.494394 -2.095278

H $9.385704-1.6029991 .666606$

O $3.400999-4.7163760 .807488$

O $3.851003-3.5090162 .719335$

C $2.147149-5.1274941 .361982$

H $1.608396-5.6953940 .598882$

H $2.315532-5.7726502 .229309$

H $1.545176-4.2567361 .639294$

SCF Energy (B3LYP/6-31G**//MMFF) $=-3245.92231689$

0800100

MM̄FF Geometry

C $3.4967063 .507069-0.977469$

C $4.4863683 .765909-0.108057$

C $5.1997202 .773600 \quad 0.784767$

$\begin{array}{lllll}\text { O } & 4.674340 & 1.436096 & 0.632836\end{array}$

C 5.0147363 .1898762 .256900

C 5.7728132 .3156823 .238225

C 5.2082051 .4101484 .062652

C 7.2618452 .5465453 .283391

C 3.7557911 .0336214 .164802

C $3.556626-0.4695003 .926953$

C $2.070753-0.8430813 .809656$

C $1.912226-2.3551563 .636422$

C $0.457216-2.7619323 .364368$

O $1.545007-0.1557442 .670708$

O $0.482179-4.1242262 .910906$

C $-0.097903-1.8899602 .217171$

C $-0.377363-2.7535404 .649894$

O $-1.485028-2.1491832 .014624$

C $0.153383-0.3814342 .415609$

C $-0.229721 \quad 0.463787 \quad 1.176228$

O $-0.652471 \quad 0.0797053 .506877$

C $5.2396030 .644974-0.320061$

O $6.1190820 .955320-1.107350$

C $4.592816-0.683977-0.239141$

C $4.873804-1.620395-1.157283$

C $4.343364-2.986080-1.192304$

C $3.365423-3.384689-0.118769$

C $0.6041070 .202343-0.082348$

C $-0.004105-0.800061-1.045870$

C $0.437054-1.862433-3.256821$

O $-1.091174-1.339969-0.866806$

C $0.920080-3.253391-2.993496$

C $1.725055-3.928411-3.828075$

C $2.155983-5.349079-3.604009$

C $3.656954-5.521993-3.642959$

C $4.272908-5.972078-4.749224$

C $4.429595-5.268694-2.369149$

C $4.764624-3.816628-2.169248$
N $\quad 0.811495-1.001775-2.151958$

H $3.1380432 .490354-1.109689$

H $4.8268684 .795818-0.011787$

H 6.2674362 .7873860 .533370

H 3.9463573 .2061312 .496331

H 5.3525484 .2262272 .395538

H 5.8593020 .8649694 .745353

H $7.752788 \quad 1.9481234 .058341$

H 7.7216172 .2834562 .325963

H 7.4770003 .5986853 .496735

H 3.3979541 .2914635 .168795

H 3.1423281 .6003623 .461599

H $4.073461-0.7535733 .001496$

H $4.019483-1.0348734 .745466$

H $1.548698-0.5005494 .710769$

H $2.304949-2.8898864 .510434$

H $2.525274-2.6869642 .787824$

H $-0.425219-4.3662872 .657183$

H $\quad 0.411049-2.2210101 .304347$

H $\quad 0.040897-3.4577125 .379244$

H $-1.401301-3.0943344 .461396$

H -0.421766 -1.771743 5.125885

H -1.980231 -1.674247 2.703661

H -1.304692 0.3803440 .977312

H $-0.066811 \quad 1.518521 \quad 1.441023$

H -0.7709061 .0400123 .420848$

H $3.909944-0.8601960 .583155$

H $5.572018-1.371277-1.955550$

H $3.860453-3.4022940 .857786$

H $2.921139-4.369605-0.276240$

H $2.527376-2.680916-0.075831$

H $0.6914651 .145788-0.634842$

H $1.618238-0.1177340 .171842$

H $0.874356-1.435441-4.164892$

H $-0.652788-1.863955-3.362966$

H $0.560914-3.740571-2.089876$

H $2.060572-3.449882-4.745306$

H $1.690303-5.963195-4.386063$

H $1.768630-5.740741-2.655227$

H $5.345999-6.129811-4.773785$

H $3.718100-6.186919-5.656678$

H $5.381828-5.816195-2.398435$

H $3.891650-5.705046-1.522749$

H $5.473566-3.432646-2.904407$

H $1.727307-0.562843-2.181680$

C $1.3031574 .545874-1.561201$

O $0.725476 \quad 3.438617-2.281856$

C $-0.5760043 .832831-2.777551$

C $-0.8409505 .218621-2.206965$

C $0.5638485 .775764-2.078456$

H $-0.4780013 .881178-3.869195$

C -1.583986 2.750431-2.376761

H -1.485358 $5.821811-2.853460$

H -1.315734 $5.152137-1.220047$

C $0.6496956 .985874-1.164828$

H $0.9323146 .045103-3.077431$

C $-3.0609803 .127772-2.579077$

O $-1.299971 \quad 1.581611-3.154188$

H -1.413750 $2.480335-1.328294$

H -3.320033 $3.966099-1.921498$

O $-3.2246203 .596471-3.922057$

C $-4.0541651 .967623-2.346602$

C $-3.8216691 .233288-1.021048$

H $-4.0046531 .263440-3.184857$

O $-5.3794912 .504419-2.330432$

H $-2.8349490 .758517-1.021487$

H -3.792910 $1.965952-0.202404$

H $0.0302527 .802455-1.549915$

H $0.3038046 .750268-0.152745$

H $1.6789597 .350307-1.092060$

C $2.8137524 .571442-1.796143$

H $-0.3639341 .358353-3.012976$

H $-4.1114483 .987779-3.991399$

H -5.581887 $2.815495-3.228685$

H $3.2193295 .557044-1.542297$

H $3.0346004 .381432-2.853334$

H $1.0803504 .389822-0.496604$

C $-5.227867-1.434481 \quad 1.145859$ 
O

C $-4.8742470 .174880-0.648623$

C $-5.104215-0.888660-1.733158$

C $-6.000360-2.022163-1.225025$

C $-5.536388-2.5506600 .135908$

H -4.619994 -1.873812 1.947783

C $-6.482225-0.8244751 .789590$

H $-5.8152040 .691480-0.431399$

O $-3.849807-1.420465-2.151977$

H -5.571059 -0.416780 -2.605535

H $-5.994293-2.838716-1.957264$

O $-7.336620-1.537125-1.130589$

H $-6.284198-3.2295700 .561954$

O $-4.337292-3.310221-0.041762$

C $-\begin{array}{llll}6.146061 & 0.049670 & 2.980934\end{array}$

H -7.043314 -0.2068541 .083791$

H -7.131845 -1.630122 2.150951

H $-4.023543-2.063527-2.860115$

H -7.895455 -2.273632 -0.829223

H $-4.538614-4.039023-0.652900$

O $-7.308723 \quad 0.519155 \quad 3.524438$

$\begin{array}{lllll}\text { O } & -5.017917 & 0.294550 & 3.385641\end{array}$

C -7.1549791 .3687404 .665759$

H -8.1504871 .6712795 .001200$

H -6.6607420 .8267095 .477809$

H -6.5875892 .2649324 .397092$

SCF Energy (B3LYP/6-31G**//MMFF) $=-3245.89437931$

0800101

MM̄FF Geometry

C 1.4294113 .5238930 .403814

C $2.0734144 .278917-0.500494$

C $3.2531933 .841381-1.338662$

O $3.3392312 .400062-1.395435$

C $4.5547964 .380161-0.724163$

C $5.7803234 .052662-1.553177$

C $6.6500393 .063285-1.267587$

C $6.0146974 .952329-2.738466$

C $6.5606322 .070114-0.141161$

C $6.8827910 .646811-0.619488$

C $6.759820-0.3694530 .524728$

C $7.313288-1.7313550 .105054$

C $7.136174-2.7797651 .213272$

O $5.373458-0.4943490 .862196$

O $7.388158-4.0725010 .642059$

C $5.665850-2.7685321 .677135$

C $8.154641-2.5982202 .344715$

O $5.480461-3.6183892 .807239$

C $5.115143-1.3534031 .981226$

C $3.584951-1.4354742 .189647$

O $5.719442-0.8543813 .175312$

C $2.6279261 .776638-2.373504$

O $1.9436702 .303345-3.236480$

C $2.7933840 .318983-2.184820$

C $1.979132-0.517767-2.844662$

C $1.946100-1.975243-2.716189$

C $3.030654-2.632969-1.906334$

C $2.871298-0.0795612 .251465$

C $1.368663-0.2877842 .255956$

C $-0.510023-0.9545930 .745855$

O $0.713096-0.242520 \quad 3.293814$

C $-0.512536-2.3779990 .281615$

C -1.131732 -2.795797 -0.832805

C -1.121431 -4.223172 -1.305039

C $-0.806739-4.357707-2.780978$

C $-1.751825-4.743261-3.655180$

C $0.619180-4.113537-3.235514$

C $0.937608-2.645519-3.311407$

N $0.866280-0.565436 \quad 0.994293$

H 1.7659992 .5096000 .596420

H $1.7422615 .303273-0.658588$

H $3.1307754 .243498-2.352152$

H 4.6723963 .9931660 .295011

H $4.4923275 .471013-0.609876$

H $7.5228712 .949629-1.909150$

H $6.9330324 .698236-3.278631$

H $5.1848264 .876514-3.447887$

H $6.1014635 .994115-2.413289$
H 7.2824432 .3621090 .631018

H 5.5707142 .0728510 .323379

H $6.1954110 .375248-1.430961$

H $7.9017860 .624842-1.025708$

H 7.3117440 .0150331 .390674

H $8.369506-1.652807-0.181447$

H $6.783290-2.080210-0.792522$

H $8.291638-4.0699680 .281964$

H $5.067454-3.2024530 .863443$

H $9.177079-2.6715051 .954843$

H $8.061668-3.3948903 .091193$

H $8.063547-1.6364902 .853873$

H $5.822782-4.4968572 .568367$

H $3.146479-2.0148271 .369360$

H $3.363016-1.9845083 .114023$

H $5.139864-0.1791903 .562207$

H $3.538819-0.021367-1.475669$

H $1.230797-0.093573-3.513453$

H $4.020337-2.300154-2.239793$

H $3.036666-3.721661-1.991056$

H $2.917656-2.386894-0.847716$

H 3.1441570 .4746653 .155449

H 3.1345000 .5513281 .395285

H $-0.909529-0.272829-0.011458$

H -1.102965 -0.855608 1.660008

H $\quad 0.021585-3.1010300 .894191$

H -1.689568 -2.076959-1.428776

H -2.109453 -4.650672 -1.091072

H $-0.403066-4.824364-0.733752$

H -1.530241 -4.863536 -4.710378

H $-2.769606-4.943120-3.336744$

H $0.776404-4.540003-4.234581$

H $1.289115-4.663376-2.569054$

H $\quad 0.219436-2.076017-3.903902$

H $1.501966-0.5674180 .202756$

C $-0.915807 \quad 3.0252391 .151427$

O $-1.4300082 .954726-0.192859$

C $-2.8655173 .085245-0.152325$

C -3.2606552 .8422611 .295059$

C -2.0884423 .4509742 .035046$

H $-3.0767184 .122494-0.441423$

C $-3.4990432 .110554-1.152140$

H -4.2162383 .3081691 .551910$

H -3.3326101 .7676811 .505739$

C -1.9699012 .9856973 .476377$

H -2.1938984 .5440912 .019636$

C $-5.0379982 .138915-1.136112$

O $-3.0515542 .464647-2.464550$

H $-3.1306401 .097437-0.958347$

H $-5.4128051 .844327-0.153221$

O $-5.4753483 .493959-1.320002$

C $-5.7147041 .285143-2.222590$

C $-5.345303-0.207968-2.245585$

H $-5.4905231 .708782-3.209344$

O $-7.1349451 .385866-2.068711$

H $-5.826893-0.642406-3.131924$

H -4.262953 -0.306102 -2.384246

H -1.088467 3.4221393 .956504

H -2.850472 3.2879464 .051661

H -1.8836221 .8956873 .540530$

C 0.2584534 .0050641 .213405

H -2.078914 2.452597 -2.451610

H $-5.114793 \quad 3.805321-2.168058$

H -7.359493 2.332148 -2.059806

H 0.5880504 .1210852 .252107

H -0.0644084 .9905040 .855219$

H -0.5842612 .0150221 .417425$

C $-5.303376-1.3099461 .342619$

O $-4.995688-0.6602190 .101375$

C $-5.805895-1.025690-1.019230$

C $-5.713924-2.540708-1.283838$

C $-6.028121-3.350786-0.023883$

C $-5.249763-2.8402791 .191895$

H -4.491959-1.010764 2.017852

C $-6.629586-0.8228861 .941986$

H $-6.848667-0.771107-0.805862$

O $-4.410335-2.898426-1.733799$

H $-6.422826-2.811738-2.076068$ 
H $-5.774686-4.402951-0.204349$

O $-7.428416-3.276265 \quad 0.226755$

H -5.624258 -3.317385 2.105130

O $-3.886075-3.2411421 .046524$

C -6.5188710 .5781812 .494397$

H $-7.449002-0.8187131 .218063$

H $-6.950259-1.4705742 .767127$

H -4.305084 -2.566475 -2.641062

H -7.615499 -3.8212881 .009901$

H -3.407578 -2.9581691 .844351$

O $-5.823622 \quad 0.5629363 .667692$

O $\quad-7.0109111 .5626891 .958845$

C -5.6993021 .8246314 .330911$

H -5.3243152 .5910523 .647165$

H -6.6674132 .1209804 .744838$

H -4.9854441 .7088795 .150858$

SCF Energy $\left(B 3 L Y P / 6-31 G^{* *} / / M M F F\right)=-3245.91405670$

0800102

MM̄FF Geometry

C $-1.283372-4.184561-0.361360$

C $-2.479167-3.588692-0.255764$

C $-3.356508-3.239113-1.429608$

O $-3.358677-1.797135-1.540006$

C $-4.794621-3.721237-1.167839$

C $-5.782102-3.320074-2.247301$

C $-6.744153-2.386025-2.099610$

C $-5.645932-4.062521-3.551401$

C $-7.031377-1.551757-0.881491$

C $-6.574680-0.101295-1.082586$

C $-6.358704 \quad 0.6262900 .253036$

C -6.0238002 .0989470 .009930$

C $-5.6491392 .834883 \quad 1.305283$

O $-5.269993-0.0234660 .919807$

$\begin{array}{lllll}\text { O } & -5.039010 & 4.082748 & 0.939468\end{array}$

C -4.5796352 .0119882 .056810$

C -6.8895533 .1871402 .134366$

O $-4.2969322 .589153 \quad 3.329325$

C -4.9404540 .5193192 .204550$

C $-3.781277-0.3292152 .785815$

O $-6.043746 \quad 0.397422 \quad 3.110848$

C $-2.334025-1.233618-2.239446$

O $-1.458480-1.815282-2.860481$

C $-2.4318360 .233273-2.077871$

C $-1.4401041 .015502-2.528576$

C $-1.3643462 .472186-2.402391$

C $-2.4733233 .166540-1.656686$

C $-2.508768-0.3812861 .933966$

C - -1.4579890 .6505132 .302588$

C 0.7127981 .5707221 .491142

O $-1.485898 \quad 1.2998193 .344041$

C 0.4392332 .9147070 .891995

C $1.2434603 .494993-0.011838$

C $1.0277614 .874392-0.567570$

C $1.1883974 .936623-2.071234$

C $2.3350185 .365269-2.625358$

C $-0.0061964 .585874-2.928648$

C $-0.3004023 .111146-2.932140$

$\begin{array}{llll}N & -0.453624 & 0.721707 & 1.345477\end{array}$

H $-0.910578-4.470711-1.341519$

H $-2.833919-3.2885680 .727573$

H -2.982733 -3.688596 -2.357986

H $-5.122002-3.353197-0.190894$

H -4.804052 -4.816093 -1.077709

H -7.398132 -2.181181-2.946196

H $-6.428857-3.791553-4.267842$

H $-4.681815-3.843936-4.020413$

H $-5.715775-5.142100-3.382766$

H -8.112372 -1.567203 -0.694839

H $-6.579993-1.9806110 .015372$

H $-5.634022-0.090378-1.646707$

H -7.320104 $0.430399-1.686782$

H -7.2664420 .5308670 .860488$

H $-6.8548762 .607298-0.495004$

H -5.173696 2.165916 -0.680972

H -5.6756784 .5811890 .399128$

H -3.656159 2.0982091 .472693

H -7.5705893 .8209061 .553419$
H -6.6204943 .7739963 .019424$

H -7.4530902 .3092012 .456693$

H -5.0238862 .3529123 .930237$

H -3.566736 -0.020932 3.816090

H -4.146371 -1.363557 2.863040

H $-6.056928-0.5087493 .460839$

H $-3.2995690 .627394-1.563060$

H $-0.5934850 .546593-3.028729$

H -3.419491 $3.065168-2.199062$

H -2.301949 $4.234104-1.506590$

H $-2.5908592 .736939-0.656140$

H -2.030035 -1.356347 2.091436

H -2.746139 -0.3014920 .869836$

H 1.5457221 .0587051 .000982

H 0.9487511 .6822852 .554376

H -0.4358043 .4523981 .248956$

H $2.1322222 .964002-0.342093$

H $1.7598255 .540182-0.091513$

H $0.0423615 .268433-0.289853$

H $2.4508235 .439761-3.701384$

H $3.1871325 .644800-2.014452$

H $0.1743424 .881770-3.970816$

H $-0.8609545 .190902-2.613890$

H $0.4600502 .519199-3.443600$

H -0.5372240 .1755010 .493115$

C $0.852060-3.5577350 .792360$

O $1.689343-3.929464-0.322032$

C $2.989808-4.2646940 .174995$

C $3.116484-3.5542411 .510939$

C $1.709125-3.7150002 .054161$

H $2.974468-5.3472470 .367256$

C $4.081095-3.987882-0.859710$

H $3.877830-4.0042662 .154374$

H $3.352579-2.4936331 .387124$

C $1.359684-2.7212423 .149664$

H $1.604735-4.7323082 .454999$

C $4.476941-2.519360-1.121481$

O $5.257424-4.676962-0.409859$

H $3.791514-4.456835-1.808663$

H $5.034444-2.143090-0.256639$

O $5.419416-2.535299-2.206391$

C $3.339891-1.540966-1.469555$

C $3.813905-0.186273-2.037725$

H $2.719559-1.367951-0.584083$

O $2.504570-2.144065-2.462379$

H $4.246965-0.346226-3.033736$

H $2.9157250 .425437-2.200349$

H $2.033216-2.8373104 .004925$

H $1.440140-1.6883912 .794714$

H $0.334509-2.8755583 .501193$

C $-0.391107-4.4532360 .817277$

H $5.948194-4.538767-1.080448$

H $4.941655-2.819961-3.004546$

H $1.743737-1.554712-2.602905$

H $-0.940279-4.2992091 .753759$

H $-0.098355-5.5096010 .780409$

H $\quad 0.568877-2.5083620 .640276$

C 4.9079871 .6989071 .003104

O 4.1231850 .9898310 .033003

C $4.8089790 .582956-1.153544$

C $5.4046661 .799612-1.881371$

C $6.3146172 .599362-0.946708$

C 5.6242582 .9129270 .383460

H $4.1771102 .087043 \quad 1.724598$

C 5.8600050 .7545651 .754678

H $5.629605-0.087013-0.884541$

O $4.3766342 .660014-2.363097$

H $5.9845551 .455624-2.746709$

H $6.6026433 .533880-1.443606$

O $7.5013631 .846988-0.710865$

H $6.344663 \quad 3.3162401 .104577$

O $4.6442073 .932200 \quad 0.172213$

C $5.118603-0.1541012 .713937$

H 6.4293750 .1167661 .074060

H $6.558711 \quad 1.3474792 .356457$

H $3.9326102 .210991-3.101624$

H $8.0786792 .381237-0.139160$

H $5.1015614 .711113-0.187633$ 
O $6.029856-0.895416 \quad 3.411875$

O $3.903632-0.2075772 .843218$

C $5.481498-1.7823164 .391913$

H $4.854265-2.5371363 .910567$

H $6.310495-2.2852424 .896806$

H $4.907806-1.2200175 .134860$

SCF Energy (B3LYP/6-31G**//MMFF) $=-3245.90080648$

0800103

MM̄FF Geometry

C -1.099895 1.7683792 .558070

C -1.6503800 .5720842 .818096$

C $-0.956575-0.7672562 .912074$

O $0.476458-0.6094702 .811462$

C -1.453106-1.677382 1.773875

C $-1.146659-3.1468961 .969345$

C $-0.256284-3.8553821 .248449$

C $-1.974457-3.8398103 .023146$

C $0.645856-3.3781660 .144811$

C $2.108970-3.7154530 .456018$

C $3.042387-3.394509-0.718502$

C $4.481960-3.780324-0.370794$

C $5.460408-3.411133-1.493886$

O $2.965816-1.992834-1.000959$

O $6.792641-3.500674-0.966275$

C $5.228909-1.939463-1.889497$

C $5.389056-4.399525-2.663666$

O $6.012192-1.590974-3.029139$

C $3.741178-1.586679-2.137842$

C $3.601780-0.057619-2.302450$

O $3.299736-2.226813-3.335804$

C $1.250341-1.3457683 .650803$

O $0.859380-2.1549594 .482523$

C 2.695265-1.068559 3.477039

C $3.210603-0.2232092 .571073$

C 4.6366640 .0737012 .386413

C $5.622372-0.6306173 .281710$

C $2.1608210 .464188-2.385920$

C $2.1972321 .977065-2.482597$

C $2.7991363 .957223-1.092012$

O $2.1587532 .558293-3.564218$

C $4.2811573 .925071-0.883233$

C 4.8675704 .1496930 .302328

C 6.3404783 .9856540 .540940

C 6.6455472 .8578171 .505848

C 7.2082683 .1021372 .701108

C 6.3694531 .4372511 .056181

C 4.9919150 .9684651 .441000

N $2.3581802 .582137-1.246414$

H -0.0317591 .8523262 .383235$

H $-2.725154 \quad 0.5330512 .992304$

H -1.226810 -1.180842 3.890967

H $-1.046809-1.3171380 .823687$

H -2.542497 -1.589702 1.679285

H $-0.162819-4.919206 \quad 1.466217$

H -1.779602 -4.9168333 .064790$

H -1.761932 -3.431034 4.015181

H -3.041047 -3.7091612 .814138$

H $\quad 0.342645-3.880889-0.781517$

H $0.552752-2.304036-0.032252$

H $2.419889-3.1454521 .339117$

H $2.201035-4.7797260 .707720$

H $2.695485-3.957653-1.593053$

H $4.553846-4.849740-0.136208$

H $4.789412-3.2522470 .543026$

H $6.926867-4.408481-0.644062$

H $5.596122-1.317811-1.060543$

H $5.629654-5.413775-2.322844$

H $6.132713-4.157855-3.431070$

H $4.405047-4.435876-3.135526$

H $6.936588-1.812839-2.824137$

H $4.0917000 .433883-1.453371$

H $4.1351140 .266451-3.205853$

H $2.520038-1.760356-3.674923$

H $3.333763-1.6212484 .159705$

H $2.5348990 .306544 \quad 1.902031$

H $5.403042-0.4229024 .334946$

H $6.657601-0.3240113 .119475$
H $5.581365-1.7133593 .119453$

H $1.6393640 .081042-3.268526$

H $1.5767630 .168866-1.507159$

H $2.2743034 .377842-0.230329$

H $2.5510834 .543239-1.981895$

H $4.8926423 .646677-1.738486$

H 4.2547544 .4159721 .160481

H 6.7278134 .9388260 .922386

H $6.8759713 .799391-0.398539$

H 7.4519592 .2954113 .384505

H 7.4345524 .1126493 .024636

H 7.1492010 .7691691 .435404

H $6.4657761 .372588-0.035472$

H $4.197456 \quad 1.4198120 .850537$

H $2.3925751 .993114-0.419664$

C -2.0368293 .6628551 .119202$

O -2.5341862 .6950880 .170648$

C $-1.7165732 .763440-1.011900$

C -1.043796 $4.128130-0.957147$

C -0.7487194 .2358910 .526970$

H $-0.9451681 .991872-0.900691$

C $-2.5346252 .494047-2.278398$

H $-0.1507854 .181391-1.584767$

H -1.725694 $4.929520-1.265177$

C -0.4371555 .6498120 .986446$

H $\quad 0.105448 \quad 3.5899370 .762459$

C $-3.3405841 .178457-2.255937$

O $-3.4665313 .567757-2.459390$

H -1.869243 $2.518845-3.149835$

H $-4.1934561 .300797-1.580490$

O $-3.900597 \quad 0.990693-3.561767$

C $-2.545530-0.079197-1.865739$

C $-3.317568-1.403099-2.002994$

H $-2.1937390 .012429-0.832949$

O $-1.390232-0.209833-2.699651$

H $-3.445803-1.652451-3.064692$

H -2.659282 -2.185632 -1.601741

H $\quad 0.452377 \quad 6.0340020 .476821$

H $-1.265794 \quad 6.333367 \quad 0.773487$

H -0.2471525 .6753112 .063925$

C -1.9098603 .0383732 .512031$

H -3.973089 $3.378955-3.267909$

H $-3.1645590 .820650-4.174530$

H $-0.7990470 .538771-2.519248$

H -2.9211812 .8338932 .885802$

H -1.4683023 .7621753 .206706$

H -2.7987544 .4517541 .171298$

C $-5.707056-1.0129950 .859494$

O $-4.513838-1.0531780 .062992$

C $-4.683772-1.457001-1.296978$

C $-5.299569-2.864783-1.369850$

C $-6.599716-2.950202-0.565171$

C $-6.432992-2.3693200 .842268$

H $-5.352659-0.8444131 .884642$

C $-6.617010 \quad 0.1634310 .470523$

H $-5.351155-0.750971-1.801014$

O $-4.380799-3.839692-0.877016$

H -5.494724 -3.112047 -2.419712

H $-6.911855-3.998875-0.487428$

O $-7.632175-2.237802-1.239604$

H $-7.407376-2.2674961 .334510$

O $-5.658759-3.276647 \quad 1.632164$

C $-5.992416 \quad 1.5017320 .802365$

H $-6.8409310 .157211-0.599378$

H $-7.560826 \quad 0.1074821 .024472$

H $-4.123303-3.5777790 .023674$

H -7.740987 -2.639609 -2.118284

H $-6.140451-4.1206851 .665836$

O -6.6395302 .4816540 .104937$

O -5.0744571 .6726091 .591917$

C $-6.166576 \quad 3.8134920 .329182$

H $-6.8142274 .501063-0.221163$

H -6.2141774 .0639421 .393372$

H $-5.1454553 .916955-0.047238$

SCF Energy (B3LYP/6-31G**//MMFF) $=-3245.91450500$

0800104

MM̄MF Geometry 
C -2.1094314 .3276791 .435336$ C $-3.026102 \quad 3.640177 \quad 0.737711$ C -4.3129643 .1061131 .310163$ O -4.2327991 .6631861 .237060$ C $-5.504300 \quad 3.580256 \quad 0.459753$ C $-6.842026 \quad 3.046890 \quad 0.938100$ C $-7.562178 \quad 2.095798 \quad 0.308762$ C $-7.368961 \quad 3.675858 \quad 2.202256$ C $-7.206652 \quad 1.359749-0.953119$ C $-6.864886-0.107012-0.661630$ C $-6.095980-0.757670-1.821119$ C $-5.866193-2.244771-1.547078$ C $-4.973270-2.901051-2.610840$ O $-4.839531-0.079377-1.932708$ O $-4.546387-4.174963-2.103025$ C $-3.703015-2.044105-2.799841$ C $-5.745370-3.186667-3.904162$ O $-2.917876-2.530837-3.885258$ C -3.998749 -0.543661-2.997009 C $-2.7205860 .332055-2.989227$ O $-4.630678-0.353313-4.268647$ C -3.7652391 .0131492 .338082$ O -3.5012321 .5022423 .425135$ C $-3.581561-0.4107581 .979941$ C $-2.854797-1.2022132 .782915$ C $-2.564255-2.6222242 .571965$ C $-3.116780-3.2725991 .331821$ C $-1.9084020 .303035-1.689615$ C $-0.759734-0.692130-1.689201$ C $0.963292-1.653011-0.161160$ O $-0.360842-1.263336-2.699949$ C $0.526266-3.0010370 .316393$ C $0.958705-3.5518961 .460936$ C $0.583275-4.9302421 .922580$ C $0.041352-4.948898 \quad 3.334004$ C $0.838404-5.2374524 .376842$ C $-1.441416-4.7276043 .524139$ C -1.819796 -3.2717903 .491348$ $\mathrm{N}-0.201023-0.829151-0.425257$ H -2.283728 4.5513172 .484187 H -2.826506 $3.408175-0.306670$ H $-4.449615 \quad 3.4351292 .347383$ H $-5.330716 \quad 3.308609-0.585985$ H $-5.547762 \quad 4.6778770 .466246$ H $-8.513818 \quad 1.7969370 .746527$ H $-8.369715 \quad 3.3136362 .461036$ H -6.7105013 .4530973 .047418$ H -7.433907 4.7627432 .087063 H -8.064501 $1.402295-1.635697$ H $-6.386963 \quad 1.846043-1.485507$ H $-6.252582-0.1621940 .246549$ H -7.790141 -0.662053 -0.463533 H -6.668006 - $0.618675-2.745965$ H $-6.822244-2.776701-1.465563$ H -5.378726 -2.360773 - 0.570306 H $-5.340756-4.700760-1.906402$ H -3.096907 -2.170219-1.895196 H $-6.583133-3.866693-3.707469$ H $-5.112445-3.697221-4.638234$ H $-6.155202-2.286397-4.366543$ H $-2.735378-3.470319-3.712563$ H -2.098371 $0.100328-3.861971$ H -3.043161 $1.373713-3.132245$ H $-4.5051490 .570712-4.541104$ H -4.027806 -0.7621501 .057836$ H -2.426140 - 0.7700823 .686534 H $-4.210469-3.3056681 .373983$ H $-2.767168-4.295968 \quad 1.181047$ H -2.814033 -2.7171630 .438277$ H $-1.4487881 .290162-1.551548$ H -2.552971 $0.121317-0.825051$ H $1.568125-1.1283890 .584644$ H $1.554405-1.757988-1.076547$ H $-0.148047-3.563188-0.325266$ H $1.657067-2.9988442 .084349$ H $1.483779-5.5559441 .864150$ H $-0.142360-5.3968711 .244996$ H $\quad 0.450700-5.2835295 .389053$
H $1.897915-5.4279964 .241358$ H $-1.755408-5.1197734 .501142$ H $-1.995304-5.3301872 .798837$ H - $-1.455275-2.7110754 .353325$ H $-0.634074-0.3568660 .362816$ C $0.4245424 .091646 \quad 1.418175$ $\begin{array}{llllllll}\text { O } & 1.579624 & 4.580291 & 0.697298\end{array}$ C $2.1422073 .486714-0.051059$ C 1.8300762 .2657220 .789581 C 0.4202562 .5632501 .257998 H $1.6050213 .454957-1.007923$ C $3.6380323 .728377-0.284518$ H $1.897476 \quad 1.3322830 .224959$ H 2.5146902 .2043701 .643637 C 0.0663951 .8171142 .535778 H -0.2791612 .2646820 .467655$ C $4.3236212 .551699-1.008029$ O $3.7717274 .914082-1.071364$ H 4.1412133 .9214810 .670496 H $4.1423741 .643351-0.426075$ O $3.7046462 .356212-2.280308$

C $5.8481562 .695685-1.190902$

C $6.4931831 .552319-1.998529$

H $6.3250662 .757569-0.205600$

O $6.1684203 .901216-1.887340$

H $6.1603411 .604910-3.043333$

H $7.5729551 .752613-2.024584$

H -0.9455372 .0613862 .865610$

H $\quad 0.1214730 .7348492 .381446$

H 0.7502602 .0749953 .351296

C $-0.813103 \quad 4.791546 \quad 0.839046$

H $3.3188265 .631108-0.595024$

H $3.8664783 .158151-2.806658$

H $5.8554774 .646343-1.347306$

H $-0.8196204 .669877-0.252575$

H -0.7188235 .8733660 .998740$

H 0.5596354 .3838322 .466463

C $6.538971-1.1429980 .593627$

$\begin{array}{lllll}\text { O } & 6.733929 & 0.095778 & -0.103153\end{array}$

C $6.2400530 .141146-1.444502$

C $6.910185-0.940918-2.305968$

C $6.754707-2.326997-1.671738$

C $7.145739-2.320696-0.191633$

H $7.112264-1.0327511 .523329$

C $5.065871-1.3623800 .977013$

H $5.161476-0.040685-1.441652$

O $8.295187-0.652863-2.486396$

H $6.451047-0.934521-3.301470$

H $7.386791-3.046486-2.206728$

O $5.402099-2.759698-1.786959$

H $6.858668-3.269756 \quad 0.276073$

O $8.571704-2.232140-0.114373$

C $4.635144-0.4391572 .098469$

H $4.377528-1.2014770 .144166$

H $4.945377-2.3916731 .336494$

H $8.702413-0.585865-1.605808$

H $5.181709-2.785768-2.733695$

H $8.820200-2.300970 \quad 0.823217$

O $3.566914-0.9922902 .744414$

$\begin{array}{lllll}\text { O } & 5.156140 & 0.635566 & 2.363498\end{array}$

C $3.054765-0.2551803 .858924$

H $3.718261-0.3855254 .718554$

H $2.9465580 .805776 \quad 3.617704$

H $2.069441-0.6579574 .107906$

SCF Energy (B3LYP/6-31G**//MMFF) $=-3245.91580805$

08_00105

MM̄FF Geometry

C $-0.886494-4.018799-1.211986$

C $-2.108175-3.629751-0.818002$

C $-3.274228-3.413295-1.747634$

O $-3.611979-2.009069-1.673018$

C $-4.485023-4.238118-1.276943$

C $-5.715960-4.061088-2.147272$

C $-6.829064-3.392350-1.782511$

C $-5.645775-4.720056-3.501189$

C $-7.088049-2.682119-0.483007$

C $-7.058914-1.159410-0.667320$ 
C $-6.848297-0.4248190 .664858$ C $-6.928671 \quad 1.0891920 .463927$ C -6.5695381 .8683151 .737889$ O $-5.549986-0.7873381 .146988$ O C $-5.233377 \quad 1.3303972 .297317$ C $-7.729574 \quad 1.8704162 .740087$ O $-4.9386961 .908306 \quad 3.566324$ C -5.193015 -0.207452 2.407744 C $-3.791164-0.7509942 .781520$ O $-6.095715-0.626693 \quad 3.438417$ C $-3.054507-1.187793-2.604320$ O $-2.383036-1.516243-3.569514$ C $-3.3594230 .206668-2.215567$ C $-2.6626091 .209683-2.769849$ C $-2.8147722 .637046-2.479602$ C $-3.8484043 .037236-1.460788$ C $-2.678166-0.4493861 .771848$ C -1.8592170 .7897472 .090968$ C -0.0624112 .2184021 .114514$ O C -0.6656483 .4715570 .565305$ C $-0.0790484 .214579-0.385896$ C $-0.6363545 .512484-0.896344$ C $-0.7496585 .552182-2.403771$ C $0.2079426 .123380-3.153794$ C $-2.0153165 .014992-3.031600$ C $-2.0212293 .514613-3.128899$ $\mathrm{N}-1.0238231 .1358221 .037006$ H - $0.696765-4.215330-2.263895$ H $-2.281219-3.424580 \quad 0.236475$ H -3.014739 -3.696864 -2.774768 H -4.706542 -3.985337 -0.235539 H -4.222410 -5.304694 -1.264561 H -7.651802 -3.334757 -2.494101 H $-6.587706-4.636088-4.053816$ H -4.863949 -4.261092 -4.113866 H -5.424098 $-5.786931-3.393197$ H $-8.075954-2.982096-0.111947$ H $-6.383146-2.9920190 .291009$ H $-6.244722-0.892449-1.352165$ H -7.996104 -0.833353 -1.135049 H $-7.609042-0.761840 \quad 1.378691$ H $-7.922437 \quad 1.3808620 .101703$ H $-6.2280811 .384362-0.328318$ H -6.1890593 .7573112 .139457$ H -4.4447081 .6589401 .610662$ H -8.6088842 .3587742 .303220$ H -7.479299 2.4497233 .635691 H -8.028542 0.8674473 .051751 H -4.848403 2.8676593 .440290 H -3.518971 $-0.431593 \quad 3.794647$ H -3.874038 -1.846202 2.836203 H -5.829946 -1.510700 3.741255 H -4.123772 $0.367129-1.465148$ H -1.897091 $0.964378-3.505252$ H -4.853804 2.798875 -1.823856 H -3.836489 4.101839-1.218909 H $-3.6804072 .511566-0.515138$ H -1.968354 -1.286630 1.783471 H $-3.076180-0.3752200 .756059$ H $\quad 0.8215351 .9056330 .550714$ H 0.2270152 .3748412 .158605 H -1.607781 3.8025790 .995711 H $0.8795433 .896482-0.790445$ H $0.0295036 .315989-0.554529$ H -1.614156 $5.732226-0.449976$ H $0.1199336 .183831-4.233528$ H $1.1043106 .538084-2.704608$ H -2.121139 $5.404008-4.053555$ H $-2.8823705 .413036-2.497054$ H -1.294437 $3.125629-3.843564$ H $-1.092420 \quad 0.6265520 .160887$ C $1.434281-3.236373-0.516703$ O $2.464752-3.550747 \quad 0.447324$ C $2.763869-2.3693551 .214987$ C $2.355322-1.2132050 .324569$ C $1.101209-1.750106-0.334572$
H $2.133023-2.4165802 .111697$

C $4.248207-2.3786561 .596051$

H $2.176516-0.2908380 .884432$

H $3.128112-1.015056-0.428385$

C $0.783786-1.020282-1.631088$

H $\quad 0.260152-1.6337040 .360511$

C $4.710824-1.0878952 .293395$

O $4.478956-3.4806332 .478996$

H $4.846979-2.5671490 .698730$

H $4.542033-0.224247 \quad 1.646294$

O $3.885620-0.8625923 .445443$

C $6.177217-1.0937902 .761806$

C $7.234837-1.4219151 .692735$

H $6.292016-1.8069453 .587288$

O 6.4872310 .1929703 .308660

H $8.206655-1.4734282 .201829$

H $7.033509-2.4201161 .288777$

H $-0.119115-1.418224-2.097059$

H $0.6250660 .047044-1.446125$

H $1.601404-1.114555-2.353940$

C $0.263072-4.194283-0.265464$

H $4.174433-4.2845802 .023935$

H $3.970234-1.6410644 .022644$

H 5.8175210 .3893893 .986194

H $-0.066854-4.0961310 .777502$

H $0.625936-5.227052-0.348428$

H $1.865318-3.426119-1.507245$

C $6.1137500 .506731-1.327844$

O $6.174375-0.422578-0.238058$

C $7.368307-0.3945140 .546293$

C $8.588358-0.703834-0.339498$

C $8.6532190 .234105-1.549712$

C $7.3075380 .317232-2.277634$

H $5.2041010 .231428-1.877109$

C $5.9511121 .953027-0.841564$

H 7.4880500 .6001430 .987526

O $8.519674-2.056144-0.788956$

H $9.497655-0.6039160 .265256$

H $9.418255-0.123041-2.249810$

O $9.032221 \quad 1.540251-1.126325$

H $7.318438 \quad 1.128717-3.014835$

O $7.090645-0.899520-2.997913$

C $4.6229562 .150624-0.146510$

H $6.7533162 .251474-0.161021$

H $5.9752952 .653689-1.684393$

H $9.316904-2.231009-1.317363$

H $9.8986921 .468695-0.691039$

H $7.818481-0.996673-3.635239$

O $4.8257452 .601101 \quad 1.124777$

O $3.5371441 .936202-0.669674$

C 3.6424262 .8361581 .893992

H 3.9445763 .2035242 .878285

H 3.0205693 .5959571 .411233

H 3.0841061 .9046972 .024599

SCF Energy (B3LYP/6-31G**//MMFF) $=-3245.90923750$

0800106

MM̄MF Geometry

C $1.391923-1.715754-1.442053$

C $2.167407-2.771756-1.155171$

C $2.867220-2.9989550 .166877$

O $2.350198-2.0895571 .167925$

C $4.374124-2.7394650 .028852$

C $5.160051-3.2562581 .216994$

C $5.630392-2.4898632 .220038$

C $5.446141-4.7362871 .202048$

C $5.450678-1.0103742 .420610$

C $6.765918-0.2387762 .241934$

C $7.211931-0.1365950 .774833$

C 8.5970910 .5044510 .674483

C $9.0236900 .710042-0.786380$

O $6.2512520 .669450 \quad 0.085108$

O $10.166370 \quad 1.579043-0.794494$

C $7.8921711 .442938-1.535317$

C $9.470336-0.600954-1.444260$

O $8.177979 \quad 1.531794-2.929758$

C $6.4934410 .816031-1.319855$

C $5.4107631 .762763-1.890779$ 
O $6.430632-0.437954-2.000122$

C $1.351176-2.5271331 .975012$

O $0.920668-3.6698122 .051816$

C $0.782700-1.4254082 .787023$

C $0.978105-0.1243892 .517179$

C $0.379790 \quad 1.006660 \quad 3.233676$

C $-0.5205830 .695367 \quad 4.401096$

C $3.9666591 .354060-1.565831$

C $2.9721172 .410042-2.020915$

C $0.5751213 .049422-1.782867$

O $3.2868353 .374567-2.713454$

C $0.4602593 .946597-0.590392$

C -0.6515344 .0572200 .151200$

C $-0.763616 \quad 4.928432 \quad 1.367723$

C $-1.037373 \quad 4.1325182 .625241$

C -2.2872824 .0084653 .103157$

C $0.1445903 .560852 \quad 3.377931$

C 0.6378772 .2596172 .801472

N $1.6872222 .141001-1.569822$

H $1.279410-0.925527-0.705511$

H $2.290792-3.553333-1.901352$

H $2.687323-4.0369940 .470170$

H $4.550879-1.667231-0.108890$

H $4.765577-3.222020-0.876658$

H $6.203410-2.9761273 .008978$

H $6.056101-5.0511942 .055421$

H $4.515005-5.3100151 .235127$

H $5.990574-5.0078860 .291635$

H $4.670056-0.5928291 .778207$

H $5.097556-0.8617213 .448955$

H 6.6132110 .7730072 .639603

H $7.554265-0.7084182 .843428$

H $7.230088-1.1384290 .332723$

H $9.343465-0.0946301 .211023$

H $8.583734 \quad 1.4837941 .173211$

H $10.865038 \quad 1.159947-0.263295$

H $7.8688502 .475249-1.158449$

H $10.315571-1.035140-0.896909$

H $9.831645-0.427856-2.463981$

H $8.680261-1.353711-1.481484$

H $9.0548001 .942881-3.019993$

H $5.5701852 .769212-1.481012$

H $5.5165521 .834634-2.980802$

H $5.504554-0.645003-2.199696$

H $\quad 0.130257-1.7500873 .591248$

H $1.6143180 .149671 \quad 1.677303$

H $\quad 0.033430 \quad 0.1629915 .182112$

H -1.364691 0.0742394 .081346

H $-0.952744 \quad 1.5822864 .868685$

H $3.7092890 .415338-2.066760$

H $3.8445241 .215229-0.485644$

H $-0.3209842 .438888-1.931775$

H $0.7500643 .645860-2.683627$

H $1.3400624 .524969-0.317402$

H $-1.5383903 .496251-0.132678$

H $-1.575357 \quad 5.6463791 .191620$

H $0.137616 \quad 5.537596 \quad 1.509529$

H -2.490962 3.4662244 .020252

H -3.1359914 .4399652 .582843$

H -0.0944993 .4549244 .441865$

H 0.9699204 .2843923 .361631

H 1.2933832 .3853401 .939474

H $1.5407401 .361648-0.935478$

C $-0.890244-1.442229-2.492114$

O $-1.189012-0.436641-1.498937$

C $-1.966480-1.054478-0.451038$

C $-2.658945-2.223025-1.127197$

C $-1.541694-2.743842-2.011557$

H $-1.245606-1.4145100 .293801$

C -2.886875 -0.0058090 .175244$

H -3.021924 -2.972541-0.418527

H -3.503765 -1.876271-1.735020

C $-2.025862-3.636656-3.141082$

H $-0.847022-3.319636-1.387617$

C -3.897482 -0.5826641 .178304$

O -2.0645730 .9439230 .859675$

H -3.401621 $0.544746-0.618942$

H $-4.574063-1.2752610 .671159$
O $-3.176953-1.3726662 .135812$

C -4.7052250 .4713351 .956421$

C $-5.439221 \quad 1.5313201 .114414$

H $-4.034576 \quad 0.9831112 .658120$

O $-5.659839-0.2264502 .764331$

H $-5.8769762 .257528 \quad 1.812294$

H $-4.7059402 .075454 \quad 0.508794$

H -2.522476 $-4.527042-2.741568$

H $-2.740495-3.115506-3.786966$

H $-1.186607-3.965832-3.761922$

C $0.626246-1.518416-2.719435$

H -1.3613591 .2159320 .245779$

H -3.829389 -1.7392982 .757145$

H -6.1284750 .4354863 .300966$

H $0.965794-0.569317-3.152503$

H $0.855784-2.305362-3.446776$

H $-1.363290-1.102046-3.421701$

C $-6.950464-0.430432-1.705244$

O $-6.0178120 .185683-0.807756$

C $-6.581726 \quad 0.9969090 .224141$

C $-7.3900072 .149141-0.397911$

C $-8.4411221 .631888-1.385611$

C $-7.8469490 .624285-2.375377$

H $-6.325773-0.881187-2.487386$

C $-7.740756-1.556829-1.026461$

H -7.2458250 .3859840 .843908$

$\begin{array}{lllll}\text { O } & -6.520345 & 3.059810 & -1.069252\end{array}$

H -7.8814682 .7108320 .404973$

H -8.864134 $2.477071-1.942509$

O $-9.503657 \quad 1.005931-0.672991$

H $-8.6417390 .122329-2.939488$

O $-7.038948 \quad 1.327699-3.323966$

C $-6.828251-2.691352-0.617874$

H $-8.283793-1.205016-0.145063$

H -8.486594 -1.978335 -1.710356

H $-6.0186852 .556682-1.733590$

H $-9.8889571 .670138-0.076389$

H $-7.6156301 .956742-3.790334$

O $-6.943456-2.9228940 .721180$

O $-6.103688-3.301050-1.393881$

C $-6.128196-3.979467 \quad 1.237949$

H $-5.071740-3.7859711 .029874$

H $-6.269219-4.0181882 .321223$

H -6.437286 -4.9366510 .807718$

SCF Energy (B3LYP/6-31G**//MMFF)= -3245.91814997

0800107

MM̄FF Geometry

C $0.928814-2.7572982 .351817$

C $-0.126777-2.2130842 .976091$

C $-0.310418-0.7730353 .396642$

O $-1.460209-0.2548642 .681724$

$\begin{array}{llll}C & 0.874926 & 0.166686 & 3.109843\end{array}$

C $0.689081 \quad 1.5454853 .715800$

C $0.3194372 .642693 \quad 3.026414$

C $1.001497 \quad 1.648517 \quad 5.186764$

C $-0.0276302 .727542 \quad 1.566807$

C $-1.274618 \quad 3.589483 \quad 1.326621$

C $-1.5996623 .700001-0.170519$

C $-2.7050254 .729338-0.408820$

C $-3.0970894 .807724-1.891249$

O $-2.0254552 .413659-0.634130$

O $-4.3235345 .548019-1.984749$

C $-3.387923 \quad 3.382985-2.402871$

C $-2.0609415 .575966-2.720045$

O $-3.6153493 .382771-3.810618$

C $-2.2894922 .352804-2.043011$

C $-2.7935180 .930774-2.380967$

O $-1.1163162 .624071-2.811199$

C -2.684284 -0.4187693 .251025$

O $-2.935442-0.948072 \quad 4.321829$

C -3.6877910 .1332212 .315224$

C $-4.973099-0.2226012 .456380$

C $-6.0809570 .177726 \quad 1.587922$

C $-5.822347 \quad 1.2663390 .582008$

C $-1.910690-0.208041-1.856241$

C $-2.623621-1.531886-2.048902$

C $-4.491190-2.895820-1.102135$ 
O $-2.393819-2.252723-3.016784$

C $-5.863201-2.356660-1.367838$

C $-6.945495-2.685090-0.645524$

C -8.320057 -2.136599-0.910003

C $-8.997272-1.6045350 .336153$

C $-10.041113-2.2507730 .883062$

C $-8.515253-0.2851570 .906110$

C $-7.265728-0.452346 \quad 1.729120$

N $-3.561805-1.781409-1.058847$

H $1.782639-2.1435882 .081070$

H $-0.955527-2.8704793 .235634$

H $-0.509850-0.7680354 .475655$

H 1.0357730 .2451882 .028962

H $1.801835-0.2566723 .518489$

H $\quad 0.2662443 .5888643 .563541$

H 0.9011522 .6721785 .562945

H 0.3240721 .0172535 .769938

H 2.0302021 .3271315 .379850

H 0.8232693 .1773261 .042725

H -0.1943691 .7373871 .135022$

H -2.1252903 .1462181 .859894$

H $-1.1086404 .591581 \quad 1.741467$

H $-0.6865903 .992355-0.702422$

H $-2.4042715 .718032-0.040204$

H $-3.595217 \quad 4.4500640 .172390$

H $-4.1721706 .428340-1.599943$

H -4.329763 $3.058564-1.937953$

H -1.946561 $6.597558-2.338210$

H $-2.3868445 .680575-3.760806$

H -1.073905 $5.108872-2.713218$

H -4.318893 $4.029726-3.990690$

H -3.799143 $0.803166-1.965834$

H -2.889117 $0.817862-3.468954$

H $-0.6152041 .801172-2.925270$

H $-3.3380370 .775299 \quad 1.515341$

H $-5.237254-0.9069843 .261894$

H $-5.1606370 .903182-0.207395$

H -5.3540952 .1345001 .059996$

H $-6.729493 \quad 1.6412270 .103458$

H $-0.956454-0.251717-2.391136$

H -1.679086 $-0.085051-0.792178$

H -4.428898 -3.408342 -0.137059

H -4.204815 -3.593508-1.894773

H -5.971702 -1.663972 -2.199665

H $-6.843885-3.3992690 .168415$

H -8.919593 -2.945609 -1.347296

H -8.291924 -1.341252 -1.665449

H $-10.547394-1.8642901 .761433$

H -10.409833 -3.183029 0.468390

H $-9.2855340 .147927 \quad 1.557470$

H $-8.395450 \quad 0.4222010 .081260$

H -7.357525 -1.206794 2.512265

H -3.674861 -1.107504 -0.307775

C $1.224110-4.5120590 .526431$

O $2.463226-3.942046 \quad 0.064924$

C $2.253803-3.401787-1.254229$

C $0.909289-3.945181-1.726068$

C $0.138716-3.999403-0.419200$

H $2.161259-2.316756-1.134794$

C $3.447905-3.720521-2.159860$

H $0.441061-3.300053-2.475485$

H $1.002988-4.950058-2.153710$

C -1.095129-4.882643 -0.471626

H $-0.162141-2.977754-0.155030$

C $4.812392-3.297130-1.574324$

O $3.490553-5.137399-2.373310$

H $3.293919-3.266899-3.145957$

H $5.057739-3.951534-0.728636$

O $5.795238-3.553744-2.584666$

C $4.895808-1.821968-1.135877$

C $6.275406-1.453127-0.556788$

H $4.140169-1.623317-0.368549$

O $4.627321-0.970247-2.248001$

H $\quad 6.596815-2.2361370 .139966$

H $7.002018-1.394414-1.376676$

H - $1.789423-4.530885-1.240286$

H -0.836646 -5.920719 -0.706253

H - $-1.619867-4.8741930 .488961$
C $1.027270-4.2209432 .015552$

H $4.250098-5.319698-2.952967$

H $6.671359-3.491575-2.168568$

H $3.705957-1.112934-2.519952$

H $1.889969-4.6165052 .566322$

H $\quad 0.144035-4.7618082 .375653$

H $1.333924-5.5985110 .408845$

C $5.8851782 .257992-0.227270$

O $6.011246 \quad 0.935128-0.764913$

C $6.250917-0.102590 \quad 0.189339$

C 7.5648670 .1635410 .939010

C 7.5663431 .5548421 .580102

C 7.1298752 .6415530 .590063

H $5.8496242 .912505-1.108026$

C 4.5697882 .4372270 .539355

H $5.425666-0.1315520 .909382$

O 8.6752690 .0456810 .051789

H $7.691756-0.5998321 .715563$

H $8.5753601 .774967 \quad 1.949425$

O 6.6864391 .5390762 .700500

H 6.9463523 .5892111 .109599

O $8.1934712 .873238-0.338832$

C $3.3924052 .297211-0.396635$

H 4.4508601 .7283621 .363568

H $4.510223 \quad 3.437490 \quad 0.984348$

H $8.5343430 .664984-0.684794$

H 6.7207762 .4189293 .112868

H $8.968353 \quad 3.1666230 .170132$

O $2.8196521 .069155-0.247818$

O $3.0425233 .167440-1.183680$

C $1.6995560 .799066-1.095957$

H $2.0464850 .590375-2.111425$

H $1.184944-0.083374-0.707213$

H $0.9964121 .636125-1.098374$

SCF Energy $\left(B 3 L Y P / 6-31 G^{* *} / / M M F F\right)=-3245.90580315$

08_00108

MM̄FF Geometry

C -0.345205 -2.257299 2.911010

C $-0.393683-1.1119652 .216365$

C -0.5775040 .2368152 .865262$

$\begin{array}{llll}\text { O } & -1.762839 & 0.845424 & 2.305639\end{array}$

C 0.6012291 .1557782 .511589

C $0.7494662 .330566 \quad 3.455197$

C 0.2567673 .5656483 .238315

C 1.5656002 .0495984 .690889

C -0.6178344 .0374162 .109900$

C 0.1216614 .9281931 .102140

C 0.9236324 .1216090 .070029

C $1.6976105 .050002-0.866066$

C $2.4380304 .260898-1.956339$

O $\quad-0.0095703 .347984-0.691437$

O $2.8653725 .192061-2.963096$

C $1.4489683 .288650-2.637386$

C $3.7113703 .603999-1.410357$

O $2.1278652 .412454-3.533411$

C $0.5880222 .465942-1.648409$

C $-0.5550981 .753233-2.403311$

O $1.3477191 .438998-1.012796$

C -2.9746280 .4638842 .782550$

O $-3.204464-0.331973 \quad 3.677246$

C -3.9865861 .1721031 .969398$

C $-5.2497380 .725452 \quad 1.940813$

C $-6.331807 \quad 1.2718001 .119170$

C -6.0224142 .4780280 .270087$

C $-1.5268670 .998766-1.483502$

C $-2.5627370 .215869-2.274296$

C $-4.531506-1.282377-1.959263$

O $-2.572374 \quad 0.166741-3.501776$

C $-5.786985-0.482827-2.118952$

C $-6.994333-0.923816-1.731465$

C $-8.269889-0.150407-1.920166$

C $-9.107870-0.078436-0.661156$

C $-10.164983-0.889855-0.488213$

C -8.7683880 .9862540 .359118$

C -7.5291090 .6499601 .139048$

N -3.468909 -0.434715 -1.447205

H $-0.419016-2.1932453 .995917$ 
H $-0.304562-1.1206751 .135267$ H -0.6876880 .1498603 .953447$ H $0.4880091 .518007 \quad 1.485205$ H 1.5427230 .5927562 .526556 H 0.4692544 .3355393 .979640 H 1.6224352 .9152495 .359217 H 1.1271921 .2236375 .260059 H 2.5890351 .7773684 .414481 H -1.112629 $3.207621 \quad 1.599400$ H -1.427098 4.6262722 .561017 H -0.6348035 .5212720 .571458$ H $0.7770005 .633173 \quad 1.627978$ H 1.6125773 .4526880 .596474 H $2.3992185 .676822-0.301733$ H $0.9990935 .744409-1.353810$ H $3.4253895 .861754-2.534327$ H $\quad 0.7712923 .896807-3.252950$ H 4.407564 4.366360-1.040099 H $4.2480033 .068461-2.200590$ H $3.5190392 .911894-0.587884$ H $2.6360972 .965594-4.151062$ H -1.134804 2.489628 -2.975193 H $-0.1294091 .042751-3.123082$ H $2.2536981 .455526-1.358959$ H -3.648770 2.0124041 .372994 H $-5.509091-0.1412432 .547387$ H -5.7335113 .3257020 .901562$ H $-6.8624892 .812515-0.341098$ H $-5.2027112 .263026-0.424476$ H $-0.9800510 .289563-0.853384$ H -2.053199 $1.705363-0.831986$ H -4.656573 -2.108198-1.251939 H $-4.230377-1.691494-2.928960$ H $-5.7059350 .489673-2.598653$ H -7.077542 -1.911866 -1.283848 H $-8.840877-0.643757-2.717777$ H $-8.0716280 .866269-2.281677$ H $-10.786791-0.8276560 .398633$ H -10.429214 -1.638388 -1.227879 H -9.5881691 .0954701 .081665$ H $-8.7097571 .954844-0.144353$ H -7.649644 -0.2215661 .784349$ H -3.407101 $-0.304937-0.442031$ C $-0.441654-3.9822710 .915880$ O $0.521769-3.3507660 .057153$ C $-0.095093-3.149223-1.234486$ C -1.498724 -3.750138-1.147995 C $-1.806542-3.6279430 .334052$ H $-0.195660-2.066791-1.366658$ C $0.794293-3.732281-2.341816$ H -2.211894 -3.217286-1.782974 H -1.516926 -4.802420 -1.450675 C $-2.937500-4.5277920 .798304$ H -2.071429 -2.5862140 .545607$ C $2.221864-3.146160-2.366019$ O $0.938844-5.144385-2.133409$ H $\quad 0.320262-3.605987-3.322457$ H $2.737254-3.442790-1.445843$ O $2.914272-3.786028-3.446128$ C $2.286960-1.617176-2.560687$ C $3.716689-1.046742-2.640633$ H $1.756716-1.112240-1.748111$ O $1.631254-1.259118-3.779774$ H $4.282949-1.553069-3.430834$ H $3.636544 \quad 0.000742-2.961657$ H $-3.865107-4.2819920 .271111$ H -2.714440 -5.5835720 .611715$ H $-3.117011-4.4050181 .870980$ C $-0.188792-3.6599432 .391260$ H $0.137442-5.577553-2.471235$ H $3.867260-3.670190-3.295160$ H $0.677742-1.401332-3.656571$ H $0.834078-3.9714082 .639155$ H $-0.857831-4.2941042 .987038$ H $-0.274926-5.063028 \quad 0.798713$ C $4.311994-0.2262660 .938688$ O $3.806120-0.223502-0.402048$ C $4.470524-1.112809-1.305394$
C $5.942248-0.701639-1.447771$

C $6.637071-0.665427-0.081980$

C 5.8163160 .1011130 .963709

H 3.7818400 .6018041 .426279

C $3.912907-1.5268711 .651100$

H $4.422556-2.133358-0.912826$

O $6.044150 \quad 0.578310-2.067421$

H $\quad 6.449371-1.417710-2.104661$

H $7.619972-0.190082-0.187542$

O $6.848453-1.9954560 .382593$

H $6.217043-0.0738401 .968164$

O $5.9425391 .503450 \quad 0.701027$

C $3.804077-1.3623913 .150888$

H $2.927566-1.8379001 .280548$

H $4.612451-2.3405931 .444443$

H $5.5582231 .213689-1.514668$

H $7.401763-2.451805-0.274031$

H $6.883903 \quad 1.7330830 .783849$

O $3.115463-2.4235653 .665266$

O $4.271333-0.4337373 .795285$

C $2.906459-2.3935185 .080501$

H $2.339030-1.5008225 .360878$

H $2.326480-3.2780235 .356835$

H $3.865980-2.4235935 .605143$

SCF Energy (B3LYP/6-31G**//MMFF)= -3245.91040764

08_00109

MM̄MF Geometry

C - $0.629545-2.5382221 .488746$

C $0.002221-1.6431992 .263493$

C $-0.550582-0.3568372 .824698$

O $-1.987859-0.2834672 .684102$

C 0.0534090 .8618972 .106408

C 0.1469142 .0686063 .020128

C -0.7254193 .0946823 .044898$

C 1.3571312 .0885293 .920600

C -1.9756723 .2742812 .229163$

C -1.8492204 .4380181 .232968$

C $-0.898652 \quad 4.122240 \quad 0.069417$

C $-0.6779575 .345904-0.820060$

C $0.2065935 .008886-2.032136$

O $-1.4928563 .071217-0.698533$

O $0.1103546 .096213-2.964555$

C $-0.3628503 .758107-2.734978$

C $1.6874344 .895354-1.648636$

O $0.5074543 .313957-3.773483$

C $-0.6724442 .590534-1.769143$

C $-1.4561081 .485670-2.515132$

O $0.5519192 .045131-1.281201$

C $-2.741605-0.6945723 .735867$

O $-2.330182-1.1675584 .787381$

C $-4.189094-0.491313 \quad 3.498115$

C $-4.724086-0.0868732 .335368$

C $-6.153616 \quad 0.116207 \quad 2.076527$

C $-7.104218-0.0392723 .235635$

C $-2.0382250 .398886-1.600488$

C $-2.896001-0.585335-2.376948$

C $-4.609141-2.360090-2.017160$

O $-2.915664-0.639069-3.603715$

C $-5.926906-1.659901-2.115918$

C $-6.993818-1.962644-1.361865$

C $-8.290878-1.209684-1.429611$

C $-8.675570-0.592413-0.101875$

C $-9.683465-1.1065460 .623301$

C $-7.9613290 .668160 \quad 0.340887$

C -6.5553550 .4231420 .824877$

N -3.627485 -1.408124 -1.531671

H -1.654064 -2.359423 1.175690

H $1.026774-1.8556862 .561992$

H $-0.284054-0.3305513 .888709$

H -0.5367311 .0995091 .215173$

H 1.0660470 .6415951 .741780

H -0.5312553 .9119393 .739043$

H 1.3961172 .9858854 .547255

H 1.3574661 .2213674 .587911

H 2.2733632 .0657383 .321596

H -2.269151 2.3571491 .710380

H -2.7936493 .4981292 .925334$ 
H $-2.850503 \quad 4.645414 \quad 0.833763$ H -1.514362 5.3406701 .758799 H $0.059941 \quad 3.7816220 .474784$ H - $-0.2425256 .172792-0.245548$ H -1.647075 $5.711658-1.187258$ H $0.4008316 .906113-2.511085$ H -1.300130 $4.058641-3.224290$ H $2.0431255 .836838-1.212927$ H $2.3116704 .719916-2.531874$ H $1.8851104 .101996-0.924777$ H $0.6667194 .071731-4.361844$ H -2.294631 $1.945364-3.055135$ H $-0.8087501 .010098-3.262973$ H $\quad 0.4000351 .128619-1.003602$ H -4.811533 -0.7132394 .359798$ H $-4.066705 \quad 0.106778 \quad 1.490070$ H -7.085791-1.067637 3.613081 H -8.1417990 .1847042 .980118$ H -6.8324950 .6410424 .050568$ H -1.240617 -0.167124-1.109180 H -2.663396 $0.855101-0.824443$ H -4.632597 -3.196145 -1.311347 H -4.308544 -2.736114 -2.999992 H $-5.997686-0.844860-2.832861$ H -6.930085 -2.780494 -0.647921 H $-9.066129-1.911249-1.763883$ H -8.256970 -0.421211-2.191994 H -9.996186 -0.655632 1.558960 H -10.219731 $-1.991940 \quad 0.298151$ H $-8.540404 \quad 1.1983801 .106583$ H -7.921642 $1.370298-0.502209$ H $-5.799410 \quad 0.5192530 .045372$ H -3.587858 -1.245574 -0.529643 C $0.408663-3.916920-0.408898$ O $1.431535-2.946578-0.709529$ C $1.201901-2.441855-2.039893$ C $0.127771-3.333683-2.656969$ C $-0.696110-3.695561-1.436671$ H $0.775859-1.440092-1.917985$ C $2.513173-2.357950-2.833654$ H $-0.449638-2.813479-3.427345$ H $0.550759-4.238743-3.105756$ C $-1.606287-4.892168-1.643600$ H -1.302478 -2.826998 -1.163262 C $3.594954-1.460443-2.193325$ O $3.086541-3.666339-2.949542$ H $2.309467-2.014283-3.855215$ H $3.984351-1.951507-1.295776$ O $4.696715-1.387367-3.108278$ C $3.139483-0.032165-1.851142$ C $4.2573280 .930119-1.415039$ H $2.384476-0.066711-1.058530$ O $2.536296 \quad 0.570208-3.001517$ H $4.8928831 .179844-2.274999$ H $3.7611671 .871814-1.143497$ H -2.320171 -4.697280 -2.450567 H -1.036782 -5.789240 -1.908494 H -2.175846 $-5.109733-0.734645$ C $-0.000981-3.8392241 .063375$ H $2.585006-4.151971-3.625287$ H $4.390587-0.909355-3.898173$ H $1.678630 \quad 0.140807-3.154005$ H $0.887079-4.0304511 .678882$ H - $0.708445-4.6460071 .289489$ H $0.871663-4.899477-0.573630$ C $4.971431-0.4681971 .987200$ $\begin{array}{lllll}\text { O } & 4.295040 & 0.052745 & 0.832676\end{array}$ C $5.1396010 .450412-0.250596$ C $6.1196481 .549596 \quad 0.199168$ C $6.909576 \quad 1.1243881 .440912$ C 5.9937550 .5484502 .525761 H $4.185856-0.5819922 .745255$ C $5.587678-1.8531711 .725564$ H $5.710195-0.416511-0.598468$ O $5.3954622 .743780 \quad 0.495009$ H $6.801871 \quad 1.774631-0.629362$ H 7.4451041 .9892811 .851229 O $7.8788890 .144968 \quad 1.081428$
H $\quad 6.594502 \quad 0.079679 \quad 3.313387$

O $\quad 5.278464 \quad 1.620777 \quad 3.146317$

C $4.538618-2.9072831 .452684$

H $6.264542-1.8344540 .867570$

H $\quad 6.150680-2.1818542 .606593$

H 6.0438053 .4297760 .728720

H 8.4665650 .5428190 .416664

H 4.6871512 .0090592 .478832

O $5.143183-4.0159690 .932632$

O $3.344402-2.7852801 .682326$

C $4.269024-5.1050520 .620375$

H $3.604212-4.826385-0.201751$

H $4.882214-5.9528780 .303366$

H $3.691827-5.4008361 .501765$

SCF Energy (B3LYP/6-31G**//MMFF) $=-3245.90419569$

0800110

MM̄FF Geometry

C $2.724783-2.220468-2.450279$

C $1.942942-1.527097-1.608779$

C $0.548302-1.929151-1.211974$

O $-0.281526-0.778429-1.520108$

C $0.428240-2.2218180 .298251$

C $0.677768-3.6692950 .681487$

C $-0.265333-4.5097301 .154233$

C $2.096343-4.1566210 .545488$

C -1.713709-4.205698 1.411287

C $-2.589351-4.6066180 .219199$

C $-4.024208-4.0771030 .353158$

C $-4.864882-4.469943-0.863959$

C $-6.272339-3.852777-0.818397$

O $-3.960830-2.6506520 .455199$

O $-6.854426-3.982562-2.124589$

C $-6.152230-2.340529-0.527229$

C -7.193962 -4.611969 0.143501

O $-7.435783-1.754772-0.323682$

C $-5.228616-2.0186560 .667493$

C $-4.991134-0.4933980 .776794$

O $-5.850014-2.4682001 .875369$

C -1.553962 -0.992827 -1.936201

O $-2.108345-2.075316-2.063148$

C $-2.2546580 .274199-2.246749$

C $-1.6528541 .473033-2.283150$

C $-2.2855442 .746910-2.634517$

C $-3.7698522 .746251-2.890378$

C $-3.823097-0.1316281 .703888$

C $-3.944442 \quad 1.2766812 .244837$

C -3.7480403 .6587731 .646207$

O -4.1617451 .4886063 .436693$

C -3.8265254 .5145340 .425321$

C -2.8938215 .4233590 .102848$

C $-2.9946856 .348207-1.074812$

C $-1.8141596 .235246-2.011145$

C $-0.7572687 .057142-1.895955$

C $-1.9067435 .235902-3.140016$

C $-1.5095953 .847730-2.719662$

N -3.787355 2.2573331 .277495

H $2.363054-3.149523-2.882376$

H $2.323024-0.601550-1.181154$

H $\quad 0.214275-2.789737-1.805359$

H $-0.561906-1.8984140 .634659$

H $1.120493-1.5888400 .867436$

H $\quad 0.025341-5.5324591 .390648$

H $2.351882-4.312636-0.505393$

H $2.792437-3.4313560 .976836$

H $2.259260-5.1064631 .066428$

H -2.033530 -4.762398 2.301033

H -1.848435 -3.151672 1.669396

H -2.142210 $-4.212236-0.700325$

H -2.603982 -5.6994530 .123674$

H $-4.458012-4.4875781 .272624$

H -4.925288 -5.561368 -0.958783

H -4.366452 -4.118366 -1.778280

H $-6.865604-4.927395-2.355058$

H -5.733238 -1.865797-1.425736

H $-7.299103-5.657028-0.172358$

H $-8.206478-4.1938260 .134045$

H $-6.830348-4.6155281 .173073$ 
H -7.980565 -1.974652 -1.098680 H $-4.763630-0.081934-0.214672$ H $-5.908284-0.0029251 .126877$ H -5.566356 -1.904469 2.612277 H $-3.3072210 .157564-2.485115$ H $-0.5888791 .534411-2.062231$ H $-4.0029342 .175779-3.795871$ H $-4.1888643 .746678-3.018740$ H -4.308298 $2.299882-2.046810$ H -3.771236 -0.8051582 .566701$ H -2.867805 -0.2286921 .174862$ H -2.827753 3.8275582 .215842 H -4.594286 3.8704832 .308573 H -4.709964 4.409327 -0.199556 H -2.026192 5.5445310 .747542 H $-3.0627787 .373518-0.687614$ H -3.925133 $6.186652-1.633003$ H $0.0789197 .002195-2.585155$ H -0.702779 $7.798442-1.105557$ H -1.229086 $5.530530-3.953242$ H $-2.9030495 .279473-3.589563$ H $-0.4438023 .747248-2.511343$ H -3.4363351 .9840430 .363843$ C $5.196035-2.548614-2.088905$ O $5.097153-2.243982-0.681384$ C $6.270544-1.514562-0.291083$ C $7.357282-2.051301-1.206987$ C $6.606422-2.122696-2.525614$ H $6.087771-0.458816-0.531080$ C $6.525642-1.6483711 .214100$ H $8.234136-1.398557-1.247846$ H $7.682947-3.053802-0.906738$ C $7.237486-3.066169-3.535811$ H $6.577540-1.115864-2.964222$ C $5.281220-1.3897712 .091608$ O $6.976724-2.980708 \quad 1.485133$ H $7.346362-0.9800121 .501266$ H $4.613342-2.2583442 .046311$ O $5.705078-1.2801773 .455254$ C $4.484153-0.1245541 .729819$ C 3.2841120 .0961262 .672342 H $4.117072-0.2125280 .702259$ O 5.3569201 .0024091 .780455 H $2.800939-0.8660262 .879994$ H 3.6445680 .5180703 .618389 H $8.250725-2.737764-3.788958$ H $7.302214-4.087310-3.144960$ H $6.649054-3.094681-4.458590$ C $4.106615-1.776957-2.841236$ H $7.306940-3.0000002 .398905$ H $6.045081-2.1483783 .729152$ H 4.8414361 .7859811 .524598 H $4.222472-1.914818-3.922543$ H $4.212213-0.703185-2.642512$ H $5.049306-3.630222-2.194391$ C 2.0086163 .3207651 .267618 O 2.8212882 .3428181 .930812 C 2.2396561 .0433672 .051653 C 0.9524221 .1181442 .883779 C -0.0288772 .1295032 .283519$ C 0.6476293 .4704481 .969532 H 2.5536774 .2660931 .386818 C $1.8884423 .037656-0.237989$ H 1.9988250 .6625841 .054079 O 1.2519661 .4794964 .229685 H 0.4871180 .1258912 .912053 H -0.8527892 .2866182 .989774$ O -0.5749851 .5724851 .091708$ H -0.0072954 .1018531 .358648$ O $\quad 0.876171 \quad 4.1690593 .196974$ C $3.2370543 .014248-0.925882$ H $1.4077732 .073802-0.423980$ H $1.2896573 .821145-0.714733$ H 1.7340662 .3238834 .211046 H -1.1964672 .2223040 .722023$ H 0.0112474 .3125583 .617771 O $3.1002612 .431087-2.153661$ O $4.2744123 .464275-0.458902$
C $4.3019642 .336157-2.924617$

H $5.0623121 .769489-2.378093$

H $4.0697191 .805899-3.852083$

H $4.6693753 .336106-3.173510$

SCF Energy $(B 3 L Y P / 6-31 G * * / / M M F F)=-3245.90283763$

$08 \quad 00111$

MM̄MF Geometry

C $3.1051672 .658234-1.324205$

C $3.3675263 .884144-0.843139$

C 2.6851994 .5328870 .340592

O 1.9313563 .5617631 .100737

C $1.7161055 .631749-0.129466$

C 1.0284526 .3545701 .015572

C -0.2998736 .3345851 .245933$

C 1.9323927 .1477161 .924454

C -1.3638585 .6604230 .426268$

C -1.7245844 .2819270 .981592$

C -2.6662313 .4918940 .061150$

C $-4.0516934 .134270-0.053014$

C $-5.0141053 .284615-0.896491$

$\begin{array}{llll}\text { O } & -2.781056 & 2.169826 & 0.610089\end{array}$

O $-6.3342483 .804793-0.675735$

C $-4.9818841 .832131-0.375887$

C $-4.7379813 .444249-2.396149$

O $-5.7615350 .978146-1.210010$

C $-3.5522931 .271868-0.199369$

C $-3.546540-0.1211670 .468103$

O $-2.9597391 .110549-1.494659$

C 2.6139812 .8222712 .017274

O 3.7989902 .9060482 .297197

C 1.6601501 .8571862 .605460

C 2.1158110 .8835593 .407316

C $1.306117-0.1514524 .053320$

C $-0.180047-0.1193673 .813692$

C $-4.042946-0.1664081 .913677$

C $-4.197922-1.6029802 .377084$

C $-2.874607-3.6326992 .952559$

O $-5.304052-2.1287252 .480798$

C $-1.890125-3.8057164 .063321$

C $-0.654778-4.3001023 .893455$

C $0.320684-4.5210625 .013479$

C $1.501954-3.5796464 .940082$

C $2.656853-3.9817524 .381575$

C $1.317648-2.2186695 .582683$

C $1.928824-1.0781274 .811739$

N -2.987329 -2.221411 2.638942

H $2.3042022 .065105-0.890861$

H $4.1672174 .465034-1.297897$

H 3.4600804 .9728750 .980420

H $0.9780795 .177738-0.798793$

H $2.2556346 .373941-0.732511$

H $-0.680521 \quad 6.8764432 .110845$

H 1.3699657 .7630552 .635042

H 2.5778556 .4826522 .506124

H 2.5627697 .8234421 .337236

H -2.246533 6.3107520 .433357

H -1.066468 $5.582785-0.624190$

H -0.8128653 .6892911 .127124$

H $-2.1710424 .377048 \quad 1.980317$

H $-2.1918663 .421393-0.925025$

H -3.990495 $5.146779-0.468626$

H -4.479736 4.2490360 .952893

H $-6.9606753 .227018-1.144890$

H -5.4871651 .8447890 .596608$

H $-4.8556904 .493061-2.694658$

H -5.462243 $2.881968-2.995652$

H $-3.732943 \quad 3.129337-2.684204$

H $-5.240806 \quad 0.789207-2.009200$

H $-4.122630-0.823551-0.147579$

H $-2.517987-0.5074530 .453186$

H -2.215427 $0.491010-1.416053$

H $0.612206 \quad 1.9656442 .351153$

H 3.1853110 .8321493 .607570

H -0.6121990 .8109364 .198554$

H $-0.715551-0.9392094 .294254$

H $-0.398147-0.1901562 .742152$

H $\quad-3.3501530 .3556802 .583347$ 
H -5.0197830 .3133552 .023296$ H -2.554740 -4.143338 2.038252 H -3.847469 -4.041433 3.243087 H -2.221440 -3.527246 5.060771 H $-0.326590-4.5817582 .895539$ H $0.659774-5.5640924 .963571$ H $-0.164555-4.4225635 .992829$ H $3.531259-3.3415914 .345303$ H $2.756411-4.9717773 .947948$ H $1.770126-2.2450636 .582659$ H $0.255081-2.0318565 .765839$ H $3.012280-1.0124684 .919705$ H $-2.131626-1.7270742 .406488$ C $4.5828480 .710505-1.979786$ O $3.607281-0.303185-1.659373$ C $4.042780-1.001977-0.478249$ C $5.500969-0.604466-0.264070$ C $5.4988790 .827065-0.759688$ H $3.444276-0.6123720 .353936$ C $3.821991-2.512134-0.618039$ H $5.805852-0.6970980 .782810$ H $6.183213-1.212378-0.869398$ C $6.8804721 .371016-1.076790$ H 5.0439101 .4502860 .019076 C $2.378154-2.945169-0.946277$ O $4.663960-3.015477-1.664985$ H $4.159325-3.0102120 .298594$ H $2.152673-2.694967-1.988505$ O $2.339625-4.377684-0.871175$ C $1.286187-2.366556-0.028698$ C $-0.093050-3.043421-0.161479$ H $1.187167-1.291478-0.217425$ O $1.706307-2.5187391 .330224$ H $-0.042913-4.0615300 .245810$ H $-0.779345-2.4972890 .498585$ H $7.5103241 .366612-0.181205$ H $7.3824020 .771116-1.843230$ H $6.8181422 .401204-1.441451$ C $3.8889072 .003352-2.426335$ H $4.432010-2.540145-2.481321$ H $2.493238-4.6268610 .056540$ H $1.017700-2.1316121 .896732$ H $3.1895121 .776471-3.239235$ H $4.6383582 .695021-2.828840$ H $5.1494430 .318210-2.834583$ C $-1.290119-1.583952-3.385617$ O $-0.815480-1.734762-2.040236$ C $-0.665555-3.082028-1.586196$ C $-2.010644-3.824971-1.640817$ C $-2.630545-3.746210-3.040130$ C $-2.632276-2.314062-3.583036$ H -1.471637 -0.508068 -3.501255 C $-0.227206-1.987977-4.420007$ H $\quad 0.046459-3.605370-2.230827$ O $-2.908840-3.261750-0.689084$ H -1.846021 -4.871561-1.357445 H -3.662970 -4.115453 -3.005968 O $-1.901687-4.580134-3.935815$ H -2.900250 -2.317335 -4.646076 O $-3.655708-1.587771-2.897687$ C $0.990917-1.089901-4.377189$ H $0.122041-3.012038-4.265876$ H $-0.653628-1.908624-5.426854$ H -3.738377 $-3.767050-0.730457$ H -1.940572 -5.488035 -3.589745 H $-3.687505-0.693341-3.277623$ O $1.887364-1.524207-5.312502$ O $1.142157-0.137314-3.625561$ C $3.109241-0.784454-5.394252$ H $3.649516-1.118657-6.283913$ H $2.9113520 .287621-5.489049$ H $3.723090-0.989852-4.513541$ SCF Energy $(B 3 L Y P / 6-31 G * * / / M M F F)=-3245.91511850$

\section{8_00112}

\section{MM̄FF Geometry}

C -4.5539193 .8070741 .366892$

C -4.3692042 .8029640 .495911$
C -5.3168751 .6372910 .352703$

O -4.5554540 .4134810 .472320$

C $-5.9694731 .670191-1.041247$

C $-6.9451730 .533540-1.284439$

C $-6.721322-0.516226-2.101064$

C $-8.2613750 .652703-0.558994$

C $-5.487198-0.809702-2.908109$

C $-4.808043-2.102342-2.435738$

C $-3.386979-2.239831-3.002553$

C $-2.771043-3.578825-2.597169$

C $-1.299604-3.692161-3.023346$

O - $-2.612606-1.163595-2.466865$

O $-0.728890-4.814479-2.331397$

C $-0.538251-2.434556-2.549795$

C $-1.162477-3.991227-4.520830$

O $0.789595-2.451256-3.068883$

C $-1.250741-1.113494-2.911216$

C $-0.6117520 .131103-2.245399$

O $-1.181078-0.919652-4.328628$

C $-4.310578-0.0421331 .731955$

O $-4.648261 \quad 0.4815002 .781779$

C -3.547904-1.305074 1.613607

C $-3.093827-1.9149982 .718304$

C $-2.331327-3.1651382 .765878$

C $-1.959895-3.8077901 .455506$

C $-0.6527510 .141321-0.713782$

C $0.600514-0.397407-0.046971$

C $1.502335-0.9207112 .221703$

O $1.628255-0.668893-0.660611$

C $1.361600-2.3803572 .513165$

C $1.316057-2.8883853 .753805$

C $1.238040-4.3586074 .049547$

C $0.068164-4.7178594 .937167$

C $0.233207-4.9029906 .257881$

C $-1.271807-4.9532504 .277920$

C $-2.000064-3.6719463 .972153$

N $0.438813-0.503293 \quad 1.328717$

H -5.4349143 .7733122 .005793$

H $-3.5045312 .798730-0.161472$

H $-6.097124 \quad 1.6630001 .123675$

H $-5.1867261 .680118-1.806190$

H $-6.5088272 .618283-1.173009$

H -7.508836 -1.261840 -2.205422

H $-8.960323-0.145593-0.830796$

H -8.1112050 .6022310 .523677$

H $-8.7431831 .605731-0.800661$

H $-5.776323-0.917822-3.960680$

H $-4.7778810 .019172-2.876630$

H -4.758053 -2.101370 -1.339577

H $-5.417766-2.964300-2.733888$

H $-3.431018-2.148184-4.094127$

H $-3.354973-4.414070-3.003665$

H $-2.821448-3.684980-1.505274$

H -1.242957 -5.605554 -2.567802

H $-0.453403-2.517750-1.459552$

H - $-1.665377-4.933252-4.770695$

H -0.112732 -4.130074 -4.802072

H - $1.590818-3.211863-5.154533$

H $1.297532-1.761186-2.610409$

H $\quad 0.4021270 .295898-2.628786$

H $-1.1889231 .006359-2.577604$

H $-1.3019210 .025463-4.518277$

H -3.379373 -1.6998990.618888

H $-3.304408-1.461023 \quad 3.685958$

H -2.857071 -4.166148 0.939641

H - $1.284660-4.6592511 .561888$

H -1.442088 -3.0934210 .806312$

H $-0.756907 \quad 1.180647-0.379563$

H -1.522398 $-0.405267-0.340145$

H $1.425587-0.3084393 .125794$

H $2.471951-0.7276601 .753055$

H $1.327412-3.0556301 .662299$

H $1.377414-2.2156034 .606139$

H $2.178419-4.6489464 .536366$

H $1.190744-4.9514543 .127395$

H $-0.595350-5.1908076 .896365$

H $1.199914-4.7649156 .730785$

H $-1.918621-5.5392234 .945145$ 
H -1.136324 -5.590438 3.399515 H -2.310809 -3.1277614 .865227$ H $-0.464079-0.286017 \quad 1.740579$ C $-2.4065205 .117916 \quad 0.731789$ O -1.4486234 .1435101 .191641$ C $-0.130666 \quad 4.7403381 .187669$ C $-0.281558 \quad 6.074517 \quad 0.473292$ C $-1.696051 \quad 6.4653640 .852095$ H 0.1269824 .9031822 .241892 C 0.8518863 .7534830 .542916 H $0.465798 \quad 6.8079550 .790438$ H $-0.1988845 .947957-0.613545$ C $-2.2765587 .557071-0.030259$ H -1.701949 6.8028941 .897227 C 2.2513994 .3363680 .270077 O 0.9510572 .6334301 .423971 H $0.4162933 .378082-0.389764$ H $2.1195835 .225315-0.358707$ O $2.829624 \quad 4.774668 \quad 1.496873$ C $3.2320693 .407322-0.480948$ C 3.7591892 .2076330 .318498 H $4.0927424 .022689-0.767305$ O $2.6272452 .936840-1.682863$ H 4.1988632 .5394991 .266814 H 2.9288021 .5489630 .591524 H $-1.677158 \quad 8.4704060 .044338$ H -2.297334 7.254035 -1.082599 H -3.3001737 .8009530 .270119$ C -3.683466 5.0219011 .572025 H 1.4026852 .9319342 .232100 H 3.6922525 .1728651 .289814 H $2.3504003 .714310-2.196929$ H -4.3117375 .8991681 .378247$ H -3.412542 5.0451082 .635410 H $-2.6152934 .902208-0.323858$ C $6.034688-0.725365-0.069365$ O $\quad 5.1215150 .2546190 .443992$ C $4.7724641 .326220-0.445913$ C $6.0309922 .082400-0.900858$ C $7.0845481 .134979-1.480043$ C $7.332205-0.066592-0.565324$ H $6.297633-1.3440430 .798415$ C $5.373715-1.633421-1.119930$ H $4.2624360 .902164-1.317161$ O $\quad 6.5923212 .808618 \quad 0.190991$ H $5.7564812 .815070-1.667500$ H $8.0203411 .689051-1.623166$ O $6.6416570 .689628-2.758601$ H $7.951847-0.816540-1.070574$ O $8.060837 \quad 0.3670310 .587052$ C $4.377658-2.594999-0.504735$ H $4.843706-1.054848-1.880702$ H $6.139680-2.246228-1.609459$ H 6.7783832 .1746920 .904762 H $7.3316240 .109116-3.122625$ H $8.903621 \quad 0.7404250 .277136$ O $3.757044-3.288429-1.505528$ O $4.178791-2.7377170 .693315$ C $2.798762-4.262646-1.079833$ H $1.937194-3.765641-0.625730$ H $2.461005-4.814237-1.961170$ H $3.252539-4.969063-0.378166$ SCF Energy $\left(B 3 L Y P / 6-31 G^{* \star} / / M M F F\right)=-3245.91846651$

\section{8_00113}

MM̄FF Geometry

C $2.192514-3.6874162 .552151$

C $2.027862-2.3615202 .428396$

C $0.963122-1.5789403 .148967$

O $-0.128674-1.3810142 .218639$

C $1.499409-0.1945343 .552831$

C 0.5334090 .5692914 .435581

C -0.3112751 .5265954 .004809$

C $0.584258 \quad 0.209845 \quad 5.898512$

C -0.5255702 .0092772 .597007$

C -0.1660183 .4950102 .457210$

C -0.4248754 .0166831 .036540$

C -0.0213775 .4865620 .907602$
C $-0.3609986 .054398-0.480469$

O

O $-0.2639807 .485173-0.407809$

C -1.830488 $5.718154-0.816652$

C $0.6492145 .605547-1.543596$

O $-2.1469236 .090229-2.155967$

C $-2.1914184 .234068-0.580591$

C $-3.7185114 .020764-0.723204$

O $-1.5188183 .429538-1.553562$

C $-1.097870-2.3364402 .176519$

O $-1.191289-3.3250572 .886118$

C $-2.020587-1.9873261 .073694$

C $-2.962213-2.8627280 .689017$

C $-3.918535-2.676895-0.406729$

C $-3.781489-1.424873-1.232742$

C $-4.1865332 .658850-0.195296$

C $-5.6639622 .400172-0.433501$

C -7.4212370 .7137950 .100971$

O $-6.3944383 .158107-1.063756$

C $-7.416861-0.7473530 .416805$

C $-7.924905-1.695085-0.385266$

C $-7.882476-3.159673-0.049418$

C $-7.284598-4.009965-1.151721$

C -7.964101 -5.059577-1.644596

C $-5.912501-3.659502-1.695332$

C $-4.843366-3.634397-0.633571$

N -6.0528891 .1938950 .133565$

H $1.552437-4.2476903 .228561$

H $2.653316-1.8160621 .725587$

H $\quad 0.613907-2.1181554 .038306$

H 1.7380490 .3918792 .657086

H $2.450361-0.3018874 .092151$

H -0.9516132 .0107354 .741561$

H -0.1131860 .8044116 .498118$

H $0.327905-0.843866 \quad 6.045037$

H $1.5896790 .382083 \quad 6.296342$

H $\quad 0.0432141 .425377 \quad 1.867962$

H $-1.583710 \quad 1.8555402 .352719$

H -0.7666514 .0733543 .171606$

H 0.8893083 .6443842 .717635

H $0.146707 \quad 3.4015360 .334997$

H 1.0452405 .6193411 .126936

H $-0.557967 \quad 6.0781491 .662664$

H $0.6387117 .710227-0.123832$

H $-2.4647406 .335562-0.165074$

H $1.6583605 .944024-1.279797$

H $\quad 0.4272176 .058842-2.515893$

H $0.6880024 .521549-1.668859$

H $-1.9171537 .029837-2.257426$

H $-4.2539164 .794081-0.157088$

H $-4.0114604 .124924-1.775326$

H -2.033291 2.624187-1.718013

H - $1.884569-1.0251780 .594606$

H -3.039881 -3.810922 1.219555

H $-3.893737-0.538676-0.602377$

H $-4.518559-1.336570-2.032249$

H -2.796546 -1.389610 -1.711722

H $-3.6300761 .849408-0.678089$

H -4.0056692 .5983630 .884594$

H $-7.8566800 .935529-0.878840$

H -7.9850461 .2645990 .860500$

H -6.964709 -1.042709 1.361414

H -8.396769 -1.408626 -1.322019

H $-8.911244-3.478107 \quad 0.162627$

H $-7.315674-3.3332750 .873980$

H $-7.554310-5.679255-2.435353$

H -8.946764 -5.325407 -1.269186

H -5.996822 -2.711127 -2.231288

H $-5.604347-4.399571-2.445481$

H $-4.833482-4.5220230 .000702$

H -5.4073020 .7410580 .775150$

C $2.747669-4.7412720 .294959$

O $2.658980-3.496863-0.431376$

C $1.405654-3.466368-1.136527$

C $0.971040-4.921626-1.244449$

C $1.402150-5.4490280 .112805$

H $\quad 0.691445-2.939708-0.492610$

C $1.541954-2.715963-2.465302$ 
H $-0.103318-5.024605-1.423907$

H $1.506745-5.447309-2.043131$

C $1.492714-6.9632540 .181812$

H $0.674784-5.106906 \quad 0.859331$

C $2.122261-1.289338-2.331914$

O $2.411859-3.466989-3.320117$

H $0.566082-2.685214-2.965221$

H $3.191936-1.348227-2.103375$

O $2.013680-0.644563-3.606373$

C $1.416777-0.406651-1.288407$

C $1.8424961 .072172-1.280700$

H $1.582493-0.823219-0.290048$

O $0.006927-0.447870-1.529445$

H $1.4608361 .577626-2.177369$

H $1.3146081 .537417-0.439360$

H $0.514604-7.416123-0.010585$

H $2.196791-7.357938-0.558433$

H $1.827356-7.2870261 .172546$

C $3.182696-4.4786221 .743421$

H $2.338951-3.094090-4.214559$

H $2.622163-1.094306-4.216189$

H $-0.4230910 .103431-0.853784$

H $4.140161-3.9448491 .745172$

H $3.360351-5.4333872 .252299$

H $3.542321-5.316202-0.197918$

C 5.2458050 .7104320 .241459

O $3.8345010 .613416-0.002402$

C $3.3524331 .327626-1.144137$

C $3.6625902 .829066-1.012280$

C $5.1534703 .069455-0.760388$

C 5.6862702 .1799470 .367545

H $5.393620 \quad 0.2334321 .218654$

C $6.066673-0.071515-0.797710$

H $3.8399630 .940250-2.044118$

O $2.910022 \quad 3.3793710 .067135$

H $3.3461093 .337887-1.930807$

H $5.3077154 .123252-0.497048$

O $5.8663252 .815616-1.966696$

H 6.7803022 .2378750 .407708

O 5.1968402 .6994551 .607263

C $5.836424-1.563569-0.702730$

H $5.8198670 .237635-1.816505$

H $7.1372000 .096518-0.633536$

H 3.1072644 .3304370 .108856

H $6.8064622 .995632-1.795524$

H 5.5831072 .1665732 .323082

O $6.260483-2.156021-1.857736$

O $5.369913-2.1407370 .268990$

C $6.117069-3.578785-1.911912$

H $5.057483-3.845678-1.945542$

H $\quad 6.597050-3.933736-2.827845$

H $6.608178-4.049828-1.054799$

SCF Energy $\left(B 3 L Y P / 6-31 G^{* *} / / M M F F\right)=-3245.90090812$

\section{8_00114}

MM̄FF Geometry

C $-0.822914-3.520184-0.386322$

C $-1.847760-2.655333-0.384122$

C $-2.570305-2.220541-1.632371$

O $-2.475404-0.780889-1.728858$

C $-4.064523-2.559628-1.517625$

C $-4.777629-2.577239-2.853619$

C $-5.478498-1.549487-3.370781$

C $-4.705149-3.889079-3.592589$

C $-5.627888-0.155246-2.828576$

C $-7.0189980 .118846-2.242001$

C $-7.169375-0.383765-0.797840$

C $-8.594568-0.155744-0.291979$

C $-8.747142-0.5380631 .187420$

O -6.2422750 .3493540 .011353$

O

C $-7.6297520 .153051 \quad 1.998240$

C $-8.823455-2.058063 \quad 1.373104$

O $-7.630822-0.2879053 .354454$

C $-6.218130-0.0338081 .390721$

C $\begin{array}{llll}-5.209936 & 0.885105 & 2.123099\end{array}$

O $-5.817360-1.3952161 .556313$

C $-1.327345-0.233373-2.203193$
O $-0.333676-0.818387-2.599823$

C $-1.4930721 .233969-2.111740$

C $-0.4223872 .036560-2.190189$

C $-0.4339163 .494161-2.044593$

C $-1.7684754 .165744-1.845644$

C $-3.8237420 .976714 \quad 1.464329$

C -2.8841371 .8685432 .262293$

C -0.5270072 .6795172 .304782$

O -3.2302242 .4655283 .278923$

C -0.4382174 .0246231 .653080$

C 0.7235424 .5904531 .290240

C 0.8392535 .9521660 .665616

C $1.6770855 .951526-0.595548$

C $2.9738736 .302283-0.562246$

C $0.9903455 .627083-1.904095$

C $0.7437424 .152194-2.065905$

N -1.611073 1.9172301 .709390

H $-0.490351-3.952611-1.326869$

H $-2.172630-2.2149170 .555638$

H -2.135404 -2.677677 -2.529877

H $-4.551931-1.843781-0.847534$

H -4.200935 -3.544175 -1.050140

H -5.979719 -1.704592 -4.325855

H $-5.235460-3.858431-4.550344$

H -3.663629 -4.152942 -3.801782

H -5.152768 -4.688288 -2.992650

H $-4.8483120 .089400-2.102890$

H $-5.4676660 .530713-3.670570$

H $-7.1693231 .206577-2.250182$

H -7.794227 -0.320727 -2.881521

H $-6.922614-1.450138-0.763180$

H $-9.318966-0.704615-0.906841$

H $-8.8539140 .906842-0.403028$

H - $10.154190-0.2855122 .540518$

H $-7.858138 \quad 1.227958 \quad 2.019117$

H $-9.690990-2.4635900 .838613$

H $-8.970273-2.3218752 .426387$

H -7.935910 -2.580081 1.009475

H $-8.491373-0.053683 \quad 3.740444$

H $-5.619018 \quad 1.9033812 .165627$

H $-5.0863180 .541515 \quad 3.158285$

H -4.849415 -1.441879 1.591302

H $-2.4944291 .611427-1.936433$

H $0.5566391 .585023-2.345041$

H -2.408186 $4.010212-2.721383$

H $-1.6981495 .244092-1.691342$

H -2.278849 $3.763030-0.963783$

H $-3.364561-0.0139171 .388602$

H -3.9108201 .3953840 .455305$

H 0.3897292 .0961642 .173536

H -0.7160152 .8017043 .376096$

H -1.3660604 .5687131 .493359$

H 1.6519534 .0609371 .488321

H $\quad 1.2922276 .620687 \quad 1.409592$

H -0.1477196 .3784460 .447485$

H $3.5756776 .330602-1.464606$

H 3.4649396 .5610610 .370133

H $1.6156665 .946149-2.748875$

H $0.0810236 .227826-1.992212$

H $1.6571563 .575257-2.215725$

H -1.4290251 .4334070 .835079$

C $1.329388-3.3006730 .849794$

O $2.133544-3.900287-0.183354$

C $3.516173-3.8876360 .242907$

C $3.528175-3.2668781 .635905$

C $2.120847-3.5359822 .131149$

H $3.829101-4.9376890 .282372$

C $4.328656-3.117396-0.806079$

H $4.300953-3.7001512 .278061$

H $3.705499-2.1861731 .581631$

C $1.714305-2.6440633 .291433$

H $2.046736-4.5890172 .433189$

C $5.781214-2.830786-0.393777$

O $4.341508-3.882873-2.015536$

H $3.804814-2.184460-1.038038$

H $5.807716-2.2582390 .535596$

O $6.432117-4.071370-0.087786$

C $6.624851-2.102885-1.457678$ 
C $6.015735-0.823589-2.060731$

H $6.845364-2.794598-2.280061$

O $7.889979-1.760774-0.881331$

H $6.694539-0.477207-2.851621$

H $5.070227-1.083564-2.548919$

H $0.694780-2.8677873 .619746$

H $2.383350-2.7952214 .144988$

H $1.754931-1.5844563 .016689$

C $-0.066548-3.9211990 .849643$

H $3.415497-4.050236-2.261787$

H $6.377550-4.633498-0.879856$

H $8.270261-2.575843-0.511459$

H $-0.618046-3.6136981 .745226$

H $\quad 0.000749-5.0156160 .865589$

H $1.267663-2.2252340 .631024$

C 4.5852780 .9398530 .932551

O $4.868766-0.030700-0.081916$

C $5.8090010 .356875-1.084091$

C $5.3051291 .609501-1.825002$

C $4.9608252 .740550-0.849472$

C 4.0829352 .2493940 .304667

H 3.7586360 .5044411 .508578

C 5.7674591 .1372201 .888526

H $6.7703430 .579388-0.609627$

O $4.1463131 .276838-2.589355$

H $6.0791041 .938143-2.528756$

H $4.4305623 .536901-1.385567$

O $6.1577913 .299077-0.317404$

H 4.0028403 .0266341 .073521

O $2.7671692 .024097-0.207512$

C $6.017487-0.1149712 .696940$

H 6.6888611 .4123831 .367905

H 5.5616241 .9416192 .604534

H $3.8641222 .079091-3.060658$

H $6.6838673 .632327-1.064231$

H $2.200363 \quad 1.7611170 .537038$

O $7.220834-0.6585942 .357399$

O $5.232258-0.5611653 .523670$

C $7.570858-1.8629073 .046718$

H $7.720856-1.6553884 .110228$

H $6.797554-2.6245312 .907352$

H $8.507710-2.2355972 .624230$

SCF Energy (B3LYP/6-31G**//MMFF) $=-3245.90711098$

$08 \_00115$

MM̄FF Geometry

C -1.149093 -3.274561 0.616087

C $-0.651928-2.6578601 .698613$

C -1.295213-1.458554 2.337409

O $-0.293668-0.4128402 .327767$

C $-1.739660-1.7323193 .785499$

C -2.795674 -2.804211 3.943778

C $-4.095212-2.6459163 .624562$

C $-2.313997-4.0975644 .546739$

C $-4.746259-1.4372773 .009542$

C $-5.530899-1.8307751 .749799$

C $-6.022681-0.6035880 .967843$

C $-7.135890-1.003718-0.002814$

C $-7.5855460 .175702-0.875085$

O $-4.906610-0.075104 \quad 0.239627$

O $-8.396709-0.345547-1.938693$

C $-6.3403270 .808855-1.524223$

C $-8.4663651 .162404-0.100060$

O $-6.6857721 .995625-2.236364$

C $-5.1953971 .104122-0.524640$

C $-3.9231751 .498496-1.316392$

O $\quad-5.5779712 .191183 \quad 0.319316$

C $-0.722017 \quad 0.8700402 .466501$

O -1.8611291 .2476972 .688805$

C 0.4461551 .7527852 .260605

C 0.2541803 .0707232 .099954

C 1.2954414 .0712241 .858371

C 2.7194903 .5852961 .802780

C $-2.6554461 .607613-0.464432$

C -1.439835 $1.884321-1.326272$

C $-0.3686143 .675290-2.692999$

O $-0.6115541 .010580-1.571689$

C $0.7419004 .284202-1.896569$
C $0.9878925 .602865-1.864267$

C $2.1300726 .237414-1.122257$

C 1.6672097 .1020320 .029593

C $1.2092618 .348057-0.179625$

C 1.8137356 .5485801 .429332

C $0.9296865 .358147 \quad 1.679456$

N $-1.3982873 .185917-1.796927$

H -2.078737 -2.913743 0.184243

H $\quad 0.267050-3.0235212 .149305$

H -2.140379-1.127954 1.723442

H $-2.110827-0.8071034 .244776$

H $-0.858575-1.9823374 .393299$

H $-4.774235-3.4752273 .819407$

H $-3.116040-4.8369714 .642913$

H -1.530061 -4.541348 3.925105

H -1.905735 -3.920046 5.547156

H -5.438756 -1.006634 3.743002

H -4.021101 -0.654936 2.774115

H -4.894406 -2.445046 1.099129

H -6.385599-2.448492 2.054169

H -6.3860640 .1500561 .676255$

H -7.992138 -1.425024 0.538858

H $-6.774384-1.805777-0.661794$

H $-9.150433-0.812767-1.539248$

H $-5.967430 \quad 0.096738-2.273941$

H -9.3619780 .6575340 .281692$

H $-8.8300031 .963615-0.752875$

H $-7.955773 \quad 1.6164220 .751651$

H -7.394779 $1.762806-2.860107$

H $-3.7322760 .743658-2.091156$

H $-4.0910712 .452318-1.831603$

H -4.7793742 .6067690 .680200$

H 1.4260631 .2904052 .222199

H -0.7636913 .4573022 .135852$

H 3.0188653 .1633792 .768430

H $3.4427204 .364911 \quad 1.557237$

H 2.8279222 .8142851 .032412

H $-2.7401272 .401406 \quad 0.284481$

H -2.4723560 .6695800 .068546$

H $-0.8408594 .401608-3.362516$

H $0.0169902 .850882-3.301118$

H $1.3891443 .603711-1.349233$

H $0.3422636 .273654-2.427250$

H $2.6995176 .850164-1.833505$

H $2.8394695 .481109-0.764558$

H $0.901401 \quad 8.984626 \quad 0.643181$

H $1.1311908 .758007-1.181377$

H $\quad 1.547321 \quad 7.3122782 .172187$

H $2.8703296 .335878 \quad 1.614614$

H -0.1310225 .6084391 .725246$

H $-2.0960293 .850397-1.475255$

C $-0.044440-4.233731-1.472876$

O $0.980763-3.221131-1.524614$

C $0.812648-2.467669-2.741733$

C $-0.240870-3.206945-3.564614$

C $-1.111743-3.802484-2.476517$

H $0.403698-1.493498-2.451750$

C $2.149636-2.282862-3.466955$

H $-0.787215-2.535918-4.234645$

H $0.203401-4.001800-4.175107$

C $-2.004665-4.932437-2.956649$

H -1.737333 -2.999934 -2.068029

C $3.241109-1.558124-2.651916$

O $2.657287-3.576969-3.818593$

H $1.975576-1.755454-4.412975$

H $3.598841-2.218018-1.854537$

O $4.360184-1.359825-3.527374$

C $2.820444-0.201324-2.059494$

C $3.9786990 .665742-1.529968$

H $2.092335-0.368747-1.257595$

O $2.1592960 .555896-3.078376$

H $4.5977561 .009505-2.368741$

H $3.5258531 .574699-1.111649$

H $-2.690776-4.577193-3.732585$

H $-1.418870-5.756249-3.378124$

H $-2.604433-5.331612-2.132848$

C $-0.507920-4.468550-0.032137$

H $3.500884-3.440670-4.283047$ 
H $4.082724-0.736830-4.221084$ H $1.8133721 .363710-2.662041$ H $\quad 0.357223-4.7847110 .563296$ H -1.225265 -5.297135 -0.009387 H $\quad 0.432138-5.160087-1.821987$ C $4.685866-1.0846181 .693519$

O $4.028683-0.3936550 .622387$

C $4.865482-0.005827-0.470041$

C $5.9909190 .929803 \quad 0.006857$

C 6.7721880 .3196241 .175556

C $5.838805-0.2361312 .256664$

H $3.923822-1.1770912 .478174$

C $5.124197-2.5016311 .289881$

H $5.315465-0.898443-0.914797$

O $5.437401 \quad 2.178457 \quad 0.416334$

H $6.6617551 .130193-0.837024$

H $7.419817 \quad 1.082787 \quad 1.624275$

O $7.607359-0.7303440 .697939$

H $6.411651-0.8310602 .977014$

O $5.275766 \quad 0.856807 \quad 2.988358$

C $3.953414-3.3982240 .951867$

H $5.780650-2.4888350 .416190$

H $5.659651-2.9677472 .125153$

H 6.1753382 .7591450 .668561

H $8.212169-0.3485910 .039128$

H 4.7053381 .3575172 .380523

O $4.432953-4.6099560 .541350$

O $2.775110-3.0835191 .034450$

C $3.443374-5.5739950 .169894$

H $2.740382-5.7395100 .991758$

H $2.918803-5.242063-0.730201$

H $3.952532-6.516289-0.049028$

SCF Energy (B3LYP/6-31G**//MMFF)= -3245.91334397

08_00116

MM̄FF Geometry

C $0.036982-3.2386940 .851589$

C $0.974587-2.281060 \quad 0.808613$

C $1.444613-1.4768241 .990181$

O $1.118985-0.1012771 .692345$

C $2.973461-1.6097932 .116376$

C $3.532993-1.0944193 .424924$

C 4.1527830 .0917023 .581334

C $3.402048-2.0388654 .591681$

C 4.3427231 .1803092 .561397

C 5.8078651 .3590872 .141769

C $6.3091550 .264474 \quad 1.186311$

C 7.7888880 .4781090 .857994

C $8.296163-0.521409-0.192211$

O $5.529178 \quad 0.341674-0.011429$

O $9.566594-0.053345-0.670168$

C $7.330503-0.514222-1.395791$

C $8.544620-1.9081430 .412973$

O $7.679366-1.529172-2.334904$

C $5.843286-0.652183-0.995265$

C $4.939594-0.400484-2.227899$

O $5.614832-1.974543-0.505001$

C 0.7292490 .7116022 .704894

$\begin{array}{lllll}\text { O } & 0.595596 & 0.403993 & 3.881216\end{array}$

C 0.4471582 .0830472 .217080

C 0.6790252 .4853840 .957876

C $0.3751153 .802890 \quad 0.394444$

C -0.3385954 .7969051 .271588$

C $3.443638-0.307381-1.896728$

C $2.593595-0.009067-3.120004$

C $0.2504630 .582897-3.749143$

O $3.0400060 .004008-4.263806$

C $0.2918752 .061744-3.975216$

C $-0.7016892 .902297-3.648113$

C $-0.6355044 .387363-3.869944$

C $-0.7460775 .182152-2.585855$

C -1.874706 $5.844870-2.281665$

C $0.4773045 .286633-1.699383$

C $0.7148194 .043327-0.889068$

N $1.2677320 .225394-2.776745$

H $-0.457014-3.4749051 .789095$

H $1.446623-2.062618-0.145412$

H $\quad 0.933725-1.8107852 .900980$
H $3.453486-1.095773 \quad 1.276317$

H $3.268643-2.6637742 .019661$

H 4.5424030 .3382164 .568470

H $3.848244-1.6348465 .506635$

H $2.348067-2.2439944 .803240$

H $3.903946-2.9867894 .371313$

H 3.7066921 .0416371 .682526

H 4.0014942 .1155073 .023905

H 5.8859042 .3306111 .636145

H $6.448801 \quad 1.4050393 .030992$

H $6.161120-0.7151751 .653978$

H $8.4005530 .424078 \quad 1.767276$

H $7.931110 \quad 1.4939580 .462666$

H $\quad \begin{array}{llll}10.165853 & 0.018409 & 0.092518\end{array}$

H $7.4611050 .445887-1.914593$

H $9.296742-1.8489041 .208934$

H $8.953360-2.597787-0.333647$

H $7.646350-2.3562320 .842594$

H $8.614593-1.399119-2.567694$

H $5.233206 \quad 0.544741-2.703238$

H $5.088772-1.197752-2.966937$

H $4.681039-2.205379-0.625338$

H 0.0214222 .7494352 .960419

H $1.124073 \quad 1.7826590 .255622$

H 0.2852845 .0679872 .130186

H -1.2819574 .3801811 .640493$

H -0.5939085 .7257050 .756973$

H $3.089536-1.254415-1.481532$

H $3.2677950 .483258-1.158361$

H $-0.7139050 .250791-3.351840$

H $0.4431240 .056294-4.689324$

H $1.1939372 .461843-4.433180$

H -1.609567 2.507409 -3.198624

H -1.450106 $4.656765-4.554788$

H $0.2892444 .672465-4.387269$

H $-1.9542126 .441581-1.379100$

H $-2.7475295 .800191-2.924464$

H $0.4143686 .170147-1.054402$

H $1.3607595 .470275-2.324465$

H $1.2356453 .259562-1.438720$

H $1.0123510 .264699-1.794048$

C $-1.623037-3.553275-1.022960$

O $-2.774780-3.930500-0.235691$

C $-3.705307-4.613090-1.090259$

C $-3.333631-4.221934-2.513082$

C $-1.823690-4.174233-2.410097$

H $-3.495560-5.685703-0.970226$

C $-5.165884-4.374632-0.689187$

H $-3.691753-4.940377-3.256187$

H -3.716796 -3.231156-2.778317

C $-1.162378-3.390278-3.532051$

H -1.439265 -5.203215 -2.420881

C $-5.757400-2.979806-0.983030$

O $-5.947764-5.339122-1.408666$

H $-5.286708-4.6040890 .376515$

H $-5.797367-2.818067-2.065686$

O $-7.127587-2.992450-0.552131$

C $-5.041977-1.802952-0.300715$

C $-5.690270-0.452458-0.664602$

H -3.993865 -1.776069 -0.610269

O $-5.081622-2.0125251 .107828$

H -5.725654 -0.370043 -1.757629

H $-6.718845-0.433193-0.283145$

H $-1.376479-3.851893-4.501524$

H - $1.523131-2.356735-3.565381$

H $-0.075896-3.362829-3.402685$

C -0.340365 -4.051892 -0.354655

H $-6.875534-5.214560-1.144332$

H -7.127825 -3.0756020 .417209$

H $-4.605430-1.2782671 .530092$

H $0.485882-4.034304-1.076054$

H $-0.459114-5.094333-0.033961$

H -1.626058 -2.457691-1.087705

C -4.5870471 .8844492 .041822$

O $\quad \begin{array}{llll}-5.222903 & 0.826779 & 1.313264\end{array}$

C $-4.9233450 .754257-0.085379$

C $-5.3158782 .061762-0.790272$

C $-4.6585243 .269484-0.116123$ 
C $-4.8709093 .253478 \quad 1.399586$

H $-5.071212 \quad 1.8737783 .027142$

C - -3.0944321 .6123012 .246753$

H $-3.8498900 .580323-0.218728$

O $-6.7340352 .209100-0.765786$

H -5.008499 2.002069-1.841322

H $-5.0783954 .195808-0.526736$

O $-3.2598923 .264582-0.385262$

H -4.2439104 .0168861 .874007$

$\begin{array}{lllll}\text { O } & -6.226400 & 3.612884 & 1.682078\end{array}$

C -2.887874 0.5012603 .247508

H -2.5674871 .3563991 .323516$

H -2.5951702 .4970162 .657866$

H $-6.952212 \quad 3.027822-1.242919$

H -3.145970 $3.295346-1.350173$

H -6.799792 2.9075111 .336215

O $-2.705056-0.6849682 .602223$

O -2.9088340 .6686624 .460330$

C -2.543851 -1.826906 3.449763

H $-1.569579-1.7875983 .944729$

H $-3.350865-1.8807154 .187019$

H -2.589215 -2.722292 2.824738

SCF Energy (B3LYP/6-31G**//MMFF) $=-3245.91452076$

08_00117

MM̄FF Geometry

C -2.205149 $3.770270 \quad 0.472170$

C $-2.3459453 .849291 \quad 1.805228$

C -2.8293592 .7484282 .721223$

O $-2.683906 \quad 1.4544222 .094330$

C -4.310956 2.9620523 .066841

C $-4.807073 \quad 2.013946 \quad 4.140721$

C -5.5595360 .9205553 .908228$

C -4.4441232 .3955015 .552824$

C $-6.0097310 .388596 \quad 2.576217$

C $-5.798185-1.1291852 .477948$

C $-6.256031-1.6683391 .115966$

C $-6.235407-3.1968761 .096488$

C $-6.619857-3.749830-0.284218$

O $-5.364464-1.1606640 .117972$

O $-6.279110-5.144136-0.311630$

C $-5.759514-3.052071-1.357359$

C $-8.129904-3.666378-0.536204$

O $-6.181167-3.419819-2.668908$

C $-5.723669-1.510404-1.224787$

C $-4.648151-0.937633-2.175616$

O $-6.996482-0.972221-1.585430$

C $-1.481144 \quad 0.833628 \quad 2.243342$

O $-0.524411 \quad 1.2293212 .890591$

C -1.509031 -0.4042461 .434252$

C $-0.350089-1.0213941 .159778$

C $-0.172538-2.1998960 .311092$

C $-1.408123-2.933460-0.135381$

C $-4.330080 \quad 0.547726-1.961135$

C -3.136922 $0.947684-2.807049$

C $-0.6584160 .654930-2.940481$

O $-3.2704251 .553846-3.867101$

C $-0.142239-0.714881-3.257159$

C $1.109955-1.115589-2.989071$

C $1.632232-2.491709-3.290281$

C $2.353331-3.120252-2.115731$

C $3.689170-3.264212-2.124052$

C $1.521729-3.650168-0.964787$

C $1.082864-2.552300-0.036654$

N -1.935178 $0.519455-2.265870$

H -2.482465 $2.858704-0.049757$

H -2.084955 4.781510 2.302469

H -2.230101 2.7802993 .639767

H -4.9206942 .8683492 .160698$

H -4.4716703 .9908793 .416996$

H -5.8958090 .3405404 .766886$

H $-4.868931 \quad 1.7080796 .292048$

H -3.358013 2.3897555 .686328

H $-4.818263 \quad 3.3983185 .783438$

H -7.0771340 .6119562 .461877$

H $-5.4874840 .875248 \quad 1.748127$

H -4.733622 -1.353135 2.626059

H -6.357448 -1.6277173 .279396$
H -7.269613 -1.298938 0.920293

H $-6.896025-3.6067411 .870583$

H -5.225111 -3.5517621 .344678$

H $-6.763431-5.5850850 .407360$

H $-4.733648-3.430868-1.245236$

H $-8.677424-4.2288520 .229548$

H $-8.396493-4.127735-1.493616$

H -8.510230 -2.642773 -0.529121

H $-6.174961-4.391270-2.713631$

H $-3.722035-1.508267-2.045139$

H $-4.958482-1.077669-3.219287$

H $-6.880747-0.050137-1.864443$

H -2.463586 $-0.739264 \quad 1.045674$

H $\quad 0.570568-0.598708 \quad 1.560427$

H $-1.971436-2.330130-0.851388$

H -2.056162 $-3.158640 \quad 0.719345$

H $-1.196204-3.892553-0.612096$

H $-5.1778811 .181487-2.241501$

H $-4.099626 \quad 0.764075-0.911811$

H $0.0040331 .195569-2.259498$

H $-0.7763091 .235191-3.860272$

H $-0.831456-1.410346-3.730867$

H $1.806288-0.411252-2.542645$

H $2.313869-2.406384-4.146584$

H $\quad 0.825576-3.161457-3.613350$

H $4.210684-3.736639-1.298577$

H $4.291863-2.909311-2.953267$

H $2.107540-4.366380-0.373999$

H $0.687982-4.224706-1.376660$

H $1.912596-1.9681160 .364854$

H $-1.9412200 .046402-1.367641$

C $-0.523254 \quad 4.436288-1.273062$

O $0.5395093 .881875-0.478161$

C $1.8042514 .343124-0.982512$

C $1.5033135 .025257-2.313178$

C $0.1104935 .573977-2.071459$

H $2.1647955 .089992-0.263844$

C $2.8289793 .198386-1.064946$

H $2.2355705 .801619-2.555154$

H $1.4865144 .310054-3.143622$

C $-0.6364455 .924014-3.346609$

H $0.194516 \quad 6.472671-1.445718$

$\begin{array}{llll}\text { C } 3.020029 & 2.447517 & 0.273471\end{array}$

$\begin{array}{lllll}\text { O } & 2.423772 & 2.291524 & -2.087423\end{array}$

H $3.7883773 .628537-1.377540$

H 3.2300593 .1812131 .061497

$\begin{array}{lllll}\text { O } & 1.824866 & 1.757700 & 0.638452\end{array}$

$\begin{array}{llll}\text { C } & 4.171866 & 1.420813 & 0.248641\end{array}$

$\begin{array}{llll}\text { C } & 4.370609 & 0.749079 & 1.622337\end{array}$

H $5.0964641 .932867-0.041420$

$\begin{array}{lllll}\text { O } & 3.915447 & 0.420924 & -0.733462\end{array}$

H 4.4451451 .5271562 .391332

H 3.4918580 .1299871 .843758

H $-0.0987026 .695123-3.907884$

H -0.749192 $5.050900-3.998087$

H $-1.6365476 .306681-3.120127$

C $-1.669124 \quad 4.896910-0.368770$

H 3.138667 1.643883 -2.207695

H 1.1211672 .4190670 .747952

H $3.083202-0.020531-0.491725$

H $-2.4858555 .295513-0.981014$

H $-1.3186495 .708940 \quad 0.280724$

H $-0.8693543 .638903-1.942898$

C $6.518614-2.1873210 .736054$

O $5.416879-1.2756970 .840470$

C $5.629092-0.1367241 .679880$

C $5.934068-0.5903353 .116402$

C $7.108495-1.5734633 .155289$

C $6.948012-2.6942322 .123082$

H $6.118969-3.0442840 .179308$

C $7.672140-1.598125-0.083146$

H $6.4729100 .446742 \quad 1.296186$

O $4.784239-1.202176 \quad 3.698655$

H $6.1687240 .291213 \quad 3.724056$

H $7.181897-2.0136124 .157212$

O $8.324822-0.8794772 .894266$

H $7.876927-3.2689552 .029897$

O $5.937666-3.6007312 .575780$ 
C $7.254681-1.406752-1.521934$

H $8.037519-0.6431170 .304692$

H $8.533102-2.277125-0.086967$

H $4.514947-1.9381473 .122484$

H $8.425298-0.1955063 .578116$

H $6.231734-3.9652413 .427965$

O $6.859510-0.118182-1.726239$

O $7.255254-2.303497-2.355309$

C $6.3966670 .183238-3.046238$

H $6.0927351 .233223-3.067711$

H $7.2013920 .034448-3.772264$

H $5.530178-0.436646-3.295668$

SCF Energy (B3LYP/6-31G**//MMFF)= -3245.92594666

08_00118

MM̄FF Geometry

C $0.146509-3.3165651 .434439$

C $0.922720-3.5995542 .492226$

C $1.855244-2.6588853 .220564$

O $1.952899-1.3789562 .558561$

C $3.264715-3.2809993 .261332$

C $4.296293-2.4193863 .963860$

C $5.271600-1.7279163 .340084$

C $4.199879-2.4010815 .467461$

C $5.511106-1.6162401 .859483$

C $5.550891-0.1493731 .410014$

C $5.492397-0.021737-0.119757$

C $5.6489271 .438521-0.543741$

C $5.4779881 .620570-2.058089$

O $4.219272-0.517264-0.544956$

O $5.3268263 .030659-2.287473$

C $4.1728800 .922006-2.506398$

C $6.7405521 .192538-2.814931$

O $4.0603420 .909110-3.926587$

C $4.017332-0.517644-1.964666$

C $2.618179-1.125676-2.227881$

O $4.927551-1.417078-2.611207$

C $1.110168-0.3901512 .965352$

O $0.199929-0.4816853 .772802$

C 1.5111110 .8478202 .259900

C 0.8388761 .9906402 .460759

C 1.1602293 .2934671 .870642

C 2.3336913 .3650710 .929102

C $1.443751-0.408367-1.555583$

C $0.8084170 .680432-2.401990$

C $-0.7999472 .577566-2.222860$

O $1.019701 \quad 0.808056-3.604722$

C $-0.2186263 .862453-1.726117$

C $-0.9205714 .774505-1.036872$

C $-0.3647486 .090777-0.578626$

C $-0.513561 \quad 6.3023290 .910681$

C -1.550505 6.9957331 .410684

C 0.5921905 .7980481 .808532

C 0.4075884 .3611922 .209234

N $-0.0538521 .471578-1.655955$

H $\quad 0.121448-2.3099041 .028276$

H $0.893491-4.6139992 .887980$

H $1.489432-2.5277224 .246531$

H $3.587777-3.5162142 .241642$

H $3.224880-4.2527373 .772918$

H $5.977048-1.1692483 .953932$

H $5.002128-1.8153725 .928768$

H $3.248939-1.9629155 .785949$

H $4.265906-3.4189845 .865079$

H $6.470499-2.0917921 .622956$

H $4.750060-2.1506551 .287436$

H 4.6998820 .3852301 .849527

H 6.4648620 .3226521 .791430

H $6.286388-0.639654-0.554970$

H $6.6166621 .838203-0.215397$

H $4.8893132 .046813-0.035458$

H $5.2650193 .175341-3.246757$

H $3.3462201 .533962-2.126036$

H $7.5883571 .827391-2.530049$

H $6.6193731 .321972-3.895886$

H $7.0259320 .156324-2.620156$

H $4.0251841832127-4.228580$

H $2.452538-1.241270-3.306125$
H $2.633012-2.150110-1.827490$

H $5.361444-0.948431-3.341945$

H 2.3728650 .7905901 .606121

H $-0.014766 \quad 1.9792483 .137001$

H $2.2306102 .632243 \quad 0.121488$

H 3.2657323 .1648181 .468233

H 2.4429774 .3358010 .440816

H $0.651820-1.142963-1.364785$

H $1.7422500 .005486-0.589118$

H -1.842621 2.450425 -1.921039

H $-0.7523852 .547031-3.315700$

H $0.8224374 .061727-1.968846$

H $-1.9685974 .584056-0.819214$

H $-0.8979216 .883251-1.120415$

H $0.6890146 .201969-0.862583$

H -1.6458467 .1798692 .475686$

H $-2.330013 \quad 7.3886300 .766137$

H 0.6156356 .3905532 .733718

H 1.5629495 .9899431 .342248

H -0.4233724 .2109292 .899562$

H $-0.1700291 .282938-0.664301$

C $-0.755503-4.172920-0.753094$

O $-1.587198-3.040846-1.087070$

C $-2.378162-3.368506-2.247406$

C $-1.839966-4.696144-2.768188$

C $-1.377761-5.359472-1.486707$

H -3.405286 -3.521299-1.897479

C $-2.330976-2.226390-3.267240$

H $-2.603518-5.271941-3.300108$

H $-0.993438-4.552412-3.449968$

C $-0.422422-6.517618-1.717760$

H $-2.258655-5.720491-0.938826$

C $-2.847361-0.879611-2.721709$

O $-0.966248-2.046113-3.669309$

H -2.881680 -2.510485 -4.171249

H $-2.193019-0.552961-1.907958$

O $-2.6943760 .092808-3.765021$

C $-4.319476-0.878973-2.255412$

C $-4.8134690 .508031-1.794184$

H $-4.469892-1.598602-1.444316$

O $-5.159946-1.279069-3.340837$

H $-4.5501471 .263674-2.543836$

H $-5.9113510 .486579-1.760755$

H $-0.904478-7.300503-2.312519$

H $0.477095-6.194885-2.252713$

H $-0.107795-6.961827-0.768454$

C $-0.720286-4.3490600 .765826$

H $-0.942880-1.300088-4.292889$

H $-3.303823-0.150176-4.483264$

H $-5.010783-2.226969-3.495816$

H $-0.358417-5.3527731 .016041$

H -1.731132 -4.247656 1.169280

H $0.248925-3.962542-1.144129$

C $-4.556326 \quad 0.297500 \quad 1.921785$

$\begin{array}{llll}\text { O } & -4.969199 & 0.120053 & 0.559071\end{array}$

C $-4.2673830 .902718-0.414482$

C $-4.4569812 .395156-0.117324$

C -3.9795042 .7210921 .300114$

C -4.5996801 .7791962 .336016$

H $-5.321731-0.2235372 .512495$

C $-3.208874-0.3945552 .186850$

H -3.200705 $0.667026-0.372865$

O $-5.8229642 .769153-0.274028$

H -3.882182 $2.980068-0.842481$

H -4.2415123 .7595811 .535538$

O -2.5605162 .6033271 .339093$

H -4.1149641 .9004903 .311605$

O -5.9761462 .1310572 .512122$

C $-3.401601-1.8776202 .432654$

H $-2.484930-0.2851001 .375459$

H $-2.7549280 .013644 \quad 3.097564$

H -6.3569502 .1963530 .302782$

H -2.274081 2.8254052 .241099

H -6.0055963 .0520032 .822852$

O $-4.091059-2.4441341 .403300$

O $-2.975293-2.4554363 .424393$

C $-4.460660-3.8241441 .521154$

H $-5.528814-3.8792721 .747194$ 
H $-4.280292-4.3075950 .557283$

H $-3.894545-4.3541132 .292120$

SCF Energy (B3LYP/6-31G**//MMFF)= -3245.90808137

08_00119

MM̄FF Geometry

C $2.656243 \quad 3.717861 \quad 1.131525$

C 3.5079614 .2509050 .241614

C $4.1989703 .522098-0.889985$

O $3.7142752 .166472-1.034443$

C $5.7146693 .474237-0.631659$

C $6.5074752 .949141-1.814002$

C $7.0667441 .725006-1.888473$

C $6.6859573 .924913-2.949818$

C $7.0092030 .620921-0.870883$

C $6.344232-0.633216-1.452619$

C $6.149596-1.720050-0.385966$

C $5.552028-2.984542-1.004371$

C $5.230268-4.0493090 .055181$

O $5.265890-1.1982910 .611756$

O $4.385096-5.037001-0.555408$

C $4.412396-3.3977831 .191606$

C $6.490705-4.7870260 .522174$

O $4.228868-4.3102342 .271065$

C $5.025440-2.0825981 .714595$

C $4.107411-1.3338602 .712755$

O $6.243766-2.3783362 .409061$

C $2.5459931 .999253-1.716897$

O $1.8528652 .875578-2.209803$

C $2.2425380 .550901-1.755350$

C $1.0719920 .137108-2.264030$

C $0.634598-1.251486-2.427490$

C $1.567155-2.338810-1.965224$

C $2.744559-0.8989022 .162093$

C $1.622234-1.8865452 .433245$

C $-0.782182-2.2743581 .906978$

O $1.744260-2.8631453 .167145$

C $-0.981115-3.1127980 .685801$

C $-2.034743-2.982091-0.134201$

C $-2.295190-3.875101-1.310934$

C $-2.432817-3.114444-2.608418$

C $-3.642525-2.808088-3.105888$

C $-1.172659-2.807702-3.382121$

C $-0.562036-1.485878-3.005892$

N $0.451513-1.5242771 .781540$

H 2.4377092 .6539201 .108320

H 3.7052225 .3203400 .291080

H $4.0025764 .073010-1.818302$

H 5.9115822 .8795840 .266350

H $6.0868094 .480904-0.396088$

H $7.6341001 .475477-2.784615$

H $7.3246693 .525969-3.745162$

H $5.7205014 .174858-3.399792$

H $7.1518184 .847413-2.588193$

H $8.0340440 .379496-0.563663$

H 6.4817880 .9309060 .033665

H $5.369053-0.356279-1.872095$

H $6.954759-1.025411-2.275594$

H $7.122688-1.9379160 .069798$

H $\quad 6.219925-3.395135-1.771806$

H $4.621685-2.726758-1.527032$

H $4.859015-5.405262-1.320742$

H $\quad 3.415932-3.1887180 .784317$

H $6.975365-5.289611-0.323559$

H $6.243445-5.578658 \quad 1.238079$

H $7.230366-4.1261070 .978850$

H $3.818477-5.1126761 .905530$

H $4.003413-1.9134953 .637935$

H $4.633398-0.4136233 .006116$

H $6.443248-1.6419503 .010522$

H $2.994055-0.133510-1.381017$

H $\quad 0.3689770 .888604-2.620932$

H $1.125698-3.337104-2.002994$

H $1.861635-2.180013-0.922798$

H $2.468215-2.355821-2.587178$

H 2.4535580 .0351102 .660415

H $2.799204-0.6924251 .088941$

H -1.589209-1.550992 2.044835
H $-0.744324-2.9156282 .793256$

H $-0.245565-3.8899690 .492942$

H $-2.780276-2.2197040 .077815$

H -3.220224 -4.430325 -1.105798

H -1.514934 -4.639200-1.414673

H $-3.750161-2.292407-4.054306$

H $-4.551552-3.059813-2.570886$

H -1.404932 -2.760337 -4.455250

H -0.472264 -3.643412 -3.295893

H -1.168340 -0.630358 -3.306434

H $0.450599-0.7095281 .174381$

C 0.4189164 .6014331 .928283

O -0.1687843 .2876491 .983451$

C -1.1165003 .1509610 .894575$

C -1.3681104 .5676650 .401233$

C -0.0074715 .2072880 .591712$

H -0.5887772 .5623450 .135316$

C -2.3422142 .3893571 .411651$

H -1.699283 4.604141 -0.639959

H -2.1171445 .0717101 .024191$

C -0.0367536 .7255950 .585952$

H $0.6414404 .864815-0.222844$

C -3.5361722 .1778560 .454823$

O -1.8817741 .1059491 .848912$

H -2.703242 2.9109672 .307735

H -4.2935961 .6450911 .040323$

O $-4.1239713 .425644 \quad 0.103274$

C $-3.2521551 .328670-0.801908$

C $-4.4804931 .099881-1.706424$

H $-2.8351200 .361331-0.500055$

O $-2.2643641 .983147-1.599027$

H -4.787435 $2.049535-2.163227$

H $-4.1451730 .469494-2.540820$

H $-0.4099917 .099349-0.373114$

H -0.6869747 .1180151 .374915$

H 0.9669497 .1339580 .740780

C 1.9299234 .5197392 .175537

H -2.6382910 .6395932 .243078$

H -3.691086 $3.743997-0.706213$

H $-2.0635371 .404870-2.354171$

H 2.1103784 .0350443 .142851

H 2.3433355 .5330762 .242999

H -0.0310525 .1657742 .755588$

C $-6.236412-1.5033960 .317298$

O $-5.248515-0.783402-0.432002$

C $-5.6859730 .444703-1.017667$

C $-6.8449580 .191776-1.995613$

C $-7.986872-0.568115-1.312573$

C $-7.479136-1.793595-0.544538$

H $-5.760878-2.4629590 .557324$

C $-6.574569-0.8040871 .644261$

H $-6.0351581 .119659-0.230547$

O $-6.374723-0.557139-3.114055$

H $-7.2054581 .156676-2.372120$

H -8.710084 -0.887994-2.072726

O $-8.656791 \quad 0.321890-0.424711$

H -8.279154 -2.202254 0.083828

O $-7.147279-2.802949-1.501902$

C $-5.389748-0.7441972 .585225$

H -6.9134370 .2227251 .484649$

H -7.367635 -1.360435 2.157268

H -7.123459-0.679232 -3.722052

H $-9.395763-0.165825-0.022696$

H $-6.876605-3.597616-1.011188$

O $-5.744791-0.0339053 .697074$

O $-4.296574-1.2525042 .380690$

C -4.7217110 .1141814 .686877$

H $-4.361131-0.8655565 .014886$

H $-3.8994440 .716240 \quad 4.289636$

H -5.1533290 .6337325 .546318$

SCF Energy (B3LYP/6-31G**//MMFF)= -3245.90632765

08_00120

MM̄FF Geometry

C $-2.613306-3.6368612 .631630$

C $-1.397582-3.4056122 .114823$

C $-0.199190-3.0432662 .956152$

O $0.321032-1.7668152 .522152$ 
C $0.912284-4.0891542 .757224$

C $2.138615-3.8215613 .608981$

C $3.321505-3.4076993 .112398$

C $1.983525-4.0858375 .083376$

C $3.616564-3.1268541 .663574$

C $5.025852-2.5500401 .466986$

C $5.371167-2.367865-0.018360$

C $6.829986-1.932828-0.177503$

C $7.194039-1.665993-1.645999$

O $4.497335-1.375827-0.569505$

O $8.450968-0.971265-1.667002$

C $6.138706-0.714175-2.247482$

C $7.407021-2.967004-2.427973$

O $6.354414-0.527880-3.644300$

C $4.681133-1.149536-1.974682$

C $3.654735-0.085318-2.427102$

O $4.403823-2.338759-2.725146$

C $-0.150684-0.6535643 .144852$

O $-1.062112-0.5929293 .954172$

C 0.6696360 .4919542 .687889

C 0.3795151 .7292793 .118023

C 1.1093012 .9574272 .789500

C 2.3383452 .8233491 .932183

C $3.7838161 .275694-1.741500$

C $2.6709162 .230708-2.141633$

C $1.9784724 .603781-1.870125$

O $1.7392581 .914485-2.875978$

C $2.0292095 .581953-0.740819$

C $0.9431746 .003651-0.076756$

C 0.9805926 .9987101 .045188

C 0.3934286 .4526182 .326930

C -0.8096696 .8646012 .762786$

C 1.2490575 .5068393 .142770

C 0.6529754 .1294863 .279129

N $2.8448373 .481530-1.563500$

H $-2.735496-3.5280983 .708577$

H -1.230347 -3.503502 1.047166

H $-0.468194-2.9951144 .018671$

H $1.189943-4.1240581 .696415$

H $0.533345-5.0911232 .999103$

H $4.146568-3.2636113 .807782$

H $2.928125-3.9738785 .626402$

H $1.265477-3.3900225 .527566$

H $\quad 1.628987-5.1082285 .250837$

H $3.535128-4.0622501 .097064$

H $2.878802-2.4199141 .267140$

H $5.086704-1.5823441 .982005$

H $5.762021-3.2186101 .931289$

H $5.202212-3.322279-0.531059$

H $7.508432-2.6788810 .254889$

H $6.998129-1.0091500 .394343$

H $9.110390-1.530835-1.222035$

H $6.313060 \quad 0.263499-1.782652$

H $8.204620-3.563310-1.968381$

H $7.740716-2.762632-3.451204$

H $6.513440-3.592900-2.472831$

H $6.033107-1.323346-4.102074$

H $3.7019250 .040169-3.516886$

H $2.648003-0.473019-2.225400$

H $3.440992-2.421631-2.825615$

H 1.4980470 .2818622 .020579

H -0.474496 1.8600123 .781790

H 2.8167593 .7764431 .700050

H 2.0833082 .3601330 .973894

H 3.0903492 .2024612 .431510

H $3.7520281 .157004-0.652712$

H $4.7339841 .747300-2.013148$

H $0.964946 \quad 4.238510-2.064879$

H $2.3556275 .068379-2.786788$

H $3.0063005 .969848-0.462457$

H $-0.0351465 .636524-0.372934$

H 0.4236067 .8870540 .719955

H 2.0030167 .3470981 .237858

H -1.222768 6.5087923 .700856

H -1.408777 7.5658722 .191564

H 1.3634185 .9235534 .152816

H 2.2665205 .4795252 .743062

H -0.2489414 .1094693 .893199$
H $3.7295433 .677042-1.102913$

C $-3.788121-4.3266050 .403042$

O $-3.890687-3.070953-0.309345$

C $-4.965577-3.173145-1.261963$

C $-5.157599-4.664413-1.492823$

C $-4.954070-5.193437-0.090335$

H $-5.862699-2.778586-0.770223$

C $-4.682563-2.379170-2.541592$

H $-6.143565-4.901311-1.903413$

H $-4.395743-5.071894-2.167662$

C $-4.674321-6.686316-0.039929$

H -5.856784 -4.981722 0.499093

C $-4.456446-0.864989-2.355005$

O $-3.517005-2.913184-3.182414$

H $-5.506702-2.535997-3.248113$

H $-3.461760-0.687001-1.936295$

O $-4.452130-0.268590-3.659203$

C $-5.513763-0.151008-1.497153$

C $-5.4732421 .388256-1.543680$

H $-5.408766-0.480981-0.457082$

O $-6.815385-0.555257-1.934820$

H -5.848116 $1.742288-2.512966$

H $-6.207797 \quad 1.739087-0.806531$

H -5.519202 -7.249701-0.449293

H -3.783392 -6.947626 -0.621001

H $-4.513504-7.0188910 .990450$

C -3.862669-4.079753 1.916385

H -2.784302 -2.861344 -2.544912

H $-3.769214-0.722116-4.182550$

H -7.465502 -0.149424 -1.336313

H $-4.177560-5.0070252 .411437$

H -4.636086 -3.327753 2.118984

H -2.835531 -4.786167 0.115272

C -2.2889281 .9397130 .343005$

O $-3.597271 \quad 1.489824-0.036734$

C $-4.1040102 .027604-1.259748$

C $-4.1972553 .561688-1.177761$

C $-2.8556924 .180160-0.770767$

C -2.2497023 .4725040 .445762$

H -2.1385641 .5337611 .350865$

C $-1.1866991 .372498-0.568226$

H $-3.4307301 .755688-2.078568$

O $-5.1908153 .930963-0.222354$

H $-4.5168593 .948700-2.152639$

H -2.994926 $5.241577-0.531150$

O $-1.9427554 .096507-1.860542$

H -1.215172 $3.796790 \quad 0.593611$

O -2.9672323 .8662281 .618745$

C $-1.064313-0.129567-0.448390$

H -1.369236 $1.596946-1.621932$

H $-0.2209561 .802881-0.279563$

H $-5.2524944 .901405-0.223151$

H $-2.3305594 .583213-2.607598$

H -3.8727643 .5193651 .543592$

O $-0.036811-0.556701-1.239147$

O $-1.780651-0.8390440 .242991$

C $\quad 0.198489-1.968675-1.251699$

H $0.735578-2.257132-0.344518$

H $-0.738034-2.527238-1.341071$

H $0.821907-2.202109-2.118720$

SCF Energy $\left(B 3 L Y P / 6-31 G^{* *} / / M M F F\right)=-3245.90756022$

08_00121

MM̄FF Geometry

C $-2.6062372 .465953-1.403371$

C $-3.3880313 .200417-0.597777$

C -3.8350622 .8276590 .798460$

O -3.2092531 .6068791 .253696$

C $-5.3516642 .575700 \quad 0.816983$

C -5.9676742 .7779832 .186230$

C -6.2049081 .8006193 .082421$

C -6.3688984 .1979522 .495362$

C -5.8413460 .3446722 .994955$

C $-7.057548-0.5618342 .764354$

C $-7.463204-0.6467391 .285220$

C $-8.744226-1.4660591 .121630$

C $-9.109600-1.661800-0.357072$

O $-6.386254-1.2771290 .581361$ 
O $-10.120494-2.678593-0.430974$

C $-7.873141-2.197778-1.107334$

C $-9.720774-0.396073-0.969278$

O $-8.118042-2.264352-2.510772$

C $-6.580163-1.392562-0.832644$

C $-5.364630-2.149552-1.420649$

O $-6.685213-0.113064-1.458656$

C $-1.9482381 .664638 \quad 1.754092$

O -1.2476232 .6530571 .891419$

C $-1.556560 \quad 0.2724072 .064438$

C $-0.259615-0.0563822 .145076$

C $0.269118-1.3964572 .410731$

C $-0.725416-2.4984182 .673680$

C -3.992376 -1.582000 -1.023040

C $-2.859458-2.374798-1.657808$

C $-0.379139-2.398864-1.891288$

O $-3.047958-3.373724-2.348321$

C $0.257377-3.272919-0.855680$

C $1.567921-3.239713-0.567804$

C $2.233752-4.1399060 .433807$

C $3.069386-3.3833651 .443779$

C $4.400900-3.2751571 .297387$

C $2.376187-2.8411492 .673013$

C $1.606235-1.5785342 .400041$

N -1.612471 -1.837019-1.367933

H -2.264455 $1.486615-1.081016$

H -3.716950 4.177782 -0.946207

H -3.5691313 .6463301 .478993$

H -5.5591961 .5608300 .460794$

H $-5.866963 \quad 3.2459280 .115895$

H -6.6883292 .0759834 .019514$

H -6.808838 4.2996993 .493032

H -5.4979114 .8596042 .451008$

H -7.1101314 .5495481 .770118$

H -5.0718290 .1562872 .242248$

H $-5.3848270 .072828 \quad 3.955495$

H $-6.788853-1.5683203 .111650$

H -7.904275 $-0.223173 \quad 3.373904$

H -7.6117040 .3659300 .895460$

H -9.576204 -1.000541 1.664524

H -8.605677 -2.456294 1.577985

H -10.880641 -2.384429 0.099682

H -7.712994 -3.232421 -0.772180

H -10.630805 -0.110257 -0.428072

H -10.031157 -0.568859-2.005689

H $-9.0435960 .460512-0.949959$

H -8.927173 -2.788410 -2.638936

H -5.396692 -3.193838-1.081972

H -5.435908 -2.161398 -2.515969

H $-5.7959910 .220292-1.655303$

H -2.353874 -0.4528272 .183813$

H $\quad 0.484516 \quad 0.723080 \quad 1.988833$

H -1.295199 -2.2896873 .585885$

H $-0.267347-3.4813052 .799837$

H -1.426632 -2.595925 1.837411

H $-3.897595-0.540397-1.344867$

H -3.864625 -1.6216140 .064567$

H $0.262706-1.561025-2.178442$

H $-0.598497-2.990935-2.785548$

H $-0.381828-3.984923-0.339050$

H $2.211279-2.554951-1.114196$

H $2.870708-4.839975-0.122982$

H $1.501161-4.7633080 .961150$

H $5.011712-2.7607602 .031913$

H $4.909870-3.6932520 .435575$

H $3.117040-2.6034843 .448483$

H $1.761037-3.6289353 .116551$

H $2.245359-0.7202892 .190496$

H -1.545770 -1.036293 -0.746451

C $-0.6228343 .191771-2.801543$

O $0.1133202 .044082-2.329780$

C $1.0507602 .486098-1.323451$

C $1.2535423 .966056-1.597412$

C $-0.1566934 .386735-1.964351$

H $0.5474032 .343747-0.358519$

C $2.3073451 .616443-1.389557$

H $1.6467994 .508667-0.732809$

H $1.9343604 .116846-2.444135$
C $-0.2271215 .713891-2.699505$

H $-0.7414084 .468393-1.040127$

C $3.4515782 .106664-0.477989$

O $1.8939400 .301771-1.009439$

H $2.6569761 .550302-2.426820$

H $3.7492593 .110967-0.800104$

O 2.9752312 .2298990 .862354

C $4.7051331 .207398-0.499466$

C 5.8354351 .7769400 .380105

H $5.0496551 .113653-1.536100$

O $4.356948-0.094160-0.034195$

H 5.9667042 .8423770 .157159

H $5.541745 \quad 1.6775381 .432584$

H $0.1667956 .521554-2.073952$

H $0.3566755 .691894-3.625875$

H -1.261977 $5.959972-2.958126$

C $-2.1293422 .906957-2.759098$

H $2.654455-0.290893-1.130135$

H $2.7253411 .339391 \quad 1.162216$

H $5.143648-0.658835-0.116870$

H $-2.3569472 .092505-3.458178$

H -2.683432 $3.787190-3.105260$

H $-0.3286223 .330693-3.849601$

C $8.185822-1.140508 \quad 0.416443$

O $7.040375-0.2999230 .605436$

C $7.1808351 .053540 \quad 0.164793$

C $8.326627 \quad 1.7398390 .924187$

C 9.6308260 .9436510 .805809

C $9.422280-0.5442341 .109931$

H $7.930882-2.0762620 .931200$

C $8.416189-1.470662-1.062545$

H $7.3986291 .064815-0.908780$

O 7.9888191 .8922112 .301711

H $8.4684472 .747481 \quad 0.516782$

H $\quad 10.372008 \quad 1.353558 \quad 1.502656$

O $10.154578 \quad 1.073664-0.512624$

H $10.310840-1.123016 \quad 0.831818$

O $9.239054-0.7079262 .519477$

C $7.281006-2.304936-1.607207$

H $8.529753-0.584895-1.693798$

H $9.334532-2.056424-1.190134$

H $7.784507 \quad 1.0102702 .657219$

H $10.3136672 .019372-0.673519$

H $10.043516-0.3831632 .958938$

O $6.358258-1.501372-2.208604$

O $7.213927-3.521769-1.491084$

C $5.215848-2.169346-2.753097$

H $4.518905-1.409047-3.115754$

H $5.517616-2.801998-3.592921$

H $4.714633-2.762779-1.983303$

SCF Energy (B3LYP/6-31G**//MMFF) $=-3245.92618344$

08 00122

MM̄MF Geometry

C 0.4277174 .5560860 .422284

C 1.6171993 .9400440 .490928

C $2.5389743 .783030-0.690776$

O $2.8380942 .373849-0.835207$

C $3.8566234 .531504-0.440623$

C $4.7321164 .598170-1.676350$

C $5.8142213 .826062-1.894469$

C $4.3457095 .655206-2.679757$

C $6.3558052 .731345-1.018346$

C $6.6398131 .458190-1.828583$

C $7.2760950 .368173-0.955479$

C $7.712757-0.826240-1.804329$

C $8.291994-1.954645-0.937671$

O $6.307761-0.0538730 .010350$

O $8.380687-3.134302-1.750816$

C $7.301910-2.2644600 .203438$

C $9.717618-1.643138-0.467287$

O $7.859991-3.1989661 .124544$

C $6.804572-1.0077070 .958591$

C $5.647372-1.4001921 .904528$

O $7.873863-0.4671451 .736606$

C $1.9060681 .606686-1.464000$

O $0.8728641 .987836-1.990846$

C $2.3394190 .196625-1.358735$ 
C $1.430695-0.774678-1.533904$

C $1.668206-2.215347-1.423288$

C $3.094494-2.675581-1.287978$

C $4.884169-0.2148322 .507397$

C $3.691927-0.7248503 .292117$

C $1.408341-1.6816162 .970645$

O $3.740260-0.8773174 .510362$

C $1.354342-3.0638532 .397050$

C $0.268367-3.5793701 .800957$

C $0.213246-4.9670441 .227258$

C $-0.323022-5.004053-0.188102$

C $-1.542525-5.504157-0.450351$

C $0.584941-4.543369-1.309975$

C $0.605126-3.046352-1.449990$

N 2.609185-1.030010 2.481434

H $0.1190845 .006431-0.517203$

H $1.9333943 .487757 \quad 1.428237$

H 2.067344 4.152285-1.610049

H 4.3988244 .0671750 .391153

H $3.6493615 .559965-0.114690$

H $6.3875964 .000392-2.804457$

H $5.0371305 .696389-3.528241$

H $3.3464805 .460369-3.080548$

H $4.3453446 .643676-2.208638$

H $7.2887413 .089337-0.566678$

H $5.6743212 .488568-0.198758$

H $5.6977841 .090005-2.255158$

H $7.3112911 .696411-2.663110$

H $8.1397370 .802627-0.438307$

H $8.438416-0.519021-2.567653$

H $6.846014-1.219483-2.354199$

H $8.947918-2.930115-2.514037$

H $\quad 6.431307-2.759951-0.249574$

H $10.380003-1.490737-1.327967$

H $10.142005-2.4850430 .090774$

H $9.777856-0.7498230 .157872$

H $8.143934-3.9759120 .612995$

H $4.931599-2.0200451 .353953$

H $6.031052-2.0201762 .725378$

H 7.5009880 .0489652 .468779

H $3.3740980 .000218-1.102410$

H $0.401939-0.489401-1.751861$

H $3.213370-3.759243-1.341987$

H $3.506660-2.353168-0.329069$

H $3.713297-2.261119-2.092179$

H 5.5170370 .3671603 .185314

H $4.527340 \quad 0.471667 \quad 1.731119$

H $\quad 0.556367-1.0709252 .657892$

H $1.425727-1.7319644 .063447$

H $2.251523-3.6729772 .482748$

H $-0.637980-2.9820201 .743713$

H -0.423952 -5.571196 1.886154

H $1.200979-5.4446441 .245942$

H -1.924571 -5.559094 -1.464484

H -2.190182 -5.8620130 .343051$

H $\quad 0.237790-4.956381-2.266161$

H $1.576366-4.975083-1.150767$

H $-0.386194-2.609218-1.579208$

H $2.677588-0.861743 \quad 1.482108$

C -1.8021723 .8113431 .383293$

O -1.4591472 .4211651 .207690$

C -2.264886 1.8803920 .137676

C $-3.3956442 .876734-0.068325$

C $-2.6901494 .191410 \quad 0.198032$

H -1.614529 $1.857165-0.745570$

C -2.7017110 .4580840 .506881$

H -3.829901 2.822237 -1.071074

H -4.1958442 .7139040 .662690$

C -3.634483 5.3462450 .482169

H $-2.0875684 .437477-0.684012$

C $-3.716413-0.146234-0.483309$

O $-1.527904-0.3567260 .548494$

H -3.1270690 .4479851 .517683$

H $-4.5978630 .500892-0.487350$

O $-3.174241-0.115114-1.803691$

C $-4.165679-1.582944-0.146663$

C -5.233157 -2.142861-1.107338

H $-4.547200-1.6164010 .880351$
O $-3.059432-2.483530-0.214715$

H -4.828132 -2.194136 -2.125971

H $-5.418362-3.183948-0.809782$

H $-4.2928185 .524519-0.374347$

H -4.2655525 .1448021 .353937$

H $-3.074936 \quad 6.2666280 .676416$

C -0.5319204 .6471041 .576114$

H $-0.8980820 .071547 \quad 1.153194$

H -2.386265 -0.685747 -1.806239

H $-2.398856-2.1879800 .433588$

H -0.0307604 .3074532 .491767$

H -0.7964175 .6990951 .732168$

H -2.3851863 .8618002 .312471$

C $-8.273749-0.6318230 .444306$

O $-7.070256-1.3796050 .228694$

C $-6.557020-1.362924-1.105926$

C $-7.585566-1.956780-2.079478$

C $-8.931171-1.230490-1.966085$

C $-9.389447-1.097875-0.510262$

H $-8.580790-0.8861421 .467044$

$\begin{array}{llll}\text { C } & -8.020971 & 0.884231 & 0.400749\end{array}$

H $-6.352454-0.332094-1.407334$

O $-7.771414-3.347465-1.825783$

H -7.199291 -1.866898 -3.101314

H -9.691913 -1.781875 -2.532349

O

H -10.245443 -0.415924-0.446318

O $-9.845987-2.383800-0.078968$

C -7.1722011 .3509481 .566949$

H -7.514181 $1.199862-0.514420$

H $-8.983857 \quad 1.4045300 .467476$

H -8.050613 -3.446313 -0.899378

H -8.564869 -0.032891 -3.463819

H $-10.207119-2.2817510 .818208$

O -7.2606012 .7107541 .665002$

O -6.5065260 .6242362 .291845$

C -6.5350033 .2997842 .749187$

H $-6.984613 \quad 3.0040583 .701675$

H -5.4810223 .0085792 .715150$

H -6.6008194 .3865912 .651180$

SCF Energy $\left(B 3 L Y P / 6-31 G^{* *} / / M M F F\right)=-3245.92163789$

$08 \_00123$

MMFF Geometry

C -2.2086862 .9762111 .787588$

C -1.3796641 .9486111 .554700$

C $-0.642687 \quad 1.2068512 .638811$

O 0.7403251 .0683612 .238239

C -1.228919-0.204784 2.797134

C $-0.670565-0.9391773 .999107$

C $0.262372-1.9095793 .940489$

C -1.273592 -0.551489 5.325234

C $0.971038-2.4334112 .722209$

C $2.482221-2.5558382 .964044$

C $3.196513-3.1777531 .755929$

C $4.654024-3.4940812 .091371$

C $5.407582-4.0587630 .878631$

O $3.141891-2.2452050 .671234$

O $6.802834-4.0350431 .215334$

C $5.184476-3.120933-0.325787$

C $5.044680-5.5252530 .617663$

O $5.726397-3.680009-1.520575$

C $3.700065-2.740425-0.553797$

C $3.620092-1.617906-1.613539$

O $2.985458-3.881640-1.031447$

C 1.5658002 .1242202 .466855

O $\quad 1.276753 \quad 3.1841672 .998213$

C 2.8953131 .7728741 .920538

C $3.8034912 .742771 \quad 1.738704$

C 5.1397112 .5803901 .162737

C $5.6292831 .178250 \quad 0.919743$

C $2.235559-0.980036-1.766898$

C $2.3291810 .224994-2.680995$

C $3.0686822 .610223-2.699109$

O $2.0798200 .143674-3.881374$

C $4.5548642 .796325-2.693182$

C $5.1605373 .934101-2.319563$

C $6.6523774 .117336-2.307452$ 
C $7.1689224 .692293-1.004909$

C $7.6482835 .946437-0.946563$

C 7.2008073 .7911050 .213364

C $5.8528493 .689408 \quad 0.875696$

N $2.7589341 .365219-2.019704$

H -2.3681103 .3108592 .809857$

H -1.218646 1.6154090 .534229

H $-0.697210 \quad 1.746787 \quad 3.592291$

H -1.055714 -0.7835171 .883368$

H -2.320828 -0.1511312 .905660$

H $0.548513-2.3949464 .872987$

H $-0.876681-1.1480126 .153536$

H -1.0676630 .4993195 .550331$

H -2.358555 -0.6973985 .305932$

H $0.562129-3.4251272 .495043$

H $\quad 0.799640-1.8030781 .845257$

H $2.893713-1.5587203 .167455$

H $2.659850-3.1756793 .851913$

H $2.663937-4.0938211 .474761$

H $4.719096-4.1885642 .938310$

H $5.159292-2.5741392 .418620$

H $7.298073-4.4370710 .481787$

H $5.750295-2.199329-0.128420$

H $5.298198-6.1411251 .488847$

H $5.624501-5.934404-0.217179$

H $3.984303-5.6730290 .402657$

H $6.680589-3.802684-1.383231$

H $4.332342-0.828213-1.350052$

H $3.931057-2.006099-2.592197$

H $2.199600-3.581323-1.514692$

H 3.0748340 .7390511 .648440

H 3.5324753 .7638932 .004920

H 6.6794051 .1265660 .625835

H 5.0457250 .7044990 .126776

H $5.5393030 .573523 \quad 1.829493$

H $1.516864-1.683005-2.200623$

H $1.830347-0.659605-0.800510$

H $2.5502063 .410631-2.162228$

H $2.7010922 .576950-3.729179$

H $5.1625581 .954180-3.016925$

H $4.5531844 .786304-2.022974$

H $6.9101204 .783211-3.141193$

H $7.1705303 .171114-2.507528$

H $8.0424196 .358069-0.023286$

H $7.6528496 .591824-1.818608$

H 7.9041894 .1882750 .956785

H $7.6069112 .821452-0.086170$

H 5.4161904 .6592381 .120464

H $2.9505641 .315098-1.023710$

C $-4.463598 \quad 3.511050 \quad 0.847738$

O $-4.8100652 .165747 \quad 0.461106$

C -6.096582 $2.188014-0.193165$

C $-6.5964813 .627698-0.111337$

C $-5.2961774 .406703-0.065156$

H $-5.9210781 .940453-1.245484$

C $-7.032237 \quad 1.1573640 .450450$

H -7.226450 $3.893998-0.965699$

H $\quad-7.1813593 .803140 \quad 0.799164$

C -5.4637375 .8297700 .438086$

H -4.859965 4.426011-1.073040

C $-6.509249-0.2924240 .366842$

O $\quad-7.1781541 .497707 \quad 1.835813$

H $-8.031199 \quad 1.2302850 .005261$

H $-5.545270-0.359788 \quad 0.881422$

O $-7.410548-1.1248751 .110709$

C $-6.399361-0.845556-1.069732$

C $-5.905328-2.306146-1.123021$

H $-5.739045-0.213686-1.671029$

O $-7.696829-0.787060-1.668588$

H -6.437024 -2.906617 -0.375767

H $-6.174988-2.729246-2.100023$

H -6.140031 $6.391257-0.214895$

H $-5.8797905 .850963 \quad 1.450960$

H $-4.503475 \quad 6.3543790 .457915$

C $-2.954970 \quad 3.7155320 .711401$

H $-7.759904 \quad 0.8300212 .237950$

H -8.266183 -1.115055 0.647965

H -7.604305 -1.065400 -2.595800
H -2.7038324 .7780660 .801239$

H $-2.6215413 .382475-0.278323$

H -4.7759873 .6372541 .893294$

C $-2.339825-1.924636-2.142640$

O $-3.772990-1.948767-2.133062$

C $-4.385162-2.414684-0.923361$

C $-3.938243-3.850638-0.619429$

C $-2.410346-3.940856-0.557768$

C $-1.753536-3.300432-1.784999$

H $-2.071307-1.712091-3.185975$

C $-1.801830-0.780200-1.279274$

H $-4.089441-1.767241-0.092971$

O $-4.439342-4.730313-1.622700$

H -4.371765 -4.159265 0.339507

H -2.115611 -4.994867 -0.489045

O $-1.963898-3.2859430 .625980$

H -0.670754 -3.208180 -1.640275

O $-1.948886-4.154862-2.915790$

C $-2.0642830 .544178-1.958812$

H $-2.258031-0.726713-0.288024$

H $-0.723157-0.886833-1.121864$

H -4.158321 -5.631424 -1.389475

H $-0.994839-3.3580910 .652404$

H -1.538348 $-5.012084-2.710385$

O $-0.905678 \quad 1.043576-2.474305$

O $-3.1703121 .062826-2.037070$

C -1.025191 $2.292470-3.162177$

H $-1.3177453 .080307-2.462089$

H $-0.0491792 .543364-3.585125$

$\mathrm{H}-1.7491412 .212034-3.978828$

SCF Energy $($ B3LYP/6-31G**//MMFF $)=-3245.89776028$

08_00124

MMFF Geometry

C -1.990794 3.701235-1.193634

C $-2.1724064 .338819-0.026699$

C -2.3812633 .6834061 .322343$

O -2.2401092 .2452651 .241703$

C -3.7885954 .0075131 .851900$

C -4.0117793 .5456083 .280273$

C -4.7602822 .4846373 .642843$

C -3.3501614 .3919784 .338565$

C -5.5002641 .5299032 .749301$

C -4.9242240 .1123672 .857082$

C $-5.534472-0.8292451 .809321$

C $-5.003903-2.2525341 .984208$

C $-5.501226-3.1939280 .876520$

O $-5.175851-0.3306390 .516493$

O $-4.707160-4.3896500 .925268$

C $-5.242477-2.537819-0.497543$

C $-6.953241-3.6284371 .108603$

O $-5.834954-3.302506-1.544166$

C $-5.722493-1.074318-0.580733$

C $-5.283624-0.359606-1.883032$

O $-7.155101-1.049553-0.552463$

C -0.9699421 .7503131 .245523$

O 0.0657162 .3889241 .350010

C -1.0515870 .2838241 .063464$

C $0.071097-0.4077640 .818301$

C $0.183785-1.8586850 .658849$

C $-1.080416-2.6723410 .710585$

C $-3.770283-0.258658-2.107331$

C $-3.198202-1.364608-2.978412$

C $-1.024878-2.211737-3.853689$

O $-3.891878-2.192686-3.562015$

C $-0.470813-3.287623-2.975223$

C $0.842193-3.519153-2.824993$

C $1.402242-4.656116-2.021027$

C $2.369934-4.204385-0.952511$

C $3.696165-4.219845-1.168541$

C $1.803651-3.8379250 .399245$

C $1.412087-2.3906940 .493010$

N -1.813575 -1.295180 -3.053544

H -2.035565 2.616487 -1.233415

H $-2.1198095 .425788-0.015357$

H -1.6283394 .0809312 .014230$

H -4.5358303 .5757241 .178589$

H -3.9616705 .0918021 .816077$ 
H -4.8565942 .2645474 .705526$ H -3.604700 4.0647565 .352379 H -2.2610564 .3474694 .244102$ H -3.666547 5.4357694 .242610 H -6.5543231 .5176463 .052251$ H $-5.4889281 .858740 \quad 1.708261$ H -3.8375990 .1571212 .714912$ H $-5.105940-0.2821343 .864543$ H -6.625397 -0.8091201 .916882$ H -5.268697 -2.647917 2.972735 H $-3.906520-2.2354891 .955148$ H $-4.801220-4.7759531 .812890$ H $-4.159184-2.566562-0.663442$ H -7.046734 -4.153905 2.066670 H -7.279815 -4.341653 0.343885 H $-7.654298-2.7914481 .123235$ H -5.488471 -4.208512 -1.473082 H $-5.792174-0.804177-2.747152$ H -5.660292 $0.672129-1.826425$ H -7.457226 $-0.210730-0.938350$ H $-2.024585-0.1850201 .143148$ H 1.0106590 .1396280 .748930 H $-0.932318-3.7233610 .453045$ H -1.816424 -2.288043 -0.003148 H -1.513234 -2.639287 1.715871 H $-3.562707 \quad 0.681374-2.634984$ H -3.229159 -0.225938 -1.156634 H $-0.239789-1.628500-4.343004$ H -1.650897 -2.659406 -4.632329 H -1.187551-3.933787 -2.473809 H $1.556433-2.888679-3.349624$ H $1.911187-5.334833-2.718266$ H $\quad 0.604527-5.254979-1.564254$ H $4.397597-3.931815-0.393048$ H $4.108957-4.515637-2.127248$ H $2.560805-4.0160781 .175493$ H $0.987314-4.5189440 .656126$ H $2.265229-1.7135220 .468565$ H -1.318329 -0.597896 -2.504552 C $-0.2336994 .179900-2.949014$ O $0.0768732 .774285-3.030130$ C $1.3744532 .545485-2.422160$ C $2.0254463 .917596-2.354867$ C $0.8335124 .803625-2.050722$ H $1.1582202 .169971-1.414914$ C $2.1080591 .478099-3.239849$ H $2.7991353 .989216-1.586434$ H $2.4660894 .189304-3.321923$ C $1.0821536 .277168-2.321457$ H $0.5783184 .678138-0.991638$ C $3.5653491 .138669-2.866799$ O $1.3319530 .277503-3.144219$ H $2.078527 \quad 1.785376-4.293354$ H $3.8864040 .352651-3.563006$ O $4.4160952 .252207-3.120984$ C $3.7870500 .584456-1.442307$ C $5.2409690 .103839-1.255913$ H $3.102104-0.252965-1.273842$ O $3.4882191 .596293-0.488014$ H $5.495302-0.573730-2.079907$ H $5.9068580 .974564-1.308029$ H $1.8986226 .650816-1.694901$ H $1.3545876 .453249-3.367515$ H $0.1883486 .869302-2.100915$ C -1.684292 $4.384527-2.495852$ H $1.737579-0.380734-3.733594$ H $4.4712932 .780082-2.307042$ H 3.5664381 .2025870 .397452 H -2.357684 $3.957592-3.249442$ H -1.908797 $5.455926-2.439852$ H $-0.1365914 .571218-3.970202$ C $5.616131-0.1528562 .464657$ O 5.4249290 .3450641 .133539 C $5.482685-0.6243940 .082415$ C $6.839631-1.3483640 .085553$ C $7.150894-1.9448281 .460244$ C $6.943358-0.9212872 .580005$ H 5.7009190 .7449303 .090896
C $4.404758-0.9602402 .957370$

H $4.682092-1.3569600 .222673$

O $7.868620-0.430895-0.280963$

H $6.818035-2.140708-0.672533$

H $\quad 8.189978-2.2956681 .473925$

O $6.305971-3.0725851 .668052$

H $6.996639-1.4149353 .556751$

O 8.0170410 .0237932 .549486

C $3.205915-0.0765343 .237154$

H $4.089231-1.7212432 .240433$

H $4.668045-1.4527103 .901364$

H $8.705579-0.925075-0.310091$

H $6.533007-3.4540092 .533230$

H 7.9443730 .5302641 .722230

O $2.297195-0.7883613 .966807$

O 3.0813541 .0803422 .860847

C $1.103101-0.1059004 .368790$

H 0.8434110 .7171893 .698004

H $0.282706-0.8285684 .366218$

H $\quad 1.2383450 .2713385 .385887$

SCF Energy $(B 3 L Y P / 6-31 G * * / / M M F F)=-3245.91585420$

08_00125

MMFF Geometry

C -1.6037653 .9394100 .952892$

C -1.1314312 .6928991 .102776$

C -0.6105032 .1371612 .401476$

O 0.7368091 .6657912 .169604

C -1.4780480 .9462012 .841013$

C $-1.0179120 .331528 \quad 4.148072$

C $-0.333978-0.8256564 .246688$

C -1.4161901 .0854405 .390060$

C $0.140191-1.6967203 .117609$

C $1.595383-2.1468883 .308925$

C $2.088930-2.9409482 .090625$

C $3.427781-3.6175592 .378074$

C $3.961364-4.3532961 .141060$

O $2.236242-2.0229441 .002158$

O $5.324420-4.7062901 .421097$

C $3.955711-3.383463-0.059041$

C $3.215244-5.6706300 .897641$

O $4.316968-4.085841-1.246441$

C $2.615373-2.626292-0.241688$

C $2.788446-1.497360-1.283942$

O $1.618611-3.535392-0.711690$

C 1.7391362 .5756662 .309310

O 1.6312343 .7408072 .658086

C 3.0045271 .9166861 .916828

C 4.0807012 .6790121 .671751

C 5.3870572 .2162011 .200326

C 5.6229060 .7315741 .129737

C $1.654190-0.464011-1.302457$

C $1.9515730 .608913-2.331808$

C $3.4931372 .535335-2.744699$

O $1.3873070 .633073-3.422561$

C $4.9853082 .419478-2.697685$

C $5.8061473 .428044-2.366862$

C $7.3018303 .293015-2.301200$

C $7.8836973 .776827-0.989074$

C $8.6284204 .894231-0.935570$

C 7.6659952 .9282260 .249136

C 6.3028243 .1422710 .846510

N $2.9355231 .487935-1.909321$

H -1.6204204 .6162991 .802869$

H -1.1220162 .0223610 .245892$

H -0.6122122 .9007893 .189332$

H -1.502742 0.1889312 .048809

H -2.521918 1.2689342 .956431

H $-0.091091-1.1949975 .242153$

H -1.1070830 .5708316 .306080$

H -0.9571772 .0787565 .401096$

H -2.503957 1.2035495 .430302

H $-0.504266-2.5828353 .080887$

H $0.050599-1.1908872 .152739$

H $2.231354-1.2646053 .457459$

H $1.665189-2.7651004 .212446$

H $1.332638-3.6916441 .834744$

H $3.344431-4.3073253 .227263$ 
H $4.163886-2.8584162 .678592$

H $5.694046-5.1132730 .618551$

H $4.753951-2.6505100 .125490$

H $3.316487-6.3315011 .766980$

H $3.645899-6.2201410 .053170$

H $2.149000-5.5314810 .707311$

H $4.487916-3.429285$-1.942079

H $3.725355-0.966658-1.080633$

H $2.883143-1.927366-2.289244$

H $0.960199-3.041728-1.226330$

H 3.0026790 .8401081 .792796

H 3.9874753 .7583861 .787891

H 5.3669180 .2525432 .081805

H 6.6640530 .4671920 .931660

H $5.014697 \quad 0.2843500 .339343$

H $0.694853-0.925624-1.551675$

H $1.5323320 .015494-0.324927$

H $3.138743 \quad 3.491806-2.347838$

H $3.1386102 .427019-3.773978$

H $5.4116241 .449966-2.946337$

H $5.3846834 .404641-2.139449$

H $7.7226593 .868053-3.136041$

H $7.6145242 .254106-2.464718$

H $9.0689475 .235405-0.004453$

H $8.8107465 .495842-1.819781$

H 8.4086113 .1847401 .015588

H $7.8557371 .883937-0.014338$

H $6.0447294 .195010 \quad 0.973244$

H $3.2688931 .424542-0.952303$

C $-3.6497534 .692765-0.283811$

O $-4.308174 \quad 3.442450 \quad 0.000995$

C $-5.3291253 .214140-0.982979$

C $-5.6236394 .582305-1.581456$

C $-4.2345505 .192170-1.607816$

H $-4.8826242 .575957-1.756054$

C $-6.5248582 .490463-0.356874$

H -6.083216 4.514881 -2.571951

H $-6.2818885 .176818-0.937656$

C -4.233059 $6.704624-1.746497$

H $-3.6807464 .759141-2.451777$

C $-6.146222 \quad 1.2516090 .481597$

O

H -7.240462 2.223218-1.143375

H -5.6756241 .5760941 .417662$

O

C $-5.229040 \quad 0.234636-0.222339$

C $-4.981922-1.0087610 .654304$

H $-4.2690610 .708579-0.454116$

O $-5.836824-0.146246-1.454702$

H -4.719024 -0.6848721 .668677$

H -5.910431 -1.591059 0.705750

H -4.705469 $7.005123-2.687420$

H $-4.7804877 .183283-0.927686$

H -3.210070 $7.094283-1.741260$

C $-2.1386344 .462542-0.351240$

H -7.9659482 .9208840 .895703$

H $-7.772491 \quad 0.243630 \quad 0.058335$

H -5.231835 -0.760032 -1.904404

H -1.622994 $5.398892-0.592365$

H -1.910128 $3.751447-1.155513$

H $-3.897951 \quad 5.3758770 .538274$

C -3.323064 -3.302350 -1.794971

O $-4.291062-2.484057-1.122001$

C -3.854972 -1.9131390 .115941$

C $-3.499215-3.0246191 .117072$

C $-2.472027-3.996506 \quad 0.530579$

C $-2.850868-4.449833-0.883360$

H $-3.875818-3.745293-2.633286$

C -2.199286 -2.434510 -2.380077

H -2.973021 -1.287639-0.064523

O $-4.681516-3.7411361 .469550$

H -3.105366 -2.564434 2.031448

H $-2.391217-4.8724611 .185836$

O $-1.203378-3.3532160 .508228$

H -2.007345 -4.971512 -1.347648

O $-3.910810-5.407090-0.786531$

C -1.367363 -3.158699-3.415461

H $-2.635291-1.552181-2.863964$
H -1.535537 -2.070926-1.591336

H -4.434659-4.404746 2.136067

H $-0.552200-4.0014800 .189979$

H $-4.692972-4.949878-0.432519$

O $-0.217591-2.454702-3.632267$

O $-1.687501-4.196996-3.977522$

C $0.686630-3.023361-4.584062$

H $1.029999-4.003193-4.238979$

H $\quad 0.206402-3.103348-5.563877$

H $1.550990-2.359450-4.670131$

SCF Energy $(B 3 L Y P / 6-31 G * * / / M M F F)=-3245.92897028$

08_00126

MMFF Geometry

C $-0.093752-1.8011082 .750201$

C $0.299920-2.8856392 .062842$

C $-0.299941-3.379480 \quad 0.767155$

O $-0.945316-2.2966330 .059966$

C -1.341482 -4.472377 1.049564

C $-1.872546-5.118970-0.214254$

C $-3.068155-4.839883-0.769820$

C $-0.980538-6.169469-0.823286$

C $-4.073105-3.822357-0.305246$

C $-4.620340-2.996535-1.478540$

C $-5.630465-1.946311-0.996579$

C $-6.339082-1.285101-2.178775$

C -7.301615 -0.181408 -1.715097

O $-4.916384-0.957237-0.246997$

O $\quad-7.682256 \quad 0.584819-2.868025$

C $-6.5459470 .771764-0.767205$

C $-8.596065-0.756658-1.128683$

O $-7.4323731 .727879-0.189897$

C -5.7499970 .0468360 .345930$

C -4.8414421 .0653201 .072108$

O $-6.660216-0.5178641 .290887$

C $-0.165048-1.562525-0.779917$

O $1.012196-1.751305-1.042699$

C $-0.984988-0.445060-1.296641$

C $-0.3684820 .572500-1.915760$

C $-0.995508 \quad 1.799667-2.406354$

C $-2.4983441 .849644-2.453316$

C -3.7990780 .4406632 .008126$

C -2.8744601 .5164752 .544864$

C -1.0259213 .0372571 .824360$

O -2.9976661 .9651953 .681718$

C -1.2700794 .0668770 .764749$

C -0.3019324 .6055630 .007646$

C $-0.5529005 .635596-1.057602$

C $0.0763865 .276888-2.387540$

C $1.1543605 .938341-2.841982$

C $-0.5870144 .202078-3.225593$

C $-0.1940262 .819735-2.777066$

N -1.9428641 .9338811 .607664$

H - $0.936351-1.2134402 .397348$

H $1.129036-3.4765982 .446349$

H $\quad 0.507279-3.7929620 .149501$

H -2.165698 -4.056929 1.641126

H $-0.901724-5.2566671 .680772$

H -3.364113 $-5.402152-1.654478$

H $-1.430449-6.636707-1.705743$

H $-0.027043-5.731372-1.134139$

H -0.778960 -6.963972 -0.097381

H -4.901406 -4.355082 0.177322

H $-3.647911-3.1438800 .439337$

H $-3.784999-2.500127-1.989373$

H $-5.100927-3.667795-2.201240$

H -6.360002 -2.440752 -0.344355

H $-6.873303-2.030422-2.781049$

H $-5.591558-0.836912-2.848533$

H -8.098043 -0.020392 -3.505827

H $-5.835217 \quad 1.341796-1.382337$

H -9.109831 -1.378244 -1.871790

H $-9.3002010 .041459-0.868372$

H -8.429456 -1.371780 -0.242054

H -7.898409 2.169039 -0.920612

H -4.3112131 .6631030 .322687$

H -5.4554081 .7651871 .653826$

H $-6.204726-0.6252192 .140764$ 
H -2.051513 -0.460968 -1.105125 H $0.7132860 .526952-2.037067$ H -2.885958 $2.705286-3.010375$ H -2.909621 $1.901893-1.442427$ H -2.902002 $0.960056-2.950216$ H -4.274987 -0.0584812 .858521$ H -3.193844 -0.3116401 .490156$ H -0.0137832 .6277181 .776539$ H -1.184952 3.4724862 .815270 H -2.298586 4.3886870 .615963 H 0.7304024 .3083870 .172951 H $-0.1491506 .590134-0.695955$ H -1.627253 $5.802190-1.205456$ H $1.5970485 .703026-3.804208$ H $1.6220766 .725525-2.259908$ H $-0.2871074 .306333-4.276475$ H -1.667355 4.368561-3.210336 H $0.8853732 .668361-2.731823$ H $-1.892811 \quad 1.4512900 .715793$ C $\quad 0.9504290 .153968 \quad 3.934911$ $\begin{array}{lllll}\text { O } & 1.787654 & 0.392699 & 2.788641\end{array}$ C 2.9684461 .0940573 .204288 C 2.6183981 .7134764 .549525 C $1.746453 \quad 0.6286415 .153463$ H $3.739540 \quad 0.3266543 .346957$ $\begin{array}{llll}\text { C } & 3.464736 & 2.070761 & 2.126274\end{array}$ H $3.504767 \quad 1.9382495 .150077$ H 2.0380982 .6360594 .434157 C $0.876972 \quad 1.1111416 .301616$ H $2.397768-0.1777945 .516688$ C $3.477210 \quad 1.4386350 .716781$ $\begin{array}{lllll}\text { O } & 2.647460 & 3.239687 & 2.128265\end{array}$ H 4.4776572 .3847452 .405835 H 3.8598600 .4166680 .799690 $\begin{array}{lllll}\text { O } & 2.142293 & 1.342212 & 0.222356\end{array}$ $\begin{array}{llll}\text { C } 4.350386 & 2.192747 & -0.302351\end{array}$

C $4.2216551 .698078-1.755261$ H 5.3964642 .1200590 .018878 O $4.0107813 .581285-0.292320$ H $3.2704482 .043510-2.180241$ H $5.0001122 .217025-2.330170$ H $1.4968151 .486127 \quad 7.122582$ H $\quad 0.209974 \quad 1.920754 \quad 5.986809$ H $\quad 0.257795 \quad 0.296068 \quad 6.689462$ C $0.579899-1.3304634 .009248$ H $3.0160133 .857726 \quad 1.474679$ H 1.8023202 .2473880 .123064 H $4.6155624 .036673-0.902744$ H $-0.095625-1.4992484 .855521$ H $1.484081-1.9258314 .187069$ H 0.0476950 .7615913 .797668 C $5.807036-1.678193-1.406511$ O $5.562024-0.264892-1.347398$ C $4.3477690 .178952-1.957598$

C $4.315181-0.198805-3.448748$

C $4.576575-1.692985-3.656994$

C $5.797523-2.172974-2.864873$ H $6.825579-1.802167-1.017297$

C $4.855919-2.477868-0.499724$ H $3.500428-0.299011-1.457636$ O $5.2860020 .552303-4.176339$ H $3.3333640 .066063-3.858593$ H $4.737159-1.880791-4.725802$

O $3.417913-2.417549-3.255929$

H $5.855041-3.267515-2.888221$

O $6.969182-1.680041-3.521046$

C $5.117312-2.2486320 .974227$

H $3.807849-2.230304-0.681830$

H $5.002152-3.548803-0.685371$

H $6.1564940 .375416-3.780472$

H $3.589175-3.360315-3.420946$

H $7.741855-2.040588-3.053560$

O $4.298330-3.0721841 .695333$

O $5.925168-1.456021 \quad 1.437498$

C $4.438152-2.9962193 .117055$

H $3.769693-3.7369803 .563977$

H $5.465861-3.2279523 .412299$

H $4.148291-2.0043043 .472441$
SCF Energy (B3LYP/6-31G**//MMFF)= -3245.91615989

08_00127

MMFF Geometry

C $-3.109045 \quad 4.3841180 .243554$

C $-4.151486 \quad 3.5411290 .217120$

C $-4.837578 \quad 3.010827 \quad 1.449151$

O -4.4504041 .6226381 .581115$

C $-6.364540 \quad 3.0898591 .276694$

C -7.1407602 .4882752 .433056$

C $-7.810091 \quad 1.3181912 .383762$

C $-7.147123 \quad 3.3078193 .697474$

C -7.9173230 .3719391 .219793$

C $-7.107704-0.9074251 .467193$

C $-6.802199-1.6543970 .160289$

C $-6.104326-2.9835710 .451361$

C $-5.644790-3.695730-0.829276$

O $-5.946878-0.812904-0.621887$

O $-4.743641-4.735879-0.418904$

C $-4.857726-2.696635-1.706586$

C $-6.815448-4.387089-1.537782$

O $-4.552497-3.262143-2.978796$

C $-5.583863-1.349664-1.900458$

C $-4.709727-0.288706-2.616092$

O $-6.743850-1.556892-2.715918$

C $-3.282767 \quad 1.3739142 .238785$

O -2.5705472 .1879942 .805660$

C -2.986627 -0.0697042 .112275$

C $-1.791271-0.5319192 .507961$

C -1.324467 -1.916875 2.420907

C $-2.240774-2.9238781 .778602$

C $-3.4252580 .114698-1.883371$

C $-2.185030-0.648123-2.318253$

C $0.219428-0.940122-1.722979$

O $-2.152330-1.373869-3.308060$

C $0.372602-2.221384-0.964878$

C $1.393674-2.466674-0.129290$

C $1.604336-3.7750530 .578503$

C $1.807662-3.6147842 .068782$

C $3.040752-3.6098052 .602379$

C $0.580386-3.5566092 .948974$

C $-0.099767-2.2149742 .902423$

N $-1.101261-0.386184-1.488736$

H $-2.7306114 .746915 \quad 1.195438$

H $-4.4912863 .155201-0.741816$

H -4.5423453 .5730682 .343775$

H $-6.645112 \quad 2.610787 \quad 0.333687$

H $-6.667616 \quad 4.139597 \quad 1.163129$

H -8.3355380 .9873193 .278629$

H -7.7782312 .8663494 .476107$

H -6.1353103 .3950854 .105042$

H -7.5303814 .3135003 .496167$

H -8.9734140 .1094581 .079711$

H $-7.610990 \quad 0.8461280 .285504$

H -6.161445 -0.6496381 .958224$

H $-7.662688-1.5584502 .154060$

H $-7.741865-1.820527-0.379436$

H $-6.752080-3.6406801 .045239$

H $-5.221451-2.7964261 .076750$

H - $4.476839-5.233569-1.210046$

H $-3.900579-2.515507-1.204095$

H $-7.256893-5.150766-0.886112$

H $-6.478928-4.917745-2.435155$

H $-7.611082-3.696334-1.824642$

H -3.989836 -4.040191-2.828966

H $-4.506891-0.599314-3.648144$

H $-5.3180070 .623417-2.703196$

H $-6.997517-0.707570-3.113801$

H $-3.744821-0.7061341 .672972$

H -1.0770630 .1720532 .933802$

H $-1.786097-3.9067871 .640880$

H -2.539744 -2.588452 0.780038

H $-3.139407-3.0651832 .388304$

H -3.222196 $1.169033-2.108696$

H $-3.5467860 .029766-0.800372$

H $0.949425-0.186351-1.413156$

H $0.349357-1.128872-2.793632$

H $-0.367917-2.997484-1.143031$ 
H $2.148688-1.699247 \quad 0.021693$

H $2.488238-4.2518750 .134205$

H $\quad 0.773156-4.4675510 .396251$

H $3.194585-3.5260763 .673049$

H $3.924720-3.6867191 .979170$

H $0.858398-3.7401543 .995741$

H $-0.085183-4.3853932 .692307$

H $0.494119-1.4124523 .342352$

H -1.233295 $0.191098-0.663288$

C -1.222375 $3.843831-1.299196$

O $-0.2021503 .950238-0.288259$

C $1.0915063 .805880-0.916127$

C $0.819933 \quad 3.451897-2.372312$

C $-0.5129604 .128284-2.618925$

H $1.5682714 .791314-0.843708$

C $1.9055862 .760037-0.142510$

H $1.6099743 .803084-3.042966$

H $0.7245852 .366143-2.499255$

C -1.239728 $3.597334-3.842675$

H $-0.3467225 .207734-2.732843$

C $3.2516842 .415296-0.808271$

O 2.1415413 .2752061 .170436

H $1.3130191 .846202-0.016561$

H $3.0562942 .036662-1.817843$

O $4.0260503 .605408-0.958619$

C $4.0772841 .346791-0.060352$

C $5.3456110 .973751-0.842328$

H 3.4539540 .4579630 .080402

O 4.4727521 .8117941 .226839

H $5.0819640 .664253-1.861743$

H $5.9769821 .865074-0.961811$

H -0.642275 $3.761540-4.745389$

H -1.433915 2.522609-3.759370

H -2.200556 4.103249 -3.978143

C -2.370895 4.807006-0.995037

H 1.2750323 .4839491 .560215

H $4.2223883 .937489-0.065691$

H 3.6648281 .9955241 .735251

H -3.065093 4.852392 -1.842199

H -1.989568 $5.822570-0.834567$

H $-1.5793252 .805991-1.278680$

C $8.359252-1.193658-0.579416$

O $7.360876-0.269694-1.030951$

C $6.217814-0.109882-0.179094$

C $5.500907-1.4558450 .008678$

C $6.462498-2.5481630 .486474$

C $7.749285-2.584610-0.344298$

H $9.062817-1.277837-1.418043$

C $9.136626-0.6549950 .631900$

H 6.5543230 .2699490 .791110

O $4.889589-1.865313-1.213189$

H $4.699784-1.3378260 .745915$

H $5.956768-3.5192670 .421944$

O $6.774249-2.3104841 .855970$

H $8.495940-3.235106 \quad 0.125823$

O $7.455953-3.144490-1.627918$

C 9.9473710 .5789950 .292508

H $8.471057-0.388344 \quad 1.456731$

H $9.840523-1.4202330 .979480$

H $5.584360-1.911314-1.892253$

H $7.358598-3.0285742 .153041$

H $7.116680-4.044429-1.484513$

O $10.609088 \quad 0.993234 \quad 1.414295$

O $9.999248 \quad 1.113377-0.806454$

C 11.4213802 .1603891 .253117

H 11.8890572 .3846782 .215436

H 12.2078651 .9754910 .515195

H 10.8039993 .0133140 .955404

SCF Energy (B3LYP/6-31G**//MMFF) $=-3245.91581361$

0800128

MM̄FF Geometry

C $1.5598762 .980469-0.718030$

C $\quad 0.5914543 .729797-0.172990$

C $-0.333483 \quad 3.2231690 .899173$

O $-1.673068 \quad 3.2275840 .353677$

C -0.3304894 .1215992 .148460$

C 1.0097284 .2158142 .849875
C 1.5480353 .2320953 .597417

C 1.7406955 .5206882 .673729

C 0.9478681 .8852773 .888260

C 1.7099660 .7694063 .161757

C $1.063149-0.6061653 .379700$

C $1.846306-1.6912272 .636294$

C $1.165962-3.0653132 .732416$

O $-0.281690-0.5514952 .889318$

O $1.761133-3.9271541 .752070$

C $-0.322300-2.9155172 .353213$

C $1.412124-3.7264444 .094008$

O $-1.027024-4.1364372 .566144$

C $-1.028075-1.7585743 .093404$

C $-2.451618-1.5456052 .526775$

O $-1.135811-2.0901994 .481273$

C $-2.1251792 .058127-0.177284$

O $-1.557246 \quad 0.977385-0.170392$

C $-3.4347072 .328513-0.810721$

C $-4.0975881 .322698-1.400419$

C $-5.3656671 .426889-2.122561$

C $-5.9712572 .795029-2.292523$

C $-3.121159-0.2554293 .022599$

C $-4.618769-0.4273483 .163321$

C $-6.716620-0.7119571 .895813$

O $-5.161726-0.4386574 .266715$

C $-7.141655-1.1533000 .533133$

C $-8.095006-0.527519-0.173556$

C $-8.571480-0.969769-1.528249$

C $-8.4488120 .124505-2.564863$

C $-9.4573890 .987418-2.775152$

C $-7.1954350 .178017-3.408319$

C $-5.9279560 .300595-2.607704$

N $-5.272975-0.5829961 .951044$

H $1.6773541 .948900-0.395097$

H $0.4567504 .756626-0.503872$

H $-0.0634282 .205274 \quad 1.198785$

H -1.091904 3.7616232 .853392

H -0.6832035 .1273391 .879534$

H 2.5206863 .4032344 .056310

H 2.7110145 .5265473 .181577

H $1.9241585 .719743 \quad 1.613545$

H 1.1468226 .3441983 .083547

H $\quad 0.991278 \quad 1.7139604 .970829$

H -0.1126851 .8602853 .620982$

H 1.7328070 .9915302 .087520

H 2.7511620 .7461863 .507359

H $1.046730-0.8119494 .456490$

H $2.879776-1.7461433 .000371$

H $1.918767-1.4166501 .575963$

H $2.711014-3.9934691 .948784$

H $-0.369285-2.7142351 .273927$

H $2.485904-3.8807454 .255742$

H $0.957445-4.7222584 .138299$

H $1.036608-3.1367614 .932631$

H -0.558462 -4.830365 2.071306

H $-2.408488-1.4923281 .431217$

H -3.075625 -2.410919 2.784793

H -1.942030 -1.693875 4.847642

H $-3.8018283 .348401-0.800448$

H $-3.6655150 .323172-1.364788$

H $-6.2055773 .235375-1.317060$

H -6.901357 $2.787196-2.865784$

H $-5.2797693 .459569-2.822212$

H $-2.737094 \quad 0.055128 \quad 4.000662$

H $-2.919424 \quad 0.5732672 .335235$

H -7.1465890 .2559832 .175705$

H -7.028165 -1.449767 2.642980

H $-6.664039-2.038490 \quad 0.120426$

H -8.5861180 .3425830 .257365$

H -9.622867 -1.271795 -1.432392

H $-8.039417-1.865870-1.870772$

H $-9.3925001 .760857-3.533168$

H $-10.3702700 .940635-2.190427$

H $-7.2460310 .983662-4.150995$

H $-7.136715-0.744772-4.000448$

H $-5.428483-0.652433-2.430354$

H -4.765728 -0.3819821 .093511$

C $3.9819383 .267478-1.405712$ 
O $4.267061 \quad 1.859887-1.298154$

C $5.2193431 .664774-0.232911$

C 5.7041543 .0560580 .166067

C $4.4644543 .891453-0.097722$

H $4.664778 \quad 1.2375090 .610497$

C $6.3178870 .696989-0.684829$

H 6.0360693 .0955861 .207962

H $6.5337973 .395335-0.464993$

C $4.7351305 .382121-0.185763$

H 3.7486043 .7048870 .712315

C $5.776382-0.657113-1.192200$

O $7.0408681 .308680-1.760958$

H 7.0386230 .5463000 .127259

H $5.185008-0.490999-2.098560$

O $6.898316-1.452749-1.597182$

C $4.955977-1.443118-0.151877$

C $4.468255-2.814599-0.657691$

H $4.096650-0.8500410 .175371$

O $5.779620-1.6554760 .998025$

H $5.304654-3.378827-1.087358$

H $4.137366-3.3924590 .214215$

H $5.143517 \quad 5.7548790 .759264$

H $5.4550145 .613958-0.977763$

H $3.8129165 .933076-0.395808$

C $2.5106633 .477383-1.768404$

H $7.7249500 .678412-2.045115$

H $7.418560-1.651582-0.799713$

H $5.229243-2.0795201 .678486$

H $2.2950032 .924549-2.690455$

H $2.3355974 .538905-1.979107$

H $4.5916993 .636302-2.241430$

C $1.040854-1.880958-1.787436$

O $2.198227-2.134351-0.981531$

C $3.312701-2.711479-1.669847$

C $2.922455-4.082538-2.241324$

C $1.688452-3.971048-3.143700$

C $0.561087-3.167421-2.482407$

H $0.270682-1.581135-1.064715$

C $1.294714-0.689695-2.722098$

H $3.612182-2.050518-2.489888$

O $2.652039-4.987497-1.172961$

H $3.771820-4.484041-2.806732$

H $1.318819-4.975596-3.383376$

O $2.048107-3.343586-4.370832$

H $-0.214496-2.924214-3.217023$

O $-0.054771-3.981853-1.478537$

C $0.021613 \quad 0.015400-3.137352$

H $1.9202760 .040464-2.193942$

H $1.833259-0.986986-3.625556$

H $2.432142-5.849233-1.565929$

H $2.738530-3.886590-4.787761$

H $-0.416834-4.768495-1.920659$

O $0.3483351 .187088-3.758707$

O $-1.112621-0.404402-2.954998$

C $-0.757443 \quad 1.981767-4.198269$

H $-1.3762191 .418269-4.903153$

H $-1.3496682 .310060-3.339518$

H $-0.3601712 .863767-4.707520$

SCF Energy (B3LYP/6-31G**//MMFF) $=-3245.90518239$

$08 \quad 00129$

MMFF Geometry

C -3.158002 $4.855633-0.093955$

C $-3.8535753 .711446-0.012502$

C -4.2693213 .0717481 .287110$

O $-3.756903 \quad 1.717151 \quad 1.295427$

C $-5.803866 \quad 3.016887 \quad 1.377791$

C -6.3106712 .4296952 .682192$

C -6.8689801 .2095842 .815619$

C $-6.185193 \quad 3.3318573 .883657$

C -7.0889250 .1751501 .747639$

C $-6.229610-1.0706011 .997703$

C $-6.220816-2.0091470 .782335$

C $-5.438056-3.2860691 .091393$

C $-5.293628-4.189950-0.141573$

O $-5.601508-1.304205-0.298733$

O $-4.295072-5.171953 \quad 0.175685$

C $-4.770393-3.345502-1.324856$
C $-6.585436-4.966647-0.420418$

O $-4.750671-4.115750-2.523942$

C $-5.546904-2.028861-1.533796$

C $-4.895325-1.097171-2.585399$

O $-6.863790-2.338918-2.005898$

C -2.4665621 .5477951 .698506$

$\begin{array}{llll}\text { O } & -1.700308 & 2.414080 & 2.089346\end{array}$

C $-2.133597 \quad 0.111047 \quad 1.572488$

C $-0.867320-0.2945361 .752694$

C $-0.381145-1.6755661 .696126$

C $-1.381451-2.7558151 .381517$

C $-3.476048-0.621027-2.254743$

C $-2.376567-1.465498-2.876748$

C $0.101253-1.746433-2.844740$

O $-2.584828-2.323117-3.730191$

C $0.486649-2.822333-1.879688$

C $1.704492-2.910462-1.324791$

C $2.121347-4.000963-0.382326$

C $2.629456-3.467602 \quad 0.937517$

C $3.944025-3.2900021 .150490$

C $1.624561-3.2414382 .042482$

C $0.924291-1.9138331 .943160$

$\mathrm{N}-1.123688-1.114727-2.391500$

H $-2.896092 \quad 5.385124 \quad 0.817925$

H $-4.1116843 .179149-0.925500$

H -3.8682183 .6244542 .145621$

H -6.1971872 .4608810 .521130$

H -6.2167764 .0299961 .276511$

H $-7.2068820 .905543 \quad 3.805766$

H -6.6394892 .8963524 .780113$

H -5.1330753 .5276354 .111676$

H -6.6854594 .2874313 .694877$

H -8.148423 -0.1088161 .751571$

H -6.8909700 .5746250 .751124$

H $-5.201476-0.7581772 .218476$

H $-6.605979-1.6023852 .880291$

H -7.257204 -2.246706 0.514833

H -5.901367 -3.8361601 .920001$

H $-4.432836-3.0163851 .440945$

H $-4.139832-5.703046-0.624550$

H $-3.724170-3.111508-1.097565$

H $-6.846315-5.5928180 .441454$

H $-6.460489-5.657139-1.261815$

H $-7.438777-4.318225-0.629234$

H $-5.662186-4.166852-2.858450$

H $-4.948792-1.554960-3.580438$

H $-5.520839-0.194897-2.648440$

H -7.226309 -1.552607 -2.446913

H $-2.939794-0.5744271 .340733$

H -0.1104950 .4545381 .981262$

H $-0.929897-3.7384701 .229294$

H -1.918297 -2.526799 0.455033

H -2.107121 -2.851637 2.195782

H -3.347270 $0.390228-2.662106$

H $-3.322750-0.558022-1.173472$

H $0.862157-0.962882-2.916994$

H $-0.052304-2.178197-3.838687$

H $-0.263014-3.573627-1.643043$

H $2.461695-2.176609-1.589978$

H $2.910394-4.583865-0.875736$

H $1.304054-4.710199-0.202259$

H $4.319794-2.938727 \quad 2.105579$

H $4.672398-3.4862660 .370771$

H $2.133942-3.2613873 .015988$

H $0.931038-4.0863322 .084883$

H $1.575952-1.0644142 .153176$

H -1.055838 -0.441372 -1.634112

C $-1.1756875 .332240-1.610683$

O $-0.772227 \quad 3.949421-1.520434$

C $0.3536673 .862601-0.621739$

C $0.9555235 .257182-0.605154$

C $-0.2976686 .112236-0.629527$

H $-0.063352 \quad 3.6195700 .362925$

C $1.2836252 .738798-1.085402$

H 1.5780145 .4414700 .275153

H $1.5602495 .431932-1.503463$

C $-0.0468127 .551893-1.043670$

H -0.7352476 .1091670 .375763$ 
C $2.5976662 .656870-0.284612$

O $0.5653641 .509001-0.955454$

H $1.5119582 .854418-2.151717$

H $3.1556843 .586438-0.445038$

O $2.3073312 .586297 \quad 1.111882$

C $3.5058741 .474162-0.680325$

C 4.8745451 .5608890 .009867

H $3.6394331 .479776-1.767336$

O $2.9093880 .233311-0.314684$

H $5.3525892 .520918-0.223679$

H 4.7368521 .5612471 .099905

H $0.6283868 .043572-0.335740$

H $0.4089367 .610730-2.037691$

H $-0.9834818 .117783-1.067490$

C -2.689194 5.447380-1.395676

H $-0.2646371 .606054-1.453470$

H 1.8317161 .7521841 .266786

H $2.0531250 .170556-0.770167$

H -3.194126 $4.936109-2.225622$

H $-2.9970176 .498216-1.440246$

H $-0.9522985 .645048-2.638883$

C $8.070558-0.3212350 .317928$

O $7.037960 \quad 0.6673840 .417233$

C $5.8382080 .403757-0.322087$

C $6.1526260 .266808-1.820748$

C $7.269812-0.750120-2.086461$

C $8.465857-0.556309-1.148618$

H $8.934810 \quad 0.126826 \quad 0.825781$

C $7.710367-1.6111781 .069179$

H $5.393719-0.5231050 .053793$

O $6.538616 \quad 1.533280-2.354431$

H $5.249630-0.046720-2.355725$

H $7.611444-0.631404-3.122021$

O $6.780298-2.083507-1.972090$

H $\quad 9.149257-1.411109-1.211353$

O $9.198380 \quad 0.598136-1.574399$

C $7.559547-1.3896942 .560090$

H $6.771371-2.0362270 .711240$

H $8.507938-2.3499210 .928491$

H $7.3087601 .845902-1.849168$

H $6.068029-2.105575-1.313261$

H $9.5113590 .429072-2.479398$

O $7.175162-2.5647903 .142321$

O $7.753531-0.3322853 .143384$

C $6.976666-2.5138174 .558570$

H $6.666270-3.5069514 .894194$

H $7.910844-2.2486115 .062587$

H $6.186828-1.7977034 .804762$

SCF Energy (B3LYP/6-31G**//MMFF) $=-3245.92858442$

0800130

MMFF Geometry

C $-0.570857-3.803590 \quad 0.925195$

C $-1.841583-3.6366940 .531651$

C $-2.278730-3.673419-0.909562$

O $-2.588740-2.308418-1.280197$

C $-3.544389-4.533169-1.062720$

C $-4.082342-4.569543-2.481309$

C -5.201980 -3.942022 -2.895905

C $-3.279570-5.395914-3.452760$

C $-6.128344-3.087680-2.076602$

C $-5.975910-1.601934-2.427448$

C $-6.535757-0.697777-1.319962$

C $-6.5029820 .769523-1.747963$

C $-6.9343871 .711282-0.613416$

O $-5.715660-0.886107-0.160269$

O $-6.5636693 .047998-0.985863$

C $-6.137121 \quad 1.3620630 .662110$

C $-8.4562051 .719511-0.427955$

O -6.6181712 .1034951 .780173$

C $-6.133617-0.1444590 .993443$

C -5.170872 -0.511735 2.151075

O $-7.449584-0.5371721 .403059$

C $-1.542320-1.533036-1.682815$

O $-0.392662-1.894846-1.880185$

C $-2.022919-0.138314-1.790116$

C -1.130386 $0.848772-1.957835$

C $-1.4232172 .280395-2.043960$
C $-2.8530052 .715446-1.865735$

C $-3.692534-0.1776321 .917592$

C $-3.259790 \quad 1.1631502 .487531$

C -1.301926 2.7034422 .553350

O $-3.967191 \quad 1.8409773 .227880$

C -1.4456863 .7736841 .518009$

C -0.4033624 .4172590 .969981$

C $-0.5392665 .554558-0.001814$

C $0.2464135 .340880-1.275504$

C $1.4834735 .846040-1.418907$

C $-0.4401654 .623833-2.415013$

C $-0.3997843 .131832-2.262385$

N -1.967909 1.4964582 .101537

H $0.197215-4.0253140 .188667$

H -2.592977 -3.367651 1.270794

H $-1.486480-4.069539-1.556908$

H $-4.308627-4.172909-0.367588$

H $-3.331201-5.563160-0.745903$

H -5.491181 -4.039964 -3.941432

H $-3.752051-5.453500-4.439232$

H $-2.282003-4.967898-3.591164$

H -3.171872 -6.420185 -3.081176

H $-7.160225-3.401672-2.277248$

H $-5.982284-3.248600-1.006548$

H $-4.914829-1.368217-2.575820$

H $-6.488812-1.400787-3.376068$

H -7.562045 -1.011657 -1.095671

H $-7.1250920 .929143-2.637541$

H $-5.4813351 .036812-2.048260$

H -7.000294 $3.256053-1.829677$

H $-5.105447 \quad 1.6896710 .490240$

H -8.951983 $2.049707-1.348903$

H -8.7564362 .4346880 .345676$

H -8.862126 $0.739329-0.169660$

H -6.5817493 .0454491 .540701$

H $-5.536727-0.0878773 .094189$

H -5.227566 -1.602020 2.283003

H -7.379955 -1.354887 1.922969

H -3.086876 $0.041544-1.697732$

H $-0.0756250 .587241-2.034474$

H $-3.2596632 .324890-0.926938$

H -3.468751 $2.352254-2.695308$

H $-2.9777103 .799241-1.820108$

H $-3.083964-0.9332392 .430842$

H $-3.441210-0.222540 \quad 0.854210$

H -0.2552322 .4494652 .743942$

H -1.749164 3.0404553 .493972

H $-2.4566394 .062178 \quad 1.239797$

H 0.6056014 .1471811 .273890

H -0.1845336 .4639900 .501319$

H $-1.5907055 .748520-0.247299$

H $2.0410805 .722543-2.341429$

H $1.9680386 .388563-0.613732$

H $\quad 0.058798 \quad 4.864997-3.363590$

H -1.455060 $5.013775-2.533810$

H $\quad 0.6023362 .717227-2.374687$

H -1.4692570 .8941061 .452552$

C $0.311685-2.1299652 .537122$

O $1.465093-1.8412101 .726216$

C $2.293542-0.8903192 .419595$

C $1.561516-0.5401043 .713005$

C $0.734500-1.7869143 .960560$

H $3.226444-1.4114892 .664888$

C 2.6028870 .3249171 .530499

H $2.254488-0.3159634 .530000$

H $0.9104680 .332186 \quad 3.583848$

C $-0.424032-1.5622824 .916645$

H $1.390281-2.5726344 .358579$

C $3.435261-0.0439690 .282754$

O $1.3688840 .929544 \quad 1.146917$

H 3.1606321 .0557072 .128877

H $4.297503-0.6266890 .622698$

O $2.679586-0.888753-0.583386$

C $3.9479181 .176205-0.510861$

C $4.7503790 .813622-1.776703$

H $4.561351 \quad 1.7964660 .153105$

O $2.8387631 .977653-0.920472$

H $4.1025010 .290337-2.491192$ 
H $5.0276801 .757258-2.265495$ H $-0.056755-1.2369665 .895574$ H -1.110039 -0.7945294 .542972$ H $-0.996857-2.4834265 .061073$ C $-0.115946-3.5853012 .340438$ H $1.576667 \quad 1.7325210 .640317$ H $1.880859-0.398400-0.843269$ H $3.1926252 .773915-1.352334$ H $-0.919140-3.8458153 .039015$ H $0.721669-4.2645082 .538359$ H $-0.487731-1.4525402 .206712$ C $8.0466880 .084373-0.186927$ O $6.8479730 .739291-0.622100$ C $6.013818-0.015905-1.504414$ C $6.777914-0.357225-2.791941$ C $8.089102-1.086285-2.475292$

C $8.896474-0.366555-1.388626$ $\begin{array}{llll}\text { H } & 8.612267 & 0.860131 & 0.345917\end{array}$ C $7.745467-1.0428170 .813458$ H $5.715646-0.949685-1.019307$ O $7.0600820 .826297-3.535477$ H $6.143760-0.989345-3.424166$ H $8.694565-1.157792-3.387153$ O $7.807915-2.411610-2.034589$ H $9.718986-0.999635-1.035699$ O $9.4855830 .808355-1.955081$ C $7.097974-0.5211912 .080860$ H $7.072682-1.7903440 .385169$ H $8.679564-1.5368641 .103935$ H $7.5595091 .428584-2.957744$ H $7.321442-2.861045-2.746568$ H $\quad 10.070801 \quad 0.523408-2.677498$ O $6.689008-1.5814662 .839192$

O $6.976386 \quad 0.6590692 .378628$

C $6.063340-1.2453464 .081843$

H $5.652131-2.1613594 .514180$

H $6.805596-0.8315514 .770644$

H $5.245432-0.5354583 .925768$

SCF Energy $\left(B 3 L Y P / 6-31 G^{* *} / / M M F F\right)=-3245.91786264$

\section{1}

MM̄FF Geometry

C -0.8790044 .0210621 .017646$

C $-0.4822702 .746163 \quad 1.146441$

C 0.0175992 .1443112 .433743

O 1.2698621 .4737612 .162275

C -1.0000341 .1036032 .935765$

C -0.5996520 .4363594 .237982$

C $-0.187200-0.8422474 .355333$

C -0.7186691 .3023475 .465929$

C $-0.011674-1.855383 \quad 3.259224$

C $1.468972-2.1987873 .055041$

C $1.710385-2.9173881 .719486$

C $3.174341-3.3408961 .594503$

C $3.493219-3.9334750 .213088$

O $1.375676-1.9964110 .676226$

O $4.922327-3.9864890 .078615$

C $2.971944-2.971417-0.877381$

C $2.993324-5.377396 \quad 0.088210$

O $3.123393-3.543996-2.174017$

C $1.515947-2.514188-0.652146$

C $1.068549-1.398963-1.628379$

O $0.643880-3.630903-0.863102$

C 2.3923522 .2447992 .145261

O 2.4613623 .4469052 .347194

C 3.5385631 .3668671 .819030

C 4.7533091 .9027061 .627663

C 5.9795231 .1729641 .295849

C $5.874014-0.3153411 .094236$

C $1.859658-0.089363-1.544494$

C $3.0078810 .012390-2.533885$

C $4.9543371 .467258-3.096647$

O $3.165551-0.769297-3.467216$

C $6.2009440 .898184-2.496030$

C $7.2894221 .633806-2.223082$

C $8.5679471 .067141-1.675387$

C $9.0482411 .791681-0.437886$

C $9.9841142 .752704-0.519393$
C 8.5119191 .3374410 .900269

C 7.1324891 .8649221 .181766

N $3.8141831 .112920-2.272522$

H -0.8405514 .6880201 .874737$

H -0.5395372 .0826450 .285835$

H $\quad 0.1684372 .9143543 .200497$

H -1.169151 0.3588822 .151864

H -1.976002 $1.585473 \quad 3.086264$

H $0.063292-1.2123535 .348846$

H $\quad-0.500670 \quad 0.750857 \quad 6.386808$

H $-0.0203892 .143187 \quad 5.414286$

H $-1.735794 \quad 1.697847 \quad 5.554482$

H $-0.559442-2.7650893 .534134$

H $-0.455195-1.5156932 .321646$

H $2.059046-1.2740883 .074304$

H $1.814969-2.8236513 .887651$

H $1.048190-3.7893191 .661819$

H $3.442778-4.0490372 .388572$

H $3.817780-2.4651301 .749316$

H $5.272301-4.5300090 .805289$

H $3.628714-2.094291-0.854571$

H $3.451147-6.0088930 .859300$

H $3.289902-5.817463-0.870290$

H $1.909977-5.4663170 .191640$

H $2.414783-4.198924-2.293632$

H $1.027932-1.785063-2.653849$

H $\quad 0.030970-1.147408-1.370673$

H $-0.254306-3.293988-1.017237$

H $3.3506540 .303076 \quad 1.736602$

H $4.8665862 .981678 \quad 1.726554$

H $6.793615-0.7739950 .725163$

H $5.103314-0.5502080 .352399$

H $5.619311-0.8101202 .037403$

H $1.1807880 .739457-1.784449$

H $2.2328380 .078943-0.530623$

H $4.9845352 .559772-3.155222$

H $4.8155961 .067467-4.106245$

H $6.219860-0.172662-2.307383$

H $7.2770902 .699347-2.441667$

H $9.3276421 .139453-2.464981$

H $8.471530-0.003814-1.457703$

H $\quad 10.356577 \quad 3.256318 \quad 0.366536$

H $10.3944843 .061159-1.475258$

H $9.164995 \quad 1.6966511 .707351$

H 8.5739740 .2473540 .963825

H 7.1068052 .9444311 .337591

H $3.6340031 .677233-1.447211$

C $-2.9373814 .768066-0.192136$

$\begin{array}{lllll}\text { O } & -3.556070 & 3.480581 & 0.006732\end{array}$

C $-4.5425183 .271704-1.013039$

C $-4.8964334 .663044-1.514181$

C $-3.5355105 .334110-1.482986$

H $-4.0462472 .712058-1.815622$

C $-5.7178252 .436483-0.489090$

H $-5.3455184 .647476-2.511636$

H $-5.5861485 .175006-0.833282$

C -3.600730 $6.851107-1.513238$

H -2.956434 $4.987941-2.349899$

C $-5.2579071 .085790 \quad 0.107890$

O -6.4305443 .1939700 .486763$

H $-6.3997802 .255210-1.328761$

H $-4.5019740 .653212-0.556447$

O $-4.641058 \quad 1.285716 \quad 1.379726$

C -6.3980190 .0612970 .263824$

C $-5.990940-1.2459050 .971502$

H $-6.797772-0.168877-0.731148$

O $-7.466913 \quad 0.6425401 .013646$

H -5.801707 -1.049527 2.034785

H $-6.869967-1.9037960 .948385$

H $-4.0776027 .197330-2.436021$

H $-4.1767327 .245020-0.669130$

H -2.596393 $7.283975-1.467612$

C -1.419933 $4.585299-0.266817$

H -7.1777872 .6523870 .791886$

H $-3.902317 \quad 1.9052251 .255432$

H $-8.192434-0.0042871 .041842$

H $-0.9281735 .544039-0.465684$

H $-1.1685303 .914558-1.098591$ 
H $-3.2089745 .387260 \quad 0.672100$

C $-4.007705-2.816777-1.782911$

O $-5.058532-2.195983-1.030094$

C $-4.782832-1.9707290 .355292$

C $-4.483366-3.2973101 .072515$

C $-3.358614-4.063096 \quad 0.367924$

C $-3.593293-4.154275-1.143347$

H $-4.454875-3.039620-2.760319$

C $-2.821608-1.867505-2.012215$

H -3.909646-1.318009 0.450132

O $-5.659445-4.1039961 .099256$

H $-4.205957-3.0825492 .111482$

H -3.288846 -5.0776530 .779015$

O $-2.115263-3.4105530 .604658$

H -2.692960 -4.533531-1.639896

O $-4.626340-5.111815-1.394016$

C $-3.164320-0.713874-2.931670$

H -2.452346 -1.435251-1.079050

H -2.008694 -2.426581-2.490437

H $-5.447182-4.9162341 .589945$

H $-1.966625-3.4039931 .565406$

H -5.453872 -4.760706 -1.022717

O $-2.015021-0.036404-3.229722$

O $-4.285712-0.433142-3.330609$

C $-2.163599 \quad 1.078640-4.113749$

H $-2.5350350 .741958-5.086238$

H -2.837148 $1.822632-3.680282$

H $-1.1799381 .535320-4.252170$

SCF Energy (B3LYP/6-31G**//MMFF) $=-3245.92061142$

\section{$08 \_00132$}

MMFF Geometry

C $-0.764592-1.4486800 .766938$

C $0.468717-1.332344 \quad 0.254472$

C $0.748897-1.206479-1.218239$

O $2.025942-1.844572-1.440589$

C $0.865538 \quad 0.280197-1.594172$

C $0.8409990 .537137-3.085907$

C $1.915046 \quad 0.881450-3.822853$

C $-0.5187730 .445301-3.729988$

C $3.3438771 .012323-3.372933$

C $3.8227012 .470901-3.340714$

C $3.2279923 .287757-2.181847$

C $3.6970124 .742883-2.250790$

C $3.2144255 .558730-1.041520$

O $3.6710562 .693922-0.958287$

O $3.9491546 .791984-1.019354$

C $3.572767 \quad 4.7961420 .251472$

C $1.7336345 .938080-1.162635$

O 3.0180385 .4420691 .395257

C $3.1523003 .308740 \quad 0.228226$

C 3.7602642 .5692541 .444969

O $1.727608 \quad 3.2255590 .291045$

C $2.213475-2.578042-2.564126$

O $1.392317-2.796562-3.443438$

C $3.584543-3.141870-2.607220$

C $4.565462-2.753965-1.776455$

C $5.933275-3.276111-1.737679$

C $6.280499-4.404978-2.671610$

C $3.5879461 .044562 \quad 1.398585$

C 4.3041450 .3491232 .542950

C $4.994763-1.9290153 .291386$

O $4.797752 \quad 0.9507003 .492790$

C $6.402673-2.1170452 .819006$

C $6.912254-3.2978462 .436299$

C $8.326260-3.4788171 .961280$

C $8.406959-4.0575320 .564687$

C $8.702060-5.3544140 .373531$

C $8.235292-3.118975-0.610184$

C $6.798837-2.742940-0.849709$

N $4.310828-1.0302962 .379373$

H -1.621616 -1.4761370 .098374$

H $1.317828-1.2785720 .931319$

H $-0.040282-1.711715-1.787664$

H $1.7771450 .692935-1.151325$

H $0.0390850 .850390-1.147836$

H $1.7663081 .073863-4.884760$

H $-0.4937160 .705793-4.793566$
H $-0.915695-0.571273-3.650590$

H -1.218139 $1.131245-3.240486$

H $3.5222650 .526678-2.409002$

H $3.9616650 .461738-4.094140$

H $4.9157622 .453925-3.238076$

H $3.5952582 .957546-4.297310$

H $2.1348253 .241240-2.234027$

H $3.3703105 .212828-3.186764$

H $4.7956854 .771297-2.269501$

H $3.7915597 .249979-1.862773$

H 4.6646514 .8447530 .367435

H $1.5651956 .538387-2.064738$

H $1.4167396 .565774-0.322447$

H $1.0712465 .071543-1.215742$

H 3.3169306 .3674541 .378596

H 4.8367362 .7806381 .491110

H 3.3133252 .9467132 .373439

H 1.4739632 .3734720 .677252

H $3.745387-3.892141-3.374606$

H $4.356052-1.977548-1.042270$

H $5.607673-5.255625-2.515147$

H $7.294908-4.785729-2.534910$

H $6.198909-4.078905-3.714376$

H 2.5291860 .7778201 .463970

H 3.9874140 .6469810 .458601

H $4.433102-2.8681623 .307202$

H $4.993841-1.5023844 .299421$

H $7.035894-1.2331132 .795159$

H $6.285469-4.1859252 .472776$

H $8.833384-4.1422412 .674076$

H $8.883416-2.5341171 .992366$

H $8.792496-5.773025-0.623152$

H $8.855390-6.0281911 .210036$

H $8.685594-3.542910-1.514775$

H $8.810217-2.202169-0.426137$

H $6.451584-1.927439-0.214959$

H $3.936840-1.4269311 .522011$

C -1.616220 -0.188343 2.794995

$\begin{array}{llll}\text { O } & -2.817957 & 0.181776 & 2.087729\end{array}$

C -2.7508911 .5870061 .782783$

C -1.6820812 .1592362 .706570$

C $-0.680666 \quad 1.0214312 .721385$

H $-2.393747 \quad 1.6680950 .748934$

C $-4.127245 \quad 2.244155 \quad 1.907771$

H $-1.262643 \quad 3.0965152 .328901$

H -2.0697112 .3381173 .716223$

C $\quad 0.324477 \quad 1.099182 \quad 3.856539$

H -0.1368931 .0374481 .770043$

C $-5.226781 \quad 1.6061081 .031852$

O $-4.552700 \quad 2.156115 \quad 3.273425$

H -4.0377013 .3139641 .683136$

H $-5.5080970 .635388 \quad 1.454045$

$\begin{array}{llll}\text { O } & -6.392654 & 2.433446 & 1.142854\end{array}$

C $-4.8632831 .442668-0.454957$

C $-6.0427111 .079993-1.377214$

H $-4.0792490 .683174-0.553324$

O

H $-6.7382871 .926692-1.440472$

H $-5.6289800 .966745-2.388393$

H 0.9212172 .0139993 .778841

H -0.1710561 .1029134 .832991$

$\begin{array}{llll}H & 1.010020 & 0.246316 & 3.828269\end{array}$

C $-1.049829-1.4999402 .241530$

H $-5.425672 \quad 2.5807793 .332858$

H $-6.185635 \quad 3.2888180 .728575$

H $-4.0166462 .532683-1.843784$

H $-1.778034-2.3037502 .403204$

H $-0.149034-1.7772622 .801863$

H $-1.918571-0.3599573 .836291$

C $-6.412826-2.547314-0.549097$

O $-5.876074-1.278494-0.949543$

C $-6.808003-0.194363-0.986341$

C $-7.949721-0.495961-1.972184$

C $-8.621370-1.835459-1.651909$

C $-7.592765-2.952888-1.449523$

H $-5.600275-3.264362-0.723880$

C $-6.756856-2.5795440 .948456$

H $-7.232088-0.0465570 .011446$ 
O $-7.435907-0.533018-3.302740$ H $-8.6820990 .318807-1.924139$ H -9.294173 -2.115774 -2.471500 O $-9.405894-1.708181-0.469981$ H -8.081081 -3.841520 -1.033474 O $-7.072030-3.337357-2.725760$ C $-5.531506-2.4182961 .822068$ H $-7.455609-1.7832201 .217677$ H -7.209855 -3.545085 1.200924 H -8.185733 -0.686174 -3.902629 H $-10.071216-1.017691-0.631637$ H -6.570769 -2.583606 -3.081495 O $-5.931102-2.2909293 .122090$ O $-4.376126-2.4120561 .422001$ C $-4.884790-2.1192974 .083246$ H $-4.092485-2.8603753 .939901$ H $-4.484389-1.1046954 .011626$ H -5.313595-2.257593 5.079392

SCF Energy (B3LYP/6-31G**//MMFF) $=-3245.91288273$

0800133

MM̄FF Geometry

C $0.621947-1.439206-2.293168$

C $-0.419068-2.050396-1.708972$

C $-1.610869-2.598286-2.450303$

O $-2.765053-1.852944-1.999482$

C $-1.811988-4.082453-2.096297$

C $-3.016303-4.712015-2.772087$

C $-4.157217-5.062175-2.143625$

C $-2.865141-4.963624-4.250429$

C $-4.488790-4.898249-0.686436$

C $-5.519729-3.782323-0.474231$

C $-5.516548-3.2663290 .972289$

C $-6.630193-2.2383301 .178899$

C $-6.567328-1.5830142 .566669$

O $-4.239351-2.6610481 .203189$

O $-7.415657-0.4243212 .545737$

C -5.129124-1.082457 2.821521

C $-7.121676-2.5066503 .657536$

O $-4.986606-0.603174 \quad 4.156126$

C $-4.049322-2.1425902 .525744$

C $-2.608869-1.5758112 .595083$

O $-4.137636-3.1870143 .502478$

C $-3.125708-0.765600-2.734467$

O $-2.643438-0.399274-3.794455$

C $-4.192629-0.056196-1.994838$

C $-4.5150821 .197445-2.345872$

C $-5.5216682 .046115-1.704543$

C $-6.273007 \quad 1.475288-0.531308$

C -2.287409 -0.4662851 .587718$

C $-2.462411 \quad 0.9428162 .127600$

C -2.424655 3.3200921 .372839

O $-2.618977 \begin{array}{lll}1.194737 & 3.318931\end{array}$

C $-3.830633 \quad 3.809131 \quad 1.223231$

C -4.1711554 .8467590 .443272$

C $-5.566278 \quad 5.3939130 .339057$

C $-6.0428695 .523432-1.090326$

C $-5.9522036 .693124-1.745816$

C $-6.7082694 .323934-1.725212$

C $-5.7170963 .291078-2.186992$

N -2.3739021 .8929681 .118294$

H $0.643558-1.326803-3.373823$

H $-0.431887-2.148906-0.625517$

H $-1.481645-2.494770-3.534433$

H $-1.880428-4.184175-1.008887$

H $-0.918496-4.653962-2.382602$

H $-4.954531-5.510291-2.735281$

H $-3.724810-5.495797-4.671668$

H $-2.765210-4.019359-4.794369$

H - $1.976542-5.573690-4.442830$

H $-4.899378-5.844652-0.313119$

H $-3.593367-4.717073-0.088589$

H -5.295312 -2.947152 -1.148839

H $-6.516422-4.155481-0.740660$

H $-5.644060-4.1172511 .651823$

H -7.614425 -2.693763 1.012412

H $-6.537743-1.4488120 .421747$

H -8.313416 -0.7143482 .309381$
H $-4.970969-0.2213102 .161759$

H $-8.171001-2.7522083 .454142$

H $-7.114620-2.0126754 .635431$

H $-6.574772-3.4481203 .741172$

H -5.6694010 .0752694 .294918$

H $-2.370288-1.2735643 .621853$

H -1.924539 -2.4072152 .371925$

H -3.285178 -3.651986 3.535987

H $-4.661836-0.571881-1.166102$

H $-3.9863541 .654299-3.181779$

H $-5.577097 \quad 1.1258210 .238501$

H $-6.8975920 .634634-0.851366$

H -6.929051 $2.195321-0.038169$

H $-1.228577-0.5501491 .311407$

H -2.872742 -0.5840500 .671846$

H -1.7415713 .8003270 .665083$

H -2.071103 3.5210782 .389192

H -4.5972023 .3114651 .812601$

H $-3.3961875 .362842-0.119409$

H -5.5716896 .3808780 .820403$

H $-6.279468 \quad 4.7854580 .908943$

H $-6.319686 \quad 6.802877-2.760665$

H -5.502881 $7.565698-1.283034$

H $-7.2831694 .636433-2.607559$

H $-7.458493 \quad 3.920845-1.039177$

H $-5.1155283 .618698-3.036145$

H -2.2990351 .5868960 .152600$

C $1.9689830 .626119-1.656693$

O $3.1548520 .987291-0.911093$

C $2.7970531 .955566 \quad 0.085727$

C $1.5528912 .636163-0.458246$

C $0.8183571 .464858-1.083308$

H $2.529344 \quad 1.3986480 .992018$

C 3.9653562 .9002380 .384175

H $0.973757 \quad 3.1363090 .323886$

H $1.8014913 .377640-1.226402$

C $-0.202448 \quad 1.913257-2.117732$

H $0.3041830 .907930-0.289605$

C $5.2785572 .198734 \quad 0.789827$

O $4.2390063 .678004-0.788602$

H 3.6605023 .6141151 .159040

H $5.7265091 .734553-0.095113$

$\begin{array}{lllll}\text { O } & 6.197127 & 3.219769 & 1.202226\end{array}$

C $5.1451901 .156347 \quad 1.913083$

C 6.4808440 .6581792 .497297

H 4.5697750 .2998251 .543944

$\begin{array}{lllll}O & 4.396178 & 1.734732 & 2.986628\end{array}$

H 6.9617981 .4652253 .065016

H $6.232015-0.1116523 .240068$

H $-0.9524992 .568166-1.662646$

H $0.2721002 .466884-2.934901$

H $-0.724271 \quad 1.059243-2.553317$

C $1.786692-0.890536-1.525098$

H $4.9770984 .274304-0.575068$

H 5.8489363 .6140192 .020441

H 4.2615691 .0428153 .656619

H $1.719779-1.156045-0.461580$

H $2.696257-1.389467-1.881705$

H $2.1666390 .889489-2.703000$

C $7.590192-1.589611-0.279863$

O $6.819185-0.971594 \quad 0.761141$

C $7.470185 \quad 0.085967 \quad 1.469727$

C $8.754651-0.427352 \quad 2.142105$

C $9.680024-1.1135171 .132059$

C $8.924255-2.1271840 .266122$

H $6.991028-2.452961-0.596999$

C $7.762859-0.663679-1.494949$

H 7.7337710 .8832070 .768154

O $8.439715-1.3452163 .188098$

H 9.2734300 .4193842 .606390

H $10.486508-1.6282841 .668519$

$\begin{array}{lllll}\text { O } & 10.276820 & -0.136737 & 0.284494\end{array}$

H $9.550651-2.462843-0.568775$

O $8.625969-3.2818591 .056654$

C $6.444615-0.354373-2.171819$

H $8.216736 \quad 0.289259-1.211130$

H $8.402629-1.148022-2.241489$

H $7.910447-2.0646212 .803023$ 
H $\quad \begin{array}{lll}10.775384 & 0.477764 & 0.849634\end{array}$

H $9.471263-3.6571901 .357255$

O $6.636924 \quad 0.649114-3.078304$

O $5.386464-0.924728-1.947651$

C $5.4744611 .062014-3.804122$

H $5.7965861 .726287-4.610523$

H $4.9623380 .201342-4.245105$

H $4.8029601 .614615-3.141699$

SCF Energy $\left(B 3 L Y P / 6-31 G^{* *} / / M M F F\right)=-3245.91582812$

\section{$08 \_00134$}

MM̄FF Geometry

C $-0.902009-3.4452551 .547491$

C -0.028401 -3.000242 2.464301

C $0.085908-1.5889382 .991820$

O $-0.980723-0.7508282 .495674$

C $1.416401-0.9786122 .519547$

C $1.7155570 .354153 \quad 3.173960$

C 1.5242651 .5497082 .583194

C 2.3117770 .2740774 .554810

C 0.9354911 .8020391 .222868

C 0.0489273 .0548421 .212820

C $-0.4199023 .395073-0.207926$

C -1.178975 4.721730-0.228098

C $-1.7070925 .048477-1.632737$

O $-1.2718862 .341174-0.667471$

O $-2.655466 \quad 6.119052-1.509800$

C $-2.4737733 .823840-2.173181$

C $-0.5954275 .556584-2.558665$

O $-2.8562374 .021276-3.532672$

C $-1.7018892 .489777-2.027808$

C $-2.641217 \quad 1.311054-2.368275$

O $-0.5972192 .480564-2.933475$

C $-2.135343-0.7067363 .212018$

O $-2.380737-1.2914634 .254498$

C $-3.078066 \quad 0.1596442 .470483$

C $-4.386746 \quad 0.0966972 .755805$

C -5.4552320 .8353562 .080082$

C $-5.052117 \quad 1.9038701 .099723$

C $-2.064406-0.075823-2.061263$

C $-3.142887-1.122441-2.253611$

C $-5.086512-2.166855-1.086334$

O $-3.308877-1.683518-3.334047$

C $-6.303716-1.294257-1.076789$

C -7.317841-1.440924 -0.210029

C $-8.536486-0.561091-0.199691$

C -8.872623 -0.0284991 .177760$

C $-9.957083-0.4653601 .840455$

C -7.9969131 .0649341 .756189$

C -6.7346020 .5136312 .363481$

N $-3.910974-1.316120-1.115912$

H -1.634776 -2.7682301 .119198$

H $0.695972-3.7039542 .871458$

H $\quad 0.057070-1.6215054 .087858$

H $1.408786-0.8712111 .427743$

H $2.248607-1.6622452 .736341$

H $1.8282712 .441893 \quad 3.128637$

H 2.5669301 .2608714 .955556

H $1.607325-0.1922605 .250345$

H $3.230225-0.3215604 .538790$

H $1.756398 \quad 1.9389520 .513413$

H $\quad 0.347441 \quad 0.948783 \quad 0.873110$

H $-0.8189322 .881275 \quad 1.862158$

H 0.6064793 .9058121 .623714

H $0.4630843 .453618-0.855061$

H -0.5485895 .5390240 .143751$

H -2.033684 4.6640610 .460660

H -2.201114 $6.876480-1.102592$

H -3.407238 $3.745451-1.597699$

H $-0.135017 \quad 6.461112-2.143209$

H $-0.9974105 .847313-3.535510$

H $0.1998494 .825148-2.716784$

H -3.348492 $4.858964-3.576417$

H -3.574820 $1.424632-1.805318$

H $-2.9097691 .344167-3.432309$

H $-0.3405441 .561690-3.108658$

H $-2.6761250 .788811 \quad 1.684301$

H $-4.715327-0.5893453 .535800$
H $-4.5694841 .456421 \quad 0.227332$

H -4.3532892 .6100381 .562521$

H -5.8894722 .5021180 .735382$

H $-1.229512-0.322105-2.725749$

H -1.684716 -0.136497 -1.035031

H $-5.015701-2.787444-0.187565$

H -5.100018 -2.816727 -1.966537

H $-6.355634-0.505729-1.824197$

H -7.280600 -2.247003 0.519314

H $-9.374680-1.153579-0.588708$

H $-8.4213630 .285282-0.888507$

H -10.218954 -0.071515 2.816946

H -10.604721 -1.2299371 .424526$

H -8.5378271 .6006362 .547277$

H $-7.810228 \quad 1.8071690 .975879$

H $-6.916886-0.2593953 .111905$

H $-3.704255-0.774558-0.282236$

C $-0.401769-5.009128-0.387315$

O $0.951384-4.519935-0.474441$

C $1.099266-3.796875-1.712564$

C $-0.128314-4.141460-2.549912$

C -1.189343 -4.280638 -1.474816

H $1.060636-2.730073-1.462058$

C $2.442420-4.133027-2.366642$

H $-0.360309-3.364888-3.285172$

H $-0.003006-5.089547-3.085713$

C -2.434676 -5.018575 -1.932559

H $-1.471418-3.275480-1.143166$

C $3.662939-3.868358-1.460819$

O $2.442371-5.527188-2.701451$

H $2.540108-3.589792-3.313631$

H $3.639418-4.556119-0.607283$

O $4.837057-4.213847-2.208876$

C $3.803905-2.418932-0.952900$

C $5.055530-2.245649-0.068364$

H $2.927568-2.139226-0.358358$

O $3.880817-1.526944-2.061661$

H $4.970740-2.9296050 .784857$

H $5.944768-2.528525-0.645499$

H -2.909115 -4.494461 -2.768239

H $-2.199690-6.034976-2.265656$

H -3.164667 -5.089828 -1.120176

C $-0.920528-4.862827 \quad 1.045633$

H $3.303773-5.727656-3.106112$

H $4.918547-3.574235-2.937297$

H $3.009334-1.510798-2.491373$

H $-0.302320-5.4880661 .702778$

H $-1.943696-5.2489231 .116726$

H $-0.352345-6.080791-0.622130$

C $5.9187511 .377613-0.377762$

O $5.746208-0.019994-0.651102$

C $5.244884-0.8017790 .439428$

C $6.234482-0.7421031 .613558$

C 6.5283390 .7029342 .029306

C 6.8543691 .5945290 .824605

H $6.4224601 .766925-1.271875$

C $4.5542122 .069262-0.265944$

H $4.272500-0.4071940 .756675$

O $7.456434-1.3905731 .263346$

H $5.811661-1.2930322 .461706$

H 7.3764640 .7145412 .724977

O $5.402748 \quad 1.2428942 .714751$

H 6.8443632 .6507881 .115768

O $8.1823951 .294950 \quad 0.381153$

C $4.6082843 .557204-0.534221$

H $3.8697521 .628422-1.001486$

H $4.1184601 .916808 \quad 0.723894$

H $7.805321-0.9561690 .466150$

H 5.2419430 .6888353 .497269

H $8.787977 \quad 1.493079 \quad 1.115712$

O $3.3496404 .072666-0.401146$

O $5.6134334 .189536-0.826309$

C $3.2281995 .479303-0.631883$

H $3.4992495 .716934-1.664897$

H $2.1846285 .761330-0.469077$

H 3.8556376 .0353780 .071081

SCF Energy (B3LYP/6-31G**//MMFF) $=-3245.91302139$ 
0800135

MM̄FF Geometry

C $-0.202975-0.8289511 .649604$

C $-0.209609-1.9321640 .884456$

C $-0.703958-2.039603-0.541993$

O $-1.117683-0.743588-1.025157$

C -1.944094 -2.949304 -0.591120

C $-2.202752-3.553912-1.955287$

C -3.088381-3.085319-2.855859

C $-1.420994-4.809021-2.249249$

C $-3.908286-1.827414-2.791528$

C $-5.396516-2.075013-2.507443$

C $-5.713484-2.164577-1.006356$

C $-7.202576-2.429587-0.778209$

C $-7.573786-2.3932920 .712746$

O $-5.351409-0.910621-0.417431$

O $\quad-9.007099-2.3420110 .777688$

C $-7.015979-1.0952931 .338598$

C -7.149696-3.680819 1.429501

O $-7.195692-1.0859622 .752996$

C $-5.528723-0.8408741 .001231$

C $-5.109258 \quad 0.577387 \quad 1.464000$

O $-4.725170-1.8066621 .683998$

C $-0.540926-0.229006-2.138560$

O $0.345598-0.711896-2.821113$

C $-1.1879381 .077207-2.372325$

C $-0.4595852 .101468-2.834761$

C $-0.9431393 .469893-3.022966$

C $-2.4305763 .700617-2.947810$

C $-3.757226 \quad 1.0376180 .897678$

C -3.2876062 .3531831 .496713$

C -1.3294913 .9057351 .467202$

O -3.9549773 .0030982 .296844$

C $-1.515778 \quad 4.993644 \quad 0.455887$

C $-0.5025455 .700868-0.068738$

C $-0.6876346 .836971-1.035651$

C $0.2088496 .736333-2.251042$

C $1.3629707 .421792-2.315994$

C $-0.2764795 .916792-3.426103$

C $-0.0366374 .446221-3.234077$

N -2.0290872 .7113891 .029244$

H $-0.568097 \quad 0.1105941 .247609$

H $\quad 0.156377-2.8615921 .317454$

H $\quad 0.111470-2.446892-1.150785$

H -2.822058 -2.383017 -0.262878

H -1.845564 -3.777305 0.124162

H -3.212008 -3.641536 -3.784722

H -1.647461 -5.219549-3.238908

H $-0.345844-4.606290-2.216061$

H -1.652198 -5.582939-1.509910

H -3.492765 -1.108166 -2.082047

H -3.822419 -1.347021 -3.775120

H -5.953102 -1.226897 -2.928101

H -5.742178 -2.977831 -3.025524

H -5.112560 -2.966095 -0.563724

H -7.500819 -3.385899-1.225832

H -7.791559-1.660816-1.298355

H $-9.268705-2.3753131 .713230$

H -7.609042 -0.2592080 .942316$

H -7.658401 $-4.546690 \quad 0.989025$

H -7.446098 -3.6636412 .484105$

H $-6.074680-3.8662501 .378121$

H -8.149882 -1.1375902 .929776$

H $-5.863233 \quad 1.3071081 .141209$

H -5.0732860 .6098842 .560328$

H $-3.850643-1.4279271 .864186$

H -2.229489 $1.174126-2.090308$

H $0.595806 \quad 1.935409-3.046218$

H -2.949445 $3.089519-3.695026$

H -2.723313 $4.735988-3.131937$

H $-2.8159583 .440159-1.956515$

H -2.987504 0.2864341 .093476

H $-3.8356291 .173129-0.186881$

H -0.2763423 .6354411 .594427$

H -1.726298 4.2291022 .434739

H $-2.536461 \quad 5.2337370 .167034$

H 0.5145485 .4837040 .249438

H $-0.4711487 .768172-0.495242$
H -1.732452 $6.920393-1.359725$

H $1.9969137 .379379-3.195506$

H $1.7024158 .035733-1.488299$

H $0.2557006 .215640-4.339316$

H -1.323535 $6.158316-3.627708$

H $1.0193244 .173696-3.265034$

H -1.5789002 .1329980 .326222$

C 1.2526620 .3012213 .379223

O $2.529518-0.0332292 .795855$

C 3.5341320 .0078413 .823356

C 2.9515090 .8869834 .920413

C 1.4965740 .4687834 .883309

H $3.633833-1.0166244 .203039$

C $4.885386 \quad 0.4797393 .273415$

H 3.4249800 .7133685 .891247

H 3.0413041 .9526184 .680467

C 0.5593381 .4641945 .546582

H $1.399505-0.5007765 .390710$

C $5.381324-0.2566182 .012381$

O $4.807971 \quad 1.8723522 .941125$

H 5.6394090 .4081284 .066002

H 4.8236330 .0978391 .139621

O $6.745620 \quad 0.126021 \quad 1.795113$

C $5.309394-1.7893702 .079350$

C $6.007855-2.5241810 .919593$

H $4.258553-2.0988222 .113835$

O $\quad 5.914397-2.2281003 .299229$

H $7.097452-2.4469931 .030061$

H $5.780357-3.5909891 .046912$

H $0.809719 \quad 1.5812846 .606019$

H 0.6229722 .4506815 .074908

H -0.4794861 .1258315 .479466$

C $0.235998-0.8074663 .086910$

H 4.0834461 .9791642 .301577

H 6.7692651 .0969761 .741893

H $5.807688-3.1933423 .349057$

H -0.660664 -0.651499 3.698451

H $0.661300-1.7797183 .365248$

H 0.9359751 .2499462 .927652

C $3.622352-1.642224-1.831164$

O $4.173826-2.090800-0.584731$

C $5.600182-2.067713-0.492817$

C $6.230586-2.953201-1.582040$

C $5.720667-2.571515-2.974803$

C $4.192277-2.454458-3.006788$

H $2.552783-1.872365-1.757895$

C $3.769181-0.122347-2.024146$

H $5.950780-1.039486-0.626905$

O $5.921902-4.322286-1.326090$

H $7.321027-2.845888-1.535429$

H $6.045660-3.330697-3.696450$

O $6.314848-1.333777-3.353727$

H $3.859451-2.008845-3.951410$

O $3.620175-3.763669-2.940277$

C $2.9420740 .670575-1.036047$

H $4.8058530 .204549-1.911622$

H $3.4189240 .149839-3.026697$

H $\quad 6.367039-4.855954-2.006104$

H $5.995915-1.116104-4.246174$

H $3.944553-4.264711-3.707807$

O $3.1117012 .003743-1.279562$

O $2.2397810 .189740-0.159197$

C $2.3948142 .885873-0.409605$

H $1.3352582 .617261-0.367105$

H 2.8355502 .8601550 .590374

H $2.4826543 .900254-0.807326$

SCF Energy (B3LYP/6-31G**//MMFF)= -3245.89101287

\section{6}

MM̄FF Geometry

C $-2.458599-3.7238340 .574306$

C $-2.032849-4.3423391 .686338$

C $-0.999455-3.8099152 .654734$

O $-0.639750-2.4452342 .343046$

C $0.279742-4.6604322 .553065$

C $1.321644-4.2975323 .592338$

C $2.389016-3.5079003 .360466$

C $1.118875-4.9215954 .948203$ 
C $2.728156-2.7747252 .092535$

C $3.961299-3.3709331 .401847$

C $4.282989-2.6356040 .092345$

C $5.474236-3.273540-0.624346$

C $5.863302-2.489835-1.889938$

O $4.586345-1.274166 \quad 0.409823$

O $7.143180-2.965382-2.333465$

C $5.999985-0.990108-1.539851$

C $4.901544-2.781716-3.047618$

O $6.202210-0.206421-2.713060$

C $4.803120-0.437483-0.734059$

C $5.1004510 .999679-0.236956$

O $3.650253-0.394062-1.579161$

C -1.412351-1.442818 2.841437

O $-2.398565-1.5498373 .550739$

C $-0.843262-0.1741692 .334129$

C $-1.468461 \quad 0.9931982 .550481$

C -1.0123852 .3033632 .074075$

C 0.2944752 .3390831 .328106

C 4.1468811 .4563970 .873780

C 4.2764712 .9302841 .220261

C 3.3970404 .5771262 .891501

O $4.935158 \quad 3.7287600 .562145$

C 2.0728854 .6715933 .583166

C 1.1331995 .5877823 .303855

C -0.2080965 .6138343 .984198$

C - -1.3683015 .7830633 .023503$

C -2.2420566 .7917323 .185622$

C -1.524883 4.8109501 .872506

C -1.7698243 .3937472 .317608$

N 3.5165813 .2399852 .339352

H -2.044758 -2.756798 0.303366

H $-2.451069-5.3158801 .933867$

H - $-1.406250-3.8598313 .672297$

H $0.707612-4.5689931 .546986$

H $0.034793-5.7253192 .665845$

H $3.088891-3.3357764 .177242$

H $1.910551-4.6492925 .654448$

H $0.166883-4.5980415 .380584$

H $1.111387-6.0134064 .866362$

H $1.887324-2.7609851 .393099$

H $2.915788-1.7261552 .355068$

H $4.818577-3.3011632 .084029$

H $3.789934-4.4342941 .193221$

H $3.392063-2.668849-0.544947$

H $5.267300-4.322390-0.870311$

H $6.337616-3.2904710 .055264$

H $7.785299-2.823363-1.617307$

H $6.906651-0.874868-0.929901$

H $4.917459-3.849982-3.295657$

H $5.209803-2.259077-3.959968$

H $3.867099-2.509743-2.828068$

H $6.964139-0.582948-3.185761$

H 6.1192831 .0510110 .168100

H $5.0504471 .698977-1.080953$

H $3.0306150 .264506-1.229771$

H $0.082123-0.2425631 .772189$

H $-2.4020890 .992096 \quad 3.110874$

H 1.0923461 .9375301 .959938

H 0.6086953 .3372261 .021241

H 0.2402431 .7334550 .419854

H 3.1061581 .2860870 .578593

H $4.3396570 .872991 \quad 1.782440$

H 3.5111485 .3159792 .091850

H 4.2111354 .7122393 .610323

H 1.8683363 .9270074 .350074

H 1.3342066 .3499772 .555083

H $-0.192514 \quad 6.4387014 .708281$

H -0.3681904 .6983834 .567437$

H -3.0767476 .9329982 .507136$

H -2.1448277 .5003794 .001458$

H -0.6625084 .9065441 .208469$

H $-2.384577 \quad 5.098679 \quad 1.251974$

H -2.703167 3.2640772 .866866

H 3.1558282 .4702932 .896576

C $-4.732425-3.424474-0.504014$

O $-4.395424-2.098415-0.961280$

C $-5.230576-1.151598-0.266213$
C $-6.310254-1.9724340 .433868$

C $-5.548468-3.237640 \quad 0.774428$

H $-4.603294-0.6811610 .499140$

C $-5.778961-0.099061-1.234640$

H $-6.713173-1.4619731 .313970$

H -7.146618 -2.198442 -0.238239

C $-6.441364-4.4165331 .117319$

H -4.893894 -3.024699 1.628850

C $-4.6981860 .780087-1.899961$

O $-6.503252-0.782001-2.265825$

H $-6.5077820 .533423-0.713339$

H $-4.0709260 .170494-2.560715$

O $-5.3718091 .728223-2.738615$

C $-3.8055681 .563613-0.914518$

C $-2.9628552 .676961-1.562863$

H $-3.1434460 .875618-0.377307$

$\begin{array}{lllll}\text { O } & -4.620846 & 2.219772 & 0.063661\end{array}$

H -3.623813 $3.412620-2.038958$

H -2.471331 $3.218972-0.744186$

H -7.055034 -4.191197 1.995635

H $-7.115836-4.6638480 .290780$

H $-5.843315-5.3052181 .342548$

C $-3.475187-4.287392-0.376511$

H $-7.024079-0.117076-2.746863$

H $\quad-5.733468 \quad 1.241257-3.498122$

H $-4.954227 \quad 1.5446710 .677589$

H -2.992258 -4.354632 -1.359306

H $-3.755692-5.307272-0.089115$

H $-5.365087-3.862264-1.287814$

C $0.1513640 .885023-2.585935$

O $-0.9640571 .393022-1.842501$

C -1.899132 $2.199540-2.563655$

C $-1.1989783 .398430-3.230024$

C $-0.0087762 .951807-4.081044$

C $0.9135272 .011344-3.302525$

H $0.8181110 .466420-1.822409$

C $-0.266501-0.252003-3.523797$

H $-2.379746 \quad 1.589891-3.334765$

O $-0.7518294 .311918-2.228634$

H $-1.9300703 .927671-3.853224$

H $0.5567543 .835624-4.400783$

O $-0.5007232 .302025-5.248754$

H $1.6679001 .583004-3.972838$

O $1.6167382 .785885-2.327012$

C $-0.528175-1.508555-2.730461$

H $-1.173320-0.032803-4.094403$

H $0.518794-0.475294-4.256057$

H $-0.3444745 .069259-2.682357$

H $0.2674262 .045485-5.786988$

H $2.2531682 .198863-1.885479$

O $0.641309-2.180490-2.527222$

O $-1.633555-1.834151-2.319273$

C $0.544370-3.381071-1.755139$

H $-0.255805-4.025969-2.131062$

H $1.492658-3.918131-1.841177$

H $\quad 0.378189-3.128097-0.704883$

SCF Energy $(B 3 L Y P / 6-31 G * * / / M M F F)=-3245.89001909$

0800137

MM̄FF Geometry

C $2.918628-0.7524703 .474002$

C 3.7461110 .2723193 .215453

C 4.8243350 .3466422 .160505

O 4.3262231 .1585581 .069121

C $5.242583-1.0003391 .542191$

C $6.637143-0.9702360 .947157$

C $6.927717-0.748181-0.349249$

C $7.746336-1.2685881 .924729$

C $5.994279-0.385171-1.469528$

C $5.769205-1.538520-2.456817$

C $4.658795-2.496293-2.000709$

C $4.541853-3.688883-2.950551$

C $3.362051-4.599386-2.577362$

O $3.433968-1.753625-1.996923$

O $3.134858-5.498628-3.673125$

C $2.093139-3.734228-2.431461$

C $3.674952-5.472202-1.356303$

O $0.998782-4.509695-1.946610$ 
C $2.291342-2.484460-1.540803$

C $1.056657-1.560090-1.661676$

O $2.436142-2.898393-0.180964$

C 4.3506902 .5103571 .180490

O $4.747475 \quad 3.1713872 .124974$

C $3.7724293 .045919-0.071592$

C $3.3191834 .306380-0.115649$

C $2.6927454 .952447-1.271930$

C $2.6211424 .167463-2.556580$

C $1.230147-0.168427-1.034954$

C $-0.0591420 .636662-1.095058$

C $-1.0448942 .794672-0.328031$

O $-1.0792950 .230001-1.646074$

C $-0.9276873 .854830-1.376576$

C $-1.0032605 .167882-1.109339$

C $-0.9285666 .240723-2.158596$

C $0.0753697 .322806-1.824998$

C $-0.3235928 .504154-1.323954$

C $1.5295317 .067536-2.153952$

C $2.2066616 .202748-1.124895$

N $0.060894 \quad 1.862433-0.454174$

H $2.963851-1.6607152 .880699$

H 3.6491101 .1684323 .826772

H 5.7012170 .8336182 .606464

H $4.517038-1.2980590 .777867$

H $5.232149-1.7958062 .298253$

H $7.974169-0.792340-0.649969$

H $8.735954-1.2354321 .456735$

H $7.743986-0.5385102 .740285$

H $7.616652-2.2682552 .352366$

H $5.0411980 .001675-1.100774$

H $6.4591310 .448483-2.011838$

H $5.476220-1.097013-3.418500$

H $6.704352-2.087297-2.622749$

H $4.880290-2.843914-0.986144$

H $5.477238-4.261938-2.973149$

H $4.388100-3.325766-3.976371$

H $\quad 3.953890-6.001570-3.822732$

H $1.811594-3.398540-3.439572$

H $4.547405-6.106750-1.553041$

H $2.848710-6.158439-1.139594$

H $3.886335-4.891463-0.456133$

H $\quad 0.900571-5.274439-2.539297$

H $0.818421-1.410834-2.723283$

H $0.191756-2.052091-1.202461$

H $2.133438-2.1870250 .404394$

H $3.7081812 .371633-0.918430$

H $3.395606 \quad 4.9176790 .782588$

H $3.6287693 .934485-2.918476$

H $2.1073684 .692972-3.363580$

H $2.0773023 .228203-2.408495$

H $1.531160-0.2492360 .014128$

H $2.0035940 .392340-1.571702$

H -1.005339 3.2097520 .683501

H -1.992027 2.259628 -0.448855

H $-0.8009013 .527640-2.405910$

H -1.162319 $5.491284-0.082870$

H -1.930285 $6.679739-2.254564$

H $-0.6935905 .820567-3.144438$

H $0.3865569 .295727-1.108721$

H -1.368713 8.704069-1.112381

H $2.0794178 .017880-2.188207$

H $\quad 1.6039476 .669759-3.169600$

H $2.3051026 .684620-0.150930$

H $0.9558722 .130474-0.056168$

C $0.444369-0.6935184 .087229$

O $0.099826-1.9308483 .436591$

C $-0.756816-1.6334972 .310755$

C -1.162716 -0.1730802 .470795$

C 0.0806520 .4175643 .104044

H $-0.131261-1.7660721 .420261$

C -1.935617 -2.614462 2.284722

H -1.432589 $0.298343 \quad 1.523371$

H -2.018751 -0.080779 3.150732

C $-0.132248 \quad 1.770398 \quad 3.757046$

H 0.8433580 .5169712 .322705

C -2.873886 -2.385718 1.082089

O $-1.400294-3.9393712 .220779$
H $-2.500908-2.541618 \quad 3.221446$

H -3.156109 -1.328778 1.051442

O $-2.158859-2.655044-0.124067$

C $-4.173050-3.2162141 .110893$

C $-5.001110-3.146586-0.187448$

H $-4.781254-2.8794921 .958972$

O $\quad-3.867782-4.592757 \quad 1.342001$

H $-4.474877-3.684456-0.986785$

H $-5.922359-3.717137-0.007855$

H -0.4639442 .5067863 .018964$

H $-0.887901 \quad 1.7205894 .547952$

H 0.7977002 .1372914 .202843

C $1.894123-0.7218664 .575625$

H $-0.805315-4.0488572 .982332$

H -1.891835 -3.590173 -0.100177

H $-4.710014-5.0714801 .425863$

H $2.048905-1.6188215 .188114$

H 2.0667860 .1407555 .230806

H $-0.204435-0.6334824 .971497$

C -6.4798010 .2752350 .116226$

O $-6.093004-1.0817440 .376316$

C $-5.355441-1.728955-0.663886$

C $-6.162893-1.755213-1.972029$

C $-6.611969-0.345374-2.367995$

C $-7.2761580 .383392-1.196531$

H -7.1621980 .5336450 .936557$

C $\quad-5.280370 \quad 1.235638 \quad 0.180471$

H $-4.424925-1.182129-0.842648$

O $-7.304635-2.595280-1.816507$

H -5.539001 -2.190325 -2.762084

H $-7.320752-0.406270-3.202794$

O $\quad-5.4888620 .413624-2.805890$

H $-7.4320811 .440450-1.441870$

O $-8.568336-0.183835-0.962307$

C -4.8118631 .4536221 .604198$

H $-4.4189400 .883915-0.392054$

H $-5.5888892 .211506-0.213545$

H $-7.775734-2.610943-2.666911$

H $-5.090343-0.057281-3.557421$

H $-9.087992-0.075809-1.776957$

O $-4.1305002 .635291 \quad 1.671586$

$\begin{array}{llll}\text { O } & -5.004440 & 0.682128 & 2.533807\end{array}$

C -3.6538663 .0088402 .967915$

H -3.1655092 .1679503 .467631$

H -2.9232993 .8123692 .842618$

$\mathrm{H}-4.4877333 .3792883 .570918$

SCF Energy (B3LYP/6-31G**//MMFF) $=-3245.89549250$

0800138

MM̄FF Geometry

C $-2.7114734 .392540 \quad 0.802769$

C $-3.680428 \quad 3.469048 \quad 0.725097$

C -4.2692812 .7475331 .908738$

O $\quad-3.8634401 .3625061 .821444$

C -5.8059322 .8266611 .842780$

C -6.5128781 .9752822 .878651$

C $-7.147643 \quad 0.8150872 .612528$

C -6.4896492 .5222854 .281821$

C -7.2580790 .1133541 .285727$

C $-6.508414-1.2250861 .304448$

C $-6.191829-1.730934-0.111869$

C $-5.556964-3.120504-0.049563$

C $-5.080897-3.607324-1.425501$

O $-5.280268-0.799954-0.706693$

O $-4.220326-4.738556-1.215438$

C $-4.223696-2.503910-2.084123$

C $-6.248710-4.105558-2.285678$

O $-3.898042-2.883349-3.419369$

C $-4.889462-1.110868-2.051885$

C $-3.9562580 .030925-2.531907$

O $-6.024975-1.117736-2.925658$

C -2.677324 1.0297522 .404686

O -1.9210391 .7719353 .011146$

C -2.440777 -0.4098462 .158670$

C $-1.285450-0.9744422 .540164$

C $-0.904506-2.3746322 .339461$

C -1.855826 -3.252051 1.569290

C $-2.6918710 .251069-1.695132$ 
C $-1.462837-0.492682-2.189376$

C $0.886223-1.011554-1.519155$

O $-1.409432-1.056782-3.278611$

C $0.919942-2.393633-0.944674$

C $1.872270-2.821381-0.101267$

C $1.952737-4.224410 \quad 0.431125$

C $2.102316-4.2799681 .936191$

C $3.301173-4.4938942 .504325$

C $0.850800-4.1940552 .780090$

C $0.275232-2.8038212 .834874$

N $-0.415518-0.415992-1.279152$

H -2.2961414 .6631621 .769478$

H $-4.0615103 .192252-0.256225$

H -3.9266773 .1829722 .855882$

H -6.1426472 .5596360 .836176$

H -6.1225953 .8708871 .971926$

H -7.633160 0.2936933 .436673

H -7.0554341 .8975964 .981188$

H -5.4619122 .5834944 .652931$

H -6.9297143 .5245664 .305943$

H $-8.318466-0.0693101 .073160$

H -6.8953360 .7361810 .466180$

H -5.568620 -1.105673 1.857167

H -7.109685 -1.967047 1.844444

H -7.119546 -1.754654 -0.695522

H $-6.250885-3.8443170 .395877$

H -4.690175 -3.0908530 .622810$

H $-4.727770-5.417008-0.737987$

H $-3.280200-2.468859-1.526540$

H -6.759343 -4.940578 -1.790959

H -5.894412 $-4.498996-3.244854$

H -6.996142 -3.334196 -2.482124

H $-3.221923-2.267867-3.748080$

H $-3.721449-0.088383-3.596099$

H $-4.5344680 .964608-2.469386$

H $-6.236425-0.200405-3.165669$

H -3.218895 -0.9660261 .650649$

H $-0.544826-0.3562653 .046036$

H $-2.781082-3.3997722 .136143$

H -1.454936 -4.241237 1.339926

H -2.100364 -2.798272 0.602984

H -2.435294 $1.316772-1.738186$

H $-2.8716990 .001207-0.647214$

H $1.633250-0.354799-1.062237$

H $1.072343-1.056189-2.596993$

H $0.144324-3.085110-1.265507$

H $2.663580-2.1368660 .192673$

H $2.815710-4.708228-0.045342$

H $1.078980-4.8166100 .131998$

H $3.412699-4.5686323 .580987$

H $4.198672-4.6024521 .905282$

H $1.072029-4.4890243 .814862$

H $0.133795-4.9414792 .429371$

H $0.904509-2.0914243 .370191$

H $-0.5696700 .033102-0.381040$

C $-0.9251194 .342148-0.956196$

O $0.1892864 .492346-0.056513$

C $1.3950674 .665001-0.833884$

C $1.0018934 .428669-2.285945$

C $-0.4412064 .886069-2.297836$

H $1.6983545 .708604-0.684490$

C $2.4726573 .707588-0.308301$

H $1.6336834 .982090-2.987103$

H $1.0654853 .362761-2.539523$

C -1.225093 4.374175 -3.494524

H $-0.4641655 .984004-2.290315$

C $3.7552383 .682586-1.159249$

O $2.7953854 .082547 \quad 1.034143$

H $2.0465282 .699838-0.246243$

H $3.5234753 .311178-2.164126$

O $4.2293105 .022503-1.340341$

C $4.9094542 .844273-0.575886$

C $4.5039501 .400083-0.261649$

H 5.3136213 .3302860 .319099

O $5.9639812 .815717-1.543094$

H 3.7550261 .3776100 .539200

H $4.0043330 .967778-1.140008$

H $-0.7796534 .733719-4.427860$
H -1.237495 $3.279351-3.527808$

H -2.262503 $4.720839-3.461403$

C $-2.1469845 .079173-0.409212$

H 3.0930015 .0078151 .024784

H $4.5090445 .360767-0.472986$

H $6.2011623 .738074-1.740951$

H -2.922077 $5.152521-1.181186$

H -1.880912 $6.104577-0.124708$

H -1.127019 $3.265950-1.033514$

C $5.981785-1.8830550 .661081$

O $5.073016-0.8150070 .372178$

C 5.6695650 .4609470 .104658

C 6.4941010 .9272131 .312786

C $7.521081-0.1334071 .724945$

C $6.891985-1.5244321 .846510$

H $5.339119-2.7149880 .970349$

C $6.746374-2.334296-0.587565$

H $6.3186200 .367704-0.772426$

O 5.6182491 .2029972 .403534

H 7.0124661 .8589411 .059456

H 7.9650050 .1521632 .686491

O $8.563515-0.1548530 .753754$

H $7.673822-2.2845601 .958717$

O $6.111462-1.5492453 .045289$

C $5.807487-2.994235-1.570157$

H $7.269535-1.523463-1.102166$

H $7.509604-3.076756-0.324919$

H 6.1630641 .5134653 .146604

H $9.221469-0.8093911 .043993$

H $5.758328-2.4493693 .148413$

O $5.227716-2.052148-2.367320$

O $5.600630-4.200248-1.607702$

C $4.295267-2.547532-3.332741$

H $3.533030-3.168886-2.853419$

H $3.802902-1.690695-3.800173$

H $4.825017-3.114693-4.103514$

SCF Energy $(B 3 L Y P / 6-31 G * * / / M M F F)=-3245.90001322$

0800139

MM̄FF Geometry

C $-1.7503973 .660057-0.306282$

C -2.5145094 .3140920 .582919$

C -3.7486223 .7587991 .258622$

O $-3.7065862 .313773 \quad 1.305578$

C $-5.007230 \quad 4.1956440 .488972$

C $-6.3026283 .795917 \quad 1.169744$

C $-7.1139872 .797888 \quad 0.765000$

C -6.6812814 .6320202 .365869$

C $-6.9164891 .857149-0.391468$

C -6.7466060 .4144980 .100604$

C $-6.334071-0.541804-1.029057$

C $-6.208443-1.969880-0.494533$

C $-5.642134-2.943292-1.538040$

O $-5.075247-0.092250-1.538558$

O $-5.270489-4.135203-0.828181$

C $-4.363304-2.332886-2.153438$

C $-6.711026-3.357384-2.555924$

O $-3.889740-3.124647-3.239870$

C $-4.548947-0.874359-2.617952$

C $-3.223545-0.210426-3.065909$

O $-5.431910-0.853402-3.746413$

C -2.9431991 .7608512 .290378$

O -2.3072542 .3566593 .146042$

C -2.9961890 .2898852 .140813$

C $-2.234494-0.4765802 .935613$

C $-2.169175-1.9391202 .933010$

C -3.017996 -2.676652 1.932874

C $-2.168744-0.036113-1.968904$

C $-1.142777-1.151651-1.887152$

C $0.783963-1.897131-0.491451$

O $-1.021064-2.022580-2.743554$

C $0.332110-3.0942820 .283581$

C $0.890086-3.477967 \quad 1.441975$

C $0.505879-4.7271742 .181752$

C $0.230681-4.4823353 .647992$

C $1.163853-4.7418494 .579465$

C -1.154764 -4.022762 4.038835

C $-1.355807-2.5486873 .820558$ 
N $-0.343003-1.025430-0.757562$ H -2.030640 $2.658527-0.616568$ H -2.2445115 .3305030 .861372$ H -3.7891384 .1485602 .283461$ H $-4.9655103 .796028-0.529435$ H $-5.008811 \quad 5.287631 \quad 0.367713$ H -8.0285642 .6207771 .329969$ H -7.655435 4.3500132 .779605 H -5.9415134 .5202893 .164326$ H -6.7375525 .6895402 .087464$ H $-7.7952541 .918068-1.044777$ H $-6.0610832 .145783-1.005292$ H -5.9785850 .3971870 .883848$ H -7.6828360 .0689140 .556796$ H -7.085696 -0.491095 -1.825526 H -7.173880 -2.329290 -0.116613 H $-5.535554-1.9683810 .372615$ H -4.956151 -4.787223 -1.476907 H -3.587916 -2.363758 -1.379180 H -7.533521 -3.879882 -2.052467 H $-6.309114-4.066707-3.287666$ H -7.139224 -2.510553 -3.096283 H -3.663790 -4.002336 -2.889150 H $-2.816651-0.727596-3.943122$ H $-3.4731280 .801199-3.417879$ H $-5.288316-0.027085-4.236712$ H -3.654365 -0.120503 1.384699 H -1.5951720 .0128013 .669781$ H -4.078997 -2.551858 2.172231 H -2.817103 -3.749339 1.894795 H $-2.836786-2.3017290 .920253$ H - $-1.5961250 .871982-2.195441$ H $-2.6340210 .106772-0.989028$ H $1.528949-1.3081150 .051790$ H $1.220266-2.219531-1.442160$ H $-0.459211-3.699738-0.151932$ H $1.702978-2.8884161 .859721$ H $1.332986-5.4415392 .074987$ H $-0.364363-5.213247 \quad 1.723450$ H $0.963996-4.5965605 .635853$ H $2.150925-5.0996144 .305356$ H -1.327269 -4.216932 5.106291 H -1.900109-4.637049 3.525715 H $-0.772842-1.9273874 .501880$ H $-0.547508-0.296198-0.080472$ C $0.6513183 .244901-0.947233$ O $1.0094102 .862591 \quad 0.395080$ C 2.4417492 .9456040 .543514 C $2.9989613 .052310-0.867285$ C $1.9149683 .838127-1.571352$ H 2.6277023 .8699351 .104265 C 2.9595201 .7284941 .322870 H $3.9711403 .551585-0.897259$ H $3.1073332 .058913-1.320106$ C $1.9699083 .722359-3.084938$ H $2.0137474 .895185-1.290045$ C 4.4884971 .7495411 .515435 O $2.317318 \quad 1.7347782 .600987$ H 2.6375230 .8165000 .809441 H 4.9922391 .7878090 .547096 O 4.8484522 .9715652 .177712 C $5.066760 \quad 0.5872652 .341837$ C $4.786543-0.8308851 .812608$ H 4.6968650 .6516543 .372266 O $6.487780 \quad 0.7494862 .428939$ H $5.173960-1.5349112 .561138$ H $3.703712-0.9860941 .753380$ H $1.1629474 .297031-3.549994$ H $2.9217914 .106989-3.465731$ H $1.8741992 .681293-3.411256$ C $-0.512530 \quad 4.241677-0.927335$ H $2.468026 \quad 0.8667303 .011314$ H 4.3707222 .9967123 .024869 H 6.6575571 .6454512 .767131 H $-0.7521904 .542883-1.953519$ H $-0.2130075 .142835-0.377546$ H $0.3572452 .325194-1.468507$ C $5.288436-0.637590-1.900541$
O $4.774408-0.459455-0.573200$

C $5.453573-1.1743110 .461781$

C $5.410230-2.6878700 .182613$

C 5.924601-3.025186-1.219495

C $5.294312-2.125553-2.288834$

H $4.565002-0.126761-2.548942$

C $6.6544250 .036463-2.089337$

H $6.498792-0.8517860 .496515$

O $4.080793-3.1812610 .324289$

H $6.022702-3.2021890 .932432$

H $5.694432-4.074923-1.439186$

O $7.341339-2.880100-1.231743$

H $5.806765-2.249276-3.249947$

O $3.933825-2.520682-2.486295$

C $6.5482271 .540170-1.978806$

H $7.391773-0.318176-1.364330$

H $7.061111-0.178746-3.084298$

H $3.519778-2.704047-0.310147$

H $7.653725-3.124276-2.119527$

H $3.937020-3.447861-2.779368$

O $7.3989281 .996529-1.015339$

O $5.8088932 .227609-2.671073$

C $7.4119843 .414135-0.821186$

H $8.1427833 .643198-0.041090$

H $7.7121683 .920799-1.743268$

H $6.4286043 .761449-0.492098$

SCF Energy $(B 3 L Y P / 6-31 G * * / / M M F F)=-3245.91249002$

0800140

MM̄FF Geometry

C $1.6832405 .027269-0.708073$

C $2.7030014 .667970-1.502691$

C $4.0779614 .218192-1.059230$

O $4.3880212 .925655-1.648576$

C 4.3671364 .2322670 .460233

C 5.7776523 .8188620 .839928

C 6.0912242 .8018981 .668450

C 6.8813424 .6695150 .262408

C 5.1697051 .8610772 .395482

C 5.3807860 .4155331 .931123

C $4.433594-0.5700192 .632578$

C $4.710555-1.9983642 .157331$

C $3.709309-3.0118562 .728789$

O $3.086352-0.1980242 .322214$

O $3.865134-4.2178771 .964467$

C $2.276238-2.4820542 .502737$

C $4.040469-3.3710344 .181754$

O $1.316522-3.3086593 .155650$

C $2.087139-1.0165182 .949011$

C $0.698499-0.4388722 .571287$

O $2.189026-0.9458834 .377240$

C $3.6598541 .826470-1.317618$

O $2.7058771 .771073-0.559481$

C $4.2494430 .674834-2.039598$

C $3.860340-0.571726-1.732502$

C $4.377441-1.810650-2.317431$

C $5.365406-1.693082-3.448112$

C $0.384502-0.4046921 .073529$

C $-0.298456-1.6580740 .554828$

C $-0.631952-2.957237-1.545770$

O $-0.940997-2.418947 \quad 1.273297$

C $0.416272-4.024264-1.535782$

C $1.035627-4.465062-2.640853$

C $2.090138-5.533939-2.637308$

C $3.413994-5.042917-3.180323$

C $3.759107-5.266414-4.459676$

C $4.373859-4.375971-2.221164$

C $3.945898-2.989518-1.821991$

N $-0.136419-1.806214-0.815799$

H 1.8019275 .0133120 .371432

H $2.5522694 .696369-2.580532$

H 4.774714 4.917419-1.537796

H 3.6322563 .6060320 .970376

H 4.2066045 .2484790 .845258

H 7.1467682 .6218211 .872164

H 7.8584794 .4371230 .699556

H $6.9615324 .516778-0.818108$

H 6.6840415 .7294410 .453858 
H $5.388041 \quad 1.932107 \quad 3.468185$ H 4.1208722 .1415392 .281031 H $5.217103 \quad 0.3677540 .848671$ H 6.4207440 .1172122 .114918 H $4.589623-0.4884143 .714746$ H $5.738468-2.2961312 .399813$ H $4.645026-2.0315631 .061791$ H $3.273031-4.8915052 .339197$ H $2.079973-2.5551251 .426219$ H $5.030756-3.8384584 .241726$ H $3.335936-4.1102994 .578289$ H $4.042454-2.5055434 .847626$ H $1.371555-4.1944092 .759654$ H $-0.089813-0.9519343 .135274$ H $0.674566 \quad 0.6033762 .920639$ H $1.753277-0.1312604 .677780$ H $5.0105410 .883650-2.782980$ H $3.108397-0.704472-0.956890$ H $4.951577-1.083988-4.259744$ H $5.630749-2.654463-3.893600$ H $6.295055-1.230253-3.099622$ H $-0.311860 \quad 0.4198580 .881557$ H $1.289661-0.2040030 .495252$ H -0.867176 -2.623159 -2.560609 H -1.548705 -3.327369-1.075967 H $0.675291-4.454848-0.571220$ H $0.768071-4.040959-3.606069$ H $1.721124-6.369894-3.245726$ H $2.239105-5.947824-1.632034$ H $4.718954-4.946420-4.851219$ H $3.086732-5.770949-5.145666$ H $5.390513-4.366527-2.630483$ H $4.454788-4.987883-1.313200$ H $3.225744-2.975205-1.003766$ H $0.463957-1.157491-1.316558$ C $-0.7602264 .495170-0.787778$ O $-0.6802643 .300665-1.594107$ C $-2.0137312 .925962-2.008248$ C $-2.9583363 .759790-1.156791$ C -2.170152 $5.043009-0.999904$ H $-2.0941613 .224560-3.061593$ C -2.171087 $1.405602-1.878246$ H $-3.931723 \quad 3.912519-1.632217$ H $-3.1232193 .290347-0.179903$ C -2.6698245 .9244880 .131635$ H $-2.2183525 .604679-1.942606$ C -3.629756 $0.919607-2.004800$ O $-1.3487850 .822536-2.891675$ H -1.760689 $1.079247-0.916038$ H $-4.1918311 .366012-1.180517$ O $-4.204559 \quad 1.419167-3.212075$ C -3.800428 -0.611558-1.950605 C $-5.272137-1.069907-1.890582$ H -3.254339 -1.004488 -1.085510 O $-3.213818-1.187976-3.119470$ H $-5.817375-0.691490-2.764037$ H -5.279202 -2.164107 -1.983968 H $-2.063572 \quad 6.831370 \quad 0.218332$ H -3.706811 $6.227508-0.046865$ H -2.633892 5.4021501 .093350 C $0.3377725 .472716-1.209878$ H $-1.360057-0.140705-2.762722$ H $-3.7037461 .033746-3.951653$ H -3.287859 -2.154322 -3.040383 H $0.142603 \quad 6.467350-0.793213$ H $\quad 0.3517495 .570568-2.302315$ H -0.6245294 .1844310 .256555$ C $-5.746421-0.8947621 .804994$ O $\quad-5.277184-1.244478 \quad 0.497050$ C $-5.993054-0.662868-0.595989$ C $-7.458332-1.118294-0.566786$ C $-8.104663-0.7887410 .784922$ C $-7.237818-1.2443001 .964125$ H -5.176388 -1.538190 2.487482 C $-5.4112080 .563674 \quad 2.158280$ H $-5.9690210 .426668-0.512434$ O $-7.551671-2.519329-0.813206$ H -8.003198 -0.613885 -1.372955
H $-9.083708-1.2793590 .849536$

$\begin{array}{lllll}\text { O } & -8.311942 & 0.617214 & 0.886804\end{array}$

H -7.622619 -0.8217332 .899785$

O $-7.359278-2.6669092 .065796$

C $-3.9155960 .803703 \quad 2.224565$

H -5.8207931 .2667461 .428650$

H $-5.8249360 .798656 \quad 3.145725$

H -7.020977 -2.976256 -0.138275

$\begin{array}{llll}H & -8.893458 & 0.881949 & 0.153653\end{array}$

H $-6.873428-2.9471582 .860256$

O -3.6887152 .0962862 .604736$

O $-3.053895-0.0279251 .975890$

C -2.3111442 .4667312 .724157$

H -1.828506 1.8761803 .508609

H $-1.7958512 .332717 \quad 1.768579$

$\mathrm{H}-2.2655933 .5232853 .001113$

SCF Energy (B3LYP/6-31G**//MMFF) $=-3245.90254008$

0800141

MM̄FF Geometry

C -3.1597621 .3691372 .868724$

C -3.5782802 .6136272 .590181$

C $-4.0550323 .141281 \quad 1.256419$

O -4.1697392 .0928530 .271296$

C -3.0197294 .1400560 .708658$

C $-3.5950955 .123290-0.288993$

C $-3.5102845 .008228-1.628487$

C $-4.250965 \quad 6.3351190 .322943$

C $-2.9555293 .868250-2.435771$

C -1.588672 $4.178246-3.063937$

C $-0.4145773 .858542-2.125789$

C $0.9201534 .241308-2.767874$

C $2.1154923 .823095-1.895822$

O $-0.4457392 .449457-1.874813$

O $3.3032003 .930048-2.695647$

C $1.9571932 .335725-1.516167$

C $2.3091484 .768069-0.704219$

O $2.9725441 .931241-0.600235$

C $0.5548941 .977172-0.968638$

C $0.4150610 .440730-0.869097$

O 0.4073852 .5495610 .332272

C $-5.324114 \quad 1.381866 \quad 0.208781$

O $-6.306008 \quad 1.4951230 .922749$

C $-5.1582450 .407867-0.892058$

C $-5.970700-0.654630-0.975019$

C $-5.896144-1.712909-1.984887$

C $-4.871451-1.562508-3.080030$

C $-1.002545-0.056525-0.551464$

C $-1.047862-1.570671-0.424926$

C $-2.540340-3.4171820 .337599$

O $-0.102912-2.296482-0.724469$

C $-3.180826-4.053905-0.855663$

C $-4.288562-4.808470-0.784575$

C $-4.909235-5.497162-1.967570$

C $-6.395677-5.240000-2.087713$

C $-7.290499-6.150335-1.667734$

C $-6.841877-3.967496-2.772765$

C $-6.731461-2.766760-1.874189$

N -2.268559 -2.017314 0.062992

H -3.1565900 .6053252 .097150$

H -3.5574483 .3526093 .390074$

H -5.028432 3.6269051 .396532

H -2.1908413 .5878750 .253373$

H -2.5708794 .7223561 .524981$

H $-3.9285215 .811080-2.235257$

H $-4.6426327 .024089-0.432954$

H -5.0890056 .0359300 .960378$

H $-3.530456 \quad 6.8895920 .933052$

H -2.922101 $2.939410-1.860059$

H $-3.6715253 .680560-3.246774$

H $-1.4932483 .554796-3.962841$

H -1.544210 $5.224572-3.389474$

H $-0.5495704 .403230-1.185631$

H $0.9558315 .317456-2.978776$

H $1.0128793 .742370-3.742802$

H $3.3774744 .850397-3.000927$

H $2.1314061 .754644-2.432211$

H $2.4580645 .797267-1.052537$ 
H $3.2121194 .511801-0.139146$ H $1.4599974 .771675-0.017637$ H 2.7328942 .2717910 .278267 H $0.718412-0.011623-1.822490$ H $1.0971960 .065621-0.098883$ H $-0.2359002 .030576 \quad 0.839571$ H -4.346745 $0.579180-1.590504$ H $-6.750216-0.773167-0.223580$ H $-5.078455-0.666931-3.676393$ H -4.842594 -2.405677 -3.772722 H -3.863288 -1.477285 -2.659952 H -1.3573600 .3741710 .389284$ H -1.696194 $0.233833-1.348259$ H -3.183659 -3.4548821 .222398$ H -1.600551 -3.9301960 .566077$ H -2.693316 -3.915372 -1.817847 H $-4.755281-4.9739770 .183975$ H -4.727255 -6.574189-1.855768 H -4.414522 $-5.209118-2.903359$ H -8.357460 -5.986348 -1.775952 H $-6.975755-7.077892-1.200812$ H -7.894864 -4.050500 -3.074114 H $-6.294774-3.854214-3.712525$ H $-7.434853-2.784573-1.040270$ H $-3.014989-1.347900 \quad 0.225393$ C $-1.130880 \quad 0.702235 \quad 4.150507$ O $-0.883564-0.572313 \quad 3.524412$ C $0.299968-1.1572474 .121903$ C $0.823556-0.1308735 .117406$ C $-0.443647 \quad 0.6046225 .507802$ H $-0.042823-2.0579804 .645701$ C $1.264842-1.5270452 .990303$ H $1.331649-0.5949665 .968002$ H 1.5287730 .5585614 .636348 C -0.1789201 .9438196 .173781$ H -1.030909 -0.0311406 .183799$ C $2.690569-1.8868653 .437401$ O $0.713460-2.6523972 .298177$ H $1.298731-0.7002742 .272069$ H $3.164480-1.0174103 .906926$ O $2.626337-2.896044 \quad 4.451331$ C $3.609358-2.4046852 .309190$ C $3.626095-1.4871071 .081490$ H $3.314056-3.4199652 .022235$ O $4.939793-2.4944192 .826262$ H $2.634429-1.4595240 .618667$ H $3.827428-0.4563001 .405408$ H -1.116010 $2.444486 \quad 6.435448$ H 0.3977241 .8074537 .094602 H 0.3884242 .6130435 .518081 C -2.6352680 .9621274 .220161$ H -0.198234 -2.422335 2.050682 H $2.161634-3.6622664 .073047$ H $4.907450-3.0676193 .611340$ H -2.851988 1.7453764 .955085 H $-3.168171 \quad 0.0577204 .536981$ H -0.6374691 .4659633 .532845$ C $5.389291-0.936892-2.141113$ O $4.466751-0.874524-1.047260$ C $4.663420-1.8361800 .000487$ C $4.543977-3.261549-0.559567$ C $5.506582-3.468812-1.734785$ C $5.403947-2.341093-2.766351$ H $4.982375-0.242862-2.888338$ C $6.778993-0.430095-1.740941$ H $5.659862-1.6908640 .430616$ O $3.217294-3.513697-1.018238$ H $4.770986-3.9912190 .225956$ H $5.281656-4.423110-2.227078$ O $6.845802-3.529340-1.252503$ H $6.228162-2.412592-3.484915$ O $4.197827-2.509682-3.517949$ C $6.7472661 .059196-1.490107$ H $7.188775-0.920230-0.853479$ H $7.499629-0.602017-2.549569$ H $2.632860-3.551209-0.242847$ H $6.899423-4.271909-0.626981$ H $3.448951-2.392249-2.908409$
O $6.369744 \quad 1.309591-0.204168$

O $7.0089161 .897690-2.343114$

C 6.2768152 .6922510 .152732

H $5.6213963 .227545-0.540925$

H $5.8483462 .755874 \quad 1.156451$

H 7.2741563 .1414430 .165122

SCF Energy $(B 3 L Y P / 6-31 G * * / / M M F F)=-3245.91872845$

0800142

MM̄FF Geometry

C $-0.753645 \quad 1.754072 \quad 2.884814$

C -1.594770 2.7172853 .295479

C -2.6452523 .4117352 .460585$

O $-3.0279722 .615241 \quad 1.317532$

C -2.0984264 .7665031 .969786$

C -3.0719045 .5752681 .129604$

C $-2.8404125 .942428-0.148189$

C -4.3464145 .9906601 .815319$

C -1.604432 $5.639265-0.949725$

C $-1.6748874 .247794-1.579311$

C $-0.3078203 .560056-1.664825$

C $0.6087334 .199836-2.707235$

C $1.9266093 .421085-2.856205$

O $-0.5619102 .197195-2.015056$

O $2.569443 \quad 3.865823-4.060808$

C $1.6118381 .918929-3.042951$

C $2.8957953 .734730-1.709671$

O $2.801446 \quad 1.137787-3.022164$

C $0.6036921 .362929-2.012315$

C $0.148027-0.057375-2.398541$

O $1.183274 \quad 1.259432-0.709247$

C -3.9543211 .6396111 .534893$

O -4.4355751 .3144792 .609256$

C -4.3114651 .0440960 .226549$

C -5.2884770 .1255380 .172399$

C $-5.813006-0.527351-1.030587$

C $-5.147416-0.213389-2.343845$

C $-0.926872-0.604657-1.450306$

C -1.320697-2.029883-1.783896

C $-2.783605-3.872493-0.963979$

O $-0.857534-2.651567-2.735989$

C $-3.990220-3.868660-1.847824$

C $-5.199608-4.297546-1.457624$

C $-6.397160-4.336594-2.362027$

C $-7.584169-3.592045-1.795736$

C $-8.608675-4.253135-1.230570$

C -7.626212 -2.090523 -1.971850

C $-6.867970-1.359647-0.899804$

$\mathrm{N}-2.249373-2.527463-0.879820$

H -0.7692451 .4199651 .850867$

H -1.5352033 .0535884 .329009$

H -3.528112 3.5825583 .089603

H -1.1754914 .5785241 .410540$

H -1.805114 5.3797252 .832705

H -3.601388 $6.515044-0.675106$

H $-4.936418 \quad 6.6852891 .207810$

H -4.9751125 .1194402 .022654$

H -4.1218936 .4944412 .761153$

H $-1.5001486 .394359-1.738488$

H $-0.7140765 .755368-0.321582$

H $-2.3466883 .594897-1.009659$

H -2.129038 $4.311545-2.577061$

H $0.1535413 .583527-0.670607$

H $0.8108485 .250089-2.465060$

H $0.1015824 .208759-3.682068$

H $2.7070554 .826058-3.990368$

H $1.1784431 .798386-4.045777$

H $3.1487344 .802051-1.703519$

H $3.8451833 .204237-1.838056$

H $2.4899763 .490436-0.725752$

H $3.4083531 .511903-3.683569$

H $-0.260272-0.052878-3.417470$

H $1.006839-0.739233-2.391935$

H $2.1441101 .374745-0.787631$

H $-3.7884361 .399116-0.653559$

H $-5.776380-0.164526 \quad 1.102357$

H $-5.525153-0.808717-3.177468$

H $-4.073416-0.416382-2.290833$ 
H $-5.2921930 .840463-2.605677$ H - $0.566720-0.585753-0.415872$ H -1.826309 $0.016120-1.508275$ H -3.014374 -4.1968660 .055345$ H -2.022255 -4.540094 -1.380203 H $-3.855227-3.518845-2.868746$ H $-5.330912-4.671828-0.445147$ H $-6.657112-5.391150-2.522408$ H -6.161336 -3.937708 -3.356685 H $-9.478460-3.728331-0.849698$ H $-8.597390-5.333615-1.131185$ H $-8.667287-1.742747-1.923286$ H $-7.297305-1.835604-2.982644$ H -7.280589-1.515157 0.098335 H -2.629994-1.909621 -0.168583 C $0.216610-0.4225693 .818011$ O $0.867177-0.9652172 .654201$ C $0.306670-2.2684912 .394320$ C $-0.772322-2.5072253 .454783$ C $-1.150846-1.0957303 .859966$ H $-0.174905-2.2107751 .412932$ C $1.403922-3.3422582 .379901$ H -1.617170 -3.0778103 .055719$ H $-0.383182-3.0620364 .316288$ C $-1.843723-1.0095425 .207747$ H $-1.812585-0.6907083 .084139$ C $2.551429-3.1011781 .376772$ O $1.989875-3.4126863 .687704$ H $0.943812-4.3213912 .198828$ H $3.181266-2.2805551 .735811$ O $3.382615-4.2704161 .396174$ C $2.109015-2.825553-0.068824$ C $3.231803-2.879012-1.122213$ H $1.619808-1.846957-0.111643$ O $1.133738-3.806671-0.436447$ H $3.543701-3.920689-1.273916$ H $2.783549-2.569716-2.075863$ H $-2.778009-1.5799495 .197092$ H $-1.214058-1.4110176 .008737$ H -2.0842830 .0287395 .456813$ C $0.249538 \quad 1.108207 \quad 3.799524$ H $2.675379-4.1021333 .661287$ H $2.861343-5.0064391 .032442$ H $0.859857-3.623202-1.351264$ H $1.244461 \quad 1.4429283 .485095$ H 0.0996391 .4678964 .825196 H $0.819281-0.752407 \quad 4.675540$ C $5.0780780 .274163-0.307300$ O $4.041956-0.659207-0.648632$ C $4.464762-2.013229-0.823192$

C $5.507323-2.109577-1.949383$

C $6.689848-1.174086-1.685111$

C $6.2241030 .243451-1.335064$

H $4.6008371 .259997-0.372260$

C $5.5682830 .083840 \quad 1.136945$

H $4.915261-2.3744380 .106127$

O $4.899370-1.771975-3.194674$

H $5.855776-3.146987-2.020872$

H $7.328702-1.142578-2.576212$

O $7.464158-1.711157-0.616841$

H $7.0626360 .842137-0.960481$

O $5.758395 \quad 0.886689-2.523810$

C 4.4978940 .4090752 .156046

H $5.892805-0.9430771 .322838$

H $6.4101250 .759567 \quad 1.328174$

H $5.571770-1.878806-3.888816$

H $8.219466-1.115821-0.473148$

H $6.4982320 .910907-3.154071$

$\begin{array}{lllll}\text { O } & 4.994008 & 0.162864 & 3.405275\end{array}$

O 3.3778730 .8256531 .897180

C 4.0954130 .4131554 .489808

H 3.7085911 .4356604 .443717

H $3.278952-0.3133594 .468910$

H 4.6482680 .2917645 .425063

SCF Energy (B3LYP/6-31G**//MMFF) $=-3245.90868436$

0800143

MM̄FF Geometry
C $-0.738921-3.160316-0.708802$

C $-1.622562-4.000080-1.270475$

C $-3.055684-3.666468-1.629894$

O $-3.323885-2.254584-1.457791$

C $-4.021778-4.462382-0.736407$

C $-5.475527-4.318214-1.148535$

C $-6.400961-3.597631-0.483723$

C $-5.870200-5.082923-2.386765$

C $-6.212102-2.784000 \quad 0.764853$

C $-6.375443-1.2857640 .480523$

C $-5.956974-0.4320921 .685405$

C -6.2058991 .0519421 .414259$

C $-5.686261 \quad 1.9410462 .554702$

O $-4.561441-0.6577151 .911371$

O -5.6579303 .2958932 .078735$

C -4.2283141 .5466992 .877748$

C $-6.629624 \quad 1.926262 \quad 3.763235$

O $-3.7612782 .233563 \quad 4.035889$

C $-4.0252880 .027308 \quad 3.051136$

C -2.536134 -0.3817813 .171419$

O $-4.674518-0.3979194 .255782$

C -2.924206 -1.426621 -2.464565

O $-2.426650-1.755271-3.530283$

C $-3.149316-0.031544-2.024358$

C $-2.6049370 .971733-2.729133$

C $-2.7364722 .403631-2.451941$

C $-3.5279412 .812829-1.238779$

C $-1.650336-0.0135641 .975520$

C -0.9053441 .3001432 .142060$

C 0.5680322 .8655590 .885383

$\begin{array}{llllll}\text { O } & -0.876207 & 1.930794 & 3.195114\end{array}$

C -0.2134303 .9463750 .210196$

C $0.1825374 .541346-0.925312$

C $-0.5398425 .692265-1.561750$

C $-0.9030725 .434350-3.005168$

C $-0.1115845 .859140-4.004886$

C $-2.2344174 .780251-3.289211$

C $-2.1576113 .278253-3.301121$

N $-0.243593 \quad 1.6679910 .978738$

H - $-1.048452-2.158321-0.425529$

H $-1.290449-4.999412-1.544333$

H $-3.216396-3.939347-2.680188$

H $-3.877433-4.1612550 .305907$

H $-3.765546-5.530272-0.766054$

H -7.417259 $-3.580820-0.875982$

H $-6.943203-5.012688-2.595128$

H $-5.340588-4.699068-3.263817$

H -5.628973 -6.144637 -2.270151

H $-6.963158-3.0979691 .500058$

H -5.242646 -2.975865 1.228933

H $-5.758275-1.016320-0.385072$

H $-7.419031-1.0746730 .215959$

H $-6.525842-0.7643872 .561796$

H $-7.271387 \quad 1.2413031 .233959$

H -5.691193 1.3421710 .488696

H -6.5587593 .5345101 .800312$

H $-3.613793 \quad 1.894072 \quad 2.038958$

H -7.624275 2.2886313 .476689

H $-6.278617 \quad 2.606277 \quad 4.547271$

H $-6.7545090 .932353 \quad 4.197739$

H -3.8833843 .1852583 .877385$

H -2.118129 -0.0104384 .114768$

H -2.509035 -1.4780243 .255538$

H $-4.276270-1.2367924 .541607$

H -3.744666 $0.130630-1.134209$

H $-2.0108560 .724062-3.608312$

H $-4.5838052 .552141-1.366128$

H $-3.4792473 .883240-1.027757$

H $-3.1466002 .311880-0.342747$

H $-0.879853-0.7873851 .866687$

H $-2.2268970 .006779 \begin{array}{lll}1.045180 \\ \text { H }\end{array}$

H 1.4727052 .6069050 .330010

H $\quad 0.8651163 .195194 \quad 1.886108$

H $-1.121954 \quad 4.2841610 .703069$

H $1.1069364 .218488-1.399189$

H $0.1158196 .570903-1.497343$

H $-1.4413675 .961320-0.997401$

H $-0.3845655 .708113-5.044067$ 
H $0.8293656 .361138-3.805289$ H -2.592494 $5.091302-4.280478$ H -2.990696 $5.163582-2.598200$ H -1.595475 2.882276 -4.148425 H -0.3510191 .1000240 .143075$ C $1.658831-2.690478-1.360232$

O $1.452097-1.271165-1.184628$ C $1.346044-0.669856-2.484460$ C $1.990543-1.649634-3.453955$ C $1.535945-2.971068-2.862124$ H $0.272399-0.628324-2.714510$ C $1.8809370 .763476-2.510055$ H $1.656306-1.504206-4.485446$ H $3.083552-1.583924-3.432096$ C $2.350977-4.164134-3.330172$ H $0.489009-3.129494-3.147357$ C $3.4056600 .956871-2.371189$ O $1.4765361 .337128-3.760780$ H $1.3725241 .339126-1.729142$ H $3.9070380 .595578-3.276100$ O $3.6500692 .371978-2.339902$ C $4.0526890 .315917-1.131348$ C $5.5458230 .680505-0.996565$ H $3.959208-0.772167-1.194216$ O 3.3401040 .7533520 .022805 H $6.0464780 .508679-1.956729$ H $5.6265041 .746112-0.748369$ H $2.271725-4.281859-4.415836$ H $3.411312-4.048773-3.081483$ H $1.992555-5.086605-2.862486$ C $0.705923-3.485741-0.458743$ H $1.7688212 .264980-3.761258$ H $3.2747652 .715228-1.510646$ H 3.7265930 .3109130 .796679 H $0.914333-3.2385380 .589315$ H $0.893146-4.559020-0.578061$ H $2.685749-2.889796-1.029566$ C $6.195575-0.4960362 .491271$ O $\quad 5.709854 \quad 0.2360341 .360949$ C $6.260580-0.1398610 .096597$ C 7.7779150 .0975750 .097927 C $8.450927-0.6387571 .262744$ C $7.727775-0.3945272 .593598$ H $5.770317 \quad 0.019706 \quad 3.361713$

C $5.672834-1.9403022 .502842$ H $6.056645-1.200634-0.088843$ $\begin{array}{lllll}\text { O } & 8.039721 & 1.495720 & 0.201347\end{array}$ H $8.190999-0.246331-0.857909$ H $9.491646-0.3047451 .353898$ O $8.471543-2.038184 \quad 0.996917$ H $8.087047-1.0897413 .361513$ O $8.034430 \quad 0.927642 \quad 3.047653$ C $4.170954-2.0417672 .347472$ H $6.106778-2.5208471 .683527$ H $5.950271-2.4411743 .437150$ H $9.003848 \quad 1.6172260 .171901$ H $8.962461-2.1730580 .168442$ H 8.9973920 .9789283 .172696 O $3.529597-1.1527253 .155242$ O $3.630304-2.8578031 .610657$ C $2.101070-1.1556103 .063077$ H $1.784999-0.9584132 .034143$ H $1.703006-2.1129073 .412309$ H $1.716780-0.3587543 .705283$

SCF Energy (B3LYP/6-31G**//MMFF) $=-3245.90958062$

0800144

MM̄FF Geometry

C -3.590582 -3.445296 1.290965

C $-2.310695-3.0459301 .264956$

C $-1.529519-2.6435442 .485680$

O $-0.933936-1.3461172 .264045$

C $-0.376920-3.6386572 .708124$

C $0.507735-3.2632953 .880102$

C $1.702448-2.6504573 .758718$

C $-0.017913-3.6509395 .236330$

C $2.358621-2.1739692 .490403$

C $3.478141-3.1271882 .064495$
C $4.141526-2.702138 \quad 0.745489$

C $5.186907-3.742610 \quad 0.333814$

C $5.968020-3.316402-0.914996$

O $4.759108-1.4259220 .941660$

O $7.115051-4.171903-1.033147$

C $6.494259-1.883274-0.701115$

C $5.147420-3.510803-2.195675$

O $7.116032-1.392813-1.887721$

C $5.408233-0.890494-0.222470$

C 6.0762890 .4473620 .189540

O $4.487142-0.670718-1.287860$

C -1.679923 -0.239144 2.509765

O $-2.872067-0.1903092 .761932$

C -0.7552380 .9163702 .454029$

C -1.2331842 .1677302 .499513$

C -0.4308003 .3944112 .497634$

C 1.0661953 .2484592 .580967

C 5.1188861 .5343120 .705422

C $4.6293682 .483497-0.378127$

C $3.4789974 .648658-0.701322$

O $4.8115042 .289708-1.577290$

C 2.7395595 .6655550 .106750

C $1.5016476 .090597-0.188332$

C 0.7792987 .1649840 .571212

C -0.5313356 .6892251 .154758$

C -1.6978396 .9803880 .553174$

C -0.4796295 .9673662 .483561$

C -1.0691924 .5817282 .439795$

N 3.9383343 .5672580 .150269

H -4.116390 -3.485544 2.241021

H $-1.782307-3.0229380 .315872$

H -2.166718 -2.605095 3.378308

H $0.228755-3.717743 \quad 1.796428$

H $-0.778866-4.6472202 .873960$

H $2.272841-2.4472344 .663976$

H $0.675114-3.3859896 .041923$

H $-0.967048-3.1448745 .438742$

H $-0.182108-4.7323625 .285008$

H $1.635910-2.0603111 .676339$

H $2.763433-1.1711352 .673220$

H $4.234284-3.1591452 .860139$

H $3.073220-4.1416061 .956227$

H $3.365577-2.611141-0.023884$

H $4.719014-4.7227750 .177545$

H $5.903145-3.8828241 .156021$

H $6.799482-5.090049-1.091236$

H $7.281699-1.9310990 .064321$

H $4.881899-4.567343-2.322921$

H $5.729743-3.239961-3.083181$

H $4.218473-2.936807-2.202653$

H $7.794731-2.039139-2.147312$

H 6.7770990 .2304961 .007180

H $6.6663560 .847278-0.643384$

H $4.0231310 .165531-1.134979$

H $\quad 0.3058810 .7008802 .389493$

H -2.3121332 .3105202 .544834$

H 1.3498552 .7159263 .495573

H 1.5981084 .2012432 .595375

H 1.4487492 .6913861 .719498

H $4.251701 \quad 1.0986401 .213007$

H 5.6631102 .1434091 .438747

H $2.8676334 .220125-1.502881$

H $4.3607945 .108635-1.160643$

H 3.2620436 .1002350 .955702

H $0.9916975 .679388-1.056008$

H $0.6019927 .998190-0.121402$

H 1.4047037 .5811751 .370900

H $-2.647483 \quad 6.6811840 .984058$

H $-1.7290427 .526331-0.383948$

H -1.0463706 .5525393 .220650$

H 0.5426085 .9610132 .872647

H -2.1586184 .5759662 .381513$

H 3.9455043 .7055891 .157186

C $-5.477992-2.963892-0.355541$

O $-4.959806-1.667371-0.706615$

C $-5.923176-0.669703-0.308809$

C -7.180389 -1.429021 0.106451

C $-6.585016-2.7089350 .664730$ 
H $-5.513948-0.180940 \quad 0.582862$ C $-6.1109360 .361964-1.428163$ H $-7.775035-0.8751370 .839173$ H -7.826754 -1.657379 -0.747674 C $-7.584355-3.8444560 .792161$ H -6.164945 -2.486912 1.653272 C $-4.7969501 .039822-1.871617$ O $-6.644748-0.290336-2.588294$ H $-6.8432191 .121797-1.130662$ H -4.163346 $0.291479-2.361254$ O $\begin{array}{llll}-5.143770 & 2.001582 & -2.876148\end{array}$ C $-4.024373 \quad 1.745640-0.740138$ C $-2.7549692 .486165-1.196024$ H $-3.740358 \quad 1.0236930 .032477$ O $-4.8632082 .720945-0.114465$ H $-3.0106273 .285623-1.902207$ H -2.356699 $3.001472-0.312279$ H -8.394517 -3.568841 1.475156 H -8.031829 $-4.096911-0.174936$ H -7.102159-4.744722 1.186060 C $-4.340810-3.894000 \quad 0.068086$ H -7.596436 -0.421857 -2.442050 H $-4.3283852 .246035-3.344960$ H -5.5588092 .2471770 .371414$ H -3.640802 -3.986812 -0.772735 H $-4.737156-4.8983640 .257338$ H -5.915872 -3.372537-1.276753 C $-0.402180-0.409354-1.236980$ O $-1.358240 \quad 0.575003-0.820632$ C $-1.6728471 .573994-1.794842$ C $-0.4095002 .365041-2.168946$ C $0.7185191 .437825-2.629501$ C $0.9137000 .256833-1.670168$ H $-0.195396-0.985701-0.327377$ C -1.026454-1.366429-2.260878 H -2.071651 $1.088690-2.692004$ O $0.044263 \quad 3.127451-1.053204$ H $-0.6578153 .076838-2.964496$ H $1.6536642 .007738-2.693638$ $\begin{array}{lllll}\text { O } & 0.430132 & 0.938797 & -3.931317\end{array}$ H $1.583467-0.487729-2.113453$ O $1.541842 \quad 0.728849-0.472859$ C $-0.341825-2.715795-2.295139$ H -2.075386 -1.537521-1.987061 H $-1.016317-0.947015-3.269830$ H $0.1947112 .512150-0.315680$ H $0.3499251 .702593-4.527923$ H $2.3127531 .259141-0.731586$ O $-1.051822-3.549402-3.112316$ O $0.679369-3.008297-1.688451$ C $-0.533013-4.877311-3.234669$ H $0.473569-4.853057-3.663014$ H $-0.526739-5.372287-2.258844$ H -1.187507 -5.437365 -3.907768 SCF Energy (B3LYP/6-31G**//MMFF) $=-3245.90492572$

\section{$08 \_00145$}

MM̄FF Geometry

C $1.656593-2.549614-3.515517$

C $2.076912-1.670024-2.594974$

C $3.160774-0.654849-2.838051$

O $4.054544-0.765916-1.707045$

C $2.5782230 .766778-2.865480$

C $3.5269941 .783068-3.467826$

C $4.2922112 .637983-2.761582$

C $3.5441261 .824552-4.974557$

C $4.434172 \quad 2.737882-1.268338$

C $3.8761474 .053052-0.707494$

C $2.342412 \quad 4.134345-0.750865$

C $1.8591805 .483803-0.215176$

C $0.3267415 .553478-0.137265$

O 1.8262223 .0764840 .062036

O -0.0233576 .6940860 .661463$

C -0.1935414 .3049520 .605672$

C $-0.306145 \quad 5.778217-1.515765$

$\begin{array}{lllll}\text { O } & -1.617187 & 4.255544 & 0.576609\end{array}$

C 0.3963742 .9778640 .074510

C 0.0122451 .8107671 .016285
O $-0.1285712 .720104-1.228905$

C $5.393740-0.759650-1.909987$

O $5.973829-0.646811-2.980274$

C $6.122303-0.952104-0.631661$

C $5.522511-0.9045670 .568798$

C $6.152383-1.1310661 .871043$

C $7.609595-1.5084051 .890524$

C $0.751274 \quad 0.497596 \quad 0.721024$

C $0.403977-0.591257 \quad 1.720837$

C $1.205401-2.8147802 .518765$

O $-0.538034-0.5088372 .503825$

C $2.050323-2.5248493 .720287$

C $3.142411-3.2277924 .057396$

C $3.988373-2.9223875 .261466$

C $5.432297-2.6339464 .908358$

C $6.386571-3.5594645 .104763$

C $5.786135-1.2483954 .411124$

C $5.387359-1.0286812 .978389$

N $1.264716-1.6766001 .619835$

H $2.089831-2.532394-4.512300$

H $1.631493-1.691005-1.603207$

H $3.687070-0.889787-3.770975$

H $2.2932751 .069734-1.852973$

H $1.6488820 .786202-3.450843$

H $4.917767 \quad 3.338766-3.313284$

H $3.8690130 .862529-5.382541$

H $2.5431022 .045532-5.359166$

H $4.2224692 .591748-5.362509$

H $3.9877971 .884845-0.749860$

H $5.5066202 .692616-1.039620$

H 4.2071714 .1299590 .336486

H $4.3113764 .902764-1.248044$

H $2.0058733 .995870-1.784260$

H $2.2512046 .307566-0.824749$

H 2.2632105 .6406770 .795076

H 0.3612747 .4828800 .242162

H 0.0910694 .4107331 .662104

H $0.0524996 .718147-1.952658$

H $-1.3941995 .880246-1.439277$

H $-0.0831194 .979491-2.226077$

H $-1.944977 \quad 5.0993520 .932267$

H 0.2409682 .0930902 .052424

H -1.066689 1.6279450 .959223

H $-0.140176 \quad 1.763256-1.387062$

H $7.186003-1.139770-0.735418$

H $4.455853-0.6904370 .614534$

H $7.782311-2.4110791 .293761$

H $7.989518-1.7228222 .891816$

H $8.221615-0.6957561 .484135$

H $0.4913280 .123143-0.273595$

H 1.8345760 .6600920 .756640

H $1.558758-3.6892291 .963420$

H $\quad 0.168816-2.9881042 .825061$

H $1.743427-1.6900494 .346398$

H $3.444873-4.0713283 .441086$

H $3.930404-3.7869855 .935628$

H $3.582411-2.0776065 .831894$

H $7.428396-3.3532834 .883672$

H $6.146785-4.5460585 .487335$

H $6.852783-1.0437704 .556615$

H $5.279061-0.5010975 .035117$

H $4.340477-0.7489592 .858677$

H $2.058199-1.6193920 .988041$

C $-0.735448-3.236255-3.846843$

O $-1.338359-2.189067-3.055158$

C $-2.684224-2.573218-2.725564$

C $-3.047116-3.671546-3.714432$

C $-1.725455-4.402805-3.828861$

H -2.649842 -3.003680 -1.716482

C $-3.635968-1.369272-2.724421$

H $-3.860749-4.307123-3.352357$

H -3.338246 -3.263863 -4.688679

C $-1.633581-5.307050-5.045932$

H $-1.579036-5.007522-2.923554$

C $-3.341846-0.383399-1.571382$

O $-3.556067-0.687096-3.973313$

H $-4.664874-1.731984-2.606693$

H - $-3.332343-0.960747-0.641475$ 
O $-2.0424800 .184061-1.718654$

C -4.385786 $0.745165-1.446766$

C $-4.150328 \quad 1.697456-0.258200$

H $-5.3838140 .298082-1.366967$

O $-4.3766891 .535285-2.637906$

H -3.205461 $2.235772-0.396390$

H $-4.9349282 .464417-0.302887$

H -2.400632 -6.087223 -5.003873

H $-1.776681-4.745301-5.975019$

H $-0.655874-5.796519-5.095333$

C $0.620927-3.604509-3.245892$

H $-3.980431-1.244058-4.646753$

H -2.034317 $0.672582-2.559972$

H $-5.0907712 .190848-2.562469$

H $0.986309-4.545710-3.672591$

H $0.519044-3.759399-2.164063$

H $-0.619806-2.841058-4.864169$

C -5.572378 -0.4599112 .439485$

O $-5.407262 \quad 0.3054001 .238430$

$\begin{array}{llll}\text { C } & -4.172608 & 1.017581 & 1.120537\end{array}$

$\begin{array}{llll}C & -4.031157 & 2.025219 & 2.272369\end{array}$

C $-4.184137 \quad 1.339136 \quad 3.634712$

C $-5.4187710 .432406 \quad 3.683524$

H $-6.613025-0.8084902 .408983$

C $-4.664213-1.7001732 .456110$

H -3.3377450 .3122431 .166573$

O $-5.006072 \quad 3.0599532 .155033$

H -3.048697 2.5043102 .208719

H $-4.265526 \quad 2.1023104 .418237$

O $-3.029927 \quad 0.5533363 .913140$

H -5.401292 -0.1952894 .582172$

O -6.5899381 .2504893 .766401$

C -5.013384-2.680472 1.354093

H -3.611269-1.433933 2.333335

H $-4.785013-2.2270843 .409519$

H -5.8832732 .6406512 .133548$

H -2.256179 1.1411223 .878650

H -6.522929 1.7758494 .581918

O $-4.119597-3.7136101 .382731$

O $-5.949559-2.566376 \quad 0.575135$

C $-4.329073-4.740738 \quad 0.408034$

H $-4.446540-4.313823-0.592356$

H -3.451693 -5.3928810 .407661$

H $-5.208894-5.3315280 .678739$

SCF Energy (B3LYP/6-31G*//MMFF) $=-3245.91053377$

0800146

MM̄FF Geometry

C $0.428925-3.1114282 .175895$

C $-0.755215-3.1756621 .550466$

C $-0.919334-3.539460 \quad 0.097482$

O $-1.351622-2.341481-0.593167$

C $-2.002318-4.620957-0.056034$

C $-2.298775-4.986051-1.498608$

C $-3.425291-4.650535-2.160569$

C $-1.233005-5.797759-2.188543$

C $-4.590679-3.855624-1.639896$

C $-4.618257-2.446115-2.244865$

C $-5.442735-1.472689-1.389490$

C $-5.563951-0.115299-2.083876$

C $-6.2602320 .929018-1.199368$

O $-4.761319-1.324578-0.137945$

$\begin{array}{llll}0 & -6.035480 & 2.209307 & -1.809414\end{array}$

C $-5.5854420 .939596 \quad 0.190219$

C -7.779173 $0.723406-1.181335$

O -6.2865701 .7999411 .084482$

C $-5.415521-0.4651200 .804233$

C $-4.563715-0.4615652 .098472$

O $-6.707533-0.9769551 .153421$

C $-0.381600-1.437918-0.911315$

O $0.821447-1.566874-0.747332$

C -1.038609 $-0.228460-1.452873$

C $-0.3062530 .875727-1.661467$

C $-0.796045 \quad 2.165789-2.150786$

C -2.279237 2.316271-2.356264

C $-3.1199260 .027101 \quad 1.937697$

C -2.913968 1.4974172 .258333

$\begin{array}{llll}\text { C }-1.152715 & 3.261147 & 2.200920\end{array}$ $\begin{array}{llll}\text { O } & -3.768577 & 2.197612 & 2.793699\end{array}$

C $-1.394848 \quad 4.1466101 .019927$

C -0.4387014 .8989990 .453896$

C $-0.6832225 .839575-0.691849$

C $0.2490155 .598101-1.857771$

C $1.3536116 .344329-2.026716$

C $-0.1454474 .546745-2.870886$

C $0.098856 \quad 3.151419-2.369933$

$\mathrm{N}-1.6367451 .9238001 .915390$

H $1.336217-3.3794801 .641211$

H $-1.652228-2.8688432 .083685$

H $\quad 0.022700-3.897306-0.336529$

H -2.912405 -4.2904360 .453645$

H $-1.691628-5.5306220 .475646$

H -3.528529 -4.969171 -3.196991

H $-1.529738-6.100995-3.198226$

H $-0.306864-5.221730-2.277694$

H $-1.023638-6.710267-1.620827$

H $-5.516870-4.380159-1.905719$

H -4.590555 -3.808964 -0.549094

H $-3.593373-2.064453-2.328028$

H $-5.028344-2.498192-3.260890$

H $-6.433684-1.908017-1.215181$

H $-6.084770-0.214796-3.044561$

H $-4.5599690 .254951-2.329474$

H $-6.4047362 .886608-1.216747$

H $-4.596177 \quad 1.3895460 .049942$

H -8.185536 $0.802085-2.197036$

H $-8.279417 \quad 1.505770-0.600145$

H $-8.075603-0.247988-0.780374$

H $-7.082946 \quad 1.3299681 .384946$

H -5.0886850 .0797962 .894767$

H -4.503828 -1.502765 2.447266

H $-6.592769-1.6746821 .819764$

H $-2.105212-0.267860-1.635848$

H $\quad 0.7600350 .838376-1.444397$

H $-2.623566 \quad 1.654407-3.158035$

H -2.585389 $3.329573-2.622875$

H $-2.8194382 .068260-1.436646$

H -2.489598 -0.5235022 .647803$

H $-2.743255-0.1847470 .933761$

H -0.0893913 .1768772 .445769$

H -1.679797 3.6595103 .073891

H $-2.410607 \quad 4.1978550 .634595$

H 0.5700954 .8682220 .859718

H $-0.5504056 .862496-0.315558$

H $-1.7225605 .786829-1.039470$

H $2.0130656 .194848-2.875245$

H $1.6260917 .118057-1.316406$

H $0.4446934 .668509-3.789129$

$\mathrm{H}-1.1802224 .718799-3.178659$

H $1.1526952 .942660-2.185106$

H $-1.007398 \quad 1.2832331 .440151$

C $1.094629-1.1347803 .518314$

O $2.481395-1.1217723 .121065$

C $3.153040-0.0820993 .851487$

C $2.0783850 .688274 \quad 4.610064$

C $1.053502-0.4032924 .857469$

H $3.777622-0.5874744 .601203$

$\begin{array}{llll}\text { C } & 4.090358 & 0.733156 & 2.947429\end{array}$

H 2.4579401 .1344525 .534496

H 1.6465781 .4895124 .001668

C -0.3156410 .1301995 .240058$

H $1.421659-1.0619275 .655544$

C $3.562813 \quad 1.0104931 .518981$

O 4.4378501 .9329203 .631653

$\begin{array}{llll}\text { H } & 5.013648 & 0.149468 & 2.833916\end{array}$

H 3.3444180 .0434461 .055950

$\begin{array}{llll}\text { O } & 2.334479 & 1.729645 & 1.561303\end{array}$

C 4.5734551 .7635710 .627294

C $4.1432101 .919440-0.845136$

H 5.5364521 .2420400 .664980

$\begin{array}{llllll}\text { O } & 4.807477 & 3.074537 & 1.148078\end{array}$

H $3.2037532 .481623-0.903986$

H $4.9008122 .550387-1.327950$

H -0.259356 $0.694176 \quad 6.176844$

H $-0.7143930 .798763 \quad 4.471388$

H - $-1.030769-0.6859235 .380811$ 
C $0.586317-2.576415 \quad 3.571710$

H 5.1238312 .3837993 .111150

H 2.4886082 .5455972 .067104

H 3.9870273 .5863841 .047843

H $-0.371716-2.6238194 .101730$

H $1.292907-3.2183554 .110503$

H $0.540841-0.5606992 .762159$

C $5.307801-1.405208-2.077922$

O $5.266811-0.090464-1.509366$

C $4.0130510 .591046-1.607732$

C $3.6286280 .778671-3.084631$

C $3.618918-0.564682-3.821896$

C $4.894833-1.374867-3.559023$

H $6.365122-1.697501-2.032957$

C $4.509567-2.415377-1.238795$

H $3.236811-0.003652-1.119209$

O $4.547748 \quad 1.646491-3.743536$

H $2.6353651 .235951-3.149619$

H $3.517371-0.377654-4.897953$

O $2.477122-1.305917-3.402964$

H $4.783429-2.401755-3.926098$

O $5.975865-0.786971-4.289246$

C $5.070594-2.5713740 .160150$

H $3.463993-2.116708-1.138622$

H $4.548091-3.400280-1.717836$

H $4.3675012 .552709-3.442091$

H $2.472408-2.143131-3.897154$

H $5.736501-0.802853-5.231591$

$\begin{array}{llllll}\text { O } & 4.239877 & -3.381177 & 0.881886\end{array}$

O $\quad 6.106052-2.0637810 .568064$

C $4.647194-3.6400112 .229265$

H $5.531189-4.2841742 .231274$

H $4.851230-2.7057702 .760883$

H $3.830621-4.1594522 .737670$

SCF Energy (B3LYP/6-31G**//MMFF)= -3245.90936514

0800147

MM̄FF Geometry

C -2.140394 4.3331051 .541215

C -3.0504113 .3529501 .635605$

C -3.0591332 .3303012 .742747$

O -2.7145391 .0535582 .154403$

C -4.4642782 .2323573 .360838$

C $-4.559321 \quad 1.224753 \quad 4.491683$

C -5.1808720 .0306844 .410473$

C $-3.916764 \quad 1.6500615 .787247$

C -5.879295 -0.5602723 .218255$

C $-5.096924-1.7508042 .649592$

C $-5.555719-2.1027661 .227564$

C $-4.859236-3.3703230 .731837$

C $-5.192999-3.676441-0.735984$

O $-5.216142-0.996280 \quad 0.383507$

O $-4.259788-4.663284-1.203119$

C $-4.962798-2.404334-1.580558$

C $-6.588233-4.294105-0.886038$

O $-5.409905-2.596964-2.920029$

C $-5.619128-1.142099-0.984538$

C $-5.2200170 .160678-1.723444$

O $-7.043423-1.261832-1.087187$

C -1.3856420 .7674502 .051989$

$\begin{array}{lllll}\text { O } & -0.455439 & 1.447729 & 2.455757\end{array}$

C -1.246003 -0.5174101 .331881$

C $-0.027118-0.9314180 .954336$

C $0.287338-2.1655010 .231611$

C $-0.857913-3.064662-0.149882$

C $-3.7225580 .490721-1.725593$

C -2.978317 -0.012061 -2.951482

C $-0.670300-0.251343-3.859429$

O $-3.542715-0.455411-3.947792$

C $-0.126122-1.613835-3.568045$

C $1.185025-1.881067-3.468502$

C $1.741795-3.260331-3.261764$

C $2.709681-3.340892-2.103601$

C $4.034107-3.237568-2.304128$

C $2.150178-3.646076-0.733587$

C $1.578227-2.432114-0.056249$

N -1.603505 $0.129220-2.815769$

H -1.369142 4.4240172 .302076
H -3.7977913 .2460050 .852613$

H -2.336045 2.5905103 .525731

H -5.1882432 .0025752 .573216$

H -4.7657743 .2147323 .749657$

H $-5.188793-0.6068185 .293708$

H $-4.0839660 .925966 \quad 6.591867$

H -2.8350151 .7592515 .664072$

H -4.3291742 .6085686 .118892$

H $-6.875389-0.8982283 .530043$

H $-6.054703 \quad 0.1869752 .442082$

H $-4.027794-1.5067582 .630298$

H -5.221830 -2.615940 3.312424

H $-6.643870-2.2369271 .232178$

H $-5.109928-4.2268001 .370154$

H -3.772396 -3.243269 0.818898

H $-4.340017-5.445961-0.631171$

H -3.878386 -2.250712 -1.630989

H $-6.653676-5.232893-0.322882$

H $-6.794214-4.556944-1.929401$

H -7.387275 -3.640885 -0.529553

H $-4.956035-3.384201-3.266743$

H $-5.6349680 .160432-2.738612$

H $-5.7220390 .991196-1.205975$

H -7.432262 -0.372723-1.041339

H -2.147275 -1.079657 1.119963

H $0.832297-0.308752 \quad 1.200275$

H -1.612405 -2.509285 -0.716928

H -1.327347 -3.4840390.746098

H $-0.562499-3.903116-0.784067$

H $-3.6085711 .582480-1.729238$

H $-3.2362790 .115098-0.821058$

H $0.1144260 .509602-3.893023$

H -1.181435 -0.259544 -4.827438

H $-0.844341-2.425409-3.476492$

H $1.902022-1.072506-3.592810$

H $2.252655-3.552681-4.189026$

H $\quad 0.942667-3.999250-3.123842$

H $4.737437-3.334540-1.484729$

H $4.443723-3.051884-3.291529$

H $2.946013-4.031199-0.081307$

H $1.435571-4.471004-0.805900$

H $2.337488-1.7142920 .255911$

H -1.221035 $0.472531-1.939350$

C -1.219438 $4.754174-0.743964$

O $0.1224194 .520162-0.285799$

C $1.0263354 .684235-1.394160$

C $0.1775155 .128859-2.586551$

C $-1.0563965 .709053-1.920484$

H $1.7174955 .489462-1.117287$

C $1.8224093 .391450-1.646137$

H $0.7036635 .856128-3.213316$

H $-0.0936264 .283518-3.229959$

C $-2.2631455 .779796-2.839336$

H $-0.8157646 .716562-1.556004$

C $2.6798962 .959441-0.435465$

O $0.8995952 .357347-1.987902$

H $2.4754013 .552027-2.512617$

H $3.2884643 .814502-0.117240$

O 1.8363542 .6230430 .665133

C $3.6327321 .778856-0.730243$

C 4.4364841 .3787810 .516532

H $4.3041922 .080890-1.541168$

O $2.8817340 .656216-1.182880$

H 4.9445192 .2587670 .931563

H $3.7461141 .041611 \quad 1.302349$

H $-2.0484276 .410516-3.708182$

H -2.546989 $4.787391-3.205735$

H $-3.126526 \quad 6.206164-2.319244$

C -2.0768935 .3034730 .395588$

H $1.414057 \quad 1.559496-2.195645$

H 1.2409331 .9137010 .368376

H $3.5051080 .008236-1.551557$

H -3.0877305 .5289850 .036715$

H $-1.653734 \quad 6.2416750 .774645$

H -1.619379 $3.788936-1.084469$

C $6.992182-1.064627 \quad 1.663915$

O 6.0512920 .0133231 .592548

C 5.4569680 .2431240 .306670 
C $6.541240 \quad 0.561575-0.733341$

C $7.629032-0.518892-0.758158$

C $8.128098-0.8644660 .648120$

H $7.425256-0.9894932 .670099$

C $6.305339-2.4303401 .554657$

H $4.911187-0.6580770 .009840$

O $7.1293461 .825117-0.431089$

H $6.0789170 .640371-1.723597$

H $8.475366-0.172256-1.363578$

O $7.118735-1.700314-1.368226$

H $8.756032-1.7627840 .623677$

$\begin{array}{llll}\text { O } & 8.946704 & 0.206148 & 1.127704\end{array}$

C $5.413309-2.6762042 .748070$

H $5.704713-2.5466710 .648917$

H $7.048528-3.2363331 .535760$

H $7.7920832 .013148-1.117231$

H $6.838764-1.468679-2.270073$

H 9.6958970 .2995940 .514891

O $4.162457-2.1921982 .502632$

O $5.791152-3.2136453 .781055$

C $3.222803-2.3383363 .571555$

H $2.287826-1.8583753 .271018$

H $3.029776-3.3986623 .759398$

H $3.593404-1.8458394 .475643$

SCF Energy (B3LYP/6-31G*//MMFF) $=-3245.90053279$

0800148

MM̄FF Geometry

C 2.3433785 .1759380 .624649

C 3.1622394 .1166100 .707793

C $4.0342153 .641209-0.424190$

O $3.6964042 .259535-0.694885$

C $5.5122453 .701970-0.011004$

C $6.453468 \quad 3.367738-1.150531$

C $7.0884012 .188297-1.295435$

C $6.6989644 .484955-2.131964$

C $6.9652070 .970482-0.421671$

C $6.870804-0.312169-1.260513$

C $6.815129-1.561382-0.370583$

C $6.967809-2.831633-1.208383$

C $6.856535-4.095742-0.343856$

O $5.553405-1.5759370 .307363$

O $6.698968-5.219364-1.223398$

C $5.574302-4.002820 \quad 0.506530$

C $8.132695-4.3534400 .465903$

O $5.499720-5.0806641 .437364$

C $5.407812-2.6515001 .244857$

C $3.991592-2.5804501 .860804$

O $6.371979-2.5642752 .295822$

C $2.6104242 .032715-1.482734$

O $1.9115162 .870730-2.030100$

C $2.3932010 .570409-1.543499$

C $1.2035730 .111178-1.959727$

C $0.801119-1.291990-2.072592$

C $1.868263-2.338615-1.894525$

C $3.574759-1.1915852 .361569$

C $2.182921-1.2570422 .962154$

C $-0.207984-1.5879562 .326868$

O $2.001842-1.2291854 .177017$

C $-0.766787-2.6208531 .399143$

C $-1.842313-2.4309090 .620521$

C $-2.391951-3.475432-0.307579$

C $-2.446389-3.015160-1.747737$

C $-3.621291-2.779563-2.354825$

C $-1.144817-2.913736-2.516008$

C $-0.489339-1.568057-2.356257$

N $1.193398-1.3980292 .002687$

H $2.315196 \quad 5.758260-0.292187$

H $3.1839983 .530337 \quad 1.623827$

H $3.8747564 .243502-1.327331$

H 5.6904463 .0336950 .839728

H $5.7587654 .708140 \quad 0.354445$

H $7.7811202 .082723-2.129573$

H $7.4258034 .209166-2.903397$

H $5.7706514 .762345-2.640455$

H $7.0891715 .366954-1.613466$

H $7.8554490 .919456 \quad 0.216606$

H 6.0945091 .0284310 .237486
H $5.973136-0.263427-1.890577$

H $7.741149-0.375084-1.925802$

H $7.623189-1.4954390 .367512$

H $7.917982-2.826683-1.756813$

H $6.178974-2.861519-1.973293$

H $7.470922-5.247577-1.814365$

H $4.723412-4.126985-0.178327$

H $8.994791-4.463153-0.202892$

H $8.063907-5.2951361 .021813$

H $8.362458-3.5526711 .171752$

H $5.592084-5.9065520 .932255$

H $3.260489-2.9004641 .109317$

H $3.914846-3.2910042 .694499$

H $6.039783-1.9613052 .979602$

H $3.200333-0.079069-1.225160$

H $0.4356640 .835298-2.230272$

H $2.714105-2.144699-2.563975$

H $1.528793-3.351507-2.119996$

H $2.234461-2.341773-0.864913$

H $4.260063-0.8205403 .130511$

H $3.573174-0.4577101 .547676$

H $-0.704237-0.6186442 .224082$

H $-0.309145-1.9214373 .364302$

H $-0.255769-3.5809251 .369709$

H -2.371598 -1.4835800 .663209$

H $-3.397841-3.7359760 .046943$

H $-1.808460-4.403135-0.253048$

H -3.670395 -2.476338 -3.395330

H $-4.559412-2.881911-1.822855$

H -1.337092 -3.063262 -3.586651

H $-0.498379-3.742057-2.213836$

H $-1.171768-0.729772-2.506269$

H $1.437899-1.2582991 .026703$

C $-0.051515 \quad 5.2978121 .455043$

O $\begin{array}{llll}-0.226135 & 3.893420 & 1.175246\end{array}$

C -1.1152903 .7572760 .043598$

C $-1.8083075 .104090-0.090729$

$\begin{array}{llll}\text { C }-0.685306 & 6.051985 & 0.286445\end{array}$

H $-0.470703 \quad 3.576039-0.825476$

C $-2.0345012 .555276 \quad 0.279042$

H -2.196079 $5.281901-1.098019$

H $-2.639107 \quad 5.1886830 .620933$

C -1.1599517 .4502800 .640886$

H $0.0084856 .117294-0.560396$

C -3.174965 $2.427946-0.746758$

O $-1.201326 \quad 1.393611 \quad 0.219519$

H $-2.4342152 .611966 \quad 1.297763$

H $-3.8235383 .309335-0.682253$

O $-2.6147542 .447756-2.066437$

C $-4.0522951 .163389-0.627612$

$\begin{array}{llll}\text { C }-4.603951 & 0.938502 & 0.792277\end{array}$

$\begin{array}{llll}\mathrm{H} & -3.488495 & 0.284324 & -0.960492\end{array}$

O $-5.132812 \quad 1.329802-1.550330$

H $-3.7666970 .804497 \quad 1.484834$

H -5.1568751 .8377821 .093500$

H $-1.661357 \quad 7.914359-0.214701$

H $-1.8675407 .434927 \quad 1.476545$

H $-0.316108 \quad 8.087144 \quad 0.924581$

C $1.4241145 .596173 \quad 1.737829$

H -1.7294630 .6332590 .509735$

H $-1.9954781 .700850-2.132664$

H $-5.6452860 .504164-1.559710$

H 1.7106055 .0715792 .658638

H $1.5634636 .665867 \quad 1.930479$

H $-0.619775 \quad 5.4962622 .373477$

C -7.801417 -0.9590500 .333855$

$\begin{array}{lllll}\text { O } & -6.794390 & 0.061967 & 0.325828\end{array}$

$\begin{array}{llll}C & -5.536415 & -0.284608 & 0.913075\end{array}$

C $-5.728663-0.693483 \quad 2.382794$

C $-6.774641-1.8021052 .527185$

C $-8.059439-1.4721901 .761116$

H $-8.714547-0.453910-0.007017$

C $-7.488435-2.083405-0.664571$

H $-5.091659-1.1171940 .357293$

$\begin{array}{lllll}\text { O } & -6.120870 & 0.433591 & 3.164867\end{array}$

H -4.767221 -1.0382782 .781339$

H -7.013464 -1.941152 3.588599

O $-6.244770-3.0295482 .035432$ 
H $-8.718705-2.347170 \quad 1.720485$

O $-8.768601-0.4460012 .461575$

C -7.518269-1.604948 -2.101410

H -6.502405 -2.517475 -0.485652

H -8.244291 $-2.871753-0.570527$

H -6.9328950 .7989142 .773582$

H -5.447127 -3.2338712 .552713$

H -8.980906 -0.7885013 .346660$

O $-7.262602-2.665400-2.924286$

O $-7.731341-0.455659-2.461560$

C -7.234078 -2.365098 -4.323233

H $-6.452673-1.629646-4.537218$

H -7.004833 -3.287055 -4.864091

H -8.211328 -1.998479-4.651299

SCF Energy (B3LYP/6-31G**//MMFF) $=-3245.90347075$

08_00149

MM̄MF Geometry

C -2.259450 4.8395461 .327150

C $-3.4014894 .310421 \quad 1.792702$

C $-4.559897 \quad 3.7703260 .985282$

O

C $-4.4948743 .923095-0.551576$

C $-5.7536793 .470240-1.271929$

C $-5.8173862 .482378-2.187638$

C $-7.0036464 .248757-0.941052$

C $-4.7117491 .610757-2.715232$

C $-4.9391690 .141562-2.339241$

C $-3.851154-0.785406-2.902419$

C $-4.092161-2.227857-2.450093$

C $-2.964826-3.175583-2.885888$

O $-2.579237-0.329317-2.432800$

O $-3.132326-4.386307-2.131534$

C -1.604653 -2.553201-2.498315

C $-3.096920-3.561304-4.363780$

O $-0.523236-3.314870-3.028699$

C -1.462495-1.079240 -2.933387

C $-0.173366-0.407279-2.399450$

O $-1.399683-1.015687-4.363999$

C -3.9097231 .4219801 .139520$

O $-2.841331 \quad 1.5365510 .561967$

C $-4.439893 \quad 0.162879 \quad 1.712087$

C $-3.779133-0.9859911 .505735$

C $-4.170808-2.3127021 .984725$

C $-5.378507-2.4052472 .879507$

C $-0.039188-0.367746-0.875556$

C $0.742667-1.530190-0.289926$

C $1.131073-2.7082081 .873048$

O $1.483128-2.248009-0.955851$

C $0.247117-3.9133371 .805211$

C $-0.443386-4.3946882 .849654$

C -1.318992 -5.612783 2.777120

C -2.769040 -5.309963 3.083587

C $-3.279014-5.5454574 .304132$

C $-3.649948-4.8262711 .953100$

C $-3.428777-3.3771971 .613700$

N $0.541706-1.6469001 .078886$

H -2.0866694 .9156930 .257791$

H -3.5354804 .2519772 .871947$

H -5.4322434 .3341891 .338096$

H $-3.6174243 .398925-0.936298$

H $-4.3352354 .980776-0.801192$

H $-6.7875042 .272446-2.638083$

H -7.835482 4.005598-1.611159

H -7.3334684 .0353410 .080194$

H $-6.8178975 .323961-1.033013$

H $-4.6950731 .707873-3.807798$

H $-3.730506 \quad 1.939383-2.366165$

H -4.956816 $0.060842-1.246861$

H -5.920675 -0.189904 -2.701571

H $-3.876280-0.716318-3.996352$

H -5.062000 -2.591729 -2.812137

H -4.157405 -2.255423 -1.354279

H -2.456911 -5.020872 -2.424998

H -1.528686 -2.612077 -1.405533

H -4.041460 -4.091289-4.535510

H -2.305040 -4.255792 -4.665000

H $-3.073729-2.700391-5.035164$
H $-0.568730-4.203712-2.638461$

H $0.709918-0.853241-2.872396$

H $-0.1890150 .637855-2.741196$

H $-0.986924-0.173556-4.617490$

H $-5.364396 \quad 0.214128 \quad 2.275949$

H $-2.866071-0.9600550 .914275$

H -5.262003 -1.753836 3.753057

H $-5.554203-3.4101533 .269628$

H $-6.282088-2.1073092 .336668$

H $\quad 0.5161730 .534410-0.600565$

H -1.020483 $-0.299759-0.398791$

H $1.241806-2.3309532 .894491$

H $2.123111-2.9537831 .481278$

H $0.179486-4.4191180 .844636$

H $-0.369765-3.8958963 .813211$

H $-0.928036-6.3471893 .493644$

H -1.248746 -6.0986881 .795891$

H $-4.325387-5.3628164 .524934$

H -2.659812 -5.9220635 .111585$

H $-4.705420-5.0207272 .174332$

H $-3.448314-5.4270471 .056553$

H -2.571139 -3.208006 0.962601

H $-0.143220-1.0480031 .531052$

C 0.1584764 .5946972 .100928

O $0.7320654 .783325 \quad 0.792864$

C 1.2711993 .5212840 .339250

C 1.3310722 .6344961 .577355

C 0.0940303 .0856232 .327049

H $0.5429363 .129598-0.382208$

C $2.6261523 .752383-0.341818$

H 1.3229381 .5669081 .344114

H 2.2335542 .8491002 .162727

C 0.0700382 .6796413 .788830

H -0.7783112 .6508991 .827111$

C $3.2461012 .450414-0.892490$

O $2.4019334 .679696-1.407720$

H $3.310976 \quad 4.234747 \quad 0.365905$

H $3.253051 \quad 1.700954-0.095148$

O $2.4103141 .940732-1.932413$

C $4.6893732 .593992-1.416048$

C $5.2340081 .337906-2.124675$

H $5.3409342 .860902-0.575620$

O $4.7590483 .670664-2.352893$

H $4.6917111 .182065-3.066372$

H $6.2693421 .560697-2.416102$

H $-0.843403 \quad 3.0360894 .275593$

H 0.1008791 .5893533 .885500

H 0.9252513 .0914944 .334735

C -1.1646515 .3563892 .221697$

H $3.2662234 .892326-1.798329$

H $2.3898262 .606712-2.641209$

H $5.6908903 .778482-2.609414$

H $-0.9960746 .406761 \quad 1.952681$

H -1.489354 5.3446323 .269161

H 0.8711905 .0458122 .804345

C $6.027976-0.8149720 .825517$

O $5.973070 \quad 0.276373-0.103551$

C $5.2003460 .050733-1.285571$

C $5.744509-1.154683-2.070032$

C $5.846396-2.399138-1.182006$

C $6.548340-2.0940900 .143611$

H $6.772973-0.5095551 .571604$

C $4.689459-1.0171801 .554059$

H $4.162427-0.155687-1.008564$

O $7.028018-0.858581-2.617601$

H $5.076729-1.355416-2.915863$

H $6.404880-3.180636-1.711673$

O $4.543774-2.902961-0.904309$

H $6.450345-2.9475190 .824896$

O $7.944151-1.931665-0.124087$

C 4.4236160 .0806622 .562761

H $3.835199-1.0420640 .874291$

H $4.727956-1.9639352 .106258$

H $7.616644-0.616772-1.882075$

H $4.116640-3.102519-1.754776$

H $8.394853-1.8095240 .728855$

O $3.504294-0.3643103 .469695$

O 4.9424961 .1882972 .551526 
C 3.1763400 .5454494 .524196

H 3.9957110 .5773705 .247780

H $2.972464 \quad 1.5461254 .134110$

H 2.2770540 .1760045 .024078

SCF Energy $\left(B 3 L Y P / 6-31 G^{* *} / / M M F F\right)=-3245.91258454$

08_00150

MM̄FF Geometry

C -0.4510111 .6362853 .462501$

C -0.5402992 .9703673 .345223$

C -1.2902663 .7403372 .280960$

O -2.0803502 .8710541 .439964$

C $-0.2668384 .470014 \quad 1.390456$

C -0.8911635 .3542670 .329341$

C $-0.9235685 .071859-0.988550$

C $-1.465880 \quad 6.6524730 .835318$

C $-0.4175013 .836416-1.681511$

C $-1.5527943 .134927-2.438139$

C $-1.1322401 .757825-2.973236$

C $-2.2781121 .127290-3.768307$

C $-1.970289-0.313686-4.197417$

O $\quad-0.8009780 .940814-1.846571$

O $-3.208779-0.892446-4.637050$

C -1.486412 -1.108128 -2.964953

C $-1.028114-0.347915-5.405974$

O $-1.068772-2.417768-3.342407$

C $-0.377764-0.389408-2.169106$

C $-0.037768-1.098278-0.836423$

O $0.817821-0.374160-2.958391$

C -3.3373632 .5680191 .867905$

O -3.8604482 .9135822 .915319$

C $-3.978079 \quad 1.732835 \quad 0.826975$

C -5.1982811 .2187061 .042385$

C -5.9747790 .4059080 .102430$

C $-5.3406890 .069323-1.221002$

C $-1.172749-1.1553460 .190248$

C $-1.965383-2.4502860 .180521$

C $-3.957639-3.499326 \quad 1.255211$

O $-1.652137-3.431306-0.487303$

C $-5.136951-3.3602050 .345657$

C $-6.405912-3.3311950 .780460$

C -7.598677 -3.253803 -0.127959

C $-8.529565-2.1150660 .220841$

C $-9.640839-2.3276460 .946063$

C $-8.223163-0.751337-0.353952$

C -7.2147650 .0114880 .460977$

N -3.052768 -2.3862861 .042713$

H -0.9702510 .9891752 .761763$

H 0.0038813 .5874174 .058692

H -1.942122 4.4739422 .771181

H 0.4018103 .7329150 .933946

H $\quad 0.3860005 .0976062 .012961$

H -1.374387 $5.804673-1.657111$

H -1.841989 7.2854700 .024565

H -2.3000236 .4638951 .518059$

H -0.6999307 .2244901 .369306$

H $\quad 0.3662204 .128468-2.390594$

H $0.0445503 .138837-0.980142$

H -2.406287 $3.010904-1.759570$

H -1.886948 $3.770204-3.267965$

H $-0.244127 \quad 1.879956-3.604278$

H -2.530843 $1.743423-4.640417$

H $-3.182491 \quad 1.113289-3.145807$

H -3.040387 -1.828966 -4.839322

H -2.361564 -1.243134 -2.318782

H $-1.4843450 .170213-6.258246$

H $-0.851032-1.374937-5.743270$

H $-0.062774 \quad 0.123684-5.210585$

H $-0.186003-2.343545-3.743370$

H $\quad 0.378359-2.091965-1.035283$

H $0.776873-0.529219-0.367261$

H $1.583212-0.298440-2.365372$

H $-3.4328911 .574232-0.095605$

H $-5.687257 \quad 1.4241751 .993951$

H -5.221405 $0.974554-1.825445$

H -5.917174 -0.644584 -1.813002

H -4.357052 -0.388839-1.074135

H $\quad-0.733199-1.0796881 .192157$
H -1.857135 -0.3109320 .071484$

H $-4.244840-3.4870752 .311287$

H -3.438772 -4.440305 1.046160

H $-4.937906-3.313140-0.722436$

H $-6.603282-3.4066811 .847436$

H $-8.135098-4.208873-0.052475$

H -7.297304 -3.169478 -1.179582

H $-10.330386-1.5212831 .173063$

H $-9.882321-3.3117631 .333911$

H $-9.139363-0.145506-0.380206$

H $-7.935872-0.854545-1.404082$

H -7.5858940 .3016851 .445014$

H -3.262676 -1.510404 1.512847

C 1.5621870 .2275593 .834319

O $1.097396-1.0128873 .266078$

C $2.174083-1.9807573 .328233$

C $3.363604-1.2463633 .935597$

C $2.685628-0.1833774 .778500$

H $1.828675-2.7705624 .006695$

C $2.390045-2.5402511 .916108$

H $4.011032-1.9093224 .517298$

H $3.974472-0.7741023 .155650$

C 3.6159980 .9494945 .176081

H $2.270658-0.6527795 .680202$

C $3.709970-3.3101901 .728884$

O $1.281858-3.4048001 .641544$

H $2.319618-1.7121381 .204852$

H $4.561467-2.6613911 .959184$

O $3.764726-4.3729702 .688417$

C $3.923469-3.9152430 .328139$

C $3.729053-2.946412-0.849120$

H $3.249280-4.7701100 .195317$

O $5.245543-4.4597950 .284400$

H $4.015754-3.449511-1.782254$

H $2.657119-2.745913-0.962481$

H 4.4528450 .5682435 .770522

H 4.0308521 .4555124 .297716

H 3.0900241 .6970685 .777544

C 0.3981630 .9566774 .503880

H $1.302377-3.6290450 .696604$

H $2.992149-4.9442022 .537523$

H $5.363067-4.877372-0.585876$

H $\quad 0.772971 \quad 1.6963555 .219935$

H -0.2328560 .2508615 .056611$

H 1.9679960 .8292663 .008650

C $4.4025670 .544490-1.923217$

O $3.962399-0.819836-1.880819$

C $4.458254-1.589159-0.773400$

C $5.988832-1.673084-0.841744$

C $6.616972-0.275423-0.901780$

C $5.9393080 .622241-1.945207$

H $4.0329450 .915327-2.887771$

C $3.7257591 .365214-0.813703$

H $4.164810-1.0817550 .149496$

O $6.371702-2.422112-1.992331$

H $6.364432-2.1997120 .041902$

H $7.683101-0.373991-1.139739$

O $\quad 6.520284 \quad 0.317784 \quad 0.390155$

H $6.2576821 .663307-1.822080$

O $6.363260 \quad 0.208570-3.248519$

C $3.7222762 .851790-1.093599$

H $2.6801721 .050339-0.716364$

H $4.206728 \quad 1.1958250 .153509$

H $7.341768-2.486475-1.991381$

H $\quad 6.950247 \quad 1.1889590 .346107$

H $7.3293750 .311220-3.289089$

O $3.2596543 .509027 \quad 0.011174$

O $4.0663193 .374302-2.144709$

C $3.2054764 .934729-0.100567$

H 2.8465435 .3374000 .850165

H $4.2034185 .338533-0.296900$

H $2.5083645 .225925-0.891678$

SCF Energy (B3LYP/6-31G**//MMFF) $=-3245.90683940$

08_00151

MM̄FF Geometry

C $3.6001822 .950405-0.413199$

C 3.5148753 .9085600 .521689 
C 2.5182493 .9511901 .657939

O 1.6202752 .8191501 .621762

C 1.6605735 .2263521 .561300

C 0.6621675 .3626192 .696481

C $-0.677462 \quad 5.2849852 .565013$

C 1.2636005 .6291074 .053770

C $-1.4844995 .068058 \quad 1.315034$

C $-2.228317 \quad 3.7302331 .350047$

C -2.973554 $3.421142 \quad 0.042056$

C $-4.126275 \quad 4.395045-0.220925$

C $-4.9297374 .016607-1.474501$

O -3.4766532 .0805340 .149147$

O $-6.1411164 .786908-1.442776$

C $-5.3124642 .524348-1.385960$

C -4.196108 4.427111-2.756678

O $-5.9549242 .092338-2.582660$

C $-4.1219411 .603727-1.040595$

C -4.549934 $0.138189-0.805826$

O $-3.2140351 .591824-2.150256$

C 1.9910021 .6928002 .289554

$\begin{array}{lllll}\text { O } & 3.037771 & 1.497250 & 2.884900\end{array}$

C $0.868552 \quad 0.7350802 .182310$

C $0.883943-0.3994482 .897263$

C $-0.194316-1.3872802 .952818$

C $-1.389175-1.1743982 .061175$

C $-5.539929-0.0913590 .336566$

C $-5.940721-1.553328 \quad 0.403732$

C $-5.077841-3.7992651 .012861$

O $-7.010386-1.949927-0.053666$

C $-4.262702-4.3372672 .144069$

C $-3.102249-4.9915391 .982917$

C $-2.285707-5.5440993 .117221$

C $-0.997845-4.7743853 .328125$

C $0.171205-5.2722732 .890519$

C $-1.130197-3.4752334 .096773$

C $-0.086002-2.4303993 .803078$

N $-4.978928-2.3515591 .000860$

H $2.9000602 .120812-0.401661$

H $4.234137 \quad 4.7247390 .495374$

H 3.0768283 .9545372 .601955

H 1.1532185 .2376910 .591787

H 2.3080506 .1134981 .564328

H $-1.284116 \quad 5.405417 \quad 3.462424$

H 0.5021495 .8412004 .811986

H 1.8374714 .7639664 .399105

H 1.9298686 .4968534 .010089

H -2.201196 5.8940261 .243503

H -0.8715545 .1175350 .412036$

H -1.5162352 .9180661 .544545$

H -2.933244 3.7058852 .191788

H - $-2.2466553 .457103-0.776885$

H -3.766708 $5.426480-0.310769$

H -4.8065104 .3896340 .642493$

H -6.691556 $4.501367-2.192324$

H -6.067146 $2.450908-0.594306$

H -4.035938 $5.512047-2.771835$

H $-4.795164 \quad 4.203205-3.646126$

H -3.221326 $3.947863-2.866890$

H $-5.267967 \quad 1.968555-3.259661$

H $-4.949802-0.285094-1.736826$

H -3.645639 $-0.445964-0.590930$

H -2.650828 $0.803477-2.080090$

H $0.0356691 .012368 \quad 1.546329$

H $1.745106-0.6074593 .530548$

H $-1.983390-0.3218522 .406601$

H -2.044519-2.045625 2.021802

H $-1.080461-0.9897621 .027687$

H $-5.109060 \quad 0.210514 \quad 1.297931$

H -6.4588170 .4862230 .197572$

H $-4.726591-4.1594940 .040070$

H -6.125553 -4.097865 1.122146

H $-4.653531-4.1837413 .147125$

H $-2.713445-5.1445970 .979220$

H $-2.074485-6.5988252 .898396$

H $-2.858505-5.5497374 .053371$

H $1.113535-4.7612533 .053549$

H $\quad 0.214835-6.2166052 .357406$

H -1.095903 -3.705893 5.169622
H -2.125613 -3.045170 3.938753

H $0.817879-2.5267074 .405141$

H $-4.070409-1.9397671 .189142$

C $5.6338961 .778961-1.357986$

O $4.9571390 .505206-1.312302$

C $5.571078-0.299857-0.287893$

C $6.893626 \quad 0.382828 \quad 0.044879$

C $6.5187821 .842215-0.111972$

H $4.918265-0.248308 \quad 0.591465$

C $5.719730-1.749505-0.754400$

H $7.2479500 .134461 \quad 1.049888$

H $7.6803270 .112563-0.669371$

C $7.7128322 .770241-0.249289$

H 5.9366872 .1414330 .768269

C $4.393362-2.465886-1.085501$

O $6.539437-1.778698-1.930658$

H $6.267395-2.3214010 .003949$

H $3.954480-2.029236-1.989172$

O $4.722518-3.819941-1.427745$

C $3.349306-2.4700160 .051846$

C $2.214809-3.496569-0.130281$

H $2.930881-1.4637830 .166710$

O $4.014050-2.792941 \quad 1.278293$

H $2.644946-4.504114-0.190969$

H $1.614107-3.4896890 .788802$

H 8.3445372 .7188070 .643643

H $8.3309712 .504694-1.113495$

H $7.3869323 .807819-0.373271$

C $4.6241492 .919981-1.511091$

H $6.102013-1.231480-2.605460$

H $5.093165-4.241506-0.633238$

H $3.364675-2.7010071 .996493$

H $4.0801182 .789599-2.454838$

H $5.1538073 .877309-1.579212$

H $6.2608931 .755198-2.259049$

C $-0.473009-1.697383-1.987377$

O $0.477646-2.107018-0.995724$

C $1.297041-3.230739-1.333624$

C $0.431511-4.460023-1.656008$

C $-0.603412-4.143293-2.738864$

C $-1.378311-2.864677-2.416780$

H $-1.102004-0.956970-1.477473$

C $0.213063-0.995985-3.163579$

H $1.904991-2.985704-2.209019$

O $-0.230501-4.902074-0.473596$

H $1.088040-5.273578-1.987442$

H $-1.310871-4.977135-2.826135$

O $0.045492-3.989585-3.997067$

H - $-1.986555-2.566519-3.278890$

O $-2.281679-3.161182-1.348645$

$\begin{array}{llll}\text { C } & 0.821238 & 0.309147 & -2.711433\end{array}$

H $1.017186-1.588345-3.609310$

H $-0.499999-0.783016-3.968873$

H $-0.746799-5.693186-0.703270$

H $0.510270-4.821240-4.191730$

H $-2.841986-2.380017-1.211271$

O $-0.118458 \quad 1.296131-2.741029$

$\begin{array}{lllll}\text { O } & 1.984420 & 0.425221 & -2.349527\end{array}$

C $0.3176622 .587669-2.305724$

H $-0.4450013 .317069-2.591044$

H $0.4195642 .592735-1.216994$

H $1.2602442 .866961-2.786148$

SCF Energy (B3LYP/6-31G**//MMFF) $=-3245.90944673$

08_00152

MM̄FF Geometry

C -3.618340 -3.671757 0.572303

C $-2.294257-3.4701390 .489540$

C $-1.385568-3.4726201 .690002$

O $-0.542322-2.2986611 .642554$

C $-0.466092-4.7084401 .668709$

C $0.441401-4.7972662 .882585$

C $1.787933-4.7760012 .833335$

C $-0.252211-4.9359804 .214058$

C $2.668474-4.7219711 .616850$

C $2.998430-3.2859891 .207910$

C $4.020256-3.2039990 .062561$

C $5.434061-3.5660430 .530477$ 
C $6.487357-3.350905-0.568000$

O $3.993410-1.852467-0.417171$

O $7.782689-3.3940190 .051508$

C $6.312149-1.933330-1.151637$

C $6.466573-4.482333-1.602327$

O $7.185258-1.726173-2.259512$

C $4.854059-1.603618-1.537249$

C $4.677147-0.126660-1.956245$

O $4.479955-2.404529-2.664986$

C $-1.021509-1.1565402 .203556$

O $-2.141725-0.9700582 .650452$

C $0.087335-0.1743482 .221192$

C $-0.089544 \quad 1.0090342 .827111$

C 0.9122912 .0679052 .981025

C 2.3018041 .7950162 .468950

C $4.9578140 .897111-0.853587$

C $4.5900262 .306983-1.284366$

C $4.3249544 .603284-0.385682$

O $4.3424692 .611814-2.448202$

C 3.8768165 .1908450 .913232

C 2.6620735 .7274241 .102533

C 2.2164116 .3651292 .386284

C 1.0489935 .6401823 .017184

C $-0.203963 \quad 6.1037712 .865086$

C $1.374854 \quad 4.4379803 .880297$

C $0.5393213 .221597 \quad 3.574419$

N $4.578748 \quad 3.186738-0.210198$

H -4.058174 -3.8674201 .546449$

H -1.835444 -3.297331-0.480501

H -1.974141 -3.465799 2.615834

H $0.126523-4.6881960 .747321$

H - $1.071410-5.6234911 .625399$

H $2.335910-4.8265883 .773755$

H $0.446967-5.1742605 .022930$

H $-0.762252-4.0067064 .484915$

H $-0.990251-5.7439674 .175586$

H $3.584541-5.2734581 .856106$

H $2.215131-5.2593270 .776833$

H $2.077270-2.7796880 .892269$

H $3.364349-2.7113412 .069389$

H $3.693532-3.869157-0.745332$

H $5.482079-4.5986350 .894406$

H $5.701973-2.9353971 .389982$

H $7.877310-4.2571150 .489758$

H $6.642783-1.235214-0.372994$

H $6.661819-5.446885-1.118087$

H $7.263448-4.355654-2.343315$

H $5.513123-4.566506-2.127566$

H $6.806539-2.189330-3.026076$

H $5.294050 \quad 0.089724-2.838160$

H $3.6404120 .012065-2.292514$

H $3.722551-1.984016-3.104667$

H $1.026288-0.4633791 .763062$

H $-1.063787 \quad 1.2255543 .264372$

H 2.7396660 .9341672 .986418

H 2.9904342 .6303432 .606622

H 2.2833181 .5840881 .395534

H 4.3828100 .6502350 .045968

H $6.0216280 .901642-0.594579$

H $3.5870714 .742013-1.183431$

H $5.2607915 .071750-0.707821$

H 4.5948345 .1939321 .729858

H 1.9519565 .7374410 .278685

H 1.9425477 .4051332 .164935

H 3.0389846 .4300743 .109626

H $-1.052247 \quad 5.623693 \quad 3.340566$

H $-0.410163 \quad 6.9817902 .261578$

H 1.2132884 .7130564 .931141

H 2.4426154 .2097353 .817282

H $-0.493365 \quad 3.3112253 .914727$

H 4.9714872 .8743290 .673671

C $-5.650353-2.623457-0.559563$

O $-5.087006-1.297754-0.512553$

C $-5.897250-0.4890530 .362160$

C $-7.164311-1.2947560 .636005$

C $-6.627995-2.7125160 .611965$

H $-5.339556-0.3903931 .301072$

C $-6.1488510 .895603-0.243445$
H -7.628281-1.025841 1.589896

H $-7.912748-1.158311-0.153361$

C -7.703751 -3.770475 0.441640

H $-6.098800-2.8907721 .555391$

C $-4.8741181 .687192-0.602157$

O $-6.9105050 .735013-1.447553$

H $-6.777093 \quad 1.4792480 .440397$

H -4.404442 $1.236788-1.482819$

O $-5.2869192 .999149-1.008012$

C -3.8442391 .8121110 .533403$

C $-2.709003 \quad 2.818400 \quad 0.268992$

H -3.4140160 .8259200 .739448$

$\begin{array}{llll}\text { O } & -4.520742 & 2.231373 & 1.722536\end{array}$

H $-3.109577 \quad 3.8403050 .254937$

H -2.047144 $2.780801 \quad 1.143655$

H -8.415947 -3.7319021 .272359$

H $-8.264741-3.627729-0.488079$

H $-7.264331-4.7726600 .420088$

C $-4.544199-3.681783-0.611755$

H -7.064120 $1.622916-1.813587$

H $-5.6616043 .442938-0.227626$

H $-3.871307 \quad 2.225422 \quad 2.446383$

H -3.962794 -3.530405 -1.529492

H $-4.995795-4.677773-0.687375$

H -6.208169 -2.679065 -1.504376

C $-0.6660900 .784975-2.112565$

O $-1.412981 \quad 1.215400-0.965845$

C $-1.8962752 .560522-1.010272$

C $-0.7343773 .560339-1.159495$

C $0.198743 \quad 3.191789-2.316096$

C $0.550093 \quad 1.700040-2.326164$

H $-0.289887-0.211448-1.846113$

C -1.543131 $0.638491-3.365876$

H $-2.5672332 .671228-1.867623$

$\begin{array}{lllll}\text { O } & 0.025908 & 3.636302 & 0.042989\end{array}$

H $-1.1534294 .559611-1.327314$

H $1.1189583 .783862-2.235467$

O $-0.440515 \quad 3.546731-3.539086$

H $1.0438281 .437305-3.268371$

O $1.5028421 .451646-1.289667$

C $-2.586952-0.444371-3.207129$

H $-2.0681801 .567774-3.600798$

H $-0.9191940 .363124-4.223705$

H 0.3003952 .7364300 .281228

H $0.1781193 .346296-4.262053$

H $1.0373431 .083072-0.520723$

O $-3.479411-0.356082-4.237109$

O $-2.611614-1.269733-2.305644$

C $-4.541542-1.315068-4.220697$

H -5.079496 -1.241785 -5.169551

H $-4.148016-2.331238-4.120559$

H $-5.233775-1.085450-3.406341$

SCF Energy (B3LYP/6-31G**//MMFF) $=-3245.91402660$

08_00153

MM̄FF Geometry

C -0.154187 -2.869226 1.531111

C $-0.573455-1.612604 \quad 1.317077$

C $-1.121905-0.7236822 .405883$

O $-2.474873-0.3620482 .041470$

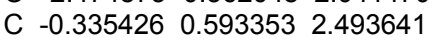

C $-0.488567 \quad 1.288347 \quad 3.831311$

C $-1.3776812 .263614 \quad 4.102015$

C $0.488988 \quad 0.845697 \quad 4.889943$

C $-2.459752 \quad 2.812417 \quad 3.214915$

C -2.1691954 .2372012 .725382$

C $-1.192117 \quad 4.2753851 .540703$

C $-0.8837895 .719136 \quad 1.140844$

C $0.0002975 .787801-0.113338$

O $-1.814415 \quad 3.591146 \quad 0.447326$

O $-0.0226517 .139770-0.595923$

C $-0.6239864 .904063-1.214096$

C 1.4651635 .4650850 .202582

$\begin{array}{lllll}\text { O } & 0.236971 & 4.817125 & -2.347530\end{array}$

C $-1.0050753 .485721-0.729313$

C $-1.8382952 .763017-1.815902$

O $0.1894912 .741797-0.485119$

C $-3.475000-1.2501452 .267573$ 
O $-3.392401-2.345413 \quad 2.796508$

C $-4.699890-0.6503291 .695725$

C $-5.738669-1.4344601 .376439$

C $-6.973921-0.993897 \quad 0.724657$

C -7.1290710 .4792530 .445894$

C $-2.4965581 .454686-1.348067$

C $-3.2462650 .764271-2.476827$

C $-4.565423-1.295187-2.965693$

O $-3.3103791 .219226-3.616177$

C -6.026384 -0.973731-2.901157

C $-6.984006-1.911563-2.827215$

C $-8.456229-1.607084-2.808104$

C $-9.197132-2.340713-1.710219$

C $-9.892978-3.459284-1.975542$

C $-9.198409-1.733136-0.324463$

C $-7.885708-1.9269520 .380574$

N $-3.829597-0.426623-2.063528$

H -0.184936 -3.271398 2.541153

H $-0.575266-1.2154100 .304816$

H -1.128582 -1.237144 3.375502

H $-0.647873 \quad 1.2666101 .688305$

H $0.7330810 .416326 \quad 2.332461$

H -1.360165 2.706464 5.097516

H $\quad 0.341266 \quad 1.369390 \quad 5.840294$

H $\quad 0.381360-0.2258885 .085012$

H 1.5157311 .0393344 .562667

H $-2.674000 \quad 2.1539602 .369748$

H -3.381184 2.833296 3.811129

H -3.124172 4.6754452 .406686

H -1.7899474 .8513253 .551343$

H -0.2699323 .7542501 .820582$

H -0.4154716 .2615451 .971566$

$\begin{array}{llll}H & -1.823877 & 6.248295 & 0.929972\end{array}$

H $\quad 0.3008547 .7176470 .116365$

H -1.538065 $5.408703-1.557343$

H $1.859663 \quad 6.1662120 .947929$

H $2.095508 \quad 5.587892-0.684821$

H 1.6079084 .4554380 .593071

H $0.4406935 .726005-2.627157$

H -2.642372 $3.428828-2.156783$

H $-1.2019472 .551113-2.684742$

H $0.0187151 .799187-0.636229$

H $-4.6883840 .416108 \quad 1.499503$

H -5.665245 -2.502500 1.577078

H -7.1200161 .0481801 .382270$

H -8.057176 $0.733850-0.068845$

H $-6.3145140 .840686-0.191618$

H - $-1.7433830 .756231-0.970153$

H -3.211762 $1.658904-0.543087$

H $-4.356561-2.326779-2.664758$

H $-4.201041-1.145899-3.987224$

H $-6.3124240 .074467-2.947194$

H -6.696699 -2.960797-2.817730

H $-8.865669-1.893412-3.785733$

H -8.640829 $-0.529846-2.712661$

H $-10.448886-3.976933-1.200797$

H $-9.917930-3.885508-2.973007$

H $-9.973703-2.2030750 .295311$

H $-9.497646-0.684205-0.395660$

H -7.675464 -2.970171 0.620937

H -3.767091 -0.698579-1.087016

C $1.595700-4.5189890 .730559$

O $2.697083-3.709787 \quad 0.258569$

C $3.557919-4.530272-0.553977$

C $3.210067-5.968213-0.196474$

C $1.713962-5.860397-0.000753$

H $3.272039-4.350812-1.597231$

C $5.036817-4.182535-0.351226$

H $3.492261-6.674055-0.983510$

H $3.693429-6.2833800 .735677$

C $1.116789-7.0317650 .761330$

H $1.234616-5.794351-0.987164$

C $5.428292-2.725671-0.671653$

O $5.399409-4.4365501 .012094$

H $5.651439-4.863483-0.952309$

H $5.052551-2.0593720 .111019$

O $6.858921-2.643401-0.612812$

C $4.969606-2.219733-2.049947$
C $5.600752-0.888116-2.501672$

H $3.878036-2.120192-2.048801$

O $5.298924-3.203829-3.035852$

H $6.664511-1.037621-2.728011$

H $5.133706-0.630307-3.461706$

H $1.279769-7.9675710 .217002$

H $1.568913-7.1371861 .753374$

H $0.038381-6.9022200 .895365$

C $0.262675-3.8183370 .441280$

H $\quad 4.814277-3.9007351 .574307$

H $7.131247-2.9596360 .265701$

H $4.941031-2.898054-3.886834$

H $-0.536288-4.5659440 .365704$

H $0.316051-3.295884-0.521728$

H $1.745564-4.6576601 .808899$

C $3.7208531 .446408-0.270995$

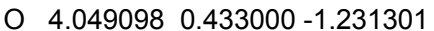

C $5.4408730 .278780-1.514784$

C $6.0223171 .586840-2.077777$

C $5.7382202 .768717-1.143840$

C $4.2675902 .813760-0.713368$

H $2.6254631 .501361-0.288072$

C $4.143703 \quad 1.053398 \quad 1.154013$

H $5.9717350 .022208-0.592793$

O $5.4544711 .851468-3.359460$

H $7.1029111 .461327-2.215960$

H $5.9914923 .707136-1.652183$

$\begin{array}{lllll}\text { O } & 6.558167 & 2.672883 & 0.016821\end{array}$

H 4.1262783 .5406240 .095071

O $3.4715313 .252811-1.817838$

C $3.430584-0.1902891 .636050$

H $\begin{array}{llll}5.216197 & 0.855177 & 1.222402\end{array}$

H 3.8894771 .8673231 .842889

H $5.8653652 .663792-3.701021$

H $7.4850472 .672294-0.277209$

H $3.7773054 .142062-2.064634$

O $3.811174-0.4543102 .920693$

O $2.646064-0.8526060 .972078$

C $3.226578-1.6214543 .507266$

H $3.530689-2.5147402 .954925$

H $3.589749-1.7034564 .535113$

H $2.136677-1.5338163 .527198$

SCF Energy (B3LYP/6-31G**//MMFF) $=-3245.89330700$

08_00154

MM̄FF Geometry

C -1.771039-2.339705 1.684576

C $-1.280580-1.6622132 .734188$

C $-1.615234-0.2402983 .126389$

$\begin{array}{lllll}\text { O } & -2.734317 & 0.270943 & 2.370082\end{array}$

C $-0.4280100 .694527 \quad 2.831484$

C $-0.372679 \quad 1.885879 \quad 3.766880$

C -0.9429123 .0850713 .538864$

C $0.4277451 .661590 \quad 5.024245$

C $-1.822043 \quad 3.5008912 .393295$

C -1.1712604 .5552451 .488198$

C $-0.182310 \quad 3.948895 \quad 0.482024$

C $0.4833295 .046460-0.349996$

C $1.3947214 .463031-1.440216$

O $-0.924546 \quad 3.077896-0.378165$

O $1.7152095 .517887-2.359889$

C $0.6128643 .393926-2.232554$

C $2.7259413 .972925-0.863829$

O $1.4653402 .709067-3.147828$

C $-0.1319512 .373527-1.339857$

C $-1.0836691 .513292-2.207261$

O $0.821813 \quad 1.515086-0.710323$

C $-3.993621-0.0084132 .792108$

O $-4.325082-0.6707343 .760315$

C $-4.9078530 .617678 \quad 1.813104$

C $-6.1511120 .141303 \quad 1.661528$

C -7.1306780 .6089390 .678258$

C $-6.7542661 .794857-0.172545$

C $-2.1219530 .715751-1.403259$

C $-2.957325-0.189522-2.295149$

C $-4.848933-1.813847-2.193131$

O $-2.771935-0.295760-3.504823$

C $-6.138895-1.121706-2.506572$ 
C $-7.343050-1.636398-2.211372$

C $-8.645724-0.969568-2.554867$

C $-9.607196-0.913495-1.386567$

C $-10.626630-1.783617-1.289409$

C $-9.4363190 .200884-0.377242$

C $-8.298135-0.0584640 .567684$

N $-3.928680-0.874446-1.577125$

H -2.473911-1.859334 1.010650

H $-0.573411-2.1673263 .389963$

H -1.862651 -0.2262894 .195546$

H $-0.470785 \quad 1.042247 \quad 1.792565$

H $0.523206 \quad 0.1533832 .921897$

H -0.8007973 .8649424 .286707$

H 0.4143332 .5313845 .689470

H 0.0259840 .8119145 .585695

H $1.472367 \quad 1.4522284 .774833$

H -2.158669 2.6485221 .799665

H -2.7304703 .9353142 .830061$

H -1.977307 5.0519710 .932181

H -0.6750955 .3225242 .094889$

H 0.5718813 .3701461 .026822

H $1.0450055 .735740 \quad 0.292687$

H $-0.2910975 .655464-0.837458$

H $2.1451686 .232149-1.858781$

H $-0.1320203 .924857-2.842062$

H $3.2579724 .794697-0.369919$

H $3.3926043 .620043-1.657730$

H $2.6014223 .173835-0.131849$

H $1.9024123 .382785-3.696484$

H -1.639990 2.164586 -2.894230

H $-0.4930670 .822723-2.822916$

H $0.4070760 .656782-0.529783$

H $-4.509510 \quad 1.420572 \quad 1.203044$

H $-6.469327-0.6902382 .288931$

H $-7.5226702 .080454-0.893213$

H $-5.8495561 .584273-0.753487$

H -6.5696302 .6729690 .456427$

H $-1.6308600 .092548-0.649933$

H $-2.8031871 .402509-0.887779$

H -4.985860 -2.641094-1.489525

H $-4.407073-2.204997-3.115038$

H $-6.081818-0.166173-3.022668$

H -7.398408 -2.608494 -1.726098

H -9.098191 -1.534194 -3.380746

H -8.486149 $0.045071-2.940732$

H -11.336423 -1.734092 -0.470284

H -10.771148 -2.568864 -2.024071

H -10.3460180 .3050230 .229277$

H $-9.3463751 .151980-0.908256$

H -8.472855 -0.9088111 .228654$

H $-4.037904-0.685520-0.585555$

C -0.406897 $-3.951179 \quad 0.249812$

O $0.885459-3.4917270 .686380$

C $1.543406-2.843387-0.415712$

C $0.704946-3.150504-1.653555$

C $-0.690160-3.231980-1.066709$

H $1.505519-1.765884-0.209564$

C $3.011304-3.280765-0.532232$

H $\quad 0.803340-2.377820-2.422170$

H $0.984531-4.110318-2.103514$

C -1.690431 -3.947010-1.956430

H -1.037673 -2.209567 -0.887055

C $3.855571-2.9805200 .721830$

O $3.083604-4.677566-0.819169$

H $3.433504-2.758566-1.396262$

H $3.745535-1.931777 \quad 1.007847$

O $3.367120-3.743881 \quad 1.833417$

C $5.348935-3.3331320 .579670$

C $6.096096-2.716841-0.617119$

H $5.456216-4.4236360 .523651$

O $6.030184-2.9324471 .774292$

H $7.111240-3.136034-0.621975$

H $5.615179-3.045376-1.544496$

H -1.811888 -3.413537 -2.904829

H -1.367248 -4.968136 -2.184683

H - $2.669645-4.004822-1.471589$

C -1.430025 -3.771193 1.373821

H $2.688907-5.149054-0.065343$
H $2.452569-3.4619532 .003980$

H $5.560243-3.3352062 .524553$

H -1.046880 -4.257499 2.280271

H -2.359697 -4.289289 1.110007

H $-0.293743-5.0283480 .067700$

C $4.8759350 .829406-0.623145$

O $4.933970-0.599898-0.693276$

C $6.235052-1.178760-0.593978$

C $7.120941-0.675607-1.746944$

C $7.1574610 .856308-1.794856$

C $5.7526831 .464789-1.714600$

H $3.8299971 .072062-0.847957$

C 5.1881421 .3520590 .784874

H $6.688756-0.8905070 .360007$

O $6.620449-1.177132-2.985270$

H $8.133547-1.074928-1.614718$

H $7.6368051 .182526-2.725866$

O $7.9387591 .352156-0.711715$

H $5.8116982 .547035-1.549875$

O $5.0864951 .266723-2.964803$

C 4.1644980 .8699511 .787502

H 6.1831271 .0548351 .127836

H 5.1673012 .4479210 .807824

H $7.216639-0.870221-3.689213$

H $8.8312650 .975503-0.796148$

H $5.609590 \quad 1.716286-3.650242$

O $4.760684 \quad 0.0581172 .706681$

O $2.9793241 .173711 \quad 1.755504$

C $3.900947-0.4834063 .714903$

H $2.988481-0.8982933 .275602$

H $4.438812-1.2891524 .221422$

H 3.6595440 .2932424 .445252

SCF Energy (B3LYP/6-31G**//MMFF) $=-3245.91164578$

08 00155

MM̄MF Geometry

C $-0.8951331 .779122-2.139708$

C $-1.3646763 .032346-2.041460$

C $-0.9296224 .095198-1.061528$

O $0.3034323 .743557-0.391553$

C -1.9766684 .3177460 .046450$

C $-3.2838424 .902841-0.442466$

C $-4.3977974 .187410-0.693501$

C $-3.2839026 .397155-0.635262$

C $-4.5740122 .698738-0.582074$

C -5.7774912 .3448760 .298949$

C $-5.926790 \quad 0.8256330 .451850$

C $-7.2616850 .478141 \quad 1.112201$

C $-7.401716-1.0321241 .348888$

O $-4.8420620 .354472 \quad 1.261440$

O $-8.513512-1.2396952 .233067$

C $-6.141647-1.5362342 .079649$

C $-7.727773-1.7886950 .055596$

O $-6.154293-2.9577462 .194500$

C $-4.815574-1.0677081 .432705$

C $-3.635719-1.4277402 .365267$

O $-4.640085-1.7431680 .185136$

C $1.4676324 .091838-0.996875$

O $1.590614 \quad 4.581152-2.111944$

C $2.6382033 .847369-0.122662$

C 2.5619983 .3314681 .114192

C 3.6835473 .1412582 .038117

C 5.0660143 .4852531 .546997

C $-2.294680-0.7759621 .993866$

C -1.148095-1.425723 2.752402

C $1.325951-1.6541422 .739777$

O $-1.322301-2.1882693 .700615$

C $1.910723-0.8161593 .831724$

C $3.235147-0.7086974 .016041$

C 3.8794920 .0783255 .119181

C 4.8717011 .0940864 .599667

C 6.1548950 .7573304 .384389

C 4.4007802 .5170314 .418963

C 3.4287912 .6844923 .282474

N $0.094028-1.0585082 .254412$

H $-0.1119671 .435368-1.470144$

H -2.146644 $3.340535-2.733424$

H $-0.7787345 .035388-1.606819$ 
H $-1.553720 \quad 4.9930860 .803888$ H -2.132662 3.3849960 .601980 H -5.278462 4.720235-1.050318 H $-4.2557846 .773836-0.971444$ H -3.0435116 .9010210 .306734$ H -2.541203 $6.687652-1.384743$ H -4.737206 $2.300160-1.591208$ H -3.678695 2.205084 -0.196448 H -5.6484522 .8043851 .287678$ H $-6.6874162 .765216-0.147366$ H $-5.863287 \quad 0.367222-0.542312$ H -8.1021280 .8507160 .513291$ H -7.3339090 .9906962 .081898$ H -9.304646 -0.859362 1.814022 H $-6.181930-1.1429143 .105280$ H -8.666352 -1.421254 -0.376362 H -7.886213 -2.8550410 .251458$ H $-6.953036-1.689690-0.707440$ H -6.993316 -3.206670 2.618798 H -3.871141 -1.116097 3.391560 H $-3.514743-2.5188702 .378871$ H -3.691799 -1.8491520 .010168$ H $3.5865884 .152884-0.553654$ H 1.5881503 .0469811 .508828 H 5.2910922 .9503860 .617580 H 5.8556793 .2115532 .250612 H 5.1516054 .5616251 .362605 H -2.097413 -0.8668260 .921539$ H $-2.310617 \quad 0.2905712 .245362$ H $2.004428-1.7373561 .885623$ H $1.111105-2.6581193 .120140$ H $1.233070-0.3046534 .510325$ H $3.902220-1.2585763 .355936$ H $4.393567-0.6299485 .782266$ H 3.1303980 .5761655 .747211 H 6.8817691 .4857144 .041339 H $6.504586-0.2576884 .542181$ H 5.2510553 .1996894 .307400 H 3.9098482 .8479865 .343657 H 2.3989222 .4472773 .550933 H $0.152432-0.383887 \quad 1.497095$ C -1.925271 $-0.487396-2.505742$ O $-0.813825-1.267018-2.018215$ C -1.003557 -2.644146 -2.396550 C $-2.446014-2.755935-2.874228$ C $-2.668699-1.390623-3.489966$ H $-0.334948-2.834075-3.244348$ C $-0.653472-3.575563-1.232072$ H $-2.583522-3.575751-3.586060$ H $-3.140461-2.916573-2.042326$ C $-4.136249-1.035867-3.662103$ H $-2.175090-1.363927-4.470843$ C $0.788585-3.413908-0.708371$ O $-1.551017-3.297644-0.150365$ H $-0.839398-4.615792-1.524009$ H $0.921536-2.400665-0.317020$ O $0.940148-4.2903610 .416574$ C $1.889790-3.724705-1.744150$ C $3.313780-3.758241-1.152696$ H $1.851787-2.993781-2.557929$ O $1.631980-5.002230-2.335102$ H $3.324860-4.387812-0.254893$ H $3.968577-4.261439-1.877537$ H $-4.630073-1.753486-4.325403$ H -4.666780 -1.044019-2.704015 H $-4.249365-0.038974-4.099527$ C -1.407148 $0.795930-3.157943$ H -1.307024 -3.8812390 .588309$ H $\quad 0.758595-5.196810 \quad 0.116283$ H $1.763318-5.680607-1.651338$ H $-2.2040341 .255558-3.754255$ H $-0.5846130 .559546-3.842614$ H -2.559144 -0.253801-1.640535 C $4.804941-0.453135-2.028112$ O $4.243918-1.771001-2.091173$ C $3.879715-2.363653-0.838447$ C $5.098780-2.4436770 .094044$ C 5.769861-1.077958 0.258026
C $6.024360-0.406810-1.093722$

H $5.162627-0.255478-3.047336$

C $3.7404910 .596807-1.694058$

H $3.104577-1.755561-0.364201$

O $6.047798-3.384660-0.404774$

H $4.768748-2.8108561 .073110$

H $6.718542-1.2093460 .792546$

O $4.929136-0.2482971 .051966$

H $6.3386610 .634368-0.955749$

O $7.097851-1.081650-1.755512$

C $2.8131430 .776790-2.872206$

H $3.1204630 .340836-0.830848$

H $4.2141891 .559531-1.470963$

H $6.288348-3.114444-1.307586$

H 5.4034580 .5859371 .207052

H $7.882341-1.014449-1.184880$

O $3.0726581 .961363-3.494466$

O $1.979976-0.052274-3.213762$

C $2.2877342 .235276-4.659086$

H $2.3750531 .419469-5.383039$

H $1.2432242 .393259-4.378179$

H $2.6695633 .151399-5.117356$

SCF Energy (B3LYP/6-31G**//MMFF) $=-3245.91654848$

$08 \_00156$

MM̄MF Geometry

C $0.3410713 .270799-0.955237$

C $-0.8792653 .665001-0.560167$

C -1.7783372 .8276010 .315050$

O $-2.9935392 .446950-0.394577$

C -2.1198833 .4523521 .683497$

C -0.9220073 .8400212 .524691$

C -0.1502002 .9706193 .205428$

C -0.6415595 .3185672 .596061$

C $-0.303458 \quad 1.478066 \quad 3.272510$

C 0.9488370 .7612502 .746862

C $0.767062-0.7625562 .771002$

C $2.070798-1.4829972 .428149$

C $1.876254-3.0063632 .363467$

O $-0.233729-1.0943221 .804510$

O $3.037837-3.5773621 .742936$

C $0.673584-3.3190431 .448604$

C $1.783313-3.6305933 .760750$

O $0.358979-4.7094321 .475938$

C $-0.588175-2.4826461 .768823$

C $-1.637104-2.6717400 .644264$

O $-1.133284-2.9182773 .012888$

C $-3.8745873 .381917-0.830724$

O $-3.7783104 .596054-0.712218$

C $-5.0286382 .772178-1.531178$

C $-5.3221381 .462270-1.525089$

C $-6.4744710 .841406-2.188131$

C $-7.3432451 .721505-3.049705$

C $-2.856257-1.7415250 .730022$

C $-3.997960-2.2799751 .573897$

C $-6.320812-1.6888152 .258325$

O $-3.996810-3.3985052 .079510$

C $-7.182663-2.273651 \quad 1.184317$

C $-8.251449-1.6548360 .661778$

C $-9.062580-2.224251-0.465534$

C $-9.067129-1.331103-1.688140$

C -10.171957-0.646601-2.029622

C $-7.831561-1.303466-2.564746$

C $-6.712305-0.473103-1.993191$

N $-5.035030-1.3614091 .669863$

H $0.7094152 .295085-0.652057$

H -1.233690 $4.647985-0.857103$

H $-1.278148 \quad 1.8711380 .507043$

H -2.7556202 .7535812 .243890$

H $-2.7618134 .332176 \quad 1.553018$

H 0.6768663 .3674223 .792866

H 0.2656395 .5441883 .166439

H $-0.5113855 .734887 \quad 1.592782$

H -1.476228 5.8373913 .078943

H $-0.465194 \quad 1.200147 \quad 4.321324$

H -1.1883131 .1387132 .726570$

H 1.1482631 .0920591 .719263

H 1.8146571 .0426603 .359066 
H $\quad 0.421134-1.0548083 .769619$

H $2.860085-1.2265983 .145418$

H $2.430219-1.1373501 .450024$

H $3.815302-3.3185172 .267047$

H $0.979965-3.0946330 .419012$

H $2.694713-3.4223004 .334087$

H $1.710342-4.7222493 .701413$

H $0.936393-3.2594314 .341449$

H $\quad 1.171676-5.1952831 .253627$

H -1.148326 -2.451714 -0.312979

H $-1.966026-3.7169450 .606197$

H -2.051759 -2.614171 3.074975

H $-5.6620443 .490772-2.042857$

H $-4.6776450 .779375-0.974925$

H -7.823196 $2.498769-2.444852$

H $-8.1426201 .179726-3.558904$

H $-6.7472712 .201813-3.833858$

H $-2.577865-0.7457731 .090884$

H -3.259298 -1.619296 -0.284099

H $-6.738815-0.7655622 .671083$

H $-6.187798-2.4108413 .069927$

H $-6.891224-3.2478680 .797960$

H -8.547705 -0.6815681 .045733$

H -10.085630 -2.374567-0.097133

H -8.703230 -3.220122 -0.753935

H - $10.204178-0.030852-2.922029$

H -11.071175 -0.685109-1.423516

H $-8.080390-0.966988-3.578136$

H -7.467873 -2.329851-2.704144

H -6.029330 -1.034507 -1.355982

H $-4.972489-0.496741 \quad 1.140249$

C $2.6035924 .410948-1.125842$

O $3.3848073 .207186-0.986389$

C 4.0687383 .2518610 .280267

C 3.8915944 .6712090 .816867

C 2.5293555 .0363470 .266527

H 3.5403072 .5564940 .943408

C 5.5318322 .8251850 .132353

H 3.9384304 .7082361 .909637

H 4.6529785 .3555460 .425469

C 2.2482206 .5280680 .259019

H $1.776046 \quad 4.533550 \quad 0.883194$

C $5.7570581 .441801-0.509560$

O $6.2058853 .790338-0.688405$

H 6.0189542 .8687501 .114413

H $5.5400951 .498242-1.582796$

O $7.1640771 .185133-0.389120$

C 4.9670690 .2857780 .134509

C $5.433352-1.127015-0.263230$

H $3.9043230 .395817-0.104342$

O $5.074910 \quad 0.373899 \quad 1.559922$

H $6.461478-1.2728620 .091076$

H $4.841794-1.8446470 .316854$

H $2.270628 \quad 6.928527 \quad 1.277811$

H $2.9902057 .074075-0.333079$

H $1.2603256 .735667-0.164201$

C $1.2622224 .105715-1.799159$

H $7.1374583 .517643-0.749728$

H $7.3942150 .482099-1.019029$

H $\quad 6.0194790 .325738 \quad 1.787115$

H $1.446414 \quad 3.552232-2.728094$

H $0.7759325 .048698-2.075956$

H $3.1789735 .061752-1.797893$

C $3.119472-2.403497-1.812174$

O $4.001739-1.371239-2.268985$

C $5.340672-1.445742-1.768944$

C $5.967957-2.794013-2.152017$

C $5.126593-3.962226-1.651445$

C $3.681080-3.802508-2.114336$

H $2.957724-2.286126-0.736175$

C $1.773026-2.219355-2.531257$

H $5.907503-0.675633-2.303506$

O $7.287840-2.910838-1.631266$

H $6.046042-2.863952-3.244801$

H $5.186345-4.065540-0.561761$

O $5.639806-5.179378-2.197910$

H $3.632729-3.998851-3.193047$

O $2.874894-4.794702-1.479221$
C $0.983488-1.033629-2.023581$

H $1.942078-2.068448-3.604085$

H $1.142903-3.103799-2.383204$

H $7.805901-2.164971-1.978911$

H $6.572504-5.245589-1.929890$

H $2.908631-4.636867-0.520106$

O $-0.204522-0.986105-2.698163$

O $1.349579-0.257831-1.153014$

C $-1.0999610 .064164-2.322060$

H $-1.1934380 .126740-1.234562$

H $-0.7483821 .011436-2.738591$

H $-2.084250-0.161188-2.740962$

SCF Energy (B3LYP/6-31G**//MMFF) $=-3245.91656968$

08 00157

MM̄FF Geometry

C $0.845181-2.3660081 .945651$

C $-0.378192-2.7406421 .544377$

C $-0.694519-3.2815190 .174916$

O $-1.469404-2.258523-0.491379$

C $-1.545711-4.5578230 .288436$

C $-1.955376-5.133943-1.054348$

C $-3.203334-5.086190-1.564104$

C $-0.853152-5.813543-1.825253$

C $-4.421741-4.460323-0.944438$

C $-4.802284-3.156989-1.658775$

C $-5.680952-2.257244-0.777600$

C $-6.141293-1.023527-1.555870$

C $-6.901622-0.027467-0.667406$

O $-4.888893-1.8598840 .348051$

O $-6.9956341 .215920-1.380223$

C -6.0724320 .2459490 .606146$

C $-8.340781-0.485008-0.402491$

O -6.8087691 .0435591 .529629$

C $-5.573133-1.0367961 .301604$

C $-4.584972-0.7549162 .461759$

O $-6.693306-1.7302141 .865152$

C $-0.807799-1.448333-1.361653$

O $0.337452-1.575351-1.763703$

C $-1.709274-0.327624-1.705460$

C $-1.1893230 .794676-2.223509$

C -1.926408 $2.006323-2.586709$

C $-3.4214191 .995568-2.407941$

C $-3.288915-0.0362302 .069955$

C -3.3312621 .4722222 .244436$

C -1.9680853 .5112641 .799342$

O -4.2417622 .0604012 .820877$

C -2.4771774 .1799660 .561249$

C -1.732835 $5.005206-0.190657$

C $-2.2500235 .743335-1.391941$

C $-1.4011485 .529356-2.624730$

C $-0.4125196 .383288-2.940137$

C $-1.7634184 .383870-3.541105$

C -1.233152 $3.064542-3.056226$

N -2.202276 2.0822841 .712373

H $1.689468-2.4682811 .268842$

H -1.217860 -2.610601 2.223769

H $\quad 0.226149-3.503991-0.377721$

H $-2.425534-4.3484850 .904539$

H $-0.986476-5.3261030 .839330$

H $-3.379073-5.535535-2.540609$

H -1.216532 -6.277812 -2.748340

H $-0.076661-5.094292-2.103159$

H - $0.397155-6.603234-1.219051$

H -5.255322 -5.169738 -1.020082

H $-4.285567-4.2908190 .125595$

H -3.891310 -2.608902 -1.927992

H $-5.323818-3.396774-2.593726$

H $-6.542929-2.838513-0.429443$

H $-6.753538-1.314830-2.418382$

H $-5.265013-0.511444-1.974545$

H $-7.449387 \quad 1.044730-2.223285$

H $\quad-5.2055790 .8427350 .298731$

H $-8.892125-0.578800-1.345992$

H -8.8883490 .2552610 .191050$

H $-8.397426-1.4486830 .107771$

H $-7.084613 \quad 1.849677 \quad 1.060758$

H $-5.101413-0.2399153 .280657$ 
H $-4.287745-1.7297332 .874951$ H -6.370742 -2.3196902 .566919$ H $-2.764134-0.441439-1.489193$ H $-0.1137340 .838235-2.390853$ H -3.683630 $1.749736-1.373611$ H $-3.8801791 .256659-3.073536$ H -3.894645 2.956824 -2.618391 H -2.486324 -0.3907912 .729561$ H -2.999042 -0.2774121 .043637$ H -0.8924943 .6570141 .938461$ H -2.488367 3.9132892 .674710 H $-3.517038 \quad 4.0078560 .293436$ H -0.7037185 .2023650 .100379$ H -2.270236 $6.812258-1.141305$ H -3.291157 5.475181-1.609986 H $0.1799876 .255076-3.839862$ H $-0.1702837 .226258-2.301259$ H -1.338886 4.555091 -4.539904 H -2.844772 4.374939 -3.704731 H $-0.1484212 .979242-3.137360$ H -1.5221381 .5194091 .209542$ C $1.444937-0.2681493 .185763$ O $2.736589-0.0940212 .581974$ C $3.732582-0.1388993 .614898$ C 3.0198540 .1854334 .928397 C $1.564166 \quad 0.4120754 .548176$ H 4.096971-1.171649 3.667241 C 4.9095420 .7826203 .269888 H $3.096366-0.6834255 .594533$ H 3.4649051 .0242385 .473504 C 1.2410961 .9040324 .449825 H $0.885412-0.0253485 .288940$ C 5.5302950 .5435451 .876087 O 4.4605042 .1418513 .315196 H 5.6771330 .6898774 .047958 H 4.8517890 .9302551 .108553 O $\quad 6.7142181 .347611 \quad 1.790654$ C $5.890173-0.9191701 .565110$ C $6.730365-1.1265170 .291264$ H $4.970144-1.5099481 .489319$ O $6.639148-1.4478852 .664253$ H $7.739866-0.7220410 .440435$ H $6.866152-2.2105710 .179761$ H 1.4000762 .3981295 .413992 H 1.8583512 .4112173 .703017 H 0.1925532 .0493354 .168531 C $1.117551-1.7670633 .298648$ H 5.2222852 .7064623 .099186 H 7.3572310 .9905802 .427214 H $6.796698-2.3907322 .485436$ H $0.245013-1.9015963 .949623$ H $1.945967-2.3243443 .750731$ H 0.6979940 .2260532 .552667 C $4.074090-0.600771-2.292308$ O $4.806759-1.077291-1.153796$ C $6.124505-0.545547-0.997111$ C $6.988073-0.874922-2.227423$ C $6.315731-0.420646-3.527008$ C $4.850053-0.864198-3.594273$ H $3.164907-1.215013-2.316779$ C $3.6467140 .867313-2.134964$ H $6.0640490 .541482-0.885650$ O $7.241290-2.277578-2.300481$ H $7.960782-0.381298-2.118666$ H $6.858000-0.840342-4.383155$ O $6.3750210 .998504-3.629764$ H $4.337394-0.372752-4.429460$ O $4.800712-2.272418-3.842028$ C $2.6878571 .070741-0.983067$ H $4.5053831 .520234-1.958809$ H $3.1336551 .198095-3.045462$ H $6.382464-2.733925-2.318091$ H $7.3127151 .255577-3.615450$ H $5.241788-2.435364-4.693181$ O $2.4468202 .407736-0.841548$ O $2.207167 \quad 0.180592-0.296894$ C 1.5685242 .7601960 .230225 H 1.9948822 .4456201 .186783
H 1.4606573 .8476390 .236896

H 0.5845782 .3083660 .077830

SCF Energy (B3LYP/6-31G**//MMFF) $=-3245.90681655$

08 00158

MM̄FF Geometry

C $0.329484-3.3479881 .214397$

C $0.796916-3.7924100 .037896$

C $0.164748-3.532568-1.310131$

O $-0.592679-2.297890-1.315316$

C $-0.771708-4.693760-1.682408$

C $-1.362098-4.565867-3.073072$

C $-2.619457-4.158788-3.338983$

C $-0.444346-4.969124-4.198312$

C $-3.664689-3.707348-2.356432$

C $-4.169603-2.298072-2.693616$

C $-5.112353-1.755481-1.609554$

C $-5.677099-0.394124-2.018004$

C $-6.5376890 .229349-0.909106$

O $-4.362974-1.629869-0.395413$

O $-6.7443871 .612294-1.237278$

C -5.7488440 .1947790 .417719$

C $-7.928728-0.411478-0.838005$

O $-6.567156 \quad 0.6189181 .504764$

C $-5.130833-1.1840250 .732030$

C $-4.185866-1.1640781 .960753$

O $-6.182596-2.1106061 .031823$

C $0.133990-1.146282-1.379069$

O $1.350650-1.052226-1.351566$

C $-0.797760-0.006511-1.514824$

C $-0.2998381 .234451-1.626683$

C $-1.0652902 .463858-1.844406$

C $-2.5666612 .357426-1.872412$

C $-2.976229-0.2297401 .852432$

C $-3.208624 \quad 1.1582682 .426470$

C -2.1481863 .4147482 .522878$

O $-4.167511 \quad 1.4401593 .139437$

C -2.3719034 .3132951 .349807$

C -1.462162 5.1983310 .915406

C $-1.6982196 .151045-0.219016$

C $-0.6773776 .009148-1.323398$

C $0.3761636 .839573-1.404103$

C $-0.9359014 .971566-2.390546$

C $-0.3890043 .616416-2.035399$

N -2.192041 2.0358242 .076552

H $-0.602195-2.7882671 .244523$

H $1.715853-4.374306 \quad 0.021757$

H $0.963647-3.451610-2.058711$

H $-1.567255-4.779970-0.934362$

H $-0.225455-5.645481-1.625872$

H $-2.941585-4.136908-4.379439$

H -0.931034 -4.901301 -5.177085

H $0.438218-4.322705-4.225075$

H $-0.114162-6.004682-4.066390$

H -4.504258 -4.411816 -2.393582

H $-3.288774-3.722738-1.331078$

H $-3.309358-1.625926-2.798252$

H -4.687815 -2.317455 -3.660647

H -5.922976 -2.478758 -1.461723

H $-6.250603-0.474528-2.949925$

H $-4.8479250 .291591-2.237297$

H -7.174282 $1.652534-2.108672$

H -4.9429840 .9325850 .324100$

H -8.459303 $-0.280736-1.788872$

H $-8.5507680 .076713-0.079668$

H $-7.898077-1.481601-0.623706$

H -6.9160911 .4981511 .279044$

H -4.760945 -0.973363 2.875019

H $-3.785913-2.1817812 .076678$

H $-5.808439-2.8473831 .542873$

H $-1.858553-0.219118-1.554056$

H $0.7810441 .364980-1.588625$

H -2.936453 $1.857907-0.970516$

H $-2.8924501 .786526-2.748425$

H -3.070945 $3.325525-1.906551$

H -2.150852 -0.661885 2.432905

H -2.638273 -0.1478580 .816011$

H -1.1695543 .5784332 .984136$ 
H -2.9167993 .5954753 .280692$ H $-3.338827 \quad 4.2525980 .856253$ H $-0.504378 \quad 5.269895 \quad 1.426127$ H -1.666188 7.1694190 .190511 H -2.706814 $6.036866-0.635605$ H $1.0941386 .763770-2.214036$ H $0.5472037 .608845-0.658320$ H $-0.4485855 .278789-3.326344$ H -2.001679 $4.952485-2.634911$ H $\quad 0.7010393 .589329-1.993107$ H -1.4342641 .7065981 .484732$ C $1.192871-2.2410863 .273255$ O $2.026667-1.3345372 .527423$ C $2.578628-0.3740143 .454727$ C $2.277187-0.9038254 .860563$ C $1.902310-2.3547794 .615528$ H $3.661865-0.3603803 .297312$ C 1.9716791 .0121153 .181827 H $3.142603-0.8024825 .523910$ H $1.451066-0.3581065 .331527$ C $1.057348-2.9549105 .725084$ H $2.825327-2.9389204 .503091$ C 2.2731171 .5587131 .770395 O $0.549597 \quad 0.905355 \quad 3.324473$ H 2.3027301 .7221903 .947826 H $1.9173580 .845801 \quad 1.019252$ O $1.4922392 .748140 \quad 1.584068$ C 3.7577461 .9024521 .525845 C 4.0229282 .4607810 .113040 H 4.3940391 .0285011 .696293 O 4.1666212 .9103492 .455291 H $3.2196213 .147258-0.179056$ H 4.9413003 .0625440 .147338 H $1.592941-2.9174656 .679423$ H $\quad 0.112966-2.4136995 .847214$ H $0.820539-4.0022535 .514162$ C $1.031690-3.5632382 .525726$ H $\quad 0.177327 \quad 1.786198 \quad 3.152612$ H 1.8224483 .4161442 .209270 H 4.2552102 .4891423 .326684 H $0.436996-4.2616493 .124627$ H $2.012636-4.0209122 .359169$ H $\quad 0.209937-1.7657973 .396965$

C $5.800795-0.368926-1.532884$

O $5.453955 \quad 0.731357-0.683687$

C $4.1855741 .348138-0.934378$

C $4.1344501 .892250-2.369010$

C $4.467714 \quad 0.800840-3.390407$

C $5.7427780 .036834-3.015037$

H $6.849026-0.588908-1.291171$

C $4.977069-1.622349-1.203532$

H $3.3903560 .610978-0.795419$

O $5.0434972 .980849-2.521338$

H $3.1295922 .285015-2.563045$

H $4.5920681 .264295-4.376671$

O $3.368152-0.100537-3.469517$

H $5.859104-0.855114-3.641576$

O $6.8743340 .875403-3.269993$

C $5.277680-2.1532970 .183611$

H $3.904197-1.430904-1.249769$

H $5.227207-2.413460-1.919780$

H $5.9300122 .664521-2.276831$

H $3.585036-0.771302-4.139146$

H $6.8702201 .093024-4.217822$

O $4.664201-3.364130 \quad 0.337747$

O $5.954911-1.5837321 .028251$

C $4.880137-4.0011571 .600697$

H $4.517669-3.3665052 .414876$

H $4.318661-4.9391131 .609007$

H $5.942497-4.2275001 .731905$

SCF Energy (B3LYP/6-31G**//MMFF) $=-3245.91513455$

08_00159

MM̄FF Geometry

C $0.473293-3.279513-0.521857$

C $-0.387154-3.890850-1.352247$

C $-1.474813-3.246243-2.179652$

O $-1.695142-1.881936-1.761704$
C $-2.800496-4.008642-2.006934$

C $-3.857976-3.592449-3.011675$

C $-4.930084-2.828754-2.724322$

C $-3.679282-4.147415-4.401977$

C $-5.268673-2.193223-1.404940$

C $-5.831950-0.776561-1.592254$

C $-6.193756-0.135029-0.245952$

C $-6.9182881 .195502-0.451560$

C -7.2183661 .8894320 .885723$

O

O -7.6029253 .2436040 .603646$

C -5.9208071 .9511931 .717302$

C $-8.403034 \quad 1.243872 \quad 1.614088$

O -6.1775302 .4515903 .027711$

C -5.1695680 .6004231 .803103$

C -3.7759780 .8242942 .431938$

O $-5.910191-0.3051792 .621972$

C $-1.450778-0.890981-2.658111$

O $-1.039854-1.004144-3.801343$

C $-1.7593500 .391245-1.992679$

C $-1.0769551 .488199-2.351155$

C $-1.2316912 .823419-1.772801$

C $-2.4627043 .086173-0.947091$

C $-2.846106-0.3947182 .373431$

C -1.4389710 .0152092 .759402$

C 0.5464031 .2370611 .863371

O $-1.003416-0.1613153 .894346$

C 0.4131492 .7184771 .697655

C 1.2326953 .4513300 .928610

C 1.1120494 .9390950 .759479

C $1.0958045 .371684-0.691522$

C $2.1508436 .001117-1.236044$

C $-0.1731395 .148260-1.488638$

C $-0.2732313 .741997-2.013446$

N $-0.7620540 .630518 \quad 1.718166$

H $0.438659-2.201440-0.392988$

H $-0.288418-4.967641-1.483122$

H -1.153800 -3.296184 -3.226396

H $-3.168789-3.881016-0.982265$

H -2.635040 -5.088368 -2.123197

H -5.650082 -2.642306 -3.520195

H $-4.507014-3.878641-5.066869$

H $-2.759042-3.766060-4.854747$

H $-3.625725-5.240562-4.370845$

H $-6.021411-2.816141-0.907276$

H $-4.395596-2.145192-0.747970$

H $-5.084810-0.159631-2.108126$

H $-6.723793-0.819548-2.229918$

H $-6.830109-0.833406 \quad 0.310302$

H $-7.842721 \quad 1.052632-1.024831$

H -6.289607 1.863867 -1.056779

H $-8.380666 \quad 3.2188170 .020161$

H $-5.2594002 .682595 \quad 1.231224$

H -9.3045481 .2863420 .991191$

H -8.647742 1.7904732 .531522

H $-8.2293640 .197211 \quad 1.872125$

H -6.6344163 .3042542 .927838$

H -3.2862431 .6580231 .915923$

H -3.8825441 .1247323 .482436$

H $-5.306838-0.9813182 .968513$

H $-2.5056540 .387120-1.207057$

H $-0.3051141 .394408-3.114069$

H -2.4004332 .5625060 .009474$

H $-3.3629602 .746991-1.472589$

H -2.623307 $4.144232-0.730382$

H $-3.176559-1.1806823 .060675$

H -2.817571 -0.8363651 .370978$

H 1.1908780 .7946631 .097728

H 0.9527861 .0142442 .852695

H -0.3876173 .2129962 .242522$

H 2.0512592 .9581690 .411249

H 1.9617465 .4000911 .279731

H $\quad 0.2128685 .3243121 .256177$

H $2.1373636 .337139-2.267612$

H $3.0546866 .187600-0.665503$

H $-0.1972325 .823918-2.353802$

H -1.027190 $5.439465-0.871658$

H $\quad 0.5666173 .455444-2.648301$ 
H -1.2152010 .7067410 .813252$

C $1.594545-3.603927 \quad 1.709597$

O $2.402646-2.4116491 .825671$

C $3.333272-2.5846582 .914293$

C $2.813085-3.7683113 .717322$

C $2.259497-4.6447332 .612354$

H $4.295003-2.8633772 .468396$

C $3.480272-1.2853803 .711446$

H $3.601062-4.2550254 .299968$

H $2.012940-3.4723274 .405984$

C $1.312064-5.7223443 .111447$

H $3.097892-5.1195042 .084729$

C $3.964472-0.0934072 .860169$

O $2.199294-0.9541504 .260479$

H $4.147651-1.4444394 .565895$

H 3.2248610 .1087782 .081933

O 3.9845931 .0686623 .698430

C $5.356741-0.2724362 .224873$

C 5.8237130 .9477541 .407333

H $5.378173-1.1573101 .581251$

$\begin{array}{lllllll} & \text { O } & 6.326507 & -0.477742 & 3.255831\end{array}$

H 5.7565641 .8588822 .014216

H 6.8927620 .8078661 .196411

H $1.829054-6.4002573 .798600$

H $0.458346-5.2904713 .644676$

H $0.922855-6.3170032 .279216$

C $1.534438-4.0302830 .240419$

H $2.299433-0.118547 \quad 4.748014$

H $4.6714190 .930506 \quad 4.373211$

H $6.175460-1.3611073 .631918$

H $1.339791-5.1070490 .172872$

H $2.499571-3.835004-0.242875$

H $0.595673-3.3516102 .089036$

C $4.639958-0.073044-1.972847$

O $5.326554-0.028794-0.716618$

C $5.0624101 .128851 \quad 0.083289$

C $5.5018212 .398148-0.665054$

C $4.8589322 .486534-2.053155$

C $4.9694481 .165581-2.824586$

H $5.064978-0.947275-2.483203$

C $3.145593-0.336761-1.760798$

H $3.9888991 .186767 \quad 0.289660$

$\begin{array}{lllll}\text { O } & 6.921617 & 2.426412 & -0.804427\end{array}$

H $5.2201923 .275031-0.070199$

H $5.3502893 .286795-2.620218$

O $3.4904762 .844999-1.900874$

H $4.331987 \quad 1.183205-3.715100$

O $6.3179551 .014947-3.281943$

C $2.520544-0.927934-2.998815$

H $2.990776-1.030528-0.924732$

H $2.5860490 .562455-1.487453$

H $7.1903201 .616124-1.270456$

H $3.1071872 .930396-2.790348$

H $6.5140861 .765755-3.867930$

O $2.699713-2.279466-3.009840$

O $1.971146-0.263408-3.867433$

C $2.195428-2.960528-4.162556$

H $2.329096-4.034468-4.008049$

H $1.129373-2.756856-4.295104$

H $2.758493-2.659359-5.050740$

SCF Energy (B3LYP/6-31G**//MMFF) $=-3245.91252236$

0800160

MMFF Geometry

C $3.006226 \quad 3.756226 \quad 0.241837$

C $3.5932624 .124273-0.907815$

C $4.4010253 .214175-1.802042$

O $3.950551 \quad 1.846199-1.684828$

C $5.8816993 .269746-1.390525$

C $6.7655012 .359903-2.218593$

C $7.2002411 .152603-1.805311$

C $7.1847612 .908675-3.556016$

C $6.8480570 .462160-0.514308$

C $6.682319-1.051928-0.708808$

C $6.251568-1.7418510 .594289$

C $6.431886-3.2565100 .485906$

C $5.950272-3.9753841 .753291$

O $4.871666-1.4324090 .831321$
O $5.871373-5.3788111 .461543$

C $4.521564-3.4987342 .076924$

C $6.942031-3.8272492 .912843$

O $4.079621-4.0263713 .326285$

C $4.352498-1.9585282 .061433$

C $2.844928-1.6203862 .147086$

O $5.020351-1.3965563 .191883$

C $2.9840251 .429162-2.545305$

O $2.4004312 .100989-3.380435$

C $2.759565-0.011480-2.292954$

C $1.783316-0.654404-2.950120$

C $1.412612-2.061561-2.785941$

C $2.295555-2.924428-1.923790$

C $2.492418-0.1464961 .913857$

C $0.9838540 .021536 \quad 1.870943$

C $-0.991082-0.475756 \quad 0.414804$

$\begin{array}{lllll}\text { O } & 0.354197 & 0.460477 & 2.830150\end{array}$

C $-1.319408-1.864948-0.036813$

C $-1.993840-2.146776-1.162280$

C $-2.274433-3.546936-1.632100$

C $-1.815754-3.797565-3.054505$

C $-2.702579-4.014473-4.040416$

C $-0.325325-3.874030-3.325534$

C $0.303762-2.509032-3.411323$

N $0.435651-0.411466 \quad 0.674915$

H 3.1080252 .7318130 .589879

H $3.5245965 .161951-1.226562$

H $4.295616 \quad 3.550337-2.841037$

H $5.9826863 .017268-0.327555$

H $6.2548264 .299541-1.474203$

H $7.8748770 .600490-2.457965$

H $7.8458192 .223696-4.097560$

H $6.3089613 .088675-4.187288$

H $7.7221603 .853821-3.427614$

H $7.656440 \quad 0.6433020 .204035$

H $5.9282700 .865678-0.080970$

H $5.935819-1.239240-1.491354$

H $7.636641-1.470275-1.052789$

H $6.858570-1.3458031 .416826$

H $7.478403-3.5116190 .277702$

H $5.854523-3.632767-0.370437$

H $\quad 6.755309-5.6779301 .187081$

H $3.858389-3.9244061 .310463$

H $7.922061-4.2316112 .632158$

H $6.618606-4.4060903 .785137$

H $7.088812-2.7905513 .222614$

H $4.185763-4.9923493 .286640$

H $2.306690-2.2215781 .404671$

H $2.454022-1.9142973 .130149$

H $4.624487-0.5340723 .393627$

H $3.389958-0.499587-1.558802$

H $\quad 1.174750-0.091437-3.656879$

H $3.343063-2.839900-2.235267$

H $2.052141-3.987636-1.980693$

H $2.218528-2.626450-0.875017$

H 2.8852400 .4902952 .713227

H 2.9132750 .2209890 .972009

H $-1.2106570 .268322-0.356681$

H $-1.553241-0.2282161 .319966$

H $-0.970311-2.6826460 .590106$

H $-2.364138-1.331668-1.779994$

H $-3.354559-3.716843-1.545900$

H $-1.801459-4.287307-0.974836$

H $-2.379307-4.218106-5.056030$

H -3.770963 -3.992238 -3.857840

H $-0.142043-4.385272-4.279350$

H $0.127681-4.504122-2.554996$

H $-0.243732-1.813079-4.048988$

H $1.060108-0.690786-0.074789$

C 0.9007324 .1096421 .557721

\begin{tabular}{llllll}
\hline & 0.013943 & 4.040375 & 0.424397
\end{tabular}

C -1.308866 4.4448650 .841887

C $-1.2611414 .537803 \quad 2.359997$

C $0.173416 \quad 4.9587792 .598148$

H -1.4698895 .4360800 .400082$

C $-2.3336813 .442100 \quad 0.299669$

H $-1.989355 \quad 5.2492282 .760788$

H -1.452512 3.5587462 .817284 
C 0.6413574 .7283154 .024781

H $\quad 0.2733196 .0244952 .352117$

C $-3.765422 \quad 3.6812490 .807060$

O $-2.3333093 .530734-1.128082$

H -2.0116292 .4267230 .553280$

H -3.8009933 .5493861 .894618$

O

C -4.8462962 .7910950 .162660$

C $-4.5297351 .293403 \quad 0.248746$

H $-5.0035133 .086248-0.880761$

O $\quad-6.0786793 .033108 \quad 0.849367$

H -3.645210 $1.057162-0.353948$

H -4.2554451 .0409961 .282602$

H 1.6833185 .0383314 .150078

H $\quad 0.0311635 .3055754 .727247$

H 0.5681213 .6721124 .305409

C 2.2470664 .6972481 .134340

H $-1.4219613 .369940-1.427641$

H $-4.0542305 .203456-0.392408$

H -6.2598663 .9869350 .791604$

H 2.8631874 .8867652 .020487

H 2.0900215 .6561420 .625371

H 1.0293413 .0841791 .925845

C -6.125637 -2.029074-0.273429

O $-5.184730-0.977686-0.021756$

C $-5.6856470 .359006-0.160557$

C $-6.1976970 .588834-1.589373$

C $-7.235470-0.468001-1.981108$

C $-6.752820-1.887512-1.671271$

H -5.526673 -2.948695-0.266595

C $-7.166848-2.1447190 .850828$

H $-6.5024870 .502466 \quad 0.554436$

O $\quad-5.097897 \quad 0.548237-2.496163$

H -6.648352 $1.585449-1.657687$

H -7.448006 -0.380512 -3.053760

O $-8.443977-0.199505-1.275787$

H -7.579204 -2.599364-1.781865

O $-5.770268-2.236645-2.649695$

C $-6.541685-2.5444472 .171896$

H -7.695692 -1.202119 1.013549

H $-7.897527 \quad-2.9189700 .589908$

H $-5.4465440 .714102-3.388521$

H $-9.100803-0.858649-1.557701$

H $-5.530048-3.166950-2.501377$

O $-7.522964-2.6133213 .120549$

O $-5.353479-2.7703122 .354565$

C $-7.084927-2.9805654 .432642$

H $-6.641198-3.9805294 .417491$

H $-6.369624-2.2451614 .813165$

H -7.957716 -2.994027 5.090945

SCF Energy $\left(B 3 L Y P / 6-31 G^{* *} / / M M F F\right)=-3245.90711170$

08_00161

MM̄FF Geometry

C -1.813915 -0.521621 -2.037845

C $-0.589470-1.003792-1.781482$

C $-0.278155-1.859909-0.579604$

O $0.948165-1.3779620 .019664$

C $-0.066963-3.319068-1.017437$

C $0.164244-4.2696760 .142996$

C $1.341891-4.8565460 .438261$

C -1.054534 -4.570841 0.979149

C $2.655613-4.693497-0.273681$

C $3.673660-3.9577720 .606563$

C $4.885374-3.473715-0.203450$

C $5.929525-2.8416280 .717947$

C $7.090004-2.209708-0.064967$

O $4.408904-2.506034-1.144600$

O $7.825182-1.3696860 .838663$

C $6.513408-1.291451-1.164043$

C $8.076367-3.267606-0.573232$

O $7.548844-0.802785-2.012899$

C $5.413652-1.963495-2.010903$

C $4.705115-0.980243-2.976217$

O $6.004802-2.990781-2.815798$

C $0.847924-0.3242360 .875991$

O $-0.170754 \quad 0.2646861 .200164$

C 2.2090190 .0045591 .354656
C 2.3986971 .0955312 .111904

C 3.6734641 .5545992 .668561

C 4.9070360 .7552592 .343624

C $3.9549430 .176590-2.305863$

C $4.7601531 .459561-2.185436$

C $4.6340643 .735136-1.171835$

O $5.834767 \quad 1.637967-2.751455$

C 5.4281653 .7518210 .096014

C 5.2120164 .6270851 .089940

C 6.0411254 .6958332 .340361

C 5.2074304 .6490043 .600998

C 4.8736505 .7768024 .251175

C 4.8423123 .2918784 .156809

C 3.6805282 .6633173 .438050

N $4.1089932 .402018-1.399535$

H -2.631804 -0.779003 -1.369403

H $0.231094-0.773190-2.456553$

H - $1.089701-1.8080700 .156830$

H $0.760292-3.362796-1.732286$

H $-0.947164-3.671182-1.572580$

H $1.379794-5.5304721 .293350$

H $-0.858784-5.3350451 .738988$

H -1.398952 -3.672199 1.499814

H -1.868091 -4.9406660 .346393$

H $3.043893-5.689250-0.520911$

H $2.536583-4.178073-1.228543$

H $3.186527-3.0923371 .072772$

H $4.002182-4.6222321 .415387$

H $5.312531-4.326582-0.744048$

H $6.307208-3.5771971 .439156$

H $5.452274-2.0565011 .318390$

H $8.131444-1.9212491 .578888$

H $6.087151-0.418110-0.656643$

H $8.506961-3.8245270 .267838$

H $8.923901-2.802967-1.088904$

H $7.616802-3.993831-1.246763$

H $8.208575-0.369228-1.444973$

H $5.409976-0.621288-3.735726$

H $3.952523-1.559295-3.530873$

H $5.417899-3.169868-3.568981$

H $3.018029-0.6571051 .069626$

H $1.535896 \quad 1.7145002 .355753$

H 5.0117460 .6300571 .260755

H $4.852562-0.2338052 .810560$

H $5.833981 \quad 1.2232742 .681233$

H $3.0825760 .425466-2.924088$

H $3.581578-0.114943-1.320292$

H $3.7795094 .418127-1.134292$

H $5.2744374 .023771-2.011466$

H 6.2395343 .0332800 .185657

H 4.4194905 .3645710 .983519

H 6.6119215 .6332572 .308242

H 6.7915053 .8959842 .368672

H 4.3021175 .7501915 .172940

H 5.1600356 .7524683 .872359

H 4.5551913 .3847255 .213153

H 5.7311622 .6547644 .171396

H 2.7356023 .1808273 .610671

H $3.2461682 .146395-0.927893$

C $-2.6919141 .713503-2.783534$

O $-3.9246841 .542101-2.054551$

C $-4.9632462 .281204-2.713597$

C $-4.235723 \quad 3.376397-3.477660$

C $-3.0210352 .616304-3.978167$

H $-5.4285031 .594160-3.432089$

C $-6.0253242 .754169-1.714405$

H -4.841505 $3.798465-4.284682$

H -3.920776 $4.192899-2.817851$

C $-1.8698503 .513619-4.399554$

H $-3.3232232 .005299-4.839467$

C $-6.5229621 .665888-0.737467$

O $-5.4793823 .816364-0.922096$

H $-6.8662773 .189926-2.267075$

H $\quad-5.767050 \quad 1.518258 \quad 0.039475$

O $-7.6759982 .182092-0.061269$

C $-6.8744380 .315697-1.379919$

C -7.511986 -0.713358 -0.429765

H $-5.972070-0.126715-1.815951$ 
O $-7.788687 \quad 0.546001-2.455763$ H -8.543922 $-0.420694-0.195586$ H -7.603874 -1.644721 -1.003980 H $-2.1719414 .164390-5.226624$ H -1.535756 4.151720 -3.574526 H -1.014322 $2.917318-4.732191$ C $-2.1567630 .343793-3.217005$ H $-6.1733854 .103331-0.303910$ H $-8.3870912 .267400-0.719459$ H -7.952900 -0.308865 -2.889345 H -1.282526 $0.475760-3.865040$ H -2.920801 - $0.186133-3.799475$ H -1.985892 $2.202453-2.100827$ C $-4.504447-1.4041321 .710299$ O $-5.363479-1.1909440 .578737$ C $-6.745653-0.979508 \quad 0.879817$ C $-7.325246-2.1874611 .638498$ C $-6.487156-2.5404242 .871400$ C $-4.991178-2.6028132 .543327$ H $-3.532398-1.6734311 .277236$ C $-4.311874-0.1317162 .554880$ H -6.841006 -0.086103 1.505201 O $-7.391369-3.329772 \quad 0.784803$ H -8.353325 -1.955355 1.939524 H -6.809201 -3.512596 3.264285 O $-6.698265-1.5694363 .891396$ H -4.398934 -2.672081 3.463285 O $-4.726132-3.7860951 .784617$ C $-3.5420320 .944803 \quad 1.823308$ H -5.2670890 .3047582 .856622$ H $-3.736638-0.3783153 .454972$ H $-6.496319-3.4968240 .442570$ H $-7.646812-1.5686514 .104994$ H $-4.983732-4.5479472 .331231$ O -3.4551302 .0508392 .620233$ O $-\begin{array}{llll}-3.073065 & 0.838173 & 0.700145\end{array}$ C -2.752784 3.1602382 .050780 H -2.7774323 .9834572 .769660$ H -1.7094512 .8899541 .861900$ $\mathrm{H}-3.2410863 .4860471 .127372$

SCF Energy (B3LYP/6-31G**//MMFF) $=-3245.91212246$

0800162

MMFF Geometry

C -2.056298 -0.6398232 .672493$

C -2.0999380 .1755153 .736891$

C $-2.692507 \quad 1.566173 \quad 3.764675$

O -3.6982881 .7208232 .731864$

C -1.647461 2.6713553 .513714

C -0.3224822 .5086384 .229020$

C 0.8382612 .2262083 .602353

C -0.3575112 .7277065 .717478$

C 1.0228001 .9872252 .127850

C 1.4694163 .2703301 .417519

C $1.3221153 .176220-0.107898$

C $1.8786694 .438779-0.771107$

C $1.6234834 .464622-2.284931$

O $-0.073713 \quad 3.048130-0.398995$

O $1.8635795 .802380-2.749074$

C $0.1349904 .156191-2.547369$

C $2.5999383 .562926-3.047040$

O $-0.1163804 .020595-3.944678$

C $-0.3829202 .908851-1.791597$

C -1.924370 $2.821844-1.926660$

O $\quad 0.2173101 .743693-2.350587$

C $-4.906816 \quad 1.1295072 .930375$

O $\quad-5.300016 \quad 0.5690263 .940154$

C $-5.6404761 .208874 \quad 1.648855$

C $-6.544376 \quad 0.2612451 .360795$

C $-7.2854650 .123742 \quad 0.104803$

C $-7.073137 \quad 1.177623-0.949848$

C -2.595252 $1.704919-1.112459$

C $-2.7120660 .378217-1.844792$

C $-3.692789-1.900093-1.576441$

O $-2.2443490 .180400-2.962538$

C $-5.015548-1.892337-2.278618$

C $-6.022033-2.729864-1.984507$

C $-7.333453-2.744085-2.718661$
C $-8.531198-2.681767-1.795840$

C $-9.265379-3.778468-1.542278$

C $-8.928851-1.332467-1.235967$

C $-8.095458-0.945133-0.045426$

N $-3.403409-0.563046-1.092868$

H $-2.454770-0.3059531 .719798$

H -1.674010 -0.172196 4.675966

H -3.1655591 .7332024 .740917$

H -2.0879533 .6350613 .805703$

H -1.500386 2.7841832 .432489

H 1.7487802 .1570044 .194804

H 0.6337362 .6351526 .173666

H -0.7331883 .7307445 .944126$

H -1.011754 1.9946516 .199460

H $1.781453 \quad 1.207021 \quad 1.988727$

H $\quad 0.1089941 .5828661 .681817$

H $0.8616104 .111461 \quad 1.777453$

H 2.5124413 .4848221 .681981

H $1.8485592 .282803-0.461764$

H $2.9492374 .550084-0.559196$

H $1.3960415 .321927-0.328612$

H $2.7805476 .037053-2.525356$

H $-0.4444505 .027780-2.211805$

H $3.6350763 .873231-2.860674$

H $2.4530753 .646236-4.129551$

H $2.5147802 .512482-2.767629$

H $0.2110364 .827645-4.377599$

H -2.345472 $3.767685-1.559864$

H -2.206673 $2.727879-2.981814$

H $-0.2789370 .964649-2.054396$

H -5.3654091 .9953420 .956195$

H $-6.734463-0.5140582 .102415$

H $-7.3377772 .167584-0.561623$

H -7.673177 $1.026245-1.848843$

H $-6.026628 \quad 1.195872-1.271580$

H $-2.0828031 .541033-0.158749$

H -3.619281 $2.026585-0.885955$

H -3.686133 -2.568746 -0.709959

H -2.906279 -2.214206 -2.270057

H -5.142674 -1.175297 -3.086568

H $-5.886668-3.467469-1.196657$

H -7.368255 -3.666127 -3.313836

H -7.394926 -1.921799 -3.442632

H -10.142013 -3.736457 -0.904163

H -9.003376 -4.743830 -1.962322

H $-9.976341-1.357313-0.906710$

H -8.903587 -0.594345 -2.041552

H $-8.182106-1.6462670 .786473$

H $-3.827743-0.278246-0.215332$

C $-0.106467-2.0538682 .010571$

O $-0.267590-1.8215810 .598083$

C $0.686581-2.636929-0.119221$

C $1.545197-3.3156570 .939708$

C $0.596006-3.4035932 .115157$

H $0.091822-3.377053-0.667865$

C $1.470998-1.741481-1.086067$

H $1.918014-4.2914960 .615446$

H $2.408286-2.6908891 .199079$

C $1.301714-3.6398673 .439360$

H $\quad-0.118837-4.2168341 .932229$

C $2.664594-2.431773-1.770819$

O $0.564724-1.265979-2.085515$

H $1.805398-0.855866-0.536311$

H $3.365859-2.826262-1.031791$

O $2.190430-3.565061-2.511118$

C $3.429654-1.539855-2.764643$

C $3.944394-0.194475-2.222871$

H $2.792250-1.338384-3.634686$

O $4.555511-2.271616-3.262808$

H $4.3843280 .349725-3.069244$

H $3.0899280 .393394-1.872947$

H $1.856017-4.5839163 .416015$

H $2.013577-2.838133 \quad 3.663315$

H $0.583042-3.6917424 .262957$

C -1.465233 -2.019694 2.705657

H $0.278260-2.031572-2.612540$

H $1.878049-4.227186-1.872053$

H $4.219662-3.111871-3.619633$ 
H -1.368372 -2.362754 3.742066 H -2.167104 -2.697036 2.204104 H $0.540760-1.2547162 .396494$ C $5.281484-0.9815911 .194829$ O $4.415007-0.8073360 .066213$ C $5.019251-0.278979-1.116543$

C $5.6307211 .105515-0.829452$ C 6.5852571 .0654870 .367140 C 5.9607550 .3461651 .567610 H $4.612242-1.2570652 .020029$ C $6.277707-2.1294900 .987402$ H $5.809505-0.957737-1.452602$ O $4.5870802 .040158-0.563497$ H $6.1620061 .449071-1.725251$ H $6.8461702 .091526 \quad 0.653738$ O $7.7840300 .407546-0.030501$ H $6.719810 \quad 0.1645432 .336640$ O 4.9800721 .2022642 .161846 C $5.569353-3.4625560 .915323$ H $6.878601-2.0000150 .083020$ H $6.982381-2.1911951 .824957$ H $4.9977902 .913088-0.442341$ H 8.3879860 .4120150 .731588 H 4.2619051 .3242051 .517093 O $5.793873-4.045306-0.296864$ O $4.899447-3.9310171 .826740$ C $5.181169-5.323806-0.491268$ H $5.595171-6.0504610 .214005$ H $4.095838-5.249277-0.376732$ H $5.402954-5.655048-1.509195$ SCF Energy (B3LYP/6-31G**//MMFF) $=-3245.91561880$

\section{8_00163}

MM̄FF Geometry

C $1.0660503 .728604-0.934916$

C $1.8936784 .613673-0.357695$

C 2.6694504 .3478840 .911659

O 3.1145262 .9709240 .949522

C 1.7758014 .6272362 .133461

C 2.4275884 .2853963 .457676

C 2.1636743 .1721614 .171664

C 3.4053315 .3074373 .973993

C 1.2440942 .0412953 .795177

C 1.9926360 .7019393 .781198

C $1.156239-0.4194593 .145552$

C $1.890854-1.7575813 .250315$

C $1.154463-2.8871602 .514318$

O $0.940570-0.0821431 .769306$

O $2.065510-3.9895382 .380153$

C $0.808930-2.4103021 .086814$

C $-0.038755-3.4088513 .324255$

O $0.012360-3.378930 \quad 0.410790$

C $0.134569-1.0231751 .046982$

C $-0.059930-0.484170-0.392239$

O $-1.170039-1.1258861 .627928$

C 4.2537242 .6739120 .262460

O $4.9310473 .441858-0.402622$

C 4.5438571 .2364090 .461980

C $5.6312010 .688692-0.101562$

C $6.062895-0.7057420 .027248$

C $5.165975-1.6476480 .785851$

C $1.223204-0.241279-1.190362$

C $1.659842-1.405127-2.061816$

C $3.561783-2.117972-3.512157$

O $0.963671-2.394462-2.268764$

C $4.547223-2.935850-2.739435$

C $5.852119-3.001200-3.044617$

C $6.840568-3.861163-2.311995$

C $8.034618-3.082365-1.809049$

C $9.188021-3.063368-2.498323$

C $7.921352-2.409661-0.460581$

C $7.236534-1.073588-0.528586$

N $2.913763-1.180482-2.615726$

H $0.9204632 .756749-0.470529$

H $2.0174635 .597424-0.803451$

H 3.5454755 .0080440 .944162

H 0.8262664 .0904422 .031695

H 1.4911595 .6885762 .144292
H 2.6704293 .0429235 .127212

H 3.8237605 .0286474 .946955

H 4.2413905 .4233813 .277063

H 2.9131456 .2781264 .092757

H $0.429500 \quad 1.9935564 .527617$

H 0.7774862 .2067912 .821745

H 2.9262660 .8191813 .218257

H 2.2647530 .4280584 .808227

H $0.190264-0.4700073 .661720$

H $2.057180-2.0277504 .300749$

H $2.891051-1.6556192 .809436$

H $2.352119-4.2521123 .271594$

H $1.757055-2.3607990 .538851$

H $\quad 0.296479-3.7900144 .296522$

H $-0.518214-4.2562932 .822177$

H $-0.795255-2.6451033 .515105$

H $-0.898771-3.2963830 .740610$

H $-0.765156-1.118870-0.942696$

H $-0.5567250 .491716-0.306660$

H $-1.687123-0.3503351 .353434$

H $3.858593 \quad 0.6664301 .076791$

H $6.2820031 .324178-0.700885$

H $5.474263-2.6931120 .719305$

H $4.144982-1.6112080 .390974$

H $5.143520-1.3795211 .847382$

H $1.0452830 .594536-1.879029$

H $2.0448090 .048401-0.530121$

H $4.040383-1.531591-4.303022$

H $2.813980-2.773327-3.969575$

H $4.162798-3.522746-1.908516$

H $6.227545-2.432851-3.892758$

H $7.173798-4.646363-3.003387$

H $6.369251-4.389610-1.474002$

H $10.059273-2.533617-2.127796$

H $9.284362-3.572387-3.451655$

H $8.924394-2.234683-0.047748$

H $7.451396-3.0953600 .250150$

H $7.813987-0.319558-1.065491$

H $3.431062-0.347102-2.350895$

C $-1.1793434 .252153-1.929514$

O $-1.7927003 .114279-1.292938$

C $-3.1009552 .910953-1.855696$

C $-3.3779924 .119925-2.743489$

C $-1.9844044 .490229-3.205647$

H $-3.0301972 .012975-2.481124$

C $-4.1629152 .688366-0.767372$

H $-4.0553903 .879681-3.568793$

H $-3.8222104 .946039-2.175774$

C $-1.8819355 .903965-3.751456$

H -1.674408 $3.780117-3.984046$

C -3.8998901 .4304270 .089481$

O -4.2211083 .8520200 .056554$

H $-5.1367482 .585340-1.262230$

H $-3.7030380 .597697-0.593452$

O $-2.726693 \quad 1.6094410 .880254$

C -5.0805241 .0510861 .007660$

C $-4.839257-0.2063591 .866056$

H -5.9769850 .9119970 .392123$

O -5.3523772 .1343401 .900080$

H -4.006741 -0.0311742 .559090$

H $-5.726895-0.3381182 .499129$

H -2.538074 $6.029525-4.619062$

H $-2.1723246 .646108-3.000217$

H $-0.8586206 .128137-4.068135$

C $0.3012273 .980938-2.201081$

H -4.9466973 .7280830 .691333$

H -2.879328 2.3778931 .456734

H $-6.143448 \quad 1.9002872 .415038$

H $0.7418104 .821923-2.749064$

H $0.4116903 .092048-2.834389$

H $-1.2886465 .099193-1.239297$

C $-5.615861-2.801154-0.703709$

O $-5.713782-1.6876880 .193962$

C $-4.590703-1.4887251 .057018$

C $-4.386796-2.7157851 .959344$

C $-4.255660-3.9938581 .124313$

C $-5.365112-4.1063340 .071801$

H $-6.607506-2.876486-1.168371$ 
C $-4.591857-2.545305-1.821269$

H $-3.691440-1.3435780 .452943$

O $-5.492297-2.8419812 .851526$

H -3.486382 -2.565501 2.566603

H $-4.294387-4.864264 \quad 1.790545$

O $-2.982504-3.9889340 .487378$

H $-5.136217-4.911804-0.634869$

O $-6.581710-4.4786910 .727218$

C $-4.992425-1.384272-2.709125$

H -3.600311 -2.321965 -1.418997

H -4.519243 -3.433015 -2.459951

H -5.315668 -3.602299 3.431255

H -2.900302 -4.818663 -0.012976

H $-6.841887-3.7449811 .310418$

O $-3.988532-1.145582-3.604518$

O $-6.049035-0.771924-2.639665$

C $-4.227780-0.084186-4.534474$

H $-3.2902150 .129949-5.054286$

H $-4.975085-0.398975-5.268384$

H $-4.5552940 .823301-4.018262$

SCF Energy (B3LYP/6-31G**//MMFF) $=-3245.92848870$

0800164

MM̄FF Geometry

C $-4.295577-2.8904221 .278761$

C $-2.965679-2.8936681 .105812$

C -1.977579 -2.808655 2.238092

O $-1.138131-1.6482382 .036741$

C -1.066218 -4.048872 2.248742

C $-0.086954-4.0552553 .407446$

C $1.254300-3.9959293 .292230$

C $-0.707060-4.1706414 .778408$

C $2.099165-3.9423372 .049473$

C $2.836268-2.6054341 .928821$

C $3.895920-2.5889110 .813129$

C $5.105843-3.4623531 .162920$

C $6.222438-3.3500320 .115773$

O $4.316188-1.2264750 .663615$

O $7.413839-3.9262950 .674101$

C $6.524655-1.859213-0.138810$

C $5.896293-4.166746-1.140504$

O $7.441929-1.704936-1.219418$

C $5.262623-1.001865-0.392206$

C $5.6276890 .498881-0.375492$

O $4.698762-1.269838-1.677955$

C -1.618849 -0.4514252 .469450$

O $-2.720156-0.2298792 .947098$

C -0.5485820 .5531912 .280566$

C -0.7657451 .8247282 .648516$

C 0.1910842 .9295182 .555373

C 1.5643182 .6185072 .023277

C $4.4198711 .414624-0.606344$

C $4.8038922 .884090-0.644648$

C $3.8022135 .118553-1.150429$

O $5.936106 \quad 3.292270-0.402815$

C 3.2305195 .7861920 .059384

C $2.035026 \quad 6.3949410 .069927$

C 1.4694847 .1273891 .252122

C $\quad 0.290374 \quad 6.4056041 .864881$

C -0.9617946 .6834481 .461695$

C 0.6077525 .4288652 .978921

C -0.2026224 .1594002 .947554$

N $3.7210703 .681019-0.986296$

H -4.699382 -2.846906 2.286820

H -2.560511 -2.943677 0.100329

H -2.498201 -2.722234 3.199691

H -0.536605 -4.1070121 .292563$

H -1.676958 -4.959456 2.311006

H $1.840145-4.0126804 .211610$

H $\quad 0.040916-4.3418775 .560285$

H -1.247755 -3.255575 5.037941

H -1.406412 -5.0126314 .809782$

H $2.812796-4.7701402 .118161$

H $1.520662-4.1188241 .139069$

H $2.104940-1.8083371 .737778$

H $3.312269-2.3373442 .881729$

H $3.427362-2.918663-0.121179$

H $4.820947-4.5130041 .286638$
H $5.511711-3.1469392 .134417$

H $7.216043-4.8463270 .919803$

H $7.029855-1.4693530 .756066$

H $5.809614-5.231774-0.893174$

H $6.701812-4.096345-1.879627$

H $4.960004-3.865578-1.615437$

H $8.227989-2.238390-1.010417$

H $\quad \begin{array}{llll}6.079887 & 0.757476 & 0.590733\end{array}$

H $6.3728470 .708687-1.153314$

H $5.304922-1.846050-2.170513$

H $\quad 0.390522 \quad 0.216570 \quad 1.856250$

H -1.7382402 .0864613 .063899$

H $2.079611 \quad 1.9078512 .678686$

H 2.2084543 .4946491 .932823

H 1.4958122 .1844341 .021854

H $3.9416641 .168617-1.561225$

H $3.684628 \quad 1.2766830 .193564$

H $3.2453815 .371208-2.058795$

H $4.8429195 .427005-1.291630$

H 3.8427915 .7910880 .957773

H $1.4298396 .389958-0.834093$

H $\quad 1.167127 \quad 8.1280870 .916283$

H 2.2357227 .3026192 .017928

H -1.827930 6.2069541 .907193

H -1.150106 7.4053670 .673365

H 0.4293565 .9304463 .939117

H 1.6779835 .2001632 .982356

H -1.220803 4.2821133 .318870

H $2.8133833 .245386-1.124104$

C $-6.057043-1.646001-0.070718$

O $-5.155456-0.587531-0.447695$

C $-5.6217180 .643657 \quad 0.142110$

C -7.0057820 .3470430 .713857$

C $-6.861160-1.1099281 .112382$

H $-4.9435150 .867840 \quad 0.973506$

C $-5.5809431 .779384-0.886898$

H -7.2543561 .0009501 .555349$

H $-7.7925650 .457800-0.041220$

C -8.185096 -1.819473 1.332305

H $-6.274983-1.1504572 .038279$

C $-4.2048281 .969680-1.557502$

O $-6.5274061 .481214-1.922799$

H $-5.9181932 .713554-0.423192$

H $-4.0000231 .092546-2.181224$

O $-4.3288923 .084085-2.449641$

C $-3.0408222 .226556-0.580724$

C -1.677279 2.457740 -1.259284

H $-2.940574 \quad 1.3865790 .113927$

O -3.3128663 .3901990 .204659$

H -1.716265 $3.345310-1.902285$

H $-0.9620092 .707661-0.464733$

H -8.747529 -1.341971 2.141297

H $-8.807728-1.7968180 .431595$

H -8.024168 -2.867159 1.605042

C $-5.283689-2.9490700 .148274$

H $-6.4920212 .211043-2.564861$

H -3.570145 $3.062370-3.056619$

H -4.0503023 .1796320 .801521$

H $-4.757548-3.201165-0.781430$

H $-5.985478-3.7677990 .344769$

H $-6.723144-1.793995-0.931724$

C $-0.699407-1.105935-1.738270$

O $-1.0715330 .143664-1.142495$

C $-1.164717 \quad 1.244419-2.051804$

C $0.2037761 .509705-2.696183$

C $0.7594870 .249822-3.369271$

C $0.651388-0.984196-2.463788$

H $-0.558397-1.779545-0.883745$

C - $-1.852236-1.656173-2.590317$

H $-1.8863921 .004824-2.839577$

O $1.1315621 .961859-1.714218$

H $\quad 0.0998152 .313767-3.433822$

H $1.8128290 .412145-3.629563$

O $\quad 0.0500190 .000707-4.578675$

H $0.850853-1.897945-3.034224$

O $1.655143-0.893954-1.447305$

C -1.812639-3.161357-2.736666

H $-2.801319-1.399199-2.103209$ 
H $-1.867564-1.214120-3.589599$ H $1.2082761 .265992-1.039207$ H $0.1665340 .776628-5.153072$ H $2.521284-0.901262-1.889462$ O $-2.966826-3.575682-3.339130$ O $-0.894983-3.884433-2.374332$ C $-3.087788-4.988504-3.530158$ H $-2.287441-5.352894-4.181263$ H -3.067049 -5.503931-2.565182 H $-4.049184-5.185381-4.011866$

SCF Energy (B3LYP/6-31G**//MMFF) $=-3245.92981654$

08_00165

MM̄FF Geometry

C $-1.056219 \quad 1.3652083 .415622$

C $-2.1863982 .004713 \quad 3.756656$

C -3.3832402 .2730302 .875075$

O -3.3243791 .5254681 .641399$

C $-3.436173 \quad 3.7745012526105$

C $-4.6343794 .180198 \quad 1.683784$

C $-4.550304 \quad 4.6930890 .438383$

C -5.9814214 .0003912 .333467$

C $-3.2923904 .968943-0.338711$

C $-2.8475773 .735931-1.123954$

C $-1.3557063 .749111-1.470286$

C $-1.0067864 .774641-2.548076$

C $0.4747184 .689175-2.957082$

O $-1.0328172 .431976-1.932068$

O $\quad 0.6352045 .422672-4.181142$

C $0.833554 \quad 3.216162-3.260223$

C $1.384225 \quad 5.367514-1.924851$

O $2.2308783 .072414-3.505782$

C $0.3650912 .226136-2.168947$

C $0.5599300 .768145-2.644776$

O $1.1374442 .440236-0.988286$

C -3.8609450 .2744851 .639300$

$\begin{array}{lllll}\text { O } & -4.274942 & -0.349348 & 2.603177\end{array}$

C $-3.906765-0.1883120 .232475$

C $-4.572999-1.311375-0.074909$

C $-4.793962-1.850337-1.420434$

C $-4.195537-1.101214-2.581917$

C $-0.031539-0.280716-1.691983$

C $0.058509-1.685643-2.260850$

C $-0.813211-3.990466-1.876880$

O $0.749004-1.972510-3.234969$

C $-1.963603-4.186098-2.812692$

C -2.976042 -5.033367 -2.573400

C $-4.100453-5.279946-3.536676$

C $-5.462530-5.073263-2.915586$

C $-6.221572-6.119401-2.547459$

C $-5.983878-3.658042-2.812717$

C -5.543091 -2.965860 -1.550485

$\mathrm{N}-0.705398-2.586397-1.529816$

H -0.9223220 .9876672 .405986$

H -2.278074 2.382717 4.773716

H -4.2870511 .9930863 .430399$

H -2.502956 4.0392952 .017293

H -3.454143 4.3694593 .449125

H $-5.4717224 .942401-0.085369$

H $-6.7910554 .433287 \quad 1.736160$

H -6.2060882 .9382452 .470008$

H -5.9995864 .4913183 .311969$

H -3.479887 $5.800690-1.028647$

H $-2.500138 \quad 5.3203530 .330685$

H $-3.0478872 .827537-0.546647$

H -3.449590 $3.629572-2.036145$

H $-0.7932353 .944900-0.549632$

H -1.253766 $5.791787-2.221385$

H -1.621937 4.588308 -3.439519

H $\quad 0.345647 \quad 6.337583-4.022882$

H $0.3341712 .953273-4.202788$

H $1.1039616 .420473-1.801318$

H $2.4270275 .375429-2.260443$

H $1.3398854 .897979-0.940169$

H $2.687753 \quad 3.108655-2.648388$

H $0.0766510 .643704-3.622927$

H $1.6277230 .557523-2.779712$

H $1.0747941 .657448-0.419567$
H -3.436408 $0.434662-0.518949$

H $-5.034443-1.8773220 .733660$

H $-4.642356-0.104608-2.667063$

H $-4.335002-1.600485-3.542805$

H -3.114608 -0.989284 -2.454101

H $0.501591-0.281868-0.735762$

H - $1.084461-0.055682-1.494841$

H $-0.942111-4.547828-0.943787$

H $0.113921-4.319149-2.357258$

H $-1.945639-3.627800-3.745772$

H $-2.979243-5.611792-1.652256$

H $-4.006096-6.312756-3.897542$

H $-4.009707-4.647796-4.428741$

H $-7.213453-5.978312-2.130936$

H -5.865862 -7.139302 -2.648856

H $-7.082480-3.670586-2.805077$

H $-5.723375-3.107817-3.720724$

H $-5.937534-3.429950-0.645083$

H $-1.295129-2.240463-0.778417$

C $0.441337-0.3154564 .620201$

O $1.103183-0.8778123 .472590$

C $0.835672-2.2944453 .446811$

C $-0.031573-2.6024664 .669943$

C $-0.707426-1.270440 \quad 4.929727$

H $0.248438-2.4849482 .541591$

C $2.147470-3.0906683 .408860$

H $-0.742059-3.4118614 .474398$

H $0.573245-2.8999695 .534433$

C $-1.270584-1.1314786 .332317$

H -1.519441 -1.157701 4.200652

C $3.076395-2.7798922 .215744$

O $2.890271-2.8317904 .608570$

H $1.917866-4.1625753 .428665$

H $3.519715-1.7866432 .342035$

O $4.171077-3.705132 \quad 2.278735$

C $2.412478-2.8802450 .831391$

C $3.391132-2.915301-0.357141$

H $1.708411-2.0506140 .706162$

$\begin{array}{llllll}\text { O } & 1.650771 & -4.091660 & 0.786199\end{array}$

H $3.970277-3.847425-0.323654$

H $2.783498-2.983918-1.269345$

H -2.030865 -1.896941 6.518992

H $-0.489150-1.2415627 .091727$

H - $1.737869-0.1512736 .469331$

C $\quad 0.079472 \quad 1.151295 \quad 4.378152$

H $3.074650-1.8772034 .638772$

H $3.810478-4.5967962 .132990$

H $1.225640-4.137988-0.086002$

H $\quad 0.9550491 .6743203 .976601$

H -0.1534401 .6176665 .343490$

H $1.179348-0.3428635 .433940$

C $4.288907 \quad 0.692652-0.787367$

O $3.556025-0.535453-0.664834$

C $4.343777-1.713905-0.467994$

C $5.341952-1.905858-1.623689$

C $6.202993-0.656945-1.827556$

C $5.346457 \quad 0.608157-1.899901$

H $3.5443961 .429762-1.106483$

C $4.860317 \quad 1.151837 \quad 0.558326$

H $4.902050-1.6225190 .468274$

O $4.631849-2.194546-2.827088$

H $5.976901-2.771743-1.400455$

H $6.778756-0.752147-2.756213$

O $7.126263-0.529260-0.750570$

H $5.9862391 .497189-1.863852$

O $4.6768820 .646261-3.163673$

C $3.758016 \quad 1.6625651 .454418$

H $5.3793860 .358347 \quad 1.103013$

H $5.586472 \quad 1.9629870 .425266$

H $5.289495-2.344732-3.527312$

H $7.678235-1.329630-0.740493$

H $4.048092-0.095264-3.190389$

O $3.5067132 .975543 \quad 1.183940$

$\begin{array}{llll}\text { O } & 3.178562 & 0.972128 & 2.281753\end{array}$

C $2.4693363 .578253 \quad 1.964275$

H $1.5203623 .058811 \quad 1.802270$

H 2.7396723 .5676803 .024398

H 2.3596664 .6163331 .639882 
H 5.5690724 .5123181 .008364

H $6.1705606 .240093-0.834084$

0800166

MM̄FF Geometry

C $1.685385-2.5627463 .405360$

C $2.905476-2.5377182 .846564$

C $3.385848-3.2670311 .611777$

O $3.856259-2.2607560 .677755$

C 2.328016-4.085982 0.845520

C $2.935446-5.134706-0.067557$

C $3.200626-4.975082-1.378720$

C $3.201846-6.4643090 .593548$

C $3.076558-3.736260-2.219988$

C $1.938968-3.816373-3.247757$

C $0.601103-3.317273-2.682883$

C $-0.525092-3.498993-3.702472$

C $-1.847544-2.887990-3.212093$

O $0.761348-1.925590-2.380222$

O $-2.740708-2.810110-4.333700$

C $-1.585822-1.441415-2.742639$

C $-2.537521-3.775654-2.169503$

O $-2.752105-0.876596-2.150821$

C $-0.383891-1.313328-1.778883$

C $-0.0630460 .179598-1.528529$

O $-0.710042-1.927617-0.532161$

C $5.123064-1.7878380 .790583$

O $5.963176-2.1023151 .616273$

C $5.291479-0.776677-0.276241$

C $6.2647630 .140077-0.183054$

C $6.5153651 .223998-1.136534$

C $5.6727401 .264013-2.385548$

C $1.2915990 .424278-0.847085$

C $1.4914611 .886266-0.484001$

C 3.1197043 .3986370 .651375

O $0.678002 \quad 2.764307-0.759327$

C $4.0535584 .000786-0.351343$

C $5.2499104 .518188-0.031545$

C $6.1757895 .159225-1.026646$

C $7.5914284 .630424-0.942853$

C $8.5576125 .337380-0.332525$

C $7.9049463 .325394-1.641259$

C $7.4704332 .130642-0.842049$

N 2.6900592 .0924450 .186327

H $0.894088-3.1701612 .978602$

H $3.660354-1.9138653 .323805$

H $4.221368-3.9162181 .902891$

H $1.684827-3.4143310 .264756$

H $1.665066-4.6167891 .538942$

H $3.604995-5.829651-1.921011$

H $3.647392-7.191373-0.093554$

H $3.892149-6.3407901 .434140$

H $2.268357-6.8950800 .970165$

H $2.983944-2.834445-1.611205$

H $4.024456-3.629095-2.763083$

H $2.217097-3.177916-4.096651$

H $1.836534-4.838999-3.630504$

H $\quad 0.374655-3.869465-1.764109$

H $-0.659430-4.559752-3.948626$

H - $0.246346-3.006306-4.644704$

H -2.856925 -3.708915 -4.686679

H - $1.373956-0.841350-3.638896$

H -2.759032 -4.762231 -2.593919

H -3.504157 -3.355274 -1.870414

H -1.935669 -3.931605 -1.271896

H -3.481103 -0.982560 -2.785561

H $-0.0461910 .715606-2.486661$

H $-0.860030 \quad 0.627249-0.920951$

H $-0.160068-1.5390510 .165595$

H $4.580751-0.793133-1.094828$

H $\quad 6.9249540 .1107580 .682767$

H $5.8246790 .354943-2.978055$

H $5.8968302 .108702-3.039502$

H $4.6095351 .343544-2.134645$

H $1.379473-0.1667050 .069231$

H $2.1056160 .130946-1.520128$

H 3.5961973 .2550041 .626297

H $2.2473004 .047190 \quad 0.779592$

H $3.7195494 .034823-1.385720$

H $5.8056725 .039011-2.052516$

H $9.580874 \quad 4.979127-0.292546$

H $8.345700 \quad 6.287990 \quad 0.145884$

H $8.9881713 .233806-1.798482$

H $7.4804633 .347269-2.648336$

H 8.0245102 .0132330 .090652

H $3.321023 \quad 1.3092240 .326649$

C $0.151428-0.778648 \quad 4.379861$

O $-1.067764-1.5160644 .160114$

C -1.666841-1.055925 2.934036

C -1.1051560 .3411622 .717218$

C 0.3235840 .1708713 .191164

H -1.317534 -1.744230 2.156079

C $-3.195573-1.1107613 .041715$

H -1.1681630 .6704321 .676270$

H -1.6323971 .0705633 .343909$

C 1.0145131 .4735863 .553647

H $0.889467-0.3051722 .383585$

C $-3.885442-0.724497 \quad 1.718306$

O $-3.566872-2.4461193 .393648$

H -3.537677 $-0.456993 \quad 3.852533$

H $-3.471354 \quad 0.227512 \quad 1.372961$

O $-3.561535-1.6999940 .726287$

C $-5.416215-0.5664371 .805851$

C -6.108021 -0.3679270 .442007$

H -5.6419790 .2803502 .464861$

O $-5.991792-1.7264252 .408734$

H $-6.057260-1.301461-0.133597$

H -7.175184 -0.2054480 .645102$

H 1.0504162 .1438562 .690023

H $0.489787 \quad 1.9960604 .360448$

H 2.0420821 .2885143 .882611

C $1.314562-1.7506144 .620181$

H $-3.103575-2.6674894 .219664$

H $-3.921643-2.5515131 .028583$

H $-6.945106-1.5635212 .509813$

H $1.033326-2.4559885 .411865$

H $2.181629-1.1853964 .982472$

H $-0.012603-0.2077335 .302475$

C $-5.2040413 .188663-0.273612$

$\begin{array}{llll}\text { O } & -5.711082 & 1.999648 & 0.348896\end{array}$

C $-5.5587840 .792682-0.402347$

C $-6.2718700 .899459-1.759499$

C $-5.7918462 .130480-2.534862$

C $-5.8258453 .391941-1.667077$

H -5.5541594 .0098830 .365622$

C $-3.6661123 .221550-0.281401$

H $-4.4962910 .613505-0.585197$

O $\quad-7.6809290 .978928-1.557367$

H $-6.072126-0.012505-2.334977$

H $-6.4283472 .281665-3.415067$

O $\quad-4.4590101 .922631-2.993249$

H $\quad-5.3182414 .220217-2.174965$

O $-7.1858353 .792310-1.475375$

C -3.1037883 .4758901 .102082$

H $-3.2188162 .293607-0.645574$

H -3.335114 $4.045878-0.924576$

H $-8.1015041 .019701-2.432939$

H -4.462188 1.131647 -3.558498

H -7.566438 $3.966680-2.352930$

O -1.8476543 .9986800 .982803$

O -3.6831463 .2340612 .152116$

C -1.1951664 .3383942 .210012$

H -1.2476033 .5132202 .925152$

H -0.1442344 .5440641 .990677$

H -1.650951 5.2389622 .631220

SCF Energy (B3LYP/6-31G**//MMFF) $=-3245.88983369$

0800167

MM̄FF Geometry

C $1.011491-1.7687060 .213511$

C $1.874216-2.5343410 .900008$

C $2.815909-2.0651001 .986323$

O $2.643148-0.6467752 .198664$

C $4.274385-2.3132071 .566657$

C $5.250638-2.2380732 .722732$ 
C $6.060084-1.1924342 .980905$

C $5.314019-3.4710413 .587617$

C 6.1349320 .1241032 .258660

C $7.442595 \quad 0.289138 \quad 1.471501$

C $7.491993-0.5603720 .191915$

C $8.851165-0.422821-0.497020$

C $8.890320-1.171564-1.838055$

O $6.457750-0.091394-0.678317$

O $10.057520-0.737185-2.552334$

C $7.665082-0.754662-2.678377$

C $9.038354-2.685221-1.644070$

O $7.577434-1.529633-3.872201$

C $6.329041-0.823978-1.903044$

C $5.204779-0.159830-2.734271$

O $5.994177-2.192901-1.668918$

C $2.544133-0.1750353 .464816$

O $2.608546-0.8272034 .497531$

C $2.311504 \quad 1.289778 \quad 3.474264$

C 2.3461362 .0552082 .371527

C 2.0991143 .4978672 .306458

C 1.7078114 .2002413 .579992

C $3.8889860 .038300-1.967986$

C $2.8534140 .778820-2.797871$

C $0.6297691 .888865-2.571549$

O $2.9953521 .019296-3.993701$

C $0.8828293 .340950-2.315180$

C $0.1247564 .100929-1.510414$

C $0.3903895 .555684-1.248058$

C $0.623500 \quad 5.853066 \quad 0.217516$

C $-0.335434 \quad 6.4252700 .964883$

C 1.9942035 .5723050 .794866

C $2.206406 \quad 4.1162601 .111339$

N $1.7420051 .123152-2.041069$

H $0.956059-0.7024760 .412199$

H $1.905139-3.6007060 .683812$

H $2.563588-2.6263542 .893748$

H $4.553824-1.5978920 .785964$

H $4.377373-3.3049981 .105975$

H $6.740275-1.2702693 .828427$

H $6.063348-3.3846854 .381664$

H $4.347949-3.6555504 .067363$

H $5.575424-4.3456382 .982824$

H 5.2713390 .2918821 .608862

H 6.0870660 .9138073 .019451

H 7.5298711 .3487371 .197609

H 8.2977240 .0492942 .115596

H $7.304152-1.6083430 .449120$

H $9.657825-0.7703420 .160534$

H $9.0574310 .639759-0.688379$

H $10.835189-0.927857-2.000047$

H $7.8265380 .285264-2.995751$

H $9.963310-2.912677-1.100497$

H $9.123440-3.200384-2.607245$

H $8.211387-3.129631-1.086511$

H $8.429635-1.448041-4.333560$

H $5.5438780 .829577-3.068798$

H $5.004477-0.756668-3.633131$

H $5.032602-2.275360-1.574624$

H $2.107358 \quad 1.7083204 .454582$

H 2.5721081 .5891911 .413796

H 2.5201594 .1465574 .312870

H $0.8116913 .741887 \quad 4.013185$

H 1.4745405 .2578863 .439800

H $3.457485-0.927267-1.686175$

H $4.0673130 .616289-1.053894$

H - $0.2772321 .531570-2.075697$

H $0.533957 \quad 1.709884-3.647056$

H $1.7368743 .787707-2.819053$

H $-0.7353993 .658921-1.013438$

H -0.474387 6.122955 -1.616501

H $1.2477075 .917317-1.829501$

H -0.1682286 .6712802 .008105$

H - 1.3115426 .6565610 .551432

H 2.1776416 .1973561 .675775

H 2.7611125 .8887740 .075935

H $2.495513 \quad 3.5200420 .245867$

H $1.7576360 .953912-1.039791$

C $-1.393970-2.029452-0.559387$
O $-1.658885-0.612918-0.534944$

C -2.600732 -0.3385050 .526702$

C $-3.136700-1.6932590 .969762$

C $-1.930730-2.5854890 .758134$

H -2.0161940 .1266901 .330292$

$\begin{array}{lll}\text { C }-3.670290 & 0.630590 & 0.009697\end{array}$

H $-3.488790-1.6883762 .005438$

H $-3.965850-2.0134690 .326642$

C $-2.266340-4.0654470 .713163$

H -1.225131 -2.404311 1.578192

C $-4.8138110 .879474 \quad 1.007008$

O $-3.0384811 .880470-0.283234$

H $-4.0656760 .252665-0.939147$

H $-5.326631-0.058700 \quad 1.232966$

$\begin{array}{llll}\text { O } & -4.238214 & 1.315805 & 2.246864\end{array}$

C $-5.8369861 .940004 \quad 0.563609$

C $-6.4793681 .735024-0.821145$

H -5.3563912 .9259890 .585810$

O

H -7.099810 $2.617273-1.027329$

H $-5.6898291 .718115-1.580397$

H $-2.712735-4.3872881 .659828$

H -2.978461 -4.291941 -0.087492

H $-1.365336-4.663350 \quad 0.543302$

C $0.086793-2.290652-0.850640$

H $-2.3222561 .700274-0.915470$

H -4.9708201 .4596922 .870346$

H -7.484876 2.6948681 .304953

H $0.357018-1.789088-1.788212$

H $\quad 0.241890-3.364980-1.004388$

H $-1.979018-2.428386-1.398799$

C $-7.264387-1.933856-0.931263$

O $-6.571672-0.680257-0.864791$

C $-7.3838150 .491301-0.958451$

C $-8.1411570 .498895-2.298189$

C $-8.937711-0.793643-2.504303$

C $-8.087615-2.037618-2.225920$

H -6.468081 -2.687697 -0.981907

C -8.088197 $-2.203070 \quad 0.334445$

H $-8.1070170 .497907-0.136817$

O $-7.2293900 .667609-3.383007$

H $-8.820187 \quad 1.359147-2.316068$

H $-9.306350-0.833078-3.536544$

O $-10.068695-0.804742-1.638470$

H -8.716924 -2.934454 -2.186812

O $-7.164260-2.219523-3.303656$

C $-7.192803-2.360844 \quad 1.542426$

H -8.813123 -1.4089950 .533071$

H $-8.659298-3.133840 \quad 0.238708$

H $-6.571360-0.046773-3.331966$

H $-10.611672-0.027400-1.854056$

H -7.683662 -2.341512 -4.116734

O $-7.507609-1.4238562 .481909$

O $-6.320373-3.2141401 .640726$

C $-6.729882-1.466684 \quad 3.682473$

H $-5.665579-1.3565943 .454492$

H $-7.038970-0.6319464 .317110$

H -6.915329-2.403308 4.216496

SCF Energy (B3LYP/6-31G**//MMFF) $=-3245.91693548$

08_00168

MMFF Geometry

C $1.836879-3.1535701 .259743$

C $1.391775-2.2829740 .340699$

C $0.598219-2.572159-0.909186$

O $-0.541822-1.680676-0.853168$

C $0.085136-4.014496-1.057021$

C $-0.592369-4.273968-2.388496$

C $-1.926349-4.305982-2.576909$

C $0.341210-4.550009-3.538835$

C $-2.999287-4.026242-1.561349$

C $-4.001973-2.995404-2.097064$

C $-5.009310-2.562819-1.022793$

C $-6.071089-1.643150-1.628950$

C $-7.036333-1.103389-0.564726$

O $-4.288834-1.8698620 .002141$

O $-7.782632-0.026065-1.150938$

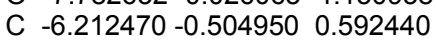


C $-8.061331-2.158031-0.132416$

O $-7.059281-0.1152621 .671839$

C $-5.091410-1.4399861 .107965$

C $-4.184384-0.6573802 .085179$

O $-5.678018-2.5462641 .794007$

C $-0.662869-0.725524-1.813280$

O $0.013746-0.591307-2.819306$

C $-1.7468580 .181823-1.381116$

C $-1.9977281 .309540-2.060419$

C $-2.9626142 .329405-1.652602$

C $-3.6995392 .962742-2.798609$

C $-2.912081-1.3947572 .526739$

C $-2.107545-0.4898833 .440140$

C $-0.7590991 .605072 \quad 3.370017$

O $-2.202018-0.5583754 .663387$

C - 1.7424622 .7271563 .230634

C - 1.5527123 .7983772 .445370

C $-2.594544 \quad 4.8584892 .225015$

C -3.0658164 .9236410 .785600$

C -2.7954445 .9918120 .016549$

C -3.9114863 .7825440 .253226$

C -3.067552 $2.699019-0.359998$

N -1.344311 0.4335542 .741406

H $1.630799-4.2147151 .162900$

H $1.643743-1.2322600 .478848$

H $1.244230-2.323429-1.759934$

H $-0.593026-4.255345-0.230532$

H $0.919525-4.722431-0.967530$

H -2.296568 -4.545936 -3.572977

H $-0.195930-4.760734-4.469833$

H $0.989818-3.687793-3.722148$

H $0.970286-5.418455-3.317769$

H $-3.525723-4.962486-1.341336$

H $-2.578520-3.668060-0.618549$

H -3.454736 -2.115190 -2.457071

H $-4.535679-3.421509-2.955935$

H -5.473839 -3.459800 -0.596493

H $-6.625215-2.158197-2.423494$

H $-5.578591-0.788152-2.113826$

H -8.250109 -0.374326 -1.929606

H $-5.746671 \quad 0.4173630 .216875$

H -8.655433 -2.489311 -0.992549

H $-8.778321-1.7416740 .583761$

H -7.603577 -3.0440490 .312441$

H -7.7365990 .4804001 .308002$

H $-3.8803990 .283153 \quad 1.608672$

H $-4.755300-0.3874312 .983270$

H -5.029601 -2.914527 2.414046

H -2.284762 -0.069696 -0.474462

H -1.443068 $1.534018-2.969103$

H $-4.2908792 .208781-3.330451$

H -2.995634 $3.411342-3.508576$

H $-4.3880143 .752128-2.489842$

H -3.144333 -2.311514 3.077888

H -2.295978 -1.681037 1.667204

H $\quad 0.185001 \quad 1.8163222 .860535$

H -0.5565141 .4074774 .426781$

H -2.6812362 .6245493 .770728$

H -0.6175723 .9028911 .900966$

H -2.164114 5.8196722 .533826

H -3.4653074 .6997372 .873579$

H -3.149395 $6.052302-1.007415$

H $-2.210637 \quad 6.8255170 .390349$

H -4.652623 4.169104 -0.453327

H -4.502822 3.3505031 .070373

H $-2.453396 \quad 2.1702710 .366088$

H $-1.382250 \quad 0.426688 \quad 1.726638$

C $4.162099-2.6897082 .190555$

O $4.446820-1.7042621 .174348$

C $5.261853-2.3192030 .163285$

C $5.940476-3.4900410 .860646$

C $4.803645-4.0014071 .727104$

H $4.578986-2.714328-0.599078$

C $6.205145-1.300473-0.479352$

H $6.313511-4.2385670 .155543$

H $6.775752-3.1594871 .488725$

C $5.256472-4.8956262 .868188$

H $4.116224-4.5709511 .089888$
C $5.508219-0.041090-1.039466$

O $7.153391-0.8679770 .504291$

H $6.786217-1.795698-1.266663$

H $5.2104350 .604538-0.206165$

O $6.493010 \quad 0.698524-1.773592$

C $4.293776-0.307146-1.947602$

C $3.7746760 .920657-2.719127$

H $3.479760-0.724062-1.345998$

O $4.652982-1.299734-2.913782$

H $4.507648 \quad 1.213277-3.482063$

H $2.8872270 .592738-3.277343$

H $5.752244-5.7919552 .481113$

H $5.962662-4.3797283 .527437$

H $4.401635-5.2151373 .472782$

C $2.653109-2.7387562 .455080$

H $7.744696-0.2265410 .074326$

H $6.7342640 .170364-2.553970$

H $3.854506-1.511456-3.426974$

H $2.316647-1.7486812 .787332$

H $2.440863-3.4293233 .279666$

H $4.657259-2.3310113 .101960$

C 2.0068362 .7498330 .025054

O $2.4158131 .738870-0.908254$

C $3.4163532 .135257-1.849748$

C $2.9117683 .312571-2.699997$

C $2.4576344 .480025-1.819610$

C $1.5195094 .018076-0.700401$

H 1.1481622 .3123280 .549360

C 3.0964593 .0449671 .068710

H $4.3168052 .448135-1.312767$

O $1.8304762 .898641-3.533629$

H $3.7198313 .643390-3.362920$

H $1.9443225 .219673-2.446556$

O $3.6099345 .104716-1.262297$

H $1.3683874 .827196 \quad 0.023727$

O $0.2462803 .745044-1.288701$

C 3.3942621 .8483411 .945563

H $4.0345603 .344440 \quad 0.594442$

H 2.7637443 .8536841 .729553

H $1.1346002 .534806-2.959754$

H $3.3063885 .863412-0.735412$

H $-0.3692893 .537162-0.565895$

O 4.4995732 .1177732 .701276

O 2.7393050 .8165391 .975935

C 4.9168221 .0746163 .587878

H 4.0743470 .6979734 .175984

H 5.3797040 .2676623 .013726

H 5.6611651 .4894754 .272823

SCF Energy (B3LYP/6-31G**//MMFF)= -3245.91291109

0800169

MMFF Geometry

C $3.660516-2.751441-2.386054$

C $2.373822-2.532972-2.076146$

C $1.336921-2.161063-3.101448$

O $0.698568-0.919621-2.722005$

C $0.244033-3.233535-3.302872$

C $-0.621212-3.542189-2.094902$

C $-1.737050-2.864103-1.756721$

C $-0.195946-4.739330-1.283571$

C $-2.348733-1.686895-2.468447$

C $-3.819001-1.913585-2.856816$

C $-4.788482-1.873133-1.665213$

C $-6.229052-2.106349-2.126514$

C $-7.231052-1.963908-0.969362$

O $-4.679703-0.582529-1.057422$

O $-8.547792-1.870508-1.534412$

C $-6.960887-0.634878-0.231585$

C -7.235418 -3.200484 -0.062791

O $-7.763475-0.5286030 .942069$

C $-5.472633-0.4173790 .124121$

C -5.2536831 .0219050 .650852$

O $-5.094312-1.343818 \quad 1.146191$

C $1.3866160 .237020-2.916503$

O $2.5335020 .354310-3.316842$

C $0.4765121 .355391-2.580590$

C $0.9444872 .612085-2.579996$

C $0.1640723 .829486-2.342137$ 
C $-1.3210843 .679547-2.147828$ C $-3.776322 \quad 1.4349880 .699609$ C $-3.557472 \quad 2.748710 \quad 1.430297$ C -1.698678 $4.229805 \quad 2.200844$ $\begin{array}{llll}\text { O } & -4.468928 & 3.413546 & 1.913775\end{array}$ C -1.576446 5.3901421 .265963 C $-0.418262 \quad 6.0162851 .006634$ C $-0.314957 \quad 7.251477 \quad 0.160094$ C $0.6771707 .117434-0.970578$ C $1.8837347 .706375-0.905572$ C $0.2272136 .397607-2.220823$ C $0.8080065 .015218-2.342866$ N $-2.210133 \quad 3.0785501 .484907$ H $3.975907-2.686013-3.424652$ H $2.062002-2.578331-1.036759$ H $1.809677-2.008086-4.081479$ H $\quad 0.711397-4.163038-3.655120$ H $-0.404375-2.916076-4.129089$ H $-2.267288-3.181159-0.860531$ H $-0.844431-4.906268-0.416807$ H $-0.231863-5.643287-1.900674$ H $0.824010-4.619506-0.910601$ H $-2.257800-0.805381-1.823730$ H $-1.802043-1.450739-3.385802$ H $-4.095148-1.115329-3.558494$ H -3.920749 -2.865219-3.392958 H $-4.497690-2.641924-0.941579$ H $-6.330503-3.088708-2.604558$ H $-6.488256-1.368993-2.899404$ H -8.704108 -2.671294-2.063807 H $-7.2782600 .178848-0.898777$ H $-7.504329-4.095148-0.637259$ H -7.993250 -3.1113690 .723395$ H $-6.269138-3.3906660 .408778$ H $-8.688977-0.6583280 .672682$ H $-5.770003 \quad 1.738206-0.001444$ H $-5.6952051 .116287 \quad 1.651071$ H $-4.377679-0.9620631 .677033$ H $-0.559754 \quad 1.115807-2.372325$ H $2.0012232 .771270-2.792079$ H -1.788434 $3.284752-3.056586$ H $-1.8271904 .615906-1.904689$ H $-1.5330422 .993969-1.322192$ H -3.181776 $0.669196 \quad 1.206907$ H -3.388142 $1.545301-0.319608$ H $-0.734342 \quad 3.9427342 .632137$ H -2.380442 4.4845193 .018711 H -2.495955 5.7645910 .821932 H $\quad 0.4966845 .6593881 .474169$ H $-0.019878 \quad 8.0779370 .820347$ H -1.291805 $7.539974-0.248079$ H $2.5844397 .647239-1.731985$ H $2.1984828 .256306-0.024937$ H $0.5585686 .968978-3.099072$ H $-0.8642336 .408201-2.288603$ H $1.8882015 .012798-2.497428$ H -1.5365982 .4796381 .016828$ C $5.581208-1.872014-0.971544$

O $4.791183-0.954616-0.191902$

C $5.1845690 .392069-0.522972$

C $6.4464280 .271336-1.373465$

C $6.201458-1.041342-2.094559$

H $4.3811510 .806616-1.143088$

C $5.3350651 .232902 \quad 0.750233$

H $6.5690311 .119645-2.053783$

H $7.3507710 .200939-0.758303$

C $7.452292-1.655895-2.696023$

H $5.475276-0.853675-2.894678$

C $4.122179 \quad 1.1505101 .699667$

$\begin{array}{lllll}\text { O } & 6.476721 & 0.758273 & 1.476666\end{array}$

H $\begin{array}{llll}5.551034 & 2.273693 & 0.482343\end{array}$

H 4.0762350 .1396432 .117286

O 4.3746692 .0206562 .810401

C 2.7718121 .5290911 .065135

C 1.5908641 .5120842 .056361

$\begin{array}{lllll}\text { H } & 2.549629 & 0.856331 & 0.230237\end{array}$

$\begin{array}{lllll}\text { O } & 2.883619 & 2.848591 & 0.525130\end{array}$

H 1.7695702 .2311442 .865191
H $\quad 0.709335 \quad 1.8823391 .517341$

H $7.890440-0.984480-3.441700$

H $8.212136-1.850211-1.931600$

H $7.219898-2.604673-3.189947$

C $4.727938-3.075741-1.381244$

H $6.555214 \quad 1.301573 \quad 2.279545$

H 4.3800612 .9325012 .471507

H 2.0325803 .0713300 .111254

H $4.263439-3.503570-0.484488$

H $5.369686-3.856761-1.805814$

H $6.369584-2.233654-0.297628$

C $0.726155-2.1068521 .890479$

O $0.930831-0.730223 \quad 1.541693$

C 1.2934630 .1204252 .634829

C 0.1644310 .1479563 .675161

C $-0.194228-1.2666024 .144060$

C $-0.363908-2.2397702 .970603$

H $0.347908-2.5651490 .968718$

C $2.067796-2.7745252 .227845$

H $2.197402-0.2677753 .114085$

$\begin{array}{lllll}\text { O } & -0.996418 & 0.767652 & 3.131457\end{array}$

H 0.4827560 .7557054 .529869

H -1.129381 -1.233322 4.716973

O $0.825556-1.7569325 .008908$

H - $0.407076-3.2684853 .344478$

O $-1.630203-1.9625542 .360693$

C $2.053250-4.2715232 .014457$

H $2.838104-2.3529651 .569498$

H $2.379226-2.5798203 .256968$

H $-1.244586 \quad 0.2752532 .330631$

H $0.886350-1.1494965 .765801$

H -1.775787 -2.631319 1.669813

O $3.340864-4.7250631 .966424$

O $1.048167-4.9621311 .920424$

C $3.489846-6.1322491 .754036$

H $4.558633-6.3580811 .711846$

H $3.045161-6.6891482 .584200$

H $3.032348-6.4228900 .803397$

SCF Energy (B3LYP/6-31G**//MMFF) $=-3245.92085592$

0800170

MMFF Geometry

C $0.445676 \quad 4.686922 \quad 0.638748$

C $1.6328814 .068401 \quad 0.725921$

C $2.5711343 .910048-0.443206$

$\begin{array}{llll}\text { O } & 2.778247 & 2.494258 & -0.669868\end{array}$

C $3.9298684 .549765-0.120396$

C $4.8406434 .628419-1.329133$

C $5.8781523 .801486-1.562444$

C $4.5524655 .763620-2.278411$

C $6.3186902 .625283-0.736014$

C $6.6384751 .412367-1.621639$

C $7.1820330 .239657-0.794419$

C $7.696358-0.875059-1.706193$

C $8.188718-2.084866-0.898716$

$\begin{array}{lllll}\text { O } & 6.123262 & -0.258354 & 0.031179\end{array}$

O $8.355439-3.183799-1.807285$

C $7.091803-2.490113 \quad 0.105814$

C $9.561923-1.833006-0.265014$

O $7.555986-3.5134630 .983746$

C $6.524395-1.3052870 .925420$

C $5.282536-1.7794821 .713770$

O $7.514009-0.8532181 .851732$

C $1.8161231 .837811-1.374903$

O $0.8087852 .322061-1.867189$

C $2.1788240 .403028-1.419873$

C $1.236614-0.483793-1.774384$

C $1.380832-1.939275-1.844718$

C $2.766431-2.510273-1.715516$

C $4.446954-0.6538662 .336379$

C $3.260729-1.2447203 .074038$

C $1.087500-2.4038092 .667715$

O $3.244250-1.3387054 .299128$

C $0.891560-3.5950831 .782739$

C $-0.250324-3.8656031 .132913$

C $-0.445773-5.0687090 .256251$

C $-0.901675-4.710810-1.140897$

C $-2.169442-4.927749-1.531164$ 
C $0.132500-4.177988-2.112145$

C $0.269034-2.680970-2.033805$

N $2.268371-1.692128 \quad 2.216914$

H $0.136448 \quad 5.120295-0.309296$

H 1.9336093 .6178771 .668805

H $2.1512684 .366333-1.348515$

H 4.4181804 .0000520 .692906

H 3.7845395 .5689270 .262868

H $6.4906813 .991512-2.443312$

H $5.2698295 .807468-3.104939$

H $3.5551215 .657596-2.715833$

H $4.6010586 .721712-1.750469$

H $7.2198102 .917677-0.183729$

H 5.5640362 .3389510 .001722

H $5.7260371 .104796-2.148918$

H $7.3791871 .698876-2.378822$

H $7.9932460 .613336-0.158330$

H $8.492362-0.505806-2.364720$

H $6.885925-1.206199-2.370862$

H $8.994565-2.914458-2.489139$

H $6.269135-2.931268-0.474944$

H $10.304796-1.606393-1.039170$

H $9.929462-2.7278410 .249307$

H $9.561485-1.0031770 .444808$

H $7.887611-4.2413890 .430483$

H $4.631545-2.3502041 .041682$

H $5.590746-2.4683222 .511197$

H $7.072210-0.4223132 .600476$

H $3.1845960 .117980-1.134258$

H $0.240806-0.110180-2.012879$

H $3.443867-2.045987-2.441282$

H $2.813740-3.585786-1.897344$

H $3.160950-2.335596-0.711824$

H $5.033584-0.0659453 .049835$

H 4.0774720 .0394951 .572213

H $0.246338-1.7061782 .621270$

H $1.216306-2.7259963 .705548$

H $1.733878-4.2757301 .679068$

H $-1.102425-3.2027921 .256625$

H -1.189359 -5.7143190 .741692$

H $0.470297 \quad-5.6693140 .191788$

H -2.496522 -4.696680 -2.539554

H $-2.910357-5.331953-0.849335$

H $-0.164117-4.426981-3.139581$

H $1.076020-4.704615-1.946912$

H $-0.681059-2.156727-2.148735$

H $2.325366-1.4484561 .232524$

C -1.673606 3.7857871 .574058

$\begin{array}{lllll}\text { O } & -2.489845 & 4.183947 & 0.455262\end{array}$

C $-3.859178 \quad 3.799192 \quad 0.722782$

C -3.8375543 .0554242 .052216$

C -2.643444 $3.684158 \quad 2.745098$

H $-4.417106 \quad 4.738400 \quad 0.825018$

C $-4.3801763 .001758-0.480787$

H -4.7703203 .1730272 .612028$

H -3.666823 1.9829201 .900176

C -2.130092 $2.870317 \quad 3.919692$

H -2.922179 4.6883223 .091513

C $-5.7185822 .278954-0.234623$

O $-4.519408 \quad 3.915717-1.572139$

H -3.630431 2.264282 -0.791854

H $-5.552603 \quad 1.5429740 .556359$

O $\begin{array}{lllll}-6.686674 & 3.202232 & 0.262049\end{array}$

C $-6.2822791 .559381-1.479217$

C $-7.4923900 .655008-1.172180$

H $-5.4935850 .962422-1.951251$

O $-6.723872 \quad 2.515153-2.446302$

H -8.269136 $1.232964-0.656387$

H -7.929334 $0.353883-2.133889$

H $-1.266518 \quad 3.3554064 .385139$

H -2.908021 2.7651544 .682958

H $-1.825481 \quad 1.8652243 .610875$

C -0.5331784 .7815971 .776154$

H $-3.6536334 .336893-1.710668$

H $-6.8431733 .861249-0.436056$

H -5.953136 $3.047244-2.706358$

H $-0.023968 \quad 4.5894922 .727247$

H -0.9184535 .8074781 .811169$
H -1.2644302 .7957011 .335707$

C $-5.681609-2.537569-0.502193$

O $-6.207360-1.365135-1.136825$

C $-7.132693-0.599291-0.359190$

C -8.362610 -1.452725 -0.019359

C $-7.951706-2.751477 \quad 0.686237$

C $-6.816674-3.473292-0.048928$

H $-5.116844-3.052411-1.290041$

C $-4.692368-2.179530 \quad 0.617647$

H $-6.655734-0.2868100 .573288$

O $-9.096957-1.763740-1.201389$

H $-9.029336-0.872170 \quad 0.628613$

H -8.818756 -3.420432 0.750518

O $-7.525162-2.4609572 .014286$

H $-6.410829-4.2752170 .579004$

O $-7.379176-4.093662-1.209683$

C $-3.502139-1.4019240 .093993$

H $-5.168902-1.5720671 .391733$

H $-4.307667-3.0953881 .080471$

H -8.496256 - $2.223630-1.812625$

H -8.269776 -2.0390142 .475889$

H $-6.676805-4.615710-1.633596$

O $-2.818375-0.8695971 .149565$

O $-3.195950-1.289983-1.085065$

C $-1.647014-0.124403 \quad 0.805536$

H $-0.899693 \quad-0.787300 \quad 0.364219$

H -1.8953740 .6878740 .116549$

H -1.234241 0.3035551 .722186

SCF Energy (B3LYP/6-31G**//MMFF)= -3245.92243789

0800171

MMFF Geometry

C $0.2284862 .875270 \quad 0.812111$

C $0.618391 \quad 1.592643 \quad 0.762473$

C $0.879861 \quad 0.737514 \quad 1.974880$

$\begin{array}{llll}\text { O } & 2.236060 & 0.243002 & 1.892380\end{array}$

C $-0.030549-0.496661 \quad 1.941396$

C $-0.088635-1.248663 \quad 3.253801$

C $0.587692-2.3834253 .518086$

C $-1.026477-0.672488 \quad 4.283425$

C $1.614553-3.0750382 .665186$

C $1.082404-4.3312691 .961020$

C $0.394947-4.0294330 .619418$

C $-0.139052-5.314802-0.016576$

C $-0.712095-5.067851-1.420667$

O $1.374155-3.430356-0.237670$

O $-0.885155-6.343456-2.056820$

C $0.324822-4.285183-2.252440$

C $-2.098906-4.417497-1.369098$

O $-0.215140-3.909552-3.517788$

C $0.888500-3.041344-1.526968$

C $2.078877-2.462568-2.330631$

O $-0.137956-2.051981-1.432009$

C 3.2515101 .0525302 .283976

O 3.1692132 .1789442 .742914

$\begin{array}{llll}\text { C } 4.503341 & 0.314222 & 2.007488\end{array}$

C $5.673860 \quad 0.966141 \quad 1.980015$

C $6.9738180 .376483 \quad 1.651749$

C $7.016375-1.1022181 .362638$

C $2.882816-1.377798-1.595826$

C $3.984112-0.803191-2.473906$

C $5.6903201 .015391-2.520241$

O $4.248899-1.243015-3.590105$

C $7.0270370 .498960-2.087579$

C $8.0454041 .299871-1.736141$

C $9.4072680 .801572-1.339989$

C $9.9155601 .430323-0.059947$

C $10.7919642 .448645-0.091627$

C $9.471247 \quad 0.826041 \quad 1.253939$

C $8.051271 \quad 1.1867791 .595837$

N $4.6360860 .261018-1.864909$

H $\quad 0.076646 \quad 3.353606 \quad 1.776100$

H $\quad 0.789522 \quad 1.130273-0.206737$

H $0.745063 \quad 1.3019972 .905556$

H $0.304759-1.1667221 .143987$

H - $-1.050912-0.2079231 .670435$

H $0.427456-2.8589824 .485246$

H $-1.022570-1.2455505 .216841$ 
H $-0.742818 \quad 0.355343 \quad 4.530023$ H -2.052793 -0.6677363 .905781$ H $2.078706-2.3897151 .950597$ H $2.426609-3.3790123 .338972$ H $1.944019-4.9843201 .767614$ H $0.402191-4.8818532 .622183$ H $-0.426749-3.3255610 .788607$ H $-0.892049-5.7838160 .629079$ H $0.677728-6.045414-0.102110$ H -1.482257 -6.875392 -1.503228 H $1.158450-4.970045-2.462840$ H $-2.802080-5.059371-0.825171$ H -2.518719 -4.300183 -2.374252 H -2.095981 -3.440944 -0.880874 H $-0.547431-4.719007-3.942654$ H $2.775786-3.274742-2.577075$ H $1.712735-2.049660-3.279520$ H $0.266905-1.171732-1.392430$ H $4.418929-0.7445621 .788422$ H 5.6793832 .0350992 .188952 H $6.706294-1.6731462 .244937$ H $8.006040-1.4666661 .081369$ H $6.349472-1.3536770 .530431$ H $2.230671-0.553655-1.292325$ H $3.351192-1.795669-0.697492$ H $5.5492152 .067418-2.252886$ H $5.589890 \quad 0.912531-3.605461$ H $7.174695-0.578466-2.090154$ H $7.9050952 .378079-1.769144$ H $10.0960541 .030830-2.163772$ H $9.419655-0.290647-1.237354$ H 11.1821372 .8878250 .820455 H $11.1347652 .869571-1.031059$ H 10.1045951 .1921072 .073162 H $9.647485-0.252546 \quad 1.227918$ H $7.9142972 .246807 \quad 1.815108$ H $4.3810740 .524904-0.918034$ C $-1.2558974 .506119-0.447657$ O $-2.3704973 .614524-0.672292$ C $-3.1126384 .082198-1.811871$ C $-2.7348025 .548986-1.971894$ C -1.269941 5.517271-1.600670 H -2.739643 3.521097 -2.677199 C -4.620324 3.850019-1.660001 H -2.914192 $5.917457-2.986341$ H $-3.2837326 .191277-1.273629$ C $-0.7068446 .879276-1.230057$ H $-0.7040635 .122238-2.455452$ C -5.066439 2.413657 -1.328247 O $-5.1294584 .693000-0.615988$ H $-5.1213704 .184753-2.576449$ H $-4.8995542 .214053-0.265017$ O $-6.4875812 .360389-1.523640$ C $-4.4188831 .296343-2.161520$ C -5.100692 -0.082987 -2.040411 H $-3.3641361 .206107-1.876493$ O $-4.4500451 .668512-3.542510$ H -6.048028 $-0.072904-2.595241$ H -4.459919 -0.800989-2.569700 H $-0.7907237 .572372-2.073625$ H $-1.2422987 .317051-0.380878$ H $0.350776 \quad 6.803818-0.958623$ C $0.0618393 .724780-0.415434$ H -4.6408094 .4782040 .197173$ H $-6.8825233 .053995-0.967693$ H $-3.9833040 .977371-4.042745$ H $0.9016674 .429769-0.436164$ H $0.1356483 .100483-1.315051$ H -1.436872 5.0041640 .512983 C $-4.179831-0.971270 \quad 1.471919$ O $-4.103767-0.6133820 .084761$ C $-5.353603-0.568624-0.605128$ C $-6.028793-1.948394-0.575512$ C $-6.196562-2.4416940 .865903$ C $-4.894933-2.3206531 .664545$ H $-3.138074-1.1094491 .779967$ C -4.7743890 .1600572 .327694$ H $-6.0143020 .150594-0.112582$
O $-5.244637-2.881754-1.314424$

H -7.006342 -1.874698 -1.066936

H $-6.520211-3.4895340 .860240$

O $-7.207815-1.6796231 .519540$

H $-5.083137-2.4829362 .732308$

O $-3.991850-3.345946 \quad 1.242848$

C -3.8951731 .3936032 .344044$

H $-5.7598980 .471822 \quad 1.972723$

H $-4.866824-0.1882303 .363187$

H $-5.706488-3.736961-1.292343$

H $-8.030775-1.7879511 .013210$

H -4.420696 -4.202396 1.409457

O $-4.376021 \quad 2.272637 \quad 3.272358$

O -2.9226351 .5784161 .626574$

C -3.6440493 .4972153 .388680$

H -2.6149153 .2945343 .700603$

H -3.6597294 .0405262 .439027$

$\mathrm{H}-4.1280634 .1115814 .152324$

SCF Energy $\left(B 3 L Y P / 6-31 G^{* *} / / M M F F\right)=-3245.89619003$

\section{8_00172}

MM̄FF Geometry

C $0.445642-3.085179 \quad 1.381626$

C $-0.200894-1.9229621 .205177$

C $-0.800571-1.1330192 .339029$

O $-2.233426-1.2604272 .193021$

C -0.4310890 .3558792 .233258$

C $-0.598127 \quad 1.1008403 .542292$

C -1.666406 1.8511723 .877472

C 0.5708550 .9891564 .487578

C -2.9329852 .0695473 .096186$

C -3.1106813 .5271292 .646461$

C -2.1390983 .9349071 .530094$

C -2.3159055 .4074561 .155094$

C $-1.4181845 .805618-0.028158$

$\begin{array}{llll}\text { O } & -2.414685 & 3.113316 & 0.390694\end{array}$

O $-1.8723197 .075818-0.519673$

C $-1.6098194 .784616-1.170828$

C $0.0406746 .004190 \quad 0.402845$

O $-0.6821985 .022599-2.227542$

C $-1.5154463 .313872-0.705429$

C -1.927225 $2.364451-1.854646$

O $-0.1689993 .031390-0.332536$

C $-2.980880-1.5449853 .287796$

O $-2.579388-1.6804124 .435051$

C $-4.408968-1.7235362 .930476$

C $-4.912491-1.4232801 .722854$

C $-6.296187-1.6188201 .283778$

C -7.255964 -2.261174 2.250030

C $-2.0814740 .896376-1.433345$

C $-2.5384020 .017785-2.584755$

C $-3.464913-2.252710-3.039689$

O $-2.5510140 .393952-3.753759$

C $-4.939715-2.029361-3.165327$

C $-5.871091-2.894423-2.736261$

C $-7.348640-2.645862-2.852350$

C -8.050393 -2.638937-1.510712

C $-8.797159-3.685781-1.120512$

C $-7.959950-1.389240-0.660132$

C $-6.629014-1.2517680 .028422$

N $-2.913441-1.246713-2.149833$

H $0.551173-3.4851032 .387507$

H $-0.339665-1.5373800 .198274$

H $-0.466911-1.5515343 .296362$

H -1.0331870 .8322491 .453066$

H $\quad 0.6133870 .468592 \quad 1.912216$

H -1.652920 2.3537984 .844122

H 0.4137691 .5522465 .413590

H $0.743061-0.0557904 .763520$

H $1.478781 \quad 1.3784584 .014971$

H -3.0108901 .3981172 .236747$

H -3.7707331 .8072113 .754537$

H -4.140275 3.6358092 .281373

H -2.9986404 .1991703 .506367$

H -1.113792 3.7547751 .871619

H -2.1229846 .0556482 .018829$

$\begin{array}{llll}H & -3.362231 & 5.588714 & 0.871599\end{array}$

H $-1.827041 \quad 7.7140640 .212818$ 
H -2.610949 $4.949067-1.593576$ H 0.1114336 .7945251 .159888 H $\quad 0.659167 \quad 6.339009-0.437216$ H $0.4910345 .103557 \quad 0.825078$ H $-0.7849485 .950807-2.499246$ H $-2.8907702 .693609-2.265549$ H -1.189732 $2.423478-2.665279$ H $-0.0542922 .070536-0.278227$ H -5.028866 -2.124626 3.725782 H $-4.250515-1.0039530 .967207$ H $-6.887973-3.2448232 .562751$ H $-8.251763-2.422077 \quad 1.831487$ H -7.382430 -1.633704 3.139202 H -1.128795 $0.494206-1.076277$ H -2.816835 $0.813485-0.625032$ H $-3.227090-3.232741-2.614356$ H -2.990379 -2.171754 -4.022863 H -5.256556 -1.099922 -3.633369 H -5.560151 -3.829640 -2.276542 H -7.767588 -3.430791 -3.495417 H -7.555818 -1.700649-3.369551 H -9.329063 -3.680114 -0.174968 H -8.890346 -4.574176 - 1.736206 H $-8.780764-1.3513000 .064853$ H -8.118772 -0.508519-1.295782 H $-5.862652-0.791820-0.595467$ H -2.935606 -1.439103 -1.152462 C $2.441512-4.3409120 .525641$ $\begin{array}{lllll}\text { O } & 3.319443 & -3.207351 & 0.388747\end{array}$ C $4.602476-3.677085-0.082186$ C $4.476878-5.189477-0.254061$ C $2.985496-5.364952-0.465624$ H $4.762521-3.224706-1.066110$ C $5.704511-3.2495230 .898163$ H $5.074321-5.557716-1.094067$ H $4.805547 \quad-5.7282740 .642592$ C $2.508328-6.786825-0.226793$ H $2.736898-5.063774-1.492042$ C $5.822627-1.7209891 .065532$ O $5.417471-3.8139412 .185119$ H $6.668158-3.6701270 .589885$ H $4.865091-1.3110201 .404255$ O $6.767861-1.4541462 .110369$ C $6.302556-0.993546-0.206333$ C $6.3591350 .537201-0.033609$ H $5.656835-1.240358-1.053993$

O $7.612438-1.467241-0.528614$ H $\quad \begin{array}{llll}6.780467 & 0.789318 & 0.946478\end{array}$ H $7.0513180 .949654-0.779804$ H $3.011042-7.480287-0.909005$ H $2.716238-7.1130300 .797818$ H $1.430610-6.872858-0.395305$ C $0.996201-3.9227390 .260692$ H $4.555037-3.4671082 .471580$ H $6.470206-1.9362172 .900790$ H $7.875411-1.054006-1.368641$ H $\quad 0.361829-4.8116910 .171834$ H $0.934455-3.373481-0.684226$ H $2.548080-4.7140661 .553416$ C $3.4000791 .651424-2.003660$ O $4.6750731 .131429-1.610442$ C $4.9750541 .182462-0.210428$ C 4.9438432 .6328880 .288014 C $3.5984003 .290158-0.034770$ C $3.2105243 .095522-1.504541$ H $3.4397391 .684057-3.100658$ C $2.2548440 .705386-1.612834$ H 4.2319820 .5973790 .339840 O $6.0028513 .386869-0.296851$ H 5.1079472 .6379641 .371748 H 3.6534274 .3632580 .185227 O $2.5841282 .724503 \quad 0.790146$ H $2.1753423 .409354-1.677195$ O $4.0376853 .936800-2.315250$ C $2.279527-0.568899-2.432606$ H $2.2736850 .417348-0.558950$ H $\quad 1.3055111 .208767-1.824067$ H $5.9151383 .323658-1.263322$
H 2.8278082 .8954901 .715860

H $3.8747514 .856228-2.043476$

O $1.005501-1.040135-2.572808$

O $3.289756-1.093353-2.881312$

C $0.865413-2.221286-3.368973$

H $-0.154729-2.594429-3.246739$

H $1.028215-1.976414-4.422402$

H $1.562495-2.999481-3.043423$

SCF Energy (B3LYP/6-31G**//MMFF) $=-3245.91512188$

0800173

MMFF Geometry

C $2.172548 \quad 5.245997 \quad 0.205694$

C 3.0238874 .2118780 .281258

C $3.6902643 .595834-0.921729$

O $3.4117392 .175130-0.909502$

C $5.2122463 .786665-0.839524$

C $5.9185883 .398794-2.123262$

C $6.5994462 .251321-2.309505$

C $5.8661084 .430050-3.221997$

C $6.7673391 .113727-1.341206$

C $6.507890-0.238256-2.021344$

C $6.775625-1.408832-1.065799$

C $6.698061-2.744597-1.805976$

C $6.899953-3.932216-0.853035$

O $5.792687-1.373875-0.025702$

O $6.506832-5.126247-1.546564$

C $5.947862-3.7723750 .349224$

C $8.372954-4.117288-0.469995$

O $6.201645-4.7682571 .337659$

C $5.984324-2.3647430 .993029$

C $4.833354-2.2402582 .016462$

O $7.228105-2.1856021 .672597$

C $2.2106391 .775390-1.409117$

O $1.3554512 .480418-1.920680$

C $2.0951350 .316561-1.192086$

C $0.883017-0.255150-1.255913$

C $0.576449-1.671152-1.040052$

C $1.737081-2.620167-0.909564$

C $4.584211-0.8178282 .532152$

C $3.358928-0.8121363 .424677$

C $0.867093-0.9327073 .316377$

O $3.453054-0.8812184 .647917$

C $0.266064-2.2121622 .821235$

C $-0.982235-2.3080472 .338831$

C $-1.589336-3.5888981 .841382$

C $-2.211335-3.456546 \quad 0.467411$

C $-3.546209-3.4537520 .311939$

C -1.289303 -3.417295 -0.734064

C $-0.718520-2.046096-0.975528$

N $2.174456-0.7767072 .705427$

H $1.9699835 .694454-0.763096$

H 3.2324873 .7595091 .247956

H $3.3077174 .035985-1.851123$

H 5.6153643 .2201250 .007932

H $5.4491964 .837817-0.625896$

H $7.1042402 .109678-3.264560$

H $6.4352834 .125300-4.106785$

H $4.8337394 .602956-3.540385$

H $6.2862915 .378856-2.872372$

H $7.7972371 .138489-0.965109$

H $6.1052551 .211369-0.476674$

H $\quad 5.465817-0.269852-2.365539$

H $7.152076-0.333584-2.904523$

H $7.772043-1.274957-0.628395$

H $7.428758-2.781845-2.623487$

H $5.711100-2.842442-2.279869$

H $7.053316-5.201140-2.347502$

H $4.928358-3.955580-0.019191$

H $8.983484-4.283004-1.365719$

H $8.509787-5.0075120 .153974$

H $8.789957-3.2579910 .059121$

H $6.153561-5.6335330 .896145$

H $3.906864-2.6012771 .556200$

H $5.029313-2.8925012 .877730$

H $7.115555-1.5143182 .363972$

H $2.997788-0.235463-0.957336$

H $0.0216910 .378868-1.463638$ 
H $2.308130-2.399267-0.004765$

H $2.405167-2.534620-1.774179$

H $\quad 1.442777-3.669609-0.852898$

H $5.433855-0.4465383 .114248$

H $4.425449-0.1131101 .708038$

H $0.270224-0.0602583 .034956$

H $0.960632-0.9615514 .406089$

H $0.887130-3.1039792 .866310$

H -1.612038 -1.422809 2.325263

H -2.350550 -3.8981202 .569612$

H $-0.849297-4.398707 \quad 1.821389$

H -3.999454 -3.387378 -0.671510

H $-4.216493-3.5083001 .163262$

H -1.842146 -3.703850 -1.638538

H $-0.523273-4.186713-0.609939$

H -1.482566 -1.278933 -1.109599

H $2.213529-0.6983611 .693607$

C -0.0491585 .5379831 .404432$

O -0.2765134 .1135251 .398336$

C -1.2869663 .8151630 .411450$

C -2.0070765 .1302130 .161483$

C $-0.856438 \quad 6.1150790 .239881$

H $-0.7449283 .501096-0.488965$

C -2.166239 2.6699790 .921661

H -2.522724 5.154520 -0.803065

H -2.7402365 .3302120 .952613$

C - -1.2957617 .5534270 .451790$

H $-0.2927716 .055438-0.698520$

C $-3.3527802 .346374-0.007651$

O

H -2.5383762 .9013321 .926799$

H -3.984043 $3.238137-0.094159$

O $-2.8635162 .059097-1.318536$

C $-4.240473 \quad 1.1815640 .479803$

C $-5.4550530 .970972-0.435398$

H $-4.578709 \quad 1.3987041 .498689$

O $-3.504967-0.0369690 .513431$

H $-6.0453031 .894014-0.497724$

H $-5.1120670 .773479-1.460443$

H $-1.9165837 .892928-0.383748$

H -1.8810257 .6636611 .370908$

H -0.4288888 .2179640 .521452$

C 1.4563235 .8261021 .394187

$\mathrm{H}-0.5806131 .7596051 .615610$

H -2.320476 $1.254499-1.254750$

H $-2.751667 \quad 0.090723 \quad 1.113308$

H 1.8927175 .4160292 .314158

H 1.6302956 .9079651 .417020

H -0.4582365 .9033162 .355406$

C $-8.300975-1.378533-0.904119$

O $-7.386662-0.281776-1.030928$

C $-6.360869-0.208579-0.031556$

C $-6.988918-0.0663311 .362388$

C $-8.008180-1.1787231 .635694$

C $-8.987612-1.3604340 .471112$

H $-9.068728-1.188888-1.665824$

C -7.637658 -2.719344 -1.237894

H $-5.765065-1.125660-0.075275$

O $\quad-7.626484 \quad 1.2031451 .488829$

H -6.200536 -0.1065982 .121675$

H $-8.571320-0.9395012 .545978$

O $-7.325855-2.4095961 .858685$

H $-9.572317-2.279143 \quad 0.598340$

O $\quad-9.913347-0.269470 \quad 0.470189$

C -7.173283 -2.740983 -2.674553

H $-6.784717-2.953922-0.594912$

H -8.348651 -3.544740 -1.113306

H $-8.286740 \quad 1.2761470 .778418$

H -6.730205 -2.283164 2.616698

H -10.389704 -0.289009 1.317808

O $-5.861759-2.374072-2.744964$

O $-7.897059-3.023935-3.620337$

C $-5.302413-2.327131-4.060629$

H $-5.830371-1.587280-4.669854$

H $-4.254762-2.026926-3.973406$

H $-5.347084-3.315848-4.526997$

SCF Energy (B3LYP/6-31G**//MMFF) $=-3245.92661681$

\section{4}

MMFF Geometry

C $-0.958330-3.1139001 .932103$

C $-0.243790-2.5031172 .889882$

C $-0.370996-1.0643053 .336516$

O $-1.433099-0.3790092 .637931$

C $0.939541-0.3227463 .029878$

C 0.9863161 .0717063 .619225

C 0.8021812 .2050672 .914382

C 1.3243421 .1385425 .085893

C 0.4616762 .3280901 .454505

C -0.5776133 .4333111 .218247$

C $-0.8760233 .619644-0.275688$

C $-1.7216514 .873640-0.503232$

C $-2.0888045 .048821-1.983345$

O $-1.5832792 .465231-0.742011$

O $-3.117546 \quad 6.046561-2.067920$

C $-2.6935083 .729671-2.502157$

C $-0.9083445 .570089-2.811107$

O $-2.915740 \quad 3.789901-3.909649$

C $-1.8561662 .475285-2.150484$

C $-2.6713561 .209220-2.498501$

O $-0.6516152 .478284-2.918006$

C $-2.670871-0.3967673 .200173$

O $-3.001688-0.9439094 .239679$

C -3.5744240 .3541142 .300572$

C -4.9011310 .2349452 .456126$

C $-5.9294390 .867757 \quad 1.627867$

C -5.4820011 .8993970 .627446$

C $-2.060599-0.107725-2.007703$

C $-3.045997-1.233076-2.251874$

C $-5.082308-2.295796-1.272553$

O $-3.031453-1.886732-3.292293$

C $-6.334479-1.492909-1.444737$

C $-7.429494-1.649913-0.685140$

C $-8.683472-0.839116-0.855895$

C $-9.186355-0.2442170 .442800$

C $-10.310786-0.7001341 .020842$

C $-8.430047 \quad 0.929247 \quad 1.032735$

C -7.2134900 .4903521 .800866$

N $-3.955316-1.383084-1.216543$

H -1.734134 -2.567146 1.405116

H $0.518802-3.0804293 .410331$

H $-0.560675-1.0520264 .416822$

H $1.095748-0.2843371 .945242$

H $1.792197-0.888863 \quad 3.427487$

H 0.9161013 .1530243 .438761

H 1.3929732 .1688995 .450744

H 0.5594000 .6300325 .680683

H 2.2898980 .6578615 .274765

H 1.3800282 .5757200 .909764

H $0.081917 \quad 1.3877331 .045004$

H $-1.5013453 .176521 \quad 1.752985$

H -0.2039564 .3768331 .635898$

H $0.076973 \quad 3.702308-0.811467$

H -1.205093 $5.766708-0.129809$

H -2.650512 4.7981390 .079655

H -2.771494 6.867551-1.677576

H $-3.6841453 .622941-2.037478$

H $-0.5647926 .536113-2.421852$

H $-1.2052985 .754865-3.849383$

H -0.052595 $4.891904-2.812860$

H $-3.4565944 .579333-4.084064$

H -3.674072 $1.301301-2.065469$

H $-2.8051831 .141005-3.586178$

H $-0.3195091 .569218-2.985769$

H -3.1251630 .9533921 .516797$

H $-5.274718-0.415268 \quad 3.246484$

H $-4.8915891 .430700-0.163446$

H -4.8695722 .6684791 .111863$

H -6.3066082 .4287730 .146205$

H -1.132555 -0.345636 -2.537946

H -1.818353 -0.066988 -0.939689

H $-5.083316-2.868087-0.339594$

H $-4.964388-2.986673-2.112695$

H $-6.342048-0.748390-2.237796$

H -7.433394 -2.413168 0.089791

H $-9.447001-1.498784-1.288362$ 
H $-8.537995-0.030801-1.583583$ H $-10.691293-0.2634601 .938196$ H -10.873323 -1.5234230 .593089$ H -9.0756231 .4809361 .728620$ H $-8.200377 \quad 1.6320660 .227648$ H -7.432997 -0.2463052 .575520$ H $-3.886989-0.773352-0.407472$ C $-0.250694-4.7458660 .106639$ O $1.046235-4.138806-0.057939$ C $1.121574-3.576165-1.383395$ C $-0.114195-4.070777-2.132110$ C $-1.125936-4.181284-1.009612$ H $1.047746-2.488303-1.266616$ C $2.448472-3.942487-2.055835$ H - $0.421371-3.379595-2.922933$ H $\quad 0.051368-5.052284-2.591335$ C $-2.327801-5.044168-1.349583$ H $-1.467733-3.170496-0.763632$ C $3.708444-3.530801-1.268351$ O $2.492101-5.366062-2.224529$ H $2.474007-3.513213-3.064707$ H $3.796606-4.143511-0.363295$ O $4.841953-3.870407-2.080170$ C $3.785323-2.038612-0.888076$ C $5.147460-1.673390-0.275370$ H $2.982006-1.815969-0.178511$ O $3.566213-1.256106-2.060257$ H $5.363409-2.3357020 .572825$ H $5.942921-1.867401-1.008146$ H -2.858815 -4.641805 -2.217949 H $-2.030831-6.071349-1.586124$ H -3.030097 -5.080208-0.510887 C $-0.758389-4.5520591 .538476$ H $3.336385-5.580817-2.656983$ H $4.822317-3.296243-2.865088$ H $3.402911-0.340372-1.778560$ H $-0.042552-5.0224382 .224644$ H -1.710855 -5.0793451 .665801$ H $-0.100794-5.822336-0.052184$ C $7.0460381 .238741 \quad 1.051278$ O $6.645264-0.0781730 .650869$ C $5.302227-0.2035280 .160333$ C 4.3025580 .2346551 .239630 C 4.6186621 .6378341 .766486 C 6.0997321 .7961832 .125645 H $8.032377 \quad 1.0998421 .513044$ C $7.2220942 .173584-0.150360$ H $5.1952280 .429901-0.725809$ O $4.313622-0.6925712 .322961$ H 3.2917080 .2301030 .818435 H 4.0037791 .8314532 .653347 O 4.2574082 .5887810 .769435 H 6.3427402 .8481982 .315030 O $\quad 6.3614821 .081767 \quad 3.337255$ C $8.3701411 .712640-1.016165$ H $6.3285332 .256020-0.775606$ H $7.458046 \quad 3.1914840 .182413$ H $5.223117-0.7413772 .664116$ H 4.4262873 .4742261 .133900 H 5.7969281 .4640604 .030716 O $7.913156 \quad 0.871584-1.987347$ O $9.5351962 .040814-0.832824$ C $8.9132260 .336662-2.859483$ H $9.637836-0.252035-2.289017$ H $8.419158-0.319418-3.581120$ H $9.412727 \quad 1.144102-3.403169$ SCF Energy (B3LYP/6-31G**//MMFF) $=-3245.91681374$

\section{5}

MM̄FF Geometry

C $0.508316 \quad 5.1444330 .332878$

C 1.5937334 .3875510 .552773

C $2.5919634 .060285-0.528565$

O $2.6924742 .621864-0.661029$

C $3.9788054 .600394-0.145946$

C $4.9417154 .616482-1.315982$

C $5.9312643 .723621-1.510227$

C $4.7720205 .771515-2.270847$
C $6.2638812 .519404-0.673542$

C $6.5780491 .303414-1.557308$

C $7.0764920 .112170-0.727972$

C $7.587450-1.003468-1.641181$

C $8.038975-2.231297-0.838005$

O $5.991103-0.3714790 .070817$

O $8.202911-3.323342-1.755344$

C $6.913190-2.6247940 .138246$

C $9.403124-2.013906-0.173000$

O $7.338400-3.6675141 .013284$

C $6.352714-1.4376170 .960171$

C $5.085209-1.8975081 .715282$

O $7.331808-1.0173551 .912129$

C $1.7036811 .993870-1.354606$

O $0.7188652 .510064-1.859350$

C $2.0240000 .549419-1.398750$

C $1.069447-0.315732-1.773944$

C $1.208852-1.768155-1.906429$

C $2.593192-2.347222-1.785957$

C $4.269922-0.7672152 .355819$

C $3.045139-1.3480883 .035941$

C $0.846561-2.4110352 .514491$

O $2.997090-1.5070364 .253268$

C $0.677916-3.5853911 .601882$

C $-0.433462-3.8190750 .888406$

C $-0.610372-4.996628-0.025089$

C $-1.054670-4.597014-1.414972$

C $-2.306929-4.841262-1.837496$

C $-0.024564-3.986928-2.342543$

C $0.101394-2.497907-2.157881$

N 2.058293 -1.710988 2.134309

H $0.3417055 .556149-0.659679$

H 1.7650703 .9654561 .539822

H $2.2778224 .484961-1.490351$

H 4.3919584 .0156630 .684539

H 3.8941975 .6282150 .232668

H $6.5905323 .877736-2.363950$

H $5.5244265 .768119-3.066810$

H $3.7890025 .736283-2.749898$

H $4.8646206 .722652-1.736254$

H $7.1437232 .763177-0.066101$

H 5.4530442 .2619440 .013957

H $5.671998 \quad 1.021068-2.109139$

H $7.3437771 .576070-2.294603$

H $7.8819660 .462562-0.071657$

H $8.404087-0.643498-2.279267$

H $6.784396-1.310871-2.325906$

H $8.861039-3.059169-2.421096$

H $6.093897-3.042184-0.464436$

H $10.166670-1.794230-0.928970$

H $\quad 9.741933-2.9215160 .338871$

H $9.404381-1.1919410 .545760$

H $7.665969-4.3960410 .458399$

H $4.430907-2.432607 \quad 1.017119$

H $5.361103-2.6150712 .499240$

H $6.884179-0.5801342 .653583$

H $3.0270870 .242083-1.126353$

H $0.0788570 .075675-2.004588$

H $2.636497-3.422669-1.966764$

H $2.995869-2.177981-0.784357$

H $3.268122-1.882640-2.514015$

H $4.853265-0.2265933 .108021$

H $3.945977-0.0341831 .608343$

H $\quad 0.020264-1.7005392 .430359$

H $0.914691-2.7490153 .552952$

H $1.510865-4.2812851 .531040$

H -1.274121 -3.137601 0.978211

H -1.354530 -5.661795 0.432017

H $\quad 0.311827-5.586230-0.099564$

H -2.625282 -4.579677 -2.841105

H $-3.044361-5.299853-1.187024$

H $-0.317304-4.162449-3.386353$

H $\quad 0.921367-4.519394-2.215559$

H $-0.848431-1.969753-2.258072$

H $2.151891-1.4249351 .164541$

C -1.7643354 .5627411 .311365$

O -1.4129653 .2340151 .743722$

C $-2.054577 \quad 2.2781370 .874397$ 
C $-3.113866 \quad 3.058518 \quad 0.109683$ C -2.442078 $4.407855-0.050449$ H $-1.271881 \quad 1.9202330 .194338$ C -2.6007311 .1202811 .716800$ H -3.373535 $2.594010-0.846276$ H -4.0289303 .1534370 .705573$ C -3.399365 $5.536202-0.390104$ H - $1.7026644 .317895-0.854978$ C -3.3119850 .0453650 .872562$ $\begin{array}{llll}\text { O } & -1.496905 & 0.528620 & 2.405984\end{array}$ H -3.284606 1.5041142 .483298 H -4.1045770 .5323580 .296068$ O $-2.388832-0.494509-0.074204$ C $-3.952469-1.099774 \quad 1.686426$ C $-4.543427-2.2294030 .820932$ H $-4.736942-0.6864802 .331146$ O $-2.995534-1.7206562 .545641$ H -3.738029 -2.739064 0.277399 H $-4.953194-2.9798131 .510626$ H -3.911935 $5.335036-1.336471$ H $-4.162606 \quad 5.6628600 .384780$ H -2.860930 $6.483788-0.490596$ C -0.5283335 .4658241 .371325$ H $-1.060193 \quad 1.2331792 .915120$ H $-1.651795-0.8823800 .428073$ H -2.676265 $-1.047213 \quad 3.169445$ H -0.0779415 .3888742 .369189$ H -0.8287426 .5120981 .240333$ H -2.4888714 .9318442 .049359$ C $-7.795155-0.677444-0.160614$ O $-6.698471-1.2044850 .597867$ C $-5.633588-1.780581-0.163079$ C $-6.150982-2.947708-1.018034$ C $-7.327220-2.509629-1.896951$ C $-8.387192-1.750615-1.093683$ H $-8.558392-0.4288660 .588133$ C $-7.4163280 .623041-0.888242$ H -5.210267 -1.024026 -0.829865 O $-6.554552-4.036225-0.189941$ H $-5.332649-3.313734-1.648741$ H $-7.789272-3.392927-2.355121$ O $-6.856065-1.667545-2.944993$ H -9.117450 -1.294666 -1.772599 O $-9.096709-2.703285-0.295888$ C $-7.210912 \quad 1.773259 \quad 0.077237$ H $-6.5002670 .527185-1.475780$ H $-8.2374550 .903849-1.558800$ H $-7.234928-3.7078880 .422622$ H $-6.209409-2.177032-3.462695$ H $-9.816061-2.2305150 .156206$ O $-7.2495902 .948318-0.618638$ O $-7.028606 \quad 1.6597361 .281436$ C -7.112603 4.1396190 .162808 H -8.0308914 .3137850 .730974$ H -6.2533134 .0733000 .835996$ H $-6.9555814 .978201-0.520719$ SCF Energy (B3LYP/6-31G*//MMFF)= -3245.92356177

0800176

MM̄FF Geometry

C $-0.683594 \quad 3.0859982 .426290$

C -1.050193 2.0000211 .728469

C -1.4558350 .7037852 .382521$

O $-0.766597-0.347003 \quad 1.664355$

C $-2.9753350 .496556 \quad 2.250941$

C $-3.579260-0.3329953 .366225$

C $-4.032624-1.5945643 .233612$

C -3.7229560 .3795864 .687909$

C $-3.977664-2.4802872 .021308$

C $-5.355007-2.6832441 .372202$

C $-5.847146-1.4572840 .585999$

C $-7.229814-1.715142-0.014414$

C -7.689146 $-0.556811-0.914391$

O $-4.906579-1.216697-0.462022$

O $-8.819393-1.013474-1.673970$

C $-6.568207-0.224923-1.924525$

C $-8.1757310 .643081-0.092364$

O $-6.9210680 .936864-2.672524$
C $-5.177252-0.061961-1.266801$

C $-4.0662260 .007540-2.342772$

O $-5.163307 \quad 1.141119-0.497281$

C $-0.389012-1.4550652 .352878$

O $-0.551615-1.678676 \quad 3.541240$

C $0.266825-2.3680311 .390497$

C $0.638288-3.594127 \quad 1.783653$

C $1.320087-4.5752260 .945012$

C $2.388193-5.3412821 .678657$

C $-2.642467-0.054371-1.767861$

C $-1.590804-0.219272-2.849491$

C $0.818539-0.798455-3.120901$

O $-1.831726-0.100858-4.047584$

C $0.894860-2.273379-3.362950$

C $2.002651-2.996910-3.141767$

C $2.113054-4.467320-3.422848$

C $2.583072-5.261170-2.224416$

C $3.885701-5.547226-2.058087$

C $1.540058-5.813537-1.280626$

C $0.977964-4.779348-0.343611$

N $-0.344812-0.505962-2.307203$

H $-0.6970593 .021943 \quad 3.512920$

H -1.0655252 .0241280 .643145$

H $-1.132194 \quad 0.7085803 .430054$

H $-3.1972640 .051141 \quad 1.277352$

H -3.4960551 .4641112 .257954$

H $-4.481039-2.0700254 .105442$

H $-4.226541-0.2366685 .440594$

H -2.7426100 .6514285 .090770$

H -4.3137801 .2932114 .564454$

H $-3.248950-2.1285931 .285168$

H -3.605552 -3.4581692 .353307$

H -5.267506 -3.5332010 .682623$

H $-6.092566-2.9609042 .135302$

H $-5.879169-0.5903871 .254852$

H $-7.967300-1.9055490 .775199$

H -7.198977 -2.630860 -0.621663

H $-9.510610-1.288048-1.047404$

H $-6.526510-1.057987-2.640420$

H -9.0166040 .3521710 .548773$

H $-8.5571401 .438799-0.741748$

H $-7.401213 \quad 1.063830 \quad 0.552273$

H $-6.308206 \quad 1.006014-3.423273$

H $-4.188466-0.837395-3.033240$

H $-4.1626950 .928595-2.930413$

H $-4.253513 \quad 1.470838-0.433470$

H $0.445174-2.009814 \quad 0.382953$

H $0.462164-3.9118372 .809537$

H $3.075830-4.6515882 .182215$

H $3.000321-5.9648361 .023219$

H $1.939432-5.9936122 .435901$

H -2.410571 $0.863010-1.217004$

H -2.546376 $-0.902349-1.080490$

H $1.693336-0.430662-2.577778$

H $0.739600-0.268346-4.075083$

H $0.009356-2.762768-3.761200$

H $2.896110-2.495588-2.777584$

H $2.823000-4.594705-4.250975$

H $1.163645-4.878880-3.787058$

H $4.231660-6.146779-1.222969$

H $4.636319-5.182491-2.751617$

H $1.939828-6.667751-0.721720$

H $\quad 0.713581-6.238975-1.864853$

H $0.178168-4.178512-0.775526$

H $-0.259376-0.624729-1.302399$

C $-0.123467 \quad 4.576545 \quad 0.378012$

O $0.9883553 .794377-0.105749$

C $1.6969754 .557956-1.100514$

C $0.8338725 .779435-1.396228$

C $0.170726 \quad 6.013487-0.055462$

H $2.6325314 .892284-0.637722$

C $1.9908653 .696870-2.331698$

H $1.4275996 .632709-1.738142$

H $0.0791815 .567467-2.162781$

C $-1.0574856 .903933-0.136528$

H 0.9030726 .4696330 .624142

C $2.8620962 .452288-2.065875$

O $0.7488903 .241380-2.888577$ 
H $2.4575514 .313534-3.108605$

H $2.3041191 .729280-1.461043$

O $3.0976331 .826052-3.335466$

C $4.2284422 .741136-1.412800$

C $5.1946591 .538249-1.417915$

H $4.0799153 .094505-0.386657$

O $4.8576053 .802824-2.138173$

H $5.3162581 .169245-2.443702$

H $6.186314 \quad 1.904786-1.118528$

H $-0.7905487 .891820-0.526207$

H -1.819851 $6.477089-0.796880$

H -1.5072637 .0422560 .851429$

C -0.2453864 .4255351 .896434$

H $0.2872652 .733834-2.198770$

H $2.2339371 .704560-3.765385$

H $5.6784024 .032158-1.670129$

H -0.9540425 .1686892 .280365$

H 0.7292874 .6427832 .352242

H -1.026745 $4.210028-0.126013$

C $4.686040-0.0819041 .904474$

O 5.0003230 .8476680 .859052

C $4.7758840 .393160-0.481084$

C $5.576062-0.889282-0.763727$

C $5.284452-1.9678340 .283445$

C $5.414375-1.4204961 .705521$

H 5.0894490 .3789722 .816010

C $3.171864-0.2315112 .081389$

H $3.7116040 .183379-0.619872$

O $6.971006-0.592452-0.772011$

H $5.311536-1.253384-1.763940$

H $5.981019-2.8051860 .154579$

O $3.963551-2.467121 \quad 0.105356$

H $5.039827-2.1502482 .433181$

O $6.796558-1.2074852 .004010$

C 2.6159330 .9913662 .770815

H $2.627720-0.3454321 .140453$

H $2.938007-1.1105962 .693266$

H $7.445330-1.415511-0.979274$

H $3.923965-2.878865-0.773684$

H $7.249925-2.0636541 .920375$

O $2.367310 \quad 0.7091114 .080158$

O 2.4563262 .0686752 .213026

C 1.8861621 .7764444 .905426

H 2.3483431 .6753275 .891168

H 2.1354392 .7640454 .506248

H 0.8036551 .6758905 .015240

SCF Energy (B3LYP/6-31G**//MMFF) $=-3245.88445743$

0800177

MM̄̄FF Geometry

C $2.7499731 .647742-2.259618$

C $3.1606562 .925672-2.257953$

C $4.0009783 .601578-1.197166$

O $4.7029502 .631439-0.382525$

C $3.1668134 .443740-0.212633$

C $2.1410275 .365348-0.834456$

C $0.8151335 .120717-0.825845$

C $2.6885796 .623472-1.450595$

C $0.1403143 .893011-0.279245$

C $-0.516614 \quad 4.1619301 .077593$

C -0.8510932 .8576911 .819258$

C -1.6683153 .1618983 .076561$

C -1.8951931 .9122853 .937594$

$\begin{array}{llll}\text { O } & 0.392283 & 2.239229 & 2.173231\end{array}$

O -2.3772382 .3416265 .220099$

C $-0.536574 \quad 1.2216844 .174051$

C $-2.978147 \quad 0.999972 \quad 3.349197$

O $-0.713819-0.0157004 .861548$

C 0.2741050 .9951622 .876572

C 1.7083060 .5222313 .231007

O $-\begin{array}{llll}0.382097 & 0.006725 & 2.089426\end{array}$

C $5.8305592 .066265-0.887910$

O $6.3730112 .316688-1.951255$

C 6.2542571 .0268450 .074710

C $7.0064510 .003378-0.353531$

C $7.429231-1.1482350 .446348$

C $7.065868-1.1540301 .908462$

C 2.6160260 .2221722 .026883
C $2.518478-1.2121971 .528244$

C $3.396034-2.716344-0.256827$

O $1.818398-2.0656382 .066761$

C $4.493514-3.527770 \quad 0.359315$

C $5.445832-4.152642-0.350514$

C $6.523543-4.9999600 .266578$

C $7.911911-4.639536-0.216966$

C $8.549829-5.405699-1.117995$

C $8.592468-3.4390480 .405096$

C $8.093124-2.146846-0.172225$

N $3.311917-1.4293470 .409782$

H $3.0114750 .992660-1.434383$

H $2.8704293 .561237-3.092744$

H $4.7384514 .249445-1.688213$

H $3.8553765 .043870 \quad 0.399075$

H 2.7010543 .7793670 .526558

H $0.1451215 .853613-1.271692$

H $1.8992587 .261614-1.861680$

H $3.2307537 .208836-0.700794$

H $3.3762996 .381316-2.267155$

H $-0.6247703 .575021-0.996090$

H $0.8384473 .053989-0.212956$

H $\quad 0.1650274 .7582541 .698903$

H -1.426654 4.7552030 .924348

H -1.407519 2.1947591 .145876

H -2.626952 3.6260982 .812739

H -1.135284 3.9060003 .685371

H -3.1984762 .8432065 .078904$

H 0.0513561 .8665504 .842264

H $-3.932804 \quad 1.5349473 .278753$

H $-3.1653350 .138108 \quad 3.999021$

H -2.7334130 .6322692 .350844$

H -1.2175190 .1728335 .671793$

H $2.193170 \quad 1.3238883 .803979$

H $1.665555-0.3587993 .881863$

H $\quad 0.216501-0.2680591 .377744$

H 5.8829381 .1007261 .090340

H $7.311130-0.014157-1.399311$

H $7.507407-0.2886742 .415394$

H $7.412367-2.0398532 .443635$

H $5.978825-1.1179612 .037763$

H $2.4136250 .909677 \quad 1.198600$

H 3.6576440 .3716642 .337931

H $3.569787-2.522244-1.319769$

H $2.442498-3.242469-0.145786$

H $4.490023-3.6279571 .442386$

H $5.432415-4.080403-1.435730$

H $\quad 6.302508-6.0465040 .019041$

H $6.498700-4.9425281 .361993$

H $9.555559-5.169699-1.449711$

H $8.078599-6.283191-1.548315$

H $9.674672-3.4828280 .223545$

H $8.487763-3.4955831 .491499$

H $8.308523-2.041644-1.236871$

H $3.918643-0.6850400 .080049$

C $0.4961970 .743482-2.883606$

O $0.511602-0.348448-1.943889$

C $-0.631727-1.198227-2.194964$

C -1.486312 -0.463004-3.216475

C $-0.4400540 .298534-4.004275$

H $-0.226897-2.120013-2.631415$

C $-1.323278-1.499420-0.860604$

H $-2.078380-1.143590-3.835598$

H $-2.1746670 .232885-2.722291$

C - $-1.0147341 .440217-4.824859$

H $0.078410-0.402756-4.671846$

C -2.677631 -2.223274 -1.001447

O $-0.404653-2.292112-0.105748$

H $-1.466446-0.561994-0.310505$

H -3.342186 -1.576250 -1.580833

O $-2.504620-3.420639-1.758791$

C $-3.361098-2.5545090 .342375$

C $-4.749544-3.2083900 .192641$

H -3.441487 -1.637510 0.937425

O $-2.538924-3.4619701 .080143$

H $-4.662807-4.140502-0.379437$

H -5.080010 -3.505184 1.197115

H $-1.7408051 .063099-5.552463$ 
H -1.523912 2.173577 -4.191040 H $-0.2262821 .962313-5.375542$ C $1.9179541 .044742-3.358141$ H $-0.767788-2.3933480 .790278$ H -1.909204 -3.999527 -1.252127 H -2.945645 -3.582796 1.955206 H $1.8901871 .719995-4.221100$ H $2.4121080 .121235-3.683590$ H $\quad 0.0755851 .610566-2.359527$ C $-6.829803-0.123034-0.074238$ O $-5.935514-1.1394290 .394916$ C $-5.807298-2.292262-0.442604$ C $-7.166173-2.991172-0.599404$ C -8.226151-2.013337-1.118197 C $-8.233538-0.702791-0.321932$ H -6.9061160 .5899540 .757284$ C -6.254080 $0.630489-1.283449$ H $-5.455160-1.988517-1.432070$ O $-7.580027-3.521418 \quad 0.657877$ H -7.053787 -3.832161 - 1.294284 H -9.213891-2.485837-1.052593 O $-7.961048-1.745246-2.492103$ H -8.852551 $0.045082-0.829936$ $\begin{array}{lllll}\text { O } & -8.850565 & -0.941287 & 0.947214\end{array}$ C $-5.005003 \quad 1.410571-0.926502$ H $-5.991239-0.041993-2.103618$ H -6.999332 $1.350162-1.642284$ H -8.422202 -3.9866640 .516988$ H -8.657630 -1.147942 -2.814216 H -8.279256 -1.550673 1.445807 O $-4.6637952 .207789-1.982212$ O $-4.396573 \quad 1.3283140 .131451$ C -3.494649 $3.008900-1.788178$ H $-2.6299922 .371907-1.581323$ H $-3.3069103 .567818-2.708630$ H $-3.6541733 .719146-0.971247$ SCF Energy (B3LYP/6-31G**//MMFF)= -3245.91902139

\section{8}

MM̄FF Geometry

C $-0.562255 \quad 4.505127 \quad 0.495375$

C $0.675587 \quad 4.006794 \quad 0.636222$

C $1.6829813 .957101-0.484828$

O $2.0761162 .581115-0.698433$

C $2.9391524 .751340-0.092925$

C $3.8992264 .937966-1.250319$

C $5.044367 \quad 4.247525-1.413483$

C $3.5269556 .024484-2.226767$

C $5.5780873 .138713-0.548267$

C $6.2029612 .023607-1.399411$

C $6.8678650 .948343-0.528817$

C $7.697669-0.003084-1.392996$

C $8.330014-1.123663-0.556304$

$\begin{array}{lllll}\text { O } & 5.843173 & 0.217684 & 0.155226\end{array}$

O $8.820204-2.126283-1.459306$

C $7.229555-1.7821720 .297757$

C $9.540751-0.6308790 .244935$

O $7.787372-2.7319011 .203793$

C $6.343071-0.7711051 .067317$

C $5.142214-1.5224131 .687417$

O $7.108986-0.1762222 .116854$

C $1.314162 \quad 1.844962-1.551297$

O $0.3154552 .214144-2.149105$

C $1.8917510 .483156-1.617250$

C $1.124014-0.515354-2.078383$

C $1.493346-1.927728-2.190966$

C $2.943984-2.285119-2.011964$

C $4.031418-0.6201452 .240144$

C $2.942459-1.4600092 .883125$

C $1.150196-3.0998872 .329353$

O $2.763648-1.4698224 .098503$

C $1.066045-4.1863111 .305817$

C $-0.012532-4.4185920 .542881$

C $-0.089972-5.530511-0.463022$

C $-0.440126-5.048675-1.852542$

C $-1.633195-5.333816-2.401983$

C $0.623489-4.310696-2.641251$

C $0.520508-2.818666-2.477873$
N $2.229853-2.206348 \quad 1.959181$

H $-0.8742064 .888469-0.473220$

H 0.9893423 .6201761 .603041

H $1.2585674 .369819-1.408617$

H 3.4446224 .2595180 .746803

H 2.6562315 .7459690 .278304

H $5.6788374 .512489-2.258488$

H $4.2816636 .157491-3.009191$

H $2.5797475 .790646-2.722136$

H $3.4210706 .982419-1.706984$

H 6.3450263 .5628610 .110683

H 4.7995892 .7075350 .087848

H $5.4215901 .568900-2.022200$

H $6.9522242 .459133-2.072781$

H 7.5096201 .4475390 .206892

H $8.4724280 .544388-1.944043$

H $7.052005-0.459603-2.156364$

H $9.465758-1.705291-2.052583$

H $6.585514-2.351150-0.387921$

H $10.306466-0.227169-0.428290$

H $10.020601-1.4560210 .782732$

H $9.289840 \quad 0.1502810 .965405$

H $8.320541-3.3539020 .679582$

H $4.699535-2.1719720 .922657$

H $5.493686-2.1788112 .494395$

H 6.5088350 .1157052 .820908

H $2.9039800 .336748-1.259274$

H $\quad 0.104007-0.281640-2.382729$

H $3.567758-1.710299-2.706170$

H $3.159820-3.338169-2.203658$

H $3.274856-2.069283-0.993175$

H 4.4173440 .0694172 .997728

H $3.581501-0.012347 \quad 1.446814$

H $\quad 0.232497-2.5075032 .388746$

H $1.347986-3.5238263 .319062$

H $1.940482-4.8240971 .196742$

H $-0.895411-3.7968310 .663868$

H $-0.844452-6.244509-0.107870$

H $0.851404-6.092647-0.508840$

H -1.879998 -5.014993 -3.409397

H $-2.391486-5.885066-1.855774$

H $\quad 0.505977-4.536349-3.709472$

H $1.605513-4.703556-2.364493$

H $-0.490872-2.437301-2.629669$

H $2.321546-1.9638410 .977541$

C -2.7232693 .6161571 .416728$

O $-3.4695943 .946538 \quad 0.233162$

C -4.8476203 .5544250 .430837$

C -4.9383432 .9935441 .846689$

C -3.7554973 .6425322 .538465$

H $\quad-5.437760 \quad 4.473100 \quad 0.333753$

C $-5.2245042 .545219-0.661234$

H -5.8952473 .2262482 .324073$

H -4.8243861 .9019781 .844407$

C $-3.3247872 .915303 \quad 3.800601$

H -4.0176814 .6799532 .784680$

C $-6.6507541 .980953-0.527882$

O $-5.1125923 .193425-1.932536$

H $-4.4886121 .733689-0.659627$

H $\quad-6.7850001 .4994550 .446143$

O $-7.5831473 .069336-0.548272$

C $-7.0416810 .989918-1.642410$

C $-6.073507-0.191020-1.825360$

H -7.123799 $1.529076-2.594006$

O $-8.353691 \quad 0.499993-1.359946$

H $-6.460886-0.856632-2.607564$

H $-5.1267140 .188704-2.231558$

H -2.472889 3.4161414 .270394

H -4.1428252 .8903224 .527922$

H $-3.0319851 .881513 \quad 3.587933$

C $-1.5681444 .597301 \quad 1.610234$

H $-4.2083433 .545943-1.998912$

H -7.463859 $3.541585-1.390232$

H $-8.605242-0.099345-2.083187$

H -1.0796344 .4154942 .574236$

$\mathrm{H}-1.9480785 .6261351 .628700$

H -2.3374602 .5996581 .279338$

C $-4.098856-2.6375520 .163543$ 
O $-4.636290-1.845453-0.903272$

C -5.764490 -1.024972 -0.564269

C $-6.926814-1.904566-0.076107$

C $-6.490542-2.8414181 .057529$

C $-5.180417-3.5685520 .735963$

H $-3.330101-3.260492-0.310828$

C $-3.407301-1.782831 \quad 1.239161$

H $-5.458288-0.3445300 .233999$

O $-7.426360-2.677852-1.165124$

H $-7.741042-1.2649460 .280185$

H $-7.281825-3.5800291 .235668$

O $-6.343620-2.0717082 .247614$

H $-4.799939-4.0750301 .630719$

O $-5.466617-4.584265-0.229528$

C $-2.216920-1.0215480 .698227$

H $-4.084477-1.0457681 .677774$

H -3.041072 -2.440395 2.036393

H -8.179234 -3.197518 -0.835736

H $-6.100381-2.6825642 .963894$

H -4.643493 -5.075473 -0.393062

O $-1.612345-0.359416 \quad 1.729040$

O $-1.861799-1.003250-0.471414$

C -0.4612750 .4112671 .373690$

H $0.357093-0.2519421 .086162$

H -0.6970001 .0989490 .557628$

H - 0.1557420 .9926012 .247388

SCF Energy (B3LYP/6-31G**//MMFF) $=-3245.90026048$

0800179

MM̄FF Geometry

C $1.392809-1.3321660 .695905$

C $0.170985-1.7145930 .296599$

C $-0.295680-1.650026-1.135291$

O $-1.521976-0.883839-1.181056$

C $-0.584779-3.072349-1.647092$

C $-1.011448-3.119231-3.102411$

C $-2.263646-3.373669-3.533044$

C $0.093744-2.890447-4.102095$

C $-3.489475-3.631811-2.702191$

C $-4.522217-2.511564-2.879228$

C $-5.637333-2.589469-1.826077$

C $-6.694703-1.516230-2.083837$

C $-7.752377-1.463618-0.971525$

O $-5.031652-2.382618-0.546632$

O $-8.488184-0.239306-1.125660$

C $-7.043425-1.394996 \quad 0.399497$

C $-8.771926-2.601426-1.103950$

O $-8.008199-1.4972261 .444325$

C $-5.931875-2.454571 \quad 0.567270$

C $-5.078074-2.2606761 .845698$

O $-6.534310 \quad-3.7512680 .656669$

C -1.399352 $0.472804-1.205906$

O $-0.3621591 .116809-1.187762$

C $-2.7608591 .051632-1.253350$

C -2.918935 2.381595-1.176977

C -4.190023 3.108571-1.222408

C $-5.4555382 .299058-1.319309$

C $-4.305025-0.939767 \quad 1.927955$

C $-5.028122 \quad 0.163783 \quad 2.680527$

C -4.814496 2.5854963 .237938

O $-6.054435-0.0214933 .328292$

C $-5.664513 \quad 3.384736 \quad 2.301614$

C $-5.429570 \quad 4.672998 \quad 2.009485$

C -6.3104015 .5038531 .121273$

C $-5.546403 \quad 6.1859020 .008951$

C $-5.154661 \quad 7.4662310 .123384$

C $-5.322178 \quad 5.413598-1.270618$

C $-4.164698 \quad 4.457270-1.177017$

N $-4.363197 \quad 1.3784572 .572061$

H $2.096344-0.943896-0.036561$

H $-0.534699-2.0984231 .029783$

H $\quad 0.458047-1.175049-1.775713$

H $-1.337556-3.540878-1.005424$

H $0.313806-3.694792-1.535417$

H -2.441913 -3.395366 -4.607641

H $-0.246816-3.017560-5.135315$

H $0.492691-1.875645-4.011041$

H $0.908950-3.602405-3.936683$
H -3.931010 -4.584739 -3.018677

H $-3.243646-3.752783-1.645637$

H $-4.016130-1.541752-2.794447$

H $-4.952456-2.567724-3.886883$

H -6.084822 -3.589845 -1.859163

H $-7.170539-1.666575-3.061101$

H $-6.206572-0.534761-2.140159$

H -8.884711 $-0.237494-2.013755$

H $-6.604993-0.3925750 .471899$

H $-9.289273-2.541229-2.069082$

H $-9.554275-2.522685-0.341015$

H -8.319334 -3.592853 -1.035422

H $-7.564135-1.2852462 .282223$

H $-5.685820-2.4330792 .741809$

H -4.324608 -3.061936 1.852895

H $-5.907496-4.3548891 .088818$

H -3.596145 $0.368761-1.350938$

H -2.030741 $3.005160-1.080605$

H $-5.4956731 .548304-0.523066$

H $-5.5077321 .790428-2.287604$

H $-6.3649332 .894309-1.214795$

H -3.372614 -1.1203782 .478748$

H -4.030067 -0.5820860 .931860$

H $-3.921826 \quad 3.133062 \quad 3.556810$

H $-5.395375 \quad 2.3184604 .126554$

H -6.5318152 .8898071 .870929$

H $-4.578207 \quad 5.1733712 .465683$

H -6.7993386 .2583301 .751771$

H $-7.1250494 .907248 \quad 0.692529$

H $-4.633513 \quad 7.972298-0.682548$

H -5.3413338 .0347541 .028459$

H $-5.0988526 .108338-2.091827$

H -6.255565 $4.928928-1.570725$

H $-3.1972104 .955220-1.099065$

H -3.5487941 .4476791 .968561$

C $3.061211-2.3262032 .334039$

O $4.197718-1.8723121 .571347$

C $4.851610-3.0214961 .001159$

C $4.280351-4.2345821 .728016$

C $2.848227-3.7920821 .956214$

H $4.543395-3.069178-0.050051$

C $6.373972-2.8831201 .094736$

H $4.359252-5.1500401 .134074$

H $4.782363-4.4072452 .687206$

C $2.116290-4.6017573 .011499$

H $2.308493-3.8774671 .005001$

C $6.956637-1.622388 \quad 0.421808$

O $6.760513-2.8504232 .475062$

H $6.847498-3.7779290 .674066$

H $6.708197-0.734103 \quad 1.013420$

O $8.385152-1.7403470 .452258$

C $6.520408-1.411748-1.038859$

C $7.308901-0.338259-1.812239$

H $5.455474-1.156052-1.064464$

O $6.660940-2.639318-1.762107$

H $8.342876-0.673112-1.966234$

H $6.866794-0.288149-2.816596$

H $2.058620-5.6546212 .716613$

H $2.624119-4.5517833 .980558$

H $1.094820-4.2321183 .146089$

C $1.863574-1.3963102 .121518$

H $6.295807-2.1043562 .891688$

H $8.645596-1.8621081 .381499$

H $7.595207-2.904827-1.711116$

H $2.146649-0.3782792 .414050$

H $1.045795-1.7003652 .785672$

H $3.360666-2.2553213 .388242$

C $5.7560652 .753955-0.393957$

O $5.9488851 .479054-1.022639$

C $7.3076921 .059415-1.172459$

C $8.0974992 .071468-2.020541$

C $7.9789433 .487830-1.450412$

C $6.5233133 .856825-1.143570$

H $4.6839692 .962626-0.503559$

C 6.0785192 .7102141 .107996

H $7.7741950 .983863-0.185464$

O $7.6062032 .059723-3.359930$

H $9.1491861 .761984-2.050977$ 
H $8.3915024 .202435-2.172981$

O $8.7641743 .566439-0.264601$

H $6.4836824 .786647-0.565091$

O $5.8444544 .119614-2.375466$

C 5.1323481 .8102171 .873339

H 7.0926892 .3474431 .293753

H 5.9811973 .7164531 .531865

H $8.1529962 .674361-3.878389$

H $8.699047 \quad 4.4770910 .070405$

H $5.8022473 .286366-2.875528$

O $5.539767 \quad 1.750736 \quad 3.176019$

O 4.1619221 .2380241 .398086

C 4.7447890 .9313354 .038445

H 3.6893541 .2140493 .978262

H $4.880104-0.1215303 .777195$

H 5.0888971 .0848485 .064657

SCF Energy (B3LYP/6-31G**//MMFF) $=-3245.92212051$

0800180

MM̄FF Geometry

C $-0.826879-3.5814380 .559840$

C $-0.933595-2.902563-0.591574$

C $0.233359-2.262352-1.291404$

O $-0.045420-0.850442-1.451986$

C $0.457354-2.843559-2.698175$

C $0.940331-4.278977-2.702706$

C $2.214717-4.650825-2.468094$

C $-0.108281-5.315077-3.012789$

C $3.385876-3.754545-2.174017$

C $3.822256-3.864420-0.707924$

C $5.052671-2.998227-0.395607$

C $5.379505-3.0549161 .099436$

C $6.541033-2.1220471 .478371$

O $4.773959-1.649539-0.786555$

O $6.546051-1.9851552 .908224$

C $6.266258-0.7198010 .890546$

C $7.898353-2.7255641 .099104$

O 7.3758290 .1499851 .102553

C $5.872132-0.746387-0.601850$

C $5.4442250 .640798-1.129486$

O $7.009557-1.149099-1.375437$

C $0.195812-0.050654-0.376764$

O $0.600639-0.3978590 .721711$

C $-0.1137881 .340608-0.772082$

C 0.0450262 .3248720 .124886

C $-0.1935573 .752506-0.097393$

C $-0.6335604 .179069-1.472300$

C $4.2165231 .248130-0.449973$

C $3.7465592 .468683-1.216155$

C $4.3051024 .857731-1.646926$

O $2.7877462 .416853-1.983087$

C $3.7576125 .840789-0.661852$

C $2.5059976 .320907-0.712535$

C 1.9679487 .3657110 .221215

C 1.1225776 .8007381 .339195

C 1.4724326 .9824122 .624290

C -0.1672546 .0945220 .979353$

C -0.0000574 .5994110 .935041$

N $4.5284583 .588375-0.982625$

H $0.151802-3.6999311 .017309$

H -1.907865 -2.788968 -1.055851

H $1.154683-2.367431-0.708479$

H $1.170351-2.210239-3.242165$

H $-0.465136-2.755639-3.289380$

H $2.455991-5.712534-2.494551$

H $0.299633-6.331364-3.016036$

H -0.913003 -5.286748 -2.272359

H $-0.540599-5.130506-4.001758$

H $4.215351-4.049145-2.828518$

H $3.158442-2.713257-2.420654$

H $2.988270-3.552212-0.067469$

H $4.041925-4.910281-0.458509$

H $5.894121-3.376366-0.988300$

H $5.595080-4.0845461 .411265$

H $4.494039-2.7489941 .674991$

H $6.640390-2.8728393 .294318$

H $5.436513-0.2973431 .470598$

H $8.044014-3.6875521 .605179$
H $8.722843-2.0864001 .433481$

H $8.006143-2.9000870 .026645$

H $8.066695-0.0895260 .461689$

H $6.2906651 .337496-1.073838$

H $5.2235820 .547160-2.202747$

H $\quad 6.873336-0.864993-2.294531$

H $-0.4529061 .516863-1.786366$

H $\quad 0.3887072 .063091 \quad 1.125068$

H -1.575061 $3.690411-1.743585$

H $\quad 0.1248493 .913708-2.217254$

H $-0.8010065 .253735-1.564105$

H $3.3871850 .533337-0.415765$

H 4.4353121 .5408910 .582444

H $3.6386394 .721272-2.504965$

H $5.2720505 .208674-2.021092$

H $4.435777 \quad 6.1978890 .109377$

H $1.8346775 .969821-1.492046$

H $1.3654848 .075972-0.359176$

H $2.7989157 .956876 \quad 0.628721$

H 0.8576646 .6034003 .434271

H 2.3808347 .5097222 .895877

H -0.5666996 .5129240 .051855$

H $-0.928493 \quad 6.3178271 .739190$

H $0.308404 \quad 4.1755541 .892417$

H $5.2952023 .529139-0.318659$

C $-2.382884-3.5230882 .572029$

O $-2.835276-2.1800692 .313799$

C $-2.434791-1.3429853 .418595$

C -1.875074 -2.278794 4.487856

C -1.299515 -3.398174 3.641093

H $-1.620098-0.7082233 .050866$

C -3.608604 -0.4733733 .883381$

H -1.126833 -1.786505 5.116568

H -2.662512 -2.6652015 .145340$

C -1.040148 -4.679548 4.412543

H $-0.356961-3.0432603 .207067$

C -4.2358770 .3839692 .764713$

O $-4.634070-1.3352064 .394979$

H $-3.2904450 .158768 \quad 4.720797$

H $-4.716707-0.2780312 .037138$

O $\quad-5.291700 \quad 1.1578693 .349115$

C -3.2532811 .3340042 .055978$

C -3.9169942 .2790411 .034443$

H -2.4726190 .7509831 .557177$

O -2.6081522 .1382073 .047209$

H -4.6770672 .8956151 .529515$

H -3.144760 2.9790780 .691732

H $-0.315146-4.5055865 .214363$

H -1.957619 -5.0674074 .867578$

H $-0.636005-5.4548143 .754091$

C $-1.993074-4.2146271 .263662$

H $-5.371744-0.7693664 .680660$

H $-4.884688 \quad 1.783903 \quad 3.972623$

H - 1.9393782 .6805912 .595624

H -2.864931 -4.213182 0.596887

H $-1.748301-5.265091 \quad 1.458716$

H -3.248664 -4.057699 2.986200

C $-3.8564780 .028781-1.928920$

O $-3.4543010 .871376-0.841562$

C $-4.5225281 .550989-0.174430$

C $-5.2305082 .503529-1.151215$

C $-5.7206751 .748718-2.391476$

C $-4.6374810 .834402-2.981796$

H -2.911293 -0.293797-2.383975

C $-4.578649-1.218831-1.398897$

H $\quad-5.2497340 .816503 \quad 0.186828$

O $-4.3490893 .538966-1.578690$

H $-6.0824962 .975995-0.647151$

H $-6.0348392 .476824-3.149771$

O $-6.864527 \quad 0.981182-2.028977$

H $\quad-5.0798760 .152941-3.716058$

O $-3.7008041 .647040-3.698151$

C $-4.445463-2.409597-2.322751$

H $-4.133079-1.497220-0.435324$

H -5.642420 -1.035992-1.227455

H $-4.2232264 .148708-0.832390$

H $-7.1864210 .537432-2.832115$

H $-3.2595432 .228245-3.055007$ 
O $-4.834123-3.534832-1.652457$

O $-4.057409-2.365631-3.481826$

C $-4.731151-4.756356-2.390528$

H -3.694374 -4.929012 -2.695122

H $-5.045085-5.575233-1.737862$

H $-5.391493-4.728203-3.262302$

SCF Energy (B3LYP/6-31G**//MMFF) $=-3245.91974948$

0800181

MM̄FF Geometry

C $-2.228392-2.820232-3.188940$

C $-2.711405-2.359031-2.025722$

C $-4.111452-2.647529-1.549188$

O $-4.728844-1.378824-1.221521$

C $-4.100660-3.517760-0.278078$

C $-3.784557-4.981523-0.514802$

C $-2.612834-5.568050-0.197054$

C $-4.913198-5.800346-1.084042$

C $-1.390740-4.880046 \quad 0.338145$

C $-1.410469-4.7429861 .864677$

C $-0.306721-3.7966762 .360811$

C $-0.365393-3.648013 \quad 3.883647$

C $0.622771-2.5932174 .405847$

O $-0.512871-2.5262801 .731120$

O $0.252957-2.309588 \quad 5.764007$

C $0.444765-1.2972003 .584038$

C $2.053605-3.1402004 .457737$

O $1.406507-0.3178303 .963067$

C $0.465660-1.5353532 .059341$

C $0.141191-0.2628111 .249658$

O $1.777160-1.9537881 .664276$

C $-6.083216-1.315017-1.313650$

O $-6.827169-2.201848-1.713285$

C $-6.618358-0.013121-0.855417$

C $-5.8944680 .931489-0.234776$

C -6.4063452 .2094390 .272961$

C -7.869004 2.5074400 .060868

C $-1.2559460 .315891 \quad 1.469213$

C -1.4805401 .5087260 .561275$

C -0.9052943 .9249830 .321870$

O $-2.1479451 .422446-0.466629$

C -1.9829134 .7570740 .939435$

C $-3.011565 \quad 5.2692860 .247238$

C $-4.079890 \quad 6.1263110 .860173$

C -5.4674345 .5556720 .674896$

C $-6.3433336 .131915-0.165505$

C -5.8713124 .3790831 .534705$

C -5.5559843 .0506510 .899290$

N -0.8379602 .6509631 .011329$

H -2.898384 -3.396601 -3.825545

H $-2.078025-1.780878-1.359089$

H -4.685458 -3.130973 -2.349318

H $-5.081035-3.4604420 .214365$

H $-3.419930-3.072856 \quad 0.458477$

H $-2.500823-6.637648-0.364472$

H $-4.652053-6.859953-1.176237$

H -5.794788 -5.733702 -0.438330

H -5.184238 $-5.440891-2.081449$

H $-0.504652-5.4514010 .034420$

H -1.281898 $-3.904077-0.142421$

H -2.384706 -4.3495792 .181504$

H -1.295358 -5.730824 2.327402

H $0.663853-4.2003292 .048784$

H -0.194820 -4.613909 4.375376

H -1.379471 -3.341222 4.177258

H $0.824566-1.5887706 .080274$

H - $0.530810-0.8842923 .868172$

H $2.106474-4.0014965 .134910$

H $2.748445-2.3979684 .865670$

H $2.426106-3.4635113 .483591$

H $2.251768-0.5543293 .545240$

H $\quad 0.908491 \quad 0.4993041 .433618$

H $\quad 0.230948-0.5048970 .181828$

H $\quad 1.882720-1.7861470 .712985$

H -7.682756 $0.112204-1.029523$

H $-4.8353720 .753795-0.060799$

H -8.172876 3.4916630 .422497

H $\quad-8.4908201 .7717770 .582869$
H $-8.1154822 .484041-1.006595$

H -2.030507 -0.4276941 .249817$

H -1.4005590 .6378402 .505669$

H -1.076285 $3.757101-0.745769$

H 0.0624844 .4191860 .448379

H -1.9025144 .9610282 .004608$

H -3.082488 $5.080118-0.821434$

H -4.0125237 .1202890 .399050$

H $-3.8989006 .285426 \quad 1.930684$

H -7.352539 $5.751096-0.278813$

H $-6.0702866 .996168-0.761760$

H -6.9309174 .4539881 .802564$

H -5.3498954 .4332802 .499253$

H -4.5129882 .7511111 .000864$

H -0.3494852 .6243691 .901801$

C $0.235738-2.054960-2.830548$

O $0.030200-0.629630-2.750448$

C $1.2482020 .041412-3.132699$

C $2.347618-0.989990-2.954324$

C $1.646384-2.254641-3.396679$

H $1.1272850 .304907-4.191008$

C $1.4364651 .302149-2.283101$

H $3.234830-0.764461-3.552067$

H $2.647863-1.064457-1.901739$

C $2.325453-3.524038-2.909243$

H $1.613114-2.269168-4.494479$

C $2.7424042 .054660-2.590697$

O $0.3349032 .179333-2.530390$

H $1.3931431 .031704-1.224355$

H $3.6037181 .398464-2.446187$

O $2.7398092 .394240-3.984731$

C $2.9334093 .350587-1.784570$

C $2.9326873 .198623-0.251684$

H $2.1520604 .065462-2.070679$

O $4.1742803 .938721-2.191429$

H 2.9549794 .2113540 .172486

H 1.9906282 .7407740 .064817

H $3.341136-3.596000-3.311211$

H $2.394447-3.547280-1.816165$

H $1.770926-4.411208-3.230736$

C $-0.832188-2.684442-3.734378$

H $-0.4797351 .682591-2.340780$

H $3.5687612 .869211-4.167455$

H $4.2632864 .786570-1.723677$

H $-0.508047-3.697215-4.005101$

H $-0.904186-2.108376-4.665758$

H $0.174058-2.451034-1.809729$

C 5.0502970 .1744440 .444946

O $3.9949341 .037703-0.001871$

C 4.1282612 .4200420 .339938

C 4.1955242 .5834591 .868255

C 5.3135741 .7266322 .468575

C 5.2531620 .2817001 .967317

H $4.687861-0.8378400 .225883$

C $6.3501480 .396371-0.340190$

H $5.0486022 .815294-0.100540$

O 2.9428942 .2158812 .441835

H 4.3684333 .6408322 .102853

H 5.2278571 .7388413 .562053

O 6.5667972 .3098072 .123042

H $6.162147-0.2564202 .260282$

O $4.162712-0.3657012 .624456$

C $6.249423-0.133843-1.750769$

H $6.6315881 .450552-0.411021$

H $7.193126-0.1164150 .139236$

H 3.0111992 .3409683 .403633

H 7.2642031 .7716722 .534628

H $4.159354-1.2969132 .344119$

O $6.325582-1.495794-1.743086$

O $6.1342940 .577493-2.740050$

C $6.296464-2.124551-3.028000$

H $5.405218-1.824813-3.585801$

H $7.202637-1.872518-3.586718$

H $6.265831-3.206720-2.876153$

SCF Energy (B3LYP/6-31G**//MMFF) $=-3245.92066850$

0800182

MM̄MF Geometry 
C $0.856225-2.8951410 .603751$

C $-0.419960-3.2111920 .340169$

C - $1.020127-3.142135-1.040549$

O $-1.731404-1.885970-1.120986$

C $-2.011846-4.295477-1.256932$

C $-2.662406-4.265374-2.625806$

C $-3.909327-3.813235-2.866913$

C $-1.825750-4.821635-3.747956$

C $-4.866745-3.215507-1.873644$

C $-5.432705-1.876520-2.367557$

C $-6.285958-1.204732-1.282572$

C $-7.0278430 .010302-1.838174$

C $-7.8192580 .738903-0.741072$

O $-5.405514-0.792348-0.231199$

O $-8.2239892 .014003-1.262173$

C -6.8805521 .0142630 .451537$

C $-9.105990-0.007175-0.369474$

O -7.6019851 .5548431 .556428$

C $-6.065265-0.221150 \quad 0.904810$

C $-4.9833850 .218748 \quad 1.918406$

O $-6.936408-1.1619451 .533685$

C $-1.075225-0.849582-1.711988$

O $-0.011173-0.892942-2.309483$

C $-1.838250 \quad 0.384973-1.430850$

C $-1.1770161 .551861-1.445946$

C $-1.7120152 .858965-1.067417$

C $-3.1941182 .980295-0.844340$

C -3.940061 -0.8554252 .249862$

C -2.831354 -0.2589333 .096002$

C -0.8429791 .2475442 .933545$

$\begin{array}{lllll}\text { O } & -2.796589 & -0.412527 & 4.314467\end{array}$

C $-1.1523302 .704907 \quad 2.778301$

C -0.2800043 .6039982 .296167$

C $-0.590540 \quad 5.067278 \quad 2.141918$

C $-0.223877 \quad 5.6131240 .776838$

$\begin{array}{lllll}\text { C } & 0.829093 & 6.433694 & 0.621963\end{array}$

C -1.117477 $5.264962-0.397781$

C $-0.843773 \quad 3.879167-0.909700$

N -1.926699 0.4793102 .348538

H $1.497203-2.568410-0.211489$

H $-1.077843-3.4881191 .160764$

H $-0.234654-3.199659-1.804467$

H -2.776426 -4.284874 -0.471883

H $-1.494752-5.256973-1.135104$

H $-4.288988-3.872610-3.886074$

H $-2.356212-4.812893-4.706078$

H $-0.911468-4.233343-3.873304$

H - $1.546632-5.858837-3.535564$

H $-5.692338-3.921426-1.722559$

H -4.396503 -3.065705 -0.898635

H $-4.603337-1.213371-2.645635$

H $-6.037006-2.048732-3.266763$

H -6.997988-1.942107 -0.893491

H $-7.692358-0.280281-2.661346$

H $-6.3034290 .714704-2.270682$

H -8.761247 $1.855808-2.057455$

H -6.1746531 .7941350 .132904$

H $-9.750905-0.119663-1.249225$

H $-9.693700 \quad 0.5592300 .361363$

H -8.921913 -1.0047260 .034841$

H -8.085650 2.3341691 .233200

H -4.4560721 .0900171 .516092$

H $-5.456993 \quad 0.5426732 .854201$

H $-6.415437-1.7238222 .128808$

H -2.876948 $0.293928-1.138096$

H $-0.1177391 .543094-1.701490$

H $-3.7537802 .509279-1.660378$

H -3.539210 $4.016312-0.807508$

H -3.475959 2.5023730 .096783

H -4.386836 -1.686213 2.805928

H -3.494587 -1.279113 1.342769

H 0.0726100 .9642142 .407083

H -0.7352900 .9984513 .993353$

H -2.1410853 .0381603 .085895$

H $0.718248 \quad 3.2764702 .016096$

H -0.0363995 .6057732 .921736$

H -1.6515685 .2706962 .332884$

H $1.0863616 .845740-0.348221$
H $1.462105 \quad 6.7063301 .459801$

H $-0.9459435 .965185-1.225290$

H -2.157671 $5.411355-0.093601$

H $0.2087513 .697804-1.127915$

H $-2.0583000 .546807 \quad 1.344270$

C $2.891343-3.4033342 .044951$

O $3.744881-2.5431461 .261682$

C $4.649648-3.3670150 .504289$

C $4.599336-4.7442821 .156449$

C $3.134785-4.8271441 .545467$

H $4.231403-3.449945-0.505912$

C $6.040972-2.730396 \quad 0.442530$

H $4.908713-5.5404070 .472850$

H $5.233942-4.7941582 .049008$

C $2.833259-5.9010142 .575722$

H $2.548050-5.0291330 .640476$

C $6.065001-1.309828-0.165475$

O $6.556837-2.6507931 .776869$

H $6.717717-3.391252-0.112876$

H $5.606670-0.5974730 .530482$

O $7.436636-0.918161-0.304311$

C $5.390011-1.193211-1.545416$

C $5.6641980 .123266-2.297391$

H $4.308277-1.326025-1.430486$

O $5.857675-2.261972-2.375704$

H $6.7264010 .182226-2.567527$

H $5.1244370 .059615-3.252016$

H $3.089075-6.8913562 .184816$

H $3.404420-5.7459193 .497115$

H $1.769634-5.9036772 .834260$

C $1.445375-2.9036671 .986660$

H $7.503644-2.4407701 .713335$

H $7.796837-0.7891770 .589222$

H $5.365001-2.220505-3.213146$

H $1.422064-1.8701682 .351339$

H $\quad 0.826395-3.5018612 .665558$

H $3.241470-3.3077703 .081234$

C $3.2729282 .412242-0.560649$

O $3.840576 \quad 1.309926-1.282479$

C $5.2413541 .399116-1.554865$

C $5.5513012 .661032-2.379686$

C $4.9933283 .922010-1.714204$

C $3.5313713 .740705-1.290783$

H $2.1913042 .228676-0.576174$

C 3.7239632 .4345920 .907886

H $5.7914901 .447167-0.610240$

O $5.007143 \quad 2.546332-3.693972$

H $6.6381622 .751909-2.490810$

H $5.0719514 .759792-2.417859$

O $5.7954414 .231595-0.578811$

H $3.2061264 .573779-0.657206$

O $2.704700 \quad 3.755497-2.458019$

C $3.211783 \quad 1.2393701 .681179$

H 4.8127552 .4364921 .001372

H 3.3256323 .3321751 .395053

H $4.0520052 .384150-3.607057$

H $5.4433615 .047822-0.184635$

H $2.8240374 .618199-2.890531$

O $3.624977 \quad 1.3457952 .978832$

\begin{tabular}{lllll}
\hline & 2.541329 & 0.330545 & 1.212908
\end{tabular}

$\begin{array}{lllll}C & 3.230487 & 0.277009 & 3.844266\end{array}$

H $3.711019-0.6541633 .531615$

H 3.5627210 .5198344 .857055

H $2.1418750 .169756 \quad 3.850381$

SCF Energy (B3LYP/6-31G*//MMFF)= -3245.90741204

0800183

MM̄FF Geometry

C $-0.835802-1.6861371 .499905$

C $0.313801-1.6139720 .814626$

C $0.365704-1.629426-0.691401$

O $1.715593-2.003914-1.049025$

C $0.078678-0.226557-1.253215$

C $-0.238237-0.211686-2.734010$

C $0.591060 \quad 0.247977-3.691331$

C $-1.619298-0.692273-3.100426$

C $1.9932870 .767832-3.532510$

C $2.0830352 .290391-3.717668$ 
C $1.4962553 .084775-2.539691$

C $1.5438414 .588799-2.816364$

C $1.0622075 .407247-1.607328$

O $2.2785802 .783054-1.380452$

O $1.4329166 .777043-1.825201$

C $1.8159294 .930218-0.347302$

C $-0.4655675 .386417-1.475413$

$\begin{array}{lllll}\text { O } & 1.299515 & 5.556100 & 0.825207\end{array}$

C $1.8127283 .395119-0.171383$

C 2.7769672 .9872740 .969020

$\begin{array}{lllll}\text { O } & 0.490781 & 2.968578 & 0.163262\end{array}$

C $1.913236-2.832903-2.102596$

O $1.052923-3.336618-2.811018$

C $3.357013-3.107563-2.301735$

C $4.330985-2.421974-1.681905$

C $5.772328-2.653800-1.803225$

C $6.228621-3.804230-2.660978$

C 3.0666881 .4809501 .026238

C 3.9968091 .1121662 .168080

C $5.223028-0.8538303 .095584$

$\begin{array}{lllll}\text { O } & 4.433878 & 1.929320 & 2.973362\end{array}$

C $6.580466-0.8430812 .464983$

C $7.261720-1.9511152 .135875$

C $8.615034-1.9312941 .483683$

C $8.632696-2.6444340 .148161$

C $9.166952-3.8719130 .031270$

C $8.114609-1.904405-1.066803$

C $6.612646-1.849792-1.118098$

N $4.280381-0.2475682 .173594$

H -1.770434 -1.764355 0.949646

H $1.249907-1.5118601 .357826$

H $-0.348098-2.374460-1.063101$

H $0.9312540 .424009-1.039116$

H $-0.7753270 .227199-0.733230$

H $\quad 0.2362530 .241599-4.721419$

H $-1.808981-0.632411-4.177412$

H -1.761468 -1.735024-2.801236

H -2.375866 -0.079833 -2.600193

H $2.4416960 .467095-2.580910$

H $2.606183 \quad 0.288527-4.306688$

H $3.1454732 .545959-3.823940$

H $1.5848242 .580906-4.650950$

H $0.4612022 .766684-2.374208$

H $0.9565384 .839402-3.708497$

H $2.5773564 .885318-3.044760$

H $1.0227147 .069916-2.657013$

H $2.8583805 .265151-0.444033$

H $-0.9331925 .805558-2.374524$

H $-0.7990896 .016188-0.643385$

H $-0.8702384 .382444-1.332145$

H 1.3289956 .5168550 .677432

H 3.7377423 .5009370 .833208

H 2.3677493 .3110851 .934522

H $\quad 0.541405 \quad 2.150590 \quad 0.681391$

H $3.575283-3.908972-3.000133$

H $4.056428-1.610155-1.010462$

H $5.785662-4.744114-2.312867$

H $7.310752-3.951138-2.651237$

H $5.937753-3.643455-3.704850$

H $2.1410120 .914743 \quad 1.154800$

H 3.5395301 .1537560 .092719

H $4.869997-1.8685393 .304020$

H $5.241469-0.2885764 .032642$

H 7.0219740 .1306792 .265098

H $6.827283-2.9260992 .344548$

H $9.323856-2.4054312 .175009$

H $8.978893-0.905161 \quad 1.347647$

H $9.213167-4.379957-0.926208$

H $9.566809-4.3985890 .891348$

H $8.523547-2.335261-1.987624$

H $8.500791-0.876954-1.054384$

H $6.185156-1.042209-0.523640$

H $3.926021-0.8265941 .417676$

C $-1.362829-0.2777813 .548310$

$\begin{array}{lllll}\text { O } & -2.472436 & 0.251064 & 2.792437\end{array}$

$\begin{array}{llll}\text { C }-2.159095 & 1.608519 & 2.422228\end{array}$

C -1.106521 2.0618263 .421793

$\begin{array}{llll}\text { C } & -0.272692 & 0.798081 & 3.528545\end{array}$
H $-1.692717 \quad 1.5700761 .431485$

C -3.4288212 .4582402 .359649$

H $-0.5356562 .925442 \quad 3.068073$

H $-1.5501852 .311491 \quad 4.392721$

$\begin{array}{llll}\text { C } & 0.649602 & 0.771508 & 4.734072\end{array}$

H $\quad 0.3359700 .7075192 .622570$

C -4.4794151 .9068751 .370848$

O -4.0165042 .4902163 .664672$

H -3.1712913 .4937942 .108292$

H -4.8457550 .9438851 .741619$

O -5.6072092 .7900111 .385233$

C $-3.9712881 .767285-0.076936$

C $-5.0349461 .285205-1.080384$

H $-3.1212211 .079372-0.108537$

O $-3.4915493 .042322-0.512701$

H $-5.879427 \quad 1.984802-1.104983$

H $-4.5820021 .334262-2.079513$

H 1.3689621 .5956084 .685019

H $\quad 0.0900850 .868518 \quad 5.670317$

H $1.212907-0.1662484 .772151$

C $-0.944572-1.6458642 .997520$

H -4.8303573 .0191293 .602672$

H -5.3149473 .6433551 .021182$

H $-3.0996742 .922762-1.394685$

H $-1.707122-2.383698 \quad 3.277139$

H $-0.011457-1.9741893 .470145$

H $-1.727443-0.4155934 .574366$

C $-4.667304-2.375607-0.474330$

O $-4.393686-1.010143-0.811737$

C $-5.532973-0.143746-0.810739$

C $-6.546546-0.612775-1.865534$

C $-6.939188-2.078732-1.645478$

C $-5.716322-2.978079-1.427070$

H $-3.716324-2.894376-0.650459$

C $-4.999257-2.5020041 .016129$

H $-6.006935-0.168753 \quad 0.176054$

O $-6.005469-0.462099-3.177133$

H $-7.4366980 .024490-1.811154$

H -7.496295 -2.440469-2.518637

O $-7.795962-2.179896-0.512319$

H $-6.033969-3.963377-1.067813$

O $-5.096874-3.178370-2.702752$

C $-4.798187-3.909838 \quad 1.516861$

H $-4.367010-1.8311191 .611497$

H $-6.024776-2.1997471 .247104$

H $-5.179819-0.974647-3.218875$

H $-8.582695-1.636355-0.688892$

H $-4.370092-3.812626-2.579994$

O $-3.471065-4.1467111 .719904$

O $-5.710448-4.706307 \quad 1.694603$

C -3.142916 -5.4572002 .190598$

H $-2.057871-5.5119322 .313014$

H $-3.615379-5.6408443 .160190$

H -3.452607 -6.210381 1.459938

SCF Energy (B3LYP/6-31G**//MMFF) $=-3245.91160607$

0800184

MM̄FF Geometry

C $1.948271-4.2512860 .202999$

C $0.654895-3.8937370 .197365$

C $-0.181094-3.818926-1.053903$

O $-0.938716-2.586143-1.045884$

C $-1.178300-4.991204-1.075817$

C $-1.994621-5.067020-2.351846$

C $-3.296328-4.732866-2.458030$

C $-1.257675-5.605980-3.551801$

C $-4.201309-4.180785-1.391010$

C $-4.814287-2.844327-1.829430$

C $-5.666604-2.203660-0.722837$

C $-6.282872-0.895272-1.220844$

C $-7.018910-0.132526-0.110439$

O $-4.813780-1.9552340 .397950$

O $\begin{array}{llll}-7.251594 & 1.205751 & -0.579004\end{array}$

C $-6.098058-0.023903 \quad 1.125259$

C $-8.399376-0.737405 \quad 0.172502$

$\begin{array}{llll}\text { O } & -6.820027 & 0.553214 & 2.210762\end{array}$

C $-5.466806-1.3723261 .534673$

C $-4.404279-1.2456892 .654673$ 
O $-6.497626-2.2337092 .033878$ C $-0.293423-1.445602-1.414899$ O $0.890982-1.322203-1.681983$ C -1.303204 -0.363499-1.464498 C $-0.9482820 .876830-1.832245$ C $-1.8544142 .017181-1.997729$ C $-3.3108551 .806152-1.680275$ C $-3.155901-0.433295 \quad 2.298647$ C -3.229014 1.0387812 .662341 C $-1.951512 \quad 3.1635972 .379201$ O -4.1209661 .5196013 .355154$ C $-2.574542 \quad 3.937546 \quad 1.261188$ C -1.9119754 .8501990 .534765$ C $-2.5423155 .671212-0.552393$ C $-1.7894095 .594034-1.861216$ C $-0.9392856 .568449-2.227102$ C $-2.1021894 .444809-2.790877$ C $-1.3540803 .186664-2.448423$ N -2.147898 1.7451612 .149849 H $2.415060-4.516532-0.740433$ H $\quad 0.160737-3.6593291 .137205$ H $0.449468-3.846555-1.951387$ H $-1.834270-4.930368-0.200658$ H $-0.637402-5.940955-0.962840$ H $-3.778707-4.865203-3.426143$ H $-1.907257-5.709517-4.427590$ H $-0.435583-4.939998-3.830918$ H -0.845186 -6.595962 -3.331360 H $-5.003349-4.905394-1.206659$ H -3.675048 -4.046905 -0.443482 H $-4.002509-2.159146-2.104403$ H $-5.428730-2.996754-2.725696$ H -6.450618 -2.911771 -0.429592 H $-6.952691-1.081116-2.069886$ H -5.487543 -0.247670 -1.610038 H $-7.7712131 .150387-1.399179$ H -5.3035530 .6845620 .860630$ H -9.025610 $-0.699178-0.727131$ H $-8.935929-0.1609750 .933909$ H -8.352934 -1.7796730 .494631$ H -6.1809270 .7803472 .906441$ H $-4.866487-0.8924443 .584014$ H $-4.053716-2.2637012 .879969$ H $-6.087773-2.9229372 .582329$ H -2.327318 $-0.628389-1.229063$ H $0.097877 \quad 1.072213-2.064519$ H -3.433729 $1.372324-0.682417$ H -3.762439 $1.133524-2.416638$ H $-3.8957102 .728441-1.676936$ H -2.315411 -0.8398942 .875119$ H -2.905667 -0.5368921 .238597$ H $-0.872833 \quad 3.3349312 .451635$ H -2.415893 3.4474803 .329006 H -3.628219 3.7593281 .059008 H $-0.867583 \quad 5.0498960 .760450$ H -2.575419 $6.711946-0.203682$ H -3.588343 5.383967 -0.716535 H $-0.420746 \quad 6.537791-3.179678$ H $-0.7363797 .413956-1.578291$ H $-1.8220584 .713057-3.819056$ $\mathrm{H}-3.1850554 .297059-2.837649$ H $-0.2851003 .247074-2.654831$ H -1.490795 1.2719451 .536222 C $4.255684-4.0026211 .299338$ O $4.444329-2.654620 \quad 0.823239$ C $5.595514-2.642220-0.045580$ C $6.247281-4.0198930 .073344$ C $5.054341-4.9051470 .359321$ H $5.214880-2.522442-1.066035$ C $6.557576-1.5015790 .305355$ H $6.784096-4.301946-0.837932$ H $\quad 6.960150-4.0616700 .905441$ C $5.424546-6.2523560 .955168$ H $4.521394-5.068741-0.583952$ C $6.002859-0.0706170 .168598$ O $6.998589-1.661666 \quad 1.661385$ H $7.460256-1.594796-0.310996$ H 5.3023090 .1452280 .981622 $\begin{array}{llll}\text { O } & 7.112381 & 0.822230 & 0.351099\end{array}$

C $5.3376580 .253261-1.181466$

C $5.1932101 .753789-1.499752$

H $4.352433-0.224916-1.219620$

O $6.107807-0.316429-2.246674$

H $6.1893942 .186723-1.661703$

H $4.6842931 .823341-2.471106$

H $6.068296-6.8123550 .268864$

H $5.962349-6.1389561 .902349$

H $4.530369-6.8542311 .144657$

C $2.770116-4.3454521 .459192$

H $6.208081-1.6500632 .228391$

H 7.5034130 .6239791 .219393

H $7.0018250 .063927-2.199708$

H $2.345009-3.6745502 .215884$

H $2.684246-5.3668441 .848395$

H $4.708859-4.0307622 .300049$

C 2.2176162 .7990530 .507722

O $3.0809492 .125834-0.420148$

C $4.4340882 .587434-0.457743$

C $4.4941434 .085882-0.808395$

C $3.6212104 .917357 \quad 0.133047$

C $2.218876 \quad 4.3192840 .271309$

H 1.2120132 .4315330 .268305

C 2.5342402 .4243891 .961599

H 4.8929312 .4349050 .523711

O $4.0578704 .279716-2.153277$

H $5.5372854 .419733-0.748503$

H $3.5445375 .940051-0.256065$

$\begin{array}{lllll}\text { O } & 4.256746 & 4.983326 & 1.405314\end{array}$

H 1.6762924 .8147571 .083989

O $1.488458 \quad 4.589422-0.926958$

$\begin{array}{llll}\text { C } & 2.337029 & 0.952819 & 2.231733\end{array}$

H 3.5687862 .6592652 .227955

H 1.8920322 .9766812 .657442

H $4.1512275 .225646-2.358368$

H 3.7005875 .5332501 .983096

H $1.9071714 .092620-1.650710$

$\begin{array}{lllll}\text { O } & 1.049803 & 0.590045 & 1.972301\end{array}$

$\begin{array}{lllll}\text { O } & 3.219941 & 0.219860 & 2.659381\end{array}$

C $0.752961-0.7924512 .175984$

H $-0.196430-1.0132071 .683654$

H $1.526325-1.4234991 .731372$

H $0.655137-0.9961923 .245771$

SCF Energy (B3LYP/6-31G**//MMFF) $=-3245.92025698$

0800185

MM̄FF Geometry

C $2.3583423 .684234 \quad 0.209089$

C $2.7107994 .197956-0.979873$

C $3.5162543 .484607-2.041907$

O $3.4696852 .052538-1.851673$

C $4.9844763 .932779-1.965522$

C $5.8538113 .300718-3.033646$

C $6.6822632 .258963-2.820302$

C $5.7774093 .947119-4.391720$

C $6.8655101 .491157-1.539163$

C $6.937278-0.021499-1.794304$

C $7.089316-0.804972-0.482271$

C $7.422846-2.270927-0.760789$

C $7.513944-3.0866410 .537048$

O $5.852618-0.718743 \quad 0.235702$

$\begin{array}{llll}\text { O } & 7.521309 & -4.478078 & 0.183783\end{array}$

C $6.240970-2.8374341 .369908$

C $8.821901-2.822808 \quad 1.292054$

O $6.340658-3.4541922 .651872$

C $5.878025-1.3402091 .528344$

C $4.469398-1.2220742 .156360$

O $6.824711-0.7105692 .392856$

C $2.4279891 .383580-2.415876$

O $1.5226591 .856869-3.083522$

C $2.565274-0.042208-2.046669$

C $1.540190-0.878418-2.266300$

C $1.487120-2.293998-1.897483$

C $2.751239-2.933200-1.387921$

C 3.8744680 .1908932 .139516

C $2.431994 \quad 0.1477862 .607171$

C $0.157437-0.5667201 .848635$ 
O $2.116933 \quad 0.443255 \quad 3.757234$ C $-0.062639-2.0373941 .669504$ C -1.017526 -2.562209 0.885927 C -1.200890 -4.040592 0.680560 C $-1.277099-4.434986-0.780944$ C $-2.417631-4.910810-1.308493$ C $-0.012728-4.358537-1.615151$ C $0.312651-2.946427-2.023337$ N $1.564787-0.2912301 .618996$ H 2.6772182 .6809890 .476355 H $2.4104555 .215252-1.222043$ H $3.1037923 .737274-3.026733$ H $5.389463 \quad 3.711227-0.970600$ H $5.0486715 .025183-2.062762$ H $7.2969901 .916741-3.651778$ H $6.4509463 .475808-5.115376$ H $4.7618673 .876422-4.793450$ H $6.0530615 .004759-4.325098$ H $7.8013151 .821282-1.072687$ H $6.0594801 .692781-0.828089$ H $6.025854-0.343707-2.314335$ H $7.788419-0.234680-2.453060$ H $7.886407-0.3395380 .109430$ H $8.354991-2.358015-1.332760$ H $\quad 6.639098-2.709892-1.394263$ H $8.283217-4.634910-0.400041$ H $5.412036-3.338608 \quad 0.850144$ H $9.684450-3.0713680 .661991$ H $8.903196-3.4641592 .176587$ H $8.932720-1.783578 \quad 1.608231$ H $6.552209-4.3922912 .506384$ H $3.783931-1.8868291 .618964$ H $4.494373-1.5734023 .196230$ H $6.416703 \quad 0.0779302 .784367$ H $3.484913-0.354133-1.565208$ H $\quad 0.634552-0.481697-2.723710$ H $3.586046-2.741678-2.071918$ H $2.682934-4.019516-1.297622$ H $3.009121-2.536674-0.402905$ H 4.4292130 .8665972 .798673 H $3.904240 \quad 0.6251421 .134308$ H $-0.4106230 .025667 \quad 1.124992$ H $-0.127527-0.2625552 .860127$ H $0.607578-2.7058332 .205717$ H -1.704942 -1.897785 0.367186 H $-2.120996-4.3357011 .200753$ H - $0.391364-4.6091401 .155415$ H -2.475173 -5.219699 -2.347107 H $-3.320167-5.003006-0.714663$ H - $0.128971-4.950580-2.532046$ H $0.793329-4.834735-1.050130$ H - $0.536367-2.403498-2.441803$ H $1.936569-0.4968780 .696978$ C $0.3439703 .600328 \quad 1.692423$ O -0.5904253 .4619930 .604720$ C -1.9301733 .6454901 .111502$ C -1.807676 3.6358032 .628233 C -0.4385554 .2509562 .830233$ H -2.247932 4.6343420 .757881 C -2.8399522 .5514950 .538502$ H $-2.607203 \quad 4.1991493 .118592$ H -1.826017 2.6087213 .014305 C 0.1439533 .9912204 .208849 H -0.5115435 .3344412 .666602$ C -4.2675132 .5775501 .115022$ O $-2.8905572 .747578-0.877996$ H $-2.367457 \quad 1.578326 \quad 0.709247$ H -4.2356452 .3970372 .195584$ O -4.8056923 .8970910 .951689$ C $-5.263640 \quad 1.588588 \quad 0.477915$ C -4.8041190 .1224020 .558098$ H $-5.4662881 .866035-0.562947$ O H -3.8059120 .0185390 .121379$ H -4.747705 -0.1615221 .616631$ H -0.4976864 .4217844 .984555$ H $\quad 0.2390622 .9186074 .408648$ H 1.1371104 .4408104 .304870
C 1.5517004 .4221301 .240227

H -3.281482 $1.950980-1.274233$

H $-4.802144 \quad 4.0989110 .000061$

H -6.7758112 .6457981 .128660$

H 2.2039304 .6285662 .096240

H 1.2154815 .3855410 .837480

H 0.6544462 .5870431 .975351

C $-8.028559-1.680144-0.091762$

O $-7.007871-0.8689520 .503646$

C $-5.750634-0.850693-0.177262$

C $-5.162989-2.270021-0.238744$

C $-6.154870-3.255816-0.864123$

C $-7.550781-3.133193-0.243185$

H $-8.850705-1.6714040 .635674$

C $-8.552726-1.074252-1.398169$

H $-5.893689-0.471285-1.195066$

O $-4.829433-2.7002441 .079798$

H $-4.236229-2.243715-0.824180$

H $-5.784380-4.278297-0.724185$

O $-6.218992-3.004732-2.264752$

H $-8.279763-3.697343-0.835719$

O $-7.530624-3.7372771 .053903$

C $-9.2353010 .246858-1.134609$

H -7.770414 -0.921561-2.147070

H -9.295830 -1.733428 -1.862493

H $-4.444023-3.5899711 .010343$

H -6.831098 -3.653850 -2.651449

H $-6.944030-3.2066081 .619980$

O $-8.3979091 .275966-1.448570$

O $-10.367930 \quad 0.349355-0.681497$

C $-8.9188802 .587877-1.214764$

H -8.137604 $3.312022-1.460785$

H $-9.7839942 .769993-1.859162$

H $-9.1886572 .708789-0.161154$

SCF Energy (B3LYP/6-31G**//MMFF) $=-3245.90539021$

0800186

MM̄FF Geometry

C -3.009317 $3.740894-0.984769$

C $-3.9907514 .251080-0.224683$

C -4.8855253 .4820420 .720727$

$\begin{array}{lllll}\text { O } & -4.419650 & 2.128376 & 0.925774\end{array}$

C -6.3116853 .4271840 .145698$

C -7.3086892 .7493381 .065633$

C -7.7940511 .5038330 .889413$

C -7.7848093 .5876522 .223903$

C -7.432662 $0.522504-0.190959$

C $-6.930164-0.7991260 .405005$

C $-6.367067-1.733212-0.676096$

C $-5.967556-3.079819-0.071030$

C $-5.286407-3.995969-1.098500$

O $-5.221943-1.094522-1.252011$

O $-4.659409-5.071392-0.382053$

C $-4.160695-3.209328-1.804342$

C $-6.302369-4.642275-2.047611$

O $-3.604573-3.970710-2.873090$

C $-4.607352-1.826250-2.321755$

C $-3.434754-0.966730-2.859676$

O $-5.523092-2.007737-3.409336$

C -3.4327021 .9475441 .848568$

O -2.8503312 .8152162 .479904$

C -3.1894540 .4928361 .972465$

C -2.2431430 .0439292 .810678$

C $-1.931558-1.3599403 .094128$

C -2.699751 -2.413278 2.340808

C $-2.340140-0.624566-1.843313$

C $-1.183503-1.609211-1.818204$

C $0.843920-2.179211-0.483213$

O $-1.004069-2.460303-2.684771$

C $0.600971-3.0995010 .668990$

C $1.315944-3.0632021 .803621$

C $1.128900-4.0249652 .939943$

C $0.845706-3.3331224 .252944$

C $1.824550-3.1225925 .149387$

C $-0.589333-2.9814824 .568424$

C $-0.997668-1.6380204 .028195$

N $-0.351981-1.396691-0.727610$

H $-2.8148402 .672066-0.977697$ 
H $-4.1680115 .324527-0.258171$ H -4.9011354 .0054871 .685117$ H $-6.2923282 .935400-0.832687$ H -6.670339 $4.445831-0.057541$ H -8.5312511 .1358961 .602119$ H -8.543872 3.0750722 .824541 H -6.9513363 .8336092 .888974$ H $-8.2284004 .520617 \quad 1.860819$ H $-8.3247640 .329400-0.798740$ H $-6.6767980 .926927-0.867402$ H $-6.147370-0.584091 \quad 1.142693$ H -7.751637 -1.2961810 .936011$ H -7.131856 -1.871429-1.449457 H -6.837289 $-3.580000 \quad 0.373078$ H $-5.267002-2.9112810 .757344$ H $-5.346903-5.5322540 .128740$ H -3.361357 -3.071881-1.066157 H -7.020462 -5.249444 -1.483308 H $-5.810912-5.330982-2.743509$ H $-6.872877-3.913326-2.626748$ H - $-3.312840-4.820993-2.502055$ H -3.015763 -1.424506 -3.764031 H $-3.862326-0.009296-3.191101$ H -5.539213 -1.193330 -3.938763 H $-3.816026-0.172351 \quad 1.391053$ H $-1.656070 \quad 0.7723353 .368855$ H -3.754164 -2.399164 2.636203 H $-2.327549-3.4265232 .506587$ H $-2.634601-2.243107 \quad 1.260873$ H -1.904099 $0.345064-2.116899$ H $-2.758344-0.522212-0.838140$ H $1.656528-1.477003-0.280721$ H $1.106666-2.759521-1.373109$ H $-0.176525-3.8500990 .549308$ H $2.108815-2.3256971 .908357$ H $2.046834-4.6216113 .023700$ H $\quad 0.330586-4.7469172 .727043$ H $1.621419-2.6576956 .108579$ H $2.851272-3.4048504 .941068$ H $-0.727399-2.9441375 .657876$ H -1.246994 -3.791882 4.241449 H $-0.492143-0.8045234 .518102$ H - $0.581853-0.664262-0.061902$ C - $0.6519734 .558970-1.443084$ O $-0.1054883 .233021-1.580307$ C $0.7462942 .965547-0.444361$ C $0.966870 \quad 4.308830 \quad 0.237730$ C $-0.3610334 .995998-0.007222$ H $\quad 0.1714912 .293704 \quad 0.205988$ C $2.0266512 .274229-0.927226$ H 1.2058084 .2070951 .300563 H $1.7804544 .863726-0.246024$ C $-0.317858 \quad 6.501706 \quad 0.181472$ H -1.093080 4.5720360 .690556 C 3.0519882 .0329130 .199336 O $1.6498581 .028337-1.515293$ H $2.4929982 .865945-1.724184$ H $3.340473 \quad 3.0035050 .618794$ O 2.4387941 .2966391 .258550 C $4.3378981 .311793-0.255593$ C $5.357956 \quad 1.1993260 .895720$ H $4.7773861 .872597-1.088925$ O $4.0111470 .007884-0.727638$ H 5.4507462 .1725761 .392415 H $4.9805800 .472847 \quad 1.626543$ H -0.0383546 .7524821 .209964$ H $\quad 0.4111206 .970100-0.488185$ H -1.297247 $6.946907-0.020919$ C $-2.1233454 .574238-1.868401$ H $0.9979821 .218681-2.211692$ H 2.1928560 .4262670 .901709 H $4.829788-0.404666-1.050468$ H $-2.2066104 .172309-2.885607$ H -2.478860 $5.610919-1.903255$ H $-0.0896485 .187655-2.146042$ C $7.859215-1.149828-0.606209$ O $6.656798-0.592040-0.060589$ C $6.7551550 .752370 \quad 0.417880$
C 7.7969630 .8407001 .544196

C 9.1502230 .2811931 .091301

C $9.005400-1.0871140 .417436$

H $7.621589-2.207658-0.779166$

C $8.215964-0.524105-1.959007$

H $7.0544621 .410088-0.405493$

O 7.3338220 .1090862 .677712

H 7.9019781 .8891251 .848019

H 9.8165510 .1829921 .957068

O $9.758390 \quad 1.185206 \quad 0.173644$

H $9.946023-1.368350-0.069526$

O $8.760696-2.0729351 .425423$

C $7.165575-0.859502-2.991238$

H $8.3302790 .562817-1.917612$

H $9.169340-0.918329-2.330454$

H $7.9958150 .210237 \quad 3.382625$

H 9.8742982 .0351500 .631599

H $7.893286-1.8831661 .822579$

O $6.2771990 .168454-3.103843$

O $7.125927-1.919457-3.602458$

C $5.205670-0.040104-4.029095$

H $4.620053-0.918235-3.740630$

H $4.5557310 .838644-3.999010$

H $5.599504-0.153003-5.043376$

SCF Energy (B3LYP/6-31G**//MMFF) $=-3245.91878768$

0800187

MM̄MF Geometry

C $2.263173 \quad 0.304778 \quad 3.210842$

C $2.880892-0.8461473 .521276$

C $3.987754-1.5306242 .751345$

O $4.394046-0.7655921 .596389$

C $3.500586-2.8903762 .219484$

C $4.620798-3.8969462 .051926$

C $5.275554-4.1474160 .901722$

C $4.952374-4.6846533 .294116$

C $5.139474-3.449560-0.422471$

C $4.437402-4.307676-1.484198$

C $2.906240-4.253288-1.375202$

C $2.252145-5.188968-2.393209$

C $0.720469-5.062814-2.382606$

O $2.508182-2.902018-1.633133$

O $0.215603-5.718022-3.556083$

C $0.340864-3.571365-2.499066$

C $0.095198-5.781584-1.181056$

O $-1.065216-3.389546-2.347174$

C $1.103133-2.659254-1.509703$

C $0.840528-1.173660-1.854833$

O $0.636850-2.919736-0.185241$

C 5.3515450 .1847501 .743922

O 5.9166870 .5191242 .771180

C 5.5646890 .7738350 .404371

C $6.100288 \quad 1.996628 \quad 0.288132$

C $6.3096152 .722096-0.967064$

C $5.9958911 .999117-2.251911$

C $1.755411-0.176558-1.127090$

C $1.3785201 .264289-1.435885$

C $2.0050953 .606589-0.845501$

O $\quad 0.4512601 .570764-2.181022$

C $2.9573094 .184489-1.844917$

C $3.7250755 .257536-1.598189$

C $4.6564975 .870160-2.606234$

C $6.0466716 .109651-2.057007$

C $6.4319897 .335732-1.664168$

C $7.0151494 .947322-2.042463$

C $6.7487643 .996513-0.906902$

N $2.1940442 .168873-0.769117$

H 2.5510880 .8503602 .317792

H $2.577901-1.3600384 .432457$

H $4.843390-1.6649193 .424919$

H $2.982864-2.7482091 .265137$

H $2.755807-3.3317962 .895292$

H $6.037924-4.9261260 .909848$

H $5.766238-5.398953 \quad 3.130885$

H $5.261310-4.0130154 .101340$

H $4.077469-5.2514803 .628860$

H $4.650547-2.477033-0.325752$

H $6.156972-3.232094-0.772573$ 
H $4.731857-3.919058-2.468005$

H $4.787545-5.345501-1.426847$

H $2.610475-4.535333-0.359005$

H $2.553355-6.228919-2.215998$

H $2.612251-4.940472-3.401581$

H $0.509126-6.644948-3.531542$

H $0.585724-3.251810-3.521783$

H $\quad 0.347064-6.848797-1.200309$

H -0.998801 -5.731967-1.216605

H $\quad 0.427963-5.382340-0.220787$

H $-1.504087-3.969347-2.993064$

H $0.987251-1.021092-2.932339$

H $-0.204531-0.926137-1.633207$

H $0.849212-2.1598180 .378104$

H $5.2309540 .197074-0.451009$

H 6.3843872 .5283281 .195292

H $6.6296741 .111274-2.354923$

H $6.1529742 .604471-3.146353$

H $4.9469441 .683959-2.274403$

H $1.694456-0.313809-0.042942$

H $2.796080-0.327995-1.435071$

H $2.168316 \quad 4.007437 \quad 0.159899$

H $0.9755113 .825209-1.146382$

H $2.9992193 .715088-2.825111$

H $3.6567215 .745437-0.628350$

H $4.2153286 .822724-2.927756$

H $4.7265605 .254799-3.511800$

H $7.4350107 .521977-1.295020$

H $5.7508248 .179604-1.697624$

H $8.0435665 .313930-1.923219$

H $7.0063384 .462086-3.022031$

H $6.943327 \quad 4.4309720 .074877$

H $2.9571591 .820994-0.196690$

C $-0.185902 \quad 1.0133183 .395669$

O $-0.652776-0.2977063 .024189$

C $-1.326565-0.2008941 .748661$

C -1.5136701 .2861951 .484586$

C -0.2744621 .8680272 .134411$

H $-0.635237-0.626497 \quad 1.013847$

C -2.614535 -1.030613 1.801474

H -1.5859851 .5183490 .417745$

H -2.4168151 .6612721 .979864$

C -0.3665053 .3599322 .397971$

H 0.5784631 .6748041 .476962

C -3.506752 -0.8613370 .556123$

O $-2.234226-2.402361 \quad 1.939840$

H -3.186904 -0.771895 2.699624

H -3.7473310 .2007440 .456883$

O $-2.772098-1.226243-0.611304$

C $-4.829594-1.6535260 .605611$

C $-5.708130-1.483849-0.650111$

H $-5.391411-1.3498881 .496823$

O $-4.549186-3.048167 \quad 0.741222$

H -5.182341 -1.886061 -1.525484

H $-6.590712-2.123787-0.515909$

H $-0.5018063 .908346 \quad 1.459910$

H -1.210725 3.6010953 .052644

H 0.5474503 .7278762 .874892

C 1.1875010 .9209804 .065803

H -1.674345 -2.470793 2.732179

H $-2.537586-2.166625-0.525203$

H -5.397828 -3.5104450 .848338$

H 1.0781040 .3352504 .987821

H 1.5175061 .9216044 .367937

H $-0.899464 \quad 1.3872774 .142306$

C $-7.387395 \quad 1.7599390 .147720$

$\begin{array}{lllll}\text { O } & -6.916676 & 0.405540 & 0.204744\end{array}$

C $-6.159422-0.040995-0.924765$

C $-6.9942960 .064600-2.210326$

C $-7.5369201 .485075-2.397785$

C $-8.2153772 .003843-1.126658$

H -8.0679341 .8533261 .004441$

C -6.2454472 .7690840 .340554$

H $-5.2708470 .585614-1.040176$

O $-8.079006-0.860526-2.183168$

H $-6.363883-0.207526-3.064704$

H -8.259486 $1.493693-3.222888$

O $-6.4706922 .366402-2.738648$
H $-8.4384213 .073292-1.218327$

O $-9.4667341 .330684-0.958023$

C -5.8872122 .9197401 .801654$

H $-5.3305932 .517282-0.201725$

H $-6.5604453 .761920-0.002988$

H $-8.601843-0.682755-1.382550$

H $-6.0693262 .037319-3.561063$

H $-10.011498 \quad 1.525687-1.739539$

O -5.3581151 .7606092 .285075$

O $-\begin{array}{llll}-6.048381 & 3.955897 & 2.434342\end{array}$

C -4.9946591 .7814873 .669095$

H -5.8559782 .0528724 .287311$

H $-4.6671830 .777633 \quad 3.950608$

H -4.1678502 .4797503 .829260$

SCF Energy $\left(B 3 L Y P / 6-31 G^{* *} / / M M F F\right)=-3245.91068929$

$08 \quad 00188$

MM̄FF Geometry

C -3.792651 2.4938420 .162946

C $-3.8205383 .548091-0.667607$

C $-2.7608813 .905686-1.685747$

O $-1.7041192 .920855-1.708546$

C $-2.1305835 .268377-1.347638$

C $-1.0788495 .703668-2.352886$

C $0.2476105 .752476-2.115028$

C $-1.6130926 .124732-3.698752$

C $0.9767325 .406275-0.846905$

C $1.9013504 .199375-1.032860$

C 2.4439993 .6586280 .299040

C 3.3497524 .6625961 .017959

C 3.9417974 .0801022 .310279

O $3.1771332 .456763 \quad 0.012434$

O 4.9965104 .9634892 .722093

C 4.5680492 .7052671 .995469

C 2.9151844 .0813593 .449170

O 5.0124702 .0655493 .189448

C 3.6381851 .7729741 .187024

C 4.3427320 .4654310 .761982

O 2.5349521 .3870372 .017513

C $-1.8453931 .866469-2.557286$

O $-2.7828931 .638814-3.304220$

C $-0.6359831 .027283-2.416063$

C $-0.4328330 .002600-3.256598$

C $0.733842-0.879135-3.261648$

C $1.755559-0.698814-2.169347$

C $5.5296900 .627316-0.189444$

C $6.219731-0.707528-0.404044$

C $5.871663-2.949556-1.407799$

O $7.289528-0.9773680 .137400$

C $5.129570-3.499976-2.581839$

C $4.050109-4.289769-2.473730$

C $3.268750-4.807751-3.647774$

C $1.941067-4.091991-3.802072$

C $0.803652-4.676079-3.388640$

C $2.014701-2.731975-4.468482$

C $0.846461-1.812210-4.230905$

N $5.506083-1.556092-1.233860$

H $-2.937224 \quad 1.823810 \quad 0.164238$

H -4.691486 $4.200414-0.649666$

H -3.240269 $3.961017-2.671015$

H $-1.7125255 .227252-0.337395$

H -2.910678 $6.040576-1.307103$

H $0.8989906 .090136-2.920584$

H $-0.8288196 .521899-4.352095$

H -2.071287 $5.276057-4.215551$

H $-2.3668306 .909830-3.579150$

H $1.5661146 .283170-0.554778$

H $0.2903815 .211287-0.019849$

H $1.3624953 .389656-1.541070$

H $2.7350934 .456460-1.699481$

H 1.5827623 .4009610 .927235

H 2.8174815 .5940381 .242768

H 4.1769754 .9436850 .350926

H 5.4283954 .5651193 .497411

H 5.4715072 .9090371 .409044

H 2.5760365 .1040703 .654140

H 3.3590023 .7229674 .384424

H $2.031813 \quad 3.4772703 .232426$ 
H 4.2305611 .7033193 .639754 H $4.656074-0.0904691 .655758$ H $3.601052-0.1824710 .275324$ H 2.0775390 .6480521 .582019 H $0.0682531 .290970-1.635309$ H -1.174841 -0.196240 -4.028625 H $2.3039670 .239624-2.302870$ H $2.484303-1.510892-2.136914$ H $1.278431-0.690270-1.182802$ H $5.2061641 .026025-1.157504$ H $\quad \begin{array}{llll}6.279398 & 1.315976 & 0.211207\end{array}$ H $5.624878-3.474482-0.478342$ H $6.953030-3.027808-1.559821$ H $5.487780-3.215263-3.568168$ H $3.688007-4.564801-1.486186$ H $3.116446-5.885480-3.507019$ H $3.840923-4.712366-4.579452$ H $-0.164943-4.202942-3.502748$ H $0.813956-5.656576-2.923222$ H $2.116615-2.884960-5.550985$ H $2.935749-2.218090-4.170476$ H $0.052658-1.901638-4.972365$ H $4.568459-1.276283-1.503665$ C -5.6387520 .8557710 .731901$ O $-4.724033-0.2588210 .663596$ C $-5.023137-1.020514-0.520807$ C $-6.414724-0.577859-0.959272$ C $-6.3758490 .895671-0.608518$ H $-4.300500-0.711863-1.285701$ C $-4.895758-2.523099-0.255702$ H $-6.594480-0.763048-2.022600$ H -7.201222 -1.083815 -0.387167 C $-7.7463401 .545858-0.538862$ H $-5.7786491 .411226-1.370297$ C $-3.510380-2.986104 \quad 0.242423$ O $-5.861362-2.9096340 .731348$ H -5.163854 -3.073575 -1.165325 H -3.367859 -2.660676 1.278400 O $-3.531650-4.4199890 .282384$ C -2.316698 -2.524822 -0.614640 C $-0.986565-3.240502-0.310822$ H -2.185446 -1.443118 -0.497648 O $-2.624480-2.766272-1.991653$ H -1.051686 -4.287953 -0.632957 H $-0.224293-2.785051-0.954620$ H $-8.2509841 .484065-1.508564$ H -8.3858791 .0573350 .203970$ H -7.661677 2.603146 -0.268449 C $-4.8975482 .140107 \quad 1.117870$ H -5.686139 -2.386732 1.532728 H $-3.615098-4.737719-0.633165$ H -1.883313 -2.429120 -2.522669 H -4.4399072 .0064512 .105631$ H -5.613482 2.9652891 .209441 H -6.3455270 .6156641 .537011$ C $-0.014484-1.5632652 .886446$ O $-0.374135-1.8023901 .517667$ C $-0.528972-3.1758531 .152452$ C $0.789862-3.933641 \quad 1.367730$ C $1.267471-3.7911552 .816282$ C $1.264447-2.3305523 .274848$ H $0.209894-0.4899882 .933734$ C - $-1.188235-1.8375683 .840411$ H -1.304539 -3.633766 1.773280 O $1.783793-3.4249360 .480232$ H $0.636316-4.991117 \quad 1.121804$ H $2.283893-4.1929042 .909417$ O $0.421259-4.5469963 .677743$ H $1.413985-2.2804824 .359949$ O $2.386179-1.6826332 .670847$ C $-2.350841-0.8938323 .613650$ H -1.571009 -2.855440 3.730554 H $-0.854364-1.6964854 .875061$ H $2.603973-3.9209180 .642359$ H $\quad 0.458270-5.4739723 .386688$ H $2.476884-0.8099253 .090032$ O $-3.349834-1.2128094 .489616$ O $-2.384330 \quad 0.002156 \quad 2.782191$
C $-4.532629-0.4121674 .403347$

H $-5.167972-0.6517165 .260237$

H $-4.2862720 .653318 \quad 4.439742$

H -5.075438 -0.654055 3.485895

SCF Energy (B3LYP/6-31G**//MMFF) $=-3245.91691158$

0800189

MM̄FF Geometry

C $0.0764194 .765454 \quad 0.995427$

C -0.9899743 .9569320 .900611$

C -1.7052053 .3898292 .101741$

O -1.9674031 .9868341 .854744$

C -3.0507624 .1071602 .303397$

C -3.7667173 .6922623 .575910$

C -4.8710652 .9208613 .628162$

C -3.1781044 .2411734 .851528$

C -5.6153462 .2949802 .483075$

C -5.5278100 .7646052 .536273$

C $-6.1097870 .118147 \quad 1.271148$

C $-6.081368-1.4067771 .380452$

C $-6.531852-2.0889790 .079728$

$\begin{array}{llll}\text { O } & -5.310719 & 0.545015 & 0.163561\end{array}$

O $-6.160286-3.4742000 .157561$

C $-5.751196-1.479662-1.105250$

C $-8.056281-2.057564-0.080997$

O $-6.248871-1.970580-2.347230$

C $-5.7512130 .062839-1.112412$

C $-4.8064800 .668151-2.180053$

O $\quad-7.072990 \quad 0.530199-1.408527$

C -0.9282031 .1223092 .021187$

O 0.2074451 .3983492 .374666

C -1.412237-0.235985 1.685585

C $-0.546985-1.2609721 .666555$

C $-0.864884-2.6613541 .377578$

C $-2.289376-3.0007441 .030212$

C $-3.3229560 .313705-2.025390$

C $-2.879710-0.876788-2.858593$

C $-0.895468-2.330760-3.252071$

O $-3.586965-1.405654-3.711435$

C $-0.976155-3.580827-2.435527$

C $0.100906-4.259419-2.011404$

C $0.028524-5.563681-1.271835$

C $0.781378-5.5427580 .037972$

C $2.034347-6.0209620 .124316$

C $0.043863-5.0624361 .265929$

C $0.126599-3.5738641 .445602$

$\mathrm{N}-1.577468-1.254844-2.560441$

H 0.4135835 .0562451 .988920

H -1.370698 $3.663502-0.073329$

H -1.089698 3.4868323 .004719

H -3.6832843 .9426621 .425577$

H -2.890994 5.1932532 .345804

H $-5.2999912 .705828 \quad 4.606320$

H -3.781374 3.9895935 .730421

H -2.173947 3.8404695 .018686

H -3.1144915 .3331674 .801257$

H -6.6669612 .6006362 .545291$

H $-5.2537432 .657781 \quad 1.519007$

H -4.4757150 .4713122 .640786$

H $-6.059551 \quad 0.396693 \quad 3.422569$

H $-7.1374310 .475870 \quad 1.136700$

H -6.692428 -1.747372 2.225703

H $-5.057377-1.7335281 .603701$

H $-6.585618-3.8548360 .945063$

H -4.717588 -1.835218 -1.019652

H $-8.539847-2.5770170 .755246$

H -8.369697 -2.590962 -0.985208

H -8.462925 -1.044876 -0.117737

H -6.210962 -2.941772 -2.311175

H $-5.1763830 .437976-3.186395$

H $-4.877127 \quad 1.761874-2.089956$

H -7.011360 $1.438812-1.746525$

H -2.466819 -0.3565081 .468092$

H $0.499116-1.0604291 .896086$

H $-2.423400-4.0324380 .698058$

H $-2.648503-2.3716690 .209177$

H -2.937662 -2.850670 1.899574

H $-2.7264861 .166547-2.375058$ 
H $-3.063050 \quad 0.143087-0.976185$ H $0.135346-2.008266-3.417641$ H -1.361789 -2.498765 -4.228240 H -1.969423 -3.968747 -2.221494 H $1.093304-3.885320-2.251931$ H $0.447873-6.338802-1.927103$ H $-1.010844-5.865683-1.092568$ H $2.567464-6.039776 \quad 1.069007$ H $2.556491-6.397406-0.749114$ H $\quad 0.482695-5.5205872 .163084$ H $-0.984721-5.4337661 .248334$ H $1.128293-3.2300601 .703494$ H $-1.084458-0.788846-1.804374$ C $0.8712494 .541252-1.456531$ O $1.417298 \quad 3.223856-1.232940$ C $2.5202393 .020550-2.128797$

C $2.2710893 .978817-3.282734$ C $1.7437225 .190183-2.538052$ H $3.4247573 .330897-1.588414$ C $2.6666211 .546298-2.528217$ H $3.1784114 .188456-3.856889$ H $1.507903 \quad 3.594599-3.969449$ C $0.9929016 .171399-3.421877$ H $2.5925835 .711834-2.075670$

C $2.853034 \quad 0.622212-1.304149$

O 1.520488 1.150827 -3.280184

H $3.5381731 .461237-3.188122$

H $3.5615751 .098747-0.619449$

O $1.6186850 .499253-0.598545$

C $3.393835-0.778633-1.657393$

C $3.437333-1.755218-0.466922$

H $4.395019-0.670845-2.091104$

O $2.569319-1.363955-2.664886$

H $2.421036-1.915154-0.087615$

H $3.763990-2.729513-0.855181$

H $1.6517386 .570140-4.200033$

H $\quad 0.1394135 .694866-3.915777$

H $0.6149937 .013450-2.833230$

C $0.8955955 .324377-0.138104$

H $1.6556530 .229755-3.559722$

H $0.9760540 .094490-1.205796$

H $2.972452-2.211064-2.921104$

H $0.5845896 .361653-0.307721$

H $1.933443 \quad 5.3548280 .220262$

H $-0.1538414 .409822-1.821405$

C $6.726980-0.881621 \quad 1.073449$

O $5.703268-1.289617 \quad 0.155736$

C $4.369294-1.3205570 .675800$

C $4.273485-2.2701021 .880860$

C $5.312475-1.9150922 .947401$

C $6.709558-1.7505422 .342453$

H $7.668861-1.0855120 .547451$

C $6.663756 \quad 0.6227251 .364259$

H $4.083245-0.316647 \quad 1.001503$

O $4.470262-3.6145621 .449537$

H $3.264257-2.2012452 .303202$

H $5.333499-2.7055633 .707456$

O $4.912950-0.705025 \quad 3.584270$

H $7.394829-1.3263463 .084907$

O $7.226796-3.0430652 .012244$

C $7.219437 \quad 1.410996 \quad 0.201868$

H 5.6575050 .9917951 .580519

H 7.2819890 .8745872 .234503

H $4.352978-4.1908972 .223904$

H $5.568081-0.5065954 .274774$

H $6.676181-3.4109001 .299874$

O $6.2592031 .590065-0.748309$

$\begin{array}{lllllll}\text { O } & 8.377523 & 1.804081 & 0.140863\end{array}$

C $6.6749022 .312057-1.911542$

H $5.8244802 .378503-2.594449$

H $6.9839763 .325212-1.637599$

H $7.4881681 .782086-2.416303$

SCF Energy (B3LYP/6-31G*//MMFF) $=-3245.90426524$

08_00190

MM̄FF Geometry

C $-1.007513-3.416881-0.242362$

C $-0.182344-2.3897340 .015786$
C $0.484254-2.1891731 .350909$

O $0.403930-0.7788881 .662368$

C $1.970184-2.5863061 .278238$

C $2.568139-2.8557282 .644807$

C $3.383444-2.0181773 .313878$

C $2.223489-4.1986153 .239319$

C $3.857099-0.6492332 .909331$

C $5.377978-0.6157482 .699867$

C $5.813220-1.2758361 .382531$

C $7.331373-1.4570041 .344398$

C $7.800734-2.024394-0.002903$

O $5.397551-0.417327 \quad 0.314849$

O $9.227647-1.881312-0.069179$

C $7.205859-1.164190-1.135332$

C $7.507815-3.524031-0.129350$

O $7.494174-1.731609-2.411638$

C $5.684244-0.914954-0.998034$

C $5.2494820 .162023-2.021311$

O $4.981819-2.129688-1.262457$

C $0.126456-0.4172632 .939887$

O $-0.157465-1.1639663 .866721$

C $0.210464 \quad 1.050410 \quad 3.124324$

C 0.6886551 .8971872 .198792

C $0.8064453 .351938 \quad 2.337627$

C $0.3520543 .967764 \quad 3.635832$

C $3.8080180 .658283-1.851735$

C $3.4865321 .795771-2.808765$

C $1.6417223 .393086-3.349838$

$\begin{array}{lllll}\text { O } & 4.275451 & 2.213400 & -3.651787\end{array}$

C $1.7635524 .630797-2.520587$

C $0.7202015 .235617-1.932793$

C $0.8400836 .480388-1.103712$

$\begin{array}{llll}\text { C } & 0.496073 & 6.247141 & 0.349832\end{array}$

C $-0.647120 \quad 6.717601 \quad 0.876178$

C 1.5221885 .5486141 .214710

C $1.2961124 .063027 \quad 1.299803$

N $2.2044872 .282084-2.606633$

H $-1.187330-4.1459490 .542329$

H $0.047511-1.674709-0.769041$

H $-0.048595-2.7775822 .107322$

H $2.538422-1.8104920 .752259$

H $2.093101-3.4985410 .678721$

H $3.755175-2.3410314 .286227$

H $2.719377-4.3683804 .200952$

H $1.145838-4.2833203 .407629$

H $2.534475-5.0026602 .564171$

H $3.339401-0.2678652 .024860$

H 3.6036350 .0376303 .726535

H 5.6923680 .4360572 .694487

H $5.876544-1.0993933 .549330$

H $5.317359-2.2469781 .281290$

H $7.667959-2.0990012 .168048$

H $7.820169-0.4852841 .502068$

H $9.612238-2.3616350 .684163$

H $7.718124-0.192076-1.106714$

H $8.006868-4.0803840 .673251$

H $7.908355-3.926692-1.066245$

H $6.442543-3.759159-0.080635$

H $8.457365-1.856846-2.461935$

H $5.912108 \quad 1.032241-1.922864$

H $5.369173-0.224192-3.041507$

H $4.073692-1.918384-1.529230$

H $-0.126444 \quad 1.3988984 .095479$

H 1.0308861 .4976491 .245746

H $-0.694863 \quad 3.7150873 .838061$

H 0.4085995 .0579373 .647457

H $0.9677213 .606926 \quad 4.467018$

H $3.095886-0.151826-2.041039$

H $3.6498941 .025040-0.830990$

H $0.5977053 .146239-3.566691$

H $2.1711273 .521847-4.298975$

H $2.7605715 .048421-2.402342$

H $-0.2767384 .817903-2.053213$

H $0.1741927 .236624-1.539191$

H $1.8487316 .907410-1.169663$

H -0.8829316 .5800181 .926164$

H $-1.3745327 .245503 \quad 0.268560$

H 1.5510826 .0132372 .205718 
H 2.5271045 .7227560 .808799

H 1.5922093 .5236490 .400203

H $\quad 1.6459671 .895691-1.850577$

C $-3.128561-4.064123-1.531337$

O $-3.932294-3.037412-0.923516$

C $-5.043697-3.669193-0.250616$

C $-4.960003-5.160522-0.574562$

C $-3.470136-5.351176-0.783656$

H $-4.872833-3.5356490 .823715$

C $-6.367932-3.010440-0.658688$

H $-5.363273-5.7799650 .232651$

H -5.508284 -5.411843 -1.489800

C $-3.117714-6.621349-1.537313$

H -2.992095 -5.3745260 .202209$

C $-6.406544-1.485837-0.435757$

O $-6.574932-3.245737-2.058537$

H -7.200585 -3.498458 -0.139107

H -5.692843 -1.018596-1.121503

O $-7.700113-1.016391-0.837280$

C $-6.138936-1.0258771 .009913$

C $-6.248767 \quad 0.4993491 .209150$

H $-5.145504-1.3509531 .336170$

O $-7.090349-1.6280821 .890937$

H -7.2606940 .8419920 .960133$

H -6.1248200 .6956942 .282727$

H $-3.468403-7.502716-0.990289$

H $-3.576003-6.639078-2.531858$

H $-2.034357-6.711935-1.663180$

C -1.651631 -3.657359-1.579108

H -7.414209 -2.819067-2.302897

H -8.350682 -1.382075 -0.213281

H $-6.896401-2.5794841 .931106$

H -1.568128 -2.747772 -2.186634

H -1.084153 -4.441180 -2.094344

H -3.478464 -4.145019-2.570108

C $-2.818035 \quad 1.3947280 .087614$

$\begin{array}{lllll}\text { O } & -3.913177 & 0.853101 & 0.836666\end{array}$

C -5.2059331 .2955510 .412155$

C -5.3264472 .8111770 .620363$

C $-4.2085073 .555201-0.122566$

C -2.8289072 .9334660 .135664$

H -1.9289971 .0454470 .626266$

C $-2.7897890 .784597-1.321181$

H $-5.3366361 .078262-0.652772$

O

H -6.3040993 .1451730 .254496$

H -4.1961594 .6041600 .198281$

O $-4.4681103 .536404-1.522881$

H -2.087162 $3.329733-0.567034$

O -2.4099413 .3115851 .450783$

C $-1.4008800 .731334-1.912796$

H -3.157071 -0.247673 -1.265059

H -3.436595 $1.328954-2.013818$

H -4.4324842 .7752612 .360035$

H -5.328583 $3.965344-1.668342$

H $-2.329560 \quad 4.280440 \quad 1.465831$

O $-1.4455830 .009482-3.071508$

O $-0.4072681 .252380-1.427253$

C $-0.198129-0.174340-3.748350$

H $-0.327898-0.970871-4.485916$

H $\quad 0.0751680 .746209-4.270201$

H $0.589884-0.474046-3.050866$

SCF Energy (B3LYP/6-31G**//MMFF) $=-3245.92354514$

08 00191

MM̄MF Geometry

C 0.4004914 .3574511 .858502

C $-0.6734703 .578636 \quad 1.658637$

C - -1.4133332 .8750372 .768011$

O $-1.562871 \quad 1.4817022 .408170$

C -2.8129233 .4927572 .926168$

C -3.6783082 .7912033 .955396$

C -4.7834212 .0813793 .650342$

C -3.2666302 .9790965 .391484$

C -5.3131391 .8182162 .267534$

C -6.1209910 .5145762 .188329$

C $-6.619380 \quad 0.258896 \quad 0.758001$

C $-7.567644-0.9399300 .712568$
C $-8.002708-1.268839-0.724517$

O $-5.4745940 .011488-0.065418$

O $-8.620705-2.565106-0.712093$

C $-6.743083-1.377165-1.610131$

C $-9.061700-0.289851-1.244037$

O $-7.090781-1.543869-2.982178$

C $-5.773827-0.184054-1.454564$

C $-4.429328-0.404853-2.188904$

O $-6.373131 \quad 0.988217-2.018566$

C -0.5015180 .6647422 .651568$

O $0.585147 \quad 0.9886603 .104691$

C $-0.895566-0.7128482 .277749$

C $0.005775-1.7015212 .380403$

C $-0.212580-3.1188902 .078618$

C $-1.593645-3.5352191 .649955$

C $-3.667978-1.658422-1.755343$

C $-2.255078-1.719426-2.310286$

C $-0.318441-3.273390-2.461226$

O $-1.725381-0.800003-2.926611$

C $0.105619-4.551427-1.812209$

C $1.222476-4.685764-1.081683$

C $1.642037-5.980263-0.445878$

C $1.978674-5.8471101 .022968$

C $3.209107-6.1394031 .478166$

C $0.872114-5.4558961 .980461$

C $0.825894-3.9694762 .217281$

N -1.652524 -2.933274 -2.006988

H 0.7510524 .4949462 .880006

H -1.059103 3.4227600 .655233

H -0.8714572 .9503113 .719436$

H $-3.3164603 .510407 \quad 1.952621$

H -2.717186 $4.547722 \quad 3.217381$

H $-5.366241 \quad 1.6599144 .467628$

H -3.983068 2.5342116 .090238

H -2.294249 2.5127705 .577245

H -3.1944604 .0452295 .630143$

H -5.9596872 .6554881 .978601$

H $-4.4908621 .763803 \quad 1.546627$

H -5.486574 -0.3200282 .514085$

H -6.9753220 .5715492 .874260$

H $-7.131741 \quad 1.1610560 .403169$

H -8.444898 -0.7673021 .348378$

H -7.061827 -1.820922 1.132119

H $-9.374375-2.531840-0.098074$

H -6.229886 -2.297503 -1.305507

H $-9.955127-0.320162-0.608898$

H $-9.398923-0.569657-2.248192$

H $-8.7138880 .744672-1.272626$

H -7.696533 -2.302768 -3.037738

H $-4.590434-0.426053-3.274510$

H $-3.7920160 .471585-2.005296$

H $-5.6681831 .621023-2.234464$

H - $-1.913012-0.8752851 .940888$

H $1.009853-1.4521632 .722275$

H -1.670501 -4.590445 1.381694

H $-1.912739-2.9674420 .771700$

H -2.312876 -3.357005 2.457114

H -3.594456 -1.699057 -0.662768

H $-4.198704-2.552529-2.100658$

H $0.352053-2.439405-2.230412$

H $-0.354007-3.393132-3.548877$

H $-0.536180-5.416701-1.962161$

H $1.876617-3.828281-0.952634$

H $2.511426-6.353785-1.002088$

H $0.860964-6.743131-0.555533$

H $3.450449-6.0709912 .533976$

H $4.003432-6.4485510 .807148$

H $1.043743-5.9376002 .952452$

H $-0.074823-5.8672691 .621671$

H $1.775603-3.5614922 .567335$

H $-2.230644-3.671570-1.614601$

C $0.8569854 .824550-0.651930$

O $1.1990213 .463257-0.987235$

C $1.9511823 .461358-2.212600$

C $1.7027734 .820227-2.851308$

C $1.6461335 .707351-1.623154$

H $3.0089883 .399534-1.932528$

C $1.5832512 .255902-3.081068$ 
H $2.4945065 .103491-3.551291$

H $\quad 0.746107 \quad 4.850108-3.385677$

C $1.0105817 .062634-1.881308$

H $2.6683865 .857505-1.250415$

C $1.7130510 .891327-2.372375$

O $\quad 0.222004 \quad 2.393991-3.507012$

H $2.1923002 .270469-3.993108$

H $0.8858590 .763020-1.663671$

O $1.533099-0.124318-3.368587$

C $3.0488280 .656385-1.643557$

C $3.185769-0.789211-1.127929$

H $3.1245021 .354393-0.802617$

O $4.115140 \quad 0.943442-2.548017$

H $2.239280-1.108338-0.675086$

H $3.399234-1.444827-1.981292$

H $1.5871067 .621812-2.625449$

H $-0.0131216 .961096-2.256765$

H $0.9752717 .658445-0.963870$

C $1.2048465 .091970 \quad 0.816654$

H $\quad 0.006317 \quad 1.614180-4.046937$

H $2.292771-0.078419-3.974340$

H $4.9496400 .843226-2.059408$

H 1.1083126 .1639301 .025641

H $2.255577 \quad 4.8225110 .984233$

H $-0.2191044 .943428-0.828255$

C $6.690624-0.6023880 .193950$

O $5.552713-0.583921-0.678832$

C $4.305826-0.944543-0.078727$

C $4.380558-2.3785990 .465865$

C $5.548877-2.5373641 .442484$

C $6.853491-1.9790060 .862034$

H $7.551900-0.448599-0.469162$

C $6.657334 \quad 0.5573201 .195212$

H $4.085338-0.2527640 .741741$

O $4.544601-3.287622-0.620918$

H $3.434907-2.6182940 .964054$

H $5.679653-3.6007721 .677536$

O $5.222560-1.8606412 .652405$

H $7.619682-1.9173681 .643014$

O $7.350122-2.896853-0.117232$

C $6.757609 \quad 1.8809700 .474655$

H 5.7573020 .5594801 .816594

H $7.5074070 .502392 \quad 1.885468$

H $4.558678-4.186975-0.252476$

H $5.961680-1.9916523 .270548$

H $6.717810-2.914231-0.856166$

O $5.5482242 .510004 \quad 0.478941$

O $7.7846392 .294493-0.048420$

C $5.5107903 .775334-0.187510$

H $5.6644613 .636519-1.261731$

H $4.5232494 .214575-0.026811$

H $6.264426 \quad 4.451545 \quad 0.227536$

SCF Energy (B3LYP/6-31G**//MMFF) $=-3245.92317683$

08_00192

MM̄FF Geometry

C -1.7718923 .3471221 .168839$

$\begin{array}{llll}C & -1.094314 & 2.191098 & 1.127548\end{array}$

C $-0.474756 \quad 1.5438232 .339752$

O 0.8880591 .1770692 .021230

$\begin{array}{llll}C & -1.254894 & 0.265908 & 2.695895\end{array}$

C $-0.753611-0.4251493 .948899$

C $-0.042097-1.5702973 .966138$

C $-1.141154 \quad 0.2389945 .245425$

C $0.438715-2.3761532 .791050$

C $1.970030-2.4574032 .770966$

C $2.499759-3.1141811 .486886$

C $4.026089-3.2030601 .529160$

C $4.619897-3.7114090 .207636$

O $2.083476-2.3068210 .382580$

$\begin{array}{llll}0 & 6.030822 & -3.441369 & 0.229410\end{array}$

C $4.022522-2.897648-0.961699$

C $4.472021-5.2313580 .067962$

O $4.452426-3.453886-2.202138$

C $2.483247-2.792324-0.906484$

C $1.891826-1.818156-1.955145$

O $1.920614-4.083611-1.167949$

C 1.8250812 .1630352 .091261 $\begin{array}{llll}\text { O } & 1.636944 & 3.335477 & 2.375132\end{array}$

C 3.1388331 .5651161 .761977

C 4.2248992 .3494851 .690443

C 5.5949441 .9128721 .409613

C $5.820580 \quad 0.4507131 .131932$

C $2.319126-0.353739-1.808671$

C $3.5071330 .039996-2.669115$

C $5.0276451 .970212-3.097299$

O $3.987654-0.689262-3.531977$

C $6.3151881 .737514-2.372178$

C $7.1313292 .728005-1.981025$

C $8.4573152 .504014-1.313348$

C $8.5967793 .263618-0.013814$

C $9.254062 \quad 4.434651 \quad 0.035791$

C 8.0602732 .6174261 .242378

$\begin{array}{llll}\text { C } 6.581861 & 2.833328 & 1.422862\end{array}$

N $3.943561 \quad 1.326642-2.380338$

H -1.8812953 .8664112 .117949$

H $-0.982814 \quad 1.673397 \quad 0.178961$

H -0.4767372 .2284103 .197309$

H -1.234139 -0.4213591 .846001$

H -2.3168930 .5079852 .841286$

H $0.239520-1.9829744 .934332$

H $-0.818640-0.3359116 .120154$

H $-0.688363 \quad 1.2321615 .320619$

H -2.2291810 .3459705 .306880$

H $0.020410-3.3869712 .865753$

H $0.081249-1.9635321 .846118$

H $2.377660-1.4417342 .850477$

H $2.320380-3.0179493 .646689$

H $2.055683-4.1120731 .392638$

H $4.354977-3.8315702 .366343$

H $4.439437-2.2059851 .728437$

H $6.410499-3.8912691 .003484$

H $4.459172-1.893731-0.898310$

H $4.992018-5.7432340 .886703$

H $4.940644-5.591734-0.854459$

H $3.432019-5.5640350 .081596$

H $4.211964-2.830561-2.907719$

H $2.065529-2.194764-2.969969$

H $0.800572-1.838324-1.829048$

H $\quad 1.009395-3.966673-1.482655$

H $3.1789260 .494818 \quad 1.598854$

H 4.1061493 .4173951 .870049

H $\begin{array}{llll}5.154142 & 0.100391 & 0.336848\end{array}$

H $5.634263-0.1394702 .035014$

H $\begin{array}{lllll}6.833636 & 0.220726 & 0.795024\end{array}$

H $1.4857910 .282131-2.134504$

H $2.527938-0.104637-0.764285$

H $4.7808783 .033964-3.172718$

H $5.0939601 .555631-4.108393$

H $6.6056090 .705063-2.192299$

H $6.852423 \quad 3.758718-2.188752$

H $9.2394682 .822261-2.015331$

H $8.6410651 .437397-1.133926$

H 9.3861784 .9695990 .970457

H $9.6697284 .887241-0.858539$

H 8.5572593 .0471982 .122971

H 8.3442281 .5613631 .259143

H 6.3188813 .8719391 .628799

H $3.5210331 .833506-1.607799$

C -3.9363704 .0299410 .132828$

O $-4.4809452 .701885-0.009384$

C $-5.7770652 .800317-0.635989$

C $-6.055508 \quad 4.289426-0.822043$

C $-4.6544914 .860252-0.925857$

H $-5.6906522 .335844-1.623494$

C -6.8240612 .0665810 .209894$

H $-6.6659974 .486351-1.708865$

H $-6.580713 \quad 4.7139750 .041804$

C $-4.5960176 .359444-0.691663$

H $-4.2519324 .631229-1.921507$

C -6.5503950 .5572840 .382616$

O $-6.859383 \quad 2.658716 \quad 1.515782$

H $-7.8222722 .218013-0.216041$

H -5.602601 0.4077320 .911030

$\begin{array}{llll}0 & -7.576433 & 0.022553 & 1.229598\end{array}$

C $-6.560116-0.239482-0.938015$ 
C $-6.434845-1.765389-0.756188$ H $-5.7575520 .109744-1.594344$ O $-7.7886830 .014075-1.626975$ H -7.170070 -2.116705 -0.022476 H -6.711032 -2.236422 -1.709599 H $-5.2035996 .888167-1.433498$ H -4.9719796 .6254460 .301996$ H $-3.5693406 .729321-0.773016$ C $-2.4171643 .987067-0.029163$ H -5.9704102 .5719861 .900946$ H -7.5677290 .5368652 .055042$ H -8.514466 - $0.273739-1.046680$ H $-2.0137105 .000945-0.124960$ H -2.152343 $3.439754-0.941650$ H $-4.2047954 .382971 \quad 1.138044$ C $-2.769686-2.180762-1.205748$ O $-4.165536-2.013212-1.484302$ C $-5.022293-2.212428-0.356581$ C $-4.958668-3.690900 \quad 0.068202$ C $-3.527171-4.2437750 .027253$ C $-2.498069-3.116711-0.015102$ H -2.333398 -2.618975 -2.112340 C $-2.140644-0.803992-0.986250$ H $-4.664498-1.5893480 .471578$ O $-5.760607-4.488092-0.806518$ H -5.401001 -3.790115 1.066715 H -3.386704 -4.916848 -0.827656 O $-3.320831-5.0359831 .197424$ H $-2.524405-2.5663850 .933391$ O $-1.183378-3.658562-0.126081$ C $-2.064708-0.035313-2.283735$ H -2.716610 -0.220635 -0.258506 H -1.118185 -0.883173 -0.600985 H -5.728053 -5.402934 -0.477951 H $-2.396107-5.337421 \quad 1.184491$ H -1.153337 $-4.206171-0.928944$ O $-3.0312850 .924757-2.318651$ O $-1.253518-0.274885-3.169021$ C -3.066574 $1.716369-3.509872$ H $-3.2543511 .082185-4.381425$ H $-3.8844522 .435439-3.417985$ $\mathrm{H}-2.1279842 .266564-3.625577$ SCF Energy (B3LYP/6-31G**//MMFF)= -3245.91604895

08_00194

MM̄FF Geometry

C $1.753617 \quad 4.022800 \quad 0.849400$

C 2.2293342 .8481440 .411347

C $2.5052072 .547438-1.036960$

O $1.7013531 .394562-1.381204$

C $3.9800772 .172580-1.242649$

C $4.3806762 .170837-2.704346$

C $4.5121421 .067839-3.466620$

C $4.6888543 .527829-3.284654$

C $4.246936-0.361727-3.084630$

C $5.530852-1.202916-3.033230$

C $6.434601-0.865255-1.836868$

C $7.721868-1.691327-1.878769$

C $8.593941-1.454324-0.636625$

O $5.704749-1.167291-0.644815$

O $9.597179-2.481152-0.627472$

C $7.721911-1.628256 \quad 0.625737$

C $9.345095-0.120703-0.723899$

O $8.458077-1.2939671 .800259$

C $6.388436-0.8473240 .572662$

C $5.479311-1.2756571 .748902$

$\begin{array}{lllll}\text { O } & 6.666064 & 0.550031 & 0.675843\end{array}$

C $0.4833381 .606268-1.939759$

O $0.0193572 .680416-2.297021$

C $-0.2807760 .343506-2.072385$

C $0.077126-0.805491-1.477616$

C $-0.659574-2.071035-1.521161$

C $-1.940894-2.114536-2.311326$

C $4.052513-0.7130041 .673167$

C $3.152427-1.3003022 .745481$

C $0.741770-1.5766293 .312881$

O $3.580840-1.9163573 .717805$

C $0.402540-2.9484722 .818637$
C $-0.802596-3.2955352 .343228$

C $-1.133633-4.6727091 .842217$

C $-1.703664-4.6707930 .439520$

C $-3.013020-4.8916480 .232450$

C $-0.752019-4.509757-0.726682$

C $-0.170391-3.126030-0.836248$

N $1.811819-1.0375302 .493846$

H 1.5900734 .8288760 .139181

H 2.3798542 .0391491 .121741

H $2.2403773 .402962-1.669792$

H $4.1793921 .194427-0.793605$

H $4.6310212 .880500-0.712400$

H $4.8313091 .196393-4.500464$

H $5.0125103 .470282-4.329459$

H $3.8054064 .172507-3.250248$

H $5.4928974 .008715-2.717832$

H $3.695944-0.446168-2.143526$

H $3.585629-0.786201-3.850791$

H $5.229877-2.256596-2.965726$

H $\quad 6.089887-1.085458-3.969997$

H $6.6719900 .203918-1.857265$

H $8.290347-1.482074-2.793587$

H $7.466892-2.759706-1.923642$

H $\quad 10.107732-2.386917 \quad 0.195204$

H $7.494070-2.6997490 .708886$

H $9.985881-0.102871-1.613745$

H $10.018574 \quad 0.012527 \quad 0.129926$

H $8.6796970 .743555-0.776501$

H $8.498596-0.3240141 .857750$

H $5.404987-2.3712721 .761084$

H $5.929386-0.9684332 .701327$

H 5.8818991 .0058511 .019100

H -1.192580 $0.425471-2.655284$

H $0.988322-0.827982-0.882230$

H -2.434247 -3.088712 -2.284815

H $-1.750991-1.884754-3.365592$

H -2.660610 -1.389740 -1.917008

H 4.0602450 .3727841 .809176

H $3.609091-0.9361910 .696150$

H $-0.105104-0.8868323 .242781$

H $1.069705-1.6274534 .356071$

H $1.191502-3.6961532 .858691$

H -1.594787 -2.552390 2.317698

H -1.855624 -5.113692 2.541965

H $-0.255104-5.3294511 .869138$

H $-3.430918-4.925048-0.768069$

H $-3.699573-5.0378681 .059789$

H -1.231956-4.795685 -1.670196

H $\quad 0.072687-5.225483-0.612752$

H $\quad 0.762992-3.001719-0.286615$

H $1.552254-0.5809121 .624144$

C $-0.120648 \quad 4.2241902 .552547$

O -0.6126082 .9010672 .249905$

C -1.7287643 .0240851 .338557$

C -2.2670274 .4234361 .584245$

C $-0.9819485 .210077 \quad 1.758742$

H -1.288622 2.9458680 .338419

C -2.7039131 .8680051 .581403$

H -2.8776254 .8009590 .759653$

H -2.8619954 .4557912 .505199$

C -1.1724346 .5479862 .452557$

H -0.5593015 .3940360 .764276$

C $-4.0248521 .867307 \quad 0.777560$

O

H -2.9428141 .8501652 .652642$

H -4.5942670 .9948381 .116213$

O -4.8122693 .0106371 .091944$

C $-3.8699911 .747098-0.752029$

C $-5.2001321 .690604-1.529244$

H $-3.2781140 .854979-0.982351$

O $-3.1465492 .871561-1.253140$

H -5.727178 2.649284-1.440467

H $-4.9379281 .600683-2.591989$

H $-1.8333677 .194711 \quad 1.866281$

H -1.617542 6.4260923 .445887

H -0.2134977 .0619612 .572640$

C 1.3889804 .2877512 .284795

H $-2.589865-0.0886971 .510901$ 
H -4.938579 3.0243932 .055906 H -2.971065 2.713888 -2.196489 H 1.8890073 .5502882 .925940 H 1.7792495 .2711912 .570718 H -0.2829284 .3642663 .628703$ C $-6.111991-1.858433-0.786606$ O $-5.402937-0.680663-1.194247$ C $-6.1416550 .541131-1.132271$ C $-7.3757540 .464608-2.046923$ C $-8.234226-0.764998-1.728829$ C $-7.389514-2.040808-1.623912$ H -5.433858 -2.689025 -1.022483 C $-6.374120-1.8839630 .727559$ H $-6.4731380 .714368-0.103767$ O $\quad-6.980950 \quad 0.414145-3.417292$ H -7.968634 $1.377585-1.918852$ H -8.987209 $-0.896243-2.515722$ O $-8.923634-0.565184-0.498617$ H -7.983695 -2.862833 -1.207862 O $-6.983949-2.438786-2.936936$ C $-5.096010-1.9158981 .537371$ H -6.940134 -1.010008 1.059286 H $-6.943051-2.7871620 .977403$ H -6.386954 -0.348043 -3.527700 H $-9.4771990 .228136-0.597462$ H $-7.790148-2.607929-3.453652$ O $-5.417869-2.0180612 .861181$ O $-3.965226-1.8470911 .077649$ C $-4.311798-2.0441183 .768438$ H $-4.705903-1.9790364 .786157$ H $-3.768294-2.987123 \quad 3.662111$ H -3.648365 -1.190896 3.596455 SCF Energy (B3LYP/6-31G**//MMFF)= -3245.91782165

08_00195

\section{MM̄FF Geometry}

C $-0.709811-1.7371672 .931608$

C $-0.971161-1.0458654 .052608$

C -1.8267450 .1947014 .163938$

O -2.6514890 .3702932 .991102$

C -0.9206431 .4280554 .307099$

C -1.6911882 .7244174 .452821$

C -1.8646033 .6160253 .456931$

C -2.234189 3.0019755 .829390

C -1.404909 3.4732082 .031517

C -2.4400734 .0279611 .042664$

C -2.002983 $3.793533-0.410418$

C $-2.8846264 .583965-1.377610$

C $-2.5138894 .302288-2.840921$

O $-2.1182192 .392224-0.683954$

O $-3.5611864 .827370-3.670623$

C $-2.4849862 .776657-3.059636$

C $-1.2265135 .023211-3.257252$

O $-2.0041232 .456317-4.363334$

C -1.672039 2.006361 -1.990679

C $-1.9184340 .488648-2.158363$

O $-0.2804712 .273720-2.169836$

C -3.900206 -0.168466 3.024755

O $-4.398908-0.8149163 .932362$

C $-4.563110 \quad 0.153570 \quad 1.741418$

C $-5.728462-0.4397031 .442976$

C $-6.490139-0.2850720 .202199$

C $-6.030710 \quad 0.759962-0.779157$

C $-1.397268-0.375346-1.003581$

C $-1.781767-1.826376-1.223959$

C $-3.770728-3.341271-1.212601$

O $-0.970274-2.658118-1.621961$

C $-4.999006-3.087869-2.030543$

C $-6.224613-3.518298-1.694722$

C $-7.456449-3.231848-2.507396$

C $-8.578510-2.627443-1.688498$

C $-9.702004-3.321721-1.439886$

C $-8.431150-1.193264-1.216904$

C -7.554815 -1.0898910 .002497$

N -3.121678 -2.066082 -0.969846

H -1.130844 -1.418116 1.983207

H $-0.518167-1.3789164 .984769$

H -2.4597730 .1030425 .055287$
H $-0.242791 \quad 1.488040 \quad 3.448335$

H $-0.263756 \quad 1.3113105 .179906$

H -2.3910744 .5420803 .683621$

H -2.7451853 .9689205 .887391$

H -2.955005 2.2312126 .119697

H -1.4218723 .0136716 .563278$

H -0.4655414 .0264841 .914098$

H -1.2031512 .4276711 .780993$

H -3.407841 3.5411431 .220426

H -2.5691275 .1023181 .224184$

H $-0.9550134 .099043-0.514027$

H -2.826894 $5.659515-1.169075$

H -3.936695 4.304296 -1.224648

H -3.636804 $5.780212-3.490288$

H -3.525872 2.425078 -3.019281

H $-1.3360006 .106871-3.129019$

H -1.013659 4.868089 -4.320723

H $-0.3532854 .709250-2.681609$

H $-2.5488912 .947665-5.001893$

H $-2.9962440 .313306-2.254459$

H $-1.4583820 .136910-3.091110$

H $0.2324671 .523760-1.830196$

H -4.0615360 .8382051 .067385$

H -6.153305 -1.138495 2.162843

H $-5.9059651 .729282-0.282879$

H $-6.7379390 .929612-1.593852$

H -5.076299 $0.472836-1.228073$

H $-0.308058-0.315656-0.921872$

H -1.808392 $-0.047520-0.042753$

H $-4.005040-3.778278-0.236869$

H $-3.093832-4.013943-1.747745$

H -4.871927 -2.513512 -2.945689

H $-6.351597-4.111571-0.792072$

H -7.783297 -4.178103 -2.957394

H -7.231602 -2.561848 -3.346676

H -10.520018 -2.888823 -0.873603

H -9.827811-4.340140 -1.792336

H $-9.413958-0.780479-0.955326$

H -8.068336 $-0.593206-2.055974$

H $-7.827717-1.7879730 .795819$

H $-3.681959-1.324257-0.562658$

C $1.517087-2.6618682 .238112$

O $1.351402-2.5262370 .811491$

C $2.452325-3.1900780 .147561$

C $3.449698-3.5402871 .241615$

C $2.536777-3.7834582 .424294$

H $2.029150-4.100841-0.294098$

C $3.006044-2.270615-0.947704$

H $4.064018-4.4093380 .988242$

H $4.119826-2.6950111 .444501$

C $3.260463-3.7508673 .759653$

H $2.049507-4.7599512 .299744$

C $4.312407-2.773960-1.587388$

O $2.021017-2.152336-1.978268$

H $3.142760-1.266253-0.535059$

H $5.099607-2.862627-0.831426$

O $4.104017-4.101965-2.085281$

C $4.825828-1.906743-2.752628$

C $5.012295-0.417808-2.427902$

H $4.141401-1.990005-3.605749$

O $6.072698-2.435398-3.210132$

H $5.4742150 .082375-3.289453$

H $4.0221130 .047639-2.331045$

H $2.565107-3.9214884 .587217$

H $4.028731-4.5299653 .799358$

H $3.751645-2.7861483 .926206$

C $0.169520-2.9567962 .900034$

H $1.183395-1.902867-1.553128$

H $3.373513-4.063061-2.726372$

H $5.931539-3.375159-3.417195$

H $0.324736-3.3269493 .920107$

H $-0.361667-3.7416102 .347876$

H $1.926371-1.7104362 .603827$

C 6.2959341 .8830370 .195295

O $5.708961 \quad 1.344334-0.995698$

C $5.825456-0.077233-1.161185$

C $7.306213-0.479380-1.213950$

C 8.0677810 .0387410 .013221 
$\begin{array}{llll}\text { C } & 7.787587 & 1.519766 & 0.282723\end{array}$

H 6.2183202 .9712010 .074455

C 5.4997631 .4988341 .448462

H $5.348210-0.560534-0.304725$

O $7.897290 \quad 0.043432-2.400989$

H $7.385503-1.570779-1.253774$

H $9.145527-0.097467-0.140203$

O $7.695619-0.7184841 .161580$

H $8.183116 \quad 1.8045191 .264957$

O $8.5006712 .284060-0.693998$

C 4.0987812 .0598391 .379639

H 5.4382090 .4176481 .600804

H 5.9697851 .9091812 .350009

H $8.825045-0.246864-2.420099$

H $7.915464-1.6489050 .983965$

H $8.3729133 .224439-0.482501$

O 3.2059241 .0592841 .133005

O 3.8347563 .2487381 .505996

C 1.8391551 .4668401 .021395

H $1.5402922 .053677 \quad 1.894473$

H 1.6978132 .0442230 .103575

H 1.2177900 .5689990 .974315

SCF Energy (B3LYP/6-31G**/MMFF) $=-3245.91140819$

08_00196

MM̄FF Geometry

C -2.799050 4.061473 -1.239487

C $-3.4022642 .895086-0.966065$

C $-4.2801822 .680208 \quad 0.238436$

O -3.7082251 .6063401 .019652$

C $-5.6801302 .217380-0.193377$

C -6.7354412 .4785530 .861769$

C $-7.149406 \quad 1.5869331 .782815$

C $-7.366143 \quad 3.846460 \quad 0.799314$

C $-6.6242980 .203195 \quad 2.046904$

C $-7.599864-0.8964801 .607865$

C $-7.492121-1.2192990 .110144$

C $-8.576859-2.212635-0.308648$

C $-8.425227-2.633367-1.777472$

O $-6.195388-1.786927-0.114225$

O $-9.269499-3.773133-1.999675$

C $-6.972331-3.093278-2.013373$

C $-8.906441-1.541701-2.740487$

O $-6.741253-3.362234-3.394790$

C $-5.908699-2.101786-1.481343$

C $-4.512206-2.767003-1.538704$

O $-5.906597-0.933259-2.302673$

C $-2.660073 \quad 1.8879831 .834542$

O -2.1412992 .9744652 .028481$

C -2.231721 0.6045732 .430640

$\begin{array}{llll}\text { C } & -1.011827 & 0.499340 & 2.975299\end{array}$

C $-0.439578-0.7097443 .569984$

C $-1.314660-1.9346023 .642827$

C $-3.389284-1.993500-0.828537$

C $-2.051702-2.704457-0.979567$

C $0.380429-2.413597-0.528758$

O $-1.935834-3.800994-1.522584$

C $0.809109-3.0066650 .776278$

C $1.909471-2.614876 \quad 1.437493$

C $2.406853-3.2610312 .699118$

C $2.750655-2.2624733 .782283$

C $4.023722-1.8950964 .006790$

C $1.629880-1.7625814 .664668$

C $0.835229-0.6683364 .009558$

N $-1.000767-1.973547-0.442532$

H $-2.965503 \quad 4.912762-0.584544$

H -3.231773 2.038819-1.614612

H -4.3441293 .5866570 .853699$

H $-5.6581411 .150697-0.442464$

H -5.990137 2.731140 -1.113392

H -7.9424941 .8920782 .465137$

H -8.118419 3.9954221 .581066

H $-6.605332 \quad 4.6237660 .922239$

H $-7.8599983 .992703-0.166878$

H -5.6390390 .0431801 .602568$

H -6.4751820 .1212453 .131336$

H -7.357155 -1.801795 2.179791

H -8.628310 -0.615017 1.865716
H -7.589726 -0.292128 -0.464376

H $-9.576139-1.796513-0.130012$

H -8.510397 -3.1127930 .318503$

H -10.182587 -3.516260 -1.784646

H -6.845514 -4.049978 -1.487176

H $-9.962489-1.308850-2.558019$

H $-8.850440-1.880621-3.780947$

H -8.343326 $-0.610587-2.650081$

H $-7.418848-3.997468-3.683395$

H $-4.570127-3.758884-1.070644$

H $-4.224604-2.919652-2.587107$

H $-5.032024-0.516206-2.261435$

H -2.924038 -0.2281362 .375868$

H $-0.364762 \quad 1.3753762 .972693$

H -2.182529-1.746302 4.284455

H $-0.805535-2.8134084 .043475$

H $-1.671614-2.2172782 .646435$

H -3.287710 -0.986165 -1.245389

H $-3.606477-1.9049460 .241764$

H $0.983584-1.545245-0.806704$

H $0.480552-3.163408-1.320179$

H $\quad 0.214550-3.827743 \quad 1.169409$

H $2.516380-1.8147961 .021958$

H $3.299889-3.8448832 .439451$

H $1.682251-3.9864253 .089481$

H $4.280518-1.2019534 .800904$

H $4.837084-2.2761343 .398478$

H $2.040089-1.3522555 .597440$

H $1.015428-2.6082084 .985770$

H $1.3890530 .265666 \quad 3.907937$

H $-1.192723-1.0927340 .025841$

C $-0.3923414 .310581-2.012334$

O $-0.002028 \quad 3.049321-1.433215$

C $0.8557723 .308429-0.299735$

C $1.2758244 .765120-0.423637$

C $0.0234955 .388036-1.010949$

H 0.2282893 .1653340 .588263

C $2.0043612 .296026-0.306940$

H 1.5624665 .2043710 .536317

H $2.1196344 .870801-1.116937$

C $0.2532266 .752755-1.635208$

H $-0.7158645 .485346-0.206952$

C 3.0643242 .5401410 .779985

$\begin{array}{lllll}\text { O } & 1.440391 & 0.997108 & -0.100760\end{array}$

H $2.4709952 .284575-1.298132$

H 3.5607723 .5025190 .612216

O 2.4095252 .6484542 .050361

C 4.1321401 .4330480 .897160

C $4.9065951 .202592-0.406429$

H $3.670098 \quad 0.502386 \quad 1.242718$

$\begin{array}{llll}\text { O } & 5.069011 & 1.825098 & 1.906002\end{array}$

H $4.230150 \quad 0.893327-1.211274$

H $5.3407552 .156300-0.737379$

H $0.6123347 .463849-0.884080$

H $0.9974846 .707869-2.437273$

H $-0.6757597 .147767-2.058411$

C $-1.872665 \quad 4.268672-2.404642$

H $\quad 0.7587350 .866236-0.782201$

H $1.922497 \quad 1.8197412 .197335$

H 4.5655501 .9803042 .723672

H $-2.0183243 .457985-3.130256$

H -2.151968 $5.199201-2.911912$

H $0.1977784 .413449-2.932662$

C $7.890110-0.638950-1.651211$

O $6.7124860 .177080-1.574083$

C $6.0591270 .185212-0.296026$

C $5.592682-1.2347840 .057136$

C $6.750761-2.2369690 .007946$

C $7.576854-2.098051-1.275701$

H $8.164204-0.622340-2.714010$

C $9.0381470 .000943-0.863303$

H 6.7697830 .5449530 .456233

O $4.568306-1.654370-0.842747$

H $5.158722-1.2345351 .061602$

H $6.350651-3.2564020 .070065$

O $7.603846-2.0393361 .131253$

H $8.508240-2.669482-1.197548$

O $6.832793-2.661028-2.362004$ 
C $10.379860-0.554597-1.269435$

H $9.0526401 .087275-1.018800$

H $8.929393-0.1258580 .217867$

H $4.924361-1.597758-1.746058$

H $7.068876-2.1662671 .933287$

H $6.682559-3.599931-2.158401$

O $10.7818390 .007354-2.445032$

O $10.991450-1.395981-0.623915$

C $12.041751-0.450481-2.945515$

H $12.002381-1.525872-3.143651$

H $12.2448580 .071878-3.884118$

H $12.839559-0.216999-2.233994$

SCF Energy (B3LYP/6-31G**//MMFF)= -3245.92150505

08_00197

MMFF Geometry

C -2.179966 $5.159483-0.657879$

C $-2.9790924 .137763-0.997729$

C $-4.2058123 .750797-0.212576$

$\begin{array}{llll}\text { O } & -4.051962 & 2.384263 & 0.239599\end{array}$

C $-5.4493173 .833294-1.114554$

C $-6.7436063 .519643-0.388105$

C $-7.4426092 .373898-0.516857$

C $-7.261694 \quad 4.6201810 .502376$

C $-7.094774 \quad 1.170872-1.348511$

C $-6.943273-0.080835-0.474588$

C $-6.387039-1.269583-1.272378$

C $-6.328208-2.523075-0.398800$

C $-5.659036-3.703085-1.120126$

O $-5.072232-0.917625-1.715128$

O $-5.350607-4.700564-0.133497$

C $-4.316138-3.228949-1.719164$

C $-6.610083-4.367039-2.122506$

O $-3.727338-4.253564-2.516364$

C $-4.425486-1.913085-2.518504$

C $-3.049821-1.342432-2.945751$

O $-5.165704-2.162798-3.720199$

C -3.2838822 .1846071 .347732$

O $-2.6699553 .031531 \quad 1.977925$

C -3.3305750 .7433841 .681486$

C $-2.630127 \quad 0.2798792 .727763$

C $-2.618956-1.1000063 .220610$

C $-3.417088-2.1220422 .456179$

C $-2.106482-0.963015-1.799277$

C $-1.133620-2.059728-1.401632$

C $0.571370-2.5919110 .338005$

O $-0.962023-3.081827-2.059933$

C $-0.015002-3.3662931 .474195$

C $0.470204-3.3190422 .723981$

C $-0.068811-4.1361573 .861341$

C $-0.450895-3.294876 \quad 5.057412$

C $0.380437-3.165537 \quad 6.105375$

C $-1.834918-2.6892595 .080342$

C $-1.915658-1.3824034 .337805$

N $-0.448935-1.738397-0.237803$

H -2.425395 5.7602170 .214410

H $-2.729670 \quad 3.528997-1.863667$

H -4.3410254 .4053730 .657662$

H $-5.3198383 .172706-1.978073$

H $-5.5320274 .843353-1.538912$

H $-8.373708 \quad 2.2768850 .040310$

H -8.230851 4.3728190 .948727

H $-6.563352 \quad 4.8143201 .321938$

H -7.391664 $5.543484-0.071580$

H -7.897553 $1.008572-2.077863$

H $-6.1820571 .328877-1.926258$

H -6.2654110 .1455090 .357818$

H $-7.915899-0.344499-0.041127$

H -7.030557 -1.437771-2.144106

H $-7.331118-2.802538-0.052507$

H $-5.752040-2.303844 \quad 0.509447$

H $-6.180237-4.953787 \quad 0.306216$

H -3.637977 -3.076192 -0.871827

H $-7.513985-4.723928-1.614017$

H $-6.153062-5.254850-2.573109$

H -6.926815 -3.694924 -2.922624

H $-4.193759-4.270804-3.369313$

H -2.566311 -2.018186-3.661674
H -3.244883 -0.417641 -3.507937

H $-4.957344-1.463517-4.361758$

H $-3.9609870 .106274 \quad 1.073037$

H -2.023551 0.9819723 .298659

H $-4.488152-1.9154742 .551969$

H -3.249977 -3.147447 2.792457

H $-3.148729-2.1084841 .394556$

H $-1.489439-0.115570-2.125252$

H $-2.668826-0.636637-0.920080$

H $1.387988-1.9428400 .663663$

H $0.956945-3.279362-0.421529$

H $-0.856512-4.017877 \quad 1.250688$

H $1.328898-2.6856822 .936176$

H $\quad 0.706985-4.8586534 .147251$

H $-0.929794-4.7399613 .549029$

H $0.099937-2.5909666 .981843$

H $1.362670-3.6266866 .107062$

H $-2.132480-2.4858546 .118343$

H $-2.562882-3.4265644 .730158$

H - $1.363470-0.5749424 .820668$

H $-0.676942-0.8784090 .252798$

C $0.3549855 .022560-0.752252$

$\begin{array}{lllll}\text { O } & 0.412946 & 3.584974 & -0.834457\end{array}$

C $0.995673 \quad 3.075088 \quad 0.386046$

C $1.5486354 .286767 \quad 1.125515$

C 0.5678755 .3694170 .719882

H $\quad 0.1648602 .6255550 .943593$

C 2.0429632 .0139720 .030108

H 1.5945824 .1344972 .207790

H 2.5578794 .5333820 .772419

C $1.078018 \quad 6.7802410 .951513$

H $-0.352083 \quad 5.222708 \quad 1.298780$

C 2.8283841 .4673261 .235690

$\begin{array}{llll}\text { O } & 1.373263 & 0.924161 & -0.610540\end{array}$

H $2.7341902 .434713-0.708783$

H 3.3893012 .2721851 .725473

$\begin{array}{llll}\text { O } & 1.909581 & 0.953638 & 2.209488\end{array}$

C 3.7983860 .3172800 .898589

C $4.8086610 .665556-0.199867$

H $3.232051-0.5770150 .622698$

O $4.527691-0.0091802 .086452$

H $4.2940160 .890098-1.141343$

H $\quad 5.335619 \quad 1.589764 \quad 0.074934$

H 1.2824286 .9461432 .014292

H 2.0039836 .9684120 .397757

H $0.3367247 .518707 \quad 0.630110$

C $-0.932345 \quad 5.532762-1.406082$

H $0.8810891 .288895-1.365976$

H 1.4347921 .7073372 .597707

H $3.875797-0.236526 \quad 2.771647$

H $-0.9900535 .137941-2.428584$

H $-0.897576 \quad 6.625144-1.491914$

H $1.2025175 .389294-1.346961$

C $7.831882-0.718436-1.876808$

O $6.7165810 .100408-1.501565$

C $5.877925-0.415440-0.458188$

C $5.294356-1.777862-0.865533$

C $6.390789-2.756995-1.295568$

C $7.365426-2.123178-2.293410$

H $8.249723-0.231411-2.767403$

C $8.923758-0.733549-0.795308$

H $6.478815-0.525800 \quad 0.450414$

O $4.358149-1.616268-1.929766$

H $4.746698-2.205316-0.018365$

H $5.921070-3.638740-1.748426$

O $7.095661-3.187827-0.134954$

H $8.239349-2.767609-2.443811$

O $6.715314-1.998330-3.561744$

C $9.5682390 .625016-0.609640$

H $8.531257-1.0411040 .177206$

H $9.716463-1.429986-1.092742$

H $4.817470-1.176051-2.665286$

H $7.766948-3.830372-0.421094$

H $6.459711-2.891354-3.849385$

$\begin{array}{llllll}\text { O } & 10.549490 & 0.520731 & 0.335950\end{array}$

O $9.258057 \quad 1.645333-1.208568$

C $\quad 11.254656 \quad 1.731594 \quad 0.627298$

H $11.997940 \quad 1.5144901 .398971$ 
H $\quad 11.7720652 .092978-0.266550$

H 10.5645912 .4904831 .008311

SCF Energy (B3LYP/6-31G**//MMFF) $=-3245.91272150$

08_00198

MM̄FF Geometry

C $2.3810931 .524815-2.368670$

C $2.7351520 .549941-3.220588$

C $3.056387-0.880498-2.851549$

O $2.507066-1.220127-1.559242$

C $4.578163-1.081160-2.754702$

C $5.001580-2.510388-3.025907$

C $5.190448-3.457203-2.086609$

C $5.255902-2.827914-4.477580$

C $4.943656-3.368453-0.606566$

C $6.238334-3.3818430 .217035$

C $6.897675-1.9976770 .311680$

C $8.234437-2.0859241 .050512$

C $8.860746-0.7003201 .267615$

O $5.999276-1.1436771 .029946$

O $9.923286-0.8405822 .222900$

C $7.804437 \quad 0.2298661 .899681$

C $9.499325-0.153837-0.014678$

O $8.291413 \quad 1.567078 \quad 1.990211$

C 6.4431690 .2107861 .166795

C 5.3879330 .9779652 .001292

O $6.5888350 .849828-0.103364$

C $1.202216-1.589002-1.489113$

O $0.411845-1.700845-2.410927$

C $0.897652-1.787953-0.055654$

C $-0.375995-1.7712340 .360128$

C $-0.826960-1.8706001 .748865$

C $0.211927-2.1539122 .802855$

C 3.9419010 .7962651 .514977

C 2.9673081 .6727512 .285797

C 0.5664702 .3481842 .321835

O 3.3061092 .3815383 .230149

C -0.1902031 .5299573 .320716$

C $-1.5253571 .390263 \quad 3.308284$

C $-2.2925490 .626694 \quad 4.351495$

C $-3.308025-0.329990 \quad 3.765287$

C -4.6084120 .0012403 .699902$

C $-2.833103-1.7027613 .343853$

C -2.136603 -1.680095 2.010804

N 1.6735691 .5732701 .790431

H $2.3360621 .317321-1.304240$

H $2.7991410 .781813-4.281870$

H $2.624682-1.542156-3.613209$

H $4.932749-0.770364-1.764981$

H $5.101752-0.434855-3.471924$

H $5.527575-4.439652-2.416133$

H $5.550925-3.871317-4.631647$

H $4.353167-2.650096-5.070723$

H $6.059701-2.195792-4.869183$

H $4.329069-2.505350-0.340722$

H $4.349774-4.249344-0.330373$

H $5.979983-3.7161061 .230507$

H $6.943492-4.113334-0.196321$

H $7.048091-1.602607-0.698971$

H $8.931604-2.7432860 .516225$

H $8.077959-2.5520392 .033615$

H $10.562445-1.4821861 .868258$

H $7.645294-0.1120422 .932054$

H $10.287826-0.829523-0.367476$

H 9.9899110 .8086780 .167607

H $8.784540-0.028212-0.830622$

H $9.138608 \quad 1.5350422 .466647$

H 5.4266220 .6329093 .042946

H 5.6328812 .0478802 .004032

H $5.7387331 .233205-0.369563$

H $1.735423-1.8880260 .625079$

H -1.163325 -1.639614 -0.380180

H $0.691858-3.1208582 .615131$

H -0.191335 -2.192199 3.816622

H $0.983716-1.3765972 .807676$

H 3.8606951 .0549280 .455928

H $3.630000-0.2469091 .639486$

H -0.0561522 .6472831 .474247$
H $\quad 0.952893 \quad 3.2516222 .804602$

H $\quad 0.3845401 .0601024 .115562$

H $-2.104028 \quad 1.8906402 .535215$

H $-2.8035301 .361804 \quad 4.987542$

H $-1.6191750 .075386 \quad 5.019519$

H -5.347863 -0.690405 3.312180

H -4.9619140 .9729054 .028401$

H $-3.687808-2.3863383 .250667$

H $-2.221728-2.1336634 .141391$

H -2.807895-1.476554 1.175339

H $1.4763040 .915740 \quad 1.041978$

C $0.7539883 .408791-2.101879$

O $-0.3572272 .575026-2.473204$

C -1.546349 $3.383179-2.546176$

C $-1.1352814 .795148-2.134224$

C $0.3361574 .820610-2.499865$

H $-1.8563673 .385983-3.598350$

C $-2.6711952 .783417-1.686038$

H -1.719307 $5.561204-2.653842$

H -1.263805 $4.957279-1.057623$

C $1.1151925 .921078-1.801354$

H $0.4241954 .946984-3.587211$

C $-3.0836501 .356768-2.112883$

O $-2.2620992 .784289-0.319040$

H -3.546305 $3.439793-1.758325$

H $-3.3134871 .361826-3.185627$

O $-2.0047950 .444557-1.915073$

C $-4.3194460 .821408-1.358015$

C $-4.691893-0.602688-1.817693$

H $-5.1588251 .503543-1.536922$

O -4.0570300 .8134560 .042796$

H -4.677092 -0.644374 -2.913300

H -3.936994 -1.301070-1.434996

H $0.7126786 .904486-2.065769$

H $1.0650265 .819049-0.712072$

H $2.1697315 .899528-2.093235$

C $2.0291302 .923545-2.793864$

H -1.545076 2.132541 -0.232765

H -1.261229 $0.737920-2.467918$

H $-4.8625150 .510670 \quad 0.494576$

H $2.8643913 .583601-2.535013$

H $1.8966092 .960970-3.882086$

H $0.8630113 .332752-1.011551$

C -7.286478 -1.502109 0.731624

O $-6.046689-1.1518530 .101862$

C $-6.082225-1.051121-1.324343$

C $-6.494681-2.398184-1.938893$

C $-7.819609-2.897967-1.353201$

C $-7.828323-2.8302940 .177679$

H $-7.027526-1.6627431 .785867$

C $-8.302218-0.3559890 .672108$

H $-6.805467-0.281915-1.616139$

O $-5.480510-3.376814-1.717054$

H $-6.590888-2.278306-3.024161$

H -7.989257 -3.934469 -1.669197

O $-8.894290-2.108581-1.854330$

H -8.839159 -3.001899 0.565797

O $-6.997761-3.8754230 .692797$

C $\quad \begin{array}{llll}-7.817270 & 0.828017 & 1.474581\end{array}$

H $-8.520240-0.019819-0.345554$

H $-9.261546-0.6656821 .103748$

H -5.325376 -3.432446 -0.758519

H -8.893032 -2.191907 -2.823153

H -7.368683 -4.7212050 .388442$

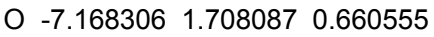

O $\quad \begin{array}{llll}-7.975277 & 0.942310 & 2.683149\end{array}$

C -6.6312652 .8672691 .305039$

H -5.8844322 .5754122 .048987$

H -6.1444043 .4832970 .544263$

H -7.4333703 .4491141 .768870$

SCF Energy (B3LYP/6-31G**//MMFF) $=-3245.92360393$

08_00199

MM̄MF Geometry

C $1.7395065 .059102-0.563574$

C $2.8847314 .385443-0.377738$

C $3.4320573 .869050 \quad 0.933488$

O 3.5269152 .4263360 .820987 
C $2.569866 \quad 4.1642992 .175376$

C 3.1977503 .6770073 .466448

C 2.8001302 .5779934 .137538

C 4.2967244 .5472694 .018484

C $1.734877 \quad 1.5954473 .728546$

C 2.1134520 .1642634 .137671

C $1.019360-0.8481393 .767544$

C $1.302603-2.2000954 .426185$

C $0.274161-3.2621184 .015337$

O $1.002571-0.9923932 .342827$

O $0.778378-4.544604 \quad 4.417759$

C $0.170600-3.2786462 .478411$

C -1.065891 -3.075940 4.736870

O $-0.874379-4.1501202 .050762$

C $-0.016219-1.8757541 .850484$

C $0.139345-1.9843010 .316568$

O $-1.323875-1.3930932 .159354$

C 4.6942791 .8942780 .373505

$\begin{array}{lllll}\text { O } & 5.693547 & 2.498422 & 0.019439\end{array}$

$\begin{array}{llll}\text { C } & 4.535099 & 0.423065 & 0.373619\end{array}$

C $5.431578-0.334689-0.274777$

C $5.396290-1.792524-0.407614$

C $4.378688-2.5432590 .408972$

C $0.097913-0.647165-0.425687$

C $0.433543-0.863837-1.888000$

C $2.371582-1.098604-3.441356$

O $-0.438269-1.109342-2.718730$

C $3.002028-2.455667-3.377945$

C $4.245231-2.718546-3.809578$

C $4.881826-4.078168-3.735517$

C $6.265001-4.052317-3.118689$

C $7.358185-4.281545-3.865818$

C $6.377715-3.835145-1.622971$

C $6.267095-2.379558-1.254785$

N $1.793540-0.797381-2.144127$

H 1.1096845 .3073150 .285609

H $3.4883214 .154827-1.254083$

H 4.4294184 .3025401 .078354

H $1.576613 \quad 3.7157802 .052777$

H $2.400103 \quad 5.2451602 .267697$

H 3.2889832 .3587305 .086084

H 4.6716294 .1835844 .981107

H $5.144794 \quad 4.5801303 .327695$

H 3.9324535 .5682844 .172243

H 0.7994501 .8757734 .227249

H 1.5564261 .6219442 .649423

H $3.055004-0.1116913 .645012$

H 2.2854910 .1352805 .221317

H $0.051943-0.456578 \quad 4.103515$

H $1.334738-2.1027895 .518690$

H $2.301177-2.5489914 .126881$

H $0.919293-4.5226645 .379846$

H $1.107082-3.7061502 .092793$

H $-0.927526-3.1381565 .823026$

H - $1.768165-3.8763154 .478472$

H $-1.541370-2.1171534 .519821$

H $-0.707358-5.0206832 .450980$

H $1.092549-2.4752010 .088370$

H $-0.650490-2.625037-0.094830$

H $-1.538638-0.6626371 .558185$

H 3.6703770 .0078410 .879138

H $6.2558120 .160157-0.787181$

H $4.452634-2.2683161 .467449$

H $4.500215-3.6274610 .369649$

H $3.368336-2.3170410 .059676$

H $-0.898922-0.199751-0.373164$

H $\quad 0.7993720 .0760950 .005160$

H $3.102054-0.313966-3.661506$

H $1.592654-1.084368-4.209693$

H $2.404862-3.262650-2.959043$

H $4.832943-1.918598-4.254533$

H $4.932673-4.475394-4.757667$

H $4.255141-4.779848-3.171124$

H $8.350647-4.288480-3.427417$

H $7.286639-4.462218-4.933274$

H $7.351543-4.192027-1.263166$

H $5.634503-4.463809-1.125695$

H $6.983573-1.744151-1.778095$
H $2.432816-0.620875-1.375489$

C $-0.048170 \quad 4.731383-2.335087$

O $-1.0699094 .850728-1.318146$

C $-1.4086213 .521173-0.874387$

C $-1.1546792 .655392-2.094279$

C $0.157645 \quad 3.231483-2.585659$

H $-0.7034463 .277124-0.068923$

C $-2.8434063 .478596-0.339034$

H $-1.0923341 .591883-1.865608$

H $-1.9400202 .804013-2.843898$

C $0.4690852 .889246-4.032462$

H $\quad 0.9644202 .833800-1.958369$

C $-3.219075 \quad 2.084727 \quad 0.208351$

$\begin{array}{lllll}\text { O } & -2.941822 & 4.441870 & 0.713121\end{array}$

H $-3.5495853 .781704-1.120758$

H $-3.0326521 .344717-0.574971$

O $-2.347256 \quad 1.7585491 .293779$

C -4.6858391 .9339820 .661323$

C $-4.993070 \quad 0.5952591 .363276$

H $-5.3369402 .057849-0.211946$

$\begin{array}{llll}\text { O } & -5.019048 & 2.975941 & 1.580432\end{array}$

H -4.4545940 .5515662 .318828$

H -6.0593950 .6068991 .626935$

H $0.5590881 .805484-4.160468$

H $-0.3175493 .242505-4.707487$

H $1.4132123 .347437-4.343646$

C $1.2151705 .491585-1.906464$

H -2.6862375 .3038120 .342064$

H -2.5014742 .4152541 .994842$

H -5.9663302 .8965011 .785150$

H $0.9774356 .559997-1.828715$

H $1.9851535 .391659-2.680424$

H $-0.4554975 .214875-3.231596$

C $-5.320956-1.706175-1.564058$

O $-5.502632-0.622602-0.640660$

C $-4.683130-0.6590980 .531634$

C $-4.931634-1.9483881 .330408$

C $-4.734600-3.1837800 .446962$

C $-5.510306-3.065208-0.867525$

H $-6.137442-1.592421-2.289478$

C $-3.992116-1.586287-2.325049$

H $-3.629865-0.632970 \quad 0.240401$

O $-6.256021-1.940148 \quad 1.858188$

H -4.239109-1.976169 2.180342

H $-5.070328-4.0783660 .985046$

$\begin{array}{llll}0 & -3.351782 & -3.347582 & 0.149037\end{array}$

H $-5.224613-3.869546-1.555647$

O $-6.907613-3.221146-0.603809$

C $-4.104453-0.593254-3.459157$

H -3.146678 $-1.286526-1.701053$

H $-3.730003-2.548276-2.781804$

H $-6.371104-2.755643 \quad 2.374997$

H $-2.876498-3.4353690 .993044$

H -7.034522 $-4.098708-0.204633$

O $-4.277007 \quad 0.667121-2.970935$

O $-4.041985-0.905356-4.641565$

C $-4.436927 \quad 1.691635-3.956537$

H $-5.3287281 .499420-4.560524$

H $-4.5644572 .645372-3.437473$

H -3.546773 $1.751888-4.589822$

SCF Energy (B3LYP/6-31G**//MMFF)= -3245.91464720

08_00200

MMFF Geometry

C -0.065595-2.905190 1.194425

C $0.621049-2.3147972 .185516$

C $0.386220-0.9254692 .735590$

$\begin{array}{lllll}\text { O } & -0.893608 & -0.403454 & 2.312151\end{array}$

C 1.4799050 .0356652 .243829

C 1.4629171 .3613522 .978825

C 0.9344362 .5032722 .497219

C $2.140028 \quad 1.3539864 .325681$

C $\quad 0.2283372 .7103501 .186894$

C -1.0967613 .4616191 .374020$

C $-1.775707 \quad 3.7417250 .025791$

C -2.9814714 .6644140 .206395$

C $-3.7146114 .904243-1.121406$

O $-2.1997272 .493016-0.530840$ 
O - $-4.9824775 .507740-0.822650$

C -4.005892 $3.541508-1.780679$

C $-2.9642305 .892098-2.022390$

O $-4.552812 \quad 3.712308-3.086506$

C $-2.7783152 .599152-1.838964$

C $-3.236252 \quad 1.190832-2.280765$

O $-1.838913 \quad 3.101325-2.790482$

C $-1.978081-0.7473283 .059321$

O $-1.987198-1.4156544 .081013$

C -3.176959-0.207729 2.382508

C $-4.376247-0.7285102 .681362$

C $-5.653420-0.3710812 .063040$

C -5.6831770 .8401191 .171566$

C $-2.1722820 .095646-2.144295$

C $-2.816180-1.258399-2.364288$

C $-4.241722-2.953229-1.212813$

O $-2.819428-1.798277-3.468020$

C $-5.682692-2.543962-1.186163$

C $-6.588768-3.063750-0.343501$

C $-8.034386-2.651336-0.318705$

C $-8.542678-2.3474311 .075641$

C $-9.407917-3.1726101 .689242$

C $-8.108920-1.0508071 .729834$

C $-6.726533-1.1494162 .313965$

N $-3.421209-1.755705-1.219563$

H $-0.862592-2.365682 \quad 0.690234$

H $1.422167-2.8738032 .665677$

H $0.411265-0.9845773 .830911$

H 1.3798270 .1922411 .163799

H $2.471162-0.4150602 .382393$

H $1.023493 \quad 3.4013153 .107448$

H $2.1518632 .345027 \quad 4.791685$

H 1.6253150 .6744155 .011632

H $3.179877 \quad 1.0258994 .226286$

H $0.884406 \quad 3.305645 \quad 0.542273$

H $\quad 0.0371901 .7659190 .670563$

H - 1.7618132 .8625462 .009097

H -0.908205 4.4098591 .893014

H $-1.0395944 .204899-0.641981$

H -2.679302 5.6193280 .654370

H -3.686461 4.2089410 .916167

H $-4.8139856 .336853-0.342727$

H $-4.786788 \quad 3.052922-1.180725$

H -2.840562 $6.855961-1.514034$

H -3.533504 6.105629-2.933922

H $-1.9712135 .541086-2.310403$

H -5.327021 4.294981-3.002582

H -4.106258 $0.896748-1.684236$

H $-3.5703261 .217955-3.326252$

H $-1.3162112 .361998-3.139003$

H -3.035805 0.5567761 .627516

H $-4.426102-1.5267123 .421373$

H -5.1347930 .6418360 .248180$

H -5.2281541 .7028201 .671483$

H $-6.691974 \quad 1.1523650 .893939$

H -1.373096 $0.220462-2.882069$

H $-1.7014820 .106198-1.154844$

H -3.961129 -3.534649-0.329111

H $-4.039843-3.547184-2.109327$

H -5.998055 -1.787690 -1.901488

H $-6.281836-3.8420150 .351617$

H -8.618241 -3.469187 -0.760745

H -8.209194 -1.777319 -0.958365

H $-9.795991-2.9504602 .677923$

H $-9.744030-4.091127 \quad 1.219813$

H -8.794879 -0.7924332 .547066$

H -8.208389 -0.2459780 .996729$

H -6.604882 -1.988761 3.000566

H $-3.396265-1.201350-0.368968$

C $1.022807-4.444717-0.529149$

O $2.375884-4.033841-0.256661$

C $2.864683-3.285497-1.384775$

C $1.870227-3.529097-2.516588$

C $0.566334-3.647541-1.750150$

H $2.812447-2.227501-1.103858$

C $4.312666-3.671860-1.705124$

H $1.866828-2.714536-3.247472$

H $2.080447-4.462194-3.051708$
C $-0.545004-4.319533-2.536654$

H $0.245555-2.637697-1.467189$

C $5.301482-3.583239-0.521179$

O $4.323588-5.032212-2.163363$

H $4.670697-3.068263-2.547488$

H $5.059117-4.3826370 .190207$

O $6.612746-3.890872-1.013715$

C $5.376843-2.2472630 .243735$

C $5.572620-1.021699-0.659191$

H $6.226059-2.3002420 .935355$

O $4.206640-2.0686691 .034176$

H $6.500919-1.111665-1.235802$

H $4.773341-0.982511-1.409834$

H $-0.774239-3.749801-3.442506$

H $-0.264894-5.333255-2.842218$

H $-1.457467-4.389272-1.936803$

C $0.174100-4.317904 \quad 0.737477$

H $5.245051-5.259202-2.376731$

H $6.821713-3.265425-1.727674$

H $4.113493-2.8485421 .607486$

H $0.661636-4.8795021 .544857$

H $-0.801975-4.7904950 .574791$

H $1.084226-5.511019-0.785063$

C $5.5011872 .684191-0.490824$

O $5.6529141 .332188-0.938654$

$\begin{array}{llll}\text { C } & 5.538449 & 0.328526 & 0.079004\end{array}$

C $6.639200 \quad 0.5201041 .131995$

C 6.6072151 .9432361 .705025

C 6.5279073 .0104770 .605718

H $5.7368183 .297155-1.370675$

C $4.0552482 .991426-0.088471$

H $4.557647 \quad 0.422873 \quad 0.556655$

$\begin{array}{llll}\text { O } & 7.925791 & 0.293398 & 0.561610\end{array}$

H $6.503772-0.1996221 .947391$

H 7.5096322 .1128112 .305089

O $\quad 5.4821352 .0878872 .567353$

H 6.2985523 .9917921 .037587

O $7.805758 \quad 3.119471-0.028410$

C $3.1129342 .748625-1.244026$

H 3.7116682 .4008490 .765166

H 3.9502084 .0412870 .209241

H $8.005040-0.6568190 .373984$

H 5.5664881 .4262553 .274672

H 8.4507593 .3731720 .653483

O $2.5841541 .494454-1.153387$

O $2.8904473 .565680-2.127546$

C $1.668907 \quad 1.128771-2.189485$

H $0.8185221 .816115-2.201521$

H $2.1772691 .122954-3.158068$

H $1.3019670 .120698-1.978141$

SCF Energy (B3LYP/6-31G**//MMFF) $=-3245.89921124$

08_00201

MMFF Geometry

C $-0.261563 \quad 3.194750 \quad 0.143937$

C $-0.9098693 .787647 \quad 1.157343$

C $-0.926753 \quad 3.323037 \quad 2.593464$

$\begin{array}{lllll}\text { O } & 0.124464 & 2.360851 & 2.851190\end{array}$

C -2.2501132 .6315072 .966738$

C -3.473704 3.5163102 .874468

C $-4.397216 \quad 3.440041 \quad 1.896309$

C -3.6383704 .5070923 .997043$

C -4.3905052 .5316600 .698390$

C $-5.5049331 .484486 \quad 0.797014$

C $-5.5121350 .541968-0.414654$

C $-6.729814-0.383330-0.363736$

C $-6.723050-1.403470-1.510016$

O $-4.305379-0.227798-0.383565$

O $-7.722340-2.386882-1.200433$

C $-5.350413-2.108991-1.532398$

C $-7.147606-0.764636-2.837088$

O $-5.248605-2.978686-2.658275$

C $-4.149740-1.134646-1.480716$

C $-2.803239-1.882874-1.313267$

O $-4.083971-0.427699-2.726266$

C $1.3572422 .841953 \quad 3.160511$

O $1.6952094 .016407 \quad 3.209596$

C 2.2758901 .7435793 .531183 
C $2.1347950 .486217 \quad 3.083563$

C $2.951290-0.6638653 .481484$

C $4.003531-0.4393604 .537106$

C $-2.588164-2.573408 \quad 0.037263$

C $-2.070169-1.6484971 .128955$

C $-1.774899-1.5706213 .610336$

O $-1.501892-0.5853480 .894424$

C $-0.598572-2.3066544 .185761$

C $-0.068016-3.4640443 .757807$

C $1.129372-4.1228664 .386882$

C $2.268392-4.3020673 .404620$

C $2.396292-5.4409732 .703147$

C $3.301192-3.1966523 .293413$

C $2.699387-1.8651252 .919808$

N -2.271626 -2.184997 2.393235

H 0.2937522 .2771030 .318483

H -1.469852 4.6980850 .952605

H $-0.7756124 .190513 \quad 3.247888$

H -2.171470 2.2518273 .995387

H -2.367290 1.7188962 .370865

H $-5.250034 \quad 4.1162901 .940619$

H -4.5663495 .0825613 .911176$

H -3.661715 3.9884844 .961048

H -2.808836 5.2207474 .005005

H $-4.5452893 .148314-0.195829$

H -3.4222472 .0429980 .560590$

H -5.3612010 .8984441 .714587$

H -6.4751281 .9908710 .876792$

H $-5.5305951 .148574-1.327890$

H -7.661359 $0.196511-0.372505$

H $-6.729000-0.9319920 .588970$

H -7.674069 $-3.080157-1.881152$

H -5.326655 -2.759566 - 0.650941

H $-8.156848-0.344218-2.750882$

H -7.204278 -1.511168 -3.636973

H $-6.4811080 .037456-3.160621$

H -5.077495 -2.428724 -3.441745

H -2.707558 -2.626380 -2.114997

H $-1.983989-1.174601-1.483714$

H -3.191344 -0.056134-2.821993

H 3.0816162 .0330184 .197546

H 1.3286910 .2649992 .385268

H 4.6999690 .3471924 .225162

H $4.620175-1.3189324 .734049$

H $3.540924-0.1454035 .485518$

H $-3.494518-3.0815550 .376640$

H -1.817981 -3.344997 -0.089993

H -2.596187-1.568395 4.333655

H $-1.489011-0.5337313 .408249$

H $-0.137021-1.8180505 .042211$

H $-0.508520-3.9852032 .911687$

H $0.811098-5.1008334 .771156$

H $1.484009-3.5652105 .262725$

H $3.223477-5.5961452 .019327$

H $1.673384-6.2447282 .797081$

H $4.056181-3.4491892 .540277$

H $\quad 3.822590-3.1545374 .254135$

H $1.957268-1.9175842 .121955$

H $-2.734340-3.0851412 .479757$

C $1.1387704 .079866-1.775673$

O $1.9910872 .916471-1.778426$

C $3.2950323 .305405-1.307194$

C $3.3194514 .829834-1.349070$

C $1.8842955 .156595-0.986428$

H $3.3622142 .983990-0.261119$

C $4.3919952 .632533-2.136577$

H $4.0474005 .252916-0.650395$

H $3.5533625 .205011-2.352375$

C $1.4709376 .575143-1.335407$

H 1.7624405 .0066240 .093520

C $4.3476351 .090278-2.141386$

O $4.287026 \quad 3.070670-3.497858$

H $5.3745932 .972251-1.788774$

H $3.4821710 .742315-2.716807$

O $5.5104780 .630044-2.842848$

C $4.3386680 .442278-0.744714$

C $4.593396-1.076950-0.730495$

H $3.3806010 .648399-0.255080$ $\begin{array}{llll}\text { O } & 5.348613 & 1.046317 & 0.070356\end{array}$

H $5.614316-1.288690-1.073125$

H $4.569140-1.3893010 .322069$

H $2.0871497 .299661-0.793039$

H $1.5825296 .774493-2.406411$

H $0.4249196 .752589-1.066131$

C $-0.2584543 .721310-1.262842$

H $3.3939372 .843928-3.810133$

H $5.4979341 .040800-3.724366$

H $6.2041100 .906464-0.370905$

H $-0.6844992 .938429-1.899790$

H $-0.9146954 .594948-1.353390$

H $1.0461264 .385872-2.826093$

C $1.200272-2.324176-1.671151$

O $2.283116-1.647835-1.020604$

C $3.587256-1.911290-1.542820$

C $3.911639-3.414584-1.481895$

C $2.813272-4.258162-2.134760$

C $1.417344-3.845319-1.653781$

H $\quad 0.324351-2.106072-1.049179$

C $0.931362-1.773591-3.080294$

H $3.630451-1.583290-2.585808$

O $4.066869-3.817523-0.124208$

H $4.870334-3.585773-1.986443$

H $2.984190-5.315346-1.898326$

O $2.907901-4.112292-3.548407$

H $\quad 0.642259-4.329929-2.258985$

O $1.231515-4.287867-0.307165$

C $0.424287-0.348779-3.059312$

H $1.828514-1.784360-3.703991$

H $0.158098-2.384021-3.561698$

H $4.324383-4.754996-0.122725$

H $2.218753-4.670045-3.947842$

H $1.333840-5.254732-0.298852$

\begin{tabular}{lllll}
\hline & 0.114685 & 0.023426 & -4.336961
\end{tabular}

$\begin{array}{llll}\text { O } & 0.315377 & 0.348780 & -2.061286\end{array}$

C $-0.385848 \quad 1.354566-4.490508$

H $-1.2927241 .491550-3.894146$

H $0.3817492 .079529-4.205580$

H $-0.6351051 .504415-5.544208$

SCF Energy (B3LYP/6-31G**//MMFF) $=-3245.91311718$

08_00202

MMFF Geometry

C $-2.044150-4.017157 \quad 0.668449$

C $-1.870793-3.401758-0.511919$

C $-2.877699-3.203170-1.623209$

O $-3.095819-1.770849-1.719053$

C $-4.247121-3.875995-1.412223$

C $-5.201507-3.689785-2.580110$

C $-6.315077-2.929217-2.552523$

C $-4.847275-4.452031-3.831653$

C $-6.842207-2.120589-1.400975$

C $-6.574498-0.623316-1.600126$

C $-6.633007 \quad 0.145195-0.272651$

C $-6.4880321 .649095-0.508699$

C -6.3981992 .4353910 .808189$

$\begin{array}{lllll}\text { O } & -5.551813 & -0.329579 & 0.537961\end{array}$

$\begin{array}{lllll}0 & -5.937502 & 3.760336 & 0.499585\end{array}$

C -5.3269631 .7855911 .710834$

C $-7.7719572 .597182 \quad 1.469256$

$\begin{array}{lllll}\text { O } & -5.318599 & 2.386713 & 3.003033\end{array}$

$\begin{array}{llll}\text { C } & -5.480089 & 0.256621 & 1.843828\end{array}$

C $-4.294109-0.4133872 .581983$

O $-6.662093-0.0329172 .599913$

C $-2.197086-1.044461-2.438809$

O $-1.289034-1.475385-3.131878$

C $-2.4645370 .386870-2.175046$

C $-1.537208 \quad 1.296524-2.508422$

C -1.606807 $2.739595-2.269930$

C $-2.8413093 .278573-1.597815$

C -2.920761 -0.2337301 .925156$

$\begin{array}{llll}\text { C }-2.115826 & 0.930533 & 2.476731\end{array}$

C $-0.0107702 .198692 \quad 2.065538$

$\begin{array}{llll}\text { O } & -2.425801 & 1.543048 & 3.494687\end{array}$

C $-0.322524 \quad 3.458514 \quad 1.321043$

C $0.583587 \quad 4.125285 \quad 0.589922$

C $0.3094745 .430163-0.100698$ 
C $0.6656265 .404716-1.569831$

C $1.8724575 .814489-1.995792$

C $-0.4099704 .995820-2.549587$

C $-0.5619033 .504166-2.650397$

N $-0.979281 \quad 1.1762841 .717365$

H -3.002216 -4.480712 0.889349

H $-0.905269-2.947821-0.722020$

H $-2.433737-3.576550-2.554044$

H -4.690328 -3.492460 -0.488951

H -4.111948 -4.954353 -1.255776

H $-6.922375-2.870409-3.454911$

H -5.623149 -4.372049 -4.600617

H -3.916542 -4.073759-4.264897

H -4.720986 -5.515863 -3.605563

H -7.924315 -2.286157 -1.325497

H $-6.434941-2.469369-0.449342$

H -5.582288 $-0.485397-2.046500$

H -7.307857 $-0.215847-2.306923$

H -7.585429 -0.0781770 .222417$

H -7.312170 2.026391 -1.126819

H $-5.5735231 .838974-1.085955$

H -6.564118 4.156718 -0.130001

H -4.3538672 .0066321 .256612$

H -8.4558873 .1370230 .803252$

H -7.703594 3.2014092 .380616

H -8.2421091 .6440851 .720309$

H -5.1974473 .3433582 .876902$

H -4.283111 -0.1064093 .634682$

H $-4.493824-1.4947812 .598125$

H -6.580672 -0.9275762 .969997$

H $-3.3899690 .646814-1.675938$

H $-0.6237600 .951047-2.991626$

H $-3.7205563 .123439-2.232103$

H $-2.7885544 .346443-1.376196$

H $-3.0057242 .777671-0.637853$

H $-2.323335-1.1312582 .127480$

H -3.010504 -0.138673 0.838894

H 0.9806321 .8055791 .820880

H -0.0512702 .3889013 .142825$

H $-1.3279993 .860681 \quad 1.419643$

H 1.5973803 .7380160 .523082

H $0.899635 \quad 6.204674 \quad 0.406911$

H -0.7374725 .7353770 .019210$

H $2.1250385 .828168-3.050894$

H $2.637166 \quad 6.137942-1.297348$

H $-0.1599355 .363370-3.554342$

H -1.344419 $5.505222-2.298040$

H $0.2937653 .015506-3.118952$

H -0.8293910 .6511060 .860878$

C $0.234842-3.2694951 .639198$

O $1.114382-3.9181470 .698900$

C $2.479183-3.7577091 .148574$

C $2.418685-2.8031192 .332072$

C $1.056819-3.1254812 .916954$

H $2.802163-4.7519641 .482712$

C $3.330341-3.298176-0.041891$

H $3.237933-2.9589073 .040167$

H $2.450985-1.7590951 .995861$

C $0.545239-2.0576783 .868980$

H $1.111858-4.0883343 .442033$

C $4.737901-2.8031740 .340163$

O $3.435005-4.402303-0.945372$

H $2.808831-2.505383-0.588873$

H $4.619043-1.9342170 .993087$

O $5.407992-3.8030001 .107680$

C $5.615151-2.401803-0.865738$

C $6.948044-1.746381-0.456754$

H $5.059040-1.722091-1.521610$

O $5.951636-3.556358-1.638557$

H $7.462828-2.3808380 .275278$

H $7.593849-1.725067-1.345114$

H -0.448164 -2.313717 4.249885

H $\quad 1.218317-1.9536594 .726146$

H $0.475683-1.0816133 .377346$

C - $-1.044679-4.0968621 .800061$

H $2.530283-4.676774-1.174887$

H $5.525873-4.5787420 .532812$

H $5.122341-3.940302-1.969677$
H $-1.571244-3.7674532 .703683$

H $-0.786331-5.1538301 .938857$

H $\quad 0.004473-2.271853 \quad 1.243212$

C $6.0748281 .878685-0.663838$

O $6.3204600 .503180-0.984556$

C $6.790266-0.3179210 .090612$

C 8.1130250 .2316300 .645504

C 7.9723101 .7011921 .056029

C $7.3192282 .540613-0.045112$

H $5.8908002 .358744-1.633468$

C 4.8091502 .0414150 .186754

H $6.047474-0.3226560 .892550$

O $9.1534090 .102673-0.321164$

H $8.408378-0.3689481 .513679$

H 8.9636572 .1136351 .280817

O 7.1799391 .7963922 .236102

H $7.060693 \quad 3.5333260 .341895$

O $8.2898352 .734749-1.078688$

C $3.5799011 .700354-0.624705$

H 4.8256411 .4220251 .087768

H 4.6890463 .0789860 .519435

H $8.8750320 .576530-1.123488$

H $7.628596 \quad 1.2871812 .932642$

H $7.9000973 .332294-1.739597$

O $2.9483970 .615636-0.092562$

O $3.2249012 .324348-1.616923$

C $1.7505650 .216413-0.765834$

H $1.0668641 .063383-0.867226$

H $1.995347-0.202292-1.746224$

H $1.260857-0.553105-0.164333$

SCF Energy $($ B3LYP/6-31G**//MMFF $)=-3245.91691567$

08_00203

MMFF Geometry

C -3.0029020 .8677193 .149465$

C -3.3127982 .1731313 .173130$

C -3.8029943 .0094752 .013706$

O -4.1648382 .1941960 .878515$

C -2.676042 3.9436791 .538885

C -3.1854225 .1827610 .832545$

C $-3.2972575 .324923-0.502255$

C -3.5320896 .3323961 .744313$

C $-3.0619404 .298670-1.574841$

C $-1.7795444 .552226-2.379258$

C $-0.5330473 .939717-1.721825$

C $0.7263214 .296704-2.511624$

C $1.9713993 .598082-1.945596$

O $-0.7122932 .519422-1.711305$

O $3.0274403 .734697-2.909330$

C $1.6793632 .089345-1.803462$

C $2.4684974 .269190-0.659466$

O $2.7714021 .449461-1.148552$

C $0.3426951 .781060-1.084392$

C $0.0001080 .276817-1.216420$

O 0.4706082 .1119860 .299658

C -5.4108911 .6579020 .833759$

O -6.2911511 .7537501 .672000$

C $-5.4971360 .899578-0.433223$

C $-6.454905-0.023230-0.597457$

C $-6.631534-0.873562-1.776776$

C $-5.716086-0.638554-2.950819$

C -1.427447 -0.090366 -0.780953

C $-1.682695-1.584579-0.893096$

C $-3.324358-3.335795-0.219871$

O $-0.899703-2.358616-1.438075$

C $-4.163565-3.680968-1.410122$

C $-5.353074-4.296731-1.322513$

C $-6.179646-4.692270-2.513999$

C -7.620552 -4.241378 -2.409758

C $-8.587984-5.099269-2.043840$

C $-7.950606-2.821014-2.812118$

C -7.586194 -1.826674 -1.743885

N $-2.887081-1.954226-0.309597$

H $-3.100181 \quad 0.2994742 .229240$

H -3.189975 2.7150224 .109366

H -4.6753713 .5877582 .342605$

H -1.995635 3.3922260 .880475

H -2.0606974 .2735302 .386973$ 
H $-3.6416606 .285661-0.884114$ H $-3.894057 \quad 7.2074311 .194081$ H -4.3174196 .0386232 .447948$ H -2.651244 6.6428902 .315918 H -3.077927 $3.280087-1.179586$ H $-3.9166824 .358845-2.261265$ H $-1.9145204 .089749-3.366142$ H $-1.6369295 .627545-2.542169$ H $-0.4524894 .307545-0.693498$ H $0.8740385 .383478-2.538636$ H $0.6015953 .985715-3.558510$ H $3.1789484 .684218-3.055641$ H $1.6383281 .675873-2.821105$ H $2.7142055 .321320-0.847035$ H $3.3952723 .804983-0.304279$ H 1.7358354 .2411930 .149785 H $2.6600670 .490023-1.251058$ H $0.113905-0.027624-2.265214$ H $\quad 0.708645-0.316859-0.625714$ H -0.1385841 .5637240 .818309$ H $-4.744418 \quad 1.101550-1.187099$ H -7.162335 -0.1884140 .214093$ H $-5.8563520 .373031-3.347782$ H $-5.879048-1.329849-3.779761$ H $-4.667565-0.755781-2.655893$ H -1.5977120 .2084830 .257677$ H -2.162662 $0.420502-1.412470$ H $-3.875648-3.4413260 .719859$ H -2.446528 -3.989507 -0.186687 H $-3.761157-3.441436-2.391514$ H $-5.739166-4.566526-0.341866$ H $-6.138262-5.786403-2.596245$ H $-5.747006-4.308525-3.446303$ H $-9.627318-4.791856-1.993772$ H -8.361460 -6.127235 -1.780784 H $-9.029231-2.721165-2.993998$ H -7.486648 -2.604508 -3.778013 H $-8.194808-1.918811-0.842998$ H $-3.500687-1.2365710 .064438$ C $-0.995279-0.1662244 .220170$ O $-0.764196-1.1253983 .171135$ C $0.342956-1.9746783 .553453$ C $0.869305-1.4224744 .870808$ C $-0.373030-0.7896985 .466168$ H $-0.084775-2.9744563 .697263$ C $1.360200-1.9821112 .407279$ H $1.302935-2.2003865 .506490$ H $1.638987-0.6601134 .696272$ C $-0.0712230 .200966 \quad 6.576999$ H -1.023309 -1.585219 5.854171 C $2.678231-2.7076162 .725501$ O $\quad 0.751865-2.6314521 .286289$ H $1.559321-0.9488702 .101790$ H $3.207930-2.1929823 .534766$ O $2.378676-4.0192353 .219160$ C $3.626223-2.8632641 .517796$ C $4.008692-1.5236430 .878091$ H $3.178625-3.5348940 .777948$ O $4.829153-3.4932481 .969916$ H $3.118078-0.9687550 .566202$ H $4.494832-0.8925111 .635152$ H $-0.992850 \quad 0.6358396 .975725$ H $0.451120-0.2943327 .402011$ H 0.5622221 .0209136 .222178 C $-2.4916880 .116501 \quad 4.348444$ H $-0.104604-2.1981811 .132926$ H $1.870284-4.4824732 .531343$ H $4.575337-4.3300872 .395854$ H -2.691370 0.6871665 .262602 H -3.052972 -0.8228944 .422395$ H $-0.456626 \quad 0.7502853 .942670$ C $6.210052-0.127503-1.775776$ O $5.262888-0.287468-0.712933$ C $4.972181-1.636250-0.320417$ C $4.404406-2.426946-1.510904$ C $5.335170-2.345194-2.726131$ C $5.767593-0.906821-3.024927$ H $6.1775150 .941708-2.022985$
C $7.639706-0.461721-1.322763$

H $5.897163-2.1065200 .028954$

O $3.127792-1.915593-1.890704$

H $4.272660-3.478212-1.230760$

H $4.822392-2.752394-3.606401$

O $6.497129-3.135963-2.493100$

H $6.578032-0.903880-3.762610$

O $4.672689-0.209157-3.626530$

C $8.1484280 .501246-0.269615$

H $7.708410-1.468000-0.901656$

H $8.317081-0.390749-2.181705$

H $2.486604-2.181239-1.210050$

H $\quad 6.204847-4.051633-2.346511$

H $3.960423-0.148222-2.967345$

O $9.426548 \quad 0.151881 \quad 0.065384$

O $7.516095 \quad 1.436310 \quad 0.201325$

C 10.0481020 .9729301 .059162

H 10.1238862 .0051110 .704318

H $9.4849090 .925957 \quad 1.996115$

H $11.0559890 .588044 \quad 1.236167$

SCF Energy (B3LYP/6-31G**//MMFF) $=-3245.91874945$

0800204

MMFF Geometry

C $0.658751-2.6636924 .298956$

C $0.182656-1.5552163 .711325$

C -1.099851-0.880754 4.124146

O $-1.951867-0.7710212 .961979$

C -0.7998720 .5415924 .623151$

C -2.0323941 .2539315 .142085$

C -2.7082632 .1991924 .459853$

C $-2.455517 \quad 0.876520 \quad 6.537926$

C -2.4281712 .6897253 .065527$

C -3.7260682 .9396432 .285586$

C -3.4445903 .5025070 .884426$

C -4.7373084 .0154370 .247669$

C $-4.5018294 .530512-1.178062$

O -2.8832072 .4585470 .078784$

O $-5.7828244 .703593-1.802366$

C $-3.7520693 .446131-1.974619$

C $-3.8229445 .905166-1.189983$

O $-3.3809403 .928809-3.264543$

C $-2.5110232 .874358-1.243913$

C $-1.9999201 .639507-2.021308$

O $-1.4830403 .865446-1.211753$

C $-2.791202-1.8079572 .701253$

O $-2.886995-2.853597 \quad 3.323214$

C $-3.582130-1.4455731 .504083$

C $-4.357997-2.3720760 .922327$

C $-5.169286-2.187979-0.282944$

C $-5.282151-0.794610-0.841259$

C $-0.8881090 .844338-1.329051$

C $-0.628321-0.422003-2.121552$

C $-1.433085-2.767631-2.389730$

O $0.143543-0.435188-3.077843$

C $-2.709118-2.840514-3.170264$

C $-3.537262-3.896019-3.142273$

C $-4.823742-3.965997-3.916277$

C $-6.006040-4.376120-3.062881$

C $-6.577661-5.583452-3.210074$

C $-6.573406-3.360608-2.091349$

C $-5.760036-3.271189-0.829341$

N -1.390764 -1.495748 -1.690050

H $0.077605-3.1040235 .107710$

H $0.730131-1.0855882 .899331$

H -1.611222 -1.4481024 .911840$

H $-0.333707 \quad 1.1265193 .821636$

H $-0.053783 \quad 0.5077995 .428565$

H -3.5502332 .6803994 .955937$

H -3.3185341 .4565306 .882123$

H $-2.731921-0.1813086 .583693$

H $-1.637628 \quad 1.053691 \quad 7.243953$

H -1.870278 3.6302583 .143159

H -1.8093081 .9835572 .504710$

H -4.2837551 .9977542 .203263$

H -4.3502803 .6474572 .845626$

H -2.717807 4.3183560 .976561

H $-5.1930134 .799406 \quad 0.865385$ 
H -5.4728853 .1996360 .207227$ H $-6.2940645 .334656-1.267255$ H -4.459717 $2.622242-2.145271$ H $-4.4358496 .639280-0.653164$ H -3.724626 $6.287889-2.211950$ H -2.833912 $5.899711-0.727346$ H $-4.1883784 .260963-3.693109$ H $-2.8410160 .959476-2.200774$ H -1.632554 $1.950353-3.008232$ H $-0.6219453 .425021-1.136687$ H $-3.485333-0.4359861 .120867$ H $-4.381950-3.373157 \quad 1.351343$ H $-5.624107-0.095310-0.069585$ H -5.993479 -0.708763-1.664675 H -4.312888 -0.455242 -1.214851 H $0.0469511 .411916-1.280861$ H -1.160935 $0.584397-0.300403$ H -1.365740 -3.556324 -1.633849 H - $0.577350-2.849691-3.066677$ H -2.962395 -1.984218 -3.791486 H -3.271049-4.763894-2.542914 H -4.680089 -4.686403 -4.731951 H -5.050193 -3.006970-4.398806 H -7.439721 -5.876145 -2.619812 H $-6.194032-6.308410-3.920196$ H -7.595340 -3.642476 -1.806100 H -6.668931-2.403024 -2.609574 H $-5.635485-4.232618-0.328266$ H -2.036981 -1.364299-0.918461 C $2.837913-2.7874512 .902998$ O $2.225675-2.9668801 .610815$ C $3.255284-3.2703150 .642670$ C $4.580985-3.0990791 .370252$ C $4.203831-3.4618242 .791903$ H $3.102938-4.3200340 .362865$ C $3.065705-2.356947-0.573088$ H $5.370271-3.7355570 .959184$ H $4.922926-2.0572811 .320676$ C $5.217634-2.9923323 .821197$ H $4.093284-4.5522292 .861477$ C $4.215233-2.424736-1.594122$ O $1.852115-2.738279-1.228267$ H $2.919505-1.328445-0.227550$ H $5.158074-2.123958-1.125264$ O $4.400441-3.789271-1.992809$ C $3.992883-1.582416-2.864147$ C $3.647720-0.106394-2.617985$ H $3.193572-2.034724-3.464088$ O $5.173788-1.672018-3.665248$ H $3.598340 \quad 0.419476-3.580713$ H $2.629023-0.047933-2.215183$ H $4.905406-3.2674094 .833185$ H $6.194625-3.4499063 .634325$ H $5.345043-1.9048633 .791871$ C $1.942073-3.3916163 .987553$ H $1.136994-2.677689-0.571704$ H $3.563289-4.096966-2.380939$ H $5.014682-1.166876-4.480771$ H $2.505587-3.4551534 .925790$ H $1.670696-4.4157293 .701488$ H $2.967235-1.7086223 .060440$ C $4.5535482 .826961-0.532109$ O $3.9366281 .955841-1.486518$ C $4.571240 \quad 0.680536-1.667275$ C $6.0082790 .876866-2.168643$ C $6.7993891 .803551-1.236845$ C $6.0257823 .082539-0.898339$ H $4.0109763 .775345-0.632262$ C 4.3584532 .3274960 .905227 H $4.5905870 .168162-0.702004$ O $5.980291 \quad 1.425713-3.483842$ H $6.511051-0.094568-2.221357$ H $7.7489132 .068587-1.718063$ O $7.1053801 .088514-0.042692$ H $6.5232223 .621609-0.083581$ O $6.0674843 .932348-2.048676$ C 2.8942262 .1851031 .250461 H 4.8221191 .3518061 .076816
H 4.8144423 .0167341 .625573

H $6.9005181 .514424-3.784650$

H $7.637186 \quad 1.6748630 .522055$

H $5.6310804 .768779-1.813100$

O 2.3140693 .4147051 .349510

O 2.3378561 .1073111 .418443

C 0.9213663 .4175471 .676598

H 0.5339374 .4263891 .510942

H $0.3659982 .723148 \quad 1.039078$

H 0.7928653 .1590362 .731199

SCF Energy (B3LYP/6-31G**//MMFF) $=-3245.90613543$

08_00205

MMFF Geometry

C -3.2149843 .2623860 .235709$

C -2.9168664 .1631731 .185171$

C -1.6080654 .2517351 .934849$

O -0.5895013 .4571541 .291426$

C -1.805532 3.6788013 .351798

C -0.5655713 .7658774 .219652$

C 0.3447842 .7789044 .346659

C -0.4115025 .0507184 .988465$

C 0.3764081 .4712233 .604579

C 0.1457130 .2645054 .519301

C $0.080384-1.0369393 .703473$

C $-0.229825-2.2348794 .602576$

C $-0.217006-3.5549573 .814452$

O $1.343004-1.2212093 .052843$

O $-0.238549-4.6376174 .756965$

C $1.097350-3.6426943 .006922$

C $-1.488481-3.7246202 .975929$

O $1.097214-4.7800262 .148210$

C $1.402235-2.3670922 .190562$

C $2.809843-2.3955751 .548958$

O $0.453873-2.2543601 .122472$

C 0.2357964 .0420620 .387172

O $0.1924175 .186645-0.029386$

C 1.2264243 .0107710 .004958

C $2.1705333 .265380-0.912125$

C $3.1967562 .317161-1.356522$

C $3.2598450 .988885-0.647683$

C $3.966684-2.5199922 .541121$

C $5.306941-2.3031311 .865281$

C $6.862195-0.5225101 .110031$

O $6.051140-3.2422051 .592331$

C 6.7189520 .8292210 .487672

C $7.1882531 .143246-0.729747$

C $7.0384242 .505455-1.347930$

C $6.4970222 .457208-2.761959$

C $7.2245762 .925670-3.790329$

C $5.1226641 .865889-2.994297$

C $4.0263922 .676725-2.358304$

N $5.568644-0.9670861 .599160$

H -2.472702 $2.528342-0.065781$

H -3.681622 4.8748361 .489550

H -1.2881955 .2994341 .989431$

H -2.131429 2.6321933 .288499

H -2.627686 4.2032283 .857507

H 1.1878232 .9366675 .017715

H 0.4979895 .0643965 .598289

H -0.3614455 .9015534 .301485$

H -1.263383 5.1957035 .660999

H -0.3601201 .4463612 .797117$

H 1.3575381 .3883643 .120919

H 0.9648720 .2075635 .247630

H $-0.788816 \quad 0.3952305 .078546$

H $-0.701187-0.9231212 .942878$

H -1.191833 -2.102640 5.112931

H $0.525777-2.2942705 .398402$

H $0.539110-4.5541985 .334595$

H $1.904294-3.8013763 .732466$

H -2.373041 -3.741442 3.624188

H -1.488355 -4.683326 2.445624

H -1.631989 -2.926947 2.244539

H $0.896477-5.5579322 .696404$

H $2.870658-3.2033480 .808034$

H $2.940633-1.4705230 .973042$

H $0.838944-1.7027460 .421299$ 
H 1.1484812 .0491170 .500879 H $2.1903494 .245547-1.385568$ H $3.456626 \quad 1.1365630 .420037$ H $4.0394070 .323722-1.021428$ H $2.3148770 .446964-0.752895$ H $3.869688-1.7965603 .358845$ H $3.984871-3.5162532 .996131$ H $7.260824-1.2664700 .412276$ H $7.534034-0.4678601 .972847$ H 6.2104021 .5934321 .071339 H $7.7222350 .390926-1.305536$ H $8.0272712 .981906-1.336904$ H $6.3891053 .145845-0.738443$ H $6.8505652 .896762-4.808311$ H $8.2116113 .350030-3.638983$ H $5.122504 \quad 0.821393-2.673328$ H $4.9054301 .824217-4.070195$ H $3.9015813 .664093-2.804812$ H $4.958875-0.2716702 .020641$ C $-5.260797 \quad 1.8670050 .000728$ O $-4.5809740 .722694-0.554031$ C $-5.556515-0.301274-0.833866$ C $-6.9028240 .243930-0.363088$ C $-6.6986011 .741146-0.491188$ H -5.589711 -0.421070 -1.922088 C $-5.145267-1.615176-0.158925$ H -7.733110 -0.129531-0.970609 H $-7.107123-0.0289830 .679175$ C -7.7056122 .5561850 .301140$ H -6.759162 2.017267 -1.552382 C $-3.809182-2.202413-0.665320$ O $-5.023482-1.3926741 .252768$ H -5.944398 -2.356104 -0.276611 H $-2.987638-1.522570-0.415198$ O $-3.563794-3.406147 \quad 0.074773$ C $-3.788850-2.530262-2.172746$ C $-2.561644-3.344420-2.626391$ H $-3.856056-1.605126-2.754666$ O $-4.954415-3.304417-2.476852$ H -2.467379 -4.248158 -2.012109 H $-2.760000-3.701469-3.646349$ H -8.723869 2.349622 -0.044244 H $-7.6591582 .322321 \quad 1.370070$ H -7.521896 3.6284900 .183224 C $-4.558248 \quad 3.154217-0.430964$ H $-4.331418-0.722157 \quad 1.384697$ H -4.267437 -4.038320 -0.151488 H -4.980103 -3.430350 -3.440720 H -5.174710 $4.025472-0.183076$ H $-4.4015193 .158597-1.515994$ H -5.2243281 .7643401 .094214$ C $-0.202911-0.717918-3.831178$ O $-1.341906-1.569870-3.657774$ C -1.245680 -2.550934 -2.618808 C $-0.035342-3.470832-2.851863$ C $1.255453-2.670551-3.039517$ C $1.079821-1.538311-4.054368$ H $-0.409774-0.164275-4.756301$ C $-0.0791990 .303498-2.695953$ H -1.133459 -2.048145-1.653807 O $-0.248974-4.297809-3.994891$ H $0.068299-4.140106-1.989515$ H $2.047875-3.348855-3.379515$ O $1.647575-2.134438-1.780818$ H $1.954430-0.877360-4.037140$ O $1.025317-2.122483-5.359124$ C $-1.1427701 .368527-2.828818$ H $-0.176255-0.128758-1.696652$ H $0.9011740 .789592-2.741169$ H -0.403746 -3.715724 -4.758422 H $2.506027-1.695383-1.905950$ H $\quad 0.984845-1.396661-6.004962$ O $-0.5700732 .556204-3.176058$ O $-2.3378771 .163796-2.662280$ C -1.469556 $3.652942-3.363137$ H $-1.8644773 .977628-2.397307$ H $-0.9082674 .479105-3.807542$ H $-2.2828993 .378870-4.041998$
SCF Energy (B3LYP/6-31G**//MMFF) $=-3245.91150350$

08_00206

MM̄FF Geometry

C $1.0261164 .343795-0.371738$

C $0.799124 \quad 4.6990180 .902121$

C 1.1263413 .8891552 .135808

O 1.8651002 .6884001 .817055

C -0.1889233 .4722312 .820033$

C 0.0157102 .6598224 .083029

C $-0.1559981 .325340 \quad 4.168582$

C 0.3985843 .4553475 .303474

C $-0.5149450 .377742 \quad 3.056632$

C $0.439086-0.8234793 .016847$

C $0.198537-1.7126571 .786829$

C $1.110825-2.9406161 .831208$

C $0.995873-3.795210 \quad 0.561657$

O $0.474935-0.9323740 .619066$

O $2.097815-4.7155630 .575691$

C $1.157522-2.879623-0.671150$

C $-0.276255-4.6498020 .576707$

O $\quad 0.928765-3.611304-1.873085$

C $0.270471-1.618462-0.623330$

C $0.578783-0.620251-1.766296$

O $-1.098301-2.012876-0.772940$

C $3.2233602 .778237 \quad 1.781190$

O $3.897323 \quad 3.7893621 .896364$

C 3.7566101 .4085271 .601451

C 5.0808431 .2042371 .537986

C $5.751902-0.0951701 .431886$

C $4.886504-1.3238661 .334782$

C $1.9759720 .006056-1.738241$

C $3.014537-0.707913-2.584204$

C $5.449509-0.603124-3.126828$

O $2.755426-1.651241-3.324827$

C $6.239571-1.482349-2.210703$

C $7.503941-1.222374-1.845456$

C $8.324328-2.127703-0.974166$

C $8.882157-1.424170 \quad 0.241522$

C $10.149779-0.9783120 .261808$

C $8.004221-1.3318681 .467952$

C $7.101212-0.1290141 .453033$

N $4.269500-0.132661-2.429194$

H $1.5165153 .400083-0.590055$

H 0.3085265 .6520271 .092792

H 1.7130544 .5130382 .821655

H -0.8148612 .9209292 .108905$

H $-0.7750524 .366013 \quad 3.074923$

H -0.0195870 .8528095 .140417$

H $0.4890392 .828717 \quad 6.197127$

H 1.3620553 .9515885 .151229

H -0.3578944 .2188955 .512141$

H -1.5360520 .0212963 .225261$

H -0.5079250 .8761592 .084588$

H $1.472793-0.4568263 .002514$

H $0.316851-1.4167023 .931840$

H -0.853982 -2.016604 1.774223

H $0.904582-3.5463212 .722518$

H $2.154109-2.6126931 .931555$

H $2.078618-5.210402-0.261513$

H $2.210867-2.575533-0.694066$

H $-0.267940-5.3291041 .437782$

H $-0.335639-5.292432-0.308268$

H - $-1.189218-4.0547760 .634824$

H $-0.032325-3.716938-1.977514$

H $\quad 0.357698-1.074718-2.739494$

H $-0.1361390 .208179-1.665446$

H -1.610924 -1.246522 -1.078778

H $3.0411370 .596417 \quad 1.557893$

H 5.7424062 .0675271 .598309

H $5.448463-2.2396971 .140056$

H $4.170881-1.2313460 .511162$

H $4.331714-1.4729642 .267221$

H $1.908508 \quad 1.021161-2.147293$

H $2.3461370 .094305-0.713625$

H $6.0139850 .280525-3.441312$

H $5.156929-1.164146-4.019901$

H $5.756011-2.389000-1.854905$ 
H $7.992325-0.326720-2.222784$

H $9.146355-2.521566-1.586512$

H $7.750210-3.006648-0.655963$

H $10.567409-0.5033461 .143407$

H $10.795868-1.073183-0.604801$

H $8.633638-1.2487542 .364895$

H $7.464171-2.2729951 .603484$

H 7.6405850 .8165881 .525116

H $4.3898770 .618454-1.755551$

C $-0.5140174 .588555-2.383490$

O $-1.7122314 .484089-1.588045$

C $-2.3687403 .231428-1.900146$

C $-1.6969202 .723434-3.168814$

C $-0.2733273 .199666-2.968071$

H -2.148073 2.566616-1.056166

C $-3.8793473 .474361-2.007816$

H - $1.7704361 .638266-3.283641$

H $-2.1296713 .196610-4.058893$

C $0.5635303 .192043-4.233817$

H $0.1953152 .545682-2.227220$

C $-4.7288282 .256886-2.414733$

O $-4.3600463 .973323-0.758128$

H $-4.0471214 .277325-2.737532$

H $-5.7889932 .526831-2.322200$

O $-4.5028822 .012765-3.808284$

C $-4.4974210 .936455-1.645251$

C $-4.7653251 .042232-0.132019$

H $-3.4847810 .562138-1.831169$

O $-5.397972-0.015382-2.225834$

H $-4.087623 \quad 1.7787850 .310117$

H $\quad-5.795523 \quad 1.3966900 .000484$

H $0.6415722 .176757-4.636468$

H $0.1250723 .828632-5.009545$

H $1.5766733 .553672-4.032086$

C $0.6271615 .185875-1.553076$

H $-3.8148044 .743904-0.523477$

H $-5.0627331 .259856-4.064879$

H -5.205181 - $0.883155-1.833154$

H $0.3192866 .180176-1.205106$

H $1.5092985 .332430-2.186946$

H $-0.7497245 .294914-3.190671$

C $-5.563820-2.5076660 .786306$

O $-5.592055-1.2030330 .193930$

C $-4.593589-0.2839150 .644374$

C $-4.720478-0.0656822 .163239$

C $-4.717179-1.3920322 .930725$

C -5.683800 -2.410529 2.315834

H $-6.466339-3.0067580 .410392$

C $-4.347918-3.3270130 .326866$

H -3.600813 -0.6834130 .411436$

O $\begin{array}{llll}-5.931682 & 0.636050 & 2.442061\end{array}$

H -3.890206 0.5674152 .497796

H $-4.999114-1.2022843 .973547$

O $-3.394821-1.9184852 .932001$

H -5.531242 -3.402732 2.756189

O $-7.026782-2.0220112 .616902$

C $-4.357787-3.602581-1.162015$

H $-3.411513-2.8127990 .553129$

H $-4.348554-4.296896 \quad 0.837730$

H $-5.961630 \quad 0.801473 \quad 3.399616$

H $-3.406553-2.7433673 .446776$

H -7.116009-2.000138 3.584852

O $-3.217183-4.279452-1.490522$

O $-5.242285-3.271295-1.938801$

C $-3.059829-4.588025-2.878556$

H $-3.026696-3.667621-3.469160$

H -2.111986 -5.118329 -3.002886

$\mathrm{H}-3.873145-5.236788-3.217200$

SCF Energy (B3LYP/6-31G**//MMFF) $=-3245.90838528$

0800207

MM̄MF Geometry

C $2.530249-0.0161863 .162346$

C $2.280872-1.3316323 .250885$

C $2.793747-2.4220582 .340756$

O $3.935848-1.9555521 .586889$

C $1.692012-2.8530911 .354861$

C $1.877635-4.2609230 .825434$
C $2.313150-4.575024-0.410129$

C $1.476632-5.3584761 .779293$

C $2.766048-3.650932-1.506510$

C $1.787131-3.633914-2.691331$

C $0.528533-2.800502-2.409513$

C $-0.495098-2.940487-3.537187$

C $-1.715598-2.031046-3.317113$

O $0.938716-1.433893-2.301781$

O $-2.446296-2.015933-4.552635$

C -1.224555 -0.595207-3.029495

C $-2.662780-2.604379-2.256385$

O $-2.3043230 .255820-2.652417$

C $-0.113526-0.527799-1.956448$

C $0.4805590 .898828-1.897602$

O $-0.676181-0.841458-0.681438$

C $4.982778-2.8068461 .434816$

O $5.074482-3.9414101 .885123$

C $6.082813-2.2003030 .650725$

C 5.980891-1.052963-0.038276

C $7.051552-0.422279-0.818215$

C $8.407780-1.079189-0.805356$

C $1.8085910 .980990-1.133427$

C $2.2443492 .417058-0.903084$

C $4.0090383 .734003 \quad 0.269757$

O $1.7018663 .379880-1.438270$

C $5.1042923 .929736-0.729257$

C $6.4094873 .843755-0.432794$

C $7.4992923 .975108-1.456549$

C $8.3225622 .711911-1.593503$

C $9.5853332 .662451-1.136894$

C $7.7175521 .539571-2.337746$

C $6.7832270 .719284-1.486643$

N $3.3294082 .490109-0.041258$

H 3.1584510 .3656352 .362990

H $1.630997-1.6763994 .053830$

H $3.089636-3.2497572 .996420$

H $1.641571-2.1355790 .528573$

H $\quad 0.706330-2.8135631 .838609$

H $2.375054-5.630589-0.673975$

H $1.574607-6.3542071 .333891$

H $2.101896-5.3388202 .676909$

H $\quad 0.431010-5.2356902 .080330$

H $2.955056-2.636137-1.144151$

H $3.735051-4.023050-1.862973$

H $2.314584-3.202407-3.552005$

H $1.509764-4.660484-2.960829$

H $\quad 0.089076-3.126935-1.460945$

H $-0.811298-3.985102-3.649639$

H $-0.024622-2.665473-4.491902$

H -3.248160 -1.482250 -4.423072

H $-0.822800-0.193042-3.970057$

H -3.047843 -3.579884 -2.576773

H $-3.541453-1.964891-2.121359$

H -2.184961 -2.739436 -1.284029

H $-2.9368720 .277108-3.389861$

H $\quad 0.669349 \quad 1.262249-2.916262$

H -0.247896 $1.580499-1.439756$

H $-0.147109-0.4158030 .010819$

H $7.002519-2.7772140 .658481$

H $5.031184-0.521091-0.038169$

H $9.165924-0.525707-1.363138$

H $8.354394-2.079138-1.249869$

H $8.782901-1.1683860 .220473$

H $1.732497 \quad 0.478251-0.165220$

H $2.6005650 .482823-1.705580$

H 4.3882693 .6528851 .293141

H 3.3019454 .5682240 .222369

H $4.8034554 .126519-1.755836$

H 6.7135743 .6466270 .592655

H $8.1361744 .818386-1.159919$

H $7.0933534 .244027-2.439892$

H $10.196614 \quad 1.774638-1.258126$

H $10.0363493 .508270-0.628563$

H $8.5042160 .912665-2.772655$

H $7.1600791 .911789-3.207137$

H $5.7697351 .115879-1.440595$

H $3.761278 \quad 1.6280810 .278816$

C 0.5335311 .4539353 .828231 
$\begin{array}{llll}\text { O } & -0.382180 & 0.361301 & 4.046984\end{array}$

C -1.186712 0.1907432 .863231

C $-1.155714 \quad 1.5451992 .172786$

C $0.279438 \quad 1.970671 \quad 2.411459$

H $-0.675177-0.5673212 .258085$

C -2.587074 -0.2990113 .248722$

H -1.4100831 .4916041 .110429$

H -1.8483682 .2412372 .660938$

C 0.5147393 .4626592 .256079

H 0.9108141 .4443271 .691211

C -3.479857-0.560944 2.017295

O $-2.411821-1.507153 \quad 3.994574$

H -3.0580550 .4294653 .919402$

H -3.4662040 .3278581 .379065$

O $-2.914251-1.6268091 .253242$

C $-4.952146-0.8841832 .341377$

C $-5.786763-1.3268081 .123044$

H $-5.408665-0.0023812 .806487$

O $-5.016486-1.9396793 .302389$

H -5.444416 -2.3121930.780698

H $-6.813841-1.4812781 .480134$

H $\quad 0.2791003 .7858601 .237068$

H -0.1095524 .0416092 .944630$

H 1.5608203 .7130312 .458466

C $1.9692300 .998509 \quad 4.125625$

H $-3.287859-1.7870484 .309217$

H -2.911209-2.419059 1.817579

H $-5.951690-2.069443 \quad 3.535437$

H 2.0066610 .5773425 .138730

H 2.6352931 .8696534 .126184

H $\quad 0.2600702 .219426 \quad 4.565327$

C $-6.3396661 .971765-0.546417$

O -6.2991840 .9098710 .417335$

C $-5.792872-0.341110-0.055522$

C $-6.641281-0.860967-1.227507$

C $-6.7493120 .184507-2.341559$

C -7.143947 1.558481-1.792059

H $-6.8947402 .778541-0.049820$

C $-4.9344952 .501582-0.875637$

H -4.763856 -0.209619-0.402586

O $-7.949814-1.213862-0.782413$

H $-6.180863-1.775251-1.619343$

H -7.498306 -0.139870-3.074197

O $-5.500664 \quad 0.300142-3.016728$

H -7.038581 $2.327229-2.566524$

O $-8.5247131 .530773-1.417255$

C $-4.348943 \quad 3.2976010 .272713$

H $-4.2243291 .706277-1.111008$

H -5.004932 $3.176473-1.737085$

H -8.341027 $-0.430877-0.358339$

H $-5.285819-0.573379-3.385741$

H $-9.0405941 .322791-2.214859$

O $-3.3612254 .109576-0.207697$

O $-4.700092 \quad 3.210156 \quad 1.441369$

C -2.737661 4.9669080 .753357

H -3.427337 5.7684311 .033190

H -2.425699 $4.405141 \quad 1.637768$

H -1.851552 5.4089910 .290260

SCF Energy (B3LYP/6-31G**/MMFF) $=-3245.90773455$

$08 \_00208$

MMFF Geometry

C $0.248552-2.477810 \quad 0.486444$

C $0.830740-2.161643 \quad 1.655127$

C $0.297811-1.1856562 .683745$

O $-1.012746-0.7032282 .313015$

C $1.243100 \quad 0.0231252 .776316$

C $0.793681 \quad 1.061465 \quad 3.784137$

C 0.1914012 .2222713 .456780

C 1.1056760 .7419955 .221783

C $-0.1989952 .677623 \quad 2.077337$

C -1.5877393 .3313802 .062667$

C -2.0050363 .7030250 .632563$

C $-3.247258 \quad 4.5932840 .637569$

C $-3.7161664 .914278-0.789451$

O $-2.2835422 .488296-0.073784$

O $-5.0318735 .482228-0.704046$

C $-3.8419953 .595926-1.578921$
C $-2.8302465 .968827-1.462851$

O $-4.130945 \quad 3.848323-2.952444$

C -2.605178 $2.671917-1.458718$

C $-2.9410651 .290047-2.066910$

O $-1.511883 \quad 3.244224-2.177893$

C $-2.090071-1.3652212 .813478$

O $-2.081886-2.3142123 .580763$

C $-3.299922-0.7548732 .218883$

C $-4.467508-1.4089642 .303667$

C $-5.738810-0.9957431 .706482$

C -5.8061770 .3680951 .072972$

C $-1.9040880 .196607-1.785021$

C $-2.394551-1.138190-2.311836$

C $-4.086666-2.918404-1.843238$

O $-1.969242-1.613236-3.361910$

C $-5.552506-2.612214-1.835067$

C $-6.461759-3.324825-1.152323$

C $-7.929130-2.998236-1.133021$

C $-8.500020-2.916766 \quad 0.267825$

C $-9.356787-3.848890 \quad 0.718561$

C $-8.136535-1.717728 \quad 1.122201$

C $-6.773222-1.8614291 .744695$

N $-3.369622-1.702417-1.505648$

H $-0.700076-2.0114040 .234048$

H $1.778405-2.6228581 .920765$

H $0.255681-1.6906963 .656663$

H $1.347290 \quad 0.480643 \quad 1.787503$

H $2.255242-0.310303 \quad 3.042956$

H $-0.047005 \quad 2.9205314 .257727$

H $0.7971531 .542923 \quad 5.902070$

H $0.588479-0.1711945 .532465$

H 2.1826520 .5947325 .353098

H 0.5416773 .4088331 .732936

H -0.1927461 .8488491 .364009$

H -2.320471 2.6371772 .493842

H -1.568748 4.2300122 .691738

H $-1.168008 \quad 4.2209230 .149368$

H -3.062822 5.5190591 .196632

H -4.0630844 .0799771 .165948$

H $-4.9785686 .279601-0.149695$

H $-4.7120793 .059645-1.174176$

H $-2.8275496 .896479-0.878055$

H -3.220205 $6.239242-2.450346$

H -1.792852 $5.647883-1.578051$

H $-4.9196094 .416675-2.983063$

H -3.906068 $0.953915-1.670399$

H -3.062467 $1.380867-3.154291$

H $-0.8931662 .538748-2.425215$

H -3.1871630 .1824951 .686469$

H -4.489171 -2.364566 2.826512

H -5.1940320 .4010380 .167831$

H $-5.446048 \quad 1.135942 \quad 1.767187$

H -6.8186700 .6675750 .793951$

H $-0.9498930 .419964-2.269265$

H $-1.7062600 .099215-0.712146$

H $-3.817146-3.672170-1.096975$

H -3.782917 -3.274793-2.831954

H $-5.881677-1.756905-2.421256$

H $-6.139016-4.194947-0.585226$

H -8.448381 $-3.776100-1.707696$

H $-8.132869-2.053942-1.653258$

H $-9.788545-3.7859901 .711966$

H $-9.642110-4.6985200 .107050$

H -8.861804 -1.6033251 .938193$

H $-8.234170-0.8181200 .508623$

H $-6.629321-2.8121492 .261006$

H $-3.598150-1.252155-0.624927$

C $2.131668-4.047239-0.356638$

O $3.148231-3.028663-0.399515$

C $4.360680-3.607624-0.923095$

C $4.042659-5.065371-1.252669$

C $2.539068-5.033809-1.447579$

H $4.589904-3.077014-1.853348$

C $5.501788-3.4286960 .085000$

H $4.580959-5.410465-2.141214$

H $4.310138-5.737407-0.428972$

C $1.888111-6.401097-1.332554$

H $2.322971-4.610109-2.437551$ 
C $5.780982-1.9599720 .465962$

O $5.151084-4.130561 \quad 1.286267$

H $6.411841-3.905898-0.297041$

H $4.906514-1.5429280 .976474$

O $6.834562-1.9679901 .440785$

C $6.198167-1.055214-0.712920$

C $6.6019690 .366478-0.272687$

H $5.391421-0.998403-1.450985$

O $7.322942-1.658099-1.358364$

H $7.2754830 .303800 \quad 0.590056$

H $7.1904990 .829036-1.076525$

H $2.293293-7.084342-2.086231$

H $2.061496-6.846386-0.347101$

H $\quad 0.806607-6.334975-1.486464$

C $0.753258-3.421518-0.575415$

H $5.879203-4.003606 \quad 1.918467$

H $7.639084-2.2898090 .998834$

H $7.531699-1.123626-2.143548$

H $0.005041-4.215220-0.684328$

H $0.763469-2.859580-1.517154$

H $2.183040-4.5306240 .627895$

C $3.7523372 .614647-1.137086$

O $4.8969751 .753044-1.203216$

C $5.3890691 .255438 \quad 0.048303$

C $5.766672 \quad 2.418427 \quad 0.976091$

C $4.586673 \quad 3.372812 \quad 1.167754$

C $3.9765143 .791546-0.172290$

H $3.6667203 .035415-2.147861$

C $2.4767931 .817121-0.850510$

H 4.6113540 .6540290 .527975

$\begin{array}{lllll} & \text { O } & 6.886513 & 3.130438 & 0.454651\end{array}$

H 6.0694682 .0105871 .947726

H 4.9315204 .2600591 .712386

O 3.6016922 .7226341 .965069

H $3.0297284 .322275-0.018028$

O $4.8653034 .708741-0.818376$

C $2.040174 \quad 1.090205-2.099207$

H $2.5921351 .089957-0.043769$

H $1.6492312 .478011-0.568019$

H $6.659325 \quad 3.427173-0.443174$

H 2.8870643 .3619572 .125529

H $4.9688205 .478180-0.232878$

O $2.538424-0.178329-2.107328$

O $1.366740 \quad 1.607521-2.981820$

C $2.237767-0.941740-3.279504$

H $1.155831-1.067029-3.383290$

H $2.657427-0.456124-4.165696$

H $2.696288-1.927850-3.173018$

SCF Energy (B3LYP/6-31G**//MMFF) $=-3245.92237303$

0800209

MMFF Geometry

C $1.174534 \quad 3.242963 \quad 0.915914$

C $1.780800 \quad 4.153168 \quad 0.137623$

C $2.8392123 .866165-0.904760$

O $2.9870782 .444947-1.121937$

C $4.1912734 .421155-0.427453$

C $5.3128094 .193154-1.420889$

C $6.2487903 .230634-1.300066$

C $5.3585075 .159767-2.574712$

C $6.3354252 .181460-0.225241$

C $6.6699550 .800387-0.807301$

C $6.688907-0.274552 \quad 0.288734$

C $7.258769-1.588200-0.244530$

C $7.247079-2.6800230 .835075$

$\begin{array}{llll}\text { O } & 5.343066 & -0.484720 & 0.726931\end{array}$

O $7.494687-3.9403510 .191805$

C $5.834226-2.7626531 .451791$

C $8.380805-2.4855851 .848912$

O $5.818039-3.6306492 .582441$

C $5.215772-1.3922161 .830188$

C $3.709642-1.5702082 .115962$

O $5.789051-0.8529453 .021829$

C $2.1656401 .861813-2.036651$

$\begin{array}{llll}\text { O } & 1.323369 & 2.410440 & -2.728547\end{array}$

C $2.4439080 .409204-2.013746$

C $1.580791-0.434970-2.597884$

C $1.670789-1.896401-2.595857$
C $2.924677-2.519655-2.042277$

C $2.962433-0.2576272 .380777$

C $1.476640-0.5314322 .516556$

C $-0.501869-1.2212461 .153413$

O $0.927804-0.5921793 .613851$

C $-0.443434-2.628803 \quad 0.644420$

C $-1.125536-3.060496-0.426777$

C $-1.036079-4.461623-0.963293$

C $-0.886470-4.510375-2.470903$

C $-1.883382-4.968873-3.246625$

C $0.441524-4.099042-3.076968$

C $0.626668-2.604802-3.074247$

N $0.860754-0.740741 \quad 1.292228$

H 1.4430812 .1945800 .833029

H $1.4998365 .199144 \quad 0.243327$

H $2.5516924 .355611-1.843592$

H 4.4500783 .9821490 .543426

H $4.1064765 .499873-0.236884$

H $7.0380613 .186769-2.049389$

H $6.2098814 .975205-3.238460$

H $4.4484935 .080657-3.177478$

H $5.4445026 .187301-2.206495$

H 7.1236852 .4749090 .478368

H 5.4042242 .1091230 .343418

H $5.9245230 .538815-1.569296$

H $7.6487220 .845327-1.300883$

H 7.2912330 .0934801 .127560

H $8.274801-1.446006-0.632950$

H $6.654991-1.931484-1.096497$

H $8.346886-3.878005-0.272760$

H $5.185077-3.2283300 .696376$

H $9.355071-2.5402781 .348128$

H $8.384985-3.2855592 .597341$

H $8.333908-1.5255602 .366983$

H $6.180607-4.4865562 .296227$

H $3.239249-2.0742491 .264614$

H $3.569172-2.2245592 .986420$

H $6.500521-1.4394853 .321994$

H $3.3216980 .071549-1.474737$

H $0.700389-0.021800-3.088776$

H $3.814122-2.081035-2.508879$

H $2.992663-3.594457-2.222946$

H $2.983785-2.363859-0.962154$

H 3.3147770 .2167303 .303252

H $3.1160730 .461174 \quad 1.568337$

H $-1.014159-0.5517910 .455487$

H -1.014650 -1.190300 2.119185

H $0.199820-3.3242781 .178864$

H - $1.785956-2.369842-0.944862$

H $-1.946545-4.993262-0.658831$

H $-0.200187-5.007031-0.507594$

H $-1.778777-5.029855-4.324847$

H $-2.829172-5.288758-2.821913$

H $0.504133-4.438788-4.118677$

H $1.234349-4.627905-2.541006$

H $-0.221729-2.057353-3.488322$

H $1.417133-0.667610 \quad 0.445863$

C $-1.2243742 .949758 \quad 1.541439$

\begin{tabular}{lllll}
\hline & -1.733277 & 3.624691 & 0.371198
\end{tabular}

$\begin{array}{llll}\text { C }-3.072017 & 4.059858 & 0.638261\end{array}$

C $-3.561330 \quad 3.2155341 .804545$

C -2.2922943 .1030732 .628251$

H -2.9928115 .1001570 .984621$

C $-3.9338494 .049181-0.626235$

H -4.3869503 .6838352 .348294$

H -3.8835182 .2211691 .479949$

C $-2.3148091 .955373 \quad 3.624125$

H -2.1426604 .0448413 .173178$

C $-4.3618162 .674517-1.181483$

O $-5.1265094 .784779-0.318769$

H $-3.4149784 .610409-1.413410$

H $-5.0874392 .228880-0.493712$

$\begin{array}{lllll}\text { O } & -5.087623 & 2.917308 & -2.396772\end{array}$

C $-3.228998 \quad 1.674712-1.476214$

C $-3.6876220 .400943-2.215667$

H $-2.7377701 .387565-0.541945$

O $-2.248888 \quad 2.322233-2.291164$

H - $4.0687010 .666619-3.209671$ 
H -2.793204 -0.210866 -2.395691 H -3.1229662 .0917304 .349988$ H -2.471529 0.9933543 .124046 H -1.3702801 .8986894 .174398$ C 0.1185343 .5675841 .933503 H $-5.6681974 .808187-1.126340$ H $-4.4574073 .272037-3.047414$ H $-1.4999271 .710024-2.392205$ H $\quad 0.447416 \quad 3.1763122 .903018$ H 0.0138974 .6548752 .034784 H -1.0989001 .8946131 .270008$ C $-4.998284-1.5865280 .652308$ O $-4.124961-0.878098-0.238001$ C $-4.732425-0.426894-1.452211$ C $-5.232901-1.625610-2.270406$ C $-6.202009-2.482046-1.447528$ C $-5.652778-2.792302-0.049777$ H $-4.331246-1.9727331 .433736$ C $-5.985668-0.6127231 .312201$ H $-5.5874020 .213892-1.215319$ O $-4.123082-2.419361-2.681018$ H -5.728336 -1.255032 -3.175685 H $-6.394197-3.426206-1.971756$ O $-7.446096-1.800796-1.314746$ H $-6.449124-3.2006120 .581729$ O $-4.668216-3.823964-0.170795$ C $-6.560532-1.1365422 .609779$ H -5.4638420 .3239841 .541527$ H $-6.813386-0.3698470 .640163$ H $-4.468537-3.147844-3.224471$ H $-7.795123-1.648599-2.209497$ H -3.949994 -3.481885 -0.729470 O $-7.465876-0.2313143 .087235$ O $-6.255646-2.1932863 .144630$ C $-8.097244-0.5852994 .321786$ H -8.7967240 .2119424 .586779$ H -8.656183 -1.519043 4.207842 $\mathrm{H}-7.350458-0.6756075 .116411$

SCF Energy (B3LYP/6-31G**//MMFF) $=-3245.91535224$

0800210

MMFF Geometry

C -1.079107 -0.2823265 .189749$

C -0.9641850 .5326574 .129768$

C $-1.826566 \quad 1.743840 \quad 3.892774$

O -2.4160391 .6115562 .576383$

C -0.9679233 .0214703 .904867$

C -1.7461874 .2597213 .500841$

C -1.802095 4.7444002 .242838

C -2.4862264 .9443944 .618244$

C -1.1532324 .1545891 .020415$

C $-0.307378 \quad 5.1806770 .255472$

C $0.7667044 .499339-0.605051$

C $1.4756425 .501690-1.515887$

C $2.5276574 .814935-2.403060$

O $0.1597113 .469335-1.392353$

O $2.8963805 .733695-3.442847$

C $1.8871813 .588294-3.089755$

C $3.8127774 .503038-1.625073$

O $2.8676852 .829295-3.793831$

C $1.1069812 .672921-2.117943$

C $0.3352961 .589930-2.906649$

O $2.0359402 .027033-1.244089$

C -3.556675 0.8816702 .460762

O

C -4.0170371 .0039701 .058136$

C -5.1202050 .3521840 .662562$

C $-5.7305260 .405950-0.668828$

C $-5.1081251 .325575-1.686447$

C $-0.6839520 .806551-2.065053$

C $-1.243710-0.386789-2.822227$

C -2.776024 -2.330396-2.529876

O $-0.931191-0.656027-3.979296$

C -4.052401-1.972469 -3.223895

C $-5.235386-2.538933-2.940890$

C $-6.506864-2.215538-3.671960$

C -7.642456 -1.842647 -2.745769

C $-8.656357-2.694240-2.516437$
C -7.647725 -0.449409-2.156855

C $-6.821706-0.352725-0.904832$

N -2.129530 -1.123777 -2.047741

H -1.817276 -0.036505 5.951005

H $-0.2341120 .318914 \quad 3.354157$

H -2.6221581 .8311764 .643715$

H $-0.0978942 .898773 \quad 3.250394$

H $-0.543068 \quad 3.172590 \quad 4.906437$

H -2.400632 5.6349872 .057604

H -3.034213 5.8279544 .274330

H -3.2117634 .2607525 .070567$

H - 1.7851355 .2707965 .393178

H -0.5376713 .2867901 .270298$

H -1.945612 3.7841670 .358183

H $-0.9782445 .772300-0.380647$

H $\quad 0.1823845 .8785240 .946277$

H 1.4884764 .0437560 .081208

H $1.9339286 .307315-0.928884$

H $0.7365065 .987415-2.168060$

H $3.2343536 .542764-3.021975$

H $1.1845903 .965771-3.846140$

H $4.2527385 .425675-1.227674$

H $4.5751634 .066624-2.279787$

H $3.6528423 .826407-0.783149$

H $3.3202893 .436296-4.404283$

H $-0.2152472 .058643-3.732843$

H $1.0525910 .887571-3.350636$

H $1.6877731 .160496-0.982175$

H -3.4466351 .6439840 .394751$

H $-5.637437-0.276877 \quad 1.386126$

H $-5.1631662 .365446-1.345893$

H $-5.5884191 .285243-2.665764$

H $-4.057241 \quad 1.068647-1.850471$

H $-0.2270230 .432626-1.143108$

H -1.519186 $1.459989-1.788647$

H $-2.942875-2.980508-1.665580$

H -2.106668 -2.841699 -3.229404

H $-3.995931-1.231830-4.018337$

H $-5.288668-3.301625-2.167227$

H $-6.780303-3.098270-4.264749$

H $-6.355687-1.406647-4.397694$

H -9.488758 -2.421711-1.876245

H $-8.674256-3.684766-2.959133$

H $-8.674375-0.163080-1.890946$

H $-7.3509580 .267818-2.926376$

H $-7.199419-0.977500-0.093706$

H -2.379073 -0.788535 -1.121786

C $0.526757-2.088598 \quad 4.273924$

O $-0.347120-2.432773 \quad 3.183034$

C $0.202902-3.5682382 .479426$

C $1.521832-3.8963313 .167779$

C $1.288487-3.3760094 .574598$

H $-0.519125-4.3826042 .612833$

C $0.327623-3.1902191 .000251$

H $1.752754-4.9654563 .137418$

H $2.354100-3.3586562 .695590$

C $2.569922-3.1720205 .363174$

H $0.639548-4.0827645 .108639$

C $1.006355-4.2572650 .124758$

O $-0.988961-2.9555050 .489373$

H $0.862889-2.2385960 .923874$

H $2.017396-4.4657640 .489929$

O $0.288509-5.4905890 .255517$

C $1.068968-3.893037-1.372917$

C $1.689543-2.519895-1.678831$

H $0.056728-3.922764-1.794172$

O $1.800145-4.917054-2.048371$

H $1.712246-2.367472-2.765458$

H $\quad 1.015154-1.740166-1.304997$

H $2.355467-2.7894846 .365754$

H $3.109072-4.1188845 .471753$

H $3.237864-2.4600544 .866545$

C $-0.306035-1.5550585 .442972$

H $-1.399243-2.2729711 .047908$

H $-0.628092-5.327369-0.026170$

H $1.808191-4.695860-2.995348$

H $0.337095-1.3898486 .314802$

H $-1.043277-2.3186845 .723176$ 
H $1.223236-1.3200613 .915070$

C $4.528445-0.353421-0.637571$

O $3.314077-0.846508-1.217678$

C $3.097936-2.261277-1.100179$

C $4.230258-3.026880-1.802641$

C $5.608809-2.579413-1.300730$

C $5.744430-1.053326-1.265784$

H $4.5623570 .706866-0.918514$

C $4.512756-0.4264570 .894109$

H $3.089693-2.513883-0.037213$

O $4.144453-2.815443-3.210063$

H $4.111560-4.099689-1.618801$

H $6.381878-2.998272-1.956893$

O $5.811282-3.118003 \quad 0.002829$

H $6.656619-0.767077-0.729272$

O $5.889817-0.595099-2.612979$

C $3.405922 \quad 0.423616 \quad 1.469335$

H $4.364451-1.4387181 .280776$

H $5.469566-0.0835091 .306262$

H $4.851903-3.334220-3.629290$

H $6.701075-2.8552340 .293668$

H $6.0217660 .367878-2.584241$

O 3.8798641 .6629141 .779930

O 2.2547090 .0325861 .613270

C $2.9290352 .562032 \quad 2.359204$

H 2.7815702 .3086213 .412872

H 3.3329183 .5754752 .290805

H 1.9763852 .5301071 .823310

SCF Energy (B3LYP/6-31G**//MMFF) $=-3245.90806931$

0800211

MM̄FF Geometry

C -0.471841 -3.474965 1.342526

C $0.146000-3.0744162 .465444$

C $-0.021496-1.7478253 .172380$

O $-1.104383-0.9766522 .605844$

C $1.266944-0.9168683 .056886$

C 1.2794710 .2724943 .997165

C 1.0329921 .5457283 .632745

C $1.647409-0.0431905 .424794$

$\begin{array}{llll}\text { C } & 0.665687 & 2.057343 & 2.268838\end{array}$

C -0.5460852 .9973152 .326851$

C -0.8840753 .5556850 .937531$

C $-1.940443 \quad 4.656586 \quad 1.034131$

C $-2.340808 \quad 5.179336-0.353468$

O $-1.3789242 .479598 \quad 0.133213$

O $-3.5311965 .967148-0.201841$

C $-2.7035723 .978765-1.250571$

C $-1.2758526 .109785-0.946301$

O $-2.9392144 .397693-2.593124$

C $-1.6533122 .841750-1.226586$

C -2.219945 $1.602831-1.955307$

O $-0.4729473 .273768-1.902390$

C $-2.352487-1.2117473 .095292$

O $-2.660519-1.9868253 .986764$

C $-3.301898-0.3870162 .317290$

C $-4.601664-0.7181282 .324971$

C $-5.667533-0.059474 \quad 1.568904$

C $-5.342923 \quad 1.246341 \quad 0.896303$

C $-1.3928880 .322719-1.785280$

C $-2.162112-0.853156-2.353576$

C $-4.083484-2.362332-1.831829$

O $-1.960533-1.264888-3.493445$

C $-5.425721-1.710945-1.965248$

C $-6.540934-2.177287-1.382335$

C $-7.886843-1.520758-1.516542$

C $-8.588935-1.327097-0.188431$

C $-9.666160-2.0622370 .135949$

C $-8.090813-0.2306310 .731885$

C $-6.874749-0.6591101 .508256$

N $-3.112637-1.345192-1.471593$

H $-1.163972-2.8095930 .835166$

H $0.833427-3.7662472 .949712$

H $-0.231747-1.9545054 .229124$

H $1.408116-0.5909482 .020034$

H $2.141840-1.5390733 .289049$

H 1.1144882 .3184214 .396495

H 1.6878860 .8533726 .052663
H $0.915236-0.7229065 .871092$

H $2.633680-0.5173335 .466052$

H 1.5257652 .6122281 .878059

H $\quad 0.4593421 .2446941 .567332$

H - 1.4078092 .4483252 .727535

H $-0.331303 \quad 3.8257273 .013633$

H 0.0360993 .9503420 .490252

H -1.589032 $5.481326 \quad 1.666715$

H $-2.8377814 .259342 \quad 1.529107$

$\begin{array}{llll}H & -3.335148 & 6.689784 & 0.418894\end{array}$

H $-3.6568453 .574954-0.881183$

H $-1.1093306 .969931-0.286794$

H -1.605539 $6.527120-1.904265$

H $-0.3122955 .618792-1.097786$

H $-3.6127415 .098596-2.563887$

H $-3.2323541 .406560-1.587162$

H $-2.3143211 .812355-3.028991$

H $0.0090612 .494126-2.220989$

H -2.9033650 .4304101 .727891$

H $-4.913820-1.5824052 .910286$

H $-6.222015 \quad 1.7712390 .516926$

H $-4.670703 \quad 1.0788050 .052067$

H -4.8603721 .9366101 .597652$

H $-0.4339210 .392653-2.309016$

H $-1.1698840 .120493-0.731539$

H $-4.068728-3.119546-1.041677$

H $-3.801357-2.830875-2.779303$

H $-5.483491-0.814031-2.578043$

H $-6.490094-3.090448-0.793570$

H $-8.497417-2.151645-2.175425$

H $-7.806642-0.549524-2.020645$

H -10.189969-1.913407 1.074405

H -10.045800 -2.832822 -0.526683

H -8.8702600 .0351871 .457811$

H -7.9253890 .6703010 .135193$

H -7.015285 -1.590985 2.058325

H $-3.205041-0.913932-0.556982$

C $0.524509-4.872280-0.549229$

O $1.860299-4.396174-0.300789$

C $2.277142-3.593920-1.422891$

C $1.260318-3.856293-2.529545$

C $-0.009634-4.052606-1.721834$

H $2.184044-2.545531-1.117945$

C $3.732225-3.906654-1.784456$

H $1.194904-3.025454-3.238560$

H $1.496550-4.765977-3.093553$

C $-1.125079-4.736224-2.491949$

H $-0.355693-3.068896-1.384207$

C $4.743805-3.781782-0.622564$

O $3.810945-5.256528-2.267032$

H $4.047615-3.278264-2.624560$

H $4.557312-4.587788 \quad 0.098603$

O $6.057069-4.009215-1.150325$

C $4.781025-2.4404790 .135399$

C $5.014583-1.226131-0.775366$

H $5.608845-2.4860340 .853305$

O $3.590694-2.2555450 .890854$

H $5.988905-1.309414-1.273410$

H $4.277678-1.224608-1.588542$

H $-1.413830-4.139934-3.363276$

H $-0.818905-5.724885-2.850099$

H $-2.010025-4.865806-1.861244$

C $-0.292290-4.8439920 .743734$

H $3.468100-5.838585-1.567028$

H $6.038920-4.863815-1.614727$

H $3.475370-3.0363361 .458859$

H $\quad 0.200229-5.495963 \quad 1.476894$

H $-1.284955-5.2732050 .563498$

H $0.632466-5.921990-0.854084$

C $5.0293002 .490908-0.696742$

O $5.202557 \quad 1.121903-1.084609$

C $4.8891630 .143123-0.082681$

C $5.793978 \quad 0.335676 \quad 1.142377$

C $5.677387 \quad 1.7661641 .682214$

C 5.8328302 .8127530 .574145

H $5.4637353 .070695-1.521532$

C $3.5461022 .860112-0.595621$

H 3.8417890 .2651220 .214377 
$\begin{array}{llll}\text { O } & 7.156905 & 0.090674 & 0.803646\end{array}$ H $5.512105-0.3699761 .932060$ H 6.4508071 .9336752 .441932 O $4.407472 \quad 1.942172 \quad 2.303820$ H 5.5447313 .8020390 .947386 $\begin{array}{llllll}\text { O } & 7.215938 & 2.903718 & 0.217671\end{array}$ C $2.8861512 .759855-1.949617$ H 2.9883752 .2076920 .080282 H $3.4150903 .881652-0.219902$ H $7.250748-0.8549660 .601731$ H 4.3355221 .2871013 .018850 H $7.4808082 .049945-0.165559$ O $3.0724623 .916941-2.646253$ O $2.298957 \quad 1.761847-2.347534$ C $2.530786 \quad 3.933234-3.970562$ H $2.9756393 .135413-4.573124$ H $1.4424363 .830583-3.936840$ H $2.7779464 .895543-4.426589$ SCF Energy (B3LYP/6-31G**//MMFF) $=-3245.92615741$

0800212

MMFF Geometry

C $0.453528 \quad 4.715972 \quad 2.200574$

C $-0.2341393 .964752 \quad 1.326955$

C $-1.722586 \quad 4.092616 \quad 1.122169$

O $-2.3160742 .774245 \quad 1.156299$

C $-2.0053684 .697006-0.262764$

C $-3.4615645 .070959-0.452732$

C $-4.348782 \quad 4.357284-1.172586$

C $-3.879538 \quad 6.371627 \quad 0.185015$

C $-4.1191773 .048615-1.877344$

C $-5.2958112 .086295-1.657901$

C $-5.1202490 .786852-2.455548$

C $-6.392042-0.060436-2.397218$

C $-6.217788-1.395407-3.135835$

O $-4.0251090 .056790-1.892381$

O $-7.316816-2.245818-2.775441$

C $-4.931745-2.078256-2.629534$

C $-6.282574-1.223242-4.657759$

O $-4.643102-3.249790-3.389581$

C $-3.696591-1.145117-2.603214$

C $-2.540493-1.851191-1.859468$

O $-3.283886-0.866436-3.942104$

C -2.590434 2.241560 2.376856

O -2.3632332 .7419353 .466638$

C -3.2185420 .9199542 .153631$

C -3.3722910 .0809503 .188773$

C $-3.950434-1.2638333 .134452$

C $-4.616810-1.6846301 .852072$

C -1.343422 $-0.950602-1.534284$

C $-0.353655-1.715728-0.678190$

C $-0.034056-2.5368811 .660627$

O $0.617888-2.285440-1.168598$

C $-0.978518-3.600643 \quad 2.128185$

C -1.237601 -3.8441803 .422117$

C $-2.200144-4.8962043 .895997$

C $-3.201239-4.3691484 .902991$

C $-3.143560-4.7415406 .192977$

C $-4.314819-3.4706614 .402681$

C -3.859538 -2.046967 4.229710

N $-0.711632-1.7324520 .660782$

H -0.0985515 .4452182 .791431$

H 0.2765313 .2295250 .713508

H -2.174884 4.7160321 .903595

H -1.683652 4.000915-1.046517

H $-1.4003705 .602011-0.410628$

H -5.356011 4.759051-1.277866

H $-4.9243356 .622596-0.027343$

H $-3.769522 \quad 6.321268 \quad 1.272505$

H -3.261712 7.194129 -0.189935

H $-4.0156313 .254283-2.949394$

H -3.195578 $2.564447-1.547276$

H $-5.375138 \quad 1.857947-0.587047$

H $-6.2290592 .576759-1.962498$

H $-4.8861921 .048594-3.494307$

H -7.248733 $0.491759-2.803161$

H $-6.641287-0.272808-1.347820$

H $-8.140441-1.793534-3.026503$
H $-5.128362-2.417144-1.602316$

H $-7.243956-0.786046-4.952945$

H $-6.225589-2.191528-5.167399$

H $-5.492821-0.579731-5.051014$

H $-5.435823-3.812668-3.366822$

H $-2.921020-2.265178-0.918941$

H $-2.181521-2.702637-2.452580$

H -2.343995 -0.625454 -3.938416

H $-3.517414 \quad 0.666598 \quad 1.142702$

H $-3.0179560 .395923 \quad 4.169604$

H -5.361766 -0.9431191 .541560$

H $-5.149682-2.6344911 .927041$

H -3.876624 -1.7894911 .055538$

H $-0.831790-0.623646-2.444201$

H $-1.652619-0.047036-0.996731$

H $0.254464-1.8650972 .473637$

H $0.865227-2.9919961 .235705$

H - $1.473878-4.1969871 .365242$

H $-0.722225-3.2646744 .184921$

H $-1.610370-5.7085204 .340130$

H $-2.744616-5.3446013 .055558$

H -3.871117 -4.3878626 .916024$

H -2.365011 -5.4039526 .556704$

H $-5.144698-3.4665145 .121325$

H $-4.722183-3.9058073 .486400$

H $-3.385087-1.6318375 .120496$

H $-1.534679-1.2163230 .956146$

C 2.7795053 .6756121 .699206

$\begin{array}{lllll}\text { O } & 2.478261 & 2.349453 & 2.177198\end{array}$

C 3.7138701 .6414122 .413078

C 4.7917392 .4519491 .713002

C 4.2825693 .8638351 .914069

H 3.8644841 .6545783 .499913

C 3.5664850 .2029851 .909010

H $5.7859842 .290628 \quad 2.140211$

H $4.832328 \quad 2.2066500 .644037$

C $4.918398 \quad 4.875206 \quad 0.975549$

H 4.4802694 .1638512 .952041

C $4.864337-0.6201171 .995781$

$\begin{array}{lllll}\text { O } & 2.567547 & -0.443854 & 2.703129\end{array}$

H 3.1858350 .2189570 .882106

H $5.654787-0.1492151 .401909$

O $5.335390-0.599137 \quad 3.349725$

C $4.712950-2.0915501 .565538$

C $4.134242-2.299887 \quad 0.157517$

H $4.078739-2.6185082 .288920$

O $5.996966-2.7134821 .654841$

H $4.151629-3.370106-0.087294$

H $3.069624-2.0346020 .174297$

H 4.5101155 .8763611 .144976

H 6.0007444 .9203531 .135279

H $4.7445124 .613556-0.073729$

C $1.936803 \quad 4.6995502 .466423$

H 1.7607450 .0970282 .649696

H $4.637247-0.981738 \quad 3.908615$

H $5.888486-3.6484461 .410237$

H $2.3110805 .707762 \quad 2.252562$

H 2.0640854 .5283753 .543300

H $2.546873 \quad 3.6983670 .627295$

C $4.364128-1.033768-3.332507$

O $3.995016-1.759455-2.152895$

C $4.802647-1.515457-0.990998$

C $6.261646-1.901306-1.274742$

C $6.793621-1.196550-2.528391$

C $5.827734-1.315955-3.711146$

H $3.726670-1.445814-4.125206$

C $4.044047 \quad 0.464465-3.206691$

H $4.746409-0.449688-0.754687$

O $6.353118-3.314089-1.442110$

H $6.884652-1.624341-0.417644$

H $7.761105-1.635582-2.801984$

O $7.015417 \quad 0.175814-2.215262$

H $6.137595-0.646122-4.521815$

O $5.919091-2.651830-4.215124$

C $2.5630370 .709373-3.008887$

H $4.5736930 .914559-2.362903$

H $4.3429960 .993058-4.118589$

H $7.288621-3.531998-1.592837$ 
H $7.3834390 .601717-3.008185$

H $5.348166-2.710334-4.999974$

O $2.3837661 .914130-2.391846$

O $1.674212-0.047326-3.374757$

C $1.0236472 .285990-2.146636$

H $\quad 0.4350982 .237088-3.067891$

H $0.5941241 .636505-1.378470$

H $1.0148163 .316839-1.783127$

SCF Energy (B3LYP/6-31G**//MMFF)= -3245.89878865

0800213

MM̄FF Geometry

C - $-1.657420-2.565925-0.969256$

C $-0.463887-2.960432-0.503368$

C $-0.052379-2.7864790 .937770$

O $0.945523-1.7401710 .994672$

C $0.562085-4.0905731 .475416$

C $0.910455-4.0285272 .951289$

C $2.160443-3.937748 \quad 3.448484$

C $-0.265531-4.1112893 .891645$

C $3.452689-3.8528332 .685661$

C $4.130147-2.4931882 .897699$

C $5.307731-2.2835561 .934514$

C $6.001078-0.9504802 .215223$

C $7.095179-0.6366571 .183987$

O $4.780301-2.2890070 .605023$

O $7.448558 \quad 0.748324 \quad 1.329105$

C $6.516620-0.808929-0.238309$

C $8.373775-1.4401321 .451475$

O $7.558793-0.669364-1.201275$

C $5.758011-2.139902-0.433206$

C $4.995986-2.227939-1.778928$

O $6.699017-3.219880-0.410885$

C $0.480102-0.4605101 .065265$

O $-0.687629-0.1050041 .110908$

C 1.6423170 .4545081 .059846

C 1.4351201 .7733890 .926494

C 2.4590082 .8201930 .921192

C 3.8961832 .3944901 .057683

C $3.903234-1.173260-1.985605$

C $4.3563350 .053540-2.758277$

C $3.5214742 .284670-3.498950$

O $5.4429190 .132621-3.324037$

C $4.0270283 .346894-2.575416$

C $3.3951714 .514020-2.377252$

C $3.9315395 .618798-1.513553$

C $2.9419356 .083028-0.469470$

C $2.1777397 .169110-0.674968$

C 2.9116715 .3472930 .849846

C $2.065114 \quad 4.1056130 .804173$

N $3.376898 \quad 1.037769-2.772641$

H -2.352822 -2.090983 -0.281140

H $\quad 0.261224-3.396263-1.186207$

H $-0.914221-2.5115271 .558816$

H $1.441879-4.3498910 .878217$

H $-0.142069-4.9206501 .326149$

H $2.281239-3.9156834 .531143$

H $0.043079-4.1448164 .941930$

H $-0.919567-3.2425263 .770145$

H -0.847706 -5.017135 3.692796

H $4.118879-4.6492193 .039427$

H $3.304909-4.0370301 .619912$

H $3.390752-1.6982272 .739710$

H $4.476819-2.4142243 .935693$

H $6.009963-3.1175282 .050508$

H $6.413696-0.9335823 .231848$

H $5.257641-0.1431972 .183907$

H 7.7506780 .8865422 .243094

H $5.8276070 .028976-0.396577$

H $8.768248-1.2107662 .448847$

H $9.167291-1.1673240 .747182$

H $8.220361-2.5199461 .399406$

H $7.147832-0.617697-2.080276$

H $5.701521-2.254184-2.617621$

H $4.492039-3.205389-1.799142$

H $\quad 6.302097-3.985835-0.857846$

H 2.6296350 .0222081 .165714

H 0.4107362 .1290550 .819880
H 4.1491651 .6412360 .304414

H 4.0749111 .9731322 .052328

H 4.6086193 .2099240 .916380

H $3.098051-1.624366-2.580405$

H $3.467128-0.863023-1.031570$

H $2.5408662 .532281-3.917572$

H $4.2266702 .148819-4.325285$

H $4.9759783 .162434-2.077512$

H $2.4611784 .706904-2.900267$

H $4.1943016 .456558-2.173051$

H $4.8691535 .326379-1.024676$

H 1.4890837 .5270470 .083226

H $2.2196417 .720611-1.608411$

H 2.4880175 .9970511 .628110

H 3.9347045 .1550111 .185422

H $0.998801 \quad 4.3139780 .702790$

H $2.5337390 .906076-2.221835$

C $-3.549216-3.095133-2.581475$

O $-4.410549-2.170373-1.883069$

C $-5.340709-2.929412-1.091003$

C $-5.376542-4.309444-1.731872$

C $-3.909766-4.494271-2.078645$

H $-4.907600-3.026574-0.088363$

C -6.686201-2.207817-1.010467

H -5.757966 -5.077116 -1.052143

H $-5.988994-4.316376-2.641239$

C $-3.654078-5.595415-3.092522$

H $-3.360777-4.728907-1.157861$

C $-6.618401-0.804963-0.364070$

O $-7.220075-2.059031-2.331629$

H $-7.405587-2.830116-0.465597$

H $-6.094757-0.106863-1.027146$

O $-7.962747-0.321066-0.249125$

C $-5.971357-0.7798051 .035051$

C $-6.1754580 .520879 \quad 1.832165$

H $-4.898175-0.9795360 .945211$

O $-6.518391-1.8411641 .826821$

H -7.2472430 .6868532 .000201$

H -5.7463600 .3507202 .829413$

H -3.983284 -6.563209 -2.700344

H $-4.191741-5.412536-4.028971$

H -2.586933 -5.669102 -3.324730

C $-2.086434-2.675864-2.405630$

H $-6.568067-1.563100-2.856299$

H -8.354096 -0.340093 -1.139313

H $-7.478330-1.6965891 .888135$

H -1.961749 -1.682421 -2.852662

H -1.435868 -3.364495 -2.956979

H -3.806947 -3.001864 -3.644406

C -3.3461832 .6648830 .647889$

O $-4.122371 \quad 1.5799851 .177348$

C -5.5406881 .7765901 .212065$

C -5.9039983 .0337962 .024635$

C -5.1411594 .2638461 .529623$

C -3.6438963 .9770761 .390651$

H -2.3051652 .3930750 .864474$

C $-3.5022272 .790867-0.870927$

H $-5.913918 \quad 1.8911260 .189991$

O $\quad-5.6053362 .8173503 .403245$

H -6.9849203 .2023831 .944783$

H -5.2904795 .0898152 .235633$

$\begin{array}{llll}\text { O } & -5.682976 & 4.659173 & 0.273127\end{array}$

H -3.1475914 .8080540 .876905$

O -3.0579013 .9044672 .693377$

C $-2.7331011 .698858-1.571590$

H $-4.5392992 .724378-1.210905$

H -3.119764 $3.754054-1.230039$

H -5.8889663 .6081553 .892682$

H $-5.2035235 .454276-0.015832$

H $-3.4233993 .122146 \quad 3.141184$

O $-1.4579652 .114080-1.817516$

O $-3.2060760 .605355-1.848653$

C $-0.6204711 .177207-2.500828$

H $-0.3926910 .332027-1.846446$

H $-1.0966980 .834321-3.424478$

H $\quad 0.3117041 .685186-2.758405$

SCF Energy (B3LYP/6-31G**//MMFF) $=-3245.92156391$ 
0800214

MM̄FF Geometry

C $-0.9121934 .708332-0.099861$

C $-1.8749873 .812936 \quad 0.164419$

C -2.2720493 .3795411 .551196$

O -2.3749311 .9363121 .556060$

C -3.6516453 .9694031 .894136$

C -4.1938953 .5234853 .238569$

C -5.2232212 .6684793 .408019$

C -3.5128964 .1297524 .438456$

C $-6.020410 \quad 1.9679602 .342572$

C $-5.764797 \quad 0.455551 \quad 2.363449$

C $-6.191710-0.2145741 .048925$

C $-6.058203-1.7344291 .153682$

C $-6.331279-2.435983-0.184720$

O $-5.332703 \quad 0.2857620 .017366$

O $-5.864416-3.789906-0.075691$

C $-5.490682-1.758052-1.287955$

C $-7.831748-2.518260-0.488395$

O $-5.826711-2.277079-2.572157$

C $-5.610668-0.220544-1.294840$

C $-4.6213410 .461607-2.274454$

O $-6.9320040 .143321-1.713362$

C -1.221434 $1.224370 \quad 1.685717$

O $-0.091098 \quad 1.6672341 .810571$

C $-1.580507-0.2106731 .633663$

C $-0.623890-1.1499931 .662728$

C $-0.842783-2.5982771 .609483$

C $-2.253134-3.0850741 .406085$

C $-3.1339060 .242423-1.975146$

C $-2.508451-0.927688-2.715566$

C $-0.399125-2.255509-2.813895$

O $-3.069347-1.524843-3.630058$

C $-0.589116-3.510745-2.021746$

C $0.425150-4.229221-1.516026$

C $0.241689-5.516273-0.762803$

C $0.952859-5.5251850 .572269$

C $2.144718-6.1295610 .714639$

C $0.239743-4.9220291 .761181$

C $0.220231-3.4195251 .733394$

N -1.226355 -1.202076 -2.255509

H $-0.385987 \quad 5.181866 \quad 0.723710$

H -2.415142 $3.354528-0.661316$

H -1.534094 3.6979902 .297949

H -4.3550013 .7254371 .092099$

H -3.5920415 .0665851 .893678$

H -5.5311102 .4294914 .425204$

H -3.9898023 .8363485 .379815$

H -2.466477 3.8134754 .488155

H -3.5451835 .2227004 .381632$

H -7.0859742 .1565332 .523244$

H -5.8155012 .3735491 .350270$

H -4.6959340 .2707332 .526434$

H -6.3047980 .0102113 .208126$

H -7.2277710 .0676810 .828053$

H $-6.718143-2.1288691 .936462$

H -5.038567 -1.985882 1.472458

H $-6.331833-4.2094310 .666920$

H -4.445016 -2.031514 -1.103257

H -8.350207 -3.0842520 .294934$

H $-8.017367-3.061488-1.421556$

H -8.309881 -1.538986 -0.556642

H -5.715818 $-3.242430-2.532706$

H $-4.869070 \quad 0.194754-3.308945$

H -4.795601 $1.545309-2.205297$

H $-6.9114671 .057039-2.042475$

H -2.632957 -0.458593 1.562438

H $0.415681-0.8337811 .737956$

H -2.325933 -4.161069 1.235901

H -2.699188 -2.612996 0.524121

H $-2.867319-2.8513382 .282092$

H -2.581656 $1.133887-2.299577$

H $-2.9650690 .127452-0.901504$

H $0.636393-1.902722-2.791192$

H $-0.686324-2.430320-3.855791$

H -1.609414 -3.860806 -1.881960

H $1.446896-3.896099-1.683174$

H $0.627864-6.327051-1.394731$
H $-0.820708-5.742937-0.609293$

H $2.648743-6.1685161 .674560$

H $2.647580-6.591695-0.128443$

H $0.741956-5.2196672 .691599$

H $-0.759940-5.3586841 .836731$

H $1.203682-2.9702041 .865716$

H $-0.859839-0.682944-1.462778$

C $0.9449234 .908402-1.833039$

O $1.3538283 .553132-1.564280$

C $2.7195833 .574148-1.099892$

C $3.2128905 .006100-1.292970$

C $1.9371695 .798490-1.089511$

H $2.6959993 .347742-0.026360$

C $3.5710382 .529102-1.832890$

H $4.0018875 .273467-0.583862$

H $3.6049015 .161062-2.305642$

C $2.0147007 .220903-1.615123$

H $1.7136065 .823293-0.016127$

C $3.2413561 .078875-1.410674$

O $3.3993872 .715108-3.236248$

H $4.6273392 .718942-1.605258$

H $3.4059401 .015987-0.330245$

O $1.8632170 .787087-1.631544$

C $4.1094960 .020408-2.119989$

C $3.929406-1.408472-1.572889$

H $5.1632590 .313635-2.049019$

O $3.774516-0.000583-3.510248$

H $2.878289-1.710386-1.649525$

H $4.482029-2.082561-2.240749$

H $2.8040547 .778308-1.100104$

H $2.2341377 .241952-2.687806$

H $1.0696217 .748624-1.453730$

C $-0.5344305 .107897-1.498987$

H $4.0043912 .106499-3.692487$

H $1.6950650 .886652-2.584412$

H $4.385022-0.616110-3.950890$

H -1.123909 $4.518808-2.213741$

H $-0.8114336 .156956-1.653068$

H $1.0651135 .054711-2.915503$

C $6.420129-1.2190551 .200956$

O $5.800352-1.191037-0.090447$

C $4.428780-1.594743-0.130079$

C $4.289299-3.0464030 .352128$

C $4.920191-3.2364581 .736969$

C $6.322985-2.6244801 .817681$

H $7.481008-1.0120611 .008980$

C $5.883137-0.1072502 .115485$

H $3.837491-0.9480410 .524393$

O $4.922208-3.922785-0.578309$

H $3.225376-3.3028990 .377501$

H $4.986791-4.3067721 .968057$

O $4.097286-2.6287592 .727291$

H $6.659372-2.5894832 .859984$

O $7.236450-3.4813061 .124824$

C 6.1506501 .2738711 .553197

H $4.803694-0.1974512 .262973$

H $6.374213-0.1635303 .093470$

H $4.782030-4.832937-0.266317$

H $3.254114-3.1119172 .741696$

H $6.994295-3.4739060 .182881$

O 5.4370812 .1906822 .272070

O $\quad 6.898860 \quad 1.523477 \quad 0.618266$

C 5.5918653 .5526701 .861577

H 4.8497584 .1547782 .392452

H 6.5915673 .9080702 .127446

H 5.4200863 .6557930 .786385

SCF Energy (B3LYP/6-31G**//MMFF) $=-3245.91053372$

0800215

MM̄FF Geometry

C $1.167891-2.2203300 .047371$

C $0.732631-2.655898-1.146257$

C $1.336740-2.372326-2.504572$

O $2.646237-1.764731-2.396912$

C $0.510842-1.395423-3.360546$

C $-0.968737-1.684737-3.452469$

C $-1.897148-1.008513-2.748394$

C $-1.365219-2.765507-4.419085$ 
C -1.622096 $0.050107-1.718405$ C -1.930636 $1.452412-2.249150$ C $-1.2750172 .545563-1.390878$ C -1.755065 $3.926984-1.837396$ C -1.030227 $5.055479-1.093112$ O $0.1445632 .434307-1.556217$ O $-1.3043126 .285268-1.781393$ C $0.4893264 .813324-1.187185$ C $-1.5558265 .230940 \quad 0.336240$ O $1.2005655 .765058-0.397366$ C $0.9170753 .376965-0.798502$ C $2.4068033 .166771-1.177145$ O 0.7364053 .1982350 .604060 C $3.724897-2.571659-2.224296$ O $3.733776-3.785035-2.103568$ C $4.910309-1.688227-2.185658$ C $6.055595-2.125086-1.643306$ C $7.281909-1.339047-1.485782$ C $7.3005560 .049883-2.070999$ C $3.0042781 .803507-0.793062$ C 3.7191351 .8139250 .551706 C 5.2663510 .4341481 .942874 O 3.6433412 .7477301 .345742 C $6.6995530 .701267 \quad 1.602448$ C $7.702368-0.1249101 .939441$ C $9.1480300 .139368 \quad 1.624439$ C $9.840077-1.0499620 .992485$ C $10.654966-1.8378951 .714230$ C $9.651897-1.277085-0.491895$ C $8.314076-1.886500-0.810847$ N $4.442363 \quad 0.6481600 .765869$ H $2.038872-1.5745850 .099480$ H $-0.127582-3.323674-1.161960$ H $1.430607-3.322338-3.046632$ H $0.931462-1.383629-4.376341$ H $\quad 0.689417-0.369002-3.014517$ H -2.947199 -1.250175 -2.896996 H -2.449432 -2.917340 -4.448954 H -1.038089 -2.508768 -5.431833 H $-0.905920-3.717768-4.135551$ H $-2.244784-0.162758-0.844458$ H $-0.593469-0.005134-1.352098$ H -1.559201 $1.541255-3.278804$ H -3.018263 $1.593422-2.277637$ H -1.527923 2.374760 -0.339994 H -2.841159 4.019952 -1.711607 H -1.567991 4.047605 -2.913925 H $-2.2683106 .413843-1.792685$ H $0.7872544 .991902-2.230061$ H -2.627962 5.4609720 .324297 H - $-1.073262 \quad 6.0798410 .833068$ H -1.415961 4.3434570 .956594 H $0.9134816 .649782-0.681614$ H $2.4867573 .253346-2.269306$ H $3.0219233 .966234-0.748029$ H 1.2346632 .4156670 .886272 H $4.792626-0.681510-2.570855$ H $6.090954-3.140596-1.251074$ H $7.1483850 .009338-3.155323$ H $8.2374620 .584396-1.904692$ H $6.5108990 .667844-1.630015$ H $2.2422741 .017158-0.787600$ H $3.753711 \quad 1.537542-1.549303$ H $5.109945-0.6001202 .263626$ H $4.946615 \quad 1.1068742 .744423$ H 6.9255151 .6232451 .071832 H $7.476459-1.0304262 .498486$ H 9.6485010 .4044962 .564982 H 9.2614381 .0126320 .970086 H $11.175868-2.6763711 .263847$ H $10.815474-1.6653152 .773323$ H $\quad 10.420517-1.964925-0.869496$ H $9.834492-0.338535-1.021452$

H $8.199425-2.900077-0.423528$ H $4.462422-0.0619910 .040949$ C $0.087920-1.5701882 .310938$ O $-1.115813-0.9322611 .848407$ C -1.1935600 .3691592 .472693$
C 0.1046190 .5608823 .263650

C $1.048533-0.4274732 .608035$

H $-1.2217151 .104973 \quad 1.663607$

C -2.4500270 .4900633 .351395$

H $0.465846 \quad 1.5929743 .209914$

H $-0.0279690 .322747 \quad 4.325735$

C $2.219125-0.8188583 .490664$

H 1.4178900 .0232881 .679690

C -3.7943490 .3370462 .610450$

O $-2.394469-0.5256874 .362665$

H $-2.425726 \quad 1.4502453 .881366$

H -3.910003 -0.699150 2.277832

O $-4.839974 \quad 0.5631123 .565544$

C -3.9878331 .2977551 .428090$

C $-5.406630 \quad 1.343241 \quad 0.835114$

H -3.2826841 .0338720 .638939$

O -3.6571292 .6237751 .852433$

H -6.0858221 .8512901 .532072$

H $-5.352527 \quad 1.999115-0.044308$

H 2.8117280 .0620093 .756383

H $1.881528-1.2869224 .421591$

H $2.873642-1.5293542 .976779$

C $0.566377-2.6661121 .353506$

H -3.190081-0.429641 4.913780

H $-4.787351 \quad 1.4937593 .843525$

H -3.7227253 .2051821 .075632$

H $-0.274517-3.3417471 .156712$

H $1.331007-3.2619801 .867870$

H $-0.183620-2.0708983 .251510$

C $-5.471556-2.000644-0.853149$

O $-5.076619-0.689463-0.421465$

C $-6.003208-0.0140960 .432724$

C $-7.3620540 .155807-0.270648$

C -7.899455 -1.178154 -0.795143

C $-6.830333-1.954467-1.572776$

H $-4.716474-2.294190-1.592509$

C $-5.433382-3.0267880 .290099$

H $-6.144611-0.6022891 .344724$

O $-7.2503831 .071648-1.359793$

H -8.0759560 .5892030 .439553$

H $-8.759927-0.983376-1.446939$

O $\quad-8.358179-1.9506700 .309779$

H -7.168260 -2.976648 -1.778654

O $-6.621208-1.320590-2.838228$

C $-4.032375-3.2667310 .809702$

H $-6.044622-2.7056871 .137406$

H -5.811348 -3.991446 -0.067687

H $-6.5585160 .739601-1.957301$

H $-8.718954-2.781856-0.042838$

H -7.468363 -1.333087 -3.315443

O $-4.113580-4.002468 \quad 1.958481$

O $-3.003032-2.8769530 .277236$

C $-2.866605-4.3144292 .585772$

H -2.209893 -4.845969 1.890768

H $-2.392380-3.3984922 .947763$

$\mathrm{H}-3.069834-4.9646913 .440852$

SCF Energy (B3LYP/6-31G**//MMFF)= -3245.91224859

\section{6}

MM̄FF Geometry

C $-1.778043 \quad 4.467183-0.611915$

C $-0.6535703 .796713-0.317424$

C $0.471326 \quad 3.598583-1.300942$

O $0.7869962 .187523-1.264409$

C $1.7116694 .403088-0.874783$

C $2.6896914 .638548-2.010132$

C $3.8827634 .029415-2.155127$

C $2.2653595 .688075-3.008795$

C $4.5121012 .977932-1.285228$

C $4.7462961 .689135-2.081238$

C $5.4942190 .617640-1.275988$

C $5.581660-0.681112-2.082699$

C $6.231781-1.818997-1.283266$

$\begin{array}{lllll}\text { O } & 4.794238 & 0.387153 & -0.049574\end{array}$

O $5.957980-3.051937-1.966630$

C $5.554179-1.9107910 .099613$

C $7.757965-1.679587-1.234938$

O $6.225415-2.8541270 .932100$ 
C $5.441866-0.5484360 .823455$

C $4.575992-0.7007092 .093441$

O $6.749518-0.111776 \quad 1.198749$

C $0.9339981 .516535-2.432510$

O $0.905978 \quad 1.992587-3.558924$

C $1.1352460 .064359-2.201779$

C $1.068912-0.514517-0.992313$

C $1.216822-1.944220-0.699281$

C $1.519545-2.864585-1.850937$

C 4.2479000 .6174292 .808562

C 3.4198530 .3236664 .043014

C $1.131650-0.4071874 .702606$

O $3.934974 \quad 0.194915 \quad 5.151065$

C $1.059461-1.8781324 .432948$

C $0.011830-2.4728953 .843646$

C $-0.016185-3.922836 \quad 3.458826$

C $-0.063207-4.1180291 .957089$

C $-1.134037-4.6695871 .362478$

C $1.165868-3.7421941 .151229$

C $1.075055-2.3500410 .580345$

N $2.074280 \quad 0.1604853 .755451$

H -1.863992 $4.909717-1.602897$

H -0.5197513 .3517380 .664149$

H $\quad 0.1340253 .890097-2.301845$

H $2.2015543 .906071-0.030846$

H $1.4090115 .389923-0.498065$

H $4.4974974 .317881-3.007763$

H $3.0357525 .881760-3.763039$

H $1.3606685 .376615-3.539230$

H $2.061896 \quad 6.636107-2.499794$

H $5.4714313 .364099-0.920109$

H $3.9046082 .760675-0.403330$

H $3.7737911 .295024-2.394534$

H $5.3106551 .907605-2.997052$

H $6.4966561 .000344-1.050223$

H $6.117962-0.517963-3.025774$

H $4.568854-0.998823-2.367837$

H $\quad 6.313326-2.979573-2.869095$

H $4.539670-2.303310-0.056917$

H $8.176856-1.705698-2.248151$

H $8.215280-2.519812-0.701080$

H $8.088999-0.750265-0.766941$

H $6.260661-3.6957730 .445951$

H $3.628397-1.1828751 .823275$

H $5.080201-1.3650322 .807160$

H $\quad 6.678687 \quad 0.5189991 .931444$

H $1.318358-0.513762-3.101846$

H $\quad 0.8684980 .109574-0.122927$

H $0.724769-2.815945-2.603033$

H $2.466816-2.584714-2.325182$

H $1.613634-3.912776-1.561806$

H $5.150578 \quad 1.1452453 .131846$

H 3.6941981 .2977562 .151625

H 0.1704260 .0917734 .548639

H $1.464648-0.2251085 .728878$

H $1.934997-2.4699374 .690005$

H $-0.856095-1.8772213 .576670$

H $-0.890390-4.3816603 .937693$

H $0.856683-4.4554323 .856958$

H -1.157115 -4.8369880 .291123$

H -2.010072 -4.962976 1.931053

H $1.324921-4.4984290 .377284$

H $2.054296-3.796843 \quad 1.792927$

H $0.881034-1.5823931 .328730$

H 1.7710930 .2309792 .788204

C $-3.0402363 .911591 \quad 1.584425$

O -3.2981042 .5179451 .305438$

C -4.2511042 .0222772 .265651$

C -4.3932513 .1112903 .323557$

C -4.1812694 .3619602 .497068$

H $-5.208127 \quad 1.9214991 .741506$

C $-3.806607 \quad 0.6718402 .833721$

H -5.3682753 .0824243 .819526$

H -3.619784 3.0268324 .096310

C -3.8639545 .5906413 .332123$

H -5.0862584 .5514491 .904319$

C -3.761563 -0.483198 1.809650

O $-2.494696 \quad 0.829574 \quad 3.388505$
H $-4.4609520 .398493 \quad 3.670562$

H -2.965461 -0.3019961 .079384$

O $-3.374555-1.6639212 .526925$

C $-5.096075-0.7547511 .083625$

C $-5.181276-2.1223270 .378094$

H -5.2898560 .0498360 .365692$

O $\quad-6.152210-0.7184332 .051039$

H $-5.053831-2.9241811 .116385$

H $-6.208653-2.2318530 .003817$

H $-4.6888305 .813000 \quad 4.016917$

H -2.9592165 .4456723 .932220$

H -3.7088156 .4676062 .696232$

C -2.9745064 .6995710 .273190$

H $-2.242797-0.0249473 .777503$

H -4.081493 -1.866187 3.163692

H $-6.993686-0.7967801 .569857$

H $-3.013775 \quad 5.7745460 .484236$

H $-3.8619574 .454595-0.325159$

H -2.0894263 .9675812 .128498$

C $-3.799655-1.443455-3.021624$

O $-4.593079-1.412762-1.827516$

C $-4.196829-2.310836-0.785713$

C $-4.201952-3.764922-1.289216$

C $-3.354142-3.920722-2.553517$

C $-3.709476-2.863577-3.602394$

H $-4.358141-0.829197-3.739918$

C -2.431341-0.791772 -2.801355

H $-3.189077-2.053242-0.446396$

O $-5.541446-4.175128-1.559327$

H -3.814607 -4.412913 -0.493991

H $-3.513163-4.922280-2.971063$

O $-1.979782-3.811561-2.199158$

H $-2.982753-2.874927-4.423003$

O $-4.983531-3.176996-4.171878$

C $-2.5867380 .697928-2.619359$

H $-1.910720-1.173940-1.919567$

H - $1.765608-0.961781-3.655878$

H $-5.512830-5.105978-1.838733$

H -1.456954 -3.940942 -3.008651

H -4.915489 -4.058952 -4.575475

O $-2.6811251 .303966-3.837119$

O $-2.6390091 .247685-1.527689$

C $-2.8756002 .721185-3.820188$

H -1.926727 $3.216946-3.601931$

H -3.211086 $3.028795-4.814236$

$\mathrm{H}-3.6404333 .008074-3.092027$

SCF Energy (B3LYP/6-31G**//MMFF) $=-3245.89460608$

0800217

MM̄FF Geometry

C $2.223384-3.339299-0.053018$

C $2.297173-2.9942791 .243222$

C $1.157132-2.5206242 .118457$

O $-0.039632-2.2842331 .344671$

C $1.521215-1.1942342 .811975$

C $0.513988-0.8090913 .878814$

C -0.5189340 .0374603 .696448$

C $0.735604-1.4505475 .224687$

C -0.9117930 .7582072 .435851$

C -1.1437782 .2510382 .699022$

C -1.4967503 .0275111 .420042$

C -1.7481474 .5016411 .751933$

C -2.2259215 .3020730 .531391$

$\begin{array}{llll}\text { O } & -2.664496 & 2.440108 & 0.837975\end{array}$

O $-2.721908 \quad 6.5677650 .994740$

C $-3.3911334 .543994-0.141721$

C - $-1.0652335 .647073-0.406466$

O $-3.7717645 .174331-1.362080$

C $-3.0856853 .051018-0.390282$

C $-4.3200042 .277355-0.908882$

O $-2.0725052 .941858-1.396920$

C $-0.981328-3.2637091 .302226$

O $-0.920844-4.3712601 .809994$

C $-2.115267-2.7413000 .507635$

C $-3.111391-3.5608140 .140278$

C $-4.286516-3.184129-0.650401$

C $-4.447362-1.727294-0.995209$

C -5.4830662 .1828650 .080848$ 
C $-6.6022371 .293565-0.435581$

C $-8.606876 \quad 0.0447380 .378579$

O $-6.7114550 .964430-1.613390$

C -8.164544 -1.364709 0.622936

C $-8.532792-2.400841-0.146166$

C $-8.105747-3.817760 \quad 0.115909$

C $-7.634407-4.533792-1.132144$

C $-8.293445-5.609407-1.595886$

C $-6.407026-4.011043-1.848433$

C $-5.152938-4.149226-1.024380$

N $-7.480053 \quad 0.9375020 .578094$

H $1.261507-3.279294-0.556636$

H $3.250402-3.0771071 .758614$

H $\quad 0.975265-3.2896872 .879584$

H $1.612380-0.3960232 .069129$

H $2.511463-1.2678693 .281284$

H $-1.183503 \quad 0.2172144 .541126$

H $-0.006634-1.1335535 .964999$

H $0.676579-2.5405145 .145958$

H $1.723328-1.1825695 .613782$

H $-0.161186 \quad 0.6474811 .650767$

H -1.836090 0.3022222 .062185

H -1.964018 2.352744 3.422144

H $-0.2490312 .691373 \quad 3.157082$

H -0.6641872 .9301680 .715805$

H -0.8509304 .9612312 .185346$

H -2.520381 4.5662962 .531494

H -3.4497026 .4004931 .617398$

H -4.2548564 .6214050 .529978$

H $-0.317925 \quad 6.2547210 .118400$

H -1.405596 $6.255921-1.251331$

H $-0.5556834 .766931-0.800038$

H -3.951881 6.108958-1.162616

H -4.661900 2.708711-1.858610

H -4.006207 $1.254099-1.156675$

H -2.096827 2.041893 -1.762266

H -2.092081 -1.6918970 .235749$

H $-3.059733-4.6084480 .434166$

H $-4.479240-1.119869-0.083608$

H -5.361647 -1.505159-1.546703

H -3.613741 -1.384236 -1.618238

H -5.1288251 .7765931 .035024$

H $-5.915061 \quad 3.1718700 .265388$

H $-9.0053150 .183010-0.631725$

H $-9.372826 \quad 0.326393 \quad 1.107608$

H $-7.525427-1.5460411 .484179$

H -9.199722 -2.227661 -0.987644

H $-8.965241-4.3444040 .550492$

H -7.313428 -3.8573130 .873442$

H -7.967072 -6.132141 -2.489010

H $-9.175024-5.993779-1.093589$

H -6.598870 -2.985190 -2.171169

H $-6.243429-4.575393-2.776338$

H $-4.936894-5.177832-0.731848$

H $-7.266644 \quad 1.2124381 .532515$

C $4.760067-3.765664-0.375495$

O $5.163302-2.385399-0.283495$

C $6.547391-2.276223-0.671183$

C $7.064721-3.703350-0.804005$

C $5.813969-4.432150-1.258948$

H $\quad 6.562738-1.809972-1.662613$

C $7.301029-1.4029510 .338375$

H $7.891314-3.779966-1.517003$

H $7.411037-4.1011590 .156967$

C $5.899767-5.939687-1.096401$

H $5.628288-4.191587-2.314369$

C $6.703047 \quad 0.012290 \quad 0.496459$

O $7.263235-2.0419601 .621237$

H $8.359482-1.3391990 .062382$

H $5.684353-0.0694640 .889100$

O $7.456115 \quad 0.7023301 .502128$

C $6.7162070 .850720-0.797257$

C $6.1335832 .266634-0.621976$

H $6.1687330 .331807-1.589756$

O $8.0723370 .972395-1.234955$

H 6.5921832 .7575950 .244474

H $6.4266772 .862914-1.496510$

H $6.721484-6.344586-1.696104$
H $\quad 6.075108-6.221750-0.052669$

H $4.973771-6.421968-1.424500$

C $3.339343-3.838324-0.939422$

H $6.328732-2.1431461 .871458$

H 8.3623600 .8107301 .165802

H $8.065095 \quad 1.439620-2.087721$

H $3.100954-4.874004-1.207701$

H $3.297566-3.246293-1.863077$

H $4.805492-4.1926930 .634174$

C $2.647551 \quad 1.691901-1.801235$

O $4.0724361 .837095-1.755297$

C $4.6018072 .269428-0.495909$

C $4.0537953 .661431-0.151164$

C $2.5212983 .664226-0.149531$

C $1.9422703 .002637-1.406096$

H $2.4279191 .496361-2.858606$

C $2.2104050 .462617-1.000731$

H 4.3023541 .5619810 .284112

O $4.5319684 .630194-1.081930$

H 4.4271193 .9549120 .836729

H $2.1586844 .697879-0.090541$

O 2.0476952 .9715091 .001327

H $0.8694652 .826723-1.278448$

O $2.086543 \quad 3.932190-2.485393$

C $0.878770-0.063880-1.475836$

H $2.954363-0.339276-1.088944$

H 2.1373260 .6634640 .070454

H $4.2537164 .351164-1.971016$

H $2.3819423 .439748 \quad 1.785163$

H $1.6343633 .555247-3.259476$

O $1.064566-0.939085-2.504865$

O $\quad-0.1985460 .276789-1.005447$

C $-0.130232-1.506594-3.050636$

H $\quad 0.152334-2.161748-3.878898$

H $-0.645332-2.102869-2.291858$

$\mathrm{H}-0.785600-0.718610-3.433843$

SCF Energy $(B 3 L Y P / 6-31 G * * / / M M F F)=-3245.92209846$

0800218

MM̄FF Geometry

C -1.043083 -1.337171-1.071728

C $0.171451-1.886693-0.927927$

C $0.628957-2.6261600 .302406$

O $1.806760-1.9468760 .799587$

C $1.012187-4.068882-0.069978$

C $1.540057-4.8810611 .098021$

C $2.825920-5.2570111 .255340$

C $0.506894-5.2896482 .116344$

C $3.986454-4.9536140 .349071$

C $4.925528-3.9162630 .977474$

C $5.813927-3.236934-0.075114$

C $6.826988-2.3079050 .595466$

C $7.635746-1.495180-0.426481$

O $4.951715-2.484537-0.936660$

O $8.301832-0.4368880 .279932$

C $6.661959-0.828015-1.421597$

C $8.735809-2.338241-1.081612$

O $7.374224-0.188461-2.477594$

C $5.618360-1.801373-2.006143$

C $4.529972-1.089263-2.849238$

O $6.279105-2.731106-2.873280$

C $1.602783-0.8531341 .585361$

O $0.530878-0.4182571 .975244$

C $2.920833-0.2404671 .862660$

C 2.9787920 .9436312 .490279

C 4.1925691 .7000652 .805416

C 5.5087021 .1320672 .345305

C $3.669700-0.069606-2.094244$

C $4.1646201 .363381-2.190513$

C $3.7247633 .631758-1.252946$

O $5.0337331 .725435-2.978582$

C $4.7552663 .922491-0.207168$

C 4.5864494 .8380030 .759425

C 5.6346685 .1841931 .778687

C 5.1140325 .1313063 .197983

C 4.6988496 .2458963 .823372

C 5.1585543 .8032033 .919096

C 4.0695462 .8679693 .470259 
N $3.4840312 .202398-1.317012$ H -1.775965 -1.443591-0.278906 H $0.903475-1.768870-1.724033$ H -0.149327 -2.637239 1.075568 H $1.740415-4.044820-0.886298$ H $0.136208-4.587874-0.482568$ H $3.087575-5.8422572 .136020$ H $0.922542-5.9423252 .891542$ H $0.089469-4.4105932 .616637$ H $-0.308781-5.8359351 .631299$ H $4.542478-5.8826430 .171336$ H $3.652123-4.625418-0.637102$ H $4.330954-3.1490291 .488378$ H $5.546339-4.4055811 .738112$ H $\quad 6.326210-4.011123-0.658458$ H $7.497404-2.8748551 .253462$ H $6.294890-1.6054601 .249812$ H $8.862972-0.8401910 .964486$ H $6.139580-0.036342-0.871825$ H $\quad 9.436087-2.709467-0.323475$ H $9.335942-1.736985-1.773473$ H $8.348018-3.204209-1.622151$ H $8.0036120 .430576-2.069411$ H $4.975864-0.653698-3.751455$ H $3.843410-1.867709-3.212548$ H $5.622836-3.102531-3.485789$ H $3.804547-0.7647281 .519845$ H $2.045851 \quad 1.4137622 .799487$ H $5.487903 \quad 0.9389791 .267664$ H 5.7232920 .1943262 .868809 H $6.357579 \quad 1.7972612 .514816$ H $2.666446-0.068829-2.539446$ H $3.560094-0.350609-1.043477$ H $2.7660134 .112917-1.035154$ H $4.0794113 .983819-2.227042$ H $5.6958213 .379999-0.268115$ H 3.6569815 .4018550 .797771 H 5.9934976 .1974331 .554162 H $\quad 6.513521 \quad 4.533874 \quad 1.687416$ H 4.3513096 .2222034 .850826 H 4.6922487 .2065593 .318868 H 5.0278033 .9558384 .998952 H 6.1597373 .3747883 .821028 H 3.0696373 .2007683 .752972 H $2.8115951 .805730-0.666984$ C $-1.7199860 .924578-1.894706$ O $-3.0967731 .064668-1.474451$ C $-3.6631352 .210881-2.137044$ C -2.480395 $3.008160-2.672607$ C $-1.5333781 .893946-3.062951$ H $-4.2362301 .830321-2.990502$ C -4.577575 $3.009695-1.203669$ H $-2.7580753 .651959-3.512530$ H -2.030332 $3.636865-1.895119$ C $-0.0978272 .356136-3.253425$ H $-1.8913641 .435226-3.994564$ C $-5.8014792 .240713-0.662835$ O $-3.8051273 .452457-0.080316$ H $-4.9103263 .917757-1.721885$ H $-5.474471 \quad 1.5119090 .085828$ O -6.6149683 .1880350 .043489$ C $-6.6579681 .540814-1.733498$ C $-8.0488201 .073797-1.264182$ H -6.101436 $0.684818-2.132219$ O $-6.8653092 .449729-2.820601$ H $-8.692937 \quad 1.945703-1.090804$ H -8.502655 $0.543141-2.112073$ H -0.035133 $3.091602-4.062105$ H $\quad 0.2980602 .822057-2.344529$ H $0.5537421 .515215-3.510372$ C -1.439551 $-0.531765-2.276943$ H -4.3990713 .9621570 .497081$ H -6.963580 $3.819685-0.609066$ H -7.337531 $1.967163-3.520525$ H - $0.639413-0.578226-3.025083$ H $-2.330669-0.990494-2.721430$ H -1.098313 $1.236487-1.044707$ C $-7.085172-1.8891170 .804301$
O $-7.224927-0.960794-0.281411$

C $-8.060437 \quad 0.169147-0.022738$

C $-9.487202-0.2870420 .330470$

C $-9.487924-1.2974591 .482026$

C $-8.457875-2.410949 \quad 1.262237$

H $-6.531942-2.7365480 .378903$

C $-6.247396-1.3091841 .955031$

H $-7.6505450 .737393 \quad 0.817713$

O $-10.119717-0.875390-0.805607$

H -10.0812510 .5919450 .606011$

H -10.485661 -1.7435531 .574175$

O $-9.195951-0.6320772 .706919$

H $-8.335089-3.0070592 .174076$

O $-8.938186-3.2945770 .244829$

C $-4.826961-1.004497 \quad 1.533375$

H $-6.678796-0.3809052 .338230$

H $-6.194674-2.0352392 .774347$

H $-9.560536-1.610936-1.109316$

H -9.8777410 .0480072 .841868$

H $-9.779804-3.6693030 .555990$

O $-4.206612-0.3185932 .538690$

$\begin{array}{llll}\text { O } & -4.321766 & -1.332176 & 0.469118\end{array}$

C $-2.843741 \quad 0.038614 \quad 2.291648$

H $-2.4807510 .611176 \quad 3.149259$

H $-2.234600-0.863797 \quad 2.188877$

H -2.7685570 .6633241 .396950$

SCF Energy (B3LYP/6-31G**//MMFF) = -3245.91390956

0800219

MM̄FF Geometry

C $-0.726866 \quad 4.840001 \quad 1.595543$

C $-1.548396 \quad 3.7915451 .434341$

C -2.0760982 .9590182 .575345$

O -1.8470501 .5631862 .269914$

C $-3.590456 \quad 3.185858 \quad 2.719253$

$\begin{array}{llll}\text { C }-4.238310 & 2.327203 & 3.788398\end{array}$

C $-5.109474 \quad 1.3314453 .528164$

C $-3.8988662 .688815 \quad 5.210069$

C -5.5431220 .8664942 .165157$

C $-5.953300-0.6133862 .156099$

C $-6.354097-1.0680260 .744622$

C $-6.927797-2.484490 \quad 0.767141$

C $-7.249558-2.991137-0.647614$

O $-5.182206-1.028471-0.075663$

O $-7.483963-4.405832-0.562616$

C $-6.008073-2.789166-1.543770$

C $-8.538696-2.371826-1.200411$

O $-6.322029-3.123498-2.893475$

C $-5.405680-1.366898-1.452163$

C $-4.043083-1.234215-2.174894$

O $-6.301713-0.435487-2.069016$

C $-0.620452 \quad 1.0572332 .574674$

$\begin{array}{lllll}\text { O } & 0.329374 & 1.667958 & 3.038833\end{array}$

C $-0.635855-0.3904252 .260680$

C $0.487914-1.1090922 .408646$

C $0.639586-2.5502082 .185872$

C $-0.599792-3.3322221 .841889$

C $-2.973962-2.215348-1.692072$

C $-1.592012-1.913487-2.246205$

C $0.707592-2.869683-2.286135$

O $-1.333345-0.928961-2.931102$

C $1.433597-3.984861-1.605823$

C $2.544364-3.826529-0.870648$

C $3.238300-4.967297-0.182821$

C $3.472916-4.7272061 .292059$

C $4.714789-4.7585811 .804470$

C $2.268958-4.5465672 .193263$

C $1.864200-3.0996152 .333467$

$\mathrm{N}-0.679146-2.885959-1.861400$

H $-0.428705 \quad 5.1062902 .608299$

H $-1.875607 \quad 3.4992040 .440711$

H $-1.577872 \quad 3.2153403 .518816$

H $-4.078148 \quad 3.0227701 .751156$

H $-3.782193 \quad 4.2403822 .961281$

H -5.5601630 .8090794 .370222$

H -4.4712962 .1014915 .935904$

H -2.8373452 .5120835 .409193$

H $-4.118368 \quad 3.7451305 .396678$ 
H $\quad-6.398317 \quad 1.474645 \quad 1.846841$ H -4.738482 1.0105191 .436723 H -5.111985 -1.220227 2.515401 H $-6.792163-0.7643552 .846698$ H -7.095882 -0.3634020 .350476$ H -7.819930 -2.533832 1.403939 H $-6.196541-3.1671611 .222385$ H -8.222074 -4.5510180 .053877$ H -5.267811 -3.523513-1.202952 H $-9.387621-2.610173-0.548452$ H $-8.788579-2.790713-2.181447$ H $-8.488907-1.284963-1.291390$ H -5.490630 -3.147311 -3.395685 H -4.175249 -1.330884 -3.260110 H -3.672167 -0.211199-2.018944 H $-5.798607 \quad 0.357907-2.316839$ H -1.573445 $-0.825576 \quad 1.933812$ H $1.391339-0.5956902 .734805$ H $-0.416380-4.3940101 .668335$ H -1.061192 -2.942307 0.930637 H -1.330660 -3.265839 2.655781 H -2.904211 -2.190822 -0.598769 H -3.237424 -3.233257-1.999948 H $1.128712-1.887016-2.053588$ H $\quad 0.729755-3.005679-3.372128$ H $1.018772-4.983605-1.724882$ H $2.981137-2.837046-0.766477$ H $4.191977-5.135938-0.698273$ H $2.666264-5.897389-0.293313$ H $4.890793-4.6170612 .865807$ H $5.583498-4.9219981 .175810$ H $2.508471-4.9153173 .199703$ H $1.461731-5.1905141 .835288$ H $2.688287-2.4430842 .617308$ H -1.030570 -3.716153-1.391771 C $-0.4306385 .332385-0.934421$ O $0.2334374 .086459-1.227249$ C $0.9808254 .230080-2.446871$ C $0.4019955 .455775-3.138390$ C $0.1131276 .346724-1.945179$ H $2.0158294 .450314-2.157610$ C $0.9438882 .932753-3.259745$ H $1.1015915 .902916-3.850757$ H -0.527549 $5.223020-3.670564$ C $-0.8450397 .484501-2.252983$ H $1.0610966 .767551-1.583597$ C $1.3496811 .673693-2.464452$ O $-0.3914002 .726493-3.737787$ H $1.5743213 .044972-4.149966$ H $0.5470021 .406407-1.766429$ O $1.436804 \quad 0.588145-3.396148$ C $2.6781451 .792101-1.695342$ C $3.0569830 .481331-0.990981$ H $2.5826242 .590854-0.954216$ O $3.7055682 .161635-2.612267$ H $2.2238920 .140837-0.362342$ H $3.203671-0.309197-1.739334$ H -0.425455 8.143410 -3.020181 H $-1.8068207 .111228-2.620551$ H $-1.036498 \quad 8.085758-1.358688$ C -0.1503335 .7259790 .520035$ H $-0.3947891 .892686-4.238685$ H $2.1895120 .767346-3.985443$ H $4.4839722 .427042-2.094539$ H $-0.520034 \quad 6.742410 \quad 0.699709$ H $0.936113 \quad 5.7434300 .676544$ H -1.504126 5.176319-1.097751 C $5.703672-0.9644571 .185208$ O $4.509543-0.7603120 .421285$ C $4.348250 \quad 0.546367-0.149001$ C $4.325071 \quad 1.6120780 .957594$ C 5.5492781 .5004371 .872193 C 5.7966840 .0588522 .327205 H 5.581118-1.955718 1.636911 C $6.952804-1.0082250 .298812$ H $5.1856720 .737098-0.827760$ O $3.137147 \quad 1.4595991 .729557$ H 4.3014932 .6084710 .502156
H 5.4005052 .1394532 .751234

O 6.6856891 .9900531 .165864

H $6.776201-0.0228102 .811391$

O $4.824764-0.2844403 .319532$

C $6.920565-2.207716-0.618350$

H $7.088105-0.113155-0.314965$

H $7.856312-1.0967260 .914288$

H 3.1197982 .1737262 .389317

H 7.4482761 .9408311 .767196

H $3.949861-0.2744752 .894573$

O $6.224546-1.908952-1.752239$

O $7.440849-3.283136-0.351520$

C $6.112863-2.968175-2.707176$

H $7.095394-3.191771-3.132916$

H $5.681281-3.860605-2.245130$

H $5.448744-2.634194-3.508729$

SCF Energy (B3LYP/6-31G**//MMFF) $=-3245.91143839$

0800220

MM̄MF Geometry

C -4.4747503 .8759481 .370068$

C -4.3291942 .7943360 .589112$

C $-5.269791 \quad 1.6150410 .609488$

$\begin{array}{lllll}\text { O } & -4.484589 & 0.406122 & 0.730417\end{array}$

C $-6.0500121 .557080-0.716275$

C $-7.0229420 .395954-0.800478$

C $-6.850910-0.701808-1.564883$

C -8.2741970 .5470410 .026436$

C $-5.682441-1.034464-2.451131$

C $-4.972333-2.309222-1.976661$

C $-3.615482-2.501686-2.671239$

C $-2.977503-3.825347-2.245929$

C $-1.553519-3.988839-2.798046$

O $-2.780478-1.405184-2.286257$

O $-0.928750-5.072027-2.091415$

C $-0.742282-2.715381-2.476785$

C $-1.558280-4.383091-4.279372$

O $0.539782-2.759315-3.097964$

C $-1.469021-1.411367-2.864771$

C $-0.741760-0.139245-2.361655$

O $-1.537668-1.320723-4.292751$

C $-4.0944390 .041328 \quad 1.982879$

O $-4.3125640 .639213 \quad 3.024543$

C $-3.347699-1.2313671 .867451$

C $-2.796621-1.7845252 .958054$

C $-2.047936-3.0432573 .004645$

C $-1.816429-3.7672611 .704546$

C $-0.628941-0.008687-0.839202$

C $0.671588-0.536113-0.259559$

C $1.777904-0.9489771 .936835$

O $1.627329-0.880547-0.948350$

C $1.635113-2.3881612 .315104$

C $1.688222-2.8252203 .582404$

C $1.602789-4.2726313 .972153$

C $0.527097-4.5397345 .000739$

C $0.830204-4.6504496 .305322$

C $-0.881931-4.7717374 .505314$

C -1.608031 -3.4894754 .200079$

N $0.644339-0.5439631 .129052$

H -5.3195623 .9011412 .056309$

H $-3.5044302 .732336-0.114983$

H -5.9733351 .6812891 .448825$

H $-5.3434501 .532057-1.551914$

H $-6.6164582 .488900-0.851227$

H -7.632736 -1.460392 -1.555292

H $-8.977249-0.278441-0.127709$

H $-8.030503 \quad 0.575418 \quad 1.092776$

H $-8.7943491 .473685-0.237756$

H $-6.051512-1.185188-3.472913$

H $-4.969365-0.210049-2.506062$

H $-4.814680-2.248085-0.892569$

H -5.616512 -3.177127 -2.164228

H $-3.767856-2.477627-3.756732$

H $-3.606240-4.673214-2.545361$

H -2.927242 -3.866655 -1.149958

H - $1.472241-5.868001-2.221770$

H $-0.566634-2.716579-1.394344$

H $-2.077587-5.338860-4.419286$ 
H $-0.539320-4.539749-4.650204$ H -2.049867 -3.646310 -4.917918 H $0.973958-3.580087-2.808861$ H $\quad 0.233809-0.039650-2.852225$ H -1.327590 $0.725522-2.705635$ H -1.665816 $-0.389465-4.538004$ H $-3.276174-1.6841460 .885694$ H $-2.912489-1.2754233 .914069$ H -2.764448 -4.139914 1.302782 H - $-1.142358-4.6216301 .793584$ H -1.358510 -3.1002330 .966112$ H $-0.6691651 .056477-0.580789$ H -1.471424 $-0.493855-0.339061$ H $1.807933-0.2896052 .809783$ H $2.699029-0.8127051 .365611$ H $1.514712-3.1062651 .507339$ H $1.838187-2.1065984 .385286$ H $2.581957-4.5663444 .372860$ H $1.438752-4.9147263 .097833$ H $0.068850-4.8727937 .045678$ H $1.845734-4.5152476 .662293$ H -1.465849 -5.2971155 .273391$ H $-0.859301-5.4641803 .659163$ H -1.820088 -2.8900015 .086613$ H $-0.210411-0.2821401 .611442$ C -2.3384235 .0900900 .544557$ O $\quad-1.384030 \quad 4.166613 \quad 1.106675$ C -0.0626754 .7485991 .022430$ C $-0.2078095 .982637 \quad 0.146071$ C $-1.613323 \quad 6.4340710 .491025$ H 0.2012365 .0398222 .047041 C 0.9075893 .6784090 .508635 H $\quad 0.551048 \quad 6.741406 \quad 0.358259$ H $-0.1419275 .717939-0.916681$ C -2.194822 $7.416065-0.511274$ H $-1.602216 \quad 6.8967601 .486936$ C 2.3036474 .2131630 .131123 O 1.0370522 .6960291 .537467 H $0.4640533 .172033-0.356152$ H $2.1624724 .905892-0.707819$ O $2.852554 \quad 4.983291 \quad 1.196795$ C $3.3240033 .154433-0.348717$ C 4.0146892 .3446210 .768563 H $4.1126123 .692929-0.890475$ O $2.6765052 .299941-1.286233$ H $4.4761963 .038346 \quad 1.479967$ H $3.264543 \quad 1.7523021 .301211$ H -1.586657 8.325600 -0.554942 H -2.231082 $6.987626-1.518542$ H -3.212676 $7.704786-0.231411$ C $-3.6028755 .106748 \quad 1.408080$ H $\quad 0.1469442 .3513151 .724619$ H 2.7818454 .4549632 .010079 H $3.3413791 .688869-1.643945$ H -4.2330875 .9553121 .117143$ H -3.314294 5.2608842 .455787 H $-2.5634274 .752076-0.474741$ C $5.275843-0.719705-0.971856$

O $4.435140 \quad 0.281426-0.386919$

C $5.100437 \quad 1.382686 \quad 0.237644$

C 6.0134040 .8810991 .367672

C $6.995902-0.1767130 .856873$

C $6.287580-1.2680740 .048215$

H $4.592229-1.538481-1.229860$

C $5.925147-0.219996-2.267180$

H $5.7017461 .915118-0.507529$

O $5.216494 \quad 0.3307912 .414776$

H $6.562475 \quad 1.7347971 .783234$

H $7.511030-0.6316391 .711658$

O $7.976286 \quad 0.468614 \quad 0.049655$

H $7.023402-1.893882-0.469191$

O $5.592306-2.1321680 .952798$

C $4.8796750 .103520-3.308448$

H $6.5196870 .685424-2.114360$

H $6.601523-0.972892-2.688531$

H $5.8179030 .056578 \quad 3.127991$

H $8.606838-0.210419-0.245099$

H $4.899833-1.6089721 .391107$
O $4.305617-1.043296-3.771779$

O $4.6028711 .242905-3.660713$

C $3.262034-0.863009-4.734019$

H $2.446886-0.273526-4.303279$

H $3.655728-0.380077-5.633372$

H $2.875368-1.849009-5.005309$

SCF Energy (B3LYP/6-31G**//MMFF) $=-3245.91799870$

0800221

MM̄̄F Geometry

C $1.849755-4.1052070 .084386$

C $0.527411-3.8874070 .039879$

C $-0.276119-3.867690-1.233930$

O $-1.050438-2.646596-1.271808$

C $-1.247072-5.061980-1.241470$

C $-2.158565-5.099043-2.452531$

C $-3.467567-4.774981-2.437683$

C $-1.511007-5.571479-3.728312$

C $-4.275587-4.273490-1.272124$

C $-4.927063-2.920688-1.588660$

C $-5.529542-2.268285-0.334900$

C $-6.264433-0.978361-0.702871$

C $-6.769277-0.2219770 .535119$

O $-4.453053-1.9822790 .565415$

O $\quad-7.132860 \quad 1.1042810 .119932$

C $-5.606495-0.0773461 .541810$

C $-8.040794-0.8588601 .107849$

O -6.0641690 .4991442 .762682$

C $-4.854164-1.3977461 .811696$

C $-3.579056-1.2085262 .672929$

O $-5.719011-2.2808892 .537461$

C $-0.437321-1.528406-1.750404$

O $0.729251-1.414997-2.090442$

C $-1.457821-0.457500-1.812982$

C $-1.1458760 .751490-2.303000$

C $-2.0688871 .880549-2.457595$

C $-3.4807241 .701907-1.964810$

C $-2.482440-0.3339142 .055642$

C -2.5600461 .1343122 .437681$

C -1.5762783 .3454921 .834841$

O $-\begin{array}{lllll}-3.287301 & 1.561713 & 3.329687\end{array}$

C -2.3523094 .0131520 .743960$

C $-1.8226214 .925197-0.084870$

C $-2.5902625 .624531-1.168272$

C $-1.9622985 .452187-2.533194$

C $-1.1190216 .374606-3.027254$

C $-2.3900444 .263665-3.363666$

C -1.619506 $3.013948-3.036054$

N -1.688047 1.9072861 .682289

H $2.394954-4.279647-0.839798$

H $-0.015991-3.7164020 .966701$

H $\quad 0.372854-3.917547-2.117589$

H $-1.836068-5.063098-0.318254$

H $-0.675627-5.999946-1.209350$

H -4.029477 -4.868907 -3.366245

H $-2.222764-5.632362-4.558467$

H -0.712399-4.886991-4.030295

H - $1.081638-6.569075-3.589568$

H -5.058311 -5.007898-1.047846

H $-3.668391-4.182528-0.369313$

H $-4.171516-2.250443-2.016573$

H -5.706149 -3.061259-2.348339

H $-6.216345-2.9813430 .136208$

H -7.093452 -1.186807 -1.390712

H $-5.582319-0.318283-1.253601$

H $-7.8139901 .026595-0.569839$

H $-4.9093050 .647148 \quad 1.104879$

H -8.835873 -0.8713590 .352572$

H $-8.435458-0.271577 \quad 1.944318$

H -7.891509-1.886857 1.444274

H $-6.524022-0.1950943 .264614$

H -3.850170 -0.8654653 .678691$

H -3.136891 -2.205006 2.816588

H $-5.173100-2.9474292 .986369$

H -2.455827 -0.702253 -1.470025

H $-0.1257040 .928988-2.641108$

H $-4.0056310 .963265-2.579728$

H $-4.0704092 .620889-1.985464$ 
H $-3.4890271 .364263-0.922989$ H -1.509968 -0.6898072 .420066$ H -2.476682 -0.4277370 .966691$ H $-0.5116783 .593461 \quad 1.795634$ H -1.969504 3.6493212 .809916 H $-3.401353 \quad 3.7451780 .642206$ H -0.7813125 .2124180 .040967$ H -2.631616 $6.691485-0.911483$ H -3.634916 5.291446-1.198965 H $-0.6897506 .274503-4.018683$ H $-0.8344587 .246721-2.447889$ H $-2.2218274 .475876-4.428575$ H -3.472792 4.130566 -3.284048 H $-0.5791583 .051334-3.361818$ H -1.151130 1.4681860 .939746 C $3.640632-2.9807181 .415094$ O $4.697438-3.205068 \quad 0.455633$ C $5.945117-2.8341761 .056659$

C $5.603829-2.1064092 .351791$

C $4.339192-2.8339732 .765820$ H $6.442828-3.774817 \quad 1.331428$ C $6.871883-2.0879570 .090358$ H $6.402234-2.1762403 .096739$ H 5.391473-1.046474 2.175992 C $3.526609-2.0954503 .814548$ H $4.613046-3.8237623 .155721$ C $6.357642-0.752731-0.493849$ O $8.088038-1.8303820 .805387$ H $7.133471-2.759939-0.736070$ H $6.186016-0.0435250 .320592$ O $7.428124-0.191535-1.271456$ C $5.111185-0.853128-1.395229$ C $4.7278120 .469750-2.089504$ H $4.254695-1.206761-0.818203$ O $5.368104-1.825809-2.411770$ H $5.5857360 .865458-2.646006$ H $3.962844 \quad 0.237654-2.842643$ H $4.113378-1.9581224 .728687$ H $3.218765-1.1066533 .462226$ H $2.622985-2.6548574 .075352$

C $2.642410-4.1352721 .361612$

H $8.690681-1.3709430 .195884$

H $7.579295-0.782709-2.029213$

H $4.545187-1.948027-2.915384$

H $1.968569-4.0909762 .224857$

H $3.171005-5.0952111 .407154$

H $3.148522-2.0381811 .139933$

C $2.312645 \quad 1.8428470 .382896$

O $2.9284981 .032450-0.625376$

C $4.1810181 .522535-1.117521$

C $3.9846592 .894274-1.781303$

C $3.3433743 .882580-0.802472$

C $2.1111673 .286113-0.109673$

H 1.3196101 .3955230 .520544

C 3.0726681 .7132331 .705015

H $4.8791451 .632338-0.281631$

O $3.1470382 .787160-2.930015$

H $4.9563003 .282477-2.110213$

H $3.0549594 .787733-1.351235$

O $4.3159884 .259351 \quad 0.167424$

H 1.7922933 .9211850 .723804

O $1.0324613 .257479-1.046942$

C 2.1734301 .9922862 .883220

H $3.4574940 .693411 \quad 1.825934$

H 3.9572692 .3550821 .751158

H $3.6486412 .323317-3.621269$

H $3.900868 \quad 4.9056290 .763714$

H $0.8899514 .165876-1.362661$

O 2.4248793 .2365913 .379454

O 1.3328081 .2049323 .298652

C 1.6240523 .6248874 .499890

H $0.5635603 .618724 \quad 4.231275$

H 1.8116492 .9580615 .346899

H 1.9074324 .6417774 .783996

SCF Energy (B3LYP/6-31G $\left.{ }^{* *} / / M M F F\right)=-3245.90280302$

0800222

MM̄FF Geometry
C $2.032019-1.3212564 .288244$

C $2.266718-1.5036002 .979774$

C $3.560746-2.0450512 .428709$

O $4.050776-1.1321461 .422633$

C $3.281378-3.3761841 .712668$

C $4.526191-4.1849231 .416579$

C $5.169431-4.208040 \quad 0.232744$

C $5.000350-5.0564362 .551167$

C $4.901867-3.378523-0.992828$

C $4.199330-4.153958-2.116481$

C $2.668836-4.145001-1.981046$

C $2.017008-4.986903-3.078250$

C $0.483948-4.901618-3.029620$

O $2.236122-2.783616-2.091970$

O $0.002399-5.471485-4.256006$

C $0.068234-3.415125-2.991437$

C $-0.095004-5.753444-1.893744$

O $-1.337322-3.278877-2.793136$

C $0.827821-2.586070-1.927219$

C $0.527731-1.080501-2.132134$

O $0.388465-2.979497-0.626474$

C $4.750073-0.038074 \quad 1.814475$

O $5.012351 \quad 0.3077162 .953598$

C 5.1318450 .6770910 .576495

C 5.6168261 .9250420 .636079

C $5.9988822 .748137-0.514294$

C $5.8920752 .126724-1.883709$

C $1.395275-0.127604-1.294445$

C $1.0551541 .329102-1.574086$

C $1.7014753 .644399-0.903869$

O $0.1605391 .671379-2.343480$

C $2.7925944 .241793-1.735251$

C $3.5047415 .312578-1.350150$

C $4.5776325 .952875-2.185214$

C $5.8595396 .188640-1.415331$

C $6.1505837 .401000-0.913790$

C $6.8398915 .042729-1.300656$

C $6.4058914 .015483-0.293414$

N $1.8597302 .201139-0.852878$

H $2.830732-1.5564324 .989839$

H $1.495286-1.2811792 .248314$

H $4.316034-2.1745583 .213539$

H $2.742345-3.1753380 .781639$

H $2.608209-4.0013622 .315095$

H $6.028924-4.8705600 .133823$

H $5.895111-5.6313682 .289787$

H $5.246269-4.4453323 .425367$

H $4.219926-5.7704142 .833973$

H $4.358222-2.461295-0.752885$

H $5.879665-3.045546-1.365173$

H $4.465699-3.669124-3.065063$

H $4.573300-5.183893-2.162633$

H $2.396744-4.539738-0.997025$

H $2.345667-6.031922-3.015110$

H $2.353120-4.627045-4.061099$

H $-0.969176-5.479452-4.225067$

H $\quad 0.286149-2.990217-3.981380$

H $\quad 0.171660-6.807750-2.034675$

H $-1.190158-5.718962-1.891214$

H $0.259096-5.450078-0.906188$

H - $-1.784376-3.713605-3.538488$

H $0.689120-0.825936-3.188162$

H $-0.528360-0.881826-1.908695$

H $0.531330-2.246631-0.007597$

H $4.9785800 .157330-0.363034$

H 5.7294292 .3943221 .612305

H $6.5707701 .270890-1.968912$

H $6.1396562 .811329-2.697104$

H $4.869478 \quad 1.783432-2.075503$

H $1.244862-0.306466-0.224776$

H $2.455697-0.278497-1.525776$

H 1.7279064 .0076820 .128456

H $0.7285613 .894829-1.337959$

H $2.9879893 .792400-2.705863$

H $3.2815325 .779051-0.393015$

H $4.1844746 .910147-2.552177$

H $4.7958475 .358646-3.081140$

H $7.0794367 .586252-0.384715$ 
H $5.4633838 .234451-1.016544$

H $7.8219825 .415644-0.979852$

H $7.0167824 .621319-2.293847$

H 6.4419904 .3766490 .735332

H $2.5860281 .824867-0.251088$

C $-0.435997-0.594764 \quad 4.001059$

O $-0.196406 \quad 0.5880093 .214677$

C -1.4453741 .2954313 .040724$

C -2.5210910 .4091953 .650955$

C $-1.748736-0.3212854 .731611$

$\mathrm{H}-1.3461502 .223178 \quad 3.618034$

C -1.6122161 .6113671 .549940$

H -3.366396 0.9845424 .040455

H $-2.907238-0.3039392 .913253$

C $-2.455277-1.5681365 .234822$

H $-1.577896 \quad 0.3653685 .571340$

C -3.0038372 .1518341 .171528$

O $-0.6147112 .577540 \quad 1.212144$

H -1.3953420 .7173190 .954240$

H -3.749016 1.4139021 .480157

O -3.2724983 .3423361 .910831$

C $-3.1828512 .420027-0.337506$

C $-4.5817912 .940805-0.720180$

H $-2.9651651 .498236-0.889979$

O $-2.2342473 .399598-0.765197$

H $-4.7598583 .910369-0.237971$

H $-4.5655353 .145658-1.799084$

H -1.863363 -2.070155 6.006254

H $-3.426140-1.3099645 .670478$

H $-2.630557-2.2846854 .425048$

C $0.753916-0.8425184 .931938$

H $0.2502412 .193722 \quad 1.437713$

H -2.614141 4.0052151 .639728

H -2.298742 $3.469425-1.732940$

H $0.475152-1.5943405 .679552$

H $\quad 0.990126 \quad 0.082581 \quad 5.472738$

H $-0.566894-1.4339883 .305234$

C $-6.456661-0.290107-0.901625$

O $\quad-5.497686 \quad 0.756283-1.112958$

C $-5.7339091 .974741-0.400093$

C $-7.1004412 .566984-0.778625$

C $-8.2207941 .542002-0.574791$

C $-7.8826380 .195824-1.220159$

H -6.194633 -1.058966 -1.640111

C $-6.322771-0.9156840 .494807$

H $\quad-5.731737 \quad 1.7748000 .674767$

O $-7.0988373 .008974-2.134292$

H $-7.2873833 .449686-0.156094$

H $-9.1511251 .925797-1.011362$

O -8.4385531 .3395560 .818640$

H $-8.613548-0.561550-0.913164$

O $-8.0065690 .343750-2.637684$

C -5.185991-1.910935 0.544237

H $-6.164579-0.1898661 .296426$

H $-7.232227-1.4757810 .744107$

H $-6.8674282 .248778-2.695098$

H -8.6836752 .1975901 .204976$

H -7.863902 $-0.530867-3.038001$

O $-3.989397-1.274794 \quad 0.401271$

O $\quad-5.346698-3.114290 \quad 0.705481$

C $-2.836657-2.1220740 .429229$

H $-2.719275-2.5629841 .423540$

H $-2.914565-2.902739-0.333364$

H $-1.959629-1.5084690 .211944$

SCF Energy (B3LYP/6-31G**//MMFF) $=-3245.90847774$

\section{$08 \_00223$}

MM̄FF Geometry

C $1.522357-2.129680-0.563546$

C $2.165699-2.9285500 .300592$

C $2.720438-2.4916821 .638718$

O $2.223770-1.1761241 .983323$

C $4.253021-2.4150511 .583242$

C $4.877278-2.2983442 .959148$

C $5.349213-1.1561083 .495107$

C $4.995905-3.5934013 .721587$

C 5.3207750 .2271622 .905895

C 6.7230160 .7232022 .524550
C 7.2821850 .0440201 .264473

C 8.7311120 .4671761 .019516

C $9.283058-0.113867-0.290374$

O $6.466405 \quad 0.444180 \quad 0.159100$

O $10.512850 \quad 0.576300-0.559128$

C $8.2923290 .201922-1.431042$

C $9.636459-1.598928-0.146463$

O $8.677464-0.447448-2.641016$

C $6.823498-0.153463-1.093187$

C $5.8877250 .430103-2.178933$

O $6.680347-1.574194-1.060476$

C $1.097614-1.0945742 .736393$

O $0.512387-2.0214823 .279585$

C 0.6074720 .3004552 .833279

C $0.984387 \quad 1.277846 \quad 1.993187$

C 0.4903722 .6581021 .993112

C -0.5097983 .0427343 .052008$

C $4.3901880 .320295-1.857387$

C $3.5421221 .051235-2.885893$

C $1.2151661 .864041-3.256944$

O $3.998231 \quad 1.503443-3.933002$

C $1.1375093 .255286-2.710576$

C $0.0138323 .801875-2.223883$

C $-0.0600255 .184624-1.645132$

C $-0.4803725 .182263-0.191489$

C -1.7485925 .4558280 .158238$

C 0.5851824 .9576110 .859177

C 0.9367873 .5067261 .042538

N $2.2109891 .127917-2.499494$

H $1.416523-1.073732-0.332487$

H $2.280823-3.9824120 .057703$

H $2.403993-3.2226492 .392284$

H $4.554918-1.5707090 .954078$

H $4.664847-3.3087501 .095651$

H $5.799345-1.2048294 .486185$

H $5.497493-3.4641324 .686555$

H $4.006787-4.0165803 .920636$

H $5.576529-4.3210423 .145012$

H 4.6402560 .3043772 .053058

H 4.9111420 .8984493 .671455

H 6.6545821 .8042562 .346213

H 7.4091630 .5761313 .367876

H $7.223880-1.0429661 .384993$

H $9.369470 \quad 0.180546 \quad 1.864613$

H $8.785123 \quad 1.563580 \quad 0.962748$

H $\quad \begin{array}{llll}0.909783 & 0.187860 & -1.356840\end{array}$

H $8.351127 \quad 1.282300-1.624293$

H $10.402799-1.734525 \quad 0.626251$

H $10.070322-1.994389-1.071511$

H $8.779392-2.2199040 .122808$

H $9.557861-0.117016-2.886190$

H $6.121103 \quad 1.495117-2.311382$

H $6.076382-0.066423-3.139338$

H $5.754825-1.804450-1.235716$

H -0.1378250 .4648643 .604746$

H $1.7083451 .050503 \quad 1.212504$

H -0.0761642 .9239924 .051108$

H -1.406331 2.4175922 .980545

H -0.8503024 .0773772 .975744$

H $4.073672-0.727505-1.848374$

H $4.1799990 .754191-0.873236$

H $\quad 0.2672831 .325610-3.160399$

H $1.4988651 .892628-4.313546$

H $2.0560843 .837772-2.705887$

H $-0.9098913 .229696-2.245794$

H $-0.7771605 .759893-2.245089$

H $0.8959295 .713541-1.746354$

H $-2.0544495 .488076 \quad 1.198528$

H $-2.5118185 .646618-0.588819$

H 0.2895815 .4143831 .810364

H 1.4949695 .5017040 .573843

H 1.6561963 .1347970 .312855

H $\quad 1.9466110 .797257-1.576451$

C $-0.620932-2.351704-1.881762$

O $-0.924949-0.975843-1.564615$

C $-1.862677-0.959686-0.467039$

C $-2.571433-2.299621-0.544214$

C $-1.418216-3.217750-0.900446$ 
H $-1.260955-0.9010830 .449291$ C $-2.750588 \quad 0.282113-0.585874$ H $-3.059588-2.5786190 .394126$ H -3.323544 -2.295766 -1.340728 C $-1.858126-4.550396-1.481996$ H $-0.837833-3.4106460 .010123$ C -3.938333 0.2852010 .395456 O -1.920305 1.419265 -0.338340 H $-3.1230090 .381601-1.612488$ H $-4.554769-0.5892460 .168457$ $\begin{array}{llllll} & \text { O } & -3.458518 & 0.118101 & 1.729798\end{array}$ C $-4.825344 \quad 1.5449990 .321801$ C $-6.038178 \quad 1.511005 \quad 1.274264$ H $-5.1672421 .678865-0.711079$ $\begin{array}{llll}\text { O } & -4.050952 & 2.697126 & 0.661703\end{array}$ H -5.6927351 .4835882 .315410$ H -6.5631622 .4691641 .162079$ H -2.465103 -5.104383 -0.758625 H $-2.457017-4.415107-2.388787$ H $-0.990429-5.166258-1.739258$ C $0.898132-2.571209-1.856605$ H $-1.143806 \quad 1.345214-0.918799$ H -2.897842 0.8865851 .932345 H $-4.611075 \quad 3.4799770 .523981$ H $1.354861-1.972230-2.654259$ H $1.127259-3.621327-2.070494$ H $-0.977968-2.513742-2.906514$ C -8.344697 $-0.576361-0.790354$ O $-7.4702430 .465230-0.337839$ C $-7.016890 \quad 0.3550351 .013911$ C $-8.208748 \quad 0.370117 \quad 1.982221$ C $-9.215340-0.7283751 .620994$ C $-9.570873-0.7063850 .130468$ H -8.702356 $-0.235639-1.770962$ C $-7.591594-1.899860-1.003424$ H $-6.479338-0.5882311 .144401$ O $-8.849362 \quad 1.642877 \quad 1.937157$ H -7.836498 0.2234333 .003116 H -10.130223 -0.596399 2.211368 O $-8.671242-2.0048901 .944291$ H $-10.137449-1.604282-0.142747$ O $-10.4190650 .416514-0.128651$ C $-6.619911-1.819851-2.164454$ H -7.018762 -2.202917 -0.123768 H -8.318275 -2.686284 -1.239610 H $-9.582601 \quad 1.6254962 .575304$ H -8.474978 -2.008029 2.896674 H -11.219399 0.3105340 .413021 O $-6.226916-3.084032-2.502352$ O $-6.242158-0.787024-2.700210$ C $-5.328800-3.176658-3.613208$ H $-4.453596-2.537780-3.461853$ H $-4.993853-4.214174-3.693561$ H $-5.849842-2.901662-4.534897$ SCF Energy (B3LYP/6-31G**/MMFF) $=-3245.89364015$

0800224

MM̄FF Geometry

C -3.5665311 .1467022 .854794$

C $-2.471598 \quad 0.6083103 .414039$

C $-1.071876 \quad 0.9706662 .999559$

O $-0.317815-0.265968 \quad 2.938258$

C $-0.367917 \quad 1.8894184 .016311$

C $-0.942126 \quad 3.284422 \quad 4.115254$

C $-0.652630 \quad 4.286323 \quad 3.262573$

C $-1.848407 \quad 3.5321195 .293280$

C 0.2513504 .2469702 .060453

C $-0.516470 \quad 4.6175870 .784967$

C $0.417093 \quad 4.841320-0.414843$

C $-0.3860625 .311464-1.629889$

C $0.4978975 .472896-2.874020$

O $1.084887 \quad 3.612378-0.715455$

O $-0.364198 \quad 5.597618-4.015278$

C $1.3213084 .184310-3.068351$

C $1.334716 \quad 6.756433-2.819561$

O $2.262913 \quad 4.339950-4.128297$

C $2.0415733 .705844-1.784431$

C $2.6292042 .293043-2.022855$
O $3.0980534 .612068-1.469891$

C $0.710632-0.318847 \quad 2.050864$

$\begin{array}{lllll}\text { O } & 0.986277 & 0.513496 & 1.202412\end{array}$

C $1.487901-1.5483682 .326031$

C $2.638878-1.7499331 .666854$

C $3.564164-2.8708521 .839489$

C $3.252240-3.8771372 .914953$

C $3.2764371 .651456-0.790032$

C $4.7815541 .831540-0.687727$

C 6.6845491 .1694120 .795917

O $5.4597112 .385802-1.546900$

C $7.126794-0.2283590 .494642$

C $7.468058-1.1208271 .436698$

C $7.888478-2.5319971 .138787$

C $6.977273-3.558194 \quad 1.778415$

C $7.306082-4.1381412 .945067$

C $5.733405-3.979168 \quad 1.026117$

C $4.653773-2.9323381 .045176$

N $5.271231 \quad 1.272102 \quad 0.485663$

H $-3.439321 \quad 1.8979282 .080645$

H $-2.581747-0.1154564 .217802$

H -1.0913001 .4384082 .007245$

H $0.7004601 .955183 \quad 3.771223$

H -0.3708661 .4136745 .007220$

H -1.1028605 .2607253 .450401$

H -2.2231844 .5609345 .320924$

H $-2.7170802 .868577 \quad 5.263930$

H -1.3080263 .3534706 .228863$

H $1.0595914 .969506 \quad 2.226760$

H $\quad 0.7254003 .269607 \quad 1.933963$

H -1.2260403 .8111010 .558089$

H -1.1020705 .5297410 .956878$

H $1.1613755 .596392-0.135160$

H $-0.9124826 .248860-1.410259$

H $-1.1698044 .574522-1.855513$

H $-0.9413096 .367419-3.872118$

H $0.6246343 .395572-3.386498$

H $0.6829527 .635670-2.749584$

H $1.9164296 .888153-3.738582$

H $2.0198416 .786516-1.969759$

H $1.7679084 .623097-4.916049$

H $1.8030521 .635686-2.320420$

H $3.3422052 .313063-2.854855$

H $3.7249624 .168528-0.877304$

H $1.112183-2.2316753 .078725$

H $2.944337-1.0147610 .925261$

H $3.149610-3.3825743 .887400$

H $4.028466-4.6358523 .037198$

H $2.318926-4.4043812 .688991$

H 2.8179992 .0160830 .134291

H $3.1044640 .569336-0.847388$

H $6.8049951 .421376 \quad 1.854188$

H 7.2587931 .8839920 .198511

H $7.156033-0.520079-0.552696$

H $7.443917-0.8248462 .483074$

H $8.914810-2.6596481 .507504$

H $7.941300-2.7152860 .058397$

H $6.675898-4.8967513 .397398$

H $8.211185-3.8634943 .476653$

H $5.356721-4.9369131 .402139$

H $5.999480-4.184719-0.018822$

H $4.791431-2.1531790 .295032$

H 4.6234720 .8022261 .111829

$\begin{array}{llll}\text { C }-5.923229 & 0.476016 & 2.129027\end{array}$

O $-5.440544-0.6554511 .379854$

C $-5.735439-0.435155-0.014064$

C $-6.6734120 .767599-0.070460$

C $-6.183725 \quad 1.5843581 .108974$

H $-4.789149-0.159031-0.493360$

C $-6.295547-1.704683-0.661720$

H $-6.604505 \quad 1.302047-1.022884$

H -7.7199320 .4764050 .077441$

C $-7.1731602 .637149 \quad 1.576080$

H -5.2509342 .0786890 .814433$

C $-5.393015-2.946543-0.522288$

O $-7.553606-2.013338-0.048000$

H $-6.510345-1.506948-1.718949$

H $-5.400132-3.2944170 .517436$ 
O $-5.994854-3.999156-1.288642$

C $-3.938143-2.749796-0.986971$

C $-3.153637-4.076837-1.005676$

H -3.440200 -2.038173 -0.318986

O $-3.955283-2.181489-2.295920$

H $-3.347597-4.626878-0.077157$

H $-3.512100-4.681513-1.847994$

H -7.3779003 .3545910 .774729$

H -8.1261882 .1885751 .876051$

H -6.7767243 .1916342 .432290$

C $-4.969823 \quad 0.8136753 .279149$

H -7.894597 -2.817764 -0.475288

H $-5.942670-3.745237-2.226164$

H -3.033637 -2.015858 -2.557280

H $-4.945213-0.0424783 .965533$

H -5.3706381 .6642803 .842597$

H -6.8773550 .1581002 .571424$

C $-0.009473-2.925107-2.680538$

O $-1.378466-3.256316-2.415006$

C -1.632266 -3.872126 -1.150048

C $-0.865031-5.199265-1.045716$

C $0.630785-4.995402-1.303525$

C $0.888642-4.167945-2.568612$

H $-0.000878-2.599954-3.728939$

C $0.463077-1.745877-1.822159$

H -1.309862 -3.199902 -0.347234

O $-1.389895-6.128519-1.991787$

H -1.021551 -5.620744 -0.045302

H $1.114589-5.975006-1.399856$

O $1.198503-4.344020-0.172768$

H $1.939781-3.861823-2.623829$

O $0.630458-4.984344-3.715046$

C $-0.344440-0.507156-2.134432$

H $0.391850-1.954167-0.751657$

H $1.514049-1.508419-2.023422$

H $-0.910036-6.966455-1.877915$

H $2.151960-4.250030-0.338461$

H $1.233200-5.746319-3.674342$

O $-1.096811-0.152365-1.054318$

O $-0.3201620 .067441-3.215473$

C $-1.9091211 .012419-1.227662$

H -2.649297 $0.842365-2.015238$

H $-2.4345971 .203941-0.288946$

H -1.284807 $1.879363-1.463195$

SCF Energy (B3LYP/6-31G**//MMFF)= -3245.91003691

08_00225

MM̄MF Geometry

C $-0.7996973 .615474-0.941762$

C $-1.9134053 .887862-0.245092$

C -2.6189872 .8649910 .602201$

O -3.9923312 .7643850 .151837$

C -2.6847143 .2605542 .089287$

C -1.354444 3.5207562 .760131

C -0.4821962 .5616653 .125462$

C $-1.0583984 .967983 \quad 3.056327$

C -0.6283581 .0775302 .940831$

C 0.5403840 .5058042 .126906

C $0.383346-1.0036931 .896724$

C $1.662332-1.5974441 .303992$

C $1.497997-3.0902780 .985744$

O $-0.709607-1.1993920 .993046$

O $2.603201-3.4963290 .167207$

C $0.222505-3.2796490 .141978$

C $1.557585-3.9557682 .249531$

O $-0.050036-4.664971-0.061049$

C - $-1.024359-2.574223 \quad 0.727937$

C $-2.166364-2.605635-0.319158$

O $-1.434869-3.2555181 .911609$

C $-4.2743481 .860780-0.823415$

O $-3.4844771 .152378-1.425839$

C $-5.7420181 .861291-1.010930$

C $-6.3355260 .793758-1.564469$

C $-7.7719430 .624448-1.787784$

C $-8.6658671 .812281-1.546348$

C $-3.436942-1.8402840 .073199$

C $-4.470027-2.668690 \quad 0.816994$

C $-6.777097-2.478066 \quad 1.750325$
O $-4.318945-3.856812 \quad 1.085421$

C -7.760736 -2.814343 0.673115

C $-8.984360-2.2710370 .588352$

C $-9.976997-2.606211-0.489023$

C $-10.432671-1.384044-1.256124$

C $-11.547807-0.725964-0.896302$

C $-9.650069-0.971727-2.483723$

C $-8.228520-0.579988-2.190302$

N -5.591496 -1.913219 1.134191

H $-0.3899062 .608352-0.916273$

H $-2.3304574 .891521-0.267943$

H $-2.138187 \quad 1.8831710 .514951$

H -3.2241882 .4780952 .640927$

H -3.3316034 .1425032 .201540$

H $\quad 0.4358782 .8667843 .626297$

H $-0.0637765 .109563 \quad 3.492383$

H -1.104381 5.5661372 .141367

H -1.792591 5.3650463 .765078

H -0.6392530 .6112943 .933763$

H -1.579562 0.8172032 .468641

H 0.5918021 .0220821 .159332

H 1.4797540 .7034212 .658301

H $\quad 0.150691-1.4806662 .856131$

H $2.512131-1.4429521 .979482$

H $1.916688-1.0678390 .374981$

H $3.422739-3.2959170 .650286$

H $0.423618-2.855293-0.851972$

H $2.517292-3.8178062 .762269$

H $1.501910-5.0214442 .001389$

H $\quad 0.765344-3.7265102 .965254$

H $0.750628-5.065014-0.441648$

H -1.790864 -2.132917-1.236894

H -2.421726 -3.641930 -0.569233

H -2.347975 -3.0011872.116706

H $-6.2945922 .723817-0.655990$

H $-5.714732-0.049421-1.864140$

H -8.644806 $2.100958-0.489777$

H $-9.711217 \quad 1.625980-1.803431$

H -8.342890 $2.666699-2.151504$

H -3.201303 -0.9470520 .661293$

H $-3.924912-1.511201-0.852543$

H -7.167216 -1.732503 2.450235

H $-6.513255-3.3808082 .309929$

H -7.450247 -3.545540 -0.069731

H $-9.299160-1.5525021 .341956$

H -10.839562 -3.088822 -0.010827

H -9.576182 -3.349437-1.189445

H $-11.9030490 .134220-1.453881$

H -12.129092 -1.031696 -0.032545

H -10.156197 -0.164650 -3.027260

H -9.636470 -1.813524 -3.188561

H -7.512731 -1.390838 -2.329442

H $-5.643559-0.9508250 .812723$

C $1.3891164 .849785-1.305912$

O $2.1578373 .639942-1.457502$

C $3.0153893 .494824-0.310209$

C 2.9947484 .8416570 .405016

C 1.5713365 .2913390 .145765

H 2.5484402 .7390420 .334707

C $4.4171803 .016918-0.710654$

H 3.2303664 .7496841 .469684

H $3.7045855 .548576-0.040505$

C 1.3418026 .7738750 .375579

H $0.913806 \quad 4.7214560 .811354$

C $4.4059931 .634414-1.400503$

O $5.0114013 .997473-1.559200$

H 5.0243642 .9602370 .200142

H $3.7720840 .960185-0.813129$

O $3.8370141 .728824-2.704987$

C $5.8073861 .002672-1.516832$

C $5.848060-0.350353-2.250553$

H $6.2210410 .881041-0.509967$

O $6.6949131 .883643-2.209404$

H $5.625703-0.196078-3.314471$

H $6.888470-0.699296-2.233517$

H 1.5501297 .0378781 .417552

H $1.9899357 .384458-0.261881$

H $\quad 0.303388 \quad 7.044490 \quad 0.159679$ 
C $-0.0544214 .625624-1.767574$

H $5.9357853 .736476-1.707472$

H $2.9400872 .091553-2.610494$

H $6.3264342 .031213-3.097869$

H $-0.0435614 .255650-2.800245$

H $-0.5856105 .584728-1.774692$

H $1.8503925 .583694-1.980023$

C $6.216463-2.0644470 .247567$

O $4.989418-1.530092-0.257844$

C $4.913709-1.435122-1.681072$

C $5.098328-2.821080-2.316943$

C $6.370049-3.511877-1.824243$

C $6.443787-3.479936-0.296555$

H $7.049981-1.412135-0.040785$

C $6.118981-2.0675601 .776559$

H $3.889095-1.117356-1.910895$

O $5.117343-2.702420-3.735562$

H $4.235180-3.447332-2.059234$

H $7.266736-3.064682-2.269285$

O $6.355557-4.880390-2.236730$

H $5.691672-4.1663570 .111560$

O $7.718166-3.964853 \quad 0.122288$

C $6.208865-0.6623782 .323088$

H $5.180895-2.5184552 .122158$

H $\quad 6.934564-2.6403382 .232352$

H $5.107442-3.600213-4.107663$

H $6.421463-4.895976-3.206368$

H $8.397153-3.364081-0.229573$

O $4.973144-0.0869042 .343355$

O $7.257697-0.1355222 .671347$

C 4.9217141 .2582602 .828499

H 5.0644921 .2646393 .912720

H 5.6760001 .8804632 .337610

H 3.9330211 .6648042 .599343

SCF Energy (B3LYP/6-31G**//MMFF) $=-3245.91526472$

08_00226

MM̄MF Geometry

C $5.279500-13382052.435246$

C $4.719560-0.2180951 .954161$

C 4.0751890 .8389612 .810342

O $2.669454 \quad 0.8434722 .473656$

C 4.6536312 .2265372 .471185

C 3.9938163 .3644683 .229467

C 3.2362094 .3271902 .664189

C 4.2444773 .3827554 .715463

C 2.9006794 .5000921 .207498

C 1.5238443 .9210320 .880608

C $1.2587193 .793791-0.625347$

C $1.1254295 .152054-1.316686$

C $0.7533235 .007852-2.802631$

O $0.0411143 .048005-0.771389$

O $0.327667 \quad 6.295961-3.274993$

C $-0.4581054 .057180-2.925395$

C $1.9659954 .615865-3.655326$

O $-0.7606543 .785298-4.291525$

C $-0.2938292 .742236-2.132627$

C $-1.5749881 .878579-2.124761$

O $\quad 0.730731 \quad 1.951833-2.748514$

C $1.7883190 .351183 \quad 3.384720$

O $2.040779-0.1441314 .470576$

C $0.432131 \quad 0.557738 \quad 2.830634$

C $-0.641370 \quad 0.473353 \quad 3.629856$

C $-2.025640 \quad 0.728566 \quad 3.227414$

C -2.2860311 .0320551 .775164$

C $-2.8319382 .572579-1.594501$

C -3.982092 $1.597087-1.406471$

C $-6.3643691 .522620-0.674519$

O $-3.8849940 .394016-1.631602$

C $-6.538701 \quad 1.413737 \quad 0.806687$

C $-6.439727 \quad 0.261967 \quad 1.488102$

C $-6.652216 \quad 0.1488272 .971698$

C $-5.370896-0.1638053 .715729$

C -5.125238-1.413335 4.145142

C $-4.458093 \quad 1.0192253 .971038$

C -2.9990230 .6909234 .161939$

N $-5.1303312 .232241-0.954680$

H $5.282496-1.4885273 .513413$
H $4.687277-0.0415380 .882892$

H $4.228120 \quad 0.6241313 .874454$

H 4.5678542 .3876751 .391812

H 5.7299322 .2414662 .688386

H 2.8026255 .0871803 .313030

H 3.8425814 .2825435 .193524

H 3.7772532 .5188125 .197499

H 5.3199923 .3578174 .919058

H 2.9180945 .5728530 .980758

H 3.6655324 .0525930 .566293

H 1.4284482 .9245611 .324422

H $\quad 0.7352164 .5224281 .351768$

H $2.0788313 .214389-1.065826$

H $2.0445015 .741078-1.215536$

H $0.3390835 .736065-0.818297$

H $1.0576716 .923084-3.135116$

H -1.319642 $4.607982-2.528456$

H $2.7587825 .367795-3.561291$

H $1.7082064 .587519-4.719789$

H $2.3941543 .651090-3.375802$

H $-0.1091203 .140990-4.616700$

H -1.769642 $1.488822-3.132740$

H -1.380509 $0.992689-1.503956$

H $0.6074111 .025085-2.483324$

H $\quad 0.3576380 .814004 \quad 1.779689$

H $-0.4933840 .230105 \quad 4.680975$

H -1.8359181 .9915361 .499412$

H $-3.3485801 .085174 \quad 1.531154$

H $-1.868513 \quad 0.249623 \quad 1.132217$

H -2.628246 $3.048983-0.629612$

H $-3.1621093 .343143-2.299568$

H -6.350649 $0.541054-1.157403$

H -7.186442 2.103529-1.104000

H -6.7761522 .3303921 .341493$

H $-6.204625-0.6557380 .953652$

H $-7.401115-0.6346703 .145561$

H -7.091221 1.0671283 .382191

H $-4.233728-1.6660044 .707852$

H -5.821320 -2.2221163 .946559$

H $-4.814732 \quad 1.5402204 .869479$

H -4.5624491 .7542403 .165306$

H -2.7308710 .4467805 .190057$

H $-5.1028703 .230006-0.765030$

C $5.946383-2.3019190 .119063$

O $4.609913-2.252334-0.412349$

C $4.575486-2.943779-1.673416$

C $6.015414-3.337590-1.988502$

C $6.612028-3.474090-0.600115$

H $3.987971-3.855226-1.511605$

C $3.893877-2.070476-2.735226$

H $6.071579-4.261215-2.572859$

H $6.540295-2.557299-2.551212$

C $8.130105-3.432512-0.587360$

H $6.272217-4.423629-0.165436$

C $2.524990-1.493547-2.316804$

O $4.739116-0.953735-3.045933$

H $3.794795-2.638023-3.667700$

H $2.662775-0.711246-1.559779$

O $1.958398-0.845510-3.462827$

C $1.518256-2.532616-1.792366$

C $0.154386-1.899424-1.474578$

H $1.930738-2.990766-0.888600$

O $1.359852-3.557314-2.770461$

H $\quad 0.290765-1.042028-0.802360$

H $-0.283841-1.485666-2.393101$

H $8.541924-4.251811-1.185840$

H $8.509314-2.491455-0.999496$

H $8.515952-3.5334660 .431778$

C $5.897510-2.4636121 .642210$

H $4.897574-0.466369-2.219068$

H $2.601270-0.182554-3.768359$

H $0.870249-4.288663-2.358378$

H $6.915835-2.6091772 .021285$

H $5.328468-3.3700031 .885874$

H $6.441871-1.359589-0.149471$

C $-3.203850-2.767626-0.128826$

O $-2.063070-2.069103-0.643364$

C $-0.889393-2.861408-0.874415$ 
C $-0.434110-3.5399090 .427283$

C $-1.573097-4.3206291 .091458$ C $-2.856179-3.4904751 .182004$ H $-3.930212-1.9790330 .106684$ C $-3.833708-3.687210-1.180004$ H -1.126133 -3.621762 -1.625537 O $\quad 0.067777-2.566837 \quad 1.341224$ H $0.389702-4.2275490 .208316$ H -1.266936 -4.620573 2.101155 O $-1.841105-5.5061590 .349139$ H $-3.701552-4.1175491 .488594$ O $-2.686580-2.4896742 .187825$ C $-4.389147-2.888330-2.334201$ H -3.127504 -4.407022 -1.603473 H -4.648174 -4.278403 -0.744244 H $-0.641372-1.9219311 .505415$ H $-1.025994-6.0360740 .336795$ H $-2.517051-2.9444323 .030334$ O $-5.684502-2.543738-2.084310$ O $-3.742620-2.596125-3.332086$ C $-6.334766-1.803168-3.121981$ H $-5.782271-0.885712-3.345271$ H $-6.432138-2.422031-4.018966$ H -7.334373 -1.532949 -2.771163 SCF Energy (B3LYP/6-31G**//MMFF) $=-3245.91668294$

08_00227

MM̄FF Geometry

C -1.775217 4.8812560 .764686

C -2.8217534 .2866991 .357723$

C -4.0291643 .6714960 .689740$

O -3.9108722 .2304420 .793235$

C $-4.2025874 .009967-0.802044$

C $-5.5592603 .599660-1.341076$

C $-5.7862622 .513995-2.105765$

C $-6.6913724 .540773-1.014898$

C $-4.7970791 .467080-2.539475$

C $-5.3939540 .057594-2.412669$

C $-4.457572-1.012880-2.990160$

C $-5.163053-2.368907-3.049059$

C $-4.229979-3.471809-3.568204$

O $-3.298909-1.103975-2.153946$

O $-4.851944-4.738027-3.301588$

C $-2.918803-3.431819-2.759182$

C $-4.036949-3.393947-5.087210$

O $-1.959338-4.338417-3.298874$

C $-2.301745-2.016747-2.635930$

C $-1.140998-2.057091-1.616621$

O $-1.788848-1.610858-3.905780$

C -4.3149561 .6370041 .947346$

O -4.7842442 .1824402 .933066$

C $-4.056852 \quad 0.186388 \quad 1.812033$

C $-4.028349-0.5791032 .913076$

C -3.754837-2.017182 2.959684

C $-3.700232-2.7567011 .650159$

C $-0.581732-0.686053-1.223553$

C $0.409495-0.861874-0.091395$

C $0.527567-1.0888892 .390147$

O $1.599901-1.077618-0.306082$

C $0.153643-2.4612652 .857131$

C $-0.256941-2.7344414 .105340$

C $-0.630560-4.1119854 .575329$

C $-1.961610-4.1520585 .296092$

C $-2.022040-4.3603616 .622374$

C $-3.226115-4.0314674 .470250$

C $-3.568465-2.5972004 .163729$

N $-0.187463-0.8088051 .158666$

H -1.724511 $4.953294-0.317535$

H -2.8208014 .2247732 .445060$

H $-4.9188154 .007793 \quad 1.237200$

H -3.404897 $3.539557-1.388609$

H $-4.0935905 .091117-0.960342$

H $-6.7997322 .358856-2.474597$

H -7.636509 4.230266 -1.473137

H $-6.852927 \quad 4.5908880 .066149$

H $-6.4651715 .547840-1.380214$

H $-4.5406231 .658592-3.588219$

H $-3.8695001 .516021-1.961893$
H $-5.590969-0.151712-1.353272$

H $-6.3548480 .020123-2.941409$

H $-4.154747-0.699413-3.996316$

H $-6.068056-2.312381-3.666815$

H -5.503912 -2.646634 -2.041515

H $-5.715069-4.748110-3.749767$

H -3.151276 -3.794281-1.747620

H $-5.001486-3.485550-5.600835$

H $-3.424485-4.226668-5.450559$

H -3.576663 -2.459213 -5.414126

H $-2.380971-5.213614-3.343729$

H -1.483285 -2.559079 -0.704806

H $-0.316950-2.662790-2.016217$

H -1.108178 $-0.933195-3.768149$

H -3.867374 -0.2032550 .818277$

H $-4.192616-0.1043863 .879860$

H -2.840660 -2.423960 1.064037

H $-4.611200-2.5790971 .067039$

H $-3.615756-3.8393401 .760290$

H $-0.063681-0.206938-2.060476$

H $-1.376759-0.002555-0.904900$

H $0.243862-0.3177873 .112317$

H $1.606456-1.0339132 .216851$

H $\quad 0.231902-3.2708722 .134979$

H $-0.307529-1.9299894 .835675$

H $0.168590-4.4611445 .242122$

H -0.656973 -4.823299 3.740354

H -2.972639 -4.416230 7.142437

H -1.122935 -4.474441 7.218883

H -4.074274 -4.460587 5.019674

H $-3.117380-4.6505813 .576089$

H -3.656059-1.972541 5.054270

H -1.190081 -0.6607331 .222487$

C 0.6812464 .6793761 .395948

O 1.1198514 .6401960 .024090

C $1.5775923 .302424-0.272553$

C 1.7695302 .6281891 .078955

C 0.6293373 .2282351 .875144

H $0.7625652 .824452-0.830926$

C $2.8458333 .370052-1.131980$

H 1.7261041 .5385911 .022957

H $2.7341612 .910371 \quad 1.517581$

C 0.7734663 .0749983 .378395

H $-0.2988042 .738376 \quad 1.556455$

C $3.3847181 .974366-1.505790$

O $2.5264384 .090393-2.325412$

H $3.6206883 .943460-0.609376$

H $3.5014161 .389331-0.588517$

O $2.4137251 .299098-2.306557$

C $4.7434491 .982596-2.235284$

C $5.1955170 .599467-2.744322$

H $5.5005232 .396022-1.558357$

O $4.6815052 .849866-3.368946$

H $4.5357460 .274528-3.559222$

H $\quad 6.1838870 .736062-3.203614$

H 0.8225602 .0179583 .656442

H 1.6823773 .5619263 .746618

H -0.0807173 .5212983 .897373$

C $-0.6202755 .474621 \quad 1.524452$

H $2.1822844 .959318-2.056195$

H $2.3031911 .815586-3.123479$

H $5.5713242 .888768-3.759166$

H -0.4613826 .4864931 .131112$

H -0.8742955 .5855672 .585440$

H 1.4631405 .2229301 .942770

C $6.363299-0.9521890 .451029$

O $6.194354-0.065270-0.663830$

C $5.272871-0.496392-1.669481$

C $5.704960-1.846622-2.263756$

C $5.897401-2.894107-1.162639$

C $6.767573-2.360104-0.021493$

H $7.210253-0.5377771 .013449$

C $5.136245-0.952077 \quad 1.377278$

H $4.280796-0.615845-1.225589$

O $6.924129-1.685386-2.986029$

H $4.940648-2.179590-2.976140$

H $\quad 6.370754-3.789430-1.583712$

O $4.630023-3.275763-0.635782$ 
H $\quad 6.739154-3.0527220 .827182$

O $8.129263-2.314831-0.458789$

C $5.020416 \quad 0.3379302 .162634$

H $4.196580-1.0867150 .837697$

H $5.243676-1.7659162 .104517$

H $7.150614-2.547209-3.375043$

H $4.099778-3.628062-1.370692$

H $8.193068-1.648652-1.164541$

O $4.220851 \quad 0.124093 \quad 3.249338$

O $5.5500501 .397871 \quad 1.858937$

C 4.0474721 .2400244 .128200

H 4.9491991 .3717884 .732936

H 3.8251812 .1538523 .570893

H 3.2058441 .0224854 .791092

SCF Energy (B3LYP/6-31G**//MMFF) $=-3245.91405339$

0800228

MM̄FF Geometry

C 2.1338753 .4295201 .296491

C 2.7311504 .2795990 .446551

C $3.7882513 .895927-0.564579$

O $3.6380222 .507889-0.950875$

C 5.1917374 .1053630 .020731

C $6.2924753 .902054-1.001517$

C $7.0726162 .805991-1.075780$

C $6.5188695 .056688-1.943120$

C $6.9874881 .569584-0.225274$

C $7.1009220 .292988-1.071148$

C $7.006155-0.961262-0.192365$

C $7.327496-2.221942-0.994888$

C $7.208635-3.484371-0.128208$

O $5.669371-1.0463120 .310305$

O $7.204455-4.606925-1.026289$

C $5.848575-3.4677880 .605008$

C $8.442217-3.6690550 .762658$

O $5.759102-4.5274571 .553243$

C $5.465217-2.1100881 .247572$

C $3.963153-2.1211821 .606102$

O $6.155559-1.8693022 .473625$

C $2.6416012 .235381-1.840523$

O $1.9218383 .046086-2.403989$

C $2.5301970 .766229-1.976159$

C $1.4146780 .262565-2.526281$

C $1.092227-1.151263-2.725850$

C $2.163955-2.161700-2.418868$

C $3.428769-0.7692192 .097657$

C $1.966983-0.8873402 .483667$

C $-0.286144-1.3211941 .505547$

O $1.602472-0.8389643 .656055$

C $-0.656008-2.4318140 .573540$

C $-1.626835-2.336161-0.346584$

C $-2.015525-3.450339-1.274393$

C $-2.048799-3.020843-2.724875$

C $-3.215213-2.879169-3.377159$

C $-0.727079-2.827142-3.439550$

C $-0.138965-1.465751-3.181364$

N $1.139523-1.0834681 .390222$

H 2.4573952 .3924891 .334839

H 2.4107675 .3187010 .424288

H $3.6617924 .526165-1.454014$

H $5.338573 \quad 3.4414510 .880310$

H 5.2814575 .1229280 .425001

H $7.8644112 .792039-1.823592$

H $7.3600204 .880843-2.622255$

H $5.6314575 .231724-2.558860$

H $6.7374665 .969428-1.379270$

H 7.8104291 .5958140 .498876

H $6.055415 \quad 1.5347610 .345297$

H $6.296830 \quad 0.284413-1.818494$

H $8.0568520 .296720-1.609489$

H $7.704781-0.8517630 .645373$

H $8.327192-2.158569-1.442347$

H $\quad 6.625750-2.307014-1.836646$

H $7.062292-5.409076-0.494610$

H $5.093902-3.696016-0.160904$

H $9.337439-3.8108610 .144517$

H $8.360011-4.5730031 .375864$

H $8.633366-2.8191521 .419807$
H $\quad 6.266432-4.2702802 .341197$

H $3.386156-2.4206390 .723958$

H $3.771586-2.8738102 .382283$

H $7.022090-2.2982982 .442798$

H $3.3383290 .151240-1.598423$

H $\quad 0.6404920 .957351-2.850529$

H $3.083509-1.927460-2.967289$

H $1.894575-3.182409-2.697463$

H $2.388928-2.169195-1.349196$

H $3.987207-0.4237072 .974645$

H $3.5256420 .001872 \quad 1.324978$

H $-0.795777-0.3853091 .259427$

H $-0.537689-1.5959062 .534290$

H $-0.104839-3.3644670 .669690$

H -2.193599-1.412584-0.421499

H $-3.005926-3.808113-0.964475$

H -1.340729 -4.309296 -1.170998

H -3.246015 -2.592695 -4.423203

H $-4.165584-3.036440-2.877873$

H $-0.872343-2.922632-4.523600$

H $-0.057760-3.645389-3.161428$

H $-0.829197-0.649042-3.400811$

H $1.523678-0.9582170 .458351$

C -0.2181422 .8860331 .880360$

$\begin{array}{lllll}\text { O } & -0.615681 & 3.029876 & 0.503712\end{array}$

C -2.0552572 .9668190 .419754$

C -2.5416982 .5914141 .813083$

C -1.4683303 .2036272 .693581$

H -2.382281 3.9809110 .158828

C -2.437136 $1.978812-0.690101$

H -3.5434032 .9769912 .025666$

H -2.563745 1.5020021 .936482

C -1.4434232 .6491704 .106372$

H -1.626886 4.2895752 .733854

C $-3.9584161 .771927-0.837049$

O $-1.9011832 .482160-1.917764$

H -1.949564 $1.014449-0.510107$

H $-4.351736 \quad 1.4450930 .131425$

O $-4.5567603 .033267-1.135937$

C $-4.3520650 .725753-1.904205$

C $-5.8668290 .553665-2.117816$

H -3.921289 -0.242746 -1.628698

O $-3.815274 \quad 1.069320-3.183436$

H $-6.2874121 .471307-2.548824$

H -5.989711 -0.214596-2.893726

H -0.6715023 .1435904 .704382$

H -2.407179 2.8079724 .600884

H -1.2328461 .5749314 .112317$

C 0.9729553 .7995402 .176297

H $-0.9468892 .616458-1.784992$

H $-5.5172972 .943014-1.021765$

H -2.846247 $1.066618-3.107523$

H 1.2759333 .7003203 .224172

H 0.6904574 .8456812 .007373

H $\quad 0.072014 \quad 1.8365362 .019324$

C $-6.743646-1.6174130 .798554$

O $-6.133535-1.091192-0.388552$

C $-6.6616200 .149731-0.865594$

C $-8.1649870 .025301-1.166877$

C $-8.929188-0.5088550 .047091$

C $-8.265042-1.7634500 .622953$

H -6.327306 -2.628069 0.902671

C $-6.351974-0.8133672 .049525$

H $-6.5210950 .920069-0.101899$

O $-8.362835-0.847270-2.277388$

H $\quad-8.549578 \quad 1.012639-1.450114$

H -9.959023 -0.740723 -0.250277

O

H -8.717996 -2.032252 1.584209

O $-8.491326-2.863049-0.263457$

C $-4.909521-1.0491052 .445199$

H -6.4779940 .2633851 .914317$

H -6.973910 -1.1404912.891379

H -9.316879 $-0.875168-2.462831$

H -9.4895050 .1650411 .791402$

H $-9.452956-2.981744-0.344365$

O $-4.712445-0.5759883 .711405$

O $-4.060747-1.5720051 .737277$ 
C $-3.396387-0.7633164 .241180$

H $-2.640843-0.3798693 .550377$

H $-3.325146-0.2082895 .180305$

H -3.226573 -1.824764 4.443933

SCF Energy $\left(B 3 L Y P / 6-31 G^{* *} / / M M F F\right)=-3245.89385466$

08_00229

MM̄FF Geometry

C $-0.457097 \quad 4.6023210 .074347$

C $0.790837 \quad 4.1421690 .244444$

C $1.7812214 .014467-0.885554$

O $2.2005732 .631532-0.978440$

C $3.0174884 .884189-0.598834$

C $4.0157104 .907969-1.741414$

C $5.2034294 .270252-1.744949$

C $3.6165775 .754817-2.923618$

C $5.7829563 .391031-0.672509$

C $5.9913451 .960326-1.185163$

C $6.3999211 .001737-0.057090$

C $6.662838-0.399064-0.611516$

C $6.944189-1.4218950 .500010$

O $5.325391 \quad 0.962645 \quad 0.886863$

O $6.842800-2.733756-0.076172$

C $5.838495-1.3127921 .573440$

C $8.374705-1.2946711 .036513$

O $6.133385-2.1465102 .691476$

C 5.5634510 .1345522 .031886

C 4.3250780 .2560502 .953918

O $\quad 6.691327 \quad 0.6045202 .780560$

C $1.3505551 .771325-1.607588$

O $0.2542342 .034556-2.076236$

C $1.9912620 .437395-1.641014$

C $1.321799-0.607307-2.152006$

C $1.831036-1.972354-2.307368$

C $3.222473-2.260701-1.810390$

C $2.989361-0.1500042 .321343$

C $2.583190-1.5890592 .588754$

C $0.848379-3.2600471 .944726$

O $3.162109-2.3169313 .390345$

C $1.368736-4.1350130 .848788$

C $0.575708-4.776049-0.022725$

C $1.078747-5.700488-1.092382$

C $0.580756-5.326910-2.469835$

C $-0.485586-5.941070-3.010012$

C $1.379704-4.315205-3.257841$

C $1.042747-2.893865-2.900367$

N $1.464892-1.9507881 .848939$

H $-0.7767214 .918060-0.916229$

H 1.1125383 .8138441 .229803

H $1.3313214 .318128-1.839204$

H 3.4935594 .5463610 .327231

H $2.7050265 .918750-0.400306$

H $5.8424624 .383657-2.620042$

H $4.4020055 .802605-3.685668$

H $2.7198225 .351246-3.402990$

H $3.4094116 .780992-2.602910$

H $6.7483223 .810111-0.363621$

H $5.156546 \quad 3.3768340 .221603$

H $5.0573661 .606603-1.639487$

H $6.7571871 .959540-1.970729$

H $7.297772 \quad 1.3979790 .431735$

H $7.486410-0.381824-1.336278$

H $5.781916-0.735851-1.173010$

H $7.484080-2.791897-0.805095$

H $4.927951-1.7225101 .121252$

H $9.101091-1.4399760 .227674$

H $8.591629-2.0753801 .773777$

H $8.577633-0.322166 \quad 1.489372$

H $6.813043-1.6994143 .223959$

H $4.504020-0.2704953 .899043$

H 4.2300601 .3168533 .227795

H $6.4044881 .346583 \quad 3.338124$

H $3.0036590 .355261-1.264389$

H $0.307412-0.446549-2.515579$

H $3.960872-1.732039-2.422019$

H $3.481645-3.321540-1.829125$

H $3.335012-1.944044-0.768129$

H 2.1981390 .4731352 .758184
H $2.9906040 .034484 \quad 1.243114$

H $-0.233437-3.1127381 .880277$

H $1.081354-3.7071382 .916538$

H $2.446126-4.2701740 .788631$

H $-0.502105-4.6631280 .059148$

H $\quad 0.743271-6.714821-0.839027$

H $2.174807-5.747720-1.099437$

H $-0.828992-5.701149-4.010879$

H -1.043109-6.691716 -2.459823

H $1.173426-4.434112-4.330535$

H $2.446584-4.537100-3.162199$

H $0.045329-2.592310-3.222959$

H $1.084384-1.2973371 .170359$

C -2.5589833 .6205410 .990162$

O $-3.283521 \quad 3.855047-0.235522$

C -4.6874723 .7089690 .021278$

C -4.8049563 .0199531 .372502$

C -3.6095683 .6085932 .101408$

H -5.0821934 .7287710 .131830$

C $-5.4129533 .052180-1.157681$

H $-5.7571353 .227877 \quad 1.869230$

H -4.6928251 .9344411 .284877$

C -3.1959072 .8186973 .330921$

H -3.850394 4.6376762 .400161

C $-5.1587591 .551248-1.417389$

O $-6.8185083 .222475-0.928269$

H -5.177155 $3.615777-2.069297$

H $-5.6733010 .967973-0.648767$

O $-5.8159641 .218043-2.649812$

C $-3.6919181 .105447-1.506910$

C $-3.483222-0.351612-1.953201$

H -3.202823 $1.231721-0.537637$

O $-2.9913561 .918989-2.450901$

H -3.734202 $-0.458993-3.016725$

H -2.402263 -0.537501-1.894415

H $-3.9989842 .817420 \quad 4.074705$

H $-2.967024 \quad 1.777216 \quad 3.080699$

H $-2.307803 \quad 3.2569823 .796634$

C -1.481556 4.6905911 .170294

H -7.285609 2.832726 -1.687254

H $-5.3396701 .670866-3.367053$

H -2.917859 2.812278 -2.074589

H -1.0038884 .5831322 .150745$

H -1.928525 5.6913261 .131594

H -2.0889432 .6327010 .900944$

C $-4.635915-2.1009751 .161867$

O $-3.983399-1.199140 \quad 0.254825$

C $-4.241909-1.414122-1.136321$

C $-3.834544-2.836753-1.560502$

C $-4.484330-3.894670-0.666189$

C $-4.305728-3.5639690 .818579$

H -4.182766 -1.883981 2.136935

C $-6.147570-1.8446861 .257398$

H - $5.311921-1.280712-1.323953$

O $-2.415783-2.969339-1.487733$

H $-4.128051-2.990949-2.605886$

H $-4.034307-4.872124-0.878912$

O $-5.869565-3.972320-0.987370$

H $-4.923803-4.2311721 .429808$

O $-2.950097-3.8201431 .192891$

C $-6.478151-0.5886942 .029617$

H -6.617284 -1.750284 0.274555

H -6.654342 -2.671032 1.770744

H -2.187617 -3.858211-1.808396

H $-6.262071-4.664185-0.428064$

H $-2.386974-3.1855330 .717173$

O $-6.085014-0.7150753 .329080$

O

C $-6.3949530 .393170 \quad 4.179326$

H -6.0116121 .3254623 .755766$

H -7.4768670 .4552524 .329142$

H $-5.9139230 .224926 \quad 5.146369$

SCF Energy (B3LYP/6-31G**//MMFF)= -3245.92255036

0800230

MM̄FF Geometry

C $-0.2983634 .358316-0.454321$

C $-1.4940923 .817275-0.730588$ 
C $-2.6975873 .958660 \quad 0.165830$ O $-3.0801772 .627241 \quad 0.586974$ C $-3.8693794 .588410-0.605428$ C $-5.077719 \quad 4.8718320 .268938$ C $-6.231073 \quad 4.173628 \quad 0.247286$ C -4.937343 6.0545201 .194092 C -6.572963 $2.985308-0.606069$ C -6.6832761 .7096730 .238401$ C $-6.7794770 .453359-0.638674$ C $-6.954121-0.796830 \quad 0.222674$ C $-6.922829-2.084354-0.615079$ O $-5.5651140 .360297-1.388430$ O $-6.763844-3.1899530 .288514$ C -5.677867-2.065545 -1.530489 C $-8.248155-2.314221-1.351693$ O $-5.720260-3.176740-2.422716$ C $-5.506890-0.740635-2.305131$ C $-4.153456-0.626575-3.049434$ O $-6.540120-0.641159-3.292493$ C $-2.3888582 .098861 \quad 1.635578$ $\begin{array}{llll}\text { O } & -1.538726 & 2.658726 & 2.310231\end{array}$ C -2.8200150 .6942701 .808358$ C $-2.107133-0.1129272 .608434$ C $-2.395523-1.5161822 .911554$ C $-3.590018-2.1452342 .246121$ C $-2.906350-0.692295-2.160162$ C $-2.287953-2.076229-2.060287$ C $-0.448225-3.290823-0.897865$ O $-2.650133-3.029079-2.744501$ C $-0.990705-4.039177 \quad 0.277043$ C $-0.260668-4.335706 \quad 1.362988$ C $-0.766157-5.1638502 .508396$ C $-0.609665-4.4779143 .845491$ C $0.442105-4.7411504 .639565$ C $-1.723932-3.5668794 .304114$ C $-1.587872-2.1667113 .775072$ N -1.251958 -2.108374 -1.136675 H -0.1859314 .9711140 .436168$ H $-1.6071743 .194361-1.615055$ H -2.463261 4.571295 1.045136 H -4.137685 $3.941080-1.446075$ H $-3.5491075 .536185-1.059377$ H -7.030352 4.4817910 .920324 H -5.8642816 .2713191 .736019$ H -4.1574445 .8728871 .939479$ H -4.6744936 .9524040 .625302$ H -7.533317 $3.177436-1.100028$ H $-5.8487252 .846527-1.411206$ H $-5.799951 \quad 1.6310370 .883957$ H $-7.560882 \quad 1.7778510 .893219$ H -7.624446 $0.570669-1.327411$ H $-7.880937-0.740048 \quad 0.807170$ H $-6.141174-0.8455030 .959119$ H -7.507169 -3.174097 0.915545 H $-4.810969-2.219571-0.877482$ H $-9.075937-2.387654-0.636057$ H -8.238844 -3.265353 -1.895385 H $-8.491651-1.515393-2.055134$ H $-4.861432-3.229796-2.874160$ H $-4.107079-1.350746-3.871333$ H $-4.1370820 .359425-3.536583$ H -6.251583 -0.019079-3.980660 H -3.702313 $0.364072 \quad 1.273717$ H -1.226493 0.2949663 .103738 H -3.535932 -2.019649 1.159793 H $-4.515125-1.6854592 .608876$ H -3.670581-3.220422 2.419798 H $-2.135143-0.046825-2.600357$ H $-3.114186-0.311996-1.155229$ H $0.580032-2.957371-0.734821$ H $-0.465508-3.933205-1.784225$ H $-2.017712-4.3904630 .211524$ H $0.775403-4.007844 \quad 1.411685$ H $-0.203655-6.1070542 .508194$ H $-1.815249-5.4504152 .363774$ H $0.543100-4.2775805 .615447$ H $1.228238-5.4212564 .328291$ H $-1.717279-3.4971975 .400682$
H -2.690759-4.020849 4.068413

H $-0.740550-1.6259094 .199426$

H $-1.060640-1.283124-0.575951$

C $1.9458303 .185174-0.661681$

O $1.3614181 .874956-0.494100$

C $1.701797 \quad 1.391906 \quad 0.814569$

C 2.8767622 .2368541 .283153

C 2.5056783 .5901260 .704384

H $\quad 0.8441841 .6277451 .458372$

C $1.894274-0.1274240 .840875$

H 2.9758222 .2508392 .372167

H 3.8200841 .8878100 .852865

C 3.6660414 .5661750 .626165

H 1.7236554 .0247911 .337929

C $3.152711-0.7098520 .161641$

$\begin{array}{lllll}\text { O } & 1.907633 & -0.543719 & 2.214100\end{array}$

H $1.010204-0.5987390 .396960$

H $4.044115-0.4491390 .743007$

O $3.046545-2.1399630 .226243$

C $3.361027-0.315323-1.310122$

C $4.429056-1.132535-2.061328$

H $3.6244580 .744745-1.369099$

O $2.133843-0.475031-2.029042$

H $4.090020-2.171037-2.170660$

H $4.470933-0.730668-3.082930$

H $4.084214 \quad 4.7488801 .621432$

H $4.4694364 .185109-0.012360$

H 3.3375195 .5259640 .214769

C $0.928294 \quad 4.141038-1.295042$

H $2.699055-0.1651592 .633279$

H $2.920032-2.3795051 .160354$

H $1.876623-1.410724-1.968347$

H $0.631598 \quad 3.736259-2.271183$

H $1.3978145 .112842-1.485738$

H $2.7706193 .055933-1.374552$

C $7.5963150 .461915-0.856661$

$\begin{array}{llll}\text { O } & 6.290280 & 0.247134-1.412617\end{array}$

C $5.834930-1.109661-1.438481$

C $6.806702-2.003777-2.229662$

C $8.237666-1.872552-1.704574$

C $8.652006-0.405590-1.564239$

H $7.8310661 .508946-1.087148$

$\begin{array}{llll}\text { C } 7.604930 & 0.291746 & 0.671570\end{array}$

H $5.766431-1.486116-0.413685$

O $6.773791-1.644529-3.610000$

H $6.469753-3.044605-2.151482$

H $8.922982-2.381464-2.393444$

O $8.320662-2.526917-0.442216$

H $9.604011-0.333163-1.026652$

$\begin{array}{llll}\text { O } & 8.885715 & 0.138515 & -2.866524\end{array}$

C $6.926068 \quad 1.4430201 .383483$

H $7.101818-0.6275100 .981855$

H $8.6398840 .269371 \quad 1.032157$

H $7.362504-2.254717-4.086076$

H $9.240268-2.454855-0.134492$

H $8.0353700 .157352-3.338438$

$\begin{array}{lllll}\text { O } & 6.905127 & 1.180721 & 2.724410\end{array}$

$\begin{array}{llllll}\text { O } & 6.488575 & 2.449741 & 0.844866\end{array}$

C 6.3296252 .2031403 .543688

H 6.8781173 .1420703 .421863

H 5.2738902 .3377293 .294334

H $6.4058401 .885608 \quad 4.587081$

SCF Energy (B3LYP/6-31G**//MMFF) $=-3245.91536108$

08_00231

MM̄FF Geometry

C -3.017409 -3.253226 -1.109063

C $-2.105019-4.060262-0.548959$

C $-0.970992-3.5394250 .292848$

O $0.243378-3.748127-0.461603$

C $-0.861790-4.2646591 .646880$

C -2.015932 -3.977264 2.583821

C $-2.104901-2.882086 \quad 3.363838$

C $-3.095318-5.0277342 .630544$

C $-1.106358-1.767328 \quad 3.502528$

C $-1.604713-0.4666662 .861251$

C $-0.733178 \quad 0.736042 \quad 3.254245$

C -1.2075322 .0044512 .543046$ 
C -0.3128493 .2117282 .862316$

O $0.625991 \quad 0.4606142 .900100$

O -0.6165304 .2500691 .918918$

C 1.1630102 .8164772 .641803

C -0.6124853 .7903374 .250278$

O 2.0397043 .8537213 .076225

C 1.5501421 .4783553 .311999

C 2.9686241 .0478362 .868144

O $1.547418 \quad 1.655513 \quad 4.732189$

C $1.241744-2.842596-0.270773$

O $1.262917-1.9323980 .542369$

C $2.297761-3.118165-1.269233$

C $3.268589-2.211913-1.458501$

C $4.348424-2.291608-2.442803$

C $4.444576-3.539036-3.281263$

C $3.325553-0.3914493 .262408$

C $4.824385-0.6080233 .264429$

C $6.794263-0.8708341 .797154$

O $5.448586-0.7618634 .312581$

C $7.213454-0.4034520 .441694$

C $7.816908-1.195409-0.457642$

C $8.275605-0.730828-1.810552$

C $7.676126-1.542905-2.936968$

C $8.340662-2.586139-3.462183$

C $6.350948-1.096625-3.511402$

C $5.202763-1.252647-2.550261$

N $5.383848-0.5953151 .995878$

H -2.904497 -2.178547 -0.991231

H $-2.186488-5.136358-0.677465$

H -1.108104 -2.463677 0.456974

H $0.086613-4.0010792 .131098$

H $-0.771643-5.3478181 .484269$

H -2.982895 -2.775027 3.999808

H $-3.904046-4.7629913 .319989$

H -3.542755 -5.174873 1.644016

H $-2.676492-5.9825012 .965253$

H $-0.935238-1.6089064 .574660$

H $-0.135384-2.0399063 .078562$

H -1.596006 -0.5871141 .770918$

H -2.643204 -0.2699683 .156432$

H $-0.802603 \quad 0.8592544 .341688$

H -2.253192 2.2239032 .791734

H $-1.185377 \quad 1.8380311 .457957$

H -1.566802 4.4467171 .984203

H 1.3187772 .7169951 .559977

$\mathrm{H}-1.657150 \quad 4.1186884 .310378$

H -0.0072694 .6824414 .445735$

H -0.4456523 .0755645 .058679$

H 1.7690384 .6703322 .622533

H 3.0497081 .1196371 .775900

H $3.709714 \quad 1.7366903 .293574$

H 2.2148401 .0769745 .132871

H $2.223908-4.032492-1.846633$

H $3.260575-1.307061-0.851715$

H $4.526245-4.426315-2.643421$

H $5.316619-3.552645-3.939057$

H $3.559561-3.643338-3.918265$

H $2.959629-0.6410384 .264041$

H $2.864091-1.1081582 .574294$

H $6.941811-1.9476701 .933370$

H $7.370287-0.3493002 .569086$

H 7.0317660 .6403880 .197101

H $8.017537-2.233246-0.200408$

H $9.370396-0.809091-1.836876$

H $8.0542320 .332121-1.967212$

H $7.934728-3.157598-4.290098$

H $9.305405-2.894139-3.072505$

H $\quad 6.135217-1.613092-4.454055$

H $6.425139-0.037684-3.792013$

H $5.061744-0.391750-1.896089$

H $4.762768-0.654771 \quad 1.193510$

C -5.528164 -3.078780 -1.445535

O $-5.441444-1.647864-1.613086$

C $-5.758414-1.027090-0.353933$

C $-6.656622-2.0297210 .352225$

C $-5.937861-3.3257160 .011630$

H -4.820763 $-0.946401 \quad 0.211279$

C $-6.3269260 .376752-0.571250$
H $-6.726221-1.8476831 .428522$

H -7.668812 -2.034953 -0.068344

C $-6.787296-4.5693680 .204836$

H $-5.054232-3.4061510 .655867$

C $-5.4691791 .256603-1.508477$

O $-7.6297170 .261119-1.154089$

H -6.4656690 .8689230 .398828$

H $-5.5894830 .906029-2.541179$

O $-6.0188682 .577564-1.459653$

C $-3.9728941 .301470-1.142269$

C $-3.1398922 .168920-2.094528$

H $-3.5803810 .283451-1.172953$

O $-3.814412 \quad 1.7745380 .192860$

H $-3.3486801 .890077-3.135278$

H -3.441482 $3.220834-2.001992$

H $-7.087596-4.6709101 .252842$

H -7.697239 -4.533244 -0.403392

H $-6.227814-5.467368-0.076134$

C $-4.209729-3.725159-1.894023$

H $-7.9710471 .163065-1.281108$

H $-5.6479903 .079535-2.204809$

H -4.1962242 .6679490 .235194$

H -4.030117 -3.457265 -2.942641

H $-4.302338-4.816106-1.844289$

H -6.323134 -3.413083 -2.124012

C $0.3963313 .125701-2.750274$

O $-1.0259872 .968908-2.825316$

C $-1.6180872 .110487-1.839976$

C -1.019205 $0.696253-1.929179$

C $0.5109490 .720790-1.881275$

C $1.1013961 .763738-2.833952$

H $0.6610963 .696806-3.649787$

C $0.8162153 .953353-1.531502$

H -1.425608 $2.531073-0.848014$

O $-1.4484670 .076833-3.139888$

H $-1.3935660 .091703-1.095291$

H $0.890012-0.272319-2.147402$

O $0.9178270 .995333-0.545182$

H $2.1732051 .896556-2.645063$

O $0.969271 \quad 1.293766-4.178251$

C $0.3167035 .372888-1.652346$

H $0.4577223 .542028-0.585123$

H $1.9088854 .000412-1.451566$

H -1.103945 -0.832485 -3.142387

H $1.8892520 .961980-0.520950$

H $1.4549080 .453914-4.244320$

O $-0.9130775 .484853-1.074213$

O $0.9359876 .266846-2.214477$

C $-1.5089586 .783507-1.138884$

H -1.660969 $7.078954-2.181419$

H -2.481883 $6.735702-0.642461$

H $-0.8835697 .513741-0.616675$

SCF Energy (B3LYP/6-31G**//MMFF) $=-3245.90027033$

08_00232

MM̄FF Geometry

C -0.8896832 .8910961 .154555$

C -1.2498342 .3390142 .325119$

C -0.3854021 .4787693 .222298$

O 0.9804991 .5122502 .755660

C -0.9074550 .0301673 .187231$

C $-0.323195-0.8570374 .267246$

C $0.582703-1.8334604 .063693$

C $-0.866237-0.6177855 .654126$

C $1.234321-2.2468372 .774034$

C $2.757877-2.0988712 .859349$

C $3.457930-2.5544551 .571962$

C $4.975188-2.4128801 .713409$

C $5.708545-2.7738940 .414028$

O $2.991329-1.7419150 .489849$

O $7.061701-2.3074060 .526242$

C $5.060567-2.003271-0.754152$

C $5.788057-4.290698 \quad 0.205013$

O $5.611041-2.416845-2.003030$

C $3.517642-2.113966-0.791475$

C $2.956320-1.132290-1.844065$

O $3.149364-3.446629-1.148639$

C 1.9752241 .6872843 .661486 
O $1.864195 \quad 1.6828874 .879829$

C 3.2761751 .9518872 .997889

C 3.4360351 .9820911 .665686

C 4.6601642 .3306730 .938861

C 5.8868632 .6677931 .741843

C $1.426198-1.041100-1.885628$

C $1.006958 \quad 0.061724-2.837294$

C $0.9923852 .546562-3.025532$

O $0.716094-0.170638-4.008342$

C $2.3931092 .901769-3.422144$

C $3.0795223 .935607-2.911543$

C $4.5227254 .207895-3.230027$

C $5.4184714 .120471-2.009466$

C $6.0281965 .213826-1.521264$

C $5.6710452 .757035-1.395446$

C $4.6038682 .372733-0.408207$

N $1.055231 \quad 1.318333-2.252641$

H $\quad 0.1240752 .7364580 .791963$

H -2.2608772 .4876672 .695612$

H $-0.455078 \quad 1.9020984 .231382$

H $-0.728531-0.3941362 .193653$

H -1.999685 0.0220993 .309665

H $0.900922-2.4185034 .926320$

H $-0.466375-1.3274696 .386342$

H $-0.612724 \quad 0.388026 \quad 6.001849$

H $-1.956145-0.7231915 .657978$

H $0.980321-3.2968932 .585890$

H $\quad 0.858040-1.676248 \quad 1.921872$

H $2.998889-1.0472953 .051959$

H $3.140576-2.6806113 .707527$

H $3.184158-3.5993351 .383317$

H $5.347728-3.0220572 .546455$

H $5.220836-1.3726361 .970330$

H $7.460832-2.7302731 .305752$

H $5.327548-0.943739-0.634341$

H $6.322166-4.7641591 .037535$

H $6.361304-4.536089-0.695826$

H $4.807558-4.7656120 .130579$

H $6.576586-2.316537-1.941334$

H $3.354784-0.131948-1.641336$

H $3.307444-1.417159-2.844318$

H $2.233681-3.446638-1.468072$

H 4.0963492 .1637543 .676345

H 2.5792851 .7623291 .031073

H 6.1968601 .8078582 .345791

H 5.6906903 .5141702 .409465

H 6.7448802 .9494431 .127707

H $0.985738-1.977283-2.241143$

H $1.006036-0.838775-0.894121$

H $\quad 0.5330983 .311564-2.392034$

H $0.3714922 .400739-3.914633$

H $2.8822022 .239620-4.133238$

H $2.5966294 .596674-2.195680$

H $4.5826345 .206765-3.680775$

H $4.8987203 .513693-3.992116$

H $6.6893645 .154640-0.663023$

H $5.8768286 .192211-1.964952$

H $6.6680822 .725477-0.944814$

H $5.7037381 .999559-2.189100$

H $3.6606892 .103619-0.878863$

H $1.3750551 .394299-1.291395$

C -3.2304693 .8713440 .586261$

O $-3.8565112 .581014 \quad 0.692335$

C $-5.0876462 .581752-0.045884$

C $-5.4190514 .047676-0.286134$

C $-4.0311634 .634992-0.472326$

H $-4.8702102 .104895-1.009367$

C -6.1492281 .7567640 .690889$

H -6.068049 4.191390 -1.155032

H -5.9054594 .5053650 .582604$

C $-3.9805446 .144389-0.312293$

H -3.674191 4.371595-1.477042

C $-5.636600 \quad 0.401200 \quad 1.224295$

O $-6.6167822 .503487 \quad 1.821314$

H -7.0180861 .6166370 .037189$

H -4.9998740 .5764902 .100230$

O $-6.766439-0.3352151 .708107$

C $-4.868649-0.4566540 .204749$
C $-4.432069-1.8085170 .803668$

H -3.980192 $0.087271-0.132288$

O $-5.703928-0.668529-0.931552$

H -4.034736 -1.648565 1.813160

H $-5.308269-2.4649140 .873125$

H -4.614455 $6.631661-1.060309$

H -4.3298526 .4549310 .678056$

H -2.958983 $6.515559-0.441042$

C -1.7541413 .6942940 .213346$

H $-7.292766 \quad 1.9634612 .265794$

H $-7.316394-0.5650640 .939303$

H -5.194784 -1.184071 -1.579601

H -1.2907974 .6802080 .090224$

H -1.697093 $3.188836-0.759496$

H -3.3226554 .3575681 .565513$

C $-3.023672-3.411049-2.266869$

O $-3.923970-2.837929-1.310432$

C $-3.346950-2.495042-0.048199$

C $-2.780759-3.7495390 .632555$

C $-1.790372-4.480191-0.282270$

C $-2.350051-4.671743-1.697622$

H -3.663668 -3.719183 -3.103771

C $-2.027776-2.373430-2.795241$

H $-2.537146-1.772804-0.200341$

O $-3.837430-4.6373140 .992600$

H $-2.279693-3.4540201 .561789$

H -1.554612 -5.4612840 .147666$

O $-0.575588-3.740229-0.364815$

H -1.561252 $-5.003525-2.382890$

O $-3.341191-5.703431-1.665629$

C $-2.742672-1.229097-3.476015$

H -1.403901 -1.966719-1.996326

H -1.347812 -2.818625 -3.530392

H $-4.336263-4.8490580 .184962$

H $-0.218943-3.6595910 .535896$

H $-2.905641-6.518547-1.362910$

O $-2.480462-0.061803-2.820907$

O $-3.451663-1.357205-4.465632$

C $-3.0930111 .106798-3.373096$

H $-4.1826271 .011189-3.347598$

H $-2.8013151 .964482-2.761589$

H -2.742103 $1.270716-4.396350$

SCF Energy $\left(B 3 L Y P / 6-31 G^{* *} / / M M F F\right)=-3245.92266444$

08_00233

MM̄FF Geometry

C $2.814890-1.0356703 .287121$

C 3.0664260 .2652463 .498716

C 4.1494541 .1077822 .868892

O 3.5712001 .8285241 .749774

C 5.3435680 .3158562 .302587

C 6.6069901 .1458902 .186969

C 7.0133381 .7936901 .077954

C 7.4603001 .1732673 .429899

C $6.2942631 .941274-0.232792$

C $6.9476991 .151274-1.374012$

C $6.565636-0.337276-1.374509$

C $7.268154-1.069834-2.520181$

C $6.805119-2.529309-2.641946$

O $5.146089-0.415589-1.542536$

O $7.254668-3.031116-3.909978$

C $5.262262-2.563256-2.664800$

C $7.443693-3.421533-1.571029$

O $4.781784-3.905562-2.635977$

C $4.610960-1.743763-1.527907$

C $3.084713-1.634043-1.760755$

O $4.853410-2.402709-0.283873$

C $2.6999362 .842598 \quad 1.979805$

O $2.3272843 .273643 \quad 3.057890$

C 2.2505123 .3256100 .655555

C 1.0755573 .9610950 .543393

C $0.5029614 .501565-0.691904$

C $1.3419784 .428122-1.941346$

C $2.368428-0.677124-0.798821$

C $0.884913-0.653354-1.102179$

C $-0.8121440 .425892-2.542978$

O $0.116627-1.480294-0.615771$

C $-1.0632291 .800087-3.075208$ 
C $-2.1277932 .539122-2.728962$

C $-2.4386933 .891445-3.300246$

C $-2.6746904 .937656-2.234747$

C $-3.9156865 .211100-1.797453$

C -1.485239 $5.734032-1.752772$

C $-0.7303175 .047355-0.646967$

N $0.5269760 .349750-1.990054$

H $3.426043-1.6119072 .599708$

H 2.4040260 .8043554 .174532

H 4.5029451 .8310083 .614732

H $5.084350-0.0997341 .322683$

H $5.576174-0.5440262 .944197$

H 7.9634052 .3259991 .118999

H 8.3586591 .7873963 .306369

H 6.8945091 .5835974 .272441

H $7.7856650 .160463 \quad 3.689497$

H $5.2342321 .690449-0.151924$

H $6.3230273 .007655-0.491961$

H $6.6054541 .599329-2.316434$

H $8.0382751 .263797-1.339755$

H $6.843389-0.779195-0.411234$

H $8.357805-1.020020-2.402675$

H $7.047116-0.559492-3.468417$

H $8.223115-2.946368-3.939601$

H $4.939291-2.139121-3.625955$

H $8.536113-3.410944-1.666098$

H $7.142627-4.467378-1.697153$

H $7.199870-3.111533-0.552739$

H $5.207080-4.381130-3.369948$

H $2.900029-1.279165-2.783280$

H $2.623962-2.626528-1.675965$

H $4.135225-2.198830 \quad 0.334340$

H $2.8950753 .126297-0.193151$

H $\quad 0.4730884 .099204 \quad 1.440472$

H $2.2568875 .019275-1.823791$

H $0.8311614 .799120-2.832351$

H $1.6210353 .391812-2.160637$

H $2.484180-0.9852870 .243849$

H $2.7748840 .337405-0.876819$

H -1.537193 $0.136434-1.775303$

H $-0.870780-0.305356-3.355913$

H $-0.3576752 .190927-3.804358$

H -2.848224 2.129653 -2.026874

H -3.338715 $3.790406-3.921370$

H -1.649361 4.229981 -3.982549

H -4.096554 5.982825 -1.056688

H -4.777022 $4.662585-2.164729$

H -1.822786 $6.702270-1.357602$

H $-0.8493545 .998955-2.602526$

H $-1.264648 \quad 5.0429300 .304217$

H $\quad 1.269416 \quad 0.845749-2.475267$

C $0.475963-1.8513532 .951587$

O $-0.015271-0.5247342 .664928$

C - $1.445547-0.5022112 .839782$

C -1.875966 -1.959122 2.819252

C $-0.711044-2.6256223 .523583$

H -1.621402 -0.063982 3.830507

C $-2.064825 \quad 0.381901 \quad 1.751801$

H -2.831645 -2.125640 3.325004

H -1.962555 -2.323398 1.789193

C $-0.635744-4.1246803 .292057$

H -0.799860 -2.435699 4.601787

C -3.6051490 .3665691 .752929$

O

H -1.7004490 .0724750 .766211$

H $-3.926767-0.6682391 .604892$

O $-4.088416 \quad 0.770178 \quad 3.033731$

C -4.2507901 .2405010 .658502$

C $-5.790018 \quad 1.1797480 .635388$

H $-3.8646290 .934611-0.319534$

O $-3.906227 \quad 2.6153610 .838791$

H -6.1936031 .5488111 .586693$

H $-6.1275051 .894664-0.127304$

H $\quad 0.226435-4.5556413 .810776$

H -1.538119 -4.618044 3.667420

H $-0.541035-4.3622152 .227438$

C $1.655016-1.7530193 .921723$

H -0.6235111 .6870991 .947683$
H -3.8076331 .6914523 .171385$

H -2.942109 2.6926320 .739425

H $1.986890-2.7512294 .228310$

H $1.345430-1.2227424 .831376$

H $\quad 0.799959-2.285158 \quad 1.997288$

C $-6.211641-1.934235-1.379157$

O $-5.844191-0.619057-0.945993$

C $-6.360237-0.2133580 .324892$

C -7.896000 -0.2252770 .308231$

C $-8.425253-1.597884-0.121898$

C $-7.742752-2.097787-1.399966$

H -5.852935 -1.997665 -2.414653

C $-5.490450-3.026118-0.571951$

H -6.016701-0.907104 1.097047

O $\quad-8.3739880 .777295-0.585476$

H $-8.2620620 .023317 \quad 1.311592$

H -9.507274 -1.528938 -0.288230

O $-8.205888-2.5223710 .939827$

H $-8.002723-3.147341-1.580261$

O $-8.267224-1.345517-2.498241$

C $-4.000888-3.046564-0.852557$

H -5.613072 -2.906775 0.507167

H $-5.894611-4.002712-0.864270$

H $-9.3456540 .756811-0.556963$

H $-8.571921-3.3794820 .662794$

H $-7.878377-1.704704-3.313809$

O $-3.498005-4.267310-0.501679$

O $-3.355028-2.109358-1.301131$

C $-2.097689-4.447822-0.735296$

H $-1.873076-4.332810-1.800001$

H $-1.518377-3.736993-0.140512$

H -1.829615 -5.461986 -0.427570

SCF Energy $\left(B 3 L Y P / 6-31 G^{* *} / / M M F F\right)=-3245.90488488$

08_00234

MM̄FF Geometry

C -3.727518 -2.155005 -3.187602

C $-3.851933-1.611478-1.967360$

C $-5.189305-1.374055-1.312970$

O $-5.2623630 .029518-0.961720$

C $-5.339638-2.200272-0.021019$

C $-5.607100-3.677029-0.237400$

C $-4.710584-4.6552600 .001275$

C $-7.004316-4.021711-0.681944$

C $-3.279714-4.4678590 .414182$

C $-3.115248-4.3383201 .933829$

C $-1.702573-3.8668152 .309766$

C $-1.566826-3.6909503 .823854$

C $-0.215487-3.0735254 .221878$

O $-1.483852-2.6153961 .652380$

O $-0.338233-2.6650255 .593066$

C $0.033483-1.8105873 .364391$

C $0.911645-4.1120554 .176753$

O $1.341386-1.2867933 .580049$

C $-0.185430-2.0530771 .856469$

C $-0.111632-0.7589791 .021044$

O $0.840864-2.9245231 .366058$

C $-6.5045730 .579013-0.900449$

O $-7.5603420 .020919-1.172061$

C $-6.477721 \quad 1.988789-0.450113$

C -5.3997392 .6035630 .060742$

C -5.3512393 .9851920 .551794$

C $-6.615398 \quad 4.801604 \quad 0.462747$

C $-1.1780050 .290674 \quad 1.333604$

C $-1.102277 \quad 1.4295800 .335292$

C $0.2292083 .472455-0.206755$

O $-1.8858811 .517853-0.606850$

C $-0.317354 \quad 4.677876 \quad 0.487980$

C $-1.1781065 .534519-0.082013$

C -1.7257696 .7460260 .613555$

C $-3.237157 \quad 6.7565990 .667864$

C $-3.9457757 .633680-0.062970$

C -3.9213775 .8188151 .637743$

C $-4.1901554 .459113 \quad 1.051847$

N -0.0584512 .3001670 .597575$

H -4.640034 -2.396466 -3.731279

H -2.969641 -1.351279-1.389702

H $-5.994529-1.608466-2.019974$ 
H $-6.166286-1.7947780 .578455$ H -4.460549 -2.0377110 .614909$ H -5.015309 -5.690615 -0.140201 H -7.164178 -5.103175 -0.747834 H $-7.738161-3.6236740 .026466$ H $-7.209370-3.599057-1.670170$ H $-2.695538-5.3248600 .056222$ H $-2.863305-3.599061-0.104536$ H -3.845564 -3.613845 2.316581 H -3.331893 -5.299834 2.415002 H $-0.979773-4.6026071 .937929$ H -1.722032 -4.6457994 .341351$ H $-2.365086-3.0256794 .182845$ H $0.523449-2.3225295 .884587$ H $-0.672305-1.0449653 .710126$ H $0.714105-4.9190424 .892644$ H $1.868829-3.6720194 .477595$ H $1.036580-4.5672543 .192045$ H $1.413597-1.0495504 .519674$ H $0.891811-0.3238151 .098819$ H $-0.228112-1.034872-0.035743$ H $0.903156-2.8178570 .402241$ H $-7.4338252 .496691-0.531625$ H -4.4688932 .0476200 .146333$ H -6.4982355 .8296410 .810915$ H -7.4079854 .3503481 .069802$ H $-6.9595584 .866031-0.575608$ H -2.185323 -0.1372091 .278791$ H -1.054146 0.6988432 .342218 H $-0.1941373 .348408-1.208087$ H $\quad 1.3171213 .552150-0.292586$ H 0.0177364 .8575621 .506843 H -1.500142 5.364609-1.106853 H -1.357256 7.6307050 .078105 H -1.332081 6.8323891 .634045 H $-5.0281307 .673754-0.005868$ H $-3.4599268 .330860-0.737593$ H -4.840908 6.2746222 .021204 H -3.2942385 .6945612 .530169$ H -3.3266703 .7945351 .071691$ H $0.5138362 .148507 \quad 1.423375$ C -1.137948 -2.432009 -3.159082 O $-0.714349-1.054918-3.082549$ C $0.665826-0.963217-3.502974$ C $1.203128-2.383325-3.449402$ C $-0.009830-3.179261-3.876332$ H $0.641047-0.595738-4.536614$ C $1.4120160 .025199-2.599808$ H $2.065418-2.534437-4.105495$ H $1.496628-2.651178-2.426465$ C $0.078081-4.652890-3.515111$ H $-0.126739-3.083542-4.964290$ C $2.9242090 .097535-2.868611$ O $\quad 0.8491531 .324226-2.794113$ H $1.232098-0.244707-1.556888$ H $3.376705-0.890386-2.721736$ O $3.116533 \quad 0.429924-4.249093$ C $3.6884861 .128695-2.011482$ C $3.5171790 .888225-0.499133$ H $3.3734982 .144769-2.276528$ O $5.0654841 .013229-2.375999$ H $2.4608251 .014359-0.247119$ H $3.777191-0.153400-0.282122$ H $0.936124-5.119470-4.010177$ H $0.195461-4.794692-2.435290$ H $-0.824038-5.187437-3.828406$ C -2.466406 -2.530356 -3.918997 H $-0.1069361 .253719-2.628412$ H $4.0755110 .476196-4.404560$ H $5.5449651 .742410-1.947553$ H -2.605742 -3.567389-4.249142 H -2.416067 -1.908583 -4.821854 H -1.240369 -2.799523 -2.130563 C $6.324504 \quad 0.4675240 .658736$ $\begin{array}{lllll}\text { O } & 5.758129 & 1.702713 & 0.203115\end{array}$ C 4.3470001 .8342670 .400306 C 4.0325941 .7126301 .898855 C 4.5752740 .4125012 .487050
C $\quad \begin{array}{lll}6.059987 & 0.261150 & 2.155461\end{array}$

H $5.899316-0.364852 \quad 0.085624$

C 7.8308130 .5211830 .375209

H 4.0868972 .8560690 .095358

$\begin{array}{lllll}\text { O } & 2.630433 & 1.800812 & 2.121734\end{array}$

H 4.4930632 .5580222 .426743

H $3.994039-0.4497502 .139198$

$\begin{array}{lllll}\text { O } & 4.410612 & 0.466155 & 3.905819\end{array}$

H $6.641027 \quad 0.9832362 .743806$

O $6.496930-1.0451762 .524337$

C $8.1099270 .469973-1.107684$

H $8.266017 \quad 1.451896 \quad 0.759346$

H $8.371101-0.3061340 .849163$

H 2.4786421 .6824463 .075096

H $4.656859-0.403797 \quad 4.262925$

H $6.428753-1.1169983 .491178$

O $7.967548-0.806846-1.565300$

O $8.4075041 .449571-1.778877$

C $8.189001-0.985480-2.967628$

H $7.474904-0.389547-3.543808$

H $9.216916-0.713950-3.225926$

H $8.035124-2.041740-3.204145$

SCF Energy (B3LYP/6-31G**//MMFF) $=-3245.91864868$

08_00235

MMFF Geometry

C $2.286103 \quad 3.8383901 .418548$

C 3.0273214 .4770590 .500622

C $3.7064353 .854729-0.699661$

O $3.4141402 .442548-0.817258$

C $5.2305404 .013781-0.579306$

C $5.9554423 .714332-1.876533$

C $6.6380942 .581184-2.130247$

C $5.925571 \quad 4.821131-2.900039$

C $6.7834871 .379475-1.238636$

C $6.6335500 .074829-2.034553$

C $6.878843-1.154660-1.149922$

C $6.939266-2.428014-1.994535$

C $7.122168-3.678017-1.121373$

O $5.807427-1.249510-0.204871$

O $6.858344-4.829076-1.937534$

C $6.060272-3.661882-0.003805$

C $8.563506-3.822794-0.619093$

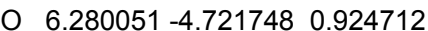

C $5.961538-2.3112500 .746608$

C $4.719050-2.3284331 .665405$

O $7.128392-2.1299561 .550826$

C $2.2457072 .095282-1.423990$

O $1.4088392 .849290-1.894603$

C $2.139503 \quad 0.619552-1.387384$

C $0.9357540 .049414-1.548909$

C $0.644886-1.386828-1.550351$

C $1.817751-2.331164-1.543101$

C $4.332743-0.9639052 .250988$

C $3.093697-1.1116793 .112788$

C $0.644353-1.5647522 .944108$

O $3.158827-1.1308814 .339410$

C $0.034466-2.6961042 .177171$

C -1.179996 -2.650568 1.609165

C -1.784364 -3.7966690 .849628$

C $-2.248603-3.409867-0.537467$

C $-3.557553-3.351266-0.836271$

C $-1.197204-3.184580-1.604579$

C $-0.645344-1.783044-1.587217$

N $1.940063-1.2706642 .362515$

H 2.1608292 .7606731 .362900

H 3.1284385 .5580150 .581719

H $3.3486644 .374464-1.597036$

H $5.607093 \quad 3.3759850 .229098$

H $5.4839215 .040487-0.281657$

H $7.1618512 .506775-3.082578$

H $6.5127194 .579140-3.792440$

H $4.8998795 .017717-3.226511$

H $6.3386565 .742094-2.475783$

H $7.7810121 .412816-0.784363$

H $6.0535101 .386118-0.424357$

H $5.6230910 .031029-2.461683$

H $7.3469100 .072073-2.868326$ 
H $7.824279-1.010382-0.613799$

H $7.739621-2.363676-2.742293$

H $6.004418-2.533809-2.562960$

H $7.477276-4.812708-2.687605$

H $5.089244-3.863287-0.478147$

H $9.258999-3.884891-1.464778$

H $8.693778-4.753671-0.056210$

H $8.883989-2.9909000 .011374$

H $6.319853-5.5494310 .415490$

H $3.861749-2.7066991 .096544$

H $4.880200-3.0310312 .493652$

H $6.914998-1.5355052 .287291$

H $3.0455950 .050304-1.214780$

H $\quad 0.0708080 .697750-1.684258$

H $2.489940-2.115921-2.381580$

H $1.536729-3.381541-1.637371$

H $2.382436-2.234693-0.612372$

H $5.133923-0.5523712 .873177$

H $4.127851-0.2325541 .460823$

H $0.046015-0.6515962 .889301$

H $0.758588-1.8442573 .995925$

H $0.624494-3.6060122 .092075$

H -1.784241 -1.7535741 .713994$

H $-2.629602-4.1748251 .439319$

H -1.080583 -4.6345330 .767504$

H $-3.898825-3.102302-1.835659$

H -4.318086 -3.541834 -0.086706

H -1.637381 -3.348649-2.597126

H $-0.426169-3.952086-1.499279$

H -1.417587 -1.013236 -1.623496

H $1.982509-1.1144391 .359901$

C $0.044228 \quad 4.5650372 .353825$

O $-0.465233 \quad 3.2325632 .139213$

C -1.284304 3.2448260 .950051

C -1.7163914 .6908240 .783844$

C -0.4457915 .4181431 .179260$

H -0.6247322 .9450550 .125800$

C -2.4159762 .2274931 .108957$

H $-2.0393384 .923980-0.234960$

H $-2.533017 \quad 4.936106 \quad 1.474038$

C $-0.662507 \quad 6.8793181 .533327$

H $0.2543845 .364216 \quad 0.337144$

C $-3.4336682 .248178-0.048402$

$\begin{array}{lllll}\text { O } & -1.807404 & 0.936861 & 1.189717\end{array}$

H -2.938022 2.3886092 .059768

H $-3.9043683 .237295-0.081367$

O $-2.7491272 .077322-1.290162$

C -4.5494891 .1893950 .074392$

C $-5.5757591 .311126-1.069632$

H $-5.051027 \quad 1.3189931 .040816$

O $-3.970520-0.112048 \quad 0.051452$

H -5.876672 2.360325-1.174246

H $-5.0949200 .994750-2.003852$

H - 1.0604637 .4283380 .673668

H -1.371485 6.9913672 .360526

H 0.2799117 .3505941 .829800

C 1.5656214 .5242412 .546746

H -1.168822 $0.965275 \quad 1.922233$

H -2.345174 $1.192787-1.276897$

H $-4.683930-0.7557530 .196663$

H 1.7935693 .9616843 .460758

H 1.9472805 .5408212 .696930

H $-0.411323 \quad 4.916628 \quad 3.288247$

C -7.501366 -1.872633 -0.626364

O $-6.455593-0.926296-0.882179$

C $-6.8370630 .452090-0.840699$

C $-7.9180880 .736828-1.895388$

C $-9.113561-0.208490-1.732949$

C $-8.671556-1.669709-1.603334$

H -7.051075 -2.850405 -0.841684

C $-7.929517-1.8687080 .845212$

H $\quad-7.2283890 .690410 \quad 0.154272$

O $-7.361880 \quad 0.581058-3.199716$

H -8.242494 $1.779748-1.797349$

H $-9.778938-0.113384-2.599666$

O $-9.855460 \quad 0.153654-0.571948$

H -9.518341 -2.292717 -1.293797

O $-8.268573-2.145309-2.891311$
C $-6.810022-2.3693811 .726381$

H $-8.242131-0.8846611 .205607$

H -8.783983 -2.537894 1.002086

H $-8.0569340 .800311-3.843296$

H -10.157404 $1.070359-0.690453$

H -7.472027 -1.652707 -3.154365

O $-6.085656-1.3176232 .203545$

O $-6.593026-3.5544681 .943516$

C $-4.971943-1.6603103 .034016$

H -4.311420 -2.364259 2.520085

H $-4.410841-0.7458723 .244430$

H -5.327076 -2.083138 3.978207

SCF Energy $\left(B 3 L Y P / 6-31 G^{* *} / / M M F F\right)=-3245.91811587$

08 00236

MM̄FF Geometry

C -4.195958 -0.8641902 .952419$

C $-2.892171-1.0613302 .703479$

C $-1.796252-0.6501633 .648212$

O $-\begin{array}{llll}0.992832 & 0.380260 & 3.028107\end{array}$

C $-0.861410-1.8065144 .068039$

C $-0.088919-2.4923102 .953532$

C $1.087618-2.0615762 .452692$

C $-0.689259-3.7801082 .449822$

C $1.863527-0.8374082 .856671$

C $3.315277-1.1346223 .262774$

C $4.237429-1.5097422 .091097$

C $5.672509-1.7149622 .586220$

C $6.653887-1.9662881 .430853$

O $4.199948-0.4418261 .139904$

O $7.986909-1.8104421 .941598$

C $6.452997-0.876727 \quad 0.355763$

C $6.557024-3.4033760 .904820$

O $7.238246-1.151764-0.802098$

C $4.974782-0.672901-0.043075$

C $4.8322090 .567549-0.958601$

O $4.525874-1.828230-0.754228$

C $-1.453726 \quad 1.6588923 .053489$

O -2.5194302 .0527113 .498264$

C -0.4116552 .5122122 .440269$

C -0.6970413 .7773682 .099759$

C 0.2337694 .7423641 .507352

C 1.6443384 .2839941 .246723

C $3.3782541 .012676-1.167872$

C $3.2498982 .155683-2.159591$

C $1.5157833 .651598-3.157383$

O $4.1924622 .589302-2.815941$

C $1.7153744 .996288-2.532652$

C $0.7266355 .883116-2.340572$

C $0.9521377 .277025-1.830308$

C $0.0913437 .624981-0.638977$

C $-0.9976688 .400961-0.776025$

C 0.5575627 .1714750 .725096

C -0.2132235 .9876531 .243166$

N $1.9413262 .613612-2.239779$

H $-4.498894-0.4036633 .889399$

H -2.599791-1.490964 1.749553

H -2.224314 -0.2406344 .573399$

H -1.446524 -2.559488 4.612956

H $-0.143765-1.4218104 .804053$

H $1.538559-2.6419161 .649631$

H $-0.069763-4.2527751 .681084$

H $-0.790017-4.4961923 .272207$

H -1.678398 -3.611948 2.016512

H $1.841823-0.1238052 .025096$

H $1.390632-0.3332293 .704228$

H $3.706917-0.2235163 .734756$

H $3.336104-1.9247374 .023493$

H $3.864103-2.4266071 .622410$

H $5.718406-2.5332943 .315503$

H $6.002570-0.8158213 .125929$

H $8.103290-2.4409112 .672976$

H 6.8322220 .0679390 .770534

H $6.794353-4.1189931 .701261$

H $7.291091-3.5844120 .111907$

H $5.565436-3.6549880 .522842$

H $8.158819-1.261821-0.508188$

H $5.3734921 .414420-0.517027$ 
H $5.2912730 .359860-1.933380$ H $3.793706-1.582082-1.340452$ H $\quad 0.5668702 .0729242 .284908$ H -1.705732 4.147526 2.278924 H 2.1517664 .0485392 .188519 H 2.2555625 .0244510 .726748 H 1.6469363 .3897120 .615358 H $2.7781300 .183749-1.553762$ H $2.9447561 .335123-0.214287$ H $0.4662363 .460192-3.402257$ H $2.1056433 .582219-4.077197$ H $2.7354255 .274889-2.278190$ H $-0.2891265 .618487-2.625312$ H $0.7387277 .966960-2.657647$ H $2.0064377 .445109-1.576800$ H $-1.596038 \quad 8.6882210 .082391$ H -1.316436 $8.762193-1.748088$ H 0.4166937 .9929331 .441297 H 1.6382057 .0044820 .716951 H $-1.255657 \quad 6.218342 \quad 1.468621$ H $1.2425332 .226925-1.612724$ C $-5.532832-0.1587440 .895011$ O $-4.410643-0.140401-0.009576$ C $-4.0068671 .222645-0.215281$ C -5.2148922 .0645290 .174648$ C $-5.739604 \quad 1.2829851 .366418$ H -3.196611 1.4203590 .496552 C $-3.4853071 .426018-1.640264$ H -4.9432093 .0949340 .422216$ H -5.968445 $2.088740-0.620832$ C -7.1783601 .6061691 .728876$ H -5.1025251 .5117472 .228910$ C $-2.4255270 .394729-2.088542$ O $-4.5857211 .331565-2.553470$ H -3.095052 2.446266 -1.737959 H -2.920532 $-0.558941-2.301577$ O $-1.8920810 .845190-3.340632$ C $-1.2709880 .168288-1.099226$ C $-0.104457-0.671270-1.645072$ H -1.655693 -0.301900 -0.188810 O $-0.740758 \quad 1.442507-0.723752$ H $0.411164-0.111143-2.435300$ H $0.622265-0.766373-0.826958$ H -7.277804 2.6609142 .005339 H $-7.857081 \quad 1.4133530 .891247$ H -7.5092521 .0001492 .578228$ C -5.294544 -1.209136 1.986276 H $-4.2320081 .469683-3.448882$ H -1.400309 $1.667001-3.170539$ H $-0.048376 \quad 1.284541-0.059805$ H $-5.055506-2.1693831 .513711$ H $-6.217687-1.3618832 .557737$ H $-6.390982-0.4841260 .292990$ C -1.473787 -4.150975-1.401389 O $-1.067785-2.808826-1.088951$ C $-0.475982-2.069606-2.162104$ C $0.762931-2.797880-2.711931$ C $0.439172-4.241839-3.100994$ C $-0.307366-4.970542-1.982630$ H -1.736128 -4.591901-0.431653 C -2.724001-4.179916-2.289805 H -1.205599 -1.958761 -2.969563 O $1.814023-2.793830-1.747963$ H $1.133023-2.252038-3.587695$ H $1.370016-4.779333-3.320174$ O $-0.363686-4.254863-4.277257$ H - $0.674542-5.937684-2.345826$ O $0.625519-5.247156-0.934450$ C -3.962797 -3.901336-1.473492 H -2.699876 -3.448587 -3.102136 H $-2.854770-5.161850-2.760863$ H $1.476702-3.211261-0.936841$ H $\quad 0.142168-3.816642-4.982828$ H $0.165678-5.784169-0.266825$ O $-4.411558-5.056848-0.904607$ O $-4.458663-2.790582-1.344801$ C -5.587351-4.941963 -0.096741 H $-5.325949-4.501148 \quad 0.868661$
H $-6.356225-4.346739-0.598989$

H -5.982068 -5.947479 0.071901

SCF Energy (B3LYP/6-31G**//MMFF) $=-3245.91599389$

08_00237

MMFF Geometry

C 0.5212433 .4171882 .872016

C 1.5244352 .7049213 .406716

C 1.4712541 .2788153 .903828

O $2.093591 \quad 0.4271722 .911661$

C $0.059003 \quad 0.726813 \quad 4.174687$

C $0.081363-0.5654624 .970451$

C $-0.129542-1.7922994 .455342$

C $0.306413-0.4034376 .453142$

C $-0.385389-2.1551693 .020465$

C $0.458825-3.3608982 .583458$

C $0.142523-3.7630711 .136772$

C $0.824628-5.0807440 .770064$

C $0.570275-5.457700-0.697392$

O $0.611109-2.7208520 .275894$

O $1.476982-6.514959-1.044875$

C $0.920104-4.249189-1.589648$

C $-0.844145-6.009461-0.911828$

O $0.560147-4.494607-2.947258$

C $0.297055-2.916517-1.108613$

C $0.904886-1.745472-1.911914$

O $-1.113746-2.944126-1.329142$

C 3.4486540 .3182112 .932730

$\begin{array}{llll}\text { O } & 4.220542 & 0.859191 & 3.708226\end{array}$

C $3.841418-0.5641241 .812349$

C $5.110078-0.5373331 .378064$

C $5.658077-1.3046710 .258291$

C $4.801126-2.394410-0.326968$

C $0.588411-0.354800-1.349808$

C $1.3678140 .690862-2.121730$

C $3.6715051 .637233-2.339425$

O $\quad 0.8675491 .303577-3.061822$

C $4.7059650 .733909-2.936422$

C $6.0261530 .887925-2.751094$

C $7.061727-0.019746-3.353613$

C $8.074692-0.517619-2.344093$

C $9.344390-0.077800-2.366281$

C $7.638467-1.582609-1.358068$

C $6.893053-0.994464-0.187743$

N $2.6745780 .817235-1.675883$

H -0.4544672 .9628422 .725765$

H 2.4898103 .1945973 .525838

H 2.0480031 .2379834 .836555

H -0.4766560 .5845953 .229372$

H $-0.530017 \quad 1.457787 \quad 4.744579$

H $-0.133124-2.6379945 .142377$

H $\quad 0.245928-1.3562346 .989987$

H 1.2952040 .0207976 .651014

H $-0.450418 \quad 0.262004 \quad 6.881416$

H -1.446265 -2.411857 2.924492

H $-0.191567-1.3168722 .345931$

H $1.522535-3.1060522 .675768$

H $0.260423-4.2080293 .251930$

H $-0.944879-3.8581831 .034874$

H $0.496941-5.8892691 .435299$

H $1.908963-4.9859600 .922559$

H $1.314071-7.260020-0.441306$

H $2.014136-4.142782-1.574402$

H -1.006674 -6.898232 -0.290199

H $-0.986481-6.338878-1.947097$

H $-1.627720-5.288832-0.668581$

H $0.982115-5.329764-3.212601$

H $1.993975-1.860078-1.941441$

H $0.557913-1.787333-2.952704$

H -1.443465 -2.032404-1.370673

H $3.073763-1.1780091 .355522$

H $5.8115310 .138621 \quad 1.865977$

H $3.948596-1.963219-0.856277$

H $4.425381-3.0581910 .460208$

H $5.332894-3.036156-1.032398$

H $-0.477816-0.124391-1.431603$

H $0.850982-0.277689-0.288626$

H $4.0943902 .307971-1.584967$ 
H $3.2036752 .236955-3.125748$

H $4.349861-0.083786-3.559217$

H $\quad 6.384828 \quad 1.721707-2.151718$

H $7.5686060 .540740-4.149775$

H $6.595963-0.883340-3.844873$

H $10.082705-0.447897-1.662615$

H $9.6732600 .665833-3.084668$

H $8.516261-2.107819-0.959301$

H $7.065330-2.340016-1.899100$

H $7.443296-0.2054470 .327641$

H $2.9792090 .262754-0.881661$

C 0.6195605 .1312430 .981854

O -0.5835564 .6046880 .388782$

C $-0.2430724 .037489-0.892964$

C $1.1588974 .541555-1.214343$

C $1.779398 \quad 4.5542240 .170221$

H $-0.1901082 .952403-0.749574$

C -1.312562 4.373232 -1.935763

H $1.6849173 .893992-1.919695$

H $1.1410755 .554510-1.633244$

C 3.0628195 .3595950 .257526

H 1.9858173 .5182470 .467590

C $-2.7379483 .941105-1.532023$

O $-1.3248835 .793234-2.130164$

H -1.037419 $3.934082-2.901960$

H $-3.0807644 .567079-0.698668$

O $-3.5909604 .238276-2.643831$

C $-2.8729752 .450952-1.159641$

C $-4.3035912 .064910-0.762824$

H $-2.2070842 .229781-0.321464$

O $-2.483967 \quad 1.630360-2.259345$

H -4.6830332 .7532440 .002870$

H $-4.9723132 .181239-1.626399$

H $3.8255104 .939489-0.406431$

H $2.9031796 .403454-0.032505$

H $3.4603795 .349287 \quad 1.277217$

C 0.6434574 .8659952 .487258

H $-2.0077495 .987767-2.794992$

H $-4.5095794 .211254-2.327608$

H $-1.5411501 .787693-2.432994$

H $-0.206504 \quad 5.3855812 .948043$

H 1.5484195 .3072672 .921829

H $0.576174 \quad 6.2190510 .835252$

C $-6.245631-0.893756 \quad 0.384413$

O

C $-4.4516180 .608799-0.274813$

C $-3.630881 \quad 0.282928 \quad 0.984532$

C $-3.971143-1.1023571 .542211$

C $-5.482569-1.3286781 .644567$

H $-7.305850-0.7957300 .652201$

C $-6.146872-1.900364-0.767176$

H -4.152664 -0.056753 -1.091460

O -3.8589691 .2668751 .991584$

H $-2.562998 \quad 0.313171 \quad 0.741529$

H -3.518125 -1.204019 2.535565

O $-3.389574-2.0867510 .693375$

H -5.703713 -2.380576 1.859648

O $-5.992832-0.5609772 .738719$

C -7.141552 -1.562495-1.852007

H -5.151124 -1.956322 -1.216103

H $-6.382591-2.912638-0.417507$

H -4.813929 1.2883442 .174729

H -3.575809 -2.958104 1.082761

H -5.546922 -0.8692923 .545979$

O $-6.562825-0.744339-2.776723$

O $-8.300044-1.957784-1.860065$

C -7.414944 -0.323811-3.846146

H $-8.2629660 .245229-3.453305$

H $-6.8345780 .324203-4.508384$

H $-7.761483-1.190209-4.417582$

SCF Energy (B3LYP/6-31G**//MMFF) $=-3245.91814702$

08_00238

MM̄FF Geometry

C 2.5880953 .7673800 .970913

C 1.4375743 .3192470 .444625

C $\quad 0.1693323 .137381 \quad 1.241471$

O -0.3014801 .8062250 .926353$
C -0.8718854 .1900990 .811947$

C -1.8803184 .5396901 .890816$

C -3.1927334 .2342241 .862131$

C -1.3320315 .3524783 .038705$

C -3.9461103 .4586850 .818978$

C -4.7625172 .3232921 .453594$

C -5.5877911 .5579190 .410609$

C -6.3803090 .4282251 .069286$

C -7.156089 -0.3978910 .032318$

O $-4.6872181 .009149-0.551904$

O $-7.593543-1.6091720 .668461$

C $-6.197442-0.812172-1.106409$

C $-8.4253120 .328830-0.428070$

O $-6.910101-1.427161-2.176635$

C $-5.3258860 .345512-1.650754$

C $-4.203478-0.208168-2.549478$

O $-6.080377 \quad 1.252513-2.455288$

C -1.0443891 .1428951 .848640$

O -1.4580611 .5676872 .914403$

C - $-1.232495-0.2345681 .344888$

C $-2.085308-1.0694361 .956213$

C $-2.256231-2.4776931 .605089$

C $-2.416017-3.3735732 .803165$

C $-3.2783200 .882414-3.107922$

C $-2.0372230 .255419-3.706627$

C $0.041228-0.942092-3.025413$

O $-1.9120030 .086909-4.916649$

C $-0.362180-2.379233-2.915880$

C $0.090345-3.217320-1.971182$

C $-0.379726-4.637621-1.837826$

C $-1.019513-4.926862-0.495352$

C $-0.438946-5.7742340 .371233$

C $-2.369577-4.308721-0.188607$

C -2.256172 -2.8982720 .324169$

$\mathrm{N}-1.122681-0.126914-2.736613$

H 2.6018364 .0163922 .030869

H $1.3779283 .072232-0.611432$

H $\quad 0.398973 \quad 3.1956962 .311760$

H -1.370688 $3.858554-0.104612$

H -0.3674585 .1293340 .544309$

H -3.8127804 .5876972 .685830$

H -2.114897 5.6694213 .736250

H -0.6007954 .7738913 .610673$

H $-0.843736 \quad 6.2581372 .664038$

H $-4.624643 \quad 4.1485100 .303180$

H $-3.276713 \quad 3.0428880 .062001$

H -4.0747531 .6314491 .954459$

H -5.4331712 .7286812 .221676$

H $-6.2607352 .267132-0.085412$

H -7.0607620 .8223721 .834308$

H $-5.689031-0.2426441 .598938$

H -8.130690 -1.365126 1.441679

H $-5.525421-1.581033-0.699076$

H -9.1031370 .4890050 .419072$

H -8.987500 -0.273216 -1.150292

H $-8.2221231 .305652-0.872159$

H -7.421326 -2.163276 -1.798832

H -3.609206 -0.928113 -1.974488

H $-4.632087-0.754846-3.399280$

H $-6.9929070 .929443-2.520114$

H -0.623635 -0.5491820 .503481$

H -2.661216 -0.7285902 .813932$

H -2.467895 -4.434222 2.548615

H $-3.334088-3.124223 \quad 3.346792$

H -1.567585 -3.252299 3.486299

H $-3.785914 \quad 1.465385-3.884485$

H -2.967948 $1.588475-2.329088$

H $0.811653-0.671619-2.298518$

H $0.408166-0.727356-4.033639$

H -1.087581 -2.740305 -3.641641

H $0.818578-2.862481-1.246233$

H $0.488301-5.290106-1.997565$

H -1.095287 -4.894399 -2.629234

H $-0.900404-6.0138941 .323289$

H $0.513883-6.2441560 .151466$

H $-2.928066-4.9366310 .514825$

H -2.984091 -4.305306 -1.098050

H $-2.158383-2.152674-0.462406$ 
H $-1.368427-0.000020-1.759294$

C $3.9955033 .542175-1.197136$

O $4.0233772 .101322-1.252863$

C $4.9790381 .691829-2.252347$

C $5.381002 \quad 2.958468-2.998217$

C $5.2756573 .996916-1.898081$

H $5.8568681 .315847-1.714918$

C $4.386600 \quad 0.587912-3.134749$

H $6.3849282 .885968-3.427599$

H $4.6827713 .190429-3.810954$

C $5.2284235 .423517-2.417347$

H $6.1348143 .885532-1.223022$

C $3.945376-0.663565-2.347364$

O $3.2326711 .118472-3.799703$

H $\quad 5.1006970 .315307-3.920501$

H $3.137418-0.386656-1.663246$

O $3.375652-1.590049-3.281308$

C $5.079664-1.370032-1.581525$

C $4.628821-2.649355-0.848570$

H $5.533742-0.681942-0.861916$

O $6.097694-1.726983-2.520652$

H $4.114230-3.322243-1.544953$

H $5.532031-3.184801-0.526776$

H $6.1410465 .659876-2.974176$

H $4.3763165 .578892-3.087597$

H $5.1426926 .137931-1.592874$

C $3.9003893 .995654 \quad 0.262641$

H $2.8577470 .404303-4.343160$

H $4.087538-1.879367-3.877842$

H $6.838952-2.107890-2.019647$

H 4.1161135 .0684190 .328163

H 4.6706893 .4733940 .844918

H $3.1231803 .877978-1.771711$

C $3.858858-1.2063282 .495800$

O $4.548059-1.6442551 .317970$

C $3.744426-2.3621130 .374546$

C $3.208274-3.6488201 .018748$

C $2.437141-3.3412252 .307360$

C $3.214053-2.3949303 .231030$

H $4.651062-0.8063293 .142173$

C $2.908811-0.0542542 .156208$

H $2.899434-1.7373150 .068335$

O $4.279005-4.544975 \quad 1.309416$

H $2.551657-4.157050 \quad 0.303672$

H $2.230842-4.2776942 .839748$

O $1.187350-2.7408241 .986804$

H $2.568939-2.0271844 .036185$

$\begin{array}{lllllll} & \text { O } & 4.274722 & -3.130539 & 3.850867\end{array}$

C $2.6354810 .805887 \quad 3.363811$

H 3.3560420 .5942311 .392180

H $1.965217-0.3915531 .718983$

H $4.906639-4.0800231 .888938$

H $0.700014-3.3593601 .417490$

H $3.870159-3.8475444 .368543$

O $1.447385 \quad 0.454494 \quad 3.930213$

$\begin{array}{lllll}\text { O } & 3.401398 & 1.670873 & 3.769182\end{array}$

C 1.0826961 .1922135 .100544

H 0.1208440 .8127565 .455423

H 1.8288211 .0475835 .887586

H $0.974105 \quad 2.254084 \quad 4.859894$

SCF Energy (B3LYP/6-31G**//MMFF) $=-3245.91281612$

0800239

MMFF Geometry

C -2.688385 $5.435314-1.104468$

C $-3.3142304 .252245-1.014915$

C $-4.069916 \quad 3.8012910 .207836$

O -\begin{tabular}{lllll}
\hline & -3.440283 & 2.602636 & 0.718890
\end{tabular}

C $-5.5219853 .462665-0.166080$

C -6.3623613 .0249601 .018346$

C $-6.8350651 .773976 \quad 1.188540$

C -6.7136334 .1076322 .005245$

$\begin{array}{llll}\text { C } & -6.586946 & 0.595441 & 0.286868\end{array}$

C $-6.739846-0.7401661 .030296$

C $-6.558883-1.9363300 .083860$

C $-6.893856-3.2484760 .796307$

C $-6.648216-4.467365-0.105335$

O $-5.197926-1.952336-0.361618$ $\begin{array}{llll}\text { O } & -6.700590 & -5.628108 & 0.738142\end{array}$

C $-5.223245-4.366987-0.690742$

C $-7.764718-4.640056-1.140934$

O $-4.983142-5.410319-1.631488$

C $-4.902037-2.987821-1.310160$

C $-3.415244-2.846366-1.712686$

O $-5.675211-2.823035-2.505373$

C $-2.348676 \quad 2.773346 \quad 1.514804$

$\begin{array}{llll}\text { O } & -1.822406 & 3.831843 & 1.821322\end{array}$

C $-1.899117 \quad 1.4364821 .965211$

C -0.7542811 .3318892 .657385$

C $-0.178905 \quad 0.1061913 .218792$

C $-0.957777-1.1693173 .046587$

C $-2.417739-3.070605-0.574710$

C $-0.991601-2.724921-0.969060$

C $1.335974-2.882670-0.120931$

O $-0.691695-2.200614-2.037894$

C $2.000804-3.013597 \quad 1.210794$

C $2.727270-2.0416901 .782397$

C $3.431801-2.1975913 .098007$

C $3.166854-1.0552874 .051567$

C $4.165513-0.2584454 .468770$

C $1.762874-0.8883594 .591341$

C $0.9967520 .191976 \quad 3.876183$

N $-0.093881-3.0583020 .037336$

H $-2.7330586 .128154-0.268210$

H $-3.2544883 .553835-1.846497$

H $-4.062730 \quad 4.5777740 .983137$

H $-5.5281802 .689829-0.943896$

H $-6.0016424 .339730-0.621479$

H $-7.472993 \quad 1.5818552 .049841$

H -7.4139173 .7591272 .771945$

H -5.8173094 .4670472 .519519$

H $-7.184652 \quad 4.952188 \quad 1.491754$

H $-7.3120740 .631467-0.534840$

H $-5.5821310 .645575-0.145657$

H $-5.994704-0.7869071 .835122$

H -7.733195 -0.7886271 .493953$

H -7.219771 -1.795462 -0.779549

H -7.929502 -3.2432101 .158600$

H $-6.264531-3.3458991 .692365$

H $-6.470514-6.3995800 .192031$

H $-4.540716-4.5527840 .147080$

H $-8.731627-4.768563-0.639612$

H -7.615990 -5.547106 -1.737155

H $-7.854721-3.791174-1.821775$

H $-5.435993-5.173059-2.458532$

H $-3.184729-3.525259-2.544295$

H $-3.264829-1.833687-2.112807$

H -5.247596 - $2.146893-3.056897$

H $-2.5270760 .584904 \quad 1.730005$

H -0.1790962 .2382962 .844558$

H $-0.436006-2.0549283 .414361$

H -1.164969-1.351271 1.988115

H $-1.911979-1.1100193 .581884$

H $-2.683801-2.4532600 .290412$

H $-2.430406-4.122302-0.268647$

H $1.520381-1.912392-0.591221$

H $1.695823-3.661849-0.800781$

H $1.900025-3.9698621 .719003$

H $2.847924-1.0933191 .267407$

H $4.506496-2.2752362 .888895$

H $3.149416-3.1376373 .588332$

H $3.9867160 .544743 \quad 5.176287$

H $5.181748-0.3888054 .113225$

H $1.817953-0.6058055 .651658$

H $1.250597-1.8539814 .587613$

H 1.4677191 .1735793 .953757

H $-0.426581-3.6281290 .810563$

C $-0.385123 \quad 5.603391-2.162703$

O $-0.1458344 .183232-2.090225$

C $0.8149093 .929816-1.042974$

C $1.4765555 .270377-0.764321$

C $0.3095406 .221887-0.948696$

H $0.231643 \quad 3.603418-0.173104$

C $1.7582302 .811036-1.494267$

H 1.9167025 .3245910 .235873

H $2.2627975 .478334-1.500668$ 
C $0.7233637 .668859-1.150094$ H $-0.3243146 .157388-0.056172$ C $2.9013612 .530150-0.499610$ O $\quad 0.969327 \quad 1.628427-1.659799$ H $2.1780903 .048655-2.479156$ H $3.4927513 .444228-0.375123$ $\begin{array}{llll}\text { O } & 2.348786 & 2.219798 & 0.780942\end{array}$ C $3.8584861 .400563-0.936947$ C 5.0205211 .2241740 .060904 H $4.2716801 .638632-1.924431$ O $3.1586600 .168165-1.049519$ H 5.5505002 .1798460 .152254 H 4.6080410 .9666991 .044700 H $1.2672708 .038638-0.274746$ H $1.3749987 .782965-2.022914$ H $-0.1542678 .306276-1.297854$ C -1.887956 $5.866329-2.301360$ H $0.2543151 .840585-2.284213$ H 1.8410311 .3952660 .685755 H $2.4812200 .272148-1.738472$ H $-2.2580775 .338464-3.189794$ H -2.066752 $6.934111-2.473197$ H $0.1049085 .941751-3.085073$

C $6.140148-2.302789-0.503467$ O $5.381629-1.144242-0.137544$ C $6.0133630 .120582-0.358019$ C $7.320357 \quad 0.2009220 .445621$ C $8.243946-0.9804330 .130946$ C $7.502894-2.3191870 .207836$ H $5.555237-3.149160-0.119880$ C $6.240596-2.454930-2.024883$ H $6.2325550 .239449-1.424662$ O $7.041853 \quad 0.226017 \quad 1.844445$ H 7.8273241 .1422340 .204062 H $9.078875-0.9889280 .842136$ O $8.787465-0.830463-1.177219$ H $8.119751-3.126356-0.204279$ O $7.260904-2.6365381 .582164$ C $4.879210-2.735779-2.617993$ H $6.665305-1.573301-2.513281$ H $6.886099-3.299522-2.292956$ H $6.520342-0.5670692 .056559$ H $9.2795740 .008000-1.196454$ H $8.124565-2.7013592 .024260$ O $4.428817-1.643277-3.297425$ O $4.281503-3.795261-2.477828$ C $3.134220-1.778471-3.892227$ H $2.386897-2.023947-3.131763$ H $2.867785-0.820610-4.346760$ H $3.157357-2.544924-4.672516$ SCF Energy $\left(B 3 L Y P / 6-31 G^{* *} / / M M F F\right)=-3245.93482590$

08_00240

MM̄FF Geometry

C $2.311409-0.012443 \quad 3.269264$

C $2.939045-1.1830813 .462088$

C $4.100051-1.7406842 .674089$

O $4.634757-0.734897 \quad 1.787425$

C $3.617433-2.9264091 .820249$

C $4.732574-3.8471201 .370629$

C $5.239447-3.8924270 .123457$

C $5.233751-4.8051972 .421376$

C $4.902552-3.017131-1.051263$

C $4.074736-3.750746-2.116150$

C $2.575215-3.801065-1.782112$

C $1.817935-4.640346-2.812851$

C $0.299439-4.598153-2.582150$

O $2.092349-2.454014-1.800584$

O $-0.339409-5.135441-3.750114$

C $-0.144361-3.125605-2.461453$

C $-0.126989-5.485070-1.406463$

O $-1.523892-3.035660-2.115044$

C $0.707233-2.302449-1.467252$

C $0.357810-0.799591-1.595908$

O $0.432898-2.740224-0.136411$

C $5.981857-0.5983021 .700416$

O $6.824308-1.2036882 .348491$

C 6.3571420 .4161700 .689091
C $5.4876310 .936034-0.190139$

C $5.8294661 .900637-1.228624$

C $5.2570071 .555260-2.576409$

C $1.2857500 .128186-0.799042$

C $1.0347421 .595835-1.100654$

C $1.8534163 .867611-0.464797$

O $0.1936141 .983163-1.907016$

C $2.9152904 .304881-1.424033$

C $3.8975835 .161096-1.103998$

C $4.9310235 .649709-2.078608$

C $6.3467075 .420864-1.598482$

C $7.0406666 .403435-0.999504$

C $6.9870504 .084149-1.896804$

C $6.5446043 .007644-0.946349$

N $1.8689632 .421015-0.357929$

H 2.6244480 .6556892 .472530

H $2.584543-1.8284134 .264864$

H $4.856774-2.0539743 .402850$

H $3.066963-2.5438940 .953896$

H $2.895037-3.5350922 .381114$

H $6.016207-4.627612-0.084813$

H $5.999361-5.4856082 .033632$

H $5.674902-4.2601533 .261368$

H $4.409745-5.4194562 .799166$

H $4.412776-2.088139-0.746218$

H $5.854521-2.710647-1.503776$

H $4.204230-3.209798-3.062950$

H $4.465341-4.764831-2.266043$

H $2.442385-4.223630-0.780375$

H $2.178299-5.676571-2.811016$

H $2.024623-4.253743-3.820849$

H -0.011312 -6.041636 -3.880935

H $-0.045925-2.672793-3.458093$

H $\quad 0.154579-6.528551-1.592527$

H -1.216149 -5.485757 -1.286932

H $\quad 0.324098-5.187026-0.457699$

H -2.022331 -3.553003 -2.770537

H $0.422254-0.511557-2.653390$

H $-0.675599-0.623541-1.271317$

H $0.633033-2.0222170 .484010$

H 7.4070240 .6938480 .673092

H $4.4509750 .613115-0.195642$

H $5.7324260 .649208-2.968054$

H $5.3881192 .344648-3.319471$

H $4.1789051 .371371-2.505026$

H $1.147151-0.0252710 .275543$

H $2.331571-0.082273-1.045563$

H 2.0106644 .2697960 .540912

H $0.8760994 .201903-0.826170$

H $2.8527983 .922018-2.439989$

H $3.9423575 .564061-0.094689$

H $4.7600016 .723322-2.232588$

H $4.8016315 .188299-3.065515$

H $8.0669616 .257095-0.679642$

H $6.5952647 .374706-0.810585$

H $8.0791654 .163389-1.809014$

H $6.8124913 .825588-2.945276$

H $6.8750773 .163476 \quad 0.081227$

H 2.5622552 .0021010 .254523

C $-0.1690390 .333139 \quad 3.343284$

O $-0.281541 \quad 1.4351162 .419429$

C -1.6441371 .9228272 .440338$

C -2.4390710 .9004663 .237830$

C $-1.404651 \quad 0.4249514 .236009$

H -1.610395 2.8771312 .981938

C -2.1136372 .1498970 .998458$

H $-3.325091 \quad 1.3326653 .712181$

H $-2.762610 \quad 0.0705042 .598628$

C - $-1.779527-0.8788304 .919149$

H $-1.2621151 .204306 \quad 4.996779$

C -3.6319712 .3823000 .862760$

O -1.3906243 .2851590 .518086$

H $-1.814219 \quad 1.2954300 .379599$

H -4.1373001 .4940961 .250554$

O $-4.0273663 .476597 \quad 1.689072$

C $-4.1116912 .625720-0.583003$

C $-5.6419222 .768758-0.718922$

H $-3.7573021 .805452-1.218175$ 
O $-3.5214353 .827792-1.082521$

H $-5.9898493 .630338-0.135796$

H -5.848864 $3.013893-1.769011$

H -2.716153 -0.765902 5.474835

H -1.914713 -1.687642 4.194100

H -1.003779 -1.188210 5.626404

C 1.1476690 .4328614 .113859

H $-1.5775903 .372701-0.431840$

H -3.5732224 .2696141 .355288$

H $-3.7700363 .910724-2.018941$

H $1.098616-0.1767105 .023744$

H $1.330828 \quad 1.4676494 .427576$

H $-0.200452-0.5887072 .746881$

C $-6.520562-0.850976-0.877013$

O $-5.9801210 .443596-1.167444$

C $-6.4245361 .507998-0.321689$

C $-7.9464951 .681087-0.439584$

C $-8.6644390 .361109-0.131760$

C $-8.059364-0.817179-0.903914$

H -6.186643 -1.487640 -1.706797

C $-5.938745-1.4356950 .420365$

H $-6.188991 \quad 1.2721310 .719346$

O $-8.3103282 .095593-1.753849$

H -8.2809852 .4563430 .260306$

H $-9.7255670 .452294-0.394633$

O

H -8.452531-1.763305 -0.514990

O $-8.480537-0.736329-2.269453$

C $-4.453007-1.713910 \quad 0.303154$

H $-6.075701-0.7714351 .277100$

H $-6.436718-2.3894080 .631948$

H -8.048494 $3.026272-1.854170$

H $-9.0040620 .822411 \quad 1.734865$

H -8.097461 $0.072042-2.651212$

O $-4.065926-2.5064961 .346455$

O $-3.720501-1.281693-0.575960$

C $-2.682400-2.8728491 .362617$

H $-2.477807-3.3868132 .305405$

H $-2.471043-3.5583600 .537170$

H -2.044855 -1.985748 1.302252

SCF Energy $\left(B 3 L Y P / 6-31 G^{* *} / / M M F F\right)=-3245.91066370$

08_00241

MM̄MF Geometry

C 1.9618530 .4219213 .250339

C 3.2926870 .4767643 .414165

C 4.3073040 .9311052 .391257

O 3.6970851 .7392541 .360315

C $4.949344-0.2778961 .689057$

C $6.394125-0.0306271 .306054$

C 6.8130560 .4438400 .117253

C $7.399198-0.3959692 .368697$

C $5.983914 \quad 0.915981-1.043787$

C $6.075329-0.017162-2.258015$

C $5.134690-1.227124-2.152654$

C $5.348049-2.177062-3.333036$

C $4.337845-3.333579-3.328324$

O $3.792607-0.726930-2.162643$

O $4.401534-3.980971-4.608192$

C $2.916923-2.750480-3.186997$

C $4.691946-4.399211-2.284563$

O $1.951761-3.789783-3.037466$

C $2.777912-1.729014-2.033955$

C $1.403447-1.024042-2.126794$

O $2.871095-2.420496-0.786989$

C 3.4617503 .0504811 .625930

O 3.6952263 .6514832 .661165

C 2.8261253 .6221340 .419755

C 2.0919054 .7384980 .523626

C $1.3566015 .387434-0.564288$

C $1.5062154 .811660-1.948791$

C $1.2362070 .173166-1.178021$

C $-0.1759910 .733097-1.225674$

C -1.697574 $2.287998-0.011327$

O $-1.0086550 .379694-2.056636$

C $-1.8580393 .481514-0.899912$

C $-2.2639374 .683563-0.462546$

C $-2.4723785 .870678-1.360499$
C -1.761956 $7.114394-0.871777$

C $-2.4353938 .092154-0.242067$

C $-0.2896147 .261697-1.188456$

C $0.5749746 .446400-0.266745$

N $-0.3995011 .676955-0.232700$

H 1.5185080 .6949692 .297185

H 3.7093710 .1804934 .375230

H 5.0696591 .5297262 .906439

H $4.369010-0.5478450 .798871$

H $4.923473-1.1652672 .335884$

H $7.8865500 .547812-0.039155$

H $8.428901-0.1887792 .058814$

H 7.2095390 .1738003 .284110

H $7.332774-1.4633972 .603198$

H $4.9407311 .083589-0.766274$

H 6.374117 1.899938 -1.334126

H $5.7950070 .567606-3.144057$

H $7.110483-0.349926-2.403384$

H $5.322609-1.747851-1.207323$

H $\quad 6.374695-2.564097-3.340122$

H $5.231945-1.621191-4.274188$

H $5.313999-4.289613-4.742723$

H $2.678356-2.240336-4.130967$

H $5.683571-4.820513-2.489344$

H $3.993939-5.242401-2.328563$

H $4.703345-4.012972-1.263321$

H $2.065163-4.400155-3.786157$

H $1.255930-0.652361-3.149601$

H $0.606511-1.751798-1.930696$

H $2.357789-1.946280-0.115002$

H $2.9473043 .083143-0.512792$

H 1.9954295 .2069691 .502299

H $2.5517774 .863086-2.272727$

H $0.9156165 .330426-2.706054$

H $1.1849863 .764762-1.971075$

H $1.451650-0.117537-0.145273$

H $1.9258950 .975879-1.460273$

H -1.7593102 .5490071 .049905$

H -2.476757 $1.553550-0.237055$

H -1.660669 $3.343710-1.960436$

H -2.4934264 .8145950 .592564$

H -3.553062 $6.056707-1.416355$

H -2.155793 $5.654976-2.388650$

H -1.939041 8.9991690 .086845

H -3.497147 $8.004945-0.036400$

H $0.0167328 .310616-1.078077$

H -0.126943 $7.034487-2.245235$

H $\quad 0.535810 \quad 6.784546 \quad 0.770000$

H $\quad 0.365594 \quad 1.951067 \quad 0.376345$

C $0.478580-1.3972074 .171280$

O $-0.345338-1.4796332 .992305$

C -1.539449 -2.219078 3.305422

C $-1.288365-2.8721804 .658739$

C $-0.411929-1.8382175 .333482$

H $-2.342273-1.4785363 .405626$

C $-1.906656-3.2116742 .191949$

H -2.217120 -3.070452 5.202427

H $-0.747396-3.8202874 .557149$

C $0.352226-2.3786756 .529992$

H -1.045225 -1.002304 5.660083

C $-2.177165-2.5181010 .839621$

O $-0.850981-4.1631612 .064414$

H -2.806564 -3.755885 2.503700

H -2.871822 -1.691631 1.021244

O $-0.971634-1.9472290 .339337$

C $-2.785664-3.446464-0.231155$

C $-3.018467-2.773577-1.598472$

H $-3.732248-3.8490170 .148637$

O $-1.916008-4.560450-0.445437$

H -2.055026 -2.507013 -2.051254

H $-3.452637-3.535178-2.259912$

H $-0.340441-2.7395397 .297323$

H $1.003924-3.2120536 .246735$

H $0.976954-1.6005106 .979308$

C 1.0105130 .0257394 .344826

H $-1.126016-4.822347 \quad 1.405457$

H -0.336502 -2.673077 0.213251

H $-2.352801-5.158303-1.075945$ 
H 1.5023360 .1231825 .319982 H $0.179616 \quad 0.742214 \quad 4.333868$ H $\quad 1.308327-2.1022584 .030541$ C $-6.141233-0.890107-0.786487$ O $-5.180971-1.938729-0.974915$ C -3.936394-1.542887-1.557355 C $-4.171581-0.947246-2.953821$ C $-5.1720170 .212451-2.895502$ C $-6.434453-0.163349-2.111402$ H -7.061493-1.404921 -0.480755 C -5.7352250 .0567310 .354272$ H -3.463111 $-0.784556-0.927356$ O $-4.655494-1.946224-3.849478$ H -3.216553 - $0.594262-3.357833$ H $-5.4531180 .501545-3.915491$ O $-4.565893 \quad 1.342152-2.274544$ H $-7.0436590 .726075-1.912240$ O $-7.229925-1.044460-2.910903$ C $-5.717060-0.6409501 .699392$ H -4.7415910 .4822420 .195563$ H -6.4623430 .8742060 .422740$ H -5.463086 - $2.326250-3.462798$ H $-3.7718271 .569859-2.787546$ H $-7.464932-0.568502-3.725641$ O $-5.338043 \quad 0.2571142 .656928$ O $-6.001487-1.8147851 .892865$ C $-5.294518-0.2485753 .995212$ H $-6.310805-0.4407214 .351383$ H -4.690317 -1.158912 4.048555 H $-4.8353660 .512803 \quad 4.631441$

SCF Energy (B3LYP/6-31G**//MMFF) $=-3245.92145390$

08_00242

MMFF Geometry

C -0.964036 -0.6565862 .822884$

C $-0.222600-1.4970262 .084168$

C $-0.685474-2.403236 \quad 0.972737$

O $-0.261333-1.759329-0.251957$

C -2.203770 $-2.648960 \quad 0.893325$

C $-2.556315-3.8613920 .053027$

C $-3.027719-3.819424-1.208094$

C $-2.387411-5.1907230 .745355$

C $-3.268216-2.614014-2.073750$

C $-4.760533-2.412337-2.372852$

C $-5.550711-1.893490-1.161805$

C $-7.050619-1.877435-1.460275$

C $-7.853225-1.267993-0.301359$

O $-5.094278-0.565517-0.886792$

O $-9.183799-1.008622-0.774151$

C $-7.231390 \quad 0.093356 \quad 0.070290$

C $-7.992289-2.240907 \quad 0.875417$

$\begin{array}{lllll}\text { O } & -7.850485 & 0.635837 & 1.234971\end{array}$

$\begin{array}{llll}C & -5.696122 & 0.046977 & 0.260182\end{array}$

C $-5.146986 \quad 1.489322 \quad 0.367936$

O $-5.391156-0.6665101 .459099$

C $0.365408-2.490543-1.205133$

O $0.635095-3.682245-1.156586$

C $0.735198-1.646931-2.367400$

C $0.308274-0.384121-2.525781$

C $0.6596770 .526668-3.617583$

C $1.6088830 .022164-4.671773$

C -3.6155351 .5913010 .354668$

$\begin{array}{llll}\text { C }-3.148944 & 3.037119 & 0.332777\end{array}$

C $-1.071043 \quad 4.3994610 .154089$

O

C $-0.8234454 .715341-1.287275$

C $0.3948464 .753445-1.848056$

C $0.641498 \quad 5.099552-3.288048$

C $1.2663043 .962659-4.065314$

C $2.5807513 .953406-4.344016$

C $0.3514772 .881859-4.597501$

C $0.1380631 .771760-3.604802$

N $-1.767473 \quad 3.1304930 .243460$

H -2.030891 -0.5618252 .648859$ H $0.848523-1.5368762 .279961$

H $-0.156278-3.3541771 .110043$

H -2.705847 -1.7554780 .506095$

H $-2.616575-2.8156011 .896922$
H -3.259775 $-4.765374-1.696740$

H $-2.700739-6.0308930 .116370$

H $-1.340204-5.356647 \quad 1.014916$

H $-2.991297-5.2220581 .658123$

H $-2.834827-1.702172-1.653156$

H $-2.743229-2.783323-3.022439$

H $-4.840305-1.684283-3.190615$

H $-5.195456-3.353377-2.732202$

H $-5.348010-2.533670-0.296494$

H $-7.413506-2.887449-1.688379$

H $-7.237424-1.280231-2.363985$

H $-9.562610-1.850753-1.079869$

H $-7.4564680 .788036-0.751294$

H $-8.495618-3.1608030 .554166$

H -8.620964 -1.8165321 .666133$

H $-7.033225-2.5255681 .312921$

H $-8.808397 \quad 0.6612581 .068512$

H $-5.5253522 .076309-0.479607$

H $-5.521271 \quad 1.9627101 .284746$

H $-4.517588-0.3921221 .778331$

H $1.383985-2.128268-3.091703$

H $-0.3565690 .043296-1.776635$

H $1.8705310 .772689-5.420436$

H $1.170055-0.825689-5.209076$

H $2.552413-0.299615-4.218503$

H $-3.191032 \quad 1.118122 \quad 1.245147$

H $-3.207122 \quad 1.092284-0.531435$

H $-0.146007 \quad 4.305467 \quad 0.727319$

H $-1.675178 \quad 5.1911210 .608692$

H -1.696160 4.938155 -1.896863

H $1.2704314 .542071-1.240120$

H $1.2988165 .978645-3.310674$

H $-0.2829245 .416030-3.787180$

H $3.0285223 .154921-4.926194$

H $3.2389234 .739672-3.989611$

H $0.7309592 .504936-5.552755$

H $-0.6242253 .316842-4.849099$

H $-0.5381632 .038657-2.792519$

$\begin{array}{llll}H & -1.217938 & 2.281449 & 0.144473\end{array}$

C $-0.1044261 .634073 \quad 3.446642$

\begin{tabular}{lllll}
\hline & 0.869376 & 1.602135 & 2.381974
\end{tabular}

C $2.1181832 .068998 \quad 2.906794$

C 1.7031843 .1468723 .891517

C 0.5134742 .4850164 .569061

H $2.571556 \quad 1.2252563 .442573$

C 3.0887372 .5051751 .800310

H $2.5015013 .409178 \quad 4.591719$

H 1.3802294 .0584793 .375448

C $-0.4654863 .479091 \quad 5.173019$

H $0.885037 \quad 1.8383535 .375170$

C 3.2293991 .4519350 .679013

$\begin{array}{lllll}\text { O } & 2.677177 & 3.750227 & 1.244604\end{array}$

H 4.0669392 .6767872 .264577

H 3.3453450 .4723161 .154102

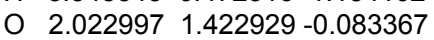

C $4.4282381 .705102-0.256190$

C $4.5906680 .679652-1.394128$

H $\quad \begin{array}{lllll}\text { H } & 4.348717 & 1.717706 & 0.339889\end{array}$

$\begin{array}{lllll}\text { O } & 4.320444 & 2.985235 & -0.883402\end{array}$

H $3.8011170 .821907-2.143193$

H $5.5255400 .935616-1.910617$

H $\quad 0.024542 \quad 4.083774 \quad 5.943108$

H $-0.8655384 .159573 \quad 4.413687$

H -1.3090172 .9569185 .636263$

C $-0.378903 \quad 0.1981993 .913287$

H 1.8605953 .5807670 .746317

H $2.0536330 .639500-0.655865$

H $4.3120543 .659280-0.183133$

H -1.0727540 .2043094 .762184$

H $0.552060-0.2611584 .269693$

H -1.0159382 .0876693 .040917$

C $5.735409-2.2416570 .647290$

O $5.609756-0.931768 \quad 0.076156$

C $4.624916-0.793693-0.951565$

C $4.926975-1.740446-2.124865$

C $5.080095-3.187204-1.644154$

C $6.025098-3.288385-0.442846$

H $6.621848-2.1826481 .292540$ 
C $4.531325-2.6060201 .532956$

H $3.641456-1.046192-0.544003$

O $6.126275-1.329064-2.778146$

H $4.113359-1.668279-2.855344$

H $5.470205-3.806428-2.461066$

O $3.808340-3.709635-1.274137$

H $5.981292-4.292190-0.004291$

O $7.369968-3.085166-0.885643$

C $4.489622-1.7965972 .813043$

H $3.576280-2.4522451 .024572$

H $4.610873-3.6605591 .823043$

H $6.270254-1.925991-3.531884$

H $3.235658-3.674053-2.059080$

H $7.570477-3.775331-1.540500$

O $3.545558-2.3347693 .642052$

O $5.181340-0.8176943 .055951$

C $3.403616-1.6978224 .915483$

H $3.157851-0.6398894 .791126$

H $2.584363-2.1860625 .450039$

H $4.322749-1.8140435 .497310$

SCF Energy $\left(B 3 L Y P / 6-31 G^{* *} / / M M F F\right)=-3245.89698613$

08_00243

MMFF Geometry

C $1.268719-3.9789430 .334848$

C $1.460611-3.3823771 .522224$

C $0.475355-2.5166372 .271877$

O $-0.715593-2.293131 \quad 1.485210$

C $1.099179-1.1414172 .571850$

C $0.274414-0.3314873 .552374$

C $-0.5202190 .704336 \quad 3.220657$

C $0.421873-0.7319254 .998530$

C $-0.7949631 .262851 \quad 1.852488$

C -0.1993972 .6693651 .719371$

C -0.4724573 .2911590 .343103$

C $0.206803 \quad 4.6569010 .230299$

C $-0.1188365 .345808-1.102214$

O $-1.884906 \quad 3.4422320 .197970$

O $\quad 0.2750636 .722897-0.996675$

C $-1.6489615 .332670-1.315688$

C $0.6988974 .750987-2.254957$

O $-1.9855825 .809589-2.616291$

C $-2.3055893 .952552-1.076102$

C -3.842855 $4.090000-1.012715$

O $-2.0166623 .037782-2.134963$

C $-1.852242-2.9530471 .833528$

O -2.002358 -3.732669 2.759840

C $-2.898223-2.5628530 .860371$

C $-4.118500-3.1172320 .925819$

C $-5.239925-2.843898 \quad 0.020887$

C $-5.014091-1.824362-1.065526$

C $-4.5484052 .742661-0.814946$

C $-6.0265452 .888278-0.498169$

C -8.0093491 .4774510 .057320$

O $-6.6051873 .967798-0.428550$

C $-8.153908 \quad 0.1804370 .786828$

C $-8.971500-0.8119880 .405092$

C $-9.065865-2.1222501 .135173$

C $-8.906564-3.3212910 .223616$

C $-9.863895-4.262803 \quad 0.162957$

C $-7.655323-3.439379-0.625222$

C $-6.393127-3.5260210 .191043$

N $-6.6094831 .648697-0.279204$

H $\quad 0.316827-3.871609-0.175306$

H $2.415043-3.5316132 .024058$

H $0.234219-3.0283683 .211066$

H $1.243261-0.5872891 .637292$

H $2.104144-1.2646862 .996648$

H -1.051863 1.2168484 .021989

H $-0.141988-0.0772445 .671521$

H $0.061175-1.7527485 .156240$

H $1.473588-0.6831625 .299795$

H $-0.4089030 .619056 \quad 1.056947$

H -1.882464 1.2984741 .715292

H $-0.630518 \quad 3.3111722 .499423$

H 0.8818462 .6238851 .894642

H -0.102903 $2.608679-0.429203$

H 1.2922294 .5653410 .359358
H $-0.1378815 .305146 \quad 1.048218$

H $1.2224806 .748105-0.778234$

H -2.083690 $6.045688-0.601100$

H $1.7718554 .890498-2.075326$

H $0.4845745 .263674-3.198948$

H $\quad 0.5284203 .681669-2.393117$

H -1.576632 $6.686144-2.718090$

H $-4.1112854 .758476-0.184578$

H -4.224092 $4.542365-1.936768$

H -1.517730 $3.504217-2.824157$

H $-2.627124-1.8344890 .104120$

H $-4.316801-3.8483781 .708417$

H $-4.678897-0.873702-0.637841$

H $-5.905259-1.600678-1.654356$

H -4.249779 -2.178036-1.766611

H $-4.4514102 .134335-1.721933$

H -4.0890252 .1925320 .014765$

H $-8.5803831 .501354-0.876455$

H -8.3362262 .3112870 .686620$

H $-7.5396310 .047391 \quad 1.675041$

H $-9.602691-0.679156-0.470222$

H -10.042015 -2.150715 1.636217

H -8.314359 -2.183062 1.932148

H $-9.768684-5.127125-0.485970$

H $-10.766179-4.1927780 .761459$

H -7.644223 -2.608698 -1.334730

H -7.703301-4.349482 -1.237785

H $-6.440114-4.2665310 .990867$

H $-6.0823730 .820862-0.538963$

C $2.707851-4.316424-1.741049$

O $3.104578-2.930945-1.686310$

C $2.492570-2.240142-2.790765$

C $2.066574-3.325325-3.772180$

C $1.625889-4.418877-2.818233$

H $1.589065-1.757836-2.398859$

C $3.436781-1.182678-3.370450$

H $1.268880-2.991477-4.442467$

H $2.906921-3.671480-4.385261$

C $1.527448-5.789702-3.464920$

H $0.643087-4.148366-2.414209$

C $3.929642-0.136230-2.347421$

O $4.585118-1.850378-3.907226$

H $2.945123-0.684052-4.214874$

H $4.662420-0.593361-1.673161$

O $4.624644 \quad 0.888333-3.069039$

C $2.8127270 .529459-1.523900$

C $3.2496231 .758247-0.705252$

H $2.371556-0.213008-0.849120$

O $1.7769550 .954573-2.415248$

H $3.4922742 .589932-1.379318$

H $2.3675012 .088438-0.143091$

H $0.785991-5.782242-4.270656$

H $2.485654-6.101721-3.893812$

H $1.223468-6.544453-2.732884$

C $2.316953-4.817548-0.346899$

H $5.077381-1.205012-4.441618$

H $5.4431040 .497888-3.418704$

H $\quad 1.0311791 .260444-1.872245$

H $3.222314-4.8405560 .272842$

H $1.955332-5.849983-0.412496$

H $3.598029-4.873029-2.062916$

C 5.0622350 .0323462 .070540

O $4.0605280 .429698 \quad 1.124166$

C 4.4157321 .5152450 .263914

C 4.7473152 .7738021 .084885

C 5.8116912 .4838162 .147629

C 5.4836541 .2211872 .952005

H $4.563977-0.7024372 .715273$

C $6.251428-0.6688631 .393531$

H $5.2944821 .235764-0.325305$

O $3.566044 \quad 3.257734 \quad 1.720181$

H 5.0982503 .5567510 .401653

H 5.8820543 .3410892 .828466

O $7.0726162 .340707 \quad 1.501152$

H $6.3404860 .937476 \quad 3.574275$

O 4.4069861 .5332693 .841150

C $5.849266-1.9708340 .735340$

H $6.702433-0.0393180 .622365$ 
H $7.015239-0.9073272 .142345$

H 3.8012604 .0699182 .200178

H 7.7398232 .1838232 .190924

H $4.231997 \quad 0.744067 \quad 4.381433$

O $6.881926-2.413846-0.041214$

O $4.775902-2.535368 \quad 0.889281$

C $6.648697-3.640669-0.740387$

H $\quad 6.303835-4.420750-0.054636$

H $5.920606-3.478172-1.539626$

H $7.593706-3.961846-1.186604$

SCF Energy $\left(\mathrm{B} 3 L Y P / 6-31 G^{* *} / / \mathrm{MMFF}\right)=-3245.90221788$

\section{8_00244}

MMFF Geometry

C $-2.686104 \quad 4.124755-1.283397$

C $-3.0119862 .935836-0.755363$

C $-3.5872492 .773056 \quad 0.624598$

O $-2.6366231 .994922 \quad 1.389397$

C -4.9058541 .9884780 .574029$

C -5.6748182 .0656661 .877901$

C -5.7232651 .0872762 .802463$

C -6.4660413 .3327912 .081668$

C $-5.013320-0.2381112 .790598$

C $-5.982791-1.4118652 .589364$

C $-6.505473-1.5219211 .148376$

C $-7.569885-2.6141061 .038821$

C $-8.033419-2.817458-0.411167$

O $-5.392336-1.844247 \quad 0.309709$

O $-8.788755-4.038085-0.433175$

C $-6.791752-3.000952-1.309834$

C $-8.986760-1.704475-0.862185$

O $-7.155218-3.038008-2.688341$

C $-5.700384-1.925562-1.087117$

C $-4.407163-2.337416-1.830356$

O $-6.162012-0.675302-1.600443$

C -1.674407 2.6660652 .072017

O -1.5674443 .8800372 .181684$

C -0.7161781 .7383602 .714699$

C -0.6035690 .4417992 .385630$

C $0.334217-0.5182522 .973072$

C $1.231387-0.0325814 .081563$

C $-3.185742-1.458417-1.521981$

C -1.919105 -2.029205-2.138363

C $0.559228-1.815443-2.032182$

O $-1.924276-2.954663-2.946421$

C $1.015613-2.807546-1.008973$

C $2.063664-2.614036-0.194749$

C $2.512866-3.6130260 .831816$

C $2.519228-3.0510862 .237115$

C $3.676391-2.7553972 .852365$

C $1.201609-2.9322072 .971018$

C $0.353834-1.7813772 .497585$

N $-0.780502-1.375746-1.685832$

H -2.876132 $5.032485-0.716592$

H -2.801013 2.027826-1.315420

H -3.7472053 .7487251 .099418$

H -4.7038830 .9446340 .311234$

H $-5.5511712 .376916-0.225174$

H -6.3338851 .2526873 .689476$

H -7.043259 3.3182943 .012534

H -5.8019264 .2014902 .121512$

H -7.174519 3.4736321 .258851

H -4.207050 -0.2731252 .052140$

H -4.524558 -0.3542553 .766369$

H -5.443892 -2.333732 2.844146

H $-6.822909-1.3227413 .289283$

H -6.924704 $-0.557550 \quad 0.842279$

H -8.426438 -2.3905591 .687043$

H -7.156014 -3.5635601 .407206$

H $-9.139448-4.160797-1.331362$

H $-6.361235-3.984479-1.074098$

H -9.880346 -1.688019-0.226759

H -9.347274 -1.880589-1.881671

H $-8.534487-0.711092-0.825830$

H -7.753565 -3.793083 -2.815772

H -4.152861 -3.368553 - 1.550055

H $-4.580262-2.328112-2.914080$

H $-5.397492-0.123715-1.828406$
H $-0.0727832 .198213 \quad 3.457972$

H $-1.2502330 .037150 \quad 1.608733$

H 1.8836440 .7717623 .723273

H $1.884794-0.8092204 .483952$

H $\quad 0.6370600 .3428824 .922017$

H $-3.325522-0.448301-1.920096$

H -3.033517 - $1.385711-0.439143$

H $1.200383-0.930381-2.068606$

H $\quad 0.552247-2.280332-3.023241$

H $\quad 0.453232-3.735808-0.938745$

H $2.635423-1.693573-0.272882$

H $3.520999-3.9448420 .550832$

H $1.888906-4.5152940 .810954$

H $3.699954-2.3887703 .872742$

H $4.628984-2.8777212 .350576$

H $1.361210-2.8687634 .054085$

H $\quad 0.633170-3.8619072 .837160$

H $-0.329352-2.0436921 .689510$

H $-0.871627-0.670448-0.960084$

C $-0.4874444 .442646-2.499497$

O $0.0810793 .260830-1.898508$

C $1.0263213 .665241-0.884268$

C $1.3533965 .117171-1.195952$

C $0.0081925 .630393-1.673145$

H $0.496133 \quad 3.5895150 .072883$

C $2.2158062 .699558-0.907163$

H $1.7304335 .662429-0.325807$

H $2.0986725 .185463-1.998155$

C $0.0903606 .929101-2.455928$

H $-0.6252245 .790544-0.792425$

C 3.3380673 .0859510 .074704

O $1.7134241 .406692-0.555620$

H $2.6094272 .625736-1.927481$

H $3.5904494 .138609-0.097482$

O 2.8658072 .9969701 .417925

C $4.6584402 .298358-0.067600$

C $4.5532590 .780756 \quad 0.125107$

H $5.3566232 .693248 \quad 0.679125$

O $5.2170792 .551669-1.356485$

H 4.0725610 .5409621 .080890

H $3.9079370 .359693-0.655006$

H $0.5049377 .728271-1.832823$

H $0.7296936 .826480-3.339085$

H $-0.9029647 .242828-2.792456$

C $-2.0073804 .280941-2.615181$

H $0.9750531 .209073-1.157040$

H 2.4870692 .1096091 .540008

H $5.3436553 .512243-1.439177$

H $-2.2215923 .399848-3.233836$

H -2.441026 $5.143498-3.133778$

H $-0.0714814 .493200-3.514073$

C $6.677842-2.272335-0.014305$

O $5.581923-1.3636720 .151089$

C 5.8954270 .0291310 .013136

C 6.9391470 .4391361 .061212

C $8.182241-0.4555730 .991113$

C $7.819085-1.9431100 .962060$

H $\quad 6.270555-3.252760 \quad 0.265464$

C $7.128849-2.358148-1.476316$

H $6.2945790 .201084-0.991918$

O 6.3761350 .3734532 .369715

H 7.2377931 .4790480 .891338

H $8.821974-0.2572321 .859659$

O $8.930884-0.145098-0.180843$

H $8.697501-2.550008 \quad 0.713195$

O $7.393220-2.3400192 .268747$

C $6.049041-2.981515-2.328804$

H $7.396194-1.391149-1.911519$

H $8.018304-2.993006-1.568925$

H $6.045427-0.5310832 .506066$

H $9.1816730 .793007-0.129600$

H $8.135257-2.1860672 .878203$

O $5.196548-2.018085-2.780979$

O $5.964620-4.182962-2.548041$

C $4.114156-2.482922-3.592925$

H $4.497520-2.850918-4.549078$

H $3.552021-3.266497-3.076349$

H $3.445004-1.639428-3.782240$ 
C $4.108350-0.700301-2.659547$

O $6.455647-0.478258-2.735867$

C $1.951806-1.2013402 .139199$

O $0.964605-1.6089822 .730068$

C 2.2771540 .2236141 .909908

C 1.3695131 .1634342 .213682

C 1.5101272 .6067192 .008232

C 2.7751623 .1009051 .358852

C $2.748948-0.437823-2.002751$

C $1.9854530 .737218-2.588244$

C $-0.0812532 .082200-2.217043$

O $2.3247081 .317019-3.615855$

C $0.2814403 .351449-1.512505$

C $-0.6025934 .086088-0.820831$

C $-0.2732625 .384424-0.142549$

C $-0.6313645 .383376 \quad 1.326947$

C -1.8149415 .8580201 .750807$

C 0.4185244 .9146862 .309042

C 0.4994603 .4138482 .393260

N $0.852298 \quad 1.036689-1.842115$

H $0.142401-4.3680441 .126300$

H $2.422043-3.432020-0.695756$

H $2.140037-3.7108452 .391135$

H $4.560693-3.8457270 .517068$

H $3.899825-5.184971 \quad 1.409241$

H $6.614466-2.8651443 .514854$

H $5.253483-4.2476624 .745068$

H $3.563908-4.0674134 .266562$

H $4.471741-5.5273113 .813665$

H $7.684654-2.4909411 .344175$

H $6.191700-2.6679010 .468398$

H $5.397690-0.583276 \quad 1.954779$

H $7.129994-0.3527742 .206853$

H $7.415703-0.352861-0.328051$

H $7.192174 \quad 1.8209020 .935136$

H 5.4495801 .6827070 .870038

H $6.5255293 .919468-0.165990$

H $4.2807031 .809098-1.512336$

H $8.3952792 .831197-1.014599$

H $7.6935592 .792682-2.615494$

H $8.1828541 .278799-1.823955$

H $4.5335351 .754447-3.733321$

H $4.092402-0.457051-3.728811$

H $4.274117-1.786939-2.617854$

H $\quad 6.8767190 .293431-3.146805$

H 3.2373920 .4586821 .467667

H $\quad 0.4226080 .8473292 .648848$

H 3.6348342 .9192312 .012459

H $2.762276 \quad 4.168677 \quad 1.131425$

H 2.9431762 .5912140 .404153

H $2.113802-1.318164-2.162932$

H $2.854612-0.302732-0.923493$

H - $-1.083781 \quad 1.732046-1.951153$

H -0.040002 2.236557 -3.300021

H $1.3096263 .695254-1.596602$

H $-0.8287206 .179415-0.657602$

H $0.7861185 .642625-0.262778$

H -2.068019 5.8864062 .805394

H -2.560200 6.2213851 .050965

H 0.1793035 .2813123 .316545

H 1.3766045 .3836162 .067903

H -0.3798522 .9596062 .851153$

H $\quad 0.6775120 .532762-0.977499$

C $-1.215758-3.323985-1.227898$

O $-2.370254-3.773831-0.486725$

C $-3.554946-3.548562-1.287178$

C $-3.108778-2.657806-2.437156$

C $-1.695178-3.147040-2.666862$

H $-3.853365-4.535542-1.662522$

C $-4.656969-2.955943-0.399763$

H $-3.743107-2.757454-3.322647$

H $-3.103529-1.602762-2.134532$

C $-0.852748-2.186077-3.488342$

H -1.733614 -4.120521 -3.174150

C $-5.867534-2.392419-1.168552$

O $-5.098680-3.9742410 .503569$

H $-4.211125-2.1800170 .227258$

H $-5.554590-1.601026-1.858395$

O $-6.438971-3.430682-1.975908$

C $-7.002555-1.857117-0.276444$

C -6.596713 -0.8346090 .792059$

H -7.502755 -2.695547 0.224239

O $-8.000179-1.260779-1.111586$

H -7.502897 -0.4268491 .260082$

H $-6.075929-1.3644591 .601358$

H -1.282991 -2.054936 -4.486590

H $-0.796470-1.199848-3.016354$

H $\quad 0.168230-2.561331-3.607848$

C $-0.082045-4.342056-1.088050$

H $-5.546743-4.659011-0.022054$

H $-5.796850-3.659223-2.668336$

H -8.266235 -1.928203 -1.767109

H $0.611846-4.251877-1.932133$

H $-0.478860-5.364153-1.100990$

H $-0.924571-2.352508-0.805564$

C -4.4482032 .1246481 .411237$

O $-5.406161 \quad 1.068744 \quad 1.548591$

C -5.7007740 .3383950 .348162$

C $-6.3110991 .285344-0.691787$

C $-5.3805422 .477251-0.956274$

$\begin{array}{llll}\text { C } & -4.894932 & 3.134228 & 0.342433\end{array}$

H -4.4579042 .6364052 .382635$

C -3.0333831 .5746691 .196285$

H -4.767352 -0.071020 -0.045382

O

H $-6.4774830 .742074-1.628076$

H $-5.9139293 .216971-1.565603$

O $-4.2639642 .018858-1.714680$

H -4.0773063 .8354510 .141062$

O -5.9696903 .8960140 .901562$

C $-2.660621 \quad 0.6366752 .320981$

H $-2.926674 \quad 1.047706 \quad 0.243635$

H -2.298696 2.3854581 .184937

H $-7.9494092 .323803-0.915374$

H $-3.7029522 .789670-1.905682$

H -6.219844 4.5726430 .249670

O $-2.575648-0.639697 \quad 1.851006$

O $-2.503991 \quad 0.9935013 .481397$

C $-2.333816-1.6596872 .825413$

H $-1.934944-2.5359102 .307301$

H -1.607042 -1.337374 3.576767

H $-3.279023-1.9321263 .303015$

SCF Energy (B3LYP/6-31G**//MMFF) $=-3245.90458305$

0800246

MMFF Geometry

C $-2.598806 \quad 4.938717-0.252800$

C $-3.116302 \quad 4.654857 \quad 0.952321$

C -4.3636503 .8602801 .250050$

$\begin{array}{llll}\text { O } & -3.988712 & 2.656619 & 1.980242\end{array}$

C $-5.3080513 .541456 \quad 0.071293$

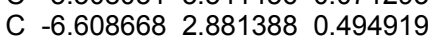


C $-7.0149531 .650607 \quad 0.124130$ C $-7.5102793 .723212 \quad 1.364641$ C $-6.3266700 .660690-0.772775$ C $-5.908825-0.595170 \quad 0.000929$ C $-5.310103-1.668369-0.919022$ C $-4.876620-2.893604-0.112634$ C $-4.201852-3.955788-0.993574$ O $-4.180715-1.113051-1.601778$ O $-3.557893-4.879097-0.101351$ C $-3.110977-3.280138-1.855541$ C $-5.234001-4.765416-1.787413$ O $-2.582501-4.191133-2.815301$ C $-3.595842-1.999207-2.569196$ C $-2.463182-1.224709-3.290122$ O $-4.551219-2.357593-3.577180$ C -3.189022 1.7194031 .400050 O -2.7628321 .7102380 .256523$ C -2.9042680 .6853912 .421515$ C -2.235770 -0.4251192 .074665$ C $-1.898650-1.5421912 .960016$ C $-2.186270-1.3761604 .429455$ C $-1.269906-0.824341-2.416726$ C $-0.156259-1.859104-2.408740$ C $1.795689-2.606300-1.056811$ O $-0.024552-2.710363-3.284372$ C $1.331926-3.631711-0.072848$ C $1.760569-3.6855341 .196765$ C $1.302145-4.7107322 .190705$ C $0.502951-4.1037643 .321510$ C $1.082592-3.8078534 .497088$ C $-0.986713-3.935852 \quad 3.123641$ C -1.348139-2.652435 2.424232 N $0.698072-1.697932-1.328811$ H -3.097919 4.614193 -1.160479 H -2.560379 4.9703671 .834475 H -4.920181 4.4679511 .973763 H $-4.7846372 .928623-0.665550$ H -5.562826 $4.473916-0.450329$ H $-7.979744 \quad 1.3033340 .494125$ H $-8.503898 \quad 3.2786651 .487121$ H -7.0812993 .8451242 .363724$ H -7.6520814 .7135730 .919553$ H -7.030351 $0.381995-1.567136$ H -5.460076 $1.095030-1.276245$ H -5.173112 -0.3105890 .760523$ H $-6.773804-1.0142000 .530563$ H $-6.075710-1.945496-1.653283$ H $-5.727973-3.3268240 .427149$ H $-4.161546-2.5825890 .661085$ H $-3.158820-5.586403-0.635432$ H $-2.290334-3.021108-1.175526$ H -5.913831 -5.288782 -1.104282 H $-4.751239-5.546344-2.385269$ H -5.841965 -4.149511-2.453211 H -2.169897 -4.925202 -2.330228 H -2.138201-1.779783 -4.178520 H -2.901862 -0.292644 -3.674747 H $-4.604407-1.632617-4.221841$ H -3.2669420 .8604083 .428048$ H -1.921472 -0.5417191 .038756$ H $-1.726396-0.4572214 .810616$ H -1.791376 -2.187467 5.044697 H -3.266228 -1.3291204 .606598$ H $-0.8256800 .092164-2.825614$ H -1.592274 -0.602717 -1.394634 H $2.627165-2.012087-0.670657$ H $2.121959-3.093956-1.980754$ H $0.607554-4.362330-0.424871$ H $2.488989-2.9572351 .544601$ H $2.193322-5.2155572 .586380$ H $0.713898-5.5010321 .707756$ H $0.508374-3.4044255 .324319$ H $2.145601-3.9572344 .655643$ H - $1.508155-4.0296324 .082279$ H $-1.372344-4.7741432 .528897$ H - $1.175701-2.6756421 .348310$ H $0.487682-0.991361-0.629705$ C $-0.1668814 .562922-0.631774$ $\begin{array}{llll}\text { O } & -0.087184 & 3.715633 & 0.532939\end{array}$

C 1.2958083 .5146820 .883036

C $2.0945454 .006734-0.314061$

C $1.2301105 .153986-0.803318$

H 1.4810214 .1563371 .754070

C 1.4940262 .0415341 .256818

H $3.1081804 .318430-0.045720$

H $2.1660873 .226405-1.080769$

C $1.5443815 .596488-2.220974$

H $1.3639336 .006558-0.123837$

C $2.967473 \quad 1.6690401 .513350$

$\begin{array}{lllll}\text { O } & 0.704908 & 1.810663 & 2.427548\end{array}$

H 1.0783721 .4051620 .465685

H 3.5301991 .8916640 .602330

O 3.5012462 .5014182 .542493

C 3.1926250 .1886781 .878224

C $4.670491-0.1878062 .099947$

H $2.765045-0.4340151 .086303$

O $2.485877-0.1232903 .080636$

H 5.0668540 .3545552 .967718

H $4.692463-1.2495812 .379973$

H $0.893716 \quad 6.423040-2.523622$

H $2.5819275 .937530-2.296977$

H $1.4051614 .780477-2.937293$

C $-1.2619515 .610313-0.416514$

H $\quad 0.7283690 .8570742 .614886$

H 2.9980062 .3163093 .354173

H $2.579750-1.0781533 .239319$

H $-1.3121616 .300903-1.265317$

H $-1.036734 \quad 6.208796 \quad 0.475328$

H $-0.4204493 .913126-1.479885$

C $5.710260-0.569896-1.467667$

O $5.034434-0.711011-0.211832$

C $5.575130 \quad 0.040438 \quad 0.877407$

C $7.029253-0.3763251 .144112$

C $7.877611-0.258622-0.127434$

C $7.202012-0.926127-1.329527$

H $5.244811-1.320448-2.119735$

C $5.4658490 .809076-2.102421$

H $5.559911 \quad 1.1056580 .630351$

O $7.085609-1.7153311 .631310$

H $7.443498 \quad 0.266822 \quad 1.929095$

H $8.854293-0.7289060 .040893$

O $8.0987101 .115648-0.431192$

H $7.735533-0.667618-2.251700$

O $7.315777-2.342919-1.163640$

C $4.0303380 .965795-2.561640$

H $5.6789681 .638321-1.423828$

H $6.1104900 .907705-2.984159$

H $6.662334-2.290400 \quad 0.970944$

H $8.559565 \quad 1.5133170 .327247$

H $6.953566-2.762410-1.962756$

O $3.981998 \quad 1.839732-3.609337$

$\begin{array}{llll}\text { O } & 3.067397 & 0.410245 & -2.051178\end{array}$

C $2.6828372 .060721-4.168388$

H $2.3460751 .159680-4.689495$

H $1.9663392 .344145-3.391910$

H $2.7576122 .878589-4.889906$

SCF Energy (B3LYP/6-31G**//MMFF) $=-3245.90082340$

08_00247

MMFF Geometry

C -2.745792 $3.702894-0.294508$

C $-2.998791 \quad 4.153111 \quad 0.944144$

C $-3.659621 \quad 3.342476 \quad 2.037047$

\begin{tabular}{llll}
\hline & -3.434878 & 1.927118 & 1.825632
\end{tabular}

C $-5.172202 \quad 3.6062142 .054378$

C $-5.8806292 .901963 \quad 3.194205$

C $-6.6070321 .774798 \quad 3.058543$

C -5.7695803 .5762004 .536820$

$\begin{array}{llll}C & -6.814276 & 0.973435 & 1.802803\end{array}$

C $-6.696412-0.5346892 .068040$

C $-6.898526-1.3418090 .778224$

C $-7.018498-2.8353691 .078183$

C $-7.184999-3.651415-0.212176$

O $-5.766095-1.118080-0.066687$

O $-6.976111-5.0355640 .109916$

C $-6.074860-3.251629-1.208482$ 
C $-8.611634-3.550258-0.765442$

O $-6.294650-3.842859-2.486725$

C $-5.870064-1.723040-1.362558$

C $-4.549050-1.451759-2.112961$

O $-6.903041-1.118079-2.142223$

C $-2.216428 \quad 1.4408162 .193455$

O -1.3163782 .0569682 .742632$

C -2.1409980 .0262691 .765463$

C $-0.940812-0.5725531 .731942$

C $-0.660108-1.9442681 .303590$

C $-1.833161-2.8402731 .010591$

C $-4.1590440 .030358-2.166322$

C $-2.8561520 .211413-2.921152$

C $-0.411314-0.271939-2.753400$

O $-2.8245810 .710657-4.043300$

C $0.171415-1.632976-2.542388$

C $1.350876-1.854618-1.942914$

C $1.920492-3.226796-1.720947$

C $2.292070-3.486913-0.277011$

C $3.576571-3.6361850 .086990$

C $1.173811-3.6546080 .733358$

C $0.628460-2.3330301 .202867$

N -1.760497 $-0.265188-2.219526$

H -3.082373 $2.709633-0.580367$

H -2.674277 5.1531521 .222112

H -3.2225563 .6312803 .001222$

H -5.611781 3.3124321 .093995

H -5.3635044 .6846092 .139055$

H -7.1124181 .3833843 .940312$

H -6.3383083 .0535305 .313239$

H -4.7256873 .6131714 .863367$

H -6.1537024 .5999714 .480699$

H $-7.817646 \quad 1.1935891 .418990$

H $-6.099972 \quad 1.2522051 .023188$

H $-5.705797-0.7499002 .489386$

H -7.446785 -0.8311082 .811481$

H -7.803506 -0.9771220 .278553$

H -7.851407 -3.031745 1.764716

H -6.112314 -3.179689 1.596155

H -7.615447 -5.2830730 .799799$

H -5.137178 -3.680669-0.827225

H -9.332078 -3.946628 -0.039769

H -8.731129 -4.159838-1.667802

H -8.911185 -2.525885 -0.996037

H $-6.385048-4.801782-2.350928$

H -3.739208 -2.007033-1.627444

H -4.619529 -1.828995 -3.141605

H -7.554834 -1.795493-2.380119

H -3.057595 -0.465689 1.461406

H -0.0650840 .0070792 .023501$

H - $-2.535783-2.8458311 .851725$

H -1.552941 -3.8828410 .845636$

H -2.361735 -2.4988920.117004

H $-4.9328800 .619671-2.670531$

H -4.038849 $0.446229-1.159762$

H $\quad 0.1520310 .500468-2.222783$

H - $0.425339-0.027873-3.819838$

H $-0.408339-2.480040-2.902492$

H $1.943653-1.008848-1.602089$

H $2.804225-3.326003-2.364468$

H $1.220719-4.005177-2.050353$

H $3.850469-3.8502481 .114794$

H $4.383949-3.543647-0.631901$

H $1.545099-4.1919471 .615603$

H $\quad 0.407081-4.2968470 .291072$

H $1.402920-1.6159321 .477440$

H -1.885428 -0.551923 -1.253287

C $-0.7294293 .649121-1.727184$

O $0.1732473 .567496-0.607304$

C $1.5313393 .622692-1.096598$

C $1.4329853 .582269-2.615581$

C $0.0925794 .250199-2.860506$

H $1.9249894 .593632-0.770098$

C $2.3282382 .482314-0.449870$

H $2.2649424 .099370-3.102930$

H $1.4166912 .547729-2.979796$

C $-0.4713883 .983092-4.244750$

H $0.2033025 .332768-2.713512$
C $3.7373342 .288513-1.046174$

O 2.4066782 .7888710 .945603

H $1.7607991 .547295-0.530518$

H $3.6304292 .017807-2.102615$

O $4.4485013 .526161-1.009197$

C $4.5742731 .192698-0.353439$

C $5.9000880 .940898-1.098030$

H $3.9876800 .266913-0.319651$

O 4.8461421 .5908250 .987746

H $5.6883310 .797011-2.164326$

H $6.536750 \quad 1.828057-0.988957$

H $\quad 0.2023374 .371377-5.015552$

H $-0.6045312 .910913-4.424104$

H -1.443930 $4.468754-4.371266$

C $-1.9653924 .457859-1.333311$

H 2.8126022 .0273561 .392932

H $4.5543573 .770952-0.073669$

H 5.3434460 .8733461 .414949

H -2.610272 $4.620754-2.203402$

H -1.665698 $5.439496-0.947171$

H $-1.0191412 .621572-1.978101$

C $7.845272-1.0473861 .413277$

O 7.1384240 .0066940 .746146

C $6.664327-0.291105-0.570568$

C $7.839754-0.661339-1.488915$

C $8.682409-1.793376-0.892463$

C $9.041475-1.5264800 .572023$

H $8.238653-0.5820252 .326193$

C $6.910952-2.1856941 .837373$

H $5.962778-1.130981-0.522214$

O $8.6680170 .476963-1.719462$

H $7.443357-0.968841-2.463465$

H $9.606691-1.904873-1.472892$

O $7.963253-3.020415-0.974170$

H $9.478496-2.4258101 .021811$

O $10.050276-0.5124640 .598654$

C $5.976511-1.7322612 .933282$

H $6.313695-2.5875341 .015434$

H $7.486000-3.0287202 .239091$

H $8.9764920 .800457-0.855665$

H $7.778789-3.190355-1.913667$

H $\quad 10.327069-0.3973101 .523687$

O $4.825220-1.2404302 .394141$

O $6.249327-1.7899434 .125507$

C $3.862572-0.7514073 .333555$

H $4.316655-0.0164084 .005341$

H $3.061700-0.2603882 .774652$

H $3.439080-1.5856253 .900242$

SCF Energy (B3LYP/6-31G**//MMFF) = -3245.91301046

0800248

MMFF Geometry

C $-1.3303414 .621666 \quad 0.556150$

C -2.3658253 .9401390 .042873$

C $-3.6502183 .673300 \quad 0.783802$

O $-3.752621 \quad 2.2371910 .932366$

C $-4.8536164 .168680-0.031744$

C -6.1609054 .0521620 .727386$

C -7.0750733 .0808330 .536146$

C -6.4241385 .1509231 .724926$

C $-6.9849821 .911059-0.403441$

C -7.3143560 .5917760 .310266$

C $-7.198350-0.602381-0.646728$

C -7.700101-1.886996 0.012534

C $-7.515937-3.104053-0.907246$

O $-5.819382-0.749269-1.000572$

O $-7.709541-4.287305-0.117521$

C $-6.059011-3.131396-1.413744$

C $-8.569971-3.147238-2.019832$

O $-5.875344-4.154137-2.390049$

C $-5.565630-1.774014-1.969443$

C $-4.040702-1.838137-2.215612$

O $-6.222588-1.500308-3.208032$

C -3.3356551 .7064682 .113074$

O -3.0219932 .3121023 .125826$

C -3.2391650 .2410841 .939326$

C $-2.328100-0.4192822 .669919$

C $-2.012714-1.8454302 .591646$ 
C $-2.955354-2.7317051 .824858$ C $-3.382074-0.477938-2.476373$ C $-1.879110-0.633811-2.601537$ C $0.169253-1.056784-1.238336$ O $-1.316918-0.598733-3.693514$ C $0.393761-2.346003-0.513199$ C $1.155694-2.4590650 .585973$ C $1.410251-3.7622161 .289183$ C $1.119911-3.7078652 .773681$ C $2.115920-3.7604373 .674237$ C $-0.328630-3.6819643 .218427$ C $-0.898034-2.2888153 .209746$ N -1.258049 -0.840859-1.379667 $\mathrm{H}-1.4038265 .0376151 .557618$ H -2.277413 $3.513715-0.954167$ H -3.641292 4.1673091 .762896 H $-4.9116193 .625870-0.982117$ H $-4.7096695 .221745-0.309298$ H -7.9968013 .1257501 .115105$ H -7.399672 5.0462612 .211815 H -5.6631745 .1479122 .511321$ H $-6.407907 \quad 6.1266411 .228202$ H -7.702462 2.070205-1.217471 H $-5.9953711 .833032-0.861098$ H $-6.6238430 .458532 \quad 1.153239$ H $-8.331590 \quad 0.6412880 .718471$ H -7.784005 -0.382069-1.547042 H $-8.751005-1.788110 \quad 0.311619$ H -7.138718 -2.0643470 .940778$ H -8.602295 -4.2484620 .266448$ H $-5.424788-3.403696-0.558129$ H $-9.578329-3.185477-1.590550$ H -8.468240 -4.054946 -2.625039 H -8.527005 -2.282528 -2.685173 H -6.182238 -4.988896-1.996419 H $-3.558965-2.285371-1.339988$ H -3.826238 $-2.500147-3.064549$ H -5.669507 $-0.900024-3.732482$ H $-3.854606-0.2310491 .183253$ H $-1.7144990 .150043 \quad 3.367792$ H -3.996606 -2.525713 2.097544 H -2.801821 -3.795432 2.019875 H -2.835961 -2.5717970 .751146$ H -3.756558 $-0.025936-3.400873$ H $-3.5885590 .230493-1.666000$ H $0.568484-0.202684-0.686948$ H $0.647528-1.092695-2.221707$ H $-0.077037-3.234592-0.927484$ H $1.648602-1.5750330 .983424$ H $2.462778-4.0257901 .120900$ H $0.824198-4.5754750 .842843$ H $1.914396-3.7498054 .740304$ H $3.155172-3.8089783 .366653$ H $-0.411716-4.0660464 .243484$ H $-0.894434-4.3808152 .596162$ H $-0.297232-1.5684473 .767458$ H -1.810713 -0.778274 -0.529909 C $1.159754 \quad 4.1254690 .486750$ O $2.3352044 .415882-0.305396$ C $2.8757953 .175206-0.798002$ C 2.4490162 .1436860 .226821 C 1.0398392 .5965670 .548903 H $2.3925882 .990804-1.766110$ C $4.3943333 .298955-0.972216$ H $2.4892301 .119648-0.153724$ H 3.0847182 .2047821 .118271 C 0.5586542 .0635661 .888934 H $0.3664292 .238641-0.240624$ C $5.0488111 .978510-1.426599$ O $4.6426284 .313306-1.948094$ H $4.8530143 .638222-0.035828$ H $4.7755551 .198803-0.709657$ O $4.4994051 .590646-2.686660$ C $6.5878742 .009401-1.519944$ C $7.2085770 .711437-2.072776$ H $7.0045222 .216120-0.527087$ O $7.0273433 .057924-2.384860$ H $6.9520870 .603358-3.134810$
H $8.2984810 .845338-2.047025$

H -0.4559232 .4012322 .107499$

H 0.5582200 .9686661 .889319

H 1.20190724004982 .708390

C $-0.0352384 .827890-0.171985$

H $4.2052635 .125622-1.639874$

H $4.7429422 .277093-3.331502$

H $6.7287263 .900603-2.003155$

H $-0.1155814 .504432-1.218285$

H $\quad 0.1692605 .904840-0.225498$

H 1.3408274 .5503061 .481585

C $6.897217-1.5057530 .923882$

O $7.221153-0.4142740 .050569$

C $6.824638-0.569500-1.314666$

C $7.478012-1.816636-1.931209$

C $7.186823-3.064999-1.092086$

C $7.475654-2.8304570 .392706$

H $7.412958-1.2720181 .864384$

C $5.389968-1.5763611 .222671$

H $5.738674-0.689353-1.366780$

O $8.888143-1.639088-2.047331$

H $7.089456-1.953379-2.946966$

H $7.804291-3.898023-1.450158$

O $5.819349-3.436230-1.240317$

H $7.094420-3.6697030 .986171$

O $8.895669-2.8081780 .565799$

C $4.949987-0.4605392 .148515$

H $4.771505-1.5136230 .324270$

H $5.175612-2.5246521 .730377$

H $9.238129-1.447159-1.160523$

H $5.663370-3.610717-2.184132$

H $\quad 9.074737-2.7312961 .518472$

O $3.792862-0.8306242 .771307$

$\begin{array}{llll}\text { O } & 5.536264 & 0.602891 & 2.296363\end{array}$

C 3.2620110 .0993693 .720706

H 3.8274420 .0285724 .653989

H $3.288600 \quad 1.1223063 .335458$

H $2.221228-0.1723043 .916329$

SCF Energy $\left(B 3 L Y P / 6-31 G^{* *} / / M M F F\right)=-3245.91627311$

0800249

MM̄FF Geometry

C $-2.509046 \quad 4.911921-0.743550$

C $-3.3251483 .849505-0.800673$

C -4.2651123 .4685690 .314771$

O -3.9686842 .1057030 .703167$

C -5.721706 $3.535372-0.175056$

C -6.7390443 .2763430 .921554$

C -7.484869 2.159211 1.038497

C -6.9154494 .3996991 .912169$

C -7.4715180 .9452670 .153418$

C $-6.899770-0.2713970 .891497$

C $-6.618351-1.437531-0.066669$

C $-6.130083-2.6646870 .704131$

C $-5.703479-3.805595-0.232279$

O $-5.610187-1.001950-0.984077$

O $-4.963121-4.7599030 .544976$

C $-4.736254-3.246861-1.298509$

C $-6.912550-4.555863-0.802659$

O $-4.436840-4.234916-2.281003$

C $-5.251707-1.964809-1.983162$

C $-4.196733-1.298179-2.900787$

O $-6.377661-2.292926-2.806081$

C -2.8871891 .9198361 .511280$

O -2.1549152 .7837851 .967848$

C -2.7327130 .4660081 .738735$

C -1.652991 0.0122332 .392885

C $-1.360487-1.3848212 .720669$

C $-2.329387-2.4334662 .243377$

C $-2.915362-0.831794-2.200094$

C $-1.776126-1.835079-2.256866$

C $0.522359-2.205298-1.369748$

O $-1.795342-2.840102-2.961887$

C $0.454898-3.149647-0.211702$

C $1.349782-3.1470950 .787295$

C $1.356714-4.1333401 .917725$

C $1.485047-3.4696393 .269860$

C $2.677001-3.3753573 .883394$ 
C $\quad 0.219555-2.996896 \quad 3.945847$ C $-0.247975-1.6616073 .432796$ N $-0.712008-1.449694-1.452290$ H $-2.547597 \quad 5.5616800 .126897$ H -3.293427 $3.194658-1.668327$ H -4.1353824 .1284631 .181568$ H $-5.8541792 .835240-1.005704$ H $-5.9256244 .528639-0.597857$ H $-8.1900762 .097158 \quad 1.866653$ H -7.724271 4.2032142 .624280 H -5.999874 4.5528652 .491046 H -7.1590855 .3310681 .390633$ H -8.501451 $0.730166-0.157031$ H $-6.915915 \quad 1.125017-0.769091$ H $-5.965766 \quad 0.0181421 .388923$ H -7.601295 -0.5882211 .673235$ H $-7.536992-1.668477-0.618608$ H -6.896126 -3.011132 1.408959 H $-5.265714-2.3837041 .319826$ H $-5.536110-5.0660331 .268798$ H -3.794379 $-3.021328-0.784817$ H $-7.508387-4.9936410 .007329$ H -6.596387 -5.397301-1.428872 H $-7.575816-3.917179-1.389707$ H $-4.095293-5.015567-1.812297$ H $-3.978813-1.945440-3.758746$ H $-4.663532-0.399034-3.328545$ H -6.481872 -1.599407 -3.478379 H $-3.510596-0.1894651 .366188$ H -0.9122130 .7338822 .735958$ H - $1.981525-3.4559522 .405231$ H $-2.497633-2.3414341 .165199$ H -3.288712 -2.329030 2.760883 H $-2.5505790 .068496-2.711755$ H -3.110663 -0.557459-1.159003 H $1.339838-1.484969-1.270197$ H $0.671426-2.769201-2.296118$ H $-0.338033-3.893434-0.218391$ H $2.152187-2.4153330 .770447$ H $2.204546-4.8130141 .758771$ H $0.462121-4.7679101 .903096$ H $2.772852-2.9261904 .866392$ H $3.584552-3.7389953 .412692$ H $0.391710-2.8849715 .025086$ H $-0.546116-3.7745763 .872639$ H $0.399942-0.8341223 .725632$ H $-0.805168-0.628479-0.861372$ C $-0.0581205 .046885-1.401925$ O $0.191447 \quad 3.647972-1.158499$ C $1.1048033 .529007-0.043027$ C $1.617532 \quad 4.937822 \quad 0.230141$ C $0.419828 \quad 5.783355-0.152495$ H $0.501828 \quad 3.1702900 .800272$

C $2.1941292 .505097-0.392051$

H 1.9238375 .0785831 .271120 H $2.4739755 .173679-0.414020$ C $0.7519467 .246158-0.386671$ H $-0.315804 \quad 5.7091770 .656959$ C 3.2869752 .3865020 .689331 O $1.555327 \quad 1.236581-0.554132$ H $2.6429832 .762588-1.358427$ H 3.6843203 .3915340 .875232 $\begin{array}{lllll}\text { O } & 2.717648 & 1.943101 & 1.919729\end{array}$ C $4.504071 \quad 1.5100050 .329142$ $\begin{array}{llll}\text { C } & 4.204262 & 0.020661 & 0.075789\end{array}$ H 5.2122891 .5796951 .164520 O $5.1303292 .088104-0.815380$ $\begin{array}{llll}\text { H } & 3.532043 & -0.359310 & 0.851747\end{array}$ H $3.711514-0.076746-0.898677$ H 1.1591437 .6984380 .523396 H $1.4942137 .367519-1.182770$ H $-0.1434817 .807433-0.671471$ C $-1.5172415 .264942-1.815280$ H $\quad 0.874197 \quad 1.339914-1.240670$ H $2.175878 \quad 1.1580351 .730738$ H $5.9392301 .581086-0.998125$ H -1.719044 4.664049-2.711221 H $-1.6702096 .313320-2.096239$
H $0.5758205 .319591-2.256592$

C $7.542806-1.131026-1.184114$

O $6.281990-0.461002-1.054084$

C $5.482310-0.8487780 .066712$

C $5.155020-2.348030-0.008459$

C $6.429295-3.189159-0.135168$

C $7.355079-2.656583-1.233489$

H $7.927997-0.809539-2.160781$

C $8.543163-0.675357-0.116022$

H $6.032410-0.6452320 .991957$

O $4.307086-2.594587-1.128144$

H $4.604768-2.634136 \quad 0.895433$

H $6.161494-4.228095-0.362907$

O $7.132160-3.1856561 .103851$

H $8.334981-3.145617-1.181233$

O $6.795573-2.976764-2.510728$

C $8.905136 \quad 0.777094-0.315438$

H $8.177097-0.8062950 .906245$

H $9.474433-1.250329-0.184397$

H $4.080919-3.540202-1.122859$

H $6.537667-3.5523731 .780355$

H $6.722978-3.944949-2.564666$

O 8.1414441 .5636880 .494889

O $9.746633 \quad 1.165230-1.115375$

C $8.365086 \quad 2.971904 \quad 0.376147$

$\begin{array}{llll}\mathrm{H} & 9.392316 & 3.216918 & 0.662190\end{array}$

H $8.1573713 .304273-0.645450$

H 7.6789163 .4829661 .056641

SCF Energy (B3LYP/6-31G**//MMFF) $=-3245.91090369$

0800250

MMFF Geometry

C -2.323055 2.410996-1.295233

C $-3.263406 \quad 3.180390-0.727262$

C $-3.814068 \quad 2.993458 \quad 0.668977$

O -2.9899882 .0703531 .420378$

C -5.2352192 .4154490 .610828$

C -5.9603552 .5219391 .937205$

C $-6.144121 \quad 1.5018862 .797669$

C -6.5413923 .8788232 .243993$

C -5.6473950 .0873932 .681108$

C $-6.788862-0.9072992 .425371$

C $-7.338859-0.8398920 .991944$

C $-8.565325-1.7409470 .839460$

C $-9.071346-1.771224-0.610373$

O $-6.300376-1.2782490 .110540$

O $-10.006500-2.854131-0.728838$

C $-7.886173-2.092434-1.543581$

C $-9.836347-0.494368-0.977952$

O $-8.273517-1.992045-2.912393$

C $-6.633503-1.221834-1.282265$

C $-5.433429-1.790654-2.076440$

$\begin{array}{llll}\text { O } & -6.892413 & 0.115443 & -1.710363\end{array}$

C -1.944487 2.5796932 .121179

$\begin{array}{llll}\text { O } & -1.680689 & 3.763355 & 2.283367\end{array}$

$\begin{array}{llll}\text { C }-1.100737 & 1.507647 & 2.698028\end{array}$

$\begin{array}{llll}C & -1.128614 & 0.232701 & 2.277814\end{array}$

C $-0.291752-0.8604492 .781639$

C $0.690118-0.5407773 .878856$

C $-4.084170-1.131075-1.754812$

C $-2.931455-1.868393-2.416867$

C $-0.449097-2.060472-2.338862$

O $-3.093242-2.740656-3.266686$

C $-0.128846-3.126907-1.338130$

C $0.997783-3.158315-0.610940$

C $1.301301-4.2183640 .407772$

C $1.502801-3.6530201 .796966$

C $2.737544-3.5094062 .307441$

C $0.278487-3.3587292 .635604$

C $-0.431026-2.0898852 .241149$

N $-1.698457-1.429227-1.953423$

H -1.943964 $1.547268-0.756426$

H -3.646784 $4.036037-1.278607$

H -3.8227993 .9715501 .164750$

H -5.1914301 .3716490 .281819$

H $-5.8323932 .939835-0.147094$

H $-6.7067901 .698281 \quad 3.709844$

H $-7.101566 \quad 3.8889203 .185154$ 
H -5.7492494 .6291702 .326230$ H -7.229839 4.1849661 .449422 H $-4.865237-0.0194571 .923712$ H -5.169907 -0.169308 3.635255 H $-6.396383-1.9159412 .609126$ H $-7.596897-0.7366103 .147572$ H -7.6036940 .1960890 .755159$ H $-9.367090-1.4262361 .518988$ H -8.304914 -2.766485 1.137283 H -10.725336 -2.700219-0.092020 H -7.616107 -3.144576 -1.375341 H $-10.699807-0.361386-0.315002$ H -10.245324 -0.557412 -1.992400 H $-9.2250950 .407687-0.907867$ H -9.051122 -2.563034-3.035893 H $-5.340880-2.862357-1.854741$ H -5.619904-1.696112 -3.153740 H $-6.0508180 .552678-1.912983$ H -0.4073951 .8427643 .462782$ H -1.819153 -0.044952 1.483249 H $\quad 0.166523-0.1807294 .771355$ H $1.3971680 .229073 \quad 3.549603$ H $1.290867-1.3982254 .189256$ H $-4.066785-0.096175-2.110942$ H $-3.909896-1.128140-0.672862$ H $0.315908-1.279467-2.376510$ H $-0.549250-2.502548-3.335089$ H $-0.868985-3.912175-1.200814$ H $1.750920-2.388932-0.758429$ H $2.206543-4.7455800 .079193$ H $0.513359-4.9812490 .439435$ H $2.895306-3.1414123 .315540$ H $3.620170-3.7589541 .728602$ H $0.533214-3.3424813 .701880$ H $-0.427779-4.1942132 .543198$ H -1.161141 -2.2267611 .443331$ H -1.668787 -0.758165 -1.191556 C $-0.1992102 .941677-2.553721$ O $\quad 0.4647351 .861019-1.863647$ C $1.2189772 .419565-0.766645$ C $1.5017253 .851858-1.181745$ C $0.1784384 .233123-1.818490$ H 0.5524422 .3959430 .104573 C $2.4492441 .547645-0.510580$ H $1.7678474 .495218-0.338143$ H $2.311573 \quad 3.891218-1.920519$ C $0.2644655 .447927-2.726518$ H $-0.5376704 .450641-1.016714$ C 3.4176122 .1178630 .538716 O $1.9890530 .272205-0.051280$ H $2.9713331 .372698-1.457531$ H $3.850433 \quad 3.0580720 .176797$ O 2.6615902 .4433681 .713075 C 4.5452831 .1520590 .956911 C $5.4147950 .697294-0.222847$ H $4.110036 \quad 0.2983491 .487815$ O $\quad 5.3677811 .8510911 .897147$ H $4.8024720 .224013-0.998492$ H $5.8602141 .581444-0.699778$ H $\quad 0.5818216 .329708-2.160343$ H $0.9836285 .293184-3.537754$ H $-0.7097135 .665730-3.175615$ C $-1.7048692 .656257-2.642303$ H $1.324024-0.041867-0.686794$ H 3.2849952 .8006162 .368583 H 5.9261241 .1943742 .346108 H -1.859928 $1.747848-3.237601$ H -2.208441 $3.476701-3.166232$ H $0.2117742 .950278-3.571004$ C $8.444316-1.338717-0.960976$ O $7.281801-0.506805-1.071711$ C $6.566457-0.2589900 .147109$ C $6.087549-1.5844930 .759592$

C $7.240570-2.5772300 .933378$ C $8.088033-2.698581-0.337458$ H $8.757736-1.521696-1.997263$ C $9.598671-0.622438-0.242316$ H 7.2350860 .2569750 .842960
O $5.079225-2.169778-0.062894$

H $5.633242-1.3900441 .737077$

H $6.826808-3.5585001 .195831$

O $8.052858-2.1430042 .020040$

H $9.007822-3.260337-0.137823$

O $7.351612-3.437479-1.316840$

C $10.1131740 .568491-1.024748$

H $9.300752-0.2574380 .743812$

H $10.436919-1.318640-0.122746$

H $5.452442-2.288163-0.952981$

H $8.761858-2.7983652 .136564$

H $7.165889-4.315818-0.942964$

O $11.146871 \quad 1.135498-0.333486$

O $9.6728190 .956039-2.097999$

C $11.7417622 .282934-0.947933$

H $11.0013433 .081219-1.056538$

H $12.5468342 .637318-0.298644$

H $12.1671272 .014401-1.919647$

SCF Energy (B3LYP/6-31G**//MMFF)= -3245.91333402

08_00251

MM̄FF Geometry

C $-0.011544-3.9313870 .890414$

C $0.551528-2.7147710 .867163$

C $1.030667-1.9974622 .099565$

O $0.436557-0.6822562 .130534$

C $2.553940-1.8052922 .032760$

C $3.140388-1.1327613 .258584$

C 3.6927310 .0971633 .269416

C $3.138457-1.9655634 .514924$

C 3.8040111 .0852702 .138489

C 5.2336271 .1805021 .580506

C $5.611494-0.0238440 .706133$

C 7.0890240 .0017960 .318686

C $7.443797-1.189244-0.587237$

O $4.8177260 .037100-0.481892$

O $8.736149-0.937591-1.161968$

C $6.437844-1.254831-1.759361$

C $7.578340-2.4892610 .215315$

O $6.604448-2.449679-2.517973$

C $4.954212-1.099617-1.341383$

C $4.066167-0.886977-2.591740$

O $4.450164-2.284409-0.717141$

C $-0.815510-0.5515322 .635031$

O $-1.562794-1.4558482 .981086$

C -1.1930810 .8763662 .764064$

C $-0.360862 \quad 1.8827402 .453591$

C -0.6205933 .3136802 .608053$

C -1.9611783 .7339823 .147407$

C $2.710996-0.223931-2.295971$

C $2.7579551 .294080-2.416335$

C $1.2464283 .262661-2.539631$

O $3.7774461 .917022-2.703617$

C $1.5921014 .169418-1.400283$

C $0.8351025 .227055-1.068025$

C $1.1894336 .230016-0.006457$

C $0.1198516 .364131 \quad 1.054659$

C -0.9640297 .1322860 .852989$

C 0.3490495 .6766592 .379518

C 0.3552484 .1791342 .262606

N $1.5146041 .875994-2.203419$

H -0.112342 -4.447945 1.840820

H $\quad 0.674238-2.200305-0.082602$

H $0.757837-2.5451873 .009922$

H $2.796924-1.2277951 .135815$

H $3.048365-2.7776841 .906113$

H 4.1126130 .4580234 .207992

H $3.682507-1.4835945 .334312$

H $2.115176-2.1413254 .859974$

H $3.616699-2.9331804 .330456$

H 3.0864840 .8818401 .337700

H 3.5225012 .0676142 .539086

H 5.2959462 .0946530 .976032

H 5.9465251 .2862402 .407551

H $5.385599-0.9448401 .252839$

H 7.7280840 .0082971 .210297

H $7.3123460 .932239-0.221465$

H $9.368399-0.809265-0.434133$ 
H $6.686006-0.423091-2.434123$ H $8.379334-2.3982780 .959023$ H $7.865060-3.326106-0.430905$ H $6.665920-2.7606870 .750367$ H $7.537492-2.492774-2.789124$ H $4.602132-0.290658-3.339563$ H $3.874370-1.863441-3.056493$ H $5.163371-2.939476-0.664854$ H $-2.185867 \quad 1.0530543 .164541$ H 0.6265401 .6463702 .061442 H -2.772093 3.2735762 .574178 H -2.129055 4.812366 3.095633 H -2.062338 3.4340374 .196073 H $1.988770-0.572738-3.044683$ H $2.330682-0.504368-1.308285$ H $0.189387 \quad 3.331643-2.813820$ H $1.8497613 .531692-3.413423$ H $2.5219563 .989054-0.866984$ H $-0.0733455 .420640-1.634779$ H $1.3363547 .201755-0.496314$ H 2.1531885 .9918040 .460277 H -1.722014 7.2545941 .619338 H -1.118787 $7.651832-0.086992$ H $-0.376586 \quad 6.013171 \quad 3.127926$ H 1.3213305 .9941982 .778546 H 1.2924503 .7781321 .874412 H $0.726073 \quad 1.284341-1.959688$ C $-2.002394-4.925750-0.350579$ O $-2.759112-3.698697-0.297542$ C $-3.947158-3.9345540 .484943$ C $-4.007164-5.4404510 .736849$ C -2.537175 -5.8014300 .780768$ H -3.796536 -3.427007 1.444706 C $-5.193511-3.387349-0.217175$ H $-4.538050-5.6833091 .662533$ H $-4.505850-5.970100-0.083576$ C $-2.266479-7.2844720 .599526$ H -2.139145 -5.480666 1.750591 C $-5.198779-1.864329-0.460220$ O $-5.314512-4.034196-1.491377$ H $-6.083610-3.6746660 .356237$ H $-4.464457-1.612630-1.233368$ O $-6.480524-1.536508-1.016772$ C $-4.957048-0.998742 \quad 0.791704$ C -5.3538780 .4831150 .642603$ H $-3.902844-1.0741271 .081478$ O $-5.729241-1.5312281 .873475$ H -6.4431720 .5611870 .531751$ H -5.1208140 .9745901 .597325$ H -2.745873 -7.862870 1.396183 H $-2.651676-7.649384-0.358558$ H $-1.192279-7.4912040 .632209$ C $-0.497586-4.644523-0.338896$ H $-6.113810-3.682715-1.919788$ H -7.150538 -1.711022 -0.333422 H -5.496952 -1.030087 2.673885 H $-0.249245-4.049713-1.225441$ H $0.053205-5.587827-0.430883$ H -2.246575 $-5.386805-1.317505$ C $-2.4294851 .871236-1.208513$ O $-3.2561421 .239408-0.222143$ C $-4.657604 \quad 1.229842-0.501679$ C $-5.1935212 .664364-0.650767$ C $-4.3835733 .467214-1.674323$ C $-2.8761293 .325752-1.443237$ H $-1.4272491 .891615-0.762223$ C -2.346706 $1.051459-2.505530$ H $-4.8374130 .689340-1.435448$ O $-5.167597 \quad 3.341003 \quad 0.604550$ H $-6.2434142 .616517-0.962358$ H $-4.6572664 .527341-1.604669$ O $-4.695032 \quad 3.023086-2.990955$ H $-2.3249403 .754263-2.288224$ O $-2.5414714 .097614-0.287634$ C $-1.691046-0.296418-2.298626$ H $-3.3334140 .861502-2.935360$ H $-1.744112 \quad 1.600724-3.238077$ H $-4.243303 \quad 3.3713190 .903791$
H $-5.6488243 .154310-3.127420$

H $-1.5823784 .022200-0.150350$

O $-1.536104-0.898022-3.515412$

O $-1.370841-0.769166-1.217548$

C $-0.954644-2.204351-3.491066$

H $-0.770266-2.513924-4.523178$

H $-0.001310-2.193745-2.954522$

H $-1.651357-2.911051-3.031580$

SCF Energy (B3LYP/6-31G**//MMFF)= -3245.90788906

\section{2}

MMFF Geometry

C 2.4586165 .4702530 .594073

C $3.096515 \quad 4.306018 \quad 0.788497$

C $3.9976503 .682703-0.246436$

$\begin{array}{llll}\text { O } & 3.540318 & 2.338965 & -0.529599\end{array}$

C $5.431828 \quad 3.588070 \quad 0.297845$

C $6.4422553 .225927-0.772029$

C $7.0017232 .009103-0.916061$

C $6.8605644 .360189-1.672931$

C $6.7154030 .768990-0.114528$

C $6.716601-0.480851-1.007039$

C $6.546808-1.765723-0.184917$

C $6.802777-2.997703-1.054971$

C $6.581546-4.299823-0.273208$

O $5.210921-1.8014540 .329901$

O $6.536041-5.379792-1.218105$

C $5.201611-4.2370490 .409816$

C $7.743981-4.6043720 .678998$

O $5.006911-5.3581991 .269395$

C $4.945931-2.9214821 .186787$

C $3.462354-2.8694231 .617820$

O $5.769640-2.8952242 .353834$

C $2.4964982 .205343-1.392362$

O $1.8586323 .103681-1.918398$

C $2.2671210 .757960-1.605055$

C $1.1501080 .357071-2.230936$

C $0.783268-1.022492-2.562041$

C $1.827756-2.088077-2.357316$

C $2.994886-1.5135412 .162356$

C $1.531988-1.5915932 .558141$

C $-0.748407-1.8250681 .572591$

O $1.180417-1.6214173 .734883$

C -1.202431-2.761728 0.498093

C $-2.101569-2.440514-0.443673$

C $-2.552002-3.379337-1.524053$

C $-2.440410-2.774983-2.906723$

C $-3.539198-2.494441-3.627479$

C $-1.055444-2.568460-3.486341$

C $-0.446930-1.256571-3.066878$

N $0.688600-1.6679691 .462199$

H $2.5987336 .000853-0.344687$

H 2.9605163 .7682081 .723520

H $3.9890784 .267708-1.174748$

H 5.4709612 .8651111 .121586

H 5.7339334 .5488710 .736360

H $7.7595851 .888848-1.689492$

H $7.6366434 .061254-2.385688$

H $6.0084834 .728092-2.252293$

H $7.2618045 .188326-1.079449$

H 7.4979350 .6688990 .647008

H 5.7552520 .8349010 .405456

H $5.903978-0.397068-1.740529$

H $7.661665-0.528076-1.562836$

H $7.256682-1.7384060 .650072$

H $7.814843-2.974209-1.478075$

H $6.117635-2.986176-1.914569$

H $7.376629-5.385442-1.707319$

H $4.444582-4.320705-0.383020$

H $8.683663-4.6911620 .120432$

H $7.602663-5.5693171 .178246$

H $7.884431-3.8378371 .443958$

H $5.161128-6.1594810 .740135$

H $2.834805-3.1216560 .754506$

H $3.271237-3.6357392 .380603$

H $5.357403-2.3220423 .019129$

H $3.0246640 .067532-1.251913$

H $0.4260001 .113645-2.532118$ 
H $\quad 2.748189-1.831145-2.894385$ H $1.526212-3.071855-2.721791$ H $2.066850-2.198982-1.296673$ H $3.569695-1.2169103 .045516$ H $3.115152-0.7205401 .415575$ H -1.191701-0.831244 1.473606 H -1.007801 -2.2250042 .557608$ H $-0.769643-3.7595070 .500379$ H -2.546598 -1.450439 -0.440396 H -3.593820 -3.652868 -1.312012 H -1.982708 -4.316964 -1.499430 H -3.467952 -2.083391 -4.629072 H -4.533885 -2.666511 -3.231355 H -1.110808 -2.569370 -4.582944 H $-0.437850-3.430900-3.222443$ H - $-1.110855-0.402901-3.213861$ H $1.069411-1.4631850 .543680$ C 0.0564355 .8811601 .323530 O $-0.2549174 .486768 \quad 1.511181$ C -1.076255 4.0446840 .410961 C $-1.6290575 .313779-0.219557$ C $-0.4519006 .258097-0.069085$ H $-0.3961693 .536509-0.283927$ C -2.131210 3.0638180 .930432 H -1.930159 5.171932 -1.261696 H -2.494546 5.6844020 .343644 C $-0.8184877 .724787-0.210404$ H $0.2829656 .003694-0.841824$ C $-3.0881362 .565846-0.172540$ O $-1.4133281 .967188 \quad 1.503228$ H -2.6995343 .5311461 .743675$ H -3.588134 $3.428756-0.626596$ O $-2.3229091 .943050-1.207374$ C -4.1799391 .5923230 .319077$ C $-5.1020291 .145765-0.834292$ H -4.7756802 .0921041 .091996$ O -3.5640530 .4609820 .919543$ H -5.542954 2.039135 -1.292318 H -4.495895 $0.635306-1.593593$ H -1.229468 $7.924453-1.205408$ H -1.569072 8.0246710 .528619 H $0.0628758 .359560-0.073899$ C 1.5451716 .1140971 .598955 H $-2.0617181 .388557 \quad 1.938835$ H -1.880597 $1.170065-0.815725$ H $-4.266351-0.115031 \quad 1.263933$ H 1.7836665 .7325312 .600081 H 1.7551667 .1900001 .613422 H -0.5181516 .4189122 .088930$ C $-6.511302-2.1183800 .332928$ O $-5.626421-1.086977-0.121697$ C $-6.2239720 .187411-0.385852$ C $-7.3132030 .050639-1.461173$ C -8.343141 -1.018199-1.083130 C -7.673207 -2.326346 -0.651799 H $-5.900557-3.0306650 .322429$ C $-6.969758-1.877750 \quad 1.775524$ H -6.6675590 .5803300 .535542$ O $-6.730041-0.276050-2.721144$ H -7.812314 $1.018871-1.583035$ H -8.996894 -1.213927 -1.941646 O $-9.156914-0.544456-0.014232$ H -8.408595 -3.008514 -0.209391 O $-7.139816-2.978702-1.808011$ C $-5.811389-2.0413972 .731515$ H -7.415065 -0.8900811 .925160$ H -7.730925 -2.609073 2.072347 H -6.221403 -1.096969-2.608244 H $-9.5996910 .265850-0.319051$ H -7.881456 -3.160217 -2.410160 O $-5.371816-0.8204023 .148960$ O $-5.339739-3.1256623 .049433$ C $-4.253327-0.8408754 .041811$ H -3.399595 -1.3372813 .570876$ H -3.9772670 .1934824 .263625$ H -4.525499 -1.341473 4.975706

SCF Energy (B3LYP/6-31G**//MMFF)= -3245.92487123
08_00253

MMFF Geometry

C $-1.059078 \quad 1.8244982 .555741$

C -1.619234 0.6394172 .844376

C $-0.941805-0.7076412 .942579$

O $0.492143-0.5688052 .829305$

C $-1.459483-1.6168421 .813097$

C $-1.179036-3.0903342 .018194$

C $-0.303694-3.8198801 .299899$

C $-2.016503-3.7602513 .079058$

C $0.603213-3.3672790 .189817$

C $2.061971-3.7200040 .503854$

C $2.999378-3.422486-0.673783$

C $4.434765-3.819701-0.319902$

C $5.417890-3.470646-1.445754$

O $2.938180-2.023349-0.973086$

O $6.751422-3.600165-0.930134$

C $5.201300-2.001426-1.862633$

C $5.331844-4.474086-2.600724$

O $5.987822-1.677353-3.007238$

C $3.716743-1.639102-2.115635$

C $3.591440-0.111254-2.300628$

O $3.269269-2.290904-3.305037$

C $1.263513-1.3109553 .665801$

O $0.869169-2.1109144 .504823$

C $2.710451-1.0537103 .477833$

C $3.228047-0.2189982 .563477$

C 4.6558990 .0583252 .362889

C $5.641478-0.6581853 .248603$

C $2.1554990 .423382-2.389230$

C $2.2070911 .934141-2.509298$

C $2.8375823 .928380-1.151897$

O $2.1698522 .499286-3.599452$

C $4.3202583 .882806-0.950394$

C 4.9156084 .1169160 .228861

C 6.3878973 .9399930 .462024

C 6.6856692 .8203711 .438708

C 7.2570163 .0726822 .628315

C 6.3915751 .3978911 .007207

C 5.0124490 .9466121 .412016

N $2.3804042 .556396-1.283246$

H 0.0066501 .8920062 .360305

H $-2.6909980 .616063 \quad 3.038206$

H -1.209185 -1.111660 3.926129

H -1.050764 -1.2709390.858742

H $-2.547604-1.5095741 .721803$

H $-0.228461-4.8835561 .525322$

H -1.842156-4.840522 3.127017

H -1.792873 -3.349167 4.067803

H -3.081199 -3.610369 2.872799

H $0.292614-3.876449-0.730404$

H $0.523646-2.2940610 .000439$

H $2.380181-3.1455421 .381322$

H $2.140665-4.7829590 .765884$

H $2.646627-3.992204-1.541586$

H $4.497005-4.887270-0.074402$

H $4.742305-3.2856980 .590440$

H $6.845403-2.996454-0.173782$

H $5.572869-1.369328-1.043982$

H $5.571513-5.484051-2.246465$

H $6.070398-4.247511-3.377886$

H $4.344401-4.512509-3.065185$

H $\quad 6.909010-1.912792-2.802468$

H $4.0869950 .387180-1.458891$

H $4.1267510 .195655-3.208841$

H $2.493642-1.822014-3.650083$

H $3.348049-1.6122154 .156596$

H 2.5526370 .3172851 .899030

H $5.437125-0.4453214 .303926$

H $6.679097-0.3668473 .073973$

H $5.583461-1.7406313 .088909$

H $1.6283870 .031838-3.264762$

H $1.5704750 .147536-1.504677$

H $2.3222374 .367731-0.293843$

H $2.5911444 .503697-2.049057$

H $4.9241153 .586384-1.805008$

H 4.3102424 .4013121 .086615

H 6.7874524 .8933310 .830223 
H $6.9163913 .736974-0.477946$ H 7.4952572 .2712903 .320019 H 7.4958904 .0843232 .938655 H 7.1682970 .7266521 .386774 H $6.4770551 .320444-0.084516$ H 4.2172041 .4074110 .829785 H $2.4118551 .979791-0.447550$ C -1.987356 $3.707461 \quad 1.097874$ O $-2.503007 \quad 2.731652 \quad 0.167191$ C -1.696493 2.776131-1.023965 C $-1.0065724 .133306-0.992272$ C $-0.697703 \quad 4.257430 \quad 0.487551$ H $-0.9336841 .996584-0.911087$ C $-2.5296302 .505115-2.280364$ H $-0.1182834 .167202-1.627886$ H -1.680985 4.938925 -1.305701 C $-0.365926 \quad 5.6735380 .925296$ H $\quad 0.151035 \quad 3.6047720 .724181$ C $-3.3439811 .194949-2.246475$ O $-3.456403 \quad 3.584198-2.456490$ H - $1.8729212 .522114-3.158541$ H $-4.1898911 .324551-1.563507$ O $-3.9168441 .008658-3.547041$ C $-2.555243-0.068075-1.860829$ C $-3.331252-1.390233-2.003606$ H -2.203026 $0.019542-0.827837$ O $-1.400635-0.201511-2.695768$ H -3.451790 -1.639099 -3.066113 H $-2.675315-2.170023-1.594075$ H $0.522496 \quad 6.0412390 .401971$ H $-1.189147 \quad 6.363455 \quad 0.711833$ H -0.1647215 .7109182 .000595$ C - $-1.855846 \quad 3.1022612 .498916$ H -3.971639 $3.395864-3.259738$ H $-3.187400 \quad 0.832041-4.165958$ H $-0.8046340 .542244-2.512257$ H -2.8664632 .9144052 .883455$ H -1.4005803 .8313143 .179261$ H $-2.739826 \quad 4.505587 \quad 1.144428$ C -5.718134 -1.030021 0.858007 O $-4.524647-1.0804170 .063211$ C $-4.700783-1.441717-1.307782$ C $-5.358909-2.828908-1.428106$ C $-6.660783-2.896232-0.625837$ C $-6.477554-2.3660700 .800638$ H $-5.362890-0.897757 \quad 1.888179$ C $-6.597541 \quad 0.178770 \quad 0.498749$ H $-5.349648-0.704954-1.791606$ O $-4.487588-3.849660-0.944905$ H -5.567915 -3.040296 -2.484061 H $-7.002458-3.938029-0.589394$ O $-7.656222-2.137695-1.305801$ H $-7.448241-2.253728 \quad 1.297428$ O $-5.728478-3.316807 \quad 1.562703$ C $-5.938592 \quad 1.4932800 .857262$ H $-6.8243800 .203964-0.570156$ H -7.5405790 .1330531 .055352$ H $-3.806072-4.002355-1.620782$ H $-8.480920-2.212091-0.795998$ H $-6.221423-4.1548301 .553232$ O $-6.601568 \quad 2.5089490 .229121$ O $-4.982107 \begin{array}{llll}1.619411 & 1.608247\end{array}$ C $-6.097854 \quad 3.8248450 .479597$ H $-6.784877 \quad 4.5435180 .025069$ H -6.0472554 .0216891 .554959$ H -5.1136203 .9407970 .018159$ SCF Energy (B3LYP/6-31G*//MMFF) $=-3245.90140504$

\section{4}

MMFF Geometry

C $-1.331887-4.187360 \quad 0.917416$ C $-2.407300-3.6288720 .344952$ C $-2.561465-3.447225-1.142792$ O $-2.439001-2.029189-1.406295$ C $-3.954310-3.920831-1.591604$ C $-4.222093-3.708358-3.069999$ C $-5.065359-2.783878-3.573488$ C $-3.481219-4.636171-3.998070$
C $-5.888550-1.784649-2.809414$

C $-5.314305-0.368765-2.946050$

C $-5.7917590 .550951-1.812947$

C $-5.3254501 .987804-2.050568$

C $-5.6644732 .909433-0.869614$

$\begin{array}{llll}\text { O } & -5.232309 & 0.049527 & -0.592786\end{array}$

O $-4.913624 \quad 4.123476-1.028618$

C -5.1797402 .2488170 .439269$

C $-7.143792 \quad 3.311932-0.867940$

$\begin{array}{llll}\text { O } & -5.611745 & 2.995088 & 1.574140\end{array}$

C $-5.614200 \quad 0.7758330 .583100$

C -4.9648990 .0649151 .798059$

$\begin{array}{llllll}\text { O } & -7.033004 & 0.722614 & 0.777139\end{array}$

C $-1.175245-1.540449-1.555855$

O $-0.137141-2.182257-1.587809$

C $-1.264463-0.065718-1.630269$

C $-0.1423240 .667019-1.573208$

C $-0.0599462 .128707-1.607115$

C $-1.3514642 .900391-1.664692$

C -3.433402 -0.0043881 .787640$

C $-2.752396 \quad 1.1293352 .535216$

C -0.5007912 .1203292 .929906$

O $-3.351864 \quad 1.902621 \quad 3.277109$

C -0.3028723 .2814872 .006515$

C $0.9060513 .758004 \quad 1.671652$

C 1.1231584 .9624790 .800613

C $2.0743344 .698901-0.345708$

C $3.3786315 .006857-0.245518$

C $1.4960864 .171505-1.638812$

C $1.1588372 .706908-1.573473$

N $-1.382214 \quad 1.1502942 .307706$

H $-0.529090-4.5713540 .293208$

H -3.186793 -3.211573 0.978640

H $-1.791709-4.000906-1.694446$

H $-4.714512-3.419986-0.984725$

H $-4.069094-4.990494-1.368968$

H $-5.178113-2.722907-4.655119$

H -3.771628 -4.496079-5.044836

H $-2.402125-4.467716-3.931342$

H -3.688998 $-5.679274-3.737707$

H $-6.911245-1.798881-3.206580$

H $-5.985917-2.063650-1.758260$

H $-4.218718-0.414741-2.928029$

H $-5.6059440 .045476-3.918953$

H $-6.8859160 .504354-1.758542$

H $-5.7487452 .384514-2.981970$

H $-4.2369211 .996036-2.192700$

H $-5.1516834 .512860-1.887602$

H -4.0845042 .2964010 .431076$

H -7.396260 $3.845454-1.792378$

H $-7.3630784 .008964-0.051607$

H -7.820482 $2.458931-0.785932$

H -5.2983083 .9082131 .457019$

H -5.3424880 .4963542 .732959$

H -5.324469 -0.9744321 .792803$

H $-7.255693-0.1249501 .196493$

H $-2.2498990 .377650-1.704061$

H $0.8128900 .149914-1.491086$

H $-2.0113262 .607183-0.841239$

H $-1.8660362 .713932-2.613330$

H $-1.2212273 .980629-1.574416$

H $-3.127019-0.9258622 .299033$

H $-3.050048-0.0543030 .764971$

H $0.438293 \quad 1.608605 \quad 3.160331$

H $-0.9451802 .468741 \quad 3.867837$

H -1.1940333 .7740581 .624881$

H 1.7920323 .2822752 .084575

H 1.5257285 .7621991 .436388

$\begin{array}{lllll}\text { H } & 0.174721 & 5.349276 & 0.407847\end{array}$

H $4.0625124 .849333-1.072995$

H $3.793393 \quad 5.4141150 .670637$

H $2.2213214 .297320-2.454230$

H $0.6446624 .792193-1.932073$

H $2.0363462 .061545-1.521747$

H -0.9759680 .4979001 .643020$

C $-0.291146-3.0398372 .867707$

O $1.015786-3.0904292 .271480$

C $1.986911-2.6028463 .207487$ 
C $1.194883-2.1230974 .424948$

C $-0.041151-2.9996714 .370142$

H $2.604481-3.4592203 .502249$

C $2.865908-1.5079242 .584992$

H $1.760603-2.2369285 .354966$

H $0.916931-1.0659924 .334171$

C -1.206337-2.454413 5.177665

H $0.220332-4.0012414 .736623$

C $3.826027-1.9158031 .442078$

O $2.038888-0.4284272 .152166$

H $3.492531-1.1223663 .400841$

H $4.534355-1.0846911 .358486$

O $4.592793-3.0532921 .826734$

C $3.211467-2.1267740 .043440$

C $4.248583-2.345774-1.077707$

H $2.578176-1.270404-0.212439$

O $2.379743-3.2855020 .057631$

H $4.787096-3.287287-0.909538$

H $3.680987-2.496232-2.005974$

H $-0.936114-2.3730906 .235575$

H -1.505644 -1.460019 4.829416

H -2.076891 -3.113262 5.101752

C $-1.121809-4.2377382 .404303$

H 2.6214600 .2815391 .832909

H $4.024744-3.8365091 .728506$

H $1.905325-3.318774-0.790762$

H -2.086654 -4.255045 2.923909

H $-0.608111-5.1771622 .641218$

H $-0.757919-2.1034522 .533031$

C $5.3111301 .208167-1.540286$

O $4.5206520 .016620-1.428138$

C $5.254435-1.199952-1.271893$

C $6.167289-1.428647-2.487747$

C $7.078852-0.220873-2.734799$

C $6.2982371 .099048-2.715318$

H $4.5914412 .001284-1.781031$

C $5.9868871 .581753-0.210972$

H $5.876312-1.139020-0.373777$

O $5.389919-1.675010-3.658298$

H $6.773957-2.324525-2.312269$

H $7.573883-0.331845-3.707219$

O $8.092429-0.170912-1.735115$

H $6.9856341 .952815-2.694320$

O $5.5349991 .204259-3.921084$

C 4.9828361 .8826740 .881097

H $\quad \begin{array}{llll}6.632194 & 0.781262 & 0.159170\end{array}$

H $6.5931672 .483105-0.358088$

H $4.787217-0.921270-3.779033$

H $8.595442-1.001613-1.784864$

H $6.1604991 .190823-4.665614$

O 5.6533022 .2908021 .998948

$\begin{array}{lllll}\text { O } & 3.770241 & 1.765458 & 0.778028\end{array}$

C 4.8330592 .5976443 .130930

H 4.2292233 .4858062 .924218

H 4.1951401 .7460063 .386829

H 5.4897452 .8090553 .978831

SCF Energy (B3LYP/6-31G**//MMFF)= -3245.91390497

0800255

MM̄FF Geometry

C $2.377726-3.975089-1.698752$

C $1.095761-3.762835-1.364175$

C $0.028411-3.442675-2.379022$

O $-0.655507-2.234104-1.970527$

C $-1.003418-4.582569-2.438751$

C $-1.998041-4.422168-3.573565$

C -3.275771-4.016424 -3.433518

C $-1.474895-4.790448-4.939345$

C -3.986971 -3.616114 -2.171752

C $-4.417253-2.144834-2.224060$

C $-5.083760-1.694597-0.916666$

C -5.509054 -0.227623-1.000899

C -6.1032810 .2747090 .325056$

O $-4.143471-1.866650 \quad 0.149579$

$\begin{array}{lllll}\text { O } & -6.165621 & 1.708228 & 0.263364\end{array}$

C $-5.136761-0.0879151 .475100$

C -7.544655 -0.209865 0.520843

O $\quad \begin{array}{llll}-5.708928 & 0.235600 & 2.739566\end{array}$
C $-4.667130-1.5593931 .449699$

C $-3.558589-1.8731872 .485705$

O $-5.778927-2.4070501 .767767$

C $-0.091051-1.049812-2.334568$

O $0.983623-0.885570-2.890173$

C - $-1.0442120 .027161-1.990385$

C $-0.9366851 .208562-2.614620$

C $-1.8681212 .322636-2.470322$

C -2.229430 $2.957002-3.787200$

C $-2.284395-1.0310922 .367284$

C -2.3109010 .2247773 .222963$

C $-1.2988202 .496153 \quad 3.368314$

O $-3.031475 \quad 0.350357 \quad 4.209613$

C -2.2142773 .4170282 .627805$

C -1.7805814 .4103591 .838707$

C -2.6901645 .3218861 .071193$

C $-2.6004365 .094738-0.420243$

C $-2.0137695 .995565-1.226217$

C $-3.2707423 .860499-0.981761$

C $-2.3002582 .743399-1.264449$

N $-1.403903 \quad 1.1747872 .778250$

H $2.645759-3.919443-2.752887$

H $0.779868-3.813128-0.326456$

H $\quad 0.467638-3.292145-3.373249$

H -1.519817 -4.661384-1.476387

H $-0.490472-5.544935-2.571647$

H -3.894889 -3.960111-4.328365

H $-2.246622-4.721335-5.713517$

H $-0.656859-4.125887-5.232624$

H -1.103998 -5.820720 -4.938382

H $-4.873673-4.251788-2.059290$

H -3.370851 -3.789914 -1.286952

H $-3.533849-1.525764-2.416174$

H $-5.108978-1.990362-3.061748$

H $-5.953971-2.337593-0.738903$

H -6.219185 -0.074270 -1.822986

H $-4.6337920 .391377-1.242881$

H $-6.7063941 .952473-0.507132$

H $-4.2653000 .567206 \quad 1.357181$

H $-8.1784290 .131472-0.306545$

H $-7.9854780 .217344 \quad 1.428195$

H -7.629063 -1.297080 0.572294

H $-6.359054-0.4543072 .956192$

H -3.973144 -1.833561 3.500478

H -3.260435 -2.920642 2.334568

H $-5.435655-3.2643002 .069913$

H -1.843101-0.190283 -1.290551

H $-0.1434891 .367188-3.342982$

H -2.870904 $3.834993-3.689980$

H -2.757435 $2.236832-4.422294$

H -1.327269 $3.285242-4.316367$

H -1.435335 -1.623504 2.731497

H $-2.079162-0.7775941 .321778$

H -0.2523882 .8078603 .293956$

H $-1.5776502 .458550 \quad 4.425930$

H -3.2830603 .2492982 .739362$

H -0.712405 4.5769821 .730453

H $-2.420577 \quad 6.355772 \quad 1.322446$

H -3.7335985 .2054401 .390523$

H $-1.9753005 .850794-2.300524$

H $-1.5563726 .895763-0.829428$

H -3.853245 $4.130468-1.868796$

H $-4.0207483 .490530-0.271505$

H -1.942751 2.225799 -0.374782

H -0.9105911 .0119291 .905806$

C $3.263483-4.2854030 .723476$

O $2.885388-2.9612371 .148887$

C $3.721092-2.5657182 .247902$

C $4.291071-3.8594202 .810415$

C $4.507854-4.6553231 .538488$

H $4.540359-1.9745491 .821977$

C $2.943072-1.7016403 .242980$

H $5.211262-3.6980333 .379659$

H $3.572810-4.3750433 .458065$

C $4.667639-6.1477901 .772968$

H $5.410973-4.2769101 .040611$

C $2.158610-0.5351462 .609203$

O $1.992312-2.528413 \quad 3.927503$ 
H $3.631452-1.3289964 .011250$ H $1.263873-0.9282222 .109759$

O $1.693656 \quad 0.2706393 .699165$

C $2.961066 \quad 0.3348541 .623147$

C 2.1562561 .5501551 .122527

H $3.255339-0.2788970 .764757$

$\begin{array}{lllll}\text { O } & 4.153045 & 0.778636 & 2.268794\end{array}$

H 1.1309371 .2376550 .894358

H 2.1238242 .3023481 .920238

H $5.541725-6.3468522 .401581$

H $3.790392-6.5717052 .273164$

H $4.804372-6.6783630 .825484$

C $3.534162-4.281853-0.784759$

H $1.524262-1.9631824 .566082$

H 1.0075110 .8651503 .354493

H 4.6666811 .2886561 .619771

H $3.938203-5.257094-1.081351$

H $4.306872-3.533362-1.001990$

H $2.430163-4.955450 \quad 0.968025$

C $4.7874773 .251007-0.933985$

O 4.0604532 .6867140 .165988

C $2.7579172 .186376-0.146082$

C $1.8716693 .318281-0.687340$

C $2.5232083 .998166-1.894110$

C $3.9829174 .373066-1.612867$

H $5.6748923 .706776-0.475800$

C $5.2660932 .171589-1.911046$

H $2.8431961 .398014-0.902238$

O $1.649136 \quad 4.281900 \quad 0.339566$

H $0.8981852 .903336-0.966768$

H $1.9557444 .901895-2.147979$

O $2.4514883 .111548-3.006493$

H $4.4829754 .670070-2.541579$

O $4.0053955 .521128-0.758762$

C 6.267848 1.261119-1.241089

H $4.4498761 .564949-2.313483$

H $5.7695752 .621604-2.774808$

H $1.0557014 .962437-0.021627$

H $2.8444683 .566854-3.770578$

H 3.6303955 .2603790 .100147

O $5.6891370 .062208-0.947331$

O $7.4204091 .587984-0.988270$

C $6.537895-0.885820-0.293083$

H $7.455355-1.044697-0.867990$

H $6.769482-0.5418570 .719179$

H $6.000736-1.835039-0.228142$

SCF Energy (B3LYP/6-31G**//MMFF) $=-3245.91293750$

0800256

MM̄FF Geometry

C $-1.959016-4.139056-1.894006$

C -2.948717 -3.500950-1.251824

C $-4.131004-2.856091-1.926133$

O $-4.053858-1.440677-1.634021$

C $-5.438694-3.413452-1.337679$

C $-6.687234-2.776748-1.919540$

C $-7.487987-1.911975-1.263626$

C $-7.022651-3.191968-3.328951$

C -7.320139-1.386629 0.135071

$\begin{array}{llll}\text { C } & -6.918640 & 0.093977 & 0.126848\end{array}$

C $-6.3032680 .527774 \quad 1.465321$

C -6.0236542 .0313951 .467130$

C -5.2736462 .4815132 .729859$

O $-5.079651-0.1991731 .626809$

O -4.7694593 .8055082 .494266$

C $-4.049131 \quad 1.5651872 .941620$

C -6.2082982 .5828683 .940871$

O $-3.412042 \quad 1.847927 \quad 4.184749$

C $-4.383576 \quad 0.0625602 .852445$

C $-3.125429-0.8417012 .870582$

O $-5.182040-0.307577 \quad 3.983095$

C -3.438054 -0.648169-2.553724

O $-3.030737-0.978063-3.656163$

C $-3.3007310 .703412-1.967056$

C $-2.4584611 .584951-2.527081$

C $-2.2036612 .952920-2.068542$

C $-2.9427813 .428956-0.846281$

C $-2.142320-0.6316861 .713224$
C $-1.001110 \quad 0.3221102 .024831$

C 0.9078341 .4854820 .918697

O -0.7469850 .7228713 .157111$

C 0.5160002 .8971370 .616850

C $1.1199003 .643109-0.320409$

C $0.7774665 .077105-0.602714$

C $0.4678855 .328396-2.061295$

C $1.4159795 .777155-2.901255$

C $-0.9613195 .154535-2.520502$

C -1.328116 $3.714512-2.758005$

N -0.2697830 .6384520 .887110$

H - $1.990957-4.237175-2.975595$

H -2.891900 -3.397064 -0.169985

H -4.111443 -3.030886 -3.008535

H $-5.418667-3.302636-0.249290$

H $-5.489275-4.496706-1.513537$

H -8.361814 -1.522764 -1.784697

H -7.971414 -2.766752 -3.673195

H $-6.244157-2.862371-4.023792$

H -7.109742 -4.281504 -3.393239

H $-8.272780-1.4995700 .667376$

H -6.598950 -1.977095 0.703440

H $-6.1885220 .267493-0.672884$

H -7.800284 $0.706191-0.100148$

H $\quad-6.994070 \quad 0.2619372 .274023$

H -6.9549122 .5995591 .349672$

H -5.4095392 .2856490 .593412$

H -5.5247914 .3807382 .283234$

H -3.3262841 .8145272 .156141$

H -7.0053893 .3113103 .749121$

H $-5.6735162 .950740 \quad 4.823491$

H -6.6851701 .6340104 .195285$

H -3.197595 2.796465 4.191406

H -2.625232 -0.7735853 .844191$

H -3.475386 -1.881656 2.797347

H $-5.104571-1.2668724 .115624$

H -3.879683 $0.928934-1.079892$

H -1.898681 $1.278779-3.410210$

H -4.017106 3.489511-1.049759

H -2.623282 $4.412609-0.496462$

H -2.779682 $2.744661-0.007267$

H -1.671727 -1.596504 1.484807

H -2.661902 -0.3005680 .809821$

H $1.613814 \quad 1.0874640 .183396$

H 1.3692341 .4333691 .910040

H $-0.2795503 .334464 \quad 1.215601$

H $1.9377423 .213103-0.892755$

H $1.6354055 .689961-0.295841$

H -0.0622915 .4185020 .015076$

H $1.1951515 .990694-3.941757$

H $2.4366615 .933758-2.567902$

H -1.115990 $5.685073-3.469990$

H -1.632098 $5.656547-1.817339$

H $-0.8321153 .278060-3.625955$

H $-0.5885120 .304567-0.017643$

C $0.554306-4.000486-1.519813$

O $1.589296-4.596758-0.702896$

C $2.160328-3.5773290 .139043$

C $1.937975-2.285503-0.623132$

C $0.562056-2.496356-1.215031$

H $1.585052-3.5860891 .073648$

C $3.635973-3.8995410 .412118$

H $1.986619-1.4008960 .017671$

H $2.684495-2.175797-1.419837$

C $0.328467-1.613811-2.432455$

H $-0.188517-2.260178-0.450800$

C $4.361708-2.7637571 .157450$

O $3.700863-5.0969911 .187416$

H $4.149016-4.105932-0.534951$

H $4.199860-1.8452020 .592234$

O $3.766217-2.5668542 .438669$

C $5.881985-2.9570601 .309076$

C $6.595946-1.7681781 .985817$

H $6.325804-3.1197620 .319700$

O $6.169833-4.1140012 .094181$

H $6.317733-1.7211593 .046633$

H $7.670705-1.9930481 .973623$

H $-0.645503-1.810928-2.883525$ 
H $\quad 0.365421-0.555529-2.154105$

H $1.089329-1.783340-3.202033$

C $-0.765485-4.710002-1.186614$

H $3.219598-5.7862230 .698040$

H $3.909803-3.3805062 .952179$

H $5.780088-4.8816181 .642694$

H $-0.914940-4.700568-0.098369$

H -0.675489 -5.772297-1.447260

H $0.827109-4.194309-2.564208$

C $6.3403380 .648028-0.851054$

O $6.674276-0.506861-0.064766$

C $6.338549-0.4043391 .322511$

C 7.1466030 .7299431 .967067

C 6.9017852 .0560221 .235603

C $7.0193781 .911198-0.287515$

H $6.7754930 .437253-1.836194$

C $4.8171680 .750985-1.025674$

H $5.273977-0.1684141 .424924$

$\begin{array}{lllll}\text { O } & 8.538914 & 0.423918 & 1.946091\end{array}$

H $\begin{array}{llll}6.853215 & 0.824533 & 3.018824\end{array}$

H 7.6291732 .8011601 .581005

O 5.6001622 .5422931 .551742

H $6.6224842 .806347-0.779410$

O $8.4146961 .844085-0.602941$

C $4.4046371 .473269-2.288676$

H $4.409617-0.264669-1.090650$

H $4.3376701 .244887-0.176679$

H $8.7975760 .278475 \quad 1.019972$

H 5.5591802 .6668652 .515258

H $8.4965961 .843791-1.571986$

O $3.084546 \quad 1.214244-2.530919$

O $5.1327542 .182493-2.968445$

C $2.5341421 .835046-3.696738$

H $1.4632501 .616982-3.723682$

H $2.6697652 .919650-3.653632$

H $3.0012711 .422784-4.595753$

SCF Energy (B3LYP/6-31G*//MMFF) $=-3245.92085900$

0800257

MM̄FF Geometry

C $-0.577535 \quad 2.072837 \quad 0.964711$

C $-1.241716 \quad 1.9185252 .120572$

C -1.6825950 .6169152 .750154$

O $-1.160280-0.5041992 .004497$

C -3.2211250 .5436212 .713955$

C $-3.810486-0.5262443 .608347$

C $-4.304918-1.7024213 .175495$

C -3.872536 -0.1738575 .072254$

C $-4.305618-2.2503941 .774980$

C $-5.705858-2.2760751 .147065$

C $-6.176938-0.897706 \quad 0.656290$

C $-7.611941-0.975120 \quad 0.130952$

C -8.072266 $0.357448-0.478938$

O $-5.295794-0.489397-0.395405$

O $-9.278271 \quad 0.111745-1.218138$

C $-7.0110150 .835012-1.492115$

C $-8.4267391 .387850 \quad 0.599636$

O $-7.3227932 .137751-1.981136$

C $-5.568260 \quad 0.809649-0.936058$

C $-4.5592541 .067045-2.082546$

O -5.4303631 .8334200 .050887$

C $-0.721673-1.5888692 .690941$

O $-0.637612-1.7007363 .906413$

C $-0.340942-2.694808 \quad 1.780360$

C $-0.511129-2.660927 \quad 0.449425$

C $-0.152665-3.720330-0.497755$

C $0.448819-4.9851040 .057046$

C $-3.0927010 .841013-1.689664$

C $-2.1449901 .034587-2.862301$

C $0.2387920 .648115-3.502006$

O $-2.498843 \quad 1.488114-3.947011$

C $0.275766-0.689346-4.171668$

C $1.260448-1.585275-4.003923$

C $1.267357-2.938560-4.655980$

C $1.258023-4.074160-3.654418$

C $2.360493-4.812411-3.440846$

C $-0.048832-4.414759-2.968533$

C $-0.355370-3.501046-1.814006$
N $-0.849614 \quad 0.650899-2.541376$

H -0.2898851 .2017200 .384808$

H -1.537126 2.8139842 .665571

H -1.305172 0.6082753 .779189

H -3.5472220 .3918081 .679299$

H -3.6558291 .5058323 .017804$

H -4.735680 -2.378067 3.913854

H -4.346789 -0.959511 5.669996

H -2.866439-0.018589 5.473693

H $-4.4510390 .744275 \quad 5.217765$

H $-3.600073-1.7257731 .124473$

H $-3.935741-3.2821651 .834848$

H $-5.668043-2.9607460 .289510$

H $-6.428657-2.6904081 .860871$

H $-6.114571-0.1814261 .482700$

H $-8.300174-1.2923130 .924407$

H -7.674338 -1.749882 -0.646281

H $-9.935077-0.260444-0.604764$

H $-7.0593070 .159635-2.358011$

H $-9.2361181 .011508 \quad 1.236835$

H -8.8044032 .3139920 .152210$

H -7.585132 1.6361551 .249472

H $-8.2295282 .106159-2.331542$

H $-4.7850270 .391835-2.918442$

H $-4.6716402 .093857-2.453102$

H -4.5000652 .1007450 .107512$

H $0.085864-3.5597062 .278455$

H $-0.950450-1.773279-0.002140$

H $\quad 0.741970-5.701696-0.713033$

H $-0.268212-5.4940330 .710650$

H $1.353686-4.765790 \quad 0.634123$

H -2.795185 $1.544390-0.907188$

H -2.957123 $-0.177459-1.307868$

H $1.1611520 .861735-2.952871$

H $0.0816891 .436414-4.244714$

H $-0.562103-0.935794-4.819829$

H $2.102260-1.340105-3.360741$

H $2.161429-2.996863-5.290157$

H $\quad 0.415563-3.057411-5.337441$

H $2.356405-5.647300-2.748218$

H $3.294171-4.594527-3.948566$

H $-0.056556-5.464314-2.654343$

H $-0.868046-4.345231-3.696007$

H $-0.804045-2.554406-2.114022$

H $-0.6808890 .200199-1.647149$

C $1.0547283 .489160-0.356206$

$\begin{array}{lllll}\text { O } & 2.137051 & 3.437638 & 0.591921\end{array}$

C $3.1595234 .359246 \quad 0.172847$

C $2.7496384 .895453-1.194112$

C $1.2400544 .799498-1.123015$

H 3.1196105 .2020850 .876530

C $4.558578 \quad 3.729547 \quad 0.284610$

H $3.1035145 .918008-1.360009$

H $3.1259364 .281922-2.015755$

C $0.5693564 .818402-2.486797$

H $0.8649355 .640973-0.524945$

C $4.7096932 .321849-0.337653$

O $5.5183674 .639352-0.244248$

H 4.7775393 .6238421 .354940

H $3.923523 \quad 1.6935790 .092122$

O $4.4853382 .386234-1.742495$

C $6.0851641 .667397-0.070960$

C $6.2386330 .246148-0.651512$

H 6.2667081 .6330941 .009761

O $7.1343532 .448111-0.647663$

H $6.1876840 .280925-1.746776$

H $7.257092-0.088574-0.412839$

H $0.7994435 .749356-3.015182$

H $0.9077073 .984895-3.111496$

H $-0.5183334 .746044-2.390931$

C -0.2910323 .4230450 .367577$

H $5.4398884 .620403-1.213391$

H $4.3334961 .481379-2.062468$

H $7.1368763 .312159-0.202250$

H - $-1.0950533 .638856-0.345760$

H -0.3232114 .1909451 .150611$

H $1.1763602 .629990-1.028009$

C $4.496367-1.6741172 .021830$ 
O $5.436666-0.864498 \quad 1.306201$

C $5.220143-0.761036-0.102708$

C $5.323977-2.145681-0.756376$

C $4.323955-3.108117-0.106114$

C $4.421233-3.0916311 .424610$

H $4.913754-1.7590453 .033957$

C $3.128615-0.9833412 .145813$

H $4.215897-0.373442-0.292786$

O $6.635551-2.682504-0.617200$

H $5.109368-2.059625-1.828527$

H $4.505873-4.126569-0.469998$

O $3.001425-2.737791-0.481587$

H $3.575486-3.6292681 .869057$

O $5.609470-3.7830031 .821203$

$\begin{array}{llll}\text { C } & 3.209569 & 0.295988 & 2.954771\end{array}$

H $2.698311-0.7245821 .175197$

H $2.443008-1.6612852 .666707$

H $7.229668-2.167477-1.188311$

H $2.949861-2.771153-1.451645$

H $5.545898-4.6928431 .483992$

O $1.952588 \quad 0.659614 \quad 3.345810$

$\begin{array}{lllll}\text { O } & 4.237667 & 0.914030 & 3.196094\end{array}$

C $1.8773201 .856406 \quad 4.127247$

H $2.5460301 .796127 \quad 4.991610$

H $2.1247592 .722558 \quad 3.506802$

H $0.852313 \quad 1.9635384 .490997$

SCF Energy (B3LYP/6-31G*//MMFF) $=-3245.90078124$

0800258

MM̄FF Geometry

C $-0.531400 \quad 4.618981-1.278026$

C $-1.6972293 .955517-1.281611$

C $-2.6975684 .023123-0.158409$

O $-2.8015102 .684998 \quad 0.383158$

C $-4.082378 \quad 4.444782-0.677678$

C $-5.089113 \quad 4.669328 \quad 0.437820$

C $-6.106724 \quad 3.8398600 .745886$

$\begin{array}{llll}\text { C } & -4.906798 & 5.947887 & 1.217127\end{array}$

C $-6.473326 \quad 2.541166 \quad 0.084520$

C $-6.113801 \quad 1.3437550 .972880$

C -6.3095120 .0034440 .249039$

C $-5.902061-1.164916 \quad 1.150807$

C $-5.958675-2.5137580 .416732$

O $-5.496342 \quad 0.019039-0.928361$

O $-5.255853-3.4630421 .233432$

C $-5.201749-2.388751-0.925602$

C $-7.398102-3.0290510 .305796$

O $-5.341801-3.578012-1.697861$

C $-5.615594-1.150475-1.748340$

C $-4.739929-0.923353-3.004127$

O $-6.961942-1.324347-2.207568$

C $-1.9440292 .352729 \quad 1.386475$

O -1.1431273 .0860851 .944235$

C -2.1348790 .9168361 .683197$

C -1.482114 $0.365048 \quad 2.715589$

C $-1.604297-1.0275053 .132678$

C $-1.723566-1.1893774 .624186$

C $-3.243848-0.736740-2.733898$

C $-2.437482-2.017275-2.871317$

C $-0.232434-2.995593-2.243057$

O $-2.835641-3.002115-3.487387$

C $-0.549106-3.906014-1.099915$

C $0.242916-4.058198-0.028926$

C $-0.070538-4.9782951 .113383$

C $-0.339390-4.2325852 .399874$

C $0.533133-4.2709723 .420766$

C -1.663234 -3.512387 2.530315

C $-1.552458-2.0402752 .243975$

$\mathrm{N}-1.195314-1.911115-2.262981$

H $-0.301583 \quad 5.287823-0.453082$

H - $-1.9193883 .272221-2.098482$

H -2.364743 4.7217370 .618712

H $-4.4469283 .694662-1.386602$

H -3.997663 5.376429-1.253299

H $-6.7616274 .115991 \quad 1.571637$

H -5.7045706 .1053431 .950918$

H -3.958198 5.9381951 .762012

H $-4.911767 \quad 6.8086690 .540330$
H -7.553715 $2.541885-0.105657$

H $-6.0009452 .447984-0.895935$

H -5.0667101 .4354751 .280889$

H $-6.720693 \quad 1.3636661 .886616$

H -7.363521 $-0.081484-0.040695$

H $-6.521467-1.1918932 .056045$

H $-4.872540-1.0104351 .502039$

H $-5.214940-4.3014470 .741759$

H $-4.137094-2.313274-0.672613$

H $-7.831171-3.1650411 .304149$

H $-7.431899-4.015667-0.169141$

H $-8.053355-2.355019-0.249695$

H $-6.226421-3.567447-2.101225$

H $-4.925547-1.715209-3.739683$

H $-5.0922480 .002801-3.481044$

H $-7.106877-0.735930-2.967019$

H $-2.8096410 .346006 \quad 1.054716$

H -0.8307810 .9756503 .337770$

H $-1.738972-2.2307854 .951691$

H $-2.646943-0.7212154 .983320$

H $-0.876944-0.7131415 .131991$

H -2.836789 $-0.046045-3.483397$

H -3.072744 -0.285145-1.750585

H $0.763154-2.548809-2.156127$

H $-0.285150-3.549531-3.185788$

H $-1.474755-4.473581-1.162490$

H $1.170641-3.4965210 .031693$

H $0.779147-5.6626291 .234498$

H $-0.931710-5.618504 \quad 0.884074$

H $\quad 0.328958-3.761580 \quad 4.356514$

H $1.472358-4.8082613 .342127$

H -2.092054 -3.706345 3.518900

H -2.391057 -3.944049 1.831582

H $-1.429557-1.7985681 .188551$

H $-0.989954-1.089996-1.702789$

C $1.7230393 .615097-1.865887$

O $1.2783542 .316506-1.417822$

C $1.8971892 .034672-0.143951$

C $3.0996302 .960881-0.070086$

C $2.547790 \quad 4.212617-0.723914$

H 1.1571932 .3066820 .618105

C $2.2179230 .539761-0.058789$

H 3.4408823 .1320520 .954818

H $3.9372492 .558792-0.653290$

C $3.6218825 .182356-1.185091$

H 1.9030984 .7222020 .002039

C $3.014978 \quad 0.1500201 .196882$

O $0.980347-0.178852-0.051851$

H $2.7504650 .233365-0.965060$

H $3.9774780 .666543 \quad 1.208546$

$\begin{array}{lllll} & \text { O } & 2.316093 & 0.625675 & 2.356424\end{array}$

C $3.240987-1.3613841 .380498$

C $3.870579-2.1085720 .191964$

H $2.285513-1.8430991 .620854$

O $4.078361-1.5630402 .524385$

H $3.876336-3.1771570 .445648$

H $3.222931-1.991749-0.683961$

H $4.2152875 .531844-0.333895$

H $4.3059954 .713847-1.900599$

H $3.1742436 .056598-1.668279$

C $0.5221484 .445226-2.337347$

H $0.4671220 .128903-0.818177$

H 1.4356810 .2126012 .355928

H $3.665241-1.0973693 .271798$

H $0.0784593 .946776-3.208916$

H $0.8547675 .433256-2.674896$

H $2.3698893 .428879-2.732982$

C $6.6039370 .176075-0.990278$

O $5.321771-0.369832-0.653333$

C $5.320535-1.709464-0.157064$

C $5.920666-2.659339-1.209216$

C $7.307072-2.195322-1.665013$

C $7.319868-0.705591-2.026438$

H $6.3783091 .137423-1.469702$

$\begin{array}{llll}\text { C } 7.453817 & 0.458522 & 0.255270\end{array}$

H $5.921831-1.7580360 .756420$

O $5.058703-2.752138-2.342804$

H $5.992482-3.665054-0.778550$ 
H $7.611569-2.788575-2.535818$

O $8.238702-2.450669-0.618354$

H $8.347146-0.347800-2.161863$

O $6.646644-0.526069-3.276244$

C $6.822116 \quad 1.531765 \quad 1.112735$

H $7.604597-0.4370360 .864355$

H $8.4500250 .820142-0.024829$

H $4.923170-1.852735-2.687269$

H $9.116929-2.177387-0.933941$

H $7.126596-1.044113-3.944704$

O $6.579127 \quad 1.0461382 .363546$

O $\quad 6.5765192 .665135 \quad 0.719912$

C 5.9814831 .9690033 .279618

H 5.7908991 .4406514 .217609

H $6.6655192 .799847 \quad 3.475599$

H 5.0293232 .3373222 .886464

SCF Energy (B3LYP/6-31G**//MMFF) $=-3245.92385240$

08_00259

MMFF Geometry

C -1.047092 4.6348890 .768562

C $-2.229892 \quad 4.181976 \quad 0.328257$

C -3.3771853 .8372311 .244518$

O $-3.542522 \quad 2.3981241 .235630$

C $-4.674641 \quad 4.4872070 .733517$

C $-5.866977 \quad 4.2440331 .639660$

C $-6.873452 \quad 3.3854381 .378881$

C $-5.896288 \quad 5.0758212 .896691$

C $-7.026698 \quad 2.476403 \quad 0.191285$

C $-7.039358 \quad 1.0036750 .621030$

C $-6.9911330 .053037-0.584610$

C $-7.067278-1.403635-0.123859$

C $-6.882306-2.392015-1.285238$

O $-5.757797 \quad 0.286804-1.271838$

O $-6.633149-3.690186-0.723428$

C $-5.621306-1.998858-2.084957$

C $-8.155441-2.522120-2.129391$

O $-5.496808-2.793605-3.261543$

C $-5.571828-0.502523-2.454156$

C $-4.221603-0.077736-3.082581$

O $-6.591263-0.224782-3.422048$

C -2.735652 1.6863472 .073166

O $\begin{array}{llll}-1.890271 & 2.134305 & 2.832591\end{array}$

$\begin{array}{llll}C & -3.046720 & 0.248750 & 1.908098\end{array}$

C $-2.282668-0.6618092 .530621$

C $-2.464238-2.1151852 .511398$

C $-3.595746-2.6684401 .687794$

C $-2.993648-0.248628-2.181589$

C $-2.232549-1.546036-2.397559$

C $-0.318481-2.837666-1.449376$

O $-2.444976-2.306231-3.337631$

C $-0.793232-3.895948-0.506151$

C $-0.051950-4.3648870 .508714$

C $-0.491440-5.468586 \quad 1.425338$

C $-0.450887-5.0700672 .882480$

C $0.588735-5.4083593 .663852$

C $-1.659998-4.3670103 .454218$

C $-1.624136-2.8790823 .241041$

$\mathrm{N}-1.250149-1.726839-1.433125$

H -0.9010904 .7900591 .834987$

H -2.369756 $4.004226-0.735463$

H -3.179118 4.1787882 .268323

H $-4.883366 \quad 4.137159-0.282597$

H $-4.532272 \quad 5.5723870 .638453$

H -7.686518 3.3154942 .100713

H -6.8077774 .9137293 .482009$

H $-5.045740 \quad 4.8322273 .540510$

H -5.8518716 .1413032 .648094$

H -7.971835 $2.716261-0.310647$

H -6.239003 $2.639748-0.546876$

H $-6.1717480 .814571 \quad 1.265157$

H -7.9379680 .8030481 .217769$

H $-7.8281640 .290854-1.251630$

H $-8.012536-1.596576 \quad 0.398739$

H $-6.276844-1.5900740 .614653$

H -7.387739 -3.920504 -0.154659

H $-4.757252-2.238030-1.454021$

H -8.987429 -2.884635 - 1.513656
H -8.028156 -3.263850 -2.925616

H $-8.468659-1.578797-2.581367$

H $-5.511734-3.725820-2.984591$

H $-4.085233-0.567851-4.054035$

H -4.295202 $0.995953-3.308668$

H $-6.3527970 .588016-3.897765$

H -3.895872 -0.0196761 .292013$

H $-1.444670-0.3104513 .132081$

H $-4.558293-2.3781902 .121456$

H -3.587406 -3.7576671 .610477$

H $-3.544867-2.2946220 .659943$

H $-2.2844340 .556905-2.412449$

H -3.261095 -0.152407-1.125467

H $0.661890-2.440759-1.167053$

H $-0.248110-3.248055-2.461689$

H $-1.779873-4.316436-0.686111$

H $\quad 0.945079-3.9610300 .669044$

H $\quad 0.173376-6.325784 \quad 1.254884$

H $-1.497509-5.8245541 .171329$

H $0.609079-5.1537524 .718463$

H $1.445049-5.9419763 .264849$

H $-1.706580-4.5359104 .538960$

H -2.571379 -4.8324643 .068322$

H $-0.830616-2.3838183 .802770$

H $-1.196180-1.078914-0.652787$

C 1.2493683 .8918660 .062225

$\begin{array}{lllll}\text { O } & 2.512213 & 4.498921 & -0.282671\end{array}$

C $3.3373483 .516025-0.946601$

C $2.5833542 .197797-0.853159$

C $1.1401002 .658414-0.833714$

H $3.4098293 .844903-1.990629$

C $4.7261873 .496287-0.293646$

H $2.8078731 .526614-1.687914$

H $2.827157 \quad 1.6735370 .079225$

C $0.1899851 .580622-0.347579$

H $0.8596532 .953385-1.853707$

C $5.6485912 .398728-0.861902$

O $5.3240674 .778013-0.502148$

H 4.6256603 .3666790 .790820

H $5.1422351 .436001-0.745298$

O $5.8227472 .605546-2.263766$

C $7.0271122 .296009-0.177253$

C $7.953578 \quad 1.228217-0.789818$

H 6.8868932 .0852480 .889523

$\begin{array}{llllll}\text { O } & 7.735269 & 3.533577 & -0.270494\end{array}$

H $8.2243691 .516657-1.813741$

H $8.8916621 .257619-0.218997$

H $-0.8505721 .868912-0.511990$

H $\quad 0.362190 \quad 0.648315-0.893660$

H $0.321003 \quad 1.3781720 .719410$

C $0.1318114 .924043-0.112505$

H $4.7156165 .444568-0.139330$

H $6.2776673 .458099-2.375761$

H 7.2138334 .2079710 .196498

H $-0.1636074 .991221-1.166841$

H 0.5075305 .9220990 .147195

H $1.3360533 .597922 \quad 1.117007$

C $6.523400-1.8571120 .767482$

O $7.100456-0.5584550 .570352$

C $7.389681-0.201894-0.784259$

C $8.371193-1.205041-1.411405$

C $7.854650-2.641362-1.280293$

C $7.408629-2.9557030 .149953$

H $6.527066-2.0012921 .855666$

C $5.061387-1.9154840 .293287$

H $6.464957-0.212033-1.368366$

O $9.654025-1.103772-0.796712$

H $8.504765-0.953609-2.469987$

H $8.646929-3.342243-1.570928$

O $6.750721-2.837250-2.159023$

H $6.883828-3.9179870 .176158$

O $8.582792-3.0991730 .954726$

C $4.136576-1.1549341 .222280$

H $4.921921-1.504057-0.709127$

H $4.732593-2.9617370 .293531$

H $9.539927-1.2562460 .157061$

H $7.061865-2.664618-3.063981$

H $8.298476-3.3704991 .844169$ 
O $2.858994-1.6035441 .042796$

O $4.485844-0.2732521 .995169$

C $1.859493-1.0124581 .880109$

H $1.887396-1.4836852 .866470$

H 1.9986780 .0687791 .969290

H $0.882972-1.2007291 .426426$

SCF Energy (B3LYP/6-31G**//MMFF) $=-3245.91623764$

08 00260

MM̄FF Geometry

C $1.091430 \quad 3.2732220 .989542$

C 1.7278554 .1745030 .224188

C $2.8376253 .909282-0.768497$

O $3.0953122 .495731-0.917959$

C $4.1387504 .581845-0.300577$

C $5.1752284 .677818-1.403288$

C $6.2498763 .874676-1.524828$

C $4.9668725 .806853-2.381315$

C $6.6338892 .709262-0.656868$

C $6.9921701 .479644-1.504608$

C $7.4870910 .318414-0.631913$

C $7.978096-0.843689-1.495783$

C $8.409654-2.043819-0.639242$

O $6.400343-0.1166930 .190274$

O $8.549271-3.178485-1.507565$

C $7.282162-2.3746650 .360063$

C $9.781089-1.8217730 .009266$

O $7.694300-3.3836991 .279403$

C $6.749188-1.1422891 .129705$

C $5.473198-1.5319111 .908852$

O $7.740054-0.6942152 .055539$

C $2.3193571 .814873-1.804890$

O $1.4423942 .271572-2.520760$

C $2.6892530 .386276-1.714064$

C $1.806626-0.535047-2.127200$

C $1.971707-1.987159-2.050054$

C $3.329450-2.516896-1.676245$

C $4.719710-0.3476732 .526723$

C $3.360331-0.8011813 .018913$

C $1.096137-1.4371762 .182879$

O $3.157094-1.0686994 .200478$

C $1.049075-2.8287631 .631177$

C $0.076164-3.2670380 .817674$

C $0.017153-4.6579430 .253046$

C $-0.300870-4.679953-1.228036$

C $-1.493148-5.113319-1.671280$

C $0.785379-4.262417-2.198843$

C $0.899536-2.766706-2.301779$

N $2.432084-0.9057531 .994313$

H 1.3801392 .2264520 .962159

H $1.406557 \quad 5.2135260 .280048$

H $2.5307284 .329205-1.734472$

H 4.5436974 .0456200 .565400

H 3.9343085 .6001240 .057212

H $6.9458124 .078018-2.338187$

H $5.7674845 .865735-3.126456$

H $4.0245845 .680328-2.922739$

H $4.9392056 .765893-1.853582$

H $7.5048333 .004207-0.059377$

H 5.8378252 .4424190 .043560

H $6.1044131 .167830-2.070138$

H $7.7702081 .746781-2.230805$

H 8.2991800 .6887040 .005040

H $8.799081-0.524776-2.149928$

H $7.168848-1.167778-2.165354$

H $9.208832-2.956422-2.186990$

H $6.452303-2.804276-0.218735$

H $10.544771-1.651852-0.759283$

H $10.105466-2.7107420 .561401$

H $9.801135-0.9672290 .688838$

H $8.005836-4.1422870 .756489$

H $4.792563-2.0588721 .231393$

H $5.724240-2.2369362 .711885$

H $7.305770-0.1897712 .761525$

H $3.6440330 .134050-1.267278$

H $0.849703-0.196195-2.522786$

H $3.553705-2.282944-0.632917$

H $4.106792-2.074305-2.309546$
H $\quad 3.424111-3.598184-1.794994$

H $5.269316 \quad 0.074877 \quad 3.374443$

H $4.5734200 .462671 \quad 1.803579$

H $0.410664-0.7648941 .659727$

H $0.844469-1.4472883 .247592$

H $1.846445-3.5094651 .920143$

H $-0.733744-2.5926360 .553231$

H $-0.752481-5.2083940 .809672$

H $0.957796-5.1961950 .423644$

H -1.721849 -5.151924 -2.731200

H $-2.270015-5.433146-0.984684$

H $0.562327-4.646156-3.203022$

H $1.719454-4.746688-1.902811$

H $-0.034807-2.280897-2.588392$

H $2.717549-0.6826131 .045652$

C -1.3874242 .9946631 .505706$

O -1.3708311 .5771431 .756326$

C $-2.1648990 .921280 \quad 0.740903$

C $-2.8300112 .031187-0.065786$

C -1.8181753 .1529080 .048903$

H -1.4493230 .3707510 .117432$

C $-3.146570-0.0500391 .412642$

H -3.026734 $1.735795-1.100831$

H -3.7827432 .3273200 .388616$

C $-2.3771824 .523594-0.286430$

H $-0.9906902 .926483-0.634450$

C -4.114818 $-0.722708 \quad 0.417377$

O $-2.382308-1.0434502 .095447$

H -3.7260310 .4775192 .180169$

H $-4.7058740 .069556-0.050291$

O $-3.372641-1.351727-0.627942$

C $-5.075698-1.7491451 .052249$

C $-6.087553-2.3524780 .055916$

H $-5.608813-1.2731721 .883448$

O $-4.321027-2.8327501 .599736$

H $-5.553024-2.887699-0.738996$

H $-6.658943-3.1184090 .597124$

H -2.746554 $4.547226-1.316766$

H -3.2075314 .7918390 .374415$

H -1.605440 $5.293214-0.186281$

C $-0.0504073 .630391 \quad 1.901771$

H -1.810148 -0.5823022 .732571$

H $-2.873563-2.084109-0.227333$

H $-4.942393-3.4194752 .063446$

H $\quad 0.220083 \quad 3.3017402 .912717$

H $-0.1748954 .719345 \quad 1.942420$

H -2.161115 3.4039212 .169658

C $-8.674491 \quad 0.334414 \quad 0.153232$

$\begin{array}{lllll}\text { O } & -7.773082 & -0.717018 & 0.521257\end{array}$

C $-7.055406-1.327538-0.554962$

C $-8.031822-1.966837-1.552794$

C $-9.049296-0.934224-2.052872$

C $-9.687407-0.155629-0.897380$

H -9.2321670 .5606561 .071609$

C $-7.9232811 .609699-0.261878$

H $-6.470386-0.569859-1.082733$

O $-8.716505-3.049786-0.927452$

H -7.460707 -2.377396 -2.394103

H -9.839099 -1.440638 -2.620962

O -8.405206 -0.011713 -2.926881

H $-10.2528150 .698675-1.286295$

O -10.636782 -1.003346 -0.242422

C -7.1765222 .2316160 .901663$

H -7.192895 $1.426408-1.053669$

H $-8.6507382 .349017-0.617587$

H $-9.303943-3.448195-1.592093$

H -8.034532 $-0.519658-3.668604$

H $-10.146354-1.7334760 .172951$

O $-6.7887893 .495440 \quad 0.556825$

O -6.9483291 .6817521 .970440$

C -6.0931344 .2243561 .573511$

H -6.7614214 .4178042 .417919$

H -5.2057023 .6759201 .902933$

H -5.7765655 .1808521 .149522$

SCF Energy (B3LYP/6-31G**//MMFF) $=-3245.91268975$

0800261

MM̄MF Geometry 
C $-0.948424-1.4572542 .606006$ C -1.521943 -2.350073 1.782515 C $-0.899451-2.942410 \quad 0.538536$ O $0.480078-2.5326850 .431015$ C - $1.656685-2.416405-0.697208$ C $-1.067636-2.852531-2.024833$ C $-0.465518-2.024656-2.904012$ C $-1.204666-4.319294-2.339107$ C $-0.262798-0.539025-2.771985$ C $1.140052-0.211553-2.261019$ C $1.2761751 .217793-1.718469$

C $1.1515812 .278040-2.814347$ C $1.4164923 .697019-2.280642$ O $2.5674111 .299489-1.096852$ O $1.6165554 .560043-3.411564$ C $2.7354363 .692230-1.476996$ C $0.2073384 .256250-1.521957$ O $2.9607844 .953667-0.852689$ C $2.8227712 .549835-0.442583$ C 4.2083492 .4603310 .236190 $\begin{array}{lllll}\text { O } & 1.868491 & 2.789503 & 0.599929\end{array}$ C $1.452717-3.4606490 .631039$ O $1.311504-4.6414260 .901233$ C $2.747502-2.770516 \quad 0.439304$ C $3.881944-3.4745750 .315387$ C $5.208082-2.9092560 .059729$ C $5.321305-1.410101-0.039563$ C $5.3945852 .262998-0.711205$ C $6.687336 \quad 1.9970750 .044584$ C $9.107507 \quad 1.544807-0.347557$ $\begin{array}{lllll}\text { O } & 6.752346 & 1.952287 & 1.270345\end{array}$ C $9.3826660 .079527-0.473477$ C $9.475166-0.7526130 .575053$

C $9.792112-2.2173190 .462845$ C $8.589068-3.0882470 .757753$ C $8.403932-3.5942621 .989235$ C $7.678308-3.357478-0.423380$ C $6.262228-3.740028-0.080784$ N $7.762246 \quad 1.820163-0.816346$ H $0.069570-1.1278842 .421167$ H -2.542427 -2.667249 1.986320 H $-0.983330-4.0340620 .592522$ H - $1.692926-1.325212-0.637970$ H $-2.703276-2.746398-0.657515$ H - $0.067899-2.450335-3.824249$ H $-0.838541-4.565312-3.341550$ H -0.636286 $-4.922170-1.624434$ H -2.256382 -4.620029-2.291598 H $-0.418000-0.080970-3.756175$ H -1.018217 $-0.094593-2.120277$ H $1.412872-0.902461-1.456915$ H $1.881890-0.386668-3.051345$ H $0.5079981 .365028-0.949719$ H $0.1680722 .234540-3.297182$ H $1.8831242 .065811-3.606700$ H $0.8204324 .514574-3.968229$ H $3.5406743 .570664-2.211845$ H $-0.6672994 .304734-2.181481$ H $0.386320 \quad 5.286293-1.194701$ H $-0.0674713 .658027-0.650834$ H $2.3834055 .005405-0.071972$ H $4.377334 \quad 3.3503590 .856767$ H 4.1863901 .6168700 .940698 H 2.1318492 .2772351 .382505 H $2.726555-1.6882580 .374492$ H $3.834546-4.5605830 .378968$ H $4.745004-1.037605-0.893265$ H $6.347763-1.061457-0.167711$ H $4.949357-0.9334930 .874252$ H $5.2109141 .417372-1.382506$ H $5.5468673 .161836-1.318315$ H $9.220251 \quad 1.8953920 .683764$ H $9.8016492 .109259-0.977677$ H $9.531919-0.308954-1.477980$ H $9.325967-0.3609021 .578851$ H $10.608468-2.4418891 .161626$ H $10.186526-2.464129-0.531152$ H $7.567810-4.241046 \quad 2.229738$
H $9.097546-3.3747252 .794849$

H $8.120186-4.164421-1.022522$

H $7.665755-2.488574-1.091424$

H $6.104705-4.8137190 .021553$

H $7.6006321 .825584-1.819396$

C - $-1.9143130 .661792 \quad 3.623560$

O $-2.6958150 .909673 \quad 2.435980$

C -2.1662252 .0754801 .776299$

C -1.2728002 .7641812 .801172$

C $-0.684903 \quad 1.5662893 .523412$

H $-1.532597 \quad 1.7129540 .957726$

C $-3.293102 \quad 2.947822 \quad 1.213174$

H -0.5155373 .4000852 .332947$

H -1.8532743 .3810333 .497015$

C $-0.058321 \quad 1.9078604 .863433$

H 0.0807001 .1197722 .877652

C $-4.2428562 .215546 \quad 0.239764$

$\begin{array}{llll}\text { O } & -4.080825 & 3.438077 & 2.304858\end{array}$

H -2.8586653 .8302160 .727771$

H $-4.869346 \quad 1.5165240 .802816$

O $-5.138278 \quad 3.190546-0.308685$

C $-3.5351741 .480066-0.909019$

C $-4.457130 \quad 0.927431-2.008755$

H $-2.9427590 .657823-0.496159$

O $-2.6209602 .387294-1.531269$

H $-4.8807771 .754177-2.593528$

H $-3.8094910 .382443-2.708622$

H 0.7702512 .6114054 .731905

H $-0.7849762 .367904 \quad 5.541515$

H 0.3350091 .0094295 .349274

C $-1.646264-0.8390903 .785966$

H -4.7901073 .9859801 .927397$

H $-4.6108923 .797671-0.855867$

H $-2.156127 \quad 1.904676-2.235395$

H -2.604930 -1.346857 3.948205

H $-1.043436-1.0153984 .684025$

H -2.5386810 .9844664 .467414$

C $-5.955482-1.936533-0.117196$

O $-5.021547-1.010492-0.691915$

C $-5.5874940 .006295-1.523199$

C $-6.349659-0.618902-2.705563$

C $-7.386248-1.640502-2.227510$

C $-6.788994-2.621487-1.213768$

H $-5.332608-2.7076820 .354061$

C $-6.819159-1.2858090 .976029$

H $-6.2808840 .610580-0.929735$

O $-5.426646-1.266759-3.580826$

H $-6.8364990 .182104-3.274692$

H -7.769458 -2.206206 -3.085472

O $-8.486223-0.963537-1.627649$

H $-7.586287-3.213462-0.750208$

O $-5.946110-3.548160-1.905567$

C $-6.001890-0.8681742 .178364$

H $-7.334022-0.3971970 .602245$

H -7.570796 -1.999010 1.332936

H $-5.931872-1.613884-4.335686$

H $-8.868664-0.372042-2.298048$

H $-5.185550-3.053850-2.256781$

$\begin{array}{llll}\text { O } & -6.728337 & 0.010303 & 2.930306\end{array}$

O $-4.879692-1.276272 \quad 2.440343$

$\begin{array}{llll}\text { C } & -6.081326 & 0.491911 & 4.112455\end{array}$

H -6.7965861 .1077424 .664233$

H $-5.772990-0.3423974 .749913$

H $-5.221217 \quad 1.1105293 .842179$

SCF Energy (B3LYP/6-31G**//MMFF)= -3245.91722643

0800262

MM̄FF Geometry

C $0.094943-2.7309010 .874272$

C $0.572249-2.5045862 .108914$

C $0.073606-1.4680643 .095225$

O $-1.140726-0.8362322 .629283$

C $1.156082-0.386013 \quad 3.269134$

C $0.875645 \quad 0.576071 \quad 4.406678$

C 0.5077331 .8640204 .258850

C $1.080526 \quad 0.011523 \quad 5.790143$

C $0.2628172 .616873 \quad 2.981847$

C -1.2035543 .0497902 .881960$ 
C -1.5273113 .6990051 .528305$ C -2.977796 4.1870231 .520831 C $-3.402197 \quad 4.7197020 .147208$ O $-1.325523 \quad 2.7090360 .517012$ $\begin{array}{lllll}\text { O } & -4.830585 & 4.865317 & 0.152428\end{array}$ C $-3.0607773 .658615-0.916989$ C -2.823473 $6.111256-0.132314$ O $-3.3129834 .159944-2.228789$ C -1.608623 $3.132596-0.821513$ C $-1.3970021 .914237-1.755696$ O $-0.7142414 .167848-1.238753$ C -2.310424 -1.472736 2.918928 O $-2.451751-2.4695353 .610298$ C $-3.399088-0.7882732 .185105$ C $-4.532532-1.4662711 .947222$ C $-5.693986-1.0016801 .186647$ C -5.7292180 .4376100 .748552$ C $-1.9685360 .609383-1.195562$ C -2.311911 -0.417537-2.256877 C $-3.555880-2.571881-2.479023$ O $-2.085777-0.260704-3.452515$ C $-5.015742-2.252110-2.581754$ C $-5.992503-3.049099-2.122511$ C $-7.453376-2.712196-2.223278$ C $-8.169215-2.778601-0.891079$ C $-9.049697-3.759633-0.629554$ C $-7.929183-1.6635670 .107893$ C $-6.680602-1.8841670 .920986$ N -2.923404 -1.530684 -1.692252 H $-0.746062-2.1391180 .521417$ H $1.412217-3.0929602 .469848$ H - $0.110641-1.9673244 .054039$ H 1.2835170 .1500752 .323112 H $2.129344-0.8573403 .465123$ H $0.3572852 .458645 \quad 5.159535$ H 0.9579350 .7692026 .571537 H $0.360305-0.7867555 .992851$ H $2.091643-0.3970265 .887798$ H $0.905727 \quad 3.5051052 .977108$ H 0.5401182 .0323122 .102405 H -1.842294 2.167184 3.019219 H -1.4346173 .7504363 .694359$ H $-0.8360654 .532581 \quad 1.358057$ H -3.1345524 .9498492 .293871$ H -3.643802 3.3534691 .786395 H -5.0668595 .4774190 .870381$ H -3.754830 $2.821488-0.772356$ H -3.167297 6.8275110 .623527 H -3.175568 $6.499676-1.094342$ H -1.731742 $6.130406-0.130609$ H $-4.2343754 .470769-2.248143$ H - $1.794308 \quad 2.141592-2.752184$ H $-0.3239561 .754519-1.912214$ H $0.137233 \quad 3.763337-1.474062$ H $-3.2199570 .216232 \quad 1.821507$ H $-4.608057-2.4881232 .318702$ H $-5.0678410 .592449-0.107624$ H $-5.4203491 .106061 \quad 1.559929$ H $-6.729436 \quad 0.7706330 .459543$ H -1.245966 $0.161420-0.503078$ H -2.885232 $0.807845-0.635155$ H $-3.373994-3.523313-1.969917$ H -3.108754 -2.611953 -3.477004 H $-5.280035-1.311590-3.060169$ H -5.732466 -3.998844 -1.660734 H - $-7.907070-3.417449-2.931759$ H -7.603218 -1.715901-2.657778 H $-9.587047-3.7996290 .312304$ H $-9.249376-4.547432-1.348239$ H -8.772649 -1.6026610 .807752$ H $-7.919607-0.713460-0.434362$ H $-6.586826-2.8964641 .317443$ H -3.100906 -1.534934 -0.692292 C $1.919888-4.4468740 .184972$ O $2.974484-3.4880190 .389269$ C $4.101849-3.839728-0.427000$ C $3.900639-5.305674-0.781658$ C $2.393308-5.357755-0.952713$
H $4.023089-3.239062-1.342152$

C $5.411551-3.4945170 .290411$

H $4.446759-5.595948-1.684060$

H $4.208345-5.9697120 .034043$

C $1.819827-6.762854-0.892956$

H $2.138007-4.911976-1.923694$

C $5.441799-2.0806420 .911067$

O $5.616885-4.4289591 .357518$

H $6.250360-3.633352-0.401845$

H $4.820558-2.0681451 .814999$

O $6.780258-1.8281691 .356667$

C $4.994529-0.945720-0.024780$

C 5.1417140 .4422440 .631173

H $3.947986-1.102324-0.307842$

O $5.778907-1.004041-1.215443$

H $4.823227 \quad 0.392020 \quad 1.679198$

H 6.1978960 .7368420 .601493

H $2.231077-7.380571-1.698006$

H $2.053611-7.2516660 .058816$

H $0.730983-6.743687-1.003409$

C $0.617710-3.709242-0.147161$

H $6.455557-4.194298 \quad 1.790773$

H $7.344138-1.7647050 .566631$

H $5.458107-0.307716-1.813428$

H $-0.174160-4.439197-0.352055$

H $0.770003-3.133585-1.069078$

H $1.814196-5.0156361 .116921$

C $3.9961992 .494197-2.275750$

O $4.759647 \quad 1.623963-1.430823$

C $4.2911551 .508317-0.085765$

C 4.3519462 .8763180 .607850

C $3.5826383 .937569-0.189448$

C $3.9384253 .911736-1.681703$

H $4.5637202 .544938-3.213988$

C $2.6146071 .904750-2.595812$

H $3.2550431 .151912-0.088140$

$\begin{array}{lllll}\text { O } & 5.707298 & 3.292368 & 0.763587\end{array}$

H 3.9261202 .7848341 .613811

H $3.807510 \quad 4.9312920 .216922$

O $2.1821993 .719650-0.049348$

H $3.2306434 .519037-2.258368$

O $5.2335294 .495442-1.855026$

C $2.7145160 .542100-3.249662$

H $2.0203401 .792196-1.685893$

H $2.0699682 .563866-3.280688$

H $6.1171613 .306558-0.118321$

H 1.9664553 .8039700 .894545

H $5.1856515 .413576-1.538018$

O $1.546332-0.137395-3.052075$

$\begin{array}{lllllll}0 & 3.685373 & 0.131410 & -3.870727\end{array}$

C $1.497961-1.454371-3.609231$

H $1.615362-1.410671-4.696035$

H $2.272612-2.083528-3.160509$

H $0.519889-1.885245-3.379426$

SCF Energy (B3LYP/6-31G**//MMFF) $=-3245.92742829$

0800263

MM̄FF Geometry

C $3.004066-1.4138022 .200179$

C $3.850785-2.4480402 .074769$

C $4.876159-2.6702010 .986150$

O $4.961598-1.533793 \quad 0.095123$

C $4.503640-3.8608320 .078314$

C $4.265841-5.180258 \quad 0.780415$

C $3.040827-5.6763461 .048667$

C $5.504911-5.951799 \quad 1.143383$

C $1.727548-4.9899870 .793671$

C $0.986824-5.585516-0.406211$

C $-0.140430-4.654163-0.877483$

C $-1.023827-5.360298-1.905881$

C $-2.084646-4.417956-2.488814$

O $0.476311-3.498742-1.460690$

O $-2.658199-5.056444-3.639603$

C $-1.384449-3.137505-2.983948$

C $-3.237357-4.169966-1.508001$

O $-2.344726-2.179718-3.423415$

C $-0.436755-2.500486-1.938651$

C $0.407342-1.391663-2.621362$ 
O $-1.211481-1.949253-0.877253$

C $5.762558-0.4934260 .440344$

O $6.460639-0.390507 \quad 1.434597$

C $5.5892470 .533234-0.610213$

C $5.897341 \quad 1.811277-0.350307$

C $5.7020692 .940776-1.261984$

C $5.1943362 .639457-2.648622$

C $1.388003-0.641606-1.704045$

C $0.819010 \quad 0.653235-1.141803$

C 1.5139922 .7349620 .044132

O $-0.3707080 .948700-1.214302$

C $2.0722373 .806948-0.838997$

C $2.7343954 .876026-0.369541$

C $3.2642585 .981289-1.240087$

C $4.6997766 .345663-0.925414$

C $4.9909167 .434971-0.194174$

C $5.7978075 .494603-1.525246$

C $5.9569184 .184747-0.805699$

N $1.7943651 .433221-0.535207$

H $3.034173-0.5899891 .493813$

H $3.789602-3.2433022 .816064$

H $5.856961-2.8513781 .442891$

H $5.303571-3.996562-0.663554$

H $3.638806-3.588170-0.541348$

H $2.964554-6.6498051 .529701$

H $5.272893-6.9031241 .633905$

H $6.089952-6.1761050 .245607$

H $\quad 6.128671-5.370077 \quad 1.829438$

H $\quad 1.105182-5.0933371 .690983$

H $1.862830-3.9138130 .658778$

H $1.693064-5.741511-1.232343$

H $0.581007-6.565675-0.126909$

H $-0.734431-4.347378-0.009253$

H -1.497839 -6.247555 -1.468028

H $-0.399015-5.731753-2.730558$

H $-3.040238-5.903661-3.352862$

H -0.790662 -3.408384 -3.868416

H -3.736088 -5.113913-1.257475

H $-4.008389-3.533685-1.956569$

H -2.913715 -3.710707 -0.571624

H $-2.907094-2.615388-4.086506$

H $1.010579-1.870203-3.404821$

H $-0.249989-0.670458-3.120575$

H $-0.685616-1.278440-0.415504$

H $5.1593210 .214168-1.553232$

H 6.2965572 .0605930 .631948

H $5.8997951 .991743-3.181137$

H $5.0535793 .527178-3.267729$

H $4.2220442 .136602-2.604985$

H $1.725739-1.271835-0.874857$

H $2.270457-0.371183-2.298260$

H 1.9606202 .7483651 .043050

H $\quad 0.4334192 .8729590 .134617$

H $1.9005253 .716065-1.909002$

H 2.8721344 .9826230 .704326

H $2.6186176 .856833-1.091914$

H $3.1811295 .726701-2.303996$

H 6.0172517 .7186620 .014104

H 4.2089218 .0645110 .217396

H $6.7606236 .019442-1.463668$

H $5.6130515 .377687-2.596257$

H $\quad 6.317411 \quad 4.297017 \quad 0.217884$

H $2.756100 \quad 1.107323-0.525417$

C $0.569603-1.0511902 .642787$

O $0.4001380 .382632 \quad 2.564929$

C $-0.876247 \quad 0.728746 \quad 3.147723$

C -1.679943 -0.5584373 .129168$

C $-0.605784-1.5692253 .472089$

H $-0.6626661 .026348 \quad 4.182923$

C -1.4879391 .9027992 .376514$

H -2.505314 -0.5563003 .846302$

H -2.084496 -0.750399 2.127940

C -1.000704-3.000483 3.152736

H -0.374652 -1.491382 4.543070

C -2.9659342 .1820432 .701734$

O -0.7076243 .0665792 .674473$

H -1.369100 $1.723843 \quad 1.304425$

H -3.5839701 .3408252 .374625$
O $-3.0970302 .244072 \quad 4.128985$

C -3.5147753 .4997682 .124896$

C -3.3074123 .7335300 .618362$

H -3.0625474 .3431412 .662222$

O $-4.919616 \quad 3.5729832 .390489$

H $-3.651954 \quad 4.7542990 .404347$

H -2.2352703 .7048060 .394206$

H -1.878070 -3.296678 3.736760

H -1.247802 -3.1208672 .092898$

H $-0.186614-3.6915843 .391297$

C $1.937063-1.3609243 .261377$

H -0.8371603 .2748583 .615638$

H -4.0445342 .1887074 .338639$

H -5.0342913 .6427833 .353073$

H $1.899483-2.3197893 .791715$

H $2.210436-0.5861883 .987424$

H $0.504429-1.4344011 .616537$

C $-4.0871190 .461109-1.046214$

O $-3.4806511 .482703-0.243338$

C $-4.0762282 .779652-0.321503$

C $-4.0443723 .295559-1.771848$

C $-4.6902412 .296535-2.734778$

C $-4.1481070 .880318-2.524839$

H $-3.403853-0.392869-0.973376$

C $-5.4469120 .027020-0.485176$

H -5.1163822 .7203940 .014558$

O $-2.6920423 .530021-2.162439$

H $-4.5728914 .255558-1.817018$

H -4.494455 $2.613381-3.766401$

O $-6.0998802 .317168-2.533402$

H $-4.7514800 .156781-3.084494$

O $\quad-2.828627 \quad 0.813417-3.072533$

C $-5.290671-0.6496880 .858105$

H $-6.1333410 .871472-0.378122$

H -5.931809 -0.696926-1.150166

H $-2.7053693 .886392-3.066977$

H $-6.4958051 .694106-3.166332$

H $-2.2467421 .360833-2.517478$

O $-6.079378-0.0323331 .784015$

O $-4.565810-1.6153471 .059963$

C $-6.034341-0.5872343 .102169$

H -6.403212 -1.617107 3.090712

H $-5.015965-0.5462173 .498639$

$\mathrm{H}-6.6842160 .0120203 .745321$

SCF Energy $\left(B 3 L Y P / 6-31 G^{* *} / / M M F F\right)=-3245.91332241$

08_00264

MM̄FF Geometry

C $1.094185-1.3186860 .883671$

C $-0.158138-1.1485780 .437105$

C $-0.502853-1.002572-1.020385$

O $-0.9219330 .369563-1.189164$

C $-1.672013-1.926713-1.395185$

C $-1.843479-2.091690-2.891079$

C $-2.759684-1.445062-3.637663$

C $-0.922860-3.098623-3.531781$

C $-3.739945-0.393331-3.197817$

C $-5.194913-0.878127-3.260723$

C $-5.554416-1.872828-2.146062$

C $-6.993826-2.366253-2.307749$

C $-7.422946-3.272804-1.144031$

O $-5.411771-1.193116-0.895129$

O $-8.849736-3.422741-1.204015$

C $-7.098818-2.5633000 .187398$

C $-6.832865-4.682379-1.270166$

O $-7.345064-3.424127 \quad 1.297270$

C $-5.657545-2.007580 \quad 0.258011$

C $-5.499574-1.1125951 .511271$

$\begin{array}{lllll}\text { O } & -4.739856 & -3.099441 & 0.340896\end{array}$

C $-0.4411691 .073461-2.243355$

O $0.2941110 .658597-3.128355$

C $-0.8866592 .486558-2.193347$

C $-1.7717172 .953276-1.298726$

C $-2.2055334 .344013-1.149987$

C $-1.5629705 .378042-2.036345$

C $-4.181640-0.3263421 .559319$

C $-4.098846 \quad 0.5794752 .775998$

C -2.6811542 .3829543 .754847$ 
O $-4.930565 \quad 0.5708213 .679546$

C -3.355809 3.6674193 .389328 C $-2.701894 \quad 4.802442 \quad 3.099021$ C -3.391663 6.0873002 .736854 C -3.0117696 .5913931 .361261$ C $-2.1514657 .613321 \quad 1.216109$ C $-3.694566 \quad 5.9738520 .159587$ C -3.123662 $4.631799-0.203890$ N -2.9719451 .3903852 .736498$ H $1.909835-1.3538470 .165851$ H $-0.971690-1.075668 \quad 1.153705$ H $0.380373-1.220296-1.632969$ H -2.597795 -1.548286 -0.948883 H $-1.521817-2.924331-0.960557$ H -2.801022 -1.669415 -4.703011 H $-1.105647-3.205716-4.606296$ H $\quad 0.122257-2.799778-3.404760$ H - $1.061277-4.083855-3.074655$ H $-3.510745-0.000964-2.202983$ H -3.629879 $0.457920-3.881749$ H $-5.8391150 .006070-3.168072$ H -5.396937-1.324471-4.242350 H $-4.858731-2.718315-2.182025$ H -7.122071 -2.884698 -3.266194 H -7.673522 -1.503046 -2.341328 H $-9.074929-3.802035-2.070833$ H $-7.798637-1.7215830 .287427$ H -7.162138 -5.152835 -2.204512 H -7.190551 -5.333948 -0.465270 H -5.741032 -4.692815 -1.263970 H $-8.261798-3.7396571 .221133$ H $-6.320210-0.3832821 .537780$ H -5.579129 -1.725857 2.417719 H -3.929037 -2.8041480 .783048$ H - $0.4245623 .133096-2.932489$ H -2.216467 2.259760 -0.587038 H -0.474009 5.367801-1.913645 H -1.887890 6.398212 -1.821024 H $-1.8004735 .182982-3.087955$ H -3.329142 -1.010500 1.601655 $\begin{array}{llll}\text { H } & -4.080460 & 0.298206 & 0.664257\end{array}$ H -1.5934912 .4859923 .811494$ H -3.049327 2.0328374 .724503 H -4.4433063 .6620943 .369008$ H $-1.614906 \quad 4.815514 \quad 3.129727$ H -3.127114 6.8319573 .499012 H -4.4827445 .9853562 .793559$ H - 1.8959128 .0028780 .236341 H -1.679737 8.0811192 .073933 H -3.666473 $6.657792-0.695980$ H $-4.764435 \quad 5.8531710 .374021$ H $-3.529316 \quad 3.8115130 .388287$ H -2.3822391 .3772411 .909132$

C $2.466362-2.5093912 .663482$

O $3.698541-2.2663491 .957999$

C $4.231516-3.5310411 .516750$

C $3.425114-4.6034842 .242093$

C $2.060419-3.9412032 .321792$

H $4.016640-3.6078390 .444768$

C $5.744512-3.5701161 .752821$

H $3.411989-5.5524571 .697528$

H $3.815871-4.7950633 .248205$

C $1.127875-4.577674 \quad 3.336479$

H $1.594737-3.9873551 .329281$

C $6.499120-2.4014791 .080373$

O 5.980669 -3.492176 3.164077

H $6.149254-4.5337131 .422517$

H $6.176641-1.4582181 .532980$

O $7.890022-2.5342451 .400375$

C $6.342396-2.345978-0.451531$

C $7.126954-1.198406-1.116766$

H $5.285009-2.261446-0.719974$

O $6.813977-3.584006-0.991186$

H $8.171407-1.212361-0.783249$

H $7.154499-1.396771-2.196653$

H $0.932040-5.6228683 .076084$

H $1.555982-4.5571964 .344332$

H $\quad 0.168596-4.051337 \quad 3.366513$
C $1.455480-1.4083022 .339433$

H $6.944103-3.5007373 .297013$

H $8.212118-3.3462700 .972345$

H $6.641878-3.571584-1.948257$

H $1.899566-0.4448982 .618412$

H $0.558358-1.5427512 .954860$

H $2.711635-2.4383933 .731790$

C $4.4405601 .377548-1.312421$

O $5.2149990 .186064-1.500163$

C $6.5016730 .185470-0.871598$

C $7.3665521 .316063-1.448693$

C $6.6669142 .672176-1.305051$

C $5.2111722 .623008-1.787526$

H $3.5784091 .240870-1.977973$

C 3.9155291 .4548910 .128987

H 6.3773780 .3391860 .205512

O $7.6316091 .059425-2.826449$

H $8.3291931 .327561-0.923600$

H $7.2110833 .427578-1.885082$

$\begin{array}{lllll}\text { O } & 6.691492 & 3.081161 & 0.059045\end{array}$

H $4.6755343 .527620-1.478775$

O $5.2037832 .601025-3.218938$

C 2.6356832 .2558250 .242993

H $3.7027790 .438895 \quad 0.481950$

H 4.6549231 .8882220 .807184

H $8.2003871 .776298-3.154714$

H $7.6228113 .136627 \quad 0.333143$

H $5.6311043 .418283-3.526919$

$\begin{array}{lllll}\text { O } & 2.157895 & 2.147963 & 1.517728\end{array}$

O $2.1194862 .897032-0.661508$

$\begin{array}{lllll}\text { C } & 0.930083 & 2.835345 & 1.780194\end{array}$

H 0.1059162 .3288521 .270337

H 0.7495192 .8039662 .857720

H $0.9939723 .882271 \quad 1.468173$

SCF Energy (B3LYP/6-31G**//MMFF)= -3245.90560943

0800265

MM̄FF Geometry

C $-0.4189495 .056473-1.222352$

C $0.6522094 .276721-1.008877$

C $1.4897663 .705159-2.124256$

O $1.7478892 .306386-1.851533$

C $2.8397114 .438964-2.189947$

C $3.7053043 .994565-3.353276$

C $4.7849393 .194423-3.248425$

C $3.3088824 .551532-4.696851$

C $5.3338442 .546056-2.008047$

C $5.4577921 .026680-2.183612$

C $5.9369950 .342918-0.894852$

C $6.141347-1.155641-1.122059$

C $6.514088-1.890037 \quad 0.174247$

$\begin{array}{lllll}\text { O } & 4.943607 & 0.555519 & 0.114267\end{array}$

O $6.358723-3.298949-0.057667$

C $5.506795-1.5019471 .278468$

C $7.984591-1.6730810 .550081$

O $5.905739-2.0371632 .537554$

C 5.2831330 .0199901 .401461

C $4.139400 \quad 0.3942992 .378486$

$\begin{array}{lllll}\text { O } & 6.477686 & 0.628340 & 1.909276\end{array}$

C $0.745111 \quad 1.421732-2.108536$

O $-0.388798 \quad 1.682493-2.477808$

C $1.285240 \quad 0.059167-1.897007$

C $0.512728-1.010152-2.140740$

C $0.930979-2.413455-2.074579$

C $2.344616-2.704404-1.645848$

C $2.752290-0.1492272 .019232$

C $2.431822-1.4939302 .650311$

C $0.769978-3.3485982 .516089$

O $3.087126-1.9808913 .567338$

C $0.959081-4.3459361 .418554$

C $-0.057576-4.946801 \quad 0.782164$

C $0.121182-6.010032-0.261285$

C $-0.529319-5.660162-1.578993$

C $-1.741549-6.141543-1.902779$

C $0.274848-4.848352-2.567018$

C $0.047239-3.367336-2.435501$

N $1.300970-2.0688582 .088856$

H $-0.6751575 .304332-2.251142$ 
H 0.9534614 .0184690 .001931 H $0.9724113 .789296-3.088275$ H $3.3731114 .315373-1.241205$ H $2.6713675 .520584-2.284285$ H $5.3465552 .973042-4.155512$ H $4.0004314 .252908-5.492002$ H $2.3119794 .200825-4.980894$ H $3.2977985 .645944-4.666107$ H $6.3251432 .969689-1.807068$ H $4.7170902 .759417-1.132141$ H $4.4800690 .620731-2.471521$ H $6.1568320 .807082-3.000292$ H $6.8765540 .814058-0.582493$ H $6.899601-1.332113-1.895206$ H $5.212748-1.593435-1.510910$ H $6.933885-3.545380-0.802255$ H $4.555239-1.9815891 .018748$ H $8.641665-2.043481-0.246040$ H $8.251808-2.2429411 .446728$ H $8.234189-0.623700 \quad 0.719627$ H $6.011877-2.9972512 .423903$ H 4.4204880 .1311973 .405490 H 4.0561761 .4908312 .372825 H 6.2454601 .4884392 .296720 H $2.320505-0.027532-1.589658$ H $-0.516736-0.845462-2.457110$ H $2.545844-3.768114-1.501178$ H $2.567927-2.223486-0.688018$ H $3.052199-2.336634-2.396391$ H 1.9962480 .5497472 .399732 H $2.624423-0.2087770 .934263$ H -0.285602 -3.200719 2.759374 H $1.281413-3.6919623 .420659$ H $1.982151-4.6113021 .163349$ H $-1.078800-4.6954131 .058073$ H $-0.314640-6.937278 \quad 0.133805$ H $1.182233-6.235809-0.425064$ H $-2.197213-5.923581-2.862920$ H $-2.307582-6.757349-1.211682$ H $-0.015900-5.127459-3.589449$ H $1.330910-5.126162-2.504137$ H $-0.953663-3.056131-2.737842$ H $0.815832-1.5812031 .341133$ C - $1.236692 \quad 5.085274 \quad 1.233944$ O $-1.586238 \quad 3.6863291 .222263$ $\begin{array}{llll}\text { C }-2.572389 & 3.440648 & 2.235788\end{array}$ C -2.482097 4.6267723 .184910 C -2.2065515 .7556492 .210962$ H -3.5486373 .4542851 .733218$ C -2.3716872 .0696662 .894262$ H -3.400188 4.770915 3.762306 H -1.647757 4.5198953 .887838 C $-1.6504217 .006712 \quad 2.868700$ H -3.1433506 .0109251 .697207$ C -2.4472230 .9088231 .876609$ O -1.1151532 .0601523 .569703$ H -3.1549101 .9418243 .651354$ H -3.314007 1.0819041 .230101 $\begin{array}{lllll}\text { O } & -1.293906 & 0.912641 & 1.036098\end{array}$ C -2.603146 -0.4763682 .532644$ C $-2.600145-1.6545291 .541225$ H -3.533169 -0.4864183 .113392$ O $-1.534961-0.6866963 .458116$ H -1.608557 -1.752071 1.082500 H -2.742066 -2.568289 2.133445 H -2.3648117 .4098833 .593743$ H -0.7146716 .7996203 .398746$ H $-1.450742 \quad 7.7826232 .122886$ C -1.345728 $5.642345-0.188920$ H -1.0301401 .2039834 .021954$ H -0.5167670 .7945531 .608694$ H -1.703657 -1.527966 3.915896 H - $1.1922486 .727666-0.175613$ H -2.366412 $5.464808-0.552935$ H $-0.208616 \quad 5.161513 \quad 1.608400$ C $-6.043503-1.2507770 .152609$ O $-4.948017-1.4399111 .061110$ C $-3.667388-1.5798130 .439221$
C $-3.651619-2.829201-0.454817$

C $-4.771227-2.771388-1.497310$

C $-6.121055-2.402909-0.867327$

H $-6.935577-1.304697 \quad 0.789635$

C $-5.990167 \quad 0.154588-0.465217$

H $-3.463890-0.698078-0.177704$

O $-3.814799-3.994040 \quad 0.351938$

H -2.676314 -2.899263 -0.948106

H $-4.853320-3.747648-1.990632$

O $-4.414244-1.814720-2.490276$

H $-6.845755-2.157602-1.650832$

O $-6.634853-3.556954-0.192714$

C $-7.3204060 .610582-1.023520$

H -5.6963870 .8749930 .307881$

H $-5.2438610 .208513-1.262626$

H $-3.771902-4.766876-0.236163$

H $-5.119765-1.808974-3.159431$

H $-6.037454-3.7582950 .548044$

O $-7.1456691 .809572-1.655136$

O $-8.378673 \quad 0.007392-0.914153$

C $-8.324472 \quad 2.377295-2.234930$

H $-8.048512 \quad 3.322795-2.709462$

H -8.731895 $1.707157-2.998090$

H $-9.0690702 .578163-1.458721$

SCF Energy (B3LYP/6-31G**//MMFF)= -3245.91199349

0800266

MM̄FF Geometry

C $1.077522 \quad 4.108653 \quad 0.713151$

C 2.3279623 .6332150 .803162

C $3.3400753 .725819-0.309537$

O $3.601626 \quad 2.375482-0.756714$

C $4.648937 \quad 4.3386950 .211501$

C $5.7077504 .471666-0.865295$

C $6.7580993 .639377-1.009850$

C $5.5455905 .654285-1.784512$

C $7.0682072 .415485-0.194049$

C $7.3501111 .197098-1.084843$

C $7.553570-0.067435-0.238984$

C $8.039875-1.234563-1.097127$

C $8.171169-2.524423-0.272220$

O $6.294858-0.400050 \quad 0.356888$

O $8.325376-3.619532-1.187591$

C $6.855774-2.760102 \quad 0.498568$

C $9.424585-2.5163620 .610895$

O $6.978470-3.8615341 .395777$

C $6.343638-1.5123471 .258260$

C $4.910917-1.7748001 .776473$

O $7.198459-1.2488292 .371631$

C $2.7983431 .895601-1.745720$

O $1.9555552 .514958-2.375950$

C $3.0677530 .448160-1.883146$

C $2.129649-0.321161-2.455387$

C $2.155641-1.778601-2.577766$

C $3.428415-2.493637-2.215708$

C $4.185375-0.5335692 .311635$

C $2.740980-0.8757462 .621646$

C $0.571326-1.3830421 .493038$

O $2.365808-1.1128753 .767386$

C $0.512501-2.7201710 .820260$

C $-0.380959-3.036329-0.130006$

C $-0.433131-4.375706-0.811520$

C $-0.494578-4.271646-2.321894$

C $-1.621258-4.575767-2.988353$

C $0.768231-3.883240-3.066968$

C $1.031374-2.403797-2.984911$

N $1.948073-0.924771 \quad 1.485180$

H $0.759316 \quad 4.609542-0.197815$

H $2.634733 \quad 3.113344 \quad 1.708113$

H $2.9605334 .335590-1.138979$

H $5.030097 \quad 3.7481201 .052165$

H $4.4513835 .334756 \quad 0.630735$

H $7.4782723 .854570-1.798139$

H $6.3601185 .730149-2.512546$

H $4.6092125 .579307-2.346016$

H $5.5323806 .584689-1.207469$

H 7.9530312 .6260160 .418564

H 6.2560032 .1720890 .495369 
H $6.506748 \quad 1.049064-1.771858$ H $8.243817 \quad 1.389407-1.691239$ H 8.2823620 .1562400 .549143 H $8.992911-0.992056-1.583481$ H $7.323368-1.413474-1.911282$ H $9.116221-3.449351-1.727547$ H $6.095799-3.048309-0.241442$ H $10.325222-2.400641-0.004095$ H $9.542668-3.4697871 .137353$ H $9.423701-1.7124041 .349890$ H $7.286320-4.6246510 .877288$ H $4.310457-2.1926990 .961444$ H $4.933449-2.5329532 .570110$ H $6.703225-0.7435463 .035644$ H $3.9776540 .051036-1.449159$ H $1.2218860 .156567-2.823363$ H $4.291602-2.025551-2.702780$ H $3.440279-3.539531-2.530832$ H $3.581671-2.471680-1.134133$ H $4.654658-0.161044 \quad 3.228044$ H 4.2003630 .2874731 .585770 H $-0.024793-0.6309510 .967003$ H $0.211311-1.4672672 .522666$ H $1.239012-3.4667661 .133447$ H - $1.126863-2.300722-0.422952$ H $-1.319224-4.903066-0.434832$ H $0.427318-4.996934-0.533436$ H -1.668371 -4.525619-4.071169 H $-2.523770-4.876204-2.466401$ H $0.677998-4.146985-4.128582$ H $1.593070-4.486684-2.677793$ H $0.170624-1.791202-3.258357$ H $2.368843-0.7238970 .583385$ C $-1.0048292 .925601 \quad 1.446451$ $\begin{array}{lllll}\text { O } & -1.779417 & 3.378416 & 0.318992\end{array}$ C -3.1758313 .1084150 .567359$ C $-3.2131102 .191874 \quad 1.780244$ C -2.013149 2.6781992 .567272 H $-3.628924 \quad 4.0790280 .805445$ C -3.805463 $2.523092-0.703000$ H -4.1511692 .2679462 .338002$ H -3.076865 1.1461401 .479927 C $-1.537015 \quad 1.693708 \quad 3.621230$ H $-2.275093 \quad 3.6274573 .053870$ C $-5.2844392 .123155-0.525885$ O $-3.6988913 .513120-1.729904$ H -3.227406 $1.654386-1.040890$ H $-5.345017 \quad 1.3968450 .290518$ O $-6.0365373 .266199-0.117250$ C $-5.9369781 .495904-1.776883$ C $-7.433523 \quad 1.170382-1.610972$ H $-5.393640 \quad 0.583837-2.049959$ O $-5.8538492 .383148-2.894502$ H -8.001362 $2.099400-1.470795$ H -7.776666 $0.759013-2.570113$ H -0.6744482 .0907754 .165315$ H -2.331273 1.4925244 .347512 H -1.240603 0.7396473 .174199 C $0.054333 \quad 3.9700001 .805336$ H -2.757583 $3.743581-1.814564$ H $-5.9909763 .915818-0.840060$ H -4.912208 2.522617 -3.091083 H 0.5424503 .6999212 .748975 H -0.4139254 .9510491 .951100$ H -0.5260571 .9843331 .145426$ C $-7.210970-2.0691490 .238644$ O $-7.056216-1.033371-0.740933$ C $-7.7616090 .183047-0.480290$ C $-9.272814-0.080017-0.374120$ C $-9.576517-1.1699050 .658952$ C $-8.697727-2.4068940 .455121$ H -6.730519-2.949591-0.207376 C $-6.467661-1.7417771 .543633$ H -7.4149460 .6101030 .465029$ O $-9.805132-0.466276-1.639844$ H $-9.7741190 .852201-0.089343$ H -10.630916 -1.4628580 .581163$ O $-9.358427-0.661698 \quad 1.971721$
H -8.806112 -3.088404 1.307113

O $-9.178579-3.101951-0.699253$

C $-4.963578-1.8028871 .373566$

H $-6.708209-0.7475811 .927670$

H $-6.738375-2.4875592 .300782$

H $-9.321268-1.255574-1.937897$

H -9.9486660 .1016552 .092819$

H $-8.665212-3.923556-0.782806$

O $-4.382887-1.8443602 .609741$

$\begin{array}{lllll}\text { O } & -4.371819 & -1.802688 & 0.303034\end{array}$

C -2.955662 -1.937373 2.621291

H -2.649973 -2.940379 2.310655

H $-2.509810-1.1804591 .970275$

H $-2.610828-1.7640733 .644103$

SCF Energy (B3LYP/6-31G**//MMFF)= -3245.91982770

08_00267

MM̄FF Geometry

C $2.752505-1.3261043 .246769$

C $3.187659-0.0650823 .393159$

C $4.387850 \quad 0.5661372 .725977$

O $3.9117381 .407644 \quad 1.648471$

C $5.376115-0.4283142 .088555$

C 6.7438010 .1653221 .816946

C $7.158178 \quad 0.651541 \quad 0.630733$

C 7.6930600 .1288002 .987987

C $6.3669450 .834683-0.634067$

C $6.693956-0.207928-1.712428$

C $5.827285-1.471156-1.596869$

C $6.240598-2.514764-2.634793$

C $5.313765-3.739317-2.613850$

O $4.468166-1.076687-1.818140$

O $5.607633-4.496321-3.797471$

C $3.849459-3.257214-2.699399$

C $5.613345-4.655769-1.421763$

O $2.939622-4.342633-2.530893$

C $3.507204-2.130038-1.694298$

C $2.114497-1.542617-2.029299$

O $3.483823-2.672686-0.373736$

C 3.4672952 .6591251 .922332

O $3.3672493 .190644 \quad 3.014890$

C $3.1156453 .270157 \quad 0.621032$

$\begin{array}{llll}C & 2.390748 & 4.396311 & 0.571642\end{array}$

C $1.9748395 .090628-0.649852$

C $2.4628254 .541035-1.966392$

C $1.736842-0.276567-1.243111$

C $0.3813830 .263429-1.675557$

C -1.248535 $2.080104-1.166535$

O $-0.297429-0.260749-2.555279$

C -1.040094 $3.287959-2.023889$

C $-1.5214824 .500592-1.708363$

C $-1.3649785 .718108-2.574253$

C $-0.8482486 .918904-1.811917$

C $-1.6875357 .873663-1.376221$

C $0.6469517 .055619-1.633505$

C $1.1784026 .173985-0.537444$

N $0.0162491 .396697-0.961779$

H $3.265265-2.0088762 .576053$

H 2.6328720 .5926974 .060096

H 4.9119091 .1798463 .469624

H $4.950301-0.8162571 .157668$

H $5.522761-1.2982032 .741972$

H $8.185242 \quad 1.0084840 .558776$

H 8.6707320 .5594172 .746762

H 7.2835280 .6952993 .830311

H $7.858222-0.9043373 .311057$

H $5.2922100 .872504-0.440400$

H $6.6261851 .827326-1.025294$

H $6.4995550 .256961-2.688087$

H $7.758678-0.469306-1.684133$

H $5.931697-1.884373-0.588762$

H $7.283890-2.820368-2.486484$

H $\quad 6.200387-2.066515-3.637747$

H $5.071164-5.306665-3.779393$

H $3.691873-2.873201-3.717103$

H $6.645808-5.021724-1.473021$

H $4.977797-5.548158-1.438483$

H $5.483507-4.161012-0.456746$ 
H $3.105085-4.980093-3.245499$

H $2.085329-1.287422-3.097141$

H $1.343687-2.303918-1.856374$

H $2.901048-2.1317320 .181121$

H $3.4579002 .760371-0.272903$

H 2.0641284 .8471571 .507695

H $2.0722895 .073658-2.835646$

H $2.1580573 .495852-2.088167$

H $3.5556894 .595297-2.021546$

H $1.689276-0.486763-0.169528$

H $2.4809240 .510051-1.410182$

H -1.634638 $2.347795-0.179553$

H -1.960078 $1.402592-1.648609$

H $-0.4963943 .155043-2.956061$

H $-2.0941204 .619531-0.790930$

H -2.349089 $5.947702-3.003689$

H $-0.7078215 .519616-3.429914$

H -1.322822 8.752183 -0.854290

H -2.759157 $7.795101-1.526821$

H $0.9012368 .090700-1.367291$

H $\quad 1.1419256 .891737-2.594694$

H 0.8714806 .4904630 .460515

H $0.6591721 .782215-0.276453$

C $0.338317-1.9609893 .054083$

O $-0.186105-0.6287712 .871623$

C -1.611202 $-0.650453 \quad 3.100990$

C -2.012187 -2.113466 3.024422

C $-0.805314-2.7848753 .646016$

H -1.758634 -0.2688834 .119730$

C $-2.284552 \quad 0.2787112 .086417$

H -2.941873 -2.325985 3.560683

H -2.135079 -2.4317191 .982977$

C $-0.714276-4.2704993 .343213$

H $-0.846518-2.6435164 .734603$

C -3.8196790 .1584442 .052682$

O

H -1.887795 0.0806651 .083803

H $-4.058822-0.868464 \quad 1.764727$

$\begin{array}{lllll}\text { O } & -4.349656 & 0.352267 & 3.363581\end{array}$

C $-4.5100391 .126246 \quad 1.068695$

C -6.0299030 .8926800 .936241$

H $-4.034467 \quad 1.0367090 .085429$

O $-4.3151392 .471493 \quad 1.509237$

H $-6.5102440 .994674 \quad 1.917199$

H -6.4353541 .7045330 .317914$

H -1.586874 -4.798319 3.741693

H - 0.671525 -4.458677 2.265351

H $\quad 0.181110-4.7069153 .796814$

C $1.561344-1.8916913 .968263$

H $-0.937624 \quad 1.6586262 .407476$

H -4.1384331 .2643443 .628221$

H -4.6812213 .0601560 .827313$

H $1.831493-2.8919524 .325940$

H $1.334836-1.2821464 .852128$

H $\quad 0.611483-2.3362052 .059107$

C $-5.948093-1.725442-1.715599$

O $-5.809469-0.491224-1.002376$

C $-6.389541-0.4610850 .304559$

C -7.903559 $-0.693270 \quad 0.213874$

C $-8.203177-2.010654-0.512262$

C -7.429608 -2.129678-1.831107

H -5.579695 -1.507354 -2.726482

C $-5.049150-2.824512-1.125289$

H $-5.956390-1.2554030 .917463$

O

H -8.319982 $-0.717801 \quad 1.228034$

H -9.279926 -2.072055 -0.712885

O $-7.859230-3.0918890 .349861$

H -7.506977 -3.149986 -2.224349

O $-8.062408-1.267791-2.782448$

C $-3.577435-2.477496-1.242370$

H -5.258040 -3.001360 -0.067318

H -5.212230 -3.756988 -1.677854

H $-9.4709710 .221317-0.506949$

H $-8.085401-3.917824-0.110834$

H -7.614180 -1.392134 -3.636262

O $-2.834209-3.526371-0.779496$

O $-3.131456-1.425760-1.679604$
C $-1.415251-3.349753-0.849084$

H -1.111128 -2.450303 -0.304832

H $-0.940695-4.217362-0.383339$

H -1.097069 -3.293947-1.894197

SCF Energy $\left(B 3 L Y P / 6-31 G^{* *} / / M M F F\right)=-3245.89491925$

0800268

MM̄FF Geometry

C $4.004402-2.257908-0.066822$

C $3.854436-3.218691-0.990233$

C $2.669957-3.405006-1.910027$

O $1.664249-2.384655-1.715669$

C $2.003256-4.764302-1.638779$

C $0.988040-5.139361-2.700346$

C $-0.348273-5.054325-2.552078$

C $1.570035-5.688509-3.978424$

C $-1.117326-4.542732-1.363990$

C $-2.233115-3.588646-1.802242$

C $-3.065243-3.015470-0.642594$

C $-3.800108-4.1057500 .137857$

C $-4.736971-3.5210381 .199948$

O $-4.015348-2.124149-1.238331$

O $-5.573930-4.5959471 .654320$

C $-5.643054-2.4548110 .548834$

C $-3.954679-3.0390162 .428042$

O $-6.416319-1.8145001 .560678$

C -4.879062 -1.428304 -0.319807

C $-5.816185-0.591064-1.228888$

$\begin{array}{llll}\text { O } & -4.126135 & -0.558238 & 0.521024\end{array}$

C $1.782398-1.234814-2.433211$

O $2.691395-0.927558-3.187168$

C $0.587292-0.409836-2.145093$

C $0.4442050 .783470-2.740603$

C $-0.6925041 .694783-2.589776$

C $-1.8130161 .269316-1.677268$

C $-6.9042260 .254500-0.567912$

C -6.3760521 .4063720 .262053$

C -5.4357013 .6905880 .078671$

O -6.4180831 .3930791 .490162$

C $-4.7391964 .511237-0.957260$

C $-3.4901054 .979908-0.820624$

C $-2.7842705 .787814-1.872142$

C $-1.5344695 .097364-2.377406$

C $-0.3217805 .563184-2.032051$

C $-1.7548433 .918319-3.306482$

C $-0.6818242 .862171-3.268106$

N $-5.8578382 .433653-0.511596$

H $3.215012-1.5285640 .090505$

H $4.665260-3.932136-1.127446$

H $3.034596-3.370538-2.944183$

H $1.536547-4.756447-0.646704$

H $2.760973-5.558582-1.597712$

H $-0.972020-5.400263-3.375918$

H $\quad 0.796407-5.997982-4.689344$

H $2.189023-4.935529-4.475565$

H $2.190681-6.565380-3.766994$

H -1.544866 -5.412982 -0.855169

H $-0.473044-4.025929-0.647208$

H -1.800934 -2.751846 -2.368040

H $-2.904947-4.094758-2.509401$

H -2.400914 -2.440809 0.013383

H -3.098177 -4.802502 0.610366

H $-4.398106-4.710125-0.558828$

H $-6.221355-4.2221412 .276775$

H $-6.359020-2.985431-0.093115$

H $-3.372907-3.8634012 .857789$

H -4.628342 -2.705303 3.225007

H -3.262825 -2.227182 2.199293

H $-5.849927-1.1519681 .992189$

H $-5.1909850 .066969-1.848677$

H $-6.303331-1.272543-1.939815$

$\begin{array}{llll}H & -3.921931 & 0.249373 & 0.021342\end{array}$

H $-0.151385-0.816232-1.463254$

H $1.2284691 .125479-3.414794$

H $-2.3131290 .378930-2.073778$

H $-2.5778002 .036543-1.540468$

H -1.431204 $1.039920-0.677066$

H $-7.5411230 .682179-1.353862$ 
H $-7.578707-0.3502620 .043990$ H -4.8024143 .4765440 .946572$ H -6.3320124 .2110240 .431938$ H $-5.2913354 .730994-1.868151$ H -2.940973 4.7760320 .094692 H -2.538598 $6.764805-1.436667$ H $-3.4429075 .998954-2.724013$ H $0.5940945 .115647-2.399872$ H - $0.2141926 .418438-1.372627$ H $-1.829540 \quad 4.299282-4.333347$ H $-2.7292373 .459032-3.110477$ H $0.1789513 .091555-3.897278$ H $-6.0298192 .409162-1.513243$ C $6.040742-0.8501490 .489440$ $\begin{array}{llllll}\text { O } & 5.211367 & 0.322877 & 0.600533\end{array}$ C $5.5006471 .194803-0.510445$ C $6.8258430 .708803-1.082982$ C $6.684870-0.790949-0.897010$ H $4.7180331 .025472-1.259547$ C $5.4783372 .656887-0.054127$ H $6.9612161 .002525-2.128188$ H $7.6799561 .085127-0.508068$ C $7.998008-1.546436-0.999224$ H $5.997240-1.169564-1.663315$ C 4.1597683 .0676220 .636241 O $6.545247 \quad 2.871987 \quad 0.878341$ H $5.6832183 .316121-0.905005$ H 4.0660732 .5184971 .578721 O 4.2592994 .4507150 .996883 C $2.9011412 .865255-0.228478$ C 1.5963653 .3149900 .456172 H $2.8101411 .813312-0.515926$ O $3.0582613 .615916-1.435070$ H 1.6789494 .3585020 .782967 H $\quad 0.8046543 .300704-0.302748$ H $8.442750-1.411679-1.990502$ H $8.722044-1.196246-0.255999$ H $7.843249-2.618334-0.841066$ C $5.224924-2.1086610 .797853$ H 6.4128112 .2598991 .622623 H 4.3123624 .9646340 .172737 H $2.3038743 .404362-2.011009$ H $4.876475-2.0576901 .836369$ H $5.868620-2.9930870 .724911$ H $6.814196-0.7482361 .262231$ C 0.6756170 .0906932 .067619 O $0.968870 \quad 1.102224 \quad 1.097346$ C 1.1880702 .4123641 .630628 C -0.0820382 .9092782 .334991$ C $-0.523558 \quad 1.923557 \quad 3.423867$ C $-0.551373 \quad 0.477347 \quad 2.914024$ H $0.412801-0.7873091 .464024$ C $1.934278-0.2528962 .877426$ H 2.0072632 .3769602 .355913 O H $\quad 0.1171613 .8949962 .772018$ H -1.525948 $2.194513 \quad 3.778020$ O 0.3622122 .0098954 .535758 H -0.662110 -0.215842 3.755584 O -1.7194720 .3386622 .100173$ C $1.910355-1.6595503 .433589$ H $2.811043-0.1733742 .223934$ H $2.0815080 .442848 \quad 3.707433$ H -1.916262 3.3781731 .853876 H 0.3352122 .9251754 .863014 H - $-1.753580-0.5802621 .785522$ O $3.086264-1.8932754 .088864$ O $0.988820-2.4544543 .311632$ C $3.225117-3.1994714 .656356$ H $2.447586-3.3713835 .406653$ H $3.180587-3.9598793 .870678$ H $4.202009-3.2561225 .143757$

SCF Energy $(B 3 L Y P / 6-31 G * * / / M M F F)=-3245.92250364$

\section{8_00269}

MM̄FF Geometry

C $-0.7627053 .435167 \quad 1.204176$

C -0.8209712 .1302961 .508257$
C -0.6263161 .5969222 .901398$

O 0.2939020 .4812912 .844449

C -1.9369161 .0493193 .493313$

C -2.954882 2.1210933 .814729

C -4.0231972 .4200123 .050687$

C -2.7162592 .8652885 .103365$

C -4.4179471 .8089161 .735262$

C $-5.827126 \quad 1.2095401 .808467$

C -6.2704380 .6390260 .454703$

C -7.7458010 .2397710 .499947$

C $-8.202994-0.372027-0.830520$

O $-5.468866-0.5111010 .165575$

O $-9.476716-0.999858-0.615074$

C -7.216063-1.487930-1.232867

C $-8.4296080 .703832-1.899394$

O $-7.495487-1.965611-2.546996$

C $-5.720846-1.091753-1.121389$

C $-4.844080-2.355707-1.253030$

O $-5.323765-0.215391-2.178643$

C $1.624700 \quad 0.7476812 .846505$

O 2.1479331 .8533312 .831418

C $2.423139-0.4985822 .925495$

C $1.892237-1.7260832 .809635$

C $2.605243-2.9981942 .940921$

C $4.073683-2.9502633 .272622$

C $-3.341008-2.102990-1.069325$

C $-2.524644-3.333975-1.429662$

C $-0.178989-4.115691-1.682716$

O $-3.030792-4.380521-1.828714$

C $0.088862-5.077039-0.567290$

C $1.307330-5.585481-0.327461$

C $1.599712-6.6184520 .722724$

C $2.743176-6.2248751 .630487$

C $4.011523-6.5409051 .318687$

C $2.410334-5.5542622 .941705$

C $1.912971-4.1450802 .775473$

N -1.160204 -3.126402 -1.275694

H -0.5867594 .1503872 .002644$

H - -1.0281591 .4049270 .725506$

H -0.2124122 .3605523 .571937$

H $-1.7078080 .496654 \quad 4.415317$

H $-2.352260 \quad 0.2780882 .833433$

H -4.6850043 .2166773 .389160$

H -3.498729 3.6033975 .309272

H -2.6938332 .1666465 .946101$

H -1.7618183 .3996365 .068372$

H -4.3987892 .6018330 .977276$

H -3.708906 1.0469021 .401729

H $-5.838414 \quad 0.4153782 .566323$

H -6.5349951 .9842812 .129720$

H $-6.1021391 .400936-0.315390$

H -8.3761331 .0989170 .761479$

H -7.900794 -0.500906 1.297063

H -10.091037 -0.324977 -0.278776

H -7.392953 -2.333281 -0.552755

H $-9.2162141 .398996-1.581640$

H -8.782842 $0.262349-2.837711$

H -7.536260 $1.295930-2.108710$

H $-8.429678-2.235666-2.564548$

H $-5.152577-3.099750-0.506817$

H $-5.006202-2.801195-2.243303$

H -6.086634 -0.054083 -2.755627

H $3.483243-0.3465923 .100819$

H $0.825079-1.8217002 .616506$

H $4.617614-2.3459932 .539422$

H $4.551587-3.932463 \quad 3.269498$

H $4.227776-2.5161634 .266570$

H -3.011327 -1.284263 -1.717753

H $-3.126386-1.831207-0.029686$

H $0.722531-3.576039-1.984221$

H $-0.568568-4.660814-2.549049$

H $-0.753202-5.4070130 .036075$

H $2.136799-5.285508-0.963413$

H $1.849671-7.5559290 .208235$

H $\quad 0.709378-6.8428851 .323011$

H $4.835814-6.2916751 .978399$

H $4.253314-7.0514260 .392216$

H $3.264619-5.5895983 .627230$ 
H $\quad 1.631861-6.136574 \quad 3.452094$ H $0.852848-4.0833692 .527001$ H $-0.826065-2.245664-0.895026$

C $0.1969714 .808450-0.719151$ O $1.3980984 .014211-0.793671$ C $2.5129784 .842437-0.409905$ C $1.9895746 .276487-0.374148$ C $0.559056 \quad 6.0633160 .075507$ H 2.7862924 .5449480 .609249 C $3.7030294 .644526-1.352708$ H 2.5653296 .9102860 .307245 H $2.0101586 .743007-1.366020$ C $-0.3500477 .249687-0.192626$ H 0.5709075 .8610911 .152624 C $4.2321143 .199385-1.448069$ O $3.3102505 .049307-2.671347$ H $4.5129585 .321829-1.054911$ H $3.5145782 .584565-2.002970$ O $5.4171263 .241967-2.256428$ C $4.5625692 .526045-0.102502$ C $5.4276691 .255387-0.212148$ H 3.6284452 .2947920 .421209 O $5.2836203 .457107 \quad 0.711202$ H $6.4285241 .526550-0.571991$ H 5.5755150 .8757310 .808216 H $\quad 0.006333 \quad 8.1358990 .342564$ H - $0.3875857 .494479-1.259459$ H -1.3710997 .0412740 .141960$ C $-0.9723453 .979028-0.180552$ H $4.0801284 .927075-3.253148$ H $6.0896653 .744052-1.764552$ H 5.4078373 .0476941 .584644 H $-1.1532803 .148312-0.872527$ H -1.882035 $4.590485-0.176090$ H $-0.0413405 .087346-1.754587$ C $2.918833-1.337559-1.175519$ O $3.633095-0.311935-0.471041$ C $4.8365630 .146066-1.092514$

C $5.826918-1.015455-1.277662$

C $5.184134-2.177943-2.037443$

C $3.821496-2.553306-1.447428$

H $2.130472-1.657546-0.481474$

C $2.236328-0.794840-2.440496$

H $4.6018600 .563284-2.075442$

O $6.299674-1.476468-0.013762$

H $6.699691-0.650886-1.832144$

H 5.855858 -3.044081-1.997902

O $5.034504-1.802977-3.403433$

H $3.298397-3.256707-2.104979$

O $4.026568-3.220710-0.200265$

C $1.079725 \quad 0.118944-2.097497$

H $2.912172-0.228052-3.085122$

H $1.838176-1.637701-3.017501$

H $5.525507-1.7360980 .514088$

H $4.650104-2.561108-3.875493$

H $4.550062-4.020770-0.377522$

O $\quad 0.143347 \quad 0.052153-3.088436$

O $1.0214380 .826684-1.101419$

C $-1.0078580 .882943-2.910382$

H -1.746301 $0.607955-3.668121$

H -1.450361 $0.728919-1.921463$

$\mathrm{H}-0.7311831 .931579-3.051784$

SCF Energy (B3LYP/6-31G**//MMFF) $=-3245.90673593$

08_00270

MM̄MF Geometry

C 3.4594960 .4020052 .436118

C $4.665057-0.0922642 .115156$

C $5.5399850 .378066 \quad 0.972445$

$\begin{array}{lllll}\text { O } & 4.921644 & 1.535862 & 0.360287\end{array}$

C $5.690492-0.719695-0.102536$

C $6.385309-1.9812950 .370273$

C $5.751515-3.1215740 .712807$

C $7.888545-1.9036730 .414882$

C $4.266459-3.3519560 .761258$

C $3.813076-4.373985-0.287692$

C $2.288455-4.344696-0.472000$

C $1.831352-5.468260-1.403189$
C $0.333305-5.370841-1.728715$

O $1.965321-3.073399-1.041966$

O $0.062526-6.249647-2.831263$

C $0.020087-3.937327-2.205977$

C $-0.538336-5.853727-0.563219$

O $-1.382042-3.760569-2.395666$

C $0.570471-2.842089-1.265115$

C $0.449710-1.455205-1.936567$

O $-0.174386-2.850403-0.048554$

C $5.6813862 .397397-0.357881$

O $6.8739532 .292361-0.604391$

C $4.8549943 .516086-0.874291$

C $3.7114523 .898702-0.282597$

C $2.8261764 .988177-0.703336$

C $3.1141475 .644711-2.026840$

C $1.033658-0.300320-1.110124$

C $1.3676700 .889057-1.987074$

C $0.3173292 .608358-3.454332$

O $2.5298271 .244482-2.169446$

C $-0.2463173 .870683-2.875332$

C $-0.7551994 .059867-1.647616$

C -1.318781 $5.373571-1.186247$

C -0.6403295 .9052460 .055509$

C -1.303780 5.9934591 .220777

C $0.7779646 .420148-0.073825$

C 1.8049595 .3333630 .110016

N $0.247324 \quad 1.487617-2.537632$

H $3.057475 \quad 1.2477661 .885558$

H $5.045652-0.9307842 .694055$

H $6.5148020 .669557 \quad 1.382001$

H $6.251693-0.318439-0.957117$

H $4.707692-0.943809-0.536316$

H $6.352376-3.9754411 .022317$

H $8.344205-2.8439370 .743121$

H $8.286482-1.673155-0.578755$

H $8.210807-1.1212251 .109038$

H $4.005769-3.7216541 .760864$

H $3.714867-2.4163790 .642026$

H $4.294786-4.144551-1.247567$

H $4.140231-5.3760820 .015558$

H $1.808220-4.4355910 .509542$

H $2.069253-6.449598-0.973972$

H $2.392998-5.411271-2.346347$

H $0.325964-7.148521-2.569392$

H $\quad 0.484532-3.811043-3.194089$

H $-0.317784-6.902296-0.329530$

H -1.601579 $-5.822738-0.825696$

H $-0.391505-5.2752460 .351375$

H -1.683054 -4.459714 -3.000929

H $\quad 0.965517-1.484431-2.905542$

H $-0.602969-1.232762-2.134609$

H -0.150535 -1.965714 0.347038

H $5.2592314 .030858-1.739137$

H 3.3886173 .3743560 .615116

H $4.0734136 .172503-1.992993$

H $2.3586226 .373515-2.326565$

H $3.1536604 .896356-2.826602$

H $0.3292730 .023771-0.336220$

H $1.957196-0.599624-0.602350$

H $-0.2598412 .328554-4.341559$

H $1.3544622 .777934-3.760514$

H -0.231892 4.719013 -3.557691

H $-0.795823 \quad 3.238043-0.938083$

H $-2.3909155 .225823-1.002222$

H -1.255028 $6.133465-1.975412$

H -0.8322096 .4006872 .109040$

H -2.329979 5.6539691 .315042

H $\quad 0.964727 \quad 7.176890 \quad 0.700089$

H $0.8778116 .953375-1.022692$

H 1.6978894 .7955941 .053676

H $-0.6726351 .123787-2.307524$

C $1.192818-0.5059272 .867679$

O $0.504493 \quad 0.7307042 .580163$

C $-0.8371590 .651883 \quad 3.103876$

C $-1.096113-0.8311283 .300994$

C $0.264332-1.2993303 .784412$

H $-0.807074 \quad 1.1637164 .074704$

C -1.7864351 .3847272 .149583$ 
H -1.893137 -1.037175 4.019680 H $-1.352942-1.308722 \quad 2.348660$ C $0.456142-2.803833 \quad 3.704206$ H $\quad 0.399732-0.9770884 .825714$ C -3.2830941 .2463692 .483566$ O $\begin{array}{llll}-1.422808 & 2.770583 & 2.175421\end{array}$ H $-1.598814 \quad 1.046556 \quad 1.125257$ H -3.619282 0.2165582 .327370 O -3.4839851 .5317603 .876510$ C -4.2033332 .2200001 .724699$ C $-4.089102 \quad 2.2441330 .192541$ H -4.0248333 .2392092 .090863$ O -5.5633391 .9224672 .063906$ H $-4.7216503 .071942-0.155766$ H -3.059524 $2.485450-0.090484$ H $-0.270823-3.3181044 .341273$ H $0.325808-3.1730622 .682143$ H $1.459303-3.0869934 .037999$ C $2.556820-0.1861453 .485245$ H -1.620708 3.1113873 .064710 H -3.129937 0.7866054 .389168 H -5.6301141 .9384843 .034179$ H $3.001457-1.0924463 .910987$ H 2.4450050 .5488004 .291127 H $1.320500-1.0297051 .912178$ C $-3.832666-1.310037-0.975398$ O $-3.548819-0.037540-0.376131$ C $-4.5565110 .965088-0.531676$ C $-4.8073921 .239883-2.024914$ C $-5.163477-0.046448-2.773699$ C $-4.165119-1.167227-2.472386$ H -2.892622 -1.869211-0.896835 C $-4.909870-2.076871-0.195572$ H $-5.4869730 .618708-0.071510$ O $-3.640803 \quad 1.825663-2.601085$ H $-5.6243631 .965718-2.119317$ H $-5.1722240 .155824-3.851651$ O $-6.476880-0.445451-2.394450$ H -4.541358 -2.122814 -2.854298 O $-2.956388-0.899043-3.187090$ C $-4.426759-2.4574571 .186462$ H -5.827428 -1.490471-0.096851 H $-5.171720-3.010016-0.707325$ H $-3.8422052 .026351-3.530990$ H $-6.695866-1.248914-2.896499$ H $-2.579007-0.075691-2.833202$ O $-5.400334-2.1913662 .104429$ O $-3.332457-2.9519141 .423510$ C $-5.079508-2.5220193 .459024$ H $-4.853838-3.5890083 .547802$ H $-4.236068-1.9182103 .805097$ H -5.950517-2.295079 4.079450

SCF Energy (B3LYP/6-31G**//MMFF)= -3245.90987711

\section{8_00271}

\section{MMFF Geometry}

C $0.622541-2.3014961 .751534$

C $-0.558903-2.7865151 .344902$

C $-0.812751-3.305278-0.047254$

O $-1.525894-2.264754-0.754321$

C $-1.693768-4.5657330 .009972$

C -2.072894 -5.109033-1.354940

C $-3.305613-5.033390-1.897853$

C $-0.959059-5.790117-2.107832$

C $-4.534425-4.407803-1.297290$

C $-4.867920-3.070141-1.971412$

C $-5.741134-2.179647-1.074514$

C $-6.166243-0.914259-1.819291$

C $-6.9212210 .067401-0.910639$

O $-4.954156-1.8302980 .069709$

O $-6.984848 \quad 1.331967-1.589981$

$\begin{array}{llll}\text { C } & -6.105746 & 0.290707 & 0.382362\end{array}$

C $-8.371791-0.373072-0.679441$

$\begin{array}{llll}\text { O } & -6.863018 & 1.077853 & 1.298482\end{array}$

C $-5.629815-1.0219891 .042727$

C $-4.635282-0.8019902 .211326$

O $-6.764556-1.7115961 .580985$

C $-0.768576-1.328076-1.391546$
O $0.444307-1.332532-1.532029$

C $-1.671902-0.248457-1.844943$

C $-1.1454680 .905393-2.281195$

C $-1.8894302 .088556-2.717691$

C $-3.3923212 .033868-2.648005$

C $-3.336900-0.0743121 .845041$

C -3.3778591 .4287652 .063348$

C -2.0322473 .4847701 .645342$

O -4.2674091 .9917872 .694976$

C -2.5025234 .2004900 .418720$

C $-1.7207495 .010842-0.312351$

C $-2.2131115 .814232-1.483442$

C $-1.3754335 .627591-2.728637$

C $-0.4102576 .504681-3.052594$

C $-1.7209504 .476900-3.645950$

C $-1.1930273 .164372-3.139892$

N -2.2740602 .0612641 .506518$

H $1.460814-2.2948381 .061292$

H -1.406426 -2.751947 2.025784

H $0.128443-3.541938-0.559321$

H $-2.589485-4.3496530 .600675$

H $-1.169315-5.3551960 .565112$

H -3.459699 -5.460066 -2.887866

H - $-1.303234-6.232326-3.048937$

H $-0.165336-5.077248-2.350473$

H $-0.531188-6.597231-1.504078$

H -5.377040 -5.097855 -1.431065

H $-4.434372-4.285525-0.216780$

H -3.938450 -2.535646 -2.202437

H $-5.376318-3.263348-2.924213$

H $-6.618459-2.753157-0.752831$

H -6.770641 -1.165964 -2.699868

H $-5.274078-0.405723-2.207360$

H $-7.4337271 .193558-2.441627$

H -5.2336010 .8907450 .097421$

H $-8.908719-0.437787-1.633522$

H $-8.9184670 .363172-0.079734$

H -8.451457 -1.346090 -0.190426

H -6.2784521 .3262252 .034134$

H $-5.141624-0.3254213 .059025$

H $-4.342588-1.7978762 .575086$

H $-6.457964-2.3189432 .274486$

H $-2.739230-0.416522-1.772568$

H $-0.0606031 .001578-2.313497$

H $-3.7215291 .785531-1.633405$

H $-3.779186 \quad 1.277691-3.339693$

H -3.878137 $2.978362-2.900439$

H $-2.538856-0.4479342 .498952$

H $-3.042796-0.2895920 .814177$

H $-0.961023 \quad 3.6184821 .817004$

H -2.5691293 .8653912 .520255$

H -3.5496094 .0857780 .149140$

H $-0.6815205 .149012-0.021393$

H -2.200007 $6.871358-1.186194$

H -3.262171 $5.587132-1.711886$

H $\quad 0.1730496 .393351-3.961018$

H $-0.1788737 .350994-2.414793$

H -1.280029 $4.641300-4.638555$

H $-2.8000224 .468538-3.822797$

H $-0.1033573 .106848-3.145086$

H -1.6039891 .5166380 .971128$

C $0.876406-0.1700383 .012901$

O $2.074680 \quad 0.2638402 .340318$

C 2.5137821 .5019002 .932910

C 1.4584481 .8858773 .967147

C $0.9015270 .533804 \quad 4.365617$

H 3.4540591 .2884263 .453147

C 2.7322252 .5602091 .846254

H 1.8874032 .4389584 .808722

H 0.6703612 .5097093 .529897

C -0.4526750 .6193635 .048833$

H 1.6177420 .0381845 .034363

C 3.7864792 .1876710 .784693

O $1.4945972 .785362 \quad 1.157614$

H 2.9950383 .5175472 .310869

H $3.428998 \quad 1.3445610 .183450$

O $3.9000413 .302203-0.111570$

C 5.1879181 .8778521 .349475 
C $\quad \begin{array}{llll}6.298460 & 1.791966 & 0.283423\end{array}$

H 5.1591990 .9435451 .919608

O 5.5632292 .9073142 .270327

H $6.3267442 .724838-0.292512$

H 7.2613671 .7417500 .810973

H -0.3803801 .2011975 .973403$

H $-1.196071 \quad 1.1024624 .406351$

H $-0.827510-0.3762135 .305813$

C $0.849139-1.6954333 .106910$

H 1.2092591 .9354130 .780370

H $3.0115933 .483707-0.463234$

H 5.5648963 .7494581 .783566

H $0.062469-2.0196413 .797270$

H $1.801482-2.0748583 .496284$

H 0.0276710 .1897642 .416230

C $6.441523-1.830615-0.597518$

O $6.513067-0.5803250 .100650$

C $6.1657790 .584820-0.657281$

C $7.072798 \quad 0.723406-1.889859$

C $7.045748-0.550813-2.737066$

C $7.282292-1.800218-1.884786$

H $6.909182-2.5558110 .082091$

C $4.987998-2.265046-0.820792$

H $5.1284270 .503499-0.993154$

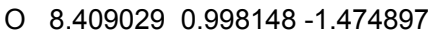

H $6.729412 \quad 1.578223-2.485311$

H $7.818078-0.484570-3.512869$

O $5.779533-0.637868-3.384503$

H $7.082324-2.702602-2.473236$

O $8.665159-1.858377-1.521781$

C $4.405140-2.7764870 .477109$

H $4.334267-1.463495-1.174991$

H $4.939487-3.068387-1.565361$

H $8.9417451 .133651-2.277271$

H $5.789241-1.434802-3.941606$

H $8.849700-1.108339-0.930732$

O $4.193429-4.1206230 .394709$

O $4.188356-2.0688711 .452778$

C $3.695274-4.7521131 .578394$

H $4.274912-4.4525212 .456642$

H $2.638424-4.5089361 .711467$

H $3.792036-5.8334501 .451058$

SCF Energy (B3LYP/6-31G*//MMFF)= -3245.92132198

08_00272

MM̄FF Geometry

C $0.2296224 .505379-0.350758$

C $-1.0734664 .190576-0.359082$

C $-1.887129 \quad 4.029373 \quad 0.899935$

O $-2.220552 \quad 2.6244501 .033397$

C $-3.1894104 .840652 \quad 0.800963$

C $-4.0165554 .800706 \quad 2.072234$

C -5.1465314 .0839292 .237458$

C -3.5101255 .6718593 .193876$

C $-5.811366 \quad 3.1677631 .248699$

C $-5.8290911 .721397 \quad 1.759450$

C $-6.350420 \quad 0.7460480 .693809$

C $-6.418451-0.676596 \quad 1.250228$

C $-6.813510-1.703074 \quad 0.177906$

O $-5.449803 \quad 0.792765-0.417257$

O $-6.531155-2.9993630 .727718$

C $-5.918666-1.503455-1.066147$

C $-8.320107-1.667728-0.104807$

O $-6.360227-2.316773-2.150260$

C -5.829527 $-0.032919-1.526108$

C $-4.7833010 .192511-2.645788$

O $-7.1002210 .369429-2.053178$

C $-1.251913 \quad 1.812946 \quad 1.547176$

O $-\begin{array}{llll}0.142573 & 2.149123 & 1.932737\end{array}$

C $-1.762546 \quad 0.4238381 .552886$

C $-0.928592-0.5774751 .874019$

C $-1.268920-1.9986481 .975868$

C $-2.680844-2.4058251 .651210$

C $-3.335752-0.151429-2.277301$

C $-2.901236-1.547302-2.691933$

C $-0.961076-3.102851-2.504621$

O $-3.581542-2.285135-3.398890$

C $-1.148802-4.051250-1.364041$
C $-0.130516-4.612495-0.695124$

C $-0.304479-5.6144740 .407905$

C $0.360013-5.1888601 .696362$

C $1.586127-5.6320252 .022008$

C $-0.440765-4.3361232 .652612$

C $-0.311953-2.8630782 .373859$

N $-1.636986-1.855035-2.206976$

H 0.7213704 .7045110 .598780

H $-1.5639813 .963976-1.302508$

H $-1.3195824 .353662 \quad 1.781295$

H $-3.7752464 .489163-0.054513$

H $-2.956147 \quad 5.890700 \quad 0.577558$

H $-5.661363 \quad 4.153583 \quad 3.195001$

H $-4.177275 \quad 5.6622014 .062496$

H $-2.526525 \quad 5.3326873 .532650$

H -3.4244776 .7107872 .858738$

H -6.8416003 .5120391 .096113$

H $-5.328738 \quad 3.2092220 .270242$

H -4.8103241 .4337332 .046772$

H -6.4527091 .6570292 .659775$

H $-7.342927 \quad 1.0815530 .370945$

H -7.106108 -0.7283262 .103633$

H -5.433098 $-0.954761 \quad 1.646848$

H $-6.817537-3.669093 \quad 0.084110$

H $-4.915564-1.854142-0.796556$

H $-8.883534-1.9134280 .803450$

H $-8.602724-2.423574-0.845863$

H -8.667381-0.694165 -0.457000

H $-6.287751-3.244924-1.871900$

H $-5.098993-0.314005-3.565859$

H $-4.797305 \quad 1.265126-2.887947$

H $-6.964743 \quad 1.134923-2.635993$

H $-2.8051130 .264721 \quad 1.306667$

H $0.107573-0.3342132 .107758$

H $-3.373520-2.0049282 .398396$

H -2.825827 -3.4878621 .618420$

H -2.972791 -2.0337720 .663935$

H $-2.6727190 .541638-2.811147$

H $-3.155464-0.015375-1.206666$

H $\quad 0.092778-2.867147-2.680378$

H $-1.374194-3.539361-3.419586$

H $-2.170367-4.311957-1.097814$

H $0.890255-4.372443-0.982567$

H $\quad 0.125743-6.5644040 .064181$

H $-1.364784-5.8261680 .593831$

H $2.055000-5.3569632 .961010$

H $\quad 2.151132-6.2726201 .352954$

H -0.083992 -4.5012453 .678784$

H - $-1.479582-4.6780712 .670771$

H $0.683578-2.4704242 .583974$

H $-1.173282-1.204718-1.579025$

C $2.035848 \quad 3.304487-1.572648$

O $3.3391313 .673544-2.063137$

C $3.9781172 .475069-2.570072$

C $2.9105281 .384284-2.564220$

C $1.6139952 .165484-2.494477$

H $4.2937532 .710166-3.593077$

C $5.1930812 .164977-1.681730$

H $2.9748220 .739414-3.445732$

H $3.0054760 .747268-1.678040$

C $0.450503 \quad 1.324948-2.002247$

H $1.3876742 .553420-3.496528$

C $5.8345350 .790808-1.942103$

O $6.1788693 .178432-1.906060$

H $4.8958152 .252709-0.631719$

H $5.114412-0.012352-1.766641$

$\begin{array}{llll}\text { O } & 6.175657 & 0.695289 & -3.331969\end{array}$

C $7.1061290 .504690-1.123370$

$\begin{array}{llll}\text { C } 7.000218 & 0.695408 & 0.400191\end{array}$

H $7.9239561 .137394-1.490077$

O $7.513855-0.845497-1.371118$

H 8.0024820 .5256320 .816273

H $6.741741 \quad 1.7396660 .608211$

H $-0.4846981 .888881-2.036198$

H $0.3251210 .440964-2.636011$

H $0.606797 \quad 0.982817-0.974091$

C $1.1077784 .522621-1.568192$

H $5.7561514 .036868-1.731295$ 
H $6.7802581 .429425-3.536047$

H $7.590479-0.953161-2.334698$

H $0.5095934 .559586-2.486289$

H $1.6939195 .449370-1.550165$

H $2.1905682 .933491-0.549152$

C $3.647656-0.7115841 .363284$

$\begin{array}{lllll}\text { O } & 4.687305 & 0.076419 & 0.766651\end{array}$

C $6.027725-0.2522321 .139885$

C $6.201591-0.1328622 .665780$

C $5.151813-0.9550283 .419106$

C $3.739106-0.6812032 .896970$

H $2.717402-0.2029511 .078914$

C $3.608886-2.1350550 .792592$

H $6.245694-1.2799120 .832457$

$\begin{array}{lllll}\text { O } & 6.098284 & 1.237066 & 3.051726\end{array}$

H $7.209522-0.4733942 .931982$

H $5.192692-0.7119984 .487758$

O $5.436210-2.343673 \quad 3.279770$

H $3.027729-1.4009423 .318624$

O 3.3209860 .6174953 .325627

C $3.090119-2.133979-0.625817$

H $4.589393-2.6180700 .812342$

H $2.930002-2.7721721 .369740$

H $6.246128 \quad 1.2840394 .011470$

H $6.326445-2.4989543 .638841$

H $3.3313880 .622793 \quad 4.297957$

O $4.014479-2.684201-1.462825$

O $1.991023-1.702806-0.949837$

C $3.634571-2.753118-2.840440$

H $2.757825-3.396931-2.958240$

H $3.434024-1.751424-3.230762$

H $4.466902-3.186584-3.400976$

SCF Energy (B3LYP/6-31G**//MMFF) $=-3245.90451525$

$08 \quad 00273$

MM̄FF Geometry

C -1.580740 -2.822530 2.855452

C $-2.001949-2.2196993 .978251$

C $-2.967786-1.0609114 .076263$

O $-3.442356-0.6524062 .774649$

C -2.2501070 .1461514 .704690$

C $-3.185603 \quad 1.2991295 .005877$

C -3.2739142 .4225894 .267462$

C $-4.011573 \quad 1.148276 \quad 6.257182$

C -2.537296 2.7399902 .994485

C $-3.4530093 .447708 \quad 1.985344$

C $-2.696298 \quad 3.837068 \quad 0.707721$

C $-3.5539694 .755434-0.164274$

C $-2.8481335 .109786-1.480269$

O $-2.3784302 .640143-0.011672$

O $-3.8188655 .702566-2.356190$

C $-2.3692603 .807157-2.149756$

C $-1.747136 \quad 6.157472-1.278886$

O $-1.5786384 .088641-3.303020$

C -1.599905 2.858340 -1.197337

C $-1.3913141 .498288-1.901127$

O $-0.325358 \quad 3.425057-0.890556$

C $-4.592207-1.2133932 .315484$

O $-5.283072-2.0473992 .877864$

C $-4.851029-0.6473370 .973059$

C $-5.740840-1.2468860 .168660$

C $-6.075220-0.847804-1.199951$

C $-5.5201410 .458509-1.701011$

C $-0.8152250 .392236-1.011815$

C $-0.844436-0.919891-1.769782$

C $-2.356621-2.823671-2.333193$

O $0.096883-1.277487-2.474224$

C $-3.336540-2.491077-3.415619$

C $-4.473793-3.172032-3.624546$

C $-5.460095-2.831990-4.706624$

C $-6.884743-2.734957-4.201672$

C $-7.799212-3.661714-4.534740$

C $-7.270038-1.525448-3.373616$

C $-6.851179-1.670700-1.935893$

N $-2.038313-1.605907-1.610680$

H $-1.961761-2.4995801 .891690$

H -1.606691 -2.571016 4.930003

H $-3.810322-1.3637084 .709664$
H $-1.4371480 .476501 \quad 4.046544$

H -1.759985 -0.150475 5.642089

H -3.9456703 .2066774 .614941$

H -4.6274482 .0314206 .458272$

H -4.685932 $0.290796 \quad 6.172080$

H -3.3619500 .9962007 .125323$

H -1.696569 3.3987123 .242188

H $-2.123621 \quad 1.8402442 .529784$

H -4.2900282 .7833001 .733658$

H -3.873172 4.3489732 .449531

H -1.7680204 .3446940 .995310$

H -3.827141 5.6681090 .380013

H -4.501962 4.252321 -0.401818

H $-4.1821546 .488313-1.912824$

H -3.264339 $3.280372-2.510325$

H $-2.1667007 .076122-0.851592$

H -1.300598 $6.449624-2.235865$

H $-0.9489765 .819240-0.614971$

H -2.103103 $4.671342-3.878647$

H $-2.3529641 .150905-2.296635$

H $-0.7246621 .625336-2.764156$

H $0.2741612 .716886-0.607078$

H -4.2771930 .2216530 .671298$

H -6.251814 -2.137549 0.532551

H $-5.9166310 .758610-2.672775$

H $-4.4334710 .397089-1.798926$

H -5.763471 $1.273167-1.009391$

H $0.2225280 .599624-0.731376$

H - $-1.3837330 .285300-0.081149$

H -2.766676 -3.533658 -1.608138

H $-1.447359-3.247866-2.769741$

H -3.095995 -1.650209 -4.062448

H $-4.701997-4.030384-2.996483$

H -5.386160 -3.610136 -5.477316

H $-5.195250-1.890327-5.203810$

H $-8.827873-3.586999-4.197519$

H -7.539614 -4.518440 -5.147632

H $-8.359666-1.391703-3.388028$

H $-6.859932-0.633224-3.853844$

H -7.224795 -2.581955 -1.465829

H $-2.765978-1.203113-1.028361$

C $\quad 0.775109-3.5339852 .223411$

O $1.319788-2.3964432 .925561$

C $1.729748-1.4121591 .952222$

C $1.893484-2.1829060 .652633$

C $0.738092-3.1586720 .741354$

H $0.898075-0.6995971 .873601$

C $2.990394-0.6988942 .452496$

H $1.845226-1.541252-0.231007$

H $2.849407-2.7184160 .637905$

C $0.862973-4.344451-0.198791$

H $-0.183827-2.6196550 .501199$

C $3.5996170 .259644 \quad 1.410546$

O $2.633411 \quad 0.0329893 .627757$

H $3.743208-1.4361292 .756642$

H $3.850222-0.3312040 .524829$

O 2.6172651 .2139121 .006144

C 4.8716950 .9969661 .877887

C 5.4804341 .9272440 .810065

H 5.6237670 .2638402 .191846

O 4.5909761 .8226193 .010061

H 4.7745942 .7333930 .572538

H 6.3468102 .4199401 .271720

H $0.897058-4.008433-1.240192$

H $1.773437-4.919797-0.001719$

H $0.007323-5.018316-0.088624$

C $-0.567938-3.9364992 .843780$

H $2.247179-0.5994894 .257790$

H 2.3892351 .7437061 .789385

H 4.2905661 .2429883 .730041

H $-0.385138-4.2711503 .873012$

H $-0.988828-4.7920612 .303538$

H $1.485764-4.3554752 .381742$

C $7.351462-0.604068-1.199836$

O $6.8749930 .215324-0.124430$

C $5.9173871 .216033-0.480063$

C $6.5166562 .189687-1.505817$

C $7.0534831 .437645-2.729067$ 
C $7.946030 \quad 0.259006-2.328725$

H $8.169286-1.191090-0.762213$

C $6.278928-1.593375-1.685082$

H $5.0401710 .740888-0.927802$

O $7.5666772 .954414-0.917736$

H $5.7404192 .898644-1.816445$

H $7.6309882 .127450-3.356814$

O $5.9651760 .944871-3.505297$

H $8.159214-0.365269-3.204469$

O $9.1965220 .791221-1.880642$

C $5.983547-2.660292-0.649519$

H $5.331398-1.105504-1.925345$

H $\quad 6.650222-2.102817-2.582500$

H $8.2319582 .332857-0.575655$

H $5.4290181 .708988-3.777375$

H $9.7871990 .040552-1.699080$

O $5.344028-3.704181-1.255995$

O $6.262444-2.5862780 .539365$

C $5.032449-4.815674-0.409586$

H $5.954027-5.325061-0.113218$

H $4.473342-4.490407 \quad 0.472581$

H $4.411705-5.512570-0.978883$

SCF Energy (B3LYP/6-31G**//MMFF) $=-3245.92431838$

08_00275

MM̄FF Geometry

C -1.519370 4.114757 -0.083453

C -1.9068952 .8436690 .102520$

C -2.307991 2.2829461 .442243

O -1.5212661 .0866831 .648107$

C -3.7961921 .8943381 .424861$

C -4.3780551 .6754052 .806429$

C $-4.6367780 .471026 \quad 3.351849$

C -4.7217832 .9334073 .562827$

C $-4.354739-0.8887362 .775904$

C $-5.635246-1.6406412 .386708$

C $-6.300467-1.0910631 .115017$

C $-7.608598-1.8303120 .826565$

C $-8.238455-1.381250-0.500894$

O $-5.385960-1.2818230 .031416$

O $-9.262551-2.326733-0.845585$

C -7.167908-1.449948-1.609974

C $-8.930513-0.019064-0.373591$

O $-7.663256-0.906018-2.831432$

C $-5.834437-0.765204-1.227736$

C $-4.752352-1.096809-2.283584$

O $-6.0310790 .648739-1.181492$

C -1.0286390 .8409412 .888311$

O -1.1378291 .5563803 .874685$

C $-0.281533-0.4385432 .932672$

C $-0.336760-1.3657671 .963388$

C $0.398673-2.6322591 .930714$

C $1.327914-2.9332453 .076861$

C $-3.336353-0.642102-1.899948$

C $-2.297117-1.118664-2.900372$

C $0.169688-1.347138-3.173734$

O $-2.587545-1.625800-3.980736$

C $0.469983-2.758688-2.776920$

C $1.597688-3.140397-2.159129$

C $1.885217-4.557582-1.754510$

C $2.090417-4.711258-0.262881$

C $3.323383-4.8613240 .249240$

C $0.866829-4.7918800 .623916$

C $0.229083-3.4505130 .870686$

N -1.003380 -0.897876-2.445904

H $-1.494557 \quad 4.7900010 .768015$

H -1.942130 $2.169126-0.749137$

H -2.099383 3.0210902 .225644

H -3.9313280 .9991370 .809102$

H -4.3879672 .6802070 .935848$

H -5.0841790 .4454704 .344920$

H -5.1821712 .7227824 .533982$

H -3.8233003 .5296343 .748614$

H -5.429599 3.5400792 .988588

H $-3.662619-0.8461801 .930128$

H -3.835511 -1.4674403 .550649$

H -5.361072 -2.690339 2.218342

H $-6.346760-1.6216253 .221582$
H $-6.493051-0.0205381 .244587$

H -8.319345 -1.700722 1.652200

H -7.411937 -2.910159 0.767455

H -9.908293 -2.347527 -0.118580

H $-6.966730-2.513079-1.803677$

H $-9.724642-0.0624160 .381545$

H $-9.4239050 .262354-1.310452$

H $-8.2482140 .784013-0.087619$

H -8.491106 -1.371357-3.040908

H -4.722562 -2.184259-2.433211

H $-5.018684-0.642360-3.246304$

H $-5.1854941 .094757-1.343889$

H $0.317951-0.5807253 .826136$

H $-0.973602-1.1842361 .098990$

H $2.077487-2.1419373 .181730$

H $1.881219-3.8668552 .955664$

H $\quad 0.767433-3.0137344 .014679$

H $-3.2808750 .450038-1.862178$

H -3.065136 -1.037042 -0.914321

H $\quad 0.988900-0.663477-2.932660$

H $-0.023924-1.296233-4.249872$

H -0.284567 -3.506297 -3.011071

H $2.360620-2.399483-1.936237$

H $2.784033-4.881330-2.295730$

H $1.087041-5.236473-2.079549$

H $3.481934-5.0052371 .312596$

H $4.204074-4.839465-0.383937$

H $1.105903-5.2905501 .570166$

H $\quad 0.124452-5.4498640 .153705$

H $-0.462879-3.1444030 .085954$

H $-0.864177-0.537244-1.506210$

C $0.3502854 .965570-1.601662$

O $1.0749373 .719713-1.593484$

C $2.2186913 .862473-0.732327$

C $2.4464505 .364475-0.606185$

C $1.0157425 .868845-0.560372$

H 1.9194913 .4752480 .247916

C $3.4120253 .062692-1.265187$

H 3.0236065 .6253600 .285939

H $2.9630485 .774195-1.481670$

C $0.8768247 .351640-0.857299$

H $0.6208895 .673646 \quad 0.443443$

C $3.1204441 .576949-1.572718$

O $3.8490353 .673805-2.487450$

H $4.2498553 .150877-0.563974$

H $2.4295321 .541712-2.424976$

O $4.3147320 .946225-2.046211$

C $2.5223760 .723121-0.439459$

C 3.1551890 .8553030 .959162

H $2.565774-0.329201-0.741245$

O $1.1354741 .055109-0.336422$

H 3.1162291 .8958081 .298387

H 2.5065070 .3034861 .652208

H $1.4203727 .945040-0.114844$

H $1.2763027 .603152-1.845430$

H $-0.1743487 .655717-0.830400$

C -1.146821 $4.695802-1.420396$

H $4.6079963 .162246-2.815850$

H $5.0772951 .338484-1.591359$

H $\quad 0.7292350 .4357310 .290952$

H - $1.4765544 .013985-2.215108$

H -1.706240 $5.629027-1.554616$

H $0.4974665 .386277-2.605020$

C $5.837034-1.7420130 .778345$

O $4.569597-1.0719500 .747126$

C 4.5873190 .3153001 .094952

C 5.1348060 .5065252 .519969

C $6.504176-0.1606582 .686211$

C $6.505269-1.5978692 .157720$

H $5.597259-2.8026760 .636809$

C $6.742219-1.310483-0.385941$

H 5.2324170 .8592030 .402730

O $4.228306-0.0290023 .482154$

H 5.2173091 .5804652 .724225

H $6.778539-0.1702253 .748408$

O 7.4922540 .5891291 .986103

H $7.530928-1.9830092 .118174$

O $5.788651-2.4090513 .093931$ 
C $6.181612-1.726439-1.730419$

H $6.878012-0.226193-0.408812$

H $7.724208-1.786727-0.285411$

H $4.116539-0.9753583 .289059$

H 7.5029151 .4850962 .363846

H $5.847818-3.3309592 .790374$

O $6.935132-1.158005-2.718417$

O $5.217696-2.459894-1.899645$

C $6.517425-1.452991-4.055037$

H $7.182761-0.925068-4.743304$

H $6.594314-2.527438-4.246627$

H $5.493662-1.102978-4.219026$

SCF Energy (B3LYP/6-31G**/MMFF) $=-3245.90436524$

08_00276

MMFF Geometry

C $1.925966-4.242054-0.360286$

C $0.915024-3.362032-0.423398$

C $0.046393-3.178229-1.643429$

O $0.184429-1.837486-2.189902$

C $-1.429318-3.543721-1.376811$

C $-2.200675-3.838431-2.648549$

C $-3.105384-3.019416-3.218512$

C $-1.939429-5.195671-3.255379$

C $-3.537560-1.643864-2.791672$

C $-5.015394-1.616764-2.374960$

C $-5.232280-2.098277-0.931641$

C $-6.716625-2.348567-0.666332$

C $-6.976246-2.7243260 .798937$

O $-4.751803-1.066737-0.062995$

O $-8.392484-2.651036 \quad 1.025390$

C $-6.314678-1.6686001 .708374$

C $-6.561824-4.1691631 .102784$

O $-6.428248-2.0713373 .072122$

C $-4.841178-1.3623611 .337558$

C $-4.356287-0.1084192 .107318$

O $-4.025560-2.4799151 .688716$

C $-0.169713-0.747150-1.461950$

O $-0.575298-0.721017-0.311426$

C $-0.0191860 .441893-2.327847$

C $-0.7484411 .530192-2.041830$

C $-0.7420142 .805044-2.760132$

C $0.2771552 .985687-3.852831$

C $-2.963801 \quad 0.3828491 .697953$

C $-2.669061 \quad 1.744867 \quad 2.294347$

C -3.150846 4.1689661 .983368

O $-1.990743 \quad 1.873395 \quad 3.310747$

C -3.2470635 .0379060 .769653$

$\begin{array}{llll}C & -2.245791 & 5.814457 & 0.328235\end{array}$

C -2.324087 $6.664471-0.907260$

C $-1.3595056 .215750-1.984633$

C $-0.1658826 .813518-2.137813$

C $-1.8144555 .135303-2.942362$

C -1.625847 $3.752957-2.382960$

N -3.2415442 .7798131 .570408$

H $2.134853-4.869664-1.222360$

H $0.691980-2.7587110 .451369$

H $0.434393-3.831624-2.433761$

H $-1.931510-2.761851-0.799853$

H $-1.479898-4.440982-0.744745$

H $-3.601184-3.367940-4.124619$

H -2.575281 -5.392799 -4.125158

H $-0.899883-5.281228-3.584986$

H -2.138084 -5.983594 -2.521392

H $-2.910172-1.231121-1.998897$

H $-3.407513-0.981942-3.657127$

H $-5.368825-0.580811-2.460226$

H $-5.608718-2.220391-3.072978$

H $-4.656579-3.014671-0.762261$

H -7.106190 -3.125911-1.335529

H $-7.288584-1.439081-0.898577$

H -8.826551 -3.2677340 .411206$

H $-6.904204-0.746601 \quad 1.604264$

H -7.108230 -4.8676290 .457711$

H $-6.821044-4.4463892 .130651$

H $-5.495040-4.3498690 .955199$

H $-6.215361-1.3045113 .629319$

H $-5.073806 \quad 0.705771 \quad 1.943044$
H $-4.337921-0.3088403 .185597$

H $-3.103582-2.1870091 .759872$

H $0.6592240 .381992-3.170290$

H $-1.4289141 .485027-1.192296$

H $1.2838972 .755218-3.486890$

H $0.3219044 .005736-4.240362$

H $0.0531662 .324783-4.697186$

H -2.184669 $-0.308702 \quad 2.035850$

H $-2.874820 \quad 0.459134 \quad 0.609043$

H -2.2203664 .3264852 .538723$

H -3.9881684 .3701042 .659078$

H $-4.184891 \quad 5.015270 \quad 0.219541$

H -1.310497 5.8430840 .882367

H $-2.1069927 .700351-0.614982$

H $-3.3427596 .685622-1.314062$

H $0.5179506 .514740-2.925424$

H $\quad 0.1567127 .608344-1.473589$

H - $1.3134685 .252778-3.908695$

H $-2.878973 \quad 5.275831-3.169428$

H $-2.3238473 .509438-1.582373$

H -3.9051612 .5454230 .837099$

C $4.254221-4.043517 \quad 0.657649$

O $4.362870-2.6326430 .380174$

C $5.387105-2.442220-0.614094$

C $6.130902-3.771098-0.719232$

C $5.012748-4.760437-0.459078$

H $4.869374-2.256481-1.562655$

C $6.281713-1.249003-0.266111$

H $6.605313-3.904232-1.696320$

H $6.907483-3.866090 \quad 0.048734$

C $5.497649-6.149352-0.083145$

H $4.405597-4.829654-1.369150$

C $5.5405050 .089286-0.068881$

O $6.975349-1.5401930 .954840$

H $7.054001-1.142786-1.037784$

H 4.9947630 .0644150 .880681

$\begin{array}{lllll}\text { O } & 6.541699 & 1.105753 & 0.082957\end{array}$

C $4.5845730 .488971-1.207389$

C $4.1058941 .953351-1.165478$

H $3.717168-0.181107-1.201234$

O $5.255173 \quad 0.297291-2.457259$

H $4.9479772 .622960-1.383344$

H $3.4058222 .082384-2.001978$

H $6.096578-6.579724-0.892519$

H $6.118247-6.1282820 .818885$

H $4.652195-6.8186990 .104236$

C $2.788524-4.4412110 .854844$

H $7.544183-0.7767311 .154086$

H $7.0096471 .185509-0.766162$

H $4.6148750 .483879-3.165044$

H $2.380228-3.8597921 .690831$

H $2.730706-5.4959721 .147744$

H $4.773987-4.200607 \quad 1.612452$

C $1.549197 \quad 1.7683451 .550872$

$\begin{array}{lllll}\text { O } & 2.287960 & 1.539393 & 0.342969\end{array}$

C 3.4276032 .3789200 .145437

C 3.0163393 .8612810 .117903

C 2.2009574 .2428381 .357991

C 1.0844903 .2323691 .634976

H $\quad 0.656594 \quad 1.1370901 .458007$

C 2.3186621 .3060792 .797895

H 4.1341292 .2280600 .966959

O $2.2535814 .148031-1.052575$

H 3.9219014 .4766860 .064152

H 1.7556905 .2347201 .209757

$\begin{array}{lllll}\text { O } & 3.055329 & 4.314225 & 2.494598\end{array}$

H 0.6332563 .4273382 .614787

$\begin{array}{llllll}\text { O } & 0.068528 & 3.436653 & 0.652347\end{array}$

C $2.564065-0.1871812 .803164$

H 3.2943951 .7924822 .875391

H 1.7338061 .5434273 .694022

H $1.4615853 .584312-1.034365$

H 3.7344824 .9852302 .310032

H -0.6608842 .8254330 .848240$

O $3.297386-0.5084903 .910189$

$\begin{array}{lllll}\text { O } & 2.169548 & -0.971518 & 1.952328\end{array}$

C $3.628772-1.8930814 .052600$

H $2.728698-2.5136534 .005162$ 
H $4.342992-2.1847573 .277784$

H $4.096575-2.0314615 .030913$

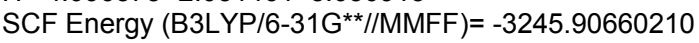

$08 \_00277$

MM̄FF Geometry

C $0.988278-2.7577642 .245396$

C $-0.042657-2.1928472 .892080$

C $-0.198498-0.7444043 .297262$

O $-1.379066-0.2311422 .629505$

C 0.9757880 .1833552 .935757

C $\quad 0.841191 \quad 1.5666553 .544497$

C 0.4525532 .6691062 .874387

C 1.2319041 .6685124 .997229

C 0.0332432 .7699431 .434902

C -1.2575843 .5863481 .279696$

C -1.670586 $3.707101-0.194177$

C $-2.8209254 .700799-0.357011$

C -3.295373 $4.782352-1.815305$

O $-2.0796372 .413107-0.649331$

O $-4.5479105 .483415-1.833223$

C $-3.5682583 .354420-2.328514$

C $-2.3307815 .592377-2.689447$

O $-3.8713303 .361640-3.721917$

C -2.420506 2.356627 -2.040980

C $-2.8959220 .922208-2.365005$

O $-1.3013342 .670091-2.870666$

C $-2.577288-0.3973843 .251196$

O $-2.778809-0.9110744 .340058$

C -3.624996 $0.123072 \quad 2.346606$

C $-4.894498-0.2677822 .532122$

C -6.0414830 .0945661 .698478$

C -5.8488651 .1859310 .680813$

C $-1.959950-0.191736-1.879274$

C $-2.642078-1.534422-2.053791$

C $-4.448203-2.945500-1.056409$

O $-2.421191-2.247962-3.029127$

C $-5.841548-2.439260-1.270916$

C $-6.889398-2.797529-0.512702$

C -8.286494 -2.286204 -0.729223

C $-8.937602-1.7792090 .541066$

C $-9.939545-2.4606851 .122243$

C $-8.481008-0.4448831 .096715$

C -7.201188 -0.5706671 .879833$

N -3.545227 -1.808971 -1.037702

H $1.841447-2.1576221 .943550$

H - $0.870335-2.8378633 .184074$

H $-0.346733-0.7203384 .384136$

H $1.070606 \quad 0.252913 \quad 1.846889$

H $1.922119-0.2448203 .291130$

H 0.4396113 .6148503 .415340

H 1.1689942 .6947055 .374632

H 0.5755501 .0507405 .617576

H $2.263873 \quad 1.3302245 .137322$

H $\quad 0.838078 \quad 3.2713630 .885924$

H $-0.106640 \quad 1.7856370 .980301$

H -2.059627 3.1023391 .851699

H -1.106475 4.5875391 .702342

H $-0.7996694 .037269-0.772420$

H -2.5334145 .6947190 .007803$

H $-3.668075 \quad 4.384516 \quad 0.267924$

H -4.404143 $6.363679-1.445137$

H -4.472927 2.995206 -1.817848

H -2.226765 $6.612403-2.300525$

H $-2.7171155 .698975-3.709007$

H $-1.3310805 .156310-2.743371$

H -4.603945 $3.987177-3.855819$

H -3.879712 $0.763695-1.910349$

H -3.032528 $0.810186-3.448700$

H $-0.772710 \quad 1.867177-3.002448$

H -3.3191810 .7681951 .531315$

H -5.112388 -0.9543803 .349639$

H $-5.2067830 .837087-0.130938$

H -5.387152 2.0682861 .138510

H $-6.782609 \quad 1.534487 \quad 0.234613$

H - $-1.023178-0.202965-2.446159$

H -1.695270 -0.068429 -0.823093

H $-4.339746-3.462380-0.097877$
H $-4.173037-3.630733-1.863612$

H $-5.996668-1.747582-2.096039$

H $-6.741132-3.5113690 .294478$

H -8.877355 -3.109695 -1.151017

H -8.305135 -1.487353 -1.481113

H -10.428085 -2.092384 2.018294

H -10.290775 -3.4045810 .718605$

H -9.243656 -0.037405 1.773098

H -8.4112710 .2663860 .269347$

H -7.244691 -1.324611 2.667623

H $-3.651528-1.140540-0.280641$

C $1.236080-4.5307630 .431723$

O $2.476137-3.975899-0.045752$

C $2.247142-3.392931-1.342850$

C $0.902184-3.934610-1.814101$

C $0.142102-4.013526-0.502240$

H $2.146681-2.312181-1.185884$

C $3.433486-3.671508-2.272447$

H $0.425201-3.279945-2.549449$

H $0.996588-4.932574-2.257239$

C $-1.084348-4.906766-0.558618$

H $-0.166827-2.998706-0.222361$

C $4.804872-3.282425-1.679535$

O $3.470616-5.076550-2.555243$

H $3.272463-3.170007-3.234040$

H $5.059033-3.983266-0.874506$

O $5.776483-3.482216-2.713169$

C $4.893125-1.835255-1.160097$

C $6.278345-1.496095-0.573928$

H $4.146001-1.682409-0.373888$

O $4.608094-0.923023-2.219102$

H $6.604551-2.3121080 .081333$

H $\quad 6.999891-1.394326-1.393809$

H -1.784827 -4.553157-1.320647

H $-0.818106-5.939839-0.805839$

H $-1.604455-4.9135220 .404498$

C $1.059611-4.2258941 .921154$

H $4.223933-5.231612-3.150737$

H $6.657088-3.440846-2.304247$

H $3.669623-1.018289-2.451919$

H $1.922505-4.6302912 .465362$

H $0.171714-4.7498032 .294612$

H $1.334638-5.6188540 .320426$

C $5.8547492 .184054-0.065316$

O $6.0204690 .895924-0.670439$

C $6.250111-0.1847210 .236722$

C 7.5550140 .0557281 .008100

C 7.5291051 .4108041 .724685

C 7.0756062 .5459450 .796565

H $5.8215082 .884118-0.910283$

C 4.5182562 .2908200 .679003

H $5.419101-0.2487870 .947971$

O $8.671030 \quad 0.003656 \quad 0.122104$

H $7.688214-0.7471891 .742428$

H $8.532853 \quad 1.6272712 .110173$

O 6.6465131 .3165352 .839352

H 6.8596863 .4550951 .369696

O $8.1467722 .859909-0.099342$

C $3.3644581 .989761-0.247721$

H 4.4441661 .5950971 .519764

H 4.3782063 .2941571 .097756

H $8.5256780 .662986-0.577972$

H 6.6652952 .1718413 .301588

H $8.905628 \quad 3.1427130 .439097$

O $3.1286393 .054675-1.065271$

O $2.7603260 .924815-0.256096$

C $2.0828672 .873153-2.024898$

H $1.1793052 .483256-1.548139$

H $1.8520203 .846204-2.466201$

H $2.4211432 .196346-2.814771$

SCF Energy (B3LYP/6-31G**//MMFF) $=-3245.91026212$

08_00278

MMFF Geometry

C $-0.6062762 .981560-0.834209$

C $-1.2154273 .995707-0.198952$

C -2.4452983 .9188660 .677036$

O -2.8907732 .5568090 .855302$ 
C $-3.596528 \quad 4.7116990 .036250$ C $-4.762273 \quad 4.9221930 .982768$ C $-5.9212394 .236329 \quad 0.939474$ C -4.5777736 .0261931 .992869$ C $-6.288086 \quad 3.1116210 .011914$ C -6.9533301 .9552330 .772970$ C $-7.3826730 .829884-0.178114$ C $-8.201552-0.2281390 .562065$ C $-8.578371-1.398736-0.358431$ O $-6.202010 \quad 0.232743-0.723027$ O $-9.057891-2.471706 \quad 0.466034$ C $-7.305353-1.905864-1.066200$ C $-9.725020-1.035382-1.309153$ O $-7.623817-2.892751-2.044887$ C $-6.455764-0.780896-1.704824$ C $-5.097188-1.358417-2.161991$ O $-7.141685-0.252841-2.840919$ C $-2.3406551 .850751 \quad 1.879905$ O $-1.509600 \quad 2.2422422 .683613$ C -2.902056 0.4836651 .827437 C $-2.264027-0.5051712 .470402$ C $-2.637741-1.9201482 .467416$ C $-3.970035-2.2910301 .873982$ C $-4.070057-0.305284-2.595755$ C $-2.713001-0.955105-2.777897$ C $-0.801653-1.877541-1.461133$ O $-2.296992-1.282859-3.886265$ C $-1.069697-3.211116-0.834607$ C $-0.356572-3.707230 \quad 0.188406$ C $-0.631834-5.0388890 .828894$ C $-0.670354-4.971915 \quad 2.342123$ C $0.300379-5.5339723 .082101$ C $-1.867538-4.3148463 .000353$ C $-1.765795-2.8129332 .980501$ N $-2.055746-1.158141-1.574426$ H $-1.0042941 .973182-0.769772$ H $-0.7824484 .990870-0.288959$ H -2.194001 4.3565171 .651172 H $-3.9292034 .207918-0.878729$ H -3.242382 $5.699471-0.289033$ H -6.7031934 .5148911 .645138$ H $-5.467464 \quad 6.1751722 .614284$ H -3.743892 5.7998352 .664194 H -4.3695306 .9740301 .485872$ H $-6.9884903 .501909-0.736177$ H $-5.4170272 .729723-0.527671$ H $-6.246314 \quad 1.5637431 .515957$ H $-7.8302292 .330577 \quad 1.315198$ H -7.974628 $1.270377-0.989070$ H $-9.1015600 .214527 \quad 1.006409$ H -7.613666 -0.6221191 .403141$ H $-9.820177-2.1427820 .972671$ H $-6.692243-2.410952-0.306304$ H -10.615059 -0.740415 -0.740317 H -10.027917 - $1.899346-1.910951$ H $-9.478177-0.213908-1.984959$ H -8.142367 -3.584942 -1.600215 H $-4.666461-1.941627-1.340603$ H $-5.248141-2.056132-2.995869$ H $-6.4952270 .160777-3.434468$ H -3.789284 $0.321133 \quad 1.226217$ H -1.353618 -0.2650993 .018808$ H $-4.257141-3.3275912 .062596$ H -3.957335 -2.1463920 .791173$ H $-4.770248-1.6751542 .300352$ H -4.356602 $0.164267-3.542675$ H -3.974554 $0.497190-1.855397$ H $-0.138506-1.263590-0.846357$ H $-0.353407-2.009980-2.450259$ H - $1.888297-3.798560-1.244358$ H $0.479011-3.1325520 .581092$ H $\quad 0.155271-5.7312300 .503200$ H $-1.576865-5.4645410 .468916$ H $0.269787-5.5100324 .166405$ H $1.150468-6.0260532 .621199$ H $-1.940180-4.6272454 .050150$ H -2.773937 -4.6902532 .518296$ H $-0.837951-2.435973 \quad 3.414024$
H $-2.501730-0.857612-0.713137$

C $1.8711692 .438871-1.099271$

O $1.7353431 .013128-1.240046$

C $2.4303680 .375958-0.142825$

C $3.107624 \quad 1.4936130 .644921$

$\begin{array}{llll}\text { C } & 2.199347 & 2.675068 & 0.372672\end{array}$

H $1.652470-0.1043100 .462773$

C $3.409010-0.667428-0.700827$

H 3.1979821 .2581811 .709563

H 4.1140501 .6913340 .255792

C 2.8447024 .0193530 .656817

H 1.3056672 .5634360 .998822

C $4.269196-1.3469540 .379094$

O $2.660237-1.663028-1.402980$

H $4.043449-0.183725-1.450419$

H $4.860205-0.6016460 .916682$

O $3.384111-1.9194071 .353117$

C $5.185803-2.468713-0.139345$

C $6.130455-2.100022-1.298485$

H $4.564497-3.318393-0.448620$

O $5.966597-2.9340330 .967597$

H $6.637422-3.022188-1.612553$

H $5.531826-1.761075-2.150929$

H $3.1285124 .094144 \quad 1.711646$

H $3.747923 \quad 4.1663860 .055123$

H 2.1534104 .8381480 .433569

C $0.6404543 .159549-1.657133$

H $2.146512-2.160221-0.744443$

H $3.937476-2.3362442 .036033$

H $6.503608-3.6812760 .653256$

H $0.4263462 .778970-2.663585$

H $0.8735224 .226115-1.763461$

H $2.7315062 .718975-1.722571$

C $7.4741151 .274939-0.352633$

O $6.599576 \quad 0.199873-0.719936$

C $7.223329-1.062501-0.961861$

C $8.240533-0.934233-2.109761$

C $9.2512900 .186630-1.846386$

C $8.5622681 .486575-1.418570$

H $6.8325292 .165618-0.350940$

C 8.0391091 .0986031 .062694

H $7.743442-1.389584-0.055965$

O $7.568520-0.681713-3.343034$

H $8.767705-1.888579-2.223639$

H $9.8352030 .371196-2.756425$

O $10.157139-0.213525-0.822555$

H $9.2984742 .210272-1.049899$

O $7.9307602 .074569-2.559907$

C 6.9421131 .1807982 .099700

H 8.5684690 .1493891 .182253

H 8.7582501 .8916861 .298299

H $7.0310440 .121040-3.229665$

H $10.608168-1.019163-1.127650$

H $8.624798 \quad 2.260441-3.215136$

O $6.883874 \quad 0.0185382 .810451$

$\begin{array}{llll}\text { O } & 6.224482 & 2.159684 & 2.260007\end{array}$

C $5.881907-0.0321053 .831012$

H $5.903853-1.0290164 .279089$

H $6.0986740 .708048 \quad 4.607054$

H 4.8894590 .1366923 .402771

SCF Energy (B3LYP/6-31G**//MMFF) $=-3245.91802577$

08_00279

MM̄FF Geometry

C $3.527140-0.8051432 .587156$

C $3.240413-1.1310173 .857655$

C $1.851624-1.2758674 .440020$

O $0.853229-1.2981853 .394254$

C $1.548570-0.0987295 .381350$

C $0.193927-0.2103596 .054301$

$\begin{array}{llll}C & -0.889686 & 0.524560 & 5.733290\end{array}$

C $0.116075-1.2137027 .176708$

C $-1.012502 \quad 1.574392 \quad 4.664046$

C $-2.062827 \quad 1.187582 \quad 3.619902$

C -2.070744 2.1218732 .400245

C -2.4784293 .5528562 .761284$

C $-2.579860 \quad 4.4526301 .521048$

O -3.0013251 .5701811 .458812$ 
O -3.2509445 .6545031 .929301$ C -3.4613343 .7501600 .467592$ C -1.1936054 .8828041 .029623$ O $-3.4784034 .485546-0.753693$ C $-3.068746 \quad 2.2784340 .212595$ C $-4.0920501 .550690-0.687378$ O $-1.8081842 .239125-0.464628$ C $0.610371-2.4984912 .797016$ O $1.102862-3.5775583 .085840$ C $-0.340344-2.2617951 .688531$ C $-0.660162-3.2699380 .863148$ C - $-1.559082-3.188702-0.290788$ C $-2.221675-1.863327-0.554756$ C $-5.5142791 .500959-0.125787$ C $-6.3277490 .399374-0.775963$ C $-6.697596-2.057455-0.709509$ O $\quad-7.1907860 .639500-1.616483$ C $-5.830773-3.252263-0.472706$ C $-5.571488-4.182356-1.404151$ C $-4.702331-5.382415-1.154089$ C $-3.600706-5.534878-2.181550$ C $-3.545285-6.620362-2.971771$ C $-2.564753-4.436416-2.301969$ C -1.732640 -4.287868-1.055303 N -5.971881 $-0.862201-0.321969$ H $2.724605-0.5753711 .891257$ H $4.059767-1.3477044 .540054$ H $1.810281-2.2160545 .003906$ H $1.630646 \quad 0.8411104 .826073$ H $2.318703-0.037626 \quad 6.162429$ H $-1.8040120 .362528 \quad 6.303362$ H - $0.859143-1.2060047 .675061$ H $0.283986-2.2274426 .800664$ H $0.873470-0.9931617 .936142$ H -1.2987342 .5127195 .152524$ H -0.0618541 .7633524 .159943$ H -1.881458 0.1626433 .270633 H -3.0616731 .1643724 .075766$ H -1.0694732 .1050981 .953604$ H -1.7842253 .9965353 .484241$ H -3.4580903 .5346713 .259234$ H -3.384456 6.2012591 .135845 H -4.4877583 .7843850 .853481$ H -0.6653055 .4353321 .815984$ H $-1.2684885 .572787 \quad 0.182147$ H $-0.563997 \quad 4.0403190 .737760$ H -2.634626 4.323197-1.208934 H -4.108426 2.004874 -1.686795 H $-3.7340420 .527514-0.851797$ H -1.727899 $1.385609-0.921660$ H $-0.733279-1.258768 \quad 1.568623$ H - $0.213470-4.2479501 .037885$ H -2.815536 -1.553274 0.312535 H -2.901637 -1.872020 -1.407902 H -1.469993 -1.092729 -0.759523 H $-5.515251 \quad 1.3287340 .956799$ H $-6.0293722 .452945-0.296613$ H -7.014648 -1.966288 -1.753736 H $-7.593698-2.113333-0.083038$ H $-5.401258-3.3638970 .520562$ H $-6.018043-4.088525-2.391399$ H -5.352542 -6.266805-1.159148 H $-4.255629-5.345210-0.152849$ H -2.762048 -6.742464 -3.712620 H -4.281848 -7.413643 -2.896784 H -3.065532 -3.511184 -2.596082 H -1.872607 -4.659196 -3.125112 H -1.191447 $-5.195716-0.784338$ H $-5.364863-0.9206350 .491288$ C $5.153396-1.7895050 .917507$ O $4.301044-1.519374-0.213305$ C $3.828935-2.774607-0.743003$ C $4.661884-3.861357-0.069096$ C $4.905498-3.2503131 .296638$ H $2.785707-2.879010-0.421587$ C $3.907396-2.773042-2.273492$ H $4.134295-4.819046-0.024646$ H $5.614569-4.025193-0.585800$
C $6.049103-3.8918342 .061849$

H $3.983707-3.3547441 .881760$

C $3.167857-1.599194-2.948092$

O $5.283623-2.698665-2.670946$

H $3.535062-3.727600-2.662596$

H $3.689544-0.666727-2.708563$

O $3.291981-1.765320-4.366917$

C $1.674320-1.476816-2.589283$

C $0.909457-0.423713-3.417463$

H $1.567200-1.249341-1.523130$

O $1.054083-2.745447-2.815894$

H $0.920764-0.708727-4.476937$

H $-0.144523-0.464832-3.110645$

H $5.844705-4.9524572 .241030$

H $6.992296-3.8200611 .509853$

H $6.188144-3.4057983 .032717$

C $4.918352-0.7547432 .022979$

H $5.655728-1.884739-2.289882$

H $2.812524-2.576240-4.609548$

H $0.113112-2.657192-2.589235$

H 5.0677520 .2508861 .610647

H $5.663151-0.8926172 .815531$

H $6.182126-1.6682790 .553031$

C $1.7696662 .669561-1.525073$

O $1.2197051 .395020-1.885254$

C $1.4387141 .009033-3.246014$

C $0.7459182 .003519-4.187142$

C $1.2622593 .425869-3.940128$

C $1.2515003 .788531-2.450682$

H $1.3782092 .861983-0.517978$

C $3.2955322 .572974-1.424866$

H $2.5116441 .016504-3.460582$

O $-0.6633051 .956906-3.975422$

H $0.937413 \quad 1.705460-5.224833$

H $0.6382624 .143457-4.487033$

O $2.5930223 .544688-4.434244$

H $1.8282044 .704154-2.282482$

O $-0.0964204 .092554-2.079374$

C $3.8571293 .648880-0.529824$

H $3.5915941 .607796-0.994163$

H $3.7844652 .608859-2.403094$

H -1.074128 $2.578049-4.600696$

H $2.5725393 .344693-5.385532$

H -0.613553 $3.270896-2.139338$

O $4.4635044 .611782-1.280090$

O $3.750373 \quad 3.6433580 .689800$

C $5.0194055 .705514-0.543748$

H $5.796725 \quad 5.347215 \quad 0.137917$

H $5.4708686 .400697-1.256481$

H 4.2327046 .2295420 .007405

SCF Energy (B3LYP/6-31G**//MMFF $)=-3245.92871463$

08_00280

MM̄FF Geometry

C $-3.900518-2.7110951 .402958$

C $-2.713770-2.0915141 .315550$

C -1.891381-1.655695 2.498879

O $-1.654302-0.2317302 .416759$

C $-0.525668-2.3640242 .428124$

C $0.337707-2.1642363 .656531$

C $1.401837-1.3391483 .719469$

C -0.021504 -3.025682 4.839206

C $1.884325-0.3802832 .670573$

C $3.224067-0.7400782 .021862$

C $3.142942-1.8893831 .004116$

C $3.365632-3.2607281 .646185$

C $3.388705-4.3858040 .599707$

O $4.172076-1.6533940 .031862$

O $3.906202-5.567077 \quad 1.230372$

C $4.375061-4.004961-0.524223$

C $1.976984-4.7354560 .115066$

O $4.317991-4.945890-1.594076$

C $4.169065-2.572902-1.066960$

C $5.330139-2.195366-2.024313$

O $2.937644-2.525862-1.794387$

C -2.6398080 .6050732 .833966$

$\begin{array}{llll}\text { O } & -3.758594 & 0.282411 & 3.210002\end{array}$

C -2.2143572 .0251202 .774566$ 
C -0.9344592 .4018592 .631887$ C -0.4138583 .7711402 .583720$ C -1.412045 4.8975352 .597218 C $5.377527-0.710036-2.417809$ C $6.0448750 .182851-1.382554$ C $6.3520792 .587537-0.796768$ O $6.744646-0.251629-0.471363$ C 5.3892132 .9138930 .301228 C 4.9935344 .1618290 .597189 C 4.0682684 .4929941 .736105 C 2.7677765 .1845091 .364361 C 2.5210405 .7773150 .183325 C 1.7163685 .2221772 .461274 C 0.9251073 .9405482 .532794 N $5.8016321 .529188-1.624141$ H -4.341163 -2.8970532 .378749$ H -2.285893 -1.9059700 .334126$ H $-2.393230-1.8900663 .445666$ H $0.011429-2.0321991 .531979$ H $-0.671220-3.4447892 .291878$ H $1.975724-1.3113774 .644919$ H $0.662715-2.8817445 .682171$ H - $-1.030818-2.7924135 .192169$ H $\quad 0.015232-4.0846334 .562982$ H $1.135488-0.2156821 .890197$ H $2.005091 \quad 0.5910373 .167463$ H 3.5834350 .1583831 .503204 H $3.980542-0.9542692 .787327$ H $2.175093-1.8425680 .494329$ H $2.612651-3.4787482 .409286$ H $4.330665-3.2626822 .172444$ H $3.335718-5.7755171 .990012$ H $5.389181-4.072539-0.105875$ H $1.356172-5.0734830 .953566$ H $1.996860-5.567234-0.597430$ H $1.466264-3.891514-0.351458$ H $4.462783-5.828266-1.211453$ H $6.289106-2.470735-1.568765$ H $5.229558-2.781480-2.946853$ H $3.018578-1.896752$-2.527732 H -3.0144162 .7483922 .892002$ H -0.1751821 .6298232 .550412$ H -2.0927674 .8206191 .742721$ H -0.9553665 .8877252 .546646$ H -2.004089 $4.874133 \quad 3.519017$ H $5.976010-0.615070-3.332733$ H $4.377666-0.327924-2.641438$ H $6.5433803 .445518-1.448844$ H $7.3020132 .253805-0.366842$ H 5.0225082 .0849050 .901487 H 5.3930124 .9934270 .021602 H 4.6101855 .1391492 .438314 H 3.8349993 .5718812 .284525 H $1.5710466 .266928-0.007819$ H $3.2432735 .796418-0.623997$ H 1.0732036 .0947922 .316904 H 2.2141605 .3807653 .426219 H 1.5486563 .0460592 .559304 H $5.1639941 .794409-2.369074$ C $-5.668425-2.248608-0.380571$ O $-5.004697-1.047890-0.819970$ C $-5.8383340 .078316-0.491769$ C $-7.202580-0.499276-0.132580$ C $-6.797983-1.7977670 .542296$ H -5.4138600 .5331970 .410882$ C $-5.8381741 .091170-1.639449$ H -7.7768290 .1649610 .520337$ H -7.804521 -0.703455 - 1.025928 C -7.930332 $-2.800806 \quad 0.668872$ H $-6.413477-1.5633121 .542923$ C $-4.4338311 .619540-2.011956$ O $-6.3967150 .465960-2.801001$ H $-6.5043901 .925498-1.389279$ H -3.857031 $0.825737-2.498863$ O $-4.6071972 .641360-3.001850$ C $-3.6404402 .192653-0.825568$ C $-2.3667792 .963062-1.202828$ H $-3.3828391 .382348-0.135589$
O $\quad-4.4902243 .099154-0.112701$

H -2.626430 $3.848279-1.797552$ H -1.957211 $3.359102-0.265727$ H -8.739576 -2.391472 1.282368 H $-8.348988-3.060165-0.309352$ H $-7.580799-3.7239661 .141895$ C $-4.648620-3.2239300 .203726$ H $-6.3786021 .121228-3.519504$ H $-5.0766143 .381446-2.579872$ H -4.0081613 .3954220 .677877$ H $-3.924656-3.478268-0.580376$ H $-5.142849-4.1632720 .478037$ H $-6.091755-2.706137-1.284972$ C $0.0716610 .145694-1.630308$ O $-0.963248 \quad 1.003497-1.124251$ C $-1.2802682 .147346-1.925334$ C $-0.0317603 .016049-2.175940$ C $1.1497372 .199922-2.701729$ C $1.3688580 .934830-1.869423$ H $0.261531-0.568301-0.818589$ C $-0.375702-0.639172-2.870564$ H $-1.6832341 .809537-2.885133$ O $0.3669223 .671979-0.973918$ H $-0.2907623 .802898-2.893883$ H $2.0598552 .811774-2.670907$ O $\quad 0.917511 \quad 1.832152-4.057256$ H $2.1042120 .284519-2.353673$ O $1.9016201 .292294-0.592318$ C -1.257899-1.802776 -2.493175 H $-0.933736-0.035779-3.591549$ H $0.487966-1.044129-3.412721$ H $0.5378442 .987805-0.304110$ H $0.8065562 .651249-4.569438$ H $2.7492441 .744040-0.744045$ O $-0.490156-2.900055-2.237957$ O $-2.477791-1.737337-2.425840$ C $-1.188741-4.103151-1.900713$ H -1.422454 -4.099034 -0.833205 H -2.099814 -4.221659-2.495366 H -0.527415 -4.947927 -2.111199 SCF Energy $(B 3 L Y P / 6-31 G * * / / M M F F)=-3245.90234570$

08_00281

MM̄FF Geometry

C 1.5169683 .5073020 .825618

C 2.2250354 .3445850 .051083

C $3.4274473 .968238-0.784626$

O $3.4531612 .545706-1.039427$

C $4.7175504 .352373-0.043536$

C $5.9684154 .085530-0.856476$

C $6.7812393 .025553-0.676669$

C $6.2989675 .129699-1.890733$

C 6.5954101 .8904740 .293012

C $6.8822390 .534116-0.367359$

C $6.664220-0.6239440 .616771$

C $7.180526-1.9390590 .033492$

C $6.908767-3.123410 \quad 0.972224$

O $5.259739-0.7300030 .875249$

O $7.157170-4.3151150 .211439$

C $5.419064-3.1079171 .374348$

C $7.885159-3.1462982 .154103$

O $5.144694-4.0884112 .372760$

C $4.915502-1.7224131 .851014$

C $3.375465-1.7583501 .986476$

O $5.485929-1.4232973 .126065$

C $2.7431562 .098057-2.110665$

O $2.1095452 .771700-2.907441$

C $2.8360730 .621932-2.123261$

C $2.001172-0.074431-2.908650$

C $1.902272-1.532407-2.991972$

C $2.935357-2.347272-2.261386$

C $2.717067-0.3906222 .204318$

C $1.208113-0.5313712 .139264$

C $-0.642108-0.9247330 .503736$

O $0.520768-0.5939383 .155679$

C $-0.671504-2.264503-0.163468$

C $-1.284673-2.501456-1.332984$

C $-1.289823-3.844093-2.009380$ 
C $-0.935540-3.766452-3.480119$

C $-1.866341-3.978435-4.425884$

C $0.511422-3.513601-3.855922$

C $0.885999-2.064045-3.702910$

N $0.737553-0.621342 \quad 0.838590$

H $1.816147 \quad 2.4663640 .906572$

H 1.9304725 .3907770 .003749

H $3.3748744 .509931-1.737334$

H $4.767858 \quad 3.8214950 .914561$

H $4.695074 \quad 5.4182120 .221387$

H $7.6794652 .960855-1.289337$

H $7.2315744 .909508-2.421038$

H $5.5031385 .194234-2.639201$

H $6.4127576 .110542-1.417652$

H 7.2893952 .0373251 .129284

H 5.5847121 .8754850 .710151

H $6.2235930 .410212-1.236714$

H $7.9176090 .521022-0.730244$

H $7.189401-0.3890241 .549975$

H $8.251275-1.871875-0.196360$

H $6.679648-2.132519-0.925984$

H $7.041564-5.0797070 .800352$

H $4.840792-3.3978170 .485641$

H $8.917335-3.2307261 .793524$

H $7.717228-4.0227382 .789879$

H $7.822796-2.2532172 .779387$

H $5.365948-4.9586682 .001150$

H $2.950232-2.2005841 .078520$

H $3.089238-2.4156772 .817968$

H $4.917336-0.7829923 .582136$

H $3.5491780 .150959-1.456712$

H $1.2890440 .474610-3.523999$

H $2.809777-2.241045-1.180934$

H $3.946879-2.021849-2.529971$

H $2.891900-3.413644-2.492055$

H 2.9812770 .0296523 .180212

H 3.0349610 .3324741 .445243

H $-0.999970-0.128032-0.155412$

H $-1.256457-0.9406031 .408690$

H $-0.158895-3.0808590 .340618$

H -1.822668 -1.693081-1.823291

H -2.290954 -4.276117-1.885371

H $-0.599938-4.539694-1.515370$

H $-1.616756-3.946974-5.481319$

H $-2.899723-4.183292-4.167142$

H $0.683743-3.790997-4.903816$

H $1.140368-4.184287-3.264573$

H $0.209365-1.383015-4.221852$

H $1.397625-0.5419850 .071230$

C $-0.878348 \quad 2.9986701 .364421$

$\begin{array}{lllll}\text { O } & -1.303166 & 3.118272 & -0.007647\end{array}$

C $-2.7381183 .252655-0.044449$

C -3.2263092 .8482921 .338377$

C -2.098366 $3.343800 \quad 2.218040$

H $-2.9330254 .317331-0.224234$

C $-3.3085752 .399319-1.185505$

H $-4.1935013 .291757 \quad 1.592339$

H -3.3172151 .7578491 .414929$

C -2.084208 2.7074093 .597560

H -2.188816 4.4330492 .324808

C $-4.8458382 .441542-1.263124$

O $-2.7565392 .859827-2.422589$

H $-2.9571801 .369767-1.068894$

H $-5.2842522 .089901-0.325220$

O $-5.2641663 .808858-1.385432$

C $-5.4556351 .652792-2.434614$

C $-5.1488810 .145028-2.471736$

H $-5.1412482 .096897-3.386697$

O $-6.881472 \quad 1.796822-2.386527$

H -5.680590 $-0.265313-3.340235$

H $-4.076082-0.002186-2.642921$

H -3.009393 $2.936904 \quad 4.136440$ H -1.9936021 .6174113 .535788$ H -1.244945 3.0820004 .191724 C $0.314291 \quad 3.920737 \quad 1.626272$ H -2.930951 $3.813310-2.493591$ H -4.970363 $4.134922-2.252807$ H $-7.0764032 .749570-2.371538$
H $\quad 0.5826573 .8870502 .688167$

H $0.041096 \quad 4.9554901 .384864$

H -0.5906101 .9520321 .516707$

C $-4.866258-1.0926761 .046350$

O $-4.580570-0.489354-0.222687$

C $-5.600529-0.633389-1.216158$

C $-5.815827-2.123713-1.522294$

C $-6.146702-2.910110-0.249724$

C $-5.175854-2.5929050 .893350$

H $-3.926195-0.9986331 .604869$

C $-5.938701-0.2872651 .794795$

H $-6.536005-0.204908-0.839533$

O $-4.641792-2.656737-2.128579$

H $-6.633405-2.220426-2.246838$

H $-6.109856-3.985182-0.464320$

O $-7.472901-2.6030380 .170600$

H $-5.559344-2.9890651 .840214$

O $-3.934722-3.2568310 .637598$

C $-5.901236-0.4985673 .292600$

H -5.7744220 .7814961 .615361$

H $-6.942596-0.5283551 .435410$

H $-4.813892-3.591657-2.332243$

H $-8.074573-2.842570-0.554647$

H $-4.113915-4.211616 \quad 0.599534$

O $-6.8952520 .242826 \quad 3.866700$

O $-5.105444-1.2109143 .888423$

C $-6.983258 \quad 0.153974 \quad 5.292225$

H $-7.189679-0.8769225 .595402$

H $-6.0583130 .515017 \quad 5.752007$

H -7.809900 $0.789008 \quad 5.621922$

SCF Energy (B3LYP/6-31G**//MMFF) $=-3245.89830474$

08_00282

MMFF Geometry

C $0.651954 \quad 4.9891250 .230665$

C 1.8543284 .5607640 .646084

C $2.1908613 .875494 \quad 1.952346$

$\begin{array}{llll}\text { O } & 2.714763 & 2.557688 & 1.643827\end{array}$

C 1.0107693 .6938332 .927967

C 1.4084473 .0614564 .250238

C 1.1275121 .7940874 .615717

C $2.133397 \quad 3.974967 \quad 5.205487$

C $0.422306 \quad 0.739414 \quad 3.809330$

C $1.284605-0.5208823 .658379$

C $0.677252-1.5109962 .652734$

C $1.522219-2.7833382 .571809$

C $1.032710-3.7376431 .472273$

O $0.637716-0.8613191 .378550$

O $2.058676-4.717757 \quad 1.250567$

C $0.874600-2.9466190 .155639$

C $-0.219190-4.510217 \quad 1.905283$

O $0.282196-3.757906-0.855616$

C $0.069087-1.6429170 .320331$

C $0.069698-0.761314-0.952708$

O $-1.295534-1.9719750 .600911$

C 4.0415102 .4592021 .352126

O $4.847332 \quad 3.3731551 .276676$

C 4.3547661 .0274811 .143961

C $5.5766310 .667053 \quad 0.723214$

C $6.053262-0.7027170 .512177$

C $5.083199-1.8270630 .759267$

C $1.440609-0.236100-1.387472$

C $2.139038-1.076547-2.441118$

C $4.299295-1.196432-3.685280$

O $1.614992-2.033907-3.002239$

C $5.219985-2.158115-3.003874$

C $6.557259-2.062896-3.054108$

C $7.489604-3.058537-2.427664$

C $8.491506-2.418387-1.494535$

C $9.743169-2.149103-1.903061$

C $8.072543-2.185442-0.061486$

C $7.333563-0.8888160 .127265$

N $3.417542-0.599244-2.701438$

H $-0.225094 \quad 4.858506 \quad 0.857138$

H $2.7031634 .719778-0.017492$

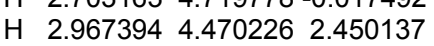

H $\quad 0.223578 \quad 3.106477 \quad 2.444981$

H 0.5556604 .6695013 .145751 
H 1.4399411 .4655545 .606296 H 2.3301703 .4965706 .170940 H 3.0978954 .2810814 .789179 H 1.5366334 .8721835 .400168 H $-0.513431 \quad 0.478146 \quad 4.317709$ H $\quad 0.147673 \quad 1.1108582 .820844$ H $2.285650-0.2285083 .318034$ H $1.400910-1.0053744 .635809$ H $-0.344678-1.7490142 .968621$ H $1.547702-3.2945263 .542204$ H $2.563131-2.5129062 .352506$ H $2.223843-5.1745862 .093053$ H $1.885210-2.700290-0.191837$ H $-0.005939-5.1165032 .794053$ H $-0.535597-5.216831 \quad 1.130232$ H -1.063078 -3.8605302 .147031$ H $0.828413-4.557980-0.942091$ H $-0.449181-1.273406-1.771890$ H $-0.5432920 .123798-0.729728$ H -1.850859 -1.2091150 .372208$ H $3.575326 \quad 0.306613 \quad 1.358270$ H 6.3100411 .4490660 .529930 H $5.464178-2.8063900 .462221$ H $4.161954-1.6780220 .186449$ H $4.831658-1.8831231 .823508$ H $\quad 1.3006610 .752962-1.837969$ H $2.105186-0.109364-0.528647$ H $4.840567-0.379893-4.173579$ H $3.707563-1.722484-4.441250$ H $4.763703-2.989243-2.471045$ H $7.010692-1.245492-3.610292$ H $8.016108-3.576029-3.240437$ H $\quad 6.939057-3.841294-1.890973$ H $10.477507-1.720400-1.229127$ H $10.059076-2.345724-2.922261$ H $8.962503-2.1445910 .581682$ H $7.515313-3.0532690 .302588$ H $7.959618-0.009266-0.029987$ H $3.7829680 .168466-2.145253$ C $-0.4879124 .891311-2.044038$ O $-1.8083324 .763891-1.485353$ C -2.308395 $3.432295-1.764870$ C - $1.3571852 .848402-2.800230$ C $-0.0377073 .476964-2.397561$ H $-2.2217002 .893439-0.814431$ C $-3.7783923 .545109-2.191476$ H -1.320694 $1.756113-2.779245$ H -1.637338 $3.166337-3.812080$ C $1.0245843 .421689-3.481364$ H $0.3305342 .951548-1.508376$ C $-4.5306912 .256465-2.587796$ O $-4.5074574 .178893-1.132101$ H $-3.8255044 .239542-3.040163$ H -5.559087 $2.559199-2.824733$ O $-3.9920191 .718496-3.791916$ C $-4.6275581 .167688-1.499170$ C $-5.522853-0.007956-1.941456$ H $-5.0328321 .610101-0.582816$ O $\quad-3.3227910 .675491-1.212880$ H $-6.4413050 .384143-2.394002$ H $-4.985635-0.592867-2.698360$ H $1.2461382 .384695-3.753253$ H $0.700513 \quad 3.943081-4.388212$ H $1.9539993 .887207-3.138075$ C $0.4237645 .670900-1.091849$ H $-4.3321553 .690052-0.310582$ H -3.274080 $1.108696-3.553360$ H $-3.4055140 .026759-0.494198$ H $-0.0355456 .644055-0.877717$ H $1.3789105 .872840-1.591297$ H $-0.6065525 .485183-2.960163$ C $-4.890215-2.4010200 .873549$ O $-4.719817-1.560031-0.275265$ C $-5.907964-0.931982-0.766524$ C $-6.936091-1.993737-1.186233$ C $-7.223452-2.967245-0.038610$ C -5.933384 -3.496699 0.597931 H $-3.918426-2.8933341 .006812$
C $-5.178656-1.5879462 .140751$

H $-6.338195-0.3047030 .021856$

O $-6.440432-2.714634-2.312588$

H -7.860266 -1.490193 -1.494608

H -7.814500 -3.808843 -0.420139

O $-8.007420-2.2921970 .940918$

H $-6.160788-4.0265191 .529669$

O $-5.349284-4.461926-0.282205$

C $-3.983442-0.7441882516446$

H $-6.050270-0.9334892 .051668$

H $-5.381044-2.2533332 .988590$

H -7.125567 -3.351763 -2.577577

H $-8.206370-2.9298381 .647596$

H $-5.069777-3.997567-1.089913$

O $\quad-4.077647 \quad 0.484174 \quad 1.932864$

O $-3.064463-1.1409673 .220874$

C -2.9786381 .3695452 .169025$

H $-2.0761320 .977376 \quad 1.691671$

H -3.2192932 .3369551 .720129$

H -2.8214821 .5105143 .242462$

SCF Energy $(B 3 L Y P / 6-31 G * * / / M M F F)=-3245.91455819$

$08+00283$

MMFF Geometry

C $-2.2678073 .710230-0.010428$

C -2.3406714 .0202141 .293349$

C -2.8170013 .0838562 .382300$

O -2.6888311 .7057381 .956099$

C -4.2882233 .3665182 .718086$

C $-4.7874512 .558828 \quad 3.899378$

C -5.5646651 .4625883 .799615$

C -4.3952423 .0887435 .254187$

C -6.0418170 .7970462 .538825$

C $-5.870991-0.7271742 .607512$

C $-6.350405-1.3967731 .312482$

C $-6.399408-2.9167061 .466484$

C $-6.814099-3.6034720 .156527$

O $-5.436222-1.0454690 .267332$

$\begin{array}{lllll}\text { O } & -6.539054 & -5.006374 & 0.287537\end{array}$

C $-5.925533-3.072155-0.986375$

C $-8.319374-3.478749-0.106711$

O $-6.368170-3.564210-2.249365$

C $-5.814680-1.528211-1.027964$

C $-4.719901-1.120377-2.040662$

O $-7.062321-0.974224-1.449816$

C -1.4509031 .1490802 .073581$

O $-0.468870 \quad 1.6504582 .598519$

C $-1.470560-0.1654571 .395615$

C $-0.302479-0.6933300 .998585$

C $-0.104036-1.9663620 .302541$

C $-1.309419-2.8447370 .101958$

C $-4.2826090 .348535-1.957063$

C $-3.2222410 .636497-3.003436$

C $-0.8726500 .054866-3.612649$

O $-3.4682321 .299120-4.008363$

C $-0.248646-1.305485-3.599359$

C $1.055507-1.527863-3.379624$

C $1.675687-2.894850-3.354636$

C $2.421869-3.175622-2.068850$

C $3.764956-3.198344-2.039483$

C $1.611826-3.513944-0.834106$

C $1.143863-2.286325-0.101368$

N $-2.0075240 .039665-2.708891$

H -2.619566 $2.739854-0.350998$

H $-2.0027714 .999746 \quad 1.623826$

H -2.1983263 .2447793 .274036$

H -4.9127283 .1821231 .836367$

H -4.422732 4.4324962 .946778

H $-5.901051 \quad 0.9890894 .721026$

H -4.8219772 .4977516 .071687$

H $-3.307476 \quad 3.075100 \quad 5.372807$

H -4.746193 4.1187725 .375350

H -7.1042411 .0351592 .408097$

H $-5.515867 \quad 1.173157 \quad 1.657347$

H -4.812380 -0.963464 2.777489

H $-6.440782-1.1184283 .459502$

H -7.346620 -1.007723 1.070598

H -7.075580 -3.205819 2.280630 
H $\quad-5.405445-3.287077 \quad 1.754761$ H -7.041085 -5.340088 1.050787 H $-4.918629-3.484858-0.829849$ H $-8.890079-3.9243260 .717005$ H -8.610171 -4.033166 -1.005820 H -8.651619 -2.444491 -0.217025 H $-6.408752-4.533706-2.184664$ H $-3.834512-1.744856-1.875347$ H $-5.063843-1.327401-3.062537$ H $-6.901685-0.106922-1.854364$ H $-2.429379-0.6382361 .219826$ H $0.609526-0.1278951 .188832$ H -2.038964 -2.353149 -0.547035 H -1.789704 -3.064023 1.062290 H -1.077434 -3.809351 -0.353337 H $-5.1247631 .026474-2.129584$ H $-3.8723950 .585145-0.968984$ H $-0.1852130 .831301-3.265775$ H -1.204770 $0.307632-4.624231$ H -0.907222 -2.152495 -3.778691 H $1.721089-0.682760-3.227751$ H $2.358523-2.965075-4.211356$ H $0.923148-3.678004-3.510208$ H $4.303986-3.427171-1.126196$ H $4.355171-2.983295-2.924022$ H $2.227528-4.096226-0.135765$ H $0.793922-4.178280-1.124519$ H $1.950705-1.5881430 .126290$ H $-1.869489-0.354047-1.782723$ C $-0.5065013 .904820-1.742743$ O $0.5784223 .770695-0.805585$ C $1.8252923 .872042-1.517768$ C $1.4643814 .029432-2.993961$ C $0.0876504 .662569-2.922989$ H $2.317514 \quad 4.787232-1.166266$ C $2.7263332 .660466-1.225630$ H $2.1918004 .645379-3.531718$ H $1.4151163 .058454-3.501212$ C $-0.7063694 .527706-4.210425$ H $0.199730 \quad 5.725474-2.671442$ C 3.2002312 .5690530 .241105 O $2.0151091 .474063-1.577067$ H $3.5972472 .734393-1.886223$ H 3.7678083 .4715530 .499056 O $2.0708172 .533187 \quad 1.120905$ C 4.0436591 .3213970 .580213 C $5.2833361 .132450-0.313022$ H 3.4113340 .4277600 .536438 O 4.4607371 .4396601 .945959 H $4.9935201 .148343-1.367919$ H $5.9689521 .969655-0.132340$ H $-0.1842455 .019718-5.037538$ H $-0.8537883 .477108-4.483211$ $\mathrm{H}-1.6930184 .990900-4.112003$ C -1.685725 4.605025 -1.067796 H $2.6420340 .732249-1.559939$ H 1.5752013 .3611021 .004669 H 5.0479852 .2117112 .012628 H -2.468863 4.828188 -1.800308 H $-1.3574485 .553770-0.626603$ H $-0.7936062 .890422-2.048898$ C $7.226493-1.3600461 .705419$ O $6.561205-0.1649201 .276146$ C $6.021148-0.197001-0.046484$ C $7.144690-0.435459-1.068739$ C $7.961112-1.687706-0.729907$ C $8.372270-1.7214730 .746022$ H $7.670774-1.0972202 .674398$ C $6.243050-2.5122881 .940508$ H $5.286411-1.005919-0.119115$ O $8.0153580 .694018-1.119228$ H $6.700679-0.540763-2.065444$ H $8.861830-1.714483-1.355175$ O $7.197138-2.853649-1.020444$ H $8.773928-2.7062291 .012517$ O $9.419345-0.7694360 .956848$ C $5.295784-2.1810833 .068822$ H $5.655522-2.7663941 .054074$
H $\quad 6.776582-3.4268192 .225621$

H $8.3615440 .842513-0.222429$

H $\quad 6.979162-2.833807-1.967890$

H $10.168830-1.0295220 .394541$

O $4.095032-1.7743372 .566941$

O $5.597469-2.2528274 .253199$

C $3.118150-1.3947563 .540861$

H $2.237991-1.0211903 .012143$

H $2.827755-2.2630704 .139458$

H $3.505525-0.5954944 .180090$

SCF Energy $\left(B 3 L Y P / 6-31 G^{* *} / / M M F F\right)=-3245.90018244$

08_00284

MMFF Geometry

C $-2.234108-2.8694642 .203763$

C $-1.641962-1.9126871 .472942$

C $-0.236872-1.9908290 .943101$

O $0.410353-0.7705641 .382864$

C $-0.198723-2.017329-0.597199$

C $-0.185327-3.415008-1.182130$

C $0.915046-4.039345-1.647412$

C $-1.521583-4.105473-1.261614$

C $2.323194-3.517906-1.693512$

C $3.202459-4.193448-0.635496$

C $4.643264-3.663498-0.667044$

C $5.494380-4.3406410 .409051$

C $6.918735-3.765880 \quad 0.460211$

O $4.611460-2.249695-0.441079$

O $7.528146-4.2032111 .684499$

C $6.837492-2.2254420 .521592$

C $7.793087-4.308883-0.676438$

O $8.133768-1.6411620 .414470$

C $5.891859-1.614105-0.537170$

C $5.709323-0.098793-0.278085$

O $6.469863-1.785239-1.834898$

C $1.716690-0.8176831 .742662$

O $2.435951-1.8063241 .758456$

C 2.2245460 .5205282 .127232

C 1.4545631 .6181122 .175460

C 1.8842492 .9703842 .536050

C 3.3431263 .1882472 .836787

C $4.5346510 .524411-1.046888$

C $4.6042132 .042179-1.087359$

C $3.2615534 .012614-1.813794$

O $5.6315062 .673760-0.853915$

C $3.1533994 .848936-0.578365$

C $2.1104735 .649775-0.312185$

C $2.054476 \quad 6.5862130 .860441$

C 0.8855846 .3174341 .778458

C -0.2879746 .9503501 .611188$

C 1.1137805 .3889792 .948638

C 0.9518873 .9447992 .571916

N $3.3962822 .608018-1.474363$

H -1.696252 -3.784841 2.434586

H -2.198622 -1.005705 1.244153

H $0.284810-2.8584061 .366450$

H $\quad 0.667591-1.446065-0.946747$

H -1.052018 -1.466482 -1.013878

H $\quad 0.802433-5.045921-2.048826$

H -1.471301 -5.044918 -1.822699

H $-1.897118-4.342620-0.263470$

H -2.248456 -3.464983 -1.769380

H $2.729396-3.721155-2.692170$

H $2.356283-2.432318-1.574437$

H $2.765991-4.0137040 .354533$

H $3.207937-5.279282-0.793622$

H $5.055997-3.860547-1.663605$

H $5.523146-5.4268740 .257000$

H $5.025878-4.1884061 .391686$

H $7.517739-5.1757111 .693818$

H $6.457881-1.9574091 .517621$

H $7.868214-5.401168-0.613036$

H $8.819686-3.934123-0.599058$

H $7.408933-4.062923-1.668604$

H $8.690020-2.0421661 .104202$

H 5.5236420 .0771230 .789399

H $6.6419270 .420002-0.532076$

H $\quad 6.168880-1.069543-2.416518$ 
H $3.281506 \quad 0.5520262 .371609$ H 0.3972891 .5279151 .932381 H 3.9659382 .8411152 .004824 H 3.6333082 .6438243 .741817 H 3.6025574 .2375612 .992733 H $4.522676 \quad 0.182547-2.087507$ H $3.5893090 .217960-0.586111$ H $2.3818084 .111065-2.457444$ H $4.1425634 .320628-2.387057$ H 4.0034424 .8438720 .099591 H $1.2741825 .681001-1.006505$ H 1.9854927 .6086720 .465645 H 2.9885906 .5629601 .435349 H -1.120574 6.7844872 .286670 H $-0.443357 \quad 7.6423770 .790104$ H $0.3830745 .602363 \quad 3.740761$ H 2.0831555 .6045423 .406684 H -0.0784623 .6807592 .332749$ H $2.6024542 .001531-1.659474$ C $-4.643888-3.5453231 .910379$ O $-4.692978-3.0086120 .570834$ C $-5.999049-2.4546190 .349296$ C $-6.911304-3.3025851 .219537$ C $-6.067156-3.4285712 .475839$ H $-5.980823-1.4305030 .742618$ C $-6.329432-2.398290-1.146210$ H -7.881400 -2.830540 1.399167 H -7.079962 -4.2955510 .788108$ C $-6.463944-4.5962123 .363392$ H -6.168285 -2.501860 3.056756 C $-5.202143-1.813975-2.023285$ O $-6.578455-3.719779-1.641039$ H -7.255507 -1.830700 -1.299949 H -4.407641 -2.561732 -2.129499 O $-5.752996-1.622525-3.332691$ C $-4.602907-0.487512-1.518755$ C $-3.507706 \quad 0.060819-2.454258$ H -4.162605 $-0.644595-0.529336$ O $\quad-5.6400280 .479047-1.367874$ H -2.844492 -0.760346 -2.750781 H $-3.9798230 .469371-3.356134$ H -7.495976 -4.482406 3.710904 H $-6.392067-5.5493432 .828753$ H $-5.813772-4.6526014 .242291$ C -3.633533 -2.742705 2.736349 H $-7.460789-3.987376-1.333049$ H -5.013174 -1.483859 -3.947687 H $-6.0569790 .601446-2.237889$ H -3.640855 -3.0837293 .778234$ H -3.922229 -1.684211 2.745979 H $-4.328774-4.5921131 .823985$ C -2.876068 $3.417772-0.920982$ O $-3.4930702 .311398-1.591927$ C $-2.6626351 .164498-1.787322$ C $-1.4378911 .534681-2.638267$ C $-0.6704302 .711045-2.028559$ C -1.600297 $3.869684-1.653265$ H -3.611814 4.229114 -0.992592 C $-2.648147 \quad 3.127851 \quad 0.566174$ H $-2.3255150 .791411-0.814266$ O $-1.838478 \quad 1.863402-3.966995$ H $-0.777797 \quad 0.662125-2.709097$ H $0.0766083 .060128-2.751626$ O $0.0216722 .247819-0.873856$ H -1.062914 4.605274-1.044066 O $-1.9821314 .529745-2.863931$ C -3.9667702 .9471941 .279403$ H -2.0261202 .2455040 .741468$ H -2.137268 3.9681141 .050092 H $-2.4723532 .598972-3.913187$ H $0.5232992 .997647-0.511233$ H -2.512766 $5.307161-2.620173$ O -4.1703561 .6300001 .566003$ O -4.7335643 .8679961 .531495$ C -5.4016381 .3321642 .230940$ H -5.4032740 .2701422 .487188$ H -5.4899661 .9123853 .154539$ H -6.2450581 .5342441 .564289$
SCF Energy (B3LYP/6-31G**//MMFF) $=-3245.90337222$

$08 \_00285$

MM̄FF Geometry

C $-3.432943-3.0754472 .189958$

C -2.141734 -2.770769 1.987688

C -1.243128 -2.216284 3.060385

O $-0.772016-0.9137462 .651531$

C -0.005505 -3.112369 3.246012

C $0.924061-2.5961814 .328443$

C $2.081022-1.9420944 .102052$

C $0.484165-2.8825605 .741395$

C $2.694128-1.5647852 .779831$

C $4.004804-2.3304042 .565246$

C $4.632754-2.0632861 .189715$

C $5.889988-2.9177021 .015834$

C $6.603761-2.628920-0.310463$

O $4.965592-0.6747721 .093304$

O $7.911686-3.218953-0.241713$

C $6.800357-1.105666-0.450951$

C $5.893628-3.290091-1.497975$

O $7.333103-0.813613-1.742048$

C $5.513574-0.283503-0.177313$

C $5.864817 \quad 1.225241-0.096437$

O $4.587595-0.506689-1.238013$

C -1.5392160 .1604522 .974618$

O -2.6641650 .1520613 .446655$

C -0.7396821 .3675982 .674949$

C - -1.3180612 .5768572 .705189$

C -0.6532323 .8524892 .430372$

C 0.8466493 .8365962 .320271

C 4.6982472 .1634240 .246058

C $3.9496852 .694174-0.967958$

C $2.1073614 .248275-1.569508$

O $4.1247902 .276202-2.108960$

C $1.5347925 .515002-1.020513$

C $0.2187625 .749011-0.895574$

C $-0.3414697 .048548-0.391043$

C -1.402209 6.8732890 .672235

C -2.6773567 .2285600 .437873$

C -0.9704016 .3759812 .034698$

C -1.4104874 .9607012 .297360$

N $3.0442153 .686001-0.615348$

H -3.870472 -2.917092 3.172223

H -1.705052 -2.924011 1.003573

H $-1.778058-2.1318044 .014963$

H $\quad 0.532872-3.2053392 .295664$

H $-0.316598-4.1332723 .504897$

H $2.665165-1.6258294 .965755$

H $1.214910-2.5409986 .482212$

H $-0.463343-2.3808215 .959935$

H $\quad 0.350143-3.9595845 .885836$

H $2.014281-1.7457381 .942428$

H $2.888078-0.4854042 .788172$

H $4.713631-2.0391563 .351873$

H $3.820163-3.4070922 .673949$

H $3.893734-2.3112170 .420828$

H $5.649843-3.985327 \quad 1.096917$

H $6.589509-2.7062231 .837191$

H $7.802591-4.172781-0.085949$

H $7.568237-0.8136520 .279493$

H $5.843260-4.376592-1.357507$

H $6.451162-3.135241-2.428306$

H $4.873917-2.929576-1.642669$

H $7.7021350 .084633-1.717805$

H $6.612403 \quad 1.3502190 .698396$

H $6.3258611 .559656-1.032474$

H $3.8999190 .176396-1.198142$

H $\quad 0.307007 \quad 1.2244452 .430258$

H -2.381682 2.6404492 .931554

H 1.2959643 .4780593 .253194

H 1.2829274 .8158062 .115422

H 1.1582513 .1742811 .506934

H 3.9810901 .6815670 .919246

H $5.110048 \quad 3.0360670 .769584$

H $1.3372763 .494539-1.760723$

H $2.6340314 .444084-2.509350$

H $2.2414596 .290924-0.735133$ 
H $-0.4911344 .986899-1.208793$ H $-0.7581167 .583523-1.254519$ H 0.4521547 .6934220 .007187 H -3.437535 7.1386971 .206960 H $-2.9904177 .615177-0.526360$ H -1.422886 7.0128162 .807252 H $0.106386 \quad 6.5172602 .161558$ H -2.4919314 .8563862 .399992$ H 2.8985173 .8749610 .371722 C $-5.298546-2.6610400 .498356$ O $-4.568827-1.623065-0.187253$ C $-5.194285-0.358630 \quad 0.104374$ C $-6.577507-0.6985120 .645520$ C $-6.270733-1.9535051 .443281$ H -4.6152730 .0911830 .919949$ C $-5.1547760 .569998-1.116852$ H $-6.997430 \quad 0.1076401 .254305$ H -7.287480 -0.930705 -0.155682 C -7.499430 -2.775055 1.790986 H $-5.768698-1.6554472 .371888$ C $-3.7471810 .747417-1.728592$ O $\quad-5.9856430 .037653-2.155415$ H $-5.5736351 .549697-0.856627$ H -3.469439 -0.177684 -2.243777 O $-3.854366 \quad 1.758242-2.738090$ C $-2.6584401 .147634-0.718380$ C $-1.2563621 .373757-1.304531$ H $-2.569523 \quad 0.3800050 .057419$ O $-3.0273592 .368300-0.070591$ H -1.231573 2.304467-1.886569 H $-0.5943391 .565535-0.450194$ H -8.187884 -2.192847 2.412170 H -8.041286 -3.0881190 .892305$ H -7.220135 -3.675252 2.347770 C $-4.329025-3.660347 \quad 1.134952$ H $-6.9100360 .217010-1.915156$ H -3.026713 $1.760304-3.247005$ H -3.8030372 .1910160 .486645$ H -3.708550 -4.1012120 .346090$ H -4.892029 -4.488144 1.581776 H -5.862836 -3.191906 -0.279695 C $-0.516715-2.199069-2.215008$ O $-0.946334-1.025956-1.504328$ C $-0.6933710 .221930-2.157876$ C $0.8136420 .398109-2.411937$ C $1.397775-0.792129-3.176497$ C $0.984150-2.124244-2.543899$ H $-0.655251-3.021814-1.502445$ C - $-1.381782-2.481925-3.454928$ H $-1.2240330 .235678-3.114914$ O $1.5108470 .541624-1.174460$ H $0.9734421 .320660-2.981271$ H $2.491811-0.720517-3.183671$ O $0.952903-0.762296-4.528893$ H $1.257628-2.960920-3.197395$ O $1.696816-2.302273-1.317220$ C -2.766672 -2.967150 -3.087644 H -1.485002 -1.593973 -4.083672 H $-0.931121-3.273217-4.065007$ H $1.302755-0.231881-0.622615$ H $1.2555180 .075422-4.919040$ H $2.645427-2.279647-1.527953$ O $-3.667553-2.516132-4.008485$ O $-3.018768-3.693215-2.136197$ C $-5.019053-2.942892-3.810788$ H -5.365391 -2.677590 -2.808197 H -5.647774 -2.430956 -4.544080 H -5.096654 -4.022187 -3.971836 SCF Energy (B3LYP/6-31G**//MMFF) $=-3245.91160117$

0800286

MM̄FF Geometry

C -4.246131 -0.0754802 .250595$

C -4.5519261 .2247362 .130273$

C $-4.561943 \quad 1.9227040 .797058$

O $-3.6254443 .021410 \quad 0.908944$

C $-5.9355962 .482758 \quad 0.397133$

C $-7.025717 \quad 1.4524870 .200302$
C $-7.0603990 .554520-0.804062$

C -8.1383891 .4992011 .213965$

C $-6.0373910 .350919-1.888970$

C $-5.398016-1.036576-1.750361$

C $-4.174500-1.229141-2.657764$

C $-3.745449-2.699659-2.635053$

C $-2.466442-2.942654-3.443161$

O $-3.114859-0.397986-2.164537$

O $-2.007776-4.262798-3.113971$

C $-1.395229-1.936849-2.977572$

C $-2.743687-2.948390-4.950566$

O $-0.227269-2.039717-3.790620$

C $-1.898221-0.474237-2.920310$

C $-0.8451110 .455198-2.266759$

O $-2.098109-0.011195-4.261625$

C $-2.9775613 .403358-0.223817$

O $-3.1600082 .989466-1.357192$

C -1.9505404 .3894280 .169151$

C $-0.8528304 .517153-0.590100$

C $0.2880235 .390746-0.313977$

C $0.1484776 .379200 \quad 0.814575$

C $-0.6842790 .289614-0.752451$

C $0.5641950 .978359-0.222938$

C $1.7124281 .450671 \quad 1.939128$

O $1.4989761 .317185-0.943778$

C 2.0900652 .8877851 .765611

C 3.2405663 .2730661 .191437

C 3.6982684 .6972221 .056185

C $3.8122855 .137325-0.387105$

C $4.8958614 .831978-1.120879$

C $2.7010785 .995266-0.950502$

C $1.4021415 .247882-1.062323$

N $0.532287 \quad 1.1176961 .160497$

H -4.053266 -0.6532941 .349832$

H $-4.738996 \quad 1.822446 \quad 3.018219$

H -4.1892641 .2323220 .033038$

H $-5.8384193 .070420-0.525740$

H -6.2514573 .2247521 .143778$

H -7.908821 $-0.126993-0.854200$

H $-8.900040 \quad 0.733279 \quad 1.033699$

H $-7.741561 \quad 1.3393832 .221721$

H -8.6355772 .4744121 .185779$

H $-6.5396330 .424556-2.861124$

H $-5.2760841 .134599-1.883252$

H -5.094072 -1.192812 -0.708057

H $-6.155445-1.796233-1.983571$

H $-4.435809-0.917812-3.675717$

H $-4.554228-3.347210-2.996774$

H -3.561151 -3.007172 -1.595433

H -1.152102 -4.396349 -3.556838

H -1.088102 -2.251867 -1.973643

H -3.467983 -3.732924 -5.200871

H $-1.838671-3.187103-5.520042$

H $-3.141904-2.000183-5.317406$

H $-0.408026-1.578602-4.627437$

H $\quad 0.1151310 .329706-2.782758$

H -1.123193 $1.501202-2.451555$

H -2.101855 $0.960267-4.253834$

H -2.1006124 .9362251 .092558$

H $-0.7608853 .896260-1.480388$

H $-0.711928 \quad 7.0360140 .644057$

H 1.0164547 .0292550 .938741

H 0.0088145 .8586361 .768319

H $-1.5645770 .692679-0.240089$

H $-0.593292-0.766305-0.481173$

H 2.5219430 .7787191 .639489

H 1.4800441 .2551622 .990757

H 1.4082063 .6341372 .163198

H 3.9188882 .5109700 .813507

H 4.6786704 .7853291 .543108

H 3.0410875 .3803941 .607673

H $4.9964975 .164736-2.148466$

H $5.7050394 .237773-0.708799$

H $2.9624476 .350516-1.956031$

H $2.6153756 .902828-0.346575$

H $1.4062664 .492716-1.849716$

H $-0.2455750 .701857 \quad 1.664543$

C $-2.632316-1.3048383 .661136$ 
O $-2.381812-2.2903112 .642331$ C -1.314899-3.155410 3.094234 C $-1.009574-2.7408834 .531296$ C -2.284463 -2.028318 4.954177 H $-1.716640-4.1746463 .056613$ C $-0.148942-2.9907152 .111868$ H $-0.767852-3.5988295 .166285$ H $-0.157837-2.0502424 .568786$ C -2.096545-1.115006 6.152039 H -3.050635 -2.781078 5.182556 C $1.141774-3.7296562 .502432$ $\begin{array}{llll}\text { O } & -0.579057 & -3.471746 & 0.832488\end{array}$ H $0.054672-1.9210361 .990760$ H $1.530920-3.3292923 .446024$ O $\quad 0.814266-5.1020222 .743793$ C $2.245947-3.673037 \quad 1.422683$

C $2.590866-2.238611 \quad 1.006835$ H $1.947034-4.2667900 .551920$ O $3.431403-4.2728731 .950824$ H $1.708095-1.7330100 .602004$ H $2.874325-1.667737 \quad 1.901885$ H $-1.755579-1.6864457 .021525$ H -1.354466 -0.3352535 .949588$ H -3.036663 -0.6232316 .420453$ C $-4.074473-0.8096093 .551056$ H $-1.390865-2.9896030 .598713$ H $1.588295-5.5202933 .156731$ H $3.261206-5.2252012 .045098$ H $-4.322114-0.1600044 .397312$ H $-4.770902-1.6561123 .559617$ H -1.935386 -0.4717593 .489703$ C $4.913974-0.280226-1.128626$ O $3.869736-0.685562-0.238103$ C $3.736052-2.096326-0.012056$ C $3.477177-2.826927-1.337794$ C $4.544411-2.483443-2.381850$ C $4.770026-0.971921-2.492951$ H $4.7485290 .794713-1.280148$ C $6.296732-0.453253-0.492659$ H $4.657084-2.4691910 .448049$ O $2.188666-2.466262-1.830594$ H $3.473992-3.908209-1.158810$ H $4.232931-2.880796-3.355421$ O $5.759693-3.132048-2.018686$ H $5.653686-0.756534-3.104839$ O $3.648858-0.380418-3.156282$ C $6.470157 \quad 0.506557 \quad 0.660857$ H $6.488505-1.466754-0.129041$ H $7.087548-0.230472-1.218807$ H $2.037030-2.961966-2.653182$ H $6.419316-2.923291-2.701830$ H $3.577052-0.790686-4.034856$ O $6.004580-0.058142 \quad 1.811522$ O $6.9402501 .631062 \quad 0.546111$ C 6.0899810 .7628592 .979974 H 5.6544010 .2095083 .816262 H 7.1365950 .9826863 .210430 H 5.5231231 .6876702 .839279

SCF Energy (B3LYP/6-31G**/MMFF) $=-3245.90229523$

0800287

MMFF Geometry

C $0.026288-1.707703 \quad 1.916242$

C $0.640178-2.235993 \quad 2.986545$

C $2.036386-1.9033263 .468657$

O $2.611230-0.8299972 .689005$

C $2.943171-3.1360003 .322918$

C $4.312485-2.9355413 .941341$

C $5.435703-2.6710843 .245497$

C $4.370876-3.0975675 .438468$

C $5.567891-2.4711601 .761223$

C $6.385154-1.2122881 .437057$

C $6.523739-1.009916-0.078119$

C $7.491533 \quad 0.131404-0.391156$

C $7.5854530 .395697-1.901635$

O $5.228589-0.702842-0.604886$

O $8.249967 \quad 1.654505-2.088874$

C $6.1599540 .547059-2.470609$
C $8.440254-0.657291-2.616642$

O $6.1850820 .643644-3.893013$

C $5.189331-0.576562-2.032474$

C $3.747574-0.201673-2.444765$

O $5.553520-1.797471-2.678064$

C 2.3810420 .4417313 .114074

$\begin{array}{lllll}\text { O } & 1.820938 & 0.786693 & 4.142133\end{array}$

C 2.8658521 .3556342 .056686

C 2.3577552 .5954771 .997991

C $2.6371303 .599800 \quad 0.971087$

C 3.7566583 .3211490 .005722

C $2.659121-1.103523-1.851189$

C $1.290192-0.530859-2.160119$

C $-0.2637101 .299923-1.470822$

O $0.609347-0.953833-3.091380$

C $0.1282622 .670824-1.928527$

C $-0.3139903 .801871-1.357935$

C $0.0850475 .176985-1.814922$

C $0.5295606 .074180-0.678364$

C $-0.2363717 .100186-0.270038$

C $1.8940685 .830962-0.064429$

C $1.870417 \quad 4.7096860 .936676$

N $0.9429820 .509413-1.311791$

H $0.537614-0.966564 \quad 1.309074$

H $\quad 0.103285-2.9723073 .581695$

H $1.976525-1.6108324 .524040$

H $3.035617-3.4052222 .264321$

H $2.474769-4.0074083 .800589$

H $6.370950-2.5899213 .798430$

H $5.388328-2.9900095 .829497$

H $3.748783-2.3444905 .931791$

H $4.011149-4.0905015 .727403$

H $6.074666-3.3487811 .342307$

H $4.592942-2.3993661 .271930$

H $5.890629-0.3397701 .883267$

H $7.380820-1.2972551 .890133$

H $6.883466-1.946551-0.520164$

H $8.486051-0.0744410 .024068$

H 7.1440281 .0503520 .101810

H $9.1267441 .595407-1.671843$

H $5.7652141 .504927-2.103009$

H $9.455445-0.671892-2.202269$

H $8.554644-0.417496-3.679540$

H $8.035144-1.667898-2.532836$

H $6.7914701 .368290-4.123520$

H $3.5451410 .828204-2.132327$

H $3.654639-0.221215-3.538449$

H $4.777292-2.378387-2.714044$

H $3.562640 \quad 0.965788 \quad 1.323944$

H 1.6259432 .8916172 .748687

H $3.4780852 .511668-0.672862$

H $4.6689143 .032844 \quad 0.540404$

H $4.0280994 .184042-0.605997$

H $2.709317-2.115015-2.267326$

H $2.760032-1.197999-0.763841$

H $-0.7688041 .316988-0.500665$

H $-0.9253840 .833172-2.206760$

H $0.8072512 .735811-2.775910$

H - $-1.015873 \quad 3.739397-0.530272$

H $-0.7804065 .619050-2.325543$

H $0.8848005 .130409-2.564639$

H $\quad 0.084875 \quad 7.762627 \quad 0.526955$

H -1.204061 $7.297832-0.719189$

H $2.237427 \quad 6.733199 \quad 0.458293$

H $2.6113355 .667983-0.873299$

H 1.1030024 .8292211 .703164

H $1.5873220 .781147-0.575292$

C $-1.431108-2.534840 \quad 0.030677$

O $-2.790039-2.395468-0.427662$

C $-3.142784-3.548130-1.213028$

C $-1.843784-4.304126-1.462269$

C $-1.061796-4.001148-0.196114$

H $-3.792535-4.165391-0.581091$

C $-3.905756-3.120842-2.473246$

H $-2.011184-5.374321-1.618378$

H $-1.306802-3.917899-2.336291$

C $0.425685-4.271646-0.345103$

H $-1.461665-4.6209250 .617265$ 
C $-5.068352-2.144476-2.199377$ O $-2.993185-2.454075-3.356176$ H -4.257898 -4.008772 -3.010842 H -4.642843 -1.178502 -1.908445 O $-5.750762-1.912386-3.437669$ C $-6.088038-2.610577-1.145094$ C $-7.254173-1.628515-0.924136$ H $-5.592730-2.780068-0.183228$ O $-6.664667-3.855507-1.547557$ H -7.853385 -1.541486 -1.839253 H -7.919170 -2.087563 -0.180284 H $\quad 0.599019-5.315980-0.625792$ H $0.872688-3.638794-1.118302$ H $0.958727-4.0891550 .591493$ C -1.364672 -2.056405 1.482028 H $-3.495090-2.188772-4.146018$ H $-6.182792-2.745286-3.694131$ H $-5.965357-4.529710-1.522727$ H -1.822146 -2.800946 2.145192 H -1.983222 -1.1566631 .588190$ H $-0.830104-1.881217-0.612148$ C $-5.470728 \quad 0.811076 \quad 1.267442$ O $-6.042946-0.3965420 .746165$ C $-6.820647-0.233460-0.444179$ C $-8.0211440 .684534-0.166268$ C -7.5679522 .0306180 .408992$ C -6.5627611 .8577151 .555995$ H -5.0340950 .5128352 .229392$ C $-4.329148 \quad 1.2967280 .367723$ H $\quad-6.200767 \quad 0.224542-1.222002$ $\begin{array}{llll}\text { O } & -8.924375 & 0.062251 & 0.745472\end{array}$ H -8.568820 $0.847112-1.101828$ H -8.4484982 .5760210 .770052$ O $-6.9844262 .798453-0.639129$ H $-6.0986212 .816541 \quad 1.811198$ O -7.2703691 .4163342 .720205$ C -3.405845 2.2413521 .095226 H -3.7370650 .4474690 .003438$ H $-4.6866281 .797450-0.536647$ H -8.428176 -0.1558631 .553038$ H $-6.7254313 .657652-0.264616$ H -7.925181 2.1003872 .941676 O $-2.523547 \quad 1.5351701 .857392$ O $-3.4712063 .460521 \quad 1.007045$ C -1.5897122 .3171072 .607440$ H -0.9234761 .6322883 .138495$ H -0.9908322 .9404631 .937566$ H -2.1175982 .9340913 .340437$

SCF Energy (B3LYP/6-31G**//MMFF) $=-3245.91839201$

0800288

MM̄FF Geometry

C $3.347949-0.6425493 .025985$

C 4.229925-1.650419 2.942250

C $5.109282-1.9900681 .760469$

O $5.060795-0.9664180 .743866$

C $4.594517-3.2822681 .103271$

C $5.640836-4.0003210 .277002$

C $5.771159-3.906129-1.060467$

C $6.541504-4.9222201 .058958$

C $5.024410-3.013685-2.012055$

C $3.979255-3.762003-2.851953$

C $2.614508-3.863276-2.153352$

C $1.630111-4.678991-2.992960$

C $0.224715-4.691380-2.371794$

O $2.122613-2.528659-1.986785$

O $-0.688624-5.199337-3.356185$

C $-0.198959-3.239134-2.068953$

C $0.138042-5.638292-1.169068$

O $-1.437871-3.204255-1.363112$

C $0.870300-2.428186-1.301046$

C $0.466864-0.934698-1.271511$

O $0.957664-2.918956 \quad 0.037763$

C $5.884431 \quad 0.1058840 .860116$

$\begin{array}{lllll}\text { O } & 6.681494 & 0.336637 & 1.753500\end{array}$

C $5.6102710 .974850-0.305243$

C $6.0077622 .254805-0.294253$

C $5.7700853 .235349-1.356039$
C $5.0784632 .757025-2.606845$

C $1.5573850 .013320-0.752398$

C $1.0771841 .455022-0.726091$

C 1.6579713 .6843600 .225641

O $0.0482201 .827620-1.284976$

C $2.2640864 .515621-0.861300$

C $3.0217735 .598782-0.628550$

C $3.5883236 .471589-1.713095$

C $5.0708276 .728060-1.551301$

C $5.5192317 .880355-1.025298$

C $6.0322105 .686663-2.079403$

C $6.1656244 .509467-1.154093$

N $1.9372352 .279805-0.013756$

H 3.2212710 .0439582 .194738

H $4.323996-2.324273 \quad 3.792411$

H $6.141226-2.1147152 .110820$

H $3.724185-3.0483410 .481143$

H $4.231170-3.9868631 .863829$

H $6.539507-4.511806-1.540304$

H $7.275631-5.4276040 .422478$

H $7.095681-4.3618621 .818587$

H $5.950459-5.6961651 .559659$

H $4.576774-2.156858-1.502037$

H $5.771736-2.587054-2.693872$

H $3.848457-3.201311-3.787132$

H $4.346487-4.759823-3.121151$

H $2.748922-4.331090-1.172592$

H $1.996384-5.703195-3.136066$

H $1.559403-4.242967-3.999391$

H $-0.390240-6.089473-3.610685$

H $-0.379671-2.745262-3.034186$

H $0.385691-6.663184-1.470393$

H $-0.882756-5.681350-0.773244$

H $\quad 0.812342-5.362085-0.355746$

H -2.083549 -3.708864 -1.886779

H $0.207483-0.613166-2.288833$

H $-0.428897-0.809347-0.650603$

H $1.269304-2.2088380 .620107$

H $5.0579500 .541206-1.131440$

H 6.5394072 .6264130 .580743

H $5.6843101 .995878-3.110990$

H $4.8918603 .548675-3.334897$

H $4.1006502 .324213-2.368775$

H $1.854329-0.2692290 .261050$

H $2.442320-0.030631-1.396835$

H $2.0631693 .932679 \quad 1.211695$

H $0.5738883 .837480 \quad 0.244192$

H $2.0425704 .233377-1.887909$

H 3.2142765 .8998960 .398760

H $3.0424937 .424132-1.689515$

H $3.4001276 .047614-2.707416$

H $6.5808518 .084045-0.932030$

H $4.8342888 .643520-0.670815$

H $7.0338306 .122349-2.195233$

H $5.7357985 .404709-3.093280$

H $6.6539004 .756423-0.210113$

H 2.8003751 .8964850 .360012

C $1.017267-0.7599893 .922436$

O 0.4524350 .2079723 .015032

C -0.8961890 .5142803 .437634$

C $-1.236247-0.5038424 .515661$

C $0.116946-0.7363685 .155282$

H $-0.8514221 .522753 \quad 3.868314$

C -1.8193040 .4995712 .213314$

H $-1.985830-0.1343135 .221798$

H -1.613067 -1.433184 4.070241

C $0.178229-2.0010115 .994498$

H $\quad 0.3633550 .1287425 .785291$

C -3.3163740 .6211692 .563575$

O $-1.411864 \quad 1.594973 \quad 1.390523$

H $-1.642517-0.4158561 .635637$

H -3.590058 -0.206101 3.227864

O $-3.537890 \quad 1.826646 \quad 3.295752$

C $-4.2591480 .571762 \quad 1.342963$

$\begin{array}{llll}\text { C } & -5.739916 & 0.621562 & 1.769932\end{array}$

H $-4.061882-0.3527210 .787526$

$\begin{array}{llllll}0 & -3.975797 & 1.669714 & 0.479758\end{array}$

H $-5.901633-0.0792182 .597620$ 
H $-5.966175 \quad 1.6346062 .125764$ H $-0.543475-1.9519856 .816413$ H $-0.052062-2.8900475 .397803$ H $1.173815-2.1354776 .428342$ C $2.473651-0.4047254 .225924$ H $-1.904340 \quad 1.5355320 .554281$ H -3.3074132 .5675842 .709039$ H -4.550195 $1.588956-0.300197$ H $2.835706-0.9932465 .076802$ H 2.5584410 .6533934 .501509 H $0.956444-1.7370163 .424238$ C -7.415209 $1.070203-1.551764$ O $-6.6153861 .277066-0.379652$ C $-6.708676 \quad 0.263196 \quad 0.624329$ C $-8.157534 \quad 0.142926 \quad 1.123044$ C $-9.132075-0.086738-0.037164$ C $-8.8991090 .904294-1.182784$ H $-7.3144262 .002110-2.123600$ C $-6.873106-0.069930-2.421072$ H $-6.389595-0.6959890 .201847$ O H -8.220494 -0.691610 1.830872 H -10.1617330 .0178500 .326232$ O $-8.979570-1.411731-0.537214$ H $-9.4649280 .604967-2.072799$ O $-9.3861812 .190796-0.789259$ C $-5.5145830 .285737-2.976336$ H $-6.792580-1.022132-1.888882$ H -7.533098 -0.252814 -3.277516 H -8.411249 2.0797501 .240476 H -9.164310 -2.0250250 .194429$ H -10.337546 2.101251 -0.609129 O $-4.537083-0.224634-2.175223$ O $-5.345621 \quad 0.958469-3.984951$ C $-3.1946370 .076182-2.567607$ H $-3.0503641 .158014-2.647086$ H -2.525907 -0.307600 -1.794319 H -2.960419-0.417919-3.515037 SCF Energy $\left(B 3 L Y P / 6-31 G^{* *} / / M M F F\right)=-3245.92909141$

\section{9}

MM̄FF Geometry

C -1.048992 $-3.595731 \quad 1.507396$

C -1.570507 -3.7142122 .739100$

C $-2.393919-2.6786823 .469647$

O $-2.920530-1.6905532 .556717$

C $-1.520193-1.9591254 .509459$

C $-2.307081-1.0002155 .380810$

C $-2.318545 \quad 0.338323 \quad 5.223491$

C $-3.072493-1.6378646 .511028$

C -1.6266321 .1332344 .150744$

C -2.5412142 .2280483 .581612$

C -1.843047 3.0060852 .457855

C -2.6540694 .2377352 .055487$

C -2.0005614 .9858670 .883416$

O $-1.707107 \quad 2.130483 \quad 1.333053$

O

C $-1.7042393 .978938-0.246618$

C -0.7842665 .8052051 .330987$

O $-0.9593404 .593215-1.296166$

C -0.9836732 .6959380 .232744$

C $-0.9580541 .662994-0.916174$

O $\quad 0.358077 \quad 3.0155840 .598614$

C -4.132795 -1.951265 1.996163

O $-4.835188-2.9321502 .182878$

C $-4.450411-0.8373461 .076198$

C $-5.442558-0.9922190 .186959$

C $-5.840857-0.031524-0.843013$

C $-5.2342241 .344625-0.791704$

C $-0.4989620 .257564-0.508828$

C $-0.703157-0.705637-1.662749$

C -2.509962 -1.880230 -2.927576

O $0.225151-1.051139-2.388937$

C $-3.444974-1.027273-3.728963$

C $-4.671104-1.418983-4.108612$

C $-5.618406-0.549652-4.888160$

C $-7.002834-0.483818-4.275409$

C $-8.051764-1.069099-4.878120$
C $-7.1873780 .319288-3.002089$

C $-6.715958-0.433574-1.788086$

N $-2.025523-1.092498-1.808686$

H -1.195073 -2.682427 0.938436

H -1.388433 -4.633646 3.292761

H -3.220651 -3.191002 3.977476

H $-0.701960-1.4315194 .005039$

H -1.028558 -2.694377 5.161089

H -2.8831620 .9325085 .940783$

H -3.585690 -0.898556 7.135315

H -3.832093 -2.323112 6.122527

H -2.393578 -2.201359 7.159206

H $-0.737207 \quad 1.6005974 .590092$

H -1.285322 0.4941553 .331837

H $-3.459917 \quad 1.766556 \quad 3.196962$

H $-2.8264732 .916148 \quad 4.387077$

H -0.8482803 .3043572 .809685$

H -2.7919094 .9115132 .910269$

H -3.6636513 .9269311 .751176$

H -3.206142 6.5247661 .094784

H $-2.6706673 .688644-0.682492$

H -1.075822 6.5375842 .093319

H -0.3728326 .3873440 .499016$

H 0.0156225 .1919671 .751204

H -1.452816 $5.382576-1.577842$

H $-1.9639841 .583498-1.342676$

H $-0.3058152 .020096-1.722692$

H 0.8838632 .2000900 .598093

H -3.8336250 .0525371 .121904$

H $-5.991091-1.9334180 .180079$

H -5.3260341 .7732260 .212830$

H -5.717217 $2.056375-1.464780$

H $-4.1757091 .304397-1.059071$

H $0.5607410 .248574-0.234385$

H -1.058236 -0.1132380 .357231$

H $-3.008764-2.761496-2.512249$

H -1.672337 -2.206269 -3.551208

H $-3.096231-0.035024-4.006684$

H -5.011262 -2.420231-3.853885

H $-5.675866-0.954282-5.906942$

H $-5.2280240 .470602-4.990781$

H -9.050585 -1.008146-4.458757

H -7.935159-1.626565 -5.801623

H $-8.2492650 .553718-2.853950$

H $-6.684177 \quad 1.281559-3.129947$

H -7.126143 -1.442282 -1.714525

H -2.712163 -0.755476 -1.141174

C $1.251585-4.3665400 .862176$

O $1.548330-3.298827-0.060480$

C $2.797415-3.594462-0.729540$

C $3.374786-4.812340-0.023581$

C $2.120719-5.5393660 .414085$

H $2.528668-3.842599-1.763973$

C $3.689627-2.346560-0.692258$

H $4.013218-5.413242-0.678085$

H $3.966948-4.5134970 .850550$

C $2.372818-6.5776241 .493960$

H $1.671695-6.028059-0.461027$

C $5.125825-2.581768-1.181924$

O $3.090058-1.356628-1.532510$

H $3.699467-1.9441670 .326508$

H $5.622492-3.319057-0.540875$

O $5.072685-3.171877-2.488565$

C $6.020122-1.326486-1.272065$

C $6.195313-0.5621830 .054057$

H $5.682109-0.661063-2.073987$

O $7.330340-1.774829-1.657713$

H $6.114078-1.2613530 .895467$

H $7.217649-0.1615340 .085831$

H $1.442584-7.0747111 .785615$

H $3.066000-7.3452141 .134335$

H $2.808595-6.1257192 .391369$

C $-0.244732-4.6804470 .844693$

H $2.175318-1.231602-1.227892$

H $4.601006-2.550800-3.070215$

H $7.234910-2.256095-2.497606$

H $-0.434144-5.6373531 .344801$

H $-0.600704-4.780494-0.187899$ 
H $\quad 1.560050-4.0230711 .858741$

C $4.6283892 .715232-0.808390$

O $5.501846 \quad 1.578821-0.770621$

C $5.2099750 .604356 \quad 0.235995$

C $5.314826 \quad 1.239458 \quad 1.629947$

C 4.4084752 .4706181 .742000

C $4.588786 \quad 3.4262490 .557595$

H $5.0916863 .399633-1.530662$

C $3.2363982 .349107-1.346400$

H 4.1930750 .2380540 .095145

$\begin{array}{lllll}O & 6.662613 & 1.610443 & 1.910563\end{array}$

H 5.0246880 .4955012 .380803

H 4.6350693 .0067092 .671876

O 3.0465072 .0565031 .797110

H 3.7961294 .1837250 .561725

O $5.828903 \quad 4.116370 \quad 0.743231$

C $3.2830721 .861532-2.779826$

H $2.7676961 .561165-0.752242$

H $2.5947373 .237503-1.324976$

H $6.9544482 .221415 \quad 1.212494$

H $2.944267 \quad 1.4857192 .577696$

H $5.906977 \quad 4.776190 \quad 0.033228$

O $2.015047 \quad 1.547396-3.178717$

O $4.292394 \quad 1.764526-3.463808$

C $1.8941251 .051550-4.515394$

H $2.2559331 .795619-5.231446$

H $2.4456200 .112321-4.620153$

H $0.8357460 .861159-4.712601$

SCF Energy (B3LYP/6-31G**/MMFF) $=-3245.92860774$

0800290

MMFF Geometry

C -2.947598 $4.234694 \quad 0.947337$

C $-2.9698692 .895026 \quad 1.015795$

C $-3.843862 \quad 2.132857 \quad 1.978074$

O -3.0376661 .0861652 .565536$

C $-5.003057 \quad 1.4590901 .227239$

C $-6.093826 \quad 0.964163 \quad 2.156174$

C $-6.287110-0.3234142501812$

C -7.0386622 .0265822 .658481$

C $-5.466020-1.5222222 .117129$

C $-6.210193-2.4561191 .150876$

C $-6.326039-1.891868-0.274104$

C $-7.137828-2.832070-1.167195$

C $-7.168099-2.350283-2.626314$

O $-5.000211-1.757988-0.792079$

O $-7.662116-3.427463-3.436786$

C $-5.723675-2.062666-3.088501$

C $-8.138782-1.179692-2.821746$

O $-5.710772-1.475412-4.387791$

C $-4.922188-1.182228-2.102113$

C $-3.429288-1.156040-2.509088$

O $-5.4422780 .147506-2.130108$

C $-2.458172 \quad 1.3178363 .768821$

O -2.5689712 .3265894 .451905$

C - 1.6353020 .1647224 .204863

C $-1.335371-0.8793743 .415890$

C $-0.502297-2.0279893 .780841$

C $0.021451-2.0962955 .191501$

C $-2.515134-0.471680-1.482273$

C $-1.045740-0.684939-1.799507$

C $1.197505-0.606457-0.714731$

O $-0.643253-1.058823-2.897778$

C $1.474955-2.010473-0.278409$

C $2.230394-2.3250770 .784586$

C $2.479290-3.735814 \quad 1.236114$

C $2.073180-3.9675802 .675888$

C $2.999510-4.0444533 .646353$

C $0.608069-4.1971972 .982205$

C $-0.229619-2.9556472 .839147$

N $-0.236933-0.388151-0.710745$

H -3.599842 4.7910061 .618913

H -2.348306 2.3012200 .352949

H -4.232461 2.7988812 .757635

H -4.6166330 .6348530 .619588$

H -5.4589472 .1624770 .517385$

H $-7.126888-0.556063 \quad 3.155934$

H -7.8416491 .6110553 .276728$
H -6.5050142 .7629953 .266733$

H -7.5083642 .5451611 .816304$

H $-4.489640-1.2440941 .709767$

H $-5.250812-2.0779323 .038746$

H -5.653111 -3.401367 1.114405

H -7.207886 -2.683162 1.546993

H $-6.802411-0.906438-0.233114$

H -8.157569 -2.952067 -0.780726

H $-6.689066-3.835227-1.144172$

H $-8.545357-3.668594-3.108620$

H $-5.212048-3.030989-3.181292$

H $-9.156678-1.475652-2.540595$

H $-8.193705-0.883825-3.875209$

H $-7.876174-0.300736-2.229346$

H $-6.212975-2.064374-4.976745$

H $-3.078030-2.188995-2.634240$

H $-3.311062-0.651324-3.476224$

H $-4.7497190 .767054-1.852907$

H -1.2579310 .2398855 .219876$

H $-1.718025-0.8975332 .396921$

H $-0.806328-2.0997405 .909365$

H $0.668739-1.2382145 .403765$

H $0.612236-2.9925935 .392260$

H $-2.6956830 .607903-1.465820$

H $-2.708043-0.868359-0.479173$

H $1.6326040 .127087-0.031813$

H $1.593661-0.439400-1.721033$

H $1.025405-2.808725-0.864558$

H $2.695708-1.5298601 .362689$

H $3.548786-3.9440861 .106231$

H $1.958663-4.4591330 .596111$

H $2.724831-4.2416744 .677103$

H $4.054567-3.9102803 .433723$

H $0.477331-4.6330063 .979847$

H $0.217104-4.9615632 .298059$

H -0.649697 -2.8239811 .841674$

H $-0.670971-0.1470850 .175683$

C -1.026178 $4.413359-0.792452$

$\begin{array}{lllll}\text { O } & 0.040579 & 4.014593 & 0.094351\end{array}$

C $1.2951544 .491463-0.438625$

C $1.0199804 .799381-1.900206$

C $-0.389173 \quad 5.349058-1.823706$

H $1.524308 \quad 5.412513 \quad 0.112629$

C $2.3807833 .439016-0.189598$

H $1.7333025 .511470-2.325572$

H $1.0431743 .883052-2.503240$

C $-1.1061995 .368229-3.162800$

H $-0.345506 \quad 6.372694-1.427798$

C $3.7235553 .745448-0.876541$

$\begin{array}{lllll}\text { O } & 2.594383 & 3.351006 & 1.222732\end{array}$

H $2.0124742 .460134-0.513888$

H $3.5918563 .715085-1.964982$

O $4.0978525 .089081-0.548718$

C $4.8873032 .815595-0.473391$

C $4.5447181 .326879-0.599232$

H 5.2116293 .0444060 .548213

$\begin{array}{llll}\text { O } & 5.996612 & 3.090928 & -1.333764\end{array}$

H 3.7569381 .0610740 .113813

H $4.1196301 .135987-1.594441$

H $-0.5754636 .009919-3.873840$

H $-1.1699604 .365302-3.598408$

H $-2.124788 \quad 5.753845-3.054603$

C $-2.132288 \quad 5.0968810 .018710$

H 1.7358183 .1510851 .633880

H $4.8335665 .340108-1.131904$

H $6.3294163 .976231-1.110000$

H $-2.8348315 .591353-0.662805$

H -1.6763245 .8809990 .637466$

H $-1.3961353 .507454-1.287204$

C $6.129486-2.036163-0.481607$

O $5.186381-0.959444-0.548166$

C $5.7302780 .361007-0.412604$

C $6.440805 \quad 0.504327 \quad 0.941274$

C $7.491856-0.5919531 .141721$

C $6.937788-1.9844090 .825696$

H $5.511947-2.943057-0.459776$

C $7.005350-2.100189-1.737154$

H $6.4419070 .532539-1.227100$ 
O $5.490640 \quad 0.4550482 .004129$

H 6.9304231 .4831190 .992060

H $7.840248-0.5638642 .181655$

O $8.606154-0.3102430 .299847$

H $7.752597-2.7168440 .789905$

O $6.080025-2.366106 \quad 1.904859$

C $6.161601-2.371177-2.959631$

H $7.555372-1.173727-1.924521$

H $7.757733-2.893603-1.653812$

H $5.012484-0.3892171 .936786$

H $9.279055-0.9921560 .465881$

H $5.801535-3.2839441 .746150$

O $5.898633-3.705490-3.058936$

O $5.766707-1.497471-3.720790$

C $5.078187-4.088571-4.166904$

H $4.096468-3.610885-4.093979$

H $5.567666-3.825754-5.109566$

H $4.943999-5.173034-4.131715$

SCF Energy (B3LYP/6-31G**//MMFF) $=-3245.90258343$

\section{1}

MM̄FF Geometry

C $-2.132268-2.7274503 .220329$

C -2.607106 -2.144023 2.109941

C $-3.536444-2.8153791 .135862$

O $-2.841086-2.812839-0.134588$

C $-4.832886-2.0110340 .972608$

C $-5.882932-2.7628120 .179721$

C $-6.175346-2.538360-1.116065$

C $-6.657054-3.7955230 .958694$

C $-5.523397-1.565183-2.058775$

C $-6.472649-0.433146-2.478045$

C $-6.7874940 .553205-1.342243$

C $-7.8029161 .600763-1.801828$

C $-8.0604832 .662344-0.722155$

O $-5.564961 \quad 1.195411-0.969378$

O $-8.7719283 .733179-1.360986$

C $-6.7046863 .210876-0.227318$

C -8.9753652 .1296620 .387012$

O

C -5.6835892 .1042910 .132276$

C -4.2924892 .7376370 .375357$

O $-6.1126091 .440091 \quad 1.322099$

C -2.000067 -3.846667 -0.393889

O $-1.927269-4.9015910 .221171$

C - $-1.104378-3.544565-1.533863$

C $-0.868288-2.295626-1.966283$

C $0.068153-1.907370-3.023903$

C $0.870953-2.996679-3.685384$

C $-3.153530 \quad 1.720604 \quad 0.534280$

C -1.7987412 .4006020 .630229$

C 0.6368431 .8949150 .504542

O

C $1.0347202 .158301-0.913638$

C $2.0116351 .493176-1.547298$

C $2.4115011 .754329-2.971134$

C $2.3969750 .503969-3.823641$

C $3.542881-0.118207-4.149010$

C $1.071188 \quad 0.028860-4.376843$

C $0.186989-0.598982-3.333677$

N $-0.755840 \quad 1.489172 \quad 0.530917$

H -2.491510 -3.717480 3.490931

H -2.251454 -1.156457 1.829778

H -3.760901 -3.843169 1.445377

H -4.615176 $-1.048590 \quad 0.498382$

H $-5.254921-1.7667891 .956617$

H -6.968294 -3.131145 -1.571113

H -7.433647 -4.2766830 .354665$

H $-5.991243-4.583027 \quad 1.324491$

H -7.151063 -3.330275 1.817941

H $-4.593113-1.153222-1.657205$

H -5.233511 -2.124662 -2.957238

H $-5.9921850 .111416-3.301313$

H -7.403931 -0.859320 -2.871667

H -7.182896 -0.002068 -0.484916

H $-8.7442191 .125060-2.104322$

H -7.421683 2.106314 -2.700460

H $-8.9938754 .394831-0.684422$
H $-6.2829313 .820178-1.039041$

H $-9.9475471 .838563-0.028504$

H -9.1878872 .9046181 .131885$

H $-8.561828 \quad 1.2613270 .904374$

H -7.4330814 .8274790 .595312$

H $-4.0425463 .384709-0.476036$

H -4.3234753 .3731641 .269590$

H $-5.344340 \quad 1.040810 \quad 1.758741$

H - $0.586767-4.403602-1.947820$

H -1.388104 -1.466466 -1.489031

H $1.574900-2.624842-4.432966$

H $\quad 0.209743-3.705617-4.195607$

H $1.466078-3.540602-2.943258$

H -3.2898711 .1279841 .444424$

H $-3.1322531 .038845-0.323606$

H 1.2208421 .0880400 .955566

H $\quad 0.7675672 .8013651 .103318$

H $0.4940002 .939641-1.442792$

H $2.5624840 .726244-1.009635$

H $3.4195192 .188500-2.960199$

H $1.7688332 .513232-3.434462$

H $3.549584-0.996483-4.785531$

H $4.5013150 .233721-3.781597$

H $1.221303-0.656419-5.219038$

H $0.5400960 .883750-4.815329$

H $-0.4294380 .120707-2.794401$

H -0.9665310 .5172550 .323456$

C $0.128930-1.5550653 .299873$

O $0.667827-2.5491552 .406807$

C $2.102944-2.4123162 .356610$

C $2.435497-1.1577723 .153705$

C $1.305157-1.1123014 .164274$

H $2.505605-3.3011322 .857668$

C $2.535141-2.3883730 .883906$

H $3.424369-1.2087623 .619565$

H $2.410867-0.2685002 .513031$

C 1.1214920 .2518944 .805668

H $1.502355-1.8583594 .945513$

C $4.034122-2.0925950 .689519$

O $2.217919-3.6616520 .316303$

H $1.942366-1.6449630 .336559$

H $4.242586-1.1291341 .160289$

O $4.813814-3.0661361 .382175$

C $4.479058-2.024543-0.784906$

C $5.961681-1.643580-0.978673$

H $3.843851-1.304389-1.310509$

O $4.274681-3.292859-1.409581$

H $6.605765-2.429833-0.565645$

H $6.149784-1.640594-2.060233$

H 2.0239260 .5443995 .352348

H 0.9181841 .0242234 .055805

H $0.286248 \quad 0.240974 \quad 5.512901$

C -1.039200 -2.159109 4.088743

H $1.267802-3.8142600 .458197$

H $4.626815-3.929200 \quad 0.974140$

H $4.473823-3.190071-2.355804$

H -1.467687-1.427362 4.782332

H $-0.654621-2.9896114 .695167$

H $-0.212700-0.7168952 .680413$

C $5.6200712 .020106-0.406597$

O $5.5037690 .710773-0.980477$

C $6.345451-0.280902-0.384852$

C $7.8188090 .086473-0.614253$

C $8.1188811 .480035-0.047057$

C $7.0746252 .520883-0.474845$

H $5.0156332 .657878-1.063340$

C 4.9873202 .0437550 .993038

H $\quad 6.165710-0.3136160 .694597$

O $8.1278680 .092049-2.005733$

H $8.462642-0.657394-0.129596$

H $9.1084541 .807604-0.389214$

O 8.1513731 .4182061 .376030

H 7.1809153 .4293950 .127823

O $7.3455342 .899675-1.829428$

C 4.4143153 .3949131 .357853

H 4.1599311 .3238121 .015791

H 5.6985751 .7532201 .770441

H $8.162735-0.831902-2.305212$ 
H $8.8500450 .790314 \quad 1.626984$

H $7.2125382 .115218-2.388967$

O 3.5748743 .2440512 .425643

O $4.676933 \quad 4.4489910 .795999$

C $2.944621 \quad 4.4424192 .888119$

H 3.6970325 .1482153 .252216

H $2.347648 \quad 4.8910792 .088231$

H 2.2798564 .1789593 .715150

SCF Energy (B3LYP/6-31G**/MMFF) $=-3245.90483281$

0800292

MM̄FF Geometry

C $-2.7272302 .617869-1.413290$

C $-3.527886 \quad 3.303489-0.583694$

C $-3.969028 \quad 2.8704350 .796053$

O -3.2926521 .6670901 .224605$

C $-5.4725262 .549366 \quad 0.793243$

C -6.1110522 .6999722 .158830$

C -6.3073001 .6999203 .039620$

C $-6.586198 \quad 4.0936832 .483275$

C $-5.870093 \quad 0.2650132 .939099$

C $-7.036826-0.6963842 .676158$

C $-7.381285-0.8110961 .183785$

C $-8.635085-1.6618690 .979558$

C $-8.937480-1.876585-0.510480$

O $-6.262005-1.4252270 .532543$

O $-9.924478-2.913779-0.615417$

C -7.661443 -2.393696-1.205252

C $-9.547686-0.628295-1.158649$

O $-7.848422-2.476736-2.616802$

C $-6.395033-1.562363-0.886554$

C $-5.145801-2.308512-1.415037$

O $-6.493765-0.292543-1.532304$

C $-2.040800 \quad 1.768290 \quad 1.740594$

O -1.3790892 .7813651 .890626$

C $-1.607041 \quad 0.3913682 .063958$

C $-0.305515 \quad 0.1187802 .231679$

C $0.261273-1.1962412 .540550$

C $-0.699602-2.3386882 .749810$

C $-3.795511-1.713032-0.983727$

C $-2.633326-2.515611-1.551698$

C $-0.148522-2.504352-1.733885$

O $-2.788474-3.551363-2.194805$

C $0.514117-3.267114-0.629569$

C $1.804951-3.114008-0.294390$

C $2.498122-3.9177820 .769079$

C $3.209457-3.0591421 .791628$

C $4.532194-2.8365301 .710324$

C $2.406364-2.5434042 .963672$

C $1.602516-1.3190512 .621766$

N $-1.402380-1.943383-1.260181$

H -2.364215 $1.634666-1.126908$

H -3.879959 4.284601 -0.897213

H -3.7463453 .6802991 .502169$

H $-5.629702 \quad 1.5324580 .417326$

H -6.0107863 .2074460 .097765$

H -6.8130291 .9386153 .974996$

H -7.0390304 .1595653 .478296$

H -5.7492684 .7986172 .455616$

H $-7.338032 \quad 4.4172281 .756041$

H $-5.080247 \quad 0.1258602 .197105$

H -5.4165780 .0033863 .903890$

H $-6.738390-1.6871703 .043421$

H -7.919154 -0.388909 3.250551

H -7.5383420 .1920910 .774205$

H $-9.498376-1.2115231 .485290$

H -8.491447 -2.644881 1.449979

H -10.711251-2.629957-0.119045

H -7.495687 -3.422246 -0.854750

H -10.481197 -0.352484 -0.653656

H -9.817383 $-0.817990-2.203454$

H $-8.8861140 .239854-1.125444$

H $-8.641760-3.017188-2.772630$

H $-5.174593-3.346212-1.056413$

H $-5.177432-2.343451-2.511725$

H -5.602509 $0.061761-1.675306$

H -2.377481 -0.3684302 .133490$

H $\quad 0.412956 \quad 0.9303462 .126413$
H -1.331933 -2.150757 3.624556

H $-0.208305-3.3005302 .909537$

H -1.344614 -2.469653 1.874066

H $-3.698606-0.680631-1.333993$

H -3.708080 -1.719039 0.108363

H $0.465046-1.673069-2.091083$

H $-0.341048-3.175833-2.576746$

H $-0.087117-4.005014-0.103567$

H $2.415185-2.403521-0.846304$

H $3.222703-4.573376 \quad 0.268077$

H $1.799089-4.5902631 .281456$

H $5.054120-2.2468852 .456740$

H $5.122438-3.2314930 .890849$

H $3.079876-2.2663353 .786190$

H $1.798616-3.3552593 .372666$

H $2.216066-0.4344052 .448765$

H $-1.363922-1.105368-0.687203$

C $-0.7561643 .429018-2.782429$

O $-0.0073872 .262705-2.383336$

C $0.9303212 .652143-1.355902$

C $1.1203454 .146980-1.542697$

C $-0.2950964 .576800-1.878954$

H $0.4319912 .448340-0.399191$

C $2.1922371 .797201-1.480010$

H $1.5131144 .640733-0.649076$

H $1.7963484 .352983-2.381739$

C $-0.379643 \quad 5.943916-2.535155$

H $-0.8757884 .599793-0.948890$

C $3.3345172 .231066-0.538103$

O $1.7851890 .458443-1.187105$

H $2.5412921 .801719-2.519670$

H $3.6326623 .253259-0.797383$

$\begin{array}{lllll}\text { O } & 2.853971 & 2.271719 & 0.805892\end{array}$

C $4.5876471 .333074-0.609160$

C $5.714657 \quad 1.8473350 .308904$

H $4.9374511 .302348-1.647912$

$\begin{array}{lllll}\text { O } & 4.235872 & 0.005541 & -0.227486\end{array}$

H 5.8603202 .9201610 .135932

H $5.4076931 .703597 \quad 1.352542$

H $0.0101776 .717062-1.865013$

H $0.2006085 .980831-3.463277$

H $-1.4175316 .195545-2.775198$

C $-2.259040 \quad 3.125235-2.749138$

H $2.541218-0.123864-1.370242$

H $2.597001 \quad 1.3653151 .045831$

H $5.018266-0.556804-0.354073$

H $-2.4795502 .341301-3.484441$

H -2.823186 $4.014625-3.053076$

H $-0.4694383 .631948-3.822196$

C $8.024373-1.097894 \quad 0.256268$

O $6.892461-0.2472660 .474983$

C 7.0529861 .1165690 .075768

C $8.205075 \quad 1.7626450 .860303$

C $9.4981930 .954398 \quad 0.709192$

C $9.271893-0.541606 \quad 0.962007$

H $7.757680-2.0450230 .742855$

C $8.241599-1.383933-1.233753$

H $7.274407 \quad 1.157501-0.996545$

$\begin{array}{llll}\text { O } & 7.851576 & 1.848303 & 2.239238\end{array}$

H $8.3542502 .784897 \quad 0.492204$

H $\quad 10.244451 \quad 1.3378831 .415597$

O $10.0068341 .154819-0.606439$

H $10.150332-1.122980 \quad 0.658325$

O $9.096428-0.7529992 .366185$

C $7.034611-2.059753-1.839435$

H $8.426181-0.477676-1.817728$

H $9.113838-2.030378-1.387435$

H 8.5830682 .2900972 .703032

H $10.838970 \quad 0.657109-0.678561$

H $9.904116-0.4460522 .812262$

O $7.029687-3.385737-1.523117$

O $6.190430-1.472494-2.503995$

C $5.921537-4.134270-2.033334$

H $4.974658-3.680788-1.724683$

H $5.982664-4.194429-3.123867$

H $5.975190-5.145142-1.620603$

SCF Energy (B3LYP/6-31G**//MMFF) $=-3245.91274850$ 
0800293

MM̄FF Geometry

C $-0.997760 \quad 5.2689690 .889079$

C $-1.655086 \quad 4.2332490 .344617$

C -3.1152683 .9565960 .602694$

O -3.2749682 .5440820 .876800$

C $-3.9447564 .291312-0.646727$

C $-5.4363544 .281000-0.376574$

C $-6.276093 \quad 3.290408-0.734687$

C -5.9747005 .5173810 .298190$

C $-5.9344761 .993911-1.415059$

C $-6.6291860 .807339-0.731100$

C $-6.382890-0.503770-1.489805$

C -7.226907-1.637225 -0.905537

C $-6.947131-2.972863-1.610585$

O $-4.993456-0.830777-1.384177$

O $-7.524637-4.020410-0.816629$

C $-5.424076-3.211770-1.636718$

C $-7.620439-3.048383-2.985960$

O $-5.100976-4.363552-2.412730$

C $-4.608175-1.992498-2.131767$

C $-3.103748-2.250108-1.889059$

O $-4.826433-1.812177-3.532034$

C -2.9503232 .1103082 .124433$

O -2.5593362 .7888453 .060823$

C -3.1264680 .6411942 .146502$

C $-2.572867-0.0658863 .142955$

C $-2.618387-1.5205143 .305596$

C $-3.516262-2.2974612 .380989$

C $-2.204765-1.023508-2.085519$

C $-0.782377-1.365157-1.688356$

C $0.637352-1.763214 \quad 0.327933$

O $0.066482-1.661205-2.525618$

C $0.353668-2.9713661 .164057$

C $0.706511-3.0801822 .454157$

C $0.423169-4.2927523 .294571$

C $-0.235106-3.9561724 .615661$

C $0.433659-4.0893925 .773709$

C -1.692122 -3.5394174 .603080$

C -1.861299-2.081525 4.271637

N $-0.594062-1.347171-0.315327$

H - 1.5578005 .9537321 .523854

$\mathrm{H}-1.1401733 .537738-0.311196$

H -3.477434 4.5334921 .462854

H -3.695339 $3.597512-1.457953$

H -3.676134 5.288053 -1.022586

H -7.336941 $3.424554-0.525323$

H -7.0624965 .4842330 .421816$

H -5.5356675 .6367201 .293320$

H $-5.7389246 .406851-0.295340$

H $-6.2714082 .059758-2.456535$

H -4.856162 $1.813927-1.434229$

H -6.2533430 .7192070 .296587$

H -7.708409 $0.997398-0.674325$

H -6.639393 -0.344350 -2.543894

H $-8.295647-1.394952-0.955620$

H $-6.994342-1.7521520 .162511$

H -8.477198 $-3.842607-0.733178$

H $-5.114435-3.436877-0.606121$

H $-8.706081-2.927945-2.887848$

H -7.469202 -4.031194 -3.446142

H -7.265270 -2.284206 -3.680319

H -5.620880 -5.105882 -2.059747

H $-2.965832-2.612927-0.864412$

H -2.750543 -3.049265 -2.553873

H -4.071624 -1.333699-3.909673

H -3.676656 0.1829991 .332619

H -2.0056590 .4685303 .904467$

H -4.535337 -1.8946842 .402763$

H -3.602508 -3.354386 2.640373

H -3.142929 -2.2454701 .355468$

H -2.199019 -0.693528 -3.129390

H -2.544046 -0.174544 -1.481065

H $0.983168-0.9213960 .932524$

H $1.396873-1.998958-0.422743$

H $-0.160355-3.799708 \quad 0.681870$

H $1.243799-2.2624972 .928889$

H $1.378586-4.8043413 .468929$
H $-0.207988-5.0109502 .756148$

H $-0.038631-3.8739016 .726571$

H $1.469432-4.4114235 .795594$

H -2.137336 -3.705798 5.592771

H -2.235147 -4.201077 3.923229

H $-1.269703-1.4217244 .908514$

H -1.360728 -1.0558190 .283657$

C 1.3819924 .5074780 .211349

O $1.439863 \quad 3.4426981 .182495$

C 2.8154883 .0408441 .361049

C $3.5783343 .668126 \quad 0.204977$

C 2.8266234 .9700780 .018501

H 3.1370763 .4858822 .311673

C 2.8763291 .5112171 .446150

H $4.638733 \quad 3.8159410 .430275$

H $3.5020923 .049213-0.696328$

C $3.0899845 .636114-1.320823$

H $3.1129175 .659248 \quad 0.824320$

C 4.3078420 .9426811 .377691

O $2.243051 \quad 1.1480892 .675666$

H 2.2712351 .0812090 .639274

H 4.7303501 .2415510 .415093

O 5.1159541 .5473012 .386935

C $4.393237-0.5929301 .500761$

C $5.815494-1.1524031 .285683$

H $3.702822-1.0489000 .782598$

O $3.962848-0.9861142 .805340$

H $6.507415-0.7009632 .007387$

H $5.788777-2.2238611 .525074$

H $4.1512495 .885357-1.423869$

H $2.8163084 .982209-2.155279$

H $2.5148236 .562305-1.416065$

C 0.4602245 .6150690 .730189

H 2.2059120 .1778072 .715277

H $4.740271 \quad 1.2943493 .247988$

H $3.949002-1.9582062 .829873$

H $\quad 0.525564 \quad 6.493968 \quad 0.078497$

H $0.8166715 .922106 \quad 1.722408$

H $0.9992444 .078973-0.722802$

C $5.705675-1.502397-2.422920$

O $5.447528-1.657915-1.022378$

C $6.337630-0.960126-0.146513$

C $7.770142-1.475914-0.333671$

C $8.199977-1.344994-1.800911$

C $7.149348-1.915489-2.761135$

H $5.031948-2.216782-2.913970$

C $5.327304-0.095527-2.914617$

H $6.3236820 .107138-0.380962$

O $7.844236-2.8407650 .070277$

H $8.442817-0.9003770 .313305$

H $9.148524-1.873660-1.954991$

O $8.409761 \quad 0.028621-2.116818$

H $7.381084-1.622480-3.791291$

O $7.224967-3.344506-2.720260$

C $3.8454600 .181084-2.750018$

H $5.8663040 .686071-2.373309$

H $5.561957-0.011906-3.981932$

H $8.766442-3.129196-0.038836$

H $9.1027700 .364007-1.522998$

H $6.953852-3.630956-1.831219$

O $3.5605291 .415889-3.259947$

O $3.037300-0.581540-2.238646$

C $2.185118 \quad 1.806326-3.181582$

H $1.8381631 .785347-2.143847$

H $2.0997812 .828708-3.558545$

H $1.5725621 .148681-3.805548$

SCF Energy (B3LYP/6-31G**//MMFF) $=-3245.90906008$

0800294

MM̄FF Geometry

C 0.4789094 .6824940 .452227

C 1.6529234 .0362090 .512463

C $2.5530523 .838060-0.679574$

O $2.8133012 .419938-0.812288$

C $3.8940174 .553104-0.455681$

C $4.7520054 .588554-1.704659$

C $5.8120093 .788921-1.931938$

C $4.3777935 .647603-2.710413$ 
C $6.3388182 .687143-1.055142$

C $6.6082281 .411466-1.866207$

C $7.2395240 .315523-0.997085$

C $7.670568-0.876812-1.851529$

C $8.253271-2.008640-0.991149$

O $6.269826-0.107005-0.032838$

O $8.336895-3.184599-1.810867$

C $7.266502-2.3201210 .152914$

C $9.682517-1.698289-0.531438$

O $7.821267-3.2695641 .061121$

C $6.763806-1.0664270 .910629$

C $5.606423-1.4668491 .852189$

O $7.832333-0.5319681 .694074$

C $1.8564721 .671092-1.425499$

O $0.8218442 .072309-1.934344$

C $2.2695950 .253310-1.338596$

C $1.346010-0.703885-1.515223$

C $1.567658-2.149665-1.440088$

C $2.990887-2.627882-1.333530$

C $4.840905-0.2877172 .464265$

C $3.663910-0.8107153 .263518$

C $1.388292-1.7947392 .967263$

O $3.731930-0.9703434 .479905$

C $1.317159-3.1518992 .337611$

C $0.210175-3.6470611 .764007$

C $0.135245-5.0043521 .125138$

C $-0.433149-4.966008-0.277592$

C $-1.667438-5.430863-0.535486$

C $0.459453-4.470457-1.396523$

C $0.495309-2.968525-1.478755$

N $2.573106-1.1223802 .466924$

H $0.1648665 .122809-0.490216$

H 1.9727323 .5932331 .452756

H $2.0791734 .209938-1.596750$

H $4.436786 \quad 4.0802170 .370887$

H $3.7192315 .589001-0.134294$

H $6.3770293 .943564-2.850550$

H $5.0578325 .665607-3.568845$

H $3.3685265 .474755-3.095845$

H $4.4086306 .639125-2.246777$

H $7.2755753 .033509-0.602319$

H $5.6528122 .451903-0.236862$

H $5.661311 \quad 1.051580-2.289284$

H $7.2782611 .644174-2.703447$

H $8.1054810 .743581-0.478453$

H $8.392607-0.568314-2.617765$

H $6.800710-1.267559-2.398452$

H $8.903271-2.978268-2.574148$

H $6.399699-2.815847-0.305878$

H $10.332569-1.519165-1.396238$

H $10.120842-2.551327-0.001782$

H $9.743630-0.8206120 .115410$

H $8.462116-2.8015911 .623149$

H $4.891373-2.0831131 .296076$

H $5.990285-2.0931192 .668167$

H $7.458152-0.0122892 .423049$

H $3.305810 \quad 0.039634-1.103597$

H $0.317961-0.401895-1.713076$

H $3.426358-2.312926-0.382361$

H $3.598289-2.218396-2.148760$

H $3.095821-3.712763-1.389902$

H $5.476290 \quad 0.2998763 .134793$

H $4.469210 \quad 0.395121 \quad 1.691884$

H $0.526914-1.1724322 .706846$

H $1.440306-1.8905054 .055922$

H $2.220510-3.7576222 .356735$

H - $0.700606-3.0545301 .773135$

H $-0.490533-5.6378331 .767049$

H $1.120210-5.4870081 .098005$

H -2.071935 -5.431632 -1.542266

H $-2.304378-5.8136870 .254933$

H $0.089483-4.841886-2.361400$

H $1.448602-4.919087-1.275090$

H $-0.492748-2.517406-1.582364$

H $2.619680-0.9336361 .469901$

C $-1.7340313 .988178 \quad 1.487558$

O -1.3988542 .5911321 .362779$

C $-2.2092882 .014148 \quad 0.315465$
C $-3.3476732 .998506 \quad 0.093730$

C -2.6433624 .3240950 .305188$

H $-1.567623 \quad 1.971677-0.573158$

C -2.631532 0.6001220 .727627

H $-3.7970402 .909338-0.899616$

H $-4.1353662 .859638 \quad 0.843337$

C -3.5896785 .4824990 .567934$

H $-2.0564904 .544811-0.594111$

C -3.632791-0.047706 -0.251164

O $-1.430959-0.1729280 .802681$

H -3.0550410 .6258891 .738059$

H $-4.5007040 .612274-0.334200$

O $-3.044509-0.120671-1.549871$

C $-4.131043-1.4443790 .173519$

C $-5.102372-2.097338-0.829363$

H $-4.606340-1.366747 \quad 1.158374$

O $-3.014833-2.323790 \quad 0.315576$

H -4.591698 -2.255755-1.787696

H $-5.334290-3.101203-0.448639$

H $-4.2565755 .635684-0.286811$

H -4.2123865 .3006151 .450450$

H -3.0321346 .4098850 .733006$

C -0.4560854 .8236391 .621148$

H -1.670308 -1.0535511 .135891$

H -2.265254 -0.698860 -1.482012

H $-3.347152-3.1681900 .664748$

H 0.0631084 .5173562 .538402

H -0.7117945 .8825301 .741888$

H -2.298147 4.0791352 .425164

C $-8.317585-0.5002070 .210819$

O $-7.086818-1.2372860 .208548$

C $-6.409489-1.319672-1.050216$

C $-7.304162-1.989047-2.105469$

C $-8.650717-1.268063-2.215730$

C $-9.293083-1.056175-0.841364$

H $-8.755646-0.692627 \quad 1.199202$

C -8.0770381 .0111830 .085925$

H $-6.160845-0.313388-1.397570$

O $-7.518390-3.354932-1.757086$

H $-6.785344-1.970508-3.071624$

H $-9.324934-1.858282-2.848046$

O $-8.440772-0.010427-2.851601$

H $-10.157379-0.388285-0.927815$

O $-9.800696-2.308051-0.369853$

C $-7.6613001 .608158 \quad 1.411074$

H $-7.3211311 .283783-0.654981$

H $-9.0059731 .517729-0.202970$

H -8.057903 -3.755498 -2.459962

H $-9.3071080 .422625-2.938150$

H -9.042352 -2.896587 -0.212738

O $-6.421856 \quad 1.1652671 .764415$

O -8.3580172 .3852742 .051637$

C -5.9309271 .6504963 .017931$

H -5.7715192 .7315552 .965611$

H $-6.626921 \quad 1.4002813 .824453$

H -4.9739121 .1632593 .220793$

SCF Energy $\left(B 3 L Y P / 6-31 G^{* *} / / M M F F\right)=-3245.91778364$

0800295

MM̄FF Geometry

C -2.6406431 .4088132 .309198$

C $-1.708500 \quad 0.446588 \quad 2.228792$

C $-0.6750970 .214530 \quad 3.294924$

O $0.615928 \quad 0.1719402 .648827$

C $-0.908271-1.0733574 .110050$

C $-0.911341-2.3820633 .339119$

C $-2.010743-3.1293363 .114906$

C $0.437561-2.8953222 .904051$

C $-3.425592-2.7936183 .491070$

C $-4.488274-3.3716002 .541124$

C $-4.467846-2.7713471 .124916$

C $-5.761241-3.1336350 .386229$

C $-5.755748-2.637773-1.066781$

O $-3.342825-3.3017760 .413455$

O $-6.853894-3.256113-1.754507$

C $-4.445860-3.089902-1.744334$

C $-6.025718-1.131399-1.149343$

O $-4.326184-2.523396-3.047602$ 
C $-3.181825-2.776147-0.910880$

C $-1.949334-3.443489-1.566675$

O $-2.983925-1.364476-0.891987$

C 1.6940220 .5892633 .363656

O $1.693431 \quad 1.0844494 .479078$

C 2.8983130 .3010412 .556201

C 4.1017360 .6804943 .007187

C 5.3582030 .4221002 .315632

C 6.2062971 .6510572 .138958

C $-0.677431-3.424063-0.707721$

C $0.512624-4.011216-1.450180$

C $2.857658-4.722335-1.112073$

O $0.451699-4.404215-2.612528$

C $4.026872-4.305648-0.278445$

C $5.157020-3.800951-0.797881$

C $6.368293-3.4354840 .014378$

C $6.734902-1.966418-0.040128$

C $6.922897-1.310099-1.197815$

C $6.977059-1.2733891 .282495$

C $5.697828-0.8270151 .938076$

N $1.657182-4.042113-0.661531$

H -2.681066 2.0245293 .203480

H -1.665757 -0.1872891 .348015$

H -0.6916671 .0633293 .991881$

H -1.839392 -0.9612344 .678358$

H $-0.122122-1.1509134 .873514$

H -1.881149 -4.091718 2.621788

H $\quad 0.396675-3.9392872 .574861$

H $\quad 0.824461-2.3044832 .069472$

H $1.153469-2.8476423 .730967$

H $-3.611206-3.2071084 .490061$

H -3.574970 -1.710998 3.555926

H -4.364223 -4.460859 2.483760

H -5.467761 -3.180881 2.998004

H $-4.367893-1.682844 \quad 1.207534$

H $-6.637783-2.7424380 .917931$

H $-5.878320-4.2265750 .382369$

H $-6.739429-4.220444-1.706233$

H $-4.503525-4.178385-1.883840$

H -7.010509-0.897238 -0.727059

H $-6.058499-0.790864-2.190160$

H -5.287282 $-0.531596-0.614457$

H $-5.135793-2.748273-3.537214$

H -2.179740 -4.494809-1.784032

H -1.737257 -2.952659-2.525348

H $-2.042796-1.174784-0.756989$

H $2.776182-0.2321871 .619544$

H 4.1939171 .2389923 .936106

H 5.6063912 .4783821 .742069

H 7.0331061 .5093231 .439685

H 6.6268921 .9652383 .100245

H -0.419689-2.403857 -0.409513

H $-0.835409-4.019140 \quad 0.198153$

H $3.007725-4.513739-2.176906$

H $2.685508-5.798157-1.000563$

H $3.952194-4.4544500 .796032$

H $5.230858-3.680431-1.876258$

H $7.223395-4.023651-0.342198$

H $6.216405-3.7405861 .057617$

H $7.211309-0.263909-1.211319$

H $6.796387-1.799018-2.157438$

H $7.677263-0.4440381 .144057$

H $7.494435-1.9639911 .960814$

H $5.002844-1.6382222 .157960$

H $1.555157-3.8655270 .333688$

C $-3.560346 \quad 3.1119040 .652421$

O $-2.2488223 .347498 \quad 0.099183$

C -1.8121894 .6587320 .499026$

C $-3.0702305 .389650 \quad 0.954881$

C -3.8355454 .2626121 .622466$

H -1.1648294 .5151641 .371934$

C $-1.0235805 .348585-0.620852$

H $-2.847693 \quad 6.2196801 .632069$

H -3.647168 5.7821850 .110511

C -5.3115464 .5588641 .822724$

H -3.3786174 .0733292 .600528$

C $0.2058654 .563113-1.126563$

O $-1.8769135 .538462-1.757317$
H $-0.7121686 .348564-0.294887$

H $-0.1366123 .694170-1.697924$

O $0.8917415 .401453-2.067831$

C $1.2062084 .111450-0.045898$

C $2.5330243 .546639-0.591231$

H $0.741666 \quad 3.3604200 .603233$

O $1.5684395 .224016 \quad 0.779005$

H $3.1198724 .352768-1.050742$

H $3.115728 \quad 3.2271790 .283597$

H -5.4439505 .4313582 .470821$

H -5.8124824 .7682720 .871580$

H $-5.818373 \quad 3.7094402 .291271$

C $-3.6524991 .696504 \quad 1.235424$

H -2.459961 $6.292276-1.567527$

H $1.2523686 .159547-1.576519$

H 0.7913095 .4793851 .302165

H -3.5238670 .9804180 .416135$

H $-4.6552521 .530488 \quad 1.645475$

H -4.257058 $3.171533-0.194255$

C $1.3866300 .182703-1.750191$

O $1.6408151 .346156-0.950647$

C $2.4012312 .377335-1.582350$

C $3.7783321 .847520-2.024057$

C $3.6591050 .572793-2.865049$

C $2.707026-0.443896-2.225712$

H $0.905052-0.523642-1.063235$

C $0.4052790 .460758-2.900376$

H $1.8603922 .733217-2.464706$

O $4.5974421 .577203-0.888386$

H $4.2860822 .627728-2.603140$

H $4.6538290 .123469-2.972771$

O $3.2008120 .925692-4.165952$

H $2.502908-1.264463-2.923323$

O $3.368000-1.008516-1.091535$

C $-0.9547120 .885289-2.398572$

H $\quad 0.765318 \quad 1.254692-3.559188$

H $0.267976-0.453592-3.489245$

H $4.1387890 .915362-0.343256$

H $3.1576850 .109663-4.693018$

H $2.775456-1.676779-0.707492$

O $-1.765161 \quad 1.145434-3.465810$

O $-1.2669100 .981762-1.220863$

C $-3.0856891 .592290-3.142436$

H $-3.598408 \quad 0.857117-2.515857$

H $-3.0401392 .563533-2.641208$

$\mathrm{H}-3.6439671 .705423-4.075503$

SCF Energy $\left(B 3 L Y P / 6-31 G^{* *} / / M M F F\right)=-3245.89755469$

0800296

MM̄̄FF Geometry

C 3.1815590 .5612052 .500770

C 2.1351691 .1193103 .127406

C 0.7032070 .7892172 .804850

O $\quad 0.1337501 .9869192 .222285$

C $-0.127810 \quad 0.4329104 .051715$

C $0.227060-0.8834984 .707461$

C $-0.377716-2.0563124 .434499$

C $1.271555-0.8152575 .792603$

C $-1.453906-2.3313333 .418720$

C $-1.116431-3.5803932 .592217$

C $-2.275012-4.0199441 .683071$

C $-1.896940-5.2995630 .932137$

C $-2.989416-5.732872-0.053711$

O $-2.554913-2.9702250 .753208$

O $-2.412667-6.735682-0.903647$

C $-3.369705-4.524305-0.932813$

C $-4.163840-6.4037890 .667663$

O $-4.467708-4.847074-1.783846$

C $-3.657315-3.233736-0.128641$

C $-3.782641-2.037943-1.104937$

O $-4.880196-3.3959520 .588301$

C -0.8343111 .8259261 .281445$

$\begin{array}{llll}\mathrm{O} & -1.269586 & 0.771393 & 0.849411\end{array}$

C -1.2861403 .1729630 .866671$

C $-2.2590753 .288949-0.049168$

C $-2.8389524 .537179-0.547836$

C $-2.2756725 .831531-0.023332$

C $-3.971549-0.674413-0.427312$ 
C $-5.420555-0.251323-0.252493$ C -6.7678841 .6618810 .623211$ O $-6.372007-0.902325-0.673443$ C $-6.9027762 .697874-0.448393$ C $-6.9092564 .018259-0.211407$ C -7.021429 5.059220-1.289055 C $-5.8434116 .009999-1.302711$ C $-5.9259747 .213527-0.710680$ C $-4.6037595 .605977-2.071485$ C $-3.8564704 .468761-1.431801$ $\begin{array}{llll}\mathrm{N} & -5.514613 & 0.958776 & 0.422474\end{array}$ H $3.004260-0.1614791 .707981$ H $2.312966 \quad 1.8474963 .915170$ H $0.661231-0.0345882 .080909$ H $-1.192730 \quad 0.4370403 .785082$ H -0.0538201 .2428194 .791333$ H $-0.076082-2.9275725 .015521$ H $1.395106-1.772426 \quad 6.310949$ H $2.246371-0.5412845 .380851$ H $0.989585-0.0724746 .546123$ H -2.394972 -2.494566 3.957090 H $-1.605032-1.4816492 .746548$ H $-0.227580-3.3657741 .985266$ H $-0.858047-4.409997 \quad 3.262828$ H $-3.158710-4.1899712 .309204$ H -1.669862 -6.111679 1.634100 H $-0.969852-5.1273320 .366677$ H -3.073687 -6.964683 -1.579628 H $-2.517757-4.334672-1.600335$ H -3.817855 -7.287865 1.216912 H $-4.910684-6.770621-0.044922$ H $-4.662260-5.7440541 .380959$ H $-5.277603-4.814700-1.246647$ H $-2.851107-1.977313-1.682254$ H $-4.593625-2.215948-1.820364$ H $-5.197775-2.5221150 .864453$ H -0.8149314 .0299381 .334230$ H -2.684003 $2.380315-0.472357$ H -2.387029 $5.886961 \quad 1.065385$ H -2.768846 $6.716595-0.431718$ H $-1.2123035 .916939-0.273350$ H $-3.475623-0.6447850 .547824$ H -3.504604 $0.087691-1.064059$ H $-6.7374702 .102778 \quad 1.624409$ H -7.6058680 .9598110 .574490$ H -6.992487 2.339628 -1.471371 H $-6.829702 \quad 4.3752730 .812948$ H -7.952130 $5.616354-1.119405$ H -7.129767 4.601547-2.280229 H $-5.1000137 .916236-0.738572$ H $-6.821427 \quad 7.521647-0.180897$ H -3.939195 $6.463952-2.226754$ H $-4.8975795 .302220-3.084681$ H $-4.2030613 .485453-1.751101$ H $-4.660843 \quad 1.4432480 .684037$ C $5.331388 \quad 1.682803 \quad 1.789090$ O $5.2951331 .012133 \quad 0.513625$ C 6.6436340 .7788530 .075994 C $7.461090 \quad 1.867971 \quad 0.751938$ C $6.813016 \quad 1.9002712 .123822$ H $6.941239-0.1969550 .481178$ C $6.7280560 .729971-1.454500$ H 8.5286391 .6317810 .786160 H $7.336270 \quad 2.837576 \quad 0.256456$ C 7.0813623 .1807462 .896251 H 7.1955931 .0527572 .708678 C $5.649676-0.142629-2.130845$ O $6.5915852 .053748-1.987334$ H $7.7277920 .393417-1.751947$ H $4.7003590 .397576-2.121120$ O $5.999630-0.303518-3.509658$ C $5.452758-1.531325-1.509140$ C $4.406208-2.411593-2.214666$ H $5.153275-1.426063-0.460850$ O $6.703804-2.222310-1.515078$ H $4.793964-2.768378-3.177741$ H $4.306487-3.316753-1.601734$ H 8.1545513 .3063093 .072567
H $\quad 6.724934 \quad 4.0606682 .350211$

H 6.5778503 .1602133 .868065

$\begin{array}{llll}\text { C } 4.617474 & 0.836163 & 2.847687\end{array}$

H $5.7334372 .403467-1.691000$

H $6.1101740 .586536-3.886126$

H $6.567741-3.080818-1.079396$

H 4.6884311 .3307943 .823934

H $5.115591-0.1367272 .945932$

H 4.8143082 .6403861 .660867

C $1.414571-0.318897-1.318835$

O $2.664416-1.020806-1.259066$

C $3.023425-1.761842-2.429180$

C $1.946070-2.811796-2.762299$

C $0.546358-2.194114-2.831333$

C $0.265097-1.291895-1.624405$

H $1.2654370 .057867-0.301567$

C $1.4409450 .880140-2.279116$

H $3.117641-1.065154-3.268818$

O $1.938306-3.849930-1.781642$

H $2.194254-3.280497-3.721663$

H $-0.197142-3.000053-2.867370$

O $0.435128-1.446956-4.038107$

H $-0.661978-0.726200-1.771101$

O $0.082809-2.105718-0.464508$

C $2.2960512 .029113-1.796118$

H $1.8141200 .604136-3.269108$

H $\quad 0.430513 \quad 1.282330-2.422157$

H $1.800221-3.436403-0.912229$

H $-0.466223-1.084113-4.076493$

H $-0.675235-2.690897-0.632159$

O $1.8543712 .483478-0.590839$

O $3.2104842 .510665-2.453427$

C $2.5202173 .641781-0.077414$

H $2.132645 \quad 4.533899-0.577288$

H $2.303098 \quad 3.7147030 .991421$

H $3.6040073 .571674-0.209015$

SCF Energy (B3LYP/6-31G**//MMFF)= -3245.93283940

0800297

MM̄FF Geometry

C $-4.143776 \quad 4.905784-0.121013$

C $-4.4708313 .653810-0.474498$

C $-5.175767 \quad 2.694294 \quad 0.449158$

$\begin{array}{lllll}\text { O } & -4.337476 & 1.530927 & 0.651959\end{array}$

C $-6.4973432 .232086-0.187913$

C $-7.330662 \quad 1.3602790 .731821$

C $-7.4498330 .021737 \quad 0.625946$

C -8.0950452 .1018871 .798728$

C $-6.772005-0.889417-0.359680$

C $-6.026388-2.0239210 .356259$

C $-5.273280-2.927526-0.631320$

C $-4.604223-4.089543 \quad 0.105342$

C $-3.748877-4.953548-0.832222$

O $-4.285487-2.131956-1.295042$

O $-2.934297-5.7930330 .000909$

C $-2.809255-4.033311-1.642389$

C $-4.617259-5.897320-1.671859$

O $-2.092412-4.782251-2.620501$

C $-3.530386-2.833551-2.292871$

C $-2.558776-1.817541-2.943569$

O $-4.385010-3.318607-3.336412$

C -3.3315251 .6444821 .563866$

$\begin{array}{lllll}\text { O } & -2.999676 & 2.651959 & 2.168805\end{array}$

C $-2.7205750 .306677 \quad 1.737306$

C -1.7094110 .1486112 .605210$

C $-1.069948-1.1170102 .976899$

C $-1.564849-2.3737352 .311882$

C $-1.555166-1.159843-1.991085$

C $-0.216953-1.875275-1.906623$

C $1.875425-1.904870-0.546441$

O $0.147006-2.721076-2.718659$

C $1.851927-2.6628470 .740554$

C $2.522460-2.2728311 .834670$

C $2.573167-3.061637 \quad 3.108921$

C $2.081626-2.2775254 .302218$

C $2.945555-1.6955315 .151323$

C $0.594612-2.2611284 .568178$

C $-0.107512-1.0983853 .923403$ 
N $0.539883-1.421077-0.836212$ H -4.4020825 .2637040 .872664$ H -4.200499 3.288787 -1.462484 H $-5.3808233 .162047 \quad 1.420470$ H $-6.2917281 .711296-1.129635$ H $-7.100648 \quad 3.106191-0.469167$ H -8.109303 -0.4884891 .327371$ H -8.7262221 .4365072 .397532$ H -7.408204 2.6068512 .484843 H -8.7492342 .8533581 .344465$ H -7.537747 -1.317511 -1.017469 H $-6.073313-0.345344-0.999501$ H $-5.315117-1.5868461 .067925$ H -6.739384 -2.624969 0.934762 H -5.989794 -3.304513 -1.370780 H $-5.351172-4.7057020 .621350$ H -3.953605 -3.6902250 .894688$ H $-2.332632-6.289524-0.580328$ H -2.060033 -3.660068 -0.934413 H -5.196066 -6.562519-1.019583 H $-4.001712-6.555116-2.295353$ H -5.322963 -5.370370 -2.317436 H $-2.695910-4.953981-3.363243$ H -2.057826 -2.274125 -3.805735 H -3.172133 -1.006133 -3.361339 H -4.573142 -2.586122 -3.946235 H $-3.151319-0.5194201 .184724$ H -1.3353881 .0272303 .129962$ H -0.967737 -3.2566532 .549779$ H -1.533986 -2.275304 1.222054 H -2.595658 -2.583574 2.616258 H -1.333051-0.151971-2.364695 H -1.980861 $-1.048292-0.989607$ H $2.532261-1.033450-0.489991$ H $2.233845-2.550807-1.353778$ H $1.286052-3.5910910 .759808$ H $3.104993-1.3548441 .802332$ H $3.615300-3.3689053 .267501$ H $2.004604-3.9961683 .025156$ H $2.599439-1.1613566 .030006$ H $4.016627-1.7350954 .982609$ H $0.420390-2.1818785 .650382$ H $\quad 0.159110-3.2267914 .296124$ H $\quad 0.180075-0.1353754 .348144$ H $0.144151-0.724393-0.211973$ C -1.887367 $5.837922-0.832047$ O $-1.3704274 .584207-1.320224$ C $-0.4115114 .077044-0.369701$ C -0.0373615 .2629590 .506292$ C -1.3602875 .9996160 .595095$ H $-0.943384 \quad 3.3177240 .216587$ C $0.7544583 .438648-1.130064$ H 0.3481104 .9603351 .484498 H $\quad 0.718538 \quad 5.887967 \quad 0.014977$ C -1.230009 7.4455751 .039466 H $-1.9938365 .466848 \quad 1.314183$ C $1.8603832 .896691-0.205171$ O $0.2217802 .362479-1.908628$ H $1.1814654 .159630-1.837440$ H 2.2307313 .7123110 .426179 O $1.295626 \quad 1.9230700 .675095$ C $3.0729222 .292046-0.947377$ C 4.1191031 .7410480 .033816 H $3.5251243 .071401-1.570465$ O $2.6741611 .231670-1.810310$ H 4.4554432 .5364010 .710999 H 3.6537680 .9865620 .682426 H -0.7954607 .5020062 .042964$ H -0.5867158 .0177780 .362708$ H -2.209919 7.9326061 .066403 C $-3.4086565 .867546-1.011360$ H $-0.4827862 .730508-2.469414$ H 0.9760431 .1888010 .124011 H $2.0691821 .601268-2.475277$ H -3.649361 $5.643065-2.058449$ H -3.786170 $6.877164-0.811291$ H -1.453887 $6.610098-1.480895$ C $7.306257-0.1804430 .048908$
O $\quad 6.144161 \quad 0.562611 \quad 0.437356$

C $5.3325601 .063775-0.633121$

C $6.1505922 .003772-1.532385$

C $7.4536001 .352588-2.012028$

C $8.2075670 .654852-0.875354$

H $7.857906-0.3429640 .984373$

C $6.946689-1.561300-0.522062$

H $4.9647860 .214156-1.216357$

O $6.4622103 .204135-0.825635$

H $5.5502082 .289439-2.402922$

H $8.0981372 .132100-2.436178$

O $7.1966200 .424902-3.062464$

H $9.0103260 .023845-1.273906$

O $8.8281151 .655482-0.059440$

C $6.372892-2.4876550 .531489$

H $6.209837-1.501272-1.325477$

H $7.856244-2.035552-0.909214$

H $6.9511272 .956797-0.021818$

H $6.377612-0.056805-2.866815$

H $9.4543492 .143927-0.620522$

O $6.249215-3.738554-0.004748$

O $6.062325-2.1621941 .668746$

C $5.724486-4.7408720 .871646$

H $4.665259-4.5500471 .062672$

H $5.822292-5.7102440 .375606$

H $6.288153-4.7705161 .809256$

SCF Energy (B3LYP/6-31G**//MMFF) = -3245.92721124

\section{8}

MM̄FF Geometry

C $-0.820676-3.2458872 .987710$

C $-0.027834-2.3728012 .348167$

C $0.657523-1.2119703 .016882$

O $0.198370-0.0128132 .353052$

C $2.181671-1.3113082 .814740$

C $2.954708-0.2924583 .625582$

C 3.3644500 .9068403 .167816

C $3.277194-0.7151895 .035457$

C 3.1040331 .5309371 .823080

C 4.3912801 .9236281 .091297

C 5.1109090 .7360470 .433795

C $6.4992461 .180217-0.032749$

C $7.2331940 .063322-0.779626$

O $4.3284390 .310207-0.688908$

O $8.3721810 .642531-1.435293$

C $6.307420-0.478275-1.885827$

C $7.782019-0.9965200 .182622$

O $6.887114-1.623133-2.509307$

C $4.866888-0.807352-1.411660$

C $3.969880-1.042634-2.653891$

O $4.833242-2.002122-0.630632$

C $-0.3161170 .992880 \quad 3.108002$

O $-0.576410 \quad 0.9705504 .299292$

C -0.4494282 .1689512 .220805$

C $-0.795374 \quad 3.3620812 .723429$

C $-0.8769654 .587724 \quad 1.936326$

C -2.1358315 .3758432 .163547$

C $2.582443-1.622781-2.368961$

C $1.619882-0.672364-1.693216$

C $0.1953691 .321870-2.095788$

O $1.230523-0.868746-0.544826$

C $0.8357902 .629415-1.757243$

C $0.4281503 .794640-2.281805$

C $1.0002215 .136668-1.925297$

C $0.0115845 .997537-1.166870$

C $-1.0076336 .603791-1.799317$

C $\quad 0.247320 \quad 6.217717 \quad 0.310116$

C $0.1314794 .958216 \quad 1.121489$

N $1.2133990 .373316-2.502952$

H $-0.987162-3.1026154 .053921$

H $\quad 0.154327-2.4850241 .284166$

H $\quad 0.416178-1.1912434 .086394$

H $2.433940-1.2033201 .753855$

H $2.532434-2.3140973 .094171$

H 3.9194731 .5478953 .852405

H 3.8357500 .0509705 .583417

H $2.357545-0.9150415 .594432$

H $3.885520-1.6255185 .028763$ 
H $2.4805280 .906495 \quad 1.179484$

H 2.5326642 .4487622 .006563

H 4.1214932 .6486370 .311797

H 5.0648132 .4367891 .789213

H $5.190321-0.0874411 .151905$

H 7.1012001 .5321770 .814637

H $6.3998142 .042411-0.707432$

H $8.9261021 .065591-0.756955$

H $6.2399850 .296730-2.662680$

H $8.510021-0.5487470 .869995$

H $8.324276-1.779832-0.358009$

H $7.006231-1.4667710 .790651$

H $7.773888-1.367827-2.816559$

H $3.867634-0.102489-3.209645$

H $4.466273-1.760727-3.319054$

H $5.725221-2.382460-0.598930$

H $-0.2166902 .039607 \quad 1.169087$

H -1.039453 3.4683453 .777844

H -3.015066 4.7261922 .083959

H $-2.276273 \quad 6.176731 \quad 1.432984$

H -2.1308455 .8254233 .162314$

H $2.123061-1.928150-3.318226$

H $2.652668-2.534373-1.764756$

H $-0.3430220 .940106-1.222678$

H $\quad-0.514007 \quad 1.409547-2.924924$

H $1.6334252 .622885-1.018795$

H - $0.3808463 .790137-3.009450$

H $1.2922745 .645549-2.853031$

H $1.9275555 .029827-1.349063$

H -1.705714 7.241028 -1.266962

H -1.166680 $6.473255-2.864731$

H -0.4167326 .9971970 .699994$

H $1.258822 \quad 6.6220060 .447217$

H 1.0062294 .3115181 .052966

H $1.5833210 .448510-3.445900$

C $-1.246639-4.752617 \quad 0.925229$

O $-1.842717-3.7454350 .084884$

C $-2.270281-4.361308-1.145831$

C $-1.865855-5.830730-1.057805$

C $-1.855098-6.0661470 .440374$

H $-3.363657-4.299323-1.170083$

C -1.662589-3.616223 -2.339963

H -2.566111 -6.482560 -1.589490

H $-0.869236-6.002594-1.481250$

C -1.078848 -7.3062120 .848330$

H $-2.892021-6.1528480 .791541$

C $-2.083380-2.133811-2.447144$

O $-0.233345-3.662583-2.234210$

H -1.910812 -4.142101 -3.268970

H -1.708677 -1.580615 -1.579494

O $-1.413291-1.579376-3.587669$

C -3.602451-1.913734 -2.604749

C $-3.998717-0.462290-2.940626$

H -4.121010 -2.238335 -1.696588

O $-4.064751-2.745623-3.674196$

H -3.434872 $-0.116399-3.815377$ H -5.052481 -0.468120 -3.251577 H -1.515933 -8.2003590 .391730$ H $-0.031629-7.2456530 .533484$ H -1.096626 -7.440846 1.933969 C -1.528834 -4.440807 2.397486 H $0.012535-3.226892-1.399910$ H - $1.747908-2.040106-4.376278$ H -5.033736 -2.669827 -3.707050 H -1.250141 -5.3076413 .007881$ H -2.605452 -4.279972 2.534069 H $-0.165549-4.7491740 .732161$ C $-4.818237 \quad 0.9714140 .404781$ O $-4.8113720 .174498-0.786893$ C $-3.8250290 .516085-1.768549$ C $-3.9917291 .975980-2.221802$ C -3.989868 2.931371-1.025352 C -4.9492742 .4668690 .071809$ H -5.7312030 .6709420 .935850$ C $-3.620973 \quad 0.648111 \quad 1.304427$ H $-2.8274720 .386674-1.340507$ O $\quad-5.2146882 .117137-2.942263$ H $-3.1742082 .226677-2.908505$
H $-4.2825513 .936410-1.353106$

O $-2.6773153 .011352-0.482322$

H -4.7948363 .0585230 .981134$

O $-6.2929052 .719574-0.349393$

C -3.825462 -0.6894571 .975099$

H $-2.6695680 .596551 \quad 0.769281$

H -3.5090091 .4119282 .082837$

H -5.272765 $3.039905-3.243406$

H $-2.1007403 .387390-1.168008$

H $-6.4777192 .147139-1.113665$

O $-4.163580-0.5080303 .283283$

O $\quad-3.727141-1.7631851 .395985$

C $-4.438233-1.7005404 .024490$

H -4.835188-1.409449 5.000595

H $-5.187108-2.3120043 .511646$

$\mathrm{H}-3.515225-2.2660514 .175823$

SCF Energy $(B 3 L Y P / 6-31 G * * / / M M F F)=-3245.90885054$

0800299

MM̄FF Geometry

C $2.532550-2.1946933 .327676$

C $3.406911-1.2439403 .691347$

C $4.679355-0.8637962 .966610$

O 4.6781050 .5596112 .664090

C $5.043342-1.6877001 .711211$

C $6.443174-1.4292481 .189740$

C $6.734711-0.8253670 .021288$

C $7.560486-1.9747002 .044300$

C $5.800938-0.211672-0.985272$

C $5.645398-1.083774-2.239590$

C $4.490278-2.090839-2.117901$

C $4.535098-3.104793-3.260926$

C $3.328742-4.052648-3.229835$

O $3.269245-1.344073-2.174377$

O $3.328474-4.757259-4.480508$

C $2.040106-3.208864-3.149548$

C $3.465542-5.111230-2.129202$

O $0.894778-4.039832-2.972939$

C $2.073203-2.123277-2.045252$

C $0.867344-1.168515-2.234735$

O $1.991396-2.749692-0.765036$

C 3.7210391 .0741731 .847089

O $2.771763 \quad 0.485327 \quad 1.355650$

C 4.0452032 .4976061 .612537

C 3.5564673 .0900900 .513273

C 3.7536824 .4814970 .108304

C 4.4715695 .3968491 .063499

C $0.8418790 .026836-1.274470$

C $-0.2136791 .052370-1.656171$

C $-1.0157783 .328753-1.015683$

O $-1.0016590 .895405-2.584580$

C $-0.2720814 .350239-1.817365$

C $0.0301455 .575228-1.360174$

C $0.7819396 .605738-2.155073$

C $2.0569667 .051007-1.470353$

C $2.0857138 .172476-0.730752$

C $3.3152276 .243734-1.705874$

C $3.2558264 .878392-1.081214$

N $-0.1670622 .166581-0.829118$

H $2.705666-2.7824292 .430898$

H $3.203482-0.6734784 .596034$

H $5.479108-0.9924513 .705783$

H $4.305609-1.5123350 .923203$

H $4.978297-2.7596411 .940894$

H $7.785703-0.737874-0.254535$

H $8.538835-1.8803771 .560731$

H $7.614583-1.4408842 .997574$

H $7.399761-3.0385782 .247661$

H $4.8274150 .033266-0.554718$

H $6.2420030 .748892-1.281454$

H $5.438977-0.416542-3.086819$

H $6.586503-1.603505-2.457789$

H $4.559262-2.607324-1.155169$

H $5.471409-3.676538-3.238610$

H $4.533840-2.570942-4.221895$

H $2.603988-5.405016-4.462761$

H $1.916332-2.709941-4.121142$

H $4.362952-5.719189-2.295252$ 
H $2.622286-5.810823-2.145497$

H $3.534710-4.682531-1.127147$

H $0.834759-4.628118-3.744241$

H $\quad 0.890307-0.776924-3.260383$

H $-0.072455-1.721398-2.119881$

H $1.709915-2.091538-0.110435$

H 4.6810973 .0035312 .329080

H $2.9537072 .493018-0.169606$

H 3.9825185 .3916382 .044212

H 4.4885046 .4384900 .734150

H 5.5127855 .0804561 .188663

H $0.626667-0.309176-0.254547$

H $1.8129120 .533322-1.279864$

H -1.276124 $3.702155-0.021543$

H -1.934559 $3.040685-1.535845$

H $0.0292254 .066982-2.823167$

H $-0.2846325 .860933-0.358876$

H $\quad 0.1156267 .465790-2.301714$

H $1.0177746 .241942-3.162983$

H $2.9998538 .515214-0.257584$

H $1.1934478 .770622-0.578082$

H $4.2026396 .789354-1.365586$

H $3.4641846 .125383-2.787063$

H $2.7492654 .139677-1.703215$

H $0.5512902 .219696-0.112878$

C $0.035629-2.273873 \quad 3.240411$

O $-0.154992-0.8544903 .065237$

C $-1.553445-0.5449113 .251735$

C $-2.278546-1.8814703 .243764$

C $-1.249443-2.7858903 .888569$

H -1.626514 -0.0807734 .243685$

C -1.991779 0.4501642 .170955

H -3.224715 -1.851545 3.792128

H -2.486816 -2.207714 2.218063

C -1.513662 -4.264013 3.662332

H -1.232217 -2.585157 4.968333

C -3.516976 0.6646432 .107740

O -1.3363351 .6920542 .436877$

H $-1.636587 \quad 0.1155131 .188704$

H -3.963740 -0.2928391 .827956$

O $-\begin{array}{llll}-4.020013 & 0.989371 & 3.403273\end{array}$

C $-3.966871 \quad 1.7423301 .100330$

C $-5.492742 \quad 1.7995580 .891192$

H -3.4755341 .5729350 .135503$

O

H -5.9921722 .0313301 .840475$

H -5.6950582 .6545650 .232181$

H $-0.741080-4.8764634 .137447$

H -2.480836 -4.551075 4.087745

H -1.529444 -4.510684 2.595902

C $1.280855-2.5224824 .092215$

H -0.3789421 .5191772 .443738$

H -3.6249731 .8394623 .662953$

H -2.6180643 .0275511 .702941$

H $1.334151-3.5765024 .387136$

H $1.229603-1.9250485 .010831$

H $\quad 0.158333-2.7051742 .238150$

C $-5.791650-0.923609-1.643582$

$\begin{array}{lllll}\text { O } & -5.426331 & 0.284232 & -0.964290\end{array}$

C $\begin{array}{llll}-6.091126 & 0.523788 & 0.278801\end{array}$

C -7.6063940 .6319870 .056533$

C $-8.144847-0.614320-0.657140$

C -7.310799 -0.974802 -1.891228

H -5.294784 -0.856409 -2.620083

C $-5.241700-2.169417-0.929627$

H $-5.902650-0.3084520 .961970$

O $\quad-7.918636 \quad 1.792957-0.709990$

H $-8.100422 \quad 0.7473301 .028165$

H -9.182716 $-0.437622-0.965385$

O $-8.136886-1.7214890 .239614$

H -7.595720 -1.966763 -2.261263

O $-7.632482-0.035457-2.921857$

C $-3.726689-2.221789-0.958977$

H $-5.546570-2.2160850 .118643$

H -5.609294 -3.065096-1.444141

H -7.431706 $1.734935-1.549805$

H -8.699261 -1.488866 0.997894

H $-7.160625-0.309201-3.726948$
O $-3.316151-3.436271-0.486464$

O $-2.994301-1.319090-1.339391$

C $-1.901142-3.652247-0.489769$

H -1.699237 -4.5950360 .025043$

H -1.543989 -3.731953-1.520008

$\mathrm{H}-1.382198-2.8462010 .037736$

SCF Energy (B3LYP/6-31G**//MMFF) $=-3245.91551902$

0800300

MM̄FF Geometry

C $-2.176045-2.2688902 .557217$

C $-0.940118-2.5737682 .979851$

C $0.252477-1.6789552 .781495$

O $1.177222-2.4317011 .961191$

C $0.942457-1.3054134 .105588$

C $0.166355-0.3005564 .932486$

C 0.2670891 .0366984 .795709

C $-0.744258-0.8816875 .983257$

C 1.1540691 .8002213 .853156

C 0.3723072 .3608802 .659719

C 1.2385753 .2706641 .775359

C $0.4719503 .694600 \quad 0.521103$

C $1.3387934 .521858-0.440538$

O $2.4181412 .553765 \quad 1.401757$

O $0.6369194 .570148-1.691932$

C $2.6783883 .787039-0.670455$

C $1.477021 \quad 5.9717760 .038030$

O $3.5848134 .591000-1.421894$

C 3.3549523 .3248200 .640045

C 4.5727462 .4234620 .329399

O 3.8080284 .4754341 .359965

C $1.965525-1.7395901 .095651$

O $2.057477-0.5280060 .987191$

C $2.675048-2.7346990 .259704$

C $3.476164-2.327807-0.736291$

C $4.180274-3.197935-1.681608$

C $3.973712-4.685186-1.553285$

C 5.1107641 .6780161 .559280

C 6.5384691 .2160321 .353015

C $7.940416-0.3632080 .060680$

O 7.4634971 .6706162 .023166

C $7.928821-0.859242-1.348098$

C $8.136790-2.140943-1.683970$

C $8.144691-2.641881-3.099420$

C $7.127214-3.736123-3.331415$

C $7.485329-5.029928-3.271245$

C $5.722709-3.334727-3.721786$

C $4.966736-2.637139-2.624160$

N $\begin{array}{llll}6.666113 & 0.263742 & 0.354787\end{array}$

H -2.353697 -1.321549 2.056185

H $-0.767567-3.5239903 .480029$

H $-0.048621-0.7740002 .242906$

H $1.949177-0.9241013 .897193$

H $1.132840-2.2098054 .700072$

H -0.3433521 .6670755 .441124$

H - $-1.225131-0.1070096 .590329$

H -1.538582 -1.478298 5.527459

H $-0.174902-1.5226536 .664448$

H $1.6113852 .624607 \quad 4.414295$

H 1.9841111 .1813793 .500363

H 0.0005011 .5208232 .061624

H -0.5048132 .9210723 .007130$

H 1.5234984 .1475622 .368623

H -0.4397664 .2432030 .787969$

H $0.1349092 .795541-0.012562$

H $1.1407985 .137429-2.299453$

H $2.4657892 .898991-1.282168$

H $0.492723 \quad 6.4538510 .079635$

H $2.0721426 .566529-0.663710$

H 1.9289626 .0551181 .028730

H $3.1729184 .774428-2.282761$

H $4.2889311 .668530-0.415378$

H $5.3748213 .028761-0.111958$

H 4.5982584 .2452031 .873219

H $2.488573-3.7827770 .466307$

H $3.615177-1.258388-0.890598$

H $4.299302-5.034873-0.567248$

H $4.533893-5.266516-2.288916$ 
H $2.916653-4.940321-1.686963$ H 5.1003912 .3139222 .451141 H $4.487706 \quad 0.8055581 .787424$ H $8.085271-1.1689100 .788077$ H 8.7453860 .3666800 .196000 H $7.753684-0.124373-2.130232$ H $8.330149-2.871219-0.901271$ H $9.153602-3.017267-3.315220$ H $7.975673-1.828181-3.815627$ H $6.773762-5.824746-3.468037$ H $8.499399-5.323600-3.020522$ H $5.152024-4.197444-4.086189$ H $5.775518-2.659882-4.586182$ H $5.067968-1.551694-2.641908$ H $5.823001-0.178750-0.000501$ C -3.830334 -3.765170 1.411560 O $-4.360597-2.7292550 .556500$ C $-5.636132-3.1570430 .049560$ C $-5.687279-4.662826 \quad 0.272794$ C $-4.955489-4.7866181 .592398$ H $-6.397314-2.6836070 .681045$ C $-5.830643-2.728155-1.408472$ H $-6.712156-5.0439330 .310750$ H $-5.148922-5.209171-0.510362$ C $-4.468921-6.1950131 .887933$ H $-5.629252-4.4654342 .398367$ C $-5.663725-1.220385-1.689540$ O $-4.887546-3.419999-2.238096$ H $-6.819830-3.057093-1.748826$ H $-4.600549-0.955044-1.684075$ O $-6.128899-0.984213-3.025491$ C $-6.425679-0.287805-0.734346$ C $-6.5376331 .177192-1.195036$ H $-5.947596-0.321144 \quad 0.251545$ O $-7.759353-0.781739-0.569700$ H -7.258475 $1.248992-2.020430$ H $-6.9952691 .729885-0.363422$ H -5.313546 -6.889265 1.946487 H $-3.790136-6.556851 \quad 1.108326$ H $-3.934921-6.2317252 .842619$ C $-3.365938-3.1654492 .740621$ H -3.997565 -3.211369 -1.905895 H $-5.621347-1.566922-3.616077$ H $-8.202148-0.2183470 .087631$ H -3.128352 -3.966729 3.450111 H $-4.169371-2.5656043 .185448$ H $-2.983847-4.2144740 .875864$ C -2.992117 $2.318810-0.765352$ O $-4.2885561 .746554-0.528362$ C $-5.2214211 .856069-1.606899$ C $-5.450843 \quad 3.330243-1.987090$ C $-4.131764 \quad 4.050640-2.272304$ C $-3.1028823 .800124-1.166173$ H $-2.492113 \quad 2.2831130 .210645$ C -2.168476 $1.488309-1.764015$ H $-4.8247491 .326635-2.478211$ O $-6.142473 \quad 4.015742-0.943986$ H $-6.0923513 .369962-2.875337$ H $-4.3249225 .126729-2.360012$ O $-3.6238843 .595372-3.522672$ H $-2.1173384 .170692-1.467942$ O -3.4821914 .5332530 .001904$ C $-1.7001180 .183810-1.158058$ H $-2.740297 \quad 1.262779-2.667667$ H $-1.2651702 .030441-2.065889$ H $-5.6225413 .917185-0.127954$ H $-2.7983394 .078572-3.697473$ H $-3.5009865 .475608-0.237210$ O $-1.679556-0.788139-2.115388$ O -1.3524620 .0468890 .006571$ C $-1.215536-2.069971-1.679585$ H $-1.801429-2.421809-0.825829$ H $-1.342109-2.775909-2.504641$ H $-0.153882-2.014938-1.422598$

SCF Energy (B3LYP/6-31G*//MMFF) $=-3245.91777217$

0800301

MM̄FF Geometry
C $-2.709575 \quad 4.329968 \quad 0.163731$

C $-3.738818 \quad 3.4873800 .331604$

C $-4.2007392 .975941 \quad 1.671344$

O -3.8149001 .5832161 .747713$

C $-5.733163 \quad 3.0765601 .770164$

C $-6.3043592 .484253 \quad 3.044485$

C $-6.994468 \quad 1.3271003 .111913$

C $-6.0711593 .297071 \quad 4.291638$

C $-7.323120 \quad 0.3894531 .982833$

C $-6.495780-0.8990782 .072522$

C $-6.418974-1.6279060 .722403$

C $-5.699463-2.968756 \quad 0.877652$

C $-5.465037-3.663716-0.471702$

O $-5.694532-0.781570-0.178604$

O $-4.511010-4.717304-0.264966$

C $-4.819646-2.658514-1.449638$

C $-6.741081-4.331916-0.996695$

O $-4.723781-3.213874-2.758785$

C $-5.550868-1.302301-1.506862$

C $-4.792510-0.239956-2.342814$

O $-6.830537-1.485797-2.124906$

C -2.550102 $1.320612 \quad 2.182876$

O -1.7310412 .1280592 .593062$

C $-2.309354-0.1314072 .033523$

C $-1.074005-0.618642 \quad 2.222914$

C $-0.662903-2.0171192 .081431$

C $-1.703809-3.0085251 .633705$

C $-3.4006440 .140707-1.826117$

C $-2.258680-0.638001-2.456833$

C $0.197794-1.005652-2.227486$

O $-2.392813-1.342164-3.453656$

C $0.408634-2.310027-1.523809$

C $1.550026-2.635185-0.897342$

C $1.795945-3.959704-0.232227$

C $2.291654-3.8179001 .190000$

C $3.603737-3.8765091 .472833$

C $1.263041-3.7004012 .291401$

C $0.621049-2.3419502 .340474$

N $-1.056596-0.416509-1.795830$

H $-2.172507 \quad 4.706504 \quad 1.030283$

H $-4.2410623 .089322-0.547626$

H -3.7442713 .5399922 .494213$

H $-6.1810282 .603103 \quad 0.891229$

H $-6.036445 \quad 4.130763 \quad 1.710182$

H -7.3598451 .0008774 .084762$

H -6.5632012 .8632185 .168763$

H $-5.001748 \quad 3.3628954 .514168$

H -6.4644554 .3107694 .163023$

H -8.3896230 .1379672 .037199$

H -7.1890760 .8669541 .010293$

H $-5.478316-0.6565042 .402516$

H $-6.934660-1.5567952 .833062$

H $-7.436611-1.7770570 .342585$

H -6.250902 -3.628137 1.559546

H $-4.724193-2.8019641 .352557$

H $-4.870625-5.3195670 .408617$

H -3.792432 - $2.493211-1.103807$

H -7.093688 $-5.091999-0.289098$

H $-6.551813-4.863164-1.935882$

H -7.559830 -3.627283-1.156032

H $-4.238556-4.053336-2.682558$

H $-4.764676-0.538655-3.397582$

H $-5.3955930 .679414-2.318115$

H $-7.134748-0.628558-2.466095$

H $-3.146343-0.755341 \quad 1.744970$

H $-0.2800690 .072412 \quad 2.503811$

H -2.484727 -3.1117302 .394612$

H $-1.306307-4.006928 \quad 1.441938$

H -2.164746 -2.6831340 .695141$

H $-3.2190401 .192755-2.078790$

H $-3.3486980 .052286-0.737838$

H $0.989090-0.282625-2.007089$

H $0.166047-1.171587-3.309071$

H $-0.403307-3.032866-1.557428$

H $2.372595-1.924598-0.893332$

H $2.541322-4.498810-0.831527$

H $0.897154-4.588356-0.244927$

H $3.967994-3.8042112 .492194$ 
H $4.343893-3.993830 \quad 0.688904$ H $1.735470-3.8687113 .268624$ H $\quad 0.538620-4.5135502 .192677$ H $1.305140-1.5499602 .648779$ H $-1.0495050 .143604-0.948319$ C -1.054600 $3.824454-1.633639$ O $0.0964424 .030915-0.793870$ C $1.2889683 .936635-1.604438$ C $0.8268513 .506335-2.990375$ C $-0.5693554 .091406-3.054656$ H $1.7081124 .949791-1.636415$ C $2.2718582 .971087-0.927550$ H $1.4832503 .875986-3.783956$ H $0.7855872 .412207-3.065778$ C -1.435292 $3.465574-4.134542$ H $-0.4920315 .172685-3.229815$ C $3.5476482 .720774-1.755942$ $\begin{array}{lllll}\text { O } & 2.632218 & 3.542953 & 0.332499\end{array}$ H $1.7670292 .021655-0.713494$ H $3.2388892 .369207-2.747708$ O $4.2498173 .944923-1.962702$ C $4.5134321 .653046-1.203131$ C 5.1123751 .9498610 .185006 H $5.3386731 .557698-1.920496$ O $3.8163350 .409108-1.182270$ H $5.4548892 .989116 \quad 0.228322$ H 4.3340451 .8007840 .942391 H - $0.9921063 .625667-5.122855$ H -1.5441392.385836 -3.986213 H -2.436638 $3.907159-4.138962$ C $-2.1992144 .736674-1.189867$ H $1.810193 \quad 3.6814780 .833921$ H $4.3536524 .377336-1.098061$ H $4.447711-0.280149-0.915673$ H -3.015386 4.705228 -1.920832 H -1.860399 $5.777731-1.127133$ H $-1.3418402 .770584-1.523020$ C $6.789266-1.3153750 .919176$ O $5.802083-0.306194 \quad 0.673183$ C $6.301634 \quad 1.026103 \quad 0.525655$ C $7.0195501 .465973 \quad 1.811053$ C $8.121209 \quad 0.472806 \quad 2.195647$ C $7.621102-0.9762452 .166595$ H $6.212098-2.220797 \quad 1.141933$

C $7.636392-1.596635-0.326547$ H $7.0058361 .060324-0.312956$ O $6.072895 \quad 1.5644812 .873215$ H $7.4480112 .463407 \quad 1.654477$ H 8.4862860 .7127873 .201678 O $\quad 9.2085680 .6307691 .288904$ H $8.465500-1.6706552 .241197$ O $6.807881-1.2032933 .322338$ C $6.770285-2.066558-1.470984$ H $8.181578-0.715544-0.677047$ H $8.391028-2.365957-0.125196$ H $6.547273 \quad 1.882522 \quad 3.660220$ H 9.9108950 .0167291 .563228 H $6.014433-0.6464453 .241679$

O $6.424196-3.372895-1.292044$

O $6.428792-1.349864-2.402993$

C $5.565516-3.922972-2.295959$

H $4.637360-3.346923-2.360664$ H $6.078535-3.939951-3.262178$ H $5.320757-4.948992-2.008797$

SCF Energy (B3LYP/6-31G**//MMFF) $=-3245.92086754$

0800302

MM̄FF Geometry

C 4.0436941 .7712162 .074046

C 5.1946991 .8789591 .393654

C $5.4079622 .713606 \quad 0.148267$

O $4.2232223 .513534-0.086763$

C $5.6388331 .829666-1.095319$

C $6.870565 \quad 0.950921-1.027074$

C $6.868498-0.347727-0.663069$

C $8.1601401 .633576-1.400727$

C $5.686237-1.167736-0.223680$

C $5.664931-2.551655-0.886255$
C $4.373856-3.306653-0.537719$

C $4.458878-4.771106-0.966496$

C $3.137328-5.512844-0.713798$

O $3.300741-2.659772-1.229727$

O $3.194965-6.766808-1.410362$

C $1.983878-4.700607-1.337849$

C $2.941390-5.8481580 .769503$

O $0.719596-5.271001-1.006183$

C $2.002418-3.200699-0.959102$

C $0.978828-2.433617-1.829371$

$\begin{array}{lllll}\text { O } & 1.650304 & -3.057890 & 0.416691\end{array}$

C $4.3315454 .681691-0.762574$

O $5.3509465 .151491-1.247702$

C $3.0162645 .363850-0.867969$

C $1.9067354 .941976-0.238714$

C $0.5787565 .563084-0.288629$

C $0.4090336 .768942-1.174369$

C $1.061107-0.905640-1.706697$

C $0.116042-0.211105-2.672484$

C $-0.6414052 .071229-3.334498$

O $-0.603529-0.816425-3.462287$

C -1.805364 $2.503991-2.501350$

C $-2.0273413 .778609-2.149470$

C $-3.182565 \quad 4.223936-1.302450$

C $-2.823857 \quad 4.360785 \quad 0.160822$

C $-3.404727 \quad 3.576573 \quad 1.084911$

C $-1.8532995 .452517 \quad 0.564029$

C -0.4144645 .0175410 .446780$

N $0.182161 \quad 1.169488-2.551040$

H $3.1781462 .347773 \quad 1.760267$

H 6.0508381 .3026291 .736792

H 6.2624103 .3783290 .325048

H $5.7176372 .470263-1.984334$

H $4.7363741 .239806-1.296276$

H $7.822396-0.872699-0.634081$

H $9.0215330 .960378-1.337079$

H $8.1076592 .006524-2.428813$

H $8.3524012 .479311-0.732956$

H $5.749597-1.3008720 .863568$

H $4.741728-0.655085-0.421380$

H $5.740717-2.434036-1.975102$

H $6.537982-3.125037-0.550633$

H $4.205630-3.2349560 .543425$

H $5.287155-5.279340-0.457082$

H $4.688278-4.825218-2.040027$

H $3.963011-7.260685-1.075487$

H $2.082928-4.782412-2.429516$

H $3.764746-6.4752191 .132011$

H $2.028668-6.4340170 .924573$

H $2.895630-4.9632891 .407592$

H $0.744774-6.206023-1.273102$

H $\quad 1.147020-2.691057-2.883534$

H $-0.040617-2.751240-1.574826$

H $1.315105-2.1600910 .564734$

H $3.0174416 .254516-1.488296$

H 1.9631134 .0516440 .384605

H $1.0478327 .590162-0.831041$

H -0.612354 $7.153251-1.200186$

H $0.674696 \quad 6.525995-2.209346$

H $0.803987-0.585519-0.692348$

H $2.076550-0.558933-1.930050$

H $-0.996777 \quad 1.567408-4.238824$

H $-0.0079842 .913463-3.630788$

H $-2.4944901 .733541-2.166370$

H $-1.3422494 .550437-2.489833$

H $-4.0215893 .526707-1.429620$

H -3.547408 $5.187863-1.678430$

H -3.1738543 .6812452 .139780$

H -4.1182102 .8071570 .808144$

H -2.023546 $5.729023 \quad 1.613230$

H -2.079116 $6.356962-0.006616$

H -0.1792574 .1473851 .062489$

H $0.7742761 .575488-1.831856$

C $2.689643-0.1286032 .933358$

$\begin{array}{lllll}\text { O } & 1.468281 & 0.607731 & 2.737054\end{array}$

C $0.377298-0.1020163 .355748$

C $0.937804-1.4520453 .776824$

C $2.390761-1.1148574 .058320$ 
H $\quad 0.0989790 .4894624 .236589$

C $-0.780008-0.1606422 .353482$

H $0.417983-1.8678264 .645263$

H $0.863079-2.1792812 .960002$

C $3.303788-2.3285244 .070365$

H $2.450857-0.6064345 .029885$

C -1.998598 -0.9682722 .826350$

O -1.1834741 .1847252 .073996$

H $-0.404129-0.5665221 .409421$

H -1.725824 -2.023701 2.942704

O $-2.365191-0.495594 \quad 4.129081$

C $-3.236854-0.8411011 .915216$

C $-2.985844-1.2688450 .457157$

H $-3.611140 \quad 0.188097 \quad 1.946823$

O $-4.271527-1.6565412 .476839$

H $-2.100237-0.7639320 .059344$

H -2.798553 -2.3497380 .442248$

H $2.999549-3.031044 \quad 4.853124$

H $3.277575-2.8597843 .113119$

H $4.340354-2.0351994 .263816$

C 3.8232170 .8528603 .244270

H -1.5469201 .5589542 .894855$

H -3.141943 -1.006743 4.414612

H $-3.980769-2.5831662 .427973$

H 4.7403440 .3065403 .490305

H $3.558758 \quad 1.4717974 .110013$

H $2.895305-0.6626271 .995806$

C $-6.525869-1.424686-0.768380$

O $-5.313904-1.685102-0.048685$

C $-4.170817-0.941416-0.475680$

C $-3.847206-1.269206-1.941616$

C $-5.063176-1.038885-2.845286$

C $-6.331075-1.681600-2.272386$

H $-7.239990-2.167394-0.389459$

C -7.089523 -0.033598 -0.456519

H $-4.377823 \quad 0.130389-0.381121$

O $-3.427453-2.628486-2.044072$

H -3.011694 -0.641119-2.266202

H -4.868354 -1.459813 -3.839295

O $\quad-5.283508 \quad 0.359978-3.001515$

H $-7.212626-1.329749-2.820168$

O $-6.262295-3.095969-2.479454$

C -7.4304660 .0864811 .009989$

H $-6.4045310 .775888-0.724283$

H -8.012034 $0.148100-1.020481$

H $-3.177157-2.788418-2.970101$

H $-4.4854890 .739283-3.407297$

H $-5.523003-3.438503-1.948170$

O $-6.476936 \quad 0.8205501 .650911$

O $-8.412737-0.4306651 .526099$

C $-6.6634430 .980373 \quad 3.060370$

H -7.584964 1.5357443 .258357

H -6.6857290 .0042793 .554350$

H -5.817182 1.5512423 .451848

SCF Energy (B3LYP/6-31G**//MMFF)= -3245.91852121

0800303

MM̄MF Geometry

C -1.094127 3.9655800 .495976

C $-2.275394 \quad 4.573558 \quad 0.298770$

C $-3.640670 \quad 4.054196 \quad 0.692277$

O -3.5939602 .6363390 .963924$

C $-4.6410474 .268771-0.457787$

C $-6.0717413 .955665-0.062737$

C $-6.7445112 .848184-0.432730$

C -6.7591465 .0160300 .758879$

C $-6.2257631 .693434-1.242953$

C $-6.6577270 .346402-0.644447$

C $-6.118373-0.828372-1.471676$

C $-6.698974-2.156084-0.985157$

C $-6.107702-3.347464-1.754470$

O $-4.694217-0.845694-1.334007$

O $-6.453832-4.546492-1.044911$

C $-4.569039-3.238775-1.736658$

C $-6.712842-3.482397-3.156718$

O $-3.976357-4.237150-2.564578$

C -4.037236 -1.839552 -2.130498

C $-2.521770-1.761500-1.838039$
O $-4.253209-1.623097-3.525479$

C -3.517684 2.2369402 .260562

O $-3.5014932 .943782 \quad 3.254802$

C -3.4017670 .7626002 .255085$

C $-2.8397770 .152316 \quad 3.308270$

C $-2.574148-1.2815493 .433155$

C $-3.185638-2.1987612 .408250$

C $-1.921983-0.353718-1.953803$

C $-0.504634-0.350605-1.417284$

C $0.763092-0.489770 \quad 0.728182$

O $0.469973-0.379887-2.164938$

C $0.723172-1.7893891 .468864$

C $1.024970-1.9117862 .770747$

C $0.995030-3.2198693 .510565$

C $0.217739-3.1515894 .808473$

C $0.848199-3.2101565 .993979$

C -1.295075 -3.091755 4.734558

C $-1.788931-1.6997984 .447474$

N $-0.463107-0.351328-0.031606$

H -1.0515773 .0056501 .001845$

H -2.267965 $5.556506-0.170161$

H -3.9744634 .6128231 .574915$

H $-4.3399253 .670997-1.325789$

H $-4.6057925 .312077-0.799798$

H -7.786159 $2.758223-0.126677$

H -7.8076354 .7716720 .960475$

H -6.260124 5.1390251 .724976

H $-6.741605 \quad 5.9758560 .232350$

H $-6.6270791 .780738-2.259632$

H $-5.135397 \quad 1.714329-1.321623$

H -6.2852040 .2761030 .385606$

H $-7.7531870 .298327-0.605649$

H $-6.380484-0.660325-2.522938$

H -7.793400 -2.155524 -1.061706

H $-6.472371-2.2846400 .082619$

H $-7.423824-4.591604-0.988132$

H $-4.241598-3.454846-0.709742$

H -7.799847 -3.613485 -3.093574

H $-6.333314-4.375069-3.666280$

H -6.521340 -2.615116 -3.792025

H $-4.324268-5.097822-2.274486$

H $-2.339227-2.131562-0.822790$

H -1.973864 -2.427035 -2.517603

H -3.623954 -0.955617 -3.841384

H $-3.7250010 .231008 \quad 1.367400$

H $-2.497410 \quad 0.765302 \quad 4.141232$

H -4.258799 -2.002276 2.302631

H $-3.098496-3.2566742 .664548$

H -2.707772 -2.057204 1.435719

H -1.896670 -0.016921 -2.995519

H $-2.5057250 .383725-1.391290$

H 0.8208570 .3659461 .407012

H $1.622416-0.4733710 .052624$

H $0.446324-2.6760680 .902960$

H $1.334400-1.0311583 .329368$

H $2.034855-3.5093383 .710358$

H $\quad 0.575597-4.0194072 .887136$

H $0.297334-3.1862986 .928433$

H $1.929236-3.2779316 .057150$

H -1.732085 -3.405962 5.691355

H -1.634128 -3.828555 4.001731

H -1.418662 -0.954144 5.153203

H -1.334061 -0.3009520 .487949$

C $1.2340453 .499260-0.343369$

O $1.8231812 .936880 \quad 0.850833$

C 3.2614512 .9731760 .724086

C $3.5256173 .135234-0.762248$

C $2.3972254 .063551-1.160201$

H 3.5898383 .8653351 .272865

C 3.8583371 .7125321 .357749

H $4.5147903 .551732-0.973112$

H $3.4342802 .173233-1.281921$

C $2.1346054 .095978-2.655975$

H $2.6391325 .078913-0.817824$

C 5.3633091 .5331481 .099316

O $3.638267 \quad 1.7783262 .770295$

H 3.3122180 .8367231 .001436

H 5.5519101 .4290220 .027758 
O $\quad 6.0474032 .731743 \quad 1.491792$

C 6.0183090 .3585861 .849019

C $5.350773-1.0190541 .693772$

H 6.0636110 .5942172 .919661

O $7.378510 \quad 0.242011 \quad 1.417378$

H $5.885097-1.7125752 .356938$

H $4.320281-0.9529132 .059902$

H $1.2841984 .744215-2.889487$

H $3.0084904 .481127-3.190891$

H $1.9141753 .096725-3.046598$

C 0.2138914 .5695270 .058407

H $2.680611 \quad 1.8777732 .909385$

H 5.8536232 .8847952 .432626

H 7.7940231 .1144021 .527986

H $0.0361155 .248800-0.783660$

H $\quad 0.6118195 .1580900 .894001$

H $0.7497482 .674283-0.881305$

C $4.406211-1.270695-1.931732$

O $4.508763-0.855163-0.564246$

C $5.375649-1.6251190 .271561$

C $4.946716-3.1065040 .285772$

C $4.793903-3.668103-1.129744$

C $3.965026-2.740902-2.023302$

H $3.602097-0.651531-2.348463$

C $5.688703-0.985356-2.723922$

H $6.397340-1.557398-0.115646$

O $3.702728-3.2692920 .962772$

H $5.697237-3.692340 \quad 0.830879$

H $4.307121-4.649647-1.072791$

O $6.090590-3.857686-1.687168$

H $4.013075-3.079457-3.064805$

O $2.597277-2.837883-1.617400$

C $5.8915830 .492531-2.956053$

H $6.588248-1.356425-2.225273$

H $5.659161-1.469076-3.707961$

H $3.863947-3.1517391 .913868$

H $5.975622-4.241642-2.573126$

H $2.071954-2.271678-2.208336$

O $5.0321850 .936660-3.917088$

O $6.7281691 .165167-2.367747$

C $5.1629722 .315832-4.275213$

H $5.1539402 .953911-3.387105$

H $6.0860272 .463141-4.843443$

H $4.3126282 .584894-4.907185$

SCF Energy (B3LYP/6-31G**//MMFF) $=-3245.91541402$

0800304

MM̄FF Geometry

C -3.767872 4.782261 -0.818036

C $-4.265593 \quad 3.540270-0.719947$

C -4.7792942 .9377550 .561352$

$\begin{array}{lllll}\text { O } & -4.048959 & 1.710824 & 0.804483\end{array}$

C -6.2719792 .5972470 .414058$

C -6.8799032 .0046931 .670673$

C -7.1790250 .7009491 .840055$

C -7.1846782 .9960402 .764289$

C $-6.947713-0.4292650 .875573$

C $-6.069784-1.5215101 .500573$

C -5.686189-2.600062 0.476577

C $-4.890529-3.7212241 .146869$

C $-4.389195-4.7603630 .133952$

O $-4.893742-1.977208-0.540627$

O $-3.410314-5.563993 \quad 0.810546$

C $-3.681655-4.028463-1.028393$

C $-5.510828-5.716439-0.288248$

O $-3.324921-4.948088-2.057233$

C $-4.497654-2.852350-1.605467$

C $-3.706378-2.006719-2.634603$

O $-5.642297-3.377443-2.290298$

C -2.8472341 .8193751 .436671$

O $-2.3071502 .846676 \quad 1.815242$

C $-2.297510 \quad 0.4553691 .607468$

C -1.0703950 .2899832 .124443$

C $-0.423114-0.9893852 .429579$

C $-1.178310-2.2502382 .102840$

C $-2.440765-1.324441-2.103382$

C -1.164837 -2.121120 -2.319712

C $1.259720-2.089800-1.736546$
O $-1.113456-3.143608-2.997118$

C $1.529857-2.818320-0.458148$

C $2.553299-2.5204080 .355545$

C $2.875186-3.2642731 .616875$

C $2.883734-2.3706702 .835905$

C $4.032707-1.8763483 .327135$

C $1.574085-2.1467433 .554436$

C $0.791155-0.9774213 .019417$

N $-0.077909-1.530638-1.688736$

H -3.7438135 .4196560 .061527$

H $-4.2806372 .902837-1.601412$

H -4.6404263 .6236541 .406400$

H $-6.4139461 .923669-0.437765$

H -6.8343843 .5049210 .154848$

H -7.6401050 .3997222 .780096$

H -7.6766532 .5290553 .624270$

H $-6.2645703 .464246 \quad 3.127162$

H -7.8513603 .7803002 .391089$

H -7.919895 -0.857465 0.603070

H $-6.490629-0.081728-0.053157$

H $-5.158883-1.060478 \quad 1.901694$

H $-6.601591-1.9808272 .343092$

H -6.604934 -2.996014 0.028242

H $-5.484578-4.2050001 .932308$

H -4.019875 -3.289835 1.657995

H $-3.029897-6.1764790 .157340$

H -2.738658 -3.644784 -0.622213

H $-5.908984-6.2451510 .586341$

H $-5.136833-6.495916-0.961050$

H -6.345192 -5.209273 -0.776986

H $-4.128219-5.149385-2.566702$

H -3.497253 -2.602554 -3.531384

H $-4.375808-1.202266-2.972330$

H $-5.962378-2.706046-2.915423$

H $-2.930959-0.3785611 .330654$

H $-0.493027 \quad 1.1764592 .384020$

H -0.587045 -3.159162 2.233014

H -1.497313 -2.250317 1.055441

H -2.063157 -2.339562 2.741489

H -2.304306 -0.379977 -2.646292

H $-2.541948-1.079106-1.042470$

H $1.954095-1.259244-1.896275$

H $1.347489-2.780245-2.581242$

H $\quad 0.866840-3.641794-0.204140$

H $3.228361-1.7151030 .080581$

H $3.862589-3.7267021 .486419$

H $2.181525-4.0987531 .777763$

H $4.047471-1.2650844 .223319$

H $4.983837-2.0697542 .843534$

H $1.770831-1.9379104 .615426$

H $0.998603-3.0768123 .568216$

H $1.263896-0.0133623 .212776$

H $-0.224592-0.704589-1.115334$

C -1.678152 $5.405324-2.119934$

O $-1.1384974 .089014-1.877781$

C $-0.1339714 .192417-0.846872$

C $0.3037545 .646535-0.866507$

C -1.024567 $6.342144-1.101248$

H -0.6432693 .9655130 .097291$

C $0.9582143 .154652-1.110007$

H $\quad 0.787600 \quad 5.957790 \quad 0.063768$

H $0.9920705 .836027-1.699276$

C $-0.8866797 .774442-1.587317$

H -1.583203 $6.345188-0.157496$

C $2.1785113 .288423-0.177861$

O $0.3650371 .862586-0.944703$

H $1.2896603 .212987-2.153689$

H $2.6468874 .261878-0.362861$

O 1.7506873 .2885631 .184231

C $3.2435642 .191902-0.380517$

C 4.5179802 .4741170 .438945

H $3.5044542 .133724-1.443937$

$\begin{array}{lllll}\text { O } & 2.729423 & 0.924422 & 0.017063\end{array}$

H 4.8341463 .5101390 .269269

H 4.2868292 .3524951 .504705

H $-0.3669508 .385134-0.841836$

H $-0.3177077 .829017-2.521531$

H $-1.8705598 .220486-1.764501$ 
C $-3.2092635 .347241-2.095876$ H $-0.4032181 .816604-1.539527$ H 1.3566252 .4175651 .363737 H $1.9509430 .733700-0.531942$ H -3.543930 4.727641 -2.938051 H -3.628379 $6.346779-2.257255$ H -1.362019 $5.679761-3.134671$ C $6.269779-0.8112280 .089374$ $\begin{array}{lllll}\text { O } & 5.341135 & 0.204924 & 0.486471\end{array}$ C 5.6782631 .5270090 .059965 C 7.0024461 .9521610 .713125 C 8.1270770 .9510310 .417615 C $7.683749-0.5058530 .612796$ H $5.917210-1.7142070 .602922$ C $6.177098-1.068194-1.417770$ H $5.7837301 .539496-1.030977$ O 6.8344432 .0627962 .126381 H 7.2841692 .9445600 .342706 H 8.9655521 .1583391 .093599 O $8.6245361 .132310-0.905265$ H $8.403651-1.1955430 .157992$

O $7.669077-0.7833302 .019210$ C $6.502152-2.504360-1.744475$ H $5.168316-0.850969-1.791799$ H $\quad 6.845073-0.429269-2.000878$ H 6.5222881 .2026472 .456302 H $7.8854361 .349923-1.495225$ H $8.577442-0.6736352 .348748$ O $5.386256-3.275283-1.602583$ O $7.617067-2.895846-2.063279$ C $5.564547-4.672281-1.854029$ H $6.290586-5.093376-1.152173$ H $4.602562-5.169686-1.704101$ H $5.886203-4.833227-2.887319$ SCF Energy (B3LYP/6-31G $\left.{ }^{* *} / / \mathrm{MMFF}\right)=-3245.92052333$

0800305

MM̄FF Geometry

C $1.641331-3.063856-0.381285$

C $0.726169-3.548045-1.232743$

C $-0.154234-2.669329-2.078890$

O $-1.528764-2.929025-1.708709$

C $-0.021501-2.982525-3.579314$

C $1.357813-2.737320-4.152334$

C $1.861696-1.516407-4.417707$

C $2.167150-3.973407-4.444565$

C $1.187036-0.189851-4.207726$

C $1.9161030 .637233-3.141166$

C $1.2517082 .005180-2.931806$

C $2.0791852 .863517-1.973571$

C $1.4014214 .207772-1.672813$

O $-0.0573561 .792085-2.389985$

O $2.1001844 .785716-0.561540$

C $-0.0559433 .938530-1.242264$

C $1.5558195 .192125-2.838239$

O $-0.7794415 .158016-1.091005$

C $-0.8147352 .996523-2.206963$

C $-2.1877892 .617489-1.603420$

O $-1.033936 \quad 3.677832-3.446358$

C $-2.063426-2.155654-0.724797$

O $-1.523327-1.231703-0.137672$

C $-3.433717-2.649870-0.463730$

C $-4.179014-2.0562650 .480047$

C $-5.525121-2.453127 \quad 0.895174$

C $-6.156733-3.634297 \quad 0.206523$

C $-2.9152591 .500258-2.366229$

C $-4.4119281 .733657-2.398374$

C $-6.3938412 .037197-0.957938$

O $-4.9979421 .985268-3.449774$

C -6.9075561 .4982450 .337540$

C $-8.001542 \quad 0.726987 \quad 0.428352$

C $-8.573996 \quad 0.2195501 .721530$

C $-8.613513-1.2907461 .781413$

C $-9.658730-1.9726221 .283050$

C $-7.483742-2.0061162 .485180$

C $-6.134612-1.7487561 .871111$

N $-5.0120751 .638968-1.150749$

H $1.746142-1.986882-0.272662$
H $\quad 0.602218-4.623453-1.333430$

H $\quad 0.072200-1.609318-1.915307$

H $-0.762358-2.393962-4.137175$

H $-0.325130-4.022000-3.767838$

H $2.864450-1.452393-4.837856$

H $3.162581-3.736735-4.835162$

H $2.304132-4.569040-3.537089$

H $1.656408-4.591012-5.190562$

H $1.1977410 .351516-5.161778$

H $\quad 0.133932-0.308882-3.936361$

H $1.9174050 .081099-2.194658$

H $2.9625490 .778310-3.440057$

H $1.1634672 .498317-3.907061$

H $3.0911713 .023798-2.365494$

H $2.2064682 .321660-1.026759$

H $1.7129695 .658507-0.380181$

H $-0.0249053 .467415-0.249848$

H $2.6166195 .404194-3.018461$

H $1.0926606 .157472-2.605544$

H $1.1287804 .823651-3.773061$

H $-0.3430035 .675174-0.393535$

H -2.049253 $2.270253-0.571025$

H -2.820766 $3.513122-1.562322$

H -1.861138 $3.364554-3.844959$

H -3.781088 -3.502145 -1.036457

H $-3.759047-1.2059261 .016293$

H -6.225383 -3.458912 -0.872969

H -7.172271 -3.843764 0.551196

H $-5.566962-4.5412860 .379387$

H -2.573956 $1.423375-3.404468$

H $-2.7192500 .528214-1.902227$

H $-6.9865841 .700707-1.815581$

H $-6.4195413 .132075-0.941682$

H -6.3760831 .7810581 .242834$

H $-8.5410430 .466048-0.479720$

H $-9.5921750 .619027 \quad 1.819279$

H -8.0240670 .6119012 .585849$

H -9.710564 -3.054565 1.344053

H $-10.485308-1.4620770 .799776$

H -7.672385 -3.084348 2.549525

H -7.453287 -1.668173 3.529436

H $-5.610498-0.8975172 .307123$

H $-4.411803 \quad 1.585241-0.332426$

C $4.023143-3.5834970 .319630$

O $4.259708-2.2254170 .737251$

C $5.231017-1.635025-0.149919$

C $5.829159-2.790556-0.945088$

C $4.623544-3.704199-1.079994$

H $4.675609-0.994368-0.844869$

C $6.230896-0.7951150 .652553$

H $6.234004-2.466376-1.908562$

H $6.629878-3.294809-0.391705$

C $4.970800-5.125193-1.484472$

H $3.949949-3.273659-1.831202$

C 5.5519550 .2785341 .532127

O $6.963700-1.6761031 .511987$

H $6.961036-0.338004-0.025385$

H $4.936636-0.2103462 .295343$

O $6.569596 \quad 1.0026802 .232791$

C $4.707446 \quad 1.2911830 .736353$

C 4.0559612 .3856661 .603026

H $3.924806 \quad 0.7689630 .177752$

O $5.5521731 .925416-0.228279$

H 4.8200512 .9175462 .182700

H 3.6339693 .1325870 .920453

H $5.459017-5.137249-2.464423$

H $5.650308-5.593477-0.764595$

H $4.068978-5.742223-1.548817$

C $2.538242-3.9234890 .462685$

H $7.723768-1.1794871 .859049$

H $\begin{array}{llll}6.965766 & 0.396617 & 2.881117\end{array}$

H $4.9957352 .516569-0.763333$

H $2.244259-3.7678491 .507932$

H $2.383527-4.9852890 .238900$

H $4.583980-4.2166731 .020211$

C 0.9006550 .5772812 .442875

O $1.964033 \quad 1.212747 \quad 1.720540$

C 2.9503161 .8593862 .533656 
C 2.3001272 .9841203 .354806

C 1.1247372 .4545204 .185044

C $0.174963 \quad 1.587965 \quad 3.348467$

H $0.193596 \quad 0.257528 \quad 1.666774$

C $1.413978-0.679895 \quad 3.151907$

H 3.3919291 .1274593 .217490

O 1.8351394 .0094112 .479406

H 3.0597573 .4280204 .009364

H $0.5634573 .298426 \quad 4.604278$

O $1.616910 \quad 1.683010 \quad 5.276451$

H $-0.534568 \quad 1.0621853 .996558$

O -0.5961532 .4400382 .494416$

C $0.292099-1.5984523 .565189$

H $2.096339-1.2359362 .497056$

H $2.009563-0.4515854 .040720$

H 1.4622404 .7190353 .029454

H 2.1895752 .2598975 .810133

H $-1.1006313 .046063 \quad 3.063277$

O $-0.100239-2.3638582 .508223$

O $-0.185151-1.6242864 .692343$

C $-1.153953-3.2932702 .778007$

H $-2.077163-2.7554893 .012725$

H $-1.314225-3.8957921 .880150$

H $-0.875214-3.9601813 .599607$

SCF Energy (B3LYP/6-31G*//MMFF)= -3245.89117277

0800306

MMFFF Geometry

C $-0.528073 \quad 3.280572-0.555778$

C $-0.973633 \quad 3.832022 \quad 0.584142$

C -1.3556873 .0708631 .836372$

O $-1.677271 \quad 1.7029661 .493569$

C -2.6083303 .6977142 .473191$

C -3.0401133 .0025333 .749692$

C $-4.0807112 .150142 \quad 3.838895$

C -2.240676 $3.355775 \quad 4.976624$

C -4.9656151 .6783092 .718434$

C -5.1642630 .1559592 .755357$

C $-5.948787-0.3297001 .528371$

C $-6.326721-1.8043041 .664055$

C $-7.040938-2.3224890 .406074$

O $-5.114392-0.150942 \quad 0.378515$

O $-7.081102-3.7556790 .480811$

C $-6.197896-1.957199-0.833063$

C -8.497854 -1.849869 0.337304

O $-6.889847-2.282320-2.036679$

C $-5.743922-0.478238-0.866268$

C $-4.706580-0.281460-1.996250$

O $-6.8720780 .359936-1.120204$

C $-0.791180 \quad 0.728881 \quad 1.825069$

$\begin{array}{llll}\text { O } & 0.234001 & 0.844343 & 2.475153\end{array}$

C -1.258031 -0.5163181 .180078$

C $-0.346163-1.4106340 .772765$

C $-0.621897-2.638642 \quad 0.026102$

C $-2.051738-3.096131-0.072789$

C $-3.9878381 .073461-1.975050$

C $-2.864608 \quad 1.080671-2.993613$

C $-0.5665390 .173606-3.374547$

O $-2.996272 \quad 1.607672-4.095607$

C $-0.503798-1.289166-3.686110$

C $0.594232-2.041572-3.516864$

C $0.641091-3.514617-3.811738$

C $1.217278-4.331449-2.673709$

C $2.408049-4.941540-2.800948$

C $0.382339-4.509487-1.419608$

C $0.414384-3.291530-0.538328$

$\mathrm{N}-1.7356320 .411237-2.547720$

H $-0.3853132 .205813-0.616540$

H $-1.075296 \quad 4.9146290 .626645$

H -0.5189093 .1236742 .543002$

H -3.427705 3.7079651 .745362

H -2.420621 4.7554852 .702991

H $-4.326704 \quad 1.7418274 .818243$

H -2.623060 $2.864758 \quad 5.877817$

H -1.196328 3.0520174 .855053

H -2.271476 4.4360545 .152277

H -5.9406792 .1699992 .817451$

H -4.5624751 .9600901 .742357$
H $-4.183670-0.3363572 .783431$

H $-5.699196-0.1171813 .673317$

H $-6.8489410 .287652 \quad 1.424964$

H $-6.948479-1.9682302 .552972$

H -5.417249 -2.401946 1.818734

H $-7.551401-4.0000541 .296198$

H $-5.301111-2.592286-0.811572$

H $-9.052812-2.1904541 .219662$

H $-9.013368-2.284494-0.526353$

H $-8.594368-0.7634690 .285669$

H $-7.143466-3.219624-1.981100$

H $-3.948962-1.069453-1.924115$

H -5.191264 -0.401006 -2.973868

H $-6.5629351 .196068-1.503089$

H $-2.318511-0.6219140 .985731$

H $0.706563-1.1972070 .954449$

H -2.532891 -3.0880390 .911915$

H $-2.152379-4.118205-0.445632$

H $-2.614899-2.441935-0.742604$

H $-4.6739641 .892297-2.215389$

H $-3.5630381 .290001-0.988312$

H $\quad 0.3067250 .511958-2.808104$

H $-0.6360620 .752587-4.300174$

H $-1.409983-1.753389-4.069321$

H $1.509340-1.575143-3.159241$

H $1.245405-3.650593-4.717972$

H $-0.356182-3.902686-4.054014$

H $2.818400-5.552246-2.003530$

H $3.002099-4.841592-3.703236$

H $0.762567-5.354766-0.831684$

H $-0.631882-4.783799-1.723400$

H $1.415177-2.892114-0.379461$

H $-1.7395600 .006490-1.616483$

C $1.2454814 .230711-2.122216$

O $1.9516712 .973327-2.093938$

C $3.2630843 .208933-1.540441$

C $3.4295984 .723289-1.460100$

C $2.0061335 .161221-1.180100$

H $3.2499392 .816172-0.518924$

C $4.3431302 .507630-2.367362$

H $4.1341955 .021893-0.678019$

H $3.7814305 .141367-2.410866$

C $1.7640336 .638832-1.431068$

H $1.7713714 .930547-0.133322$

C $4.2606970 .966400-2.352988$

O $4.230793 \quad 2.952733-3.724631$

H $5.3335482 .827980-2.021160$

H $3.3609090 .634064-2.884876$

$\begin{array}{llll}\text { O } & 5.381888 & 0.497946 & -3.114458\end{array}$

C $4.3036710 .348692-0.938806$

C $4.509374-1.179011-0.944574$

H $3.3669320 .585410-0.421833$

O $5.3690510 .964243-0.212886$

H $3.831299-1.636118-1.674889$

H $5.542025-1.388758-1.249611$

H $2.4031327 .248099-0.783478$

H $1.9810916 .911163-2.469319$

H $0.7230536 .904923-1.223063$

C $-0.2406604 .042646-1.817181$

H $4.9241902 .500483-4.235141$

H $5.221376-0.435442-3.333104$

H 5.3289310 .6397410 .702361

H $-0.6949043 .473154-2.637929$

H $-0.7452425 .015854-1.805464$

H $1.3310344 .603947-3.152030$

C $5.051412-1.7256202 .727189$

O $5.199071-1.2832771 .371240$

C $4.262708-1.8246440 .435648$

C $4.401698-3.353861 \quad 0.386712$

C $4.251045-3.9718131 .780655$

C $5.125887-3.2591502 .817484$

H $5.927184-1.3171913 .248336$

C $3.799772-1.1360713 .386734$

H $3.247710-1.5580010 .747175$

O $5.665599-3.722329-0.162289$

H $3.637066-3.760402-0.281138$

H $4.529776-5.0320731 .741076$

O $2.890708-3.8941072 .196159$ 
H $4.860244-3.578473 \quad 3.831940$

O $6.491318-3.6308552 .604898$

C $3.9323340 .361536 \quad 3.532376$

H $2.877149-1.3549812 .841735$

H $3.662872-1.5440964 .395350$

H $6.357927-3.3030810 .377209$

H $2.358466-4.3921951 .552584$

H $6.553122-4.5948102 .716847$

O $3.266810 \quad 0.9907322 .523211$

O $4.5719410 .903473 \quad 4.424630$

C 3.2965292 .4213582 .555556

H 4.2875292 .7764302 .259607

H $2.5583242 .792307 \quad 1.839639$

H 3.0312392 .7958973 .549061

SCF Energy (B3LYP/6-31G**//MMFF) $=-3245.91316479$

0800307

MM̄FF Geometry

C -3.1751672682748 2.042546

C -2.8479431 .7618772 .961974$

C $-3.5161630 .432271 \quad 3.203819$

O $-2.596060-0.5684542 .699378$

C $-4.869717 \quad 0.2234552 .499190$

C -5.637780 -0.969595 3.033297

C $-5.732240-2.1643692 .418479$

C $-6.372071-0.7310724 .328436$

C $-5.073023-2.610177 \quad 1.142744$

C $-6.083755-2.8013850 .003409$

C $-6.543612-1.474166-0.619617$

C $-7.667070-1.712598-1.630050$

C $-8.069915-0.417527-2.350383$

O $-5.411930-0.894445-1.276762$

O $-8.889070-0.773926-3.474304$

C $-6.7996810 .253893-2.911089$

C $-8.9249300 .492071-1.460280$

O $-7.101627 \quad 1.531796-3.467463$

C $-5.6558740 .383491-1.877001$

C $-4.3563290 .823266-2.594213$

O $-6.013344 \quad 1.364378-0.902995$

C -2.234205 -1.596184 3.505062

O $-2.563804-1.7623254 .671454$

C -1.365011-2.565950 2.794550

C $-1.029380-2.471173 \quad 1.497624$

C $-0.213649-3.4286570 .742902$

C $0.353052-4.6073051 .490888$

C $-3.0988710 .787078-1.713556$

C -1.840873 $1.075046-2.516999$

C $0.6414920 .945623-2.341833$

O $-1.8614801 .458425-3.683754$

C $1.025768-0.404091-2.859252$

C $1.983463-1.171697-2.317256$

C $2.348547-2.528744-2.845497$

C $2.136554-3.635773-1.835583$

C $3.184689-4.270793-1.284989$

C $0.721297-4.092367-1.555684$

C $-0.013126-3.215108-0.575239$

N $-0.6900630 .859077-1.770723$

H $-4.0085102 .512781 \quad 1.368360$

H -1.988510 1.9607933 .600851

H -3.6412870 .3373364 .289113$

H -4.7112900 .1186941 .420280$

H -5.5063141 .1088192 .626703$

H $-6.338504-2.9354142 .893221$

H $-6.950644-1.6050254 .646461$

H -5.669318 -0.4934035 .132753$

H -7.0722790 .1037214 .219561$

H -4.265524 -1.940226 0.834050

H -4.593952 $-3.576246 \quad 1.347507$

H -5.597689 -3.407452 -0.772353

H $-6.949208-3.370390 \quad 0.365448$

H $-6.887559-0.8021180 .173941$

H $-8.538763-2.167440-1.143103$

H -7.332289 -2.438703 -2.384181

H -9.660032 -1.263516 -3.139833

H $-6.443134-0.369157-3.743510$

H -9.840532 $-0.026326-1.150946$

H -9.255554 $1.381938-2.007239$

H $-8.4078840 .815744-0.554662$
H $-7.8119101 .403288-4.119332$

H -4.181069 $0.156479-3.449035$

H $-4.4757691 .838776-2.992661$

H -5.206092 $1.743506-0.523070$

H -1.031150 -3.396859 3.408272

H -1.398473 -1.627027 0.917576

H $-0.452600-5.2498321 .862647$

H $0.955951-4.2705732 .341583$

H $1.008122-5.2342260 .882351$

H $-3.1647851 .534490-0.917674$

H $-2.986803-0.200461-1.251815$

H $1.3180531 .293445-1.557575$

H $0.6512681 .675911-3.157010$

H $0.481300-0.767719-3.727826$

H $2.535448-0.810354-1.453236$

H $3.401309-2.490400-3.154701$

H $1.783415-2.766515-3.755650$

H $3.048541-5.093685-0.591685$

H $4.204790-3.981705-1.514363$

H $\quad 0.713739-5.137642-1.225536$

H $0.156004-4.107074-2.496753$

H $-0.464350-2.332885-1.029069$

H $-0.7752980 .477474-0.832976$

C -1.168962 3.7066390 .967926

O -0.1542273 .0793371 .777790$

C 1.1352633 .6233051 .421787

C 0.8995114 .4648940 .177207

C $-0.513707 \quad 4.965690 \quad 0.410710$

H 1.4350334 .2632342 .261380

C 2.1116672 .4538791 .270002

H 1.6297435 .2727720 .071616

H $0.9396833 .846122-0.727976$

C $-1.1780845 .503805-0.844594$

H $-0.4912865 .751407 \quad 1.177645$

C 3.4609602 .8298530 .631647

$\begin{array}{llll}\text { O } & 2.332131 & 1.907314 & 2.574444\end{array}$

H $1.6439581 .655848 \quad 0.683048$

H $3.2709143 .234862-0.368558$

O 4.0850803 .8665001 .385986

C 4.4234041 .6341330 .486382

C $5.6820132 .001750-0.321497$

H $3.8983150 .816445-0.020671$

O 4.8349221 .1647621 .767942

H $5.3901852 .560565-1.218522$

H 6.3225712 .6448530 .294728

H $-0.6191686 .358744-1.238926$

H -1.226701 $4.742683-1.630871$

H -2.199485 $5.836117-0.634786$

C -2.4053293 .9589461 .829945$

H 1.4630771 .6673382 .939815

H 4.2770123 .5069832 .269304

H 4.0405300 .8724882 .245345

H -3.0750944 .6794251 .347364$

H -2.1181774 .3814102 .800871$

H -1.4027873 .0114590 .151765$

C $7.689459-1.1156330 .204202$

O 6.9940350 .1182730 .419820

C $6.4771390 .752157-0.753050$

C $7.6235251 .098616-1.714053$

C $8.455984-0.144935-2.045820$

C $8.852045-0.923257-0.785761$

H $8.115607-1.3685221 .183575$

C $6.732962-2.246908-0.189641$

H $5.7795800 .073101-1.255896$

O $8.4552892 .093691-1.121684$

H $7.2000761 .524036-2.632028$

H $9.3604500 .162405-2.584961$

O $7.695529-0.980136-2.914433$

H $9.272144-1.897927-1.059967$

O $9.891835-0.190672-0.130517$

C $5.773263-2.5486800 .937759$

H $6.153844-2.031420-1.092063$

H $7.287703-3.169812-0.396580$

H $9.1596122 .304605-1.757779$

H $8.247054-1.748868-3.139436$

H $\quad 10.183170-0.7140860 .635461$

O $4.591623-1.9009460 .731646$

O $6.051157-3.2570861 .896718$ 
C $3.595917-2.0917541 .741700$

H $3.346261-3.1526871 .833851$

H $3.947636-1.6936872 .698085$

H $2.698617-1.543677 \quad 1.441723$

SCF Energy (B3LYP/6-31G**//MMFF) $=-3245.89883501$

0800308

MM̄MF Geometry

C $\quad 0.394460 \quad 0.2071762 .579229$

C 0.9356231 .3112953 .117840

C 0.6360202 .7406132 .727681

O

C 1.8631503 .4278992 .097044

C 3.0247213 .6372863 .041899

C 4.1568742 .9073463 .032126

C 2.8660814 .7793674 .011293

C 4.4821421 .7246542 .162497

C 5.5612302 .0755291 .133187

C 6.0258540 .8411030 .347595

C $7.1849951 .205029-0.583201$

C $7.6297930 .010450-1.439295$

O $4.919560 \quad 0.339616-0.412178$

O $8.4806680 .508341-2.483703$

C $6.386079-0.597230-2.121247$

C $8.470819-0.989994-0.638124$

O $6.719199-1.798252-2.813694$

C $5.211722-0.855267-1.148456$

C $3.923086-1.310368-1.878482$

O $5.579246-1.922987-0.263272$

C -1.2193663 .8609831 .674008$

O -1.2047664 .8403142 .399888$

C -2.0938383 .6651520 .501054$

C $-2.946114 \quad 4.6174600 .097482$

C $-3.7744954 .466290-1.092768$

C $-5.2442094 .651846-0.851500$

C $3.315419-0.320274-2.872459$

C $2.3777420 .679794-2.224780$

C $-0.0026850 .958792-1.535386$

O $2.7341251 .826087-1.962656$

C $-0.727717 \quad 1.487549-2.735739$

C $-1.9883691 .161072-3.061631$

C $-2.7047801 .697857-4.271567$

C $-3.9763942 .448431-3.930424$

C $-5.1741911 .844184-4.004111$

C $-3.8754673 .922436-3.597057$

C $-3.2044074 .168531-2.277211$

N $1.1161840 .154197-1.987580$

H $-0.347357 \quad 0.293616 \quad 1.790379$

H 1.6621611 .1872083 .918553

H $\quad 0.303618 \quad 3.271322 \quad 3.628141$

H 1.5655004 .4082431 .699938

H 2.1689252 .8762461 .199008

H 4.9393293 .1646653 .745261

H 3.7527634 .9186994 .638864

H 2.6955575 .7158823 .470104

H 2.0162124 .6026434 .677614

H 4.8414750 .9191032 .815042

H $3.596103 \quad 1.3291571 .657823$

H 5.1559062 .8236860 .439386

H 6.4221492 .5288361 .640747

H 6.3435490 .0775001 .067521

H $8.0333111 .602756-0.012218$

H $6.8705612 .015532-1.255849$

H $9.2305520 .966128-2.066503$

H $6.0702430 .121486-2.885756$

H $9.375136-0.505330-0.250743$

H $8.820653-1.810432-1.274220$

H $7.937285-1.4165970 .213567$

H $7.453995-1.591191-3.416153$

H $4.132069-2.252296-2.402182$

H $3.168403-1.558408-1.120646$

H $4.768522-2.3347180 .077097$

H -1.999661 2.729917 -0.041879

H -3.063125 5.5435600 .653086

H -5.582557 4.010747 -0.029339

H $-5.8568114 .398009-1.719991$

H -5.458697 $5.692506-0.585596$

H $4.0648870 .227922-3.448267$
H $2.733132-0.881986-3.615066$

H $\quad 0.3644041 .790261-0.925962$

H $-0.6389230 .321956-0.913538$

H $-0.1859272 .194031-3.360763$

H -2.528629 $0.451202-2.440700$

H $-2.9390200 .844843-4.922030$

H $-2.0508282 .347318-4.866876$

H $-6.0928922 .380247-3.790495$

H $-5.2626360 .797548-4.275658$

H $-4.8618704 .398337-3.630009$

H $-3.2971604 .426881-4.381660$

H -2.117868 $4.090365-2.317714$

H $0.913274-0.793013-2.293644$

C $1.210273-2.0455451 .828688$

O $0.097188-2.3007860 .946565$

C $0.157738-3.6712170 .500404$

C $1.512236-4.2029230 .956079$

C $1.718680-3.4258072 .241746$

H $-0.627672-4.2113991 .040472$

C -0.096155 -3.757477 -1.009369

H $1.503077-5.2869221 .104124$

H $2.306358-3.9668890 .238074$

C $3.152289-3.4403912 .740926$

H $1.065027-3.8497013 .016184$

C $-1.421161-3.102881-1.451149$

O $0.972062-3.082594-1.683003$

H $-0.055017-4.803559-1.333769$

H -1.401260 -2.040143 -1.191832

O $-1.480650-3.152194-2.883567$

C $-2.685185-3.776024-0.878599$

C $-3.986973-3.074692-1.316269$

H -2.638929 $-3.809407 \quad 0.213812$

O $-2.716738-5.128730-1.339749$

H $-3.936746-2.833553-2.384540$

H -4.822521 -3.777779-1.200722

H $3.481161-4.4662782 .936384$

H $3.836917-3.0015992 .009391$

H $3.248446-2.8731853 .671917$

C $0.754059-1.1876543 .010097$

H $0.802236-3.152766-2.638087$

H - $-1.544657-4.088175-3.140827$

H -3.480051 -5.563047 -0.922043

H $1.538807-1.1576953 .774473$

H -0.134645 -1.629696 3.469903

H $1.970810-1.5140901 .240979$

C $-5.027818-1.2109431 .742607$

O $-4.749748-2.2453750 .789202$

C $-4.263520-1.809569-0.487068$

C $-5.286985-0.886836-1.157391$

C $-5.6181910 .298829-0.245511$

C $-5.993001-0.1591671 .164744$

H -5.551996-1.723173 2.560068

C $-3.730846-0.6213432 .317458$

H $-3.327519-1.260311-0.350620$

O $-6.469690-1.620582-1.461687$

H $-4.868068-0.530707-2.104451$

H $-6.4511290 .871401-0.671617$

O $-4.4900971 .162481-0.155249$

H -6.0498640 .7045361 .837543$

O $\quad-7.305269-0.7263561 .106117$

C $-3.172989-1.5253793 .394949$

H -2.945646 -0.4595211 .574543$

H $-3.931976 \quad 0.3451362 .794718$

H -7.093149 -1.008176 -1.887862

H $-4.2955231 .483813-1.051726$

H $-7.570520-0.9487112 .014832$

O $-2.780924-2.7159402 .858818$

O $-3.102365-1.2096114 .575820$

C $-2.327538-3.6998083 .794114$

H $-1.504840-3.3115784 .400899$

H $-3.157936-4.0165074 .431891$

$\mathrm{H}-1.968479-4.5647573 .230177$

SCF Energy (B3LYP/6-31G**//MMFF)= -3245.89786157

08 00309

MM̄FF Geometry

C 1.6969034 .5975240 .976590

C 2.7768973 .8134141 .106194 
C 3.8570643 .7053370 .061924

O $3.7166882 .401855-0.551895$

C 5.2435613 .8023250 .715301

C $6.3755563 .707906-0.288202$

C $7.1181652 .602790-0.497526$

C $6.6713364 .975536-1.046603$

C $6.9575001 .260330 \quad 0.159664$

C $6.9389690 .124356-0.872688$

C $6.699267-1.232039-0.195900$

C $6.888852-2.380455-1.183987$

C $6.613610-3.736936-0.518723$

O $5.355116-1.2483890 .291254$

O $6.504056-4.721735-1.559600$

C $5.244417-3.685594 \quad 0.196290$

C $7.785803-4.1788530 .366273$

O $5.059370-4.866004 \quad 0.974114$

C $5.014731-2.4128031 .055305$

C $3.522194-2.2895651 .428853$

O $5.731852-2.4596692 .289407$

C $2.8509722 .317035-1.600193$

O $2.2779253 .240658-2.157290$

C $2.6559350 .885923-1.917103$

C $1.5516530 .531804-2.591877$

C $1.134385-0.828483-2.933567$

C $2.103490-1.946818-2.662675$

C $3.157991-0.9773002 .135314$

C $1.662640-0.9265902 .381322$

C $-0.502796-0.6772891 .162821$

O $1.180030-1.1815863 .482126$

C $-0.878458-1.8181730 .268524$

C $-1.765041-1.709508-0.732100$

C $-2.129386-2.838544-1.654564$

C $-2.126514-2.429579-3.112695$

C $-3.278667-2.253315-3.781681$

C $-0.788974-2.293599-3.812365$

C $-0.096363-1.005151-3.458675$

N $0.944992-0.6143921 .237058$

H $1.587150 \quad 5.2213350 .093197$

H 2.8573703 .1620251 .973801

H $3.7572944 .493885-0.694439$

H 5.3499753 .0293551 .484839

H $5.3340574 .754998 \quad 1.254882$

H $7.9332942 .662018-1.217687$

H $7.5244464 .865473-1.724344$

H $5.8085845 .272580-1.650686$

H $6.9064425 .787933-0.351231$

H 7.8006201 .1111290 .844965

H $6.045917 \quad 1.2088310 .760508$

H $6.1454330 .316690-1.606346$

H $7.8944090 .108336-1.411674$

H $7.394104-1.3284630 .646583$

H $7.896897-2.363715-1.616392$

H $6.195772-2.257287-2.028209$

H $7.332217-4.713480-2.069533$

H $4.481554-3.710753-0.594966$

H $8.697778-4.287456-0.233291$

H $7.600515-5.1643360 .807421$

H $8.006669-3.4736781 .170133$

H $4.124543-4.9091721 .235344$

H $2.916730-2.3744300 .520428$

H $3.228395-3.1189522 .084662$

H $6.298573-3.2462612 .294922$

H $3.3796790 .172070-1.542449$

H $0.8560021 .314898-2.892706$

H $1.797100-2.902165-3.093583$

H $2.219628-2.096180-1.586480$

H $3.085866-1.722823-3.093929$

H $3.671737-0.8940613 .099293$

H $3.445756-0.1043541 .538344$

H -0.8476290 .2822430 .767410$

H $-0.925457-0.8256872 .160138$

H $-0.395675-2.7758280 .449326$

H -2.266166 - $0.757887-0.889086$

H $-3.126699-3.196290-1.371096$

H -1.455679 -3.694106 -1.521291

H -3.286569-1.981502 -4.831949

H $-4.240270-2.369329-3.292273$

H -0.929412 -2.305124 -4.901084
H $-0.189763-3.178731-3.583554$

H $-0.710607-0.121549-3.640621$

H $1.449721-0.4172970 .378197$

C $-0.509780 \quad 3.595653 \quad 1.567210$

$\begin{array}{lllll}\text { O } & -1.113493 & 3.973724 & 0.315733\end{array}$

C -2.517027 3.6313840 .350496

C -2.7451112 .9098001 .673899$

C -1.6657613 .5018572 .556903$

H -3.0572914 .5859250 .338002$

C $-2.8589412 .806788-0.899723$

H -3.7542923 .0673412 .066894$

H $-2.5927591 .829572 \quad 1.562423$

C -1.3664232 .6600463 .785610$

H -1.9774384 .5072712 .869670$

C -4.293156 $2.241267-0.880808$

O $-2.6732593 .664466-2.027912$

H $-2.1350371 .988798-1.001029$

H -4.4072861 .6620420 .037377$

O $\quad-5.232850 \quad 3.312421-0.809550$

C $-4.6364551 .324940-2.071517$

C $-6.0494420 .709252-2.003502$

H $-3.8919790 .523729-2.126840$

O $-4.5411132 .065471-3.289006$

H $-6.8046541 .498220-2.110603$

H $-6.1640070 .068166-2.887644$

H -2.2599882 .5608784 .410870$

H $-1.0355841 .652474 \quad 3.511699$

H $-0.5797703 .118908 \quad 4.392336$

C 0.5640894 .6100531 .963241

H $-2.8105683 .130990-2.828866$

H $-5.1306543 .841065-1.619754$

H $-4.6997891 .447212-4.022586$

H 0.9411244 .3923312 .969162

H 0.1459515 .6235361 .986536

H -0.0569452 .6064261 .416761$

C $-5.350011-1.8768820 .591368$

O $-5.321605-1.124881-0.630362$

C $-6.324192-0.109116-0.730070$

C $-7.716044-0.755447-0.731021$

C $-7.916485-1.6031450 .531914$

C $-6.726719-2.534801 \quad 0.799825$

H $-4.619241-2.6792520 .434177$

C $-4.855435-1.0094801 .758283$

H -6.2551490 .5607110 .133532$

O $-7.858988-1.577555-1.887017$

H $-8.4761170 .032964-0.785886$

H $-8.825789-2.2079440 .428022$

O $-8.093490-0.7465541 .656499$

H $-6.790749-2.9442031 .813823$

O $-6.820248-3.649985-0.094420$

C $-4.179967-1.8123202 .847431$

H $-4.112090-0.3005421 .373814$

H $-5.663679-0.4292492 .210746$

H $-8.756694-1.951248-1.871716$

H -8.875195 -0.1941491 .485188$

H $-6.702548-3.314661-0.999983$

O $-3.471733-0.9601133 .647270$

O $-4.268456-3.0235792 .991476$

C $-2.753205-1.5750144 .720957$

H -2.209944 -0.7926135 .257632$

H $-3.448919-2.0589015 .412817$

H -2.030350 -2.297448 4.329444

SCF Energy (B3LYP/6-31G**//MMFF)= -3245.91265186

08_00310

MM̄FF Geometry

C -1.297869 $3.889650-1.632730$

C $-1.7034182 .633132-1.396553$

C $-2.3948061 .777203-2.423097$

O $-3.6486991 .371406-1.830799$

C $-1.5572270 .521162-2.719413$

C $-1.937186-0.148161-4.023887$

C $-2.732582-1.229836-4.133435$

C $-1.3177120 .460453-5.255997$

C $-3.459832-1.958845-3.039013$

C $-2.954352-3.394724-2.848739$

C $-1.564714-3.477164-2.196747$

C $-1.121060-4.937918-2.079970$ 
C $0.197359-5.082669-1.306689$

O $-1.658027-2.885247-0.897252$

O $0.358038-6.467583-0.963171$

C $0.083250-4.2997290 .017197$

C $1.410299-4.705310-2.165223$

O $1.322962-4.3023350 .717625$

C $-0.426664-2.851338-0.164488$

C $-0.711222-2.2160531 .217722$

O $0.570939-2.079105-0.834819$

C $-4.7732351 .410144-2.587359$

O $-4.858359 \quad 1.752314-3.758532$

C $-5.9626101 .005265-1.800235$

C $-5.8870860 .440052-0.584750$

C -7.0145980 .0484330 .264189$

C $-8.4045360 .350456-0.230368$

C $-1.455376-0.8742721 .152663$

C $-1.791246-0.3459712 .536991$

C $-3.249872 \quad 1.327407 \quad 3.673764$

O $-1.287933-0.7904503 .565502$

C $-4.428246 \quad 0.534778 \quad 4.146928$

C -5.6830431 .0074284 .191402$

C $-6.8584490 .195440 \quad 4.657454$

C -7.9308250 .0523103 .598342$

C -9.0673680 .7655113 .670059$

C -7.727494 -0.977594 2.507125

C $-6.751356-0.5271031 .456084$

$\begin{array}{llll}\mathrm{N} & -2.706848 & 0.697050 & 2.483867\end{array}$

H -1.476696 4.336148 -2.607861

H $-1.5181122 .187537-0.422107$

H -2.567549 2.361070 -3.335243

H - $-1.643022-0.188488-1.889523$

H $-0.490697 \quad 0.778685-2.775248$

H $-2.913673-1.631721-5.129781$

H -1.629168 $1.503607-5.367964$

H $-0.2254890 .429147-5.186174$

H -1.604589 $-0.068143-6.171285$

H -3.433724 -1.419016 -2.088525

H $-4.518425-2.001477-3.325492$

H $-3.678569-3.911935-2.205616$

H -2.947469 -3.918442 -3.812741

H $-0.848022-2.910775-2.801821$

H -1.037911 -5.399005 -3.071994

H -1.894492 -5.511025 -1.549111

H $0.355201-6.983093-1.787909$

H $-0.631609-4.8390210 .654678$

H $1.470366-5.353343-3.047820$

H $2.345782-4.860270-1.616608$

H $\quad 1.382890-3.672365-2.518095$

H $1.602318-5.2292950 .810667$

H -1.327034 -2.906034 1.809774

H $\quad 0.231190-2.0724591 .761346$

H $0.444886-1.141067-0.622919$

H $-6.9145551 .196246-2.284989$

H $-4.9054950 .248027-0.154780$

H -8.518260 $1.421700-0.431068$

H -9.1871650 .0854230 .483880$

H -8.612917 $-0.204338-1.151727$

H $-0.845577-0.1160970 .650983$

H -2.391021 -0.9900190 .593747$

H -3.519318 2.3539953 .406882

H $-2.483172 \quad 1.3566874 .454444$

H $-4.235373-0.4857404 .470429$

H -5.8797842 .0305523 .880035$

H -7.269440 0.6877985 .548429

H -6.548298 -0.8029224 .990513$

H -9.8557050 .6490022 .934028$

H -9.2337081 .4852614 .464739$

H -8.685111 -1.2598532 .055375$

H -7.354639 -1.908028 2.954581

H -5.710239 -0.7064091 .724458$

H $-3.1212010 .945351 \quad 1.590047$

C $0.8760514 .962343-1.050967$

O $1.5842503 .708161-0.992629$

C $2.8685003 .921875-0.383177$

C $3.0690945 .431339-0.354675$

C $1.6469675 .913018-0.137575$

H 2.7877243 .5590260 .649558

C $3.9627683 .126012-1.110352$
H 3.7555485 .7447920 .437664

H $3.4548985 .810508-1.307306$

C $1.4458247 .382644-0.462511$

H 1.3710145 .7350320 .910592

C $3.7691451 .599359-0.962175$

O $3.9784443 .475089-2.492155$

H $4.9391993 .391268-0.686523$

H $3.691614 \quad 1.3885320 .109128$

O $2.536614 \quad 1.188807-1.552394$

C $4.9194060 .759190-1.554054$

C $4.745278-0.761291-1.359781$

H $5.8651721 .084382-1.104999$

O $5.015808 \quad 1.004788-2.958384$

H $3.851064-1.106228-1.894482$

H $5.594221-1.251168-1.855341$

H $2.073693 \quad 8.0078980 .180640$

H $1.7063727 .603124-1.503062$

H $0.4034367 .677695-0.306614$

C $-0.5745284 .739062-0.625016$

H $4.3496354 .369302-2.571007$

H $2.5777881 .418558-2.496777$

H $5.7901950 .518972-3.289851$

H -1.101000 $5.696035-0.539175$

H $-0.603773 \quad 4.2588950 .361104$

H $0.9235335 .307170-2.092439$

C $5.977507-1.0230352 .152759$

O $5.892861-0.7750120 .743317$

C $4.683218-1.2007210 .110518$

C $4.513122-2.7179110 .263095$

C $4.560998-3.1269411 .740263$

C $5.766725-2.5154862 .463695$

H $7.012301-0.7722072 .420062$

C $5.050797-0.0894162 .947703$

H $3.827792-0.7091010 .582003$

O $5.528494-3.412486-0.457662$

H $3.553503-3.009838-0.175899$

H $4.610533-4.2200661 .813580$

O $3.372256-2.6990902 .398753$

H $5.676447-2.6518963 .547823$

O $6.950608-3.2058452 .051539$

C 5.4235881 .3689062 .769741

H $4.008401-0.2017202 .638051$

H $5.124445-0.3172084 .017032$

H $\quad 6.390417-3.102084-0.131025$

H $2.618723-3.1080611 .939957$

H $6.844982-4.1406672 .297534$

O 4.4293002 .1484933 .289229

O $6.457621 \quad 1.7764052 .258442$

C 4.6450153 .5608833 .204137

H 4.8883883 .8558892 .178947

H 3.7214234 .0644143 .501659

H 5.4450603 .8552703 .889455

SCF Energy (B3LYP/6-31G**//MMFF) $=-3245.91511618$

08_00311

MM̄MF Geometry

C $3.783885-2.0135221 .567370$

C $4.362406-3.1014531 .034429$

C $4.809892-3.289544-0.397430$

O $4.864674-2.030059-1.108326$

C $3.848731-4.178934-1.210681$

C $3.437524-5.480022-0.556807$

C $2.224405-5.688123-0.006155$

C $4.469392-6.574015-0.584114$

C $1.120416-4.6748560 .119990$

C $0.036398-4.882316-0.942358$

C $-0.876578-3.652961-1.062597$

C $-2.075989-3.963774-1.959016$

C $-2.946167-2.723190-2.199472$

O $-0.097264-2.594948-1.635236$

O $-3.852388-3.019929-3.272892$

C $-2.042038-1.568345-2.676518$

C $-3.812547-2.387527-0.980378$

O $-2.785365-0.354982-2.779907$

C $-0.792698-1.349956-1.789729$

C $0.174470-0.360144-2.490500$

O $-1.202468-0.822964-0.532236$

C $5.962530-1.246699-0.946116$ 
O $6.952176-1.485557-0.274573$

C $5.711813-0.003583-1.706613$

C $6.3796121 .113518-1.386405$

C $6.1801812 .434680-1.986003$

C $5.2043472 .542712-3.129036$

C $1.455321-0.024921-1.708275$

C $1.3292511 .208712-0.825123$

C 2.6956852 .6906280 .647559

O $\quad 0.280057 \quad 1.831155-0.684624$

C $3.2065863 .841776-0.162593$

$\begin{array}{llll}\text { C } 4.229424 & 4.622088 & 0.220050\end{array}$

C $4.7202685 .802112-0.571676$

C $6.2211765 .799735-0.770334$

C $7.0152006 .602540-0.041755$

C $6.7921934 .928108-1.868008$

C $6.8549913 .481583-1.467123$

N $2.5324531 .530143-0.210055$

H $3.591903-1.1392390 .953123$

H $4.530187-3.9600481 .682787$

H $5.808579-3.744411-0.400140$

H $4.319834-4.404254-2.178138$

H $2.968890-3.589569-1.499219$

H $2.002022-6.6661370 .416771$

H $4.107367-7.497237-0.119290$

H $4.745883-6.809950-1.616922$

H $5.369810-6.263510-0.044686$

H $0.674887-4.7678421 .118452$

H $1.515033-3.6557120 .075024$

H $\quad 0.509489-5.071754-1.915158$

H $-0.553196-5.770350-0.683583$

H -1.209236 -3.357979 -0.060281

H -2.677258 $-4.778211-1.535913$

H - $1.719345-4.330188-2.932045$

H -4.385385 -3.790083 -3.010731

H -1.705041-1.812918 -3.693689

H $-4.468907-3.230336-0.732595$

H $-4.477412-1.545029-1.194019$

H -3.229629 -2.151501-0.087895

H $-3.557335-0.534565-3.343267$

H $0.491233-0.818251-3.437105$

H $-0.3524140 .567500-2.742976$

H $-0.427117-0.449540-0.085571$

H $4.942324-0.029829-2.469811$

H $7.1156241 .066364-0.584758$

H $5.5248601 .911668-3.965596$

H $5.0991053 .555183-3.522712$

H $4.2035012 .226280-2.815861$

H $1.783306-0.872342-1.096910$

H $2.2499390 .190111-2.434118$

H $3.3856752 .408901 \quad 1.449105$

H 1.7310612 .9572891 .087919

H $2.6940354 .060657-1.096539$

H 4.7204844 .4241241 .170302

H $4.4177666 .709801-0.032976$

H $4.2279815 .859042-1.550471$

H $8.0889426 .626807-0.195750$

H $6.610000 \quad 7.249651 \quad 0.729144$

H $7.8160645 .244728-2.108220$

H $6.2246785 .096396-2.786723$

H $7.5390783 .295395-0.637619$

H $3.3611390 .986854-0.430842$

C $1.826949-1.8247483 .118268$

O $1.418536-0.4729372 .827417$

C $0.362922-0.0969333 .743690$

C $-0.025794-1.3699394 .479758$

C $1.292080-2.1160394 .518026$

H $\quad 0.812730 \quad 0.623471 \quad 4.438182$

C -0.7680920 .5576402 .944661$

H $-0.436752-1.1716305 .474164$

H $-0.770482-1.9397413 .909625$

C $1.137365-3.5956764 .826263$

H $1.940601-1.6526385 .273634$

C -2.067106 0.8043253 .728882

O $-0.280822 \quad 1.8039132 .439559$

H $-0.979936-0.0666252 .072092$

H -2.501327 -0.1534224 .038441$

O $-1.740377 \quad 1.5102204 .931445$

C $-3.123751 \quad 1.6304022 .964207$
C $-3.451493 \quad 1.0701401 .574519$

H -2.783646 2.6685192 .887490

O $-4.318141 \quad 1.6595093 .751828$

H $-2.559611 \quad 1.0813090 .938139$

H -3.731466 $0.011591 \quad 1.668804$

H $0.677542-3.7371615 .809803$

H $\quad 0.505249-4.0961054 .084710$

H $2.109104-4.0988314 .833430$

C $3.347831-1.9404763 .006380$

H -0.1261362 .3901063 .200086$

H -2.5716961 .6618775 .413165$

H -4.6579810 .7501553 .809627$

H $3.698184-2.8281263 .545200$

H $3.830354-1.0683063 .463810$

H $1.332524-2.4724292 .382742$

C $-5.7778811 .564429-1.282005$

O $-4.726011 \quad 1.108587-0.424033$

C $-4.595504 \quad 1.787070 \quad 0.833000$

C $-4.344258 \quad 3.285570 \quad 0.607906$

C $-5.4179353 .902173-0.296544$

C $-5.647503 \quad 3.070914-1.561077$

H $-5.613728 \quad 1.031222-2.227124$

C $-7.1573121 .158896-0.751657$

H -5.5174891 .6455591 .406535$

$\begin{array}{llll}\text { O } & -3.057975 & 3.466045 & 0.018638\end{array}$

H -4.3391873 .7998301 .575526$

H $-5.1148054 .915711-0.586820$

O -6.6459964 .0049190 .418353$

H $-6.5382253 .429866-2.090096$

O $-4.531694 \quad 3.278947-2.431721$

C $-7.307606-0.343605-0.749855$

H -7.3422901 .5012800 .270359$

H $-7.9581701 .582850-1.369249$

H $-2.9163724 .421404-0.092762$

H $-6.4856924 .561618 \quad 1.199347$

H $-4.7055342 .792428-3.255400$

O $-7.650185-0.779796-1.995670$

O $-7.122642-1.0448610 .236521$

C $-7.807653-2.195454-2.130973$

H $-8.598528-2.554752-1.465620$

H $-8.094944-2.408206-3.164069$

H -6.862806 -2.703862 -1.918407

SCF Energy (B3LYP/6-31G**//MMFF) $=-3245.89459713$

08_00312

MM̄FF Geometry

C -3.727842 4.4391751 .191768

C $-3.965499 \quad 3.198246 \quad 0.740654$

C -4.8849582 .2250291 .436516$

O $-4.2232050 .939303 \quad 1.497083$

C $-6.1834912 .065274 \quad 0.626078$

C $-7.205478 \quad 1.157438 \quad 1.286155$

$\begin{array}{lllll}C & -7.550644 & -0.068948 & 0.843932\end{array}$

C $-7.880483 \quad 1.7292502 .507286$

C -7.010179 $-0.801030-0.351794$

C $-6.143790-1.9924710 .074549$

C $-5.288579-2.518697-1.086717$

C $-4.521607-3.772932-0.665521$

C $-3.539888-4.244311-1.748724$

O $-4.372442-1.478018-1.442911$

O $-2.647547-5.197127-1.149943$

C $-2.679076-3.045275-2.202112$

C $-4.257201-4.978320-2.887352$

O $-1.857025-3.399729-3.310942$

C $-3.506401-1.788969-2.541572$

C $-2.632852-0.536297-2.804225$

O $-4.260650-2.032879-3.734989$

C $-3.296446 \quad 0.7633832 .478981$

O -2.9658851 .5724973 .331139$

C $-2.735153-0.5980322 .329008$

C $-1.720960-0.9894993 .114833$

C $-1.062170-2.2979233 .091606$

C $-1.533075-3.2993672 .070434$

C $-1.766951-0.079243-1.624906$

C $-0.359325-0.649843-1.632760$

C $1.657560-0.846078-0.185776$

O $0.148392-1.176537-2.619066$

C $1.702898-2.1714750 .502848$ 
C $2.391672-2.3808691 .634822$

C $2.511642-3.7154242 .310819$

C $2.167192-3.657393 \quad 3.782020$

C $3.131059-3.5574794 .713029$

C $0.714638-3.8040014 .173458$

C $-0.070912-2.5382053 .975553$

N $0.281037-0.457181-0.416470$

H -4.2433314 .7626942 .094714$

H -3.488744 2.843220 -0.168104

H -5.1186662 .5623452 .453869$

H $-5.9342501 .709832-0.378319$

H -6.6508353 .0484990 .477753$

H $-8.305271-0.6228551 .401173$

H -8.6798741 .0811712 .882318$

H $-7.160390 \quad 1.864626 \quad 3.319675$

H -8.3301372 .6998702 .273332$

H -7.855494 -1.163994 -0.949563

H -6.449926 -0.136360 -1.012037

H $-5.482109-1.6826280 .893098$

H $-6.788887-2.7910870 .461163$

H -5.943598 $-2.734177-1.939003$

H -5.214552 -4.579838 -0.396524

H -3.949793 -3.5570160 .246265$

H $-3.183470-5.924307-0.789464$

H -2.001930 -2.811586 -1.372078

H $-4.777836-5.863899-2.503372$

H -3.542998 -5.350401 -3.630043

H $-4.997040-4.358588-3.398267$

H -1.337109-4.178781-3.048805

H -2.037528 $-0.675041-3.714649$

H $-3.317470 \quad 0.294074-3.029352$

H -4.495332 -1.178270 -4.132714

H $-3.168505-1.2407191 .571954$

H $-1.334798-0.2874943 .852833$

H -2.559656 -3.611815 2.288952

H $-0.921370-4.2028632 .028506$

H -1.501432 -2.868509 1.064042

H -1.648968 $1.010874-1.682607$

H $-2.253298-0.302500-0.670841$

H $2.114580-0.0561280 .416416$

H $2.187189-0.913021-1.141027$

H $1.181018-3.0006010 .031473$

H $2.935591-1.5517402 .082286$

H $3.547030-4.0588442 .185768$

H $\quad 1.888677-4.4724571 .818657$

H $2.895571-3.5443655 .771982$

H $4.177298-3.4804274 .435741$

H $0.637467-4.0674005 .237116$

H $0.282608-4.6604713 .648112$

H $0.206715-1.7453214 .671485$

H $-0.228141-0.0364230 .355249$

C -1.785577 $4.989065-0.437361$

O $-0.807203 \quad 4.1641200 .231077$

C $0.5135844 .641752-0.102646$

C $0.3307965 .476643-1.358154$

C $-1.0025776 .138681-1.077540$

H $0.8239445 .277163 \quad 0.737183$

C $1.4644973 .445942-0.238092$

H $1.1398476 .196926-1.510618$

H $\quad 0.2631234 .836041-2.246229$

C $-1.6645816 .720373-2.314896$

H $-0.8474916 .942915-0.345517$

C $2.8645113 .830375-0.762965$

O 1.5857842 .8522701 .055419

H $1.0144282 .688875-0.891070$

H $2.7241964 .246545-1.768322$

O $3.4265214 .874561 \quad 0.028336$

C $3.8809822 .676862-0.913295$

C 4.5004072 .1576660 .401438

H $4.7064343 .056629-1.529746$

O $3.2564741 .629474-1.646322$

H 4.9436863 .0006340 .943164

H 3.7154521 .7205401 .025620

H -1.035565 $7.500066-2.756738$

H -1.833829 $5.953085-3.077843$

H $-2.6322347 .167217-2.065644$

C -2.8197685 .4763730 .583684$

H 0.6912782 .6049631 .347031
H $\quad 3.357370 \quad 4.608377 \quad 0.961034$

H $3.9252880 .947239-1.820333$

H -3.455316 $6.247097 \quad 0.131846$

H -2.283873 5.9485611 .417889

H -2.252241 $4.375046-1.216514$

C $5.768715-1.258225-0.452684$

O $4.922659-0.138240-0.167042$

C 5.5857941 .0818420 .177690

C 6.4459330 .8756361 .434185

C $7.432633-0.2803291 .244532$

C $6.741094-1.5372080 .706040$

H $5.083621-2.112698-0.520422$

C $6.466530-1.104178-1.809273$

H $6.2239641 .399063-0.654577$

O 5.5987260 .6059522 .549589

H 6.9901681 .8037791 .647089

H $7.907712-0.5086162 .206382$

O 8.4529780 .1386950 .343295

H $7.487332-2.2729180 .385668$

O $6.008075-2.1480281 .772369$

C $5.465945-0.980568-2.934335$

H $7.103120-0.215252-1.846679$

H $7.111813-1.964237-2.022213$

H $\quad 6.166340 \quad 0.5179873 .334215$

H $9.085959-0.5942050 .255237$

H $5.314173-1.5259292 .049956$

O $4.764029-2.140745-3.074398$

O $5.3267210 .032330-3.608171$

C $3.747697-2.126232-4.081539$

H $3.022826-1.331574-3.879569$

H $4.198635-1.996762-5.069946$

H $3.228450-3.087863-4.054459$

SCF Energy $\left(B 3 L Y P / 6-31 G^{* *} / / M M F F\right)=-3245.92026392$

08_00313

MM̄FF Geometry

C $2.673123-3.600255-0.803633$

C $2.320589-4.137588-1.981635$

C $1.318395-3.548600-2.948665$

O $0.958598-2.202417-2.565336$

C $0.032090-4.391901-2.944689$

C $-0.976281-3.925223-3.974996$

C $-2.040692-3.144817-3.702435$

C $-0.739430-4.423771-5.376636$

C $-2.423070-2.534397-2.381338$

C $-3.680109-3.200276-1.811774$

C $-4.126052-2.573275-0.481221$

C $-5.354605-3.3098370 .058954$

C $-5.905023-2.649627 \quad 1.330237$

O $-4.446389-1.201355-0.721107$

O $-7.197072-3.2126811 .604286$

C $-6.085842-1.1361941 .076329$

C $-5.046870-3.0015522 .550689$

O $-6.443757-0.4600372 .279260$

C $-4.848164-0.4603920 .441991$

C $-5.1989260 .965278-0.041989$

O $-3.782206-0.3310181 .383666$

C $1.733978-1.178033-3.010846$

O $2.710897-1.249087-3.737316$

C $1.1839730 .060844-2.417888$

C $1.7873631 .241237-2.622697$

C $1.3555972 .525964-2.063676$

C $0.1470972 .506833-1.166240$

C $-3.9742481 .731536-0.554924$

C $-4.3290623 .009694-1.296481$

C $-3.1881185 .024407-2.260712$

O $-5.4527943 .257294-1.721874$

C $-1.9425285 .005213-3.091222$

C $-0.9701995 .927095-3.021449$

C $0.2913195 .853131-3.836628$

C $1.5475346 .022446-3.005786$

C $2.4034457 .027079-3.260217$

C $1.8180595 .046748-1.878416$

C $2.0552813 .640576-2.363744$

N -3.206932 $3.803591-1.477190$

H $2.204918-2.677598-0.472345$

H $2.787585-5.069241-2.293825$

H $1.759443-3.540538-3.953065$ 
H $-0.419746-4.378519-1.945481$ H $0.270229-5.446119-3.140081$ H $-2.711213-2.888227-4.521797$ H $-1.510311-4.082581-6.075618$ H $0.225444-4.069385-5.752323$ H $-0.739428-5.518615-5.395115$ H -1.611568 -2.592937 -1.650586 H -2.603317 -1.465309-2.547263 H -4.491110 -3.106301 -2.546423 H -3.492325 -4.271662 -1.666325 $\begin{array}{lllll}\text { H } & -3.294347 & -2.628881 & 0.230237\end{array}$ H $-5.126591-4.3672860 .243013$ H $-6.144239-3.304321-0.705552$ H -7.771313 -3.045033 0.837668 H $-6.932319-1.0136460 .386373$ H $-5.071497-4.0827082 .734227$ H -5.440275 -2.535383 3.460656 H -3.999316 -2.713415 2.437879 H -7.231947 -0.9031462 .637537$ H $-5.9503880 .899200-0.839000$ H $-5.639890 \quad 1.5491610 .775482$ H -4.100509 -0.6079632 .257543$ H $\quad 0.293155-0.039696-1.806869$ H $2.6804381 .272300-3.244740$ H $-0.7325922 .170351-1.725158$ H $-0.0976763 .477524-0.733764$ H $\quad 0.3026451 .826339-0.323751$ H -3.3204981 .9828270 .287705$ H -3.400037 $1.110138-1.252389$ H -3.209968 5.864948 -1.560194 H -4.073922 $5.076043-2.900991$ H -1.829631 $4.169760-3.778984$ H -1.083509 $6.773985-2.349021$ H $\quad 0.2339416 .637133-4.602783$ H $\quad 0.352884 \quad 4.902642-4.381062$ H $3.3060197 .164420-2.673939$ H $2.2230687 .736086-4.061465$ H $1.0079455 .118631-1.149111$ H $2.7188385 .348317-1.326931$ H $2.9238813 .545002-3.016619$ H $-2.3713453 .581291-0.945777$ C $4.867505-3.2493950 .404025$ O $4.408042-2.0163160 .996677$ C $5.152481-0.9267170 .418327$ C $6.323055-1.563867-0.325162$ C $5.697207-2.853344-0.817098$ H $4.492935-0.448885-0.314934$ C $5.5733750 .077141 \quad 1.495786$ H $6.694354-0.928817-1.135311$ H $7.161713-1.7791980 .347592$ C $6.708752-3.902278-1.243419$ H $5.050525-2.615030-1.670701$ C $4.399697 \quad 0.7608542 .228867$ O $6.362365-0.6230212 .466598$ H $\quad 6.231137 \quad 0.834741 \quad 1.052659$ H 3.8423920 .0212512 .815426 O $4.963308 \quad 1.685262 \quad 3.169627$ C 3.4263861 .5404121 .318093

C 2.4744652 .4906842 .069893 H $2.838097 \quad 0.843444 \quad 0.710877$ O 4.1662542 .3722690 .416772 H 3.0613653 .2367592 .620933 H 1.9236383 .0621481 .310327 H $7.320173-3.532118-2.072848$ H $7.382718-4.168172-0.422119$ H $6.205261-4.815462-1.576194$ C $3.687710-4.1878160 .134071$ H 6.8074650 .0431593 .016938 H 5.3712891 .1648083 .881991 H $4.5639491 .800423-0.260316$ H $3.173003-4.3916931 .081130$ H $4.058873-5.147565-0.243203$ H $5.513003-3.7225831 .156002$ C $-0.461240 \quad 0.3324242 .903535$ O 0.5767551 .0346632 .206965 C 1.4747391 .8038403 .012503 C 0.7060952 .8410503 .849932 C $-0.4026222 .181374 \quad 4.672414$
C $-1.277172 \quad 1.2713303 .807966$

H -1.126939 -0.0251482 .108578$

C $0.086534-0.8899513 .647225$

H 2.0198201 .1349923 .685084

O 0.1417163 .8293772 .989441

H 1.4149973 .3494954 .514696

H -1.024862 2.9611595 .128420

O $\quad 0.1957591 .4296395 .723825$

H $-1.9515490 .684047 \quad 4.442323$

O -2.1020192 .0997582 .984590$

C $0.443366-1.9776512 .664651$

H $0.983342-0.6734794 .234266$

H $-0.653967-1.2986534 .345189$

H -0.3118464 .4820003 .549559$

H -0.5223351 .0388336 .250297$

H -2.7129301 .5162302 .503087$

O $-0.672162-2.6912162 .337250$

O $1.571469-2.1512742 .223981$

C $-0.480455-3.7314881 .374524$

H $-1.417353-4.2872271 .282532$

H $-0.235565-3.2945640 .402930$

H $\quad 0.305078-4.4200161 .700315$

SCF Energy (B3LYP/6-31G**//MMFF) $=-3245.90780565$

08 00314

MM̄MF Geometry

C $-0.084028 \quad 4.1267960 .052966$

C -0.8611883 .0383820 .149883$

C -2.1085883 .0136490 .996013$

O -1.9887661 .9523631 .969773$

C -3.3416192 .7180960 .126599$

C -4.6406813 .1286450 .792450$

C -5.5252752 .2803441 .351519$

C -4.9471074 .6048960 .743876$

C -5.4191240 .7886821 .498083$

C -6.4007610 .0427270 .581313$

C $-5.9856940 .066898-0.898280$

C $-7.037254-0.618323-1.772562$

C $-6.586590-0.711844-3.239133$

O $-4.742070-0.629555-1.002997$

O $-7.462782-1.628471-3.912421$

C $-5.169326-1.321366-3.289598$

C $-6.7221460 .631523-3.965559$

O $-4.648938-1.295469-4.616788$

C $-4.171703-0.649965-2.317111$

C $-2.868337-1.479842-2.239262$

O $-3.868071 \quad 0.663991-2.786090$

C -1.4166432 .2610553 .161490$

O -0.9561113 .3464423 .490158$

C -1.4214111 .1087654 .091370$

C $-1.786373-0.1371133 .750648$

C -1.799940-1.300321 4.641805

C $-1.479933-1.0751716 .096916$

C $-1.888313-1.005021-1.155178$

C $-0.783705-2.017931-0.914600$

C $0.896617-2.6817180 .798295$

O $-0.518857-2.920129-1.704926$

C $0.235188-3.7454661 .615469$

C $0.533151-3.9959632 .898936$

C $-0.148782-5.0580613 .713687$

C $-0.790802-4.5082774 .968926$

C $-0.211516-4.6874736 .168140$

C $-2.139769-3.8304444 .849606$

C $-2.086566-2.5136204 .124918$

N $-0.119416-1.7844340 .282465$

H -0.3605135 .0185880 .611347$

H - $0.6049732 .140116-0.405248$

H -2.241605 3.9730001 .511146

H $-3.3618021 .654513-0.130490$

H $-3.2745683 .254961-0.829310$

H -6.4392202 .6997881 .771301$

H $-5.9247784 .841817 \quad 1.177132$

H -4.196616 5.1757621 .298790

H $-4.9550594 .956402-0.293141$

H -4.3973380 .4284311 .347122$

H -5.6644780 .5457202 .539955$

H -6.440745 -1.000155 0.922169

H $\quad-7.4088880 .4600510 .695982$ 
H $-5.8498821 .105791-1.217556$ H -8.001946 -0.101040 -1.698971 H -7.214400 -1.637092 -1.400071 H -8.371522 -1.292480 -3.826129 H -5.260760 -2.381302 -3.013561 H $-7.7645440 .971961-3.949450$ H $-6.4549520 .536952-5.023964$ H $-6.1109451 .421581-3.524304$ H -5.298587 -1.735910 -5.191173 H -3.129449 -2.524312 -2.022591 H -2.352906 -1.466134 -3.207761 H $-2.9960690 .927464-2.453365$ H -1.0850301 .3469225 .095797$ H -2.095222 -0.3363642 .726184$ H $-0.451690-0.7153116 .213348$ H -1.570675 -1.977168 6.705711 H $-2.163705-0.3378216 .532036$ H -1.417038 -0.061395-1.448533 H -2.415418 -0.846236 -0.207926 H $1.598303-2.0809671 .384071$ H $1.429596-3.143273-0.035983$ H $-0.528569-4.3435291 .123224$ H $1.308353-3.4129143 .390860$ H $\quad 0.605015-5.8128173 .973109$ H $-0.907946-5.5892353 .126004$ H $-0.675154-4.3283077 .080736$ H $\quad 0.741738-5.1965306 .265050$ H -2.606780 -3.700516 5.833471 H -2.823584 -4.497001 4.307866 H -2.305362 -2.588756 3.059443 H $-0.453822-1.0494800 .898961$ C $2.4759714 .249606-0.072251$ O 2.9402202 .9025440 .137000 C 3.7943862 .8941641 .298267 C 3.8165454 .3247171 .845756 C 2.5272844 .9077981 .303020 H 3.3113302 .2397932 .032442 C 5.1908462 .3585390 .952081 H 3.8639454 .3409982 .939509 H 4.6780554 .8931181 .477357 C 2.5122866 .4255511 .265127 H 1.7100984 .5503581 .941653 C 5.2311380 .9630390 .298728 $\begin{array}{lllll}\text { O } & 5.813991 & 3.269951 & 0.034338\end{array}$ H 5.8099412 .3688771 .857633 H $4.8795401 .036502-0.736213$ O $\quad 6.613221 \quad 0.584108 \quad 0.213837$ C $4.451684-0.1410461 .032609$ C $4.787052-1.5759190 .577922$ H $3.3771080 .042000 \quad 0.918601$ O $4.745307-0.0596582 .430897$ H $5.804388-1.8353650 .898671$ H $4.126108-2.2497341 .139127$ H $2.627473 \quad 6.8365172 .273415$ H 3.3260886 .8193400 .646968 H 1.5685256 .7975990 .854510 C $1.1348144 .247329-0.814038$ H $6.7039732 .926668-0.155503$ H $6.9396810 .460817 \quad 1.121784$ H $4.190041-0.7161052 .885335$ H $1.1343213 .450324-1.566658$ H $1.0375715 .193116-1.362250$ H $3.2176914 .715687-0.735561$ C $2.968589-1.709549-2.684863$ O $3.286011-1.551943-1.295448$ C $4.637355-1.835687-0.929427$ C $5.012000-3.282834-1.294124$ C $4.712500-3.589973-2.765355$ C $3.307812-3.131282-3.169301$ H $1.879750-1.585925-2.739587$ C $3.605946-0.611651-3.550266$ H $5.305511-1.156950-1.467479$ O $4.310008-4.208300-0.466274$ H $6.079976-3.428911-1.094219$ H $4.798728-4.670126-2.937002$ O $5.668466-2.939236-3.596628$ H $3.191803-3.191654-4.257773$ O $2.368722-4.045922-2.596950$
C $3.0634390 .765114-3.231216$

H $4.690044-0.568618-3.417875$

H $3.386927-0.809405-4.606002$

H $3.359629-4.098340-0.636818$

H $6.548029-3.271725-3.348875$

H $1.480309-3.778051-2.888023$

O $3.7029481 .678637-4.021042$

O $2.198568 \quad 1.011776-2.402578$

C $3.3153863 .042611-3.832095$

H $3.8099033 .645636-4.598189$

H $2.2328813 .156035-3.943327$

H $3.6442753 .387361-2.848302$

SCF Energy (B3LYP/6-31G**//MMFF)= -3245.92031186

08_00315

MM̄FF Geometry

C -1.6574902 .9178152 .536950$

C -1.0024211 .8089622 .165824$

C $-0.376320 \quad 0.842568 \quad 3.138621$

O 0.9762820 .6028402 .680689

C $-1.133104-0.4909553 .137287$

C $-0.573864-1.4851814 .136100$

C $0.216249-2.5302713 .818907$

C $-0.991875-1.2611175 .566418$

C $0.735444-2.9062502 .459132$

C $2.260568-3.0791452 .463767$

C $2.787298-3.4019071 .058124$

C $4.283574-3.7129751 .089970$

C $4.844167-3.949541-0.321244$

O $2.550534-2.2595070 .229840$

O $6.277004-3.918610-0.237754$

C $4.418869-2.778458-1.231653$

C $4.470255-5.334982-0.861099$

O $4.786504-3.024511-2.587195$

C $2.911985-2.438571-1.144590$

C $2.630054-1.113247-1.887128$

O $2.157702-3.482891-1.759345$

C 1.9170401 .5190953 .036615

O $1.7860082 .442806 \quad 3.823977$

C 3.1222051 .2562172 .221122

C 3.9752132 .2680332 .003356

C 5.1472502 .2412071 .128489

C 5.5748550 .9131290 .566901

C $1.225593-0.537484-1.656622$

C $1.1405410 .860823-2.235809$

C $1.9926193 .178219-1.861447$

O $\quad 0.598574 \quad 1.079954-3.316416$

C $3.4312963 .318977-2.258179$

C $4.2200504 .328264-1.857296$

C $5.6655524 .461431-2.250751$

C $6.5758794 .773579-1.080022$

C $7.1339695 .988797-0.946420$

C $6.8986543 .659573-0.102996$

C 5.7654103 .4085720 .853759

N $1.7660051 .808026-1.437316$

$\mathrm{H}-1.8031633 .1258243 .593973$

H -0.8559071 .6071621 .106686$

H $-0.357544 \quad 1.2578944 .153895$

H -1.125865 -0.9137712 .129406$

H $-2.192888-0.321503 \quad 3.371010$

H $0.529010-3.1973794 .621230$

H -0.616485 -2.041602 6.236782

H $-0.611900-0.3025465 .932525$

H $-2.083644-1.2562425 .647690$

H $\quad 0.266249-3.8513922 .160647$

H $0.463792-2.165774 \quad 1.703618$

H $2.724329-2.1528002 .826751$

H $2.536814-3.8826243 .157682$

H $2.225969-4.2580900 .665756$

H $4.489392-4.5767221 .734337$

H $4.822626-2.8672841 .539938$

H $6.555467-4.6075110 .389800$

H $4.990842-1.896454-0.912191$

H $4.856356-6.120374-0.200163$

H $4.929798-5.514927-1.839250$

H $3.392294-5.482771-0.953959$

H $5.739244-3.219746-2.598115$

H $3.362951-0.365849-1.565068$ 
H $2.774866-1.249968-2.966611$

H $\quad 1.292078-3.131269-2.021887$

H $3.228087 \quad 0.280837 \quad 1.761109$

H 3.7641333 .2320222 .465543

H 5.6458120 .1593251 .359340

H 6.5583870 .9403200 .092511

H $4.8561940 .568960-0.180514$

H $0.460691-1.155741-2.137300$

H $0.976630-0.485839-0.590627$

H $1.7318803 .826952-1.019501$

H $1.3458203 .416952-2.711112$

H $3.8439122 .553830-2.912263$

H $3.8011095 .111148-1.228894$

H $5.7309335 .261585-2.999513$

H $6.0268933 .552986-2.748754$

H $7.8052476 .215138-0.124605$

H $6.9287496 .786646-1.652344$

H $7.784503 \quad 3.9209160 .490044$

H $7.1748372 .771503-0.678334$

H 5.3984234 .3134841 .340764

H $2.2061461 .508003-0.572499$

C -3.651229 3.9191121 .349661

$\begin{array}{llll}\text { O } & -3.971229 & 2.842618 & 0.436098\end{array}$

C $-4.6034733 .408945-0.723804$

C $-5.2392654 .700034-0.230639$

C $-4.157345 \quad 5.2117490 .695140$

H $-3.8038723 .653803-1.433643$

C $-5.5893212 .434701-1.372472$

H $-5.4701755 .392067-1.045873$

H $-6.159784 \quad 4.5104930 .333412$

C $-4.647508 \quad 6.2479861 .692707$

H -3.3623125 .6622130 .085282$

C $-5.0146401 .062676-1.777219$

O $-6.6776162 .189099-0.471237$

H $-6.0373372 .915075-2.250693$

H $-4.891848 \quad 0.434843-0.887295$

O $\quad-6.0070390 .417686-2.588964$

C $-3.689907 \quad 1.097195-2.560574$

C $-3.316250-0.219823-3.270227$

H $-2.8814401 .387381-1.879203$

O $-3.7721832 .109697-3.569026$

H -3.972278 -0.362011-4.139143

H - $-2.307868-0.084722-3.684227$

H -5.0321697 .1312671 .172460$

H -5.451796 5.8509952 .321009

H -3.8336126 .5694432 .350269$

C -2.138036 3.9620331 .571400

H -6.3007881 .8354890 .352960$

H $-6.8278630 .380987-2.068530$

H -2.904754 2.158945 -4.006167

H -1.8437094 .9363871 .980564$

H -1.618091 3.8495740 .611215

H $-4.185148 \quad 3.7145692 .285760$

C $-2.416570-2.377286-0.356113$

O $-2.411737-1.314616-1.323567$

C $-3.350506-1.477188-2.389553$

C $-3.017920-2.742824-3.194968$

C $-3.004562-3.976221-2.288718$

C $-2.173639-3.745014-1.021479$

H -1.552194 -2.163916 0.281860

C $-3.677977-2.2921450 .516181$

H $-4.356757-1.582562-1.971816$

O $-1.744949-2.590601-3.819347$

H -3.765700 -2.866663 -3.987583

H -2.595835 -4.826878 -2.847552

O $-4.348065-4.297057-1.939672$

H -2.351447 -4.547980 -0.298681

O $-0.786918-3.802394-1.367534$

C $-3.534037-2.9884521 .851585$

H -3.886720 -1.233872 0.718103

H $-4.552728-2.7069800 .008216$

H -1.577294 -3.392226 -4.343238

H -4.325004 -5.097204-1.387589

H - $0.609933-4.688812-1.726004$

O $-4.581969-2.6301542 .651733$

O $-2.634931-3.7591992 .155810$

C $-4.590820-3.2217363 .954697$

H $-4.708135-4.3064403 .874125$
H $-3.672632-2.9717474 .494191$

H $-5.442679-2.8157874 .506627$

SCF Energy (B3LYP/6-31G**//MMFF) $=-3245.91127402$

08 00316

MM̄FF Geometry

C $1.8331293 .882836-0.919608$

C $1.2918902 .661297-1.040132$

C $0.7069962 .122862-2.318968$

O $-0.6588361 .727718-2.055753$

C $1.4953600 .879972-2.764541$

C $1.0137730 .321260-4.088649$

C $0.244307-0.777333-4.219189$

C $1.4960211 .057639-5.311609$

C $-0.320992-1.627873-3.116453$

C $-1.799067-1.965042-3.355665$

C $-2.374372-2.794912-2.198239$

C $-3.756989-3.339202-2.554044$

C $-4.378433-4.111593-1.381580$

O $-2.470822-1.945068-1.050472$

O $-5.765934-4.324713-1.684097$

C $-4.318033-3.231816-0.116016$

C $-3.751738-5.501013-1.213095$

O $-4.759194-3.9814471 .014492$

C -2.924612 -2.602695 0.140670

C $-3.027966-1.5389691 .258880$

O $-2.015166-3.6226240 .557949$

C $-1.6114042 .693069-2.166278$

O $-1.4437683 .857488-2.492809$

C $-2.9082242 .092911-1.782113$

C $-3.9454942 .903280-1.523798$

C $-5.2764332 .495305-1.070849$

C $-5.5938781 .024403-1.061001$

C -1.801790 -0.626056 1.387506

C $-2.048040 \quad 0.4185362 .458510$

C -3.3890972 .4858192 .889102$

$\begin{array}{lllll}\text { O } & -1.569171 & 0.314008 & 3.584887\end{array}$

C -4.8853352 .4583742 .831438$

C -5.6430073 .5282602 .545598$

C -7.1438053 .4867862 .469468$

C -7.6889164 .0790041 .186210$

C -8.3636765 .2413081 .195454$

C $-7.5184373 .290025-0.097620$

C $-6.1416543 .455245-0.682187$

N -2.886289 1.4332552 .025737

H $1.8539084 .550465-1.776914$

H $1.2791201 .997534-0.178158$

H $0.7317212 .878164-3.114427$

H $1.4515730 .110041-1.985334$

H $2.561318 \quad 1.128157-2.860877$

H $-0.003960-1.111509-5.225691$

H $\quad 1.1656170 .581837-6.241076$

H $1.1186132 .084785-5.316778$

H $2.5904501 .088291-5.329211$

H $\quad 0.255552-2.559507-3.080248$

H $-0.221628-1.144304-2.141181$

H -2.368995 -1.032915 -3.461809

H -1.894209-2.522532 -4.295762

H $-1.684428-3.619402-1.983610$

H -3.709060 -3.974012 -3.447546

H $-4.423134-2.504283-2.813663$

H -5.817781-4.826058 -2.515795

H -5.049114 -2.423468 -0.259385

H -3.872656 -6.089339 -2.130701

H $-4.258030-6.069564-0.425074$

H $-2.685048-5.465489-0.981590$

H -4.902176 -3.360415 1.747896

H -3.903173 -0.9076481 .070288$

H -3.196308 -2.028292 2.226842

H -1.368246 -3.239569 1.171654

H $-2.9615731 .015163-1.680947$

H -3.797751 $3.978914-1.615515$

H $-5.3541150 .568891-2.028709$

H $-6.6496310 .809548-0.882381$

H $-5.0192240 .515229-0.283641$

H $-0.908992-1.1946171 .661686$

H -1.579129 $-0.114240 \quad 0.444741$

H -2.9783843 .4315342 .522156$ 
H -3.049393 2.3273443 .916878 H -5.3694821 .5053413 .033568$ H -5.1630194 .4876242 .366417$ H -7.5339404 .0374813 .335191$ H -7.518469 2.4606972 .572498 H -8.7782735 .6609650 .284838$ H -8.512917 5.8021032 .112102 H -8.240927 $3.634080-0.848859$ H -7.7736412 .2463610 .105798$ H -5.825669 $4.496633-0.764756$ H -3.1750201 .4485801 .052373$ C $3.966377 \quad 4.5482340 .210664$ O $4.5493383 .270264-0.112730$ C $5.5912752 .977122 \quad 0.830798$ C 5.9925994 .3245151 .412548 C 4.6427175 .0124011 .503919 H 5.1376762 .3675531 .622468 C $6.7108172 .180607 \quad 0.153824$ H $6.493784 \quad 4.2295362 .380343$ H 6.6522164 .8805950 .736769 C 4.7330476 .5223831 .638985 H 4.1063804 .6116612 .374685 C $6.2233480 .966373-0.665524$ O $7.428976 \quad 3.034946-0.745932$ H 7.4405461 .8611990 .906612 H $5.7374841 .309440-1.587526$

O $7.3776320 .223010-1.077092$ C 5.2690250 .0178630 .082166 C $4.903652-1.214685-0.768633$ H 4.3519220 .5576880 .342224 O $5.891770 \quad-0.392066 \quad 1.297742$ H $4.643192-0.887784-1.782559$ H $5.780284-1.871706-0.829362$ H 5.2651376 .7954062 .556006 H 5.2677286 .9696640 .794285 H 3.7345506 .9691871 .680675 C $2.451179 \quad 4.386863 \quad 0.354944$ H $6.7896623 .389441-1.387766$ H $7.9639140 .837133-1.551516$ H $5.265421-0.9644821 .771884$ H 1.9906085 .3463300 .615856 H 2.2310213 .6889471 .172838 H $4.2027975 .223278-0.621495$ C $3.109453-3.2956471 .770512$ O $4.131986-2.5864081 .056932$ C $3.718372-2.011809-0.187120$ C $3.248163-3.114148-1.149791$ C $2.155982-3.980655-0.515674$ C $2.525522-4.4251180 .903746$ H $3.633482-3.7617422 .614831$ C $2.079238-2.3162422 .341868$ H $2.894794-1.311194-0.006199$ O $4.358456-3.936279-1.505913$ H $2.873795-2.645988-2.068293$ H $1.989162-4.864318-1.143941$ O $0.945168-3.234036-0.486492$ H $1.653323-4.8624701 .400535$ O $3.501461-5.4687520 .815136$ C $1.218366-2.9520873 .403687$ H $2.581403-1.4429162 .777594$ H $1.421570-1.9024231 .572757$ H $4.042025-4.594245-2.148185$ H $0.246016-3.826197-0.161073$ H $4.310222-5.0876080 .431764$ O $1.893994-2.9981864 .586783$ O $0.085367-3.3693753 .200968$ C $1.182559-3.5924115 .676844$ H $\quad 0.270108-3.0262775 .887600$ H $0.946479-4.6365355 .450249$ H $1.826257-3.5618686 .559910$

SCF Energy (B3LYP/6-31G**//MMFF) $=-3245.92401286$

08_00317

MM̄FF Geometry

C -2.212221 $5.466109-0.327157$

C $-2.9009694 .373724-0.690027$

C $-4.005143 \quad 3.7866320 .151087$

$\begin{array}{lllll}\text { O } & -3.682959 & 2.409661 & 0.461831\end{array}$
C $-5.325843 \quad 3.800884-0.634208$

$\begin{array}{llll}\text { C } & -6.527508 & 3.485068 & 0.233374\end{array}$

C $-7.1781342 .305641 \quad 0.240249$

C $-7.024726 \quad 4.6259161 .084667$

C $-6.835493 \quad 1.065847-0.538805$

C $-7.039289-0.196902 \quad 0.310720$

C $-6.807035-1.474013-0.508029$

C $-7.248704-2.7070120 .282109$

C $-6.971555-4.004480-0.489895$

O $-5.411575-1.566726-0.816725$

O $-7.119230-5.102107 \quad 0.423535$

C $-5.502676-3.997438-0.955754$

C $-7.989062-4.234204-1.613554$

O $-5.231691-5.111357-1.804332$

C $-5.072366-2.682530-1.652015$

C $-3.540108-2.695844-1.854225$

O $-5.708273-2.594985-2.928505$

C $-2.7887102 .194426 \quad 1.465255$

O $-2.191903 \quad 3.0418102 .110891$

C -2.661270 0.7318391 .656244

$\begin{array}{lll}\text { C }-1.664227 & 0.260221 & 2.420383\end{array}$

C $-1.406515-1.143252 \quad 2.753917$

C $-2.438794-2.1576762 .338611$

C $-2.932581-1.356485-2.291380$

C $-1.433505-1.510329-2.467440$

C $0.655014-1.884750-1.151990$

O $-0.917219-1.586069-3.579721$

C $0.853869-2.878210-0.051575$

C $1.654309-2.6718211 .004479$

C $1.846573-3.6695392 .109556$

C $1.627361-3.0703673 .480904$

C $2.659007-2.8612104 .316223$

C $0.203601-2.7869843 .913594$

C $-0.283056-1.4421203 .440421$

N $-0.764175-1.606805-1.258871$

H -2.4626615 .9660480 .605462$

H -2.651619 $3.863929-1.617238$

H -4.1226184 .3460251 .087755$

H $-5.266414 \quad 3.101226-1.476513$

H $-5.4861534 .789562-1.085825$

H $-8.0645792 .219901 \quad 0.867843$

H -7.928869 4.3640961 .644914

H -6.2644604 .9255241 .812188$

H $-7.266347 \quad 5.4903290 .457598$

H $-7.4945161 .022323-1.414205$

H $-5.8045751 .087278-0.903996$

H $-6.347972-0.1694411 .163064$

H $-8.060739-0.2034740 .711823$

H $-7.380029-1.396459-1.439563$

H $-8.311125-2.6410990 .548126$

H $-6.702222-2.7447871 .235089$

H $-8.023926-5.0757200 .779869$

H $-4.879014-4.132262-0.060584$

H $-9.005419-4.284826-1.204913$

H -7.818228 $-5.195778-2.110071$

H $-7.976884-3.448088-2.371261$

H $-5.500808-5.913284-1.324493$

H $-3.061747-2.991773-0.912764$

H $-3.271949-3.458754-2.596965$

H $-5.173312-2.033216-3.510856$

H -3.3904870 .0906531 .174619$

H -0.9599320 .9730402 .848522$

H -3.419122 -1.8992022 .754979$

H $-2.217345-3.1718272 .675997$

H -2.525967 -2.198551 1.249759

H $-3.352935-1.015110-3.242650$

H $-3.123745-0.570865-1.551614$

H $1.160618-0.937651-0.949152$

H $1.032044-2.286935-2.097288$

H $\quad 0.311659-3.817738-0.131490$

H $2.213405-1.7426931 .075534$

H $2.866914-4.0652872 .028473$

H $1.181436-4.5333971 .987205$

H $2.506536-2.4549725 .310572$

H $3.679116-3.0845804 .022127$

H $0.143560-2.7889065 .010150$

H $-0.432147-3.6139653 .587468$

H $0.379661-0.6212453 .719927$ 
H $-1.265296-1.378973-0.405637$

C $0.3037895 .635968-0.636998$

O $0.5079694 .237313-0.917278$

C $1.163557 \quad 3.6291710 .215249$

C 1.6820294 .7810491 .064276

C $0.602550 \quad 5.824303 \quad 0.850939$

H $\quad 0.381377 \quad 3.075903 \quad 0.749365$

C $2.2469922 .672137-0.291372$

H 1.8092864 .5053032 .115278

H 2.6459955 .1431250 .685149

C 1.0290507 .2345331 .217378

H $-0.259648 \quad 5.544067 \quad 1.467523$

C 3.0536892 .0007580 .833286

O $1.6103931 .652985-1.066411$

H $2.9151973 .213103-0.970326$

H 3.6060672 .7600261 .398625

$\begin{array}{llll}\text { O } & 2.138243 & 1.404524 & 1.764019\end{array}$

C 4.0333390 .9018930 .373763

C $5.052191 \quad 1.386377-0.673893$

H $3.4742350 .041323-0.010139$

$\begin{array}{lllll} & & 4.725162 & 0.458551 & 1.545950\end{array}$

H $4.5255501 .827884-1.525615$

H $5.6765042 .162178-0.212751$

H 1.2900227 .2938722 .278961

H 1.9023577 .5536090 .638784

H 0.2193727 .9463341 .027301

C - $1.0852246 .059697-1.123295$

H $1.0934282 .096070-1.761082$

H 1.6075820 .7513921 .276204

H $5.273493-0.3032721 .293864$

H - $1.1963825 .773584-2.176998$

H -1.175035 $7.151522-1.081739$

H $1.0505886 .171237-1.238331$

C $7.697993-1.262047-0.464993$

O $6.819697-0.172566-0.151056$

C $5.9595130 .258172-1.209096$

C $6.7963210 .728168-2.410119$

C $7.788191-0.349665-2.860022$

C $8.578114-0.926621-1.680448$

H $8.354733-1.348840 \quad 0.410531$

C $6.938055-2.585036-0.611154$

H $5.316109-0.573598-1.516195$

O $7.508927 \quad 1.919828-2.082080$

H $6.1208860 .977837-3.236622$

H $8.4882110 .080412-3.586640$

O $7.087090-1.408351-3.505655$

H $9.131878-1.821633-1.987679$

O $9.5439930 .038254-1.252665$

C $6.276636-2.9657840 .690745$

H $6.151915-2.549319-1.370686$

H $7.614029-3.396071-0.907486$

H $8.0562971 .733203-1.299966$

H $6.619291-1.028727-4.268817$

H $\quad 10.1369840 .211714-2.003684$

O $7.152189-3.6492631 .480702$

O $5.125117-2.666877 \quad 0.978979$

C $6.643583-4.0435602 .758830$

H $5.773377-4.6960182 .637370$

H $7.427724-4.5983703 .280535$

H $6.384895-3.1602043 .350654$

SCF Energy (B3LYP/6-31G**//MMFF) $=-3245.92139533$

08_00318

MM̄FF Geometry

C -3.724015 $4.736330-0.106123$

C $-4.1548513 .467626-0.032199$

C $-4.3561042 .718757 \quad 1.258003$

O -3.5541821 .5136561 .195042$

C -5.8325712 .3130291 .406674$

C -6.1272811 .5925192 .709177$

$\begin{array}{llll}\text { C } & -6.339151 & 0.266148 & 2.827641\end{array}$

C -6.2049292 .4775983 .927029$

C $-6.304484-0.7729201 .741663$

C $-5.160486-1.770177 \quad 1.965737$

C $-4.956584-2.6906800 .753899$

C $-3.845312-3.7072831 .023711$

C $-3.551186-4.577020-0.208062$

O $-4.604540-1.873062-0.368940$
O $-2.314876-5.2601800 .047849$

C $-3.342992-3.656524-1.432080$

C $-4.611104-5.669754-0.389451$

O $-3.196958-4.426375-2.622183$

C $-4.450820-2.593998-1.599671$

C $-4.151655-1.559192-2.713367$

O $-5.671807-3.250567-1.964795$

C $-2.305107 \quad 1.560395 \quad 1.735277$

O $-1.7627492 .523682 \quad 2.253234$

$\begin{array}{llll}\text { C }-1.710419 & 0.209861 & 1.619539\end{array}$

C $-0.588309-0.0791812 .294523$

C $0.027722-1.4012632 .357080$

C $0.410580-1.8025053 .756814$

C $-2.846533-0.772345-2.554732$

C $-1.667217-1.400023-3.280785$

C $0.825494-1.420751-3.276753$

O $-1.793132-2.211829-4.193529$

C $1.185399-2.614944-2.451591$

C $2.210695-2.645109-1.588264$

C $2.533412-3.830956-0.729647$

C $2.260639-3.5749180 .735854$

C $3.267518-3.4854691 .620377$

C $0.818442-3.5359051 .190948$

C $0.246346-2.1442391 .253159$

N $-0.453862-0.915797-2.813695$

$\mathrm{H}-3.5224335 .2878970 .808000$

H $-4.3425252 .917013-0.951745$

H -4.0552703 .3304702 .117362$

H -6.1319551 .7020260 .549111$

H $-6.4694353 .206813 \quad 1.356896$

H $-6.557030-0.1319333 .818130$

H $-6.483433 \quad 1.9216024 .828686$

H -5.2385782 .9529594 .120604$

H -6.9560083 .2605993 .780075$

H -7.260437 -1.3106161 .744079$

H $-6.215810-0.3196830 .752570$

H $-4.236130-1.2148612 .158329$

H -5.368536 -2.371754 2.859542

H $-5.904419-3.2013880 .547360$

H $-4.092820-4.3358881 .888260$

H $-2.924204-3.1741091 .297731$

H $-2.075038-5.747830-0.759006$

H $-2.382270-3.151282-1.274873$

H $-4.641774-6.3196830 .493512$

H $-4.365731-6.325451-1.232051$

H $-5.616398-5.272001-0.542493$

H $-4.082119-4.723206-2.894109$

H -4.214164 -2.039875 -3.697129

H $-4.968150-0.822835-2.698065$

H $-6.260547-2.597537-2.378250$

H $-2.231090-0.5329331 .025879$

H -0.1118170 .6800732 .911330$

H $0.920320-2.7664293 .811986$

H $-0.481626-1.8702474 .389394$

H $1.088656-1.0624204 .196931$

H $-2.9792780 .221390-3.001492$

H -2.605093 -0.623395 -1.497326

H $1.554326-0.610334-3.176961$

H $0.753278-1.701769-4.332093$

H $\quad 0.553816-3.494278-2.557349$

H $2.847889-1.771687-1.480556$

H $3.589626-4.083057-0.889590$

H $1.969214-4.716808-1.047820$

H $3.080377-3.3404092 .678816$

H $4.302633-3.5584281 .305860$

H $0.717637-4.0534622 .151433$

H $0.202463-4.1296550 .504173$

H $-0.046881-1.7348640 .287350$

H $-0.447589-0.312110-1.997015$

C $-1.972325 \quad 5.492173-1.784807$

O $-1.4367094 .151292-1.785773$

C $-0.3477864 .107974-0.842219$

C $0.2062085 .519445-0.844590$

C $-1.0818216 .325525-0.854502$

H $-0.794307 \quad 3.8791590 .133205$

C $0.6155372 .990858-1.239619$

H 0.8330725 .7352250 .025147

H $0.7891535 .708099-1.754290$ 
C $-0.8978547 .761380-1.316078$ H $-1.489421 \quad 6.341410 \quad 0.163624$ C $1.9011362 .952295-0.391564$ O $-0.0934691 .756896-1.093798$ H $0.8788563 .073485-2.300821$ H $2.4722843 .865502-0.595809$ $\begin{array}{lllll}\text { O } & 1.566947 & 2.978231 & 0.996847\end{array}$ C $2.8052361 .735290-0.674748$ C 4.1530961 .8527680 .048818 H $2.969421 \quad 1.657407-1.754666$ O $2.1810020 .533033-0.231911$ H $4.6496392 .791736-0.226989$ H $3.982781 \quad 1.9187691 .132431$ H $-0.2243798 .299387-0.640958$ H $-0.4709197 .808121-2.323444$ H $-1.8569618 .288897-1.330517$ C -3.459551 5.441148-1.410588 H $-0.9148621 .831700-1.609795$ H 1.0654622 .1674531 .188959 H $1.3483640 .433036-0.722348$ H -3.999099 $4.920265-2.212432$ H -3.873503 $6.454703-1.362155$ H $-1.8873975 .855397-2.816545$ C $7.273757-0.0903540 .611593$ $\begin{array}{lllll}\text { O } & 6.269714 & 0.932227 & 0.610447\end{array}$ C $5.1081320 .666240-0.187618$ C $5.5087450 .470422-1.657991$ C $6.597416-0.597121-1.809949$ C $7.749533-0.389529-0.819417$ H $\quad 8.115730 \quad 0.347418 \quad 1.163500$ C $6.818147-1.339151 \quad 1.372534$ H $4.624788-0.2419580 .187615$ O $5.9716541 .701105-2.210938$ H $4.6294040 .167969-2.236941$ H $6.983905-0.565585-2.835913$ O $6.006317-1.877737-1.608962$ H $8.412698-1.262301-0.807695$ O $8.533726 \quad 0.727126-1.249562$ C $6.534327-1.0137402 .819377$ H $5.927569-1.8011020 .939814$ H $7.598221-2.1095871 .357554$ H $6.7149832 .010802-1.665502$ H $6.701448-2.545579-1.736796$ H $8.8793570 .519052-2.134326$ O $5.199795-0.7932892 .992879$ O $7.398050-0.9403143 .683675$ C $4.796241-0.4521834 .322103$ H $3.714670-0.2932014 .318031$ H $5.028567-1.2709175 .009674$ H 5.2857340 .4727324 .641451

SCF Energy $\left(B 3 L Y P / 6-31 G^{* *} / / M M F F\right)=-3245.92692038$

$08 \_00319$

MM̄FF Geometry

C $3.040505-2.8140942 .163743$

C $2.532257-2.6608093 .396608$

C $1.091112-2.3716943 .750237$

O $0.260527-2.4453352 .571322$

C $0.951929-0.9603354 .346365$

C $-0.405647-0.7036534 .973744$

C $-1.3357840 .136272 \quad 4.477824$

C $-0.658881-1.4145016 .279128$

C -1.2756640 .9384543 .208100$

C -0.8165112 .3753823 .483292$

C $-0.878763 \quad 3.2508612 .223485$

C -0.1635604 .5817752 .457995$

C -0.2727155 .5071791 .238059$

O $-2.255543 \quad 3.492894 \quad 1.917917$

$\begin{array}{lllll}0 & 0.132483 & 6.823667 & 1.644683\end{array}$

C -1.7534225 .6044510 .812557$

C 0.6887645 .0875390 .120089

O $-1.8851996 .314216-0.416986$

C -2.4748674 .2360520 .709856$

C -3.999592 4.4609520 .554828

O $-2.0419663 .506504-0.436992$

C $-0.752848-3.3504732 .551185$

O $-1.072277-4.1272273 .436345$

C -1.419745 -3.229837 1.235249
C $-2.475646-4.0057230 .947213$

C $-3.248232-3.979668-0.297591$

C $-2.757879-3.070093-1.393706$

C -4.8580013 .1948090 .666882$

C $-4.8473732 .298052-0.559490$

C $-5.5479480 .026294-1.289547$

O $-4.4163292 .644888-1.654901$

C $-5.846992-1.269956-0.609808$

C $-6.817110-2.115467-0.989115$

C $-7.110952-3.402292-0.270893$

C $-6.767102-4.632281-1.085084$

C $-7.713281-5.535366-1.393276$

C $-5.331369-4.835316-1.535287$

C $-4.348803-4.755775-0.394747$

N $-5.4112901 .060327-0.280763$

H $2.401289-2.7166721 .291835$

H $3.202828-2.7710044 .247300$

H $0.791414-3.1269634 .485742$

H $1.159762-0.2170003 .568947$

H $1.715185-0.8009725 .120011$

H $-2.259975 \quad 0.267474 \quad 5.039667$

H -1.602054 -1.107565 6.743776

H $-0.706264-2.4969716 .127966$

H $0.142608-1.1961266 .992537$

H -0.6194210 .4667762 .469552$

H -2.2771090 .9392402 .760312$

H -1.451694 2.8145884 .263833

H $\quad 0.2101622 .3524203 .869354$

H -0.4149752 .7074671 .394428$

H 0.8891224 .4180642 .720494

H -0.6130955 .0906813 .322260$

H 1.0393756 .7648751 .991153

H -2.269072 6.2058931 .574537

H 1.7261745 .1181750 .474786

H $0.6409845 .782940-0.725047$

H $0.4993024 .078874-0.250609$

H -1.445141 $7.174116-0.303533$

H -4.3332845 .1330021 .356316$

H $-4.2103064 .958217-0.399906$

H -1.481339 $4.082145-0.981417$

H $-1.027333-2.4944540 .541757$

H -2.822416 -4.714407 1.698128

H -1.724127 -3.318264 -1.660545

H -2.794641 -2.023295 -1.073136

H -3.334291 -3.142417 -2.317531

H -4.5689752 .6108631 .547931$

H -5.901858 3.5021920 .811945

H $-4.608420-0.042144-1.847981$

H $-6.3408480 .332589-1.979532$

H $-5.224792-1.5313380 .243591$

H -7.440556 -1.869955 -1.845168

H -8.177110 -3.397455 -0.009264

H $-6.578605-3.4461980 .687620$

H -7.481288 -6.423261 -1.972108

H -8.742754 -5.408200-1.075096

H $-5.123605-4.105980-2.322126$

H -5.214226 -5.822683 -1.999926

H $-4.599305-5.4045410 .445997$

H -5.9035340 .9419640 .600804$

C $5.327156-1.9649701 .495488$

O $4.894000-1.4255050 .228241$

C $6.046153-1.234420-0.612615$

C $7.257098-1.3119110 .309604$

C $6.799871-2.3436711 .316465$

H $6.071412-2.087252-1.301346$

C $5.9606780 .073034-1.409409$

H $8.167237-1.603459-0.223443$

H $7.450403-0.3569290 .811678$

C $7.603140-2.3305932 .606220$

H $6.879603-3.3382880 .857027$

C $4.6891530 .267782-2.258469$

O $6.0374561 .186356-0.508230$

H $6.8451820 .155361-2.052546$

H $3.8447710 .523930-1.609892$

O $4.9118711 .411548-3.095463$

C $4.302455-0.921392-3.151824$

C $3.252758-0.609745-4.236686$

H $3.939631-1.739098-2.518112$ 
O $5.474122-1.399161-3.821031$ H $3.712032-0.004783-5.029587$ H $3.001184-1.567529-4.711914$ H $8.657515-2.5460482 .404531$ H $7.548303-1.3560673 .102964$ H $7.232487-3.0862403 .305737$ C $4.473727-3.1718531 .893027$ H 5.3186351 .0879310 .138902 H $5.1323432 .158484-2.512522$ H $5.220826-2.189208-4.328400$ H $4.906020-3.6578332 .776031$ H $4.479245-3.9167601 .087328$ H $5.220790-1.1607122 .234650$ C $0.188241-0.207677-2.126450$ O $1.380847-0.734203-2.726428$ C $1.9687630 .077767-3.745931$ C $0.9765460 .310380-4.899952$ C $-0.3524090 .876458-4.390501$ C $-0.8834230 .081241-3.193172$ H $-0.187052-1.018202-1.489573$ C $0.4798051 .006829-1.232435$ H $2.2436571 .046838-3.319372$ O $0.734476-0.922066-5.577306$ H $1.4301961 .000632-5.621413$ H $-1.0900250 .848637-5.201881$ O $-0.1586372 .239959-4.028040$ H -1.724948 $0.609600-2.730691$ O $-1.400249-1.165347-3.666560$ C $1.3439210 .653176-0.043796$ H $0.9830591 .801179-1.789565$ H $-0.456827 \quad 1.408290-0.833635$ H $0.142672-0.734053-6.325625$ H - $-1.0145762 .587167-3.723785$ H $-0.653462-1.688522-4.004723$ O $2.017068 \quad 1.7654590 .370731$ O $1.388638-0.4501250 .479899$ C 2.8477461 .6128421 .526036 H 3.3796490 .6576881 .517940 H 3.5844172 .4206811 .522026 H 2.2349851 .7012982 .426299

SCF Energy $\left(\mathrm{B} 3 \mathrm{LYP} / 6-31 \mathrm{G}^{* *} / / \mathrm{MMFF}\right)=-3245.91835906$

0800320

MMFF Geometry

C $2.944935-4.027654-0.861029$

C $1.698903-3.537290-0.773614$

C $0.806624-3.333834-1.966365$

O $0.101936-2.076686-1.848426$

C $-0.244845-4.452962-2.142867$

C $-1.188223-4.675298-0.972876$

C $-2.332920-3.992628-0.765775$

C $-0.794967-5.781207-0.025465$

C $-2.906689-2.887091-1.609634$

C $-4.404244-3.055562-1.913338$

C $-5.320952-2.824360-0.700228$

C $-6.793114-2.926817-1.108285$

C -7.738117 -2.5882960 .055649$

O $-5.050622-1.517642-0.184175$

O $-9.051660-2.383917-0.487160$

C $-7.297916-1.2500360 .687244$

C $-7.856435-3.7495281 .049907$

O $-8.052227-0.967721 \quad 1.863893$

C $-5.785770-1.1834180 .999608$

C $-5.3880550 .254701 \quad 1.411846$

O $-5.492969-2.0765252 .076421$

C $0.744883-0.929609-2.191523$

O $1.919966-0.800952-2.491200$

C $-0.2737110 .146583-2.188909$

C $0.0449541 .391173-2.572918$

C $-0.8910172 .513705-2.702722$

C $-2.3447762 .246915-2.412811$

C $-3.872198 \quad 0.476410 \quad 1.492376$

C -3.5436801 .8632432 .005592$

C $-3.491460 \quad 4.2517001 .349049$

O -3.1592662 .0489123 .158171$

C $-3.3179375 .018510 \quad 0.077234$

C $-2.1866895 .656832-0.260090$

C $-2.0444816 .510473-1.486743$
C $-0.9072496 .080421-2.384231$

C $0.2677006 .733393-2.368019$

C $-1.1764954 .976451-3.381982$

C $-0.4141653 .713028-3.095884$

N -3.7139042 .8510991 .048285$

H $3.330460-4.308056-1.837252$

H $\quad 1.291740-3.280537 \quad 0.200885$

H $1.403332-3.302923-2.888030$

H $\quad 0.271239-5.396600-2.365774$

H $-0.836877-4.237383-3.041005$

H $-2.910637-4.2393520 .123036$

H $-1.529136-5.9244470 .774635$

H $-0.712666-6.729745-0.566524$

H $0.166778-5.5690420 .449002$

H -2.736904 -1.935981-1.093007

H $-2.387305-2.815189-2.569793$

H $-4.658218-2.318474-2.686940$

H $-4.591034-4.048447-2.340513$

H -5.091524 -3.5711920.066994

H -7.017014 -3.923382 -1.508728

H $-6.993329-2.223045-1.928431$

H $-9.312898-3.196502-0.953433$

H -7.537030 -0.454321 -0.032258

H -8.247764 -4.6433530 .549439$

H -8.570958 -3.516264 1.846880

H $-6.903250-4.0209071 .508443$

H -8.993236 -1.010144 1.621708

H -5.7940260 .9646860 .679988$

H -5.8351720 .5016152 .383138$

H -4.718063 -1.754484 2.561952

H -1.284425 -0.129860 -1.911255

H $1.0782561 .604654-2.842828$

H $-2.753037 \quad 1.526350-3.129909$

H -2.971762 $3.139157-2.461628$

H -2.467229 $1.842114-1.403320$

H $-3.393852-0.2439862 .164120$

H -3.4040420 .3486480 .509329$

H -2.6254694 .3437442 .013825$

H -4.3690254 .6187141 .891256$

H $-4.1830235 .085922-0.578143$

H $-1.329917 \quad 5.6097110 .408024$

H -1.883384 $7.543550-1.150823$

H $-2.9757386 .537481-2.066293$

H $1.0738916 .460475-3.040760$

H $0.4502497 .551849-1.679677$

H $-0.8768295 .324895-4.380165$

H $-2.2522234 .800772-3.472129$

H $0.6570243 .803482-3.282624$

H -4.1906542 .6132050 .183083$

C $5.180080-3.5460670 .280141$

O $4.994761-2.1183680 .228554$

C $6.027028-1.556703-0.605721$

C $7.018497-2.684649-0.882228$

C $6.105264-3.894588-0.884415$

H $5.547041-1.278841-1.551384$

C $6.651179-0.3209780 .050128$

H $7.550767-2.544903-1.828089$

H $7.766612-2.773695-0.085819$

C $6.838717-5.214037-0.721200$

H $5.557764-3.904530-1.834161$

C $5.6542120 .807710 \quad 0.384861$

O $7.277369-0.7236431 .275615$

H $7.4535790 .061977-0.592109$

H 5.0262270 .4993591 .227616

O $6.419817 \quad 1.925403 \quad 0.855237$

C $4.7691961 .261844-0.788936$

C $3.9904452 .568025-0.546596$

H $4.0662470 .460128-1.040402$

O $5.6001331 .466888-1.936034$

H $4.6886143 .412998-0.489101$

H $3.3897062 .747207-1.448654$

H $7.544439-5.365864-1.544432$

H $7.404342-5.2464130 .216074$

H $6.135627-6.052842-0.720248$

C $3.831920-4.2702050 .327852$

$\begin{array}{llll}\text { H } & 7.670699 & 0.071443 & 1.674708\end{array}$

H $6.9503892 .252467 \quad 0.108335$

H $5.0174561 .673419-2.686630$ 
H $3.307249-3.962151 \quad 1.240427$

H $4.002966-5.3497170 .413115$

H $5.697597-3.747462 \quad 1.228170$

C 1.2453411 .2841231 .636916

O 2.1669621 .4662270 .553755

C 3.0768562 .5605690 .688903

C 2.3187283 .8917730 .842222

C 1.2678713 .8269821 .954287

C 0.4138202 .5586231 .855259

H $\quad 0.565874 \quad 0.492219 \quad 1.296418$

C 1.9381840 .7908362 .916912

H 3.6973772 .4043991 .576505

O $1.6781454 .244296-0.381706$

H 3.0424524 .6853671 .061507

H 0.6201894 .7093901 .883196

O 1.9310863 .8764303 .213105

H -0.2090562 .4505542 .750437$

O -0.4680932 .7142430 .741683$

C $2.553615-0.5804212 .747922$

H 2.7386821 .4660483 .229166

H 1.2021050 .7191143 .725910

H $1.0573903 .530366-0.605624$

H 1.2481233 .8615923 .905132

H -1.029029 1.9218110 .694920

O $3.246422-0.8949043 .882535$

O $2.440873-1.2870551 .756852$

C $3.902190-2.1664433 .874803$

H $4.331607-2.3331534 .866214$

H $3.186937-2.9674163 .664733$

H $4.710742-2.1639333 .138759$

SCF Energy $\left(B 3 L Y P / 6-31 G^{* *} / / M M F F\right)=-3245.90790198$

$08 \_00321$

MMFF Geometry

C $-0.724804-3.6978760 .943699$

C $-0.267400-4.132867-0.241265$

C $-0.757135-3.681033-1.596740$

O $-1.234824-2.317023-1.549787$

C $-1.898822-4.596137-2.071614$

C $-2.395058-4.269844-3.466874$

C $-3.547335-3.626727-3.743591$

C - $1.513833-4.754399-4.589505$

C $-4.544395-3.064135-2.768359$

C $-4.730485-1.555786-2.978913$

C $-5.584758-0.921447-1.871018$

C $-5.8119580 .564497-2.155526$

C $-6.5420861 .272847-1.005079$

O $-4.883917-1.085042-0.633465$

O $-6.4089162 .688989-1.204491$

C $-5.830214 \quad 0.937386 \quad 0.323203$

C $-8.0456270 .973420-1.015265$

O $-6.562147 \quad 1.449387 \quad 1.433882$

C $-5.569919-0.5701790 .515740$

C $-4.695807-0.882251 \quad 1.756502$

O $-6.822184-1.2398110 .707866$

C $-0.289013-1.337326-1.618836$

O $0.921921-1.488127-1.668541$

C $-0.985495-0.031924-1.637590$

C $-0.2684591 .099772-1.702478$

C $-0.8009042 .462949-1.759944$

C $-2.2951412 .635190-1.703235$

C $-3.277711-0.3032031 .729468$

C -3.1354471 .0479732 .408767$

C -1.4641092.869936 2.745815

O $-4.010611 \quad 1.547918 \quad 3.109409$

C -1.6004033 .9536861 .724623$

C $-0.577316 \quad 4.726083 \quad 1.328235$

C $-0.714165 \quad 5.866648 \quad 0.361961$

C $0.2289925 .756741-0.813795$

C $1.3985596 .418330-0.828365$

C $-0.2290364 .955886-2.010316$

C $0.0680813 .489209-1.869471$

N $-1.887417 \quad 1.6079132 .170984$

H -1.539349 -2.9795970 .976466$

H $0.526832-4.876450-0.256784$

H $0.077634-3.740568-2.306602$

H -2.722995 -4.557397-1.351405

H -1.563908 $-5.642607-2.061838$
H $-3.810046-3.474658-4.789846$

H $-1.942143-4.544217-5.575324$

H $-0.534347-4.268289-4.547133$

H -1.369383 $-5.837398-4.518412$

H $-5.504274-3.572628-2.918682$

H $-4.251018-3.256091-1.734276$

H -3.744597 -1.074757 -2.995185

H $-5.196197-1.377294-3.956206$

H $-6.541489-1.454126-1.818159$

H $-6.3561260 .700590-3.098388$

H $-4.8415041 .056666-2.300212$

H $-6.7853312 .905877-2.074788$

H $-4.869031 \quad 1.4653170 .310265$

H $-8.4976901 .316188-1.953828$

H $-8.5629801 .518499-0.218174$

H $-8.272143-0.089687-0.911642$

H -6.6852122 .4025341 .285143$

H $-5.228621-0.6053442 .674207$

H -4.583958 -1.975251 1.805505

H -6.654806 -2.086709 1.153467

H -2.068192 $-0.036875-1.612459$

H $0.8180851 .024517-1.730212$

H -2.611310 3.676891-1.616181

H $-2.7131272 .120247-0.831944$

H -2.757257 $2.228181-2.608552$

H $-2.618083-0.9849912 .281340$

H $-2.898027-0.2412210 .705876$

H -0.4294062 .7448873 .076383$

H -2.079194 3.1061463 .619900

H -2.594253 4.1342281 .321732

H $0.4091834 .559923 \quad 1.754695$

H -0.5123656 .7943520 .913744$

H -1.744603 $5.962182-0.002318$

H $2.0659006 .367016-1.682325$

H 1.7175167 .0196490 .016565

H $0.2922665 .305771-2.911962$

H -1.284284 $5.162316-2.209814$

H $1.1340743 .263182-1.902730$

H -1.2501501 .1405351 .533261$

C $0.146396-2.9927413 .197605$

O $1.061012-2.0794512 .566207$

C $2.060699-1.6760373 .516695$

C $1.580115-2.1746084 .875628$

C $0.828047-3.4333954 .492757$

H $2.977657-2.2108173 .237544$

C $2.330227-0.1633783 .448426$

H $2.409084-2.3612515 .565169$

H $0.899345-1.4618735 .354978$

C $-0.133273-3.9193215 .563360$

H $1.560415-4.2245704 .283132$

C 2.6614170 .3249052 .020627

O $1.187570 \quad 0.5311883 .947810$

H 3.1691670 .0592304 .118945

H $3.382662-0.3714041 .579446$

O $1.486461 \quad 0.2768581 .211529$

C 3.2623541 .7450721 .961749

C 3.4138162 .3123260 .536981

H 4.2367821 .7332312 .464404

O 2.4321812 .6497602 .692381

H $2.4212122 .499516 \quad 0.109842$

H 3.8823863 .3011030 .633123

H $0.406638-4.1568946 .485782$

H $-0.886675-3.1605915 .800376$

H $-0.656843-4.8231385 .236374$

C $-0.165965-4.1619672 .260070$

H 1.4129321 .4759363 .985842

H 0.8487930 .9026001 .594680

H 2.8656853 .5201492 .684722

H $-0.895590-4.8280102 .734445$

H $\quad 0.747316-4.7450302 .086799$

H $-0.768714-2.4258333 .412903$

C $6.4153800 .399578-0.576767$

O $5.542543 \quad 1.277986 \quad 0.148340$

C $4.2389361 .438316-0.418938$

C $4.3424692 .058805-1.821089$

C $5.2547531 .224682-2.725568$

C $6.5771520 .866488-2.035114$

H $7.3867280 .511391-0.078283$ 
C $5.965349-1.059528-0.410100$

H $3.755797 \quad 0.459280-0.503152$

O $4.8559403 .385370-1.719434$

H $3.3382622 .123194-2.255921$

H $5.4640521 .788721-3.642761$

O $4.5586310 .038969-3.097611$

H $7.1068280 .101937-2.613170$

O $7.4172162 .026313-2.037919$

C $7.062631-2.058446-0.704546$

H $5.647877-1.2193940 .627611$

H $5.109475-1.286793-1.051639$

H $4.8674043 .764416-2.614841$

H $5.137180-0.466445-3.693927$

H $6.9937772 .700917-1.479568$

O $6.540651-3.319390-0.636676$

O $8.227635-1.778907-0.950710$

C $7.465372-4.382355-0.887759$

H $6.924509-5.329238-0.809112$

H $7.876720-4.295998-1.898010$

H $8.265633-4.372064-0.141602$

SCF Energy (B3LYP/6-31G**//MMFF) $=-3245.90959952$

08_00322

MMFF Geometry

C $2.286894-1.0023873 .490294$

C $3.293515-0.1166783 .429181$

C $4.568276-0.2249562 .624828$

$\begin{array}{lllll}\text { O } & 4.454134 & 0.660102 & 1.484627\end{array}$

C $4.880071-1.6231382 .058523$

C $6.350388-1.8251821 .746394$

C $6.918881-1.6695290 .535309$

C $7.185645-2.2880872 .913755$

C $6.292277-1.171248-0.736143$

C $6.065478-2.282784-1.770201$

C $4.741417-3.031023-1.555032$

C $4.594137-4.178947-2.554854$

C $3.223717-4.864167-2.438398$

O $3.684481-2.083389-1.742683$

O $3.048657-5.699855-3.592738$

C $2.121161-3.785776-2.489388$

C $3.147524-5.788343-1.217416$

O $0.837370-4.355047-2.241827$

C $2.367857-2.600819-1.526979$

C $1.359701-1.465261-1.825115$

O $2.187660-3.047734-0.181890$

C $4.710107 \quad 1.982916 \quad 1.642752$

O $5.027058 \quad 2.562076 \quad 2.667604$

$\begin{array}{llll}\text { C } 4.503670 & 2.610113 \quad 0.319217\end{array}$

C $4.302098 \quad 3.9317390 .226785$

C $4.0351614 .671803-1.008926$

C $4.072626 \quad 3.903070-2.305035$

C $1.667877-0.137261-1.115751$

C $0.5858300 .900907-1.367959$

C $-0.0993913 .201435-0.690042$

O $-0.369447 \quad 0.707004-2.115497$

C $0.3702264 .194690-1.706269$

C $0.4798725 .509040-1.457347$

C $0.9130226 .521489-2.480106$

C $2.0030847 .441787-1.974039$

C $1.7167248 .686236-1.555538$

C $3.4324056 .949710-2.032890$

C $3.7532315 .988422-0.921519$

N $0.8113452 .071195-0.655568$

H $2.319807-1.9119732 .897870$

H 3.2122610 .7866634 .031920

H $5.3986280 .104316 \quad 3.262655$

H $4.280807-1.7987371 .159161$

H $4.593251-2.4049982 .773320$

H $7.982664-1.8873660 .441529$

H $8.238769-2.4244702 .645813$

H $7.145615-1.5566413 .726967$

H $6.814182-3.2471513 .289860$

H $5.367787-0.619904-0.548176$

H $6.987668-0.437921-1.165150$

H $6.041287-1.809531-2.760791$

H $6.907257-2.985896-1.767089$

H $4.710902-3.416930-0.530635$

H $5.401819-4.910698-2.429081$
H $4.699607-3.789050-3.577046$

H $3.779499-6.341303-3.611822$

H $2.094082-3.396414-3.516960$

H $3.913608-6.570521-1.281167$

H $2.186619-6.313446-1.178851$

H $3.291843-5.262551-0.271393$

H $\quad 0.717260-5.085107-2.872970$

H $1.350792-1.262893-2.904472$

H $0.350405-1.793202-1.549672$

H $1.905789-2.3012640 .369154$

H $4.4863821 .954517-0.544390$

H $4.3080554 .523852 \quad 1.140845$

H $5.0714073 .484571-2.471934$

H $3.8322074 .507869-3.181395$

H $3.3473703 .082273-2.290951$

H $1.751114-0.284411-0.034488$

H $2.617147 \quad 0.271880-1.479740$

H -0.1271493 .6260550 .318135$

H $-1.1034732 .852164-0.951186$

H $0.6064933 .820465-2.699642$

H $0.2113135 .885558-0.472756$

H $0.0289657 .110519-2.757361$

H $1.2490836 .037001-3.405408$

H $2.4949469 .362230-1.216970$

H $0.6966579 .055173-1.536637$

H $4.1269577 .795420-1.938797$

H $3.6294066 .539903-3.027150$

H 3.7464936 .4518650 .066243

H $1.6537542 .157700-0.095120$

C $-0.237385-0.657167 \quad 3.624360$

O $-0.534633-1.8277982 .839491$

C $-1.154590-1.4029791 .603670$

C $-1.4680110 .077046 \quad 1.779235$

C -0.3222780 .5262052 .664051$

H $-0.390919-1.5392200 .829704$

C $-2.364303-2.3005981 .313262$

H -1.5153900 .6146350 .828530$

$\begin{array}{llll}\text { H } & -2.425987 & 0.213474 & 2.294542\end{array}$

C $-0.5574891 .861865 \quad 3.345079$

H 0.5821790 .5941032 .047885

C $-3.215138-1.818796 \quad 0.120003$

O $-1.868484-3.617245 \quad 1.059008$

H -2.997945 -2.3712732 .205716$

H $-3.576963-0.8147880 .361962$

O $-2.375719-1.702975-1.027669$

C $-4.425548-2.722972-0.197315$

C $-5.322054-2.211184-1.344001$

H $-5.026070-2.8378140 .712903$

O $-3.969068-4.026294-0.565154$

H $-4.760321-2.210523-2.286267$

H $-6.122563-2.950874-1.480921$

H $-0.7063232 .652482 \quad 2.603133$

H $-1.442367 \quad 1.8350743 .988666$

H 0.3011382 .1379053 .965152

C $1.083403-0.8294344 .376249$

H $-1.340205-3.8838841 .830873$

H -2.859721 -1.197371 -1.701692

H $-4.751638-4.592649-0.675577$

H $1.017500-1.7275265 .003445$

H 1.2249940 .0192235 .056310

H $-1.040173-0.5857764 .370922$

C $-7.3299690 .309390 \quad 0.538431$

$\begin{array}{lllll} & \text { O } & -6.722480 & -0.912374 & 0.100226\end{array}$

C $-5.941059-0.827251-1.094240$

C $-6.808690-0.355683-2.270211$

C $-7.4964210 .973410-1.937144$

C $-8.1964230 .925662-0.574805$

H $-8.0021590 .011242 \quad 1.353964$

C $-6.2929901 .282321 \quad 1.122964$

H $-5.131787-0.105790-0.952476$

O $-7.790810-1.344095-2.570621$

H $-6.171943-0.242334-3.155783$

H -8.234626 $1.209023-2.713218$

O $-6.5330872 .023077-1.921581$

H $-8.517627 \quad 1.929284-0.272409$

O $-9.3780370 .126493-0.687010$

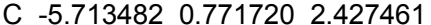

H -5.4544551 .4623730 .446047$ 
H -6.7863132 .2384041 .334741$

H $-8.307642-1.023906-3.329262$

H $-6.1192722 .055000-2.800956$

H $-9.9509570 .541961-1.353731$

O -5.1189101 .8094213 .087681$

O $-5.759400-0.3879912 .814017$

C $-4.553200 \quad 1.4854794 .361907$

H -4.0303982 .3695734 .736264$

H -5.3480191 .2220375 .066120$

$\mathrm{H}-3.8341480 .666124 \quad 4.269946$

SCF Energy (B3LYP/6-31G**//MMFF) $=-3245.87954113$

08_00323

MM̄FF Geometry

C -4.251891-2.949502 1.313602

C $-3.071562-2.3168201 .374789$

C $-2.434616-1.8284322 .646359$

O $-2.127066-0.4235812 .508655$

C -1.118554 -2.592484 2.872610

C $-0.288696-2.0886794 .037378$

C $0.990287-1.6778833 .920624$

C $-0.952785-2.1253695 .387996$

C $1.804136-1.6160312 .656644$

C $2.397040-2.9869372 .308779$

C $3.173487-2.9666830 .985486$

C $3.605853-4.3854690 .607543$

C $4.484645-4.403517-0.649902$

O $4.320731-2.130684 \quad 1.158215$

O $5.095668-5.699282-0.744126$

C $5.623753-3.378901-0.471288$

C $3.656132-4.219660-1.927399$

O $6.388999-3.260466-1.669079$

C $5.133461-1.984341-0.014993$

C $6.348824-1.0994200 .361425$

O $4.409782-1.380121-1.083159$

C $-3.118940 \quad 0.4549402 .804855$

$\begin{array}{lllll}\text { O } & -4.284786 & 0.178940 & 3.056436\end{array}$

C -2.6262361 .8501302 .855528$

C -1.385786 2.2337422514700

C -0.8563553 .5982562 .609645$

C -1.7529734 .6549593 .203968$

C 5.9961640 .2956320 .899642

C $5.814671 \quad 1.350638-0.179443$

C $5.2981973 .753728-0.473573$

O $6.0020571 .135443-1.373728$

C 4.5078844 .7946330 .250071

C $3.3523135 .296914-0.209771$

C 2.5743126 .3793650 .480819

C 1.2121975 .9075430 .936746

C $0.109867 \quad 6.2245120 .235838$

C 1.1559975 .1453792 .243474

C $0.397126 \quad 3.8470232 .173514$

N 5.4148042 .5692330 .354679

H -4.819637 -3.119954 2.224386

H -2.516665 -2.1433880 .457034$

H $-3.096951-1.9811823 .508036$

H -0.532406 -2.558701 1.947490

H -1.340222 -3.654814 3.043608

H $1.519421-1.3627974 .818402$

H $-0.256497-1.8858756 .199000$

H -1.772520 -1.401946 5.434759

H -1.355043 -3.1242045.586296

H $1.199173-1.2317541 .827580$

H $2.608399-0.8839142 .800080$

H $3.065481-3.2976153 .122714$

H $1.595377-3.7327812 .243656$

H $2.529128-2.5423060 .206513$

H $2.732259-5.0358970 .473964$

H $4.179993-4.8239741 .436119$

H $4.386016-6.363341-0.781160$

H $\quad 6.305886-3.7737850 .294729$

H $2.920209-5.026849-2.025452$

H $4.288066-4.280911-2.820163$

H $3.110482-3.274141-1.954321$

H $6.683638-4.154362-1.914210$

H $6.904512-1.6126741 .157693$

H $7.025057-1.000866-0.495820$

H $4.274197-0.443740-0.872105$
H $-3.3611542 .563326 \quad 3.216070$

H -0.6842951 .4889202 .142948$

H -2.676011 4.7482792 .620626

H -1.303376 5.6501633 .222186

H -2.0122454 .4026444 .238006$

H 5.0989860 .2629521 .527086

H $\quad \begin{array}{llll}6.827721 & 0.638481 & 1.528683\end{array}$

H $4.8472713 .476409-1.432791$

H $6.3105484 .121151-0.672041$

H 4.9196455 .1709871 .183478

H $2.9532194 .932127-1.153333$

H $2.4731997 .218055-0.220436$

H 3.1243586 .7832371 .340252

H -0.8807445 .9276880 .561198$

H $\quad 0.173620 \quad 6.792707-0.686522$

H 0.7812055 .8079763 .030293

H 2.1748384 .8909362 .564820

H 0.9723203 .0273031 .742026

H 5.4655832 .6908601 .362598

C $-5.746960-2.483955-0.694048$

O $-4.983222-1.337109-1.120962$

C $-5.756559-0.152977-0.857072$

C $-7.184102-0.631712-0.614456$

C $-6.933991-1.9436540 .103470$

H -5.3695420 .2705150 .076550$

C $-5.6095380 .857076-1.997246$

H $-7.7660470 .079944-0.021026$

H -7.718923 -0.808832 -1.554919

C -8.135480 -2.871689 0.123795

H $-6.643043-1.7175071 .136725$

C $-4.1585451 .302050-2.278333$

O $-6.1245480 .263169-3.196218$

H $-6.2408521 .730521-1.794054$

H -3.597741 $0.464182-2.707325$

O $-4.2160542 .300773-3.306735$

C $-3.3957041 .872353-1.067053$

C $-2.0790442 .584170-1.420406$

H $-3.1933581 .077116-0.341412$

O $-4.1992542 .859615-0.410030$

H -2.292128 $3.459157-2.048400$

H $-1.6814782 .994276-0.482782$

H -8.973627 -2.4041150 .650757$

H $-8.471001-3.117349-0.889388$

H -7.894008 -3.8084390 .635985$

C $-4.837023-3.474800 \quad 0.032472$

H $-6.0209400 .915396-3.910232$

H -4.667967 $3.078051-2.935576$

H -4.9229322 .4032370 .049577$

H $-4.018901-3.763040-0.639912$

H $-5.390080-4.3945150 .256362$

H $-6.104527-2.963862-1.614613$

C $0.404414-0.214236-1.604262$

O $-0.6403100 .672888-1.174370$

C $-1.0061151 .710159-2.091365$

C $0.2122152 .566802-2.485427$

C $1.3840781 .713619-2.968116$

C $1.6697220 .564597-1.999857$

H $0.648066-0.802977-0.710906$

C $-0.067208-1.172109-2.706277$

H $-1.4278081 .257839-2.993816$

O $0.6432053 .359395-1.382142$

H -0.090347 $3.263708-3.275776$

H $2.2803732 .339597-3.058752$

O $1.0929331 .177478-4.254582$

H $2.399820-0.125229-2.437393$

O $2.2482531 .084546-0.799798$

C $-0.837867-2.335100-2.132069$

H $-0.711778-0.690958-3.446916$

H $0.781193-1.595052-3.258313$

H $0.8468742 .758324-0.645528$

H $0.942773 \quad 1.925438-4.857548$

H $3.0485121 .577047-1.049590$

O $0.026305-3.233201-1.576903$

O $-2.056529-2.433842-2.180131$

C $-0.562946-4.417463-1.031997$

H $0.241061-5.125030-0.812395$

H -1.083179-4.179358 -0.101170

H - $-1.247228-4.879204-1.750208$ 
H -7.204411 -1.161675 -4.676102

H $-6.6012230 .325935-3.975089$

H $-9.949368-1.375431-2.246081$

H $-9.256413-1.988453-3.850441$

H $-8.8242680 .349121-1.040569$

H -7.449620 $1.123979-1.782836$

H -7.366046 -1.520177 -0.159717

H $-2.838830-0.691267-1.060219$

C $0.684723-4.702716-0.460800$

O $1.790094-3.9275760 .044123$

C $2.120105-2.917713-0.927773$

C $1.394817-3.317459-2.207837$

C $0.123497-3.923531-1.648499$

H $1.701066-1.973533-0.555866$

C $3.637302-2.762803-1.096598$

H $1.215608-2.464526-2.868075$

H $1.959464-4.069356-2.772068$

C $-0.639598-4.775437-2.646479$

H $-0.523892-3.108133-1.308680$

C $4.354476-2.2721740 .175442$

O $4.210039-4.000456-1.517074$

H $3.800605-2.047775-1.908461$

H $3.880739-1.3602490 .546872$

O $4.211443-3.2433721 .220614$

C $5.866711-2.0365140 .003632$

C $6.300266-1.108487-1.146189$

H $6.368330-3.003116-0.130180$

O $6.383748-1.490827 \quad 1.223391$

H $7.398451-1.107672-1.167914$

H $5.963919-1.539345-2.095469$

H $-0.940845-4.176628-3.512079$

H $-0.031227-5.609728-3.011290$

H -1.544384 -5.190648 -2.191626

C $-0.297988-5.0336410 .665289$

H $4.048163-4.649466-0.810729$

H $3.262270-3.3335911 .410626$

H $6.121280-2.0904801 .942885$

H $\quad 0.224961-5.6579541 .401294$

H $-1.121573-5.6383510 .268466$

H $1.117926-5.647824-0.815185$

C $3.8143721 .698116-1.025897$

O $4.4179800 .406941-1.165310$

C $5.8383560 .360991-1.031560$

C $6.4956041 .241931-2.110677$

C $5.9361992 .671021-2.102164$

C $4.4044432 .692931-2.036944$

H 2.762977 1.537661 -1.293428

C 3.8549052 .2027210 .422061

H $6.1190550 .728152-0.039536$

O $6.2678340 .658285-3.393789$

H $7.5792211 .259789-1.943382$

H $6.2550423 .180453-3.019535$

O $6.4754413 .422737-1.018561$

H $4.0405613 .702281-1.811834$

O $3.8855142 .337783-3.323381$

C 3.1133551 .2661901 .349620

H 4.8800912 .3113830 .784248

H $3.379893 \quad 3.1868280 .506404$

H $6.7321151 .203488-4.051337$

H $6.6694602 .823676-0.280080$

H $4.1994473 .002415-3.959839$

O 3.9148620 .9298362 .400916

O $1.966103 \quad 0.881406 \quad 1.165420$

C 3.3542200 .0189383 .351400

H 2.5959120 .5300983 .950009

H $2.928448-0.8559292 .851239$

H $4.157061-0.3169244 .013011$

SCF Energy (B3LYP/6-31G**//MMFF) $=-3245.91630098$

0800325

MM̄FF Geometry

C $-0.8637072 .598933-0.297492$

C -1.7560512 .8241120 .678952$

C -2.022556 1.9506501 .884143

O $-1.309297 \quad 0.6995101 .761295$

C -3.5280221 .6321351 .956665$

C -3.9819351 .0788203 .291399$ 
C $-4.308227-0.2086163 .518398$

C -4.1196712 .0968874 .394223$

C $-4.215505-1.3707712 .569182$

C $-5.588085-1.8552172 .080838$

C $-6.215508-0.9412121 .015838$

C $-7.597894-1.4547660 .608947$

C -8.200469 $-0.639307-0.545373$

O $-5.346509-0.943000-0.120206$

O $-9.323655-1.387831-1.034366$

C $-7.159448-0.533561-1.681815$

C $-8.750720 \quad 0.705984-0.056771$

O $-7.6097480 .343497-2.712094$

C $-5.760335-0.089791-1.194577$

C $-4.726584-0.244898-2.337010$

O $-5.819366 \quad 1.280361-0.794455$

C -0.7211740 .1746812 .865023$

O -0.6424630 .6955873 .969293$

C $-0.158872-1.1711342 .596904$

C $-0.394155-1.8667791 .473059$

C $0.086009-3.2178421 .169652$

C $0.967669-3.8964602 .184336$

C $-3.271954-0.017668-1.898808$

C $-2.282580-0.359937-2.999561$

C $0.133877-0.887187-3.333116$

O $-2.610526-0.515241-4.172831$

C $0.168064-2.383688-3.338320$

C $1.161903-3.120429-2.819038$

C $1.147512-4.623291-2.804684$

C $1.259470-5.207575-1.411704$

C $2.381268-5.837901-1.023640$

C $0.046529-5.166110-0.506078$

C $-0.282788-3.787106 \quad 0.002537$

N $-0.984461-0.455566-2.513582$

H $-0.2282441 .718405-0.263984$

H -2.360352 3.7279630 .617349

H -1.6909552 .5072432 .768518$

H -3.7814470 .9350241 .152112$

H -4.120115 2.535928 1.757902

H $-4.658621-0.4790654 .513934$

H -4.5082821 .6582695 .319577$

H -3.149921 2.5467894 .627092

H -4.8094562 .8920884 .093131$

H -3.556275 -1.159938 1.722338

H $-3.733565-2.1927903 .114327$

H -5.445852 -2.854132 1.647775

H $-6.271268-1.9660172 .931951$

H -6.2911380 .0757741 .416014$

H -8.278588 -1.463794 1.469424

H -7.514636 -2.5017790 .284121$

H -9.756518 $-0.864251-1.729548$

H $-7.068018-1.529878-2.136801$

H -9.5482550 .5462790 .678880$

H $-9.2087591 .268842-0.877716$

H -7.990846 1.3365790 .409498

H -8.433948 -0.023611 -3.073095

H $-4.797123-1.261792-2.745389$

H $-4.9609380 .451499-3.152116$

H -4.939033 $1.677533-0.880429$

H $0.435148-1.5810143 .407602$

H -1.017592 -1.420469 0.700564

H $0.414251-4.0779073 .112079$

H $1.842197-3.2780442 .414300$

H $1.353475-4.8605081 .845068$

H -3.113389 $1.030674-1.629637$

H -3.039029 $-0.637318-1.025563$

H $1.042435-0.451077-2.906194$

H $\quad 0.006990-0.512305-4.353554$

H $-0.684497-2.889191-3.786800$

H $2.022958-2.623605-2.378861$

H $1.981971-4.967213-3.429633$

H $0.240227-5.017275-3.279971$

H $2.464258-6.294438-0.042980$

H $3.246066-5.906705-1.675265$

H $0.158855-5.8652610 .330979$

H $-0.821617-5.545108-1.061315$

H $-0.930368-3.220078-0.666371$

H $-0.828402-0.385400-1.512500$

C $0.6153844 .261658-1.544993$
O $1.7266693 .344603-1.560760$

C $2.7025893 .802435-0.608716$

C $2.3811905 .269903-0.365575$

C $0.8646135 .232818-0.388252$

H $2.512563 \quad 3.2437590 .315925$

C $4.1341993 .502830-1.071195$

H 2.7887195 .6360520 .581363

H $2.7545425 .908928-1.173408$

C $0.2194726 .594169-0.578138$

H 0.5184114 .8081490 .562006

C $4.3790351 .986809-1.231416$

O $4.4071794 .164040-2.302826$

H $4.8303883 .893007-0.318247$

H $3.9669561 .487495-0.348296$

O $3.6856211 .476915-2.369497$

C $5.8691601 .610659-1.345557$

C $6.123987 \quad 0.102167-1.539434$

H $6.3878151 .955253-0.442782$

O $6.4571092 .297950-2.450863$

H $5.783346-0.206627-2.536096$

H $7.212486-0.043471-1.541126$

H 0.4841937 .2635690 .246867

H $0.5445387 .066772-1.511112$

H $-0.871134 \quad 6.506315-0.606254$

C $-0.7069403 .492874-1.498543$

H $4.5029825 .112811-2.117172$

H $2.7433531 .689985-2.262178$

H $7.4053052 .083016-2.460639$

H $-0.7721442 .848290-2.384413$

H $-1.5415754 .201073-1.564645$

H $0.6602134 .801922-2.499598$

C $5.402408-1.0482701 .944067$

O $5.965641-0.3720440 .810996$

C $5.479787-0.790816-0.467639$

C $5.774943-2.279368-0.692490$

C $5.175016-3.1175730 .441554$

C $5.567838-2.5743001 .819077$

H $6.010452-0.7206242 .797352$

C $3.951793-0.6070722 .204326$

H $4.396235-0.647419-0.512002$

O $7.182577-2.488656-0.759843$

H $5.348327-2.583411-1.655978$

H $5.513727-4.156397 \quad 0.349729$

O $3.754810-3.110330 \quad 0.340711$

H $4.986541-3.0681812 .606648$

O $6.944196-2.8773332 .064314$

C 3.8625440 .8456402 .628853

H $3.325463-0.7265551 .316463$

H $3.527827-1.2057063 .018640$

H $7.331370-3.436693-0.915595$

H $3.521547-3.383046-0.562185$

H $7.041488-3.8442672 .033644$

O 2.5462201 .1920652 .742709

$\begin{array}{lllll}\text { O } & 4.817413 & 1.577886 & 2.847976\end{array}$

C 2.2781522 .5317093 .173131

H 2.9732693 .2483862 .725402

H 1.2642152 .7929032 .858468

H 2.3336652 .5775234 .264059

SCF Energy (B3LYP/6-31G**//MMFF) $=-3245.88769729$

08_00326

MMFF Geometry

C $-3.790484-1.3977202 .936814$

C $-2.495350-1.5326472 .610943$

C -1.370013 -0.9400153 .415572$

$\begin{array}{llll}\text { O } & -0.703151 & 0.082383 & 2.639639\end{array}$

C $-0.317212-2.014686 \quad 3.746519$

C $0.787440-1.4748334 .635278$

C $2.020244-1.1303214 .212335$

C $0.423489-1.3241836 .090925$

C $2.585873-1.2303962 .819318$

C $4.085945-1.5555142 .871894$

C $4.705156-1.8018861 .487219$

C $6.203084-2.0913241 .637308$

C $6.910157-2.237557 \quad 0.283407$

O $4.504720-0.640688 \quad 0.674247$

O $8.325695-2.163067 \quad 0.512563$

C $6.533984-1.034461-0.605095$ 
C $6.646033-3.608254-0.350971$

O $7.053070-1.196097-1.923605$

C $5.012475-0.766094-0.662799$

C $4.7387300 .557161-1.418023$

O $4.389011-1.838836-1.372720$

C -1.214743 1.3408512 .699524

O $-2.2887331 .676762 \quad 3.172360$

C -0.1990962 .2612302 .139313$

C $-0.488446 \quad 3.5653732 .018589$

C $0.421352 \quad 4.621017 \quad 1.566805$

C $1.861427 \quad 4.2489881 .334409$

C $3.2894011 .051659-1.313923$

C $3.0108802 .221850-2.242995$

C $1.1580893 .743425-2.935810$

O $3.8440872 .677814-3.021515$

C $1.4832845 .073158-2.332424$

C $0.5620306 .001993-2.034124$

C $0.9043007 .369210-1.516077$

C $0.1671397 .727308-0.246875$

C $-0.838788 \quad 8.618403-0.258148$

C $0.662211 \quad 7.129662 \quad 1.050531$

C $-0.068290 \quad 5.8700051 .421341$

N $1.7071822 .682323-2.113293$

H $-4.062970-0.8245453 .819305$

H -2.227272 -2.097411 1.722683

H $-1.750309-0.5013214 .346931$

H $0.099670-2.4141362 .815397$

H $-0.790357-2.8676984 .250610$

H $2.705424-0.7142544 .950777$

H $1.271793-0.9942176 .700174$

H $-0.376559-0.5885806 .216887$

H $0.083396-2.2825646 .496965$

H $2.078106-2.0018492 .234409$

H $2.436923-0.2706922 .311597$

H $4.602991-0.7143823 .353188$

H $4.250495-2.4399763 .500858$

H $4.192075-2.6545681 .027030$

H $6.365902-2.9844802 .253789$

H $6.678405-1.2635272 .182745$

H $8.566159-2.8696861 .136271$

H $7.026195-0.146287-0.184505$

H $7.023560-4.408706 \quad 0.296887$

H $7.181988-3.714628-1.300590$

H $5.586225-3.802936-0.527466$

H $8.009797-1.349879-1.841266$

H $5.3836861 .349847-1.016694$

H $5.0005840 .429645-2.476172$

H $3.566790-1.521710-1.776600$

H $\quad 0.767434 \quad 1.846802 \quad 1.876812$

H -1.4877003 .9012342 .293723$

H 2.3248813 .9137112 .268945

H $2.4696475 .070002 \quad 0.949943$

H 1.9391163 .4419370 .600967

H $2.5900840 .255309-1.582412$

H $3.0741881 .363869-0.285825$

H $0.0794273 .573983-3.011363$

H $1.5927973 .678995-3.938648$

H $2.5351015 .300874-2.175262$

H $-0.4884335 .790081-2.219853$

H $0.6609548 .090552-2.307311$

H $1.9832587 .472964-1.345335$

H -1.348688 8.9043810 .656116

H $-1.1782679 .077437-1.180672$

H $0.5043967 .848907 \quad 1.866095$

H 1.7470107 .0009061 .006515

H -1.127273 6.0342081 .627565

H $1.1097612 .286095-1.393470$

C $-5.551048-1.087773 \quad 1.112735$

O $-4.575205-0.7523040 .106056$

C -4.604233 $0.672006-0.099788$

$\begin{array}{llll}C & -5.973761 & 1.115512 & 0.392508\end{array}$

C $-6.122997 \quad 0.236865 \quad 1.624428$

H -3.8486381 .0997000 .571197$

C $-4.2337391 .019350-1.547283$

H $-6.0128952 .184960 \quad 0.618670$

H $-6.7641550 .882251-0.329438$

C $-7.549613 \quad 0.1313332 .133515$

H -5.4951830 .6529262 .421882$
C $-2.9520960 .310861-2.043124$

O $-5.288324 \quad 0.620298-2.429375$

H $-4.1312752 .105276-1.659437$

H $-3.182947-0.745822-2.211935$

O $-2.6276690 .863586-3.323360$

C $-1.7443600 .453016-1.101687$

C $-0.466001-0.267930-1.549025$

H -1.994673 $0.072152-0.107141$

O $-1.406247 \quad 1.834195-0.951225$

H $-0.0205330 .242152-2.412415$

H $0.257094-0.126335-0.734969$

H $-7.924931 \quad 1.1154482 .432615$

H -8.222417 -0.2652931 .365736$

H $-7.602730-0.5307873 .003655$

C -4.918378 -2.013045 2.156339

H $-6.016614 \quad 1.255383-2.324282$

H $-1.9241630 .317848-3.712526$

H -2.143944 $2.277480-0.500759$

H $-4.544623-2.9124191 .651737$

H $-5.684020-2.3515642 .864202$

H $-6.334179-1.6532030 .591467$

C $-1.682755-3.791540-1.050081$

O $-1.428765-2.388991-0.868347$

C $-0.616300-1.766328-1.867465$

C $0.757389-2.454048-1.947392$

C $0.623898-3.965021-2.151284$

C $-0.365810-4.580334-1.157316$

H $-2.177660-4.106015-0.122707$

C $-2.636641-4.064183-2.224807$

H -1.119353 -1.848508 -2.836141

O $1.499350-2.217451-0.750796$

H $1.328019-2.013603-2.772985$

H $1.605016-4.438073-2.021600$

O $\quad 0.180802-4.235986-3.476973$

H $-0.583884-5.620536-1.426199$

$\begin{array}{lllll} & \text { O } & 0.227048 & -4.600788 & 0.144717\end{array}$

C $-4.063165-3.675653-1.903025$

H -2.327580 -3.531444 -3.127636

H $-2.659297-5.133687-2.464188$

H $0.956746-2.5129180 .000357$

H $\quad 0.838406-3.863109-4.088594$

H $1.036493-5.136794 \quad 0.089410$

O $-4.643466-3.106882-2.999330$

O $-4.614669-3.891198-0.832354$

C $-6.019246-2.742310-2.850746$

H $-6.154265-2.084193-1.987963$

H $-6.327614-2.204930-3.751544$

H -6.633949 -3.641391-2.748713

SCF Energy (B3LYP/6-31G**//MMFF) $=-3245.91234155$

0800327

MMFF Geometry

C $-3.9998973 .286065-0.045466$

C $-4.992503 \quad 3.175010-0.942521$

C $-5.5639311 .897284-1.518341$

O $-4.892496 \quad 0.725955-1.003377$

C $-5.391408 \quad 1.907203-3.049520$

C $-6.0242100 .714836-3.742576$

C $-5.343540-0.312489-4.290065$

C $-7.5286200 .751599-3.833444$

C $-3.855956-0.533112-4.301556$

C $-3.490528-1.869850-3.641855$

C $-1.974841-2.015384-3.436074$

C $-1.645511-3.383835-2.835744$

C $-0.160014-3.519139-2.472236$

O $-1.561953-0.972868-2.548293$

O $-0.037012-4.686712-1.645350$

C $0.260902-2.294809-1.630206$

C $0.699368-3.780935-3.714171$

O $1.663424-2.317563-1.375185$

C $-0.160604-0.947905-2.252229$

C $0.088640 \quad 0.256714-1.312015$

O $0.612918-0.725812-3.437663$

C $-5.385520 \quad 0.172811 \quad 0.138833$

$\begin{array}{llll}\text { O } & -6.314612 & 0.585027 & 0.814651\end{array}$

C $-4.585540-1.0378190 .431061$

C $-4.774494-1.7009481 .581441$

C $-4.086606-2.9259611 .998035$ 
C $-3.044072-3.4932221 .071011$ C $-0.7399380 .266996-0.023340$ C $-0.040210-0.3352031 .180919$ C $-0.411505-0.7792973 .605270$ O $1.113011-0.7532381 .156185$ C $-0.709956-2.2372603 .754900$ C $-1.443721-2.7436104 .757441$ C $-1.689046-4.2119294 .952525$ C -3.157277 -4.554042 5.061552 C $-3.735773-4.745908 \quad 6.259299$ C $-3.928253-4.7709283 .779918$ C $-4.427558-3.4879173 .176868$ N $-0.858273-0.3233842 .303272$ H -3.5268792 .3955440 .358336$ H -5.449897 $4.090083-1.315822$ H $-6.6320681 .855069-1.271949$ H -4.326102 $1.977822-3.292302$ H $-5.8394092 .818950-3.468297$ H -5.914095 -1.096494 -4.786869 H -7.931736 $-0.088265-4.409472$ H $-7.9760690 .709395-2.835806$ H $-7.8572871 .673210-4.325009$ H $-3.511774-0.537489-5.342734$ H -3.324343 $0.279913-3.803373$ H -3.993699 -1.934484 -2.668865 H -3.864494 -2.697226 -4.257848 H - $1.473447-1.886687-4.402580$ H -1.951032 -4.189979 -3.514446 H -2.236507 -3.528014 -1.921544 H $0.886162-4.736695-1.342710$ H $-0.228282-2.408262-0.655679$ H $\quad 0.383914-4.709038-4.205967$ H $1.751134-3.929590-3.445790$ H $0.641116-2.978904-4.452966$ H $2.116923-2.009958-2.178357$ H $1.1605420 .364666-1.108382$ H $-0.191874 \quad 1.163822-1.867017$ H $\quad 0.6098730 .225306-3.635598$ H $-3.868114-1.357486-0.315031$ H -5.514828 -1.3202822 .284143$ H -3.511401 -3.8486840 .146622$ H -2.488883 -4.330983 1.498078 H -2.295512 -2.735194 0.817880 H $-0.952787 \quad 1.3110450 .235868$ H -1.701869 $-0.233266-0.164579$ H $-0.924376-0.1707034 .356753$ H $0.666540-0.6116043 .698670$ H $-0.271460-2.9112613 .022711$ H -1.857867 -2.070711 5.504958 H -1.167304 -4.515427 5.869857 H - $-1.235458-4.8046064 .148398$ H -4.781198 -5.0237116 .343784$ H -3.178221 -4.623457 7.181876 H -4.808685 -5.3976043 .978610$ H -3.324390 -5.363243 3.086600 H -5.193565 -2.998903 3.780687 H -1.822925 -0.0178702 .212938$ C $-1.961984 \quad 4.7079300 .206950$ O -1.2688573 .9126361 .189883$ C -0.0435024 .5888561 .558147$ C 0.0657555 .7929650 .633993 C $-1.392454 \quad 6.113966 \quad 0.368744$ H -0.1827564 .9151162 .596314$ C 1.1058583 .5793881 .462666 H 0.6130726 .6232601 .090183 H $0.5727225 .524088-0.301291$ C -1.600822 $7.014013-0.836765$ H $-1.818677 \quad 6.5932281 .260195$ C 2.5141264 .1885741 .565154 O 0.9418882 .6296682 .521802 H 1.0110473 .0229600 .523307 H 2.6899864 .8560520 .713309 O 2.5648035 .0044012 .740822 C 3.6546053 .1502461 .646782 C 3.5833932 .0909620 .540503 H 3.6518812 .6715352 .632483 O 4.9010263 .8400661 .521199 H 2.6673371 .4987520 .641739
H $3.5004432 .592141-0.433930$ H -1.097050 $7.975118-0.690081$ H -1.200759 $6.560792-1.750002$ H -2.664620 $7.213197-0.998481$ C -3.4692174 .6083250 .443664$ H $\quad 0.0430332 .2652242 .449747$ H 3.3881165 .5195352 .706343 H 5.0205064 .3792672 .320812 H $-3.9864185 .430238-0.063585$ H -3.6885994 .6868721 .515221$ H $-1.6983794 .303227-0.779899$ C $5.479273-0.758257-0.896010$ O $4.4588520 .196784-0.581396$ C 4.7853941 .1308970 .459228 C 5.0818680 .3876501 .771148 C $6.157572-0.6860431 .578618$ C $5.882802-1.5619950 .350898$ H $4.997303-1.453144-1.596011$ C $6.635993-0.078858-1.635487$ H $5.6660901 .705193 \quad 0.149755$ O $3.884860-0.2169432 .254265$ H 5.4165421 .1086902 .525820

H $6.198451-1.3154872 .475892$

O $7.421864-0.0427841 .449385$

H $6.751909-2.1883050 .122228$

O $4.799978-2.4494060 .650018$

C $7.514736-1.075911-2.347426$

H $6.2517390 .634012-2.376359$

H $7.2662770 .521012-0.972527$

H $4.092364-0.6466813 .101169$

H $8.095390-0.7365491 .346358$

H $5.066024-2.9910941 .412357$

O $6.917399-1.483523-3.503356$

O $8.597326-1.455406-1.920053$

C $7.649570-2.447107-4.266650$

H $7.066122-2.688264-5.159251$

H $8.612445-2.031186-4.578347$

H $7.793599-3.361811-3.683687$

SCF Energy $\left(B 3 L Y P / 6-31 G^{* *} / / M M F F\right)=-3245.89717700$

0800328

MMFF Geometry

C $2.880003-1.7960883 .620974$

C $2.199049-1.5314592 .496808$

C $0.802916-0.9701322 .469393$

O $0.026834-1.8909961 .667019$

C $0.760256 \quad 0.426139 \quad 1.821158$

C 1.3824141 .5120792 .671170

C 0.7471442 .1314993 .685057

C 2.7860481 .9120982 .299100

C $-0.659633 \quad 1.8933624 .162645$

C $-1.493555 \quad 3.182402 \quad 4.189292$

C -1.7160113 .8032432 .801301$

C -2.4180815 .1570392 .929592$

C -2.7095845 .7772931 .556191$

O -2.5199212 .9064582 .023853$

O $-3.626713 \quad 6.8651141 .747905$

C -3.4269874 .7286940 .683798$

C $-1.451450 \quad 6.377716 \quad 0.917515$

O $-3.588367 \quad 5.202993-0.651722$

$\begin{array}{llll}\text { C }-2.735347 & 3.343486 & 0.674848\end{array}$

C $-3.6482042 .322621-0.043184$

O $-1.5060373 .442295-0.045818$

C $-1.309337-1.968782 \quad 1.900237$

O $-1.964770-1.2888162 .672384$

C $-1.836413-3.0707391 .064959$

C $-3.145187-3.3614701 .089460$

C $-3.792975-4.4660760 .380081$

C $-2.929779-5.358558-0.473273$

$\begin{array}{llll}C & -3.232027 & 0.854060 & 0.120094\end{array}$

C $-3.886788-0.014321-0.939071$

C $-6.124103-0.620019-1.861612$

O $-3.220589-0.606404-1.785417$

C $-6.768650-1.859124-1.329350$

C $-6.773229-3.022985-1.996456$

C $-7.475740-4.264244-1.525225$

C $-6.539479-5.443522-1.396990$

C $-6.284385-6.237655-2.450719$ 
C $-5.965470-5.752084-0.034636$ C $-5.121899-4.6436120 .530123$ N $-5.268760-0.034906-0.846825$ H $2.391472-1.5830544 .570875$ H $2.652548-1.7033911 .528364$ H $0.386279-0.9416293 .484443$ H -0.2750840 .6950351 .580618$ H 1.2524250 .3959750 .840442 H 1.2885822 .8978344 .238365 H 3.1879782 .6840122 .963813 H $3.463974 \quad 1.0552542 .345961$ H 2.8050152 .3135071 .281150 H -1.1779871 .1384463 .566304$ H $-0.603816 \quad 1.4952225 .183105$ H -2.465899 2.9403074 .637245 H -1.006559 3.9126634 .847957 H -0.7454513 .9349482 .310823$ H -1.825176 5.8477243 .542182 H -3.370775 5.0256663 .461697 H -3.214262 7.5007752 .357634 H -4.439785 4.6046031 .092286 H -1.0306977 .1587021 .562324$ H -1.687134 $6.869377-0.032780$ H $-0.666413 \quad 5.6403470 .738539$ H -4.039992 $6.062886-0.601020$ H $-4.6685502 .418468 \quad 0.350075$ H -3.692026 2.565939-1.112988 H $-1.2654502 .564316-0.379578$ H -1.123765 -3.6396210 .478633$ H -3.803596 -2.751076 1.706122 H -2.420033 -4.774194 -1.247659 H -3.493492 -6.136189-0.994131 H -2.176076 -5.8658510 .138860$ H -2.1493870 .7311150 .018770$ H -3.5120150 .4791591 .110526$ H $-5.546711-0.809840-2.772322$ H $-6.8952460 .121940-2.092743$ H -7.287608 -1.786773 -0.376633 H -6.275462 -3.079929-2.962235 H -8.265794 -4.499524 -2.250526 H -7.995014 -4.095869 -0.573577 H -5.636832 -7.103668 -2.364184 H $-6.716607-6.036049-3.425501$ H -5.399812 -6.691603 -0.043953 H $-6.795156-5.9373200 .660441$ H $-5.676578-3.942714 \quad 1.154484$ H $-5.7208910 .449700-0.077426$ C $4.988793-2.9917752 .567735$

O $5.141285-2.0877201 .459392$

C $4.974835-2.838570 \quad 0.237595$

C $4.999994-4.3105490 .635428$

C $4.346327-4.2578872 .005576$

H $3.973219-2.610388-0.142666$

C $6.018334-2.416334-0.799499$

H $4.458546-4.942142-0.075499$

H $6.024614-4.691844 \quad 0.715573$

C $4.587151-5.5019092 .842570$

H $3.266470-4.1230191 .872111$

C $6.033461-0.900153-1.089287$

O $7.315713-2.781741-0.313198$

H $5.865261-2.981671-1.726588$

H $6.424133-0.366017-0.214368$

O $6.977265-0.692800-2.147459$

C $4.664417-0.319109-1.494663$

C $4.7343091 .179835-1.842676$

H $3.959177-0.453091-0.667266$

O $4.170968-1.056464-2.611293$

H $5.3100291 .699125-1.067308$

H $5.2514631 .298711-2.802681$

H $4.173810-6.3854582 .345280$

H $5.656011-5.6772923 .004564$

H $4.107750-5.4110843 .822293$

C $4.285135-2.3229203 .754746$

H $7.963452-2.508159-0.985309$

H $7.1658820 .259502-2.193655$

H $3.294229-0.701753-2.835984$

H $4.899974-1.4723904 .077851$

H $4.283142-3.0367574 .588244$
H $\quad 6.011571-3.2272312 .894087$

C $1.3480661 .768509-3.334094$

O $2.6883531 .310052-3.110927$

C $3.3378701 .827206-1.946142$

C $3.4278073 .358444-2.020938$

C $2.0439563 .979009-2.228053$

C $1.2838613 .304614-3.376026$

H $1.0917541 .396421-4.334713$

C $\quad 0.3617041 .146375-2.339967$

H $2.7649851 .540377-1.058257$

O $4.2919303 .733654-3.091045$

H $3.8713373 .732390-1.090006$

H $2.1581615 .048876-2.440405$

O $1.3105803 .854539-1.013904$

H $0.2352663 .624349-3.385651$

O $1.8573873 .721021-4.619066$

C $0.268571-0.346483-2.549032$

H $0.6256141 .342691-1.297948$

H $-0.6467001 .551886-2.484865$

H $4.3478854 .704226-3.096767$

H $0.4390984 .262983-1.152661$

H $1.7725554 .688271-4.671902$

O $0.976981-1.001269-1.585988$

O $-0.339583-0.865811-3.476179$

C $0.988013-2.428000-1.693257$

H $1.549905-2.730596-2.581575$

H $1.485218-2.832352-0.807856$

H $-0.032891-2.819631-1.731132$

SCF Energy (B3LYP/6-31G**//MMFF) $=-3245.90211307$

0800329

MMFF Geometry

C - -1.0744212 .8627682 .940236$

C -0.4168751 .7048343 .110910$

C $-1.0381420 .342456 \quad 3.311328$

O $-2.467411 \quad 0.425572 \quad 3.094565$

C $-0.787774-0.2277194 .719043$

C $0.654747-0.5501885 .044357$

C $1.293632-1.6602734 .624711$

C 1.3486940 .4278835 .955153

C $0.746357-2.7299183 .720241$

C $1.773282-3.1374502 .654467$

C $1.137478-4.0066051 .558355$

C $2.205847-4.8291420 .836068$

C $1.618650-5.628111-0.334142$

O $0.486316-3.1237900 .634009$

O $2.711852-6.114535-1.127755$

C $0.804462-4.671178-1.224806$

C $0.843150-6.8614840 .142487$

O $0.120411-5.389492-2.249990$

C $-0.193752-3.782286-0.442740$

C $-0.760408-2.703606-1.399814$

O $-1.265760-4.5945230 .038593$

C $-3.110879-0.6922412 .665766$

O $-2.644799-1.8119512 .533781$

C $-4.489312-0.2935802 .311127$

C $-5.149296-1.0118771 .391082$

C $-6.458532-0.6880580 .824149$

C $-7.2609300 .393378 \quad 1.498903$

C $-1.593571-1.609388-0.721019$

C $-2.132636-0.602420-1.725291$

C $-3.4436401 .516405-1.838788$

O $-1.973236-0.714789-2.938009$

C $-4.9292831 .421467-1.693979$

C $-5.7443200 .930655-2.640437$

C $-7.2429290 .879280-2.521491$

C -7.780482 -0.535441-2.472463

C $-8.040393-1.216041-3.601397$

C $-8.090515-1.127022-1.112634$

C $-6.849772-1.341283-0.290386$

N $-2.821966 \quad 0.430140-1.104657$

H -2.160299 2.8848782 .973114

H 0.6704921 .7189313 .063292

H $-0.610751-0.3109752 .541047$

H -1.389775 -1.135338 4.860765

H -1.1935250 .4653265 .469573$

H $2.315570-1.8239324 .964144$

H $2.3831040 .137070 \quad 6.167019$ 
H 1.3736351 .4242505 .504079 H $\quad 0.8212140 .493340 \quad 6.912496$ H $0.498834-3.6077294 .329463$ H -0.177638 -2.411831 3.234229 H $2.207245-2.2370642 .201159$ H $2.585029-3.6844983 .150323$ H $0.392848-4.6705562 .012853$ H $2.719070-5.5001201 .536394$ H $2.981716-4.1594730 .443263$ H $3.272313-6.668801-0.557998$ H $1.521974-4.014906-1.736085$ H $1.497749-7.5263960 .718615$ H $\quad 0.485727-7.455542-0.705984$ H $-0.012331-6.6127020 .773933$ H $\quad 0.784004-5.917667-2.726043$ H $0.072765-2.207646-1.915246$ H -1.375775 -3.186248 -2.169947 H -2.046527 -4.0371830 .181032$ H -4.8832860 .6170282 .746999$ H -4.659601 -1.8857250 .963361$ H -7.3929850 .1647652 .562732$ H -8.2635730 .5169101 .085599$ H $-6.7570581 .361848 \quad 1.409025$ H -2.451840 -2.048947 -0.205572 H $-0.982789-1.0693450 .010853$ H $-3.1334951 .494143-2.888398$ H -3.091037 $2.454864-1.400336$ H $-5.3540531 .805535-0.770292$ H $-5.3135620 .559389-3.567872$ H -7.667812 $1.405369-3.386540$ H $-7.5922031 .439082-1.644988$ H -8.450732 -2.220023 -3.575918 H -7.844389 $-0.783488-4.577062$ H -8.586496 -2.100418 -1.220654 H -8.823512 -0.487113 -0.614539 H $-6.189173-2.105438-0.702909$ H -2.883485 $0.446476-0.091153$ C $-0.7369294 .699751 \quad 1.280088$ O $-0.194592 \quad 3.803702 \quad 0.289835$ C $0.473556 \quad 4.574437-0.724335$ C $0.0004576 .008777-0.533157$ C -0.1337336 .0716470 .976990$ H $1.5450854 .527673-0.497370$ C $0.2192723 .969609-2.108397$ H $0.7106256 .738516-0.933404$ H $-0.9723176 .184344-1.006552$ C -0.9753737 .2372491 .466030$ H $0.870486 \quad 6.1462501 .415693$ C $0.5143832 .456595-2.190294$ O $-1.1589774 .161387-2.447709$ H $\quad 0.798926 \quad 4.518293-2.860089$ H - $0.2346561 .906197-1.610532$ O $0.3314772 .048939-3.551645$ C $1.9228692 .049595-1.722277$ C $2.1827880 .542249-1.911082$ H $2.0407392 .301945-0.662981$ O $2.8766382 .809993-2.461693$ H $1.332059-0.021659-1.511178$ H $2.2663120 .331670-2.984436$ H -0.5243598 .1890871 .167040$ H -1.989152 7.2013961 .053198 H -1.055538 7.2291672 .557561 C -0.3929384 .1780152 .676562$ H -1.301747 $3.757006-3.320772$ H $1.0286842 .473772-4.080383$ H $3.7607202 .574835-2.133236$ H -0.7154634 .8940933 .440467$ H 0.6941824 .0633912 .771945 H -1.824706 4.7150651 .132131 C $5.8692890 .411093-1.282222$ O $4.5840390 .690098-1.850481$ C $3.4697920 .068814-1.204889$ C $3.616526-1.459674-1.243482$ C $4.962130-1.905220-0.658804$ C $6.131631-1.103160-1.240035$ H $6.5861570 .856963-1.984137$ C $6.053477 \quad 1.101866 \quad 0.073987$ H $3.4229170 .401513-0.161974$
O $3.507296-1.915002-2.590518$

H $2.790287-1.904121-0.677483$

H $5.118061-2.970547-0.867932$

O $4.952150-1.7401100 .755758$

H $7.050323-1.296601-0.673749$

O $6.368776-1.533121-2.583515$

C $5.9767352 .603283-0.076600$

H $\begin{array}{llll}5.313801 & 0.785682 & 0.814992\end{array}$

H 7.0358380 .8683900 .500987

H $3.578870-2.884607-2.579995$

H $4.241002-2.2980781 .111784$

H $6.585006-2.480626-2.554438$

O $4.809267 \quad 3.066470 \quad 0.454582$

O $6.8413083 .279080-0.619258$

C $4.6082744 .480480 \quad 0.367103$

H $4.5003884 .779540-0.679577$

H 3.6861054 .7259000 .900451

H 5.4376725 .0157180 .839220

SCF Energy (B3LYP/6-31G**//MMFF) $=-3245.90837357$

0800330

MMFF Geometry

C -1.248135 -2.733067 2.509677

C $-2.173205-1.9038812 .004991$

C $-3.042521-1.0027592 .840033$

O $-4.354309-1.0428492 .234405$

C -2.5156110 .4397572 .775317$

C $-3.142846 \quad 1.3647273 .797580$

C -4.0543162 .3164213 .516051$

C -2.6325911 .2042035 .206773$

C -4.6947992 .6260032 .191267$

C $-4.212223 \quad 3.9580781 .598495$

C -2.7776563 .8990971 .049665$

C -2.3339695 .2714790 .539766$

C $-0.9471485 .219626-0.120147$

O $-2.7637182 .958082-0.027261$

O $-0.7780046 .462753-0.817982$

C $-0.9280394 .076483-1.158875$

C $0.1721885 .149246 \quad 0.925534$

O $\quad 0.3833643 .880680-1.684167$

C $-1.4734572 .737257-0.610068$

C $-1.6490461 .726554-1.768956$

O $-0.546466 \quad 2.2113600 .340055$

C $-5.451283-1.0682223 .028896$

O $-5.471739-1.0855484 .251402$

C $-6.693205-1.0996812 .218637$

C $-6.713493-0.8776870 .894469$

C $-7.889532-0.9121470 .022233$

C $-9.209598-1.2933500 .638304$

C $-2.330793 \quad 0.414196-1.358407$

C $-2.639985-0.470198-2.553688$

C $-3.962834-2.492423-3.175135$

O $-2.187605-0.264668-3.676673$

C $-5.247370-1.984324-3.751775$

C $-6.423886-2.618333-3.632317$

C $-7.708523-2.105304-4.219102$

C $-8.788331-1.897723-3.178809$

C $-9.783377-2.788827-3.032703$

C $-8.767484-0.617628-2.371588$

C $-7.725386-0.631583-1.287741$

N -3.467495 -1.530639 -2.207261

H -1.092247 -2.774719 3.584533

H -2.306622 -1.858640 0.926856

H -3.080112 -1.375169 3.870457

H $-2.6673020 .832723 \quad 1.765445$

H -1.429035 0.4531762 .936851

H -4.4086172 .9424244 .334342$

H -3.0691901 .9370715 .893613$

H -2.8725400 .2091955 .593630$

H -1.545983 1.3358595 .234242

H -4.5686251 .8141081 .468949$

H -5.7765262 .6962182 .363493$

H -4.8949604 .2184580 .779070$

H -4.293376 4.7514232 .351918

H -2.1048583 .5558751 .842901$

H $-2.345957 \quad 6.0109871 .350218$

H -3.057374 $5.633126-0.204785$

H $0.1158456 .475900-1.199869$ 
H $-1.5595204 .388603-2.002447$ H 0.1482046 .0369921 .568855 H 1.1593885 .1502590 .450375 H 0.1028184 .2703751 .569923 H $0.6536714 .706286-2.119716$ H -2.260188 2.190130-2.554721 H $-0.6738831 .490148-2.212328$ H -0.7367691 .2706570 .476433$ H $-7.598460-1.3065062 .779852$ H $-5.778563-0.6492420 .385348$ H $-9.140211-2.2738531 .122636$ H - $10.022999-1.365327-0.086873$ H $-9.512246-0.5522131 .386128$ H -1.681209 $-0.158334-0.689108$ H -3.271333 $0.622438-0.835897$ H $-4.090516-3.445735-2.652810$ H -3.224226 -2.625160 -3.972134 H $-5.203020-1.047926-4.303393$ H -6.468338 -3.562757-3.094767 H -8.043954 -2.832741-4.969906 H -7.552033 - $1.168321-4.768121$ H -10.579355 -2.634814 -2.311947 H -9.822074 -3.695138 -3.627958 H $-9.759909-0.401446-1.960593$ H -8.562681 $0.227780-3.041294$ H -6.727868 $-0.366629-1.638871$ H -3.847164 -1.573627 -1.265848 C $1.027564-3.0464831 .509931$ O $0.978174-1.8716910 .672431$ C $2.050385-1.936379-0.292765$ C $2.980047-3.033845 \quad 0.198552$ C $1.997984-4.0051530 .820673$ H $1.582003-2.230727-1.240765$ C $2.682780-0.545386-0.424871$ H $3.569461-3.480118-0.607976$ H $3.671860-2.6489810 .956504$ C $2.647218-5.0083111 .758182$ H $1.483263-4.5473960 .015989$ C $3.974968-0.528114-1.263561$ O $1.7103750 .311213-1.027358$ H $2.890326-0.1329840 .570136$ H $4.699290-1.177089-0.763628$ O $3.723248-1.101631-2.546209$ C $4.6050570 .870065-1.439897$ C $5.9424350 .856200-2.206751$ H $4.7549511 .331064-0.456817$ O $3.7309041 .727255-2.177640$ H $5.7881320 .462289-3.219327$ H $6.2483091 .902931-2.339032$ H $1.898854-5.6806692 .189246$ H $3.380211-5.6187121 .220653$ H $3.165983-4.5095472 .583231$ C $-0.381690-3.6207121 .660223$ H $0.9088140 .269840-0.478935$ H $3.081008-0.527894-2.998640$ H $2.9098221 .822018-1.665764$ H $-0.346563-4.6098812 .130655$ H -0.838076 -3.7496030 .670472$ H $1.424084-2.7286042 .483239$ C 8.2292450 .0267120 .623888 $\begin{array}{lllll}\text { O } & 7.266247 & 0.672192 & -0.218588\end{array}$ C $7.0639810 .076891-1.502958$ C $8.3742460 .083895-2.304107$ C $9.501015-0.596946-1.519241$ C $9.594254-0.075420-0.080698$ H $8.3450490 .700666 \quad 1.482561$ C $7.711505-1.3186321 .158584$ H $6.740888-0.960151-1.379507$ O $8.756194 \quad 1.418938-2.627651$ H $8.213272-0.442791-3.252032$ H $10.452047-0.419569-2.036431$ O $9.262874-2.001567-1.509335$ H $10.271705-0.7056930 .507069$ O $10.177577 \quad 1.230488-0.129543$ C $6.538003-1.1414092 .102005$ H $7.385280-1.9885310 .359468$ H $8.514506-1.8076581 .722815$ H $8.8372011 .915172-1.795218$
H $10.004731-2.421285-1.041309$

H $10.302336 \quad 1.5315930 .786608$

O $6.307240-2.3216892 .750438$

O $5.897049-0.1096732 .247476$

C $5.239855-2.301978 \quad 3.703960$

H $5.106770-3.3175324 .086093$

H $5.496855-1.6427764 .538369$

H $4.306354-1.9799213 .232888$

SCF Energy (B3LYP/6-31G**//MMFF) $=-3245.91508945$

\section{1}

MMFF Geometry

C -1.622623 4.5266432 .092407

C $-2.3831293 .529154 \quad 1.615884$

C -3.2508652 .6496822 .480015$

$\begin{array}{llll}\text { O } & -3.046041 & 1.274394 & 2.073726\end{array}$

C $-4.730628 \quad 3.0087132 .260765$

C $-5.6892112 .164186 \quad 3.078986$

C -6.4890091 .2013422 .577551$

C $-5.7328652 .486744 \quad 4.550721$

C -6.5905610 .7414601 .149928$

C $-6.115752-0.7092850 .999120$

C $-5.890392-1.081993-0.473418$

C $-5.545799-2.565515-0.608541$

C $-5.181735-2.945887-2.051479$

O $-4.806683-0.280356-0.957900$

O $-4.562955-4.241736-2.022417$

C $-4.123359-1.954092-2.581327$

C $-6.426455-3.079789-2.936602$

O $-3.871069-2.173985-3.966676$

C $-4.491776-0.475090-2.343069$

C $-3.344627 \quad 0.505102-2.698587$

O $-5.607698-0.131098-3.173831$

C -1.9293410 .6447772 .534328$

O $\begin{array}{lllll}-1.066886 & 1.114125 & 3.259582\end{array}$

C $-1.935941-0.733707 \quad 1.993764$

C $-0.916775-1.5646662 .259148$

C $-0.798842-2.9568191 .816443$

C $-1.884293-3.5003800 .925505$

C $-2.0474390 .325662-1.901405$

C $-1.027002-0.579952-2.570551$

C $1.126824-1.748995-2.109667$

O $-1.115116-0.946732-3.739074$

C $0.879228-3.139094-1.614153$

C $1.772474-3.830926-0.891637$

C $1.561033-5.233162-0.402120$

C $1.750525-5.3586901 .092900$

C $2.931759-5.7362551 .610779$

C $0.545634-5.1386301 .977564$

C $0.261858-3.6820492 .229212$

N $0.017738-0.901113-1.714542$

H $-1.6582214 .735914 \quad 3.160008$

H -2.387116 3.3034460 .553299

H -2.993856 2.7562413 .541251

H $-4.9643742 .939817 \quad 1.193841$

H -4.8964114 .0634242 .520503$

H $-7.1468740 .670766 \quad 3.264933$

H -6.494843 1.9060235 .081601

H -4.7689352 .2709865 .021656$

H -5.9654493 .5460944 .700686$

$\begin{array}{llll}H & -7.638697 & 0.813416 & 0.834614\end{array}$

H -6.0312551 .3909220 .474096$

H $-5.176722-0.8408791 .550568$

H $-6.856099-1.3821701 .449388$

H $-6.799427-0.844738-1.038818$

H $-6.369400-3.190970-0.242321$

H $-4.687883-2.7975430 .035731$

H -5.191852 -4.865067 -1.620051

H $-3.188885-2.177416-2.052727$

H $-7.093610-3.856357-2.543414$

H $-6.159095-3.400521-3.949297$

H $-7.004062-2.155745-3.007158$

H -3.619269 -3.107530 -4.070824

H $-3.1590400 .492241-3.779393$

H $-3.7077551 .519559-2.479185$

H $-5.6231050 .833723-3.286508$

H -2.787436 -1.0319291 .393770$

H $-0.096118-1.1994912 .874143$ 
H -2.829251 -3.568982 1.474678 H -1.666004 -4.493351 0.526706 H -2.028259 -2.8530790 .053749$ H -1.563435 $1.305868-1.801998$ H -2.256272 -0.038378 -0.891541 H $2.036569-1.313561-1.684592$ H $1.218797-1.754943-3.200280$ H $-0.069490-3.602111-1.874899$ H $2.731175-3.375376-0.661468$ H $2.279524-5.880126-0.922516$ H $0.569961-5.611303-0.681358$ H $3.067380-5.8574842 .680421$ H $3.791480-5.9244660 .976207$ H $\quad 0.712791-5.6065332 .957609$ H $-0.312817-5.6762721 .564768$ H $1.003032-3.2001792 .867176$ H $-0.009603-0.577127-0.751972$ C $-0.2898364 .909553-0.090237$ O $\quad 0.4026913 .651944 \quad 0.036934$ C $1.6263793 .704328-0.726584$ C $1.4456584 .868401-1.685243$ C $0.6762125 .846741-0.817485$ H 2.4157463 .9274850 .000723 C $1.8566222 .328734-1.360907$ H $2.3934865 .276390-2.046756$ H $0.8414354 .571632-2.551414$ C $-0.0110416 .948999-1.604353$ H $1.3725496 .301806-0.100189$ C $3.0934352 .194379-2.267547$ O $1.943581 \quad 1.363227-0.311967$ H $0.9654152 .064086-1.944188$ H $3.232548 \quad 1.137517-2.527005$ O $2.7973672 .866587-3.498448$ C $4.4219482 .754771-1.721786$ C $4.9172372 .174818-0.389196$ H $4.3329333 .842024-1.616085$ O $5.4224062 .558279-2.726608$ H $5.8816452 .638452-0.139997$ H 4.2436512 .4981510 .414287 H $-0.5689837 .613711-0.937428$ H $\quad 0.7258717 .552245-2.144797$ H $-0.7136736 .540248-2.338311$ C -0.6832025 .4056701 .305345$ H $1.148087 \quad 1.4573140 .240008$ H $3.5711592 .762305-4.078548$ H $6.2494302 .951132-2.399104$ H -1.127647 6.4051721 .236929 H 0.2301935 .4986421 .907748 H -1.178150 $4.731690-0.708310$ C $5.524302-0.9926351 .453688$

O $5.426811 \quad 0.3860791 .078020$

C $5.0779670 .643783-0.292387$

C $6.1307380 .031297-1.226110$

C $6.330775-1.460929-0.933736$

C $6.531080-1.7343780 .559576$

H $5.928446-0.9744352 .473905$

C $4.147075-1.6614121 .511252$

H $4.1104000 .181031-0.496065$

O $7.365708 \quad 0.724741-1.069235$

H $5.8074860 .150306-2.265624$

H $7.204563-1.820611-1.490990$

O $5.190751-2.170713-1.409767$

H $\quad 6.480814-2.8116070 .756873$

O $7.851556-1.308076 \quad 0.907752$

C $3.311337-1.0620292 .616320$

H $3.575481-1.5508510 .587036$

H $4.240104-2.7386471 .691707$

H $8.0049190 .329491-1.686284$

H $5.343064-3.116612-1.244105$

H $7.998596-1.5375801 .841065$

O $3.535534-1.7285823 .784470$

O $2.567556-0.1018342 .463085$

C $2.815639-1.2376934 .919356$

H $3.093940-0.1995615 .124549$

H $\quad 1.737947-1.3229104 .751565$

H $3.085056-1.8504815 .783770$

SCF Energy (B3LYP/6-31G**//MMFF) $=-3245.89941519$
0800332

MM̄FF Geometry

C $-0.386028-4.2709510 .015892$

C $-1.591640-3.711242-0.155461$

C $-2.241708-3.481159-1.494082$

O $-2.488489-2.059172-1.612607$

C $-3.587484-4.225334-1.545939$

C $-4.392452-3.954512-2.802425$

C $-5.522418-3.219125-2.846175$

C $-3.857536-4.591744-4.058467$

C $-6.200422-2.513551-1.704032$

C $-6.029592-0.993066-1.811205$

C $-6.233486-0.296032-0.457627$

C $-6.2073551 .223191-0.629164$

C $-6.264981 \quad 1.9624980 .716089$

O $-5.165254-0.7124530 .402170$

$\begin{array}{lllll}\text { O } & -5.902942 & 3.332243 & 0.478897\end{array}$

C -5.1958131 .3691921 .659835$

C $-7.6868931 .984207 \quad 1.289148$

O

C $-5.217987-0.1721421 .729005$

C $-4.023526-0.7614312 .522372$

O $-6.412563-0.5876032 .403129$

C -1.444822 -1.275465 -2.005458

O $-0.327523-1.645032-2.329758$

C $-1.8928520 .133824-1.936488$

C - $-1.0220161 .130419-2.156947$

C $-1.3215092 .563842-2.090111$

C $-2.7051122 .966052-1.651012$

C $-2.633585-0.4624731 .950050$

C -1.9636380 .7691252 .534339$

C 0.0020242 .2610862 .176196

O $-2.366420 \quad 1.3404873 .543698$

C -0.4666673 .4789801 .443479$

C 0.3550814 .2973690 .768648

C -0.0994145 .5430940 .062383$

C $0.3878615 .619083-1.368093$

C $1.4792716 .336233-1.685249$

C $-0.4415794 .948876-2.440427$

C $-0.350628 \quad 3.447711-2.402929$

N -0.8312401 .1295961 .814502$

H $\quad 0.171315-4.626740-0.846741$

H $-2.133025-3.3473110 .715035$

H -1.600411 -3.815630 -2.318871

H $-4.168953-3.974343-0.653778$

H $-3.410896-5.307228-1.473512$

H $-6.018981-3.093981-3.807488$

H -4.514013 -4.428186-4.919716

H -2.875503 -4.179986 -4.311010

H -3.758189 -5.673642 -3.922695

H $-7.269558-2.758984-1.727673$

H $-5.844254-2.873930-0.737036$

H $-5.022324-0.763797-2.179741$

H -6.741542 $-0.602981-2.549020$

H -7.190653 -0.620416 -0.032797

H $-7.0227291 .553748-1.284880$

H $-5.2809371 .512097-1.141579$

H -6.524152 $3.698254-0.173706$

H -4.2249481 .6939561 .268471$

H -8.3748762 .4703170 .586918$

H -7.7338642 .5771752 .209096$

H -8.0803430 .9871501 .497283$

H $-6.056114 \quad 1.5064993 .410315$

H -4.094669 -0.475751 3.578921

H -4.136919-1.855195 2.509671

H $-6.278792-1.4890422 .740057$

H -2.929892 $0.315547-1.680987$

H $\quad 0.007550 \quad 0.878387-2.407608$

H $-3.4467252 .659711-2.396417$

H -2.823653 $4.040651-1.499372$

H $-2.9533902 .500147-0.691453$

H -1.971824 -1.303449 2.193642

H $-2.670323-0.3798700 .860795$

H 1.0331351 .9972461 .923147

H $-0.0640742 .430353 \quad 3.255708$

H $-1.5277983 .712817 \quad 1.490324$

H $1.420643 \quad 4.0817360 .751285$

H $\quad 0.2814846 .402800 \quad 0.629328$ 
H -1.192073 5.6389670 .080126 H $1.8201556 .424694-2.711397$ H $2.0611526 .845081-0.923718$ H -0.100162 $5.266094-3.435168$ H -1.470202 $5.314627-2.374540$ H $\quad 0.6256123 .062492-2.699529$ H -0.6023230 .6272900 .961779$ C $1.522283-3.4873301 .398305$ O $2.598578-4.1409120 .691512$ C $3.792029-4.0384471 .481158$ C $3.518413-2.9628882 .520205$ C $2.049212-3.2135522 .806612$ H $3.889443-4.9964442 .011452$ C $5.044951-3.8563460 .619997$ H $4.153590-3.0547783 .405804$ H $3.650200-1.9619622 .098500$ C $1.358712-2.0527553 .502336$ H $1.955701-4.1150053 .426839$ C $5.203378-2.533427-0.162581$ O $6.173635-3.9860091 .495484$ H $5.103242-4.694191-0.086335$ H $5.418270-1.7196460 .538010$ O $6.386061-2.666649-0.967330$ C $4.029451-2.139150-1.073308$ C $4.332259-1.016352-2.081657$ H $3.175534-1.845152-0.455695$ O $3.634786-3.285277-1.834583$ H $5.042766-1.380197-2.835289$ H $3.396256-0.830958-2.626129$ H $\quad 1.826107-1.8520804 .471799$ H $1.415200-1.1366662 .904614$ H $0.300994-2.2742163 .675883$ C $0.278597-4.3752011 .361039$ H $6.974610-3.9019660 .950207$ H $\quad 6.209533-3.350757-1.636040$ H $2.824966-3.050333-2.319294$ H $-0.421790-4.0778342 .150143$ H $0.547022-5.4239571 .534046$ H $1.330972-2.5373430 .881404$ C 4.1391232 .0400420 .070972 O $3.8466090 .793767-0.578121$ C $4.8441830 .302052-1.478737$ C $5.168671 \quad 1.332312-2.578929$ C $5.5135542 .697540-1.983326$ C $4.4697423 .137222-0.953675$ H 3.1973072 .3203070 .557088 C 5.2195761 .8932481 .151388 H $5.7592210 .094669-0.915735$ O $4.0633851 .501091-3.464955$ H $6.0162560 .968583-3.173193$ H $5.5660923 .436566-2.792331$ O $6.8002552 .622613-1.378118$ H $4.8033554 .039380-0.427874$ O $3.2556263 .473963-1.627043$ C 4.6773591 .2560702 .408170 H 6.0668871 .2857810 .821552 H 5.6260052 .8696691 .441882 H $4.0273650 .722841-4.046172$ H $7.0099413 .503931-1.025021$ H $3.4602294 .172846-2.271148$ O 3.8223922 .1127253 .036975 O $4.9976190 .141128 \quad 2.798075$ C 3.2578931 .6335494 .261162 H 2.6055722 .4134644 .662778 H $2.6592460 .737676 \quad 4.075855$ H 4.0482871 .4271954 .988851 SCF Energy (B3LYP/6-31G**//MMFF)= -3245.89891397

0800333

MM̄FF Geometry

C $-2.7970140 .097093 \quad 3.232056$

C -2.8950831 .4256003 .399466$

C -3.6410112 .4087182 .525846$

O -4.3457461 .7494831 .452189$

C -2.6522893 .3831261 .860943$

C -3.2662394 .7347261 .557008$

C -3.7865125 .1029510 .370246$

C -3.2190415 .7199732 .697280$
C $-3.9761244 .269332-0.865932$

C $-3.0079244 .644159-1.996830$

C $-1.6282723 .987374-1.841317$

C $-0.6729474 .456184-2.940066$

C $0.6759323 .722659-2.875098$

O $-1.8173682 .570919-1.935161$

O $1.3775753 .979833-4.100982$

C $0.4159162 .202567-2.813486$

C $1.5646044 .257561-1.745737$

O $1.6307091 .485563-2.605221$

C $-0.6287891 .795818-1.747756$

C $-0.9983670 .302447-1.916043$

O $-0.0682701 .994213-0.449323$

C -5.6023671 .2901551 .679434$

O

C -6.0636690 .6894740 .409303$

C $-7.047625-0.2201170 .417944$

C $-7.557859-0.938524-0.752180$

C -7.009381 -0.552466 - 2.101914

C $-2.221289-0.149316-1.103088$

C $-2.466726-1.644539-1.236553$

C $-3.970240-3.450218-0.396618$

O $-1.760721-2.382241-1.919248$

C $-5.091365-3.709291-1.353421$

C $-6.220164-4.349947-1.011159$

C $-7.336811-4.649986-1.971724$

C -8.696518 -4.248796-1.440559

C $-9.533850-5.164653-0.924663$

C $-9.117614-2.802699-1.584260$

C $-8.468914-1.914991-0.559557$

N $-3.561726-2.060274-0.490850$

H -3.289353 -0.381264 2.391301

H $-2.400570 \quad 1.8708634 .261636$

H -4.3552012 .9547243 .155035$

H -2.259295 2.9379470 .940945

H -1.7771703 .5528682 .502689$

H $-4.163727 \quad 6.1207520 .274150$

H -3.6730056 .6820872 .437057$

H -3.757546 5.3261353 .565066

H -2.181701 5.9127372 .989811

H $-3.9247263 .199049-0.651303$

H $-5.0009244 .448987-1.215867$

H $-3.4547564 .300289-2.939124$

H -2.906136 $5.734228-2.064507$

H -1.222312 4.238841-0.855709

H $-0.5229605 .541852-2.888335$

H -1.124372 $4.262747-3.923335$

H $1.4859084 .942349-4.188063$

H $\quad 0.040379 \quad 1.895897-3.799866$

H $1.7673435 .325453-1.891527$

H $2.5432543 .764811-1.745901$

H $1.1189334 .139733-0.755919$

H $2.2526401 .760498-3.300609$

H $-1.2192380 .101359-2.972841$

H $-0.138353-0.320103-1.641562$

H $-0.557739 \quad 1.4546130 .190351$

H $-5.5450340 .982565-0.496689$

H -7.500953 $-0.486628 \quad 1.371905$

H -7.234772 $0.497498-2.319475$

H -7.417488 -1.139817 -2.926438

H $-5.923216-0.690758-2.133861$

H -2.084738 $0.074012-0.040454$

H $-3.1186670 .374030-1.452112$

H $-4.263980-3.6308630 .642349$

H -3.121363 -4.097985 -0.637811

H $-4.958852-3.377850-2.380672$

H -6.336067 -4.710031 0.008857

H -7.319968 -5.729511 -2.171041

H -7.170445 -4.166055 -2.942168

H $-10.520697-4.890542-0.566886$

H $-9.248832-6.208305-0.843644$

H -10.203998 -2.710604-1.451782

H -8.935498 -2.477942 -2.611992

H $-8.799444-2.1205440 .459755$

H -4.105907 -1.371089 0.018981

C $-0.834402-1.5041173 .581369$

O $0.117455-0.5354703 .100425$

C $0.680297-1.0226931 .860889$ 
C $0.267487-2.4838721 .760306$ C -1.098990 -2.453977 2.416359 H $0.197423-0.4425331 .067208$ C $2.190330-0.7600801 .858898$ H $\quad 0.237487-2.8429220 .727128$ H $0.953244-3.1241582 .328988$ C -1.609928 -3.8217992 .830387$ H -1.806845 -2.009427 1.710249 C $2.924791-1.3845530 .655931$ O 2.3771220 .6579621 .854008 H $2.635299-1.1310332 .789988$ H $2.781268-2.4705460 .687829$ O $2.332230-0.928683-0.559546$ C $4.442246-1.1071100 .625977$ C $5.128302-1.852667-0.536847$ H $4.875500-1.4279101 .580687$ $\begin{array}{lllll}\text { O } & 4.667418 & 0.293531 & 0.489238\end{array}$ H $4.779426-2.892008-0.556015$ H $4.836943-1.372802-1.479665$ H -1.707907 -4.475692 1.957547 H $-0.930641-4.3071473 .539084$ H -2.593280 -3.742618 3.304620 C -2.053452 -0.801391 4.184726 H 1.9028311 .0167802 .623585 H $2.4528340 .036043-0.598118$ H 5.6271570 .4434820 .517717 H -1.715304 -0.2129485 .047603$ H $-2.754837-1.5497034 .571974$ H $-0.327614-2.0487014 .389500$ C $8.541535-0.311190-0.443723$ O $7.127645-0.505861-0.580838$ C $6.666849-1.850536-0.425502$ C $7.315817-2.757241-1.483085$ C $8.844230-2.652910-1.446782$ C $9.317325-1.195206-1.434818$ H $\quad 8.705811 \quad 0.735948-0.729908$ C $9.002286-0.4744001 .008944$ H $6.931889-2.2120670 .574082$ O $6.848553-2.413813-2.786508$ H $7.010360-3.793358-1.297096$ H $9.264680-3.161227-2.323153$ O $9.342707-3.307127-0.283717$ H $10.389095-1.139185-1.211603$

O $9.130711-0.636912-2.738916$

C 8.4036310 .6050741 .879204 H $8.749492-1.4482291 .437818$ H $10.092289-0.3805431 .083875$ H $7.042255-1.472160-2.933223$ H $9.062061-4.237157-0.326654$ H $9.664453-1.160378-3.360741$ O $7.287621 \quad 0.1341002 .504957$ O 8.8590581 .7378561 .968956 C 6.6014701 .0757953 .335720 H 6.2780011 .9382602 .745131 H 5.7168830 .5829843 .748233 H 7.2464341 .3913314 .161126 SCF Energy (B3LYP/6-31G**//MMFF) $=-3245.91787798$

0800334

MM̄FF Geometry

C $1.126961-2.7724922 .684081$

C $1.578753-1.6501662 .104521$

C $2.069917-0.4593262 .886253$

$\begin{array}{lllll}\text { O } & 1.559632 & 0.744427 & 2.276597\end{array}$

C $3.600942-0.3905012 .763244$

C 4.2592930 .5432213 .755775

C 4.6566481 .8020673 .485276

C $4.522051-0.0573235 .112963$

C 4.4333772 .6028812 .232295

C 5.6760562 .7089381 .336433

C 5.7963871 .5460580 .338944

C $7.0691301 .671548-0.498675$

C $7.1539360 .572922-1.570359$

O $4.6473121 .593328-0.515134$

O $8.1941690 .937431-2.490607$

C $5.8305650 .550223-2.365023$

C $7.559597-0.778518-0.970566$

O $5.809519-0.535530-3.289700$
C $4.5638160 .534969-1.475244$

C $3.3104150 .782139-2.350665$

O $4.453141-0.743626-0.848358$

C $\quad 0.3322121 .1996222 .627804$

$\begin{array}{lllll}\text { O } & -0.474168 & 0.670835 & 3.372868\end{array}$

C 0.1613322 .4905521 .924243

C -1.0145523 .1321851 .935685$

C -1.2966124 .3962971 .250221$

C -0.1496745 .0849180 .554018$

C $2.0097261 .015658-1.564996$

C $0.8446131 .332812-2.491006$

C $-1.5692871 .962636-2.441704$

O $0.9400091 .316868-3.715793$

C -1.816223 $3.434989-2.329860$

C $-2.9979753 .957293-1.965953$

C $-3.2817155 .430448-1.881619$

C $-3.9609545 .823529-0.586542$

C $-5.2948445 .974357-0.525004$

C -3.0875356 .1202100 .611989$

C -2.5601924 .8683501 .258098$

N $-0.3160151 .627394-1.788481$

H $1.069281-2.7983593 .771145$

H $1.666329-1.5857781 .024276$

H $1.761931-0.5065693 .937863$

H $3.861756-0.0886941 .744556$

H $4.042190-1.3876062 .897418$

H 5.1658662 .3553564 .273956

H 5.0138600 .6467485 .792574

H $3.582721-0.3660405 .582771$

H $5.170142-0.9351665 .021334$

H 3.5727742 .2375301 .666336

H 4.1562043 .6154462 .554339

H 5.5888543 .6439170 .767001

H $6.5843082 .784878 \quad 1.946582$

H 5.8071850 .6021030 .892607

H 7.9601351 .6542320 .141200

H $7.0811602 .648454-1.002412$

H $9.0225411 .023185-1.988215$

H $5.813274 \quad 1.463667-2.975321$

H $8.523886-0.694538-0.454964$

H $7.705946-1.530407-1.754022$

H $6.833427-1.163957-0.251833$

H $5.622417-1.347360-2.788216$

H $3.4821561 .671101-2.972369$

H $3.161595-0.066410-3.030771$

H $3.522343-0.913043-0.634772$

H 1.0236592 .8770001 .391271

H -1.847113 2.6864422 .477170

H -0.4381615 .9983280 .030729$

H $0.2980514 .429265-0.200676$

H $\quad 0.623843 \quad 5.363950 \quad 1.278153$

H $1.7434520 .126757-0.984013$

H $2.1308831 .857966-0.874780$

H $-2.3512971 .376848-1.951622$

H -1.525971 $1.676961-3.497065$

H $-0.9957614 .101705-2.583933$

H -3.826053 $3.288181-1.748520$

H -3.927077 $5.693032-2.730372$

H $-2.3674716 .023047-2.009658$

H $-5.787724 \quad 6.2814850 .391256$

H $-5.9241725 .788144-1.389129$

H -3.662894 6.6606611 .375911

H $-2.298153 \quad 6.8181460 .319579$

H -3.3247394 .2983221 .786782$

H $-0.2962081 .625487-0.773276$

C $0.881051-4.1299670 .487115$

O $-0.214868-3.425656-0.133262$

C $-0.604182-4.140323-1.325598$

C $0.419022-5.254994-1.511779$

C $0.810586-5.547937-0.077556$

H $-1.576406-4.599624-1.118069$

C $-0.706304-3.180991-2.515157$

H $-0.003327-6.122064-2.028794$

H $1.288669-4.913432-2.086062$

C $2.104458-6.3351030 .041756$

H $-0.001654-6.1068890 .406057$

C -1.801699 -2.106243 -2.357199

O $0.556260-2.516480-2.660069$ 
H $-0.865614-3.749624-3.438937$ H -1.565475 -1.472948 -1.496093 O $-1.733650-1.252637-3.507453$ C -3.234861 -2.670079 -2.243192 C $-4.329880-1.584731-2.211186$ H -3.321841 -3.300556 -1.352886 O $-3.475609-3.507584-3.377886$ H -4.181719 -0.883253 -3.040950 H -5.298404 -2.068517 -2.396049 H $2.011693-7.304313-0.459296$ H $2.943659-5.799698-0.415152$ H $2.355716-6.5220501 .090180$ C $0.744278-4.0653192 .010656$ H $0.471484-1.891225-3.400218$ H -1.978578 -1.785805 -4.283267 H -4.348953 -3.918599 -3.259171 H $1.370535-4.8442262 .461433$ H $-0.290851-4.2814962 .291811$ H $1.811821-3.6528760 .154613$ C $-4.995336-1.240077 \quad 1.443572$ O $-4.920595-1.739276 \quad 0.100598$ C $-4.382446-0.835781-0.872398$ C $-5.245260 \quad 0.429015-0.949414$ C -5.3348621 .0926840 .427126$ C $-5.7602940 .093661 \quad 1.506867$ H $-5.599374-1.9830041 .981588$ C -3.604051-1.202704 2.097838 H $-3.366103-0.553745-0.585397$ O $-6.5469340 .097081-1.424002$ H $-4.7980881 .118594-1.672977$ H -6.0528021 .9203650 .381992$ $\begin{array}{llll}\text { O } & -4.059016 & 1.635266 & 0.752961\end{array}$ H -5.6479210 .5309002 .505681$ O $-7.150721-0.2029321 .341444$ C -3.230931 -2.566619 2.641662 H $-2.803334-0.8829391 .426249$ H -3.611803 -0.519077 2.955105 H -7.061195 $0.920864-1.469725$ H -4.1331652 .0505461 .628760$ H -7.6412080 .6321861 .428404$ O $-3.267709-3.499496 \quad 1.649157$ O $-2.942956-2.7680643 .814541$ C $-3.064448-4.8671352 .024111$ H $-2.534585-5.3676451 .209493$ H $-2.475456-4.9708372 .939696$ $\mathrm{H}-4.039561-5.3439272 .154150$

SCF Energy (B3LYP/6-31G**//MMFF) $=-3245.89837040$

0800335

MMFF Geometry

C $2.2290071 .720770-2.206561$

C $2.2876193 .060783-2.243414$

C $2.8206853 .962323-1.152350$

O $3.7325713 .245608-0.283464$

C $1.7143614 .522332-0.238083$

C $0.4779225 .042531-0.939221$

C $-0.7139434 .411868-0.918103$

C $0.6399746 .358047-1.650610$

C -1.010304 $3.091509-0.261264$

C -1.6472663 .2894441 .118023$

C -1.6275272 .0002621 .951549$

C -2.409339 2.1984783 .251460

C $-2.307016 \quad 0.979078 \quad 4.177522$

O $-0.259046 \quad 1.6945602 .243103$

O -2.7797651 .3688745 .475796$

C $-0.8216310 .593446 \quad 4.333162$

C $-3.211723-0.1661963 .711822$

O $-0.688862-0.6217815 .068153$

C -0.0617490 .4853772 .988676$

C $1.458170 \quad 0.3480563 .263176$

O $-0.532620-0.6553992 .275261$

C $4.9901323 .005625-0.741431$

O $5.488453 \quad 3.405168-1.780757$

C 5.6328582 .0909860 .226501

C $6.6196261 .286604-0.194215$

C 7.2957860 .2588880 .600556

C 6.9031640 .1255912 .048665

C 2.3482460 .2822932 .012468
C $2.593403-1.1283511 .500405$

C $3.838777-2.358116-0.276743$

O $2.106426-2.1282412 .020565$

C $5.101569-2.8622780 .350936$

C $6.205581-3.178137-0.343581$

C $7.456906-3.7233640 .286120$

C $8.704863-2.973586-0.127180$

C $9.558192-3.494950-1.024998$

C $9.007038-1.6603960 .562611$

C $8.209761-0.523173-0.010927$

N $3.426448-1.1364210 .389343$

H $2.5683041 .188145-1.323915$

H $1.9262673 .571117-3.134255$

H $3.3612354 .798286-1.614625$

H 2.1434375 .3329850 .367703

H 1.4528613 .7665510 .513090

H - $1.5590304 .873333-1.425327$

H $-0.2940536 .698704-2.109969$

H $0.9643097 .132232-0.947580$

H $1.3875626 .274929-2.445679$

H $-1.6950332 .525389-0.904231$

H $-0.1075402 .477012-0.192827$

H $-1.0984904 .069907 \quad 1.662068$

H -2.678432 3.6419260 .991211

H $-2.059003 \quad 1.185770 \quad 1.359847$

H -3.4601122 .4337443 .040287$

H -2.009886 3.0715803 .786801

H -3.6958561 .6811235 .379734$

H -0.3416841 .3753294 .938596$

H $-4.2592270 .157205 \quad 3.685747$

H $-3.175637-1.0100104 .409530$

H -2.953824 -0.528990 2.716303

H $-1.168091-0.5070415 .906678$

H $1.776464 \quad 1.2343773 .828514$

H $1.650243-0.5256803 .896869$

H $0.093911-0.8597571 .563641$

H 5.2326982 .0586911 .232989

H $6.9401411 .360673-1.232865$

H 7.0906871 .0631262 .584053

H $7.451054-0.6530572 .582216$

H $5.840479-0.1228322 .139390$

H 1.9404690 .8936511 .200555

H 3.3313050 .6920092 .275825

H $3.967008-2.123749-1.338138$

H $3.049933-3.110094-0.174748$

H $5.098698-2.9982821 .430096$

H $6.200767-3.072103-1.426090$

H $7.540911-4.778120-0.007199$

H $7.385069-3.7250541 .380965$

H $10.469045-2.976742-1.306125$

H $9.362349-4.448823-1.503306$

H $10.069001-1.4080740 .441734$

H $8.868559-1.7860531 .639395$

H $8.423358-0.338545-1.065073$

H $3.850341-0.2660320 .082785$

C $0.3698040 .234680-3.001222$

O $0.547112-0.766226-1.978989$

C $-0.230119-1.933479-2.325834$

C $-1.123575-1.512411-3.483685$

C $-0.255456-0.493744-4.189489$

H $0.494953-2.688885-2.653340$

C $-0.994815-2.417550-1.088373$

H -1.405045 -2.353077 -4.124865

H -2.043378 -1.040322 -3.115447

C $-1.0312550 .410544-5.132021$

H $0.520790-1.025424-4.756271$

C -1.974913 -3.570306 -1.374475

O $-0.041455-2.841543-0.110207$

H $-1.520081-1.564283-0.646969$

H -2.709729 -3.268642 -2.128759

O $-1.231833-4.650722-1.953661$

C $-2.704670-4.103931-0.128624$

C $-3.470352-3.0457510 .679256$

H -1.981286 -4.5997410 .530220$

O $-3.605795-5.129961-0.552374$

H $-4.033047-3.5391051 .482736$

H -2.741425 -2.410844 1.198722

H -1.514011 -0.178339-5.918906 
H $-1.8119230 .966865-4.602165$ H $-0.3682411 .136376-5.612948$ C $1.7128220 .881285-3.340326$ H $0.420309-3.619787-0.466661$ H -1.858775 -5.375352 -2.120995 H $-4.040722-5.4852120 .241599$ H $1.6168791 .491812-4.245702$ H $2.4620570 .107591-3.549306$ H $-0.3266390 .981931-2.599315$ C $-5.682881-0.091151 \quad 0.270831$ O $-4.892457-1.1478280 .831584$ C $-4.428595-2.127839-0.109808$ C $-5.632183-2.839323-0.743847$ C $-6.602766-1.835774-1.378984$ C $-6.919215-0.658853-0.448210$ H $-6.0254610 .478071 \quad 1.144154$ C $-4.8101590 .838337-0.586451$ H -3.857034 -1.610150 -0.886216 $\begin{array}{lllll}\text { O } & -6.310848 & -3.597747 & 0.254566\end{array}$ H - $-5.281589-3.534843-1.513496$ H -7.533934 -2.355021 -1.637708 O $-6.026266-1.360518-2.592411$ H $-7.4292850 .135122-1.005371$ O $-7.838831-1.1302750 .542519$ C $-5.3698592 .238331-0.705227$ H $-3.8202250 .920850-0.124798$ H $-4.6667950 .440184-1.594314$ H -7.050248 -4.057753-0.177700 H $-6.666968-0.755937-3.004167$ H -8.095390 -0.3694411 .091036$ O $-4.5118902 .991911-1.455596$ O $-6.4165662 .627971-0.207162$ C $-4.8961334 .356776-1.647137$ H -4.149133 $4.833461-2.287331$ H $-5.8698714 .412497-2.143123$ $\mathrm{H}-4.9200184 .879263-0.686152$ SCF Energy (B3LYP/6-31G**//MMFF) $=-3245.90479427$

0800336

MM̄FF Geometry

C -1.6003334 .5965651 .256826$

C -1.0064203 .7562642 .118760$

C -1.6767542 .7891913 .067631$

O $-1.188236 \quad 1.4595702 .758329$

C -3.2186522 .7536383 .018193$

C -3.8573901 .8660224 .074205$

C -4.7036230 .8460643 .825153$

C -3.5441852 .2278315 .505422$

C -5.2050770 .3459742 .499800$

C $-4.858343-1.1347492 .296544$

C $-5.290605-1.6350290 .909568$

C $-5.032848-3.137676 \quad 0.777540$

C $-5.284776-3.653962-0.645585$

O $-4.531528-0.905522-0.058025$

O $-4.703171-4.964690-0.723758$

C $-4.537891-2.743257-1.645023$

C $-6.782354-3.833755-0.917806$

O $-4.839188-3.117123-2.987008$

C $-4.805336-1.241119-1.422743$

C $-3.923710-0.327961-2.310070$

O $-6.170103-0.962598-1.759383$

C $-0.2251450 .943048 \quad 3.565289$

O $0.311191 \quad 1.5008704 .513890$

C $0.108806-0.4525723 .209779$

C $-0.217683-1.0495112 .053393$

C $0.083331-2.4492251 .760840$

C -1.087929-3.213604 1.211857

C $-2.422198-0.362497-2.008941$

C -1.640762 -1.390634-2.807204

C $0.629848-2.422900-2.913362$

O $-2.092942-1.963880-3.793832$

C $0.880856-3.584701-2.008733$

C $2.052080-3.799656-1.391171$

C $2.342219-5.012332-0.558262$

C $2.782258-4.6741880 .845954$

C $4.080200-4.7133981 .191017$

C $1.710730-4.4202051 .878676$

C $1.305450-2.9742151 .983398$
N $-0.357356-1.553900-2.305284$

H $-2.6820734 .615119 \quad 1.164870$

H 0.0804543 .7787292 .186253

H -1.369589 3.0879714 .077151

H -3.524056 2.4465322 .014232

H -3.6146893 .7667953 .167428$

H -5.1122440 .3065644 .679594$

H $-4.180586 \quad 1.693156 \quad 6.218876$

H -2.505479 1.9843295 .748017

H -3.702436 3.2986595 .670674

H -6.2953690 .4655692 .480568$

H -4.8189960 .9355941 .666205$

H -3.772984 -1.261718 2.406715

H -5.342091 -1.736588 3.076047

H $-6.356072-1.4167910 .770047$

H $-5.634215-3.6990891 .503652$

H -3.988576 -3.349573 1.037053

H $-4.789272-5.270335-1.643273$

H -3.468802 -2.941828 -1.504623

H -7.211298 -4.560647 -0.217374

H $-6.958638-4.245362-1.917508$

H -7.350639 -2.906557-0.819816

H -5.721678 -2.769197 -3.200831

H $-4.129239-0.515373-3.370912$

H $-4.2485840 .707501-2.132263$

H $-6.257765-0.011165-1.935178$

H $0.668850-0.9887573 .971778$

H $-0.781689-0.5180761 .291558$

H $-0.809835-4.1770330 .778237$

H -1.582715 -2.649826 0.413308

H $-1.817765-3.3994892 .006723$

H -1.999880 $0.612481-2.283446$

H -2.235678 $-0.504532-0.939538$

H $1.533731-1.827966-3.073564$

H $\quad 0.279735-2.777598-3.887694$

H $0.072873-4.302825-1.891757$

H $2.863610-3.088756-1.527904$

H $3.129697-5.582168-1.069348$

H $1.477140-5.685941-0.516595$

H $4.400474-4.5137172 .208257$

H $4.851369-4.9412650 .463041$

H $2.083399-4.7170562 .869139$

H $0.861392-5.0868521 .700632$

H $2.092355-2.3191112 .357480$

H $-0.075971-1.026113-1.484116$

C $-1.0160195 .299682-1.086664$

O $-0.2854614 .101777-1.420638$

C $0.6037124 .390958-2.513548$

C $0.0333615 .634150-3.178842$

C $-0.4386036 .413095-1.966921$

H $1.5767874 .641517-2.073123$

C $0.753858 \quad 3.169357-3.423765$

H $0.7791896 .170834-3.772414$

H $-0.8168345 .395635-3.828405$

C $-1.4341537 .511661-2.298124$

H $0.4360236 .863556-1.478233$

C $1.1224051 .867128-2.686424$

O $-0.4823502 .931987-4.110017$

H $1.4932893 .382009-4.204301$

H $0.2632671 .509500-2.107270$

O $1.3890820 .869810-3.678971$

C $2.3441681 .971856-1.758107$

C $2.6702770 .637390-1.072319$

H $2.1397202 .721827-0.989310$

O $3.4773052 .419022-2.498055$

H $1.7880030 .269903-0.532624$

H $2.890575-0.126246-1.829903$

H $-0.9824078 .251277-2.967227$

H $-2.3247817 .110305-2.793479$

H $-1.7585188 .029925-1.390192$

C -0.8533865 .5881130 .406693$

H -1.173671 $2.810848-3.436558$

H $0.5937760 .793204-4.233736$

H $3.642528 \quad 1.768977-3.202532$

H -1.241582 6.5863010 .640315

H $\quad 0.2117305 .5825980 .670861$

H $-2.0679005 .111784-1.336277$

C $5.098599-0.8540961 .317801$ 
O $3.990856-0.634947 \quad 0.436199$

C $3.8807760 .684141-0.117980$

C $3.7564821 .729007 \quad 1.002092$

C 4.8780661 .5923022 .036241

C 5.0681110 .1419122 .487801

H $4.933603-1.857477 \quad 1.728330$

C $6.435797-0.8733180 .567977$

H $4.7746040 .890838-0.715969$

$\begin{array}{lllll}\text { O } & 2.493990 & 1.603798 & 1.651862\end{array}$

H 3.7957692 .7334990 .566692

H 4.6383462 .2179252 .905009

O 6.0853942 .0849361 .462520

H 5.9841990 .0475143 .082197

O $3.974879-0.1966773 .346348$

C $6.543453-2.095767-0.312383$

H $6.6066490 .009154-0.054755$

H $7.270380-0.9169921 .278283$

H 2.4353070 .7007132 .007336

H 6.7784652 .0256322 .142003

H $4.127989-1.0984223 .676463$

O $5.877927-1.889308-1.484266$

O $7.140742-3.1146950 .009639$

C $5.897124-2.983681-2.405613$

H $5.276741-2.715062-3.264714$

H $6.919030-3.165159-2.751458$

H $5.481176-3.882991-1.941625$

SCF Energy (B3LYP/6-31G*//MMFF) $=-3245.91661111$

0800337

MM̄FF Geometry

C $2.207719-0.471132-2.685868$

C $2.983197-1.512589-3.025722$

C $3.924772-2.252371-2.103174$

O $3.492043-2.144208-0.727583$

C $5.344265-1.665574-2.177599$

C $6.416772-2.699366-1.899864$

C $6.965455-2.946743-0.694808$

C $6.891620-3.456346-3.114095$

C $6.605121-2.3488430 .636239$

C $7.710444-1.4463141 .201046$

C $7.700432-0.0377680 .589018$

C $8.883018 \quad 0.784676 \quad 1.104746$

C $8.832428 \quad 2.236302 \quad 0.604303$

$\begin{array}{lllll}\text { O } & 6.469884 & 0.586602 & 0.972570\end{array}$

O $9.7728893 .001631 \quad 1.373094$

C $7.432507 \quad 2.8157890 .896302$

C $9.2725632 .352392-0.859768$

$\begin{array}{lllll}\text { O } & 7.286682 & 4.112821 & 0.322164\end{array}$

C 6.2750201 .8997610 .436200

C 4.9280512 .4409620 .974725

O $6.2318931 .885063-0.992674$

C $2.460003-2.928111-0.319594$

O $1.858566-3.762779-0.974782$

C $2.154124-2.5396211 .073562$

C $0.938822-2.8009081 .574466$

C $0.451572-2.4118862 .898617$

C $1.431592-1.7607763 .839765$

C 3.7572701 .4560710 .837370

C 2.4267962 .0934291 .203865

C -0.0200221 .6106091 .203349$

$\begin{array}{llll}\text { O } & 2.324709 & 3.246500 & 1.614626\end{array}$

C $-0.478822 \quad 1.2023702 .567702$

C \begin{tabular}{llll}
\hline & 1.650773 & 0.588678 & 2.795627
\end{tabular}

C $-2.150660 \quad 0.2123194 .161633$

C $-2.626779-1.2222504 .239133$

C $-3.936535-1.5190294 .188854$

C $-1.589671-2.3024434 .455368$

C $-0.846421-2.634092 \quad 3.192641$

N $1.364014 \quad 1.2216091 .008018$

H $2.269444-0.062363-1.682278$

H $2.927431-1.898144-4.041694$

H $3.927517-3.312036-2.389230$

H $5.442575-0.829350-1.475503$

H $5.538281-1.240768-3.171661$

H $7.749868-3.701206-0.638705$

H $7.656045-4.201641-2.869407$

H $6.056246-3.983971-3.585403$

H $7.324662-2.766420-3.845875$
H $5.652758-1.8141690 .606201$

H $6.453520-3.184897 \quad 1.331045$

H $7.544203-1.3602132 .282968$

H $8.691393-1.9163451 .059632$

H $7.742951-0.120895-0.502596$

$\begin{array}{llll}\text { H } & 9.834622 & 0.315190 & 0.825674\end{array}$

H $8.866874 \quad 0.797622 \quad 2.203751$

H $\quad \begin{array}{llll}\text { H } & 10.651800 & 2.601745 & 1.255828\end{array}$

H 7.3553212 .9487951 .984591

H $10.300414 \quad 1.989351-0.980075$

H $9.2845193 .397957-1.186864$

H $8.6404461 .783167-1.544512$

H 8.0245494 .6574530 .646015

H 5.0285172 .6786312 .041975

H $4.678013 \quad 3.3752200 .455928$

H $5.3210431 .725172-1.285065$

H $2.916116-2.0006951 .624910$

H $0.218428-3.3201670 .943956$

H $2.262760-2.4414174 .055786$

H $0.995977-1.4793184 .800178$

H $1.837645-0.8416863 .403537$

H $3.6826421 .092888-0.191459$

H $3.9124000 .592766 \quad 1.494694$

H $-0.6003391 .125226 \quad 0.413667$

H $-0.1122542 .695703 \quad 1.091855$

H 0.1652241 .4479093 .408745

H $-2.3091370 .375781 \quad 1.956776$

H -2.976899 $0.891348 \quad 4.409892$

H -1.3852760 .3824614 .928867$

H -4.288004 -2.542365 4.267986

H $-4.686360-0.7459374 .057261$

H -2.072477 -3.2260374 .801974$

H $-0.931408-2.0082475 .277088$

H $-1.473373-3.1103962 .437572$

H 1.5507770 .2687450 .710681

C $-0.138323 \quad 0.373797-2.908791$

O $-0.642272-0.877601-2.410336$

C $-2.081757-0.886237-2.526610$

C $-2.4769160 .483869-3.062428$

C $-1.2360830 .906637-3.824309$

H $-2.315917-1.669667-3.257171$

C $-2.682378-1.230047-1.156361$

H $-3.3730330 .444113-3.689235$

H $-2.6733941 .181913-2.240900$

C -1.161861 $2.401920-4.078202$

H $-1.2174820 .373654-4.784322$

C $-4.224037-1.255502-1.146663$

O $-2.185628-2.517682-0.778589$

H -2.320337 $-0.520071-0.404564$

H $-4.591488-0.295682-1.522161$

O $-4.677727-2.253469-2.062390$

C $-4.853862-1.4972350 .244047$

C $-6.381153-1.6918570 .221990$

H $-4.600109-0.6616710 .906691$

O $-4.323188-2.681510 \quad 0.843504$

H $-6.626078-2.590358-0.359278$

H $-6.689348-1.9158591 .252621$

H $-0.2491122 .660631-4.623792$

H $-2.0174442 .733440-4.675594$

H -1.166725 $2.968662-3.140822$

C $1.2056750 .161688-3.611373$

H -1.214695 -2.477652 -0.821407

H $-4.360192-3.112533-1.734487$

H $-3.372593-2.5382110 .987098$

H $1.6000191 .125098-3.952948$

H $1.063947-0.473465-4.494494$

H $-0.0062681 .034023-2.041458$

C $-7.653036 \quad 1.824750 \quad 0.265529$

$\begin{array}{llll}\text { O } & -6.974557 & 0.603356 & 0.593211\end{array}$

C $-7.187190-0.492905-0.302440$

C $-8.684320-0.831007-0.410040$

C $-9.505520 \quad 0.404335-0.786453$

C $-9.167188 \quad 1.5982490 .109217$

H $-7.5014972 .468201 \quad 1.141857$

C $-7.0198552 .521550-0.946054$

H $-6.816448-0.226151-1.296097$

O $-9.149036-1.3570280 .831371$

H $-8.813459-1.613431-1.167882$ 
H $-10.5728430 .168566-0.694671$ O $-9.244367 \quad 0.726755-2.149187$ H $-9.6413382 .506760-0.279942$ O $-9.731861 \quad 1.3578311 .400932$ C $-5.7625873 .252125-0.540554$ H $-6.7706871 .846694-1.769333$ H $-7.7001873 .279311-1.353385$ H $-10.087676-1.5849180 .720944$ H $-9.7931661 .495925-2.378880$ H $-9.563928 \quad 2.144570 \quad 1.947194$ O $-4.7357422 .369980-0.388904$ O $-5.706768 \quad 4.462821-0.367321$ C $-3.4920842 .945865 \quad 0.018525$ H $-3.589697 \quad 3.3954431 .011091$ H $-2.7532602 .144604 \quad 0.067533$ H $-3.1557073 .686576-0.713085$ SCF Energy (B3LYP/6-31G*//MMFF) $=-3245.90275803$

0800338

MM̄FF Geometry

C $-2.449974 \quad 4.436143-0.847975$

C $-2.8423203 .209545-0.474433$

C -3.4563752 .9063540 .864270$

O $-2.521347 \quad 2.075452 \quad 1.590889$

C $-4.756944 \quad 2.1071770 .689146$

C -5.5712082 .0301141 .964515$

C $-5.590650 \quad 0.9753932 .802448$

C $-6.438540 \quad 3.2310892 .242631$

C $-4.791510-0.2948682 .712873$

C $-5.665483-1.5205072 .412528$

C $-6.170248-1.5701560 .961520$

C -7.088124 -2.7767490 .753065$

C $-7.518128-2.923560-0.713667$

O $-5.026698-1.6759390 .108623$

O $-8.106136-4.226090-0.849028$

C $-6.260685-2.872672-1.607679$

C $-8.614178-1.916818-1.080994$

O $-6.620740-2.882652-2.987439$

C $-5.322455-1.684037-1.293603$

C $-3.984236-1.857817-2.050028$

O $-5.952723-0.473261-1.712409$

C -1.596806 2.6959122 .367283

O -1.4815223 .9007202 .546446$

C $-0.7100991 .720263 \quad 3.040445$

C $-0.573207 \quad 0.4441992 .647138$

C $0.289940-0.5615913 .273184$

C $1.043902-0.1602734 .514769$

C -2.934122 -0.789620 - 1.717925

C $-1.568615-1.179687-2.246196$

C $0.322149-2.732331-1.835491$

O $-1.096791-0.673573-3.261424$

C $0.856745-3.507219-0.676181$

C $1.933392-3.1396600 .034136$

C $2.474963-3.9307251 .188310$

C $2.467423-3.1562052 .486966$

C $3.614318-2.7213833 .034704$

C $1.147693-2.9788443 .204050$

C $0.365449-1.7890902 .716367$

N $-0.951529-2.146003-1.466924$

H -2.589259 $5.274089-0.169891$

H - $2.6740852 .371901-1.146458$

H -3.656970 3.8290521 .422855

H -4.5264261 .0993500 .328356$

H $-5.3832922 .563723-0.089004$

H -6.2386501 .0294083 .676752$

H $-7.027898 \quad 3.1164793 .158592$

H $-5.825793 \quad 4.130642 \quad 2.356897$

H -7.140675 3.3909351 .417719

H -3.973812 -0.2227131 .990292$

H $-4.309585-0.4440843 .687547$

H $-5.055722-2.4126632 .606520$

H -6.514454 -1.551655 3.106881

H $-6.707316-0.6424670 .735533$

H -7.966824 -2.715943 1.407310

H $-6.557710-3.6925411 .050836$

H $-8.314448-4.358980-1.789924$

H -5.708997 -3.806776 -1.434440

H $-9.493122-2.061223-0.441186$
H -8.965836 -2.069270 -2.107364

H $-8.294770-0.877814-0.976529$

H $-6.926883-1.989367-3.219560$

H $-3.571084-2.845579-1.809823$

H $-4.158157-1.838742-3.133390$

H $-5.2757510 .208800-1.842847$

H -0.1428182 .1268903 .871412$

H -1.137571 $0.096141 \quad 1.783394$

H $1.661775-0.9595144 .928864$

H 0.3482290 .1419605 .305498

H 1.7203240 .6748514 .303342

H $-3.2038030 .173725-2.163194$

H -2.847447 -0.630341 -0.637031

H $1.000766-1.937279-2.159317$

H $0.155191-3.395852-2.690283$

H $0.332445-4.423334-0.414458$

H $2.461201-2.227209-0.231639$

H $3.498175-4.2340830 .930638$

H $1.917899-4.8658541 .327164$

H $3.627134-2.1964833 .983627$

H $4.567870-2.8821762 .544866$

H $1.305625-2.9309154 .287249$

H $0.533872-3.878343 \quad 3.065157$

H $-0.216913-1.9852341 .816220$

H $-1.493999-2.594338-0.733582$

C $-0.251174 \quad 4.553397-2.116528$

O $0.077595 \quad 3.157879-1.967953$

C $1.1829733 .033440-1.040156$

C $1.7499144 .438398-0.897886$

C $0.4970235 .286835-1.004679$

H $0.7264042 .706021-0.099348$

C $2.1437041 .965118-1.577309$

H 2.2829664 .5870010 .045487

H $2.4323454 .670887-1.724449$

C $0.7688746 .749349-1.308126$

H $-0.0407755 .216015-0.051407$

C $3.4780011 .733823-0.836670$

O $1.4418010 .719949-1.647544$

H $2.3801472 .230711-2.615924$

H $4.0377301 .004621-1.433298$

O $4.2561672 .925971-0.815503$

C 3.3768951 .1568160 .588968

C 4.7329950 .9868961 .303246

H 2.8662120 .1883330 .552030

$\begin{array}{llll}\text { O } & 2.579929 & 2.020454 & 1.398829\end{array}$

H 5.1493961 .9735231 .544788

H 4.5224260 .5136412 .271953

H $1.3636927 .203737-0.509046$

H $1.3201036 .869521-2.246716$

H $-0.1681817 .308760-1.392601$

C $-1.7726044 .727991-2.156408$

H $1.0697640 .532239-0.769179$

H $4.385643 \quad 3.207818-1.737110$

H 2.4884891 .6041822 .272408

H -2.189239 $4.069677-2.929560$

H $-2.021345 \quad 5.754277-2.450551$

H $0.148545 \quad 4.850114-3.095194$

C $6.041562-2.018249-0.508454$

$\begin{array}{llll}\text { O } & 5.211275 & -1.122579 & 0.245145\end{array}$

$\begin{array}{llll}\text { C } 5.778357 & 0.157338 & 0.536425\end{array}$

C 7.0826300 .0059681 .336725

C $8.069301-0.9244510 .624322$

C $7.400868-2.2299800 .181812$

H $5.509046-2.977496-0.484860$

C $6.171398-1.580411-1.975831$

H $6.0054930 .674123-0.400687$

$\begin{array}{lllll}\text { O } & 6.815364 & -0.500341 & 2.642857\end{array}$

H 7.5337850 .9967421 .465952

H $8.902693-1.1564571 .298563$

O $8.605890-0.270723-0.521813$

H $8.063235-2.794228-0.485098$

O $7.172248-3.0480701 .333057$

C $4.858689-1.705310-2.719937$

H $6.514382-0.545344-2.053835$

H $6.893656-2.212670-2.504592$

H $6.366963-1.3570792 .543282$

H $9.0518550 .537913-0.217207$

H $8.036746-3.2301901 .739306$ 
O $4.743906-0.685151-3.619411$

O $4.051286-2.610366-2.558164$

C $3.552442-0.692141-4.412316$

H $3.5343220 .225277-5.006540$

H $3.559857-1.551313-5.089196$

H $2.664427-0.712181-3.774017$

SCF Energy (B3LYP/6-31G*//MMFF) $=-3245.91385296$

08_00339

MM̄FF Geometry

C $-0.773383-3.773154-1.017230$

C $-1.788376-2.913416-0.847173$

C $-2.581867-2.312214-1.977468$

O $-2.455245-0.871745-1.933223$

C $-4.074043-2.624733-1.765620$

C $-4.918721-2.475965-3.013130$

C $-5.628598-1.379174-3.342284$

C $-4.978714-3.704019-3.885045$

C $-5.647524-0.039959-2.660703$

C $-6.9335010 .240690-1.871524$

C $-6.910445-0.328833-0.443259$

C -8.2052650 .0193280 .294955$

C -8.165144 -0.4008761 .772439$

$\begin{array}{lllll}0 & -5.794236 & 0.255841 & 0.235815\end{array}$

O $\begin{array}{llll}-9.271505 & 0.250810 & 2.414928\end{array}$

C -6.8599340 .1263312 .410200$

C $-8.393245-1.9085921 .932976$

O $-6.700617-0.3659503 .738802$

C $-5.598797-0.2010931 .579131$

C $-4.367386 \quad 0.545022 \quad 2.152289$

O $-5.362113-1.6096731 .634522$

C $-1.301825-0.286883-2.345646$

O $-0.287999-0.837571-2.739169$

C $-1.505798 \quad 1.175038-2.222619$

C $-0.4654872 .019650-2.262501$

C $-0.5462513 .480192-2.153890$

C -1.915148 $4.095310-2.011384$

C -3.1287450 .4666091 .250918$

C -1.9322651 .1354561 .895195$

C $-0.911362 \quad 3.3563212 .319320$

O $\begin{array}{llll}-1.106680 & 0.493650 & 2.540901\end{array}$

C -0.7416194 .6081321 .519458$

C 0.4496365 .0710501 .110416

C $0.629640 \quad 6.351427 \quad 0.346848$

C $1.4571836 .180634-0.907608$

C $2.7525996 .537469-0.932770$

C $0.7593575 .695356-2.157963$

C $0.5960594 .197770-2.190776$

$\mathrm{N}-1.8987122 .5045591 .682063$

H $-0.476582-4.065642-2.020970$

H -2.067006 $-2.621190 \quad 0.162269$

H -2.238876 -2.675913 -2.953823

H $-4.473032-1.979574-0.975380$

H $-4.201115-3.652220-1.397536$

H -6.235895-1.418793 -4.246202

H $-5.599703-3.554498-4.774696$

H $-3.975367-3.978156-4.226382$

H $-5.398986-4.546831-3.326417$

H $-4.7679240 .109252-2.031018$

H $-5.5633220 .714774-3.454008$

H $-7.0377421 .332008-1.805022$

H -7.808756 -0.133679 -2.416269

H $-6.779563-1.415238-0.495344$

H $-9.071823-0.426712-0.209003$

H -8.3644091 .1063320 .252803$

H $-9.303405-0.0484953 .339149$

H $-6.949608 \quad 1.2187792 .489671$

H -9.382684 -2.186593 1.550252

H -8.387649 -2.200462 2.988968

H -7.652379 -2.512970 1.405219

H -7.457568 -0.0536924 .262171$

H $-4.615747 \quad 1.6053982 .288835$

H -4.1132660 .1423243 .140887$

H $-4.413144-1.7831531 .539129$

H $-2.5260281 .519238-2.092179$

H $0.5351901 .607515-2.384689$

H -2.513540 $3.909418-2.909990$

H $-1.8959255 .175622-1.855296$
H -2.441474 $3.675442-1.147201$

H $-2.847969-0.5686191 .041921$

$\begin{array}{llll}H & -3.324324 & 0.943126 & 0.284294\end{array}$

H 0.0269992 .8037372 .435911

H -1.2895933 .5998933 .317419$

H $-1.6396865 .175634 \quad 1.286458$

H 1.3505494 .5213061 .371039

H 1.1170427 .0704381 .018462

$\begin{array}{llll}H & -0.336452 & 6.800803 & 0.084982\end{array}$

H $3.3455876 .449370-1.837109$

H $3.2513806 .917370-0.047388$

H $1.3464765 .973754-3.043924$

H $-0.184576 \quad 6.234274-2.277548$

H $1.5394613 .661072-2.291017$

H $-2.7223492 .947779 \quad 1.284260$

C $1.339067-3.6441430 .306751$

O $2.223457-3.963442-0.783760$

C $3.585782-3.923375-0.295894$

C $3.498076-3.5883531 .189161$

C $2.107010-4.0631371 .554991$

H $3.988705-4.932376-0.443078$

C $4.362317-2.900518-1.135437$

H $4.286237-4.0734501 .772716$

H $3.577423-2.5070451 .351493$

C $1.587569-3.4523562 .845260$

H $2.117389-5.1574931 .643768$

C $5.756102-2.559721-0.583611$

O $4.511156-3.425155-2.458666$

H $3.756122-1.994061-1.231521$

H $5.677933-2.1631840 .430733$

O $6.515325-3.769509-0.456870$

C $6.572005-1.575951-1.442020$

C $5.865163-0.271107-1.850167$

H $6.905391-2.082885-2.356197$

O $7.763391-1.227847-0.728445$

H $6.5481600 .277854-2.512467$

H $4.982129-0.524809-2.447013$

H $\quad 0.583000-3.8193383 .076900$

H $2.242108-3.7120363 .683812$

H $1.540651-2.3599002 .781853$

C $0.004233-4.3623050 .126635$

H $3.619836-3.627863-2.791237$

H $6.557625-4.181833-1.336838$

H $8.207230-2.057577-0.482464$

H $-0.586706-4.301023 \quad 1.047640$

H $0.165081-5.426318-0.084398$

H $1.191122-2.5550850 .297533$

C 4.0430620 .7853111 .262964

$\begin{array}{lllll}\text { O } & 4.501881 & 0.043595 & 0.126441\end{array}$

C $5.4764080 .683836-0.698094$

C $4.9168102 .008573-1.248183$

C $4.3820652 .906925-0.128346$

C 3.4661202 .1428080 .831090

H 3.2206450 .1847441 .672177

C 5.1214640 .9051382 .346904

H $6.3730420 .888257-0.103901$

O $3.8655431 .733005-2.173122$

H $5.7123552 .523421-1.800262$

H $3.8200133 .739290-0.566325$

O $5.469874 \quad 3.4576920 .607251$

H 3.2532442 .7552991 .715065

$\begin{array}{lllll}\text { O } & 2.219578 & 1.917155 & 0.167247\end{array}$

C $5.427569-0.4431932 .956599$

H 6.0505181 .3415971 .969936

H 4.7831891 .5521703 .164836

H $3.5863532 .580613-2.558599$

H $6.016313 \quad 3.968081-0.014415$

H 1.6180381 .4946760 .803818

O $6.700904-0.8185262 .646229$

O $4.627842-1.0877443 .623042$

C $7.113676-2.0878333 .162491$

H $7.144998-2.0559354 .255577$

H $6.441095-2.8791802 .818628$

H $8.118927-2.2962382 .786825$

SCF Energy (B3LYP/6-31G*//MMFF)= -3245.90132346

0800340

MM̄FF Geometry 
C $-3.145905-1.8803572 .598711$ C $-2.527781-2.5797363 .563089$ C $-1.051919-2.5538323 .885077$ O $-0.349924-1.6122613 .043936$ C $-0.435958-3.9419323 .629401$ C $1.010668-4.0247534 .075465$ C $2.074276-3.8309393 .270307$ C $1.211817-4.3566155 .531127$ C $2.069991-3.4642721 .811179$ C $2.875031-4.4704560 .980761$ C $2.811487-4.167297-0.525113$ C $3.676477-5.163490-1.303446$ C $3.742054-4.828776-2.801304$ O $3.276806-2.830741-0.737584$ O $4.788972-5.616247-3.389478$ C $4.109877-3.337639-2.965641$

C $2.462294-5.253723-3.529023$ O $4.046677-2.944503-4.334192$ C $3.239615-2.397066-2.104769$ C $3.734096-0.932159-2.150861$ O $1.900005-2.403109-2.612178$ C $-0.072277-0.3791093 .542247$ O -0.4078190 .0772794 .622315$ C $\quad 0.734436 \quad 0.3239872 .519429$ C 1.0962191 .6021432 .703036 C 1.8728192 .4138251 .761579 C $2.453957 \quad 1.7130250 .562417$ C $5.119192-0.700696-1.542538$ C $5.4613530 .777417-1.450298$ C $7.0064112 .330696-0.249413$ O $4.8766401 .644575-2.093828$ C $6.2573922 .843470 \quad 0.941612$ C 5.8122814 .1047301 .051445 C 5.0772584 .6200922 .257162 C 3.8570975 .4421151 .898987 C 3.7885876 .7451282 .221167 C $2.7185314 .766621 \quad 1.166084$ C 2.0189203 .7327882 .007917 N $6.5188671 .004353-0.581046$ H -2.575184 -1.208928 1.963488 H -3.126588 -3.240259 4.187741 H $-0.931980-2.2846714 .941654$ H $-0.518941-4.1984562 .566565$ H -1.010162 -4.714863 4.157938 H $3.068268-3.9112163 .709107$ H $2.271147-4.4099715 .803955$ H $0.746240-3.595796 \quad 6.165417$ H $0.762065-5.3272455 .764375$ H $1.057384-3.3885871 .405958$ H $2.518529-2.4678951 .717382$ H $3.920544-4.4387761 .315337$ H $2.504979-5.4874851 .162422$ H $1.764937-4.235579-0.845085$ H $3.318984-6.190046-1.154349$ H $4.698415-5.143455-0.899389$ H $5.622922-5.400024-2.938766$ H $5.157740-3.229032-2.659704$ H $2.322714-6.339019-3.451182$ H $2.525665-5.037165-4.601238$ H $1.562831-4.777905-3.133307$ H $4.601687-3.562546-4.839903$ H $3.703566-0.556380-3.181678$ H $3.016730-0.309971-1.598619$ H $1.450451-1.596551-2.310544$ H $1.001872-0.2335091 .628465$ H $0.7757382 .110742 \quad 3.611077$ H $3.0900910 .877707 \quad 0.876730$ H $3.0775882 .355778-0.059792$ H $1.6566731 .325579-0.080939$ H $5.161328-1.130283-0.535227$ H $5.893517-1.176447-2.153184$ H $6.8970952 .986183-1.119455$ H $8.0686892 .230870-0.006776$ H 6.0862322 .1490981 .761283 H 6.0119684 .8107940 .248518 H 5.7835255 .2281942 .837303 H 4.7749383 .7972852 .916375 H 2.9205827 .3456871 .969928
H 4.5984867 .2425612 .744265

H 3.0900514 .3690890 .218720

H 1.9615315 .5092080 .879348

H 1.5510844 .1460972 .902671

H $6.8959410 .223827-0.051286$

C $-4.883642-2.4457090 .895288$

O $-4.539141-1.404643-0.041892$

C $-5.483007-1.430397-1.130969$

C $-6.370419-2.649934-0.901436$

C $-6.347647-2.7682560 .609467$

H $-6.098298-0.528484-1.041020$

C $-4.739685-1.442307-2.470847$

H -7.377243 -2.510030 -1.306954

H $-5.947822-3.550427-1.363015$

C $-6.797894-4.1280701 .114378$

H $-6.994651-1.9894801 .035092$

C $-3.868182-0.193228-2.727295$

O $-3.885978-2.593877-2.515571$

H $-5.456645-1.569626-3.289977$

H -3.060457 -0.149810-1.988947

O $-3.229086-0.371621-3.998888$

C $-4.6478291 .138704-2.741160$

C $-3.8311022 .343381-3.248728$

H $-5.0379631 .350647-1.739992$

O $-5.7756800 .987088-3.609911$

H $-3.4077822 .114013-4.234203$

H $-4.5305873 .174310-3.414973$

H $-7.828077-4.3312130 .803764$

H $-6.164378-4.9313370 .723377$

H -6.761343 -4.172625 2.207160

C $-4.622603-1.9639262 .322123$

H -3.258089-2.521598 -1.776196

H $-3.926030-0.388029-4.677371$

H $-6.3079151 .798268-3.544526$

H -5.103105 -2.636328 3.041382

H $-5.050823-0.9648362 .466958$

H $-4.250185-3.3122870 .661411$

C $-2.5162443 .890698-0.118899$

O $-3.3760223 .389379-1.150172$

C $-2.7268732 .800366-2.282935$

C $-1.7793623 .811521-2.949501$

C $-0.7886464 .399050-1.941671$

C $-1.4951764 .897020-0.678267$

H $-3.185970 \quad 4.4366280 .558295$

C -1.8693812 .7560250 .681220$

H $-2.1490211 .930329-1.958093$

O $-2.5217594 .866010-3.559256$

H $-1.2305793 .303365-3.751191$

H $-0.2507565 .230531-2.413607$

O $0.1634663 .395874-1.602286$

H $-0.757188 \quad 5.148678 \quad 0.092391$

O $-2.1817796 .108183-1.006885$

C -2.8932122 .0944091 .573549$

H -1.423759 1.9729660 .062265

H -1.071013 3.1578421 .313468

H $-3.0632895 .286263-2.869408$

H $0.8071513 .797263-0.994232$

H $-2.5695896 .458312-0.186778$

O -2.6528432 .4179222 .876016$

O -3.808008 1.394876 1.159143

C -3.5782241 .8910193 .831648$

H -3.3837602 .3735184 .793138$

H -4.6096222 .1063703 .536123$

$\mathrm{H}-3.4243730 .8149043 .942551$

SCF Energy $\left(B 3 L Y P / 6-31 G^{* *} / / M M F F\right)=-3245.91378356$

$08 \_00341$

MM̄FF Geometry

C $1.256624-1.837540 \quad 0.072764$

C $2.051156-2.5267740 .906696$

C $2.835668-1.9574082 .067477$

O $2.592329-0.5369662 .167478$

C $4.342242-2.1718061 .845899$

C $5.163379-1.9905173 .106215$

C $5.901857-0.9012683 .394544$

C $5.155681-3.1663844 .049365$

C 6.0269030 .3716012 .604176

C 7.4169070 .5372091 .973616 
C $7.649142-0.3848810 .766316$

C $9.078823-0.2409970 .241925$

C $9.308342-1.064193-1.034504$

O $6.719696-0.005011-0.252585$

O $10.554649-0.619595-1.591280$

C $8.185844-0.742553-2.045152$

C $9.476395-2.555788-0.721599$

O $8.260742-1.594690-3.186085$

C $6.765823-0.814133-1.433913$

C $5.733826-0.243249-2.436598$

O $6.445171-2.178068-1.154544$

C $2.3184540 .002593 \quad 3.379758$

O $2.273566-0.5857204 .451020$

C 2.0371221 .4544543 .269121

C 2.1844512 .1565302 .134002

C 1.8986663 .5817761 .950117

C 1.3240334 .3379793 .118873

C $4.328063-0.048224-1.850460$

C $3.382210 \quad 0.602629-2.846070$

C $1.1153101 .639228-2.964998$

O $3.6650150 .777997-4.028020$

C $1.2882833 .112273-2.768696$

C $0.4093113 .887230-2.115443$

C $0.5926235 .363660-1.911344$

C $0.6236815 .752225-0.448799$

$\begin{array}{llll}\text { C } & -0.443240 & 6.324707 & 0.134074\end{array}$

C $1.9169315 .561466 \quad 0.314157$

C 2.1367034 .1355980 .742401

N $2.1750790 .948332-2.254574$

H $1.145060-0.7639620 .193583$

H $2.140425-3.6024060 .765698$

H $2.490787-2.4743112 .970783$

H $4.693732-1.4928161 .062028$

H $4.533477-3.1839901 .464592$

H $6.472622-0.9024844 .322690$

H $5.798348-3.0050434 .921380$

H $4.143667-3.3575974 .418875$

H $5.515479-4.0652793 .538151$

H $5.245964 \quad 0.468017 \quad 1.844271$

H $5.8616801 .203103 \quad 3.301178$

H $7.506721 \quad 1.5809771 .645522$

H $8.191934 \quad 0.3676652 .731387$

H $7.459243-1.4214701 .064817$

H $9.807061-0.5191691 .014025$

H 9.2765340 .8156810 .012135

H $10.748737-1.164909-2.372142$

H $8.3559520 .280963-2.407780$

H $10.343594-2.712247-0.068897$

H $9.678441-3.131391-1.631652$

H $8.605569-2.991309-0.226963$

H $9.121272-1.443535-3.611568$

H $6.0825870 .736131-2.790157$

H $5.663677-0.899940-3.313024$

H $5.482052-2.289678-1.170746$

H 1.6967241 .9199134 .188670

H 2.5453371 .6464871 .242317

H 2.0379974 .3612433 .949435

H 0.3969803 .8668653 .464738

H 1.0728115 .3748752 .885599

H $3.893363-1.010645-1.562482$

H $4.3745080 .589597-0.960257$

H $\quad 0.1646221 .275558-2.564854$

H $\quad 1.161757 \quad 1.396168-4.031157$

H $2.1860513 .562122-3.186455$

H $-0.4936833 .441601-1.705804$

H $-0.2344115 .874813-2.421151$

H $1.5062155 .725060-2.399573$

H -0.4211046 .6354601 .173281$

H -1.364907 $6.491252-0.413666$

H 1.9632536 .2419861 .171656

H $2.7590805 .869063-0.319178$

H $2.5541233 .503983-0.041769$

H $2.0706680 .838615-1.250432$

C -1.029197 -2.236937 -0.958522

O $-1.338399-0.832374-1.056082$

C $-2.393688-0.526791-0.116846$

C -2.945955 -1.870006 0.340413

C $-1.700815-2.7335280 .320557$
H $-1.906397-0.0107700 .720408$

C $-3.4116920 .401946-0.791071$

H -3.414681 -1.818804 1.327696

H -3.687768 -2.249121 -0.371921

C $-1.986426-4.2243960 .328609$

H -1.099993 -2.482381 1.203363

C -4.6727520 .6513600 .060022$

O $-2.7523261 .642665-1.052730$

H -3.705456 -0.009665 -1.764450

H $-5.149141-0.3169410 .237241$

O $-4.294873 \quad 1.1653641 .337259$

C $-5.7071191 .593967-0.589447$

C -6.9826251 .7962780 .254400$

H $-5.9732901 .202642-1.578331$

O $-5.1216522 .882006-0.790719$

H -6.7248842 .2701421 .209842$

H -7.610320 $2.523940-0.277156$

H -2.538064 -4.505900 1.231524

H $-2.585079-4.523984-0.537550$

H -1.054616 -4.798207 0.308597

C $0.483243-2.457933-1.057371$

H -1.978689 $1.447236-1.608127$

H -3.8666792 .0259061 .186756$

H $-5.7666253 .426491-1.273531$

H $\quad 0.847908-2.003285-1.986857$

H $0.689168-3.532758-1.122335$

H -1.497940 -2.707409-1.833153

C $-8.850031-1.276617-0.746435$

O $-8.149636-0.026581-0.771592$

C -7.7863410 .5100750 .503131$

C -9.0390430 .7601071 .355356$

C -9.870206 -0.521659 1.486704

C $-10.114598-1.1831200 .126079$

H $-9.174524-1.436883-1.783181$

C $-7.921910-2.441168-0.364320$

H $-7.146449-0.2017411 .031695$

O $-9.828911 \quad 1.7868930 .760247$

H $-8.727967 \quad 1.1141592 .345513$

H -10.835375 -0.2869461 .951578$

O $-9.195191-1.4480082 .332873$

H $-10.541267-2.1845840 .256901$

O $-11.076794-0.411819-0.599696$

C $-6.880668-2.709741-1.432936$

H -7.385531 -2.2615240 .570570$

H $-8.523341-3.351661-0.256638$

H $-10.599792 \quad 1.9342591 .334175$

H -9.071127 -1.020059 3.197182

H $-11.895025-0.394687-0.074748$

O $-6.319147-3.934894-1.208889$

O $-6.584497-1.943366-2.339390$

C $-5.333838-4.345085-2.162582$

H -4.537186 -3.599532 -2.242158

H $-4.898420-5.286669-1.817737$

H -5.804255 -4.510424 -3.136341

SCF Energy (B3LYP/6-31G**//MMFF) $=-3245.89421654$

0800342

MM̄FF Geometry

C -1.104685 3.8895671 .325493

C -0.7747355 .0503740 .736847$

C $-0.0580835 .198604-0.587163$

O $-0.0448833 .951081-1.317219$

C $1.3971395 .642552-0.357968$

C $2.1913585 .815628-1.640465$

C $3.3066565 .124923-1.952271$

C $1.6710056 .860459-2.594624$

C $4.0198234 .097291-1.119521$

C $3.7855512 .678483-1.641429$

C $4.2697751 .592267-0.668550$

C $5.7736641 .664394-0.395371$

C $6.2362780 .527530 \quad 0.528113$

O $3.9514550 .324539-1.258133$

O 7.6703010 .4691250 .474834

C $5.712429-0.815218-0.021641$

C 5.8709200 .8069591 .991425

O $5.968040-1.8756730 .896822$

C $4.212273-0.798478-0.403378$

C $3.856481-2.085316-1.178478$ 
$\begin{array}{llll}\text { O O } & 3.373970 & -0.777158 & 0.753455\end{array}$

C -1.159956 $3.648631-2.038642$

O $-2.1689884 .325948-2.153560$

C $-0.9484132 .319656-2.651768$

C $-1.974894 \quad 1.703278-3.256778$

C $-1.9326790 .393502-3.910363$

C $-0.611478-0.327033-3.927152$

C $2.408954-2.116571-1.680718$

C $2.044490-3.461203-2.289859$

C $0.070433-4.698954-3.154032$

O $2.828151-4.402828-2.370257$

C $-1.052057-4.349478-4.075780$

C $-2.325099-4.209918-3.676393$

C $-3.463152-3.886718-4.600664$

C $-3.973515-2.475370-4.409628$

C $-5.039376-2.239259-3.625025$

C $-3.262618-1.392409-5.197847$

C $-3.067380-0.097441-4.452266$

N $0.727559-3.479088-2.727627$

H $-0.810267 \quad 2.947357 \quad 0.870858$

H -1.069763 5.9821361 .215114

H $-0.5850845 .958677-1.177423$

H $1.876635 \quad 4.9112800 .299754$

H $1.412656 \quad 6.595482 \quad 0.187474$

H $3.7856395 .328916-2.909335$

H $2.3640717 .050071-3.421429$

H $0.7181396 .546037-3.031291$

H $1.5213217 .810827-2.071937$

H $5.0903754 .333195-1.154695$

H $3.7393534 .161561-0.064868$

H $2.7148522 .517935-1.827845$

H $4.2726792 .548851-2.616993$

H 3.7017531 .7010740 .263020

H 6.0573482 .6298130 .039033

H $6.3203401 .588440-1.345496$

H $8.016461 \quad 1.3378040 .742269$

H $6.291489-1.043392-0.927657$

H $6.360686 \quad 1.7244842 .339633$

H 6.2307350 .0086102 .649506

H $4.7972450 .929657 \quad 2.149061$

H $6.921719-1.8696731 .087640$

H $4.519299-2.190585-2.047118$

H $4.022316-2.957458-0.532868$

H $3.925314-0.9108591 .540988$

H $\quad 0.0418321 .884167-2.580805$

H $-2.9431452 .202175-3.276364$

H $-0.658631-1.312622-4.392164$

H $-0.248071-0.480891-2.905308$

H $\quad 0.1356330 .251113-4.481853$

H $1.716208-1.925338-0.853090$

H $2.258464-1.342363-2.441606$

H $-0.284437-5.212363-2.253822$

H $0.787481-5.358818-3.653041$

H $-0.802116-4.213886-5.125136$

H -2.569726 -4.341745 -2.624582

H $-4.265672-4.612511-4.414733$

H -3.179863 -4.034754 -5.650483

H $-5.449388-1.243794-3.496847$

H $-5.533607-3.045142-3.091953$

H -3.847634 -1.186352 -6.103882

H - $2.304287-1.768385-5.568682$

H $-3.9736620 .505831-4.384732$

H $0.131304-2.703879-2.454556$

C -3.236233 3.0509302 .405186

O -3.0050211 .6649462 .077679$

C -3.9548691 .2680261 .070782$

C $-5.001526 \quad 2.378196 \quad 1.012013$

C $-4.1613213 .600844 \quad 1.318292$

H $-3.410113 \quad 1.2475550 .119281$

C -4.533471 -0.1184031 .369859$

H -5.4964072 .4289520 .037329$

H $-5.7760082 .250821 \quad 1.777121$

C $-4.969914 \quad 4.810712 \quad 1.751456$

H $-3.599018 \quad 3.8568020 .412536$

C $-3.493164-1.2442591 .538944$

O $-5.284464-0.0441992 .589815$

H $-5.252727-0.3831380 .585005$

H -2.971900 -1.114747 2.493968
O $-4.223625-2.4740771 .656303$

C $-2.466078-1.3768310 .400372$

C -1.658076 -2.6898930 .412558$

H $-1.781068-0.5217880 .430050$

O $-3.159481-1.316828-0.849626$

H $-2.320482-3.5314770 .171932$

H $-0.946121-2.630884-0.419673$

H $-5.661755 \quad 5.1145590 .959093$

H $-5.559963 \quad 4.5992102 .649550$

H $-4.3132425 .658687 \quad 1.970747$

C -1.9058803 .7895302 .592491$

H $-5.654493-0.9280892 .756455$

H $-4.655840-2.6407930 .800774$

H -2.494004 -1.358509 -1.557277

H $-1.2942513 .252513 \quad 3.326331$

H -2.101581 4.7869053 .003898

H -3.7587963 .0453003 .370960$

C $0.812388-2.0005493 .127304$

O $\quad 0.038312-1.9195581 .922417$

C $-0.899728-2.9784571 .716095$

C $-0.182173-4.3381451 .670105$

C $0.677072-4.5549052 .918574$

C $1.565257-3.3406203 .212554$

H $1.560292-1.2024393 .035734$

C $-0.035407-1.7078974 .375490$

H $-1.619270-2.9911442 .539937$

$\begin{array}{lllll}\text { O } & 0.641250 & -4.407122 & 0.509385\end{array}$

H $-0.935756-5.1308621 .588356$

H $1.304612-5.4424592 .772246$

O $-0.182754-4.8125514 .024443$

H $2.029632-3.4332044 .201208$

O $2.628129-3.3007902 .256870$

C $-0.561351-0.288298 \quad 4.400410$

H $-0.900653-2.3719784 .444587$

H $0.583008-1.8403105 .270981$

H $1.051056-5.2885090 .489652$

H $\quad 0.378753-4.967484 \quad 4.802949$

H $3.129652-4.1297252 .338768$

O $-1.287273-0.1129425 .544791$

O $\begin{array}{llll}0.368344 & 0.554809 & 3.536026\end{array}$

C $-1.867813 \quad 1.182723 \quad 5.720415$

H $-1.106973 \quad 1.9639045 .630131$

H -2.667298 1.3317484 .990015

H -2.297348 $1.229148 \quad 6.724622$

SCF Energy (B3LYP/6-31G**//MMFF) = -3245.90361297

08_00343

MM̄FF Geometry

C -2.083278 -3.2235671 .719272$

C $-1.948854-4.1122380 .722564$

C $-2.336360-3.865874-0.719072$

O $-2.387195-2.445981-0.996839$

C $-3.716840-4.474581-1.005647$

C $-4.120389-4.360958-2.462109$

C $-4.994909-3.454656-2.941824$

C $-3.505588-5.383133-3.382446$

C $-5.689657-2.365497-2.172296$

C $-5.658610-1.030687-2.930838$

C $-6.3518170 .079411-2.128924$

C $-6.5287001 .339411-2.976114$

C $-7.159786 \quad 2.481219-2.164806$

$\begin{array}{llll}0 & -5.538818 & 0.374883 & -0.987717\end{array}$

O $-7.007744 \quad 3.693907-2.917755$

C $-6.3657532 .658618-0.854800$

C -8.666529 $2.279931-1.965030$

$\begin{array}{llllll} & \text { O } & -7.000141 & 3.604033 & 0.003814\end{array}$

C $-6.1124461 .335503-0.091568$

C $-5.1102401 .590081 \quad 1.058270$

$\begin{array}{llll}\text { O } & -7.341781 & 0.865167 & 0.464042\end{array}$

C $-1.200776-1.833613-1.267035$

O $-0.108845-2.367585-1.381743$

C $-1.448633-0.377517-1.348774$

C $-0.4069450 .462046-1.246569$

C $-0.461978 \quad 1.925533-1.269444$

C $-1.7873902 .567960-1.581055$

C $-4.5349710 .319649 \quad 1.698155$

C $-3.531185 \quad 0.683700 \quad 2.775872$

$\begin{array}{lll}\text { C }-1.270027 & 1.703429 & 3.074735\end{array}$ 
$\begin{array}{llll}\text { O } & -3.795154 & 0.567800 & 3.970273\end{array}$

C -0.8572713 .0257702 .509614$

C $0.404308 \quad 3.335007 \quad 2.174335$

C $0.805475 \quad 4.6618161 .595848$

C $1.595124 \quad 4.533185 \quad 0.310977$

C 2.8975754 .8607120 .267653

C $0.859025 \quad 4.108000-0.944023$

C $0.6717182 .615233-1.021898$

N -2.346247 1.1782192 .255579

H -2.546382 -2.261050 1.519781

H -1.499909 -5.0799470 .934365$

H $-1.584958-4.333614-1.367503$

H $-4.470709-4.004714-0.363167$

H $-3.723820-5.536721-0.725446$

H -5.244991 -3.496740 -4.001193

H $-3.866504-5.286309-4.412032$

H $-2.416984-5.274890-3.404806$

H -3.748467 -6.395184 -3.042491

H $-6.732493-2.667659-2.018270$

H -5.245336 -2.221672 -1.183763

H $-4.614880-0.751115-3.124828$

H $-6.156628-1.153292-3.900670$

H -7.327200 -0.293219-1.794129

H $-7.1285791 .128458-3.870134$

H $-5.5481361 .673381-3.343511$

H $-7.4463413 .570043-3.776965$

H -5.391771 $3.091260-1.124319$

H $-9.1762202 .217555-2.934088$

H $-9.1138493 .135455-1.446822$

H -8.908146 1.373604 -1.406067

H -7.125731 $4.421429-0.507897$

H -4.2734152 .1841370 .673798$

H -5.587851 2.192078 1.842407

H $-7.146564 \quad 0.303794 \quad 1.231006$

H -2.473090 -0.040280 -1.454436

H $0.5878940 .039243-1.108013$

H -2.503757 2.377158 -0.777762

H -2.198458 2.171861-2.516770

H $-1.7283143 .650276-1.711933$

H $-5.322518-0.2878552 .155882$

H $-4.033301-0.3083950 .953691$

H -0.4559280 .9739933 .053036$

H -1.6066001 .8232974 .108749$

H -1.6399793 .7681762 .369931$

H 1.1935072 .6044172 .334419

H 1.3999545 .1870552 .354717

H $-0.0706895 .295807 \quad 1.410218$

H $3.4637054 .801102-0.656018$

H $3.4287385 .191922 \quad 1.153753$

H $1.4277124 .413613-1.832043$

H $-0.0833034 .660364-0.991110$

H $1.5886792 .050654-0.844172$

H -2.190792 $1.121742 \quad 1.253436$

C $-0.569687-2.3706523 .487610$

O $0.622382-2.564413 \quad 2.702639$

C $1.772076-2.2791503 .518795$

C $1.233227-1.7045394 .824702$

C $-0.107114-2.4057984 .940097$

H $2.258725-3.2413243 .722419$

C $2.754977-1.3594842 .778297$

H $1.897826-1.9050225 .670637$

H $1.092439-0.6190034 .758951$

C -1.061722 -1.731723 5.910044

H $0.062131-3.4435685 .257007$

C $3.339899-1.9962641 .498267$

$\begin{array}{llll}\text { O } & 2.105301 & -0.129776 & 2.462861\end{array}$

H $3.580640-1.1208293 .459045$

H $3.759749-2.9765941 .755343$

O $2.315919-2.2119770 .528522$

C $4.452660-1.1490400 .846719$

C $5.087334-1.873732-0.358371$

H $5.229619-0.9519451 .594331$

$\begin{array}{lllll}\text { O } & 3.937645 & 0.113121 & 0.432275\end{array}$

H $5.392108-2.879161-0.044465$

H $4.334738-1.974095-1.150579$

H $-0.639485-1.7213906 .920200$

H $-1.263548-0.6951135 .619546$

H -2.018242 -2.262232 5.949520
C $-1.600421-3.439948 \quad 3.125734$

H $1.394289-0.3302201 .829913$

H $1.654062-2.8057620 .921140$

H $3.218010-0.056071-0.199746$

H -2.462244 -3.384087 3.799397

H $-1.158314-4.4381873 .227588$

H $-0.955574-1.3746453 .238551$

C $6.8583430 .901347-2.138895$

O $5.8432710 .045646-1.597429$

C $6.310505-1.127282-0.925776$

C $7.110915-2.004365-1.900760$

C $8.251247-1.214985-2.552741$

C $7.7712490 .130487-3.107318$

H $6.3101311 .649491-2.726371$

C $7.6241651 .644497-1.033140$

H $6.952449-0.838718-0.086407$

O $6.254703-2.528815-2.913908$

H $7.519844-2.861272-1.353107$

H $8.684129-1.807674-3.367924$

O $9.279097-0.976498-1.595593$

H $8.6245980 .760968-3.383694$

O $7.019331-0.104664-4.302044$

C $6.7186322 .558803-0.234350$

H $8.0902340 .947856-0.331471$

H $8.4066862 .268466-1.479349$

H $5.822831-1.777944-3.356210$

H $9.592495-1.841155-1.279515$

H $7.610393-0.541178-4.938961$

O $7.3925713 .001564 \quad 0.868249$

O $5.5739752 .861272-0.541350$

C 6.6555603 .8792951 .725416

H 7.2926724 .1390082 .574959

H 6.3920514 .7965481 .190378

H 5.7577003 .3784502 .100533

SCF Energy (B3LYP/6-31G**//MMFF) $=-3245.91583381$

08_00344

MM̄FF Geometry

C $1.394236-0.0738052 .531906$

C $2.461576 \quad 0.362325 \quad 3.218398$

C $3.546775 \quad 1.2803862 .700935$

O 3.2147691 .8215201 .402314

C 4.8665050 .5092372 .537768

C 6.0847011 .4049372 .637038

C 6.7427991 .9378191 .589621

C 6.5795851 .6469514 .040383

C $6.3941591 .856676 \quad 0.129663$

C $7.3572860 .961298-0.661338$

C $7.031616-0.533241-0.521381$

C $8.083766-1.386401-1.231426$

C $7.720307-2.878428-1.204214$

O $5.746784-0.748425-1.116442$

O $8.571809-3.557410-2.139716$

C $6.271483-3.046399-1.706519$

C $7.991097-3.5093490 .166666$

O $5.836592-4.397389-1.567027$

C $5.262498-2.092634-1.025150$

C $3.903340-2.160190-1.762164$

$\begin{array}{lllll}\text { O } & 5.079341 & -2.498572 & 0.332367\end{array}$

C 2.3554092 .8719631 .342378

$\begin{array}{lllll}\text { O } & 1.835305 & 3.459694 & 2.275913\end{array}$

C $2.1138593 .141086-0.091318$

C $0.9836943 .758407-0.463224$

C $0.5739894 .048616-1.838226$

C $1.5454713 .716212-2.941030$

C $2.894008-1.076502-1.350403$

C $1.574472-1.236125-2.089030$

C $-0.705364-0.247551-2.247673$

O $1.367138-2.136392-2.899266$

C $-0.8508960 .844196-3.259615$

C $-1.915721 \quad 1.659757-3.304009$

C $-2.1171652 .732953-4.334627$

C $-2.4408844 .077474-3.721165$

C $-3.713514 \quad 4.486705-3.584264$

C $-1.2865664 .978623-3.346043$

C $-0.6476134 .582940-2.045100$

N $0.650729-0.264041-1.729685$

H 1.2753940 .1939061 .486151 
H $2.557501 \quad 0.0759524 .264162$ H 3.6677002 .1063953 .413237 H $4.867960-0.0199251 .578775$ H $4.960813-0.2700633 .305923$ H 7.6275342 .5394351 .796906 H 7.4602632 .2974934 .066169 H 5.8008342 .1237264 .644023 H $\quad 6.8554740 .6990574 .514100$ H $5.3585361 .545728-0.031217$ H $6.4606282 .876234-0.271620$ H $7.2735681 .241560-1.719661$ H $8.3923731 .156088-0.354645$ H $6.990042-0.7935280 .541730$ H $9.076456-1.224616-0.793225$ H $8.164380-1.068921-2.280582$ H $9.494180-3.398408-1.875330$ H $6.274603-2.832501-2.784702$ H $9.051248-3.4119820 .430010$ H $7.783838-4.5851940 .154022$ H $7.408992-3.0550390 .971030$ H $6.488543-4.959630-2.019569$ H $4.074005-2.052802-2.841693$ H $3.449349-3.146818-1.602096$ H $4.196774-2.2290090 .630565$ H $2.8508752 .781359-0.800315$ H 0.2789024 .0613330 .310272 H $2.4645244 .302192-2.830084$ H $1.1574913 .917218-3.941577$ H $1.8049112 .651942-2.922054$ H $2.686491-1.129880-0.277184$ H $3.291878-0.080736-1.577045$ H -1.371899 -0.106860 -1.392290 H $-0.933640-1.211001-2.713896$ H $-0.066796 \quad 0.949748-4.005158$ H $-2.7155481 .522720-2.580475$ H -2.942139 2.416941 -4.986669 H -1.243073 $2.828940-4.990492$ H -3.952871 $5.460347-3.169851$ H $-4.5444053 .853113-3.876507$ H -1.637725 $6.013264-3.233405$ H $-0.5745505 .020531-4.175174$ H -1.281692 4.777496-1.178734 H $\quad 0.9161600 .456166-1.064329$ C -1.056486 -0.1808163 .165657$ O C $-1.842117 \quad 1.5818081 .854038$ C -2.1622721 .9004783 .306413$ C -1.0859661 .0971864 .013094$ H -0.9421842 .1498251 .580470$ C -2.9136581 .8873750 .801759$ H -2.1144302 .9710443 .525996$ H -3.1453451 .5268373 .604818$ C -1.3713240 .8486525 .484100$ H -0.1413201 .6478893 .930022$ C $-4.245768 \quad 1.1222960 .965629$ O H -2.488508 $1.614175-0.169988$ H -4.0204630 .0768251 .199287$ O -4.9798411 .6522882 .067967$ C $-5.1405071 .156459-0.294072$ C $-6.5370250 .533276-0.094667$ H -4.617452 $0.647840-1.112091$ O $-5.3327522 .512836-0.702425$ H -7.0916811 .1057230 .659473$ H -7.090888 $0.667835-1.033726$ H $-1.438866 \quad 1.796322 \quad 6.028110$ H $-2.315737 \quad 0.3123605 .625298$ H $-0.573407 \quad 0.252951 \quad 5.938894$ C $0.293714-0.9084063 .125628$ H -3.7254513 .5003170 .056535$ H -5.1576112 .5890211 .875420$ H -5.820291 2.500338 -1.543796 H $\quad 0.199083-1.8079902 .507105$ H $0.558880-1.2424964 .135550$ H -1.815904 -0.887342 3.524587 C $-5.777612-3.080287-0.632070$ O $-5.935432-1.667346-0.816190$ C $-6.517615-0.9562850 .279940$
C $-7.925304-1.4929360 .580490$ C -7.891248 -3.0049880 .828281$ C $-7.117842-3.743291-0.268328$ H -5.478762 -3.460097 -1.618101 C $-4.644343-3.4014000 .355484$ H -5.898094 -1.086069 1.171619 O $-8.791717-1.206444-0.515157$ H $-8.321904-0.9724511 .460453$ H $-8.915503-3.3952900 .867464$ O $-7.273872-3.2710712 .084393$ H $-6.942296-4.7829620 .030443$ O $-7.927574-3.793535-1.447493$ C $-3.289818-3.016221-0.202655$ H $-4.761450-2.8819111 .309638$ H $-4.627953-4.4819830 .539724$ H $-9.677792-1.531443-0.281047$ H -7.795817 -2.820074 2.769766 H $-8.034567-2.883535-1.773689$ O $-2.311953-3.5707810 .573009$ O $-3.108157-2.310262-1.184917$ C $-0.971054-3.3051080 .149727$ H -0.812699 -3.670094 -0.869685 H $-0.762934-2.2336380 .212526$ H -0.290462 -3.8354900 .820976$ SCF Energy $\left(B 3 L Y P / 6-31 G^{* *} / / M M F F\right)=-3245.92440931$

0800345

MM̄MF Geometry

C $1.8204403 .505233-0.980501$

C $2.9108324 .142893-0.526404$

C 3.8127563 .6391050 .577761

O 3.8402722 .1918230 .590499

C 3.3070154 .1397631 .939750

C 4.1751523 .6949693 .099854

C 3.8480362 .7033173 .952337

C $5.4473924 .477103 \quad 3.292480$

C $2.628287 \quad 1.8260883 .883807$

C 2.9480140 .3706854 .256051

C $1.716325-0.5271614 .073436$

C $1.945879-1.9100764 .682417$

C $0.750808-2.8429884 .432175$

O $1.474529-0.6526232 .667789$

O $1.161219-4.1811244 .751396$

C $0.408328-2.8208552 .928544$

C $-0.433354-2.5207365 .351371$

O $-0.783541-3.5567982 .665264$

C $0.296003-1.3965002 .335163$

C $0.196208-1.4816490 .794638$

O $-0.882915-0.7635982 .836786$

C $4.7007221 .590350-0.277199$

O $5.4709552 .143342-1.046949$

C $4.5220610 .125574-0.159737$

C $5.040165-0.663892-1.112734$

C $4.926742-2.121054-1.202105$

C $4.284370-2.848241-0.051373$

C $0.443829-0.1513030 .075320$

C $0.226092-0.287314-1.418176$

C $1.179179-1.404774-3.434716$

O $\quad-0.716157 \quad 0.255702-1.988512$

C $1.779804-2.764486-3.601606$

C $2.865370-3.021969-4.346503$

C $3.478916-4.388405-4.467006$

C $4.951426-4.408837-4.113869$

C $5.880339-4.674055-5.048132$

C $5.346968-4.194038-2.664763$

C $5.408111-2.732304-2.305231$

N $1.181183-1.087933-2.019831$

H $1.5219712 .566065-0.524842$

H $3.1898765 .095158-0.971600$

H 4.8279484 .0175910 .403980

H $2.272033 \quad 3.8125252 .094797$

H 3.2601975 .2373651 .938972

H 4.5203642 .4984554 .784158

H 6.0087394 .1472114 .173118

H 6.1029854 .3636152 .423555

H 5.2235945 .5406723 .424095

H 1.8812122 .2179884 .584240

H 2.1815511 .8443352 .885562 
H $3.7676090 .006745 \quad 3.622649$ H 3.2882220 .3314475 .298225 H $0.856636-0.0403654 .549121$ H $2.153882-1.8341335 .756938$ H $2.841219-2.3630634 .233872$ H $1.447324-4.1928665 .680888$ H $1.218660-3.3482612 .405414$ H $-0.137213-2.6124286 .403238$ H $-1.250837-3.2358225 .207447$ H $-0.827770-1.5129475 .206302$ H -0.666074 -4.444286 3.045163 H $0.932574-2.2057830 .427148$ H $-0.790942-1.8598330 .505659$ H $-1.177243-0.0920142 .201006$ H $3.947401-0.2547580 .676506$ H $5.580834-0.194342-1.934103$ H $4.360603-3.935121-0.130230$ H $3.222138-2.5993220 .019389$ H $4.769119-2.5784130 .893956$ H -0.2260720 .6271370 .444076$ H 1.4660550 .2018030 .244845 H $1.752824-0.626168-3.947621$ H $0.152951-1.389377-3.815202$ H $1.299411-3.580534-3.066055$ H $3.339745-2.212511-4.896413$ H $3.329863-4.728597-5.499749$ H $2.955794-5.115208-3.832839$ H $6.935753-4.713410-4.799581$ H $5.607919-4.852884-6.082934$ H $6.340650-4.623564-2.482970$ H $4.653141-4.756577-2.033280$ H $5.899187-2.115420-3.059707$ H $2.011783-1.326322-1.486432$ C $-0.3681064 .566632-1.645074$ O $-1.1615073 .545370-1.006056$ C $-2.4960213 .588715-1.540483$ C $-2.6147894 .928032-2.255145$ C $-1.2143405 .093989-2.806236$ H $-2.5535072 .782081-2.282321$ C -3.562325 $3.336097-0.462589$ H $-3.3855304 .918964-3.031631$ H -2.839632 $5.744951-1.561048$ C $-0.885883 \quad 6.520383-3.213034$ H $-1.1034984 .438888-3.680902$ C $-3.3830391 .980176 \quad 0.257067$ O $-3.5449804 .383705 \quad 0.505018$ H -4.545685 $3.347775-0.949572$ H $-3.1617391 .215155-0.494461$ O $-2.2784152 .031858 \quad 1.158040$ C -4.6373591 .5514431 .043305$ C -4.5042850 .2011791 .773892$ H -5.4891311 .5109950 .353986$ O -4.9473622 .5449102 .023739$ H -3.7417900 .2708032 .559769$ H -5.4524590 .0374742 .303033$ $\mathrm{H}-1.5650246 .861309-4.001286$ H $-0.9789897 .210269-2.367545$ H $0.1372296 .592082-3.594890$ C $0.9691933 .991891-2.116962$ H -3.9955375 .1530720 .118658$ H -1.5279432 .4283190 .684748$ H -5.7809012 .2863262 .452359$ H $1.5135924 .745401-2.698291$ H $0.7955803 .137587-2.783528$ H $-0.2025145 .352212-0.896483$ C $-5.045126-2.048462-1.163237$ O $-5.187090-1.013666-0.181419$ C $-4.209368-1.0027380 .862056$ C $-4.234932-2.3318501 .637509$ C $-4.113215-3.5400410 .701638$ C $-5.063241-3.434067-0.495672$ H $-5.944646-1.967251-1.787636$ C $-3.824015-1.827122-2.070365$ H $-3.215478-0.8645660 .425498$ O $-5.444486-2.450226 \quad 2.386514$ H $-3.412437-2.3340192 .361275$ H $-4.340660-4.4561831 .260530$ $\begin{array}{llll}\text { O } & -2.779583 & -3.649077 & 0.217423\end{array}$
H $-4.828516-4.201833-1.242353$

O $-6.401601-3.676304-0.051806$

C $-3.975282-0.605842-2.953834$

H $-2.899499-1.699199-1.502508$

H -3.712938 $-2.695826-2.730490$

H $-6.187963-2.3707601 .764519$

H -2.197099 -3.7625930 .987866$

H $-6.427340-4.5725810 .324474$

O $-3.020209-0.647913-3.930250$

$\begin{array}{llll}\text { O } & -4.803671 & 0.280990 & -2.800481\end{array}$

C $-3.0195630 .454493-4.842330$

H $-3.9730360 .505274-5.376526$

H $-2.825327 \quad 1.388765-4.307925$

H $-2.2182400 .293807-5.568530$

SCF Energy (B3LYP/6-31G**//MMFF) $=-3245.92496773$

0800346

MM̄FF Geometry

C $3.246364-2.487110-2.929895$

C $2.641524-1.492769-2.263760$

C $1.164272-1.209785-2.353718$

O $1.0361740 .215137-2.569841$

C $0.430570-1.578839-1.045445$

C $0.587461-3.019353-0.612249$

C $-0.045832-4.058191-1.189387$

C $1.515834-3.2488760 .551918$

C $-1.028612-4.010178-2.326996$

C $-2.273228-4.868485-2.053560$

C $-3.154362-4.335004-0.912649$

C $-4.262243-5.339250-0.586746$

C $-5.221994-4.8026610 .483456$

O $-3.727442-3.094261-1.338475$

O $-6.380081-5.6511570 .503719$

C $-5.697093-3.398747 \quad 0.060840$

C $-4.610875-4.8679841 .888080$

O $-6.480733-2.7985931 .090707$

C $-4.546582-2.448306-0.353384$

C $-5.149852-1.171389-0.992411$

O $-3.792532-2.0947240 .804510$

C $-0.1441920 .677461-3.058500$

\begin{tabular}{lllll}
\hline & -1.103664 & 0.019205 & -3.424261
\end{tabular}

C $-0.081036 \quad 2.153244-3.021386$

C $-1.2169992 .855366-2.903821$

C $-1.3184094 .310681-2.787174$

C $-0.0568045 .116737-2.955011$

C $-4.128863-0.162630-1.532704$

C $-3.5824660 .772555-0.472408$

C -4.2704712 .5972601 .088234$

O $-2.4059810 .723396-0.122856$

C $-4.472618 \quad 3.979698 \quad 0.555951$

C -3.5467664 .9483900 .621711$

C $-3.766396 \quad 6.340907 \quad 0.101936$

C $-2.7117676 .770663-0.894326$

C $-1.7218767 .602520-0.528362$

C $-2.8524686 .312082-2.329801$

C $-2.5226444 .856364-2.517120$

N $-4.534351 \quad 1.6447550 .025949$

H $2.629861-3.098013-3.588479$

H $3.199529-0.870495-1.575384$

H $0.728368-1.740891-3.209149$

H $-0.637034-1.342354-1.136894$

H $0.772088-0.912863-0.242048$

H $0.161963-5.057641-0.809548$

H $1.632737-4.3118060 .788046$

H $2.511960-2.8481460 .341670$

H $1.124148-2.7537021 .445840$

H $-1.335079-2.987666-2.566544$

H $-0.527145-4.406974-3.218093$

H $-2.863352-4.900690-2.978699$

H $-1.961477-5.897541-1.833648$

H $-2.533419-4.161257-0.027007$

H $-3.836512-6.300140-0.271638$

H $-4.841071-5.552355-1.496573$

H $-6.084290-6.5571850 .697555$

H $-6.366094-3.526238-0.802088$

H $-4.355031-5.9028342 .146148$

H $-5.329477-4.5395912 .647099$

H $-3.703453-4.2693271 .990260$ 
H -7.196012 -3.420168 1.309730 H $-5.767499-1.479171-1.846581$ H $-5.811446-0.677637-0.271647$ H -3.147348 -1.415905 0.551840 H $\quad 0.8990512 .614853-3.039266$ H -2.160718 $2.313800-2.856507$ H $0.6599034 .879256-2.161804$ H $-0.2220526 .195536-2.918265$ H $0.4082404 .906493-3.924593$ H -3.293113 -0.668140 -2.025913 H $-4.607487 \quad 0.462693-2.297305$ H -3.264290 2.4477221 .490275 H -4.9938322 .3996331 .885493$ H -5.4381034 .1991420 .105404$ H -2.587742 $4.739021 \quad 1.090248$ H -3.7675847 .0197950 .964777$ H $-4.7577226 .446013-0.356402$ H $-0.9777627 .945241-1.239438$ H -1.630824 7.9578800 .492742 H -2.247412 $6.931670-3.002029$ H -3.885296 $6.482190-2.660699$ H -3.381609 4.194363 -2.405455 H $-5.4809051 .602329-0.340506$ C $5.732260-2.131772-2.085278$ O $5.344524-1.877368-0.722840$ C $5.803458-0.555439-0.366445$ C $6.765715-0.129776-1.468366$ C $6.120025-0.780476-2.680581$ H $4.9357410 .109602-0.416438$ C $6.371948-0.5463611 .053991$ H $6.844790 \quad 0.958010-1.555101$ H $7.771750-0.533746-1.304008$ C $7.042425-0.868892-3.883579$ H $5.226224-0.207231-2.956115$ C $5.356810-0.9948312 .129603$ O $7.493463-1.4355121 .098169$ H $6.758200 \quad 0.452258 \quad 1.290382$ H $5.140239-2.0632322 .007928$ O $6.005333-0.8503173 .398467$ C $4.046767-0.1824482 .122919$ C $3.073774-0.5775673 .249997$ H $3.541197-0.3318511 .163063$ O $4.376048 \quad 1.2020132 .231278$ H 3.005933 -1.6705993.300539 H $3.461780-0.2040394 .205454$ H $7.3500560 .131413-4.205722$ H $7.947691-1.441301-3.655357$ H $6.539775-1.354236-4.725895$ C $4.677391-2.947776-2.840544$ H $7.836824-1.4217242 .008147$ H $5.462081-1.3073014 .062270$ H 3.5482811 .7086122 .177022 H $4.636846-3.940973-2.372744$ H $5.035539-3.107454-3.864958$ H $\quad 6.632749-2.758995-2.020695$ C 0.5190172 .1387372 .893421 O $1.733396 \quad 1.425143 \quad 3.160393$ C 1.6628720 .0034593 .018366 C $0.628569-0.5764383 .992341$ C $-0.738707 \quad 0.0851593 .790383$ C -0.6343461 .6152253 .766962$ H 0.7306423 .1708453 .202212 C 0.1912682 .1596791 .396422 H $1.365931-0.2408691 .993748$ O $1.072752-0.3771345 .331916$ H $0.551039-1.6574843 .825424$ H -1.410226 -0.2257764 .599650$ O $-1.291010-0.3861562 .564580$ H -1.576705 2.0625273 .430665 O -0.4045912 .0831895 .099623$ C 1.2438702 .9238830 .629137 H $0.099756 \quad 1.1590840 .966973$ H -0.7639002 .6645571 .213312$ H $0.409147-0.7718985 .922811$ H -2.1600380 .0353232 .450992$ H $-1.157293 \quad 1.7972235 .644628$ O $2.0564402 .058283-0.040774$ O $1.3443794 .143940 \quad 0.645512$
C $3.1124912 .656885-0.799096$

H $3.8965203 .010008-0.123562$

H $3.5313771 .893443-1.459657$

H $2.7379593 .479242-1.415950$

SCF Energy $(B 3 L Y P / 6-31 G * * / / M M F F)=-3245.90402598$

$08 \_00347$

MM̄FF Geometry

C -2.577284 1.1624693 .552937

C -1.3063460 .8031993 .789725$

C -0.1301751 .3416403 .022445$

$\begin{array}{lllll}\text { O } & 0.732178 & 0.232558 & 2.667639\end{array}$

C 0.7267252 .2953533 .873724

C 0.1028363 .6547374 .101779

C 0.1309274 .6552563 .199599

C -0.5368703 .8623885 .448808$

C 0.7478654 .6220011 .828908

C -0.3017414 .8724460 .737901$

C $0.3233684 .823456-0.663193$

C $-0.6939025 .227257-1.730591$

C $-0.1107895 .102393-3.146431$

O $0.7663413 .483695-0.901650$

O $-1.1979695 .186099-4.080383$

C $0.5164123 .702243-3.307103$

C $0.8379116 .259896-3.479387$

O $1.2029453 .589594-4.551875$

C $1.4574383 .301738-2.144539$

C $1.8261851 .802909-2.277044$

O $2.6442964 .090628-2.207173$

C $0.431130-0.4838471 .555783$

O $-0.534533-0.3207540 .822735$

C $1.460179-1.5147711 .279992$

C $2.497925-1.7735792 .091416$

C $3.570730-2.7418621 .845691$

C $3.613940-3.4034780 .493829$

C $2.5151391 .177077-1.057813$

C $4.0216821 .350392-1.011060$

C 5.9656390 .8834270 .489265

O $4.6742411 .821342-1.937555$

C $6.431263-0.5356900 .583146$

C $7.023663-1.0498101 .671678$

C $7.521303-2.4641221 .766446$

C $6.969181-3.1976882 .968780$

C $7.672739-3.2779694 .110983$

C $5.644011-3.9113422 .817507$

C $4.472993-2.9666232 .824278$

N 4.5467790 .8868880 .187496

H -2.7821341 .8975672 .779957$

H $-1.0917630 .088530 \quad 4.580974$

H -0.4476591 .8488792 .102793$

H 1.7103582 .4191943 .400850

H $\quad 0.966721 \quad 1.8258424 .838080$

H $-0.3279415 .605743 \quad 3.468211$

H -0.9559544 .8683185 .559062$

H -1.350856 3.1491275 .606498

H 0.2034813 .7249416 .243801

H 1.5160385 .4036071 .782093

H 1.2596283 .6745921 .637922

H -1.0877374 .1097920 .816468$

H $-0.7717005 .850613 \quad 0.899687$

H $1.1854745 .500613-0.681190$

H -1.060245 $6.246400-1.555182$

H -1.575819 $4.575021-1.659512$

H -1.647828 $6.037065-3.940976$

H $-0.3110922 .979890-3.345348$

H $0.3117147 .219221-3.406289$

H $1.1951346 .192963-4.513055$

H $1.7047586 .308036-2.817191$

H $0.574098 \quad 3.833517-5.252453$

H $0.892599 \quad 1.246099-2.409797$

H $2.4340901 .639997-3.174699$

H $3.3459613 .639083-1.712921$

H $1.321079-2.0487150 .345882$

H $2.584165-1.2251983 .027841$

H $3.635795-2.648022-0.299635$

H $2.736614-4.0430750 .350025$

H $4.495149-4.0288410 .338970$

H $2.077806 \quad 1.544445-0.123807$ 
H $2.3411090 .094344-1.098930$ H $6.0965601 .424327 \quad 1.431950$ H $6.5222121 .407839-0.293353$ H $6.288383-1.163618-0.293057$ H $7.186128-0.4118632 .537667$ H 8.617171-2.428255 1.824671 H $7.293602-3.0319870 .855781$ H $7.298390-3.8263614 .969094$ H $8.637349-2.7919784 .214823$ H $5.497163-4.6115313 .650834$ H $5.681625-4.5431761 .926306$ H $4.356962-2.4299423 .767151$ H 3.9155380 .5212740 .894541 C $-4.8722640 .049548 \quad 3.481258$ O $-4.375898-0.9735382 .596648$ C $-5.113010-0.8965151 .358978$ C $-6.257868 \quad 0.083877 \quad 1.599237$ C -5.6312791 .0374512 .597709$ H -4.437043 -0.458564 0.615982 C $-5.562571-2.2906970 .912848$ H -6.5808280 .5766970 .677111$ H -7.131120 -0.412022 2.039246 C $-6.6429291 .889703 \quad 3.343207$ H -4.9384211 .6938812 .057525$ C $-4.403939-3.2777990 .660989$ O $-6.401442-2.8439251 .934476$ H $-6.186538-2.2037380 .015277$ H $-3.898722-3.5103161 .605514$ O $-4.977036-4.5134910 .213321$ C $-3.368052-2.793104-0.372564$ C $-2.338877-3.881245-0.742670$ H $-2.831471-1.9307300 .036601$ O $-4.048123-2.341119-1.542838$ H -1.886078 -4.2729410 .175710$ H -2.861014 -4.703841 -1.246771 H -7.2112632 .5115012 .643643$ H -7.356322 1.2725893 .899521 H -6.1436942 .5538354 .055531$ C -3.7418320 .6251144 .336766$ H $-6.670601-3.7307971 .639581$ H $-5.459822-4.337150-0.611903$ H -4.416418 -3.118641-1.995169 H $-3.388315-0.1678735 .008600$ H $-4.134773 \quad 1.4266174 .972867$ H -5.579717 $-0.450206 \quad 4.157363$ C $-0.956367-2.441619-3.904239$ O $-1.829909-3.025522-2.931744$ C $-1.229100-3.357798-1.677229$ C $-0.113179-4.393241-1.889241$ C $0.906159-3.912040-2.929256$ C $0.227693-3.376819-4.197170$ H -1.562443 -2.366682 -4.816260 C $-0.531080-1.024307-3.506369$ H - $0.807462-2.454625-1.223879$ O $-0.686508-5.626119-2.320759$ H $0.384246-4.577444-0.929466$ H $1.569250-4.745405-3.191522$ O $1.709026-2.892821-2.342910$ H $0.952005-2.855654-4.833890$ O $-0.277326-4.480939-4.953802$ C $-1.731056-0.121496-3.325686$ H $0.051298-1.019845-2.581101$ H $0.104739-0.575154-4.278011$ H $\quad 0.035895-6.270823-2.409410$ H $2.366759-2.618573-3.004380$ H $0.477748-5.048841-5.183406$ O $-1.7524250 .371999-2.054162$ O $-2.5483760 .116792-4.204969$ C -2.836682 $1.252690-1.745782$ H $-2.7307781 .569712-0.704722$ H $-2.7995022 .137804-2.387846$ H $-3.7921050 .731679-1.858790$ SCF Energy (B3LYP/6-31G**//MMFF) $=-3245.90135811$

0800348

MM̄MF Geometry

C -2.740192 $2.619289-3.415434$

C $-3.0811691 .663384-2.537858$
C $-3.7007520 .349920-2.934289$

O $-4.7203590 .070506-1.948344$

C $-2.647036-0.769559-2.884209$

C $-3.067337-2.024856-3.620960$

C $-3.472120-3.166666-3.031581$

C $-2.953790-1.953929-5.122805$

C $-3.661044-3.439744-1.565516$

C $-2.627996-4.434077-1.015245$

C $-1.217159-3.838792-0.883956$

C $-0.225778-4.895502-0.395102$

C $1.167986-4.299369-0.151803$

O $-1.285521-2.7705240 .063009$

O $1.922220-5.2825690 .573434$

C $1.033563-3.0469160 .742284$

C $1.918196-4.068651-1.468867$

O $2.300981-2.4022450 .856098$

C $-0.056813-2.0588440 .259029$

C $-0.322453-0.9783601 .334982$

O $0.389792-1.429402-0.943035$

C $-5.894783-0.473287-2.350321$

O $-6.221689-0.749450-3.495900$

C $-6.805937-0.684837-1.199524$

C $-6.407930-0.6036060 .080256$

C -7.249917 -0.7733301 .266801$

C $-8.723244-1.0018411 .055085$

C $-1.550938-0.1008031 .051032$

C - $1.798736 \quad 0.914527 \quad 2.152779$

C -3.5064072 .5717462 .903784$

O -1.0249621 .0956813 .088832$

C $-4.3476731 .856753 \quad 3.914312$

C -5.6644872 .0600024 .071948$

C -6.5027591 .3277595 .081219$

C -7.6361840 .5487984 .448400$

C $-8.8988811 .005407 \quad 4.502297$

C -7.323381 -0.7985093 .832923$

C $-6.671466-0.6845762 .482917$

N -2.989885 1.6013231 .955804

H -2.908696 $2.457630-4.477261$

H -2.885441 $1.822418-1.479901$

H $-4.1463770 .442137-3.931900$

H $-2.409281-1.000452-1.840883$

H -1.704595 -0.424442 -3.330999

H -3.721619 -4.011437 -3.672939

H -3.210550 -2.903254 -5.605027

H -3.625226 -1.189821 -5.525753

H - $1.928052-1.705176-5.414367$

H -3.668056 -2.523176 -0.968463

H -4.660461 -3.876356 -1.442871

H -2.974258 -4.750440 -0.022571

H -2.599705 -5.329541-1.648641

H $-0.901931-3.444670-1.856170$

H -0.168018 -5.733177 -1.101367

H $-0.587774-5.3257520 .549401$

H $2.782653-4.8877040 .797215$

H $\quad 0.770462-3.4012551 .748884$

H $2.027489-5.013463-2.015085$

H $2.937175-3.711583-1.287232$

H $1.417187-3.358704-2.130252$

H $2.235912-1.7356531 .559965$

H -0.484859-1.469275 2.303719

H $0.556173-0.3323651 .443157$

H $-0.073020-0.584593-1.051850$

H -7.829580 -0.924872 -1.467814

H $-5.362156-0.3867910 .292508$

H -9.293565 -1.051554 1.985109

H $-8.892718-1.9458820 .525552$

H $-9.159706-0.1858700 .468357$

H $-1.4243180 .452720 \quad 0.115813$

H $-2.447329-0.7254230 .961653$

H -4.0786253 .3117142 .335693$

H $-2.6742443 .078740 \quad 3.402612$

H -3.843222 1.1318354 .549225

H -6.1701002 .7924293 .446825$

H -6.900662 2.0711405 .784228

H $-5.8937310 .646171 \quad 5.688335$

H -9.7230870 .4408554 .079501$

H -9.1365311 .9565304 .967175$

H $-8.224160-1.4202673 .782201$ 
H -6.650162 -1.352364 4.499981 H $-5.595369-0.5180732 .531835$ H $\quad-3.597000 \quad 1.3250651 .189417$ C $-0.5738483 .828554-3.101373$ O $-0.1011533 .010445-2.010993$ C $1.0153373 .669819-1.376896$ C $1.4632794 .741987-2.354467$ C $0.1341855 .173914-2.945097$ H $0.6130394 .127122-0.463793$ C $2.0622162 .610111-1.025481$ H $2.0021795 .559958-1.867508$ H $2.1099924 .316519-3.131878$ C $0.2702785 .952719-4.241782$ H $-0.3944165 .793468-2.208065$ C $3.3943403 .166606-0.498978$ O $1.4935571 .764509-0.020108$ H $2.2419891 .976891-1.901673$ H $3.9144233 .701414-1.302463$ O $3.107342 \quad 4.1385800 .515120$ C 4.3297092 .1079890 .122959 C $4.6427210 .921767-0.806908$ H 3.8949421 .7438361 .061172 O 5.5501212 .7650180 .482065 H $3.7148980 .499395-1.204425$ H $5.2422241 .288568-1.649334$ H $0.8357706 .875885-4.078108$ H $0.7933545 .369639-5.007205$ H $-0.7128786 .224364-4.638699$ C -2.098115 $3.921530-3.022723$ H $0.6467751 .436430-0.366675$ H $3.9588534 .475748 \quad 0.842529$ H $5.9777033 .068030-0.337031$ H -2.473786 $4.702125-3.693782$ H $-2.4069704 .193233-2.005337$ H $-0.2612223 .334463-4.030632$ C $7.515539-0.6598261 .010435$ $\begin{array}{lllll}\text { O } & 6.703489 & 0.261451 & 0.269849\end{array}$ C $5.404597-0.212479-0.091137$ C $5.527752-1.458507-0.981687$ C $6.376030-2.543379-0.309808$ C $7.690828-1.9812670 .240622$ H $8.496941-0.1726611 .075603$ C $6.999811-0.8557072 .440623$ H $4.845520-0.4695790 .815324$ O $6.104734-1.113655-2.239888$ H $4.524154-1.845891-1.188147$ H $6.600526-3.332945-1.037542$ O $5.642609-3.132116 \quad 0.760223$ H $8.179167-2.7249130 .881411$ O $8.562961-1.746097-0.869132$ C 7.0766920 .4390203 .214430 H $5.972167-1.2278322 .481259$ H $7.612053-1.5896502 .978023$ H $6.973058-0.710518-2.068291$ H $4.821786-3.497390 \quad 0.388483$ H $9.421190-1.459509-0.512668$ O 5.8440451 .0178163 .278966 O $8.113360 \quad 0.8920303 .682417$ C 5.7874962 .2750043 .959753 H 6.0604402 .1469125 .011385 H 6.4468213 .0008393 .474235 H 4.7600402 .6446403 .904913 SCF Energy (B3LYP/6-31G**//MMFF) $=-3245.91103121$

0800349

MM̄FF Geometry

C -2.485171 -2.458859 1.929813

C - $1.282207-2.7429542 .451405$

C $-0.048041-1.9134662 .229372$

O $0.910574-2.8003001 .599687$

C $0.565768-1.4010663 .546822$

C $-0.228895-0.3216014 .249636$

C $-0.008697 \quad 1.0002474 .106365$

C -1.262567 -0.8023425 .236637$

C $0.993908 \quad 1.6831873 .215972$

C 0.3512452 .8575882 .463955

C 1.3866143 .7153711 .720074

C 0.7059964 .9233271 .072683
C $1.680075 \quad 5.7595920 .233230$

O 2.0106292 .9091290 .718052

O $0.8794726 .652700-0.556019$

C $2.4415454 .820306-0.725126$

C 2.5749436 .6376951 .115580

O $3.4682245 .524944-1.420647$

C $3.0429303 .578335-0.023408$

C 3.571922 2.586885-1.089666

O 4.1156143 .9934210 .820873

C $1.867242-2.2389140 .815368$

O $2.003471-1.0537390 .560981$

C $2.741689-3.3280580 .324464$

C $3.768413-3.035782-0.487797$

C $4.741296-3.983490-1.034547$

C $4.587831-5.436348-0.668912$

C $4.1492941 .284035-0.520532$

C $5.6465801 .320336-0.260680$

C $7.478814-0.1280130 .624875$

O $6.3671962 .260316-0.583025$

C $8.004596-0.974815-0.491232$

C $8.416588-2.241865-0.334807$

C $8.919256-3.100374-1.460503$

C $8.101496-4.361988-1.637966$

C $8.524422-5.531226-1.128508$

C $6.842284-4.289199-2.474850$

C $5.735251-3.510882-1.815646$

$\begin{array}{llll}\mathrm{N} & 6.080630 & 0.157567 & 0.361816\end{array}$

H -2.604950 -1.576429 1.306658

H -1.171922 -3.6242813 .079597$

H $-0.268288-1.0752311 .556378$

H $1.584823-1.0419083 .354936$

H $0.725878-2.2436594 .234471$

H $-0.606509 \quad 1.6803374 .712799$

H -1.698169 0.0197715 .814808

H -2.084774 -1.311132 4.727402

H $-0.811321-1.4973075 .952477$

H 1.8070622 .0580653 .848696

H 1.4348200 .9900932 .493743

H -0.3777032 .4541971 .750289$

H -0.1986053 .4940193 .169026$

H 2.1441374 .0450762 .440869

H 0.2167315 .5488051 .829813

H -0.0976024 .5724620 .411996$

H $1.4776857 .235284-1.053533$

H $1.7274934 .475453-1.486340$

H 1.9649647 .3398291 .696497

H 3.2467067 .2551670 .509113

H 3.1797156 .0624531 .819719

H $3.0440976 .228046-1.940229$

H $2.7273812 .306417-1.732514$

H $4.3161643 .076177-1.728347$

H $4.6387423 .214547 \quad 1.066237$

H $2.525029-4.3372830 .655821$

H $3.920073-1.997544-0.778191$

H $4.634626-5.5670160 .418062$

H $5.366517-6.074926-1.091980$

H $3.628544-5.823894-1.029045$

H 3.6359330 .9966740 .402288

H $3.9857880 .487606-1.257291$

H $7.531153-0.6377511 .592014$

H 8.0478710 .8046200 .688765

H $8.038664-0.521673-1.479459$

H $8.391220-2.6914170 .655210$

H $9.964603-3.356738-1.243914$

H $8.943041-2.547566-2.407850$

H $7.963463-6.448282-1.273851$

H $9.440385-5.596839-0.550640$

H $6.495636-5.291370-2.752597$

H $7.079509-3.808386-3.432820$

H $5.769983-2.440827-2.022404$

H $5.411179-0.5860720 .536987$

C -4.299556-3.868591 0.900014

O $-4.876254-2.8050910 .113480$

C $-6.247889-3.131658-0.171570$

C $-6.336609-4.642630-0.025567$

C $-5.432632-4.8646591 .171005$

H $-6.853925-2.6636310 .614393$

C $-6.667679-2.577513-1.536528$ 
H $-7.361397-4.9882210 .138731$ H $-5.934342-5.161432-0.903326$ C $-4.961693-6.301236 \quad 1.320774$ H -5.978041-4.575142 2.079527 C $-6.351621-1.081246-1.739545$ O $-5.990122-3.298756-2.573662$ H $-7.736479-2.762073-1.694773$ H $-5.271485-0.957463-1.871625$ O $-6.956849-0.663812-2.968977$ C $-6.847295-0.158197-0.613745$ C $-6.6527761 .345684-0.885119$ H $-6.335650-0.4149580 .320403$ O $\quad-8.241908-0.394743-0.404664$ H -7.317729 $1.670706-1.695492$ H -7.0009901 .8779290 .010026$ H $-5.814477-6.9725381 .465405$ H $-4.414979-6.6387170 .433951$ H $-4.298348-6.4052772 .185238$ C -3.712424 -3.288498 2.189164 H $-5.034179-3.211962-2.415618$ H -6.632048 -1.256942 -3.668014 H $-8.5252530 .159637 \quad 0.342290$ H -3.478148 -4.100827 2.887410 H -4.451527 -2.640757 2.676797 H -3.513486 -4.3278640 .287455$ C $-2.9549201 .485321-0.353059$ O $-4.3615021 .279809-0.144732$ C $-5.2035841 .751288-1.202126$ C $-5.0488543 .271554-1.362384$ C $-3.5831503 .659689-1.581672$ C $-2.6508032 .978984-0.570701$ H $-2.490687 \quad 1.1806830 .593962$ C $-2.4347540 .545835-1.451139$ H $-4.9124011 .267654-2.140389$ O $-5.547536 \quad 3.947347-0.209335$ H -5.651771 $3.602922-2.215813$ H $-3.4883414 .749007-1.494561$ O $-3.2103673 .299268-2.908124$ H -1.604918 $3.104781-0.869574$ O $-2.805916 \quad 3.6254680 .697726$ C $-0.9302020 .392119-1.458211$ H -2.863798 $-0.452149-1.299023$ H $-2.7488360 .885142-2.442081$ H -5.0680393 .6067660 .565285$ H $-2.2889643 .580479-3.040407$ H -2.5659924 .5606520 .581844$ O $-0.570112-0.315507-2.570136$ O $-0.1705780 .818993-0.600883$ C $0.830652-0.553512-2.742030$ H $\quad 1.142775-1.375579-2.093692$ H $\quad 0.999522-0.844238-3.782351$ H $1.4156760 .346936-2.534904$ SCF Energy (B3LYP/6-31G**//MMFF) $=-3245.91495625$

\section{8_00350}

MM̄MF Geometry

C -0.710805 -3.692076 1.412241

C $-1.979826-3.5530001 .002706$

C $-2.422488-3.758605-0.422538$

O $-2.706521-2.443563-0.958246$

C $-3.705371-4.605013-0.467446$

C $-4.249035-4.805065-1.870237$

C $-5.357223-4.210493-2.357748$

C $-3.466459-5.761179-2.733238$

C $-6.263004-3.242757-1.648976$

C $-6.080668-1.814679-2.179180$

C $-6.615260-0.770069-1.189157$

C $-6.5531120 .633109-1.793365$

C $-6.9581711 .715674-0.781186$

O $-5.793789-0.833359-0.016939$

O $-6.5600572 .988365-1.314777$

C -6.1625951 .5070570 .525754$

C $-8.4786081 .780514-0.594720$

O $\quad-6.6218972 .389971 \quad 1.545901$

C -6.1903020 .0523341 .038574$

C $-5.230455-0.1924322 .230620$

O $-7.512522-0.2583571 .495404$

C $-1.646853-1.746898-1.458048$
O $-0.506878-2.155653-1.615233$

C $-2.098054-0.365238-1.732758$

C $-1.1852110 .573987-2.021535$

C $-1.4461941 .990853-2.279983$

C $-2.8653802 .476208-2.156021$

C $-3.7466030 .080501 \quad 1.956191$

C -3.2852061 .4715482 .358550$

C -1.2981922 .9698372 .232167$

O -3.9753552 .2474793 .013998$

C $-1.4216303 .907921 \quad 1.073384$

C -0.3670774 .4562750 .450552$

C $-0.4804945 .469254-0.652905$

C $0.2954075 .083715-1.891684$

C $1.5432395 .538443-2.096470$

C $-0.4118144 .249786-2.934962$

C $-0.4045672 .786447-2.599537$

N $-1.989396 \quad 1.730544 \quad 1.931277$

H $\quad 0.050917-4.0153650 .707377$

H -2.724206 $-3.182647 \quad 1.704477$

H -1.640393 -4.247625 -1.016521

H $-4.460000-4.1466080 .178831$

H -3.511752 -5.592209 -0.026494

H -5.652221 -4.430591 -3.382877

H -3.942699 -5.929095 -3.705185

H -2.460501 -5.374871-2.923206

H $-3.379425-6.734279-2.238964$

H -7.301936 -3.557064 -1.809226

H $-6.117156-3.274657-0.567432$

H -5.015559-1.624196 -2.357480

H -6.593774 -1.719851-3.144136

H -7.647026 -1.031399 -0.925985

H -7.176251 $0.696601-2.694085$

H $-5.5275360 .839163-2.126710$

H $-6.9959213 .101552-2.176845$

H $-5.124792 \quad 1.7881950 .312774$

H $-8.9716872 .006932-1.547799$

H -8.7592902 .5914140 .086454$

H $-8.9046110 .848586-0.217525$

H -6.5645973 .2946551 .193461$

H $-5.5838250 .349702 \quad 3.116042$

H $-5.308858-1.2574022 .493358$

H -7.458173 -1.008290 2.110752

H -3.157314 -0.151920-1.659599

H $-0.1368990 .281425-2.068874$

H -3.277715 2.212690 -1.176560

H $-3.4914302 .028196-2.934861$

H -2.965959 $3.560051-2.243382$

H $-3.151391-0.6193582 .556729$

H $-3.500428-0.0981760 .905851$

H -0.2566972 .7198622 .453483$

H $-1.7365793 .428876 \quad 3.124179$

H $-2.426866 \quad 4.1823370 .762172$

H $0.636577 \quad 4.2032190 .785142$

H $-0.1034346 .424934-0.264905$

H -1.528262 $5.655914-0.919303$

H $2.0944335 .290628-2.997579$

H $2.0433846 .163306-1.363774$

H $0.0887254 .362865-3.906350$

H -1.418001 $4.645694-3.098685$

H $0.5880022 .338900-2.660336$

H -1.5054001 .0442321 .359278$

C $0.219760-1.8547682 .803307$

O $1.384314-1.7033071 .971339$

C $2.229715-0.6855272 .537659$

C $1.506275-0.1602843 .776087$

C $0.647580-1.3453754 .174313$

H $3.156134-1.1846062 .847621$

C 2.5564870 .4001201 .499448

H $2.204681 \quad 0.145628 \quad 4.561311$

H 0.8778820 .7058843 .539254

C $-0.507197-0.9730155 .087706$

H $1.282508-2.0904424 .671872$

C $3.355152-0.1440930 .294175$

O $1.3337780 .989497 \quad 1.059053$

H $3.143526 \quad 1.1825131 .995870$

H $4.194404-0.7268690 .685431$

O $2.549913-1.042783-0.466835$

C $3.9095330 .959510-0.630796$ 
C $4.6843170 .426324-1.851367$

H $4.5524971 .625120-0.043059$

O $2.8272031 .753070-1.121287$

H $4.018470-0.181986-2.476406$

H $4.9564791 .294446-2.466991$

H $-0.134465-0.5355446 .019637$

H -1.172738 -0.2420714 .616051$

H -1.103384 -1.854047 5.344290

C $-0.247691-3.3110282 .789860$

H $1.556267 \quad 1.7160800 .453111$

H $1.769692-0.547767-0.770101$

H $3.2069662 .485836-1.635481$

H -1.056832 -3.459771 3.514002

H $\quad 0.571301-3.9829993 .072737$

H $-0.559139-1.2032892 .383744$

C $8.056532-0.072240-0.323279$

O $6.8261420 .506599-0.778107$

C $5.952249-0.368158-1.498632$

C $6.651374-0.918838-2.751398$

C $7.975697-1.597139-2.385311$

C $8.838678-0.706093-1.486583$

H 8.6431230 .7795800 .044798

C $7.829153-1.0289720 .852753$

H $5.670924-1.209411-0.859014$

O $6.8943230 .142171-3.672344$

H $5.981648-1.634747-3.242575$

H $8.532636-1.832731-3.300345$

O $7.717828-2.823004-1.706899$

H $9.688805-1.271665-1.087475$

O $9.379650 \quad 0.362130-2.269623$

C $7.386021-0.2660102 .079022$

H $7.067692-1.7878250 .652050$

H $8.748209-1.5718141 .104130$

H $7.313693-0.243059-4.460439$

H $7.187645-3.381590-2.300410$

H $9.916412-0.035086-2.976358$

O $8.473146 \quad 0.2256192 .739843$

O $6.215183-0.1086392 .399253$

C 8.1836300 .9887773 .915229

H 7.5889051 .8702703 .657266

H 7.6595320 .3696904 .649617

H $9.131617 \quad 1.3193764 .347793$

SCF Energy (B3LYP/6-31G**//MMFF)= -3245.90695253

08_00351

MM̄FF Geometry

C $-1.447527-3.7028561 .275242$

C $-0.605991-3.4951882 .300161$

C $-0.383050-2.1912823 .029777$

O $-1.285301-1.1662862 .560242$

C $1.050424-1.7069912 .748465$

C $1.417063-0.4544673 .516294$

C 1.4314250 .7778432 .971598

C $1.829128-0.6656034 .947882$

C 1.0389381 .1203011 .560359

C 0.6814142 .6042791 .409253

C $0.4245132 .980234-0.056445$

C $0.1214274 .474220-0.183436$

C $-0.2275634 .866446-1.626499$

O $-0.6851862 .211852-0.532055$

O $-0.809166 \quad 6.178897-1.599482$

C -1.309692 $3.903751-2.157667$

C $1.0221894 .964136-2.509441$

O $-1.5610804 .135704-3.542037$

C $-0.9819852 .410421-1.920881$

C $-2.2119461 .544538-2.277168$

O $0.1145482 .034794-2.755997$

C $-2.471713-1.0099673 .202446$

O $-2.890965-1.6548584 .149153$

C -3.1921310 .0866092 .516806$

C $-4.499198 \quad 0.2691382 .753461$

C -5.3600461 .2708522 .119405$

C $-4.7043592 .288388 \quad 1.223453$

C $-2.0675840 .058880-1.928219$

C $-3.353413-0.666161-2.270851$

C $-5.515468-1.415113-1.279575$

O $-3.606275-1.012359-3.423080$

C $-6.519195-0.306260-1.209680$
C $-7.583420-0.317843-0.392375$

C $-8.5865120 .798737-0.316453$

C $-8.848584 \quad 1.263670 \quad 1.100678$

C $-10.0236891 .013808 \quad 1.702753$

C -7.7790742 .0839441 .793307$

C -6.6863981 .2219412 .363982$

N $-4.185384-0.842406-1.177823$

H $-2.078828-2.8954370 .917386$

H $0.015713-4.3238692 .634885$

H $-0.521146-2.3548154 .105387$

H $1.172272-1.5322221 .671741$

H $1.775179-2.4933862 .999056$

H 1.7589221 .6095053 .592995

H 2.1187140 .2696605 .438682

H $1.006085-1.0991575 .524183$

H $2.686951-1.3441994 .999590$

H $1.8717270 .876666 \quad 0.894727$

H $\quad 0.1828130 .517683 \quad 1.241588$

H -0.2148572 .8115222 .008530$

H 1.4944173 .2264331 .804246

H $1.3170292 .722087-0.638191$

H 0.9625755 .0749610 .184870

H -0.7326694 .7281540 .460037$

H $-0.1638886 .784423-1.196106$

H -2.244777 4.138918-1.629468

H $1.7064515 .726641-2.118974$

H $0.7652505 .286162-3.524528$

H $1.5770344 .025841-2.574348$

H -1.774652 $5.079113-3.643610$

H -3.090801 $1.931506-1.746190$

H $-2.4249321 .628846-3.350803$

H $0.1066931 .073151-2.878921$

H -2.6342980 .6905991 .809740$

H -4.996987 -0.3974873 .456746$

H $-4.274292 \quad 1.8042160 .342091$

H -3.9050202 .8140721 .758176$

H -5.3874523 .0597620 .862956$

H -1.261197 -0.416832 -2.495691

H $-1.833075-0.077818-0.866778$

H $-5.626627-2.126023-0.454844$

H $-5.618361-1.952790-2.227103$

H $-6.3649580 .546450-1.867254$

H -7.753624 -1.1820400 .245814$

H $-9.5174360 .438310-0.773222$

H $-8.2699081 .659159-0.919184$

H $-10.228713 \quad 1.3645912 .708914$

H -10.8055170 .4489161 .205897$

H -8.2193802 .6494062 .625165$

H -7.4120592 .8379071 .092400$

H $-7.0546150 .457008 \quad 3.049874$

H $-3.897934-0.484709-0.272254$

C $-0.998938-4.948018-0.886129$

O $0.373683-4.504402-0.871571$

C $0.574393-3.595712-1.973359$

C $-0.644855-3.756891-2.875745$

C $-1.734069-4.022314-1.854205$

H $\quad 0.566037-2.582480-1.554308$

C $1.918054-3.871784-2.653976$

H $-0.831719-2.867059-3.484297$

H $-0.540466-4.613690-3.551668$

C $-2.997765-4.621376-2.444819$

H $-1.984323-3.073621-1.367791$

C $3.131624-3.774946-1.706782$

O $1.883034-5.202711-3.186669$

H $2.045201-3.200774-3.511306$

H $3.063290-4.560919-0.945566$

O $4.303764-4.076775-2.477077$

C $3.327285-2.405859-1.022330$

C $4.561935-2.407463-0.097364$

H $2.450600-2.155841-0.415749$

O $3.475195-1.390009-2.010516$

H $4.403761-3.1650070 .679861$

H $5.446072-2.697675-0.678867$

H $-3.434007-3.946056-3.187788$

H $-2.796150-5.577782-2.938663$

H -3.746761 -4.794619-1.665626

C $-1.547633-5.0122210 .542674$

H $2.744966-5.369897-3.605088$ 
H $\quad 4.425045-3.356299-3.119374$

H $2.615710-1.275425-2.449544$

H $-0.988045-5.7798631 .092504$

H -2.594983 -5.3338960 .530744$

H $-0.984312-5.968914-1.290605$

C $5.7453231 .137480-0.013038$

O $5.458342-0.203958-0.430820$

C $4.841980-1.0390350 .556791$

C $5.773794-1.1796031 .771138$

C 6.1696370 .1868632 .339021

C 6.6368601 .1512121 .241486

H $6.3284401 .561686-0.840799$

C 4.4525571 .9459580 .112305

H $3.892221-0.5921610 .871496$

O $6.951077-1.900618 \quad 1.410328$

H $5.262924-1.7673702 .542770$

H $6.977151 \quad 0.0554953 .069674$

O 5.0602880 .7614543 .022786

H 6.7096302 .1724641 .631750

O 7.9525920 .7684240 .825963

C $4.6911253 .423436-0.067695$

H $3.7175831 .620140-0.634503$

H 3.9653931 .8039581 .079470

H $7.374946-1.4279060 .673558$

H $4.797450 \quad 0.1462293 .728460$

H 8.5355620 .8355281 .601337

O $4.6464623 .744844-1.391916$

O 4.9073654 .1955900 .857205

C $4.8708965 .124620-1.696935$

H $4.1563685 .757193-1.161404$

H $5.8981755 .401770-1.442530$

H $4.7239945 .263585-2.771270$

SCF Energy (B3LYP/6-31G**//MMFF) $=-3245.90881746$

\section{8_00352}

MM̄FF Geometry

C $1.775397-2.015437-0.655721$

C $2.403470-2.9020090 .131302$

C $3.219630-2.5705231 .363010$

O $3.057462-1.1862101 .747349$

C $4.716051-2.7730681 .072274$

C $5.537398-2.9960202 .325512$

C $6.203630-2.0370942 .997482$

C $5.614708-4.4301922 .783473$

C $6.215824-0.5552962 .743834$

C $7.558522-0.0514532 .198507$

C $7.704011-0.2600780 .683509$

C 9.0931670 .1722520 .210296

C $9.2292800 .088411-1.317463$

O $6.700696 \quad 0.536186 \quad 0.041897$

O $10.4194320 .797489-1.694262$

C $8.0393580 .826899-1.964967$

C $9.414639-1.356231-1.796693$

O $8.0423190 .656115-3.380599$

C $6.6652040 .416520-1.384753$

C $5.5656051 .367637-1.918064$

O $6.366214-0.919287-1.796000$

C $1.944741-0.8259602 .437249$

O $1.055994-1.5530692 .846993$

C 1.9753230 .6495482 .535626

C 0.8305461 .3258662 .705191

C 0.6919512 .7838032 .734317

C 1.9465103 .6070822 .595239

C $4.2119081 .235894-1.203021$

C $3.1374052 .090100-1.857702$

C $0.7014632 .607808-1.703790$

O $3.3537642 .813845-2.826385$

C $0.4659443 .841482-0.889732$

C $-0.7369224 .177936-0.399213$

C $-1.003728 \quad 5.4292560 .388326$

C -1.7510275 .1644621 .677555$

C $-3.0785175 .357773 \quad 1.757987$

C -0.9475954 .7578342 .893292$

C -0.5446823 .3096322 .856190$

N $1.903611 \quad 1.939609-1.238129$

H $1.856637-0.955005-0.440484$

H $2.317644-3.961043-0.103313$

H $2.890830-3.2157022 .187184$
H $5.105071-1.9087220 .522214$

H $4.869531-3.6368330 .411504$

H $6.787052-2.3375693 .867413$

H $6.216139-4.5467533 .691183$

H $4.613675-4.8164323 .000037$

H $6.067410-5.0532782 .005109$

H $5.395747-0.2403712 .094165$

H $6.030100-0.0664773 .708981$

H 7.6160721 .0244742 .410386

H $8.388285-0.5318102 .731161$

H $7.536073-1.3170480 .450032$

H $9.873616-0.4243760 .698949$

H $9.275580 \quad 1.211948 \quad 0.516527$

H $11.1713350 .387158-1.233588$

H $8.184321 \quad 1.901109-1.782486$

H $10.316660-1.794020-1.352340$

H $9.565912-1.396285-2.880959$

H $8.574186-2.004691-1.540991$

H $8.9154410 .936715-3.704439$

H $5.8953562 .408329-1.800474$

H $5.4221951 .191819-2.991958$

H $5.404294-1.033254-1.843265$

H $2.933640 \quad 1.1393672 .403664$

H -0.0966420 .7620032 .800972$

H 2.6225353 .4187593 .436586

H 1.7642834 .6831292 .568299

H 2.4696633 .3622341 .664037

H $3.8697980 .197443-1.220994$

H $4.3053831 .549407-0.156881$

H $-0.1213381 .891602-1.613250$

H $0.8191002 .877560-2.758088$

H $1.3140214 .499415-0.716208$

H -1.589583 $3.536276-0.607168$

H -1.591731 $6.102633-0.249185$

H -0.0745875 .9682930 .610912$

H -3.6184195 .1962962 .685046$

H -3.656133 5.6742790 .895775

H -1.5423444 .9060683 .804764$

H -0.0975375 .4362063 .005179$

H -1.3904752 .6264182 .946373$

H $1.8361801 .376147-0.395987$

C $-0.514674-1.912890-1.609321$

O $-1.144470-2.792239-0.655510$

C $-2.514657-3.014173-1.059712$

C $-2.768673-2.058993-2.217382$

C $-1.395672-1.969852-2.852964$

H -2.559666 -4.053568 -1.408254

C $-3.417489-2.8249040 .165441$

H -3.536603 -2.426920 -2.904381

H -3.081899-1.073539-1.851076

C $-1.237140-0.777032-3.780007$

H -1.201451 -2.894971 -3.411764

C $-4.920406-2.774303-0.166182$

O $\quad-3.158697-3.9057561 .065266$

H -3.133666 -1.908753 0.694604

H $-5.077656-1.945214-0.860670$

O $-5.303404-3.964751-0.852925$

C $-5.832332-2.563778 \quad 1.060570$

C $-7.323623-2.4034770 .706969$

H $-5.497473-1.6824621 .619860$

O $-5.747192-3.6818371 .946746$

H -7.687615 $-3.311450 \quad 0.209814$

H -7.874160 -2.343291 1.655647

H $-0.227457-0.738616-4.200196$

H -1.946533 -0.840147 -4.611753

H $-1.4192860 .166746-3.254852$

C $0.926218-2.366688-1.843670$

H $-2.205368-3.9005701 .258853$

H $-5.170909-4.707990-0.239426$

H $-4.825444-3.7488712 .248185$

H $1.349596-1.863269-2.719707$

H $0.957123-3.446719-2.031043$

H $-0.537922-0.903121-1.176670$

C $-7.3694311 .244785-0.110011$

O $-7.207557-0.0095500 .566491$

C $-7.641813-1.168663-0.152586$

C $-9.136644-1.066060-0.490592$

C $-9.4330410 .231699-1.249280$ 
C $-8.8326491 .451525-0.543613$ H -7.1370542 .0020000 .649971$ C $-6.3606101 .408063-1.257646$ H -7.084298 -1.246926 -1.089443 O $-9.905208-1.1123220 .708845$ H -9.421071 -1.932291 -1.100098 H $-10.5191840 .356661-1.336734$ O $-8.897550 \quad 0.121337-2.565110$ H -8.909306 $2.335059-1.187932$ O -9.6215451 .7184810 .619569$ C $-4.9983161 .819096-0.744060$ H -6.227732 $0.510019-1.866163$ H -6.689479 2.208827 -1.931301 H -10.843398 -1.0619060 .458596$ H $-9.1202940 .939050-3.041862$ H -9.2762312 .5294361 .030031$ $\begin{array}{lllll}\text { O } & -4.467873 & 0.839697 & 0.041199\end{array}$ O $-4.4624862 .883136-1.027955$ C $-3.184318 \quad 1.125442 \quad 0.606656$ H -3.168302 2.1227381 .055347 H -2.986674 0.3935011 .393821 H -2.413693 $1.033972-0.163741$

SCF Energy (B3LYP/6-31G*//MMFF)= -3245.90528941

08_00353

MMFF Geometry

C $1.640251-0.4503311 .694248$

C $0.467997-1.0693931 .496117$

C $0.131667-1.7976990 .219436$

O $-1.110521-1.269499-0.299389$

C $-0.054743-3.2961510 .514700$

C $-0.282935-4.133814-0.729424$

C $-1.458965-4.692444-1.080235$

C $0.939199-4.356478-1.584688$

C $-2.774208-4.600335-0.358251$

C $-3.802633-3.812167-1.179028$

C $-5.040717-3.448677-0.345954$

C $-6.087284-2.750208-1.215382$

C $-7.278514-2.236849-0.392932$

O $-4.608710-2.5748110 .702203$

O $-8.021884-1.324584-1.216420$

C $-6.747702-1.4265780 .809285$

C $-8.242919-3.369025-0.020780$

O $-7.811291-1.0597541 .684326$

C -5.643584-2.156500 1.601314

C $-4.980935-1.2644422 .681022$

O $-6.218776-3.2801102 .279019$

C $-1.033173-0.116461-1.021329$

O $-0.026421 \quad 0.528781-1.268221$

C $-2.400690 \quad 0.236007-1.464453$

C $-2.6114731 .396629-2.103528$

C $-3.8934751 .884474-2.618020$

C $-5.1108741 .029877-2.386649$

C $-4.249947-0.0241252 .154081$

C $-5.085518 \quad 1.244886 \quad 2.157197$

C -4.9936313 .6225481 .408991$

O $-6.177641 \quad 1.3333192 .710776$

C -5.7617913 .7626620 .132937$

C $-5.5408514 .742191-0.757083$

C $-6.3460614 .931414-2.010453$

C $-5.4875635 .025626-3.251521$

C $-5.1609596 .218316-3.777212$

C $-5.089169 \quad 3.737394-3.934212$

C -3.920426 $3.065517-3.270578$

N -4.4404852 .2846461 .499180$

H $2.383832-0.4681610 .901433$

H $-0.282216-1.0665952 .282994$

H $0.924220-1.666864-0.527953$

H $-0.874265-3.4232261 .228653$

H $\quad 0.835248-3.6874431 .025864$

H $-1.493033-5.285329-1.993536$

H $0.744931-5.041134-2.417268$

H $1.290225-3.412340-2.011818$

H $1.747482-4.790188-0.986693$

H -3.149388 -5.616878 -0.187752

H -2.661264 -4.1555580 .632207$

H -3.335702 -2.890636 -1.548330

H $-4.099126-4.401812-2.055406$
H $-5.452506-4.3652910 .092363$

H $-6.431376-3.414469-2.018006$

H -5.622865 -1.892850 -1.719345

H $-8.299986-1.801670-2.016922$

H $-6.337994-0.4928370 .406891$

H -8.643667 $-3.842999-0.924937$

H $-9.111666-2.985246 \quad 0.525417$

H -7.774277 -4.1514480 .579581$

H $-8.473005-0.5847951 .152819$

H $-5.709750-1.0067973 .458826$

H $-4.223507-1.8803863 .187240$

H -5.640526 -3.5234203 .020800$

H -3.196247 -0.469161 -1.255687

H $-1.7611522 .055064-2.277341$

H $-5.2152490 .785699-1.324440$

H $-5.0351760 .098913-2.957946$

H $-6.0465481 .513398-2.675070$

H -3.3946170 .1768012 .812263$

H $-3.852862-0.1976591 .150004$

H $-4.155678 \quad 4.324402 \quad 1.467174$

H $-5.6578873 .801522 \quad 2.260520$

H $-6.5584823 .045540-0.050570$

H $-4.7633785 .475872-0.555693$

H $-6.9301025 .853860-1.892923$

H $-7.0849264 .130580-2.137538$

H $-4.5714396 .294028-4.684934$

H $-5.471103 \quad 7.146077-3.307857$

H $-4.796713 \quad 3.940136-4.973581$

H $-5.963653 \quad 3.086154-4.019917$

H -2.986274 $3.617790-3.382013$

H $-3.5609582 .103502 \quad 1.024130$

C $2.440551 \quad 1.7000132 .696024$

O 3.6427381 .7176541 .901903

C 4.4857642 .7949682 .350723

C $3.682046 \quad 3.565628 \quad 3.394324$

C 2.7869732 .4809503 .961319

H 5.3503762 .3335712 .841636

C 4.9470263 .6340251 .154539

H 4.3247534 .0300424 .148763

H 3.0771104 .3591112 .940593

C 1.5806153 .0206644 .709556

H $3.382317 \quad 1.8540354 .638671$

C 5.6452462 .8209530 .044054

$\begin{array}{lllllllll}\text { O } & 3.795018 & 4.258356 & 0.570689\end{array}$

H 5.5946594 .4472691 .502504

H $4.9181442 .148090-0.422426$

O $6.0309303 .747665-0.981320$

C 6.8882622 .0302980 .500972

C $7.6588371 .370738-0.660342$

H 6.6005841 .2683411 .233177

O $7.7750352 .939362 \quad 1.159431$

H $7.8503282 .115560-1.442074$

H $8.6480281 .069673-0.288855$

H $1.898126 \quad 3.634578 \quad 5.558734$

H 0.9517973 .6413274 .062387

H 0.9628562 .2043965 .096586

C $2.023543 \quad 0.254282 \quad 2.964726$

H $4.1034514 .783675-0.187635$

H $6.7137444 .330389-0.606696$

H $8.5170622 .420177 \quad 1.513554$

H 1.1950330 .2279593 .681424

H $2.862172-0.2957343 .408499$

H 1.6649832 .2212742 .119114

C $6.546961-2.193476-0.685281$

O $7.137101-0.936660-0.324416$

C $6.9423750 .143162-1.246596$

C $7.494149-0.224255-2.632070$

C $6.886045-1.534747-3.138505$

C $6.986336-2.643550-2.089176$

H $6.967712-2.9112610 .031669$

C $5.024979-2.174710-0.490581$

H $5.8728230 .352696-1.335452$

O $8.915175-0.336259-2.591860$

H $7.258066 \quad 0.586324-3.331269$

H $7.407170-1.850575-4.050453$

O $5.513209-1.335585-3.462763$

H $6.398182-3.516928-2.394037$

O $8.347978-3.072748-1.993289$ 
C $4.697376-2.3145090 .979999$

H $4.544182-1.259156-0.844477$

H $4.566322-3.013192-1.028069$

H $9.142926-0.994611-1.913145$

H $5.469818-0.644957-4.145925$

H $8.612690-3.404955-2.868116$

O $3.956700-3.440507 \quad 1.187855$

O $5.066862-1.5260801 .840821$

C $3.628848-3.7229582 .551750$

H $3.017324-2.9200122 .971753$

H $3.052105-4.6514212 .576805$

H $4.541324-3.8607143 .139701$

SCF Energy (B3LYP/6-31G**//MMFF) $=-3245.91228320$

08_00354

MM̄FF Geometry

C $0.640146-2.8849331 .616804$

C $-0.667249-2.820077 \quad 1.909831$

C -1.535337 -1.586863 1.828518

O $-0.861696-0.4992861 .147448$

C - $-1.914775-1.0496883 .220691$

C $-2.908838-1.8996423 .981141$

C $-4.240154-1.8675403 .774766$

C $-2.327791-2.7770155 .058331$

C $-4.984123-1.0360892 .766493$

C $-5.804837-1.9195631 .817335$

C $-6.568032-1.0826670 .780859$

C $-7.493884-1.966942-0.055936$

C $-8.197948-1.168724-1.163661$

O $-5.606592-0.448116-0.067937$

O $-8.779340-2.106605-2.082292$

C -7.140843 -0.363714 -1.948290

C $-9.355085-0.325788-0.614291$

O $\quad-7.760737 \quad 0.507724-2.891735$

C $-6.1707490 .436576-1.045385$

C $-5.0127171 .006532-1.905630$

O $-6.8849431 .509466-0.433345$

C $-0.881646-0.488197-0.210360$

O $-1.370132-1.341520-0.938826$

C $-0.2571090 .739460-0.756480$

C $0.3676631 .662180-0.008316$

C $0.9361492 .924174-0.491378$

C $0.6784273 .297673-1.928014$

C $-3.8392061 .602586-1.115616$

C $-4.0343693 .037676-0.661583$

C -2.9936674 .7490370 .830962$

O $-4.9349323 .758194-1.082402$

C -1.804536 5.4615350 .267998

C -0.8218575 .9714721 .025755$

C 0.3595926 .7180960 .475815

C 1.6796836 .1429010 .938270

C 2.3299946 .6652641 .991977

C 2.2873445 .0214290 .125991

C 1.6218233 .6970460 .377386

N -3.061565 3.4211250 .251043

H $1.173616-1.9864681 .318330$

H -1.176581-3.734855 2.205644

H -2.446772 -1.8326951 .269124$

H -2.314260 -0.031343 3.118094

H -1.006899 -0.9059403 .823466$

H -4.873005 -2.4976674 .398302$

H -3.096486 -3.351748 5.585828

H -1.616555 -3.492068 4.635222

H -1.804875 -2.166493 5.801867

H -5.657362 -0.3620813 .310030$

H $-4.308248-0.4006202 .187037$

H -5.127145 -2.612942 1.302006

H $-6.514024-2.5214482 .399448$

H $-7.146649-0.3170761 .311109$

H -8.231150 -2.471957 0.580654

H $-6.905933-2.767596-0.526348$

H -9.395916 -2.670549 -1.584546

H -6.552124 -1.084195 -2.533575

H -10.101017 -0.968656-0.131531

H $-9.8845210 .192308-1.421517$

H -9.0348960 .4158570 .120343$

H -8.348522 -0.034908 -3.444917

H $-4.5925970 .181353-2.496072$
H $-5.3997691 .750036-2.612529$

H $-6.2487582 .167947-0.115203$

H $-0.3603010 .853279-1.831053$

H $0.466167 \quad 1.4944821 .062668$

H $-0.3972923 .293587-2.138881$

H $1.1700282 .590873-2.604775$

H $1.0349704 .294828-2.192459$

H $-3.5896190 .976183-0.253113$

H -2.963644 $1.616184-1.776549$

H $-2.9245634 .623688 \quad 1.916263$

H -3.9057875 .3089950 .602978$

H -1.761361 $5.579086-0.812268$

H -0.8813805 .8728442 .107438$

H $\quad 0.274923 \quad 7.7627810 .803029$

H $\quad 0.3391906 .750910-0.620505$

H $3.291103 \quad 6.2772182 .312781$

H 1.9120607 .4892472 .561033

H 3.3467724 .9066450 .387723

H $2.2921325 .306731-0.929210$

H 1.7470573 .3386881 .400320

H -2.3479372 .7519900 .524989$

C $1.927622-4.617998 \quad 0.290517$

O $2.683134-3.579133-0.368225$

C $2.229683-3.483403-1.730765$

C $1.496306-4.789191-2.013526$

C $0.819051-5.033392-0.679720$

H $1.502552-2.663936-1.761933$

C $3.392950-3.191666-2.681782$

H $\quad 0.790505-4.699236-2.844675$

H $2.192495-5.604716-2.241811$

C $0.337250-6.460932-0.489601$

H $-0.041775-4.357814-0.603804$

C $4.163997-1.890259-2.377862$

O $4.323309-4.279727-2.603993$

H $3.023838-3.178697-3.714419$

H $4.729929-2.016351-1.448377$

O $5.143126-1.725664-3.413239$

C $3.304791-0.614027-2.295675$

C $4.1083770 .698767-2.261180$

H $2.654604-0.657809-1.414825$

O $2.458885-0.519943-3.446916$

H $4.6170340 .848610-3.222533$

H $3.3746281 .512120-2.193533$

H $-0.405391-6.720738-1.251041$

H $1.162119-7.177209-0.567066$

H $-0.127935-6.5865300 .493240$

C $1.453669-4.1474151 .669046$

H $5.055138-4.079211-3.212409$

H $4.665652-1.558842-4.244219$

H $1.776031-1.207040-3.377358$

H $2.328492-3.9408152 .296816$

H $0.896534-4.9528492 .161743$

H $2.625684-5.4525450 .436072$

C 5.2225270 .7234661 .307145

$\begin{array}{llll}\text { O } & 4.418594 & 0.665555 & 0.120379\end{array}$

C $5.1222820 .823083-1.113914$

C $5.8555142 .175517-1.156514$

C 6.7527322 .3674220 .070100

C 6.0131132 .0414031 .371758

H 4.5012910 .7280262 .134769

C $6.114229-0.5190791 .462844$

H $5.857100 \quad 0.018820-1.216264$

O $4.9038873 .235761-1.215515$

H $6.4529132 .221343-2.074962$

H 7.1053793 .4052800 .108342

O $7.8980041 .527135-0.035656$

H 6.7164322 .0021372 .211802

O 5.0801323 .0865161 .657439

C $5.312464-1.7834121 .681484$

H $6.739604-0.6824230 .581563$

H $6.763195-0.3944712 .337470$

H $5.3967034 .071532-1.277093$

H $8.3661191 .769051-0.852912$

H 5.5825423 .9146681 .740636

O $6.172626-2.8436391 .734538$

O $4.096616-1.8378541 .796612$

C $5.570285-4.1250921 .939396$

H $4.908934-4.1093042 .810969$ 
H $\quad 5.023333-4.4243351 .041529$ H $6.368225-4.8498682 .121267$

SCF Energy (B3LYP/6-31G**//MMFF) $=-3245.90833029$

$08 \_00355$

MM̄FF Geometry

C $1.673433-2.738764-0.611613$

C $2.363872-3.4525250 .290160$

C $2.918436-2.9147331 .588759$

O $2.228723-1.7016101 .974445$

C $4.412256-2.5880631 .444616$

C $5.102654-2.4240622 .783718$

C $5.402614-1.2424123 .356551$

C $5.501948-3.7112783 .459585$

C 5.0860720 .1390542 .855592

C 6.3463410 .9118602 .440557

C $6.960997 \quad 0.411243 \quad 1.123762$

C 8.2605351 .1572280 .816539

C $8.8392930 .765028-0.551016$

O 6.0081880 .6500690 .084661

O $9.8597591 .727718-0.856160$

C $7.7280100 .885058-1.616327$

C $9.529006-0.603471-0.500862$

O $\quad 8.168338 \quad 0.384865-2.877152$

C $6.4071880 .187713-1.211908$

C $5.2850740 .574661-2.204360$

O $6.596416-1.227291-1.235713$

C $1.074090-1.8359592 .677120$

O $0.580691-2.8824923 .076191$

C $0.435891-0.5274412 .943917$

C 0.7363360 .6050522 .288351

C 0.1182591 .9164272 .514073

C -0.9368272 .0128593 .586619$

C $3.9002490 .032379-1.825148$

C $2.8047840 .729468-2.606661$

C $1.6350972 .910630-2.810728$

O $2.236722 \quad 0.188544-3.552133$

C $1.2529774 .008321-1.871586$

C $-0.0020654 .223292-1.451264$

C $-0.3925975 .320870-0.507173$

C $-0.930548 \quad 4.7938260 .804041$

C -2.2516374 .7664091 .047419$

C 0.0665544 .3994311 .870338

C 0.5163832 .9651781 .762456

N $2.5452322 .007137-2.133705$

H $1.531741-1.672791-0.453574$

H $2.519456-4.5146110 .113878$

H $2.772963-3.6777982 .363201$

H $4.534645-1.6830940 .840399$

H $4.928121-3.384398 \quad 0.891447$

H $5.923712-1.2563724 .313364$

H $6.030942-3.5395994 .403065$

H $4.620371-4.3194113 .683798$

H $6.167713-4.2896122 .810332$

H $4.355796 \quad 0.1307592 .041327$

H 4.6029380 .6807253 .678672

H 6.0616381 .9655592 .322037

H $7.091740 \quad 0.866083 \quad 3.244380$

H $7.152618-0.6644331 .201884$

H 9.0015590 .9917991 .608602

H 8.0656202 .2392020 .811932

H $10.2813211 .467132-1.692321$

H $7.5250641 .955861-1.758511$

H $10.359836-0.5848170 .214766$

H $9.973282-0.859153-1.469152$

H $8.856399-1.412339-0.207952$

H $8.9336150 .915736-3.154502$

H $5.2335121 .669070-2.264991$

H $\quad 5.528217 \quad 0.210465-3.210605$

H $5.734759-1.661226-1.334505$

H $-0.338695-0.557818 \quad 3.703787$

H 1.4997190 .5787991 .512577

H $-0.512081 \quad 1.7674894 .566385$

H $-1.7628101 .323733 \quad 3.377726$

H -1.3841263 .0047733 .673916$

H $3.835263-1.040794-2.032756$

H $3.6904510 .166162-0.757972$

H $0.7714992 .345493-3.175976$
H $2.1593783 .322941-3.678896$

H $2.0507664 .658540-1.519987$

H $-0.8021533 .583702-1.812971$

H -1.150553 $5.937672-1.007615$

H $0.4481685 .997853-0.310562$

H -2.6449484 .4229311 .998157$

H -2.970707 5.0807750 .298340

H -0.3380894 .6190812 .864389$

H 0.9523905 .0438971 .798772

H 1.2804592 .7979051 .003307

H $3.1778472 .399907-1.441897$

C $-0.466342-3.021293-1.929902$

O $-0.671168-1.597634-2.039667$

C -1.621222 -1.191509-1.033916

C $-2.400534-2.450389-0.694424$

C $-1.307339-3.499937-0.741728$

H $-1.028257-0.859483-0.172395$

C -2.462544 -0.032585-1.576091

H -2.894665 -2.395762 0.279982

H -3.160097 -2.650777 -1.458418

C $-1.826638-4.919066-0.895877$

H $-0.740233-3.4393690 .194773$

C $-3.5412550 .445937-0.584937$

O $-1.5672861 .041788-1.866394$

H -2.931667 -0.320141 -2.524536

H $-4.176832-0.409814-0.338385$

$\begin{array}{llll}\text { O } & -2.917178 & 0.861222 & 0.630602\end{array}$

C $-4.4425731 .579787-1.115911$

C $-5.4762772 .090459-0.092132$

H $-4.9547641 .228543-2.019328$

O $-3.6397512 .697381-1.499414$

H -4.9589272 .5639600 .752056$

H $-6.0412912 .895806-0.580209$

H $-2.468003-5.188552-0.050398$

H -2.413941 -5.034911-1.812882

H $-0.997247-5.632373-0.934005$

C $1.036823-3.317716-1.844030$

H $-0.8976330 .702862-2.484977$

H -2.3389511 .6137910 .416527$

H $-4.2284573 .361809-1.896061$

H $1.538215-2.871579-2.711519$

H $1.197123-4.401040-1.892686$

H $-0.849107-3.450912-2.864122$

C $-8.060287-0.586065-0.425045$

O $-7.1397410 .476690-0.705481$

C $-6.448676 \quad 1.0181790 .423541$

C -7.4448471 .5860811 .445963$

C -8.4738010 .5252591 .851509$

C $-9.096432-0.1564940 .628984$

H $-8.597815-0.747392-1.368783$

C $-7.331985-1.893560-0.072121$

H $-5.867441 \quad 0.2290490 .908948$

O

H -6.8886611 .9314872 .325670$

H $-9.267780 \quad 0.9924462 .446558$

O $-7.849647-0.4659582 .662315$

H -9.686924 -1.027945 0.934840

O $-9.9980470 .755482-0.005282$

C $-6.632528-2.494277-1.275387$

H $-6.577235-1.7602850 .706574$

H $-8.070641-2.6262150 .274484$

H -8.717014 3.0633641 .568901

H -7.481630 -0.017987 3.442986

H -10.6829090 .9898310 .643881$

O -6.353219 -3.806505-1.016888

O $-6.354575-1.892303-2.303292$

C $-5.727563-4.531150-2.081007$

H $-4.859797-3.990680-2.469382$

H $-5.390299-5.492703-1.684808$

H -6.453764 -4.712686 -2.878688

SCF Energy $\left(B 3 L Y P / 6-31 G^{* *} / / M M F F\right)=-3245.89444919$

08 00356

MM̄FF Geometry

C 3.2714254 .9556690 .935708

C 3.8537163 .7476580 .962745

C $4.6276413 .182045-0.201250$

O $4.0145201 .926884-0.585159$ 
C $6.084806 \quad 2.9119790 .209316$

C $6.9714232 .529086-0.961589$

C $7.4249131 .285788-1.217303$

C $7.3784573 .672061-1.857805$

C $7.1519750 .025362-0.446955$

C $6.376813-0.986621-1.299744$

C $5.985883-2.231599-0.491015$

C $5.258629-3.245114-1.375688$

C $4.742236-4.448754-0.572689$

O $5.129306-1.8079020 .573854$

O $3.805300-5.159278-1.397471$

C $3.960886-3.9367720 .657164$

C $5.866646-5.437774-0.243685$

O $3.601420-5.0161891 .515390$

C $4.716535-2.8572291 .459470$

C $3.855621-2.2065472 .570146$

O $5.846628-3.4582902 .103978$

C $2.8997742 .004974-1.365551$

O $2.3731913 .021993-1.790051$

C $2.4152660 .629260-1.619606$

C $1.232997 \quad 0.454467-2.229837$

C $0.624577-0.822623-2.611127$

C $1.387161-2.082263-2.300487$

C $2.595724-1.4784482 .088157$

C $1.334096-2.3234322 .138835$

C $-1.075668-2.240597 \quad 1.512534$

O $1.283644-3.4324562 .663019$

C -1.299633 -2.8769610 .178291$

C $-2.301137-2.530350-0.643536$

C $-2.590620-3.206412-1.949959$

C $-2.627453-2.239803-3.110097$

C $-3.789668-1.720361-3.540485$

C $-1.330642-1.959295-3.831625$

C $-0.568180-0.805382-3.242999$

N $0.256098-1.6700431 .557370$

H 3.3868535 .5784300 .052051

H 3.7390323 .1156391 .840150

H $4.6071733 .870530-1.055308$

H 6.1084362 .1416740 .986773

H 6.5131173 .8092910 .676781

H $8.0714551 .145277-2.083022$

H $8.0801803 .359426-2.638527$

H $6.5054924 .103428-2.356494$

H $7.8693404 .456728-1.272879$

H $8.112662-0.410960-0.147498$

H 6.6074540 .2261460 .478057

H $5.470129-0.503947-1.685204$

H $6.983066-1.281525-2.165386$

H $6.896919-2.672498-0.069712$

H $5.905126-3.579016-2.196905$

H $4.399685-2.756240-1.853812$

H $4.264444-5.423994-2.212985$

H $3.023459-3.5094880 .281702$

H $6.322419-5.819369-1.165318$

H $5.478845-6.3163170 .283679$

H $6.663577-4.9964230 .358274$

H $3.106930-5.6600620 .979798$

H $3.625831-2.9426083 .349994$

H $4.483847-1.4505773 .063280$

H $6.110839-2.8970752 .851670$

H $3.047532-0.195848-1.315039$

H $\quad 0.656127 \quad 1.338196-2.500897$

H $0.822729-2.994693-2.504912$

H $1.651022-2.123035-1.238639$

H $2.306115-2.126359-2.894107$

H $2.415209-0.6246082 .754143$

H $2.728880-1.0805571 .077275$

H $-1.780911-1.4290231 .709077$

H -1.186024 -2.9907272 .302151$

H - $0.629551-3.684480-0.106533$

H $-2.986385-1.743042-0.342129$

H -3.562517 -3.708961-1.855600

H $-1.868724-4.005809-2.157371$

H $-3.827793-1.050692-4.393312$

H $-4.727199-1.945713-3.042578$

H $-1.542352-1.704578-4.879422$

H $-0.734313-2.874362-3.893913$

H $-1.0471460 .159064-3.418262$
H $\quad 0.401729-0.7709641 .106692$

C $0.926677 \quad 5.446185 \quad 1.785289$

$\begin{array}{lllll}\text { O } & 0.497052 & 4.069858 & 1.762704\end{array}$

C $-0.4019663 .884006 \quad 0.649258$

$\begin{array}{llll}\text { C } & -0.835862 & 5.282131 & 0.234232\end{array}$

$\begin{array}{llll}\text { C } & 0.430680 & 6.077091 & 0.482959\end{array}$

H $0.1952863 .415943-0.142272$

C $-1.5462792 .954316 \quad 1.067325$

H $-1.1700645 .329369-0.806504$

H $-1.649583 \quad 5.6445650 .874815$

$\begin{array}{lllll}\text { C } & 0.207486 & 7.576212 & 0.574386\end{array}$

H $1.1199775 .873866-0.344956$

C -2.569823 $2.714076-0.059864$

O $-0.969891 \quad 1.703248 \quad 1.455099$

H $-2.053214 \quad 3.3583601 .951677$

H -2.949098 $3.679819-0.412072$

O $-1.905016 \quad 2.106590-1.169255$

C -3.7885851 .8581290 .348213$

C $-4.734043 \quad 1.616528-0.846119$

H -4.3373422 .3743151 .145050$

$\begin{array}{llll}\text { O } & -3.373957 & 0.598958 & 0.862107\end{array}$

H $-4.9932412 .582357-1.295900$

H -4.203372 $1.015973-1.595791$

H $-0.2031927 .962184-0.364439$

H $-0.4936117 .829972 \quad 1.376455$

H $1.149616 \quad 8.0974300 .771546$

C $2.435078 \quad 5.5158862 .051543$

H -0.3166021 .8878122 .151730$

H $-1.591171 \quad 1.233592-0.875819$

H -2.859849 $0.763022 \quad 1.670417$

H 2.6552574 .9721142 .979088

H $2.7343296 .557516 \quad 2.216198$

H 0.4113705 .9032322 .640245

C $-6.771868-1.193706 \quad 0.515150$

O $-5.703423-0.474144-0.114936$

C $-6.0250040 .876193-0.453107$

C $-7.0754530 .865339-1.579999$

C $-8.119679-0.244184-1.386244$

C $-8.162463-0.7102070 .065595$

H -6.646458 - 2.2423110 .216845

C $-6.620799-1.1039652 .040865$

H -6.4509661 .3698610 .430076$

O $-6.4423430 .651511-2.843466$

H $-7.5444501 .855061-1.634991$

H -7.928281 -1.100657-2.044780

$\begin{array}{llll}\text { O } & -9.406606 & 0.259561 & -1.746146\end{array}$

H -8.5233310 .1052510 .705195$

O $-9.087062-1.786373 \quad 0.211591$

C $-5.377764-1.8084352 .542550$

H $-6.552080-0.0525372 .342880$

H $-7.480916-1.5659262 .537767$

H $-7.1330000 .685611-3.526985$

H $-9.3605420 .537639-2.676902$

H $-9.945473-1.477989-0.126021$

O $-5.139417-1.421185 \quad 3.830855$

O $-4.712166-2.609397 \quad 1.901325$

C -3.992472 -2.011506 4.450372

H $-4.058737-3.1034174 .421529$

H -3.080771 -1.6653953 .955428$

H -3.967713 -1.690338 5.495141

SCF Energy (B3LYP/6-31G**//MMFF) $=-3245.90980976$

08_00357

MM̄FF Geometry

C $2.6277693 .763522-0.690065$

$\begin{array}{llll}\text { C } 2.350940 & 4.765625 & 0.157425\end{array}$

C 1.4181354 .6834731 .344679

$\begin{array}{lllllll}\text { O } & 0.950220 & 3.333154 & 1.564504\end{array}$

C $0.1864555 .577252 \quad 1.115907$

C $-0.766293 \quad 5.5948572 .296653$

$\begin{array}{llll}C & -2.031754 & 5.130984 & 2.280408\end{array}$

C $-0.220778 \quad 6.218377 \quad 3.557651$

C -2.8006504 .5259251 .138560$

C $-3.185142 \quad 3.072211 \quad 1.427447$

C $-4.0169262 .423073 \quad 0.307880$

C $-5.392857 \quad 3.079172 \quad 0.151046$

C $-6.2729672 .351168-0.876396$

$\begin{array}{llll}\text { O } & -4.172583 & 1.039843 & 0.657513\end{array}$ 
O $\quad-7.6194202 .824009-0.715563$ C $-6.2795560 .845640-0.546325$ C $-5.8633952 .692466-2.314344$ O $-6.9812690 .108841-1.545997$ C $-4.869110 \quad 0.255943-0.321334$ C $-4.987263-1.1909550 .217248$ O $-4.1766930 .232811-1.572531$ C 1.7503532 .5036102 .289673 O 2.8447182 .7633642 .763243 C 1.0499991 .2057472 .406634 C 1.6190320 .2007053 .089066 C $1.026132-1.1158173 .332816$ C $-0.321123-1.3929072 .720971$ C $-3.659119-1.7809290 .708289$ C $-3.774360-3.263197 \quad 1.026912$ C $-2.329955-5.2560561 .473241$ O -4.849748 -3.843026 1.147691 C $-1.384868-5.3766072 .625847$ C $-0.077301-5.6439402 .491105$ C $0.884263-5.7489603 .639525$ C $1.787118-4.5367713 .735231$ C $3.006585-4.5520773 .170665$ C $1.242156-3.3724094 .538305$ C $1.699754-2.0059024 .092259$ N -2.527457 $-3.846621 \quad 1.191929$ H $2.1338312 .804191-0.568047$ H 2.8547295 .7201190 .020014 H 1.9657335 .0285282 .230266 H $-0.324516 \quad 5.2451430 .207125$ H 0.5059296 .6091870 .917643 H -2.611653 5.2126223 .199967 H -0.990969 6.3452854 .326015 H 0.5701645 .5958253 .986734 H $\quad 0.1906707 .210328 \quad 3.343719$ H -3.700401 $5.136176 \quad 1.004609$ H $-2.255527 \quad 4.5735860 .193209$ H -2.274239 2.4766151 .577989 H -3.7358453 .0029322 .375407$ H $-3.4479722 .487426-0.626426$ H $-5.3030664 .135256-0.126770$ H $-5.9109153 .067707 \quad 1.120213$ H -7.617402 $3.787429-0.848868$ H -6.8589520 .7235370 .379047$ H -5.940388 $3.772689-2.488010$ H $-6.5394752 .226614-3.039615$ H $-4.8399432 .394471-2.551429$ H $-6.3971050 .028124-2.319046$ H $-5.688623-1.2117511 .061668$ H -5.400385 -1.840384 -0.565246 H -3.643291 -0.574236 -1.633367 H $\quad 0.0725381 .1208291 .944809$ H 2.6012150 .3600283 .531866 H - $-1.077184-0.7093983 .122604$ H -0.676833 -2.406189 2.909342 H $-0.281688-1.2759741 .632041$ H - $-2.875374-1.646536-0.042473$ H -3.338104 -1.269353 1.623108 H -1.929174 $-5.716150 \quad 0.564365$ H $-3.281250-5.742871 \quad 1.708448$ H -1.799702 -5.2242483 .619286$ H $\quad 0.336275-5.7894081 .496656$ H $1.479312-6.6612633 .503269$ H $0.355260-5.8886444 .590920$ H $3.688421-3.7131363 .250253$ H $3.357466-5.4169432 .616801$ H $1.537825-3.5108615 .586644$ H $\quad 0.147909-3.4096074 .554543$ H $2.676331-1.7215734 .485507$ H -1.701169 -3.296839 0.974574 C $4.7941942 .886313-1.647286$ O $4.3270901 .521402-1.614403$ C $5.0483080 .815167-0.587009$ C $6.2353471 .701388-0.222308$ C $5.6372413 .083558-0.386007$ H 4.3795350 .7441050 .278893 C $5.441350-0.583584-1.068413$ H 6.6015501 .5076730 .790541 H $7.0726901 .565219-0.916707$
C $6.6705014 .190014-0.502913$

H 5.0022173 .2832330 .485558

C $4.258945-1.490721-1.468253$

O $6.299352-0.458425-2.211072$

H $6.043361-1.078469-0.297346$

H $3.785556-1.096170-2.373986$

O $4.807492-2.762332-1.843804$

C $3.188415-1.700899-0.373979$

C $2.238321-2.889323-0.629326$

H $2.603002-0.782423-0.256447$

O $3.830998-1.9348200 .882825$

H $2.829811-3.808951-0.719058$

H $1.621452-3.0224730 .270298$

H 7.2874114 .2370350 .400424

H $7.3371624 .029009-1.356661$

H $6.1854525 .162769-0.631845$

C $3.6154883 .850235-1.817760$

H $5.8078790 .029591-2.894036$

H $5.278116-3.123691-1.073797$

H $4.327262-2.7679550 .815092$

H $3.0788563 .600005-2.741124$

H $3.9906884 .873970-1.930131$

H $5.4306122 .964464-2.538615$

C $-0.661494-1.486796-2.507067$

O $0.287305-1.808966-1.481788$

C $1.330520-2.715254-1.855716$

C $0.735973-4.051980-2.328237$

C $-0.266480-3.835304-3.465347$

C -1.288931 -2.749732 -3.120577

H -1.455557 -0.943126 -1.982566

C $-0.060171-0.542411-3.552367$

H $1.909749-2.281934-2.675492$

O $0.068385-4.713684-1.257343$

H $1.545310-4.707629-2.671849$

H $-0.796773-4.773708-3.669838$

O $0.425992-3.455115-4.650612$

H -1.861265 -2.474852 -4.013814

O $-2.233722-3.285005-2.189965$

C $\quad 0.2557110 .794336-2.928749$

H $0.866316-0.923622-3.991340$

H $-0.754135-0.374852-4.384624$

H $0.746044-5.022514-0.633317$

H $1.044894-4.171700-4.872034$

H $-1.752663-3.514687-1.376586$

O $-0.8803631 .540927-2.822246$

O $1.375806 \quad 1.120684-2.559860$

C $-0.7243362 .822937-2.206376$

H $-0.5016612 .698569-1.142626$

H $\quad 0.0617513 .398723-2.703963$

H $-1.6684413 .365139-2.306322$

SCF Energy (B3LYP/6-31G**//MMFF) $=-3245.91371441$

08_00358

MM̄FF Geometry

C 1.1280974 .4106011 .069264

C 2.3369193 .8420950 .951655

C $3.1015443 .772665-0.344520$

O $3.1284492 .379025-0.739570$

C $4.5440394 .258405-0.141973$

C 5.348345 4.259555-1.426902

C $6.2541053 .319643-1.762253$

C $5.1065435 .436590-2.336058$

C $6.6178312 .084829-0.986207$

C $6.5786990 .830352-1.870634$

C $6.899717-0.429916-1.055599$

C $7.064516-1.647536-1.964751$

C $7.313543-2.928190-1.152933$

O $5.817283-0.651821-0.145025$

O $7.146684-4.048730-2.034691$

C $6.229871-3.045589-0.061380$

C $8.750329-3.002287-0.622971$

O $6.503046-4.135880 \quad 0.816021$

C $6.021226-1.7476560 .755395$

C $4.759591-1.8909411 .637185$

O $7.152655-1.5294391 .599706$

C $2.0516321 .923935-1.439207$

O $1.1295572 .590142-1.883639$

C $2.1425490 .448533-1.507993$ 
C $1.027701-0.244602-1.785185$

C $0.884160-1.700741-1.802569$

C $2.135519-2.529959-1.713619$

C $4.270400-0.5835402 .272986$

C $2.966299-0.8236623 .008602$

C $0.543758-1.2731612 .595385$

O $2.929580-0.9515004 .230082$

C $0.163605-2.5721601 .954843$

C $-0.994509-2.7713811 .307148$

C $-1.378254-4.0725770 .661754$

C $-1.834061-3.907745-0.772774$

C $-3.129684-4.041069-1.104318$

C $-0.779250-3.661282-1.833667$

C $-0.359151-2.219123-1.885508$

N $1.879392-0.9168962 .153492$

H $0.672788 \quad 4.890977 \quad 0.206872$

H 2.7654423 .3254121 .807770

H $2.618275 \quad 4.375350-1.123667$

H $5.040942 \quad 3.6483470 .620716$

H $4.539596 \quad 5.2783320 .265987$

H $6.8006723 .445738-2.696146$

H $5.7455095 .413677-3.225372$

H $4.0675125 .451242-2.678804$

H $5.3122886 .373234-1.807483$

H $7.6322682 .215948-0.590639$

H $5.9586481 .935749-0.127322$

H $5.5806990 .736766-2.318297$

H $7.3017420 .938846-2.688465$

H $7.821296-0.250210-0.489287$

H $7.874029-1.489040-2.687922$

H $6.151127-1.785034-2.560616$

H $7.778993-3.950100-2.767223$

H $5.285632-3.287661-0.569587$

H $9.466396-2.969631-1.452858$

H $8.932523-3.952141-0.108053$

H $8.998536-2.1868910 .059501$

H $6.611792-4.930413 \quad 0.265887$

H $3.944666-2.295468 \quad 1.027678$

H $4.944055-2.6202062 .436754$

H $6.881346-0.9838852 .354852$

H $3.088721-0.020745-1.266555$

H $\quad 0.109110 \quad 0.309104-1.975951$

H $1.970954-3.587192-1.932887$

H $2.562736-2.464347-0.710256$

H $2.883771-2.186130-2.436887$

H $5.000346-0.1900422 .988082$

H $4.106366 \quad 0.1944551 .518643$

H $-0.121696-0.4579872 .299529$

H $0.519225-1.3717883 .684654$

H $\quad 0.874950-3.3913082 .032787$

H $-1.718689-1.9625371 .253255$

H -2.183043 -4.5148611 .263488$

H $-0.550928-4.7924240 .693068$

H -3.462396 -3.945929 -2.132654

H $-3.888495-4.238122-0.354395$

H $-1.172913-3.927378-2.823196$

H $0.056341-4.343365-1.654577$

H $-1.198779-1.529250-1.977742$

H $2.020478-0.7681511 .158901$

C -0.6135593 .1023662 .251894$

$\begin{array}{llll}\text { O } & -1.577181 & 3.263618 & 1.195277\end{array}$

C -2.743942 2.4828681 .519765

C -2.5098501 .9171752 .921394$

C -1.4620282 .8568843 .491359$

H -3.596594 3.1717611 .542183

C $-2.977226 \quad 1.4029080 .450286$

H -3.430651 1.8973743 .512902

H -2.1296260 .8898652 .879935$

C -0.7008112 .2697014 .666519$

H -1.9542913 .7883413 .801017$

C $-3.3987181 .956694-0.927415$

$\begin{array}{llll}\text { O } & -1.792440 & 0.620542 & 0.302161\end{array}$

H -3.756202 0.7285400 .817766

H $-4.3462742 .500978-0.838052$

O $-2.4321492 .906200-1.394581$

C -3.512256 $0.889538-2.032736$

C $-4.365685-0.339817-1.699038$

H $-2.5080910 .541504-2.303025$
O $-4.029998 \quad 1.500875-3.216904$

H $-4.419040-0.986811-2.585059$

H $-3.837881-0.940035-0.946200$

H $-1.386007 \quad 2.025716 \quad 5.485055$

H -0.1723131 .3526784 .385447$

H $0.0388612 .980257 \quad 5.047948$

C $\quad 0.297019 \quad 4.327186 \quad 2.319891$

H $-1.0923141 .213124-0.021589$

H $-2.4108923 .643992-0.762130$

H -3.459962 $2.261364-3.423932$

H 0.9462484 .2743463 .201076

H -0.2934795 .2470822 .402093$

H -0.0193072 .2075502 .018633$

C $-7.601174-1.397551-0.215075$

O $-6.306982-1.382116-0.829683$

C $-5.797161-0.087600-1.186832$

C $-6.7415070 .584110-2.193431$

C $-8.1800360 .637330-1.663088$

C $-8.643132-0.716681-1.116079$

H $-7.862291-2.461683-0.145608$

C $-7.561119-0.8363331 .210403$

H $-5.7390740 .522951-0.282120$

O $-6.721156-0.114694-3.435960$

H $-6.3987441 .605048-2.389258$

H -8.853401 $0.948716-2.471053$

O -8.271905 1.607579 -0.623608

H $-9.585662-0.609761-0.566662$

O $-8.894905-1.597668-2.215145$

C $-6.689160-1.6984812 .092444$

H $-7.1972770 .193928 \quad 1.261198$

H -8.564157 -0.8284121 .653308$

H $-6.959877-1.041022-3.260234$

H -8.011144 $2.465810-0.998854$

H $-9.593052-1.195060-2.759259$

O $-5.440445-1.1584482 .184532$

O $-7.065832-2.7412462 .611505$

C $-4.503104-1.9006852 .970084$

H $-4.882829-2.0436563 .986274$

H $-4.296069-2.8640752 .494844$

H -3.574373 -1.326764 3.022246

SCF Energy (B3LYP/6-31G**//MMFF) $=-3245.92007790$

08_00359

MMFF Geometry

C -1.057222 -4.1950560 .550104$

C $0.230986-3.8283570 .617939$

C $0.912630-3.396381 \quad 1.891721$

O $1.552328-2.121817 \quad 1.653183$

C $1.994921-4.4248572 .267964$

C $2.747097-4.0836333 .540755$

C $4.027888-3.6644193 .591287$

C $1.968102-4.2730414 .817292$

C $4.961877-3.4209792 .438763$

C $5.285829-1.9285322 .296638$

C $5.887949-1.6024560 .921935$

C $6.308566-0.1339150 .856282$

C $6.7794200 .275681-0.547551$

O $4.878141-1.869729-0.057302$

O $6.827903 \quad 1.710578-0.591858$

C $5.722274-0.172862-1.580528$

C $8.204121-0.214152-0.831604$

O $6.1789600 .066027-2.909596$

C $5.279374-1.641714-1.413639$

C $4.081791-2.022255-2.320244$

O $6.369482-2.497870-1.776768$

C $0.797173-1.0036331 .837313$

O $-0.373504-0.9544752 .179666$

C $1.6486810 .168454 \quad 1.531825$

C 1.1222931 .4014051 .567823

C 1.8183502 .6521081 .256086

C 3.2739332 .5677550 .881801

C $2.782295-1.253159-2.056458$

C $2.594437-0.022931-2.927615$

C $1.1010091 .956063-3.189922$

$\begin{array}{llllll}\text { O } & 3.291064 & 0.221056 & -3.908649\end{array}$

C $1.7188223 .149763-2.533565$

C $1.0125234 .209026-2.109539$

C $1.6314265 .441975-1.516327$ 
C $1.0275895 .823799-0.183759$

C $0.0577956 .750550-0.106993$

C 1.6189685 .2074751 .063378

C $\quad 1.1232853 .8076921 .307662$

N $1.5221490 .750710-2.501195$

H -1.661461 -4.1831891 .453971$

H $\quad 0.840432-3.852150-0.282793$

H $\quad 0.192481-3.3046092 .714407$

H $2.685622-4.5420521 .427445$

H $1.533758-5.4135912 .398711$

H $4.463810-3.4612294 .568773$

H $2.580199-4.0960975 .708319$

H $1.121325-3.5812934 .858859$

H $1.586231-5.2972194 .880853$

H $5.890254-3.9770122 .618019$

H $4.560130-3.8132641 .502769$

H $4.366500-1.3451172 .430315$

H $5.979209-1.6309123 .093052$

H $6.748052-2.2593680 .746485$

H 7.0850810 .0811431 .601012

H $\begin{array}{llll}5.453913 & 0.497904 & 1.129519\end{array}$

H 7.4350842 .0119780 .105595

H $4.8536950 .479891-1.435901$

H $8.9031480 .192344-0.090745$

H $8.5597530 .145035-1.803525$

H $8.296046-1.301916-0.807838$

H $6.818349-0.631401-3.133523$

H $4.375423-1.972343-3.375621$

H $3.859071-3.082544-2.131767$

H $\quad 6.013472-3.374844-1.996268$

H $2.682478-0.015847 \quad 1.265672$

H 0.0744451 .5092651 .844838

H 3.8646512 .2179141 .734976

H 3.7008973 .5204940 .562742

H 3.4186651 .8773360 .044175

H $1.936992-1.915445-2.284505$

H $2.699857-0.972650-1.003392$

H $0.0081601 .983814-3.156911$

H $1.4152281 .908718-4.237592$

H $2.8020663 .158837-2.436746$

H $-0.067574 \quad 4.212235-2.237881$

H $1.4899346 .260938-2.234034$

H $2.7176635 .332862-1.407653$

H -0.3558007 .0589150 .847055$

H $-0.3471847 .219179-0.997837$

H 1.3459075 .8053691 .943153

H 2.7095295 .2733061 .017710

H 0.0682753 .7645921 .579015

H $1.0303270 .495313-1.649764$

C -2.924224 -3.805326 -1.094816

O $-3.954046-3.931789-0.092679$

C -5.191893 -4.262254 -0.736794

C $-5.022654-3.884896-2.200176$

C -3.562382 -4.234672 -2.420805

H -5.281573 -5.356403 -0.679737

C $-6.388111-3.652431-0.000339$

H -5.699630 -4.439051 -2.857193

H -5.183321 -2.815773 -2.371379

C $-2.953349-3.559423-3.638053$

H -3.479836 -5.323349-2.541022

C $-6.611088-2.131118-0.132071$

O $-7.562306-4.307691-0.499455$

H $-6.314460-3.9128221 .063071$

H $-6.982680-1.904208-1.138314$

O $\quad-7.684027-1.7815470 .755691$

C $-5.397834-1.2367730 .173881$

C $-5.7649920 .254563 \quad 0.163981$

H -4.621061 -1.435590 -0.565969

O $-4.879866-1.5804291 .457714$

H $-6.4362510 .479733-0.673813$

H -6.3366340 .4946521 .071266$

H -3.476760 -3.864965 -4.549794

H -3.015571 -2.468393 -3.564138

H -1.898282 -3.829994 -3.746560

C -1.724669 -4.674432 -0.709312

H $-8.323572-3.949737-0.011103$

H -7.362663 -1.905477 1.665475

H $-4.021990-1.1356061 .560555$
H $-1.005341-4.700989-1.536328$

H -2.056632 $-5.704889-0.530967$

H $-2.634343-2.747442-1.125106$

C -4.0978043 .5642240 .400561$

O

C $-4.551603 \quad 1.2038620 .115687$

C $-3.7418291 .128150-1.190737$

C $-2.6538262 .204641-1.238656$

C $-3.1725633 .588533-0.828043$

H -4.6853934 .4912960 .398650$

C $-3.3747493 .463808 \quad 1.752347$

H -3.9024120 .9799270 .969223$

O $-4.592919 \quad 1.306559-2.321037$

H -3.267895 $0.144410-1.282499$

H -2.253003 $2.253282-2.258325$

O $-1.5919631 .805717-0.380112$

H $-2.3381874 .275198-0.646753$

O $-3.9308374 .131617-1.914118$

C -2.7928264 .7813772 .214559$

H -4.090927 3.1386792 .517206

H -2.5697012 .7244861 .720068$

H $-5.1157990 .494410-2.428739$

H $-0.8845852 .467041-0.465236$

H $-3.3351114 .206923-2.678844$

O -2.1454224 .5835793 .401600$

O -2.8954995 .8474901 .624350$

C - -1.5517665 .7489253 .981884$

H -0.8107916 .1781843 .301698$

H -2.324432 6.4843304 .225237

H -1.047865 5.4498854 .904785

SCF Energy $($ B3LYP/6-31G**//MMFF $)=-3245.90121082$

08_00360

MMFF Geometry

C $-0.235924 \quad 4.9275680 .884493$

C 0.8871204 .1949920 .839831

C $1.6132443 .880076-0.442479$

O $1.8516822 .452789-0.499760$

C $2.9750804 .590432-0.469127$

C $3.6167144 .566298-1.841994$

C $4.6234593 .749423-2.207494$

C $3.0858765 .588470-2.815669$

C $5.2881952 .678927-1.387508$

C $5.4299221 .376558-2.188643$

C $6.2416920 .325019-1.420339$

C $6.527951-0.890114-2.303666$

C $7.287721-1.981927-1.536359$

O $5.488965-0.077226-0.271207$

O $7.239503-3.185411-2.317544$

C $6.545118-2.268880-0.216001$

C $8.771228-1.635797-1.362852$

O $7.290163-3.1668200 .603808$

C $6.180549-0.9957160 .586460$

C $5.240029-1.3785851 .750874$

O $7.370890-0.4173001 .124437$

C $0.790316 \quad 1.659337-0.809133$

O $-0.3463182 .020889-1.069321$

C $1.2478940 .253495-0.753009$

C $0.332523-0.726627-0.724566$

C $0.597368-2.165015-0.662124$

C $2.029535-2.613304-0.773288$

C $4.596592-0.1900382 .474997$

C $3.600778-0.7054773 .494812$

C $1.314880-1.6960763 .655741$

O $3.910979-0.8676574 .672701$

C $1.158264-3.0670203 .071887$

C $-0.029305-3.6016342 .750190$

C $-0.200168-4.9706302 .155327$

C $-1.063396-4.9713270 .910921$

C $-2.320668-5.4452620 .942355$

C $-0.443657-4.500455-0.388276$

C $-0.449434-3.003158-0.511783$

N 2.371089-1.011732 2.933007

H $-0.6240625 .353456-0.036739$

H 1.2905353 .7712901 .756321

H $1.0152874 .175453-1.313838$

H $3.643963 \quad 4.146118 \quad 0.277087$

H $2.8599105 .641250-0.170064$ 
H $5.0296523 .864823-3.211942$ H $3.6145905 .564458-3.774495$ H $2.0258935 .411934-3.021930$ H $3.1971756 .597354-2.404827$ H $6.2836233 .041054-1.103752$ H $4.7429282 .472410-0.462426$ H $4.4283540 .985506-2.410733$ H $5.9232731 .586259-3.146086$ H $7.1814410 .786985-1.095112$ H $7.084048-0.597667-3.203032$ H $5.578347-1.312716-2.661605$ H $7.643215-2.997664-3.182251$ H $\quad 5.612447-2.789540-0.476230$ H $9.249700-1.501733-2.340411$ H $9.312924-2.453516-0.874754$ H $8.934451-0.722179-0.787416$ H $7.483489-3.9553860 .068329$ H $4.436997-2.0164611 .365110$ H $5.788000-1.9783422 .489537$ H 7.1383060 .1188921 .898709 H $2.3151650 .067179-0.720472$ H $-0.721114-0.450694-0.732709$ H $2.153087-3.697586-0.775122$ H $2.614232-2.2300330 .066229$ H $2.476135-2.248001-1.705006$ H 5.3433430 .4140853 .000340 H 4.0779770 .4749191 .775092 H $0.405886-1.0959363 .548530$ H $1.571089-1.7689394 .716714$ H $2.063537-3.6468382 .906406$ H $-0.932532-3.0317552 .949401$ H -0.651256 -5.611173 2.924346 H $\quad 0.769599-5.4260311 .918664$ H $-2.936982-5.4718060 .049665$ H -2.764912 -5.809097 1.862833 H -1.010591 -4.899949 -1.239636 H $0.553694-4.937816-0.480134$ H -1.450614 -2.571802 -0.468753 H $2.219535-0.8267661 .945960$ C -2.426859 4.6067332 .128867 O $-2.381397 \quad 3.1851321 .895780$ C $-3.4773992 .823641 \quad 1.032887$ C -4.3916134 .0440840 .986114$ C -3.3887975 .1763181 .087882$ H -3.055862 2.6511950 .034570 C $-4.178761 \quad 1.5484091 .519897$ H -4.9898694 .0792510 .070502$ H -5.0788044 .0629521 .840610$ C $-4.0069606 .506741 \quad 1.479918$ H -2.897137 5.2851230 .114072 C -3.3195970 .2749421 .395736$ O $-4.586074 \quad 1.7233962 .877869$ H $-5.092613 \quad 1.4201050 .931389$ H -3.0583430 .1064410 .345898$ O -2.0778520 .4803522 .082092$ C $-3.967776-0.9848391 .994028$ C $-5.367532-1.3575511 .477996$ H $-4.013189-0.8744733 .084718$ O $-3.084801-2.0830011 .748767$ H -5.701901 -2.222992 2.065765 H $-6.062730-0.537851 \quad 1.690953$ H -4.741495 6.8245730 .732612 H -4.517672 6.4437062 .446584 H -3.241112 7.2852341 .553110 C -1.0107205 .1906722 .144880$ H -3.7802371 .8597743 .405660$ H -1.545472 -0.3252181 .963903$ H -3.457516 -2.8646412 .191128$ H -0.4713754 .7591102 .997996$ H -1.055233 6.2725972 .312913 H -2.8546824 .7354003 .132309$ C $-5.426645-0.722558-2.222151$ O $-5.358109-0.554386-0.798487$ C $-5.438922-1.748136-0.013508$ C $-6.744084-2.505699-0.315961$ C $-6.904759-2.771437-1.813911$ C $-6.690047-1.498387-2.633668$ H $-5.5330300 .296206-2.617356$
C $-4.130312-1.324213-2.791001$

H $-4.590199-2.396080-0.253404$

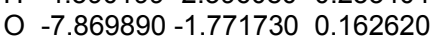

H $-6.732135-3.4582510 .226804$

H $-7.910265-3.162860-2.010120$

O $-5.961264-3.753288-2.232564$

H $-6.643604-1.732885-3.703587$

O $-7.808886-0.626329-2.448385$

C $-3.061445-0.266869-2.979892$

H $-3.704163-2.105985-2.158096$

H $-4.338618-1.747456-3.781174$

H $-7.844048-0.888654-0.244147$

H $-6.131026-4.561395-1.719230$

H $-8.602211-1.099151-2.753069$

O $-2.206459-0.667307-3.966974$

O $-2.9776720 .769715-2.335924$

C $-1.1697680 .258836-4.306944$

H $-0.673803-0.104461-5.211011$

H $-1.5865921 .249655-4.512485$

H $-0.4351270 .309177-3.500439$

SCF Energy (B3LYP/6-31G**//MMFF) $=-3245.91215302$

08 00361

MMFF Geometry

C $1.453752-3.720450-0.134646$

C $2.436649-2.813278-0.033122$

C $3.139070-2.4925901 .260325$

O $2.882982-1.1031641 .570146$

C $4.659258-2.6376161 .092355$

C $5.385926-2.8047762 .410926$

C $5.955607-1.8070753 .114490$

C $5.486856-4.2266592 .901149$

C $5.926117-0.3302692 .833976$

C 7.2757720 .2133052 .347430

C $7.4919010 .003628 \quad 0.841032$

$\begin{array}{llll}\text { C } & 8.896364 & 0.447835 & 0.429662\end{array}$

C $9.1016150 .349893-1.088903$

$\begin{array}{lllll}\text { O } & 6.509116 & 0.787227 & 0.152433\end{array}$

O $10.304426 \quad 1.061169-1.418399$

C $7.9384521 .076725-1.793990$

C $9.313351-1.097738-1.547419$

$\begin{array}{llll}\text { O } & 8.006648 & 0.895643 & -3.207003\end{array}$

C $6.5392660 .665900-1.274099$

C $5.4719011 .621682-1.860202$

O $6.257750-0.668882-1.697356$

C $1.699841-0.7799132 .151947$

O $0.810315-1.5405092 .495321$

C 1.6650310 .6953612 .253856

C $0.492399 \quad 1312468 \quad 2.453810$

C $0.2758562 .759427 \quad 2.503042$

C 1.4871033 .6519122 .432421

C $4.0650151 .481271-1.255655$

C $3.0810882 .428741-1.927657$

C $0.6551692 .984960-2.028247$

O $3.421226 \quad 3.257439-2.769235$

C $0.273527 \quad 4.088831-1.092655$

C $-0.989062 \quad 4.316137-0.697727$

C $-1.402318 \quad 5.4613340 .182415$

C $-2.221300 \quad 5.0224661 .376117$

C $-3.5645595 .051874 \quad 1.342531$

C -1.4801144 .6320112 .635180$

C -0.9925443 .2115722 .585490$

N $1.7788902 .242111-1.483694$

H $1.146085-4.2783740 .746424$

H $2.726468-2.239551-0.910580$

H $2.778137-3.1259352 .080489$

H $5.055982-1.767757 \quad 0.557496$

H $4.896377-3.5066840 .463883$

H $6.480807-2.0681244 .032893$

H $6.017234-4.3023163 .856251$

H $4.488829-4.6530533 .044320$

H $6.026989-4.8414322 .173746$

H $5.128865-0.0582472 .138154$

H 5.6733640 .1674103 .779162

H 7.2919651 .2911952 .556513

H $8.094993-0.2407892 .918275$

H $7.347890-1.0554420 .602543$

H $9.660039-0.1359800 .958631$ 
H $9.053515 \quad 1.4922160 .733953$

H $11.0370820 .656843-0.922732$

H $8.0712042 .152894-1.613774$

H $10.192554-1.530308-1.055059$

H $9.519219-1.145037-2.622463$

H $8.462019-1.745961-1.329967$

H $8.8926501 .177672-3.492669$

H $5.7942002 .658905-1.697241$

H $5.4038371 .470227-2.945372$

H $5.297309-0.789366-1.755279$

H $2.595931 \quad 1.2327532 .113527$

H $-0.403574 \quad 0.7014352 .558114$

H 1.2459814 .7167352 .438712

H $2.051292 \quad 3.469254 \quad 1.511308$

H 2.1466783 .4686523 .287825

H $3.6902700 .459793-1.377047$

H $4.0855511 .717890-0.186007$

H $-0.1605662 .273163-2.183490$

H $0.930297 \quad 3.408342-2.999658$

H $1.0646144 .754815-0.756374$

H -1.782611 $3.670101-1.066225$

H -1.990532 $6.155733-0.432106$

H -0.5335536 .0369790 .525212$

H $-4.157860 \quad 4.7632112 .203862$

H -4.1002015 .3562190 .449399$

H -2.146030 4.7182443 .504560

H $-0.682185 \quad 5.3543302 .828323$

H -1.8000052 .4791642 .626074$

H $1.5930061 .562941-0.751279$

C $-0.668558-3.299958-1.364530$

O $-1.519893-3.937832-0.392508$

C $-2.884035-3.898755-0.874748$

C $-2.851538-3.092738-2.167043$

C $-1.448418-3.367886-2.672638$

H -3.151905 -4.940881-1.089894

C $-3.781103-3.3374340 .238160$

H $-3.629981-3.400459-2.871747$

H -2.979843 -2.022424 -1.966349

C $-0.985077-2.385178-3.733362$

H $-1.411893-4.387156-3.079821$

C $-5.205566-2.955726-0.216448$

O $-3.831124-4.3182821 .278239$

H -3.301521 -2.4574560 .682337$

H $-5.109192-2.149769-0.949472$

O $-5.789994-4.071222-0.887811$

C $-6.117292-2.4933750 .941738$

C $-7.462272-1.8889690 .487575$

H -5.575542 -1.760528 1.551274

O $-6.407556-3.610597 \quad 1.785473$

H -8.004071 -2.605766 -0.140541

H -8.081567 -1.7531981 .384527$

H - $-1.641326-2.427912-4.608758$

H $-0.991488-1.356131-3.359719$

H $0.032000-2.616386-4.064405$

C $0.695248-3.987716-1.404981$

H $-4.349897-5.0710450 .946315$

H -6.600819 $-3.763486-1.325616$

H -6.896912 -3.277540 2.556942

H $1.273038-3.635567-2.267041$

H $0.577203-5.072562-1.511125$

H $-0.550223-2.255284-1.049285$

C $-6.466562 \quad 1.693626 \quad 0.175055$

$\begin{array}{lllll}0 & -6.750183 & 0.386986 & 0.692221\end{array}$

C $-7.319733-0.540709-0.236780$

C -8.664398 $-0.008480-0.752889$

C $-8.506328 \quad 1.390031-1.359557$

C $-7.7265822 .332499-0.433699$

H -6.1861242 .2881831 .054397$

C $-5.2613941 .673509-0.777349$

H -6.644385 -0.667964 -1.086568

O -9.6229120 .0344910 .301772$

H $-9.051460-0.698678-1.511626$

H $-9.5019481 .805856-1.556534$

O $-7.830091 \quad 1.269847-2.607881$

H -7.449078 $3.252999-0.960316$

O -8.5754582 .7132620 .653982$

C $-4.001318 \quad 1.205341-0.078816$

H $-5.4370961 .007999-1.626778$
H -5.068858 $2.682311-1.159196$

H $-9.2562770 .585166 \quad 1.014713$

H $-7.7675792 .159945-2.994380$

H $-9.348573 \quad 3.167278 \quad 0.277520$

O $-3.0658790 .876006-1.017475$

$\begin{array}{llll}\text { O } & -3.846117 & 1.152922 & 1.133567\end{array}$

C $-1.812490 \quad 0.425957-0.496815$

H $-1.316538 \quad 1.2408850 .034382$

H $-1.954423-0.4341560 .163504$

H - $1.1835620 .124082-1.337315$

SCF Energy (B3LYP/6-31G**//MMFF) $=-3245.89841763$

$08 \_00362$

MMFF Geometry

C $3.761577-1.9016602 .864019$

C $4.601493-2.8365172 .392191$

C $5.227682-2.8557911 .015209$

$\begin{array}{lllll}\text { O } & 4.824519 & -1.703206 & 0.241408\end{array}$

C $4.763072-4.1190340 .262801$

C $5.199491-4.147189-1.189168$

C $4.440658-3.708488-2.214602$

C $6.574512-4.705802-1.436333$

C $3.074958-3.084586-2.119057$

C $2.049408-3.784233-3.018408$

C $0.607305-3.484202-2.579094$

C $-0.399728-3.976501-3.619570$

C $-1.844843-3.625644-3.229125$

O $0.460962-2.073966-2.388967$

O $-2.671579-3.803635-4.389447$

C $-1.919005-2.130448-2.847541$

C $-2.390042-4.579923-2.159958$

O $-3.203007-1.802571-2.321750$

C $-0.815145-1.690346-1.858305$

C $-0.819821-0.152644-1.697453$

O $-1.068798-2.290492-0.584687$

C $5.615215-0.598468 \quad 0.280215$

$\begin{array}{llll}\text { O } & 6.614757 & -0.430421 & 0.959421\end{array}$

C $5.0437120 .381831-0.671344$

C $5.566747 \quad 1.614183-0.748968$

C $5.1413912 .681260-1.658328$

C $4.0630572 .358779-2.659247$

C $0.3894680 .400498-0.926325$

C $0.251598 \quad 1.887366-0.649994$

C $1.3790923 .751647 \quad 0.559048$

O $-0.6962432 .559233-1.047709$

C $1.9538214 .620295-0.515499$

C $2.9791895 .464060-0.322171$

C $3.5040346 .387837-1.384140$

C $4.9990246 .278158-1.580396$

C $5.8296347 .226663-1.115109$

C $5.5220445 .117631-2.396714$

C $5.7460693 .883815-1.561486$

N 1.3124302 .3763220 .100850

H $3.500446-1.0458382 .247690$

H $4.850177-3.6816533 .031342$

H $6.320114-2.8773581 .115082$

H $3.672600-4.2145640 .326495$

H $5.146454-5.0143710 .771047$

H $4.849535-3.763533-3.222211$

H $6.841429-4.698537-2.498279$

H $7.326679-4.116584-0.902073$

H $6.631246-5.742034-1.087632$

H $2.706141-3.069366-1.090276$

H $3.160370-2.034731-2.426232$

H $2.206903-3.438456-4.048359$

H $2.202347-4.870719-3.016293$

H $\quad 0.444135-3.990628-1.619991$

H $-0.295393-5.056218-3.783080$

H $-0.181351-3.504276-4.587845$

H $-2.579203-4.725646-4.684937$

H $-1.804613-1.549109-3.773273$

H -2.368497 -5.614381-2.523527

H $-3.440550-4.364770-1.937726$

H $-1.824435-4.547829-1.226521$

H $-3.865390-2.077841-2.978580$

H $-0.8211520 .319220-2.688890$

H $-1.7409900 .156872-1.190205$

H $-0.729790-1.7167080 .119151$ 
H $4.2148300 .058140-1.290402$ H $6.3932921 .868573-0.086472$ H $4.4021951 .576498-3.347259$ H $3.7620753 .212678-3.269050$ H $3.1568472 .009309-2.154900$ H $0.504159-0.1054620 .037862$ H $1.3067340 .244533-1.505371$ H $1.9839353 .763627 \quad 1.471162$ H 0.3677014 .0923450 .804523 H $1.4772624 .583006-1.492420$ H 3.4372535 .5285650 .662041 H $3.2395037 .411863-1.089558$ H $3.0020696 .218287-2.345052$ H $6.9005857 .168939-1.279295$ H $5.4583568 .078280-0.554832$ H $6.4894565 .385364-2.843206$ H $4.8619884 .948516-3.251461$ H $6.5219024 .010856-0.804743$ H 2.0918121 .7628520 .321020 C $1.579864-1.9385294 .064378$ O $1.157513-0.626693 \quad 3.635972$ C $-0.049985-0.2695154 .344365$ C $-0.501751-1.5335395 .060047$ C $0.824668-2.2016955 .364081$ H $\quad 0.2471210 .4953515 .073059$ $\begin{array}{llll}\text { C }-1.052811 & 0.312091 & 3.341137\end{array}$ H $-1.090846-1.3217905 .957367$ H -1.104413 -2.164955 4.395245 C $0.693073-3.6723185 .720035$ H $1.307357-1.6694016 .194705$ C -2.4672040 .5173853 .908472$ O -0.5491381 .5777592 .900822$ H - $1.096486-0.3332012 .455821$ H -2.908933 -0.4546194 .156503$ O $-2.3801591 .235725 \quad 5.145595$ C -3.4379891 .2885082 .991066$ C $-3.4924190 .738116 \quad 1.562277$ H -3.173635 2.3516582 .978023 $\begin{array}{llll}\text { O } & -4.747121 & 1.199360 & 3.562018\end{array}$ H -2.524714 0.8732631 .065349 H -3.648700 $-0.349241 \quad 1.600635$ H $1.673485-4.1163505 .918379$ H $0.078778-3.7967986 .617784$ H $0.223459-4.2408354 .910273$ C $3.102266-1.963518 \quad 4.214389$ H $0.336351 \quad 1.4238782 .528832$ H -1.9386212 .0828244 .962263$ H -4.691536 1.5440714 .469889 H $3.415583-2.8667294 .749536$ H $3.438181-1.0964654 .795502$ H $1.259030-2.6462983 .287814$ C $-5.4206091 .037480-1.601311$ O $-4.4296750 .719946-0.617519$ C $-4.606103 \quad 1.323657 \quad 0.671632$ C $-4.592252 \quad 2.855547 \quad 0.549665$ C $-5.6261123 .335750-0.475784$ C -5.533326 $2.558514-1.793069$ H $-5.0333070 .609941-2.535320$ C $-6.7589610 .356930-1.297870$ H $-5.5682440 .999101 \quad 1.082278$ $\begin{array}{llll}\mathrm{O} & -3.310116 & 3.319295 & 0.135997\end{array}$ H -4.8126663 .3085251 .522920$ H $-5.4688294 .401996-0.680748$ $\begin{array}{lllllll} & \text { O } & -6.938052 & 3.183038 & 0.058048\end{array}$ H -6.400109 $2.783756-2.424694$ O $-4.3802413 .005341-2.513338$ C $-6.613058-1.144714-1.364814$ H $-7.1698820 .623008-0.319737$ H $-7.5169240 .642829-2.036833$ H -2.678680 3.1444430 .853167 H -6.9874393 .7127270 .871902$ H -3.593284 $2.762903-1.995397$ O $-6.373831-1.644698-0.118945$ O $-6.674771-1.789894-2.403489$ C $-6.181304-3.060270-0.049314$ H $-7.075763-3.582942-0.401181$ H -5.306574 -3.351555 -0.637312 H $-6.006021-3.3285770 .995957$
SCF Energy (B3LYP/6-31G**//MMFF) = -3245.92141014

08_00363

MMFF Geometry

C -2.064338 $2.333194-0.840494$

C $-3.1156403 .008996-0.351048$

C -3.8137632 .7406960 .963368$

O -3.1625791 .6450851 .643415$

C $-5.279646 \quad 2.348487 \quad 0.715849$

C $-6.1386782 .433815 \quad 1.960842$

C $-6.534824 \quad 1.3776322 .697384$

C -6.5950113 .8222802 .329460$

C $-6.176688-0.0708112 .511469$

C $-7.371328-0.9159802 .047398$

C -7.741007 -0.6864990 .573586$

C -8.992659-1.482499 0.198960

C $-9.320476-1.360977-1.297133$

O $-6.633916-1.120448-0.221991$

O $-10.292463-2.367429-1.619343$

C $-8.050993-1.680921-2.113770$

C $-9.965196-0.011330-1.634020$

O $-8.264162-1.441382-3.503251$

C $-6.794832-0.918195-1.631287$

C $-5.536561-1.481345-2.335286$

O $-6.9349590 .466004-1.955277$

C $-2.860591 \quad 1.7689692 .958306$

O $-\begin{array}{llll}\text { O } & -1.107877 & 2.724301 & 3.680576\end{array}$

C $-2.1362160 .574564 \quad 3.456864$

C $-1.937129-0.5342702 .726319$

C -1.209535 -1.734213 3.148385

C $-0.556853-1.7168994 .505604$

C $-4.209390-0.959397-1.765852$

C $-3.009827-1.621859-2.422645$

C $-0.538070-1.840348-2.161489$

O $-3.097703-2.344648-3.411626$

C $-0.283262-3.074284-1.354589$

C $0.717533-3.194344-0.469367$

C $0.963463-4.4300900 .347673$

C $0.907281-4.1658371 .836866$

C $2.034455-4.0717382 .562416$

C $-0.451668-4.1005762 .499950$

C -1.138155-2.777885 2.295224

N $-1.825638-1.292569-1.777812$

H $-1.650817 \quad 1.497493-0.283523$

H $-3.5079543 .845005-0.927064$

H $-3.7450583 .660761 \quad 1.555881$

H $-5.318506 \quad 1.3380300 .295480$

H $-5.7276793 .000749-0.045928$

H -7.1764031 .5665103 .557576$

H -7.2488843 .8264293 .208036$

H -5.7371604 .4624982 .556367$

H -7.1546304 .2701461 .501652$

H $-5.325270-0.205851 \quad 1.838067$

H -5.842434 -0.4493733 .485877$

H -7.100910 -1.971061 2.185471

H -8.239228 -0.7173782 .688612$

H -7.9112320 .3828680 .408120$

H $-9.850420-1.1682610 .806700$

H -8.833386 -2.544686 0.432693

H -11.072737-2.221142 -1.057450

H $-7.860652-2.758644-2.011186$

H -10.896695 $0.119142-1.070072$

H -10.246699 $0.038863-2.691647$

H $-9.3211520 .840762-1.407320$

H $-9.051681-1.947695-3.766712$

H $-5.530837-2.575059-2.236291$

H $-5.575652-1.251795-3.407697$

H $-6.0552560 .863804-2.045801$

H -1.7698190 .6612624 .474867$

H $-2.338980-0.5783501 .715458$

H $-1.312718-1.6313975 .293943$

H $\quad 0.136317-0.8721924 .589167$

H $0.027020-2.6146834 .719518$

H $-4.1193340 .119828-1.924952$

H $-4.156977-1.154939-0.688508$

H $0.212215-1.064609-1.982828$

H $-0.540801-2.085232-3.228341$

H $-0.960694-3.911345-1.507905$ 
H $1.402279-2.363190-0.322202$ H $1.950909-4.8212340 .069967$ H $\quad 0.251355-5.2255890 .095261$ H $2.004900-3.9133783 .635188$ H $3.012612-4.1462102 .098857$ H $-0.372826-4.3424473 .565606$ H -1.091953 -4.893023 2.091015 H -1.643926 -2.693894 1.333329 H -1.867575 $-0.755276-0.917116$ C $0.0968472 .986708-2.000526$ O $0.8169251 .851222-1.480279$

C $1.7631822 .319418-0.493367$ C $1.8575003 .825143-0.693560$ C 0.433768 4.165349-1.087131 H $\quad 1.3137672 .0883810 .480991$ C $3.0846511 .561369-0.665056$ H 2.1896114 .3507360 .206600 H $2.5497024 .068017-1.509474$ C $0.2887405 .521954-1.753333$ H $-0.1831084 .148264-0.180169$ C 4.2230792 .0751710 .232918 O $2.8459860 .184362-0.357866$ H $3.3872481 .600691-1.717305$ H $4.4917133 .097336-0.059566$ O $3.7293902 .155758 \quad 1.577376$ C $5.488781 \quad 1.1944780 .254084$ C $6.0925620 .946191-1.139920$ H $5.269640 \quad 0.2444320 .755563$ O $\quad 6.4436541 .8824601 .070581$ H $5.3248270 .559433-1.817042$ H $6.4499031 .905791-1.534308$ H $0.6066826 .319786-1.074228$ H $0.8978965 .591243-2.660853$ H $-0.7533275 .709057-2.030854$ C -1.390912 $2.651696-2.146352$ H $2.126253-0.119101-0.936716$ H 4.4553422 .4895952 .132252 H 7.2266251 .3122861 .152083 H -1.493244 $1.766807-2.786764$ H -1.901714 $3.477919-2.654595$ H $0.5043413 .164372-3.004512$ C $9.502694-0.327172-0.238455$ O $8.3493960 .510099-0.397319$ C $7.260476-0.063019-1.125018$ C $7.710689-0.428424-2.548881$ C $8.948262-1.331793-2.532111$ C $10.039538-0.787857-1.603775$ H $10.2589590 .326476 \quad 0.215388$ C $9.242052-1.4833940 .733656$ H $6.915278-0.966285-0.610170$ O $7.9970810 .752156-3.297000$ H $6.886595-0.938388-3.061190$ H $9.349962-1.417758-3.549184$ O $8.583307-2.638138-2.097157$ H $10.825752-1.536628-1.452564$ O $10.653240 \quad 0.346051-2.224105$ C $8.984489-0.9594422 .126437$ H $8.400917-2.1166270 .437904$ H $10.115195-2.1439640 .795291$ H $8.6755461 .253560-2.813027$ H $7.915997-2.978705-2.717055$ H $11.0432870 .045391-3.062689$ O $7.643022-0.9072422 .364349$ O $9.869618-0.6145072 .898598$ C $7.263021-0.3991903 .646908$ H $7.6368160 .620936 \quad 3.777723$ H $6.170908-0.3801183 .694119$ H $7.638825-1.0539594 .438670$

SCF Energy (B3LYP/6-31G**//MMFF) $=-3245.92157089$

0800364

MM̄FF Geometry

C $-2.855007-1.3164873 .557198$

C -3.341197-0.070770 3.667804

C $-4.272150 \quad 0.6260972 .703794$

O $-4.600100-0.2460641 .598036$

C -3.623569 1.8996012 .127684

C -4.6570752 .9412801 .746462$
C $-5.1194113 .163100 \quad 0.501214$

C -5.1573213 .7863172 .891950$

C $-4.7643742 .447647-0.772830$

C $-4.2025013 .412008-1.828105$

C $-2.7636333 .856932-1.526447$

C $-2.3438774 .995326-2.457537$

C $-0.8746435 .391030-2.252056$

O $-1.9135292 .723975-1.730139$

O $-0.5199236 .239359-3.354274$

C $-0.0047404 .118643-2.321638$

C $-0.6843356 .223960-0.978984$

O $1.3512084 .402225-1.982054$

C $-0.5303252 .958806-1.441399$

C $0.2503331 .665998-1.778086$

O $-0.3320513 .288557-0.065798$

C $-5.901077-0.3736801 .237607$

O

C $-6.060710-1.2754860 .072325$

C $-5.040168-1.759568-0.653809$

C $-5.154524-2.638781-1.822693$

C $-6.533112-3.110319-2.208048$

C $-0.3303570 .394502-1.146465$

C $0.482903-0.836230-1.508778$

C $0.450090-3.315296-1.243834$

O $1.507213-0.793779-2.184412$

C $-0.272590-3.890045-2.419599$

C $-1.154375-4.896958-2.330512$

C $-1.892274-5.460382-3.509307$

C $-3.385200-5.232746-3.423805$

C $-4.227231-6.250512-3.178046$

C $-3.908101-3.843737-3.718255$

C $-4.030971-2.989203-2.484413$

N $-0.078717-1.990494-0.982869$

H -3.114605 -1.926816 2.696876

H -3.0718040 .5085234 .549592$

H $-5.173926 \quad 0.8715313 .278034$

H $-2.992766 \quad 1.6467611 .267304$

H -2.9472062 .3580692 .861729$

H -5.8651093 .9474180 .371291$

H -5.8886584 .5351592 .570040$

H -5.6391393 .1622613 .650974$

H $-4.3240614 .321163 \quad 3.359542$

H $-4.0730421 .616066-0.611411$

H $-5.6880642 .005673-1.166917$

H $-4.2221512 .895717-2.796724$

H $-4.8592514 .286777-1.913580$

H -2.695212 $4.179880-0.481973$

H $-2.9964685 .867487-2.325975$

H $-2.4756784 .678152-3.501908$

H $0.3918096 .547441-3.217839$

H $-0.0005283 .783996-3.368545$

H -1.271873 $7.148040-1.037347$

H $0.3588346 .538792-0.863930$

H $-0.9843715 .695951-0.071433$

H $1.6857485 .053291-2.621266$

H $0.2486921 .519224-2.866433$

H $1.2977301 .773770-1.470265$

H -0.2722982 .4710920 .452558$

H -7.092742 -1.504579 -0.174565

H $-4.022492-1.483219-0.383190$

H $-6.535866-3.828151-3.030821$

H -7.155455 -2.263900 -2.518507

H -7.018027 -3.613818 -1.363914

H $-0.352210 \quad 0.473227-0.055621$

H $-1.3564360 .235040-1.498043$

H $\quad 0.303237-3.911042-0.338944$

H $1.522633-3.258056-1.453283$

H $-0.061888-3.448607-3.390995$

H -1.363986 -5.339626 -1.359439

H -1.669728 -6.534079 -3.560242

H $-1.519417-5.039131-4.451428$

H -5.301254 -6.100055 -3.148974

H -3.861746 -7.255880 -2.997317

H $-4.857106-3.908988-4.261021$

H $-3.233318-3.338874-4.421624$

H -3.079125 -2.598364 -2.125086

H $-0.963921-1.926753-0.488086$

C $-0.486113-1.9839754 .234376$ 
O $0.037153-0.6408064 .230701$

C $0.799947-0.4489633 .023059$

C $1.144790-1.8520782 .542787$

C $-0.125490-2.6050462 .884069$

H $\quad 0.1216990 .0471372 .317882$

C 2.0186700 .4384823 .304823

H $1.392774-1.8911741 .479522$

H $1.993110-2.2564193 .108517$

C $0.040341-4.1134922 .940955$

H $-0.874981-2.3631852 .121702$

C 2.8157740 .7523222 .022558

O $1.529698 \quad 1.6498523 .887027$

H $2.659265-0.0447164 .051985$

H $3.020212-0.1856591 .497515$

O $1.997507 \quad 1.5397351 .157257$

C 4.1636361 .4655192 .245280

C 4.8332011 .9652550 .948886

H $4.836944 \quad 0.7807572 .774289$

O 3.9782982 .6009993 .091839

H 4.2570122 .8051540 .539057

H 5.8060852 .3879791 .233603

H $0.351863-4.5063571 .968952$

H $0.794911-4.4073293 .678199$

H $-0.903362-4.596883 \quad 3.213219$

C -1.974269-1.961632 4.593692

H 2.3004142 .1915114 .126988

H 1.8194452 .3811421 .611864

H 4.8540242 .9884383 .260823

H -2.096579 -1.437174 5.550211

H -2.330106 -2.986718 4.751459

H $0.047745-2.5048945 .039723$

C $6.112145-1.254828-0.454505$

O $5.854023-0.1398240 .410822$

C $5.0306250 .893971-0.134976$

C $5.6645401 .479117-1.406789$

C $5.9702140 .378739-2.427642$

C $6.724819-0.790932-1.788466$

H $6.875359-1.8447710 .069971$

C $4.869852-2.143800-0.630458$

H $4.0529310 .480070-0.397616$

O $6.8646202 .182401-1.092205$

H $4.9722282 .208442-1.843438$

H $6.5731380 .795531-3.243569$

O $4.755234-0.110903-2.987352$

H $\quad 6.776718-1.639300-2.480890$

O $8.071254-0.387053-1.522198$

C $4.563136-2.935000 \quad 0.624473$

H $3.970969-1.576688-0.881170$

H $5.064082-2.866284-1.432357$

H $7.4583331 .565894-0.630280$

H $4.2958030 .640998-3.398605$

H $8.476839-0.142185-2.371490$

O $3.814169-4.0270440 .289962$

O $4.919279-2.6286711 .753980$

C $3.475701-4.9042081 .368618$

H $4.361071-5.4687771 .674497$

H $3.065979-4.3471202 .215427$

H $2.715883-5.6043241 .011403$

SCF Energy $\left(B 3 L Y P / 6-31 G^{* *} / / M M F F\right)=-3245.91581897$

08_00365

MM̄FF Geometry

C $0.874566-0.3017520 .747258$

C $-0.389597-0.4794480 .336978$

C $-0.787928-0.565431-1.112737$

O $-1.8631720 .371278-1.344839$

C -1.347738 -1.965056 -1.412792

C $-1.341450-2.307488-2.887750$

C $-2.392789-2.168917-3.718672$

C $-0.048114-2.891938-3.395941$

C $-3.737489-1.562895-3.427586$

C $-4.861635-2.602901-3.330778$

C $-4.933660-3.283207-1.954865$

C $-6.024114-4.356177-1.939367$

C $-6.201462-4.971594-0.543263$

O $-5.233570-2.269180-0.988354$

O $-7.427367-5.718851-0.542637$

C $-6.365248-3.833471 \quad 0.485517$
C $-5.085093-5.968854-0.211297$

O $-6.392093-4.348072 \quad 1.815264$

C $-5.288410-2.7297320 .366456$

C $-5.670007-1.527061 \quad 1.263957$

O $-4.032553-3.2561030 .799482$

C $-1.5591391 .681611-1.522318$

O $-0.4522442 .192448-1.538947$

C $-2.8483612 .395057-1.652447$

C -2.899685 $3.721587-1.469275$

C $-4.1130354 .541581-1.490366$

C $-5.4161233 .851793-1.803587$

C $-4.819564-0.2660881 .041067$

C -5.2055180 .8494802 .000545$

C -4.546732 3.1598862 .669917

O

C -5.4336224 .1539451 .986629$

C -5.1467585 .4628091 .905252$

C -6.0464126 .4801101 .260324$

C -5.3154397 .3832690 .289664$

C -4.9337668 .6200950 .651124$

C $-5.1003286 .883410-1.122085$

C $-4.0055555 .854730-1.199338$

N $-4.406879 \quad 1.974570 \quad 1.842074$

H $1.666723-0.2013300 .009418$

H -1.180656 -0.582714 1.075749

H $0.053835-0.331484-1.776312$

H -2.366062 -2.044730-1.018211

H $-0.765159-2.734029-0.887239$

H $-2.270551-2.490526-4.752526$

H $-0.088186-3.127237-4.464815$

H $0.775442-2.186920-3.244765$

H $\quad 0.185164-3.818893-2.861955$

H -3.719424 -0.931049 -2.536151

H -3.966428 $-0.885139-4.260292$

H -5.809270 -2.077928 -3.510262

H $-4.752558-3.354634-4.122100$

H -3.960659 -3.730699-1.725775

H -5.811678 -5.137550 -2.679755

H $-6.981026-3.908657-2.243154$

H -7.366364 -6.395346 -1.238618

H $-7.349274-3.3757560 .311071$

H -5.072795 -6.786417 -0.942180

H $-5.254119-6.4410620 .762762$

H -4.091595 -5.515868 -0.207979

H $-7.088308-5.0262201 .849271$

H $-6.716080-1.2526431 .072991$

H -5.598942 -1.821072 2.318952

H $-3.485681-2.5354441 .148625$

H -3.731686 $1.796730-1.847080$

H -1.974222 $4.253061-1.252034$

H $-5.3881983 .421448-2.810852$

H $-6.2834584 .513425-1.766922$

H $-5.6118793 .048436-1.084659$

H $-3.757580-0.4843761 .190234$

H $-4.955017 \quad 0.1054770 .019017$

H -3.5406803 .5552602 .842219$

H -4.9839462 .8784503 .633247$

H -6.3666523 .7877861 .564684$

H -4.229683 5.8321682 .359206

H -6.4927367 .0826472 .062413$

H $-6.8882486 .000596 \quad 0.745535$

H -4.433185 $9.283992-0.045775$

H -5.1087248 .9944221 .654366$

H -4.810835 $7.714596-1.778870$

H $-6.0536946 .530685-1.524286$

H $-3.0156226 .257286-0.979152$

H -3.7086311 .9893421 .104730$

C $2.183559-1.3837432 .635350$

O $3.410806-1.3878741 .878243$

C $3.737734-2.7508731 .548603$

C $2.856036-3.6213822 .438189$

C $1.591569-2.7873182 .501580$

H $3.436036-2.9028490 .505437$

C $5.240967-3.0034161 .700006$

H $2.694590-4.6176372 .015736$

H $3.283000-3.7417623 .440728$

C $0.656530-3.1737203 .633537$

H $1.060315-2.9001101 .548450$ 
C $6.144365-2.078276 \quad 0.856067$

O $5.611088-2.8329563 .074369$

H $5.457246-4.0513641 .461461$

H $6.137256-1.0707491 .285626$

O $7.490597-2.552370 \quad 0.993774$

C $5.785760-2.007175-0.639292$

C $6.842645-1.327415-1.530392$

H $4.826181-1.490568-0.755664$

O $5.607495-3.339908-1.130159$

H $7.744719-1.951104-1.577513$

H $6.438091-1.323019-2.551567$

H $0.323441-4.2105213 .519952$

H $1.146751-3.0833004 .608602$

H $-0.230940-2.5328523 .640837$

C $1.280239-0.2296442 .192262$

H $5.372129-1.9258903 .331808$

H $7.535649-3.4319540 .580922$

H $5.309590-3.275236-2.053539$

H $1.815590 \quad 0.7171552 .331127$

H $0.396824-0.1901872 .840593$

H $2.475435-1.2029303 .678499$

C $6.1864192 .258100-0.697134$

O $6.0273660 .892724-1.107655$

C $7.220513 \quad 0.104388-1.118355$

C $8.2634310 .712387-2.072268$

C $8.5333402 .182378-1.737950$

C $7.2321742 .974603-1.569085$

H $5.2133762 .726447-0.894071$

C $6.4727712 .377397 \quad 0.808032$

H $7.6414610 .070754-0.108928$

O $7.7973220 .612585-3.417088$

H $9.1902930 .130727-1.999132$

H $9.1311192 .630367-2.541166$

O $9.3013222 .241218-0.539761$

H $7.4443683 .962866-1.145899$

O $6.6606683 .203776-2.860615$

C $5.3102991 .903136 \quad 1.653443$

H 7.3476341 .7916261 .101151

H 6.6507543 .4282931 .063718

H $8.4951970 .963870-3.996160$

H $9.4852773 .177995-0.354905$

H $6.3953922 .342037-3.225554$

O 5.6752971 .9514802 .969095

O $4.2242921 .542707 \quad 1.222825$

C 4.6795871 .5286243 .905701

H 3.7390062 .0632153 .741340

H $4.531105 \quad 0.4485353 .825404$

H 5.0401761 .7590394 .911668

SCF Energy (B3LYP/6-31G*//MMFF) $=-3245.91066768$

0800366

MM̄FF Geometry

C $-2.240881-4.361387-0.026238$

C $-3.295130-3.592806-0.333238$

C $-3.616321-3.123552-1.728077$

O $-3.392343-1.692729-1.755112$

C $-5.095425-3.405382-2.043997$

C $-5.553491-2.851865-3.379726$

C $-6.373285-1.791346-3.530620$

C $-5.040930-3.587640-4.590411$

C $-6.978248-0.940740-2.447936$

C $-6.3194130 .443493-2.396128$

C $-6.5197591 .123812-1.033658$

C $-5.9783052 .552865-1.064417$

C $-6.026135 \quad 3.2217990 .316886$

O $-5.8061410 .350886-0.061493$

O $-5.212007 \quad 4.4040090 .258623$

C $-5.382040 \quad 2.280340 \quad 1.358766$

C $-7.4430693 .688845 \quad 0.671985$

O

C $-5.9118020 .831514 \quad 1.286277$

C $-5.128315-0.1600682 .184761$

O -7.2739630 .8110491 .730466$

C -2.108745 -1.271236 -1.941314

O $-1.130264-1.967054-2.162695$

C $-2.0884840 .200389-1.787310$

C $-0.917421 \quad 0.855330-1.768381$

C $-0.7408252 .299926-1.596203$
C $-1.9736173 .124325-1.332516$

C $-3.637705-0.3128651 .862897$

C $-2.724140 \quad 0.6011732 .660253$

$\begin{array}{llll}\text { C } & -0.348984 & 1.349920 & 2.776687\end{array}$

O -3.1012211 .2455163 .635123$

C -0.2589042 .6869902 .110396$

C $0.8849823 .202523 \quad 1.633971$

C 0.9880014 .5685701 .016143

C $1.7117114 .564767-0.312426$

C $3.0077284 .911588-0.388926$

C $0.9126434 .262515-1.559935$

C $0.5043862 .816750-1.663825$

N -1.4223150 .5807202 .175211$

H $-1.584410-4.717212-0.816040$

H $-3.920775-3.2107430 .470579$

H $-2.980453-3.613553-2.475873$

H $-5.715761-3.014240-1.232057$

H $-5.271344-4.489688-2.037911$

H $-6.636972-1.483285-4.541522$

H $-5.457461-3.193611-5.523645$

H $-3.951621-3.507483-4.657300$

H $-5.310160-4.647361-4.532847$

H $-8.049419-0.822878-2.654210$

H $-6.925687-1.429667-1.473258$

H $-5.2441010 .343361-2.587563$

H $-6.7342281 .068886-3.196404$

H $-7.5885501 .119691-0.789647$

H $-6.5193353 .157157-1.803312$

H $-4.9344232 .536527-1.403195$

H $-5.5652274 .977300-0.443177$

H $-4.304576 \quad 2.284068 \quad 1.155370$

H -7.804797 4.411661 -0.069406

H -7.457251 4.2150631 .632836

H $-8.1660952 .871676 \quad 0.713377$

H $-5.006402 \quad 2.306187 \quad 3.278802$

H -5.2911490 .0740663 .243524$

H $-5.580731-1.1517942 .037866$

H -7.496294 -0.094102 2.004480

H $-3.0398290 .704794-1.669659$

H $\quad 0.002090 \quad 0.283718-1.888112$

H $-2.6300073 .122022-2.209220$

H $-1.7621874 .167298-1.089420$

H -2.525669 $2.724170-0.475313$

H $-3.334495-1.3353312 .120724$

H $-3.452913-0.1755730 .794518$

H $0.571690 \quad 0.7690882 .666923$

H $-0.553523 \quad 1.483406 \quad 3.843894$

H $-1.172646 \quad 3.2734642 .045484$

H 1.8051512 .6300201 .729132

H $1.522898 \quad 5.212508 \quad 1.726814$

H -0.0005895 .0270150 .889077$

H $3.5295874 .946762-1.339303$

H 3.5785875 .1616800 .499340

H $1.5074324 .492018-2.454505$

H $0.0631434 .949265-1.611752$

H $1.3393522 .138867-1.845298$

H -1.2123800 .0546691 .331855$

C $-0.787036-3.6795991 .862659$

O $0.427064-3.8682111 .115125$

C $1.547371-3.5430451 .957339$

C $0.963023-3.1127453 .302019$

C $-0.372301-3.8332253 .319907$

H $2.123211-4.4669642 .090576$

C $2.435251-2.4748481 .298616$

H $1.610685-3.3901984 .139566$

H $0.814574-2.0269213 .345812$

C $-1.359694-3.2482914 .314571$

H $-0.200456-4.892376 \quad 3.553345$

C $3.129338-2.9451490 .003990$

O $1.653606-1.3115881 .020539$

H $3.199436-2.1816852 .026461$

H $3.780491-3.797904 \quad 0.232023$

O $2.138645-3.425803-0.912439$

C $3.931692-1.850334-0.728123$

C $4.981741-1.1660730 .155336$

H $3.232878-1.119920-1.150868$

O $4.589309-2.485016-1.830290$

H $4.507325-0.6945521 .023686$ 
H $5.657198-1.9285120 .567411$ H $-0.964485-3.3165645 .333265$ H -1.564470 -2.193321 4.103974 H -2.311233 -3.787943 4.285571 C $-1.844680-4.6765371 .389359$ H $0.970042-1.5684320 .377651$ H $2.604455-3.731033-1.709813$ H $4.899336-1.787488-2.431894$ H -2.722344 -4.643299 2.044630 H -1.453552 -5.7003351 .416387$ H -1.124105 -2.651138 1.672884 C $7.6992041 .370140-0.010097$ O $\quad 6.743646 \quad 0.4020320 .440324$ C $5.853943-0.114796-0.559803$ C $5.0440701 .023770-1.198547$ C $5.9545912 .138542-1.721150$ C $6.9978812 .560837-0.681953$ H $8.1758331 .737406 \quad 0.908350$ C $8.7899200 .734316-0.878965$ H $6.449019-0.623848-1.324733$ O $4.1390681 .554849-0.233675$ H $4.4482660 .622626-2.026386$ H $5.3392063 .003252-1.997031$ O $6.6032501 .675986-2.902394$ H $7.7506963 .217046-1.134207$ O $\quad 6.351655 \quad 3.314471 \quad 0.347701$

C $9.632291-0.214565-0.060347$ H $8.3993450 .191109-1.744030$ H $9.4643531 .501759-1.277341$ H $3.5755162 .201814-0.689582$ H $7.1581312 .401560-3.235877$ H $5.9570834 .099716-0.068315$ O $9.114746-1.474331-0.127743$ O $10.615925 \quad 0.129863 \quad 0.581915$ C $9.807818-2.4662940 .635327$ H $9.784505-2.210127 \quad 1.698863$ H $9.296790-3.4223840 .493733$ H $10.839180-2.5636390 .283062$

SCF Energy (B3LYP/6-31G**//MMFF $)=-3245.90314710$

0800367

MM̄FF Geometry

C $-0.498616-3.3559990 .875629$

C $-0.032529-2.2177921 .408972$

C $-0.870650-0.9877191 .615328$

$\begin{array}{lllll}\text { O } & -0.269357 & 0.105527 & 0.882478\end{array}$

C $-0.915498-0.5594743 .092656$

C -1.613012 -1.539752 4.011910

C -2.949495-1.705741 4.065075

C $-0.708012-2.3424484 .908283$

C $-3.978241-0.9785963 .244799$

C $-4.588117-1.9029492 .184023$

C $-5.513134-1.1476971 .220210$

C $-6.094135-2.106548 \quad 0.178790$

C $-6.964568-1.371851-0.850484$

O $-4.748578-0.1315050 .561419$

O $-7.186877-2.259328-1.957567$

C $-6.182545-0.158142-1.399808$

C $-8.348856-1.040945-0.279364$

O $\quad-7.015680 \quad 0.651340-2.226222$

C $-5.5214260 .711719-0.303140$

C $-4.5704301 .748838-0.940633$

O

C $-0.5839250 .230504-0.432868$

O $-1.309280-0.509746-1.083696$

C $0.0773621 .406278-1.043382$

C $0.7988482 .298011-0.346988$

C $1.4943913 .464515-0.893602$

C $1.3636203 .728467-2.370921$

C -3.7805712 .5698990 .090443$

C $-3.2638853 .880791-0.483101$

C $-2.363103 \quad 6.071733 \quad 0.286282$

O $-3.3990334 .203353-1.660456$

C -1.041576 $6.251580-0.390062$

C $-0.036313 \quad 6.9641940 .141530$

C $1.2502687 .272874-0.569829$

C 2.4641556 .7228360 .142252

C 3.0442107 .3987731 .148321
C $3.0560135 .434759-0.378869$

C $2.2110884 .237093-0.050460$

N -2.6575194 .6654010 .490539$

H - $1.541738-3.4170030 .576784$

H $1.008745-2.1583401 .708035$

H $-1.890856-1.1331171 .246589$

H -1.398442 0.4240453 .169718

H $\quad 0.106067-0.3706723 .452738$

H $-3.355448-2.4381974 .760917$

H -1.264771 -3.032386 5.551128

H $-0.007963-2.9378014 .314164$

H $-0.132067-1.6761545 .558781$

H $-4.767488-0.6173423 .915124$

H -3.550255 -0.0871592.776821

H -3.782018 -2.376722 1.610305

H -5.146266 -2.709021 2.676979

H $-6.311504-0.6776941 .806364$

H -6.665181 -2.908769 0.662501

H -5.272908 -2.604249 -0.356318

H -7.603042 -3.068741-1.614625

H $-5.386490-0.549145-2.049157$

H -8.883604 -1.961252 -0.014696

H -8.974629 -0.535686 -1.023165

H $-8.304512-0.4201660 .618049$

H $-7.3874270 .074790-2.915675$

H $-3.8468051 .245687-1.594462$

H $-5.1610772 .427545-1.568773$

H $-7.3560061 .368023-0.014965$

H $-0.0648121 .497897-2.115321$

H 0.9094972 .1628110 .727501

H $1.8486764 .651432-2.694438$

H $\quad 0.3086503 .824012-2.652213$

H $1.8075522 .912044-2.950250$

H -4.418828 2.8203650 .946006

H $-2.930573 \quad 1.987950 \quad 0.462922$

H -2.3922686 .5541701 .268734$

H $-3.1523116 .511474-0.333137$

H $-0.9317765 .835812-1.388277$

H $-0.164414 \quad 7.4043841 .128225$

H $1.3371438 .364979-0.647427$

H $1.2286456 .913488-1.605853$

H 3.9342777 .0245361 .643028

H 2.6378038 .3410861 .501223

H 4.0454375 .2642430 .066603

H $3.2513845 .535210-1.450586$

H 2.2332743 .9704931 .007026

H -2.596957 4.3086321 .439695

C $0.743041-4.822390-0.788475$

O $1.591295-3.754457-1.252801$

C $1.278972-3.489628-2.635437$

C $0.359467-4.620024-3.089982$

C $-0.389494-4.932030-1.807832$

H $\quad 0.711672-2.551688-2.653138$

C $2.565694-3.339037-3.454111$

H $-0.298318-4.314075-3.909388$

H $0.925140-5.497905-3.422811$

C -1.085125 -6.281122 -1.816160

H -1.137014 -4.144648 -1.652226

C $3.537729-2.276004-2.902094$

O $3.253347-4.597397-3.449873$

H $2.316575-3.129155-4.500793$

H $3.927897-2.623120-1.939532$

O $4.666450-2.218645-3.784084$

C $2.946488-0.861164-2.755484$

C $3.9666470 .196470-2.293071$

H $2.105637-0.870723-2.054750$

O $2.439935-0.418445-4.017576$

H $4.8033460 .249565-3.000394$

H $3.4685501 .172596-2.352925$

H -1.832057 -6.323676 -2.615589

H $-0.374621-7.098865-1.976547$

H - $-1.597152-6.460311-0.865439$

C $0.344438-4.5819150 .668998$

H $4.070315-4.482651-3.964992$

H $4.355468-1.863030-4.634363$

H $1.651053-0.947533-4.222824$

H $1.257918-4.4998501 .271945$

H $-0.206904-5.4484251 .051903$ 
H $\quad 1.359108-5.731268-0.821721$

C $3.655383-0.2853311 .386062$

$\begin{array}{lllll}\text { O } & 3.362972 & 0.009984 & 0.014753\end{array}$

C $4.489699-0.026825-0.866174$

C $5.5161461 .038096-0.451132$

C 5.9313110 .8615401 .013562

C $4.7224490 .676713 \quad 1.940649$

H $2.711715-0.085327 \quad 1.908877$

C $3.982413-1.777207 \quad 1.553044$

H $4.960410-1.013896-0.812069$

O $4.9592562 .336724-0.637238$

H $6.3931060 .957388-1.104721$

H 6.5027501 .7421391 .332012

O $6.796439-0.2665781 .103587$

H 5.0551840 .3406042 .929020

O 4.1190101 .9625292 .119578

C $3.651734-2.2994642 .934120$

H $3.385854-2.3507970 .832351$

H $5.034676-1.990551 \quad 1.349478$

H $5.6375442 .987148-0.388148$

H $7.079162-0.3460392 .030580$

H $3.389878 \quad 1.8600272 .754581$

O $3.739474-3.6627982 .922937$

O $3.359179-1.6092623 .900560$

C $3.434545-4.3089574 .162568$

H $2.405499-4.0882414 .461574$

H $3.533095-5.3879804 .016621$

H $4.139285-3.9932974 .937794$

SCF Energy (B3LYP/6-31G**/MMFF) $=-3245.90767243$

0800368

MMFF Geometry

C $-4.3216801 .515711-0.194432$

C $-4.5547322 .498944-1.077856$

C $-3.547572 \quad 3.075886-2.046051$

O $-2.3417342 .280212-2.084279$

C $-3.1563544 .507442-1.638098$

C $-2.2590085 .190636-2.655780$

C $-0.9662195 .517295-2.455349$

C $-2.9152875 .528540-3.971338$

C $-0.1367255 .320985-1.217442$

C $0.816985 \quad 4.133125-1.362147$

C $1.599120 \quad 3.833344-0.075230$

C $2.649747 \quad 4.9044690 .231460$

C 3.4953044 .5492421 .466207

$\begin{array}{lllll}\text { O } & 2.239516 & 2.559469 & -0.251986\end{array}$

O $4.644408 \quad 5.4112341 .474007$

C 4.0154713 .1031051 .316678

C 2.7390784 .8291222 .770589

$\begin{array}{lllll}\text { O } & 4.691030 & 2.678260 & 2.497854\end{array}$

C 2.9181692 .0911040 .922323

C $3.481173 \quad 0.681596 \quad 0.639035$

O 2.0044721 .9473632 .017241

C -2.320659 $1.209202-2.924458$

O $-3.2223710 .819560-3.648157$

C $-0.9804400 .594095-2.814867$

C $-0.613553-0.370500-3.670688$

C $0.700615-1.011571-3.719244$

C $1.722657-0.595548-2.693477$

C $4.5076380 .584082-0.491345$

C $4.932742-0.856538-0.730163$

C $6.428862-2.238061-2.159729$

O $4.499785-1.801349-0.075111$

C $5.611193-2.814637-3.271932$

C $4.727956-3.811088-3.103974$

C $3.921276-4.418268-4.216407$

C $2.486695-3.931868-4.212562$

C $1.526175-4.658393-3.615992$

C $2.255099-2.624832-4.944043$

C $0.945268-1.934043-4.673221$

N $5.862231-0.964841-1.755550$

H -3.320828 $1.105092-0.091452$

H -5.563492 2.898744 -1.159973

H -4.007907 $3.086773-3.041622$

H -2.674712 $4.473185-0.655756$

H -4.057525 5.121483 -1.508988

H -0.428163 $5.997422-3.272306$

H -2.270315 6.138778-4.613009
H -3.162698 $4.619079-4.526769$

H $-3.835605 \quad 6.097073-3.801604$

H $0.4320746 .243285-1.051655$

H $-0.7603085 .193301-0.328295$

H $0.2456013 .239091-1.636408$

H $1.5149504 .298054-2.193560$

H $0.878473 \quad 3.751728 \quad 0.747156$

H 2.1877255 .8890190 .367150

H $3.3235815 .006433-0.630847$

H 4.3292946 .3312041 .491558

H 4.7755293 .1303210 .526537

H $2.4575145 .887503 \quad 2.829934$

H 3.3737334 .6417533 .643646

H 1.8250184 .2407642 .872348

H 4.0164152 .4611713 .163537

H 3.9109230 .2673301 .560611

H $2.6389380 .020023 \quad 0.391644$

H 1.5079981 .1218511 .890549

H $-0.3160780 .975201-2.048053$

H -1.326553 $-0.701432-4.424514$

H $1.9967160 .456216-2.828192$

H $2.643908-1.180283-2.741249$

H $1.329274-0.730890-1.679933$

H $4.0905420 .982593-1.422068$

H $5.4082091 .154051-0.240520$

H $6.471016-2.910010-1.295790$

H $7.452721-2.057630-2.501268$

H $5.766135-2.398815-4.264339$

H $4.571600-4.221011-2.108682$

H $3.958895-5.509989-4.108360$

H $4.375693-4.209846-5.193541$

H $0.486171-4.352774-3.609366$

H $1.761144-5.592384-3.115232$

H $2.318811-2.818809-6.022893$

H $3.076767-1.930471-4.733903$

H $0.148600-2.200165-5.367914$

H $6.111776-0.138593-2.291362$

C -5.590441 -0.6012150 .353899$

O $-4.381997-1.3413640 .624501$

C $-4.152031-2.257291-0.461011$

C $-5.475677-2.351719-1.212456$

C $-5.989143-0.931539-1.086441$

H $-3.405611-1.790306-1.114871$

C $-3.625497-3.5978050 .058653$

H -5.342153 - $2.671011-2.250587$

H $-6.169962-3.048681-0.729086$

C $-7.475899-0.788584-1.360233$

H $-5.438534-0.308999-1.801693$

C $-2.341649-3.5147230 .911066$

O $-4.635920-4.2180220 .866222$

H $-3.465849-4.275476-0.788322$

H -2.579964 -3.075001 1.885506

O $-1.929397-4.8620991 .182824$

C $-1.167230-2.747942 \quad 0.271287$

C $0.194071-2.971507 \quad 0.960230$

H $-1.400685-1.6773430 .248228$

O $-1.031202-3.180314-1.086558$

H $\quad 0.490643-4.0214370 .838339$

H $\quad 0.941819-2.3896880 .403704$

H $-7.709979-1.098987-2.383787$

H $-8.071382-1.404576-0.678178$

H $-7.7942530 .251896-1.241059$

C -5.3836160 .8863140 .662633$

H $-4.844958-3.6070641 .593802$

H $-1.675073-5.2673110 .335908$

H $-0.316484-2.660022-1.490850$

H $-5.0707320 .994899 \quad 1.707185$

H $-6.336403 \quad 1.4176300 .555138$

H $-6.343202-0.9930131 .050325$

C $0.139694-0.6038563 .856551$

O $0.120893-1.1556942 .531275$

C $0.242584-2.5793042 .446750$

C $1.547679-3.0555203 .106590$

C $1.668996-2.5416364 .543658$

C $1.396621-1.0389214 .631072$

H 0.2040110 .4802753 .703276

C -1.164053 -0.8906564 .610464$

H $-0.598619-3.0454342 .966963$ 
O $2.676977-2.6258502 .350392$

H $1.563006-4.1518103 .103991$

H $2.678793-2.7457514 .920561$

O $\quad 0.741332-3.2270365 .379641$

H $1.312505-0.7331575 .680503$

O $2.529264-0.3557634 .087379$

C $-2.297612-0.0844854 .024701$

H $-1.461140-1.9423724 .576455$

H $-1.077206-0.6252235 .671008$

H $2.643950-1.6560142 .292896$

H $0.950035-4.1756625 .333666$

H 2.3812500 .5984844 .201693

O -2.2571691 .1916244 .504709$

O $-3.106485-0.5305393 .222538$

C -3.2619402 .0745653 .997296$

H -3.1112372 .2353392 .925822$

H $-4.260557 \quad 1.6725384 .193285$

H -3.1635703 .0336814 .512781$

SCF Energy (B3LYP/6-31G**//MMFF) $=-3245.91815862$

\section{8_00369}

MM̄FF Geometry

C -3.352285 -0.812307 2.700902

C $-4.497114-0.1558862 .456546$

C -5.511906 -0.5286421 .396047$

$\begin{array}{llll}0 & -5.053562 & -1.709553 & 0.694567\end{array}$

C $-5.684038 \quad 0.5916410 .351861$

$\begin{array}{llll}\text { C } & -6.377734 & 1.834946 & 0.862089\end{array}$

C -5.7617743 .0127551 .080652$

C -7.8649121 .7072641 .065162$

C $-4.295274 \quad 3.3132140 .950625$

C $-3.9798904 .015186-0.374408$

C $-2.5180654 .478716-0.438636$

C $-2.2390235 .218912-1.748388$

C $-0.7623125 .625831-1.872869$

O $-1.6711803 .328466-0.335580$

O $-0.5230926 .006761-3.236916$

C $0.119504 \quad 4.390419-1.588230$

C $-0.4317586 .854841-1.018079$

O $1.4992324 .744960-1.545248$

C $-0.2724693 .635914-0.297798$

C $0.5213952 .320904-0.099531$

O $0.0339074 .463597 \quad 0.833960$

C -5.962964 $-2.524878 \quad 0.108062$

O $-7.177647-2.3850920 .108324$

C $-5.291518-3.634332-0.613002$

C $-3.993178-3.935560-0.448819$

C $-3.237215-4.972728-1.155237$

C $-3.957239-5.750503-2.224706$

C $0.4640411 .285914-1.223382$

C $-0.8310610 .498064-1.315330$

C $-2.007397-1.081751-2.856893$

O $-1.6771720 .477173-0.427105$

C -1.562989 -2.475174 -3.193454

C $-0.384412-3.048410-2.898817$

C $-0.023214-4.458907-3.277306$

C $0.240460-5.334536-2.070931$

C $1.487660-5.503456-1.600878$

C $-0.932704-6.073476-1.459301$

C $-1.935515-5.141446-0.837917$

N $-0.894302-0.223953-2.499185$

H -3.110793 -1.7024882 .126671$

H $-4.7273550 .722503 \quad 3.055305$

H $-6.459424-0.756758 \quad 1.898526$

H $-6.2632610 .209826-0.500113$

H $-4.7085130 .827048-0.092237$

H $-6.365007 \quad 3.857248 \quad 1.410925$

H $-8.3264262 .654543 \quad 1.363708$

H -8.348952 1.3838370 .137733

H $-8.0851690 .974141 \quad 1.847013$

H $-4.009672 \quad 3.9592521 .790347$

H -3.690273 2.4071261 .055071

H -4.181956 $3.317673-1.197833$

H $-4.6424324 .879853-0.505700$

H -2.3350375 .1396420 .416928$

H -2.888552 $6.097832-1.845646$

H -2.490176 $4.565452-2.595827$

H - $1.1304766 .734189-3.455740$
H $-0.0039863 .719217-2.446328$

H $-1.0381867 .713055-1.331869$

H $0.6114967 .160929-1.153249$

H $-0.610956 \quad 6.699058 \quad 0.047486$

H $1.7059185 .205368-2.376666$

H 1.5725382 .5773350 .079617

H $\quad 0.182201 \quad 1.845752 \quad 0.828042$

H 0.0850763 .8983561 .622314

H $-5.929941-4.191459-1.290682$

H $-3.406333-3.3570540 .262361$

H $-4.797636-6.306043-1.794070$

H $-3.328235-6.481954-2.735086$

H $-4.341668-5.075588-2.997818$

H $0.6920911 .738996-2.191781$

H $1.2496160 .543330-1.034947$

H -2.472678 $-0.630168-3.739283$

H $-2.750705-1.108116-2.053944$

H -2.302633 -3.068766 -3.727895

H $0.373835-2.486329-2.359656$

H $0.872131-4.415926-3.911257$

H $-0.799951-4.919713-3.900352$

H $1.691006-6.147147-0.751700$

H $2.333348-4.995331-2.052130$

H $-0.585711-6.752515-0.669575$

H $-1.367900-6.718749-2.226541$

H $-1.514883-4.527175-0.039861$

H $-0.158400-0.101161-3.188989$

C $-0.988453-0.0902223 .046463$

O $-0.510545-1.2534012 .340580$

C $0.859946-1.4988132 .706604$

C $1.358255-0.1913183 .298050$

C $0.119058 \quad 0.292233 \quad 4.029000$

H $\quad 0.826835-2.2791993 .477582$

C $1.627149-1.9950791 .475914$

H $2.218998-0.326858 \quad 3.959553$

H 1.6396630 .5131952 .505800

C $\quad 0.153330 \quad 1.771188 \quad 4.371574$

H $0.012695-0.2857094 .956983$

C $3.128269-2.2191101 .725310$

O $1.027874-3.2259401 .055486$

H $1.480044-1.2897420 .651894$

H $3.611591-1.2677081 .962168$

O $3.266108-3.0349372 .898010$

C $3.869480-2.9321210 .578417$

C $3.774722-2.283414-0.815400$

H $3.483834-3.9557300 .499352$

O $\quad 5.247619-3.0832190 .936524$

H $4.191583-3.002365-1.533147$

H $2.719649-2.147756-1.075096$

H 0.9925751 .9918875 .039230

H $0.2675292 .388147 \quad 3.474407$

H $-0.7695252 .075948 \quad 4.874952$

C $-2.325362-0.409736 \quad 3.722312$

H $1.168889-3.8790991 .762203$

H $4.217787-3.1659913 .049938$

H $5.577571-2.2211181 .241304$

H -2.668801 0.4533934 .303548

H -2.203129 -1.250009 4.416552

H -1.1323950 .6982602 .299231$

C $4.5739201 .324930-0.195299$

O $3.9140020 .051096-0.210097$

C $4.547999-0.963065-0.990662$

C $4.615073-0.524461-2.463687$

C $5.337700 \quad 0.819596-2.596847$

C $4.7886591 .865752-1.621584$

H 3.8729291 .9952920 .316324

C $5.863770 \quad 1.283358 \quad 0.635095$

H $5.569535-1.115938-0.629900$

O $3.306363-0.396331-3.014321$

H $5.150328-1.284148-3.046412$

H $5.225603 \quad 1.183131-3.625878$

$\begin{array}{llll}\text { O } & 6.727049 & 0.614146 & -2.356348\end{array}$

H $5.4516742 .738338-1.591952$

O $3.5335572 .318173-2.135188$

C 5.5612710 .9734562 .084376

H $\begin{array}{llll}6.573242 & 0.543637 & 0.254038\end{array}$

H $\quad 6.3741112 .2531790 .614217$

H $2.935712-1.289398-3.112496$ 
H $7.175613 \quad 1.467665-2.482271$ H $3.2350443 .055327-1.575675$ O $6.279914-0.1128412 .488681$ O $4.783473 \quad 1.6189662 .775391$ C $6.083886-0.5113953 .849218$ H $6.479546 \quad 0.2539594 .523239$ H $5.023364-0.6911274 .049875$ H $6.632218-1.4429454 .012416$

SCF Energy (B3LYP/6-31G**//MMFF) $=-3245.91065235$

0800370

MM̄FF Geometry

C $-2.259473 \quad 3.864111-0.887123$

C $-3.2432734 .408778-0.154153$

C -4.1718773 .6893150 .795256$

O $-3.7821672 .312043 \quad 0.988258$

C -5.5982143 .7023930 .220340$

C -6.6180003 .0619661 .140630$

C -7.1293141 .8282700 .958651$

C -7.0886683 .9216362 .283684$

C $-6.7496630 .850169-0.119078$

C $-6.766025-0.5966760 .394262$

C $-6.309549-1.576359-0.695868$

C $-6.619322-3.018725-0.295188$

C $-6.110150-4.017173-1.344323$

O $-4.896875-1.413863-0.873980$

O $-6.162572-5.330071-0.765965$

C $-4.628874-3.710782-1.639696$

C $-7.005694-4.053783-2.588130$

O $-4.146088-4.514589-2.714339$

C $-4.340424-2.215284-1.924768$

C $-2.810116-1.993477-1.949682$

O $-4.883799-1.861212-3.197107$

C -2.924809 2.0435672 .009399

O -2.3806012 .8516642 .744710$

C -2.7648430 .5736872 .083209$

C - 1.8688870 .0533972 .934586

C $-1.572872-1.3684013 .124109$

C -2.450681-2.368185 2.418690

C $-2.363542-0.524800-1.976229$

C $-0.849107-0.442768-1.899390$

C $1.048239-0.921916-0.345042$

O $-0.166135-0.133229-2.872375$

C $1.233151-2.1372340 .507500$

C $1.815298-2.1179101 .715618$

C $1.999722-3.3393852 .570013$

C $1.507552-3.1469493 .988685$

C $2.369871-3.1068945 .018670$

C $0.011390-3.0713934 .222465$

C $-0.537137-1.703174 \quad 3.922151$

N $-0.362994-0.795120-0.651284$

H -2.065888 $2.796439-0.837803$

H $-3.4144995 .480925-0.237132$

H -4.1618574 .2166931 .757461$

H $-5.6062603 .205869-0.757601$

H $\quad-5.912895 \quad 4.7355150 .018953$

H -7.897409 1.4844661 .649846

H -7.858646 3.4274942 .885698

H -6.2552744 .1634852 .950524$

H -7.5152974 .8563961 .905798$

H $-7.4700000 .945739-0.940366$

H -5.757405 $1.069505-0.524503$

H $-6.106004-0.6797011 .267556$

H $-7.783125-0.8488380 .719485$

H -6.824823 -1.323518-1.630030

H -7.694966 -3.155792 -0.128360

H -6.134727 $-3.243673 \quad 0.665325$

H -7.084116 $-5.508276-0.510731$

H $-4.055028-4.008059-0.750310$

H -8.031584 -4.327215 -2.313293

H $-6.670170-4.822178-3.293503$

H -7.048442 -3.098138 -3.114591

H $-4.331411-5.442213-2.488245$

H -2.375299 -2.466253 -1.060891

H $-2.375390-2.501367-2.820570$

H -4.402346 -1.092694 -3.542167

H -3.378937 -0.0370791 .431359$

H $-1.2745600 .735644 \quad 3.541483$
H -3.506771 -2.181003 2.644678

H -2.257707 -3.4012212 .715074$

H $-2.311613-2.3101801 .335621$

H $-2.686406-0.027688-2.896797$

H $-2.7860540 .039210-1.137744$

H $\quad 1.356237-0.0069140 .168428$

H $1.625148-1.020042-1.269488$

H $\quad 0.873977-3.0817740 .105367$

H $2.194569-1.1762862 .105687$

H $3.069704-3.5840532 .570778$

H $1.492225-4.2090312 .134111$

H $2.024164-2.9911926 .040486$

H $3.441014-3.1825684 .863263$

H $-0.215926-3.2955705 .272711$

H $-0.467840-3.8624663 .639689$

H $0.010604-0.8993024 .417049$

H -1.020437 -0.8844420 .117104$

C $0.0903244 .657985-1.474147$

O $0.6544523 .349038-1.668311$

C $1.7292153 .169049-0.715555$

C 1.8712614 .4945360 .026309

C $0.4640285 .053559-0.049510$

H $1.3894542 .383962-0.030254$

C $2.9877482 .718893-1.472155$

H 2.2252614 .3619941 .053251

H $2.5742815 .159504-0.491037$

C $\quad 0.3809506 .5443390 .222680$

H -0.1537824 .5175570 .681831$

C $4.2312192 .581132-0.571244$

O $2.6935241 .457536-2.075616$

H $3.1926423 .420403-2.289465$

H $4.3651173 .537091-0.049648$

O $3.984173 \quad 1.604607 \quad 0.434881$

C $5.5610912 .296535-1.302679$

C $5.6395841 .070729-2.231072$

H $6.3525842 .209077-0.548014$

O $5.8642893 .457054-2.087107$

H $4.9213241 .174000-3.052045$

H $6.624978 \quad 1.108808-2.717106$

H $\quad 0.739630 \quad 6.771347 \quad 1.232056$

H $\quad 0.988237 \quad 7.116787-0.486369$

H $-0.6521056 .897566 \quad 0.144083$

C $-1.3979834 .662273-1.826050$

H $1.8969891 .573065-2.621634$

H 4.7520401 .5999961 .030983

H $6.7541233 .336316-2.460176$

H -1.528144 $4.235039-2.828236$

H -1.748754 $5.700203-1.875165$

H $0.6031465 .315100-2.190089$

C $6.612389-1.6504860 .135120$

O $6.579970-0.436579-0.627784$

C $5.486802-0.293034-1.540314$

C $5.479076-1.445474-2.558627$

C $5.488178-2.808429-1.859570$

C $6.575457-2.885038-0.782869$

H $7.588553-1.6403520 .637499$

C $5.526141-1.6708051 .219781$

H $4.544463-0.312108-0.988270$

O $6.605253-1.354271-3.429436$

H $4.584126-1.355533-3.185229$

H $5.659977-3.597091-2.602031$

O $4.222071-3.049284-1.253511$

H $6.450493-3.788274-0.174065$

O $7.853883-2.982780-1.417673$

C $5.766658-0.6367822 .299480$

H $4.537861-1.4727640 .801167$

H $5.518359-2.6525761 .707120$

H $7.407224-1.352726-2.879171$

H $3.547764-3.009030-1.952977$

H $7.851395-3.791359-1.957997$

O $4.810300-0.7794673 .264775$

O $\quad 6.660951 \quad 0.1974592 .296100$

C 4.8808880 .1549204 .345905

H $4.055147-0.0551485 .030821$

H 5.8248070 .0370914 .886247

H $4.773771 \quad 1.1772773 .970959$

SCF Energy (B3LYP/6-31G**//MMFF) $=-3245.92120467$ 
08_00371

MM̄FF Geometry

C $2.3943124 .577913-0.158399$

C $3.5431233 .889965-0.089814$

C $4.3319573 .449735-1.294668$

O $4.1755732 .015573-1.398571$

C $5.8212803 .774486-1.100775$

C $6.6892473 .278030-2.239592$

C $7.4395302 .159047-2.188658$

C $6.7023504 .149491-3.467403$

C $7.5238121 .177242-1.052449$

C $7.389495-0.271933-1.541321$

C $7.342764-1.249818-0.358523$

C $7.467378-2.696356-0.835382$

C $7.349697-3.6869420 .332865$

O $6.089234-1.0689620 .311242$

O $7.168769-4.999921-0.218768$

C $6.078249-3.3556741 .140176$

C $8.631316-3.7410411 .172644$

O $6.001649-4.1499042 .322041$

C $5.935966-1.8561301 .498950$

C $4.523331-1.5998712 .074342$

O $6.908230-1.5087452 .486043$

C $3.1315501 .566806-2.148260$

O $2.3625742 .238682-2.817662$

C $3.0416210 .102319-1.964567$

C $1.897342-0.516496-2.291340$

C $1.586437-1.930976-2.084801$

C $2.700034-2.838945-1.638354$

C $4.143992-0.1205752 .219051$

C $2.695528-0.0006122 .655013$

C $0.366913-0.3025781 .792531$

O $2.390273 \quad 0.229578 \quad 3.822613$

C $-0.067699-1.6959451 .456754$

C $-1.068128-1.9837830 .609370$

C $-1.480214-3.3864940 .256806$

C $-1.560466-3.627516-1.237454$

C $-2.743456-3.838133-1.838873$

C $-0.267239-3.705035-2.026467$

C $0.318046-2.343211-2.289392$

N $1.805556-0.2187451 .615126$

H $1.9938084 .863888-1.127393$

H 3.9132893 .5784390 .884809

H $3.9722503 .941633-2.207200$

H $6.1750373 .361977-0.148753$

H $5.9535574 .860485-1.002836$

H $8.0584951 .915738-3.051110$

H $7.3716203 .764040-4.243913$

H $5.6997174 .216213-3.900976$

H $7.0398915 .159463-3.212788$

H $8.4964161 .301917-0.561279$

H $6.7577931 .367629-0.296396$

H $6.473404-0.370523-2.137796$

H $8.238732-0.512312-2.192800$

H $\quad 8.161223-1.0033580 .328277$

H $8.410413-2.850518-1.374407$

H $6.668378-2.913115-1.558468$

H $7.933690-5.193844-0.787311$

H $5.218540-3.6437360 .518933$

H $9.485246-4.0330790 .549532$

H $8.558049-4.503757 \quad 1.955737$

H $8.876620-2.7867401 .643156$

H $6.078962-5.0808792 .051017$

H $3.783095-2.0764231 .422537$

H $4.429140-2.0795643 .057402$

H $6.595541-0.7314472 .975248$

H $3.881338-0.409096-1.509500$

H $\quad 1.084840 \quad 0.080253-2.704812$

H $3.585746-2.709107-2.270709$

H $2.443461-3.899204-1.699967$

H $2.974813-2.624212-0.602902$

H 4.7694380 .3807322 .964770

H $\quad 4.270480 \quad 0.4217521 .275303$

H $-0.083330 \quad 0.439037 \quad 1.126762$

H $0.098631-0.0629022 .825690$

H $\quad 0.473017-2.5107301 .933491$

H -1.627095 -1.171580 0.150288

H -2.457458 -3.569989 0.721856
H $-0.795160-4.1227510 .695775$

H -2.809912 -4.036223 -2.903463

H $-3.672300-3.817724-1.280209$

H $-0.445749-4.181912-2.998696$

H $\quad 0.418510-4.364790-1.487145$

H $-0.408351-1.615341-2.654453$

H $2.172106-0.3896850 .683970$

C 0.5085793 .8919421 .333732

$\begin{array}{lllll}\text { O } & -0.467501 & 3.885071 & 0.274637\end{array}$

C -1.7793503 .6912850 .848428$

C -1.5553933 .3850582 .322593$

C -0.2825374 .1536362 .612501$

H $-2.299544 \quad 4.6501670 .731934$

C -2.5064312 .5916640 .063600$

H -2.396842 3.6980112 .947955

H -1.3968662 .3110712 .479468$

C 0.4191943 .7009163 .881111

H -0.5256155 .2216652 .693063$

C -3.8589242 .1859180 .679454$

O $-2.713128 \quad 3.072303-1.267357$

H $-1.8644371 .707194-0.021320$

H -3.6847101 .8262061 .699872$

O $-4.698568 \quad 3.3356640 .786267$

C $-4.5968061 .070922-0.091717$

C -5.8665780 .6255940 .648167$

H $-3.9195300 .218369-0.208743$

O $-4.9750811 .511674-1.392647$

H $-5.616676 \quad 0.316428 \quad 1.671139$

H -6.5458631 .4820600 .759519$

H $-0.2287653 .847046 \quad 4.751640$

H $0.6850422 .639776 \quad 3.835159$

H 1.3383424 .2714754 .045799

C 1.5828524 .9432631 .052327

H -1.843001 $3.320058-1.624898$

H -4.873592 $3.651152-0.117049$

H $-4.1627491 .743648-1.873363$

H 2.2409375 .0544751 .922057

H 1.1233335 .9219340 .868490

H $\quad 0.9560212 .8895151 .361299$

C $-8.746072-1.6999480 .274652$

O $-7.809390-0.7360640 .772551$

C $-6.662839-0.491489-0.054125$

C $-5.867468-1.789439-0.258336$

C $-6.757648-2.920651-0.785329$

C $-8.057269-3.0487620 .014713$

H $-9.460610-1.8420391 .096144$

C $-9.525476-1.168785-0.933345$

H $-7.003227-0.110674-1.022815$

O $-5.259615-2.1954720 .966402$

H $-5.057821-1.606848-0.972742$

H $-6.209707-3.869365-0.733620$

O $-7.080400-2.679568-2.151782$

H $-8.757235-3.723655-0.491687$

O $-7.761589-3.6285351 .288928$

C $-10.404166-0.009623-0.527946$

H $-8.885626-0.845658-1.759396$

H -10.183958 -1.945075 -1.341154

H -5.965051 -2.299288 1.628011

H -6.245184 -2.637169 -2.647945

H $-7.377870-4.5076371 .128628$

O $-9.7344581 .165782-0.699965$

O $-11.538985-0.137455-0.086635$

C $-10.4508012 .344253-0.319227$

H -10.6938812 .3113250 .747122$

H $-9.8071853 .208024-0.505626$

H $-11.3592492 .446425-0.920342$

SCF Energy (B3LYP/6-31G**//MMFF)= -3245.92647042

0800372

MM̄FF Geometry

C $-0.7154935 .089895-0.144165$

C $-1.8110794 .318921-0.203915$

C -2.6813274 .0224700 .990548$

O -2.6604712 .5939501 .223874$

C $-4.1291734 .453883 \quad 0.707720$

C $-5.0696754 .219091 \quad 1.874473$

C $-6.080650 \quad 3.327333 \quad 1.865994$

C $-4.859855 \quad 5.1109763 .070828$ 
C $-6.4346152 .382344 \quad 0.750772$

C $-7.103503 \quad 1.1017301 .272224$

$\begin{array}{llll}\text { C }-7.508126 & 0.171282 & 0.119435\end{array}$

C $-8.315103-1.0203590 .637856$

C $-8.656654-2.011742-0.485646$

O $-6.312300-0.285543-0.521941$

O $-9.123541-3.2251810 .124244$

C $-7.360827-2.359101-1.248501$

C $-9.799975-1.505748-1.372360$

O $-7.636309-3.166738-2.389879$

C $-6.539305-1.119316-1.667467$

C $-5.156487-1.488659-2.254765$

O $-7.249441-0.405578-2.686739$

C -1.5815912 .1026751 .895697$

$\begin{array}{lllll}\text { O } & -0.630839 & 2.740377 & 2.321687\end{array}$

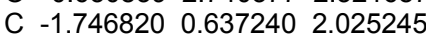

C $-0.714818-0.0920722 .476654$

C $-0.693726-1.5396492 .704180$

C -1.968092 -2.301006 2.460161

C $-4.265607-2.313315-1.324199$

C $-2.852076-2.477168-1.856509$

C $-0.758487-3.734852-1.404975$

O $-2.425012-1.885247-2.843300$

C $-0.172603-4.509243-0.268803$

C $0.955962-4.1625310 .367132$

C $1.555384-4.9590551 .488624$

C $1.881981-4.1181532 .701534$

C $3.153247-3.9531923 .105276$

C $0.731491-3.5371893 .495348$

C $0.453495-2.1016343 .140710$

N $-2.117778-3.356916-1.072251$

H $-0.441656 \quad 5.5553730 .799417$

H -2.076026 $3.839288-1.143398$

H $-2.313067 \quad 4.5429821 .883587$

H $-4.4942903 .940703-0.189249$

H $-4.153261 \quad 5.524186 \quad 0.460714$

H $-6.728970 \quad 3.282152 \quad 2.739814$

H -5.641665 4.9821503 .827042

H $-3.901073 \quad 4.893583 \quad 3.550965$

H $-4.868926 \quad 6.1629852 .767509$

H -7.1255512 .8953800 .071277$

H -5.5434462 .1049290 .178736$

H $-6.4046650 .582181 \quad 1.940700$

H -7.992076 1.3646891 .859710

H -8.104103 $0.749005-0.597055$

H $-9.229470-0.6824911 .141250$

H -7.730478 -1.5507881 .402774$

H $-9.897875-3.0070650 .670856$

H -6.758406 -2.975061 - 0.569465

H -10.707437 -1.352403-0.775912

H $-10.065043-2.246140-2.135183$

H -9.571916 -0.561854 -1.871659

H -8.149081 -3.934803 -2.084769

H -5.281492 -2.015039-3.209994

H $-4.630798-0.555729-2.502824$

H -6.615032 $0.142411-3.177900$

H $-2.704376 \quad 0.206886 \quad 1.755979$

H 0.2164490 .4227522 .712407

H -2.743891 -1.982291 3.165071

H $-1.859480-3.3820252 .566976$

H -2.332152 -2.126938 1.443520

H $-4.196002-1.834914-0.340804$

H $-4.689016-3.315163-1.194102$

H - $0.189936-2.828582-1.638788$

H $-0.794719-4.355661-2.306258$

H $-0.695408-5.4136290 .033906$

H $1.492021-3.2734200 .050222$

H $2.464451-5.439043 \quad 1.103717$

H $\quad 0.889271-5.775437 \quad 1.795024$

H $3.392262-3.3734323 .990944$

H $3.982543-4.3887222 .557928$

H $0.981486-3.5661994 .564733$

H $-0.144979-4.1826843 .395321$

H $1.312853-1.4480683 .301106$

H $-2.611056-3.901169-0.369587$

C $1.4331214 .421891-1.320061$

O $1.0310983 .056254-1.548535$

C $1.8154412 .193602-0.696104$
C $2.9583193 .053503-0.174763$

C $2.290127 \quad 4.408762-0.054581$

H $1.150771 \quad 1.8999490 .125585$

C $2.2479550 .959019-1.495944$

H 3.3603332 .6898490 .775488

H $3.7775123 .092010-0.902152$

C 3.2637035 .5695720 .040253

H 1.6657824 .3955050 .846713

C $3.1787830 .017972-0.707044$

$\begin{array}{llll}\text { O } & 1.063363 & 0.249779 & -1.870560\end{array}$

H $2.7391181 .269959-2.425904$

H $4.0451440 .597394-0.374590$

O $2.508084-0.4311890 .470800$

C $3.698001-1.195948-1.507512$

C $4.560020-2.168596-0.679155$

H $4.276499-0.839178-2.367703$

O $2.616073-1.968802-2.028390$

H $3.954040-2.6147010 .118948$

H $4.832561-3.000566-1.342503$

H 3.9066465 .4624650 .920002

H $3.9086965 .626608-0.842537$

H 2.7267686 .5195280 .126467

C $0.2010375 .332806-1.309314$

H $0.4931610 .871510-2.354776$

H $1.710497-0.9093420 .185546$

H $2.107754-1.399230-2.630041$

H $-0.3555895 .187319-2.244047$

H $0.5142246 .383012-1.288905$

H $2.0504844 .698966-2.185009$

C $7.839703-0.389514-0.802980$

O $6.627170-1.061218-1.167967$

C $5.830747-1.544551-0.083071$

C $6.625396-2.5501010 .763691$

C $7.944633-1.9356451 .243914$

C $8.717104-1.2809910 .094245$

H $8.375830-0.244973-1.750344$

C $7.5633611 .000208-0.206401$

H $5.538870-0.7076390 .557683$

O $6.891589-3.721599-0.003817$

H $6.012737-2.8502081 .621862$

H $8.568121-2.7147161 .699196$

$\begin{array}{llll}\text { O } & 7.682632 & -0.952069 & 2.240762\end{array}$

H $9.556707-0.6922750 .482000$

O $9.274293-2.304871-0.735452$

C $7.028796 \quad 1.965819-1.245238$

H 6.8383000 .9716260 .610450

H $8.505123 \quad 1.415702 \quad 0.171725$

H $7.371437-4.3446950 .567903$

H $7.212675-1.3909072 .970229$

H $9.869140-2.839165-0.182231$

O $7.150267 \quad 3.240879-0.770164$

O $6.5541691 .647835-2.326939$

C $6.720228 \quad 4.276727-1.659380$

H $7.4184834 .357689-2.497403$

H $5.7063574 .085112-2.022350$

H $6.7209255 .220263-1.107269$

SCF Energy (B3LYP/6-31G**//MMFF) $=-3245.91001939$

0800373

MM̄FF Geometry

C $-4.272201 \quad 4.3112751 .330176$

C $-4.394966 \quad 3.0923190 .782908$

C -5.3318002 .0309501 .302429$

$\begin{array}{llll}\text { O } & -4.631436 & 0.763632 & 1.292521\end{array}$

C $-6.551248 \quad 1.919893 \quad 0.370486$

$\begin{array}{llll}\text { C }-7.548652 & 0.858256 & 0.794758\end{array}$

C $-7.745858-0.313350 \quad 0.156658$

C $-8.374800 \quad 1.201678 \quad 2.007934$

C $-7.030051-0.829412-1.060392$

C $-6.190567-2.069067-0.726209$

C $-5.176905-2.384339-1.835785$

C $-4.448003-3.696489-1.544047$

C $-3.328096-3.974995-2.557874$

O $-4.240643-1.301145-1.879060$

O $-2.502373-5.022493-2.025063$

C $-2.438033-2.719215-2.680562$

C $-3.881531-4.494258-3.889967$

O $-1.477716-2.877535-3.721815$ 
C $-3.236949-1.417343-2.896350$ C $-2.357399-0.142692-2.825019$ O $-3.831043-1.442973-4.200036$ C $-3.7894190 .504418 \quad 2.330677$ O $-3.549594 \quad 1.2302953 .282101$ C -3.198384 -0.834207 2.103672 C $-2.279087-1.3175672 .952559$ C -1.625221 -2.626454 2.862351 C $-1.955929-3.4917711 .674961$ C $-1.6397940 .092478-1.491033$ C $-0.233297-0.479658-1.433528$ C $1.598949-0.9641760 .184552$ O $0.385395-0.846309-2.428780$ C $1.518919-2.3540900 .729309$ C $2.055159-2.7083051 .907190$ C $2.027976-4.1044282 .456921$

C $1.454586-4.1688493 .854497$ C $2.259395-4.1911384 .930532$ C $-0.043824-4.3030803 .998631$ C $-0.768265-2.9928323 .838888$ N $0.266451-0.492420-0.138572$ H -4.9007144 .5563242 .184560$ H -3.802339 2.819674 -0.085591 H -5.6618142 .2575262 .323890$ H $-6.2064461 .743746-0.653026$ H -7.0749042 .8851230 .332054$ H -8.502296 -0.9914810 .550019$ H -9.1331520 .4413662 .223594$ H -7.7393831 .2917322 .894167$ H $-8.8976942 .151379 \quad 1.854777$ H $-7.777497-1.093138-1.818800$ H -6.402792 $-0.061899-1.517384$ H -5.651246 -1.8975950 .213430$ H $-6.856967-2.926101-0.568692$ H -5.709136 -2.443486 -2.792397 H $-5.155018-4.534912-1.512037$ H $-4.001439-3.646837-0.542628$ H -3.066665 -5.799264 -1.869657 H -1.874426 -2.639465 -1.743386 H -4.432822 -5.429806 -3.736409 H $-3.071900-4.735802-4.587300$ H -4.559302 -3.790023 -4.376492 H $-0.982966-3.694369-3.538072$ H -1.658147 -0.119371 -3.669433 H $-3.0240840 .718429-2.977075$ H $-4.032163-0.530297-4.465708$ H -3.540116 -1.391230 1.239429 H - $1.983822-0.7053973 .803690$ H -3.000216 -3.818626 1.720726 H -1.336953 -4.388337 1.600790 H -1.798178 -2.941783 0.740986 H -1.531547 $1.174449-1.340808$ H $-2.230850-0.294379-0.656456$ H $2.018506-0.266606 \quad 0.913947$ H $2.226435-0.952125-0.712012$ H $1.023303-3.1046960 .118605$ H $2.575269-1.9579812 .499003$ H $3.059654-4.4796292 .462214$ H $1.471365-4.7843501 .800187$ H $1.857988-4.2684385 .935581$ H $3.337777-4.1263834 .829914$ H -0.289632 -4.685535 4.998856 H $-0.405677-5.0770223 .315801$ H $-0.583865-2.2938254 .655863$ H $-0.328328-0.1915790 .628232$ C $-2.1690775 .009575-0.004282$ $\begin{array}{lllll}\text { O } & -1.279226 & 4.161257 & 0.749491\end{array}$ C 0.0779254 .6148150 .557929 C $0.0311015 .529676-0.654139$ C $-1.3199136 .193399-0.468916$ H $0.331603 \quad 5.1907621 .457550$ C 0.9919183 .3922230 .438960 H $0.8588786 .244425-0.677321$ H $0.0492274 .947515-1.583981$ C -1.840461 $6.872200-1.723790$ H $-1.239203 \quad 6.938206 \quad 0.334347$ C 2.4383273 .7306300 .020812 O $0.9693712 .753546 \quad 1.719218$
H $\quad 0.5584572 .679937-0.273438$

H $2.4116934 .187203-0.974984$

O 2.9827394 .7073630 .907665

C $3.3738482 .506659-0.035975$

C $4.7557592 .859885-0.620591$

H $2.9009731 .735603-0.655439$

O $3.5335111 .979131 \quad 1.279097$

H $4.6196483 .423573-1.550969$

H 5.2915773 .4951420 .095633

H -1.160893 $7.670626-2.038892$

H -1.934143 $6.164056-2.553982$

H -2.824992 $7.316455-1.546965$

C $-3.346075 \quad 5.4166140 .887701$

H 1.4670751 .9225431 .644810

H 3.0150854 .3075691 .793926

H 4.0811491 .1788341 .214247

H -3.9512626 .1807970 .386493$

H -2.943822 5.8713741 .802573

H -2.510383 $4.435257-0.873880$

C $6.645284-0.2495790 .231215$

O 5.9640631 .0085520 .335299

C $5.6004591 .603844-0.914746$

C $6.8674551 .930608-1.716968$

C $7.7226040 .674427-1.922762$

C $7.921098-0.111163-0.620106$

H $6.949604-0.4782001 .260704$

C $5.669661-1.343020-0.227711$

H $4.9794520 .902852-1.485265$

O $7.6255232 .918553-1.022149$

H $6.5766732 .354942-2.685316$

H $8.7049030 .959520-2.319271$

O $7.098981-0.173352-2.883041$

H $8.329839-1.103315-0.839762$

$\begin{array}{lllll}\text { O } & 8.906081 & 0.566641 & 0.168483\end{array}$

C $6.085531-2.7326100 .200595$

H $4.688731-1.1444490 .220726$

H $5.541810-1.342659-1.313309$

H $8.4046793 .126338-1.565568$

H $7.0221730 .328249-3.712416$

H $8.539363 \quad 1.4307750 .422929$

O $5.081959-3.606599-0.110180$

O $7.148572-3.0225450 .731264$

C $5.324291-4.9725490 .240025$

H $4.437567-5.553960-0.026407$

H $6.180548-5.359271-0.320428$

H $5.493085-5.0650221 .317165$

SCF Energy $(B 3 L Y P / 6-31 G * * / / M M F F)=-3245.91368551$

0800374

MM̄FF Geometry

C $1.945145-0.5384614 .420136$

C $2.379107-0.2082473 .193484$

C 3.7661290 .3230462 .935701

O $3.622461 \quad 1.5120562 .124186$

C $4.595690-0.7068062 .150413$

C $6.086411-0.4403772 .212142$

C 6.8210270 .0742461 .207064

C $6.757816-0.8494463 .498976$

C $6.3520550 .544132-0.141596$

C $6.821750-0.381154-1.273405$

C $6.056887-1.713267-1.320448$

C $6.649257-2.642711-2.380488$

C $5.836129-3.937780-2.525116$

O $4.695459-1.416099-1.642879$

O $6.294711-4.578383-3.725111$

C $4.347264-3.573612-2.710291$

C $6.114746-4.912089-1.374552$

O $3.526219-4.739534-2.693772$

C $3.825207-2.553653-1.669103$

C $2.419738-2.058328-2.085571$

O $3.743565-3.187092-0.391666$

C 4.3928222 .5884792 .421663

O 5.1754742 .6928103 .356549

C $4.1838203 .695317 \quad 1.459337$

C 3.5128113 .5704110 .303287

C $3.3022664 .628965-0.689270$

C $3.8191596 .006393-0.364220$

C $1.882117-0.891902-1.243675$ 
C $0.588433-0.337559-1.815828$

C $-0.9794711 .581560-1.560420$

O $-0.016858-0.865181-2.745322$

C $-0.5657852 .581444-2.592272$

C $-0.6240203 .908584-2.406098$

C $-0.1930844 .910801-3.437048$

C $0.9364405 .794222-2.954124$

C $0.7051677 .064749-2.582906$

C $2.3434155 .238267-2.991707$

C $2.6498254 .327459-1.831918$

N $0.1842550 .814768-1.156035$

H $2.633780-0.4136845 .254743$

H $1.732690-0.3272142 .329093$

H 4.2437780 .5662953 .892139

H $4.253063-0.7353621 .110684$

H $4.423072-1.7168572 .546269$

H 7.8923750 .1924881 .366626

H $7.841597-0.6942253 .470898$

H $6.366276-0.2711834 .341084$

H $6.584319-1.9125283 .695903$

H $5.2676390 .681563-0.181008$

H $6.7813131 .540555-0.306414$

H $6.6677620 .151566-2.220971$

H $7.898649-0.568045-1.177447$

H $\quad 6.102439-2.189686-0.335464$

H $7.698430-2.871994-2.155738$

H $6.657284-2.128156-3.351781$

H $5.831913-5.428916-3.809938$

H $4.241671-3.131633-3.711053$

H $7.176240-5.187196-1.357305$

H $5.564232-5.850070-1.507528$

H $5.861572-4.502417-0.394483$

H $3.806725-5.308690-3.429824$

H $2.459405-1.723534-3.130742$

H $\quad 1.700975-2.886225-2.035850$

H $3.084397-2.7266300 .150358$

H 4.6514164 .6315991 .747505

H 3.0847432 .6036030 .043851

H $3.5660026 .755900-1.116959$

H $4.9113365 .996351-0.278276$

H $3.394323 \quad 6.3644790 .580389$

H $1.682126-1.216161-0.217086$

H $2.613109-0.076016-1.212422$

H -1.381078 $2.061682-0.664770$

H -1.740413 $0.910484-1.971239$

H $-0.1975612 .191920-3.538496$

H -1.001406 4.299715-1.463926

H -1.068742 $5.520485-3.695261$

H $\quad 0.1082384 .420271-4.371089$

H $\quad 1.5117127 .717947-2.267297$

H $-0.2972377 .479974-2.582943$

H $3.0769566 .049663-3.057531$

H $2.4839924 .674355-3.922936$

H $2.3122503 .301604-1.978709$

H $\quad 0.790926 \quad 1.217345-0.447274$

C $-0.472224-1.1842603 .726538$

$\begin{array}{llll}\text { O } & -0.876747 & 0.141845 & 3.334111\end{array}$

C -2.3173210 .1893543 .238866$

C -2.783527 -1.256370 3.295756

C $-1.747251-1.8774644 .208375$

H -2.6593100 .7355934 .127096$

C $-2.7012250 .949670 \quad 1.964738$

H -3.804246 -1.355413 3.677039

H -2.742354 -1.719857 2.303767

C -1.697897 -3.393384 4.121652

H -1.969285 -1.583091 5.242932

C $-4.2114480 .917181 \quad 1.654510$

O -2.2559522 .2965142 .149324$

H -2.136990 0.5405431 .119661

H -4.526446 -0.1285331 .597768$

O $\begin{array}{llll}-4.933619 & 1.502512 & 2.737317\end{array}$

C -4.6032911 .6023020 .328063$

C -6.1166541 .6113480 .039795$

H $-4.0795181 .102634-0.494331$

O -4.1631242 .9619850 .316816$

H -6.6395532 .1730310 .824393$

H -6.263209 $2.186920-0.884295$

H $-2.659655-3.8265384 .415634$
H -1.475359 -3.730006 3.103397

H $-0.928267-3.7983104 .786043$

C $0.599961-1.0893454 .815417$

H -2.407679 2.7750411 .317226

H -4.5796152 .3972002 .877252$

H -4.6513623 .4392111 .009093$

H $0.771969-2.0836435 .244481$

H $0.223586-0.4495475 .624358$

H $-0.082654-1.6870782 .832649$

C $-6.559813-1.762276-1.512099$

O $-6.115147-0.428211-1.225649$

C $-6.7474320 .218361-0.115985$

C $-8.2669980 .304364-0.331639$

C $-8.863477-1.075738-0.623227$

C $-8.083610-1.806945-1.719176$

H $-6.090610-2.009859-2.473429$

C $-6.056063-2.767369-0.467432$

H $-6.558467-0.3556090 .795383$

O $-8.5731661 .191297-1.405724$

H -8.7281410 .7245110 .569737$

H $-9.908394-0.963153-0.936633$

O $-8.843091-1.8685330 .560219$

H -8.411441 -2.850412 -1.793857

O $-8.362283-1.191891-2.980506$

C $-4.616851-3.142139-0.732820$

H $-6.128436-2.4139700 .564535$

H $-6.632365-3.698840-0.524068$

H $-8.1023230 .875444-2.195973$

H -9.366422 -1.4029381 .234687$

H $-9.319363-1.264343-3.136420$

O $-3.787171-2.126445-0.362757$

O $-4.273969-4.213281-1.217059$

C $-2.393326-2.367993-0.577324$

H $-2.063918-3.2330440 .006157$

H $-2.195213-2.520457-1.642277$

$\mathrm{H}-1.840077-1.489128-0.239076$

SCF Energy $(B 3 L Y P / 6-31 G * * / / M M F F)=-3245.91006155$

0800375

MM̄FF Geometry

C -3.907658 -3.401595 1.402747

C $-2.705414-2.806246 \quad 1.384644$

C $-1.934616-2.4350802 .624056$

O $-1.864364-0.9937492 .704810$

C - $0.497252-2.988598 \quad 2.540947$

C $0.398972-2.6042023 .705456$

C $1.660028-2.1417633 .583472$

C $-0.177303-2.8131215 .083909$

C $2.474325-1.9487892 .332350$

C $3.756216-2.7914142 .399430$

C $4.564350-2.7453881 .094373$

C $5.802327-3.639808 \quad 1.208027$

C $6.696197-3.551556-0.037105$

O $4.957843-1.3919650 .848786$

$\begin{array}{lllll}\text { O } & 7.964580 & -4.142019 & 0.285953\end{array}$

C $6.958871-2.065582-0.356778$

C $6.126333-4.362342-1.206969$

O $7.662625-1.926583-1.589093$

C $5.675730-1.203670-0.379270$

$\begin{array}{llll}\text { C } & 6.049551 & 0.293676 & -0.498453\end{array}$

O $4.889664-1.575483-1.514756$

C $-2.875540-0.367092 \quad 3.361647$

$\begin{array}{llll}\text { O } & -3.875422 & -0.894426 & 3.832342\end{array}$

C $-2.645032 \quad 1.0891193 .486275$

C $-1.584777 \quad 1.7379942 .980790$

C -1.3352413 .1792983 .081382$

C $-2.312238 \quad 4.006921 \quad 3.876851$

C $4.8752301 .245250-0.240605$

C $5.2107332 .689100-0.568733$

C $4.1120334 .907669-0.778605$

O $6.3145123 .055626-0.960957$

C 3.1591285 .6647670 .086682

C $1.9101475 .980827-0.286102$

C 0.9507896 .7528020 .571853

C -0.1398535 .8700591 .135244$

C -1.3416915 .8054090 .536680$

C $0.187774 \quad 5.133936 \quad 2.419157$

$\begin{array}{llll}\text { C } & -0.265739 & 3.700291 & 2.443870\end{array}$ 
N $4.114713 \quad 3.517896-0.367229$ H -4.377462 -3.6348352 .354597$ H -2.251412 -2.558082 0.428226 H -2.423842 -2.840799 3.518794 H $-0.059682-2.6462371 .597830$ H $-0.535622-4.0845962 .488976$ H $2.197417-1.8910164 .498162$ H $0.574591-2.6944605 .871752$ H $-0.976622-2.0933365 .284041$ H -0.583527 -3.826002 5.174998 H $1.919678-2.2178041 .431226$ H $2.728318-0.8859862 .243958$ H $4.376136-2.4195013 .226124$ H $3.499527-3.8341582 .626899$ H $3.918436-3.0874190 .276986$ H $5.513894-4.6808571 .400048$ H $6.395592-3.3290912 .079780$ H $7.805520-5.0601870 .564555$ H $7.624066-1.6743300 .425959$ H $6.042770-5.421155-0.933684$ H $6.795296-4.329477-2.073876$ H $5.136219-4.025439-1.521032$ H $8.465800-2.471555-1.526920$ H $\quad 6.8335390 .537577 \quad 0.230273$ H $6.4616090 .488376-1.496855$ H $4.353005-0.818655-1.796760$ H -3.422776 1.6139924 .032505 H $-0.832850 \quad 1.1746162 .431207$ H -3.3225243 .9133973 .462636$ H -2.081497 5.0743673 .877520 H $-2.327774 \quad 3.6824264 .923074$ H $4.0138020 .966277-0.852785$ H $4.575991 \quad 1.1955200 .812788$ H $3.8205494 .935041-1.834156$ H $5.1196525 .328050-0.698911$ H 3.5198595 .9709381 .065526 H $1.5508335 .667427-1.263847$ H $0.5193717 .555946-0.039525$ H 1.4699547 .2628221 .393348 H $-2.151597 \quad 5.2057990 .936422$ H -1.546186 $6.360222-0.373397$ H -0.1869845 .7124103 .269237$ H 1.2765095 .1099852 .559467 H $\quad 0.3789923 .0304841 .874087$ H $3.2133083 .080970-0.192737$ C $-5.609630-2.699962-0.361331$ O $-4.863586-1.526341-0.743392$ C $-5.600605-0.361173-0.327370$ C -7.002355 -0.8529440 .016098$ C $-6.696671-2.2204440 .599381$ H $-5.127804-0.0028330 .594901$ C -5.539793 $0.730838-1.400568$ H $-7.515104-0.1897330 .719159$ H -7.628892 -0.951476 -0.877993 C -7.903555 -3.138502 0.673751 H $-6.291695-2.0808381 .609506$ C $-4.1068581 .148162-1.799638$ O $-6.198961 \quad 0.251222-2.578743$ H $-6.1109171 .604133-1.063366$ H $-3.6395530 .338792-2.369073$ O $-4.2118452 .254110-2.704973$ C $-3.2087431 .549372-0.618756$ C -1.848837 2.153482 -1.006744 H -3.037799 0.6771990 .021893 O H -1.988883 $3.147659-1.450528$ H -1.314529 $2.335303-0.067588$ H -8.675024 -2.7075381 .320213$ H -8.346406 -3.301644 -0.314546 H $-7.624925-4.1143631 .083884$ C $-4.663160-3.7829840 .160906$ H -6.143506 $0.954441-3.248339$ H -4.579337 $3.005326-2.208409$ H -3.344509 2.7454550 .928134 H -3.943707 -4.036420 -0.626793 H $-5.225488-4.7014640 .366509$ H -6.071732 -3.074302 -1.284573 C $-0.199436-0.988645-2.216449$
O $-0.941917-0.044708-1.429357$

C $-0.9771601 .292636-1.935631$

C $0.4480071 .863986-2.060521$

C $1.3621490 .937086-2.867747$

C $1.253142-0.518007-2.401337$

H $-0.178177-1.902074-1.608779$

C $-0.894975-1.317133-3.548046$

H $-1.4506021 .288293-2.922536$

O $1.0234372 .066943-0.769889$

H $0.3934022 .847581-2.541369$

H $2.4024191 .268478-2.762914$

O $1.0209451 .008537-4.248417$

H $1.774459-1.177026-3.105621$

O $1.921646-0.633823-1.142874$

C $-2.188213-2.074698-3.345599$

H $-1.122770-0.410715-4.114376$

H $-0.250164-1.953524-4.164667$

H $1.0102301 .215040-0.300806$

H $1.1320741 .932262-4.531162$

H $1.936411-1.576399-0.904349$

O $-2.998127-1.882728-4.427960$

O $-2.442876-2.775949-2.376895$

C $-4.256544-2.562614-4.384337$

H $-4.104179-3.642315-4.294423$

H -4.859156 -2.186960 -3.553044

H $-4.784884-2.359863-5.319547$

SCF Energy $(B 3 L Y P / 6-31 G * * / / M M F F)=-3245.92936870$

0800376

MM̄FF Geometry

C $0.633978 \quad 3.7746010 .500894$

C 0.0638793 .6888701 .712642

C 0.1934652 .5308952 .676043

O 1.2378001 .6150462 .274170

C -1.1404631 .7606952 .705666$

C -1.1391300 .5826313 .658554$

C - $-1.022467-0.7065713 .282094$

C -1.3231000 .9335855 .111697$

C $-0.824420-1.2407171 .890052$

C $0.386970-2.1792081 .822083$

C $0.684594-2.6299890 .383665$

C $1.883207-3.5801170 .359859$

C $2.313445-3.939312-1.068409$

O $0.965494-1.459784-0.388559$

O $3.607917-4.553923-0.972150$

C $2.464982-2.640931-1.891791$

C $1.383824-4.990807-1.685801$

O $2.730347-2.968953-3.253525$

C $1.245722-1.701857-1.774025$

C $1.461469-0.319300-2.437698$

O $0.125192-2.310176-2.427249$

C 2.5083581 .9083012 .667522

O $2.8752752 .888374 \quad 3.295768$

C 3.3884740 .8140432 .198299

C 4.7102810 .8783832 .418088

C $5.699514-0.1408452 .056733$

C $5.209926-1.3632741 .326800$

C $2.5694700 .541727-1.822890$

C $3.9197840 .415803-2.504441$

C $6.2726701 .215601-2.279107$

O $4.099282-0.219214-3.539353$

C $7.0885210 .179636-1.573429$

C $8.1712890 .468610-0.835686$

C $9.034598-0.568092-0.177696$

C $9.210819-0.3307161 .304406$

C $10.3152390 .267242 \quad 1.782706$

C $8.154942-0.8775492 .236886$

C 6.9876440 .0553132 .409158

N $4.897506 \quad 1.133077-1.827020$

H 1.2773512 .9743540 .146627

H $-0.591761 \quad 4.4950782 .036339$

H $\quad 0.4153532 .922847 \quad 3.676427$

H -1.398516 1.4313821 .692554

H -1.9553342 .4388982 .994814$

H -1.072133 -1.471751 4.055791

H -1.3565260 .0464655 .753243$

H -0.4983531 .5617945 .462207$

H -2.2619591 .4785015 .254589$ 
H $-1.722205-1.7935141 .594751$ H $-0.699749-0.4337581 .164673$ H $1.261606-1.6562062 .227878$ H $\quad 0.210215-3.0574572 .455553$ H -0.207697 -3.122695 -0.020526 H $1.671489-4.4879050 .938792$ H $2.733210-3.1011550 .863323$ H $3.919819-4.725842-1.877520$ H $3.357796-2.131596-1.508843$ H $1.413067-5.916154-1.097898$ H $1.710586-5.267362-2.694306$ H $\quad 0.342528-4.665727-1.737840$ H $2.991371-2.152384-3.711211$ H $1.583384-0.427272-3.521835$ H $\quad 0.5253720 .242780-2.311960$ H -0.519420 -1.617102 -2.645306 H $2.921489-0.0256611 .698154$ H 5.1089591 .7521212 .932317 H $6.013864-2.0129460 .974557$ H $4.640962-1.0810090 .434695$ H $4.566872-1.9625591 .979932$ H $2.2809821 .595385-1.921733$ H $2.6832880 .336255-0.754711$ H $6.6223172 .231623-2.070309$ H $6.316651 \quad 1.047972-3.360002$ H $6.792718-0.858699-1.703046$ H $8.4805431 .506080-0.730284$ H $10.012721-0.549263-0.676439$ H $8.642427-1.579523-0.341514$ H 10.4636910 .4108042 .847872 H 11.0925410 .6316891 .119227 H $8.588973-1.0363683 .233648$ H $7.859184-1.8777671 .908171$ H $7.246000 \quad 0.9762762 .933906$ H $4.6629531 .582088-0.946322$ C $-0.6748734 .612013-1.504856$ O $-1.9627964 .511611-0.863449$ C $-2.6510283 .350456-1.378159$ C $-1.9282122 .993705-2.669185$ C $-0.4933603 .329180-2.316788$ H $-2.5082422 .565717-0.625465$ C $-4.1407403 .680334-1.531648$ H $-2.0594401 .946016-2.954711$ H -2.271882 $3.625684-3.497378$ C $0.4146893 .489728-3.523109$ H $-0.1076512 .517847-1.690345$ C -5.016548 $2.545582-2.091293$ O $-4.6642884 .084640-0.265533$ H $-4.2346534 .554531-2.189177$ H $-6.0689732 .851787-2.026148$ O $-4.7249832 .418828-3.488171$ C $-4.8745381 .152953-1.435378$ C $-5.223467 \quad 1.1303800 .065326$ H -3.867289 $0.756895-1.604168$ O $\quad-5.7767530 .294948-2.146243$ H -4.5818241 .8300500 .608608$ H -6.2637561 .4644020 .167868$ H $0.4663772 .555940-4.092471$ H $0.0532454 .274000-4.196711$ H $1.4304213 .753625-3.211987$ C $0.4017514 .911237-0.455318$ H -4.0963604 .8000290 .068867$ H -5.297476 $1.716108-3.840855$ H -5.643519 $-0.609823-1.817558$ H 0.1111195 .8069910 .108299 H $1.3512015 .143649-0.951708$ H $-0.7444815 .476287-2.178006$ C $-5.927093-2.5175610 .582645$ O $-5.973176-1.1664070 .109330$ C $-5.073885-0.2526760 .740076$ C $-5.365961-0.1786172 .250514$ C $-5.384888-1.5672642 .899188$ C $-6.216370-2.5698012 .090515$ H $-6.752383-3.0220600 .063260$ C -4.626088 -3.224439 0.186179 H $-4.043984-0.5903570 .581403$ O H $-4.603540 \quad 0.4507542 .725696$
H $-5.797092-1.4826603 .912055$

O $-4.046941-2.0346653 .020250$

H $-6.054932-3.5907372 .455892$

O $-7.604431-2.2752642 .270221$

C $-4.497982-3.312189-1.315917$

H $-3.732765-2.7340760 .581358$

H $-4.607314-4.2496940 .574525$

H $-6.7642310 .532037 \quad 3.417488$

H -4.075491 -2.8998523 .462971$

H -7.800223 -2.351854 3.219519

O $-3.671524-2.327173-1.770818$

O $-5.081844-4.140457-2.002395$

C $-3.490790-2.276920-3.189267$

H $-4.456614-2.195715-3.697284$

H $-2.898465-1.388616-3.424578$

H $-2.947829-3.163884-3.528539$

SCF Energy $\left(B 3 L Y P / 6-31 G^{* *} / / M M F F\right)=-3245.91222500$

0800377

MM̄FF Geometry

C $-2.2031602 .780363-0.365375$

C -3.2913523 .2051120 .296035$

C -4.0379472 .4789291 .390738$

O $\quad-3.3756601 .2561891 .775388$

C -5.4354372 .0772540 .887472$

C -6.4421361 .8901402 .003924$

C -6.7701580 .7092922 .563099$

C $-7.136543 \quad 3.1527712 .447119$

C -6.167094 -0.6437782 .309077$

C -7.092392 -1.578117 1.517595

C $-7.016269-1.351167-0.000143$

C $-8.003544-2.260457-0.734359$

C $-7.861254-2.146480-2.259904$

O $-5.678251-1.653812-0.410571$

O $-8.579477-3.238923-2.853165$

C $-6.375621-2.324632-2.637560$

C $-8.501758-0.862072-2.799405$

O $-6.170268-2.086383-4.028438$

C $-5.414730-1.445861-1.802464$

C $-3.948156-1.862175-2.074503$

O $-5.589947-0.078142-2.177160$

C -2.3678671 .3134902 .682615$

O -1.9043642 .3045093 .219981$

C -1.907953 -0.0781392.877064

C $-0.694518-0.3235183 .389865$

C $-0.105340-1.6508763 .579720$

C $-0.957541-2.8469463 .237326$

C $-2.925831-1.241966-1.109288$

C $-1.500439-1.646960-1.449662$

C $0.851311-1.305383-0.688991$

O $-1.217763-2.380288-2.393614$

C $1.253833-2.4516290 .185540$

C $2.316066-2.4141841 .004823$

C $2.749313-3.5666471 .867399$

C $3.101517-3.1502393 .280292$

C $4.383627-3.0182193 .660155$

C $1.976331-2.9788914 .276410$

C $1.166135-1.7357294 .022994$

N $-0.579265-1.085343-0.575140$

H $-1.7680521 .811507-0.142536$

H -3.6969554 .1832570 .041363$

H -4.1153293 .1429602 .260751$

H $-5.360901 \quad 1.1583840 .296029$

H -5.8362852 .8363460 .202050$

H $-7.5413730 .707727 \quad 3.332951$

H -7.8568822 .9725603 .252165$

H -6.407124 3.8810532 .815496

H -7.682329 3.5999191 .609741

H $-5.187063-0.5730951 .830795$

H -5.979757 -1.095994 3.291712

H $-6.777940-2.6081541 .731997$

H -8.126920 -1.475716 1.867220

H -7.236899-0.300293 -0.216170

H $-9.034255-2.044086-0.427036$

H $-7.819279-3.305625-0.448157$

H $-9.506123-3.180940-2.563485$

H -6.119085 -3.379111-2.462909

H -9.571194 -0.837055 -2.557677 
H -8.441445 -0.819666 -3.892521 H $-8.0513110 .045824-2.392850$ H $-6.782706-2.664614-4.514770$ H -3.859958 -2.953235 -1.987139 H -3.674765 -1.594967-3.103272 H $-4.7632670 .405138-2.024322$ H -2.574651 -0.8690482 .551309$ H -0.0664850 .5203333 .672050$ H -1.847626 -2.877368 3.875420 H $-0.445670-3.802728 \quad 3.362321$ H -1.277981 -2.807670 2.190162 H -2.980192 -0.150144 -1.143186 H -3.131737 -1.565729 -0.082645 H $1.345144-0.373517-0.397397$ H $1.102371-1.529576-1.730450$ H $0.660548-3.3611330 .126617$ H $2.924083-1.5136181 .034209$ H $3.622853-4.0287211 .390216$ H $1.980846-4.3488281 .903187$ H $4.648666-2.7493944 .677150$ H $5.197994-3.1763602 .961164$ H $2.379099-2.8992345 .295191$ H $1.372193-3.8900134 .289813$ H $1.706447-0.8117674 .233234$ H $-0.912545-0.5349060 .210342$ C $-0.0821313 .974143-1.120669$ O $0.7868722 .830446-1.200418$ C $1.9342253 .076858-0.360022$ C $1.6967994 .427830 \quad 0.321824$ C $0.189814 \quad 4.576816 \quad 0.252899$ H 1.9490522 .2828280 .395881 C $3.2376793 .045126-1.177465$ H 2.0796364 .4400811 .347433 H $2.1899155 .244860-0.218389$ C $-0.291893 \quad 6.007210 \quad 0.412092$ H $-0.244803 \quad 3.9565991 .046668$ C $3.6020991 .648746-1.731379$ O $3.1428453 .996795-2.236819$ H $4.0589163 .375144-0.529508$ H $3.6827360 .972868-0.873260$ O $2.5439431 .167395-2.556399$ C $4.9254341 .649934-2.527785$ C $5.4288280 .255294-2.947978$ H $5.6973962 .151150-1.932008$ O $4.7558252 .419475-3.721794$ H $4.686905-0.240958-3.585187$ H $6.3057230 .413681-3.590202$ H $0.0084426 .408177 \quad 1.385657$ H $\quad 0.1243046 .659522-0.362977$ H $-1.383040 \quad 6.0610300 .347170$ C -1.527772 $3.586480-1.438750$ H $2.4594763 .678103-2.851459$ H $2.7159850 .229734-2.744015$ H $5.6245232 .489958-4.153020$ H -1.545515 2.989409-2.358859 H $-2.1010434 .499725-1.640082$ H $0.2537664 .665268-1.906367$ C $7.254130-0.6347680 .182064$ O $6.8058290 .033327-1.002424$ C $5.818539-0.656853-1.773571$ C $6.367121-2.011722-2.250324$ C $6.885772-2.845627-1.073560$ C $7.805236-2.032084-0.153837$ H $8.084681-0.0209770 .553484$ C $6.172076-0.6596511 .270251$ H $4.929776-0.828612-1.159036$ O $7.425072-1.798674-3.183045$ H $5.568356-2.550709-2.773984$ H $7.434870-3.711864-1.462834$ O $5.768646-3.337300-0.340225$ H $7.999850-2.5869750 .771417$ O $9.061854-1.877419-0.819874$ C 5.7230090 .7258991 .682779 H $5.288018-1.2014990 .929028$ H $6.562408-1.1562172 .165340$ H $7.729026-2.670852-3.487149$ H $6.111684-3.8841670 .386844$ H $9.662482-1.413920-0.211617$
O $4.569558 \quad 0.617227 \quad 2.407202$

O 6.3132901 .7655331 .424982

C 4.0151961 .8470672 .884030

H 4.6224872 .2290423 .709543

H 3.9517802 .5860122 .080079

H 3.0053531 .6447243 .251176

SCF Energy (B3LYP/6-31G**//MMFF) $=-3245.88968392$

0800378

MM̄FF Geometry

C $2.920557-0.0925154 .464312$

C 3.8852570 .5384753 .777361

C $4.869103-0.0706882 .806498$

O 4.4562490 .3167381 .471491

C $4.959279-1.6076812 .829008$

C $5.995976-2.1560851 .864618$

C $5.683733-2.8084180 .726690$

C $7.433987-1.9720712 .269823$

C $4.287948-3.0644330 .228137$

C $4.264955-3.608644-1.207183$

C $2.830686-3.922928-1.654757$

C $2.820414-4.650727-2.999164$

C $1.387401-4.923756-3.481301$

O $2.112795-2.688088-1.767538$

O $1.452881-5.312994-4.861678$

C $0.581449-3.610173-3.418544$

C $0.742058-6.094602-2.730528$

O $-0.795958-3.841141-3.706924$

C $0.722418-2.852240-2.075942$

C $0.069816-1.455402-2.203080$

O $0.042479-3.579193-1.049655$

C 4.8769551 .5174700 .994431

O 5.5755312 .3360131 .570066

C $4.3129611 .674160-0.365109$

C $4.3360712 .881521-0.947996$

C $3.8047853 .219485-2.270553$

C $3.2899422 .092605-3.126421$

C $0.442674-0.470469-1.086203$

C $-0.3648390 .811702-1.202182$

C $-0.2808443 .243543-0.679956$

O $\begin{array}{llll}-1.486970 & 0.845027 & -1.702579\end{array}$

C $0.0055743 .881963-2.003924$

C $0.5697145 .090901-2.146657$

C $0.8181945 .734767-3.481724$

C $2.2501166 .187185-3.666352$

C $2.5667447 .493116-3.675713$

C $3.3029045 .129054-3.922684$

C $3.8091444 .515680-2.646785$

N $0.2872921 .907463-0.654693$

H $2.786580-1.1654624 .367560$

H 3.9667591 .6184293 .892231

H $5.8607920 .344448 \quad 3.025731$

H $3.977243-2.0351242 .595443$

H $5.207870-1.9549813 .840319$

H $6.495275-3.1927860 .111826$

H $8.125251-2.4691321 .580948$

H $7.697031-0.9102452 .289483$

H $7.604728-2.3928443 .266118$

H $3.809911-3.7968900 .889922$

H $3.703552-2.1380670 .267409$

H $4.711759-2.865207-1.879785$

H $4.875716-4.518366-1.262006$

H $2.361272-4.546715-0.884646$

H $3.389269-5.586948-2.941701$

H $3.330598-4.034290-3.752503$

H $2.018172-6.102005-4.923798$

H $\quad 0.959988-2.960533-4.220366$

H $1.335314-7.007400-2.862357$

H $-0.250780-6.323009-3.133840$

H $0.645540-5.915861-1.657690$

H $-0.839332-4.302757-4.561784$

H $\quad 0.376780-0.991582-3.150016$

H -1.020870 -1.571980 -2.242306

H $-0.230387-2.960102-0.353713$

H $3.8779470 .800599-0.836801$

H $4.7706553 .713943-0.395528$

H $2.4270141 .611964-2.656359$

H $4.0702791 .337997-3.276881$ 
H $2.9716912 .408498-4.121559$

H $0.244476-0.893940-0.097261$

H $\quad 1.511280-0.237117-1.143728$

H $\quad 0.155320 \quad 3.802927 \quad 0.152791$

H -1.363033 $3.173572-0.531227$

H $-0.2891493 .330011-2.893753$

H $0.8406645 .660957-1.261062$

H $0.137698 \quad 6.592220-3.565881$

H $0.5501595 .060579-4.304813$

H $3.5873407 .825386-3.834478$

H $1.8142848 .258969-3.519890$

H $4.1642405 .576501-4.436429$

H $2.9003334 .394788-4.625136$

H $4.2271595 .249514-1.955479$

H $1.2384741 .804144-0.314074$

C $0.535667 \quad 0.723224 \quad 4.782934$

O $-0.029697-0.5866174 .574444$

C $-0.671745-0.6080623 .280766$

C -0.8460780 .8521772 .888986$

C $0.421117 \quad 1.4633573 .448606$

H $0.034349-1.1080452 .605360$

C -1.982191-1.401040 3.363030

H -0.9496720 .9935591 .810638$

H $-1.728475 \quad 1.2808863 .378778$

C 0.3713122 .9740073 .589252

H $\quad 1.2452301 .1994122 .775194$

C $-2.723730-1.4598462 .011975$

O $-1.663466-2.7280873 .789279$

H $-2.634550-0.9660544 .129390$

H -2.851817 -0.4379321 .642732$

O $-1.907474-2.1441941 .060881$

C $-4.115770-2.1201332 .064638$

C $-4.778158-2.2983430 .683384$

H $-4.763804-1.5206582 .714849$

O $-4.016218-3.4164542 .656702$

H $-4.218973-3.0416910 .100365$

H $-5.766508-2.744610 \quad 0.858006$

H $\quad 0.1993583 .4467432 .617539$

H -0.4321203 .2886944 .263089$

H 1.3152513 .3573843 .989437

C 1.9480050 .6153925 .367766

H -1.191123 -2.6548904 .636324$

H $-1.796470-3.0563841 .380749$

H -4.917228 -3.7727662 .739078$

H $1.906574 \quad 0.050021 \quad 6.306941$

H 2.3151301 .6179305 .618105

H -0.1042431 .2069775 .532668$

C $-5.946107 \quad 1.1940420 .044600$

O $-5.729634-0.096102 \quad 0.632231$

C $-4.932262-1.004815-0.132658$

C $-5.570817-1.268327-1.505563$

C $-5.832507 \quad 0.045083-2.249743$

C $-6.5554931 .062893-1.363218$

H $-6.698407 \quad 1.6720440 .685649$

C -4.6767482 .0604630 .081131$

H $-3.939344-0.574288-0.291275$

O $-6.799088-1.973012-1.336655$

H -4.899823 -1.909551 -2.089816

H $-6.442987-0.150748-3.139650$

O $-4.5972730 .607534-2.681902$

H $-6.5652962 .044473-1.850457$

O $-7.9240610 .667944-1.226105$

C -4.3519472 .5275941 .485239$

H $-3.7953341 .537817-0.295378$

H $-4.8427412 .956111-0.529435$

H $-7.158020-2.152748-2.222332$

H $-4.158850-0.046341-3.252515$

H $-7.943957-0.167815-0.729051$

O -3.5296423 .6157291 .411366$

O -4.7536782 .0006762 .513555$

C -3.1613964 .2080252 .660903$

H -4.005399 4.7796993 .057170

H -2.8425293 .4494803 .380942$

H -2.326069 4.8898252 .480120

SCF Energy (B3LYP/6-31G**//MMFF) $=-3245.91938055$

0800379

MM̄FF Geometry
C $3.302037-2.049449-2.990364$

C $2.213158-2.650422-2.484384$

C $0.971489-1.973302-1.960232$

O $0.932064-0.606263-2.439337$

C $0.974761-1.925499-0.418176$

C $0.827599-3.2475470 .304615$

C $-0.340716-3.7396350 .761097$

C $2.108496-3.9825980 .613125$

C $-1.713790-3.1414240 .638961$

C $-2.702337-4.1336270 .010843$

C $-4.153823-3.6466360 .132133$

C $-5.122016-4.663831-0.473909$

C $-6.573738-4.161786-0.438026$

O $-4.270175-2.397620-0.553144$

O $-7.353591-5.003160-1.301248$

C $-6.626169-2.736416-1.027911$

C $-7.187568-4.2893940 .961347$

O $-7.926631-2.170910-0.877045$

C $-5.560736-1.780602-0.439266$

C $-5.530603-0.464298-1.255634$

O $-5.890609-1.4915230 .918443$

C $-0.2802990 .004452-2.507356$

O $-1.370759-0.500234-2.295619$

C $-0.0412941 .428257-2.834831$

C $-1.0141512 .323119-2.608882$

C $-0.9312493 .771089-2.807660$

C $0.3433954 .328777-3.382548$

C $-4.3897700 .495022-0.889405$

$\begin{array}{llll}\text { C } & -4.705120 & 1.467998 & 0.233235\end{array}$

C -3.6050003 .2617181 .579270$

O -5.8304691 .6219090 .698170$

C -3.6932364 .5422620 .811064$

C -2.6816415 .4163950 .699454$

C $-2.7637606 .685404-0.099844$

C $-1.7627846 .724412-1.234930$

C $-0.6213847 .424309-1.121446$

C $-2.1218226 .027708-2.530381$

C $-1.9850484 .532749-2.445248$

N $-3.571677 \quad 2.165510 \quad 0.629813$

H $3.326734-0.969478-3.095940$

H $2.218941-3.734687-2.399478$

H $0.096385-2.515711-2.339196$

H $\quad 0.197163-1.232425-0.076002$

H $1.900722-1.438686-0.086672$

H $\quad-0.313878-4.6782151 .314279$

H $1.956288-4.794187 \quad 1.333191$

H $2.538370-4.425823-0.287676$

H $2.844766-3.3019191 .050891$

H $-2.052411-2.8776781 .648290$

H -1.708509 -2.217503 0.054081

H $-2.439608-4.263717-1.047087$

H -2.611847 -5.1144420 .494455$

H $-4.375026-3.4970721 .195624$

H $-5.038175-5.6328760 .033586$

H -4.846188 -4.849533 -1.521503

H $-7.270124-5.918466-0.982824$

H $-6.450453-2.824163-2.109422$

H $-7.180821-5.3363511 .288158$

H $-8.239642-3.9836500 .960904$

H $-6.658433-3.7055691 .717264$

H $-8.560826-2.794131-1.270933$

H -5.386386 -0.727103 -2.312231

H $-6.4936610 .053056-1.176259$

H $-5.399769-0.7036831 .198290$

H $\quad 0.938773 \quad 1.705070-3.205593$

H $-1.9619711 .965053-2.212435$

H $1.1894314 .109682-2.723814$

H $0.3244705 .412746-3.514217$

H $0.5428963 .894935-4.368611$

H $-3.475308-0.053566-0.642950$

H $-4.1766551 .113221-1.770585$

H -2.692395 3.2035012 .179040

H -4.4677743 .1605202 .244756$

H -4.6358664 .7504940 .310322$

H -1.7396165 .2091641 .202182$

H -2.5897947 .5239920 .587100$

H $-3.7725456 .841020-0.502044$

H $0.0893257 .484133-1.938823$ 
H $-0.3694717 .949408-0.206003$

H -1.533324 $6.428096-3.363662$ H $-3.1599156 .269234-2.793011$ H -2.866599 $4.036967-2.038839$ H -2.699835 2.0078630 .132526

C $5.797881-2.321920-2.600253$

O $5.525476-2.191382-1.186769$

C $6.079833-0.939714-0.734855$

C $7.085998-0.527832-1.801112$

C $6.367669-0.978053-3.058761$

H $5.263483-0.210091-0.740112$

C $6.657107-1.0634510 .677300$

H $7.2944800 .546115-1.785982$

H $8.035584-1.064537-1.689101$

C $7.273079-1.073242-4.274585$

H $5.560145-0.267212-3.273791$

C $5.622833-1.4476181 .757549$

O $7.678845-2.0674760 .659205$

H $7.152817-0.1246440 .952770$

H $5.285177-2.4780551 .593412$

O $6.318557-1.4400023 .010648$

C $4.411134-0.4962711 .837161$

C $3.470033-0.8068403 .018403$

H $3.846666-0.5685820 .902825$

O $4.8914550 .843153 \quad 1.949885$

H $3.313021-1.8897313 .082666$

H $3.946760-0.4681223 .946643$

H $7.701133-0.093749-4.512511$

H $8.101743-1.769208-4.105385$

H $\quad 6.715267-1.419551-5.150158$

C $4.551890-2.804593-3.354575$

H $8.035649-2.1326331 .561644$

H $5.753963-1.8837943 .665532$

H $4.119876 \quad 1.4344601 .949908$

H $4.410722-3.868370-3.123024$

H $4.714689-2.737444-4.435929$

H $6.571425-3.096056-2.690626$

C 1.1133872 .0984682 .762633

O 2.2904981 .3006792 .950904

C $2.097320-0.1141482 .880257$

C $1.106621-0.5767433 .958776$

C $-0.2118750 .198607 \quad 3.865758$

C 0.0227891 .7113223 .774942

H 1.4342763 .1233522 .990728

C $0.6296132 .075723 \quad 1.307197$

H $1.693385-0.3658991 .897444$

O $1.686200-0.3888125 .247836$

H $\quad 0.921319-1.6502513 .831441$

H $-0.823022-0.0266314 .748094$

O $-0.926170-0.2535642 .718734$

H -0.9074502 .2317053 .522872$

O 0.4364252 .1959325 .055718

C 1.6630482 .6892160 .392845

H $\quad 0.395609 \quad 1.0705780 .946627$

H -0.2893822 .6624071 .192638$

H $1.050072-0.7159855 .906369$

H -1.767689 0.2325312 .685298

H -0.2727751 .9931525 .689341$

O $2.3586661 .708066-0.248426$

O 1.8455293 .8949200 .286067

C $3.3941952 .146194-1.133572$

H $4.2818282 .412023-0.553273$

H $3.6416651 .317372-1.801423$

H $3.0687582 .993204-1.743825$

SCF Energy (B3LYP/6-31G**//MMFF) $=-3245.89564212$

08_00380

MM̄FF Geometry

C $0.141723-1.1284452 .275254$

C $0.678893-2.1550541 .596753$

C $0.005665-2.9013270 .468096$

O $-0.969202-2.056408-0.187308$

C $-0.702443-4.153407 \quad 1.004204$

C -1.281236 -5.018687 -0.097309

C $-2.586296-5.056781-0.431066$

C $-0.292053-5.908413-0.804451$

C $-3.701116-4.2367230 .156978$

C $-4.615046-3.664207-0.936194$
C $-5.742859-2.817703-0.330322$

C $-6.777385-2.442403-1.391372$

C $-7.876206-1.537558-0.813652$

O $-5.159981-1.6297850 .217152$

O $-8.616069-0.985079-1.912886$

C $-7.212029-0.357526-0.075774$

C $-8.881977-2.3246640 .034816$

$\begin{array}{lllll}\text { O } & -8.184082 & 0.430575 & 0.607747\end{array}$

C $-6.090636-0.7822950 .903128$

C $-5.335484 \quad 0.474274 \quad 1.394571$

O $-6.668955-1.4430922 .030105$

C $-0.486080-1.159711-1.091957$

O $0.672038-1.040590-1.460938$

C $-1.610041-0.300538-1.524574$

C $-1.3324860 .849299-2.157392$

C $-2.2981041 .849153-2.616605$

C $-3.7599751 .504549-2.522229$

C -4.0161940 .1840352 .121898$

C -3.3356911 .4833502 .508785$

C -2.1783073 .4567741 .505010$

O $-3.325021 \quad 1.8862823 .669278$

C $-2.7678194 .314824 \quad 0.429957$

C $-2.0399094 .992051-0.470822$

C $-2.6413855 .843992-1.552454$

C $-2.1224695 .499944-2.932290$

C $-1.3119316 .342699-3.594755$

C $-2.6034904 .215306-3.577484$

C $-1.8266093 .017479-3.100750$

N -2.787685 2.1427841 .420462

H -0.876509-0.815107 2.061935

H $1.693966-2.4693591 .829918$

H $\quad 0.771129-3.194895-0.260350$

H -1.486382 -3.862657 1.713118

H $\quad 0.001622-4.7614021 .588445$

H -2.894239 -5.752861 -1.210309

H $-0.771362-6.557118-1.545333$

H $0.456594-5.308078-1.329563$

H $\quad 0.221029-6.554709-0.084829$

H $-4.290725-4.8845900 .816645$

H -3.320255 -3.413939 0.768120

H -4.016062 -3.048751-1.620005

H $-5.043872-4.488848-1.519223$

H $-6.215184-3.3950210 .473373$

H -7.218946 -3.339976 -1.841944

H $-6.280557-1.906653-2.212575$

H -8.983182 -1.723694 -2.428276

H $-6.7698170 .294383-0.842680$

H -9.356199 -3.110916-0.564911

H -9.697279 -1.679475 0.380292

H -8.427873 -2.802314 0.905508

H $-8.8582760 .688520-0.044079$

H $-5.113438 \quad 1.1138280 .533046$

H -5.9816611 .0600942 .061395$

H $-6.069180-1.3568092 .787936$

H -2.617927 -0.613729-1.279080

H $-0.2878951 .102553-2.335446$

H $-3.9630360 .543393-3.008193$

H $-4.4114642 .232597-3.009830$

H -4.070954 $1.438700-1.476578$

H $-4.183271-0.3988903 .033539$

H $-3.331385-0.3925221 .490266$

H -1.099690 3.3245661 .384058

H -2.369644 3.8993132 .487264

H -3.8529564 .3831450 .393426$

H $-0.9548624 .946025-0.422449$

H $-2.4142886 .890991-1.313360$

H -3.735981 5.768521-1.556111

H $-0.9554016 .114854-4.593854$

H $-0.9820827 .276335-3.151437$

H $-2.4820714 .276247-4.666947$

H $-3.6791184 .124736-3.403914$

H $-0.7447343 .150127-3.158401$

H $-2.758524 \quad 1.6672020 .523458$

C 0.9052821 .1567972 .923078

O $1.516612 \quad 1.313875 \quad 1.627114$

C 2.6166882 .2288711 .738532

C 2.3021023 .0679152 .968786

C 1.7202702 .0149403 .894550 
H $3.5114801 .628173 \quad 1.940575$

C 2.8043823 .0075650 .432845

H 3.1895183 .5584363 .379273

H 1.5501393 .8366242 .756483

C $0.8971512 .590465 \quad 5.034248$

H 2.5459461 .4267024 .317040

C $2.8587252 .152616-0.852537$

O 1.7132223 .9269800 .277496

H 3.7027613 .6308350 .506644

H $1.8564601 .748975-1.043291$

O $3.1457413 .044882-1.937687$

C $3.8809300 .999904-0.889163$

C $5.3221791 .440917-0.565375$

H $3.8848050 .577940-1.902404$

O $3.478775-0.045021-0.011719$

H $5.5958852 .281893-1.212892$

H $5.367393 \quad 1.780537 \quad 0.475697$

H 1.5158783 .2313645 .670654

H $0.061530 \quad 3.192706 \quad 4.661496$

H 0.4842951 .7905615 .657201

C $0.886589-0.326823 \quad 3.304712$

H $0.895793 \quad 3.402758 \quad 0.226880$

H $3.0859042 .532707-2.762101$

H $2.580543-0.316905-0.266317$

H $\quad 0.402355-0.457555 \quad 4.278759$

H $1.914811-0.6991593 .392769$

H -0.1196881 .5350982 .834991$

C $6.949536-1.8340010 .227836$

O $6.133270-0.6577390 .287894$

C $6.343502 \quad 0.300818-0.752787$

C $7.791396 \quad 0.811533-0.704033$

C $8.792404-0.346819-0.768444$

C $8.442993-1.4641090 .223039$

H $6.741642-2.3677491 .164350$

C $6.540212-2.752835-0.933377$

H $6.155214-0.166179-1.725486$

O $8.011242 \quad 1.5658620 .486460$

H $7.9576911 .489409-1.549433$

H $9.7957660 .039159-0.551061$

O $8.803934-0.863172-2.096123$

H $9.039952-2.3615480 .023300$

O $8.775525-1.0264251 .544582$

C $5.108547-3.233174-0.804327$

H $6.630569-2.241189-1.895219$

H $7.182954-3.640055-0.950144$

H 7.7922850 .9961591 .243722

H $9.464818-1.575610-2.128241$

H $9.730038-0.8419951 .560681$

O $4.713498-3.774202-1.995126$

O $4.427112-3.1610240 .209156$

C $3.368892-4.261861-2.032694$

H $3.203099-4.728800-3.007151$

H $3.210669-5.013222-1.253004$

H $2.667128-3.430739-1.917972$

SCF Energy (B3LYP/6-31G**/MMFF) $=-3245.91028893$

0800381

MM̄FF Geometry

C -2.820598 2.7630140 .814354

C -3.191131 2.6866602 .101403

C -3.699901 1.4310352 .773198

O $-3.107034 \quad 0.256023 \quad 2.167973$

C $-5.223111 \quad 1.2989882 .612933$

C $-5.856426 \quad 0.473134 \quad 3.713889$

C -6.086906 -0.8528833 .657020$

C $-6.271545 \quad 1.2586474 .931881$

C $-5.708402-1.8232102 .572717$

C $-6.926830-2.3958781 .836808$

C $-7.446832-1.4623630 .733429$

C -8.738007 -2.0146190 .126407$

C $-9.220804-1.167692-1.060183$

O $-6.430318-1.380116-0.273260$

O $-10.231229-1.915169-1.754330$

C -8.047314 -0.973925 -2.042366

C $-9.8841470 .136025-0.600836$

O $-8.403108-0.075256-3.090967$

C -6.739875 -0.503395-1.362500

C $-5.572021-0.566255-2.378008$
O $-6.9037290 .846485-0.921863$

C -1.799131-0.011625 2.422207

$\begin{array}{llll}\text { O } & -1.052533 & 0.587881 & 3.177594\end{array}$

C $-1.421045-1.1453271 .551218$

C $-0.130114-1.3491161 .252149$

C $0.391770-2.3888210 .362207$

C $-0.600887-3.345077-0.247841$

C $-4.179692-0.387502-1.754253$

C $-3.091261-0.278339-2.810485$

C $-0.6590490 .216224-3.056028$

O $-3.305999-0.421237-4.011601$

C $0.107317-1.062659-3.174180$

C $1.429701-1.150792-2.962600$

C $2.216578-2.419214-3.130632$

C $3.106021-2.723775-1.946198$

C $4.426598-2.482226-1.995732$

C $2.482926-3.392975-0.742010$

C $1.719389-2.426357 \quad 0.123108$

$\mathrm{N}-1.8502090 .002156-2.253318$

H -2.9484331 .8945350 .176000$

H -3.0735703 .5575702 .741481$

H $-3.4166921 .457866 \quad 3.833084$

H -5.4616680 .8605231 .636301$

H -5.6995242 .2885722 .617134$

H $-6.575184-1.3203404 .511849$

H -6.7134250 .6243505 .707544$

H $-5.4061501 .763730 \quad 5.372733$

H -7.0150372 .0152754 .660295$

H $-4.999646-1.3950201 .860217$

H $-5.177449-2.6518493 .058691$

H -6.618922 -3.3443671 .377040$

H -7.726725 -2.6259112 .551259$

H -7.621247 -0.4675571 .157765$

H $-9.524152-2.0915230 .887925$

H $-8.564445-3.040186-0.229022$

H $-10.946452-2.109088-1.124408$

H -7.857634 -1.946093 -2.519202

H -10.750769 -0.0792410 .035685$

H -10.273187 $0.703058-1.453750$

H $-9.2120940 .783082-0.033275$

H $-9.218490-0.414124-3.498732$

H $-5.579003-1.541085-2.883025$

H $-5.7203970 .200325-3.149413$

H $-6.0398201 .286637-0.906214$

H -2.222204 -1.751794 1.143992

H $0.614752-0.6802281 .681496$

H $-1.096682-3.931470 \quad 0.533614$

H $-0.154178-4.056548-0.944872$

H - $-1.364562-2.801680-0.815167$

H $-4.1501580 .520915-1.147886$

H $-3.942295-1.240089-1.107929$

H $-0.0724841 .000753-2.568678$

H $-0.9504000 .564975-4.052057$

H -0.443171 -1.949932 -3.478095

H $1.985008-0.255405-2.691597$

H $2.827362-2.311053-4.036518$

H $1.558339-3.276881-3.317650$

H $5.074631-2.729057-1.162425$

H $4.887890-2.028582-2.866483$

H $3.264791-3.840761-0.114842$

H $1.879666-4.241210-1.076168$

H $2.354765-1.6841540 .609061$

H $-1.7557890 .044153-1.242788$

C $-0.8425093 .512512-0.467104$

$\begin{array}{llllll}\text { O } & 0.059555 & 3.016799 & 0.540803\end{array}$

$\begin{array}{llll}\text { C } & 1.403848 & 3.422718 & 0.206009\end{array}$

C $1.3211324 .040836-1.182909$

C $-0.0804004 .623169-1.179927$

H 1.6743264 .1873240 .945122

C 2.3321322 .2079500 .334068

H $2.0975814 .792243-1.354870$

H $1.4135253 .270358-1.958868$

C $-0.6063444 .944332-2.567651$

H $-0.0836305 .535594-0.568769$

C $3.7712272 .478396-0.151196$

$\begin{array}{llll}\text { O } & 2.325585 & 1.842272 & 1.717024\end{array}$

H $1.9058701 .360179-0.216182$

H $3.7361092 .725884-1.218207$ 
O 4.2905153 .6282040 .519438

C 4.7439551 .2940890 .033807

C $6.1221231 .599168-0.589185$

H $4.3128840 .405494-0.441829$

$\begin{array}{lllll}\text { O } & 4.889605 & 1.018061 & 1.423698\end{array}$

H $5.9761661 .902845-1.632741$

H $6.5748242 .438640-0.046584$

H $0.0285415 .690789-3.056045$

H $-0.628747 \quad 4.053525-3.204551$

H -1.622670 5.347281-2.516232

C -2.1554123 .9527120 .183123$

H 2.8219511 .0110711 .804798

H 4.3370413 .4135291 .467114

H 5.4618680 .2379851 .514242

H -2.829159 $4.376342-0.569105$

H -1.961004 4.7239820 .937736

H $-1.0303872 .682657-1.159370$

C $8.371848-0.8924271 .068429$

$\begin{array}{lllll}\text { O } & 7.495009 & 0.213485 & 0.817927\end{array}$

C $7.0913390 .400322-0.541764$

C $8.3212580 .629233-1.433973$

C $9.338735-0.504601-1.276960$

C $9.636408-0.8027090 .197122$

H $8.680457-0.7740322 .115285$

C $7.642427-2.2389810 .946136$

H $6.551927-0.487825-0.885828$

O $8.9307201 .873082-1.094206$

H $7.9920230 .696695-2.478015$

H $10.267050-0.226393-1.790362$

O $8.823513-1.668636-1.916533$

H $10.211875-1.7305270 .295340$

$\begin{array}{lllll}\text { O } & 10.447799 & 0.248731 & 0.729119\end{array}$

C $6.535702-2.3886101 .969852$

H $7.193663-2.360777-0.042024$

H $8.352321-3.0572721 .112470$

H $9.6839912 .004852-1.694533$

H $9.489797-2.371356-1.828145$

H $\begin{array}{llll}11.268821 & 0.277529 & 0.209247\end{array}$

O $5.844444-3.538317 \quad 1.710791$

O $6.307776-1.6087722 .884249$

C $4.760085-3.8157102 .602568$

H $5.135679-3.9605113 .619926$

H $4.026399-3.0044792 .572841$

H $4.275679-4.7385722 .272446$

SCF Energy (B3LYP/6-31G**/MMFF) $=-3245.89877426$

0800382

MM̄FF Geometry

C -0.699322 1.0919023 .244251

C -1.6479180 .3178023 .792483$

C $-1.986645-1.0572963 .287030$

O $-3.320450-0.9611172 .734026$

C $-1.977105-2.1200364 .399171$

C $-0.614173-2.4197854 .985563$

C $0.274128-3.2656564 .427006$

C $-0.313418-1.7653416 .307600$

C $0.128343-3.9761783 .109691$

C $1.462078-4.0215112 .350786$

C $1.263940-4.387343 \quad 0.873347$

C $2.589322-4.823880 \quad 0.246626$

C $2.443418-5.097411-1.256437$

O $\quad 0.767267-3.219848 \quad 0.203972$

O $3.759006-5.236134-1.814049$

C $1.763054-3.883084-1.918576$

C $1.745783-6.434541-1.525532$

O $1.477592-4.143383-3.290937$

C $0.482971-3.406572-1.188797$

C $0.036440-2.049683-1.787591$

O $-0.556395-4.366617-1.383920$

C -3.662007 -1.852168 1.765444

O $-3.026775-2.8255691 .394807$

C -4.923662 -1.375179 1.162357

C $-5.147122-1.636964-0.133616$

C $-6.251449-1.113630-0.937403$

C $-7.389675-0.449475-0.208653$

C $-1.062852-1.326464-1.001081$

C $-1.421407 \quad 0.011357-1.629177$

C $-2.8622622 .010691-1.251489$
O $-0.9493220 .399099-2.694896$

C $-4.2931111 .863637-1.663751$

C $-4.7074631 .853359-2.940246$

C $-6.1488121 .762621-3.361909$

C $-6.4662840 .479812-4.101518$

C $-6.2581240 .374490-5.424710$

C $-7.097861-0.651399-3.315832$

C $-6.161253-1.209561-2.280564$

N $-2.3398370 .714542-0.861818$

H -0.1402250 .7211972 .389321$

H -2.2178140 .6901704 .640293$

H $-1.292008-1.3335602 .484454$

H -2.413107 -3.052976 4.017925

H $-2.671202-1.8258355 .198981$

H $1.202550-3.4629144 .960900$

H $0.670625-2.0480046 .696756$

H $-0.326131-0.6758366 .210466$

H -1.059457 -2.057194 7.053960

H $-0.207475-5.0029893 .297883$

H -0.631043 -3.500253 2.485583

H $1.956129-3.0452592 .416207$

H $2.114649-4.7555872 .839797$

H $0.523198-5.1922070 .801589$

H $2.991646-5.7075530 .757610$

H $3.336527-4.0305700 .389335$

H $4.249262-4.413247-1.646512$

H $2.489020-3.059435-1.905150$

H $2.318480-7.259048-1.083743$

H $1.697503-6.646405-2.599571$

H $\quad 0.732722-6.480970-1.120543$

H $2.307606-4.426365-3.711359$

H $\quad 0.899879-1.375717-1.831143$

H $-0.307781-2.198894-2.819082$

H $-1.414772-3.929961-1.267240$

H $-5.569805-0.7454441 .762278$

H $-4.414408-2.230434-0.678850$

H -7.792936 -1.116941 0.561467

H -8.229358 $-0.188331-0.855435$

H $-7.053486 \quad 0.4759130 .271259$

H $-1.971757-1.933491-0.974303$

H $-0.732403-1.1414070 .026867$

H -2.250608 $2.439821-2.051686$

H -2.795211 $2.669295-0.380147$

H $-5.0257291 .794249-0.863872$

H $-3.9697381 .936762-3.735439$

H $-6.3655962 .623367-4.008394$

H $-6.8223421 .872281-2.502735$

H $-6.507161-0.531524-5.967046$

H $-5.8302221 .193289-5.993842$

H $-7.377596-1.475890-3.984343$

H $-8.039622-0.294070-2.891249$

H $-5.289199-1.706976-2.708135$

H -2.6752660 .3090530 .006806$

C $-0.663316 \quad 3.5450872 .719210$

O $0.261207 \quad 3.4692151 .614143$

C $0.617365 \quad 4.8070331 .212142$

C -0.2938305 .7460991 .995795$

C $-0.517742 \quad 4.961907 \quad 3.271849$

H 1.6513454 .9704111 .536135

C $0.5048034 .961879-0.307547$

H $0.168496 \quad 6.7232432 .166549$

H $-1.246195 \quad 5.913165 \quad 1.478685$

C $-1.714553 \quad 5.444105 \quad 4.073592$

H $0.3860405 .032636 \quad 3.892058$

C $1.4316514 .029802-1.116225$

O $-0.848798 \quad 4.679369-0.685156$

H $0.6860556 .007190-0.585441$

H $1.1394072 .987420-0.943928$

O $1.1676574 .305082-2.498780$

C $2.937158 \quad 4.231180-0.832979$

C $3.8830633 .545873-1.839187$

H 3.1664113 .8866680 .181356

O $3.2202805 .634431-0.873494$

H $3.6609803 .902679-2.852163$

H $4.9048643 .890298-1.627351$

H $-1.581472 \quad 6.4893494 .371551$

H -2.640530 5.3754843 .492799

H -1.8432674 .8491424 .983077$ 
C -0.3414452 .4612043 .749269$ H -0.905832 4.777127-1.651249 H $1.4831173 .544233-3.015086$ H $4.1466095 .755599-0.603331$ H $-0.8733292 .663563 \quad 4.685947$ H $\quad 0.7315902 .464770 \quad 3.976319$ H -1.669946 3.3963672 .305879 C $4.5740350 .206106-0.330233$ O $4.5234711 .616287-0.586118$ C $3.8446362 .012450-1.783140$ C $4.5222201 .383525-3.009272$

C $4.595202-0.140055-2.875299$ C $5.168032-0.566521-1.521742$ H $5.2706420 .104600 \quad 0.512275$ C $3.212568-0.3222730 .129979$ H $2.8040521 .678582-1.742166$ O $5.833587 \quad 1.915178-3.184100$ H $3.9481021 .648681-3.904792$ H $5.224540-0.547615-3.676072$ O $3.291148-0.693261-3.016872$ H $5.027629-1.644316-1.376217$ O $6.579361-0.330519-1.554531$ C 2.9044360 .2099951 .508944 H $2.386198-0.027077-0.521314$ H $3.207635-1.4175740 .168818$ H $6.3354041 .744070-2.368835$ H $2.957203-0.440988-3.894597$ H $6.955053-0.678175-0.727760$ O $3.254415-0.7076332 .453626$ O $2.4455451 .324851 \quad 1.719182$ C $3.038501-0.3133133 .812069$ H $1.967535-0.2179244 .008492$ H $3.444557-1.0943014 .460515$ H $3.557260 \quad 0.625756 \quad 4.027267$ SCF Energy (B3LYP/6-31G**//MMFF)= -3245.90691049

0800383

MM̄FF Geometry

C -1.618015 -2.725583 1.465554

C $-0.319790-2.7635551 .799799$

C $0.614558-1.5893391 .687395$

O $1.725888-1.9798410 .842531$

C $1.219337-1.2077343 .051294$

C $0.317624-0.3449573 .908631$

C 0.3423631 .0027163 .909218

C $-0.601401-1.0830134 .846184$

C 1.2017881 .8983303 .060291

C 0.3587512 .9477532 .323407

C 1.2290303 .8565351 .443181

C 0.3872564 .9576510 .798052

C $1.2071525 .815569-0.176532$

$\begin{array}{lllll}\text { O } & 1.824269 & 3.043858 & 0.427797\end{array}$

O $0.2642516 .585074-0.938322$

C $1.9655554 .884100-1.147604$

C 2.0934626 .8216830 .567034

O $2.8648525 .622630-1.971636$

C $2.7291993 .740135-0.436948$

C $3.2592192 .733374-1.491040$

O $3.826254 \quad 4.296095 \quad 0.286729$

C $1.567973-1.871847-0.501319$

O $0.563863-1.496888-1.090715$

C $2.810721-2.228323-1.222899$

C $3.813129-2.929700-0.670857$

C $5.070583-3.299652-1.326321$

C $5.371764-2.666400-2.659672$

C $3.7819481 .403736-0.927569$

C $5.2054701 .454171-0.403974$

C $6.821364 \quad 0.1193740 .948887$

O $5.9814432 .370451-0.660947$

C $7.585371-0.8379130 .089520$

C $8.119142-1.9794240 .549589$

C $8.905018-2.937602-0.298938$

C $8.359770-4.347246-0.241796$

C $8.922122-5.2734250 .553031$

C $7.218274-4.703867-1.168443$

C $5.896886-4.161420-0.696608$

$\begin{array}{llll}\mathrm{N} & 5.513137 & 0.338304 & 0.361281\end{array}$

H -2.047504 -1.800050 1.090146
H $\quad 0.102945-3.6898662 .182620$

H $0.122002-0.7130761 .246130$

H $2.178716-0.6989252 .891134$

H $1.506138-2.1101213 .609384$

H $-0.320174 \quad 1.5280744 .595963$

H -1.152166 -0.4047765 .506740$

H -1.339688 -1.665647 4.289105

H $-0.027824-1.7640125 .483409$

H $1.9208752 .405148 \quad 3.714952$

H $1.782615 \quad 1.3263302 .331457$

H -0.3798082 .4295821 .699533$

H -0.1925013 .5566103 .050910$

H 2.0171464 .2928482 .068159

H -0.0849565 .5874291 .562410$

H $-0.4382994 .499420 \quad 0.238009$

H $0.7624557 .179665-1.524183$

H $1.2202144 .437098-1.820580$

H 1.4761947 .5058391 .161544

H $2.6505287 .453211-0.133816$

H 2.8075096 .3467251 .243063

H $2.3401536 .258899-2.485499$

H $2.4281402 .472967-2.157888$

H $4.0339273 .206327-2.106405$

H 4.4634463 .5918910 .480200

H $2.845741-1.896090-2.255503$

H $3.708017-3.2791530 .354863$

H $4.683965-3.039783-3.425962$

H $6.386912-2.856512-3.013733$

H $5.273365-1.576261-2.599930$

H $3.1140701 .016885-0.150698$

H $3.7889790 .671019-1.744357$

H $6.661765-0.2685121 .959936$

H 7.3620981 .0683401 .019600

H $7.717580-0.571162-0.956434$

H $8.005561-2.2322531 .601485$

H $9.943296-2.9236430 .057977$

H $8.947801-2.606465-1.343996$

H $8.555324-6.2941900 .579918$

H $9.758970-5.0297911 .199214$

H $7.113553-5.795477-1.230918$

H $7.474045-4.389176-2.183646$

H $5.586640-4.5611780 .270057$

H $4.817835-0.393697 \quad 0.473439$

C $-3.179761-4.3171190 .291732$

O $-4.108394-3.297526-0.133248$

C $-5.412320-3.888579-0.263011$

C $-5.147977-5.362340-0.524833$

C $-3.990093-5.6131380 .421514$

H -5.907035 -3.781942 0.710731

C $-6.236729-3.165638-1.332072$

H $-6.019453-5.988967-0.313917$

H -4.833964 -5.542745 -1.559167

C $-3.194272-6.8650320 .093335$

H $-4.387541-5.7074341 .441350$

C $-6.270760-1.630751-1.180787$

O $-5.696599-3.451582-2.628767$

H -7.256760 -3.566490 -1.340360

H $-5.301803-1.219013-1.483268$

O $-7.232722-1.117223-2.109905$

C $-6.631499-1.1248580 .226119$

C -6.8120670 .4011730 .333151$

H $-5.856590-1.4368310 .935012$

O $-7.851430-1.7450230 .640581$

H -7.723383 $0.708422-0.195628$

H -6.9982490 .6262001 .391698$

H $-3.829640-7.7540620 .162184$

H $-2.782103-6.824154-0.920523$

H $-2.360406-6.9894610 .791427$

C $-2.542599-3.9007721 .620426$

H -4.764913 -3.171415 -2.627316

H $-6.985698-1.442974-2.992411$

H -8.035703 -1.452656 1.549389

H -2.003895 -4.751828 2.054054

H $-3.325243-3.6111162 .332911$

H $-2.419040-4.404415-0.493960$

C $-3.212166 \quad 1.3800040 .067243$

$\begin{array}{lllll}\text { O } & -4.438178 & 0.778803 & 0.514286\end{array}$

C $-5.6136641 .221426-0.172588$ 
C $-5.813822 \quad 2.7292200 .042828$ C $-4.5746483 .517950-0.389876$ C -3.2828802 .9142530 .175576$ H $-2.457151 \quad 1.025845 \quad 0.781013$ C $-2.8271250 .844056-1.319989$ H $-5.5001191 .032169-1.244911$ O -6.0931563 .0041931 .414316$ H -6.684065 $3.059704-0.536250$ H -4.677176 $4.555361-0.048621$ O $-4.5207863 .534578-1.813360$ H -2.410772 $3.359447-0.313016$ O $-3.1952893 .242803 \quad 1.566210$ C $-1.3944691 .138876-1.704606$ H $-2.953518-0.245352-1.333620$ H -3.482332 $1.254277-2.093542$ H -5.3591412 .6486121 .944161$ H $-3.7480704 .065367-2.071691$ H $-3.158510 \quad 4.212102 \quad 1.635939$ O $-1.237250 \quad 0.893391-3.039445$ O $-0.5200221 .518921-0.939162$ C $0.069041 \quad 1.146648-3.565424$ H $0.0744340 .852168-4.618201$ H $\quad 0.2974812 .214323-3.498333$ H $\quad 0.8199280 .555486-3.033152$ SCF Energy (B3LYP/6-31G**//MMFF)= -3245.91781337

0800384

MM̄FF Geometry

C -0.356808 2.7650911 .507075

C -0.0771913 .8556550 .776882$

C $0.7567783 .897360-0.484713$

O $1.1040462 .551982-0.887583$

C $2.0390374 .706073-0.225404$

C $2.8805814 .931420-1.464859$

C $4.0453904 .306859-1.727857$

C $2.3532435 .973657-2.418019$

C $4.7392033 .260395-0.901329$

C $5.0106631 .992475-1.720055$

C $5.7254350 .921891-0.884622$

C $6.067624-0.294619-1.745704$

C $6.718936-1.411773-0.917743$

O $4.857802 \quad 0.5305020 .185798$

O $6.712226-2.609212-1.709786$

C $5.845664-1.6855360 .323310$

C $8.187895-1.107995-0.601084$

O $6.484025-2.6100801 .201484$

C 5.442961-0.407083 1.099201

C $4.386694-0.7687342 .166869$

$\begin{array}{lllll}\text { O } & 6.593749 & 0.135343 & 1.747991\end{array}$

C $0.9092052 .191971-2.182417$

O $0.5516712 .921034-3.098016$

C $1.182396 \quad 0.750822-2.403618$

C $1.351360-0.134264-1.409384$

C $1.577074-1.576911-1.549449$

C $1.718480-2.139714-2.937100$

C $3.743070 \quad 0.4272042 .883277$

C $2.638413-0.0823203 .789927$

C $0.383205-1.1360863 .634524$

O $2.834208-0.3231824 .978565$

C $0.652668-2.5444423 .197920$

C $-0.059689-3.1865062 .259448$

C $0.310024-4.5387501 .720233$

C $0.604184-4.5190110 .231443$

C $-0.175250-5.193423-0.630324$

C $1.847871-3.799972-0.256223$

C $1.636446-2.319110-0.424542$

N $1.454136-0.3094363 .104597$

H $\quad 0.044575 \quad 1.798331 \quad 1.217079$

H -0.5002444 .8091551 .087841$

H $0.1438784 .382148-1.254184$

H 2.6248494 .2140000 .558135

H 1.7801145 .6900440 .189736

H $4.5748914 .578927-2.640455$

H $3.0211586 .133498-3.271261$

H $1.3786945 .677104-2.816676$

H $2.2410486 .934335-1.904662$

H $5.6912603 .678104-0.551936$

H $4.1666022 .996074-0.009609$
H $4.0572721 .595093-2.087189$

H $5.6211772 .242966-2.596625$

H $6.6376721 .364904-0.467638$

H $6.716406-0.010217-2.583454$

H $5.147825-0.690494-2.199504$

H $7.199680-2.427562-2.531578$

H $4.928199-2.177670-0.028632$

H $8.761856-0.985699-1.527563$

H $8.657242-1.941389-0.066550$

H $8.320169-0.200129-0.009029$

H $6.701966-3.4016630 .679906$

H $3.588431-1.3472531 .688237$

H $4.835988-1.4214222 .926814$

H $\quad 6.3051260 .6893612 .490187$

H $1.1973410 .447483-3.445558$

H $1.3012210 .221617-0.382033$

H $1.811450-3.227784-2.956113$

H $2.610428-1.730443-3.424200$

H $\quad 0.844422-1.894451-3.549206$

H 4.4682930 .9689523 .498671

H $3.321824 \quad 1.1438392 .169300$

H -0.557102 -0.7510283 .229884$

H $0.358322-1.0722484 .726276$

H $1.519771-3.0346313 .635133$

H $-0.920071-2.6950461 .813700$

H $-0.519136-5.2232371 .939910$

H $1.183672-4.9477342 .243452$

H $\quad 0.044522-5.212543-1.692661$

H -1.052810 -5.736656 -0.295967

H $2.210608-4.260041-1.180953$

H $2.659688-3.9556700 .466035$

H $1.536305-1.7927600 .521236$

H $1.429704-0.1146852 .108144$

C -2.5603382 .0714802 .398938$

O $-3.3371412 .915271 \quad 1.519785$

C -4.7204342 .7835451 .868751$

C -4.8041341 .6529062 .887343$

C -3.4689331 .8099513 .595907$

H -4.9931993 .7102342 .393448$

C -5.6328222 .6711040 .643093$

H -5.6607091 .7541453 .560621$

H -4.8700610 .6784462 .391598$

C -3.0761810 .6135874 .441566$

H -3.5110892 .7019574 .235806$

C $-5.3012341 .594763-0.417740$

O -6.9623422 .4416841 .127811$

H -5.6555993 .6488460 .145311$

H $\quad \begin{array}{llll}-5.416770 & 0.597133 & 0.019123\end{array}$

O $-6.3170271 .693448-1.430647$

C $-3.9226891 .712539-1.101041$

C $-3.7733770 .932374-2.421311$

H $-3.1429111 .386720-0.407117$

O $-3.6748563 .089690-1.404023$

H $-4.4470501 .354204-3.178100$

H -2.758290 $1.129106-2.793435$

H -3.8239110 .4305025 .220145$

H -2.989757 -0.2949543 .839578$

H $-2.113007 \quad 0.782767 \quad 4.932877$

C -1.242080 2.7676652 .723919

H -7.5492232 .3947240 .353656$

H $-6.2187822 .560742-1.860169$

H -2.760062 $3.159076-1.726960$

H -0.7157642 .2469723 .531242$

H -1.428847 3.7953343 .057903

H $-2.370718 \quad 1.1322291 .862176$

C $-3.051533-2.511856-1.174187$

O $-2.996885-1.100324-1.421058$

C $-3.992681-0.582865-2.307680$

C $-3.903228-1.263821-3.684483$

C $-3.964073-2.788604-3.555946$

C $-2.990701-3.302771-2.492069$

H -2.136187 -2.731406 -0.610655

C $-4.250667-2.899531-0.295375$

H $-4.984763-0.764458-1.883849$

O $-2.682846-0.892033-4.324058$

H $-4.726944-0.901253-4.311036$

H -3.714841 -3.250041-4.519351

O $-5.285698-3.189945-3.209524$ 
H $-3.178930-4.363672-2.291930$

O $-1.659982-3.217536-3.008062$

C $-4.109510-2.3796031 .118135$

H $-5.197977-2.518067-0.683786$

H $-4.307278-3.993274-0.238170$

H $-2.674122-1.306820-5.203511$

H $-5.881290-2.878418-3.912221$

H $-1.442353-2.275147-3.109541$

O $-4.976344-3.0552721 .928818$

O $-3.354880-1.482061 \quad 1.464469$

C $-4.958031-2.6749353 .308019$

H $-5.324591-1.6505913 .418991$

H $-5.624741-3.3471363 .854580$

H -3.948776 -2.773081 3.719438

SCF Energy (B3LYP/6-31G**//MMFF) $=-3245.90892440$

08_00385

MM̄FF Geometry

C $1.213340-3.579763-0.258219$

C $1.387573-3.2297391 .026606$

C $0.370397-2.5513491 .920899$

O $-0.841951-2.2490251 .194068$

C $0.937324-1.2227102 .452066$

C $0.095448-0.6257483 .562260$

C $-0.774437 \quad 0.3914683 .407152$

C $0.314808-1.2285184 .926483$

C -1.1420741 .1093252 .138991$

C -0.7173592 .5834912 .190717$

C -1.0840213 .3297880 .899722$

C -0.6321034 .7898550 .960124$

C $-1.0797605 .579064-0.281356$

$\begin{array}{lllll}\text { O } & -2.503339 & 3.270340 & 0.743739\end{array}$

O $-0.9134916 .977178-0.000013$

C $-2.5887265 .342049-0.511449$

C $-0.1988465 .274216-1.499136$

O $-3.0188185 .931750-1.736151$

C $-2.9903943 .850582-0.473232$

C $-4.5322753 .706670-0.480568$

O $-2.4554203 .193131-1.624580$

C -1.877957 -3.126231 1.281338

O $-1.929160-4.1463661 .948499$

C $-2.943237-2.6358040 .376566$

C $-4.039387-3.380393 \quad 0.164417$

C $-5.151531-3.044197-0.730910$

C $-5.055755-1.744799-1.487572$

C $-5.0071322 .285397-0.152467$

C $-6.5197442 .149785-0.144445$

C -8.2914520 .5272730 .519679$

O $-7.2828383 .021965-0.547276$

C $-8.348672-0.947190 \quad 0.757405$

C $-9.044965-1.8085230 .000854$

C $-9.052096-3.2910640 .247898$

C $-8.691262-4.102515-0.979280$

C $-9.506474-5.079838-1.411568$

C $-7.402763-3.794121-1.717756$

C $-6.177343-3.914648-0.849836$

$\begin{array}{llll}\mathrm{N} & -6.902663 & 0.908573 & 0.345599\end{array}$

H $0.258261-3.368388-0.732946$

H $2.334751-3.4514281 .511658$

H $\quad 0.156544-3.2243042 .760035$

H $1.038125-0.5098611 .628076$

H $1.955434-1.3687052 .837296$

H -1.311417 0.7434124 .287502

H $-0.282177-0.7349735 .700748$

H $\quad 0.042432-2.288274 \quad 4.928244$

H $1.366947-1.1386245 .216222$

H -0.7107280 .6320611 .255109$

H -2.230152 1.0372592 .021250

H -1.215407 3.0636533 .043487

H 0.3642092 .6524932 .361242

H -0.6079432 .8168200 .057169$

H $\quad 0.4550414 .857387 \quad 1.088687$

H -1.067729 5.2688961 .848251

H 0.0212557 .1296450 .221243

H -3.1280535 .8654450 .290688$

H $0.8407305 .560444-1.300521$

H $-0.5082115 .864079-2.368766$

H $-0.2021374 .218322-1.778379$
H $-2.7583956 .868673-1.713767$

H -4.9659484 .3808750 .269604$

H -4.931633 4.004870 -1.458033

H -3.004882 $2.423684-1.839447$

H -2.781210 -1.680792 -0.111123

H $-4.129617-4.3334820 .683875$

H $-4.945617-0.905756-0.794238$

H -5.928232 -1.525153 -2.104856

H $-4.189652-1.756638-2.158930$

H $-4.619666 \quad 1.571294-0.886451$

H $-4.6389431 .990407 \quad 0.837125$

H $-8.8600320 .839255-0.362596$

H -8.6804771 .0680651 .388340$

H -7.775256 -1.329248 1.599537

H -9.636199-1.435301-0.831703

H $-10.054941-3.5609090 .603162$

H -8.361888 -3.556316 1.058651

H $-9.267428-5.670448-2.289783$

H $-10.432898-5.313827-0.897340$

H -7.502687 -2.805229 -2.171499

H $-7.273382-4.492820-2.554694$

H $-6.132351-4.845392-0.282410$

H -6.1947410 .3403920 .803302$

C $3.604928-4.517419-0.631427$

O $4.311108-3.266812-0.500156$

C $5.685366-3.467472-0.885882$

C $5.855739-4.967143-1.106114$

C $4.467949-5.368151-1.562350$

H $5.823168-2.956730-1.845419$

C $6.628164-2.8826650 .169586$

H $6.632847-5.190762-1.843399$

H $6.118034-5.486544-0.176740$

C $4.209138-6.861749-1.463111$

H $4.331512-5.044891-2.603095$

C $6.477252-1.3618770 .383817$

O $6.360810-3.5334441 .419132$

H $7.665551-3.127221-0.088388$

H $5.489917-1.1492830 .807417$

O $7.426408-0.9806131 .389708$

C $6.711923-0.506075-0.878842$

C $6.8429231 .004833-0.603328$

H $5.906350-0.683139-1.599677$

O $7.931131-0.937237-1.492701$

H $7.647137 \quad 1.1770420 .122615$

H $7.1771241 .486848-1.532485$

H $4.913927-7.416198-2.091508$

H $4.321449-7.221092-0.434762$

H $3.196279-7.107441-1.796645$

C $2.197345-4.262406-1.174622$

H $6.961066-3.1479812 .080239$

H $8.315672-1.1244551 .022742$

H $8.025240-0.452799-2.330571$

H $1.744396-5.214383-1.475374$

H $2.274165-3.641829-2.076802$

H $3.563558-4.9803950 .362559$

C $3.3670352 .299504-1.019963$

O $4.6527761 .708740-1.254052$

C $5.5405091 .666353-0.129815$

C 5.7919123 .0802950 .417847

C 4.4762063 .7909980 .741921

C $3.4937423 .712962-0.428759$

H $2.9234332 .403397-2.019196$

C $2.4709791 .371359-0.194475$

H 5.0962261 .0571360 .661874

O $6.5380633 .855188-0.518401$

H 6.4007583 .0030391 .326380

H 4.6864864 .8405610 .980566

O 3.9007753 .1842081 .895622

H $2.5037674 .069284-0.124706$

O $3.9419934 .578321-1.475650$

C $1.8595570 .318695-1.089056$

H 2.9944780 .8476890 .609495

H 1.6573541 .9324220 .276492

H $6.0469633 .861947-1.357764$

H 3.0776113 .6605262 .098313

H $3.9752095 .480947-1.115478$

O $0.5689050 .651114-1.377361$

O $2.465700-0.661458-1.500854$ 
C $-0.132162-0.247470-2.241377$

H $-1.082500 \quad 0.218620-2.515497$

H $0.440955-0.436223-3.154166$

H $-0.340165-1.181842-1.714427$

SCF Energy (B3LYP/6-31G**//MMFF) $=-3245.90636155$

0800386

MM̄FF Geometry

C $2.778932-1.522610-2.758685$

C $1.948583-0.863364-1.935469$

C $0.455241-1.042023-1.930176$

O $\quad-0.1090250 .292905-1.936754$

C $-0.073025-1.747521-0.668296$

C $0.298465-3.208046-0.561617$

C $-0.330327-4.200680-1.220181$

C $1.425527-3.5222240 .383869$

C -1.485271-4.078585-2.176117

C $-2.662283-4.986063-1.788277$

C -3.382682 -4.550711-0.501892

C $-4.453247-5.574986-0.121454$

C $-5.266471-5.1258271 .100364$

O $-3.992940-3.280302-0.746208$

O $-6.423677-5.9715251 .191678$

C $-5.772913-3.6874770 .863441$

C $-4.486269-5.3161362 .407002$

O $-6.410801-3.2047672 .044781$

C $-4.667131-2.711186 \quad 0.386275$

C $-5.303058-1.372633-0.071749$

O $-3.766355-2.4658881 .464986$

C $-1.2256050 .517999-2.676727$

O $-1.790199-0.263019-3.424494$

C $-1.6687771 .897348-2.379845$

C $-2.7745892 .391929-2.954109$

C $-3.3455923 .705982-2.667185$

C $-4.8502403 .720529-2.690621$

C $-4.322555-0.380661-0.709109$

C $-3.705763 \quad 0.556251 \quad 0.310110$

C -4.1777052 .6325931 .608805$

$\begin{array}{lllll}\text { O } & -2.602671 & 0.332043 & 0.802429\end{array}$

C -4.3616953 .9928621 .014723$

C -3.3830484 .9062520 .921639$

$\begin{array}{llll}C & -3.586422 & 6.292557 & 0.379696\end{array}$

C $-2.6359096 .634476-0.745768$

C -1.538563 $7.377410-0.522281$

C $-3.0067896 .193900-2.144663$

C $-2.5710044 .783892-2.431042$

N $-4.521041 \quad 1.6331330 .615134$

H $2.377820-2.257323-3.451823$

H $2.360711-0.136004-1.239788$

H $\quad 0.142574-1.583168-2.831614$

H $-1.165528-1.650425-0.629353$

H $\quad 0.263508-1.2072450 .227979$

H $\quad 0.024442-5.219618-1.069523$

H $1.646094-4.5937420 .431026$

H $2.339015-3.0125110 .070505$

H $1.173540-3.1920151 .396919$

H -1.832590 -3.046766 -2.280519

H -1.126981 -4.385043 -3.166632

H -3.377636 -4.971080 -2.621001

H -2.308415 -6.019664 -1.685745

H $-2.649373-4.452246 \quad 0.305997$

H $-4.003753-6.5597530 .058149$

H -5.144220 $-5.711246-0.965391$

H $-6.117472-6.890857 \quad 1.274281$

H $-6.550577-3.7468250 .088731$

H $-4.213324-6.3701122 .539687$

H -5.100737 $-5.055423 \quad 3.275919$

H $-3.565613-4.7304182 .446653$

H -6.928353 -2.418299 1.805157

H $-6.061186-1.600774-0.832248$

H $-5.810889-0.8899580 .770942$

H -3.200272 -1.714369 1.229452

H -1.092075 2.468515 -1.660594

H -3.342824 $1.781729-3.653198$

H -5.215339 $3.619436-3.718533$

H $-5.2809784 .632015-2.270174$

H -5.253635 $2.888832-2.101937$

H $-3.521626-0.883105-1.258676$
H $-4.8582840 .230956-1.446283$

H $-3.1593672 .472443 \quad 1.974207$

H -4.865582 2.5016982 .450268

H -5.3606954 .2483010 .668855$

H -2.3895984 .6611371 .289626$

H -3.4467476 .9956081 .211450$

H -4.6190626 .4422910 .040459$

H $-0.8683527 .652050-1.329993$

H -1.282579 7.7215520 .474454

H -2.516043 $6.843337-2.882296$

H $-4.0768326 .352158-2.307022$

H -1.487666 $4.665585-2.464777$

H $\quad-5.453236 \quad 1.6712370 .212617$

C $5.060992-2.359589-2.071779$

O $4.787192-2.249279-0.659498$

C $5.979334-1.795363-0.002026$

C $7.100249-2.402894-0.826274$

C $6.577121-2.161936-2.232604$

H $6.008840-0.702412-0.102982$

C $5.952991-2.1606511 .484936$

H $8.066689-1.925255-0.641201$

H $7.201676-3.478513-0.641956$

C $7.197156-3.075692-3.276389$

H $6.794319-1.121864-2.511886$

C $4.643059-1.7834552 .211290$

O $6.131329-3.5739111 .638303$

H $6.809390-1.6969001 .987661$

H $3.855256-2.4979501 .946150$

O $4.855929-1.9348633 .619567$

C $4.138314-0.3568081 .938470$

C $2.863366-0.0239072 .738411$

H $3.925918-0.2491890 .869773$

O 5.1738670 .5675482 .267686

H $2.193787-0.8922152 .742776$

H 3.1441540 .2059953 .773540

H $8.279508-2.920232-3.330552$

H $7.019996-4.130760-3.042064$

H $\quad 6.774978-2.874317-4.266349$

C $4.262056-1.280693-2.809984$

H $5.420485-4.0153211 .142120$

H $5.169583-2.8431353 .769295$

H $4.849718 \quad 1.4599942 .058317$

H $4.564781-1.240535-3.862998$

H $4.483006-0.297972-2.375921$

H $4.746918-3.361985-2.385520$

C 2.4249703 .5177081 .610229

O 2.9413692 .3289812 .222984

C 2.1037631 .1738202 .133192

C $0.765018 \quad 1.4332572 .837511$

C 0.0870692 .6892482 .276715

C 1.0502113 .8782732 .199848

H 3.1284224 .3105061 .895838

C 2.4287453 .4164240 .076274

H 1.9142280 .9433351 .079325

O $0.987422 \quad 1.5939514 .236660$

H $\quad 0.1173710 .5586682 .702684$

H -0.7664032 .9606852 .909518$

O $-0.4050982 .422010 \quad 0.968228$

H 0.6024494 .6883921 .612777

O 1.2484284 .3946723 .519297

C $3.8257853 .230212-0.479177$

H $1.8235672 .575229-0.271917$

H $2.0239194 .338526-0.356230$

H $\quad 0.120870 \quad 1.7238914 .658281$

H -1.0126841 .6656531 .029401$

H 1.7078073 .7131854 .039391

O $3.7376062 .897789-1.801380$

O $4.862375 \quad 3.373588 \quad 0.154375$

C $4.9873642 .699788-2.469922$

H $4.7788322 .334684-3.479030$

H $5.5261983 .649014-2.542349$

H $5.5911261 .952575-1.945446$

SCF Energy (B3LYP/6-31G*//MMFF)= -3245.92409584

08_00387

MM̄MF Geometry

C -1.678225 $5.035197-1.112294$

C $-2.5831864 .048268-1.192109$ 
C $-3.6797563 .848675-0.175760$

O $-3.5610522 .507203 \quad 0.358724$

C $-5.0528743 .994211-0.851980$

C $-6.204313 \quad 4.061461 \quad 0.132774$

C -7.1344363 .1020760 .305023$

C $-6.310216 \quad 5.3492280 .912405$

C $-7.2335531 .767311-0.378120$

C -7.3442620 .6325220 .648113$

C -7.509496 $-0.737001-0.026779$

C -7.696971-1.828295 1.028786

C $-7.761719-3.228900 \quad 0.403768$

O $-6.334215-0.989206-0.800272$

O $-7.628344-4.1888751 .463505$

C $-6.544144-3.407540-0.527064$

C $-9.119373-3.498013-0.254888$

O $-6.620889-4.646345-1.230180$

C $-6.336244-2.231802-1.512617$

C $-4.986517-2.349218-2.263106$

O $-7.380263-2.266681-2.491684$

C $-2.5937902 .314952 \quad 1.299871$

O $-1.869073 \quad 3.168006 \quad 1.789647$

C -2.5303390 .8705451 .616663$

C $-1.419027 \quad 0.3922402 .198023$

C -1.161221 -0.9939132 .595575$

C $-2.307452-1.9660772 .521577$

C $-3.767894-2.015988-1.399547$

C $-2.451039-2.419067-2.036904$

C $0.001974-2.301764-1.570036$

O $-2.372369-3.130786-3.033542$

C $0.447142-3.182211-0.444004$

C $1.447792-2.8696360 .392224$

C $1.891785-3.7437161 .528690$

C $1.911789-3.0174622 .856181$

C $3.069765-2.7641713 .489430$

C $0.584172-2.6580393 .492805$

C $0.073458-1.3170383 .035948$

N -1.373644 -1.919762 -1.317675

$\mathrm{H}-1.7481425 .753618-0.299685$

H $-2.5179293 .324498-2.001021$

H -3.5879914 .5732240 .642518$

H $-5.1953493 .177967-1.567699$

H -5.076391 4.915011-1.451093

H -7.9354823 .2955101 .018319$

H -7.223763 5.3972541 .514922

H -5.4628825 .4602911 .595272$

H -6.3248806 .2062030 .230933$

H -8.128640 $1.773686-1.011784$

H $-6.3807061 .577994-1.034143$

H $-6.4368620 .629781 \quad 1.266887$

H -8.1967210 .8165651 .314243$

H -8.377819 $-0.695931-0.694928$

H -8.592940 -1.636016 1.632387

H $-6.852561-1.8032071 .732364$

H -8.350518 -4.0383802 .097356$

H -5.666622 -3.4907620 .124614$

H $-9.927557-3.3965380 .479477$

H -9.181935 -4.527169-0.625378

H -9.338075 -2.818151 -1.080844

H -7.266931 -4.538974 -1.948973

H -4.899672 -3.346394 -2.711756

H -4.981255 -1.654145 -3.114110

H -7.089219-1.766065 -3.271492

H $-3.3781380 .249509 \quad 1.352166$

H -0.6008311 .0848422 .395398$

H -3.186863 -1.568401 3.040908

H $-2.092048-2.9292692 .988598$

H -2.576313 -2.162981 1.481040

H -3.744106 -0.938482 -1.196653

H -3.822777 -2.540254 -0.441489

H $\quad 0.590184-1.381769-1.635129$

H $0.082678-2.837246-2.520604$

H -0.083994 -4.121987 -0.312200

H $1.985986-1.9372850 .246910$

H $2.894315-4.1192341 .286069$

H $1.253134-4.6310911 .619895$

H $3.088138-2.2663744 .453320$

H $4.024990-3.0428213 .057604$

H $\quad 0.699810-2.6131774 .583777$
H $-0.122106-3.4710413 .304383$

H $0.821841-0.5248813 .089890$

H - $1.552520-1.335527-0.506306$

C $0.8230874 .824438-1.499741$

O $0.8411883 .421143-1.170124$

C 1.5409723 .2499080 .082937

C 2.2525124 .5698870 .344002

C $1.2679765 .564760-0.238902$

H $\quad 0.7626903 .0754920 .835087$

C $2.4640472 .031957-0.024547$

H 2.4524464 .7388531 .406223

H $3.2066294 .612697-0.196068$

C $1.8669286 .933799-0.507644$

H 0.4384485 .6729390 .469811

C 3.3687911 .8084521 .200577

O $1.6456020 .871748-0.202139$

H $3.0725642 .129232-0.930286$

H $4.0348862 .666693 \quad 1.349073$

O 2.5584651 .7080272 .378556

C $4.218506 \quad 0.5228791 .137514$

C $5.1350310 .460823-0.090641$

H $3.568952-0.3566421 .179874$

O 5.0432530 .4797812 .307246

H $4.5472050 .469546-1.015509$

H $5.7497921 .371176-0.124228$

H 2.2280757 .3848020 .422396

H $2.7119056 .873790-1.201659$

H $1.1202447 .606098-0.941955$

C $-0.5416685 .210118-2.079635$

H $1.0737541 .034461-0.971856$

H 2.1756932 .5840502 .552537

H 4.4513080 .5135303 .078354

H $-0.7273494 .598521-2.971934$

H $-0.5241776 .254871-2.410727$

H $1.5740764 .956102-2.290135$

C $7.946821-1.559005-1.462613$

O $6.917334-0.577718-1.279342$

C $6.095834-0.744526-0.115007$

C $5.380548-2.102537-0.165479$

C $6.372292-3.253612-0.356934$

C $7.354913-2.977394-1.500319$

H $8.361675-1.342984-2.455898$

C $9.078161-1.398879-0.441046$

H $6.733839-0.6926460 .773039$

O $4.426275-2.118740-1.225266$

H $4.829776-2.2557560 .767734$

H $5.812092-4.173304-0.565576$

O $7.085225-3.4452230 .861349$

H $8.168584-3.711962-1.498402$

O $6.667106-3.118926-2.746831$

C $9.805132-0.093318-0.657428$

H $8.739053-1.4389260 .597983$

H $9.817174-2.201295-0.552937$

H $4.896026-1.914832-2.052131$

H $7.688402-4.1970910 .733156$

H $6.332108-4.030652-2.794670$

O $9.243753 \quad 0.8854750 .108045$

O $10.7417520 .043507-1.433915$

C $9.8252792 .185164-0.030709$

H 9.2889332 .8698860 .631542

H $\quad \begin{array}{llll}10.878450 & 2.162800 & 0.265271\end{array}$

H $9.7197922 .538867-1.060777$

SCF Energy (B3LYP/6-31G**//MMFF) $=-3245.92069012$

08_00388

MM̄MF Geometry

C $1.0566450 .960489-2.912042$

C $1.709615-0.211051-2.981120$

C $1.164912-1.578208-2.632066$

O $-0.197331-1.491792-2.158493$

C $2.017742-2.204672-1.514456$

C $1.688484-3.658140-1.238657$

C $0.947293-4.098604-0.202578$

C $2.298062-4.643923-2.201983$

C $0.250906-3.2770130 .846634$

C $-1.255519-3.5715450 .859291$

C $-2.020217-2.6380841 .808842$

C $-3.511459-2.9702861 .805345$ 
C $-4.313651-1.9869302 .668364$

O $-1.831508-1.297878 \quad 1.352778$

O $-5.705245-2.1686892 .364655$

C -3.949602 -0.5386222 .271871$

C $-4.152224-2.2860034 .164345$

O $-4.5559490 .377054 \quad 3.177852$

C $-2.429928-0.2829262 .178557$

C $-2.086524 \quad 1.0463181 .462723$

O $-1.851064-0.2819583 .483203$

C -1.191871-1.734164-3.054656

O $-1.069281-2.007120-4.237909$

C $-2.478971-1.622003-2.334771$

C $-3.601287-2.059981-2.921897$

C $-4.919155-2.072022-2.296224$

C $-5.654725-3.369863-2.497407$

C -2.4082012 .3484882 .200232$

C -3.7561662 .9686811 .881703$

C $-5.176637 \quad 3.7983770 .010356$

O -4.5351413 .2954332 .776169$

C -6.2930342 .8025950 .000786$

C $-6.8794962 .338647-1.112843$

C $-8.0342981 .378353-1.096342$

C $-7.6979520 .047575-1.726588$

C $-8.278770-0.343901-2.873291$

C $-6.748978-0.861639-0.977967$

C $-5.412623-0.991077-1.659074$

N -3.9743983 .1739620 .529600$

H $0.0204590 .984582-2.589811$

H $2.743456-0.203392-3.322904$

H $1.221568-2.206723-3.529010$

H $1.917441-1.611243-0.600882$

H $3.080958-2.147987-1.781894$

H $\quad 0.810089-5.173747-0.092872$

H $2.066214-5.681444-1.938810$

H $1.924861-4.471476-3.216121$

H $3.388330-4.542544-2.209876$

H $\quad 0.679075-3.5242231 .825342$

H $\quad 0.415187-2.2078110 .695179$

H -1.647540 $-3.454238-0.158319$

H -1.424230 -4.615347 1.152688

H -1.598146 -2.743392 2.815032

H -3.682255 -4.002086 2.136776

H -3.895606 -2.919557 0.777026

H -5.933264 -3.096357 2.546850

H -4.389180 -0.342348 1.285143

H -4.471841 -3.311176 4.386604

H -4.794058 -1.636368 4.769806

H -3.123209 -2.176953 4.513199

H -5.5135240 .2077953 .165303$

H -1.000429 1.0439491 .300809

H -2.5145301 .0264600 .453619$

H -1.016966 $0.215087 \quad 3.448091$

H -2.474556 -1.216634 -1.329321

H -3.561411 -2.493888 -3.918941

H -5.127965 -4.185413 -1.989293

H -5.720462 -3.615029 -3.563712

H -6.678434 -3.356940 -2.117805

H -2.3325962 .2187143 .285084$

H -1.658996 $3.103071 \quad 1.931001$

H $-4.9467734 .173245-0.992143$

H -5.4431064 .6480990 .647359$

H -6.6570512 .4656440 .968798$

H -6.531493 2.679819-2.084696

H -8.871264 $1.854703-1.622938$

H -8.390798 $1.204103-0.072980$

H -8.065053 -1.312673 -3.312150

H -8.980876 $0.294882-3.398659$

H -7.230210 -1.831105 -0.815237

H $-6.577681-0.4713630 .033067$

H -4.797719 -0.092527-1.603965

H -3.247485 $2.934662-0.137304$

C $1.7359523 .281744-2.105965$

O $2.3485852 .679983-0.945930$

C 1.5107852 .9463500 .193307

C $0.6772284 .158473-0.197107$

C $0.3836253 .837537-1.650618$

H $\quad 0.8411632 .085030 \quad 0.295829$

C 2.3383523 .1015991 .471257
H $\quad-0.2256884 .2639640 .409739$

H $1.2536345 .087993-0.122119$

C $-0.0935775 .033803-2.455589$

H $-0.3903663 .061157-1.684413$

C 3.2727451 .9099301 .773054

O $3.1513894 .274677 \quad 1.352704$

H 1.6641583 .2799552 .317662

H 4.1171901 .9314991 .076609

O 3.8339292 .1173823 .074957

C 2.5942770 .5308121 .729141

C $3.459333-0.6414782 .222571$

H 2.2670850 .3255950 .704730

O $1.4193680 .569636 \quad 2.544431$

H $3.603575-0.5696873 .308483$

H $2.864053-1.5517632 .070695$

H -1.031851 $5.422519-2.046240$

H $0.6406075 .846361-2.441967$

H $-0.2693304 .754864-3.499253$

C $1.6893862 .280654-3.265795$

H 3.6688454 .3527952 .172513

H 3.1098432 .0473713 .720669

H $0.967376-0.2857662 .444723$

H $2.7169662 .108509-3.609415$

H $1.1469492 .717675-4.111957$

H $2.3937084 .110446-2.399213$

C $5.798085-0.900490-0.691646$

O $4.615237-0.804570 \quad 0.114778$

C $4.823288-0.7915531 .528795$

C $5.548262-2.0713951 .979796$

C $6.842182-2.2925241 .191672$

C $6.620944-2.145485-0.317122$

H $5.428243-1.043501-1.714794$

C $6.6197720 .398279-0.665025$

H 5.4337230 .0772521 .794269

O $4.701344-3.2089001 .817674$

H $5.775062-1.9913663 .049389$

H $7.223890-3.2977891 .409318$

O $7.813182-1.350741 \quad 1.637258$

H $7.584132-2.127130-0.840176$

O $5.920351-3.307081-0.772467$

C $5.8851371 .549611-1.317970$

H $\quad \begin{array}{llll}6.862171 & 0.696361 & 0.358224\end{array}$

H $7.5555270 .261637-1.218818$

H $4.428662-3.246650 \quad 0.884741$

H $8.636733-1.5260501 .150851$

H $5.832357-3.238532-1.738448$

O $6.4656072 .725015-0.935064$

O $4.9402161 .434889-2.085884$

C $5.8808563 .909739-1.484840$

H $6.4849744 .764605-1.169323$

H $5.8800743 .866135-2.578271$

H $4.8648544 .038406-1.102099$

SCF Energy (B3LYP/6-31G**//MMFF) $=-3245.90698230$

08_00389

MM̄MF Geometry

C 2.2484553 .6195041 .206332

C 2.9859924 .2651010 .289704

C $3.6733173 .643283-0.907553$

O $3.4281662 .219275-0.976519$

C $5.1916353 .863607-0.815145$

C $5.9151593 .504701-2.098447$

C $6.6287992 .377924-2.288288$

C $5.8376854 .540527-3.191114$

C $6.8209421 .241654-1.323883$

C $6.577029-0.114463-2.001309$

C $6.805520-1.273778-1.022607$

C $6.739834-2.620823-1.742171$

C $6.897741-3.793113-0.761855$

O $5.787772-1.212939-0.018063$

O $6.516277-4.995773-1.446721$

C $5.907601-3.6033430 .405585$

C $8.355194-3.984878-0.326239$

O $6.119337-4.5829301 .419868$

C $5.935307-2.1848701 .024702$

C $4.750195-2.0277722 .003403$

O $7.156101-2.0034111 .743772$

C $2.2890471 .811843-1.601526$ 
O $1.4772942 .511864-2.185595$

C $2.1612070 .353056-1.389133$

C $0.944801-0.208068-1.466106$

C $0.620412-1.617206-1.230267$

C $1.770361-2.569918-1.042429$

C $4.496419-0.5919322 .480292$

C $3.232441-0.5594973 .315605$

C $0.750435-0.6887063 .089843$

O $3.268960-0.6314114 .541755$

C $0.224877-2.0056442 .604802$

C $-1.025933-2.1823912 .153893$

C $-1.555306-3.4894451 .638552$

C $-2.181293-3.3673440 .265332$

C $-3.516462-3.358580 \quad 0.115303$

C $-1.263489-3.346103-0.939701$

C $-0.678692-1.981927-1.191057$

N $2.082558-0.5050322 .542648$

H 2.1323192 .5408711 .150555

H $3.075164 \quad 5.347162 \quad 0.368711$

H $3.2848714 .129000-1.811332$

H 5.5975313 .2965450 .030246

H $5.4084774 .916179-0.587606$

H $7.1411372 .253714-3.241751$

H $6.4224974 .259579-4.073579$

H $4.8025194 .682175-3.515802$

H $6.2251345 .500010-2.832924$

H $7.8523541 .280369-0.953150$

H $6.1636001 .329780-0.454848$

H $5.546765-0.145274-2.379159$

H $7.249903-0.220517-2.861322$

H $7.787816-1.140633-0.553908$

H $7.497366-2.678908-2.533625$

H $5.768582-2.717994-2.247610$

H $7.088807-5.090305-2.227249$

H $4.899428-3.7845730 .006583$

H $8.993863-4.170834-1.198175$

H $8.462551-4.8658380 .316197$

H $8.762169-3.1207320 .202787$

H $6.077879-5.455776 \quad 0.992818$

H $3.837117-2.3893761 .517737$

H $4.908329-2.6637302 .884399$

H $7.025348-1.3182012 .418071$

H $3.054707-0.201985-1.128381$

H $0.0934230 .431916-1.694947$

H $2.454803-2.521973-1.896968$

H $1.466781-3.614005-0.949382$

H $2.326251-2.319081-0.135753$

H $5.323316-0.2192153 .093586$

H $4.3826330 .098987 \quad 1.637274$

H $\quad 0.1386290 .1508212 .746098$

H $0.789403-0.6809694 .183022$

H $0.907300-2.8526822 .616403$

H $-1.718612-1.3460382 .177133$

H -2.299893 -3.8529922 .358835$

H $-0.770716-4.2557521 .609918$

H -3.972909 $-3.302930-0.866998$

H $-4.182990-3.4013000 .970370$

H $-1.823297-3.631118-1.840614$

H $-0.506911-4.125037-0.816969$

H -1.434661 -1.213465 - 1.360452

H $2.169717-0.4461741 .532307$

C $0.000860 \quad 4.3742532 .107442$

O $\begin{array}{lllll}-0.532571 & 3.069185 & 1.797697\end{array}$

C $-1.295658 \quad 3.1700740 .575718$

C $-1.699348 \quad 4.6315290 .486614$

$\begin{array}{llll}\text { C } & -0.434920 & 5.310065 & 0.974938\end{array}$

H $-0.6011612 .914220-0.234664$

C -2.454728 2.1706930 .609523

H -1.979439 $4.935472-0.526088$

H -2.538155 4.8462931 .160178

C $-0.645095 \quad 6.7481361 .417027$

H $0.295006 \quad 5.301577 \quad 0.156873$

C $-3.4203012 .304005-0.583388$

$\begin{array}{llll}0 & -1.866464 & 0.8677449 & 0.603138\end{array}$

H -2.9888742 .2769051 .559948$

H -3.922222 $3.277754-0.544100$

O $-2.6531042 .304483-1.796440$

C -4.484705 1.195906 -0.712289
C -5.3626221 .0338310 .542172$

H $-4.0028190 .246376-0.972736$

$\begin{array}{llll}\text { O } & -5.312066 & 1.555917 & -1.825037\end{array}$

H $-4.7347340 .933162 \quad 1.432496$

H -5.974076 1.9382220 .651939

H $-1.003638 \quad 7.3586630 .581857$

H -1.3823996 .8168912 .223811$

H 0.2920387 .1841091 .777688

C 1.5162044 .2909722 .335205

H -2.5706470 .2222510 .779426$

H -2.171732 $1.460431-1.838953$

H $-5.9508970 .836659-1.962723$

H $1.707863 \quad 3.6968383 .237612$

H 1.9197025 .2923772 .525604

H -0.4723234 .6796143 .049206$

C -8.126586 - $1.097024-0.825677$

O $-7.231698-0.004737-0.580429$

C $-6.286857-0.2010640 .474045$

C $-7.018564-0.4398031 .804219$

C -8.017068 -1.596212 1.688709

C $-8.908096-1.4618520 .448629$

H $-8.840444-0.709707-1.563998$

C $-7.410040-2.294802-1.459214$

H $-5.662673-1.0703690 .242722$

$\begin{array}{lllll}\text { O } & -7.704001 & 0.750425 & 2.188379\end{array}$

H $-6.276613-0.6598042 .581444$

H -8.644745 -1.6192072 .588098$

O $-7.288511-2.8194791 .637771$

H $-9.472059-2.3879160 .286685$

O $-9.864932-0.4304130 .707888$

C $-6.839108-1.925909-2.807902$

H $-6.604302-2.692094-0.835942$

H -8.108321 -3.125049-1.619292

H $-8.1377690 .577636 \quad 3.041231$

H -7.935091 -3.544412 1.593417

H $-10.466193-0.387906-0.055158$

O $-5.504055-1.674081-2.694759$

O $-7.505225-1.848010-3.831934$

C $-4.840008-1.291399-3.902975$

H -3.792522 -1.088292 -3.664137

H $-4.883943-2.106259-4.631461$

H $-5.289015-0.381421-4.312105$

SCF Energy (B3LYP/6-31G**/MMFF)= -3245.90285449

08_00390

MM̄FF Geometry

C -0.106191 -2.696956 0.798773

C $-0.496151-1.415290 \quad 0.721577$

C $-0.843557-0.5694061 .920053$

O $-2.242807-0.2163791 .822355$

$\begin{array}{llll}\text { C } & -0.064243 & 0.753976 & 1.884829\end{array}$

C $-0.0441331 .476004 \quad 3.215873$

C -0.8714892 .4811193 .562533$

C $1.039678 \quad 1.0323154 .163838$

C -2.0386703 .0363092 .794637$

C -1.7548504 .4100002 .171716$

C $-1.008800 \quad 4.318641 \quad 0.831376$

C $-0.695473 \quad 5.7138740 .289524$

C $-0.0572365 .653143-1.104956$

O $-1.858307 \quad 3.620603-0.085912$

O $-0.077916 \quad 6.987968-1.632935$

C $-0.9296554 .764770-2.017981$

C $1.4179545 .241012-1.032292$

O $-0.283168 \quad 4.592937-3.277573$

C $-1.293598 \quad 3.399909-1.383998$

C $-2.3608362 .676289-2.241813$

O $-0.1183332 .589568-1.321904$

C -3.174534 -1.108882 2.241507

O $-2.983664-2.2058202 .738241$

C $-4.491874-0.508798 \quad 1.937778$

C $-5.582903-1.2848391 .880471$

C $-6.929736-0.8405481 .514820$

C -7.1291340 .6274891 .236676$

C $-2.9997201 .450840-1.567556$

C $-3.9840040 .749038-2.490813$

C $-5.438963-1.273521-2.612793$

O $-4.267183 \quad 1.167045-3.610875$

C $-6.842724-0.923272-2.227969$ 
C $-7.770375-1.841274-1.913683$

C $-9.194885-1.509536-1.565929$

C $-9.671862-2.202786-0.307321$

C $-10.424714-3.313867-0.374494$

C $-9.345310-1.5627711 .024300$

C $-7.906466-1.7660531 .415929$

N $-4.507243-0.402432-1.917379$

H $-0.023469-3.1702361 .773939$

H $-0.612275-0.954178-0.256566$

H $-0.659465-1.1058772 .859168$

H -0.4924531 .4067601 .117446$

H 0.9735050 .5829651 .578309

H -0.7259592 .9475154 .536566$

H $\quad 0.990974 \quad 1.552507 \quad 5.126269$

H $0.957946-0.0399824 .366843$

H $2.0239601 .232023 \quad 3.730337$

H -2.402751 2.3364172 .038458

H -2.864309 3.1466063 .509857

H $-2.723648 \quad 4.8969261 .998694$

H -1.197010 5.0390712 .876027

H $-0.082223 \quad 3.7531670 .976074$

H $-0.050696 \quad 6.266667 \quad 0.984168$

H -1.625776 6.2952990 .219227

H $0.2629036 .949526-2.543268$

H -1.855592 $5.323273-2.214793$

H $1.9860715 .962576-0.432844$

H $1.8817655 .245952-2.024972$

H $1.5646814 .253722-0.589849$

H $-0.9264684 .203140-3.892735$

H $-3.1704803 .379321-2.478557$

H $-1.9153882 .359395-3.193244$

H $-0.3717561 .653858-1.352374$

H $-4.517020 \quad 0.552843 \quad 1.718213$

H -5.476471 -2.348644 2.088147

H -8.1467930 .8837930 .936592$

H -6.4758930 .9597760 .422036$

H -6.9035751 .2198212 .130446$

H -2.232442 $0.725661-1.279646$

H $-3.542917 \quad 1.754797-0.665590$

H -5.182444 -2.302871 -2.343007

H $-5.312223-1.151142-3.693223$

H -7.117838 $0.128831-2.234904$

H -7.501827 -2.895121 -1.942203

H -9.821611 -1.811843 -2.415169

H -9.339368 -0.427016-1.460463

H $-10.793588-3.802557 \quad 0.521240$

H -10.684656 -3.763044 -1.327329

H -9.962140 -2.003862 1.818657

H $-9.640375-0.5106400 .995559$

H -7.658220 -2.806401 1.631682

H $-4.252134-0.643748-0.964528$

C $1.395999-4.431931-0.277328$

O $2.589144-3.638434-0.461516$

C $3.438001-4.296502-1.420375$

C $2.933801-5.731232-1.503213$

C $1.443017-5.524206-1.350686$

H $3.250290-3.809723-2.385032$

C $4.923588-4.165368-1.062077$

H $3.207993-6.215791-2.445060$

H $3.308660-6.348328-0.679589$

C $0.690914-6.790796-0.977488$

H $1.043699-5.138389-2.298522$

C $5.444931-2.723069-0.906525$

O $5.178176-4.8176880 .189101$

H $5.528873-4.688065-1.813692$

H $5.097674-2.3065220 .044175$

O $6.874891-2.797380-0.809103$

C $5.087337-1.761930-2.052326$

C $5.861620-0.428305-2.051218$

H $4.011139-1.555702-2.021932$

O $5.355218-2.409178-3.300382$

H $6.904636-0.606297-2.344171$

H $5.4342950 .184770-2.856075$

H $0.814124-7.553526-1.753430$

H $1.054852-7.210106-0.033459$

H $-0.379631-6.592226-0.866964$

C $0.143261-3.559297-0.406819$

H $5.212778-5.7744140 .022732$
H $7.083486-3.386381-0.063643$

H $5.064179-1.810761-4.009435$

H $-0.736523-4.203087-0.524410$

H $0.221311-2.935991-1.306399$

H $1.461267-4.8743310 .724934$

C $4.243131 \quad 1.239106 \quad 0.878736$

O $4.4575390 .580492-0.378267$

C $5.8240190 .351867-0.727453$

C $6.5798181 .687098-0.826609$

C 6.4361542 .5054460 .461349

C 4.9770002 .5907880 .922606

H 3.1658091 .4458160 .905500

C 4.5757150 .3284502 .072952

H $6.297874-0.2627530 .043753$

O $6.0962202 .454632-1.927614$

H $7.6388201 .481644-1.020875$

H 6.8210743 .5187950 .293571

O 7.2121921 .9118671 .497855

H 4.9162943 .0058641 .935365

O 4.2705073 .4860750 .059256

C $3.660476-0.8747392 .144042$

H $5.599985-0.0500482 .032908$

H 4.4474740 .8964423 .001712

H $5.1390932 .577901-1.806522$

H 8.1372721 .8934821 .198771

H 4.6950574 .3581620 .129801

O $3.875765-1.5341053 .320139$

O $2.868546-1.2083301 .274519$

C $3.083655-2.7103923 .513125$

H $2.021587-2.4493863 .543827$

H $3.280892-3.4363882 .718442$

H $3.363375-3.1551494 .471788$

SCF Energy $\left(B 3 L Y P / 6-31 G^{* *} / / M M F F\right)=-3245.90567932$

08_00391

MM̄MF Geometry

C $0.1989940 .177615-1.845314$

C $0.275012-1.150305-2.027399$

C $0.671749-2.166517-0.979460$

O $0.575089-1.5613210 .328803$

C $2.124613-2.616029-1.213389$

C $2.507670-3.859315-0.438788$

C $3.179191-3.8611150 .729464$

C $2.114476-5.160834-1.087857$

C $3.611830-2.6775701 .549559$

C $5.135945-2.5527331 .666709$

C $5.820310-2.0874990 .371498$

C $7.335760-2.0069850 .575220$

C $8.053917-1.422601-0.649356$

O $5.301916-0.7953240 .040008$

O $9.392433-1.082211-0.256157$

C $7.355639-0.108554-1.056689$

C $8.182236-2.450794-1.779561$

O $7.8995470 .398365-2.273635$

C $5.819447-0.237006-1.175346$

C $5.1877561 .164588-1.353885$

O $5.506660-1.048940-2.307523$

C $0.014136-2.2658071 .341522$

O $-0.402395-3.4146791 .293333$

C $-0.086918-1.4449832 .572628$

C $0.495973-0.2436522 .712685$

C 0.4014860 .6364323 .879803

C $-0.542013 \quad 0.2348604 .982792$

C $3.6574801 .157977-1.419584$

C $3.1168902 .573077-1.397671$

C 2.1156304 .2790210 .090562

O $3.0545763 .255855-2.418019$

C 2.1655674 .6085371 .547017

C 1.0736434 .7915402 .304358

C 1.1166905 .1178593 .769030

C 0.4341374 .0707174 .621902

C -0.7931394 .2829295 .125907$

C 1.2099472 .8190354 .969145

C 1.1341621 .7701303 .891859

N $2.7519462 .993835-0.128399$

H $0.4546510 .603446-0.878536$

H $\quad 0.047241-1.556248-3.010909$

H $-0.025450-3.008872-1.062426$ 
H $2.807612-1.797367-0.963290$

H $2.292554-2.817137-2.280288$

H $3.417103-4.823857 \quad 1.180614$

H $2.423862-6.030562-0.498708$

H $1.028066-5.215283-1.209011$

H $2.579960-5.248453-2.075112$

H $3.183669-1.7391231 .186903$

H $3.201600-2.8175162 .557950$

H $5.341259-1.8195502 .457843$

H $5.564478-3.5081981 .994269$

H $5.580758-2.791335-0.433354$

H $7.746582-2.9925750 .828011$

H $7.552665-1.3632361 .439511$

H $9.822258-1.8896600 .074338$

H $7.5809050 .637897-0.281894$

H $8.757774-3.321031-1.441635$

H $8.736915-2.037332-2.628972$

H $7.218061-2.815024-2.140046$

H $8.8615310 .473601-2.151657$

H $5.4930091 .804502-0.515472$

H $5.5658881 .632112-2.271765$

H $4.577445-0.913456-2.548230$

H $-0.680223-1.8893903 .365325$

H $1.101196 \quad 0.1464691 .896120$

H -1.5489480 .0635504 .585360$

H $-0.648060 \quad 0.9925365 .761987$

H -0.194935 -0.6830325 .469528$

H $3.2994820 .686685-2.340977$

H $3.2310620 .591523-0.584862$

H $1.0902864 .208696-0.288705$

H $2.6408585 .042831-0.492323$

H 3.1502054 .7007771 .999385

H 0.0907174 .7133431 .846616

H $\quad 0.631124 \quad 6.092848 \quad 3.906893$

H 2.1478975 .2531274 .118548

H -1.276243 3.5504395 .763933

H -1.344757 5.1910564 .906745

H 0.8807592 .4232215 .935918

H 2.2646033 .0773225 .130187

H 1.7839131 .9717243 .040351

H 2.6846772 .2983280 .609440

C -1.199006 2.187341-2.405430

O $-2.471121 \quad 1.555238-2.189647$

C $-3.2233921 .605561-3.412267$

C $-2.5910192 .697470-4.276325$

C $-1.4812873 .290348-3.422740$

H -3.076914 $0.644903-3.919548$

C $-4.7200881 .782890-3.116035$

$\mathrm{H}-2.1528732 .227477-5.166437$

H -3.303772 $3.437926-4.651802$

C - $-1.9294134 .577089-2.728525$

H $-0.5992043 .530070-4.027273$

C $-5.3174880 .720647-2.167066$

O $-4.9480803 .051941-2.492786$

H $-5.2834291 .794254-4.057272$

H $-4.958142 \quad 0.902737-1.148217$

O $-6.7355820 .934098-2.124487$

C $-5.043058-0.741868-2.561386$

C $-5.872759-1.786240-1.789659$

H -3.976240 -0.957164 -2.433614

O $-5.341229-0.902662-3.952122$

H -6.931205 -1.695880 -2.065800

H -5.559153 -2.773225 -2.156012

H $-2.2381175 .327711-3.463424$

H -2.763130 $4.407665-2.041218$

H -1.104182 $4.999953-2.145174$

C $-0.1819611 .155988-2.921498$

H $-4.8239053 .741889-3.165367$

H -7.088324 $0.736571-3.009301$

H -5.093786 -1.809253-4.201977

H $0.7290881 .676154-3.238379$

H $-0.5768580 .620921-3.792942$

H $-0.8521912 .601524-1.451467$

C $-4.010146-1.8576501 .449167$

O $-4.344610-1.9229860 .056249$

C $-5.725767-1.733249-0.260687$

C $-6.590399-2.8015530 .429885$

C $-6.335071-2.8302321 .940490$
C $-4.838058-2.8672602 .262491$

H -2.962507 -2.175031 1.499157

C $-4.093062-0.4245981 .997462$

H -6.044324 -0.744666 0.083230

O $-6.299092-4.084240-0.122679$

H -7.646371 $-2.586630 \quad 0.226210$

H $-6.815620-3.7134312 .378920$

O $-6.909818-1.6763042 .546089$

H -4.679485 -2.692184 3.332631

O $-4.338839-4.1803531 .989861$

C -3.0689590 .4918641 .363672$

H -5.0753220 .0229981 .826426$

H -3.893211 -0.4398023 .075386$

H $-6.885281-4.7294660 .308165$

H -7.863280 -1.688257 2.355488

H $-4.397338-4.327378 \quad 1.030117$

O $\quad-3.139097 \quad 1.7155741 .965733$

O -2.3135830 .1835690 .453251$

C -2.247048 2.7096331 .452901

H -1.2218702 .3301561 .405141$

H -2.5852293 .0332780 .464544$

H -2.271051 3.5673562 .129942

SCF Energy (B3LYP/6-31G**//MMFF) $=-3245.89706716$

08 00392

MM̄FF Geometry

C 0.4137983 .8005860 .588001

C $1.4423353 .932067-0.263300$

C $2.0889242 .768692-0.965585$

O $3.4996082 .783050-0.629232$

C $2.0194442 .867176-2.499457$

C $0.6285042 .831585-3.088899$

C $-0.1024931 .710878-3.246630$

C $0.1029234 .156269-3.575429$

C $0.2797640 .307153-2.862888$

C $-0.790325-0.326320-1.964339$

C $-0.426649-1.762426-1.558207$

C $-1.607085-2.434671-0.854901$

C $-1.252766-3.838019-0.347644$

O $0.696669-1.701099-0.673811$

O $-2.291020-4.2589290 .550186$

C $0.048015-3.7489570 .474142$

C $-1.218252-4.866678-1.483624$

O $0.498516-5.0486070 .851356$

C $1.185733-2.979658-0.241649$

C $2.341038-2.7278730 .759918$

O $1.657555-3.758596-1.339230$

C 3.9148582 .0324000 .424666

O $3.2264531 .343497 \quad 1.159287$

C 5.3835922 .1895490 .521680

C 6.1034871 .3009561 .221989

C 7.5565881 .3184681 .399435

C 8.3088302 .5298130 .913930

C $3.503658-1.8858940 .217349$

C $4.607725-2.691098-0.446010$

C $6.833289-2.378329-1.528632$

O $4.603903-3.916730-0.513596$

C $7.882686-2.437373-0.463226$

C $9.035767-1.754140-0.517542$

C $10.090130-1.8067170 .551598$

C $10.419214-0.4376841 .105332$

C $11.447651 \quad 0.2716590 .610138$

C $9.617406 \quad 0.0754642 .281451$

C 8.1535300 .2590161 .984561

N $5.608631-1.866633-0.942652$

H 0.0080572 .8104330 .781417

H $1.8586384 .918836-0.450919$

H $1.6449081 .818459-0.644839$

H $2.6191952 .055721-2.934682$

H $2.5409423 .775576-2.833417$

H $-1.0792871 .800075-3.720664$

H $-0.9404044 .096310-3.902718$

H $0.1566544 .909262-2.784179$

H $0.7005024 .508535-4.422808$

H $0.374763-0.282798-3.782498$

H $1.2533220 .270508-2.365874$

H $-0.9054940 .291128-1.064582$

H $-1.752389-0.325821-2.492277$ 
H $-0.149434-2.322196-2.459142$ H -2.479659 -2.478478 -1.516981 H -1.910998 -1.8252770 .007315$ H $-3.129516-4.2657820 .057211$ H $-0.190985-3.2212291 .408285$ H -2.194651 -4.921477 -1.980003 H -1.021554 -5.873409-1.098731 H $-0.473031-4.637451-2.248049$ H $-0.238424-5.4892581 .307978$ H $1.927981-2.1745891 .614297$ H $2.723003-3.6806941 .144483$ H $2.520532-3.414507-1.617061$ H $5.8341683 .023154-0.005379$ H 5.5848430 .4710491 .699676 H $8.2313672 .619605-0.175132$ H 9.3738502 .5025401 .155438 H 7.9088493 .4412941 .372183 H $3.150049-1.117693-0.478404$ H $3.971590-1.3750741 .068202$ H $7.109414-1.705500-2.346548$ H $6.661028-3.378215-1.939101$ H $7.682833-3.0769510 .393437$ H $\quad 9.241297-1.127634-1.382608$ H $10.989737-2.257710 \quad 0.112627$ H 9.798608 -2.472922 1.373202 H $\quad \begin{array}{lll}11.714668 & 1.241408 & 1.016438\end{array}$ H $12.044528-0.101318-0.215736$ H $10.037175 \quad 1.0101142 .673410$ H $9.715011-0.6397483 .108694$ H $7.535064-0.5829402 .297292$ H $5.549094-0.865873-0.778371$ C -1.6889975 .1671141 .057030$ O $-2.450818 \quad 3.9885451 .387337$ C -3.4086493 .7582160 .335539$ C $-3.4926345 .067102-0.441156$ C $-2.0462575 .520243-0.386066$ H $-2.9726103 .001608-0.325924$ C -4.7370743 .2541420 .907106$ H $-3.8606714 .923207-1.461400$ H -4.1399385 .7976230 .057835$ C $-1.8454086 .983523-0.734749$ $\mathrm{H}-1.4709684 .905157-1.086503$ C -4.6231851 .9850631 .779826$ O $\quad-5.291324 \quad 4.2971121 .720357$ H $-5.449018 \quad 3.0925190 .089385$ H -4.0694582 .2345592 .694082$ O $-5.934674 \quad 1.6004962 .204606$ C $-3.964623 \quad 0.755326 \quad 1.123410$ C $-4.4552310 .392206-0.291774$ H $-4.118431-0.1131491 .773865$ O H -4.289615 $1.233489-0.973956$ H -3.794984 $-0.393367-0.675269$ H -2.179355 $7.183941-1.758037$ H -2.410272 $7.638031-0.062595$ H $-0.7882757 .258245-0.664536$ C -0.2025464 .9431631 .347181$ H -6.2201414 .0700501 .895073$ H -6.2553372 .2800782 .821151$ H $-2.228527 \quad 1.1204431 .950836$ H $-0.086616 \quad 4.7127612 .413841$ H 0.3482235 .8728741 .161752 H -2.0499295 .9553421 .731173$ C $-5.588219-2.3400490 .359687$ O $-6.233407-1.080480 \quad 0.581024$ C $-5.921423-0.072301-0.383487$ C $-6.345887-0.547876-1.780859$ C $-5.751803-1.912184-2.126245$ C $-5.997688-2.916258-0.999243$ H $-4.500596-2.2093930 .397640$ C $-5.987649-3.2705261 .508936$ H $-6.5478870 .793764-0.141677$ O $\quad-5.962830 \quad 0.393679-2.777684$ H -7.439999 -0.629622 -1.818307 H -4.683303 -1.835484 -2.356428 O $-6.377513-2.410594-3.311199$ H -7.058033 -3.199071 -0.985423 O $-5.240999-4.098126-1.253524$
C $-5.295749-2.8530702 .785227$

H $-7.071488-3.2658911 .675678$

H $-5.697443-4.3086251 .311683$

H $-6.3650491 .247443-2.543027$

H $-6.244105-1.746498-4.009424$

H $-5.501647-4.426171-2.131359$

O $-6.050126-1.9289513 .445774$

O $-4.199871-3.2734163 .133468$

C $-5.472923-1.4080384 .646769$

H -5.326510 -2.210592 5.375826

H $-4.525003-0.9080454 .425528$

H -6.166084-0.674047 5.066138

SCF Energy (B3LYP/6-31G**//MMFF) $=-3245.90722787$

08 00393

MM̄MF Geometry

C $-1.516752-3.083275-0.708695$

C $-0.552666-2.860975-1.613015$

C $-0.452722-1.664220-2.535290$

O $0.794790-0.992680-2.232836$

C $-1.584029-0.627237-2.390268$

C $-1.4789660 .527722-3.364147$

C $-1.0428391 .759680-3.036187$

C -1.962502 $0.238529-4.760893$

C $-0.5202962 .216104-1.700878$

C $0.7200523 .105374-1.864149$

C $1.2697643 .566457-0.506699$

C $2.3253954 .656356-0.700189$

C 2.9502115 .0861770 .634039

O $1.8525362 .433693 \quad 0.148712$

O 4.1198105 .8660600 .342893

C 3.4215323 .8266921 .386116

C 2.0137895 .9915281 .442647

O $3.880397 \quad 4.157728 \quad 2.695277$

C $2.3536902 .707771 \quad 1.464801$

C 3.0106651 .4202862 .013330

O 1.3095993 .1132092 .350461

C $1.895577-1.353674-2.944499$

O $1.959416-2.186654-3.834268$

C $3.036853-0.571653-2.421364$

C $4.285311-0.963108-2.715752$

C $5.521018-0.351509-2.224138$

C $5.4086730 .954625-1.485140$

C 2.1284640 .1685651 .959853

C $2.962032-1.0454642 .321470$

C $4.598423-2.6569591 .346109$

O $3.054602-1.4412183 .481214$

C $5.967831-2.0639191 .214472$

C $6.919059-2.5606930 .408486$

C $8.289022-1.9578810 .266956$

C $8.703765-1.759308-1.176680$

C $9.662857-2.521193-1.729619$

C $8.060756-0.630807-1.958524$

C $6.685739-0.995976-2.444200$

N $3.620989-1.5894841 .230227$

H $-2.325847-2.366733-0.603280$

H $\quad 0.241522-3.598590-1.710924$

H $-0.443840-2.033868-3.568204$

H $-1.607435-0.245191-1.363705$

H -2.556296 -1.112257 -2.547870

H -1.062792 $2.529066-3.807045$

H -1.908998 $1.118879-5.410188$

H -1.356297 $-0.547294-5.222096$

H -3.006131 $-0.092301-4.740547$

H $-1.3100502 .790352-1.202920$

H $-0.2705001 .372344-1.051547$

H $1.4943792 .547331-2.406321$

H $0.4560013 .980845-2.471054$

H 0.4366973 .9457350 .097007

H $1.9002395 .524237-1.219682$

H $3.1248704 .278068-1.353028$

H $3.8449636 .629522-0.193511$

H 4.2929973 .4325200 .844030

H $1.766526 \quad 6.8941480 .871096$

H $2.499046 \quad 6.3420902 .360237$

H 1.0744045 .5050221 .713448

H 4.5568384 .8498992 .598949

H 3.9243581 .2171121 .443539 
H $3.319740 \quad 1.5759863 .055259$ H 0.8654322 .3238262 .697299 H $2.8208930 .269816-1.773023$ H $4.419153-1.843485-3.343473$ H $4.9236580 .802283-0.518130$ H $4.8213511 .678206-2.062133$ H $6.3720041 .432974-1.296357$ H 1.2975390 .2341412 .669215 H $1.692143 \quad 0.0223620 .965355$ H $4.384467-3.380760 \quad 0.553477$ H $4.497048-3.1515502 .316870$ H $6.188693-1.1834981 .813962$ H $\quad 6.709106-3.457716-0.169752$ H $8.997721-2.6259170 .773335$ H $8.355880-0.9960030 .790567$ H $9.982895-2.370469-2.755330$ H $10.146377-3.315995-1.171296$ H $8.668329-0.387709-2.839832$ H $8.072242 \quad 0.267956-1.336287$ H $\quad 6.660595-1.924213-3.017507$ H $3.511731-1.1520820 .320738$ C - $-1.539051-3.9241741 .684682$ O $-2.353921-2.7621961 .964247$ C $-1.534211-1.8042112 .657418$ C $-0.463835-2.640403 \quad 3.337894$ C $-0.136371-3.6092682 .215165$ H -1.045620 -1.184924 1.896224 C $-2.373512-0.9266013 .584206$ H $0.392884-2.0455693 .661757$ H $-0.863383-3.1774424 .206503$ C $0.641682-4.8306612 .670975$ H $0.451994-3.0825571 .452416$ C $-3.397528-0.0345952 .844775$ O $-3.081974-1.776814 \quad 4.492650$ H -1.712886 -0.3039964 .199582$ H $-4.180220-0.6654142 .407859$ $\begin{array}{llll}\text { O } & -4.030212 & 0.769046 & 3.848734\end{array}$ C $-2.7817020 .883076 \quad 1.766834$ C $-3.722170 \quad 1.9487881 .183215$ H -2.3906530 .2787220 .941691$ O -1.6765621 .6076342 .320028$ H -4.0251292 .6522881 .969224$ H -3.117852 2.5438180 .485360 H $1.611162-4.5352563 .085545$ H $\quad 0.100656-5.3863143 .444282$ H $0.824883-5.5099551 .832567$ C -1.609473 -4.2801960 .195124$ H -3.616842 -1.203137 5.067770 H -4.832271 1.1542463 .457693 H -0.9519920 .9790062 .469929$ H $-2.584707-4.743183-0.000301$ H - $0.852363-5.032902-0.051847$ H $-1.976161-4.7508862 .259128$ C -5.557324 -0.029498-1.416337 O $-4.5248590 .544566-0.600256$ C -4.9625301 .4135520 .449880$ C $-5.8101022 .573953-0.107521$ C $-6.9477342 .074349-0.999120$ C $-6.4476521 .062603-2.032759$ H -5.018468 -0.519291-2.236926 C $-6.362688-1.097471-0.665147$ H $\quad-5.564154 \quad 0.839547 \quad 1.160952$ O $-4.995917 \quad 3.477818-0.854820$ H -6.2229803 .1420410 .734442$ H -7.400425 2.930738 -1.513978 O H -7.297003 $0.603139-2.551769$ O $-5.6898551 .768480-3.019143$ C $-5.602566-2.399214-0.600679$ H -6.608348 -0.8151570 .362003$ H -7.316551 -1.302381 -1.166491 H -4.561660 $2.971386-1.562562$ H -8.673259 $1.196930-0.753406$ H -5.418163 $1.126862-3.697573$ O $-5.768106-3.087249-1.766506$ O $-4.938348-2.7525550 .363908$ C -5.112939-4.356612 -1.851576 H $-5.211947-4.921888-0.919829$
H $-5.585979-4.928229-2.654565$

H -4.060083 -4.205592 -2.102371

SCF Energy (B3LYP/6-31G**//MMFF) $=-3245.90073212$

08_00394

MM̄FF Geometry

C $2.565800-3.5425570 .690851$

C $1.267928-3.2016430 .689441$

C $0.389243-3.281790-0.530032$

O $-0.515125-2.155086-0.457175$

C $-0.437812-4.577172-0.504704$

C -1.083664 -4.896425 -1.838005

C $-2.385017-4.706633-2.129793$

C $-0.166428-5.519132-2.859952$

C $-3.443882-4.093041-1.256913$

C $-4.221053-3.014727-2.023281$

C $-5.291666-2.343966-1.152154$

C $-6.091397-1.335666-1.979782$

C $-7.108380-0.567638-1.125050$

O $-4.636510-1.678569-0.067029$

O $\quad-7.567570 \quad 0.557585-1.889414$

C $-6.389176-0.0058420 .117268$

C $-8.346822-1.414768-0.810850$

O $\quad-7.3202850 .5933471 .016029$

C $-5.527800-1.0527150 .864466$

C $-4.679023-0.3412361 .941828$

O $-6.385978-2.0035101 .495897$

C $-0.637128-1.336819-1.533951$

O $-0.076548-1.436716-2.612353$

C $-1.587958-0.269166-1.153215$

C $-1.8455930 .747295-1.988303$

C $-2.7556391 .853948-1.694973$

C $-3.5727662 .289291-2.879198$

C $-3.638142-1.2255042 .643318$

C $-2.871246-0.3818773 .643590$

C $-1.2509861 .509028 \quad 3.705362$

O $-3.181873-0.3459774 .831835$

C -2.0469742 .7219013 .330068$

C -1.6133103 .6661882 .481927$

C -2.4626734 .8078832 .003058$

C -2.7343544 .7402260 .513238$

C $-2.2768955 .689819-0.319826$

C $-3.598803 \quad 3.606635-0.004817$

C $-2.7787772 .434527-0.477963$

N $-1.8695200 .370616 \quad 3.049020$

H $3.008521-3.918287-0.226875$

H $\quad 0.808146-2.8560651 .612742$

H $1.009639-3.225507-1.432270$

H -1.193158 -4.5225900 .287841$

H $\quad 0.202543-5.428654-0.235437$

H $-2.737230-5.022549-3.111307$

H $-0.688825-5.774012-3.788294$

H $0.645101-4.832355-3.118856$

H $0.271109-6.442104-2.465351$

H $-4.134941-4.883677-0.941943$

H $-3.017665-3.656860-0.349986$

H -3.515301 -2.256785 -2.385010

H -4.696521 -3.462670 -2.905201

H -5.951633 -3.123066 - 0.752731

H $-6.593331-1.831808-2.819874$

H -5.401885 -0.608992 -2.431555

H -7.957881 $0.220381-2.714093$

H $-5.7284560 .802195-0.226715$

H $-8.847041-1.719779-1.737943$

H $-9.088057-0.837463-0.247264$

H $-8.114629-2.321589-0.248510$

H -7.827456 1.2520210 .511176

H $-4.1483090 .497867 \quad 1.475289$

H $-5.338494 \quad 0.0870702 .708011$

H -5.910513 -2.421742 2.230290

H $-2.059868-0.340052-0.179571$

H $-1.3709490 .783197-2.966652$

H $-4.1087591 .435014-3.307800$

H -2.925145 $2.712232-3.655115$

H $-4.3268893 .040364-2.635407$

H $-4.105841-2.0521223 .187277$

H -2.936803 -1.664851 1.925379

H $-0.215793 \quad 1.567906 \quad 3.357384$ 
H -1.2552371 .3719064 .790827$ H -3.053374 2.7928893 .736529 H -0.6125963 .5904832 .066652$ H -1.9478905 .7418312 .261531$ H -3.422518 4.8367052 .534034 H -2.488768 $5.654508-1.383557$ H -1.6760856 .5181030 .040400$ H -4.256964 $3.990310-0.790103$ H $-4.272165 \quad 3.2652420 .791351$ H -2.121055 2.0242740 .286331 H -1.740122 0.2962612 .044546 C $4.883664-3.0398451 .644773$ O $4.943746-1.8132030 .889440$ C $5.993687-1.937244-0.089997$ C $6.806625-3.1647350 .310203$ C $5.730227-4.0617460 .884521$ H $5.505102-2.142527-1.049693$ C $6.821455-0.653007-0.184244$ H $7.330365-3.610789-0.540811$ H $7.551927-2.9255921 .077945$ C $6.273388-5.1846261 .751750$ H $5.165902-4.4990950 .052441$ C $6.0260170 .608195-0.576410$ O $7.426075-0.4081361 .092571$ H $7.647841-0.808412-0.888761$ H $\begin{array}{llll}5.383634 & 0.908646 & 0.258965\end{array}$ O $\quad 6.977521 \quad 1.669475-0.740659$ C $5.1857780 .480609-1.861508$ C $4.6868841 .817431-2.443216$ H $4.332277-0.179515-1.669185$ O $5.984592-0.146733-2.870377$ H $5.5420332 .398306-2.812336$ H $4.0893251 .577941-3.333311$ H $6.932156-5.8362211 .168436$ H $6.849654-4.7961282 .598312$ H $5.459627-5.798023 \quad 2.150811$ C $3.433281-3.4529631 .915580$ H 7.9516130 .4063421 .012657 H $7.5314831 .450385-1.509854$ H $5.417237-0.288544-3.647268$ H $2.994616-2.7222862 .605822$ H $3.423676-4.4231232 .425397$ H $5.357114-2.8224112 .611753$ C $1.7933122 .563115-0.200087$ O $2.6766821 .930988-1.137643$ C $3.8529102 .670863-1.476088$ C $3.4793424 .028043-2.094485$ C $2.5378574 .815707-1.179693$ C $1.3656153 .956265-0.698258$ H $0.899908 \quad 1.925946-0.181350$ C $2.3862592 .581997 \quad 1.217592$ H $4.4429702 .849762-0.573000$ O $2.8634513 .844466-3.367792$ H $4.3967814 .604185-2.263185$ H $2.1545125 .685630-1.727332$ O $3.281147 \quad 5.294215-0.062919$ H 0.8125124 .4809780 .088845 O $0.4702043 .791222-1.800936$ C $2.4829351 .194717 \quad 1.816936$ H 3.3898493 .0132421 .242290 H $1.7358443 .173077 \quad 1.871751$ H $2.0820703 .279428-3.241569$ H $2.675800 \quad 5.8172800 .489819$ H -0.308658 $3.311204-1.474775$ O $2.845432 \quad 1.310468 \quad 3.129176$ $\begin{array}{llll}\text { O } & 2.279438 & 0.148002 & 1.218425\end{array}$ C $2.981777 \quad 0.079210 \quad 3.844339$ H $2.070704-0.5208913 .757527$ H $3.848650-0.4726493 .470248$ H 3.1443060 .3152064 .899316

SCF Energy (B3LYP/6-31G**//MMFF) $=-3245.90294270$

\section{8_00395}

MM̄FF Geometry

C $0.1499803 .567890-0.835744$

C $-0.950634 \quad 4.326209-0.967092$

C -2.385942 $3.911495-0.742546$

O $-2.4728402 .541002-0.292535$
C -3.198792 $4.029902-2.045771$

C $-4.6269394 .469604-1.786085$

C $-5.6847203 .646499-1.655971$

C $-4.8202265 .962567-1.689795$

C $-5.7034122 .145896-1.707383$

C $-6.1291871 .561999-0.354262$

C $-6.2847840 .036101-0.403488$

C $-6.659576-0.5114100 .975489$

C $-6.732633-2.045748 \quad 0.983912$

O $-5.044672-0.531792-0.831712$

O $-6.753469-2.4797702 .352383$

C $-5.445233-2.612590 \quad 0.350495$

C $-8.029300-2.5580430 .346016$

O $-5.531679-4.0276430 .197709$

C $-5.077090-1.954520-0.999893$

C $-3.668577-2.412458-1.437918$

O $-6.027202-2.351294-1.989935$

C -2.474916 $2.312671 \quad 1.048768$

O $-2.463145 \quad 3.1454361 .940674$

$\begin{array}{llll}\text { C }-2.457383 & 0.846939 & 1.244898\end{array}$

$\begin{array}{lll}\text { C }-2.406114 & 0.339982 & 2.484996\end{array}$

C $-2.339873-1.0842382 .799798$

C $-3.232397-1.4919583 .938163$

C $-3.106984-1.665127-2.656186$

C $-1.703063-2.152579-2.950501$

C $0.591736-2.121498-1.983792$

O $-1.474313-2.975577-3.833568$

C $0.581511-3.219082-0.965845$

C $1.102832-3.095288 \quad 0.263019$

C $0.999020-4.152572 \quad 1.323243$

C $0.225405-3.6767052 .535301$

C $0.812736-3.5788773 .739765$

C $-1.249964-3.370802 \quad 2.361844$

C $-1.501858-1.9039162 .133839$

N $-0.761897-1.610379-2.088915$

H $0.0695712 .518603-0.567960$

H $-0.8200445 .375524-1.228735$

H -2.8033454 .5791440 .021853$

H $-3.1785803 .082516-2.596469$

H $-2.7476114 .767559-2.723429$

H $-6.6624344 .096322-1.485143$

H $-5.8614896 .236914-1.489875$

H $-4.209807 \quad 6.377139-0.881298$

H $-4.5267136 .443697-2.628618$

H $-6.416203 \quad 1.839476-2.482488$

H $-4.7322231 .736987-1.998367$

H $-5.374251 \quad 1.8310180 .394810$

H $-7.0773522 .010803-0.032012$

H -7.063589 $-0.203596-1.137191$

H -7.606388 -0.0791181 .322331$

H $-5.902294-0.1991401 .708464$

H -7.520029 -2.0671512.786067

H $-4.625900-2.4286841 .058403$

H $-8.900970-2.1748230 .890354$

H -8.095416 -3.6499340 .407185$

H $-8.138471-2.262936-0.699565$

H $-5.746910-4.4002371 .069836$

H $-2.973791-2.269429-0.601124$

H $-3.678278-3.486585-1.663490$

H -5.624627 - $2.270727-2.868434$

H -2.4643060 .2136900 .364260$

H -2.417839 1.0015423 .348677

H $-2.865241-1.0727404 .881219$

H $-3.309791-2.5744874 .060812$

H $-4.252578-1.1258193 .773712$

H $-3.716431-1.832151-3.549956$

H $-3.077173-0.583456-2.482707$

H $1.233087-1.286059-1.687911$

H $0.928735-2.503976-2.951882$

H $0.082221-4.144319-1.243483$

H $1.592219-2.1652650 .539952$

H $2.016453-4.449357 \quad 1.605977$

H $0.518407-5.0584300 .933012$

H $0.254673-3.2569504 .613028$

H $1.862889-3.813358 \quad 3.878345$

H - $1.791849-3.7481923 .234443$

H $-1.645016-3.9328731 .506486$

H $-0.911712-1.4719951 .326666$ 
H $-1.079282-0.991369-1.348409$

C 2.3918204 .1221340 .252495

$\begin{array}{llll}\text { O } & 2.859068 & 2.798380 & 0.573359\end{array}$

C 3.0272392 .7124682 .003242

C 2.6674224 .0861632 .575479

C 1.7248434 .6374931 .525478

H 2.2937491 .9794542 .357758

C 4.4468582 .2576712 .367713

H 2.2068824 .0085603 .565554

H 3.5472584 .7322702 .674826

C $1.566965 \quad 6.1466151 .576032$

H $0.746146 \quad 4.165771 \quad 1.677269$

C 4.8944750 .9147021 .757806

O $5.373660 \quad 3.257167 \quad 1.918442$

H 4.5408572 .2245203 .460176

H 5.0819091 .0466230 .686961

$\begin{array}{lllll}\text { O } & 6.167521 & 0.595450 & 2.338769\end{array}$

C $3.933099-0.2658821 .974861$

C $4.534123-1.6506721 .663347$

H $3.031797-0.1100421 .371234$

O $3.521645-0.279613 \quad 3.345469$

H $5.300937-1.8979852 .409147$

H $3.735383-2.3859121 .821664$

H 1.1576456 .4580642 .542604

H $2.526864 \quad 6.655474 \quad 1.437751$

H 0.8858126 .4945590 .793155

C $1.5399524 .113149-1.021468$

H 6.2645192 .9617122 .173829

H 6.0225420 .4206153 .284684

H $2.857912-0.9835503 .444878$

H $2.0350713 .506346-1.788333$

H $1.4885755 .136901-1.412518$

H 3.2945644 .7117840 .041526

C $4.491197-1.537821-2.078647$

O $4.098454-1.487103-0.699786$

C $5.118973-1.8081710 .247728$

C $5.650713-3.2322040 .009023$

C $6.100674-3.430782-1.441354$

C $5.052143-2.925059-2.438193$

H $3.560783-1.396849-2.642248$

C $5.438504-0.387377-2.452705$

H $5.946293-1.1003660 .138975$

$\begin{array}{llllll}\text { O } & 4.651226 & -4.199368 & 0.326077\end{array}$

H $6.494179-3.4118730 .685896$

H $6.284217-4.498479-1.613824$

O $7.331304-2.740360-1.634263$

H $5.471703-2.906677-3.450757$

O $3.971522-3.862621-2.449971$

C $4.7791040 .968594-2.316945$

H $6.331098-0.378242-1.821958$

H $5.748483-0.494265-3.498612$

H $3.880576-4.026542-0.241018$

H $7.618588-2.903162-2.548774$

H $3.338095-3.573633-3.128664$

O $5.717017 \quad 1.936122-2.542739$

O $3.6004461 .158861-2.052281$

C $5.255105 \quad 3.285550-2.431835$

H $4.3844923 .451400-3.073382$

H $5.0181353 .510324-1.388567$

H $6.0605533 .948391-2.758914$

SCF Energy (B3LYP/6-31G**/MMFF) $=-3245.91079732$

08_00396

MM̄FF Geometry

C 1.4730553 .9823880 .604614

C $2.0575104 .506837-0.483778$

C $2.858646 \quad 3.768717-1.530187$

$\begin{array}{llll}\text { O } & 2.763279 & 2.334730 & -1.379817\end{array}$

C $4.3407284 .160333-1.413268$

C $5.191373 \quad 3.605792-2.538538$

C $6.0222722 .552405-2.413078$

C $5.1040594 .352951-3.843785$

C $6.2185881 .699368-1.190047$

C $6.458244 \quad 0.228808-1.561232$

C $6.645189-0.639629-0.309519$

C $7.162505-2.028331-0.685993$

C $7.293378-2.9356620 .545454$

O $5.376596-0.754152 \quad 0.345930$ $\begin{array}{llll}\text { O } & 7.482963 & -4.281670 & 0.083646\end{array}$

C $5.963453-2.9067861 .323763$

C $8.525091-2.5878181 .389242$

O $6.077916-3.6166092 .555467$

C $5.418027-1.4799451 .582114$

C $3.977811-1.5822382 .135331$

O $6.242949-0.8253242 .546858$

C $1.7156251 .716814-1.990045$

O $0.794708 \quad 2.253126-2.585352$

C $1.8889930 .256468-1.818261$

C $0.908107-0.569155-2.212335$

C $0.908495-2.031026-2.124625$

C $2.191662-2.707444-1.720055$

C $3.213753-0.2531382 .208258$

C $1.799093-0.4987882 .700612$

C $-0.347713-1.5690422 .005065$

O $1.449477-0.2007153 .839861$

C $-0.524774-2.918174 \quad 1.383607$

C $-1.524726-3.2215070 .542673$

C $-1.693616-4.568067-0.100021$

C $-1.767661-4.495120-1.610248$

C $-2.908940-4.778702-2.260526$

C $-0.498048-4.171211-2.373156$

C $-0.229760-2.689870-2.428812$

N $1.007972-1.122647 \quad 1.750269$

H 1.5651942 .9209640 .817111

H $1.9539195 .576527-0.659094$

H $2.4799794 .057738-2.518694$

H $4.7331133 .832919-0.442689$

H $4.4440455 .254064-1.412434$

H $6.6283042 .274660-3.274311$

H $5.7804963 .945033-4.602401$

H $4.0887484 .302914-4.248647$

H $5.3698395 .404943-3.697972$

H $7.0908902 .077545-0.643802$

H $5.3569071 .759661-0.518538$

H $5.604763-0.139907-2.145000$

H $7.3508780 .159692-2.195657$

H $7.355513-0.1388510 .358877$

H $8.122612-1.958339-1.212230$

H $6.465285-2.498568-1.393807$

H $8.283429-4.298995-0.468536$

H $5.224666-3.4530780 .720258$

H $9.438481-2.6803070 .789496$

H $8.642861-3.2886672 .223208$

H $8.496660-1.5735691 .792919$

H $6.406564-4.5078882 .346749$

H $3.406195-2.2687961 .499043$

H $3.996731-2.0257273 .139495$

H $5.724535-0.1339302 .987891$

H $2.812085-0.097416-1.373846$

H $0.002175-0.132874-2.630246$

H $3.020596-2.371460-2.353803$

H $2.159299-3.794601-1.817546$

H $2.438027-2.482461-0.679115$

H 3.6969210 .4479452 .896458

H $3.1642190 .236151 \quad 1.229349$

H $-1.020875-0.8260161 .569093$

H $-0.527103-1.6242183 .082947$

H $0.207845-3.6812281 .636373$

H $-2.267822-2.463627 \quad 0.310024$

H -2.610817 -5.0153860 .304443$

H $-0.879502-5.2475250 .181766$

H $-2.963323-4.752158-3.343837$

H $-3.815927-5.038093-1.725116$

H $-0.582973-4.529395-3.407399$

H $0.324131-4.738917-1.929267$

H -1.090949 - $2.103554-2.753320$

H $1.351952-1.1841200 .797313$

C $-0.777246 \quad 4.3701051 .732448$

\begin{tabular}{lllll}
\hline & -0.855227 & 3.073114 & 2.347380
\end{tabular}

C $-1.964697 \quad 2.3425891 .766081$

C $-2.709732 \quad 3.347614 \quad 0.899814$

$\begin{array}{llll}\text { C }-1.592367 & 4.268788 & 0.448578\end{array}$

H -1.5052591 .5709591 .136593$

C -2.7557651 .6833822 .902561$

H -3.216719 2.8812630 .051596

H $-3.449103 \quad 3.9023741 .489446$ 
C $-2.0762285 .604433-0.085884$ H -1.026909 $3.752845-0.337354$ C -3.9057710 .7444562 .476665$ O $-1.8009550 .885007 \quad 3.627405$ H -3.102655 2.4401843 .615703 H -3.509739 -0.0119001 .791340$ O -4.3255020 .0294123 .650949$ C -5.1491771 .4196311 .868399$ C $-6.342537 \quad 0.4651951 .654401$ H -4.9005001 .8890410 .915688$ O $\begin{array}{llll}-5.573661 & 2.457811 & 2.756115\end{array}$ H $-6.601150-0.0287302 .598809$ H -7.2151041 .0800981 .394282$ H -2.714220 $5.458419-0.963638$ H $-2.656898 \quad 6.1510850 .664609$ H $-1.2310936 .233433-0.382962$ C 0.6836454 .8030371 .587919 H $-2.275636 \quad 0.4623304 .363329$ H $-4.701793 \quad 0.679757 \quad 4.269004$ H -6.3087322 .9287942 .327627$ H 1.1806894 .7106092 .561588 H $0.7149535 .864046 \quad 1.312182$ H -1.265296 5.0627272 .431422 C $-5.797543-0.662739-1.859907$ O $-6.038427 \quad 0.133174-0.692743$ C $-6.108739-0.5766360 .548392$ C $-7.235222-1.6205980 .503518$ C $-7.073346-2.562012-0.693957$ C $-6.835994-1.789802-1.995877$ H $-5.9489420 .027010-2.699914$ C $-4.349327-1.162075-1.912934$ H -5.157684 -1.0859290 .729917$ O $-8.508220-0.980657 \quad 0.435923$ H -7.217824 -2.198801 1.434664 H -7.975802 -3.176531 -0.798404 O $-5.973241-3.438771-0.471036$ H -6.530384 -2.471412 -2.798437 O $-8.066824-1.185566-2.405759$ C -3.362396 -0.019113-1.962186 H -4.095064 -1.769186-1.040969 H -4.179964 -1.789022 -2.796058 H -8.509930 -0.403690 -0.347056 H $-6.154636-3.9376580 .343685$ H -8.710329 -1.900428 -2.549384 O $-3.5060780 .675086-3.126088$ O $-2.5484110 .211541-1.077651$ C $-2.6356561 .796752-3.307335$ H -1.668463 $1.446768-3.675878$ H $-3.0815772 .452790-4.059713$ H $-2.5132752 .366493-2.381336$

SCF Energy $\left(\mathrm{B} 3 \mathrm{LYP} / 6-31 \mathrm{G}^{* *} / / \mathrm{MMFF}\right)=-3245.91402390$

08_00397

MM̄FF Geometry

C $0.541527-2.7380942 .845547$

C $-0.590999-2.9616472 .162935$

C $-0.615132-3.4983950 .755076$

O $-1.060536-2.417055-0.102309$

C $-1.609114-4.6653640 .643040$

C $-1.701756-5.245378-0.756502$

C $-2.747998-5.079324-1.591244$

C $-0.519671-6.075007-1.188526$

C $-4.007773-4.300656-1.335603$

C $-4.029690-2.994309-2.139428$

C $-5.052057-1.998547-1.573685$

C $-5.155960-0.760570-2.465008$

C $-6.0734030 .311921-1.857894$

O $-4.602897-1.622221-0.266298$

O $-5.8540321 .536650-2.575240$

C $-5.6518940 .565952-0.394111$

C $-7.555237-0.037079-2.039741$

O $-6.574857 \quad 1.4359490 .255844$

C $-5.478669-0.7258810 .430394$

C $-4.864774-0.4785361 .831873$

O $-6.763761-1.3256350 .636817$

C $-0.127638-1.476217-0.423844$

O $1.057830-1.492489-0.131881$

C $-0.798687-0.380513-1.154328$
C $-0.0950890 .723094-1.447735$

C $-0.5923961 .922640-2.121856$

C $-2.0479791 .958943-2.502232$

C -3.4735810 .1664961 .843552$

C -3.4925351 .6789051 .992052$

C -1.9741613 .6541991 .938980$

O

C -1.9985354 .2918350 .585451$

C -0.9875515 .0264720 .096388$

C $-1.0354295 .742931-1.223196$

C $0.1439085 .421760-2.113228$

C $1.2314126 .210897-2.133098$

C $0.0213904 .239613-3.046794$

C $0.2728732 .932103-2.351277$

N -2.2321082 .2322901 .810340$

H $1.498538-3.0062792 .404853$

H -1.539764 -2.642938 2.589054

H $\quad 0.380775-3.8316210 .437305$

H $-2.591511-4.3339860 .992838$

H -1.314629 -5.4692761 .331303$

H $-2.701960-5.544977-2.574811$

H $-0.672156-6.536227-2.170211$

H $\quad 0.382358-5.458747-1.252242$

H $-0.340911-6.883024-0.471769$

H -4.865120 -4.919225-1.628786

H $-4.147618-4.101183-0.271123$

H -3.034152 -2.535051-2.116702

H -4.260356 -3.219157 -3.188068

H $-6.023840-2.500102-1.495114$

H -5.496925 -1.033504 -3.471427

H $-4.156986-0.324962-2.597985$

H $-6.043126 \quad 1.371641-3.514902$

H $-4.6928301 .095937-0.428542$

H -7.799260 -0.126797 -3.105177

H $-8.1996480 .759682-1.652126$

H $-7.837119-0.975537-1.557989$

H $-6.6369592 .245248-0.280001$

H -5.5721010 .0760862 .460230$

H -4.758036 -1.460904 2.314471

H $-6.709593-1.9135441 .408462$

H -1.846654 -0.495272 -1.400974

H $0.9551170 .760160-1.159776$

H $-2.3709672 .920902-2.905148$

H -2.678384 $1.770482-1.626713$

H $-2.263571 \quad 1.200292-3.262121$

H $-2.922988-0.2151052 .713260$

H -2.904961 -0.1078460 .950719$

H -1.0051863 .7684712 .434040$

H -2.739985 4.1112572 .573786

H -2.907592 $4.178702-0.000428$

H -0.0921285 .1630770 .698951$

H -1.055326 $6.820843-1.014022$

H $-1.9685865 .532241-1.760277$

H $2.0686116 .003214-2.791271$

H $1.3105357 .078564-1.486477$

H $0.7593864 .322926-3.856191$

H $-0.9468214 .275948-3.553735$

H $1.3072712 .810936-2.026619$

H -1.4571321 .6324801 .541825$

C $0.657183-0.4848383 .871423$

O $1.883980-0.172646 \quad 3.186942$

C 2.2636511 .1761353 .522420

C 1.2353191 .6817804 .535297

C 0.6815490 .3925665 .115645

H $3.241421 \quad 1.1146404 .012852$

C 2.3641432 .0208282 .245160

H 1.6913892 .3276015 .292452

H 0.4392002 .2605624 .053421

C $-0.6716300 .564821 \quad 5.783241$

H $1.401953-0.0050955 .842616$

C 3.3191751 .4447021 .178209

O $1.0624932 .099548 \quad 1.650058$

H 2.6475673 .0479142 .503731

H 2.8913750 .5163100 .789011

O 3.3383602 .3654710 .079301

C 4.7600791 .2037951 .660323

C $5.783666 \quad 0.8784380 .556547$

$\begin{array}{llll}\text { H } & 4.770146 & 0.422920 & 2.424461\end{array}$ 
O 5.2100322 .4033972 .307525 H $5.8019031 .726564-0.142288$ H $\quad \begin{array}{llll}6.786376 & 0.873720 & 1.005893\end{array}$ H -0.6032841 .2764536 .612395$ H -1.421959 0.9397695 .078585 H -1.033374 -0.3865406 .185166$ C $0.578707-1.9876374 .146676$ H 1.1435782 .6380580 .844095 H 3.7459893 .1901710 .394989 H 6.0839112 .2191432 .692166 H $-0.307720-2.2247234 .745688$ H $1.456183-2.3241764 .711362$ H $-0.166709-0.1795963 .210916$

C $4.141082-1.495173-1.848678$

O $4.345225-0.439640-0.898807$

C $5.626401-0.408789-0.272507$

C $5.874861-1.7073350 .506624$

C $5.753591-2.915976-0.429544$

C $4.453545-2.875752-1.240743$

H $3.067287-1.464101-2.073900$

C $4.893597-1.221440-3.155920$

H $6.390647-0.332531-1.055083$

O $4.931441-1.8367891 .564174$

H $6.877202-1.6721750 .949782$

H $\quad 5.785287-3.836674 \quad 0.165854$

O $6.874288-2.926102-1.309151$

H $4.483157-3.626097-2.038655$

O $3.373572-3.252544-0.381832$

C $4.301563-0.023169-3.860521$

H $5.964943-1.048499-3.019950$

H $4.802469-2.074137-3.839392$

H $5.137701-2.6565982 .044526$

H $\quad 6.794270-3.711511-1.877030$

H $3.249354-2.5416040 .270452$

O $4.8517221 .124984-3.372182$

O $3.435696-0.100249-4.722495$

C $4.3359302 .339580-3.925011$

H $3.2525062 .394716-3.783626$

H $4.7998593 .177135-3.397207$

H $4.5909142 .406617-4.986663$

SCF Energy $\left(\mathrm{B} 3 \mathrm{LYP} / 6-31 \mathrm{G}^{* *} / / \mathrm{MMFF}\right)=-3245.91565516$

08_00398

MM̄FF Geometry

C $-1.705224-3.1424790 .433856$

C $-1.163386-3.0698371 .659786$

C -1.805775 -2.503556 2.906145

O $-3.164580-2.0871822 .627124$

C -1.052992 -1.290922 3.485780

C $0.406442-1.5351133 .803749$

C $1.439225-0.9235643 .191496$

C $0.669865-2.5222594 .912356$

C 1.3883410 .1070782 .098702

C 2.0773551 .4008382 .551028

C 1.9072292 .5384201 .535323

C 2.6862563 .7755181 .986553

C 2.4355894 .9843411 .073833

O $0.5120542 .840642 \quad 1.451378$

O $2.918283 \quad 6.155103 \quad 1.751323$

C 0.9145585 .1654820 .887259

C $3.2264604 .886919-0.235091$

O $0.6397686 .183463-0.073228$

C 0.1762823 .8617680 .502667

C - $-1.355075 \quad 4.082180 \quad 0.586374$

O $0.5395943 .490144-0.825040$

C $-4.080527-2.2186643 .620791$

O $-3.885615-2.6792594 .737729$

C $-5.421838-1.7580123 .194821$

C $-5.635932-0.9577702 .138754$

C $-6.938995-0.4584621 .687169$

C $-8.169323-1.0019582 .366176$

C -2.2038322 .8374580 .282499$

C $-2.6103862 .704817-1.176937$

C $-3.9776551 .244134-2.668413$

O $-2.327753 \quad 3.535008-2.035807$

C $-5.4164781 .643288-2.586637$

C $-6.4309860 .767051-2.637690$

C $-7.874041 \quad 1.157375-2.506009$
C $-8.5314920 .543820-1.288639$

C $-9.459252-0.419563-1.415705$

C -8.1783831 .1081110 .070838$

C -6.9746460 .4414380 .681750$

N $-3.3456221 .548177-1.397641$

H $-2.696526-2.7365350 .255787$

H -0.184679 -3.5227681 .809689$

H -1.826176 -3.318067 3.641442

H -1.553294 -0.9732544 .411180$

H -1.195741 -0.438299 2.814359

H $2.447718-1.1876393 .508066$

H $1.731869-2.5803005 .174007$

H $0.126142-2.2304055 .816786$

H $0.348721-3.5267204 .620471$

H $1.901395-0.2952181 .219824$

H $\quad 0.366811 \quad 0.317861 \quad 1.777114$

H 1.6478271 .7124153 .512749

H 3.1450231 .2072932 .715079

H 2.2605642 .1947040 .558447

H 3.7597043 .5562472 .049121

H 2.3778674 .0461903 .006295

H 3.8633706 .0237241 .939741

H 0.5069665 .5232271 .843360

H $4.3013494 .814934-0.029705$

H $3.0976525 .789104-0.843031$

H $2.9482024 .022111-0.838307$

H 1.1086916 .9860380 .213016

H -1.5940764 .3834051 .615162$

H -1.655692 $4.906914-0.069872$

H $-0.0913042 .827916-1.147428$

H $-6.234329-2.087393 \quad 3.834711$

H -4.782124 -0.6188521 .554075$

H -8.175672 -2.097452 2.333487

H -9.102791 -0.6799551 .899897$

H -8.203489 -0.680879 3.412905

H -1.6971561 .9197650 .600336$

H -3.137520 2.9141070 .854980

H $-3.8629180 .171050-2.840913$

H -3.480762 $1.785616-3.479073$

H -5.629962 $2.702330-2.462102$

H $-6.216461-0.291619-2.765312$

H $-8.3903770 .841975-3.421905$

H $-7.9896472 .247687-2.462174$

H $-9.956477-0.842308-0.549192$

H -9.740102 -0.809827 -2.388352

H $-9.046901 \quad 1.059550 \quad 0.736256$

H -7.971926 2.182297 -0.022244

H -6.0270880 .7726600 .258149$

H $-3.5676310 .948177-0.608229$

C $-0.767334-3.058566-1.967954$

O $0.343537-2.163163-1.777219$

C $0.209214-1.086116-2.730678$

C $-1.100875-1.326230-3.487466$

C -1.887377 -2.182993 -2.514721

H $0.113264-0.162210-2.149704$

C $1.431982-1.016236-3.657139$

H -1.602233 -0.387207 -3.739446

H $-0.934903-1.865901-4.427541$

C $-3.025103-2.953762-3.158854$

H $-2.286042-1.526531-1.731717$

C $2.777874-0.751206-2.952141$

O $1.545539-2.264163-4.354993$

H $1.256980-0.252393-4.424959$

H $3.069049-1.632956-2.372399$

O $3.772633-0.609544-3.975947$

C $2.7985770 .498367-2.057430$

C $4.1974310 .956326-1.606274$

H $2.1702550 .319172-1.178599$

O $2.2084211 .584399-2.779143$

H $4.7581141 .347484-2.465351$

H $4.0474731 .821062-0.948797$

H -3.745405 -2.268337 -3.616813

H -2.660679 -3.628894 -3.940427

H $-3.557950-3.555081-2.415604$

C $-1.057688-3.875853-0.708851$

H $2.319130-2.199110-4.940890$

H $3.5643800 .197322-4.477880$

H $2.1236602 .333258-2.164978$ 
H $-0.120778-4.333270-0.368672$

H - $-1.727653-4.702259-0.978087$

H - $0.447229-3.770202-2.742555$

C $4.892049-1.6813950 .956338$

O $4.268026-0.6389450 .194032$

C $5.041704-0.104954-0.881157$

C $6.3584360 .488441-0.349826$

C $7.134771-0.5268350 .493437$

C $6.236865-1.2078001 .533324$

H $4.214042-1.8576971 .800383$

C $5.002191-2.9916770 .162214$

H $5.268573-0.904330-1.593519$

O 6.1005051 .6491620 .440624

H $6.9709410 .809917-1.200334$

H $7.959455-0.0111651 .000558$

O $7.701635-1.502053-0.376023$

H $6.754443-2.0565421 .995283$

O $5.946190-0.2755082 .579043$

C $3.648052-3.573159-0.185620$

H $5.545201-2.847033-0.775382$

H $5.531248-3.7421290 .760842$

H $5.483561 \quad 1.3955251 .148574$

H $8.212312-2.1222860 .171775$

H $6.792179-0.0100772 .978307$

O $3.828745-4.605712-1.062421$

O $2.575783-3.1862090 .256814$

C $2.636332-5.262552-1.501459$

H $2.926045-6.105049-2.134983$

H $2.072916-5.646068-0.645706$

H $2.026931-4.573591-2.092449$

SCF Energy $\left(B 3 L Y P / 6-31 G^{* *} / / M M F F\right)=-3245.91676360$

08_00399

MMFF Geometry

C $2.453669-1.990136-3.267935$

C $1.580542-1.687599-2.295027$

C $0.359015-0.832738-2.481015$

O $0.7150450 .468177-1.958584$

C $-0.855322-1.390397-1.712094$

C $-1.511851-2.563322-2.409026$

C $-2.538469-2.451521-3.274966$

C $-0.973843-3.925488-2.055196$

C $-3.245742-1.196065-3.705791$

C $-4.766564-1.283900-3.511472$

C $-5.196931-1.551153-2.060571$

C $-6.716852-1.705581-1.975725$

C -7.181425 -1.934012 -0.531190

O $-4.767620-0.457968-1.238820$

O $-8.605442-1.756378-0.491593$

C $-6.563377-0.8426780 .364180$

C $-6.909851-3.367119-0.058472$

O $-6.841991-1.0972421 .739748$

C $-5.039742-0.6462050 .157408$

C $-4.587216 \quad 0.613150 \quad 0.933188$

O $-4.347082-1.7837310 .677861$

C $0.032014 \quad 1.541810-2.434869$

O $-0.9505221 .531972-3.158342$

C $0.7201862 .756510-1.949986$

C $0.0887783 .937615-2.004630$

C $0.6493855 .229206-1.610616$

C $2.1266535 .299454-1.325867$

C -3.1586551 .0919960 .639686$

C -2.7484672 .2001201 .598527$

C -0.8068373 .6186232 .230674$

O -3.4826252 .6214642 .489827$

C -1.1116815 .0109061 .774463$

C -0.1973565 .9928081 .780493$

C $-0.473803 \quad 7.4073801 .353675$

C 0.5134467 .8964930 .317011

C 1.6324448 .5416620 .689131

C $0.1841907 .705954-1.147488$

C $-0.1731916 .295547-1.530880$

N -1.458622 $2.654086 \quad 1.362039$

H $2.296938-1.607244-4.272579$

H $1.766653-2.058418-1.290109$

H $0.127591-0.734582-3.550058$

H -1.586123 -0.592320 -1.544021

H $-0.566061-1.678844-0.692482$
H $-2.927738-3.362966-3.727457$

H $-1.553013-4.732681-2.516610$

H $0.061644-4.037132-2.387444$

H $-1.012433-4.078247-0.971793$

H -2.872413 -0.309212 -3.189476

H -3.040356 -1.039704 -4.771680

H $-5.199926-0.334383-3.851091$

H $-5.166490-2.071617-4.162507$

H -4.715189-2.472228 -1.714961

H -7.063661 -2.521788 -2.621772

H $-7.198922-0.794558-2.357099$

H -9.003952 -2.393239-1.109446

H -7.0672130 .1026960 .116997$

H -7.422631 -4.087624-0.707070

H -7.310246 -3.533480 0.947791

H $-5.848682-3.624730-0.053752$

H -7.805945 -1.192531 1.827289

H $\quad-5.260768 \quad 1.4474350 .695829$

H -4.6814830 .4181232 .009507$

H -3.445331 -1.523159 0.922895

H $1.7321982 .647451-1.578331$

H $-0.9386603 .963611-2.365560$

H $2.3688494 .744955-0.412755$

H $2.4886696 .321973-1.193161$

H $2.7020794 .877108-2.157304$

H -2.4406730 .2734730 .751276$

H $-3.0911291 .474219-0.385069$

H $0.266408 \quad 3.4052042 .207705$

H -1.172989 3.4779253 .253005

H -2.1254695 .2304991 .448862$

H 0.8072995 .7745672 .136866

H -0.417497 8.0434222 .246919

H -1.4960857 .5231840 .972966$

H $2.3382718 .919458-0.043048$

H $1.8688868 .703451 \quad 1.735726$

H $0.9978788 .059246-1.793370$

H $-0.6731438 .348992-1.387330$

H -1.231280 $6.157329-1.755191$

H -0.9316352 .2790340 .579055$

C $4.851200-2.121798-2.386543$

O $4.468433-1.609589-1.092728$

C $4.925586-0.249368-0.988058$

C $6.019414-0.110472-2.037797$

C $5.433057-0.941269-3.166212$

H $4.0866920 .393051-1.282209$

C 5.3413630 .0737320 .449436

H $6.1991990 .931206-2.319226$

H $6.966212-0.544823-1.696058$

C $6.447992-1.342696-4.221961$

H $4.634939-0.360753-3.645876$

C $4.227053-0.1612311 .495009$

O $6.455842-0.7545530 .800259$

H $5.699047 \quad 1.1090840 .500800$

H $4.066150-1.2375061 .616529$

O 4.7099650 .3087402 .759348

C 2.8952600 .5374041 .174451

C 1.8371190 .4861412 .288592

H $2.4679390 .103050 \quad 0.265120$

O $3.161849 \quad 1.916157 \quad 0.896155$

H $2.145758 \quad 1.117440 \quad 3.131759$

H 0.9422280 .9700311 .878190

H $6.872764-0.455901-4.703593$

H $7.273468-1.917009-3.788122$

H $5.979431-1.957526-4.996934$

C $3.669443-2.846491-3.039746$

H $6.697844-0.541251 \quad 1.717803$

H $4.814607 \quad 1.2735002 .692762$

H 2.3145222 .3446480 .687805

H $3.379431-3.695492-2.408304$

H $3.980642-3.274243-3.999984$

H $5.637663-2.863022-2.193600$

C $0.835082-3.1087371 .971930$

O $1.173044-1.7467751 .672297$

C $1.473359-0.9182292 .798578$

C $0.283809-0.8680503 .775965$

C $-0.185262-2.2727064 .167922$

C $-0.357603-3.1741452 .942122$

H $\quad 0.508917-3.5326901 .014522$ 
C $2.049692-3.9154642 .460545$

H $2.347477-1.3235343 .317994$

O $-0.802980-0.1660503 .174192$

H $0.584872-0.3037624 .666612$

H -1.144008 -2.206076 4.696859

O $0.757666-2.8652465 .055369$

H $-0.522282-4.2104973 .259847$

O $-1.535881-2.7567552 .246170$

C $3.082380-4.1146031 .372995$

H $2.542545-3.423180 \quad 3.302675$

H $1.735404-4.9146252 .783216$

H $-1.521547-0.1179783 .827429$

H $0.825291-2.2919735 .837770$

H -1.670510 -3.367366 1.501462

O $4.302874-4.3308451 .946142$

$\begin{array}{llll}\text { O } & 2.843971 & -4.117692 & 0.173767\end{array}$

C $5.381906-4.5642891 .035674$

H $5.594709-3.6551460 .467500$

H $6.267733-4.8277941 .619782$

H $5.146663-5.3949040 .363355$

SCF Energy (B3LYP/6-31G**//MMFF) $=-3245.91241380$

08_00400

MMFF Geometry

C $0.4992860 .536968 \quad 2.887649$

C $0.934221-0.7091323 .132810$

C $0.419420-1.9647482 .466969$

O $-0.211423-1.5934941 .220271$

C $-0.589444-2.6870553 .371697$

C $-0.873408-4.1092102 .930344$

C $-1.996950-4.5206892 .310807$

C $0.203711-5.1098543 .268575$

C $-3.183038-3.6984621 .891826$

C $-3.387301-3.768290 \quad 0.373798$

C $-4.621267-2.979868-0.084235$

C $-4.771786-3.061782-1.605111$

C $-5.942210-2.208094-2.113816$

O $-4.462754-1.6152900 .317180$

O $-5.806777-2.075515-3.537052$

C $-5.823839-0.791318-1.515193$

C $-7.293735-2.888778-1.867959$

O $-6.968322-0.004854-1.839840$

C $-5.589401-0.7822360 .014034$

$\begin{array}{llll}C & -5.264789 & 0.653847 & 0.481027\end{array}$

O $-6.774795-1.2308230 .672772$

C $0.146776-2.2482190 .088719$

O $0.780331-3.2922390 .019097$

C $-0.276404-1.506147-1.125116$

C $-0.939383-0.339298-1.090055$

C $-1.3252150 .482953-2.240918$

C $-0.8859700 .027958-3.606483$

C $-4.824330 \quad 0.7712001 .946786$

C -4.5094242 .2210152 .256486$

C -2.802177 3.9682001 .768394

O -5.3474452 .9737142 .747270$

C $-3.0425794 .417213 \quad 0.359849$

C $-2.0629674 .653478-0.525529$

C $-2.3105135 .003946-1.964624$

C $-1.8007403 .945260-2.922365$

C $-0.7681724 .195439-3.745009$

C - $-2.5431532 .626576-2.996019$

C -2.025145 $1.613580-2.010853$

N $-3.2307832 .584391 \quad 1.863314$

H $-0.3346440 .692496 \quad 2.208307$

H $1.751801-0.8509853 .836039$

H $1.293722-2.5962092 .271295$

H -1.514075 -2.103019 3.428287

H $-0.208598-2.727643 \quad 4.401479$

H -2.085215 -5.5795062 .068808$

H $-0.072743-6.1312462 .985711$

H $1.135092-4.8671342 .748103$

H $\quad 0.396825-5.1098404 .346310$

H $-4.070779-4.0943712 .399472$

H -3.086123 -2.6550612 .200468$

H -2.495633 $-3.365228-0.119403$

H $-3.486539-4.8146670 .057959$

H $-5.501896-3.4027190 .413764$

H $-4.885901-4.103457-1.929947$
H $-3.849370-2.699604-2.081426$

H $-5.804197-2.968684-3.922265$

H $-4.965726-0.302888-1.998452$

H $-7.334302-3.856201-2.383146$

H -8.116304 -2.295253 -2.281755

H $-7.496434-3.070848-0.810600$

H $-7.069641-0.026158-2.806913$

H $-4.4608411 .057350-0.146177$

H $-6.140066 \quad 1.2992980 .330472$

H $-6.778235-0.8981251 .583606$

H $0.024392-1.963694-2.062158$

H $-1.2223900 .074337-0.123914$

H $0.201988-0.100701-3.638234$

H $-1.1328560 .735215-4.401026$

H $-1.360818-0.925081-3.864080$

H -5.6084020 .4429102 .636427$

H -3.9392130 .1568912 .147259$

H -1.743972 4.0035102 .040899

H $-3.368778 \quad 4.5913042 .466792$

H $-4.079313 \quad 4.5076780 .043822$

H $-1.0276624 .560373-0.209026$

H $-1.8244345 .967967-2.161848$

H $-3.3790445 .165978-2.154502$

H $-0.4180893 .451178-4.452640$

H $-0.2456495 .145999-3.724651$

H -2.520858 $2.242080-4.020837$

H $-3.608327 \quad 2.796159-2.792078$

H -2.285078 $1.844076-0.979282$

H $-2.642241 \quad 1.8871131 .416688$

C $1.4262272 .787496 \quad 2.365137$

$\begin{array}{llll}\text { O } & 2.376244 & 2.242567 & 1.430501\end{array}$

C 3.0828253 .3393550 .813787

C 2.6465424 .6095421 .548798

C $2.084577 \quad 4.0717292 .852012$

H 4.1503913 .1727310 .991449

C $2.7882673 .367246-0.693040$

H $3.484275 \quad 5.297793 \quad 1.702227$

H $1.878523 \quad 5.1585130 .991241$

C 1.1428225 .0386123 .547114

H 2.9217113 .8324463 .521334

C $3.1970512 .080477-1.439187$

O $1.3770803 .543306-0.864281$

H $3.2683754 .242684-1.146269$

H $2.635602 \quad 1.226157-1.044888$

O $2.8024932 .226292-2.810210$

C $4.7117551 .784810-1.403643$

C $5.140690 \quad 0.626788-2.327596$

H $5.0260391 .574325-0.376051$

O $5.4104132 .960100-1.825597$

H $4.7567840 .802026-3.339850$

H $6.2352920 .649635-2.418409$

H $1.6634425 .969073 \quad 3.796557$

H 0.2879695 .2927122 .911924

H 0.7568324 .6099314 .476909

C 1.1185801 .7761723 .470366

H $1.2269523 .793325-1.791186$

H $1.8319892 .175823-2.840925$

H $6.3621972 .788184-1.725033$

H $0.4161562 .210343 \quad 4.189934$

H $2.037074 \quad 1.5224324 .011644$

H 0.5102863 .0142321 .802692

C $5.308914-2.338182-0.072854$

O $5.564840-1.083708-0.718506$

C $4.702774-0.752464-1.814229$

C $4.793609-1.823821-2.909611$

C $4.490973-3.213489-2.342331$

C $5.307781-3.501200-1.081367$

H $6.167522-2.4834100 .595789$

C $4.046423-2.2693050 .795252$

H $3.669440-0.696322-1.461563$

O $6.086198-1.817098-3.510863$

H $4.072809-1.582584-3.699628$

H $4.717338-3.974561-3.099112$

O $3.106647-3.308468-2.021450$

H $4.943999-4.415545-0.597910$

O $6.657879-3.753011-1.484057$

C $4.357307-1.6032762 .115433$

H $3.216210-1.7302670 .332222$ 
H $3.684770-3.276893 \quad 1.032037$

H $6.742397-1.964549-2.808288$

H $2.603666-3.153750-2.839150$

H $7.156469-4.016716-0.691952$

O $4.519913-0.261151 \quad 1.940577$

O $4.461244-2.2112433 .173248$

C $4.8996110 .468078 \quad 3.111578$

H 4.9066471 .5327632 .864504

H $4.183611 \quad 0.3004373 .921158$

H 5.9063490 .1739053 .422668

SCF Energy (B3LYP/6-31G**//MMFF) $=-3245.90108927$

08_00401

MM̄FF Geometry

C $2.013247-2.999791-1.128653$

C $1.270600-3.207217-2.225922$

C $0.231388-2.222599-2.696040$

O $-1.036748-2.918987-2.676193$

C $0.516239-1.682108-4.109881$

C $1.689843-0.723750-4.172944$

C $1.6742940 .522519-3.657094$

C $2.913776-1.245583-4.874368$

C $0.5004371 .174951-2.979591$

C $0.5718592 .706670-3.005400$

C $-0.7641233 .351314-2.602202$

C $-0.6062064 .863450-2.429825$

C -1.909449 $5.527893-1.959165$

O $-1.2205322 .766314-1.377303$

O $-1.5896046 .848786-1.495756$

C $-2.4657724 .747617-0.748413$

C $-2.9058415 .703509-3.111547$

O $-3.7534585 .234838-0.377371$

C $-2.5206223 .217814-0.970928$

C -2.9019392 .5033430 .347622$

O $-3.5171712 .926588-1.954424$

C $-2.148790-2.152567-2.504017$

O $-2.213743-0.934146-2.537098$

C $-3.275553-3.055913-2.185401$

C $-4.399753-2.528612-1.677709$

C $-5.568904-3.267990-1.199048$

C $-5.552666-4.766248-1.350691$

C $-2.735697 \quad 0.977080 \quad 0.308078$

C $-3.2248680 .311473 \quad 1.583682$

C $-3.276423-1.9087412 .717716$

O -3.8252830 .9164622 .467971$

C $-4.412918-2.7921682 .309244$

C -5.674089-2.629529 2.738152

C $-6.816665-3.5375352 .375164$

C -7.859316 -2.850245 1.519712

C -8.823681-2.097573 2.075603

C $-7.824178-3.0996050 .026193$

C -6.567337 -2.574041 -0.612247

N $-2.921195-1.0432851 .608660$

H $1.864011-2.082634-0.562321$

H $1.395566-4.122996-2.797454$

H $0.171021-1.399874-1.974448$

H $-0.374553-1.183289-4.511773$

H $\quad 0.675573-2.522424-4.799536$

H $2.5785121 .123414-3.731222$

H $3.763738-0.559700-4.795917$

H $3.221905-2.203742-4.448436$

H $2.700568-1.396281-5.937683$

H $-0.4308830 .859757-3.461133$

H $0.4739260 .848053-1.933708$

H $1.3591813 .026091-2.311812$

H $0.8563263 .064188-4.002950$

H -1.489625 $3.129825-3.394111$

H $-0.2508505 .326749-3.358526$

H $0.1714515 .063192-1.678950$

H -1.168881 $7.330009-2.228942$

H -1.8011084 .9473440 .103978$

H -2.473581 $6.333267-3.898508$

H -3.810341 $6.223055-2.776773$

H -3.200130 $4.758371-3.572365$

H -3.669989 $6.193071-0.233236$

H $-2.2730792 .883570 \quad 1.162368$

H -3.9420772 .7425170 .603389$

H -3.829702 2.016546-1.834956
H $-3.126408-4.121618-2.312750$

H $-4.458087-1.447565-1.558162$

H $-5.444846-5.043537-2.405330$

H $-6.461236-5.254242-0.993595$

H $-4.719981-5.200593-0.786297$

H $-3.3093000 .547934-0.518224$

H -1.679498 0.7199680 .166680

H -3.522277 -1.307716 3.599390

H $-2.399410-2.5182272 .957102$

H $-4.175790-3.6221581 .648597$

H $-5.901483-1.8029053 .408025$

H -7.278727 -3.889645 3.306895

H $-6.459588-4.4443031 .871284$

H -9.589830 -1.622009 1.472392

H $-8.865308-1.9390383 .148252$

H $-8.672797-2.606521-0.465618$

H -7.972388 -4.168441 -0.147796

H -6.477457-1.488211 -0.550356

H -2.449812 -1.4613690 .811501$

C $4.458253-3.411845-0.559362$

O $4.482575-2.079670-0.004535$

C $5.179065-1.220994-0.924472$

C $6.064928-2.150297-1.740371$

C $5.131129-3.330497-1.931256$

H $4.425397-0.787744-1.593567$

C $5.912378-0.103866-0.181013$

H $6.387235-1.700640-2.684057$

H $6.955877-2.455926-1.179608$

C $5.839448-4.608680-2.345490$

H $4.395507-3.074347-2.701132$

C 4.9967050 .7821070 .692310

O $\quad 6.899247-0.6940530 .673118$

H $6.4605590 .513426-0.902954$

H $4.6709560 .212510 \quad 1.569186$

O $5.797729 \quad 1.857942 \quad 1.198137$

C $3.7735841 .364630-0.039553$

C 3.0047262 .4527320 .732177

H $3.0838120 .553128-0.297348$

O $4.2202811 .946112-1.268880$

H $3.617203 \quad 3.3610720 .800995$

H 2.1416292 .7296750 .112170

H $6.342218-4.474580-3.308841$

H $6.595334-4.905372-1.610593$

H $5.126090-5.432603-2.447572$

C $3.026669-3.960148-0.571607$

H 7.3495670 .0310221 .139411

H 6.0581992 .4103440 .441068

H $3.4364202 .268578-1.744042$

H $2.726107-4.1659060 .463368$

H $2.997592-4.919604-1.100790$

H $5.060461-4.0346350 .114421$

C 1.2176360 .3241123 .237890

O $1.707706 \quad 0.8754912 .006439$

C 2.5234052 .0435982 .132608

C 1.7381803 .1772612 .813319

C 1.1738412 .7228224 .162351

C 0.4449241 .3799164 .048682

H $0.500665-0.4496482 .933893$

C $2.333582-0.3545794 .048272$

H $3.403274 \quad 1.8080382 .738740$

O $0.668402 \quad 3.5934331 .965555$

H 2.4053314 .0365092 .952435

H 0.4791873 .4846434 .536590

O 2.2478272 .6185805 .091885

H 0.2232880 .9843195 .046148

O -0.8166251 .5980583 .413450$

C $2.899107-1.5674113 .341236$

H 3.1639510 .3296944 .240385

H $1.933664-0.6997945 .008651$

H 0.2119104 .3290432 .408115

H 1.8701892 .3545005 .948050

H -0.6420121 .8685862 .495800$

O $3.999928-2.002148 \quad 4.022857$

O $2.432515-2.0758872 .331910$

C $4.657723-3.1519363 .480454$

H $3.938881-3.9396703 .234581$

H $5.231967-2.8620842 .596658$

H $5.347729-3.5355864 .236623$ 
C 5.5609010 .6283421 .921549

C $4.662228-0.3928132 .635586$

C 5.004279-1.812201 2.176491

C $4.049784-2.8640162 .759390$

O $3.299423-0.0868152 .322025$

O $4.260531-4.0700582 .008387$

C $2.593895-2.4027362 .527639$

C $4.396521-3.1913664 .216235$

O $1.671935-3.2647253 .189146$

C $2.338769-0.9425692 .958352$

C $0.926441-0.4317152 .573517$

O $2.436436-0.8513874 .385511$

C $3.7514701 .905057-1.338830$

O $2.8035261 .811645-0.577027$

C $4.3965500 .775714-2.048682$

C $4.073352-0.484928-1.723601$

C $4.650264-1.703757-2.294392$

C $5.626142-1.551585-3.431159$

C $0.611620-0.4378311 .075451$

C $-0.035580-1.7214680 .586319$

C $-0.295274-3.097339-1.476104$

O $-0.669693-2.4748521 .320007$

C $0.807901-4.107247-1.453560$

C $1.442140-4.536464-2.554863$

C $2.552798-5.546613-2.539386$

C $3.844398-4.995647-3.102479$

C $4.189707-5.220722-4.381397$

C $4.776427-4.264447-2.162737$

C $4.280777-2.896049-1.780962$

N $0.148114-1.906493-0.776871$

H 1.7440685 .0461680 .313645

H $2.4999134 .697245-2.633385$

H $4.7099625 .043922-1.600742$

H 3.6441843 .7079180 .928006

H 4.1314375 .3764830 .780590

H 7.2119102 .9233321 .824764

H 7.8201834 .7595140 .627025

H $6.916014 \quad 4.766698-0.888510$

H $6.577094 \quad 5.982678 \quad 0.365434$

H 5.5021152 .1621543 .440488

H 4.2178852 .2936152 .260730

H 5.3986800 .5604290 .839953

H $\quad \begin{array}{lll}6.614158 & 0.382443 & 2.107210\end{array}$

H $4.814888-0.2916713 .716585$

H $6.044765-2.0599302 .422061$

H 4.940895 -1.860484 1.081386

H $3.700569-4.7660032 .391648$

H $2.401576-2.4966701 .452129$

H $5.407163-3.6124894 .281266$

H $3.726352-3.9576774 .621180$

H $4.358891-2.3194434 .872421$

H $1.769939-4.1521832 .805722$

H $0.161882-0.9687483 .147985$

H $\quad 0.8578780 .6144572 .904764$

H $1.964339-0.0536614 .676654$

H $5.1427331 .013333-2.798508$

H $3.332835-0.645480-0.942413$

H $5.178958-0.974298-4.248327$

H $5.936991-2.504337-3.865872$

H $\quad 6.533183-1.038696-3.093017$

H $-0.106508 \quad 0.362448 \quad 0.863692$

H $1.510996-0.225176 \quad 0.493010$

H $-0.557460-2.798348-2.495485$

H -1.186459-3.504913 -0.988658

H $1.096586-4.503553-0.482748$

H $2.225291-6.413001-3.129058$

H $2.730765-5.932329-1.527754$

H $5.127893-4.857022-4.786762$

H $3.538706-5.770395-5.053172$

H $5.787501-4.208739-2.581811$

H $4.896841-4.856932-1.246306$

H $3.564097-2.906821-0.959640$

H $0.737183-1.256461-1.289425$

C $-0.8210614 .459934-0.788400$

O $-0.7139063 .207407-1.496676$

C $-2.0334052 .790958-1.914618$

C $-3.0056793 .669184-1.143046$

C $-2.2359914 .969924-1.057917$

H -2.090996 $3.015982-2.987563$

C $-2.1830701 .282029-1.683139$

H -3.970062 $3.776124-1.648383$

H $-3.1864713 .266622-0.139487$

C -2.7655065 .9163680 .005956$

H -2.277099 $5.468309-2.035876$

C $-3.6270100 .769555-1.868028$

O $-1.2934880 .641880-2.601231$

H $-1.8295091 .030177-0.677967$

H $-4.2623481 .293640-1.148860$

O $-4.096031 \quad 1.127609-3.168303$

C $-3.801572-0.748016-1.655434$

C $-5.258284-1.231141-1.794212$

H -3.405844 -1.022496 -0.670805

O $-3.029202-1.448193-2.633235$

H -5.639905 -0.968879-2.789135

H $-5.242776-2.329064-1.762145$

H -2.1718846 .8350390 .042928$

H $-3.8030286 .193328-0.208209$

H -2.7389155 .4577251 .000140$

C $0.2635095 .422029-1.277079$

H $-1.301844-0.309799-2.403693$

H $-3.5327550 .668685-3.815179$

H -3.105715 -2.398861 -2.443661

H $0.0562996 .437082-0.919380$

H $0.2654865 .454041-2.373711$

H -0.6911234 .2346150 .278348$

C $-6.512740-0.8200371 .702819$

O $-5.753278-1.2146600 .551736$

C $-6.209920-0.707126-0.706810$

C $-7.662024-1.136422-0.972120$

C $-8.578982-0.7376130 .187893$

C $-8.002677-1.1689681 .537969$

H $-6.116370-1.4387112 .518343$

C -6.2693690 .6499082 .065241$

H $\quad-6.167608 \quad 0.385077-0.693753$

O $-7.739895-2.545376-1.179081$

H -8.006508 -0.659964 -1.897426

H $-9.562972-1.2030550 .052552$

$\begin{array}{lllll}\text { O } & -8.757722 & 0.675710 & 0.195341\end{array}$

H -8.583263 -0.7224602 .353838$

O $-8.152862-2.5874451 .647442$

$\begin{array}{llll}\text { C } & -4.930520 & 0.805827 & 2.746547\end{array}$

H $-6.309705 \quad 1.3264191 .207203$

H $-7.021136 \quad 1.0080272 .778774$

H -7.376707 -2.982765 -0.390068

H $-9.1548350 .925694-0.656346$

H -7.855724 -2.846848 2.536251

O $-3.986454 \quad 1.212521 \quad 1.851804$

O $-4.744813 \quad 0.568505 \quad 3.933697$

C -2.6732701 .3911582 .392146$

H -2.6990092 .0540943 .262528$

H -2.246778 0.4199022 .658505

H -2.046848 1.8542911 .626209

SCF Energy $\left(B 3 L Y P / 6-31 G^{* *} / / M M F F\right)=-3245.90589250$

0800403

MM̄FF Geometry

C $-2.060672-0.5564562 .720406$

C $-2.1138720 .292972 \quad 3.757225$

C -2.7197231 .6783773 .738600$

O -3.7192041 .7921622 .694148$

C -1.684509 2.7869913 .463775

C -0.3620492 .6560864 .189771$ 
C 0.8041332 .3659213 .577108

C -0.4069162 .9164795 .671292$

C 0.9989882 .0882362 .110735

C 1.4589523 .3506381 .372172

C $1.3079233 .224220-0.150443$

C $1.8827124 .461495-0.843466$

C $1.6433024 .438099-2.359399$

O $-0.0899263 .120146-0.436953$

O $1.9000135 .757053-2.867612$

C $0.1539804 .135412-2.631291$

C $2.6273743 .505842-3.073209$

O $-0.0776783 .931105-4.023334$

C $-0.4048872 .937711-1.823990$

C $-1.9472532 .898483-1.939741$

O $0.0926631 .695449-2.314319$

C -4.9209881 .1897542 .899450$

$\begin{array}{llll}\text { O } & -5.316121 & 0.654442 & 3.922169\end{array}$

C -5.6429341 .2178051 .609385$

C -6.5125800 .2347841 .335288$

C -7.2388640 .0449940 .077576$

C $-7.0546481 .082674-0.998212$

C $-2.6210641 .770718-1.144249$

C $-2.7057950 .445012-1.883803$

C $-3.564467-1.879177-1.591643$

O $-2.292874 \quad 0.278075-3.027864$

C $-4.892656-1.923719-2.281887$

C $-5.865501-2.796178-1.975915$

C $-7.178095-2.865961-2.704775$

C $-8.374441-2.835682-1.778865$

C $-9.063526-3.956274-1.504089$

C $-8.824910-1.493855-1.242333$

C $-8.011730-1.053244-0.054449$

N $-3.320310-0.532371-1.110624$

H $-2.458557-0.2567161 .756186$

H -1.689957 $-0.021144 \quad 4.708964$

H $-3.202346 \quad 1.8694204 .705825$

H -2.136058 3.7536213 .728293

H -1.532988 2.8736062 .380849

H 1.7121452 .3207654 .175878

H 0.5826542 .8455326 .134932

H $-0.792403 \quad 3.9222985 .867596$

H -1.057590 2.1916806 .170291

H 1.7543551 .3007971 .997736

H 0.0869091 .6774711 .666780

H 0.8616874 .2066491 .714229

H 2.5048963 .5579621 .630757

H $1.8153292 .312769-0.485309$

H $2.9522834 .569385-0.624362$

H $1.4031335 .362211-0.434595$

H $2.8132155 .996317-2.633590$

H $-0.4163235 .032093-2.350295$

H $3.6598643 .835575-2.905903$

H $2.4790673 .526836-4.158320$

H $2.5538412 .472579-2.733040$

H $0.2620224 .712976-4.491348$

H $-2.3427223 .845326-1.548698$

H -2.246498 $2.830003-2.992393$

H $0.577973 \quad 1.855387-3.139404$

H -5.3860101 .9972090 .902084$

H -6.683724 -0.5294012 .092888$

H $-7.3586662 .070506-0.634154$

H -7.639441 $0.889595-1.899141$

H $-6.0063331 .132053-1.310658$

H -2.125254 $1.613284-0.180367$

H -3.653912 $2.077255-0.938217$

H -3.522989 -2.547621 -0.725969

H -2.772766 -2.161900 -2.292974

H -5.051779 -1.217895 -3.094035

H -5.698080 -3.523005 -1.184355

H -7.181707 -3.795880 -3.288591

H -7.271261 -2.055662 -3.438658

H -9.939515 -3.938240 -0.864133

H -8.763844 -4.917589-1.908181

H -9.871498 -1.553674 -0.914707

H $-8.826636-0.768133-2.059496$

H $-8.080217-1.7393360 .791436$

H -3.722374 -0.272398 -0.215194

C $-0.116728-1.9941752 .087917$
O $-0.298599-1.8121870 .670682$

C $0.648817-2.648490-0.031286$

C $1.517421-3.2966531 .038733$

C $0.583769-3.3417162 .228961$

H $0.049092-3.403538-0.553344$

C $1.425097-1.780689-1.029558$

H $1.881448-4.2843370 .741473$

H $2.386922-2.6687181 .266722$

C $1.306699-3.5349813 .550847$

H $-0.135276-4.1586242 .083223$

C $2.611299-2.490946-1.706471$

O $\quad 0.511020-1.329817-2.033447$

H $1.765991-0.881633-0.506035$

H $3.315912-2.871935-0.963554$

O $2.128043-3.638630-2.417959$

C $3.373208-1.623739-2.724296$

C $3.896994-0.269303-2.214112$

H $2.730417-1.437955-3.593865$

O $4.492558-2.370551-3.214437$

H $4.3333220 .255438-3.074396$

H $3.0470560 .329133-1.870823$

H $1.858756-4.4805173 .551926$

H $2.023247-2.7278893 .738098$

H $0.599175-3.5574164 .385352$

C -1.465342 -1.932747 2.801084

H $0.213511-2.109200-2.533254$

H $1.814340-4.283611-1.762078$

H $4.151228-3.217500-3.549574$

H -1.352455 -2.233828 3.848750

H -2.173198 -2.630789 2.337546

H $0.537345-1.1831232 .435575$

C $5.258398-0.9987681 .206984$

O $4.383403-0.8419260 .082520$

C $4.979305-0.335335-1.113794$

C $5.5941101 .053104-0.854787$

C $6.559170 \quad 1.0313390 .333808$

C 5.9437740 .3336451 .551620

H $4.595108-1.2583702 .042076$

C $6.250067-2.1526881 .011487$

H $5.766479-1.020773-1.443794$

O $4.5533931 .992346-0.594165$

H $6.1177311 .381976-1.760675$

H 6.8245992 .0616300 .600712

O $7.7530220 .364360-0.063223$

H $\quad 6.709328 \quad 0.1636202 .316994$

O 4.9700831 .2020792 .139908

C $5.537300-3.4847490 .967818$

H $6.844557-2.0404500 .100714$

H $6.960853-2.2022701 .844804$

H $4.9657212 .866566-0.488843$

H 8.3635560 .3799040 .693588

H 4.2451241 .3124631 .500850

O $5.744773-4.085640-0.238536$

O $4.877336-3.9375681 .894270$

C $5.124798-5.364293-0.407168$

H $5.328030-5.708326-1.424726$

H $5.548633-6.0837160 .299523$

H $4.041816-5.285180-0.274846$

SCF Energy (B3LYP/6-31G**//MMFF) $=-3245.90313621$

0800404

MM̄FF Geometry

C $-0.297303-4.1608890 .117610$

C $-1.572558-3.821083-0.117901$

C $-2.125082-3.504669-1.482464$

O $-2.455451-2.096409-1.487239$

C -3.405899-4.316511-1.732434

C $-4.091054-3.960108-3.036265$

C $-5.172154-3.159895-3.128598$

C $-3.503933-4.595816-4.268335$

C $-5.859733-2.436950-2.002897$

C $-6.122758-0.964305-2.347824$

C $-6.677899-0.205525-1.133936$

C $-7.1850751 .178674-1.536248$

C $-7.6778751 .973986-0.317959$

O $-5.619035-0.072428-0.177598$

O $-7.8450983 .341245-0.722418$

C $\begin{array}{llll}-6.581440 & 1.953078 & 0.766088\end{array}$ 
C $-9.050471 \quad 1.4928000 .166639$

O

C -6.0113340 .5428011 .055841$

C $-4.759561 \quad 0.673378 \quad 1.954893$

O $-6.994486-0.2358241 .739148$

C -1.480855 -1.237055 -1.892537

O $-0.378282-1.526243-2.329915$

C $-1.9677430 .138780-1.652211$

C $-1.0788731 .142962-1.633688$

C -1.356147 2.540289-1.299695

C $-2.7933522 .954321-1.136779$

C -3.939499 -0.612805 2.116391

C $-2.672864-0.3108472 .895272$

C $-0.458438 \quad 0.8242602 .645567$

O $-2.591821-0.5255824 .102243$

C -0.5342292 .3155802 .522599$

C 0.4380183 .0723801 .990412

C $0.3417984 .566113 \quad 1.843719$

C 0.6751415 .0508730 .446852

C 1.8051425 .7376920 .208296

C $-0.3319314 .806931-0.661387$

C $-0.3111963 .377440-1.134393$

N $-1.6860790 .263322 \quad 2.108527$

H $\quad 0.402598-4.237567-0.710275$

H $-2.254728-3.7188440 .723876$

H -1.396216 -3.729043 -2.271436

H -4.101266 -4.190295 -0.894468

H -3.167949 -5.389014-1.738700

H -5.611595 -3.000274 -4.112240

H -4.055096 -4.329177 -5.176402

H -2.466531 -4.276048 -4.407106

H -3.523545 -5.686882 -4.178918

H $-6.816304-2.935861-1.805894$

H -5.278959 -2.484687 -1.078196

H -5.187432 -0.495390 -2.679168

H $-6.834595-0.912171-3.180878$

H -7.490972 $-0.796986-0.696445$

H $-7.9794471 .100716-2.288926$

H -6.371248 $1.741696-2.014487$

H -8.484914 $3.360756-1.454811$

H -5.7603862 .5884020 .405483$

H $-9.7926701 .582768-0.635730$

H -9.4203592 .1172640 .987484$

H -9.0477530 .4527530 .499116$

H -7.379385 3.4356871 .751811

H -4.1005891 .4422091 .536569$

H -5.0542761 .0230882 .953006$

H $-6.547866-0.9363342 .240445$

H -3.018651 $0.277079-1.428590$

H $-0.0323610 .911368-1.826862$

H $-3.3933082 .623207-1.992134$

H -2.926504 4.037332 -1.080810

H -3.213893 $2.521373-0.226023$

H -4.500579 -1.3810562 .658411$

H -3.663763 -1.039309 1.145503

H $\quad 0.3715680 .4048232 .068425$

H -0.3427440 .5408753 .695770$

H -1.439648 2.7969902 .886198

H 1.3552542 .5968231 .651031

H 1.0303485 .0137922 .572023

H -0.6583324 .9276972 .113618$

H $2.0433306 .108316-0.783233$

H 2.5225795 .9385030 .997082

H $-0.1074905 .446553-1.524403$

H -1.315530 $5.119472-0.299104$

H $0.6918522 .992086-1.318008$

H -1.871899 $0.409917 \quad 1.121611$

C $1.005130-3.1878832 .013773$

O $2.228604-3.0230871 .270125$

C $3.276106-2.6188602 .180356$

C $2.589875-2.3243903 .506117$

C $1.432268-3.3018333 .474645$

H $3.937021-3.4892652 .277484$

C $4.034713-1.4373061 .564109$

H $3.252662-2.4711714 .364151$

H $2.218369-1.2922543 .533431$

C $0.336616-2.9724894 .473105$

H $1.815539-4.3110083 .676963$
C $5.056495-0.7891122 .509071$

O $4.729678-1.9247040 .411932$

H $3.315588-0.6881031 .214661$

H $4.544063-0.3830803 .388711$

O $5.935103-1.8095973 .004068$

C 5.9326980 .3213921 .893213

C 5.1495311 .5092231 .302839

H $6.636288-0.0951221 .164256$

O $\quad 6.7387290 .8546732 .957593$

H 4.1924951 .6209891 .827245

H 5.7185752 .4267901 .504682

H -0.487394 -3.689115 4.402765

H $\quad 0.726293-3.0060745 .495763$

H $-0.072127-1.9716914 .303330$

C $0.242616-4.4086941 .498657$

H $4.067453-2.319320-0.181264$

H $6.377105-2.2117452 .236440$

H 7.2426840 .1141543 .336812

H $-0.578231-4.6573392 .181328$

H $\quad 0.902797-5.283237 \quad 1.452958$

H $0.413349-2.2754741 .860972$

C $6.184607 \quad 1.291712-2.278439$

O $6.194503 \quad 1.440697-0.852535$

C $4.9137741 .406964-0.214179$

C $4.0320722 .549136-0.739934$

C $3.9241812 .510231-2.268838$

C $5.2958382 .364179-2.936047$

H $7.2214241 .478524-2.586399$

C $5.825711-0.141318-2.702328$

H $4.4273320 .456201-0.435653$

O $4.5573333 .810790-0.333091$

H $3.0353162 .458411-0.294551$

H $3.4485853 .432987-2.623437$

O $3.1045841 .415232-2.666523$

H $5.1736512 .144411-4.003223$

O $5.9633963 .626142-2.841139$

C $6.829084-1.158296-2.198123$

H $4.844178-0.437958-2.323560$

H $5.813877-0.208307-3.796114$

H $5.467813 \quad 3.876590-0.668431$

H $2.2350851 .530259-2.248263$

H $6.8027103 .552026-3.326546$

O $6.358325-2.412688-2.466841$

O $7.888993-0.894819-1.647535$

C $7.192904-3.489234-2.028336$

H $8.160663-3.449275-2.537375$

H $7.324139-3.448980-0.942680$

H $6.698677-4.429416-2.286956$

SCF Energy (B3LYP/6-31G**//MMFF) $=-3245.91852079$

0800405

MM̄FF Geometry

C $4.019870-1.641916-2.844368$

C $2.916516-2.076727-2.219691$

C $1.532499-2.018643-2.806240$

O $0.737117-1.149259-1.963636$

C $0.900340-3.422850-2.778072$

C $-0.210540-3.585710-3.795099$

C $-1.508709-3.311214-3.566036$

C $0.228830-4.106056-5.139100$

C $-2.107321-2.734501-2.317410$

C $-3.265885-3.582623-1.773469$

C $-3.903239-2.898258-0.557122$

C $-5.044296-3.7215580 .035571$

C $-5.714181-2.9823251 .208373$

O $-4.418278-1.642001-1.000188$

O $-6.959606-3.6393741 .492008$

C $-6.059568-1.5351830 .778304$

C $-4.873658-3.0860112 .487286$

O $-6.500689-0.7582331 .888176$

C $-4.909437-0.8028850 .048541$

C $-5.4078450 .504372-0.605317$

O $-3.865898-0.418430 \quad 0.947974$

C $0.7833260 .187761-2.212121$

O $1.3718010 .744534-3.125387$

C $0.0261970 .872717-1.137089$

C $0.1119762 .207216-1.024311$

C -0.4860463 .0372210 .024208$ 
C -1.3181542 .3600751 .078859$

C -4.386155 $1.094967-1.588965$

C $-4.7113712 .518039-2.001013$

C -3.772066 $4.392003-3.350277$

O $-5.6856443 .136076-1.581407$

C -2.813514 5.170066 -2.505402

C $-3.1943585 .923169-1.463124$

C $-2.2489296 .688297-0.582133$

C $-2.029540 \quad 6.0041850 .749418$

C -2.9916545 .9908411 .687908$

C $-0.675195 \quad 5.392274 \quad 1.023904$

C $-0.249697 \quad 4.365786 \quad 0.009848$

N $-3.768938 \quad 3.015013-2.894193$

H $3.940844-1.215888-3.840839$

H $3.008136-2.494746-1.221888$

H $1.552327-1.631569-3.833025$

H $\quad 0.526209-3.654554-1.773537$

H $1.660043-4.188208-2.988879$

H -2.217915 -3.468069-4.377689

H $-0.602274-4.185894-5.847716$

H $0.975750-3.438201-5.580062$

H $0.671139-5.102231-5.034103$

H -1.366063 -2.609786-1.523642

H -2.465341 -1.728623 -2.568412

H $-4.019155-3.710360-2.561639$

H $-2.901231-4.578935-1.496122$

H -3.119514 - 2.7301970 .191251

H $-4.689828-4.7095220 .354112$

H $-5.803121-3.907175-0.737289$

H -6.769212 -4.5735531 .684390$

H $-6.911755-1.6008340 .087139$

H $-4.765402-4.1347042 .790129$

H -5.364330 -2.582019 3.327009

H $-3.868802-2.6745332 .373366$

H -7.240802 -1.236001 2.300390

H $-6.3346130 .318346-1.162797$

H $-5.638168 \quad 1.2392580 .176183$

H -4.121499 $-0.668751 \quad 1.849696$

H $-0.537743 \quad 0.259652-0.443418$

H $0.7128442 .749697-1.753626$

H $-2.200868 \quad 1.8967730 .630491$

H -1.6813293 .0438921 .849892$

H -0.7418501 .5846881 .589819$

H -3.386470 $1.098237-1.143621$

H -4.348281 $0.480023-2.496247$

H $-4.7870354 .799622-3.298224$

H -3.449038 4.403674 -4.395539

H $-1.7591005 .097249-2.759326$

H $-4.2513795 .982103-1.212171$

H -2.661986 $7.691813-0.416294$

H $-1.2900236 .853961-1.088554$

H -2.834965 5.5216882 .653491

H -3.958529 6.4501781 .510132

H $-0.625078 \quad 4.9668212 .033581$

H $0.0726526 .196492 \quad 1.025376$

H $0.3540964 .779023-0.798768$

H -2.960954 $2.443936-3.125474$

C $5.917108-0.396920-1.690210$

O $5.105614 \quad 0.051587-0.587384$

C $5.1398931 .494788-0.558343$

C $6.0930711 .930811-1.671526$

C $5.972944 \quad 0.782476-2.655644$

H $4.130727 \quad 1.839744-0.809638$

C $5.541996 \quad 1.9853730 .837707$

H $5.8084472 .896251-2.101462$

H $7.1245772 .020943-1.311021$

C $7.1116130 .713464-3.656836$

H $5.0234430 .893325-3.193966$

C $4.584397 \quad 1.5362051 .962100$

O $6.845226 \quad 1.466973 \quad 1.135906$

H $5.638215 \quad 3.0774300 .832348$

H 4.6083990 .4446372 .045345

O 5.1055402 .0379103 .200383

C 3.1347662 .0301341 .796456

C 2.2250601 .7323883 .004252

H 2.6972681 .5962060 .891895

O 3.1631993 .4486541 .610038

H 2.6542272 .1669483 .915384
H $\quad \begin{array}{lll}1.281333 & 2.268967 & 2.842424\end{array}$

H $7.1486221 .626116-4.260577$

H $8.0797990 .601463-3.157301$

H $6.982117-0.135386-4.335683$

C $5.391244-1.724323-2.237537$

H 7.0837851 .7782382 .025828

H 5.0587563 .0088563 .165433

H 2.2519783 .7411931 .439522

H $5.381963-2.458006-1.420697$

H $6.080256-2.112938-2.996415$

H $6.917563-0.582713-1.275299$

C $0.981038-1.6415832 .025855$

O $1.264998-0.2365772 .041585$

C $1.935365 \quad 0.2377213 .213144$

C $1.064798-0.0091254 .456159$

C $0.661149-1.4827314 .574930$

C $0.136294-2.045827 \quad 3.247473$

H $0.363477-1.7807841 .128969$

C $2.275024-2.4457181 .837438$

H $2.884103-0.2969553 .328302$

$\begin{array}{lllll} & \text { O } & -0.111610 & 0.796665 & 4.411959\end{array}$

H $\begin{array}{llll}1.626481 & 0.296770 & 5.346314\end{array}$

H $-0.117503-1.5849635 .340867$

O $1.781759-2.254294 \quad 4.995626$

H $0.058114-3.137393 \quad 3.299543$

O $-1.185473-1.5408483 .026476$

C $2.036634-3.807224 \quad 1.221855$

H $2.939377-1.8888581 .165002$

H $2.809581-2.5842602 .780448$

H $-0.6170740 .541253 \quad 3.621741$

H $2.074582-1.9043755 .854331$

H $-1.743017-1.8483993 .761403$

O $3.244190-4.3802820 .938197$

O $\quad 0.940720-4.3090051 .014102$

C $3.182277-5.668817 \quad 0.319070$

H $4.205340-6.0062120 .133666$

H $2.690876-6.3851420 .984245$

H $2.655449-5.604312-0.637782$

SCF Energy (B3LYP/6-31G**//MMFF) $=-3245.92084986$

\section{6}

MMFF Geometry

C $2.502368-1.7347303 .367524$

C $3.806728-1.458563 \quad 3.214748$

C $4.763799-2.0581942 .210214$

O $5.019375-1.0645211 .188876$

C $4.257665-3.3141151 .475828$

C $5.377375-4.1681710 .913145$

C $5.827940-4.126085-0.355566$

C $5.960044-5.1644741 .883838$

C $5.431671-3.186502-1.459043$

C $4.564973-3.853841-2.536088$

C $3.072243-3.864425-2.174229$

C $2.257106-4.599960-3.239082$

C $0.747822-4.515545-2.962153$

O $2.646991-2.499688-2.092358$

O $0.057376-4.944786-4.145484$

C $0.365492-3.039200-2.726157$

C $0.317858-5.466805-1.839238$

O $-0.997671-2.921815-2.323954$

C $1.280344-2.317896-1.709713$

C $0.984951-0.799227-1.724421$

O $1.021779-2.834433-0.402993$

C $5.943479-0.0986261 .419464$

$\begin{array}{lllll}\text { O } & 6.614609 & 0.064682 & 2.424256\end{array}$

$\begin{array}{llll}\text { C } & 5.967205 & 0.754588 & 0.211490\end{array}$

C 6.4719991 .9943220 .275619

C $6.5067052 .961555-0.824011$

C $6.0148612 .502887-2.172961$

C $1.9997920 .055073-0.949114$

C $1.5923301 .519510-0.912799$

C 2.2697453 .7038890 .082756

O $0.6045561 .955893-1.498322$

C $3.0753754 .484819-0.907961$

C $3.8708735 .510730-0.566701$

C $4.6542936 .327834-1.555440$

C $6.1082966 .490001-1.166639$

C $6.5482477 .624655-0.596724$ 
C $7.0659265 .371821-1.515185$ C $6.9540414 .208725-0.568924$ N $2.4612002 .282727-0.144361$ H $2.011161-2.4436172 .707694$ H $4.255481-0.7346253 .893317$ H $5.698547-2.2941922 .734882$ H $3.573326-3.0207630 .673200$ H $3.676831-3.9532002 .153108$ H $6.613213-4.826550-0.639426$ H $6.763204-5.7611911 .438500$ H $6.377590-4.6503642 .755328$ H $5.185249-5.8579372 .227096$ H $4.949163-2.284515-1.074859$ H $6.361594-2.841853-1.929815$ H $4.694271-3.279606-3.463223$ H $4.917739-4.873283-2.734241$ H $2.943813-4.348049-1.199911$ H $2.575551-5.646815-3.318559$ H $2.453094-4.151775-4.223328$ H $0.344668-5.851740-4.347596$ H $\quad 0.448417-2.522841-3.692975$ H $0.556343-6.503879-2.104067$ H $-0.767169-5.437812-1.690931$ H $0.802724-5.251485-0.884772$ H -1.537350 -3.376217 -2.993299 H $0.983190-0.440602-2.762308$ H -0.017696 -0.620141-1.319375 H $1.230560-2.1560050 .257367$ H $5.528420 \quad 0.348327-0.693013$ H $6.8722482 .344518 \quad 1.226156$ H $6.6305551 .675348-2.542636$ H $6.0393513 .281547-2.937461$ H $4.9745262 .164942-2.112821$ H $2.094610-0.2952710 .083350$ H $2.985186-0.008279-1.424258$ H 2.5728563 .9110831 .113623 H $1.2088253 .950142-0.027590$ H $2.9806814 .211728-1.956346$ H 3.9381065 .8034710 .478913 H $4.1758927 .313865-1.622371$ H $4.5950035 .900229-2.564037$ H $7.5936157 .760275-0.340167$ H $5.8714908 .441556-0.368915$ H $8.1021915 .732427-1.464172$ H $6.9222665 .090818-2.561735$ H 7.2842374 .4430580 .444268 H $3.278091 \quad 1.8421940 .267781$ C $0.506826-0.2507063 .942181$ O $-0.402184-1.011148 \quad 3.124239$ C -0.860327 -0.1673902 .042057$ C $-0.394046 \quad 1.2414542 .384946$ C 0.9083140 .9633513 .109569 H $-0.343234-0.5297971 .146359$ C $-2.375649-0.3345401 .880967$ H -0.2681921 .8713871 .500024$ H $-1.107197 \quad 1.7353993 .056883$ C 1.4154192 .1356033 .929562 H 1.6669290 .6916362 .366233 C -2.9994760 .6454020 .865387$ O $-2.591691-1.688171 \quad 1.471895$ H $-2.860477-0.2187942 .857959$ H -2.791226 1.6695941 .194691 O $-2.3673850 .486234-0.404157$ C -4.5273340 .5059950 .703716$ C $-5.0944241 .569167-0.258490$ H -4.9933890 .6098281 .690572$ O $-4.833594-0.7951940 .209154$ H -4.676985 $2.549824-0.000870$ H -4.782183 $1.317863-1.279862$ H 1.6028793 .0022483 .288082 H $\quad 0.6903132 .4351374 .693737$ H $2.352274 \quad 1.8804654 .434673$ C $1.647481-1.1343274 .450207$ H -3.551546 -1.839567 1.448640 H $-2.547919-0.420775-0.706278$ H $-5.801010-0.8705410 .152469$ H $1.218064-1.9708245 .016034$ H $2.262824-0.5615655 .154357$
H -0.0764150 .0758324 .813905$

C $-8.6014730 .322715-0.685441$

O $\quad-7.1723960 .434105-0.714203$

C $-6.6336091 .659425-0.211013$

C $-7.1608592 .846390-1.032894$

C $-8.6915622 .854936-1.084608$

C $-9.2586771 .479668-1.456133$

H $-8.817687-0.606703-1.228615$

C -9.1340830 .1619370 .742721$

H $-6.930211 \quad 1.7829420 .836313$

O $-6.6416032 .800876-2.360750$

H $-6.8022603 .778252-0.580218$

H $-9.0180303 .600075-1.820242$

O -9.1865663 .2553980 .189788$

H $-10.3433081 .452074-1.299711$

O $-9.031259 \quad 1.246332-2.849339$

C $-8.661171-1.1416721 .341023$

H $-8.8419740 .977799 \quad 1.409806$

H -10.230343 0.1396980 .748302

H $-6.886673 \quad 1.942445-2.746740$

H $-10.156704 \quad 3.286378 \quad 0.131185$

H -9.488192 $1.948948-3.342594$

O $-7.544380-0.9311332 .094044$

O $-9.204542-2.2199641 .139285$

C $-6.971080-2.0994822 .689063$

H $-6.070606-1.7973793 .230731$

H -7.675278 -2.546809 3.396918

$\mathrm{H}-6.688534-2.8202581 .915808$

SCF Energy (B3LYP/6-31G**//MMFF) $=-3245.91885305$

\section{7}

MMFF Geometry

C $-2.1233094 .483258 \quad 1.337943$

C -3.3156873 .9288961 .606051$

C $-4.391708 \quad 3.524376 \quad 0.626639$

O $-4.610122 \quad 2.1050100 .832128$

C $-4.0775093 .757533-0.866241$

C $-5.2668263 .537950-1.786622$

C $-5.3455342 .590045-2.741952$

C $-6.4075074 .511549-1.617398$

C $-4.3083201 .578928-3.140369$

C $-4.6351050 .191934-2.579162$

C $-3.620342-0.871881-3.024654$

C $-3.970243-2.230489-2.413950$

C $-2.905655-3.295330-2.709163$

O $-2.325500-0.451856-2.589580$

O $-3.172292-4.405303-1.837979$

C $-1.514175-2.729905-2.346656$

C $-3.032223-3.834073-4.138895$

O $-0.504705-3.656590-2.739072$

C $-1.252269-1.332848-2.951077$

C $0.054596-0.674477-2.447068$

O $-1.139784-1.452117-4.374064$

C $-5.8551411 .690372 \quad 1.177749$

O -6.8504962 .3908451 .307290$

C -5.9169780 .2247691 .381573$

C $-4.846550-0.5826001 .451677$

C $-4.881415-2.0367401 .647644$

C $-6.235499-2.6874561 .777210$

C $0.113046-0.423766-0.938273$

C $0.803570-1.522702-0.150588$

C $1.071432-2.3404432 .191329$

O $1.521258-2.374734-0.666309$

C $0.165105-3.5264612 .265240$

C $-0.673207-3.7631273 .284932$

C $-1.569371-4.9644313 .355350$

C -3.030375 -4.606895 3.212336

C $-3.878073-4.7213144 .248342$

C $-3.522047-4.2047001 .839581$

C $-3.716484-2.7182241 .698992$

N $0.549891-1.4110051 .209353$

H -1.828280 4.6839430 .312551

H -3.5706903 .7538262 .650616$

H -5.2815014 .0998450 .907668$

H $-3.2415983 .110870-1.150480$

H $-3.7384214 .789910-1.020975$

H $-6.2583492 .537151-3.334755$

H -7.161503 $4.409164-2.405375$ 
H $-6.9139014 .360078-0.660357$ H -6.036214 $5.541018-1.657152$ H $-4.2828161 .533993-4.236452$ H -3.306132 $1.892324-2.835174$ H $-4.6328240 .255925-1.484934$ H -5.643675 -0.110869-2.887294 H -3.631321 $-0.926979-4.119614$ H $-4.958099-2.567241-2.752457$ H $-4.052015-2.123649-1.324628$ H -2.460624 -5.056301-1.964750 H -1.479641 -2.668830 -1.251501 H -4.009784 -4.310938 -4.280118 H $-2.288307-4.613560-4.337207$ H -2.930567 -3.058428 -4.900660 H $0.338833-3.356487-2.361471$ H $0.928229-1.232079-2.804447$ H $\quad 0.127270 \quad 0.310797-2.930529$ H $-0.659294-0.678557-4.712514$ H -6.925776 -0.1614501 .490691$ H -3.852135 -0.1510231 .352028$ H $-6.792165-2.2535852 .615587$ H -6.191344 -3.761446 1.967435 H $-6.816364-2.5503660 .858438$ H $0.7026820 .482857-0.766842$ H $-0.885442-0.241044-0.526261$ H $1.134486-1.8085693 .145826$ H $2.077963-2.6576391 .900758$ H $0.208608-4.2296851 .436712$ H $-0.719103-3.0621794 .114995$ H - $1.384570-5.4644374 .314812$ H $-1.306044-5.7016572 .586153$ H $-4.934216-4.4983394 .140480$ H -3.535088 -5.0390705 .227322$ H $-4.430017-4.7658501 .596541$ H -2.799696 -4.5255021 .077990$ H - $2.784735-2.1606471 .600480$ H $-0.112415-0.7119341 .532933$ C 0.2148024 .1736932 .305851 O $\quad 0.893395 \quad 4.5488231 .089347$ C $1.461814 \quad 3.359601 \quad 0.499122$ C 1.4798782 .3285811 .619518 C 0.1800312 .6457292 .329326 H $0.7640793 .057873-0.292479$ C $2.8387943 .673008-0.098902$ H 1.5315961 .2999371 .259357 H 2.3333662 .4998662 .286579 C $0.075093 \quad 2.050328 \quad 3.721519$ H -0.6446602 .2599381 .717216$ C $3.4879652 .436829-0.756332$ O $2.6458434 .705087-1.069423$ H 3.4918904 .0814520 .681311 H $3.4882721 .619982-0.028598$ O $2.6783102 .012167-1.854196$ C $4.9390672 .638810-1.235311$ C $5.5151551 .438991-2.014966$ H $5.5687612 .854951-0.364184$ O $5.010535 \quad 3.778096-2.093941$ H $4.9967881 .342561-2.977745$ H $6.5554521 .687999-2.264484$ H -0.8821012 .3118464 .183620$ H 0.1437280 .9582823 .679682 H $\quad 0.874748 \quad 2.413125 \quad 4.375368$ C - $1.138498 \quad 4.8821132 .404381$ H $3.5219834 .960670-1.403850$ H $2.6650812 .737923-2.501840$ H $5.9450923 .914502-2.325371$ H -0.9792695 .9633282 .307083$ H -1.561085 4.7104823 .401735 H $0.8484214 .543632 \quad 3.122989$ C $6.252360-0.8949610 .808529$ O $6.2229860 .249551-0.055609$ C $5.470138 \quad 0.100109-1.262168$ C $6.028605-1.054412-2.108352$ C $6.089535-2.352474-1.296777$ C $6.764497-2.1396530 .061618$ H $6.994282-0.6447521 .578302$ C $4.903225-1.1151871 .511825$ H $4.428418-0.126285-1.017293$
O $7.330445-0.735994-2.595485$

H $5.384938-1.194440-2.984560$

H $6.645281-3.110262-1.862157$

O $4.771512-2.847826-1.079494$

H $6.638145-3.0241900 .696665$

O $8.170772-1.967892-0.139288$

C $4.645788-0.0615862 .569738$

H $4.054068-1.0962980 .824864$

H $4.923758-2.0888612 .016093$

H $7.893183-0.531289-1.829083$

H $4.364449-2.994547-1.950206$

H $8.506674-2.775757-0.563652$

O $3.775467-0.561763 \quad 3.495309$

O 5.1311801 .0614962 .575651

C $3.456706 \quad 0.306561 \quad 4.587304$

H 4.3048030 .3555285 .275995

H $3.193667 \quad 1.306923 \quad 4.233200$

H $2.596200-0.1134275 .114981$

SCF Energy (B3LYP/6-31G**//MMFF)= -3245.92693330

\section{8}

MM̄FF Geometry

C $0.161038 \quad 4.320794 \quad 0.077256$

C 1.4313333 .8923090 .092315

C $2.3210183 .830493-1.120728$

O $2.6964242 .446691-1.309557$

C $3.5956084 .653561-0.873821$

C $4.6127804 .527844-1.990198$

C $5.704460 \quad 3.738496-1.932345$

C $4.3534655 .386944-3.198764$

C $6.0875682 .809343-0.812646$

C $6.524483 \quad 1.433371-1.335975$

C $6.7600920 .452276-0.178255$

C $7.445623-0.822486-0.668898$

C $7.626346-1.8387870 .468811$

$\begin{array}{lllll}\text { O } & 5.485258 & 0.125664 & 0.389358\end{array}$

O $7.986618-3.098296-0.118376$

C $6.271045-2.0399611 .176456$

C $8.774920-1.4527271 .408030$

O $6.415611-2.8570742 .336278$

C $5.553290-0.7186221 .545727$

C $4.109690-1.0314802 .007802$

O $6.255114-0.0833482 .615933$

C $1.8544721 .666983-2.040400$

$\begin{array}{llll}\text { O } & 0.824926 & 2.012542 & -2.597177\end{array}$

C $2.3886960 .288467-2.006539$

C $1.595836-0.731418-2.366384$

C $1.938373-2.152654-2.292429$

C $3.369847-2.513754-1.998462$

C $3.192357 \quad 0.1931342 .122712$

C $1.807023-0.2206422 .585601$

C $-0.226317-1.4587791 .832901$

$\begin{array}{lllll}\text { O } & 1.400264 & 0.040667 & 3.715297\end{array}$

C $-0.383960-2.8550631 .300882$

C $0.545433-3.6153090 .699073$

C $0.295883-5.0033000 .177520$

C $-0.026396-5.048019-1.302846$

C $-1.186617-5.570478-1.735582$

C $1.012559-4.552282-2.291900$

C $0.951038-3.056832-2.459674$

N $1.106605-0.9257541 .619707$

H $-0.2797554 .669229-0.853028$

H 1.8553693 .5298141 .026760

H $1.8112894 .207186-2.016466$

H 4.0498594 .3658490 .081674

H $3.3328045 .713075-0.750186$

H $6.3952533 .753677-2.774067$

H $5.1341705 .280633-3.959461$

H $3.4010245 .115179-3.664286$

H $4.3129466 .443037-2.912702$

H $6.9182113 .261902-0.257889$

H $5.2660702 .672675-0.104445$

H $5.7509291 .035594-2.004896$

H $7.4442801 .548581-1.922769$

H 7.3783500 .9500300 .577967

H $8.412606-0.592510-1.133315$

H $6.835399-1.285892-1.457150$

H $8.805631-2.968372-0.626596$ 
H $5.627359-2.6002040 .484402$ H $9.716134-1.3770320 .850430$ H $8.939252-2.2243502 .168393$ H $8.613053-0.4989541 .914351$ H 6.853742 -3.679769 2.058492 H $3.652055-1.7279001 .295759$ H $4.133501-1.5439232 .978369$ H 5.6384160 .4833293 .105677 H $3.3948560 .144666-1.631413$ H $\quad 0.580083-0.511189-2.692782$ H $4.046603-2.005804-2.695186$ H $3.578567-3.580773-2.100295$ H $3.637601-2.222947-0.979342$ H 3.5868110 .9185892 .841688 H 3.0964470 .7109841 .161997 H $-0.921710-0.7905141 .315001$ H -0.468351-1.443099 2.900292 H -1.382926 -3.271117 1.419273 H $1.556083-3.2396010 .570197$ H $-0.507092-5.4732330 .760803$ H $\quad \begin{array}{llll}1.188447 & -5.612840 & 0.366298\end{array}$ H -1.421643 -5.625349 -2.793399 H -1.931177 -5.948699 -1.042903 H $\quad 0.840570-5.005031-3.276981$ H $1.994165-4.909633-1.969664$ H $-0.045761-2.688490-2.705766$ H $1.474776-0.9432040 .673136$ C -1.7991373 .2462011 .191019$ $\begin{array}{lllll}\text { O } & -2.802729 & 3.661197 & 0.242668\end{array}$ C -4.1059683 .2938390 .751000$ C -3.8467152 .4213251 .970583$ C -2.5513383 .0043862 .496276$ H -4.5861524 .2350831 .045775$ C $-4.8968592 .616445-0.374177$ H -4.6613052 .4625442 .699229$ H $-3.702045 \quad 1.3737181 .677908$ C - 1.8283182 .0905223 .470677 H -2.7675483 .9618262 .989048$ C -6.2162971 .9617790 .072970$ O $-5.189503 \quad 3.603497-1.369090$ H $-4.2510781 .876212-0.857508$ H $-6.029611 \quad 1.1866780 .821959$ O $\quad \begin{array}{lllll}-7.038110 & 2.945617 & 0.717882\end{array}$ C -7.058958 $1.369332-1.071678$ C $-6.3361820 .422316-2.046776$ H $-7.4971202 .193579-1.648859$ O $-8.1625710 .672131-0.482002$ H $-7.0470230 .170934-2.845522$ H $-5.5110250 .966349-2.519516$ H $-2.440891 \quad 1.9188214 .361543$ H -1.6101851 .1168153 .020720$ H -0.8807822 .5313843 .794852$ C -0.7165734 .3201271 .298163$ H -4.344177 4.000491-1.641056 H -6.6128683 .1852411 .557975$ H -8.731956 $0.359614-1.205670$ H -0.108304 4.154252 2.194784 H -1.170677 5.3139201 .391411 H -1.3743412 .3042410 .817653$ C $-4.205433-1.7450620 .161111$ O $-4.795520-0.621124-0.502679$ C $-5.828844-0.909820-1.446423$ C $-5.303121-1.844984-2.552104$ C $-4.652661-3.101286-1.965765$ C $-3.655858-2.754445-0.857933$ H $-3.350327-1.3249090 .704466$ C -5.157075 -2.379106 1.182118 H $-6.662075-1.396940-0.929675$ O $-4.346043-1.148598-3.349925$ H -6.138346 -2.120488 -3.207106 H $-4.127736-3.649413-2.757725$ O $-5.657375-3.958903-1.433689$ H -3.342981 -3.665512 -0.336219 O $-2.478440-2.198396-1.448788$ C $-5.349486-1.4875472 .385529$ H $-6.149066-2.5832960 .770069$ H -4.766636 -3.3378281 .544581$ H $-4.063083-1.749321-4.060296$
H $-6.261035-4.190430-2.159883$

H -2.719345 -1.346175 -1.850585

O $-4.237131-1.5222443 .174173$

O $-6.370011-0.8476582 .602892$

C $-4.304065-0.7416984 .371325$

H $-4.5814660 .292007 \quad 4.146198$

H -5.020428 -1.189135 5.066480

H -3.313749 -0.744976 4.834540

SCF Energy (B3LYP/6-31G**//MMFF) $=-3245.90026727$

\section{9}

MMFF Geometry

C $-2.141996 \quad 4.348287 \quad 1.499382$

C -3.0529643 .3705341 .608382$

C -3.0624652 .3630472 .729199$

O -2.7186621 .0782352 .157960$

C -4.4682232 .2750003 .347631$

C -4.5667991 .2795954 .488795$

C -5.1908290 .0861044 .418869$

C $-3.924868 \quad 1.717040 \quad 5.780507$

C $-5.888277-0.5155773 .231328$

C $-5.107786-1.7142682 .677688$

C $-5.563106-2.0803991 .258015$

C $-4.861913-3.3493820 .775525$

C $-5.209035-3.679066-0.683123$

$\begin{array}{lllll}\text { O } & -5.217683 & -0.985778 & 0.403468\end{array}$

O $-4.266857-4.674237-1.114115$

C $-5.000898-2.417083-1.553345$

C $-6.596414-4.322574-0.790230$

O $-5.492077-2.618747-2.875513$

C $-5.632213-1.136671-0.960466$

C $-5.2173110 .155107-1.704725$

O $-7.061945-1.165730-1.060190$

C -1.3898870 .7911362 .056730$

O -0.4589081 .4762912 .450023$

C $-1.251449-0.5021461 .351202$

C $-0.033640-0.9189360 .972892$

C $0.279026-2.1601180 .261640$

C $-0.867245-3.061622-0.111564$

C $-3.7167670 .469294-1.711784$

C $-2.980064-0.052022-2.934605$

C $-0.678116-0.296848-3.856108$

O $-3.550111-0.519744-3.916576$

C $-0.130928-1.654084-3.546890$

C $1.180772-1.917841-3.445383$

C $1.740044-3.293326-3.220976$

C $2.704841-3.358146-2.059254$

C $4.029515-3.252388-2.256852$

C $2.142152-3.650407-0.687727$

C $1.569650-2.430881-0.023997$

N $-1.6049800 .099083-2.812954$

$\mathrm{H}-1.3696734 .4490112 .257917$

H $-3.801104 \quad 3.253674 \quad 0.827397$

H -2.3394142 .6331503 .508999$

H -5.1923972 .0388672 .562082$

H -4.7674973 .2620493 .725908$

H -5.201546 -0.5422005 .308519$

H $-4.093567 \quad 1.001196 \quad 6.592166$

H -2.842795 1.8237735 .657145

H -4.3364142 .6791886 .102202$

H $-6.886221 \quad-0.8472203 .543977$

H -6.0592950 .2235732 .446471$

H -4.037815 -1.473415 2.659218

H $-5.237287-2.5718453 .349402$

H $-6.651241-2.2131121 .259802$

H $-5.099080-4.1986881 .428425$

H -3.775324 -3.211883 0.850238

H -4.494955 -4.931963 -2.023143

H $-3.917633-2.270362-1.636304$

H $-6.615556-5.277247-0.250598$

H $-6.844991-4.561646-1.830025$

H -7.391375 -3.696612 -0.379267

H $-4.984248-3.346645-3.271573$

H $-5.6317890 .154691-2.720302$

H $-5.7137250 .992308-1.192428$

H -7.323459-1.951714 -1.565675

H -2.152755 -1.067911 1.149546

H $0.825968-0.292827 \quad 1.208766$ 
H $-1.624045-2.510061-0.679452$ H -1.333582 -3.4762170 .788372$ H $-0.573372-3.903381-0.742074$ H -3.591692 $1.559878-1.723881$ H $-3.2323130 .094882-0.805890$ H $\quad 0.1057390 .464180-3.906971$ H -1.195383 -0.320616 -4.820709 H $-0.847515-2.465524-3.443331$ H $1.896224-1.109886-3.581134$ H $2.254014-3.595545-4.143225$ H $0.942129-4.032190-3.076688$ H $4.730760-3.337513-1.434356$ H $4.441349-3.075835-3.245005$ H $2.936408-4.030701-0.030706$ H $1.426488-4.474911-0.754191$ H $2.329681-1.7110840 .281675$ H -1.217546 $0.461717-1.946321$ C $-1.2289704 .732559-0.795551$ O $0.1150274 .503878-0.340664$ C $1.0137084 .652345-1.455463$ C $0.1586425 .075937-2.651043$ C -1.071202 $5.668050-1.988178$ H $1.7039225 .463722-1.194650$ C $1.8117213 .357916-1.690468$ H $\quad 0.6814085 .791533-3.293528$ H $-0.1164074 .219256-3.277396$ C -2.282637 5.724111-2.902029 H $-0.8280796 .681282-1.641657$ C $2.6778312 .948811-0.478105$ O $0.8897072 .315645-2.009221$ H $2.4589593 .505843-2.563491$ H $3.2857913 .810845-0.178048$ O 1.8422342 .6280480 .633170 C $3.6322721 .766516-0.760029$ C $4.447277 \quad 1.3902010 .486715$ H $4.2964382 .056571-1.581269$ O $2.881521 \quad 0.633737-1.187428$ H 4.9564382 .2785210 .882149 H 3.7646841 .0647001 .284021 H -2.071866 6.339719-3.782602 H -2.569281 $4.725888-3.249662$ H -3.142988 $6.160191-2.384977$ C -2.0795655 .3017830 .339617$ H $1.4053191 .516570-2.208783$ H $1.246923 \quad 1.9125970 .351574$ H $3.504005-0.016461-1.553697$ H -3.091239 $5.525680-0.017903$ H $-1.651423 \quad 6.2438360 .703212$ H -1.631956 $3.762501-1.118307$ C $7.024828-1.0254101 .647558$ O $6.078540 \quad 0.047857 \quad 1.569283$ C $5.468746 \quad 0.2541270 .286886$ C $6.5417940 .558514-0.769296$ C $7.638875-0.511081-0.787313$ C $8.151808-0.8336520 .620419$ H $7.464952-0.9349532 .649412$ C $6.345287-2.3962361 .560290$ H $4.923142-0.6538850 .011244$ O $7.129306 \quad 1.834560-0.522007$ H $6.0729120 .606324-1.758227$ H $8.469521-0.156317-1.409695$ O $7.114759-1.688166-1.393807$ H $8.787767-1.7264100 .606560$ O $8.964277 \quad 0.251903 \quad 1.076891$ C $5.458497-2.6297082 .759955$ H $5.743145-2.5288580 .657923$ H $7.093095-3.1982451 .550448$ H 7.4957021 .8234520 .378871 H $7.829976-2.346070-1.425340$ H $9.7075600 .342593 \quad 0.456670$ O $4.201450-2.1654632 .508077$ O $5.844946-3.1420313 .802358$ C $3.266051-2.3016843 .581764$ H $3.632656-1.7873794 .475276$ H $2.324896-1.8385073 .274256$ H $3.085915-3.3603303 .790705$ SCF Energy $\left(B 3 L Y P / 6-31 G^{* *} / / M M F F\right)=-3245.91283368$
0800410

MM̄FF Geometry

C $-3.296376-3.439357-1.748338$

C $-4.539277-3.842440-1.440943$

C $-5.681158-2.980622-0.945998$

O $-5.278944-1.603768-0.768019$

C $-6.168845-3.5123610 .415428$

C $-7.387290-2.7852830 .953531$

C -7.379070 -1.924803 1.991649

C $-8.686673-3.1158860 .263772$

C $-6.198208-1.4668952 .801833$

C -6.0253830 .0552212 .712479$

C $-4.6911490 .518886 \quad 3.316675$

C -4.584762 2.0440473 .272672

C -3.2054842 .5493723 .720571$

O $-3.642765-0.071127 \quad 2.542655$

$\begin{array}{lllll}\text { O } & -3.127908 & 3.929348 & 3.330658\end{array}$

C -2.1123891 .7716792 .953975$

C -3.06510925186155 .246821$

O -0.8142982 .0904453 .448779$

C -2.3167330 .2429682 .985906$

C - $-1.333114-0.5191432 .063365$

O $-2.095587-0.2306664 .319846$

C $-5.403961-0.770661-1.837846$

O $-5.785539-1.065308-2.959205$

C $-4.9852910 .581469-1.404205$

C $-4.8808081 .569492-2.305304$

C $-4.5130302 .960430-2.027045$

C $-4.1998213 .329783-0.601348$

C $-1.463212-0.2094510 .568850$

C -0.5158040 .8638530 .062913$

C $-0.0252042 .189140-1.993528$

O $0.412436 \quad 1.315143 \quad 0.727341$

C $-0.6855413 .528823-1.919868$

C $-1.0448844 .234796-3.002750$

C $-1.6504625 .607249-2.943166$

C -2.947635 $5.710901-3.712277$

C $-2.9752656 .219502-4.955913$

C $-4.2226095 .318633-3.002567$

C $-4.4866073 .839208-3.051125$

N $-0.8089841 .224915-1.246010$

H -3.027471 -2.390343 -1.665437

H $-4.777486-4.899020-1.554152$

H $-6.499125-3.040663-1.674513$

H $-5.343309-3.4740281 .133639$

H $-6.422252-4.5780940 .329649$

H -8.327720 -1.486339 2.299936

H -9.545652 -2.630115 0.739023

H -8.662596 -2.790039 -0.780506

H $-8.866213-4.1957780 .288756$

H $-6.360845-1.7520763 .848138$

H -5.277310 -1.962302 2.488611

H -6.0666080 .3564911 .658256$

H -6.8599310 .5492863 .225370$

H $-4.6270890 .156486 \quad 4.349359$

H -5.3778512 .5062253 .873894$

H -4.7572572 .3862782 .243940$

H -2.2822984 .2862853 .650537$

H -2.1472742 .1149381 .913173$

H $-3.809933 \quad 3.1764605 .710749$

H -2.0887202 .9015865 .563579$

H -3.196944 1.5203155 .669547

H -0.6677143 .0400163 .303097$

H $-0.303091-0.3921292 .417557$

H -1.547582 -1.591613 2.178040

H $-1.886590-1.1785314 .281249$

H $-4.7830840 .729821-0.350335$

H $-5.099340 \quad 1.345472-3.348784$

H $-3.8109094 .343948-0.487770$

H $-3.4323292 .666998-0.187928$

H $-5.101047 \begin{array}{lll}3.254389 & 0.015834\end{array}$

H -1.214384 -1.118757 0.009081

$\begin{array}{llll}\text { H } & -2.490916 & 0.059220 & 0.308427\end{array}$

H $0.0483351 .819335-3.021247$

H $0.9839952 .250013-1.573530$

H $-0.8457683 .950500-0.930337$

H $-0.8571703 .821730-3.991519$

H $-0.916346 \quad 6.311293-3.356687$ 
H -1.813453 $5.929017-1.907054$ H $-3.9067336 .327523-5.501738$ H $-2.0660456 .533437-5.457897$ H -5.079999 $5.813102-3.479792$ H -4.212019 $5.718336-1.984576$ H -4.724235 $3.475573-4.051808$ H -1.625484 $0.826389-1.700943$ C $-0.939207-4.201585-1.385327$ O $-0.286089-2.973451-1.767713$ C $1.137076-3.205444-1.858416$ C $1.373798-4.572176-1.236800$ C $0.097714-5.299632-1.611866$ H $1.368129-3.229576-2.930945$ C $1.866477-2.038372-1.184497$ H $2.274322-5.059668-1.621823$ H $1.464006-4.494965-0.146044$ C $-0.142927-6.557610-0.795386$ H $0.140721-5.562575-2.677358$ C $3.385670-2.245543-1.043744$ O $1.621327-0.870966-1.973687$ H $1.431211-1.847130-0.196094$ H $3.562742-3.144947-0.443149$ O $3.954646-2.490490-2.329944$ C $4.119382-1.069443-0.362616$ C $5.605774-1.385182-0.141912$ H $3.638396-0.8676930 .600634$ O $4.032307 \quad 0.114558-1.150948$ H $5.711569-2.3121370 .436262$ H $6.085422-1.587899-1.109376$ H $0.669335-7.275793-0.947865$ H $-0.198870-6.3361430 .275729$ H -1.079927 -7.041230 -1.088397 C $-2.212692-4.375275-2.214945$ H $0.657297-0.775672-2.060383$ H $3.819595-1.688571-2.863642$ H $3.0921410 .349813-1.226589$ H -2.560695 -5.412778 -2.155968 H $-2.004846-4.151304-3.268324$ H -1.180642 -4.117555 -0.317193 C $8.7182760 .196194 \quad 1.145206$ O $7.761479-0.7300260 .614839$ C $6.410106-0.2556270 .530576$ C 5.8958140 .1320491 .925252 C $6.836711 \quad 1.1252282 .616917$ C 8.2993620 .6760692 .543993 H $9.640095-0.3902051 .254703$ C 9.0087321 .3405000 .167933 H $6.3856050 .618674-0.128061$ O $5.751666-1.0305192 .739054$ H 4.9023090 .5838151 .832860 H 6.5441681 .2305333 .668725 O 6.7186762 .4053272 .002763 H 8.9672551 .4830432 .867391 O $8.494391-0.4173343 .445845$ C $9.6861270 .815863-1.075568$ H $8.1162411 .896408-0.133075$ H 9.6880272 .0728940 .620359 H $6.615551-1.4757662 .776573$ H 5.7919992 .6874812 .086587 H $8.283421-0.0999134 .340438$ O $8.752276 \quad 0.506186-2.019723$ O $10.8973850 .674183-1.182554$ C $9.266130-0.034676-3.240394$ H $8.421160-0.242361-3.902360$ H $9.9247760 .690045-3.728267$ H $9.797517-0.971180-3.045814$ SCF Energy (B3LYP/6-31G**//MMFF) $=-3245.92436754$

\section{1}

MMFF Geometry

C -2.7126014 .3832431 .830570$

C -3.0468553 .0872001 .740187$

C -3.0724632 .1360932 .906922$

O -2.2114841 .0212652 .579690$

C -4.5015551 .5916143 .107508$

C -4.6015140 .5121824 .170126$

C $-4.969499-0.7624853 .924866$

C $-4.269751 \quad 0.9442975 .574459$
C $-5.379413-1.3576812 .604168$

C $-4.171303-1.7551111 .757591$

C $-4.540541-2.2842440 .365888$

C $-5.133159-3.6938080 .416161$

C $-5.354705-4.280135-0.988983$

O $-3.322777-2.288730-0.390197$

O $-5.587009-5.690218-0.844250$

C $-4.055925-4.117346-1.810335$

C $-6.610885-3.702297-1.651416$

O $-4.247778-4.531305-3.160741$

C $-3.471119-2.690314-1.757335$

C $-2.079936-2.586644-2.421562$

O $-4.341311-1.800679-2.466823$

C -0.9050411 .0824902 .948560$

O -0.3351862 .0108843 .497433$

C $-0.291050-0.2163492 .585566$

C $0.956794-0.5058162 .983959$

C $1.666285-1.7683902 .752149$

C $0.952225-2.8287041 .957492$

C $-0.999243-3.466836-1.789755$

C $0.394568-3.089251-2.261654$

C $2.786951-3.561232-1.807644$

O $0.606608-2.312794-3.188825$

C $3.589795-4.034509-0.639449$

C $4.398238-3.2394650 .077079$

C $5.256828-3.7264841 .207315$

C $5.068674-2.9351422 .481051$

C $6.051004-2.1494342 .955217$

C $3.786299-3.1406263 .256259$

C $2.900855-1.9225823 .275105$

N $1.376685-3.732123-1.520906$

H -2.454762 4.7808192 .810511

H -3.309622 2.6573360 .777785

H $-2.7279172 .622833 \quad 3.827434$

H $-4.872231 \quad 1.2112892 .148775$

H -5.1743162 .4149903 .381861$

H -4.993553 -1.462004 4.759142

H -4.4785270 .1599936 .309959$

H -3.209438 1.2010065 .660063

H -4.8657401 .8196565 .852579$

H $-6.001955-2.2366652 .808379$

H -6.022299 -0.660548 2.054895

H $-3.520625-0.8854601 .619273$

H -3.556226 -2.493796 2.288691

H -5.240370 -1.582006 -0.101825

H $-6.070746-3.7088850 .984120$

H $-4.445064-4.3568640 .959412$

H $-6.360255-5.809187-0.266420$

H -3.329220 -4.817869-1.380711

H -7.496835 -3.912388 -1.039921

H $-6.800412-4.175988-2.620970$

H -6.561291 -2.621238-1.795909

H $-4.736033-3.826981-3.620194$

H $-2.155072-2.797875-3.496021$

H -1.752170 -1.539780 -2.353252

H -3.834909 -1.011951 -2.722697

H $-0.900241-0.9165402 .024724$

H $1.505103 \quad 0.2405673 .557217$

H $0.052500-3.1651892 .484173$

H $1.559658-3.7145761 .764074$

H $0.658796-2.4352910 .978753$

H -1.024799 -3.374108 -0.698385

H -1.165771 -4.517555 -2.050831

H $2.977523-2.509625-2.049683$

H $3.022697-4.158888-2.694231$

H $3.523900-5.091015-0.390670$

H $4.479407-2.190167-0.191245$

H $\quad 6.301684-3.6670370 .875791$

H $5.071943-4.7873401 .418193$

H $5.939523-1.6092093 .889537$

H $6.985882-2.0255532 .418873$

H $4.041412-3.3797994 .298034$

H $3.266398-4.0317742 .893907$

H $3.324656-1.0901443 .838965$

H $1.097173-4.485125-0.897903$

C $-2.9011774 .857980-0.711413$

O $-1.8774703 .910840-1.073153$

C $-1.4433294 .175179-2.418111$ 
C $-2.4706835 .131624-3.008857$

C $-2.852005 \quad 5.945657-1.786257$

H $-0.4830404 .699325-2.340095$

C -1.250974 2.859712 -3.181215

H -2.055866 $5.739147-3.818732$

H -3.346777 $4.600316-3.397944$

C $-4.1516016 .714302-1.949612$

H -2.040932 $6.653523-1.568662$

C $-0.3626631 .822734-2.460011$

O $-2.527897 \quad 2.243758-3.396675$

H $-0.8484903 .070628-4.178690$

H $-0.9021741 .394739-1.605847$

O $-0.129702 \quad 0.741679-3.370407$

C $0.9960792 .364162-1.981266$

C $1.8661551 .270701-1.341924$

H $0.8139883 .158048-1.251357$

O $1.6806582 .938304-3.092528$

H $1.3000360 .756256-0.554510$

H $2.0992600 .498895-2.088182$

H -4.074410 7.428655 -2.775909

H $-4.9907056 .043657-2.162648$

H -4.390762 $7.274633-1.040348$

C -2.634248 $5.383650 \quad 0.703437$

H -2.938331 $2.107283-2.525321$

H $-0.9977480 .417253-3.665366$

H $2.4362343 .442853-2.747947$

H -3.339228 6.1918240 .930398

H -1.625045 5.8142680 .735428

H -3.863022 $4.331083-0.753114$

C $5.1640010 .823477 \quad 0.323508$

O $3.8533420 .610405-0.216580$

C $3.2092131 .765759-0.771856$

C 3.0668272 .8630920 .295326

C $4.405356 \quad 3.180586 \quad 0.970447$

C $5.140548 \quad 1.9111931 .408033$

H $5.420518-0.1237310 .811353$

C $6.202168 \quad 1.076637-0.774422$

H $3.8169382 .135831-1.603773$

$\begin{array}{lllll}\text { O } & 2.121105 & 2.466742 & 1.285851\end{array}$

H $2.6788493 .774839-0.171206$

H 4.2287323 .8149001 .847614

$\begin{array}{lllll}\text { O } & 5.236853 & 3.906225 & 0.069589\end{array}$

H 6.1676712 .1453681 .711797

O 4.4795451 .3603912 .550721

C $6.370786-0.146084-1.644088$

H $5.9387351 .906031-1.436578$

H $7.1777491 .328463-0.341321$

H 2.4299981 .6283971 .669680

H $4.7699854 .726061-0.165921$

H $4.5056592 .029448 \quad 3.255738$

O $7.295198-0.985167-1.095629$

O $5.743260-0.337351-2.677671$

C $7.558128-2.182069-1.834777$

H $6.629636-2.719209-2.050272$

H $8.084036-1.936945-2.761995$

H $8.198987-2.824203-1.224639$

SCF Energy (B3LYP/6-31G*//MMFF)= -3245.90712571

0800412

MM̄FF Geometry

C $-2.723286 \quad 3.829103-0.556282$

C $-3.304750 \quad 2.786763 \quad 0.055032$

C $-3.8383062 .832341 \quad 1.463038$

O -3.2266351 .7442032 .200029$

C -5.3559352 .5891421 .524001$

C -6.1912183 .5256360 .676960$

C $-6.8058343 .155332-0.464663$

C $-6.342898 \quad 4.923844 \quad 1.211309$

C $-6.714503 \quad 1.807212-1.123787$

C $-7.902480 \quad 0.912785-0.754117$

C $-7.624871-0.560898-1.086054$

C $-8.882281-1.403682-0.868350$

C -8.609638 -2.901575-1.062245

O $-6.578833-1.004585-0.213043$

O $-9.733934-3.626197-0.540321$

C $-7.384024-3.296847-0.213530$

C -8.501569 -3.276410 -2.544883

O $-7.008463-4.649371-0.467009$
C $-6.164974-2.363500-0.407606$

C $-5.095129-2.6807340 .669611$

O $-5.623176-2.566374-1.710429$

C $-1.939548 \quad 1.8986352 .609328$

O $\begin{array}{llll}-1.259882 & 2.909897 & 2.549665\end{array}$

C - 1.4767230 .5723823 .072560

C $-0.1617820 .315216 \quad 3.117314$

C $0.454758-0.9735743 .439732$

C $-0.461445-2.1065573 .823223$

C $-3.823488-1.8184680 .611895$

C $-2.708257-2.427529-0.224561$

C $-0.303220-2.102629-0.794461$

O $-2.848946-3.449339-0.891151$

C $0.511096-2.9199370 .160997$

C $1.813642-2.7082600 .405538$

C $2.633388-3.5572001 .336143$

C $3.393896-2.7486532 .364567$

C $4.710133-2.5195052 .224087$

C $2.655080-2.2919963 .603676$

C $1.796926-1.0834763 .350696$

N $-1.533851-1.689630-0.142664$

H -2.645132 $4.777812-0.032520$

H -3.379968 $1.836772-0.469120$

H -3.6006223 .7812961 .960309$

H -5.6818412 .6745672 .570293$

H -5.5657321 .5391301 .283239$

H -7.415992 $3.885827-0.992376$

H -6.991004 5.5410750 .580255

H -6.7819664 .9017312 .213964$

H -5.3690545 .4201351 .268336$

H $-6.6894821 .950151-2.211248$

H $-5.7667721 .319469-0.877314$

H -8.105937 1.0019910 .321276

H $-8.795546 \quad 1.261049-1.287554$

H $-7.285257-0.633000-2.126032$

H $-9.692415-1.071608-1.529770$

H $-9.251420-1.2499890 .155542$

H -10.528177-3.332089-1.018675

H $-7.687916-3.2526440 .841902$

H -9.432951 -3.032584 -3.070075

H $-8.361304-4.355724-2.670365$

H -7.688855 -2.760002 -3.059939

H -7.796254 -5.200816 -0.321925

H -5.551116 -2.504092 1.653265

H $-4.820510-3.7412770 .628299$

H $-4.731089-2.186871-1.739833$

H -2.230159 -0.1748953 .292865$

H $\quad 0.530918 \quad 1.1145682 .858231$

H $-1.031488-1.8498694 .723068$

H $\quad 0.060323-3.040745 \quad 4.038177$

H $-1.165399-2.3251403 .012797$

H $-4.037782-0.8098880 .242992$

H $-3.433850-1.7243871 .633256$

H $0.217532-1.196579-1.116382$

H -0.543697 -2.701711 -1.678566

H $0.007654-3.744606 \quad 0.660773$

H $2.330646-1.907232-0.117433$

H $3.336960-4.1342520 .721472$

H $2.012192-4.3007741 .850983$

H $5.271306-1.9694432 .971811$

H $5.254879-2.8758361 .356415$

H $3.372176-2.0192224 .389710$

H $2.098669-3.1378194 .015549$

H $2.370106-0.2008763 .061875$

H $-1.493119-0.8892360 .481321$

C $-0.634140 \quad 3.780717-2.003481$

O $-0.098073 \quad 2.644734-1.295491$

C $1.0744583 .070626-0.564654$

C $1.4307474 .444057-1.116144$

C $0.0576575 .011393-1.419828$

H $\quad 0.758767 \quad 3.1524580 .482958$

C $2.1677142 .007128-0.711989$

H $1.9965045 .051040-0.403020$

H $2.0212804 .354536-2.036526$

C $0.0829906 .207091-2.355189$

H $-0.4048045 .310344-0.471519$

C $3.5367692 .419156-0.146183$

O $1.7171040 .827034-0.041744$ 
H $2.263206 \quad 1.741139-1.770512$

H $3.9338413 .263099-0.722539$

O 3.3432672 .8969311 .191646

C $4.5859911 .290139-0.090142$

C $4.7794070 .552391-1.426985$

H 4.3152540 .5720690 .692770

$\begin{array}{lllll}\text { O } & 5.822566 & 1.878978 & 0.323443\end{array}$

H $3.8320370 .090614-1.724469$

H $5.0589891 .287605-2.192361$

H $0.6526827 .030325-1.911609$

H $0.5468245 .956872-3.315132$

H $-0.9315776 .566663-2.553964$

C $-2.164013 \quad 3.763149-1.949685$

H 1.7116161 .0165710 .911479

H 4.2151683 .1585651 .534684

H $6.1306382 .459181-0.393643$

H -2.516767 $2.845188-2.437842$

H -2.568376 $4.601136-2.528896$

H $-0.3330173 .650931-3.051691$

C $8.271713-0.783146-1.195897$

O $7.1407480 .091386-1.300941$

C $5.861237-0.545436-1.363593$

C $5.790024-1.475489-2.585064$

C $6.943898-2.483258-2.583642$

C $8.295073-1.799411-2.349927$

H $9.142869-0.125214-1.312511$

C $8.361192-1.4353570 .188111$

H $5.701174-1.129892-0.452034$

O $5.834226-0.695972-3.778805$

H $4.828330-2.002467-2.571301$

H $6.970090-3.011966-3.544128$

O $\quad 6.732819-3.453532-1.562124$

H $\quad 9.076077-2.545390-2.161722$

O $8.670010-1.091072-3.534956$

C $8.590255-0.3929091 .257245$

H $7.467513-2.0110220 .441841$

H $9.203596-2.1355380 .237245$

H $5.760717-1.307006-4.531405$

H $5.881826-3.888825-1.740704$

H $8.736901-1.740392-4.255700$

O $7.469152-0.2515792 .019249$

$\begin{array}{lllll}\text { O } & 9.635883 & 0.230208 & 1.388772\end{array}$

C 7.5507360 .7210603 .065435

H 8.3030440 .4203023 .800564

H 7.7852901 .7070652 .653061

H 6.5762390 .7710663 .558770

SCF Energy (B3LYP/6-31G**//MMFF) $=-3245.90375931$

0800413

MM̄FF Geometry

C - $-1.587544-4.0712632 .396923$

C $-0.460092-3.519847 \quad 1.923481$

C $0.611615-2.9507592 .820849$

O $0.922241-1.6041542 .401123$

C $1.893048-3.7929552 .691051$

C $3.008623-3.3383363 .612732$

C $4.163739-2.7908303 .185522$

C $2.789042-3.5918585 .081423$

C $4.533861-2.4991101 .756631$

C $5.686786-1.4891971 .659052$

C $6.127391-1.2645850 .205058$

C $7.374709-0.3797660 .152097$

C $7.796966-0.067699-1.292013$

O $5.049080-0.639365-0.498887$

O $8.7569150 .999620-1.247331$

C $6.5663860 .459913-2.059727$

C $8.504453-1.257180-1.951087$

O $6.8663020 .651304-3.440218$

C $5.305780-0.418834-1.893205$

C $4.0450470 .232817-2.507669$

O $5.510825-1.660142-2.578999$

C $0.204935-0.5953892 .965044$

O $-0.744099-0.702357 \quad 3.725148$

C 0.7926620 .6825132 .502426

C 0.1888891 .8382632 .818004

C 0.6397323 .1844362 .453938

C 1.9527553 .3040331 .729655

C $3.6698481 .590896-1.912763$
C $2.3393332 .108942-2.431729$

C $0.8501004 .089998-2.272441$

O $1.6297391 .490078-3.218971$

C $0.6919885 .267166-1.365189$

C $-0.3892285 .482226-0.601293$

C $-0.558904 \quad 6.6902250 .273492$

C $-0.937828 \quad 6.346607 \quad 1.695912$

C -2.1452976 .6761062 .186413$

C 0.1124725 .7048242 .576803

C -0.1352204 .2352922 .794570$

N $2.0402653 .353833-1.895161$

H -1.735841 -4.070451 3.476283

H $-0.260092-3.5042850 .857187$

H $0.282008-2.947453 \quad 3.867365$

H $2.231587-3.7800921 .647989$

H $1.671814-4.8448882 .916826$

H $4.917573-2.5430353 .931200$

H $3.676858-3.3607935 .679817$

H $1.968598-2.9759065 .461594$

H $2.544455-4.6452715 .253244$

H $4.840207-3.4370281 .278556$

H $3.668256-2.1053781 .212331$

H $5.362041-0.5372572 .099455$

H $6.540763-1.8511892 .245543$

H $6.335863-2.241265-0.247653$

H $8.204266-0.8425010 .701241$

H 7.1690060 .5698880 .665989

H $9.5065430 .706077-0.701760$

H $6.363317 \quad 1.458362-1.654542$

H $9.393581-1.540394-1.374709$

H $8.868914-0.995565-2.950510$

H $7.870543-2.142252-2.033746$

H $6.874223-0.223102-3.865329$

H $4.1597550 .320962-3.595896$

H $3.199606-0.452621-2.359724$

H $4.643198-2.055283-2.766333$

H $1.7045080 .635276 \quad 1.917979$

H $-0.732624 \quad 1.7992553 .397919$

H 2.1989154 .3252921 .433331

H 1.9372362 .7096320 .811351

H 2.7726842 .9457612 .361871

H $3.5999081 .520053-0.821394$

H $4.4312082 .336723-2.164828$

H $-0.0090223 .411072-2.239429$

H $0.9774284 .424998-3.307027$

H $1.5022225 .992763-1.354194$

H -1.209202 $4.771150-0.632518$

H $-1.3333177 .320509-0.182548$

H $0.352968 \quad 7.3004110 .288664$

H -2.412405 6.4612943 .215953

H -2.8943617 .1620361 .570324$

H 0.0972406 .1915413 .561698

H 1.1094165 .9160552 .180837

H -1.0656534 .0313603 .327560$

H $2.7658943 .841167-1.376082$

C $-2.585435-4.8340220 .104114$

O $-3.106576-3.612802-0.473336$

C $-4.159221-3.959413-1.392165$

C $-3.875685-5.397394-1.797188$

C $-3.435103-5.976445-0.470858$

H $-5.096979-3.935647-0.824013$

C -4.242355 -2.985631-2.571288

H $-4.755041-5.897201-2.214034$

H $-3.061962-5.459788-2.529196$

C $-2.685627-7.292050-0.601073$

H $-4.324215-6.1365940 .154244$

C -4.502799-1.511032 -2.202778

O $-3.018278-3.035466-3.314879$

H $-5.022474-3.327865-3.261970$

H -3.588896 -1.061617 -1.802677

O $-4.792156-0.810855-3.420426$

C $-5.663842-1.280484-1.220805$

C $-6.1306410 .180758-1.079351$

H $-5.374597-1.661482-0.234260$

O $-6.793999-2.046838-1.650835$

H $-6.6824050 .483280-1.979030$

H $-6.8717310 .193036-0.268995$

H $-3.324742-8.055149-1.056925$ 
H $-1.792508-7.185079-1.225860$ H -2.368140 -7.6585530 .380266$ C $-2.683831-4.767536 \quad 1.634431$ H -2.295832 -2.812531 -2.703219 H -4.043481 -0.957899-4.023758 H -7.491192 -1.943044 -0.980832 H -2.718939 -5.7892922 .033541$ H $-3.628947-4.2823681 .910283$ H -1.548912 -4.936544 -0.235340 C $-3.150823 \quad 1.5760190 .699887$ $\begin{array}{lllll}\text { O } & -4.280155 & 0.764469 & 0.347239\end{array}$ C $-5.0231421 .206148-0.790334$ C $-5.5988942 .612308-0.545274$ C $-4.5077093 .607225-0.133889$ C $-3.577918 \quad 3.0336880 .941294$ H -2.8068691 .1705981 .660353$ C $-1.9968141 .433610-0.306040$ H $-4.3635731 .236209-1.662003$ O H -6.102712 2.955217 -1.456807 H $-4.982896 \quad 4.5114250 .265597$ O $\quad-3.7539844 .017604-1.270102$ H $-2.689033 \quad 3.6626751 .044524$ O -4.2573153 .0890692 .201332$ C $-1.4014600 .041938-0.296459$ H $-2.3151001 .635691-1.330466$ H -1.195381 2.132471-0.042566 H -6.9648663 .4328570 .587346$ H $-3.5639973 .243509-1.822546$ H -5.0211802 .4886372 .153044$ O $-0.368745-0.002905-1.188232$ O $-1.792615-0.8869840 .395532$ C $0.289356-1.266645-1.325239$ H $1.011500-1.390901-0.513836$ H $-0.427069-2.093508-1.334575$ H $0.824842-1.268079-2.278078$

SCF Energy (B3LYP/6-31G**//MMFF) $=-3245.90716880$

0800414

MM̄FF Geometry

C $-1.5308782 .517672-0.781855$

C $-2.6120593 .118725-0.262555$

C -3.2659432 .7799161 .059117$

O -2.5633521 .6817051 .683894$

C -4.7187982 .3352890 .818525$

C -5.5586592 .3018202 .078376$

C -5.8940241 .1824092 .748949$

C -6.0720033 .6411042 .541135$

C $-5.463188-0.2309242 .469695$

C $-6.605485-1.1038911 .931816$

C $-6.936890-0.8300260 .456168$

C $-8.134678-1.6696860 .007301$

C $-8.416910-1.508592-1.494235$

O $-5.783752-1.177265-0.316972$

O $-9.325812-2.548482-1.886726$

C $-7.105945-1.735619-2.275892$

C $-9.116850-0.180361-1.805023$

O $-7.284134-1.456333-3.662876$

C $-5.907581-0.928947-1.722850$

C $-4.597934-1.399230-2.402196$

O $-6.1098520 .457561-2.000871$

C -2.2522051 .7561093 .000277$

O -2.4941782 .6848773 .758440$

C -1.546552 0.5330393 .457446

C -1.331774 -0.540195 2.679358

C $-0.650565-1.7777923 .070354$

C $-0.092278-1.8620804 .466292$

C $-3.321521-0.828913-1.767161$

C $-2.063037-1.392153-2.406513$

C $0.404094-1.511048-2.039585$

O $-2.072207-2.060659-3.436442$

C $0.643833-2.812717-1.342304$

C $1.542883-2.984359-0.361555$

C $1.751070-4.2914050 .348158$

C $1.506714-4.2029171 .839991$

C $2.529135-4.2879102 .707569$

C $0.077991-4.1303242 .335955$

C $-0.547598-2.7719812 .162892$
N $-0.924685-1.040802-1.693129$

H $-1.0491571 .711953-0.235418$

H $-3.0776643 .924914-0.826368$

H -3.2234423 .6787371 .685191$

H -4.7192041 .3511930 .338112$

H $-5.212221 \quad 3.0094180 .105233$

H $-6.532258 \quad 1.284243 \quad 3.626169$

H $-6.7082313 .559173 \quad 3.428685$

H -5.2401664 .3056522 .793952$

H -6.6673014 .1126011 .752253$

H $-4.596779-0.2764061 .803777$

H -5.122001 -0.6569433 .422084$

H -6.293478 -2.151593 2.034655

H -7.501351-0.9703142.550855

H -7.1535850 .2361230 .328611$

H $-9.026880-1.4200280 .594901$

H -7.931597 -2.731121 0.208188

H -10.130754 -2.462016-1.347706

H -6.864430 -2.805460 -2.204256

H -10.073727 -0.118382 -1.272766

H $-9.361802-0.102754-2.870065$

H -8.525901 $0.693034-1.521817$

H -8.035538 -1.991086 -3.971717

H $-4.535864-2.494027-2.342107$

H -4.613644 -1.131586 -3.466239

H $-5.2499510 .903106-2.049641$

H -1.218452 0.5622854 .491655

H -1.691810 -0.5255761 .652018$

H -0.898910 -1.809792 5.205705

H $0.608675-1.0409494 .652703$

H $0.458246-2.7856864 .658063$

H -3.289758 $0.258832-1.880554$

H -3.294486 -1.065200 -0.697174

H $1.115565-0.738371-1.732905$

H $\quad 0.478724-1.646718-3.123076$

H $0.035392-3.657041-1.658546$

H $2.156900-2.143934-0.048125$

H $2.781286-4.6145320 .149089$

H $1.109231-5.077456-0.069254$

H $2.365761-4.2641363 .779732$

H $3.554556-4.3817282 .366244$

H $\quad 0.012419-4.4553073 .380819$

H $-0.527887-4.8636841 .787595$

H $-0.988187-2.6183451 .177815$

H $-1.031925-0.561408-0.804240$

C $0.5277443 .306929-2.040887$

O $1.3262762 .313206-1.366284$

C $2.1914382 .985823-0.423500$

C $2.2101184 .441585-0.860666$

C $0.7741114 .627789-1.311875$

H 1.7006502 .8905060 .554183

C $3.5467472 .272683-0.404334$

H $2.4950085 .123138-0.053993$

H $2.9003414 .588783-1.700769$

C $0.5597045 .857911-2.175976$

H $0.1381874 .705073-0.420980$

C 4.6459613 .0279070 .357476

O $3.342972 \quad 1.0028650 .222515$

H $3.8702752 .071552-1.431891$

H $4.8392623 .988812-0.134507$

O $4.1476773 .338346 \quad 1.666123$

C 5.9802362 .2721490 .528002

C $6.6180351 .792079-0.791749$

H 5.8583811 .4530331 .245775

O 6.8884083 .2014321 .140529

H $6.2368152 .387754-1.629696$

H $7.7000391 .974418-0.751473$

H $0.8137936 .766784-1.620627$

H $1.1820615 .830058-3.076610$

H $-0.4859575 .934880-2.490330$

C $-0.9314222 .851582-2.119190$

H $2.6506750 .537880-0.277755$

H 4.8479923 .8242752 .134713

H $7.6996472 .712247 \quad 1.360848$

H $-0.9791801 .937441-2.724279$

H - $1.5284393 .608718-2.640698$

H $\quad 0.9216763 .367622-3.063882$

C $6.946410-1.853171-0.078096$ 
O $7.114376-0.429395-0.057327$

C $6.3817270 .295100-1.051789$

C $6.832224-0.138946-2.452545$

C $6.684742-1.655200-2.629041$

C $7.299812-2.432608-1.458758$

H $7.685888-2.2314270 .639720$

C $5.555411-2.2609170 .427880$

H $5.3172210 .081609-0.943396$

O $\quad 8.187358 \quad 0.240766-2.680690$

H $6.2223410 .382837-3.199018$

H $7.172983-1.961474-3.562319$

O $5.304442-1.994937-2.728913$

H $7.003775-3.487497-1.496772$

O $8.725515-2.392086-1.580911$

C $5.324683-1.7662401 .840704$

H $4.767381-1.858363-0.214027$

H $5.448494-3.3511410 .437966$

H $8.730429-0.159486-1.980102$

H $4.938884-1.518212-3.493545$

H $8.959314-2.814931-2.424772$

O $4.044708-1.3134911 .965371$

O $\quad 6.166489-1.7884362 .728985$

C $3.700974-0.7663523 .241846$

H 4.3609560 .0716563 .486094

H $2.673951-0.3963303 .185484$

H $3.755934-1.5403704 .012739$

SCF Energy (B3LYP/6-31G*//MMFF) $=-3245.91467253$

0800415

MMFF Geometry

C $-0.7560493 .858346-1.188489$

C $-1.1887352 .592825-1.084267$

C $-1.8713401 .861594-2.209782$

O $-3.222747 \quad 1.602208-1.768499$

C -1.173376 $0.517592-2.482568$

C $-1.463553-0.024788-3.866851$

C $-2.388189-0.961834-4.151691$

C $-0.5962670 .536822-4.964560$

C $-3.360740-1.619106-3.213639$

C $-3.115865-3.126721-3.072275$

C $-1.859178-3.469934-2.256609$

C $-1.658677-4.986195-2.205927$

C $-0.496250-5.385446-1.285869$

O $-2.044531-2.953780-0.934980$

O $-0.592174-6.798204-1.045901$

C $-0.674916-4.6810610 .075295$

C $0.866647-5.150026-1.948917$

O $0.450830-4.9500660 .905849$

C $-0.935170-3.158969-0.050432$

C $-1.310138-2.5605551 .326653$

O $0.248153-2.515545-0.526211$

C $-4.2427351 .837222-2.631492$

O $-4.1511282 .241972-3.782358$

C $-5.5573781 .574212-1.999468$

C $-5.7033150 .952637-0.818741$

C $-6.9675260 .695201-0.124469$

C $-8.233026 \quad 1.215321-0.753772$

C $-1.855288-1.1258091 .264043$

C -2.245976 -0.6091082 .638437$

C -3.5113051 .2444313 .726038$

O $-1.947333-1.1835153 .682154$

C $-4.844444 \quad 0.6385704 .035527$

C $-6.007847 \quad 1.3025653 .961399$

C $-7.340193 \quad 0.6722864 .253135$

C $-8.280647 \quad 0.712682 \quad 3.067212$

C -9.3102501 .5755093 .035950$

C -8.083114 -0.3021341 .960488$

C $-6.9324320 .040600 \quad 1.055229$

N -2.955334 0.5822602 .559569

H $-0.9164324 .399926-2.117804$

H $-1.0444832 .052201-0.151991$

H $-1.8698102 .490708-3.108338$

H $-1.460412-0.214972-1.720282$

H $-0.0841310 .626804-2.388971$

H -2.487243 -1.282576 -5.188292

H $-0.8325400 .107080-5.943714$

H $-0.7270781 .620669-5.041215$

H $0.4587290 .327452-4.758671$
H -3.376746 -1.144732 -2.228855

H $-4.365099-1.470634-3.630706$

H -3.993293 -3.553696 -2.568651

H $-3.056597-3.589630-4.065275$

H - $0.988887-2.987661-2.715245$

H $-1.508110-5.391991-3.214256$

H $-2.574759-5.461560-1.827400$

H $-0.553286-7.251720-1.905414$

H $-1.541488-5.147893 \quad 0.564764$

H $0.953995-5.746109-2.865326$

H $1.684966-5.478942-1.298911$

H $1.038629-4.106240-2.219433$

H $\quad 0.225143-4.6816531 .812061$

H -2.082003 -3.1865591 .793266$

H $-0.434353-2.5738971 .987629$

H $0.236676-1.584386-0.254715$

H $-6.4113181 .922321-2.571729$

H $-4.8151640 .601589-0.296244$

H $-8.1739112 .299852-0.898947$

H $-9.1255401 .032174-0.151644$

H -8.402338 $0.738551-1.725556$

H $-1.103636-0.4417640 .857261$

H -2.741408 -1.0882740 .619981$

H -3.5828932 .3117313 .494833$

H $-2.835907 \quad 1.1136194 .577562$

H $-4.850550-0.4078504 .332642$

H $-6.0075192 .351275 \quad 3.673920$

H -7.780442 1.2057785 .105620

H -7.226838 -0.3676314 .584151$

H -10.009091 1.5929762 .206428

H $-9.476100 \quad 2.286930 \quad 3.838061$

H $-9.006701-0.4358381 .385938$

H $-7.895850-1.2882422 .404864$

H $-5.966687-0.3002481 .428110$

H -3.2188300 .9403021 .646099$

C $1.3166305 .104745-0.568006$

O $2.1860913 .982442-0.812545$

C $3.5132014 .313961-0.361295$

C $3.4861115 .796085-0.004524$

C $2.0504745 .969787 \quad 0.454134$

H 3.6857183 .7388310 .555950

C $4.5434063 .921690-1.426751$

H 4.2186016 .0494110 .767989

H $3.6860826 .429220-0.876766$

C $1.594915 \quad 7.418116 \quad 0.485041$

H 1.9464075 .5363191 .457786

C $4.4585642 .448069-1.877666$

O $4.3514134 .736184-2.591142$

H $5.5532204 .147599-1.065865$

H $3.5365742 .281056-2.448148$

O $5.5410492 .201743-2.784094$

C $4.5414871 .427075-0.729631$

C $4.543979-0.026558-1.243229$

H $3.6850321 .565405-0.060698$

O $5.726792 \quad 1.6860910 .020422$

H $3.728612-0.152112-1.965687$

H $5.495169-0.220017-1.754541$

H 2.2090097 .9994151 .180589

H $1.6730047 .884625-0.502752$

H $0.553116 \quad 7.4933740 .811693$

C $-0.0466264 .602566-0.091234$

H $3.4378754 .600861-2.896564$

H $5.4751002 .857889-3.498988$

H $5.740087 \quad 1.0720910 .773693$

H $-0.675523 \quad 5.447367 \quad 0.210295$

H 0.0809193 .9535680 .783822

H $1.2148935 .643359-1.519860$

C $5.541929-1.8798001 .851076$

O $5.550522-1.0252590 .700160$

C $4.369284-1.053119-0.106209$

C $4.129927-2.471635-0.644840$

C $4.087797-3.4963360 .493648$

C $5.275788-3.3398311 .448700$

H $6.564711-1.8230632 .245593$

C $4.591688-1.3612842 .936700$

H $3.508705-0.7458930 .498237$

O $5.151526-2.831598-1.572484$

H $3.181128-2.487150-1.193253$ 
H $4.091196-4.5094330 .073679$

O $2.884870-3.334187 \quad 1.237498$

H $5.127700-3.9444422 .351135$

O $6.456542-3.827803 \quad 0.805245$

C $5.027229-0.0021503 .428587$

H $3.559688-1.2587032 .589376$

H $4.561257-2.0450673 .793337$

H $6.008055-2.744808-1.119960$

H $2.141033-3.4727690 .626391$

H $6.309146-4.7657570 .595294$

O $6.037519-0.1338724 .334580$

O $4.538410 \quad 1.0503583 .038155$

C 6.5546551 .0928324 .859215

H 7.3531110 .8507265 .565572

H 6.9724391 .7033694 .052869

H 5.7691761 .6386855 .390572

SCF Energy (B3LYP/6-31G**//MMFF) $=-3245.92658859$

08_00416

MM̄FF Geometry

C $-2.156049-3.349572-0.674815$

C $-1.877703-2.468920-1.649126$

C $-0.504248-1.907115-1.895326$

O $-0.662033-0.467402-1.964814$

C $0.109032-2.388007-3.223569$

C $0.431943-3.863798-3.278391$

C $1.550540-4.417204-2.770748$

C $-0.568611-4.718805-4.012491$

C $2.668900-3.738018-2.025087$

C $2.888982-4.407838-0.662376$

C $4.139317-3.8889330 .066955$

C $4.362040-4.7015611 .346070$

C $5.542352-4.1734442 .168947$

O $3.947719-2.5075950 .385584$

O $5.469129-4.8017023 .457586$

C $5.360803-2.6562802 .367235$

C $6.885568-4.5926681 .561335$

O $6.493523-2.0990123 .031754$

C $5.056356-1.8874531 .058881$

C $4.644419-0.4350271 .406873$

O $6.228774-1.8733390 .245514$

C $0.4286810 .283020-1.660738$

O $1.521252-0.131348-1.309694$

C $0.074461 \quad 1.709043-1.829338$

C $0.9233102 .638505-1.366624$

C $0.7771984 .090351-1.465129$

C $-0.3963084 .626540-2.240185$

C 4.2944730 .4362990 .194585

C $5.4136871 .351081-0.276742$

C $5.8252303 .160935-1.955304$

O 6.5188091 .4017830 .254210

C $5.3405494 .479669-1.437971$

C $4.7439575 .407565-2.202203$

C $4.2418146 .725139-1.681184$

C $2.7556496 .913360-1.904565$

C $2.3011197 .612176-2.958537$

C $1.7997006 .367419-0.865624$

C $1.7165544 .866938-0.885990$

N $5.0118922 .113077-1.366732$

H -1.338977 -3.685799 -0.038723

H $-2.661254-2.121424-2.315814$

H $\quad 0.154861-2.157713-1.054586$

H $\quad 1.020079-1.813915-3.437567$

H - $0.558262-2.120992-4.055232$

H $1.692791-5.488922-2.908040$

H $-0.274151-5.773409-4.040551$

H -1.550520 -4.666295 -3.533434

H $-0.669559-4.377886-5.048159$

H $3.580273-3.825804-2.628416$

H $2.480490-2.670213-1.885871$

H $2.000824-4.233165-0.041048$

H $2.982021-5.492893-0.800016$

H $5.000872-3.986519-0.604083$

H $4.503358-5.7646531 .113366$

H $3.456944-4.6515081 .968498$

H $6.168015-4.4158204 .013457$

H $4.513560-2.5224623 .053991$

H $6.956900-5.6861251 .514055$
H $7.723874-4.2671872 .187325$

H $7.038922-4.2061380 .551612$

H $7.205498-2.0072042 .375838$

H $3.743456-0.4855602 .033304$

H 5.4250040 .0488812 .004249

H $\quad 6.152202-1.156938-0.403952$

H $-0.8579121 .951399-2.325239$

H $1.8279062 .304219-0.861856$

H $-0.4034894 .221384-3.258308$

H $-0.3874865 .714157-2.341914$

H $-1.3370694 .356153-1.748493$

H $3.971575-0.177056-0.651930$

H 3.4607981 .0905290 .478482

H $5.7225783 .080816-3.042030$

H $6.8774933 .017465-1.691388$

H $5.4843134 .674648-0.377693$

H $4.6089595 .214578-3.264201$

H $4.8008647 .520529-2.191284$

H $4.4674116 .846591-0.614421$

H $\quad 1.2400407 .777359-3.112922$

H $2.9806478 .030798-3.693535$

H $0.806936 \quad 6.818588-0.972511$

H $2.136568 \quad 6.682740 \quad 0.130497$

H $2.5282944 .379499-0.345401$

H $4.0604672 .015466-1.709248$

C $-4.715969-3.353589-1.040423$

O $-4.938396-2.023516-0.530025$

C $-6.356827-1.808984-0.393970$

C -7.033702 -3.010471-1.043635

C $-6.019273-4.106269-0.776907$

H $-6.579398-1.8250830 .678766$

C $-6.739167-0.453546-0.996986$

H $-8.016078-3.217715-0.608594$

H -7.167389-2.866262 -2.122358

C $-6.215183-5.336149-1.646080$

H $-6.080673-4.3958800 .280741$

C $-6.0258360 .749574-0.341662$

O $-6.403479-0.453826-2.391142$

H -7.825366 $-0.316091-0.949288$

H $-4.948740 \quad 0.692038-0.533735$

O $-6.489491 \quad 1.939776-0.991668$

C $-6.2883580 .890477 \quad 1.170260$

C -5.7349872 .1865921 .793349$

H $-5.871970 \quad 0.027197 \quad 1.699781$

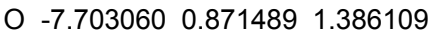

H -6.1445093 .0589121 .269385$

H -6.1243302 .2482492 .818792$

H -7.198191 -5.782545 -1.463785

H $-6.150612-5.089569-2.711206$

H $-5.455781-6.093662-1.429343$

C $-3.494777-3.962774-0.348075$

H $-5.449403-0.629202-2.462737$

H $-6.3277821 .831410-1.944588$

H -7.8542800 .8828382 .346612$

H -3.430082 -5.031859 -0.580641

H $-3.626910-3.8737660 .738205$

H -4.553837 -3.270321 -2.122379

C -2.3215801 .0644352 .785526$

O $\quad-3.7453411 .1972912 .681845$

C -4.1999542 .2551381 .829252$

C -3.7044003 .6126512 .355296$

C -2.1822123 .6214382 .522782$

C -1.6819752 .3788193 .269501$

H -2.1710880 .3119753 .570727$

C -1.7426370 .5017591 .485158$

H -3.8101642 .0979180 .818237$

O -4.3206873 .8908623 .611585$

H $-4.0124764 .397341 \quad 1.653795$

H -1.886812 4.5213943 .075960

O

H -0.5917672 .3123043 .199126$

O -1.9826612 .5323984 .661231$

C $-0.382249-0.1115831 .705220$

H -2.412088 -0.2620121 .068703$

H $-1.656234 \quad 1.2547240 .697612$

H -4.0226224 .7718293 .895515$

H -0.6213563 .7189841 .354772$

H -2.9505472 .5326994 .757892$ 
O $-0.504640-1.447707 \quad 1.945709$

$\begin{array}{lllll}0 & 0.663515 & 0.524709 & 1.684711\end{array}$

C $0.726236-2.146342 \quad 2.154886$

H $\quad 0.500567-3.2105692 .262498$

H $1.387196-2.0146301 .293894$

H $1.209572-1.793083 \quad 3.070425$

SCF Energy (B3LYP/6-31G*//MMFF) $=-3245.93173483$

08_00417

MMFF Geometry

C $1.056796-2.971267-0.218242$

C $-0.048320-3.2895560 .471850$

C $-0.412576-2.8401651 .869139$

O $-1.658865-2.1040991 .768731$

C $0.615613-1.911846 \quad 2.554204$

C $0.208167-1.447727 \quad 3.942097$

C $0.012822-0.1572764 .283636$

C $0.045181-2.5316974 .975385$

C 0.1741221 .0530353 .403433

C $-1.093773 \quad 1.3270272 .598145$

C $-0.9385552 .450511 \quad 1.566896$

C $-0.8770803 .836867 \quad 2.209306$

C -0.8914024 .9625751 .160120$

$\begin{array}{lllll}\text { O } & -2.085579 & 2.360599 & 0.714661\end{array}$

O $-1.184756 \quad 6.1936041 .839223$

C $-2.042104 \quad 4.7141310 .159526$

C $0.482797 \quad 5.148110 \quad 0.506014$

O $-1.9926705 .645618-0.918539$

C $-2.0758793 .271398-0.390188$

C -3.368405 $3.028441-1.205539$

O $-0.9457363 .066657-1.240422$

C -2.824516 -2.801784 1.823566

O $-2.953462-4.0100931 .937503$

C $-3.933918-1.8253201 .717822$

C $-5.200296-2.2662291 .682608$

C $-6.410015-1.4413181 .614277$

C $-6.2355360 .053487 \quad 1.566855$

C -3.610290 $1.548801-1.544407$

C $-4.9070871 .341636-2.306003$

C -6.520552 -0.451665 -2.937542

O $-5.4924522 .248795-2.891568$

C $-7.694424-0.243816-2.032428$

C -8.524539-1.225423-1.648349

C $-9.749802-0.992761-0.812047$

C $-9.804703-1.8679400 .418469$

C $-10.625971-2.930283 \quad 0.475757$

C -8.984389-1.452991 1.619030

C -7.608709-2.061657 1.616075

N $-5.3129850 .013365-2.282741$

H $1.824225-2.349667 \quad 0.233493$

H $-0.789092-3.918695-0.018991$

H $-0.556113-3.7351782 .486827$

H $0.785227-1.0444491 .907092$

H $1.580552-2.4281402 .640795$

H -0.2898090 .0671095 .305393$

H $-0.106328-2.1261905 .981512$

H $-0.818449-3.1618264 .742247$

H $0.940252-3.1613855 .009259$

H 0.4080681 .9123514 .042916

H 1.0376270 .9343852 .739641

H $-1.389423 \quad 0.4195782 .060124$

H -1.933867 1.5480543 .270196

H $-0.041223 \quad 2.2550680 .969639$

H $0.002146 \quad 3.9334342 .857171$

H -1.748205 3.9702542 .866093

H -0.5047686 .3288682 .521331$

H $-2.985375 \quad 4.9037270 .690858$

H 1.2404095 .3807911 .263916

H $0.4801485 .998332-0.184967$

H $0.8204234 .263955-0.036720$

H $-1.9854136 .537441-0.530533$

H $-4.2338953 .379551-0.628259$

H -3.337843 $3.611180-2.134694$

H $-1.1736062 .421529-1.926887$

H -3.679404 -0.772166 1.683966

H -5.374476 -3.3411221 .721254$

H $-7.173400 \quad 0.6002551 .451688$

H -5.6088880 .3415450 .717379$
H -5.7633700 .4140612 .487391$

H $-2.8009241 .152389-2.166523$

H $-3.6497590 .956846-0.623574$

H $-6.371494-1.506289-3.189496$

H -6.666494 $0.112756-3.864330$

H $-7.8957950 .775634-1.711084$

H $-8.344414-2.241192-1.992477$

H - $10.623666-1.186145-1.448277$

H $-9.8341970 .058762-0.509980$

H -10.694563 -3.542481 1.369039

H $-11.242911-3.214750-0.370227$

H $-9.485429-1.7888602 .537339$

H -8.974322 $-0.362537 \quad 1.694375$

H $-7.622808-3.1520181 .661557$

H $-4.800690-0.652630-1.711728$

C $1.339807-2.225126-2.620356$

O $2.310166-1.241228-2.200414$

C $1.6798420 .052124-2.219468$

C $0.497306-0.095758-3.166963$

C $0.010598-1.482723-2.793033$

H $1.2959460 .234005-1.207731$

C $2.6805461 .147517-2.591463$

H $-0.2597760 .678532-3.020288$

H $0.812695-0.074653-4.216601$

C $-0.914648-2.106056-3.823039$

H $-0.525875-1.412464-1.838743$

C $3.9371881 .200358-1.695059$

O $3.1170720 .930354-3.938970$

H $2.1699672 .118220-2.587529$

H $4.5874890 .352092-1.934757$

O $4.6737392 .373819-2.064195$

C $3.6607781 .232965-0.180927$

C 4.8820241 .5665680 .696449

H 3.2413130 .2699350 .131516

$\begin{array}{lllll}\text { O } & 2.666866 & 2.228161 & 0.078847\end{array}$

H 5.1847872 .6085340 .530353

H 4.5413581 .5250151 .739963

H - $-1.819484-1.500896-3.942611$

H $-0.431132-2.184655-4.802579$

H -1.218017 -3.111338 -3.514322

C $1.315241-3.400333-1.636383$

H $3.7484281 .637477-4.156496$

H $4.1383273 .146467-1.813816$

H 2.4588752 .1954241 .028076

H $2.291502-3.898921-1.650460$

H $0.577349-4.141219-1.965408$

H $1.688050-2.598274-3.591949$

C $6.665645-1.7176790 .606712$

$\begin{array}{llll}\text { O } & 5.666412 & -0.702351 & 0.777023\end{array}$

C $6.091386 \quad 0.636579 \quad 0.511116$

C $7.256472 \quad 1.0282751 .436390$

C 8.4048450 .0196411 .341086

C $7.904363-1.4237451 .469734$

H $6.202331-2.6345810 .993463$

C $7.002102-1.948782-0.875201$

H $6.4244700 .711141-0.528485$

$\begin{array}{lllll}\text { O } & 6.795946 & 1.097231 & 2.784640\end{array}$

H 7.6078322 .0286621 .155777

H 9.1322550 .2284622 .134962

$\begin{array}{llll}\text { O } & 9.066096 & 0.199304 & 0.092447\end{array}$

H $8.700876-2.1323351 .214544$

O $7.545754-1.6734192 .831862$

C $5.816929-2.475570-1.655292$

H $7.328232-1.026034-1.362072$

H $7.802458-2.692778-0.959555$

H 7.5442261 .3819113 .336365

H $9.803203-0.4339160 .056856$

H $8.339952-1.5326683 .374823$

O $6.132660-2.486692-2.984051$

O $4.756566-2.838125-1.166233$

C $5.106408-2.953745-3.865691$

H $4.674450-3.892913-3.506405$

H $4.334480-2.186622-3.968242$

H $5.556671-3.131231-4.846031$

SCF Energy (B3LYP/6-31G**//MMFF)= -3245.90313186

0800418

MM̄FF Geometry 
C 2.8275865 .1233510 .519008

C $3.553137 \quad 4.000410 \quad 0.629463$

C $4.3961003 .434876-0.482886$

O $3.9477322 .081916-0.737602$

C $5.8685383 .378154-0.050001$

C $6.7921182 .942703-1.169607$

C $7.3258811 .710854-1.283014$

C $7.1446374 .015242-2.167975$

C $7.0871990 .525930-0.388027$

C $6.908363-0.763271-1.202728$

C $6.735585-1.984788-0.289344$

C $6.811461-3.280854-1.097371$

C $6.584064-4.513712-0.210915$

O $5.460288-1.8891320 .356042$

O $6.363469-5.641516-1.071348$

C $5.292194-4.3060120 .603852$

C $7.816894-4.8484150 .636728$

O $5.113373-5.354687 \quad 1.553623$

C $5.210385-2.9302701 .310399$

C $3.788746-2.7401261 .887913$

O $6.152412-2.8931872 .384293$

C $2.858727 \quad 1.932283-1.539249$

O $2.2361342 .816252-2.106324$

C $2.529170 \quad 0.490734-1.589689$

C $1.314786 \quad 0.119787-2.022154$

C $0.808719-1.249822-2.130114$

C $1.791082-2.372702-1.929285$

C $3.464853-1.3127262 .347355$

C $2.061124-1.2603692 .921472$

C $-0.335704-1.4389762 .251144$

O $1.860316-1.1707154 .130331$

C $-0.966903-2.4269791 .321117$

C $-1.993815-2.1438460 .506027$

C $-2.619244-3.144079-0.423045$

C $-2.579481-2.710528-1.872052$

C $-3.706300-2.397315-2.533240$

C $-1.243612-2.726516-2.587127$

C $-0.494383-1.430591-2.432119$

N $1.081371-1.371217 \quad 1.948123$

H $2.8589195 .690456-0.407217$

H $3.5147063 .430007 \quad 1.555039$

H $4.2992584 .033550-1.397368$

H $5.978398 \quad 2.7137180 .815186$

H $6.195094 \quad 4.366781 \quad 0.300569$

H $8.0180121 .530186-2.104530$

H $7.8546323 .662955-2.923941$

H $6.2497514 .360674-2.694614$

H $7.6025094 .870713-1.660699$

H $7.9568420 .418126 \quad 0.271238$

H $6.2099920 .667340 \quad 0.249525$

H $6.030607-0.657714-1.853379$

H $7.784470-0.908948-1.846982$

H $7.528214-1.9641360 .468036$

H $7.772165-3.359548-1.621283$

H $6.041291-3.268058-1.881537$

H $7.145280-5.740609-1.641522$

H $4.451235-4.380598-0.100354$

H $8.684253-5.037763-0.007156$

H $7.663430-5.7695001 .209973$

H $8.089500-4.0515181 .331629$

H $5.155115-6.1961411 .067656$

H $3.054480-3.0227431 .124607$

H $3.639081-3.4232512 .734403$

H $5.849527-2.2532853 .047820$

H $3.278717-0.215911-1.252539$

H $0.6091130 .897841-2.311785$

H $2.659313-2.247375-2.586311$

H $1.379498-3.358393-2.155687$

H $2.140340-2.397237-0.894090$

H $4.161379-0.9735773 .120929$

H $3.531742-0.6013651 .516531$

H $-0.746106-0.4316422 .136049$

H -0.480020 -1.754639 3.289152

H $-0.554845-3.4338221 .322881$

H $-2.423239-1.1465210 .516811$

H -3.657417 $-3.292575-0.098190$

H $-2.136080-4.125108-0.333700$

H $-3.686800-2.113155-3.580187$
H $-4.672423-2.416291-2.043120$

H $-1.403563-2.888267-3.661304$

H $-0.674885-3.592874-2.238249$

H $-1.109063-0.544768-2.600708$

H $1.352036-1.2778040 .973618$

C $0.4426665 .456047 \quad 1.320194$

$\begin{array}{lllll}\text { O } & 0.157853 & 4.066101 & 1.059929\end{array}$

C $-0.722387 \quad 3.983610-0.083681$

C $-1.3061155 .378363-0.245391$

$\begin{array}{llll}\text { C } & -0.116119 & 6.240185 & 0.133254\end{array}$

H $-0.081204 \quad 3.741407-0.940332$

C -1.734766 2.8593420 .152291

H -1.666901 $5.571517-1.259857$

H $-2.135807 \quad 5.5381680 .454396$

$\begin{array}{llll}\text { C }-0.482597 & 7.676668 & 0.462428\end{array}$

H $0.5905576 .238394-0.705279$

C -2.867216 $2.806083-0.889089$

O -0.9922301 .6365050 .119571$

H -2.143260 $2.959851 \quad 1.164016$

H -3.451736 $3.732296-0.840716$

O $-2.2884562 .773953-2.200605$

C $-3.8329151 .607725-0.773140$

C $-4.424496 \quad 1.4368970 .638183$

H $-3.325607 \quad 0.688422-1.087715$

O $-4.884718 \quad 1.836844-1.716299$

H -3.6111891 .2719341 .352107$

H -4.9387822 .3669180 .912583$

H $-0.9357468 .166310-0.405738$

H $-1.198996 \quad 7.729547 \quad 1.289133$

H $\quad 0.405698 \quad 8.2486970 .748164$

C 1.9348455 .6383841 .613936

H -1.5791680 .9233830 .416638$

H $-1.7226441 .984514-2.251513$

H $-5.4499411 .046406-1.727558$

H $2.168440 \quad 5.109598 \quad 2.547201$

H 2.1594396 .6965341 .789022

H $-0.116396 \quad 5.7143912 .229091$

C $-7.664663-0.3524820 .091890$

\begin{tabular}{lllll}
\hline & -6.644166 & 0.655789 & 0.130456
\end{tabular}

C $-5.417000 \quad 0.261784 \quad 0.752675$

C $-5.673445-0.1114582 .221239$

C $-6.751010-1.1924562 .345668$

C $-7.991292-0.8674191 .506259$

H $-8.5478910 .174191-0.290984$

C -7.298912 -1.446654 -0.920347

H $-5.000962-0.6020370 .221693$

O $-6.068741 \quad 1.0421642 .961951$

H $-4.737063-0.4700962 .664456$

H -7.047548 -1.291033 3.397322

O $-6.220693-2.446057 \quad 1.925610$

H $-8.643769-1.7458821 .450264$

O $-8.722472 \quad 0.1527832 .195182$

C $-8.491131-2.253450-1.384731$

H $-6.854480-0.979795-1.807756$

H -6.557819 - $2.134643-0.505498$

H $-6.868337 \quad 1.4045332 .543512$

H $-5.458577-2.6487602 .494515$

H -9.5515670 .2987301 .708498$

O $-8.050192-3.269914-2.184370$

O $-9.657622-2.022467-1.098514$

C $-9.069790-4.125182-2.710663$

H $-9.756689-3.552052-3.340542$

H $-8.587722-4.891163-3.323825$

H $-9.610099-4.616481-1.895823$

SCF Energy (B3LYP/6-31G**//MMFF) = -3245.90497474

0800419

MM̄FF Geometry

C $3.033664-0.6170553 .478195$

C 3.6928690 .5349793 .278777

$\begin{array}{llll}\text { C } 4.760551 & 0.821917 & 2.249245\end{array}$

O 4.1581691 .5964321 .183551

C $5.389353-0.4161011 .583041$

C $6.767427-0.1456261 .010741$

C $7.0317330 .165336-0.272842$

C $7.900593-0.3016301 .993730$

C $6.063748 \quad 0.417991-1.393992$

C $6.026970-0.722208-2.421036$ 
C $5.071324-1.853214-2.013302$

C $5.139755-3.012502-3.008200$

C $4.106824-4.102710-2.683650$

O $3.748833-1.303765-1.995412$

O $4.022674-4.981927-3.815647$

C $2.721757-3.443298-2.519340$

C $4.539839-4.966708-1.493395$

O $1.753160-4.391812-2.076731$

C $2.726478-2.214280-1.579549$

C $1.368680-1.478898-1.676638$

O $2.925261-2.655464-0.235080$

C 3.9805062 .9310011 .350062

O 4.2636153 .6026202 .327542

C 3.3435553 .4279870 .110619

C 2.7054954 .6066650 .109985

C $1.9993345 .198689-1.029291$

C $2.0616914 .467161-2.345585$

C $1.336578-0.100487-0.998790$

C $-0.0552070 .511443-1.038933$

C $-1.3467312 .466417-0.187996$

O $-1.003470-0.014839-1.616026$

C -1.397005 $3.567637-1.198594$

C $-1.6765474 .842286-0.884973$

C -1.771306 $5.950870-1.894264$

C $-0.9472127 .162650-1.516724$

C $-1.5254458 .251157-0.981377$

C $0.5307727 .144836-1.838918$

C $1.3249296 .352050-0.839327$

N $-0.115782 \quad 1.714028-0.347520$

H $3.228565-1.4791352 .847315$

H $3.450370 \quad 1.3765053 .926377$

H 5.5460191 .4163562 .733402

H $4.725459-0.7899500 .796589$

H $5.496343-1.2347872 .306098$

H $8.075136 \quad 0.296152-0.559337$

H $8.877020-0.0983121 .541275$

H 7.7770010 .3900662 .833033

H $7.924993-1.3235722 .385960$

H $5.0595850 .642593-1.025996$

H $6.4001601 .330593-1.903058$

H $5.679206-0.297592-3.372133$

H $7.036393-1.116342-2.591076$

H $5.337409-2.202191-1.009979$

H $6.150336-3.438263-3.041117$

H $4.939774-2.637050-4.021750$

H $4.908389-5.350821-3.974953$

H $2.398511-3.113730-3.516795$

H $5.498159-5.456186-1.704499$

H $3.824054-5.776082-1.311998$

H $4.657865-4.396803-0.569413$

H $1.773889-5.138963-2.699054$

H $1.113706-1.326741-2.733936$

H $0.584083-2.109158-1.242340$

H $2.514427-2.022426 \quad 0.373850$

H $3.3906572 .788567-0.763813$

H 2.6784915 .1835841 .033570

H $3.0972024 .402330-2.697823$

H $1.4856074 .944264-3.140626$

H $1.6625143 .452022-2.245324$

H $1.644227-0.175340 \quad 0.049016$

H $2.0214290 .586324-1.508954$

H -1.364542 2.8468550 .837783

H $-2.2024101 .799360-0.327519$

H -1.221929 $3.300982-2.238153$

H -1.8839625 .0995280 .151422$

H $-2.8294046 .231479-1.978358$

H -1.474982 $5.608907-2.893814$

H $-0.9465569 .134610-0.733573$

H -2.589936 $8.280728-0.773510$

H $0.9298788 .168126-1.830300$

H $\quad 0.669340 \quad 6.803635-2.868222$

H $1.339146 \quad 6.8014770 .154812$

H 0.7292192 .0914760 .070507

C $0.577136-0.9848454 .055881$

O $0.449817-2.2164673 .319913$

C $-0.446251-1.9956022 .207891$

C -1.081838 -0.630709 2.447688

C $0.0383790 .113824 \quad 3.142439$
H $\quad 0.187977-1.976076 \quad 1.314375$

C -1.455314 -3.148204 2.120232

H -1.410846 -0.149590 1.523893

H -1.951959-0.717095 3.110963

C $-0.416179 \quad 1.361248 \quad 3.877985$

H $\quad 0.779967 \quad 0.395828 \quad 2.385871$

C -2.461581-2.965714 0.967227

O $-0.728416-4.3635611 .928264$

H -1.990424 -3.241941 3.072924

H -2.885852 -1.961032 1.036649

O $-1.773197-3.035743-0.280678$

C $-3.632472-3.9663060 .974043$

C $-4.584405-3.836838-0.232630$

H $-4.199437-3.8302151 .902904$

O $-3.129931-5.3027950 .986159$

H $-4.094229-4.226816-1.134190$

H -5.428990 -4.512686-0.043611

H -0.8643232 .0773613 .182241$

H $-1.162627 \quad 1.1266434 .644300$

H $0.429496 \quad 1.8524894 .369580$

C $2.008670-0.8014454 .564248$

H $-0.092587-4.4400762 .660127$

H -1.381155 -3.923668 -0.345739

H -3.892081 -5.9032741 .050296$

H $2.300851-1.6902855 .137346$

H $2.0354590 .045210 \quad 5.260974$

H -0.077179 -1.093658 4.931515

C $-6.140365-0.5492580 .628772$

O $-5.705213-1.9159530 .694423$

C $-5.103197-2.414898-0.503715$

C $-6.112249-2.375242-1.661629$

C $-6.668800-0.959504-1.854633$

C $-7.132351-0.340915-0.530328$

H $-6.686819-0.3893941 .567386$

C -4.9352190 .3990250 .626851$

H $-4.246508-1.786312-0.768267$

O $-7.184126-3.276519-1.392822$

H $-5.614603-2.716180-2.577283$

H -7.514746 -0.990782 -2.552032

O $-5.668886-0.123400-2.429601$

H $-7.3336720 .728744-0.657128$

O $-8.367483-0.952717-0.143513$

C $-5.333404 \quad 1.8210170 .937445$

H -4.2089850 .0926231 .389383$

H $-4.3859960 .365509-0.318584$

H $-7.789214-3.248378-2.153377$

H -5.404585 -0.520416 -3.277000

H $-9.015028-0.775814-0.846902$

O $-5.0399912 .628645-0.121352$

O -5.8468662 .1718621 .991926$

C -5.3681924 .0110000 .047669$

H $-4.790716 \quad 4.437638 \quad 0.873233$

H $-5.1055464 .537894-0.873465$

H $-6.441648 \quad 4.127790 \quad 0.224658$

SCF Energy (B3LYP/6-31G**//MMFF $)=-3245.89644160$

08_00420

MM̄FF Geometry

C 0.6466203 .3876070 .944085

C 1.3140034 .0914020 .015005

C $2.0531553 .538031-1.184084$

O $2.2052482 .107521-1.052258$

C $3.4545334 .170443-1.268803$

C $4.2134503 .803185-2.528973$

C $5.2333842 .923243-2.576627$

C $3.7917234 .541808-3.772648$

C $5.7721412 .098881-1.440546$

C $6.0851960 .661428-1.881809$

C $6.543132-0.197896-0.695053$

C $7.028016-1.569088-1.165193$

C $7.406399-2.4725290 .018637$

O $5.425982-0.3567510 .185005$

O $7.545038-3.813068-0.476020$

C $6.242790-2.4818331 .031327$

C $8.762753-2.0892820 .622068$

O $6.603042-3.1863372 .217533$

C 5.717851-1.071669 1.391791

C $4.405774-1.1986902 .199527$ 
O $6.690101-0.3902442 .184887$

C $1.8753271 .325366-2.112876$

O $1.4826741 .681800-3.211482$

C $2.035737-0.078414-1.685972$

C $1.151801-0.987434-2.120419$

C $1.104470-2.393106-1.718749$

C $2.332726-2.978002-1.074847$

C 3.6643610 .1257092 .424600

C $2.282323-0.1382952 .990057$

C $0.021381-0.9628492 .313852$

O $2.039225-0.0083754 .187236$

C $-0.138614-2.4087511 .958501$

C -1.151561-2.887512 1.219611

C $-1.317497-4.3396560 .871401$

C $-1.547534-4.573877-0.606987$

C $-2.762829-4.912298-1.069941$

C $-0.353711-4.500237-1.537146$

C $-0.033461-3.084633-1.935480$

N $1.384134-0.5611242 .023147$

H 0.5872282 .3052850 .872661

H 1.3273595 .1761150 .110606

H $1.4648763 .794319-2.073340$

H $4.0337553 .898064-0.378755$

H $3.3736545 .265650-1.232179$

H $5.7389122 .776847-3.530120$

H $4.4014644 .274522-4.642267$

H $2.750198 \quad 4.315717-4.020844$

H $3.8872685 .622291-3.623541$

H $6.6929062 .570410-1.077000$

H $5.0696262 .068419-0.603117$

H $5.1873530 .219013-2.331980$

H $6.867090 \quad 0.679678-2.651184$

H $7.3467500 .332868-0.171050$

H $7.873936-1.468403-1.856503$

H $6.229598-2.062453-1.737346$

H $8.228805-3.807939-1.167815$

H $5.419760-3.0487190 .573373$

H $9.552655-2.161197-0.135173$

H $9.049975-2.7825991 .420288$

H $8.782132-1.0739521 .023557$

H $6.911276-4.0686541 .948351$

H $3.733358-1.8849361 .672762$

H $4.610622-1.6492283 .179322$

H 6.2468380 .3019692 .700107

H $2.821862-0.306081-0.976550$

H $0.352108-0.659548-2.783737$

H $2.330249-4.070114-1.054699$

H $2.426173-2.627972-0.044166$

H $3.236781-2.692635-1.625215$

H $4.205427 \quad 0.768212 \quad 3.127225$

H 3.5537150 .6922921 .493193

H $-0.634191-0.3185251 .720818$

H -0.195096 -0.8127823 .375463$

H $0.612729-3.0984412 .336812$

H -1.921489 -2.205600 0.868409

H $-2.170470-4.7209091 .448000$

H $-0.450922-4.9288961 .196164$

H -2.931772 -5.107318 -2.123815

H $-3.618027-4.993239-0.407135$

H $-0.558814-5.062650-2.457477$

H $0.487630-5.015342-1.065854$

H $-0.860620-2.574795-2.431620$

H $1.686844-0.6028511 .055657$

C -1.549404 $3.997946 \quad 2.111854$

O -2.0470392 .6470462 .133128$

$\begin{array}{llll}\text { C }-3.188742 & 2.568282 & 1.259767\end{array}$

C $-3.575964 \quad 4.0072610 .938528$

C $-2.216504 \quad 4.6792190 .918135$

H -2.8381422 .0859110 .341386$

C -4.3237601 .7229211 .857269$

H $-4.1122914 .091261-0.011509$

H -4.2049634 .4402621 .725270$

C -2.278350 6.1922161 .023188

H $-1.7148104 .406584-0.018435$

C -3.905211 $0.269878 \quad 2.144706$

$\begin{array}{llll}\text { O } & -4.805179 & 2.325163 & 3.058167\end{array}$

H $-5.154247 \quad 1.7400971 .146424$

H $-3.414585-0.1585201 .265607$ $\begin{array}{llll}\text { O } & -2.924388 & 0.282627 & 3.190462\end{array}$

C $-5.044988-0.6532732 .600775$

C $-6.239144-0.7984691 .642147$

H $-5.408965-0.3226923 .581298$

O $-4.477965-1.9555572 .807236$

H $-6.918475-1.5334492 .093720$

H -6.7793920 .1542681 .591971$

H $-2.830826 \quad 6.6139560 .177190$

H -2.7787516 .5112271 .943555$

H $-1.2725106 .623971 \quad 1.018558$

C $-0.0189194 .017382 \quad 2.138518$

H $-4.0666852 .337974 \quad 3.691411$

H -2.689216 -0.6422073 .377898$

H $-5.175237-2.5242073 .176033$

H $0.326383 \quad 3.466368 \quad 3.022632$

H 0.3240135 .0518262 .262182

H $-1.904042 \quad 4.4587403 .043726$

C $-5.198153-0.342636-1.931403$

O $-5.531848-0.124791-0.554305$

C $-5.877847-1.280036 \quad 0.217911$

C $-7.061287-2.020046-0.428791$

C $-6.782710-2.353888-1.895812$

C $-6.297168-1.126635-2.667586$

H $-5.1799960 .664172-2.366373$

C $-3.802016-0.962580-2.086084$

H $-5.015916-1.9535140 .265976$

O $-8.250204-1.236959-0.333876$

H $-7.244593-2.9454610 .129866$

H $-7.698415-2.736160-2.363062$

O $-5.790301-3.371351-1.980074$

H $-5.936900-1.413596-3.662440$

O $-7.398647-0.234929-2.865990$

C $-2.7061620 .038681-1.794288$

H $-3.652961-1.811750-1.413615$

H $-3.637802-1.325544-3.107438$

H $-8.073316-0.375760-0.749572$

H $-6.125861-4.149978-1.503942$

H $-8.073630-0.712743-3.377510$

O $-2.767457 \quad 1.074291-2.676938$

O $-1.882167-0.103647-0.900995$

C $-1.8235922 .143110-2.528768$

H $-1.1244211 .990825-1.702428$

H $-1.2644712 .237639-3.462931$

H $-2.3798553 .068654-2.357757$

SCF Energy (B3LYP/6-31G**//MMFF)= -3245.91486953

08 00421

MM̄FF Geometry

C -1.669047 $0.494429 \quad 4.230713$

C $-2.306850 \quad 0.552768 \quad 3.051746$

C -3.0860341 .7594902 .599605$

O $-4.267427 \quad 1.270515 \quad 1.926805$

C -2.243052 2.5691851 .598765

C $-2.753120 \quad 3.976626 \quad 1.367988$

C $-3.3486894 .403238 \quad 0.237511$

C -2.4917884 .9479642 .491229$

C $-3.697366 \quad 3.604495-0.987129$

C $-2.7934323 .940731-2.182469$

C $-1.3754843 .359144-2.059639$

C $-0.505653 \quad 3.799611-3.238222$

C $0.8804443 .137390-3.211301$

O $-1.494790 \quad 1.934347-2.052793$

O $1.475152 \quad 3.379970-4.495434$

C $0.7029221 .612750-3.041539$

C $1.7994233 .792553-2.173690$

O $1.9587070 .964065-2.852626$

C $-0.2584571 .228846-1.892150$

C $-0.577837-0.283808-1.957866$

O $0.366342 \quad 1.528085-0.642530$

C -5.4337321 .9407792 .099312$

\begin{tabular}{llll}
\hline & -5.619867 & 2.916009 & 2.813974
\end{tabular}

C -6.5302241 .3318291 .310251$

$\begin{array}{llll}C & -6.334516 & 0.419598 & 0.344933\end{array}$

C $-7.374918-0.220005-0.464925$

C $-8.8103020 .117284-0.156462$

C $-1.689342-0.730302-0.996997$

C $-2.029965-2.201696-1.158353$

C $-3.619480-3.923905-0.305141$ 
O $-1.441815-2.948531-1.935563$

C $-4.699559-4.013024-1.336548$

C $-5.990166-4.241811-1.050868$

C -7.069049-4.319217 -2.092993

C $-8.150607-3.279336-1.894093$

C $-9.346835-3.619951-1.385615$

C $-7.880617-1.867281-2.367581$

C -7.001331 -1.092558-1.424354

N $-3.067993-2.581483-0.317836$

H $-1.727641 \quad 1.342527 \quad 4.908912$

H $-2.225792 \quad-0.2813252 .359598$

H -3.3629782 .3605503 .473620$

H -2.184607 2.0213580 .653038

H -1.206955 2.6523151 .955068

H -3.641406 5.4510150 .178425

H -2.802193 5.9678432 .239880

H -3.0362434 .6533293 .393224$

H -1.422388 4.9796782 .724475

H $-3.7021252 .527616-0.793835$

H $-4.7318803 .856259-1.253814$

H $-3.2698703 .525400-3.080306$

H -2.743028 $5.028434-2.316598$

H $-0.9290923 .692423-1.116474$

H $-0.4086994 .892320-3.263772$

H -1.002293 $3.522669-4.179143$

H $2.3747873 .012057-4.486480$

H $\quad 0.289878 \quad 1.224781-3.983174$

H $1.9319974 .857183-2.401153$

H $2.8030873 .354643-2.201870$

H $1.4188823 .715986-1.152902$

H $2.5022551 .135935-3.639529$

H -0.899197 -0.537409-2.976806

H $\quad 0.328407-0.865428-1.748989$

H $\quad 0.023244 \quad 0.9319310 .040708$

H $\quad-7.5212841 .6985501 .557620$

H $-5.317434 \quad 0.1058730 .115575$

H $-9.041977-0.1055160 .891242$

H $-9.528383-0.445651-0.756584$

H $-9.0033331 .179594-0.341818$

H -1.384265 -0.5715330 .041855$

H -2.604128 $-0.154694-1.179416$

H -3.992909 -4.1149140 .705689$

H -2.829582 -4.647705 -0.529469

H -4.400934 -3.881999-2.374107

H -6.290999-4.384262 -0.015560

H $-7.500327-5.327861-2.050532$

H -6.656358 -4.219136 -3.104683

H -10.140696 -2.889974 -1.268529

H -9.558518 -4.636678 -1.071135

H -8.820021 -1.334724 -2.553806

H $-7.392456-1.903316-3.350213$

H -5.936537 -1.271485-1.573684

H $-3.543868-1.8712220 .231283$

C $0.540882-0.6356023 .923722$

O $0.395713-1.3419832 .672572$

C $1.577471-2.1466022 .451920$

C $2.588326-1.6821713 .487572$

C $1.681157-1.3387434 .653669$

H $1.278196-3.1818462 .661893$

C $1.986651-2.0067710 .981274$

H $3.326698-2.4514863 .731658$

H $3.122611-0.7886273 .142481$

C $2.358441-0.4853105 .711968$

H $1.319553-2.2690665 .111931$

C $3.413900-2.4869140 .664528$

O $1.049442-2.7617420 .209271$

H $1.881560-0.9626910 .662175$

H $4.101464-1.8533731 .230489$

O $3.597342-3.8197361 .139599$

C $3.781566-2.417097-0.833740$

C $5.274748-2.682104-1.113474$

H $3.506082-1.435815-1.237015$

O $3.047490-3.395922-1.572806$

H $5.566015-3.655835-0.700654$

H $5.394397-2.768172-2.201840$

H $1.663629-0.2407696 .521245$

H $3.210098-1.0179746 .147576$

H 2.7302600 .4553845 .291816
C $-0.795908-0.6498404 .667628$

H $\quad 0.158633-2.4736550 .470888$

H $2.990868-4.3916840 .638390$

H $2.102332-3.192705-1.471031$

H $-0.637521-0.5457275 .747114$

H -1.323892 -1.5975224 .506747$

H 0.8361360 .3925683 .676145

C $6.5107060 .817541-0.769373$

O $5.828382-0.357075-1.224092$

C $6.201260-1.578417-0.579854$

C $7.685844-1.869862-0.834491$

C $8.557305-0.692328-0.380056$

C $8.0354960 .650860-0.906680$

H $6.1946461 .609406-1.460749$

C 6.0552441 .2290150 .640290

H $6.047680-1.4867980 .498113$

O $7.913708-2.130086-2.217391$

H $7.970421-2.774675-0.284761$

H $9.582940-0.852020-0.734993$

O $8.585485-0.6733881 .044539$

H $8.547797 \quad 1.479977-0.404917$

O $8.374166 \quad 0.729114-2.295235$

C 4.5760911 .5595600 .685151

H 6.2345190 .4378751 .372825

H 6.6036622 .1260300 .950189

H $7.603948-1.357573-2.720410$

H 9.1733870 .0518851 .316405

H $8.1116891 .609892-2.612879$

O 4.2335881 .9029651 .962148

O $3.8146051 .524790-0.271355$

C 2.8574212 .2478202 .153018

H 2.6929812 .4140613 .220815

H 2.6237113 .1695351 .611837

H 2.2079251 .4320631 .821732

SCF Energy $\left(B 3 L Y P / 6-31 G^{* *} / / M M F F\right)=-3245.90944998$

0800422

MM̄FF Geometry

C -1.290825 2.670012 -0.001459

C -2.4140802 .9664040 .671812$

C -3.0125362 .2008831 .828550$

O -2.2462341 .0039962 .081405$

C -4.4541081 .7824641 .489876$

C -5.2648611 .3636162 .699203$

C -5.5780910 .0913093 .012792$

C -5.7810352 .4969543 .548535$

C $-5.150520-1.1690432 .314002$

C $-6.303117-1.8486031 .560894$

C $-6.695166-1.1241590 .263144$

C $-7.883921-1.817256-0.406176$

C $-8.224491-1.186214-1.764631$

O $-5.561867-1.157966-0.608614$

O $-9.126578-2.084077-2.428515$

C $-6.935140-1.094543-2.609454$

C $-8.9815770 .135971-1.595109$

O $-7.178417-0.401195-3.831450$

C $-5.740142-0.469957-1.852596$

C $-4.441937-0.646608-2.676134$

O $\quad-5.991137 \quad 0.923082-1.660844$

C $-1.9397730 .686817 \quad 3.363463$

O $-2.204141 \quad 1.3391934 .363829$

C -1.200953 -0.595436 3.440931

C $-1.054271-1.4328282 .402168$

C $-0.342592-2.7125902 .415924$

C $0.376720-3.1027553 .680321$

C $-3.164592-0.242014-1.925142$

C -1.909856 -0.607064 -2.699374

C $0.540908-0.930206-2.369422$

O $-1.920735-0.920275-3.886812$

C $0.700992-2.396572-2.113587$

C $1.624112-2.916872-1.291421$

C $1.759503-4.388161-1.018505$

C $1.665962-4.7209810 .455392$

C $2.766937-5.0120921 .168770$

C $0.292073-4.8109151 .082455$

C $-0.349561-3.4669231 .297080$

N $-0.773133-0.523827-1.905664$

H $\quad-0.7065891 .7934040 .261989$ 
H -2.9589503 .8652350 .386195$ H -2.990975 2.8698092 .696977 H -4.4285640 .9753530 .751356$ H -4.9890212 .6092731 .002868$ H $-6.197055-0.0805203 .892774$ H -6.4085002 .1459234 .374821$ H -4.9513443 .0615913 .984506$ H -6.3869903 .1793342 .943571$ H -4.298389-1.005008 1.648312 H -4.787915 -1.859503 3.086483 H $-5.975982-2.8668011 .312619$ H -7.176321 -1.9406692 .218668$ H $-6.945565-0.0833070 .494681$ H -8.761251 -1.8095130 .252506$ H -7.642325 -2.877412 -0.568595 H $-9.288625-1.732452-3.320950$ H $-6.668311-2.121361-2.894321$ H $-9.913531-0.027811-1.040684$ H $-9.2814470 .549355-2.564403$ H $-8.4059250 .894164-1.059899$ H -7.216647 $0.549378-3.629691$ H -4.341621 -1.702767 -2.959489 H -4.504479 $-0.063953-3.603974$ H -5.146348 $1.389455-1.565207$ H $-0.794975-0.8241924 .420988$ H -1.502769 -1.1696321 .445692$ H $-0.335398-3.2388814 .501454$ H $\quad 1.100530-2.3311093 .965697$ H $0.940013-4.0340613 .589377$ H $-3.1445570 .839036-1.755907$ H $-3.122754-0.744331-0.952154$ H $1.279606-0.329609-1.829277$ H $0.633913-0.724788-3.440442$ H $\quad 0.014005-3.063940-2.629105$ H $2.318631-2.251454-0.785891$ H $2.726787-4.716197-1.419257$ H $1.004540-4.966966-1.565190$ H $2.706565-5.2837512 .217268$ H $3.754843-4.9850460 .722658$ H $\quad 0.326516-5.3758282 .021253$ H $-0.360141-5.4080220 .431883$ H $-0.895433-3.1005990 .427261$ H $-0.879935-0.336328-0.913047$ C $0.5707524 .190015-0.854178$ O $1.6435703 .230427-0.874508$ C 2.6456413 .6485410 .072257 C 2.2074695 .0143900 .599958 C 0.6998504 .9282530 .476692 H 2.6097592 .9234180 .893174 C $4.0529603 .631717-0.543936$ H 2.5455305 .1863701 .626636 H $2.5872025 .836323-0.015878$ C $0.002298 \quad 6.2761480 .507071$ H $\quad 0.3320834 .313568 \quad 1.307415$ C $4.4899672 .233990-1.040039$ O $4.1133434 .552182-1.633234$ H $4.7655493 .967783 \quad 0.220121$ H $4.2506461 .496418-0.266620$

O $3.767327 \quad 1.866888-2.213526$

C $6.0033652 .154335-1.332743$

C $6.4822160 .825125-1.941998$

H $6.5464942 .344334-0.398997$ O $6.376626 \quad 3.192776-2.242414$ H $\quad 6.107097 \quad 0.728919-2.969225$ H $7.5733310 .902523-2.039569$ H $\quad 0.196474 \quad 6.7883121 .455116$ H $0.3490836 .926279-0.303062$ H -1.080786 6.1567430 .403051 C $-0.7718833 .510445-1.136180$ H $4.2288395 .443688-1.264609$ H $2.8195651 .907602-2.001234$ H $7.3434593 .165049-2.341574$ H $-0.6701572 .856471-2.011165$ H -1.507467 4.280641-1.399091 H $0.7757274 .879507-1.684677$

C $6.249745-1.3298051 .110121$

O $6.556970-0.256048 \quad 0.209075$

C $6.123283-0.436290-1.141496$
C $6.767235-1.692884-1.751706$

C $6.503122-2.926717-0.884607$

C $6.817210-2.6633650 .591854$

H $6.783461-1.0803872 .036460$

C $4.748559-1.3975661 .434848$

H $5.034319-0.547679-1.159859$

O $8.174969-1.499794-1.878949$

H $6.362596-1.841888-2.759970$

H $7.117466-3.763442-1.238785$

O $5.138897-3.310611-1.007170$

H $6.443572-3.4840881 .215473$

O $8.236496-2.6193210 .765935$

C $4.262371-0.1827022 .197964$

H $4.147722-1.4712950 .526230$

H $4.546646-2.2728272 .062833$

H $8.543941-2.294931-2.299645$

H $4.967824-3.497862-1.945910$

H $\quad 8.593057-3.4799150 .487351$

O $2.900432-0.2369242 .283502$

O 4.9811370 .6872042 .669264

C 2.2417800 .8263852 .983226

H 2.8605951 .7236953 .073133

H 1.3361581 .0859412 .428641

H $1.9595520 .469813 \quad 3.977244$

SCF Energy (B3LYP/6-31G**//MMFF) $=-3245.89607028$

0800423

MM̄FF Geometry

C 2.4560821 .0444292 .884111

C 1.1771041 .3190943 .179617

C 0.0277820 .4501302 .744891

O $\quad-0.7459961 .2484611 .813131$

C $-0.8969020 .049125 \quad 3.910317$

C $-0.424946-1.1678694 .683868$

C $-0.940793-2.4063804 .544145$

C $0.675309-0.9276415 .684504$

C $-2.060320-2.8291603 .635119$

C $-1.530757-3.3344792 .289296$

C -2.657321 -3.5820641 .274520$

C $-2.074906-4.018144-0.070955$

C $-3.159863-4.171422-1.146087$

O $-3.381065-2.3604631 .108261$

O $-2.508505-4.245351-2.424289$

C $-4.037813-2.900127-1.164699$

C $-3.931504-5.485441-0.978721$

O $-5.158986-3.066587-2.028677$

C $-4.514751-2.4494230 .235798$

C $-5.147889-1.0436490 .164966$

O $-5.519295-3.3208240 .758585$

C -1.1714240 .6513180 .668404$

$\begin{array}{llll}\text { O } & -1.007733 & -0.511077 & 0.335706\end{array}$

C $-1.8656331 .671341-0.144389$

C $-2.2742911 .352413-1.381036$

C $-2.9928392 .247263-2.279825$

C $-4.1475051 .586444-2.982596$

C $-5.582256-0.5134571 .534757$

C -6.2384910 .8470541 .418190$

C $-5.714483 \quad 3.2770501 .237649$

O $-7.460691 \quad 0.9755251 .443821$

C $-5.7590263 .744833-0.182953$

C $-5.1436444 .859206-0.608370$

C $-5.2393785 .390013-2.010827$

C $-3.8869085 .670643-2.627292$

C $-3.3454856 .899488-2.576287$

C $-3.2075004 .549335-3.378822$

C $-2.6036633 .524960-2.459617$

N $-5.325056 \quad 1.8802571 .283967$

H 2.6901340 .1440952 .320454

H 0.9394192 .2238943 .733555

H $0.407195-0.4479872 .241623$

H $-1.903825-0.1163833 .512054$

H -1.0287240 .8906944 .604239$

H $-0.520780-3.2126085 .143915$

H $0.843855-1.7963006 .330370$

H $1.619633-0.7094955 .179003$

H $0.421874-0.0851986 .336322$

H -2.625569 -3.629662 4.128614

H $-2.775416-2.0131533 .499060$ 
H $-0.835012-2.5924581 .880911$ H $-0.958191-4.2584052 .439616$ H -3.328972 -4.3483841 .678615$ H -1.500261 -4.9467700 .034237$ H -1.359344 -3.260174 -0.416637 H $-1.881414-4.988375-2.399345$ H -3.430991 -2.090942 -1.595334 H -3.256886 -6.342814 -1.092699 H -4.693983 -5.599965 -1.756803 H -4.412861 -5.577107 -0.002722 H -4.816144 -3.325268 -2.901424 H $-4.427253-0.341346-0.266613$ H $-6.023992-1.057863-0.495737$ H -5.768686 -3.9580220 .070360$ H -2.029812 2.6531380 .286283 H $-2.1081480 .350141-1.771221$ H $-3.785917 \quad 0.812345-3.668268$ H $-4.7599982 .281216-3.561051$ H $-4.8183081 .114836-2.255846$ H -6.302058 -1.190853 2.008294 H $-4.726851-0.4338812 .215965$ H -4.9854793 .8353861 .833436$ H -6.7020193 .3968591 .695061$ H -6.357200 $3.165431-0.882025$ H -4.5746135 .4532200 .103584$ H $-5.8267256 .317147-1.975178$ H -5.804777 $4.709514-2.659405$ H -2.389606 7.116526 -3.041491 H -3.844446 $7.714782-2.062907$ H $-2.3939154 .947708-3.999999$ H $-3.9132174 .115135-4.093155$ H -1.736023 $3.888161-1.908020$ H -4.3340751 .6639061 .241785$

C 4.1856532 .6883602 .089251

O 4.6324491 .7811751 .061828

C 5.9854272 .1050490 .705541

C 6.1974983 .5358411 .180794

C 5.4033773 .5348602 .472395

H $6.6274471 .437172 \quad 1.293605$

C $6.2434701 .864295-0.786900$

H $7.2551893 .775377 \quad 1.325597$

H 5.7733254 .2658400 .481942

C 5.0515574 .9247602 .974154

H 5.9928653 .0191943 .242508

C $5.7540860 .502701-1.325310$

O $5.5737242 .880695-1.544631$

H $7.3138201 .994439-0.987310$

H $4.6696830 .546828-1.463794$

O $\quad 6.304622 \quad 0.335829-2.638426$

C $6.114087-0.718318-0.466486$

C $5.792797-2.081830-1.105216$

H $5.595783-0.6502170 .496354$

O $7.517224-0.685994-0.189910$

H $6.507672-2.295544-1.910749$

H $5.995716-2.837451-0.334849$

H 5.9597825 .4997943 .182167

H 4.4631385 .4806202 .236424

H 4.4675094 .8689123 .898231

C $3.621017 \quad 1.9047203 .278859$

H $5.7640342 .715165-2.484060$

H $7.2666840 .230497-2.541021$

H $7.716237-1.4298380 .404099$

H 3.3322472 .5983484 .077485

H $4.390867 \quad 1.2412363 .692075$

H 3.4020763 .3109991 .638637

C $2.045967-1.901518-0.984667$

O $3.434728-1.847862-0.622145$

C $4.357043-2.249746-1.638235$

C $4.083933-3.700319-2.074919$

C $2.620503-3.905030-2.478880$

C $1.656163-3.318312-1.443547$

H $1.507927-1.690190-0.052206$

C $1.659321-0.819169-2.008766$

H $4.242673-1.590588-2.504469$

O $4.405118-4.608641-1.021569$

H $4.741291-3.945181-2.917283$

H $2.418401-4.977992-2.586293$

O $2.378629-3.287984-3.739361$
H $\quad 0.636851-3.313315-1.846282$

O $1.653230-4.181669-0.303086$

C $1.7581490 .580835-1.445227$

H $2.295610-0.854086-2.896409$

H $0.617005-0.967343-2.315250$

H $3.877401-4.359643-0.243453$

H $2.975299-3.699744-4.387434$

H $1.003366-3.8316960 .329969$

O $1.4315051 .478564-2.421619$

O $2.0792600 .859652-0.299719$

C $1.5024472 .854966-2.035849$

H $2.5372573 .127088-1.807437$

H $1.1557693 .461942-2.876373$

H $0.8552253 .047112-1.174897$

SCF Energy (B3LYP/6-31G**//MMFF)= -3245.91092864

$08 \quad 00424$

MM̄FF Geometry

C -1.912193 -2.052898 -3.164689

C $-2.840917-1.598875-2.309728$

C $-3.964374-0.671701-2.695266$

O $-3.722804 \quad 0.581512-2.017678$

C $-5.305150-1.211669-2.174126$

C $-6.503987-0.607237-2.875171$

C $-7.2057860 .454704-2.433608$

C $-6.926510-1.320831-4.133826$

C $-6.9197461 .335104-1.249258$

C $-7.9013611 .129752-0.088474$

C -7.554877 -0.0863290 .784411$

C $-8.595255-0.2675001 .891797$

C $-8.214574-1.4002572 .856804$

$\begin{array}{llll}\text { O } & -6.264527 & 0.148698 & 1.360944\end{array}$

O $-9.052380-1.2918204 .017772$

C $-6.760736-1.1864103 .327413$

C $-8.488055-2.7822752 .251877$

O $-6.312304-2.2882604 .113741$

C $-5.768263-0.9224002 .171735$

C $-4.395981-0.4919212 .745115$

O $-5.601997-2.1258141 .418899$

C $-3.0529711 .556848-2.680698$

O $-2.6921231 .554436-3.845585$

C $-2.7621882 .613977-1.689533$

C $-1.6444893 .340037-1.829964$

C -1.145233 $4.344444-0.890045$

C -2.0145244 .6892530 .290921$

C -3.4078320 .0497421 .699138$

C -2.0394620 .3196602 .305138$

C 0.2903510 .9265841 .665067

O

C 0.5360472 .3841371 .895698

C 1.5529893 .0551231 .332099

C 1.8621524 .4985981 .611930

C 2.0472355 .3192910 .354523

C $3.2751075 .586325-0.121737$

C $0.8131245 .900872-0.298924$

C $0.0713064 .881922-1.116964$

N -1.108123 0.7004691 .347727

H -1.954620 -1.758718 -4.210160

H $-2.778684-1.886077-1.262374$

H -4.003199 -0.525572 -3.781696

H $-5.380634-1.033385-1.096276$

H -5.354416 -2.301941 -2.298952

H $-8.0739510 .767576-3.013132$

H -7.793722 $-0.850463-4.609389$

H $-6.110951-1.321741-4.864051$

H -7.194622 -2.358669 -3.910147

H $-5.8895611 .234568-0.900672$

H -7.009877 2.372572 -1.596680

H -7.8602532 .0313150 .537080$

H $-8.9268491 .045651-0.468594$

H $-7.515017-0.9799010 .152080$

H -9.590585 -0.444083 1.465201

H -8.6786530 .6638682 .469585$

H $-9.978355-1.3505463 .725770$

H $-6.758031-0.3106563 .991543$

H $-9.552320-2.8903882 .010212$

H -8.261911 -3.579082 2.969022

H $-7.922616-2.9700651 .336739$ 
H $-6.955388-2.4114824 .832914$ H $-4.546508 \quad 0.299761 \quad 3.491040$ H -3.935641 -1.342942 3.263359 H -4.714872 -2.141001 1.027623 H $-3.4278872 .706889-0.839336$ H -1.009771 $3.150962-2.695188$ H -2.972889 $5.099030-0.046982$ H -1.571863 5.4302310 .959198 H -2.210197 3.8005410 .901038 H -3.277539 -0.6673930 .883174$ H $-3.7825380 .988602 \quad 1.275897$ H $\quad 0.8790910 .5394390 .829512$ H $\quad 0.5541330 .3631402 .565920$ H -0.1239002 .9005382 .588533$ H 2.2323852 .5245970 .668897 H 2.7820724 .5287982 .210811 H 1.0879594 .9599932 .237349 H $3.4178616 .196639-1.007135$ H 4.1648015 .1929750 .359055 H $1.0943736 .720082-0.974406$ H $\quad 0.190054 \quad 6.3739190 .464828$ H $0.6238184 .557108-2.000108$ H $-1.402967 \quad 0.8182270 .382713$ C $0.597514-2.287913-2.832355$ O $1.588115-3.289030-2.504649$ C $2.333620-2.846035-1.354674$ C $2.230143-1.334473-1.389500$ C $0.799003-1.132288-1.843574$ H $1.817121-3.254903-0.476731$ C $3.768437-3.381754-1.431656$ H $2.437311-0.870271-0.421224$ H $2.928461-0.919620-2.126533$ C $0.5781130 .245173-2.446821$ H $0.135070-1.255080-0.978091$ C $4.653006-2.894489-0.265980$ O $3.703314-4.809755-1.413562$ H $4.223538-3.096014-2.387568$ H $4.617326-1.801232-0.242083$ O $4.098363-3.3521710 .967978$ C $6.134745-3.313124-0.356496$ C $6.978292-2.9159360 .870055$ H $6.577759-2.874037-1.258301$ O $6.259919-4.731267-0.477614$ H $6.664793-3.5071781 .740421$ H $8.009257-3.2308940 .658834$ H $-0.455707 \quad 0.370114-2.770206$ H $\quad 0.799679 \quad 1.028292-1.714699$ H $\quad 1.2190760 .408419-3.319359$ C $-0.783278-2.952840-2.756943$ H $3.130805-5.083540-2.150733$ H $4.132358-4.3244140 .956943$ H $5.822482-4.997046-1.303928$ H $-0.941432-3.350561-1.745584$ H $-0.791107-3.835749-3.409136$ H $\quad 0.804630-1.970868-3.861809$ C 7.4149530 .7440890 .227315 O $7.407160-0.6836810 .086688$ C $6.955017-1.4205021 .226045$ C $7.826824-1.1151852 .455126$ C 7.8903610 .3919732 .722450 C 8.2386701 .1760541 .455040 H $7.9359321 .109523-0.667103$ C 5.9932291 .3313630 .214011 H $5.923444-1.137603 \quad 1.455051$ O $9.143801-1.6214352 .248345$ H $7.406506-1.6368043 .323329$ H 8.6470370 .5982693 .489453 O $\quad \begin{array}{lll}6.633626 & 0.846524 & 3.215892\end{array}$ H 8.1085912 .2500871 .632253 O 9.6252630 .9663801 .173190 C $5.3837021 .297440-1.172550$ H 5.3065620 .8049320 .880867 H 6.0435572 .3831570 .520876 H 9.662082 -1.427863 3.047896 H $6.4443530 .355504 \quad 4.033713$ H 9.8559901 .5158930 .404839 O $4.3930752 .234761-1.244836$ O $5.7214800 .538869-2.070847$
C $3.7367602 .361526-2.510233$

H $4.3675202 .940856-3.190130$

H $3.5091091 .383106-2.942219$

H $2.7980592 .899529-2.353778$

SCF Energy $\left(B 3 L Y P / 6-31 G^{* *} / / M M F F\right)=-3245.90338573$

08 00425

MM̄MF Geometry

C -0.141850 $4.784039-2.029001$

C $0.4045924 .043962-1.052076$

C $1.8274834 .209851-0.583552$

O $2.4603382 .908290-0.603518$

C 1.8528904 .7348050 .862820

C 3.2468655 .1079201 .334891

C 3.9957524 .3866202 .193108

C $3.777266 \quad 6.4126870 .795235$

C 3.6485213 .0811522 .850671

C 4.5230431 .9415332 .312775

C 4.0742820 .5728732 .843731

C $4.958019-0.5440422 .286713$

C $4.462229-1.9355352 .710980$

$\begin{array}{llll}\text { O } & 2.718863 & 0.366151 & 2.435766\end{array}$

O $5.133981-2.9078411 .894866$

C $2.950990-2.0518772 .403923$

C $4.842852-2.2556854 .161795$

O $2.447384-3.2776802 .929127$

C $2.130205-0.8493222 .920283$

C $0.653346-0.8465892 .455074$

O $2.112683-0.8756844 .353134$

C $3.0437532 .518032-1.769557$

O $3.0757883 .138679-2.820446$

C $3.6677521 .196397-1.541654$

C $4.5422290 .719577-2.438950$

C $5.281615-0.531113-2.302429$

C $6.739683-0.396219-2.650955$

C $0.436605-0.8494530 .938991$

C $0.272834-2.2402490 .351615$

C $0.364885-3.451200-1.824549$

O $-0.025504-3.2238331 .023766$

C $1.684521-4.152708-1.786166$

C $2.516977-4.238555-2.834114$

C $3.847447-4.930305-2.775525$

C $5.007379-3.972474-2.929032$

C $5.795186-4.006879-4.016767$

C $5.306570-3.035948-1.778737$

C $4.672077-1.681049-1.951025$

N $0.454851-2.245610-1.023279$

H $0.4750535 .549976-2.496182$

H $-0.1769463 .272193-0.557610$

H $2.3756244 .899105-1.237510$

H 1.4005843 .9919671 .527636

H 1.2172425 .6267490 .948314

H 4.9799594 .7695272 .460934

H 4.7511526 .6747721 .222480

H $3.8994926 .361898-0.290829$

H $3.0869517 .229244 \quad 1.030833$

H 3.8082003 .1839453 .930905

H 2.5913252 .8377272 .725989

H $4.4684601 .940898 \quad 1.218436$

H 5.5712342 .1193662 .583917

H 4.1241570 .5956053 .938760

H $6.003274-0.3994512 .586986$

H $4.954641-0.4984501 .188965$

H $6.091774-2.7941592 .019226$

H $2.861314-2.1137591 .312046$

H $5.932321-2.2305424 .285865$

H $4.534869-3.2704704 .436928$

H $4.414719-1.5564134 .882731$

H $1.535894-3.3878162 .611224$

H $\quad 0.097977-1.6545672 .946042$

H $\quad 0.2044690 .0824172 .833277$

H $1.353635-0.3543634 .663097$

H $3.4265640 .661600-0.629654$

H $4.7864161 .302696-3.324807$

H $6.857954-0.011451-3.670465$

H $7.289606-1.338162-2.604730$

H $7.2307920 .300202-1.961894$

H $-0.499186-0.3198080 .718273$ 
H $1.241858-0.3140930 .425412$ H $\quad 0.082115-3.152102-2.838705$ H $-0.417646-4.102480-1.421975$ H $1.970273-4.609795-0.841324$ H $2.236169-3.783626-3.780902$ H $3.864904-5.690055-3.567346$ H $3.966304-5.483038-1.834852$ H $6.647884-3.343826-4.117051$ H $5.604264-4.696560-4.832180$ H $6.389248-2.962053-1.631057$ H $4.936939-3.473429-0.842996$ H $3.601536-1.657803-1.750539$ H $0.766193-1.396548-1.485892$ C $-2.4293433 .560423-2.052035$ O $-1.8910692 .289353-2.463610$ C $-2.9702871 .435591-2.898585$ C $-4.2539502 .145499-2.495730$ C $-3.8516123 .601304-2.611372$ H $-2.8849331 .376370-3.990555$ C $-2.787127 \quad 0.052857-2.266379$ H $-5.0991381 .881819-3.138524$ H $-4.5251701 .904684-1.459895$ C $-4.7817954 .545307-1.869091$ H -3.831281 $3.876313-3.674490$ C $-3.926161-0.929786-2.589814$ O $-1.560685-0.502085-2.751217$ H $-2.6695680 .169143-1.183632$ H $-4.890950-0.515790-2.279720$ O $-4.014051-1.086097-4.011852$ C $-3.749024-2.326769-1.964662$ C $-3.587491-2.336520-0.437814$ H -2.879814 -2.826023 -2.409354 O $-4.875318-3.137611-2.306562$ H -3.574659-3.374266 -0.079621 H -2.594936 -1.943171 -0.187088 H $-5.7984414 .477078-2.269817$ H $-4.8254114 .307910-0.800717$ H $-4.4485405 .582820-1.970175$ C -1.540537 $4.689158-2.583010$ H $-0.8541170 .134547-2.546763$ H $-3.152549-1.412263-4.324221$ H -4.964319 -3.117808-3.274980 H -2.029293 5.653599-2.401778 H -1.440384 $4.576405-3.670402$ H -2.454902 $3.566850-0.955152$ C $-4.937995-0.7918952 .665077$ O $-4.184465-1.5844301 .737634$ C $-4.628775-1.5375200 .373327$ C $-6.075696-2.0450760 .270561$ C $-7.002084-1.2849211 .226145$ C $-6.421849-1.1911222 .641773$ H -4.537522 -1.058613 3.651702 C -4.7064490 .7134692 .454905$ H -4.587512 -0.4972630 .040443$ O $-6.144617-3.4333070 .585161$ H $-6.446460-1.912803-0.751662$ H -7.972459 -1.795462 1.259744 $\begin{array}{lllll}\text { O } & -7.220044 & 0.022070 & 0.701571\end{array}$ H -6.997644 -0.483789 3.249881 O $-6.529454-2.469603 \quad 3.274643$ C -3.2574621 .0990242 .665340$ H -4.9896841 .0233261 .445523$ H $-5.303716 \quad 1.2891843 .170867$ H -5.733380 -3.922228 -0.147652 H $-7.8342980 .479619 \quad 1.300656$ H -7.471490 -2.709523 3.294008 O -3.0160392 .2930012 .047427$ O $-2.4466640 .450636 \quad 3.312192$ C - -1.6822832 .7954682 .176540$ H -1.409804 2.896088 3.231482 H -0.9815672 .1352331 .657632$ H -1.645065 3.7837141 .710631 SCF Energy $(B 3 L Y P / 6-31 G * * / / M M F F)=-3245.90869653$

\section{8_00426}

MM̄FF Geometry

C -1.298691-2.383889-1.326325

C $-0.297008-2.355232-0.436261$
C $-0.281152-1.4225430 .748731$

O $0.905744-0.6007190 .669852$

C $-0.231551-2.2411632 .050534$

C $-0.283390-1.3875453 .303748$

C $0.759465-1.1603854 .127784$

C $-1.634284-0.7987083 .621889$

C $2.171589-1.6558813 .985301$

C $3.145078-0.4911713 .764807$

C $4.531438-0.9794853 .319223$

C 5.4996670 .1967843 .195797

C $6.856349-0.2257712 .613357$

O $4.372241-1.6178652 .048779$

O 7.5593810 .9677462 .232082

C $6.621699-1.0434751 .323777$

C $7.729541-0.9269463 .660979$

O $7.862140-1.5680010 .855715$

C $5.575257-2.1660521 .493004$

C $5.181551-2.8521130 .160380$

O $6.116708-3.1826942 .344832$

C $0.8255000 .518067-0.103988$

O $\quad-0.1378520 .895143-0.752719$

C $2.1279121 .216902-0.030113$

C $2.3490112 .290654-0.803284$

C $3.5699483 .099435-0.836721$

C 4.7114102 .6804380 .050823

C $4.532308-1.938598-0.885154$

C $5.503776-1.347666-1.892334$

C $5.580140 \quad 0.285587-3.776394$

O $6.674567-1.703972-1.990019$

C $6.1118541 .592892-3.280159$

C $5.8408812 .769450-3.865576$

C $6.4189944 .080581-3.416328$

C $5.3633575 .134890-3.171574$

C $5.0725826 .052807-4.109094$

C $4.7163095 .182896-1.806748$

C 3.610793 4.174211-1.651957

N $4.890234-0.397302-2.698370$

H -2.127976 -1.690990 -1.204526

H $\quad 0.545315-3.032456-0.553081$

H -1.171816 -0.7818430 .757005$

H $0.661262-2.8742492 .046385$

H -1.078505 -2.940577 2.079697

H $0.591965-0.5407205 .008107$

H $-1.638856-0.2566174 .573508$

H -1.939913 -0.0949232 .842493$

H -2.387364 -1.590385 3.692061

H $2.447886-2.1903594 .902462$

H $2.265785-2.381698 \quad 3.175555$

H 2.7358550 .1762392 .996218

H 3.2319130 .0918254 .690120

H $4.899945-1.7095754 .049322$

H 5.6342180 .6924354 .165371

H 5.0630260 .9555752 .533584

H $7.6548571 .525367 \quad 3.023094$

H $6.273386-0.3325890 .565073$

H $7.917474-0.2598494 .510928$

H $8.715477-1.1747203 .252740$

H $7.277571-1.8400544 .053942$

H $7.716359-1.930107-0.034022$

H $6.034761-3.402231-0.253740$

H $4.435864-3.6228120 .404379$

H $5.633125-4.0101002 .185585$

H $2.868600 \quad 0.831590 \quad 0.659989$

H $1.5557572 .619682-1.473730$

H $4.9688201 .629857-0.120242$

H 4.4410232 .8113491 .103808

H $5.6302113 .244691-0.122053$

H $3.818669-2.535634-1.467949$

H $3.967920-1.133125-0.407218$

H $4.8609630 .413658-4.591582$

H $6.406419-0.336348-4.135319$

H $6.7771951 .565917-2.420363$

H $5.1990852 .789404-4.743524$

H $7.1104764 .422868-4.197683$

H $7.0319573 .960767-2.514358$

H $4.3390626 .831710-3.929461$

H $5.5579046 .041659-5.079548$

H $4.2724106 .173793-1.638765$ 
H $5.4867315 .096266-1.035447$

H $2.7352354 .394010-2.264451$

H $3.929101-0.128931-2.507433$

C $-2.587381-4.231293-2.501280$

O $-3.790100-3.447420-2.357470$

C $-4.527821-3.961151-1.233317$

C $-4.104018-5.416572-1.126898$

C $-2.611971-5.283829-1.386683$

H -4.169361 $-3.433002-0.340774$

C $-6.023918-3.695780-1.409235$

H $-4.332404-5.851413-0.149579$

H $-4.572971-6.032988-1.902494$

C -1.938835 -6.591147-1.766893

H -2.133568 $-4.897198-0.477995$

C $-6.363188-2.214662-1.689997$

O $-6.519650-4.471822-2.505881$

H $-6.567572-4.044134-0.523624$

H -6.042756 -1.942284 -2.703073

O $-7.787808-2.073536-1.665620$

C $-5.754355-1.224584-0.679715$

C $-6.1197200 .235223-0.977935$

H $-4.666309-1.319759-0.709267$

O $-6.174438-1.5611630 .640569$

H $-5.8338620 .488207-2.007017$

H -7.209803 $0.363356-0.935687$

H -2.027766 -7.320062 -0.954747

H -2.390516 -7.030081-2.662876

H $-0.874051-6.434782-1.967273$

C $-1.364001-3.306173-2.512175$

H -6.006108 -4.226965 -3.294969

H $-8.152137-2.703534-2.310998$

H $-7.145366-1.5063240 .661495$

H -1.416852 -2.669253 -3.404265

H $-0.449282-3.903750-2.599506$

H -2.658738 -4.719748 -3.481273

C $-5.515507 \quad 3.671516 \quad 0.320833$

O $-5.9226062 .547523-0.469355$

C -5.5041401 .2583990 .000274$

C $-3.970606 \quad 1.1912640 .097818$

C -3.4072612 .3486330 .927656$

C -3.9879793 .6974860 .493435$

H $-5.7959124 .549742-0.275201$

C -6.282071 3.7432311 .650867

H $-5.943306 \quad 1.0853640 .988643$

O $-3.4107501 .222942-1.213301$

H -3.679211 0.2415060 .560706

H $-2.315640 \quad 2.366280 \quad 0.822668$

$\begin{array}{lllll}\text { O } & -3.704750 & 2.110460 & 2.300443\end{array}$

H -3.716423 4.4828421 .208367

O $-3.4200104 .066196-0.766529$

C $-7.760356 \quad 4.006721 \quad 1.448941$

H -6.1949732 .8154552 .221897$

H -5.8848994 .5687242 .253105$

H -2.446560 $1.133984-1.122851$

H -3.314005 2.8365422 .815700

H $-2.4560094 .116905-0.650347$

O $-8.364210 \quad 4.0864562 .672408$

$\begin{array}{lllll}\text { O } & -8.318462 & 4.126617 & 0.367222\end{array}$

C $-9.774051 \quad 4.330956 \quad 2.647607$

H -10.130336 4.3713383 .680330

H -9.983692 5.2908602 .166267

H -10.290504 3.5171552 .129650

SCF Energy (B3LYP/6-31G**//MMFF) $=-3245.91942887$

$08 \_00427$

MM̄FF Geometry

C $-3.007157-1.756790-2.326118$

C $-1.965971-1.474885-3.122320$

C $-0.580810-1.250093-2.578423$

O $-0.2177190 .102880-2.944541$

C $0.464854-2.231911-3.136223$

C $0.165730-3.691761-2.883246$

C $0.241659-4.294187-1.680639$

C $-0.226791-4.483891-4.103082$

C $0.604977-3.683568-0.353358$

C $1.719685-4.4688760 .353759$

C $3.125664-4.038952-0.094985$

C $4.160132-5.075710 \quad 0.345555$
C $5.587191-4.634747-0.001827$

O $3.405314-2.7813160 .529902$

O $6.499528-5.496882 \quad 0.696463$

C $5.812335-3.2097390 .543473$

C $5.886689-4.809947-1.495146$

O $7.065999-2.689214 \quad 0.106930$

C $4.679703-2.2092320 .203642$

C $4.843306-0.9367081 .068185$

O $4.730064-1.790585-1.160892$

C $0.734230 \quad 0.708685-2.187307$

$\begin{array}{llll}\text { O } & 1.366612 & 0.211577 & -1.270174\end{array}$

C $0.8597192 .107860-2.645091$

C $1.9216812 .829050-2.257271$

C $2.1599704 .245491-2.540998$

C $1.0505515 .011407-3.213385$

C $3.7673350 .121898 \quad 0.805563$

C 3.8880851 .3216671 .729653

C $2.745053 \quad 3.4789512 .248420$

O $4.750878 \quad 1.4178872597742$

C $2.5281344 .633397 \quad 1.322504$

C $3.4222845 .619187 \quad 1.151797$

C $3.2307376 .789708 \quad 0.227838$

C $4.2238616 .785485-0.915180$

C $5.4492767 .316604-0.766624$

C $3.7734776 .224576-2.249188$

C $3.3328574 .789475-2.152516$

N $2.910907 \quad 2.2712301 .460917$

H $-2.839238-1.860244-1.255839$

H $-2.117116-1.361119-4.192325$

H $-0.609967-1.312350-1.484581$

H $1.454562-1.997723-2.722680$

H $\quad 0.586080-2.054718-4.214049$

H $-0.012371-5.352617-1.629250$

H $-0.440886-5.532253-3.869491$

H $-1.124260-4.058799-4.563273$

H $0.583004-4.468290-4.840125$

H $\quad 0.887323-2.630967-0.439375$

H $-0.294387-3.7193130 .273932$

H $1.623736-4.2899541 .432826$

H $1.576888-5.5443870 .191608$

H $3.141432-3.915846-1.183633$

H $3.944474-6.055161-0.099681$

H $4.094736-5.219757 \quad 1.433312$

H $6.312508-6.4127030 .427677$

H $5.875905-3.2907381 .637941$

H $5.806118-5.866018-1.780087$

H $6.915133-4.514858-1.730400$

H $5.207568-4.246289-2.138224$

H $7.751228-3.3292670 .365280$

H $4.806650-1.2129362 .130032$

H $5.824109-0.4807170 .882159$

H $5.458375-2.251796-1.605144$

H $0.0646222 .510689-3.261320$

H $2.6882332 .341639-1.656466$

H $0.8341844 .586634-4.200062$

H $1.2792836 .065966-3.376240$

H $0.1379314 .975878-2.607877$

H $3.8457970 .481976-0.225984$

H $2.772684-0.3122130 .952055$

H 3.6123213 .6277412 .899632

H 1.8645183 .3398042 .883938

H 1.5906104 .6583920 .772372

H 4.3547315 .5887761 .711585

H 3.3466317 .7096570 .815943

H $2.2077926 .825043-0.167363$

H $6.1605117 .339311-1.585548$

H 5.7684117 .7393490 .180529

H $4.5917976 .270670-2.979074$

H $2.9886476 .880473-2.633964$

H $4.0775474 .136208-1.694441$

H 2.1866852 .0449560 .784926

C $-5.282180-0.709460-2.388572$

O $-5.185681-0.474212-0.969776$

C $-6.505488-0.404613-0.412308$

C $-7.421981-0.097203-1.588680$

C $-6.769706-0.914210-2.688471$

H $-6.735422-1.409380-0.035956$

$\begin{array}{llll}\text { C } & -6.561688 & 0.592694 & 0.751239\end{array}$ 
H $-8.459643-0.385452-1.396310$ H -7.404655 $0.966170-1.853131$ C $-7.176894-0.491189-4.089187$ H -7.041422 -1.968736 -2.544854 C $-5.4262370 .455692 \quad 1.790289$ $\begin{array}{lllll}\text { O } & -6.489666 & 1.924852 & 0.225657\end{array}$ H -7.5408830 .5133991 .238948$ H -4.5173290 .9111641 .384874$ O -5.7850351 .2550642 .924959$ C $-5.118644-0.975425 \quad 2.255677$ C $-4.140056-1.0824233 .439826$ H $-4.718366-1.5523751 .414567$ O $-6.344143-1.6014032 .647823$ H $-4.638579-0.7631494 .364565$ H -3.940874 -2.153649 3.577102 H $-8.255957-0.612777-4.229349$ H $-6.9294280 .558609-4.279314$ H $-6.666908-1.099541-4.842826$ C $-4.426219-1.918241-2.783937$ H -6.5459442 .5373980 .979159$ H -6.555249 $0.836103 \quad 3.346362$ H -6.141575 -2.524189 2.878450 H $-4.473217-2.070841-3.868345$ H $-4.817631-2.828315-2.312566$ H $-4.9036130 .195771-2.880477$ C $-0.976824-0.0038551 .728670$ O $-2.213352-0.6681552 .035421$ C $-2.809275-0.3212443 .288303$ C -1.848576 -0.6337094 .450903$ C $-0.4741090 .007114 \quad 4.241524$ C $0.066362-0.2531062 .832169$ H $-0.618789-0.4905100 .813514$ C $-1.168373 \quad 1.492167 \quad 1.422991$ H $-3.0343490 .749757 \quad 3.291192$ O $-1.684800-2.0439834 .598472$ H $-2.291241-0.2645935 .383466$ H $0.225431-0.4000594 .982268$ O $-0.584881 \quad 1.4084064 .471180$ H 0.9535880 .3632892 .653529 O $0.486734-1.6181892 .763362$ C $-1.937562 \quad 1.734066 \quad 0.143819$ H -1.7055962 .0090672 .221728$ H -0.1857101 .9608211 .298436$ H $-1.352130-2.3943983 .754496$ H 0.3020941 .7933214 .367855 H $0.911048-1.7507261 .898632$ O $-1.9655313 .075677-0.111630$ O $-2.4601440 .867262-0.540723$ C -2.694258 $3.460414-1.281665$ H -2.286940 $2.965268-2.168095$ H -3.754516 $3.219445-1.158945$ H -2.590967 $4.541558-1.406345$ SCF Energy (B3LYP/6-31G**/MMFF) $=-3245.91729119$

08_00428

MMFF Geometry

C -1.476608 -3.440235 -0.460686

C $-0.669770-4.0252010 .435845$

C $0.070272-3.2467091 .489605$

O $1.479215-3.4565221 .234741$

C $-0.251811-3.7192622 .916684$

C -1.676323 -3.4651903 .353543$

C $-2.128103-2.2748553 .793934$

C $-2.583918-4.666773 \quad 3.339254$

C -1.353188 -0.9888863 .900786$

C $-2.177507 \quad 0.1985853 .385011$

C -1.413321 1.5258593 .519025

C -2.3950002 .6996953 .548103$

C -1.670288 4.0478973 .619426

O -0.5230851 .6540922 .400361$

$\begin{array}{lllll} & -2.629366 & 5.080091 & 3.343782\end{array}$

C -0.619996 4.0943512 .496304

C -1.114554 4.3330755 .019179

$\begin{array}{lllll}\text { O } & 0.178089 & 5.272424 & 2.599637\end{array}$

C 0.2881002 .8390622 .428315

C $1.1206602 .910961 \quad 1.125487$

O 1.1706992 .8397433 .551157

C $2.341540-2.4946511 .659946$
O $2.068791-1.4813182 .282563$

C $3.694645-2.8823331 .206450$

C $4.718934-2.0362231 .388686$

C $6.095561-2.2387550 .932557$

C $6.418626-3.5487440 .261509$

C $1.976917 \quad 1.6793130 .805950$

C $2.746027 \quad 1.878872-0.493347$

C $4.1809550 .685970-2.126253$

O $2.7245792 .932670-1.126372$

C $5.414589-0.142195-1.964637$

C $6.6504150 .378888-1.915271$

C $7.912148-0.428813-1.786955$

C $8.627804-0.180873-0.475671$

C $9.4732790 .853940-0.334726$

C $8.415593-1.1750470 .646688$

C $6.980628-1.2325521 .095162$

N $3.4696700 .752794-0.862053$

H $-1.608326-2.361604-0.420149$

H $-0.511769-5.0999020 .398362$

H $-0.151510-2.1776701 .379900$

H $0.432413-3.2378373 .627944$

H $-0.012966-4.7873683 .016206$

H -3.161304 -2.216303 4.133996

H $-3.610357-4.4167913 .627655$

H -2.623081 -5.1117972 .341065$

H $-2.216628-5.4244074 .039491$

H $-1.115034-0.8270324 .959113$

H -0.403601 -1.0334693 .362608$

H -2.4452580 .0249902 .335030$

H $-3.1096740 .248283 \quad 3.962161$

H $-0.827117 \quad 1.5057424 .445219$

H -3.096498 $2.601494 \quad 4.386152$

H -3.010474 2.6853802 .638933

H -3.3386815 .0146404 .006152$

H -1.1660954 .1746811 .546092$

H -1.9244194 .3382465 .758668$

H -0.6591825 .3284835 .067723$

H -0.3745463 .5995615 .345659$

H -0.4282896 .0325362 .616724$

H $0.438677 \quad 3.0613300 .277943$

H 1.7818463 .7864081 .168017

H 1.9374492 .2814163 .348975

H $3.802612-3.8358350 .702234$

H $4.528062-1.0869871 .887997$

H $6.220178-4.3859270 .940126$

H $7.461207-3.640001-0.047736$

H $5.812959-3.677459-0.642581$

H $2.706991 \quad 1.4914701 .599447$

H 1.3381730 .7967250 .702238

H $4.4121191 .696960-2.478594$

H $3.5119650 .225047-2.860141$

H $5.282595-1.219479-1.910288$

H $6.770007 \quad 1.458251-1.983347$

H $8.571600-0.161529-2.623319$

H $7.714201-1.501113-1.907858$

H $10.009199 \quad 1.0258120 .592775$

H $9.6456511 .554081-1.145610$

H $9.021772-0.9036761 .520925$

H $8.798365-2.1470980 .325020$

H $6.644080-0.3187351 .587051$

H $3.299872-0.116099-0.363607$

C $-3.689649-3.850087-1.629129$

O $-3.889328-2.425687-1.750023$

C $-4.879736-2.025996-0.784732$

C $-5.647420-3.296114-0.446726$

C $-4.514918-4.306383-0.424101$

H $-4.339867-1.7011820 .113291$

C $-5.721907-0.871874-1.327367$

H $-6.178087-3.2232300 .507169$

H $-6.372371-3.552119-1.228251$

C $-4.977224-5.750103-0.505176$

H $-3.950415-4.1691470 .505736$

C $-4.8920060 .370398-1.720740$

O $-6.419990-1.325808-2.492746$

H $-6.490689-0.601267-0.593694$

H $-4.2933080 .138724-2.608192$

O $-5.8138301 .391256-2.124132$

C $-3.9864580 .919446-0.599621$ 
C $-3.3579102 .293459-0.895226$ H $-3.1925870 .197229-0.379365$ O -4.7795531 .0537790 .584260$ H $-4.1522073 .037090-1.037256$ H -2.8173272 .6054470 .008730$ H -5.607885 -6.000546 0.354097 H -5.559001 -5.935538 -1.414238 H $-4.121086-6.432141-0.506751$ C $-2.191743-4.160539-1.567822$ H $-6.939964-0.575735-2.829145$ H -6.329159 $1.647081-1.339703$ H -4.1858351 .3161721 .307210$ H $-1.731739-3.829765-2.507764$ H $-2.039636-5.243500-1.496725$ H -4.094955 -4.291270 -2.548809 C $-0.3107631 .345203-2.832677$ O $-1.2524751 .515998-1.764263$ C $-2.3968802 .307080-2.092893$ C -1.958043 3.736633 -2.464609 C $-0.754013 \quad 3.748125-3.413594$ C $-0.4803912 .357074-3.977934$ H $0.6769491 .504842-2.387005$ C $-0.378676-0.104071-3.334811$ H $-2.8942601 .854756-2.958474$ O $-1.5957964 .457450-1.284615$ H -2.814936 $4.266324-2.898350$ H $0.1419364 .137758-2.914285$ O $-1.037144 \quad 4.648419-4.485670$ H $-1.2981102 .063167-4.647205$ O $0.7028402 .383126-4.775726$ C $0.464118-1.018681-2.470229$ H - $-1.404755-0.487199-3.327409$ H $0.011578-0.161165-4.357600$ H $-1.370908 \quad 5.364472-1.554223$ H $-0.2839194 .613640-5.099994$ H $1.4414462 .662676-4.208215$ O $1.043358-1.979363-3.247212$ O $0.587290-0.908894-1.257905$

C $1.913663-2.889100-2.566143$ H $2.632928-2.349637-1.942340$ H $1.324894-3.584765-1.963140$ H $2.465964-3.457622-3.319026$ SCF Energy (B3LYP/6-31G*//MMFF) $=-3245.91564669$

$08 \quad 00429$

MM̄FF Geometry

C $-2.795171 \quad 4.379698 \quad 0.769548$

C -3.7723813 .4692430 .649494$

C $-4.4191502 .752921 \quad 1.806134$

O -3.9870991 .3741671 .759998$

C -5.9504782 .8043431 .650258$

C -6.7023311 .9746352 .672442$

$\begin{array}{llll}\text { C } & -7.308716 & 0.798648 & 2.409471\end{array}$

C -6.7618942 .5641704 .057412$

C $-7.342047 \quad 0.0552831 .101411$

C $-6.564793-1.2639011 .196777$

C $-6.181526-1.812543-0.186755$

C $-5.504344-3.175632-0.046311$

C $-4.979680-3.702704-1.388781$

O $-5.275244-0.878954-0.781706$

O $-4.086836-4.794538-1.112608$

C $-4.140279-2.600921-2.075734$

C $-6.113985-4.280299-2.243797$

O $-3.784421-3.015616-3.392121$

C $-4.832090-1.217797-2.103618$

C $-3.907190-0.069677-2.574199$

O $-5.924834-1.203334-3.030544$

C $-2.837408 \quad 1.0683382 .424960$

$\begin{array}{llll}\text { O } & -2.142864 & 1.827360 & 3.082297\end{array}$

C $-2.547719-0.3637092 .193338$

C $-1.395513-0.8902022 .633840$

C $-0.955250-2.2751142 .452322$

C $-1.833478-3.1854081 .635490$

C $-2.6861050 .205861-1.691338$

C $-1.416355-0.510378-2.119133$

C $0.932491-0.898094-1.364082$

O $-1.306760-1.110738-3.184405$

C $1.017555-2.266736-0.763589$
C $1.943007-2.6202660 .141833$

C $2.088513-4.0085640 .698216$

C $2.153035-4.0381182 .210051$

C $3.326717-4.1657782 .851692$

C $0.850744-4.0200692 .977678$

C $0.213701-2.6597113 .005720$

N $-0.403779-0.363252-1.178608$

H -2.415233 4.6420621 .752949

H $-4.1160563 .200180-0.347664$

H -4.1391993 .2071492 .764973$

H -6.2239742 .4985620 .635659$

H $-6.2902213 .846451 \quad 1.727371$

H -7.8308220 .2962683 .222855$

H $-7.3603601 .955096 \quad 4.743379$

H $-5.7570272 .647246 \quad 4.482914$

H -7.2113273 .5619874 .026473$

H $-8.387245-0.1586020 .846452$

H -6.9560910 .6622280 .280552$

H -5.650214 -1.102529 1.780278

H -7.169725 -2.0004111 .740135$

H $-7.084747-1.886226-0.803357$

H $-6.185999-3.9027150 .412781$

H -4.656171 -3.0883410 .644218$

H $-4.578507-5.464347-0.607071$

H -3.206831 - $2.525006-1.505258$

H $-6.582517-5.131127-1.734131$

H $-5.734974-4.673409-3.193384$

H $-6.901788-3.554135-2.455822$

H $-3.115191-2.396496-3.728599$

H -3.622039 $-0.212722-3.623264$

H $-4.5129000 .848632-2.567476$

H $-5.912308-2.029822-3.539292$

H $-3.280680-0.9432901 .645849$

H $-0.702903-0.2477753 .176000$

H -2.778140 -3.370623 2.157555

H $-1.384153-4.1576951 .424883$

H $-2.049302-2.7385040 .659191$

H -2.460095 $1.277720-1.748135$

H $-2.899869-0.027330-0.645954$

H $1.630226-0.196599-0.895872$

H $1.157578-0.951084-2.434182$

H $\quad 0.310530-3.011751-1.120670$

H $2.664983-1.8793660 .474602$

H $3.009646-4.4356240 .279673$

H $1.276805-4.6630720 .357071$

H $3.377233-4.2190313 .934206$

H $4.264363-4.2211782 .310176$

H $1.023445-4.3168494 .021133$

H $0.190287-4.7941682 .577836$

H $0.786401-1.924953 \quad 3.573354$

H $-0.6038290 .112106-0.303471$

C $-0.9702494 .297572-0.954854$

$\begin{array}{llllll}\text { O } & 0.137907 & 4.404479 & -0.040427\end{array}$

C $1.3512304 .600983-0.799823$

C $0.9778174 .373563-2.257986$

C $-0.4603964 .843125-2.286780$

H $1.6372515 .647582-0.637187$

C $2.4404613 .655814-0.274909$

H $1.6229004 .925402-2.948200$

H $1.0361113 .308018-2.514348$

C $-1.2326474 .345066-3.496797$

H $-0.4737445 .941275-2.274416$

C $3.7311743 .671256-1.114435$

$\begin{array}{llll}\text { O } & 2.742696 & 4.016541 & 1.075753\end{array}$

H $2.0342962 .638942-0.232728$

H $3.5143953 .315481-2.128381$

O $4.1806405 .023631-1.265560$

C $4.8982552 .844354-0.540992$

C $4.5209281 .385908-0.259719$

H 5.2880973 .3183160 .366721

O $5.9582112 .859492-1.502998$

H 3.7856681 .3309970 .552419

H $4.0125150 .969969-1.140930$

H $-0.772175 \quad 4.706898-4.422037$

H $-1.253173 \quad 3.250608-3.537317$

H $-2.2676234 .699788-3.474701$

C $-2.1774155 .065570-0.416355$

H 3.0196754 .9482081 .083708 
H $4.4471915 .349854-0.389596$ H $6.1780313 .790674-1.677729$ H -2.933667 5.181262 -1.201995 H -1.881720 $6.075678-0.107430$ H -1.201760 3.228755 -1.046069 C $6.067649-1.8962190 .548035$

$\begin{array}{lllll}\text { O } & 5.136436 & -0.834541 & 0.310742\end{array}$ C 5.7070810 .4565680 .061097 C 6.5535780 .9042231 .260666 C $7.609146-0.1492871 .614306$ C $7.004987-1.5539001 .715813$ H $5.446158-2.7448520 .857684$ C $6.799754-2.306966-0.733739$ H $6.3366450 .394283-0.832657$ O 5.7019261 .1317842 .381168 H $7.048621 \quad 1.8522311 .021478$ H 8.0777250 .1200302 .568587 O $8.622474-0.1290790 .612616$ H $7.794664-2.3112741 .782518$ O $6.237575-1.6447412 .919978$ C $5.840344-2.965865-1.697173$ H $7.289263-1.476438-1.249614$ H $7.584677-3.039579-0.510791$ H 6.2596051 .4366063 .116998 H $9.295830-0.7842510 .863033$ H $6.838846-1.4735243 .664642$

O $5.178869-2.017608-2.420128$ O $5.683346-4.177195-1.779003$ C $4.208265-2.513567-3.346873$ H $3.478264-3.149124-2.836755$ H $3.683638-1.657642-3.779845$ H $4.705984-3.065519-4.149520$ SCF Energy (B3LYP/6-31G**//MMFF) $=-3245.90014873$

08_00430

MM̄FF Geometry

C $2.4703932 .851566 \quad 0.416924$

C $3.5270483 .336839-0.254717$

C $4.3984792 .592409-1.240603$

O $3.849517 \quad 1.297060-1.568596$

C $5.8031292 .351000-0.662036$

C $6.8805352 .394602-1.727232$

C $7.3678631 .324234-2.383710$

C $7.4353333 .768107-2.008026$

C $6.927078-0.109043-2.284551$

C $7.984911-1.007740-1.629613$

C $8.021566-0.869169-0.100516$

C $9.157313-1.7043340 .492994$

C $9.145000-1.6749242 .028963$

O $6.763429-1.3324990 .401375$

O $10.019869-2.712403 \quad 2.497447$

C $7.725319-2.0236882 .522643$

C $9.700298-0.3567042 .580908$

O $7.623693-1.8685033 .936425$

C $6.608698-1.2164861 .819954$

C $5.224765-1.7973332 .196507$

$\begin{array}{lllll}\text { O } & 6.675124 & 0.144891 & 2.249897\end{array}$

C $2.8660921 .237536-2.503932$

O $2.3831362 .162785-3.135263$

C $2.440021-0.174613-2.584057$

C $1.185788-0.457170-2.962472$

C $0.589988-1.792070-3.032434$

C $1.489903-2.973244-2.776646$

C $4.061682-1.2702671 .341588$

C $2.722615-1.7996631 .828142$

C $0.278560-1.5901881 .393639$

O $2.609536-2.5600572 .786254$

C $-0.238129-2.691306 \quad 0.522327$

C - $-1.434555-2.643076-0.084089$

C -2.003131-3.751100 -0.924094

C $-2.541194-3.260832-2.251163$

C $-3.851338-3.011231-2.415535$

C $-1.570301-3.132488-3.403397$

C $-0.728705-1.891431-3.299861$

N $1.665756-1.3103241 .073249$

H 2.1923551 .8063300 .319085

H $3.7771934 .389316-0.131409$

H $4.460513 \quad 3.195183-2.156135$
H $\quad 5.833735 \quad 1.389668-0.137401$

H $6.0522623 .107136 \quad 0.094571$

H $8.162358 \quad 1.491003-3.110630$

H $8.2009983 .757450-2.790897$

H $6.6377694 .442174-2.336497$

H $7.8924394 .184449-1.104290$

H $5.966639-0.212415-1.773161$

H $6.756811-0.464748-3.308753$

H $7.737877-2.046719-1.884329$

H $8.974005-0.795741-2.054064$

H $8.1520730 .186603 \quad 0.161080$

H $\quad 10.128967-1.3680590 .110038$

H $9.052076-2.7485030 .166412$

H $10.905701-2.5447542 .132556$

H $7.562109-3.0907522 .315865$

H $10.733803-0.2071342 .246203$

H $9.739832-0.3723883 .675682$

H $\quad 9.123747 \quad 0.5164902 .268521$

H $8.332325-2.4000724 .337714$

H $5.245064-2.8889822 .079398$

H $5.016669-1.5883523 .253744$

H 5.7895310 .5380582 .206876

H $3.152384-0.932805-2.279881$

H $\quad 0.5212190 .368681-3.214988$

H $2.291042-3.011221-3.523020$

H $0.973312-3.934151-2.816645$

H $1.942962-2.908786-1.781305$

H $4.021426-0.1765781 .373680$

H $4.189567-1.5795860 .297948$

H $-0.270409-0.6575241 .245684$

H $0.197288-1.8826072 .445218$

H $\quad 0.380307-3.5784770 .410661$

H -2.064884 -1.769643 0.060597

H $-2.811768-4.221688-0.349413$

H -1.263478 -4.542215 -1.098615

H $-4.249551-2.681071-3.369175$

H -4.553755 -3.121649-1.595864

H $-2.116996-3.077156-4.354472$

H $-0.976379-4.047350-3.479608$

H -1.294253 -0.973145 -3.464084

H $1.858047-0.7169110 .271795$

C 0.1326813 .7783420 .806393

O $-0.5424942 .527596 \quad 1.041740$

C $-1.3974822 .243519-0.086724$

C $-1.4559183 .525703-0.907481$

C $-0.0767284 .106260-0.672733$

H $-0.8879591 .448047-0.644613$

C $-2.769874 \quad 1.7651350 .407463$

H -1.667860 $3.338242-1.964464$

H $-2.2269794 .203290-0.519940$

C $0.0329855 .585361-0.996071$

H $0.6265643 .552519-1.305162$

C $-3.7482861 .427886-0.732693$

$\begin{array}{llll}\text { O } & -2.583267 & 0.599931 & 1.212148\end{array}$

H -3.204171 2.5283621 .063106

H $-4.0003842 .341395-1.284292$

O $-3.0728810 .569278-1.662892$

C $-5.0413630 .709446-0.298371$

C -5.8374821 .4723750 .775533$

H $-4.803521-0.3043840 .045623$

O $\quad-5.8395690 .566144-1.478485$

H -5.1926871 .6817851 .634914$

H -6.1647622 .4282470 .347184$

H $-0.1905185 .765771-2.052521$

H $-0.6660586 .180083-0.398670$

H $1.0448375 .952480-0.796706$

C 1.5869743 .7008811 .290399

H -1.9802040 .8425851 .935601$

H $-3.7007390 .363895-2.376864$

H $-6.6249420 .044175-1.242855$

H 1.6103853 .2750012 .300759

H 1.9906124 .7182671 .359347

H -0.3902654 .5151331 .430486$

C $-9.225628-0.1080990 .500408$

$\begin{array}{lllll}\text { O } & -8.028456 & 0.629580 & 0.220936\end{array}$

C $-7.0710300 .694052 \quad 1.281162$

C -7.6930401 .3597352 .518790$

C -8.9844740 .6501922 .939135$ 
C $-9.930461 \quad 0.441317 \quad 1.752331$ H -9.880672 $0.084016-0.359471$ C $-8.964633-1.6217530 .561374$ H -6.744971 -0.319326 1.539332 O H $-6.9640181 .333933 \quad 3.337700$ H -9.4962441 .2436513 .706370$ O $-8.672710-0.6175643 .509295$ H -10.753158 -0.2282082 .029816$ O $-10.515938 \quad 1.697175 \quad 1.396445$ C -8.548465 -2.188203 -0.780926 H -8.176899 -1.8721551 .276207$ H $-9.887033-2.1352200 .857017$ H -8.336336 3.1294513 .040479 H -8.096237 -0.4609444 .276570$ H -11.005134 2.0227892 .171061 O $-8.378306-3.538381-0.655787$ O $-8.391405-1.539969-1.806157$ C -7.989396 -4.222605 -1.851041 H $-8.780980-4.146163-2.602298$ H $-7.050667-3.814630-2.236715$ H $-7.838179-5.277086-1.605499$ SCF Energy (B3LYP/6-31G**//MMFF)= -3245.91130397

08_00431

MM̄FF Geometry

C $0.8818360 .377382-3.704181$

C $1.0095201 .678140-4.011260$

C $1.6978072 .739718-3.184615$

O $2.3828552 .166225-2.050049$

C $0.6368513 .721162-2.653460$

C $1.1978934 .777213-1.722315$

C $1.0530284 .746173-0.382079$

C $1.9090085 .921490-2.391991$

C 0.3885003 .6528090 .408474

C 1.0229713 .4601021 .792753

C 0.4420702 .2186022 .485289

C 0.7824112 .2073703 .974758

C 0.2730520 .9294824 .658250

O 1.0073901 .0657161 .848859

O $0.8781150 .852975 \quad 5.957641$

C $0.766886-0.2909043 .855603$

C -1.2434190 .9625574 .885095$

O $0.204064-1.4985124 .364486$

C $0.504894-0.1856322 .333744$

C $1.257751-1.3235151 .604609$

O $-0.894379-0.3175342 .086832$

C $3.7236381 .969096-2.159382$

O $4.4158302 .143003-3.149333$

C $4.2150561 .504831-0.842021$

C $5.4842211 .088078-0.724471$

C 6.1210090 .5709750 .488854

C $5.342331 \quad 0.6394351 .776337$

C $1.283788-1.1954030 .076189$

C $2.085415-2.326834-0.541535$

C $4.416239-3.191113-0.748434$

O $1.546909-3.245101-1.154531$

C $5.512324-3.2572450 .267559$

C $6.813528-3.104583-0.020856$

C $7.900872-3.1389161 .016214$

C $8.786487-1.9107500 .984541$

C $10.074003-1.9982820 .609428$

C $8.201349-0.592418 \quad 1.454544$

C $7.368527 \quad 0.0658390 .387724$

N $3.443939-2.210647-0.302954$

H $1.325717-0.016781-2.795263$

H $0.5385862 .040951-4.923442$

H $2.4041743 .282396-3.824720$

H $-0.1604983 .161915-2.148598$

H $0.1383354 .221815-3.494870$

H $1.446827 \quad 5.5774250 .200152$

H $2.2567546 .671497-1.673642$

H $2.7843395 .559640-2.940235$

H $1.2393546 .424742-3.096895$

H $-0.666703 \quad 3.9151150 .542124$

H $0.4266492 .701445-0.131122$

H 2.1100853 .3510151 .686789

H 0.8330964 .3549992 .398160
H -0.6455202 .2148132 .345056$

H 0.3773043 .0958104 .474730

H 1.8728242 .2610334 .101802

H $\quad 0.628844 \quad 1.6528706 .451784$

H $1.852009-0.3649394 .014556$

H -1.5184651 .8222575 .507827$

H -1.5797700 .0758435 .433680$

H -1.8159041 .0308973 .957704$

H $\quad 0.399549-1.5307165 .316592$

H $2.295036-1.3440011 .961635$

H $0.811828-2.2923101 .864481$

H -1.028312 -0.6294601 .178278$

H $3.5179721 .504007-0.012013$

H $6.1168491 .100320-1.611399$

H 4.9849841 .6599421 .956068

H $\begin{array}{llll}5.933152 & 0.369119 & 2.654267\end{array}$

H $4.478295-0.0307761 .746764$

H $\quad 0.273288-1.224465-0.341736$

H $1.728911-0.245127-0.234892$

H $4.775861-2.873557-1.732405$

H $3.934128-4.168334-0.851088$

H $5.216235-3.4328591 .299556$

H $7.115979-2.948085-1.053768$

H $8.497943-4.0429860 .840463$

H $7.483709-3.2460192 .025348$

H $10.721487-1.1276680 .605138$

H $10.508762-2.9407600 .293632$

H 9.0083710 .1017601 .722047

H $7.644271-0.7751232 .377596$

H $7.8605190 .104925-0.585708$

H $3.794635-1.3316260 .063316$

C -1.192059-0.996256 -3.789051

O $-0.884558-2.005817-2.806697$

C $-2.028246-2.887025-2.676004$

C $-3.099250-2.323878-3.601674$

C $-2.268054-1.629896-4.663548$

H -1.693104 -3.868298 -3.034044

C $-2.407364-2.958648-1.190593$

H -3.752652 -3.102090 -4.007001

H -3.727336 -1.592545 -3.077320

C $-3.064804-0.638594-5.493949$

H -1.823312 -2.387897 -5.321927

C $-3.800407-3.544484-0.903201$

O $-1.435674-3.770882-0.521794$

H -2.317989-1.958171 -0.755865

H $-4.577303-2.926603-1.365457$

O $-3.904233-4.829128-1.529390$

C $-4.124539-3.7188930 .594225$

C $-3.908371-2.4736131 .465655$

H -3.522556-4.536411 1.009521

O $-5.484530-4.1386600 .728293$

H -4.264474 -2.677506 2.483988

H $-2.828615-2.3115991 .577923$

H $-2.431264-0.154854-6.243546$

H -3.879883 -1.146350 -6.020120

H $-3.5054450 .145714-4.869146$

C $0.079537-0.589266-4.535173$

H $-0.556683-3.426378-0.753461$

H $-3.189636-5.386254-1.175412$

H $-5.599045-4.9324280 .177871$

H $-0.173563-0.128740-5.496638$

H $0.703211-1.466384-4.743900$

H -1.610452 -0.136341 -3.246673

C -4.3817531 .2091301 .466076$

O $-4.054191-0.1330281 .852589$

C $-4.538164-1.1576660 .968927$

C $-6.073376-1.1325480 .934626$

C -6.5946320 .2594890 .554765$

C -5.9067361 .3774601 .347004$

H -4.0389731 .8259482 .306768$

C $-3.581427 \quad 1.6246470 .221411$

H $-4.156392-0.948818-0.033601$

O $-6.608242-1.4799532 .209154$

H -6.444398 -1.859579 0.204399

H $\quad-7.6750790 .3063110 .739942$

O $-6.384148 \quad 0.483910-0.836621$

H -6.1399222 .3498960 .900399$

O $\quad-6.4537151 .3995002 .670131$ 
C -3.5419853 .1218860 .011475$ H $-2.545282 \quad 1.2803490 .322291$ H -3.988249 $1.159877-0.681056$ H $-6.458456-2.4312202 .341945$ H $-6.867938-0.205720-1.322136$ H -6.2207350 .5593083 .101568$

O $-3.076193 \quad 3.380482-1.246675$

O $-3.857223 \quad 3.9602820 .844502$

C $-2.9869464 .764805-1.599243$

H $-3.9827305 .218089-1.597954$

H $-2.3216115 .292975-0.910053$

H -2.571433 $4.832410-2.608136$

SCF Energy (B3LYP/6-31G**//MMFF) $=-3245.92873541$

08_00432

MM̄FF Geometry

C -1.364214 -3.573105 1.955844

C $-0.993309-2.2842121 .956376$

C $-1.050028-1.3988903 .172855$

O $-1.750036-0.1745062 .853356$

C $0.352743-1.0330063 .706782$

C $1.265540-0.3283562 .719875$

C $2.214178-0.9304871 .976589$

C 1.0833501 .1637442 .605845

C $2.592795-2.3828661 .931353$

C $4.096036-2.6383242 .142913$

C $4.968862-2.3133300 .915343$

C $6.378213-2.8851221 .094999$

C $7.295208-2.530940-0.085894$

O $5.052559-0.8938230 .753974$

O $8.647139-2.8184610 .302867$

C $7.212930-1.012375-0.345550$

C $7.003974-3.399472-1.315839$

O $7.927064-0.657846-1.527715$

C $5.764636-0.473858-0.418727$

C $5.7846541 .075078-0.459419$

O $5.146676-0.957706-1.613262$

C -3.106699 -0.1930722 .812669$

O $-3.828467-1.1622363 .003666$

C -3.658486 1.1403502 .467015

C -2.9165752 .2580392 .424103$

C -3.3700013 .6042712 .065928$

C -4.840146 3.7988381 .806416

C $4.4110401 .729885-0.263235$

C $4.4591913 .241621-0.401402$

C $2.9830015 .237316-0.347921$

O $5.4860103 .862123-0.662386$

C 1.8281025 .6613750 .502843

C $0.796977 \quad 6.3874450 .043091$

C $-0.322606 \quad 6.894471 \quad 0.911701$

C $-1.7104936 .450866 \quad 0.493084$

C -2.141996 $6.490977-0.779343$

C -2.649606 6.0276571 .605795

C -2.448101 4.5883491 .989694

N $3.2105253 .814121-0.184959$

H $-1.725219-4.0240772 .876405$

H $-0.631598-1.839798 \quad 1.033260$

H - $1.587871-1.8971083 .990636$

H $0.833995-1.9378824 .094846$

H $0.233599-0.3779164 .580489$

H $2.758755-0.3019831 .279864$

H 1.9033161 .6434702 .061288

H $\quad 0.160564 \quad 1.3955892 .071791$

H 1.0447501 .6257593 .597520

H $2.049720-2.9523052 .691239$

H $2.283338-2.7947050 .963737$

H $4.443629-2.0676003 .013521$

H $4.213832-3.7030172 .381784$

H $4.494270-2.7532750 .031165$

H $6.343474-3.9725071 .237245$

H $6.821789-2.4766012 .014016$

H $8.696793-3.7602220 .540714$

H $7.725206-0.5106830 .487456$

H $7.148534-4.460285-1.078075$

H $7.702629-3.178154-2.130233$

H $5.985907-3.282732-1.693166$

H $8.832312-1.000291-1.432776$

H $6.441969 \begin{array}{lll}1.451075 & 0.335671\end{array}$
H $6.206448 \quad 1.410843-1.415378$

H $4.418352-0.365820-1.857652$

H $-4.721034 \quad 1.150367 \quad 2.247167$

H -1.8598132 .1919712 .668211$

H -5.1526893 .2414130 .917987$

H $-5.1226984 .840968 \quad 1.646047$

H $-5.430605 \quad 3.4527972 .662343$

H $3.6906881 .353124-0.995491$

H 4.0388391 .5062300 .741333

H $2.8069665 .420546-1.413136$

H $3.8848255 .782659-0.050701$

H 1.8635115 .3928371 .556071

H $0.7912406 .683716-1.003050$

H -0.2973017 .9914710 .915737$

H -0.1402276 .5888191 .949989$

H $-3.1524846 .192024-1.038992$

H $-1.5078596 .829248-1.590956$

H -3.6799406 .2356201 .303925$

H -2.473766 6.6571722 .487066

H $-1.412274 \quad 4.339717 \quad 2.226719$

H $2.3999673 .202634-0.145723$

C -2.631995 -4.7678550 .098846$

O $-3.211993-3.557528-0.422988$

C $-4.636007-3.599873-0.203847$

$\begin{array}{llll}\text { C } & -4.957961 & -5.028939 & 0.222621\end{array}$

C $-3.701030-5.400376 \quad 0.988047$

H $-4.842001-2.9308420 .640248$

C $-5.381336-3.106899-1.448599$

H $-5.865671-5.0853890 .831200$

H $-5.087385-5.693500-0.639378$

C $-3.527228-6.8943091 .194404$

H $-3.741558-4.9067331 .966528$

C $-4.903846-1.729829-1.955391$

O $-5.173972-4.050924-2.507113$

H $-6.459834-3.095597-1.252163$

H $-3.889228-1.832382-2.354214$

O $-5.727297-1.363445-3.069602$

C $-4.951727-0.606958-0.904309$

C $-4.5754130 .783150-1.450107$

H $-4.290054-0.855725-0.068836$

O $-6.280073-0.534156-0.380085$

H $-5.2660861 .073842-2.251143$

H $-4.7425951 .504390-0.641296$

H $-4.360017-7.3014461 .777077$

H -3.491372 $-7.430558 \quad 0.240142$

H $-2.599906-7.1064351 .735906$

C $-1.280447-4.4619280 .746842$

H -5.650306 -3.719625 -3.287813

H $-6.629070-1.223188-2.733032$

H -6.2790910 .1291550 .330957$

H $-0.632077-3.991483-0.004093$

H $-0.790204-5.397277 \quad 1.041218$

H -2.448326 $-5.411679-0.771924$

C $-0.8711580 .505623-1.148593$

O $-2.2687780 .571399-0.830816$

C $-3.1221180 .885981-1.936535$

C $-2.785510 \quad 2.284593-2.473507$

C $-1.3014312 .397155-2.842912$

C $-0.386168 \quad 1.841041-1.745065$

H $-0.378407 \quad 0.369115-0.178832$

C $-0.581350-0.740224-1.999350$

H $-2.9683260 .154327-2.736022$

O $-3.107273 \quad 3.277364-1.502757$

H $-3.4057452 .485589-3.354487$

H -1.049562 $3.450872-3.017009$

O $-1.054853 \quad 1.687443-4.052928$

H $0.6359421 .738686-2.126054$

O $-0.3460382 .811849-0.692732$

C $0.853504-1.208753-1.910936$

H -1.216395 -1.560916-1.642015

H $-0.821379-0.577380-3.053161$

H $-2.582175 \quad 3.092987-0.705363$

H $-1.6128092 .082660-4.744340$

H $0.2847602 .495203-0.024732$

O $0.959366-2.418753-2.537048$

O $1.767714-0.596150-1.378541$

C $2.269561-2.993226-2.553535$

H $2.968847-2.329665-3.070758$ 
H $\quad 2.606772-3.193179-1.532941$

H $2.218711-3.940339-3.097114$

SCF Energy (B3LYP/6-31G**//MMFF)= -3245.91717752

08 00433

MM̄FF Geometry

C -2.254533 -1.014726 3.347705

C -2.6082140 .2391253 .668946$

C -3.6881871 .0851833 .039796$

O -4.5119210 .2968232 .150489$

C -3.0744182 .2454372 .234760$

C -3.9985763 .4436912 .138395$

C -4.7254923 .7802091 .055456$

C $-4.033743 \quad 4.3213873 .364889$

C $-4.8362963 .056576-0.257438$

C $-4.2216203 .861793-1.412301$

C $-2.6859103 .862022-1.388118$

C $-2.1186734 .793674-2.459616$

C $-0.5844864 .727187-2.519647$

O $-2.2547402 .521563-1.638585$

O $-0.1922775 .391251-3.730370$

C $-0.1510673 .248171-2.617229$

C $0.0612515 .503586-1.365721$

O $1.2651093 .117613-2.512938$

C $-0.8371562 .332962-1.574731$

C $-0.5394010 .850686-1.902937$

O $-0.3259172 .636984-0.276286$

C $\begin{array}{llll}-5.858187 & 0.455187 & 2.235317\end{array}$

O -6.4626751 .1650213 .028815$

C $-6.581495-0.359550 \quad 1.233013$

C $-6.002175-0.9764610 .191359$

C $-6.698921-1.765706-0.830987$

C $-8.185102-1.960575-0.672094$

C -1.394990 -0.152415 -1.117602

C $-1.075509-1.588196-1.498447$

C $-1.881442-3.916159-1.100227$

O $-0.170374-1.896848-2.268644$

C $-2.850405-4.239106-2.191668$

C $-3.978549-4.939108-2.002359$

C $-4.974249-5.212582-3.091071$

C $-6.327245-4.596647-2.806979$

C $-7.373659-5.366459-2.463559$

C $-6.491327-3.105502-3.012067$

C $-5.985847-2.287556-1.851359$

N -1.923058 -2.485234 -0.863865

H $-2.747621-1.5302132 .528712$

H $-2.083813 \quad 0.719747 \quad 4.494183$

H $-4.289801 \quad 1.4636413 .874878$

H -2.7932011 .8932201 .236161$

H -2.139780 2.5876442 .699569

H -5.3353174 .6815131 .112100$

H -4.677301 5.1975853 .232325

H -4.4113983 .7655594 .228431$

H -3.028083 4.6860983 .598683

H $-4.4063702 .051700-0.217858$

H $-5.9050552 .915427-0.462664$

H $-4.5642703 .409078-2.351877$

H -4.600105 4.891304-1.390275

H $-2.3425774 .177173-0.396915$

H -2.451442 $5.826161-2.295052$

H $-2.5175494 .503796-3.442016$

H $0.7790565 .408344-3.764987$

H $-0.4242292 .894415-3.621460$

H -0.227569 $6.560563-1.409465$

H $1.1542985 .488393-1.440396$

H $-0.2197465 .122623-0.381662$

H $1.6624923 .614762-3.247193$

H $-0.727770 \quad 0.674130-2.970359$

H $0.5207660 .634895-1.721583$

H $-0.473304 \quad 1.8754020 .305097$

H $-7.655853-0.3950531 .386163$

H -4.925058 -0.8904840 .059987$

H -8.625480 -2.603106-1.437251

H $-8.706823-0.998624-0.727003$

H -8.411109 -2.4304880 .291690$

H -1.231953 -0.044438 -0.041119

H -2.458528 $0.019481-1.321150$

H $-2.136707-4.410591-0.157843$
H $-0.871183-4.218781-1.392645$

H -2.619368 -3.858241 -3.183856

H $-4.210481-5.322334-1.011399$

H -5.063008 -6.301590 -3.196566

H -4.614221 -4.851064 -4.062426

H $-8.356592-4.941472-2.290927$

H -7.270956 -6.440024 -2.344666

H -7.535281 -2.860930 -3.238654

H $-5.944207-2.802429-3.914271$

H -4.914919-2.090163-1.885412

H $-2.679777-2.125995-0.288905$

C $0.188964-1.7696243 .478643$

O $0.696339-0.4214573 .430052$

C $1.315944-0.2072522 .143114$

C $1.517472-1.5933581 .549438$

C $0.292142-2.3229082 .060856$

H $\quad 0.5840490 .3545351 .552113$

C 2.5912570 .6228242 .327575

H $1.580817-1.5799550 .457255$

H $2.429576-2.0571961 .942317$

C $0.410005-3.8349741 .994587$

H $-0.569216-2.0068061 .465422$

C 3.4199300 .7630301 .033912

$\begin{array}{llll}\text { O } & 2.173032 & 1.906711 & 2.799254\end{array}$

H $3.2037190 .178823 \quad 3.120513$

H $3.661737-0.2423020 .678439$

$\begin{array}{lllll}\text { O } & 2.620916 & 1.374707 & 0.022385\end{array}$

C $4.740076 \quad 1.544275 \quad 1.194753$

C $5.5372051 .703638-0.115337$

H $5.358091 \quad 1.0445141 .949848$

O $4.4608902 .857511 \quad 1.684440$

H $4.9533352 .294009-0.832863$

H 6.4224222 .3112970 .116234

H $0.557170-4.1647610 .961064$

H $1.257625-4.1981502 .585440$

H $-0.497629-4.3125112 .376802$

C -1.205710 -1.783914 4.109658

H 2.9728012 .4200453 .003318

H 2.3845132 .2647560 .335861

H 5.3120393 .3004451 .842292

H $-1.130264-1.3724145 .124833$

H -1.551986 -2.818190 4.222257

H $0.867548-2.3207744 .142993$

C $7.301718-1.589542-0.237206$

O $6.823721-0.3008390 .174740$

C $5.9794010 .380243-0.758847$

C $6.7156470 .606677-2.088205$

C $7.259238-0.712369-2.647010$

C $8.034810-1.500508-1.588681$

H $8.041729-1.8665770 .524895$

C $6.187044-2.645929-0.213986$

H $5.090944-0.224989-0.957402$

O $7.7869881 .532145-1.918123$

H $6.0187591 .054461-2.806182$

H $7.921357-0.504156-3.496383$

O $6.180198-1.516082-3.115809$

H $8.259140-2.507381-1.959837$

O $9.289000-0.843308-1.383046$

C $5.933385-3.1419371 .191530$

H $5.234221-2.299861-0.622391$

H $6.488100-3.522671-0.800210$

H $8.3815301 .177188-1.235274$

H $5.718520-1.012836-3.808162$

H $9.808654-1.387858-0.767428$

O $5.399710-2.1491921 .957783$

O $6.174791-4.2846081 .559867$

C $5.133779-2.5001083 .319363$

H $4.348243-3.2603433 .364420$

H $6.045799-2.8562803 .808089$

H $4.786814-1.6043843 .840633$

SCF Energy (B3LYP/6-31G**//MMFF $)=-3245.90821831$

08_00434

MM̄FF Geometry

C $-1.865683-2.696508-2.906037$

C $-2.760977-3.670174-2.676995$

C $-4.005974-3.581430-1.820257$

O $-4.120907-2.296055-1.169494$ 
C $-3.945023-4.663435-0.724425$

C $-5.188790-4.728918 \quad 0.142326$

C $-5.258206-4.3265891 .427641$

C $-6.400457-5.333402-0.520715$

C $-4.172440-3.7023652 .259849$

C $-4.577736-2.2994952 .730770$

C $-3.394045-1.5310213 .337592$

C $-3.852423-0.1648273 .850803$

C $-2.6810420 .686451 \quad 4.364039$

O $-2.422186-1.3607582 .303060$

O -3.1436802 .0439984 .477576$

C $-1.5564730 .692594 \quad 3.302161$

C $-2.262268 \quad 0.266877 \quad 5.777567$

O $-0.383322 \quad 1.335307 \quad 3.791603$

C -1.220994 -0.6942762 .708200$

C $-0.323475-0.6057661 .450178$

O $-0.465131-1.5035993 .615474$

C $-4.788121-1.312583-1.833997$

O $-5.275466-1.378902-2.951009$

C $-4.818230-0.120691-0.956460$

C $-5.286117 \quad 1.045121-1.426619$

C $-5.4061962 .296877-0.673843$

C -4.9352332 .3017410 .756508$

C $-0.944650 \quad 0.103917 \quad 0.243664$

C -0.5695641 .5691440 .110789$

C -1.076372 $3.566404-1.297265$

$\begin{array}{lllll}\text { O } & 0.270923 & 2.120062 & 0.815476\end{array}$

C $-2.1509884 .398620-0.673455$

C $-2.9833995 .179466-1.378622$

C $-4.0249526 .065062-0.758551$

C $-5.4078665 .828090-1.321248$

C $-5.9121846 .628114-2.275934$

C $-6.2413384 .727516-0.706604$

C $-5.9408463 .372244-1.289817$

N $-1.2685662 .178789-0.923160$

H -2.008464 -1.711607 -2.471320

H -2.593519 -4.639272 -3.144680

H $-4.879334-3.758794-2.459597$

H -3.051357 -4.504472 -0.112672

H -3.802449 -5.650879-1.185085

H $-6.205458-4.4492691 .951734$

H $-7.245354-5.4344250 .168781$

H $-6.732667-4.711120-1.357181$

H $-6.166467-6.333319-0.900479$

H -3.991829 -4.339408 3.134095

H $-3.226356-3.652687 \quad 1.717787$

H $-4.970114-1.7362371 .874833$

H -5.388018 -2.380651 3.466096

H -2.960943 -2.127535 4.148918

H -4.615430 -0.2788474 .630974$

H $-4.3419320 .381493 \quad 3.034187$

H -3.9198832 .0496475 .063437$

H -1.9262381 .3307332 .490628$

H -3.076854 0.4545086 .488111

H $-1.4147530 .860880 \quad 6.135511$

H -2.008939 -0.7918735 .856606$

H $0.081484 \quad 0.7142714 .376646$

H $0.655145-0.1841861 .709645$

H $-0.117113-1.6385811 .133876$

H $-0.649575-1.2303884 .524341$

H $-4.460607-0.2372830 .059439$

H -5.629186 $1.087697-2.459607$

H $-5.577751 \quad 1.6604081 .369147$

H -4.9351283 .2936551 .212963$

H $-3.904060 \quad 1.9391730 .828119$

H $-0.578490-0.386239-0.665551$

H $-2.033880 \quad 0.0096810 .245617$

H -1.097872 $3.613073-2.390758$

H $-0.0956043 .911189-0.954359$

H -2.226288 4.3803620 .411282

H $-2.8870575 .216028-2.461430$

H -3.724047 7.105701-0.937800

H -4.0503955 .9514010 .332349$

H $-6.9161836 .484487-2.661398$

H -5.329692 $7.440620-2.697551$

H -7.307035 $4.925164-0.887121$

H -6.1412504 .7579210 .381981$

H $-6.2484743 .272078-2.331745$
H $-1.9728711 .647480-1.427267$

C $0.620588-2.444597-2.980191$

O $0.681053-1.003401-2.960403$

C $2.051477-0.587380-3.156198$

C $2.887074-1.854946-3.064037$

C $1.931544-2.891670-3.620648$

H $2.096902-0.181254-4.174885$

C $2.3815700 .508002-2.135023$

H $3.820775-1.784961-3.630233$

H $3.136381-2.085382-2.021626$

C $2.330311-4.319175-3.289032$

H $1.877116-2.774242-4.711295$

C $3.8821750 .848236-2.042029$

O $1.6269211 .658286-2.524487$

H $2.0127290 .207452-1.146905$

H $4.404623-0.058708-1.726803$

O $4.382724 \quad 1.183310-3.335780$

C $4.2145911 .982209-1.049833$

C $5.7272972 .220536-0.863997$

H $3.7508901 .758259-0.082113$

O $3.6377273 .203206-1.518427$

H $6.1888672 .461989-1.829544$

H $5.8442173 .119141-0.243609$

H $3.312064-4.552372-3.713988$

H $2.386506-4.479548-2.207298$

H $1.608143-5.032673-3.697548$

C $-0.628773-2.886970-3.744140$

H $1.7388672 .331934-1.832646$

H $3.9165201 .986030-3.627032$

H $3.7884643 .880062-0.836733$

H $-0.535827-3.937370-4.042131$

H $-0.741919-2.288599-4.656106$

H $\quad 0.573845-2.778971-1.935178$

C $6.350775-0.2939131 .817243$

O 5.8650990 .8348121 .081126

C $6.4604941 .042918-0.202713$

C $7.9673611 .292962-0.051896$

C 8.6328420 .1378980 .707330

C $7.877669-0.2132021 .994632$

H $5.894820-0.1988482 .811452$

C $5.866447-1.6198551 .209235$

H $6.3187200 .153729-0.822303$

O 8.1823832 .5150540 .649751

H $8.411207 \quad 1.399741-1.048847$

H 9.6641950 .4109240 .962195

O $8.680506-1.017411-0.125334$

H $8.248612-1.1602252 .402463$

O $\quad 8.1602880 .7887202 .977216$

C $4.357573-1.7577861 .270398$

H $\quad 6.158497-1.7229640 .161138$

H $6.296203-2.4522961 .778643$

H 9.1428802 .6562240 .705231

H $9.191270-0.785836-0.919723$

H 7.7769391 .6266502 .665977

O $4.009169-3.0085750 .844733$

O $3.580444-0.8858751 .634136$

C $2.605697-3.2892890 .854093$

H $2.458830-4.3046810 .476897$

H $2.215832-3.2334251 .874954$

H $2.074814-2.591746 \quad 0.199879$

SCF Energy (B3LYP/6-31G**//MMFF) $=-3245.90804806$

08_00435

MM̄FF Geometry

C $-1.110962-3.559220-0.862066$

C $-2.055082-2.614821-0.746226$

C -2.791402 -2.018862 -1.916995

O $-2.401022-0.630701-2.021639$

C $-4.305671-2.050210-1.655506$

C $-5.130942-1.815287-2.903606$

C $-5.672557-0.633983-3.258741$

C $-5.373629-3.041299-3.746013$

C $-5.5148660 .700663-2.585343$

C $-6.7969091 .176363-1.887991$

C $-7.0437390 .489800-0.535302$

C -8.3577040 .9732570 .082670$

C $-8.572930 \quad 0.4054281 .494081$

$\begin{array}{llll}\text { O } & -5.950016 & 0.826403 & 0.324401\end{array}$ 
$\begin{array}{llll}\text { O } & -9.635322 & 1.148240 & 2.111627\end{array}$

C $-7.3014100 .654102 \quad 2.332817$

C $-9.031199-1.057316 \quad 1.456955$

O

C $-5.9985750 .220902 \quad 1.621731$

C -4.7676650 .7169992 .419797$

O $-5.963243-1.2056601 .544276$

C $-1.243635-0.344075-2.673907$

O $-0.501183-1.126173-3.244637$

C -1.014078 1.108333 -2.526571

C $0.1829281 .625351-2.837279$

C $0.555506 \quad 3.028661-2.683963$

C $1.4798663 .529744-3.759505$

C -3.4344650 .5608641 .672936$

C $-2.253358 \quad 1.055345 \quad 2.490334$

$\begin{array}{llll}C & 0.198994 & 1.472491 & 2.299329\end{array}$

O $-2.352147 \quad 1.4316503 .655180$

C 0.3346752 .9412672 .042627

C 1.3565543 .4911871 .369194

C 1.4812844 .9666921 .108731

C $1.6757835 .301601-0.356033$

C $2.8494145 .780207-0.802491$

C $0.4909685 .176193-1.289560$

C $0.1548893 .749962-1.617719$

N -1.0701901 .0136111 .762572$

H -0.849616 -3.939575 -1.846350

H $-2.292559-2.2268140 .240454$

H -2.552711 -2.545224 -2.849517

H $-4.562280-1.305251-0.894671$

H -4.600698 -3.019729-1.231862

H $-6.277433-0.601754-4.164453$

H -5.987337-2.826842 -4.627395

H $-4.425285-3.457885-4.099300$

H -5.893374 -3.807092 -3.160820

H $-4.6705510 .716167-1.890781$

H $-5.2606501 .425487-3.369530$

H $-6.6945762 .256637-1.719588$

H -7.660930 $1.032408-2.548411$

H $-7.070254-0.595240-0.683983$

H -9.206948 $0.724446-0.565794$

H -8.345861 2.0702290 .151612

H $-10.426498 \quad 1.060717 \quad 1.552761$

H $-7.245351 \quad 1.7340852 .529074$

H $-9.978187-1.1478440 .911473$

H $-9.230267-1.4345612 .466112$

H $-8.310081-1.7209940 .975534$

H -8.221128 0.3262304 .021456

H -4.8919291 .7832322 .651164$

H -4.7059640 .1812043 .375518$

H -5.041012 -1.504645 1.529352

H - $-1.8228821 .714868-2.132095$

H $0.9655120 .982242-3.234142$

H $0.9902353 .470661-4.737938$

H $2.3934032 .925242-3.794779$

H $1.7899654 .567061-3.615589$

H $-3.250635-0.4903101 .436068$

H $-3.458644 \quad 1.1271380 .734871$

H $0.9894640 .890368 \quad 1.814914$

H $\quad 0.229262 \quad 1.279783 \quad 3.376407$

H -0.4468943 .5862542 .437889$

H 2.1457782 .8479210 .989106

H 2.3325095 .3341381 .696578

H 0.6041725 .5107481 .481217

H $2.9904206 .053158-1.842983$

H $3.6977065 .910163-0.139101$

H $0.6520485 .755087-2.206396$

H $-0.3853805 .643329-0.821860$

H $-0.490357 \quad 3.273872-0.880447$

H -1.1024860 .7582770 .779503$

C $1.041561-3.4993190 .414086$

O $1.823258-3.848742-0.745931$

C $3.099925-4.334993-0.312636$

C $3.258660-3.8874801 .131190$

C $1.829266-4.0011801 .626666$

H $3.023431-5.431520-0.316848$

C $4.217047-3.939143-1.280390$

H $3.957881-4.5165611 .689336$

H $3.599174-2.8500591 .199329$
C $1.558716-3.2171662 .899256$

H $1.606053-5.0614101 .807139$

C $4.670747-2.463013-1.299613$

O $5.359779-4.737808-0.939659$

H $3.922500-4.236449-2.294854$

H $5.231119-2.250529-0.382578$

O $5.624783-2.338611-2.366997$

C $3.572042-1.402459-1.493301$

C $4.0932000 .011565-1.820413$

H $2.945401-1.358085-0.597285$

O $2.735598-1.805175-2.581654$

H $4.5412970 .008699-2.822594$

H $3.2172840 .670041-1.892685$

H $2.208232-3.5618903 .710355$

H $1.736341-2.1463022 .756054$

H $0.519919-3.3434163 .220367$

C $-0.352623-4.1193560 .307710$

H $6.064683-4.519906-1.573362$

H $5.146351-2.470814-3.203636$

H $1.985422-1.187624-2.619441$

H $-0.909164-3.9512991 .237286$

H $-0.277692 \quad-5.204706 \quad 0.167747$

H $0.968268-2.4041730 .429196$

C $5.203709 \quad 1.2799281 .520866$

$\begin{array}{llllll}\text { O } & 4.407621 & 0.769020 & 0.440444\end{array}$

C $5.0886020 .591762-0.805592$

C $5.6753161 .924362-1.297219$

C $6.5927752 .539950-0.238091$

C $5.9160672 .589362 \quad 1.134012$

H 4.4809031 .5254202 .309682

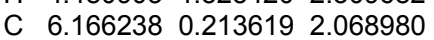

H $5.908592-0.119256-0.673777$

O $4.6162502 .828012-1.604493$

H $6.2370121 .744180-2.221883$

H $6.8697763 .555127-0.547372$

O $7.784774 \quad 1.763394-0.163360$

H 6.6486802 .8478121 .906696

O 4.9527703 .6451421 .133932

C $5.443256-0.8560092 .860943$

H $6.721182-0.2870461 .271745$

H 6.8784450 .6848482 .756582

H $5.0173923 .645657-1.945232$

H 8.3673262 .1822600 .492954

H 4.2853763 .4354090 .458825

O $6.362486-1.7461163 .340005$

O $4.235801-0.9036833 .048730$

C $5.834958-2.8012184 .150266$

H $6.669169-3.4257214 .480679$

H $5.334945-2.3886785 .031783$

H $5.144616-3.4173533 .568309$

SCF Energy (B3LYP/6-31G**//MMFF) $=-3245.91092220$

08_00436

MM̄FF Geometry

C -0.183966 -4.4294491 .618624$

C - $-1.504893-4.2518591 .776252$

C $-2.198994-3.3372662 .757451$

O $-2.747995-2.2231812 .012480$

C $-1.307808-2.7556223 .869345$

C $-2.098915-1.996844 \quad 4.918298$

C $-2.160883-0.6532115 .002600$

C -2.799352 -2.852068 5.942667

C $-1.5271690 .345464 \quad 4.073746$

C $-2.4409561 .554852 \quad 3.831674$

C -1.761446 $2.600342 \quad 2.935584$

C -2.5733943 .8958612 .906871$

C $-1.945416 \quad 4.936318 \quad 1.968823$

O $-1.6575862 .062461 \quad 1.611970$

O $-2.9079995 .980638 \quad 1.760282$

C -1.688667 4.2768800 .599805

C -0.7109995 .6005162 .589916$

O $-0.970734 \quad 5.157259-0.262653$

C -0.9629292 .9118590 .687820$

C $-0.978487 \quad 2.240839-0.704211$

O 0.3922293 .1213941 .085632

C $-3.994936-2.3588371 .489395$

O $-4.721972-3.3367091 .556939$

C $-4.319413-1.0955870 .790944$ 
C $-5.354909-1.063253-0.060515$ C $-5.778800 \quad 0.086581-0.862180$ C $-5.1236701 .412006-0.580441$ C $-0.4932630 .788245-0.729410$ C $-0.7269240 .215703-2.112349$ C $-2.458076-0.941191-3.486844$ O $0.0818680 .385091-3.022211$ C -3.494393 $0.015270-3.991457$ C $-4.716465-0.360576-4.398857$ C $-5.759670 \quad 0.599521-4.897804$ C $-7.1058550 .411653-4.229595$ C $-8.147019-0.101167-4.907333$ C $-7.2680080 .888106-2.800106$ C $-6.721165-0.104355-1.809092$ N $-1.942229-0.440281-2.225848$ H $0.522205-3.8681132 .222930$ H $-2.175240-4.8289491 .140847$ H -3.010273 -3.909976 3.224481 H $-0.543226-2.1036493 .433928$ H $-0.759356-3.5621084 .373710$ H $-2.718150-0.2200875 .832187$ H $-3.302849-2.2539416 .709750$ H -3.558219 -3.4802715 .466708$ H $-2.079360-3.5005916 .452407$ H -0.5916010 .6884644 .530843$ H $-1.278802-0.1055553 .109164$ H $-3.373107 \quad 1.2125593 .363834$ H -2.700163 2.0112204 .795203 H -0.7559012 .7917923 .328697$ H -2.684596 4.3100973 .916658 H -3.592646 3.6802592 .556326 H -3.132170 6.3590372 .627757 H -2.669069 4.1150010 .129392 H -0.9763136 .0965523 .531239$ H $-0.3165696 .387843 \quad 1.938088$ H 0.0943904 .8948532 .804051 H $-1.4677475 .992300-0.303105$ H -2.000682 2.262361 -1.099570 H $-0.3602332 .821558-1.401600$ H 0.9136592 .3430280 .833935 H -3.678836 -0.2395580 .970849$ H $-5.930807-1.975490-0.212750$ H $-4.068484 \quad 1.381120-0.862615$ H $-5.193846 \quad 1.6599950 .484929$ H -5.577758 2.247696 -1.115976 H $0.5758730 .713781-0.512284$ H -1.0151640 .1770660 .015035$ H $-2.874123-1.935067-3.295512$ H $-1.644831-1.029658-4.213535$ H -3.224086 $1.068353-4.026924$ H $-4.977848-1.416363-4.389715$ H $-5.8536190 .451606-5.981428$ H $-5.438906 \quad 1.640145-4.763417$ H $-9.121246-0.216441-4.443847$ H -8.047883 $-0.422236-5.938988$ H -8.331904 $1.031181-2.569805$ H $-6.8155081 .879181-2.712574$ H -7.151101 -1.101990 -1.913090 H $-2.559218-0.479819-1.420620$ C $1.140958-4.763097-0.536351$ O $2.266238-3.986634-0.081646$ C $2.366990-2.807751-0.903306$ C $1.426437-3.037793-2.082374$ C $0.315969-3.831440-1.422416$ H $1.977562-1.975596-0.302450$ C $3.819816-2.495681-1.294912$ H $1.083686-2.102564-2.530447$ H $1.897287-3.635187-2.870892$ C $-0.590618-4.550913-2.404339$ H $-0.285498-3.142208-0.816755$ C $4.713408-2.151401-0.087581$ O $4.400743-3.599222-1.988284$ H $3.803104-1.646171-1.985620$ H $4.256258-1.3437560 .489235$ O $4.760427-3.2807750 .794186$ C $6.165556-1.785472-0.448833$ C $6.360382-0.660032-1.482846$ H $6.676027-2.686497-0.811052$
O $6.831111-1.4165780 .765080$

H $7.437031-0.586004-1.686293$

H $5.880135-0.953177-2.422702$

H $-1.081312-3.834617-3.070889$

H $-0.030227-5.257025-3.026121$

H $-1.369509-5.110095-1.876536$

C $0.409004-5.4076680 .642002$

H $4.002435-3.639688-2.873344$

H $5.315251-3.0322761 .553376$

H $7.764047-1.2482740 .548147$

H $1.118959-6.0286641 .202907$

H $-0.364614-6.0846740 .259994$

H $1.564822-5.572173-1.146807$

C $3.8366591 .933402-0.498062$

$\begin{array}{lllll}\text { O } & 4.450161 & 0.720232 & -0.950239\end{array}$

C $5.8747410 .739124-1.045703$

C $6.3133041 .822549-2.047042$

C $5.7280813 .192588-1.690790$

C $4.2240063 .115782-1.401328$

H $2.7614091 .763172-0.626566$

C $4.1002852 .194507 \quad 0.992345$

H $6.3013920 .962084-0.062086$

O $5.909293 \quad 1.467680-3.368957$

H $7.4081041 .879751-2.050917$

H $5.9081823 .882673-2.524178$

O $6.418784 \quad 3.699125-0.552817$

H $3.8654234 .049414-0.952548$

O $3.5242752 .950162-2.638777$

C $3.660388 \quad 1.052842 \quad 1.880734$

H 5.1649892 .3507301 .188300

H 3.5765083 .0977231 .325622

H $4.9450041 .341062-3.360882$

H $6.0563734 .580144-0.357815$

H $3.7215813 .724597-3.192747$

$\begin{array}{llll}\text { O } & 2.363670 & 0.719955 & 1.632958\end{array}$

\begin{tabular}{lllll}
\hline & 4.384427 & 0.539312 & 2.724792
\end{tabular}

C $1.841029-0.3684012 .400384$

H $1.681664-0.0494143 .434005$

H $0.881531-0.6563401 .963980$

H $2.510475-1.2330792 .362333$

SCF Energy (B3LYP/6-31G**//MMFF) $=-3245.90643734$

08_00437

MMFF Geometry

C $3.484681-1.0802244 .168629$

C $3.399479-0.9658652 .834027$

C $4.602415-1.0254381 .926579$

$\begin{array}{lllll}\text { O } & 4.726128 & 0.256863 & 1.271376\end{array}$

C $4.385046-2.1055910 .852370$

C $5.648979-2.4969290 .115252$

C $5.944315-2.136419-1.148772$

C $6.580667-3.405547 \quad 0.875927$

C $5.164435-1.234269-2.064427$

C $4.467913-2.004707-3.195646$

C $3.196822-2.736905-2.736083$

C $2.610042-3.573761-3.873946$

C $1.274273-4.222538-3.480210$

O $2.252238-1.741870-2.330766$

O $0.685378-4.717787-4.692065$

C $0.343535-3.135890-2.899817$

C $1.483230-5.436194-2.566931$

O $-0.852627-3.707993-2.375059$

C $1.018168-2.258390-1.818857$

C $0.108817-1.052041-1.485673$

O $1.217833-3.038625-0.639278$

C $5.972993 \quad 0.7820101 .153118$

$\begin{array}{llll}\text { O } & 7.009295 & 0.313885 & 1.606550\end{array}$

C $5.9763772 .045621 \quad 0.381845$

C $4.9259912 .521034-0.305302$

C $4.9109613 .751880-1.103167$

C $6.1674414 .583823-1.131083$

C $0.7597020 .000698-0.577924$

C $-0.104718 \quad 1.242116-0.438914$

C $-0.054967 \quad 3.537395 \quad 0.532820$

O $-1.232913 \quad 1.336556-0.914563$

C $0.2883144 .436338-0.611463$

C $1.0657865 .523456-0.500741$

C $1.4240726 .404529-1.661923$ 
C $2.9142186 .449890-1.919942$

C $3.6352807 .534175-1.587984$

C $3.5494595 .283803-2.646576$

C $3.7823854 .087900-1.762786$

N $0.534891 \quad 2.2327760 .293990$

H $4.475757-1.1652014 .612120$

H $2.430656-0.8627732 .355194$

H $5.494872-1.2481062 .523264$

H $3.624680-1.7590880 .144850$

H $3.971394-3.0155611 .308185$

H $6.870386-2.511274-1.583382$

H $7.444866-3.7117540 .276695$

H $6.963390-2.9054371 .770494$

H $6.055956-4.3161381 .183378$

H $4.449306-0.610216-1.520383$

H $5.880820-0.533755-2.512534$

H $4.194738-1.275922-3.970005$

H $5.169214-2.714766-3.651300$

H $3.439809-3.378898-1.882826$

H $3.324815-4.337825-4.204330$

H $2.437337-2.928996-4.747519$

H $-0.138509-5.179023-4.461296$

H $\quad 0.039715-2.486681-3.733028$

H $2.081615-6.199295-3.078929$

H $\quad 0.529705-5.917330-2.322720$

H $1.990918-5.187584-1.632517$

H -1.314322 -4.150627 -3.106708

H $-0.177987-0.555333-2.422075$

H -0.812694 -1.403472 -1.005793

H $1.248243-2.4503110 .131094$

H 6.9316882 .5619080 .384796

H $3.9935131 .959726-0.299585$

H $6.0698965 .501885-1.713899$

H $6.9946274 .016216-1.571702$

H $6.4495184 .889655-0.117339$

H $0.922111-0.4064810 .425169$

H $1.7280230 .311403-0.986352$

H $0.3385153 .905572 \quad 1.485243$

H -1.142182 3.4407550 .616443

H $-0.1214094 .173207-1.584079$

H 1.4711555 .7947690 .471227

H $1.0432117 .411262-1.446057$

H $0.9111306 .087148-2.578562$

H $4.6974267 .594410-1.799109$

H $3.1797328 .386541-1.094857$

H $4.4813095 .592306-3.134568$

H $2.8996864 .980334-3.477892$

H $2.9213363 .424053-1.686741$

H 1.4995972 .0916550 .580151

C $0.975101-1.5237854 .615152$

O $0.372491-0.385706 \quad 3.968977$

C -1.038209 -0.3594194 .287266$

C $-1.333267-1.6664275 .005798$

C $-0.016393-1.9367205 .703074$

H -1.1734130 .4911974 .966986$

C $-1.830990-0.1435252 .993413$

H -2.176901 -1.5845395.697628

H -1.556712 -2.4658754 .288147$

C $0.130448-3.3751256 .169658$

H $0.071134-1.2640026 .566944$

C $-3.354548-0.2828553 .153801$

O $-1.548345 \quad 1.1784572 .526564$

H -1.463406 -0.8357662 .228034$

H -3.606612 -1.306536 3.452661

O -3.7831160 .5606414 .230992$

C $-4.1770690 .094121 \quad 1.903304$

C $-3.804664-0.7225460 .651671$

H $-4.071557 \quad 1.1652531 .697050$

O $-5.558406-0.1175772 .214062$

H $-2.731317-0.6391590 .460633$

H $-4.036805-1.7772970 .846341$

H - $0.658737-3.6290846 .884849$

H $0.063106-4.0775485 .331953$

H $1.094220-3.5320756 .663402$

C $2.354794-1.1469735 .164197$

H $-0.582111 \quad 1.2577752 .447530$

H -3.549277 1.4754643 .997405

H $-5.687159-1.0690072 .368222$
H $2.650331-1.8954005 .909359$

H $2.304584-0.1799415 .679480$

H $1.059679-2.3198533 .863668$

C -6.798494-0.142481-1.527666

O $-5.943440-0.564083-0.456992$

C $-4.554588-0.264635-0.616564$

C $-4.010005-0.955284-1.877772$

C $-4.836997-0.597207-3.117172$

C $-6.340428-0.748102-2.866111$

H -7.779839 -0.567967 -1.281397

C $-6.9523611 .382021-1.572409$

H $-4.4237040 .818937-0.710698$

O $-4.002858-2.371257-1.704112$

H $-2.969813-0.648457-2.029764$

H -4.545896 -1.249405 -3.949954

O $\quad-4.5661150 .747587-3.501409$

H -6.906621 -0.301047-3.691605

O $-6.643066-2.146504-2.857664$

C $-7.6292181 .885041-0.319476$

H $-6.0002241 .907003-1.691691$

H $-7.5775151 .685552-2.420562$

H -4.913852 -2.651291-1.510147

H -3.613106 $0.815535-3.681751$

H -7.607362 -2.237825 -2.770773

O -6.7067962 .4327110 .521821$

O $-8.8303091 .781265-0.106119$

C -7.2192112 .9215101 .765081$

H -7.9134463 .7482741 .587440$

H -7.710974 2.1154782 .318032

H -6.3775453 .2892962 .358070$

SCF Energy $($ B3LYP/6-31G**//MMFF $)=-3245.90879079$

08_00438

MMFF Geometry

C $-2.6113293 .601840-1.116834$

C $-3.3553624 .166827-0.154116$

C -3.8321423 .5036561 .120433$

O -3.4144432 .1204601 .198566$

C -5.3699153 .5468041 .179202$

C -5.9399393 .0346562 .488773$

C -6.5708631 .8538662 .647875$

C -5.7961393 .9720683 .660967$

C -6.8148170 .7929541 .611843$

C $-6.139788-0.5278742 .003266$

C $-6.180915-1.5477950 .856438$

C $-5.589308-2.8858111 .301255$

C $-5.495832-3.8902460 .142951$

O $-5.416612-1.010153-0.228382$

O $-4.641343-4.9662940 .561191$

C $-4.806119-3.209497-1.059439$

C $-6.857482-4.518068-0.177606$

O $-4.840569-4.053715-2.207177$

C $-5.397169-1.827421-1.406295$

C $-4.583522-1.065499-2.483162$

O $-6.719829-2.002847-1.929610$

C -2.1757541 .8673561 .707569$

O -1.3582542 .6880322 .092975$

C $-1.9782890 .399783 \quad 1.710001$

C $-0.777978-0.1135072 .020187$

C $-0.446000-1.5371602 .130460$

C $-1.542701-2.5328701 .860386$

C -3.130104 -0.745034 -2.115782

C $-2.127936-1.786649-2.583397$

C $0.272646-2.411830-2.347981$

O $-2.414472-2.702955-3.348915$

C $0.466382-3.351233-1.199676$

C $1.638833-3.507537-0.567846$

C $1.866229-4.4524870 .573758$

C $2.361022-3.7480811 .816428$

C $3.675456-3.6421782 .074975$

C $1.330833-3.2627282 .808318$

C $0.805540-1.8857582 .497373$

N $-0.860597-1.550616-2.067575$

H $-2.3143312 .560077-1.034448$

H $-3.6256005 .215831-0.262878$

H -3.4190604 .0605681 .970634$

H -5.7792982 .9906140 .329834$

H -5.7198194 .5789911 .039496$ 
H $-6.957702 \quad 1.6089603 .636614$ H -6.2917023 .5923734 .560928$ H $-4.740876 \quad 4.1231133 .907262$ H -6.242840 4.9439693 .427146 H $-7.8964770 .634911 \quad 1.523611$ H $-6.470505 \quad 1.1051500 .624022$ H $-5.095345-0.3295642 .273858$ H $-6.634181-0.9422892 .890658$ H -7.222714 -1.674148 0.538975 H $-6.167287-3.3093882 .132009$ H $-4.578603-2.7211331 .696325$ H $-5.024490-5.3583881 .364566$ H $-3.749979-3.087316-0.790384$ H -7.248561 -5.0508080 .697607$ H $-6.769976-5.269522-0.969932$ H $-7.608244-3.784386-0.477762$ H -4.437826 -4.902236 -1.955051 H $-4.650566-1.587130-3.445647$ H -5.084981 -0.099769-2.642043 H -6.953937 -1.215702 -2.448655 H $-2.834512-0.2207031 .474592$ H $\quad 0.0377320 .572650 \quad 2.244638$ H -1.994455 -2.3542190 .878712$ H -2.321754 -2.458082 2.626225 H -1.197158 -3.5688661 .845769$ H $-2.8467840 .192154-2.612124$ H -3.021472 $-0.589721-1.038224$ H $1.140091-1.763814-2.505743$ H $0.089804-2.980548-3.264954$ H $-0.392523-3.937767-0.882347$ H $2.503197-2.944833-0.908853$ H $2.605609-5.1968970 .249728$ H $0.960191-5.0247670 .807809$ H $4.037591-3.1664382 .980258$ H $4.418462-4.0227581 .382389$ H $1.780548-3.2122953 .809823$ H $0.537246-4.0087762 .910634$ H $1.537554-1.0937512 .662183$ H $-0.725125-0.789607-1.407896$ C $-0.6434534 .728292-2.260351$ O $0.1661103 .605326-1.853160$ C $0.9671014 .009492-0.728473$ C $1.0726875 .522237-0.848898$ C $-0.3377245 .868849-1.284173$ H $0.402397 \quad 3.7573810 .178321$ C $2.3149423 .280620-0.693270$ H 1.3608135 .9991350 .092584 H $1.7949165 .813230-1.620922$ C $-0.4651627 .251909-1.899207$ H $-0.9917455 .816903-0.405612$ C $2.1844351 .766025-0.440367$ O $3.0245193 .511207-1.908861$ H 2.9103413 .7242710 .111527 H $1.689721 \quad 1.6047710 .524583$ O $1.3221611 .178405-1.421816$ C $3.5122230 .983522-0.464489$ C $4.548174 \quad 1.4863740 .555920$ H $3.940740 \quad 0.992892-1.473528$ O $3.205866-0.379943-0.158812$ H 4.7283492 .5560430 .411565 H 4.1376001 .3374701 .562669 H $-0.1847248 .022623-1.173837$ H $0.1828537 .363578-2.775031$ H -1.495681 $7.442843-2.214996$ C $-2.1197604 .320815-2.342741$ H $2.4414393 .255360-2.643658$ H $1.7441391 .284650-2.291082$ H $2.514879-0.665090-0.780268$ H -2.249591 $3.631185-3.186578$ H $-2.7394155 .198701-2.560037$ H $-0.3043064 .998904-3.268445$ C $6.864011-1.4583750 .661691$ O $5.711887-0.6181710 .800602$ C $5.902554 \quad 0.7537590 .448373$ C $6.9662001 .382647 \quad 1.362277$ C 8.2745520 .5835261 .322936 C $8.035058-0.9209651 .500952$ H $6.560371-2.4227601 .088364$
C $7.221919-1.694016-0.811033$

H $6.2325650 .820473-0.594421$

O 6.4745231 .4279012 .700600

H 7.1433092 .4159211 .040889

H 8.9432410 .9365962 .117396

O $8.932794 \quad 0.8026720 .078678$

H $8.943014-1.4868661 .261690$

O $7.727515-1.1811062 .873985$

C $6.059745-2.306293-1.560783$

H $7.514147-0.767731-1.313369$

H $8.070954-2.381178-0.901390$

H $7.154674 \quad 1.8537863 .249407$

H $9.1030621 .756808-0.000024$

H $8.490061-0.8942873 .404670$

O $5.725468-1.516252-2.621251$

O $5.514293-3.355094-1.243493$

C $4.629507-1.976643-3.417373$

H $4.806893-2.999052-3.764801$

H $3.701985-1.918145-2.842487$

H $4.543642-1.321565-4.288342$

SCF Energy (B3LYP/6-31G**//MMFF) $=-3245.89865090$

08_00439

MMFF Geometry

C $-2.252798 \quad 4.840586-1.256042$

C $-3.1270803 .838842-1.080861$

C -4.0157073 .7184340 .130541$

O -3.6996092 .4625790 .778094$

C $-5.4991323 .699860-0.282249$

C -6.4583693 .5906560 .891260$

C -7.3503062 .5922121 .053371$

C -6.3926634 .7048741 .903806$

C -7.6051651 .4422100 .119641$

C -6.6316760 .2899330 .358852$

C $-6.764271-0.831443-0.678868$

C $-8.020175-1.680379-0.472451$

C -8.064291-2.884358 -1.429575

O $-5.598854-1.650631-0.535692$

O $-9.064603-3.795061-0.947816$

C $-6.711976-3.629401-1.373179$

C $-8.500130-2.470233-2.840416$

O $-6.652956-4.657757-2.358743$

C $-5.490207-2.694449-1.510875$

C $-4.179271-3.466284-1.231040$

O $-5.445000-2.177308-2.843217$

C -2.6161272 .4502401 .602800$

O -1.9070503 .4004061 .895167$

C -2.4242571 .0631162 .084299$

C -1.3260910 .7687972 .796738$

C $-0.984748-0.5286713 .385448$

C $-1.933685-1.6736523 .153367$

C $-2.961637-2.548525-1.045616$

C $-1.673790-3.325649-0.846297$

C $0.727354-2.966291-0.280686$

O $-1.588455-4.540706-0.999923$

C $0.879324-3.4013671 .142561$

C $1.808671-2.9038391 .971290$

C $2.028295-3.3905623 .372706$

C $2.013068-2.2743684 .390148$

C $3.157183-1.7831204 .895858$

C $0.669757-1.7972324 .892329$

C $0.145227-0.6214094 .117922$

N $-0.622125-2.486776-0.500846$

H $-2.2098425 .640101-0.521150$

H -3.169289 $3.031919-1.808700$

H $-3.8387564 .547173 \quad 0.827167$

H -5.656090 $2.872594-0.983358$

H $-5.7394414 .618278-0.834141$

H -7.9835232 .6021221 .939294$

H -7.2176024 .6603632 .623107$

H -5.4589794 .6572342 .472348$

H -6.450292 5.6769381 .403230

H $-8.636347 \quad 1.101903 \quad 0.271845$

H $-7.556467 \quad 1.779837-0.921817$

H -5.6009740 .6635850 .327111$

H $-6.759063-0.1165891 .370947$

H $-6.756960-0.381287-1.678458$

H $-8.928954-1.077094-0.581180$ 
H $-8.035074-2.0610100 .558529$ H $-9.910756-3.317168-0.907897$ H $-6.654743-4.131309-0.397047$ H -9.501563 -2.023808 -2.816139 H -8.577070 -3.340084 -3.502064 H -7.827865 -1.743336 -3.301009 H -7.430796 -5.227294 -2.230122 H -4.289064 -4.055535 -0.311197 H -3.982777 -4.171236 -2.048524 H -4.526605 -1.973822 -3.079133 H $-3.189296 \quad 0.332224 \quad 1.849031$ H -0.6056341 .5639762 .986680$ H $-2.900049-1.4738803 .628901$ H -1.570967 -2.626734 3.543924 H -2.097165 -1.827473 2.082132 H -2.821285 -1.905035 -1.920525 H -3.111366 -1.904951 -0.171447 H $1.407303-2.149590-0.540925$ H $\quad 0.924269-3.811629-0.948095$ H $0.221351-4.192551 \quad 1.494350$ H $2.483391-2.1346341 .607622$ H $2.999441-3.9025913 .395653$ H $1.289166-4.1508123 .653984$ H $3.151198-1.0037185 .650801$ H $4.124190-2.1438484 .561196$ H $0.766057-1.4783485 .939421$ H - $0.031081-2.6357754 .926891$ H $\quad 0.7630750 .271594 \quad 4.226305$ H $-0.807730-1.503348-0.326282$ C $0.1670514 .630465-1.998861$ O $0.2921113 .281399-1.501660$ C $1.1493383 .296208-0.338861$ C $1.8504964 .647014-0.360913$ C $0.7662955 .541578-0.927483$ H $\quad 0.479673 \quad 3.225676 \quad 0.526394$ C $2.0907012 .086385-0.381131$ H 2.1892034 .9627490 .630371 H $2.7191264 .625076-1.031038$ C $1.2819076 .865311-1.463878$ H $0.0435605 .741592-0.127918$ C 3.1227552 .0746580 .760064 O $1.2985820 .898055-0.287573$ H $2.5954002 .054973-1.353052$ H 3.7620352 .9626130 .696229 O 2.4311042 .1746722 .012250 C 4.0185110 .8220630 .820191 C $4.7890710 .566359-0.480407$ H $3.419848-0.0538081 .087189$ O 4.9672141 .0096141 .875372 H $4.0951830 .333148-1.296902$ H $5.2969721 .492265-0.784603$ H $1.7575487 .444555-0.665726$ H $2.0221056 .716759-2.257289$ H $0.4625557 .463844-1.874183$ C -1.284740 $4.914713-2.403377$ H $0.6421620 .930148-1.004806$ H 1.8267491 .4148302 .073380 H $4.463717 \quad 1.1747522 .691071$ H -1.579382 4.191959-3.175088 H -1.357224 $5.909793-2.857004$ H $0.7836924 .671679-2.906539$

C $7.561003-1.523842-1.828415$ O $6.470304-0.603117-1.691626$ C $5.864266-0.535002-0.393326$ C $5.311965-1.9103820 .007604$ C $6.385562-2.999276-0.090599$ C $7.134147-2.945472-1.425941$ H $7.783486-1.536537-2.903461$ C $8.820727-1.034781-1.096101$ H $6.622119-0.2144550 .328820$ O $4.208087-2.259391-0.824764$ H $4.938976-1.8644171 .036200$ H $5.914875-3.9839420 .019291$

O $7.324221-2.8454970 .969812$ H $8.015303-3.597225-1.400291$ O $6.276483-3.440837-2.458656$ C $9.3759960 .241173-1.695642$ H $8.626480-0.837578-0.038836$
H $9.603851-1.797911-1.174988$

H $4.512081-2.236218-1.748345$

H $6.835108-2.9129911 .807532$

H $6.048901-4.359419-2.234672$

O $10.497147 \quad 0.601627-1.002126$

O $8.8968490 .846249-2.644341$

C $11.1398391 .798767-1.451401$

H $10.4605242 .651459-1.358012$

H $12.0127271 .974700-0.817035$

H $11.4756411 .682829-2.486325$

SCF Energy $(B 3 L Y P / 6-31 G * * / / M M F F)=-3245.92174697$

08_00440

MMFF Geometry

C $1.3170803 .288267-1.553954$

C $1.9839534 .339276-1.051797$

C $3.2420114 .288826-0.210819$

O $3.7765232 .947600-0.127733$

C 2.9235464 .7703031 .216677

C 4.1480944 .8940572 .103844

C 4.4647064 .0519933 .108267

C 5.0245116 .0882231 .822203

C 3.7290262 .8157563 .545655

C 4.6050061 .5680283 .375394

C 3.8173300 .2700063 .609747

C $4.738777-0.9438543 .471926$

C $3.971931-2.2727473 .540276$

O 2.7763040 .2146242 .630193

O $4.845484-3.3104853 .068086$

C $2.776629-2.2137262 .565039$

C $3.600826-2.6406984 .981450$

O $1.949952-3.3663222 .704037$

C $1.927115-0.9367522 .717148$

C $0.848247-0.7953231 .616082$

O $1.242470-0.977078 \quad 3.974915$

C $4.5924962 .542589-1.140926$

O $4.9232913 .191215-2.120954$

C $5.0063891 .149209-0.860797$

C $5.6916910 .463483-1.788031$

C $6.191418-0.907922-1.662549$

C $5.901994-1.644103-0.382081$

C $1.382153-0.6414630 .187857$

C $1.441005-1.936126-0.603489$

C $2.214287-2.820180-2.803834$

O $0.949502-2.990981-0.212711$

C $3.513640-3.531222-2.594502$

C $4.427657-3.699668-3.562158$

C $5.701622-4.473775-3.382511$

C $6.928746-3.691730-3.792155$

C $7.489977-3.868837-5.000344$

C $7.560577-2.780438-2.765627$

C $6.889164-1.437584-2.689339$

N $2.077501-1.758243-1.825530$

H $1.6754352 .279378-1.370773$

H $1.6015415 .338369-1.253355$

H $3.9874634 .953004-0.665328$

H 2.1844634 .1000981 .666690

H $2.4326905 .752614 \quad 1.178115$

H $5.3625714 .262983 \quad 3.688395$

H 5.8506896 .1758812 .535945

H 5.4620436 .0181540 .821738

H 4.4394747 .0117841 .883782

H 3.4570492 .9272994 .602224

H 2.7915992 .6891953 .000659

H 5.0153281 .5609052 .357832

H 5.4545411 .6181124 .067948

H 3.3707680 .3045684 .610378

H $5.531341-0.9172134 .230200$

H $5.254306-0.8958382 .504119$

H $5.641421-3.3096773 .627044$

H $3.190230-2.2467361 .550072$

H $4.504541-2.7524205 .592652$

H $3.089991-3.6088815 .023741$

H $2.968446-1.8936215 .465411$

H $2.517759-4.1465432 .583175$

H $0.117600-1.6090941 .693701$

H $\quad 0.282566 \quad 0.1203901 .837568$

H $\quad 0.470931-0.3886143 .927480$ 
H 4.7376390 .7264400 .099712

H $5.9215710 .955965-2.732291$

H $6.444616-1.1838320 .449964$

H $6.183138-2.698903-0.410685$

H $4.830333-1.626107-0.158417$

H $\quad 0.6991630 .016389-0.363654$

H $2.366919-0.1650060 .180141$

H $2.148563-2.359457-3.794573$

H $1.385505-3.526650-2.692534$

H $3.693633-3.959378-1.611060$

H $4.231172-3.297010-4.553514$

H $5.623187-5.385253-3.989686$

H $5.817973-4.820107-2.347926$

H $8.389549-3.335439-5.289276$

H $7.058677-4.546862-5.729375$

H $8.612819-2.600437-3.025491$

H $7.603766-3.292339-1.800085$

H $7.042656-0.827715-3.580844$

H $2.505407-0.861516-2.037744$

C $-1.1094972 .623463-1.815937$

O $-2.1217672 .525268-2.839756$

C $-3.4249382 .590859-2.219826$

C $-3.1793662 .612366-0.718631$

C -1.811694 $3.259712-0.617699$

H -3.866399 $3.539569-2.549175$

C $-4.2635371 .407342-2.722390$

H -3.955039 $3.162526-0.176997$

H -3.144086 $1.594522-0.313164$

C - -1.1569783 .0102900 .730076$

H -1.922758 4.340560 -0.775231

C $-5.597807 \quad 1.227672-1.973874$

O $-4.518150 \quad 1.613947-4.114085$

H $-3.6788560 .483049-2.646869$

H $-5.3694381 .103827-0.912402$

O $-6.3796832 .415215-2.090483$

C $-6.4255300 .010295-2.435500$

C -7.718441 -0.208862 -1.625968

H $-5.807928-0.893685-2.378400$

O $-6.8255050 .160671-3.799012$

H -8.394918 $0.643608-1.767246$

H -8.232440 -1.071158 -2.071762

H -0.2035163 .5362490 .814790$

H $-1.801173 \quad 3.3663191 .541211$

H -0.9733931 .9439010 .892515$

C $0.0754093 .405006-2.386495$

H $-3.6572521 .719004-4.554499$

H -6.592739 $2.529940-3.032707$

H $-6.0187070 .214059-4.338467$

H $-0.2106094 .453024-2.541155$

H $\quad 0.3052913 .018437-3.388045$

H $-0.805706 \quad 1.593869-1.586127$

C -6.263585 -1.941127 1.352204

O $-6.638124-1.5958020 .010997$

C -7.496061 $-0.458486-0.125033$

C $-8.814600-0.6840300 .629056$

C $-8.544679-1.0320282 .097119$

C -7.508814 -2.152368 2.232147

H -5.757642 -2.9113541 .259985$

C $-5.255953-0.9387391 .939599$

H -7.0067640 .4220240 .299932$

$\begin{array}{llll}\text { O } & -9.549583 & -1.737228 & 0.010720\end{array}$

H -9.4211080 .2269890 .560321$

H -9.479119 -1.344854 2.578484

O

H -7.200891 -2.266289 3.278129

O $-8.106144-3.3912621 .838142$

C $-3.843275-1.1949571 .458461$

H $-5.490617 \quad 0.104953 \quad 1.717304$

H -5.229227 -1.046110 3.030904

H $-10.383854-1.8396410 .499464$

H $-8.7445180 .811992 \quad 2.707767$

H -8.865438 -3.550246 2.424452

O $-3.791924-1.2269710 .097305$

O $-2.886024-1.3111112 .214139$

C $-2.498112-1.465531-0.465889$

$\mathrm{H}-1.840392-0.616147-0.262237$

H -2.070233 -2.390818 -0.068350

H $-2.611722-1.570968-1.547843$
SCF Energy (B3LYP/6-31G**//MMFF)= -3245.90445461

08_00441

MMFF Geometry

C $3.367536-0.6698632 .641956$

C $2.410865-0.3040433 .507541$

C $0.946714-0.5581073 .268707$

O $0.334808 \quad 0.739385 \quad 3.084668$

C $0.267338-1.2744664 .448909$

C $0.682798-2.7219194 .600422$

C $0.131608-3.744858 \quad 3.918313$

C $1.768770-2.9872175 .610732$

C $-0.983109-3.6900802 .911031$

C $-0.471615-3.9412171 .487222$

C -1.617962 -4.197891 0.498090

C $-1.076531-4.392943-0.919659$

C -2.206672 -4.593086-1.941505

O $-2.515220-3.0833190 .524627$

O $-1.648865-4.434750-3.254794$

C $-3.255501-3.475814-1.758642$

C $-2.780803-6.013794-1.884262$

O $-4.402208-3.713112-2.572591$

C $-3.684251-3.262831-0.288532$

C $-4.555688-1.990186-0.165616$

O $-4.456134-4.391466 \quad 0.134213$

C -0.7461190 .8005042 .260927$

O $-1.336702-0.1374801 .749791$

C -1.047405 2.230349 2.027941

C -1.890782 2.5672941 .040889

C -2.2284323 .9266550 .617707$

C -1.6234395 .0722551 .385574$

C $-4.797318-1.546621 \quad 1.283262$

C $-5.991803-0.6209361 .384885$

C $-6.7757451 .669116 \quad 0.887958$

O $-7.039893-0.982073 \quad 1.916867$

C $-6.4772382 .724782-0.126045$

C -6.3391154 .0237950 .179533$

C $-6.0691825 .099515-0.833812$

C -4.816192 $5.887944-0.523402$

C -4.8829267 .0477840 .151763$

C $-3.5002895 .379448-1.066882$

C $-3.0563344 .088176-0.435431$

N $-5.772770 \quad 0.623431 \quad 0.814201$

H $3.086308-1.1719451 .718846$

H $2.6872590 .212807 \quad 4.423225$

H $0.822576-1.1431832 .349216$

H -0.822477 -1.209185 4.341899

H $0.463145-0.7284095 .382098$

H $0.509361-4.7492204 .107490$

H $1.993538-4.0547235 .708676$

H $2.696869-2.4826855 .328205$

H $1.463016-2.6255166 .598006$

H -1.714271 -4.461310 3.183892

H -1.518977 -2.737296 2.949070

H $\quad 0.104618-3.0654651 .163399$

H $\quad 0.210775-4.800573 \quad 1.475405$

H -2.151379 -5.0967180 .829917$

H $-0.372632-5.233789-0.956434$

H $-0.498361-3.505503-1.212119$

H $-0.929441-5.081508-3.355213$

H -2.807102 -2.544473 -2.126594

H -1.996671 -6.752011-2.091445

H $-3.543226-6.163470-2.656692$

H $-3.217904-6.261068-0.914591$

H -4.089879 -3.825962 -3.486738

H $-4.065791-1.157953-0.687473$

H -5.520915 -2.158314 -0.660384

H -5.114412 -4.107706 0.787556

H -0.5261192 .9629952 .633192$

H -2.3584221 .7716040 .462300$

H -1.8831345 .0036882 .447946$

H -1.969518 6.0514591 .046863

H -0.5321725 .0659281 .288435$

H -4.989732 -2.400099 1.942061

H -3.916244 -1.034159 1.684500

H -6.7716192 .0582361 .911906$

H $-7.760957 \quad 1.2307860 .695849$

H $-6.3899752 .408056-1.162630$ 
H $-6.448976 \quad 4.339732 \quad 1.214628$ H $-6.9379835 .770781-0.847563$ H $-6.0005584 .687992-1.848489$ H -3.9949237 .6369850 .354451$ H -5.8281847 .4321490 .520510$ H -2.716347 $6.140868-0.980001$ H $-3.6025835 .216474-2.147891$ H $-3.4507603 .199871-0.929712$ H -4.8198380 .8846780 .576427$ C 5.3447020 .7665072 .035342 O $5.0722690 .574720 \quad 0.640274$ C $6.147630-0.1836670 .070108$ C 7.3660210 .0504670 .963722 C 6.8632220 .9223252 .108646 H $5.868735-1.2421640 .135940$ C $6.3275500 .190893-1.406332$ H $7.710707-0.9155431 .353419$ H 8.2199120 .4833980 .432247 C 7.2823092 .3797921 .912151 H 7.2637410 .5876463 .072270 C $5.0331820 .118023-2.248233$ O $6.8262201 .530928-1.498793$ H $7.101701-0.445705-1.850032$ H $4.3837040 .959583-1.983101$ O $5.3943440 .320389-3.620025$ C $4.249594-1.199937-2.121920$ C $3.074959-1.350872-3.106480$ H $3.872463-1.302673-1.098311$ O $5.149776-2.288688-2.348187$ H $3.455853-1.452869-4.130805$ H $2.595401-2.312051-2.877520$ H $8.3736362 .470626 \quad 1.912149$ H 6.9093822 .7913580 .969963 H 6.8950333 .0024942 .725614 C $4.836194-0.4340412 .851090$ H $6.1571752 .120245-1.110412$ H $5.936503-0.439004-3.895207$ H $4.655988-3.113398-2.200593$ H $5.050691-0.2677053 .913979$ H $5.359906-1.3545362 .566578$ H 4.8239071 .6728092 .365560 C $0.6118940 .929531-1.452596$ O $1.574999-0.104038-1.699184$ C $2.030451-0.223447-3.049127$ C $0.850422-0.493515-3.998479$ C $-0.2547180 .554913-3.833850$ C $-0.6175220 .773015-2.362743$ H $0.2840360 .753770-0.423253$ C $1.2334072 .333596-1.518826$ H $2.5176680 .709297-3.348932$ O $0.304559-1.790578-3.763674$ H $1.219752-0.486415-5.030564$ H $-1.1499470 .230353-4.378491$ O $\quad 0.172126 \quad 1.794391-4.390999$ H -1.271715 $1.647097-2.263310$ O $-1.365520-0.360643-1.917847$ C $2.1589212 .606393-0.353028$ H $1.8097532 .479058-2.435820$ H $0.4408993 .089536-1.475001$ H $\quad 0.013951-1.827018-2.836536$ H $\quad 0.364709 \quad 1.642617-5.331976$ H $-1.638074-0.194676-0.999299$ O $2.7658723 .814934-0.545366$ O 2.3211131 .8694380 .608792 C 3.6725034 .2196580 .484603 H 4.0038365 .2381910 .265255 H 3.1758314 .2155411 .459689 H 4.5445083 .5607110 .491203 SCF Energy (B3LYP/6-31G**//MMFF) $=-3245.91140244$

\section{2}

MM̄̄FF Geometry

C $-0.768821-1.4352630 .773429$

C $0.459441-1.3187350 .249022$

C $0.727021-1.182956-1.225150$

O $2.003249-1.818323-1.459664$

C $0.8392330 .305869-1.593022$

C $0.8111680 .570456-3.083256$
C $1.8838440 .917349-3.820987$

C $-0.5500500 .482031-3.724520$

C $3.3137991 .044277-3.373850$

C $3.7961412 .501761-3.339874$

C $3.2062723 .317898-2.178029$

C $3.6806504 .770457-2.244406$

C $3.2016945 .585628-1.033364$

O $3.6507982 .721223-0.956968$

O $3.9408256 .816993-1.013774$

C 3.5576614 .8220870 .260794

C $1.7219415 .970182-1.154541$

O $2.9961795 .493937 \quad 1.386710$

C 3.1356383 .3324660 .233678

C 3.7424712 .5768311 .441669

O 1.7106913 .2519360 .298660

C $2.184519-2.548847-2.586024$

O $1.360186-2.761156-3.463745$

C $3.552702-3.119870-2.632780$

C $4.534862-2.743888-1.797848$

C $5.897957-3.277537-1.757120$

C $6.239841-4.402905-2.697063$

C 3.5851631 .0512471 .366773

C 4.2790690 .3425152 .516703

C $4.933437-1.9479933 .260943$

O 4.7689230 .9338383 .474889

C $6.343932-2.1528042 .803488$

C $6.841338-3.3377912 .417367$

C $8.257654-3.5344741 .955662$

C $8.345645-4.1025060 .555129$

C $8.629714-5.4007120 .355725$

C $8.193789-3.153111-0.613877$

C $6.763361-2.758634-0.860585$

N $4.272389-1.0359182 .345831$

H -1.633013 -1.453533 0.114168

H $1.315323-1.2751280 .918028$

H $-0.065594-1.685940-1.791597$

H $\quad 1.750816 \quad 0.718727-1.150484$

H $0.0126160 .871635-1.141386$

H $1.732706 \quad 1.114737-4.881744$

H $\quad-0.5270470 .745422-4.787327$

H $-0.947897-0.534236-3.647224$

H -1.247444 $1.167381-3.231607$

H $3.4932860 .556677-2.411111$

H $3.9290350 .493723-4.097259$

H $4.8893032 .482031-3.239915$

H $3.5675332 .990849-4.294944$

H $2.1128553 .274693-2.227765$

H $3.3552705 .243694-3.179297$

H $4.7794134 .794816-2.263567$

H $3.778307 \quad 7.277891-1.854558$

H 4.6495074 .8790250 .374250

H $1.5543546 .566108-2.059824$

H $1.4086386 .604154-0.317725$

H $1.0562795 .105935-1.201614$

H 3.3998295 .1216442 .188253

H 4.8163062 .7982571 .500429

H 3.2833042 .9285282 .373808

H 1.4562552 .3988150 .682557

H $3.709140-3.867241-3.403617$

H $4.329354-1.970525-1.059349$

H $5.559600-5.249287-2.549050$

H $7.250809-4.792523-2.559071$

H $6.164919-4.069391-3.737868$

H 2.5272790 .7758181 .401409

H 4.0112360 .6712820 .431314

H $4.359225-2.8797593 .265688$

H $4.926898-1.5264494 .270986$

H $6.989751-1.2776452 .793197$

H $6.201810-4.2172092 .439780$

H $8.748059-4.2105812 .668240$

H $8.827028-2.5977592 .000446$

H $8.725414-5.811821-0.643586$

H $8.768778-6.0829161 .187882$

H $8.646504-3.574758-1.518211$

H $8.777060-2.243936-0.418019$

H $6.419965-1.944033-0.222759$

H $3.903893-1.4237151 .482072$

C $-1.594619-0.1954482 .825425$ 
$\begin{array}{llll}\text { O } & -2.808820 & 0.180309 & 2.142428\end{array}$

C -2.7444341 .5874831 .844538$

C -1.6663522 .1524312 .761680$

C -0.6617451 .0164962 .747944$

H -2.388932 1.6725450 .810336

C $-4.1252212 .239641 \quad 1.965069$

H -1.2548443 .0958122 .390536$

H -2.034995 2.3137493 .780386

C $0.362374 \quad 1.0842993 .866654$

H -0.1340431 .0429341 .787825$

C $-5.210722 \quad 1.602548 \quad 1.069926$

O $-4.592993 \quad 2.1308343 .314876$

H -4.049569 3.3118611 .745914

H -5.4909630 .6258031 .479260$

O -6.3844362 .4203631 .172410$

C $-4.8301521 .454337-0.414931$

C $-5.9984001 .097152-1.353417$

H $-4.0429020 .698159-0.512032$

O $-4.2804582 .694678-0.871640$

H -6.697094 $1.941487-1.413837$

H $-5.5741530 .997336-2.361650$

H 0.9559812 .0011403 .788816

H $-0.1165541 .076514 \quad 4.851444$

H 1.0488380 .2331193 .817569

C $-1.036701-1.501208 \quad 2.250791$

H -4.1079652 .7811813 .849846$

H -6.1763853 .2829720 .773954$

H -3.973588 2.560916 -1.784709

H $-1.762951-2.3063712 .413170$

H -0.129371-1.784700 2.797524

H $-1.879443-0.3772093 .870041$

C $-6.360048-2.544278-0.587204$

O $-5.826114-1.266973-0.963731$

C $-6.761226-0.185302-0.985015$

C $-7.899355-0.473250-1.979055$

C $-8.567693-1.819874-1.683139$

C $-7.536190-2.937368-1.498122$

H -5.544908 -3.255658 -0.772758

C $-6.707684-2.6036650 .908685$

H $-7.188643-0.0553740 .013838$

O $-7.381845-0.485960-3.308526$

H $-8.6342680 .338437-1.919068$

H -9.238197 -2.087828 -2.508859

O $-9.354812-1.715408-0.500700$

H -8.022523 -3.834846 - 1.099361

O $-7.010824-3.297456-2.779562$

C $-5.483131-2.4651101 .787638$

H $-7.403438-1.8101621 .193164$

H -7.164505 -3.5726401 .140711$

H -8.129439 -0.631933 -3.912973

H $-10.021707-1.024138-0.651578$

H -6.511769 -2.535689 -3.121037

O $-5.882592-2.3989883 .092251$

O $-4.328452-2.4261931 .387699$

C $-4.836038-2.2587744 .058228$

H $-5.266013-2.4217365 .050130$

H -4.048472 -3.0006063 .894165$

H -4.429077 -1.245185 4.014768

SCF Energy (B3LYP/6-31G**//MMFF)= -3245.89985724

$08 \_00443$

MM̄FF Geometry

C -1.902671 -2.203939 1.920346

C $-1.354580-1.4712102 .902422$

C $-1.627269-0.016706 \quad 3.207677$

$\begin{array}{llll}\text { O } & -2.763844 & 0.479239 & 2.467636\end{array}$

C $-0.4245890 .852636 \quad 2.798337$

C $-0.2624962 .076284 \quad 3.677110$

C -0.7981843 .2873603 .430654$

C 0.6055711 .8685664 .891964

C -1.7275403 .6874342 .319642$

C $-1.0808394 .650702 \quad 1.316736$

C -0.1790893 .9378890 .297593$

C $0.4996774 .957291-0.619466$

C $1.3099104 .278569-1.732884$

O $-1.0090063 .061546-0.474718$

O $1.631997 \quad 5.276021-2.714435$

C $0.4184513 .230204-2.429690$
C $2.6454493 .730973-1.218372$

O $1.1657402 .476916-3.380720$

C $-0.3057592 .280108-1.447341$

C $-1.3384641 .420404-2.216538$

O $\quad 0.6524301 .414289-0.835565$

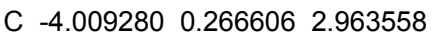

O $-4.313013-0.3220723 .987108$

C $-4.951963 \quad 0.8570491 .989704$

C $-6.212436 \quad 0.406748 \quad 1.926158$

$\begin{array}{llll}\text { C }-7.226722 & 0.839151 & 0.962002\end{array}$

C $-6.862137 \quad 1.9573980 .019530$

C $-2.3308730 .665590-1.318987$

C $-3.242960-0.246202-2.125320$

C $-5.149664-1.831707-1.849320$

O $-3.139863-0.393785-3.340557$

C $-6.434045-1.120851-2.143188$

C $-7.636610-1.581993-1.765097$

C $-8.935426-0.897813-2.089421$

C $-9.838194-0.740402-0.884254$

C $-10.873644-1.573317-0.683844$

$\begin{array}{llll}\text { C } & -9.590945 & 0.430663 & 0.041622\end{array}$

C -8.4147050 .1996950 .950428$

N $-4.177999-0.888486-1.324629$

H -2.614151 -1.7520401 .236477$

H $-0.640514-1.9529473 .568398$

H -1.8258800 .0782124 .282874$

H -0.5212761 .1594301 .750186$

H $0.509903 \quad 0.2777172 .850052$

H -0.5800924 .0922184 .132143$

H 0.6839172 .7702165 .508690

H 0.1943131 .0731085 .521806

H 1.6191441 .5850344 .590162

H -2.146664 2.8240441 .798932

H -2.5817724 .1956732 .785159$

H $-1.892746 \quad 5.1539240 .775275$

H $-0.515608 \quad 5.426454 \quad 1.847896$

H $0.573213 \quad 3.3488880 .833950$

H $1.1366455 .637690-0.040523$

H $-0.2658775 .591545-1.088390$

H $2.1338765 .981331-2.271252$

H $-0.3430743 .779572-3.001105$

H $3.2536024 .538783-0.793959$

H $3.2371503 .311272-2.038595$

H $2.5293602 .966758-0.447175$

H $1.5940033 .110606-3.981373$

H $-1.9290042 .067002-2.879107$

H $-0.809840 \quad 0.699973-2.853752$

H $0.2210350 .580137-0.592689$

H -4.5629541 .6097491 .313400$

H $-6.520701-0.3751992 .618898$

H $-6.627768 \quad 2.8686850 .580944$

H $-7.656126 \quad 2.216162-0.683317$

H -5.990695 $1.686021-0.586328$

H $-1.7998240 .048580-0.587702$

H -2.959047 $1.379412-0.774146$

H $-5.276111-2.617760-1.098425$

H $-4.759016-2.281581-2.767555$

H $-6.375735-0.198308-2.716051$

H $-7.695838-2.522641-1.221974$

H $-9.442427-1.497968-2.856472$

H $-8.7664610 .085598-2.545473$

H -11.541941 -1.4515160 .162058$

H $-11.072918-2.399263-1.358624$

H -10.4672790 .5984550 .682050$

H $-9.5030861 .343374-0.553447$

H -8.578263 -0.6016411 .672652$

H $-4.221422-0.664690-0.335253$

C $-0.683514-3.9634650 .534809$

$\begin{array}{llll}\text { O } & 0.619862 & -3.401101 & 0.772594\end{array}$

C $1.183072-2.998169-0.491670$

C $0.220272-3.493828-1.568412$

C $-1.108062-3.448266-0.836529$

H $1.180855-1.900610-0.500805$

C $2.627199-3.506271-0.633931$

H $0.239270-2.861923-2.461746$

H $0.448289-4.518791-1.880439$

C $-2.200884-4.267662-1.497463$

H $-1.427643-2.401974-0.777545$ 
C $3.598105-2.7932280 .335103$

O $2.667541-4.913225-0.406428$

H $2.974725-3.325550-1.657864$

H $3.502830-1.7181140 .153490$

O $3.204800-3.0168231 .688275$

C $5.076562-3.2027670 .161700$

C $6.043595-2.4405171 .091411$

H $5.368957-3.055708-0.884395$

O $5.217794-4.5962350 .446658$

H $5.753478-2.5978332 .137696$

H $7.035466-2.9001860 .984737$

H -2.411192 -3.887807 -2.502702

H -1.914153 -5.320560 -1.589306

H -3.127506 -4.220439-0.917262

C -1.610218 -3.6638451 .713571$

H $2.236154-5.352344-1.157633$

H $3.242104-3.9762301 .844759$

H $6.137299-4.8450680 .251327$

H -1.156473 -4.074166 2.625118

H -2.563392 -4.188092 1.577561

H $-0.539970-5.0519930 .491650$

C $6.7771630 .536509-1.031352$

O $6.676739-0.804057-0.534502$

C $6.142845-0.9384330 .786138$

C $7.031902-0.1956231 .793588$

C 7.2023231 .2747731 .394095

C $7.5973121 .424549-0.079346$

H $7.3445340 .445593-1.966643$

C $5.3953571 .110106-1.374608$

H $5.140076-0.5048140 .822138$

O $8.311840-0.8171541 .885052$

H $6.572762-0.2597162 .786824$

H 7.9733611 .7356062 .023636

O $\quad 5.9828451 .9778221 .614661$

H $7.5156302 .470662-0.396913$

O $8.9701391 .047796-0.227745$

C $4.7257020 .306950-2.470289$

H $4.738571 \quad 1.119110-0.500494$

H $5.4853772 .140357-1.735689$

H $8.698539-0.834540 \quad 0.992801$

H 5.7626001 .8953262 .558191

H $9.499616 \quad 1.6468250 .325947$

O $3.385558 \quad 0.222765-2.228394$

O $5.309147-0.166886-3.436590$

C $2.630100-0.498958-3.206375$

H $3.025577-1.511542-3.327498$

H $1.596702-0.566596-2.857171$

H $2.6450320 .035790-4.160545$

SCF Energy (B3LYP/6-31G**//MMFF) $=-3245.90112630$

\section{4}

MM̄FF Geometry

C $-2.751328-1.942583-3.217551$

C -1.498669-1.716509-2.790572

C $-0.675555-2.722500-2.031763$

O $-0.037246-1.981428-0.963206$

C $0.405547-3.325471-2.944500$

C $1.007329-4.605811-2.399944$

C $2.248110-4.729209-1.889865$

C $0.122343-5.823618-2.508264$

C $3.293559-3.666041-1.700073$

C $3.689055-3.558300-0.223203$

C $4.833265-2.5636070 .014213$

C $5.129685-2.4549611 .512718$

C $6.201046-1.3993851 .817582$

O $4.452451-1.285449-0.506419$

O $6.162609-1.1246133 .226422$

C $5.831344-0.0891361 .093262$

C $7.612084-1.9166751 .514630$

O $\quad 6.873545 \quad 0.875199 \quad 1.226063$

C $5.476626-0.286819-0.400088$

C $4.9299551 .035731-0.981560$

O $6.657776-0.656701-1.113911$

C $-0.022800-2.5094320 .283952$

O $-0.448304-3.6063820 .618780$

C $0.606816-1.5849241 .258903$

C $0.914410-0.3041880 .997727$

C 1.5261100 .6498541 .931758
C 1.8530610 .1574653 .316817

C $4.3675640 .935405-2.406298$

C $3.9727152 .321478-2.876565$

C $2.2554564 .076177-2.456281$

O $4.7441173 .027829-3.521703$

C $2.5364924 .654906-1.103803$

C $1.5852284 .935445-0.200764$

C 1.8782485 .3776521 .203156

C 1.5399084 .3083872 .222440

C $0.557723 \quad 4.4881913 .121314$

C 2.3973413 .0577112 .248806

C 1.7719761 .9058581 .500962

N $2.7146242 .698012-2.434306$

H -3.185637-2.925113 -3.043798

H -1.025745 -0.755264 -2.969962

H $-1.335643-3.495777-1.621907$

H $1.182176-2.577794-3.137603$

H $-0.023102-3.561396-3.928462$

H $2.564571-5.719986-1.563806$

H $0.629783-6.737986-2.182506$

H $-0.772812-5.709142-1.889856$

H $-0.188728-5.975767-3.547066$

H $4.172308-3.938903-2.296627$

H $2.957249-2.691049-2.060713$

H $2.809666-3.2451820 .349975$

H $3.985481-4.5436230 .158890$

H $5.715786-2.923543-0.527926$

H $5.422653-3.4295341 .922870$

H $4.209754-2.1733982 .043785$

H $6.319961-1.9600943 .699030$

H $4.9535350 .331781 \quad 1.603675$

H $7.835241-2.7996272 .125472$

H $8.371430-1.1719271 .777467$

H $7.750476-2.1978560 .468566$

H 7.0536020 .9781552 .176188

H $4.128110 \quad 1.407615-0.331994$

H $5.7231071 .794836-0.974254$

H $6.554240-0.422015-2.048862$

H $\quad 0.791303-2.0254052 .233489$

H 0.6962630 .0965880 .009044

H 2.3522680 .8999063 .941726

H $2.525724-0.7060873 .270536$

H $0.939509-0.1354653 .844680$

H $5.1027510 .540933-3.114383$

H $3.4965110 .271300-2.441329$

H $1.1873274 .064997-2.691131$

H $2.7823814 .643511-3.229144$

H $3.5818674 .789525-0.835176$

H $0.5405834 .793946-0.462540$

H $1.306725 \quad 6.294428 \quad 1.395380$

H 2.9337565 .6548851 .318619

H 0.3323653 .7321963 .866528

H $-0.0459455 .389486 \quad 3.127859$

H 2.6159962 .8077343 .290653

H 3.3735803 .2732941 .796535

H 1.5125612 .1426890 .469746

H $2.1841852 .044412-1.865362$

C $-3.2964200 .517800-3.722331$

O $\quad-3.3780030 .850336-2.324145$

C $-3.7385812 .238991-2.199607$

C $-3.9113722 .769700-3.622241$

C $-4.2400821 .505696-4.398460$

H $-4.7084262 .273134-1.691967$

C $-2.6714122 .960285-1.370020$

H -4.699633 $3.526603-3.684611$

H $-2.9885083 .226013-3.999616$

C $-4.0337141 .643268-5.896062$

H $-5.283597 \quad 1.229168-4.196840$

C -2.4978412 .3866490 .054545$

O $-1.4168192 .828778-2.052392$

H -2.890532 4.033446 -1.328202

H $-2.1452671 .351687-0.007018$

O $-1.442851 \quad 3.1224810 .685558$

C -3.7593982 .4722290 .936233$

C -3.5232212 .0757122 .407335$

H -4.5562751 .8542180 .510045$

O -4.2211663 .8269850 .918606$

H -2.6690152 .6320572 .811765$ 
H -4.3951112 .4097032 .986472$

H -4.679811 $2.428204-6.302559$

H -2.997344 $1.902734-6.136599$

H $-4.2751130 .708310-6.411126$

C $-3.661078-0.955382-3.908627$

H $-0.7342163 .217030-1.479933$

H -1.746907 4.0404110 .790914

H -5.0635463 .8560081 .403693$

H -3.697568 -1.209540 -4.973611

H -4.668069 -1.116120 -3.502869

H $-2.2674350 .707113-4.054108$

C $-4.632452-1.4852842 .481815$

O $-4.588472-0.0593892 .342082$

C -3.3282560 .5646542 .617083$

C $-2.865523 \quad 0.246748 \quad 4.048418$

C $-2.852022-1.2628214 .307502$

C $-4.162785-1.9234173 .877522$

H -5.698071 -1.738417 2.401665

C $-3.896050-2.1839451 .332377$

H $-2.578768 \quad 0.198340 \quad 1.909637$

$\begin{array}{llll}\text { O } & -3.732564 & 0.883482 & 4.985129\end{array}$

H -1.8618690 .6648474 .192789$

H -2.688122 -1.4508145 .375568$

O $-1.781188-1.8664193 .591021$

H -4.069836 -3.015656 3.903723

O $-5.191735-1.5708534 .806657$

C $-4.737949-2.117450 \quad 0.079298$

H -2.926704 -1.739599 1.093341

H -3.712640 -3.237696 1.573339

H $-3.395526 \quad 0.689708 \quad 5.876213$

H $-0.954076-1.4792783 .923581$

H -4.917592 -1.890975 5.682862

O $-5.275538-3.341514-0.188146$

O $-4.924358-1.091790-0.562144$

C $-6.155606-3.398459-1.314962$

H $-5.598548-3.215282-2.237459$

H $-6.586238-4.402412-1.358987$

H $-6.968481-2.674133-1.204071$

SCF Energy (B3LYP/6-31G**//MMFF) $=-3245.89301872$

\section{5}

MM̄FF Geometry

C $0.748479-3.267273 \quad 1.771986$

C $0.873751-1.9352631 .669599$

C $0.665735-0.9896872 .824052$

O -0.1554830 .0867192 .309731$

C $1.995379-0.4061523 .338515$

C $2.956693-1.4469563 .875575$

C $4.045375-1.8960783 .219501$

C $2.630492-1.9703885 .250041$

C $4.508311-1.4973621 .846612$

C $5.898032-0.8477561 .891232$

C $6.202922-0.0977240 .587704$

C $7.666740 \quad 0.337715 \quad 0.532711$

C $7.9604281 .189382-0.712592$

O 5.3548391 .0551170 .562827

O $9.2449341 .804913-0.530204$

C $6.9189752 .326147-0.794858$

C $8.0674040 .330326-1.977888$

O $7.0688953 .068005-2.003784$

C $5.4552401 .850556-0.622965$

C $4.5277023 .080301-0.445619$

O $5.068378 \quad 1.115758-1.782349$

C $-0.861772 \quad 0.835413 \quad 3.196525$

O $-0.896300 \quad 0.708714 \quad 4.409135$

C $-1.591428 \quad 1.8576682 .415854$

C -2.2577962 .8391073 .040305$

C -3.0269453 .8723892 .353463$

C -4.3714864 .1330092 .974269$

C $3.0742952 .758039-0.064162$

C $2.1540292 .504502-1.244995$

C $-0.2286001 .980130-1.757992$

O $2.5191222 .583010-2.414416$

C $-0.9625083 .270981-1.942372$

C $-2.291903 \quad 3.393941-1.809147$

C $-3.0262764 .693675-1.977355$

C $-3.8843795 .048386-0.781135$

C $-5.2238214 .962353-0.848672$
C $-3.2044875 .587303 \quad 0.460002$

C $-2.535955 \quad 4.515277 \quad 1.274665$

N $0.8666712 .189337-0.829754$

H $0.500140-3.6831812 .746535$

H $1.140916-1.4815350 .719555$

H $\quad 0.121609-1.4937343 .632983$

H 1.7897910 .3197814 .136786

H 2.4540640 .2089702 .555784

H $4.667695-2.6460763 .705669$

H $3.373798-2.6906655 .608038$

H $2.596124-1.1471975 .971137$

H $1.658572-2.4734605 .249250$

H $4.553100-2.3978351 .221320$

H $3.793367-0.8325681 .355799$

H $5.945723-0.1422902 .731522$

H $6.651114-1.6255312 .068265$

H $5.962345-0.748775-0.261029$

H $8.334022-0.5323710 .568963$

H $7.904321 \quad 0.934477 \quad 1.424839$

H $9.9014991 .097988-0.407201$

H 7.1525353 .0277790 .017868

H $8.851598-0.427522-1.860940$

H $8.3648600 .934940-2.841774$

H $7.139209-0.190541-2.222051$

H $6.6775172 .543483-2.722993$

H 4.9311973 .6887220 .375050

H $4.5461253 .700114-1.349824$

H $4.1004601 .066443-1.811093$

H -1.5730001 .7780391 .333636$

H -2.2811572 .8837654 .126837$

H $-4.9394613 .199843 \quad 3.063947$

H -4.9890234 .8219692 .393664$

H -4.2531734 .5605533 .976042$

H 3.0205341 .9061010 .621489

H 2.6629413 .6289210 .463262

H $-0.8669101 .201466-1.331139$

H $0.1650911 .635191-2.718762$

H $-0.3718604 .147335-2.199300$

H -2.885499 2.512992 -1.577679

H -3.649506 $4.606477-2.876881$

H $-2.3331635 .520704-2.175460$

H $-5.8518935 .242449-0.009578$

H $-5.7233704 .603544-1.742502$

H -3.9127746 .1553761 .074052$

H -2.446936 6.3247410 .165000

H $-1.533941 \quad 4.2597410 .931301$

H $\quad 0.646526 \quad 2.218657 \quad 0.161676$

C $1.135146-3.773311-0.737979$

O $-0.064995-3.125581-1.205840$

C $-0.251973-3.439354-2.599469$

C $0.997733-4.194572-3.040747$

C $1.414424-4.879292-1.754682$

H $-1.112171-4.115465-2.660389$

C $-0.522628-2.159541-3.396870$

H $\quad 0.790045-4.896823-3.854159$

H $\quad 1.784980-3.512340-3.382647$

C $2.853781-5.364208-1.767560$

H $0.745909-5.732341-1.577372$

C $-1.777780-1.380270-2.950658$

O $0.605799-1.283655-3.263310$

H $-0.591601-2.398068-4.464374$

H -1.626094 -0.996854 -1.936690

O $-1.887181-0.227824-3.797485$

C -3.097262 -2.175860 -3.016888

C $-4.360999-1.311384-2.831584$

H $-3.083174-2.978444-2.271528$

O $-3.182792-2.795129-4.304225$

H $-4.365962-0.504694-3.574979$

H -5.231812 -1.937130 -3.070892

H $3.006070-6.094288-2.569318$

H $3.554337-4.537795-1.927956$

H $3.113349-5.846269-0.819956$

C $0.919074-4.2955870 .684324$

H $0.714172-1.087128-2.316752$

H $-2.047005-0.543884-4.703522$

H $-3.976455-3.356958-4.307548$

H $1.761344-4.9352200 .972222$

H $\quad 0.017471-4.9217790 .696975$ 
H $1.942818-3.030492-0.762723$

C -5.064094-1.506099 0.828921

O $-4.862841-1.830102-0.553838$

C $-4.523442-0.737010-1.415574$

C $-5.6097260 .349560-1.367249$

C $-5.8861410 .795017 \quad 0.071302$

C $-6.117518-0.3980470 .998328$

H -5.475708 -2.423252 1.270269

C $-3.735817-1.2015381 .531724$

H -3.574821 $-0.299894-1.092202$

O $-6.816388-0.121104-1.963839$

H $-5.275196 \quad 1.207756-1.961961$

H -6.7703201 .4438860 .090960$

$\begin{array}{lllll}\text { O } & -4.778497 & 1.540678 & 0.563569\end{array}$

H -6.142790 -0.0632362 .042155$

$\begin{array}{llll}0 & -7.406459 & -0.943390 & 0.703872\end{array}$

C -2.999681-2.492686 1.802941

H $-3.063797-0.5641930 .951731$

H -3.911232 -0.6940272 .487743$

H -7.091410 -0.922753 -1.486892

H -4.670796 $2.317674-0.009401$

H -7.578302 -1.654322 1.344658

O $-3.051262-2.781805 \quad 3.133004$

O $-2.476602-3.1693280 .927596$

C -2.460055 -4.009757 3.576478

H -2.174282 -4.6715812 .754113$

H - $1.580831-3.7725194 .180906$

H -3.187894-4.528280 4.206484

SCF Energy (B3LYP/6-31G**/MMFF) $=-3245.91071919$

\section{$08 \_00446$}

MM̄FF Geometry

C $-0.521235-3.5697790 .552479$

C $-0.663567-2.903280-0.602192$

C $0.453675-2.150937-1.271346$

O $0.040007-0.773589-1.436677$

C $0.775400-2.704376-2.669899$

C $1.365473-4.098927-2.657821$

C $2.653409-4.376456-2.373632$

C $0.410471-5.210099-3.008693$

C $3.749443-3.401871-2.041503$

C $4.120976-3.453091-0.554676$

C $5.364897-2.611837-0.229903$

C $5.598043-2.560121 \quad 1.282964$

C $6.788023-1.6613081 .658348$

O $5.182918-1.286463-0.742640$

O $6.710995-1.3972623 .068269$

C $6.637546-0.303800 \quad 0.934878$

C $8.127440-2.370607 \quad 1.426920$

O $7.784961 \quad 0.5160161 .141807$

C $6.329931-0.441410-0.571807$

C $6.0431910 .912132-1.257108$

O $7.479712-0.991503-1.230416$

C $0.1926610 .043436-0.358069$

$\begin{array}{llllll}\text { O } & 0.643327 & -0.259354 & 0.735688\end{array}$

C $-0.2964341 .386833-0.736898$

C -0.3119552 .3576410 .188218$

C $-0.7648473 .736434-0.003671$

C $-1.2819314 .115187-1.365470$

C $4.8684571 .718431-0.696617$

C $4.6356242 .984348-1.508479$

C $3.2503105 .033125-1.589513$

O $5.2281223 .225146-2.557956$

C $2.7375656 .005804-0.577180$

C $1.4951996 .509807-0.609711$

$\begin{array}{llll}\text { C } & 0.958679 & 7.516049 & 0.365852\end{array}$

C $0.1388896 .902876 \quad 1.479370$

C 0.4308257 .1586052 .766114

C $-1.053966 \quad 6.0436201 .112894$

C -0.6882034 .5837991 .043825$

N $3.6896673 .818763-0.926659$

H $\quad 0.452156-3.5920551 .035558$

H -1.631333 -2.885698 -1.092885

H $1.364063-2.168299-0.663498$

H $1.458780-2.015282-3.183032$

H $-0.127886-2.683827-3.295912$

H $2.971108-5.418275-2.389577$

H $0.894688-6.192417-3.001712$
H $-0.419414-5.248638-2.297213$

H $\quad 0.000282-5.052769-4.011698$

H $4.624988-3.658404-2.650617$

H $3.471992-2.380925-2.321113$

H $3.271395-3.0864850 .033088$

H $4.298149-4.490303-0.242732$

H $6.221178-3.075834-0.733909$

H $5.734707-3.5697641 .690139$

H $4.699210-2.1598891 .773467$

H $6.725535-2.2506493 .534606$

H $5.8031540 .214711 \quad 1.422858$

H $8.182047-3.2888092 .024326$

H $\quad 8.966587-1.7498741 .759879$

H $8.292081-2.6483560 .383955$

H $8.4951150 .182148 \quad 0.567545$

H $6.951272 \quad 1.529984-1.245670$

H $5.8422230 .716157-2.320380$

H $7.417347-0.790284-2.179005$

H $-0.6346971 .536775-1.755583$

H $\quad 0.0425272 .122615 \quad 1.191272$

H $-2.1222263 .473352-1.649261$

H $-0.4932034 .009724-2.118547$

H -1.643583 $5.143522-1.425000$

H $3.9487081 .123437-0.721145$

H 5.0680592 .0148840 .338314

H $2.4851034 .746556-2.319060$

H $4.0904955 .474208-2.136319$

H 3.4239776 .3246330 .203326

H $0.8207036 .200261-1.403806$

H $0.3319048 .229978-0.183263$

H $1.7881798 .107156 \quad 0.775885$

H -0.1625186 .7398403 .572488$

H $1.269427 \quad 7.7886583 .043222$

H -1.505334 6.4226440 .192310

H $-1.834946 \quad 6.1524201 .877418$

H $-0.3118174 .192948 \quad 1.990895$

H $3.1221863 .452809-0.166712$

C $-2.124461-3.6746852 .525713$

O $-2.715561-2.3886742 .258265$

C $-2.446761-1.5206993 .379382$

C $-1.799295-2.3914194 .454790$

C -1.082727 -3.4323243 .615724$

H $-1.709684-0.785573 \quad 3.035927$

C $-3.729963-0.8098213 .824506$

H -1.124353 -1.818384 5.098059

H -2.547035 -2.869762 5.098006

C $-0.692143-4.6794294 .388056$

H $-0.179585-2.9682283 .201621$

C $-4.434593-0.0229612 .700108$

O $-4.651411-1.7990384 .302771$

H $-3.512208-0.1538074 .675578$

H -4.810585 -0.730106 1.953528

O $-5.593391 \quad 0.6025993 .266993$

C -3.5647031 .0541842 .026745$

C $-4.321171 \quad 1.9228311 .001725$

H -2.7052490 .5825381 .539655$

O -3.0491851 .9182913 .043268$

H -5.1672222 .4259601 .485491$

H -3.6395432 .7235840 .688984$

H $-0.007204-4.4274775 .204258$

H - $-1.567326-5.1726104 .824277$

H $-0.189215-5.3998143 .735172$

C -1.636269 -4.3175841 .225674$

H -5.460044 -1.3331394.576634

H -5.2836431 .2656493 .907899$

H -2.4436742 .5469992 .614959$

H -2.487163 -4.400389 0.537306

H -1.292724 -5.3392411 .425289$

H $-2.934554-4.3014812 .922951$

C $-3.892350-0.239043-1.996432$

O $-3.631310 \quad 0.630329-0.887198$

C $-4.7962761 .142790-0.232544$

C $-5.6102912 .007354-1.208548$

C $-5.9673251 .216108-2.471486$

C $-4.7572300 .470696-3.052686$

H $-2.902984-0.419707-2.436024$

C $-4.445581-1.583960-1.502342$

H $\quad \begin{array}{llll}-5.421999 & 0.308408 & 0.100091\end{array}$ 
O $-4.8731493 .163075-1.599309$ H $-6.5290852 .347520-0.715298$ H $-6.3642051 .908207-3.224556$ O $\quad-7.0011990 .291402-2.146667$ H $-5.087253-0.251648-3.806707$ O $-3.928627 \quad 1.417554-3.736846$ C $-4.146417-2.725733-2.448928$ H -3.976511 -1.822435 -0.539217 H -5.525882 -1.547616 -1.340914 H $-4.8454153 .768352-0.839401$ H $-7.243375-0.176625-2.963897$ H -3.588723 $2.045654-3.076614$ O $-4.427244-3.905590-1.819763$ O $-3.725677-2.606695-3.591091$ C $-4.163935-5.086927-2.582848$ H $-3.107069-5.130478-2.862871$ H -4.396429 $-5.953636-1.958469$ H -4.801381 -5.112199-3.471711

SCF Energy (B3LYP/6-31G**//MMFF) $=-3245.92327214$

\section{7}

MM̄FF Geometry

C $-2.2146693 .872716-0.538609$

C $-2.9467094 .285076 \quad 0.506935$

C -3.3953823 .4480961 .682084$

O -2.8725262 .1015651 .630535$

C -4.9348253 .3559581 .684705$

C -5.5058312 .5776632 .856515$

C -6.2150101 .4364552 .740614$

C -5.2541503 .1691954 .219193$

C -6.6012310 .7261941 .472277$

C $-5.559398-0.321791 \quad 1.086331$

C $-5.767364-0.907353-0.315848$

C $-7.034762-1.756900-0.419424$

C $-7.153373-2.444328-1.789563$

O $-4.615406-1.715087-0.587437$

O $-8.162939-3.460165-1.687707$

C $-5.824530-3.159786-2.112640$

C $-7.626703-1.470234-2.875252$

O $-5.837714-3.685046-3.438312$

C $-4.577242-2.269570-1.908371$

C $-3.292650-3.123787-2.025229$

O $-4.557052-1.252653-2.911124$

C -1.643240 1.8759342 .170562

O

C -1.4483700 .4077692 .229164$

C $-0.385229-0.0988732 .872183$

C $-0.121464-1.5193493 .124353$

C -1.105665 -2.521218 2.581177

C $-2.004482-2.378110-1.645501$

C $-0.798598-3.302211-1.633215$

C $1.589416-3.368570-0.924306$

O $-0.820682-4.440366-2.094825$

C $1.639836-4.0619650 .400449$

C $2.571951-3.8142621 .332736$

C $2.685608-4.5743002 .621271$

C $2.686516-3.6819613 .840141$

C $3.826922-3.4051244 .495253$

C $1.353142-3.212496 \quad 4.371857$

C $0.956432-1.854053 \quad 3.864855$

N $0.314021-2.693531-1.067954$

H -1.896053 $2.836357-0.607227$

H $-3.247218 \quad 5.3310270 .544128$

H -3.0534403 .9378442 .602210$

H -5.2520682 .9095180 .736164$

H -5.3684244 .3648841 .702704$

H -6.566753 0.9520793 .650446

H -5.8059412 .6452375 .007163$

H -4.1911043 .1173664 .473200$

H $-5.569814 \quad 4.2173504 .242305$

H -7.5742120 .2473891 .634651$

H $-6.756501 \quad 1.4368770 .654237$

H $-4.557826 \quad 0.122618 \quad 1.118101$

H -5.538646 -1.129616 1.830140

H -5.793901-0.079101-1.033753

H -7.931369 -1.158437 -0.220442

H -7.014968 -2.5346180 .356826$

H -8.991978 -3.032334 -1.412743
H $-5.746057-4.023904-1.437820$

H -8.610130 -1.058551 -2.617651

H -7.759310 -1.981757 -3.834991

H -6.946560 -0.628863 -3.022720

H -6.627204 -4.247293 -3.519150

H -3.381914 -3.993810 -1.361049

H -3.189077 -3.504115 -3.049392

H -3.649901 -0.926601 -3.015481

H $-2.219100-0.2187601 .795506$

H $\quad 0.3418640 .592236 \quad 3.297288$

H $-2.081287-2.4038593 .065437$

H $-0.795167-3.5587852 .720855$

H -1.232588 -2.388726 1.502976

H $-1.795614-1.575874-2.361220$

H $-2.106519-1.930756-0.651494$

H $2.374897-2.615220-1.027114$

H $1.707430-4.101927-1.728574$

H $\quad 0.898740-4.8369260 .581747$

H $3.329743-3.0611961 .137144$

H $3.619246-5.1511262 .580845$

H $1.886467-5.3197502 .717739$

H $3.827528-2.7975485 .394304$

H $4.784373-3.7757274 .145230$

H $1.406708-3.1411655 .467230$

H $0.592602-3.9770514 .190811$

H $1.609054-1.0537224 .216759$

H $\quad 0.217010-1.773130-0.648537$

C $-0.2866945 .060850-1.684116$

O $0.4520823 .830619-1.816442$

C $1.5535243 .858885-0.888816$

C $1.7191655 .319946-0.486748$

C $0.2732865 .774995-0.451744$

H $1.2350993 .292709-0.006677$

C $2.7972713 .216487-1.506328$

H 2.2294755 .4316250 .474588

H $2.2776305 .889816-1.238279$

C $0.1031727 .283586-0.484255$

H -0.1824045 .3885620 .467913$

C $2.5921321 .779900-2.027472$

O $3.2074664 .024055-2.619857$

H $3.624043 \quad 3.249637-0.788061$

H $1.8913201 .817754-2.871235$

O $3.8396051 .345490-2.579098$

C $2.1051590 .731319-1.006827$

C 2.7930490 .7301190 .371334

H $2.226865-0.265094-1.445790$

O $\quad 0.7040870 .901281-0.776584$

H $2.611581 \quad 1.682926 \quad 0.881457$

H $2.275246-0.0252250 .978539$

H $0.5791427 .743100 \quad 0.388158$

H $0.5547917 .719738-1.381626$

H $-0.9571047 .555331-0.473471$

C $-1.7905394 .775168-1.664402$

H $4.0018783 .610507-2.999375$

H $3.6854350 .485711-3.006611$

H $\quad 0.2538560 .838614-1.636079$

H -2.071238 $4.275634-2.600095$

H -2.342585 $5.721937-1.633299$

H $-0.0624365 .644587-2.586648$

C $5.817732-1.370086-0.261615$

O $4.467200-0.900307-0.155999$

C 4.2968880 .4212640 .361328

C 4.9041590 .5320411 .769576

C $6.3682060 .083017 \quad 1.780944$

C $6.552980-1.2691731 .086230$

H $5.724109-2.436413-0.505676$

C $6.568721-0.689374-1.415980$

H $4.8031951 .136535-0.291719$

O $4.160593-0.2500872 .701551$

H $4.833776 \quad 1.5743182 .102108$

H 6.7179100 .0069802 .817716

O 7.1741481 .0531431 .118741

H $7.617950-1.4825470 .937282$

O $6.035945-2.3003491 .931533$

C $6.007824-1.077161-2.768619$

H $6.5219680 .400297-1.344707$

H $7.619142-1.002136-1.400834$

H $4.150023-1.1671442 .378956$ 
H 7.0674441 .8961061 .591388 H $6.533817-2.2725242 .766344$

O $6.736798-0.455103-3.742626$

O $5.052430-1.818125-2.954130$

C $6.311066-0.709263-5.084981$

H $6.966351-0.152995-5.760609$

H $6.395409-1.775952-5.313268$

H $5.283109-0.362516-5.228382$

SCF Energy $\left(B 3 L Y P / 6-31 G^{* *} / / M M F F\right)=-3245.92059514$

\section{8}

MM̄FF Geometry

C $-2.795107-1.261146-3.007498$

C -1.855173 -0.984728 -2.091711

C $-0.574429-0.256197-2.383675$

O $0.474875-1.184195-2.012245$

C $-0.3965351 .015988-1.537533$

C $-1.2411202 .184155-1.992503$

C $-0.8314343 .114293-2.876629$

C $-2.5933822 .309429-1.343411$

C $0.4891453 .187628-3.593297$

C $1.2493724 .481229-3.264275$

C $1.7590704 .545239-1.814920$

C $2.3356465 .929083-1.513276$

C $2.9349985 .999961-0.102118$

O $2.777703 \quad 3.552622-1.659995$

O $3.7097317 .206044-0.015308$

C 3.9095114 .8187540 .086596

C $1.849617 \quad 6.1028730 .976724$

O $4.367744 \quad 4.788391 \quad 1.437364$

C $3.3162013 .448842-0.332947$

C $4.4374692 .377645-0.362874$

O $2.316363 \quad 3.0650350 .609499$

C $1.596774-1.239757-2.774520$

O $1.851936-0.582109-3.769292$

C $2.482500-2.250875-2.156898$

C $3.716612-2.457487-2.637473$

C $4.680915-3.381051-2.046674$

C $6.074543-2.821521-1.966413$

C $4.0117181 .013338-0.917118$

C 3.4969590 .0824000 .162008

C $4.264032-1.4456971 .975313$

O $2.293884-0.071454 \quad 0.356072$

C $5.031549-2.7078521 .738882$

C $4.468861-3.9251261 .688001$

C $5.251130-5.1947791 .504855$

C $4.742353-6.0466590 .363452$

C $3.992282-7.1372080 .596182$

C $5.178374-5.686849-1.040077$

C $4.316630-4.615900-1.647240$

N $4.510666-0.5341000 .874207$

H -2.673629 -0.913582 -4.029820

H -1.990322 -1.343572 -1.074056

H $-0.502370-0.020552-3.452949$

H $\quad 0.661910 \quad 1.301967-1.535871$

H $-0.5998890 .800293-0.480203$

H -1.518708 $3.922728-3.124249$

H -3.124761 3.213927 -1.658026

H -3.227379 $1.456256-1.594059$

H -2.485341 $2.357405-0.255515$

H $1.1229832 .318288-3.397189$

H $\quad 0.2810443 .172706-4.670376$

H $2.1049504 .546884-3.948834$

H $0.6030695 .343793-3.470582$

H $0.9322074 .326334-1.130771$

H $\quad 1.5724606 .706389-1.644387$

H $3.1277746 .161321-2.239113$

H $3.1180467 .956141-0.197556$

H $4.7890585 .029287-0.538330$

H $1.230034 \quad 6.992713 \quad 0.812247$

H 2.2919206 .2267051 .971396

H 1.1839125 .2375751 .000375

H 5.1501384 .2138751 .474892

H $5.2400262 .746028-1.015240$

H 4.8579592 .2467850 .640650

H 2.1003612 .1304360 .465390

H $2.112855-2.783173-1.285826$

H $4.077489-1.884866-3.488921$
H $\quad 6.502722-2.720129-2.969659$

H $6.755801-3.436538-1.374533$

H $6.065039-1.831279-1.497256$

H $3.2546651 .100844-1.702146$

H $4.8785020 .530649-1.386610$

H $3.190527-1.6273012 .086303$

H $4.625504-0.9580982 .886210$

H $6.109864-2.6166291 .630308$

H $3.393517-4.0200851 .821075$

H $5.191158-5.7573342 .445886$

H $6.318060-4.9888331 .352882$

H $3.648374-7.769721-0.215532$

H $3.700295-7.4182141 .602663$

H $5.107511-6.569211-1.690383$

H $\quad 6.241227-5.429844-1.034219$

H $3.277964-4.919327-1.784103$

H $5.474348-0.2762650 .681082$

C $-5.302730-1.307653-2.662289$

O $-5.279075-0.428003-1.516886$

C $-6.262683-0.885590-0.578771$

C $-7.347737-1.502659-1.447165$

C $-6.512847-2.237081-2.477179$

H $-5.787615-1.6692490 .025129$

C $-6.735514 \quad 0.2437750 .339595$

H -8.011950 -2.164087 -0.883099

H $-7.958750-0.737633-1.939725$

C -7.256513 -2.525183 -3.771031

H $-6.192676-3.194518-2.044157$

C -5.6233091 .0722331 .011640$

O $-7.5454561 .163669-0.405584$

H $-7.398954-0.1762391 .104937$

H $-5.206144 \quad 1.7906340 .295203$

O -6.2445081 .8596792 .038486$

C -4.4647160 .2735081 .636688$

C -3.5639281 .1686212 .510694$

H -3.876566 -0.1890120 .836105$

O $-5.001332-0.7748322 .442691$

H -3.4760052 .1645912 .060069$

H -4.0432701 .2809643 .491234$

H -8.126692 -3.162191 -3.581782

H -7.610892 -1.603116 -4.243972

H -6.606020 -3.041592 -4.484310

C $-3.998878-2.108664-2.710763$

H -7.006022 $1.498746-1.142651$

H -6.9530262 .3770331 .618375$

H $-4.251899-1.2793472 .802650$

H -4.064499 -2.881130 -3.486482

H $-3.853711-2.629366-1.755158$

H $-5.407630-0.676340-3.552532$

C $-0.974641-1.3858073 .438143$

O $-2.225360-0.7071493 .252808$

C $-2.139741 \quad 0.6085382 .702255$

C -1.2957751 .5231773 .607105$

C 0.0764190 .9109113 .901700

C $-0.033988-0.5557364 .329682$

H $-1.230803-2.3022833 .985518$

C $-0.328546-1.7978432 .105332$

H $-1.678006 \quad 0.5503181 .711407$

O -1.9838841 .7410754 .838450$

H -1.181593 2.4972913 .116597

H $0.569751 \quad 1.4776674 .700962$

O $\quad 0.895031 \quad 0.9953922 .741177$

H $0.959715-1.0178894 .343286$

O $-0.523898-0.6102035 .672891$

C $-1.111514-2.8791531 .391878$

H $-0.232982-0.9613471 .409757$

H $0.670550-2.2017332 .308336$

H -1.450098 2.3578095 .367860

H $\quad 0.9833811 .9360942 .510896$

H -1.433754 -0.266248 5.671553

O $-0.326362-3.4131940 .409059$

O $-2.264160-3.2014831 .643353$

C $-0.928112-4.451887-0.369348$

H $-0.224025-4.737859-1.155342$

H $-1.127221-5.3240950 .260117$

H -1.849939-4.095417 -0.838155

SCF Energy (B3LYP/6-31G**//MMFF) $=-3245.91030072$ 
08_00449

MM̄FF Geometry

C $1.578466-1.8221491 .504597$

C $0.452662-2.1977340 .880058$

C $0.154462-1.894182-0.565619$

O $-0.972627-0.989399-0.598583$

C $-0.238722-3.181378-1.307432$

C $-0.562421-2.948548-2.770054$

C -1.810971 -2.894660 -3.274549

C $0.627186-2.817197-3.683516$

C $-3.104784-2.982992-2.512800$

C $-4.120869-1.945854-3.013397$

C $-5.427445-2.009540-2.210643$

C $-6.511904-1.154764-2.867029$

C $-7.805426-1.148177-2.039301$

O $-5.160529-1.522765-0.890631$

O $-8.647218-0.100746-2.544494$

C $-7.459548-0.787426-0.580749$

C -8.594409 -2.453422 -2.194979

O $-8.603571-0.9131150 .261377$

C $-6.278757-1.6022270 .003095$

C $-5.860348-0.992506 \quad 1.360774$

O $-6.690372-2.9544830 .208776$

C $-0.6975090 .342993-0.596933$

O $0.4042430 .868814-0.594459$

C $-1.9891041 .063643-0.564209$

C $-1.9981642 .370684-0.263578$

C $-3.1830513 .218189-0.119423$

C $-4.5124322 .638571-0.522057$

C $-4.543907-1.534318 \quad 1.931171$

C $-4.166577-0.7529543 .175145$

C -3.2899541 .4786673 .884299$

O $-4.393785-1.185694 \quad 4.302341$

C -4.1344572 .6872233 .619888$

C -3.6457213 .9337973 .531083$

C $-4.4943025 .141808 \quad 3.247302$

C -3.9499755 .9976622 .122072$

C -3.4248427 .2097382 .369407$

C -4.0803995 .4828600 .701813$

C $-3.0222494 .463684 \quad 0.374304$

N $-3.602330 \quad 0.4781372 .880129$

H $2.327977-1.2595900 .953448$

H $-0.310980-2.7337531 .439381$

H $1.020553-1.439981-1.062167$

H -1.089600 -3.657017 -0.805131

H $\quad 0.578300-3.912679-1.241283$

H -1.921728 -2.771029-4.351128

H $\quad 0.336616-2.714400-4.734451$

H $1.215162-1.933741-3.418584$

H $1.266685-3.702218-3.605119$

H $-3.518430-3.988711-2.653294$

H -2.951484 -2.840996 -1.439349

H -3.683325 -0.942613 -2.928377

H $-4.329667-2.128721-4.074910$

H -5.749938 -3.056033 -2.154398

H -6.714096 -1.497359-3.889572

H -6.153790 -0.119874 -2.961876

H -8.815761 $-0.279486-3.485556$

H $-7.1825220 .276221-0.568444$

H -8.854247 -2.620251 -3.247207

H -9.546989 -2.405667 -1.655656

H -8.045227 -3.330839 -1.847437

H $-9.313128-0.376626-0.131642$

H $-5.7602290 .092693 \quad 1.246337$

H $-6.652482-1.1571442 .102933$

H $-6.128427-3.3540150 .891330$

H -2.893837 $0.493786-0.741862$

H -1.047367 2.863384 -0.062973

H -4.461259 2.214474 -1.531492

H $-5.3181573 .375207-0.547318$

H $-4.811089 \begin{array}{lll}1.850092 & 0.173153\end{array}$

H -4.630601 -2.591321 2.203264

H -3.726619 -1.454456 1.205459

H -2.220507 1.6969223 .803195

H -3.4976041 .0880244 .884857$

H -5.2030522 .5268703 .494592$

H -2.581251 4.0991203 .681629

H -4.5529555 .7290354 .172867$
H $-5.5257384 .855244 \quad 3.007199$

H -3.0542077 .8392751 .567199$

H -3.3489407 .5981613 .379542$

H -3.974999 $6.311479-0.010385$

H -5.0967125 .1032810 .567228$

H -2.0094854 .7996630 .603131$

H -3.4380370 .7229281 .908678$

C $3.229885-2.7206013 .226970$

O $4.269257-1.8173402 .800988$

C $5.243752-2.5664982 .051993$

C $5.028681-4.0209602 .446173$

C $3.513171-4.0622982 .548165$

H $4.979602-2.4573780 .993913$

C $6.648569-2.0027712 .279467$

H $5.429187-4.7189281 .705193$

H $5.481582-4.2501053 .417784$

C $2.982087-5.2612913 .313409$

H $3.099123-4.0809251 .532151$

C $6.753452-0.4785362 .049825$

O $7.035615-2.2665633 .633015$

H $7.364707-2.5375591 .644109$

H 6.2547330 .0465732 .873941

O $8.142176-0.1388222 .133188$

C $6.180106-0.0030440 .702059$

C 6.3250301 .5152850 .486411

H $5.116311-0.2589040 .658462$

O $6.839849-0.708938-0.347532$

H 6.0690922 .0402001 .414222

H $7.367085 \quad 1.742247 \quad 0.229897$

H $3.265583-6.1929742 .813224$

H $3.377806-5.2943484 .333976$

H $1.889630-5.2288933 .375506$

C $1.857571-2.0961442 .957370$

H $7.930482-1.9050443 .753296$

H 8.2085350 .8298712 .175858

H $6.440032-0.429090-1.188368$

H $1.808115-1.1312183 .477714$

H $1.078389-2.7362773 .387462$

H $3.349285-2.8204414 .313782$

C $5.0633831 .777634-3.028250$

O $5.8605181 .469921-1.876872$

C $5.4024532 .023043-0.640176$

C $5.3786153 .555338-0.722695$

C $4.5360184 .029371-1.912277$

C $4.9113663 .298211-3.205751$

H $5.6478451 .400730-3.877799$

C $3.7285681 .022950-3.009730$

H $4.3896931 .660199-0.432809$

O $6.7041194 .067428-0.839768$

H 4.9618813 .9550330 .209094

H $4.6783235 .107847-2.052307$

O $3.1551313 .802666-1.643768$

H $4.1748853 .501301-3.992162$

O $6.1691103 .798541-3.670292$

C $3.966933-0.464441-3.130457$

H $3.137626 \quad 1.214834-2.109769$

H $3.1008341 .316892-3.859225$

H $7.1123843 .660315-1.623049$

H $2.9273864 .295984-0.837337$

H $6.0610754 .749915-3.839933$

O $3.796176-1.067933-1.920442$

O $4.294894-1.015647-4.173419$

C $4.019653-2.481875-1.886333$

H $5.092281-2.677816-1.807835$

H $3.519923-2.883726-1.001338$

H $3.605245-2.977197-2.769248$

SCF Energy (B3LYP/6-31G**//MMFF)= -3245.91924157

0800450

MM̄FF Geometry

C $-1.5872603 .806575-0.686386$

C -2.1764884 .4357510 .342163$

C $-2.862728 \quad 3.793023 \quad 1.526657$

O -2.7937442 .3500731 .478538$

C -4.3466854 .2049811 .535169$

C -5.1226583 .6688632 .722950$

C -6.0194392 .6634762 .666687$

C -4.8614884 .3727884 .029828$ 
C $-6.404110 \quad 1.8409421 .468113$

C $-6.1514450 .348464 \quad 1.718611$

C $-6.283616-0.478657 \quad 0.431214$

C $-6.114766-1.9681650 .732878$

C $-6.115637-2.818698-0.545060$

O $-5.260257-0.043917-0.469300$

O $-5.622539-4.124917-0.206730$

C $-5.115998-2.212625-1.555984$

C -7.536746 -3.021005 -1.083500

O $-5.206894-2.870684-2.816327$

C $-5.272230-0.688240-1.750451$

C $-4.140250-0.055008-2.594303$

O $-6.473172-0.378313-2.469403$

C -1.6807461 .7578571 .995710$

O -0.6882312 .3145222 .437191$

C -1.8885360 .2932041 .941850$

C $-0.923674-0.5428812 .353704$

C -1.019449-2.004525 2.413547

C -2.294354 -2.641313 1.925943

C $-2.731595-0.140527-1.998148$

C $-1.941904-1.364516-2.427551$

C $0.167554-2.587340-1.908959$

O $-2.296304-2.112427-3.334346$

C $-0.088584-3.663707-0.902209$

C $0.867349-4.166290-0.106792$

C $0.644899-5.267500 \quad 0.887418$

C $1.092211-4.8905972 .281801$

C $2.301688-5.2533812 .741532$

C $0.099440-4.1846753 .174934$

C $0.018751-2.7047592 .917306$

N $-0.769465-1.500969-1.695940$

H - $1.5783672 .721800-0.732698$

H -2.159276 5.5245340 .355469

H -2.380039 4.1518212 .444330

H -4.814072 3.8951470 .594650

H -4.4274235 .3008881 .542299$

H -6.5363332 .3863243 .584728$

H -5.4930913 .9936654 .840398$

H -3.8194554 .2402754 .336585$

H -5.0627355 .4444953 .931083$

H -7.4700321 .9980961 .263509$

H -5.8678622 .1578890 .571691$

H $-5.141700 \quad 0.220353 \quad 2.127501$

H -6.857558 -0.0191842 .473506$

H -7.265832 -0.282204 -0.014109

H $-6.890745-2.3150431 .426693$

H -5.160764 -2.127361 1.251361

H $-6.191792-4.4889910 .492807$

H -4.112129 -2.416720 - 1.164394

H -8.150387 -3.560040 -0.351477

H -7.534530 -3.641711 - 1.985942

H -8.047146 -2.081721 -1.307414

H -5.069607 -3.820442 -2.658011

H -4.166451 -0.448787 -3.617963

H $-4.3757381 .015014-2.692276$

H -6.839504 -1.201999-2.829673

H -2.848270 -0.0600001 .584501$

H $\quad 0.016110-0.1204332 .706835$

H -2.532171 -2.305735 0.910586

H -3.126725 -2.380148 2.588071

H -2.246933 -3.7311051 .875269$

H -2.156296 $0.726054-2.348881$

H $-2.764596-0.089930-0.906418$

H $1.172897-2.168560-1.814113$

H $\quad 0.047980-2.987812-2.920411$

H $-1.099018-4.060349-0.839612$

H $1.882047-3.788354-0.196419$

H $1.205913-6.1458090 .542134$

H $-0.406015-5.5812470 .910902$

H $2.617832-5.0149543 .751588$

H $3.005745-5.7903302 .114291$

H $0.391636-4.3093134 .226812$

H $-0.872177-4.682603 \quad 3.105181$

H $0.906310-2.1583743 .239092$

H $-0.578898-0.854363-0.935781$

C $0.6075624 .411593-1.815959$

O $1.0160803 .038227-1.962391$

C $2.1698802 .813467-1.131148$
C $2.6301594 .189394-0.657915$

C $1.3139684 .933107-0.565486$

H $1.8263482 .230515-0.266927$

C $3.2573492 .026579-1.873282$

H 3.1660164 .1415790 .295008

H $3.2893554 .668748-1.391545$

C $1.4643646 .443259-0.523603$

H 0.8011504 .5999860 .344897

C $2.8382910 .596516-2.269220$

O $3.6620802 .742268-3.040585$

H $4.1299381 .981529-1.215621$

H $2.4736660 .051626-1.390700$

O $1.7454560 .648055-3.193632$

C $3.961844-0.209910-2.949822$

C $5.275428-0.310452-2.156977$

H $4.1761130 .230577-3.931387$

O $3.466678-1.523944-3.218994$

H $5.962221-0.989899-2.679195$

H $5.7786390 .665247-2.181726$

H 2.0435346 .7478300 .354362

H $1.9797656 .820630-1.413096$

H $0.4857516 .930281-0.468318$

C $-0.9185884 .525049-1.825194$

H $2.8875272 .803271-3.625979$

H $\quad 0.9953401 .068571-2.740128$

H $4.165017-2.011866-3.687841$

H -1.304836 $4.087777-2.754534$

H -1.204359 $5.583632-1.832309$

H $0.9896434 .934395-2.703064$

C $6.602034-0.8861191 .270865$

O $6.485385-0.630944-0.135215$

C $5.164386-0.754382-0.683551$

C $4.645702-2.183674-0.478204$

C $4.717344-2.6025930 .995018$

C $6.083303-2.2917021 .615368$

H $7.682129-0.8686441 .467017$

C 5.9601980 .2242522 .110816

H $4.509917-0.052804-0.159997$

O $5.395520-3.102658-1.269318$

H $3.606529-2.241375-0.813664$

H $4.522822-3.6787401 .074905$

O $3.710232-1.9213971 .737819$

H $6.048782-2.4108982 .704722$

O $7.041123-3.2317901 .119669$

C 6.6638771 .5403811 .879854

H 4.8939310 .3609461 .908859

H $6.040337-0.0036133 .180380$

H $6.331566-3.013614-1.021035$

H $2.848912-2.1581131 .353813$

H $\quad 6.745018-4.1191681 .385448$

O 5.9948802 .2669410 .939664

O 7.6975531 .8690642 .447220

C 6.5799983 .5275210 .600688

H 6.5894974 .1860741 .474218

H 7.5928693 .3843330 .212277

H $5.9679013 .987172-0.179929$

SCF Energy (B3LYP/6-31G**//MMFF)= -3245.91921174

0800451

MM̄FF Geometry

C $-0.6526102 .672364-1.338699$

C $-1.2520213 .801316-0.929547$

C $-2.4655293 .895752-0.028666$

O -2.8872472 .5892360 .427525$

C $-3.6373184 .540719-0.785626$

C -4.7720134 .9494840 .133530$

C -5.9268114 .2714050 .280492$

C $-4.559454 \quad 6.2430510 .878692$

C $-6.3234462 .977810-0.373266$

C $-6.854240 \quad 1.9717590 .658031$

C $-7.3153230 .672459-0.015470$

C $-7.986525-0.2602300 .992899$

C $-8.388635-1.5955870 .347711$

O $-6.165628 \quad 0.030712-0.575682$

O $-8.711752-2.515243 \quad 1.401644$

C $-7.170787-2.174944-0.400953$

C $-9.650575-1.460008-0.512600$

O $-7.532660-3.337074-1.143521$ 
C $-6.462682-1.154587-1.324607$

C $-5.130314-1.752150-1.829324$

O $-7.297865-0.871599-2.448439$

C $-2.2457512 .075574 \quad 1.513405$

O -1.4117732 .6329902 .209193$

C -2.6793020 .6694511 .664679$

C $-1.854283-0.1928462 .277605$

C $-2.078182-1.6274872 .467714$

C $-3.432690-2.1724672 .104224$

C $-4.206912-0.749808-2.532798$

C $-2.879057-1.412811-2.838350$

C $-0.777694-2.172044-1.727331$

O $-2.640808-1.899701-3.940908$

C $-0.934667-3.362576-0.831621$

C $-0.015059-3.7309070 .072520$

C $-0.163033-4.9072780 .993148$

C $0.083104-4.5465232 .442688$

C $1.230473-4.8872233 .053864$

C -1.031350 -3.860078 3.204987

C -1.065603 -2.376495 2.952851

N $-2.031632-1.441497-1.741340$

H - $1.0630831 .705978-1.060030$

H $-0.8138014 .753926-1.221659$

H -2.197285 4.5158850 .835689

H -3.995674 3.858901-1.565178

H -3.296284 $5.437986-1.319976$

H $-6.6835334 .692240 \quad 0.941763$

H $-5.427417 \quad 6.5213161 .486056$

H -3.700446 6.1643391 .551881

H $-4.376257 \quad 7.0612690 .174431$

H -7.109781 3.194807 -1.106151

H -5.492152 $2.525240-0.920075$

H $-6.059821 \quad 1.7521651 .383181$

H -7.692222 2.4169561 .208680

H $-8.0152070 .931147 \quad-0.818935$

H $-8.858853 \quad 0.221995 \quad 1.451059$

H -7.290180 -0.4682291 .817569$

H $-9.433842-2.128278 \quad 1.926039$

H $-6.450745-2.5065970 .360629$

H -10.490309-1.0998770.093950

H -9.963889-2.431832 -0.909754

H $-9.527518-0.768908-1.348951$

H -7.958540 -3.955047 -0.525062

H $-4.585523-2.176165-0.978716$

H -5.331024 -2.582870 -2.518369

H $-6.744227-0.560570-3.182080$

H -3.625486 0.3771301 .224824

H -0.9015250 .1801522 .652533$

H -4.223695 -1.622876 2.627174

H $-3.567399-3.2241642 .363375$

H -3.599588 -2.0844701 .027835$

H -4.639667 $-0.398206-3.475201$

H $-4.0242200 .134888-1.912369$

H $-0.008708-1.484079-1.364942$

H $-0.520272-2.496341-2.739952$

H -1.847012 -3.946714 -0.927976

H $\quad 0.910111-3.1667580 .144572$

H $0.553302-5.6739200 .670210$

H -1.154677 -5.3668620 .899154$

H $1.404554-4.6538754 .098972$

H $2.024126-5.4005772 .521128$

H - $0.889325-4.0041114 .284296$

H -1.974696 -4.360048 2.970943

H - $0.129136-1.8749363 .202661$

H -2.333751 -1.011331 -0.872127

C $1.8304732 .164411-1.360629$

O $1.5774730 .876501-0.758621$

C 1.9230500 .9570920 .631813

C $2.890648 \quad 2.122551 \quad 0.751557$

C $2.2724603 .101229-0.229688$

H 1.0002651 .2323351 .161238

C $2.396041-0.3829911 .195179$

H 2.9501082 .5169571 .769825

H 3.8974201 .8451130 .425223

C $3.2178684 .200613-0.681024$

H 1.4059223 .5660050 .255500

C $3.767440-0.9179290 .730630$

O $2.443296-0.2408712 .622040$
H $1.627069-1.1345650 .987137$

H $4.561065-0.3050771 .171870$

O $3.923457-2.2224501 .312001$

C $3.976997-1.025115-0.789353$

C $5.218564-1.832082-1.214155$

H $4.031548-0.021191-1.222400$

O $2.839346-1.675976-1.362218$

H $5.082979-2.885573-0.936779$

H $5.244417-1.820689-2.312392$

H 3.5665094 .7858260 .176170

H $4.0965053 .790971-1.189510$

H $2.7157944 .881772-1.375353$

C $0.6063252 .630288-2.159567$

H $2.694467-1.1053982 .990351$

H $3.259901-2.8061040 .905330$

H $2.941941-1.651025-2.328869$

H $0.429271 \quad 1.926642-2.982599$

H $0.8106783 .606438-2.614809$

H $2.6589762 .011269-2.063932$

C $7.9704050 .661938-0.743226$

O $6.7592360 .012613-1.158428$

C $6.558575-1.315860-0.664956$

C $7.718949-2.238844-1.077454$

C $9.069720-1.654683-0.656483$

C $9.205463-0.191498-1.082587$

H $8.0268891 .569805-1.357483$

C 7.9159581 .0838460 .734458

H $6.502000-1.2909670 .427096$

O $7.703725-2.427116-2.491595$

H $7.569055-3.221219-0.613533$

H $9.881271-2.235678-1.111234$

O $9.210262-1.7399140 .758201$

H $\quad 10.094218 \quad 0.252278-0.620034$

O $9.418559-0.136385-2.496255$

C 7.0044692 .2720830 .959595

H 7.5625950 .2740411 .377546

H $8.915413 \quad 1.3904281 .064565$

H $8.423826-3.041625-2.713844$

H $9.149669-2.6789161 .003487$

H $8.608653-0.453191-2.931951$

O 6.9744422 .5571532 .295693

O 6.4095542 .8842930 .084226

C 6.1830483 .6901682 .666433

H 6.2580623 .8175203 .749595

H 6.5627194 .5937462 .179947

H 5.1350003 .5226412 .404901

SCF Energy (B3LYP/6-31G**//MMFF)= -3245.91600122

0800452

MM̄MF Geometry

C $0.184407-0.9389672 .436463$

C $0.725859-2.0516651 .917914$

C $0.174345-2.8200740 .736492$

O $-0.735462-1.999980-0.034089$

C $-0.579883-4.0652871 .225327$

C $-1.060779-4.9477190 .090691$

C $-2.332386-4.992357-0.353209$

C $-0.015170-5.846860-0.515767$

C $-3.492598-4.1650120 .126293$

C $-4.319966-3.620567-1.047210$

C $-5.488800-2.759474-0.549257$

C $-6.441144-2.411965-1.693327$

C $-7.578495-1.493278-1.221780$

O $-4.946236-1.5576810 .009260$

O $-8.234271-0.969485-2.386488$

C $-6.969126-0.294323-0.467090$

C -8.645133 -2.258922 -0.430089

$\begin{array}{lllll}\text { O } & -7.987866 & 0.510588 & 0.122384\end{array}$

C $-5.923747-0.6933060 .602523$

C -5.2051800 .5756641 .116139$

O $-6.584696-1.3251331 .700305$

C $-0.172885-1.123875-0.912616$

O $1.014567-1.002147-1.169483$

C $-1.256591-0.288269-1.474988$

C $-0.9304390 .855248-2.095968$

C $-1.8575531 .831510-2.671515$

C $-3.3189741 .473430-2.703955$

C $-3.9421460 .305197 \quad 1.944746$ 
C $-3.293611 \quad 1.6146512 .352945$ C -2.0916633 .5817571 .390490$ O -3.3563342 .0349913 .505591$ C $-2.589117 \quad 4.405058 \quad 0.244223$ C $-1.7881555 .052519-0.615356$ C $-2.2970275 .867846-1.770388$ C -1.669285 $5.476774-3.091218$ C $-0.8112396 .299455-3.717918$ C $-2.0932784 .166265-3.724744$ C $-1.3529412 .991023-3.143306$ N -2.684137 2.2611941 .290128 H $-0.748049-0.5577412 .028631$ H $1.646780-2.4378102 .348052$ H $1.008740-3.1245560 .094734$ H -1.422750 -3.763490 1.857901 H $0.068208-4.6641581 .879402$ H -2.572518 -5.700162 -1.145488 H $-0.425610-6.493106-1.298945$ H $0.786407-5.254353-0.966593$ H $0.419229-6.4958820 .251472$ H -4.130669-4.800623 0.751909 H -3.163028 -3.3269920 .746655$ H $-3.670013-3.022119-1.698813$ H $-4.704438-4.459921-1.640095$ H $-6.020490-3.3161380 .231463$ H $-6.849050-3.321181-2.152443$ H $-5.884169-1.897060-2.488873$ H -8.563644 -1.721221 -2.908368 H $-6.4706220 .337848-1.215630$ H -9.074959 -3.060313 -1.042913 H $-9.482819-1.605340-0.162376$ H -8.257375 -2.7139470 .483608$ H $-8.6119380 .751097-0.583761$ H -4.9204871 .1934210 .256881$ H $-5.897678 \quad 1.177514 \quad 1.719138$ H $-6.041919-1.2198902 .497796$ H -2.280330 -0.612797 -1.329917 H $\quad 0.1237361 .121965-2.168368$ H $-3.7236391 .423553-1.689850$ H -3.468099 $0.501220-3.187694$ H -3.929733 $2.185456-3.262543$ H $-4.175169-0.2588302 .853680$ H -3.211629 -0.2823521 .377568$ H - 1.0061453 .4553361 .367319 H -2.370532 4.0495222 .339427 H -3.6674594 .4720120 .116855$ H $-0.7105745 .006751-0.477710$ H -2.086765 $6.922303-1.548855$ H -3.387941 $5.792665-1.860398$ H $-0.3756656 .037293-4.676444$ H $-0.5204297 .250691-3.284805$ H -1.885129 4.188212 -4.802305 H $-3.1791064 .075855-3.633490$ H $-0.2714943 .133072-3.105258$ H $-2.5893601 .768350 \quad 0.407082$ C 1.0197161 .3008013 .099463 O $2.142551 \quad 1.3491062 .195984$ C 2.8262432 .6052042 .383869 C 2.0234093 .3861053 .421692 C 1.3750412 .2711094 .220058 H 3.8029782 .3687802 .819752 C 3.0025963 .3183161 .036627 H 2.6605284 .0347534 .031007 H 1.2579294 .0180722 .958725 C 0.1864992 .7345335 .044475 H $2.128087 \quad 1.8233024 .882035$ C 3.8912582 .5496020 .034982 O $1.721002 \quad 3.477127 \quad 0.414358$ H 3.4044624 .3272091 .188122 H $3.4024481 .606490-0.232424$ O $3.9439183 .321090-1.173606$ C 5.3335822 .2829950 .514658 C $6.2644271 .711978-0.571758$ H 5.3312281 .6052631 .374842 O $5.928693 \quad 3.512050 \quad 0.944145$ H $6.2850972 .384270-1.438364$ H $7.2849011 .726057-0.164038$ H $0.4958193 .492848 \quad 5.771321$
H $-0.593495 \quad 3.173126 \quad 4.413215$ H -0.2573721 .9006955 .596712$ C $0.782093-0.1376793 .558030$ H 1.2563894 .1975100 .871093 H $4.4054234 .152866-0.970363$ H 5.5175243 .7614891 .788607 H $0.079931-0.1602904 .398351$

H $1.723974-0.5880443 .893209$

H 0.1478341 .6721082 .545678

C $5.792440-1.953315-0.094871$

O $6.118896-0.5734400 .115584$

C $5.912906 \quad 0.283473-1.012704$

C $6.785404-0.174874-2.193297$

C $6.539551-1.647107-2.534402$

C $6.574768-2.535957-1.285271$

H $6.147639-2.4583940 .812694$

C $4.274264-2.138856-0.167611$

H $4.8594920 .246308-1.308178$

O $8.167751 \quad 0.015551-1.895617$

H $6.5567970 .449212-3.065277$

H $7.306323-1.979488-3.244876$

O $5.275058-1.759295-3.179794$

H $6.205526-3.540946-1.516223$

O $7.935543-2.681874-0.864158$

C $3.879003-3.5785140 .041464$

H $3.773601-1.5258860 .592868$

H $3.854176-1.802642-1.119801$

H $8.366215-0.474663-1.079493$

H $5.142400-2.695604-3.406266$

H $8.424974-3.098172-1.594032$

O $3.938521-3.8912941 .367063$

O $3.565552-4.336766-0.867088$

C $3.592587-5.2395181 .697142$

H $3.670215-5.3546972 .781381$

H $2.563098-5.4514791 .395172$

H $4.287108-5.9364731 .218656$

SCF Energy $\left(B 3 L Y P / 6-31 G^{* *} / / M M F F\right)=-3245.90856828$

0800453

MM̄FF Geometry

C $-1.334440-0.0757663 .448657$

C $-1.590216 \quad 1.235123 \quad 3.304179$

C -2.7399541 .8428932 .527431$

$\begin{array}{llll}\text { O } & -3.419323 & 0.800535 & 1.793193\end{array}$

C -2.2169002 .8933521 .527690$

C -3.1692264 .0510391 .303001$

C -3.9118094 .2396890 .194760$

C -3.1998275 .0754852 .409456$

C $-4.0375633 .342073-1.003688$

C $-3.3651073 .923372-2.255625$

C $-1.8299463 .865801-2.207305$

C -1.224794 $4.463135-3.479265$

C $0.3029734 .301493-3.518711$

O $-1.455727 \quad 2.491076-2.093337$

O $0.742594 \quad 4.582816-4.856287$

C $0.6602722 .827881-3.227079$

C $1.0067845 .315026-2.608372$

O $2.0708272 .662051-3.108630$

C $-0.0471392 .258061-1.976544$

C $0.1577160 .726432-1.893448$

O $0.5030292 .872765-0.810203$

C -4.7604120 .8888051 .610878$

O

C $-5.166643-0.3276360 .875610$

C $-6.373293-0.3965910 .297186$

C $-6.874860-1.559026-0.430385$

C -8.329441 -1.842324 -0.169506

C $-0.6754470 .045726-0.797195$

C $-0.563516-1.466991-0.841533$

C $-1.602320-3.5084090 .147166$

O $0.238096-2.062768-1.554960$

C $-2.566860-4.002220-0.886285$

C $-3.696782-4.666913-0.599660$

C $-4.622597-5.221504-1.645320$

C $-6.056261-4.772594-1.469981$

C $-6.953726-5.562188-0.855845$

C -6.476012 -3.460969-2.094397

C $-6.088287-2.269674-1.264701$ 
N -1.465276 -2.069844 0.025260 H -2.000947 -0.7947992 .977415$ H -0.9501561 .9576533 .803368$ H -3.4214772 .2864483 .263323$ H -1.9787872 .4047240 .579201$ H -1.269557 $3.326201 \quad 1.877125$ H -4.530863 5.1346210 .141098 H -3.8623435 .9168562 .180043$ H -3.552425 4.6262833 .342818 H -2.1974615 .4833182 .576671$ H $-3.6702912 .331527-0.803466$ H -5.109398 $3.226186-1.209758$ H $-3.7143993 .335520-3.114584$ H -3.697114 $4.956819-2.414958$ H -1.478067 $4.413412-1.326155$ H -1.503470 $5.519203-3.583706$ H -1.646968 $3.954508-4.357507$ H $\quad 0.4596875 .485840-5.080924$ H $0.3503762 .236179-4.099933$ H $0.7680096 .339198-2.919513$ H $2.0960315 .225722-2.685214$ H $0.7250715 .216705-1.558031$ H $2.4744513 .023124-3.916387$ H $-0.1201000 .275012-2.855031$ H $\quad 1.2149830 .500506-1.721023$ H $0.4220792 .267922-0.056849$ H -4.459374 -1.148905 0.825494 H $-7.065306 \quad 0.4389360 .376696$ H -8.516258 -1.9195300 .907895$ H -8.675519 -2.778080 -0.613660 H -8.953713 $-1.035761-0.569609$ H $-0.345870 \quad 0.3698810 .195332$ H -1.733951 $0.305293-0.912047$ H -1.940128 -3.720005 1.166501 H -0.626016 -3.980053 -0.003666 H - $-2.301421-3.833372-1.927498$ H $-3.950372-4.8613480 .439832$ H -4.568028 -6.316757-1.586265 H $-4.282870-4.967207-2.657025$ H -7.992889 -5.266813 -0.756334 H $-6.668015-6.520460-0.434762$ H -7.552159 -3.461108 -2.302057 H -6.011160 -3.363617 -3.084033 H -5.051175 -1.961796 -1.395298 H -2.130924 -1.489049 0.526495 C 0.9387820 .1904734 .672029 O $1.713022 \quad 0.574495 \quad 3.514717$ C 3.1074970 .3695453 .805800 C 3.1997180 .2403355 .319599 C $1.917633-0.5097785 .618633$ H $3.385622-0.593723 \quad 3.362611$ C 3.9650081 .4855143 .204880 H $4.097869-0.2967865 .638786$ H 3.1928081 .2200215 .811575 C $1.509721-0.4561017 .080853$ H $2.044794-1.5595515 .320757$ C 3.8449181 .6295241 .673014 O 3.5745162 .7303443 .796463 H 5.0130251 .3272383 .486982 H 2.8669662 .0519191 .413196 O 4.8126332 .6034931 .258822 C 4.0666970 .3259450 .882870 C $4.1941640 .565361-0.634682$ H $3.232052-0.3562351 .080896$ O $5.260744-0.294146 \quad 1.362519$ H $3.4489881 .301344-0.956553$ H $5.1919390 .972181-0.840476$ H $2.277143-0.9170347 .711084$ H $\quad 1.3710410 .5755707 .421272$ H $0.570645-0.9939787 .244423$ C $-0.215299-0.7142254 .231441$ H 4.1298913 .4248943 .402377 H $5.6948592 .226246 \quad 1.418532$ H $5.356224-1.1448230 .901856$ H $-0.666047-1.1944645 .107836$ H $0.192752-1.5136633 .599335$ H 0.5706141 .1112155 .139532 C $4.856453-2.981917-1.625740$
O $4.984698-1.677534-1.046406$

C $3.996864-0.728031-1.452199$

C $4.098235-0.485951-2.965307$

C $3.996392-1.802570-3.746078$

C $4.903311-2.893867-3.161116$

H $5.747482-3.527623-1.289592$

C $3.624101-3.727676-1.092691$

H $3.002376-1.117531-1.210272$

O $\quad 5.343172 \quad 0.144499-3.261224$

H $3.2996830 .198432-3.271632$

H $4.276940-1.631175-4.792552$

O $2.648745-2.263595-3.731244$

H $4.646191-3.868735-3.590673$

O $6.253469-2.620329-3.549905$

C $3.604923-3.7821490 .420007$

H $2.702402-3.243036-1.423073$

H $3.609499-4.757524-1.465946$

H $5.3614550 .322865-4.216849$

H $2.093777-1.566703-4.120757$

H $\quad 6.521033-1.786294-3.126892$

O $2.310905-3.7810320 .855705$

O $4.600683-3.8360681 .129230$

C $2.136363-3.8118622 .275384$

H $2.591503-2.9281962 .731872$

H $1.063519-3.8016922 .485638$

H $2.567135-4.7277642 .690588$

SCF Energy $\left(B 3 L Y P / 6-31 G^{* *} / / M M F F\right)=-3245.91710169$

0800454

MM̄FF Geometry

C $-0.777268-4.2917821 .797022$

C $0.342010-3.5778451 .603627$

C $1.096934-2.8960412 .716592$

O $1.304464-1.5113032 .353556$

C $2.470823-3.5649142 .892065$

C $3.342618-2.9024833 .941750$

C $4.475196-2.2272573 .660235$

C $2.902511-3.0894845 .369500$

C $5.032463-1.9722692 .286644$

C $5.933937-0.7298042 .241935$

C $6.472092-0.4862810 .824056$

C 7.5054720 .6407500 .815402

C $7.9940470 .956232-0.607967$

O $5.362565-0.140347-0.012033$

O $8.7041552 .203712-0.560966$

C $6.7632161 .167932-1.516147$

C $8.992605-0.090070-1.115519$

O $7.1511461 .341368-2.876734$

C $5.6988440 .053555-1.392390$

C $4.3895650 .390326-2.145266$

O $6.215790-1.152114-1.967879$

C $0.278057-0.6495472 .592873$

O $-0.827419-0.9279273 .029938$

C $0.741573 \quad 0.7130942 .243983$

C -0.1048381 .7462862 .372552$

C 0.1898903 .1565672 .102713

C 1.5878203 .5061211 .670135

C $3.7238021 .695940-1.708008$

C $2.3312221 .876718-2.288195$

C $0.5347993 .586663-2.470200$

O $1.7403271 .008012-2.922204$

C $0.1513344 .834544-1.742070$

C $-0.9936664 .978335-1.058611$

C $-1.3799946 .245021-0.350543$

C -1.8055876 .0240821 .083719$

C -3.0563486 .3104051 .484332$

C -0.7629315 .5524972 .075475$

C -0.7982584 .0602832 .270079$

N $1.8225563 .132580-1.983508$

H -1.155479-4.385502 2.813605

H $0.755254-3.4644910 .605632$

H $\quad 0.543010-2.9465483 .662585$

H $2.990259-3.5902981 .926853$

H $2.333951-4.6183493 .172175$

H $5.058648-1.8318154 .489848$

H $3.623380-2.6766276 .083348$

H $1.944377-2.5912475 .545603$

H $2.789596-4.1546595 .596347$ 
H $5.618302-2.8475321 .981652$ H $4.219554-1.8416021 .564448$ H $5.356907 \quad 0.1430252 .574154$ H $6.771378-0.8605872 .938511$ H $6.923345-1.4170060 .460139$ H $8.354250 \quad 0.396558 \quad 1.466228$ H 7.0561231 .5506321 .237825 H 9.4411292 .1058490 .066124 H $6.3214312 .121991-1.204845$ H $\quad 9.858087-0.149700-0.444759$ H $9.3945720 .190526-2.095329$ H $8.564515-1.091771-1.190216$ H $7.3786960 .465941-3.233680$ H $4.5710960 .412102-3.227734$ H $3.681130-0.434455-1.984400$ H $5.467942-1.727323-2.199967$ H 1.7655790 .8288801 .907739 H -1.1196871 .5440062 .714356$ H 1.7159744 .5580801 .408318 H 1.8723832 .9303550 .785239 H 2.3014213 .2844522 .471370 H $3.6328201 .729124-0.616395$ H $4.3311122 .548412-2.031406$ H $-0.1956702 .781579-2.341268$ H $0.6353653 .788860-3.541354$ H $\quad 0.8463515 .669628-1.793824$ H -1.699180 4.152940 -1.030271 H -2.196756 $6.705163-0.921608$ H $-0.5565736 .970357-0.362889$ H $-3.360394 \quad 6.1798102 .517746$ H -3.8049436 .6753690 .789208$ H -0.955939 6.0159123 .052560 H 0.2192765 .9267361 .775699 H -1.767802 3.6976912 .615692 H $2.4558243 .822419-1.587820$ C $-1.183871-4.835999-0.708101$ O $-1.395219-3.468030-1.114767$ C $-2.132428-3.457708-2.349027$ C $-1.980314-4.856614-2.928247$ C -2.017149 -5.694055 -1.665080 H $-3.186024-3.303299-2.085810$ C -1.656436 -2.319267 -3.257671 H $-2.778497-5.105505-3.633947$ H - $1.018826-4.983571-3.438989$ C -1.482579 -7.103288 -1.853918 H $-3.055975-5.751559-1.312296$ C -1.662683 -0.920080 -2.604380 O $-0.315142-2.585222-3.688593$ H -2.264833 -2.306940 -4.169578 H $-0.819420-0.834418-1.907662$ O $-1.4184980 .022554-3.655579$ C -2.972144 -0.561808-1.878847 C $-3.0023620 .890825-1.363903$ H -3.104317 -1.246177-1.033301 O $-4.062301-0.768367-2.777618$ H $-2.0174791 .167114-0.968681$ H -3.241433 $1.558043-2.200943$ H -2.084400 -7.647344 -2.589335 H -0.446271 -7.095373 -2.208132 H -1.512888 -7.661313 -0.912960 C -1.601786 -5.0046820 .756642$ H $\quad 0.238702-2.662372-2.892820$ H -1.211061 $0.878284-3.245081$ H -4.884074 -0.590514 -2.289528 H -1.600794 -6.070406 1.014175 H -2.631403 -4.6440350 .872622$ H -0.116660 -5.053388 -0.838456 C $-6.396404 \quad 0.7365250 .209731$ O $-5.335296 \quad 0.739525-0.754394$ C $-4.044428 \quad 1.078609-0.243225$ C -4.0671782 .5108060 .303581$ C -5.1500912 .6659331 .376511$ C -6.5002842 .1017260 .913297$ H -7.311376 $0.592895-0.379457$ C $-6.275901-0.4486161 .180329$ H $\quad-3.777217 \quad 0.386250 \quad 0.562874$ O $-4.3195063 .420613-0.764689$ H -3.0826552 .7465600 .720380$
H -5.2635723 .7288961 .621872$

O

H -7.1901832 .0238121 .760827$

O $-7.0896883 .030927-0.002647$

C $-6.278569-1.7771960 .451630$

H $-5.352991-0.3897911 .763494$

H -7.121979 -0.4509041 .876379$

H $-4.2970914 .319745-0.395858$

H -5.3994212 .1231983 .235094$

H $-6.5266603 .064551-0.795120$

O $-5.762793-2.739776 \quad 1.271723$

O $-6.696339-1.955012-0.684612$

C $-5.696358-4.0536950 .708473$

H $-6.701136-4.4129840 .466838$

H -5.060944 -4.052574 -0.182448

H $-5.254799-4.7211361 .453211$

SCF Energy (B3LYP/6-31G**//MMFF) $=-3245.91626614$

0800455

MM̄FF Geometry

C -3.0792680 .1456722 .725710$

C -4.0927041 .0250002 .746340$

C -5.2561451 .0544661 .777349$

$\begin{array}{llll}\mathrm{O} & -5.211447 & -0.139493 & 0.959676\end{array}$

C -5.1999542 .2862130 .852887$

C -5.2872013 .6167051 .572195$

C -4.2373204 .4315341 .799605$

C -6.6699574 .0171812 .014169$

C $-2.7986204 .174498 \quad 1.447488$

C -2.3224295 .1109630 .331356$

C $-0.9690644 .666074-0.241026$

C $-0.4433235 .695927-1.241293$

C $0.8389665 .217368-1.937337$

O $-1.1820213 .413284-0.897693$

O $1.0767326 .077553-3.062219$

C $0.6035243 .798298-2.495046$

C $2.0647995 .345613-1.026937$

O $1.805223 \quad 3.254972-3.035334$

C $-0.0080522 .819700-1.465468$

C $-0.4561721 .522549-2.178168$

O $0.9617422 .502260-0.467654$

C $-6.352447-0.604206 \quad 0.396077$

O $-7.455243-0.0780490 .434901$

C $-6.098572-1.870164-0.335667$

C $-5.047311-2.660854-0.063921$

C $-4.707556-3.929047-0.714130$

C $-5.532672-4.344593-1.902335$

C $-1.1484460 .508422-1.255346$

C $-2.168117-0.321265-2.008618$

C $-2.383010-1.981727-3.855572$

O $-3.367718-0.248052-1.752117$

C $-2.202419-3.440318-3.558758$

C $-1.407274-3.999446-2.632930$

C $-1.291618-5.483382-2.430786$

C $-1.668522-5.915698-1.032114$

C $-0.731463-6.310716-0.153660$

C $-3.140391-5.973511-0.682178$

C $-3.674030-4.643563-0.219284$

N $-1.602132-1.127633-2.981383$

H $-3.061369-0.6364301 .971879$

H $-4.0951751 .791963 \quad 3.518031$

H -6.1832041 .0440392 .363746$

H -6.0219822 .2330560 .126301$

H -4.3053342 .2272280 .220586$

H -4.4161475 .3763562 .310734$

H -6.6841615 .0008322 .495742$

H -7.345525 4.0619301 .153698

H -7.0678493 .2934362 .732211$

H -2.1875814 .3381602 .343855$

H $-2.635313 \quad 3.130579 \quad 1.168214$

H -3.067492 $5.117529-0.475552$

H $-2.250083 \quad 6.133596 \quad 0.722017$

H $-0.259902 \quad 4.5295470 .583924$

H $-0.277714 \quad 6.664127-0.752181$

H $-1.2059805 .877889-2.011816$

H $1.156516 \quad 6.988610-2.730925$

H $-0.0967943 .892814-3.337116$

H $2.1945076 .385556-0.703758$ 
H $2.9828015 .085426-1.564560$ H $1.9994534 .727661-0.129101$ H $2.1519183 .898734-3.676679$ H -1.147371 $1.790080-2.988547$ H $\quad 0.4098101 .029984-2.637606$ H $0.7282641 .654410-0.057157$ H $-6.843843-2.134026-1.078167$ H $-4.362013-2.3626120 .727438$ H -6.566295 -4.545102 -1.600142 H -5.162841 -5.244580 -2.397367 H -5.536493 -3.555683-2.663139 H $-0.414285-0.164547-0.800213$ H -1.677886 $1.005333-0.434778$ H -2.056398 -1.776051-4.879969 H -3.443927 -1.723414 -3.776090 H -2.799624-4.101201-4.184909 H $-0.787461-3.375328-1.995640$ H $-0.254040-5.767415-2.649696$ H -1.906101-6.034941-3.153310 H $-0.998066-6.6484410 .842377$ H $\quad 0.323341-6.297146-0.407487$ H -3.296285 -6.6868390 .138263$ H -3.693072 -6.391493 -1.527579 H -3.148481 -4.2494180 .652392$ H - $0.593391-1.126270-3.100919$ C $-0.5846690 .217028 \quad 2.946879$ O $-0.438575-0.9533562 .118331$ C $0.841622-1.5613182 .372868$ C $1.668723-0.4887883 .061681$ C $0.6174570 .219228 \quad 3.892703$ H $0.644592-2.3994713 .052316$ C $1.426453-2.0579731 .047887$ H $2.481062-0.9049113 .664643$ H $2.103490 \quad 0.2006612 .327184$ C 1.0362641 .6072234 .345950 H $0.401238-0.3946584 .777506$ C $2.818706-2.6992771 .169878$ O $0.519732-3.0119460 .486656$ H $1.454604-1.2164890 .348885$ H $3.530549-2.0057891 .622928$ O $2.742189-3.8230522 .058171$ C $3.384192-3.224548-0.162642$ C $3.482455-2.203319-1.311671$ H $2.775663-4.070671-0.505592$ O $4.694517-3.7524850 .071785$ H $3.800529-2.752173-2.208127$ H $2.485829-1.801433-1.523581$ H $1.929641 \quad 1.5529174 .976613$ H 1.2659562 .2551763 .493132 H $\quad 0.2401202 .084073 \quad 4.926395$ C $-1.924641 \quad 0.151413 \quad 3.687276$ H $0.491196-3.7777531 .085552$ H $2.605200-3.4798982 .957206$ H $4.620287-4.4033130 .790717$ H -2.006301 0.9895224 .388790 H $-1.980239-0.776314 \quad 4.270251$ H -0.5591321 .0858872 .276523$ C 4.9067250 .8417590 .360249 O $4.033791-0.230238-0.020381$

C $4.493026-1.056932-1.091832$

C $4.667224-0.208980-2.362840$

C $5.6042660 .976365-2.109987$ C $5.222804 \quad 1.747037-0.841602$ H 4.3241521 .4327561 .078773 C $6.1640740 .333146 \quad 1.077081$ H $5.456771-1.500412-0.822256$ O $3.3929390 .269675-2.789775$ H $5.069638-0.843299-3.161735$ H $5.5694971 .652737-2.972832$ O $6.9361050 .483018-1.996540$ H $6.0200192 .448226-0.570790$ O $4.0678172 .541148-1.117812$ C $5.810009-0.3174632 .394761$ H $6.727597-0.3792020 .468194$ H 6.8460221 .1606051 .304929 H $3.5294180 .784859-3.602994$ H $7.5222941 .247987-1.867057$ H $3.3248301 .939629-1.297512$
O $6.245689-1.6095022 .409665$

O $5.218693 \quad 0.253276 \quad 3.302238$

C $5.967219-2.3416743 .607221$

H $6.540320-1.9257414 .440992$

H $4.895952-2.3249553 .829439$

H $\quad 6.274831-3.3788893 .450229$

SCF Energy (B3LYP/6-31G**//MMFF) $=-3245.91780985$

0800456

MM̄FF Geometry

C $2.542972-2.684797-3.208243$

C $3.007112-1.705104-2.418902$

C $3.693639-0.464412-2.921477$

O $4.823429-0.262181-2.043087$

C $2.7544260 .746389-2.799733$

C $3.2432551 .966524-3.552484$

C $3.8200183 .042862-2.982987$

C $2.9970741 .941905-5.039340$

C $4.1692643 .251509-1.535470$

C $3.3198144 .345254-0.872440$

C $1.8616463 .925128-0.628288$

C $1.0640615 .074173-0.009727$

C -0.3684564 .6522980 .351016$

O 1.8738552 .8124260 .269528

O -0.9016155 .6890701 .189090$

C -0.3172903 .3453641 .173221$

C -1.266146 $4.590054-0.889851$

O $-1.6254562 .820641 \quad 1.388994$

C 0.5830962 .2549050 .545258

C 0.7773821 .0912231 .547456

O $-0.0419591 .756956-0.638546$

C $6.0123560 .127311-2.561620$

O $6.2663500 .338407-3.738664$

C $7.0384680 .246143-1.496428$

C $6.7426950 .208216-0.187156$

C 7.6906350 .2793310 .926501

C 9.1582390 .3420190 .596744

C 1.8167440 .0505621 .106769

C $2.095274-0.9752712 .191732$

C $3.751352-2.7356342 .813459$

O $1.384605-1.1134383 .183622$

C $4.716992-2.0817133 .752360$

C $6.018161-2.4002503 .831020$

C $6.979969-1.7426974 .780525$

C $8.155587-1.0993844 .076277$

C $9.356442-1.7019614 .050869$

C 7.9662410 .2786143 .479406

C 7.2029100 .2486652 .184625

N $3.224303-1.7324041 .906005$

H $2.643880-2.602372-4.287103$

H $2.882125-1.795888-1.342228$

H $4.027548-0.615461-3.954813$

H $2.6062840 .988810-1.742748$

H $1.7574530 .492480-3.185086$

H $4.1049123 .871813-3.630047$

H $3.3275332 .862799-5.531493$

H $3.5342481 .110187-5.505367$

H $1.9279381 .824875-5.244957$

H $4.1180002 .324647-0.957128$

H $5.2214133 .561797-1.497666$

H $3.787514 \quad 4.5823360 .092150$

H $3.3496105 .258602-1.479511$

H $1.4124643 .621496-1.580214$

H $1.0516605 .944207-0.678109$

H $1.5659305 .410840 \quad 0.908556$

H -1.8264535 .4690301 .391676$

H 0.0854783 .5983202 .163975

H -1.302042 $5.568557-1.383588$

H $-2.3005884 .356641-0.616550$

H $-0.9302043 .857779-1.626847$

H -2.1348993 .4875551 .878784$

H 1.1070531 .5026762 .510704

H $-0.1790350 .582318 \quad 1.721041$

H $\quad 0.3432330 .894629-0.857523$

H $8.0557200 .370617-1.852699$

H 5.7009590 .1060580 .113297

H $9.451517-0.513715-0.021709$

H $9.8030400 .320461 \quad 1.478031$ 
H $9.390071 \quad 1.2644490 .053147$ H $1.464295-0.4928250 .224600$ H 2.7621000 .5440360 .854650 H $4.225604-3.5110682 .203772$ H $2.930224-3.1870933 .379216$ H $4.319759-1.3093924 .407202$ H $6.414969-3.1830083 .188597$ H $7.334792-2.5122035 .478626$ H $6.477687-0.9914615 .402783$ H $10.213055-1.2360573 .575312$ H $9.508791-2.6771584 .501190$ H 8.9303300 .7842723 .356193 H 7.4191000 .9085204 .192710 H 6.1219850 .2023462 .318756 H $3.786042-1.4936031 .093923$ C $0.345613-3.806987-2.762306$ O $-0.109387-2.801403-1.833086$ C $-1.222305-3.324699-1.078219$ C $-1.688957-4.553906-1.839706$ C $-0.375258-5.095635-2.367031$ H $-0.814148-3.610037-0.100213$ C $-2.269226-2.218285-0.915364$ H $-2.221231-5.267553-1.203917$ H -2.348633 -4.271755 -2.669674 C $-0.539511-6.084716-3.507860$ H $0.157779-5.586808-1.541608$ C $-3.570713-2.688036-0.237931$ O $-1.674683-1.177807-0.134909$ H $-2.503962-1.780094-1.893044$ H $-4.012719-3.484859-0.846784$ O $-3.270667-3.2603491 .034934$ C -4.626516 -1.575063 -0.070491 C $-5.946841-2.1227140 .506607$ H $-4.821214-1.119027-1.048242$ $\begin{array}{llll}\text { O } & -4.146729 & -0.555142 & 0.802215\end{array}$ H -6.231782 -3.032110 -0.035519 H $-5.787562-2.3840381 .560157$ H $0.434413-6.433470-3.865008$ H -1.112022 -6.958459 -3.179820 H $-1.068228-5.635542-4.355306$ C $1.868032-3.918671-2.675865$ H $-0.855949-0.910763-0.586057$ H -2.894076 -2.554365 1.588203 H $-3.352153-0.168157 \quad 0.397888$ H $2.224606-4.779282-3.253100$ H $2.169617-4.083489-1.633355$ H $\quad 0.036569-3.479907-3.763533$ C $-7.7259121 .098743 \quad 1.203132$ O $-6.791111 \quad 0.0135431 .244252$ C -7.101169-1.103761 0.408210 C $-8.446393-1.7148090 .829174$ C $-9.559625-0.6613310 .837338$ C -9.1393140 .6161361 .574251$ H -7.3850281 .7875641 .986745$ C $-7.668893 \quad 1.852715-0.130307$ H $-7.165120-0.771814-0.633932$ O $-8.339406-2.3037532 .123900$ H $-8.705120-2.5194790 .131004$ H -10.447975 -1.087094 1.320008 O $-9.901415-0.353960-0.511275$ H -9.8663361 .4159611 .391571$ $\begin{array}{lllll}\text { O } & -9.168367 & 0.336310 & 2.977324\end{array}$ C $-6.296608 \quad 2.447271-0.347249$ H $-7.9176141 .222031-0.988662$ H -8.388237 2.680075 -0.140827 H $-8.047024-1.6119952 .741751$ H $-10.632200 \quad 0.287015-0.489555$ H $-8.967423 \quad 1.1639423 .446587$ O $-5.605368 \quad 1.703450-1.257370$ O $\begin{array}{llll}-5.881907 & 3.437805 & 0.240571\end{array}$ C $-4.2682332 .136866-1.523521$ H $-3.8132711 .428476-2.220858$ H $-4.2789483 .128794-1.984824$ H $-3.6801912 .144543-0.601271$ SCF Energy (B3LYP/6-31G**//MMFF) $=-3245.91442126$

0800457

MM̄FF Geometry
C -1.6501340 .1601374 .319463$

C $-1.8644610 .556361 \quad 3.056284$

C -2.7301051 .7326982 .688140$

O -3.5652551 .3430771 .573852$

C -1.862028 2.9039792 .200142

C -2.5642544 .2413162 .319467$

C -3.2599664 .8423351 .334866$

C $-2.4034144 .922483 \quad 3.654532$

C $-3.576040 \quad 4.314824-0.036681$

C $-2.8498875 .074843-1.154060$

C $-1.3681904 .689791-1.282622$

C $-0.693898 \quad 5.510801-2.384303$

C $0.7554425 .064852-2.628924$

O $-1.309278 \quad 3.296245-1.606944$

O $1.1927515 .644701-3.867494$

C $0.7815993 .533164-2.813573$

C $1.7053025 .588426-1.545113$

O $2.1209543 .055684-2.907189$

C $0.0169572 .765258-1.710079$

C $-0.0962331 .268481-2.086788$

$\begin{array}{lllll}\text { O } & 0.732257 & 2.882785 & -0.478934\end{array}$

C $-4.631853 \quad 0.537784 \quad 1.806735$

$\begin{array}{llll}\text { O } & -5.051266 & 0.150699 & 2.883869\end{array}$

C $-5.153482 \quad 0.150091 \quad 0.478633$

C $-5.847592-0.989003 \quad 0.350003$

C $-6.321522-1.564055-0.910388$

C $-6.066610-0.782394-2.173470$

C $-1.0685770 .462866-1.210839$

C $-1.086954-1.007849-1.595117$

C $-2.174339-3.168475-0.991927$

O $-0.360966-1.481862-2.465441$

C -3.253568 -3.450516-1.991639

C $-4.179967-4.407416-1.823020$

C $-5.239447-4.737663-2.837986$

C $-6.621851-4.860130-2.231876$

C $-7.145339-6.064154-1.945372$

C $-7.426575-3.593881-2.032593$

C $-6.917946-2.773723-0.877950$

N $-2.000779-1.733764-0.842113$

H -2.0979470 .7156625 .139610$

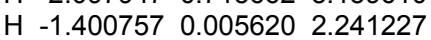

H -3.366044 2.0381193 .528596

H -1.5563422 .7362981 .161605$

H -0.9292942 .9611712 .777394$

H -3.693098 5.8207101 .540792

H -2.9314585 .8807583 .700601$

H -2.798132 4.2899354 .456061

H $-1.344973 \quad 5.1184103 .855379$

H $-3.3854593 .242456-0.121895$

H $-4.6570574 .433892-0.184841$

H $-3.3591364 .834349-2.096626$

H -2.947884 $6.156520-0.999850$

H $-0.8697314 .861163-0.322148$

H $-0.7324216 .581929-2.149677$

H - $-1.2538785 .390415-3.322477$

H $1.1111806 .610872-3.791259$

H $\quad 0.3063903 .312259-3.779698$

H $1.6950546 .684889-1.524743$

H $2.7416095 .302917-1.756470$

H $1.4479425 .239237-0.543076$

H $2.5583153 .554434-3.618466$

H $-0.441738 \quad 1.180758-3.125314$

H $0.8950220 .803063-2.033006$

H $0.5755582 .092600 \quad 0.061215$

H $-4.8843220 .773556-0.366678$

H $-6.048580-1.5790231 .243220$

H $-6.5634580 .193050-2.126444$

H $-6.429779-1.275713-3.076741$

H $-4.992297-0.622480-2.318580$

H $-0.7812740 .526934-0.156873$

H $-2.0863690 .855201-1.316531$

H -2.413724 -3.571616 -0.002826

H $-1.232321-3.608622-1.334650$

H $-3.256127-2.866051-2.908608$

H $-4.152078-5.016284-0.921894$

H $-4.958758-5.687912-3.311340$

H $-5.262530-3.997611-3.647565$

H $-8.144988-6.163625-1.535454$ 
H $-6.583388-6.977616-2.109761$

H -8.475425 -3.838938 -1.818852 H -7.454980 -3.040435 -2.974802 H $-7.049233-3.2610760 .089392$ H -2.598165 -1.247999-0.179686 C $0.629456-0.6486694 .981552$ O $1.289594-0.3210393 .739541$ C $2.516312-1.0650923 .653643$ C $2.831509-1.4851495 .081317$ C $1.440279-1.7884615 .602223$ H $2.299979-1.9633843 .061008$ C $3.615807-0.2560042 .952367$ H $3.504436-2.3466095 .124805$ H $3.280467-0.6684395 .657114$ C $1.356671-1.8481647 .117544$ H $1.112555-2.7522085 .189316$ C $3.311590-0.0304471 .453367$ O 3.7843020 .9995613 .605111 H $4.564117-0.8025453 .028850$ H $3.127741-1.0135351 .008522$ O $2.112101 \quad 0.7238981 .294054$ C 4.4564330 .6588940 .683610 C $4.1914410 .823615-0.826288$ H $5.380373 \quad 0.088616 \quad 0.835242$ O 4.6765391 .9633331 .225067 H $3.3360051 .491144-0.982135$ H $5.0573751 .350512-1.248618$ H $1.996594-2.6467857 .506785$ H $1.679102-0.9065427 .574785$ H $0.330983-2.0471817 .443562$ C $-0.819016-1.0371184 .685836$ H 4.2004360 .8354194 .467380 H 2.2539941 .5853351 .722934 H $5.4455312 .345676 \quad 0.769180$ H -1.277561 -1.510521 5.561531 H $-0.851526-1.7708483 .870036$ H 0.6736090 .2478635 .613047 C $5.034577-2.654761-1.892363$ O $5.100970-1.337555-1.330173$ C $3.972085-0.496349-1.582095$ C $3.798583-0.286049-3.094010$ C $3.696705-1.627500-3.829214$ C $4.811999-2.593254-3.413753$ H $6.030694-3.083219-1.721074$ C $4.013602-3.536502-1.155600$ H $3.066813-0.968304-1.188932$ O $4.8903280 .462902-3.624572$ H $2.8926890 .302565-3.272045$ H $3.752892-1.454320-4.910797$ O $2.439963-2.236738-3.550842$ H $4.610589-3.601768-3.793212$ O $6.041422-2.160900-4.005053$ C $4.400545-3.7716870 .290881$ H $3.016418-3.088748-1.158381$ H $3.959592-4.517657-1.641020$ H $5.710542-0.011723-3.405638$ H $1.742962-1.614836-3.821366$ H $5.922547-2.174284-4.970168$

O $3.393419-4.4535990 .913133$ O $5.450531-3.4176560 .809199$ C $3.619078-4.7691742 .291060$ H $4.372089-5.5585862 .369813$ H $3.932827-3.8822332 .849586$ H $2.679376-5.1331632 .714804$ SCF Energy (B3LYP/6-31G**//MMFF) $=-3245.91263871$

08_00458

MM̄FF Geometry

C -2.617364 $4.065938-0.772186$

C $-3.1155662 .854180-0.485856$

C -3.9578302 .5698330 .730277$

O -3.3233551 .5066801 .476539$

C -5.3402392 .0501800 .305233$

C -6.3862052 .1876501 .392142$

C $-6.741608 \quad 1.2138352 .252561$

C $-7.0831993 .523681 \quad 1.434286$

C $-6.141835-0.1555232 .409851$

C $-7.061359-1.2768051 .907960$
C $-7.011079-1.4580770 .383120$

C $-7.994736-2.541141-0.064758$

C $-7.881209-2.831705-1.568950$

O $-5.674698-1.8415940 .039323$

O $-8.589093-4.051630-1.837661$

C $-6.399388-3.083247-1.917920$

C $-8.554724-1.743833-2.413706$

O $-6.222661-3.216920-3.326599$

C $-5.440889-2.003413-1.364265$

C $-3.972305-2.455585-1.553276$

O $-5.649585-0.784796-2.081026$

C $-2.242419 \quad 1.8051872 .241574$

O -1.7463382 .9025642 .432254$

C -1.7363310 .5194342 .768136$

C -0.4773520 .4331053 .219417$

C $0.182164-0.7896313 .683109$

C $-0.631691-2.0582293 .698925$

C $-2.941953-1.596483-0.803356$

C -1.516362 -2.031636-1.103780

C $0.848954-1.405129-0.623872$

O $-1.243404-2.975826-1.840642$

C $1.383178-2.2984990 .452279$

C $2.478584-2.0061431 .169756$

C $3.065943-2.9034532 .221777$

C $3.461844-2.1531363 .476368$

C $4.745051-1.8311203 .711915$

C $2.381786-1.8399434 .488869$

C $1.483777-0.7244694 .032635$

$\mathrm{N}-0.582713-1.233315-0.456543$

H -2.847866 $4.902001-0.117037$

H $-2.8897422 .015636-1.140126$

H $-4.054223 \quad 3.4565431 .369433$

H $-5.261371 \quad 1.002182-0.002896$

H $-5.7007232 .593146-0.578917$

H -7.5368231 .4296362 .965660$

H -7.831308 3.5791022 .232100

H -6.3596754 .3270671 .605264$

H $-7.596723 \quad 3.7138960 .486173$

H $-5.155957-0.2297441 .944054$

H $-5.969704-0.3056893 .483521$

H $-6.728670-2.2096022 .382335$

H -8.092637 -1.096347 2.235541

H -7.252839 -0.504380 -0.098683

H $-9.023678-2.2642800 .196920$

H $-7.787191-3.4712970 .482743$

H $-9.511727-3.931563-1.553979$

H -6.120416 -4.051073-1.477773

H $-9.620027-1.670066-2.163828$

H $-8.514268-1.990485-3.480414$

H $-8.114329-0.754857-2.270727$

H $-6.832374-3.911014-3.630308$

H -3.861284 -3.486812 -1.192320

H $-3.725230-2.457479-2.622704$

H $-4.825009-0.274499-2.094520$

H $-2.399763-0.3367362 .715657$

H $\quad 0.139492 \quad 1.3309203 .216873$

H -1.480156 -1.959571 4.385169

H $-0.069156-2.9376654 .017546$

H -1.015039 -2.284786 2.697679

H -3.036827 -0.543639-1.086991

H $-3.099300-1.6768680 .278307$

H $1.297116-0.407163-0.593424$

H $1.048635-1.850013-1.604073$

H $0.869204-3.2406390 .626075$

H $3.004307-1.0767850 .969320$

H $3.949399-3.3892491 .787412$

H $2.376540-3.7147872 .485692$

H $5.041479-1.3138824 .618338$

H $5.527829-2.0726653 .000937$

H $2.831999-1.5265425 .440253$

H $1.839874-2.7590534 .726432$

H 1.9874140 .2419193 .978376

H $-0.903210-0.5032310 .172504$

C $-0.2840154 .638497-1.609821$

O $0.3293913 .472117-1.025194$

C $1.2698803 .911075-0.029740$

C $1.3689005 .429730-0.141791$

C $-0.0217905 .785489-0.633954$ 
H $\quad 0.8104103 .6902690 .943879$

C $2.5815093 .114545-0.109168$

H 1.6144155 .9006390 .815349

H $2.1218145 .746097-0.868209$

C $-0.1153027 .164579-1.261887$

H $-0.703762 \quad 5.7360200 .223238$

C $3.1409912 .873334-1.526679$

O 3.5731453 .7421900 .699658

H 2.3806382 .1287710 .328471

H $2.4102502 .305374-2.114686$

O $3.3098974 .117903-2.209940$

C $4.4998722 .140311-1.573773$

C $4.5109330 .828681-0.783982$

H $5.2962962 .803189-1.216985$

O $4.7928901 .843996-2.942960$

H $4.380522 \quad 1.0270940 .286708$

H $3.6402800 .229298-1.081517$

H $0.1566257 .937238-0.535197$

H $0.5561997 .260892-2.121697$

H -1.134638 $7.367366-1.605370$

C $-1.7462214 .350776-1.962767$

H 3.2126103 .8174951 .599713

H $3.9612474 .640131-1.710773$

H $4.7679062 .683813-3.432892$

H $-1.7764243 .494898-2.649270$

$\mathrm{H}-2.1710065 .204035-2.504009$

H $0.256008 \quad 4.823274-2.548402$

C $6.572097-2.208580-0.205355$

O $5.571150-1.187770-0.123646$

C $5.754261-0.057167-0.986858$

C $7.0821610 .639621-0.661972$

C $8.256498-0.344552-0.724894$

C $7.971785-1.6309440 .057264$

H $6.335723-2.8954440 .617489$

C $6.463354-2.998040-1.513420$

H $5.760510-0.403489-2.025841$

O 7.0240201 .2315020 .634309

H $7.252207 \quad 1.450760-1.378239$

H $9.1542250 .135026-0.315895$

O $8.525079-0.684577-2.082208$

H $8.727796-2.393446-0.163890$

O $8.058224-1.3477931 .457408$

C $5.122788-3.688365-1.604232$

H $6.562813-2.369774-2.403122$

H $7.251146-3.757385-1.583221$

H 6.8028410 .5306721 .271539

H $8.732046 \quad 0.138790-2.556331$

H $8.961325-1.0356131 .637724$

O $5.150011-4.856178-0.900952$

O $4.161547-3.230677-2.208956$

C $3.925601-5.596596-0.892218$

H $3.655082-5.892741-1.910144$

H $4.077973-6.498211-0.293085$

H $3.126459-5.004241-0.436891$

SCF Energy (B3LYP/6-31G**/MMFF) $=-3245.91312723$

08_00459

MM̄FF Geometry

C -0.860155 -2.731278 2.816091

C -1.553927 -2.077649 1.871273

C $-1.643073-2.5189670 .435826$

O $-0.984466-1.480747-0.329053$

C $-3.112183-2.615757-0.003697$

C $-3.299630-3.374635-1.301112$

C $-3.545206-2.805329-2.497160$

C $-3.243412-4.876047-1.179232$

C $-3.624256-1.340975-2.829170$

C $-5.051185-0.901388-3.184913$

C $-5.980962-0.819539-1.964149$

C $-7.415181-0.510756-2.398040$

C $-8.347119-0.325236-1.192257$

O $-5.4926320 .225354-1.116592$

$\begin{array}{llll}\text { O } & -9.567627 & 0.242677 & -1.690460\end{array}$

C $-7.7071730 .691777-0.224267$

C -8.724959-1.669277 -0.559348

$\begin{array}{lllll}\text { O } & -8.476767 & 0.805720 & 0.970933\end{array}$

C $-6.223819 \quad 0.395510 \quad 0.103553$

C $-5.612528 \quad 1.598296 \quad 0.860414$ $\begin{array}{llll}\text { O } & -6.155063 & -0.767478 & 0.929680\end{array}$

C $-0.213108-1.838986-1.384796$

O $-0.044644-2.970135-1.818496$

C $0.482621-0.668313-1.968172$

C $0.134327 \quad 0.603641-1.717987$

C $0.788277 \quad 1.801579-2.252924$

C $2.0428011 .608731-3.064417$

C -4.0941521 .5156481 .075076$

C -3.5493562 .7855361 .709267$

C -1.3966113 .8888792 .309170$

O -4.2662113 .7093232 .085701$

C - -1.0047114 .7542331 .152326$

C $0.266304 \quad 5.0170220 .813084$

C $0.6583085 .883040-0.348658$

C $1.4667785 .137564-1.387943$

C $2.8060745 .241535-1.423627$

C $0.7203294 .359727-2.448953$

C $0.2531863 .010024-1.976558$

N -2.165142 $2.762144 \quad 1.810398$

H $-0.353280-3.6593222 .566939$

H -2.034847 -1.1356942 .126152$

H $-1.123284-3.4763540 .310282$

H $-3.536173-1.609183-0.085683$

H $-3.705801-3.1233290 .768761$

H $-3.687391-3.461123-3.355489$

H $-3.430879-5.378700-2.134041$

H $-2.258827-5.198376-0.827136$

H $-3.999293-5.227562-0.469382$

H $-3.214106-0.710168-2.035006$

H -2.980878 -1.170482 -3.701811

H $-4.9853170 .091148-3.649346$

H $-5.472426-1.583159-3.934308$

H $-5.949327-1.770978-1.422647$

H $-7.799399-1.294202-3.063035$

H $-7.4251680 .415224-2.990242$

H $-10.1360190 .437219-0.925203$

H $-7.7654411 .673857-0.713415$

H -9.221559 -2.311109-1.297190

H $-9.447942-1.5363420 .253168$

H -7.864577 - $2.216615-0.168674$

H -8.2815530 .0295351 .523192$

H $-5.817367 \quad 2.5148490 .291192$

H $-6.0982341 .707327 \quad 1.838641$

H $-5.328447-0.7484951 .436492$

H $1.297672-0.916420-2.640490$

H $-0.7146850 .797030-1.064165$

H $2.5419492 .545106-3.322751$

H $1.8178051 .090801-4.002930$

H $2.7770721 .018880-2.504720$

H -3.8423930 .6783301 .733775$

H $-3.581593 \quad 1.3694780 .117405$

H -0.5290953 .4856762 .840728$

H -2.001583 4.4655813 .015975

H -1.811146 5.1880190 .565314

H 1.0741434 .5991121 .409060

H 1.2398446 .7264680 .046372

H $-0.2202786 .334483-0.826061$

H $3.3921654 .739361-2.185932$

H $3.3469615 .825213-0.686072$

H $1.3271594 .272349-3.357072$

H $-0.1569004 .936905-2.769109$

H $-0.6520843 .047598-1.370609$

H $-1.652246 \quad 1.981513 \quad 1.410129$

C $0.597569-1.3741274 .394182$

O $0.580421-0.2747993 .461769$

C $1.755482-0.3622342 .621547$

C $2.774882-1.0536713 .503742$

C $1.915307-2.1182384 .168251$

H $1.485343-1.0107041 .777027$

C 2.0649461 .0393092 .096851

H $3.598200-1.5034372 .947626$

H $3.182287-0.3600054 .248686$

C $2.521944-2.6806175 .442849$

H $1.780337-2.9446433 .460332$

C 3.2206601 .1514691 .084637

$\begin{array}{lllll}\text { O } & 0.866643 & 1.441119 & 1.402601\end{array}$

H $2.180177 \quad 1.751142 \quad 2.921953$

H 3.0225320 .4634890 .256243 
$\begin{array}{llll}\text { O } & 3.161008 & 2.472425 & 0.522066\end{array}$

C 4.6444220 .9386401 .627963

C $5.757094 \quad 1.290680 \quad 0.614903$

H $4.777248-0.0953031 .949153$

$\begin{array}{lllll}\text { O } & 4.817475 & 1.767446 & 2.779498\end{array}$

H 5.6685202 .3416350 .313873

H 6.7189111 .2105041 .139062

H $3.479110-3.1681355 .230799$

H $2.702777-1.8939276 .183039$

H $1.855240-3.4226895 .893872$

C $-0.681249-2.2057424 .215604$

H 1.0484352 .2981510 .982305

H 3.3426913 .1042961 .239188

H 5.6949121 .5739013 .151521

H -1.544680 -1.577399 4.470664

H $-0.687057-3.0471774 .917709$

H $0.579775-0.9251365 .394932$

C $6.142955-1.956709-1.170359$

O $6.161149-0.925455-0.175535$

C $5.764567 \quad 0.375616-0.618554$

C $6.7037760 .869487-1.727561$

C $6.738409-0.136164-2.884354$

C $6.993220-1.565332-2.392082$

H $6.630209-2.816223-0.690918$

C $4.706281-2.376749-1.521258$

H $4.7521780 .328307-1.026459$

O $8.0283501 .045629-1.233346$

H $6.3526711 .841414-2.095056$

H $7.5278290 .148506-3.590575$

O $5.497044-0.108750-3.583966$

H $6.823090-2.286532-3.200043$

O $8.366912-1.686237-2.009088$

C $4.019310-3.046460-0.347584$

H $4.083474-1.530599-1.821849$

H $4.738565-3.101925-2.342812$

H $8.0214901 .806550-0.628745$

H $5.3618950 .798085-3.907924$

H $8.908382-1.484399-2.791158$

O $2.893455-3.681364-0.787968$

$\begin{array}{lllll}\text { O } & 4.406918 & -3.007119 & 0.812122\end{array}$

$\begin{array}{llll}\text { C } 2.141775 & -4.360731 & 0.223283\end{array}$

H $1.331165-4.909406-0.263495$

H $2.773866-5.0763910 .757871$

H $1.709582-3.6334990 .915850$

SCF Energy (B3LYP/6-31G**//MMFF) $=-3245.90905036$

$08 \_00460$

MM̄FF Geometry

C -2.197761 2.9697281 .666494

C -3.532383 2.9407621 .810677

C -4.3229911 .8941512 .563002$

O

C -5.5829721 .4870241 .777077$

C $-6.633788 \quad 0.8237202 .646320$

C -6.907792 -0.4951552 .659365$

C $-7.440700 \quad 1.7644603 .505720$

C $-6.232449-1.5939271 .887599$

C $-7.150408-2.1955430 .813026$

C $-7.370617-1.260182-0.386035$

C $-8.373743-1.862410-1.371140$

C $-8.527530-0.993088-2.628946$

O $-6.106377-1.082901-1.030370$

O $-9.224753-1.766229-3.617419$

C -7.126569 $-0.687698-3.199213$

C $-9.3858920 .249652-2.364708$

O $-7.206007 \quad 0.236453-4.282183$

C $-6.122674-0.176255-2.139456$

C $-4.697143-0.144734-2.740293$

O $-6.493317 \quad 1.143617-1.738703$

C -3.495046 0.1106803 .960850

O -4.0893270 .4731404 .966668$

C -2.618443 -1.085004 3.957849

C $-2.098836-1.6253322 .843773$

C $-1.226048-2.8007682 .776136$

C $-0.790690-3.4256144 .075740$

C -3.587206 $0.121632-1.713234$

C $-2.205226-0.013607-2.329028$

C $0.196260-0.164820-1.673637$
O $-2.013280-0.108725-3.538097$

C $0.553931-1.608702-1.521329$

C $1.431102-2.067975-0.616198$

C $1.772738-3.521114-0.454406$

C $1.462702-4.0425110 .931773$

C $2.442808-4.2324821 .831275$

C $0.034255-4.4324031 .244184$

C $-0.840291-3.2499731 .563027$

N $-1.2105390 .001819-1.362094$

H -1.5888452 .1895292 .115302$

H -4.1111153 .7636451 .395028$

H $-4.5925572 .338386 \quad 3.528332$

H -5.2990800 .8318550 .947038$

H $-6.0484742 .366374 \quad 1.312216$

H $-7.706529-0.8436843 .313410$

H -8.228760 1.2469424 .063231

H -6.8002922 .2680924 .235985$

H -7.9249312 .5248472 .884143$

H $-5.284067-1.2723831 .447622$

H -5.972173 -2.383492 2.603930

H $-6.681712-3.1237360 .460654$

H -8.115005 -2.4681831 .259195$

H $-7.734435-0.292298-0.025066$

H $-9.347491-2.017305-0.890049$

H -8.029651 -2.859660 - 1.679547

H $-10.083473-2.027223-3.242674$

H $-6.735820-1.624644-3.620483$

H - $10.386867-0.041965-2.024473$

H $-9.5386450 .826572-3.283495$

H $-8.9606910 .912073-1.607973$

H $-7.834141-0.128567-4.928836$

H $-4.487650-1.115167-3.209778$

H $-4.6400030 .617111-3.528084$

H $-5.7072251 .611009-1.416032$

H -2.436373 -1.5129534 .938565$

H $-2.332861-1.1740601 .880927$

H $-1.653103-3.8359094 .612211$

H $-0.298871-2.6834484 .714724$

H $-0.075183-4.2408583 .948817$

H -3.671674 $1.133920-1.303845$

H $-3.658777-0.592064-0.884409$

H $0.7543680 .473123-0.984837$

H $\quad 0.3953080 .166225-2.697494$

H $0.061114-2.311522-2.189198$

H $\quad 1.932780-1.3653220 .045217$

H $2.841408-3.640524-0.672426$

H $1.252871-4.139649-1.196701$

H $2.235647-4.6361982 .816502$

H $3.474416-3.9847231 .604066$

H $0.005039-5.1727672 .051245$

H $-0.393308-4.9593690 .381247$

H -1.208571 -2.7293560 .679043$

H $-1.4743250 .034123-0.381363$

C $-0.3979753 .512730-0.000563$

$\begin{array}{llllll}\text { O } & 0.695113 & 2.975749 & 0.774917\end{array}$

C 1.9373203 .5062700 .265837

C $1.6106504 .029888-1.122137$

C $0.2140814 .578801-0.909935$

H $2.207997 \quad 4.332396 \quad 0.935730$

C 3.0069592 .4096910 .313262

H $2.3197854 .788088-1.466923$

H $1.5948923 .212160-1.853519$

C $-0.5546114 .796442-2.202109$

H $0.2891695 .534050-0.372753$

C $4.3365792 .796069-0.356725$

O 3.2579092 .0950531 .686814

H $2.6092841 .500646-0.147053$

H $4.1745672 .960541-1.428850$

$\begin{array}{llllllll} & \text { O } & 4.761447 & 4.050251 & 0.190478\end{array}$

C $5.4777991 .775992-0.159663$

C $5.1065900 .353506-0.617282$

H 5.7961521 .7581670 .889558

O $6.6005182 .221381-0.925936$

H $4.2230330 .011464-0.069517$

H $4.8598410 .386985-1.686160$

H -0.041289 $5.526310-2.836616$

H $-0.6530393 .866140-2.771785$

H $-1.5614265 .174157-1.998183$ 
C -1.4647564 .0689610 .944954$ H 2.4062721 .8509902 .088426 H $5.4820994 .384930-0.369201$ H $6.9637243 .007807-0.485249$ H -2.1731964 .6893920 .384246$ H $-0.988348 \quad 4.703671 \quad 1.702311$ H $-0.8054022 .687949-0.596161$ C $8.455355-1.226653-1.201122$ O $7.292361-0.395779-1.314590$ C $6.236631-0.672134-0.391109$ C $5.735678-2.112329-0.584117$ C $6.881169-3.123751-0.485354$ C $8.083305-2.713424-1.343067$ H $9.079227-0.953478-2.061741$ C $9.257974-0.9196330 .072844$ H $6.604127-0.5480410 .633317$ O $5.090128-2.245436-1.849319$ H $4.985057-2.3311120 .183944$ H $6.516851-4.106153-0.810603$ O $7.272220-3.2332980 .880032$ H $8.950682-3.339651-1.104006$ O $7.748044-2.968379-2.710678$ C 9.8019860 .4943010 .081019 H 8.646654-1.040659 0.970780 H $10.116026-1.5982290 .139709$ H $5.729103-1.991731-2.537285$ H $7.975257-3.9031620 .930846$ H $8.534169-2.770920-3.248056$ O $10.323268 \quad 0.759868 \quad 1.315842$ O $9.788588 \quad 1.263620-0.869869$ C 10.8789022 .0684621 .479856 H 10.1088312 .8294781 .321362 H 11.2508892 .1539442 .504260 H 11.7151732 .2140910 .789410 SCF Energy (B3LYP/6-31G**//MMFF) $=-3245.90336175$

0800461

MM̄FF Geometry

C $3.3687342 .914482-0.020016$

C 3.2477673 .8391740 .944593

C 2.1877903 .8681282 .021739

O 1.2308372 .8027701 .832440

C 1.4148085 .2004161 .953513

C 0.3300745 .3306333 .007564

C -0.9936865 .3678892 .751569$

C 0.8211035 .4393264 .428701

C -1.680680 5.3074201 .414781

C $-2.270023 \quad 3.9200881 .156437$

C -2.740886 $3.718630-0.290710$

C $-3.9372804 .604172-0.646490$

C $-4.4675024 .316381-2.061011$

O $-3.0963272 .334358-0.424226$

O $-5.7581044 .935634-2.179820$

C $-4.6793082 .794671-2.213203$

C $-3.5831974 .953304-3.139453$

O $-5.0488012 .460433-3.548797$

C -3.468073 $1.952301-1.756002$

C $-3.7661730 .435925-1.756586$

O $-2.3845012 .158453-2.671744$

C 1.3453921 .6849112 .598159

O 2.2150341 .4283643 .413688

C 0.1754450 .8339612 .287324

C $-0.180605-0.1547013 .120264$

C -1.374621 -0.9920292 .987320$

C $-2.275085-0.7470311 .804533$

C $-4.911785-0.008396-0.843624$

C $-5.044958-1.521627-0.803848$

C $-6.286217-3.3323120 .376512$

O $-4.389706-2.270675-1.523603$

C $-5.689996-3.7159881 .693603$

C $-4.600681-4.4885581 .823818$

C $-4.018971-4.899953 \quad 3.146987$

C $-2.712907-4.192853 \quad 3.444004$

C $-1.542275-4.8139223 .221689$

C $-2.849530-2.8023764 .031071$

C $-1.631026-1.9229323 .930239$

N -5.995504 -1.934356 0.118947

H $2.6679282 .086402-0.070502$
H $3.9708324 .652263 \quad 0.974539$

H 2.6839093 .7771092 .995225

H 0.9927865 .3058560 .949059

H 2.1107816 .0418352 .071405

H -1.676699 5.4552003 .595891

H 0.0092765 .6462455 .134253

H 1.3004514 .5078814 .744784

H 1.5466086 .2544524 .518246

H -2.478906 $6.059181 \quad 1.413412$

H -1.0078345 .5888680 .600440$

H -1.5193273 .1538751 .378909$

H -3.0978393 .7223831 .850308$

H -1.894271 $3.928131-0.955124$

H -3.687554 $5.667249-0.550790$

H $-4.7489834 .421230 \quad 0.071474$

H $-5.6507415 .887612-2.012006$

H -5.545235 $2.543399-1.588701$

H -3.528196 $6.039383-2.996702$

H $-4.0094444 .806387-4.137986$

H $-2.5608234 .570190-3.138115$

H $-4.2459312 .496710-4.096018$

H -3.954425 $0.095125-2.783455$

H -2.855473 -0.092376 -1.440732

H -1.761292 $1.418271-2.582625$

H -0.3976251 .0829441 .401517$

H $0.431385-0.3420454 .001461$

H $-2.7496860 .237077 \quad 1.881783$

H -3.070846-1.488672 1.705277

H $-1.706860-0.7894730 .870172$

H -4.7449430 .3517630 .177332$

H $-5.8654080 .392254-1.202637$

H -5.905906 -3.953142 -0.441253

H $-7.374250-3.4452860 .413278$

H -6.192059 -3.348086 2.585242

H -4.098095 -4.855634 0.931768

H $-3.874146-5.987913 \quad 3.129276$

H $-4.725410-4.7151203 .966442$

H $-0.587458-4.3504883 .442164$

H -1.511190 -5.8181872 .810840$

H -3.110286-2.902747 5.093030

H $-3.707022-2.2908773 .580297$

H -0.924296 -2.0492014.750681

H $-6.471586-1.2383290 .685647$

C $5.4831091 .864628-0.962339$

O $4.887070 \quad 0.552001-0.899128$

C $5.638063-0.2384790 .044847$

C 6.9031190 .5560920 .355748

C 6.3911351 .9782660 .260848

H $5.035282-0.2995320 .958042$

C $5.914634-1.638314-0.510897$

H $7.3155850 .310978 \quad 1.339254$

H $7.6852520 .379608-0.392498$

C $7.4919383 .015100 \quad 0.123934$

H 5.8051422 .1947211 .162728

C $4.660847-2.507795-0.744164$

O $6.607944-1.515366-1.759547$

H $6.602753-2.1655630 .160529$

H $4.075821-2.101009-1.576395$

O $5.115653-3.797374-1.177670$

C $3.757790-2.6819320 .493548$

C $2.739622-3.8332560 .398262$

H $3.234483-1.7399760 .693760$

O $4.586631-2.9509211 .630150$

H $3.271435-4.7920860 .347812$

H $2.191640-3.8532121 .350459$

H $8.1549382 .988787 \quad 0.994878$

H $8.1021382 .838059-0.768129$

H 7.0716154 .0229090 .050722

C $4.4154392 .951505-1.096186$

H $6.037728-1.006628-2.361527$

H $5.614380-4.195136-0.443366$

H $4.012148-2.9680822 .414769$

H $3.8927892 .820308-2.050565$

H $4.8942773 .936716-1.140303$

H $6.0925151 .876374-1.876226$

C $-0.017871-2.254022-1.558617$

O $0.972244-2535637-0.560977$

C $1.740757-3.724542-0.763652$ 
C $0.830378-4.963516-0.836387$

C $-0.286671-4.783837-1.868869$

C $-0.992870-3.435271-1.707613$

H $-0.580413-1.399391-1.163770$

C $0.611092-1.820440-2.891289$

H $2.298573-3.638922-1.700854$

O $\quad 0.254500-5.2100370 .444433$

H $1.445364-5.835271-1.090527$

H - $1.025141-5.587032-1.755075$

O $0.252169-4.875507-3.183685$

H -1.664414 -3.260330 -2.556575

O $-1.805396-3.505585-0.533225$

C $1.362302-0.510870-2.781751$

H $1.319352-2.561966-3.269362$

H $-0.183230-1.678950-3.633667$

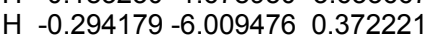

H $0.667497-5.750412-3.270893$

H $-2.336446-2.692209-0.496054$

O $1.823188-0.170512-4.022451$

$\begin{array}{llll}\text { O } & 1.534112 & 0.125390 & -1.751754\end{array}$

C $2.5806181 .040484-4.092976$

H $2.0297371 .870141-3.639442$

H $3.5469910 .899156-3.601151$

H $2.7541441 .272282-5.147132$

SCF Energy (B3LYP/6-31G**//MMFF) $=-3245.90575938$

0800462

MM̄FF Geometry

C $2.367126 \quad 1.2441932 .881028$

C 3.6991681 .0793922 .921223

C 4.7395791 .9371812 .239413

O $4.107163 \quad 2.927362 \quad 1.393243$

C 5.6856361 .1071631 .350010

C $6.704750 \quad 0.276827 \quad 2.102917$

C $6.715235-1.0702162 .132077$

C 7.8000701 .0542632 .785790

C $5.710698-1.9957381 .508044$

C $6.110430-2.3795790 .079256$

C $5.134332-3.386533-0.545825$

C $5.527413-3.685026-1.995612$

C $4.511045-4.598395-2.697292$

O $3.819598-2.824503-0.495807$

O $4.799566-4.543271-4.102929$

C $3.093481-4.023759-2.479735$

C $4.692571-6.062399-2.281101$

O $2.102990-4.901940-3.008135$

C $2.791399-3.682039-1.005213$

C $1.440747-2.956547-0.825185$

O $2.712370-4.900168-0.254419$

C $4.740649 \quad 4.121127 \quad 1.252928$

O 5.7606794 .4772741 .828144

C $4.046474 \quad 5.0073640 .291837$

C $3.1251514 .583527-0.587382$

C $2.4478375 .412826-1.589592$

C $2.730516 \quad 6.892941-1.579564$

C $1.337110-1.586628-1.499959$

C $0.023074-0.901012-1.168766$

C -1.174903 $1.246229-1.434148$

O $-0.868112-1.438110-0.516053$

C -1.300136 $2.262786-2.519728$

C -1.483933 $3.570055-2.281773$

C $-1.6135274 .601908-3.363847$

C $-0.6163845 .730441-3.217943$

C $-1.0177296 .960237-2.854551$

C $0.8271525 .458596-3.579699$

C $1.6038934 .819451-2.460009$

N $-0.046497 \quad 0.377490-1.706170$

H 1.9355852 .0590692 .306748

H 4.0939860 .2749613 .539332

H 5.2982932 .4464733 .033625

H $6.238817 \quad 1.780646 \quad 0.680524$

H $\begin{array}{lllll}5.088025 & 0.494926 & 0.663397\end{array}$

H $7.520654-1.5714662 .666838$

H 8.5708470 .4011633 .208928

H 8.2935851 .7228072 .072840

H $7.394903 \quad 1.6558433 .604933$

H $5.642405-2.8975362 .129112$

H $4.711901-1.5467891 .525730$
H $\quad 6.133594-1.469128-0.533874$

H $7.123767-2.800493 \quad 0.072329$

H $5.155627-4.3033480 .055265$

H $6.533867-4.119196-2.044547$

H $5.583806-2.740992-2.556238$

H $4.116184-5.059640-4.564114$

H $3.035884-3.107607-3.079817$

H $5.698732-6.410395-2.545163$

H $4.000339-6.717916-2.820761$

H $4.557914-6.224132-1.209674$

H $1.997554-5.639567-2.383354$

H $\quad 0.620230-3.605509-1.158327$

H $1.274182-2.8108390 .251098$

H $2.218290-4.7270890 .564077$

H $4.380126 \quad 6.0400750 .314092$

H $2.8505793 .530053-0.595796$

H $2.5064477 .321267-0.596036$

H $2.1345197 .455775-2.300696$

H $3.7829217 .084728-1.816206$

H $2.155018-0.937194-1.167907$

H $1.391135-1.689001-2.588622$

H $-1.0004911 .705455-0.455854$

H -2.084982 $0.642444-1.377931$

H $-1.2518891 .908543-3.546571$

H $-1.5592473 .918686-1.254003$

H $-2.6385554 .993030-3.327958$

H $-1.5011334 .153164-4.358756$

H $-0.3169197 .784644-2.778436$

H $-2.056273 \quad 7.170672-2.621847$

H $1.3155156 .377166-3.923633$

H $0.8640364 .793449-4.452146$

H $1.4632973 .740924-2.388505$

H $0.8032710 .795184-2.075622$

C $0.245240-0.1289092 .797249$

$\begin{array}{llll}\text { O } & -0.646686 & 0.970022 & 2.508461\end{array}$

$\begin{array}{lll}\text { C }-1.971114 & 0.629525 & 2.968173\end{array}$

C $-1.989902-0.8868093 .021829$

C $-0.594321-1.181168 \quad 3.529075$

H $-2.060329 \quad 1.0557353 .975700$

C -3.013159 $1.250592 \quad 2.032522$

H -2.771171 -1.279019 3.678907

H -2.133727 -1.309928 2.020531

C -0.147562 -2.610363 3.273198

H $-0.569842-0.9930974 .611181$

C $-4.459638 \quad 0.8379702 .350875$

$\begin{array}{llll}\text { O } & -2.906703 & 2.674178 & 2.128744\end{array}$

H -2.7694810 .9891750 .999704$

H $-4.575191-0.2427682 .232732$

O $-4.706505 \quad 1.107530 \quad 3.738438$

C $-5.5357791 .573571 \quad 1.532559$

$\begin{array}{llll}C & -5.385472 & 1.529848 & 0.000234\end{array}$

H $-5.5658672 .622814 \quad 1.851982$

O

H $-6.1625982 .181623-0.420845$

H $-4.4193531 .966252-0.276363$

H $-0.792021-3.3153883 .808378$

H $-0.193005-2.8595682 .208312$

H $0.881138-2.7649273 .613516$

C 1.4109980 .3752853 .654106

H -1.9863452 .9101001 .920820$

H -5.6192570 .8304283 .928302$

H -7.4903851 .5235301 .421320$

H $1.945879-0.4790364 .085790$

H 1.0200290 .9762884 .484771

H $0.607558-0.5118071 .835892$

C $-4.433822-2.016366-0.761190$

O $-4.428518-0.668600-0.274669$

C $-5.549726 \quad 0.136213-0.643539$

C $-5.6533390 .237938-2.176177$

C $-5.667421-1.147012-2.832362$

C $-4.549353-2.044528-2.294810$

H $-3.448058-2.413859-0.490969$

C $-5.491681-2.876735-0.057351$

H $-6.465535-0.319554-0.254029$

$\begin{array}{llll}\text { O } & -4.554243 & 0.993598 & -2.680317\end{array}$

H $-6.5693990 .784784-2.430231$

H $-5.547363-1.040685-3.917576$

O $-6.922628-1.777418-2.595805$ 
H $-4.697437-3.075258-2.637651$

O $-3.314211-1.590491-2.855961$

C $-5.174376-3.035723 \quad 1.412611$

H -6.496207-2.457016 - 0.157693

H $-5.524131-3.884762-0.486612$

H $-4.658968 \quad 1.059738-3.644594$

H -7.613549 -1.208166 -2.975466

H $-2.611200-2.184455-2.542044$

O $-6.231630-2.6157602 .164986$

O $-4.119260-3.4846521 .841684$

C $-6.062111-2.7210103 .582006$

H $-5.202908-2.1283483 .908992$

H $-6.961737-2.3265174 .061729$

H -5.940451 -3.769439 3.870192

SCF Energy (B3LYP/6-31G**//MMFF) $=-3245.91884404$

0800463

MMFF Geometry

C $1.972024-3.7704792522050$

C $1.901919-2.4329462 .442989$

C $0.872290-1.6054753 .164434$

O $-0.208062-1.3653112 .230078$

C $1.464027-0.2432713 .565201$

C $0.530496 \quad 0.559417 \quad 4.448402$

C $-0.283938 \quad 1.5414204 .014981$

C $0.578990 \quad 0.209615 \quad 5.913543$

C -0.4924412 .0166502 .603777$

C -0.0690793 .4827272 .440421$

C -0.3266653 .9979651 .017390$

C $0.138933 \quad 5.446484 \quad 0.864214$

C $-0.1835965 .999344-0.533833$

$\begin{array}{lllll} & 0 & -1.730058 & 3.920047 & 0.762382\end{array}$

O $0.004137 \quad 7.422742-0.505707$

C -1.667889 $5.717815-0.868590$

C $0.8159915 .485368-1.576302$

O $-1.9586146 .051781-2.222948$

C $-2.1070594 .262940-0.578759$

C $-3.6423424 .124900-0.672098$

O $-1.562166 \quad 3.346528-1.531894$

C -1.231538 -2.262928 2.208542

O $-1.393591-3.2161522 .953504$

C $-2.110987-1.9085631 .072538$

C $-3.087731-2.7492900 .698577$

C $-4.004010-2.560030-0.429777$

C $-3.756706-1.374035-1.325376$

C $-4.1239352 .723394-0.278970$

C -5.626811 $2.550023-0.403987$

$\begin{array}{llll}C & -7.414881 & 0.901698 & 0.137680\end{array}$

O $-6.369203 \quad 3.390385-0.901914$

C -7.461553 $-0.564146 \quad 0.425171$

C $-8.021202-1.475687-0.384132$

C -8.024076 -2.947514 -0.079622

C $-7.429272-3.788814-1.190592$

C $-8.125164-4.812212-1.714993$

C $-6.040972-3.459073-1.707022$

C $-4.989395-3.462025-0.627945$

N $-6.025853 \quad 1.315850 \quad 0.090946$

H $1.281674-4.3078653 .166850$

H $2.575719-1.9100081 .768477$

H $0.502602-2.1294084 .054633$

H $1.723545 \quad 0.332757 \quad 2.668400$

H $2.411077-0.3875284 .102779$

H -0.9019212 .0540254 .751568$

H -0.0958280 .8299916 .512708$

H $0.290440-0.834397 \quad 6.069331$

H 1.5917970 .3530086 .304292

H $\quad 0.0418181 .399094 \quad 1.876198$

H $-1.558903 \quad 1.9062962 .372581$

H -0.6316664 .0959753 .156680$

H 0.9960663 .5870692 .682594

H 0.2005813 .3468830 .313870

H 1.2113415 .5386371 .076015

H $-0.368864 \quad 6.070568 \quad 1.612706$

H $-0.586918 \quad 7.7943480 .170999$

H $-2.2781706 .386565-0.245764$

H $1.8314375 .816874-1.326537$

H $\quad 0.6031715 .898657-2.568279$

H $0.8364384 .396208-1.652306$
H -1.687056 $6.975781-2.358709$

H $-4.1236544 .852864-0.006581$

H -3.976442 $4.341610-1.694586$

H $-1.117873 \quad 3.851667-2.231400$

H - $1.913086-0.9755220 .558493$

H $-3.230124-3.6682841 .265614$

H $-3.870535-0.441755-0.765731$

H $-4.431595-1.317918-2.181256$

H $-2.741731-1.411333-1.737530$

H $-3.651993 \quad 1.971793-0.921563$

H -3.8431852 .5104820 .759270$

H $-7.8967891 .165115-0.809749$

H $-7.905844 \quad 1.4625900 .939380$

H $-6.999703-0.8945851 .353323$

H $-8.499010-1.153903-1.306173$

H $-9.064263-3.242766 \quad 0.108959$

H $-7.478228-3.1563460 .848971$

H -7.717822 $-5.424951-2.512394$

H $-9.119225-5.062575-1.359484$

H $-6.100609-2.499227-2.226854$

H $-5.734649-4.195485-2.461071$

H $-5.053677-4.3139290 .050429$

H -5.3513910 .7788720 .629488$

C $2.457853-4.789432 \quad 0.235300$

O $2.466391-3.521765-0.456344$

C $1.212238-3.366120-1.142854$

C $0.664161-4.778153-1.298052$

C $1.061035-5.3864620 .035589$

H $0.549255-2.809448-0.470378$

C $1.389671-2.578435-2.444929$

H $-0.416062-4.790349-1.471329$

H $1.152135-5.315419-2.119296$

C $1.036899-6.9048100 .049132$

H $\quad 0.363913-5.0193260 .798754$

C $2.091452-1.211842-2.273392$

O $2.177481-3.366614-3.345032$

H $0.411358-2.446679-2.922960$

H $3.158848-1.369886-2.084163$

O $1.998943-0.506225-3.516615$

C $1.497670-0.317608-1.171654$

C $2.0414831 .122070-1.124007$

H $1.665146-0.786919-0.197614$

O $0.081356-0.236005-1.358390$

H $1.6594001 .695385-1.978436$

H $1.5936651 .582690-0.235413$

H $\quad 0.026389-7.275122-0.152229$

H $1.705636-7.324167-0.709930$

H $1.350501-7.2887831 .025025$

C $2.910420-4.6001691 .690643$

H $2.120241-2.955173-4.223573$

H $2.543902-0.981641-4.165764$

H $-0.2796120 .307793-0.637459$

H $3.900986-4.130701 \quad 1.706565$

H $3.023246-5.5796182 .170272$

H $3.206559-5.409553-0.274009$

$\begin{array}{llll}\text { C } & 5.457502 & 0.456376 & 0.250577\end{array}$

$\begin{array}{lllll}\text { O } & 4.034134 & 0.491997 & 0.068564\end{array}$

C $3.5708011 .252680-1.050434$

C $4.0221332 .720326-0.932470$

C $5.5388292 .819877-0.742384$

C $6.0377551 .878006 \quad 0.357503$

H $5.602737-0.036579 \quad 1.220097$

C $6.155683-0.395537-0.822700$

H $3.9873760 .831078-1.970450$

$\begin{array}{lllllll} & \text { O } & 3.396743 & 3.357352 & 0.179794\end{array}$

H $3.7265523 .264912-1.837472$

H $5.8061193 .850236-0.476370$

O $6.1997002 .500666-1.962800$

H $7.133236 \quad 1.8350560 .344471$

O $5.6575742 .431597 \quad 1.620566$

C $5.787771-1.859460-0.720541$

H $5.897485-0.060327-1.830478$

H $7.242969-0.330507-0.701060$

H $2.4771043 .547063-0.067026$

H $5.8959313 .133644-2.635574$

H 6.0326881 .8644652 .315562

O $6.164738-2.494608-1.869407$

O $5.258899-2.3850910 .248354$ 
C $5.888703-3.897880-1.921177$

H $6.297419-4.407154-1.043063$

H $4.810437-4.061376-1.996647$

H $6.368331-4.305271-2.815210$

SCF Energy $\left(B 3 L Y P / 6-31 G^{* *} / / M M F F\right)=-3245.90188369$

08_00464

MM̄FF Geometry

C -3.316207 2.8074360 .025605

C $-3.0980833 .854330-0.785693$

C $-1.9876813 .979110-1.804310$

O $-1.0964882 .843898-1.748897$

C $-1.1437565 .241517-1.544371$

C $-0.1308225 .515934-2.643528$

C $1.2081495 .474642-2.492446$

C $-0.7137345 .881042-3.986206$

C $1.9965185 .195027-1.244208$

C $2.5116573 .755080-1.214892$

C 3.2674613 .4169480 .077882

C 4.6352624 .1023990 .153324

C 5.4282603 .6760451 .399631

O 3.4367561 .9909980 .108891

O $6.783641 \quad 4.106806 \quad 1.200201$

C 5.4282632 .1339291 .490640

C 4.9307454 .4021192 .655018

O 6.0374941 .6947382 .701779

C 4.0269701 .5096981 .325360

C $4.052589-0.0324691 .275050$

O 3.2238251 .8620972 .460383

C $-1.3417521 .796231-2.580828$

O $-2.258819 \quad 1.680768-3.376951$

C $-0.2713880 .802088-2.349015$

C $-0.153165-0.259745-3.158795$

C $0.885747-1.286106-3.071547$

C $1.880824-1.182915-1.944505$

C $4.952850-0.6609050 .210170$

C $4.812573-2.1759090 .191416$

C $5.722308-4.202596-0.931766$

O 4.035352 -2.787730 0.920155

C $4.878722-4.571100-2.110235$

C $3.677708-5.161665-2.011434$

C $2.842738-5.565331-3.193672$

C $1.649838-4.653264-3.391498$

C $0.432688-5.027591-2.961660$

C $1.945013-3.359436-4.124394$

C $0.911024-2.272341-3.992482$

N 5.663841-2.766606 -0.731994

H -2.6383901 .9584740 .015962$

H -3.806402 $4.680541-0.763517$

H -2.455038 4.045420 -2.794004

H $-0.6472105 .136008-0.574260$

H -1.796415 6.120205-1.457493

H $1.8273645 .680173-3.365243$

H $0.0509136 .224868-4.691343$

H -1.213473 $5.020059-4.439866$

H -1.441298 $6.692040-3.876851$

H $2.8370355 .898177-1.220787$

H $1.4118105 .403419-0.343483$

H $1.6651413 .065129-1.312217$

H $3.1516293 .555378-2.084466$

H 2.6382703 .7138030 .925283

H 4.5375665 .1938530 .128419

H $5.2212203 .834950-0.737386$

H 7.3100453 .7792661 .949839

H $6.075717 \quad 1.7796660 .679166$

H 5.0403835 .4868262 .535179

H 5.5323494 .1381613 .531670

H 3.8821804 .1992252 .881674

H 5.3955071 .8250543 .420411

H $4.323452-0.4287262 .263026$

H $3.025902-0.3775741 .091968$

H 2.4683631 .2526352 .500951

H $\quad 0.4104670 .985241-1.526204$

H $-0.865546-0.378344-3.973883$

H $2.497458-0.285203-2.059044$

H $2.556809-2.038747-1.887246$

H $1.367761-1.136899-0.977330$

H $4.697594-0.276340-0.782905$
H $6.003333-0.4299810 .415561$

H $5.396971-4.716507-0.020990$

H $6.766135-4.472054-1.120255$

H $5.287806-4.354668-3.094119$

H $3.267782-5.374754-1.026728$

H $2.516245-6.601992-3.039785$

H $3.440844-5.583791-4.113810$

H $-0.448332-4.413572-3.108706$

H $0.285529-5.973576-2.450028$

H $2.063899-3.588615-5.191608$

H $2.919282-2.967164-3.811473$

H $\quad 0.142257-2.293039-4.764784$

H $6.229981-2.177116-1.335350$

C -5.5293381 .6660850 .498436$

$\begin{array}{llll}\text { O } & -4.920735 & 0.366038 & 0.356014\end{array}$

C $-5.333135-0.194769-0.905464$

C $-6.5806450 .580378-1.309202$

C $-6.219478 \quad 1.974279-0.833739$

H $-4.5338800 .023379-1.624652$

C $-5.521884-1.710349-0.790613$

H $-6.7738810 .528734-2.384762$

H $-7.4724250 .220884-0.782962$

C $-7.4111872 .906996-0.705987$

H $-5.5127282 .405179-1.552711$

C $-4.301827-2.458396-0.215387$

O $-6.637463-1.9828510 .067713$

H -5.790860 -2.122029-1.769984

H $-4.204746-2.2083380 .846043$

O $-4.586668-3.862209-0.262679$

C $-2.970743-2.191769-0.943156$

C $-1.796734-3.060236-0.449819$

H $-2.702358-1.134559-0.843357$

O $-3.155967-2.449976-2.337299$

H -1.996965 -4.116800 -0.667093

H $-0.919580-2.794876-1.052625$

H $-7.8994093 .042529-1.676593$

H $-8.1569242 .512936-0.007377$

H -7.096909 $3.891449-0.344808$

C -4.4893802 .6943350 .959407$

H $-6.451423-1.5733700 .930299$

H -4.643265 -4.117785 -1.199495

H -2.319250 -2.245792 -2.788283

H -4.0948922 .3903131 .937169$

H $-4.9714573 .668847 \quad 1.100122$

H -6.2805791 .5609591 .292004$

C $-0.930785-1.1383812 .620890$

O $-1.125208-1.5054001 .247937$

C $-1.470933-2.8777671 .038211$

C $-0.316693-3.7761801 .499533$

C $-0.004155-3.5230102 .979243$

C $0.131382-2.0290753 .297211$

H -0.537538 -0.115077 2.570964

C $-2.282615-1.0867893 .348977$

H $-2.362743-3.1253341 .622995$

O $0.837922-3.5154810 .705568$

H $-0.596716-4.8250031 .345030$

H $0.929339-4.0320483 .248793$

O $-1.042939-4.071543 \quad 3.786233$

H $0.115419-1.8775844 .382472$

O $1.420617-1.6130282 .839928$

C $-2.272504-0.1697014 .552062$

H -3.043361 -0.706986 2.655376

H -2.600113 -2.080548 3.676666

H $1.552000-4.0943411 .022396$

H $-1.089416-5.0243643 .597729$

H $1.556368-0.6997803 .144112$

O $-3.537442-0.1021405 .064327$

O -1.2901090 .4110894 .992288$

C $-3.6917590 .736486 \quad 6.213525$

H $-3.0763930 .365357 \quad 7.038535$

H -3.4272151 .7693545 .967170$

H -4.741468 0.7064246 .517685

SCF Energy (B3LYP/6-31G**//MMFF) $=-3245.91627306$

$08 \_00465$

MM̄MF Geometry

C -2.252191 4.1144560 .709789

C -2.7879522 .8864860 .757304$ 
C -3.170796 2.1762172 .025949

O $-2.281306 \quad 1.0403132 .138326$

C -4.6076821 .6432041 .926275$

C $-5.134516 \quad 1.123606 \quad 3.247934$

C $-5.184468-0.1766183 .597419$

C -5.6703152 .1783734 .181764$

C $-4.683644-1.3643482 .823091$

C $-5.819340-2.2738572 .333055$

C $-6.627439-1.6745371 .170694$

C -7.771721 -2.609920 0.773065

C $-8.526930-2.100249-0.463912$

O $-5.736151-1.5012010 .065174$

O $-9.349288-3.171332-0.951503$

C -7.505481-1.773799-1.573936

C $-9.476366-0.947116-0.118141$

O $-8.145747-1.161390-2.691295$

C $-6.327961-0.895544-1.091610$

C $-5.240420-0.820642-2.191240$

O $-6.8100420 .419940-0.813870$

C -1.108224 1.2140492 .798598

$\begin{array}{lllll}\text { O } & -0.773583 & 2.195010 & 3.448551\end{array}$

C -0.2133020 .0452252 .631848$

C $-0.399520-0.8958161 .692805$

C $0.476003-2.0389051 .425287$

C $1.681862-2.2252372 .308363$

C $-3.946582-0.126047-1.743281$

C $-2.849997-0.224987-2.789013$

C $-0.376142-0.016741-3.029236$

O $-3.064790-0.530473-3.958958$

C $0.093287-1.437097-2.987569$

C $1.270930-1.830083-2.479296$

C $1.705390-3.268034-2.435139$

C $2.054879-3.738905-1.039855$

C $3.335038-3.949151-0.692524$

C $0.931870-4.063320-0.077984$

$\begin{array}{llll}\text { C } & 0.175506 & -2.853370 & 0.391828\end{array}$

N $-1.6038240 .090857-2.261752$

H -2.106535 4.6751791 .629186

H -2.898363 2.329081-0.168641

H -3.067829 2.8340062 .897421

H -4.6581860 .855668 1.167155

H -5.2828292 .4348081 .574732$

H -5.611322 -0.4291544 .567598$

H $-6.066158 \quad 1.751395 \quad 5.109375$

H -4.8818362 .8857934 .456263$

H -6.483494 2.7323093 .701452

H -4.038588 -1.075792 1.988335

H $-4.044020-1.9452843 .499775$

H -5.362974 -3.214911 1.999092

H -6.487982 -2.517373 3.168068

H $-7.023699-0.6995851 .474581$

H -8.463235 -2.7569951 .612128$

H -7.365399 -3.6050760 .542789$

H -9.950401 -3.437735 -0.234678

H -7.098685 -2.728811 -1.935170

H -10.219855-1.271791 0.619953

H -10.047313-0.628346 -0.997075

H -8.962324 $-0.076596 \quad 0.294412$

H -8.868697 -1.749623 -2.968928

H $-4.980318-1.839826-2.506574$

H -5.633758 $-0.296735-3.071509$

H $-6.0763551 .050149-0.880728$

H 0.6487030 .0343723 .291171

H $-1.262585-0.8177371 .033797$

H $1.380410-2.3232853 .357102$

H $2.361792-1.3711162 .214920$

H $2.261753-3.1186572 .066644$

H $-4.1292920 .937744-1.565563$

H $-3.577993-0.575397-0.814418$

H $0.3489950 .674360-2.588728$

H $-0.567714 \quad 0.282717-4.064501$

H $-0.578393-2.186752-3.400352$

H $1.957147-1.087592-2.078708$

H $2.574136-3.370803-3.098707$

H $\quad 0.936007-3.931122-2.850388$

H $3.601325-4.3237010 .289903$

H $4.146648-3.751691-1.383276$

H $1.300479-4.6327290 .784044$
H $\quad 0.226542-4.743014-0.573879$

H $-0.715580-2.639112-0.198961$

H $-1.5184740 .260147-1.263801$

C $-0.5294484 .005625-1.117505$

O $0.5459644 .063346-0.162352$

C $1.7925784 .228543-0.870919$

C $1.4610244 .093002-2.350479$

C $0.0360524 .610791-2.398819$

H $2.1271715 .248283-0.644276$

C $2.7977443 .201392-0.336206$

H $2.1462134 .660754-2.987226$

H $1.4979703 .042441-2.664464$

C $-0.7132964 .209817-3.657085$

H $\quad 0.0564175 .706077-2.321497$

C $4.1429923 .189823-1.083253$

O 3.0406513 .5042321 .042478

H $2.3447822 .203584-0.361507$

H $3.9929072 .845040-2.113258$

O $4.6145374 .540658-1.168348$

C $5.2405972 .347209-0.402792$

C $4.8496070 .876766-0.206085$

H 5.5195322 .8051390 .552173

O $6.4079752 .375600-1.231330$

H 3.9204900 .7860530 .367181

H $4.6364510 .427866-1.186317$

H $-0.2158574 .612617-4.545528$

H $-0.7640013 .121245-3.765181$

H -1.738008 $4.594038-3.640655$

C $-1.7523134 .741673-0.563098$

H 2.1802923 .4989221 .496240

H $5.3420164 .556760-1.812785$

H $6.7767063 .273822-1.186404$

H $-2.5610104 .765895-1.302448$

H -1.488799 $5.782917-0.339492$

H $\quad-0.7577902 .943429-1.273472$

C $6.387387-2.306740 \quad 0.955363$

O $5.478030-1.3261780 .438303$

C 5.9433100 .0302630 .471234

C 6.2436490 .4536851 .918975

C $7.192177-0.5288702 .613829$

C $6.761437-1.9846292 .411277$

H $5.817953-3.2440210 .955301$

C $7.603565-2.5063970 .043527$

H $6.8541150 .100685-0.131597$

O 5.0323210 .5498662 .666767

H 6.6997511 .4495031 .918456

H $7.216728-0.3004523 .686504$

O $8.505040-0.3333982 .096466$

H $7.550898-2.6642272 .752997$

O $5.617118-2.2216403 .237009$

C $7.186447-3.085611-1.287121$

H $8.168034-1.588508-0.143059$

H $8.310751-3.2126410 .495033$

H $4.595626-0.3185762 .637383$

H $9.100853-0.9365442 .572684$

H $5.381095-3.1610003 .150650$

O $6.905200-2.081172-2.165418$

O $7.099847-4.286321-1.509524$

C $6.480227-2.498447-3.466367$

H $5.550310-3.070356-3.397143$

H $6.296680-1.603438-4.066846$

H $7.263439-3.090691-3.949171$

SCF Energy (B3LYP/6-31G*//MMFF)= -3245.90286573

08_00466

MM̄MF Geometry

C $0.234494-3.4674630 .230716$

C $-0.237192-2.2129420 .166561$

C $-0.730395-1.4373351 .358665$

O $-2.168535-1.3809061 .225440$

C $-0.156323-0.0070111 .329718$

C -0.0066110 .6236592 .698385$

C $-0.8263721 .560448 \quad 3.213521$

C 1.2004300 .1694553 .479353

C -2.0987712 .1054102 .628864$

C -1.9854393 .5745052 .199657$

C -1.238524 $3.767700 \quad 0.870657$

C -1.1695645 .2513210 .503216$ 
C $-0.5525295 .474974-0.886168$

O $-1.962973 \quad 3.057235-0.136261$

O $-0.8295456 .828984-1.276793$

C -1.255212 4.554501-1.908905

C $0.9734575 .336850-0.857102$

O $-0.5940434 .640858-3.169636$

C $-1.3666043 .083936-1.438556$

C $-2.2878162 .275253-2.383260$

O $-0.0665472 .495322-1.439051$

C $-2.918663-1.3933702 .357873$

O $-2.521487-1.4028923 .511266$

C $-4.336911-1.3941451 .940455$

C $-5.296475-1.1123192 .832614$

C $-6.728511-1.1158722 .546716$

C $-7.556524-1.6745603 .672588$

C $-2.6516220 .880480-1.851779$

C $-3.650040 \quad 0.167978-2.746278$

C $-5.129820-1.840966-2.766702$

O $-3.9798960 .583346-3.853598$

C $-6.486422-1.336921-2.383064$

C $-7.396038-2.066687-1.719589$

C $-8.776052-1.573066-1.390189$

C $-9.105404-1.6716380 .082619$

C $-9.841346-2.6920190 .555145$

C $-8.673950-0.5403710 .988616$

C $-7.228238-0.6327511 .390689$

N $-4.118859-0.998613-2.157035$

H $0.246526-3.9889671 .184371$

H $-0.270850-1.708297-0.796512$

H $-0.455052-1.9657312 .279628$

H $-0.776843 \quad 0.6248110 .686023$

H $0.840556-0.0043320 .866614$

H $-0.587546 \quad 1.9611964 .198199$

H $\quad 1.302937 \quad 0.697126 \quad 4.433450$

H $1.135533-0.9004723 .699663$

H 2.1140050 .3494972 .905642

H -2.471391 1.4906231 .804890

H -2.8645152 .0357783 .412556$

H -3.0080753 .9581152 .086157$

H -1.505657 4.1639412 .990722

H -0.2310463 .3471490 .961456$

H $-0.6171495 .814876 \quad 1.265365$

H -2.184838 5.6725530 .498459

H $-0.4475907 .419146-0.604655$

H -2.267406 $4.956346-2.057244$

H $1.4124706 .060815-0.160200$

H $1.4116085 .560269-1.835994$

H $1.3016874 .343494-0.549722$

H -1.163826 $4.220390-3.834889$

H -3.223468 $2.829817-2.532926$

H -1.813107 2.161333 -3.365646

H $-0.1459811 .536344-1.558927$

H $-4.564019-1.6351280 .908166$

H -5.031077 -0.8883023 .863904$

H -7.196799 -2.671499 3.952526

H -8.613706 -1.783319 3.421588

H -7.491770 -1.025862 4.553120

H -1.760151 $0.248711-1.782965$

H -3.094902 $0.960581-0.852518$

H -4.954373 -2.864939-2.422596

H $-5.018816-1.812557-3.855389$

H -6.742632 -0.326874 -2.694917

H -7.151578 -3.085849-1.428658

H -9.488515 -2.172959-1.971866

H -8.922857 -0.539067-1.726555

H - $-10.115357-2.7548611 .602885$

H -10.183470 -3.490306 -0.095173

H -9.322954 -0.4847231 .870043$

H -8.8340010 .4172520 .476477$

H $-6.538141-0.2303870 .649531$

H -3.819652 -1.229270 -1.214534

C $2.093123-4.906304-0.678206$

O $3.049664-3.973058-0.144538$

C $4.320275-4.181968-0.784204$

C $4.202133-5.499273-1.543196$

C $2.737827-5.494781-1.933744$

H $4.438170-3.364266-1.505768$

C $5.460664-4.1265270 .240593$
H $4.877147-5.543662-2.403358$

H $4.416036-6.362886-0.903683$

C $2.203778-6.863034-2.319521$

H $2.608337-4.808468-2.781370$

C $5.422119-2.9229361 .203897$

O $5.432242-5.307121 \quad 1.055129$

H $6.421232-4.155209-0.286798$

H $4.642202-3.0743761 .960629$

O $6.665984-2.8962941 .916748$

C $5.208739-1.5546020 .538110$

C $5.353622-0.3949541 .544853$

H $4.210957-1.5287310 .086091$

O $6.163152-1.397733-0.511088$

H $4.910526-0.6790102 .506888$

H $6.421116-0.1958371 .700473$

H $2.747430-7.258310-3.183816$

H $2.309206-7.580810-1.499067$

H $1.143285-6.806996-2.584368$

C $0.763480-4.204454-0.967627$

H $4.557878-5.3500101 .479379$

H $6.776307-3.7651742 .339860$

H $5.991374-0.541505-0.938789$

H $0.020608-4.946426-1.282006$

H $0.895058-3.502127-1.799465$

H $1.949677-5.680707 \quad 0.086574$

C $4.6202952 .390769-0.836608$

O $5.250708 \quad 1.281882-0.183585$

C 4.6587330 .8888241 .055641

C 4.7634852 .0345982 .072041

C 4.1267673 .3150291 .517951

C 4.5977853 .6206360 .089111

H $5.2697322 .621288-1.690902$

C $3.2376432 .015382-1.389261$

H 3.6037410 .6429790 .892326

O 6.1362042 .2769012 .373664

H 4.2684081 .7339653 .002608

H 4.3820984 .1629372 .165121

O 2.7087403 .1794401 .521900

H $3.9712124 .401193-0.356150$

O 5.9250494 .1531240 .152503

C $3.2946360 .820297-2.317174$

H $2.5452201 .772658-0.578742$

H $2.8208922 .854833-1.956308$

H 6.1697262 .9842023 .040119

H 2.4292903 .0230472 .439951

H $6.5111173 .451306 \quad 0.484349$

O $2.055521 \quad 0.249657-2.387320$

O $4.2915440 .444771-2.918724$

C $1.955842-0.902542-3.229410$

H $2.587111-1.707836-2.841474$

H $0.915211-1.237431-3.224696$

H $2.237471-0.650096-4.256061$

SCF Energy (B3LYP/6-31G**//MMFF) $=-3245.90746818$

08_00467

MM̄MF Geometry

C $2.450351-2.4785653 .133923$

C $3.679274-2.2914432 .628258$

C $4.209641-2.8027971 .307547$

O $4.200271-1.7090800 .360026$

C $3.390412-3.9405200 .669120$

C $4.184582-4.774785-0.316707$

C $4.160199-4.630279-1.655610$

C $5.003272-5.8787880 .304496$

C $3.461162-3.579300-2.470839$

C $2.205486-4.104712-3.182754$

C $0.950989-4.035778-2.299309$

C $-0.262101-4.630341-3.015162$

C $-1.546987-4.462481-2.188381$

O $0.714268-2.653941-2.013513$

O $-2.663211-4.759442-3.041078$

C $-1.682099-2.983587-1.766556$

C $-1.612346-5.459069-1.024696$

O $-2.777056-2.805519-0.869908$

C $-0.393649-2.396946-1.145376$

C $-0.522558-0.861147-1.006984$

O $-0.200520-2.9737780 .147575$

C $5.215849-0.8098760 .379990$ 
O $6.175506-0.7888141 .131859$

C $4.9257050 .175634-0.684256$

C $5.5312191 .371191-0.672759$

C $5.3032462 .455906-1.630392$

C $4.3730782 .183962-2.784725$

C $0.791097-0.150720-0.636158$

C $0.5740651 .333362-0.397730$

C 1.6533463 .3255800 .636824

O $-0.4448671 .922415-0.748478$

C $2.185196 \quad 4.174574-0.474868$

C $3.1297665 .112361-0.302900$

C $3.6373185 .998860-1.405270$

C $5.1480306 .021842-1.495861$

C $5.8542427 .049612-0.995108$

C $5.831884 \quad 4.896384-2.240544$

C $5.9187673 .638140-1.419828$

N $1.6548881 .930353 \quad 0.235402$

H $1.702806-3.0211412 .562988$

H $4.398670-1.7348723 .226560$

H $5.241359-3.1415001 .466742$

H $2.507025-3.5216370 .177310$

H $3.014041-4.6267661 .438767$

H $4.746355-5.328212-2.253085$

H $5.549996-6.464094-0.442480$

H $5.739679-5.4649881 .000488$

H $4.354945-6.5690860 .854012$

H $3.234827-2.688560-1.878490$

H $4.175784-3.246037-3.234799$

H $2.042498-3.474935-4.067423$

H $2.366712-5.129321-3.539339$

H $1.140237-4.578162-1.367327$

H - $0.093782-5.687769-3.254205$

H $-0.402888-4.123330-3.980118$

H $-2.553131-5.666895-3.373285$

H -1.925174 -2.408242 -2.670820

H $-1.574274-6.488785-1.400176$

H -2.561121 -5.373279-0.483582

H $-0.795535-5.338703-0.309996$

H $-3.550424-3.243658-1.263996$

H $-0.864678-0.439413-1.961519$

H - $1.286851-0.621922-0.257440$

H $\quad 0.247458-2.3344300 .722280$

H $4.190062-0.100230-1.431540$

H $\quad 6.241141 \quad 1.5867550 .124674$

H $4.7656031 .373131-3.408452$

H $4.2240193 .043715-3.440453$

H $3.3792271 .897372-2.423829$

H $1.228720-0.5878410 .266925$

H $1.519085-0.251864-1.448919$

H $2.2597183 .403232 \quad 1.544807$

H $0.628142 \quad 3.6286540 .873276$

H $1.7530644 .032266-1.462626$

H 3.5378235 .2774330 .691752

H $3.2606487 .012290-1.214317$

H $3.2212525 .706544-2.377416$

H $6.9352357 .087605-1.080173$

H $5.3676067 .873585-0.483970$

H $6.8592355 .185101-2.500268$

H $5.3340354 .749636-3.202676$

H $6.5714613 .734145-0.550764$

H $2.4832881 .378497 \quad 0.436520$

C $0.965560-0.9232444 .511144$

O $-0.213601-1.3397293 .794178$

C $-0.735094-0.1947013 .078083$

C 0.0023861 .0135523 .635127

$\begin{array}{llll}\text { C } & 1.371031 & 0.418934 & 3.907574\end{array}$

H $-0.450023-0.3504082 .032685$

C -2.264142 -0.1798673 .199081$

H $\quad 0.031127 \quad 1.8526882 .934892$

H -0.4593191 .3581084 .568795$

C 2.2389701 .2743534 .812035

H $1.885396 \quad 0.2741222 .949176$

C -2.916187 1.1408002 .743540

O $-2.748354-1.2609832 .398958$

H -2.557959 -0.3985054 .232504$

H -2.5531801 .9192613 .427359$

O -2.4412761 .4916161 .451232$

C $-4.457764 \quad 1.1986152 .797364$
$\begin{array}{llll}C & -5.269258 & 0.247370 & 1.894937\end{array}$

H -4.7588002 .2263082 .557092$

$\begin{array}{llll}\text { O } & -4.847206 & 0.963812 & 4.155135\end{array}$

H $-5.084668-0.7949542 .174354$

H $-6.326550 \quad 0.4157642 .145294$

H 2.4245872 .2513194 .353659

H $1.762757 \quad 1.4439725 .783587$

H 3.2070970 .7956114 .989425

C $2.028824-2.0216384 .502771$

H $-2.324340-2.0753512 .720147$

H -2.7956302 .3715191 .238671$

H -5.8059841 .1136774 .217151$

H $1.623128-2.9030505 .015848$

H $2.897136-1.6946575 .087090$

H $0.647935-0.7755085 .552504$

C $-5.4106412 .170869-1.306433$

$\begin{array}{llll}\text { O } & -5.500459 & 1.778229 & 0.069317\end{array}$

C -5.0907340 .4422530 .378552$

C $-5.909659-0.580821-0.427657$

C $-5.855968-0.273767-1.927916$

C $-6.178104 \quad 1.194981-2.214362$

H $-5.926143 \quad 3.139145-1.354432$

C $-3.9548552 .394064-1.744973$

H $-4.0369890 .312557 \quad 0.121376$

$\begin{array}{llll}\text { O } & -7.266232 & -0.568892 & 0.012681\end{array}$

H $-5.509754-1.582776-0.230949$

H $-6.574928-0.909677-2.458656$

O $-4.558873-0.565933-2.437778$

H $-5.9713721 .435015-3.263895$

O $-7.5747701 .420196-2.003740$

C $-3.3669523 .645662-1.125536$

H $-3.3002921 .561759-1.475141$

H $-3.9287172 .524118-2.833419$

H $-7.745595-1.246047-0.494118$

H $-4.387314-1.509411-2.279645$

H $-8.0607740 .833823-2.608329$

O $-2.3091354 .065340-1.880901$

O $-3.761964 \quad 4.171437-0.093682$

C $-1.623706 \quad 5.222505-1.392108$

H $-1.2527795 .046684-0.377931$

H $-0.7705125 .414643-2.048026$

H -2.288703 $6.090882-1.415227$

SCF Energy (B3LYP/6-31G**/MMFF)= -3245.90070277

08_00468

MM̄FF Geometry

C $1.675860-3.2434650 .618015$

C $2.451678-3.9163021 .482092$

C $3.739025-3.4156732 .103479$

O $3.951869-2.0135321 .818769$

C $4.927463-4.2132731 .537002$

C $6.255419-3.8632492 .182149$

C $7.233340-3.142387 \quad 1.597391$

C $6.457986-4.4151783 .570493$

C $7.225075-2.5115860 .232755$

C $7.289501-0.9824820 .333908$

C $7.037153-0.303828-1.020730$

C $7.1712321 .214482-0.891356$

C $6.7846291 .946535-2.185043$

O $5.711298-0.647341-1.435988$

O $6.6148853 .337672-1.870133$

C $5.4141091 .424767-2.668127$

C $7.8995601 .872738-3.234747$

O $5.0869331 .964215-3.946184$

C $5.324689-0.113792-2.709200$

C $3.895931-0.630108-3.010610$

O $6.176660-0.604815-3.751293$

C $3.345421-1.1168642 .646182$

O $2.662970-1.3681723 .626846$

C $3.6262620 .241256 \quad 2.128279$

$\begin{array}{llll}C & 2.981146 & 1.293171 & 2.654512\end{array}$

C 3.1456522 .6965502 .268750

C 4.1070423 .0082221 .153305

C $2.828934-0.265034-1.973070$

C $2.0315660 .984988-2.302319$

C $0.2848652 .471253-1.320698$

O $2.0869391 .556913-3.387027$

C $0.9326503 .700143-0.766871$ 
C $0.403711 \quad 4.4294860 .227468$

C 1.0044445 .7078830 .736412

C 1.2065435 .7087602 .234339

C $\quad 0.3105906 .2863543 .052705$

C 2.4940995 .1302592 .772905

C 2.4380853 .6349502 .932004

N $1.2111121 .358353-1.245807$

H $1.971057-2.2525660 .288084$

H $2.145839-4.9157871 .785582$

H $3.680244-3.5649893 .188679$

H $4.971962-4.0711520 .452479$

H 4.754465 -5.289179 1.677138

H $8.150829-2.9727742 .159936$

H $7.457374-4.1990243 .962876$

H $5.732371-3.9838384 .266700$

H $6.335502-5.5033043 .568339$

H $8.096499-2.875777-0.324879$

H $6.349251-2.811501-0.345859$

H $6.534044-0.6419581 .052751$

H $8.269476-0.6821280 .725145$

H $7.757689-0.694594-1.748894$

H $8.1874231 .489506-0.582220$

H $6.5116921 .566883-0.087623$

H $7.4494693 .663280-1.491452$

H $4.6628261 .805776-1.966344$

H $8.8161802 .338169-2.852735$

H $7.6322312 .435167-4.136040$

H $8.1477260 .849919-3.525435$

H $5.1338542 .932779-3.872437$

H $3.593747-0.338867-4.023722$

H $3.947880-1.728518-3.029464$

H $5.875995-1.493374-4.004029$

H $4.347178 \quad 0.337671 \quad 1.325591$

H 2.2644281 .1138913 .455251

H 4.0778754 .0507850 .829770

H 3.8746392 .4122450 .264707

H 5.1337902 .7916761 .465879

H $2.097181-1.082409-1.935922$

H $3.263513-0.170053-0.973264$

H $-0.6113242 .186460-0.760442$

H $0.0015012 .642396-2.364250$

H $1.8594864 .026513-1.232825$

H $-0.539284 \quad 4.117210 \quad 0.670811$

H $\quad 0.328924 \quad 6.5262200 .453496$

H $1.956627 \quad 5.9297770 .238679$

H 0.4651936 .3198454 .126111

H -0.6001616 .7328412 .667469$

H 2.7038635 .5517353 .765738

H 3.3325115 .4640322 .154796

H 1.7590953 .3098753 .721674

H $1.2803330 .859701-0.363650$

C $-0.863669-3.1015610 .542877$

O $-1.986924-3.752502-0.092995$

C $-2.781797-2.761027-0.771796$

C $-2.468797-1.459818-0.059656$

C $-0.986050-1.6068250 .223587$

H -2.418120 -2.732229 -1.807207

C $-4.255569-3.184632-0.734322$

H -2.696737 $-0.577244-0.664949$

H -3.033463 -1.3901580 .877336$

C $-0.525993-0.6870501 .345435$

H $-0.428284-1.367319-0.691126$

C $-5.212538-2.106131-1.284332$

O $-4.351674-4.384848-1.505779$

H $-4.534999-3.4446250 .293925$

H $-5.064684-1.195003-0.697304$

O $-4.851568-1.793801-2.629766$

C $-6.708217-2.477307-1.222177$

C -7.647011 -1.402586-1.806739

H $-6.978417-2.681296-0.179358$

O $-6.933699-3.682474-1.956188$

H -7.456590 -1.289552 -2.881667

H -8.670610 -1.792842 -1.727939

H $-0.761677 \quad 0.3560841 .111103$

H -1.015249 -0.9350202 .293034$

H $0.552436-0.757461 \quad 1.499850$

C $0.409408-3.7829560 .027208$

H $-5.260507-4.718369-1.418765$
H $-4.975030-2.601889-3.157293$

H -7.866937 -3.929131-1.837941

H $\quad 0.462217-3.687903-1.065294$

H $\quad 0.328354-4.8627600 .209632$

H $-0.970063-3.2825651 .619597$

C -7.8008020 .9390681 .098855$

O $-7.883301-0.226950 \quad 0.269086$

C -7.563524 -0.034214-1.111933

C $-8.5111220 .995260-1.747539$

C $-8.4988632 .309058-0.959821$

C -8.6817912 .0720640 .543152$

H -8.2293800 .6299332 .061217$

C $-6.344143 \quad 1.3624861 .348161$

H $-6.5389000 .337376-1.201536$

O $\quad-9.836707 \quad 0.470678-1.780919$

H $-8.197906 \quad 1.172021-2.783696$

H $-9.3031182 .957428-1.328278$

O $-7.2598922 .970292-1.199591$

H -8.482413 2.9956561 .098133

O

C $\begin{array}{llll}-5.612065 & 0.374098 & 2.233707\end{array}$

H $-5.767631 \quad 1.4580340 .424971$

H -6.3415582 .3288911 .866414$

H -10.401179 $1.128482-2.221631$

H -7.279516 $3.813906-0.716581$

H $-10.232543 \quad 0.8842910 .370498$

$\begin{array}{llll}\text { O } & -4.489774 & 0.971763 & 2.733470\end{array}$

O $-5.967348-0.7761132 .451079$

C -3.7079590 .1743883 .628779$

H -3.487802 -0.8043623 .192915$

H -2.7642080 .6952803 .811292$

H $-4.237976 \quad 0.060148 \quad 4.578742$

SCF Energy $\left(B 3 L Y P / 6-31 G^{* *} / / M M F F\right)=-3245.91046074$

08_00469

MM̄FF Geometry

C -0.3135524 .4470172 .318525$

C -1.3116513 .8262141 .670941$

C -2.511188 3.2390902 .374064

O -2.6457481 .8505761 .990081$

C -3.7837653 .9878891 .939343$

C -5.0283693 .5472062 .686775$

C -6.0127252 .7849162 .169473$

C -5.1431744 .0624714 .099356$

C -6.0891122 .1802920 .795135$

C -6.1812140 .6507480 .871173$

C $-6.103078-0.001217-0.517620$

C $-6.247486-1.519803-0.403485$

C -6.033501-2.230759-1.748578

O $-4.8316670 .332510-1.081698$

O $-5.851052-3.630159-1.480389$

C $-4.722144-1.717738-2.383841$

C $-7.269919-2.120761-2.648339$

O $-4.552287-2.249205-3.695720$

C $-4.607048-0.179210-2.401127$

C $-3.214614 \quad 0.319347-2.858828$

O $-5.5660370 .346366-3.327194$

C -1.8698410 .9492472 .653872$

O $-1.032512 \quad 1.190576 \quad 3.509140$

C $-2.226966-0.4028852 .167993$

C $-1.570423-1.4715302 .643988$

C -1.820352 -2.872929 2.297807

C $-2.906074-3.1651881 .297699$

C $-2.043780-0.073494-1.951933$

C $-1.302178-1.325693-2.387466$

C $0.525210-2.848551-1.635744$

O $-1.500925-1.890653-3.458835$

C $-0.058036-4.051612-0.965901$

C $0.588980-4.761526-0.029266$

C $0.042181-6.0100210 .599440$

C $0.013812-5.9444192 .109149$

C $0.991000-6.5016362 .844458$

C $-1.196415-5.3211772 .765385$

C - $-1.078723-3.8273552 .898205$

N $-0.358106-1.714460-1.445265$

H -0.3772054 .5193953 .403251$

H -1.291049 3.7305250 .589361

H -2.4004033 .3049953 .463666$ 
H -3.9209173 .8782780 .858611$ H -3.653837 5.0663752 .104051 H -6.870162 2.559147 2.802766 H -6.0913493 .7808824 .569607$ H -4.3374103 .6632774 .722783$ H -5.0842885 .1557264 .110876$ H -6.9805042 .5733750 .291710$ H -5.234585 2.4674990 .179066 H $-5.357376 \quad 0.277886 \quad 1.492729$ H -7.117944 $0.362311 \quad 1.364316$ H -6.898631 $0.415711-1.146571$ H -7.223246 -1.785895 0.021882 H -5.502214 -1.899736 0.306801 H $-6.640516-3.951498-1.011948$ H -3.904020 -2.129667 -1.781642 H $-8.144185-2.559634-2.152336$ H -7.137499-2.690424 -3.574659 H $-7.521966-1.090124-2.906223$ H $-5.137304-1.754228-4.294314$ H -3.034189 $0.036238-3.902777$ H -3.250645 $1.418555-2.860642$ H -5.285567 $1.238862-3.589112$ H $-3.030218-0.4838151 .445653$ H $-0.776727-1.3092903 .372355$ H -2.944355 -4.2117630 .988024$ H $-2.756049-2.586246 \quad 0.380690$ H $-3.884630-2.9128961 .718845$ H $-1.3080180 .739631-1.977853$ H $-2.367004-0.185531-0.913215$ H $1.499726-2.577135-1.218964$ H $0.648602-3.043991-2.705663$ H - $1.044521-4.372539-1.292258$ H $1.587419-4.4543850 .274202$ H $0.674228-6.8477500 .276096$ $\begin{array}{llll}\text { H } & -0.962647 & -6.240912 & 0.224423\end{array}$ H $0.960323-6.4865823 .928919$ H $1.846252-6.9792522 .377925$ H -1.316016 -5.7254413 .779982$ H -2.102611 -5.6360802 .240195$ H -0.312894 -3.515849 3.610070 H $-0.295482-1.208848-0.566237$ C 1.0765465 .0388660 .206608 O $1.3594343 .686855-0.207055$ C $2.4373483 .704965-1.161261$ C $2.5972345 .159956-1.584821$ C $2.2517035 .880147-0.297279$ H $3.3424843 .404442-0.622451$ C $2.1606232 .731103-2.310795$ H $3.6072955 .379486-1.943788$ H $1.8896205 .435808-2.375291$ C $1.9225387 .349858-0.495431$ H 3.1009915 .7923920 .393728 C $1.8883701 .274198-1.881302$ O $\quad 0.999377 \quad 3.198507-3.012410$ H $2.9890402 .768751-3.028165$ H $0.948908 \quad 1.236115-1.316562$ O $1.6921390 .489131-3.064917$ C $2.9859710 .572714-1.061345$ C $4.3907360 .684713-1.686097$ H $2.722104-0.489271-0.994755$ O $2.977712 \quad 1.088503 \quad 0.265290$ H $4.3298980 .501804-2.765216$ H $4.7635871 .703420-1.529079$ H $2.7804017 .881886-0.919746$ H $1.0747007 .483125-1.175779$ H 1.6681297 .8258780 .456615 C $0.9156995 .089148 \quad 1.728898$ H $0.9625152 .728958-3.862674$ H $0.8463440 .760244-3.459528$ H 3.6388310 .5951010 .779353 H 0.9154196 .1357712 .056178 H 1.7831894 .6044262 .194979 H $0.1510725 .347856-0.295477$ C $6.441793-0.8657931 .040581$ $\begin{array}{lllll}\text { O } & 5.591551 & 0.020776 & 0.300301\end{array}$ C $5.396954-0.313652-1.076566$ C $6.741797-0.297195-1.821571$ C $7.779296-1.186340-1.129827$
C $7.831040-0.9619930 .385785$

H $6.564000-0.3827592 .018910$

C $5.786878-2.2320811 .274548$

H $4.957536-1.314493-1.148185$

O $7.2493621 .030898-1.928580$

H $6.580235-0.654656-2.845501$

H $8.766744-0.989312-1.564930$

O $7.447216-2.547027-1.398717$

H $8.401773-1.766640 \quad 0.863073$

$\begin{array}{lllll}\text { O } & 8.555275 & 0.243383 & 0.647696\end{array}$

C $4.570216-2.0929102 .157817$

H $5.492618-2.7387320 .351025$

H $6.479208-2.9109871 .786912$

H $7.1130611 .478680-1.077842$

H $8.137713-3.102224-0.998053$

H 7.9336930 .9888460 .616786

O $3.446181-1.9999501 .392526$

O $4.618797-2.0447993 .380006$

C $2.223602-1.8239932 .113705$

H $2.026441-2.7005622 .737351$

H $2.266747-0.9163192 .723246$

H $1.414374-1.7171141 .387262$

SCF Energy (B3LYP/6-31G**//MMFF) $=-3245.90355175$

08_00470

MM̄FF Geometry

C -3.002790 5.0675810 .002842

C $-3.526200 \quad 4.2755300 .951630$

C $-4.723243 \quad 3.3620080 .821645$

$\begin{array}{lllll}\text { O } & -4.265126 & 1.994201 & 0.960982\end{array}$

C $-5.484795 \quad 3.447417-0.514264$

C $-6.7668052 .636594-0.515477$

C $-6.9152311 .446502-1.129812$

C $-7.937298 \quad 3.2787150 .183576$

C $-5.8614150 .663650-1.865161$

C $-6.042647-0.847369-1.657884$

C $-5.008336-1.657925-2.452021$

C $-5.380909-3.141540-2.459707$

C $-4.331014-3.986025-3.194884$

O $-3.726349-1.481332-1.837708$

O $-4.587565-5.366742-2.896973$

C $-2.940118-3.650056-2.623667$

C $-4.439074-3.842365-4.717408$

O $-1.912521-4.298966-3.369997$

C $-2.650386-2.131263-2.529892$

C $-1.348535-1.917582-1.724658$

O $-2.476817-1.599895-3.844259$

$\begin{array}{llll}\text { C } & -4.244626 & 1.449272 & 2.205667\end{array}$

$\begin{array}{lllll}\text { O } & -4.491167 & 2.010591 & 3.260784\end{array}$

C -3.8765350 .0229162 .057687$

C $-3.590402-0.7029243 .148482$

C $-3.211522-2.1177913 .171576$

C $-3.358115-2.9001601 .893475$

C $-1.042573-0.460481-1.359088$

C $0.157557-0.424064-0.433715$

C $0.795914-0.870763 \quad 1.941566$

O $1.294964-0.242938-0.861160$

C $0.631669-2.2545542 .487971$

C $0.481330-2.5270023 .792985$

C $0.306531-3.916767 \quad 4.336588$

C $-0.901297-4.0501135 .239676$

C $-0.756814-4.2405506 .562145$

C $-2.275872-4.040596 \quad 4.601084$

C $-2.767442-2.6440534 .332175$

N $-0.180707-0.679547 \quad 0.885554$

H $-3.4298375 .083106-0.995658$

H $-3.063162 \quad 4.2863161 .937116$

H $-5.4149643 .607891 \quad 1.637335$

H $-4.8348883 .121429-1.335035$

H $-5.7462054 .490793-0.734795$

H $-7.9017470 .984797-1.109645$

H -8.8509512 .6804290 .100058$

H $-7.726408 \quad 3.407478 \quad 1.249511$

H $-8.1473604 .261290-0.251426$

H $-5.9497170 .892223-2.934033$

H $-4.8537480 .948348-1.547970$

H $-5.953270-1.076837-0.588061$

H $-7.053116-1.134374-1.975480$ 
H $-4.975841-1.270414-3.477142$ H $-6.372080-3.293527-2.905046$ H -5.457660 -3.503894 -1.424792 H -5.491724 -5.573408 -3.189780 H $-2.897245-4.071122-1.608893$ H -5.434253 -4.149135 -5.060982 H $-3.729758-4.503466-5.227462$ H $-4.269879-2.821203-5.065353$ H -2.124158 -5.248053 -3.389819 H -1.406954 -2.497581-0.795949 H $-0.494288-2.315697-2.288210$ H -1.953916 -0.784665 -3.787135 H $-3.844169-0.388707 \quad 1.055186$ H -3.616068 -0.2115784 .120445$ H $-4.368399-2.7855721 .483781$ H -3.203533 -3.973597 2.018559 H -2.639314 -2.553976 1.147125 H $-0.809494 \quad 0.137257-2.246324$ H - $1.8933300 .018059-0.861543$ H $0.610583-0.1032392 .699373$ H $1.807452-0.7445011 .547511$ H $\quad 0.639014-3.0737131 .772722$ H $0.497918-1.7111914 .512047$ H $1.220676-4.1740564 .887121$ H $0.227074-4.6532823 .527218$ H -1.616833 -4.3618647 .212434$ H $0.223633-4.2743937 .025380$ H -3.002011 -4.5221295 .269090$ H -2.244002 -4.6647113 .704239$ H -2.733770 -1.996555 5.209984 H $-1.160955-0.7708101 .132966$ C $-0.5773305 .458578-0.475981$ $\begin{array}{lllll}\text { O } & -0.056817 & 4.339487 & 0.270491\end{array}$ C 1.3752364 .4845350 .395523 C $1.7669195 .561900-0.601542$ C $0.5598796 .476668-0.539065$ H 1.5535054 .8319621 .421418 C 2.0222153 .1137380 .180360 H $2.7003646 .065548-0.333890$ H $1.8779635 .140933-1.608669$ C $0.4657717 .436629-1.712383$ H 0.6015427 .0528840 .395131 C $3.559607 \quad 3.1497940 .084478$ O 1.6292062 .2881821 .279859 H $1.6136422 .648255-0.724706$ H $3.8407903 .781395-0.765695$ O $4.1016203 .765608 \quad 1.252458$ C $4.2026181 .761760-0.118907$ C $5.7243771 .864543-0.340927$ H $3.7345201 .284232-0.987782$ O 3.9462320 .9472391 .021903 H $5.9302122 .648792-1.078951$ H 6.1990012 .1473650 .607129 H $1.3388508 .096779-1.739608$ H $\quad 0.419680 \quad 6.900985-2.666544$ H $-0.4285068 .062805-1.634257$ C $-1.8302575 .984540 \quad 0.223008$ H 0.6569022 .2633191 .292170 H 3.8698543 .2009622 .010082 H $4.2846860 .056070 \quad 0.832035$ H -2.102017 $6.971522-0.168157$ H -1.638856 $6.101549 \quad 1.297030$ H $-0.8168115 .088856-1.481744$ C $6.699473-1.745388-0.102735$ O $6.231353-0.4271330 .209384$ C $6.3393900 .540125-0.839118$ C $7.8079460 .713113-1.256183$ C $8.436355-0.631886-1.633662$ C $8.163374-1.706179-0.575103$ H $6.673944-2.2864810 .852111$ C $5.761576-2.472652-1.079679$ H $5.7553070 .205262-1.703186$ O $8.5405561 .299230-0.182034$ H $7.8557701 .404034-2.106403$ H $9.519094-0.502638-1.751739$ O $7.906288-1.042095-2.890852$ H $8.451260-2.692981-0.954735$ O $8.995826-1.4536280 .561246$
C $4.410287-2.775141-0.463345$

H $5.580286-1.891641-1.987297$

H $6.211692-3.432656-1.359145$

H $9.4544521 .430027-0.487399$

H $8.333972-1.882140-3.129545$

H $8.707933-0.6144890 .960128$

O $3.690828-3.543760-1.334822$

O $4.028635-2.3890220 .632660$

C $2.386799-3.928021-0.887402$

H $2.458812-4.5151680 .032993$

H $1.763900-3.043365-0.733091$

H $1.929251-4.546912-1.663745$

SCF Energy (B3LYP/6-31G**//MMFF) $=-3245.91859783$

08_00471

MM̄FF Geometry

C -3.6506723 .3681340 .020764$

C -2.3580793 .1072070 .273140$

C $-1.2551503 .491679-0.681166$

O $-0.2846142 .421690-0.773033$

C $-0.5194394 .741514-0.164734$

C $0.2760975 .441136-1.249686$

C $1.6151195 .395121-1.388648$

C $-0.5429216 .285676-2.195653$

C $2.6217354 .647319-0.559717$

C $3.4678823 .714902-1.437003$

C $4.6722233 .134894-0.680601$

C $5.4820032 .216714-1.596427$

C $6.6559341 .560787-0.857489$

O $4.1962922 .398984 \quad 0.447847$

O $7.1426320 .483627-1.675044$

C 6.1406530 .9291570 .456267

C $7.8278012 .536167-0.691363$

O $7.244583 \quad 0.4711581 .232833$

C 5.2350551 .8683961 .288512

C 4.5407411 .1706182 .481020

O $5.9982362 .923297 \quad 1.889423$

C $-0.6018381 .346975-1.544424$

O $-1.6659531 .130396-2.101753$

C $0.6006400 .485644-1.624462$

C $0.538805-0.682353-2.281411$

C $1.650956-1.607799-2.517065$

C $3.021558-1.184221-2.063022$

C 3.6002830 .0172712 .123586

C $4.253997-1.3561252 .137287$

C $3.720041-3.7411781 .605388$

O $5.393512-1.5599692 .545287$

C $3.937814-4.1291540 .177499$

C $3.142621-4.981434-0.486510$

C $3.399277-5.451650-1.887911$

C $2.277870-5.110598-2.841299$

C $1.281270-5.981948-3.075140$

C $2.380519-3.815766-3.613501$

C $1.400358-2.768652-3.157768$

N $3.388847-2.3325891 .666622$

H $-3.9023333 .891610-0.897572$

H -2.074249 2.6215751 .202467

H -1.664438 $3.677682-1.682032$

H 0.1253994 .4713910 .678934

H -1.238675 $5.468556 \quad 0.236555$

H $2.0579265 .977520-2.196630$

H $0.0785116 .821019-2.921540$

H -1.244880 $5.665833-2.761238$

H -1.112864 $7.035818-1.637357$

H $3.2718405 .385587-0.074872$

H 2.1510554 .0661930 .237541

H $2.8268692 .899723-1.795686$

H $3.8298514 .255455-2.320908$

H $5.2903723 .970231-0.330719$

H $5.8401132 .762498-2.478314$

H $4.8304631 .422297-1.982590$

H $7.3971290 .852867-2.538088$

H 5.5661830 .0413930 .163508

H $8.2060182 .850527-1.671832$

H $8.6737002 .058267-0.185214$

H $7.5577973 .440399-0.141685$

H $6.893650-0.0666911 .962055$

H 5.2822710 .8652643 .229406 
H 3.9281991 .9337612 .983419 H 6.9417412 .7402521 .753930 H $1.5118140 .850493-1.164585$ H -0.416070 -0.985760 -2.709136 H $3.023505-0.962643-0.991596$ H $3.340062-0.290170-2.608345$ H $3.788352-1.946156-2.217061$ H $2.805018-0.0201292 .879443$ H $3.120422 \quad 0.186670 \quad 1.154244$ H $2.886076-4.2878992 .057498$ H $4.622412-3.9457962 .189828$ H $4.813443-3.716885-0.318231$ H $2.281676-5.4089360 .022516$ H $3.533981-6.541075-1.852254$ H $4.348274-5.059544-2.274001$ H $0.497012-5.761979-3.791923$ H $1.227032-6.931018-2.552328$ H $2.173287-4.019594-4.673468$ H $3.411477-3.450361-3.611586$ H $0.370541-2.986931-3.444701$ H $2.467096-2.0565381 .341328$ C -5.9125352 .2149200 .331269$ O $-5.4286480 .920676-0.068454$ C $-6.0992770 .535171-1.286368$ C $-7.2213421 .547790-1.496725$ C $-6.6120742 .800671-0.895152$ H $-5.3660150 .651746-2.093380$ C $-6.551091-0.928969-1.207409$ H $-7.4840491 .660893-2.552985$ H $-8.1313451 .271300-0.952057$ C $-7.6280883 .881085-0.570104$ H -5.886103 $3.198359-1.613502$ C $-5.443426-1.903772-0.760904$ O $-7.620477-1.044272-0.258851$ H -6.969171-1.242013 -2.170732 H $-5.204653-1.7118450 .290370$ O $-5.965405-3.236332-0.818395$ C $-4.165651-1.851089-1.614380$ C $-3.068630-2.838930-1.174089$ H -3.745897 -0.841018 -1.594418 O $-4.491004-2.125633-2.978706$ H -3.414738 -3.873062 -1.295041 H -2.234384 -2.723441 -1.878263 H -8.148652 4.204841-1.477298 H $-8.381412 \quad 3.5238260 .139905$ H $-7.1383754 .756006-0.131216$ C -4.782824 3.0423170 .953922 H $-7.295419-0.7057030 .593234$ H $-6.776854-3.252957-0.282641$ H -4.897607 -3.008806 -3.010628 H -4.3918022 .4948141 .820634$ H -5.1949343 .9856501 .330967$ H -6.6554282 .0256451 .118307$ C - $-1.788682-0.8253051 .690848$ O $-2.175042-1.2418750 .375947$ C $-2.577725-2.611170 \quad 0.264794$ C -1.417942 -3.543565 0.646179 C $-0.873977-3.2056532 .039020$ C $-0.639621-1.6997022 .214969$ H $-1.3956620 .189997 \quad 1.553872$ C $-3.008852-0.7308422 .610586$ H -3.413234 -2.798356 0.946890 O $-0.371326-3.418475-0.312318$ H -1.772603 -4.5808300 .617399$ H $\quad 0.070874-3.7408352 .194733$ O $-1.802159-3.6722503 .013098$ H -0.441621 -1.467292 3.266438 O $0.538998-1.3362401 .491924$ C $-2.7143050 .095580 \quad 3.837344$ H -3.844646 -0.2509422 .086818$ H -3.389886 -1.712602 2.906522 H $\quad 0.324501-4.053185-0.072404$ H -1.434206 -3.470062 3.890191 H $0.370922-1.5018110 .548477$ O $-2.637169-0.7100434 .934559$ O $-2.551445 \quad 1.308670 \quad 3.819740$ C -2.337305 -0.045302 6.165571 H -1.3591980 .4411556 .104565$
H $-3.1181130 .683561 \quad 6.402997$

H -2.306209 -0.796958 6.958887

SCF Energy (B3LYP/6-31G**//MMFF) $=-3245.93278012$

08_00472

MMFF Geometry

C 5.0803730 .8340821 .946228

C 4.4051100 .9909440 .797618

C $5.0207881 .505815-0.476581$

O $4.1981502 .613217-0.909322$

C $5.0112620 .414001-1.565298$

C $5.973152-0.722281-1.292691$

C $5.622049-1.932883-0.816607$

C $7.414789-0.429116-1.620854$

C $4.254717-2.411646-0.413365$

C $3.794038-3.571594-1.302664$

C $2.460453-4.169606-0.829869$

C $2.090728-5.380818-1.689345$

C $0.721929-5.961956-1.307487$

O $1.451276-3.161371-0.936413$

O $0.326821-6.873289-2.344668$

C $-0.311840-4.816976-1.291204$

C $0.791683-6.782728-0.014601$

O $-1.573100-5.281094-0.811764$

C $0.151336-3.572240-0.496640$

C $-0.823277-2.383472-0.682379$

O $\quad 0.160038-3.8994920 .898877$

C $4.7401643 .504697-1.779696$

O $5.8699153 .482818-2.246268$

C $3.7337824 .509602-2.187474$

C $2.7520264 .905384-1.363052$

C $1.6649675 .832183-1.686615$

C $1.5571556 .307918-3.111110$

C $-0.809835-1.738263-2.072042$

C $0.097603-0.523501-2.103409$

C $0.2140031 .830966-1.302446$

O $1.240064-0.578131-2.551202$

C $-0.6656422 .986473-1.656221$

C -1.289586 $3.749500-0.746071$

C $-2.1156654 .958789-1.086255$

C $-1.6815356 .188450-0.313524$

C $-2.3764406 .609351 \quad 0.756747$

C $-0.475886 \quad 6.961051-0.815219$

C $0.7929896 .157952-0.709263$

N $-0.5039350 .598449-1.554584$

H $\quad 6.128072 \quad 1.128702 \quad 1.970204$

H $3.3579660 .707708 \quad 0.736380$

H $6.0379871 .872091-0.290097$

H $5.2668210 .856934-2.536948$

H $3.9881250 .049180-1.717175$

H $6.410209-2.671092-0.671418$

H $8.066134-1.291553-1.443610$

H $7.515122-0.152768-2.675647$

H $7.7887960 .396589-1.007810$

H $4.310431-2.7482520 .629228$

H $3.513379-1.608837-0.437148$

H $3.687363-3.202751-2.331448$

H $4.560776-4.356641-1.312747$

H $2.565504-4.4638040 .221085$

H $2.866100-6.154511-1.625749$

H $2.055476-5.079824-2.745944$

H $1.011235-7.560526-2.417018$

H $-0.479144-4.534127-2.337091$

H $1.512880-7.602288-0.120269$

H $-0.170180-7.2597170 .203161$

H $1.090261-6.1917690 .853653$

H -1.513220 -5.3545210 .156009$

H -1.840820 -2.716138 -0.445202

H $-0.587422-1.6199620 .070342$

H $\quad 0.142155-3.0731391 .409400$

H $3.8547514 .911273-3.187341$

H $2.7212764 .492399-0.355436$

H $2.4621226 .852559-3.402964$

H $0.7216626 .988605-3.284285$

H $1.4231025 .459121-3.790752$

H $-0.500840-2.425968-2.864081$

H -1.823922 -1.408758 -2.330693

H $1.1383581 .864850-1.887927$ 
H $0.4893941 .833407-0.242231$ H -0.756486 $3.229942-2.711908$ H -1.196870 $3.495870 \quad 0.307883$ H -3.164131 4.724817 -0.862550 H -2.081863 $5.177990-2.160703$ H -2.086225 7.4985821 .306340 H -3.249477 6.0706231 .110329 H $-0.3372107 .877619-0.227889$ H - $0.6885027 .289081-1.835818$ H $\quad 0.978930 \quad 5.774517 \quad 0.295504$ H -1.417658 $0.494223-1.122018$ C $3.161251-0.3687613 .201684$ $\begin{array}{llll}\text { O } & 2.175685 & 0.681531 & 3.156634\end{array}$ C 1.0521700 .3067853 .985164 C $1.304310-1.1331414 .408505$ C $2.818151-1.1854334 .445708$ H 1.0880710 .9730834 .856018 C -0.2401360 .5265993 .189781$ H $0.842122-1.3749745 .370364$ H $0.917444-1.8347513 .658595$ C $3.370138-2.6008364 .430951$ H $3.169244-0.6662155 .347477$ C $-1.506611-0.0299653 .864624$ O $-0.393884 \quad 1.933598 \quad 2.976187$ H -0.1177010 .0792972 .196952$ H $-1.424295-1.1192443 .958375$ O $-1.574057 \quad 0.4872625 .198355$ C -2.827978 0.3240913 .150410 C -2.805737 0.0012291 .654006 H -3.056496 1.3835693 .309120 O $-3.881408-0.4118773 .778873$ H $-2.067110 \quad 0.632244 \quad 1.145947$ H $-2.454143-1.0302621 .518980$ H $3.021011-3.1582815 .306263$ H $3.051801-3.1465883 .536123$ H $4.464263-2.5954794 .449795$ C $4.5662750 .241303 \quad 3.234361$ H -0.5452102 .3475813 .843288$ H -2.3776120 .1249705 .609680$ H -3.719897 -1.357601 3.619885 H $5.284295-0.5330003 .529684$ H $4.605608 \quad 1.0297043 .996414$ H $3.024586-0.9867332 .304691$ C $-4.948649-0.114657-1.370360$ O $-3.840834-0.114906-0.464857$ C -4.1516220 .1158050 .916748$ C -4.8531851 .4696341 .095925$ C -6.0683371 .5997020 .171570$ C $-5.7385571 .200780-1.270136$ H $-4.490685-0.157535-2.366822$ C $-5.816545-1.366186-1.201009$ H $-4.804995-0.6892871 .270064$ O -3.9292982 .5192640 .824584$ H -5.1732951 .5732242 .139004$ H -6.4223942 .6378290 .190146$ $\begin{array}{llll}0 & -7.113880 & 0.776412 & 0.679736\end{array}$ H -6.658391 $1.127771-1.861938$ O $-4.9531122 .249778-1.844727$ C -5.023824 -2.616982 -1.497841 H -6.213981-1.472829-0.187672 H $-6.682728-1.340559-1.872696$ H $-4.387798 \quad 3.3628100 .978123$ H -7.8868880 .8937900 .101768$ H -4.791472 $2.021090-2.775824$ O $-4.882877-2.774023-2.844812$ O $-4.573962-3.357214-0.632630$ C $-4.130029-3.919721-3.255122$ H -3.093573 -3.827592 -2.919272 H -4.583594 -4.835758 -2.864700 H -4.142226 -3.960964-4.347475

SCF Energy (B3LYP/6-31G**//MMFF) $=-3245.90084217$

08_00473

MM̄FF Geometry

C $1.960971-0.5147523 .564640$

C $3.263204-0.2155103 .692753$

C $4.449432-0.9563063 .122056$

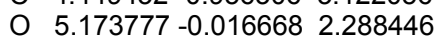

C $4.143859-2.2077982 .275889$

C $5.336298-3.1375082 .142668$

C $6.105236-3.2636341 .044048$

C $5.605839-4.0072013 .345772$

C $6.017831-2.503517-0.249161$

C $5.529280-3.387926-1.405936$

C $4.015502-3.645600-1.361325$

C $3.595303-4.634112-2.449778$

C $2.069607-4.814150-2.496747$

O $3.367697-2.388529-1.572149$

O $1.743051-5.495236-3.717579$

C $1.404979-3.423086-2.562851$

C $1.561892-5.701927-1.354613$

O $-0.013587-3.527890-2.462039$

C $1.938420-2.430208-1.504162$

C $1.417361-1.006360-1.809325$

O $1.481341-2.831271-0.211221$

C 6.5118930 .1066912 .481591

O $7.183495-0.4626953 .331987$

C 7.1285401 .0495701 .520297

C $6.496698 \quad 1.5785150 .460691$

C $7.0863642 .498526-0.518364$

C $8.5143102 .929635-0.302108$

C $2.1008020 .096953-0.989294$

C $1.5840471 .477669-1.353203$

C $1.9913423 .885840-0.847383$

O $0.6970151 .670757-2.180023$

C $2.9248414 .374044-1.908449$

C $3.9291805 .232824-1.679649$

C $4.8909795 .681865-2.740641$

C $6.3190265 .284315-2.435655$

C $7.2185306 .202904-2.044649$

C $6.7274243 .846010-2.673740$

C $6.3291392 .923870-1.551767$

N $2.2371382 .471468-0.637396$

H $1.646541-1.3541242 .952351$

H $3.528900 \quad 0.6272994 .330175$

H $5.056295-1.2393533 .990717$

H $3.780148-1.9026661 .289160$

H $3.336560-2.7961192 .729030$

H $6.916306-3.9908831 .070905$

H $6.436669-4.7015723 .180688$

H $5.859327-3.3960834 .217044$

H $4.721650-4.606708 \quad 3.586244$

H $5.402913-1.603110-0.161069$

H $7.028974-2.148727-0.485805$

H $5.774064-2.871394-2.343305$

H $6.075769-4.339113-1.406721$

H $3.744232-4.034983-0.374170$

H $4.091985-5.602218-2.309400$

H $3.930477-4.264248-3.429002$

H $2.214534-6.345906-3.721399$

H $1.614652-3.010353-3.559700$

H $2.026013-6.694140-1.405969$

H $0.482688-5.872009-1.436653$

H $1.772354-5.290694-0.365140$

H $-0.307746-4.149389-3.149764$

H $1.587927-0.780243-2.870269$

H $0.334996-0.962621-1.638081$

H $1.406026-2.0502980 .358797$

H $8.1715581 .270472 \quad 1.725908$

H $5.456788 \quad 1.313022 \quad 0.279813$

H $9.1874542 .066609-0.348837$

H 8.6268393 .4128270 .674949

H $8.8702383 .648129-1.043126$

H $1.935888-0.0565860 .081557$

H $3.1816630 .089040-1.172461$

H 2.1508134 .3917570 .109639

H $0.9535114 .041465-1.158216$

H $2.774523 \quad 3.985509-2.913097$

H $4.0794765 .625327-0.676649$

H $4.8072376 .773087-2.826583$

H $4.6125935 .287126-3.725907$

H $8.2533265 .937660-1.856460$

H $6.9401667 .241661-1.901455$

H $7.8032053 .778989-2.872284$

H $6.2617893 .486834-3.600747$

H $5.3064372 .555427-1.627775$ 
H $3.0084192 .213795-0.028142$

C -0.2189640 .8211513 .425323$

O $-0.901317-0.2019662 .675788$

C $-1.209034 \quad 0.321763 \quad 1.363564$

C -0.9218641 .8158941 .431651$

C 0.2387231 .8591662 .406189

H $-0.511655-0.1738300 .678694$

C $-2.654525-0.0411471 .004053$

H -0.6835282 .2459500 .455486$

H -1.7833972 .3593691 .839919$

C 0.4923473 .2350892 .994858

H 1.1427521 .5185651 .888772

C $-3.1505040 .594765-0.306609$

O $-2.734840-1.4651840 .882141$

H $-3.313821 \quad 0.2408991 .832639$

H $-3.1741941 .686084-0.201761$

O $\quad-2.1941120 .303073-1.332279$

C $-4.5284570 .092556-0.783811$

C -5.6291350 .2529810 .272128$

H -4.454279 -0.952715 - 1.103912

O $\quad-4.9080490 .854031-1.934388$

H $-5.403123-0.3489131 .160236$

H $-5.643498 \quad 1.2951670 .620615$

H $0.731248 \quad 3.9543172 .205195$

H -0.3842483 .6092613 .534338$

H 1.3336723 .2096553 .694470

C $0.869670 \quad 0.2100914 .307608$

H -2.414491 $-1.846373 \quad 1.717735$

H -2.388569 $0.881050-2.089060$

H $-4.3138990 .602335-2.661312$

H $0.401324-0.5262434 .973334$

H 1.2932490 .9913114 .950110

H $-0.976741 \quad 1.262244 \quad 4.087073$

C $-9.319145-0.046704 \quad 0.662654$

$\begin{array}{lllll}\text { O } & -7.918748 & 0.142788 & 0.901492\end{array}$

C $-7.051528-0.086353-0.218619$

C $-7.202895-1.527767-0.729063$

C $-8.667443-1.865848-1.025928$

C $-9.593667-1.4604370 .125061$

H $-9.7862900 .027801 \quad 1.653305$

C $-9.9076861 .065577-0.219156$

H -7.314871 $0.618622-1.013896$

O $-6.693865-2.4307850 .250767$

H -6.605989 -1.653800 -1.639456

H -8.758072 -2.944336 -1.203752

O $-9.052772-1.194099-2.221654$

H -10.641028 -1.534663 -0.188472

O $-9.427477-2.390886 \quad 1.199361$

C -9.8650592 .4200290 .458243$

H -9.371621 $1.160816-1.166896$

H $-10.9598320 .841104-0.429526$

H -6.759409 -3.328944 -0.116079

H $-9.974066-1.439876-2.412370$

H $-8.522232-2.2913911 .541373$

O $-10.4243123 .346975-0.375948$

O $-9.4042172 .643303 \quad 1.569095$

C -10.455436 4.6868510 .125857

H -11.054942 4.7350941 .039935

H $-9.4384875 .046046 \quad 0.310429$

H $-10.9199055 .323153-0.632228$

SCF Energy (B3LYP/6-31G**//MMFF) $=-3245.90626186$

08_00474

MM̄FF Geometry

C 1.0145692 .9285021 .120564

C 1.9069733 .4598060 .271001

C $2.4462952 .706346-0.914178$

O $3.8856002 .663023-0.764647$

C $2.0924513 .368240-2.258791$

C $0.6170943 .568482-2.529529$

C $-0.2711362 .575957-2.733732$

C $0.1746385 .006777-2.602918$

C $-0.0179601 .093852-2.714266$

C $-0.9125670 .405255-1.674348$

C $-0.494779-1.051559-1.423238$

C $-1.645950-1.826380-0.778756$

C -1.240744 -3.261590-0.422834

O $0.640240-1.040060-0.545435$
O $-2.256347-3.808271 \quad 0.431099$

C $0.060676-3.2140570 .397644$

C $-1.175969-4.166548-1.658778$

O $0.555641-4.5298250 .640443$

C $1.168749-2.338386-0.236098$

C $2.321123-2.1767720 .787065$

O $1.669945-2.987701-1.405508$

C $4.5460301 .672270-1.424067$

O $4.068890 \quad 0.857911-2.198504$

C $5.9627841 .719607-1.003209$

C $6.7639150 .682819-1.290635$

C $8.1752050 .549658-0.924014$

C $8.8096721 .693928-0.177059$

C $3.400880-1.1694870 .383517$

C $4.428398-1.0103701 .487308$

C $6.311621-2.2096372 .600529$

O $4.430357-0.0314082 .230322$

C $7.600899-1.6100562 .135789$

C $8.690470-2.3361121 .842352$

C $10.019774-1.752570 \quad 1.453974$

C $10.399942-2.0610460 .023113$

C $10.916514-3.254302-0.315744$

C $10.257622-0.952251-0.995548$

C $8.822059-0.588145-1.255305$

N $5.305432-2.0796441 .564551$

H $\quad 0.669717 \quad 1.909856 \quad 0.960377$

H $2.260525 \quad 4.4759190 .425494$

H $2.0724411 .678748-0.880834$

H $2.5197762 .784468-3.084484$

H $2.6167764 .332174-2.326568$

H -1.303249 $2.851315-2.944972$

H $-0.9056315 .104454-2.754376$

H $0.4260515 .531510-1.675656$

H $0.6754165 .513681-3.434228$

H $-0.2532210 .692406-3.707806$

H $1.0335220 .857129-2.538109$

H $-0.8753590 .960040-0.728590$

H $-1.9483470 .442706-2.034963$

H $-0.212362-1.512129-2.377172$

H -2.523180 -1.832680 -1.436253

H $-1.963118-1.3139780 .140531$

H $-3.097483-3.787788-0.056775$

H $-0.190840-2.7926481 .381318$

H -2.148756 -4.197517 -2.163357

H $-0.951580-5.201623-1.378409$

H $-0.436156-3.839468-2.392223$

H $-0.163809-5.0381641 .052674$

H $1.904861-1.8417471 .746735$

H $2.793278-3.1511340 .965267$

H $2.559843-2.651890-1.595291$

H $6.2973162 .588269-0.448304$

H $6.344757-0.161626-1.836738$

H $8.7901392 .605870-0.783952$

H 9.8518841 .5166760 .094907

H $8.275711 \quad 1.8839780 .760898$

H $3.914768-1.471469-0.534566$

H $2.955928-0.187177 \quad 0.204154$

H $6.411083-3.2769282 .823203$

H $5.973347-1.7011503 .509274$

H $7.648664-0.5255032 .080726$

H $8.638337-3.4202331 .916444$

H $10.781402-2.1624352 .130551$

H $\quad 10.040976-0.669196 \quad 1.623741$

H $11.217779-3.472625-1.334852$

H $11.047363-4.0403400 .420779$

H $10.693817-1.257676-1.955899$

H $10.860181-0.098151-0.674336$

H $8.269438-1.363591-1.787617$

H $5.258233-2.8105430 .860698$

C -1.0523953 .9256892 .205346$

O -1.8005282 .7026142 .055994$

C -2.9300252 .9603401 .195893$

C -2.9598264 .4704110 .971111$

C -1.4871624 .8176201 .046127$

H -2.7231202 .4619750 .240785$

C -4.2289072 .4062631 .796644$

H -3.416605 4.7372670 .013179

H -3.5167424 .9840801 .764041$ 
C $-1.214928 \quad 6.295403 \quad 1.257915$ H -1.0152604 .4950170 .111592$ C $-4.283597 \quad 0.861236 \quad 1.800929$ O -4.3728692 .9336413 .113911$ H -5.0717472 .7783141 .200786$ H -4.1513250 .5313810 .765416$ $\begin{array}{llll}\text { O } & -3.195461 & 0.319926 & 2.545717\end{array}$ C $-5.609611 \quad 0.294312 \quad 2.347713$ C -5.742903 -1.236263 2.224499 H -6.4455990 .7800641 .831165$ O H -4.940631-1.731383 2.785804 H $-6.675163-1.5182812 .731629$ H -1.623213 6.8837910 .429719 H -1.669945 6.6590112 .185272 H -0.1390686 .4894911 .310689$ C 0.4474373 .6402612 .313821 H $-5.2371182 .648010 \quad 3.454614$ H -3.275863 0.6498363 .457115 H -6.6008830 .3286374 .035839$ H 0.6230892 .9964053 .184834 H 0.9866974 .5767392 .498433 H -1.379767 4.3675223 .156300 C $-6.933374-1.339864-1.312177$ O $-6.810110-1.053150 \quad 0.085913$ C $-5.770757-1.752380 \quad 0.776470$ C $-6.012739-3.2681770 .699880$ C $-6.161362-3.728287-0.755081$ C $-7.142433-2.847241-1.538730$ H -7.849810 -0.823215 -1.625840 C $-5.767356-0.754591-2.123775$ H $-4.806077-1.5240940 .314220$ O $-7.196204-3.6009491 .423269$ H -5.174667 -3.786309 1.180555 H -6.513342 -4.766981 -0.769037 O $-4.880005-3.695308-1.374954$ H -7.076755 -3.067649 -2.610021 O $-8.475859-3.179546-1.139236$ C $-5.707130 \quad 0.757078-2.041562$ H $-4.802431-1.133192-1.780518$ H $-5.894919-1.022267-3.179214$ H -7.297275 -4.567558 1.391429 H -4.985230 -4.020045 -2.285468 H -8.577988 -2.927546 -0.205314 O $-4.7026121 .202787-2.853557$ O $-6.433600 \quad 1.459362-1.352352$ C $-4.5172832 .621468-2.881115$ H $-5.4482463 .125712-3.157772$ H $-4.1627072 .970981-1.907423$ H $-3.7602052 .850432-3.635474$

SCF Energy (B3LYP/6-31G**//MMFF) $=-3245.91540396$

08_00475

MM̄FF Geometry

C $-4.009553-2.004683-2.742836$

C $-4.319299-0.705781-2.880488$

C $-5.264755 \quad 0.100637-2.016845$

O $-4.4826131 .095166-1.312252$

C $-6.004164-0.703942-0.931564$

C $-7.1496760 .039986-0.275201$

C -7.0853840 .6712040 .913574$

C $-8.456068-0.021823-1.025119$

C $-5.882596 \quad 0.912090 \quad 1.782934$

C $-5.793901-0.0262732 .996376$

C $-4.944408-1.2752612 .716007$

C $-4.908459-2.2078603 .926368$

C $-3.979517-3.4088463 .684918$

O $-3.617789-0.8292342 .410254$

O $-3.760579-4.0574244 .946805$

C $-2.609065-2.8921843 .197451$

C $-4.620883-4.4542762 .764569$

O $-1.759314-3.9737452 .821121$

C $-2.703183-1.8668532 .042593$

C -1.316196 -1.219787 1.804553

O $-3.116182-2.5366040 .850935$

C $-4.2424872 .293194-1.901225$

O $-4.5428022 .639417-3.030891$

C $-3.5449373 .123194-0.893350$
C $-2.9748154 .284673-1.242725$

C $-2.2682725 .195691-0.337835$

C -2.2603684 .8545841 .130750$

C -1.325659-0.0059320.861446

C 0.0597010 .6082750 .722154

C $1.2456382 .501998-0.392240$

$\begin{array}{lllll}\text { O } & 1.061868 & 0.122871 & 1.241054\end{array}$

C 1.1974313 .8417540 .270068

C $1.4301354 .992388-0.380204$

C 1.4314246 .3420830 .279058

C $0.5676717 .351191-0.445793$

C $1.1121408 .256019-1.276839$

C $-0.9125467 .368396-0.137891$

C $-1.6660126 .285127-0.858951$

N $0.045146 \quad 1.753566-0.062898$

H -4.444527 -2.588977 -1.937358

H -3.857208 $-0.154294-3.697557$

H $-5.994750 \quad 0.594157-2.670598$

H -5.283601 -1.015170 -0.169917

H -6.419054 -1.627820-1.354695

H -7.9992961 .1230321 .298648$

H $-9.2600130 .512373-0.507656$

H $-8.3486780 .427607-2.017294$

H -8.776077 -1.061837-1.146662

H -4.9573870 .8932021 .201690$

H -5.9679451 .9425552 .152638$

H -5.3187230 .5361133 .811220$

H $-6.794959-0.3122913 .340771$

H $-5.365876-1.8027801 .855491$

H -5.918253 -2.548603 4.186403

H $-4.541982-1.6564894 .803571$

H $-4.626167-4.3259685 .299782$

H -2.124653 -2.402272 4.053940

H $-5.559106-4.8212063 .197733$

H -3.976680 -5.333974 2.657465

H $-4.846411-4.0686471 .768130$

H -1.715687 -4.584304 3.577071

H $-0.910729-0.8819242 .767502$

H -0.622486 -1.970927 1.405453

H -2.811552 -2.029966 0.082024

H -3.5175982 .7469850 .123333$

H -3.024030 $4.596467-2.285079$

H -3.281076 4.8587021 .529118

H -1.677229 5.5487191 .738973

H -1.824251 3.8639301 .298347

H $-1.666487-0.295091-0.138178$

H $-1.999574 \quad 0.767247 \quad 1.247207$

H $1.2837422 .584746-1.483142$

H $2.1311541 .956485-0.053600$

H 0.9904003 .8650381 .337222

H $1.6692604 .961766-1.441057$

H 2.4706216 .6954380 .307565

H 1.1126946 .2765351 .326685

H $0.5039379 .001905-1.777806$

H $2.1780568 .267417-1.479120$

H $-1.3499298 .326954-0.448996$

H $-1.056607 \quad 7.342000 \quad 0.945656$

H -1.723208 $6.442827-1.936977$

H $-0.8327192 .065718-0.467073$

C -1.806293 -3.169917-2.894109

O $-0.954236-2.016441-2.740087$

C $0.402886-2.391000-3.067358$

C $0.414452-3.910176-3.082917$

C $-0.965801-4.209997-3.632786$

H $0.586279-2.001260-4.077128$

C $1.348808-1.726682-2.064345$

H $1.219274-4.320296-3.700087$

H $0.518568-4.310877-2.066823$

C $-1.412161-5.643001-3.400375$

H $-0.970425-4.001343-4.711176$

C $2.807363-2.217904-2.152942$

O $1.277106-0.323222-2.329573$

H $\quad 0.967147-1.877769-1.047468$

H $2.824460-3.297618-1.966660$

O $3.298664-2.020595-3.478630$

C $3.758966-1.547397-1.138945$

C $5.175242-2.152415-1.204810$

H $3.345790-1.679178-0.131855$ 
O $3.823822-0.148572-1.404043$

H $5.099926-3.246043-1.179989$

H $5.638062-1.859030-2.155374$

H $-0.735018-6.342517-3.901335$

H - $-1.423221-5.891821-2.333872$

H $-2.419690-5.807490-3.794761$

C $-3.072801-2.759381-3.645578$

H $1.8309850 .127456-1.671142$

H $3.298218-1.062571-3.647537$

H $4.368451 \quad 0.259952-0.710167$

H -3.605486 -3.645092 -4.009941

H -2.811627 -2.149664 -4.519204

H $-2.047950-3.525154-1.883657$

C 7.1318840 .3248050 .810982

O $6.344389-0.293992-0.214902$

C $6.077034-1.688484-0.043475$

C $7.394640-2.4777940 .005016$

C $8.338249-1.9241671 .076597$

C $8.477770-0.4003470 .982245$

H $7.344186 \quad 1.3328140 .431205$

C 6.3480610 .4755622 .124475

H $5.527571-1.841150 \quad 0.891770$

O $8.043088-2.440516-1.265034$

H $7.170396-3.5292510 .219554$

H $9.322856-2.3930070 .959520$

O $7.832885-2.2866792 .358267$

H $8.996334-0.0033381 .862535$

O $9.282427-0.078926-0.156478$

C 5.1808841 .4326811 .991230

H $5.945391-0.4783262 .473685$

H 7.0140710 .8812282 .895162

H $8.176328-1.507124-1.503735$

H $8.457808-1.9542243 .024981$

H $10.151637-0.495728-0.027996$

O $4.596803 \quad 1.587873 \quad 3.216827$

O 4.8206521 .9709430 .953695

C 3.4695992 .4687483 .255785

H 3.1551032 .5725964 .297607

H 3.7402933 .4571222 .871659

H 2.6430672 .0430902 .680679

SCF Energy (B3LYP/6-31G**//MMFF) $=-3245.93162929$

08_00476

MM̄MF Geometry

C $-1.498842-2.789859-2.823782$

C $-2.563476-3.607572-2.796527$

C -3.973991-3.263038 -2.375837

O $-4.054784-1.927934-1.831681$

C $-4.443571-4.237792-1.281646$

C $-5.916150-4.090449-0.951546$

C $-6.394136-3.4860840 .153786$

C $-6.864178-4.728580-1.934085$

C $-5.600127-2.7989521 .229709$

C $-6.280263-1.5004871 .687389$

C $-5.473360-0.8083932 .794146$

C -6.2631370 .3512663 .401330$

C -5.4433781 .0972484 .464578$

O $-4.258555-0.3171432 .217355$

O $\quad-6.1170112 .331124 \quad 4.755638$

C -4.0694631 .4611283 .867004$

C $-5.3699010 .320225 \quad 5.784135$

O -3.2135062 .0227684 .859664$

C $-3.359847 \quad 0.279455 \quad 3.161754$

C -2.129618 0.8101322 .391974

O $-2.923414-0.6654964 .139832$

C $-4.416469-0.922950-2.672610$

O $-4.662658-1.009011-3.864716$

C $-4.4366240 .327016-1.881347$

C $-4.4310191 .505880-2.520301$

C $-4.4042682 .828670-1.893326$

C $-4.6133982 .900344-0.404348$

C -1.472094 -0.203928 1.447252

C $-0.399430 \quad 0.4934820 .634627$

C $-0.1282522 .087481-1.269090$

O $0.783340 \quad 0.4573940 .964352$

C $-0.6571793 .478627-1.105209$

C $-0.9676354 .284799-2.132049$

C $-1.4926395 .682917-1.961034$
C $-2.7480415 .948370-2.765152$

C $-2.7209426 .751300-3.842356$

C $-4.0504285 .347026-2.276364$

C -4.191794 3.904898-2.679119

N $-0.9186671 .195519-0.441726$

H $-1.598730-1.748278-2.533047$

H $-2.423440-4.636950-3.124162$

H $-4.623895-3.359134-3.254234$

H $-3.832310-4.104696-0.381236$

H $-4.269050-5.275158-1.598408$

H $-7.472080-3.4875980 .309963$

H -7.909373 -4.645936 -1.617542

H $-6.780509-4.252176-2.915570$

H $-6.637743-5.794321-2.043387$

H -5.518129 -3.482119 2.083577

H $-4.584221-2.5691680 .896072$

H $-6.384481-0.8266460 .827143$

H -7.288587 -1.729080 2.054944

H $-5.235726-1.5509533 .565149$

H -7.210735 -0.001504 3.827053

H -6.5354701 .0624922 .608857$

H -7.012712 2.1169125 .068343

H -4.2402282 .2524243 .123115$

H -6.3768560 .1429986 .180573$

H -4.8432410 .8967426 .552781$

H -4.878951 -0.6500395 .684035$

H -3.688784 2.7689615 .263717

H -2.4315791 .6817131 .799673$

H -1.369941 1.1625983 .102088

H -2.187544 -1.180656 3.772969

H $-4.4199590 .243007-0.800635$

H -4.409993 $1.505741-3.609498$

H -5.520772 2.358480 -0.114053

H $-4.7377673 .918256-0.029628$

H -3.7620982 .4618180 .122146$

H $-1.004584-1.0249272 .000804$

H -2.201368 -0.6506110 .762116$

H $-0.2080801 .732003-2.300959$

H $0.9195782 .055951-0.959017$

H $-0.7818303 .844021-0.088331$

H $-0.8174673 .931161-3.149635$

H $-0.6967086 .374125-2.267091$

H -1.693245 $5.906053-0.905660$

H -3.620743 $6.966545-4.409146$

H -1.798133 $7.207206-4.185257$

H $-4.9007905 .894394-2.703492$

H -4.117562 $5.499607-1.196049$

H $-4.0818193 .744094-3.752889$

H -1.912678 $1.131304-0.638326$

C $0.958224-2.787289-2.288961$

O $1.286631-1.406993-2.562740$

C $2.722444-1.281255-2.672744$

C $3.285789-2.535523-2.027131$

C $2.262883-3.568800-2.447829$

H $2.943304-1.279539-3.748403$

C $3.1603320 .046458-2.044083$

H $4.296464-2.772670-2.372285$

H $3.307401-2.437959-0.935696$

C $2.318834-4.845616-1.626501$

H $2.426979-3.815999-3.505500$

C $4.6813730 .156578-1.818606$

O $2.7037261 .077990-2.922593$

H $2.6379920 .186664-1.091045$

H $4.971749-0.655614-1.147121$

O $5.368640-0.059742-3.050467$

C $5.1396921 .492103-1.197868$

C $6.6468831 .539894-0.871319$

H $4.5562901 .682516-0.289552$

O $4.8589702 .557523-2.108033$

H $7.2319431 .396990-1.788211$

H $6.8748282 .557707-0.527600$

H $3.294227-5.330767-1.736248$

H $2.161480-4.645190-0.561562$

H $1.550417-5.553563-1.952500$

C $-0.132415-3.247759-3.260061$

H $2.8984231 .932154-2.501545$

H $5.1071480 .654709-3.656542$

H $5.0884063 .391750-1.663982$ 
H $-0.119181-4.340780-3.343745$

H $\quad 0.059723-2.833836-4.257418$

H $0.606499-2.832201-1.250002$

C $6.534758-0.0969092 .478599$

O $\quad \begin{array}{llll}6.336816 & 0.810355 & 1.387581\end{array}$

C 7.0819790 .5270530 .199809

C 8.5875490 .5855530 .492128

C $8.951657-0.3691051 .636201$

C $8.026540-0.1926752 .845796$

H 6.0135650 .3658823 .327001

C $5.871672-1.4584362 .213140$

H $\quad 6.840028-0.477995-0.155137$

O $8.956833 \quad 1.917270 \quad 0.841711$

H $9.1366120 .316548-0.418079$

H $9.987309-0.1896581 .949910$

O $8.859961-1.7167721 .182633$

H $8.175761-1.0131703 .556639$

O $8.395877 \quad 1.0082503 .531772$

C 4.361516-1.347689 2.129865

H $6.214981-1.9123901 .280371$

H $\quad 6.105892-2.135913 \quad 3.042754$

H 9.9170871 .9245110 .994749

H $9.476888-1.8184650 .437904$

H $8.204307 \quad 1.7580282 .942467$

O $3.823073-2.6032852 .111591$

O $3.731360-0.3005142 .077041$

C $2.393565-2.6581752 .054512$

H $2.029027-2.175461 \quad 1.143195$

H $2.094115-3.7093232 .037650$

H $1.961207-2.1840532 .940824$

SCF Energy (B3LYP/6-31G**//MMFF) $=-3245.91419591$

08_00477

MMFF Geometry

C $1.624233-3.3123891 .140324$

C $1.000258-2.1261301 .129505$

C $0.448416-1.4512842 .357794$

O $-0.912781-1.0473462 .080458$

C $1.281294-0.1941252 .664016$

C 0.8029580 .5738223 .880448

C $0.138401 \quad 1.746423 \quad 3.837745$

C $1.152799-0.0469015 .208365$

C -0.2999462 .5108772 .619314$

C -1.826061 2.6519862 .573821

C -2.3136003 .2144041 .229411$

C $-3.832193 \quad 3.3931301 .249039$

C $-4.3879903 .813971-0.118852$

O $-1.9409362 .283755 \quad 0.208528$

O $-5.8127633 .631208-0.087004$

C $-3.8351702 .863094-1.203293$

C $-4.1484395 .303324-0.394654$

O $-4.2251483 .328705-2.493266$

C $-2.3050362 .670141-1.124004$

C -1.772279 $1.569967-2.075906$

O $-1.6619353 .894559-1.497585$

C -1.879156 -1.997581 2.213404

O $-1.722209-3.1645452 .536140$

C $-3.179588-1.3677771 .892134$

C $-4.295729-2.1119641 .886047$

C $-5.653685-1.6358241 .610791$

C $-5.825529-0.1856871 .244591$

C $-2.2863860 .153228-1.798787$

C $-3.498838-0.244809-2.621996$

C $-5.141284-2.105580-2.868847$

O $-3.9358790 .429302-3.550206$

C $-6.403126-1.740531-2.153158$

C -7.271890 -2.647104-1.680366

C $-8.571449-2.293114-1.016559$

C $-8.737363-2.9480440 .335875$

C $-9.459079-4.0730960 .475340$

C $-8.148376-2.245757 \quad 1.537380$

C $-6.676508-2.5116301 .702909$

N -4.012942 -1.469700 -2.215873

H $1.743399-3.8451262 .080534$

H $\quad 0.881669-1.5970680 .188419$

H $0.463373-2.1242083 .224280$

H 1.2975860 .4536381 .783052

H $2.330844-0.4765972 .825990$
H $-0.1339292 .216184 \quad 4.782178$

H 0.8443920 .5775026 .053758

H $\quad 0.661043-1.0178675 .320953$

H $2.235173-0.1926835 .285953$

H $0.157673 \quad 3.5070162 .648004$

H 0.0502142 .0380261 .700575

H -2.278405 1.6657442 .736122

H -2.1587563 .3016153 .393055$

H -1.812778 4.1723581 .046122

H -4.1282734 .1126012 .022838$

H -4.3034772 .4432101 .532746$

H -6.1682874 .1716350 .639283$

H $-4.3321831 .897134-1.052795$

H -4.6377935 .9177430 .370703$

H $-4.5933675 .605894-1.348914$

H $-3.0904115 .573011-0.404210$

H $-4.0240942 .628662-3.136474$

H $-1.9236791 .861701-3.121880$

H $-0.6817671 .535760-1.948172$

H $-0.7501213 .694956-1.766604$

H $-3.184912-0.3064111 .674810$

H -4.213395 -3.173096 2.117980

H -5.1681040 .0832360 .411094$

H -5.5883520 .4530902 .101758$

H -6.8372630 .0665540 .920193$

H $-1.494499-0.558662-2.064559$

H $-2.505640 \quad 0.013168-0.736482$

H $-4.956280-3.184284-2.858847$

H -5.197796 -1.771339-3.909845

H $-6.627987-0.681647-2.048948$

H -7.058491 -3.705575 -1.812303

H $-9.382407-2.611232-1.685118$

H $-8.686138-1.206974-0.912748$

H -9.609327 -4.531495 1.447205

H -9.910862 -4.563737 -0.380583

H $-8.642964-2.5967342 .453534$

H -8.388930 -1.179998 1.489920

H $-6.450957-3.5455081 .968435$

H -3.619064 -1.928303 -1.399420

C $3.713153-4.0832350 .006652$

O $4.314082-2.775597-0.078075$

C $5.608007-2.906345-0.704917$

C $5.785069-4.387297-1.036442$

C $4.349843-4.868770-1.134415$

H $5.559220-2.348883-1.646247$

C $6.692741-2.3183070 .209379$

H $6.348027-4.534021-1.963759$

H $6.316359-4.926790-0.245428$

C $4.210016-6.375959-1.013291$

H $3.933097-4.539752-2.095838$

C $6.522441-0.8074290 .477468$

O $6.636356-2.9707931 .485428$

H $7.690602-2.511254-0.201617$

H $5.560963-0.6232970 .968952$

O $7.537482-0.4096081 .409942$

C $6.6653460 .075975-0.778417$

C $6.6022681 .590276-0.491143$

H $5.894950-0.181070-1.511394$

O $7.925276-0.196872-1.399619$

H $7.2827551 .847575 \quad 0.329118$

H $6.9851392 .112880-1.378272$

H $4.767625-6.878818-1.810304$

H $4.593751-6.738859-0.053857$

H $3.161953-6.680283-1.093562$

C $2.192870-3.965807-0.088948$

H $7.026305-3.8549721 .386505$

H $7.441794-0.9719852 .197801$

H $8.6215180 .012199-0.753185$

H $1.738402-4.957971-0.183660$

H $1.919932-3.393786-0.983103$

H $4.002888-4.5125370 .975618$

C $3.0115072 .170313-1.217889$

O $4.4215761 .986425-1.390937$

C $5.1814242 .079298-0.183440$

C 5.1317363 .5308640 .325184

C 3.7321094 .1454860 .175945

C $2.6689543 .065461-0.013510$

H $2.6549602 .655548-2.135575$ 
C $2.3371320 .796315-1.109574$

H $4.728566 \quad 1.4244680 .570211$

O $6.0478134 .342921-0.412946$

H 5.4729453 .5519141 .367137

H $3.6997034 .858669-0.657222$

O $3.446444 \quad 4.896817 \quad 1.356355$

H 2.5888482 .4739800 .906416

O $1.3907393 .665653-0.216303$

C $2.3294200 .060599-2.433804$

H $2.8610170 .160750-0.387574$

H $1.2972420 .924375-0.790338$

H $6.0131395 .238145-0.034109$

H 2.5387485 .2360751 .271878

H $1.4519014 .244700-0.995100$

O $1.399004-0.936964-2.384607$

O $3.0601880 .309234-3.382944$

C $1.288454-1.737948-3.564907$

H $2.230900-2.259907-3.756292$

H $0.503468-2.480521-3.398514$

H $1.007869-1.116595-4.420621$

SCF Energy (B3LYP/6-31G**//MMFF) $=-3245.91039129$

\section{8_00478}

MM̄MF Geometry

C 1.4196624 .5653550 .215174

C 2.5124603 .7891920 .168474

C $3.2853053 .493642-1.088899$

O $3.2281142 .059730-1.275243$

C $4.7589993 .904232-0.929539$

C $5.5901363 .648346-2.175212$

C $6.5161772 .675531-2.300150$

C $5.3352524 .591863-3.323109$

C $6.9202351 .658755-1.269950$

C $6.3297770 .277219-1.582543$

C $6.335206-0.637792-0.349962$

C $5.806829-2.031156-0.698061$

C $5.671048-2.9257750 .544970$

O $5.494492-0.0301780 .638432$

O $4.865208-4.0592810 .186560$

C $4.900914-2.1557031 .641535$

C $7.026494-3.4844720 .993661$

O $4.887819-2.8885402 .863350$

C $5.444870-0.7329451 .887206$

C 4.5734090 .1073952 .853852

O $6.747847-0.8214892 .477524$

C $2.2274081 .566438-2.053664$

O $1.4054622 .203775-2.692276$

C $2.2864710 .091310-1.971402$

C $1.475237-0.652561-2.736404$

C $1.429438-2.111007-2.726278$

C $1.355485-2.714049-4.102428$

C $3.105920 \quad 0.2767792 .446005$

C $2.183595-0.7566693 .070650$

C $-0.064067-1.7875692 .781912$

O $2.483219-1.4115494 .065677$

C $0.218554-3.0910812 .103978$

C $-0.584776-3.6429581 .183197$

C $-0.295755-4.9493370 .504417$

C $-0.028764-4.790659-0.975443$

C $-0.945841-5.154289-1.887586$

C $1.338218-4.299924-1.399362$

C $1.393379-2.805907-1.570940$

N $0.965809-0.8349672 .410539$

H $1.0795055 .062587-0.688762$

H 2.8411843 .2821891 .073137

H $2.8471294 .011373-1.950577$

H $5.1832303 .384174-0.065086$

H $4.8225604 .973419-0.686512$

H $7.0473612 .587280-3.246826$

H $6.0201844 .422117-4.160806$

H $4.3163514 .472240-3.703352$

H $5.4673725 .629193-2.998276$

H $8.0151561 .588805-1.259778$

H $6.6456921 .983981-0.264090$

H $5.2987580 .393859-1.934615$

H $6.898460-0.185551-2.398541$

H $7.360940-0.6990600 .032411$

H $6.445644-2.511097-1.449859$
H $\quad 4.816901-1.941332-1.164985$

H $5.296142-4.509540-0.560108$

H $3.858862-2.086274 \quad 1.304825$

H $7.472939-4.0912320 .196478$

H $6.913176-4.155851 \quad 1.851874$

H $7.746262-2.706177 \quad 1.255266$

H $4.517664-3.7668902 .670363$

H $4.665713-0.2819463 .874951$

H 5.0098721 .1161842 .888294

H 6.9409090 .0185762 .925880

H $2.984224-0.354428-1.271440$

H $0.797719-0.174190-3.440588$

H $1.245779-3.800314-4.097945$

H $2.264882-2.480753-4.667537$

H $0.496535-2.313080-4.652760$

H 2.7517741 .2505622 .807411

H $2.9967950 .274407 \quad 1.356458$

H -1.028337 -1.363627 2.484979

H $-0.061918-1.9249033 .867860$

H $1.133352-3.6065472 .387759$

H $-1.505528-3.1362640 .906412$

H -1.157804 -5.6087880 .669220$

H $\quad 0.555120-5.4615190 .971053$

H $-0.747164-5.072877-2.950823$

H -1.917516 -5.535246 -1.591396

H $1.656679-4.821989-2.308050$

H $2.083293-4.589965-0.647679$

H $1.418886-2.251831-0.633082$

H $\quad 0.829708-0.3055231 .554020$

C -0.7711694 .0418901 .384873$

O -0.5921812 .6495351 .046145$

C -1.468446 2.333581-0.056590

C $-2.5598533 .389157-0.009120$

C $-1.758781 \quad 4.6202360 .368374$

H $-0.8672582 .458687-0.966463$

C $-1.9347600 .879340 \quad 0.067388$

H -3.084962 $3.501661-0.962127$

H $-3.297093 \quad 3.1550270 .766908$

C -2.607691 5.7540020 .917545

H -1.234898 $4.978397-0.525940$

C $-3.0682890 .499458-0.905923$

O $-0.7956840 .044342-0.144160$

H $-2.263034 \quad 0.683373 \quad 1.094971$

H $-3.9421231 .090822-0.619555$

O $-2.7272590 .892968-2.235274$

C $-3.440459-0.997853-0.896452$

C $-4.712099-1.324422-1.704612$

H $-3.567901-1.3427620 .136027$

O $-2.388235-1.772602-1.477389$

H -4.579323 -1.019984 -2.750440

H $-4.812131-2.418113-1.722439$

H -3.329796 6.0923040 .167117

H -3.1680725 .4430081 .805644$

H -1.9820286 .6080841 .195070$

C 0.5918664 .7418531 .459734

H $-0.4626470 .225366-1.038660$

H -2.004866 $0.315896-2.536759$

H -1.595141 -1.651173 -0.929249

H 1.1400094 .3316942 .318042

H 0.4584105 .8117431 .654321

H -1.2189724 .0545182 .386890$

C $-7.235943-0.6153190 .949163$

O $-6.140486-1.1708260 .211589$

C $-5.992863-0.699174-1.130188$

C $-7.241081-1.046378-1.952995$

C $-8.501554-0.482017-1.285266$

C -8.566122 -0.8333010 .206059$

H -7.274471 -1.2003001 .877558$

C $-6.9737480 .849697 \quad 1.334623$

H $-5.874750 \quad 0.387419-1.126353$

O $-7.355148-2.461506-2.079019$

H -7.127088 -0.631820 -2.961686

H -9.391554 -0.879791-1.787687

O $-8.5243090 .936003-1.423252$

H $-9.357963-0.2603450 .702632$

O $-8.904972-2.2172280 .340126$

C $-5.783136 \quad 0.9912992 .263151$

H $\quad-6.778078 \quad 1.473140 \quad 0.458574$ 
H -7.8488601 .2466101 .861951$ H -8.141749 -2.645856 -2.619802 H -8.510872 $1.138978-2.374137$ H $-9.771983-2.348855-0.080026$ O $\begin{array}{llll}-5.619238 & 2.311442 & 2.575694\end{array}$ $\begin{array}{lllll}0 & -5.086106 & 0.070631 & 2.666861\end{array}$ C $-4.536253 \quad 2.603410 \quad 3.465449$ H -4.7478802 .1925604 .457040$ H -3.595738 2.2026523 .075172 H -4.445995 3.6899073 .545852

SCF Energy (B3LYP/6-31G**//MMFF) $=-3245.90419269$

\section{8_00479}

MM̄FF Geometry

C $0.633178-1.427908-2.294573$

C $-0.407231-2.043136-1.713547$

C $-1.597410-2.590597-2.457999$

O $-2.753293-1.847386-2.008335$

C $-1.797517-4.075577-2.107280$

C $-3.000247-4.704989-2.786024$

C $-4.142008-5.057308-2.160097$

C $-2.846758-4.953474-4.264655$

C $-4.476708-4.895829-0.703247$

C $-5.514046-3.785408-0.492631$

C $-5.518758-3.2719130 .954775$

C $-6.634540-2.2467181 .157082$

C $-6.580542-1.5958532 .547097$

O $-4.245598-2.662392 \quad 1.191775$

O $-7.434929-0.4410602 .522972$

C $-5.145350-1.0903622 .814915$

C $-7.139672-2.525568 \quad 3.630899$

O $-5.044564-0.6191824 .156830$

C $-4.059051-2.1462572 .516491$

C $-2.616228-1.5845172 .579526$

O $-4.145560-3.1927893 .490833$

C $-3.110547-0.756493-2.739900$

O $-2.622190-0.384208-3.795014$

C $-4.183323-0.052303-2.003766$

C $-4.5048251 .202751-2.350214$

C $-5.5169562 .046543-1.711180$

C $-6.272897 \quad 1.469647-0.543886$

C -2.300342 -0.4658141 .580889$

C -2.480884 0.9379212 .131966

C $-2.445777 \quad 3.3216501 .397711$

O -2.6479851 .1778243 .324449$

C -3.8521863 .8063851 .238462$

C -4.1898924 .8460920 .460048$

C -5.5861225 .3888230 .346516$

C $-6.0522595 .520205-1.086141$

C $-5.9600246 .691635-1.738301$

C $-6.7097774 .320385-1.728584$

C -5.713439 $3.292642-2.190253$

N -2.387028 1.8969121 .131959

H $0.655340-1.311122-3.374852$

H $-0.420939-2.145433-0.630454$

H - $1.466201-2.484936-3.541683$

H -1.867239-4.179943 -1.020303

H -0.903049 -4.645612 -2.393477

H -4.937887 -5.505186 -2.753679

H -3.705693 $-5.484890-4.688341$

H $-2.746152-4.008046-4.806244$

H $-1.957672-5.562854-4.456973$

H $-4.882783-5.844524-0.330811$

H -3.583459-4.709419-0.103942

H -5.290970 -2.948084 -1.165174

H $-6.507983-4.162894-0.763226$

H -5.646738 -4.1238281 .632813$

H -7.617139 -2.7032470 .983451$

H $-6.538947-1.4547700 .403009$

H $-8.330937-0.7358492 .286274$

H $-4.995960-0.2211812 .163700$

H -8.184320 -2.780184 3.414956

H $-7.148898-2.0324684 .609217$

H -6.585163 -3.462069 3.720061

H $-4.193278-0.1597634 .248737$

H -2.362458 $-1.296826 \quad 3.606623$

H -1.937366 -2.416066 2.339375

H -3.293245 -3.6577583 .522146$
H $-4.658063-0.573282-1.181386$

H $-3.9710471 .664866-3.179998$

H -6.895365 $0.629966-0.870553$

H $-6.9314342 .186926-0.050056$

H -5.5798921 .1172470 .227206$

H $-1.241506-0.5435211 .302403$

H -2.886964 -0.5784210 .665193$

H $-1.7579593 .810248 \quad 0.700339$

H -2.1027353 .5158302 .419084$

H $-4.6218803 .303447 \quad 1.819222$

H $-3.4122905 .367426-0.093897$

H -5.5986296 .3744350 .830496$

H -6.3015604 .7765830 .909489$

H $-6.320136 \quad 6.802626-2.755655$

H -5.516672 $7.564425-1.270432$

H $-7.2808544 .633667-2.613134$

H $-7.4626073 .912338-1.048198$

H $-5.1083123 .624810-3.035100$

H -2.3054641 .5991650 .164102$

C $1.9702660 .638231-1.640798$

O $3.1523790 .999130-0.889125$

C $2.780811 \quad 1.9353350 .132972$

C $1.5370842 .623373-0.403135$

C $0.8126221 .463808-1.061214$

H 2.5077191 .3506851 .019986

C 3.9416832 .8776050 .469672

H $0.949998 \quad 3.1016710 .386696$

H $1.7870733 .384159-1.151564$

C $-0.197608 \quad 1.930935-2.097877$

H $0.2903740 .889545-0.285338$

C $5.256748 \quad 2.1690940 .859065$

O $4.210876 \quad 3.692736-0.678128$

H 3.6281883 .5619311 .267687

H $5.7242211 .743552-0.036011$

O $6.159766 \quad 3.165874 \quad 1.354380$

C 5.1116411 .0770141 .933263

C 6.4396600 .5597632 .520665

H 4.5508920 .2333021 .515020

$\begin{array}{lllllll}\text { O } & 4.337572 & 1.598379 & 3.018442\end{array}$

H 6.9015411 .3404643 .138644

H $6.177195-0.2487943 .215613$

H $-0.9570022 .571010-1.637211$

H $0.2843192 .506100-2.895757$

H $-0.7083951 .084338-2.560201$

C $1.796498-0.880701-1.523306$

H $4.7968014 .414635-0.395378$

H 6.4095283 .7339790 .606536

H 4.1975250 .8751513 .653178

H $1.732365-1.156711-0.462190$

H $2.708203-1.371580-1.885580$

H $2.1685760 .911978-2.684209$

C $7.618285-1.557380-0.326513$

O $6.830669-0.9982570 .734609$

$\begin{array}{lllllll}\text { C } 7.454141 & 0.045631 & 1.487068\end{array}$

C $8.752001-0.4579942 .143853$

C $9.692274-1.0776291 .106155$

C $8.964538-2.0806810 .203217$

H $7.041485-2.422900-0.677403$

C $7.770282-0.582514-1.505552$

H 7.7035090 .8735410 .816668

O $8.471827-1.4432413 .135812$

H $9.256006 \quad 0.3806552 .639893$

H $10.514535-1.5823391 .628231$

O $10.256160-0.0321450 .319901$

H $9.599739-2.369910-0.642077$

O $8.696184-3.2720770 .948128$

C $6.446828-0.282783-2.176763$

H $8.1972860 .371294-1.184950$

H $8.425498-1.022071-2.266274$

H $8.109160-0.9864383 .913239$

H $10.869368-0.440606-0.314684$

H $9.548947-3.625407 \quad 1.253930$

O $6.6212390 .745553-3.058996$

O $5.399251-0.877490-1.967237$

C $5.4528811 .153927-3.777877$

H $4.9460620 .293260-4.224956$

H $4.7790831 .695082-3.108374$

H $5.7665041 .827939-4.579534$ 
H $\quad 1.1523876 .110596-0.253387$ 08_00480

MM̄FF Geometry

C $-2.808989-4.0475861 .063020$

C $-1.474277-3.9373541 .115908$

C $-0.707809-3.5794142 .359702$

O $-0.077657-2.2965792 .143570$

C $0.405116-4.6180772 .604091$

C $1.319287-4.2726653 .765295$

C $2.630870-3.9830753 .641119$

C $0.673022-4.2748795 .126132$

C $3.451805-3.9718332 .379426$

C $3.433435-2.5990471 .708221$

C $4.114880-2.5726950 .333583$

C $5.629432-2.7692050 .428591$

C $6.321027-2.598621-0.933493$

O $3.815906-1.291855-0.240503$

O $7.732171-2.469996-0.698790$

C $5.848348-1.275163-1.571308$

C $6.141934-3.834220-1.823394$

O $6.353721-1.136473-2.896875$

C $4.313940-1.109263-1.572679$

C $3.8673460 .289786-2.056363$

O $3.738483-2.063544-2.473297$

C $-0.767066-1.1750712 .485736$

O $-1.917256-1.1071862 .886498$

C $0.158035-0.0302492 .319830$

C -0.1889191 .1897022 .756211$

C 0.6539442 .3888102 .714313

C 2.0168462 .2581492 .086806

C $4.3606471 .460685-1.202907$

C $3.7589502 .781630-1.652291$

C $3.5975715 .171113-0.965707$

O $3.1447102 .913904-2.707373$

C 3.1401755 .7650850 .328436

C 1.8867506 .1914030 .544770

C 1.4172856 .8115651 .828282

C 0.3789685 .9645662 .531106

C $-0.927894 \quad 6.2591922 .421961$

C $0.896514 \quad 4.8507863 .417647$

C 0.1823013 .5332503 .253367

N $4.002443 \quad 3.796137-0.736851$

H -3.399301 -3.880130 1.960149

H $-0.896457-4.0834190 .206028$

H -1.367535 -3.531085 3.234946

H $0.989543-4.7272421 .684075$

H $-0.042722-5.6029982 .791698$

H $3.190255-3.7308974 .541036$

H $1.401889-4.1354235 .931865$

H $-0.063046-3.4692185 .206054$

H $\quad 0.167879-5.2297525 .304609$

H $4.479211-4.2471542 .645372$

H $3.111027-4.7451981 .683306$

H $2.397612-2.2723621 .570614$

H $3.888399-1.8460442 .365516$

H $3.660370-3.350772-0.291181$

H $5.876514-3.7488920 .853818$

H $6.047857-2.0273081 .123236$

H $8.031453-3.266376-0.227451$

H $6.294616-0.464536-0.981937$

H $6.580027-4.717792-1.343681$

H $6.672674-3.716579-2.774612$

H $5.095722-4.062311-2.036566$

H $7.319311-1.243998-2.851874$

H $4.1653010 .435472-3.102968$

H $2.7679980 .316878-2.062695$

H $2.860676-1.745399-2.741723$

H $1.122842-0.2316141 .868789$

H -1.169652 1.3200463 .211288

H 2.6518321 .5924662 .681597

H 2.5444983 .2084391 .990625

H 1.9377461 .8511091 .073353

H $4.0989901 .298022-0.151832$

H $5.4485081 .559291-1.278023$

H $2.8217325 .209837-1.737458$

H $4.4726345 .713766-1.337113$

H 3.8792925 .8578861 .120468

H 1.0047827 .8010021 .591132

H 2.2546347 .0031112 .511250

H -1.687544 5.6937072 .950243

H $-1.2698597 .080747 \quad 1.800686$

H 0.7904435 .1691334 .463433

H 1.9748554 .7312273 .277216

H -0.8187273 .5220413 .686316$

H 4.6344793 .6107440 .037248

C $-4.113503-3.087387-0.833371$

O $-5.118111-2.5083350 .026706$

C -6.206858 -2.055935 -0.787294

C $-5.723893-2.111365-2.230940$

C $-4.800593-3.313048-2.179558$

H $-7.001898-2.806886-0.677729$

C $-6.772252-0.712108-0.321581$

H $-6.544023-2.229985-2.945342$

H $-5.159331-1.213005-2.503593$

C $-3.852600-3.394780-3.362460$

H $-5.409696-4.226314-2.141848$

C $-5.8309900 .512109-0.370014$

O $-7.908420-0.438214-1.153728$

H -7.154874 -0.8262050 .700070$

H $-5.5358660 .711275-1.404456$

O

C $-4.5846670 .447500 \quad 0.539413$

C $-3.7868201 .766900 \quad 0.565801$

H $-3.922443-0.3635930 .228084$

O $\quad-5.0167520 .1604581 .871149$

H -4.4817782 .6085830 .667077$

H -3.1657401 .7883191 .471132$

H $-4.413441-3.484894-4.298486$

H $-3.223377-2.501737-3.434158$

H -3.196668 -4.266410 -3.278386

C $-3.558868-4.362101-0.200891$

H $-8.2908130 .404215-0.853336$

H $-6.854561 \quad 1.5306180 .960682$

H -4.2208880 .0248862 .413703$

H -2.903184 -4.881323 -0.909511

H -4.373381 -5.0516310 .051831$

H $-3.315893-2.341004-0.940407$

C $-0.6252341 .385030-1.380857$

O $-1.6382091 .321738-0.368252$

C $-2.8938671 .942648-0.674621$

C $-2.6940493 .424419-1.019756$

C $-1.6756773 .594493-2.148868$

C $-0.3865662 .827237-1.860119$

H $\quad 0.292524 \quad 1.055487-0.876371$

C $-0.9099510 .394796-2.520010$

H $\quad-3.3440901 .432138-1.531459$

O -2.2692684 .1511750 .130775$

H -3.658064 3.847293 -1.325902

H $-1.4407454 .659017-2.271364$

O $-2.2282193 .119807-3.372797$

H $\quad 0.2570382 .827164-2.747367$

O $0.3155553 .531753-0.833956$

C $-0.593513-1.024666-2.094416$

H $-1.951790 \quad 0.413560-2.849300$

H $-0.2613680 .632360-3.371348$

H -1.450882 3.7402510 .458397

H $-3.0302233 .639173-3.553381$

H $1.1820013 .105734-0.725395$

O $-0.344648-1.786227-3.199547$

O $-0.582059-1.421786-0.937531$

C $-0.011330-3.153367-2.937378$

H $\quad 0.896408-3.213101-2.329334$

H $-0.842505-3.657116-2.436034$

H $\quad 0.173617-3.647947-3.894485$

SCF Energy $($ B3LYP/6-31G**//MMFF $)=-3245.91437681$

08_00481

MM̄FF Geometry

C $1.339179 \quad 3.3820742 .096784$

C 0.7544102 .9217843 .214140

C 0.7461701 .4966183 .716393

O 1.5291670 .6284762 .868308

C $-0.695406 \quad 0.9616813 .731325$

C $-0.814924-0.3766224 .432758$ 
C $-0.921746-1.5607523 .799432$

C $-0.862907-0.3143205 .937305$

C $-0.890223-1.7995652 .316165$

C $-0.100358-3.0667231 .961580$

C $-0.148943-3.3500090 .454239$

C $0.458740-4.7155830 .134964$

C $0.464242-4.988602-1.376059$

O $0.583442-2.322045-0.221957$

O $1.308869-6.124163-1.616405$

C $1.106981-3.785225-2.094548$

C $-0.928433-5.363490-1.897447$

O $1.004970-3.923265-3.510281$

C $0.537781-2.414741-1.652530$

C $1.410692-1.285370-2.243514$

O $-0.794483-2.274193-2.144978$

C 2.8452140 .4717883 .172936

O $3.457506 \quad 0.9927984 .091099$

C $3.428528-0.4346782 .159535$

C $4.761896-0.5006822 .031766$

C $5.498333-1.3011781 .051589$

C $4.718855-2.2993610 .238526$

C $1.090198 \quad 0.118909-1.719630$

C $2.1358631 .091106-2.228319$

C $4.4646721 .870787-1.774717$

O $1.9730561 .727092-3.266933$

C $5.5326540 .895576-2.163540$

C $6.7778140 .916634-1.663442$

C $7.845966-0.067720-2.049977$

C $8.538563-0.690966-0.855872$

C $9.812345-0.382503-0.558557$

C $7.782015-1.728445-0.050315$

C $6.826372-1.0975390 .926194$

N $3.2677851 .125938-1.428888$

H 1.8827202 .7029681 .447408

H $\quad 0.2265473 .6322613 .848401$

H $1.152209 \quad 1.4905084 .735571$

H $-1.076148 \quad 0.8911082 .705155$

H -1.360655 1.6740894 .237901

H -1.055544 -2.454578 4.407321

H $-0.996426-1.3024826 .390505$

H $\quad 0.065700 \quad 0.1061396 .335020$

H -1.698058 0.3131396 .265031

H -1.922475 $-1.913247 \quad 1.973074$

H $-0.460632-0.9504281 .777093$

H $\quad 0.941319-2.9419172 .284500$

H $-0.517858-3.9219162 .507845$

H -1.196053 -3.320855 0.133226

H $-0.073664-5.5147010 .665815$

H $1.494818-4.7495440 .500218$

H $\quad 0.952076-6.874930-1.111431$

H $2.180626-3.803238-1.859125$

H -1.300944 -6.258258 -1.384460

H $-0.895148-5.621016-2.961934$

H -1.667428 -4.571789-1.757594

H $\quad 1.384593-4.787197-3.745365$

H $2.463446-1.498905-2.026466$

H $1.312703-1.273587-3.337069$

H -1.025243 -1.332010 -2.163098

H $2.747859-0.9902541 .524649$

H 5.3770870 .1180042 .684283

H $4.123416-2.9495600 .889776$

H $5.347715-2.964123-0.357002$

H $4.045362-1.784438-0.450544$

H $\quad 0.108507 \quad 0.463368-2.061498$

H $1.0768830 .147910-0.624295$

H $4.7441422 .461741-0.897004$

H $4.2575822 .548861-2.608013$

H $5.2687860 .136734-2.896768$

H $7.0510431 .691854-0.951072$

H $8.5762590 .465205-2.672735$

H $7.439474-0.867477-2.681468$

H $\quad 10.323829-0.8425880 .280448$

H $\quad 10.371106 \quad 0.340454-1.143659$

H $8.485539-2.3428830 .526665$

H $7.294262-2.416375-0.745813$

H $7.299943-0.3695851 .587064$

H $3.3019720 .545031-0.596773$

C 0.5577075 .0969490 .379826
O -0.8010034 .6269150 .466078$

C -1.206585 4.164706-0.840113

C $-0.0759664 .535358-1.797854$

C $1.1316844 .462745-0.882650$

H -1.262585 $3.072215-0.789412$

C $-2.5718534 .753963-1.207017$

H $-0.0147283 .849381-2.648124$

H $-0.1941455 .550113-2.195296$

C $2.3590765 .165086-1.433409$

H $1.3675863 .407418-0.709160$

C $-3.7052844 .425650-0.210200$

O $-2.4358856 .182137-1.253968$

H $-2.8504614 .441323-2.219643$

H $-3.451024 \quad 4.8621020 .763366$

O $-4.8937155 .105594-0.638097$

C $-4.0524322 .934064-0.015315$

C $-4.3279782 .185017-1.334814$

H -4.9623982 .8759480 .595676$

O -3.0231022 .2713310 .709880$

H $-5.0706632 .742955-1.915352$

H $-3.4001972 .134571-1.917419$

H $2.6619724 .719261-2.386342$

H $2.1692096 .229639-1.606387$

H $3.2008045 .080419-0.738962$

C 1.3032844 .8293771 .688564

H $-3.3064196 .549407-1.484268$

H -5.056203 $4.877079-1.568671$

H -2.8904572 .7538381 .543522$

H 0.8220925 .4174612 .480888

H 2.3336115 .1950651 .610245

H $0.496764 \quad 6.1859280 .247337$

C $-4.067269-1.420250-0.387083$

O $-3.734684-0.065813-0.722329$

C $-4.8441140 .750028-1.111708$

C $-5.4846640 .177358-2.385753$

C $-5.913031-1.278137-2.180075$

C $-4.798981-2.120243-1.547505$

H $-3.094921-1.910484-0.262001$

C $-4.800300-1.4709540 .961423$

H $\quad-5.5867240 .766243-0.305122$

O $-4.5507950 .257305-3.460980$

H $-6.3538610 .790166-2.653548$

H -6.192299 -1.710580 -3.148678

O $-7.070698-1.293891-1.350161$

H -5.201204 -3.080135 -1.207139

O $-3.837348-2.426140-2.562547$

C -4.611717 -2.789082 1.680907

H $-4.396110-0.6810781 .607698$

H -5.872603 -1.289707 0.852033

H $-4.992591-0.080631-4.258587$

H -7.350486 -2.220297 -1.255019

H -3.413656 -1.592978 -2.831750

O $-5.013149-2.6466302 .978901$

O $-4.175253-3.8141961 .175830$

C $-4.867692-3.8110193 .797926$

H $-3.816611-4.1117973 .845410$

H -5.206551 -3.561496 4.806886

H -5.486621 -4.625896 3.410683

SCF Energy $\left(B 3 L Y P / 6-31 G^{* *} / / M M F F\right)=-3245.91081583$

0800482

MMFF Geometry

C $0.208462-2.693874-2.808288$

C $-0.963710-2.655701-2.158853$

C $-2.305043-2.512278-2.830421$

O $-2.941825-1.339268-2.272302$

C $-3.169225-3.745578-2.513601$

C $-4.585824-3.653926-3.047099$

C $-5.682828-3.465297-2.285172$

C $-4.722022-3.811018-4.539385$

C $-5.742205-3.288570-0.792973$

C $-6.113648-1.848153-0.418297$

C $-5.711439-1.5125641 .025410$

C $-6.225591-0.1283621 .419143$

C $-5.728601 \quad 0.2993742 .807707$

O $-4.280649-1.5371751 .086381$

O

C -4.1975990 .1063262 .879623$ 
C $-6.509615-0.395546 \quad 3.929772$

O -3.7449180 .3547834 .208628$

C $-3.728913-1.2805572 .385760$

C -2.188691-1.404326 2.255120

O $-4.150781-2.2769033 .325035$

C $-2.635876-0.140124-2.840828$

O $-1.923180 \quad 0.052237-3.813153$

C $-3.2975550 .917442-2.044689$

C $-3.0405452 .207306-2.307391$

C -3.589278 3.361859-1.592171

C $-4.4816913 .091292-0.409874$

C -1.527741 -0.4200441 .284252$

C -1.0296770 .8603371 .932729$

C $-0.126444 \quad 3.108104 \quad 1.333368$

$\begin{array}{llll}\text { O } & -0.917263 & 0.999973 & 3.147372\end{array}$

C $-1.208194 \quad 4.1403531 .288667$

C -1.120082 5.2682380 .566998

C -2.170868 6.3411490 .556414

C -2.607861 $6.720084-0.841012$

C $-2.0738097 .782180-1.467540$

C $-3.7270225 .924010-1.472422$

C $-3.2603904 .600108-2.016426$

N -0.6821051 .8142120 .985936$

H $0.196862-2.615502-3.893800$

H $-0.987287-2.705093-1.074434$

H $-2.202973-2.403792-3.917261$

H -3.173519 -3.912684 -1.432144

H -2.696634 -4.642916 -2.936193

H $-6.652190-3.420463-2.779827$

H $-5.768001-3.809596-4.864022$

H $-4.215346-2.992743-5.060362$

H $-4.279428-4.758555-4.863562$

H -6.499512 -3.972650 -0.390568

H $-4.805633-3.579483-0.313606$

H -5.608373 -1.152446 -1.099061

H $-7.193444-1.708466-0.553046$

H $-6.118394-2.2808731 .693215$

H -7.321365 -0.093724 1.375238

H -5.8761460 .6097180 .685459$

H -6.9372881 .8519382 .835383$

H $-3.758594 \quad 0.8858552 .245478$

H $-7.577420-0.1559383 .858141$

H $-6.189291-0.0383484 .914730$

H -6.414791-1.483208 3.908208

H -2.7737540 .3799534 .194025$

H -1.718564 -1.3736693 .245137$

H $-1.978341-2.4123661 .868613$

H -3.592746 -3.064056 3.211904

H $-3.9609870 .606362-1.246800$

H -2.359482 $2.445662-3.123292$

H -3.9795052 .4350340 .309178$

H $-5.4116192 .612861-0.735483$

H -4.7572353 .9894860 .146122$

H $-0.640186-0.9013890 .854321$

H -2.199865 -0.1775730 .457013$

H $0.674575 \quad 3.3189520 .617784$

H $\quad 0.306337 \quad 3.0680892 .337981$

H -2.089306 3.9663591 .901876

H $-0.2234735 .451479-0.021254$

H -1.752484 7.220417 1.064023

H -3.047737 6.0502191 .147912

H -2.402717 $8.078944-2.457983$

H $-1.2911088 .374870-1.005672$

H $-4.1566326 .483529-2.314619$

H $-4.5502305 .829593-0.758717$

H -2.597296 $4.693906-2.877571$

H -0.8634551 .6276240 .004234$

C $1.700961-3.216496-0.727245$

O $2.966784-2.739717-0.221478$

C $3.542807-3.7455780 .628894$

C $2.468188-4.8091690 .822436$

C $1.714308-4.728344-0.491844$

H $4.375900-4.1840890 .066775$

C $4.069999-3.1191671 .925898$

H $2.895920-5.7981671 .013277$

H $1.797736-4.5686021 .655406$

C $0.348244-5.388157-0.433117$

H $2.317293-5.224681-1.264062$
C $4.983867-1.8913751 .734380$

O $2.964580-2.6918262 .734296$

H $4.588837-3.8852982 .513694$

H $4.378233-1.0225441 .455450$

O $5.566072-1.5808213 .006869$

C $6.117479-2.0758970 .714191$

C $7.147896-0.9328690 .675721$

H $5.691072-2.194348-0.287612$

O $6.826858-3.2849330 .999903$

H $7.743907-0.9353501 .597756$

H $7.855000-1.177756-0.128204$

H $0.440144-6.434616-0.123375$

H $-0.311796-4.8840490 .280651$

H $-0.135513-5.376884-1.413837$

C $1.579644-2.790342-2.196180$

H $2.442463-2.0619862 .207954$

H $4.835301-1.4514093 .635654$

H $7.169987-3.2161931 .907472$

H $2.178666-3.474122-2.811811$

H $2.058588-1.810638-2.322502$

H $0.944338-2.726160-0.105157$

C $5.1511491 .680898-1.124898$

O $5.7878920 .446791-0.760123$

C $6.560070 \quad 0.470770 \quad 0.445253$

C $7.6677701 .536644 \quad 0.373653$

C 7.0952002 .9083590 .010231

C $6.1738852 .827329-1.209965$

H $4.7721261 .504827-2.139604$

C $3.9600272 .007877-0.212886$

H $\quad \begin{array}{llll}5.902218 & 0.707588 & 1.287061\end{array}$

$\begin{array}{llll}\text { O } & 8.638935 & 1.155938 & -0.599385\end{array}$

H $8.174378 \quad 1.583396 \quad 1.345318$

H $7.9204183 .599661-0.200261$

O $\quad 6.3765353 .412103 \quad 1.132012$

H $5.6462513 .777984-1.347280$

O $6.9694882 .638611-2.383733$

C $2.7441221 .191423-0.584907$

H 4.1634801 .8226310 .845223

H $3.6745383 .063980-0.293822$

H $9.3437431 .825917-0.587972$

H 6.0387534 .2919990 .893151

H $7.3884001 .762915-2.321970$

O $2.1629831 .717484-1.699944$

$\begin{array}{lllll}\text { O } & 2.363397 & 0.213395 & 0.043367\end{array}$

C $0.9799491 .082197-2.199987$

H $0.2438091 .861172-2.414337$

H $1.2284340 .567835-3.131832$

H $0.5430480 .371722-1.492975$

SCF Energy (B3LYP/6-31G**//MMFF) $=-3245.91859955$

0800483

MM̄FF Geometry

C $1.125633-4.069059-0.870494$

C $0.470143-3.091153-0.227335$

C $-0.834127-2.510264-0.704492$

O $-0.627838-1.134917-1.132489$

C $-1.987347-2.633047 \quad 0.310092$

C $-2.497490-4.0487890 .481673$

C $-3.474892-4.596389-0.266144$

C $-1.882711-4.8359411 .609894$

C $-4.227233-3.959582-1.401737$

C $-5.722829-3.813792-1.086472$

C $-6.015982-2.7884450 .020506$

C $-7.499180-2.812308 \quad 0.393642$

C $-7.839157-1.7375631 .435399$

O $-5.655317-1.493829-0.471946$

O $-9.268640-1.622194 \quad 1.500331$

C $-7.296821-0.3819730 .941585$

C $-7.370143-2.1305402 .841208$

O $\begin{array}{llll}-7.462338 & 0.624462 & 1.938735\end{array}$

C $-5.820337-0.430073 \quad 0.475746$

C $-5.469097 \quad 0.895676-0.238225$

O $-4.970967-0.597507 \quad 1.610650$

C $-0.345148-0.147186-0.243672$

O $-0.293256-0.228448 \quad 0.972311$

C $-0.074876 \quad 1.079648-1.027826$

C $0.0220372 .263021-0.404086$

C $0.3550163 .544548-1.031948$ 
C $0.6910383 .534006-2.500662$

C $-4.049706 \quad 0.961510-0.820515$

C $-3.9492962 .072623-1.848737$

C $-4.2278844 .537552-2.095847$

O $-3.7715141 .833911-3.041254$

C $-2.8842015 .164247-2.278077$

C $-2.5183906 .309705-1.684002$

C $-1.2108017 .004585-1.927216$

C $-0.324057 \quad 7.028843-0.704757$

$\begin{array}{llll}\text { C } & -0.487740 & 7.956870 & 0.253107\end{array}$

C $0.8161696 .040572-0.649960$

C $0.377086 \quad 4.654931-0.264796$

N $-4.1077003 .333668-1.298374$

H $0.708036-4.473816-1.788956$

H $0.881010-2.6956810 .697122$

H $-1.141715-3.026802-1.622808$

H $-2.805400-1.9714940 .002566$

H - $1.689028-2.2444741 .291635$

H $-3.776925-5.619847-0.047319$

H -2.341911 -5.823717 1.725375

H $-0.813723-4.9912001 .442486$

H $-2.012578-4.3032772 .557749$

H -3.805938 -2.992298 -1.691438

H $-4.117535-4.610370-2.278134$

H -6.228224 -3.501252 -2.009424

H $-6.133634-4.792414-0.807503$

H $-5.407398-3.0228010 .900373$

H -7.793889 -3.8045650 .757236$

H $-8.105441-2.629620-0.504863$

H -9.628799 -2.492792 1.741776

H $-7.917108-0.0751470 .087441$

H -7.837219 -3.0731513 .150815$

H -7.679463 -1.385787 3.582979

H $-6.288222-2.2593672 .912599$

H -8.401792 0.6309952 .190396

H $-6.1817931 .051951-1.059411$

H -5.5927951 .7327850 .460198$

H -4.075855 -0.3031091 .379999$

H $0.0596990 .968910-2.097928$

H $-0.1343852 .297192 \quad 0.673484$

H $1.5847312 .928803-2.686422$

H $0.8840224 .527051-2.911327$

H $-0.1395403 .120325-3.083385$

H $-3.307367 \quad 1.138456-0.034968$

H -3.782564 $0.024158-1.320423$

H -4.924547 5.202477 -1.574942

H $-4.6580734 .292198-3.072455$

H -2.201689 $4.676068-2.968567$

H -3.214716 $6.802027-1.008521$

H -1.428014 8.033639-2.242952

H $-0.6743976 .555035-2.771825$

H 0.1678008 .0010041 .116431

H -1.284904 8.6907830 .195277

H 1.5542596 .3616970 .097866

H $1.3646486 .064240-1.596471$

H 0.1080534 .5631530 .788419

H $-4.1691513 .430577-0.289177$

C $3.647580-4.279956-1.179954$

O $3.935231-2.880254-0.991711$

C $4.455986-2.352061-2.226450$

C $4.691949-3.551389-3.142855$

C $3.599443-4.499917-2.690106$

H $3.661375-1.733410-2.659735$

C $5.704785-1.500661-1.974939$

H $4.618103-3.283202-4.201314$

H $5.677965-4.002063-2.979941$

C $3.823337-5.940576-3.112832$

H $2.650484-4.145979-3.110506$

C $5.509343-0.330687-0.988276$

O $6.731984-2.346723-1.440446$

H $6.084603-1.127262-2.933845$

H $5.409244-0.7258970 .028322$

O $6.7242890 .432181-0.992202$

C $4.3274920 .603099-1.303198$

C $4.3224331 .926762-0.514753$

H $3.3875270 .067705-1.127028$

O $4.3661890 .936276-2.694539$

H $5.1620702 .555174-0.838534$
H $3.4192292 .471202-0.818948$

H $3.855956-6.020584-4.204318$

H $4.766880-6.333173-2.719060$

H $3.013639-6.582149-2.751001$

C $2.406195-4.683671-0.381293$

H $7.517677-1.792042-1.294978$

H $6.8128790 .837584-1.872054$

H $3.5720781 .461410-2.892490$

H $2.560617-4.4146290 .670724$

H $2.291011-5.773731-0.409861$

H $4.505436-4.820078-0.756807$

C $3.092998 \quad 0.7122912 .797872$

O 3.2144110 .9875191 .395575

C 4.3438261 .7762341 .014461

C $4.3187373 .143777 \quad 1.720388$

C 4.1595173 .0019623 .237781

C 3.0402962 .0216553 .604650

H 2.1218460 .2117682 .902014

C $4.176837-0.256904 \quad 3.298041$

H 5.2616911 .2514911 .296316

$\begin{array}{llll}\text { O } & 3.252283 & 3.945155 & 1.217591\end{array}$

H 5.2494373 .6754361 .490624

H 3.9303753 .9825423 .673193

O 5.3820102 .5482903 .809780

H 3.0720511 .8003704 .677916

O $1.7891892 .665083 \quad 3.344036$

C $4.079341-1.6193852 .649048$

H 5.1815740 .1232923 .097082

H $4.066101-0.4029364 .378711$

H 2.4225793 .4629341 .375100

H 6.0679083 .2033673 .595350

H 1.0824852 .0603813 .627703

O $5.149329-2.3723113 .042057$

O $3.184224-1.9813281 .899528$

C $5.210923-3.6934572 .496190$

H $5.406888-3.6420811 .421824$

H $6.037560-4.2219462 .978327$

H $4.283767-4.2387292 .697023$

SCF Energy (B3LYP/6-31G**//MMFF) $=-3245.91053781$

\section{$08 \quad 00484$}

MMFF Geometry

C $-0.798377-1.4687612 .177607$

C $0.309805-1.9421381 .588826$

C $1.520351-2.4324242 .338649$

O $2.630632-1.5965361 .938570$

C $1.834439-3.8844111 .947115$

C $3.025197-4.4505912 .695105$

C $4.260101-4.5855102 .172253$

C $2.741400-4.9156214 .099758$

C $4.718417-4.169576 \quad 0.802159$

C $6.040104-3.390360 \quad 0.860624$

C $6.463494-2.914523-0.535775$

C $7.877583-2.334947-0.514552$

C $8.284426-1.788935-1.891822$

O $5.536170-1.907993-0.957190$

O $9.470425-0.998286-1.720022$

C $7.178019-0.841655-2.398986$

C $8.648484-2.911696-2.870377$

O $7.434813-0.425778-3.738499$

C $5.750347-1.431691-2.291747$

C $4.712506-0.323687-2.583861$

O $5.594015-2.476667-3.252949$

C $2.927279-0.5366572 .737479$

$\begin{array}{llllll} & 2.426759 & -0.261422 & 3.816291\end{array}$

C 3.9383410 .2829222 .035836

C $4.045807 \quad 1.5805782 .357743$

C 4.9283012 .5618531 .725642

C 5.9684882 .0524570 .765409

C $3.264773-0.692330-2.235981$

C $2.3655130 .512529-2.435283$

C $1.8061302 .722601-1.412457$

O $1.6745320 .641191-3.443100$

C $2.8612993 .784384-1.378669$

C $2.8688164 .799552-0.500977$

C $3.9281975 .865607-0.471833$

C $4.507108 \quad 6.0900100 .909439$

C 4.2262567 .2068551 .602148 
C 5.4731665 .0582311 .456992

C 4.7581143 .8653762 .030179

N $2.459447 \quad 1.426799-1.397575$

H - $-0.860235-1.4388783 .262234$

H $0.365424-1.9612610 .502316$

H $1.360625-2.3732533 .422141$

H $1.994514-3.9544540 .865132$

H $0.962262-4.5213272 .148530$

H $5.030181-5.0455762 .790226$

H $3.622392-5.3570444 .577736$

H $2.414605-4.0780544 .723601$

H $1.953525-5.6759144 .096299$

H $4.860274-5.0743770 .198867$

H $3.968484-3.5590110 .292573$

H $5.919529-2.5251001 .525254$

H $6.820878-4.0318871 .287782$

H $6.412028-3.767556-1.222637$

H $8.601890-3.083609-0.170294$

H $7.924846-1.5131460 .213906$

H $10.157509-1.568679-1.334642$

H $7.2220860 .065885-1.780205$

H $9.475851-3.512072-2.473459$

H $9.004623-2.504640-3.823168$

H $7.817070-3.589489-3.074322$

H $8.331205-0.048892-3.758213$

H $4.9797920 .572343-2.013449$

H $4.755013-0.043331-3.644511$

H $4.650661-2.578097-3.456599$

H $4.515682-0.1820861 .245636$

H 3.3921211 .9775223 .134001

H 6.7089052 .8055360 .487315

H $5.4954371 .701438-0.154681$

H $6.534662 \quad 1.2243181 .207073$

H $2.895979-1.504623-2.870786$

H $3.172973-1.028460-1.196950$

H $1.1521862 .766786-0.537534$

H $1.1962032 .829323-2.314364$

H $3.6549293 .717600-2.119614$

H 2.0607094 .8823030 .222353

H $3.4733556 .792989-0.843647$

H $4.7453305 .633560-1.166308$

H 4.6565497 .3871252 .581727

H 3.5559857 .9635501 .208247

H 6.0775465 .4980982 .260850

H 6.1808774 .7959660 .666001

H 3.9944274 .1270892 .764498

H $3.0412511 .203568-0.595630$

C -2.2999290 .5152751 .654873$

$\begin{array}{llllll}\text { O } & -3.497138 & 0.833634 & 0.908274\end{array}$

C $-3.205591 \quad 1.9090110 .003798$

C -2.0337462 .6431870 .632629$

C - -1.2132121 .4886881 .177296$

H $-2.875371 \quad 1.454044-0.938088$

C $-4.4449402 .774111-0.245251$

H -1.485829 $3.254178-0.091077$

H -2.3581063 .2940641 .452876$

C -0.2477951 .9315252 .266573$

H -0.6424631 .0420070 .352478$

C $-5.6866711 .999884-0.734350$

O

H $-4.1896273 .570841-0.954326$

H -6.1017211 .4235140 .099444$

O $-6.6915582 .964409-1.073618$

C $-5.4477261 .067471-1.935325$

C $-6.7229000 .510922-2.596164$

H $-4.8212630 .227364-1.615790$

O $-4.706914 \quad 1.758876-2.945588$

H -7.262687 $1.319491-3.106076$

H $-6.391927-0.167091-3.394298$

H $\quad 0.4555442 .6765371 .880184$

H -0.7781372 .3802233 .113383$

H $\quad 0.3339411 .0895462 .646370$

C $-1.985498-0.9655381 .413859$

H -5.5853333 .9763620 .797327$

H $-6.3445823 .517344-1.793930$

H $-5.2592302 .486099-3.278279$

H -1.867173 -1.1408340 .336318$

H $-2.857246-1.5658721 .702068$
H -2.5398810 .6795312 .712527$

C -7.689464 -2.049030 -0.044454

O $-6.954433-1.283742-1.010820$

C $-7.679330-0.227815-1.646103$

C $-8.901993-0.787829-2.392171$

C $-9.785024-1.631122-1.466894$

C $-8.963201-2.647726-0.667287$

H -7.025134 -2.883370 0.215751

C -7.962574-1.246435 1.238063

H $-8.0234150 .482069-0.887852$

O $-8.489482-1.584787-3.501428$

H $-9.4818440 .048840-2.798879$

H -10.534136 -2.164262 -2.065045

O $-10.478947-0.782014-0.557984$

H -9.574843 -3.102809 0.120676

O $-8.552952-3.703518-1.541630$

C $-6.687839-0.8852841 .970328$

H -8.491378 -0.3144891 .022444$

H -8.571608 -1.843851 1.925935

H -7.906837 -2.286268 -3.163199

H $-11.017650-0.166474-1.083605$

H -9.357369-4.120509-1.894606

O -6.9811870 .0194452 .950961$

O $-5.580882-1.3441741 .726798$

C $-5.871180 \quad 0.465818 \quad 3.736434$

H $-5.297634-0.3831124 .120954$

H -5.2355151 .1231163 .137105$

H -6.2625481 .0343424 .584324$

SCF Energy (B3LYP/6-31G**//MMFF) $=-3245.91340279$

\section{8_00485}

MMFF Geometry

C $3.395093-0.5198903 .361338$

C 3.6201320 .7887073 .556006

C 4.4971401 .6948652 .724171

O 3.6701472 .7453792 .145831

C 5.3843821 .0252271 .655024

C 6.4012381 .9544731 .021726

C $6.4198952 .310745-0.277688$

C 7.5034272 .4278121 .936770

C $5.4501651 .957686-1.372720$

C $5.9918730 .849926-2.290212$

C $5.584154-0.551678-1.811803$

C $6.393810-1.636705-2.518566$

C $5.938656-3.040537-2.094841$

O $4.191560-0.714642-2.107802$

O $\quad 6.563178-3.963679-2.999202$

C $4.410496-3.139560-2.278173$

C $6.431735-3.401155-0.688376$

O $3.908114-4.368605-1.756671$

C $3.623708-1.952678-1.664426$

C $2.158610-2.016129-2.162043$

O $3.643855-2.053856-0.240659$

C 2.6750632 .4228131 .274528

O $2.400734 \quad 1.316767 \quad 0.836625$

C 1.9352483 .6624380 .953363

C $1.0601223 .648931-0.062900$

C $0.2027934 .756976-0.485356$

C 0.1149385 .9558230 .421220

C $1.288982-0.803063-1.803223$

C $-0.058844-0.867014-2.507973$

C $-2.1021620 .477620-2.940482$

O $-0.466404-1.873763-3.082904$

C $-2.3062081 .893259-3.371837$

C $-3.1488472 .745031-2.768459$

C $-3.3640134 .163810-3.210355$

C $-2.8714235 .171955-2.195533$

C $-3.7098065 .719652-1.299913$

C $-1.4263265 .612542-2.279488$

C $-0.4867064 .629813-1.638075$

N $-0.7570250 .329047-2.414151$

H $3.890333-1.0514422 .554668$

H $3.078896 \quad 1.2889454 .357992$

H 5.1479742 .2069843 .442519

H 4.7569160 .5701600 .885685

H 5.9410050 .1945452 .109823

H $7.2340562 .953268-0.613608$

H 8.2952832 .9572261 .395656 
H 7.1106283 .1133592 .693453 H 7.9700041 .5764762 .443285 H $4.4607561 .703156-0.983937$ H $5.3051022 .865976-1.971812$ H $5.5798861 .012065-3.294773$ H $7.0831370 .927283-2.369892$ H $5.740683-0.623336-0.732142$ H $7.467021-1.510258-2.328628$ H $6.267021-1.539157-3.605962$ H $6.329634-4.865252-2.720983$ H $4.216822-3.158987-3.360002$ H $7.526935-3.363719-0.647556$ H $6.156852-4.428382-0.424174$ H $6.045560-2.7350130 .086096$ H $4.335636-5.092875-2.243551$ H $2.165672-2.102394-3.257128$ H $1.673428-2.918635-1.769147$ H $2.902669-1.5409060 .119056$ H 2.1161694 .5379601 .566023 H $0.9566102 .734902-0.646534$ H $-0.1267195 .648528 \quad 1.445235$ H $-0.661315 \quad 6.6661080 .128259$ H $1.066368 \quad 6.4983390 .433040$ H $1.101395-0.768246-0.724871$ H $1.7879760 .125883-2.100550$ H $-2.7984290 .180046-2.151695$ H -2.248499 -0.198528 -3.789008 H $-1.7325462 .231409-4.231745$ H -3.729543 2.405991-1.914529 H -4.439239 4.300495 -3.385262 H $-2.8878264 .356216-4.179929$ H -3.368536 $6.464692-0.588805$ H $-4.7540625 .428788-1.253516$ H -1.310106 $6.615717-1.856733$ H $-1.1435265 .728821-3.333740$ H $-0.3441373 .720430-2.220920$ H -0.417302 $1.037709-1.770066$ C $1.090222-1.4446993 .353125$ O $0.514223-0.1384543 .147752$ C $-0.914588-0.2178743 .322107$ C $-1.240706-1.7019523 .396352$ C $0.007183-2.2566124 .057197$ H $-1.1266610 .267374 \quad 4.283162$ C - -1.5815670 .5541872 .178462$ H -2.153471 -1.904127 3.964718 H -1.361957 -2.125293 2.392491 C $0.161494-3.7593123 .906502$ H $-0.019235-2.0025395 .125378$ C -3.113501 0.4069102 .143268 O -1.2355321 .9336992 .329056$ H $-1.162403 \quad 0.2316381 .217269$ H -3.338707 -0.656062 2.022113 $\begin{array}{lllll}\text { O } & -3.669240 & 0.805487 & 3.395258\end{array}$ C $-3.792817 \quad 1.191448 \quad 1.003286$ C -5.3088630 .9437270 .888949$ H -3.3134910 .9331090 .053845$ O $-3.6262512 .598871 \quad 1.186980$ H $\quad-5.813481 \quad 1.276726 \quad 1.804667$ H -5.6801591 .6002120 .090419$ H $1.077451-4.1076844 .393936$ H $-0.684790-4.2808734 .365395$ H $\quad 0.207887-4.0553322 .853711$ C $2.384325-1.3009174 .155480$ H $-0.264696 \quad 1.9881982 .350447$ H -3.474286 1.7514893 .510229 H -2.6735282 .7896551 .151074$ H $2.805640-2.2832074 .395279$ H $2.181413-0.7917715 .105977$ H $1.299712-1.8606802 .358251$ C -5.185378 -2.254190-1.032699 O $-5.016459-0.890810-0.625434$ C -5.688544 -0.5116630 .578260$ C $-7.204629-0.7021650 .421762$ C -7.537405 -2.135098 -0.009219 C $-6.678479-2.592357-1.194195$ H $-4.717369-2.307818-2.024292$ C $-4.432219-3.231117-0.114611$ H $-5.343702-1.1410761 .403285$
O $\quad-7.7284020 .216384-0.534858$

H -7.690654 -0.481083 1.379246

H $-8.598360-2.186496-0.283742$

O $-7.329697-3.002779 \quad 1.101527$

H $-6.802255-3.669104-1.357690$

O $-7.167997-1.933648-2.366473$

C $-2.931347-3.064710-0.236745$

H $-4.683739-3.1103520 .941661$

H -4.684107 -4.255897 -0.413156

H $-7.2560670 .074759-1.373070$

H $-7.579805-3.9003180 .823260$

H $-6.667296-2.275478-3.126744$

O $-2.319065-4.264740-0.018298$

O $-2.364353-2.005954-0.468632$

C $-0.891278-4.262238-0.128035$

H $-0.516972-5.1999680 .291062$

H $-0.605760-4.208377-1.182227$

H $-0.451300-3.4309160 .431068$

SCF Energy $\left(B 3 L Y P / 6-31 G^{* *} / / M M F F\right)=-3245.91629698$

08_00486

MM̄FF Geometry

C $0.8054232 .884275-2.081671$

C $1.6326103 .939329-2.011462$

C $3.0874653 .922916-1.596739$

O $3.5518772 .575969-1.356946$

C $3.2662904 .723189-0.295929$

C 4.7223454 .9362290 .068451

C 5.3825704 .2490011 .021119

C $5.4185546 .039691-0.686478$

C $4.858123 \quad 3.1213381 .866012$

C 5.8590081 .9581991 .929402

C 5.3332040 .8141382 .807051

C $6.408959-0.2514023 .018975$

C $5.876160-1.4392863 .834618$

O 4.2023200 .2339622 .149152

O $\quad 6.823469-2.5120853 .722121$

C 4.559211-1.929503 3.198990

C $5.769462-1.1085655 .327916$

O $3.945325-2.9329354 .004880$

C $3.548317-0.7955532 .902726$

C $2.385689-1.3523622 .050364$

O $3.022789-0.2935824 .132314$

C $4.0709701 .897221-2.415498$

O $4.2291512 .312028-3.552610$

C $4.3675860 .522347-1.958134$

C $4.490960-0.450730-2.872709$

C $4.705138-1.872657-2.599986$

C $5.032883-2.272416-1.186976$

C $1.447521-0.2842551 .475911$

C $0.481658-0.9221770 .497541$

C $0.388236-1.879832-1.805757$

O $-0.661932-1.2276380 .826715$

C $1.085654-3.193850-1.977431$

C $1.482222-3.677485-3.164778$

C $2.187037-4.994781-3.332646$

C $3.436064-4.896548-4.184058$

C $3.473494-5.429070-5.417490$

C $4.666469-4.248768-3.580121$

C $4.585888-2.745320-3.622165$

N $1.051562-1.142939-0.746767$

H $1.1624971 .890890-1.826136$

H $1.2415424 .919348-2.279999$

H $3.6767614 .385614-2.397881$

H 2.7347994 .2237890 .522468

H $2.7904225 .708828-0.389564$

H 6.4164604 .5258391 .224695

H $6.4502576 .187593-0.350058$

H $5.4521145 .812725-1.756354$

H $4.8888006 .987796-0.547687$

H 4.6859983 .5049972 .878786

H 3.8999492 .7478121 .494658

H $6.044243 \quad 1.5897960 .912096$

H 6.8141692 .3202282 .330239

H $5.018361 \quad 1.2340103 .769690$

H 7.2950680 .1783103 .502491

H $6.750335-0.6247272 .043100$

H $7.679296-2.1922314 .055595$ 
H $4.820548-2.4144952 .247704$ H $6.750084-0.8237905 .727960$ H $5.454295-1.9851145 .904882$ H $5.077272-0.2904865 .537878$ H $4.606923-3.6302374 .153228$ H $2.800551-1.9277561 .215801$ H $1.787248-2.0534732 .646766$ H 2.1628590 .1216493 .960875 H $4.4197800 .340544-0.890966$ H $4.380836-0.190744-3.925091$ H $5.856205-1.665953-0.792286$ H $5.354329-3.311606-1.092105$ H $4.160449-2.138580-0.543157$ H $\quad 0.865907 \quad 0.2001612 .267170$ H 2.0015030 .5048320 .954910 H $0.434343-1.264660-2.709720$ H $-0.658828-2.048283-1.541726$ H $1.268679-3.779802-1.079273$ H $1.273857-3.106769-4.066974$ H $1.474873-5.694116-3.789481$ H $2.453608-5.429401-2.361104$ H $4.371501-5.384856-6.025002$ H $2.606251-5.916931-5.849796$ H $5.562949-4.548669-4.138285$ H $4.801856-4.644349-2.569927$ H $4.376347-2.348063-4.616648$ H $2.011385-0.853024-0.907790$ C $-1.6223122 .434334-1.486488$ O $-2.9329282 .460875-2.095864$ C $-3.8875722 .976853-1.144419$ C -3.1831232 .9411200 .199866$ C - $-1.7471613 .236596-0.189220$ H $-4.0894564 .011907-1.447329$ C $-5.1645962 .133955-1.226124$ H $-3.591370 \quad 3.667686 \quad 0.908732$ H $-3.257826 \quad 1.9430480 .650661$ C -0.7688002 .8517020 .908535$ H $-1.6517104 .309973-0.399830$ C $-6.1774322 .416838-0.103401$ O $-5.7924722 .393398-2.485804$ H $-4.8908101 .075310-1.233478$ H -5.7272702 .2167940 .874648$ O $-6.4942573 .814581-0.104021$ C -7.496932 $1.630576-0.218448$ C $-7.3473860 .108799-0.357316$ H $-8.0737321 .998916-1.075663$ O $-8.301047 \quad 1.9058970 .931302$ H -8.341442 $-0.355781-0.311906$ H -6.978061 -0.115691-1.367111 H 0.2629483 .0777850 .629752 H $-0.990326 \quad 3.4035581 .828352$ H -0.8319071 .7840151 .139006$ C $-0.6266552 .982983-2.511683$ H -5.138183 $2.203262-3.180133$ H $-6.8545164 .032781-0.980804$ H -8.392296 2.8719590 .997235 H $-0.8972264 .015857-2.766887$ H $-0.7409002 .419831-3.447317$ H $-1.4001971 .380477-1.273336$ C $-5.402615-2.7866730 .925255$ O $-6.325681-1.9593230 .206477$ C -6.421802 -0.5984970 .654499$ C $-6.899201-0.5616012 .112065$ C -5.992292 -1.413455 3.011002 C $-5.741317-2.8064122 .425261$ H $-5.567120-3.7958710 .526278$ C $-3.946269-2.407506 \quad 0.629928$ H $-5.429213-0.1464370 .589828$ O $-8.238818-1.0425762 .184747$ H -6.8956190 .4730812 .470456$ H $-6.454246-1.5209504 .000170$ O $-4.740355-0.755523 \quad 3.186999$ H -4.941315 -3.309607 2.980837 O $-6.931406-3.5783622 .612261$ C -3.641647 -2.584795 -0.839184 H $-3.701964-1.3803800 .915804$ H -3.255715 -3.051809 1.186993 H -8.520535 -0.9886943 .113779$
H $-4.9178170 .111004 \quad 3.590979$

H $-6.748726-4.4809292 .299911$

O $-3.687583-1.378160-1.472255$

O $-3.424027-3.668849-1.364436$

C $-3.489730-1.409520-2.888963$

H -2.548839 -1.908039-3.139166

H -4.332456 -1.914106 -3.370442

H $-3.445140-0.378093-3.248180$

SCF Energy (B3LYP/6-31G**//MMFF) $=-3245.90938936$

\section{7}

MMFF Geometry

C 2.4447333 .5616651 .657945

C 1.9189503 .3916552 .881117

C 1.6496462 .0734263 .571184

O 2.1350370 .9576922 .792465

C $\quad 0.131561 \quad 1.8931193 .742281$

C -0.2285260 .6987014 .601715$

C $-0.672229-0.4798094 .122978$

C $-0.1140450 .917603 \quad 6.088127$

C $-0.839816-0.8763352 .681694$

C $-0.374712-2.3200492 .446563$

C $-0.641293-2.7798061 .006606$

C $-0.348716-4.2734420 .860696$

C $-0.530781-4.751100-0.585097$

O $0.204040-2.0301890 .128887$

O $0.050864-6.061302-0.667968$

C $0.260546-3.817584-1.524227$

C $-2.011589-4.915346-0.946094$

O $-0.012707-4.162793-2.880770$

C $0.004239-2.310470-1.264632$

C $1.012402-1.462370-2.071509$

O $-1.318942-1.978277-1.685206$

C 3.4166710 .5544162 .999230

O $4.219258 \quad 1.021217 \quad 3.791116$

C $3.689558-0.5446072 .047074$

C $4.960883-0.8815831 .785422$

C $5.406730-1.8969230 .829138$

C $4.357117-2.7805990 .210710$

C $1.0170770 .030886-1.726504$

C $2.1692410 .701932-2.445627$

C $4.6208071 .124706-2.247873$

O $2.036647 \quad 1.171840-3.573260$

C $5.438012-0.073260-2.621849$

C $6.714980-0.249232-2.248191$

C $7.532242-1.454272-2.622185$

C $8.243200-2.079870-1.440290$

C $9.578457-1.994388-1.314402$

C $7.418045-2.869803-0.444365$

C $6.723973-1.9775550 .548102$

N $3.3488440 .667940-1.718161$

H 2.7457122 .6984881 .072213

H 1.6251264 .2761743 .443755

H 2.1379702 .0872694 .553181

H -0.3449481 .8111322 .757585$

H $-0.3075162 .789327 \quad 4.201569$

H -0.952851 -1.247196 4.843657

H $-0.4317970 .041540 \quad 6.663652$

H $\quad 0.921916 \quad 1.134586 \quad 6.366359$

H $-0.741725 \quad 1.759611 \quad 6.397720$

H -1.901369 -0.7924902 .428697$

H $-0.290870-0.2107102 .009548$

H $0.699091-2.3869472 .664824$

H $-0.895235-2.9892183 .143066$

H -1.689156 -2.571635 0.764186

H $-0.976530-4.8631781 .540496$

H $0.689120-4.4716131 .164513$

H $0.002620-6.346696-1.596711$

H $1.326177-4.028753-1.356005$

H $-2.489249-5.640605-0.276324$

H $-2.131705-5.318653-1.957655$

H -2.573517 -3.982591-0.880759

H $\quad 0.647127-3.720950-3.440592$

H $2.022024-1.855133-1.905986$

H $0.807987-1.559908-3.145383$

H $-1.366748-1.023830-1.853216$

H $2.842987-1.0122111 .557240$

H $\quad 5.756897-0.3371742 .292149$ 
H $4.768130-3.595354-0.388317$ H $3.705115-2.195034-0.441978$ H $3.743135-3.2530650 .985964$ H $0.0893640 .519792-2.040647$ H $1.1187000 .197654-0.648247$ H $5.1001781 .725547-1.468833$ H $4.4541921 .752528-3.128414$ H $4.956651-0.831025-3.236173$ H $7.2067960 .521090-1.658436$ H $8.264392-1.135515-3.375522$ H $6.913479-2.218496-3.108952$ H $10.098537-2.460661-0.484012$ H $10.181508-1.452461-2.035422$ H $8.064856-3.5518720 .122718$ H $6.730117-3.513470-0.998726$ H $7.404050-1.3054951 .074189$ H $3.3551090 .218492-0.807670$ C $1.6068745 .196263-0.096242$ $\begin{array}{lllll}\text { O } & 0.274257 & 5.182519 & 0.454229\end{array}$ C $-0.5616624 .340327-0.373528$ C $0.155805 \quad 4.273187-1.712087$ C $1.6068784 .223255-1.275676$ H $-0.560255 \quad 3.3624190 .121577$ C $-1.976110 \quad 4.929951-0.396798$ H $-0.1327003 .403128-2.308329$ H $-0.0322645 .178947-2.301510$ C $2.5916684 .596550-2.370299$ H $1.8236873 .203353-0.942596$ C $-3.0289164 .232048-1.282318$ O $-2.479920 \quad 4.948630 \quad 0.943627$ H $-1.9017305 .978708-0.712963$ H -3.952732 $4.821225-1.203982$ O $-2.6407994 .263120-2.651846$ C $-3.3951122 .788127-0.876203$ C $-4.5287692 .225222-1.759054$ H -3.706886 2.7805740 .174595 O $-2.2502941 .949167-0.997600$ H $-5.3032092 .989797-1.893152$ H $-4.1136541 .977628-2.744053$ H $2.5010143 .915649-3.222021$ H $2.4178445 .613778-2.737036$ H $3.6206324 .541856-2.000694$ C $2.6277994 .908051 \quad 1.010832$ H -1.822408 5.4071391 .494620 H -2.493029 $5.193026-2.894347$ H $-2.4982201 .062968-0.684602$ H 2.5443305 .6919661 .774531 H 3.6449454 .9675340 .606713 H $1.7721806 .220238-0.454928$ C $-4.645861-1.271493-0.439904$ O $-4.206516-0.071604-1.091850$ C $-5.185560 \quad 0.968021-1.156065$ C $-6.3868750 .495244-1.988672$ C $-6.975489-0.805279-1.430140$ C $-5.893716-1.850531-1.131570$ H $-3.819322-1.973345-0.591705$ C $-4.792238-1.0415971 .071418$ H $-5.5189591 .214181-0.140683$ O $\quad-5.998644 \quad 0.293432-3.346619$ H $-7.152136 \quad 1.280119-1.987673$ H $-7.685549-1.223599-2.154330$ O $-7.693505-0.526930-0.231735$ H $-6.315517-2.665268-0.532532$ O $-5.486766-2.416690-2.382361$ C $-4.580538-2.3039031 .879161$ H $-4.035815-0.3133631 .389079$ H $-5.771243-0.630648 \quad 1.330457$ H $-5.277791-0.359435-3.355877$ H $-8.4032440 .099785-0.453231$ H $-4.860744-3.135417-2.189273$ O $-4.453723-1.9692643 .197659$ O $-4.540832-3.437898 \quad 1.422327$ C $-4.231269-3.063761 \quad 4.092122$ H $-4.071963-2.6565725 .094073$ H $-5.109000-3.7163054 .111890$ H $-3.339268-3.6246393 .796752$

SCF Energy (B3LYP/6-31G**//MMFF) $=-3245.92190069$
0800488

MMFF Geometry

C $0.2928713 .272196 \quad 0.470497$

C -0.2175493 .1755091 .709061$

C 0.2674472 .2725322 .824558

O 1.3919901 .4670032 .407459

C $-0.879151 \quad 1.3213283 .222803$

C -0.5580640 .4283494 .405964$

C $-0.343099-0.9009794 .336262$

C $-0.524730 \quad 1.124058 \quad 5.743026$

C $-0.331661-1.7728403 .111296$

C $1.072837-2.3353872 .859976$

C $1.202728-2.9789161 .471677$

C $2.599489-3.5772191 .293673$

C $2.839225-4.094413-0.132735$

O $0.978575-1.9514630 .502069$

O $4.250447-4.313739-0.288190$

C $2.440401-2.991713-1.138111$

C $2.162857-5.451006-0.362036$

O $2.520562-3.473405-2.477364$

C $1.051037-2.382653-0.861967$

C $0.737131-1.153192-1.749167$

O $0.052683-3.370328-1.143517$

C 2.6354721 .9974602 .568858

O 2.9150293 .0866643 .043822

C 3.6111761 .0216002 .032869

C 4.8975691 .3761661 .895647

C 5.9808930 .5300081 .389329

C $5.634988-0.8741050 .970226$

C $1.6624310 .052772-1.555744$

C $2.8329550 .109204-2.521735$

C $4.9203081 .403723-2.956140$

O $2.921277-0.603897-3.517192$

C $6.0927110 .622465-2.453026$

C $7.2643561 .184639-2.118505$

C $8.4720250 .405569-1.682282$

C $9.0599430 .908632-0.383273$

C $10.106152 \quad 1.751974-0.378957$

C 8.4977030 .3578920 .906686

C 7.2268371 .0445831 .326188

N $3.7474261 .091196-2.161753$

H 1.1724692 .6867610 .215691

H -1.087199 3.7751171 .967589

H 0.5461222 .8952263 .683245

H -1.1658650 .7249182 .350760$

H -1.7750791 .9048963 .476460$

H -0.138682 -1.435749 5.263334

H $\quad-0.3756750 .424648 \quad 6.572659$

H 0.2909161 .8525855 .778313

H -1.469333 1.6477885 .922578

H -1.033258 -2.601744 3.265081

H -0.688049 -1.237656 2.229289

H $1.802260-1.5199722 .944292$

H $1.316311-3.0703943 .637365$

H $\quad 0.433193-3.7525101 .365655$

H $2.776475-4.3736862 .027419$

H $3.350740-2.8062051 .508393$

H $4.531986-4.9500680 .391206$

H $3.198648-2.204976-1.052547$

H $2.545793-6.1941880 .347901$

H $2.393720-5.845295-1.357596$

H $1.077885-5.414404-0.244814$

H $1.737601-4.026095-2.641294$

H $0.671432-1.451344-2.802410$

H $-0.273305-0.813014-1.480878$

H $-0.792198-2.916631-1.293830$

H 3.2428000 .0405371 .757972

H 5.1917612 .3840482 .186196

H $6.460207-1.4030740 .488905$

H $4.816464-0.8703850 .242731$

H $5.332299-1.4656981 .840525$

H $1.0786600 .963047-1.743140$

H $2.0310980 .107667-0.526985$

H $5.0814572 .484175-2.885271$

H $4.729690 \quad 1.147932-4.003433$

H $5.985168-0.458308-2.398076$

H $7.3787722 .262881-2.205758$

H $9.2214050 .482210-2.481268$ 
H $8.248050-0.664537-1.592182$

H 10.5542872 .0923030 .548716

H $10.5340852 .125126-1.303658$

H $9.222808 \quad 0.497911 \quad 1.720173$

H $8.390078-0.7274160 .823490$

H 7.3784312 .0768971 .644639

H $3.6170881 .597827-1.290781$

C $-1.6948484 .589668-0.540884$

O $-2.5670623 .468478-0.307783$

C $-3.6632093 .515106-1.230238$

C $-3.7007454 .948573-1.738589$

C $-2.2186145 .272095-1.807625$

H -3.396763 2.844199-2.056385

C $-4.9567173 .000645-0.580799$

H $-4.2058975 .036210-2.705014$

H $-4.1997795 .617961-1.028588$

C -1.919351 $6.759640-1.871668$

H - $-1.7983614 .787302-2.699043$

C $-4.7709251 .608006 \quad 0.065021$

O $-5.393297 \quad 3.941900 \quad 0.398295$

H $-5.7289932 .950615-1.357869$

H $-4.2113710 .981356-0.637441$

O $-3.9748801 .720847 \quad 1.245781$

C $-6.087530 \quad 0.8847180 .408209$

C $-5.901780-0.427871 \quad 1.196432$

H $-6.6297470 .684866-0.523656$

O $\quad-6.915751 \quad 1.7436651 .194167$

H $-5.559269-0.2017332 .214623$

H $-6.898928-0.8729111 .314321$

H -2.359163 $7.202104-2.771323$

H $-2.3265657 .288739-1.003513$

H $-0.8397516 .938095-1.899374$

C $-0.2546144 .082762-0.678819$

H -6.2378893 .6216670 .757591$

H $-4.4463102 .311006 \quad 1.858493$

H $-7.759716 \quad 1.283740 \quad 1.342314$

H $0.4229314 .923563-0.866441$

H $-0.2051083 .428655-1.559827$

H -1.788395 5.2588920 .323164

C $-4.659026-2.635543-1.549781$

O $-5.459196-1.745350-0.760435$

C $-4.952480-1.4446210 .542633$

C $-4.815003-2.7260761 .379362$

C $-3.957885-3.7662540 .651706$

C $-4.407034-3.957334-0.801618$

H $-5.275434-2.863643-2.429086$

C -3.368060 -1.963162 -2.041861

H $-3.964734-0.9833160 .452228$

O $-6.107562-3.2700001 .638038$

H -4.362099 -2.471299 2.345248

H $-4.025046-4.7231221 .183760$

O $-2.599215-3.3405850 .689816$

H $-3.667349-4.551674-1.349707$

O $-5.620280-4.716460-0.811405$

C $-3.634058-0.840629-3.023430$

H $-2.787705-1.540730-1.218008$

H -2.754997 -2.706669 -2.564361

H $-5.988379-4.0549242 .199338$

H -2.062328 $-4.037066 \quad 0.274812$

H $-6.311919-4.177036-0.391157$

O $-2.436431-0.358177-3.470925$

O $-4.739032-0.436155-3.356473$

C $-2.5126610 .702271-4.428585$

H - $1.502621 \quad 1.089368-4.587580$

H $-2.897010 \quad 0.317324-5.377642$

H $-3.1441781 .515561-4.059904$

SCF Energy (B3LYP/6-31G**//MMFF) $=-3245.91291059$

\section{9}

MM̄FF Geometry

C $-1.493398-2.192083-3.716616$

C $-0.683318-1.664032-2.787091$

C $0.425301-0.695214-3.095759$

O $1.627310-1.247638-2.507926$

C $0.1471250 .688563-2.469395$

C $-0.344547 \quad 1.707892-3.476294$

C $0.3960252 .723994-3.962293$

C $-1.7813661 .556899-3.905014$
C $1.8301623 .060208-3.657121$

C $1.9898764 .445017-3.015172$

C $1.3255724 .567984-1.635168$

C $1.4901225 .987062-1.088806$

C 0.8963546 .1263700 .319722

O $1.943494 \quad 3.629915-0.746528$

O $1.3652627 .366141 \quad 0.872683$

C 1.4490314 .9937071 .209931

C $-0.634422 \quad 6.2176250 .289082$

O 0.7958455 .0191062 .478182

C 1.3536103 .5883760 .560855

C 2.1375002 .5634321 .415659

O $-0.0194793 .197822 \quad 0.503607$

C $2.813686-0.849804-3.040947$

O $2.983674-0.101955-3.990521$

C $3.891482-1.464320-2.241385$

C $5.109464-0.905547-2.254031$

C $6.245627-1.392903-1.483581$

C $6.933816-0.320104-0.686456$

C $2.303075 \quad 1.177295 \quad 0.778317$

C 2.9552710 .1962131 .739270

C $3.374519-2.2428612 .026016$

O 3.4219790 .5285022 .825999

C $4.853348-2.4317181 .899477$

C $5.421194-3.5955501 .547705$

C $6.905105-3.8261891 .510053$

C $7.386485-4.3452240 .174459$

C $7.521908-5.663455-0.047929$

C $7.798931-3.331309-0.867168$

C $6.622701-2.685359-1.541874$

N $2.945975-1.0997251 .242556$

H -1.353181 -1.932337-4.762875

H $-0.840763-1.916745-1.740625$

H $\quad 0.567199-0.621418-4.182047$

H $1.0458691 .054974-1.965664$

H $-0.5937880 .613659-1.661027$

H $-0.0726363 .399536-4.677295$

H -2.123057 2.399144 -4.516204

H -1.913286 $0.647943-4.498059$

H -2.437083 $1.504072-3.030396$

H $2.3142932 .310369-3.027629$

H $2.3812753 .053274-4.605844$

H $3.0656984 .639871-2.916153$

H $1.5845315 .209371-3.690098$

H $0.2613274 .327275-1.732946$

H $1.0402996 .721477-1.768616$

H $2.5590456 .238290-1.040739$

H 1.0789878 .0845890 .283078

H 2.5073245 .2244931 .397588

H $-0.9545337 .077454-0.311729$

H $-1.040328 \quad 6.387438 \quad 1.292557$

H -1.109875 $5.328313-0.129624$

H 1.3142704 .4730153 .092048

H 3.1436922 .9552591 .615343

H 1.6386672 .4399612 .384849

H $-0.070877 \begin{array}{lll}2.236139 & 0.392012\end{array}$

H $3.658556-2.337099-1.640904$

H $5.295743-0.002273-2.831215$

H $7.4512910 .378860-1.352466$

H $7.666270-0.7116290 .022853$

H $6.2033340 .245491-0.095854$

H 1.3294420 .7646640 .495998

H $2.927717 \quad 1.242670-0.119396$

H $2.813091-3.1119091 .668354$

H $3.116170-2.0707873 .076201$

H $5.488301-1.5872212 .156572$

H $4.785316-4.4487621 .322366$

H $7.148164-4.5524642 .297130$

H $7.459307-2.9164061 .772320$

H $7.896141-6.042239-0.993285$

H $7.255960-6.3933690 .709659$

H $8.395796-3.816977-1.650840$

H $8.477911-2.603534-0.413115$

H $6.049226-3.365411-2.172797$

H $2.549903-1.2763500 .324202$

C $-3.970595-2.352989-3.478612$

O $-4.022157-1.370389-2.422288$

C $-5.265046-1.511742-1.715436$ 
C $-6.178724-2.287004-2.652726$

C $-5.190644-3.253875-3.278073$

H $-5.060745-2.131969-0.835211$

C $-5.783817-0.143359-1.266947$

H $-6.994214-2.787560-2.122184$

H $-6.615282-1.640081-3.422365$

C $-5.694540-3.899066-4.557239$

H $-4.960746-4.042153-2.548370$

C $-4.7687780 .690686-0.455775$

O $-6.1472790 .627859-2.418980$

H $-6.707059-0.272842-0.690498$

H $-3.9806021 .071798-1.115960$

O $-5.456453 \quad 1.8423160 .049115$

C -4.119195 -0.0519840 .725980$

C $-3.2420720 .878191 \quad 1.588067$

H $-3.503896-0.8717910 .339116$

O $\quad-5.152997-0.6230881 .526691$

H -2.6186561 .4998630 .934470$

H -3.8992151 .5362922 .169853$

H $-6.597688-4.486365-4.361761$

H $-5.939907-3.148224-5.315852$

H $-4.939274-4.569749-4.978769$

C -2.641941 -3.106063 -3.394283

H $-5.3587690 .700486-2.984061$

H -5.852182 2.300398 -0.712269

H $-4.728560-1.1261782 .241909$

H -2.627702 -3.943960 -4.100176

H -2.513345 -3.525238 -2.388498

H $-4.045247-1.803720-4.426070$

C $-2.448496-1.4582524 .406069$

O $-3.145102-0.6216193 .473950$

C $-2.323127 \quad 0.1037122 .555872$

C -1.3744191 .0408073 .319582$

C $-0.555747 \quad 0.275207 \quad 4.363634$

C -1.436189 -0.6389155 .223277$

H $-3.225401-1.8150595 .094584$

C -1.829994 -2.682568 3.721978

H -1.734160 -0.602915 1.960595

O -2.1311072 .0631533 .964336$

H -0.7062391 .5270012 .601434$

H -0.0336490 .9927855 .008370$

O $0.428737-0.4986813 .686279$

H $-0.812460-1.3170665 .816263$

$\begin{array}{llll}\text { O } & -2.160051 & 0.166699 & 6.158262\end{array}$

C -2.903599-3.546805 3.103120

H - $1.104670-2.4145532 .948368$

H -1.290929 -3.304219 4.446431

H -1.501574 2.6679824 .392625

H $0.970759-0.940747 \quad 4.361817$

H -2.7713690 .7310055 .654283$

O $-2.846042-3.4611321 .743432$

O $-3.707741-4.2034863 .751804$

C $-3.822665-4.2306681 .035673$

H -3.625857 -4.127186 -0.034471

H -3.744822 -5.2884701 .304500$

$\mathrm{H}-4.826112-3.8500221 .247898$

SCF Energy (B3LYP/6-31G**//MMFF) $=-3245.90589562$

0800490

MM̄FF Geometry

C $3.8546593 .102561-0.346810$

C $3.623978 \quad 4.236776 \quad 0.333373$

C 2.3890004 .5855741 .128776

O 1.4559613 .4846991 .163501

C 1.6684675 .7875180 .486604

C $0.477651 \quad 6.2781301 .291029$

C $-0.809788 \quad 6.202019 \quad 0.897127$

C 0.8133696 .9142262 .616904

C -1.362946 $5.648607-0.386955$

C $-2.0351004 .293663-0.160237$

C $-2.4820413 .610856-1.460803$

C $-3.6490964 .338472-2.133043$

C $-4.1654163 .584925-3.370762$

O $-2.8733132 .273739-1.115265$

O $\quad-5.4397034 .145195-3.725472$

C $-4.4102832 .108292-2.990684$

C $-3.2510543 .793799-4.583617$

O $-4.7648251 .336097-4.135140$
C $-3.226146 \quad 1.460015-2.242719$

C $-3.5472590 .040043-1.726923$

O $-2.120894 \quad 1.325384-3.146089$

C 1.3907542 .7441352 .302441

O 2.1102532 .8278723 .284252

C 0.2335521 .8332142 .163030

C -0.2850281 .2464213 .251620$

C -1.4749940 .3950323 .282440$

C -2.1825330 .1290331 .979878$

C $-4.750182-0.069533-0.787682$

C $-4.872934-1.462555-0.190739$

C $-6.308651-2.7998811 .342356$

O $-4.068090-2.363420-0.412978$

C $-5.815137-2.6766462 .748797$

C $-4.734451-3.3184393 .218812$

C $-4.248967-3.2098264 .636562$

C $-2.965704-2.4114674 .741838$

C $-1.787248-3.0428004 .877699$

C $-3.140243-0.9055804 .721849$

C $-1.894773-0.0986944 .466265$

N $-5.990644-1.5800690 .623961$

H $3.1048452 .317163-0.374377$

H 4.3982345 .0024330 .333828

H 2.7119764 .8495162 .142735

H $1.3649605 .511597-0.528236$

H 2.3688696 .6255860 .370143

H - 1.5730916 .5888101 .571980

H $-0.061808 \quad 7.3692163 .093297$

H 1.2154096 .1712253 .312151

H 1.5575807 .7058512 .480872

H $-2.0917586 .370462-0.773779$

H $-0.5932075 .558014-1.157774$

H $-1.338363 \quad 3.6242990 .356974$

H $-2.892054 \quad 4.399026 \quad 0.518417$

H -1.617835 $3.560367-2.133627$

H $-3.3736605 .363274-2.407944$

H -4.476933 $4.432973-1.416261$

H $-5.3132275 .094378-3.895789$

H $-5.2912672 .102536-2.337213$

H $-3.1765304 .860602-4.827231$

H $-3.6638183 .313553-5.477668$

H -2.236708 $3.421932-4.425994$

H $-3.9525641 .168269-4.642518$

H -3.683930 -0.641610 -2.576920

H -2.661069 -0.332726 -1.194259

H -1.517095 $0.649986-2.794990$

H -0.1892311 .7085911 .172906$

H $\quad 0.184728 \quad 1.4296634 .217078$

H -2.640136 1.0486061 .600392

H -2.970645 -0.622563 2.064152

H $-1.485125-0.2514091 .226570$

H -4.6595720 .6470800 .035688$

H $-5.676350 \quad 0.141171-1.332916$

H $-5.867101-3.6597210 .827620$

H $-7.396475-2.9186081 .336397$

H $-6.384372-2.0319923 .414090$

H -4.163496 -3.960522 2.551855

H -4.105805 -4.226791 5.024160

H $-5.010235-2.7562475 .284051$

H $-0.849879-2.5077024 .979106$

H - $1.730656-4.1265974 .894253$

H -3.549163 -0.594974 5.692484

H -3.909249 -0.6297083 .992023$

H $-1.319051 \quad 0.1255745 .364356$

H $-6.566087-0.7612280 .798365$

C $5.916517 \quad 1.628991-0.652768$

O $5.2256350 .415027-0.997488$

C $5.566433-0.592365-0.022011$

C 6.5724540 .0463310 .936662

C 6.2117091 .5168200 .839603

H $4.647644-0.8114280 .532731$

C $6.073053-1.861560-0.719328$

H $\quad 6.480707-0.3513541 .952328$

H $7.606971-0.1153340 .612172$

C 7.3042662 .4503021 .328118

H 5.3033111 .6816101 .432483

C $5.111427-2.435147-1.780262$

O $7.306908-1.551937-1.382079$ 
H $\quad 6.310722-2.6229660 .033351$

H $5.064282-1.752695-2.637843$

O $5.706680-3.648563-2.258582$

C $3.692194-2.728944-1.259628$

C $2.797472-3.392092-2.326006$

H $3.227294-1.790375-0.938573$

O $3.796494-3.576021-0.116363$

H $2.900687-2.845920-3.270949$

H $3.135111-4.424493-2.479494$

H 7.5251522 .2642802 .384249

H 8.2317522 .3147040 .761685

H 6.9971883 .4959291 .226639

C $5.1233602 .852901-1.115566$

H $7.619029-2.369206-1.807510$

H $5.252328-3.896942-3.081055$

H $2.898739-3.7251830 .224776$

H $4.8455212 .719633-2.168502$

H $5.7746043 .734219-1.067111$

H $6.8570251 .609772-1.220795$

C $-0.153234-4.374968-0.246056$

O $1.162867-4.300012-0.809755$

C $1.310233-3.411980-1.920615$

C $0.406072-3.867430-3.075093$

C $-1.053160-3.983142-2.621094$

C $-1.190029-4.768698-1.312500$

H $-0.094314-5.1915540 .484848$

C $-0.511863-3.0957220 .519401$

H $1.029213-2.397230-1.617879$

O $\quad 0.848562-5.122704-3.586919$

H $0.483428-3.142857-3.894034$

H $-1.639719-4.483168-3.401832$

O $-1.595221-2.680117-2.434192$

H -2.202687 -4.656039-0.908165

O $-1.013635-6.155177-1.621479$

C $0.469723-2.8673591 .646290$

H $-0.523621-2.213743-0.127078$

H $-1.510326-3.1707070 .965120$

H $0.840419-5.762265-2.854221$

H $-1.547751-2.216424-3.287480$

H $-1.181584-6.660914-0.807980$

$\begin{array}{llll}\text { O } & 1.177463 & -1.722520 & 1.428791\end{array}$

O $\quad 0.608393-3.6299572 .593985$

C $2.170132-1.4175362 .413494$

H $2.633084-0.4640972 .146103$

H $1.710842-1.3206143 .401782$

H $2.942827-2.1919452 .421843$

SCF Energy (B3LYP/6-31G**//MMFF) $=-3245.92041223$

\section{1}

MM̄FF Geometry

C $0.344072 \quad 1.047393-0.366354$

C $-0.6395520 .142129-0.474971$

C $-1.329210-0.188550-1.773924$

O $-2.754506-0.021877-1.589584$

C $-1.050111-1.655428-2.146609$

C $-1.667241-2.073615-3.467920$

C $-2.739691-2.880527-3.599055$

C $-0.970046-1.546405-4.696386$

C $-3.562786-3.505000-2.506919$

C $-5.001253-2.972777-2.526913$

C $-5.777489-3.371387-1.262846$

C $-7.229807-2.900472-1.354401$

C $-8.004946-3.145416-0.051152$

O $-5.127858-2.751708-0.148128$

O $-9.221366-2.384149-0.112395$

C $-7.185658-2.5886861 .133228$

C $-8.419537-4.6139710 .096686$

O $-7.794420-2.928907 \quad 2.376150$

C -5.712592 -3.044135 1.127492

C $-4.852698-2.331686 \quad 2.201567$

O $-5.656277-4.4480531 .407620$

C $-3.2476451 .242483-1.708199$

O $-2.6131902 .259183-1.941404$

C $-4.7120751 .180431-1.501123$

C $-5.4280632 .314782-1.478737$

C $-6.8786722 .422030-1.303382$

C $-7.6524881 .149911-1.082360$

C $-4.727349-0.8120162 .045460$
C $-5.732126-0.0124292 .857467$

C $-6.5079212 .332740 \quad 3.205110$

O $-6.459035-0.5098653 .712548$

C -7.7595572 .5597642 .417782$

C -8.160462 3.7711632 .002795

C -9.4449004 .0203721 .265966$

C $-9.2435794 .786413-0.021858$

C $-9.4431616 .114356-0.074801$

C $-8.905167 \quad 3.999083-1.266694$

C $-7.446564 \quad 3.645502-1.351387$

N $-5.688793 \quad 1.3387972 .537799$

H $0.6714341 .589060-1.250465$

H $-0.969210-0.3969050 .410264$

H $-0.984700 \quad 0.467736-2.582905$

H $-1.390525-2.306487-1.335197$

H $0.034517-1.820456-2.209286$

H $-3.078960-3.122784-4.605642$

H -1.403969-1.939374 -5.621993

H $-1.037628-0.455175-4.740237$

H $\quad 0.087246-1.830647-4.684306$

H $-3.576038-4.591337-2.658198$

H $-3.118131-3.343877-1.523094$

H $-4.976233-1.878580-2.602653$

H $-5.517775-3.349435-3.418520$

H $-5.731132-4.460973-1.149947$

H $-7.739976-3.377017-2.200852$

H -7.247056 -1.823833 -1.566669

H $-9.715165-2.670991-0.899691$

H $-7.219429-1.4955431 .057255$

H $-9.068178-4.914048-0.735161$

H $-9.009739-4.7687701 .006531$

H $-7.569890-5.2997320 .113903$

H -8.711481 -2.606510 2.347590

H -5.193895 -2.610134 3.205959

H $-3.833960-2.7376912 .119886$

H $-4.768643-4.6623261 .739289$

H $-5.1625680 .203232-1.376784$

H -4.905329 $3.261166-1.612524$

H $-8.700692 \quad 1.314420-0.824579$

H $-7.2262700 .577559-0.251700$

H $-7.6313950 .531255-1.985601$

H $-3.736650-0.5093902 .409215$

H -4.790970 0.5173010 .994244

H $-5.9050753 .241964 \quad 3.295971$

H -6.7631591 .9822124 .210381$

H $-8.380478 \quad 1.6918332 .208479$

H $-7.551927 \quad 4.641073 \quad 2.239970$

H -10.106200 4.5865191 .935118

H $-9.9744043 .083096 \quad 1.054383$

H $-9.3313276 .666445-1.002118$

H -9.7173006 .6796910 .809771$

H $-9.1452194 .591839-2.159967$

H $-9.5614853 .126844-1.334711$

H $-6.8010754 .510817-1.509042$

H -5.0915741 .6512991 .777583$

C 2.5355881 .0696040 .922867

O $3.2177441 .899090-0.037462$

C $4.264726 \quad 1.117982-0.658802$

C $4.370958-0.1641880 .157356$

C $2.934442-0.3677490 .595912$

H $3.9054480 .892719-1.671176$

C $5.5452261 .960516-0.726132$

H $4.763006-1.002753-0.426066$

H $5.020987-0.0198711 .028154$

C $2.776391-1.3337101 .756067$

H $2.367764-0.746964-0.263437$

C $6.781927 \quad 1.161379-1.187434$

O $5.2740563 .037664-1.626754$

H 5.7379462 .4102260 .255606

H $6.9341440 .346727-0.474050$

O $6.515867 \quad 0.556948-2.453102$

C $8.0842661 .983734-1.272637$

C $9.3186131 .156853-1.687317$

H $8.2656772 .464526-0.304348$

O $7.9247543 .027499-2.235821$

H $9.1656140 .736593-2.689226$

H $10.1599111 .856191-1.784305$

H $3.155890-2.3246111 .486470$ 
H $3.324402-0.9933022 .640473$ H $1.722529-1.4390712 .032910$ C $\quad 1.0358441 .3763540 .926684$ H $6.0407383 .634929-1.610692$ H $6.3709841 .276080-3.092006$ H $8.7304253 .571810-2.216446$ H 0.8931282 .4492341 .106098 H $0.5614650 .847008 \quad 1.761722$ H 2.9396311 .3535141 .904115

C $10.237749-0.2372201 .649835$

O $9.947421 \quad 0.657071 \quad 0.568428$

C $9.6906750 .041958-0.697179$

C $10.913629-0.765186-1.157496$

C $11.322824-1.787279-0.091456$

C $11.424966-1.1512211 .299838$

H 10.5529460 .4137222 .475856

C $8.986393-1.0021242 .109951$

H $8.840693-0.639694-0.607916$

O $12.001754 \quad 0.119111-1.416165$

H $10.668664-1.275141-2.097011$

H $12.290600-2.224718-0.365367$

O $10.358317-2.835722-0.075228$

H $11.524811-1.9292002 .064912$

O $12.623970-0.3719041 .360448$

C $7.960130-0.0818392 .741158$

H $8.489577-1.5258991 .289691$

H $9.280296-1.7359732 .869674$

H $12.745796-0.418188-1.737525$

H $10.646656-3.4896550 .584161$

H $\quad 12.5281910 .3696590 .738310$

O $7.038017-0.8361013 .409961$

O 7.9547551 .1381362 .651196

C $6.014930-0.0993844 .087576$

H $5.306001-0.8147464 .512739$

H $\quad 6.453180 \quad 0.487028 \quad 4.900530$

H 5.4800320 .5509713 .389023

SCF Energy (B3LYP/6-31G**//MMFF) $=-3245.91236629$

0800492

MM̄FF Geometry

C $1.832911-0.9096532 .892366$

C $2.797963-0.2403503 .541936$

C 3.0630421 .2461803 .495312

O $1.974757 \quad 1.9265282 .821872$

C 4.3787671 .5651292 .758181

C 5.6377961 .0641963 .441128

C 6.4099870 .0655432 .966076

C 6.0362901 .8044604 .690145

C $6.129450-0.7643021 .745600$

C $6.704124-0.1234040 .475411$

C $6.030418-0.662061-0.795278$

C $6.6249730 .000192-2.040656$

C $5.862774-0.374800-3.320275$

O $4.639017-0.351971-0.687563$

O $6.2988640 .529206-4.347119$

C $4.353565-0.137592-3.087959$

C $6.235384-1.781352-3.801879$

O $3.593898-0.581555-4.209245$

C $3.828783-0.775007-1.786538$

C $2.371813-0.371075-1.459466$

O $3.852836-2.200033-1.925632$

C 1.7666803 .2303423 .140859

O 2.4332703 .9019843 .918004

C 0.5940403 .8043902 .440697

C -0.2130043 .1279331 .607995$

C -1.4040903 .6666110 .939381$

C -1.759149 $5.107678 \quad 1.204176$

C $2.1565201 .106955-1.120986$

C $1.7376961 .964913-2.299233$

C $1.2625354 .339603-2.874721$

O $1.385937 \quad 1.502172-3.380387$

C $-0.1507224 .645103-2.491587$

C $-1.2138224 .085946-3.088002$

C $-2.6375094 .367375-2.707460$

C $-3.3077013 .172260-2.067701$

C $-3.8545642 .201285-2.818845$

C $-3.4263743 .156084-0.561255$

C -2.1269782 .8548220 .138125$
N $1.765908 \quad 3.317067-1.978323$

H $1.139198-0.3792732 .246049$

H $3.469929-0.8081814 .183431$

H 3.1020121 .5978124 .533909

H 4.4747682 .6520522 .633979

H 4.3043541 .1981361 .727603

H $7.318839-0.1972633 .503959$

H 6.9949911 .4574295 .090052

H 6.1350002 .8749904 .482715

H 5.2850891 .6690595 .474133

H $6.570370-1.7594301 .883221$

H $5.052171-0.9390441 .658872$

H 6.5528700 .9635450 .516730

H $7.786407-0.2969330 .433671$

H $6.157288-1.750356-0.832590$

H $7.690126-0.243879-2.139279$

H $6.5813101 .092782-1.925595$

H $5.7667010 .350785-5.141790$

H $4.214700 \quad 0.949053-3.036275$

H $7.308815-1.836052-4.020338$

H $5.728214-2.027525-4.741186$

H $6.006929-2.559779-3.070904$

H $3.535932-1.551139-4.163313$

H $1.694640-0.711937-2.251239$

H $2.076062-0.932620-0.564209$

H $3.284564-2.585112-1.238460$

H 0.4141684 .8493542 .674490

H -0.0052552 .0786601 .409518$

H -2.013114 5.2530482 .259870

H -2.606979 5.4655800 .616896

H -0.9195935 .7642810 .949625$

H $1.3336151 .170791-0.398682$

H $3.0403751 .543929-0.644667$

H $1.3388933 .997427-3.912184$

H $1.8869125 .230501-2.758500$

H $-0.2998855 .354050-1.681477$

H -1.054168 $3.376437-3.897097$

H $-3.1829294 .656282-3.615444$

H -2.706918 $5.236394-2.041579$

H $-4.3660631 .356799-2.369880$

H $-3.7987862 .229450-3.902044$

H $-4.1408732 .383698-0.250899$

H $-3.8809884 .093617-0.229112$

H -1.783539 $1.829815-0.005406$

H $1.9879643 .593308-1.026041$

C $1.821578-3.1089001 .671332$

O $\quad 0.706034-2.7977130 .811037$

C $0.125050-4.0253410 .338869$

C $1.216901-5.0720430 .499234$

C $1.842265-4.6341191 .809198$

H $-0.699891-4.2654081 .021283$

C $-0.423857-3.859026-1.082148$

H $\quad 0.818989-6.0906170 .530996$

H $1.956842-5.016325-0.307500$

C $3.230117-5.2067582 .042706$

H $1.186100-4.9462332 .632917$

C -1.338964 -2.632181-1.273819

O $0.663951-3.728222-2.004981$

H $-0.953314-4.772311-1.377141$

H $-0.746415-1.709776-1.235563$

O $-1.890379-2.700573-2.594710$

C $-2.496301-2.533673-0.264019$

C $-3.423283-1.344575-0.551004$

H -2.077691 -2.426466 0.740478

O $-3.256757-3.739878-0.279897$

H $-2.837070-0.418853-0.605977$

H -3.879851-1.460861-1.543449

H $3.193232-6.3002792 .083407$

H $3.921055-4.9232301 .241383$

H $3.645950-4.8463002 .988794$

C $1.641748-2.3975313 .014852$

H $1.185149-2.954338-1.733939$

H -1.144578 -2.745625 -3.217524

H $-3.602523-3.856807-1.181625$

H $2.344209-2.809593 \quad 3.748914$

H $0.628501-2.5715693 .397540$

H $2.726939-2.7530181 .163869$

C $-6.495050 \quad 0.2991340 .752956$ 
O $-5.325008-0.038000-0.000300$

C $-4.577513-1.1723160 .458038$

C $-4.114390-0.9632401 .908721$

C -5.285202 -0.5953952 .826068$

$\begin{array}{llll}\text { C } & -6.147290 & 0.522827 & 2.234427\end{array}$

H $-6.827397 \quad 1.259758 \quad 0.338862$

C -7.625987 -0.7182970 .536975$

H $-5.216023-2.0599770 .397983$

O -3.1304330 .0677231 .948282$

H -3.644084 -1.883516 2.273276

H $-4.891270-0.2759063 .798772$

O $-6.076028-1.7607493 .040610$

H -7.0674520 .6395262 .818668$

O $-5.418728 \quad 1.7480792 .355148$

C -8.139448 $-0.710299-0.888657$

H $-7.304207-1.7390660 .758150$

H -8.470384 -0.4636841 .187990$

H -2.836553 0.1597542 .870525

H $-6.796683-1.5187193 .646942$

H -5.986147 2.4632252 .020529

O $-9.166228-1.606406-0.991538$

O $-7.702588-0.016190-1.796173$

C $-9.758314-1.716924-2.289827$

H -10.186964 -0.757046 -2.593780

H -9.014919-2.056557-3.017198

$\mathrm{H}-10.560290-2.457871-2.235812$

SCF Energy (B3LYP/6-31G**//MMFF) $=-3245.91265721$

0800493

MMFF Geometry

C $2.535413 \quad 2.830911-2.697315$

C $2.618703 \quad 4.032365-2.105028$

C $3.1504324 .300317-0.712485$

O $3.5047633 .068001-0.044300$

C $2.056003 \quad 4.9873590 .130935$

C $2.4835965 .296551 \quad 1.554165$

C 1.8976684 .7797592 .653409

C 3.6312916 .2620101 .698489

C $0.715535 \quad 3.8508762 .710498$

C 1.1331232 .3912252 .543192

C -0.0457381 .4097532 .575996$

C $-0.611317 \quad 1.2212613 .985071$

C -1.682567 0.1187354 .036205

$\begin{array}{lllll}0 & 0.464143 & 0.162872 & 2.089973\end{array}$

O $-1.899351-0.2214845 .414479$

C $-1.141430-1.1491373 .339235$

C $-3.0301360 .613048 \quad 3.497873$

O $-2.156217-2.1435093 .225600$

C $-0.519105-0.8698921 .954360$

C $0.198886-2.1307361 .414855$

O $-1.555364-0.5027591 .042693$

C $4.7784232 .609986-0.170722$

O $5.6959473 .140811-0.774890$

C $4.849148 \quad 1.310532 \quad 0.537903$

C $5.946296 \quad 0.548806 \quad 0.416017$

C $6.176737-0.7528881 .049309$

C $5.072943-1.3215111 .901213$

C $1.086845-1.8490420 .193345$

C $1.801047-3.093189-0.300219$

C $3.741632-3.782989-1.706931$

O $1.421046-4.231517-0.039376$

C $4.779547-4.223613-0.722252$

C $6.100707-4.155397-0.946553$

C $7.129357-4.6932170 .006196$

C $8.194491-3.6845110 .366633$

C $9.425002-3.757032-0.169015$

C $7.867568-2.6577301 .427144$

C $7.363298-1.3630890 .845795$

N $2.891373-2.777100-1.100766$

H $2.9054331 .950768-2.181053$

H $2.2576044 .904084-2.647348$

H $4.0253884 .956298-0.792827$

H 1.1650344 .3489170 .130533

H $1.7530725 .927889-0.348085$

H 2.2953655 .0580013 .628486

H 3.8000626 .5527892 .740912

H 4.5598265 .8178631 .327232

H 3.4300597 .1779551 .133373
H $\quad 0.2218953 .9955383 .678509$

H $-0.0285374 .126311 \quad 1.954673$

H $1.6490952 .267944 \quad 1.583792$

H 1.8721852 .1120283 .306002

H -0.8189751 .7679001 .886915$

H - -1.0171122 .1607014 .378333$

H 0.2029890 .9392214 .667258

H -2.168972 0.5866545 .883906

H $-0.362944-1.5730633 .988887$

H -3.3810531 .4759194 .076528$

H -3.803892 -0.1530713 .608748$

H -2.988604 0.9150702 .450542

H -2.511947 -2.297849 4.117447

H $0.842721-2.5482602 .200053$

H $-0.540819-2.8989471 .156061$

H $-1.320466-0.8000010 .150714$

H 3.9929961 .0136631 .133186

H $6.7635220 .914860-0.204593$

H $4.894025-0.6836172 .773556$

H $5.281337-2.3261662 .274106$

H $4.143946-1.3969771 .327264$

H $\quad 0.490403-1.469836-0.641173$

H $1.837570-1.0916370 .444521$

H $4.182616-3.341102-2.605930$

H $3.127965-4.639947-2.002943$

H $4.422474-4.6660440 .205144$

H $6.461268-3.743247-1.886119$

H $7.590646-5.569007-0.469458$

H $6.664354-5.0709680 .925512$

H $\quad 10.204760-3.0571350 .113054$

H $9.678293-4.513147-0.904786$

H $8.777064-2.4226821 .997172$

H $7.188086-3.0942722 .163909$

H $8.097262-0.8604890 .213634$

H $3.164446-1.804393-1.207186$

C $0.599414 \quad 1.849577-4.010612$

O $-0.4003702 .692458-3.402161$

C $-1.127748 \quad 1.914613-2.429183$

C $-0.876765 \quad 0.463114-2.812914$

C $0.5744780 .522975-3.246810$

H $-0.6702792 .142307-1.457677$

C -2.605706 $2.323199-2.432879$

H $-1.049093-0.231580-1.988589$

H $-1.5164370 .165491-3.653074$

C $1.034574-0.665080-4.071400$

H $1.1867880 .571310-2.339452$

C $-3.4241841 .620828-1.328024$

O $-2.6732553 .742393-2.264014$

H $-3.0400912 .107253-3.415677$

H -3.235049 $0.543900-1.376837$

O $-2.9572892 .063266-0.053207$

C $-4.9467291 .838298-1.431880$

C $-5.7557201 .314802-0.229954$

H $-5.302922 \quad 1.364937-2.354575$

O $-5.228828 \quad 3.234803-1.548780$

H $-5.551084 \quad 1.9357580 .652170$

H $-6.813377 \quad 1.489996-0.468560$

H $0.981610-1.585133-3.481325$

H $0.415167-0.799732-4.964154$

H $2.072207-0.534761-4.394976$

C $1.9426482 .588189-4.056992$

H -2.193140 $3.969445-1.449699$

H -3.2523622 .9820550 .065647$

H $-6.1847393 .330908-1.699628$

H $1.8022473 .546212-4.573060$

H $2.6621872 .009028-4.647137$

H $0.2643021 .691171-5.043943$

C $-5.645296-2.366197-0.901031$

O $-5.807224-0.947878-1.045257$

C -5.546296 $-0.167290 \quad 0.124633$

C $-6.455604-0.5977241 .291131$

C $-6.378233-2.1068201 .540398$

C -6.525262 -2.9019770 .241078$

H $-6.028871-2.789975-1.838285$

C $-4.168616-2.772121-0.762592$

H $-4.502485-0.3036250 .422467$

O $-7.807579-0.2429581 .001690$

H $-6.157353-0.0466652 .190960$ 
H $-7.173585-2.408258 \quad 2.233030$

O $-5.130080-2.4327832 .141532$

H -6.290474 -3.957309 0.419758

O $-7.891073-2.858983-0.181380$

C $-3.419165-2.658246-2.072799$

H $-3.629612-2.168770-0.029320$

H $-4.119586-3.824172-0.456397$

H -8.346485 -0.4850501 .773920$

H $-5.089659-1.9765962 .998879$

H $-8.101039-1.938838-0.416734$

O $-2.264794-3.383037-1.981496$

O $-3.790120-2.014615-3.044324$

C $-1.456089-3.434638-3.160701$

H $-0.445121-3.728770-2.866195$

H $-1.859040-4.188246-3.842778$

H $-1.406128-2.461893-3.656826$

SCF Energy (B3LYP/6-31G*//MMFF) $=-3245.90062118$

0800494

MM̄FF Geometry

C $2.6705663 .904915-0.008552$

C $3.252467 \quad 4.2928621 .137512$

C $3.854386 \quad 3.3959912 .194557$

O 3.9027512 .0200961 .758668

C $2.980158 \quad 3.4632953 .460451$

C 3.4201932 .5124304 .555701

C 2.7883391 .3550604 .838028

C 4.6068862 .9587355 .365696

C 1.6153200 .7857254 .087026

C $1.600697-0.7497374 .101456$

C $0.503882-1.2940023 .174057$

C $0.255602-2.7803103 .426494$

C $-0.785177-3.3505772 .451211$

O $0.942620-1.0993161 .824261$

O $-0.741831-4.7822142 .548594$

C $-0.371278-2.981271 \quad 1.012546$

C $-2.212525-2.9326072 .822788$

O $-1.378574-3.3585950 .076809$

C $-0.011676-1.487144 \quad 0.827494$

C $0.637329-1.286367-0.563026$

O $-1.201205-0.7005260 .906829$

C 5.0887091 .5503241 .288500

O 6.1106982 .1895701 .096751

C $4.931596 \quad 0.096497 \quad 1.055111$

C $5.915912-0.5815220 .447306$

C $5.914576-2.0086810 .117822$

C $4.773923-2.848556 \quad 0.630131$

C $1.2844380 .088958-0.771592$

C $1.944405 \quad 0.172909-2.136374$

C $3.857182-0.740932-3.448901$

$\begin{array}{lllll}\text { O } & 1.481265 & 0.862448 & -3.041227\end{array}$

C $4.458852-2.110022-3.485003$

C $5.769282-2.349822-3.642776$

C $6.364672-3.729859-3.638704$

C $7.483353-3.886883-2.629939$

C $8.751064-4.082072-3.030699$

C $7.125088-3.887743-1.155749$

C $6.935732-2.493032-0.619970$

N $3.074255-0.620846-2.233345$

H $2.6267652 .852099-0.270097$

H 3.2664915 .3554861 .374863

H 4.8622903 .7580172 .432202

H 1.9332563 .2686053 .195576

H 2.9821804 .4869663 .859492

H 3.1462360 .7615735 .677512

H 4.8535222 .2514376 .164672

H 5.4905293 .0563454 .727278

H 4.4053663 .9280685 .832996

H $\quad 0.692487 \quad 1.152693 \quad 4.552284$

H 1.6185661 .1263293 .046667

H $2.579186-1.1278613 .777810$

H $1.431721-1.0941695 .129076$

H $-0.415781-0.721642 \quad 3.344164$

H - $0.056897-2.9537164 .463751$

H $1.195511-3.3354703 .298475$

H $-0.938617-5.0257843 .469409$

H $\quad 0.516663-3.5817770 .768797$

H -2.453272 -3.256590 3.842400
H -2.946404 -3.4219282 .174272$

H $-2.372593-1.8537782 .772470$

H $-1.567437-4.3021130 .218426$

H $1.412495-2.050356-0.702688$

H $-0.111088-1.443325-1.350682$

H -1.0656840 .1278120 .420430$

H $4.009237-0.3722801 .378128$

H $6.804700-0.0341730 .135094$

H $4.661189-2.7241521 .713398$

H $4.916140-3.9186060 .463791$

H $3.832624-2.5666890 .149150$

H $0.5426550 .889306-0.698240$

H $2.0437900 .290099-0.009595$

H $4.6142750 .049570-3.432068$

H $3.210140-0.586633-4.318072$

H $3.777883-2.949817-3.365277$

H $6.451413-1.514371-3.781968$

H $6.734024-3.934719-4.651679$

H $5.600326-4.491235-3.437783$

H $9.555194-4.217449-2.314966$

H $9.014804-4.106060-4.082779$

H $7.928533-4.359250-0.575144$

H $6.243626-4.520265-1.018264$

H $7.736015-1.806363-0.901371$

H $3.467837-1.001873-1.378984$

C $0.4847624 .643436-0.951634$

O $0.1560043 .473298-1.727001$

C -1.105754 $3.700624-2.400695$

C $-1.6155125 .042242-1.894047$

C $-0.3207105 .771105-1.588707$

H $-0.8652393 .765081-3.469267$

C -2.019454 $2.498091-2.130817$

H -2.236534 $5.560415-2.630705$

H $-2.206774 \quad 4.916639-0.978361$

C $-0.511366 \quad 6.985243-0.696367$

H $0.1455656 .080815-2.533541$

C -3.495159 $2.703433-2.508309$

O $-1.5201921 .388730-2.886629$

H $-1.9416722 .217910-1.074093$

H -3.942130 $3.469466-1.864565$

O $-3.571407 \quad 3.216797-3.844024$

C $-4.3596131 .426100-2.448877$

C $-4.2603090 .681519-1.111726$

H $-4.0931470 .760385-3.276959$

O $-5.724683 \quad 1.804493-2.651061$

H $-3.2318180 .352747-0.925514$

H $-4.4964881 .377673-0.294653$

H $0.4447367 .477224-0.493101$

H $-1.1697817 .715826-1.177786$

H $-0.9596416 .710763 \quad 0.264567$

C $1.9989104 .862019-0.958326$

H $-0.5704391 .309129-2.697332$

H $-3.1299782 .577706-4.429723$

H $-5.7700982 .286006-3.494997$

H $2.2344705 .893914-0.674634$

H $2.4076564 .694117-1.961734$

H $0.128126 \quad 4.4606410 .071693$

C $-5.804245-2.1610300 .726361$

O $-4.953966-1.0769240 .331279$

C $-5.205503-0.527966-0.970819$

C $-5.015731-1.610747-2.044749$

C $-5.889487-2.835588-1.749762$

C $-5.750144-3.305917-0.298841$

H $-5.368583-2.5296601 .664047$

C $-7.228363-1.6843941 .031751$

H $-6.234508-0.154630-1.002659$

O $-3.654329-2.032435-2.097755$

H $-5.281122-1.210909-3.029765$

H $-5.604644-3.656766-2.419046$

O $-7.256112-2.520703-2.001231$

H $-6.528152-4.041656-0.065630$

O $-4.498656-3.983068-0.152932$

C $-7.232411-0.7702322 .233579$

H -7.703205 -1.1618470 .196497$

H $-7.878706-2.5343701 .270762$

H -3.127887 -1.300193 -2.459529

H $-7.330856-2.261571-2.935378$

H $-3.789942-3.333088-0.299865$ 
O $-7.105420 \quad 0.527062 \quad 1.833196$

O $-7.310604-1.1658943 .389541$

C -7.0603201 .4946882 .885946$

H -7.9876221 .4671303 .466140$

H -6.1948391 .3127653 .530245$

H -6.958566 2.4846342 .433464

SCF Energy (B3LYP/6-31G*//MMFF) $=-3245.92980988$

08_00495

MM̄FF Geometry

C $-0.602343-3.5442130 .259361$

C $-1.508377-2.5901610 .002794$

C $-2.095247-2.334907-1.359578$

O $-1.816525-0.959895-1.711330$

C $-3.623238-2.488453-1.306600$

C $-4.256611-2.602476-2.677566$

C $-4.837040-1.586226-3.345066$

C $-4.252757-3.989469-3.267385$

C $-4.909742-0.137118-2.951350$

C $-6.3246040 .303351-2.551260$

C $-6.716632-0.133698-1.131029$

C $-8.1574710 .277226-0.819676$

C -8.541082 -0.0326260 .635333$

O

$\begin{array}{llll}\text { O } & -9.763548 & 0.661346 & 0.927247\end{array}$

C -7.4590170 .5459331 .571049$

C $-8.828148-1.5241370 .845091$

$\begin{array}{lllll}\text { O } & -7.695720 & 0.161487 & 2.923694\end{array}$

C $-6.017914 \quad 0.168206 \quad 1.157929$

C -4.9994580 .9786611 .996836$

O $-5.816443-1.2264851 .393970$

C $-0.588443-0.657620-2.208101$

O $0.325259-1.428977-2.448063$

C $-0.5460190 .811558-2.370175$

C $0.605303 \quad 1.423429-2.680781$

C $0.7610002 .869812-2.808102$

C $1.6215773 .290424-3.967113$

C -3.5475610 .8634371 .508340$

C -2.5925211 .6928602 .350806$

C -0.2092862 .4256912 .432508$

O $-2.937927 \quad 2.289295 \quad 3.367507$

C -0.1771183 .7988391 .837413$

C $0.907196 \quad 4.3402901 .261168$

C 0.9194725 .7090250 .640585

C $1.3260805 .694771-0.818554$

C $2.5198216 .176573-1.204104$

C $0.3210975 .208799-1.842201$

C $0.2266113 .708138-1.897009$

N $-1.301973 \quad 1.6764961 .836364$

H - $0.268220-4.197723-0.542557$

H $-1.826703-1.9334800 .808134$

H $-1.664478-3.003882-2.114919$

H $-4.058745-1.642489-0.764051$

H -3.898486 -3.382661 -0.730985

H -5.289931-1.803581-4.311955

H $-4.734830-4.026043-4.250102$

H -3.227202 -4.351044 -3.390721

H $-4.789335-4.682372-2.610883$

H $-4.1917700 .115115-2.166228$

H $-4.6014880 .447848-3.827558$

H $-6.3520051 .399869-2.601292$

H -7.055305 $-0.072351-3.278125$

H $-6.605524-1.220277-1.046650$

H -8.857867 $-0.204865-1.513133$

H -8.271523 $1.358199-0.983172$

H $-10.4378250 .359857 \quad 0.294471$

H -7.5515881 .6407761 .539597$

H $-9.655646-1.8485790 .202645$

H $-9.151491-1.7233931 .872751$

H -7.970029 -2.1618830 .622885$

H -8.6044600 .4275973 .145356$

H -5.273069 2.0416971 .966609

H -5.0473290 .6595303 .045821$

H -4.875704 -1.391626 1.560891

H $-1.462627 \quad 1.367840-2.199513$

H $1.5071580 .840213-2.854738$

H $1.122303 \quad 3.053625-4.913152$

H $2.5837322 .767165-3.948158$
H $1.8464214 .359318-3.972538$

H $-3.209021-0.1752791 .555258$

H $-3.469429 \quad 1.2060320 .470197$

H 0.7112231 .8672532 .237915

H -0.3618642 .4927143 .514348$

H -1.097403 4.3771381 .879092

H $1.8352963 .774878 \quad 1.234233$

H 1.6136866 .3293281 .222166

H $-0.0596816 .196026 \quad 0.730890$

H $2.8097206 .197499-2.249345$

H $3.2374646 .553736-0.483171$

H $0.5473325 .623833-2.830696$

H $-0.6690065 .613591-1.595656$

H $-0.360528 \quad 3.277820-1.086927$

H $-1.129076 \quad 1.2152970 .947623$

C $1.484890-3.3157171 .596843$

O $2.258857-4.2551350 .819738$

C $3.473504-4.5418271 .525384$

C $3.592148-3.4930342 .622775$

C $2.133299-3.2803332 .980273$

H $3.320065-5.5147582 .013116$

C $4.676632-4.6769440 .586308$

H $4.196488-3.8341653 .468493$

H $4.026656-2.5619772 .245301$

C $1.878747-1.9932913 .746827$

H $1.787625-4.1327803 .580179$

C $5.124341-3.412977-0.181337$

O $5.778002-5.121732 \quad 1.390098$

H $4.472678-5.478420-0.134419$

H $5.495870-2.6721590 .532799$

O $6.263572-3.782596-0.974837$

C $4.070432-2.781400-1.109272$

C $4.606444-1.637129-1.992198$

H $3.232905-2.405125-0.518332$

O $3.558800-3.799495-1.973176$

H $5.434267-1.999169-2.614151$

H $3.806735-1.361631-2.693260$

H $2.428842-1.9937034 .693391$

H $2.196107-1.1157553 .173715$

H $0.814428-1.8780553 .974592$

C $0.019405-3.7472581 .612917$

H $6.542210-5.2318810 .798656$

H $5.956465-4.409163-1.652656$

H $2.830542-3.409504-2.486308$

H $-0.532668-3.1805902 .371704$

H $-0.064947-4.8096981 .870632$

H $\quad 1.585370-2.3361341 .110897$

C $4.097133 \quad 1.248948 \quad 0.309312$

$\begin{array}{llll}\text { O } & 3.867916 & 0.126943 & -0.552113\end{array}$

C $5.036191-0.395610-1.194304$

C $5.6684330 .676390-2.094517$

C $5.984127 \quad 1.945314-1.295698$

C $4.7918392 .397807-0.445121$

H 3.0909751 .5873410 .587168

C 4.8066620 .8016791 .590722

H $5.764390-0.694769-0.433936$

$\begin{array}{lllll}O & 4.776642 & 0.987678 & -3.162692\end{array}$

H $6.5866660 .271119-2.536506$

H $6.2603782 .746675-1.992056$

O $7.1117961 .689603-0.464241$

H 5.1036513 .1781960 .257494

O $3.8417492 .995008-1.330643$

C $4.626096 \quad 1.8007182 .705386$

H $4.417150-0.1666721 .927688$

H 5.8785120 .6380021 .446687

H $5.2089701 .655358-3.721682$

H 7.3137532 .5101590 .017067

H $3.0980893 .314034-0.792691$

O $3.417183 \quad 1.614123 \quad 3.307526$

$\begin{array}{lllll}\text { O } & 5.453344 & 2.655726 & 2.993983\end{array}$

C 3.1168632 .5156854 .377056

H 2.1460122 .2361374 .794654

H 3.8723392 .4379605 .164628

H 3.0551483 .5407083 .999177

SCF Energy (B3LYP/6-31G**//MMFF) $=-3245.90501971$

0800496

MM̄FF Geometry 
C $-0.590814 \quad 0.197777-1.849945$ C $-1.156240-0.948536-1.440031$ C $-0.703827-1.820206-0.288819$ O $0.574817-1.380708 \quad 0.223848$ C $-0.548258-3.273679-0.774032$ C $-0.212238-4.2529090 .335474$ C $0.979965-4.8625410 .494172$ C $-1.342149-4.554267 \quad 1.287603$ C $2.219745-4.694854-0.339878$ C $3.340319-4.0339130 .472344$ C $4.481690-3.527265-0.422014$ C $5.612882-2.9534260 .433385$ C $6.706830-2.286556-0.413156$ O $3.939174-2.510417-1.271066$ O $7.525780-1.4993290 .465793$ C $6.045442-1.308088-1.407380$

C $7.635087-3.321232-1.059588$ O $7.008423-0.777561-2.314246$ C $4.872954-1.930803-2.190938$ C $4.097909-0.896324-3.044858$ O $5.386373-2.919393-3.091893$ C $0.576765-0.5910251 .331832$ O $-0.389713-0.2255821 .980878$ C $1.979815-0.2147331 .614602$ C $2.234015 \quad 0.793648 \quad 2.461737$ C 3.5565121 .2857152 .854815 C $4.761846 \quad 0.5818592 .290914$ C $3.3918630 .214000-2.259021$ C $4.1964901 .493489-2.108888$ C $4.0997293 .713041-0.973655$ O $5.2345191 .715285-2.725576$ C $5.004140 \quad 3.683106 \quad 0.217756$ C 4.8507684 .4869531 .281243 C 5.7965554 .5215432 .447302 C $5.097305 \quad 4.3676953 .778926$ C 4.7784835 .4421814 .520659 C 4.8582462 .9672874 .293248 C 3.6298622 .3333423 .702151 N $3.5851162 .381527-1.232350$ H $0.2783130 .595936-1.334903$ H $-2.042894-1.296846-1.967504$ H $-1.472074-1.7771780 .491528$ H $0.204009-3.305213-1.568130$ H $-1.480840-3.610136-1.247794$ H $1.093446-5.5582951 .324870$ H $-1.083485-5.3409312 .004343$ H -1.609556 -3.662369 1.862307 H -2.225562 -4.8922840 .735923$ H $2.551687-5.684452-0.676745$ H $2.022053-4.122184-1.247689$ H $2.924453-3.1875041 .032751$ H $3.728480-4.7504341 .207040$ H $4.845246-4.358269-1.037751$ H $6.044016-3.7298191 .077733$ H $5.200356-2.1990371 .115459$ H $7.888541-2.0907951 .147353$ H $5.669609-0.462086-0.819722$ H $8.135962-3.919857-0.289184$ H $8.435707-2.834487-1.627221$ H $7.110990-4.012741-1.722614$ H $7.717716-0.377594-1.782380$ H $4.747469-0.488962-3.828861$ H $3.309816-1.446137-3.579606$ H $4.736626-3.060056-3.800311$ H $2.762253-0.7690361 .111402$ H 1.3911201 .3130072 .916734 H 4.7127620 .5439161 .197873 H $4.818382-0.4404442 .678584$ H 5.7073791 .0725842 .530958 H $2.4855100 .496283-2.809989$ H $3.076432-0.138346-1.272959$ H $3.2375314 .370457-0.822706$ H $4.6560254 .064383-1.848812$ H 5.8472172 .9969270 .185596 H 4.0250895 .1950201 .295548 H 6.3246145 .4838452 .416435 H 6.5771053 .7563322 .353383 H 4.3060115 .3386295 .491881
H 4.9778096 .4493684 .169850

H 4.7164692 .9888495 .382549

H 5.7607192 .3661064 .149989

H 2.6997612 .7839014 .051707

H $2.7532702 .089555-0.727335$

C -1.944289 2.192448 -2.565206

O $-3.2015861 .714919-2.057401$

C $-4.1046431 .559862-3.162827$

C $-3.598622 \quad 2.481356-4.273205$

C -2.332253 $3.117734-3.717296$

H $-4.0168510 .524046-3.511038$

C $-5.5496401 .808986-2.715604$

H $-3.3540061 .873504-5.153402$

H $-4.3424283 .212141-4.607465$

C $-2.6071324 .536773-3.216239$

H $-1.5467503 .179164-4.478722$

C $-6.0054470 .953044-1.513231$

O $-5.685804 \quad 3.185922-2.346253$

H $-6.2218391 .653548-3.568222$

H $-5.5294161 .329462-0.601667$

O $-7.4111301 .173250-1.338627$

C $-5.744186-0.557004-1.653627$

C $-6.415785-1.437355-0.583616$

H $-4.663840-0.739451-1.642501$

O $-6.229775-0.986914-2.929558$

H $-7.504903-1.423801-0.719933$

H $-6.110018-2.471723-0.791151$

H -2.946396 $5.177110-4.037284$

H $-3.3718224 .555604-2.433827$

H $-1.6950414 .979553-2.802116$

C $-1.0878501 .000200-3.021522$

H $-6.6078873 .321846-2.068337$

H $-7.8647520 .799742-2.113906$

H $-5.991151-1.923903-3.033801$

H $-0.2128321 .358991-3.576827$

H $-1.6474320 .348298-3.702685$

H $-1.4295502 .735168-1.763588$

C $-4.145692-0.7409712 .307369$

O $-4.646261-1.1048221 .012550$

C $-6.068574-1.0784680 .869822$

C $-6.726058-2.0490431 .866002$

C $-6.273632-1.7712913 .303079$

C $-4.750440-1.6353843 .402929$

H -3.071077 -0.9558862 .262820$

C -4.3136990 .7604932 .591932$

H $-6.433500-0.0657901 .066807$

O $-6.411024-3.3998621 .530360$

H $-7.815057-1.9476341 .792171$

H $-6.605307-2.5896473 .953660$

O $-6.876677-0.5704303 .774596$

H $-4.462012-1.2503324 .388027$

O $-4.158406-2.9309903 .269502$

C $-3.5085391 .621207 \quad 1.642740$

H -5.3575701 .0727762 .505552$

H -3.962969 0.9819073 .606525

H -5.442076 -3.4836911 .514277$

H $-7.840980-0.6885543 .730752$

H -4.494916 -3.482830 3.995997

O -3.8028392 .9344371 .877352$

O $-2.7215821 .203861 \quad 0.805759$

C $-3.126323 \quad 3.875657 \quad 1.038190$

H $-3.4851093 .780266 \quad 0.009747$

H -3.3584494 .8811381 .399039$

H -2.0430503 .7282321 .085928$

SCF Energy (B3LYP/6-31G*//MMFF) $=-3245.90740709$

0800497

MM̄FF Geometry

C -3.640761 -2.308161 2.138351

C $-4.823596-2.2699211 .505576$

C $-5.134488-2.936596 \quad 0.182176$

O $-3.953483-3.625752-0.293658$

C $-5.536007-1.907686-0.893018$

C $-6.878919-1.249537-0.668343$

C $-7.0449440 .029397-0.279883$

C $-8.077424-2.112816-0.965799$

C $-5.977694 \quad 1.0261130 .075850$

C $-5.7689262 .043524-1.050616$ 
C $-4.8200773 .174746-0.630798$

C $-4.6930414 .214514-1.746398$

C $-3.6908835 .321763-1.385983$

O $-3.5372232 .609759-0.336593$

O $-3.3919546 .053363-2.585180$

C $-2.3744104 .665044-0.918078$

C $-4.2841456 .334531-0.399710$

O $-1.4583345 .643913-0.433945$

C -2.5781913 .5599220 .143669$

C -1.2655112 .8143790 .492490$

O $-3.0316424 .169273 \quad 1.361283$

C $-4.099880-4.685918-1.125032$

O $-5.153075-5.159103-1.527668$

C -2.779864 -5.205427 -1.558789

C $-1.625443-4.864747-0.963507$

C $-0.278349-5.283236-1.360129$

C $-0.151459-6.135876-2.594648$

C $-0.5517702 .085577-0.647424$

C -1.110996 $0.713417-0.987654$

C $-0.773203-1.139299-2.632598$

O $-2.0236640 .177311-0.367242$

C $0.427322-2.027758-2.775007$

C $1.553409-1.984273-2.044468$

C $2.718185-2.915382-2.241067$

C $2.999663-3.764195-1.018899$

C $3.947986-3.410779-0.135134$

C $2.238030-5.066122-0.865738$

C $0.767961-4.849231-0.624972$

N $-0.4327810 .149849-2.060715$

H -2.819569 -2.8756411 .709290$

H $-5.635984-1.7104721 .963980$

H -5.932088 -3.6696990 .351608$

H -5.561695 -2.398240-1.875789

H -4.735560 -1.165295 -1.003503

H $-8.061060 \quad 0.409647-0.182446$

H $-9.020593-1.567366-0.854538$

H -8.032260 -2.484286 -1.994874

H -8.112180 -2.970439 -0.287000

H -6.2949011 .5440440 .989620$

H -5.0316380 .5361560 .324798$

H $-5.3564511 .520668-1.923467$

H $-6.7344702 .470990-1.349054$

H -5.2261333 .6392020 .275685$

H -5.671313 $4.647721-1.989182$

H -4.347722 $3.720892-2.665698$

H -4.226949 $6.407740-2.936108$

H -1.914806 4.225095-1.810627

H $-5.1678976 .817887-0.833416$

H -3.574890 7.142511 -0.188843

H -4.5881495 .8856820 .547994$

H -1.355095 $6.314123-1.131022$

H -0.5644363 .5357260 .933415$

H -1.473578 2.0920281 .292130

H -2.860839 3.5554282 .094389

H -2.812600 -5.887661 -2.401825

H - $1.655271-4.194377-0.106323$

H $-0.688310-7.082028-2.463710$

H $\quad 0.878320-6.396157-2.845573$

H $-0.564783-5.614963-3.465619$

H $-0.5086922 .693054-1.554918$

H $0.4847341 .920339-0.331874$

H -1.184362 -0.930833 -3.626053

H -1.547590 -1.638525 -2.042182

H $\quad 0.344052-2.789318-3.548122$

H $1.655992-1.243819-1.256411$

H $3.596744-2.303229-2.483071$

H $2.567750-3.568255-3.109960$

H $4.174342-4.0255620 .729628$

H $4.518324-2.496254-0.254636$

H $2.627098-5.636616-0.012446$

H $2.439347-5.682716-1.745224$

H $\quad 0.561768-4.243478 \quad 0.259167$

H $\quad 0.2686510 .705066-2.542571$

C -2.202091 -0.5826823 .216550$

O $-1.007447-1.2688212 .796083$

C $0.135987-0.672546 \quad 3.442027$

C -0.3806030 .5769924 .140139$

C -1.8053820 .1783524 .478075$
H $\quad 0.478733-1.4092264 .178924$

C $1.215646-0.4245282 .382350$

H 0.2122860 .8394065 .021432

H -0.3739691 .4367163 .459110$

C -2.7060181 .3580954 .797909$

H - $1.784078-0.5044115 .337973$

C $2.461657 \quad 0.3137772 .900443$

O $1.606346-1.6943431 .848253$

H $\quad 0.7666380 .1284481 .551363$

H $2.188196 \quad 1.3244473 .225126$

O $2.932300-0.3754654 .065931$

C 3.6292700 .3855561 .893813

C 3.2554481 .0586850 .561383

H $4.020672-0.6214611 .712595$

O 4.6915891 .1191062 .512803

H 2.3610660 .5882870 .146216

H 3.0314652 .1137990 .761320

H $-2.329614 \quad 1.9044045 .668927$

H -2.7607802 .0597953 .959336$

H -3.7233281 .0230955 .023269$

C $-3.329789-1.5958853 .425508$

H $2.035593-2.1949552 .563156$

H 3.7229760 .0952644 .381194

H 4.3826602 .0303702 .653237

H -4.221154 -1.093936 3.818030

H $-3.024243-2.3522854 .158482$

H -2.4701330 .1164202 .414243$

C $6.6575421 .646745-0.890242$

O $5.4966461 .715524-0.052082$

C $4.3676960 .963984-0.501033$

C $3.9037561 .475661-1.875779$

C $5.0546501 .458397-2.886652$

C $6.3268482 .102425-2.322415$

H $7.3569912 .371793-0.454579$

C $7.3252660 .267631-0.833697$

H $4.647401-0.090607-0.589082$

O $3.4099522 .809744-1.777950$

H $3.0856450 .844710-2.243375$

H $4.742597 \quad 1.997210-3.789941$

O $5.3133410 .106621-3.254825$

H $7.1776951 .899473-2.983281$

O $6.1335583 .519889-2.315283$

C $7.796822-0.0398940 .567863$

H $\quad 6.667827-0.540081-1.167696$

H $8.205314 \quad 0.234277-1.486764$

H $2.5547652 .777269-1.317943$

H $6.0216920 .114399-3.920817$

H $6.9638673 .928822-2.017413$

O $6.959671-0.9425781 .153464$

O $8.7782440 .477691 \quad 1.085383$

C $7.279982-1.2982642 .501824$

H $8.248061-1.8066102 .537085$

H $7.286426-0.4092643 .139721$

H $6.508759-1.9831362 .864414$

SCF Energy $(B 3 L Y P / 6-31 G * * / / M M F F)=-3245.89273910$

0800498

MM̄MF Geometry

C $3.957887-3.317219-0.486895$

C $2.637949-3.136544-0.327065$

C $1.675936-3.027351-1.479165$

O $0.677512-2.036888-1.144231$

C $0.946686-4.365872-1.698607$

C $0.014672-4.351212-2.897347$

C $-1.328214-4.454903-2.837758$

C $0.691549-4.236860-4.240714$

C $-2.200880-4.622047-1.625022$

C $-2.969247-3.336794-1.312913$

C $-3.786742-3.417664-0.015283$

C $-4.959830-4.395723-0.124096$

C $-5.843715-4.3843871 .134133$

O $-4.274708-2.0943940 .251044$

O $-7.070398-5.0600210 .814289$

C $-6.207086-2.9230401 .474347$

C $-5.204646-5.1734542 .283033$

O $-6.919763-2.8464662 .706416$

C $-4.989921-1.9723511 .488528$

C $-5.392393-0.4909811 .666046$ 
O $-4.151952-2.3069472 .602429$

C $0.842985-0.771243-1.611918$

O $1.806233-0.319022-2.207797$

C -0.412419-0.043517 -1.320755

C $-0.7087761 .080737-1.988968$

C $-1.9648551 .827167-1.886726$

C $-3.0138411 .312418-0.934405$

C -6.3203230 .0729890 .587476$

C $-6.541450 \quad 1.5673360 .756993$

C -7.735664 $3.484893-0.291775$

O -6.0196622 .2272251 .652118$

C $-6.8093724 .153234-1.257550$

C $-5.7919724 .944162-0.884067$

C $-4.8592045 .622784-1.846209$

C $-3.4961094 .961565-1.876158$

C $-2.4842615 .470669-1.153683$

C $-3.3937653 .748323-2.779724$

C $-2.1409022 .923738-2.653864$

N $-7.3959342 .076569-0.212041$

H $4.352052-3.411024-1.494590$

H $2.222860-3.0751650 .676008$

H $2.207768-2.735258-2.392842$

H $0.402358-4.624694-0.785017$

H $1.679548-5.170133-1.848073$

H -1.883034 -4.427824 -3.775364

H $-0.007074-4.381890-5.071854$

H $1.145323-3.248872-4.363279$

H $1.473768-4.996909-4.338100$

H -2.901491 -5.438740 -1.833808

H -1.629228 -4.934544 -0.747406

H -2.263524 -2.502056 -1.225164

H -3.628293 -3.071162 -2.150386

H $-3.111024-3.7124330 .796264$

H $-4.611029-5.415480-0.323765$

H -5.582595 -4.118489 -0.986388

H $-6.849406-5.961867 \quad 0.524829$

H $-6.908765-2.5937830 .698327$

H $-5.033308-6.214476 \quad 1.983219$

H $-5.872385-5.2203583 .150238$

H -4.245339 -4.7631142 .604557$

H $-6.276383-2.9526653 .427766$

H -5.843722 -0.3442472 .656296$

H $-4.4737190 .112488 \quad 1.676186$

H -3.589782 -1.540963 2.805980

H -1.092355 -0.492420 -0.605601

H $\quad 0.012851 \quad 1.467895-2.706801$

H -3.387880 $0.338196-1.266743$

H $-3.875041 \quad 1.978141-0.839942$

H -2.603624 1.2062360 .075826

H -5.895980 -0.097817 -0.408063

H $-7.300536-0.4129800 .637253$

H -7.680215 3.9340380 .705660

H $-8.7679623 .562435-0.646207$

H $-6.9896093 .977431-2.315297$

H -5.6099975 .1132920 .174881$

H $-4.7719806 .676184-1.550460$

H -5.278352 $5.640726-2.860358$

H -1.493528 5.034279-1.159463

H -2.625904 $6.348196-0.530806$

H -3.464434 4.090069-3.820896

H $-4.2689543 .105028-2.634076$

H -1.328656 $3.247792-3.304515$

H $-7.7224401 .465967-0.955556$

C $6.163558-2.5370210 .532674$

O $5.777821-1.157158 \quad 0.374224$

C $6.606658-0.569651-0.645451$

C $7.784626-1.520697-0.828562$

C $7.113507-2.863300-0.621563$

H $6.013724-0.567994-1.567925$

C $7.0045560 .862508-0.280301$

H $8.251334-1.419701-1.813070$

H $8.557826-1.362251-0.067832$

C $8.085681-3.991062-0.319901$

H $6.557838-3.112167-1.532888$

C $5.8242791 .815433-0.002903$

O 7.8151560 .8241660 .901626

H $7.6416801 .271295-1.074125$

H $5.377711 \quad 1.5657370 .966007$
O $\quad 6.3734493 .132222 \quad 0.147284$

C $4.7308771 .843813-1.086574$

C $3.7403513 .018339-0.974825$

H $4.1812780 .896016-1.066576$

O $5.3589931 .940204-2.369183$

H $4.2614933 .959005-1.195555$

H $3.0045052 .894618-1.781278$

H $8.783157-4.130218-1.152348$

H $8.674529-3.7843680 .579976$

H $7.551978-4.933970-0.164932$

C $4.927422-3.4337460 .656975$

H 8.0656841 .7403801 .110760

H $6.7321503 .400305-0.716204$

H $4.6606271 .871178-3.042586$

H $4.412803-3.1791011 .591829$

H $5.243909-4.4797230 .741850$

H $6.713389-2.5885281 .481924$

C 1.5266611 .8468781 .796002

$\begin{array}{llll}\text { O } & 2.237982 & 1.940921 & 0.554186\end{array}$

C $3.0139823 .127848 \quad 0.373915$

C 2.1175564 .3743330 .433309

C 1.2993374 .4077281 .728078

C 0.6099833 .0671002 .001082

H $\quad 0.8859680 .9621431 .687827$

C 2.4729141 .6008122 .981672

H 3.7627223 .1980501 .168181

O $1.2357064 .381552-0.686791$

H $2.7504395 .267040 \quad 0.360602$

H $\quad 0.5368405 .1934701 .661760$

O 2.1522204 .7198602 .825513

H 0.2071663 .0528143 .020166

O $\quad-0.5114002 .9417941 .123433$

C 3.1876370 .2698462 .885281

H 3.2415632 .3740903 .056843

H 1.8914071 .5907913 .910985

H $0.7475115 .222127-0.665224$

H 2.5548935 .5870202 .647625

H -0.1728792 .9224780 .212171$

O $3.977194 \quad 0.1163463 .989857$

O $3.076322-0.5289611 .966308$

C $4.732697-1.0971714 .048960$

H $5.207716-1.1539795 .031923$

H $4.079464-1.9664353 .926725$

H $5.512331-1.0856463 .282862$

SCF Energy (B3LYP/6-31G**//MMFF) $=-3245.91443435$

08_00499

MM̄FF Geometry

C -0.0409703 .9896472 .173421$

C 1.0603153 .4061512 .670812

C 1.1539952 .1301643 .478619

O 2.0113831 .2103072 .751236

C -0.1839841 .4110743 .744110$

C -0.0570460 .2295824 .690165$

C $-0.126831-1.0676474 .329651$

C 0.1179840 .5907456 .144400

C $-0.303547-1.6402922 .951734$

C $0.899742-2.5043562 .556523$

C $0.818885-2.9830141 .098407$

C $2.034537-3.8481180 .759755$

C $2.099820-4.213715-0.728611$

O $\quad 0.786338-1.8221390 .265731$

O $3.417322-4.729935-0.974328$

C $1.929005-2.930694-1.571686$

C $1.124460-5.346413-1.069742$

O $1.852131-3.273814-2.953138$

C $0.714033-2.082898-1.142201$

C $0.641586-0.702937-1.841437$

O $-0.483604-2.793423-1.476462$

C $3.357276 \quad 1.3534502 .905094$

O $3.9330882 .174274 \quad 3.602244$

C 4.0258120 .3386462 .059800

C 5.3539940 .4038381 .880605

C $6.177370-0.5295961 .107962$

C $5.487605-1.6948160 .451754$

C $1.8153230 .238480-1.552639$

C $2.9198260 .206879-2.593649$

C $5.1756011 .173673-3.041769$ 
O $2.829740-0.399707-3.656990$

C $6.1992370 .126574-2.736529$

C $7.4612080 .408226-2.378021$

C $8.516508-0.634866-2.148886$

C $9.200929-0.497321-0.808259$

C $10.3886710 .121733-0.698214$

C $8.558237-1.1666390 .383998$

C $7.507145-0.3106481 .035978$

N $4.0095100 .975097-2.203030$

H $-1.019750 \quad 3.5490552 .335888$

H 2.0148673 .8958682 .482754

H 1.6201342 .3797894 .439883

H -0.6246231 .0989802 .791963$

H -0.9036242 .1112094 .188806$

H $-0.044490-1.8197775 .113849$

H $0.117373-0.2900406 .795475$

H $1.066501 \quad 1.1128636 .301762$

H -0.6980821 .2415836 .475265$

H $-1.206073-2.2610462 .948410$

H $-0.461849-0.8583712 .206516$

H $1.814560-1.9143202 .692884$

H $0.969197-3.3701963 .226854$

H $-0.109857-3.549550 \quad 0.962948$

H $2.052373-4.7527041 .380620$

H $2.948419-3.2974661 .016872$

H $3.491859-4.903363-1.928637$

H $2.847839-2.346756-1.442709$

H $1.382714-6.252250-0.508034$

H $1.189867-5.622467-2.127871$

H $0.085196-5.101178-0.840677$

H $1.913448-2.452111-3.468157$

H $0.469833-0.825204-2.917274$

H $-0.260299-0.198525-1.464087$

H $-1.196559-2.151653-1.622436$

H $3.410579-0.4335331 .614453$

H 5.9011811 .2167132 .356872

H $5.107680-2.3848591 .211784$

H $6.135922-2.268031-0.214423$

H $4.651316-1.352633-0.166050$

H $1.4331261 .265680-1.551974$

H $2.2427980 .051340-0.563043$

H $5.5465912 .185182-2.848464$

H $4.8821341 .106064-4.094438$

H $5.898447-0.911822-2.855413$

H $7.7683601 .448031-2.289863$

H $9.256865-0.538546-2.954130$

H $8.107204-1.647928-2.247899$

H $10.901598 \quad 0.1942850 .255131$

H $10.8711630 .574640-1.558040$

H $9.321074-1.3762711 .146701$

H $8.187266-2.1543130 .095416$

H 7.9160990 .5659131 .540634

H $4.0165841 .393502-1.277210$

C $-0.5525365 .101849-0.057797$

O $-1.9645824 .822228-0.018585$

C $-2.2544963 .762209-0.956230$

C $-1.0372303 .681332-1.867792$

C $0.0876074 .001094-0.903667$

H -2.337920 $2.853432-0.348744$

C -3.582894 $4.060994-1.663887$

H $-0.9237512 .701150-2.339772$

H -1.088308 $4.442106-2.656337$

C $1.3787704 .425337-1.581879$

H $\quad 0.2812003 .104217-0.303478$

C -4.035064 $3.005986-2.692529$

O $-4.5931024 .220184-0.665657$

H -3.491295 $5.035636-2.160726$

H -5.057449 $3.240618-3.015984$

O $-3.208937 \quad 3.144522-3.854094$

C -3.991510 $1.530269-2.237811$

C $-4.8489521 .236755-0.999656$

H -2.950349 $1.229935-2.081706$

O $-4.484146 \quad 0.748736-3.333520$

H -4.515382 $1.833614-0.144754$

H $-5.8818521 .555241-1.197253$

H $2.1530674 .642924-0.839403$

H $1.7514203 .631603-2.237064$

H $1.2352425 .322668-2.193157$
C $-0.006596 \quad 5.259856 \quad 1.364888$

H $-5.4150834 .476303-1.117452$

H $-3.5123852 .489278-4.505649$

H $-4.231440-0.176144-3.174378$

H $-0.611786 \quad 6.002428 \quad 1.899436$

H 1.0150965 .6548751 .313532

H $-0.4568856 .069811-0.566997$

C $-6.025895-1.5922941 .112416$

O $-5.777472-0.2907510 .565954$

C $-4.898088-0.241479-0.566068$

C $-3.522404-0.818892-0.199172$

C $-3.639641-2.218720 \quad 0.413188$

C $-4.708140-2.2771161 .509232$

H $-6.592753-1.4054132 .034019$

C $-6.905825-2.4481360 .187913$

H $-5.344665-0.824733-1.377164$

O -2.8478150 .0421180 .716365$

H $-2.906600-0.867575-1.102206$

H $-2.672114-2.5125310 .837311$

O $-3.971975-3.160541-0.602412$

H $-4.909725-3.3158341 .796497$

O $-4.217348-1.6061862 .673285$

C $-8.301981-1.8800500 .034431$

H $-6.480398-2.535369-0.814999$

H -7.009366 -3.452091 0.616220

H -3.4116010 .1414801 .502513$

H -3.256538 $-3.148960-1.260730$

H $-3.448317-2.1059692 .994841$

O $-9.029420-2.711737-0.769760$

O $-8.706934-0.8455510 .546093$

C $-10.379698-2.305050-1.014077$

H -10.937907-2.260455 - 0.074147

H $-10.397378-1.335639-1.521229$

H -10.846551-3.049742 -1.664297

SCF Energy (B3LYP/6-31G**//MMFF)= -3245.91519505

0800500

MM̄FF Geometry

C -2.482866 $4.963494-1.110206$

C $-3.2768553 .884418-1.173398$

C $-4.2774413 .534473-0.103208$

$\begin{array}{lllll}\text { O } & -3.910049 & 2.235447 & 0.419382\end{array}$

C $-5.6965603 .438982-0.688906$

$\begin{array}{llll}\text { C } & -6.767578 & 3.274977 & 0.376270\end{array}$

C $-7.444927 \quad 2.1368450 .629857$

C -7.0812714 .5202581 .167753$

C $-7.306263 \quad 0.807641-0.056142$

C $-6.590190-0.2095730 .840766$

C $-6.264702-1.5094250 .091376$

C $-5.537909-2.4986001 .006064$

C $-5.084100-3.758617 \quad 0.252499$

O $-5.432452-1.172265-1.022825$

O $-4.154337-4.443307 \quad 1.106050$

C $-4.329932-3.333817-1.028474$

C $-6.250860-4.7271960 .028882$

O $-4.003834-4.470269-1.823758$

C $-5.087311-2.279948-1.864069$

C $-4.260939-1.718272-3.046018$

O $-6.258140-2.885258-2.426682$

C $-2.978483 \quad 2.210377 \quad 1.412247$

O $-2.444686 \quad 3.1707581 .944531$

C -2.711441 0.7949541 .746202

$\begin{array}{llll}C & -1.918492 & 0.503316 & 2.786932\end{array}$

C -1.624089 -0.8451353 .259272$

C $-1.700369-0.9752854 .756893$

C $-2.940416-1.044539-2.659588$

C $-1.741670-1.974421-2.738931$

C $0.597830-2.200797-1.916077$

O $-1.732934-3.005457-3.406103$

C $0.507044-3.204886-0.811909$

$\begin{array}{llll}\text { C } & 1.187550 & -3.108820 & 0.339247\end{array}$

C $1.106554-4.1239391 .440190$

C $0.536356-3.5502072 .716681$

C $1.307728-3.3758073 .802757$

C $-0.947870-3.2616362 .758604$

C $-1.271802-1.833232 \quad 2.412394$

$\mathrm{N}-0.662698-1.491078-2.013311$

H $-2.5916395 .652761-0.276881$ 
H $-3.1662693 .184966-1.998743$ H $-4.260021 \quad 4.2771410 .703570$ H $-5.7339672 .617795-1.411679$ H -5.926326 4.347094 -1.262704 H $-8.1919002 .149898 \quad 1.422733$ H $-7.9223174 .375823 \quad 1.854543$ H -6.2189454 .8272801 .766892$ H -7.348172 5.3409450 .493763 H -8.309916 $0.436334-0.297888$ H $-6.7905380 .906707-1.013803$ H -5.6591640 .2364221 .207968$ H -7.210212 -0.4312141 .718306$ H -7.203397 $-1.941056-0.275556$ H -6.165416 -2.767634 1.864833 H -4.647602 -2.014369 1.430347 H -3.797049 -5.1979760 .607087$ H $-3.374486-2.910308-0.695707$ H $-6.676230-5.0383900 .990635$ H -5.914660 -5.648821 -0.458884 H -7.058605 -4.295908 -0.565916 H $-4.810441-4.746322-2.291456$ H -4.114278 -2.496276 -3.804787 H $-4.876260-0.949357-3.535169$ H -6.544740 -2.353236 -3.187612 H $-3.185697 \quad 0.025177 \quad 1.147679$ H - $1.480371 \quad 1.3034283 .380271$ H -1.431171 -1.967507 5.124390 H $-2.718594-0.7664185 .103836$ H -1.020023 -0.2645505 .239802$ H $-2.738125-0.235440-3.372991$ H $-3.004405-0.590615-1.664721$ H $1.380036-1.456860-1.739080$ H $0.809042-2.707309-2.863401$ H $-0.145415-4.059409-0.975441$ H $1.841964-2.2571320 .502448$ H $2.119250-4.5115541 .610915$ H $0.505586-4.9912331 .139015$ H $0.897237-2.9939224 .731207$ H $2.367596-3.6074403 .786878$ H -1.354840 -3.542614 3.735901 H -1.471064 -3.917632 2.051494 H -1.221163 -1.609154 1.347185 H $-0.787858-0.673329-1.424017$ C $0.0089694 .974074-1.593016$ O $0.151893 \quad 3.562047-1.332249$ C $0.8252743 .392633-0.066384$ C 1.4676524 .7374910 .238177 C $0.4309775 .696606-0.312601$ H 0.0380283 .1679830 .663060 C $1.8074962 .220596-0.165472$ H $1.6581654 .882506 \quad 1.305759$ H $2.4172894 .847279-0.300121$ C $0.9579107 .101373-0.546721$ H -0.3956935 .7461570 .405756$ C 2.6388092 .0065201 .110788 O $1.0545191 .030771-0.418375$ H $2.4635792 .370369-1.029777$ H 3.2458912 .8955471 .316455 O 1.7483591 .8688752 .226363 C $3.558395 \quad 0.769031 \quad 1.089847$ C $4.5532990 .777127-0.077344$ H $2.958004-0.1463651 .076909$ O $4.304156 \quad 0.7551712 .311783$ H $4.0231330 .725121-1.035516$ H $5.0840351 .739417-0.088187$ H 1.3003727 .5454780 .393650 H $1.8014517 .103011-1.245219$ H $0.1754987 .745521-0.960308$ C -1.404120 5.270458 -2.110600 H $0.5335561 .185891-1.224902$ H 1.1695161 .1078242 .046701 H 3.6623110 .7601263 .042643 H -1.574545 4.681271 -3.020600 H -1.483506 $6.325615-2.396570$ H $0.7189775 .201420-2.398986$ C $7.602448-1.001112-1.268317$ O $6.479409-0.116733-1.154234$ C $5.622018-0.332962-0.024543$
C $5.040133-1.753531-0.066595$

C $6.142824-2.809874-0.183674$

C $7.145460-2.469569-1.291724$

H $8.039003-0.773984-2.249753$

C $8.671866-0.713324-0.202679$

H $6.209533-0.1979660 .889223$

O $4.143188-1.884583-1.167409$

H $4.462788-1.9368520 .846195$

H $5.680844-3.783019-0.390443$

O $6.815410-2.9052841 .068414$

H $8.022329-3.125027-1.235769$

O $6.529591-2.704195-2.561815$

C $9.2987210 .655635-0.371594$

H $8.261236-0.7589110 .809119$

H $9.477167-1.451901-0.290142$

H $4.629758-1.655084-1.977554$

H $7.491363-3.5994880 .986371$

H $6.282651-3.643997-2.598620$

O 10.2438350 .8299180 .599992

O $9.004595 \quad 1.468473-1.236902$

C 10.9282592 .0864720 .573822

H 11.6487042 .0984411 .396087

H $11.4702802 .202923-0.369537$

H 10.2188332 .9071890 .716694

SCF Energy $\left(B 3 L Y P / 6-31 G^{* *} / / M M F F\right)=-3245.92410368$

0800501

MM̄FF Geometry

C $0.149524-4.199701-0.233254$

C $0.948539-3.890037-1.265390$

C $0.659872-2.913024-2.381698$

O $1.565877-1.793125-2.215052$

C $-0.767761-2.336444-2.408365$

C $-0.996731-1.352725-3.541721$

C $-1.157096-0.026884-3.354959$

C $-1.087002-1.947685-4.920959$

C $-1.1062940 .672894-2.024301$

C $-1.1998432 .198586-2.159216$

C $-1.1461502 .889975-0.788783$

C $-1.3631174 .397493-0.934437$

C -1.2127655 .1310650 .407012$

O $0.1397762 .633557-0.212725$

O $-1.104751 \quad 6.536172 \quad 0.132710$

C $0.1118724 .697127 \quad 1.067807$

C -2.4492224 .9565881 .296466$

O 0.2285875 .2395862 .381425

C 0.3105093 .1642821 .107269

C 1.7431482 .8336091 .588643

O -0.6214352 .5971042 .030834$

C $2.788852-1.867755-2.801811$

O $3.237326-2.792914-3.459142$

C $3.506828-0.614251-2.481265$

C $4.830860-0.549480-2.681815$

C $5.6986560 .593249-2.389795$

C $5.0424811 .876099-1.951882$

C 2.1674141 .3760351 .355265

C 3.5003001 .0762222 .021771

C $5.502481-0.3946301 .843020$

O 3.8788081 .6565343 .037226

C 6.5651370 .5344451 .341737

C 7.6778430 .1314990 .709323

C $8.753226 \quad 1.0747290 .246958$

C $9.1095840 .902197-1.213423$

C $10.2888710 .371638-1.579288$

C $8.1332541 .418493-2.249875$

C $7.0313180 .431016-2.526071$

N $4.201067 \quad 0.0605561 .385809$

H $-0.835036-3.749724-0.148847$

H $1.931790-4.356486-1.304946$

H $\quad 0.856965-3.418484-3.335267$

H $-0.977132-1.847140-1.449919$

H -1.499653 -3.149268 -2.501080

H -1.342296 $0.599252-4.225496$

H -1.347730 -1.200377 -5.677936

H $-0.130976-2.392057-5.213164$

H - $1.857398-2.725187-4.949001$

H $-1.9430360 .325788-1.407732$

H $-0.1731230 .411549-1.512122$ 
H $-0.3680962 .551760-2.782794$ H -2.133891 $2.464343-2.669879$ H -1.923158 $2.453290-0.150132$ H -2.343730 4.608276 -1.379203 H $-0.6221874 .805386-1.636496$ H -1.902265 $6.809016-0.352551$ H $0.929425 \quad 5.135570 \quad 0.478218$ H $-3.334665 \quad 5.3752910 .804414$ H -2.342247 5.5098432 .235926 H -2.664962 3.9124871 .532627 H $0.110612 \quad 6.2021732 .308872$ H 2.4658393 .4660431 .056131 H 1.8283063 .0763692 .655359 H $-0.256797 \quad 1.7693632 .381792$ H $2.933006 \quad 0.206889-2.067125$ H $5.337549-1.429232-3.077456$ H $4.5193271 .739906-1.000664$ H $4.3180442 .210999-2.702902$ H $5.7438302 .700657-1.811149$ H $1.433824 \quad 0.678587 \quad 1.771608$ H 2.2428161 .1829850 .279548 H $5.639309-1.4176531 .479133$ H $5.508575-0.4042612 .937960$ H $\quad 6.4250101 .596376 \quad 1.531983$ H $7.841001-0.9304990 .541412$ H $\quad 9.636921 \quad 0.8995750 .874133$ H 8.4673922 .1197590 .420966 H $10.561790 \quad 0.269041-2.624482$ H $11.0055110 .023391-0.842975$ H $8.655506 \quad 1.600148-3.198599$ H $7.7677882 .398198-1.932003$ H $7.398575-0.537361-2.870344$ H $3.840366-0.3303280 .520740$ C $0.823540-4.4031292 .205972$ O $-0.310824-3.5919162 .585502$ C $0.152811-2.2430482 .776080$ C $1.616877-2.3927193 .161391$ C $2.047099-3.4758162 .190258$ H $0.097229-1.7482341 .797808$ C $-0.728059-1.4772253 .766035$ H $2.180533-1.4637823 .049647$ H $1.735105-2.7495044 .190500$ C $3.343378-4.1662912 .577506$ H $2.181918-3.0171531 .203463$ C $-2.239376-1.5318853 .461311$ O $-0.560996-2.0158585 .083184$ H - $0.399621-0.4314023 .815529$ H -2.637721 -2.510985 3.756218 O $-2.857159-0.5673834 .324283$ C -2.625947 -1.239318 1.997714 C $-4.141494-1.0972031 .793746$ H -2.246913 -2.049648 1.369222 O $-1.992486-0.0395001 .557831$ H -4.672136 -1.919405 2.289097 H -4.491349 -0.1784002 .284198$ H $4.169425-3.4481972 .601514$ H $3.272224-4.6291463 .567428$ H $3.595798-4.9493801 .855410$ C $0.538195-5.129460 \quad 0.884425$ H $0.295854-1.7060945 .421767$ H -3.805852 -0.7744654 .367679$ H -2.298126 0.6833392 .132491 H $-0.300915-5.8207951 .032069$ H $1.405165-5.7390060 .603908$ H $0.932816-5.1567502 .995885$ C $-6.552869-0.435463-0.963317$ O $-5.954083-0.6423710 .322705$ C $-4.568509-1.0125730 .314598$ C $-4.375277-2.327134-0.460418$ C $-5.001694-2.266275-1.857830$ C $-6.425243-1.700130-1.827299$ H -7.619642 -0.281244 -0.755047 C $-6.0194690 .830716-1.652932$ H -3.996201-0.210137-0.160621 O $-4.950058-3.4161050 .260761$ H -3.305371 -2.537495 -0.555184 H -5.015536 -3.276947 -2.284162 O $-4.180928-1.456034-2.691024$
H $-6.778568-1.484116-2.842264$

O $-7.305026-2.684747-1.277249$

C $-6.3422362 .089204-0.875028$

H $-4.9339950 .794054-1.776502$

H $-6.4795040 .933296-2.642407$

H $-5.890103-3.2128380 .405500$

H -4.565684 -1.466143 -3.583791

H $-7.261400-3.469474-1.850011$

O $-5.6421093 .132296-1.412964$

O

C $-5.8534674 .401669-0.787498$

H $-5.1744265 .124627-1.247433$

H $-6.8835274 .732108-0.950666$

H -5.6317094 .3437450 .282326$

SCF Energy (B3LYP/6-31G**//MMFF) $=-3245.90970064$

$08 \quad 00502$

MM̄FF Geometry

C $2.5181492 .636431-1.399960$

C $2.7707182 .177689-2.634808$

C $3.0439140 .730811-2.980152$

O $2.431598-0.157381-2.013541$

C $4.5538750 .442275-2.961081$

C $4.939668-0.720658-3.852134$

C $5.058360-2.001325-3.451530$

C $5.238194-0.347629-5.282131$

C $4.759422-2.590797-2.101431$

C $6.018989-3.075816-1.371405$

C $6.759420-1.942604-0.645324$

C $8.065369-2.456360-0.035856$

C $8.770587-1.3779970 .800265$

O $5.894878-1.4601050 .390100$

O $9.792750-2.0200351 .577755$

C $7.756261-0.7773981 .795427$

C $9.480926-0.344096-0.081306$

O 8.3230260 .3270602 .497362

C $6.418854-0.3588501 .140832$

C 5.3891140 .0055922 .238970

$\begin{array}{lllll}\text { O } & 6.643582 & 0.784450 & 0.312855\end{array}$

C $1.082136-0.319927-2.041273$

O $0.2923950 .165404-2.833702$

C $0.733102-1.163994-0.877507$

C $-0.521433-1.167572-0.404071$

C $-0.989940-1.9036970 .773346$

C $0.008180-2.7772301 .489554$

C 3.9508960 .1687631 .724966

C 3.0100390 .6768542 .805821

C 0.6531751 .4228233 .141598

O 3.3623250 .8648643 .967401

C -0.1398160 .2891813 .711893$

C $-1.4813460 .250558 \quad 3.705708$

C $-2.285353-0.8627134 .315876$

C $-3.365808-1.3881273 .395749$

C $-4.651591-1.0409163 .574648$

C $-2.971202-2.3889482 .332593$

C $-2.272291-1.7493281 .165056$

N 1.7287450 .9003992 .317489

H $2.5679731 .951570-0.559509$

H $2.7255252 .866340-3.475268$

H $2.6154920 .518278-3.967751$

H $4.8848100 .248392-1.933743$

H $5.1204831 .322687-3.292886$

H $5.372202-2.737681-4.191089$

H $5.502204-1.216480-5.894209$

H $4.3655460 .126599-5.742762$

H $6.0779520 .353699-5.323997$

H $4.189064-1.907863-1.467342$

H $4.104054-3.455094-2.270045$

H $5.702927-3.819957-0.628315$

H $6.691974-3.583414-2.073278$

H $6.963660-1.134504-1.356379$

H $8.736017-2.836518-0.816599$

H $7.850519-3.3140310 .617200$

H $10.400531-2.466442 \quad 0.963370$

H $7.544664-1.5480272 .550145$

H $10.236403-0.831340-0.709477$

H 10.0243650 .3871130 .527256

H $8.8007280 .195217-0.743828$ 
H $\quad 9.151633 \quad 0.0183182 .902379$ H $5.374370-0.7834043 .002431$ H 5.7000830 .9333092 .736108 H 5.8286251 .3071050 .258128 H $1.535916-1.726323-0.413611$ H -1.270474 -0.558141 -0.907726 H $0.871807-2.1890331 .819297$ H $\quad 0.360161-3.5764140 .827760$ H $-0.390606-3.2589332 .384029$ H $3.923821 \quad 0.8807840 .896674$ H $3.566723-0.7924831 .364920$ H 0.0426242 .0734742 .507885 H 1.0782042 .0210983 .954005 H $\quad 0.412407-0.5245134 .176464$ H $-2.032941 \quad 1.0818153 .272547$ H -2.738632 -0.4750435 .237633$ H -1.642406 -1.694991 4.628223 H -5.437627 -1.438241 2.941859 H $-4.946744-0.3468314 .354480$ H -3.865306 -2.889639 1.940502 H -2.388853 -3.189616 2.795916 H -2.916708 -1.0913760 .580716$ H $1.5129740 .654326 \quad 1.355961$ C $0.7133743 .971937-0.384578$ O $-0.2613413 .427339-1.291725$ C - $1.5558423 .979326-0.967237$ C -1.3312254 .9328810 .201612$ C 0.1308745 .3128610 .037096 H -1.879120 $4.530336-1.858658$ C -2.504495 $2.807196-0.683964$ H -2.004683 5.7948220 .169882 H -1.486704 4.4205611 .159548 C $0.7509115 .892627 \quad 1.295567$ H $\quad 0.2179876 .037656-0.783079$ C $-3.9168303 .241902-0.250087$ O $-2.5952062 .045784-1.891942$ H -2.061629 2.1505270 .074592 H $-3.810973 \quad 3.9225850 .602754$ O $-4.5428933 .991098-1.289289$ C $-4.856202 \quad 2.105718 \quad 0.212182$ C $-5.1864041 .044570-0.845216$ H -5.7945662 .5700080 .536407$ O $-4.284376 \quad 1.456810 \quad 1.345312$ H -5.577203 $1.512020-1.756490$ H $-4.2680590 .525600-1.144224$ H $1.8039686 .142614 \quad 1.132755$ H 0.2294906 .8078751 .594675 H 0.6978195 .1867142 .131361 C $2.0733004 .035667-1.079093$ H -1.693067 $1.771091-2.131564$ H $-4.4848903 .468368-2.107183$ H -4.1405252 .1307942 .031183$ H $2.8174304 .503689-0.426214$ H $2.0020574 .633110-1.995652$ H 0.7645423 .2942510 .478200 C -7.064624 -2.136957 -1.255691 O $-6.292109-0.953377-1.488962$ C $-6.148674-0.063571-0.372188$ C -7.5266110 .4321010 .091046$ C $-8.465510-0.744238 \quad 0.385887$ C $-8.470174-1.776982-0.745814$ H -7.177415 -2.598966 -2.245242 C $-6.324066-3.138010-0.355355$ H -5.659509 -0.6033140 .444846$ O H $-7.419857 \quad 1.040276 \quad 0.996190$ H $-9.486350-0.3687890 .528475$ O $-8.064565-1.3870651 .592329$ H $-8.985525-2.688068-0.421602$ O $-9.224867-1.254634-1.843699$ C $-5.055568-3.661434-0.998772$ H $-6.042022-2.6949400 .602496$ H -6.974740 -3.999978 -0.166920 H -7.628916 $2.077049-0.963538$ H -8.102689 -0.7261222 .304552$ H $-8.754156-0.474187-2.183521$ O $-4.515268-4.621977-0.191008$ O $-4.590162-3.278373-2.063025$
C $-3.293671-5.203824-0.657365$

H $-2.537410-4.430330-0.820492$

H $-2.929877-5.8913270 .110944$ H $-3.474512-5.767222-1.577537$ SCF Energy (B3LYP/6-31G**//MMFF) $=-3245.91460292$

0800503

MM̄FF Geometry

C $4.098055-2.2434472 .310816$

C $3.281363-1.1799822 .255866$

C $2.014734-1.0660473 .063649$

O $0.890838-1.1635582 .160828$

C 1.9392790 .3106323 .748799

C 0.7291570 .4342364 .652884

C $-0.4476800 .983380 \quad 4.291869$

C $0.914382-0.1125746 .044694$

C $-0.832770 \quad 1.5605742 .955574$

C -1.3861892 .9816683 .113434$

C -1.7669543 .6297781 .771524$

C -2.3755145 .0149092 .021290$

C -2.9125885 .6615200 .736656$

O -2.7062572 .7788391 .107163$

O -3.7157016 .7928631 .107015$

C $-3.819048 \quad 4.6450140 .009344$

C -1.779045 $6.236852-0.118745$

O $-4.2378555 .150082-1.255957$

C -3.156899 $3.261768-0.167004$

C $-4.1317932 .209465-0.747520$

O $-2.0687893 .385392-1.090625$

C $0.233125-2.3516582 .090925$

O $0.536105-3.3999562 .636521$

C -0.950559-2.145150 1.228037

C $-1.644880-3.1961220 .768558$

C $-2.826238-3.129702-0.095783$

C $-3.386004-1.765272-0.400793$

C -5.3190341 .8630790 .153922$

C $-6.1411210 .712007-0.403045$

C $-7.843509-0.9707240 .315490$

O $-6.0665090 .334773-1.569179$

C -7.101316 -2.219894 0.677336

C $-7.129945-3.340586-0.060359$

C $-6.400272-4.5990250 .318876$

C $-5.661322-5.228569-0.843464$

C $-6.006708-6.445546-1.297052$

C $-4.528385-4.457509-1.486041$

C $-3.352090-4.280005-0.566636$

$\begin{array}{llll}\mathrm{N} & -6.988218 & 0.177207 & 0.557085\end{array}$

H $3.808401-3.0778712 .947636$

H $3.517677-0.3435751 .605234$

H $1.967940-1.8536993 .826055$

H 1.9292191 .1066552 .994916

H $2.845186 \quad 0.485744 \quad 4.344376$

H -1.2478460 .9993425 .031413$

H 0.0179540 .0070006 .662417

H $1.149783-1.1808776 .009399$

H $1.7344010 .408176 \quad 6.550078$

H 0.0018251 .5719732 .249594

H -1.6035980 .9122402 .522721$

H -2.2760132 .9351063 .755719$

H -0.6493593 .6135963 .625543$

H -0.8634053 .7103881 .155737$

H -1.649023 5.6763632 .509918

H -3.2093284 .9168162 .730932$

H -4.4383866 .4803931 .677503$

H -4.7269204 .5305310 .614201$

H -1.2543837 .0296540 .428416$

H -2.168753 $6.708614-1.027539$

H -1.035498 $5.491328-0.407846$

H $-4.6408026 .021745-1.101722$

H $-4.4848892 .529013-1.736427$

H $-3.5688101 .283378-0.929763$

H -1.967511 2.549056-1.571642

H -1.216651 -1.122855 0.987941

H -1.311185 -4.198079 1.034773

H $-3.615519-1.2260410 .525247$

H $-4.312191-1.791911-0.975820$

H -2.669655 -1.176785 -0.982563

H $-4.961586 \quad 1.5832901 .151392$ 
$\begin{array}{llll}\text { H } & -5.990124 & 2.722230 & 0.255433\end{array}$ H -8.165547 $-0.970661-0.730875$ H $-8.720443-0.858890 \quad 0.960279$ H -6.525220 -2.209029 1.600115 H -7.732594 -3.366035 -0.965367 H -7.143762 -5.3019550 .716716$ H -5.692930 -4.413777 1.136385 H -5.486662 -6.908485 -2.129197 H $-6.817766-7.008679-0.847283$ H -4.914374 -3.512517 -1.875630 H -4.161487 -4.993922 -2.371552 H $-2.873099-5.219993-0.288660$ H $-6.9315810 .523331 \quad 1.510523$

C $6.181818-1.1674621 .227952$

O $5.566585-0.487751 \quad 0.119455$

C $6.5896460 .203633-0.626874$

C $7.920204-0.1269380 .045486$

C $7.610422-1.4305020 .756853$

H $6.587216-0.225058-1.634684$

C $6.268198 \quad 1.704562-0.685917$

H $8.733374-0.220819-0.681363$

H 8.2167050 .6438890 .766297

C $8.592878-1.7507301 .870496$

H $7.617717-2.2438930 .018880$

C $4.8716852 .023006-1.258055$

O 6.3232522 .2446920 .642086

H $7.0418902 .229068-1.257435$

H $4.1093831 .596570-0.598360$

O $4.6846183 .443807-1.200938$

C $4.6545161 .551783-2.709503$

C $3.2463571 .880451-3.244491$

H $4.8312350 .474754-2.790066$

O $5.6206192 .201429-3.538834$

H $2.9929832 .921712-3.013299$

H $3.2654521 .808347-4.340155$

H 9.610608 -1.828802 1.474067

H $8.591331-0.9735722 .642173$

H $8.347181-2.7023912 .351153$

C $5.400550-2.4376721 .575661$

H 5.6500271 .7866851 .174198

H $5.3341683 .848395-1.801386$

H $5.5172351 .850475-4.439888$

H $6.026525-3.0647002 .221651$

H $5.192253-3.0172260 .668203$

H $6.184302-0.4769292 .082013$

C $1.527757-1.398079-2.865682$

O $2.373795-0.338988-3.331710$

C $2.1795660 .925541-2.686829$

C $0.7549351 .428023-2.947057$

C $-0.2692620 .403188-2.449612$

C $0.039550-1.011352-2.956737$

H $1.706445-2.214976-3.577113$

C $1.991453-1.873792-1.481547$

H $2.3257300 .805368-1.608404$

O $0.5755091 .661895-4.341032$

H $\quad 0.6159302 .384502-2.429237$

H -1.272188 $0.692724-2.784875$

O $-0.2745860 .393705-1.025154$

H $-0.566523-1.749933-2.421149$

O $-0.331315-1.091354-4.337357$

C $1.647706-3.325029-1.223217$

H $3.083482-1.776157-1.425837$

H $1.567915-1.267864-0.677578$

H $-0.330487 \quad 1.990675-4.469255$

H $-0.4547101 .298403-0.718786$

H $-1.286879-0.920433-4.394324$

O $2.396965-3.780486-0.177084$

O $0.818910-3.975852-1.844499$

C $2.184630-5.147900 \quad 0.186623$

H $2.507572-5.805730-0.625525$

H $2.787635-5.3608951 .073248$

H $1.132021-5.3220240 .429005$

SCF Energy (B3LYP/6-31G**//MMFF) $=-3245.90996960$

0800504

MM̄MF Geometry

C -1.774632 -3.864628 -0.344050

C $-1.384134-3.154301-1.414089$
C $0.022378-2.657366-1.609026$

O $-0.087447-1.246875-1.914671$

C $0.750081-3.348923-2.774073$

C $1.087941-4.801476-2.511310$

C $2.146496-5.215681-1.786926$

C $0.161382-5.808371-3.141543$

C $3.191324-4.362075-1.123221$

C $3.012045-4.3343190 .398805$

C $4.142845-3.5771971 .112384$

C $3.825774-3.4273232 .603291$

C $4.880257-2.5899183 .341455$

O $4.287110-2.2864270 .511430$

O $4.319572-2.2386824 .615734$

C $5.125693-1.2839362 .553882$

C $6.137649-3.414698 \quad 3.639947$

O $6.193507-0.5370613 .132552$

C $5.368743-1.5116711 .045046$

C $5.410885-0.1594180 .295434$

O $6.630142-2.166713 \quad 0.878187$

C $0.835897-0.406779-1.377244$

O $1.794162-0.706467-0.684548$

C $0.4454530 .969676-1.754651$

C $1.0816492 .015073-1.206063$

C $0.7564993 .426392-1.418586$

C $-0.3501863 .754918-2.386658$

C $5.375943-0.303517-1.232689$

C $5.9853830 .900629-1.920390$

C $5.6353953 .300176-2.399945$

O $7.0348520 .813494-2.555164$

C $4.9904704 .455111-1.705946$

C $4.2185595 .355469-2.332947$

C $3.5883436 .537851-1.653678$

C $2.0838406 .557657-1.805764$

C $1.5028157 .246898-2.802336$

C $1.2508345 .852366-0.760011$

C $1.4515914 .361780-0.738707$

N $5.2517822 .063673-1.745145$

H -1.025570 -4.117469 0.404300

H -2.095795 -2.895623 -2.192144

H $\quad 0.587618-2.783776-0.680931$

H $1.665298-2.792758-3.014001$

H $\quad 0.151297-3.263222-3.691511$

H $2.295260-6.286929-1.657879$

H $\quad 0.460048-6.839943-2.926785$

H $-0.861126-5.680268-2.775147$

H $\quad 0.157199-5.687177-4.229908$

H $4.176421-4.775553-1.371835$

H $3.180427-3.342567-1.520134$

H $2.053734-3.8548260 .629848$

H $2.958090-5.356817 \quad 0.793613$

H $5.070920-4.1434610 .969783$

H $3.711378-4.4099713 .078038$

H $2.853095-2.9279562 .716286$

H $4.950531-1.6479625 .062362$

H $4.225886-0.6646972 .671411$

H $5.885482-4.2784904 .267039$

H $6.867412-2.8338854 .214787$

H $\quad 6.627083-3.7913742 .739494$

H $7.028075-0.9585392 .865683$

H $4.546407 \quad 0.450418 \quad 0.588591$

H $\quad \begin{array}{llll}6.312432 & 0.392590 & 0.590774\end{array}$

H $7.023583-1.9022570 .032035$

H $-0.386211 \quad 1.085331-2.440701$

H $1.8960751 .822076-0.508743$

H $-0.1214273 .355383-3.380942$

H $-0.5107764 .828005-2.513280$

H -1.300264 $3.328603-2.046471$

H $5.932131-1.184877-1.569323$

H $4.347194-0.430363-1.587548$

H $5.3363923 .222287-3.450760$

H $6.7252213 .401235-2.363731$

H $5.1834474 .566184-0.641587$

H $4.0452725 .252698-3.402029$

H $4.0195747 .446175-2.094805$

H $3.8498996 .577103-0.588936$

H $0.4237857 .293409-2.904998$

H $2.0949627 .777842-3.540511$

H $0.1865186 .090882-0.873473$ 
H $1.515800 \quad 6.2496600 .228591$ H $2.2428424 .036126-0.062804$ H $4.3090301 .988165-1.373149$ C $-4.305790-3.804357-0.872811$ O $-4.505039-2.414810-0.535575$ C $-5.919914-2.144252-0.506742$ C $-6.603404-3.391695-1.056328$ C $-5.650055-4.480231-0.607171$ H $-6.201353-2.029210 \quad 0.545825$ C $-6.236506-0.868008-1.291894$ H -7.616560 -3.516371 -0.662038 H $-6.669195-3.366553-2.150664$ C $-5.843614-5.794546-1.343491$ H $-5.783257-4.6451220 .470480$ C $-5.6420210 .426733-0.695477$ O $-5.736163-1.008976-2.628360$ H $-7.322622-0.756729-1.391103$ H -4.548488 $0.411796-0.767428$ O $-6.0844521 .513971-1.519552$ C $-6.067147 \quad 0.7137120 .759881$ C $-5.828926 \quad 2.1611151 .231081$ H $-5.556392 \quad 0.0145191 .431311$ $\begin{array}{llll}\text { O } & -7.472113 & 0.461281 & 0.882267\end{array}$ H -6.3998972 .8506710 .596423$ H -6.2711342 .2536842 .232812$ H $-6.852475-6.184730-1.173900$ H $-5.707427-5.673656-2.423446$ H $-5.128792-6.546885-0.996350$ C $-3.158476-4.375401-0.036590$ H $-4.778077-1.165700-2.566323$ H $-5.8082181 .317388-2.431145$ H $-7.707356 \quad 0.5568991 .820926$ H $-3.132392-5.466918-0.133267$ H $-3.351093-4.1476891 .020023$ H $-4.076463-3.854117-1.944418$ C -2.338062 2.101082 2.557352 O $-3.728197 \begin{array}{lll}1.830417 & 2.328572\end{array}$ C $-4.3548902 .589594 \quad 1.289294$ C $-4.227900 \quad 4.098636 \quad 1.564613$ C -2.7727634 .5087001 .802411$ C $-2.0905903 .594906 \quad 2.822921$ H -2.099882 1.5583733 .481540 C $-1.461582 \quad 1.535898 \quad 1.436363$ H $-3.876812 \quad 2.3596640 .332523$ $\begin{array}{lllll}\text { O } & -5.012734 & 4.469940 & 2.697087\end{array}$ H $-4.631396 \quad 4.6472380 .705612$ H -2.739481 5.5432852 .165337 $\begin{array}{lllll} & \mathrm{O} & -2.73949489 & 4.451947 & 0.577664\end{array}$ H -1.011329 3.7866922 .849840 O $-2.599129 \quad 3.887842 \quad 4.127383$ C -1.403863 $0.030573 \quad 1.524384$ H $-1.819996 \quad 1.7886280 .434988$ H $-0.436595 \quad 1.918323 \quad 1.508795$ H -4.719692 3.9309943 .451686 H -2.478410 $5.061612-0.046753$ H -2.397894 4.8200034 .317494 O $-0.382897-0.3440642 .346824$ O $-2.186718-0.7146180 .950776$ C $-0.225782-1.7507532 .555253$ H $0.262639-2.1955671 .685365$ H $0.415977-1.8940213 .428833$ H -1.188370 -2.233849 2.748995 SCF Energy (B3LYP/6-31G**//MMFF) $=-3245.91585955$

08_00505

MM̄FF Geometry

C $4.098630-0.5625642 .654285$

C $3.525598 \quad 0.4733532 .023731$

C $2.863056 \quad 1.6166812 .748638$

$\begin{array}{lllll}\text { O } & 1.557719 & 1.823257 & 2.163808\end{array}$

C 3.6865962 .9075132 .566165

C $3.030116 \quad 4.1340773 .170783$

C 2.5811975 .1886382 .460273

C $2.902736 \quad 4.130694 \quad 4.672285$

C $2.656520 \quad 5.395846 \quad 0.971611$

C 1.4293424 .8375260 .240483

C $1.7799663 .716159-0.749373$

C $2.4658904 .252196-2.008790$
C $2.740408 \quad 3.135178-3.029598$

O $0.557725 \quad 3.058327-1.107171$

O $3.0720073 .756470-4.281395$

C $1.4388602 .332470-3.252278$

C $3.9578432 .291541-2.630878$

O $1.672171 \quad 1.203321-4.090003$

C $0.7453491 .904821-1.939562$

C $-0.632934 \quad 1.253379-2.183034$

O $1.5551690 .921393-1.284054$

C $0.4816551 .305426 \quad 2.815023$

$\begin{array}{lllllllllll}\text { O } & 0.489260 & 0.598509 & 3.809434\end{array}$

C -0.7298821 .7870862 .114395$

C -1.9486061 .5255172 .609211$

C -3.222504 1.9691442 .036693

C $-3.167707 \quad 2.854298 \quad 0.820252$

C $-1.6930952 .174720-2.791753$

C $-3.0450501 .484712-2.860177$

C $-5.4732021 .960215-3.108480$

O $-3.171101 \quad 0.263493-2.816060$

C $-6.1454582 .129800-1.781822$

C $-6.5116411 .104685-0.996785$

C $-7.302441 \quad 1.256537 \quad 0.271238$

C -6.5317750 .8438301 .504396$

C $-6.601578-0.4183671 .961997$

C $-5.781418 \quad 1.9353742 .237878$

C -4.3721651 .5681352 .618140$

N $-4.0901472 .383381-3.012176$

H $4.067532-0.577683 \quad 3.742836$

H 3.5457780 .5347190 .939754

H $2.772113 \quad 1.396666 \quad 3.819493$

H 3.8789163 .0633911 .500235

H 4.6758052 .7804653 .026152

H 2.1093986 .0084883 .000483

H 2.5354355 .0870205 .059859

H 2.2048083 .3542454 .999848

H 3.8772233 .9439595 .135222

H $2.711046 \quad 6.477907 \quad 0.794594$

H 3.5942574 .9961300 .572830

H $0.680400 \quad 4.4709120 .953748$

H $0.9265675 .648795-0.302550$

H $2.4230612 .992987-0.240048$

H $3.3958874 .778868-1.764219$

H $1.8163145 .000617-2.484351$

H $3.848967 \quad 4.323637-4.138525$

H $0.7621892 .988708-3.813417$

H $4.8483682 .925156-2.540060$

H $4.197547 \quad 1.552923-3.403604$

H $3.8283901 .770379-1.680154$

H $2.1600620 .544192-3.568184$

H $-0.5158930 .354122-2.800502$

H $-1.0174900 .887494-1.220379$

H $1.0067220 .459750-0.627939$

H $-0.5859362 .367048 \quad 1.211891$

H -2.024821 0.9373543 .522543

H -2.642172 3.7887371 .045547

H $-4.149377 \quad 3.1270410 .429543$

H -2.6448152 .3442030 .007076$

H $-1.7885383 .088710-2.195586$

H $-1.4205032 .456996-3.813703$

H $-5.521026 \quad 0.923967-3.459713$

H $-5.9626282 .593468-3.855061$

H $-6.390073 \quad 3.145780-1.481542$

H $-6.2782980 .088790-1.308290$

H $-8.206690 \quad 0.6406520 .174890$

H -7.6712212 .2831370 .391162$

H $-6.095808-0.7245732 .871158$

H -7.172595 -1.176605 1.435999

H $-6.334522 \quad 2.1796403 .154640$

H -5.7980752 .8619821 .655974$

H -4.3102100 .9204663 .493409$

H $-3.8971573 .380630-3.002231$

C $4.827860-1.8495090 .515090$

O $3.510392-2.2090140 .047097$

C $3.646247-3.136878-1.049785$

C $5.139063-3.236281-1.349258$

C $5.747831-2.9601010 .008053$

H $3.292871-4.109692-0.690180$

C $2.821644-2.676536-2.256076$ 
H $5.414013-4.215518-1.753248$

H $5.458048-2.477055-2.073383$

C $7.216056-2.575686-0.059111$

H $5.635899-3.8566780 .632636$

C $1.295984-2.649392-2.033840$

O $3.237885-1.352648-2.619194$

H $3.051609-3.307811-3.122380$

H $1.039954-1.900626-1.277133$

O $\quad 0.698532-2.207117-3.260837$

C $0.672610-4.012194-1.660550$

C $-0.866200-4.050147-1.769091$

H $0.983274-4.294817-0.648970$

O $1.188867-5.005716-2.552345$

H -1.167056 -3.739043 -2.777004

H -1.188227 -5.096737 -1.677926

H $7.803742-3.381606-0.510933$

H $7.367224-1.672392-0.659670$

H $7.619097-2.3863600 .940492$

C $4.835398-1.7244962 .041321$

H $3.091215-0.778728-1.847418$

H $1.110656-1.358908-3.496788$

H $0.851242-5.868791-2.257942$

H $5.871574-1.6596372 .394087$

H $4.412024-2.6384612 .472067$

H $5.097060-0.8962690 .042468$

C $-2.138560-3.3140451 .657853$

O $-1.533883-3.9428030 .519704$

C -1.582112 -3.204146 -0.706487

C $-3.039221-2.918258-1.095495$

C $-3.771520-2.1868540 .032258$

C $-3.592459-2.8995691 .374224$

H -2.168585 -4.100739 2.423669

C -1.263957 -2.173635 2.192792

H -1.056116 -2.253558 -0.582176

O $-3.721789-4.130047-1.409917$

H $-3.048081-2.304445-2.002478$

H -4.839888 -2.124565 -0.206994

O $-3.267866-0.860427 \quad 0.151128$

H -3.958693 -2.274111 2.196350

O $-4.384563-4.091346 \quad 1.375171$

C $-0.113839-2.7347502 .995280$

H $-0.853654-1.5223961 .416643$

H $-1.841391-1.5420412 .877056$

H -3.649605 -4.720739 -0.640616

H $-3.435008-0.406660-0.692392$

H $-5.312030-3.8259531 .252178$

O $\quad 0.791587-3.3288922 .167651$

O $-0.045701-2.6747304 .216308$

C $1.863051-4.0121362 .823209$

H $2.374997-3.3473923 .524939$

H $1.476854-4.8935983 .343720$

H $2.574691-4.3399042 .061117$

SCF Energy (B3LYP/6-31G**//MMFF) $=-3245.92176951$

08_00506

MM̄MF Geometry

C $1.4814414 .651067-0.467700$

C $2.6482863 .995263-0.384369$

C $3.3340223 .382025-1.578505$

O $3.2152751 .945166-1.433817$

C $4.8223353 .758338-1.599930$

C $5.5234623 .291805-2.860736$

C $6.3153832 .204633-2.942421$

C $5.3160924 .166113-4.071166$

C $6.6398551 .221776-1.853146$

C $6.344350-0.219276-2.292333$

C $6.661806-1.217759-1.171198$

C $6.516706-2.659081-1.659207$

C $6.753659-3.667274-0.523933$

O $5.746087-0.978291-0.097944$

O $6.296782-4.952311-0.972462$

C $5.880081-3.2730890 .685335$

C $8.243646-3.825387-0.198909$

O $6.175926-4.0874541 .817674$

C $5.982848-1.7772931 .067584$

C $4.898262-1.4327562 .113011$

O $7.269226-1.5177451 .631419$

C $2.0455391 .391495-1.860571$
O $1.160914 \quad 1.940819-2.499118$

C $1.9742800 .010551-1.337801$

C $0.758374-0.521652-1.140933$

C $0.457668-1.833709-0.567826$

C $1.606751-2.777155-0.342142$

C 4.7172290 .0679142 .372133

C 3.5286560 .2933173 .284946

C 1.0333610 .2706383 .288262

O $3.669071 \quad 0.4795504 .491306$

C $0.437798-1.1029873 .280030$

C $-0.830619-1.3530842 .921614$

C -1.442933 -2.724671 2.910492

C $-2.188308-3.0298531 .627921$

C $-3.531003-3.0769791 .606050$

C $-1.377835-3.3577820 .390167$

C $-0.826225-2.121842-0.267794$

$\begin{array}{llll}N & 2.317174 & 0.223298 & 2.613661\end{array}$

H $1.0178024 .798491-1.440081$

H 3.0860173 .8089180 .593660

H $2.8603453 .704126-2.514310$

H $5.3222753 .359189-0.710381$

H $4.9335284 .848859-1.528497$

H $6.7921941 .990433-3.898339$

H $5.8788663 .812471-4.941743$

H $4.2589414 .191079-4.352383$

H $5.6450695 .189167-3.861022$

H $7.7060591 .316570-1.614456$

H $6.0904321 .436927-0.933333$

H $5.284758-0.298663-2.568245$

H $6.938395-0.460705-3.182406$

H $7.685525-1.033708-0.824472$

H $7.196132-2.858233-2.497270$

H $5.501567-2.810948-2.052488$

H $6.791666-5.181724-1.777701$

H $4.836801-3.4882740 .414219$

H $8.794598-4.164013-1.084525$

H $8.401877-4.5954920 .564137$

H $8.708610-2.8989460 .144573$

H $6.086329-5.0149161 .538859$

H $3.937999-1.8355201 .774336$

H $5.127398-1.9259203 .066663$

H $7.212569-0.7322222 .198015$

H $2.897489-0.492195-1.076546$

H $-0.1147240 .081471-1.388699$

H $2.221136-2.866608-1.245206$

H $1.293132-3.792586-0.091131$

H $2.235890-2.4172140 .475283$

H 5.6026290 .5010382 .849098

H 4.5511830 .6208791 .440535

H 0.4108150 .9894102 .748312

H $\quad \begin{array}{llll}1.163230 & 0.615781 & 4.318342\end{array}$

H $1.075158-1.9276493 .590802$

H -1.471616 -0.521708 2.641192

H -2.128231 -2.787198 3.765841

H $-0.684683-3.5001773 .075652$

H $-4.072024-3.3231920 .699077$

H -4.120185 -2.865842 2.492340

H $-2.008744-3.868915-0.348806$

H $-0.604750-4.0796360 .666484$

H -1.589038 -1.376295 -0.497525

H 2.3157890 .0421691 .614345

C -0.2119283 .9798681 .228652$

O -1.1311653 .5858440 .195269$

C -2.3721803 .1541630 .806827$

C -2.1621583 .2788632 .311025$

C -1.0976534 .3568482 .408006$

H -3.1224003 .8703880 .455180$

C $-2.681909 \quad 1.743557 \quad 0.288224$

H -3.0802213 .5444152 .843042$

H -1.7854932 .3395582 .733351$

C $-0.3796544 .380136 \quad 3.745718$

H -1.5680945 .3328002 .229049$

C $-3.944006 \quad 1.0248330 .805020$

O $-2.7877201 .807636-1.140029$

H $-1.814430 \quad 1.106508 \quad 0.504080$

H -3.9571030 .0561050 .293206$

O $\quad-3.826460 \quad 0.7370162 .194155$

C -5.2883901 .7233300 .512999$ 
C $-6.5211790 .903757 \quad 0.949238$ H $-5.3580221 .957424-0.555135$ O H $-6.483775 \quad 0.7237162 .031021$ H $\quad-7.4052821 .5340940 .784011$ H $\quad 0.3795015 .1681043 .768087$ H -1.087723 4.5712494 .558687 H 0.1186563 .4268803 .951227 C 0.6951865 .1058680 .729156 H -1.968002 2.211121 -1.473807 H -4.1278741 .5180032 .687717$ H -6.1491403 .4227160 .959549$ H 1.3722975 .4345541 .525700 H 0.0983145 .9760250 .430657 H $0.3922183 .095612 \quad 1.472298$ C -6.942366 -1.250531-2.070065 O $-6.836365-0.122379-1.192185$ C $-6.694344-0.4256490 .198154$ C -7.909910-1.220633 0.697236 C $-8.131726-2.477930-0.151345$ C $-8.106951-2.167752-1.651616$ H -7.190148 -0.820760 -3.049160 C $-5.602430-1.989485-2.213515$ H -5.798436 -1.032499 0.350779 O $-9.083974-0.4118450 .667061$ H $-7.743116-1.502847 \quad 1.743166$ H $-9.099897-2.9250160 .105885$ O $-7.117393-3.4362490 .135428$ H -8.074163 -3.099939-2.227658 O $-9.337300-1.515074-1.981142$ C $-4.546122-1.135905-2.885412$ H -5.201135 -2.298236 -1.245992 H -5.746137 -2.879617 -2.837123 H $-9.208830-0.102441-0.246414$ H -7.167112 -3.641947 1.084522 H $-9.353034-1.387316-2.945086$ O $-3.402648-1.872057-3.019085$ O $-4.6976260 .021749-3.249908$ C -2.313019-1.201725 -3.660722 H -1.451945 -1.875089 -3.661033 H -2.574784 -0.965594 -4.696365 H -2.047843 -0.292624 -3.113151

SCF Energy (B3LYP/6-31G**//MMFF) $=-3245.91766135$

08_00507

MM̄MF Geometry

C $1.668496-3.150822-0.409786$

C $1.846455-2.9421940 .904921$

C $0.800268-2.4399441 .877877$

O $-0.468430-2.2339421 .216266$

C $1.230166-1.0882092 .475486$

C $0.296339-0.6128893 .572172$

C $-0.746548 \quad 0.2207513 .384170$

C $0.609932-1.1385294 .948399$

C -1.2218190 .8145352 .086419$

C -1.3205602 .3422782 .166045$

C -1.5929612 .9689190 .790618$

C -1.6784074 .4924070 .902786$

C -2.046409 5.147615 -0.436487

$\begin{array}{lllll}\text { O } & -2.820240 & 2.435219 & 0.286783\end{array}$

O $-2.416816 \quad 6.509964-0.174546$

C -3.295850 4.441667-1.005839

C $-0.8500485 .200467-1.394376$

O $-3.6007734 .919889-2.313652$

C $-3.1764202 .902027-1.022368$

C $-4.5015442 .212218-1.422594$

O $-2.1924742 .518991-1.990602$

C $-1.412415-3.2075361 .305069$

O $-1.313178-4.287007 \quad 1.863925$

C -2.607433 -2.715753 0.581781

C $-3.672903-3.5134570 .415866$

C $-4.908729-3.159229-0.288700$

C $-5.041693-1.742490-0.781859$

C $-5.6575602 .427135-0.442709$

C $-6.8542001 .548874-0.769057$

C $-8.907895 \quad 0.6097410 .302095$

O $-7.022117 \quad 1.021462-1.865131$

C $-8.542877-0.7499270 .812849$
C $-9.019786-1.8908970 .291591$

C -8.661562 -3.2525590 .818341$

C $-8.356119-4.249978-0.279230$

C $-9.125987-5.338219-0.450613$

C $-7.170016-3.992480-1.183240$

C $-5.853697-4.107689-0.460219$

N $-7.724313 \quad 1.4504980 .307353$

H $0.694879-2.936021-0.845300$

H $2.813309-3.1595341 .351606$

H $\quad 0.694619-3.1794002 .681277$

H $1.288605-0.3341431 .684486$

H $2.247300-1.1583592 .883873$

H -1.3523820 .4867414 .249496$

H $-0.089361-0.7677495 .705350$

H $\quad 0.560659-2.2318454 .961153$

H $1.616651-0.8317495 .250771$

H -0.5770560 .5369831 .248759$

H $-2.2121250 .397272 \quad 1.867477$

H -2.1333842 .6022632 .857094$

H -0.3967202 .7617332 .580262$

H -0.7785232 .6829980 .115710$

H -0.7386294 .9075061 .287959$

H -2.4479884 .7601671 .640695$

H -1.663054 6.9478930 .256971

H -4.140909 4.726396 -0.366408

H $-0.0349915 .789078-0.955981$

H -1.114829 $5.706190-2.329392$

H $-0.4476764 .214649-1.636252$

H -3.671219 $5.888395-2.259038$

H $-4.7980732 .520241-2.433494$

H $-4.3170841 .131513-1.494658$

H -2.348981 $1.593682-2.241838$

H -2.571888 -1.703899 0.192877

H $-3.635356-4.5244910 .819298$

H -4.928935 -1.034484 0.047267

H $-6.008454-1.527063-1.238336$

H -4.278910 -1.524751-1.537419

H -5.3294992 .2005480 .578090$

H $-5.9989673 .467243-0.472038$

H $-9.3229610 .572060-0.710273$

H -9.6356191 .0768710 .972529$

H -7.865271 -0.8003011 .662331$

H $-9.725236-1.843343-0.534818$

H -9.508642 -3.6047971 .421291$

H -7.805387 -3.198073 1.501850

H $-8.919503-6.060324-1.233654$

H $-9.980897-5.5337760 .188223$

H -7.303150 -3.030264 -1.683211

H -7.151062 -4.728991-1.998037

H -5.660537 -5.106865 -0.067005

H $-7.4636401 .879816 \quad 1.190409$

C $4.103686-3.900275-0.935614$

O $4.725925-2.637984-0.627767$

C $6.109732-2.687869-1.031631$

C $6.369150-4.112271-1.512500$

C $4.997840-4.529707-2.003815$

H $6.215388-2.004815-1.881570$

C $7.013858-2.2398170 .123243$

H $7.135061-4.150171-2.293444$

H $6.696731-4.765671-0.695680$

C $4.840276-6.033870-2.144264$

H $\quad 4.814040-4.053570-2.976437$

C $6.692623-0.8326020 .666322$

O $6.869592-3.1661791 .209246$

H $8.064308-2.295935-0.183295$

H $5.688206-0.8376521 .101376$

O $7.587460-0.5728691 .757140$

C $6.8269260 .304517-0.368572$

C 6.5523801 .7000360 .228470

H $6.1528900 .130394-1.213134$

O $8.1613480 .286549-0.880607$

H 7.1114841 .8146681 .164491

H $6.9506372 .460704-0.456233$

H $5.565735-6.430778-2.861885$

H $4.998789-6.544851-1.188631$

H $3.838766-6.291733-2.501695$

C $2.666730-3.673705-1.410673$

H $5.934463-3.1614861 .476935$ 
H $8.488515-0.5335411 .392861$

H $8.2177490 .963523-1.576370$

H $2.262178-4.613884-1.803451$

H $2.680034-2.960443-2.242190$

H $4.120270-4.509420-0.022831$

C $3.0635642 .496184-0.828019$

O $4.482518 \quad 2.283917-0.815141$

C 5.0533921 .9472090 .456167

C $4.7947883 .071127 \quad 1.465673$

C 3.2933553 .3431141 .587185

C 2.6389873 .5436090 .217784

H $2.8573682 .919779-1.819701$

C $2.3054381 .166260-0.722010$

H 4.5905151 .0297890 .828640

O $\quad 5.4822804 .257973 \quad 1.081029$

H 5.1926222 .7696692 .441861

H 3.1400194 .2365042 .204690

O 2.6915352 .2355002 .251735

H $1.5484523 .551350 \quad 0.318464$

O $3.0174874 .839163-0.258552$

C $2.2554100 .482257-2.069183$

H $2.7389370 .463703-0.006588$

H $1.2661481 .337947-0.420779$

H $5.1862294 .497644 \quad 0.186229$

H 1.7449912 .4301492 .348665

H $2.5348965 .000307-1.087226$

O $3.4889710 .005652-2.399660$

O $1.2423410 .403709-2.752141$

C $3.607653-0.579835-3.700438$

H $2.773268-1.254726-3.913464$

H $3.658120 \quad 0.212711-4.452292$

H $4.536788-1.154875-3.730539$

SCF Energy (B3LYP/6-31G**//MMFF) $=-3245.90957029$

08_00508

MM̄FF Geometry

C 1.2212994 .0219450 .954095

C $1.9415564 .589840-0.024721$

C $2.6203393 .889394-1.178605$

O $2.5219652 .450857-1.078035$

C $4.1174444 .244648-1.186780$

C $4.8407813 .731096-2.415762$

C $5.6591462 .660710-2.422059$

C $4.6390994 .540313-3.670487$

C $5.961203 \quad 1.748933-1.264327$

C $6.188852 \quad 0.304217-1.731357$

C $6.525747-0.620545-0.553417$

C $7.049825-1.965478-1.058645$

C $7.339432-2.930198 \quad 0.099129$

O $5.334733-0.8196780 .218154$

O $7.529170-4.240695-0.455180$

C $6.096228-2.9972881 .006538$

C $8.637870-2.5767810 .833670$

O $6.361589-3.7667402 .177924$

C $5.528530-1.6101161 .399564$

C $4.159442-1.8072572 .090107$

O $6.423743-0.9744952 .313453$

C $1.4804941 .837820-1.700961$

O $\quad 0.540926 \quad 2.374317-2.265550$

C $1.6930700 .376407-1.582958$

C $0.719377-0.465671-1.958956$

C $0.770898-1.929045-1.916357$

C $2.106691-2.570143-1.645082$

C $3.352205-0.5232472 .323953$

C $2.025427-0.8724632 .973593$

C $-0.131965-2.0331132 .488766$

O $1.820270-0.6830624 .169942$

C $-0.345690-3.2900291 .705188$

C -1.403441 -3.5151930 .911578$

C -1.596339-4.780778 0.126985

C $-1.777063-4.535567-1.354932$

C $-2.957438-4.766407-1.954062$

C $-0.567276-4.111523-2.162854$

C $-0.364340-2.619551-2.154969$

N $1.141291-1.4656492 .087940$

H 1.0800612 .9451770 .975340

H $2.0458345 .673908-0.016970$

H $2.1557734 .234290-2.110610$
H $4.5925163 .858839-0.276479$

H $4.2493365 .334435-1.143285$

H $6.1707372 .416018-3.351864$

H $5.2313044 .160438-4.509934$

H $3.5884244 .522289-3.975496$

H $4.9357955 .580915-3.503385$

H $6.8698522 .112752-0.769833$

H $5.1541281 .760036-0.525557$

H $5.286573-0.056739-2.242012$

H $7.0112800 .288759-2.457758$

H $7.282939-0.1293050 .069027$

H $7.947461-1.828980-1.674475$

H $6.299969-2.425459-1.717716$

H $8.268776-4.195303-1.085158$

H $5.319603-3.5410690 .449910$

H $9.487615-2.5982610 .140848$

H $8.866055-3.3159261 .609515$

H $8.614069-1.5879401 .296370$

H $6.698528-4.6312051 .886500$

H $3.550515-2.482708 \quad 1.476607$

H $4.302630-2.3056633 .057936$

H $5.928664-0.3305492 .843670$

H $2.6430680 .034052-1.188535$

H $-0.215747-0.044297-2.324890$

H $2.864161-2.189668-2.340158$

H $2.102436-3.655198-1.766187$

H $2.437629-2.360366-0.624727$

H 3.8829420 .1703512 .983793

H $3.1589190 .005737 \quad 1.384422$

H $-0.904545-1.2838192 .299317$

H $-0.120093-2.2543013 .560618$

H $0.418432-4.0589391 .798141$

H -2.181708 -2.761945 0.829429

H $-2.476025-5.2933380 .537594$

H $-0.755260-5.4705040 .271662$

H $-3.086665-4.620301-3.021284$

H $-3.822206-5.101163-1.391119$

H -0.695765 -4.420555-3.208604

H $0.302820-4.663350-1.797469$

H -1.269577 - $2.055013-2.383752$

H $1.347070-1.4221861 .094561$

C -0.9351494 .9374851 .952184$

O -1.5517863 .6394451 .854189$

C $-2.5456913 .685296 \quad 0.811821$

C $-2.826338 \quad 5.1641130 .575181$

$\begin{array}{llll}\text { C }-1.438063 & 5.748976 & 0.756363\end{array}$

H $-2.075283 \quad 3.279229-0.090612$

C $-3.764122 \quad 2.8408201 .191433$

H -3.248396 $5.355153-0.415882$

H $-3.512298 \quad 5.574683 \quad 1.325212$

$\begin{array}{llll}\text { C }-1.430735 & 7.250522 & 0.983198\end{array}$

H $-0.851938 \quad 5.524474-0.142975$

C -3.439945 $1.379648 \quad 1.563687$

$\begin{array}{llll}\text { O } & -4.389702 & 3.448283 & 2.330985\end{array}$

H -4.5049592 .8748480 .384453$

H -2.852731 1.3852732 .491099

O $-4.675173 \quad 0.736041 \quad 1.890620$

$\begin{array}{llll}\text { C }-2.699939 & 0.542878 & 0.500541\end{array}$

C $-3.2497440 .607187-0.936318$

H -2.705603 -0.5049630 .816307$

$\begin{array}{llll}\text { O } & -1.328099 & 0.939592 & 0.445765\end{array}$

H -3.242928 $1.641745-1.297761$

H $-2.5313550 .074758-1.572842$

H $-1.861133 \quad 7.7714190 .121797$

H -2.014125 7.5263611 .868089

H $-0.4092347 .617272 \quad 1.124481$

C $\quad 0.584371 \quad 4.797577 \quad 2.074915$

H -5.1626702 .9043612 .560377$

H $-4.459751-0.1486782 .231837$

H -0.9654060 .8842601 .345971$

H $0.816907 \quad 4.2613003 .003689$

H 1.0387015 .7912512 .167159

H $-1.311437 \quad 5.3807722 .883585$

C $-5.779226-2.142854-0.881260$

O $-4.557457-1.397143-0.791113$

C $-4.641866-0.011307-1.136418$

C $-5.1301370 .147475-2.585442$

C $-6.456771-0.586015-2.806244$ 
C $-6.403372-2.024611-2.282792$

H $-5.480128-3.189424-0.741556$

C $-6.751479-1.7827240 .252427$

H $-5.3495330 .491384-0.472385$

O $-4.155335-0.350189-3.500471$

H $-5.2556351 .214806-2.801563$

H $-6.691040-0.600278-3.877638$

O $-7.5073640 .107181-2.139423$

H -7.405649 -2.468724-2.273907

O $-5.606004-2.811319-3.172846$

C $-6.221556-2.1959731 .610232$

H $-6.947833-0.7082630 .292775$

H -7.699482 -2.311384 0.100688

H $-3.967600-1.274317-3.261955$

H -7.543642 $1.007471-2.505105$

H $-6.029540-2.784012-4.047736$

O $-7.129779-1.8368002 .565834$

O $-5.151833-2.7531671 .814416$

C $-6.758083-2.1454943 .912798$

H $-6.640323-3.2264854 .034722$

H $-5.834627-1.6227684 .180153$

H -7.558940 -1.802929 4.573542

SCF Energy (B3LYP/6-31G**//MMFF)= -3245.91324785

08_00509

MM̄FF Geometry

C -2.297870 -2.415355 -3.517442

C $-2.726583-2.071675-2.294070$

C $-4.151646-2.228631-1.834625$

O $-4.545455-0.952899-1.273121$

C $-4.268199-3.285687-0.721749$

C $-4.082412-4.720357-1.174022$

C $-2.983370-5.460392-0.922139$

C $-5.259251-5.326350-1.891627$

C $-1.720717-4.980909-0.265246$

C $-1.795112-5.0017351 .266207$

C $-0.665012-4.1751261 .900694$

C $-0.792610-4.1705393 .426863$

C $0.219129-3.2274104 .096215$

O $\quad-0.768895-2.8399751 .391288$

O $-0.205478-3.055756 \quad 5.457221$

C $0.150190-1.8499403 .400126$

C $1.615831-3.8555704 .160366$

O $1.143197-0.9697863 .917438$

C $0.239298-1.9407491 .862290$

C $0.021481-0.5784991 .170253$

O $1.548206-2.3896591 .492919$

C $-5.862432-0.624151-1.302213$

O $-6.764023-1.261203-1.829243$

C $-6.1099990 .662050-0.612299$

C -5.2601901 .1809130 .287015$

C $-5.4685942 .424671 \quad 1.021905$

C -5.0770522 .3363822 .472293$

C $-1.368304 \quad 0.029617 \quad 1.349849$

C $-1.474801 \quad 1.3215370 .564580$

C -0.9372663 .7536630 .710673$

O $-1.9383001 .349298-0.573114$

C $-2.0602114 .551758 \quad 1.294841$

C -2.905309 5.2889940 .557600

C -3.9743996 .1657851 .144361$

C -5.3249065 .9732890 .493198$

C $-5.7546726 .810291-0.466523$

C $-6.217393 \quad 4.875758 \quad 1.024530$

C $-5.918907 \quad 3.536900 \quad 0.408122$

N $-0.9677782 .409648 \quad 1.254349$

H -3.026960 -2.811645 -4.222330

H -2.027503 -1.677852 -1.561787

H -4.803198 $-2.472077-2.682543$

H -5.255341 -3.204956 -0.246172

H -3.574625 -3.027266 0.087796

H -2.964718 -6.497921 -1.250469

H -5.093792 -6.378691-2.145919

H $-6.154900-5.275991-1.263868$

H -5.457401 -4.789824 -2.824680

H $-0.889171-5.615753-0.595930$

H - $-1.480562-3.980855-0.636675$

H -2.758900 -4.591283 1.592197

H $-1.751065-6.037473 \quad 1.624671$
H $\quad 0.296032-4.6038661 .593010$

H $-0.697057-5.1873753 .827814$

H -1.803324 -3.837314 3.703119

H $0.385288-2.4020975 .869611$

H $-0.815340-1.4104293 .678608$

H $1.590739-4.7774304 .753910$

H $2.326342-3.1940804 .668034$

H $2.019573-4.1074113 .177608$

H $1.988474-1.2010773 .496736$

H $\quad 0.796727 \quad 0.130441 \quad 1.486526$

H $0.182973-0.712787 \quad 0.091713$

H $1.715577-2.1352960 .570159$

H $-7.054557 \quad 1.144875-0.843927$

H -4.3540120 .6438950 .551718$

H -5.7527951 .6589903 .006007$

H -5.0985993 .2986292 .988122$

H -4.0556011 .9526702 .575342$

H -2.148376 -0.6502230 .989787$

H $-1.584670 \quad 0.2365312 .402920$

H $-0.9851603 .714090-0.381761$

H $\quad 0.019597 \quad 4.196999 \quad 1.003208$

H -2.1545784 .5593042 .378213$

H -2.786233 $5.308998-0.523478$

H -3.651155 7.2077961 .018787

H $-4.066317 \quad 6.0154102 .227213$

H $-6.7363126 .699081-0.915122$

H -5.131728 $7.621240-0.829191$

H -7.2666925 .1085860 .797188$

H $-6.170614 \quad 4.8637792 .117264$

H $-6.1339643 .489169-0.659896$

H -0.6256532 .2787932 .201914$

C $0.209758-1.858668-3.099225$

O $0.060657-0.440743-2.880653$

C $1.3207390 .210892-3.142679$

C $2.365042-0.889155-3.077415$

C $1.611771-2.053059-3.684036$

H $1.2438490 .614287-4.160239$

C $1.5324211 .342588-2.131694$

H $3.275966-0.639911-3.628432$

H $2.637285-1.107506-2.037091$

C $2.228644-3.403938-3.362364$

H $1.586474-1.923172-4.774536$

C $2.8848282 .058085-2.282227$

O $0.4885282 .303417-2.313737$

H $1.4231070 .946274-1.118094$

H $3.7060001 .347761-2.161101$

O $2.9823402 .543061-3.628947$

C $3.0788353 .252444-1.331719$

C 2.9797172 .9424820 .175039

H $2.3455794 .028890-1.582470$

O $4.3640613 .822279-1.605516$

H 3.0128953 .9042580 .704122

H 2.0050312 .4930040 .390274

H $3.247394-3.465638-3.758783$

H $2.277897-3.575039-2.281633$

H $1.643304-4.215722-3.805437$

C $-0.889219-2.353459-4.048210$

H $-0.357911 \quad 1.832614-2.223051$

H $3.8400312 .994367-3.711611$

H $4.4590544 .611375-1.045278$

H $-0.636134-3.365168-4.388185$

H -0.910974 -1.709053 -4.936586

H $0.136404-2.349237-2.120772$

C $4.960790-0.2104170 .645188$

O 3.9423020 .7244820 .261397

C 4.1137312 .0605090 .740057

C 4.1185032 .0657662 .277541

C 5.1932981 .1236692 .828055

C $5.129179-0.2606692 .173798$

H $4.578226-1.1864560 .319706$

C $6.2826820 .049647-0.090889$

H 5.0685032 .4565400 .380501

O $2.835596 \quad 1.6709862 .758082$

H 4.3035803 .0887322 .627509

H 5.0623861 .0254773 .912579

O 6.4709651 .7108742 .598246

H $6.022963-0.8458272 .419997$

O $4.011555-0.9716692 .709662$ 
C $6.137322-0.178348-1.578516$

H 6.6501681 .0656510 .077080

H $7.065372-0.6335310 .258851$

H 2.8628471 .7014183 .729424

H 7.1402061 .1153692 .976468

H $4.167863-1.0808013 .663396$

O $6.570498 \quad 0.919905-2.261466$

O $5.703672-1.209771-2.075285$

C $6.4963680 .830098-3.687720$

H $7.153500 \quad 0.033771-4.049487$

H $5.4650950 .654514-4.006822$

H $6.8339021 .782213-4.105613$

SCF Energy (B3LYP/6-31G**//MMFF) $=-3245.90801073$

08_00510

MM̄FF Geometry

C $3.179181-1.494962-3.740715$

C $2.203575-1.420399-2.821801$

C $0.879821-2.126459-2.980952$

O $-0.150397-1.112451-2.930850$

C $0.667687-3.118566-1.824493$

C $-0.319566-4.222882-2.142842$

C - $1.555555-4.342061-1.621307$

C $0.194520-5.286045-3.083370$

C $-2.267118-3.433879-0.657723$

C $-3.547964-2.880240-1.292550$

C $-4.442659-2.140512-0.289355$

C $-5.653216-1.540705-1.010870$

C $-6.540985-0.720131-0.065769$

O $-3.684297-1.104168 \quad 0.341797$

O

$\begin{array}{llll}\text { C } & -5.653599 & 0.266117 & 0.719927\end{array}$

C $-7.422982-1.6174340 .810551$

$\begin{array}{lllll}\text { O } & -6.412390 & 0.946760 & 1.717284\end{array}$

C $-4.407794-0.3941841 .357948$

C $-3.484666 \quad 0.6995701 .937682$

O $-4.828085-1.2587242 .414746$

C -1.155202 -1.181067-3.840453

O $-1.305970-2.040483-4.698995$

C $-2.103344-0.049113-3.708110$

C $-2.0613550 .867502-2.728405$

C $-2.9813612 .000077-2.566597$

C $-4.0698542 .168272-3.594281$

C $-2.134534 \quad 0.1938502 .461655$

C - -1.3317861 .3717232 .975799$

C -0.1114313 .3724122 .133733$

O -1.3483941 .6993704 .159891$

C -1.1425864 .3504571 .665693$

C -1.062988 5.0112490 .500944

C $-2.1557785 .897302-0.022526$

C $-2.8075955 .332036-1.267486$

C $-2.7398195 .979736-2.442596$

C $-3.6024674 .048827-1.134495$

C $-2.8001572 .833481-1.520384$

N $-0.6671592 .043098 \quad 1.962868$

H $2.988427-2.081136-4.638696$

H $2.345463-0.846391-1.910997$

H $0.863259-2.641790-3.948101$

H $\quad 0.366120-2.570752-0.925859$

H $1.619672-3.604901-1.572133$

H -2.144898 $-5.210153-1.916957$

H -0.513902 -6.112509-3.205828

H $\quad 0.383336-4.868424-4.076627$

H $1.128331-5.711903-2.701419$

H $-2.519678-4.018243 \quad 0.235174$

H -1.635494 -2.606478 -0.324394

H -3.264208 -2.198063 -2.100993

H -4.126110 -3.693611 -1.749849

H -4.766167 -2.861621 0.470682

H -6.240164 -2.324957-1.505125

H -5.303712 -0.877914 -1.815242

H -7.947757 -0.540839-1.428362

H -5.3120101 .0350280 .012585$

H -8.081248 -2.2337860 .186413$

H -8.086776 -1.020491 1.445670

H $-6.848544-2.2925431 .448291$

H -7.172962 1.3580681 .271967

H -3.2874571 .4469181 .159512$
H $-3.997459 \quad 1.2204252 .756778$

H -4.084893 -1.406952 3.019415

H -2.868206 $-0.020424-4.478256$

H -1.285255 $0.790404-1.968948$

H $-4.7508251 .310105-3.574623$

H $-3.6411592 .255276-4.598985$

H -4.679978 $3.061108-3.445339$

H -2.255054 -0.515597 3.285933

H $-1.570447-0.3163401 .673358$

H $\quad 0.800743 \quad 3.426149 \quad 1.536173$

H $\quad 0.1397473 .5524403 .183282$

H -2.020612 4.4782942 .294662

H $-0.1911464 .873440-0.134489$

H $-1.7222006 .884970-0.223447$

H -2.931255 6.0591410 .736902

H $-3.2274205 .588191-3.329377$

H -2.194486 $6.911892-2.545015$

H $-4.5299404 .134765-1.708294$

H $-3.9353823 .926638-0.095763$

H $-1.9808902 .615835-0.836274$

H -0.7605901 .7015951 .010660$

C $4.949519-0.105686-2.436884$

O $4.1549941 .091503-2.315092$

C $5.0304322 .212304-2.134475$

C $6.3622761 .640287-1.675230$

C $6.4125880 .354610-2.477518$

H $5.1768812 .647838-3.133135$

C $4.4011193 .283229-1.240660$

H $7.2018242 .309977-1.884347$

H $6.3658501 .413142-0.604463$

C $7.392069-0.667407-1.925269$

H $6.7006010 .599298-3.508963$

C 4.3149502 .9889570 .271259

O $5.1853574 .473098-1.406640$

H $3.3980173 .514958-1.620626$

H 5.3204213 .0225710 .706635

O $3.6073424 .083090 \quad 0.875274$

C 3.6294441 .6705330 .673248

C 3.4795501 .5519562 .203055

H $4.2195190 .826787 \quad 0.304652$

O $2.346557 \begin{array}{lll}1.611498 & 0.054105\end{array}$

H 4.4120181 .8706222 .684413

H 2.6783582 .2239952 .530520

H $8.411015-0.266496-1.931976$

H $7.145884-0.942113-0.894060$

H $7.384207-1.580767-2.528297$

C $4.547481-0.867642-3.703879$

H $4.7740075 .168818-0.865608$

H 2.7069824 .0863250 .507982

H 1.9415580 .7612970 .295572

H $5.271986-1.672846-3.876878$

H $4.607606-0.188863-4.564581$

H $4.762314-0.714532-1.543882$

C $1.500615-1.6215912 .417576$

O $1.889632-0.2688662 .134161$

C 3.1561300 .1198892 .671648

C 3.1235120 .0258484 .205622

C $2.714569-1.3733654 .672868$

C $1.471127-1.8848653 .934580$

H $0.471646-1.6900672 .043192$

C $2.358395-2.6096201 .613213$

H $3.933642-0.5459572 .279222$

O $2.214400 \quad 0.986770 \quad 4.741120$

H $4.117446 \quad 0.2697594 .598656$

H $2.517073-1.3432055 .751420$

O $3.808808-2.2599414 .460136$

H $1.325867-2.9543344 .122343$

O $0.321910-1.2119324 .457695$

C $1.714359-3.9721751 .474133$

H $2.515122-2.2115810 .603493$

H $3.344875-2.7380832 .066709$

H 1.3419120 .8259544 .342555

H $3.546522-3.1349604 .793468$

H $0.265244-1.4199565 .405892$

O $2.610315-4.8279500 .898685$

O $0.570836-4.2515181 .806609$

C $2.137323-6.1616360 .685797$

H $1.931182-6.6445981 .645646$ 
H $\quad 1.242010-6.154656 \quad 0.057146$

H $2.921671-6.7223650 .170774$

SCF Energy (B3LYP/6-31G**//MMFF) $=-3245.91651310$

$08 \_00511$

MM̄FF Geometry

C $-2.595746-1.1299883 .040309$

C -2.5296340 .0471923 .682323$

C -3.1970401 .3448353 .286680$

O -4.0441041 .1840492 .128707$

C -2.1358752 .3926332 .904844$

C -2.5903683 .8140193 .167962$

C -3.1512864 .6326642 .257138$

C -2.3280904 .3113544 .567008$

C -3.5425754 .3248720 .838982$

C $-2.6416085 .017694-0.192754$

C $-1.3368544 .250324-0.451856$

C $-0.4336345 .022097-1.413082$

C $0.8368854 .228243-1.751561$

O $-1.6903332 .994713-1.037854$

O $1.4641554 .861844-2.878399$

C $0.4417122 .802822-2.201148$

C $1.8564214 .284102-0.606754$

O $1.6120872 .003742-2.353378$

C $-0.5898172 .108804-1.274076$

C $-1.1663210 .844511-1.950209$

O $\quad 0.0070901 .672879-0.052018$

C $\begin{array}{llll}-5.328706 & 0.785033 & 2.308437\end{array}$

$\begin{array}{llll}\text { O } & -5.881315 & 0.504724 & 3.358437\end{array}$

C $-5.937097 \quad 0.7185450 .962554$

C $-7.010942-0.0549470 .752392$

C -7.672723 -0.266179-0.537094

C $-7.1771930 .532718-1.715171$

C $-2.3184190 .194649-1.168231$

C $-2.754559-1.122165-1.791133$

C $-4.355733-3.003106-1.441375$

O $-2.223059-1.600855-2.789674$

C $-5.560835-2.815582-2.308942$

C $-6.726442-3.447810-2.100571$

C -7.929435 -3.299250 -2.989586

C $-9.201556-3.013209-2.220509$

C -10.095051 -3.987173-1.977346

C $-9.476819-1.583806-1.807949$

C $-8.667936-1.174647-0.606717$

N -3.800307 -1.708250 -1.090329

H $-3.179903-1.2191932 .129991$

H -1.941817 0.0996134 .597552

H -3.7974571 .6954394 .135587$

H -1.863880 2.2771571 .849845

H -1.2059812 .2319243 .466941$

H -3.4014005 .6460362 .570133$

H -2.675512 5.3389714 .717161

H -2.841758 3.6802515 .299280

H -1.254635 4.290666 4.782162

H $-3.591010 \quad 3.2499010 .649023$

H -4.5677534 .6936490 .704970$

H -3.204854 $5.078597-1.133312$

H $-2.424157 \quad 6.0455610 .122514$

H -0.8252094 .0797880 .501350$

H -0.174073 $6.005073-1.000755$

H $-0.9772745 .221814-2.347089$

H $1.6477915 .786580-2.639462$

H $-0.0021312 .901716-3.201990$

H $2.1691555 .319232-0.423247$

H $2.7706263 .737320-0.862018$

H 1.4667423 .8906940 .334555

H $1.3626901 .195369-2.831634$

H -1.541505 $1.099841-2.949814$

H $-0.3715010 .100877-2.080298$

H $0.9422631 .928918-0.047610$

H $\quad-5.451406 \quad 1.2750240 .168876$

H -7.425227 -0.6092701 .593493$

H $-7.3008181 .605254-1.528246$

H -7.701526 $0.312729-2.646864$

H $-6.117146 \quad 0.330677-1.903269$

H -2.012464 $-0.005050-0.135664$

H -3.184328 $0.865870-1.147235$

H $-4.596331-3.516640-0.505135$
H $-3.601961-3.588029-1.978293$

H $-5.462902-2.150442-3.163725$

H $-6.811999-4.138913-1.264889$

H -8.039834 -4.233990 -3.554987

H -7.777071 -2.514303 -3.740913

H $-11.022210-3.785672-1.451040$

H $-9.916695-5.009326-2.294549$

H $-10.536170-1.463678-1.544116$

H $-9.330251-0.928372-2.670509$

H -8.949454 -1.704619 0.304533

H $-4.202681-1.218842-0.296848$

C $-0.762664-2.8604362 .637634$

O $0.249595-1.8395942 .533473$

C $0.693934-1.7746981 .160229$

C $0.198836-3.0546700 .504956$

C $-1.130593-3.2491601 .207129$

H $0.184512-0.9068090 .726345$

C $2.211419-1.5652911 .133417$

H $0.098344-2.962545-0.580617$

H $0.874842-3.8912920 .720568$

C $-1.697679-4.6504961 .070416$

H -1.845933 -2.536549 0.785008

C $2.832703-1.655861-0.271361$

O $2.481245-0.2647241 .668796$

H $2.689770-2.2871931 .804518$

H $2.748300-2.681734-0.648052$

O $2.064853-0.843648-1.168775$

C $4.300274-1.194894-0.374227$

C $5.253990-1.9513340 .568262$

H $4.361820-0.114379-0.198749$

O $4.700960-1.416734-1.731538$

H $4.862311-1.9407621 .590009$

H $5.312523-2.9939890 .231672$

H -1.877302 -4.8912280 .017416$

H -1.012342 $-5.402748 \quad 1.474712$

H $-2.649460-4.7384061 .603833$

C -1.909664 -2.377917 3.529901

H $2.074145-0.2245112 .551402$

H $2.1039710 .071672-0.842262$

H $5.602178-1.067263-1.833820$

H $-1.508918-2.1991254 .536182$

H -2.660456 -3.170404 3.628447

H $-0.281136-3.7113973 .137673$

C $8.561703-0.911170-0.855945$

O $7.260790-1.490603-0.693977$

C $6.673534-1.3451220 .601071$

C $7.557767-2.0199411 .661643$

C $8.993496-1.4853101 .607431$

C $9.547796-1.4793330 .178271$

H $8.890279-1.240570-1.850388$

C $8.5072600 .620813-0.868262$

H $6.573798-0.2797620 .836442$

O $7.563912-3.430111 \quad 1.447839$

H $7.123202-1.8383332 .651838$

H $9.639617-2.1053832 .240683$

O $9.031039-0.1575802 .122416$

H $10.487460-0.9165050 .132119$

O $9.845301-2.823658-0.210552$

C $7.6998761 .116481-2.044849$

H 8.0848841 .0434310 .047802

H $9.5135651 .045217-0.964824$

H $8.107648-3.8309622 .147152$

H $8.704588-0.1879513 .037877$

H $10.510104-3.1680510 .409925$

O $6.449498 \quad 1.473411-1.634621$

O $8.1228571 .156819-3.192839$

C $5.5690671 .935952-2.663393$

H $4.6044952 .168179-2.204786$

H $5.9686032 .845032-3.122504$

H $5.4204941 .155300-3.415431$

SCF Energy (B3LYP/6-31G**//MMFF) $=-3245.90853283$

08_00512

MM̄FF Geometry

C $-0.1573104 .284547-0.309365$

C $-1.3902033 .830289-0.578517$

C -2.5625574 .0030280 .353149$

$\begin{array}{llll}\text { O } & -3.013561 & 2.679254 & 0.728544\end{array}$ 
C $-3.7122524 .732242-0.361887$

C -4.8850985 .0401510 .551654$

C -6.0746954 .4060030 .526560$

C $-4.6615846 .171331 \quad 1.523682$

C $-6.4981753 .276107-0.368709$

C -6.6755091 .9759530 .425260$

C $-6.8651040 .765207-0.499727$

C $-7.103484-0.5062110 .314136$

C $-7.166464-1.757774-0.574593$

O $-5.6719670 .627976-1.276116$

O $\quad-7.061949-2.907270 \quad 0.280884$

C $-5.937943-1.778171-1.511920$

C $-8.515439-1.874944-1.294640$

O $-6.063923-2.847487-2.446585$

C -5.698109 -0.436097-2.236916

C $-4.353061-0.375606-3.002401$

O $-6.739677-0.232755-3.199273$

C -2.3350372 .0699201 .740831$

O -1.4402842 .5515722 .418147$

C $-2.8462440 .687616 \quad 1.869389$

C $-2.170083-0.1896822 .626386$

C $-2.536697-1.5838102 .883632$

C $-3.776929-2.1162992 .217820$

C -3.097989-0.553862 -2.140542

C $-2.565516-1.976281-2.106387$

C $-0.785406-3.345831-1.025372$

O $-2.996054-2.876372-2.821562$

C $-1.345052-4.1009460 .137093$

C $-0.609972-4.4746041 .195612$

C -1.137069 -5.3107492 .325228$

C $-0.915897-4.6782653 .679431$

C $0.134317-5.0257434 .442391$

C $-1.967558-3.7213244 .189514$

C $-1.755336-2.3124233 .708264$

N -1.520184 -2.109069-1.202646 H 0.0139514 .8469080 .604603 H -1.561626 $3.257358-1.487063$ H -2.2725764 .5633241 .250461$ H -4.033468 4.141537 -1.225367 H -3.347915 $5.680966-0.778881$ H -6.8420534 .7266681 .230214$ H $-5.562816 \quad 6.4103352 .098435$ H -3.874499 5.9167962 .239758 H $-4.366027 \quad 7.0792880 .987897$ H -7.451056 $3.545754-0.840639$ H $-5.7930293 .126093-1.188643$ H $-5.7886601 .815643 \quad 1.050726$ H -7.536364 2.0717051 .098602 H -7.712612 $0.963110-1.166389$ H $-8.015267 \quad-0.4163190 .917807$ H $-6.283094-0.6350921 .032588$ H -7.792069 -2.8713140 .922281$ H -5.071674 -2.011590 -0.882042 H -9.334394 -1.927719 -0.567125 H -8.574077 -2.800985 -1.877176 H -8.720618 -1.033724 -1.960008 H -5.217937 -2.934443 -2.916429 H $-4.365810-1.067603-3.852736$ H $-4.2835960 .626385-3.450697$ H $-6.4243280 .397486-3.867976$ H $-3.7552980 .430277 \quad 1.339558$ H -1.258976 $0.146878 \quad 3.120188$ H -3.733506 -1.9535281 .136059$ H -4.667050 -1.616972 2.614259 H -3.918226 -3.190635 2.353910 H $-2.2953940 .059830-2.569946$ H -3.264841 -0.202006 -1.117660 H $\quad 0.264906-3.081392-0.877035$ H $-0.862506-3.954279-1.932253$ H $-2.391743-4.3908830 .084037$ H $0.443968-4.2076301 .230882$ H $-0.627516-6.2826572 .282877$ H -2.203094 -5.534921 2.194971 H $\quad 0.280508-4.6002825 .429729$ H $0.874141-5.7386364 .093579$ H -1.939851 -3.692623 5.287490 H -2.963039 -4.108997 3.954726 H $-0.870786-1.8383394 .136416$
H $-1.271001-1.320480-0.612829$

C $2.0024472 .984711-0.634339$

O $1.3361241 .709115-0.506604$

C 1.6714471 .1496710 .773059

C 2.9141161 .8921451 .244772

C 2.6282563 .2908410 .729357

H 0.8470901 .4158331 .447579

C $1.760431-0.3799060 .728861$

H 3.0432601 .8533952 .330330

H 3.8221691 .5011810 .775646

C 3.8607564 .1753710 .652471

H 1.8998173 .7588721 .401650

C $2.978388-1.0008410 .012549$

O $1.758123-0.8574772 .081847$

H $0.846424-0.7737660 .271535$

H $3.886167-0.7913580 .587755$

O $2.818670-2.4265150 .036908$

C $3.170172-0.572934-1.451594$

C $4.288868-1.321212-2.201908$

H $3.3837760 .497803-1.497493$

O $1.952936-0.778448-2.173596$

H $4.042853-2.387613-2.281484$

H $4.287646-0.941996-3.232770$

H 4.2988594 .3116601 .646602

H 4.6288103 .7389160 .004782

H 3.6056975 .1631460 .256019

C $1.0330304 .033498-1.192187$

H $2.573397-0.5458742 .510106$

H $2.689038-2.6882260 .964727$

H $1.748412-1.728483-2.139468$

H $0.6876773 .697200-2.178382$

H $1.5587624 .982311-1.348953$

H $2.7956212 .835552-1.378363$

C $7.2141770 .595307-0.899087$

O $5.9785320 .251489-1.541775$

C $5.686408-1.148894-1.585657$

C $6.765677-1.888825-2.391723$

C $8.161441-1.617924-1.821215$

C $8.400043-0.123108-1.569045$

H $7.3240321 .671535-1.083946$

C 7.1064210 .3851800 .618348

H $5.668985-1.547497-0.565826$

O $6.719548-1.463663-3.752505$

H $6.549582-2.963728-2.371266$

H $8.915469-1.992927-2.524069$

O $8.302516-2.348867-0.606946$

H $9.3052060 .016183-0.968792$

O $8.6497390 .513563-2.827189$

C 8.1210041 .1867501 .403825

H $\begin{array}{llll}6.111716 & 0.701500 & 0.953247\end{array}$

H $7.219556-0.6694220 .883528$

H $7.383054-1.978108-4.243084$

H $9.202929-2.193409-0.274009$

H $7.8356590 .455216-3.356352$

O $7.984726 \quad 0.889773 \quad 2.730684$

O $8.9235831 .976377 \quad 0.926242$

C 8.8794771 .5783773 .610054

H $8.672347 \quad 1.2490204 .631781$

H $9.916841 \quad 1.331106 \quad 3.365095$

H 8.7140662 .6581653 .548802

SCF Energy (B3LYP/6-31G**//MMFF) $=-3245.91801450$

08_00513

MM̄MF Geometry

C $-2.3545202 .485278-1.287195$

C $-3.2027583 .183511-0.517400$

C -3.6108482 .8193130 .893056$

O -2.8124621 .7183871 .385321$

C -5.0794122 .3696570 .925300$

C -5.6719532 .4245472 .318862$

C -5.8116041 .3661193 .140225$

C -6.1671683 .7821472 .748095$

C $-5.369697-0.0527852 .912972$

C $-6.555293-1.0071772 .712898$

C $-7.175054-0.9073841 .310372$

C $-8.431510-1.7733121 .210674$

C $-9.008158-1.784658-0.212497$

O $-6.193710-1.3660000 .375587$ 
O $-9.980348-2.840059-0.252929$ C $-7.877501-2.130661-1.204921$ C $-9.758245-0.485532-0.529268$ O $-8.321261-2.004743-2.554465$ C $-6.592970-1.291461-0.998023$ C $-5.444672-1.880161-1.852551$ O $-6.842965 \quad 0.054730-1.403908$ C $-1.655593 \quad 1.993968 \quad 2.038479$ O -1.2468093 .0981562 .368856$ C $-0.8859190 .756640 \quad 2.308565$ C $-1.171409-0.423746 \quad 1.736767$ C $-0.434133-1.6662601 .945571$ C $-1.327092-2.8727502 .057970$ C $-4.065820-1.259077-1.583059$ C $-2.964972-1.982592-2.341585$ C $-0.485241-2.033133-2.588442$ O $-3.182747-2.905761-3.122162$ C $0.099640-2.982982-1.589790$ C $1.381152-2.945475-1.194895$ C $1.984479-3.904278-0.209249$ C $2.651336-3.2029990 .953278$ C $3.970440-2.9480030 .938983$ C $1.809473-2.8849702 .168793$ C $0.913746-1.6952771 .962664$ N $-1.708449-1.471610-2.045437$ H $-1.9396901 .551947-0.917844$ H $-3.614454 \quad 4.113686-0.903157$ H -3.465008 3.7002911 .529735 H $-5.162893 \quad 1.3562330 .517449$ H $-5.692892 \quad 3.003863 \quad 0.271489$ H -6.2797691 .5316884 .110202$ H -6.6081113 .7672143 .750497$ H $-5.3458394 .505142 \quad 2.761801$ H $-6.935562 \quad 4.1417062 .055929$ H $-4.660504-0.1403162 .085117$ H $-4.818313-0.3668443 .808555$ H $-6.185434-2.0298122 .864637$ H -7.318477 -0.8208793 .478713$ H -7.4201350 .1386821 .097902$ H -9.191379 -1.442212 1.929576 H -8.184172 $-2.806627 \quad 1.492711$ H - $-10.399460-2.831824-1.130001$ H -7.626138-3.190309-1.056654 H - $-10.595576-0.3502210 .165944$ H -10.202278 $-0.518414-1.530314$ H $-9.1256350 .402303-0.464173$ H $-9.056329-2.627148-2.682870$ H $-5.369658-2.956631-1.648517$ H $-5.676726-1.766190-2.919166$ H $-6.0041560 .473574-1.651860$ H -0.0381880 .8710682 .977473$ H -2.022369 -0.5164851 .066814$ H $-1.934400-2.8105142 .967840$ H $-0.781700-3.8179892 .091123$ H -2.004159 -2.934321 1.197934 H $-4.050193-0.208059-1.888812$ H -3.827777 -1.313156 -0.515102 H $0.186102-1.197988-2.810531$ H $-0.705115-2.564356-3.519779$ H $-0.558737-3.747039-1.182812$ H $2.047261-2.202607-1.627197$ H $2.722651-4.514269-0.746684$ H $1.238251-4.6135120 .169906$ H $4.463302-2.4740361 .780756$ H $4.585458-3.2023620 .082083$ H $2.457932-2.6550843 .025284$ H $1.261611-3.7825322 .469474$ H $1.455899-0.7580641 .835238$ H $-1.622109-0.745158-1.341127$ C $-0.388772 \quad 3.231724-2.691102$ O $0.3750602 .117785-2.179814$ C $1.2208312 .601492-1.114465$ C $1.4182644 .076000-1.416908$ C $0.0262414 .463360-1.877997$ H $\quad 0.6464732 .471469-0.188219$ C $2.4987801 .761186-1.061007$ H $1.7515444 .649113-0.546928$ H $2.1457064 .214190-2.226181$
C $-0.016508 \quad 5.759277-2.669666$

H $-0.608904 \quad 4.578833-0.991427$

C $3.5482262 .281561-0.064971$

$\begin{array}{llll}\text { O } & 2.129284 & 0.433264 & -0.678727\end{array}$

H $2.9302841 .691718-2.065656$

H $3.9289963 .252319-0.404347$

$\begin{array}{llll}\text { O } & 2.894103 & 2.519491 & 1.190029\end{array}$

C $4.7275641 .325843 \quad 0.203904$

C $5.475505 \quad 0.889832-1.068496$

H 4.3732750 .4513370 .762500

O 5.6267012 .0310351 .067466

H $4.7675610 .485549-1.798620$

H $5.9558931 .774509-1.505121$

H $0.3325576 .596430-2.056368$

H $0.6191015 .707490-3.560049$

H $-1.0377895 .980591-2.995817$

C $-1.8876612 .902501-2.653129$

H $1.4244480 .143334-1.282316$

H $3.5661112 .857291 \quad 1.806828$

H 6.3490201 .4237821 .300037

H $-2.0837722 .061215-3.329610$

H $-2.4654783 .756040-3.025707$

H $-0.0871383 .350856-3.739277$

C $8.631288-0.510756 \quad 0.393084$

O $7.5827470 .383701-0.002253$

C $6.544768-0.190243-0.800340$

C $7.127188-0.749926-2.107623$

C $8.263531-1.739491-1.828266$

C $9.281770-1.171599-0.833504$

H 9.3833850 .1348440 .865012

C $8.156822-1.5190391 .445713$

H $6.066392-1.000625-0.241441$

O $7.6128200 .324475-2.910039$

H $6.324937-1.243983-2.668723$

H $8.775993-1.984217-2.766479$

O $7.727294-2.949296-1.300448$

H $9.972445-1.955354-0.500652$

O $10.067296-0.172488-1.490051$

C $7.774108-0.8147422 .725517$

H $7.308340-2.1237311 .113469$

H $8.956833-2.2263151 .694822$

H $7.950678-0.056694-3.738130$

H $7.104553-3.305980-1.956598$

H $10.522003-0.600100-2.235542$

O $6.422182-0.6492142 .779716$

O $8.582749-0.4367863 .563321$

C $5.926298 \quad 0.024300 \quad 3.940483$

H 6.3519331 .0301374 .006814

H 4.8403590 .1088813 .845059

H $6.156986-0.5542374 .839880$

SCF Energy (B3LYP/6-31G**//MMFF) $=-3245.89994590$

08 00514

MM̄FF Geometry

C $2.619742-0.6612212 .656603$

C $1.376445-0.2890512 .993021$

C $0.214613-0.3822242 .041239$

$\begin{array}{lllll}\text { O } & -0.317118 & 0.958861 & 1.921658\end{array}$

C $-0.912897-1.300247 \quad 2.544474$

C $-0.497903-2.7373002 .765389$

C $-0.339277-3.6427731 .781100$

C $-0.285384-3.136374 \quad 4.202336$

C $-0.530117-3.436547 \quad 0.303580$

C $-1.499235-4.463549-0.301388$

C $-2.956895-4.2679530 .145776$

C $-3.829296-5.419016-0.359374$

C $-5.310998-5.203616-0.019803$

O $-3.419463-3.029485-0.401151$

O $-6.080301-6.145348-0.783051$

C $-5.727178-3.794557-0.487565$

C $-5.609328-5.4935891 .455976$

O $-7.056525-3.492124-0.068055$

C $-4.760104-2.675906-0.031553$

C $-5.116810-1.356677-0.762282$

O $-4.885626-2.4892981 .377513$

C $-0.7868201 .354994 \quad 0.709607$

O $-0.8076750 .707309-0.323943$

C $-1.287110 \quad 2.7390810 .866797$ 
C $-1.9364813 .335334-0.143821$

C $-2.5106194 .683720-0.121520$

C $-2.2764115 .517573 \quad 1.111477$

C $-4.161055-0.193467-0.471621$

C $-4.665005 \quad 0.718510 \quad 0.629280$

C -6.3531432 .4981971 .079217$

O

C $-6.664905 \quad 3.7272070 .286588$

C $-6.048330 \quad 4.903258 \quad 0.475150$

C $-6.3121656 .130273-0.347607$

C $-5.4356846 .218890-1.579285$

C $-5.9752516 .191072-2.809798$

C $-3.9438776 .406890-1.391399$

C $-3.2260735 .098013-1.188627$

N $-5.689037 \quad 1.5496470 .204806$

H $2.798826-1.0725071 .665383$

H 1.1872230 .1248363 .980169

H $\quad 0.567024-0.726818 \quad 1.061875$

H -1.752817 -1.264073 1.838505

H -1.338111 -0.8875803 .470173$

H $-0.028934-4.6495972 .058202$

H $0.007816-4.1866844 .304056$

H $\quad 0.501303-2.5300044 .661132$

H -1.209233 -2.9958144 .773291$

H $-0.865870-2.423746 \quad 0.062947$

H $0.448666-3.560725-0.175745$

H -1.443060 -4.367797 -1.393661

H -1.162761 -5.476728 -0.047771

H -2.996191-4.218386 1.239348

H $-3.479731-6.378170 \quad 0.042442$

H -3.732332 -5.498608 -1.451393

H $-5.776263-7.039419-0.549934$

H -5.745468 -3.807492 -1.586537

H $-5.348436-6.5298491 .702655$

H $-6.679593-5.3960381 .668944$

H $-5.060347-4.8438712 .140616$

H -7.630485 -4.210881 -0.384141

H $-5.069407-1.544178-1.843116$

H $-6.146012-1.065816-0.521875$

H -4.480357 -1.640846 1.616214

H -1.1205493 .2248331 .821902$

H -2.081555 2.779171-1.069138

H -1.202758 5.6508331 .285515

H -2.717992 5.0365451 .991350

H $-2.707430 \quad 6.5185601 .053142$

H $-3.157790-0.541119-0.209786$

H -4.046334 $0.417934-1.376039$

H -5.7212312 .7110081 .947667$

H -7.277835 2.0335931 .435612

H -7.417096 $3.637977-0.493595$

H -5.2953004 .9891821 .254057$

H -6.1503427 .0178680 .276411$

H $-7.3742006 .160405-0.625090$

H -5.358476 $6.275148-3.698474$

H -7.044264 $6.078909-2.956898$

H $-3.7757877 .122746-0.582866$

H -3.513061 $6.881029-2.283382$

H $-3.3272514 .417128-2.035312$

H $-6.0902381 .394047-0.716006$

C 4.7485820 .5896743 .098047

$\begin{array}{llll}\text { O } & 5.159938 & 0.378216 & 1.733505\end{array}$

C $6.589290 \quad 0.477073 \quad 1.649963$

C 7.0182311 .2196172 .908116

C $6.035217 \quad 0.667147 \quad 3.923479$

H $6.973931-0.5494091 .694845$

C 7.0166891 .1192670 .323693

H 8.0625701 .0269533 .170935

H 6.8845592 .3027972 .808870

C 5.9103391 .5153605 .177151

H $6.360630-0.3422234 .209035$

C $6.341910 \quad 0.535813-0.938378$

O $\quad 6.7133592 .5205840 .349349$

H 8.1071921 .0559250 .231786

H $5.3278520 .938877-1.018998$

O $7.0474571 .037414-2.080471$

C $6.289728-0.997436-1.009840$

C $5.778730-1.571949-2.343355$

H $5.657483-1.377826-0.199957$
O $7.606713-1.510571-0.788801$

H $6.540903-1.443819-3.123277$

H $5.699658-2.657403-2.197955$

H 6.8719461 .5773745 .697029

H 5.5897442 .5355094 .941020

H 5.1791741 .0837625 .867841

C $3.820223-0.5430663 .549680$

H 5.7571682 .6107870 .503954

H $7.9393030 .649619-2.067229$

H $7.545208-2.480943-0.776246$

H $3.515717-0.3838934 .590639$

H $4.350414-1.5026653 .511273$

H $4.212616 \quad 1.5473073 .118231$

C $2.184285-0.517132-2.076847$

O $3.485435-1.045633-1.775761$

C $4.434109-1.011914-2.846039$

C $3.916100-1.802653-4.062276$

C $2.517873-1.340694-4.481589$

C $1.568304-1.247941-3.282182$

H $1.575056-0.756733-1.196722$

C $2.1896271 .012231-2.252272$

H $4.5979270 .029209-3.142003$

O $3.869693-3.193598-3.744670$

H $4.622764-1.676641-4.891430$

H $2.109593-2.047314-5.214518$

O $2.631109-0.071693-5.118111$

H $0.638396-0.748365-3.575871$

O $1.202537-2.568871-2.873383$

C $2.4353431 .749297-0.954926$

H $2.9531141 .346346-2.958678$

H $1.2062471 .330853-2.618599$

H $3.588841-3.668338-4.545486$

H $\quad 1.7392670 .197415-5.396617$

H $2.000275-3.005219-2.528179$

O $2.2113023 .082670-1.149548$

$\begin{array}{llll}\mathrm{O} & 2.793470 & 1.233424 & 0.093011\end{array}$

C $2.4264443 .913727-0.004387$

H $2.1311584 .933619-0.264181$

H 1.8144843 .5760210 .837476

H 3.4868093 .9112570 .265194

SCF Energy (B3LYP/6-31G**//MMFF) $=-3245.91507402$

08_00515

MMFF Geometry

C $-1.4062315 .074577-0.464146$

C $-2.4096414 .218392-0.221383$

C -2.8267593 .7929931 .162849$

O $-2.8007572 .346147 \quad 1.214492$

C -4.2616304 .2697041 .445606$

C -4.7478493 .9280652 .841807$

C -5.6574502 .9760373 .131947$

C -4.1630964 .7688083 .948322$

C -6.3538692 .0459372 .178572$

C -5.9685350 .5859712 .448511$

C $-6.448744-0.3456051 .326178$

C $-6.140521-1.8040611 .665493$

C $-6.478596-2.7555980 .508357$

$\begin{array}{llll}\text { O } & -5.761411 & 0.034308 & 0.129455\end{array}$

$\begin{array}{lllll}\text { O } & -5.858560 & -4.013475 & 0.817683\end{array}$

C $-5.843258-2.213703-0.792011$

C $-7.986035-3.0219150 .422304$

O $-6.268297-2.970075-1.922691$

C $-6.128598-0.716915-1.034669$

C $-5.334306-0.130355-2.228820$

O $-7.520099-0.549409-1.334218$

C -1.5929931 .7563151 .438284$

O -0.5219742 .3169491 .607745$

C $-1.7976060 .290548 \quad 1.446003$

C $-0.741136-0.5329481 .526144$

C $-0.791049-1.9958521 .601626$

C $-2.144941-2.6520141 .530443$

C $-3.807676-0.176730-2.094827$

C $-3.164173-1.398423-2.728580$

C $-0.965356-2.558963-2.904386$

O $-3.770569-2.180493-3.455145$

C $-0.878274-3.633836-1.867958$

C $0.281856-4.108640-1.390169$

C $0.379597-5.222143-0.388840$ 
C $1.218738-4.858050 \quad 0.814577$

C $2.505653-5.2353440 .896794$

C $0.538360-4.159600 \quad 1.968077$

C $0.362755-2.6805501 .751375$

N $-1.814717-1.490252-2.413901$

H $-0.874206 \quad 5.5188880 .372525$

H -2.944193 $3.771265-1.056632$

H -2.146999 4.1984551 .922700

H $-4.935678 \quad 3.8598730 .686905$

H -4.320181 5.3597001 .321502

H -5.9386652 .8363754 .175222$

H -4.6045754 .5382574 .923834$

H -3.084490 4.6038224 .029948

H $-4.340146 \quad 5.8314383 .752120$

H -7.436914 2.164249 2.305870

H -6.1439012 .3011791 .138208$

H -4.8772990 .5136922 .534465$

H -6.3938650 .2675593 .408374$

H $-7.526648-0.2010531 .187968$

H -6.663942 -2.109764 2.580175

H $-5.070470-1.9022911 .890721$

H $-6.099110-4.6479550 .121727$

H -4.760617 -2.360732 -0.701766

H -8.341816 $-3.496598 \quad 1.344717$

H $-8.219473-3.724704-0.385120$

H $-8.573664-2.114490 \quad 0.268949$

H $-5.964491-3.884640-1.797516$

H -5.672247 $-0.585294-3.167744$

H $-5.6082050 .931784-2.307438$

H -7.635199 $0.290568-1.808562$

H $-2.817597-0.0722351 .404013$

H $0.256623-0.097791 \quad 1.567253$

H $-2.099756-3.7403401 .451786$

H -2.692630 -2.3123910 .645041$

H -2.731995 -2.411732 2.423122

H -3.388380 $0.692066-2.618801$

H -3.502141 -0.103847 -1.047099

H $0.010889-2.121013-3.132381$

H -1.386019 -2.969939-3.827700

H -1.813948 -4.060455 -1.514170

H $1.217461-3.704341-1.768285$

H $0.819482-6.089041-0.899539$

H $-0.612763-5.550956-0.056077$

H $3.105338-5.0128301 .772954$

H $2.990471-5.7696390 .086420$

H $1.138977-4.2771562 .880656$

H $-0.401713-4.6698802 .197673$

H $1.299222-2.1218291 .781750$

H $-1.410286-0.828033-1.758037$

C $0.3926014 .837181-2.242201$

O $0.302633 \quad 3.397717-2.199672$

C $1.4552492 .887955-1.496915$

C $2.4816604 .008781-1.539886$

C $1.5939715 .227356-1.378802$

H $1.1264702 .712721-0.465756$

C $1.8975181 .569655-2.138291$

H $3.2349123 .924542-0.750994$

H $2.9948614 .030849-2.509302$

C $2.2560786 .527246-1.801477$

H $1.3120805 .305215-0.322228$

C $3.1973281 .002223-1.533081$

O $0.8409890 .621915-1.958982$

H $2.0204671 .702872-3.219702$

H $3.9984421 .735879-1.677099$

O $3.0200200 .842324-0.126704$

C $3.644713-0.327693-2.173283$

C $5.097027-0.725852-1.858264$

H $3.583188-0.220951-3.263745$

O $2.775492-1.396423-1.821045$

H $5.328635-1.655491-2.395897$

H $5.7616070 .028779-2.301733$

H $3.1407676 .725500-1.187614$

H $2.5751846 .494033-2.848630$

H $1.5669777 .369682-1.684904$

C $-0.9530105 .454794-1.846912$

H $0.0347111 .011404-2.339184$

H 2.3095070 .1910690 .004385

H $1.870646-1.133880-2.058387$
H -1.708760 $5.134207-2.575616$

H $-0.8969566 .547390-1.910867$

H $0.5898895 .094403-3.290943$

C $7.558524-1.2772620 .875578$

O $6.919858-1.166002-0.403293$

C $5.506539-0.902610-0.382732$

C $4.779807-2.036447 \quad 0.355479$

C $5.363153-2.2661681 .753320$

C $6.891548-2.3694881 .727530$

H $8.580183-1.6100800 .650124$

C 7.6564110 .0790901 .591670

H $5.349308 \quad 0.0477650 .133077$

O $4.864304-3.244884-0.397241$

H $3.718281-1.7909690 .456358$

H $4.939392-3.1886202 .167832$

O $4.961875-1.1887662 .594549$

H $7.299688-2.3395572 .744476$

O $7.260812-3.6329691 .166517$

C 8.5341101 .0623680 .844655

H $6.6760710 .546911 \quad 1.713025$

H $8.102192-0.0663052 .582636$

H $5.805940-3.438893-0.544327$

H $5.312672-1.3646123 .484149$

H $6.877214-4.3270261 .729277$

$\begin{array}{lllll}\text { O } & 8.561027 & 2.241216 & 1.535641\end{array}$

$\begin{array}{lllll}\text { O } & 9.121808 & 0.834236 & -0.203458\end{array}$

C 9.3525253 .2795880 .949671

H 9.2878794 .1607501 .593457

H $10.399356 \quad 2.9676520 .885528$

H $8.9644313 .538876-0.040100$

SCF Energy (B3LYP/6-31G**//MMFF) $=-3245.91630569$

08_00516

MMFF Geometry

C -1.010291 -2.226979 2.200183

C $-1.382600-2.0397103 .476463$

C -2.422174 -1.057707 3.966248

O $-3.308717-0.663966 \quad 2.894981$

C $-1.732548 \quad 0.201646 \quad 4.513874$

C -2.7030861 .1760315 .151037$

C -3.1538362 .3002544 .559841$

$\begin{array}{llll}C & -3.124040 & 0.837818 & 6.557541\end{array}$

C $-2.8486492 .782790 \quad 3.168642$

C $-4.116397 \quad 3.269516 \quad 2.451504$

C $-3.8049193 .733790 \quad 1.021940$

$\begin{array}{llll}\text { C }-5.015636 & 4.425508 & 0.395351\end{array}$

C $-4.7428094 .832982-1.060373$

O $\begin{array}{llll}-3.453882 & 2.581140 & 0.248490\end{array}$

O $-5.9978335 .182814-1.663267$

C $-4.1980463 .610186-1.825728$

C $-3.854614 \quad 6.079390-1.151347$

O $-3.786675 \quad 3.972657-3.142111$

C $-3.0480532 .875423-1.094607$

C $-2.752274 \quad 1.540633-1.816387$

O $-1.872383 \quad 3.686236-1.121241$

C $-4.399628-1.4485572 .678360$

O $-4.730966-2.439518 \quad 3.309873$

C $-5.110634-0.9130821 .497059$

C $-6.027243-1.681603 \quad 0.889969$

C $-6.766197-1.350878-0.329332$

C $-6.6520190 .052321-0.859912$

C $-1.8258990 .589259-1.049754$

C $-1.753897-0.743005-1.770628$

C $-3.096782-2.808680-2.187343$

O $-0.832447-1.014261-2.536342$

C $-4.302219-2.631092-3.058944$

C $-5.345799-3.474784-3.068377$

C $-6.564828-3.281479-3.926930$

C $-7.861113-3.398143-3.151129$

C $-8.678136-4.450310-3.328569$

C $-8.247292-2.262440-2.223164$

C $-7.495430-2.321579-0.918033$

N $-2.844339-1.554820-1.500649$

H $-1.458608-1.6318651 .410227$

H $-0.901787-2.6332374 .251667$

H $-3.001297-1.5364544 .765789$

H -1.1687100 .6943383 .712977$

H $-0.980204-0.0776375 .263995$ 
H -3.8136832 .9498885 .133558$ H -3.7892441 .5949136 .986229$ H -3.656717 -0.1178216 .579973$ H -2.247172 0.7634107 .208980 H -2.136125 3.6131963 .242712 H -2.375382 2.0037022 .564850 H -4.8501682 .4535642 .424141$ H -4.5595774 .0962573 .020660$ H -2.951437 4.4210071 .059287 H -5.3167165 .2988830 .986939$ H -5.8756123 .7410660 .410233$ H $-6.3865195 .909183-1.146103$ H $-5.0338682 .906403-1.946362$ H $-4.3259286 .923844-0.634344$ H -3.728205 $6.398797-2.191808$ H $-2.8651455 .934175-0.712796$ H -4.540109 4.414811 -3.569649 H -3.697324 $1.018462-2.001101$ H -2.307589 $1.739246-2.800354$ H -1.094147 $3.115327-1.021775$ H $-4.8263350 .066722 \quad 1.131594$ H -6.226859 -2.671652 1.298585 H $-6.8453810 .784890-0.067833$ H -7.367767 $0.275305-1.654194$ H $-5.6507510 .226730-1.260388$ H $-0.812856 \quad 0.995787-0.971087$ H -2.182358 $0.417412-0.028116$ H $-3.251606-3.573384-1.419900$ H -2.231354 -3.085939-2.796618 H $-4.317374-1.762478-3.713610$ H $-5.320234-4.357823-2.433807$ H -6.532237 -4.037775 -4.721851 H $-6.542523-2.309353-4.435182$ H -9.619321 -4.531396 -2.794679 H -8.422847 -5.257090 -4.007656 H -9.318905 -2.312309-1.991033 H $-8.101715-1.320126-2.758304$ H -7.541251 -3.297695 -0.432200 H -3.554141 -1.224620 -0.854290 C $1.362575-2.5891541 .457556$ O $1.264077-1.7904300 .261140$ C $2.465661-1.974305-0.522919$ C $3.423343-2.7675280 .354130$ C $2.467763-3.6054611 .176336$ H $2.163514-2.571187-1.392818$ C $2.985790-0.602515-0.975811$ H $4.127133-3.368703-0.229429$ H $4.002292-2.1005581 .003793$ C $3.102910-4.1992702 .421620$ H $2.080774-4.4173960 .546048$ C $4.381112-0.653515-1.629505$ O $2.043234-0.066284-1.905680$ H $3.009970 \quad 0.086567-0.122844$ H $5.079096-1.039737-0.881872$ O $4.373890-1.588744-2.707953$ C $4.9041010 .708160-2.134201$ C $6.3437420 .654286-2.684015$ H $4.8520161 .444683-1.323970$ O $4.0870181 .196281-3.200375$ H $6.392727-0.037366-3.534477$ H $6.5696651 .647574-3.095159$ H $2.379022-4.7970792 .983850$ H $3.940559-4.8509352 .152393$ H $3.484885-3.4197063 .088511$ C $0.016049-3.2396951 .775163$ H $1.170319-0.085616-1.478336$ H $3.752992-1.253217-3.377466$ H $3.1876891 .315241-2.851320$ H $\quad 0.139411-3.9942042 .560754$ H $-0.372973-3.757750 \quad 0.889900$ H $1.661717-1.9129432 .269661$ C 8.2032311 .0185640 .530243 O $7.3405431 .251677-0.589988$ C $7.401990 \quad 0.278258-1.635732$ C $8.8187770 .223446-2.224676$ C $9.857321-0.040446-1.127396$ C 9.6693820 .8886560 .076638 H 8.1201371 .9274391 .140149
C $7.716896-0.1597341 .389893$

H $7.157637-0.708450-1.233780$

O $9.1304621 .442953-2.894210$

H $8.860280-0.573828-2.975721$

H $10.8646710 .103318-1.536989$

O $9.753434-1.390396-0.683343$

H $10.2910470 .550078 \quad 0.913758$

O $10.1427892 .186174-0.298420$

C 6.3852230 .1327122 .053205

H $7.593752-1.0770940 .809134$

H $8.447518-0.3421432 .186769$

H $9.0318062 .167868-2.253491$

H $9.910131-1.964971-1.452025$

H $10.094721 \quad 2.755477 \quad 0.488437$

O $6.136542-0.8266612 .993703$

O 5.6492001 .0707241 .779143

C $4.916754-0.6671273 .725532$

H 4.9522460 .2519944 .317995

H $4.059635-0.6542953 .045652$

H $4.813623-1.5182304 .403819$

SCF Energy (B3LYP/6-31G**//MMFF) $=-3245.92184735$

0800517

MMFF Geometry

C -2.4000352 .9087241 .857304$

C -1.5289361 .9663551 .468537$

C -0.6581391 .1880032 .418174$

O $0.673721 \quad 1.2054581 .855616$

C -1.144878 -0.268514 2.494700

C $-0.616051-1.0179413 .699987$

C $0.378799-1.9255753 .670263$

C $-1.330745-0.7274534 .995591$

C $1.215991-2.3411262 .492324$

C $2.708513-2.2737382 .841485$

C $3.603402-2.6771171 .661755$

C $5.074653-2.6428852 .082450$

C $6.018767-2.9347050 .908490$

O $3.377416-1.7580450 .588043$

O $7.346387-2.557871 \quad 1.305011$

C $5.629783-2.032016-0.279102$

C $6.072124-4.4306850 .577446$

O $6.384813-2.368353-1.441242$

C $4.116969-2.052160-0.604215$

C $3.801369-0.959086-1.649939$

O $3.769866-3.329398-1.141081$

C 1.7245351 .4833952 .670183

O 1.6991381 .6968043 .870698

C 2.9336451 .5024121 .818115

C $4.107977 \quad 1.9243792 .308386$

C 5.3474362 .0076801 .537513

C 6.5658091 .5789752 .306533

C $2.308030-0.748551-1.932774$

C $2.1438320 .356895-2.956824$

C $2.3930752 .827716-3.182575$

O $2.0136330 .114545-4.154329$

C $3.8633043 .100476-3.267039$

C $4.4762314 .100596-2.615908$

C $5.9659484 .292058-2.606448$

C $6.5683384 .077826-1.232193$

C $7.1767315 .080312-0.576546$

C $6.5296792 .678576-0.646698$

C 5.3533232 .4766730 .272890

N $2.2230941 .620710-2.393612$

H -2.4915143 .1494792 .913558$

H $-1.447278 \quad 1.7242680 .412709$

H -0.6699401 .6648333 .405386$

H $-0.880524-0.7934631 .571048$

H -2.242365 -0.299821 2.543103

H $0.632552-2.4308644 .601671$

H $-0.944185-1.3252185 .828016$

H -1.2192470 .3260005 .269594$

H -2.398329 -0.9508424 .898701$

H $\quad 0.951653-3.3708402 .224577$

H $1.019190-1.7191831 .615338$

H $2.953872-1.2511553 .153433$

H $2.916028-2.9306293 .695978$

H $3.318313-3.6856131 .339648$

H $5.260081-3.3424162 .907145$ 
H $5.314799-1.6463632 .478221$

H $7.576714-3.0660632 .101654$

H $5.912188-1.003369-0.014238$

H $6.428990-5.0016531 .443121$

H $6.786575-4.632186-0.228343$

H $5.101835-4.8411450 .290175$

H $7.325448-2.328468-1.197181$

H $4.217130-0.004901-1.302459$

H $4.301434-1.197897-2.597604$

H $2.957845-3.245071-1.664182$

H $2.828663 \quad 1.1779020 .788959$

H $4.1878292 .228838 \quad 3.349757$

H $\quad \begin{array}{llll}\text { H } & \text { H96924 } & 0.605887 & 2.781481\end{array}$

H 6.7974172 .3073003 .091264

H 7.4542261 .4661391 .681502

H $1.836699-1.650644-2.335002$

H $1.766040-0.479950-1.019417$

H $1.8499573 .631151-2.675616$

H $1.9732092 .691014-4.183615$

H $4.4575952 .404984-3.855427$

H $3.8856534 .791423-2.018721$

H $6.1774805 .305627-2.969766$

H $6.4561663 .611687-3.314155$

H 7.6297034 .9223870 .396868

H $7.2324986 .079494-0.995253$

H $7.4809462 .482443-0.142384$

H $6.4718911 .942665-1.458316$

H $4.3961322 .756211-0.164293$

H $2.3869911 .698559-1.394656$

C -4.7590383 .2659331 .125169$

O $-4.975283 \quad 1.9197880 .655600$

C $-6.317588 \quad 1.8225030 .134744$

C $-6.962417 \quad 3.1905340 .342570$

C -5.7568854 .1115560 .341086$

H $-6.2296391 .637558-0.940426$

C -7.0565930 .6635990 .814997$

$\mathrm{H}-7.6844513 .428530-0.444803$

H $-7.489646 \quad 3.2488291 .302114$

C -6.0381575 .4736030 .950391$

H $-5.4110804 .239029-0.693425$

C $-6.382837-0.710906 \quad 0.616281$

$\begin{array}{lllll}\text { O } & -7.117317 & 0.921530 & 2.224708\end{array}$

H -8.0957810 .6260000 .469695$

H -5.376304 -0.696268 1.048226

O $-7.129331-1.6781621 .367724$

C $-6.330133-1.182905-0.851915$

C -5.704308 -2.583157-1.018855

H $-5.777905-0.461705-1.461987$

O $-7.667152-1.223559-1.357512$

H -6.114497 -3.265324 -0.265019

H $-6.010918-2.989098-1.992120$

H -6.8257835 .9893800 .391244$

H -6.3663075 .3874741 .991718$

H -5.1439296 .1040340 .930306$

C $-3.297827 \quad 3.6589260 .912539$

H $-6.203138 \quad 0.9912102 .549616$

H -7.168647 -1.363410 2.287145

H -7.617337 -1.450892 -2.301652

H -3.1575434 .7301931 .093116$

H -3.003641 $3.460755-0.124475$

H -5.0064663 .2796342 .195558$

C -2.283314 -1.865410 -2.318879

O $-3.703114-2.016472-2.189092$

C $-4.169115-2.550358-0.941855$

C $-3.572740-3.942917-0.699188$

C $-2.042786-3.895324-0.762421$

C $-1.550269-3.186138-2.025747$

H -2.124144 -1.633729 -3.380393

C -1.777596 -0.676855 -1.494171

H -3.865575 -1.888192 -0.125891

O $-4.071239-4.853249-1.676349$

H -3.900006 -4.3008400 .284448$

H -1.640813 -4.915653 -0.744662

O $-1.537520-3.2036380 .375269$

H $-0.472729-2.998985-1.955905$

O $-1.733642-4.059649-3.144535$

C $-2.0099440 .616437-2.240788$

H $-2.246268-0.588525-0.511267$
H $-0.697694-0.750954-1.326829$

H -3.695074 -5.728306 -1.480551

H -1.832641 -3.6837611 .167700$

H -2.690044 -4.180945 -3.273869

O $-3.3263870 .965662-2.183059$

O $-1.125575 \quad 1.234253-2.819884$

C $-3.6774562 .145479-2.912619$

H $-4.7439042 .333608-2.766710$

H -3.115138 $3.007213-2.541363$

H -3.492062 $1.995901-3.980472$

SCF Energy $(B 3 L Y P / 6-31 G * * / / M M F F)=-3245.90080543$

08_00518

MM̄FF Geometry

C $1.900573-3.8799261 .110345$

C $0.644773-3.5003970 .830081$

C $0.152512-3.237039-0.570232$

O $-0.643030-2.026753-0.556680$

C $-0.742899-4.389689-1.047093$

C $-1.115019-4.269457-2.511786$

C $-2.309868-3.845725-2.968156$

C $-0.049260-4.709704-3.483126$

C $-3.484969-3.369105-2.160763$

C $-4.029728-2.038168-2.698912$

C $-5.258038-1.573863-1.904377$

C $-5.898352-0.347006-2.554264$

C $-7.0894570 .171035-1.733824$

O $-4.834443-1.250235-0.575942$

O $\quad-7.4285191 .476918-2.224391$

C $-6.6480230 .339432-0.265778$

C $-8.337012-0.700510-1.920147$

$\begin{array}{llll}\text { O } & -7.757060 & 0.671965 & 0.566649\end{array}$

C $-5.903995-0.8901710 .308475$

C $-5.296161-0.5286591 .682672$

O $-6.828307-1.9656850 .480313$

C $0.028833-0.848866-0.451549$

O $1.238955-0.696303-0.410255$

C $-0.9722190 .233812-0.343546$

C $-0.590407 \quad 1.4140270 .165958$

C -1.4436072 .5805620 .394432$

C $-2.8504502 .532708-0.136341$

C $-4.313698-1.5657242 .240243$

C $-3.686519-1.0367373 .514671$

C -2.0085970 .6287754 .316332$

O $-4.110506-1.3565754 .622661$

C -2.4592642 .0513274 .188467$

C -1.620374 3.0983644 .176804

C -2.0704934 .5232434 .016119$

C -1.3189975 .2592032 .925918$

C -0.4567206 .2448853 .227541$

C -1.6161114 .9017211 .482309$

C -0.9250593 .6329621 .060894$

N $-2.651608-0.1466603 .270986$

H $2.587611-4.0602380 .289110$

H $-0.060419-3.3387451 .642144$

H $0.993785-3.101665-1.261768$

H -1.642260 -4.446905 -0.423497

H $-0.229052-5.350029-0.903455$

H -2.471435 -3.842781-4.045605

H $-0.385384-4.652707-4.523911$

H $0.840912-4.079877-3.391450$

H $\quad 0.237226-5.748243-3.287198$

H $-4.270287-4.132293-2.220514$

H -3.233016 -3.250300 -1.103751

H -3.239604 -1.278418 -2.638505

H $-4.298565-2.153650-3.756379$

H -5.975848 -2.401842 -1.865507

H $-6.209145-0.566959-3.583175$

H $-5.1524780 .456777-2.630718$

H -7.642420 $1.395605-3.169790$

H $-5.9665421 .201150-0.230199$

H -8.628737 -0.730450 -2.976911

H $-9.195610-0.280042-1.385025$

H $-8.194283-1.730736-1.587717$

H $-8.184203 \quad 1.4557720 .180705$

H -4.7678030 .4275021 .599401$

H -6.098493 -0.380058 2.417373

H $-6.485972-2.5704241 .157498$ 
H $-1.9914400 .019383-0.642543$ H 0.4462901 .5308070 .479669 H -2.852956 2.277479-1.202206 H $-3.3795453 .484527-0.052675$ H -3.4360971 .7840540 .402462$ H $-4.815607-2.5106732 .472074$ H -3.516352 -1.791654 1.522865 H -0.9277140 .5245624 .180164$ H $-2.2836840 .235858 \quad 5.299693$ H -3.528604 2.227874 4.095451 H -0.5529262 .9253294 .294802$ H -1.927022 5.0268264 .980767 H -3.1455754 .5789713 .804124$ H $0.069607 \quad 6.7919592 .452354$ H -0.2535836 .5240394 .256148$ H -1.270604 5.7041740 .817915 H -2.7018764 .8540321 .360649$ H 0.1217653 .5826561 .365140 H -2.3973990 .0618202 .309860$ C $3.799511-3.4475622 .755345$ O $3.830410-2.0596732 .362304$ C $5.102612-1.7948941 .736737$ C $5.984666-3.0029472 .037317$ C $4.966082-4.1244222 .035089$ H $4.920498-1.7619700 .657286$ C $5.689614-0.4639212 .215408$ H $6.773556-3.1363131 .290604$ H $6.461276-2.9221053 .021501$

C $5.458299-5.3939572 .708187$ H $4.707136-4.3519820 .994293$ C $4.825494 \quad 0.7743361 .904691$ O $5.861139-0.5361453 .637381$ H $6.693581-0.3342531 .793264$ H 3.9090880 .7420322 .506825 O 5.5735051 .9100052 .361965 C 4.4694760 .9632390 .414634 C 3.7671572 .3082850 .140110 H $3.815611 \quad 0.1477270 .089078$ O $5.6568830 .915312-0.376628$ H 3.0006692 .4840520 .904253 H 4.5129723 .1099780 .206878 H $6.341477-5.7846732 .192296$ H $5.732043-5.214758 \quad 3.753374$ H $4.686464-6.1697522 .689651$ C $2.415689-4.0606732 .510915$ H $6.241711 \quad 0.311240 \quad 3.925911$ H 4.9630582 .6644262 .416617 H $5.976925-0.001830-0.383011$ H $1.711306-3.5964523 .213259$ H $2.442771-5.1311482 .743571$ H $3.984908-3.4595923 .838020$ C $3.6146472 .143799-3.605079$ O $4.0983332 .197390-2.255859$ C $3.0988152 .368729-1.249200$ C $2.3570603 .696207-1.469276$ C $1.7728443 .780430-2.883219$ C $2.8069663 .406455-3.951312$ H $4.5209882 .145432-4.224520$ C $2.8576890 .840817-3.889461$ H $2.3857161 .541573-1.308209$ O $3.2358254 .799116-1.253518$ H $1.5536933 .782240-0.731242$ H $1.4153524 .800668-3.068971$ O $\quad 0.6566812 .902235-2.994741$ H $2.3241503 .274127-4.926521$ O $3.7402154 .482805-4.087567$ C $3.772494-0.351508-3.734511$ H $\quad 1.987784 \quad 0.705728-3.240464$ H $2.4792820 .825541-4.918270$ H $3.9981704 .692148-1.847940$ H $-0.0028143 .180964-2.336760$ H $3.2406125 .270363-4.362723$ O $3.418856-1.072096-2.632815$ O $4.700185-0.601999-4.493164$ C $4.221848-2.225399-2.366090$ H $4.185363-2.918889-3.211546$ H $5.252557-1.926093-2.153701$ H $3.813568-2.726491-1.485188$
SCF Energy (B3LYP/6-31G**//MMFF)= -3245.90158038

08_00519

MMFF Geometry

C -3.4363851 .6256702 .317647$

C $-2.871053 \quad 1.283583 \quad 3.485351$

C $-1.4296570 .893265 \quad 3.713925$

O -0.5969941 .2302952 .580533$

C $-1.295569-0.6189513 .965790$

C $-0.022681-0.9572404 .717110$

C $1.093642-1.4765334 .171191$

C $-0.074102-0.701503 \quad 6.204307$

C $1.370307-1.8374172 .737477$

C $1.718932-3.3292002 .629190$

C $2.226464-3.7331111 .236426$

C $2.522560-5.2351881 .205237$

C $3.158633-5.672039-0.121019$

O $3.420390-2.9943820 .972925$

$\begin{array}{llllll}0 & 3.688504 & -6.994983 & 0.055477\end{array}$

C $4.356533-4.747559-0.429121$

C $2.113321-5.770485-1.238719$

O $4.888225-5.021024-1.723393$

C $4.026025-3.242864-0.303947$

C $5.313794-2.393172-0.361408$

O $3.202187-2.801723-1.383040$

C -0.0696942 .4857802 .548428$

$\begin{array}{lllll}\text { O } & -0.232407 & 3.373877 & 3.370109\end{array}$

C 0.7484782 .5983901 .320294

C 1.3793003 .7504881 .045161

C $2.2249154 .019174-0.121592$

C $2.4114932 .899631-1.111769$

C $5.031430-0.888121-0.264070$

C $6.298979-0.054422-0.207542$

C $7.030727 \quad 2.322902-0.118111$

O $7.428485-0.534494-0.203980$

C 6.4173383 .5999640 .357386

C $6.4360664 .746253-0.339051$

C $5.7867196 .012123 \quad 0.144095$

C $4.8136656 .598350-0.858114$

C $4.9636867 .860282-1.295866$

C $3.6701055 .738474-1.363473$

C $2.7705955 .246969-0.258969$

N $6.0031331 .299849-0.141093$

H $-2.8535851 .597003 \quad 1.400892$

H -3.483886 $1.317976 \quad 4.384650$

$\mathrm{H}-1.0802001 .4491814 .592825$

H $-1.338820-1.1616503 .015605$

H -2.143189-0.988054 4.558721

H $1.936575-1.6641954 .836774$

H $0.843120-1.0162446 .713760$

H -0.2140220 .3633446 .412009$

H $-0.904016-1.2557316 .654972$

H $0.530516-1.6074892 .076440$

H $2.213561-1.2246912 .397825$

H $2.497585-3.563883 \quad 3.367950$

H $0.835890-3.9270342 .888262$

H $1.465931-3.471128 \quad 0.494210$

H $1.612299-5.8145461 .404827$

H $3.219038-5.4860952 .017902$

$\begin{array}{llll}\text { H } 2.960173 & -7.575578 & 0.335467\end{array}$

H $5.151417-4.9862520 .291438$

H $1.361835-6.530886-0.994158$

H $2.569454-6.093072-2.180824$

H $1.581952-4.832036-1.412157$

H $5.101975-5.969346-1.754168$

H $5.979268-2.6822920 .462169$

H $5.850411-2.581459-1.299842$

H $3.137084-3.514002-2.039153$

H $0.807321 \quad 1.7293670 .676521$

H 1.2701964 .5817541 .740623

H $1.4447532 .601204-1.531598$

H $2.8662452 .029025-0.628431$

H $3.0479353 .160550-1.959256$

H $4.448245-0.559797-1.132512$

H $4.450929-0.6689740 .639858$

H $7.4423282 .404306-1.129537$

H 7.8339202 .0073310 .555598

H 5.9237973 .5762211 .326602 
H $6.9427814 .780610-1.300446$ H 6.5872656 .7298540 .364806 H 5.2605095 .8464871 .092407 H $4.2775078 .296032-2.014549$ H $5.7759898 .488535-0.945910$ H $4.1003534 .919557-1.946009$ H $3.0465016 .313918-2.059839$ H 2.5358086 .0020980 .492509 H $5.0464161 .590203-0.320069$ C -5.8061671 .1020961 .571299$ O C $-6.4318041 .246171-0.676108$ C $-7.710734 \quad 1.1479110 .140349$ C -7.2355461 .6586111 .486320$ H -6.222005 2.299878 -0.902095 C $-6.4227620 .448703-1.985209$ H $-8.5257451 .743019-0.281814$ H $-8.053748 \quad 0.1115580 .237966$ C $-8.120878 \quad 1.2349072 .646557$ H -7.2123152 .7561951 .450941$ C $-5.0265840 .216180-2.595566$ O $-6.997141-0.844349-1.753071$ H $-7.0835480 .943867-2.706960$ H $-4.530321-0.578283-2.029650$ O $-5.215651-0.307717-3.916548$ C $-4.1098041 .447423-2.666300$ C $-2.7882631 .218275-3.424847$ H $-3.8766711 .789585-1.652162$ O $-4.8145572 .508687-3.314548$ H $-2.9844931 .153923-4.503048$ H $-2.1858452 .126561-3.291761$ H -9.1355711 .6262272 .519773$ H -8.1879890 .1443162 .722644$ H -7.726230 1.6152963 .594146 C -4.8500852 .1216272 .198539$ H -6.992989 -1.320468 -2.601269 H $-5.6008690 .401324-4.459698$ H $-4.2381023 .291745-3.305473$ H -5.2301632 .4195093 .183646$ H -4.8189053 .0303511 .583564$ H -5.7762010 .1565082 .125825$ C -1.110010-1.056103-0.964325 O $-1.7825980 .063721-1.558091$ C -1.986411-0.015121 -2.972003 C $-0.641575-0.127098-3.706024$ C $0.167957-1.315715-3.181723$ C $0.239567-1.325522-1.652531$ H $-0.902195-0.7428530 .066362$ C -2.003815 -2.307035 -0.909939 H -2.581841 -0.903799 -3.202197 O $0.102015 \quad 1.076211-3.529469$ H - $0.832553-0.244936-4.779519$ H $1.182868-1.271062-3.595429$ O $-0.438012-2.518557-3.646816$ H $\quad 0.635810-2.285346-1.308835$ O $1.170626-0.320616-1.248031$ C -3.164695 -2.145232 0.047588 H -2.412749 -2.547460 -1.894631 H $-1.423731-3.167484-0.556382$ H $\quad 0.938980 \quad 0.978983-4.014453$ H $\quad 0.105340-3.261289-3.332681$ H $1.310687-0.420011-0.290816$ O $-4.122042-3.073468-0.247373$ O $\quad-3.215540-1.3333170 .960121$ C $-5.276050-3.0566310 .598812$ H $-5.975395-3.8139220 .235088$ H $-4.993033-3.3032181 .626504$ H $-5.765830-2.079808 \quad 0.557877$

SCF Energy (B3LYP/6-31G**//MMFF) $=-3245.91412613$

0800520

MM̄FF Geometry

C $1.001740 \quad 4.5964220 .159965$

C 2.1385573 .8852040 .169882

C $3.0054083 .676009-1.046454$

O $3.1702602 .249621-1.238740$

C $4.3865724 .309829-0.806368$

C $5.3091304 .247596-2.009117$
C $6.4212963 .489917-2.092313$

C $4.9323465 .149938-3.157105$

C $6.9713112 .543310-1.062834$

C $6.8509241 .092084-1.540337$

C $7.0965040 .087780-0.404938$

C $7.072548-1.339497-0.956692$

C $7.152251-2.3925740 .155335$

O 6.0528850 .2757530 .554897

O $6.816472-3.665680-0.417858$

C $6.072971-2.0739981 .207968$

C $8.571069-2.5287540 .718062$

O $6.177165-2.9518182 .326792$

C $6.086842-0.6039731 .683921$

C $4.851000-0.2814302 .563842$

O $7.258523-0.3853052 .475098$

C $2.1311351 .586778-1.821592$

O $1.1016212 .076268-2.259578$

C $2.4405680 .139674-1.805911$

C $1.440917-0.745981-1.942360$

C $1.561588-2.207896-1.948643$

C $2.937451-2.791010-1.765594$

C $3.545510-0.1269271 .777171$

C $2.380203-0.8458302 .421620$

C $1.400495-3.1260632 .671106$

O $1.518491-0.2464173 .058912$

C $1.215847-4.2041431 .650487$

C $0.048764-4.4560201 .040389$

C $-0.147793-5.5473560 .030116$

C $-0.671979-5.036886-1.292831$

C $-1.962689-5.196214-1.632371$

C $0.319919-4.440836-2.267992$

C $0.447274-2.947118-2.137607$

N $2.409453-2.2089432 .182133$

H $0.6878835 .082783-0.759743$

H 2.4538473 .3969941 .089422

H $2.5442024 .113356-1.940438$

H 4.8475723 .8372180 .066085

H $4.2646385 .366945-0.532509$

H $7.0106573 .542646-3.007007$

H $5.6874685 .151045-3.950497$

H $3.9868634 .830902-3.605807$

H $4.8235676 .182353-2.809123$

H $8.0283072 .784362-0.895974$

H $6.4830852 .667738-0.094575$

H $5.8424890 .930873-1.944208$

H $7.5617420 .917994-2.357685$

H 8.0622620 .3061300 .065709

H $7.879953-1.487226-1.684936$

H $6.137922-1.498813-1.513463$

H $7.444528-3.844958-1.138521$

H $5.105181-2.2852020 .741512$

H $9.268100-2.834322-0.071436$

H $8.619604-3.3138191 .480727$

H $8.951705-1.6015411 .151234$

H $\quad 6.159532-3.8617671 .984100$

H $4.761926-1.0171843 .372632$

H 5.0259480 .6752693 .075839

H 7.1137020 .3961383 .033488

H $3.474343-0.153524-1.666931$

H $\quad 0.427600-0.364969-2.065841$

H $2.953278-3.882070-1.738951$

H $3.369998-2.455650-0.818580$

H $3.599232-2.481530-2.582055$

H 3.2840910 .9341261 .686950

H $3.639351-0.5042160 .755120$

H $0.473578-2.5825262 .881340$

H $1.769537-3.5542113 .608234$

H $2.085305-4.8108581 .407524$

H $-0.823652-3.8644901 .303540$

H $-0.852054-6.2722830 .458931$

H $0.780350-6.107178-0.140168$

H -2.338237 -4.863258 -2.594311

H $-2.674019-5.653726-0.952726$

H $-0.014330-4.648399-3.293934$

H $1.274415-4.964944-2.176015$

H $-0.499305-2.421528-2.272670$

H $3.245714-2.6110341 .771269$

C $-1.1975413 .962807 \quad 1.261922$ 
$\begin{array}{llll}\text { O } & -0.914768 & 2.553080 & 1.145667\end{array}$ C - $-1.788817 \quad 1.990603 \quad 0.143272$ C $-2.887131 \quad 3.021587-0.069887$ C $-2.119086 \quad 4.317378 \quad 0.094934$ H $-1.1807991 .891989-0.764109$ C -2.266447 0.6101930 .607498 H -3.366583 2.932429 -1.049290 H -3.660401 2.9313810 .701561 C $-3.003025 \quad 5.524756 \quad 0.353266$ H $-1.5452734 .489991-0.823116$ C -3.346816 $0.001113-0.308793$ $\begin{array}{llll}\text { O } & -1.125332 & -0.250892 & 0.647178\end{array}$ H -2.649911 0.6713211 .633261 H $-4.200797 \quad 0.684245-0.311851$ O $-2.859901-0.057966-1.649595$ C $-3.841122-1.3954050 .119540$ C $-4.954906-1.966939-0.779422$ H $-4.194266-1.3565931 .156467$ O $-2.771939-2.3398320 .073815$ H -4.578013 -2.098905 -1.801695 H $-5.175162-2.978795-0.412901$ H $-3.6895985 .685376-0.484365$ H $-3.603677 \quad 5.3970641 .259631$ H $-2.3988196 .429486 \quad 0.473874$ C $0.110461 \quad 4.754321 \quad 1.360340$ H -0.4662620 .1650801 .229525$ H -2.098353 $-0.663090-1.653514$ H -2.079558 -2.028912 0.680559 H 0.6493354 .4234612 .257731 H $-0.106428 \quad 5.819677 \quad 1.498084$ H -1.7383774 .0828452 .209915$ C -7.890801 -0.2345510 .742217$ O $-6.723124-1.0421430 .547019$ C $-6.244749-1.131902-0.797600$ C $-7.320289-1.740036-1.709440$ C $-8.626081-0.942120-1.614897$ C $-9.045128-0.707229-0.159637$ H -8.187639 -0.4182001 .783257$ C $-7.576065 \quad 1.2641640 .605460$ H -6.003700 -0.131733 -1.168142 O $-7.556984-3.095435-1.336720$ H -6.949525 -1.740791-2.741353 H -9.424872 -1.482670 -2.136903 O $-8.466172 \quad 0.320143-2.255906$ H $-9.8666740 .017028-0.108570$ O $-9.537149-1.934910 \quad 0.386428$ C -6.6878841 .7619791 .728656$ H $-7.0723511 .503393-0.334123$ H $-8.514936 \quad 1.8285030 .656198$ H -8.223964 -3.455459 -1.945726 H $-8.2244770 .150677-3.182524$ H -10.299767 -2.209880 -0.150571 O $-6.715866 \quad 3.1278931 .745171$ O $-6.041524 \quad 1.0515622 .486278$ C -5.9458493 .7484652 .780000$ H -6.3878773 .5274763 .756019$ H -4.9053813 .4123102 .744493$ H -5.969622 4.829545 2.619418

SCF Energy (B3LYP/6-31G**//MMFF) $=-3245.90120881$

$08 \_00521$

MM̄FF Geometry

C $1.616042 \quad 5.103226-0.366397$

C $2.771743 \quad 4.451840-0.166543$

C $3.289596 \quad 3.896332 \quad 1.140891$

$\begin{array}{lllll}\text { O } & 3.436601 & 2.463335 & 0.970156\end{array}$

C $2.373696 \quad 4.107136 \quad 2.361224$

C 2.9816833 .6031043 .655389

C 2.6220622 .4604444 .272879

C 4.0131664 .5081714 .277999

C 1.6262641 .4354733 .797635

C $2.095507 \quad 0.0126594 .136321$

C $1.065332-1.0499643 .725012$

C $1.448587-2.4124754 .307608$

C $0.487701-3.5193873 .853924$

O $1.034612-1.1207972 .295195$

O $1.083764-4.7836734 .181394$

C $0.361113-3.4628422 .319676$
C $-0.849800-3.4626704 .601508$

O $-0.630018-4.378967 \quad 1.858166$

C $0.069996-2.0455791 .770560$

C $0.207348-2.0609130 .230750$

O $-1.262580-1.671178 \quad 2.121630$

C $4.6280991 .990788 \quad 0.519193$

$\begin{array}{llll}\text { O } & 5.613783 & 2.642578 & 0.215079\end{array}$

C 4.5142260 .5176510 .439100

C $5.434870-0.175846-0.246289$

C $5.443664-1.624578-0.459064$

C $4.451163-2.4494630 .315618$

C $0.096659-0.685935-0.433436$

C $0.438882-0.803526-1.905409$

C $2.385849-0.880685-3.463739$

O $-0.423564-1.032073-2.750628$

C $3.065485-2.215050-3.472243$

C $4.318781-2.408227-3.911441$

C $5.003399-3.746251-3.911427$

C $6.383694-3.706407-3.289165$

C $7.485372-3.857291-4.043888$

C $6.486511-3.567448-1.783455$

C $6.330507-2.137899-1.337069$

N $1.795737-0.672146-2.154103$

H $0.951700 \quad 5.3042380 .468852$

H $3.4092094 .269766-1.030365$

H $4.2666724 .353287 \quad 1.340703$

H 1.4068263 .6191112 .188756

H 2.1514995 .1750262 .486219

H 3.0910882 .2335795 .229727

H 4.3779484 .1256795 .237216

H 4.8790974 .6159893 .617672

H 3.5877375 .5004584 .460164

H 0.6704811 .6281204 .299091

H 1.4551541 .5080312 .719538

H $3.048942-0.1796153 .627031$

H $2.277659-0.0576935 .216412$

H $0.079644-0.7423114 .093605$

H $1.491502-2.3702505 .403255$

H $2.463473-2.6768623 .978114$

H $1.237330-4.8027385 .141604$

H $1.318005-3.8051841 .900606$

H $-0.689889-3.5733745 .680723$

H -1.501187 -4.293717 4.308910

H -1.391454 -2.527928 4.443033

H $-0.399712-5.2560682 .209740$

H $1.178021-2.494306-0.036457$

H $-0.558719-2.713408-0.206809$

H $-1.585494-1.0169321 .483636$

H 3.6607890 .0495250 .916811

H $6.2448120 .371198-0.727691$

H $4.518858-2.2305891 .387508$

H $4.605248-3.525717 \quad 0.216911$

H $3.433875-2.234998-0.020394$

H $-0.919487-0.287589-0.352941$

H $\quad 0.7655250 .0445620 .035127$

H $3.087184-0.058800-3.638565$

H $1.607543-0.853197-4.232489$

H $2.496651-3.065395-3.102287$

H $4.878599-1.563975-4.307865$

H $5.070033-4.083156-4.954020$

H $4.401140-4.500537-3.389504$

H $8.476778-3.854253-3.603060$

H $7.421771-3.982119-5.119806$

H $7.469906-3.914018-1.439961$

H $5.761557-4.244110-1.323870$

H $7.026598-1.453269-1.824812$

H $2.427785-0.515551-1.375112$

C $-0.1074264 .791985-2.204410$

O $-1.1762404 .894243-1.235557$

C -1.502481 $3.561442-0.795458$

C $-1.1837622 .692645-1.998254$

C $0.129636 \quad 3.295634-2.452802$

H -0.8242023 .3379990 .038315$

C $-2.957308 \quad 3.486214-0.318702$

H -1.102698 1.633323 -1.755497

H $-1.9465102 .813877-2.775477$

C $0.4851812 .965372-3.892625$

H $0.9265272 .908198-1.807254$ 
C -3.3075752 .0938940 .251802$ O H -3.637460 $3.742057-1.139294$ H $-3.0455181 .341671-0.498225$ O $-2.477914 \quad 1.8611201 .392770$ C $-4.794661 \quad 1.909160 \quad 0.623803$ C $-5.1240620 .568795 \quad 1.313114$ H $-5.3955642 .012291-0.287565$ O $\quad-5.2044932 .9509211 .511632$ H -4.6499310 .5397972 .302379$ H $-6.2053550 .563187 \quad 1.507264$ H $0.5943901 .883995-4.024100$ H $-0.288136 \quad 3.310825-4.586964$ H $1.4305383 .439072-4.175570$ C $1.1252595 .565474-1.712498$ H $-2.887115 \quad 5.3337420 .314938$ H -2.5543320 .9241181 .634928$ H $-6.1606302 .853672 \quad 1.659641$ H $0.8635996 .626118-1.612610$ H $1.9214625 .501429-2.463386$ H $-0.4784805 .277030-3.115585$ C $-5.265619-1.768539-1.600172$ O $-5.515504-0.682343-0.695996$ C $-4.742820-0.6836780 .508462$ C $-4.984464-1.9723571 .309745$ C $-4.715187-3.2094150 .447429$ C $-5.440731-3.126329-0.898172$ H $-6.055960-1.686289-2.357932$ C $-3.912369-1.614886-2.310169$ H $-3.680289-0.630578 \quad 0.258185$ O $-6.328175-1.9985951 .785233$ H -4.324984 -1.971336 2.185882 H $-5.045498-4.1088320 .980792$ O $-3.317084-3.334150 \quad 0.206396$ H -5.103342 -3.927453 -1.566185 O $-6.842016-3.323127-0.688407$ C $-4.009122-0.626837-3.449786$ H $-3.099637-1.291976-1.655210$ H $-3.606879-2.570250-2.753932$ H -6.440578 -2.813486 2.303583 H $-2.873960-3.4081181 .068907$ H -6.958030 -4.201653 -0.288039 O $-4.215108 \quad 0.631895-2.969799$ O $-3.911242-0.941066-4.629226$ C $-4.3704881 .650116-3.962911$ H $-5.2402371 .436357-4.591321$ H -4.534244 $2.601836-3.450778$ H -3.464199 $1.728654-4.570798$

SCF Energy (B3LYP/6-31G**//MMFF) $=-3245.89202086$

0800522

MMFF Geometry

C $-1.7575194 .367617-1.789878$

C $-0.6260433 .836205-1.301964$

C $0.5096263 .385431-2.187821$

O $0.7406521 .987467-1.897809$

C $1.7827284 .189876-1.867744$

C $2.8333224 .118490-2.958763$

C $4.0019943 .453274-2.875625$

C $2.5210744 .918646-4.200069$

C $4.5318332 .620500-1.742282$

C $4.8019791 .183931-2.204544$

C $5.5061150 .342093-1.131932$

C $5.692686-1.094862-1.625903$

C $6.320528-1.995531-0.553133$

$\begin{array}{llll}\text { O } & 4.713477 & 0.351022 & 0.059697\end{array}$

O $6.149090-3.359912-0.966165$

C $5.538464-1.8212520 .764586$

C $7.831249-1.766152-0.428625$

O $6.175333-2.5240801 .829571$

C $5.314549-0.342371 \quad 1.162549$

C $4.346588-0.2720532 .363787$

O $6.565605 \quad 0.236637 \quad 1.536626$

C $0.9452251 .141489-2.939575$

O $0.9957001 .441312-4.125212$

C $1.093580-0.264187-2.491511$

C $1.084297-0.648300-1.205899$

C $1.201369-2.023348-0.709756$
C $1.341227-3.127686-1.722922$

C 3.9114671 .1445552 .763548

C 2.9145681 .0516253 .901619

C 0.5699470 .3346334 .342926

O $3.263724 \quad 1.1370545 .076565$

C $0.592552-1.1631904 .325040$

C $-0.302410-1.9114783 .663483$

C $-0.215679-3.4042253 .537061$

C $-0.054143-3.8466072 .097120$

C $-1.011813-4.5558231 .477388$

C $1.245241-3.5169541 .391962$

C $1.161945-2.2248340 .623529$

N 1.6266160 .7928883 .459144

H -1.850940 $4.459094-2.871303$

H $-0.4872613 .723354-0.230797$

H $\quad 0.2191683 .514369-3.236841$

H $2.1912593 .859290-0.907099$

H $1.5305675 .250475-1.729451$

H $4.6791043 .514252-3.727625$

H $3.3489004 .915314-4.917422$

H $1.6437044 .514165-4.713131$

H $2.3213545 .962994-3.938390$

H $5.4666983 .076229-1.394618$

H $3.8509472 .606836-0.887636$

H $3.845838 \quad 0.717907-2.465881$

H $5.4156551 .189606-3.114541$

H $6.4771730 .805480-0.920672$

H $6.292070-1.116494-2.544543$

H $4.713986-1.515143-1.897875$

H $6.573799-3.465781-1.834707$

H $4.555641-2.2934860 .626478$

H $8.330532-1.979886-1.381403$

H $8.276301-2.4507180 .301731$

H $8.087875-0.743362-0.145272$

H $\quad 6.280782-3.4479741 .544528$

H $3.445354-0.8498692 .125759$

H $4.807085-0.7482843 .239179$

H 6.4019391 .0162102 .089441

H $1.208601-0.981739-3.297862$

H $0.9707080 .110062-0.433078$

H $1.387491-4.124702-1.280872$

H $2.258792-2.997279-2.307411$

H $0.486367-3.134479-2.406849$

H 4.7568691 .7499443 .105206

H 3.4526421 .6738681 .920883

H $-0.373004 \quad 0.741076 \quad 3.966617$

H $\quad 0.734307 \quad 0.7050635 .359204$

H $1.417112-1.6488594 .841647$

H -1.115382 -1.424235 3.133531

H -1.126487 -3.832809 3.973882

H $0.618744-3.8051994 .125964$

H -0.893407 -4.889111 0.451552

H - $1.937846-4.8107251 .980572$

H $\quad 1.540397-4.3568190 .756080$

H $2.053647-3.4328972 .129621$

H $1.065465-1.3478731 .261757$

H 1.4688910 .6876902 .460990

C -2.8961604 .8728180 .500826$

O -2.9147453 .5124790 .978839$

C $-4.0862543 .324131 \quad 1.789078$

C -4.4059674 .7076872 .332095$

C -4.1249515 .5632731 .112812$

H -4.8913123 .0094581 .112302$

C -3.8660952 .2352012 .844400$

H -5.4370224 .7913552 .687824$

H -3.7337114 .9910143 .150028$

C -3.9002717 .0308201 .436863$

H $-4.9811545 .484348 \quad 0.428992$

C -3.2381090 .9339042 .305289$

O $-2.9857332 .747558 \quad 3.852908$

H -4.8189802 .0252683 .345308$

H -2.163424 1.0842382 .141100

O $-3.361741-0.065833 \quad 3.324189$

C -3.8699240 .3848641 .013823$

C $-3.177309-0.9055820 .547889$

H $-3.783720 \quad 1.1440690 .232409$

$\begin{array}{llll}\text { O } & -5.255941 & 0.143635 & 1.238627\end{array}$

H $-2.091446-0.7482570 .506014$ 
H $-3.331033-1.6986281 .292448$ H -4.791435 7.4626461 .903938 H -3.060364 7.1653452 .126734 H -3.684154 7.6005190 .527411 C $-2.9521164 .881775-1.032115$ H -3.0124612 .1355634 .607324$ H -2.8164050 .2113704 .079151$ H $-5.665259-0.0585660 .380741$ H -3.124684 $5.910836-1.371381$ H -3.812940 4.284770 -1.361009 H -1.9683945 .3324520 .861493$ C $-3.137411-3.326313-2.276300$ O $-2.842713-2.604826-1.074476$ C $-3.662606-1.457177-0.808464$ C $-3.570521-0.446121-1.963369$ C $-3.862624-1.101581-3.315891$ C $-3.075758-2.401686-3.502537$ H $-2.324287-4.058379-2.367365$ C $-4.456016-4.099028-2.168951$ H $-4.699331-1.789151-0.690051$ O $-2.2689330 .133915-1.981746$ H $-4.2908810 .361989-1.792972$ H -3.605858 $-0.398157-4.117288$ O $-5.260992-1.360538-3.401210$ H $-3.432425-2.945273-4.385122$ O $-1.701652-2.084812-3.736218$ C $-4.344642-5.216340-1.159492$ H $-5.307596-3.469201-1.896352$ H $-4.713610-4.562591-3.129016$ H $-2.2544440 .804120-2.685868$ H $-5.434009-1.753796-4.273548$ H -1.658635 -1.528953 -4.532797 O $-4.837048-4.808848 \quad 0.044585$ O $-3.866353-6.314763-1.411718$ C $-4.815790-5.788421 \quad 1.087672$ H $-5.587352-6.5408870 .900894$ H -3.831653 -6.259912 1.165172 H $-5.035737-5.2835312 .032022$

SCF Energy $\left(B 3 L Y P / 6-31 G^{* *} / / M M F F\right)=-3245.88924374$

\section{3}

MM̄FF Geometry

C $-5.8257362 .122413-0.036927$

C $-4.9023753 .089510 \quad 0.075132$

C -4.0541503 .4138521 .282164$

O $-2.678704 \quad 3.2134980 .864004$

C -4.2950952 .5283012 .519330$

C -3.4552592 .9350753 .713969$

C -2.3300232 .3050504 .104698$

C -3.9818764 .1055934 .502515$

C -1.6423261 .1506843 .430143$

C $-1.728170-0.1283814 .273537$

C -1.031675 -1.312489 3.585788

C -1.124205 -2.578637 4.439905

C $-0.331524-3.746353 \quad 3.827973$

O $0.339969-0.9607253 .392225$

O $-0.192603-4.7610524 .834258$

C $1.092875-3.261563 \quad 3.477128$

C -1.084415 -4.395309 2.659700

O $1.814245-4.2657652 .767121$

C $1.109145-1.9386492 .681224$

C $2.553168-1.3963882 .555466$

O $0.582145-2.1791331 .376979$

C - $-1.970886 \quad 4.290517 \quad 0.430637$

O $-2.329215 \quad 5.456794 \quad 0.424071$

C $-0.6768513 .785069-0.083122$

C $0.2014364 .632832-0.640683$

C $1.4913484 .270743-1.237040$

C $1.8533162 .808705-1.256110$

C 2.6162770 .0273441 .987062

C 4.0285040 .5828831 .931059

C 5.2239512 .7412011 .590239

O $5.034961-0.0982962 .099351$

C 4.8685364 .1053391 .093613

C 5.4176574 .6866040 .016852

C $5.0124826 .050180-0.469056$

C $4.6422556 .076537-1.937474$

C $5.2393586 .943842-2.773011$
C $3.5959005 .108309-2.454334$

C $2.2635725 .246701-1.761985$

N $4.016101 \quad 1.9399081 .638774$

H -6.0680311 .4894900 .811402$

H $-4.6878903 .691791-0.806813$

H -4.2257234 .4638121 .548841$

H $-4.104498 \quad 1.4778642 .270074$

H -5.3516862 .5750162 .814351$

H -1.818606 2.6711924 .994183

H -3.361005 4.331792 5.376035

H -4.0135515 .0051253 .879980$

H -4.9939743 .8957974 .863690$

H -2.0426850 .9597942 .430088$

H -0.5926961 .4335533 .282936$

H -1.255204 0.0597075 .246435

H $-2.779248-0.3800424 .462031$

H -1.506768 -1.471914 2.610390

H -2.171349 -2.865277 4.598570

H $-0.715872-2.3730145 .439407$

H -1.084440 -5.024641 5.119037

H $1.629430-3.1036964 .423166$

H -2.049307 -4.790935 2.999133

H $-0.532677-5.2540972 .261969$

H -1.284353 -3.7028691 .839325$

H $1.782642-5.0778583 .301222$

H $3.022336-1.3795773 .547818$

H $3.151087-2.0631981 .923570$

H $0.960922-1.5441930 .749990$

H $-0.4964002 .717939-0.013763$

H $-0.0499735 .691899-0.679750$

H $1.8342442 .391025-0.244946$

H $2.8486522 .606663-1.653742$

H $1.1434122 .249910-1.876242$

H 2.2142710 .0567800 .969059

H 2.0153190 .7013792 .608673

H 5.9571332 .2330640 .955206

H 5.6305172 .7995232 .604966

H 4.1078744 .6457761 .653524

H $6.1929514 .164651-0.538369$

H $5.8509466 .731268-0.274574$

H $4.166277 \quad 6.4376090 .112238$

H $4.9905846 .976570-3.828599$

H $5.9911797 .642906-2.421870$

H $4.0060544 .098141-2.384408$

H $3.4205325 .281684-3.524386$

H $1.893036 \quad 6.272162-1.727411$

H 3.1243132 .4257851 .677532

C $-6.0733850 .409324-1.818012$

O $-4.663886 \quad 0.436915-2.115911$

C $-4.404826-0.429497-3.235001$

C $-5.737970-1.086374-3.586104$

C $-6.736902-0.046381-3.112536$

H $-4.100290 \quad 0.215623-4.068622$

C -3.266222 -1.410485 -2.909784

H $-5.820082-1.302732-4.655758$

H $-5.884833-2.028357-3.046862$

C -8.142731 -0.591459-2.934298

H $-6.7559760 .778771-3.836970$

C $-1.895549-0.708816-2.753927$

O $-3.572555-2.124689-1.713550$

H $-3.182226-2.143041-3.722147$

H -1.694029 -0.155201-3.678822

O $-1.9358220 .252472-1.700061$

C $-0.727549-1.686573-2.505229$

C $0.636245-0.966186-2.532141$

H $-0.747992-2.462308-3.279767$

O $-0.909911-2.323068-1.244066$

H $0.686631-0.333319-3.426028$

H $0.714638-0.323871-1.645940$

H $-8.529118-0.976094-3.883922$

H $-8.167169-1.408644-2.205700$

H $-8.8237800 .191069-2.585681$

C $-6.5199331 .787666-1.329622$

H -4.286072 -2.753035 -1.911801

H $-2.149193-0.225589-0.880259$

H $-0.172802-2.942615-1.112810$

H -7.602110 $1.805589-1.161537$

H $-6.2911472 .544555-2.089863$ 
H $-6.220100-0.339708-1.027716$

C $2.937981-3.548099-1.097184$

O $1.915005-2.556628-1.255744$

C $1.824783-1.949471-2.546969$

C $3.146357-1.246824-2.891080$

C $4.330606-2.212306-2.788203$

C $4.321019-2.985442-1.464937$

H $2.944040-3.770876-0.022447$

C $2.589722-4.843181-1.837794$

H $1.619341-2.719970-3.297986$

O $3.359205-0.136734-2.022492$

H $3.082461-0.849996-3.910932$

H $5.263113-1.641440-2.876129$

O $4.271671-3.123008-3.882110$

H $5.052368-3.801591-1.486506$

O $4.714760-2.100535-0.412617$

C $1.375799-5.493887-1.216916$

H $2.402007-4.692475-2.904673$

H $3.411272-5.566093-1.768757$

H $3.357809-0.468784-1.108649$

H $5.044473-3.709633-3.817223$

H $5.612269-1.784784-0.613466$

O $0.267407-5.221754-1.962569$

O $1.406893-6.143384-0.179683$

C $-0.960129-5.760189-1.461568$

H $-1.163802-5.373000-0.458853$

H $-1.766515-5.444087-2.128581$

H $-0.917375-6.853321-1.453156$

SCF Energy (B3LYP/6-31G**//MMFF) $=-3245.91313088$

0800524

MMFF Geometry

C -2.924832 4.0995490 .849395

C $-3.880058 \quad 3.168506 \quad 0.982224$

C -4.1695192 .4245102 .259809$

O -3.7283651 .0599092 .061381$

C -5.6846322 .4266702 .530469$

C -6.0989121 .5730123 .713565$

C -6.7432210 .3915713 .619428$

C $-5.756712 \quad 2.1409545 .066408$

C $-7.159722-0.327860 \quad 2.365448$

C $-6.293189-1.5708352 .126095$

C $-6.318926-2.0185890 .656507$

C $-5.548453-3.328094 \quad 0.485022$

C $-5.413375-3.736722-0.988723$

O $-5.702906-0.981864-0.115911$

O $-4.415475-4.767318-1.067299$

C -4.885587-2.533988-1.801724

C $-6.711451-4.349550-1.528508$

O $-4.895031-2.853080-3.191327$

C $-5.658260-1.225284-1.529003$

C $-5.0105720 .021435-2.184222$

O $-6.979714-1.343824-2.069685$

C -2.413651 0.7949352 .306133

O -1.5790331 .5651702 .754182$

C $-2.146473-0.5989351 .887146$

C $-0.885365-1.0560911 .859326$

C $-0.459438-2.3904371 .429303$

C $-1.519406-3.3239910 .906827$

C $-3.594070 \quad 0.359185-1.707687$

C $-2.481019-0.223122-2.560489$

C $0.001067-0.476398-2.594023$

O $-2.672173-0.737772-3.658597$

C $0.303016-1.892345-2.215695$

C $1.493335-2.297386-1.746319$

C $1.813669-3.728608-1.418143$

C $2.432709-3.897650-0.048187$

C $3.761643-4.0348490 .092648$

C $1.509256-4.0118901 .143841$

C $0.850320-2.7087171 .501369$

N $-1.237003-0.058057-1.963226$

H -2.322764 4.3829341 .708537

H -4.4490602 .8714280 .103869$

H -3.644384 $2.870223 \quad 3.113742$

H $-6.2118862 .109348 \quad 1.625713$

H -6.0233583 .4566712 .707343$

H -6.993430 -0.1336214 .540257$

H $-6.135271 \quad 1.5204805 .885657$
H -4.6719842 .2193495 .187698$

H $-6.1939603 .138197 \quad 5.180987$

H $-8.208212-0.6334082 .470027$

H -7.1369460 .3308531 .495293$

H -5.256916 -1.3523942 .411002$

H $-6.642662-2.3833872 .775138$

H -7.361692 -2.137945 0.339855

H $-6.016679-4.1325061 .065999$

H $-4.540322-3.2109280 .902957$

H $-4.700924-5.505151-0.501549$

H -3.835442 -2.401960 - 1.515618

H $-6.980695-5.243556-0.953172$

H $-6.589668-4.686621-2.563725$

H $-7.559937-3.663539-1.484638$

H $-4.403713-2.158309-3.660477$

H $-5.064608-0.048946-3.277025$

H $-5.6395240 .884700-1.921594$

H $-7.340190-0.451803-2.204471$

H $-2.992498-1.2027761 .582286$

H $-0.082891-0.3899292 .173711$

H -2.216850 -3.5953391 .706318$

H $-1.123504-4.2540800 .494776$

H -2.079111 - 2.8506750 .093226

H $-3.468226 \quad 1.447751-1.758653$

H $-3.4505530 .061714-0.666099$

H $0.783607 \quad 0.216391-2.268041$

H $-0.105757-0.399959-3.680914$

H $-0.485497-2.626617-2.364637$

H $2.293502-1.569914-1.627813$

H $2.507435-4.095772-2.186064$

H $0.924344-4.366481-1.494615$

H $4.214860-4.1935961 .065480$

H $4.427424-3.989837-0.762884$

H $2.072029-4.3405222 .028142$

H $0.791265-4.8156870 .959657$

H $1.546941-1.9610161 .883108$

H $-1.1820430 .332174-1.026876$

C $-1.3601204 .041409-1.085653$

O $-0.181948 \quad 4.361866-0.321473$

C $0.9335984 .511406-1.228003$

C $0.420328 \quad 4.102599-2.601732$

C $-1.044503 \quad 4.482906-2.511188$

H $1.1825125 .579845-1.217556$

C $2.1085863 .678636-0.700909$

H $0.9469034 .611083-3.415019$

H $0.5253883 .020691-2.752477$

C $-1.904417 \quad 3.822833-3.574957$

H $-1.1340085 .574192-2.595600$

C $3.3051313 .580499-1.663646$

$\begin{array}{lllll}\text { O } & 2.539011 & 4.264290 & 0.533281\end{array}$

H $1.7461772 .674935-0.451867$

H $3.0118603 .027602-2.564106$

O $3.6412784 .907160-2.088120$

C $4.5686512 .943889-1.050200$

C $4.3162861 .567855-0.421648$

H $5.0046543 .627799-0.313797$

O $5.5391662 .780156-2.088975$

H $3.580647 \quad 1.6356090 .387957$

H $3.8620790 .906900-1.173070$

H $-1.5826534 .132476-4.574655$

H $-1.8371262 .731327-3.525404$

H -2.956336 $4.100994-3.458405$

C $-2.579773 \quad 4.729606-0.471732$

H 2.8968025 .1458030 .331116

H $4.2519624 .828685-2.840374$

H $5.8382023 .666034-2.354281$

H $-3.4360724 .663935-1.152815$

H $-2.3789595 .793961-0.301683$

H $-1.4771662 .950175-1.046262$

C $6.185312-1.264378 \quad 1.092338$

$\begin{array}{llll}\text { O } & 5.138687 & -0.387902 & 0.652357\end{array}$

C 5.5805290 .8643120 .108597

C 6.3453951 .6585411 .180516

C $7.5060700 .840321 \quad 1.754229$

C $7.077582-0.5803292 .142028$

H $5.655651-2.0855151 .590938$

C $6.936737-1.847942-0.113653$

H $6.2402580 .660968-0.742909$ 
O $5.4775812 .018842 \quad 2.253289$

H 6.7447032 .5825240 .747150

H $7.901644 \quad 1.3581632 .636834$

O $8.548020 \quad 0.790594 \quad 0.783995$

H $7.955074-1.2044352 .344116$

O $6.323761-0.5174693 .357580$

C $7.622165-3.1613270 .194049$

H $\quad 6.220430-2.033317-0.923561$

H $7.686713-1.150005-0.494818$

H $4.8550512 .687617 \quad 1.922535$

H $9.2894470 .297426 \quad 1.174927$

H $6.898306-0.1299204 .039542$

O $8.233516-3.615377-0.940471$

O $7.623990-3.7226411 .280740$

C $8.926025-4.860783-0.809494$

H $9.729620-4.771794-0.072061$

H $8.227750-5.654540-0.527296$

H $9.365789-5.108885-1.779101$

SCF Energy (B3LYP/6-31G**//MMFF) $=-3245.89877961$

\section{5}

MM̄FF Geometry

C $-3.4911101 .654150 \quad 0.005167$

C $-4.6650631 .135156 \quad 0.398939$

C $-4.876187-0.1966631 .081813$

O $-3.634190-0.912088 \quad 1.264421$

C $-5.819061-1.0905750 .254323$

C $-6.069114-2.442648 \quad 0.898222$

C $-5.715685-3.624728 \quad 0.355690$

C $-6.770839-2.4133332 .232232$

C $-5.082138-3.868521-0.985164$

C $-3.552873-3.963044-0.919259$

C $-2.825791-2.825436-1.655485$

C $-3.060088-2.875927-3.169431$

C $-2.260418-1.795781-3.909003$

O $-1.425220-2.969233-1.372961$

O $-2.327742-2.109961-5.308388$

C $-0.786398-1.894682-3.468883$

C $-2.899495-0.411315-3.749195$

O $-0.018468-0.840662-4.042553$

C $-0.599539-1.938839-1.934636$

C $0.866280-2.225535-1.537071$

O $-0.935934-0.654907-1.394144$

C -2.929796 -0.667014 2.402043

O $-3.192357 \quad 0.150863 \quad 3.269224$

C $-1.768688-1.5843842 .410462$

C $-0.839865-1.4625313 .370003$

C $0.353701-2.2955653 .533714$

C $0.514634-3.4704352 .606671$

C $1.389791-3.606683-1.932301$

C $2.859487-3.738493-1.589017$

C $4.424898-4.1898070 .270838$

O $3.734079-3.600651-2.442105$

C $4.420024-4.0545281 .758191$

C $4.809306-2.9434032 .400799$

C $4.881899-2.8169773 .894702$

C $3.773663-1.9555364 .455923$

C $3.956456-0.6335964 .618630$

C $2.510220-2.6773134 .881370$

C $1.236032-1.9716104 .502479$

N $3.085745-3.986688-0.244323$

H -2.573211 1.0875860 .129372

H $-5.562788 \quad 1.7380980 .274041$

H -5.3352740 .0001062 .058843$

H -5.398129 -1.210934 -0.748912

H $-6.785290-0.587571 \quad 0.116513$

H -5.924895 -4.532503 0.920464

H -7.056782 -3.4144202 .572763$

H -6.125482 -1.977414 3.000843

H -7.687916 -1.818764 2.165277

H $-5.474457-4.824883-1.355680$

H -5.425249 -3.125968 -1.710406

H -3.203181 -3.9972870 .121215$

H -3.232570 -4.919643 -1.354283

H -3.166151 -1.871217-1.242621

H $-4.122273-2.785776-3.421930$

H -2.748760 -3.859237 -3.549742

H $-1.759809-1.476255-5.779984$
H $-0.398533-2.821364-3.907104$

H -3.923434 -0.417801-4.142231

H $-2.3622000 .344348-4.332638$

H $-2.946175-0.076275-2.711437$

H $-0.241336-0.024043-3.564191$

H $1.517700-1.445554-1.946754$

H $0.962403-2.121845-0.448616$

H $-0.508122-0.561115-0.526857$

H -1.713503 -2.335973 1.631559

H $-0.959253-0.6716734 .109802$

H $-0.311909-4.1777032 .737657$

H $1.438823-4.0289612 .763651$

H $0.527400-3.1394831 .564429$

H $0.828643-4.400375-1.426177$

H $1.294966-3.783489-3.007951$

H $5.105962-3.476430-0.205761$

H $4.750366-5.195326-0.016329$

H $4.110658-4.9244562 .332042$

H $5.119772-2.0762191 .822272$

H $5.860295-2.3889884 .150417$

H $4.868380-3.8017074 .378862$

H 3.1936570 .0023395 .053867

H $4.884124-0.1546064 .322190$

H $2.530835-2.7949255 .972859$

H $2.515780-3.7000724 .493363$

H $1.019354-1.1026605 .125407$

H $2.298405-4.261806 \quad 0.335727$

C $-2.6070634 .003810 \quad 0.363421$

O $-1.2407823 .582740 \quad 0.560405$

C -0.9377673 .6598351 .964821$

C -2.0044814 .5666632 .569565$

C -3.220514 4.1724251 .757065

H -1.0634672 .6463742 .365451$

C 0.5004334 .1308202 .205020

H -2.131599 4.4006483 .643601

H -1.7766725 .6273682 .413220$

C $-4.3515965 .184347 \quad 1.810125$

H -3.5871923 .2166042 .148714$

C 1.5935693 .3494341 .448546

O 0.6219855 .5063821 .816635

H $\quad 0.705612 \quad 4.1104713 .281757$

H 1.5869773 .6516640 .396102

O $2.8586463 .780931 \quad 1.970397$

C 1.5084271 .8161041 .541459

C 2.7554051 .0660601 .029207

H $\quad 0.625674 \quad 1.469236 \quad 0.992036$

O $1.321727 \quad 1.4535372 .912075$

H 3.5771691 .1901721 .746469

H $2.512594-0.0053721 .043936$

H -4.7083495 .3090152 .837739$

H -4.0303996 .1656821 .445055$

H -5.1958774 .8550911 .196180$

C $-3.3403183 .034344-0.572535$

H $0.377396 \quad 5.568460 \quad 0.877444$

H 2.9142483 .4764112 .892632

H 1.2616040 .4845162 .951901

H -2.778042 $2.938962-1.509167$

H $-4.3210313 .452292-0.829117$

H $-2.5472034 .978255-0.137957$

C $2.4262411 .632906-2.652709$

O $2.1683201 .233208-1.298847$

C $3.2313321 .472329-0.374102$

C $4.4923110 .705908-0.798346$

C $4.8980201 .076559-2.229146$

C $3.7154070 .987860-3.196927$

H $1.5861241 .229870-3.228614$

C $2.3986293 .161595-2.809924$

H $3.4662152 .540393-0.358308$

O $4.249439-0.695071-0.711912$

H $5.3043080 .944667-0.101075$

H $5.6926500 .402912-2.572268$

O $5.4132342 .404732-2.252987$

H $3.9823051 .444630-4.157136$

O $3.465422-0.396669-3.453385$

C $1.0190063 .734635-2.556753$

H $3.0890313 .653771-2.120332$

H $2.6746843 .427392-3.836852$

H $5.059796-1.152332-0.993704$ 
H $6.1799612 .433066-1.655749$

H $2.748799-0.451668-4.108505$

O $1.0801505 .094867-2.668703$

O $\quad 0.0176613 .081637-2.298149$

C $-0.1533815 .788130-2.455954$

H $\quad 0.003824 \quad 6.842792-2.696912$

H $-0.9372825 .393377-3.109530$

H -0.448096 5.708117-1.406379

SCF Energy $\left(B 3 L Y P / 6-31 G^{* *} / / M M F F\right)=-3245.90860441$

\section{6}

MM̄FF Geometry

C $0.636277-2.005708 \quad 2.471673$

C $-0.653232-2.3196462 .282420$

C $-1.135891-3.1602081 .128755$

O $-1.874836-2.278370 \quad 0.248548$

C $-2.074937-4.2679151 .627384$

C $-2.537989-5.1880090 .515060$

C $-3.749911-5.129684-0.071344$

C $-1.548417-6.2471640 .102765$

C $-4.852815-4.1474320 .207030$

C $-5.366365-3.495525-1.084647$

C $-6.456295-2.457920-0.783431$

C $-7.105963-1.950757-2.070450$

C $-8.147709-0.858132-1.783962$

O $-5.843215-1.365255-0.090418$

O $-8.476682-0.224957-3.029680$

C $-7.507177 \quad 0.218585-0.884126$

C $-9.454810-1.441149-1.234159$

O $-8.4759031 .176870-0.464064$

C $-6.761996-0.3558200 .345239$

C $-5.954613 \quad 0.7697691 .031076$

O $-7.711456-0.876900 \quad 1.276544$

C $-1.138095-1.550373-0.637148$

O $0.057102-1.659007-0.864666$

C $-2.024454-0.531314-1.238687$

C $-1.4628930 .566545-1.767884$

C $-2.1654501 .716044-2.339890$

C $-3.6540371 .603459-2.525233$

C -4.9502000 .2885252 .085783$

C -4.1380961 .4661852 .587942$

C $-2.3211963 .024921 \quad 1.878204$

O -4.4183852 .0415503 .636797$

C -2.698652 3.9853550 .792065

C -1.8027834 .6552780 .051449$

C $-2.1714465 .601193-1.055907$

C -1.448232 $5.297922-2.351670$

C $-0.4434116 .080557-2.780487$

C -1.938424 $4.132889-3.188548$

C -1.445404 2.810874-2.662846

N $-3.117671 \quad 1.821617 \quad 1.719000$

H $1.389982-2.4040561 .797110$

H $-1.408387-1.8834942 .932501$

H $-0.295145-3.6104910 .586116$

H -2.934773 -3.8256442 .142997$

H - $-1.565193-4.8738272 .388787$

H -3.984344 -5.868492 -0.836705

H -1.948522 -6.912641 -0.669582

H $-0.638747-5.789405-0.297797$

H -1.276462 -6.8680970 .962712$

H $-5.675986-4.6861040 .691770$

H $-4.535043-3.3644380 .899960$

H -4.527445 -3.011129-1.601116

H $-5.764707-4.270720-1.750834$

H $-7.207794-2.924334-0.135680$

H $-7.561479-2.776830-2.630573$

H -6.332916 -1.530564 -2.729296

H -8.818058 $-0.908144-3.632061$

H $-6.7808080 .764859-1.502360$

H -9.884487 -2.155930 -1.946197

H $-10.211144-0.659398-1.102320$

H -9.326154 -1.960303 -0.282113

H -8.907152 $1.522774-1.264267$

H $-5.400231 \quad 1.3260980 .267780$

H $-6.6404401 .485346 \quad 1.503511$

H $-7.315682-0.8771212 .162458$

H -3.095646 -0.681616-1.177759

H $\quad-0.3768590 .654499-1.746473$
H $-4.0833232 .420306-3.109133$

H -4.156992 $1.594963-1.555429$

H -3.910742 $0.682828-3.061664$

H $-5.456139-0.1696872 .941863$

H $-4.264651-0.4622201 .676371$

H -1.2700032 .7284791 .817106$

H -2.509749 3.4738882 .857965

H -3.7593914 .1334150 .602319$

H -0.7436854 .5319800 .261785$

H -1.928522 $6.617275-0.718669$

H $-3.2529425 .597944-1.240255$

H $0.0641275 .884938-3.719180$

H $-0.1019566 .931110-2.199990$

H -1.577426 $4.233212-4.220429$

H -3.028392 $4.189755-3.250385$

H $-0.3642252 .776225-2.520194$

H -2.9793931 .2772730 .872705$

C 1.1472030 .3841742 .934363

$\begin{array}{lllll}\text { O } & 2.059130 & 0.437234 & 1.822087\end{array}$

C 2.7192201 .7160191 .822001

C 2.0838842 .5319472 .944860

C 1.6451041 .4457103 .909573

H 3.7663301 .5258212 .080976

C 2.6382202 .3533890 .429476

H 2.7900313 .2403563 .389206

H 1.2172843 .1043232 .595021

C 0.6012941 .9089304 .910437

H $2.527851 \quad 1.0768544 .448782$

C $3.1301541 .443098-0.715442$

O 1.2684202 .6754710 .161210

H 3.1878193 .3025520 .430923

H $2.4073380 .636415-0.891755$

O $3.1629702 .224169-1.916774$

C $4.5261310 .831519-0.496593$

C $5.0218020 .062189-1.737519$

H 4.4890290 .1501690 .360312

O $5.4395611 .880670-0.179729$

H $4.208545-0.562387-2.126170$

H $5.3017780 .788411-2.510722$

H 0.9938502 .7256905 .524936

H -0.3032482 .2699594 .409732$

H $\quad 0.312267 \quad 1.0926565 .579669$

C $1.108934-1.0314343 .512821$

H $1.2506223 .258677-0.615932$

H $2.2443412 .427338-2.160163$

H $6.3063261 .475299-0.008346$

H $0.456366-1.0671274 .392649$

H $2.109340-1.3422003 .837081$

H 0.1567800 .6582822 .546253

C $8.530417-0.691908-0.670560$

O $7.332253-0.006235-1.055679$

C $6.234735-0.840624-1.433838$

C $6.618839-1.707904-2.642607$

C $7.892946-2.513075-2.363083$

C $9.009180-1.634050-1.787388$

H $9.2801510 .102091-0.557203$

C $8.376460-1.3845910 .688215$

H $5.965912-1.489246-0.592789$

O $6.820783-0.874247-3.782028$

H $5.786118-2.383495-2.871658$

H $8.244620-2.977001-3.292636$

O $7.606754-3.559744-1.440164$

H $9.832059-2.253556-1.411656$

O $9.543562-0.817283-2.833442$

C $8.105625-0.3706661 .775287$

H $7.576582-2.1303160 .695321$

H $9.295388-1.9158090 .962536$

H $7.033305-1.451646-4.534768$

H $6.918874-4.121259-1.836256$

H $9.896260-1.411034-3.517862$

O $6.814671-0.4762072 .201234$

O 8.9369320 .4308612 .181972

C 6.4265310 .4412083 .228311

H 7.0855980 .3466114 .096743

H 6.4406791 .4643742 .841032

H 5.4068720 .1947813 .535971

SCF Energy (B3LYP/6-31G**//MMFF) $=-3245.90129121$ 
MM̄FF Geometry

C $0.882798 \quad 3.241795-1.219038$

C $-0.120643 \quad 3.983523-0.727891$

C $-0.9654763 .549466 \quad 0.439146$

O $-2.3310443 .523121-0.038700$

C -0.8540734 .5120691 .634508$

C 0.5328194 .6311672 .224070

C 1.1360073 .6701522 .950220

C 1.2235375 .9471161 .980095

C 0.5983832 .3108783 .309333

C 1.5369601 .2079882 .802596

C $1.057089-0.1951383 .206357$

C $2.202128-1.1990073 .053854$

C $1.759362-2.6292693 .380753$

O $-0.039427-0.5677432 .359321$

O $2.786855-3.5265992 .936109$

C $0.497804-2.9442872 .558429$

C $1.609638-2.8571324 .889109$

O $-0.037598-4.2162852 .919356$

C $-0.600435-1.8561462 .656866$

C $-1.706916-2.1758691 .622714$

O $-1.168585-1.8858663 .968384$

C -3.225437 2.7793470 .665708

O -3.0357112 .2082881 .727209$

C $-4.4823332 .745918-0.112336$

C -5.4747791 .9306790 .273648$

C $-6.7553111 .746765-0.411706$

C $-6.9868642 .536714-1.673892$

C -2.746444 -1.0692801 .421877$

C $-3.912028-1.6033390 .611397$

C $-4.497406-2.365330-1.686382$

O $-4.952519-1.9768301 .148584$

C $-5.712029-1.553286-2.011476$

C $-6.961187-1.994810-1.796000$

C $-8.210925-1.245626-2.163402$

C $-9.061183-0.908807-0.957836$

C $-9.892307-1.818109-0.420991$

C $-9.002473 \quad 0.505219-0.425077$

$\begin{array}{llll}\text { C } & -7.641380 & 0.867950 & 0.102877\end{array}$

N $-3.643921-1.661469-0.747590$

H $1.1104252 .285685-0.755638$

H $-0.3583084 .938807-1.189122$

H -0.6887562 .5291190 .730812$

H -1.548448 4.2098842 .428746

H $-1.221844 \quad 5.5039361 .335867$

H $2.129452 \quad 3.878587 \quad 3.345987$

H 2.2384295 .9681972 .390940

H 1.2992696 .1478920 .906837

H 0.6585206 .7614112 .445376

H $0.530326 \quad 2.255345 \quad 4.402710$

H -0.4134912 .1489612 .929431$

H 1.6150121 .2721531 .709355

H 2.5389681 .3850173 .214670

H $0.715546-0.1656594 .247575$

H $3.059777-0.9143703 .675987$

H $2.561457-1.1760922 .017098$

H $3.613650-3.2832673 .386831$

H $\quad 0.807080-3.0273401 .507015$

H $2.562988-2.6750595 .399721$

H $1.347792-3.8985125 .106604$

H $\quad 0.860747-2.2099725 .350186$

H $0.678800-4.867802 \quad 2.829596$

H $-1.243910-2.3721280 .646757$

H -2.225227 -3.0973791 .918985$

H -2.080907 -1.559118 3.928255

H $-4.5440403 .369698-0.996727$

H $-5.334173 \quad 1.3264571 .169158$

H $-6.9785013 .611343-1.460724$

H -7.938488 2.318348 -2.161847

H -6.207164 2.318053 -2.412453

H -3.143146 -0.697812 2.371683

H -2.301319 -0.2159460 .900081$

H $-4.754357-3.338883-1.254767$

H $-3.913878-2.535199-2.596761$

H -5.555951 - $0.581584-2.472527$

H $-7.100975-2.978216-1.351531$

H -8.790495 -1.871849-2.854449
H -10.524513 -1.5774590 .427062$

H $-9.956273-2.825750-0.818767$

H $-9.7156250 .634106 \quad 0.399924$

H -9.349217 $1.187629-1.205711$

H $-7.3745540 .334812 \quad 1.016578$

H -2.719576 -1.394539-1.072199

C $3.2196863 .663851-2.108194$

O $3.6907992 .349566-1.749141$

C $4.7028962 .492283-0.732386$

C $5.0161853 .983911-0.644583$

C $3.6693664 .591985-0.982181$

H $4.2457792 .177967 \quad 0.213195$

C $5.9123171 .610805-1.053851$

H $5.382923 \quad 4.2719860 .345371$

H $5.7677654 .286461-1.383288$

C $3.7360276 .056540-1.373894$

H $3.0201074 .484472-0.105256$

C $5.5860090 .107722-1.180502$

O $6.4697292 .044751-2.301211$

H $6.6911311 .768635-0.298015$

H $4.975283-0.059631-2.074318$

O $6.823886-0.576429-1.418257$

C $4.896909-0.507570 \quad 0.053007$

C $4.830728-2.045907 \quad 0.041879$

H $3.887422-0.0939100 .152721$

O $5.629326-0.1182571 .219709$

H $5.843700-2.460005-0.035829$

H $4.461012-2.3638541 .024654$

H $4.1353376 .656809-0.549858$

H $4.3807276 .210651-2.245591$

H $2.7402546 .439344-1.618491$

C $1.7193703 .630374-2.402910$

H $7.2334081 .473176-2.491583$

H $7.371131-0.477908-0.620037$

H $5.135514-0.4345111 .995399$

H $1.5313102 .886688-3.187532$

H $1.4032664 .602881-2.797833$

H $3.7408813 .926703-3.038649$

C $1.649307-2.593973-1.824141$

O $2.581064-2.251439-0.787934$

C $3.932809-2.633201-1.054018$

C $4.032045-4.170337-1.123443$

C $2.863033-4.796814-1.893820$

C $2.129580-3.748734-2.722714$

H $0.733674-2.912398-1.308770$

C $1.327539-1.340919-2.651604$

H $4.227917-2.214183-2.023372$

O $4.030109-4.7253300 .193922$

H $4.997373-4.439512-1.568905$

H $2.157339-5.299607-1.220734$

O $3.368959-5.804799-2.769876$

H $2.793166-3.380778-3.515110$

O $1.008678-4.337728-3.380952$

C $0.277974-0.485785-1.972731$

H $2.215213-0.718094-2.805894$

H $\quad 0.932828-1.638084-3.630373$

H $4.141473-5.6874470 .106260$

H $3.818451-6.469675-2.220470$

H $0.426980-4.717910-2.700910$

O $-0.540823 \quad 0.057106-2.918810$

O $0.206553-0.310450-0.764271$

C $-1.6304310 .878430-2.478398$

H $-1.7215780 .923128-1.390423$

H $-1.485228 \quad 1.886675-2.874087$

H $-2.5552780 .470207-2.894711$

SCF Energy (B3LYP/6-31G*//MMFF) $=-3245.89175707$

0800528

MM̄FF Geometry

C -0.2799472 .9083452 .666720$

C -0.7600331 .9247891 .889953$

C -1.2924560 .6191652 .421855$

O $-0.615829-0.4199821 .675318$

$\begin{array}{llll}\text { C }-2.812788 & 0.543646 & 2.162757\end{array}$

C -3.613829 -0.0492183 .304637$

C $-4.262633-1.229612 \quad 3.262815$

C -3.7258140 .8249104 .528946$ 
C $-4.279486-2.2452492 .154615$

C $-5.625187-2.3036191 .418263$

C $-5.868149-1.1015450 .491954$

C $-7.228648-1.218190-0.198217$

C $-7.466919-0.067684-1.189762$

O $-4.834109-1.100219-0.494498$

O $-8.573980-0.436168-2.030798$

C $-6.2354200 .069103-2.115161$

C $-7.8979501 .214195-0.467685$

O $-6.332004 \quad 1.226643-2.940802$

C $-4.8679550 .015842-1.391095$

C $-3.734025-0.210183-2.417365$

O $-4.5553431 .241389-0.729231$

C $-0.608451-1.6750132 .191352$

O $-1.076821-2.0470193 .254439$

C $0.057617-2.5488331 .207421$

C $-0.354980-3.8171581 .084502$

C $0.198804-4.7630310 .125619$

C $-0.833992-5.384109-0.771823$

C $-2.347716-0.347202-1.770318$

C $-1.262096-0.623203-2.794511$

C $1.174741-1.123881-2.941849$

O $-1.471214-0.661293-4.003974$

C $1.338620-2.608367-3.045540$

C $2.488202-3.238149-2.760604$

C $2.707670-4.713569-2.929635$

C $3.239620-5.373164-1.677757$

C $4.560579-5.444230-1.440932$

C $2.243662-6.030253-0.751032$

C $1.517044-5.0406200 .114017$

N $-0.027061-0.818334-2.191195$

H -0.2541912 .7447643 .742854$

H -0.8070642 .0519880 .812050$

H $-1.039477 \quad 0.5153103 .483707$

H -2.994976 $-0.005958 \quad 1.235488$

H -3.2250201 .5477841 .989518$

H -4.839928 -1.5255884 .138233$

H -4.3794680 .3898065 .292558$

H -2.7443870 .9786274 .987451$

H $-4.141158 \quad 1.8019494 .260787$

H $-3.460299-2.098728 \quad 1.445244$

H -4.094044 -3.225467 2.612337

H -5.626812 -3.2211600 .815170$

H $-6.444700-2.3913732 .142214$

H -5.814845 -0.1784621 .079116$

H $-8.038226-1.2614940 .540928$

H -7.276430 -2.166792 -0.751492

H $-9.337683-0.622940-1.458238$

H -6.277763 -0.786359-2.803936

H $-8.856457 \quad 1.0599290 .043290$

H -8.068870 2.031949-1.175717

H -7.1835491 .5466210 .287338$

H $-6.1040932 .001974-2.401247$

H -3.936849 -1.124827 -2.989984

H $-3.7046760 .623540-3.130493$

H $-5.3732201 .674913-0.449892$

H $\quad 0.839590-2.1274570 .586293$

H -1.181557 -4.190447 1.685295

H $-0.402878-5.974543-1.583261$

H -1.445796-4.606800 -1.244665

H -1.496781 -6.039592 -0.196538

H -2.084208 $0.574730-1.239657$

H $-2.346669-1.173279-1.049880$

H $2.012791-0.654238-2.419080$

H $1.096595-0.690798-3.944197$

H $\quad 0.490417-3.184249-3.407963$

H $3.342655-2.650821-2.434556$

H $3.426226-4.852967-3.748213$

H $\quad 1.791097-5.220109-3.256316$

H $4.951731-5.942097-0.559947$

H $5.279336-4.999057-2.121117$

H $2.760329-6.728404-0.078632$

H $1.566804-6.659257-1.336739$

H $2.156860-4.5343040 .837077$

H $0.037611-0.801864-1.177991$

C $0.145796 \quad 4.5758440 .731186$

O 1.1679643 .8338050 .036999

C $1.7095954 .660252-1.013232$
C $0.8399795 .911231-1.064394$

C 0.4144196 .0400740 .385876

H $2.7157194 .955831-0.695574$

C $1.7781083 .869945-2.324210$

H $1.3902546 .783256-1.430811$

H $-0.0352385 .771468-1.709634$

C $-0.779323 \quad 6.9582290 .583435$

H 1.2632296 .4158070 .972910

C $2.6286102 .585465-2.228555$

O $0.4448883 .487696-2.687399$

H $2.1469674 .515901-3.129404$

H $2.1514751 .892032-1.528341$

O $2.5922701 .942318-3.509165$

C $4.0989422 .824976-1.835863$

C $4.9567471 .544639-1.807173$

H $4.1514763 .314495-0.858569$

O $4.673703 \quad 3.725921-2.787099$

H $4.8816591 .018019-2.766227$

H $6.0071051 .852563-1.716662$

H $-0.545374 \quad 7.971470 \quad 0.240453$

H $-1.652758 \quad 6.6074240 .023516$

H -1.0585507 .0169231 .639881$

C 0.2156764 .2649592 .228951

H $\quad 0.5054122 .968907-3.507853$

H $3.0469182 .524352-4.142155$

H $5.5770713 .929381-2.490296$

H -0.3727525 .0080582 .779596$

H 1.2551974 .3550742 .568895

H -0.8236554 .2738540 .314160$

C 4.4943910 .6081081 .767989

O $4.865467 \quad 1.305014 \quad 0.571995$

C $4.6008850 .601158-0.647283$

C $5.415443-0.700575-0.687174$

C $5.123194-1.5674360 .542337$

C $5.195262-0.7598221 .845007$

H $4.889021 \quad 1.2302382 .581725$

C 2.9700410 .5671851 .906757

H $3.5350480 .358573-0.700718$

O $6.807074-0.391312-0.732446$

H $5.167944-1.246473-1.604756$

H $5.847748-2.3899950 .582445$

O $3.827619-2.138586 \quad 0.395277$

H $4.782106-1.3428202 .674333$

O $6.573531-0.5348242 .162278$

C 2.5535050 .4002043 .345913

H $2.530607 \quad 1.5070561 .549535$

H $2.505870-0.2099291 .293257$

H $7.292004-1.231819-0.795316$

H $3.664629-2.6995941 .172373$

H 6.9485070 .0357161 .469441

O $2.252395-0.9046513 .597639$

O $2.522879 \quad 1.317750 \quad 4.155751$

C $1.849270-1.1945564 .939409$

H $1.612728-2.2601335 .000060$

H $2.665196-0.9751505 .634364$

H $0.954230-0.6212235 .199572$

SCF Energy (B3LYP/6-31G**//MMFF) = -3245.90068755

\section{9}

MM̄FF Geometry

C $0.950263-2.8787031 .900957$

C $0.199610-1.8329762 .282116$

C $0.708885-0.5471802 .886810$

O $2.140637-0.6331533 .077111$

C $0.061845-0.2096864 .240535$

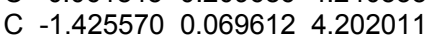

C $-1.973276 \quad 1.199437 \quad 3.712583$

C $-2.299163-0.9966154 .808417$

C -1.2539202 .3491093 .062585$

C $-1.8915452 .694261 \quad 1.709954$

C -0.9609663 .5454990 .831966$

C $-1.774726 \quad 4.396642-0.144891$

C $-0.8739185 .187926-1.100303$

O $-0.1126842 .643267 \quad 0.105190$

O $-1.7004935 .719967-2.146698$

C $0.1141634 .207931-1.757906$

C $-0.214076 \quad 6.388750-0.413648$

O $1.0658804 .907232-2.557799$ 
C $0.8428903 .281560-0.752609$

C $1.6043872 .191408-1.548190$

O $1.779696 \quad 4.0536830 .000653$

C 2.8703330 .5041542 .936145

O $2.455288 \quad 1.6410202 .783082$

C 4.2920350 .1024052 .916974

C 5.1443780 .8049112 .156705

C 6.5481150 .4750421 .910161

C $7.176866-0.5824312 .779278$

C $2.1830631 .052607-0.701173$

C $2.9930150 .078841-1.542409$

C $4.341169-2.007309-1.344858$

O $3.1816250 .230608-2.746718$

C $5.734912-1.827929-0.830548$

C $6.742886-1.325888-1.560552$

C $8.159381-1.199113-1.071486$

C $8.6181960 .239368-0.962619$

C $9.1056440 .892813-2.030763$

C 8.5868130 .8886030 .405085

C 7.1840941 .1050710 .900069

N $3.490761-0.973099-0.785777$

H $2.025917-2.8700012 .052423$

H $-0.868128-1.8671322 .074353$

H $\quad 0.499272 \quad 0.2360212 .149629$

H $\quad 0.5688900 .6596254 .681131$

H $0.272404-1.0191994 .953385$

H -3.054682 1.3150043 .774063

H $-3.363666-0.7456744 .750763$

H -2.158029 -1.9495764 .288920$

H -2.047857 -1.136118 5.864917

H $-1.323323 \quad 3.2224913 .722401$

H -0.1894472 .1453522 .935705$

H -2.1477721 .7707431 .175604$

H -2.8272333 .2319101 .908670$

H $-0.345544 \quad 4.187813 \quad 1.472546$

H $-2.447365 \quad 5.0748390 .394769$

H -2.422708 $3.745017-0.746179$

H $-2.3773216 .284812-1.736076$

H $-0.4654153 .577938-2.447905$

H $-0.9771137 .070204-0.018496$

H $0.3724856 .977809-1.127450$

H $0.4365996 .105463 \quad 0.416181$

H $\quad 0.5676175 .459269-3.184649$

H $0.9242071 .737038-2.280657$

H $2.4201792 .660531-2.113303$

H 2.4807863 .4691900 .328595

H $4.571849-0.8014743 .444876$

H 4.7653351 .6692571 .612823

H $7.070465-0.3205853 .838207$

H $8.245341-0.7171462 .601557$

H $6.701920-1.5548592 .609832$

H $2.8480801 .452376 \quad 0.067771$

H $1.3753210 .496216-0.213665$

H $4.302794-1.978815-2.438614$

H $3.950624-2.975265-1.017847$

H $5.926019-2.1604220 .186340$

H $6.547495-1.007808-2.582401$

H $8.808135-1.739288-1.773735$

H $8.294514-1.706343-0.108000$

H $9.4625001 .914471-1.955528$

H $9.1548900 .418973-3.005695$

H 9.0774981 .8703030 .375449

H $9.192997 \quad 0.290049 \quad 1.090183$

H $6.633044 \quad 1.8512290 .325533$

H $3.285260-1.0110890 .208395$

C $0.702295-3.992022-0.307490$

O $-0.021601-2.880063-0.875754$

C $-0.682075-3.319097-2.073724$

C $0.003120-4.621139-2.464353$

C $0.268018-5.225932-1.098022$

H $-1.719525-3.544253-1.797693$

C $-0.657892-2.215800-3.135052$

H $-0.627291-5.250641-3.099290$

H $0.948171-4.441083-2.989818$

C $1.299473-6.340376-1.114170$

H $-0.676392-5.617666-0.696354$

C -1.240327 -0.865549-2.661767

O $0.700846-1.988622-3.528926$
H $-1.183530-2.564645-4.032015$

H $-0.537610-0.381483-1.973637$

O $-1.320273-0.004734-3.804737$

C -2.623292 -0.954772 -1.992692

C $-3.2552720 .428401-1.737954$

H -2.524062 -1.495159-1.044351

O $-3.486521-1.715654-2.839158$

H $-2.4856701 .138074-1.412453$

H $-3.6864630 .794811-2.677522$

H $\quad 0.959139-7.170229-1.742123$

H $2.260192-5.992445-1.508411$

H $1.470194-6.727825-0.104879$

C $0.393353-4.0786031 .187234$

H $0.696632-1.275196-4.190100$

H -1.988687 -0.376920-4.405442

H $-4.339590-1.807923-2.381856$

H $\quad 0.834390-4.9815671 .623675$

H $-0.690055-4.1357951 .343407$

H $1.768261-3.790507-0.467614$

C $-6.365895-0.844505-0.084247$

O $-5.341049-0.582518-1.052002$

C $-4.353750 \quad 0.372026-0.656510$

C $-5.0195331 .733914-0.416500$

C $-6.146001 \quad 1.618506 \quad 0.616418$

C $-7.094616 \quad 0.453380 \quad 0.303997$

H $-7.084833-1.490414-0.604914$

C $-5.822963-1.6282131 .121322$

H -3.8732690 .0330910 .267936$

O $-5.5419362 .232060-1.645958$

H -4.265176 $2.444642-0.066915$

H -6.7112202 .5581380 .635610$

O $\quad-5.5620411 .4406271 .903932$

H $-7.759726 \quad 0.256241 \quad 1.152772$

O $\quad-7.925831 \quad 0.819839-0.801694$

C $-5.201986-2.9458670 .706000$

H $-5.060121-1.0553891 .655614$

H -6.636140 -1.851272 1.820998

H $-5.9288363 .106126-1.467958$

H -6.2861221 .3950422 .551231$

H $-8.427817 \quad 1.610991-0.541971$

O $-4.378352-3.3864021 .702614$

O $-5.424063-3.526977-0.347539$

C $-3.715804-4.6283191 .445428$

H $-3.109076-4.5555280 .537521$

H -3.057478 -4.843774 2.291166

H $-4.449303-5.4352251 .356346$

SCF Energy $\left(B 3 L Y P / 6-31 G^{* *} / / M M F F\right)=-3245.90037857$

0800530

MM̄FF Geometry

C $1.488336-3.322927-0.377179$

C $0.697602-3.585517-1.427797$

C $-0.176458-2.530793-2.055509$

O $-1.516650-3.078594-2.092556$

C $0.267299-2.131174-3.473492$

C $1.505017-1.255483-3.504968$

C $1.5152130 .047153-3.154102$

C $2.761268-1.924575-3.990008$

C $0.3209280 .836390-2.693449$

C $0.5984732 .343045-2.605977$

C $-0.6820643 .136444-2.302350$

C $-0.3766934 .628201-2.157139$

C $-1.6267445 .435493-1.774808$

O $-1.2568552 .636262-1.090009$

O $-1.1966446 .732775-1.334162$

C $-2.3168684 .756375-0.572375$

C $-2.5437865 .670234-2.981271$

O $-3.5649285 .382303-0.281995$

C $-2.5166583 .232700-0.752240$

C $-3.0159272 .605600 \quad 0.572180$

O $-3.4980583 .010301-1.768118$

C -2.545574 -2.187772 -2.062736

O $-2.470517-0.973626-2.163135$

C $-3.789980-2.946668-1.810616$

C $-4.893250-2.277408-1.443864$

C $-6.174798-2.864459-1.048512$

C $-6.309853-4.361878-1.138703$

C $-3.0192501 .070140 \quad 0.582785$ 
C $-3.5989690 .506972 \quad 1.869977$

C $-4.094694-1.6703362 .983580$

O $-4.036571 \quad 1.2135202 .774147$

C -5.297428 -2.428359 2.517269

C $-6.558439-2.0867702 .823641$

C $-7.773666-2.8610712 .393763$

C -8.641276 -2.091081 1.421160

C $-9.554515-1.2079061 .858799$

C $-8.499280-2.403797-0.053987$

C $-7.140029-2.041348-0.586648$

N $-3.576064-0.8815941 .881238$

H $1.528293-2.303145-0.001829$

H $0.636925-4.597143-1.820033$

H $-0.196099-1.656481-1.395394$

H $-0.548884-1.609690-3.989637$

H $\quad 0.424926-3.033017-4.080744$

H $2.457750 \quad 0.588234-3.205084$

H $3.631324-1.261042-3.945611$

H $2.985405-2.803856-3.381581$

H $2.639963-2.245918-5.029532$

H $-0.5161580 .669856-3.380713$

H $0.0269400 .487121-1.698597$

H $1.3349862 .513189-1.810711$

H $1.0378532 .703432-3.544196$

H - $1.3848442 .969422-3.127550$

H $0.0724125 .025712-3.075789$

H $0.376423 \quad 4.770222-1.370430$

H -0.695927 $7.144638-2.059146$

H $-1.677828 \quad 4.9194470 .306487$

H -2.011504 $6.222751-3.764948$

H -3.403694 $6.292182-2.709096$

H -2.913598 4.744015-3.425797

H -3.391499 $6.331642-0.161852$

H $-2.374763 \quad 2.9386351 .398318$

H -4.0312232 .9639350 .785476$

H -3.904430 $2.139977-1.636479$

H -3.746722 -4.027993 -1.869667

H -4.845405 -1.191006 -1.384022

H $-6.138328-4.700725-2.166584$

H -7.295003 -4.732701-0.850391

H $-5.584413-4.853654-0.480927$

H $-3.6219590 .681815-0.243124$

H - 1.9965320 .6908050 .474122

H $-4.325093-1.0250043 .837424$

H -3.310342 -2.369629 3.289702

H $-5.114776-3.315277 \quad 1.915770$

H $-6.732804-1.2031033 .433947$

H $-8.354905-3.1057273 .292600$

H -7.495641 -3.829234 1.958778

H -10.198232 $-0.670248 \quad 1.170799$

H $-9.675463-1.0017502 .917247$

H $-9.239501-1.841885-0.638362$

H $-8.750779-3.456190-0.209178$

H -6.937264 -0.969433 - 0.558022

H -3.188680 -1.377164 1.083239

C $3.803851-4.1822960 .259559$

O $4.194060-2.8005830 .399919$

C $5.124827-2.485557-0.651618$

C $5.649265-3.824943-1.150023$

C $4.394866-4.672618-1.062263$

H $4.547544-2.023920-1.460170$

C $6.200543-1.515840-0.159522$

H $6.056473-3.763185-2.163534$

H $6.428307-4.224189-0.489715$

C $4.667048-6.166619-1.101562$

H $3.732932-4.414271-1.896719$

C $5.653716-0.155256 \quad 0.325845$

O $6.901908-2.1279010 .929033$

H $6.940720-1.361103-0.953981$

H $5.115844-0.2919991 .270248$

$\begin{array}{llllll}\text { O } & 6.781849 & 0.674307 & 0.632543\end{array}$

C $4.7544130 .571685-0.691215$

C $4.4418212 .040660-0.357478$

H $3.8149370 .018830-0.802100$

O $5.4053710 .555104-1.966711$

H $5.3585972 .641111-0.420138$

H $3.7964922 .413863-1.163625$

H $5.145509-6.445215-2.046160$
H $5.329049-6.476148-0.285836$

H $3.735613-6.734924-1.014351$

$\begin{array}{llll}\text { C } 2.286518 & -4.336743 & 0.391252\end{array}$

H $7.574945-1.4947051 .232402$

H $7.2518590 .850131-0.200833$

H $4.7964870 .950765-2.613453$

H $2.018983-4.2011501 .447389$

H $1.986940-5.357917 \quad 0.128781$

H $4.275569-4.7186101 .093498$

C 1.8170211 .5472322 .261070

O 2.5701691 .4779011 .041803

C $3.759074 \quad 2.272486 \quad 0.999894$

C 3.4312093 .7610841 .216569

C 2.6090473 .9777302 .490625

C 1.4313383 .0021702 .578334

H $\quad 0.8907230 .9964562 .051827$

C $2.535340 \quad 0.8488513 .427604$

H 4.4427341 .9396501 .786864

$\begin{array}{lllll}\text { O } & 2.697533 & 4.257684 & 0.098404\end{array}$

H $4.370804 \quad 4.324124 \quad 1.268344$

H 2.2216765 .0036712 .509605

O $3.441146 \quad 3.8075703 .633618$

H 0.9777003 .0506213 .574649

$\begin{array}{lllll}\text { O } & 0.421757 & 3.412561 & 1.652315\end{array}$

C $2.675453-0.6418723 .212255$

H $3.540161 \quad 1.2518953 .577734$

H $1.9615610 .987028 \quad 4.351130$

H 2.5394525 .2055200 .247333

H $4.1604684 .458966 \quad 3.573402$

H $\quad 0.771673 \quad 3.2875190 .753497$

O $3.539409-1.1449894 .142919$

O $2.091762-1.2873502 .353953$

C $3.775827-2.554868 \quad 4.074486$

H $2.832877-3.1089424 .037876$

H $4.393318-2.7832303 .201766$

H $4.318976-2.8517414 .975565$

SCF Energy (B3LYP/6-31G**/MMFF)= -3245.91628258

0800531

MM̄FF Geometry

C $-3.213819-3.245274-0.779192$

C $-2.318507-4.007749-0.135147$

C $-1.157890-3.4302110 .630129$

O $0.035407-3.763002-0.114694$

C -1.046244 -4.004159 2.054487

C $-2.157602-3.5529652 .978010$

C $-2.158790-2.3847383 .649576$

C $-3.298752-4.522381 \quad 3.147592$

C $-1.090178-1.3274863 .663570$

C -1.527089 -0.0591702 .920326$

$\begin{array}{llll}\text { C } & -0.583429 & 1.120974 & 3.199442\end{array}$

C $-1.0072492 .354274 \quad 2.401968$

C $-0.044232 \quad 3.5324282 .609895$

$\begin{array}{lllll}0 & 0.748672 & 0.745819 & 2.836975\end{array}$

O $-0.324603 \quad 4.5119851 .598272$

C 1.4044153 .0452342 .385238

C $-0.277851 \quad 4.227082 \quad 3.957043$

$\begin{array}{llllll}\text { O } & 2.320294 & 4.085788 & 2.720584\end{array}$

C 1.7385351 .7386283 .146852

C 3.1157821 .1851002 .709183

$\begin{array}{lllll}\text { O } & 1.784871 & 2.022822 & 4.548684\end{array}$

C $1.072941-2.886214-0.032142$

O $1.148911-1.902948 \quad 0.686911$

C $2.092342-3.302098-1.020315$

C $3.096691-2.463349-1.316866$

C $4.144159-2.686279-2.314482$

C $4.152414-4.005160-3.042098$

C $3.385513-0.2451173 .196668$

C $4.866875-0.5596513 .190164$

C $6.782988-1.0575491 .713004$

O $5.502853-0.674653 \quad 4.236097$

C $7.194980-0.7327620 .314357$

C $7.720360-1.635188-0.527957$

C $8.167336-1.316416-1.926028$

C $7.483530-2.178427-2.963815$

C $8.067735-3.299345-3.419936$

C $6.168547-1.699437-3.535327$

C $5.047105-1.707938-2.534524$ 
N $5.397622-0.6780331 .914970$ H -3.074069 -2.167299-0.782262 H $-2.427197-5.089247-0.145962$ H -1.258161 -2.3384600 .673418$ H $-0.072628-3.7385942 .484299$ H -1.014699 -5.101882 2.014601 H -3.012861 -2.161491 4.287946 H $-4.072697-4.1405053 .822027$ H -3.778441 -4.736276 2.188938 H -2.931921 -5.464906 3.567502 H $-0.879369-1.0868374 .713196$ H $-0.149928-1.6957063 .243162$ H - $-1.543624-0.2721501 .844123$ H -2.547595 0.2202363 .211132 H -0.6175101 .3309874 .275205$ H -2.034631 2.6478092 .650431 H -1.015561 2.1055261 .332856 H -1.258271 4.7711091 .681191 H 1.5197202 .8650521 .308278 H -1.304670 4.6073724 .018476 H $\quad 0.371416 \quad 5.1025704 .068007$ H -0.119756 3.5683724 .813403 H $3.196333 \quad 3.8316342 .385967$ H 3.1778911 .1787411 .613259 H 3.9111091 .8459843 .075974 H 2.4215941 .4310684 .978937 H $1.964224-4.262544-1.506081$ H $3.144908-1.505916-0.799172$ H $4.212655-4.836776-2.331067$ H $4.999966-4.119826-3.721690$ H $3.242689-4.119330-3.641486$ H $3.024304-0.4001664 .219004$ H $2.864577-0.9745622 .567210$ H $6.868279-2.1262371 .937798$ H $7.408482-0.5101092 .426117$ H $7.073593 \quad 0.296677-0.013884$ H $7.860797-2.659153-0.188400$ H $9.253739-1.468714-1.972260$ H $8.009318-0.257921-2.167372$ H $7.601413-3.909850-4.185988$ H $9.025175-3.633626-3.033923$ H $5.892177-2.274888-4.426590$ H $6.295181-0.672721-3.903027$ H $4.969996-0.786854-1.955863$ H $4.756353-0.7568081 .130345$ C $-5.719880-3.053440-1.160154$ O $-5.593588-1.649814-1.470672$ C $-5.926009-0.896388-0.290357$ C $-6.859305-1.8037300 .494750$ C $-6.159036-3.1404910 .306271$ H $-4.999976-0.7752780 .286479$ C $-6.4610230 .487106-0.665329$ H $-6.947628-1.5098611 .544521$ H -7.862269 -1.8342190 .053651$ C -7.035634 -4.342079 0.611725 H $-5.287835-3.1680060 .971691$ C $-5.5558061 .256873-1.654503$ O $-7.746067 \quad 0.333625-1.278064$ H -6.6222331 .0754870 .245786$ H $-5.6507890 .809236-2.651833$ O $-6.0812582 .584899-1.753127$ C $-4.0704691 .307678-1.244964$ C $-3.1980652 .084378-2.240585$ H -3.701929 $0.281923-1.176507$ O H $-3.408467 \quad 1.745887-3.263268$ H -3.466588 $3.148991-2.217423$ H -7.356238 -4.3279761 .658539$ H -7.933863 -4.354084 -0.014600 H $-6.488921-5.2742770 .437140$ C $-4.412599-3.774939-1.516618$ H -8.065687 $1.223526-1.506240$ H $-5.6685273 .010048-2.523510$ H $-3.064512 \quad 1.7372190 .372317$ H -4.218327 -3.630694 -2.586907 H $-4.531293-4.851289-1.346861$ H -6.512766 -3.435915 -1.815220 C $0.3714042 .849439-2.980153$
O $-1.0570372 .745043-3.032292$

C $-1.677722 \quad 1.995457-1.977868$

C $-1.1307090 .557830-1.941877$

C $0.3993130 .527767-1.903430$

C $1.0195501 .456300-2.950414$

H $0.6531903 .327575-3.927215$

C $0.8409413 .759975-1.835573$

H $-1.4682292 .495628-1.027491$

O $-1.591955-0.152187-3.089683$

H $-1.5207550 .045482-1.054984$

H $0.738176-0.499138-2.080676$

O $0.828266 \quad 0.906312-0.599952$

H $2.0980431 .560208-2.783290$

$\begin{array}{lllll}\text { O } & 0.852022 & 0.875717 & -4.246689\end{array}$

C $0.4173525 .200874-2.029967$

H $0.4422373 .435988-0.872310$

H $1.9361423 .745640-1.788616$

H -1.282129 -1.070549 -3.011062

H $1.7977760 .836189-0.577572$

H $1.3008860 .013015-4.242029$

O $0.9380985 .952004-1.013983$

O $-0.2815875 .617948-2.942800$

C $0.6078867 .343828-1.045177$

H $-0.4757027 .477620-0.974529$

H $1.0772477 .823665-0.182308$

H $0.9965857 .804912-1.958188$

SCF Energy (B3LYP/6-31G**//MMFF) $=-3245.88486923$

0800532

MM̄FF Geometry

C $-1.200204-0.886613-1.455245$

C $-0.000478-1.461055-1.285083$

C $0.397604-2.199563-0.032488$

O $1.599520-1.5875440 .490468$

C $0.701428-3.669117-0.373538$

C $1.051215-4.514205 \quad 0.837238$

C $2.282606-4.9853131 .120884$

C $-0.107485-4.8500841 .741872$

C $3.550491-4.7730170 .341776$

C $4.531614-3.8812681 .112762$

C $5.654015-3.352461 \quad 0.207802$

C $6.673541-2.5585611 .024071$

C $7.733641-1.890488 \quad 0.136242$

O $5.046012-2.505221-0.772136$

$\begin{array}{llllll}0 & 8.429687 & -0.923254 & 0.938791\end{array}$

C $7.028452-1.113600-0.997856$

C $8.788221-2.895614-0.342513$

O $8.000092-0.614183-1.914046$

C $5.951695-1.942644-1.730945$

C $5.096072-1.111329-2.720083$

O $6.594938-2.971235-2.492905$

C $1.438854-0.467388 \quad 1.249310$

O 0.3852920 .0740181 .545913

C $2.7822880 .004881 \quad 1.652481$

C 2.9074401 .1727392 .300608

C 4.1584341 .7780222 .763835

C $5.4418291 .055027 \quad 2.453344$

C $4.2877670 .030570-2.093592$

C $4.9848811 .379978-2.121783$

C $4.7210603 .713292-1.281499$

O $6.0110811 .595553-2.760482$

C $5.573712 \quad 3.894277-0.065545$

C $5.3376694 .828804 \quad 0.868071$

C 6.2198055 .0623602 .061089

C 5.4560605 .0563083 .366350

$\begin{array}{llll}\text { C } 5.063012 & 6.204985 & 3.942417\end{array}$

C 5.2315963 .7260104 .047711

C 4.1002622 .9467543 .436019

N $4.2969832 .329082-1.376586$

H $-1.935478-0.950427-0.658431$

H $0.741287-1.396164-2.077829$

H $-0.396801-2.1533920 .722773$

H $1.501373-3.705424-1.119368$

H $-0.169990-4.123517-0.864532$

H $2.407110-5.5911292 .017578$

H $0.178922-5.5337682 .548240$

H $-0.510084-3.9452072 .206809$

H $-0.906769-5.3343761 .171232$ 
H $4.015615-5.7498050 .160248$ H $3.351606-4.354592-0.646525$ H $3.986144-3.0305071 .539467$ H $4.957459-4.4485271 .949721$ H $6.134616-4.201420-0.292361$ H $7.149899-3.1975721 .778123$ H $6.152512-1.7738081 .587470$ H $8.822494-1.3892861 .696600$ H $6.558937-0.240494-0.529525$ H $9.298911-3.3518670 .514096$ H $9.571188-2.399737-0.926813$ H $8.367782-3.704801-0.943089$ H $7.5523160 .001607-2.517784$ H $5.711018-0.762298-3.558004$ H $4.364795-1.799402-3.169049$ H $5.989469-3.260944-3.195250$ H $3.632513-0.6168401 .399266$ H 2.0062531 .7442532 .519257 H 5.5177690 .8478371 .380708 H $5.486800 \quad 0.107596 \quad 3.000698$ H $6.338198 \quad 1.6222332 .712279$ H $3.3631830 .156154-2.671985$ H $3.998836-0.209841-1.066721$ H $3.8160434 .328095-1.248180$ H $5.2928323 .982263-2.175545$ H 6.4439243 .2495260 .033963 H 4.4839715 .4924680 .749504 H 6.7117096 .0345551 .924377 H 7.0322344 .3266812 .109718 H 4.5417136 .2109164 .893962 H 5.2491617 .1648013 .471963 H 4.9790153 .8843335 .105093 H 6.1721363 .1685634 .072162 H 3.1221873 .3995703 .605161 H $3.4843132 .045055-0.837190$ C $-1.9779251 .303830-2.376986$ O $-3.2809611 .346590-1.754549$ C $-4.0118052 .467301-2.288860$ C $-3.0119473 .275820-3.108372$ C $-2.0889492 .188663-3.616961$ H $-4.7696942 .059072-2.966895$ C -4.673289 $3.269379-1.164179$ H $-3.4953213 .841088-3.911063$ H -2.458225 $3.986452-2.483131$ C $-0.7580782 .716200-4.125645$ H $-2.595457 \quad 1.643282-4.424730$ C $-5.7561922 .512263-0.366570$ O $-3.6630223 .693597-0.238954$ H -5.103992 4.189810-1.575756 H $-5.301908 \quad 1.7088860 .224368$ O $-\begin{array}{llll}6.311900 & 3.440785 & 0.575145\end{array}$ C $-6.9073871 .945612-1.223242$ C $-8.1745541 .553333-0.439360$ H -6.546706 $1.080453-1.789891$ O $-7.3088552 .925098-2.188674$ H -8.5870592 .4413570 .056603$ H -8.930630 $1.254067-1.178677$ H $-0.9125503 .403479-4.963983$ H $-0.2153783 .257432-3.343336$ H $-0.1191511 .898915-4.473941$ C -1.603134 - $0.143556-2.697186$ H -3.221621 2.8948530 .097963 H -5.5768363 .7819671 .113124$ H -7.604641 $3.713414-1.701628$ H $-0.786547-0.169576-3.427600$ H -2.460249 $-0.664988-3.140146$ H -1.267121 $1.738863-1.661454$ C $-7.501162-1.9604680 .634746$ O $-7.708402-0.777797-0.150376$ C $-7.9819860 .421806 \quad 0.581655$ C $-9.231830 \quad 0.2509681 .463433$ C $-9.111248-0.9762292 .369332$ C $-8.685136-2.217428 \quad 1.580974$ H -7.479438 -2.778937 -0.096705 C $-6.148574-1.9315751 .353348$ H -7.1263570 .6606321 .219995$ $\begin{array}{llll}\text { O } & -10.387103 & 0.120767 & 0.636471\end{array}$ H $-9.360349 \quad 1.1558702 .069738$
H -10.077662 -1.164024 2.852806

O $-8.158384-0.6952953 .389996$

H -8.432788 -3.032589 2.268441

O $-9.794148-2.6778210 .803320$

C $-5.032303-2.1195430 .355356$

H $-5.952594-0.996447 \quad 1.884792$

H $-6.083128-2.7326372 .099369$

H $-11.161038 \quad 0.058499 \quad 1.221884$

H -8.111534 -1.474454 3.969891

H $-9.989323-2.0007240 .132948$

O $-4.663391-3.4313080 .313365$

O $-4.569059-1.212311-0.322713$

C $-3.619924-3.760239-0.608836$

H $-3.595584-4.847550-0.720498$

H -3.804015 -3.313602 -1.590731

H $-2.660316-3.428276-0.205169$

SCF Energy (B3LYP/6-31G**//MMFF) $=-3245.92094026$

0800533

MM̄FF Geometry

C $3.543265-0.2978922 .646422$

C $4.507288-1.1602622 .288730$

C $5.040458-1.3977070 .894138$

O $4.530679-0.427902-0.046775$

C $4.588274-2.7783530 .389068$

C $5.522836-3.368377-0.646976$

C $5.356231-3.283932-1.980963$

C $6.686554-4.141697-0.080500$

C $4.308528-2.524657-2.745508$

C $3.252075-3.436441-3.384630$

C $2.122491-3.810426-2.412988$

C $1.147125-4.790724-3.066008$

C $-0.053102-5.090270-2.155839$

O $1.438791-2.600193-2.068313$

O $-1.051357-5.754534-2.945443$

C $-0.665725-3.755713-1.683003$

C $0.315060-6.053491-1.021083$

O $-1.690105-3.981489-0.717156$

C $0.374877-2.757096-1.123202$

C $-0.286847-1.371359-0.928655$

O $0.849752-3.2381650 .135014$

C $5.1557180 .773314-0.144161$

O $6.123871 \quad 1.160166 \quad 0.488264$

C $4.4097131 .556384-1.152836$

C $4.5266142 .891108-1.179571$

C $3.8169133 .796270-2.086083$

C $2.9298403 .179125-3.136598$

C $0.686668-0.240372-0.563897$

C $-0.0356631 .086938-0.398999$

C 0.2718853 .3674630 .554522

O

C $0.4004744 .296204-0.611719$

C $0.9352775 .524179-0.526311$

C $1.0022396 .486769-1.678547$

C $2.3871797 .056361-1.893892$

C $2.7102268 .278424-1.437822$

C $3.3668036 .246280-2.713503$

C $3.9802575 .125716-1.923281$

N 0.7659052 .0498290 .198623

H 3.0551920 .3161871 .895799

H $4.976025-1.7612723 .066263$

H $6.135467-1.3356350 .924995$

H $3.575592-2.704656-0.021161$

H $4.518073-3.4942451 .219292$

H $\quad 6.083345-3.787877-2.617195$

H $7.335987-4.550858-0.861568$

H $7.301920-3.4959070 .553874$

H $6.326690-4.9812670 .523178$

H $3.835574-1.750731-2.135948$

H $4.830110-1.986355-3.547597$

H $2.816461-2.890555-4.231892$

H $3.723392-4.340599-3.789040$

H $2.557544-4.255225-1.511929$

H $1.658530-5.720103-3.346308$

H $\quad 0.770425-4.361109-4.005034$

H $-0.655009-6.564523-3.309827$

H -1.155318 $-3.294991-2.552518$

H $\quad 0.682897-7.002682-1.429105$ 
H $-0.563572-6.307910-0.418078$

H $1.086892-5.659253-0.356766$

H -2.327109 -4.602487 -1.110023

H - $0.795853-1.085323-1.858665$

H - $1.051820-1.435775-0.146286$

H $\quad 1.145672-2.4892500 .675088$

H $3.7552711 .011497-1.823874$

H $5.1862033 .369348-0.456519$

H $3.5218222 .563100-3.822462$

H $2.3971993 .911542-3.746066$

H $2.1618782 .548738-2.675114$

H $1.202462-0.4686890 .373590$

H $1.438342-0.113683-1.350419$

H 0.8458593 .7080251 .421991

H -0.7814953 .2845350 .841817$

H $-0.0022843 .959796-1.564228$

H 1.3060245 .8757070 .433932

H $0.2948177 .300360-1.470635$

H $0.6519296 .022693-2.609012$

H $3.6918388 .706231-1.612874$

H $2.0017178 .876186-0.873977$

H $4.1882146 .887276-3.061195$

H $2.8754865 .907190-3.629435$

H $4.6415915 .472590-1.127647$

H $\quad 1.7359501 .8329970 .407536$

C $1.635843-0.5312954 .269071$

$\begin{array}{lllll}\text { O } & 0.755138 & 0.365462 & 3.558617\end{array}$

C $-0.275868 \quad 0.8039214 .458957$

C $-0.276299-0.1857625 .612484$

C $1.207334-0.4724515 .739407$

H $\quad 0.0528451 .7756844 .853754$

C $-1.597861 \quad 1.0272363 .714175$

H $-0.7151550 .228823 \quad 6.524702$

H $-0.804076-1.1103075 .364421$

C $1.517674-1.7372966 .521795$

H $1.687898 \quad 0.380695 \quad 6.237304$

C -2.177773 -0.2131493 .000026$

O $-2.560210 \quad 1.5586404 .621981$

H -1.410793 1.7877492 .945180

H -1.368443 -0.7425202 .483881$

O $-2.737647-1.1206353 .946555$

C $-3.2522040 .144872 \quad 1.952633$

C $-3.832293-1.1068361 .264473$

H $-2.799015 \quad 0.810321 \quad 1.211197$

O -4.3119240 .8675382 .577081$

H $-3.049097-1.8621201 .133528$

H -4.613587 -1.531381 1.906929

H $1.138235-1.6583927 .545917$

H $1.057902-2.6176896 .060061$

H $2.597222-1.9093736 .573533$

C $3.090245-0.1010044 .066252$

H -2.193260 2.3782824 .994677

H -3.451594 -0.6505664 .410941$

H $-4.946648 \quad 1.1153741 .883349$

H $3.741309-0.6562454 .751595$

H 3.2015230 .9643374 .303518

H $1.468417-1.5341373 .855281$

C $-6.0662580 .668998-1.155547$

O $-5.478820 \quad 0.1716490 .053666$

C $-4.424261-0.777299-0.119583$

C $-4.948838-2.024727-0.843917$

C $-5.609448-1.648516-2.174448$

C $-6.601986-0.489715-2.014730$

H $-6.9260381 .266534-0.825889$

C $-5.1104991 .603077-1.914953$

H $-3.623870-0.325864-0.715622$

O $-5.893809-2.694672-0.012416$

H -4.114208 -2.712309-1.019254

H -6.133068 -2.524561 -2.576389

O $-4.587183-1.290415-3.100079$

H $-6.899668-0.107633-2.997561$

O $-7.792835-0.990279-1.398342$

C $-4.7398272 .828356-1.104507$

H -4.179703 $1.096792-2.183582$

H $-5.5957001 .952348-2.833662$

H $-6.178602-3.497898-0.480693$

H -5.017810 -1.079948 -3.946029

H $-7.564117-1.272674-0.496074$
O $-3.8040303 .547409-1.793397$

O $-5.2114003 .126547-0.015855$

C $-3.3576244 .746635-1.152819$

H -2.593821 $5.206253-1.785625$

H -4.190565 $5.447469-1.042838$

$\mathrm{H}-2.9158724 .516140-0.178877$

SCF Energy $\left(B 3 L Y P / 6-31 G^{* *} / / M M F F\right)=-3245.91926666$

0800534

MM̄FF Geometry

C $1.3011053 .185178-1.370493$

C $0.3495424 .004068-0.899368$

C $-0.5310153 .648146 \quad 0.268222$

O $-1.8913193 .669196-0.223785$

C -0.3823674 .6432071 .434004$

C 1.0057344 .7196592 .028542

C 1.5659953 .7534862 .781554

C 1.7509955 .9997611 .755480

C 0.9697862 .4298413 .178826

C 1.8417491 .2706252 .678852

C $1.318627-0.0952823 .150115$

C $2.400741-1.1621522 .966936$

C $1.907883-2.5563863 .368750$

O $0.157136-0.4374342 .379615$

O $2.856512-3.5183132 .882918$

C $0.577864-2.8287522 .645188$

C $1.850578-2.7322124 .890051$

O $0.000902-4.0559543 .086929$

C $-0.453010-1.6786412 .768131$

C -1.641742 -1.977992 1.823805

O $-0.935491-1.6240564 .112746$

C -2.8276243 .0086160 .509095$

O -2.6716232 .4786151 .597164$

C $-4.0857703 .011638-0.267259$

C -5.1430172 .3257040 .191588$

C $-6.4415062 .195543-0.471507$

C $-6.6034452 .855689-1.816286$

C $-2.617458-0.816676 \quad 1.613557$

C $-3.871395-1.3189150 .921814$

C $-4.643488-2.277325-1.244610$

O $-4.912629-1.5234421 .542047$

C $-5.766472-1.380610-1.662128$

C $-7.052518-1.652287-1.390011$

C $-8.219413-0.825108-1.850704$

C $-9.017623-0.254463-0.699144$

C $-9.935160-0.997168-0.057027$

C $-8.8038571 .199804-0.345232$

C -7.4069321 .4764990 .138918$

N $-3.683532-1.555016-0.431356$

H $1.4410382 .213789-0.902239$

H $\quad 0.1940614 .972925-1.367265$

H -0.3065212 .6265250 .597502$

H -1.0930404 .3993592 .233723$

H -0.7039675 .6400571 .100684$

H 2.5668293 .9298103 .174581

H 2.7656105 .9878372 .167641

H 1.8357306 .1730690 .678217

H 1.2201106 .8472822 .201208

H 0.9200242 .4011844 .274322

H -0.0563872 .3114412 .821615$

H 1.8788741 .2949991 .582451

H 2.8658961 .4161103 .046443

H $1.040832-0.0177764 .207799$

H $3.306979-0.9018773 .528039$

H $2.699315-1.1955401 .911670$

H $3.724740-3.3020113 .264160$

H $\quad 0.812277-2.9638201 .581040$

H $2.843666-2.5816585 .330098$

H $1.556113-3.7527835 .158501$

H $1.164864-2.0360505 .377287$

H $0.671966-4.7500312 .969936$

H -1.254774 -2.258002 0.837031

H -2.198371 -2.844857 2.203980

H $-1.828525-1.2451344 .114247$

H $-4.0999503 .557615-1.203616$

H $-5.0501371 .798067 \quad 1.140147$

H -6.503201 $3.942818-1.724404$

H $-7.5695032 .664256-2.286879$ 
H $-5.8449162 .490018-2.517808$ H -2.917129 -0.3549862 .559469$ H $-2.155310-0.0364700 .999958$ H -4.996529 -3.144144 -0.675306 H -4.113395 -2.640068 -2.131056 H $-5.513468-0.497541-2.242915$ H $-7.291029-2.551838-0.825816$ H $-8.868873-1.466283-2.461376$ H -7.897206 -0.019474 -2.521604 H $-10.530155-0.5880170 .752655$ H -10.109205 -2.033533 -0.327392 H -9.4908651 .5046430 .455341$ H $-9.0838351 .813992-1.205412$ H -7.186760 1.0275231 .108571 H $-2.763976-1.395625-0.831414$ C $3.6872523 .454564-2.190947$ O $4.0422032 .145732-1.704250$ C $4.9290382 .292787-0.577899$ C $5.4188023 .736121-0.639266$ C $4.1732554 .445167-1.135192$ H $4.3304992 .165547 \quad 0.332458$ C $6.0530881 .252061-0.622143$ H 5.7630364 .1007770 .333258 H $6.2390673 .849780-1.358444$ C $4.4313255 .842427-1.667986$ H $3.4613484 .506645-0.304022$ C $5.665949-0.198610-0.255325$ O $6.638266 \quad 1.268858-1.925287$ H 6.8275001 .5751370 .086032 H $6.540031-0.826240-0.470099$ O $5.451983-0.2779751 .155335$ C $4.460388-0.809675-0.997609$ C $4.366676-2.330666-0.754157$ H $4.558430-0.617359-2.072243$ O $3.265641-0.187499-0.534275$ H $5.327544-2.789619-1.016124$ H $4.182910-2.5059260 .313046$ H $4.8408136 .484107-0.880984$ H $5.1459895 .830720-2.497794$ H $3.5035936 .298939-2.027234$ C $2.1958423 .505302-2.533407$ H $7.4092570 .676651-1.910067$ H 4.6183590 .1816851 .352704 H $2.530906-0.509926-1.082545$ H $1.9894992 .758252-3.309917$ H $1.9496194 .485355-2.958030$ H $4.253683 \quad 3.594562-3.121087$ C $0.821732-3.098732-1.690295$ O $1.987729-2.600069-1.021932$ C $3.245683-3.007008-1.570435$ C $3.367616-4.538304-1.537288$ C $2.182418-5.202350-2.246447$ C $0.842919-4.633796-1.769615$ H $-0.013843-2.813199-1.040727$ C $0.608761-2.417646-3.046172$ H $3.321366-2.657811-2.605923$ O $3.423513-4.980604-0.182453$ H $4.308687-4.829091-2.019098$ H $2.198611-6.282737-2.058107$ O $2.293111-5.002246-3.652472$ H $0.033829-4.976275-2.424656$ O $0.557019-5.155537-0.468262$ C $0.313951-0.948485-2.858938$ H $1.477703-2.491461-3.706225$ H $-0.230495-2.869919-3.588048$ H $3.532581-5.946807-0.193358$ H $3.132620-5.399425-3.940630$ H $1.241966-4.8295910 .140313$ O $-1.012091-0.765569-2.599017$ O $1.168760-0.073669-2.910564$ C - $-1.4152010 .587312-2.361518$ H -1.064408 $0.903331-1.375645$ H -1.034889 $1.254017-3.141491$ H -2.507658 $0.625142-2.375415$ SCF Energy (B3LYP/6-31G**//MMFF) $=-3245.91419558$

0800535

MM̄FF Geometry
C -0.3632773 .9094852 .542755$

C 0.0400612 .8083353 .194841

C -0.8348661 .7429303 .814069$

O $\quad-0.606578 \quad 0.4942843 .119809$

C -2.3531752 .0068513 .763702$

C -3.1646200 .9199274 .446562$

C $-3.862715-0.031113 \quad 3.793798$

C -3.1895120 .9802495 .951348$

C $-3.942029-0.2440092 .306753$

C $-5.262428 \quad 0.295183 \quad 1.748527$

C -5.3137720 .2429510 .214775$

C $-6.6242930 .850830-0.286388$

C $-6.7429390 .763504-1.812695$

O $-5.224412-1.121377-0.201442$

O $-8.1049031 .054333-2.165655$

C $-6.463065-0.688661-2.258080$

C $-5.8823461 .828313-2.504589$

O $-6.406667-0.746433-3.682293$

C $-5.187983-1.308397-1.626965$

C $-5.151831-2.835861-1.878759$

O $-4.003749-0.753910-2.196333$

C $0.384216-0.3201433 .566431$

O $1.238726-0.0627944 .397701$

C $0.212514-1.6183232 .879123$

C $1.170925-2.5529072 .951301$

C $1.109934-3.8849212 .344712$

C $-0.213011-4.3228741 .777407$

C $-3.914253-3.548912-1.313732$

C $-2.706777-3.526766-2.239849$

C $-0.314960-4.185920-2.310924$

O $-2.711789-3.007879-3.352493$

C $0.498230-5.321997-1.778314$

C $1.727040-5.176564-1.258851$

C $2.569172-6.327050-0.786730$

C $3.255511-6.0664780 .535538$

C $4.592305-5.9419370 .605414$

C $2.405129-6.0394021 .786289$

C $2.224402-4.6442192 .332502$

N -1.619267 -4.181819-1.676087

H $-1.422570 \quad 4.0973082 .393646$

H 1.1098622 .6626593 .336285

H $-0.534641 \quad 1.638556 \quad 4.864743$

H -2.6771712 .1124312 .721812$

H -2.5848272 .9655364 .246263$

H -4.434885 -0.7475494 .381387$

H $-3.839013 \quad 0.2136296 .387129$

H $-2.1854190 .830733 \quad 6.359656$

H -3.5608621 .9543436 .286408$

H -3.0964780 .2183621 .789822$

H -3.860163 -1.319705 2.108344

H $-6.087254-0.2974632 .165703$

H $-5.405302 \quad 1.3324862 .077307$

H -4.454332 $0.797979-0.179156$

H $-6.725206 \quad 1.8912980 .046914$

H -7.4717620 .3104270 .158587$

H $-8.3219861 .938380-1.823469$

H -7.332129 -1.288315 -1.952137

H $-6.2090372 .833752-2.212089$

H $-5.9948461 .783510-3.593376$

H $-4.8208851 .746839-2.260535$

H $-6.491706-1.676792-3.948775$

H -6.032024 -3.282059-1.397333

H -5.222803 -3.049978 -2.950988

H $-4.251572-0.171212-2.931672$

H $-0.712531-1.7706682 .333742$

H $2.086993-2.3216333 .493031$

H $-0.984195-4.3213882 .555900$

H $-0.198330-5.327440 \quad 1.351044$

H $-0.523692-3.6438870 .977577$

H $-3.628732-3.132784-0.341285$

H $-4.169814-4.605310-1.160634$

H $\quad 0.148018-3.212683-2.115621$

H $-0.444145-4.290577-3.393229$

H $0.068055-6.317727-1.856591$

H $2.164416-4.183547-1.211610$

H $3.318644-6.525140-1.564185$

H $1.973371-7.244416-0.701234$

H $5.094352-5.786267 \quad 1.554821$ 
H $5.213503-5.987603-0.282741$ H $2.894696-6.6389292 .566055$ H $1.455424-6.551471 \quad 1.607334$ H $3.132607-4.2293432 .772683$ H -1.683560 -4.473511 -0.705509 C $0.438570 \quad 5.213510 \quad 0.534599$

O $1.3383064 .328184-0.173871$ C $2.1414705 .117241-1.071439$ C $1.3774386 .419482-1.262760$ C $0.837200 \quad 6.6375190 .132824$ H $3.0787615 .336976-0.546754$ C $2.4351154 .377713-2.379896$ H $2.0203037 .234594-1.608263$ H $0.5531366 .305355-1.976465$ C -0.3072127 .6353620 .192072$ H $1.654787 \quad 6.9947450 .773810$ C $3.2022733 .047864-2.240250$ O $1.2003274 .095168-3.051007$ H $2.9902645 .044901-3.050284$ H $2.5366352 .270416-1.851550$ O $3.5749492 .632708-3.561603$ C $4.4755203 .117811-1.378940$ C $5.4239161 .909491-1.495765$ H $4.1893263 .243114-0.328726$ O $5.2347794 .278328-1.734597$ H $5.9198021 .919693-2.475355$ H $6.2263522 .070341-0.763312$ H $0.0231668 .620096-0.154272$ H -1.146003 $7.321765-0.438444$ H -0.6781797 .7438991 .215944$ C 0.5628314 .9843802 .044418 H $0.6530873 .566659-2.445232$ H $2.7588112 .574238-4.087575$ H $5.4523224 .208684-2.680096$ H 0.2912435 .8991922 .585092 H 1.6021484 .7488292 .304275 H -0.5737174 .9844650 .177780$ C $3.425148-0.6524050 .356048$ $\begin{array}{lllll}\text { O } & 4.147979 & 0.540397 & 0.017617\end{array}$

C $4.7740420 .536702-1.266601$

C $5.804665-0.600522-1.355083$

C $5.163245-1.951133-1.027724$ C $4.331674-1.8917670 .258289$ H $3.151454-0.5223511 .410887$ C $2.124401-0.787198-0.453097$ H $4.014756 \quad 0.387713-2.040268$ O $6.872725-0.350397-0.443169$ H $6.228093-0.617563-2.366601$ H $5.951443-2.706376-0.919276$ O $4.339691-2.335144-2.124114$ H $3.721874-2.795390 \quad 0.352542$ O $5.220575-1.8869631 .379586$ C $1.1476820 .327702-0.145510$ H $2.308792-0.769845-1.530089$ H $1.634051-1.732793-0.196260$ H $7.525132-1.063060-0.552885$ H $4.043716-3.245890-1.958775$ H $5.725200-1.0557681 .354819$ O $-0.0083150 .113578-0.840659$ O $1.369437 \quad 1.273440 \quad 0.597382$ C $-1.0157621 .115762-0.672020$ H -1.878415 $0.837952-1.282108$ H $-1.327677 \quad 1.1681540 .374322$ H -0.645323 $2.087256-1.012006$ SCF Energy (B3LYP/6-31G**//MMFF)= -3245.90558912

0800536

MM̄MF Geometry

C -1.450139 $4.440744 \quad 0.565224$

C $-2.502636 \quad 3.7581490 .089052$

C -3.7692703 .5095340 .869500$

O

C -4.9835694 .0424830 .091260$

C $-6.269477 \quad 4.011510 \quad 0.895669$

C $-7.284803 \quad 3.1465420 .701998$

C -6.3884945 .0769841 .956881$

C -7.370985 $2.030538-0.300008$

C -7.4207860 .6691920 .402871$
C $-7.377927-0.495956-0.596304$

C $-7.490478-1.8300600 .144236$

C $-7.319123-3.028887-0.797850$

O $-6.132953-0.416642-1.294110$

O $-7.133769-4.2015790 .009767$

C $-6.025211-2.833941-1.613664$

C $-8.572941-3.277473-1.643975$

O $-5.891880-3.852864-2.603212$

C $-5.915308-1.438174-2.273474$

C $-4.507131-1.210280-2.880542$

O $-6.870653-1.351590-3.334811$

C -3.4004471 .5305662 .161495$

O -3.0278202 .1279623 .159536$

C -3.2899860 .0677481 .964880$

C $-2.301456-0.5749332 .605764$

C $-1.948901-1.9908892 .497460$

C $-2.900017-2.9098801 .780486$

C $-3.436054-0.898784-1.831864$

C $-2.019890-1.169044-2.300902$

C $0.306463-1.228112-1.392828$

O $-1.743100-1.575975-3.424769$

C $0.561597-2.494471-0.637544$

C $1.350434-2.5730470 .445041$

C $1.619550-3.8533671 .182645$

C $1.268401-3.7798682 .652393$

C $2.228728-3.7902003 .592606$

C $-0.197433-3.7897833 .039423$

C $-0.793096-2.4072143 .057664$

N $-1.103660-0.910873-1.288961$

H -1.493488 4.8634821 .565824

H $-2.4488163 .328351-0.908898$

H -3.716990 3.9963091 .850413

H $-5.0917003 .482740-0.843045$

H $-4.8033015 .082722-0.212958$

H -8.1651553 .2409141 .337059$

H -7.3676365 .0683722 .447462$

H -5.6319104 .9356532 .734339$

H $-6.253770 \quad 6.069760 \quad 1.514987$

H -8.282733 $2.168266-0.894093$

H $-6.5402582 .054956-1.008204$

H $-6.5629280 .591603 \quad 1.083961$

H -8.3303900 .6023841 .012973$

H $-8.199910-0.379329-1.312123$

H -8.444830 -1.896657 0.681679

H -6.709426 -1.885291 0.916122

H -7.912435 -4.2954890.585098

H -5.185879 -2.971002 -0.920738

H $-9.436312-3.479292-0.998522$

H -8.455289-4.167144 -2.272451

H -8.832419 -2.432806 -2.285252

H $-5.958774-4.709685-2.147873$

H -4.231033 -2.071140 -3.501381

H $-4.538949-0.359230-3.574691$

H $-6.593571-0.647602-3.943950$

H -3.961650 -0.411623 1.264099

H $-1.6471970 .008168 \quad 3.254035$

H -3.940884 -2.688281 2.041346

H -2.750307 -3.960935 2.041593

H -2.779697 -2.814722 0.698507

H $-3.5056920 .155591-1.537524$

H $-3.595408-1.504896-0.937483$

H $0.859919-0.383145-0.977617$

H $0.591795-1.352047-2.441771$

H $0.079036-3.394653-1.011282$

H $1.846873-1.6765730 .808502$

H $2.685243-4.0864911 .058805$

H $1.078028-4.6938130 .730460$

H $1.984074-3.7677034 .649423$

H $3.280545-3.8148953 .327470$

H $-0.310905-4.2138654 .045735$

H $-0.728510-4.4705092 .367555$

H -0.186581 -1.674689 3.592701

H - $1.445591-0.600577-0.384057$

C $1.0465203 .990470 \quad 0.425167$

O $2.2020494 .345944-0.370125$

C $2.8212843 .137545-0.850824$

C 2.4292872 .0798370 .161419

C $0.9925862 .457710 \quad 0.457084$ 
H $2.3707542 .930064-1.829957$

C $4.3345743 .343996-0.990701$

H $2.5319361 .061367-0.221767$

H 3.0429342 .1699171 .065772

C $\quad 0.506933 \quad 1.875357 \quad 1.773740$

H $0.3556512 .085026-0.355680$

C $5.0683142 .062187-1.434168$

O $4.5496224 .374250-1.957774$

H $4.7533713 .703760-0.043225$

H $4.8194011 .265702-0.726868$

O $4.5699401 .652369-2.708530$

C $6.6053902 .173076-1.490498$

C $7.3051940 .912102-2.034964$

H $6.9872592 .394461-0.486767$

O $7.010310 \quad 3.248442-2.339167$

H $7.0810980 .798772-3.103656$

H $8.3859081 .100733-1.980579$

H $-0.5305892 .151463 \quad 1.967632$

H $\quad 0.5680580 .7823091 .755940$

H 1.1071342 .2333142 .616481

C $-0.1822624 .656634-0.206515$

H $4.0626905 .160715-1.656916$

H $4.7927222 .353714-3.344940$

H $6.6590044 .072034-1.960670$

H $-0.2881924 .315689-1.244850$

H $-0.0016965 .736759-0.281825$

H $1.221408 \quad 4.4011451 .427250$

C $7.033409-1.3404410 .938968$

O $7.324936-0.2282190 .080532$

C $6.967269-0.391795-1.294320$

C $7.694484-1.601016-1.903674$

C $7.443063-2.868447-1.080427$

C $7.685337-2.6324660 .412545$

H $7.516725-1.0901421 .892342$

C $5.524866-1.4831961 .203503$

H $5.890066-0.564280-1.372170$

O $9.096809-1.355104-1.984220$

H $7.337220-1.747934-2.929555$

H $8.107347-3.668583-1.429593$

O $6.098359-3.302043-1.263554$

H $7.329919-3.4932950 .990926$

O $9.098411-2.5459930 .619746$

C $5.013369-0.3952212 .125899$

H $4.924208-1.4431010 .291944$

H $5.343685-2.4438101 .700861$

H $9.415681-1.153185-1.087947$

H $5.972643-3.476118-2.211950$

H $9.251109-2.4701831 .577085$

O $3.860796-0.8219452 .720250$

O $5.547001 \quad 0.6927082 .293560$

$\begin{array}{lllll}\text { C } & 3.268529 & 0.075715 & 3.664428\end{array}$

H 3.8164520 .0228424 .609155

H 3.2575081 .1019913 .287097

H $2.236984-0.2435923 .835488$

SCF Energy (B3LYP/6-31G**//MMFF) $=-3245.91535341$

$08 \quad 00537$

MM̄FF Geometry

C -1.215042 -2.117446 2.852980

C $-0.027764-2.1679453 .474134$

C $1.059127-1.1518273 .256682$

O $2.159012-1.8199592 .590759$

C $1.628429-0.5928834 .572269$

C 0.6540070 .1905055 .422710

C 0.3566291 .4902735 .232158

C $0.055747-0.5592706 .584504$

C $0.854203 \quad 2.390565 \quad 4.134792$

C $-0.323397 \quad 3.092710 \quad 3.446302$

C $0.127717 \quad 3.976801 \quad 2.276147$

C -1.045023 4.8174021 .765452

C -0.6734585 .6285160 .517701$

O $\quad 0.605870 \quad 3.115509 \quad 1.238352$

O $-1.9008026 .115356-0.045824$

C $-0.0191294 .684876-0.511455$

C $\quad 0.155679 \quad 6.866022 \quad 0.879068$

O $0.453642 \quad 5.419569-1.638861$

C $1.101978 \quad 3.796217 \quad 0.077558$

C $1.5046262 .719666-0.961456$ $\begin{array}{llll}\text { O } & 2.224113 & 4.618921 & 0.388807\end{array}$

C $2.180883-1.800711 \quad 1.231371$

$\begin{array}{lllll}\text { O } & 1.364792 & -1.283594 & 0.485906\end{array}$

C $3.396264-2.5263280 .800278$

C $3.846923-2.378708-0.454180$

C $5.029822-3.024180-1.025611$

C $5.716336-4.085617-0.206655$

C $2.5841931 .733684-0.494237$

C $4.0074052 .195105-0.760210$

C $6.3687201 .462696-0.432633$

O $4.280312 \quad 3.229075-1.363265$

C $6.7916760 .684200-1.638811$

C $7.708454-0.294470-1.606007$

C $8.138854-1.079236-2.812948$

C $7.949370-2.569049-2.629318$

C $8.960613-3.339010-2.192723$

C $6.623117-3.180889-3.024510$

C $5.449975-2.644125-2.250319$

N $4.9383881 .300513-0.250233$

H - $-1.411879-1.3121312 .151003$

H $0.181617-2.9838854 .161611$

H $\quad 0.705367-0.3168182 .638701$

H 2.4990900 .0361074 .340101

H $2.060738-1.4114525 .165342$

H $-0.3224551 .967945 \quad 5.937471$

H $-0.6101200 .068457 \quad 7.186385$

H $-0.529673-1.4141546 .233687$

H $0.846941-0.9273327 .245828$

H 1.5171113 .1432894 .577571

H 1.4436341 .8417343 .394836

H -1.0196472 .3296963 .076904$

H -0.8611913 .7024554 .183375$

H 0.9442374 .6244732 .616279

H -1.4214695 .4801452 .554975$

H $-1.8842404 .155461 \quad 1.511539$

H $-1.6830056 .570562-0.877504$

H $-0.8133104 .031673-0.892993$

H -0.4062537 .5139951 .562526$

H $0.3637897 .477503-0.005873$

H 1.1048826 .6201421 .359714

H $1.2897745 .845952-1.384231$

H $0.6113042 .121975-1.186809$

H $1.8217793 .195579-1.896644$

H $2.989884 \quad 4.047703 \quad 0.555591$

H $3.895399-3.1500871 .533270$

H $3.309093-1.710914-1.125855$

H $6.138832-3.6529840 .706825$

H $6.536950-4.575766-0.735712$

H $5.009632-4.8754090 .071476$

H 2.4719231 .4973270 .569245

H $2.4569810 .801571-1.058888$

H $6.8559501 .109148 \quad 0.481447$

H $6.6075832 .521885-0.570449$

H $6.3246970 .944208-2.585902$

H $8.187963-0.540942-0.661331$

H $9.198601-0.857674-2.995841$

H $7.610076-0.751852-3.716741$

H $8.848879-4.412584-2.083358$

H $9.923095-2.911357-1.931600$

H $6.648896-4.275083-2.948983$

H $6.451061-2.979882-4.090154$

H $4.900848-1.859747-2.771822$

H 4.6126840 .4376980 .175580

C -2.417881-4.093717 1.891699

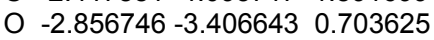

C $-3.688224-4.298522-0.066341$

C $-3.697325-5.6328460 .675901$

C $-3.445120-5.2016572 .106496$

H $-4.700531-3.879981-0.053067$

C $-3.167005-4.396592-1.504674$

H $-4.644225-6.1669770 .549351$

H -2.896184 -6.2958580 .328509$

C $-2.969021-6.332566 \quad 3.001554$

H $-4.372860-4.7798552 .515670$

C $-3.157830-3.061417-2.278026$

O $-1.820301-4.891650-1.476081$

H $-3.747230-5.142534-2.059766$

H $-2.452780-2.365382-1.810523$ 
O $-2.647549-3.331184-3.591168$

C $-4.542221-2.395481-2.424504$

C $-4.570753-1.215058-3.417489$

H $-4.897415-2.062561-1.443451$

O $-5.468794-3.379081-2.896324$

H $-4.189187-1.544995-4.391522$

H -5.621549 $-0.943857-3.589008$

H -3.716074 -7.132203 3.040508

H -2.031652 -6.765659 2.636544

H -2.801165 -5.9799864 .023808$

C $-2.315522-3.1180383 .063605$

H $-1.289898-4.266981-0.951865$

H - $1.776885-3.750955-3.483062$

H -6.350799 -2.969834 -2.908337

H -2.146337-3.666602 3.997355

H $-3.254877-2.5639913 .180285$

H $-1.431893-4.5242791 .669513$

C $-4.0124011 .776910-1.261965$

O $-4.5766390 .611655-1.878778$

C $-3.8013370 .020565-2.927928$

C $-3.572247 \quad 1.035107-4.057929$

C -2.930896 2.318645-3.524499

C -3.677982 2.859469-2.301853

H $-4.8173912 .169968-0.626544$

C -2.837389 $1.408622-0.350854$

H $-2.831297-0.292572-2.532616$

O $-4.802790 \quad 1.345920-4.708033$

H -2.916661 $0.582752-4.811372$

H $-2.928666 \quad 3.072378-4.321275$

O $-1.5770682 .041173-3.179429$

H -3.107885 $3.662688-1.821865$

O $-4.9168393 .431827-2.733098$

C -3.3560690 .6992270 .877821$

H $-2.1011450 .750932-0.820403$

H -2.296564 2.305660 -0.032049

H -5.421274 $1.674251-4.033176$

H -1.164822 $2.881601-2.916930$

H -4.706883 4.152204 -3.351751

O

$\begin{array}{llll}\text { O } & -3.722259 & -0.468685 & 0.885629\end{array}$

C $-3.8879871 .015915 \quad 3.161009$

H -3.945176 1.8266793 .892089

H -4.890812 0.6020663 .017615

H -3.203783 0.2513573 .538952

SCF Energy (B3LYP/6-31G*//MMFF)= -3245.92703362

0800538

MM̄FF Geometry

C -1.202349 2.9348162 .637458

C $-1.8072891 .851128 \quad 2.127989$

C $-2.279666 \quad 0.693148 \quad 2.969354$

O $-1.764005-0.5368862 .414134$

C -3.8107240 .6065362 .880126$

C $-4.438386-0.3033483 .914116$

C -4.846154-1.567040 3.684843

C $-4.656605 \quad 0.3272935 .265532$

C $-4.673079-2.3951032 .440948$

C $-5.952308-2.5061001 .597098$

C $-6.084487-1.3693810 .572852$

C $-7.408935-1.453275-0.184097$

C $-7.520984-0.339581-1.238300$

O $-4.999598-1.500692-0.351946$

O $-8.613562-0.672866-2.111985$

C $-6.242714-0.334924-2.107319$

C $-7.8868861 .006875-0.603031$

O $-6.2174710 .784180-2.989617$

C $-4.910306-0.453856-1.323060$

C $-3.774219-0.836945-2.300531$

O $-4.512194 \quad 0.782276-0.731609$

C $-0.475452-0.8773782 .667769$

O $0.356176-0.230603 \quad 3.281783$

C $-0.264825-2.2099012 .059635$

C $0.965503-2.7380781 .998592$

C $1.305993-4.0508681 .445688$

C $0.175203-4.9253390 .967918$

C $-2.422746-1.125439-1.625904$

C $-1.379726-1.536457-2.654857$

C $0.831610-2.642283-2.882739$
O $-1.492451-1.278750-3.851544$

C $1.324500-3.964670-2.388500$

C $2.608303-4.209488-2.083300$

C $3.132109-5.559498-1.682543$

C $3.938600-5.530777-0.403442$

C $5.279578-5.448700-0.432446$

C $3.197221-5.6996340 .902533$

C $2.605033-4.4106681 .395007$

$\mathrm{N}-0.304720-2.209376-2.088410$

H -1.0273092 .9653243 .711663$

H -2.0041831 .7804461 .062125$

H -1.9545250 .7880804 .012648$

H -4.0856680 .2704561 .876642$

H -4.2583531 .6034252 .992118$

H -5.329294 -2.101632 4.502431

H $-5.133765-0.3583565 .973661$

H -3.7020070 .6380155 .701564$

H -5.300655 1.2081525 .174366

H -3.830962 -2.046866 1.836175

H -4.392281 -3.403393 2.772772

H $-5.907076-3.4591341 .053696$

H $-6.836439-2.5433472 .244922$

H -6.015444 -0.410451 1.094260

H -8.258708 -1.4115040 .508556$

H -7.480976 - $2.424875-0.692630$

H $-9.414049-0.771304-1.568583$

H $-6.321715-1.217340-2.757966$

H -8.863121 $0.941484-0.106949$

H $-7.993071 \quad 1.788677-1.362764$

H -7.1676801 .3426710 .145769$

H $-5.9565701 .566683-2.475792$

H $-4.064440-1.736916-2.858980$

H $-3.635736-0.030898-3.032840$

H $-5.2998391 .285519-0.482160$

H $-1.139599-2.7260671 .679240$

H $1.801220-2.1581892 .388032$

H $0.508191-5.8597780 .511641$

H $-0.421287-4.4107720 .206930$

H $-0.480776-5.1914151 .803989$

H $-2.052086-0.234289-1.107442$

H $-2.531456-1.932057-0.892196$

H $1.596670-1.864591-2.812278$

H $0.535178-2.732259-3.932900$

H $0.599219-4.772675-2.327249$

H $3.336279-3.408508-2.184630$

H $3.761085-5.926058-2.504776$

H $2.320551-6.291443-1.587466$

H $5.866579-5.4597140 .479867$

H $5.819133-5.360264-1.369730$

H $3.885008-6.0590521 .680292$

H $2.457898-6.4996740 .804967$

H $3.352014-3.7096401 .768866$

H $-0.217129-2.229149-1.076386$

C $-1.021264 \quad 4.217544 \quad 0.391131$

O $-0.206925 \quad 3.241348-0.289075$

C $0.4486093 .874721-1.400873$

C $-0.3251945 .159623-1.654458$

C $-0.6655955 .569831-0.234410$

H $1.4549894 .143121-1.058452$

C $0.5637142 .910627-2.585129$

H $0.2683615 .906966-2.189049$

H -1.245509 $4.981048-2.221055$

C $-1.7781426 .600727-0.149806$

H $0.236677 \quad 5.9791130 .239801$

C $1.2004341 .550882-2.230285$

O $-0.7398872 .621537-3.104711$

H $1.126423 \quad 3.385979-3.397895$

H $0.4806060 .944257-1.667609$

O $1.4253730 .848100-3.459628$

C $2.5209431 .628294-1.441312$

C $3.1677040 .242164-1.262582$

H $2.3272972 .068565-0.457156$

O $3.4173532 .491931-2.138488$

H $2.396137-0.485075-0.983868$

H $3.606711-0.064919-2.219746$

H $-1.4883457 .522077-0.665285$

H $-2.701367 \quad 6.233963-0.610894$

H $-1.9990896 .851847 \quad 0.892332$ 
C $-0.737634 \quad 4.161811 \quad 1.895719$ H -1.025399 $3.388182-3.629243$ H $2.1135761 .329081-3.950530$ H $4.2296432 .561941-1.608908$ H -1.207056 5.0261472 .380361 H $0.343901 \quad 4.2443392 .061665$ H -2.069516 3.9695320 .182027 C $6.373698 \quad 1.263603 \quad 0.389717$ O $5.3088681 .115337-0.559688$ C $4.2599690 .223938-0.175571$ C $4.821309-1.1893290 .035897$ C $5.959433-1.1810031 .060790$ C $6.997309-0.0980210 .744967$ H $7.1351081 .852932-0.137645$ C 5.9297972 .0672771 .617253 H 3.8044840 .5793380 .755441

O $5.300696-1.701201-1.205506$ H $4.009813-1.8428580 .372319$ H $6.452146-2.1608621 .069808$ O $5.430886-0.9510452 .363463$ H 7.6902130 .0275041 .585336

O $7.776695-0.515637-0.380036$ C $5.556023 \quad 3.4763151 .220869$ H 5.0849651 .6122232 .142004 H 6.7421382 .1433132 .349656 H $5.632436-2.600509-1.044330$ H $4.790437-1.6585182 .550194$ H $8.214624-1.349849-0.139879$ O 4.2023143 .6319801 .266686 O $6.370045 \quad 4.3285680 .888920$ C 3.7185784 .9267250 .897178 H 4.1888435 .7026631 .508948 H $3.9049025 .105607-0.165790$ H $2.6405404 .949776 \quad 1.074707$ SCF Energy (B3LYP/6-31G**//MMFF) $=-3245.89564776$

0800539

MM̄FF Geometry

C $1.721553-3.1285390 .058542$

C $0.972517-2.6438741 .061445$

C $1.450684-1.8466642 .251112$

O $1.185091-0.4473911 .993308$

C $2.960183-1.9508502 .536669$

C $3.337894-1.5287713 .942560$

C $3.801409-0.3127594 .291010$

C $3.217843-2.6143094 .982397$

C 3.9468120 .9155123 .436188

C 5.4044371 .2202493 .062546

C 5.8404570 .5116461 .771635

C $7.3282590 .733408 \quad 1.499819$

C 7.7554640 .1156940 .159960

O $5.061814 \quad 1.0601520 .700826$

O $9.0615830 .617882-0.160677$

C $6.7968430 .609903-0.942814$

C $7.884904-1.4096530 .245182$

O $7.063947-0.044493-2.181550$

C $5.2986680 .466070-0.579972$

C $4.4415391 .222163-1.624576$

O $4.942383-0.916496-0.593621$

C $-0.064086 \quad 0.0444242 .181980$

O $-1.062305-0.5683782 .520329$

C 0.0131581 .4939361 .892762

C -1.1065722 .2165291 .756418$

C -1.170682 3.6510791 .468811

C 0.1294114 .4105941 .403656

C $2.9516481 .362529-1.270959$

C $2.2063312 .159186-2.333104$

C $-0.0580023 .041243-2.887922$

O $2.7480102 .581976-3.351940$

C $-0.4753364 .326929-2.247640$

C $-1.7581384 .687333-2.085476$

C $-2.1920646 .008628-1.517593$

C $-3.2126525 .869876-0.410118$

C $-4.5256446 .013625-0.657476$

C -2.7038085 .6568670 .996912$

C -2.3806644 .2159561 .277348$

N $0.8667802 .338249-2.015870$

H $2.791658-2.9481090 .033417$
H $-0.100576-2.827677 \quad 1.032695$

H $0.885084-2.1712723 .133730$

H $3.510551-1.3463831 .808601$

H $3.307905-2.9832262 .400017$

H $4.077669-0.1539945 .333085$

H $3.508108-2.2700945 .980758$

H $2.185124-2.9713315 .045291$

H $3.863522-3.4602474 .724497$

H 3.3170110 .8695912 .544305

H 3.5581351 .7565724 .025011

H 5.4864842 .3042982 .908564

H 6.0748310 .9579663 .890042

H $5.634730-0.5591291 .865593$

H 7.9382690 .3327942 .318981

H $7.538537 \quad 1.811647 \quad 1.468427$

H 9.6627250 .3733290 .563735

H $7.0114181 .675799-1.104258$

H $8.622868-1.6890851 .006810$

H $8.255248-1.827514-0.697461$

H $\quad 6.946018-1.9069220 .497534$

H $8.0075390 .083357-2.379149$

H $4.8447492 .236661-1.745813$

H $4.5246460 .721809-2.598067$

H $3.993020-0.996483-0.775328$

H 1.0015931 .9279131 .788836

H -2.0647341 .7104821 .856532$

H $\quad 0.6353784 .3856372 .375106$

H 0.0092915 .4613801 .132242

H $\quad 0.796790 \quad 3.9765330 .651407$

H $2.4766810 .379156-1.190913$

H $2.836271 \quad 1.883278-0.313730$

H $-0.9091552 .379173-3.064545$

H $0.4177453 .249126-3.851333$

H $0.3136125 .009691-1.941316$

H -2.543581 $4.015901-2.425109$

H $-2.6188866 .597479-2.340333$

H -1.335154 $6.589370-1.154174$

H -5.2611235 .9448190 .137233$

H -4.894351 $6.196592-1.661262$

H $-3.4696195 .963076 \quad 1.722647$

H -1.8636326 .3303981 .188331$

H -3.265050 3.5813131 .341079

H $0.505967 \quad 1.953753-1.147521$

C $0.928055-3.058917-2.321185$

O $-0.145094-2.132549-2.044624$

C $-1.241957-2.437621-2.916341$

C $-0.580645-3.004577-4.160275$

C $0.482064-3.888755-3.533215$

H $-1.826780-3.225984-2.425452$

C $-2.158308-1.227240-3.128618$

H $-1.278988-3.557607-4.794998$

H -0.106356 -2.220858 -4.761434

C $1.618862-4.240047-4.477966$

H $0.007417-4.821279-3.198505$

C $-2.699980-0.668645-1.793278$

O $-1.464635-0.196630-3.826269$

H -3.004571 -1.540826 -3.752421

H -3.011285 -1.513413-1.171226

O $-1.6625240 .002552-1.081080$

C $-3.9098930 .269968-1.962290$

C $-4.3835460 .952476-0.665945$

H -4.736921 -0.307507 -2.393009

O $-3.595427 \quad 1.300747-2.900109$

H $-3.6732261 .739045-0.382815$

H $-5.3152861 .479153-0.911498$

H $1.243511-4.799346-5.341159$

H $2.121974-3.341941-4.852087$

H $2.366243-4.858840-3.971129$

C $1.157850-3.928045-1.082000$

H $-1.379974-0.471319-4.754586$

H $-1.3663270 .743925-1.634656$

H $-4.3947711 .840256-3.024774$

H $1.857179-4.740770-1.310191$

H $0.213721-4.394236-0.771790$

H $1.817888-2.459845-2.550314$

C $-5.746451-2.0557181 .103569$

O $-5.476605-1.0508620 .114285$

C -4.6140470 .0120290 .529029$ 
C $-5.214497 \quad 0.754288 \quad 1.734320$

C $-5.541749-0.2125502 .876084$

C $-6.333838-1.4265302 .379923$

H -6.527077 -2.685461 0.657237

C $-4.521445-2.9451851 .378338$

H -3.644561 -0.4033920 .820225$

$\begin{array}{lllll}\text { O } & -6.398531 & 1.453541 & 1.354460\end{array}$

H $-4.499649 \quad 1.5074272 .081881$

H -6.1265040 .3120493 .641335$

O $-4.336121-0.666710 \quad 3.483632$

H $-6.400545-2.1886893 .165123$

O -7.671748 -1.019292 2.078167

C $-4.196192-3.861710 \quad 0.216433$

H -3.622490 -2.361015 1.589050

H $-4.734939-3.5886472 .240259$

H $-7.013991 \quad 0.8076200 .967232$

H -3.8515580 .1152993 .798629$

H $-8.062479-0.6684902 .896716$

O $-3.212722-4.7282070 .604581$

O $-4.724805-3.825502-0.885721$

C $-2.812254-5.690985-0.375442$

H -2.430010 -5.191358 -1.269261

H $-2.011860-6.2994690 .053746$

H $-3.653378-6.343552-0.627874$

SCF Energy (B3LYP/6-31G**//MMFF) $=-3245.90560901$

0800540

MM̄FF Geometry

C $2.340242-4.0019960 .631435$

C $2.215733-3.374000-0.548121$

C $1.244599-3.695478-1.661808$

O $0.657870-2.461735-2.163332$

C $0.162382-4.759930-1.375180$

C $-0.739144-5.067279-2.557816$

C $-2.078668-4.913923-2.576278$

C $-0.043715-5.632483-3.771777$

C $-2.985719-4.420024-1.483237$

C $-3.683566-3.118437-1.894297$

C $-4.626538-2.588722-0.803654$

C $-5.291990-1.289155-1.263040$

C $-6.158748-0.656410-0.163825$

$\begin{array}{lllll}0 & -3.860500 & -2.358146 & 0.383328\end{array}$

O $-6.4465360 .695586-0.555842$

C $-5.336333-0.5827201 .141428$

C $-7.511905-1.364875-0.032933$

O $-6.143545-0.1270462 .224001$

C -4.632914 -1.907892 1.506024

C $-3.675658-1.7801942 .718016$

O $-5.627152-2.8756381 .867206$

C $-0.104229-1.679557-1.354119$

O $-0.405404-1.889088-0.191013$

C $-0.522014-0.503472-2.151947$

C $-1.4543580 .331666-1.670185$

C $-1.9997481 .506249-2.353616$

C $-1.3830491 .899634-3.670267$

C $-2.523592-0.7865262 .547650$

C -2.8522160 .6204963 .015675$

C -2.1747582 .9927932 .656089$

O

C $-3.1284613 .551776 \quad 1.648415$

C $-2.782171 \quad 4.4485230 .713281$

C $-3.7362985 .002309-0.304690$

C $-3.3226114 .680370-1.723768$

C $-2.6519935 .577644-2.465678$

C $-3.7700403 .362440-2.315309$

C $-3.0169502 .178236-1.774692$

N -2.033440 1.5688082 .419698

H $1.694476-4.8499610 .845782$

H $2.873092-2.539700-0.771054$

H $1.868297-4.058574-2.487853$

H $-0.423935-4.451568-0.506570$

H $0.648784-5.700097-1.082705$

H -2.607101 -5.190378 -3.488655

H $-0.750744-5.992429-4.526914$

H $0.583411-4.872041-4.246871$

H $0.587306-6.481129-3.487811$

H -3.740913 -5.192486 -1.293043

H -2.452884 -4.277132 -0.540945
H $-2.918273-2.364918-2.110803$

H $-4.250830-3.277569-2.820253$

H $-5.383071-3.356043-0.600703$

H $-5.884267-1.456329-2.171396$

H $-4.515822-0.566312-1.545892$

H $-6.8919530 .669251-1.419940$

H -4.5766450 .1911580 .979939$

H -8.060111 -1.320881 -0.981998

H $-8.151419-0.8644010 .702369$

H -7.419967 -2.416984 0.244301

H $-6.696565-0.8717212 .515540$

H -4.249627 -1.577605 3.630303

H -3.218091 -2.767630 2.875249

H $-5.201344-3.5732522 .392774$

H $-0.069158-0.372947-3.128356$

H $-1.8816020 .123848-0.690956$

H -1.754542 $2.852450-4.054289$

H -1.586435 $1.139090-4.431771$

H $-0.2978462 .015053-3.570221$

H -1.679683 -1.118279 3.165215

H $-2.178040-0.7699341 .510738$

H -1.1783803 .4382442 .576732$

H -2.555951 3.1649113 .667523

H -4.1555683 .1962961 .690559$

H -1.7586304 .8127880 .678013$

H $-3.7812046 .090173-0.163550$

H $-4.7588834 .643819-0.133129$

H -2.378985 5.369949 -3.494919

H -2.354993 $6.538111-2.057611$

H -3.718662 $3.390852-3.409635$

H $-4.8369223 .218418-2.099892$

H -3.394735 $1.820165-0.817006$

H -1.3961831 .2779421 .684345$

C $4.066113-2.3464331 .641493$

O $5.027097-2.4058040 .565199$

C $6.236739-1.7678020 .993565$

C $5.918707-1.0677442 .307172$

C $4.882312-2.0087612 .890650$

H $6.944471-2.5785231 .217367$

C $6.866177-0.894260-0.095434$

H $6.799295-0.9494862 .945805$

H $5.480874-0.0772972 .140870$

C $4.082685-1.3882074 .020874$

H $5.389305-2.9102543 .260744$

C $6.0520240 .316614-0.607578$

O $8.105917-0.4067300 .437761$

H $7.126321-1.532760-0.948823$

H $5.904790 \quad 1.032690 \quad 0.206432$

O $6.8856340 .995209-1.563250$

C $4.703453-0.009468-1.283221$

C $4.0529301 .192689-1.996944$

H $4.002600-0.408770-0.547132$

O $4.930067-1.026650-2.262741$

H $4.7938331 .674568-2.645918$

H $3.2729580 .813484-2.671799$

H $4.736785-1.1442464 .864474$

H $3.596251-0.4629853 .699797$

H $3.309358-2.0730754 .381091$

C $3.303617-3.670867 \quad 1.745913$

$\begin{array}{llll}\text { H } & 8.527693 & 0.130217 & -0.255014\end{array}$

H $7.0005050 .400376-2.324508$

H $4.064409-1.289054-2.619590$

H $2.721531-3.6692362 .675270$

H $4.018999-4.5000911 .812723$

H $3.372079-1.5273621 .417463$

C 1.3768052 .5149270 .246293

O $2.1507051 .697160-0.642436$

C $3.4323272 .212780-1.029293$

C $3.2880163 .591506-1.690331$

C $2.5366574 .559562-0.774572$

C $1.2238723 .952044-0.281710$

H 0.3760752 .0648860 .237926

C 1.9164612 .4431911 .683221

H $4.0638772 .307400-0.141390$

O $2.5987583 .458414-2.931460$

H $4.2891873 .982032-1.909681$

H $2.3242225 .489022-1.316538$

O 3.3450004 .8849520 .352328 
H 0.7794944 .5817010 .497569

O $0.291882 \quad 3.915040-1.364727$

C $1.433912 \quad 1.1882292 .383560$

H 3.0079112 .4450891 .733310

H 1.5360013 .2995972 .252545

H $2.5362924 .343496-3.329072$

H 4.1654305 .2868370 .019245

H $0.1537094 .829481-1.664849$

O $1.335976 \quad 1.432598 \quad 3.722422$

$\begin{array}{lllll}\text { O } & 1.185784 & 0.129701 & 1.822616\end{array}$

C $0.8606320 .368987 \quad 4.558050$

H $-0.178927 \quad 0.573167 \quad 4.826894$

H $0.934752-0.6128364 .081910$

H $1.463808 \quad 0.359892 \quad 5.469862$

SCF Energy (B3LYP/6-31G**//MMFF) $=-3245.89144928$

0800541

MM̄FF Geometry

C $1.6236442 .745203-2.776238$

C $2.4594943 .787863-2.648756$

C $3.7897803 .808679-1.927029$

O $4.0856252 .537771-1.305611$

C $3.7531274 .881127-0.821327$

C $5.0810775 .072530-0.112301$

C 5.3498754 .6761841 .148084

C $6.1330355 .801759-0.909026$

C 4.4453053 .9419192 .098253

C 5.0456902 .5864142 .493502

C 4.0372931 .7096053 .250890

C $4.691575 \quad 0.3976513 .688251$

C $3.683020-0.5785594 .311560$

O $2.947302 \quad 1.4463792 .362171$

O $4.302957-1.8726854 .373792$

C $2.464638-0.7088463 .372143$

C $3.336565-0.1941335 .754569$

O $1.437171-1.4890543 .978004$

C 1.8940620 .6495522 .918524

C $\quad 0.7986410 .514367 \quad 1.831704$

$\begin{array}{lllll}\text { O } & 1.293451 & 1.304291 & 4.042197\end{array}$

C $4.7483191 .613395-2.054522$

O $5.0951231 .722362-3.219838$

C $4.976808 \quad 0.425217-1.201523$

C $5.487321-0.693180-1.738105$

C $5.798078-1.933897-1.022744$

C $5.508459-1.9813920 .453974$

C $1.262413-0.0961330 .505344$

C $1.035856-1.5930040 .387488$

C $1.529537-3.504415-1.137902$

O $0.365962-2.2407291 .186519$

C $2.729459-4.252269-0.650949$

C $3.528836-4.968937-1.455649$

C $4.697779-5.775957-0.969653$

C $5.981449-5.448767-1.698130$

C $6.417220-6.217817-2.710421$

C $6.807386-4.290712-1.188070$

C $6.336128-2.960821-1.713650$

N $1.639849-2.111034-0.751245$

H $1.8830391 .782222-2.346378$

H $2.1729524 .732464-3.108693$

H $4.5717034 .060917-2.653928$

H $2.9610314 .633954-0.107130$

H $3.4579545 .848688-1.250605$

H $6.3372914 .894957 \quad 1.553515$

H $7.0411115 .989483-0.326069$

H $6.4231595 .219460-1.788816$

H $5.7521106 .772542-1.242615$

H 4.3119454 .5548532 .997851

H $3.4489973 .797297 \quad 1.676559$

H $5.3671562 .061474 \quad 1.585237$

H 5.9387082 .7472423 .110172

H 3.6691322 .2663514 .120708

H 5.5205110 .5895184 .381000

H $5.143364-0.0889072 .814252$

H $5.116762-1.7903894 .900005$

H $2.801773-1.2659152 .489666$

H $4.236670-0.2083206 .380957$

H $2.649159-0.9197786 .203180$

H 2.8942570 .8004815 .839029
H $1.831285-2.3418484 .229732$

H $-0.076367-0.0080532 .236635$

H 0.4465761 .5300081 .599619

H $\quad 0.6566341 .960530 \quad 3.714430$

H $4.7255280 .505833-0.150960$

H $5.713869-0.701001-2.803660$

H $6.167522-1.2916220 .991243$

H $5.644173-2.9712600 .894663$

H $4.468128-1.7042090 .654828$

H $0.6789460 .360827-0.302646$

H 2.3148190 .1301630 .311926

H $1.438554-3.531546-2.228376$

H $\quad 0.622668-3.936727-0.702834$

H $2.929860-4.2295640 .417686$

H $3.308245-5.012872-2.519941$

H $4.450056-6.836125-1.112068$

H $4.849168-5.6528710 .109958$

H $7.353880-6.008522-3.216474$

H $5.843788-7.070293-3.059253$

H $7.851404-4.409461-1.509110$

H $6.854140-4.328427-0.096021$

H $6.504167-2.833461-2.783979$

H $2.212867-1.504012-1.330410$

C $-0.8365552 .300149-2.626670$

O $-0.769620 \quad 0.859933-2.569590$

C $-2.1039330 .321132-2.697885$

C $-3.0442991 .507584-2.554455$

C $-2.2252222 .621034-3.175323$

H $-2.164171-0.092253-3.712769$

C $-2.286974-0.792462-1.659604$

H $-4.0026031 .349112-3.057820$

H $-3.2429901 .723117-1.496932$

C $-2.7278144 .009365-2.818533$

H $-2.2358182 .501512-4.267049$

C $-3.732611-1.315173-1.562487$

O $-1.415519-1.865253-2.024509$

H $-1.959103-0.438404-0.674661$

H $-4.382640-0.464700-1.341563$

O $-4.143019-1.830602-2.828166$

C $-3.947313-2.382069-0.469636$

C $-5.398887-2.892133-0.364052$

H $-3.632606-1.965556 \quad 0.494734$

O $-3.113815-3.513151-0.728159$

H $-5.662466-3.454358-1.268881$

H $-5.421878-3.6242560 .454184$

H $-2.1003904 .781846-3.273862$

H $-3.7522704 .151184-3.177954$

H $-2.7271164 .170405-1.735137$

C $0.3051822 .824991-3.499444$

H $-0.516715-1.499942-2.091262$

H $-3.567247-2.587831-3.031715$

H $-3.211288-4.1276850 .018977$

H $0.1035943 .859503-3.799322$

H $0.3845272 .222020-4.412188$

H $-0.7379532 .666896-1.596225$

C $-6.8724860 .058303 \quad 1.380060$

O $-6.061936-1.0859151 .074163$

C $-6.444505-1.796400-0.107500$

C $-7.859983-2.3684120 .059544$

C $-8.861172-1.255715 \quad 0.392012$

C $-8.354726-0.3402881 .514051$

H $-6.5205150 .383472 \quad 2.367535$

C $-6.590811 \quad 1.1922780 .382728$

H $-6.443414-1.111634-0.961971$

O $-7.862544-3.3380091 .105451$

H $-8.149529-2.878191-0.867182$

H $-9.815827-1.7000210 .699639$

O $-9.105618-0.470257-0.771082$

H -8.9773310 .5589001 .571391$

O -8.516998 -1.024078 2.761967

C $-6.963026 \quad 2.559987 \quad 0.910444$

H $-5.516205 \quad 1.210593 \quad 0.162464$

H $-7.1166881 .033618-0.562568$

H -8.762104 -3.7023901 .165282$

H $-9.462304-1.061645-1.455624$

H $-7.925813-1.7964852 .756123$

O $-6.662548 \quad 3.493946-0.041030$

$\begin{array}{lllll} & 0 & -7.448282 & 2.788460 & 2.009560\end{array}$ 
C $-6.953940 \quad 4.8494370 .313222$

H -8.0249724 .9699860 .501403$

H $-6.3710345 .145026 \quad 1.190788$

H -6.671085 $5.488653-0.527303$

SCF Energy (B3LYP/6-31G**//MMFF) $=-3245.91255505$

0800542

MM̄FF Geometry

C -2.297793 -3.921511 -0.113042

C $-1.880323-4.6503420 .934127$

C $-0.881373-4.2361951 .990306$

O $-0.328110-2.9362531 .688778$

C $0.281537-5.2523702 .012907$

C $1.341738-4.9681583 .060619$

C $2.629050-4.6742122 .786000$

C $0.885049-5.0653204 .493674$

C $3.294325-4.6111931 .437943$

C $3.332495-3.1846180 .892418$

C $3.869900-3.088131-0.542622$

C $5.350904-3.459612-0.644833$

C $5.905829-3.240239-2.062684$

O $3.675404-1.729177-0.959835$

O $7.339221-3.294690-1.985501$

C $5.531065-1.817424-2.530417$

C $5.482895-4.362607-3.017733$

O $5.910774-1.606389-3.888007$

C $4.040392-1.471974-2.322969$

C $3.7233680 .011423-2.622059$

O $3.248511-2.255871-3.223716$

C $-0.418743-1.9598112 .631087$

O $-1.032677-1.9995613 .684723$

C $0.425937-0.8324432 .175310$

C 0.7555890 .1450973 .033003

C 1.6515721 .2701292 .752085

C $2.2780801 .335140 \quad 1.383912$

C $4.5289341 .021305-1.802241$

C $3.9706212 .430333-1.912281$

C $4.2706154 .705014-0.925451$

O $3.0626872 .737194-2.679576$

C 4.2041105 .0491670 .528391

C 3.0705105 .3822961 .163473

C 2.9971825 .7022842 .629466

C 2.1608544 .6962293 .393623

C 0.9303925 .0268353 .822033

C 2.8194023 .3597553 .679003

C 1.8837112 .1786673 .723672

N $4.6149423 .300103-1.044394$

H -1.926390 -2.911404 -0.260658

H -2.282289 -5.6558991 .049962$

H -1.401547 -4.234983 2.955113

H $\quad 0.729590-5.279368 \quad 1.013698$

H -0.110956 -6.262168 2.193244

H $3.299863-4.4752513 .620995$

H $1.715434-4.9684625 .201474$

H $0.162283-4.2794124 .728289$

H $0.414389-6.0371894 .675265$

H $4.313956-4.9971401 .554098$

H $2.807280-5.2804580 .721994$

H $2.320049-2.7649680 .900438$

H $3.921021-2.5363361 .555298$

H $3.262187-3.741774-1.179605$

H $5.523345-4.496557-0.333926$

H $5.928773-2.8375920 .053149$

H $7.587345-4.161401-1.620807$

H $\quad 6.143832-1.129095-1.935329$

H $5.834317-5.333130-2.646845$

H $5.943509-4.237115-4.003738$

H $4.400887-4.432481-3.145811$

H $5.262774-2.062860-4.451294$

H $3.8542290 .216596-3.692650$

H $2.654727 \quad 0.175732-2.422283$

H $2.387685-1.819381-3.336219$

H $\quad 0.794776-0.8648601 .156505$

H $\quad 0.355767 \quad 0.1071734 .045455$

H 2.9507850 .4841291 .231763

H 2.8598912 .2430601 .218205

H 1.5114851 .3191690 .602041

H $4.5289070 .736941-0.744291$
H $5.5667551 .049345-2.152211$

H $3.3284254 .909231-1.444046$

H $5.0621285 .283229-1.412160$

H 5.1364945 .0112761 .087050

H $2.1399365 .423256 \quad 0.603377$

H 2.5787976 .7117412 .732789

H 3.9972695 .7495353 .078777

H $\quad 0.3182254 .3427594 .399094$

H 0.5032946 .0014043 .608308

H 3.3326583 .4303194 .647090

H $3.620573 \quad 3.1716662 .956969$

H 1.3795222 .0547704 .682761

H $5.4250552 .965757-0.529811$

C $-4.604357-3.769609-1.204770$

O $-4.472421-2.380013-1.555772$

C $-5.500870-1.640816-0.865348$

C $-6.405761-2.674347-0.198478$

C $-5.424909-3.7971040 .081945$

H $-4.998540-1.068848-0.078100$

C $-6.216659-0.686661-1.826891$

H $-6.882944-2.2836360 .705598$

H -7.198012 -3.018586 -0.873541

C $-6.087983-5.1321520 .368300$

H $-4.810177-3.5074610 .943501$

C $-5.2808850 .298747-2.558380$

O $-6.891422-1.466388-2.822703$

H $-6.996684-0.138853-1.284270$

H -4.672095 -0.245981 -3.290538

O $-6.1243151 .182922-3.307709$

C $-4.3731881 .118534-1.622298$

C $-3.5551002 .193316-2.365338$

H -3.683515 $0.437599-1.111399$

O $-5.191541 \quad 1.734905-0.628389$

H -3.113156 $1.755050-3.267825$

H -4.229626 $3.004985-2.664331$

H $-6.725449-5.062174 \quad 1.255738$

H $-6.713173-5.458474-0.469492$

H $-5.337997-5.9080240 .551854$

C $-3.237341-4.452025-1.161529$

H -7.345931 -0.844934 -3.416981

H $-5.5642271 .656801-3.945150$

H $-4.6036272 .190939-0.002950$

H -2.742994 -4.313096 -2.131604

H $-3.379661-5.532226-1.035512$

H -5.179943 -4.231217 -2.019005

C -2.1213964 .0082010 .574099$

O $-3.0290443 .466565-0.392338$

C $-2.4267242 .778333-1.491633$

C $-1.5173833 .734571-2.278155$

C $-0.4848164 .403724-1.362079$

C $-1.1267314 .973603-0.092778$

H -2.754742 4.5906791 .255346

C $-1.4439462 .906273 \quad 1.394043$

H -1.829649 $1.940894-1.114673$

O $-2.2964494 .737534-2.927350$

H $-1.0046253 .169333-3.064933$

H $0.0142945 .215306-1.905802$

O $0.5080893 .454815-0.986308$

H $-0.351567 \quad 5.262947 \quad 0.625678$

O $-1.827474 \quad 6.166791-0.456322$

C -2.4563992 .1483302 .219003$

H $-0.8909232 .194446 \quad 0.775887$

H -0.7190483 .3343442 .092695$

H -2.802243 $5.209826-2.243930$

H $0.9431253 .146258-1.799507$

H $-2.171860 \quad 6.5656930 .360890$

O -2.6495120 .9044751 .695178$

O $-3.0201702 .614255 \quad 3.200831$

C $-3.586700 \quad 0.0783712 .392617$

H $-3.536567-0.9268891 .965718$

H -3.3333800 .0174353 .455550$

H $-4.5991410 .469608 \quad 2.258170$

SCF Energy (B3LYP/6-31G**//MMFF) $=-3245.92798720$

$08 \_00543$

MM̄FF Geometry

C -0.734053 -4.1862280 .244769$

C $-1.558109-3.1887490 .600290$ 
C $-2.191144-3.0904761 .961698$

O $-3.586619-2.7586241 .752954$

C - $-1.556005-1.9783782 .817118$

C $-0.115763-2.2232783 .222813$

C $0.917268-1.4325952 .869579$

C $0.113916-3.3971874 .138633$

C $0.861421-0.2176981 .988155$

C 0.5154191 .0425682 .788703

C 0.4093842 .2933121 .903787

C -0.0058503 .5034192 .746003$

C $-0.259917 \quad 4.751517 \quad 1.888771$

O $-0.5630132 .035546 \quad 0.888033$

O -0.9346565 .7040282 .724644$

C -1.213873 4.3724790 .734476

C 1.0552595 .4128481 .460937

O $-1.3933865 .470694-0.156449$

C $-0.7676553 .112145-0.034680$

C $-1.8164952 .644554-1.067528$

O $0.4316283 .415297-0.756493$

C $-4.472923-3.1503342 .703799$

O $-4.222875-3.8077513 .705646$

C -5.848475 -2.690059 2.402882

C $-6.154886-1.7991871 .446308$

C -7.497734 -1.294569 1.137942

C $-8.655516-1.8841861 .902039$

C $-3.1607802 .220491-0.472062$

C $-3.964547 \quad 1.426430-1.481945$

C $-5.2788191 .676405-3.583677$

O $-4.0982530 .208570-1.385094$

C $-6.7255291 .740837-3.209513$

C $-7.5322110 .669300-3.174433$

C $-8.9904470 .737803-2.824493$

C $-9.348833-0.120331-1.632560$

C $-10.113874-1.215966-1.772409$

C $-8.8949870 .341600-0.266034$

C $-7.633212-0.3433290 .189208$

N $-4.4827812 .213676-2.496923$

H $-0.499234-4.9662570 .964933$

H $-1.775024-2.395513-0.111314$

H -2.128060 -4.059780 2.471481

H $-2.145237-1.8523763 .735732$

H -1.676479 -1.020173 2.297216

H $1.909759-1.6735733 .246907$

H $1.145381-3.4425784 .504286$

H $-0.540513-3.3298635 .013970$

H $-0.091549-4.3386453 .620679$

H $1.841371-0.0910001 .513960$

H $0.151155-0.3771861 .169762$

H -0.4443940 .8838693 .297583$

H 1.2696491 .2071813 .568514

H 1.3813832 .4698371 .431239

H 0.7430423 .7132093 .520236

H -0.9329483 .2660803 .287148$

H -1.165432 6.4699472 .171065

H $-2.1957554 .187357 \quad 1.187541$

H 1.6266555 .7270382 .342845

H 0.8720906 .3264540 .884835

H 1.6962274 .7542040 .870995

H $-0.5974945 .535063-0.711229$

H -1.966455 $3.421384-1.828300$

H -1.403576 $1.786385-1.615620$

H $0.5354262 .763723-1.469714$

H $-6.609767-3.1106763 .052364$

H -5.354107 -1.3827450 .837899$

H $-9.630757-1.5309591 .561211$

H -8.578531 -1.637056 2.966624

H -8.673068 -2.974668 1.793605

H $-3.024628 \quad 1.5859650 .410897$

H -3.745990 $3.091422-0.157216$

H $-4.9527940 .653987-3.801883$

H $-5.0934772 .297737-4.465202$

H -7.131357 $2.721394-2.971884$

H -7.130914 $-0.309283-3.428258$

H $-9.5588520 .420966-3.708255$

H -9.303794 $1.770417-2.625519$

H $-10.400266-1.816917-0.915760$

H -10.468553 -1.538438 -2.745662

H $\quad-9.7103840 .2208340 .454624$
H $-8.7112921 .423787-0.283145$

H $-6.7357850 .015886-0.313820$

H $-4.3750693 .222390-2.444546$

C $1.242258-3.517341-1.132107$

O $0.944713-2.116895-1.313088$

C $1.859047-1.564676-2.285522$

C $2.946825-2.609387-2.464604$

C $2.158950-3.892972-2.294284$

H $1.281637-1.450884-3.211771$

C $2.328718-0.200196-1.777693$

H $3.443591-2.537164-3.436409$

H $3.707954-2.519540-1.679316$

C $3.033135-5.105644-2.025990$

H $1.565083-4.068213-3.201288$

C $3.5080300 .404444-2.553387$

O $1.2162730 .695838-1.852756$

H $2.583288-0.286014-0.717810$

H $4.389106-0.236150-2.452495$

O $3.178722 \quad 0.397159-3.949455$

C $3.8630461 .851482-2.162785$

C $4.0208402 .144875-0.659863$

H $3.0936252 .521534-2.566898$

O $5.0776902 .195619-2.837620$

H $4.1592933 .228680-0.549223$

H $3.0824111 .894281-0.155399$

H $3.709348-5.285480-2.868261$

H $3.645173-4.968545-1.128051$

H $2.423664-6.003537-1.884412$

C $-0.071037-4.298450-1.099413$

H $0.4935960 .301855-1.334056$

H $3.9397270 .769379-4.427438$

H $5.2696883 .127740-2.637998$

H $\quad 0.105050-5.362153-1.295698$

H $-0.752548-3.934196-1.878328$

H $1.778811-3.610377-0.178316$

C $6.009569-0.7649960 .630563$

$\begin{array}{lllll}\text { O } & 4.990540 & 0.042044 & 0.029107\end{array}$

C $5.206811 \quad 1.4536650 .046694$

C 5.3512501 .9545631 .496626

C 6.4222231 .1708132 .260182

C $6.248579-0.3419152 .088581$

H $5.588487-1.7784180 .643690$

C $7.293176-0.791842-0.207491$

H $6.1215191 .685080-0.508592$

O $4.101407 \quad 1.8230432 .172799$

H 5.6051793 .0212881 .477431

H $\quad 6.363573 \quad 1.4252803 .325544$

O $7.703211 \quad 1.574272 \quad 1.786525$

H $7.123589-0.8701942 .483343$

O $5.130155-0.7714052 .871342$

C $7.077562-1.467413-1.540727$

H $7.6810760 .209346-0.415661$

H $8.092450-1.332460 \quad 0.313704$

H 4.2099162 .1917403 .065915

H 8.3719531 .0860302 .296584

H $4.328971-0.3720852 .490570$

O $6.927667-2.811521-1.367412$

O $7.060205-0.870576-2.609555$

C $6.745337-3.572668-2.565398$

H $5.925406-3.165696-3.163953$

H $7.674267-3.583110-3.142977$

H $6.494641-4.598285-2.281557$

SCF Energy (B3LYP/6-31G**//MMFF)= -3245.92657067

08_00544

MM̄MF Geometry

C -1.762949 1.7881333 .579412

C -3.0462031 .9882203 .240304$

C -3.5857292 .9066872 .167236$

O -4.0690022 .0826451 .079202$

C -2.5667593 .8823561 .549684$

C -3.2122935 .0766330 .873620$

C $-3.4614805 .185538-0.445745$

C $-3.5279846 .228547 \quad 1.794543$

C $-3.2804714 .152562-1.521991$

C $-2.0764294 .432037-2.433000$

C $-0.7640293 .870447-1.867056$

C $0.4198594 .225446-2.765991$ 
C $1.722128 \quad 3.577505-2.270605$ O $-0.9063882 .447948-1.799508$ O $2.6984053 .699719-3.316845$ C $1.4825042 .068580-2.047066$ C $2.2978324 .310815-1.052671$ O $2.6330381 .484368-1.440773$ C $0.2079631 .757698-1.225204$ C $-0.1073530 .243183-1.278276$ O $\quad 0.4275642 .1446350 .132139$ C -5.3152971 .5517391 .151657$ O -6.1199181 .6631072 .060716$ C $-5.5117850 .774949-0.091615$ C $-6.465598-0.164505-0.153282$ C $-6.730047-1.040396-1.297317$ C $-5.924571-0.813630-2.551007$ C $-1.490983-0.139365-0.730781$ C $-1.685187-1.647157-0.698028$ C $-3.289121-3.3869470 .090997$ O $-0.868489-2.438602-1.162340$ C $-4.192187-3.794404-1.030992$ C $-5.363943-4.422048-0.843671$ C $-6.263174-4.868605-1.962144$ C $-7.702722-4.442759-1.767348$ C $-8.624384-5.310654-1.316687$ C $-8.089593-3.037777-2.174197$ C $-7.658780-2.010096-1.163093$ N -2.880377 -2.005349 -0.089490 H -0.9677252 .2976863 .043746$ H -3.8087451 .4505553 .802073$ H -4.4203873 .4746242 .597700$ H -1.9353543 .3468220 .833972$ H -1.8903254 .2761572 .318868$ H $-3.8912286 .119966-0.806058$ H -3.9882557 .0708881 .267215$ H -4.2234285 .9122872 .578295$ H $-2.612761 \quad 6.5970242 .269535$ H -3.232897 $3.140655-1.112122$ H $-4.1896754 .175166-2.136809$ H $-2.2753363 .946505-3.397747$ H -1.981632 $5.507726-2.624463$ H $-0.6079974 .273971-0.861259$ H $0.5370335 .313552-2.844379$ H $\quad 0.2233383 .870548-3.787484$ H $2.8120754 .645694-3.511696$ H $1.3845331 .610098-3.041157$ H $2.4962085 .361310-1.296882$ H $3.2633153 .886094-0.755631$ H $1.6319974 .293523-0.187321$ H $2.5230920 .519400-1.459880$ H -0.064895 -0.097146-2.321022 H $0.657062-0.313177-0.723887$ H $-0.148407 \quad 1.6208290 .710242$ H $-4.8336050 .973277-0.914210$ H -7.093128 $-0.326740 \quad 0.722070$ H $-6.1105730 .190511-2.948074$ H $-6.152714-1.517823-3.353016$ H -4.852966 -0.916355 -2.347955 H $-1.625211 \quad 0.2444450 .284852$ H -2.277636 $0.286137-1.364122$ H $-3.786787-3.4531421 .063446$ H $-2.403279-4.0299530 .104965$ H -3.856780 -3.586123 -2.044185 H $-5.681474-4.6587230 .169476$ H -6.204139 -5.963565 -2.016576 H -5.906011 -4.503054-2.932935 H $-9.663748-5.022312-1.200115$ H -8.358902 -6.328122 -1.048879 H -9.180854 -2.959266 -2.270376 H -7.708443 -2.838861 -3.179197 H -8.185162 -2.094419-0.211205 H $-3.515796-1.2781440 .224605$ C $-0.479182-0.3001454 .293818$ O $0.753612 \quad 0.136845 \quad 3.692399$ C $1.098029-0.7818262 .629214$ C $0.125213-1.9483092 .748812$ C $-1.112422-1.2670673 .298467$ H $0.923723-0.2344551 .696763$ C $2.581679-1.1561112 .753833$
H $-0.050005-2.4492121 .792478$ H $0.498066-2.6951903 .461003$ C $-2.113852-2.2248973 .918165$ H -1.597982 -0.721425 2481726 C $3.033861-2.2196701 .733585$ O 3.3378080 .0419502 .559356 H $2.787466-1.5010253 .773912$ H $2.353857-3.0753381 .828744$ O $2.865379-1.7142640 .413859$ C $4.459238-2.7770951 .931180$ C $5.634560-1.7846811 .984739$ H $4.655541-3.5025571 .131932$ O $4.453485-3.5109623 .162573$ H $5.509954-1.1003662 .831607$ H $6.528425-2.3747012 .232411$ H $-2.461098-2.951703 \quad 3.177298$ H -1.674284 -2.781608 4.752489 H $-2.987034-1.6837434 .296125$ C -1.331995 0.8984164 .714051 H 3.0278360 .6910573 .214126 H $3.065329-2.439243-0.202099$ H $5.309637-3.9657323 .238393$ H $-0.746621 \quad 1.5216445 .402115$ H -2.203553 0.5415855 .275824 H $-0.192189-0.8421885 .205151$ C $6.518400-1.418474-1.627930$ O $6.234753-1.954209-0.328137$ C $5.908157-1.0025110 .689533$ C 7.0540390 .0076670 .875008 C $7.4328070 .670582-0.452956$ C $7.637980-0.365175-1.563248$ H $6.897015-2.270879-2.206723$

C $5.249686-0.904254-2.323198$ H $5.004609-0.4588390 .402389$ O $8.195973-0.6532961 .417504$ H $\quad 6.7381590 .766322 \quad 1.601239$ H $8.3572501 .246695-0.324630$ O $6.4099761 .579519-0.848283$ H $7.7277350 .130024-2.537206$

O $8.866927-1.060439-1.333838$

C $4.256143-2.009488-2.610631$

H $4.739759-0.160816-1.708388$

H $5.514012-0.448151-3.284093$

H $8.8866480 .017300 \quad 1.553306$

H $6.3051382 .233647-0.136464$

H $9.582035-0.401846-1.340703$

O $3.105632-1.452867-3.094745$

O $4.454960-3.203670-2.436344$

C $2.057126-2.377553-3.399346$

H $1.744012-2.904429-2.493147$

H $1.206658-1.811845-3.788636$

H $2.386205-3.086347-4.165096$

SCF Energy (B3LYP/6-31G**//MMFF) $=-3245.90250238$

08_00545

MM̄FF Geometry

C -2.502690 -3.251750 -0.001472

C $-1.165546-3.1759370 .066194$

C $-0.431982-2.3026981 .050817$

O $0.279048-1.3155830 .269549$

C $0.575848-3.1430591 .853395$

C $1.220862-2.3790022 .991418$

C $2.470873-1.8768832 .962503$

C $0.374938-2.2332104 .229928$

C $3.445450-1.9044471 .818366$

C $4.610731-2.8583652 .106756$

C $5.617320-2.9063680 .947677$

C $6.755697-3.8782291 .262734$

C $7.825514-3.8785210 .160700$

O $6.145211-1.5917890 .766897$

O $8.989719-4.5453470 .673465$

C $8.241460-2.421394-0.141642$

C $7.371045-4.684942-1.061781$

O $9.105189-2.361937-1.274111$

C $7.049064-1.455813-0.338832$

C $7.5351490 .009779-0.341300$

O $6.393059-1.671348-1.589700$

C $0.258310-0.0252260 .698675$ 
O -0.2497440 .4091551 .719618$

C $0.9448130 .791445-0.326353$

C $0.7674012 .121214-0.337779$

C $1.3298123 .059948-1.312241$

C $2.2731052 .500454-2.345785$

C $6.3900791 .013759-0.526504$

C $6.8383892 .453172-0.346662$

C $5.9073344 .762724-0.305484$

O $8.0036802 .781962-0.146625$

C 4.5790055 .3399610 .061899

C $3.9591326 .302940-0.636324$

C $2.6034546 .836661-0.268785$

C $1.6149416 .790529-1.415929$

C $0.9442717 .897188-1.779771$

C $1.3971475 .482700-2.153934$

C $0.9570764 .356762-1.253384$

N $5.7616323 .324831-0.425639$

H -3.105675 -2.660731 0.684079

H $-0.563622-3.758730-0.627286$

H $-1.152666-1.8198281 .721951$

H $1.343430-3.5442491 .180955$

H $0.073732-4.0235732 .277324$

H $2.839258-1.3641233 .850288$

H $0.902402-1.7099685 .034715$

H $-0.534976-1.6658114 .011235$

H $0.087549-3.2185444 .611148$

H $2.964121-2.1803810 .875372$

H $3.820339-0.8834571 .676145$

H $5.120957-2.5240633 .019847$

H $4.224181-3.8674542 .297508$

H $5.086426-3.2117280 .038281$

H $6.369237-4.8923361 .424235$

H $7.234108-3.5859832 .208227$

H $8.726336-5.4400210 .949397$

H $8.829652-2.0661980 .716326$

H $7.202268-5.733475-0.787405$

H $8.144786-4.700021-1.837022$

H $6.444472-4.309933-1.501433$

H $9.852225-2.959977-1.100223$

H 8.0422210 .2254400 .608075

H $8.2639510 .166844-1.146437$

H $6.886973-2.339659-2.090785$

H $1.5435640 .270666-1.064973$

H $\quad 0.1285082 .567128 \quad 0.423646$

H $3.1267822 .010607-1.865423$

H $2.6822703 .253394-3.021991$

H $1.7578841 .765152-2.974176$

H $5.9622960 .914565-1.530903$

H 5.5954940 .8210190 .203962

H $6.2805785 .140135-1.263000$

H 6.6499804 .9853130 .467367

H 4.0956344 .9332070 .947580

H $4.4441246 .727297-1.512067$

H 2.7382717 .8689260 .078721

H 2.1818716 .2830810 .579546

H $0.2346017 .885894-2.600421$

H $1.0880148 .841625-1.265376$

H $2.3203085 .246464-2.690115$

H $0.6210015 .608967-2.919831$

H $0.229374 \quad 4.650224-0.495641$

H $4.8722392 .961424-0.755436$

C $-3.868241-3.310089-2.118965$

O $-4.791551-2.335014-1.597374$

C $-6.051218-2.469862-2.274352$

C $-5.740641-3.220930-3.561416$

C $-4.671554-4.185363-3.085610$

H -6.679603 -3.102862 -1.635203

C $-6.729398-1.105335-2.454687$

H $-6.620502-3.722721-3.974800$

H $-5.330173-2.559088-4.332440$

C $-3.841063-4.779646-4.210233$

H -5.159883 -5.001827 -2.536608

C $-6.764629-0.219181-1.190550$

O $-6.044120-0.358427-3.468882$

H $-7.744858-1.258568-2.837827$

H $-5.7763870 .226715-1.043549$

O $-7.6483650 .878704-1.449681$

$\begin{array}{llll}\text { C }-7.204828 & -0.928939 & 0.098187\end{array}$
C $-7.377298-0.009126 \quad 1.320082$

H $-6.478672-1.7095250 .350621$

O $-8.454075-1.582497-0.142052$

H $-8.287006 \quad 0.595802 \quad 1.211100$

H -7.575133 -0.6692202 .174916$

H $-4.475207-5.350893-4.896050$

H $-3.335151-4.000517-4.790297$

H -3.076311 -5.455246 -3.814445

C $-3.250868-4.118580-0.973655$

H $-5.115583-0.273267-3.192263$

H $-8.5478760 .517513-1.530707$

H $-8.690075-2.0705020 .665238$

H $-2.590767-4.893696-1.380807$

H $-4.039063-4.633252-0.409804$

H -3.088768 -2.751102 -2.652315

C $-3.763027 \quad 0.876601 \quad 1.821238$

$\begin{array}{llll}\text { O } & -4.977565 & 0.145779 & 1.592506\end{array}$

C $-6.183640 \quad 0.9134791 .634684$

C -6.3432051 .6025813 .002293$

C $-5.0975672 .408693 \quad 3.381445$

$\begin{array}{lllll}\text { C } & -3.811758 & 1.599571 & 3.179258\end{array}$

H $-2.984747 \quad 0.106291 \quad 1.879837$

C $-3.409625 \quad 1.8235700 .659892$

H $-6.144144 \quad 1.6790350 .853330$

$\begin{array}{lllll}\text { O } & -6.597945 & 0.637141 & 4.022344\end{array}$

H -7.2162942 .2643412 .964295$

H $-5.1763512 .712462 \quad 4.432704$

O $-5.061874 \quad 3.5904472 .587386$

H -2.9361532 .2502523 .287909$

$\begin{array}{llll}\text { O } & -3.734695 & 0.621237 & 4.219777\end{array}$

C $-2.976713 \quad 1.087533-0.588989$

H $-4.2503922 .462867 \quad 0.380668$

H -2.5674482 .4589320 .958832$

H $-5.8606830 .002794 \quad 4.021902$

H -4.2833164 .1045052 .861525$

H -2.887307 0.1542554 .121708

O $-2.6276462 .001464-1.542749$

O $-2.946140-0.126903-0.720326$

C $-2.189546 \quad 1.453460-2.790184$

H $-1.3621820 .754121-2.636344$

H $-3.0242820 .957483-3.293357$

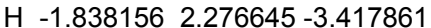

SCF Energy (B3LYP/6-31G**//MMFF) = -3245.91618542

08_00546

MM̄FF Geometry

C $2.938787-2.1913092 .530891$

C $3.441874-3.2606551 .894991$

C $3.907667-3.3300920 .460624$

O $4.128497-2.018541-0.101441$

C $2.831552-4.007550-0.407004$

C $3.410989-4.757411-1.588388$

C $3.539419-4.263655-2.834707$

C $3.817820-6.179335-1.295506$

C $3.250225-2.872226-3.322661$

C $2.002723-2.800417-4.213836$

C $0.705535-2.622033-3.409900$

C $-0.512262-2.668921-4.335422$

C $-1.816147-2.359871-3.585051$

O $0.781506-1.347188-2.761675$

O $-2.841027-2.116935-4.560820$

C $-1.631266-1.051983-2.790400$

C $-2.290162-3.550022-2.743249$

O $-2.767833-0.788297-1.970372$

C $-0.342156-1.021250-1.936848$

C $-0.1208600 .407447-1.386337$

O $-0.483235-1.934208-0.847121$

C $5.317510-1.4075520 .137523$

O $6.232148-1.8121590 .835488$

C $5.298833-0.131066-0.609452$

C $6.1767210 .834985-0.305818$

C $6.2582272 .151884-0.942035$

C $5.3445222 .421097-2.110076$

C $1.2346560 .627324-0.698205$

C $1.3289692 .012103-0.076900$

C 2.7688693 .3709131 .438807

O $0.4779342 .882113-0.245066$

C 3.5822704 .3017950 .595214 
C 4.7198134 .8780361 .013755

C 5.5157185 .8506650 .189597

C 6.9829545 .4905220 .104213

C $7.8957996 .127880 \quad 0.856913$

C $7.4006654 .454034-0.915331$

C $7.1336493 .051263-0.446418$

N $2.4757842 .161150 \quad 0.691561$

H $2.826046-1.2455132 .008791$

H $3.516112-4.1980262 .444106$

H $4.846292-3.8979560 .428685$

H $2.110163-3.259173-0.755120$

H $2.246430-4.7248080 .184684$

H $3.941114-4.923088-3.603767$

H $4.237083-6.683128-2.172806$

H $4.577263-6.204334-0.507477$

H $2.951945-6.761133-0.962644$

H $3.185091-2.153366-2.502300$

H $4.118064-2.559992-3.918085$

H $2.122390-1.933165-4.876691$

H $\quad 1.937827-3.689827-4.852530$

H $\quad 0.637666-3.412537-2.654942$

H $-0.580224-3.641265-4.839184$

H $-0.390521-1.925681-5.136056$

H -2.917110 -2.909846 -5.118901

H -1.579359 -0.233560 -3.521435

H $-2.471684-4.422503-3.382278$

H -3.246653 -3.332306 -2.255105

H -1.571162 -3.847385-1.977039

H $-3.548859-0.786939-2.549815$

H $-0.185221 \quad 1.126429-2.213698$

H $-0.9231910 .654295-0.682299$

H $0.056178-1.631588-0.100539$

H $4.544162-0.014700-1.378859$

H $\quad 6.892237 \quad 0.6535990 .495251$

H $4.2954832 .301445-1.819109$

H $5.5602351 .728092-2.930911$

H $5.4358303 .430610-2.514944$

H $1.393169-0.1102430 .094764$

H $2.0467450 .528722-1.426841$

H $3.295846 \quad 3.0702972 .349891$

H 1.8290873 .8563721 .721555

H $3.2026034 .536112-0.396664$

H 5.0822364 .6685422 .017674

H $5.402076 \quad 6.8421340 .647506$

H $5.1070295 .944310-0.824223$

H 8.9527955 .8954890 .781016

H 7.6057336 .8924991 .569967

H $8.4788264 .531203-1.110515$

H $6.9295814 .683713-1.874713$

H 7.7501042 .7580310 .404757

H $3.154898 \quad 1.4065760 .724238$

C $0.963276-2.3477094 .054667$

O $0.356076-1.1029473 .662926$

C $-0.853422-0.9162334 .425943$

C -1.002136 -2.154864 5.305473

C $0.436214-2.6086385 .461690$

H $-0.697466-0.0365265 .062326$

C -2.050115 -0.6743923 .493060$

H -1.486127 -1.926476 6.260210

H -1.598851 -2.929767 4.809059

C $0.566025-4.0499285 .922432$

H $0.939440-1.9485806 .180808$

C -1.9987320 .6678582 .734955$

O $-2.138504-1.7501152 .556704$

H -2.963594 -0.6998434 .097597$

H $-2.074514 \quad 1.4977023 .448260$

O -0.7349640 .8136772 .079678$

C $-3.0684180 .832393 \quad 1.637266$

C $-4.510707 \quad 0.6141012 .124388$

H -2.8541230 .1534050 .803642$

O

H -4.596236 -0.366971 2.602001

H -4.747902 1.3844932 .868755

H $0.087314-4.186076 \quad 6.897790$

H $0.093079-4.7411525 .216589$

H $1.617146-4.337376 \quad 6.021384$

C $2.484096-2.2348063 .965427$

H $-1.333218-1.7272042 .011424$
H -0.0477530 .8664092 .765058$

H -2.0437602 .2786860 .807708$

H $2.957450-3.0792564 .479290$

H $2.831636-1.3196014 .459761$

H $0.596498-3.1189453 .363269$

C $-6.5035202 .269611-0.585406$

$\begin{array}{llll}\text { O } & -5.632640 & 2.036442 & 0.529614\end{array}$

C $\begin{array}{llll}-5.539092 & 0.681143 & 0.975417\end{array}$

C $-6.9214350 .178503 \quad 1.419618$

C $-7.9619040 .356707 \quad 0.308800$

C $-7.9264871 .768627-0.285225$

H $-6.5514913 .362375-0.680004$

C $-5.920468 \quad 1.711582-1.893702$

H -5.1714050 .0514970 .157949$

O

H $-6.844309-0.881627 \quad 1.687627$

H $-8.962487 \quad 0.159867 \quad 0.712604$

O $-7.719297-0.585029-0.732375$

H $-8.5307361 .818277-1.198622$

O -8.5089642 .6796600 .652211$

C $-4.6517082 .429098-2.307892$

H $-5.6815110 .648362-1.812005$

H $-6.647647 \quad 1.848445-2.702488$

H $-7.343897 \quad 1.8248472 .388593$

H -7.774628 -1.474345 -0.342996

H -9.4279502 .3988050 .801028$

O $-4.1767441 .858819-3.455405$

O $-4.143891 \quad 3.370738-1.715094$

C $-2.9809402 .443365-3.980875$

H $-2.1810292 .414409-3.235128$

H $-2.6702711 .858341-4.850667$

H -3.174765 $3.471939-4.299159$

SCF Energy $\left(B 3 L Y P / 6-31 G^{* *} / / M M F F\right)=-3245.91564411$

08_00547

MM̄FF Geometry

C -1.666344 -2.256131 3.096314

C $-1.691102-1.3710304 .105151$

C -2.502025 -0.097043 4.172947

O -3.3216680 .0750192 .996264$

C -1.5494961 .1083394 .253056$

C -2.2713982 .4163964 .505398$

C -2.5068293 .3513913 .564206$

C -2.6850072 .6584885 .934057$

C -2.1732033 .2770802 .099046$

C -3.3052023 .8611611 .241437$

C $-2.9491853 .848987-0.251759$

C $-3.9706784 .656954-1.053892$

C $-3.6759674 .608359-2.559347$

O $-2.9412742 .489694-0.702818$

O $-4.8267235 .116240-3.251069$

C $-3.5063673 .136208-2.982061$

C $-2.5012225 .514784-2.945442$

O $-3.086603 \quad 3.042198-4.341843$

C $-2.5466762 .329087-2.073014$

C $-2.6474360 .830504-2.437249$

O $-1.2057532 .771980-2.287720$

C $-4.590343-0.4118993 .032905$

O $-5.129958-0.9980613 .957222$

C $-5.207600-0.1290681 .718064$

C $-6.335630-0.7667271 .372780$

C -7.043414 -0.641695 0.097012

C $-6.5862120 .433418-0.851894$

C $-1.904386-0.116176-1.489575$

C $-2.240761-1.548419-1.854098$

C $-3.973330-3.307414-1.488511$

O $-1.573911-2.178098-2.671879$

C $-5.211712-3.093986-2.302984$

C $-6.399665-3.644377-2.008821$

C $-7.643488-3.422991-2.823194$

C $-8.839288-3.024395-1.983434$

C $-9.868438-3.869536-1.803468$

C $-8.878805-1.619899-1.414949$

C $-8.055597-1.496223-0.160705$

N -3.376854 -2.013710 -1.210832

H $-2.292962-2.1004412 .223719$

H -1.056621 -1.558806 4.969824

H $-3.130873-0.1338675 .070814$ 
H $-0.955311 \quad 1.171614 \quad 3.333202$ H -0.8183200 .9603695 .059399$ H -2.988157 4.2770903 .877569 H -3.1526153 .6392786 .071380$ H $-3.406674 \quad 1.9028936 .259273$ H -1.813028 2.6147186 .594771 H -1.256793 3.8544331 .928259 H - 1.9782122 .2494861 .779003 H -4.2200973 .2783741 .410231$ H -3.504986 4.8919341 .560624 H -1.947186 4.277210 -0.373070 H -4.007173 $5.696623-0.705487$ H -4.976181 4.247168 -0.882135 H -4.987207 $6.023544-2.939626$ H -4.500270 $2.668612-2.932959$ H -2.712349 $6.554291-2.666873$ H $-2.3478475 .521379-4.030234$ H - $1.5626655 .228908-2.466243$ H -3.722837 $3.546764-4.877166$ H $-3.7038300 .537731-2.451074$ H $-2.2636420 .668803-3.453092$ H -0.594769 2.067288 -2.020523 H -4.6984980 .5649261 .058927$ H -6.767299 -1.476380 2.077808 H $-6.5548981 .406572-0.348479$ H -7.240765 $0.561562-1.716156$ H -5.587596 $0.205070-1.232180$ H $-0.8191480 .007519-1.564048$ H -2.176551 $0.064302-0.443568$ H -4.188642 -3.779110 -0.524626 H $-3.266614-3.934388-2.040336$ H -5.125324 -2.458025 -3.181278 H $-6.481095-4.302995-1.147030$ H -7.855930 -4.355566 -3.361820 H -7.482411-2.660107-3.595145 H -10.739842 -3.583304 -1.223856 H -9.861423 -4.868403 -2.227072 H $-9.910802-1.346794-1.158899$ H -8.578004 -0.921166 -2.199906 H -8.325128 -2.2213790 .608994$ H $-3.871814-1.396782-0.574081$ C $0.335820-3.4190962 .047453$ O $1.153354-2.2507692 .269883$ C $1.345908-1.5805601 .005415$ C $1.049676-2.630927-0.052735$ C $-0.110226-3.3688640 .585324$ H $\quad 0.596445-0.7787700 .973165$ C $2.758103-0.9898380 .954901$ H $0.798950-2.195441-1.023710$ H $1.906335-3.303267-0.185861$ C $-0.380813-4.732555-0.024894$ H $-1.008685-2.7532360 .476128$ C $3.122406-0.372532-0.406422$ $\begin{array}{lllll}\text { O } & 2.845165 & 0.030770 & 1.954886\end{array}$ H $3.485987-1.7620351 .227764$ H $3.138122-1.152258-1.176626$ O $2.0903830 .544445-0.795025$ C $4.4605400 .392884-0.437960$ C $5.653388-0.4510900 .024962$ H 4.3821051 .3069280 .160780 O $4.7020580 .804236-1.787646$ H $5.528616-0.7541641 .071227$ H $5.675827-1.387306-0.550188$ H -1.217760 -5.2247890 .480452$ H $-0.635735-4.636167-1.085230$ H $0.493360-5.3876990 .051613$ C $-0.787431-3.4782963 .088994$ H $2.610062-0.3770282 .806104$ H $2.0233701 .221296-0.099524$ H $3.9332251 .327044-2.073159$ H $-0.331755-3.6031544 .079699$ H -1.412203 -4.361800 2.915569 H $0.994296-4.2831182 .205688$ C $9.370282-0.3389670 .199942$ O $7.996020-0.726667 \quad 0.321870$ C $7.0292090 .224521-0.145672$ C 7.1675731 .5500980 .618675 C 8.6042642 .0801020 .564274
C 9.6285490 .9975470 .916553

H $9.929512-1.1187340 .733018$

C $9.841667-0.345517-1.262973$

H $7.1948680 .393615-1.214677$

$\begin{array}{lllll}\text { O } & 6.777678 & 1.354965 & 1.976596\end{array}$

H 6.4883662 .2910890 .182089

H 8.7035972 .9179701 .265359

O $8.8532852 .580335-0.746378$

H $10.642081 \quad 1.349896 \quad 0.692370$

$\begin{array}{llll}\text { O } & 9.566431 & 0.779082 & 2.329009\end{array}$

C $9.832693-1.736179-1.864478$

H $9.212540 \quad 0.284669-1.896610$

H $10.8732840 .022260-1.311763$

H 6.8499152 .2124772 .429169

H $9.7569062 .939127-0.756649$

H $10.256970 \quad 0.1338392 .557871$

O $10.276478-1.660291-3.154891$

O $9.484759-2.756653-1.286941$

C $10.322441-2.902798-3.863489$

H $11.011234-3.595029-3.369877$

H $9.320367-3.336834-3.932291$

H $\quad 10.688910-2.704273-4.874120$

SCF Energy (B3LYP/6-31G**//MMFF) $=-3245.90911147$

08_00548

MM̄FF Geometry

C $3.886578-1.8689941 .788852$

C $4.532154-2.9555621 .337672$

C $5.112984-3.152671-0.044439$

O $5.277894-1.889824-0.733863$

C $4.210923-4.009270-0.955301$

C $3.652230-5.272422-0.336681$

C $2.361436-5.4221430 .024466$

C $4.632499-6.401800-0.172987$

C $1.282502-4.376953-0.060974$

C $0.386178-4.597471-1.283675$

C $-0.463451-3.358072-1.605335$

C $-1.471335-3.684155-2.710271$

C $-2.236390-2.441337-3.184063$

O $0.433951-2.328454-2.038315$

O $-2.904020-2.774268-4.410826$

C $-1.217236-1.331966-3.512343$

C $-3.330984-2.028094-2.192433$

O $-1.884706-0.117294-3.844815$

C $-0.190516-1.084276-2.382435$

C $0.921549-0.127762-2.887028$

O $-0.859511-0.504873-1.266347$

C $6.348222-1.117899-0.410034$

O $7.247750-1.386325 \quad 0.368724$

C $6.1865050 .160074-1.135907$

C $6.7644841 .267195-0.649189$

C $6.6263232 .620730-1.191049$

C $5.8364392 .786274-2.463142$

C $2.0298210 .186658-1.868200$

C $1.7390901 .396927-0.993686$

C $2.7324782 .742426 \quad 0.856543$

O $\quad 0.7172762 .070650-1.094792$

C 3.3658193 .9467430 .231239

C $4.315073 \quad 4.683716 \quad 0.828752$

C 4.9198825 .9166720 .217249

C $6.4333205 .908168 \quad 0.237691$

C $7.1159866 .645602 \quad 1.129855$

C $7.1546075 .111350-0.828054$

C $7.1884053 .641734-0.511176$

N $2.760791 \quad 1.637338-0.084697$

H $3.734113-1.0153931 .135504$

H $4.652582-3.7995932 .014546$

H $6.093909-3.6362540 .047428$

H $4.784328-4.280740-1.853060$

H $3.407205-3.380662-1.359252$

H $2.041906-6.377986 \quad 0.435944$

H $4.166221-7.2977870 .250398$

H $5.058782-6.677847-1.142868$

H $5.448183-6.1073660 .494769$

H $0.673414-4.4295870 .849998$

H $1.709570-3.369764-0.065334$

H $1.012507-4.832591-2.154831$

H $-0.261549-5.463918-1.101661$ 
H $-0.979118-3.030200-0.694896$ H -2.170181 -4.463109-2.380253 H -0.939094 -4.109018 -3.573215 H -3.494843 -3.526821-4.236060 H $-0.670928-1.641451-4.414566$ H -4.066265 -2.833512 -2.076613 H -3.891824 -1.162151-2.560537 H -2.944084 -1.793013 -1.198917 H -2.513026 -0.317168 -4.559537 H $1.409585-0.606735-3.746486$ H $\quad 0.4792130 .808658-3.246653$ H $-0.196096-0.125750-0.669624$ H $5.545614 \quad 0.164668-2.009898$ H 7.3663061 .1826540 .255064 H $\quad 6.2934142 .209048-3.274784$ H $5.7748853 .819201-2.810484$ H $4.8055442 .442292-2.326716$ H $2.239090-0.677825-1.229016$ H $2.9467300 .419426-2.424225$ H 3.2605292 .4163861 .758092 H 1.6936952 .9672831 .118300 H $3.0068344 .243128-0.751805$ H 4.6523174 .4079401 .825399 H $4.544546 \quad 6.7817450 .779525$ H $4.5753856 .061018-0.814523$ H $8.2007786 .666403 \quad 1.134063$ H 6.6056327 .2408411 .879825 H $8.1959375 .450040-0.911498$ H $6.7084765 .333816-1.800631$ H 7.7423743 .4116730 .400365 H $3.604257 \quad 1.073757-0.126839$ C $1.788338-1.8239333 .136403$ O $1.264862-0.5933742 .600924$ C $0.026617-0.2806713 .282688$ C $-0.263585-1.4646714 .194624$ C $1.131686-1.9693424 .505236$ H $0.2328390 .616963 \quad 3.879172$ C $-1.044257 \quad 0.0129542 .227208$ H $-0.826121-1.1773225 .087954$ H $-0.836491-2.2362273 .664958$

C $1.150368-3.3832035 .059399$ H $1.604345-1.2879615 .225207$ C $-2.478543 \quad 0.1107432 .783245$ O $-0.662701 \quad 1.238927 \quad 1.598362$ H -1.006770 -0.7642331 .454907$ H $-2.742679-0.8555183 .226992$ O $-2.5283301 .074338 \quad 3.834050$ C $-3.5352120 .451556 \quad 1.712737$ C $-4.967730 \quad 0.3152952 .262424$ H -3.403837 -0.2270850 .861562$ O $\quad-3.321719 \quad 1.7824091 .250579$ H -5.060999 -0.647654 2.778508 H -5.1463431 .1175932 .989241$ H $\quad 0.586059-3.4359275 .996310$ H $0.703769-4.0954754 .357331$ H $2.174156-3.7106735 .264618$ C $3.314040-1.7662013 .176352$ H -1.2631331 .3883330 .848604$ H -2.303956 1.9375473 .445635 H -3.975955 $1.963018 \quad 0.554917$ H $3.707296-2.5736923 .804289$ H $3.650389-0.8183453 .613351$ H $1.453871-2.6310882 .471890$ C -7.003145 $1.988916-0.401206$ $\begin{array}{lllll}\text { O } & -6.088680 & 1.739481 & 0.675115\end{array}$ C $-6.035210 \quad 0.390626 \quad 1.151479$ C -7.414796 -0.0541461 .659631$ C $-8.4852850 .137793 \quad 0.580774$ C $-8.4272811 .539390-0.031766$ H -7.018439 $3.081845-0.504436$ C $-6.4982081 .398592-1.723625$ H -5.722571 -0.2704620 .335795$ O H -7.360047 -1.108790 1.955028 H $-9.479691-0.0215911 .014943$ O $-8.299592-0.823677-0.454188$ H -9.068201 $1.588535-0.919349$ O $\quad-8.9660702 .476088 \quad 0.906350$
C $-5.3490852 .220537-2.257128$

H $-6.1752560 .356557-1.648752$

H $-7.284927 \quad 1.426056-2.487071$

H $-8.6222450 .377527 \quad 3.133224$

H $-8.373943-1.705903-0.052055$

H -8.3689872 .5058231 .673653$

O $-4.1690891 .763290-1.751401$

O $-5.4907393 .176550-3.009041$

C $-3.0092252 .509803-2.132819$

H $-3.0776413 .532331-1.749661$

H $-2.1346312 .028647-1.688578$

H -2.896502 $2.511780-3.220994$

SCF Energy (B3LYP/6-31G**//MMFF $)=-3245.92894292$

08_00549

MM̄FF Geometry

C 1.9409622 .3628372 .289926

C 0.7377052 .5814302 .840324

C -0.4422451 .6697542 .639363$

O -1.4379422 .4479631 .934944$

C -1.0420341 .1803763 .970664$

C $-0.168603 \quad 0.1882084 .710489$

C $-0.179167-1.1421244 .495077$

C 0.7297950 .7709125 .770859

C -1.040039-1.910055 3.532274

C $-0.245519-2.3768222 .308015$

C -1.067657-3.306654 1.402801

C $-0.291045-3.6437130 .127654$

C -1.118547-4.492884 -0.848791

O $-2.294187-2.6513601 .067326$

O $-0.436682-4.463835-2.111789$

C -2.502693 -3.833882 -1.039227

C $-1.153209-5.963302-0.416637$

O $-3.352177-4.659463-1.832762$

C $-3.191770-3.4541820 .290411$

C $-4.468063-2.6235120 .021307$

$\begin{array}{lllll}\text { O } & -3.563337 & -4.655418 & 0.973798\end{array}$

C $-2.2598901 .786590 \quad 1.075747$

$\begin{array}{llll}\text { O } & -2.307777 & 0.583158 & 0.879926\end{array}$

C -3.0764152 .8043640 .376245$

C $-3.8971992 .434952-0.618611$

C $-4.7145133 .332836-1.438383$

C $-4.6554404 .807868-1.136326$

C $-5.045503-1.9664111 .283023$

C $-6.517137-1.6477741 .122189$

C $-8.105683-0.157370-0.046784$

O $-7.376415-2.2369911 .775168$

C $-8.1645740 .512140-1.380512$

C $-8.5435271 .787441-1.551066$

C $-8.6297582 .462517-2.889586$

C $-7.7654503 .700661-2.969699$

C $-8.2834984 .916717-2.728113$

C $-6.3298683 .540955-3.415610$

C $-5.4727622 .806295-2.422880$

N -6.764615 -0.6601220 .181967$

H $2.083148 \quad 1.4923851 .655092$

H 0.5896923 .4540773 .472175

H -0.1476460 .8152982 .018534$

H -2.031826 0.7454813 .786466

H -1.2620182 .0390104 .620568$

H $0.493913-1.7639635 .084020$

H $1.295447 \quad 0.0002886 .305517$

H 1.4545541 .4630975 .334427

H $\quad 0.135078 \quad 1.313217 \quad 6.513315$

H -1.445180 -2.780395 4.063307

H -1.905554 -1.321795 3.214103

H $0.066672-1.4938441 .736983$

H $0.667593-2.8964942 .623920$

H -1.291470 -4.218352 1.969393

H $0.653465-4.1467710 .368534$

H $-0.011517-2.710058-0.380886$

H $-0.991520-4.935120-2.757069$

H -2.343217 -2.916702 -1.622554

H $-0.136330-6.372683-0.380088$

H $-1.694793-6.578784-1.143266$

H $-1.604904-6.1109130 .566545$

H -3.675969 -5.381211 -1.267082

H $-4.240937-1.821381-0.692951$ 
H $-5.230340-3.263521-0.441108$ H -4.358586 -4.4928561 .504885$ H -2.9533803 .8385530 .678303$ H -3.962791 $1.378216-0.875125$ H $-4.9534985 .000436-0.099574$ H $-5.3196325 .406243-1.763778$ H $-3.6408005 .191811-1.288686$ H $-4.948267-2.6171192 .158823$ H -4.506702 -1.041869 1.519479 H -8.3458920 .5244960 .775908$ H -8.813612 $-0.992337-0.013171$ H -7.898034 -0.086213 -2.248480 H $-8.8281542 .377667-0.682775$ H $-9.6808412 .728683-3.062748$ H $-8.3638501 .777078-3.703892$ H $-7.6825715 .815714-2.813349$ H $-9.3212375 .039845-2.435919$ H $-5.8829144 .511470-3.661871$ H -6.313488 $2.986829-4.363387$ H $-5.4633691 .725689-2.567643$ H $-5.980674-0.107925-0.155057$ C 3.5812103 .9340291 .226674 O 4.0820122 .9624590 .287361 C $5.1866293 .541017-0.436035$ C 5.2929914 .9924870 .023871 C 4.7302864 .9187391 .428883 H $6.0878093 .003546-0.120857$ C $4.9704123 .380302-1.944974$ H $6.3236655 .359462-0.007270$ H $4.6851665 .661448-0.596367$ C 4.3064926 .2689451 .980605 H 5.4901894 .4790602 .088969 C $4.8189101 .919937-2.418583$ O $3.7757534 .081332-2.319906$ H $5.7863903 .867118-2.491240$ H $3.9015981 .488985-2.002723$ O $4.6408181 .947042-3.841626$ C $6.0242801 .012031-2.098092$ C $5.959608-0.372534-2.774926$ H $6.1181270 .888956-1.013783$ O $7.2133481 .664895-2.553462$ H $5.809108-0.246497-3.853914$ H $6.943220-0.850176-2.667657$ H 5.1606796 .9528952 .020619 H $3.533866 \quad 6.732106 \quad 1.357713$ H 3.9070856 .1703912 .994526 C 3.1404553 .2398192 .514710 H $3.0403543 .700285-1.809870$ H $3.8794122 .523085-4.027583$ H $7.9708561 .120644-2.278629$ H 2.9222323 .9855883 .287499 H 3.9502042 .6050692 .894668 H 2.7262474 .4326140 .750299 C $4.486762-2.608879-0.176880$ O $5.363108-1.743484-0.911558$ C $4.881363-1.292559-2.183032$ C $4.589681-2.489898-3.099035$ C $3.616644-3.465381-2.433346$ C $4.061561-3.827734-1.013726$ H $5.099835-2.9808970 .654990$ C $3.309800-1.8322240 .423734$ H $3.958608-0.724025-2.040280$ O $5.797486-3.167601-3.437483$ H $4.155552-2.120516-4.035615$ H $3.548628-4.373227-3.044854$ O $2.326469-2.863229-2.394710$ H $3.270268-4.374128-0.487453$ O $5.189645-4.705004-1.092186$ C $3.793709-0.9923151 .583873$ H $2.814853-1.159499-0.281487$ H $2.546739-2.5261800 .792307$ H $6.234609-3.428766-2.608947$ H $1.708142-3.521119-2.033580$ H $4.910042-5.498815-1.579266$ O $3.324647-1.5027432 .757885$ O $4.524451-0.0175881 .462071$ C $3.763403-0.8317843 .942758$ H $3.344666-1.3567754 .805387$
H $4.855140-0.8587644 .012586$

H 3.4020820 .1996313 .950511

SCF Energy (B3LYP/6-31G**//MMFF $)=-3245.92472053$

08_00550

MM̄̄F Geometry

C -2.5354083 .8165310 .610476$

C -2.7040213 .8533971 .941533$

C -3.1870722 .7123842 .804230$

O -2.9408461 .4346212 .175733$

C -4.7015032 .8507893 .033310$

C -5.2838151 .7338943 .875503$

C -5.9645490 .6868253 .367672$

C -5.0972501 .8790475 .361823$

C -6.2042270 .3987751 .909862$

C $-6.198448-1.1083651 .616692$

C $-6.333250-1.3824440 .111366$

C $-6.691600-2.846690-0.142585$

C $-6.768293-3.156397-1.643957$

O $-5.078631-1.074038-0.510174$

O $-6.809616-4.582967-1.798390$

C $-5.471430-2.665179-2.315231$

C $-8.052288-2.612416-2.281012$

O $-5.549922-2.796926-3.733162$

C $-5.076695-1.214750-1.937956$

C $-3.645954-0.934886-2.457006$

O $-5.984156-0.300318-2.553771$

C -1.7701770 .8065072 .468469$

$\begin{array}{llll}\text { O } & -0.841616 & 1.248070 & 3.125682\end{array}$

C $-1.826903-0.5430611 .861906$

C $-0.770863-1.3641481 .961667$

C $-0.672300-2.7170841 .407105$

C $-1.910006-3.3145830 .789670$

C $-3.0172690 .375997-1.965431$

C $-1.5694670 .468406-2.416993$

C $0.654804-0.624859-2.069289$

O $\quad-1.2119901 .253820-3.291236$

C $0.947519-2.092071-2.046493$

C $1.928901-2.645802-1.318002$

C $2.184692-4.125351-1.260457$

C $2.189257-4.6738520 .151973$

C $3.309541-5.1885410 .686628$

C $0.880073-4.7053490 .918095$

C $0.512984-3.3585801 .483368$

N $-0.751519-0.438240-1.763403$

H $-2.7626432 .905826 \quad 0.063045$

H -2.501651 4.7833422 .468989

H -2.6659822 .7542653 .768999$

H -5.2199142 .8973202 .067312$

H -4.9204463 .8112403 .519418$

H $-6.388680-0.035914 \quad 4.062988$

H $-5.567349 \quad 1.063178 \quad 5.921132$

H -4.0326601 .8814175 .615504$

H -5.540757 2.8171025 .711026

H -7.1817810 .8113821 .632566$

H $-5.4522750 .883506 \quad 1.279881$

H -5.266093 -1.550085 1.991584

H -7.030369 -1.576513 2.157812

H $-7.111127-0.725843-0.295912$

H $-7.636446-3.1091900 .349338$

H $-5.926973-3.4941630 .309483$

H -7.588474 -4.913768 -1.318910

H -4.665189-3.336819-1.987275

H -8.934259-3.039815 -1.789090

H $-8.125320-2.906313-3.334031$

H $-8.134306-1.525185-2.220466$

H -5.781432 -3.721716 -3.925822

H $-2.996925-1.763577-2.149496$

H $-3.643708-0.925100-3.554860$

H $-5.5496890 .562803-2.640658$

H $-2.737311-0.8241051 .345385$

H $0.117784-1.0068722 .480964$

H -2.176326 -2.790046 -0.131990

H $-2.756434-3.2520721 .483432$

H $-1.803981-4.3733090 .543178$

H $-3.554360 \quad 1.244537-2.360647$

H -3.043842 $0.448989-0.873290$

H $1.225505-0.085896-1.308430$ 
H $0.883862-0.203721-3.052656$ H $\quad 0.308319-2.732954-2.649819$ H $2.580986-2.004822-0.729287$ H $3.151613-4.312635-1.745157$ H $1.440844-4.678119-1.847812$ H $3.317917-5.6066131 .687704$ H $4.245865-5.1983480 .138517$ H $0.954511-5.4061501 .759560$ H $0.111312-5.111545 \quad 0.254790$ H $1.336354-2.8577271 .994514$ H $-1.126367-0.937033-0.963082$ C $-0.8297174 .636371-1.024602$ O $0.2627894 .330106-0.142784$ C $1.4961504 .734651-0.764446$ C $1.1205685 .331155-2.120252$ C $-0.3125975 .774692-1.896659$ H $1.9304885 .514185-0.127101$ C $2.468107 \quad 3.547012-0.857831$ H $1.7837116 .154703-2.403376$ H $1.1698304 .581949-2.919225$ C $-1.0915635 .977280-3.184179$ H $-0.3035596 .712541-1.325151$ C 2.8963622 .9599410 .503796 O $1.8743252 .516957-1.647873$ H $3.3597753 .887849-1.395100$ H 3.4377463 .7204931 .079499 O 1.7433112 .6067611 .279004 C 3.7447771 .6755020 .418652 C $4.9899381 .790108-0.476484$ H 3.1221090 .8380950 .081659 $\begin{array}{lllll}\text { O } & 4.170735 & 1.356767 & 1.748806\end{array}$ H $4.7035482 .139807-1.473071$ H $5.6718542 .525727-0.032093$ H $-0.6194376 .748124-3.801999$ H -1.138518 $5.055552-3.774006$ H -2.117439 $6.295374-2.974683$ C $-2.0663894 .992438-0.202780$ H $1.0541072 .247179-1.199980$ H 1.2474993 .4227241 .461633 H 3.3703211 .2506202 .291223 H -2.883036 $5.297926-0.866184$ H $-1.841307 \quad 5.8399440 .456537$ H $-1.0205353 .740858-1.631337$ C $6.962839-1.1872240 .661236$ $\begin{array}{lllll}\text { O } & 6.304612 & 0.085523 & 0.619115\end{array}$

C $5.7257750 .442605-0.638455$

C $6.818988 \quad 0.513948-1.715637$

C $7.614519-0.794414-1.789184$

C $8.069545-1.266758-0.403568$

H $7.443173-1.2186011 .647876$

C $5.964614-2.3477030 .592086$

H $4.983961-0.310546-0.926233$

O $7.708169 \quad 1.596782-1.448343$

H $6.3498620 .717117-2.685213$

H $8.494360-0.649767-2.427875$

O $6.811001-1.812287-2.379396$

H $8.455358-2.291758-0.452531$

$\begin{array}{llll}\text { O } & 9.145729 & -0.433868 & 0.038694\end{array}$

C $5.037694-2.3256721 .783526$

H $5.336041-2.325841-0.302674$

H $6.486138-3.3122960 .574103$

H $8.0710041 .469551-0.554863$

H $6.557639-1.508553-3.267608$

H $9.869757-0.524937-0.603997$

O $5.588392-3.0213702 .818441$

O $3.960405-1.7445561 .796629$

C $4.799338-3.0690184 .011168$

H $5.338343-3.6655524 .751768$

H $4.655438-2.0601274 .409546$

H $3.835487-3.5465813 .810129$

SCF Energy (B3LYP/6-31G**//MMFF) $=-3245.91672791$

08_00551

MMFF Geometry

C $1.273368 \quad 4.251587 \quad 0.471163$

C $1.7428494 .552991-0.749593$

C $2.2981173 .586792-1.772086$

O $2.2150062 .214319-1.325801$
C $3.7766003 .903473-2.050297$

C $4.2847863 .246393-3.318584$

C $5.0568522 .143374-3.359496$

C $3.9127713 .951214-4.599013$

C $5.5352331 .309318-2.204156$

C $5.338531-0.188181-2.482521$

C $5.937236-1.053071-1.365289$

C $5.889466-2.535111-1.739092$

C $6.425817-3.422376-0.605921$

O $5.181462-0.831113-0.170711$

O $6.061149-4.779856-0.897833$

C $5.716195-3.035265 \quad 0.707340$

C $7.956697-3.391155-0.527600$

O $6.286132-3.7245091 .818011$

C $5.696353-1.5116770 .981835$

C $4.760980-1.2182162 .175980$

O $7.015334-1.0701511 .307346$

C $1.0204701 .580201-1.480216$

O $-0.001792 \quad 2.040903-1.963007$

C $1.160530 \quad 0.220032-0.917298$

C $0.054288-0.453809-0.569857$

C $0.001167-1.8006720 .000975$

C $1.286643-2.5798620 .066069$

C $4.451050 \quad 0.267348 \quad 2.396580$

C 3.3878230 .4074113 .467124

C $0.9355020 .107724 \quad 3.826736$

$\begin{array}{lllll}\text { O } & 3.678678 & 0.634139 & 4.639174\end{array}$

C $0.466778-1.3152613 .830354$

C $-0.810374-1.6770073 .634949$

C $-1.284480-3.1022573 .621206$

C $-2.166166-3.4155062 .430317$

C $-3.488491-3.6008052 .581710$

C $-1.504849-3.5879691 .078341$

C $-1.190111-2.2680840 .427805$

$\begin{array}{lllll}\mathrm{N} & 2.107830 & 0.200047 & 2.976772\end{array}$

H 1.3075693 .2287860 .835223

H $1.6833705 .588374-1.081478$

H $1.7138273 .701097-2.693827$

H $4.3882123 .608108-1.189992$

H $3.9225694 .987587-2.152920$

H $5.3964331 .798492-4.335808$

H $4.3455473 .468055-5.481466$

H $2.8267773 .963261-4.732221$

H $4.2728114 .985183-4.581513$

H 6.602601 1.513757-2.058406

H $5.0252211 .570342-1.272761$

H $4.264436-0.394051-2.577928$

H $5.811991-0.449677-3.437373$

H $6.974267-0.735836-1.203301$

H $6.444155-2.722434-2.666917$

H $4.849821-2.825689-1.946321$

H $6.445372-5.014501-1.759961$

H $4.676671-3.3839070 .627965$

H $8.395420-3.731511-1.473343$

H $8.326910-4.0808160 .238983$

H $8.355472-2.396407-0.318031$

H $6.264930-4.674541 \quad 1.611260$

H $3.812955-1.7458432 .022955$

H $5.197366-1.6210793 .099373$

H $6.954574-0.2509301 .823744$

H $2.161070-0.175762-0.787336$

H $-0.9115740 .033235-0.702384$

H $1.746321-2.649042-0.926466$

H $1.161564-3.6052860 .418536$

H $1.992156-2.0930230 .743575$

H 5.3398590 .8187442 .720627

H $4.0918360 .745922 \quad 1.478468$

H 0.1858670 .7890913 .414995

H $1.1827950 .422768 \quad 4.844788$

H $1.214816-2.0857534 .003191$

H - $1.563703-0.9078553 .490216$

H - $-1.836835-3.2765804 .553772$

H $-0.440556-3.8031323 .635175$

H - $-4.123863-3.8508761 .739022$

H $-3.966804-3.5019253 .550333$

H -2.174065 -4.1339760 .400218$

H $-0.628600-4.2296671 .199263$

H $-2.063535-1.6261560 .308442$ 
H 1.9828440 .0176081 .985541 C $-0.867286 \quad 5.075167 \quad 1.553346$ O $\begin{array}{lllll} & -1.154896 & 3.798447 & 2.153828\end{array}$ C -2.295704 3.2092591 .480312 C -2.9156654 .3391380 .670867$ C $-1.6920295 .138121 \quad 0.269139$ H -1.8713052 .4429980 .820902$ C -3.2059792 .5690002 .533453$ H $-3.481860 \quad 3.985572-0.194617$ H -3.5811104 .9479831 .295286$ C -2.005288 $6.550194-0.192874$ H $-1.1948434 .601239-0.547352$ C -4.5033301 .8884272 .051705$ $\begin{array}{llll}\text { O } & -2.423598 & 1.591786 & 3.227598\end{array}$ H -3.462628 3.3414903 .270036 H -4.9690651 .4448172 .941473$ O -5.4256852 .8633361 .573900$ C $-4.325150 \quad 0.748763 \quad 1.023119$ C $-5.645323-0.0232170 .822646$ H -3.5531410 .0575261 .379140$ O $-3.890590 \quad 1.294622-0.216863$ H $-5.990770-0.3769611 .801676$ H -6.3989860 .6666530 .422198$ H $-2.6529346 .531209-1.075483$ H -2.5173157 .1238710 .586849$ H $-1.0876577 .084770-0.458075$ C $\quad 0.645664 \quad 5.261448 \quad 1.391968$ H $-2.954901 \quad 1.264188 \quad 3.973224$ H -5.2632612 .9893880 .624200$ H $-3.711006 \quad 0.555163-0.821944$ H 1.1286335 .1543542 .371202 H 0.8515256 .2810511 .045062 H - 1.2274655 .8291112 .265696 C $-5.305683-1.689648-2.515706$ O $-5.427703-0.715903-1.470238$ C $-5.520065-1.226027-0.135372$ C $-6.723924-2.1730770 .002309$ C $-6.688574-3.278782-1.054895$ C $-6.455092-2.710316-2.456922$ H $-5.422385-1.118522-3.446072$ C $-3.914974-2.343159-2.533000$ H $-4.601527-1.7689870 .108373$ O $-7.933034-1.426981-0.126462$ H $-6.712827-2.6127371 .007070$ H -7.638872 -3.826095 -1.036066 O $-5.649585-4.194055-0.721118$ H -6.259209 -3.522895 -3.165528 O $-7.653448-2.069345-2.904139$ C $-2.852897-1.396008-3.053898$ H -3.595409 -2.681134-1.545346 H -3.938462 -3.205806 -3.209624 H -8.673533 -2.043344 0.005142 H $-5.655923-4.901370-1.388372$ H $-7.806313-1.294436-2.336601$ O $-1.773795-2.124741-3.464755$ O $-2.953925-0.177517-3.086244$ C $-0.677716-1.398310-4.034062$ H $0.243992-1.937703-3.799937$ H $-0.801456-1.364666-5.119715$ H $-0.597048-0.383559-3.635214$

SCF Energy (B3LYP/6-31G**//MMFF) $=-3245.91683609$

$08 \quad 00552$

MM̄FF Geometry

C $-2.413072-4.076027 \quad 0.271184$

C $-1.410134-3.1916550 .385053$

C $-0.673966-2.9417051 .675799$

O $-0.643860-1.5188881 .937514$

C $0.779476-3.443101 \quad 1.566150$

C $1.618639-3.2270532 .814718$

C $2.871689-2.7290552 .807981$

C $0.993147-3.6342304 .124728$

C $3.706934-2.3661991 .610642$

C $5.168412-2.7993591 .806899$

C $5.998807-2.640405 \quad 0.525127$

C $7.461752-3.011708 \quad 0.778210$

C $8.339835-2.770209-0.459444$

O $5.907901-1.2808590 .092875$
O $9.713252-2.814765-0.042853$

C $8.076089-1.345276-0.992303$

C $8.168203-3.879549-1.504060$

O $8.754968-1.129341-2.227605$

C $6.574260-1.013622-1.148342$

C $6.3905090 .487952-1.471470$

O $6.035527-1.791323-2.219851$

C -1.712795 -0.994309 2.590891

O $-2.754889-1.5759762 .864942$

C $-1.4647120 .394497 \quad 3.038849$

C -0.4272501 .1569532 .657476$

C -0.1339552 .5058103 .159699$

C $-0.986326 \quad 3.018206 \quad 4.294331$

C $4.931645 \quad 0.962212-1.408693$

C $4.7920842 .436670-1.742955$

C $3.0544814 .216255-1.922828$

O $5.743993 \quad 3.157293-2.028994$

C $3.0108324 .944345-0.616805$

C $1.9024645 .517552-0.125648$

C 1.8602696 .2894431 .158760

C $0.896395 \quad 5.6994742 .161607$

C $-0.320762 \quad 6.239998 \quad 2.340943$

C 1.3889524 .5659433 .032779

C $0.878083 \quad 3.2128702 .610774$

N $3.4667522 .846902-1.684517$

H $-2.705600-4.6541491 .143730$

H $-1.095449-2.620026-0.484055$

H -1.172458 -3.458207 2.506135

H $1.241438-2.9400220 .709632$

H $0.777591-4.5179211 .342225$

H $3.366789-2.5843793 .767872$

H $1.713575-3.6247704 .949830$

H $\quad 0.178423-2.9553224 .394139$

H $0.596145-4.6523094 .054511$

H $3.326748-2.8378880 .699947$

H $3.659565-1.2803861 .469278$

H $5.605693-2.1869832 .606788$

H $5.211345-3.8452672 .136324$

H $5.565905-3.291957-0.243383$

H $7.547045-4.0527841 .113641$

H $7.854271-2.3993591 .602344$

H $\quad 9.874877-3.683058 \quad 0.364690$

H $8.512533-0.639745-0.271357$

H $8.448897-4.851225-1.079763$

H $8.834521-3.723622-2.359658$

H $7.144492-3.966334-1.873829$

H $9.693967-1.336282-2.081953$

H $6.9645451 .085900-0.751369$

H $6.7923940 .704791-2.469345$

H $5.265827-1.335868-2.593571$

H -2.2071210 .7624013 .740742$

H $\quad 0.280657 \quad 0.764126 \quad 1.930036$

H -2.031098 3.1103973 .976950

H -0.6870014 .0015274 .661263$

H -0.9359352 .3382545 .152179$

H $4.3100870 .401933-2.114935$

H $4.5289190 .808926-0.400740$

H $2.0766234 .182478-2.413321$

H $3.7660274 .704433-2.596440$

H $3.9422175 .022531-0.060867$

H $0.9772195 .460726-0.693712$

H 1.5684507 .3199160 .916747

H 2.8559746 .3705911 .612209

H -1.0077445 .8563403 .086833$

H -0.6605167 .0778951 .740995$

H 1.1527954 .7794044 .080903

H 2.4861704 .5324493 .013407

H 1.4357092 .7705291 .784987

H $2.7604992 .190269-1.365635$

C $-4.631546-3.958331-0.978993$

O $-4.773145-2.543463-0.741703$

C $-5.880982-2.340480 \quad 0.155568$

C $-6.640190-3.6629490 .194606$

C $-5.503909-4.6586090 .063077$

H $-5.449383-2.1595221 .147398$

C $-6.722652-1.133744-0.271160$

H $-7.215058-3.7847561 .117506$

H -7.330687 -3.761685 -0.651041 
C $-5.953697-6.050788-0.343207$ H $-4.991425-4.7186861 .030467$ C $-5.9337170 .184076-0.419782$ O $-7.324516-1.421199-1.540038$ H $-7.549744-1.0033550 .437281$ H -5.319285 $0.136486-1.325018$ O $-6.886993 \quad 1.230087-0.649176$ C -5.0562200 .5564130 .786946$ C -4.4761331 .9820260 .752509$ H $-4.236310-0.1650170 .869098$ $\begin{array}{lllll} & \text { O } & -5.839981 & 0.440131 & 1.978205\end{array}$ H -5.2825932 .7172920 .870622$ H -3.853343 2.0890801 .650560 H $-6.632516-6.4697270 .406793$ H -6.480686 -6.038926 -1.303210 H $-5.096259-6.724532-0.437011$ C $-3.151804-4.352673-1.008407$ H $-7.857810-0.647228-1.789686$ H $-7.413140 \quad 1.3302240 .162789$ H -5.2474290 .5920892 .734341$ H - $2.665005-3.817631-1.832597$ H $-3.061270-5.421555-1.234607$ H $-5.044573-4.136650-1.980928$ C -1.768969 $1.461474-1.772846$ O $-2.621847 \quad 1.327060-0.627531$ C $-3.6462022 .316057-0.497488$ C $-3.037573 \quad 3.727222-0.415600$ C $-2.0689793 .999347-1.571464$ C -1.088758 $2.840550-1.780484$ H $-0.9880740 .703552-1.629726$ C $-2.5006801 .141998-3.086553$ H $-4.3066522 .266556-1.368606$ O -2.3459013 .9008030 .819051$ H $-3.850708 \quad 4.462124-0.428441$ H -1.500438 4.913809-1.361869 O $-2.8016664 .209708-2.774131$ H -0.534896 $2.980220-2.716103$ O $-0.1353272 .873241-0.716021$ C $-2.945578-0.301812-3.156059$ H -3.390122 $1.764543-3.212098$ H $-1.8300721 .314446-3.935991$ H -1.6392863 .2342580 .856345$ H $-3.3892244 .971170-2.630910$ H $0.5139982 .168424-0.878484$ O $-3.817399-0.449732-4.196690$ O $-2.567339-1.189835-2.405985$ C $-4.336403-1.769643-4.387788$ H $-3.526827-2.504609-4.433866$ H -5.034154 -2.012095 -3.581906 H $-4.877479-1.787229-5.337532$

SCF Energy (B3LYP/6-31G**//MMFF)= -3245.90925916

08_00553

MM̄FF Geometry

C $-1.110773 \quad 3.358176 \quad 0.772679$

C -1.2334112 .0595791 .086089$

C -1.0665651 .5202122 .480961$

$\begin{array}{llll}\text { O } & -0.319439 & 0.283770 & 2.371242\end{array}$

C -2.425897 1.1823283 .122922

C -3.278825 2.3817023 .477332

C -4.3923032 .7429292 .809719$

C $-2.841600 \quad 3.147464 \quad 4.697937$

C $-4.936166 \quad 2.108912 \quad 1.561425$

C $-5.9715181 .020961 \quad 1.866828$

C $-6.4885310 .358496 \quad 0.581324$

C $-7.518772-0.7265140 .900140$

C $-7.971949-1.477260-0.362761$

O $-5.369664-0.221523-0.097366$

O $-8.659073-2.667410 \quad 0.053428$

C $-6.726047-1.929428-1.155309$

C $-8.979069-0.659634-1.180388$

O $-7.095495-2.494815-2.410883$

C $-5.688786-0.803807-1.366794$

C $-4.384358-1.379514-1.968281$

O $-6.2255760 .162644-2.273996$

C $0.401696-0.1112383 .451273$

O $0.614005 \quad 0.542593 \quad 4.463753$

C $0.914117-1.4925123 .297139$
C $0.525196-2.3313872 .323827$

C $0.951078-3.7252712 .163366$

C $1.958528-4.2674013 .143971$

C $-3.201274-0.402942-1.912132$

C $-2.065681-0.858431-2.805373$

C $-0.262974-2.546363-3.008151$

O $-1.794428-0.271341-3.850687$

C $-0.107382-3.977070-2.606277$

C $1.034009-4.493802-2.126863$

C $1.190226-5.931302-1.722646$

C $1.672508-6.094211-0.297535$

C $2.930562-6.490048-0.039312$

C $0.675464-5.8985210 .823391$

C $0.426475-4.4508651 .152751$

N $-1.413564-1.983434-2.326258$

H -0.8775084 .0680761 .561695$

H $-1.503740 \quad 1.3486600 .309782$

H -0.5028242 .2333353 .095014$

H -2.2597190 .6019894 .040927$

H -2.9680240 .4813562 .475094$

H $-4.962102 \quad 3.5972283 .171342$

H -3.529611 3.9628944 .945278

H -2.7929922 .4828395 .566808$

H $-1.852492 \quad 3.588464 \quad 4.540846$

H -5.3971002 .8950610 .950394$

H $-4.121746 \quad 1.7050230 .952077$

H -5.5077090 .2586742 .506331$

H -6.8109491 .4514612 .426483$

H $-6.9322351 .136461-0.051124$

H -8.382658 -0.3010621 .426012$

H $-7.076050-1.4556861 .593355$

H $-9.405917-2.4009430 .616435$

H -6.248205 -2.735604 -0.581054

H $-9.870610-0.440341-0.580541$

H $-9.334881-1.224757-2.048934$

H -8.575581 $0.293049-1.529440$

H -7.738481 -3.202265 -2.231905

H -4.093886 -2.281964 -1.414969

H $-4.558262-1.681012-3.009233$

H $-5.5013330 .579139-2.767044$

H $1.606051-1.8028324 .073823$

H $-0.190646-1.9818671 .581753$

H $1.533802-4.2957754 .153280$

H $\quad 2.859177-3.6435903 .159771$

H $2.293707-5.2795002 .907344$

H $-3.4906550 .602683-2.234966$

H $-2.827863-0.311076-0.886022$

H $0.608080-1.938327-2.741655$

H $-0.415288-2.472536-4.089990$

H $-0.975802-4.621569-2.721775$

H $1.908467-3.854801-2.034282$

H $1.901919-6.394896-2.418158$

H $\quad 0.251430-6.485499-1.847527$

H $3.277978-6.6435310 .976826$

H $3.644269-6.663329-0.837813$

H $0.984886-6.4551631 .715245$

H $-0.280273-6.358187 \quad 0.539384$

H $-0.296639-3.9703560 .493558$

H $-1.593971-2.275290-1.369416$

C $-0.1812534 .647656-1.233253$

O $0.8926373 .731563-1.515552$

C $2.1412464 .431691-1.342722$

C $1.7896175 .900714-1.114253$

C $0.4414165 .787287-0.430113$

H $2.5935504 .036667-0.426362$

C $3.0759324 .182988-2.530672$

H $2.5397196 .414646-0.505211$

H $1.6922566 .450046-2.057751$

C $-0.3661297 .072537-0.448206$

H 0.6178035 .4880750 .609816

C $3.3396482 .696391-2.841648$

O $2.4942164 .770256-3.701845$

H $4.0223054 .710536-2.360900$

H $2.4408422 .245051-3.278650$

O $4.3351852 .641953-3.872605$

C $3.7971341 .847507-1.640554$

C $4.2609040 .439165-2.062115$

H $2.9645521 .754120-0.934873$ 
O $4.8532342 .518655-0.954595$ H $3.5329190 .009439-2.760616$ H $5.2226920 .529522-2.582142$ H $\quad 0.1708007 .871557 \quad 0.073197$ H $-0.5603797 .411003-1.471520$ H $-1.330786 \quad 6.9329520 .049891$ C -1.365932 $3.914297-0.600359$ H $3.1040294 .606823-4.441689$ H $5.1447953 .055617-3.528524$ H $5.6297832 .534775-1.538805$ H -1.670429 $3.101986-1.272950$ H $-2.2174494 .601635-0.529532$ H $-0.5130975 .027229-2.209667$

C $5.656863-0.741121 \quad 1.209274$

O $5.427854 \quad 0.0045770 .006366$

C $4.427962-0.522333-0.868715$

C $4.821760-1.935327-1.328783$

C $5.107184-2.853591-0.135776$

C $6.041629-2.1944110 .884897$

H $6.525989-0.2584141 .674870$

C $4.480044-0.6280702 .184715$

H $3.469057-0.564744-0.340471$

O $5.974749-1.883248-2.167459$

H $4.007302-2.350855-1.932747$

H $5.561365-3.785589-0.493644$

O $3.886234-3.1856300 .514253$

H $6.073152-2.7784381 .812265$

$\begin{array}{lllll}\text { O } & 7.369722 & -2.175469 & 0.353999\end{array}$

C 4.2820210 .8027512 .625789

H $3.542673-0.9952751 .759362$

H $4.661384-1.2233523 .087283$

H $6.685837-1.446122-1.668234$

H $3.302901-3.597136-0.144831$

H $7.635633-3.0976360 .197596$

$\begin{array}{lllll}\text { O } & 3.177136 & 1.328389 & 2.023845\end{array}$

$\begin{array}{lllll} & 0 & 5.030280 & 1.386850 & 3.398837\end{array}$

C 2.8802392 .6868872 .359078

H 1.9668652 .9746581 .832630

H 2.7088522 .7840943 .435290

H 3.6938143 .3424622 .034805

SCF Energy (B3LYP/6-31G**//MMFF) $=-3245.90561514$

0800554

MM̄FF Geometry

C -1.882772 -3.6929851 .520231$

C $-1.760442-3.4335922 .831433$

C -2.327424 -2.247489 3.577101

O $-3.090869-1.3861322 .704624$

C $-1.170844-1.4126004 .153526$

C -1.642437 -0.2930175 .058879$

C -1.6828731 .0066064 .705565$

C -2.029937 -0.7118926 .453452$

C -1.3430841 .5988373 .364809$

C -2.320264 2.7224192 .990052

C -1.950501 3.3721521 .649286

C -2.7722344 .6425281 .424395$

C -2.472418 5.2782340 .059878

O $-2.214725 \quad 2.429628 \quad 0.603668$

O $-3.4840916 .263027-0.200217$

C -2.603783 4.195468-1.028577

C $-1.130988 \quad 6.020677 \quad 0.053055$

O $-2.193457 \quad 4.698332-2.298653$

C $-1.842722 \quad 2.885704-0.704975$

C $-2.2449861 .801817-1.730919$

O $-0.4367273 .118888-0.799026$

C $-4.428277-1.6093212 .608572$

O $-5.074977-2.4763173 .173790$

C $-4.972356-0.6151421 .657050$

C $-6.200285-0.7969941 .149438$

C -6.8638430 .0587390 .163896$

C $-6.2009551 .364100-0.185731$

C $-1.7194110 .396494-1.423789$

C $-2.327967-0.588108-2.402187$

C $-4.369395-1.974533-2.772886$

O $-1.793669-0.837221-3.480456$

C $-5.536304-1.178952-3.270761$

C $-6.810946-1.587811-3.174038$

C $-7.981956-0.783857-3.665673$
C $-9.081475-0.645728-2.633204$

C $-10.257915-1.276292-2.789553$

C $-8.847190 \quad 0.282124-1.457812$

C $-8.021975-0.369813-0.380121$

$\mathrm{N}-3.534682-1.107817-1.961527$

H $-2.461490-3.0273930 .887473$

H $-1.177176-4.1230813 .439799$

H $-2.959951-2.6191424 .392292$

H $-0.561909-1.0101413 .334737$

H -0.491012 -2.054917 4.729874

H -1.9892631 .7290095 .461101$

H $-2.308180 \quad 0.140916 \quad 7.081927$

H $-2.886338-1.3925996 .426844$

H $-1.194031-1.2221086 .943093$

H $-0.3272892 .008313 \quad 3.419774$

H -1.3503900 .8427582 .574298$

H -3.3363572 .3104272 .935429$

H -2.313176 3.4845503 .779465

H $-0.880188 \quad 3.609576 \quad 1.661616$

H -2.5990995 .3676652 .229409$

H -3.8428084 .3969001 .466585$

H $-3.4589206 .916080 \quad 0.520087$

H -3.673092 $3.957502-1.121481$

H $-1.127935 \quad 6.8122380 .811972$

H $-0.9638016 .524659-0.905272$

H -0.2782745 .3686850 .253130$

H $-2.7046185 .508156-2.468508$

H $-3.3386411 .751355-1.785940$

H $-1.8927602 .088230-2.730564$

H $0.0135832 .273748-0.954120$

H $-4.3357720 .215501 \quad 1.374091$

H $-6.764420-1.6763861 .458003$

H -5.9836591 .9425120 .719497$

H $-6.8135642 .010433-0.817215$

H $-5.2638921 .184007-0.718154$

H $-0.6307630 .343155-1.523446$

H -1.963192 $0.089131-0.400619$

H -4.679880 -2.811635-2.139633

H $-3.793885-2.363752-3.618209$

H $-5.321378-0.220083-3.737329$

H $-7.025138-2.558614-2.732728$

H $-8.369701-1.280756-4.564511$

H $-7.6694900 .217653-3.986905$

H -11.059574 -1.163674-2.066987

H -10.444753 -1.923193 -3.640211

H $-9.8067980 .569163-1.008207$

H $-8.4109401 .211545-1.833243$

H $-8.431606-1.320470-0.034593$

H $-3.903560-0.808405-1.064244$

C $-0.088734-4.468290-0.102562$

O $0.896090-3.6905610 .611856$

C $1.167994-2.493110-0.147387$

C $0.753171-2.827092-1.570503$

C $-0.500517-3.642490-1.322821$

H $0.516581-1.7154630 .272767$

C $2.638748-2.1036210 .027751$

H $0.572686-1.938292-2.180869$

H $1.517747-3.438167-2.066124$

C $-0.934756-4.478148-2.514020$

H -1.312676 -2.954234-1.067305

C $3.073655-0.917528-0.850327$

O $2.840474-1.7506391 .400304$

H $3.271311-2.975742-0.170712$

H $3.011826-1.196818-1.908443$

O $2.1420690 .159135-0.671766$

C $4.481300-0.360054-0.557358$

C $5.594326-1.418253-0.643644$

H 4.5012050 .1254170 .425615

$\begin{array}{llll}\text { O } & 4.747303 & 0.660679 & -1.525403\end{array}$

H $5.362508-2.2558380 .021834$

H $5.632003-1.794982-1.673665$

H -1.831709 -5.058058 -2.274585

H $-1.165476-3.836495-3.370674$

H $-0.150825-5.178596-2.820725$

C $-1.225420-4.8678850 .845743$

H $2.552902-2.507684 \quad 1.939308$

H 2.1444950 .3949790 .271906

H $4.0334861 .317831-1.457822$ 
H $-0.815609-5.5412951 .609483$ H $-1.987173-5.4363290 .300216$ H $0.424718-5.382449-0.426888$ C $8.6484810 .728245-1.003409$ O $7.3960300 .074581-1.246333$ C $6.983714-0.868706-0.254241$ C $8.023766-1.994836-0.134503$ C $9.427579-1.4390600 .124610$ C $9.780449-0.300263-0.838452$ H $8.8432601 .301746-1.918889$ C 8.5604801 .7241530 .158443 H $6.885077-0.3612720 .711627$ O $8.037409-2.784404-1.322724$ H $7.733515-2.6593830 .687826$ H $\quad 10.157267-2.2509190 .014757$ O $9.490150-0.975213 \quad 1.470127$ H $10.697227 \quad 0.203150-0.510203$ O $10.056713-0.880536-2.116791$ C $7.6137122 .851710-0.178084$ H 8.2384131 .2643351 .097070 H 9.5395392 .1752220 .359174 H $8.232083-2.193112-2.070004$ H $\quad 10.395687-0.658641 \quad 1.629332$ H $10.344575-0.164687-2.708615$ O 6.4120802 .6511210 .433825 O $7.9024783 .785406-0.915468$ C 5.4128933 .6408370 .170079 H 5.7273054 .6090850 .570674 H $5.2193503 .711028-0.904680$ H 4.4910013 .3344260 .671827 SCF Energy (B3LYP/6-31G**//MMFF) $=-3245.91839532$

08_00555

MMFF Geometry

C $2.546017-3.0913050 .568575$

C $1.215987-3.1943390 .430905$

C $0.438618-2.462027-0.629917$

O $-0.507482-1.5769890 .013258$

C $-0.372006-3.457074-1.479930$

C $-0.975885-2.810528-2.711260$

C $-2.212549-2.278444-2.775396$

C $-0.088073-2.810524-3.928236$

C $-3.228120-2.169168-1.671863$

C $-4.525838-2.902538-2.031178$

C $-5.563148-2.818116-0.900692$

C $-6.817522-3.617450-1.263841$

C -7.920483 -3.471006 -0.204188

O $-5.895220-1.439618-0.699024$

O $-9.137213-4.003572-0.749853$

C $-8.137773-1.9698440 .086447$

C $-7.629655-4.3229891 .035519$

O $-9.051109-1.7841561 .164523$

C $-6.825810-1.2048670 .368071$

$\begin{array}{llll}C & -7.043688 & 0.322361 & 0.476546\end{array}$

O $-6.287880-1.6396441 .622755$

C $-0.051653-0.3991580 .514528$

O $1.104880-0.0158770 .568846$

C $-1.236436 \quad 0.3620930 .969381$

C -1.091392 1.5951531 .476404

C -2.1773622 .4678961 .931990$

C -3.5860611 .9631291 .758726$

C -7.592589 $0.987949-0.785567$

C $-7.6050342 .498929-0.655714$

C $-6.1447344 .497710-0.794738$

O $-8.6508413 .123328-0.491092$

C $-4.7269254 .824107-0.451216$

C $-4.375730 \quad 5.746520 \quad 0.458058$

C -2.9488326 .0926470 .778788$

C -2.647267 6.0453272 .262104

C -2.2549917 .1510552 .917506$

C -2.7947164 .7260402 .991733$

C -1.8644233 .6683062 .463753$

N $-6.3391193 .060222-0.737775$

H $3.107573-2.462455-0.118029$

H $\quad 0.651389-3.8116831 .125411$

H $1.101687-1.871503-1.274828$

H -1.162789 -3.916549 -0.873984

H $0.269129-4.288234-1.803691$
H $-2.533426-1.844367-3.721771$

H $-0.544338-2.292399-4.778302$

H $0.860600-2.312366-3.709562$

H $0.124846-3.838168-4.240711$

H $-2.848349-2.558746-0.723544$

H -3.433688 -1.104032-1.510685

H $-4.942848-2.457170-2.944061$

H $-4.305373-3.955044-2.249063$

H $-5.108702-3.2157800 .014422$

H $-6.574423-4.675876-1.420317$

H $-7.208346-3.256444-2.225585$

H -9.349482 -3.510355-1.560436

H -8.615098 -1.540099 -0.802626

H $-7.580749-5.3847900 .764638$

H $-8.437038-4.2396471 .771404$

H $-6.688617-4.0650401 .525577$

H -9.858551 -2.281919 0.949987

H $-7.695036 \quad 0.551022 \quad 1.330141$

H -6.0806470 .7860350 .723906$

H $-5.683457-0.9548611 .954045$

H $-2.205450-0.1118450 .857417$

H -0.0877792 .0094471 .561614$

H -3.7828331 .7391260 .704268$

H -4.3533122 .6732512 .071057$

H $-3.743447 \quad 1.0521242 .346824$

H $-6.9986330 .721502-1.667292$

H -8.621075 $0.666538-0.981459$

H $-6.8621014 .985610-0.126305$

H -6.363270 $4.814688-1.819819$

H $-3.9486234 .290613-0.992254$

H $-5.150808 \quad 6.3025550 .980299$

H -2.7593957 .0998240 .385527$

H $-2.2531975 .430096 \quad 0.249347$

H $-2.034677 \quad 7.1317153 .979727$

H -2.144743 8.1025082 .408004

H -3.8444814 .4252492 .962472$

H -2.564235 4.8546404 .057746

H -0.8102483 .9359222 .550913$

H $-5.5761482 .466338-1.051216$

C $3.792474-2.7983192 .716948$

O $4.738820-1.8667452 .155868$

C $5.809639-1.6674573 .097185$

C $5.359131-2.3189244 .399538$

C $4.515869-3.4679123 .884293$

H $6.673301-2.2209242 .710074$

C $6.153066-0.1780443 .213336$

H $6.203895-2.6457775 .013390$

H $4.746581-1.6402825 .004420$

C $3.588895-4.0574554 .933205$

H $5.184652-4.2549823 .510778$

C 6.4893810 .5116521 .874082

O $5.0393190 .519896 \quad 3.786749$

H $6.981079-0.0495243 .920112$

H $5.573705 \quad 0.6169781 .281627$

O 6.9196691 .8464092 .171065

C $7.572094-0.1917461 .037085$

C $8.0747750 .615601-0.174155$

H $7.193209-1.1607340 .693158$

O $8.698807-0.456106 \quad 1.878575$

H $8.621206 \quad 1.502908 \quad 0.170235$

H $8.822159-0.008424-0.682360$

H $4.165042-4.4552605 .775126$

H $2.896648-3.3048475 .325426$

H $2.994632-4.8757794 .514983$

C $3.331207-3.7858321 .644266$

H 4.2707560 .3708993 .209297

H 7.7566411 .7820722 .662674

H $9.346305-0.9569151 .353695$

H $2.729134-4.5810362 .098793$

H $4.197176-4.2641791 .170713$

H $2.942175-2.2064713 .081599$

C $5.2161690 .064241-2.516176$

O $6.318991-0.155387-1.623872$

C $6.9819591 .029323-1.172386$

C $7.5683561 .800965-2.366601$

C $6.4986222 .102857-3.419450$

C $5.6593010 .864772-3.755724$

H $4.932717-0.943137-2.846857$ 
C $4.0158800 .658356-1.762868$

H $6.2615131 .670489-0.654263$

O $8.625588 \quad 1.056968-2.970564$

H $8.0043672 .738718-2.002749$

H $6.9896312 .469672-4.329387$

O $5.6601953 .144478-2.929423$

H $4.7888411 .155405-4.353831$

O $6.4605340 .009894-4.578333$

C $2.7264920 .561545-2.548151$

H $3.8720370 .117392-0.819779$

H $4.1931081 .706340-1.506270$

H $8.2644790 .196686-3.244918$

H $5.0088193 .347454-3.622295$

H $5.902735-0.736595-4.856488$

O $1.8106941 .423671-2.017023$

O $2.536133-0.189157-3.495079$

C $0.5263121 .424653-2.648675$

H $0.6160541 .771028-3.682612$

H $\quad 0.082216 \quad 0.425470-2.613342$

H -0.121179 2.114517-2.101018

SCF Energy (B3LYP/6-31G**//MMFF) $=-3245.91794967$

08_00556

MMFF Geometry

C $2.458162-0.9108903 .161994$

C $2.221971-2.2114902 .931020$

C $2.782802-3.0494231 .805248$

O $3.908591-2.3762671 .196663$

C $1.705847-3.277900 \quad 0.728273$

C $1.907106-4.543973-0.079992$

C $2.340312-4.591080-1.355089$

C $1.515168-5.8185720 .625026$

C $2.793556-3.458153-2.233987$

C $1.789243-3.142475-3.354802$

C $0.591623-2.317546-2.862519$

C $-0.446692-2.116037-3.967218$

C $-1.598489-1.207354-3.501606$

O $1.096747-1.044617-2.449893$

O $-2.350674-0.814405-4.659516$

C $-1.008307 \quad 0.081466-2.887574$

C $-2.573737-1.953870-2.584502$

O $-2.0262450 .883468-2.293975$

C $0.118604-0.179870-1.863809$

C $0.8224861 .145590-1.481880$

O $-0.445157-0.751166-0.682596$

C $4.980782-3.1248490 .835935$

O $5.109205-4.3337710 .977167$

C $6.053018-2.2998880 .232123$

C $5.935445-0.994982-0.061088$

C $6.975501-0.151723-0.661961$

C $8.331224-0.771949-0.884649$

C $2.2036450 .934379-0.843847$

C $2.7578492 .165863-0.153864$

C $3.3420724 .555876-0.505622$

$\begin{array}{lllll}\text { O } & 3.211899 & 2.103707 & 0.987894\end{array}$

C $4.7435204 .629687-1.022360$

C $5.8321574 .510631-0.247385$

C $7.2361724 .583280-0.772613$

C $7.9662493 .263830-0.665004$

C $8.988905 \quad 3.102502 \quad 0.191389$

C $7.5659872 .158324-1.617273$

C $6.6778241 .128663-0.970685$

N $2.7556253 .303189-0.943023$

H $3.108820-0.3509402 .496300$

H $1.550827-2.7393113 .606797$

H $3.109858-3.9933122 .257135$

H $1.668334-2.4069070 .066537$

H $0.709764-3.3421331 .187681$

H $2.405922-5.567889-1.833613$

H $1.617743-6.699192-0.018145$

H $2.142973-5.981974 \quad 1.506057$

H $\quad 0.469776-5.768196 \quad 0.946964$

H $3.032267-2.559188-1.657661$

H $3.739370-3.769523-2.695734$

H $2.321983-2.568505-4.124159$

H $1.443300-4.072114-3.823080$

H $0.137394-2.828386-2.006843$

H $-0.835593-3.080265-4.317432$
H $\quad 0.032145-1.649722-4.839609$

H $-2.662905-1.622334-5.102133$

H $-0.5918680 .672360-3.715489$

H $-3.021974-2.804417-3.111935$

H $-3.409544-1.311665-2.288553$

H -2.097091 -2.340789 -1.682261

H $-2.7126921 .028029-2.967653$

H $0.9663781 .747393-2.387594$

H $\quad 0.185248 \quad 1.727337-0.803805$

H $0.130635-0.5429020 .069984$

H $6.966982-2.8474950 .022939$

H $4.993695-0.4904220 .146090$

H $9.085692-0.063846-1.233068$

H $8.269753-1.571018-1.631529$

H $8.720974-1.1918240 .049705$

H $2.1570970 .138495-0.092353$

H $2.9330270 .620546-1.600036$

H $3.301304 \quad 4.6224040 .586694$

H $2.7439105 .370763-0.924211$

H $4.8643924 .788453-2.091294$

H 5.7123914 .3503140 .821646

H $7.7639505 .364031-0.209937$

H $7.2516954 .914312-1.818779$

H 9.5346552 .1663950 .246936

H 9.3011883 .9005140 .856555

H $8.4637321 .715468-2.059837$

H $7.0267362 .583729-2.473535$

H $5.6699111 .487462-0.764278$

H $2.4862613 .230275-1.919115$

C $0.4459200 .401141 \quad 4.060407$

O $-0.499714-0.6877254 .025171$

C -1.288699 -0.5728172 .825206$

C -1.1898150 .8919342 .427504$

C 0.2535321 .2000812 .770030

H - $0.797861-1.2137662 .083346$

C - $-2.718126-1.0642313 .083206$

H -1.4214841 .0683731 .373285$

H -1.8675311 .5015983 .037046$

C 0.5443502 .6828492 .922591

H $\quad 0.8808410 .8020891 .966279$

C $-3.588981-1.0101091 .810962$

O $-2.616919-2.410595 \quad 3.554637$

H $-3.170554-0.4727643 .887786$

H -3.520743 -0.0044721 .385412$

O $-3.044181-1.906593 \quad 0.841805$

C $-5.082322-1.327162 \quad 2.023857$

C $-5.891876-1.4487390 .716750$

H -5.517323 -0.5490182 .662275$

O $-5.220187-2.5643412 .724567$

H $-5.578557-2.3500350 .173924$

H $-6.937530-1.6259391 .002181$

H $\quad 0.359743 \quad 3.209795 \quad 1.980756$

H $-0.086942 \quad 3.1387043 .692598$

H 1.5889322 .8469843 .205147

C $1.857929-0.1446424 .311570$

H -3.512996 -2.708375 3.785199

H -3.089810 -2.802755 1.216988

H $-6.167158-2.6996972 .899728$

H $1.838398-0.7939515 .196545$

H 2.5320820 .6870084 .549995

H 0.1587351 .0097384 .927379

C $-6.2681132 .155272-0.199739$

$\begin{array}{lllll}\text { O } & -6.312221 & 0.904860 & 0.502321\end{array}$

C $-5.809393-0.227343-0.211749$

C $-6.600004-0.442670-1.511755$

C $-6.6082410 .826678-2.369134$

C $-7.0013472 .059811-1.549828$

H -6.8342392 .8512780 .433256$

C $-4.8329662 .693156-0.321961$

H $-4.760710-0.057704-0.470614$

O $-7.941676-0.831662-1.224766$

H $-6.142158-1.265988-2.072271$

H $-7.3163240 .702438-3.197478$

O $-5.3150961 .039434-2.927954$

H $-6.8265312 .975955-2.126016$

O $-8.4034922 .004580-1.269802$

C $-4.297863 \quad 3.1811841 .008950$

H $-4.1303581 .946225-0.696746$ 
H $-4.8343643 .550056-1.006411$

H $-8.335521-0.146018-0.658265$

H $-5.0955370 .259673-3.466028$

H $-8.8721971 .998750-2.121881$

O $-3.250498 \quad 4.028416 \quad 0.782716$

O -4.7307512 .8534732 .105160$

C -2.662804 4.6161391 .947725

H -3.3407635 .3678382 .362113$

H -2.431019 3.8554632 .698026

H -1.732448 5.1062191 .648816

SCF Energy $\left(\mathrm{B} 3 L Y P / 6-31 G^{* *} / / \mathrm{MMFF}\right)=-3245.91411415$

08_00557

MM̄FF Geometry

C $1.9735004 .748865-0.495685$

C $3.0781364 .017643-0.288062$

C $3.8656253 .343664-1.379363$

O $3.5939861 .931711-1.239825$

C $5.3666953 .605880-1.175323$

C $6.2466572 .969320-2.230434$

C $6.9263631 .817877-2.059317$

C $6.3642113 .739060-3.519277$

C $6.8938690 .913700-0.856765$

C $6.668640-0.546503-1.270475$

C $6.425316-1.455474-0.056828$

C $6.381703-2.920766-0.494561$

C $6.039864-3.856380 \quad 0.672291$

O $5.173922-1.081803 \quad 0.532735$

O $5.716368-5.1439200 .125642$

C $4.771115-3.3304311 .370880$

C $7.235732-4.0685411 .607529$

O $4.488562-4.0862022 .546899$

C $4.824960-1.8204941 .710163$

C $3.424087-1.3583382 .176848$

O $5.766384-1.6072402 .762101$

C $3.248612 \quad 1.231350-2.353048$

O $3.2428331 .625175-3.507509$

C $2.820976-0.110107-1.902831$

C $2.244912-0.964570-2.760142$

C $1.709877-2.274524-2.391651$

C $1.937463-3.338439-3.428328$

C 3.2657780 .1539892 .388867

C 1.8330930 .4527662 .792321

C -0.4770310 .4391631 .845667$

O $1.5066720 .580662 \quad 3.969944$

C $-0.890965-0.9951161 .716419$

C -1.569126 -1.488620 0.668667

C $-1.860782-2.9515810 .485934$

C -1.181394 -3.533998 -0.738153

C $-1.896583-3.977599-1.785394$

C $0.328476-3.670707-0.725453$

C $1.010670-2.437609-1.250530$

N 0.9690640 .4890951 .709082

H $1.5975074 .887418-1.505809$

H 3.4210123 .8635630 .733257

H $3.543003 \quad 3.714054-2.360024$

H $5.6726513 .266206-0.178556$

H $5.5548384 .688350-1.173518$

H $7.5663051 .474595-2.871098$

H $7.0263133 .246499-4.239173$

H $5.3833783 .844488-3.993389$

H $6.7682624 .738764-3.329171$

H $7.8525170 .995579-0.331253$

H $6.1153861 .211779-0.149981$

H $5.804861-0.602536-1.944548$

H $7.543396-0.899960-1.830775$

H $7.230340-1.2939510 .669616$

H $7.330837-3.217509-0.958173$

H $5.616265-3.045532-1.273804$

H $6.485454-5.455927-0.381417$

H $3.928516-3.4995790 .685084$

H $8.078800-4.5044871 .057839$

H $6.994307-4.7841382 .401238$

H $7.587316-3.1444642 .070902$

H $4.448301-5.0225252 .286841$

H $2.682997-1.6739811 .431421$

H $3.163493-1.8636853 .116050$

H $5.553620-0.7753423 .213081$
H $2.949709-0.354553-0.854938$

H $2.133731-0.694813-3.808083$

H $1.482269-4.297911-3.173586$

H $3.011055-3.513591-3.560610$

H $\quad 1.517437-3.032940-4.393225$

H 3.9235430 .5232363 .182057

H 3.5097690 .7135911 .479595

H -0.9006241 .0642651 .054883$

H -0.7817340 .8375782 .817911$

H -0.565691 -1.670799 2.504428

H $-1.890199-0.817593-0.124749$

H -2.948393 -3.0715330 .416610$

H -1.553175 -3.529275 1.366681

H -1.412811 -4.415229-2.652634

H -2.978174 -3.909284-1.803683

H $\quad 0.625979-4.566717-1.279553$

H $0.671870-3.8516150 .301123$

H $0.902171-1.573197-0.599291$

H 1.3481330 .3329600 .780123

C 0.0326094 .4974611 .068529

O $-\begin{array}{llll}0.977218 & 4.448927 & 0.042122\end{array}$

C -2.2797014 .5081710 .664829$

C -2.0386094 .3721622 .161343$

C -0.6762755 .0138182 .317936$

H -2.6777305 .5025470 .427765$

C -3.1721313 .4152610 .063704$

H -2.814181 4.8619852 .757680

H -1.9990983 .3160972 .456323$

C 0.0162334 .6461833 .619300

H -0.7909756 .1045162 .262742$

C -4.5356993 .2773230 .760746$

O $\quad-3.3940653 .726392-1.315008$

H -2.6379182 .4595840 .086975$

H -4.3924262 .9954701 .810149$

O

C -5.5042412 .2726030 .106711$

C $-4.9053940 .868962-0.043253$

H $-5.8409412 .649839-0.865228$

$\begin{array}{lllll}\text { O } & -6.665432 & 2.180527 & 0.938787\end{array}$

H $-4.0686660 .882901-0.751931$

H $-4.4691770 .559541 \quad 0.917067$

H 0.9994395 .1216263 .688246

H -0.5784924 .9759314 .477423$

H 0.1592243 .5636123 .705873

C 1.1946935 .3844110 .621835

H $-2.5217003 .810001-1.737066$

H -5.279210 $4.849961-0.141056$

H $-7.027423 \quad 3.0784891 .031404$

H 1.8623245 .5868581 .467634

H $\quad 0.823172 \quad 6.353403 \quad 0.267183$

H 0.3769493 .4671821 .225632

C $-5.924596-2.625301-0.817550$

O $-5.161896-1.445616-0.534492$

C $-5.910211-0.224084-0.461088$

C $-6.6128680 .048949-1.798575$

C $-7.473312-1.145871-2.225701$

C $-6.706905-2.468843-2.132015$

H $-5.175750-3.413029-0.964058$

C $-6.796776-3.040756 \quad 0.372353$

H $-6.655633-0.3178980 .335236$

\begin{tabular}{lllll}
\hline & -5.633797 & 0.322727 & -2.798261
\end{tabular}

H -7.245432 $0.938483-1.700654$

H -7.812242 $-0.992527-3.257593$

O $-8.627456-1.192706-1.391548$

H -7.393987 -3.313505 -2.258975

O $-5.781371-2.516256-3.221988$

C $-5.932033-3.4713961 .533811$

H -7.473534 -2.255014 0.719271

H $-7.429158-3.8961380 .105999$

H $\quad-6.1053490 .510904-3.627357$

H $-9.177930-1.935139-1.693762$

H - $-5.336715-3.380569-3.193605$

O $-5.590395-2.3881392 .288293$

O $-5.581711-4.6280051 .729243$

C $-4.735323-2.6571373 .403369$

H $-5.252300-3.2924894 .128408$

H -3.806948 -3.129522 3.068665

H -4.490155 -1.704789 3.880874 
O $7.838490 \quad 0.492732-1.930598$

C $5.969916-0.794250-1.041009$

C $4.988412-0.280947-2.121571$

O $6.606126-1.982109-1.513068$

C $1.105912-0.4817551 .601976$

O $0.293137-0.3889682 .507469$

C 1.9655690 .6188681 .124278

C 1.4997551 .8729921 .208719

C 2.1529693 .0697560 .680699

C 3.5942682 .9613380 .262696

C $3.746839-1.156492-2.332429$

C $2.759315-0.459719-3.251086$

C $1.094873 \quad 1.409567-3.281273$

O $2.653286-0.767968-4.435653$

C $1.5516512 .829264-3.138379$

C $0.7737413 .822771-2.680004$

C $1.2287185 .251641-2.563206$

C $0.9219715 .869275-1.214483$

C $-0.0710416 .763916-1.074438$

C $1.8151255 .509634-0.044153$

C 1.4215054 .1974420 .572371

N $2.0504850 .545562-2.612364$

H -1.872165 -2.244531 -0.281925

H $\quad 0.741090-3.657183-1.017456$

H $-0.370748-2.4756311 .608171$

H $2.102699-4.1777820 .951086$

H $\quad 0.649730-4.7509671 .714974$

H $3.457975-3.0172584 .240037$

H $1.353777-3.4228835 .098139$

H $0.052314-2.9563684 .000079$

H $0.488703-4.6681504 .190836$

H $4.875226-3.8861652 .197661$

H $3.797296-2.9458461 .169537$

H $4.390555-0.8910622 .588059$

H $5.627610-1.8882103 .369678$

H $6.541426-2.3890111 .017730$

H $7.617306-0.6666212 .514296$

H $6.469156 \quad 0.5350331 .958540$

H 9.1122941 .0660371 .608292

H $6.524817 \quad 1.241708-0.575722$

H $9.632352-1.1751821 .117871$

H $9.687299-0.751323-0.578011$

H $8.601832-2.041517-0.025184$

H $8.485018 \quad 1.188440-1.720976$

H $4.6544330 .724427-1.843034$

H $5.508554-0.184815-3.083422$

H $5.963044-2.503741-2.018671$

H $2.904251 \quad 0.365420 \quad 0.648642$

H 0.5162242 .0325751 .649165

H 4.1845412 .4382671 .023742

H 4.0772373 .9319410 .128505

H $3.6774172 .416772-0.680866$

H $4.012479-2.118224-2.783816$

H $3.242981-1.376394-1.385205$

H $\quad 0.1213491 .240715-2.810223$

H $1.0241061 .144560-4.340432$

H $2.5714193 .052729-3.444064$

H $0.7279685 .821926-3.356559$

H $2.3036865 .344428-2.762411$

H $-0.2815267 .228582-0.116834$

H $-0.7003997 .046349-1.911914$

H $1.7430726 .277781 \quad 0.736823$

H $2.8556035 .532699-0.379084$

H 0.3914014 .1802060 .930443

H $2.1899900 .686942-1.617395$

C $-3.064662-3.840106-2.262920$

O $-4.016719-2.793334-1.989218$

C $-4.949810-3.268214-1.002607$

C $-4.845239-4.787388-1.042841$

C $-3.353966-4.967555-1.268012$

H $-4.581557-2.917100-0.031196$

C $-6.344537-2.684379-1.246887$

H $-5.200570-5.255022-0.119860$

H $-5.406456-5.212973-1.882561$

C $-2.969757-6.345517-1.777072$

H $-2.843561-4.792467-0.313014$

C $-6.361719-1.159188-1.482642$

O $-6.905101-3.301974-2.412516$

H -7.002339-2.953719 -0.411851

H -5.965964 -0.946860 -2.483932

O $-7.737743-0.759825-1.499054$

C $-5.591765-0.334014-0.434561$

C $-5.6787651 .181101-0.702029$

H $-4.538738-0.634423-0.446102$

O $-6.112324-0.631910 \quad 0.859065$

H $-5.5529571 .365938-1.775389$

H $-6.6701821 .539355-0.398459$

H -3.250456 -7.115792 -1.051338

H $-3.469244-6.578211-2.723555$

H -1.889237 -6.410788 -1.940116

C -1.645564 -3.259319-2.247569

H $-7.793923-2.927588-2.538917$

H $-7.7801390 .145050-1.851180$

H $-5.592666-0.1287031 .508612$

H -1.620757 -2.376504 -2.898453

H $-0.953511-3.998394-2.668452$

H $-3.282632-4.175927-3.285113$

C -3.9394792 .5288292 .328557$

O -4.8857221 .8893551 .460851$

C $-4.600398 \quad 1.9741140 .062171$

C $-4.5506593 .443121-0.383745$

C -3.5512474 .2425090 .457654$

C -3.7570984 .0107981 .957872$

H -4.4056322 .4903843 .321949$

C -2.6211231 .7503942 .400307$

H -3.631314 $1.504670-0.139916$

O $-5.8430414 .037792-0.284358$

H -4.260274 $3.483552-1.439906$

H -3.6615885 .3114640 .238270$

O -2.2239023 .8599900 .112216$

H -2.921535 4.4297332 .530951

O -4.9388864 .7016912 .374772$

C $-2.829080 \quad 0.4334153 .109549$

H -2.1754151 .5560961 .420850$

H -1.870571 2.3057012 .975490

H -6.1465713 .9383360 .634192$

H $-2.0958884 .062589-0.830086$

H -4.8039545 .6477362 .194356$

O $-2.974972-0.5729832 .201398$

$\begin{array}{llll}\text { O } & -2.881466 & 0.320243 & 4.327682\end{array}$

C $-3.196221-1.8722972 .758707$

H $-4.181998-1.9114843 .231048$

H -3.165563 -2.602518 1.946541

$\mathrm{H}-2.411475-2.1225213 .479161$

SCF Energy (B3LYP/6-31G**//MMFF) $=-3245.91695238$

0800559

MM̄FF Geometry

C $2.084123 \quad 3.470723-1.726051$

C $3.3339973 .957055-1.788274$

C $4.6159253 .233227-1.439993$

O $4.3944141 .827368-1.188803$

C $5.2323323 .861713-0.176651$

C 6.5990803 .3040730 .175264 
C 6.8536162 .4714641 .204903

C $7.7299823 .780453-0.700856$

C $5.872808 \quad 1.888361 \quad 2.183481$

C $5.876656 \quad 0.3549872 .122273$

C $4.710735-0.2525132 .916545$

C $4.785751-1.7801542 .897544$

C $3.557771-2.4307363 .552077$

O 3.4987770 .1895052 .298628

O $3.555193-3.8225013 .197603$

C $2.279967-1.8103122 .947420$

C $3.630428-2.3726125 .082250$

O $1.117646-2.2750513 .629176$

C $2.296341-0.2686902 .929031$

C 1.1098990 .3395152 .140855

$\begin{array}{lllll}\text { O } & 2.204412 & 0.216470 & 4.273627\end{array}$

C $4.3958760 .999901-2.271507$

O $4.512124 \quad 1.323511-3.443089$

C $4.230122-0.387415-1.783368$

C $4.066793-1.386934-2.663088$

C $3.915821-2.809001-2.343199$

C $3.888350-3.199529-0.889389$

C $1.078567 \quad 0.0024890 .646305$

C $0.182828-1.1685400 .284406$

C $-0.557197-2.490072-1.696492$

O $-0.518818-1.7612261 .098308$

C $0.227809-3.762716-1.727508$

C $0.495213-4.439824-2.854412$

C $1.232404-5.747249-2.887411$

C $2.421428-5.727333-3.820718$

C $2.339168-6.248320-5.056681$

C $3.730130-5.192422-3.286112$

C $3.817973-3.691908-3.359365$

N $0.238628-1.449636-1.074402$

H $1.9096272 .452519-1.392377$

H $3.4700764 .982651-2.128209$

H $5.3118393 .348138-2.280437$

H 4.5363693 .7455150 .660439

H $5.3353824 .947135-0.313031$

H 7.8850102 .1613691 .369455

H $8.7040743 .414452-0.358994$

H $7.5928933 .434705-1.729903$

H $7.7739944 .874466-0.702926$

H $6.153398 \quad 2.210865 \quad 3.193329$

H 4.8618512 .2628562 .012279

H 5.8001620 .0401711 .073786

H $6.830848-0.0247182 .508241$

H 4.7510200 .1235943 .945523

H $5.708420-2.1295063 .377630$

H $4.843693-2.1266531 .857263$

H $4.391181-4.2120113 .506401$

H $2.205741-2.1775611 .916813$

H $4.518669-2.9047605 .443632$

H $2.772668-2.8790585 .538430$

H $3.677978-1.3533635 .470987$

H $1.133865-3.2470763 .599943$

H 0.1604520 .0987852 .634160

H 1.2015531 .4332392 .211046

H 1.8722921 .1290644 .251535

H $4.257057-0.550588-0.712867$

H $4.054171-1.145751-3.725482$

H $3.127901-2.626259-0.348921$

H $4.863734-3.013889-0.427663$

H $3.644116-4.250855-0.723478$

H $\quad 0.680956 \quad 0.870957 \quad 0.107399$

H $2.083769-0.1846150 .260592$

H $-0.822168-2.140499-2.699357$

H -1.479051 -2.637212 -1.124737

H $0.565939-4.160781-0.773638$

H $0.132751-4.053788-3.804511$

H $\quad 0.521249-6.520575-3.206518$

H $1.561388-6.047588-1.884707$

H $3.197065-6.266606-5.720688$

H $1.410061-6.662724-5.434134$

H $4.564359-5.591970-3.879226$

H $3.899207-5.584742-2.279514$

H $3.844411-3.309791-4.380917$

H $0.897712-0.944369-1.659695$

C $-0.0780664 .494640-0.938409$
O $-0.8031813 .277655-0.657059$

C $-2.2075833 .585209-0.565449$

C $-2.2844525 .091600-0.359098$

C $-1.1378515 .559938-1.230519$

H $-2.6464373 .346747-1.540244$

$\begin{array}{llll}\text { C }-2.882526 & 2.759216 & 0.534471\end{array}$

H $-3.2516855 .504714-0.660514$

H -2.1045705 .3694040 .685948$

C $-0.6854626 .978315-0.927493$

H $-1.4492725 .501683-2.282443$

$\begin{array}{llll}\text { C }-2.735463 & 1.230399 & 0.398277\end{array}$

$\begin{array}{lllll}\text { O } & -2.314688 & 3.128465 & 1.800464\end{array}$

H $-3.943372 \quad 3.0254110 .598377$

H -1.6810530 .9748160 .536398$

$\begin{array}{lllll}\text { O } & -3.426720 & 0.609070 & 1.486206\end{array}$

C $-3.2311370 .590281-0.913037$

C $-4.6117941 .046667-1.422812$

H -3.257114 $-0.499256-0.787085$

O $-2.289116 \quad 0.854622-1.955790$

H $-4.6100632 .129094-1.592828$

H $-4.7472360 .597856-2.416917$

H $-1.5016887 .687191-1.100916$

H -0.3650757 .0832710 .114524$

H $0.1537377 .266305-1.568210$

C $0.8715494 .271399-2.118364$

H -1.3578952 .9586251 .753197$

H -3.0843840 .9977882 .309286$

H -1.427409 $0.506972-1.669490$

H $1.183157 \quad 5.237466-2.532475$

H $0.3544983 .725853-2.917530$

H $0.4826514 .748544-0.029644$

C $-6.917226-1.3320690 .309453$

O $-5.875102-0.770147-0.501732$

C $-5.8010980 .658943-0.530782$

C $-7.1195151 .256688-1.048544$

C $-8.3131040 .747806-0.236117$

C $-8.293608-0.777735-0.098045$

H $-6.911422-2.4033560 .068340$

C $-6.616526-1.1842431 .809792$

H $-5.6253691 .038807 \quad 0.478257$

$\begin{array}{llll} & -7.308883 & 0.939432 & -2.425744\end{array}$

H $-7.0639972 .349215-0.976600$

H $-9.2403841 .063187-0.729733$

O $-8.275631 \quad 1.3506921 .054191$

H $-9.049613-1.1105240 .622471$

O $-8.635242-1.364107-1.357665$

C -5.642576 $-2.229797 \quad 2.311132$

H $-6.212735-0.2062562 .081514$

H -7.541042 -1.333565 2.380899

H $-7.276019-0.028479-2.515293$

H -9.0524641 .0364701 .547503$

H $-9.524075-1.049434-1.595810$

O $-4.544059-2.298171 \quad 1.509207$

O $-5.824133-2.8789803 .334342$

C $-3.549503-3.252755 \quad 1.892748$

H $-4.000276-4.2310682 .086750$

H $-2.843222-3.3568811 .065381$

H $-3.012741-2.8922772 .774861$

SCF Energy (B3LYP/6-31G**//MMFF) $=-3245.91188842$

0800560

MMFF Geometry

C $0.446199 \quad 4.690864 \quad 0.630812$

C 1.6339734 .0734850 .718324

C $2.5723153 .914438-0.450510$

O $2.7784892 .498593-0.677040$

C $3.9316584 .552579-0.127146$

C $4.8450294 .625842-1.334262$

C $5.8815753 .796458-1.562526$

C $4.5605975 .758389-2.287830$

C $6.3179952 .622331-0.730796$

C $6.6427011 .407044-1.611192$

C $7.1813510 .236542-0.777658$

C $7.704660-0.879006-1.683450$

C $8.192751-2.086437-0.870040$

O $6.116797-0.2615160 .040442$

O $8.368833-3.186584-1.775368$

C $7.088631-2.4920370 .126268$ 
C $9.560337-1.831158-0.225645$

O $7.547662-3.5130261 .009641$

C $6.512223-1.3066360 .939333$

C $5.264531-1.7820551 .717871$

O $7.493403-0.8517521 .873208$

C $1.817403 \quad 1.843342-1.384601$

O $0.8112322 .328758-1.878126$

C $2.1802320 .408656-1.431568$

C $1.239280-0.477360-1.791767$

C $1.383728-1.932734-1.865836$

C $2.769005-2.504152-1.733904$

C $4.421910-0.6572032 .332818$

C $3.232625-1.2499333 .064224$

C $1.071767-2.4276212 .646783$

O $3.204721-1.3334094 .289667$

C $0.876586-3.6119181 .752324$

C $-0.263236-3.8756591 .096327$

C $-0.457399-5.072390 \quad 0.210529$

C $-0.905095-4.703358-1.186376$

C $-2.171488-4.914039-1.584456$

C $0.135899-4.167051-2.148534$

C $0.272380-2.673427-2.061389$

N 2.252091-1.711582 2.201225

H $0.1362495 .122786-0.317658$

H 1.9353923 .6242151 .661701

H $2.1534724 .371494-1.355783$

H 4.4173994 .0044810 .688781

H 3.7875315 .5732350 .252867

H $6.4961613 .982372-2.442714$

H $5.2801875 .799190-3.112445$

H $3.5644005 .651941-2.727754$

H $4.6085836 .718051-1.762692$

H $7.2159832 .916295-0.174454$

H 5.5593962 .3375870 .003648

H $5.7334711 .098038-2.143358$

H $7.3879281 .691714-2.364628$

H $7.9870260 .612293-0.135778$

H $8.505109-0.509406-2.336370$

H $6.899854-1.212596-2.353708$

H $9.012792-2.917128-2.452613$

H $6.271410-2.935560-0.460348$

H $\quad 10.309119-1.604124-0.993963$

H $9.925333-2.7247770 .292586$

H $9.552548-1.0007100 .483255$

H $7.884647-4.2414570 .460296$

H $4.620190-2.3542351 .040633$

H $5.567187-2.4699862 .518342$

H $7.044944-0.4201992 .617475$

H $3.1849700 .122816-1.143497$

H $0.244305-0.103183-2.032842$

H $3.448254-2.038692-2.457218$

H $2.816469-3.579384-1.917348$

H $3.161238-2.331299-0.729000$

H $5.002336-0.0663363 .048758$

H 4.0553950 .0332491 .564744

H $0.229931-1.7302432 .606867$

H $1.201626-2.7577983 .681970$

H $1.718089-4.2933541 .647230$

H $-1.114584-3.2120941 .221177$

H -1.205088 -5.7193050 .687862$

H $0.457342-5.6748940 .146611$

H -2.492721 -4.674942 -2.592854

H $-2.916941-5.320805-0.909129$

H $-0.154386-4.411693-3.178926$

H $1.078061-4.694876-1.979419$

H $-0.677142-2.148493-2.177882$

H $2.314460-1.4706821 .216479$

C $-1.6710613 .787961 \quad 1.569257$

$\begin{array}{llll}\text { O } & -2.491140 & 4.184876 & 0.452830\end{array}$

C -3.8593793 .7986870 .724393$

C -3.832837 3.0535072 .052946

C -2.637731 3.6835252 .742939

H -4.417736 4.7373290 .829452

C $-4.3836203 .001448-0.477896$

H $-4.764116 \quad 3.1687632 .615521$

H -3.660478 1.9813951 .899262

C -2.119441 2.8692543 .915065

H -2.9170994 .6868873 .091165$
C $-5.721438 \quad 2.278722-0.227712$

O $-4.525814 \quad 3.915526-1.568636$

H $-3.6347802 .263918-0.791006$

H $-5.5527101 .542587 \quad 0.562543$

$\begin{array}{llll}\text { O } & -6.688158 & 3.201630 & 0.272310\end{array}$

C $-6.2890811 .559357-1.470756$

C $-7.4982680 .654315-1.161055$

H $-5.5016980 .962712-1.945370$

O

H $-8.2762651 .232725-0.647663$

H $-7.9337970 .350070-2.122539$

H -1.2548723 .3549564 .378016$

H -2.894833 2.7624444 .680679

H -1.814659 $1.864706 \quad 3.604296$

C -0.5321314 .7861661 .768750$

H $-3.6602164 .336432-1.709763$

H $-6.847774 \quad 3.860319-0.425538$

H -5.964323 $3.048609-2.697756$

H $-0.021874 \quad 4.596462 \quad 2.719764$

H - $0.919176 \quad 5.8113191 .802368$

H $-1.2605452 .798897 \quad 1.329430$

C -5.672531-2.524585-0.471907

O $-6.205346-1.360373-1.114161$

C $-7.137841-0.597403-0.343390$

C $-8.366539-1.455573-0.007479$

C $-7.967371-2.7659400 .685217$

C $-6.799810-3.469122-0.017822$

H $-5.104200-3.038839-1.257711$

C $-4.680380-2.1561700 .641288$

H $-6.664822-0.2768790 .587543$

O $-9.094022-1.758316-1.197323$

H $-9.036884-0.8816190 .642635$

H -8.833127 -3.4398030 .683199$

O $-7.639475-2.5336912 .052080$

H $-6.392508-4.2596180 .623173$

O $-7.333602-4.109490-1.183608$

C $-3.487611-1.3905340 .104496$

H -5.148844 -1.534542 1.407318

H $-4.298051-3.0673251 .114996$

H $-8.492249-2.223840-1.803217$

H $-7.125679-1.7139682 .121175$

H $-6.619653-4.635383-1.582946$

O $-2.794818-0.854028 \quad 1.152295$

O $-3.187358-1.289080-1.077036$

C $-1.621430-0.1181180 .795829$

$\begin{array}{llll}\mathrm{H} & -1.869728 & 0.691345 & 0.103674\end{array}$

H $-1.199783 \quad 0.312672 \quad 1.706977$

H $-0.881133-0.7884460 .353650$

SCF Energy (B3LYP/6-31G**//MMFF) $=-3245.91757248$

\section{1}

MMFF Geometry

C $1.244843 \quad 3.1663580 .663995$

C $1.7870464 .000050-0.237080$

C $2.7553843 .618404-1.337802$

O $2.9094222 .182864-1.432765$

C $4.1300154 .242114-1.038484$

C $5.1629023 .987963-2.118533$

C $6.2076653 .144005-2.002010$

C $4.9836434 .793076-3.380243$

C $6.5536522 .259071-0.836549$

C $6.589156 \quad 0.785503-1.259223$

C $6.763472-0.154066-0.056548$

C $6.902466-1.600304-0.534828$

C $6.958673-2.591483 \quad 0.635387$

O $5.605344-0.0127840 .770301$

O $6.786810-3.912760 \quad 0.099596$

C $5.759643-2.319717 \quad 1.566602$

C $8.325549-2.5791881 .328910$

O $5.833170-3.1331192 .736773$

C $5.590764-0.8279481 .947552$

C $4.241847-0.5844942 .673330$

O $6.644808-0.4636672 .843921$

C $1.9814481 .510298-2.170708$

O $1.058413 \quad 1.993505-2.807386$

C $2.2488530 .061270-2.026856$

C $1.287755-0.814779-2.358205$

C $1.355381-2.271851-2.226226$ 
C $2.680539-2.888372-1.862638$

C $3.039974-0.5635721 .725807$

C $1.712770-0.8672452 .394610$

C $-0.639093-1.4014421 .743932$

O $1.594978-1.1193143 .589443$

C $-0.640958-2.828911 \quad 1.289034$

C $-1.435676-3.3097820 .321422$

C $-1.389138-4.735104-0.153237$

C $-1.256945-4.858712-1.657389$

C $-2.259022-5.364694-2.396416$

C $0.064914-4.484410-2.300248$

C $0.237258-2.994919-2.449865$

N $0.677060-0.8570761 .466575$

H 1.4986332 .1116800 .648055

H $1.5165125 .053580-0.198342$

H $2.3734254 .010358-2.288532$

H $4.4858693 .882473-0.067602$

H $4.0256215 .329190-0.917125$

H $6.8994243 .068388-2.840319$

H $5.795794 \quad 4.630418-4.096775$

H $4.0476264 .523968-3.879187$

H $4.9607705 .862995-3.148349$

H $7.5403352 .552280-0.458082$

H $5.8548072 .385162-0.007246$

H $\quad 5.650890 \quad 0.540957-1.774447$

H $7.4064280 .630624-1.974867$

H $7.650350 \quad 0.1539270 .509546$

H $7.785057-1.714157-1.176807$

H $6.040083-1.860156-1.165267$

H $7.498493-4.070362-0.544313$

H $4.865278-2.6570431 .029765$

H $9.120109-2.8215460 .612887$

H $8.381215-3.3489562 .106533$

H $8.569895-1.6142181 .777899$

H $\quad 6.495717-2.7392793 .329633$

H $4.117243-1.3220113 .475250$

H $4.269307 \quad 0.387518 \quad 3.184779$

H $6.3742800 .332133 \quad 3.330885$

H $3.203437-0.240496-1.612252$

H $0.339819-0.428121-2.731222$

H $3.488703-2.468739-2.472551$

H $2.712154-3.967298-2.035225$

H $2.909646-2.714741-0.808326$

H $2.973973 \quad 0.419587 \quad 1.248014$

H $3.167137-1.3069820 .935134$

H - $-1.369318-0.791726 \quad 1.202686$

H $-0.851238-1.3458432 .815827$

H $\quad 0.065230-3.4986571 .775105$

H -2.153256 -2.647433 -0.156010

H -2.307767 -5.2288980 .188193$

H $-0.562330-5.2820170 .317466$

H -2.164734 -5.484379 -3.470665

H -3.199042 -5.665193-1.945783

H $\quad 0.132649-4.928326-3.301785$

H $0.865653-4.938985-1.708364$

H $-0.665740-2.467981-2.761305$

H $\quad 0.901625-0.6759740 .493124$

C -1.097449 $2.961243 \quad 1.489321$

$\begin{array}{llll}\text { O } & -1.699626 & 3.586559 & 0.336667\end{array}$

C -3.0318043 .9923260 .677056$

C -3.4065773 .2002381 .919781$

C -2.0743403 .1733392 .647764$

H -2.9632625 .0515020 .963639$

C $-3.9750263 .895485-0.523716$

H -4.2039663 .6744292 .499377$

H -3.7186732 .1809001 .671394$

C -1.9836962 .1053403 .723828$

H $-1.903627 \quad 4.1566103 .106587$

C $-4.3852602 .490557-1.013898$

O $-5.1737994 .598857-0.168345$

H $-3.5282984 .447438-1.360549$

H -5.096365 $2.059231-0.303024$

O $-5.1259362 .673757-2.231013$

C $-3.2467161 .492080-1.274476$

C $-3.6673260 .208980-2.015393$

H $-2.779421 \quad 1.216604-0.323971$

O $-2.2467272 .131908-2.072582$

H $\quad-3.8905900 .446159-3.063860$
H $-2.778600-0.435940-2.042381$

H $-0.9869722 .089427 \quad 4.175976$

H $-2.7105082 .299598 \quad 4.518911$

H -2.1856351 .1083183 .318081$

C 0.2768483 .5844891 .734069

H $-5.7659064 .570313-0.939539$

H $-4.5057883 .004238-2.903723$

H -1.492662 $1.521858-2.144530$

H $\quad 0.6771723 .2583352 .700579$

H $\quad 0.1934374 .6778261 .763139$

H -1.0019121 .8926161 .259153$

C $-5.491944-1.6376900 .683734$

O $-4.496542-0.915419-0.056256$

C -4.845864 -0.560980 -1.397298

C $-5.208961-1.805693-2.228416$

C $-6.280889-2.647831-1.535211$

C $-5.935558-2.906021-0.065453$

H $-4.974527-1.9600891 .595736$

C -6.677250 -0.746952 1.084895

H $-5.7154240 .102312-1.374388$

O $-4.064766-2.627946-2.440013$

H -5.574913 -1.487203 -3.212424

H $-6.379326-3.604246-2.063406$

O $-7.528915-1.968206-1.632459$

H $-6.788614-3.3556410 .455885$

O $-4.864267 \quad-3.8487860 .009190$

C -6.3166120 .2575872 .153750$

H $-7.076739-0.1821300 .237932$

H -7.503943 -1.348617 1.481819

H -3.507852 -2.197565 -3.110244

H $-8.201627-2.528129-1.209007$

H $-5.154750-4.660997-0.439475$

O $-5.962583-0.3816053 .304699$

O -6.3882961 .4695351 .996553$

C -5.6457690 .4684894 .411116$

H $-6.5563460 .947187 \quad 4.782729$

H -5.222380 -0.150939 5.206186

H $-4.906588 \quad 1.2207774 .123053$

SCF Energy (B3LYP/6-31G**//MMFF)= -3245.90376977

\section{2}

MMFF Geometry

C $1.741019-1.9368941 .009893$

C $0.496951-2.3614720 .746257$

C $-0.054130-2.504199-0.648548$

O $-1.119960-1.537983-0.794655$

C $-0.638406-3.911328-0.845590$

C $-1.125153-4.151799-2.260721$

C $-2.418010-4.120856-2.639654$

C $-0.050396-4.487291-3.262416$

C $-3.612497-3.800573-1.784416$

C $-4.546815-2.798015-2.476788$

C $-5.755776-2.461880-1.592852$

C $-6.784796-1.635042-2.363813$

C $-7.967849-1.230515-1.471259$

O $-5.285733-1.716914-0.464202$

O $-8.715591-0.218094-2.161789$

C $-7.420658-0.588098-0.180421$

C $-8.933269-2.397525-1.233027$

O $-8.471971-0.3316450 .748107$

C $-6.299719-1.4099840 .501483$

C $-5.657924-0.5674571 .627287$

O $-6.857796-2.595026 \quad 1.070928$

C $-0.771593-0.307253-1.259699$

O $0.3318040 .051766-1.639136$

C $-1.9668380 .559706-1.182064$

C $-1.7980631 .890516-1.191107$

C $-2.8495832 .899533-1.054697$

C $-4.2785432 .431645-1.109232$

C $-4.365703-1.1524502 .210278$

C $-3.746598-0.158703 \quad 3.173940$

C -2.4759971 .9924223 .209104$

O $-3.923506-0.2412884 .386929$

C -3.2534853 .2030052 .791247$

C -2.6824694 .3497012 .392263$

C -3.4559365 .5617421 .955444$

C -2.9782526 .1199460 .630853$

C $-2.288658 \quad 7.2720150 .576128$ 
C $-3.3630655 .382895-0.636556$ C -2.483611 4.186271-0.878682 N -3.0296980 .8365652 .527291$ H $2.397090-1.6719110 .184185$ H $-0.171625-2.5985061 .570561$ H $0.726372-2.326736-1.398996$ H $-1.449186-4.083866-0.128288$ H $\quad 0.121750-4.667956-0.607685$ H -2.651630 -4.353277 -3.678015 H $-0.459155-4.698378-4.256309$ H $0.652097-3.654880-3.366670$ H $0.505403-5.374030-2.940567$ H $-4.158874-4.732874-1.597818$ H -3.318895 -3.399557 -0.810839 H -3.986751 -1.881176 -2.701908 H $-4.890874-3.219785-3.429343$ H $-6.203828-3.401191-1.247834$ H -7.140766 -2.179096 -3.247510 H $-6.307584-0.721273-2.745016$ H -9.008930 -0.589657 -3.011402 H -7.007727 $0.392264-0.457621$ H $-9.326545-2.769805-2.186700$ H $-9.806546-2.075528-0.654871$ H -8.469173 -3.239879-0.716016 H -9.1441410 .1978170 .285921$ H $-5.4329860 .431677 \quad 1.238936$ H $-6.376450-0.4301212 .445971$ H -6.279991 -2.902070 1.787276 H $-2.9348660 .086045-1.069119$ H $-0.7847792 .284463-1.265319$ H $-4.4481651 .800696-1.989203$ H -5.0014913.246746 -1.181359 H $-4.5228571 .855705-0.213598$ H $-4.557135-2.0837702 .753435$ H -3.636359-1.381696 1.425242 H -1.4211132 .0623352 .925413$ H -2.545789 1.8588714 .292677 H $-4.338557 \quad 3.1323492 .818027$ H -1.598507 4.4295502 .393856 H -3.353536 6.3207352 .741961 H -4.529173 5.3453411 .885013 H $-1.9633417 .691335-0.370215$ H -2.029003 7.8192271 .476210 H $-3.2645656 .048349-1.503974$ H $-4.4251975 .131092-0.578777$ H $-1.4185744 .424180-0.884243$ H -2.9538250 .8098751 .515081$ C $3.632562-2.4428752 .631730$ O $4.623012-1.8967301 .740315$ C $5.466161-2.9703581 .277341$ C $5.157285-4.1631832 .175550$ C $3.674955-3.9570832 .435514$ H $5.137101-3.2133970 .260225$ C $6.929105-2.5160241 .257353$ H $5.380753-5.1180121 .690124$ H $5.718192-4.1207873 .116422$ C $3.143799-4.7479413 .617223$ H $3.122672-4.248898 \quad 1.533231$

C $7.158346-1.2182550 .451152$ O $7.341438-2.2698672 .607917$ H $7.566570-3.3245120 .880711$ H $6.641473-0.3948950 .956169$ O $8.558561-0.9238330 .520203$

C $6.728144-1.316023-1.025556$

C $6.968892-0.038739-1.852991$

H $5.666423-1.574907-1.086050$

O $7.452953-2.387932-1.635235$ H $8.0305340 .234349-1.832199$ H $6.748865-0.283655-2.900992$ H $3.266216-5.8225723 .446961$ H $3.671337-4.4921984 .542179$ H $2.078340-4.5492153 .770370$ C $2.284993-1.756938 \quad 2.399287$ H $8.268903-1.9782432 .577743$ H $8.680204-0.0012440 .239806$ H $7.120827-2.488647-2.543591$ H $2.413196-0.6784392 .555015$ H $1.564374-2.1067643 .147609$
H $3.969162-2.1908613 .646458$

C $3.7618091 .726521-1.149453$

$\begin{array}{lllll}\text { O } & 4.728228 & 0.764460 & -1.591486\end{array}$

C $6.0964331 .148673-1.414552$

C $6.3941722 .416558-2.229436$

C $5.4283703 .550325-1.865852$

C $3.9683323 .080266-1.852360$

H $2.8000171 .321242-1.487912$

C 3.7276821 .7850220 .380548

H $6.2810551 .354688-0.355486$

O $6.2985622 .145201-3.626724$

H $7.4262332 .729469-2.034109$

H $5.5368964 .366071-2.591090$

O $5.758393 \quad 4.068260-0.580760$

H $3.323725 \quad 3.838622-1.395129$

O $3.5271712 .921133-3.205491$

C $2.412706 \quad 2.327276 \quad 0.882912$

H 3.8463850 .7795380 .802568

H 4.5538262 .3641750 .803262

H $5.4072051 .797660-3.801795$

H $6.6732234 .395065-0.621389$

H $3.5949403 .788698-3.639428$

$\begin{array}{lllll}\text { O } & 2.551117 & 3.638202 & 1.230229\end{array}$

$\begin{array}{lllll}\text { O } & 1.380288 & 1.671434 & 0.936721\end{array}$

C 1.3578874 .2792361 .689925

H $0.598677 \quad 4.272995 \quad 0.902272$

H 0.9850113 .7840402 .591479

H 1.6007125 .3167651 .934170

SCF Energy (B3LYP/6-31G**//MMFF) $=-3245.91198780$

\section{8_00563}

MMFF Geometry

C $-1.5561714 .746038 \quad 1.832413$

C $-2.268174 \quad 3.9001501 .072534$

C $-3.628650 \quad 3.382125 \quad 1.473227$

O $-3.620183 \quad 1.938806 \quad 1.390728$

C $-4.691508 \quad 3.921074 \quad 0.498112$

C -6.1100563 .5189430 .858293$

C $-6.8584642 .627193 \quad 0.177764$

C $-6.693108 \quad 4.2259032 .055799$

C $-6.467161 \quad 1.830378-1.035074$

C $-6.406800 \quad 0.331567-0.714019$

C $-5.684139-0.460063-1.813633$

C $-5.724982-1.958429-1.511346$

C $-4.885663-2.776866-2.504437$

O $-4.329316-0.000426-1.844421$

O $-4.715262-4.092485-1.953659$

C $-3.479839-2.145554-2.610276$

C $-5.610485-2.958748-3.842844$

O $-2.707590-2.791807-3.619255$

C $-3.503862-0.619862-2.837457$

C $-2.1016870 .031308-2.739507$

O $-3.999730-0.355938-4.155444$

$\begin{array}{llll}\text { C } & -3.127479 & 1.262608 & 2.465457\end{array}$

O $-2.6537331 .744002 \quad 3.482523$

C $-3.270301-0.1850392 .192842$

C $-2.782562-1.0787493 .066196$

C $-2.857202-2.5368482 .945363$

C $-3.509710-3.1035601 .712397$

C $-1.418336-0.086465-1.372750$

C $-0.486542-1.278559-1.241906$

C $0.851346-2.5113240 .462910$

O $-0.102344-1.940205-2.201916$

C $0.114211-3.720068 \quad 0.943424$

C $0.354327-4.306267 \quad 2.126278$

C $-0.340353-5.5502402 .600675$

C $-0.957518-5.3898073 .971959$

C $-0.325924-5.8367015 .070673$

C $-2.344480-4.7948924 .057681$

C -2.340421 -3.2946513 .935359$

N $-0.097222 \quad-1.485324 \quad 0.075077$

H -1.981561 5.0531182 .786930

H $-1.886147 \quad 3.5676450 .111862$

H -3.881384 3.6888012 .495823

H $-4.4411743 .601258-0.518137$

H -4.647246 5.0185960 .473052

H -7.871741 2.4343870 .528320

H $-7.746150 \quad 3.971522 \quad 2.217276$ 
H -6.1483453 .9579992 .965907$ H -6.6363305 .3110091 .921160$ H -7.212614 2.002251-1.821171 H -5.515147 2.167033 -1.449449 H -5.8763460 .1893130 .235839$ H $-7.425234-0.053666-0.579797$ H $-6.162507-0.246689-2.776820$ H -6.759941 -2.322105 -1.490200 H -5.329752 -2.133500 -0.502233 H -5.598156 -4.474559-1.810594 H $-2.975433-2.356788-1.660334$ H $-6.571748-3.464774-3.691577$ H $-5.038390-3.604214-4.518270$ H -5.813042 -2.014473 -4.352316 H -3.013809 -2.464922 -4.482241 H -1.457700 $-0.331577-3.549388$ H $-2.2300791 .106404-2.931924$ H $-3.7076220 .531136-4.423360$ H -3.777146 -0.4774871 .281183$ H -2.285383 -0.7108113 .962861$ H $-3.417075-4.1881481 .626769$ H -3.050948 -2.6913250 .807376$ H $-4.578863-2.8666761 .705801$ H $-0.7944290 .803350-1.221700$ H -2.155355 -0.105281 -0.565573 H $1.494626-2.0827491 .237651$ H $1.471299-2.778118-0.396943$ H $-0.628653-4.1500710 .275662$ H $1.119449-3.8903652 .778139$ H $\quad 0.402853-6.3583902 .619726$ H -1.110387 -5.8756971 .890214$ H $-0.775014-5.7526936 .054790$ H $0.657194-6.2918085 .010823$ H -2.796637 -5.0371235 .029062$ H -2.993425 -5.2833393 .325240$ H -1.880234 -2.791684 4.787012 H $-0.490683-0.9034070 .808981$ C 0.4434245 .0172930 .190352 O $\quad 0.9768243 .676396 \quad 0.254062$ C $2.3531523 .706467-0.168333$

C $2.5140955 .008209-0.940573$

C $1.6313455 .931961-0.128584$ H 2.9600373 .7666890 .741232 C $2.7165542 .452142-0.968394$ H $3.5557755 .339081-0.988131$ H $2.1341874 .922852-1.965394$ C $1.2487577 .205871-0.863200$ H $2.159783 \quad 6.1997540 .796598$ C $2.5170361 .112428-0.231633$ O $1.9074512 .412302-2.153600$ H $3.7506482 .529329-1.321371$ H $1.4448170 .970045-0.052896$ O $2.9173800 .053204-1.107559$ C 3.2620650 .9258351 .102253 C 4.7316231 .3869701 .151575 H $3.223275-0.136247 \quad 1.374073$ O 2.5368291 .6298652 .114530 H 4.7918972 .4658490 .970667 H 5.0686591 .2510912 .188650 H $2.1414477 .789112-1.111677$ H $0.7199916 .987319-1.797065$ H $0.5969817 .832163-0.246047$ C $-0.221945 \quad 5.373214 \quad 1.524471$ H $0.9762002 .418524-1.872401$ H $2.4106810 .149997-1.932081$ H 2.9531201 .4283212 .969778 H $-0.3705316 .459003 \quad 1.571097$ H 0.4560015 .1038292 .344560 H $-0.2800235 .041630-0.633531$ C $6.558656-1.591084-0.185110$ O $5.710979-0.7319340 .590748$ C $5.697841 \quad 0.647832 \quad 0.211497$ C $7.113167 \quad 1.2486890 .284064$ C $8.1103420 .422706-0.533042$ C $8.005895-1.069175-0.207286$ H $6.563309-2.5454580 .357397$ C $5.987361-1.845859-1.588952$ H $5.3324960 .739222-0.814467$
O 7.5437621 .3016731 .643096

H $7.0771422 .278944-0.089846$

H $9.131507 \quad 0.762878-0.322151$

O $7.8653190 .611763-1.923210$

H $8.593388-1.651455-0.926173$

O $8.591152-1.3065131 .076589$

C $4.753113-2.722549-1.555035$

H $5.712598-0.918333-2.097080$

H $6.735840-2.373350-2.191966$

H $8.422473 \quad 1.7179031 .656592$

H $7.9672611 .559906-2.113457$

H $8.038589-0.8618411 .742236$

O $4.381033-3.005541-2.839088$

O $4.185044-3.106733-0.542400$

C $3.227181-3.839784-2.981004$

H $2.334212-3.294525-2.665548$

H $3.124207-4.100170-4.037839$

H $3.342269-4.762181-2.403569$

SCF Energy (B3LYP/6-31G**//MMFF)= -3245.91394718

\section{4}

MM̄FF Geometry

C $-2.7916294 .001500-0.027962$

C $-4.120769 \quad 4.180982 \quad 0.033271$

C $-5.138098 \quad 3.2532210 .660061$

O $-4.563603 \quad 1.960694 \quad 0.956595$

C $-6.310298 \quad 3.027803-0.311674$

C $-7.460578 \quad 2.261487 \quad 0.313307$

C $-7.749141 \quad 0.971532 \quad 0.051577$

C $-8.341510 \quad 3.060227 \quad 1.239063$

C $-6.9806430 .035645-0.838331$

C $-6.897543-1.376404-0.240090$

C $-6.078460-2.309188-1.141978$

C $-6.174425-3.759326-0.668801$

C $-5.297185-4.689664-1.520712$

O $-4.715189-1.875612-1.095891$

O $-5.196111-5.948400-0.837838$

C $-3.873751-4.100370-1.597357$

C $-5.929861-4.985349-2.885628$

O $-3.059373-4.845510-2.499990$

C $-3.837963-2.597801-1.968557$

C $-2.405678-2.051817-1.769434$

O $-4.209893-2.437179-3.338195$

C $-4.101731 \quad 1.7473942 .217532$

$\begin{array}{lllll}\text { O } & -4.099080 & 2.539488 & 3.146079\end{array}$

$\begin{array}{lll}\text { C }-3.561319 & 0.370371 & 2.267112\end{array}$

C -2.7147920 .0409813 .253881$

C $-2.058497-1.2544813 .440796$

C $-2.530084-2.4078512 .595886$

C $-2.291311-0.521197-1.822382$

C $-0.870007-0.088611-1.517185$

C $0.802568-0.051993 \quad 0.333404$

O $-0.1296110 .364140-2.386833$

C $1.181570-1.1917061 .224432$

C $1.612063-1.0416732 .486093$

C $1.991650-2.1921713 .374278$

C $1.257839-2.1857364 .698199$

C $1.911332-1.9455585 .847573$

C $-0.217935-2.5330754 .700507$

C -1.079188 -1.3470604 .364957$

N $-0.525311-0.297402-0.191590$

H -2.339824 3.1188410 .414716

H $-4.5285545 .100152-0.385292$

H $-5.512240 \quad 3.7286351 .575040$

H $-5.9493292 .516849-1.211953$

H $-6.6991973 .992814-0.664425$

H -8.6332230 .5402450 .519282$

H $-9.1952532 .479731 \quad 1.604589$

H $-7.776423 \quad 3.3977492 .113150$

H -8.7399713 .9387360 .720917$

H $-7.492032-0.014860-1.807073$

H $-5.9667120 .402279-1.021914$

H $-6.434016-1.3198810 .753445$

H $-7.910644-1.777879-0.113312$

H $-6.455159-2.218472-2.167630$

H $-7.215818-4.104122-0.673786$

H $-5.840260-3.8258270 .376260$

H -6.095801 -6.298878 -0.720899 
H $-3.421405-4.219846-0.602476$ H $-6.913814-5.452660-2.758319$ H $-5.328318-5.704985-3.452045$ H -6.065606 -4.091812 -3.498514 H $-3.096824-5.776941-2.222528$ H -2.034295 $-2.389112-0.794774$ H $-1.734511-2.478038-2.526425$ H $-3.827299-1.611312-3.674132$ H $-3.846474-0.3177381 .479691$ H $-2.4491430 .805066 \quad 3.983835$ H -3.620342 -2.506724 2.650613 H -2.125170 -3.3712602 .913358$ H -2.246665 -2.263040 1.550460 H -2.560165 -0.138247 -2.812268 H -2.959156 -0.042141-1.097479 H $\quad 0.7661890 .8994940 .870872$ H $1.5167940 .029751-0.490575$ H $1.104496-2.1925520 .805554$ H $1.714144-0.0405582 .898674$ H $3.074651-2.1359933 .542324$ H $1.815450-3.1538992 .876401$ H $1.397817-1.9634136 .803306$ H $2.972785-1.7210345 .859015$ H $-0.516631-2.8868135 .695904$ H $-0.374083-3.3791384 .025571$ H $-0.842796-0.4584604 .952702$ H -1.257879 -0.5315780 .471083$ C $-0.6687024 .314356-1.343672$ O $0.2585143 .883245-0.323663$ C $1.5811674 .353708-0.662731$ C $1.5205224 .684636-2.142978$ C $0.1205785 .252967-2.258267$ H $1.7395915 .263472-0.069472$ C $2.5996743 .279855-0.273756$ H $2.2959295 .391852-2.451534$ H $1.6180293 .776225-2.750538$ C $-0.3999415 .299730-3.684639$ H $0.1192656 .270370-1.844031$ C $4.0372323 .561637-0.739598$ O $2.599475 \quad 3.1696291 .153794$ H $2.2666932 .314784-0.667503$ H $4.0813453 .557383-1.834802$ O $4.4110274 .884472-0.334783$ C $5.0965962 .585206-0.186051$ C $4.7642831 .113575-0.456875$ H 5.2341702 .7553090 .887484 O $6.3472672 .883929-0.813796$ H 3.8213130 .8372940 .027251 H $4.5936350 .975744-1.533659$ H -1.419602 $5.696355-3.714798$ H $0.2324475 .944353-4.303793$ H -0.412159 4.303641-4.139984 C - $1.8634034 .997418-0.671841$ H 1.6812233 .0142501 .433840 H 4.3314994 .9277790 .633793 H $6.5381133 .822879-0.646764$ H -2.414908 $5.591957-1.409567$ H -1.502172 5.6751220 .111510 H $-0.9856203 .416410-1.889549$ C $6.226939-2.291793-0.143754$ O $5.351286-1.185188-0.391318$ C $5.8526680 .109653-0.029856$ C $6.183264 \quad 0.155617 \quad 1.470157$ C $7.123620-0.9856361 .871673$ C $6.645657-2.3382431 .335769$ H $5.618255-3.181860-0.348183$ C $7.418471-2.309134-1.114485$ H $6.7544150 .313061-0.616613$ O $4.975263 \quad 0.0705402 .223320$ H 6.6539661 .1166791 .706372 H $7.187191-1.0289982 .965971$ O $8.426349-0.6953451 .373564$ H $7.424085-3.0969371 .478274$ O $5.518881-2.7454442 .117000$ C $6.987414-2.531426-2.549966$ H $7.979054-1.371370-1.086614$ H $8.091943-3.131589-0.846795$ H $5.2107830 .123013 \quad 3.165204$
H $\quad 9.016827-1.4125441 .660832$

H $5.249791-3.6277581 .809622$

O $8.096359-2.522745-3.348737$

O $5.835565-2.693593-2.927691$

C $7.850153-2.718084-4.744900$

H $7.390457-3.696329-4.915166$

H $7.212416-1.918003-5.132760$

H $8.809975-2.685751-5.267186$

SCF Energy (B3LYP/6-31G**//MMFF)= -3245.90614650

\section{5}

MM̄FF Geometry

C 2.2340050 .0986753 .252722

C $2.803922-1.0862083 .522934$

C $3.966599-1.7264702 .803599$

O $4.594392-0.7723941 .920325$

C $3.465184-2.9093951 .956918$

C $4.548063-3.9140991 .623227$

C $5.156795-4.0239330 .426765$

C $4.891216-4.8755762 .732581$

C $4.982785-3.158793-0.789772$

C $4.230594-3.876482-1.919224$

C $2.705490-3.865079-1.728706$

C $2.019283-4.690411-2.819188$

C $0.488734-4.586951-2.739686$

O $2.279687-2.501379-1.807482$

O $-0.053777-5.117238-3.958584$

C $0.093859-3.096925-2.680426$

C $-0.085179-5.439020-1.601572$

O $-1.308858-2.948671-2.472315$

C $0.875376-2.292169-1.616474$

C $0.603504-0.779360-1.800317$

O $0.451270-2.698207-0.315201$

C $5.948800-0.6930191 .906723$

O $6.728139-1.3227162 .608386$

C 6.4200630 .2946420 .908826

C $5.6214710 .836994-0.022405$

C $6.0541841 .783284-1.043422$

C $5.5582551 .443559-2.422611$

C $1.4653430 .124976-0.908505$

C $1.3004591 .595537-1.250585$

C $2.087138 \quad 3.850733-0.526192$

O $0.577527 \quad 1.998705-2.157808$

C $3.2162044 .260853-1.418673$

C $4.2000685 .090418-1.038855$

C $5.3019755 .552134-1.949802$

C $6.6805835 .289942-1.386380$

C $7.3620346 .256923-0.748909$

C $7.3044243 .936848-1.644070$

C $6.7779382 .873762-0.721968$

N $2.0607452 .404536-0.416667$

H 2.5988930 .7107212 .433443

H $2.398310-1.6751234 .344527$

H $4.671772-2.0572433 .575086$

H $3.002705-2.5281781 .039789$

H $2.667229-3.4496432 .484688$

H $5.897347-4.813561 \quad 0.302201$

H $5.644723-5.6103302 .429192$

H $5.287852-4.3385423 .599500$

H $3.999689-5.4295293 .044224$

H $4.509441-2.201920-0.552494$

H $5.988819-2.907127-1.149335$

H $4.470451-3.355662-2.855727$

H $4.593708-4.906800-2.019236$

H $2.461207-4.267171-0.739601$

H $2.336850-5.739403-2.770233$

H $2.338546-4.327629-3.806458$

H $0.249613-6.037245-4.046267$

H $0.307151-2.663331-3.667605$

H $0.170602-6.495247-1.748175$

H $-1.179819-5.395107-1.588396$

H $\quad 0.283983-5.145098-0.616791$

H -1.760395 -3.461055 -3.164795

H $0.803889-0.510155-2.845878$

H $-0.452844-0.558095-1.603903$

H $0.626827-1.9836300 .316213$

H 7.4783310 .5350740 .952115

H $4.5760350 .551207-0.084988$ 
H $6.0337520 .523090-2.778952$ H $5.7561322 .224212-3.160100$ H $4.473517 \quad 1.287614-2.418083$ H $1.196723-0.0089020 .144143$ H $2.524088-0.125003-1.029823$ H 2.1913024 .2520320 .486852 H $1.1404354 .204635-0.946237$ H $3.2046403 .880760-2.437521$ H $4.1953615 .491376-0.027766$ H $5.1667016 .629459-2.113795$ H $5.2203945 .092414-2.942592$ H $8.3637016 .086241-0.368440$ H $6.9305457 .239405-0.588371$ H $8.3909113 .989078-1.491418$ H $7.1858463 .679562-2.700649$ H $7.047308 \quad 3.0274180 .323612$ H $2.669777 \quad 1.972993 \quad 0.271876$ C $-0.238127 \quad 0.519206 \quad 3.269351$ O -0.2973171 .5240472 .237641$ C -1.6509312 .0314462 .157631$ C -2.484353 1.1503363 .076334 C $-1.474395 \quad 0.7561334 .132685$ H $-1.609733 \quad 3.0549692 .551318$ C -2.0863872 .0439190 .686997$ H $-3.352561 \quad 1.6747003 .486859$ H -2.8431650 .2603212 .547067$ C -1.902997 -0.4500454 .951041$ H $-1.309448 \quad 1.6109194 .802145$ C -3.5925232 .3023530 .480774$ O $-1.317232 \quad 3.0679140 .051708$ H -1.8019351 .0949200 .217569$ H -4.1483331 .5533021 .050359$ O -3.9367303 .5744691 .027583$ C $-4.0527832 .219976-0.989257$ C $-5.5551832 .495177-1.196559$ H $-3.7987371 .228996-1.383884$ O $-3.333108 \quad 3.177401-1.768829$ H -5.786065 $3.526722-0.901781$ H $-5.7409122 .445536-2.277699$ H -2.838383 -0.2417025 .480824$ H $-2.064767-1.3282414 .316610$ H $-1.144676-0.7087395 .696227$ C 1.0743410 .6413934 .043330 H -1.487166 $3.013740-0.903889$ H -3.441589 4.2470280 .528941 H -3.578655 $3.043722-2.700171$ H $\quad 0.9994990 .108634 \quad 4.998547$ H $1.284576 \quad 1.6922064 .276447$ H $-0.304256-0.4571612 .770316$ C $-6.962416-0.864527-0.356189$ $\begin{array}{llll}\text { O } & -6.218553 & 0.201386 & -0.963527\end{array}$ C $-6.492747 \quad 1.517412-0.470343$ C $-7.9760491 .874397-0.664820$ C $-8.8786160 .811633-0.030052$ C -8.475599 $-0.603237-0.454662$ H $-6.739908-1.745839-0.971952$ C $-6.483801-1.1532061 .074184$ H $-6.271501 \quad 1.5584740 .599549$ O $-8.2996651 .970255-2.049890$ H $-8.1776972 .850644-0.207381$ H $-9.9196740 .990636-0.325965$ O $-8.802117 \quad 0.903336 \quad 1.389735$ H $-9.014298-1.3433380 .147874$ O $-8.882758-0.816714-1.809593$ C $-5.231740-2.0003081 .074360$ H $-6.279750-0.2575041 .666408$ H $-7.243597-1.7292521 .616263$ H $-7.9155942 .796658-2.387734$ H $-9.0963701 .794991 \quad 1.642574$ H $-8.373447-0.208894-2.372716$ O $-4.180175-1.2957350 .569913$ O $-5.193703-3.1517751 .489478$ C $-2.934850-1.9993550 .526971$ H $-2.194400-1.348758 \quad 0.056484$ H $-2.599899-2.234966 \quad 1.541540$ H $-3.031094-2.911058-0.070182$ SCF Energy (B3LYP/6-31G*//MMFF) $=-3245.91229797$
0800566

MMFF Geometry

C $0.307005-3.4867501 .783838$

C $-0.616248-2.8478112 .518176$

C $-0.498323-1.4710413 .132216$

O $-1.529120-0.6446082 .536207$

C $0.847526-0.7589962 .904071$

C 0.9435620 .5779473 .611892

C 0.8223551 .7726163 .000475

C 1.2542710 .5071215 .084095

C 0.5116582 .0247831 .550476

C -0.4698623 .1937251 .384200$

C $-0.7796523 .461090-0.095146$

C -1.556774 $4.767940-0.257532$

C $-1.9366905 .022073-1.723215$

O $-1.5590622 .367830-0.593641$

O $-2.9083296 .078536-1.750629$

C $-2.6233913 .760584-2.282679$

C $-0.7417015 .508705-2.551216$

O $-2.8639443 .889541-3.682416$

C $-1.8538302 .447937-1.995037$

C $-2.7452411 .243746-2.374353$

O $-0.6639542 .412622-2.783972$

C $-2.741229-0.5988503 .149716$

O $-3.076118-1.1812584 .168432$

C -3.6148840 .2735502 .334871$

C -4.9414150 .2342412 .527361$

C -5.9468150 .9798341 .767854$

C -5.4597452 .0391490 .816203$

C $-2.202180-0.123173-1.944035$

C $-3.258828-1.180061-2.198473$

C $-5.337393-2.144394-1.207189$

O $-3.313320-1.793416-3.261852$

C $-6.531858-1.251575-1.348014$

C $-7.632230-1.355407-0.586854$

C $-8.825869-0.451541-0.721623$

C $-9.2850650 .125906 \quad 0.601599$

C $-10.436201-0.2754541 .166927$

C $-8.449807 \quad 1.221843 \quad 1.233678$

C -7.2473560 .6706861 .951242$

N $-4.148271-1.313901-1.143839$

H $1.264825-3.0161621 .581991$

H -1.561479 -3.355523 2.703251

H $-0.669979-1.5679484 .211564$

H $1.017722-0.6265731 .829597$

H $1.672666-1.3897453 .260306$

H 0.9660322 .6695853 .601622

H 1.3668041 .4995315 .533695

H $0.452620-0.0080815 .622069$

H $2.190439-0.0362495 .248850$

H $1.4486352 .266578 \quad 1.035225$

H $0.092666 \quad 1.1378901 .066693$

H -1.398659 2.9626381 .921880

H -0.0384704 .0947591 .838188$

H $0.1680583 .511636-0.644331$

H -0.9847145 .6150230 .141273$

H $-2.479116 \quad 4.7207380 .338538$

H -2.510115 $6.862650-1.335248$

H $-3.6110173 .691226-1.804699$

H $-0.3374696 .437340-2.130522$

H $-1.0443175 .751539-3.575918$

H $0.0736774 .783819-2.595294$

H $-3.3607984 .714748-3.816307$

H $-3.7336641 .375349-1.919471$

H $-2.9006861 .224496-3.461023$

H $-0.3838671 .489765-2.889684$

H -3.1486540 .8880091 .573211$

H -5.336115 -0.438392 3.288141

H $-4.9369361 .580698-0.026570$

H -4.7733712 .7286291 .321124$

H -6.2599192 .6584810 .405844$

H -1.303246 -0.394759 -2.507032

H - $1.931170-0.134324-0.882335$

H $-5.370075-2.735399-0.286550$

H $-5.275290-2.822869-2.063267$

H $-6.490141-0.483089-2.116777$

H -7.686592 -2.1412880 .163310$

H $-9.634482-1.037962-1.176815$ 
H $-8.6236540 .372598-1.417146$

H -10.784769 0.1500062 .102302 H -11.053870 -1.041802 0.710395 H -9.0481341 .7753361 .969079$ H $-8.192270 \quad 1.9481970 .458467$ H -7.495426 -0.0948292 .688388$ H $-4.029717-0.729273-0.322385$ C $0.161982-4.885644-0.330905$ O $1.401009-4.317457-0.805037$ C $1.094266-3.374779-1.849896$ C $-0.286303-3.771794-2.352476$ C $-0.965253-4.131605-1.042741$ H $1.011865-2.390104-1.372954$ C $2.212284-3.337646-2.896058$ H $-0.782561-2.961466-2.891800$ H $-0.248905-4.650051-3.006392$ C $-2.235259-4.946654-1.212815$ H $-1.210230-3.202931-0.512479$ C $3.614139-3.077058-2.303746$ O $2.280547-4.601536-3.567928$ H $1.986668-2.587811-3.663910$ H $3.936879-3.962564-1.741408$ O $4.511972-2.949456-3.413684$ C $3.720112-1.824270-1.408722$ C $5.138583-1.608982-0.861312$ H $3.033813-1.929561-0.563556$ O $3.341665-0.659590-2.139125$ H $5.483295-2.515955-0.348398$ H $5.838810-1.454699-1.692998$ H -2.977259 -4.389212 -1.792819 H $-2.041100-5.888773-1.736226$ H -2.676033 -5.185450 -0.239755 C $0.119830-4.8603401 .200287$ H $1.538092-4.643410-4.193859$ H $5.415299-3.079065-3.079625$ H $2.396706-0.733555-2.352642$ H $0.938465-5.4821561 .584304$ H $-0.815053-5.3130841 .550531$ H $0.169602-5.934699-0.653968$ C $7.0714190 .715690 \quad 1.310564$ O $6.668392-0.3754180 .473238$ C $5.290833-0.3909740 .073746$ C $4.375689-0.4295431 .307630$ C $4.709175 \quad 0.6873772 .300993$ C 6.2118500 .7738522 .583800 H $8.097454 \quad 0.471071 \quad 1.615195$ C 7.1152202 .0453330 .541224 H $5.0847250 .512358-0.510183$ O $4.480665-1.6923381 .961914$ H $3.335095-0.3183800 .985740$ H 4.1692450 .5023643 .237357 O $4.244751 \quad 1.9235831 .767652$ H 6.4492951 .6867143 .142136 O $6.592887-0.3316713 .408137$ C $8.1759832 .049221-0.540577$ H 6.1604772 .2680540 .058221 H 7.3578532 .8575501 .236427 H $5.414710-1.8286652 .196279$ H 4.4223062 .6135282 .429329 H $6.084029-0.2699014 .234577$ O $8.1625713 .264564-1.165581$ O $8.9200611 .116006-0.807846$ C $9.1144813 .423180-2.222347$ H $8.9256382 .693067-3.015156$ H $8.9983794 .428206-2.636508$ H $\quad 10.1320783 .318700-1.834230$ SCF Energy $\left(B 3 L Y P / 6-31 G^{* *} / / M M F F\right)=-3245.90343846$

\section{7}

MM̄FF Geometry

C -2.1394040 .4970013 .694988$

C -3.4698490 .3185653 .684365$

C -4.5169241 .1636702 .997820$

$\begin{array}{llll}\text { O } & -5.236361 & 0.291157 & 2.090440\end{array}$

C -4.0106382 .3767672 .193036$

C -5.0908343 .4130971 .941606$

C -5.7248733 .6068610 .769292$

C $-5.4027304 .308828 \quad 3.115133$
C $-5.5736682 .837745-0.512427$

C $-4.8829413 .667877-1.604706$

C $-3.3645713 .778588-1.399262$

C $-2.7387224 .718000-2.431044$

C $-1.2063814 .749177-2.316938$

O $-2.8223692 .464097-1.549123$

O $\quad-0.6893085 .390855-3.492574$

C $-0.6761763 .300036-2.322564$

C $-0.7376095 .587734-1.122042$

O $0.7274083 .266993-2.073463$

C $-1.4108832 .367930-1.331485$

C $-1.0006040 .899203-1.589327$

O -1.0551912 .7282350 .004604$

C -6.5930220 .2959252 .143347$

O $\quad-7.2920790 .9330962 .920362$

C $-7.193337-0.5932841 .122421$

C $-6.508683-1.1864120 .131856$

C $-7.078213-2.055916-0.903509$

C $-8.554614-2.351238-0.835129$

C $-1.868515-0.129613-0.851012$

C $-1.448216-1.555219-1.162199$

C $-2.127209-3.911041-0.703827$

O $-0.503455-1.836463-1.894365$

C $-2.987740-4.319860-1.856206$

C $-4.085991-5.079868-1.733526$

C $-4.970272-5.447656-2.889026$

C $-6.379335-4.917723-2.733812$

C $-7.395615-5.745935-2.439242$

C $-6.625970-3.450003-3.012139$

C $-6.262267-2.558327-1.854180$

N $-2.260827-2.478379-0.518567$

H -1.6876841 .3023343 .124478$

H -3.876658 -0.4940534 .285339$

H -5.1808301 .5065403 .800851$

H -3.5773182 .0334951 .247826$

H -3.2029272 .8897142 .729394$

H -6.4628024 .4070220 .715765$

H -6.1430945 .0772022 .867862$

H -5.8024293 .7272983 .951135$

H -4.4965774 .8236883 .450710$

H $-5.0602801 .882976-0.365908$

H $-6.5833052 .580745-0.857521$

H $-5.0761103 .174743-2.566418$

H -5.332356 4.667281-1.655958

H $-3.1626834 .143501-0.386463$

H $-3.1517145 .730169-2.337231$

H -3.003654 $4.378905-3.442403$

H $-1.0734736 .283100-3.540195$

H $-0.8191982 .905761-3.338493$

H -1.094901 $6.620373-1.215588$

H $0.3556425 .651408-1.089085$

H -1.089832 $5.202963-0.162805$

H $1.1507943 .854426-2.722784$

H $-1.0809020 .685853-2.663403$

H $\quad 0.0487860 .752717-1.306743$

H -1.1155221 .9458460 .574633$

H -8.267775 -0.7155931 .220327$

H -5.435471 -1.019358 0.058666

H $-9.136418-1.429861-0.948750$

H $-8.810529-2.8144540 .124296$

H $-8.898415-3.039140-1.610125$

H -1.8053940 .0146960 .231994$

H -2.918045 -0.021442 -1.148770

H $-2.430657-4.3918980 .231037$

H -1.081836 -4.164575 -0.905933

H -2.698869 -3.956124 -2.839630

H $-4.375084-5.447801-0.751840$

H -4.979404 -6.542561 -2.966767

H $-4.556050-5.089005-3.839740$

H -8.415285 -5.384749 -2.359302

H $-7.230966-6.804726-2.268720$

H -7.664835 -3.285773 -3.320367

H $-6.036417-3.143861-3.886135$

H $-5.207338-2.286864-1.824534$

H $-3.062670-2.1453370 .009057$

C $-0.081914-1.0249073 .776809$

O $0.759205-0.0648373 .109540$

C $1.152012-0.6131941 .830508$ 
C $0.738795-2.0784651 .859615$

C $-0.515714-2.0238032 .709190$

H $0.567856-0.0633811 .083872$

C $2.650565-0.3678331 .618034$

H $0.567511-2.4900000 .861533$

H $1.506505-2.6894232 .351182$

C $-0.941024-3.3731273 .259426$

H $-1.330802-1.6109892 .103853$

C $3.224542-1.0495770 .363202$

O 2.8542221 .0446651 .507229

H $3.197733-0.6957582 .508770$

H $3.165305-2.1386640 .476833$

O $2.386687-0.707841-0.747971$

C $4.669764-0.6453790 .005596$

C $5.671372-0.8704311 .152448$

H $4.7025240 .401133-0.320396$

O $5.079205-1.441225-1.110984$

H $5.331051-0.3470082 .051414$

H $5.707920-1.9453031 .371125$

H -1.155508 -4.071425 2.444511

H $-0.158492-3.8173683 .883731$

H -1.845127 -3.2770873 .868952$

C -1.202308 -0.3215424 .543620$

H $2.478142 \quad 1.4555562 .304649$

H $2.598800-1.317352-1.474805$

H $4.567012-1.148391-1.883316$

H $-0.745923 \quad 0.370714 \quad 5.262953$

H -1.759162 -1.062864 5.129185

H $0.562133-1.5262924 .512009$

C $8.942413-0.830463-0.682543$

O $7.638989-1.196727-0.212135$

C $7.095365-0.3717070 .821790$

C $8.006491-0.4053092 .060528$

C $9.447402-0.0279391 .703070$

C $9.951747-0.7973280 .476914$

H $9.234132-1.645383-1.357636$

C $8.9135660 .466521-1.498548$

H 7.0132200 .6587620 .459151

O $7.983809-1.7132942 .629857$

H 7.6098850 .2894302 .810869

H $10.098263-0.2408582 .559945$

O $\quad 9.499514 \quad 1.3738341 .455042$

H $10.897851-0.3707890 .125162$

O $10.235837-2.145056 \quad 0.864724$

C $8.1052640 .283813-2.761193$

H $8.5078201 .315836-0.941775$

H $9.9257000 .756885-1.804465$

H $8.534892-1.6938783 .430789$

H 10.4233011 .6024371 .255242

H $\quad 9.394826-2.5668131 .111640$

O $6.875216 \quad 0.853253-2.615226$

O $8.512883-0.312155-3.749919$

C $6.001947 \quad 0.731680-3.742212$

H $6.4145801 .274222-4.597782$

H $5.844704-0.322139-3.991676$

H $5.0390431 .175034-3.474710$

SCF Energy (B3LYP/6-31G**//MMFF) $=-3245.90265117$

0800568

MM̄FF Geometry

C $-1.302113-4.2167742 .472168$

C -0.152929 -3.642079 2.085478

C $0.753304-2.891724 \quad 3.027637$

O $0.979461-1.5584372 .514254$

C $2.113418-3.6067813 .109432$

C $3.111406-2.9088674 .013989$

C $4.206869-2.2670733 .560440$

C $2.839555-3.0075325 .490941$

C $4.583437-2.1091312 .112521$

C $5.741402-1.1216981 .910205$

C $6.103142-0.9822200 .424043$

C $7.366977-0.1387180 .247382$

C $7.698970 \quad 0.089511-1.235464$

O $4.998992-0.360301-0.243240$

O $8.6886651 .127381-1.311042$

C $6.4360140 .617138-1.948434$

C $8.323255-1.151473-1.883546$

O $6.6429550 .716618-3.354915$
C $5.169092-0.219182-1.661118$

C $3.8832140 .433402-2.223279$

O $5.297424-1.499865-2.290184$

C $0.038652-0.6149662 .791855$

O $-1.055198-0.795578 \quad 3.302477$

C 0.6051890 .7025012 .419762

C -0.0855941 .8200222 .693497$

C $0.373163 \quad 3.1982642 .492697$

C 1.7545723 .3956881 .929530

C $3.5977511 .842076-1.699122$

C $2.2383692 .366593-2.130922$

C $0.8666734 .440898-2.054393$

O $1.425501 \quad 1.702541-2.767153$

C $0.7014225 .560299-1.076715$

C $-0.4188305 .769379-0.369701$

C -0.6012436 .9034310 .594690$

C $-1.122198 \quad 6.4491881 .939013$

C -2.3592616 .7787932 .348779$

C -0.1780865 .6895082 .843940$

C -0.4445634 .2091772 .855106$

N $2.0441753 .672648-1.698876$

H -1.593825 -4.122418 3.517015

H $0.176009-3.7094231 .052791$

H $0.314794-2.8238124 .031431$

H $2.528711-3.7096272 .099193$

H $1.973127-4.6333503 .473764$

H $4.883945-1.8227054 .287421$

H $3.641719-2.5639386 .090472$

H $1.911706-2.4875965 .747917$

H $2.747153-4.0563215 .791091$

H $4.882689-3.0874421 .717137$

H $3.713379-1.7627171 .542932$

H $5.450933-0.1427162 .313452$

H $6.617505-1.4671342 .473201$

H $\quad 6.256787-1.9857480 .009754$

H $8.218476-0.5995130 .763584$

H 7.2210780 .8404320 .725104

H $9.4647590 .838947-0.800905$

H $6.2800541 .639924-1.584050$

H $9.254304-1.422645-1.371546$

H $8.600805-0.955327-2.925099$

H $7.668701-2.024846-1.859327$

H $7.4437081 .250884-3.493155$

H $3.9164730 .445504-3.320508$

H $3.030917-0.210805-1.964768$

H $4.409158-1.875827-2.406985$

H $1.5977130 .717781 \quad 1.983449$

H -1.0736931 .7215123 .142031$

H 1.9948194 .4388381 .714713

H 1.8629132 .8553180 .984607

H 2.5107543 .0255572 .630465

H $3.6283251 .853016-0.603703$

H $4.3525152 .541477-2.074716$

H $-0.0025933 .776086-2.085843$

H $1.0268594 .841257-3.060693$

H $1.539556 \quad 6.242774-0.956647$

H -1.264549 $5.104333-0.507716$

H -1.3007637 .6161590 .139260$

H $0.335803 \quad 7.4545830 .743093$

H -2.726705 $6.481283 \quad 3.325575$

H $-3.0326847 .347907 \quad 1.716796$

H -0.3020436 .0526853 .873658$

H 0.8571325 .9342792 .591088

H -1.422926 3.9540083 .265485

H $2.8434014 .175040-1.321628$

C $-1.893192-5.2487690 .163035$

O $-1.891922-4.000804-0.556826$

C $-2.624376-4.164204-1.782650$

C $-2.752270-5.666299-1.993471$

C $-2.896118-6.149401-0.563830$

H $-3.623677-3.749432-1.605918$

C $-1.954568-3.396475-2.925682$

H $-3.606888-5.927050-2.624718$

H -1.850734 -6.093854 -2.446711

C $-2.628035-7.635135-0.395337$

H $-3.916358-5.929664-0.221327$

C -1.625536-1.924527-2.603028

O $-0.720291-4.044954-3.258356$ 
H $-2.584666-3.462608-3.820743$ H $-0.753897-1.881353-1.938520$ O $-1.210920-1.300179-3.825465$ C $-2.775251-1.113167-1.979833$ C $-2.4246440 .381483-1.858599$ H $-3.004608-1.518964-0.988669$ O $-3.936818-1.267049-2.794333$ H -1.387587 $0.490848-1.518713$ H -2.512280 $0.839940-2.851456$ H -3.337510 -8.223180 -0.986654 H -1.617057 -7.900072 -0.722743 H -2.731994 -7.934486 0.652285 C $-2.270878-5.0027301 .627593$ H $-0.318773-3.545706-3.990257$ H -1.986922 -1.258068 -4.410438 H -4.662637 -0.783794 -2.364195 H -2.419646 -5.9692302 .124078$ H -3.228618 -4.4688451 .666935$ H - $0.881561-5.6673220 .088988$ C $-5.6811251 .631909-0.480492$ O $-4.691147 \quad 1.036695-1.330715$ C $-3.3406271 .131354-0.869930$ C $-2.9320982 .605194-0.732211$ C -3.9007113 .3616710 .181416$ C $-5.3613703 .111971-0.209723$ H $-6.6092691 .592870-1.065461$ C $-5.897667 \quad 0.814730 \quad 0.797743$ H $-3.253747 \quad 0.6342330 .102634$ O $-2.9228023 .208531-2.025468$ H -1.913298 $2.656207-0.330294$ H $-3.687058 \quad 4.4358080 .123699$ O $-3.6764442 .944098 \quad 1.524323$ H -6.0319373 .4940990 .568014$ O $-5.6533623 .858972-1.395077$ C $-6.494297-0.532140 \quad 0.465513$ H $-4.980066 \quad 0.663111 \quad 1.373242$ H -6.6018451 .3194851 .469643$ H -2.689996 4.145538-1.916725 H -4.2668693 .4642752 .095616$ H -5.121846 $3.486657-2.119658$ O $-5.533584-1.4976740 .521087$ O $-7.669819-0.6993680 .166618$ C $-5.974526-2.8213150 .204603$ H $-6.247524-2.880711-0.853121$ H $-5.147277-3.5092750 .393567$ H $-6.817932-3.1094560 .839531$

SCF Energy (B3LYP/6-31G**//MMFF) $=-3245.91838707$

0800569

MM̄FF Geometry

C $-1.9323115 .129101-0.950860$

C -2.664388 $4.013259-1.084190$

C $-3.8121643 .660199-0.173858$

$\begin{array}{llll}\text { O } & -3.562156 & 2.346993 & 0.378725\end{array}$

C $-5.121073 \quad 3.599176-0.976271$

C $-6.3396383 .401617-0.096486$

C -6.9936652 .2334430 .056404$

C -6.8412994 .6437300 .594706$

C $-6.6455930 .900805-0.546951$

C $-6.714414-0.2211870 .499362$

C $-6.417875-1.591811-0.125127$

C $-6.679062-2.7152640 .878755$

C $-6.322216-4.0905790 .295260$

O $-5.044307-1.612357-0.527366$

O $-6.294421-5.034606 \quad 1.376458$

C $-4.896315-4.028522-0.288450$

C -7.385157 -4.589606 -0.690399

O $-4.573157-5.235246-0.976101$

C -4.650837 -2.809949-1.211595

C $-3.143264-2.708422-1.536382$

O $-5.377583-2.982313-2.429040$

C -2.7382842 .2748001 .458996$

O -2.2007413 .2044892 .039369$

C -2.5724430 .8440021 .796543$

C -1.5847810 .4796802 .627573$

C $-1.238596-0.8902073 .011561$

C -2.173163 -1.991733 2.589364

C $-2.727237-1.416096-2.249214$
C $-1.214904-1.327202-2.303048$

C $0.760319-0.843608-0.854379$

O $-0.582638-1.669388-3.299221$

C $1.095118-1.9670730 .077738$

C $1.814246-1.8082121 .199124$

C $2.142777-2.9241332 .149753$

C $1.943821-2.5379143 .601332$

C $2.996572-2.3787834 .421369$

C $0.525361-2.3982564 .118664$

C $-0.097580-1.0924943 .703162$

N $-0.666513-0.872444-1.113459$

H $-2.1844185 .836753-0.165491$

H -2.420367 3.303715 -1.871511

H $-3.909144 \quad 4.3887030 .640835$

H -5.060472 $2.805462-1.729928$

H $-5.2561294 .529259-1.545154$

H -7.8863612 .2284610 .680966$

H -7.7511744 .4584441 .175635$

H -6.0861095 .0331761 .284121$

H -7.074228 $5.419994-0.141625$

H $-7.3646530 .692379-1.348226$

H $-5.6502190 .905801-0.999179$

H $-5.988723-0.0115251 .295967$

H -7.712552 -0.2346750 .954999$

H -7.056865 -1.714562 -1.007701

H -7.722606 -2.702518 1.217109

H -6.069284 -2.549967 1.778248

H -7.170229 -5.0315431 .799426$

H -4.201742 -3.9616030 .560992$

H -8.361008 -4.664477 -0.195564

H -7.151731 -5.599695 -1.044759

H -7.504550 -3.938406 -1.558816

H $-4.724896-5.970669-0.357976$

H $-2.573921-2.783927-0.603383$

H -2.837108 -3.558984 -2.159438

H $-4.959359-2.449002-3.123271$

H -3.2349380 .1303731 .320212$

H $-0.938311 \quad 1.255138 \quad 3.037147$

H -3.201674 -1.765132 2.893094

H -1.935116 -2.960442 3.033417

H $-2.148647-2.1154411 .504067$

H -3.110194 -1.384319 -3.274572

H $-3.112027-0.528274-1.734719$

H $0.9896970 .134520-0.420874$

H $1.313897-0.954955-1.790923$

H $0.731660-2.958132-0.183711$

H $2.199418-0.8215371 .443869$

H $3.188491-3.2091061 .978462$

H $1.544651-3.8181691 .933948$

H $2.864508-2.1219815 .467235$

H $4.014593-2.4957914 .064457$

H $0.517794-2.4335925 .215669$

H $-0.046364-3.2708033 .792076$

H $0.495365-0.2206153 .984549$

H $-1.286087-0.643754-0.342184$

C $0.5915685 .401335-1.144591$

O $\quad 0.8760484 .043445-0.753641$

C 1.5974944 .0766010 .489731

C $1.9429125 .535577 \quad 0.771199$

C $0.7768546 .250387 \quad 0.113117$

H 0.8856093 .7600411 .264992

C 2.7544493 .0659750 .489841

H 2.0197545 .7441651 .842990

H 2.8846585 .8388420 .306662

C $1.0378927 .719717-0.165915$

H $-0.089994 \quad 6.1615860 .778699$

C $3.6164403 .021421-0.790002$

O $3.5880083 .290786 \quad 1.623787$

H 2.3039322 .0735600 .609177

H $2.9817702 .769918-1.647670$

O $4.1544384 .316612-1.066088$

C $4.8033662 .033013-0.734008$

C $4.3896320 .603885-0.368295$

H $5.5549952 .403319-0.028749$

O $5.4329482 .020460-2.019455$

H 3.9655970 .5795330 .641409

H $3.5805460 .284333-1.038437$

H $0.1711528 .184210-0.646702$ 
H 1.2366418 .2588850 .766074

H $1.9026497 .856231-0.823960$

C $-0.7713445 .479869-1.838286$

H 3.0245523 .2478542 .415261

H $4.7322204 .559080-0.322097$

H $4.7861001 .685791-2.664076$

H $-0.7593494 .806712-2.705144$

H $-0.9297356 .492298-2.227586$

H $1.3588875 .667542-1.884224$

C $5.736451-2.853135-0.177603$

O $4.897460-1.694942-0.100915$

C $5.506775-0.448244-0.464603$

C $6.712104-0.1613410 .442724$

C $7.700907-1.331497 \quad 0.447820$

C $6.998782-2.6737780 .679894$

H $5.140255-3.6582970 .271694$

C $6.029507-3.246604-1.628921$

H $5.831983-0.507764-1.508396$

$\begin{array}{lllll}\text { O } & 6.276319 & 0.098462 & 1.775952\end{array}$

H 7.2245900 .7403590 .089687

H $8.445374-1.1634411 .235675$

O $8.385499-1.352419-0.801332$

H $7.685916-3.5079390 .496380$

O $6.596875-2.7565282 .050696$

C $4.769514-3.729852-2.307299$

H $6.455504-2.435947-2.226688$

H $6.751721-4.070848-1.668418$

H $5.757218-0.6682032 .073793$

H $9.029374-2.080343-0.771001$

H $7.399446-2.6903502 .595730$

O $4.117259-2.680896-2.884930$

O $4.398707-4.896632-2.301042$

C $2.890345-3.007972-3.544393$

H $3.075218-3.711097-4.362006$

H $2.175338-3.425938-2.829310$

H $2.472682-2.087635-3.961077$

SCF Energy (B3LYP/6-31G*//MMFF) $=-3245.90774042$

08_00570

MM̄FF Geometry

C $2.903216 \quad 1.098746-2.551346$

C $3.2995202 .380045-2.603471$

C $4.1290043 .102233-1.565467$

O $4.8560402 .122821-0.784234$

C $3.2627753 .965281-0.622087$

C $2.2536134 .864592-1.304903$

C $0.920842 \quad 4.664946-1.273799$

C $2.8313686 .057532-2.018782$

C $0.197897 \quad 3.523560-0.615544$

C $-0.522503 \quad 3.9812260 .657175$

C $-0.9210182 .793034 \quad 1.544214$

C $-1.802785 \quad 3.2636632 .702352$

C -2.099706 2.133142 3.697641

$\begin{array}{lllll}\text { O } & 0.290577 & 2.220816 & 2.049696\end{array}$

O -2.6464732 .7233824 .886714$

C -0.7691371 .4615544 .097667$

C $-3.163821 \quad 1.1673353 .162671$

O -1.0061450 .3181994 .916925$

C 0.1138201 .0730182 .889053

C 1.5180690 .6347283 .381827

O $-0.509514 \quad 0.0007512 .188614$

C $5.9021132 .542596-0.028611$

$\begin{array}{lllll}\text { O } & 6.310452 & 3.686249 & 0.104146\end{array}$

C $6.477074 \quad 1.399328 \quad 0.707874$

C $6.7304210 .242902 \quad 0.076484$

C $7.327054-0.951493 \quad 0.675641$

C $7.345701-1.0336112 .179285$

$\begin{array}{llll}\text { C } 2.517641 & 0.275185 & 2.269747\end{array}$

C $2.419012-1.1657861 .796813$

C $3.244249-2.6825490 .001403$

O $1.739941-2.0151152 .367541$

C $4.419762-3.452456 \quad 0.513780$

C $5.302542-4.066882-0.287802$

C $6.472179-4.8643810 .213545$

C $7.783983-4.411935-0.387716$

C $8.356893-5.097552-1.391327$

C $8.469183-3.2110490 .227467$

C $7.804098-1.912193-0.143282$
N $3.188030-1.3933550 .664444$

H $3.1823920 .478209-1.705460$

H $2.9881132 .981751-3.455415$

H $4.8596413 .725144-2.097329$

H $3.9174364 .599673-0.010245$

H $2.786793 \quad 3.314270 \quad 0.120395$

H $\quad 0.2755175 .381883-1.778815$

H $2.0559766 .694695-2.457250$

H $3.4094116 .673418-1.322106$

H $3.4919555 .736789-2.830372$

H $-0.537873 \quad 3.128062-1.323843$

H $0.8718542 .688645-0.405777$

H 0.1381604 .6459541 .229821

H -1.411166 4.5607520 .377787

H -1.446961 2.0506510 .932556

H $-2.736673 \quad 3.7000632 .326513$

H -1.291993 4.0728113 .243287

H -3.448625 3.2131564 .636464

H -0.2118392 .1774644 .718080$

H -4.1043161 .6988122 .974170$

H -3.4004860 .3914293 .898782$

H -2.869602 0.6814942 .230711

H -1.5550470 .6074375 .665924$

H 1.9561451 .4734383 .939231

H $1.427316-0.2065554 .078879$

H $0.123326-0.353631 \quad 1.545297$

H 6.7437011 .5789041 .742798

H $6.5068750 .171063-0.987413$

H $8.057433-0.3120542 .594585$

H $7.622553-2.0192362 .558370$

H $6.351293-0.8208152 .588849$

H 2.4119790 .9494291 .412932

H 3.5338430 .3994842 .664930

H $3.308929-2.492062-1.074654$

H $2.324330-3.2390330 .205286$

H $4.539559-3.5261091 .592149$

H $5.165474-4.015794-1.365722$

H $6.288242-5.917404-0.036405$

H $6.543829-4.8276481 .307686$

H $9.310446-4.795032-1.811378$

H $7.882253-5.974371-1.819264$

H $9.508003-3.151868-0.123810$

H $8.541316-3.3644461 .307343$

H $7.745847-1.745849-1.220155$

H $3.784762-0.6510210 .311601$

C $0.6176780 .241587-3.090417$

O $0.607326-0.751169-2.046690$

C $-0.589996-1.552731-2.176537$

C $-1.421937-0.893710-3.267431$

C $-0.349883-0.281907-4.147576$

H $-0.250115-2.543230-2.504680$

C -1.264986-1.648871-0.803610

H -2.057890 -1.608210 -3.798777

H $-2.066029-0.111048-2.848962$

C $-0.877748 \quad 0.782601-5.093103$

H $\quad 0.126473-1.081220-4.731055$

C $-2.662484-2.301683-0.831143$

O $-0.374850-2.4002530 .024458$

H $-1.337141-0.645714-0.366824$

H -3.304941 -1.689076-1.470002

O $-2.578820-3.588523-1.442419$

C -3.326064 -2.430497 0.556133

C $-4.750834-3.0167470 .519643$

H -3.340728 -1.445653 1.035704

O $-2.534308-3.2868351 .383085$

H $-4.730410-4.0216900 .079734$

H -5.069515 -3.155557 1.561486

H - $-1.6328960 .360530-5.764231$

H -1.339038 $1.612133-4.547706$

H $-0.071948 \quad 1.193254-5.709351$

C $2.0435110 .449385-3.601326$

H $-0.730526-2.3896440 .928983$

H -2.004261 -4.138077 -0.881872

H $-2.920480-3.2738142 .275530$

H $2.032991 \quad 1.061087-4.510681$

H $2.499447-0.513581-3.861270$

H $\quad 0.231953 \quad 1.170952-2.652235$

C $-6.6527270 .124113-0.150141$ 
O $\begin{array}{llll}-5.795989 & -0.863610 & 0.435549\end{array}$

C $-5.775695-2.137790-0.214803$

C $-7.180153-2.762183-0.215566$

C $-8.205656-1.805860-0.833411$

C $-8.098020-0.394946-0.242865$

H $-6.643510 \quad 0.9612080 .560345$

C $-6.1035230 .641006-1.488807$

H -5.447042 -2.013858-1.250355

O $-7.566712-3.0743101 .121295$

H -7.149065 -3.701783 -0.780117

H $-9.214663-2.198638-0.658486$

O $-7.992783-1.761375-2.241214$

H $-8.7010870 .307065-0.829609$

O $-8.657881-0.4032991 .074043$

C $-4.8134181 .416145-1.315563$

H $-5.900911-0.164241-2.198947$

H -6.839877 1.322395 -1.931799

H -8.438665 -3.5026951 .081926$

H -8.668139-1.174235 -2.621638

H -8.095322 -0.9667951 .632742$

O $-4.5774762 .140992-2.449452$

O $-4.0919381 .377867-0.328661$

C $-3.3821242 .926312-2.436595$

H -3.310995 $3.455475-3.390427$

H $-3.4187683 .661804-1.627421$

$\mathrm{H}-2.5088372 .277795-2.329612$

SCF Energy (B3LYP/6-31G**//MMFF)= -3245.91378095

0800571

MM̄FF Geometry

C 1.9691323 .6383910 .619248

C 1.6346253 .8428221 .903129

C 1.6595862 .8165953 .014753

O 2.2698971 .5807422 .577931

C 0.2154822 .5258613 .459662

C 0.1186941 .5393554 .606039

C -0.3495730 .2803304 .497060$

C 0.5475112 .0694185 .950212

C $-0.822225-0.4202063 .254016$

C $-0.099147-1.7586173 .061322$

C $-0.457556-2.408697 \quad 1.716845$

C $0.143660-3.8121391 .629380$

C $-0.091469-4.4512490 .254711$

O $0.069321-1.5732980 .682584$

O $0.741418-5.616944 \quad 0.161490$

C $0.385667-3.466719-0.831370$

C $-1.536559-4.9340290 .084315$

O $0.063521-3.954555-2.133015$

C $-0.168327-2.032618-0.652985$

C $0.524324-1.040629-1.620886$

O $-1.564840-2.038054-0.956851$

C 3.6217691 .4836822 .718286

O 4.3682642 .3133253 .214282

C 4.0566820 .2009742 .118979

C 5.3643140 .0116111 .883456

C $5.985749-1.1582311 .258916$

C $5.114967-2.3508440 .969890$

C $1.935346-0.644503-1.177751$

C $2.823608-0.161151-2.307855$

C $5.1198180 .754606-2.666762$

O $2.540303-0.290571-3.494665$

C $6.109325-0.369018-2.637577$

C $7.377686-0.238720-2.220236$

C $8.358639-1.375964-2.180060$

C $8.996326-1.558011-0.819139$

C $10.294075-1.270486-0.621038$

C $8.152797-2.149603 \quad 0.293219$

C $7.305914-1.1107350 .980210$

N $4.0008570 .390255-1.818612$

H 2.3162912 .6613370 .297733

H 1.2885714 .8326182 .196078

H 2.2227213 .2338703 .858653

H -0.3662082 .1822432 .598054$

H -0.2701333 .4603663 .773443$

H $-0.404751-0.3242515 .401741$

H $0.374566 \quad 1.3485196 .756485$

H 1.6154992 .3079275 .947367

H -0.0126092 .9771826 .197266$
H $-1.897724-0.6029243 .353105$

H -0.6879980 .1940122 .361410$

H $0.984956-1.5884953 .104016$

H $-0.360679-2.4347803 .884883$

H -1.547739-2.452096 1.621560

H $-0.254000-4.4556102 .424150$

H $1.227638-3.7566031 .804268$

H $\quad 0.500726-6.2162450 .888715$

H $1.481062-3.437711-0.779617$

H -1.782704 -5.681827 0.847835

H -1.674764 $-5.434602-0.880395$

H -2.270301 $-4.129740 \quad 0.163893$

H $\quad 0.435080-4.850333-2.207019$

H $0.510408-1.449929-2.637927$

H -0.066246 -0.116287 -1.682047

H $-1.835863-1.136279-1.195119$

H $3.296567-0.5258571 .857959$

H 6.0569920 .8095402 .150235

H $5.684724-3.2521130 .731423$

H $4.454997-2.1447060 .123629$

H $4.501995-2.6096051 .840697$

H $1.8665130 .143026-0.417756$

H $2.449973-1.496367-0.725212$

H $5.5379621 .686323-2.273354$

H $4.7751280 .925667-3.691066$

H $5.759522-1.341784-2.976212$

H $7.7354020 .736849-1.898685$

H $9.129139-1.175129-2.935810$

H $7.886002-2.319811-2.479421$

H $\quad 10.764973-1.4227220 .344522$

H $10.913487-0.871729-1.417486$

H $8.803225-2.6019721 .053279$

H $7.563663-2.971720-0.122720$

H $7.859709-0.2148391 .265517$

H $4.1567530 .400230-0.815301$

C $0.7557304 .483667-1.449202$

O $-0.5197404 .504178-0.780027$

C $-1.3745603 .498777-1.375248$

C $-0.6640743 .055071-2.647320$

C $0.7934523 .184753-2.250637$

H $-1.4116812 .677675-0.649489$

C $-2.7706204 .101707-1.577616$

H $-0.9346822 .039844-2.951432$

H $-0.8880903 .734948-3.478580$

C $1.7470443 .220694-3.431699$

H $1.0467682 .333580-1.609530$

C -3.808452 $3.192841-2.258681$

O $-3.2860984 .517181-0.311016$

H $-2.6648905 .017558-2.173973$

H $-4.7830103 .697990-2.225442$

O $-3.4625623 .091880-3.645020$

C $-3.9875021 .764455-1.697716$

C $-4.3774041 .720924-0.208424$

H -3.082165 $1.173072-1.874939$

O $-5.0270031 .168205-2.483654$

H -3.5810112 .1736830 .390124$

H -5.288206 $2.320264-0.082976$

H $1.6538932 .309839-4.030998$

H $1.5418904 .073125-4.088172$

H $2.7843763 .299367-3.091254$

C $1.8867624 .702008-0.440491$

H -2.619827 5.0952490 .098706

H -4.139474 $2.540020-4.073256$

H $-5.1081160 .239343-2.210090$

H 1.7416715 .6797820 .036618

H $2.8489894 .746967-0.963705$

H $\quad 0.7482415 .340990-2.135645$

C $-6.195418-1.5481950 .089554$

O $-5.810225-0.220554-0.289054$

C $-4.6395840 .301740 \quad 0.345878$

C $-4.8267680 .324679 \quad 1.873683$

C $-5.248837-1.0451252 .413966$

C $-6.407295-1.6428541 .608393$

H -7.167773 -1.704345 -0.395530

C $-5.227016-2.605148-0.451451$

H $-3.778802-0.3253730 .091303$

$\begin{array}{llll}\text { O } & -5.817271 & 1.293146 & 2.216596\end{array}$

H -3.8847730 .6401282 .337848$ 
H -5.547982 $-0.939177 \quad 3.463851$

O $-4.126861-1.9198682 .371790$

H $-6.572396-2.6900381 .887715$

O $-7.609419-0.9335681 .919380$

C $-5.278068-2.661602-1.959331$

H -4.192348 -2.435998 -0.143130

H -5.495299-3.603392 -0.085302

H $-5.880150 \quad 1.318376 \quad 3.186433$

H $-4.406004-2.7751012 .740603$

H -7.766472 -1.0267202 .874379$

O $-4.238269-1.956168-2.487747$

O $-6.149729-3.245847-2.589831$

C $-4.170203-1.930097-3.916689$

H $-5.074670-1.475038-4.331581$

H -3.308344 -1.321644 -4.203549

H -4.032002 -2.942849-4.306651

SCF Energy (B3LYP/6-31G**//MMFF) $=-3245.90843928$

0800572

MM̄FF Geometry

C $0.304782-4.0157121 .433484$

C $0.609348-2.7155721 .573909$

C $0.670030-2.0525192 .926290$

O $-0.187526-0.8845072 .897735$

C $2.088362-1.5732173 .284560$

C $3.120309-2.6754613 .392880$

C $4.195257-2.7902772 .588813$

C $2.920885-3.6464334 .527732$

C $4.568871-1.9055111 .432565$

C $5.550778-0.8173071 .879690$

C 6.0449210 .0458400 .710886

C 7.0466941 .0900361 .212483

C 7.4938642 .0456740 .097299

O $4.9156050 .693900 \quad 0.116269$

O $8.1494593 .165941 \quad 0.711308$

C $6.2428652 .592934-0.619990$

C $8.5259841 .395508-0.831526$

O $6.6081523 .371146-1.757719$

C $5.2369171 .493100-1.030702$

C $3.9301732 .141095-1.545593$

O $5.8112200 .708539-2.079564$

C $-0.890271-0.6124814 .028198$

O $-0.975643-1.3226255 .022329$

C - -1.5764300 .6967813 .967855$

C -1.3251801 .6538503 .060700$

C -1.9339152 .9896093 .046058$

C -3.018092 3.2704134 .056196

C $2.7786461 .148158-1.742477$

C $1.6781601 .777473-2.570178$

C $-0.2960003 .257692-2.445643$

O $1.576803 \quad 1.558012-3.775679$

C $-0.6723964 .472081-1.663504$

C $-1.9121244 .688299-1.199961$

C $-2.3193025 .921851-0.451125$

C -2.8830425 .6160330 .917231$

C -4.2057145 .6800651 .146370$

C -1.908651 5.348666 2.040841

C -1.4989213 .9022872 .150860$

N $0.8615312 .627274-1.841017$

H $0.087457-4.5851782 .336165$

H $\quad 0.837579-2.1020010 .708027$

H $0.313128-2.7525513 .691356$

H $2.049918-1.0454544 .247664$

H $2.393928-0.7945572 .575596$

H $4.895785-3.6030012 .776149$

H $3.760851-4.3416224 .631906$

H $2.823284-3.1075075 .475803$

H $2.018125-4.2445604 .371778$

H $5.032164-2.5306020 .659240$

H $3.682005-1.4641110 .966260$

H $5.050008-0.1768122 .617958$

H $6.411974-1.2787942 .379567$

H $\quad 6.514680-0.612325-0.029545$

H 7.9161340 .6048241 .673689

H 6.5804441 .6861842 .009777

H 8.8981412 .8269361 .231406

H 5.7394773 .2789150 .075748

H $9.419874 \quad 1.104963-0.266464$
H $8.8716172 .101656-1.594567$

H $8.1497150 .501301-1.332585$

H $7.2302494 .054118-1.453909$

H $3.5910522 .901411-0.829689$

H $4.1267732 .657074-2.494424$

H $5.1087190 .380585-2.662022$

H -2.2808090 .8580394 .778560$

H $-0.585058 \quad 1.4668832 .284893$

H -3.8215932 .5295183 .973157$

H -3.4933144 .2448653 .928078$

H $-2.613783 \quad 3.2416335 .073844$

H $3.1017890 .240881-2.263277$

H $2.3711670 .826662-0.776716$

H -1.099599 $2.514890-2.465723$

H $-0.0585633 .533754-3.478328$

H $0.1010585 .216679-1.492198$

H -2.688165 $3.953223-1.398528$

H $-3.0675986 .446714-1.059365$

H -1.481976 $6.623555-0.349419$

H -4.6186085 .5021372 .133285$

H -4.9067415 .9069790 .349942$

H -2.314704 5.7185262 .989167

H -1.004042 5.9523771 .889781

H -0.7185443 .6099901 .448241$

H $\quad 0.9228372 .606790-0.826775$

C $0.625830-4.164945-1.138261$

O $-0.393485-3.226912-1.537027$

C $-0.599942-3.339103-2.958437$

C $0.500730-4.254939-3.482924$

C $0.738206-5.160458-2.294562$

H $-1.568287-3.831638-3.101601$

C $-0.602334-1.954361-3.609821$

H $\quad 0.193279-4.796774-4.382702$

H $1.413399-3.698027-3.725324$

C $2.065499-5.897656-2.355488$

H $-0.079174-5.892172-2.242134$

C $-1.694329-0.991706-3.100378$

O $0.667799-1.333310-3.371406$

H $-0.682758-2.059611-4.698089$

H -1.492782 -0.709901-2.060791

O $-1.586610 \quad 0.211763-3.873771$

C $-3.135171-1.523771-3.235796$

C $-4.225798-0.458872-3.002144$

H $-3.283490-2.365127-2.550214$

O $-3.305050-2.028152-4.564788$

H $-4.0521710 .397737-3.664914$

H $-5.185131-0.888030-3.322872$

H $2.111301-6.533114-3.246061$

H $2.909844-5.201150-2.396018$

H $2.199845-6.538048-1.478367$

C $0.231012-4.8383660 .176817$

H $\quad 0.790711-1.278062-2.407985$

H $-0.6621590 .509452-3.821587$

H -4.185480 -2.438126 -4.613029

H $0.875991-5.7104660 .338663$

H $\quad-0.798699-5.2097870 .096392$

H $1.563390-3.605184-1.027018$

C $-5.138333-0.8462310 .595365$

O $-4.918489-1.078091-0.802585$

C $-4.3459260 .006948-1.542512$

C $-5.2206351 .266556-1.425395$

C -5.4979561 .6233990 .037382$

C -5.9911070 .4111190 .828930$

H -5.727631 -1.710236 0.930099

C $-3.821971-0.8413061 .378677$

H $-3.3494440 .228168-1.149745$

O $-6.457851 \quad 1.083802-2.111752$

H $-4.7044022 .099146-1.917726$

H -6.2528942 .4177000 .083494$

O -4.3088082 .1113010 .648660$

H -6.0204460 .6378551 .901228$

$\begin{array}{lllll}\text { O } & -7.329767 & 0.102726 & 0.430991\end{array}$

C $-3.295219-2.2502781 .507222$

H $-3.033310-0.2434220 .914313$

H -3.975514 -0.4360772 .385588$

H $-6.8907680 .296191-1.740256$

H -4.028095 $2.899708 \quad 0.155080$

H $\quad-7.8787860 .8821660 .622339$ 
O $-3.559340-2.7297692 .755132$

O $-2.743784-2.8533210 .596477$

C $-3.158296-4.076397 \quad 3.030462$

H $-3.842680-4.4859663 .778389$

H $-3.200553-4.7109872 .140078$

H -2.148536 -4.069172 3.447462

SCF Energy (B3LYP/6-31G**/MMFF) $=-3245.91093212$

08_00573

MM̄FF Geometry

C $1.5318904 .910024-0.111819$

C $0.3620254 .254683-0.174938$

C $-0.4880313 .980426 \quad 1.040073$

O -1.1128202 .6814580 .911938$

C $-1.6061595 .034187 \quad 1.135633$

C -2.4455294 .9091972 .392820$

C -3.7021154 .4228942 .436090$

C -1.794927 5.4245393 .651509

C $-4.514067 \quad 3.864602 \quad 1.300371$

C $-5.0413382 .462536 \quad 1.633827$

C -5.7803841 .8300670 .444963$

C -6.3553340 .4669710 .835220$

C $-6.987771-0.261291-0.359655$

O $-4.842838 \quad 1.683235-0.626651$

O $-7.184747-1.6337990 .015016$

C $-5.988520-0.255812-1.536556$

C $-8.3745050 .298265-0.698136$

O $-6.591928-0.786539-2.713838$

C $-5.3957131 .137015-1.832134$

C $-4.2652661 .106750-2.892023$

O $-6.4297811 .982951-2.351215$

C -0.3812101 .5896791 .264457$

O $0.798847 \quad \begin{array}{llll}1.563076 & 1.574511\end{array}$

C $-1.294503 \quad 0.4226121 .251264$

C $-0.841520-0.7874331 .610635$

C $-1.634678-2.0136081 .732256$

C $-3.099370-1.935778 \quad 1.394695$

C $-3.0167630 .303556-2.512061$

C $-3.035002-1.142885-2.975892$

C $-1.756818-3.269919-2.725877$

O $-3.881052-1.597050-3.740542$

C $-2.259005-4.067830-1.564214$

C $-1.500704-4.945060-0.890422$

C $-1.992442-5.777654 \quad 0.256174$

C $-1.189251-5.5633041 .518780$

C $-0.163670-6.3732891 .832111$

C -1.642093 -4.4769342 .466492$

C -1.025593 -3.137567 2.165490

N $-1.966019-1.860414-2.454864$

H $1.876864 \quad 5.2633750 .856084$

H $-0.0231183 .931409-1.138756$

H $\quad 0.121223 \quad 3.9980851 .952643$

H -2.237488 4.9818700 .241996

H $-1.1703016 .042707 \quad 1.119002$

H -4.215196 4.4199973 .397209

H -2.465519 5.377305 4.516112

H $-0.905147 \quad 4.8359183 .894595$

H - 1.4971676 .4704753 .524375

H -5.3613714 .5359121 .116706$

H $-3.937093 \quad 3.8230950 .373820$

H -4.1956991 .8240191 .918498$

H -5.7135872 .5188882 .499133$

H -6.5824582 .5091890 .132262$

H -7.0820680 .5712261 .650594$

H $-5.551278-0.1641581 .234540$

H $-7.752553-1.6506410 .804486$

H $-5.174272-0.938502-1.264298$

H -9.0527120 .1820020 .155837$

H -8.834785 $-0.257152-1.522620$

H -8.357358 $1.357879-0.961135$

H $-6.930825-1.670785-2.492445$

H $-4.6661200 .793305-3.863640$

H -3.9331392.145002 -3.037913

H $-6.0158612 .714213-2.838798$

H -2.329899 0.6003390 .986046

H $0.209263-0.8877841 .872246$

H $-3.598133-2.9073241 .390838$

H $-3.246475-1.5182440 .393280$
H -3.621338 -1.303004 2.120222

H $-2.1487440 .766360-2.999420$

H -2.841215 $0.338045-1.433092$

H $-0.684832-3.412375-2.894643$

H -2.294066 -3.559179 -3.634395

H $-3.298584-3.929332-1.276551$

H $-0.470939-5.102050-1.201429$

H $-1.932567-6.831210-0.047207$

H $-3.054684-5.593726 \quad 0.458662$

H $0.396063-6.2450332 .752694$

H $0.144186-7.1760761 .170309$

H $-1.349397-4.7404543 .492498$

H -2.734938 -4.445691 2.499831

H $0.041576-3.0946442 .387195$

H $-1.339398-1.408197-1.794933$

C $3.8270884 .810756-1.244732$

O $3.9128743 .375097-1.211660$

C $5.0235253 .002436-0.372921$

C $5.7790144 .292258-0.061931$

C $4.6551395 .309985-0.062282$

H 4.5896582 .6089800 .555344

C $5.8866571 .911633-1.025832$

H 6.3120724 .2398060 .892317

H $6.5103294 .532766-0.842449$

C $5.1288946 .745844-0.199376$

H $4.1053615 .203147 \quad 0.879815$

C $5.0895930 .623178-1.321251$

O $6.455508 \quad 2.432512-2.226187$

H $6.7123701 .678253-0.342516$

H $4.5179010 .373823-0.421243$

O $4.1437380 .858909-2.363284$

C $5.968273-0.590241-1.690553$

C $5.172101-1.852488-2.076642$

H $6.630541-0.814113-0.845808$

O $6.805655-0.255453-2.798785$

H $4.647419-1.681007-3.025340$

H $5.902318-2.646583-2.282163$

H 5.7813957 .0169780 .637006

H $5.6917456 .896759-1.126677$

H $4.2801187 .436854-0.203260$

C $2.3656535 .263567-1.312093$

H $7.0461561 .752377-2.591338$

H $4.6440051 .124640-3.154020$

H $7.389385-1.014832-2.966612$

H $1.9110644 .827091-2.210617$

H $2.3343416 .352601-1.435936$

H $4.3103165 .116898-2.183001$

C $4.108146-2.9611741 .324005$

O $4.913508-2.619407 \quad 0.185285$

C $4.178046-2.325700-1.006960$

C $3.389287-3.563043-1.456224$

C $2.452907-4.032519-0.338687$

C $3.177549-4.148184 \quad 1.007386$

H $4.831268-3.2927702 .080223$

C $3.404309-1.7090521 .867589$

H $3.468858-1.515882-0.809025$

O $4.292979-4.609878-1.800712$

H $2.813023-3.311593-2.354681$

H $2.027414-5.008624-0.600386$

O $1.378579-3.107626-0.204961$

H $2.446843-4.273301 \quad 1.812993$

$\begin{array}{llll}\text { O } & 3.969515 & -5.341060 & 0.994127\end{array}$

C $2.930811-1.859508 \quad 3.296373$

H $4.107936-0.8678161 .842285$

H $2.547513-1.4347611 .247027$

H $3.762942-5.367876-2.100816$

H $\quad 0.931600-3.045217-1.065720$

H $4.666729-5.2270870 .325510$

O $2.295853-0.7094373 .671794$

O $3.092453-2.8487193 .997133$

C $1.755937-0.7069624 .996596$

H 1.2633420 .2550875 .160602

H $1.013668-1.5036175 .104543$

H $2.557523-0.8218425 .732190$

SCF Energy (B3LYP/6-31G*//MMFF) $=-3245.90882274$

0800574

MM̄FF Geometry 
C $-0.590002-1.8730163 .614962$ C $-0.739550-0.9000824 .527396$ C -1.5520320 .3658364 .383354$ $\begin{array}{llll}\text { O } & -2.239544 & 0.416850 & 3.115007\end{array}$ C $-0.606066 \quad 1.577764 \quad 4.441299$ C $-1.3354732 .904667 \quad 4.477444$ C -1.415259 3.7557453 .436171 C -1.9404253 .2738695 .807056$ C $-0.874402 \quad 3.5447082 .047768$ C $-1.768308 \quad 4.2154320 .995345$ C -1.197981 $4.057251-0.421073$ C -1.943072 4.968699-1.398472 C $-1.4500284 .782692-2.839332$ O $-1.3473982 .688548-0.815799$ O $-2.3877895 .427925-3.714104$ C $-1.475495 \quad 3.279924-3.177235$ C $-0.0959945 .461482-3.076461$ O $-0.892843 \quad 3.036502-4.456139$ C $-0.8002802 .387296-2.107527$ C -1.095992 $0.904321-2.427724$ $\begin{array}{llll}\text { O } & 0.611388 & 2.599861 & -2.142059\end{array}$ C -3.516365 -0.0463063 .069236$ O $-4.157491-0.5369303 .984186$ C $-3.9977590 .132517 \quad 1.681399$ C $-5.121786-0.486181 \quad 1.292141$ C $-5.709531-0.451408-0.048794$ C $-5.0814410 .468460-1.061799$ C $-0.670086-0.089390-1.341161$ C $-1.081367-1.486115-1.764449$ C $-2.680859-3.322180-1.249074$ O $-0.588934-2.027383-2.752979$ C $-3.869694-3.099355-2.132661$ C $-5.088587-3.604564-1.887654$ C $-6.272274-3.388397-2.788636$ C $-7.495266-2.884218-2.052643$ C $-8.565600-3.675708-1.868264$ C $-7.509803-1.438706-1.599313$ C $-6.767880-1.248884-0.303369$ N -2.060833 $-2.041748-0.957447$ H $-1.102326-1.8135042 .658913$ H $-0.204118-0.9948065 .470933$ H $-2.271287 \quad 0.4134255 .209889$ H 0.0804241 .5451833 .586781 H $0.034434 \quad 1.5168135 .331786$ H -1.916437 4.7093093 .597755 H -2.392552 4.2713245 .795707 H -2.723809 2.5620626 .084784 H - 1.1729493 .2701456 .587935 H $\quad 0.1274663 .9865911 .998429$ H -0.7818792 .4801411 .811449$ H - 2.7722213 .7738001 .043147 H -1.8632015 .2826131 .233303$ H $-0.1321824 .314028-0.399840$ H - $1.8534146 .020066-1.097745$ H -3.017384 4.737999-1.365412 H - $2.4342216 .366571-3.463628$ H -2.530882 $2.982508-3.257127$ H $-0.1669936 .537222-2.875232$ H $0.2117725 .373148-4.124229$ H $0.7017875 .060527-2.447775$ H - $-1.3544753 .603256-5.097577$ H -2.174565 $0.779067-2.586716$ H $-0.6020430 .627095-3.368227$ H $1.0613001 .796450-1.837718$ H -3.399471 0.7417961 .013166 H $-5.651645-1.1029502 .017150$ H $-5.0595911 .497758-0.685923$ H $-5.6130630 .500916-2.014530$ H $-4.0566830 .154896-1.279263$ H $0.414992-0.095806-1.198764$ H $-1.1216100 .168251-0.377547$ H -2.953790 -3.779118 -0.292817 H $-1.958371-3.968162-1.757866$ H $-3.716201-2.503113-3.029458$ H -5.240509 -4.224960 -1.007370 H $-6.494563-4.346340-3.276585$ H -6.032533 -2.688911 -3.599247 H $-9.455627-3.313720-1.364239$
H $-8.574433-4.704613-2.212503$

H $-8.543641-1.106501-1.437440$

H $-7.130796-0.813620-2.411749$

H $-7.156867-1.8627120 .510914$

H -2.468440 -1.484694 -0.213152

C $1.472589-2.9934442 .811705$

O $1.026624-3.4983001 .537280$

C $2.161209-4.096858 \quad 0.861301$

C $3.350104-3.9253201 .799375$

C $2.678649-3.8576553 .157999$

H $1.916913-5.1599800 .746057$

C $2.289720-3.420723-0.508074$

H $4.069794-4.7451291 .714332$

H $3.880863-2.9867041 .595996$

C $3.574550-3.2796564 .239820$

H $2.353069-4.8654783 .447644$

C $3.632871-3.640434-1.221385$

O $1.247171-3.932315-1.345751$

H $2.089875-2.350928-0.391756$

H $4.459864-3.269507-0.607671$

O $3.859478-5.047446-1.365386$

C $3.722036-2.992014-2.617251$

C $3.370717-1.496722-2.675157$

H $3.067260-3.526299-3.316691$

O $5.047796-3.166992-3.123284$

H $3.597407-1.111827-3.678298$

H $2.282870-1.389492-2.572809$

H $3.055057-3.2427655 .202129$

H $4.471040-3.8957274 .365468$

H $3.897537-2.2626243 .992823$

C $0.324569-3.0526723 .817230$

H $0.405004-3.790310-0.880623$

H $3.114643-5.413418-1.872762$

H $5.247488-4.118343-3.091565$

H $\quad 0.713517-3.051203 \quad 4.841358$

H $-0.259553-3.9709593 .685230$

H $1.792573-1.9532252 .653923$

C $3.7878161 .678500-0.786853$

O $3.4192470 .708866-1.778414$

C $4.037525-0.579690-1.629965$

C $5.559444-0.442596-1.765727$

C $6.112107 \quad 0.576711-0.760607$

C $5.3135471 .884123-0.760262$

H $3.3283192 .611303-1.136668$

C $3.154821 \quad 1.3279690 .569175$

H $3.797669-0.957126-0.632639$

O $5.881234-0.034234-3.092711$

H $6.030988-1.415114-1.591253$

H $7.1584740 .799044-1.003248$

$\begin{array}{llll}\text { O } & 6.083829 & 0.017603 & 0.550039\end{array}$

H 5.5981752 .4952260 .103635

O $5.6885772 .617695-1.931154$

C 3.1238412 .4976451 .527431

H $2.119117 \quad 1.0010790 .417544$

H 3.6855000 .4994341 .046533

H $6.8497030 .024712-3.155647$

H $6.626980-0.7888650 .537450$

H $5.2401313 .479717-1.894045$

O 2.9120332 .0300112 .793295

$\begin{array}{lllll}0 & 3.248397 & 3.672366 & 1.209658\end{array}$

$\begin{array}{llll}\text { C } 2.876946 & 3.025559 & 3.820768\end{array}$

H 3.8436243 .5338953 .885441

H 2.0750453 .7442723 .629493

H 2.6788342 .5248864 .772169

SCF Energy (B3LYP/6-31G*//MMFF) $=-3245.90986760$

0800575

MM̄FF Geometry

C $-0.601793 \quad 5.014889-1.204748$

C $0.5752784 .474543-1.558448$

C $1.9024784 .599492-0.840303$

O $2.487273 \quad 3.275044-0.728750$

C $1.837074 \quad 5.2022740 .577274$

C 3.2019745 .4236231 .204642

C 3.7362794 .6675932 .184597

C 3.9559216 .6237520 .689258

C 3.1559773 .4358672 .822493

C 4.1383852 .2604052 .732167 
C 3.5289990 .9502533 .254057

C $4.553627-0.1834463 .166869$

C $3.950690-1.5496173 .524792$

O $2.386283 \quad 0.6509742 .447768$

O $4.866236-2.564863 \quad 3.084002$

C $2.643866-1.7469382 .726729$

C $3.805917-1.7259805 .040817$

O $1.972857-2.934833 \quad 3.139545$

C $1.685841-0.5431382 .818244$

C $0.459035-0.6704531 .882973$

O $1.182029-0.4506964 .156784$

C $3.2105042 .822980-1.791798$

O $3.3740403 .388997-2.861589$

C $3.7914581 .510657-1.429045$

C $4.4550720 .804162-2.357041$

C $5.136679-0.477263-2.155884$

C $5.102571-1.079382-0.777220$

C $0.758514-0.6673990 .380838$

C $0.909248-2.045501-0.238068$

C $1.422839-3.131408-2.424516$

O $\quad 0.703672-3.089864 \quad 0.372722$

C $2.838817-3.610066-2.372431$

C $3.612201-3.751931-3.459588$

C $5.008008-4.303068-3.423945$

C $6.020078-3.384683-4.069166$

C $6.440638-3.594055-5.328277$

C $6.618428-2.287413-3.219991$

C $5.777520-1.038800-3.202636$

N $1.271861-1.964820-1.576782$

H $-0.6817635 .614194-0.303222$

H $0.6099773 .917370-2.493475$

H $2.5534265 .228728-1.460476$

H 1.2157004 .5666251 .216873

H 1.3312626 .1762100 .549543

H 4.7108444 .9564662 .577029

H $4.9004626 .782271 \quad 1.220461$

H $4.1936846 .502892-0.371937$

H 3.3554267 .5312410 .811308

H $2.9464313 .653722 \quad 3.876402$

H $2.205963 \quad 3.1546332 .362602$

H 4.4345562 .1294721 .683841

H 5.0481052 .4953703 .299167

H 3.2087471 .1010094 .291749

H $5.423786 \quad 0.031442 \quad 3.799715$

H $4.941620-0.2399082 .142115$

H $5.721476-2.4069673 .519415$

H $2.928303-1.8952551 .678097$

H $4.786613-1.6666685 .528351$

H $3.408885-2.7166035 .288103$

H $3.165600-0.9707345 .501319$

H $2.603390-3.6698313 .049235$

H $-0.147939-1.5356832 .172725$

$\begin{array}{llll}\mathrm{H} & -0.176845 & 0.205591 & 2.072147\end{array}$

H $\quad 0.358236 \quad 0.0639014 .143614$

H $3.6771291 .173414-0.406367$

H $4.5264841 .211574-3.364973$

H $4.073627-1.156972-0.411676$

H $5.678905-0.460540-0.082064$

H $5.512757-2.090496-0.731634$

H $-0.088923-0.196692-0.134591$

H $1.647152-0.0712870 .155635$

H $1.120010-2.840306-3.435310$

H $0.753696-3.924515-2.076124$

H $3.233115-3.888836-1.397910$

H $3.206371-3.499678-4.436707$

H $4.995784-5.268687-3.946681$

H $5.323547-4.530310-2.398067$

H $7.191755-2.958725-5.785885$

H $6.038616-4.401639-5.931113$

H $7.602552-2.005997-3.619849$

H $6.833777-2.673930-2.219787$

H $5.744146-0.526458-4.165274$

H $1.484237-1.056712-1.979885$

C $-3.0924884 .607451-1.205028$

O $-3.0461623 .259080-0.697984$

C $-4.3599362 .677265-0.803364$

C $-5.3002403 .812219-1.192992$

C $-4.3867674 .696785-2.013472$
H $-4.3141841 .951266-1.624219$

C $-4.773194 \quad 1.961440 \quad 0.490807$

H $-6.1705193 .455316-1.752212$

H $-5.666063 \quad 4.353301-0.312120$

C $-4.9152806 .109850-2.191197$

H $-4.2462254 .236888-3.001007$

C -3.9070090 .7204790 .795863$

$\begin{array}{lllll}\text { O } & -4.716059 & 2.899184 & 1.564247\end{array}$

H $-5.8177751 .642827 \quad 0.386095$

H $-3.9089170 .092848-0.100923$

O $-2.555118 \quad 1.1053021 .029012$

C $-4.418927-0.1131331 .988480$

C -3.597109 -1.3851112 .275350$

H $-5.465366-0.3850401 .806475$

$\begin{array}{lllll}\text { O } & -4.396567 & 0.688890 & 3.171581\end{array}$

H $-2.580970-1.1112012 .583158$

H $-4.049322-1.8672373 .152459$

H $-5.8804886 .096668-2.708347$

H $-5.0574986 .609258-1.226920$

H $-4.2238566 .715339-2.785173$

C $-1.8469674 .901582-2.043160$

H -5.0529112 .4566072 .361270$

H -2.550714 1.6974861 .800662

H $-4.788677 \quad 0.164172 \quad 3.890384$

H -1.975916 $5.855811-2.566853$

H $-1.7208044 .119122-2.801755$

H $-3.1385605 .275306-0.334584$

C $-5.048045-3.565418-0.385069$

O $-4.892701-2.7013780 .747788$

C $-3.545616-2.3835541 .108496$

C $-2.776629-3.6639981 .465562$

C $-2.839851-4.675366 \quad 0.314712$

C $-4.273800-4.880066-0.186346$

H $-6.117696-3.811532-0.406046$

C $-4.709484-2.842931-1.698984$

H $-3.043935-1.8982600 .266094$

O $-3.330423-4.2446502 .644147$

H - $1.735911-3.4028391 .686022$

H $-2.435128-5.6378330 .650280$

O $-2.036070-4.222455-0.770255$

H $-4.276216-5.448274-1.124017$

O $-4.994744-5.657511 \quad 0.774534$

C $-5.661839-1.698316-1.982755$

H -3.697853 -2.429084 -1.684848

H $-4.790586-3.548173-2.534012$

H $-2.805209-5.0348532 .856505$

H $-1.126134-4.129323-0.440248$

H $-4.536727-6.5105430 .864640$

O $-5.260201-1.061282-3.122776$

O $-6.635196-1.401848-1.303677$

C $-6.0756080 .041073-3.534015$

H $-5.560266 \quad 0.560865-4.346002$

H $-7.036519-0.328024-3.904198$

H $-6.2259630 .744237-2.709521$

SCF Energy (B3LYP/6-31G**//MMFF)= -3245.91329021

0800576

MM̄FF Geometry

C -1.658056 $3.610650 \quad 0.347776$

C -1.6276593 .8312691 .671169$

C -2.0723582 .8519792 .736166$

O $-2.244744 \quad 1.5255302 .185286$

C -3.4153093 .3069603 .328551$

C $-3.9038172 .409863 \quad 4.448202$

C -4.8634831 .4738394 .308693$

C $-3.268923 \quad 2.653595 \quad 5.792131$

C -5.5852351 .0941223 .044648$

C $-5.690845-0.4300852 .890676$

C $-6.386295-0.8101551 .575862$

C $-6.706414-2.3046081 .538931$

C $-7.341154-2.713513 \quad 0.201322$

O $-5.505844-0.478471 \quad 0.495787$

$\begin{array}{lllll}0 & -7.324853 & -4.147011 & 0.125394\end{array}$

C $-6.458307-2.188992-0.948857$

C $-8.811787-2.2898740 .109294$

O $-7.080022-2.411224-2.212803$

C $-6.063824-0.698231-0.806684$

C $-4.988895-0.350969-1.862904$ 
$\begin{array}{llll}\text { O } & -7.212797 & 0.120864 & -1.029297\end{array}$

C -1.136437 0.7406752 .091511

O -0.0064441 .0141692 .463567$

C $-1.532200-0.5076651 .402433$

C $-0.578178-1.3158660 .916903$

C $-0.790968-2.5452890 .150283$

C $-2.200141-3.0602440 .030368$

C $-4.3119131 .011294-1.665964$

C $-3.1756951 .172725-2.658358$

C $-0.8864160 .298760-3.156239$

O $-3.2968611 .853406-3.673840$

C $-0.748091-1.149819-3.506375$

C $0.389879-1.846198-3.363169$

C $0.510513-3.308559-3.686830$

C $1.129631-4.116731-2.565013$

C $2.344566-4.672534-2.710214$

C $0.311510-4.352084-1.309275$

C $0.279939-3.144696-0.410984$

N -2.050328 $0.446811-2.301542$ H $-2.0525092 .672918-0.032304$ H -1.239905 4.7800952 .035724 H -1.3116072 .8226123 .526258$ H -4.1693153 .3678622 .534914$ H $-3.326397 \quad 4.3311793 .715840$ H -5.1679680 .9174915 .194200$ H -3.686083 2.0081516 .572415 H -2.192230 2.4624915 .748288 H -3.426269 3.6912786 .103998 H -6.5939121 .5222803 .084186$ H -5.0938091 .5063712 .159045$ H $-4.683713-0.8659542 .916153$ H $-6.252309-0.8404413 .739330$ H -7.307313 -0.2218191 .488228$ H -7.360169 -2.585216 2.374091 H -5.779927 -2.880128 1.675461 H $-7.821176-4.4928530 .886976$ H -5.537637 -2.789680 -0.948206 H $-9.391530-2.7406570 .923815$ H $-9.269142-2.650919-0.818624$ H -8.950981 -1.2082620 .163949$ H -7.295850 -3.358023 -2.267028 H $-4.211691-1.123239-1.844753$ H $-5.431209-0.376157-2.867448$ H $-6.9207371 .001687-1.312385$ H -2.590450 -0.7011571 .270947$ H $0.463537-1.0322961 .060284$ H -2.801428 -2.386953 -0.585897 H -2.668231 -3.1422581 .018093$ H -2.262735 $-4.055534-0.414786$ H $-5.0187391 .833781-1.816686$ H $-3.9080571 .114393-0.652963$ H $-0.0227560 .669213-2.596742$ H - $-1.005546 \quad 0.895129-4.065266$ H -1.632178 -1.652886 -3.891892 H $1.282165-1.340527-3.000936$ H $1.118506-3.396415-4.596539$ H $-0.466673-3.741864-3.934660$ H $2.785923-5.279739-1.927081$ H $2.927194-4.532166-3.614564$ H $0.739367-5.181491-0.731567$ H $-0.686545-4.680020-1.612735$ H $1.262993-2.707964-0.232542$ H -2.050834 - $0.058130-1.420593$ C $0.032823 \quad 3.978082-1.431479$ O $1.180767 \quad 3.975735-0.558957$ C $2.3481404 .271860-1.341954$ C $1.9087774 .304460-2.800569$ C $0.4670654 .761770-2.666878$ H $2.6492265 .293409-1.071496$ C $3.508103 \quad 3.335254-0.974094$ H $2.5182814 .984168-3.404071$ H $1.9520643 .315471-3.265549$ C $-0.371513 \quad 4.487741-3.903060$ H $0.455666 \quad 5.838724-2.451694$ C $3.1436921 .836455-0.865237$ O $4.5792653 .527466-1.892639$ H 3.8692203 .6408290 .016665 H $2.2692091 .732603-0.212604$
O $2.7752321 .312598-2.137305$

C $4.2802960 .979113-0.268490$

C $3.846681-0.486049-0.064022$

H 4.5739091 .4142420 .694083

O $5.412602 \quad 1.025723-1.132892$

H $2.848298-0.5083150 .387346$

H $3.795634-0.979014-1.042913$

H $0.0332235 .023251-4.768059$

H $-0.3889803 .420655-4.147657$

H $-1.4049124 .816237-3.756603$

C $-1.1633844 .575842-0.692470$

H $4.8137074 .471074-1.878392$

H $3.5521121 .388621-2.717670$

H $6.1258330 .515492-0.713666$

H $-1.9857754 .774064-1.388374$

H $-0.882703 \quad 5.528513-0.227532$

H $-0.1648822 .932773-1.699976$

C $7.121921-2.0163860 .919502$

O $6.074773-1.377696 \quad 0.177173$

C $4.816786-1.2648870 .848579$

C $4.277103-2.6583461 .202247$

C $5.299829-3.4549862 .019530$

C $6.691648-3.4197001 .379338$

H $7.940675-2.1366200 .197996$

C $7.632768-1.1314672 .061922$

H $4.947300-0.6844851 .768600$

$\begin{array}{llllll}\text { O } & 3.975205 & -3.366489 & 0.002836\end{array}$

H $3.343275-2.5454481 .765941$

H $4.972364-4.4984732 .102418$

O $5.383318-2.9183223 .336320$

H $7.436613-3.8201432 .076237$

O $6.696367-4.2859190 .240176$

C 8.2881120 .1140661 .514312

H $6.848665-0.8337312 .764014$

H $8.389534-1.6594932 .654209$

H $3.592885-4.2236070 .257282$

H $4.498539-2.9794923 .735051$

H $6.091417-3.909578-0.422053$

O $7.401601 \quad 1.1497351 .523114$

$\begin{array}{llll}\text { O } & 9.440444 & 0.152356 & 1.102815\end{array}$

C 7.8893912 .3848330 .989865

H $8.1995492 .253321-0.051183$

H $7.0751413 .113546 \quad 1.022412$

H 8.7197672 .7563121 .597577

SCF Energy (B3LYP/6-31G**//MMFF) $=-3245.91660692$

0800577

MM̄FF Geometry

C -3.472558 4.8229590 .025296

C -4.0669293 .8959910 .792555$

$\begin{array}{llll}\text { C } & -5.068349 & 2.848476 & 0.365488\end{array}$

O $-4.463853 \quad 1.5480390 .586509$

C $-5.5170802 .908096-1.108257$

C $-6.6916541 .993839-1.409350$

C $-6.6137290 .813356-2.055069$

C $-8.0360532 .514243-0.964439$

C $-5.3890490 .118826-2.581753$

C $-5.202657-1.244343-1.903837$

C $-3.958683-1.988171-2.410497$

C $-3.786217-3.313132-1.663449$

C $-2.497347-4.043078-2.070382$

O $-2.812667-1.156369-2.200867$

O $-2.250170-5.074988-1.102680$

C $-1.313154-3.054818-1.987798$

C $-2.645328-4.748052-3.423828$

O $-0.124004-3.645440-2.505756$

C $-1.584814-1.711353-2.696741$

C $-0.470618-0.659464-2.469129$

O $-1.656814-1.934281-4.111070$

C $-4.6742540 .948201 \quad 1.789827$

$\begin{array}{llll}\text { O } & -5.284895 & 1.404533 & 2.743364\end{array}$

C $-4.055527-0.3949761 .739195$

C $-4.391740-1.3028892 .666628$

C $-3.941380-2.6908542 .683552$

C $-5.055701-3.6661362 .956035$

C $-0.211860-0.271895-1.010356$

C $0.886137-1.082883-0.343521$

C $1.855011-1.5512651 .906022$ 
O $1.665900-1.801640-0.961477$ C $1.344860-2.9244832 .200891$ C $0.801717-3.2885303 .371566$ C $0.270995-4.6673593 .635886$ C $-1.235249-4.6925883 .760496$ C $-1.830822-5.0282604 .917163$ C $-2.048628-4.4053982 .516621$ C -2.644746 -3.0220822 .519419$ N $0.912423-0.8833781 .028804$ H -3.681161 4.873612 -1.039072 H $-3.821843 \quad 3.882927 \quad 1.853742$ H -5.9514262 .9635601 .006237$ H - $4.6697892 .675190-1.761616$ H $-5.821868 \quad 3.930349-1.368460$ H -7.540353 $0.266906-2.229494$ H $-8.857708 \quad 1.853282-1.260575$ H $-8.0716182 .612440 \quad 0.124665$ H -8.229316 $3.495840-1.409570$ H $-5.510198-0.023353-3.662326$ H $-4.4899610 .724049-2.445061$ H $-5.118789-1.086901-0.823161$ H $-6.090621-1.867972-2.068497$ H $-4.082932-2.165467-3.485309$ H $-4.659026-3.960918-1.813204$ H -3.742548 -3.117122 - 0.583257 H $-3.027480-5.659029-1.081666$ H -1.133281-2.865146 -0.922089 H $-3.448657-5.493569-3.380968$ H $-1.736671-5.302870-3.682398$ H -2.877095 -4.063217 -4.242076 H $0.023325-4.474443-2.018930$ H $0.452291-0.973341-2.972031$ H $-0.7834130 .258922-2.986669$ H -1.497115 $-1.090325-4.565208$ H $-3.385574-0.6262800 .918209$ H $-5.100765-1.042533 \quad 3.450143$ H $-5.786995-3.6415422 .140325$ H $-5.572207-3.411126 \quad 3.888573$ H $-4.718574-4.6994263 .059396$ H $\quad 0.1275300 .771242-0.987371$ H $-1.130291-0.330317-0.416978$ H $1.956070-0.9411532 .809061$ H $2.831416-1.6139391 .416144$ H $1.420413-3.6564461 .399747$ H $0.719491-2.5611994 .175451$ H $0.745385-5.0404164 .552439$ H $0.570258-5.3646302 .842942$ H -2.911245 -5.0766135 .001660$ H $-1.252161-5.2612465 .804910$ H -2.809106 -5.1828442 .390774$ $\mathrm{H}-1.414970-4.5023141 .626030$ H -1.921461 -2.223136 2.357531 H $0.183454-0.3238361 .461663$ C -1.083224 5.5730120 .048176 O $-0.592171 \quad 4.3508590 .633211$ C $0.771902 \quad 4.548717 \quad 1.062326$ C $1.215687 \quad 5.864752 \quad 0.444172$ $\begin{array}{llll}\text { C } & -0.079065 & 6.652947 & 0.448224\end{array}$ H 0.7318714 .6302592 .155760 C $1.594417 \quad 3.3264370 .643641$ H 2.0147096 .3487741 .013489 H $1.5693945 .714094-0.583467$ C $-0.0606837 .855325-0.479353$ H $-0.2800196 .992381 \quad 1.473350$

C 3.0994073 .4518790 .932046 $\begin{array}{lllll}\text { O } & 1.091044 & 2.196420 & 1.362974\end{array}$ H $1.4223233 .127319-0.419364$ H $3.5240624 .284448 \quad 0.359776$ $\begin{array}{lllll}\text { O } & 3.271499 & 3.792283 & 2.314697\end{array}$ C $3.921092 \quad 2.1760490 .652313$ C $3.8489281 .717327-0.816532$ H 3.6041831 .3718991 .326203 $\begin{array}{lllllll}\text { O } & 5.279525 & 2.486184 & 0.983048\end{array}$ H $2.805827 \quad 1.636350-1.136592$ H $4.3394772 .480219-1.434128$ H $0.7214578 .560316-0.179269$ H $0.1330867 .559706-1.515841$ H $-1.0189758 .383461-0.453310$
C $-2.499438 \quad 5.840853 \quad 0.556575$

H 0.1368172 .1396301 .183056

H 2.8864873 .0723362 .843458

H $5.8004641 .671906 \quad 0.882233$

H -2.837739 6.8332010 .237865

H -2.5077625 .8315891 .653804$

H $-1.0833695 .429507-1.040406$

C $6.674666-0.746570-0.883913$

O $5.9173920 .465955-0.775219$

C $4.5230010 .352090-1.067805$

C $4.324400-0.121444-2.517128$

C $5.088314-1.423250-2.786839$

C $6.537261-1.348403-2.292754$

H $7.718896-0.435413-0.750828$

C $6.336265-1.737154 \quad 0.236050$

H $4.069399-0.370349-0.382211$

O $4.7822740 .890787-3.412711$

H $3.253246-0.262646-2.702454$

H $5.089627-1.632131-3.863667$

O $4.435964-2.508718-2.135269$

H $6.993045-2.344875-2.310124$

O $7.292128-0.540784-3.201272$

C $6.681028-1.1550001 .586101$

H $5.284031-2.0357380 .243106$

H $6.914378-2.6621770 .124561$

H $4.6125840 .579585-4.318098$

H $3.520087-2.541324-2.460635$

H $6.9460180 .366710-3.150156$

O $5.552076-0.6942932 .196082$

O $7.819486-1.0947042 .031857$

C $5.744233-0.0882353 .477638$

H 6.4177940 .7700143 .395298

H $4.7726720 .262708 \quad 3.835706$

H $6.137133-0.8223904 .187202$

SCF Energy (B3LYP/6-31G**//MMFF) $=-3245.91516518$

0800578

MM̄FF Geometry

C -1.627033 -3.931435 1.025514

C $-0.988817-3.8468112 .203688$

C $-0.880363-2.628643 \quad 3.092250$

O $-1.671520-1.5304422 .588525$

C $0.587764-2.1672453 .130563$

C $0.847633-1.098708 \quad 4.172203$

C $1.0042340 .211848 \quad 3.902765$

C $0.984751-1.600627 \quad 5.586745$

C $0.898541 \quad 0.8944292 .567091$

C $0.075407 \quad 2.1861092 .671258$

C 0.0250552 .9351131 .332920

C $-0.6199824 .310773 \quad 1.509448$

C $-0.7459495 .053723 \quad 0.171799$

$\begin{array}{lllll}\text { O } & -0.741727 & 2.154598 & 0.409863\end{array}$

$\begin{array}{lllll}0 & -1.623614 & 6.172496 & 0.367823\end{array}$

C $-1.4230214 .120693-0.851703$

C $0.596900 \quad 5.629842-0.291150$

O $-1.4287694 .708757-2.151008$

C $-0.8015702 .704274-0.913360$

C $-1.6966911 .790926-1.779584$

$\begin{array}{llll}\text { O } & 0.493117 & 2.782193 & -1.508374\end{array}$

C -2.968895 -1.457582 2.989569

O $-3.548009-2.2102813 .756020$

C $-3.584115-0.3102712 .286857$

C $-4.920848-0.236706 \quad 2.207602$

C -5.6903220 .7880551 .499945$

C $-4.943067 \quad 1.9855230 .978310$

C $-1.3187330 .306276-1.749506$

C $-2.386647-0.496968-2.463993$

C $-4.663422-1.462854-2.124322$

O $-2.288544-0.783410-3.654804$

C $-5.755116-0.440965-2.210060$

C $-6.984190-0.620277-1.701937$

C $-8.0768210 .408713-1.785291$

C $-8.7562720 .663092-0.455646$

C $-10.0183590 .256387-0.237025$

C $-8.002498 \quad 1.454847 \quad 0.594256$

C -7.0175450 .6002151 .345657$

$\mathrm{N}-3.459539-0.810173-1.643512$

H $-2.164234-3.0736930 .632804$ 
H $-0.450509-4.7235262 .560477$ H -1.214282 -2.901102 4.100722 H $0.891096-1.8151752 .137082$ H $1.247403-3.0188813 .347256$ H 1.2396330 .8758434 .733854 H $\quad 1.222306-0.7974916 .292437$ H $0.053053-2.0665035 .921994$ H $1.787738-2.3422155 .650514$ H 1.9114781 .1393422 .228661 H 0.4510390 .2445551 .810025 H -0.9431091 .9350912 .994955$ H 0.5154022 .8375553 .436966 H 1.0495553 .0401020 .956502 H $-0.057744 \quad 4.9161762 .231406$ H -1.625493 4.1902211 .936829 H -1.243031 6.7334271 .065292 H -2.476335 $4.021484-0.552758$ H $0.987346 \quad 6.336576 \quad 0.451028$ H $0.4841666 .201751-1.218809$ H $1.3577924 .863606-0.448131$ H -1.840033 $5.586394-2.069978$ H -2.734571 $1.886470-1.441922$ H -1.674873 2.129636-2.823703 H $\quad 0.7301961 .910877-1.863458$ H $-2.9245370 .410586 \quad 1.817411$ H $-5.512406-1.0244772 .673118$ H -4.339966 2.4422421 .771476 H -5.5941422 .7767380 .601628$ H -4.2803501 .6915900 .161152$ H $-0.361810 \quad 0.124997-2.249968$ H - $1.219809-0.066114-0.723681$ H -4.902312 -2.264385 -1.418186 H $-4.482567-1.900679-3.110555$ H $-5.5241390 .491235-2.720903$ H -7.225712 -1.563284 -1.216434 H $-8.8103480 .052270-2.520172$ H $-7.696897 \quad 1.360545-2.177107$ H -10.521162 0.4559230 .703506 H -10.575885 -0.289409-0.991012 H $-8.7049391 .870832 \quad 1.328217$ H -7.5404882 .3184580 .108879$ H -7.466043 -0.290397 1.788801 H $-3.450021-0.497469-0.677298$ C $-0.648311-5.013882-1.048175$ O $0.664415-4.624412-0.588757$ C $1.026121-3.393172-1.249531$ C $0.260706-3.430895-2.559195$ C $-1.075996-3.977993-2.092364$ H $0.653574-2.579828-0.611878$ C $2.551647-3.312242-1.354890$ H $\quad 0.174738-2.449064-3.032630$ H $0.734682-4.121091-3.267832$ C $-1.926900-4.553118-3.211405$ H -1.636285 -3.165310 -1.618857 C $3.045473-2.102604-2.172708$ O $3.046460-3.220969-0.015588$ H $2.949075-4.241611-1.779126$ H $2.607063-2.191166-3.174935$ O $2.519362-0.905732-1.608113$ C $4.574855-1.995201-2.369286$ C $5.477420-1.989658-1.120770$ H $4.780803-1.084420-2.945404$ O $4.970377-3.102953-3.187259$ H $5.369596-2.935788-0.579167$ H $6.516808-1.993437-1.479320$ H $-2.166781-3.780644-3.949042$ H -1.409680 -5.365775 -3.732284 H $-2.868345-4.949058-2.817071$ C $-1.587249-5.161708 \quad 0.157133$ H $2.716539-3.9963950 .470222$ H $2.792207-0.166749-2.178049$ H $5.908888-2.983326-3.411867$ H -1.246436 -6.0151430 .757499$ H -2.602805 -5.399147 -0.179090 H $-0.528640-5.998032-1.517789$ C $5.6438411 .614863-0.159531$ O $5.7503460 .373969-0.871309$ C $5.289019-0.791342-0.178802$
C $6.059631-0.9769411 .137458$

C 5.9705050 .2804002 .007310

C 6.3295911 .5402561 .215564

H $6.2113512 .330881-0.768689$

C $4.1914942 .100601-0.099434$

H $4.229996-0.6793160 .060559$

O $7.427076-1.2878800 .880492$

H $5.634053-1.8307391 .677832$

H 6.6520020 .1822992 .860944

O 4.6476710 .4225052 .516344

H $6.0880902 .440281 \quad 1.792874$

O 7.7423871 .5584430 .988136

C $3.7720002 .617453-1.456015$

H 3.4786841 .3225220 .185807

H $4.0772332 .912040 \quad 0.629142$

H $7.795861-0.5758730 .330044$

H $4.439900-0.3788053 .026536$

H $8.178708 \quad 1.565367 \quad 1.857123$

O $3.8833603 .975985-1.493353$

O $3.4198311 .897559-2.381299$

C $3.5387124 .587686-2.740384$

H $2.5027564 .356102-3.005785$

H $3.6364495 .670485-2.626017$

H $4.2230614 .253354-3.525840$

SCF Energy $\left(B 3 L Y P / 6-31 G^{* *} / / M M F F\right)=-3245.92664939$

0800579

MM̄FF Geometry

C 2.4481203 .7538670 .757856

C 2.8293544 .0288662 .015156

C 3.3369003 .0301023 .030991

O $3.656674 \quad 1.7631962 .413731$

C 2.2420992 .7884454 .085138

C 2.6037241 .7259335 .103637

C 2.1006810 .4748435 .090478

C 3.5468292 .1632476 .191355

C $1.174827-0.0953734 .050107$

C $1.396747-1.5997463 .835658$

C $0.552808-2.1163702 .660986$

C $0.522280-3.6442472 .635138$

C $-0.251236-4.1736241 .418161$

O $1.143151-1.6229431 .452529$

O $0.021287-5.5781201 .299546$

C $0.298650-3.4964670 .146971$

C $-1.767895-4.0388901 .595292$

O $-0.482995-3.840576-0.995124$

C $0.420409-1.957706 \quad 0.260801$

C $1.215540-1.416597-0.950883$

O $-0.885694-1.3781820 .259943$

C 4.9398901 .5743732 .004040

O 5.8533442 .3827892 .050665

C 5.0438780 .1946581 .476737

C $6.166392-0.1822590 .847100$

C $6.427512-1.4878780 .236754$

C $5.397813-2.5705990 .425093$

C $1.6557070 .045939-0.816929$

C $2.3829140 .498475-2.069117$

C $4.5318920 .191172-3.298136$

O $1.859611 \quad 1.244577-2.892651$

C $5.364112-1.033511-3.511625$

C $6.705428-1.035958-3.533688$

C $7.527554-2.280832-3.714813$

C $8.545765-2.481713-2.611953$

C $9.862715-2.396199-2.865188$

C $8.042423-2.852718-1.229820$

C $7.575304-1.649893-0.454827$

N $3.648701-0.053098-2.173732$

H 2.5116142 .7370050 .382461

H 2.7380785 .0523622 .373956

H 4.2276633 .4429413 .520616

H 1.3020372 .5229083 .585737

H 2.0233243 .7254244 .615148

H $2.380090-0.2026055 .895490$

H $3.738078 \quad 1.367958 \quad 6.919643$

H 4.5109272 .4588225 .765818

H 3.1297313 .0165836 .735812

H $\quad 0.1411330 .0676314 .377884$

H 1.3027030 .4179833 .091962 
H $2.459988-1.7865683 .636754$ H $1.129073-2.1345454 .755304$ H $-0.464163-1.7183472 .758141$ H $0.093530-4.0421403 .563181$ H $1.550540-4.0297672 .589552$ H -0.258947 -6.008309 2.125595 H $1.302078-3.909139-0.029932$ H -2.100111 -4.586119 2.485508 H -2.303340 -4.487665 0.751349 H -2.098106 -3.003383 1.701912 H - $0.524611-4.811313-1.036287$ H $2.117952-2.026513-1.085649$ H $\quad 0.618400-1.523738-1.865820$ H $-0.840030-0.486677-0.120342$ H $4.191867-0.4632401 .603572$ H $\quad 6.9703540 .5458250 .744385$ H $5.183038-2.7154751 .490033$ H $5.718324-3.5438110 .046897$ H $4.464531-2.315251-0.085628$ H $\quad 0.802257 \quad 0.708962-0.655627$ H $2.322124 \quad 0.1787730 .041331$ H $5.1359291 .071620-3.056646$ H $3.9388320 .409315-4.191793$ H $4.830724-1.971985-3.646865$ H $7.242537-0.097416-3.417380$ H $8.029940-2.207667-4.687943$ H $6.890518-3.172444-3.772089$ H $10.599432-2.561149-2.085915$ H $10.234417-2.154778-3.855530$ H $8.848047-3.325783-0.653518$ H $7.265427-3.614031-1.341283$ H $8.274076-0.812330-0.491688$ H $4.040411-0.514535-1.358829$ C $0.3827204 .550559-0.400122$ O $0.166813 \quad 3.324536-1.124495$ C $-0.9413053 .503782-2.036011$ C -1.519735 $4.877688-1.725993$ C $-0.2964335 .629422-1.237936$ H $-0.5023463 .490111-3.041157$ C -1.919145 2.334772 -1.856450 H -1.988785 $5.343422-2.597727$ H -2.271637 $4.814185-0.929326$ C $-0.6329496 .895794-0.470140$ H $0.3301715 .884288-2.103069$ C $-3.2515152 .471430-2.614295$ O $-1.2637641 .137432-2.287781$ H -2.110564 $2.207380-0.785353$ H $-3.8155123 .337823-2.249103$ O $-2.9865452 .706775-4.002884$ C $-4.1495821 .218355-2.545422$ C $-4.4494120 .757394-1.114013$ H -3.696539 $0.404882-3.122790$ O $-5.393447 \quad 1.530520-3.182136$ H $-3.5253500 .478418-0.594669$ H $-4.8633161 .601207-0.544781$ H $\quad 0.2762177 .401545-0.130838$ H -1.189249 $7.593493-1.104783$ H -1.247077 6.6799730 .410615 C $1.8811084 .776421-0.189089$ H -1.094978 $1.220618-3.242005$ H $-2.5964383 .592730-4.085988$ H -5.189289 $1.824631-4.086442$ H 2.0601485 .7869990 .194910 H $2.4175044 .687559-1.141524$ H -0.1253854 .4440620 .568077$ C $-6.579066-1.6656720 .743092$ O $-5.623111-0.6559440 .394906$ C $-5.454109-0.406925-1.007567$ C $-4.996806-1.687075-1.723968$ C $-5.935646-2.863163-1.431590$ C $-6.235632-3.0025260 .063148$ H $-6.461110-1.8004871 .826077$ C $-8.022872-1.2053640 .486364$ H -6.410671 -0.071497-1.421165 O $-3.669800-2.029493-1.328465$ H -4.971463 -1.507221 -2.804103 H -5.477457 -3.792579 -1.791977 O $-7.163118-2.681743-2.131508$
H -7.049709 -3.7203250 .217792$

O $-5.072461-3.5461620 .693200$

C $-8.422227-0.042861 \quad 1.371988$

H $-8.171887-0.889158-0.549126$

H $-8.709527-2.0312140 .705751$

H -3.664592 -2.137482 -0.361893

H -6.955934 -2.632935 -3.080325

H $-5.287179-3.6964211 .629515$

O $-9.717096 \quad 0.2960541 .096221$

O

C $-10.242524 \quad 1.388337 \quad 1.857069$

H -10.237141 1.1441692 .923665

H -9.6636552 .2967911 .664986$

H -11.275319 1.5571641 .540973

SCF Energy (B3LYP/6-31G**//MMFF)= -3245.91251423

0800580

MM̄FF Geometry

C 3.5585310 .2543952 .420667

C $4.724097-0.2703572 .012932$

C 5.5763530 .2587420 .877751

$\begin{array}{lllll}\text { O } & 4.964668 & 1.470151 & 0.371025\end{array}$

C $5.671816-0.755988-0.279923$

C $6.388817-2.0454690 .068070$

C $5.773994-3.2010030 .393642$

C $7.891949-1.9746850 .007913$

C $4.295314-3.4294710 .539309$

C $3.771605-4.467658-0.459990$

C $2.237940-4.436147-0.534557$

C $1.707453-5.584811-1.392508$

C $0.188140-5.489026-1.600702$

O $1.879154-3.181537-1.120450$

O $-0.172319-6.400655-2.649350$

C $-0.157531-4.069392-2.096687$

C $-0.589710-5.929101-0.354793$

O $-1.569471-3.889345-2.178112$

C $0.472915-2.946772-1.241144$

C $0.303148-1.583446-1.947929$

O $-0.173565-2.9050400 .029658$

C $5.7320342 .406351-0.233812$

O $6.9200562 .309772-0.506789$

C $4.9477443 .634815-0.523059$

C $3.6887323 .829302-0.095437$

C $2.8802395 .040408-0.266622$

C $3.4571566 .148764-1.106768$

C $1.007761-0.409871-1.250022$

C $1.1618060 .755334-2.206422$

C $-0.1741782 .419496-3.492806$

O $2.2561501 .073237-2.666628$

C $0.1201953 .742323-2.864388$

C $-0.8285924 .573150-2.408526$

C $-0.5480755 .935411-1.844118$

C $-0.5877355 .968428-0.334300$

C $-1.7417705 .823266 \quad 0.338702$

C $0.709543 \quad 6.2560230 .386529$

C $1.6755315 .102340 \quad 0.341691$

N $-0.0442581 .365739-2.507237$

H 3.1861321 .1630761 .957440

H $5.083463-1.1697142 .507354$

H 6.5696090 .4980161 .277250

H $6.191103-0.290285-1.128548$

H $4.669640-0.952281-0.681121$

H $6.390743-4.0708910 .614629$

H $8.363963-2.9317250 .254368$

H $8.218877-1.694671-0.998822$

H $8.268359-1.2294800 .715821$

H $4.099732-3.7840621 .559152$

H $3.735947-2.4964810 .438336$

H $4.185467-4.254582-1.454545$

H $4.117836-5.465108-0.163026$

H $1.830242-4.4928270 .481751$

H $1.975448-6.554012-0.953559$

H $2.193241-5.558850-2.378194$

H $0.107243-7.292493-2.380235$

H $\quad 0.225638-3.978965-3.122857$

H $-0.351789-6.969519-0.102889$

H $-1.670246-5.904251-0.534745$

H $\quad-0.372197-5.3196540 .524824$ 
H -1.921419-4.605467 -2.734216 H $0.699714-1.661740-2.969031$ H $-0.761253-1.343151-2.030401$ H -0.144489 -1.998947 0.373286 H $5.4895784 .399626-1.069995$ H 3.1973273 .0403610 .469528 H $4.3484016 .569990-0.628887$ H $2.7616686 .973066-1.277902$ H $3.7337255 .774538-2.099079$ H $0.445258-0.075344-0.371998$ H $2.009673-0.687999-0.905786$ H -1.192182 2.374554-3.892004 H $0.5217252 .237002-4.318433$ H $1.1637004 .042826-2.818037$ H -1.871399 $4.268677-2.460728$ H -1.290487 $6.638356-2.243941$ H $0.4180266 .307646-2.206746$ H -1.7758105 .8729151 .421830$ H -2.678646 $5.648120-0.179978$ H $0.5133626 .468004 \quad 1.446274$ H $1.1378057 .182639-0.006651$ H 1.3435324 .2404850 .922624 H $-0.8820891 .082423-2.007413$ C $1.272871-0.6034112 .848904$ O $0.659101 \quad 0.6651522 .532616$ C -0.6831750 .6819863 .057636$ C $-1.030351-0.776313 \quad 3.299235$ C $0.301517-1.3152263 .788539$ H -0.6206141 .2212544 .011806$ C -1.5919971 .4372742 .080918$ H $-1.834322-0.9109324 .027054$ H -1.322213 -1.263868 2.362280 C $0.400424-2.8299803 .748806$ H $\quad 0.461130-0.9749884 .820539$ C -3.0885521 .4207082 .443329$ O -1.1318382 .7941642 .035900$ H -1.446509 1.0391391 .071291 H -3.4989680 .4106542 .346993$ O -3.2424331 .7905563 .822221$ C -3.9508382 .4163501 .646129$ C -3.8870402 .3246640 .112992$ H -3.6781603 .4391901 .935493$ O -5.3187722 .2575362 .042391$ H -4.465385 $3.174399-0.274524$ H -2.851702 2.461369-0.219250 H $-0.354343-3.2813014 .401066$ H $\quad 0.244059-3.2185752 .737848$ H $1.385526-3.1649494 .088688$ C $2.657162-0.3571153 .455895$ H -1.286761 3.1874432 .911735 H -2.9363501 .0465904 .366868$ H -5.351668 2.3439993 .010582 H $3.078223-1.2955943 .832837$ H 2.5824350 .3431664 .296332 H $1.367280-1.1543151 .904743$ C $-3.932344-1.306598-0.828553$ O $-3.533707-0.021800-0.328945$ C $-4.4811661 .036284-0.498721$ C $-4.8036041 .232667-1.990593$ C $-5.274178-0.071991-2.637075$ C $-4.329540-1.232791-2.314332$ H -3.029208 -1.924610 -0.756736 C $-5.019512-1.9464470 .046901$ H -5.4037420 .7842020 .033068$ O $\quad-3.6427721 .711689-2.666017$ H $-5.5823281 .999289-2.085822$ H $-5.3279140 .064026-3.724081$ O $-6.587329-0.363846-2.169618$ H $-4.781078-2.183998-2.617445$ O $-3.143995-1.084664-3.099561$ C $-4.498426-2.2810121 .427045$ H -5.886899-1.289064 0.151835 H -5.371052 -2.886071 -0.394147 H -3.887029 $1.868893-3.594019$ H -6.879532 -1.181768 -2.606863 H $-2.703804-0.263252-2.821586$ O $-5.426387-1.9328122 .365011$ O $-3.417143-2.8096821 .648453$
C $-5.070340-2.213858 \quad 3.722005$

H -4.215560 -1.600687 4.020160

H $-5.923028-1.9582734 .356579$

H $-4.846823-3.2775903 .847654$

SCF Energy $(B 3 L Y P / 6-31 G * * / / M M F F)=-3245.91132299$

0800581

MM̄FF Geometry

C -3.715397-1.214363 3.351982

C $-4.636819-0.3108903 .721062$

C -5.3313140 .6809962 .814365$

O $-4.9425520 .500494 \quad 1.434398$

C -4.9344642 .1112053 .223109$

C -5.5701083 .1802322 .354356$

C $-4.8917623 .890821 \quad 1.430725$

C -7.0341013 .4336542 .593576$

C $-3.431733 \quad 3.7220131 .109262$

C -2.9512994 .6929930 .021785$

C -1.447876 $4.530278-0.245254$

C $-0.9447495 .606316-1.208618$

C $0.5423205 .417513-1.545064$

O $-1.2298193 .231228-0.809024$

O $0.848487 \quad 6.249173-2.674721$

C $0.7719573 .956831-1.983307$

C $1.4536925 .883782-0.403572$

O $2.1608923 .691491-2.165952$

C $0.1518302 .913182-1.022741$

C $0.2414111 .506923-1.661436$

$\begin{array}{llll}\text { O } & 0.883703 & 2.908694 & 0.205189\end{array}$

C $-5.698558-0.3300220 .668633$

O $-6.661802-0.9887281 .025564$

C $-5.133545-0.303902-0.699346$

C $-5.560343-1.198691-1.602087$

C $-5.106923-1.318742-2.989549$

C $-4.154283-0.273465-3.506858$

C $-0.5992330 .431722-0.958734$

C $-0.349545-0.941765-1.559806$

C $-1.352510-3.215179-1.736512$

O $0.673196-1.221352-2.181513$

C $-1.801241-3.287992-3.163447$

C $-2.745874-4.129885-3.609891$

C $-3.150190-4.227238-5.054211$

C $-4.641939-4.092708-5.265266$

C $-5.389032-5.152624-5.617727$

C $-5.253745-2.712869-5.149822$

C $-5.569673-2.352246-3.723635$

N -1.376427 -1.835006 -1.284916

H -3.419010 -1.292874 2.309538

H -4.914187 -0.2537534 .772078$

H -6.4158650 .5556882 .920369$

H -3.8415162 .2002153 .192274$

H -5.2142652 .2964374 .268787$

H -5.4311904 .6424970 .857665$

H $-7.416536 \quad 4.259877 \quad 1.984658$

H -7.6237502 .5447832 .349658$

H -7.2081173 .6908893 .643506$

H -2.841617 3.8986042 .016516

H -3.2519692 .6922340 .779269$

H -3.514396 $4.502781-0.900964$

H -3.1613295 .7238210 .333441$

H -0.9193024 .6025140 .712829$

H -1.120479 $6.609238-0.800089$

H -1.521409 $5.557514-2.143018$

H $0.6395217 .168411-2.435378$

H $0.3027383 .836501-2.970016$

H $1.2850376 .945786-0.188548$

H $2.5101705 .799290-0.681948$

H 1.2967455 .3307230 .524728

H $2.5058144 .361135-2.781222$

H $-0.1059371 .555546-2.702122$

H $1.2908161 .188419-1.688169$

H 0.8122372 .0329520 .616361

H $-4.3778340 .441050-0.921203$

H $-6.309042-1.927701-1.293507$

H $-4.6023450 .723570-3.429796$

H $-3.878480-0.411213-4.553812$

H -3.221956 -0.280351-2.934343

H -0.3518980 .3689120 .106013$ 
H $-1.6633400 .676756-1.048028$ H -1.998773 -3.791188 -1.066983 H $-0.329219-3.596044-1.653795$ H -1.300969 -2.634561 -3.874677 H -3.229371 -4.808365 -2.910926 H -2.802998 -5.199223 -5.428493 H $-2.635602-3.473544-5.663483$ H $-6.454966-5.057398-5.797057$ H $-4.953415-6.140031-5.729488$ H $-6.195480-2.673964-5.713439$ H $-4.598099-1.991232-5.643804$ H -6.271961 -3.042794 -3.253435 H $-2.213826-1.504366-0.814717$ C $-1.513175-1.8609874 .324681$ O $-0.935799-2.2495903 .063008$ C $0.380254-2.7986963 .300170$ C $0.658875-2.6046254 .783708$ C $-0.738080-2.653787 \quad 5.372847$ H $\quad 0.305025-3.8663443 .059593$ C $1.365610-2.1019652 .355446$ H $1.323658-3.3734205 .188767$ H $1.118029-1.6257284 .970806$ C $-0.821735-2.0802126 .776471$ H -1.080311 -3.697230 5.385059 C $2.842547-2.4473612 .603276$ O $1.032263-2.4865951 .017809$ H $1.214556-1.0186602 .418470$ H $3.151477-2.0863333 .590696$ O $2.984370-3.8736132 .641680$ C $3.822015-1.9092741 .538617$ C $3.744990-0.3842881 .347858$ H $3.658078-2.4167300 .580647$ O $5.147158-2.2465891 .961843$ H $2.712023-0.0828581 .147732$ H 4.0703620 .0951382 .279829 H -1.846110 -2.1279757 .158682$ H $-0.180201-2.6448057 .461084$ H $-0.501142-1.0332986 .802040$ C -3.016082 -2.141506 4.309264 H $0.089198-2.2884470 .886220$ H $2.680755-4.2211011 .785287$ H $5.177788-3.2117892 .077609$ H -3.431589-2.033235 5.317218 H -3.205460 -3.1705063 .980897$ H -1.327492 -0.7849824 .445748$ C $6.9336110 .280502-0.513460$ O $5.992681-0.0860650 .504694$ C 4.6170800 .1271330 .181723

C $4.3677221 .618260-0.094162$

C $5.3117632 .156518-1.173593$ C $6.7672161 .758285-0.910001$ H $7.9153810 .166200-0.036188$ C $6.885893-0.676885-1.709669$ H $4.357397-0.455224-0.709211$ O 4.5289242 .3793341 .101412 H $3.3293721 .746328-0.414631$ H $5.2402293 .250747-1.207874$ O $4.9180341 .653867-2.446982$ H $7.3835641 .981204-1.788929$ O 7.2543612 .5718490 .161576 C $7.304798-2.067024-1.294056$ H $5.899551-0.733996-2.178761$ H $7.579283-0.352849-2.494998$ H $5.4300662 .224427 \quad 1.432626$ H $4.0048561 .947260-2.608158$ H 8.1977612 .3678940 .279733 O $6.204106-2.847408-1.098952$ O $8.470030-2.408698-1.137842$ C $6.465827-4.186618-0.668593$ H $7.016393-4.730049-1.442153$ H $7.023339-4.182408 \quad 0.272914$ H $5.506119-4.683802-0.504052$

SCF Energy (B3LYP/6-31G $\left.{ }^{\star *} / / \mathrm{MMFF}\right)=-3245.92266123$

\section{00582}

MM̄FF Geometry

C -2.333417 -3.567677 0.072723

C $-3.273227-3.761207-0.865359$
C $-3.545547-2.814086-2.012861$

O $-2.370993-2.017157-2.292628$

C $-4.695532-1.852828-1.666617$

C $-5.109908-0.993663-2.845043$

C $-4.8999180 .333818-2.940869$

C $-5.817496-1.720408-3.961074$

C $-4.2734581 .259519-1.935465$

C $-2.755561 \quad 1.366720-2.105970$

C $-2.1713902 .683193-1.569874$

C $-2.4603053 .850312-2.518481$

C $-1.7857065 .147540-2.056827$

O $-0.7527112 .501243-1.457585$

O $-1.8673606 .073382-3.151208$

C $-0.2920334 .869809-1.778822$

C $-2.5534505 .788910-0.893781$

O $0.318376 \quad 6.034225-1.228871$

C $-0.0498773 .625025-0.894277$

C $1.4402183 .210110-0.840999$

O -0.4939663 .8916160 .435615$

C $-1.367767-2.603414-3.000108$

O $-1.389941-3.691172-3.552098$

C $-0.193888-1.709586-2.905025$

C $1.025636-2.192629-3.181379$

C $2.286430-1.460853-3.055460$

C $2.229397-0.059938-2.504072$

C $2.3838594 .226753-0.195338$

C 3.6219953 .5875680 .401667

C $5.7194852 .411119-0.159086$

O 3.8670123 .6735281 .604050

C $6.1425871 .372309-1.145492$

C $6.3045280 .079788-0.825306$

C $6.768310-0.978027-1.784543$

C $5.652515-1.928661-2.158925$

C $5.463843-3.059587-1.456532$

C $4.829307-1.521242-3.365368$

C $3.432294-2.081854-3.404692$

N $4.4204202 .941736-0.529296$

H -1.762213 -2.642756 0.066884

H -3.863011 -4.674346 -0.846961

H -3.795075 -3.408541-2.899806

H $-4.391300-1.222531-0.823571$

H $-5.572307-2.417705-1.323742$

H $-5.228850 \quad 0.835875-3.850369$

H -6.206870 -1.035254 -4.721754

H $-5.139190-2.416125-4.463781$

H $-6.668840-2.284918-3.566376$

H -4.751694 2.237452 -2.059953

H $-4.5108240 .955034-0.910357$

H -2.285909 $0.532338-1.570581$

H $-2.4621801 .241440-3.156711$

H $-2.5848182 .869713-0.572345$

H -3.537013 $4.011560-2.643769$

H -2.080494 $3.603993-3.520355$

H $-1.3806156 .875757-2.895055$

H $\quad 0.1941984 .702209-2.749262$

H $-3.5870146 .002260-1.192071$

H -2.120390 $6.756083-0.615615$

H -2.588688 $5.157528-0.003513$

H $0.0520506 .095508-0.295663$

H $1.5067322 .261362-0.292513$

H $1.7905842 .974698-1.854404$

H -0.0647393 .2578181 .034980$

H $-0.358056-0.695952-2.558926$

H $1.114809-3.227114-3.510386$

H $1.7127090 .606409-3.203298$

H $3.2131860 .372049-2.308554$

H $1.697619-0.043405-1.546440$

H $2.7043264 .976815-0.926957$

H 1.8847144 .7610580 .620977

H $5.6716052 .017700 \quad 0.862426$

H $6.4310753 .243376-0.162720$

H $6.3462021 .702213-2.161115$

H $\quad 6.105975-0.2436830 .194238$

H $7.595260-1.525944-1.314324$

H $7.195493-0.534401-2.692837$

H $4.689193-3.775504-1.706838$

H $6.092105-3.300682-0.604786$

H $5.358514-1.847256-4.270255$ 
H $\quad 4.794721-0.429134-3.438509$ H $3.379323-3.104885-3.779162$ H $4.2296903 .096174-1.515519$ C $-0.461769-4.8058591 .233342$ O $0.207025-3.596724 \quad 1.643987$ C $1.458023-3.4945990 .933899$ C $1.670289-4.8434340 .252307$ C $0.241449-5.259828-0.045602$ H $1.319501-2.7305680 .160552$ C $2.574344-3.0712661 .894877$ H $2.289591-4.759657-0.645992$ H $2.145618-5.5691360 .922272$ C $0.081779-6.739226-0.344942$ H -0.105990 -4.682952 -0.911068 C $2.266853-1.7699672 .666491$ O $2.753748-4.1168722 .859399$ H $3.521959-2.9845101 .350878$ H $1.391078-1.9292053 .303866$ O $3.361174-1.5239583 .560768$ C $2.066887-0.5292651 .774838$ C 1.7766950 .7554472 .576561 H $1.258414-0.7019791 .057876$ O $3.264671-0.3310031 .019256$ H 2.5007360 .8610613 .392945 H 1.9444471 .6133551 .916111 H $0.663039-7.017161-1.230330$ H $\quad 0.425826-7.357920 \quad 0.490577$ H $-0.967158-6.985361-0.538069$ C $-1.972226-4.5602431 .139191$ H $3.456485-3.8311963 .468087$ H $4.145013-1.3333143 .017085$ H 3.1207290 .4285540 .429743 H -2.322841 -4.1585262 .097087$ H $-2.488032-5.5127430 .972142$ H $-0.285854-5.5342642 .036028$ C $-1.934657 \quad 0.9003632 .300728$ O $-0.5354250 .931164 \quad 1.991155$ C $0.339770 \quad 0.8119103 .118672$ C $\quad 0.1263282 .0021514 .063534$ C -1.3376432 .0867284 .510439$ C -2.3118601 .9838793 .330501$ H -2.4215011 .1481541 .349533$ C $-2.361647-0.5216532 .691887$ H $0.120226-0.1187603 .652227$ O 0.4998893 .2085553 .401326 H $0.782177 \quad 1.8855864 .934503$ H -1.5074773 .0373375 .030927$ O -1.6152381 .0362525 .432274$ H -3.3288301 .8119493 .701213$ O -2.3218363 .2533942 .671504$ C -3.824897 -0.7909352 .414820$ H -1.776740 -1.241433 2.107921 H -2.167965 -0.722237 3.749033 H 0.3693793 .9402104 .028235 H -1.013305 1.1420756 .188607 H -2.9907973 .2141961 .966865$ O $-4.107169-2.0841562 .751030$ O -4.6212040 .0193421 .961428$ C -5.456016 -2.501265 2.519039 H $-6.142061-1.9246813 .146691$ H $-5.712784-2.3896551 .461368$ H -5.535969 -3.557577 2.788632

SCF Energy (B3LYP/6-31G**//MMFF) $=-3245.92245952$

08_00583

MM̄FF Geometry

C 2.1203453 .9187810 .642586

C $2.8746094 .387061-0.363909$

C $4.0512443 .655502-0.965291$

O $3.7849112 .230683-0.962372$

C $5.3312253 .922892-0.158457$

C $6.5871983 .615691-0.948579$

C $7.2781692 .461413-0.884766$

C $7.0689734 .734011-1.837800$

C $6.9556951 .234993-0.078719$

C $6.7360230 .028204-0.999225$

C $6.451316-1.256281-0.209847$

C $6.302223-2.443703-1.163105$
C $5.925097-3.733813-0.422571$

O $5.242168-1.0703260 .532158$

O $5.515897-4.677674-1.423885$

C $4.716520-3.4456020 .491531$

C $7.133555-4.349207 \quad 0.292277$

O $4.419672-4.5675601 .320297$

C $4.888852-2.1796901 .366744$

C $3.548805-1.8540072 .062664$

O $5.882779-2.4284612 .362295$

C $3.0008961 .747918-1.967102$

O $2.6074672 .357867-2.948946$

C $2.6472020 .350119-1.636810$

C $1.869212-0.350725-2.474723$

C $1.412702-1.717681-2.242874$

C $1.568085-2.615046-3.438785$

C $3.496385-0.4927112 .770886$

C $2.156062-0.3518683 .465769$

C $-0.269087-0.1953082 .925166$

O $2.022611-0.5826284 .665306$

C $-0.664111-1.5688202 .477030$

C $-1.455702-1.8074361 .421097$

C $-1.755367-3.1823030 .899810$

C $-1.197226-3.408027-0.490534$

C $-2.008801-3.661651-1.530733$

C $0.308035-3.416782-0.663571$

C $0.849113-2.071581-1.070600$

N $1.133052-0.0201832 .589867$

H 2.3908262 .9807361 .122172

H $2.6079975 .330326-0.834662$

H $4.1791923 .995190-2.000814$

H 5.3154523 .3499430 .775843

H $5.3755594 .977261 \quad 0.146933$

H $8.1819062 .373948-1.487308$

H $7.9796474 .468865-2.385170$

H $6.3048304 .993220-2.577277$

H $7.2908325 .624457-1.240381$

H $7.7980841 .035350 \quad 0.594374$

H $6.076894 \quad 1.3804310 .554517$

H $5.891950 \quad 0.242792-1.667221$

H $7.621225-0.121122-1.630419$

H $7.280962-1.4242380 .486982$

H $7.218377-2.591974-1.748550$

H $5.512712-2.222534-1.895823$

H $5.323354-5.522163-0.982809$

H $3.843301-3.299389-0.159722$

H $7.915114-4.605221-0.433088$

H $6.864901-5.2889290 .787562$

H $7.576176-3.6837301 .036528$

H $4.202637-5.3150870 .738661$

H $2.747871-1.8697731 .313807$

H $3.313841-2.6379092 .794561$

H $5.722466-1.8491113 .123452$

H $3.006046-0.064416-0.701807$

H $1.5382230 .089609-3.412964$

H $1.075533-2.178772-4.315298$

H $\quad 1.135115-3.607477-3.297847$

H $2.629840-2.751463-3.673440$

H $4.281768-0.394903 \quad 3.526742$

H 3.6245320 .3312812 .059668

H -0.8344530 .5845022 .407690$

H $-0.420096-0.0882464 .003493$

H -0.233075 -2.406512 3.020494

H -1.882610 -0.9708810 .875227$

H $-2.844843-3.3145490 .907534$

H $-1.353615-3.9541861 .568449$

H -1.610853 -3.847998 -2.522770

H -3.087403 -3.682398 -1.415863

H $0.592071-4.201780-1.371715$

H $0.785513-3.7127740 .279365$

H $\quad 0.770809-1.309393-0.296880$

H 1.3580520 .1018851 .606539

C -0.3100473 .5955481 .002030$

O $-0.4843113 .204448-0.372109$

C $-1.8909933 .003031-0.626955$

C $-2.597703 \quad 3.2052210 .708171$

C $-1.6606114 .160875 \quad 1.423499$

H -2.180127 $3.785472-1.338952$

C $-2.0746931 .613578-1.251010$ 
H -3.609669 3.6040570 .589311 H $-2.6703942 .258113 \quad 1.256506$ C -1.869428 4.2117052 .926108 H $-1.7972145 .166047 \quad 1.003030$ C $-3.5487911 .252721-1.521841$ O $-1.3445291 .596485-2.481824$ H $-1.6183200 .855226-0.605282$ H $-4.1134631 .367053-0.591130$ O $-4.0950872 .188895-2.451684$ C $-3.768798-0.186314-2.035739$ C $-5.214549-0.492019-2.471045$ H $-3.459881-0.885737-1.251062$ O $-2.930599-0.432697-3.166560$ H $-5.471746 \quad 0.121335-3.344058$ H -5.229682 -1.531243 -2.827003 H -1.184722 4.9284683 .390176 H -2.8927084 .5202903 .163303$ H $-1.694913 \quad 3.2349193 .388731$ C 0.8561214 .5771991 .118961 H $-0.422093 \quad 1.826155-2.275648$ H $-3.5967742 .095533-3.282047$ H -3.025413 -1.370295 -3.405911 H $0.988507 \quad 4.8893392 .160357$ H $\quad 0.649530 \quad 5.474803 \quad 0.523663$ H -0.0847312 .6824241 .567083$ C $-6.845971-1.181640 \quad 0.821145$ O $-5.968678-1.222807-0.313453$ C $-6.274681-0.312284-1.373607$ C $-7.692679-0.563428-1.912800$ C $-8.731110-0.523270-0.787838$ C $-8.311926-1.3913710 .400779$ H $-6.552224-2.0448251 .432350$ C -6.6315790 .0865451 .664098$ H $-6.2204340 .713784-0.999171$ O $-7.760976-1.823582-2.577390$ H $-7.9264500 .203307-2.660546$ H $-9.696275-0.879306-1.168763$ O $-8.9036650 .817856-0.340042$ H $-8.975699-1.2056361 .253294$ O $-8.486235-2.761495 \quad 0.027196$ C -5.3143270 .0552752 .410607$ H $-6.6398180 .995097 \quad 1.057270$ H -7.424168 0.1547272 .418583 H -7.491214 -2.509591-1.943059 H $-9.200503 \quad 1.345505-1.100986$ H -8.290608 -3.306387 0.808342 O $-5.254656 \quad 1.131145 \quad 3.250352$ O $-4.449570-0.799647 \quad 2.281984$ C $-4.078075 \quad 1.213977 \quad 4.060634$ H -4.0889162 .1763584 .579084$ H -4.0824360 .4120484 .804615$ H -3.177498 1.1591913 .442990 SCF Energy (B3LYP/6-31G**//MMFF) $=-3245.89658504$

08_00584

MM̄FF Geometry

C -3.627151 -2.918992 -0.344806

C $-3.078428-2.462053-1.481533$

C $-1.595057-2.470092-1.740336$

O $-1.245990-1.118228-2.125666$

C $-1.180619-3.431748-2.865837$

C $-1.296641-4.893605-2.489395$

C $-0.381273-5.566302-1.763665$

C $-2.524797-5.600311-3.001003$

C $0.914856-5.045081-1.205995$

C $0.837596-4.848960 \quad 0.312646$

C $2.200210-4.5090230 .936237$

C $2.036746-4.1711742 .420727$

C $3.360389-3.7401883 .069498$

$\begin{array}{lllll}O & 2.766963 & -3.392586 & 0.243537\end{array}$

O $3.024410-3.144617 \quad 4.332124$

C $4.024388-2.6590592 .188734$

C $4.251858-4.9485093 .380235$

O $5.334251-2.3466202 .657899$

C $4.083294-3.0392130 .690965$

C $4.568956-1.832989-0.147661$

O $5.009600-4.1175510 .525205$

C $-0.021300-0.658194-1.754046$
O $0.865014-1.287018-1.199584$

C $0.030323 \quad 0.782465-2.085958$

C $1.0250781 .536222-1.594908$

C $1.1686022 .984526-1.753384$

C $0.1221543 .710830-2.557167$

C $4.410463-2.029187-1.661900$

C $5.324968-1.106919-2.442048$

C $5.7146151 .263709-3.034042$

O $6.253575-1.545678-3.118136$

C $5.5426432 .576242-2.341639$

C $5.0399883 .667603-2.938204$

C $4.8832654 .996939-2.256362$

C $3.4548785 .493841-2.277710$

C $3.0387606 .349494-3.226658$

C $2.5342635 .069322-1.156332$

C $2.2177513 .599133-1.168444$

N $5.0044770 .234014-2.299016$

H $-2.966644-3.3549690 .403388$

H $-3.704633-2.032660-2.258129$

H $-1.068111-2.717366-0.812862$

H $-0.152028-3.211845-3.178774$

H $-1.772297-3.227185-3.768850$

H $-0.568320-6.617503-1.547876$

H $-2.549444-6.655563-2.708946$

H $-3.433750-5.131842-2.613315$

H $-2.554081-5.561213-4.094913$

H $1.701232-5.771573-1.445224$

H $1.209046-4.108868-1.689516$

H $\quad 0.130328-4.038794 \quad 0.525431$

H $0.439590-5.7524440 .792036$

H $2.854726-5.3793940 .808266$

H $1.598006-5.0158352 .966532$

H $1.319343-3.3443942 .525072$

H $3.851820-2.9149744 .787793$

H $3.430333-1.7412232 .290127$

H $3.750055-5.6196404 .087870$

H $5.182537-4.6400633 .869149$

H $4.506656-5.5325772 .493316$

H $5.250414-2.0111453 .566097$

H $3.995530-0.9393430 .131164$

H $5.622076-1.6277620 .083738$

H $5.436284-4.049129-0.342995$

H $-0.7802421 .192662-2.677563$

H $1.7931141 .055421-0.990502$

H $0.0662823 .304594-3.573242$

H $\quad 0.3214474 .779655-2.663150$

H $-0.861343 \quad 3.614591-2.084312$

H $4.649020-3.054520-1.963606$

H $3.376497-1.844596-1.972227$

H $5.316608 \quad 1.272455-4.054217$

H $6.7767551 .002093-3.085620$

H $5.8613202 .634999-1.303645$

H $4.7400023 .611635-3.982323$

H $5.5365195 .714785-2.769529$

H $5.2424384 .962640-1.220289$

H $2.0252276 .736375-3.235642$

H $3.7027006 .679589-4.018911$

H $1.6102585 .658925-1.160488$

H $3.0087395 .312841-0.196668$

H $2.9294632 .994132-0.606134$

H $4.1095440 .473944-1.881410$

C $-6.000050-1.946875-0.746645$

O $-5.485820-0.602948-0.679812$

C $-6.4541590 .237043-0.034639$

C $-7.787516-0.426360-0.337198$

C $-7.422051-1.891817-0.168803$

H $-6.2578340 .177444 \quad 1.043818$

C $-6.290174 \quad 1.690254-0.492393$

H $-8.582253-0.101396 \quad 0.340437$

H $-8.111349-0.238499-1.367274$

C $-8.390536-2.844751-0.848674$

H $-7.403301-2.1232990 .904663$

C $-4.8241072 .173294-0.517619$

O $-6.800524 \quad 1.816398-1.825694$

H -6.9085232 .3439590 .133506$

H $-4.3099251 .690677-1.358052$

O $-4.8249103 .574182-0.817376$

$\begin{array}{llll}\text { C } & -4.019607 & 1.929386 & 0.773880\end{array}$ 
C -2.5518162 .3391320 .599102$ H $-4.0574430 .868721 \quad 1.035045$ O -4.5795202 .6673491 .855399$ H -2.132777 $1.827877-0.276110$ H $-2.489878 \quad 3.4116090 .369150$ H $-9.394735-2.740381-0.425263$ H -8.457298 -2.649481 -1.924256 H $-8.071076-3.883143-0.713487$ C $-5.078475-2.8730790 .058428$ H $-6.6829582 .743720-2.094761$ H $-5.2124904 .035871-0.054110$ H -5.4752142 .3260422 .015512$ H $-5.474580-3.8950950 .042964$ H $-5.088398-2.5409581 .105577$ H -6.015527 -2.237281-1.803669 C 0.7112252 .3491872 .373499 O $-0.3259722 .449806 \quad 1.392317$ C -1.6459662 .0855891 .820148$ C -1.664854 0.6364002 .332475 C $-0.5848190 .391627 \quad 3.392666$ C $0.779677 \quad 0.9281852 .954404$ H 1.6395732 .5280431 .816302 C 0.5927303 .4458063 .435985 H -1.957066 2.7631402 .622535 O $-1.478918-0.2730691 .250650$ H -2.6475680 .4206632 .765892$ H $-0.499671-0.6854953 .583471$ O $-0.958398 \quad 1.023274 \quad 4.613359$ H 1.4834560 .9010393 .794412 O 1.2914240 .0502051 .947202 C 0.8428824 .8017272 .819373 H -0.3799003 .4670703 .935703$ H 1.3422123 .3060394 .224055 H $-0.608991-0.0890970 .857516$ H -1.8064240 .6400014 .894998$ H 2.1788100 .3648791 .704523 O -0.3353545 .3507662 .407400$ O 1.9549795 .2983122 .694657 C $-0.2319636 .631537 \quad 1.778698$ H $\quad 0.384109 \quad 6.563278 \quad 0.877602$ H -1.2373136 .9515651 .492063$ H 0.1843137 .3635682 .477197

SCF Energy (B3LYP/6-31G**//MMFF) $=-3245.90532062$

\section{8_00585}

MM̄MF Geometry

C -1.985540 $2.597774-1.253059$

C $-2.6155883 .161677-0.210423$

C -2.923165 2.5144951 .121685

O $-2.3528001 .190897 \quad 1.222772$

C -4.4415512 .3591491 .310934$

C -4.8531172 .4037162 .768902$

C $-5.0444881 .326578 \quad 3.554753$

C -5.0914453 .7881053 .316465$

C $-4.824268-0.1219083 .219940$

C $-6.136179-0.9112953 .114766$

C $-6.863280-0.6866701 .780538$

C $-8.205955-1.418198 \quad 1.765125$

C $-8.899573-1.3067980 .400426$

O $-6.020551-1.1977850 .742799$

O $-9.977194-2.2554630 .400473$

C -7.902732 -1.717471 -0.704831

C -9.5404190 .0724320 .203802$

O $-8.487321-1.491269-1.985676$

C $-6.526679-1.017571-0.584921$

C $-5.511844-1.657367-1.562591$

O $\quad-6.678520 \quad 0.365282-0.911577$

C $-1.0328701 .082790 \quad 1.524111$

O $-0.2464291 .987711 \quad 1.746745$

C $-0.702620-0.3562361 .473571$

C $0.546577-0.731857 \quad 1.166079$

C $1.017213-2.1100191 .020147$

C $0.036401-3.2167911 .309651$

C $-4.049632-1.249461-1.319393$

C $-3.120148-1.852490-2.360269$

C $-0.728438-1.868440-3.057796$

O $-3.513874-2.578545-3.269819$

C $0.020806-3.026894-2.478287$
C $1.359701-3.117762-2.502863$

C $2.135155-4.287852-1.967356$

C $3.285538-3.870568-1.076413$

C $4.538226-3.797649-1.557022$

C $2.995008-3.6212970 .386635$

C $2.287943-2.3151740 .616134$

N $-1.799331-1.475201-2.160473$

H -1.677676 $1.557664-1.209251$

H $-2.9068134 .207955-0.289131$

H -2.501113 3.1476521 .912704

H $-4.779573 \quad 1.4225620 .854373$

H -4.9856763 .1561910 .786611$

H $-5.369603 \quad 1.4961804 .580902$

H -5.3829803 .7751104 .372070$

H $-4.1835864 .393946 \quad 3.233419$

H -5.8925574 .2839372 .758633$

H -4.224980 -0.2508662 .314903$

H -4.225916 -0.551992 4.033542

H -5.887077 -1.976806 3.205522

H $-6.796027-0.6594473 .954160$

H $-7.0151800 .387743 \quad 1.631391$

H -8.862499 -1.046228 2.561640

H -8.043402 -2.482859 1.984929

H -10.381990 -2.241042 -0.483878

H -7.757513 -2.803162 -0.614233

H $-10.292956 \quad 0.2560920 .980146$

H -10.076006 $0.128889-0.750409$

H -8.8183620 .8907830 .241198$

H -7.930067 -1.929875 -2.649976

H -5.564938 -2.750582 -1.475033

H $-5.782689-1.399546-2.594317$

H $-5.8416250 .704049-1.266179$

H -1.508610 -1.061670 1.640950

H 1.2895270 .0419780 .976350

H $\quad 0.440599-4.216418 \quad 1.139479$

H $-0.846572-3.1301520 .666510$

H -0.287094 -3.176956 2.355646

H $-3.940120-0.160931-1.360603$

H -3.719354 -1.592249 -0.332160

H $-0.089492-0.991968-3.192681$

H -1.149005 -2.149612 -4.028473

H -0.559694 -3.838487 -2.046363

H $1.934302-2.321981-2.970146$

H $2.520033-4.851890-2.827202$

H $1.485523-4.983831-1.422380$

H $5.373714-3.528883-0.920287$

H $4.755428-4.000945-2.600543$

H $3.932241-3.5888740 .958304$

H $2.452080-4.4779540 .795160$

H $2.915500-1.4445860 .422642$

H $-1.559129-0.910095-1.351396$

C $-0.124183 \quad 3.499989-2.725259$

O $0.4970052 .220239-2.948786$

C $1.7984342 .215063-2.308767$

C $2.0588993 .656260-1.889779$

C $0.6582334 .150181-1.586250$

H $1.6813131 .566978-1.432580$

C $2.8210391 .623165-3.285510$

H $2.7307453 .736316-1.031117$

H $2.4909554 .232142-2.717618$

C $0.5374195 .662504-1.538258$

H $\quad 0.3593383 .737067-0.615297$

C $4.2871541 .535173-2.819860$

O $2.4040950 .298066-3.627818$

H $2.7861632 .206119-4.215204$

H $4.8480011 .041638-3.624930$

O $4.8454112 .836697-2.665367$

C $4.5302680 .716994-1.533351$

C $6.0308420 .418989-1.336432$

H $3.994163-0.236071-1.599530$

O $4.0242741 .411080-0.400082$

H $6.382318-0.139160-2.212859$

H $6.582487 \quad 1.366828-1.294062$

H $1.1767356 .074549-0.750528$

H $0.8365726 .119888-2.487232$

H $-0.4937855 .963781-1.328496$

C -1.633649 $3.338177-2.512935$

H $1.5125560 .368336-4.009632$ 
H $4.7427073 .302912-3.512399$ H $4.5207672 .243348-0.318961$ H $-2.0568572 .774018-3.353190$ H -2.106494 4.327453-2.520906 H $0.0248714 .069748-3.652309$ C $6.591372-0.0403982 .341953$ $\begin{array}{llll}\text { O } & 6.319824 & 0.524589 & 1.050443\end{array}$ C $6.332607-0.386293-0.055730$ C $7.694176-1.093265-0.155323$ C $8.077827-1.7610561 .167287$ C $7.930122-0.7997142 .347181$ H 6.7019870 .8275943 .004916 C $5.414010-0.8813902 .857854$ H $5.546455-1.1349940 .081762$ O $8.709323-0.166355-0.537143$ H $7.640998-1.849225-0.947517$ H $9.117411-2.1069681 .114368$ O $7.245963-2.8954301 .391778$ H $8.048736-1.3435323 .291790$ $\begin{array}{llll}\text { O } & 8.995659 & 0.152127 & 2.278801\end{array}$ C $4.359785-0.0119863 .504136$ H $4.926675-1.4794182 .085547$ H $5.758232-1.5727043 .636537$ H 8.7209480 .5498250 .120809 H $7.385702-3.5122260 .653264$ H 8.9379490 .7153753 .069360 O 3.8310790 .8510622 .593029 O $4.038602-0.1004144 .682553$ C 2.8543301 .7630183 .102987 H 2.5243002 .4012372 .279068 H 1.9920621 .2156363 .494757 H 3.2955562 .3949893 .879651

SCF Energy (B3LYP/6-31G**//MMFF)= -3245.91529755

08_00586

\section{MM̄FF Geometry}

C -3.612249 $0.046206 \quad 2.187761$

C $-3.133397-0.0116073 .439016$

C $-1.671831-0.002473 \quad 3.823422$

O -0.8427220 .5101022 .754005$

C $-1.195168-1.4312144 .137718$

C $0.127124-1.4496704 .878154$

C $1.330826-1.6591744 .310803$

C $0.024746-1.2386656 .367906$

C $1.657267-1.8726782 .858368$

C $2.304116-3.2474242 .636062$

C $2.811487-3.4263141 .196931$

C $3.378341-4.8339570 .994006$

C $4.018306-5.008474-0.393372$

O $3.836448-2.4543830 .969536$

O $4.780114-6.225578-0.382545$

C $4.987562-3.834540-0.658655$

C $2.957884-5.208775-1.481181$

O $5.482588-3.872152-1.994912$

C $4.366856-2.451856-0.362439$

C $5.446177-1.344077-0.446006$

O $3.354371-2.177621-1.334181$

C -0.7666431 .8611002 .607003$

O $\begin{array}{llll}-1.340836 & 2.708516 & 3.271323\end{array}$

C $0.1472102 .130302 \quad 1.474393$

C 0.3391623 .3896571 .052321

C $1.2229203 .805777-0.041228$

C $2.0389642 .730638-0.708747$

C $4.985655-0.0180010 .171048$

C $5.9249981 .140907-0.113050$

C $6.160603 \quad 3.5745620 .395695$

O $6.937211 \quad 1.045030-0.799362$

C $5.179224 \quad 4.6680220 .675087$

C $4.9068035 .673691-0.169922$

C 3.8939826 .7432860 .130695

C $2.8864666 .944887-0.982767$

C $2.7131528 .160056-1.530672$

C $2.0793405 .758894-1.474128$

C $1.2616915 .110039-0.388268$

N 5.4638962 .3055320 .485391

H -2.9179880 .0580871 .351441$

H -3.836791 -0.0526284 .268050$

H -1.5516570 .6406004 .704254$
H -1.120658 -2.012811 3.210925

H $-1.938145-1.9552384 .754051$

H $2.205371-1.6530924 .961367$

H $1.000380-1.2836906 .863461$

H $-0.410854-0.2595866 .589602$

H $-0.608022-2.0110086 .817480$

H $0.778113-1.7750612 .214801$

H $2.349278-1.0771592 .557034$

H $3.147960-3.3601443 .329952$

H $1.576809-4.0338802 .873464$

H $1.979567-3.2420220 .508724$

H $2.603845-5.5934411 .158222$

H $4.147075-5.0241021 .756327$

H $5.457195-6.154450 \quad 0.311623$

H $5.857970-3.968304-0.001496$

H $2.352818-6.097831-1.265671$

H $3.421076-5.392264-2.457062$

H $2.277242-4.361679-1.578321$

H $5.866466-4.754271-2.137248$

H $6.348245-1.6601540 .093526$

H $5.731389-1.185867-1.493609$

H $3.277554-1.219056-1.460502$

H $\quad 0.639476 \quad 1.278394 \quad 1.018250$

H $-0.198334 \quad 4.194753 \quad 1.551377$

H 2.6395362 .1988700 .034554

H $2.7358283 .102211-1.461327$

H $1.3878932 .006671-1.209093$

H $3.9996940 .259416-0.215048$

H $4.903717-0.126993 \quad 1.259238$

H $6.6257253 .664149-0.591332$

H 6.9523253 .5726801 .151553

H 4.6575924 .6250531 .629034

H $5.4375265 .736945-1.116787$

H 4.4451487 .6738540 .318327

H 3.3524036 .5203501 .058510

H $1.9982518 .324572-2.330094$

H $3.2817939 .020266-1.193164$

H $2.7619695 .062553-1.966536$

H $1.3767196 .079358-2.254660$

H 0.6145735 .8061220 .147242

H 4.7195242 .2234461 .172885

C -5.4503551 .2633750 .935920$

O $-4.6622601 .218101-0.269863$

C $-4.1364252 .534699-0.524458$

C $-5.028017 \quad 3.4812390 .265919$

C -5.2567802 .6622521 .526052$

H $-3.1315062 .568929-0.086854$

C $-4.0511462 .783086-2.033577$

H $-4.547346 \quad 4.4445150 .460575$

H $-5.9791823 .666337-0.246389$

C $-6.428621 \quad 3.1422242 .363879$

H -4.3461522 .7039062 .136683$

C $-3.2508551 .688890-2.776804$

O $-5.3830332 .796381-2.559433$

H -3.629237 $3.775965-2.226405$

H $-3.7938470 .740268-2.704848$

O $-3.199323 \quad 2.021786-4.168001$

C $-1.804077 \quad 1.511344-2.275887$

C $-1.0206780 .392213-2.987257$

H $-1.7884071 .301746-1.202166$

O $-1.076017 \quad 2.725626-2.476040$

H $-0.9593290 .594387-4.063719$

H $0.0125290 .442390-2.619060$

H $-6.259687 \quad 4.1673392 .709433$

H $-7.3628703 .130193 \quad 1.792480$

H -6.5629062 .5056923 .244188$

C $-5.0748050 .087236 \quad 1.843443$

H $-5.335853 \quad 3.142219-3.466494$

H $-4.0995671 .934614-4.523741$

H $-1.4326413 .389740-1.862874$

H $-5.306059-0.8464801 .317753$

H -5.6932760 .1084642 .748141$

H $-6.493619 \quad 1.1322370 .620723$

C $-2.296263-2.448822-0.928681$

O $-1.670115-1.222398-1.328188$

C $-1.586919-1.018370-2.742528$

C $-0.715210-2.114985-3.372737$

C $-1.256627-3.508603-3.034392$ 
C -1.573992 -3.663309 -1.540548 H -2.154861 -2.477995 0.159179 C $-3.805582-2.397865-1.206940$ H -2.591459-1.067004-3.175860 O $0.621832-1.994039-2.892236$ H $-0.692868-1.970470-4.459543$ H $-0.514667-4.261540-3.328381$ O $-2.429843-3.735863-3.810261$ H $-2.159842-4.574250-1.374643$ O $-0.332777-3.837873-0.851791$ C $-4.602484-3.318623-0.309492$ H $-4.163858-1.377288-1.030704$ H $-4.033190-2.649927-2.245837$ H $1.153241-2.686535-3.320741$ H -2.741110 -4.636032 -3.614375 H $-0.535897-4.0104230 .083476$ O $-5.932940-3.134622-0.560646$ O $-4.129404-4.097673 \quad 0.506137$ C $-6.823188-3.9303410 .228057$ H $-6.699004-3.6969701 .289843$ H $-7.847590-3.687811-0.066674$ H $-6.646460-4.9939070 .041915$

SCF Energy (B3LYP/6-31G**/MMFF)= -3245.90434599

08_00587

\section{MM̄FF Geometry}

C $2.8941853 .003842-2.351154$

C $3.4315213 .903213-1.511846$

C $3.9653873 .639695-0.121667$

$\begin{array}{llllll}\text { O } & 3.840094 & 2.247211 & 0.241472\end{array}$

C 3.1555594 .4575770 .899531

C $3.711390 \quad 4.369376 \quad 2.307091$

C 3.1543743 .6481353 .299985

C 4.9354455 .2066322 .572674

C 1.9439512 .7604393 .202569

C 2.0688051 .5332274 .117974

C $0.826217 \quad 0.636494 \quad 4.026445$

C $0.845178-0.4339015 .118532$

C $-0.353130-1.3871704 .998856$

$\begin{array}{lllll}\text { O } & 0.821535 & 0.013435 & 2.737215\end{array}$

O $-0.101458-2.5210795 .842403$

C $-0.429350-1.9087423 .550066$

C $-1.650755-0.7472595 .506792$

O $-1.611139-2.680600 \quad 3.348075$

C -0.334737 -0.7944602 .480631$

C $-0.179973-1.4375911 .083959$

O $-1.532124-0.0160992 .502064$

C $4.903102 \quad 1.4330760 .006017$

O $5.9682181 .738255-0.505379$

C $4.527454 \quad 0.076454 \quad 0.462712$

C $5.263441-0.9748360 .073277$

C $5.002673-2.3805850 .392340$

C $3.901184-2.6805651 .373648$

C $0.174760-0.451604-0.032950$

C $0.326571-1.207384-1.336583$

C $2.037690-2.282134-2.798268$

O $-0.652547-1.568011-1.985791$

C $2.478929-3.619355-2.289294$

C $3.656556-4.182572-2.599791$

C $4.102378-5.519318-2.077812$

C $5.484355-5.481982-1.459872$

C $6.526964-6.076593-2.064415$

C $5.644236-4.822965-0.104335$

C $5.754497-3.324743-0.211602$

N $1.646577-1.467378-1.662580$

H $2.8353431 .960347-2.058323$

H $3.4867824 .940759-1.836981$

H $5.0175653 .947863-0.091292$

H $2.107766 \quad 4.133503 \quad 0.882316$

H 3.1354855 .5158240 .605197

H 3.6097713 .7039334 .287730

H 5.2541015 .1572103 .619326

H 5.7748684 .8671581 .958167

H 4.7351596 .2575392 .339681

H 1.0652763 .3433923 .503251

H 1.7810492 .4211522 .175028

H $2.961266 \quad 0.961317 \quad 3.832325$

H 2.2052081 .8681465 .153866
H $-0.064946 \quad 1.2669024 .128955$

H $\quad 0.8706800 .024717 \quad 6.114959$

H $1.768960-1.0236045 .035485$

H $0.020020-2.1969616 .751432$

H $\quad 0.416908-2.595553 \quad 3.407303$

H $-1.546804-0.4549526 .558591$

H -2.481549-1.460948 5.475356

$\mathrm{H}-1.9399360 .1428824 .944220$

H $-1.621635-3.3773814 .026700$

H $0.609865-2.197713 \quad 1.120586$

H $-1.108171-1.9574780 .812354$

H -1.6271910 .4373251 .649964$

H $3.638774-0.0222911 .075890$

H $6.125195-0.795010-0.568468$

H $4.055011-2.1277432 .307425$

H $3.837513-3.7342911 .651884$

H $2.930773-2.3980820 .956742$

H $-0.6050210 .300842-0.171956$

H 1.0989690 .0900450 .195382

H $2.841507-1.751143-3.317747$

H $1.192942-2.402544-3.483392$

H $1.793276-4.150860-1.633023$

H $4.332435-3.663381-3.275601$

H $4.083714-6.225349-2.918120$

H $3.393823-5.914134-1.338801$

H $7.515246-6.075898-1.616374$

H $6.417692-6.573164-3.022904$

H $6.559077-5.1852010 .382733$

H $4.822768-5.1498990 .538279$

H $6.546706-2.996439-0.886552$

H $2.383180-1.149417-1.040855$

C $0.819996 \quad 3.188737-3.817181$

O $0.160148 \quad 3.991253-2.817791$

C $-0.7617013 .155250-2.077522$

C -1.066737 $2.011633-3.035811$

C $0.298627 \quad 1.763546-3.648364$

H $-0.2081082 .794298-1.201055$

C $-1.9611973 .997808-1.624334$

H $-1.4576441 .114058-2.557048$

H $-1.7804102 .330324-3.805419$

C $0.2572730 .965580-4.939635$

H $0.9034661 .211035-2.921929$

C -2.813235 $3.398232-0.485505$

O $-1.4299405 .235167-1.112158$

H -2.581174 $4.274172-2.484966$

H $-2.218377 \quad 3.3993920 .436445$

O $-3.9024494 .301480-0.244402$

C $-3.3831241 .992519-0.706816$

$\begin{array}{llll}C & -4.275421 & 1.533870 & 0.463908\end{array}$

H -2.557282 $1.286666-0.804806$

O $-4.1270021 .985038-1.921402$

H -3.7879611 .7920521 .411441$

H $-5.235606 \quad 2.0618320 .409786$

H $-0.158351-0.031291-4.761715$

H $-0.361687 \quad 1.457752-5.697197$

H $1.2637730 .842606-5.351926$

C $2.340383 \quad 3.359531-3.706155$

H $-2.1865305 .786779-0.848641$

H $-4.4795854 .277523-1.027141$

H $-4.4388741 .076287-2.071297$

H $2.5863074 .404386-3.934857$

H $2.8400632 .744195-4.463200$

H $0.5009373 .591641-4.786911$

C $-5.652281-1.664187-0.909205$

O $-5.363036-0.277241-0.688327$

$\begin{array}{llll}C & -4.528881 & 0.013287 & 0.435744\end{array}$

C $-5.188206-0.4825091 .730589$

C $-5.537765-1.9712311 .636540$

C $-6.287291-2.2990770 .340669$

H $-6.409003-1.668804-1.704928$

C $-4.425450-2.430817-1.428665$

H $-3.563465-0.4885850 .310135$

$\begin{array}{llll}\text { O } & -6.367481 & 0.269778 & 2.007808\end{array}$

H $-4.499397-0.3161982 .566842$

H $-6.158495-2.2544742 .495282$

O $-4.345401-2.7493771 .683897$

H $-6.360118-3.3841020 .201773$

O $-7.624429-1.7992500 .439204$ 
C $-4.016564-1.978968-2.816029$

H $-3.552756-2.311760-0.781794$

H $-4.674898-3.496828-1.491341$

H $-6.9566600 .193756 \quad 1.237843$

H $-3.885011-2.5350422 .513269$

H $-8.046312-2.2387891 .197085$

O $-3.140714-2.888255-3.338126$

O $-4.396100-0.954683-3.366495$

C $-2.643930-2.583881-4.645264$

H $-1.891915-3.333203-4.906178$

H $-3.457640-2.631120-5.374976$

H -2.172249 -1.596848 -4.656211

SCF Energy (B3LYP/6-31G**//MMFF)= -3245.92877529

08_00588

MMFF Geometry

C $0.436835-1.2567382 .402291$

C $0.635013-2.4283501 .776860$

C $-0.056388-2.9024770 .517121$

O $-0.920300-1.8604540 .012431$

C $-0.900497-4.1486040 .834464$

C $-1.563698-4.763766-0.381215$

C $-2.868399-4.619832-0.688482$

C $-0.669496-5.620265-1.238447$

C $-3.882689-3.7907190 .050302$

C $-4.803074-3.026686-0.912777$

C $-5.744263-2.083748-0.149771$

C $-6.804298-1.496889-1.080413$

C $-7.715848-0.509459-0.336947$

O $-4.952041-1.0250270 .394912$

O $-8.4670330 .223338-1.317750$

C $-6.840136 \quad 0.5169580 .414151$

C $-8.744837-1.2375730 .536003$

O $\quad-7.632350 \quad 1.3434891 .263275$

C $-5.674086-0.1050811 .223412$

C -4.6869961 .0035331 .647250$

O $-6.125353-0.7254032 .427880$

C $-0.848550-1.557941-1.309603$

O $-0.198633-2.126663-2.171465$

C $-1.669428-0.350779-1.528887$

C $-1.2269760 .566284-2.400457$

C $-1.8529071 .856393-2.683483$

C $-3.2851792 .048647-2.263892$

C $-3.429247 \quad 0.4783812 .350034$

C -2.4650671 .6125452 .642643$

C -0.8991013 .1952701 .508740$

O $-2.3000552 .041376 \quad 3.782087$

C -1.3084154 .1821250 .460128$

C $-0.5032784 .606938-0.526036$

C $-0.9084325 .634298-1.545689$

C $-0.5922015 .222923-2.967785$

C $0.4213595 .791761-3.642460$

C $-1.5019944 .214232-3.637058$

C -1.115032 $2.801431-3.301524$

N -1.841707 2.0929291 .501866

H $-0.275527-0.5407582 .004245$

H $1.355171-3.1255202 .201567$

H $0.727881-3.143715-0.210832$

H -1.653287 -3.8989981 .591786$

H $-0.269889-4.9156121 .304989$

H -3.251572 -5.148195 -1.560347

H -1.204721 -6.062187 -2.085620

H $0.156449-5.028186-1.644611$

H $-0.250807-6.441743-0.647956$

H $-4.490767-4.4595150 .671000$

H $-3.399394-3.0751680 .721239$

H -4.190827 -2.446580 -1.615326

H $-5.389088-3.745926-1.498363$

H $-6.214773-2.6438480 .666662$

H -7.400064 -2.291741 -1.546141

H $-6.312739-0.964534-1.906977$

H -8.962708 -0.417320 -1.856276

H $-6.4062911 .181828-0.346524$

H -9.394319 -1.867886 -0.083729

H -9.410364 -0.528059 1.039772

H -8.288604 -1.8820061 .290338$

H $-8.330819 \quad 1.738527 \quad 0.713697$

H $\quad-4.3833391 .5686870 .759391$
H $-5.185543 \quad 1.7127712 .320746$

H -7.092581 -0.6675442 .468300$

H $-2.558012-0.218683-0.924382$

H $-0.2878710 .379473-2.919840$

H $-3.7653112 .900431-2.751003$

H -3.349162 2.205565 -1.184615

H -3.891554 $1.174482-2.527672$

H -3.687739 -0.007044 3.297833

H $-2.910070-0.2673871 .738148$

H $\quad 0.0913912 .774881 \quad 1.316783$

H $-0.887303 \quad 3.6764702 .491379$

H -2.3155784 .5865840 .533230$

H $0.5125404 .224492-0.584494$

H $-0.3807656 .565598-1.301041$

H -1.977571 $5.869299-1.471096$

H $0.6367135 .521736-4.670913$

H $1.0619266 .535473-3.180084$

H -1.445259 $4.322983-4.728186$

H $-2.5375184 .450015-3.377443$

H $-0.0906382 .553116-3.584563$

H -1.9687321 .5915610 .628295$

C 1.9708610 .4134933 .492869

O 3.0715620 .1479492 .609020

C $4.162204-0.3722273 .386765$

C 3.9194260 .0639734 .832397

C 2.6294510 .8709474 .793113

H $4.089408-1.4656743 .349512$

C 5.5055140 .0472222 .772484

H $3.771521-0.8324385 .448447$

H 4.7598690 .5987645 .284360

C 2.9170442 .3726024 .771443

H 2.0013930 .6643445 .666976

C $5.651482-0.2964521 .273385$

O 5.6701901 .4659162 .876467

H $6.328966-0.4043003 .339268$

H $5.0408660 .397267 \quad 0.686453$

O $7.009532-0.0332060 .897073$

C $5.297312-1.7449850 .896875$

C $5.639532-2.142197-0.550594$

H $4.228051-1.9135441 .067306$

O $6.006221-2.6300781 .769206$

H $6.728310-2.202457-0.675719$

H $5.275771-3.169620-0.685217$

H 3.4466102 .6771505 .680198

H 3.5268882 .6630013 .911628

H 1.9814412 .9400394 .723023

C $1.130353-0.8605653 .676573$

H $5.837307 \quad 1.6808413 .808935$

H $7.572637-0.6725151 .366480$

H $5.722670-3.5367121 .560953$

H $0.360417-0.682598 \quad 4.437018$

H $1.751167-1.6891834 .036928$

H 1.3520021 .2026723 .051047

C $2.925138-0.263483-2.315906$

O $3.635602-1.118435-1.409533$

C $5.042504-1.231854-1.637768$

C $5.320289-1.776343-3.050173$

C $4.606143-0.951307-4.124934$

C $3.134022-0.710479-3.773623$

H $1.870425-0.416254-2.067002$

C $3.2465421 .222000-2.092406$

H $5.502490-0.243507-1.541360$

O $4.902751-3.137276-3.152262$

H $6.401894-1.759991-3.228324$

H $4.671125-1.482378-5.082728$

O $5.2843690 .292678-4.269348$

H $2.6978960 .028244-4.456232$

O $2.428203-1.937403-3.980526$

C $2.7739821 .713161-0.740615$

H $4.3214191 .408937-2.159944$

H $2.7424591 .835867-2.847577$

H $3.955697-3.174652-2.934437$

H $4.8374390 .790264-4.975060$

H $1.487292-1.770918-3.799089$

O $3.5043972 .812498-0.393688$

O $1.8634641 .213499-0.095220$

C $3.2216973 .434620 \quad 0.865547$

H 4.1731253 .6404361 .363052 
H $2.710706 \quad 4.3827280 .680638$

H 2.6123772 .8098581 .522117

SCF Energy (B3LYP/6-31G**//MMFF) $=-3245.91021812$

08_00589

MM̄FF Geometry

C $-0.173808-3.836561 \quad 0.623136$

C $0.693687-2.813840 \quad 0.629768$

C $1.216581-2.1800321 .891633$

O $0.863394-0.7782331 .850257$

C $2.750528-2.2920601 .936950$

C $3.338153-1.9746213 .296340$

C $3.931306-0.8104853 .625238$

C $3.270253-3.0966174 .300266$

C 4.0707200 .4283062 .785255

C 5.5223730 .7125012 .376680

C $6.047921-0.2291091 .281025$

C 7.5083860 .0928730 .954812

C $8.032187-0.738786-0.225427$

O $5.236215-0.0384260 .118532$

O $9.268359-0.154268-0.663632$

C $7.037020-0.622478-1.399262$

C $8.356309-2.1785250 .190635$

O $7.408061-1.489849-2.468594$

C $5.568991-0.882942-0.991349$

C $4.622053-0.508645-2.157464$

O $5.410995-2.266032-0.673997$

C $0.308845-0.2261482 .959162$

O $-0.013124-0.8121613 .984274$

C 0.1342981 .2391912 .821558

C $0.582911 \quad 1.9600741 .781923$

C 0.4746983 .4147731 .627205

C -0.2198524 .1853392 .719896$

C $3.132308-0.638437-1.814559$

C $2.2550210 .014791-2.862692$

C $1.5360082 .229104-3.719262$

O $1.618478-0.646011-3.679917$

C $1.5599573 .643207-3.238762$

C $0.4684374 .313511-2.841490$

C $0.4968985 .728059-2.341744$

C $0.0675575 .847567-0.895765$

C - $-1.1233356 .377818-0.570800$

C 1.0598035 .4526620 .175993

C 1.0012343 .9892550 .524585

N $2.259677 \quad 1.397544-2.777217$

H $-0.545648-4.2263151 .567964$

H $1.058808-2.422039-0.315338$

H $\quad 0.769829-2.6734782 .762849$

H $3.187988-1.6377741 .176378$

H $3.064309-3.3088961 .663986$

H $4.343599-0.7125534 .628948$

H $3.742581-2.8328075 .252512$

H $2.229777-3.3609544 .512463$

H $3.783065-3.9834683 .913558$

H 3.4237130 .4068721 .904093

H $3.712471 \quad 1.2704543 .391201$

H 5.5589791 .7442412 .002813

H $\quad 6.1749460 .664975 \quad 3.257439$

H $5.954519-1.2656771 .623286$

H $8.144205-0.0492281 .837767$

H $7.596481 \quad 1.1564920 .691763$

H $9.883066-0.1532980 .090082$

H $7.1104280 .401747-1.791456$

H $9.125481-2.1869690 .972334$

H $8.775243-2.747230-0.646866$

H $7.489800-2.7191740 .576777$

H $8.330662-1.286633-2.699391$

H $4.827510 \quad 0.525893-2.459730$

H $4.826434-1.143759-3.028628$

H $4.474084-2.504945-0.745051$

H -0.3667551 .7079633 .662952$

H 1.0932011 .4481810 .967908

H $\quad 0.3525754 .126748 \quad 3.652105$

H -1.223639 3.7860062 .900522

H $-0.354270 \quad 5.2441362 .488930$

H $2.842942-1.692200-1.747947$

H $2.900007-0.178797-0.847210$

H $\quad 0.5191391 .835426-3.824695$
H $2.0309022 .149759-4.692704$

H $2.5283654 .137421-3.210254$

H $-0.5000593 .822193-2.877815$

H $-0.1634836 .322065-2.986725$

H $1.4940326 .171346-2.457013$

H -1.4234196 .5002710 .464557$

H -1.828222 $6.695046-1.332055$

H $0.926674 \quad 6.081821 \quad 1.062749$

H $2.0766575 .685885-0.165737$

H $1.4854453 .344656-0.208543$

H $2.9138141 .842960-2.139266$

C -2.068268 -4.077902 -1.009171

O $-2.991452-4.3592200 .060910$

C $-4.176817-4.952632-0.489156$

C $-4.115331-4.732243-1.994062$

C $-2.618588-4.799144-2.241304$

H $-4.085856-6.032346-0.305457$

C $-5.434654-4.4546770 .231937$

H $-4.675530-5.490073-2.550266$

H $-4.501568-3.749661-2.283031$

C $-2.192379-4.175318-3.559201$

H $-2.309861-5.853070-2.227540$

C $-5.902266-3.010872-0.053227$

O $-6.503471-5.337410-0.135561$

H -5.289226 -4.576145 1.312855

H $-6.352232-2.970192-1.052321$

$\begin{array}{llll}\text { O } & -6.973537 & -2.721404 & 0.856771\end{array}$

C $-4.836975-1.9084550 .063983$

C $-5.419551-0.505416-0.149435$

H -4.064187 -2.083386 -0.682945

O $-4.223462-1.9402391 .350201$

H -6.081174 -0.494498 -1.024640

H $-6.052961-0.2354000 .706734$

H -2.662637 -4.694713 -4.400564

H -2.478254 -3.119286 -3.613718

H $-1.107278-4.237448-3.687294$

C $-0.659434-4.525091-0.621735$

H -7.297601 -5.0470280 .345251$

H -6.592866 -2.672443 1.750657

H -3.721351 -2.769949 1.418300

H $0.031978-4.343091-1.452950$

H $-0.646136-5.604347-0.425674$

H -2.075584 -2.991976-1.164479

C $-4.2604073 .020265-0.406040$

O $-5.0638471 .836446-0.336572$

C $-4.3467140 .594492-0.299853$

C $-3.4743040 .442758-1.559561$

C $-2.5711881 .661989-1.776418$

C $-3.3406392 .977942-1.635465$

H $-4.9787293 .837292-0.552412$

C -3.5115063 .2835680 .903776$

H -3.7132230 .5788600 .593158$

O $-4.315104 \quad 0.270113-2.699175$

H $-2.854938-0.455701-1.467907$

H $-2.1261191 .616514-2.777934$

O $-1.508351 \quad 1.642741-0.829806$

H -2.642587 $3.820217-1.594657$

O $-4.141424 \quad 3.171550-2.804905$

C -4.4803403 .5874932 .020456$

H -2.8730192 .4526341 .216228$

H -2.8539454 .1544730 .804196$

H -3.739337 $0.132385-3.470593$

H $-1.0008540 .825544-0.971923$

H $-4.8158592 .470858-2.822614$

O -4.7824612 .4441892 .699212$

O -4.9317084 .7029792 .244912$

C -5.7175322 .5936453 .771422$

H -5.3193063 .2746754 .529492$

H -6.6763502 .9570923 .389807$

H -5.8711891 .6120214 .227371$

SCF Energy (B3LYP/6-31G**//MMFF) $=-3245.91624772$

08_00590

MM̄FF Geometry

C $-0.1182732 .815614-1.325316$

C $-1.1061373 .594300-0.860211$

C $-1.7018473 .433740 \quad 0.510980$

O -3.0800353 .0405370 .310763$ 
C $-1.634647 \quad 4.732221 \quad 1.334545$ C -0.2343935 .2159781 .648189$ C 0.5883694 .6363732 .544975 C $\quad 0.197846 \quad 6.455526 \quad 0.910337$ C $0.3247473 .397340 \quad 3.356283$ C 1.4633312 .3803893 .187686 C 1.0586250 .9780113 .669905 C $2.3017160 .142978 \quad 3.980070$ C $1.946355-1.3026164 .348722$ O $0.307495 \quad 0.3652612 .613573$ O $3.157186-2.0736684 .330925$ C $1.029110-1.8740303 .252550$ C $1.380368-1.4135935 .768546$ O $0.564396-3.1754693 .604768$ C $-0.166801-0.9588142 .896321$ C $-0.852379-1.5083681 .621364$ O $-1.102037-0.958553 \quad 3.974257$ C $-3.7237642 .432637 \quad 1.340703$ O -3.3128542 .2974912 .485079$ C -5.0435241 .9133660 .921847$ C $-5.3346081 .622549-0.354992$ C $-6.6007871 .064298-0.820554$ C $-7.0813081 .684706-2.104772$ C -1.961627 -0.6179791 .058279$ C $-2.366296-1.060316-0.333598$ C $-3.588708-2.864366-1.544450$ O $-2.042351-0.422815-1.333144$ C $-4.934619-2.308407-1.888466$ C $-6.082339-2.976268-1.698587$ C $-7.439946-2.452736-2.074388$ C $-8.285162-2.112376-0.866775$ C $-8.891302-3.074703-0.151010$ C $-8.487351-0.649359-0.537421$ C $-7.2065340 .049283-0.170671$ N -3.102474 -2.232634 -0.333025 H $\quad 0.2714132 .017346-0.697791$ H -1.510669 4.382260 -1.490668 H -1.188283 2.6214611 .034170 H -2.180163 4.6078512 .278595 H -2.197548 5.5209430 .815815 H 1.5622835 .0928022 .717419 H 1.2217076 .7534791 .159690 H $0.1576626 .291112-0.170896$ H -0.4619347 .2931651 .159046$ H $0.256732 \quad 3.6824674 .413291$ H -0.6335052 .9390313 .103397$ H 1.7613392 .3273922 .131710 H 2.3296012 .7419353 .756146 H 0.4272661 .0686464 .561557 H 2.8938210 .6066334 .778997 H 2.9533130 .1238063 .096894 H $3.775246-1.6758744 .967881$ H $1.642346-1.9998092 .350239$ H 2.109143-1.046144 6.500939 H $1.188002-2.4585126 .035902$ H $0.457840-0.8462605 .908330$ H $1.347485-3.7207743 .792825$ H $-0.093428-1.6227200 .838834$ H -1.267117 -2.504376 1.819779 H -1.966855 -0.675044 3.638391 H -5.7483541 .7187311 .724256$ H -4.604256 $1.804174-1.140564$ H -7.264261 2.755750 -1.962742 H -8.006765 $1.248359-2.484621$ H $-6.3290941 .566471-2.893207$ H $-2.846466-0.6244161 .703293$ H -1.620211 0.4162730 .980797 H -3.624241 -3.942562 -1.358443 H $-2.887850-2.679959-2.365180$ H -4.958675 -1.317938 -2.335878 H $-6.048400-3.970245-1.257697$ H -7.949143 -3.218693 -2.674080 H -7.357594 -1.580531-2.734770 H -9.519761 -2.8369130 .700628$ H -8.770586 -4.123350 -0.402423 H $-9.171346-0.5405810 .314461$ H -8.999432 -0.174512 -1.378740 H $-6.741209-0.3399640 .735382$
H $-3.385591-2.6375790 .554508$

C $2.0076783 .147960-2.670981$

O $2.6530861 .988211-2.108018$

C $3.7866462 .428217-1.335326$

C $3.9704003 .910004-1.652726$

C $2.5360904 .345115-1.883394$

H $3.5035932 .335095-0.280403$

C $5.0122191 .558417-1.631687$

H $4.4585824 .451012-0.836343$

H $4.5659434 .060135-2.560836$

C $2.4053545 .672004-2.608436$

H $2.0409364 .420044-0.907372$

C $4.8181950 .055294-1.334273$

O $5.3376591 .691561-3.021187$

H $5.8751261 .945288-1.076086$

H $4.125611-0.375566-2.065129$

O $6.078459-0.590916-1.559640$

C $4.341194-0.2607280 .094298$

C $4.412376-1.7462920 .493064$

H 3.3140910 .0989030 .223615

O $5.1621870 .469221 \quad 1.013022$

H $5.461243-2.0602030 .573712$

H $4.012804-1.8151481 .512921$

H $2.8683336 .475190-2.026087$

H $2.8920775 .644028-3.589029$

H $1.3525735 .929734-2.761332$

C $0.4889452 .961881-2.690524$

H $6.1150171 .132205-3.191426$

H $6.701962-0.258662-0.891062$

H 4.8417050 .2826531 .911179

H $\quad 0.2509502 .049262-3.249219$

H $0.028373 \quad 3.796816-3.231477$

H $2.3523503 .207925-3.712177$

C $1.454738-3.044193-1.396303$

O $2.298594-2.269565-0.531465$

C $3.654279-2.712608-0.427875$

C $3.715007-4.157070 \quad 0.101183$

C $2.845737-5.097959-0.739127$

C $1.445352-4.522008-0.970670$

H $\quad 0.443673-2.649341-1.236713$

C $1.806238-2.843140-2.878379$

H $4.121482-2.675913-1.416857$

O $3.267031-4.1915111 .455500$

H $4.759069-4.4919650 .091554$

H $2.752281-6.065625-0.231112$

O $3.467402-5.327352-1.999785$

H $0.915787-5.115394-1.724481$

O $0.689723-4.6371150 .238979$

C $1.542259-1.427046-3.342135$

H $2.858858-3.059683-3.077199$

H $1.187592-3.505074-3.495094$

H $3.357204-5.1065851 .771839$

H $4.339335-5.722669-1.830011$

H $1.101787-4.0589860 .903896$

O $2.001777-1.291330-4.621560$

O $0.999450-0.556163-2.677553$

C $1.8311120 .002385-5.208141$

H $\quad 0.7838340 .314934-5.157374$

H $2.4779480 .726274-4.705257$

H $2.125801-0.059830-6.259033$

SCF Energy (B3LYP/6-31G**//MMFF) $=-3245.90687643$

08_00591

MM̄FF Geometry

C $-2.767152-3.6911892 .170610$

C $-1.625291-3.0307691 .922622$

C $-0.982250-2.1042522 .920821$

O $-0.919087-0.7686822 .366276$

C $0.460815-2.5421223 .229994$

C $1.088997-1.6922324 .320090$

C $1.935804-0.6644754 .109029$

C $0.694928-2.0646685 .727131$

C $2.459684-0.1545822 .793627$

C 3.9476120 .2068552 .900171

C 4.5962240 .4749711 .534232

C 6.0536250 .9074141 .714870

C $6.722508 \quad 1.2498660 .376268$

O 3.8587561 .4924890 .849301 
$\begin{array}{lllll}\text { O } & 7.942940 & 1.951607 & 0.658451\end{array}$

C $5.8116922 .222731-0.401022$

C $7.117557-0.011115-0.402058$

O $6.3202062 .455627-1.712865$

C $4.3376201 .759991-0.478028$

C $3.4656592 .878464-1.096770$

O $4.2626920 .602919-1.315823$

C $-2.0211320 .015546 \quad 2.509894$

O $-3.126538-0.3336432 .892643$

C $-1.629123 \quad 1.4046232 .178445$

C - $2.559746 \quad 2.3705572 .194739$

C -2.3335873 .8039771 .991605$

C $-0.908457 \quad 4.2780351 .889114$

C $1.9542492 .630711-0.985761$

C $1.1515943 .616977-1.819577$

C $-1.1994454 .142653-2.468660$

O $1.6673124 .499802-2.500294$

C -1.611542 5.342158-1.675891

C -2.884827 5.623001-1.359191

C $-3.3017466 .870629-0.635448$

$\begin{array}{llll}\text { C } & -4.144779 & 6.594270 & 0.587557\end{array}$

C $-5.465194 \quad 6.844942 \quad 0.589427$

C -3.440373 6.1226921 .839342

C $-3.405596 \quad 4.622722 \quad 1.951555$

N $-0.2134413 .382682-1.725308$

H -3.251888 -3.5667693 .136119$

H $-1.139981-3.1425570 .957207$

H -1.560588 -2.079049 3.853589

H $1.061804-2.5034272 .315598$

H $\quad 0.478187-3.592672 \quad 3.548813$

H $2.287258-0.1113204 .979755$

H $1.202769-1.4503866 .478276$

H $-0.381986-1.9391665 .873965$

H $0.954603-3.1091825 .928585$

H $2.326587-0.8889651 .997311$

H 1.8886170 .7382762 .513732

H 4.0390511 .1010843 .531073

H $4.496172-0.6010223 .401530$

H $4.547773-0.4499260 .951573$

H $6.626240 \quad 0.1349542 .243534$

H 6.0909361 .7990452 .356449

H $8.497906 \quad 1.376745 \quad 1.212974$

H $\begin{array}{llll}5.843127 & 3.189970 & 0.120017\end{array}$

H $7.824809-0.6141010 .179952$

H $7.6373410 .244899-1.331908$

H $6.266459-0.649850-0.646345$

H $7.2403212 .755812-1.617819$

H $3.6777853 .831548-0.594756$

H $3.7345203 .001167-2.153730$

H $3.4046040 .582929-1.766831$

H -0.5825391 .5998321 .974985$

H -3.5928282 .0903292 .398311$

H -0.3705884 .0748652 .821723$

H $-0.815518 \quad 5.3474351 .690228$

H -0.3888423 .7698191 .072668$

H $1.699028 \quad 1.623642-1.329834$

H 1.6384262 .7226600 .059608

H -2.040119 $3.473253-2.676264$

H $-0.7652024 .462537-3.421505$

H $-0.8263056 .035381-1.383115$

H -3.674908 $4.946696-1.677401$

H -3.863432 $7.491042-1.346223$

H -2.432089 $7.472375-0.343138$

H -6.0689336 .6797531 .475799$

H $-5.972328 \quad 7.215152-0.295314$

H -3.973378 6.5018382 .722243

H -2.450626 6.5830331 .899133

H -4.3968364 .1768812 .049555$

H $-0.5527512 .684316-1.070480$

C -4.596274 -3.9601860 .415946$

O $-4.060838-2.961825-0.473624$

C $-4.835701-1.757246-0.333675$

C $-6.162534-2.201440 \quad 0.265739$

C $-5.691474-3.271943 \quad 1.236421$

H $-4.312380-1.1326360 .400618$

C $-4.905493-1.004455-1.666369$

H $-6.696215-1.3814350 .755241$

H -6.824026 - $2.642559-0.488621$
C $-6.791638-4.2121111 .696384$

H $-5.270442-2.7695122 .115278$

C $-3.540914-0.882003-2.379614$

O $-5.786766-1.709115-2.549392$

H -5.356467 $-0.017984-1.508469$

H $-3.282601-1.860566-2.789679$

O $-3.710951-0.027954-3.517924$

C $-2.393828-0.340342-1.506637$

C $-1.025071-0.325837-2.204802$

H $-2.296498-0.909940-0.578173$

O $-2.6982491 .008723-1.137506$

H $-1.122752-0.107984-3.275403$

H $-0.4431010 .519222-1.816454$

H $-7.572335-3.6592992 .229034$

H $-7.261592-4.7253680 .850705$

H $-6.392316-4.9733612 .374019$

C $-3.448410-4.610718 \quad 1.195794$

H $-5.810982-1.217193-3.388184$

H $-3.883590 \quad 0.870037-3.186626$

H $-3.4788130 .989640-0.558762$

H $-2.706121-4.9898890 .481678$

H $-3.824241-5.4789571 .749676$

H $-5.041360-4.725921-0.232678$

C $2.123257-2.299010-2.247690$

O $1.128410-1.294439-2.495080$

C $-0.177949-1.594420-1.980338$

C $-0.716542-2.865398-2.649662$

C $0.258075-4.042823-2.521857$

C $1.699292-3.644701-2.858848$

H $3.006985-1.943289-2.792460$

C $2.473493-2.351789-0.754010$

H $-0.096029-1.751005-0.898881$

O $-0.972042-2.619531-4.031183$

H $-1.665781-3.145432-2.183911$

H $-0.058237-4.848688-3.195606$

O $0.217991-4.556431-1.193883$

H $2.397818-4.429320-2.547059$

O $1.817941-3.516277-4.279731$

C $3.824744-2.970900-0.476582$

H $2.491325-1.330920-0.356432$

H $1.719197-2.903844-0.187511$

H $-0.142006-2.315780-4.437287$

H $-0.692461-4.849724-1.019457$

H $1.614489-4.383292-4.670185$

O $3.964098-3.1341990 .872950$

O $4.661033-3.254762-1.322471$

C $5.220138-3.666876 \quad 1.303339$

H $5.204108-3.7343442 .394338$

H $5.367938-4.6692180 .890663$

H $6.035649-3.0006591 .005700$

SCF Energy (B3LYP/6-31G**//MMFF) $=-3245.92292325$

08_00592

MM̄FF Geometry

C $-0.9975670 .673679-3.059421$

C $-1.513112-0.549902-2.856321$

C $-0.791490-1.725797-2.244095$

O $0.216516-1.227961-1.337247$

C $-0.131153-2.573488-3.342090$

C $0.295944-3.947615-2.868824$

C $1.558938-4.294135-2.552191$

C $-0.806380-4.974107-2.810067$

C $2.774057-3.407906-2.516089$

C $3.524277-3.578917-1.188685$

C $4.720605-2.625342-1.062093$

C $5.470556-2.9014470 .242606$

C $6.615288-1.9066660 .471825$

O $4.229706-1.280667-1.069752$

O $7.039784-2.034078 \quad 1.838034$

C $6.073356-0.473490 \quad 0.297499$

C $7.837260-2.233574-0.394911$

O $7.151070 \quad 0.458162 \quad 0.371404$

C $5.250655-0.275574-1.001443$

C $4.559247 \quad 1.107320-0.976863$

O $6.129642-0.338467-2.125356$

C $0.321462-1.804142-0.111361$

O $-0.267552-2.789734 \quad 0.299259$

C $1.273491-0.9878490 .669486$ 
C $1.436313-1.1984671 .983183$

C $2.328118-0.4175732 .837579$

C $3.098256-1.2487023 .824757$

C $3.5531321 .354557-2.109891$

C $2.9638202 .743921-1.956046$

C $1.4307304 .033827-0.469841$

O $3.4094203 .706888-2.575593$

C 2.1455994 .2911790 .820484

C 1.5698324 .1758972 .026756

C 2.3149384 .3239523 .321901

C 2.3689253 .0291164 .107538

C 1.7481602 .9088365 .292939

C 3.2057411 .8933563 .550070

C 2.3866530 .9258772 .736743

N $1.9486352 .791842-1.014539$

H $0.0410790 .874284-2.811521$

H $-2.548891-0.734400-3.134352$

H -1.540849 -2.305892 -1.691502

H $\quad 0.723695-2.032385-3.764625$

H $-0.827674-2.711284-4.180679$

H $1.747040-5.329753-2.270516$

H $-0.443841-5.956670-2.489759$

H -1.582367 -4.664513 -2.103014

H -1.263796 -5.097168 -3.797090

H $3.435016-3.686176-3.345289$

H $2.510592-2.355901-2.652984$

H $2.828714-3.400497-0.359633$

H $3.873092-4.615888-1.099441$

H $5.378944-2.775082-1.925958$

H $5.847351-3.9317890 .264714$

H $4.771332-2.8221131 .086888$

H $7.320961-2.9541361 .980122$

H $5.424305-0.2711241 .161478$

H $8.208397-3.240497-0.168829$

H $8.668579-1.553579-0.178958$

H $7.626588-2.193536-1.465547$

H 6.7723491 .3429290 .504682

H $4.0296241 .221816-0.022569$

H $5.3164031 .900704-1.018838$

H $5.7260440 .130995-2.871701$

H $1.814181-0.2082130 .146440$

H $0.892632-1.9971562 .482782$

H $2.422748-1.6761564 .573671$

H $3.870121-0.6869444 .354960$

H $3.609621-2.0727293 .313737$

H $4.0270801 .297764-3.094928$

H $2.7457340 .614244-2.097081$

H $0.3556413 .903038-0.325436$

H $1.5972364 .858374-1.169293$

H 3.2022254 .5405420 .755408

H 0.5162293 .9166242 .093716

H 1.8217255 .1114423 .905569

H 3.3401874 .6758223 .151258

H 1.8060051 .9899205 .867314

H 1.1665173 .7226065 .712773

H $3.720217 \quad 1.396114 \quad 4.378186$

H 4.0007162 .3023352 .914161

H $\quad 1.770121 \quad 1.3989581 .974137$

H $1.6871181 .936035-0.535129$

C -1.727772 $3.053480-2.692526$

O $-2.0183092 .681584-1.332267$

C $-3.0990653 .488322-0.842876$

C $-3.0928054 .730886-1.721785$

C $-2.7473424 .132130-3.073255$

H -4.018055 2.918963-1.028559

C -2.9662913 .7277550 .665681$

H -4.054188 $5.252940-1.715557$

H -2.313619 5.440392 -1.421009

C $-2.2191505 .143784-4.075414$

H $-3.6533543 .668600-3.486226$

C -2.5535222 .4844221 .481663$

O $-1.9714344 .733103 \quad 0.895324$

H -3.9063874 .1453291 .045949$

H -1.488751 2.2782091 .312948

O -2.6806932 .8426842 .863731$

C $-3.377140 \quad 1.214061 \quad 1.197643$

C -2.988400 0.0402722 .117642

H -3.2228570 .9094060 .157781$
O $\quad-4.765913 \quad 1.502557 \quad 1.348304$

H -1.896620 -0.0033882 .211771$

H $-3.4148470 .216317 \quad 3.112801$

H -2.969960 $5.915012-4.276154$

H -1.316462 $5.641436-3.705333$

H $-1.9707914 .656831-5.023867$

C -1.802570 $1.821670-3.601915$

H -1.9230904 .8793051 .855758$

H -2.204004 2.1770503 .387642

H -4.9065451 .8077722 .261002$

H $-1.4345452 .080171-4.601281$

H -2.848167 $1.510803-3.715220$

H $-0.7077913 .456716-2.695737$

C $-5.539786-2.4788831 .022520$

O $-4.915996-1.3003511 .551425$

C $-3.487064-1.3198771 .590502$

C $-2.997792-2.4824032 .468687$

C $-3.582860-3.8183991 .998518$

C $-5.099095-3.7333651 .795710$

H $-6.611670-2.3320901 .209336$

C $-5.340369-2.597559-0.492916$

H -3.098532 -1.444252 0.573795

O $-3.355353-2.2613423 .832153$

H -1.904005 -2.521817 2.432340

H -3.360161 -4.594373 2.740825

O $-2.978657-4.2061540 .768158$

H -5.470227 -4.627606 1.281234

O $-5.737611-3.6914093 .075335$

C $-6.080118-1.492882-1.208427$

H $-4.289809-2.571606-0.795951$

H $-5.744497-3.545808-0.866835$

H $-4.320027-2.144623 \quad 3.871347$

H $-2.018127-4.2536240 .912674$

H $-5.504432-4.5098473 .545813$

O $-5.212610-0.507345-1.575115$

O $-7.290272-1.496455-1.395287$

C $-5.8035960 .608881-2.246758$

H $-5.000818 \quad 1.272037-2.576414$

H $-6.3632170 .277199-3.126828$

H $-6.4530381 .157851-1.558663$

SCF Energy $\left(B 3 L Y P / 6-31 G^{* *} / / M M F F\right)=-3245.90991121$

08_00593

MM̄FF Geometry

C $0.4138354 .153229-1.377058$

C $-0.2229583 .038141-0.989901$

C -1.156176 2.258740 -1.876162

O $-2.4193722 .177089-1.173869$

C $-0.6167420 .833452-2.126018$

C $-0.1506490 .621937-3.551618$

C $-0.782926-0.146563-4.460573$

C $1.1422301 .307053-3.914018$

C $-2.063345-0.919644-4.297189$

C $-1.852037-2.436432-4.392517$

C -1.014839-3.013769-3.240596

C $-0.781212-4.510912-3.447330$

C $-0.003499-5.130524-2.277942$

O $-1.721456-2.796788-2.013539$

O $-0.100096-6.558604-2.394742$

C $-0.691531-4.735134-0.954873$

C $1.491176-4.791123-2.340451$

O $0.099264-5.1800420 .145984$

C $-1.005758-3.220243-0.844058$

C $-1.904187-2.9583460 .389170$

O $0.219507-2.501678-0.693335$

C $-3.5282541 .944152-1.926193$

O $-3.5880411 .827294-3.139613$

C $-4.6757401 .820992-1.006519$

C $-5.7301941 .085239-1.383872$

C $-6.9086450 .853121-0.559822$

C $-7.274530-0.599377-0.432202$

C $-2.464043-1.5325890 .487635$

C $-3.204777-1.3116601 .796647$

C -4.2067620 .5102713 .171653$

O $-3.443893-2.2136532 .595213$

C $-5.6930080 .398714 \quad 3.040344$

C $-6.529672 \quad 1.431046 \quad 3.227375$

C $-8.0264331 .314128 \quad 3.185674$ 
C $-8.664323 \quad 2.280706 \quad 2.214357$ C -9.1209273 .4755582 .626230$ C $-8.857477 \quad 1.8205950 .788241$ C $-7.5912371 .880516-0.017342$ N -3.5658550 .0172601 .967830$ H $0.250854 \quad 4.543470-2.378633$ H -0.0557612 .6504350 .012615$ H -1.316340 $2.801235-2.817252$ H $-1.376067 \quad 0.094847-1.855475$ H $0.2274620 .607353-1.459851$ H $-0.339235-0.233210-5.451966$ H $1.4910571 .034098-4.915655$ H $1.0217772 .394103-3.893293$ H $1.9302861 .028731-3.207001$ H -2.585421 -0.678865 -3.368084 H $-2.739822-0.612043-5.104548$ H $-2.842830-2.908940-4.389470$ H -1.380462 -2.680188 -5.352864 H $-0.054287-2.488782-3.203046$ H $-0.260523-4.697535-4.394907$ H - $-1.749704-5.023972-3.531197$ H $0.270244-6.814733-3.256831$ H -1.636301 -5.295000 -0.907972 H $1.927152-5.153884-3.278993$ H $2.044725-5.294631-1.540063$ H $1.691443-3.719502-2.277314$ H $-0.444637-5.1211050 .948677$ H -2.759310 -3.646719 0.366586 H -1.339258 -3.1715891 .304667$ H $\quad 0.061052-1.690784-0.186305$ H -4.618595 2.304036 -0.037331 H $-5.7298020 .573856-2.344314$ H $-7.643601-0.986283-1.388117$ H $-8.040150-0.7868820 .323965$ H -6.398834 -1.190065 -0.138000 H -1.656730 -0.7955320 .435642$ H -3.161562 -1.340565 -0.335391 H $-3.879547 \quad 1.5451403 .314288$ H -3.868532 -0.0827524 .027778$ H $-6.102129-0.5858502 .826081$ H $-6.1193442 .408093 \quad 3.472532$ H -8.402748 1.5068844 .199220 H -8.3424090 .2910692 .947099$ H -9.6083084 .1616691 .941441$ H -9.012134 3.7958993 .657138 H -9.5931322 .4607420 .282765$ H -9.3111970 .8250080 .788551$ H $-7.2323972 .894479-0.197679$ H $-3.349300 \quad 0.6881841 .237353$ C $2.7900604 .875964-1.080055$ O $3.3001733 .526633-1.036278$ C $4.6434873 .554043-0.522430$ C $5.0850795 .010665-0.580623$ C $3.7817785 .724655-0.283364$ H 4.5843553 .2491850 .528239 C $5.5400402 .579945-1.291980$ H 5.8742005 .2350910 .143390 H $5.4495805 .286428-1.576991$ C $3.7907127 .193072-0.671210$ H 3.5722405 .6391110 .791568 C $5.0276861 .125868-1.344601$ O $5.6782583 .031376-2.645822$ H $6.5500952 .602014-0.866334$ H $4.1725881 .050155-2.026954$ O $\quad 6.067616 \quad 0.323807-1.920696$ C 4.6388670 .5219740 .017313 C $4.354607-0.990915-0.076190$ H 3.7512121 .0405720 .396080 O $5.704444 \quad 0.7512380 .938972$ H $3.751922-1.197150-0.968932$ H $5.311976-1.517235-0.176490$ H $4.5632927 .733039-0.114110$ H $3.9927407 .324839-1.739519$ H $2.8261867 .661034-0.450683$ C $1.3738564 .909968-0.503782$ H $4.7855063 .089430-3.028129$ H $6.2909830 .714440-2.782940$ H 5.4245720 .4069551 .803911
H $1.0214785 .944500-0.424091$

H 1.3749164 .4859340 .507951

H $2.7842365 .178325-2.135483$

C $3.818753-1.7009993 .564902$

O $4.408937-1.3150512 .316729$

C $3.606087-1.5409061 .155373$

C $3.293894-3.0391651 .013510$

C $2.640415-3.5900852 .284676$

C $3.414405-3.1844693 .544485$

H $4.623572-1.5827964 .301949$

C $2.673673-0.7647613 .967435$

H $2.669479-0.9797341 .248270$

O $4.499199-3.755006 \quad 0.749475$

H $2.632360-3.1841820 .153573$

H $2.593689-4.6839862 .220130$

O $1.305194-3.0999582 .357521$

H $2.826645-3.4143204 .440300$

O $4.603867-3.975453 \quad 3.628145$

C 3.1847970 .6413764 .175868

H $1.865328-0.7406053 .231111$

H $2.219422-1.0868964 .911765$

H $4.262058-4.6889810 .618279$

H $0.892359-3.4895413 .146926$

H $\quad 5.173019-3.7372442 .876298$

O $2.767712 \quad 1.4461603 .157293$

O 3.8870660 .9777305 .120640

C 3.2001302 .8077833 .232208

H 2.7627363 .3493982 .389730

H 2.8530873 .2680894 .162119

H 4.2903582 .8609173 .157140

SCF Energy $\left(B 3 L Y P / 6-31 G^{* *} / / M M F F\right)=-3245.90751279$

08_00594

MMFF Geometry

C $-3.193697 \quad 3.8473820 .356252$

C -3.6267712 .6372660 .738806$

C -3.9738392 .2812382 .160468$

O -3.2111251 .1045452 .533378$

C -5.4548961 .8917202 .318945$

C $-6.4540862 .948671 \quad 1.897904$

C -7.1738622 .8915410 .758760$

C -6.6461324 .0807192 .870225$

C $-7.047496 \quad 1.852274-0.319231$

C $-8.1249890 .765348-0.221619$

C $-7.704096-0.490117-1.000443$

C $-8.848329-1.498616-1.092330$

C $-8.397187-2.805251-1.765901$

O $-6.604560-1.073242-0.290985$

O $-9.426263-3.786588-1.568597$

C $-7.108431-3.311022-1.079871$

C $-8.278350-2.644306-3.285286$

O $-6.572129-4.438325-1.768691$

C $-6.021465-2.220828-0.919558$

C $-4.890598-2.7410670 .005358$

O $-5.477937-1.901824-2.199179$

C -1.8836461 .2531012 .785762$

O -1.2473142 .2934292 .812440$

C -1.325414 -0.1030452 .981876$

C $-0.013654-0.3140962 .800886$

C $0.679008-1.6000622 .923695$

C $-0.142435-2.7996113 .319241$

C $-3.842507-1.6888820 .394662$

C $-2.643198-1.673884-0.531714$

C $-0.610470-2.973855-1.126535$

O $-2.511172-0.816843-1.402346$

C $0.429632-3.700986-0.334584$

C $1.715123-3.321625-0.277835$

C $2.774224-4.0614640 .487898$

C $3.557705-3.1689601 .424837$

C $4.800886-2.7630681 .115722$

C $2.937574-2.8230612 .760407$

C $2.005184-1.6447112 .677356$

N $-1.759807-2.712237-0.280733$

H -3.0944934 .6376651 .095527$

H $-3.715718 \quad 1.847654-0.003515$

H -3.7322563 .0941512 .856800$

H $-5.637274 \quad 1.6357683 .372362$

H $\quad-5.6412090 .943625 \quad 1.798877$ 
H -7.898946 3.6777920 .557074 H -7.4054684 .7937662 .532265$ H -6.9645703 .6954063 .844247$ H -5.7107004 .6334253 .003010$ H -7.124168 2.351225 -1.293631 H $-6.0491591 .406501-0.307715$ H $-8.284847 \quad 0.4974750 .830928$ H $-9.0713491 .159150-0.611306$ H -7.374748 -0.188820 -2.001864 H $-9.707272-1.069254-1.622888$ H -9.207936 -1.732057 -0.080406 H $-9.555813-3.903387-0.612071$ H $-7.388226-3.665777-0.078150$ H -9.244922 -2.353928 -3.714438 H -8.012254 -3.593337 -3.764151 H -7.544841-1.891964 -3.582256 H $-7.283060-5.097634-1.843828$ H $-5.350696-3.0836810 .941707$ H -4.400185 -3.605194 -0.457950 H -4.640290 -1.429576 -2.071783 H -2.018286 -0.8961023 .238443$ H 0.6133650 .5323632 .523921 H $-0.554646-2.6652294 .325295$ H $0.421711-3.7341023 .326850$ H $-0.971400-2.9503842 .619720$ H $-4.261616-0.6797110 .440507$ H -3.474334 -1.911285 1.404144 H $-0.239176-2.030831-1.541372$ H -0.954704 -3.600815 -1.955396 H $\quad 0.115533-4.5927490 .202980$ H $2.032809-2.449093-0.842161$ H $3.451499-4.520437-0.244391$ H $2.346102-4.8954011 .058081$ H $5.377395-2.1439361 .794928$ H $5.265785-3.0305830 .172688$ H $3.726363-2.5594813 .478223$ H $2.468602-3.7158113 .182225$ H $2.509973-0.7184542 .401299$ H -2.056599 -3.4491080 .353146$ C $-1.3271754 .214367-1.331580$ O $-0.7650832 .903054-1.122808$ C $0.4955143 .047746-0.432906$ C $0.8661314 .518358-0.555000$ C $-0.4979545 .177214-0.482510$ H $\quad 0.2941132 .7925990 .614514$ C $1.5079692 .064068-1.028168$ H 1.5428084 .8502650 .237957 H $1.3410164 .722358-1.522841$ C $-0.5141066 .610376-0.983529$ H $-0.826656 \quad 5.1632860 .563575$ C $2.9308632 .227714-0.457972$ O $1.0330630 .741989-0.753959$ H $1.5351822 .167806-2.119511$ H $3.2810623 .243840-0.672087$ O $2.8880442 .099596 \quad 0.963423$ C $3.9663831 .240632-1.037952$ C $5.3721751 .495256-0.474667$ H $3.9785991 .346461-2.127958$ O $3.613291-0.105009-0.734526$ H $5.6697132 .535550-0.657827$ H 5.3546891 .3838630 .618231 H $0.1483717 .237045-0.377546$ H $-0.1790516 .676357-2.024024$ H -1.523281 $7.030423-0.926297$ C $-2.8340904 .186364-1.063284$ H $0.1297430 .676996-1.109105$ H 2.5850631 .1971721 .160924 H $2.749120-0.280275-1.143042$ H -3.295143 $3.452445-1.737048$ H -3.275685 5.158643 -1.310477 H $-1.1781304 .437287-2.396435$ C $8.8060770 .063916-0.597547$ O $7.6651750 .885384-0.316938$ C $6.4565050 .536538-1.005254$ C $6.6683890 .615723-2.524508$ C $7.874353-0.219233-2.970139$ C $9.1099230 .048831-2.104785$ H $9.6430970 .565251-0.094332$
C $8.668791-1.3407530 .011080$

H $6.176551-0.483487-0.723998$

O $6.8563441 .971253-2.926786$

H $5.770790 \quad 0.251327-3.035384$

H $8.1102410 .012984-4.015903$

O $7.552626-1.605120-2.895447$

H $9.892897-0.690239-2.311184$

O $9.6438301 .332184-2.444105$

C $8.598040-1.3102361 .523919$

H $7.769296-1.849399-0.344447$

H $9.543928-1.941804-0.261916$

H $7.6133692 .326393-2.430056$

H $6.787182-1.761225-3.474488$

H $9.8897411 .307580-3.384576$

O $8.424193-2.5861431 .981859$

O $8.684501-0.3068892 .218032$

C $8.322594-2.7214843 .402918$

H $7.465266-2.1539813 .777646$

H $8.170146-3.7792013 .633313$

H $9.247735-2.3869743 .881741$

SCF Energy (B3LYP/6-31G**//MMFF) $=-3245.92225688$

08_00595

MMFF Geometry

C $0.5946303 .995776-0.272547$

C 1.8448323 .5952350 .003950

C $2.9775433 .622837-0.991627$

O $3.4140312 .254789-1.172186$

C $4.1470134 .452556-0.435711$

C $5.2621014 .666389-1.441915$

C $6.4644534 .058194-1.406119$

C $4.9655695 .673387-2.524684$

C $6.9559703 .042439-0.414222$

C $7.1621531 .680018-1.085619$

C $7.4876410 .580144-0.065089$

C $7.754290-0.743950-0.782968$

C $7.955023-1.9055620 .198622$

O 6.3596790 .4548970 .804262

O $7.901365-3.112206-0.577686$

C $6.776458-1.9205371 .194728$

C $9.342663-1.8636860 .848611$

O $6.989873-2.8850572 .224111$

C $6.488311-0.5370561 .828311$

C $5.163598-0.5522742 .633887$

O $7.548864-0.2140392 .732945$

C $2.8453751 .563810-2.198409$

$\begin{array}{llll}\text { O } & 2.134776 & 2.013240 & -3.084344\end{array}$

C $3.1579410 .129261-2.014337$

C $2.280578-0.769819-2.485840$

C $2.346222-2.225792-2.349664$

C $3.598377-2.829602-1.774301$

C $3.911511-0.5174811 .752139$

C $2.689759-1.1474762 .392372$

C $0.383506-1.8791231 .778039$

O $2.673558-1.585994 \quad 3.538327$

C $0.444547-3.207127 \quad 1.090224$

C $-0.394081-3.5778750 .111004$

C $-0.343726-4.918398-0.563507$

C $-0.248514-4.818340-2.069988$

C $-1.299707-5.124550-2.849453$

C $1.088859-4.445018-2.677529$

C $1.283440-2.953226-2.754762$

N $1.618724-1.1704041 .507146$

H $0.3612614 .377456-1.263218$

H 2.0710623 .2070470 .994736

H $2.6435944 .051125-1.944181$

H 4.5267693 .9801930 .475655

H $3.7846375 .440513-0.119853$

H $7.1887354 .308918-2.180575$

H $5.8296745 .853882-3.173087$

H $4.1455755 .327567-3.161137$

H $4.6829386 .634392-2.082401$

H $7.9093003 .393518-0.001116$

H 6.2775972 .9434510 .435506

H $6.2463521 .408640-1.626753$

H $7.9692901 .753556-1.825193$

H $8.361970 \quad 0.887551 \quad 0.520134$

H $8.617624-0.655543-1.454463$ 
H $6.898192-0.984205-1.429879$

H $8.082917-3.8611220 .014722$

H $5.890996-2.2541290 .640467$

H $\quad 10.123921-1.945708 \quad 0.083327$

H $9.491387-2.7156491 .521202$

H $9.524946-0.9461611 .411980$

H $7.059231-3.7575941 .801899$

H $5.161821-1.4106973 .316103$

H $5.122596 \quad 0.3287203 .288937$

H $7.2275590 .454648 \quad 3.359877$

H $4.046129-0.137182-1.455436$

H $1.392885-0.399360-2.998751$

H $3.626419-2.688716-0.690681$

H $4.494096-2.375772-2.213053$

H $3.685467-3.901054-1.970815$

H 3.6706510 .5218371 .497199

H $4.090661-1.0532620 .817395$

H -0.437264 -1.260836 1.406196

H $0.256277-2.0199492 .855756$

H $1.212713-3.9005241 .424881$

H $-1.173108-2.890561-0.210173$

H $-1.252057-5.465298-0.278516$

H $0.494307-5.521077-0.191586$

H $-1.231280-5.081442-3.931435$

H -2.253369 -5.418261-2.423694

H $1.155076-4.841136-3.699336$

H $1.878740-4.948051-2.112334$

H $0.435584-2.424856-3.193967$

H $1.751710-0.8101010 .566641$

C $-1.688827 \quad 3.0415910 .311132$

O $-2.750825 \quad 3.225577 \quad 1.275246$

C -3.1526531 .9389231 .784692$

C $-2.6775280 .937150 \quad 0.750785$

C $-1.362006 \quad 1.544497 \quad 0.308000$

H -2.617231 1.8054302 .733265

C -4.6679061 .9361542 .014617$

H -2.564968 -0.0703381 .159643$

H -3.378741 $0.889481-0.091887$

C $-0.9068170 .996415-1.034574$

H $-0.598172 \quad 1.324623 \quad 1.065217$

C $-5.223598 \quad 0.5605292 .421857$

$\begin{array}{llll}\text { O } & -4.966871 & 2.873519 & 3.053441\end{array}$

H -5.167844 2.301336 1.111441

H $-5.007391-0.1811541 .649806$

$\begin{array}{lllll}\text { O } & -4.523242 & 0.105009 & 3.588574\end{array}$

$\begin{array}{llll}\text { C } & -6.728992 & 0.537952 & 2.741399\end{array}$

C $-7.666724 \quad 1.0875331 .651697$

H -6.9125191 .0987123 .666091$

$\begin{array}{llll}O & -7.117392 & -0.812547 & 3.017459\end{array}$

H -8.683778 1.0836572 .066243

H -7.406899 2.133263 1.454063

H $0.044103 \quad 1.436486-1.337780$

H -0.771323 $-0.088985-0.981733$

H $-1.638803 \quad 1.204197-1.822423$

C -0.5286553 .9563310 .719559$

H -4.6017603 .7330522 .781333$

H $-4.6501320 .772374 \quad 4.284894$

H -6.523377 -1.149567 3.709928

H $-0.1688643 .666724 \quad 1.715693$

H $-0.910592 \quad 4.9779700 .844228$

H $-2.0932383 .357471-0.658672$

C $-6.285919-0.307983-1.549125$

O $-6.437736 \quad 0.411356-0.318389$

$\begin{array}{llll}\text { C } & -7.703346 & 0.284477 & 0.332679\end{array}$

C -8.826238 $0.786299-0.592586$

C $-8.7872460 .081866-1.952805$

C -7.378903 $0.088680-2.555755$

H $-5.3232700 .032156-1.952311$

C $-6.193679-1.822595-1.322148$

H $-7.881664-0.7680680 .575004$

O $-8.693587 \quad 2.194160-0.781807$

H $-9.7916480 .609674-0.103160$

H $-9.4760780 .581692-2.644653$

O $-9.222853-1.266555-1.809152$

H -7.330244 -0.575649 -3.426486

O $-7.075007 \quad 1.407772-3.018637$

C $-4.927290-2.187819-0.581193$

H $-7.052170-2.211578-0.767687$
H -6.166172 -2.360214 -2.277178

H $-9.4323182 .486163-1.342327$

H $-10.126202-1.247184-1.449780$

H $-7.7360581 .641461-3.692406$

O $-5.236806-2.8145090 .589763$

O $-3.799919-1.950198-0.995296$

C $-4.121364-3.227734 \quad 1.385427$

H $-4.505550-3.6178502 .331629$

H $-3.572931-4.0233740 .873006$

H -3.463956-2.380134 1.599406

SCF Energy (B3LYP/6-31G**//MMFF) $=-3245.90765078$

08_00596

MMFF Geometry

C $0.118447 \quad 4.6992840 .931411$

C $1.2949504 .064461 \quad 1.043057$

C $2.394968 \quad 4.1421320 .018286$

O $2.6785592 .796008-0.431385$

C 3.6710594 .7053970 .661896

C $4.7989464 .884994-0.333666$

C $5.8367754 .036673-0.471572$

C $4.7260116 .136851-1.168938$

C 6.0675012 .7445340 .264339

C $6.5168921 .630674-0.692282$

$\begin{array}{llll}\text { C } & 6.812850 & 0.325808 & 0.060224\end{array}$

C $7.493038-0.686080-0.862086$

C $7.780845-2.003732-0.129577$

O $5.576934-0.2190690 .534972$

O $8.121606-2.992194-1.114498$

C $6.487528-2.486328 \quad 0.559830$

C $8.997273-1.8834650 .796250$

O $6.744672-3.6052891 .405066$

C $5.731636-1.3882171 .352697$

C $4.326084-1.9068081 .723605$

O $6.378831-1.0784282 .588847$

C $1.9006942 .291077-1.425883$

O $0.9916842 .857191-2.011197$

C $2.3364820 .898350-1.666383$

C $1.5098420 .052855-2.299118$

C $1.773062-1.358643-2.586966$

C $3.182971-1.852201-2.398851$

C $3.410478-0.8521432 .355615$

C $2.119015-1.4985512 .820885$

C $0.057128-2.6056011 .969618$

O $1.871495-1.6594974 .013895$

C $-0.241363-3.5918420 .876670$

C $0.537931-3.926410-0.164941$

C $0.171150-4.959877-1.192474$

C $-0.265295-4.371345-2.516339$

C $-1.502754-4.594509-2.991207$

C $0.750145-3.600454-3.335697$

C $0.749049-2.129995-3.008670$

N $1.313247-1.9072151 .769297$

H -0.0679625 .3346580 .069998$

H 1.4712253 .4205781 .902176

H $2.1017914 .765103-0.835999$

H 3.9933344 .0565291 .485218

H 3.4590525 .6777991 .126621

H $6.6135604 .295926-1.190033$

H $5.5831186 .237944-1.843187$

H $3.8217876 .135396-1.785451$

H $4.7073837 .022289-0.525153$

H 6.8508372 .9154721 .012614

H 5.1738682 .4137690 .800566

H $5.7319211 .459869-1.440047$

H $7.4179921 .956908-1.226956$

H 7.4586400 .5589210 .914909

H $8.418761-0.273840-1.282472$

H $6.839468-0.897278-1.720272$

H $8.887347-2.664251-1.616539$

H $5.820084-2.847918-0.235438$

H $9.895335-1.6353010 .217569$

H $9.215004-2.8366191 .290428$

H $8.879939-1.1139091 .562069$

H $7.185910-4.2822770 .863821$

H $3.832111-2.2944410 .825668$

H $4.417930-2.7497902 .421450$

H $7.190492-1.6047462 .658867$ 
H $3.3073650 .600071-1.288750$ H $0.5318380 .418435-2.610358$ H $3.883044-1.246167-2.985225$ H $3.331369-2.886323-2.714641$ H $3.472814-1.794629-1.346556$ H $3.889697-0.385303 \quad 3.223100$ H $3.172389-0.0532421 .644076$ H $-0.732093-1.8490012 .013897$ H $\quad 0.077415-3.1243422 .933849$ H -1.208015 -4.084399 0.968632 H $1.515975-3.469670-0.282330$ H $-0.609798-5.618969-0.790824$ H $1.039961-5.609103-1.359689$ H -1.817858 -4.199894 -3.951752 H -2.231629 -5.167599-2.427898 H $0.512864-3.698245-4.403489$ H $1.730030-4.069853-3.216787$ H $-0.227391-1.659855-3.135290$ H $1.507019-1.5319940 .845001$ C -2.1278333 .6447861 .464526$ O -1.5992332 .3496341 .110797$ C $-2.1582291 .959204-0.162197$ C $-3.3982102 .819395-0.335058$ C -2.926752 4.1343010 .255811 H -1.404848 $2.219630-0.915129$ C $-2.3950690 .448592-0.151893$ H -3.707148 $2.910753-1.379919$ H -4.2386392 .4148990 .242791$ C -4.0584975 .0829670 .610266$ H -2.271057 $4.624927-0.473792$ C $-3.184466-0.084813-1.358895$ O $-1.115853-0.193017-0.128129$ H $-2.896600 \quad 0.1753300 .782237$ H $-4.1788040 .367657-1.404558$ O $-2.5178220 .297378-2.570692$ C $-3.314180-1.617886-1.400015$ C $-3.864360-2.302622-0.138012$ H -2.330901 -2.050748 -1.616323 O $-4.153882-1.979480-2.501533$ H -3.798080 -3.386716 -0.301809 H $-3.207644-2.0687310 .706797$ H -4.624677 $5.357489-0.285650$ H -4.7565324 .6282971 .321395$ H -3.669058 6.0019421 .059435 C -0.9906214 .5555471 .937655$ H $-0.617496 \quad 0.1772660 .620655$ H -2.589177 $1.262165-2.659614$ H -3.778634 -1.567298 -3.298556 H -0.5795204 .1396022 .866831$ H -1.379138 5.5496992 .185633 H -2.8044493 .4744162 .312198$ C $-6.724825-0.1223060 .941635$ O $-5.420013-0.6016490 .591878$ C $-5.331001-1.9787260 .219540$ C $-5.827701-2.8775671 .367269$ C $-7.228814-2.4728101 .832789$ C -7.330774 -0.963979 2.076603 H $-6.5522940 .887781 \quad 1.334093$ C -7.646374 -0.011158 -0.279278 H -5.949186 -2.153035 -0.666931 O $\quad-4.920617-2.7905362 .465687$ H -5.831038 -3.919154 1.023676 H -7.469447 -3.008792 2.759076 O $\quad-8.169504-2.8727880 .840948$ H $-8.377031-0.6754442 .227110$ O $-6.645998-0.6429903 .291078$ C -7.202513 $1.086494-1.216749$ H $-7.689427-0.934585-0.863377$ H -8.6765720 .2123380 .023562$ H $-5.233822-3.4020943 .153772$ H $-9.054991-2.6321801 .162488$ H -5.699773 -0.8233503 .155895$ O $-7.4862012 .306097-0.676594$ O $\quad-6.6808690 .882155-2.305234$ C $-7.1487803 .435696-1.487714$ H $-6.1022003 .390666-1.801378$ H -7.809383 $3.477582-2.358488$ H $-7.2954254 .339270-0.889924$
SCF Energy (B3LYP/6-31G**//MMFF)= -3245.91474951

08_00597

MMFF Geometry

C $1.9672414 .182920-3.030358$

C $1.8700493 .739069-1.767511$

C $2.8023204 .088288-0.640421$

O $3.2594382 .844266-0.058434$

C 2.0276114 .8726030 .438173

C 2.7492584 .9528081 .768638

C 2.5665594 .0742992 .776603

C 3.7123326 .0991501 .910425

C 1.6841092 .8547232 .755473

C 0.6738152 .8399013 .908771

C -0.4932501 .8787103 .629530$

C -1.3772681 .7054334 .865100$

C $-2.5118970 .695549 \quad 4.625787$

O $0.0317330 .610067 \quad 3.227235$

O $\quad-3.049792 \quad 0.369175 \quad 5.916285$

C -1.923427 -0.5944994 .008420$

C $-3.658642 \quad 1.3214643 .822201$

O $-2.958199-1.4891903 .604911$

C $-0.973576-0.3264762 .817679$

C $-0.271006-1.6330202 .382419$

O

C $4.3407352 .235864-0.611267$

O $4.9581832 .587602-1.602829$

C 4.6554161 .0595530 .232248

C $5.6908690 .268675-0.084551$

C $6.152503-0.8983830 .672319$

C $5.420990-1.2413631 .943852$

C $0.887631-1.4207811 .394901$

C $1.401065-2.7345980 .831583$

C $2.991962-3.627973-0.866352$

O $0.974392-3.8304901 .185768$

C $4.184518-4.140865-0.122667$

C $5.391017-4.312007-0.684360$

C $6.574892-4.8778520 .046163$

C $7.808832-4.011815-0.068784$

C $8.830066-4.365000-0.867621$

C $7.896720-2.7845350 .810483$

C $7.210880-1.5910400 .201412$

N $2.387416-2.536514-0.125394$

H $2.7784084 .863648-3.279643$

H $1.0697663 .058086-1.492485$

H $3.6627844 .678852-0.978746$

H 1.0332464 .4345730 .589821

H $1.8205085 .887500 \quad 0.072393$

H 3.1349474 .2128933 .694869

H 4.2136716 .1027322 .884134

H 4.4872236 .0422451 .139274

H 3.1853347 .0529761 .805176

H 1.1524902 .7549061 .805471

H 2.3288101 .9703102 .834483

H 1.2008282 .5341104 .821840

H 0.2713903 .8447174 .088350

H -1.074667 2.3020782 .801999

H -1.783236 2.6704705 .193005

H -0.7625501 .3406295 .700266$

H -3.804078 -0.2300565 .786834$

H -1.354234 -1.107335 4.796201

H -4.082732 2.1746584 .365100

H $-4.4833150 .612477 \quad 3.689568$

H -3.3482191 .6766362 .837671$

H -3.470814 -1.724012 4.396415

H $0.140001-2.1408873 .264763$

H -1.009358 -2.309210 1.932366

H -1.281339 -0.0246350 .889705$

H $4.0367120 .888715 \quad 1.106038$

H $6.2677620 .503267-0.978271$

H $5.534213-0.4366952 .678718$

H $5.772609-2.1601352 .417171$

H $4.353553-1.3884141 .750660$

H $0.573115-0.7982850 .550872$

H $1.720605-0.9162791 .897957$

H $3.253876-3.243677-1.857244$

H $2.257896-4.431671-0.984771$

H $4.041710-4.4142850 .920167$ 
H $5.527519-4.068735-1.735681$

H $6.775267-5.873322-0.371297$

H $\quad 6.348755-5.041778 \quad 1.107218$

H $9.730771-3.763324-0.932112$

H $8.786425-5.260221-1.479095$

H $8.949904-2.5097790 .959594$

H $7.531741-3.0299681 .811302$

H $7.674398-1.255292-0.727643$

H $2.721965-1.594937-0.308596$

C $-0.0408402 .799103-3.857902$

O $0.5699421 .512835-3.641278$

C $-0.3899020 .485692-3.985305$

C $-1.6368861 .219505-4.466198$

C -1.063152 $2.540038-4.956258$

H $0.058341-0.077917-4.812851$

C $-0.553282-0.414778-2.754451$

H -2.170676 $0.667057-5.245392$

H -2.335276 $1.394731-3.638709$

C $-2.1092153 .629660-5.109254$

H $-0.5564282 .376494-5.916398$

C $-1.768417-1.359172-2.790787$

O $0.651274-1.177205-2.627633$

H $-0.6168350 .201882-1.850379$

H $-2.668367-0.739124-2.813113$

O $-1.762800-2.121408-3.995723$

C $-1.839400-2.304873-1.574776$

C $-3.154845-3.094893-1.465974$

H -1.687194 -1.724029 -0.659091

O $-0.773693-3.254839-1.653263$

H -3.289158 -3.723450 -2.355324

H $-3.038663-3.792478-0.625783$

H -1.654044 $4.563527-5.453064$

H -2.866712 $3.334475-5.842651$

H $-2.6197203 .832435-4.161653$

C $1.0440623 .829654-4.177285$

H $1.389948-0.547364-2.567529$

H -0.964806 -2.677781 -3.982007

H $-0.790656-3.785655-0.838703$

H $\quad 0.5802604 .760172-4.523409$

H $1.6714413 .450435-4.993507$

H $-0.5705423 .081180-2.939372$

C $-5.235198-0.5028560 .263239$

O $-4.175461-1.403991-0.087870$

C $-4.405587-2.227738-1.237017$

C $-5.667429-3.086879-1.051386$

C $-6.878353-2.223377-0.685565$

C $-6.560419-1.2609290 .461188$

H $-4.938786-0.0972181 .238435$

C $-5.3461080 .665611-0.726318$

H -4.546691 -1.591219-2.114899

O $-5.463775-4.069421-0.037604$

H -5.864991 -3.628537 -1.983711

H -7.713104 -2.870588 -0.389391

O $-7.288406-1.466881-1.820931$

H $-7.383121-0.5480280 .591673$

O $-6.474312-2.0223201 .669162$

C $-4.4350001 .801075-0.324312$

H $-5.1201470 .398893-1.762016$

H $-6.3612221 .081483-0.724446$

H -5.216221 -3.6060130 .780764$

H -7.518740 -2.096561 -2.525211

H -6.334373 -1.397768 2.401224

O $-3.1619541 .563426-0.748051$

O -4.8162662 .7830330 .300613$

C $-2.2103302 .584852-0.429108$

H -2.359786 $3.443972-1.089062$

H -2.291052 2.8874240 .619323

$\mathrm{H}-1.2082162 .181838-0.593130$

SCF Energy (B3LYP/6-31G**//MMFF) $=-3245.90480472$

0800598

MM̄FF Geometry

C $1.771267-3.6637831 .340749$

C $0.570848-3.7807391 .926921$

C $-0.181631-2.6998012 .667302$

O $-1.261709-2.2624021 .805568$

C $0.646632-1.4537793 .040777$

C $-0.088530-0.5219153 .984512$
C $-0.6850080 .629616 \quad 3.620001$

C $-0.087361-0.9426515 .432421$

C -0.8041121 .2184072 .242268$

C -0.0613452 .5585672 .148457$

C -0.2187753 .2081830 .766188$

C 0.5351824 .5372050 .696023

C $0.3051425 .259613-0.641324$

O -1.6119323 .4329850 .539934$

O $\quad 0.7586516 .614075-0.494452$

C $-1.2113935 .319414-0.928268$

C $1.1388174 .645111-1.771708$

O $-1.4660105 .841763-2.230058$

C $-1.924853 \quad 3.959767-0.755802$

C $-3.4609284 .142549-0.832262$

O $-1.5088423 .077026-1.800667$

C $-2.453269-2.9127181 .883405$

O $-2.747150-3.8293392 .633128$

C $-3.344183-2.3174800 .860705$

C $-4.569543-2.8255410 .657405$

C $-5.543927-2.358229-0.334559$

C $-5.135862-1.198488-1.205898$

C $-4.2403972 .901526-0.377987$

C $-5.7463313 .086002-0.447879$

C -7.8477821 .9625660 .281064$

O $-6.2897274 .043875-0.988696$

C -8.2312450 .5750980 .683577$

C $-9.076212-0.205849-0.005799$

C $-9.417835-1.6084490 .412551$

C $-9.212334-2.627257-0.689594$

C $-10.214632-3.444850-1.055031$

C $-7.863802-2.708714-1.378946$

C -6.731072 -2.995075 -0.430651

N -6.4044672 .0194580 .149611$

H $2.299473-2.7151381 .370778$

H $\quad 0.059827-4.739801 \quad 1.862827$

H $-0.587620-3.1453393 .583980$

H $\quad 0.943745-0.9149842 .134024$

H $1.582557-1.7557503 .529328$

H $-1.170123 \quad 1.2205304 .396627$

H $-0.581251-0.2099086 .079433$

H $-0.609894-1.8956945 .558677$

H $0.940068-1.0582165 .793058$

H -0.4379840 .5402541 .466087$

H -1.871669 1.3652962 .038559

H -0.4589173 .2366972 .915203$

H 1.0037912 .4068212 .364158

H 0.1611952 .5097670 .013139

H 1.6067344 .3871710 .873110

H $0.1884505 .194786 \quad 1.505718$

H $1.6973276 .589719-0.240986$

H -1.650963 $6.032605-0.216819$

H $2.2076504 .699290-1.531643$

H $1.0136275 .205202-2.704846$

H $0.8964943 .597943-1.962254$

H -1.010991 $6.699116-2.292128$

H $-3.7625174 .976309-0.184920$

H -3.756468 $4.400924-1.856834$

H -2.208045 2.426691 -1.969480

H -2.955862 -1.482058 0.288500

H $-4.890494-3.6696811 .266318$

H $-4.862505-0.335239-0.591610$

H -5.917560 -0.858512 -1.886974

H -4.274015 -1.471412 -1.825180

H -3.988372 $2.037660-1.001881$

H $-3.9803312 .659850 \quad 0.659342$

H $-8.3039152 .273369-0.664647$

H -8.141539 2.6780461 .055766

H $-7.7812930 .188494 \quad 1.595741$

H $-9.5466850 .177649-0.907790$

H -10.464817 -1.6079390.741691

H $-8.826359-1.9121971 .285454$

H -10.088058 -4.179240 -1.843484

H -11.186213 -3.400175 -0.574136

H -7.718302 -1.786817 -1.946831

H -7.870659 -3.516269-2.122715

H $-6.914493-3.8458010 .227303$

H $-5.852514 \quad 1.3757850 .710240$

C $2.489085-4.588786-0.911941$ 
O $2.964055-3.269682-1.255121$

C $2.051309-2.696509-2.208884$

C $1.323949-3.880215-2.830148$

C $1.136271-4.761111-1.608835$

H $1.318933-2.114021-1.636397$

C $2.785529-1.774869-3.185734$

H $0.380083-3.591426-3.301672$

H $1.940226-4.396062-3.574463$

C $0.794982-6.203161-1.941653$

H $0.327548-4.340202-0.998563$

C $3.535090-0.607655-2.506578$

O $3.764941-2.524229-3.914222$

H $2.081911-1.376079-3.926731$

H $4.416430-0.989462-1.978885$

O $4.0272110 .251129-3.543649$

C $2.6767360 .234438-1.545081$

C $3.3176121 .556197-1.085000$

H $2.424808-0.367537-0.664404$

O $1.444813 \quad 0.559786-2.197543$

H $3.3909682 .248980-1.933043$

H $2.6100572 .022415-0.387023$

H $-0.150434-6.257349-2.491451$

H $1.570157-6.667259-2.560669$

H $0.690354-6.798358-1.029116$

C $2.470643-4.7751680 .608486$

H $3.294341-3.076462-4.560727$

H $4.576072-0.293610-4.133696$

H $0.877657 \quad 1.008331-1.548134$

H $3.504846-4.7944710 .974409$

H $2.034959-5.7491260 .859958$

H $3.216637-5.293445-1.335106$

C $5.750631 \quad 0.242437 \quad 1.431412$

$\begin{array}{lllll}\text { O } & 4.547522 & 0.525619 & 0.703511\end{array}$

C $4.6893621 .414629-0.407544$

C $5.2359992 .776357 \quad 0.054248$

C 6.5365892 .6137950 .846417

C $6.4102091 .539971 \quad 1.932317$

H $5.418917-0.3256502 .310030$

C $6.712132-0.6505110 .629928$

H $5.3848450 .980405-1.132130$

$\begin{array}{lllll} & & 4.264308 & 3.431839 & 0.866955\end{array}$

H $5.4064813 .405031-0.828177$

H $6.796063 \quad 3.5731371 .310901$

O $7.5817042 .274648-0.059863$

H 7.3957921 .3160472 .357002

O 5.6130352 .0782112 .991292

C $6.136984-2.0266690 .371704$

H $6.953908-0.210867-0.340993$

H $7.641662-0.7900911 .193777$

H 4.6252004 .3002201 .114019

H 8.4036072 .2012590 .454392

H $5.582602 \quad 1.4163003 .702849$

O $6.950068-2.688630-0.503731$

O $5.116623-2.470560 \quad 0.878476$

C $6.540278-4.014507-0.854078$

H $5.679986-3.967852-1.526825$

H $7.369076-4.498129-1.377804$

H $6.303069-4.6014300 .038651$

SCF Energy (B3LYP/6-31G**//MMFF) $=-3245.90021692$

08_00599

MMFFF Geometry

C $-1.856370-3.8286141 .899848$

C $-1.741691-3.4991173 .196326$

C $-2.120825-2.1874473 .846349$

O $-2.630368-1.2380162 .884456$

C $-0.881365-1.558740 \quad 4.506700$

C $-1.228210-0.4172355 .442073$

C $-1.059546 \quad 0.888427 \quad 5.156634$

C $-1.741712-0.831366 \quad 6.798013$

C $-0.562532 \quad 1.492137 \quad 3.872373$

C -1.4057632 .7114703 .471667$

C $-0.851125 \quad 3.395753 \quad 2.215154$

C $-1.5844594 .711027 \quad 1.947488$

C $-1.0959425 .380565 \quad 0.654882$

O $-1.026152 \quad 2.507626 \quad 1.106583$

$\begin{array}{lllll}O & -2.028067 & 6.418271 & 0.315387\end{array}$

C $-1.1367204 .344437-0.485865$
C 0.2650616 .0621310 .839672

O $-0.552153 \quad 4.872219-1.675008$

C $-0.4749662 .992650-0.125321$

C $-0.7890751 .961415-1.230963$

O $0.939043 \quad 3.168191-0.035378$

C $-3.968745-1.2446042 .642997$

O $-4.807238-1.9713073 .151378$

C $-4.236112-0.2231841 .606877$

C $-5.379611-0.288110 \quad 0.909014$

C $-5.780900 \quad 0.605786-0.178742$

C $-4.9532451 .841747-0.405697$

C $-0.3502420 .527494-0.917398$

C $-0.871541-0.401518-1.994621$

C $-2.936913-1.611533-2.703573$

O $-0.197598-0.682584-2.982805$

C $-3.939110-0.716670-3.364880$

C $-5.240415-1.021477-3.487063$

C $-6.246046-0.125261-4.154005$

C $-7.5012430 .075388-3.330823$

C $-8.666889-0.479953-3.703561$

C $-7.4252580 .982797-2.119763$

C $-6.8593150 .272979-0.918067$

N $-2.169299-0.826081-1.754468$

H $-2.267944-3.1157571 .192670$

H $-1.341250-4.2461343 .880249$

H $-2.882188-2.3941914 .608433$

H $-0.179652-1.2240353 .733471$

H $-0.334750-2.3139715 .087871$

H $-1.2978201 .612148 \quad 5.935391$

H $-1.9181070 .026102 \quad 7.456279$

H -2.688487 -1.3717746 .703934$

H $-1.016266-1.4831437 .295948$

H $0.477424 \quad 1.8054064 .023654$

H -0.5693720 .7680113 .052755$

H -2.439013 $2.386398 \quad 3.292487$

H -1.4237753 .4311924 .299896$

H 0.2189523 .5812472 .366278

H $-1.478666 \quad 5.3971792 .796977$

H $-2.6618134 .515047 \quad 1.851875$

H -2.065502 7.0426691 .060023

H $-2.1950344 .158397-0.717860$

H $0.206938 \quad 6.826316 \quad 1.624143$

H $0.568746 \quad 6.590639-0.070709$

H 1.0598725 .3662351 .115807

H -1.004735 $5.709870-1.873169$

H $-1.8690871 .955445-1.414426$

H $-0.3162232 .267729-2.171418$

H $1.3706102 .308715-0.159352$

H -3.472595 $0.523678 \quad 1.421685$

H $-6.077422-1.0951871 .129689$

H -4.8337782 .4055140 .526682$

H $-5.3915112 .536546-1.124562$

H $-3.9634491 .569771-0.778837$

H $\quad 0.7402770 .437635-0.888395$

H -0.7263710 .1943530 .056420$

H $-3.414758-2.421484-2.143596$

H -2.269457 -2.044998 -3.454640

H $-3.5744610 .224358-3.770844$

H $-5.599611-1.975647-3.108078$

H $-6.500216-0.577428-5.121532$

H $-5.8122070 .855553-4.385596$

H $-9.576264-0.320565-3.133598$

H $-8.737535-1.110470-4.583721$

H -8.429562 $1.335491-1.850756$

H $-6.866100 \quad 1.880912-2.394317$

H $-7.405353-0.633843-0.652641$

H -2.647011 $-0.505371-0.917582$

C $-0.364935-5.1086610 .275163$

$\begin{array}{lllll}\text { O } & 0.780227 & -4.373622 & 0.752024\end{array}$

C $1.233255-3.497380-0.305959$

C $0.581009-4.024329-1.574472$

C $-0.771359-4.461556-1.047611$

H $0.837725-2.503151-0.060095$

C $2.766476-3.468184-0.307506$

H $0.509337-3.270133-2.362178$

H $1.135684-4.884362-1.970107$

C -1.522845 -5.387116 -1.987578

H $-1.379069-3.567506-0.870790$ 
C $3.367261-2.714828-1.510980$

O $3.167258-2.8339810 .909541$

H $3.158358-4.491646-0.275155$

H $3.010753-3.222058-2.416589$

O $2.841053-1.393404-1.557422$

C $4.908450-2.694278-1.600094$

C $5.708278-2.121929-0.414546$

H $5.186731-2.133004-2.500981$

O $5.337533-4.045592-1.805475$

H $5.560129-2.7457620 .473959$

H $6.771147-2.246751-0.666360$

H $-1.722688-4.886686-2.940813$

H $-0.950547-6.296419-2.199443$

H -2.483050 -5.685170 -1.555280

C $-1.447263-5.1721491 .356453$

H $2.778315-3.3402371 .643550$

H $3.161309-0.980189-2.377094$

H $6.295111-4.028526-1.973744$

H $-1.068480-5.7937452 .178090$

H -2.335817 -5.6788850 .963149$

H $-0.012583-6.1319930 .088929$

C $5.6750751 .545778-1.126946$

O $5.8564200 .126385-1.226652$

C $5.450494-0.642549-0.090362$

C $6.216034-0.1952251 .165725$

$\begin{array}{llll}\text { C } & 6.067639 & 1.311637 & 1.396921\end{array}$

C $6.368488 \quad 2.110394 \quad 0.125440$

H $6.1948881 .955253-2.003273$

C $4.1943951 .942080-1.234970$

H $4.383330-0.4947980 .088558$

O $7.599059-0.525636 \quad 1.056945$

H $5.824663-0.7432102 .030708$

H 6.7530211 .6275242 .192808

O $4.739726 \quad 1.606411 \quad 1.820624$

H $6.087003 \quad 3.161753 \quad 0.256075$

O $7.778128 \quad 2.083742-0.118851$

C $3.6626901 .741680-2.639268$

H $3.5535761 .368514-0.561551$

H $4.0939433 .007470-0.994958$

H $7.940897-0.1117370 .245906$

H $4.574871 \quad 1.1077542 .638991$

H 8.2160832 .4877970 .649586

O $2.5542302 .519458-2.815247$

O $4.1398190 .979384-3.468996$

C $1.9400912 .429750-4.104755$

H $1.5443161 .422275-4.262787$

H $1.1117803 .142597-4.137217$

H $2.6570822 .691639-4.888727$

SCF Energy (B3LYP/6-31G**/MMFF)= -3245.91814996

0800600

MM̄FF Geometry

C $0.677072-1.692204-0.049164$

C $0.057448-1.838594-1.231315$

C $0.576580-1.404914-2.583895$

O $2.017378-1.240929-2.566792$

C $0.013147-0.061490-3.077047$

C $-1.4809890 .134464-2.952125$

C $-2.040593 \quad 1.048019-2.134637$

C $-2.335118-0.720034-3.848348$

C $-1.3046701 .932744-1.169555$

C $-0.958048 \quad 3.300400-1.763970$

C $0.0655884 .033321-0.884207$

C $0.1931785 .496600-1.305663$

C $1.2889136 .221440-0.512201$

O $1.3220093 .363762-1.047257$

O $1.5476807 .475275-1.161506$

C $2.5866875 .393455-0.592502$

C $0.840392 \quad 6.5548240 .915519$

$\begin{array}{lllll}\text { O } & 3.598191 & 5.954378 & 0.242439\end{array}$

C $2.3955073 .893601-0.257626$

C $3.6817143 .119535-0.644427$

$\begin{array}{llll}\text { O } & 2.140040 & 3.748709 & 1.137699\end{array}$

C $2.792606-2.355516-2.633227$

O $2.420413-3.512691-2.733659$

C $4.196419-1.908119-2.507936$

C $5.128762-2.767573-2.073176$

C $6.537497-2.453838-1.820499$
C $7.008622-1.057648-2.135365$

C $3.6339481 .601202-0.413080$

C 4.2627291 .1649040 .901579

C $5.165972-0.8661642 .038428$

O $4.516374 \quad 1.9436661 .816553$

C $6.614697-1.0329751 .698462$

C $7.270060-2.1996731 .802459$

C $8.729287-2.3695681 .485438$

C $9.000175-3.5372770 .560957$

C $9.491815-4.6942891 .036015$

C $8.780044-3.336462-0.923127$

C $7.325997-3.417624-1.300370$

N $4.491520-0.2046900 .936054$

H $1.626579-1.1677040 .000971$

H $-0.888900-2.375074-1.255337$

H $0.327185-2.182737-3.317501$

H $0.2883380 .057071-4.135033$

H $\quad 0.5638200 .751266-2.587785$

H $-3.1234781 .157400-2.131599$

H -3.401212 -0.490312 -3.746589

H $-2.061089-0.563147-4.896623$

H -2.203341 -1.779808 -3.608670

H $-1.9428902 .092277-0.295825$

H $-0.4125931 .426496-0.785090$

H $-0.5443193 .172774-2.772675$

H $-1.8769333 .892477-1.854993$

H -0.2503763 .9622090 .163373$

H $-0.7663466 .018044-1.198524$

H $0.4449935 .549065-2.374241$

H $0.7165737 .980116-1.180178$

H $2.9594295 .471518-1.623623$

H $-0.0519647 .191926 \quad 0.897284$

H 1.6075197 .1296351 .446034

H 0.6015225 .6693551 .508267

H $3.6963656 .888713-0.009217$

H $3.8461723 .267129-1.720573$

H $4.5492183 .544943-0.126201$

H 2.3262362 .8324361 .396287

H $4.411548-0.868548-2.726188$

H $4.827163-3.789968-1.848368$

H $6.869869-0.838119-3.199843$

H $8.064594-0.890167-1.916210$

H $6.450103-0.319492-1.549820$

H $2.610857 \quad 1.214620-0.460077$

H $4.2074511 .123546-1.216901$

H $4.667471-1.8279462 .193941$

H $5.065120-0.2639122 .946631$

H $7.154324-0.1485621 .367898$

H $6.736766-3.0769102 .161824$

H $9.259165-2.5156122 .435851$

H $9.151916-1.4571501 .046433$

H $9.714255-5.5271050 .377105$

H $9.677711-4.8367262 .095439$

H $9.305710-4.115992-1.490816$

H $9.254492-2.400339-1.228090$

H $6.885763-4.397757-1.109973$

H $4.277080-0.7622130 .115192$

C $-0.365821-1.3605822 .285477$

O $-1.630732-0.8094381 .877571$

C -1.7895580 .4690242 .525615$

C -0.5448200 .6867813 .386990$

C $0.490031-0.1602802 .669577$

H $-1.7870521 .222848 \quad 1.734857$

C -3.1133570 .5298223 .299475$

H $-0.265794 \quad 1.743700 \quad 3.445599$

H -0.6933320 .3281814 .412175$

C $1.690972-0.5060293 .529512$

H $0.8221460 .383922 \quad 1.776486$

C $-4.378667 \quad 0.2736132 .450181$

O $-3.080643-0.4587454 .336924$

H $-3.1896191 .500740 \quad 3.804145$

H -4.434263 -0.788492 2.189665

O

C -4.4838611 .1299641 .178189$

C $-5.848628 \quad 1.1028520 .468716$

H -3.7104490 .8081050 .474883$

O -4.2082772 .4923081 .519793$

H $\quad-6.6059701 .5929351 .093998$ 
H $\quad-5.749430 \quad 1.741468-0.419311$ H $2.242976 \quad 0.3992693 .802119$ H $1.391523-1.0091094 .455095$ H $2.372162-1.1722472 .993585$ C $0.186523-2.316528 \quad 1.228058$ H $-3.923802-0.4013004 .818310$ H -5.5190521 .4819093 .490110$ H -4.1880403 .0039560 .693015$ H $-0.584264-3.0607140 .994379$ H $1.027125-2.8746231 .659064$ H $-0.576976-1.9599563 .182485$ C $-5.607095-2.246921-1.180377$ O $-5.322711-0.912354-0.735890$ C $-6.343467-0.2900200 .048385$ C $-7.657665-0.204433-0.749257$ C $-8.077170-1.569141-1.302021$ C $-6.911433-2.285611-1.994595$ H $-4.782054-2.497640-1.859797$ C -5.592571 -3.259702 -0.024201 H $-6.510542-0.8788350 .955630$ O H $-8.443916 \quad 0.187917-0.093707$ H -8.897311 -1.426330 -2.016278 O $-8.571730-2.362923-0.228197$ H -7.173203 -3.327330 -2.213574 O $-6.650514-1.647348-3.248168$ C $-4.232739-3.368586 \quad 0.628527$ H $-6.306572-2.9866350 .757006$ H -5.851521 $-4.255276-0.402345$ H $-6.7761490 .419971-2.381728$ H $-8.856903-3.214939-0.600093$ H $-7.459323-1.712603-3.784015$ O $-4.350065-4.105788 \quad 1.772521$ O $-3.200509-2.8817490 .190630$ C $-3.142657-4.2885332 .517763$ H $-3.358361-4.9574053 .355251$ H $-2.371162-4.748181 \quad 1.892977$ H $-2.801312-3.3288172 .915195$ SCF Energy $\left(B 3 L Y P / 6-31 G^{* *} / / M M F F\right)=-3245.91131843$

\section{8_00601}

MM̄FF Geometry

C $-0.787890-2.131404-1.556755$

C -1.415913 -1.147317 -2.219070

C $-0.847114 \quad 0.195571-2.620793$

O $0.5300520 .351079-2.211471$

C -1.670829 $1.311502-1.952082$

C $-1.3453242 .699941-2.467890$

C $-0.6578603 .638798-1.787416$

C $-1.9032733 .021856-3.831388$

C $-0.0412093 .526628-0.421339$

C $1.4727113 .765984-0.481375$

C 2.1506183 .5257000 .876055

C 3.6521753 .8007280 .777720

C 4.3994683 .4261022 .064418

O 1.9204692 .1621831 .237648

O 5.8001113 .4203581 .746870

C 4.0018981 .9925582 .481079

C 4.2104334 .4897973 .152175

O $4.5812221 .685188 \quad 3.746877$

C 2.4753661 .7677182 .499613

C 2.0724390 .2898292 .721376

O 1.9050452 .5255523 .572881

C $1.487772-0.120192-3.057472$

O $1.310526-0.703806-4.115100$

C $2.8084630 .195700-2.467417$

C $3.920361-0.306364-3.025543$

C $5.298908-0.057504-2.595342$

C $5.5105130 .846799-1.410822$

C $2.523740-0.6821531 .627128$

C $3.817749-1.4164151 .927324$

C $5.385918-3.0564920 .890011$

O $4.411424-1.3325912 .998206$

C $6.562058-2.3056290 .351202$

C $7.302243-2.733421-0.682728$

C $8.528695-2.025021-1.180308$

C $8.476720-1.725579-2.660632$

C $9.103452-2.515910-3.548692$
C $7.786400-0.455168-3.099538$

C $6.304974-0.630334-3.289164$

N $4.211619-2.207240 \quad 0.854993$

H $0.249401-2.011683-1.261200$

H -2.453741 -1.306972 -2.508894

H $-0.9212970 .286036-3.711436$

H -1.543765 $1.256495-0.865770$

H $-2.7402841 .135366-2.125193$

H $-0.5176784 .610888-2.259472$

H -1.712533 $4.059670-4.125059$

H -1.455486 2.377994 -4.594123

H $-2.9882252 .873488-3.841851$

H -0.5024864 .2785280 .230291$

H -0.2439372 .5557030 .035226$

H $1.9063863 .088114-1.227355$

H $1.6718484 .791051-0.818169$

H 1.6854664 .1804641 .622239

H 3.8390634 .8493610 .514490

H $4.0725663 .210533-0.046831$

H 6.2796473 .1191002 .537837

H 4.4628901 .3164271 .750954

H 4.6094275 .4539122 .814479

H 4.7707584 .2357404 .058602

H 3.1641734 .6428983 .425272

H 4.4613040 .7340833 .906294

H $2.375681-0.0472283 .719530$

H 0.9735920 .2567092 .732210

H 1.0409842 .1425703 .797681

H $2.8300430 .843323-1.599283$

H $3.816221-0.952704-3.896285$

H $6.5470810 .884400-1.069387$

H $4.9244580 .505035-0.551692$

H $5.2104621 .870070-1.658316$

H $1.756117-1.4569991 .524719$

H $2.608775-0.1812690 .658365$

H $5.158565-3.9500170 .300035$

H $5.585023-3.3625221 .922246$

H $6.839247-1.3856770 .860623$

H $7.040571-3.668701-1.172493$

H $\quad 9.392647-2.666497-0.961407$

H $8.707504-1.095255-0.625862$

H $9.098665-2.287894-4.609554$

H $9.629795-3.411355-3.235059$

H $8.196297-0.127088-4.064789$

H $8.0372630 .355925-2.410063$

H $6.050915-1.274170-4.132497$

H $3.687068-2.162340-0.014080$

C $-1.534274-3.668780 \quad 0.325849$

$\begin{array}{lllll}\text { O } & -2.134742 & -2.534148 & 0.978707\end{array}$

C $-1.394627-2.2404312 .175763$

C $-0.544844-3.4781392 .443858$

C $-0.204611-3.9208051 .034086$

H $-0.734136-1.3974031 .940924$

C $-2.322586-1.8620163 .338220$

H $\quad 0.338719-3.2531643 .048817$

H $-1.122430-4.2547312 .959659$

C $0.280853-5.3571210 .945944$

H $0.576924-3.2624130 .639593$

C $-3.013127-0.4897073 .206402$

O $-3.282618-2.893383 \quad 3.544813$

H -1.699995 -1.828247 4.242379

H -3.689023 -0.3537644 .060586$

O $-1.9828190 .501741 \quad 3.346416$

C $-3.790398-0.1741851 .913382$

C $-4.924860-1.1590931 .584612$

H $-3.090168-0.0890621 .074545$

O -4.3546951 .1316652 .100400$

H -4.546811 -2.183341 1.579972

H $-5.691557-1.0809602 .366147$

H $1.200631-5.4878041 .525371$

H $-0.464455-6.0583321 .336175$

H $0.493061-5.633084-0.091824$

C -1.459291 -3.426523 -1.183902

H $-3.821034-2.6453394 .315494$

H $-2.411601 \quad 1.3734443 .297163$

H -4.7924891 .3851601 .270423$

H -2.483632 -3.429431-1.578998

H $-0.932090-4.254582-1.670887$ 
H $-2.210996-4.5155100 .501692$

C $-7.0325240 .724204-0.883196$

$\begin{array}{llll}\text { O } & -6.364024 & 0.302722 & 0.313989\end{array}$

C $-5.572063-0.8849510 .208457$

C $-6.434568-2.071332-0.250841$

C $-7.177481-1.744912-1.548280$

C $-7.908324-0.402186-1.456368$

H $-7.7026631 .530389-0.556724$

C $-6.0532611 .311592-1.906890$

H $-4.769048-0.721751-0.518930$

O $-7.370516-2.4039850 .772301$

H -5.784395 -2.942067 -0.400123

H $-7.898537-2.543650-1.760051$

O $-6.235058-1.714810-2.616317$

H -8.289459 -0.102228 -2.439432

O $-9.043362-0.543578-0.597450$

C $-5.6184442 .697455-1.492612$

H $-5.1601710 .701440-2.064223$

H $-6.5347811 .416578-2.886730$

H $-7.878603-3.1743540 .466122$

H $-6.725300-1.527267-3.435003$

H -9.617939-1.227850 -0.980855

O $-4.5897532 .621126-0.602764$

O $-6.1470993 .724507-1.898621$

C $-4.1059193 .874875-0.111052$

H -4.8861824 .3759890 .469577$

H -3.253797 3.6762320 .544322

$\mathrm{H}-3.7730434 .508181-0.938802$

SCF Energy (B3LYP/6-31G**//MMFF) $=-3245.91374227$

\section{2}

MM̄FF Geometry

C $-0.6524573 .397066-0.856335$

C $-1.5977744 .303051-0.562173$

C -2.9414554 .0288270 .077435$

O -3.0703742 .6472300 .487068$

C $-4.0727514 .343273-0.914207$

C $-5.4249074 .461875-0.238582$

C $-6.3839723 .516343-0.262499$

C $-5.683607 \quad 5.7772910 .452158$

C $-6.3198902 .156547-0.898860$

C -6.8343841 .0708900 .057385$

C $-6.866707-0.304517-0.621798$

C $-7.537641-1.3429850 .277628$

C $-7.517968-2.740616-0.359671$

O $-5.518645-0.699463-0.894759$

O $-7.869240-3.693491 \quad 0.655013$

C $-6.077534-3.064098-0.805643$

C $-8.567232-2.880664-1.468785$

O $-6.031805-4.285631-1.539779$

C $-5.405843-1.931263-1.619039$

C $-3.905199-2.249537-1.802022$

O $-6.019922-1.842822-2.905889$

C $-2.5053232 .300628 \quad 1.676984$

O -1.9349363 .0404102 .463411$

C $-2.6518630 .837467 \quad 1.839190$

C -1.8142870 .1956422 .667856$

C -1.788057 -1.242746 2.942456

C $-2.911473-2.0756152 .386860$

C $-3.065609-1.085841-2.344010$

C $-1.606424-1.492440-2.387524$

C $0.341292-1.922573-0.887389$

O $-1.075857-1.878062-3.426513$

C $0.251963-3.0222560 .124701$

C $1.046173-3.1015181 .202678$

C $0.962269-4.1922262 .230158$

C $0.841746-3.6549663 .639734$

C $1.888773-3.6830564 .481762$

C $-0.513881-3.1588644 .097517$

C $-0.772650-1.7360373 .682620$

N $-0.997546-1.427030-1.144259$

H $-0.8360402 .340517-0.682361$

H -1.380539 $5.353828-0.746414$

H -3.030948 4.6751260 .959680

H $-4.0983603 .581847-1.702110$

H $-3.8731895 .291177-1.432238$

H $-7.332546 \quad 3.7430520 .223117$

H -6.6918465 .8337610 .876361$
H -4.9748725 .9297261 .271751$

H $-5.5792866 .605233-0.256822$

H $-6.9464892 .173821-1.798641$

H $-5.3051781 .901823-1.216665$

H -6.1816331 .0352250 .939444$

H -7.8430581 .3316840 .401493$

H -7.415637 -0.208746 -1.566158

H -8.566171 -1.046179 0.517755

H -7.006526 -1.391199 1.238790

H $-8.749521-3.4581580 .995123$

H $-5.486178-3.2293170 .106323$

H -9.571983 -2.694128 -1.070752

H -8.587901 -3.901496 -1.866046

H -8.408046 -2.190311 -2.299770

H $-6.447315-4.970634-0.988527$

H $-3.484777-2.549369-0.835521$

H $-3.786245-3.108494-2.475392$

H $-5.392778-1.441440-3.527878$

H -3.4131580 .3330351 .255580$

H -1.0536800 .7782883 .187148$

H $-3.880587-1.6875832 .720397$

H -2.875925 -3.122594 2.693363

H -2.889786 -2.064291 1.294334

H $-3.373281-0.805123-3.356494$

H $-3.164297-0.192258-1.717124$

H $0.935243-1.077417-0.527155$

H $0.784671-2.303332-1.811963$

H $-0.500193-3.791002-0.037725$

H $\quad 1.817797-2.3515101 .348499$

H $1.867738-4.8058542 .137787$

H $0.122104-4.8683832 .028758$

H $1.803046-3.3227055 .501577$

H $2.856691-4.0598634 .168111$

H -0.571851 -3.194759 5.193699

H -1.279707 -3.856222 3.748758

H $-0.020781-1.0372134 .053123$

H -1.516918 -1.045570 -0.359156

C $1.8358873 .500298-0.384429$

O 1.8659562 .1095190 .003570

C 1.9023522 .0473091 .439762

C 2.4401523 .3947021 .896315

C 1.7507644 .3191750 .909959

H $\quad 0.857821 \quad 1.9753751 .773801$

C 2.6252960 .7856081 .922567

H 2.1961703 .6174122 .939038

H 3.5228013 .4739091 .766310

C 2.3891525 .6936420 .806698

H $\quad 0.710077 \quad 4.442717 \quad 1.232141$

C $4.1102190 .668476 \quad 1.513441$

O $2.4762240 .714243 \quad 3.338655$

H $2.090666-0.0662721 .488802$

H 4.2023280 .9528130 .460229

O $4.899161 \quad 1.5856442 .269456$

C $4.693567-0.7518821 .689103$

C $6.207676-0.8510181 .411697$

H $4.148502-1.4412561 .034211$

O $4.474324-1.1917803 .031342$

H $6.756514-0.2262572 .127448$

H $6.511895-1.8842171 .627179$

H 2.3511536 .2087241 .772014

H $3.4392075 .627473 \quad 0.502611$

H 1.8630916 .3113660 .071969

C $0.7073513 .751050-1.392928$

H $2.839162-0.1393863 .629801$

H 4.7955081 .3531493 .208257

H $4.768611-2.1169823 .089201$

H $0.8756683 .134396-2.283098$

H $0.7366714 .796672-1.721086$

H $2.7929363 .690798-0.886917$

C $6.191219-1.223966-2.299072$

O $5.986269-1.435965-0.896431$

C $6.608383-0.487516-0.025780$

C $8.130983-0.498780-0.224855$

C $8.488203-0.248316-1.694464$

C $7.690879-1.156824-2.636181$

H $5.784488-2.123339-2.780241$

C $5.387414-0.018600-2.813156$

H $6.2361710 .515956-0.249932$ 
$\begin{array}{lrrr}\text { O } & 8.660801 & -1.754465 & 0.193337\end{array}$

H $8.576027 \quad 0.2759120 .410835$

H $9.560037-0.423855-1.846479$

O $8.2191301 .109989-2.030116$

H $7.815718-0.835958-3.677014$

O $8.208107-2.487977-2.545264$

C $3.895171-0.271257-2.743140$

H $5.5882330 .892812-2.244832$

H $5.6434300 .156568-3.864653$

H $9.625117-1.720868 \quad 0.073611$

H $8.7512011 .669668-1.439167$

H $9.143291-2.457838-2.809632$

O $3.2458290 .668188-3.492278$

O $3.362679-1.167856-2.103780$

C $1.821807 \quad 0.533695-3.541314$

H $1.548035-0.427101-3.987381$

H $1.3964240 .628400-2.538189$

H $1.4253871 .336451-4.168277$

SCF Energy (B3LYP/6-31G**//MMFF)= -3245.91213264

\section{3}

MM̄FF Geometry

C - $-1.512128-1.0353933 .632855$

C $-1.821643-0.1556894 .598581$

C -2.8838130 .9169594 .516356$

O $\begin{array}{llll} & -3.613641 & 0.859856 & 3.269241\end{array}$

C -2.320616 $2.347514 \quad 4.673149$

C -1.123226 2.6917103 .805556

C -1.1879473 .0769572 .515506$

C $0.216140 \quad 2.6143494 .495257$

C -2.4255343 .2553751 .679346$

C $-2.3201304 .400788 \quad 0.655675$

C -1.343127 $4.111312-0.495794$

C $-1.3325635 .248620-1.518731$

C $-0.4224694 .926028-2.717013$

O $-1.7585542 .900066-1.133024$

O $-0.718573 \quad 5.865560-3.761582$

C $-0.775753 \quad 3.522449-3.258122$

C $1.0606715 .113719-2.373621$

O $\quad 0.147960 \quad 3.113862-4.264460$

C $-0.8687452 .442792-2.157045$

C $-1.434228 \quad 1.126291-2.739038$

O $0.4388502 .187135-1.642107$

C $-4.6949760 .037487 \quad 3.199676$

O $-5.153957-0.6525814 .095646$

C -5.2088920 .0971901 .811878$

C $-6.180685-0.7462131 .433936$

C $-6.790666-0.8331780 .104586$

C $-6.2946260 .114271-0.955471$

C -1.883063 $0.122671-1.666955$

C $-2.298547-1.209550-2.264098$

C $-3.482769-3.320972-1.670743$

O $-2.026243-1.543119-3.414448$

C $-4.808784-3.203901-2.355788$

C $-5.908656-3.872411-1.976573$

C $-7.217200-3.794376-2.710827$

C -8.386391-3.466475-1.810098

C $-9.300388-4.400224-1.495792$

C $-8.545410-2.034926-1.345923$

C $-7.758988-1.751924-0.094604$

N $-2.983721-1.997358-1.347556$

H -2.069210 -1.024944 2.701265

H $-1.265358-0.1917355 .533154$

H -3.5898140 .7609535 .342691$

H -2.0410842 .4991565 .724609$

H -3.1226253 .0718784 .483610$

H $-0.245414 \quad 3.2682492 .007228$

H $1.0364392 .936613 \quad 3.846101$

H $0.225756 \quad 3.265723 \quad 5.375520$

H 0.4330941 .5924964 .817830

H -2.632068 $2.315127 \quad 1.156472$

H -3.291249 3.4653782 .315762

H $-3.323167 \quad 4.5515200 .235460$

H -2.037401 $5.331766 \quad 1.162167$

H $-0.3386873 .977708-0.081525$

H - $1.0306676 .192916-1.049079$

H -2.352568 $5.411927-1.893858$

H $-0.5691956 .760446-3.410853$
H -1.755986 $3.599126-3.749328$

H $1.2538696 .148172-2.065218$

H $1.6942944 .940032-3.250372$

H $1.4012464 .461048-1.567225$

H $0.1654523 .811673-4.941737$

H $-2.309493 \quad 1.345126-3.364663$

H $-0.6809980 .660052-3.386766$

H $0.4517061 .308714-1.231583$

H $-4.7651410 .820877 \quad 1.137933$

H $-6.568951-1.4503812 .169059$

H $-6.488953 \quad 1.152079-0.662640$

H $-6.763854-0.033209-1.929754$

H -5.217993 -0.008614 -1.110097

H $-1.073284-0.077503-0.959897$

H $-2.7298220 .534357-1.106143$

H $-3.545869-3.885343-0.735096$

H $-2.767991-3.817873-2.334812$

H $-4.858583-2.561488-3.232097$

H $-5.857462-4.541367-1.120565$

H -7.379187 -4.764552 -3.198581

H $-7.175376-3.057799-3.523091$

H -10.157447 -4.164134-0.873576

H $-9.209810-5.422333-1.848374$

H $-9.601176-1.835029-1.118148$

H $-8.304323-1.361582-2.172310$

H $-8.051265-2.3807490 .748194$

H $-3.218991-1.611873-0.437554$

C $0.714315-1.8926142 .741898$

O $1.420839-0.6638923 .003456$

C $1.782458-0.0615561 .740742$

C $1.524790-1.1258010 .681333$

C $0.332883-1.8566651 .262709$

H 1.0998950 .7868451 .608401

C $3.2383330 .417737 \quad 1.800367$

H $1.325568-0.699416-0.305153$

H $2.382003-1.8045760 .592676$

C $\quad 0.094497-3.2240100 .648004$

H -0.553447 -1.234488 1.098398

C $3.7405201 .012836 \quad 0.473473$

O 3.3485501 .4043532 .830166

H $3.869288-0.4203072 .113331$

H $3.6374140 .281508-0.331563$

$\begin{array}{lllll}\text { O } & 2.876249 & 2.103496 & 0.126038\end{array}$

C $5.181506 \quad 1.5523150 .514191$

C $\quad \begin{array}{llll}6.273216 & 0.575954 & 0.990101\end{array}$

H 5.2085822 .4334421 .166596

O $5.5295042 .038083-0.787615$

H 7.2140101 .1400521 .042479

H 6.0367750 .2556342 .010909

H $-0.092047-3.134996-0.427209$

H $0.959028-3.8823400 .784572$

H $-0.775145-3.7083861 .103254$

C $-0.429572-2.072602 \quad 3.745913$

H 2.8421382 .1833802 .543079

H $3.1858642 .460050-0.724346$

H $5.3344181 .338590-1.434007$

H $-0.009809-2.0546874 .759592$

H $-0.885704-3.059627 \quad 3.606702$

H $1.441390-2.6974352 .915412$

C $5.408110-2.659003-0.705088$

O $5.361880-1.4854350 .116814$

C $6.522661-0.6536130 .090320$

C $7.753705-1.4488920 .559655$

C $7.931436-2.732827-0.257762$

C $6.626508-3.529329-0.355053$

H $4.507258-3.224111-0.432540$

C $5.315829-2.316218-2.197551$

H $6.695918-0.303820-0.931990$

O $7.610750-1.7782021 .940139$

H $8.642097-0.8134990 .462968$

H $8.701358-3.3602940 .207507$

O $8.374551-2.408611-1.572195$

H $6.721708-4.335478-1.092043$

O $6.361044-4.1467310 .907864$

C $3.959095-1.742664-2.538356$

H $6.088479-1.609256-2.511783$

H $5.442758-3.213977-2.814001$

H $8.414297-2.2521722 .214058$ 
H $9.216512-1.929534-1.488801$

H $7.107647-4.736512 \quad 1.108620$

O $4.092963-0.466127-2.999019$

O $2.907304-2.356235-2.410203$

C $2.8754030 .195084-3.358939$

H $3.0930321 .257562-3.497078$

H $2.497098-0.215015-4.299505$

H $2.1272260 .093648-2.567937$

SCF Energy (B3LYP/6-31G**/MMFF) $=-3245.90529594$

\section{4}

MM̄FF Geometry

C - $-1.167337 \quad 5.2152130 .318716$

C $-1.849948 \quad 4.119823-0.047785$

C $-3.304586 \quad 3.9000690 .281749$

O $-3.371770 \quad 2.589310 \quad 0.889351$

C $-4.1576343 .913175-0.997091$

C $-5.6453404 .018228-0.722381$

C $-6.5384393 .020613-0.873694$

C $-6.121130 \quad 5.383530-0.289645$

C $-6.2941071 .603878-1.310696$

C $-6.7116720 .613535-0.216998$

C $-6.577528-0.846095-0.671403$

C $-6.964160-1.7969450 .463324$

C $-6.756926-3.2689620 .079043$

O $-5.220787-1.080029-1.059442$

O $-6.838293-4.0264251 .295950$

C -5.336560 -3.440172 -0.501367

C $-7.883059-3.777119-0.828869$

O $-5.150439-4.754138-1.023218$

C $-4.981955-2.392573-1.584222$

C $-3.482587-2.499266-1.939609$

O $-5.759189-2.646639-2.755411$

C -4.0707292 .4325482 .040235$

O $-4.780370 \quad 3.2665572 .585039$

C -3.8414831 .0907582 .632758$

C $-3.079191 \quad 0.1471902 .057288$

C $-2.742012-1.1659942 .614976$

C $-3.291072-1.5167263 .971199$

C $-2.966842-1.404124-2.883808$

C $-1.465017-1.538410-3.035215$

C $0.638022-1.202854-1.742079$

O $-0.960540-2.149878-3.973736$

C $0.743329-2.383331-0.826061$

C $1.077208-2.2925350 .469843$

C $1.080801-3.4590141 .414786$

C $0.102057-3.2817242 .558453$

C $0.534395-3.1497963 .823842$

C -1.381591 -3.325349 2.248532

C -1.927129-1.967990 1.898472

N $-0.772299-0.960085-1.982699$

H $-1.704030 \quad 5.9993150 .850549$

H - $1.352476 \quad 3.322315-0.592349$

H -3.638894 4.6689840 .987909

H -3.930980 $3.025845-1.597157$

H -3.876871 $4.770148-1.624714$

H -7.584980 $3.241152-0.663941$

H -7.210661 $5.432996-0.189957$

H $-5.692133 \quad 5.6553430 .679331$

H -5.826608 $6.138962-1.025583$

H $-6.8849541 .420514-2.216250$

H $-5.249303 \quad 1.432545-1.580113$

H $-6.081670 \quad 0.7808190 .663724$

H -7.7489410 .8040130 .086444$

H -7.231770 -0.994154 -1.538789

H $-8.000325-1.6248810 .780850$

H -6.342755 -1.580109 1.344085

H $-6.765554-4.9692921 .070806$

H $-4.626903-3.3312490 .330824$

H -8.848470 -3.707465 -0.313232

H $-7.749261-4.837099-1.071520$

H -7.965043 -3.218544 -1.763608

H $-5.269157-5.382165-0.291178$

H -2.897074 -2.456569-1.013389

H $-3.276766-3.473773-2.401041$

H -5.323952 -2.238187 -3.519645

H $-4.316786 \quad 0.9327453 .595451$

H $-2.628306 \quad 0.3572181 .088633$
H -2.976001 -0.7790534 .717610$

H -2.957509 $-2.489548 \quad 4.338075$

H $-4.385991-1.5448493 .944729$

H $-3.415565-1.483785-3.879132$

H -3.200351 -0.403532 -2.502931

H $1.056491-0.294337-1.299003$

H $1.153256-1.407599-2.685301$

H $\quad 0.489717-3.354389-1.244849$

H $1.320478-1.3201850 .889431$

H $2.102943-3.5765531 .796774$

H $0.846050-4.3944290 .891586$

H $-0.162129-3.0443054 .649040$

H $1.592694-3.1386644 .062353$

H $-1.923936-3.7799213 .083490$

H $-1.559732-4.0024421 .403359$

H $-1.597606-1.6083040 .925399$

H $-1.302095-0.533729-1.227853$

C $1.1897734 .326228-0.311863$

O $1.2219153 .355136 \quad 0.754593$

C 2.5941413 .0314921 .058223

C $3.3954773 .496642-0.145461$

C $2.6430524 .749692-0.541858$

H 2.8533393 .6240981 .944993

C 2.7024661 .5337241 .370470

H 4.4459003 .6881690 .093255

H $3.3553412 .753484-0.949751$

C $2.9323325 .207701-1.961193$

H 2.9159935 .5546690 .153842

C 4.1532281 .0566341 .581456

$\begin{array}{lllll}\text { O } & 1.935447 & 1.279761 & 2.549609\end{array}$

H 2.2416780 .9524350 .563764

H 4.7046991 .2606280 .659260

O $4.766128 \quad 1.8365742 .608700$

C $4.302159-0.4424951 .919185$

C $5.765212-0.8989412 .089177$

H $3.825956-1.0422061 .136076$

O $3.642024-0.751598 \quad 3.148065$

H $6.239026-0.3366962 .903431$

H $5.741574-1.9449552 .424165$

H $2.3561426 .105633-2.205878$

H $3.9947345 .444295-2.080075$

H $2.6768404 .433533-2.692528$

C $0.294624 \quad 5.5027600 .095488$

H 1.0280531 .5852522 .377411

H $4.283416 \quad 1.6580183 .434197$

H $2.695249-0.5671473 .028149$

H $\quad 0.367661 \quad 6.301087-0.652318$

H $0.6752565 .911847 \quad 1.041019$

H $0.7957703 .820297-1.201429$

C $6.604583-1.595104-1.477959$

O $6.003071-1.628757-0.177641$

C $6.606911-0.787795 \quad 0.808941$

C $8.069502-1.1988161 .030384$

C $8.846907-1.189289-0.290494$

C $8.102614-1.940678-1.400376$

H $6.104879-2.396675-2.037373$

C $6.313133-0.272639-2.205793$

H $\quad 6.587430 \quad 0.2506050 .468039$

O $8.141100-2.4950931 .619588$

H $8.531888-0.4989341 .736244$

H $9.828563-1.651406-0.128603$

$\begin{array}{llll}\text { O } & 9.058968 & 0.164123 & -0.682471\end{array}$

H $8.579541-1.753840-2.369545$

O $8.230475-3.341410-1.135924$

C $4.836661-0.106595-2.505465$

H $6.6264070 .598284-1.624979$

H $6.845266-0.268159-3.164275$

H $7.675469-3.1149901 .032501$

H $9.5858900 .153421-1.499543$

H $7.821931-3.819283-1.877608$

O $4.6642140 .985037-3.308309$

O $3.945624-0.833034-2.087663$

C $3.3137521 .252378-3.701067$

H $2.6693201 .359350-2.823389$

H $3.3020462 .191837-4.259965$

H $2.9482860 .451282-4.350310$

SCF Energy (B3LYP/6-31G**//MMFF) $=-3245.91243705$ 
0800605

MM̄FF Geometry

C $-0.073104-2.8651861 .437427$

C $-0.510646-1.6162641 .213258$

C -1.021640 -0.7085132 .304610$

O $-2.395814-0.3750991 .997072$

C $-0.254650 \quad 0.6230712 .323740$

C $-0.316768 \quad 1.3210553 .666939$

C -1.205146 2.275891 4.004212

C 0.7517940 .9049024 .645175

C -2.3638672 .7964103 .200449$

C $-2.154678 \quad 4.2357212 .711566$

C -1.2351334 .3242801 .484419$

C -1.010980 5.7814871 .083062

C $-0.1565895 .888947-0.187886$

O

O $-0.2547137 .238983-0.670321$

C $-0.7539374 .975887-1.283719$

C 1.3272535 .6492930 .115076

O $0.1270594 .926156-2.403792$

C $-1.1126203 .547743-0.796307$

C -1.994784 2.822355-1.837689

O $0.0529932 .739372-0.629203$

C -3.370146-1.272766 2.288089

O $-3.246762-2.3547772 .836025$

C $-4.628148-0.7043981 .758050$

C $-5.656185-1.5159561 .475010$

C $-6.920780-1.1096130 .858682$

C $-7.125700 \quad 0.3590300 .588963$

C $-2.5215951 .458941-1.361064$

C $-3.3113670 .746141-2.447526$

C $-4.566941-1.364636-2.879034$

O $-3.4584751 .203131-3.578445$

C $-6.032304-1.072710-2.779058$

C $-6.969097-2.030137-2.687800$

C $-8.446679-1.755479-2.638508$

C $-9.152524-2.511827-1.532694$

C $-9.826216-3.645085-1.792816$

C $-9.144929-1.911429-0.143724$

C -7.813104 -2.068150 0.534590

N $-3.822119-0.469365-2.010492$

H $-0.062985-3.2463712 .455989$

H $-0.553605-1.2397220 .194145$

H - $0.975156-1.1985533 .285195$

H $-0.640834 \quad 1.2867591 .542815$

H $\quad 0.8009300 .4667542 .079887$

H -1.121247 2.7225124 .994524

H 0.6657531 .4263885 .604285

H $0.687174-0.1688134 .847390$

H $1.744406 \quad 1.1238624 .238254$

H -2.6126882 .1420022 .361633$

H -3.242597 2.7792693 .857751

H -3.140669 4.6373502 .442606

H -1.762272 4.8572873 .525730

H -0.2813063 .8373921 .714282$

H -0.5518826 .3465541 .903767$

H -1.980210 6.2631640 .891748

H $0.046067 \quad 7.8338480 .037928$

H -1.674824 $5.465540-1.631090$

H $1.698898 \quad 6.3972790 .825967$

H $1.9393905 .765755-0.785584$

H 1.5221794 .6653930 .546826

H $-0.3542464 .523509-3.145434$

H -2.860358 $3.449826-2.086701$

H -1.424069 2.668769-2.761986

H $0.8381453 .272166-0.830618$

H $-4.648730 \quad 0.3608241 .556591$

H -5.548621 -2.581021 1.676069

H -7.098691 0.9269551 .525600

H -8.0786080 .5890510 .109159$

H $-6.3446380 .743265-0.076517$

H -1.686815 $0.811036-1.071373$

H -3.176996 $1.589326-0.492475$

H -4.328753 -2.387929 -2.571617

H $-4.233949-1.220152-3.912013$

H $-6.341076-0.030621-2.816025$

H -6.660351-3.073306 -2.688564

H -8.868795 -2.043274 -3.610412
H -8.650935 -0.682867 -2.531683

H $-10.357307-4.179472-1.012049$

H -9.857275 -4.066823 -2.792015

H $-9.892680-2.4080590 .488945$

H $-9.477406-0.871735-0.203582$

H $-7.566402-3.1055030 .765967$

H -3.692728 -0.744128 -1.041366

C $1.657243-4.5265650 .621113$

$\begin{array}{llll}\text { O } & 2.749641 & -3.699427 & 0.161017\end{array}$

C $3.622390-4.498260-0.658591$

C $3.281789-5.945728-0.331738$

C $1.784188-5.851727-0.137243$

H $3.341103-4.303133-1.700385$

C $5.097803-4.143462-0.444510$

H $3.570097-6.633357-1.132562$

H $3.764630-6.2780010 .594725$

C $1.193588-7.0426270 .599054$

H $1.306467-5.768047-1.122993$

C $5.481933-2.674351-0.720068$

O $5.442644-4.4326160 .917045$

H $5.714697-4.808688-1.061926$

H $5.131819-2.048403 \quad 0.106432$

O $6.915383-2.629545-0.684120$

C $4.981541-2.118224-2.064660$

C $5.548590-0.745317-2.474482$

H $3.887593-2.056285-2.039020$

O $5.323638-3.047610-3.098711$

H $6.613549-0.839420-2.722944$

H $5.056061-0.475159-3.418363$

H $1.365192-7.9660530 .036344$

H $1.643388-7.1646631 .590139$

H $0.113786-6.9241670 .732113$

C $0.317032-3.8312420 .352740$

H $6.386352-4.2245081 .026844$

H $7.186519-1.697936-0.641452$

H $4.930429-2.723516-3.927027$

H $-0.481919-4.5810970 .306422$

H $\quad 0.348643-3.322759-0.618668$

H $1.812079-4.6856431 .695928$

C $3.6141581 .418584-0.117457$

O $3.9623880 .457601-1.124668$

C $5.3519280 .383196-1.449755$

C $5.8498541 .736602-1.986948$

C $5.5288882 .876283-1.012879$

C $4.0737462 .825211-0.533891$

H $2.5169891 .415751-0.095159$

C 4.1100031 .0062121 .278799

H $5.9183490 .128293-0.549306$

O $5.2312392 .008253-3.243983$

H $6.9310301 .672880-2.158148$

H $5.7071803 .841210-1.503325$

O $\quad 6.3926292 .7982890 .116816$

H 3.9286553 .5192960 .301556

O $3.2243813 .277649-1.592696$

C $3.505645-0.3035841 .730594$

H $\begin{array}{llll}5.195704 & 0.886163 & 1.307697\end{array}$

H 3.8197571 .7729992 .006432

H $5.5947142 .847041-3.575176$

H $7.3067872 .871972-0.206368$

H $3.2824792 .631831-2.317740$

O $3.966320-0.6063342 .979223$

O $2.731642-0.9820121 .070928$

C $3.483828-1.8385463 .524633$

H $2.391804-1.8249423 .590611$

H $3.823095-2.6799742 .912938$

H $3.894192-1.9483154 .531817$

SCF Energy (B3LYP/6-31G**//MMFF) $=-3245.90409800$

0800606

MM̄FF Geometry

C $1.165616-3.5766751 .226166$

C $0.470941-2.4382761 .365759$

C $-0.119230-1.9573792 .667373$

O $-1.507474-1.6265942 .425267$

C $0.587880-0.6791643 .145421$

C $-0.080082-0.0246164 .339605$

C -0.7089601 .1680294 .310752$

C $0.003464-0.7998545 .629324$ 
C $-0.867243 \quad 2.105786 \quad 3.145989$ C -2.302360 2.1105872 .605866 C -2.453499 3.0616861 .409004 C $-3.911714 \quad 3.1451830 .956473$ C $-4.0725274 .030020-0.291060$ O $\begin{array}{lllll}-1.643681 & 2.560555 & 0.340509\end{array}$ O $-5.387496 \quad 3.802027-0.822211$ C $-3.062858 \quad 3.565110-1.364117$ C $-3.999603 \quad 5.5218570 .053743$ O $-3.0729124 .436630-2.491998$ C $-1.6243483 .386076-0.830902$ C $-0.6547842 .738849-1.855624$ O $-1.0837014 .677684-0.518151$ C -2.409009 -2.643439 2.413420 O $-2.192873-3.8291822 .602361$ C -3.721106 -2.050737 2.080255 C $-4.667527-2.8181971 .521717$ C $-5.971498-2.3529631 .045785$ C $-6.389312-0.953398 \quad 1.417269$ C $-1.1818051 .579850-2.705337$ C $-1.6454240 .357172-1.933945$ C -3.181494 -1.601560 -2.179622 O $-1.2638720 .091530-0.798543$ C $-4.642632-1.295873-2.063271$ C $-5.606716-2.016184-2.657348$ C $-7.075958-1.716461-2.537297$ C $-7.858362-2.862348-1.929011$ C $-8.457687-3.778897-2.707476$ C $-8.007501-2.899597-0.421427$ C $-6.701778-3.1816630 .270306$ N $-2.508956-0.422692-2.692159$ H $1.367673-4.1909902 .099870$ H $0.263049-1.8367040 .483849$ H $-0.056846-2.7294243 .444485$ H 0.6485810 .0199482 .307312 H $1.625153-0.9128523 .414949$ H -1.1591001 .5311925 .234059$ H -0.405458 -0.2408086 .477683$ H $-0.556109-1.7372035 .553518$ H $1.047172-1.0333085 .864265$ H -0.6026173 .1146643 .486879$ H -0.1668751 .8745892 .341375$ H -2.566541 1.0927102 .294953 H -2.995323 2.4043093 .403950 H -2.088721 4.0514901 .707864 H -4.5523493 .5084921 .769629$ H -4.2765652 .1366300 .715299$ H -6.033171 $4.019679-0.128177$ H -3.434993 2.598638 -1.724340 H -4.7748545 .7841970 .783603$ H -4.199697 $6.141000-0.827712$ H -3.037061 5.8190780 .474952 H -2.582635 $5.240321-2.248942$ H $-0.3034603 .514830-2.549257$ H $0.2388072 .394426-1.317506$ H $-0.1146014 .604451-0.496681$ H -3.848916 -0.9874652 .244847$ H -4.447535 -3.870104 1.343756 H -7.403049 -0.7003131 .101929$ H $-5.720876-0.214750 \quad 0.961994$ H $-6.364332-0.8233302 .505237$ H $-1.9885801 .937204-3.355267$ H $-0.3745961 .242806-3.368481$ H -2.776442 -1.873698 -1.200374 H -2.984927 -2.418750 -2.880688 H $-4.919347-0.444558-1.445914$ H -5.328266 -2.866056 -3.276440 H -7.455945 -1.498754 -3.544072 H -7.252582 - $0.803623-1.954735$ H $-9.041316-4.588506-2.282385$ H $-8.373880-3.741529-3.788690$ H -8.712179 $-3.687470-0.124660$ H -8.470909-1.964870 -0.095563 H $-6.307269-4.1770020 .060157$ H -2.739900 -0.128495-3.636705 C $3.118613-4.234114-0.261211$ O $3.671344-2.945734-0.620311$ C $4.354806-3.076367-1.878745$
C $4.729859-4.548115-1.972904$

C $3.490450-5.196137-1.397855$

H $3.621833-2.844022-2.661161$

C $5.549485-2.124239-1.995044$

H $4.942179-4.861419-2.999459$

H $5.601463-4.786970-1.352494$

C $3.709125-6.628000-0.938363$

H $2.706834-5.190484-2.167956$

C $5.254309-0.627816-1.774634$

O $6.551558-2.499687-1.039703$

H $6.015498-2.261480-2.978388$

H $5.148200-0.428643-0.704061$

O $6.4169740 .098859-2.198841$

C $4.032228-0.068746-2.522155$

C $3.9297711 .470917-2.542302$

H $3.121214-0.487239-2.078545$

O $4.078274-0.510999-3.882111$

H $4.6911381 .880933-3.218595$

H $2.9645001 .718471-3.004310$

H $4.012418-7.260033-1.779407$

H $4.490518-6.688789-0.173384$

H $2.790137-7.045575-0.514995$

C $1.598738-4.124768-0.104626$

H $6.141208-2.468027-0.158365$

H $7.175101-0.250553-1.699346$

H $3.266631-0.200739-4.318838$

H $1.145240-5.119848-0.192856$

H $1.188205-3.512151-0.917576$

H $3.594316-4.5345540 .680400$

C 3.0251932 .1045361 .027044

O $3.0094891 .632987-0.327435$

C $4.0390922 .152899-1.169842$

C $3.9212323 .681348-1.272196$

C 3.9430284 .3250360 .119278

C 2.9648253 .6424561 .080168

H 2.0992111 .7163521 .465712

C $4.197493 \quad 1.5174151 .831062$

H $5.016407 \quad 1.907242-0.743998$

O $2.7097864 .024863-1.941001$

H $4.7524934 .060601-1.878401$

H 3.6804165 .3868580 .037064

$\begin{array}{llllll}\text { O } & 5.256832 & 4.244047 & 0.664637\end{array}$

H 3.1442093 .9871062 .105307

$\begin{array}{lllll} & \text { O } & 1.644419 & 4.059824 & 0.726289\end{array}$

C 4.1003300 .0128911 .962767

H 5.1655461 .7377401 .374637

H 4.1804951 .9395812 .842783

H $2.6724714 .994400-2.002467$

H 5.8565464 .7080730 .056040

H 1.0318803 .6868891 .382709

O $5.012062-0.4184452 .882951$

O $3.338616-0.700698 \quad 1.325917$

C $5.038690-1.8332053 .098279$

H $5.807986-2.0493323 .844307$

H $4.072442-2.1764053 .480473$

H $5.293680-2.3540492 .170391$

SCF Energy (B3LYP/6-31G**//MMFF) $=-3245.89959904$

\section{7}

MM̄FF Geometry

C $-2.188984-2.3126312 .518274$

C $-0.941908-2.6311702 .895657$

C $0.241003-1.7232302 .698980$

O $1.138543-2.4284521 .808520$

C $0.971538-1.3997394 .015284$

C $0.233989-0.4031884 .888078$

C $0.369551 \quad 0.935517 \quad 4.798943$

C $-0.680658-0.996648 \quad 5.928548$

C $1.265477 \quad 1.7103993 .873478$

C 0.4944522 .2880182 .681273

C $1.3755413 .168677 \quad 1.780487$

C 0.6218253 .5482880 .504998

C $1.4971514 .345261-0.472329$

$\begin{array}{llll}\text { O } & 2.553005 & 2.436926 & 1.439243\end{array}$

O $0.811062 \quad 4.346405-1.734169$

C $2.8455103 .611616-0.663616$

C $1.6088115 .811825-0.039758$

O $3.7611444 .406063-1.413248$ 
C 3.5066893 .1727180 .663718

C $4.702778 \quad 2.233541 \quad 0.396783$

O $4.027770 \quad 4.292023 \quad 1.385394$

C $1.921391-1.678770 \quad 0.986938$

$\begin{array}{lllll}\text { O } & 2.036987 & -0.464158 & 0.987021\end{array}$

C $2.589040-2.6006840 .041043$

C $3.380819-2.101496-0.919817$

C $4.058746-2.868119-1.966297$

C $3.796523-4.349594-2.035631$

C $5.334174 \quad 1.702246 \quad 1.688859$

C $6.6443680 .999019 \quad 1.407358$

C $7.600916-1.1806320 .679857$

$\begin{array}{llll}\text { O } & 7.721876 & 1.577113 & 1.534666\end{array}$

C $7.757287-1.262158-0.805310$

C $7.859761-2.423169-1.469518$

C $8.018813-2.525944-2.959836$

C $6.967890-3.413262-3.589990$

C $7.231851-4.703339-3.858492$

C $5.643420-2.795285-3.979661$

C $4.876298-2.218037-2.820787$

N $6.480930-0.3122970 .993008$

H -2.379997-1.346612 2.059376

H $-0.753492-3.5991693 .353825$

H $-0.084161-0.7981472 .211626$

H $1.979495-1.0303173 .792968$

H $1.159223-2.3231854 .580425$

H $-0.217202 \quad 1.5578565 .473471$

H - $1.126071-0.2322216 .574256$

H $-1.501326-1.5468485 .461181$

H $-0.123772-1.6838076 .574082$

H 1.7216022 .5268284 .447082

H $2.096371 \quad 1.0914203 .523250$

H $0.094016 \quad 1.456142 \quad 2.090825$

H -0.3657892 .8730683 .030229$

H 1.6600344 .0623872 .348131

H $-0.2927514 .103743 \quad 0.744899$

H $0.2916582 .631789-0.003097$

H $1.3203764 .894848-2.354325$

H $2.6473352 .711123-1.261831$

H $0.622256 \quad 6.290605-0.060977$

H $2.2346736 .384745-0.732708$

H $2.010317 \quad 5.930198 \quad 0.969152$

H $3.3647834 .569812-2.285302$

H $4.3777331 .377350-0.207477$

H $5.4717642 .764654-0.178618$

H 3.9499565 .0847560 .831141

H $2.384239-3.6606280 .140287$

H $3.540456-1.024565-0.956032$

H $2.726439-4.546022-2.166457$

H $4.136114-4.843418-1.118486$

H $4.308287-4.840785-2.866525$

H $5.5452852 .514558 \quad 2.393541$

H $4.654423 \quad 1.0105032 .200254$

H $7.388338-2.1566101 .127791$

H $8.516647-0.7821321 .127456$

H $7.798288-0.326190-1.357326$

H $7.842501-3.356455-0.910763$

H $9.019411-2.930056-3.162030$

H $7.996638-1.538412-3.437241$

H $6.497768-5.344311-4.335009$

H $8.188122-5.148584-3.604471$

H $5.012859-3.510427-4.522223$

H $5.834039-1.989762-4.701202$

H $5.017006-1.144061-2.695288$

H $5.543221-0.6840640 .873254$

C $-3.876599-3.7633151 .360671$

O $-4.405270-2.692720 \quad 0.548594$

C $-5.696922-3.0832230 .052488$

C $-5.771617-4.593902 \quad 0.233357$

C $-5.015845-4.7703291 .533310$

H $-6.438020-2.6145540 .710679$

C $-5.909488-2.609703-1.389331$

H $-6.802507-4.9572310 .281430$

H $-5.259650-5.127109-0.575973$

C $-4.548773-6.195357 \quad 1.776433$

H -5.667838 -4.4623632 .362022$

C $-5.715434-1.098714-1.633862$

O $-4.997438-3.298084-2.255899$
H $-6.911781-2.908359-1.719096$

H $-4.646859-0.856183-1.644061$

O $-6.202666-0.817559-2.953137$

C $-6.436671-0.175149-0.639270$

C $-6.5276751 .303176-1.060657$

H $-5.938229-0.2435070 .334659$

O $-7.776700-0.645378-0.457691$

H -7.264933 $1.410611-1.867472$

H $-6.955125 \quad 1.843655-0.205419$

H $-5.404338-6.8764261 .830826$

H $-3.891659-6.5447870 .972964$

H $-3.997314-6.2702422 .718769$

C $-3.375076-3.2143692 .698427$

H $-4.097090-3.117833-1.935224$

H $-5.720233-1.395327-3.569245$

H -8.192964 -0.0899110 .223295$

H -3.121646 -4.0421853 .371010$

H $-4.165025-2.6301933 .186410$

H $-3.048733-4.2096510 .794466$

C -2.951246 $2.361857-0.679627$

O $-4.254056 \quad 1.811136-0.428775$

C $-5.2068971 .966641-1.484081$

C $-5.4143543 .453430-1.825822$

C $-4.084367 \quad 4.149432-2.124395$

C $-3.0401793 .854924-1.044448$

H $-2.430097 \quad 2.2888790 .282801$

C $-2.1677591 .539135-1.716610$

H $-4.8403461 .449782-2.375895$

O $-6.059898 \quad 4.110998-0.736625$

H $-6.080273 \quad 3.524819-2.694399$

H $-4.2505955 .232019-2.185913$

O $-3.614305 \quad 3.709313-3.394703$

H -2.057482 $4.218930-1.364393$

$\begin{array}{llll}\text { O } & -3.395115 & 4.594450 & 0.127251\end{array}$

C $-1.7240980 .205583-1.157356$

H $-2.7618591 .354225-2.614978$

H $-1.2562202 .066622-2.019678$

H $-6.2037705 .036996-0.995844$

H $-2.780707 \quad 4.175717-3.576753$

H $-2.704557 \quad 4.4411780 .794191$

O $-1.733693-0.734737-2.146075$

O $-1.370416 \quad 0.022675-0.000990$

C $-1.298145-2.041147-1.756301$

H $-0.230770-2.022285-1.518993$

H $-1.877033-2.402019-0.901410$

$\mathrm{H}-1.460086-2.718916-2.598614$

SCF Energy (B3LYP/6-31G**//MMFF) $=-3245.90504232$

0800608

MM̄FF Geometry

C $-2.643153-2.2484361 .758984$

C $-3.004307-3.2189570 .906336$

C $-3.410734-2.997286-0.533362$

O $-2.801954-1.793917-1.061986$

C $-4.933411-2.822415-0.652391$

C $-5.462450-3.226388-2.013328$

C $-5.645878-2.392142-3.054864$

C $-5.828293-4.683367-2.140723$

C $-5.302594-0.931953-3.154437$

C $-6.544207-0.035324-3.248451$

C $-7.1839380 .242255-1.879767$

C $-8.479717 \quad 1.039167-2.043439$

C $-9.081593 \quad 1.438664-0.688142$

O $-6.2403430 .997550-1.110862$

O $-10.091743 \quad 2.430319-0.928430$

C $-7.984613 \quad 2.108645 \quad 0.164902$

C $-9.7843120 .260053-0.004208$

$\begin{array}{llll}\text { O } & -8.454333 & 2.370083 & 1.485693\end{array}$

C -6.6653321 .3035590 .221942$

C $-5.5605392 .156952 \quad 0.892482$

O $\begin{array}{lllll} & -6.875517 & 0.119255 & 0.993861\end{array}$

C $-1.470218-1.810094-1.335355$

O $-0.706349-2.756679-1.246451$

C $-1.095276-0.429184-1.709054$

C $0.182991-0.037501-1.605939$

C $0.6953201 .305523-1.887278$

C $-0.2868382 .334104-2.387221$

$\begin{array}{llll}\text { C }-4.145928 & 1.571407 & 0.767509\end{array}$ 
C $-3.1225682 .379477 \quad 1.549413$

C -0.7093262 .3783762 .178934$

O -3.4058193 .4039562 .164988$

C 0.0419863 .2849331 .256219

C 1.3776933 .2639861 .128114

C 2.1408514 .2011710 .235489

C $3.1878563 .499515-0.601547$

C $4.4834563 .536353-0.248292$

C $2.7512352 .843159-1.892366$

C $2.0034721 .557391-1.669882$

N -1.854395 1.8160761 .484666

H -2.700562 -1.212954 1.439391

H $-2.952519-4.2557961 .230614$

H -3.066165 -3.851310 -1.130188

H $-5.209466-1.782653-0.439742$

H $-5.453312-3.4265050 .103427$

H $-6.060154-2.808762-3.972724$

H -6.195541-4.936080 -3.141086

H -4.956356 -5.314420 -1.941059

H -6.615547 -4.940803 -1.424647

H $-4.653850-0.601181-2.339884$

H -4.714398 $-0.804711-4.072348$

H $-6.2278790 .919756-3.688182$

H -7.279871 -0.478927 -3.930509

H -7.384344 -0.710459-1.377260

H $-9.2103800 .475817-2.637252$

H $-8.2744001 .956182-2.613675$

H $-10.7543502 .044171-1.526587$

H -7.772299 $3.087650-0.287551$

H -10.597112 -0.119168 -0.635142

H -10.2553710 .5718750 .934520$

H -9.114133 -0.5759770 .206453$

H -9.2742012 .8869611 .404423$

H -5.5419973 .1543280 .433677$

H $\quad-5.7990152 .292246 \quad 1.955181$

H $-6.037838-0.1533321 .399211$

H $-1.8920850 .238655-2.016584$

H $\quad 0.922917-0.760213-1.265179$

H $-0.6964052 .031723-3.357355$

H $\quad 0.1468293 .327139-2.519142$

H -1.114788 $2.455483-1.679885$

H -4.1246780 .5475351 .149169$

H $-3.8343831 .554154-0.283033$

H -0.0931741 .5426182 .524097$

H -1.0557672 .9432283 .050181$

H -0.5343664 .0111890 .687673$

H 1.9570712 .5619601 .723939

H 2.6216514 .9504790 .878119

H $1.4680204 .763132-0.424152$

H $5.2476203 .068402-0.857606$

H 4.8088014 .0326930 .659776

H $3.6276632 .601808-2.509072$

H $2.1869833 .566468-2.487501$

H $2.6330560 .748832-1.295532$

H -1.699991 1.0038970 .894606

C $-0.699938-1.8052623 .220261$

O $\quad 0.195670-2.3853742 .254073$

C $1.529356-2.4070052 .805369$

C $1.442754-1.6975264 .150803$

C $0.006076-1.9679324 .560400$

H $1.775493-3.4666542 .945905$

C $2.481702-1.7658541 .786455$

H $2.173255-2.0775534 .871372$

H $1.610443-0.6192494 .034671$

C $-0.500704-1.0353935 .645977$

H $-0.073332-3.0074404 .905278$

C $3.934122-1.6556492 .287925$

O $2.442831-2.5761460 .608459$

H $2.105831-0.7756561 .501499$

H $3.921066-1.0812963 .222226$

O $4.422574-2.9580552 .606038$

C $4.920350-0.9481181 .332815$

C $5.124227-1.643175-0.025636$

H $5.894307-0.8966601 .835417$

O $4.479838 \quad 0.389231 \quad 1.110981$

H $\quad 5.200407-2.7266750 .118534$

H $4.255878-1.430672-0.660384$

H $\quad 0.098533-1.1430946 .555989$
H $-0.448997 \quad 0.012478 \quad 5.331372$

H -1.541686 -1.259787 5.898827

C $-2.067160-2.4841153 .126535$

H $1.514757-2.6299320 .321883$

H $5.320665-2.8563262 .964439$

H $4.4189450 .828310 \quad 1.976353$

H -2.750631-2.071945 3.876353

H -1.966117 -3.559487 3.315422

H $-0.793054-0.7409892 .968514$

C $7.3439640 .845185-1.735079$

O $6.2248010 .221097-1.091830$

C $6.389902-1.161374-0.764307$

C $6.649056-1.984081-2.037543$

C $7.834966-1.424548-2.828765$

C $7.7275090 .092367-3.019252$

H $6.9808971 .837918-2.029206$

C $8.5191611 .044685-0.772241$

H $7.239336-1.273367-0.081796$

O $5.481677-1.970902-2.857446$

H $6.842660-3.025345-1.752872$

H $7.876023-1.913423-3.809726$

O $9.035506-1.744813-2.132105$

H $8.6700940 .492637-3.409247$

O $6.732794 \quad 0.362464-4.011900$

C 8.1475032 .0110640 .327161

H $8.8686300 .114471-0.315345$

H $9.382877 \quad 1.470671-1.296704$

H $5.660020-2.532944-3.630536$

H $9.779559-1.407106-2.659452$

H $5.8722780 .083273-3.654263$

O 7.7534611 .3251231 .437355

$\begin{array}{llll}\text { O } & 8.180261 & 3.228542 & 0.203305\end{array}$

C 7.3486922 .1291912 .549224

H 8.1810222 .7529712 .888604

H $\quad 6.4877742 .7465962 .277541$

H 7.0560271 .4601243 .362859

SCF Energy $\left(B 3 L Y P / 6-31 G^{* *} / / M M F F\right)=-3245.90517935$

0800609

MM̄FF Geometry

C $-0.5666901 .054738-2.032406$

C -1.232866 2.153404-2.421571

C $-1.2637653 .511156-1.762445$

O $-0.2836413 .589431-0.702323$

C $-2.6503993 .831053-1.168579$

C $-3.7460033 .990885-2.203103$

C $-4.6578573 .046568-2.509957$

C $-3.7800215 .328907-2.895192$

C $-4.7613441 .660397-1.939057$

C $-6.1283721 .423535-1.284855$

C $-6.1321980 .130685-0.457916$

C -7.545332 -0.2145550 .010332$

C $-7.556682-1.4335680 .944276$

$\begin{array}{lllll}\text { O } & -5.286214 & 0.355148 & 0.673569\end{array}$

O $-8.860349-1.4793171 .543458$

C $-6.517260-1.2124932 .064531$

C -7.385610 -2.743454 0.165656

O $-6.378593-2.3795712 .872636$

C $-5.127731-0.7723561 .542205$

C $-4.246322-0.3143302 .731961$

O $-4.509881-1.8693260 .872584$

C $0.2646944 .812089-0.466963$

O $0.0137355 .852044-1.061888$

C 1.2931604 .7594200 .593489

C 1.3230703 .8199081 .550310

C 2.3181693 .7217362 .621542

C 3.5130704 .6370522 .556334

C -2.8700110 .2485182 .342542$

C $-1.767418-0.7949662 .292668$

C $0.686111-0.9960541 .916039$

O $-1.951586-1.9829602 .542157$

C $1.441356-0.7434893 .180691$

C $2.723673-0.3522963 .206062$

C $3.476489-0.0433864 .467349$

C 4.0080631 .3742384 .491299

C 5.3215641 .6173464 .345891

C 3.0332862 .5014064 .762067

C 2.1381072 .8007783 .590534 
N $-0.546880-0.2331921 .942880$ H $0.0467991 .068260-1.136754$ H -1.821162 2.086605 -3.335912 H -1.003283 4.239475 -2.541178 H -2.590912 4.759914 -0.584780 H -2.909358 $3.078544-0.414124$ H $-5.4005943 .276799-3.272788$ H $-4.6037395 .405540-3.612881$ H -3.906586 $6.132123-2.161958$ H -2.848742 $5.501850-3.443391$ H $-4.6268100 .941406-2.756322$ H $-3.9610261 .455896-1.223903$ H $-6.3698282 .271931-0.630831$ H $-6.8981311 .376243-2.065012$ H -5.719657 -0.681173 -1.067761 H -8.210405 $-0.381135-0.846276$ H -7.9679890 .6425220 .553693$ H -8.910945 -2.276684 2.097195 H -6.906859 -0.4222262 .721579$ H -8.205772 $-2.870053-0.551169$ H -7.434781 -3.6108190 .833352$ H -6.447278 -2.792452 -0.390955 H -7.243290 -2.564362 3.275565 H -4.7784420 .4948613 .250170$ H -4.124349 -1.1332303 .450418$ H $-3.561436-1.6877130 .784351$ H 2.0128895 .5711340 .565731 H 0.5473853 .0561981 .560768 H 4.0293474 .5298911 .595620 H $4.2574314 .434593 \quad 3.329393$ H 3.2041415 .6812472 .676080 H $-2.9079680 .775851 \quad 1.383405$ H $-2.571654 \quad 0.976375 \quad 3.108434$ H $1.243257-0.6826631 .027980$ H $\quad 0.458487-2.060507 \quad 1.839592$ H $0.906105-0.8765754 .117948$ H $3.260177-0.2339902 .268815$ H $4.301824-0.7623254 .547693$ H $2.852884-0.2056935 .355269$ H 5.7188722 .6257234 .389427 H 6.0300650 .8132984 .176308 H 3.5612223 .4058995 .085844 H 2.4037922 .2318855 .620123 H 1.2382132 .1869553 .557743 H $-0.486127 \quad 0.7705331 .797622$ C -1.097973 -1.414769-1.965354 O $0.052984-2.073671-1.388856$ C $-0.009003-3.477141-1.713227$ C $-1.469868-3.747500-2.039144$ C $-1.827036-2.481508-2.789748$ H $0.586020-3.618863-2.623509$ C $0.566302-4.334674-0.582772$ H -1.605019 -4.654708 -2.635235 H -2.080362 -3.835422 -1.132932 C -3.322989-2.245471-2.908385 H - $-1.394867-2.538680-3.798045$ C $2.012805-3.961688-0.196303$ O $-0.264229-4.1746750 .572383$ H $0.503573-5.394499-0.854895$ H $2.025763-2.943857 \quad 0.201272$ O $2.408784-4.8112640 .888159$ C $3.043150-4.092595-1.334829$ C $4.484660-3.760846-0.903129$ H $2.769957-3.452149-2.179426$ O $3.053351-5.440777-1.812386$ H $4.752494-4.335334-0.008155$ H $5.154310-4.116850-1.698096$ H -3.798444 -3.060108 -3.464367 H $-3.799077-2.188632-1.924384$ H -3.528762 -1.309522 -3.436939 C $-0.627076-0.230879-2.816941$ H $0.126173-4.7059371 .287298$ H $2.455585-5.7203920 .545462$ H $2.220530-5.591687-2.290114$ H -1.300421 -0.099896-3.672756 H $\quad 0.373818-0.429085-3.219304$ H -1.725901 -1.081492 -1.129209 C $4.683309-0.178385-1.903453$
O $4.555875-1.604839-1.931019$

C $4.726327-2.260023-0.669381$

C $6.136383-1.986533-0.121441$

C $6.424930-0.484696-0.035814$

C $6.0455460 .248447-1.328648$

H $4.6661610 .114147-2.961317$

C $3.4672170 .457463-1.222620$

H $3.983501-1.8783230 .038659$

O $7.120231-2.607818-0.947156$

H $6.222525-2.4368440 .874387$

H $7.492795-0.3406720 .169392$

O $5.6993020 .055798 \quad 1.062873$

H $6.0595521 .333308-1.176988$

O $7.031781-0.044318-2.324911$

C $3.2884541 .891232-1.654029$

H $2.551991-0.096225-1.466832$

H $3.5318610 .431871-0.131734$

H $7.006654-2.267963-1.851448$

H 5.9113431 .0030061 .119473

H $7.8877740 .276189-1.992786$

O $2.6776661 .937113-2.872210$

O $3.6729662 .852022-1.000604$

C $2.4982063 .243848-3.426155$

H $1.9444683 .883946-2.734565$

H $3.4707203 .684824-3.664046$

H $1.9205793 .145126-4.349024$

SCF Energy $\left(B 3 L Y P / 6-31 G^{* *} / / M M F F\right)=-3245.91088626$

0800610

MM̄FF Geometry

C $-0.9905584 .246776-0.706099$

C $0.1811253 .610091-0.556150$

C $1.0536113 .221775-1.726704$

O $1.4863141 .850464-1.557330$

C $2.2975454 .126326-1.767985$

C $3.0907314 .008099-3.055566$

C $4.3285343 .485797-3.159488$

C $2.4290524 .599134-4.276444$

C $5.1755392 .875430-2.078404$

C $5.7141921 .505442-2.510913$

C $6.6266460 .883231-1.443692$

C $7.199448-0.443708-1.946021$

C $8.028891-1.158239-0.869773$

O $5.842820 \quad 0.674447-0.267349$

O $8.259002-2.504221-1.314803$

C $7.187822-1.2440590 .420878$

C $9.410752-0.518205-0.695721$

O $7.955977-1.7910921 .491202$

C 6.5507600 .1031290 .838691

C $5.542388-0.0783572 .000035$

O 7.5881880 .9764191 .297588

C $0.5753750 .880625-1.849674$

O $-0.5639011 .044938-2.258768$

C $1.192410-0.434348-1.561784$

C $0.397771-1.512705-1.483530$

C $0.808215-2.888566-1.195417$

C $2.281144-3.195089-1.188716$

C $4.221974-0.7243891 .575352$

C $3.343947-1.1211922 .747451$

C $1.070017-2.0642853 .184847$

O $3.744267-1.1676043 .906593$

C $0.931352-3.5035342 .798265$

C $-0.206630-4.0515652 .345995$

C $-0.328667-5.4930201 .941869$

C $-0.875333-5.6685590 .541611$

C $-2.080575-6.2264360 .335457$

C $0.004647-5.265761-0.625545$

C $-0.153253-3.810116-0.976297$

N $2.068898-1.4661102 .321694$

H $-1.3072004 .498303-1.717449$

H 0.5480683 .3530380 .432910

H $\quad 0.4965513 .305654-2.667777$

H $2.9276543 .917537-0.897253$

H $1.9963175 .178121-1.665167$

H $4.8054073 .506093-4.139205$

H $3.0958634 .613901-5.145379$

H $1.5426924 .021729-4.555381$

H $2.1258755 .633209-4.082026$ 
H $6.0167403 .550309-1.880335$ H $4.6244382 .765551-1.141393$ H $4.8624560 .838215-2.699638$ H $6.2693051 .605188-3.452288$ H $7.4343061 .589019-1.216924$ H $7.797784-0.290572-2.852880$ H $6.374480-1.107363-2.242111$ H $8.725058-2.462807-2.167348$ H $6.392719-1.9726710 .223878$ H $9.963474-0.538083-1.642745$ H $10.021346-1.0839470 .016635$ H $9.3637870 .521062-0.364113$ H $8.548580-1.0926271 .817294$ H $6.015227-0.6352622818381$ H 5.3005970 .9043682 .428252 H 7.1879621 .6677991 .850246 H $2.263722-0.475197-1.403581$ H $-0.673448-1.373949-1.625057$ H $2.500260-4.264316-1.148196$ H $2.768325-2.729109-0.328895$ H $2.757547-2.823826-2.103276$ H $3.661630-0.0302840 .937743$ H $4.407671-1.6375241 .002171$ H $\quad 0.143057-1.5048003 .038977$ H $1.371116-1.9821834 .233386$ H $1.819148-4.1248682 .892363$ H -1.100016 -3.4376492 .265138$ H $-0.983675-5.9872052 .670891$ H $0.638209-6.0074122 .009838$ H -2.468834 -6.376108 -0.666580 H $-2.708596-6.5398311 .162731$ H $-0.267921-5.851314-1.513114$ H $1.037433-5.540076-0.393025$ H $-1.193481-3.490986-1.047388$ H $1.841206-1.3847331 .335400$ C -1.723431 4.1514841 .785507 O $-1.969142 \quad 2.7286991 .806582$ C -2.983248 2.4515972 .786849 C $-2.9262873 .621633 \quad 3.756696$ C -2.697033 4.7717192 .796598 H -3.946309 2.476996 2.263018 C -2.787474 1.0702333 .417300 H -3.844801 3.7263014 .341685 H -2.082249 3.5354004 .450510 C -2.166185 6.0281113 .465888 H -3.6498495 .0109992 .304857$ C -2.653885 -0.0855582 .403815$ $\begin{array}{lllll}\text { O } & -1.590669 & 1.097792 & 4.205128\end{array}$ H -3.612306 0.8741804 .113292 H $-1.654160-0.0727881 .951891$ O $-2.759257-1.3184513 .127947$ C $-3.712115-0.1007041 .285928$ C $-3.579505-1.3467020 .386881$ H -3.6011320 .7990660 .671112$ O $-5.006917-0.0652941 .883058$ H $-2.524406-1.4939180 .130223$ H -3.925647 -2.2238970 .947543$ H -2.880985 6.4005614 .206934 H -1.217728 5.8389823 .979818 H -1.9984006 .8199912 .729054$ C -1.9514004 .6848950 .366638$ H $-1.5851590 .302524 \quad 4.763447$ H -2.020886 -1.359667 3.757501 H $-5.661914-0.0389941 .165238$ H $-1.946578 \quad 5.7813760 .382019$ H $-2.9500014 .371507 \quad 0.034692$ H -0.6873874 .3084452 .107424$ C $-6.697043-1.045134-1.661167$ O $-5.784253-1.212975-0.569450$ C $-4.396615-1.229744-0.915772$ C $-4.101415-2.386854-1.882933$ C $-5.014302-2.332944-3.111686$ C $-6.484057-2.135351-2.724061$ H $-7.690428-1.197537-1.219437$ C $-6.6501430 .377528-2.229229$ H $-4.130558-0.281072-1.394910$ O $-4.283851-3.629380-1.206995$ H $-3.052035-2.328213-2.193705$
H $-4.908880-3.265744-3.679295$

O $-4.582726-1.263796-3.948265$

H $-7.081806-1.900781-3.611886$

O $-6.992713-3.369889-2.209054$

C $-7.0944051 .379218-1.189295$

H $-5.6569500 .660379-2.589428$

H $-7.3278070 .478893-3.085015$

H $-4.036195-4.338349-1.824724$

H $-5.152624-1.259358-4.736148$

H $-6.521209-3.562997-1.380360$

O $-6.0204542 .078906-0.724032$

O $-8.254636 \quad 1.507795-0.820185$

C -6.3102493 .0571110 .279125$

H $-7.066543 \quad 3.762501-0.078556$

H $-6.6437002 .564090 \quad 1.196927$

H -5.3913483 .6098590 .490123$

SCF Energy (B3LYP/6-31G**//MMFF)= -3245.91180333

0800611

MM̄FF Geometry

C $-0.5416293 .537409-0.888089$

C $-1.5882993 .965370-0.165946$

C $-2.298025 \quad 3.119640 \quad 0.856823$

O \begin{tabular}{lllll}
\hline & -3.669466 & 3.004298 & 0.403559
\end{tabular}

C $-2.269714 \quad 3.757117 \quad 2.259060$

C $-0.894264 \quad 3.8862882 .875515$

C $-0.2029712 .860105 \quad 3.409198$

C $-0.338007 \quad 5.2846912 .922212$

C $-0.614634 \quad 1.413927 \quad 3.463285$

C $0.5093780 .504928 \quad 2.948496$

C $0.056851-0.9583162 .828688$

C $1.271621-1.8800202 .713588$

C $0.864276-3.3426962 .498483$

O $-0.753371-1.0724401 .651763$

O $2.036336-4.0765202 .113206$

C $-0.120534-3.4050691 .315520$

C $0.349825-3.9938113 .787228$

O $-0.639835-4.723644 \quad 1.155109$

C $-1.282153-2.3853851 .416168$

C $-2.058018-2.3675830 .075371$

O $-2.167538-2.7857042 .463555$

C -4.4240312 .0001800 .925596$

$\begin{array}{llll}\text { O } & -4.106088 & 1.223975 & 1.812183\end{array}$

$\begin{array}{llll}\text { C } & -5.713732 & 1.985663 & 0.200968\end{array}$

C -6.5738760 .9768340 .406649$

C $-7.8740230 .801086-0.244563$

C $-8.2896321 .838324-1.255430$

C $-3.089364-1.241213-0.044355$

C $-3.754500-1.251784-1.406610$

C $-5.392810-2.537620-2.780506$

O $-3.468815-0.426117-2.270674$

C $-6.718040-1.845077-2.737290$

C $-7.886325-2.493070-2.613301$

C $-9.232921-1.826647-2.632466$

C $-9.943537-1.908903-1.300059$

C $-10.587210-3.027385-0.925251$

C $-9.975588-0.663740-0.442391$

C $-8.617317-0.2787850 .078179$

$\mathrm{N}-4.670165-2.281124-1.549856$

H $-0.1726902 .524461-0.745757$

H $-1.9634924 .974923-0.317298$

H $-1.8580102 .115532 \quad 0.872531$

H -2.907361 3.1862382 .945771

H -2.7571514 .7417102 .221075$

H 0.7618203 .0759813 .866765

H 0.6780535 .3158703 .329512

H $-0.303315 \quad 5.7189601 .918914$

H $-0.969518 \quad 5.920846 \quad 3.551121$

H -0.8313651 .1572874 .507485$

H -1.5320791 .2277702 .900470$

H 0.8501960 .8641681 .968275

H $1.3574280 .576468 \quad 3.641302$

H $-0.540582-1.2205733 .709567$

H $1.919521-1.7883813 .594187$

H $1.880044-1.5631131 .856781$

H $2.695100-3.9828192 .822572$

H $0.452664-3.1858710 .403458$

H $1.122720-3.9658154 .564788$ 
H $\quad 0.121904-5.0538153 .629802$ H -0.539999 -3.5059814.190046 H $\quad 0.119496-5.326977 \quad 1.081925$ H -1.346328 -2.246920 -0.752398 H -2.560379 -3.331813 -0.071337 H -3.043983 -2.403755 2.300258 H $-5.9105172 .793909-0.494065$ H $-6.2998520 .196188 \quad 1.115377$ H -8.393425 $2.819098-0.778536$ H $-9.2412971 .619796-1.743456$ H $-7.545748 \quad 1.913497-2.056805$ H $-3.866293-1.3230790 .722344$ H $-2.606642-0.2662810 .079310$ H -5.498864 -3.623301 -2.873432 H $-4.812257-2.175766-3.635390$ H $-6.713565-0.763694-2.847562$ H -7.881386 -3.576832 -2.517491 H $-9.843622-2.314848-3.403523$ H $-9.152230-0.780428-2.952002$ H -11.122801 -3.084631 0.016489 H -10.590470 -3.912096 -1.553690 H -10.623498 -0.8184190 .430725$ H -10.455697 $0.140104-1.007256$ H -8.222641 -0.9791820 .815565$ H $-4.883143-2.868512-0.748941$ C $1.6586724 .552243-1.675534$ O $2.3393333 .289140-1.798650$ C $3.4081873 .248169-0.833364$ C $3.5140834 .656307-0.254119$ C $2.0714395 .118293-0.319004$ H $3.0849712 .560330-0.042000$ C $4.7114682 .728576-1.459223$ H 3.9210414 .6565840 .761695 H $4.1506585 .300042-0.872738$ C $1.9015836 .621483-0.196603$ H 1.5222094 .6262350 .492499 C $4.5994491 .260918-1.927432$ O $5.0669933 .583322-2.543752$ H $5.5068242 .800841-0.707163$ H $4.2183280 .681459-1.080859$ O $3.6373041 .145490-2.974127$ C $5.9342330 .641780-2.393046$ C $5.819407-0.831504-2.838045$ H $6.6681920 .729630-1.583440$ O $6.441408 \quad 1.388997-3.500641$ H $5.150019-0.906682-3.704137$ H $6.808833-1.143179-3.198514$ H 2.2783326 .9734230 .769206 H $2.4465437 .152937-0.983889$ H $0.845767 \quad 6.900927-0.269489$ C $0.1576704 .377844-1.919864$ H $5.9245893 .282265-2.887964$ H $3.9453751 .698174-3.712894$ H $7.3111311 .020219-3.731176$ H $0.0137723 .884070-2.889167$ H $-0.3116125 .366313-1.990691$ H $2.0573615 .186044-2.479304$ C $5.998623-2.4624870 .510347$ O $6.318854-1.715528-0.670027$ C $5.354402-1.781401-1.724728$ C $5.198715-3.229463-2.208335$ C $4.816378-4.146489-1.040741$ C $5.731211-3.9403420 .173363$ H $6.906907-2.4213431 .125385$ C $4.865113-1.7958651 .303339$ H $4.383730-1.435491-1.360022$ O $6.420859-3.673385-2.791854$ H $4.425618-3.263862-2.985437$ H $4.875319-5.191190-1.369518$ O $3.463766-3.877679-0.682638$ H $5.313960-4.4444831 .052419$ O $6.988442-4.570915-0.093519$ C $5.244259-0.4058471 .773920$ H $3.961319-1.706500 \quad 0.695931$ H $4.630332-2.3893902 .193568$ H $6.279665-4.580413-3.112978$ H $3.228417-4.4723840 .049823$ H $7.409264-4.094590-0.829840$
O $4.113244 \quad 0.271745 \quad 2.130407$

O 6.3870500 .0255521 .843740

C 4.3175811 .6047282 .610312

H 4.7850471 .5745103 .598614

H 4.9312752 .1803951 .910683

H 3.3418242 .0899822 .695718

SCF Energy (B3LYP/6-31G**//MMFF) $=-3245.91603008$

0800612

MM̄FF Geometry

C -1.002229-1.678782 2.427713

C $-1.632132-2.3783591 .469590$

C $-1.033290-2.8273520 .155380$

O $0.362052-2.4637300 .097599$

C $-1.764717-2.112549-0.997869$

C $-1.190273-2.388260-2.373902$

C $-0.558998-1.471665-3.136939$

C $-1.379631-3.793758-2.881209$

C $-0.303299-0.025538-2.806848$

C $1.1102740 .178106-2.262189$

C $1.2960071 .509032-1.521150$

C $1.1991362 .719631-2.451559$

C $1.5140374 .037470-1.721725$

O $2.593071 \quad 1.458941-0.908613$

O $1.7341095 .048284-2.718397$

C $2.8379123 .874930-0.942799$

C $0.3289364 .518172-0.876233$

O $3.1087885 .025385-0.146028$

C $2.8946302 .593029-0.084613$

C 4.2813502 .3619830 .557412

$\begin{array}{llll}\text { O } & 1.956676 & 2.710221 & 0.992817\end{array}$

C $1.296306-3.4500930 .135159$

O $1.108042-4.652146 \quad 0.217648$

C $2.617054-2.7898920 .035836$

C $3.720847-3.509484-0.212843$

C $5.066302-2.962554-0.397792$

C $5.238598-1.471377-0.267978$

C $5.4526292 .260967-0.423334$

C 6.7397631 .8426420 .270298

C $9.1366631 .356974-0.213998$

O 6.8126811 .6235601 .476700

C $9.352606-0.084277-0.552791$

C $9.418921-1.0620510 .363718$

C $9.676970-2.5063160 .037809$

C $8.442089-3.3640380 .218050$

C $8.245535-4.0365431 .365094$

C $7.512970-3.422570-0.978051$

C $6.085247-3.801416-0.679717$

N $7.7999981 .747879-0.621248$

H $0.044851-1.4170952 .308039$

H -2.681572 -2.627688 1.611908

H -1.163938 -3.9120750 .066154$

H -1.760670 -1.038625 -0.792460

H $-2.822198-2.406577-1.000815$

H $-0.176395-1.783671-4.107715$

H -1.019853 -3.916676 -3.908406

H $-0.835540-4.507077-2.254779$

H -2.441834 -4.059583 -2.871914

H $-0.4403520 .566403-3.719731$

H - $1.0430020 .354354-2.098582$

H $1.360722-0.629605-1.567030$

H $1.8449470 .093355-3.074048$

H $0.5377621 .567753-0.730981$

H $0.2115412 .777357-2.924461$

H $1.9181342 .600920-3.274340$

H $0.9327235 .109105-3.266148$

H $3.6335503 .835403-1.696847$

H $-0.5486334 .687592-1.511262$

H $0.5433405 .484258-0.406308$

H $0.0415503 .809313-0.097019$

H 2.5385234 .9820880 .640411

H 4.4859423 .1486351 .295751

H $4.235277 \quad 1.4280071 .135553$

H 2.2085282 .0827361 .690695

H $2.639774-1.7107800 .140350$

H $3.630091-4.589460-0.318287$

H $4.669321-0.950315-1.045357$

H $6.276913-1.146581-0.357429$ 
H $4.895082-1.1269060 .713804$ H $5.232877 \quad 1.527898-1.206775$ H $5.633566 \quad 3.231927-0.896812$ H 9.2706061 .5509250 .855434 H $9.8481341 .979472-0.765375$ H $9.479728-0.329249-1.604583$ H $9.291834-0.8139911 .415308$ H $10.488065-2.8607510 .687167$ H $10.055192-2.621828-0.985964$ H $7.385832-4.6787961 .519727$ H $8.953047-3.9638022 .184989$ H $7.919589-4.147338-1.695517$ H $7.528038-2.464418-1.510378$ H $5.885940-4.871299-0.740766$ H $7.6307011 .900838-1.611195$ C -1.8534320 .3262703 .734166$ $\begin{array}{lllll}\text { O } & -2.637998 & 0.768767 & 2.607976\end{array}$ C -2.0658861 .9895492 .102886$ C -1.119569 2.4868203 .189345 C $-0.580178 \quad 1.1734543 .725857$ H -1.4678291 .7172121 .224745$ C -3.1653062 .9759571 .697828$ H -0.3401483 .1444322 .793535$ H -1.654860 3.0287013 .977687 C 0.0896641 .2956835 .082691 H 0.1476650 .7828703 .004441 C $-4.1707062 .419606 \quad 0.664732$ O -3.9028693 .3448872 .869375$ H $-2.7044743 .897746 \quad 1.322448$ H $-4.8125621 .673770 \quad 1.145578$ $\begin{array}{lllll}\text { O } & -5.037485 & 3.495360 & 0.283277\end{array}$ C $-3.5246511 .821674-0.595456$ C $-4.4977051 .485391-1.736975$ H $-2.9675830 .920679-0.321311$ O $-2.5772722 .764743-1.106194$ H $-4.9186902 .410858-2.151035$ H -3.890525 $1.055985-2.545459$ H 0.9513451 .9688665 .026671 H -0.5982931 .6933085 .836397$ H $\quad 0.444464 \quad 0.320268 \quad 5.430301$ C $-1.669097-1.1939653 .684992$ H -4.596432 3.9673302 .591014 H -4.498305 $4.152355-0.189991$ H -2.146109 2.364072 -1.879596 H -2.655580 -1.664127 3.776259 H -1.081189 -1.5280224 .547284$ H -2.447050 0.5643554 .626902 C $-5.975818-1.716222-0.464161$ O $-5.054287-0.683536-0.846458$ C $-5.6344440 .513071-1.376308$ C $-6.5098550 .210100-2.606788$ C -7.544622 -0.876287 -2.304887 C $-6.900355-2.091537-1.634365$ H -5.342991 -2.588084 -0.253611 C $-6.744122-1.3582110 .814150$ H $-6.2557510 .981035-0.606581$ O $-5.685505-0.216552-3.691040$ H -7.009093 $1.135366-2.918871$ H -8.026754 -1.197238 -3.236288 O $-8.553635-0.352762-1.446890$ H -7.676940 -2.779193 -1.280754 O $-6.134940-2.813372-2.603457$ C -5.901799-1.633589 2.035889 H -7.050427 -0.3099510 .863283$ H -7.661172 -1.9531490 .906228$ H $-6.262700-0.359025-4.460400$ H -8.967538 $0.398824-1.904454$ H $-5.385027-2.253288-2.868187$ O $-6.097712-2.9170142 .453489$ O $-5.149654-0.8135612 .543875$ C $-5.369395-3.3163683 .619118$ H -5.812440 -4.242795 3.994285 H $-4.327195-3.509148 \quad 3.352091$ H -5.433639 -2.557861 4.405269 SCF Energy (B3LYP/6-31G**//MMFF) $=-3245.90885578$

0800613

MM̄MF Geometry
C $-0.470946-0.6512361 .633846$ C $-0.320105-1.8779151 .113217$ C $-0.740031-2.278640-0.283796$ O $-0.810474-1.118466-1.147692$ C $-2.136263-2.919221-0.257023$ C $-2.429845-3.726886-1.504220$ C $-3.122282-3.278361-2.569004$ C $-1.918857-5.144801-1.478476$ C $-3.680589-1.902083-2.804726$ C $-5.215085-1.881056-2.791083$ C $-5.802542-1.893712-1.371485$ C $-7.326274-2.022429-1.422197$ C $-7.955442-1.920694-0.025215$ O $-5.437526-0.659168-0.744129$ O $-9.369880-1.737285-0.190279$ C $-7.414056-0.6559780 .671983$ C $-7.775931-3.2131230 .779645$ O $-7.858946-0.5903172 .025215$ C $-5.873405-0.5254960 .614200$ C $-5.4527210 .878500 \quad 1.114181$ O $-5.288778-1.5208571 .455675$ C $0.355934-0.636763-1.653085$ O $1.466460-1.128289-1.541017$ C $0.0519460 .644551-2.323254$ C $1.0608461 .453546-2.676606$ C $0.9042952 .758551-3.310188$ C $1.9176713 .023887-4.390089$ C $-3.969527 \quad 1.2114330 .899404$ C -3.6216982 .6095031 .386173$ C -1.7054004 .2058961 .492686$ O -4.4278773 .3446291 .949995$ C -1.720244 5.1111050 .302226 C $-0.6158745 .668635-0.217603$ C $-0.6269306 .596991-1.397533$ C $0.2407616 .109463-2.536695$ C $1.4250406 .687303-2.799588$ C $-0.3033045 .015305-3.427855$ C $-0.0273623 .637756-2.888919$ N -2.295734 2.9307831 .128235 H $-0.971643 \quad 0.1121561 .045317$ H $\quad 0.159579-2.651151 \quad 1.708207$ H $0.001569-2.980826-0.685083$ H $-2.898363-2.142394-0.124294$ H $-2.238350-3.5846870 .610730$ H -3.288807 -3.968503 -3.395565 H $-2.163170-5.692793-2.394707$ H $-0.830065-5.158283-1.367657$ H $-2.360811-5.691908-0.639335$ H -3.280319 -1.163753 -2.104600 H -3.339178 -1.583697 -3.797991 H $-5.537182-0.961831-3.297703$ H -5.606202 -2.725681 -3.371827 H $-5.370683-2.729223-0.809951$ H -7.621524 -2.960568 -1.908583 H -7.739822 -1.218405 -2.047308 H $-9.713362-2.494736-0.694499$ H -7.8530930 .2116430 .159634$ H $-8.243370-4.0568140 .257900$ H -8.278501 -3.1465721 .750898$ H $-6.728585-3.4710920 .949892$ H -8.828883 -0.6614882 .014912$ H -6.0406141 .6394210 .583894$ H -5.6842610 .9744422 .182681$ H $-4.409777-1.2247561 .738172$ H $-0.9882090 .908391-2.482858$ H $2.0907021 .147009-2.505163$ H $2.9354702 .916930-3.997557$ H $1.8486804 .027281-4.815418$ H $1.7899392 .311594-5.212817$ H $-3.3387620 .505643 \quad 1.447487$ H $-3.7161521 .149206-0.164986$ H -0.6885324 .0064541 .843768$ H -2.274272 4.6578952 .311362 H $-2.6882005 .330685-0.142187$ H $\quad 0.3496415 .4645790 .239816$ H $-0.2783517 .578310-1.050158$ H -1.646669 $6.761418-1.767297$ H $2.0385256 .364031-3.633938$ 
H $1.8100947 .493395-2.183640$ H $0.0826085 .129419-4.446761$ H - $1.3888455 .136638-3.535583$ H - $0.6838693 .343987-2.070561$ H -1.7252212 .2841340 .591763$ C 0.8587321 .0585322 .828356 $\begin{array}{lllll}\text { O } & 2.072067 & 0.777327 & 2.106821\end{array}$ C $3.0671261 .744922 \quad 2.502350$ C 2.4306362 .5998113 .598656 C $1.337407 \quad 1.687438 \quad 4.129038$ H 3.9009531 .1849192 .936657 C 3.5361832 .5280661 .268044 H 3.1558672 .8865704 .366743 H 2.0014213 .5240003 .193736 C $0.260272 \quad 2.423324 \quad 4.905491$ H 1.7947780 .9229814 .771099 C $4.123591 \quad 1.632996 \quad 0.154953$ $\begin{array}{lllll}\text { O } & 2.418095 & 3.236516 & 0.714303\end{array}$ H 4.2634923 .2929441 .561478 H $3.3593140 .931663-0.195813$ $\begin{array}{lllll}\text { O } & 4.445540 & 2.469944 & -0.963947\end{array}$ C $5.3986350 .870694 \quad 0.569125$ C $5.981289-0.003333-0.560021$ H $5.1992920 .245279 \quad 1.444314$ O 6.3871501 .8290910 .955776 H $6.0032150 .564241-1.497706$ H $7.028472-0.228612-0.317287$ H 0.6916792 .9287515 .775686 H $-0.234367 \quad 3.1804844 .288363$ H $-0.505844 \quad 1.729755 \quad 5.265379$ C $0.031988-0.2167952 .981260$ H 1.7583442 .5769670 .438862 H $3.6376162 .954943-1.205153$ H 7.1603811 .3379931 .281817 H $-0.832231-0.0327743 .628349$ H $0.634939-1.0099643 .436250$ H 0.2974841 .7969022 .240823 C $4.885763-3.433508 \quad 0.421325$

O $5.526862-2.151536 \quad 0.381194$

C $5.208169-1.320766-0.743228$

C $5.574427-2.026096-2.056113$

C $4.906637-3.402292-2.142550$ C $5.141030-4.224655-0.873216$ H $5.390869-3.9701411 .235534$ C $3.400793-3.3063970 .791157$ H $4.136249-1.107142-0.742492$ O $6.990193-2.167994-2.144485$ H $5.253365-1.396667-2.894856$ H $5.304856-3.949484-3.005541$ O $3.503681-3.249687-2.333564$ H $4.517166-5.126122-0.878256$ O $6.502768-4.662937-0.845532$ C $3.227280-3.1767302 .287763$ H $2.898454-2.4563910 .325139$ H $2.855749-4.2100950 .493553$ H $7.189839-2.597693-2.993525$ H $3.370907-2.728969-3.143867$ H $6.652614-5.204605-1.638948$

O $3.762986-2.0036442 .728964$

O $2.675674-4.0219822 .981249$

C $3.717187-1.8018494 .144823$

H $4.275701-2.5912414 .656874$

H $4.187787-0.8411024 .368275$

H $2.681131-1.7754894 .493272$

SCF Energy (B3LYP/6-31G**/MMFF) $=-3245.89504364$

0800614

MM̄FF Geometry

C $-3.020571-3.2414431 .028048$

C $-2.067685-2.3774110 .645398$

C $-1.068886-2.672085-0.442538$

O $0.252866-2.5892290 .140485$

C $-1.164939-1.614527-1.559380$

C $-0.829451-2.185250-2.922412$

C $0.346617-2.044318-3.562984$

C $-1.953709-2.947100-3.581366$

C $1.580363-1.313971-3.115246$

C $2.675441-2.297432-2.688798$
C $4.060022-1.641635-2.585721$

C $5.070090-2.621217-1.982024$

C $6.451247-1.979542-1.784456$

O $3.967432-0.470523-1.770705$

O $7.212694-2.829479-0.912898$

C $6.279774-0.628427-1.059029$

C $7.232455-1.896997-3.101235$

O $7.5194470 .073143-0.992062$

C $5.1916670 .275109-1.684816$

C $4.9409601 .500565-0.778790$

O $5.6349890 .724291-2.966009$

C $0.815373-3.7374210 .597498$

O $0.352314-4.8661170 .499751$

C $2.111763-3.4888721 .271674$

C $2.575478-2.2683851 .583793$

C $3.819129-1.9746482 .305122$

C $4.704938-3.1364332 .671504$

C $3.8023122 .414315-1.251916$

C $3.4728393 .431921-0.178717$

C 2.4869213 .5808092 .104236

O $3.9020654 .582267-0.222614$

C 3.6105773 .1940153 .014289

C 3.4773202 .3602604 .056489

C 4.6382061 .8852194 .882374

C $4.880680 \quad 0.397118 \quad 4.738247$

C $4.810268-0.4227785 .800355$

C $5.272589-0.1352853 .374096$

C $4.091585-0.6907152 .619722$

N 2.6979752 .8979340 .840483

H -3.078618 -4.2193250 .556476$

H $-2.010561-1.3991751 .117502$

H $-1.236407-3.674808-0.853919$

H $-0.522102-0.760144-1.324054$

H $-2.184222-1.209616-1.621123$

H $\quad 0.448230-2.509413-4.543526$

H $-2.249967-3.805997-2.971433$

H $-2.825268-2.296751-3.711341$

H $-1.676874-3.326923-4.570588$

H $1.931796-0.705347-3.957553$

H $1.369577-0.614846-2.300880$

H $2.394471-2.717430-1.716688$

H $2.734980-3.137892-3.392223$

H $4.366846-1.348901-3.597115$

H $5.150142-3.526212-2.597185$

H $4.706318-2.958926-1.001502$

H $7.264267-3.709896-1.322969$

H $5.992403-0.847382-0.021427$

H $7.386399-2.899635-3.518353$

H $8.234309-1.483283-2.941649$

H $6.727763-1.298506-3.862380$

H $8.168080-0.523695-0.580954$

H $4.709531 \quad 1.1474350 .233165$

H $5.8528672 .106678-0.704225$

H $5.1094481 .495212-3.230218$

H $2.654749-4.3894281 .540886$

H $1.983068-1.3959601 .313254$

H $4.170553-3.8386013 .320912$

H $5.609736-2.8463913 .208709$

H $5.031094-3.6678291 .770384$

H $4.0792822 .960051-2.160048$

H $2.9012031 .838526-1.485622$

H 1.5130713 .2660052 .490694

H 2.4761414 .6642081 .951248

H 4.5957563 .5838042 .767721

H $2.4965101 .959734 \quad 4.301496$

H 4.4377442 .1485055 .928482

H $5.5592592 .415984 \quad 4.609506$

H $5.006603-1.4856625 .704579$

H $4.553408-0.0515126 .786750$

H $6.075764-0.8696313 .486798$

H 5.7209170 .6686382 .776421

$\begin{array}{llll}\text { H } & 3.381218 & 0.071757 & 2.300847\end{array}$

H $2.473788 \quad 1.9073340 .810303$

C $-5.467163-2.945151 \quad 1.475736$

$\begin{array}{llll}\text { O } & -5.550578 & -1.956758 & 0.430460\end{array}$

C $-6.635881-1.0618850 .718780$

C $-7.562226-1.8493321 .632036$

C $-6.552117-2.5840672 .496095$ 
H $-6.210979-0.2235651 .283961$ C -7.250804 -0.526542 -0.577666 H -8.231764 -1.203693 2.207784 H -8.171299 -2.568606 1.072865 C -7.129473 -3.7848223 .225204$ H -6.151946-1.880682 3.238641 C $-6.214882-0.024874-1.607407$ O $-7.999708-1.576036-1.203349$ H $-7.972876 \quad 0.263150-0.338271$ H -5.719695 -0.889154 -2.068422 O $\quad-6.949470 \quad 0.624294-2.651788$ C $-5.1552600 .936117-1.038141$ C $-4.1675201 .430426-2.112428$ H $-4.5907610 .421490-0.253447$ O $-5.8175352 .046188-0.435732$ H $-3.8898500 .593369-2.763832$ H -4.658085 2.200519-2.720286 H -7.927932 -3.473713 3.906571 H -7.550058 -4.515057 2.525684 H $-6.357370-4.2888833 .815058$ C $-4.056626-2.9297882 .072066$ H -8.389376 -1.208672 -2.015231 H $-6.342048 \quad 0.769025-3.396558$ H $-5.1358652 .623845-0.052573$ H -3.980729 -3.669232 2.877199 H -3.851862 -1.9459712 .512050$ H -5.674327 -3.916315 1.008842 C $-2.1572743 .846819-0.079610$ O $-3.2308793 .209351-0.784850$ C $-2.8844582 .015440-1.490974$ C $-1.8162302 .316154-2.552643$ C $-0.5933232 .989157-1.923491$ C $-0.9887294 .168705-1.026179$ H -2.5797414 .7991650 .266402$ C -1.7352213 .0455551 .157545$ H $-2.4897891 .278896-0.782553$ O $-2.3519143 .151820-3.575755$ H $-1.5146331 .376378-3.029857$ H $\quad 0.0739793 .338591-2.720582$ O $0.1098472 .013338-1.161189$ H $-0.1301364 .514882-0.438935$ O $-1.3972565 .262396-1.852957$ C -2.8733652 .9553562 .146688$ H -1.398876 2.0342600 .912362 H $-0.8997113 .533643 \quad 1.672025$ H $-2.6940793 .958018-3.152997$ H $0.8375482 .466605-0.704442$ H $-0.6417945 .501692-2.416496$ O $\quad-3.323820 \quad 1.6708302 .227243$ O -3.3185423 .9159392 .761710$ C -4.4114491 .4572743 .131875$ H -5.309042 1.9619592 .762474 H -4.6065020 .3833883 .181841$ H -4.153355 1.8107694 .134831 SCF Energy (B3LYP/6-31G**//MMFF)= -3245.91612575

0800615

MM̄FF Geometry

C -2.824328 -2.129253 2.404969

C $-2.283664-2.1016733 .632601$

C $-1.075551-1.2913964 .041301$

O $-0.625162-0.4498832 .957990$

C $0.094808-2.2240404 .399977$

C $1.305471-1.4733024 .924681$

C $2.388634-1.1599364 .184895$

C $1.252545-1.1114026 .385593$

C $2.594028-1.4148012 .716639$

C $3.524007-2.6087172 .481705$

C $3.620495-2.9683330 .992238$

C $4.411956-4.263928 \quad 0.805960$

C $4.551725-4.626156-0.678712$

O $4.276376-1.8990850 .302512$

O $5.525476-5.675401-0.793939$

C $5.077762-3.396703-1.450906$

C $3.253108-5.224827-1.230675$

O $5.051816-3.629761-2.857189$

C $4.341205-2.072258-1.119919$

C $5.126430-0.883816-1.724463$
O $3.038901-2.030287-1.710804$

C - -1.0925020 .8222832 .880581$

O -1.9272931 .3540103 .592164$

C $-0.354722 \quad 1.466107 \quad 1.772553$

C -0.3791172 .7984641 .627979$

C 0.3976193 .5567180 .644278

C $1.1972312 .766226-0.358511$

C $4.5580420 .499743-1.383183$

C $5.1842421 .143809-0.155171$

C 5.2992073 .3567330 .998411

O 5.9398210 .5538620 .611043

C 4.1745604 .1891271 .524450

C 4.0134655 .4935511 .253358

C 2.8918206 .3236461 .810728

C 1.9611906 .8616690 .742318

C 1.8131058 .1857470 .567141

C $1.1865725 .878428-0.117007$

C $0.381708 \quad 4.9045250 .705696$

N $4.7782012 .465129-0.019945$

H -2.372776 -1.549281 1.604970

H $-2.745757-2.6926624 .420602$

H $-1.342638-0.6751244 .908644$

H $\quad 0.376843-2.8195063 .522387$

H $-0.220686-2.9541205 .157413$

H $3.215328-0.6441034 .671119$

H $2.159910-0.5974046 .719869$

H $0.405669-0.4475256 .585587$

H $1.140346-2.0131946 .996321$

H $1.641776-1.5811742 .203988$

H $3.021564-0.5100802 .266837$

H $4.521148-2.3665582 .871877$

H $3.151304-3.4762063 .041028$

H $2.603412-3.0816100 .599066$

H $3.948755-5.0909161 .358377$

H $5.415787-4.1407991 .235524$

H $6.362503-5.357250-0.414826$

H $6.134871-3.270446-1.178180$

H $3.007941-6.154308-0.702123$

H $3.359923-5.501175-2.285352$

H $2.394604-4.556923-1.132924$

H $5.544852-4.451334-3.024171$

H $6.177715-0.933649-1.416490$

H $5.105392-0.979473-2.818186$

H $2.898138-2.845255-2.217669$

H $\quad 0.2423260 .8215891 .136390$

H -0.9778823 .3897362 .319052$

H 1.9751562 .1858910 .146778

H $1.6867553 .380273-1.116159$

H $0.5499642 .070810-0.902798$

H $4.7635211 .165881-2.231241$

H $3.4726820 .465978-1.243760$

H 6.0781483 .9659180 .528123

H 5.7595052 .7840701 .809518

H 3.4611593 .6875442 .174332

H 4.7293895 .9988750 .609511

H 3.3438047 .1487542 .376477

H 2.3079525 .7540992 .544298

H $1.1470038 .585580-0.190268$

H $2.3551218 .901784 \quad 1.176020$

H $1.8995655 .381669-0.779641$

H $0.485787 \quad 6.412121-0.771499$

H -0.2548165 .3804191 .453120$

H $4.2415542 .884375-0.774339$

C $-5.222618-2.0541021 .594958$

O $-4.842449-1.1694440 .518323$

C -5.3671320 .1410340 .802341$

C $-6.439728-0.0648061 .865953$

C $-5.817528-1.1711852 .693739$

H $-4.5480580 .718721 \quad 1.245750$

C $-5.8703610 .824338-0.471469$

H -6.6358360 .8451052 .441172$

H -7.385880 -0.402705 1.426961

C $-6.805472-1.8900903 .595565$

H $-5.025862-0.7331923 .314444$

C $-4.7945331 .061302-1.552595$

O $-6.8979780 .003926-1.043806$

H $-6.3505141 .775094-0.209350$

H $-4.4897990 .097359-1.976653$ 
O $-5.446308 \quad 1.785453-2.605625$

C $-3.5592271 .853388-1.077283$

C $-2.6111092 .326347-2.194310$

H $-2.987077 \quad 1.253051-0.360877$

O $-3.974910 \quad 3.043287-0.396965$

H -3.119192 $3.066547-2.825897$

H $-1.8012092 .879704-1.700669$

H $-7.246417-1.1928394 .315588$

H -7.621920 -2.339142 3.020230

H -6.309903 -2.687920 4.157534

C $-4.038340-2.9263992 .022689$

H $-7.2135290 .454148-1.846102$

H -4.883184 $1.734601-3.395759$

H -4.3588832 .7844210 .456835$

H -3.747482 -3.566870 1.181813

H $-4.343858-3.5932332 .837333$

H $-6.000058-2.7117251 .184466$

C $-0.699542-0.814833-2.869455$

O $-1.312510 \quad 0.306560-2.218550$

C $-2.0137861 .214988-3.071369$

C $-1.078747 \quad 1.792735-4.150886$

C $-0.346396 \quad 0.686987-4.915902$

C $0.270992-0.346051-3.967910$

H $-0.109266-1.306342-2.085555$

C $-1.736902-1.825898-3.384161$

H $-2.8346290 .683409-3.561531$

O $-0.1215782 .660621-3.546551$

H $-1.6769152 .397824-4.843167$

H $0.4448361 .137102-5.528254$

O $-1.2690890 .056656-5.798566$

H $0.632855-1.210297-4.537319$

O $1.4120860 .251557-3.347349$

C $-2.507384-2.492460-2.265716$

H $-2.470956-1.354081-4.042176$

H -1.224989 -2.620267 -3.939857

H $0.4253413 .036593-4.256972$

H $-0.782749-0.624146-6.294090$

H $1.832337-0.426786-2.791649$

O $-3.447377-3.318329-2.814965$

O $-2.311792-2.320865-1.071329$

C $-4.266181-4.033785-1.885288$

H $-4.889750-4.732197-2.449619$

H $-3.647242-4.604967-1.186761$

H -4.916826 -3.337636 -1.349449

SCF Energy (B3LYP/6-31G**/MMFF) $=-3245.90676819$

0800616

MM̄FF Geometry

C -1.873947 5.244946 -0.427142

C $-2.745572 \quad 4.229376-0.336359$

C -3.2870993 .7068890 .968515$

O $-3.032844 \quad 2.2829251 .017087$

C -4.806213 3.9227331 .037004

C $-5.407422 \quad 3.4854342 .359009$

C $-6.185500 \quad 2.3955502 .507211$

C -5.1344404 .3889903 .532766$

C -6.5482261 .4144521 .426102$

C -7.013051 0.0706352 .005100

C $-7.445135-0.8999200 .897289$

C -8.115636 -2.138876 1.493955

C $-8.496310-3.155765 \quad 0.408377$

O $-6.280795-1.2832330 .155274$

O $-8.819683-4.3946921 .057558$

C $-7.262897-3.418093-0.478593$

C $-9.750775-2.730258-0.364206$

O $-7.603192-4.242357-1.591494$

C $-6.559327-2.129439-0.968175$

C $-5.222971-2.504155-1.651966$

O $-7.395272-1.472389-1.924407$

C -1.812312 1.8835701 .465373

O -0.9057382 .5964851 .865355$

C -1.757763 0.4083531 .361152

C $-0.572292-0.2094761 .468366$

C $-0.326983-1.6497501 .364537$

C $-1.523390-2.5537341 .225039$

C $-4.285640-1.318764-1.922332$

C $-3.087496-1.763778-2.744833$

C $-0.631015-1.462394-2.986803$
O $-3.188514-2.574715-3.663979$

C $-0.046848-2.667369-2.315396$

C $1.219380-2.738414-1.878391$

C $1.807125-3.958773-1.228403$

C $2.410248-3.6750620 .129764$

C $3.742259-3.6631040 .306212$

C $1.469090-3.4933121 .301809$

C $0.950694-2.0837631 .402012$

$\mathrm{N}-1.906388-1.139596-2.370690$

H $-1.555765 \quad 5.7532670 .478986$

H -3.064893 $3.718246-1.241946$

H -2.803106 $4.199303 \quad 1.820976$

H $-5.290192 \quad 3.394838 \quad 0.206041$

H -5.0406994 .9852120 .887280$

H -6.6075082 .1968493 .491036$

H -5.6702844 .0721274 .433961$

H -4.0672264 .3985793 .773179$

H -5.450531 5.4123693 .306118

H $-7.359587 \quad 1.8442450 .826700$

H $-5.693079 \quad 1.2403270 .764221$

H $-6.193670-0.3703562 .587600$

H -7.8534380 .2420972 .689672$

H -8.142386 -0.3759340 .232602$

H $-8.998777-1.8598202 .082391$

H -7.425721 -2.6238122 .198849$

H $-9.556871-4.2283331 .670109$

H $-6.545799-3.9932020 .124299$

H - $10.601508-2.6185540 .318822$

H $-10.047393-3.497734-1.087930$

H $-9.628589-1.784065-0.895275$

H -8.023186 $-5.045746-1.239461$

H $-4.670684-3.208685-1.015665$

H -5.436733 -3.024196 -2.594382

H $-6.841683-0.941573-2.517944$

H $-2.685119-0.121738 \quad 1.177160$

H 0.3155240 .4007281 .632315

H -2.196122 -2.432564 2.081628

H - $1.268721-3.613921 \quad 1.177049$

H $-2.079527-2.3242380 .311201$

H $-4.789284-0.530322-2.490532$

H $-3.953489-0.885491-0.972788$

H $0.011661-0.581820-2.892836$

H $-0.790556-1.673260-4.049088$

H $-0.693521-3.534226-2.197499$

H $1.879522-1.887887-2.024331$

H $2.574028-4.356679-1.905835$

H $1.059547-4.755674-1.127690$

H $4.180165-3.4877401 .283194$

H $4.425013-3.819345-0.522175$

H $1.995744-3.7143602 .239794$

H $0.676412-4.2429351 .239045$

H $1.741458-1.3424471 .527085$

H -1.895102 -0.540255 -1.551240

C $0.2091795 .392135-1.874763$

O $0.419125 \quad 3.971941-1.728103$

C $1.4826473 .764500-0.775141$

C $2.2656505 .066923-0.765621$

C $1.1448296 .082267-0.879248$

H 0.9940293 .6073420 .194376

C $2.2791682 .517672-1.169538$

H 2.8648445 .1961740 .140523

H $2.9313995 .127578-1.635396$

C $1.6086317 .456378-1.330649$

H $0.670324 \quad 6.1799820 .104200$

C $3.5207072 .280674-0.287485$

O $1.3998611 .394015-1.066843$

H $2.5826762 .582770-2.221287$

H $4.1909763 .141513-0.391941$

$\begin{array}{llll}\text { O } & 3.127973 & 2.228273 & 1.084760\end{array}$

C $4.3194331 .009563-0.644517$

C 5.6106650 .9068210 .178755

H $4.5619221 .034045-1.712151$

O $3.549610-0.162234-0.395442$

H $6.221417 \quad \begin{array}{llll}1.807175 & 0.035881\end{array}$

H $5.3630100 .888721 \quad 1.248924$

H $2.3141897 .880918-0.609012$

H $2.1093857 .411806-2.303645$

H $0.7601318 .142234-1.418960$ 
C $-1.2821865 .716531-1.727623$ H $0.6202281 .582322-1.616991$ H $2.548031 \quad 1.4542441 .190022$ H $2.729793-0.092105-0.911775$ H -1.820486 $5.245413-2.560225$ H -1.442140 $6.796769-1.819804$ H $0.5118315 .637035-2.901101$ C $8.465025-1.4079260 .751912$ O $7.575711-0.2843520 .784322$ C $6.454622-0.349916-0.107909$ C $6.938634-0.458942-1.561363$ C $7.918151-1.624119-1.743857$ C $9.009685-1.630673-0.668187$ H $9.306726-1.1144011 .392994$ C $7.828427-2.6596511 .365246$ H $5.854564-1.2289010 .147952$ O $7.567437 \quad 0.756240-1.964544$ H $6.076605-0.607741-2.220323$ H $8.387595-1.552814-2.732658$ O $7.210624-2.859579-1.688204$ H $9.575229-2.569374-0.697742$ O $9.934479-0.573345-0.940224$ C $7.518707-2.4389272 .826565$ H $6.909723-2.9709130 .860860$ H $8.514205-3.5129681 .303019$ H $8.296608 \quad 0.933146-1.345546$ H $6.538979-2.845881-2.391246$ H $10.320791-0.740631-1.816774$ O $6.232476-2.0092442 .969350$ O $8.330821-2.604076 \quad 3.727463$ C $5.816616-1.7354394 .310296$ H $6.436937-0.9466744 .746558$ H $4.779775-1.3896464 .280107$ H $5.865723-2.6461444 .914664$ SCF Energy (B3LYP/6-31G**//MMFF) $=-3245.92786383$

$08 \quad 00617$

MM̄FF Geometry

C -2.045418 5.207199-0.174309

C $-2.9637134 .230183-0.188019$

C -3.6456423 .7176381 .054164$

O -3.4032432 .2953681 .164627$

C -5.1627183 .9499690 .942772$

C -5.9376043 .4927812 .163924$

C -6.7048942 .3853942 .219984$

C -5.8366294 .3997893 .363546$

C -6.9268981 .3633221 .139615$

C $-6.476886-0.0306541 .595785$

C $-6.468412-1.0371370 .435388$

C $-6.103297-2.4340650 .939112$

C $-5.941503-3.444772-0.205464$

O $-5.499367-0.589057-0.518221$

O $-5.295832-4.598642 \quad 0.354961$

C $-5.005617-2.844298-1.278256$

C $-7.301012-3.918745-0.732226$

O $-4.955256-3.675318-2.435127$

C $-5.389827-1.407850-1.689881$

C $-4.347116-0.742098-2.623871$

O $-6.630218-1.444554-2.406920$

C -2.2039931 .9102811 .684945$

O -1.2778582 .6348842 .013306$

C -2.2191930 .4355451 .811096$

C $-1.147979-0.2073932 .300271$

C -1.039994 -1.649917 2.535742

C $-2.206566-2.5126152 .132509$

C $-2.949590-0.548208-2.025637$

C $-1.981515-1.683074-2.312463$

C $0.290180-2.493512-1.680293$

O $-2.207633-2.574127-3.126034$

C $0.200569-3.494162-0.572642$

C $1.200930-3.7227490 .291291$

C $1.153247-4.7539451 .379988$

C $1.489629-4.1827202 .738807$

C $2.721824-4.3151153 .258389$

C $0.372966-3.5466083 .533126$

C $0.081746-2.1318153 .111678$

N -0.812273 -1.557732 -1.573969

H -1.7762815 .6711850 .771358$
H $-3.2315483 .761113-1.131891$

H $-3.2637184 .219643 \quad 1.952168$

H $-5.5420673 .461600 \quad 0.039225$

H -5.3620045 .0199310 .791680$

H -7.234891 2.176052 3.148462

H -6.4661714 .0630964 .194207$

H -4.806132 $4.441683 \quad 3.729351$

H -6.1553615 .4138253 .100969$

H -7.9964261 .3375970 .898374$

H -6.4102201 .6323950 .216331$

H -5.4673630 .0408822 .019052$

H -7.140747 -0.3858992 .393869$

H -7.460445 -1.042597 -0.031211

H $-6.844080-2.7913161 .665528$

H $-5.153883-2.3815091 .487760$

H $-5.226533-5.273561-0.341075$

H -3.996143 -2.834677 -0.850259

H -7.862187 -4.423841 0.063205

H -7.181045 -4.659380-1.530605

H -7.922480 -3.103902-1.109416

H $-4.589318-4.534493-2.165875$

H -4.306361 -1.271373 -3.583537

H $-4.7243340 .262673-2.863626$

H $-6.701243-0.637753-2.943427$

H -3.125462 -0.0821681 .520945$

H -0.2730790 .3777392 .580885$

H -2.002862 -3.5831802 .202227$

H -2.481528 -2.328859 1.088433

H $-3.072900-2.3014542 .768213$

H $-2.5034230 .349398-2.473793$

H $-3.005918-0.379238-0.947045$

H $1.213561-1.910561-1.631278$

H $\quad 0.251239-3.004898-2.647194$

H $-0.716028-4.073915-0.495459$

H $2.124233-3.1584780 .192251$

H $1.870649-5.5436821 .120653$

H $0.174534-5.2475721 .423131$

H $2.963082-3.935616 \quad 4.245810$

H $3.519216-4.7992152 .704083$

H $0.645865-3.5119234 .596932$

H $-0.509258-4.1924043 .501088$

H $0.878966-1.4303763 .362087$

H $-0.729819-0.805470-0.896210$

C $0.0743075 .185309-1.579173$

O $0.0386373 .776533-1.879142$

C $1.1680953 .140034-1.240553$

C $2.0492654 .268515-0.718395$

C $1.0249745 .337397-0.393801$

H $0.7508732 .560602-0.407335$

C $1.8524272 .210094-2.250076$

H 2.6447483 .9683390 .149112

H $2.7359144 .621241-1.498104$

C $1.613906 \quad 6.729085-0.249971$

H 0.5342145 .0572990 .546003

C $3.1440111 .562524-1.710775$

O $0.9214411 .182739-2.601594$

H $2.0735122 .762009-3.171631$

H $3.8435102 .356527-1.426487$

O $2.8305420 .835777-0.524703$

C $3.8527520 .653276-2.737101$

C $5.2918880 .260237-2.358637$

H $3.9236511 .201346-3.685425$

O $3.101099-0.522872-3.008721$

H $5.706758-0.364225-3.161801$

H $5.9060391 .171725-2.363957$

H 2.3414496 .7562990 .567798

H $2.1255797 .045729-1.165061$

H $0.8306047 .461456-0.030380$

C $-1.3485315 .720578-1.401702$

H $0.114407 \quad 1.619538-2.924461$

H $2.1905520 .144970-0.767507$

H $2.222559-0.248165-3.320950$

H -1.937519 $5.459813-2.290420$

H - $1.3274256 .815496-1.348778$

H $0.5060535 .668296-2.466191$

C $7.434088-1.1782030 .322215$

O $6.955349-0.616246-0.907396$

C $5.530920-0.454443-1.014707$ 
C $4.843666-1.820753-0.879310$

C $5.262150-2.5361850 .409002$

C $6.783430-2.545168 \quad 0.590537$

H $8.505631-1.3479500 .153791$

C $7.295432-0.1970111 .497252$

H $5.2074570 .213148-0.212134$

O $5.151764-2.645226-2.001271$

H $3.759012-1.684771-0.868349$

H $4.888057-3.5669190 .380180$

O $4.644407-1.8795081 .512151$

H $7.051868-2.8855531 .597381$

O $7.354712-3.478209-0.331559$

C $8.1527261 .039282 \quad 1.319633$

H $6.2637860 .139863 \quad 1.626302$

H $7.622804-0.6894192 .420298$

H $6.119803-2.716394-2.061200$

H $4.899335-2.3582092 .319136$

H $6.978788-4.352981-0.134033$

$\begin{array}{lllll}\text { O } & 7.963913 & 1.863554 & 2.393189\end{array}$

$\begin{array}{lllll}\text { O } & 8.892556 & 1.258971 & 0.370759\end{array}$

C 8.7099233 .0846112 .370003

H 8.4672973 .6485003 .274574

H 9.7833732 .8725722 .365855

H 8.4300023 .6829341 .497691

SCF Energy (B3LYP/6-31G**//MMFF) $=-3245.91616499$

0800618

MM̄FF Geometry

C $-0.988427-0.260349-1.373986$

C $0.241979-0.462596-0.881366$

C $0.537976-1.2755220 .349756$

O $1.128793-0.3758021 .315640$

C $1.574519-2.3638320 .028953$

C $1.721337-3.3832011 .139922$

C $2.704094-3.3819962 .061407$

C $0.695900-4.4872321 .133690$

C $3.809657-2.3782732 .237719$

C $5.190574-2.9639081 .907898$

C $5.416618-3.178874 \quad 0.402987$

C $6.773214-3.8370780 .147694$

C $7.055133-3.984642-1.354984$

O $5.383573-1.896945-0.227575$

O $8.446245-4.304227-1.515077$

C $6.826400-2.622926-2.048120$

C $6.272647-5.155330-1.962044$

O $6.905805-2.750734-3.465657$

C $5.500296-1.927804-1.656082$

C $5.503447-0.461915-2.141903$

O $4.370486-2.553846-2.266643$

C $0.294908 \quad 0.303193 \quad 2.142826$

$\begin{array}{lllll}\text { O } & -0.915574 & 0.158706 & 2.241976\end{array}$

C 1.0302151 .3001292 .953215

C 2.2435941 .7649162 .615987

C $2.9899562 .814927 \quad 3.312746$

C 2.4099893 .3592494 .591687

C $4.2513040 .318291-1.718063$

C $4.3276931 .779598-2.122634$

C $3.3029973 .992759-1.605946$

O $5.1326362 .205897-2.946683$

C $4.2804914 .618527-0.660151$

C 3.9392965 .4471680 .338263

C 4.9361956 .0517851 .286914

C 4.6409535 .7293662 .736631

C 4.1158196 .6597773 .551718

C 5.0314374 .3643053 .264600

C 4.1372473 .2646132 .762359

N $3.3756912 .549433-1.466186$

H - $1.846644-0.715328-0.887223$

H $1.0886670 .015901-1.367108$

H $-0.374431-1.7275350 .757123$

H $2.543085-1.899420-0.184739$

H $1.294083-2.897027-0.889373$

H $2.716793-4.1870452 .795549$

H $\quad 0.848612-5.2035151 .947988$

H $-0.311045-4.0766651 .242898$

H $0.743601-5.0431900 .191649$

H $3.636600-1.4625111 .665347$

H $3.805942-2.0720713 .291527$
H $5.944348-2.2597312 .283854$

H $5.332014-3.9085022 .447681$

H $4.609881-3.8024820 .003025$

H $6.834296-4.8119480 .647440$

H $7.568502-3.2215490 .591158$

H $8.627165-5.117435-1.013168$

H $7.655516-1.965958-1.749454$

H $6.570191-6.099814-1.490696$

H $6.495596-5.270770-3.028284$

H $5.191702-5.057183-1.841184$

H $7.761670-3.164208-3.671775$

H $6.3829120 .055325-1.736455$

H $5.580480-0.430842-3.236155$

H $4.679370-3.287933-2.820363$

H 0.4821951 .6888473 .805153

H 2.7279251 .3732871 .722938

H 3.0470734 .1031965 .074975

H 2.2634682 .5542735 .320606

H 1.4453763 .8429154 .402143

H $3.361724-0.111738-2.190258$

H $4.1250050 .266578-0.630594$

H $2.2711474 .291814-1.397228$

H $3.5501734 .271570-2.635178$

H $5.3301794 .373731-0.806914$

H 2.8936245 .7084830 .482221

H 4.9245957 .1385021 .131041

H 5.9586575 .7310901 .051170

H 3.9282956 .4536624 .600118

H 3.8584787 .6493373 .188416

H 5.0662904 .3607154 .360340

H 6.0624174 .1440062 .958473

H $4.4805212 .811784 \quad 1.831841$

H $2.8037222 .118631-0.745552$

C $-1.8934581 .932857-2.160604$

O $-3.2614911 .712653-1.761403$

C $-4.0849962 .743859-2.338144$

C $-3.1264153 .806606-2.861266$

C -1.949901 $2.955321-3.295401$

H $-4.6009792 .291292-3.193280$

C $-5.1122273 .249592-1.320126$

H $-3.5598774 .391224-3.678338$

H -2.820340 $4.503281-2.072344$

C -0.664884 $3.745212-3.478939$

H $-2.204738 \quad 2.455829-4.239768$

C $-5.9945732 .141321-0.708276$

O $-4.4118913 .887976-0.244658$

H $-5.735263 \quad 4.023745-1.783619$

H $-5.3813801 .521314-0.045337$

O $-6.9652092 .776026 \quad 0.134132$

C $-6.7224901 .255987-1.735030$

C $-7.7491450 .281673-1.127906$

H $-5.9864280 .687182-2.313644$

O $-7.416138 \quad 2.103318-2.655628$

H $-8.5811510 .842395-0.683336$

H -8.187998 $-0.278957-1.964055$

H $-0.7888334 .504933-4.257570$

H $-0.3720264 .255738-2.555089$

H $0.1594423 .089350-3.775660$

C $-1.2553670 .605788-2.573219$

H $-5.078948 \quad 4.1987790 .391409$

H $-7.5533293 .297790-0.438431$

H $-7.8148041 .533688-3.335443$

H $-0.3208350 .790300-3.115676$

H $-1.9206400 .054716-3.248853$

H $-1.3730652 .358119-1.291923$

C $-5.386282-2.320788 \quad 0.128525$

O $-6.098305-1.414259-0.725132$

C $-7.165666-0.697913-0.097274$

C $-8.227916-1.676648 \quad 0.428736$

C -7.605601 -2.715104 1.368521

C $-6.341987-3.3441290 .769420$

H $-4.719797-2.865614-0.552421$

C $-4.507266-1.548414 \quad 1.123545$

H $-6.768249-0.1200230 .743634$

O $-8.850232-2.343020-0.668209$

H $-9.004771-1.1067920 .952465$

H -8.336497-3.506415 1.574891

O $-7.275367-2.1028162 .611258$ 
H $\quad-5.807572-3.9242391 .529967$

O $-6.727669-4.262463-0.258875$

C $-3.461400-2.426176 \quad 1.773039$

H -3.983439 -0.7359630 .606224$

H -5.116024 -1.081580 1.902789

H -9.535273 -2.929895 -0.305351

H -8.094394 -1.736444 2.985869

H $-7.282868-4.946570 \quad 0.152365$

O $-3.138956-1.9168472 .997495$

O $-2.970561-3.4225591 .259721$

C $-2.152174-2.6496283 .730726$

H -2.483805 -3.679824 3.891944

H -1.195804 -2.629910 3.200493

H -2.023353 -2.166426 4.702841

SCF Energy $\left(B 3 L Y P / 6-31 G^{* *} / / M M F F\right)=-3245.90752043$

08_00619

MM̄FF Geometry

C -3.604396 2.4185353 .047685

C -2.8242361 .4227052 .599760$

C -1.4330601 .1836203 .134886$

O $-0.477315 \quad 1.3821342 .068223$

C $-1.290007-0.2669273 .630065$

C $-0.102908-0.4378694 .556845$

C $1.093851-0.9440724 .203492$

C $-0.344764-0.0161835 .985505$

C $1.547926-1.4277362 .854383$

C $1.981409-2.8990392 .930112$

C $2.589106-3.4049031 .613959$

C $2.995661-4.8745431 .741958$

C $3.703637-5.3865800 .478553$

O $3.743360-2.6132791 .328230$

O $4.338119-6.6341350 .800147$

C $4.827320-4.3994940 .089636$

C $2.700500-5.689908-0.639599$

O $5.393866-4.742382-1.172591$

C $4.383397-2.9176830 .081846$

C $5.606205-1.982986-0.045645$

O $3.538107-2.625635-1.033094$

C $-0.0304002 .650657 \quad 1.856564$

O $-0.358713 \quad 3.6593952 .460119$

C 0.9319302 .6010350 .733503

C 1.3756403 .7401450 .180836

C $2.3024773 .844995-0.949634$

C $2.7320882 .561104-1.611304$

C $5.223029-0.5014270 .053243$

C $6.409590 \quad 0.430852-0.113667$

C 6.9967952 .8420170 .080220

O $7.5305880 .052285-0.438978$

C 6.2419014 .1314970 .083192

C $6.3064755 .046135-0.895900$

C $5.5165526 .324426-0.875820$

C $4.6399386 .503046-2.098592$

C $4.7346477 .615395-2.846953$

C $3.6552585 .408954-2.468842$

C $2.6991605 .070803-1.354464$

N 6.0442551 .7494130 .122088 H -3.1925003 .0816313 .807578$ H $-3.182940 \quad 0.7428881 .832855$ H $-1.212157 \quad 1.8775373 .955708$ H -1.215842 -0.9506292 .776422$ H -2.191110 -0.574350 4.177976 H $1.862104-1.0083654 .974188$ H $0.527772-0.1885036 .624778$ H $-0.585013 \quad 1.0498306 .039831$ H $-1.179636-0.582875 \quad 6.411053$ H $0.778581-1.3101092 .086402$ H $2.393087-0.8007902 .546239$ H $2.725940-3.0066563 .730609$ H $1.119012-3.5211253 .200938$ H $1.851844-3.2729370 .815175$ H $2.125124-5.5001211 .975764$ H $3.683485-4.9896692 .591596$ H $3.654299-7.2432541 .127736$ H $5.630327-4.5112390 .831737$ H $2.000722-6.473946-0.325999$ H $3.203953-6.077134-1.532064$ H $2.108434-4.818799-0.923657$
H $5.683306-5.669302-1.117858$

H $6.328570-2.2045800 .750340$

H $6.110001-2.155507-1.005068$

H $3.509158-3.401851-1.614753$

H 1.2377191 .6229850 .381224

H $1.0270564 .687780 \quad 0.589291$

H $3.2817671 .931220-0.906124$

H $3.3759992 .706735-2.480524$

H $1.8583482 .003576-1.968229$

H $4.494050-0.248571-0.724993$

H $4.766182-0.2988141 .029118$

H $7.6337522 .724167-0.802876$

H 7.6303182 .7701420 .970203

H 5.6004454 .3200730 .941514

H $6.9558724 .873051-1.750494$

H $6.2321977 .152526-0.793184$

H $4.8861056 .381550 \quad 0.020350$

H $4.1166257 .760859-3.726791$

H $5.4324898 .408033-2.598025$

H $4.2337634 .541465-2.797628$

H $3.0522865 .720510-3.331445$

H $2.2914935 .941526-0.839204$

H $5.138713 \quad 1.9233570 .549647$

C -5.7698371 .7597761 .764819$

O

C $-6.3919211 .803720-0.490877$

C -7.6243341 .4631720 .336415$

C $-7.2649412 .079578 \quad 1.672300$

H -6.516970 $2.835507-0.848059$

C $-6.1187820 .921955-1.719551$

H $-8.541551 \quad 1.879997-0.091252$

H -7.7680950 .3850760 .444537$

C $-8.089476 \quad 1.5358912 .827304$

H -7.412260 3.1662681 .606535

C $-5.470841-0.450252-1.418321$

O $\quad-7.339270 \quad 0.789186-2.442033$

H $-5.4185811 .469926-2.363112$

H $-4.551263-0.262891-0.855891$

O $-6.315056-1.231610-0.578095$

C $-5.121023-1.271189-2.678944$

C $-4.432721-2.617251-2.373909$

H -4.486695 -0.665545 -3.336574

O $-6.320997-1.558684-3.401059$

H $-5.082475-3.233023-1.739832$

H -4.340898 -3.161575 -3.323406

H -9.1539911 .7361462 .666900$

H $-7.963914 \quad 0.4531282 .934738$

H -7.7979962 .0038373 .772586$

C -5.0154372 .7577212 .648633$

H $-7.1438210 .321849-3.271482$

H -7.131149 -1.412824 -1.075582

H -6.067767 -2.008227 -4.225402

H $-5.5741922 .891358 \quad 3.583044$

H -4.9844313 .7346162 .149413$

H -5.6227310 .7318182 .119713$

C $-0.888182-1.477036-2.217575$

O $-2.216375-1.772298-2.664697$

C $-3.045103-2.472108-1.731755$

C $-2.421676-3.828802-1.373739$

C $-0.993850-3.647554-0.846251$

C $-0.157784-2.753378-1.768372$

H -0.369738 -1.099449 -3.108411

C $-0.888408-0.360188-1.164252$

H -3.143229 -1.883800 -0.815476

O $-2.406983-4.669619-2.524765$

H $-3.047044-4.316208-0.616189$

H $-0.509150-4.627048-0.756982$

O $-1.030508-3.0634420 .452687$

H $\quad 0.785021-2.476549-1.285144$

O $0.176212-3.487172-2.950639$

C $-1.3967500 .944859-1.742940$

H -1.513711 -0.620417 -0.306008

H $0.128942-0.181603-0.801055$

H $-2.026798-5.524182-2.259522$

H $-1.539009-3.6587681 .029213$

H $0.668846-4.279371-2.678019$

O $-1.9935741 .678052-0.759441$

O $-1.2576421 .293095-2.907872$ 
C $-2.4735312 .966754-1.154506$ H $-3.2729592 .860632-1.893311$ H $-2.8755313 .466281-0.269217$ H -1.654778 $3.572184-1.554971$ SCF Energy (B3LYP/6-31G**//MMFF) $=-3245.91175013$

0800620

MM̄FF Geometry

C 2.1926085 .3132050 .769152

C $2.851640 \quad 4.1475720 .691292$

C $3.5398643 .671884-0.559385$

O $2.9250242 .404080-0.893074$

C $5.0402203 .461464-0.308328$

C $5.8232043 .205861-1.581184$

C $6.3530742 .020823-1.941558$

C $6.0372064 .415141-2.457896$

C $6.2878650 .709428-1.211766$

C $5.634914-0.375031-2.077700$

C $5.686016-1.749769-1.398091$

C $5.078695-2.823947-2.301452$

C $5.055969-4.197192-1.614164$

O $4.952804-1.676300-0.171170$

O $4.197131-5.058579-2.377008$

C $4.419528-4.045788-0.217486$

C $6.439547-4.857551-1.608396$

O $4.513022-5.2626530 .520060$

C $5.004077-2.8763860 .611923$

C $4.143952-2.6625231 .876196$

O $6.339343-3.1949831 .005662$

C $2.4001832 .250034-2.134733$

O $2.4875013 .037540-3.067415$

C $1.6446200 .980263-2.263918$

C $1.4529640 .107895-1.261915$

C $0.688293-1.142298-1.336469$

C $0.005500-1.473726-2.636654$

C $4.491817-1.4104712 .694567$

C $3.445111-1.2244913 .775622$

C $1.031441-0.6544004 .006445$

O $3.603844-1.6597524 .913411$

C $0.319989-1.8874633 .538264$

C $-0.767268-1.8753952 .752719$

C -1.388071-3.120166 2.186281

C $-1.394387-3.1378010 .670296$

C $-2.552802-3.145511-0.010149$

C $-0.071612-3.247622-0.059859$

C $0.618616-1.921185-0.236883$

N $2.300346-0.5992463 .301441$

H $2.1853125 .978558-0.090269$

H 2.8505853 .4747041 .545891

H $3.3848204 .403955-1.360303$

H 5.1800662 .6470430 .409676

H 5.4662904 .3520060 .173668

H $6.9175551 .979745-2.872985$

H $6.6895914 .200535-3.311265$

H $5.0858644 .778400-2.857680$

H $6.5056675 .221708-1.884485$

H $7.3138710 .410978-0.963919$

H $5.7494280 .789644-0.264671$

H $4.591523-0.094564-2.265912$

H $6.140025-0.431084-3.050216$

H $6.734828-1.983003-1.179215$

H $5.616321-2.881548-3.256055$

H $4.045756-2.545766-2.555089$

H $4.548073-5.105329-3.282880$

H $3.347244-3.858236-0.368582$

H $6.804700-4.990198-2.633978$

H $6.395671-5.862689-1.174424$

H $7.188723-4.279006-1.063893$

H $4.120561-5.964174-0.027475$

H $3.091712-2.5945391 .576491$

H $4.227353-3.5359952 .535935$

H $\quad 6.573855-2.663817 \quad 1.782687$

H $\quad 1.2273270 .809018-3.251374$

H $1.8892210 .322142-0.287837$

H $0.744324-1.616359-3.432980$

H $-0.677665-0.668257-2.929060$

H -0.596054 -2.384229 -2.591724

H $5.470699-1.5024483 .176144$
H $\quad 4.521370-0.5131742 .066441$

H $0.480303 \quad 0.2602063 .767205$

H $1.199822-0.6997985 .086557$

H $\quad 0.760825-2.8415383 .819211$

H -1.209143 -0.924725 2.466557

H -2.410932 -3.1917092 .577054$

H $-0.867917-4.0182782 .542822$

H -2.572937 -3.189915 -1.093753

H $-3.508284-3.1017610 .500795$

H $-0.208170-3.751549-1.023057$

H $0.595749-3.9151960 .500819$

H $1.145921-1.5862360 .653660$

H $2.267020-0.3332712 .321543$

C -0.1047725 .4981031 .821769$

O $-0.343998 \quad 4.081411 \quad 1.681287$

C -1.1449883 .8667170 .501834$

C $-1.8664585 .184993 \quad 0.274402$

C $-0.782051 \quad 6.1834370 .630694$

$\mathrm{H}-0.4393163 .664586-0.314594$

C $-2.0567092 .656900 \quad 0.724729$

H $-2.2269635 .300797-0.751874$

H $-2.7201395 .284330 \quad 0.956143$

C $-1.308913 \quad 7.5727270 .947329$

H $-0.0997486 .260264-0.223576$

C $-2.9989212 .382384-0.463101$

O -1.2198761 .5160910 .937255$

H -2.643476 2.7943481 .640767

H $-3.5871213 .284467-0.665153$

O $-2.2221382 .126468-1.634431$

C $-3.9893351 .222261-0.231959$

C $-4.9126451 .013998-1.448274$

H -4.5961651 .4436140 .653713$

O -3.2896250 .0064260 .009920$

H $-5.2924121 .984623-1.788828$

H $-4.3232960 .570386-2.260643$

H -1.8237757 .9961670 .078629$

H $-2.0185707 .553240 \quad 1.781277$

H $-0.4887898 .245796 \quad 1.217039$

C 1.4017215 .7474561 .971956

H $-0.6254471 .724793 \quad 1.678364$

H $-1.7064081 .318365-1.468524$

H -2.7676410 .1197630 .821759$

H 1.7621395 .2058172 .855953

H $1.590426 \quad 6.8116842 .153080$

H -0.5963355 .7935742 .757253$

C $-6.611135-2.168875-0.429625$

O $-5.620581-1.207401-0.815268$

C $-6.1133130 .094691-1.139237$

C $-7.0853710 .009033-2.326858$

C $-8.209514-0.998851-2.060646$

C $-7.672103-2.332807-1.531495$

H $-6.061060-3.114786-0.344916$

C $-7.208472-1.8571100 .947024$

H $-6.6368000 .510766-0.271503$

O $-6.386976-0.365098-3.513342$

H $-7.5121651 .002247-2.507989$

H -8.763398 -1.180522 -2.989955

O $-9.121255-0.459093-1.108495$

H -8.498610 -2.953075 -1.165098

O $-7.078288-3.030224-2.630798$

C $-6.142347-1.9085742 .015685$

H -7.699972 -0.8810540 .990846$

H -7.972861 -2.5958331 .215521$

H $-5.947014-1.215588-3.343469$

H $-9.4804850 .364412-1.480196$

H $-6.800743-3.904704-2.308594$

O $-5.754912-0.6440422 .346916$

O $-5.692317-2.9478692 .480449$

C $-4.720129-0.5525253 .330078$

H $-5.062361-0.9738824 .279790$

H $-3.820625-1.0658262 .980703$

H -4.4837250 .5046413 .477787$

SCF Energy (B3LYP/6-31G**//MMFF) $=-3245.91226228$

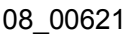

MM̄MF Geometry

C -2.563559 0.0301463 .155105

C -2.4190051 .3517793 .343527$ 
C -3.0377922 .4695242 .533957$

O

C -1.9359793 .3010641 .854369$

C -2.3507124 .7322111 .578232$

C -2.8094375 .1952960 .399219$

C -2.1611525 .6790672 .736375$

C $-3.1173044 .424282-0.854163$

C $-2.0772704 .637946-1.964243$

C $-0.8558013 .720313-1.812226$

C $0.2041754 .023927-2.871667$

C $1.3837193 .040437-2.792595$

O $-1.3160602 .371917-1.962621$

O $2.1615093 .187368-3.990380$

C $0.8333621 .598409-2.785218$

C $2.3224503 .361926-1.622992$

O $1.8777680 .653350-2.560698$

C $-0.3077691 .376061-1.765615$

C $-0.950467-0.013088-1.993565$

O $0.2307531 .431921-0.444771$

C $-5.1856001 .717642 \quad 1.735641$

O $-5.764305 \quad 1.8284602 .802891$

C $-5.785422 \quad 1.2501710 .467127$

C -6.9236650 .5432690 .484211$

C -7.595273 -0.024823 -0.687176

C $-7.0200410 .292344-2.043814$

C $-2.256971-0.247154-1.219356$

C $-2.770673-1.667881-1.395673$

C $-4.561314-3.202380-0.579317$

O $-2.224983-2.492942-2.123834$

C $-5.747867-3.203799-1.491075$

C $-6.965850-3.625924-1.116779$

C -8.155911 -3.667215 -2.033709

C $-9.386888-3.023094-1.432549$

C -10.370242 -3.771840 -0.905109

C $-9.515796-1.518122-1.519791$

C $-8.674163-0.811011-0.490920$

N -3.907774 -1.907005 -0.636121

H -3.186106 -0.3355152 .345063$

H -1.8043781 .6848414 .178788$

H -3.631039 3.0971813 .210680

H -1.6379972 .8141750 .920988$

H -1.029526 3.3321442 .473757

H $-3.033117 \quad 6.2592060 .323542$

H -2.465150 6.7026242 .492695

H -2.755684 5.3555243 .596608

H -1.1075915 .7095053 .033203$

H -3.260497 $3.359265-0.653359$

H $-4.0881884 .785487-1.217592$

H -2.565984 $4.414088-2.921547$

H -1.764800 $5.688695-1.996495$

H $-0.4327783 .856662-0.811484$

H $0.5595795 .058010-2.782291$

H $-0.2454863 .946130-3.871554$

H $2.4597314 .111654-4.042433$

H $\quad 0.442078 \quad 1.393928-3.791749$

H $2.7300414 .374639-1.726987$

H $3.1881272 .690289-1.614967$

H $1.8318793 .303047-0.649165$

H $2.5704640 .823483-3.222039$

H -1.179351 -0.135019-3.060705

H $-0.230101-0.796671-1.726435$

$\mathrm{H}-0.364574 \quad 0.964840 \quad 0.161005$

H $-5.2466491 .469541-0.447917$

H $-7.393606 \quad 0.3376841 .445068$

H -7.553313 -0.183081 -2.869152

H $-5.980736-0.046479-2.114134$

H -7.048791 $1.372119-2.227711$

H -2.110724 $-0.073706-0.148935$

H $-3.0331700 .439603-1.575245$

H -4.842501 -3.3765390 .464220$

H $-3.857757-3.982507-0.886467$

H -5.593198 -2.865212 -2.512883

H -7.110053 -3.994834 -0.103656

H $-8.361725-4.721462-2.260838$

H -7.934458 -3.193733 -2.998282

H $-11.268332-3.319863-0.497219$

H -10.296684 -4.853468 -0.864143

H -10.558038 -1.218085-1.346662
H $-9.302846-1.199242-2.543536$

H $-9.010135-0.9824460 .532912$

H $-4.306076-1.151339-0.087157$

C $-0.965788-1.9621703 .348887$

O $0.122620-1.2349972 .747772$

C $0.442331-1.8506921 .485679$

C $-0.296208-3.1845771 .467664$

C $-1.550999-2.8392842 .244983$

H $0.023900-1.2042120 .707638$

C $1.958187-1.9685291 .274677$

H $-0.501924-3.5318130 .450511$

H $0.272834-3.9658181 .985513$

C $-2.312926-4.0513322 .748787$

H -2.206696 -2.253824 1.593056

C $2.686280-0.6132201 .173861$

O $2.530798-2.7388112 .330800$

H $2.115440-2.5286370 .347004$

H $2.271925-0.032200 \quad 0.342953$

$\begin{array}{lllll}\text { O } & 2.461541 & 0.153581 & 2.362796\end{array}$

C $4.215889-0.7069041 .001838$

C $4.652751-1.545416-0.211974$

H $4.677202-1.0999261 .915641$

O $4.707803 \quad 0.6272420 .831588$

H $4.233053-2.553216-0.135144$

H $4.253583-1.072015-1.118099$

H $-2.638967-4.675806 \quad 1.910613$

H -1.694398 -4.6696853 .407806$

H -3.202780 -3.746466 3.308382

C -1.932736 -1.003972 4.050260

H $2.378615-2.2525153 .159589$

H 1.5087620 .3386892 .418666

H 4.4220411 .1424651 .605308

H -1.385246 -0.4990744 .856658$

H -2.734542 -1.5794784 .527516$

H $-0.511506-2.6001914 .119263$

C $8.154034-0.327726-0.782281$

O $6.726145-0.394901-0.676186$

C $6.185770-1.676163-0.343483$

C $6.572742-2.707191-1.416681$

C $8.090131-2.747794-1.628213$

C $8.677554-1.344328-1.810310$

H $8.3560890 .678129-1.173195$

C $8.834451-0.4418860 .586469$

H $6.579683-1.9964950 .627259$

O $5.927191-2.378992-2.645685$

H $6.207894-3.693757-1.106318$

H $8.320778-3.351544-2.514362$

O $8.714832-3.368697-0.508647$

H $\quad 9.772515-1.376457-1.764256$

O $8.327234-0.857445-3.109093$

C $8.4722710 .735378 \quad 1.460645$

H $8.580306-1.3638951 .117026$

H $9.925877-0.4398720 .481124$

H $6.167192-3.061653-3.295100$

H $8.348645-4.265765-0.427758$

H $8.701775-1.471556-3.763275$

$\begin{array}{llll}\text { O } & 7.538082 & 0.363621 & 2.381485\end{array}$

O $8.948144 \quad 1.855311 \quad 1.325718$

C 7.0904581 .4015273 .258739

H $\quad 6.3130640 .9868703 .906059$

H 7.9189531 .7508643 .881953

H 6.6628612 .2287672 .684189

SCF Energy (B3LYP/6-31G**//MMFF) $=-3245.90566329$

08_00622

MM̄MF Geometry

C $1.413230-4.322577-1.113184$

C $0.934219-3.451068-2.014435$

C $-0.504848-3.274999-2.447164$

O $-1.018099-2.016332-1.947551$

C $-1.480274-4.373378-1.979906$

C $-2.732963-4.439439-2.831355$

C $-3.940618-3.964144-2.473322$

C $-2.574470-5.165244-4.145501$

C $-4.320985-3.226316-1.218432$

C $-5.302349-2.088156-1.539792$

C $-5.968593-1.515323-0.281017$

C $-7.121092-0.588489-0.673358$ 
C $-7.788827 \quad 0.0395930 .555852$

O $-4.994325-0.7879920 .474238$

$\begin{array}{lllll}\text { O } & -8.635980 & 1.105114 & 0.098270\end{array}$

C $-6.698071 \quad 0.672977 \quad 1.440630$

C $-8.698754-0.9547841 .287198$

O $-7.279022 \quad 1.1373332 .658263$

C $-5.492691-0.2645861 .716618$

C $-4.362173 \quad 0.5435472 .393173$

O $-5.905203-1.3109402 .597780$

C $-0.742274-0.887249-2.651226$

O $-0.006074-0.782485-3.619034$

C $-1.5177090 .217296-2.042489$

C $-1.1874201 .483959-2.332694$

C -1.853460 $2.692031-1.839861$

C $-3.1966042 .525553-1.180938$

C $-3.016146-0.1829772 .496952$

C $-2.003775 \quad 0.7234793 .171739$

C -0.6417992 .7723952 .755226$

O $-1.676703 \quad 0.569784 \quad 4.345674$

C - -1.0659974 .0534132 .110347$

C -0.3126244 .7353851 .235193$

C $-0.730677 \quad 6.037440 \quad 0.616900$

C $-0.6526426 .028127-0.892756$

C $0.2804356 .747058-1.540144$

C $-1.7107365 .258696-1.657113$

C -1.246951 $3.880782-2.040956$

N -1.5597331 .7336572 .335388$

H $\quad 0.731728-4.957997-0.554949$

H $1.651195-2.841297-2.561801$

H $-0.503262-3.248482-3.544543$

H -1.740690 -4.220755 -0.925770

H -1.005933 -5.361717 -2.035874

H $-4.768105-4.138242-3.161112$

H -3.516009 -5.233192 -4.700992

H -1.853337 -4.651102 -4.787732

H -2.220937 -6.187337 -3.974138

H $-4.802545-3.942909-0.542483$

H $-3.448889-2.817175-0.699887$

H $-4.762334-1.294893-2.072974$

H $-6.084882-2.462027-2.212702$

H $-6.344515-2.3505440 .321446$

H -7.864598 -1.122803 -1.278151

H $-6.7379000 .219008-1.313337$

H $-9.2904300 .726161-0.513159$

H $-6.336393 \quad 1.5635590 .907026$

H $-9.479354-1.3269190 .612713$

H -9.226754 -0.470688 2.116309

H -8.161251 -1.8206431 .679149$

H $-6.640817 \quad 1.7314733 .086637$

H $-4.2051501 .471603 \quad 1.829537$

H $-4.669270 \quad 0.8360183 .405536$

H -5.129562 -1.649944 3.071444

H - $2.331868-0.042202-1.375509$

H $-0.3307421 .658774-2.983069$

H -3.898879 2.038003-1.866897

H $-3.6567813 .468700-0.880800$

H -3.117103 $1.911612-0.280886$

H -3.097125 -1.101306 3.087277

H -2.637383 -0.4651701 .508062$

H $\quad 0.3648812 .467122 \quad 2.454827$

H -0.6596252 .8724653 .844995$

H -2.0406154 .4452752 .392193$

H 0.6686134 .3516840 .971449

H $-0.0774606 .820180 \quad 1.024074$

H $-1.748686 \quad 6.313473 \quad 0.919077$

H $\quad 0.3233296 .768249-2.624189$

H $1.0251267 .324311-1.002351$

H -1.951489 5.798009-2.583095

H -2.637487 $5.252859-1.077675$

H $-0.2933363 .880747-2.571162$

H -1.7501941 .6539651 .341710$

C $3.307504-4.3444990 .570694$

O $3.823903-3.000804 \quad 0.719701$

C $5.114602-3.0730561 .353202$

C $5.163651-4.4381202 .024074$

C $4.441055-5.2827110 .998317$

H $5.862177-3.0508700 .551497$

C $5.343391-1.9024342 .313344$
H $\quad 6.187347-4.7735562 .214770$

H $4.617478-4.4434772 .974622$

C $3.959882-6.6181301 .540263$

H $5.119460-5.4641420 .153337$

C $5.281333-0.4990941 .678272$

O $4.360668-1.9474443 .356148$

H $6.311320-2.0314362 .812567$

H $4.242499-0.2381971 .451200$

O $5.723014 \quad 0.4368192 .671349$

C $6.149066-0.3134300 .420369$

C $6.3501741 .145089-0.035234$

H $5.709004-0.884529-0.404707$

O $7.450746-0.8638670 .647423$

H 7.0050951 .6687600 .673450

H $6.9131241 .106051-0.977480$

H $4.807328-7.2247861 .875836$

H $3.283749-6.4855162 .391569$

H $3.424057-7.1819090 .770072$

C $2.879047-4.574604-0.881905$

H $3.484898-1.9090842 .934602$

H $5.155127 \quad 0.3193903 .452409$

H $7.835587-0.4022911 .412269$

H $3.050414-5.621980-1.160264$

H $3.487179-3.959293-1.556263$

H $2.457576-4.4332981 .259029$

C $2.9469221 .843691-1.411169$

O $4.239838 \quad 1.264552-1.181458$

C $5.0582081 .949796-0.230724$

C $5.3411623 .383072-0.706953$

C $4.0372244 .136648-0.983440$

C $3.0718413 .314832-1.844732$

H $2.5339141 .282912-2.260024$

C $2.0105271 .634018-0.210388$

H $4.5402291 .995575 \quad 0.731679$

O $6.1291723 .344636-1.895185$

H 5.9239313 .9043190 .061951

H $4.2691675 .080050-1.492954$

O $3.4253344 .454345 \quad 0.262299$

H $2.0807243 .780115-1.846743$

O $3.532763 \quad 3.343120-3.199490$

C 1.7126730 .1674610 .021129

H 2.4370312 .0319930 .713691

H $1.0564072 .136014-0.398446$

H $6.3242014 .263250-2.147523$

H 2.6557885 .0170760 .070741

H $4.3835182 .872795-3.233500$

O $\quad 0.9215310 .040542 \quad 1.125595$

O $2.119934-0.751240-0.675554$

C $0.560516-1.2971771 .483278$

H $\quad 0.124127-1.2765042 .484951$

H $-0.185030-1.6794100 .781192$

H $1.438689-1.9468011 .507284$

SCF Energy (B3LYP/6-31G**//MMFF) $=-3245.90540936$

08_00623

MM̄FF Geometry

C -2.3880010 .8109912 .687332$

C $-2.8551091 .955716 \quad 3.209745$

C -3.7811092 .9444342 .538327$

O -4.4916842 .3346401 .433819$

C -3.0355094 .1534861 .943196$

C -2.0257154 .8174532 .853988$

C -0.6913674 .7005242 .698118$

C -2.6018405 .6700743 .951000$

C 0.0095713 .8630351 .664041

C 0.4300834 .7026710 .454091

C $0.7906533 .823869-0.752714$

C $1.3779554 .683622-1.873240$

C $1.6348063 .868699-3.146762$

O $-0.4148363 .190805-1.199707$

O $1.8838694 .789783-4.219251$

C $0.3534703 .090672-3.505171$

C $2.8874022 .996754-3.021883$

O $0.5868262 .210527-4.603387$

C $-0.2550522 .305862-2.317482$

C $-1.6655001 .797158-2.714290$

O $\quad 0.5907901 .203253-1.997668$

C $\begin{array}{rrrr}-5.567661 & 1.555170 & 1.720867\end{array}$ 
O $\quad \begin{array}{llll}-6.054471 & 1.338980 & 2.818223\end{array}$

C -6.0139070 .9549040 .445405$

C $-6.724410-0.1816040 .467179$

C -7.162705 -0.946370 -0.702472

C $-6.844667-0.384134-2.063569$

C $-2.4150071 .013513-1.626008$

C $-2.229117-0.493745-1.715307$

C $-3.092181-2.616870-0.725875$

O $-1.450161-1.030453-2.498192$

C $-4.202716-3.123123-1.593417$

C $-5.130872-3.998808-1.177562$

C $-6.221028-4.540171-2.059660$

C $-7.596347-4.430412-1.437215$

C $-8.196102-5.503325-0.894121$

C $-8.309706-3.097983-1.519283$

C $-7.798453-2.118875-0.498347$

N $-3.037615-1.168482-0.809404$

H $-2.663216 \quad 0.520111 \quad 1.678932$

H -2.551827 2.2220324 .220865

H -4.5129813 .3013453 .274331$

H -3.7787104 .9025891 .635043$

H -2.5747203 .8584750 .991803$

H $-0.0346545 .237647 \quad 3.379751$

H -1.824225 6.1485514 .555844

H $-3.226171 \quad 6.463267 \quad 3.527157$

H -3.2171125 .0642324 .623551$

H 0.8978293 .4109322 .122548

H $-0.615714 \quad 3.019857 \quad 1.355974$

H $-0.393150 \quad 5.372366 \quad 0.171145$

H 1.2840225 .3321350 .733719

H $1.5071003 .057429-0.434331$

H $2.2994475 .178361-1.541347$

H $0.6761755 .493678-2.117728$

H $2.6495265 .336725-3.972737$

H $-0.3886533 .824732-3.849626$

H $3.7706683 .616838-2.827285$

H $3.0942162 .466347-3.957723$

H $2.8145362 .264036-2.216161$

H $\quad 0.9537482 .743834-5.329133$

H -2.281358 $2.676036-2.948800$

H -1.606563 $1.193676-3.627520$

H $0.0891800 .568595-1.462413$

H $-5.695211 \quad 1.432641-0.473855$

H $-6.980667-0.6144301 .433505$

H $-7.3206580 .594253-2.193837$

H -7.186147 -1.011585 -2.888706

H -5.762925 -0.266754 -2.190748

H -2.131166 $1.350077-0.623856$

H -3.488037 $1.205415-1.751022$

H -3.235999 -2.8772200 .327436$

H - $2.142730-3.038271-1.067816$

H -4.229096 -2.772482 -2.622711

H $-5.086310-4.374843-0.157904$

H $-5.987797-5.593410-2.263802$

H -6.227615 -4.042892 -3.037790

H -9.192855 -5.439616 -0.470270

H -7.701692 -6.468277 -0.856240

H -9.383572 -3.232850 -1.332505

H -8.248572 -2.725011 -2.544858

H -7.980037 -2.435565 0.529978

H $-3.695995-0.639668-0.245732$

C $-0.077582-0.1821372 .839126$

O $-0.098182-0.9126531 .593364$

C $0.884390-1.9678721 .658027$

C $1.846889-1.5500342 .755961$

C $0.903549-0.9185343 .754539$

H $\quad 0.330995-2.8697241 .948689$

C $1.545740-2.1610040 .287901$

H $2.404933-2.3915273 .175224$

H $2.563973-0.8083402 .382721$

C $1.601437-0.0158324 .758166$

H $0.383268-1.7187884 .298232$

C $2.687408-3.1969170 .285087$

O $0.538778-2.574840-0.638510$

H $1.906272-1.190812-0.068933$

H $3.485626-2.8998560 .970106$

O $2.184965-4.447841 \quad 0.778443$

C $3.285335-3.487451-1.102710$
C $3.834285-2.279135-1.880128$

H $2.531194-3.983898-1.725960$

O $4.351813-4.432560-0.952394$

H $4.138963-2.644343-2.870106$

H $3.024836-1.557636-2.038274$

H $2.325913-0.5856685 .349121$

H $2.140247 \quad 0.796962 \quad 4.259437$

H 0.8792820 .4322745 .447980

C -1.485507 -0.1266673 .438405$

H $\quad 0.230930-3.455845-0.364630$

H $2.031788-4.3499761 .733092$

H $3.992668-5.200847-0.476261$

H -1.4285600 .1763994 .490755$

H -1.944376 -1.122863 3.412691

H 0.2884550 .8260362 .609835

C $5.644821-0.2069400 .668574$

O $4.625405-0.892210-0.070424$

C $5.058257-1.582913-1.244871$

C $5.714598-0.602209-2.233555$

C $6.8260930 .215009-1.569015$

C $6.3767240 .804818-0.227955$

H 5.1004430 .3594851 .435086

C $6.595640-1.1783421 .380623$

H $5.789046-2.348179-0.964754$

O $4.7230390 .281026-2.751423$

H $6.120570-1.172914-3.077755$

H $7.1264071 .027666-2.241600$

O $7.956548-0.630108-1.378022$

H 7.2373331 .2153870 .312009

O $5.4949621 .903332-0.478859$

C $5.876127-1.9677712 .450643$

H $7.065418-1.8775020 .683637$

H $7.406628-0.6350051 .879359$

H $5.1534270 .852010-3.410441$

H $8.662092-0.091154-0.981413$

H $4.687396 \quad 1.547328-0.887576$

O $6.046874-3.3038612 .236322$

O $5.247782-1.4610703 .370891$

C $5.425474-4.1684463 .192266$

H $5.839123-3.9900614 .189326$

H $4.342003-4.0199443 .191535$

H $5.636948-5.2014772 .903804$

SCF Energy (B3LYP/6-31G**//MMFF) $=-3245.91156464$

08_00624

MM̄MF Geometry

C $0.996742-2.7541392 .243956$

C $-0.035689-2.1933002 .892008$

C $-0.195231-0.7459343 .299962$

O $-1.376514-0.2339912 .632674$

C 0.9771280 .1848532 .939990

C $0.838214 \quad 1.5683293 .547109$

C 0.4493522 .6692132 .874557

C 1.2248261 .6722175 .000690

C 0.0330002 .7673321 .433921

C -1.2559623 .5856801 .274500$

C $-1.6665413 .704058-0.200346$

C $-2.8137024 .701032-0.366478$

C $-3.2877244 .780232-1.824936$

O $-2.0789032 .410103-0.652926$

O $-4.5379715 .485311-1.845096$

C $-3.5651653 .351734-2.334111$

C $-2.3203495 .584809-2.701206$

O $-3.8685603 .356025-3.727456$

C $-2.4197352 .351396-2.044389$

C $-2.8987730 .917476-2.365274$

O $-1.2998792 .660112-2.875103$

C $-2.574650-0.4029883 .253788$

O $-2.775824-0.9198184 .341227$

C -3.6227560 .1194772 .350654$

C $-4.892289-0.2709612 .536108$

C -6.0395690 .0944201 .704020$

C $-5.845757 \quad 1.1865130 .687363$

C $-1.965056-0.197554-1.877698$

C $-2.649280-1.539112-2.052679$

C $-4.455390-2.949168-1.054325$

O $-2.431252-2.251438-3.029671$

C $-5.848064-2.440410-1.267928$ 
C $-6.896163-2.797271-0.509396$

C $-8.292232-2.282821-0.724441$

C $-8.940949-1.7751850 .546815$

C $-9.944334-2.4545611 .127963$

C $-8.480408-0.4427661 .103508$

C $-7.200376-0.5686981 .886071$

N $-3.550690-1.813853-1.035235$

H $1.848317-2.151198 \quad 1.943550$

H $-0.861980-2.8408733 .182266$

H $-0.343625-0.7241214 .386862$

H $1.0729640 .253703 \quad 1.851266$

H $1.924154-0.2407993 .296847$

H $0.4334663 .615573 \quad 3.414150$

H $1.1586792 .698646 \quad 5.376894$

H 0.5682361 .0536225 .619967

H 2.2571931 .3364065 .143776

H 0.8397613 .2658180 .884898

H $-0.108171 \quad 1.7822700 .981362$

H -2.059802 $3.104612 \quad 1.846270$

H $-1.103600 \quad 4.5875261 .695139$

H $-0.7940994 .030123-0.778430$

H -2.523186 $5.695037-0.004288$

H -3.662112 4.3895370 .259152

H $-4.3914486 .366081-1.459444$

H $-4.4706302 .996710-1.822142$

$\mathrm{H}-2.2134426 .605685-2.315311$

H -2.706114 $5.689651-3.721271$

H -1.321978 5.145520 -2.753589

H $-4.5994793 .983218-3.862872$

H -3.882623 $0.762007-1.909696$

H -3.036216 $0.803614-3.448570$

H -0.774378 1.855059 -3.007072

H -3.317052 0.7663411 .536703

H $-5.110298-0.9590803 .352314$

H $-6.778585 \quad 1.5347430 .239240$

H $-5.2019780 .838549-0.123421$

H $-5.3854162 .068696 \quad 1.146769$

H -1.027317 -0.210699-2.443132

H $-1.701843-0.073764-0.821104$

H $-4.347375-3.466770-0.096133$

H $-4.181750-3.634498-1.861964$

H $-6.002332-1.747793-2.092423$

H $-6.748797-3.5120180 .297148$

H $-8.885292-3.104546-1.146334$

H -8.309906 -1.483251-1.475589

H $-10.431054-2.0858052 .024818$

H -10.298477 $-3.397010 \quad 0.723759$

H -9.241102 -0.0343641 .781532$

H -8.4104090 .2693320 .276875$

H -7.244431-1.323386 2.673116

H -3.655065 -1.146205 -0.277217

C $1.245321-4.5206990 .424395$

O $2.483214-3.961945-0.054160$

C $2.250441-3.374537-1.348579$

C $0.905227-3.917012-1.818830$

C $0.148189-4.002439-0.505733$

H $2.148615-2.294537-1.187383$

C $3.435006-3.647526-2.282259$

H $0.425266-3.260388-2.550634$

H $1.000489-4.913028-2.266148$

C $-1.076462-4.898306-0.562217$

H $-0.162548-2.989271-0.221510$

C $4.807394-3.261140-1.690165$

O $3.472255-5.050918-2.572623$

H $3.271653-3.140977-3.240808$

H $5.063492-3.966539-0.889607$

O $5.776895-3.454848-2.726933$

C $4.896581-1.817054-1.162661$

C $6.282989-1.481366-0.577569$

H $4.150913-1.669155-0.373987$

O $4.609730-0.898661-2.215699$

H $6.609404-2.3005480 .073790$

H $7.002658-1.378082-1.398775$

H $-1.779930-4.543521-1.320940$

H $-0.808527-5.929910-0.814145$

H $-1.593800-4.9098180 .402344$

C $1.071608-4.2211311 .915387$

H $4.223984-5.202298-3.171066$
H $\quad 6.658291-3.420125-2.318857$

H $3.670994-0.992968-2.447834$

H $1.936741-4.6247702 .456596$

H $\quad 0.186220-4.7487922 .289226$

H $1.345881-5.6082100 .309281$

C $5.8472412 .194294-0.044417$

$\begin{array}{llll}\text { O } & 6.033867 & 0.914296 & -0.661537\end{array}$

C $6.257521-0.1735610 .238789$

C $7.561298 \quad 0.0610051 .014467$

C $7.541117 \quad 1.4154691 .732605$

C 7.0581482 .5602390 .832086

H $5.8107162 .904040-0.880869$

$\begin{array}{llll}\text { C } 4.506156 & 2.278276 & 0.694959\end{array}$

H $5.425774-0.2399460 .948811$

O $8.689888-0.0049050 .146140$

H $7.682890-0.7407001 .752570$

H 8.5491801 .6386342 .103300

O 6.6762581 .3037862 .861646

H $6.8120503 .438241 \quad 1.439988$

O $8.1379432 .959530-0.017501$

C $3.3595391 .959818-0.234370$

H 4.4424731 .5824851 .536776

H $4.3485543 .279117 \quad 1.113708$

H $8.4493120 .408095-0.699309$

H 6.7082922 .1494813 .340536

H $8.1883062 .336930-0.760921$

O $3.1200013 .015041-1.063326$

O $2.7621090 .891180-0.234436$

C $2.0778922 .817463-2.023618$

H $1.1751502 .427884-1.544820$

H $1.8428843 .784513-2.476026$

H $2.4220502 .134209-2.805358$

SCF Energy (B3LYP/6-31G**//MMFF) = -3245.90612206

08_00625

MM̄FF Geometry

C $1.395022-3.401569-1.674261$

C $0.375347-3.723659-2.483957$

C $-0.902440-2.929646-2.663770$

O $-0.803513-1.632480-2.031822$

C $-2.072019-3.704423-2.019469$

C $-3.481509-3.212033-2.302448$

C $-4.539886-3.574347-1.546759$

C $-3.704193-2.332836-3.504470$

C $-4.546351-4.488463-0.350061$

$\begin{array}{llll}C & -5.099509 & -3.839172 & 0.929857\end{array}$

C $-4.203063-2.7281521 .498598$

C $-4.725939-2.2459282 .854899$

C $-3.913668-1.0583403 .401980$

O $-4.204004-1.6402210 .573154$

O $-4.662037-0.473314 \quad 4.479046$

C -3.792617 0.0156692 .297674

C $-2.578515-1.5121824 .001938$

O $-2.933367 \quad 1.0779492 .703193$

C -3.346978 -0.5543620 .934739$

C $-3.4144930 .488690-0.200581$

O $-1.985370-0.9854641 .028508$

C $-0.222417-0.632829-2.754239$

O $0.176127-0.696042-3.906419$

C $-0.1328580 .562588-1.885426$

C $0.2959741 .726388-2.394493$

C $0.4288472 .992405-1.672022$

C $0.0266253 .036306-0.222327$

C $-4.7974351 .091457-0.445835$

C $-4.8773561 .817809-1.776037$

C $-4.0772093 .838668-2.968652$

O $-5.6601461 .461504-2.654648$

C $-2.7399734 .479090-3.157023$

C $-2.424906 \quad 5.691765-2.677395$

C $-1.0991736 .370085-2.885788$

C $-0.1923496 .263092-1.679016$

C $-0.4839496 .901597-0.532532$

C $1.0861865 .464320-1.812021$

C $0.8833604 .072210-2.340782$

N $-4.0134962 .899193-1.864629$

H $1.334071-2.505167-1.063512$

H $0.447320-4.635032-3.074579$

H $-1.076023-2.809224-3.739725$ 
H $-1.912560-3.694947-0.933252$ H -2.029115 -4.750069-2.353362 H $-5.523696-3.197421-1.823956$ H -4.763560 -2.099959-3.658898 H -3.180361 -1.379182 -3.391496 H $-3.348507-2.830560-4.412163$ H $-5.187337-5.343571-0.599930$ H -3.556949 -4.908045 -0.143311 H -6.099466 -3.4370930 .720038$ H $-5.218327-4.6267371 .684345$ H -3.185142 -3.1229411 .600039$ H $-4.739370-3.0681983 .581218$ H $-5.772009-1.9261242 .746718$ H -4.810538 -1.161647 5.149794 H $-4.789948 \quad 0.4596412 .189839$ H -2.746783 -2.2282984 .815200$ H -2.039674 -0.6714874 .452495$ H - $-1.926053-1.9921013 .271460$ H -2.016348 0.7632352 .629716 H -2.688544 $1.285743-0.007222$ H $-3.0832910 .009533-1.131343$ H -1.660233 -1.1496820 .127772$ H $-0.4197510 .455592-0.847342$ H $\quad 0.572727 \quad 1.760865-3.447589$ H $-1.0576212 .926153-0.125728$ H $0.301516 \quad 3.9713210 .272257$ H 0.5108642 .2366040 .344501 H $-5.5643310 .308494-0.453357$ H -5.0633411 .8011310 .345364$ H $-4.8528714 .573397-2.726492$ H $-4.3838473 .322981-3.884658$ H -2.006468 $3.923688-3.735500$ H -3.166496 $6.240398-2.100705$ H -1.281122 $7.429014-3.111505$ H $-0.5999395 .972981-3.778123$ H $\quad 0.171946 \quad 6.8446770 .329647$ H -1.384849 $7.497874-0.432246$ H $1.6372285 .421309-0.864323$ H $1.7508705 .997562-2.504446$ H $1.141423 \quad 3.964407-3.394706$ H $-3.5504263 .209146-1.014830$ C $3.923122-3.461519-1.851295$ O $4.041479-2.282776-1.028799$ C $4.556101-1.210037-1.840857$ C $5.060447-1.859930-3.124951$ C $4.059205-2.985996-3.296897$ H $3.707266-0.563154-2.090770$ C $5.618631-0.422645-1.069888$ H $5.072459-1.161391-3.967076$ H $\quad 6.072887-2.263074-3.003487$ C $4.512228-4.065333-4.263714$ H $3.115620-2.557031-3.657041$ C 5.0991470 .2709860 .209551 O $6.676410-1.313339-0.692903$ H $6.0766560 .320536-1.732872$ H $4.822372-0.4822870 .955269$ $\begin{array}{lllll}\text { O } & 6.197947 & 0.998157 & 0.775681\end{array}$ C $3.9206031 .237987-0.021524$ C 3.6124632 .1701121 .163943 H $3.0244120 .666375-0.286518$ O $4.2341842 .071016-1.143670$ H 4.4936522 .7826301 .393323 H 2.8504722 .8825540 .822590 H $4.663239-3.646536-5.264019$ H $5.455623-4.520920-3.944483$ H $3.762555-4.859303-4.339555$ C $2.641029-4.224415-1.511793$ H $6.286660-2.012975-0.140861$ H 6.4462801 .6939570 .142958 H $3.4501202 .612992-1.335690$ H $2.685614-4.533730-0.460249$ H $2.587103-5.140805-2.110939$ H $4.777134-4.100227-1.588658$ C 1.2469530 .0977703 .190836 O 1.8902520 .7957902 .115325 C 3.1156781 .4639532 .434606 C 2.9109972 .4759783 .577060 C 2.2491511 .8237874 .793342
C 1.0080751 .0234834 .393542

H $0.263874-0.1799482 .789941$

C $1.993141-1.1886003 .560155$

H 3.8601500 .7227162 .740553

O $2.0967293 .556536 \quad 3.121957$

H 3.8861042 .8945303 .853666

H 1.9574822 .5969135 .514423

O 3.1756650 .9533335 .435169

H $0.652600 \quad 0.432075 \quad 5.245064$

O $-0.048523 \quad 1.9293574 .063767$

C $1.741465-2.2500982 .518738$

H $3.076124-1.0588463 .636548$

H $1.656437-1.5802814 .527723$

H 2.0222044 .1952733 .851223

H $3.942304 \quad 1.4873385 .704735$

H 0.2101032 .4089593 .258133

O $0.616959-2.9526152 .835823$

O $2.443080-2.4109791 .529763$

C $0.224605-3.9615531 .900559$

H $1.059218-4.6334111 .679010$

H $-0.583746-4.5447162 .349772$

H -0.150160 -3.490897 0.988164

SCF Energy (B3LYP/6-31G**//MMFF) $=-3245.91382311$

08_00626

MM̄MF Geometry

C $1.751968-2.0532313 .548418$

C $2.986145-2.0635163 .020969$

C $3.519647-2.9303921 .901563$

O $4.054657-2.0368310 .890685$

C $2.491117-3.8167281 .171237$

C $3.127870-4.9587360 .401063$

C $3.449234-4.940419-0.907118$

C $3.352781-6.2124071 .209517$

C $3.374482-3.796859-1.878812$

C $2.273409-3.969247-2.934925$

C $0.917648-3.420448-2.467164$

C $-0.167578-3.669502-3.515940$

C -1.504449-3.019655 -3.122636

O $1.077241-2.011252-2.266716$

O $-2.352136-3.017843-4.281616$

C $-1.254711-1.543848-2.744150$

C $-2.240122-3.831902-2.050661$

O $-2.438610-0.938582-2.229148$

C $-0.082916-1.349000-1.754504$

C $0.2463270 .155880-1.610410$

O $-0.456227-1.862593-0.475870$

C $5.326519-1.5801701 .014291$

O $6.124224-1.8314831 .901397$

C $5.559357-0.671524-0.129337$

C $6.5282950 .251900-0.061636$

C $6.8273061 .256625-1.085036$

C $6.0464551 .195211-2.372728$

C $1.5842490 .440199-0.910220$

C $1.7830791 .922518-0.641184$

C 3.3727563 .5012310 .457504

O $\quad 0.9913242 .784579-1.013271$

C $4.3283484 .042210-0.559794$

C $5.5094044 .596860-0.245199$

C $6.4566965 .176032-1.258218$

C $7.8763134 .676965-1.093262$

C $8.8133215 .441942-0.507920$

C $8.2285943 .328213-1.681939$

C $7.7643892 .188936-0.814331$

N 2.9531072 .1678600 .065448

H $0.991089-2.735653 \quad 3.184890$

H $3.711773-1.3654473 .437111$

H $4.328003-3.5518432 .307059$

H $1.886577-3.2029490 .493563$

H $1.787674-4.2687671 .880097$

H $3.867255-5.849911-1.338607$

H $3.819512-7.0105500 .622683$

H $4.008358-6.0047492 .061131$

H $2.399967-6.5957101 .589030$

H $3.276342-2.834449-1.372039$

H $4.342711-3.759246-2.394838$

H $2.584575-3.410646-3.827653$

H $2.180881-5.022367-3.227067$ 
H $\quad 0.648480-3.899336-1.519243$ H $-0.299092-4.744364-3.692022$ H $0.151742-3.245508-4.478491$ H -2.459172 -3.938737 -4.575968 H -1.013104 -1.007014 -3.672414 H $-2.429526-4.851827-2.406510$ H $-3.225679-3.403380-1.838653$ H -1.685193 -3.908404 -1.113406 H -3.142886 -1.075234 -2.885862 H $\quad 0.297171 \quad 0.616301-2.605805$ H $-0.5633780 .655834-1.063866$ H $0.063869-1.4154130 .209726$ H $4.891868-0.758316-0.979287$ H $7.140257 \quad 0.2976680 .838354$ H $6.221666 \quad 0.240190-2.880464$ H $6.3077191 .981918-3.082803$ H $4.9728031 .297237-2.180993$ H $1.644831-0.0893430 .045045$ H $2.4143340 .099642-1.540010$ H $3.8274493 .419376 \quad 1.449826$ H 2.4966844 .1539750 .525131 H $4.0240723 .998229-1.602932$ H 5.7985494 .6699750 .800813 H $6.4312226 .268167-1.147781$ H $6.1198304 .973341-2.282564$ H $9.8394415 .103511-0.410199$ H $8.5739036 .421800-0.108193$ H $9.3182193 .234441-1.783947$ H $7.8484583 .274941-2.705320$ H $8.2721942 .146510 \quad 0.150353$ H $3.571351 \quad 1.3931790 .286089$ C $0.104614-0.2528514 .285491$ O $-1.059346-1.0930184 .141615$ C -1.640836 -0.8493652 .847416$ C -1.1858890 .5515682 .470146$ C 0.2346310 .5636862 .995495 H -1.198728 -1.595285 2.176746 C -3.163724 -1.029065 2.906720 H $-1.2399540 .744107 \quad 1.395089$ H $-1.788526 \quad 1.3060922 .987910$ C $0.791831 \quad 1.9602613 .213860$ H $\quad 0.869412 \quad 0.0499742 .266289$ C $-3.832826-0.8111611 .534422$ O $-3.430879-2.3518803 .379073$ H -3.586827 -0.3420073 .647850$ H $-3.5161590 .163097 \quad 1.150520$ O $-3.335379-1.8074170 .640440$ C $-5.374801-0.8530991 .556239$ C $-6.037260-0.7547560 .166935$ H -5.740422 -0.0379522 .190947$ O $\quad-5.835091-2.0671702 .152351$ H $-5.783873-1.643336-0.425287$ H $-7.123578-0.8053660 .322733$ H 0.7895972 .5275712 .277983 H $\quad 0.1974812 .5201573 .943742$ H 1.8218691 .9126183 .581151 C $1.325023-1.1116784 .646125$ H $-3.106325-2.9748792 .706258$ H $-3.512215-1.507587-0.266879$ H $-5.444733-2.8054441 .653831$ H $1.081235-1.7237985 .523214$ H $2.153684-0.4519844 .930122$ H -0.1116780 .4076225 .134514$ C $-5.9241992 .928466-0.450346$ O $-6.201051 \quad 1.637097 \quad 0.109831$ C $-5.6854280 .518382-0.618238$ C $-6.2551820 .489965-2.043729$ C $-5.9815801 .815932-2.761292$ C $-6.4040323 .016177-1.910777$ H $-6.533715 \quad 3.6233270 .141957$ C $-4.4515123 .322417-0.264129$ H $-4.5977360 .599079-0.687851$ O $\quad-7.6577180 .241892-1.996143$ H -5.791914 -0.339036 -2.592264 H $-6.5283941 .839788-3.711763$ O $-4.5909341 .925888-3.052708$ H $-6.0416193 .944860-2.367172$ O $-7.8332843 .080001-1.919027$
C $-4.1979223 .803311 \quad 1.146428$

H -3.742454 $2.518039-0.476968$

H -4.196228 4.157831 -0.927200

H -7.984469 $0.224912-2.911631$

H $-4.3475041 .169666-3.613450$

H $-8.0949073 .881451-1.434674$

O -4.2810152 .7634932 .023566$

O $-3.9562194 .969237 \quad 1.432079$

C -4.1004013 .1052443 .401163$

H -4.8652053 .8209343 .717607$

H -4.2075352 .1933563 .994332$

H -3.0981983 .5137263 .561743$

SCF Energy (B3LYP/6-31G**//MMFF) $=-3245.89301869$

08_00627

MM̄FF Geometry

C 2.1233343 .8263721 .429792

C 2.0660993 .6182782 .754730

C 2.4155472 .3421613 .487578

O 2.8257451 .2981222 .576675

C 1.1930661 .8224554 .262551

C 1.5402020 .6792975 .197580

C $1.278364-0.6193384 .950567$

C 2.1776281 .0860806 .501485

C $0.648814-1.2026363 .716695$

C $1.331230-2.5089763 .288091$

C $0.637351-3.1233812 .063586$

C $1.147601-4.5407271 .804576$

C $0.500018-5.1537130 .554149$

O $0.908606-2.2942800 .927838$

O $1.238253-6.3366690 .212723$

C $0.657686-4.163627-0.617183$

C $-0.942346-5.6024930 .817189$

O $-0.040029-4.626128-1.772131$

C $0.247029-2.709551-0.274871$

C $0.689898-1.776719-1.423826$

O $-1.173387-2.642782-0.145025$

C $4.153887 \quad 1.1746092 .313432$

O 5.0598881 .8699632 .743291

C 4.3135440 .0413731 .375773

C $5.472471-0.1073790 .717713$

C $5.777818-1.143384-0.270891$

C $4.793753-2.271810-0.425485$

C $0.536820-0.279351-1.136237$

C $1.1895800 .508906-2.254226$

C $3.4290441 .273695-3.054167$

O $\quad 0.5507430 .889088-3.232518$

C $4.3036070 .193812-3.612287$

C $5.6383190 .293194-3.710437$

C $6.514633-0.793799-4.266789$

C $7.686113-1.129427-3.367680$

C $8.941197-0.801106-3.718060$

C $7.413169-1.913099-2.099451$

C $6.915078-1.029680-0.988053$

N $2.5507790 .684889-2.060021$

H $2.4499163 .026490 \quad 0.772479$

H 1.7584194 .4453873 .392468

H 3.2264942 .5682824 .190887

H 0.4094851 .5184023 .558530

H 0.7495832 .6306354 .859752

H $1.531746-1.3458525 .721637$

H 2.3572530 .2307007 .161593

H $3.142150 \quad 1.5714796 .324403$

H 1.5289741 .7851517 .039401

H $-0.403465-1.4100343 .942815$

H $0.675097-0.4992142 .880017$

H $2.384978-2.3066253 .057970$

H $1.303597-3.2216524 .121995$

H $-0.442223-3.1399882 .250146$

H $0.976549-5.1838332 .676931$

H $2.236703-4.5161841 .657960$

H $1.201623-6.9427690 .972493$

H $1.722262-4.165792-0.889678$

H $-0.974305-6.323847 \quad 1.642513$

H $-1.357386-6.125910-0.051336$

H -1.608930 -4.776488 1.073790

H $-0.986639-4.449708-1.635888$

H $1.742988-1.973941-1.655687$ 
H $\quad 0.120214-2.010810-2.332818$ H -1.460825 -1.728335 -0.294871 H $3.465152-0.6171521 .228693$ H 6.2661590 .6178960 .893289 H $4.572529-2.7290640 .545923$ H $5.153010-3.080793-1.064460$ H $3.860083-1.905504-0.858909$ H $-0.5160840 .014111-1.079625$ H $0.999619-0.000892-0.182908$ H $4.0073812 .056567-2.553601$ H $2.8389841 .725962-3.856737$ H $3.812671-0.713349-3.957756$ H $6.1273611 .210752-3.391037$ H $6.876707-0.456963-5.246896$ H $5.940158-1.708920-4.457525$ H $9.788545-1.059676-3.091492$ H $9.149962-0.262149-4.636183$ H $8.335329-2.395344-1.749843$ H $6.728771-2.730915-2.339543$ H $7.574865-0.186572-0.775916$ H $2.9796340 .319160-1.215533$ C $0.6038375 .118258-0.171773$ O -0.6078784 .8325120 .552096$ C -1.441527 $3.970533-0.262019$ C $-0.8133223 .983259-1.649254$ C $0.6595314 .114323-1.319480$ H $-1.3479182 .979367 \quad 0.195568$ C $-2.8927334 .467536-0.190927$ H -1.040806 $3.083376-2.226979$ H -1.149210 4.855965 -2.223006 C $1.5125474 .563007-2.492242$ H $1.0143213 .139022-0.970286$ C $-3.9503753 .737840-1.050249$ O $\quad-3.319950 \quad 4.443170 \quad 1.175644$ H -2.893108 $5.525679-0.482576$ H $-4.9047554 .252799-0.875722$ O $-3.6375303 .907311-2.429393$ C $-4.1716692 .246257-0.716512$ C $-5.3113901 .620451-1.546583$ H -4.4084832 .1562230 .348981$ O $-2.9714731 .520573-0.964019$ H $-6.1526782 .321283-1.604730$ H -4.942064 $1.436252-2.563096$ H $1.4347683 .851165-3.320119$ H $1.1994285 .544006-2.865149$ H $2.5657624 .632099-2.202594$ C 1.8046165 .1452180 .778109 H -3.150773 3.5538461 .528473 H -4.409608 3.629047 -2.949462 H $-3.1207760 .598996-0.693127$ H 1.6153895 .8992661 .552919 H $2.694323 \quad 5.4743970 .228224$ H $0.4767496 .131836-0.575493$ C $-5.034980-1.921696-0.345183$ O $-4.747008-0.651410-0.945017$ C $-5.8222530 .291927-0.948046$ C -7.019324 -0.266976-1.733159 C $-7.441685-1.638947-1.195203$ C $-6.244431-2.581239-1.030201$ H $-4.151494-2.537530-0.557662$ C $-5.179915-1.8219921 .178696$ H -6.1294020 .4930250 .084000$ O $-6.670691-0.383671-3.111366$ H -7.852329 $0.442662-1.662115$ H -8.163693 -2.097820 -1.881737 O $-8.081097-1.480780 \quad 0.067975$ H $-6.542503-3.472623-0.466654$ O $-5.834833-3.037842-2.322846$ C $-3.859679-1.4810781 .827430$ H $-5.918662-1.0846431 .504750$ H $-5.501410-2.7846501 .594511$ H -7.454826 -0.708296 -3.586039 H -8.858675 $-0.912494-0.065405$ H -5.499703 -2.270064 -2.817165 O $-3.640050-0.1378621 .758464$ O $-3.109804-2.3117852 .323201$ C $-2.396862 \quad 0.3156372 .300427$ H $-2.351647 \quad 1.4018372 .189357$
H $-2.3355870 .070708 \quad 3.364586$

$\mathrm{H}-1.562850-0.1260331 .747678$

SCF Energy (B3LYP/6-31G**//MMFF)= -3245.91138221

08 00628

MM̄̄FF Geometry

C $1.317559-3.0821980 .523600$

C $0.014579-3.3518460 .359256$

C $-0.677232-3.320084-0.978627$

O $-1.443171-2.096412-1.048315$

C $-1.641575-4.510538-1.104506$

C -2.397355 -4.525029-2.418138

C $-3.677359-4.126682-2.559606$

C $-1.633067-5.067905-3.596519$

C $-4.566212-3.547979-1.492712$

C $-5.375058-2.351284-2.013720$

C $-6.203028-1.711765-0.889917$

C $-7.209644-0.709458-1.453930$

C $-7.996703-0.010692-0.335473$

O $-5.299105-1.041986-0.002815$

O $-8.6840301 .109384-0.913364$

C -6.9989980 .5528850 .696085$

C $-9.075135-0.9202770 .264556$

O -7.6814671 .0821751 .830793$

C $-5.924316-0.4650721 .151087$

C $-4.8424660 .271692 \quad 1.975136$

O $-6.531850-1.463978 \quad 1.971359$

C $-0.847600-1.031837-1.652559$

O $0.262149-0.998861-2.160705$

C $-1.7759180 .116339-1.563389$

C $-1.3145241 .343893-1.844537$

C $-2.0601672 .598790-1.736528$

C $-3.5362002 .519857-1.455072$

C -3.581801 -0.5518602 .269354$

C -2.5322430 .3219522 .932079$

C -0.9688632 .2106822 .432532$

$\begin{array}{llll}\text { O } & -2.303640 & 0.256286 & 4.137233\end{array}$

C -1.4769783 .5372371 .959582$

C -0.7410714 .4079741 .251802$

C -1.260126 5.7304940 .760749

C $-1.0280195 .945688-0.720717$

C $-0.1345116 .850821-1.154510$

C $-1.8815365 .163703-1.701019$

C -1.381623 $3.756117-1.878803$

N -1.9236271 .1906072 .040501$

H $1.910524-2.801528-0.343109$

H -0.591257 -3.588071 1.231055

H $0.053142-3.365865-1.796396$

H $-2.346582-4.512034-0.264699$

H -1.084105 -5.452243 -1.009002

H $-4.139278-4.223624-3.541121$

H -2.238545 -5.093531-4.508650

H $-0.754649-4.448392-3.802180$

H -1.298749 -6.090642 -3.393918

H -5.258461 -4.330641-1.159900

H $-3.991100-3.231247-0.618259$

H -4.689167 -1.606389 -2.437290

H $-6.038224-2.689908-2.819433$

H $-6.724059-2.508044-0.345344$

H -7.896024 -1.197783 -2.156818

H $-6.6768160 .055589-2.036199$

H $-9.267350 \quad 0.773254-1.615294$

H $-6.488157 \quad 1.4017530 .219489$

H $-9.779851-1.241940-0.511613$

H $-9.674428-0.3839721 .008722$

H -8.667095 -1.818359 0.732976

H -8.3325811 .7260911 .503113$

H $-4.541419 \quad 1.174888 \quad 1.432765$

H -5.2660810 .6075672 .930711$

H $-5.853289-1.8554472 .543887$

H $-2.788875-0.070632-1.227334$

H $-0.2715721 .449848-2.141978$

H -4.028022 $1.828186-2.148555$

H $-4.0505543 .476284-1.572569$

H $-3.7130482 .174919-0.433438$

H $-3.802743-1.3901232 .938327$

H $-3.152943-0.9742511 .353808$

H -0.0122551 .9500391 .970381$ 
H $-0.8497142 .219263 \quad 3.519789$ H -2.503948 3.7927192 .211316 H $\quad 0.2933104 .1642941 .021809$ H -0.7589676 .5173601 .339218$ H $-2.331445 \quad 5.8396820 .970990$ H $\quad 0.0223177 .027019-2.213661$ H $0.4651097 .431273-0.461301$ H -1.865666 $5.648849-2.685563$ H -2.920643 $5.208731-1.363748$ H $-0.3200953 .701939-2.122997$ H $-2.1468521 .113771 \quad 1.053337$ C $3.468245-3.5616961 .802829$ O $4.235398-2.6115901 .036491$ C $5.057141-3.3322310 .104009$ C $5.142204-4.7511420 .652194$ C $3.732320-4.9366391 .186215$ H $4.507579-3.359447-0.844901$ C $6.393684-2.615818-0.102218$ H $5.410352-5.481104-0.117280$ H $5.869334-4.8287651 .468840$ C $3.593414-6.0851442 .169668$ H $3.064140-5.1198200 .335693$ C $6.270136-1.120381-0.475644$ O $7.150238-2.6960251 .112383$ H $\quad 6.977277-3.151587-0.860875$ H $5.984238-0.5484060 .414604$

O $7.589163-0.686477-0.831356$ C $5.296239-0.826967-1.631811$

C $5.3306290 .610027-2.180587$ H $4.274520-1.060362-1.311820$ O $5.606336-1.706508-2.718622$ H $6.2993320 .799481-2.660638$ H $4.592410 \quad 0.652051-2.993241$ H $3.848999-7.0353531 .689704$ H $4.254101-5.9568803 .033546$ H $2.565299-6.1573832 .538625$ C $2.006122-3.1083801 .860129$ H $7.997945-2.2456480 .955227$ H $7.5890220 .284987-0.847639$ H $4.932474-1.572325$-3.406712 H $1.980353-2.0858422 .253511$ H $1.456338-3.7478972 .560578$ H $3.877356-3.5218622 .820771$ C 3.2587632 .4455340 .350833 O $3.7219771 .485721-0.612650$ C $5.0259191 .718791-1.156217$ C $5.1106123 .109174-1.814562$ C $4.6460334 .216090-0.866950$ C $3.3011563 .872083-0.221253$ H 2.2044742 .1858880 .513446 C 3.9954172 .3218131 .690566 H $5.7628611 .663586-0.349690$ O $4.3229123 .151435-3.004069$ H $6.1490643 .292510-2.114728$ H $4.5559765 .153086-1.429887$ O $\quad \begin{array}{llll}5.637588 & 4.403119 & 0.137988\end{array}$ H 3.0577964 .5903270 .570300 O $2.2694653 .971901-1.205257$ C 3.4825041 .1395192 .475155 H 5.0762342 .1936401 .586024 H 3.8489633 .2189462 .304661 H $3.4120442 .907002-2.766326$ H 5.3370455 .1249740 .715887 H $2.2625634 .887934-1.531443$ O $2.4768861 .533683 \quad 3.307156$ O 3.9127340 .0016492 .348161 C 1.8912860 .5053614 .112650 H $1.233176-0.1126693 .496017$ H $2.660914-0.1068764 .592577$ H 1.2929570 .9851194 .891841 SCF Energy $\left(B 3 L Y P / 6-31 G^{* *} / / M M F F\right)=-3245.90695475$

08_00629

MM̄FF Geometry

C $-3.3386744 .386669-0.529738$

C $-3.4973143 .141679-0.057104$

C -4.4376392 .7739351 .059085$

O -3.6568602 .0421092 .032761$
C -5.5402251 .8384660 .541508$

C -6.6338421 .6009051 .562383$

C -6.7345370 .5034482 .337582$

C -7.6804972 .6821311 .643082$

C $-5.789023-0.6630122 .418144$

C $-6.406034-1.9630411 .883872$

C $-6.572685-1.9826330 .355757$

C $-7.237880-3.285188-0.094107$

C $-7.314381-3.396235-1.623875$

O $-5.270878-1.876829-0.227465$

O $-7.659812-4.757935-1.919990$

C $-5.916517-3.113448-2.216217$

C $-8.438886-2.525259-2.196500$

O $-5.965344-3.051019-3.640013$

C $-5.260789-1.827307-1.660374$

C $-3.782254-1.754091-2.112669$

O $-5.966962-0.690093-2.157614$

C $-3.0820122 .736587 \quad 3.045537$

O -3.2379063 .9227193 .300496$

C -2.1972721 .8701653 .858979$

C -1.7399290 .6791613 .439783$

C $-0.829838-0.2057434 .173156$

C -0.3763630 .2382595 .539672$

C $-3.004687-0.573094-1.517609$

C $-1.515732-0.713637-1.757559$

C $0.473911-1.999527-1.025625$

O $-0.932608-0.060090-2.619129$

C $0.887463-2.7575280 .193206$

C $1.748578-2.2856511 .106641$

C $2.187262-3.0573572 .316156$

C $1.880678-2.3391353 .610878$

C $2.852592-1.7201604 .302096$

C $0.470524-2.4201294 .152327$

C $-0.435207-1.3574773 .589131$

N $-0.924844-1.639355-0.911062$

H $-3.9499795 .180816-0.104922$

H -2.904514 2.327354 -0.462061

H -4.8806823 .6675611 .515042$

H -5.1019460 .8845390 .232595$

H $-5.9995312 .257537-0.363854$

H -7.5817790 .4345343 .018922$

H -8.4658712 .4481042 .369637$

H -7.2293203 .6332041 .942065$

H -8.1627112 .8165150 .669205$

H $-4.835488-0.4625841 .921375$

H -5.542083 - 0.8080723 .477721

H -5.741248 -2.785396 2.179128

H -7.374492 -2.140761 2.367971

H $-7.178817-1.1227040 .050394$

H $-8.236897-3.3854480 .348590$

H $-6.657251-4.1383900 .284835$

H -7.771564 -4.839435 -2.882099

H -5.273286 -3.967806 -1.962551

H $-9.408892-2.845819-1.797925$

H -8.510872 -2.635197 -3.284190

H $-8.319796-1.464520-1.966034$

H -6.277915 -3.912534 -3.962955

H -3.280478 -2.687092 -1.826286

H -3.725326 -1.682509-3.206151

H $-5.3913240 .088599-2.108583$

H -1.9003232 .2952964 .812260$

H $-2.042703 \quad 0.3145942 .459414$

H -1.233612 0.3413926 .214081

H $\quad 0.142042 \quad 1.2017505 .478700$

H $0.319555-0.4547456 .016612$

H $-3.3287440 .372153-1.965403$

H -3.165393 -0.489436 -0.436890

H $1.063434-1.090023-1.176479$

H $0.590245-2.624249-1.917373$

H $\quad 0.465988-3.751850 \quad 0.321027$

H $2.176230-1.2953510 .973332$

H $3.267853-3.2286082 .225808$

H $1.733918-4.0562232 .340530$

H $2.649189-1.2308865 .248570$

H $3.872825-1.6844463 .934275$

H $0.482592-2.3942945 .247356$

H $\quad 0.043164-3.4027343 .914007$

H $-0.813797-1.5885462 .593233$ 
H -1.527259 -2.223944 -0.337982 C -1.320971 $3.813773-2.052435$ O $-0.454213 \quad 3.487582-0.947262$ C $0.9155373 .498026-1.399777$ C $0.8467053 .517301-2.918295$ C $-0.4023564 .343101-3.153991$ H $1.3474564 .433986-1.023331$ C $1.6471962 .294012-0.795783$ H $1.7401993 .952884-3.375211$ H $0.7166922 .502964-3.315870$ C $-0.9703104 .197157-4.555020$ H $-0.1639885 .399515-2.971149$ C $3.0934882 .133479-1.303184$ $\begin{array}{llllll}\text { O } & 1.654807 & 2.458062 & 0.625057\end{array}$ H $1.0837761 .376536-0.999679$ H $3.0693812 .004997-2.391260$ O $3.8250483 .332258-1.046130$ C $3.8449070 .927820-0.699991$ C $5.2285020 .742970-1.339721$ H $3.2453800 .027456-0.864873$ $\begin{array}{lllll}\text { O } & 4.024666 & 1.085378 & 0.704615\end{array}$ H $5.1278560 .637706-2.427515$ H $5.8280111 .651179-1.188438$ H $-0.2432004 .533284-5.301436$ H -1.224023 3.155374 -4.778628 H $-1.8775854 .797690-4.673016$ C -2.369950 $4.834352-1.595770$ H 0.7297552 .5520300 .911384 H $3.8661133 .445111-0.080772$ H 3.1433431 .1408711 .110785 H -2.955791 $5.172959-2.457948$ H -1.848524 $5.712812-1.193243$ H $-1.8000832 .882251-2.378543$ C $8.252311-1.413954-1.084806$ O $7.289117-0.433122-1.490483$ C $6.046699-0.435675-0.773820$ C $5.352321-1.799035-0.909411$ C $6.282159-2.945009-0.496623$ C $7.658316-2.830182-1.158352$ H $9.050851-1.350321-1.835439$ C $8.868029-1.0801890 .283354$ H $6.251038-0.2293260 .281969$ O $4.929598-1.984192-2.258458$ H $4.457766-1.811102-0.278718$ H $5.828038-3.903499-0.775591$ O $6.448547-2.942228 \quad 0.918426$ H $8.360790-3.542739-0.710391$ O $7.540223-3.172728-2.542204$ C 9.6834340 .1963780 .251386 H $8.103246-0.9554761 .054057$ H $9.544022-1.8892520 .583528$ H $4.465680-2.837324-2.306547$ H $5.567753-3.0389541 .318693$ H $7.212909-4.086975-2.591037$ O $10.194338 \quad 0.423513 \quad 1.498594$ O $9.855004 \quad 0.904331-0.731131$ C $10.992377 \quad 1.6042021 .630658$ H 10.3967612 .4922571 .398549 H 11.3316681 .6707832 .667738 H $11.868261 \quad 1.5440280 .977772$ SCF Energy (B3LYP/6-31G**//MMFF) $=-3245.90249172$

\section{8_00630}

MM̄FF Geometry

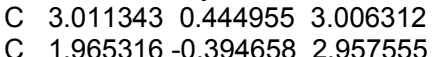
C $0.512255-0.028263 \quad 3.152729$
O 0.3933461 .4141003 .215112
C $-0.081330-0.6398844 .435493$
C $-0.269640-2.1430294 .405070$
C $-1.351183-2.751503 \quad 3.878128$
C $0.794689-2.9534645 .096759$
C $-2.501137-2.0761893 .182518$
C $-3.162200-2.9933822 .144261$
C $-4.259034-2.2590821 .357170$
C $-5.096043-3.2542240 .552458$
C -6.150387 -2.544594 - 0.307647
O $-3.624187-1.3281270 .471693$

O $-6.677093-3.501991-1.238732$

C $-5.454409-1.445682-1.133150$

C $-7.337544-2.050268 \quad 0.526853$

O $-6.409582-0.661871-1.845422$

C $-4.528404-0.525585-0.299829$

C $-3.7049270 .364837-1.264829$

$\begin{array}{lllll}\text { O } & -5.324206 & 0.312260 & 0.539838\end{array}$

C $-0.793548 \quad 1.976760 \quad 2.868777$

O $-1.845713 \quad 1.4035362 .641210$

C -0.5650773 .4323252 .738899$

C -1.4350194 .1635272 .026793$

C $-1.289766 \quad 5.5765601 .675388$

C $-0.169425 \quad 6.344023 \quad 2.326674$

C $-2.5579441 .141278-0.605724$

C $-1.7415511 .915144-1.629331$

C $0.405634 \quad 3.159137-1.872477$

O $-2.1280192 .114967-2.778317$

C $0.3334364 .587600-1.432937$

C $-0.309908 \quad 5.547620-2.115165$

C $-0.3591626 .992785-1.700811$

C $-1.7543487 .445796-1.324057$

C -2.617941 $7.874981-2.259615$

C -2.1177867 .4705830 .146804$

C -2.1295786 .0938550 .753786$

N $-0.5426782 .375662-1.102333$

H 2.8560891 .4979123 .225619

H $2.159195-1.4390662 .730245$

H $-0.028675-0.3782662 .264247$

H $-1.048709-0.1687094 .654071$

H $0.537986-0.359383 \quad 5.298829$

H $-1.424161-3.8345133 .964546$

H $0.581611-4.0274955 .069592$

H $1.769594-2.8028494 .625398$

H $0.869655-2.6590636 .148877$

H $-3.247953-1.7956873 .934779$

H -2.168792 -1.161431 2.687312

H $-2.397638-3.3667271 .451349$

H -3.592656 -3.860762 2.660426

H $-4.892658-1.7118652 .064791$

H $-5.573735-3.9875971 .214140$

H $-4.437267-3.832929-0.110712$

H -7.059526 -4.236858 -0.728967

H -4.841972 -1.951785 -1.893204

H $-7.818868-2.8898161 .042621$

H -8.110969-1.607888 -0.110856

H $-7.054025-1.3164801 .284097$

H $-6.955833-1.274378-2.367338$

H $-3.266963-0.264848-2.050227$

H $-4.3720631 .079838-1.763475$

H $-4.813011 \quad 1.1048950 .767207$

H 0.3478193 .8418833 .155437

H -2.308486 3.6684041 .605868

H $-0.2411526 .274685 \quad 3.418124$

H -0.1706447 .4090092 .087561$

H 0.8024395 .9472902 .013299

H $-2.952970 \quad 1.8636130 .114253$

H $-1.8881440 .453851-0.077999$

H $0.2019713 .051498-2.942870$

H $1.4050992 .759808-1.675296$

H $0.8583674 .843452-0.516119$

H $-0.8244975 .285543-3.037259$

H $0.0114847 .597244-2.539176$

H $0.3325627 .193914-0.873083$

H $-3.6103558 .225316-1.996610$

H -2.350497 $7.881985-3.311320$

H $-3.117196 \quad 7.9016900 .289185$

H $-1.434488 \quad 8.1532020 .658666$

H $-2.905977 \quad 5.4489200 .339081$

H $-0.3225152 .187313-0.128835$

C $4.995280 \quad 0.6758331 .509413$

$\begin{array}{lllll}\text { O } & 4.379385 & 0.088001 & 0.346207\end{array}$

C $5.3394310 .087558-0.731086$

C $6.6089350 .735625-0.182718$

$\begin{array}{llll}\text { C } & 6.490731 & 0.458614 & 1.303676\end{array}$

H $5.555690-0.960341-0.963821$

C $4.7512580 .795720-1.956786$

H $7.5130660 .307975-0.627478$

H $6.6282551 .815240-0.372850$ 
C $7.3765651 .351007 \quad 2.155012$

H $6.747401-0.5930951 .488265$

C $3.4632990 .140148-2.498233$

O $4.4370842 .144586-1.584247$

H $5.5083480 .861075-2.746987$

H $2.6799050 .190120-1.734949$

O $2.9984190 .946794-3.590088$

C $3.645255-1.310799-2.992545$

C $2.377537-1.899729-3.642134$

H $3.968223-1.953944-2.167799$

O $4.690069-1.318956-3.969595$

H $1.958449-1.177924-4.353088$

H $2.667480-2.774566-4.239692$

H $8.430098 \quad 1.2098701 .892003$

H 7.1337972 .4096002 .013921

H 7.2617251 .1181613 .218068

C 4.4370520 .0262262 .774505

H $4.0505572 .577619-2.364651$

H $3.6571270 .882051-4.302733$

H $4.850764-2.244837-4.219560$

H 5.0219660 .3290763 .649878

H $4.504400-1.0643202 .697980$

H 4.7598181 .7488931 .493832

C $0.913308-4.129115-1.030542$

O $1.762287-3.556750-2.035385$

C $1.320109-2.318880-2.608796$

C $-0.064806-2.487676-3.249148$

C $-1.066879-3.049569-2.239447$

C $-0.528678-4.298154-1.540006$

H $1.323159-5.134108-0.865023$

C $1.012286-3.358195 \quad 0.292320$

H $1.259487-1.558983-1.824398$

O $0.009169-3.340498-4.388754$

H $-0.410666-1.509071-3.602739$

H -2.004071 -3.287704 -2.757281

O $-1.333771-2.042928-1.270894$

H $-1.187510-4.580063-0.710342$

O $-0.559472-5.376467-2.479181$

C $2.256751-3.7739851 .041078$

H $1.029232-2.2712320 .176956$

H $0.163606-3.5968960 .943962$

H $0.377901-4.192595-4.099741$

H -2.024626 -2.382903 -0.677502

H $-0.286127-6.183133-2.009997$

O $3.358928-3.2045620 .476031$

$\begin{array}{llll}\text { O } & 2.247430 & -4.533011 & 2.001907\end{array}$

C $4.611324-3.5847801 .054067$

H $4.826630-4.6302660 .814860$

H $5.392787-2.9565880 .619439$

H $4.602982-3.4331242 .137573$

SCF Energy (B3LYP/6-31G**//MMFF) $=-3245.91458652$

08_00631

MM̄FF Geometry

C $3.084376-2.2980792 .311472$

C $3.531976-3.3173131 .561953$

C $3.817568-3.301408 \quad 0.079597$

O $3.917307-1.958036-0.441091$

C $2.673519-3.988472-0.688367$

C $3.150838-4.702381-1.936091$

C $3.159846-4.177989-3.176467$

C $3.603065-6.123615-1.715309$

C $2.806432-2.781186-3.603257$

C $1.498828-2.719369-4.404290$

C $0.256804-2.611663-3.506347$

C $-1.021494-2.688422-4.343398$

C $-2.278348-2.454259-3.492778$

O $0.327858-1.348361-2.835364$

O $-3.381326-2.233929-4.385199$

C $-2.091413-1.158957-2.677484$

C $-2.639756-3.684037-2.651327$

O $-3.172527-0.972613-1.767567$

C $-0.743475-1.090459-1.921309$

C $-0.540190 \quad 0.332860-1.348410$

O $-0.762394-2.034450-0.848932$

C $5.101632-1.305405-0.316147$

O $6.106410-1.694887 \quad 0.255161$

C $4.948871-0.006859-1.007439$
C $5.8128620 .987548-0.761389$

C $5.7741282 .325690-1.356205$

C $4.7446902 .584995-2.426099$

C $0.8616210 .597021-0.777466$

C $0.9537161 .969990-0.130397$

C 2.4378043 .3364841 .334262

O $0.0748572 .821658-0.236386$

C 3.1446154 .3247080 .460396

C 4.2907844 .9323610 .804325

C $4.9766875 .963211-0.047316$

C $6.4430865 .664817-0.271160$

C 7.3939006 .3142530 .421649

C $6.8080124 .678855-1.358805$

C $6.6513953 .250395-0.913561$

N 2.1378222 .1357200 .575353

H $2.877646-1.3331941 .857020$

H $3.705447-4.2760302 .048463$

H $4.767740-3.823230-0.092454$

H $1.903102-3.253070-0.949046$

H $2.167011-4.730365-0.056112$

H $3.500376-4.812961-3.994095$

H $3.949341-6.600163-2.638596$

H $4.430266-6.153726-0.998929$

H $2.778971-6.727186-1.320854$

H $2.779167-2.086219-2.760481$

H $3.623101-2.431168-4.247837$

H $1.545478-1.827230-5.042832$

H $1.417204-3.588904-5.067942$

H $\quad 0.275046-3.419791-2.767241$

H -1.085876 -3.650276 -4.867363

H $-0.989185-1.921386-5.130071$

H -3.466111 -3.017029 -4.955740

H $-2.134177-0.318802-3.384981$

H -2.825612 $-4.549297-3.298871$

H -3.569358 -3.522109-2.094588

H $-1.857315-3.964789-1.943365$

H -3.996249 -1.015424 -2.282931

H $-0.7130321 .070047-2.143850$

H -1.284919 $0.522759-0.567184$

H $-0.176480-1.728515-0.139542$

H $4.1131910 .099421-1.689800$

H $6.6147680 .813556-0.044975$

H $4.9121391 .925088-3.284738$

H $4.7553003 .608142-2.806137$

H $3.7334122 .410122-2.044025$

H $1.123596-0.153499-0.024977$

H $1.6081390 .554137-1.578223$

H 3.0444263 .0351202 .194110

H 1.5026613 .7724391 .700971

H $2.6753104 .575881-0.487929$

H $4.7435794 .705518 \quad 1.767044$

H $4.866687 \quad 6.9331160 .455464$

H $4.4785256 .075535-1.018319$

H 8.4482056 .1269610 .246753

H 7.1393307 .0437591 .183241

H $7.8587634 .812316-1.650030$

H $6.2394674 .913739-2.262505$

H $7.3572422 .961550-0.133275$

H 2.8364251 .3986660 .556613

C $1.334858-2.5657914 .079386$

O $0.665266-1.3201523 .817754$

C $-0.452759-1.1951214 .720474$

C $-0.451151-2.4544875 .584734$

C $1.004539-2.8780805 .534503$

H $-0.254110-0.3217705 .353535$

C $-1.758400-0.9819073 .937053$

H $-0.804150-2.2553466 .601427$

H -1.094419 -3.234544 5.160176

C $1.223263-4.3278575 .930043$

H $1.585316-2.225966 \quad 6.200452$

C $-1.801767 \quad 0.379323 \quad 3.204786$

O $-1.905740-2.0655303 .019707$

H -2.602426 -1.029100 4.636040

H $-1.729775 \quad 1.169573 \quad 3.961657$

$\begin{array}{llll}\text { O } & -0.663013 & 0.522912 & 2.357718\end{array}$

C -3.0764690 .6128422 .368363$

C -3.1146572 .0435631 .794479$

$\mathrm{H}-3.9564730 .4480683 .001241$ 
O $-3.113226-0.341145 \quad 1.311797$ H -3.0161602 .7554152 .622788$ H -2.256122 2.1769641 .126065 H $\quad 0.880277-4.5023356 .955092$ H $0.675729-5.0104795 .271321$ H $2.284040-4.5922205 .881487$ C $2.827659-2.4220673 .790139$ H $-2.770095-1.9711702 .585229$ H $-0.680696-0.2157231 .725218$ H $-3.923660-0.1888580 .798354$ H $3.375167-3.2823554 .192103$ H $3.228848-1.5262364 .279307$ H $0.889306-3.3210673 .417266$ C $-5.4824241 .770849-1.092569$ O $-4.3493501 .627374-0.226266$ C -4.4014732 .3555171 .004760$ C -4.5378463 .8605520 .724328$ C $-5.7350234 .154440-0.184625$ C $-5.7458863 .249389-1.420042$ H $-5.1831861 .274143-2.024837$ C $-6.7119881 .037441-0.545906$ H -5.2588892 .0139191 .594532$ $\begin{array}{llll}\text { O } & -3.347762 & 4.364344 & 0.119874\end{array}$ H -4.6588944 .3881031 .677466$ H $-5.700045 \quad 5.202717-0.505690$ O -6.9467843 .9598430 .538540$ H -6.697938 $3.339945-1.956222$ O $-4.7171103 .676054-2.318243$ C $-6.496076-0.457043-0.588468$ H $-6.968753 \quad 1.3271270 .476999$ H $-7.5970861 .247682-1.158044$ H -3.175567 $3.840295-0.681170$ H -6.9356244 .5659271 .298868$ H $-4.9088124 .596195-2.567550$ O $-6.145490-0.9150990 .646679$ O $-6.600045-1.130488-1.605728$ C $-5.879529-2.3190090 .729874$ H $-6.779509-2.8894980 .482053$ H $-5.054001-2.5894330 .064556$ H $-5.588737-2.5487011 .758458$

SCF Energy $\left(B 3 L Y P / 6-31 G^{* *} / / M M F F\right)=-3245.92053621$

0800632

MM̄FF Geometry

C $-3.492159-1.853053-1.869505$

C $-2.736765-1.174526-2.746970$

C $-1.234329-1.196718-2.747459$

O $-0.834341 \quad 0.192218-2.646583$

C $-0.636803-1.801510-4.027431$

C $-0.818551-3.300759-4.129932$

C $-0.026840-4.205143-3.519917$

C -1.959958 -3.759425 -4.999620

C $1.174428-3.942393-2.654105$

C $0.876251-4.220400-1.176098$

C $2.140405-4.179606-0.303697$

C $1.773531-4.3489611 .173224$

C $2.995922-4.2253062 .094052$

O $2.802913-2.926714-0.502887$

O $2.499399-4.0664963 .431766$

C $3.777594-2.9468091 .721654$

C $3.824913-5.5149442 .096168$

O $4.994275-2.8643662 .460820$

C $4.044167-2.8058570 .205561$

C $4.645404-1.415150-0.105950$

O $4.983914-3.811487-0.186760$

C $0.322050 \quad 0.470619-1.990870$

O $1.136810-0.330349-1.564207$

C $0.399278 \quad 1.940793-1.839133$

C $1.3330332 .475355-1.038574$

C $1.5003063 .897958-0.737511$

C $0.6215074 .879174-1.467554$

C $4.706813-1.092437-1.605284$

C $5.740952-0.026288-1.902264$

C $6.2358122 .382382-1.646925$

O $6.765450-0.283851-2.531535$

C $5.9275033 .445219-0.643550$

C $5.5406124 .685927-0.976086$

C 5.2535805 .7697270 .023561
C $3.8531156 .324956-0.109752$

C $3.6190057 .433657-0.832091$

C 2.7413425 .6500040 .661120

C 2.4310044 .2632440 .168568

N $5.4095631 .218152-1.389600$

H -3.006787 -2.511291-1.152863

H -3.213732 -0.520254 -3.472378

H $-0.874140-1.732071-1.861773$

H $0.429849-1.552291-4.090612$

H -1.066542 -1.309157 -4.910762

H $-0.251262-5.261795-3.661030$

H -2.029391 -4.851220 -5.052946

H -2.915092 -3.388909-4.616715

H $-1.828437-3.388633-6.021501$

H $1.985138-4.597012-2.997400$

H $1.539394-2.918199-2.774289$

H $0.162110-3.469858-0.816320$

H $\quad 0.394768-5.200149-1.064067$

H $2.802368-4.990366-0.630479$

H $1.259961-5.3039821 .340640$

H $1.051655-3.5706521 .454993$

H $3.263677-3.9111394 .013214$

H $3.170329-2.0932012 .049232$

H $3.215263-6.3581912 .442913$

H $4.663935-5.4475712 .797482$

H $4.217720-5.7737441 .110790$

H $5.630163-3.4767162 .053100$

H $4.037832-0.6372610 .374582$

H $5.653826-1.3497480 .322201$

H $5.523645-3.482877-0.922435$

H $-0.3398132 .540399-2.358464$

H $2.0295041 .808194-0.533077$

H $0.7338614 .763993-2.551476$

H $0.8567715 .922095-1.243268$

H -0.429689 4.724079-1.204722

H $4.969493-1.973756-2.200043$

H $3.732362-0.750304-1.969475$

H $6.0379702 .705883-2.674489$

H $7.2904762 .095144-1.578801$

H 6.0452303 .1864020 .406018

H $5.4435234 .948053-2.027358$

H $5.9888096 .570357-0.131464$

H 5.4125265 .4213241 .051657

H $2.6241377 .859229-0.910406$

H $4.4179657 .938951-1.364804$

H 1.8341856 .2659090 .667301

H 3.0326815 .5838091 .717691

H 3.0299903 .4858290 .643766

H $4.4601321 .366160-1.058632$

C $-5.448061-0.256963-1.665765$

O $-4.770406 \quad 0.412719-0.587593$

C $-5.6073351 .485076-0.112415$

C $-6.9183831 .390722-0.894476$

C $-6.925604-0.059033-1.353153$

H $\quad-5.810978 \quad 1.283373 \quad 0.944095$

C $-4.8547772 .816155-0.258997$

H -7.783320 $1.643296-0.272818$

H $-6.9288562 .068514-1.755826$

C -7.852703 -0.321214-2.525956

H -7.219835 -0.692854 -0.506216

C $-3.4607482 .821510 \quad 0.407328$

O $-4.6619113 .091516-1.653959$

H -5.4663893 .6379250 .129262$

H $-2.8173102 .077605-0.075529$

O -2.8552594 .0968160 .155665$

C -3.4855212 .5955891 .930970$

C -2.0773502 .5825112 .562365$

H -3.9985441 .6588362 .167442$

O -4.2452283 .6499742 .526924$

H -1.4694103 .3921702 .141968$

H -2.1762242 .8001333 .634236$

H -8.886998 -0.079149 -2.260133

H $-7.5789670 .283339-3.397190$

H $-7.818460-1.374228-2.822467$

C $-4.986303-1.714230-1.737206$

H $-4.1242802 .370800-2.024731$

H -2.863892 $4.236568-0.806803$

H $-4.3135583 .461013 \quad 3.478337$ 
H $-5.478169-2.244880-2.559629$ H -5.274912 -2.224995 -0.811087 H $\quad-5.1902370 .268955-2.594141$ C -1.552181-1.052112 3.217805 O $-2.0388550 .296113 \quad 3.243264$ C $-1.369080 \quad 1.2280472 .383464$ C $\quad 0.1238621 .3025892 .734207$ C $0.766831-0.0884242 .719027$ C $-0.046146-1.0999723 .527532$ H -2.073292 -1.549802 4.046269 C $-1.945798-1.7698231 .917147$ H $-1.474748 \quad 0.9046891 .343700$ O $0.307087 \quad 1.9020714 .014925$ H 0.6251471 .9500642 .006614 H $1.780211-0.0279383 .134469$ O $0.864494-0.5594991 .379712$ H $0.336975-2.1124743 .359670$ O $0.144240-0.8085424 .915134$ C $-3.366075-2.2866551 .991128$ H -1.864113 -1.147805 1.022366 H -1.307115 -2.6479581 .766028$ H -0.1855841 .3722274 .664769$ H 1.4524190 .0460860 .897841 H $-0.309356-1.5007325 .425517$ O $-4.240503-1.2578452 .175928$ O $-3.661260-3.4704921 .886052$ C $-5.611110-1.6334592 .342735$ H $-5.725397-2.247078 \quad 3.241447$ H $-6.201732-0.7218912 .464629$ H $-5.972784-2.1709061 .462027$

SCF Energy $\left(B 3 L Y P / 6-31 G^{* *} / / M M F F\right)=-3245.91655688$

08_00633

MM̄FF Geometry

C $\quad 1.3977195 .1856950 .320600$

C 2.2468694 .1484410 .268926

C $2.8982373 .685203-1.009232$

O $2.8831532 .238003-1.048068$

C $4.3630794 .154488-1.049334$

C $5.0622363 .842812-2.359849$

C $6.0126582 .901220-2.526422$

C $4.6548294 .708565-3.525679$

C $6.5626181 .951455-1.499826$

C $6.2694580 .494994-1.881837$

C $6.635849-0.476690-0.750483$

C $6.403055-1.923657-1.188049$

C $6.616944-2.920518-0.039217$

O $5.806331-0.1609460 .371745$

O $6.058727-4.181495-0.440824$

C $5.816322-2.4466641 .193143$

C $8.105776-3.1706970 .227012$

O $6.105910-3.2577192 .328715$

C $6.043725-0.9603481 .537485$

C $5.099117-0.4409542 .650264$

O $7.384708-0.7897422 .012431$

C $1.7071121 .629332-1.367927$

O $0.6340622 .168215-1.586532$

C $1.961600 \quad 0.171648-1.424137$

C $0.958452-0.680531-1.684739$

C $1.081784-2.133844-1.833464$

C $2.442201-2.744071-1.619386$

C $3.603527-0.4758202 .316392$

C $2.888895-1.7318352 .783813$

C $0.692345-2.8937682 .592392$

O $3.395084-2.5550693 .540982$

C $0.789593-3.9368851 .524326$

C $-0.275938-4.4113110 .862223$

C $-0.206227-5.491698-0.177154$

C $-0.887510-5.108136-1.471559$

C $-2.128682-5.539388-1.752704$

C $-0.090701-4.321247-2.485519$

C -0.011207 -2.850352 -2.171822

N $1.596196-1.8029612 .281183$

H $1.1979845 .740656-0.591754$

H 24789303.5961311 .176177

H $2.3572774 .068633-1.883413$

H $4.9050373 .720128-0.203307$

H $4.4095885 .241118-0.893722$
H $6.4538962 .789339-3.516271$

H $5.2505014 .506633-4.422384$

H $3.6054344 .539545-3.785017$

H $4.7872425 .766827-3.277537$

H $7.6476942 .098434-1.436744$

H $6.1693732 .160688-0.503137$

H $5.2010890 .395662-2.111119$

H $6.8246350 .235232-2.791864$

H $7.686633-0.320438-0.479718$

H $7.042006-2.178280-2.042891$

H $5.371483-2.030568-1.547430$

H $6.501266-4.457344-1.261810$

H $4.755817-2.5998630 .960398$

H $8.589607-3.585718-0.665410$

H $8.246552-3.9165971 .016908$

H $8.650015-2.2654580 .504159$

H $5.928996-4.1807042 .078215$

H $5.315334-0.9485893 .597939$

H 5.3543880 .6146402 .823179

H 7.4266390 .0285332 .534427

H $2.982082-0.160890-1.274335$

H $-0.042898-0.277876-1.831454$

H $2.434211-3.835908-1.602325$

H $2.857245-2.435326-0.653934$

H $3.127666-2.433101-2.414659$

H 3.1151150 .3595372 .834638

H $3.435571-0.3363561 .244294$

H $-0.314095-2.4698532 .668160$

H $0.962102-3.3294683 .559741$

H $1.776252-4.3385631 .305660$

H $-1.261617-4.027171 \quad 1.109923$

H $-0.684707-6.3861140 .243179$

H $\quad 0.831186-5.782596-0.383989$

H $-2.612175-5.298748-2.693742$

H -2.692983 -6.136766 -1.044082

H $-0.558107-4.409069-3.476065$

H $0.892963-4.783263-2.610278$

H $-0.959349-2.325586-2.296297$

H $1.280728-1.1015521 .617438$

C -0.8224795 .6630551 .468572$

O -1.3177434 .3551051 .118189$

C -2.328524 4.5053920 .105332

C -2.7368875 .9740320 .142097$

C -1.412545 $6.641966 \quad 0.451635$

H $-1.8443084 .290013-0.856194$

C -3.4934753 .5292190 .310785$

H -3.176685 $6.307573-0.802679$

H -3.4589756 .1729920 .942782$

C - 1.5506718 .0618700 .973320

H $-0.8192336 .662763-0.469530$

C -3.0646632 .0477730 .222229$

O -4.0999103 .8024501 .573174$

H $-4.2434073 .730922-0.464306$

H -2.464237 $1.912365-0.685584$

O $-2.228346 \quad 1.7060201 .325651$

C -4.2579951 .0728560 .152942$

C $-3.804206-0.3958880 .048395$

H $-4.8668641 .337833-0.719251$

O -5.0689841 .2357911 .314505$

H -2.979868 -0.475391 -0.670562

H $-3.438366-0.719873 \quad 1.030649$

H -2.038661 8.6991580 .228648

H -2.1497718 .0973551 .889444$

H -0.5688358 .4916931 .195085$

C 0.7064365 .6424391 .575321

H -4.8790993 .2274501 .656981$

H $-2.745947 \quad 1.8485292 .136778$

H $-5.8400880 .651305 \quad 1.220951$

H $\quad 0.983725 \quad 4.977740 \quad 2.403447$

H 1.0711596 .6432241 .833012

H -1.2277705 .8805322 .465701$

C -7.151387 -2.069637 0.283058

O $-5.974646-1.3094590 .588803$

C $-4.944950-1.332285-0.403938$

C $-4.453792-2.770502-0.618198$

C $-5.617942-3.699932-0.979294$

C $-6.797003-3.536886-0.014321$

H $-7.743382-2.0488621 .207447$ 
C $-7.985552-1.408924-0.819888$ H -5.341159 -0.939292 -1.346761 O $-3.826535-3.2346020 .574906$ H -3.700950 -2.774604 -1.415121 H $-5.279430-4.742864-0.953354$ O $-6.061346-3.418875-2.303315$ H $-7.679680-4.053649-0.407531$ O $-6.470488-4.178698 \quad 1.222413$ C -8.530184 -0.082286 -0.346165 H -7.429311-1.247530 -1.747760 H $-8.846677-2.034528-1.083737$ H $-3.544116-4.1519370 .421546$ H $-5.308591-3.563381-2.901679$ H $-5.732925-3.6894381 .625860$ O $-7.715166 \quad 0.930021-0.757620$ $\begin{array}{lllll}0 & -9.541799 & 0.034909 & 0.333041\end{array}$ C -8.096596 $2.241940-0.332351$ H $-7.3500572 .949391-0.703452$ H $-9.0720142 .506349-0.751260$ H -8.116459 2.295805 0.760241

SCF Energy (B3LYP/6-31G**//MMFF) $=-3245.91916367$

08_00634

MMFF Geometry

C -2.316985 2.404670 -1.281561

C $-3.2538453 .176768-0.711497$

C $-3.804153 \quad 2.989550 \quad 0.684844$

O -2.9831992 .0619371 .433918$

$\begin{array}{llll}C & -5.227517 & 2.417297 & 0.626531\end{array}$

C $-5.949908 \quad 2.5197151 .954725$

C -6.1395591 .4948872 .808211$

C $-6.521496 \quad 3.8782612 .271738$

C -5.6521970 .0780562 .680996$

C $-6.799657-0.9069122 .415241$

C $-7.343907-0.8271610 .980147$

C $-8.576449-1.718183 \quad 0.818002$

C $-9.077108-1.737100-0.633883$

O $-6.305399-1.2681720 .099936$

O $-10.019580-2.812447-0.761650$

C -7.890616 -2.062261 -1.564042

C $-9.831132-0.452757-0.997622$

O $-8.271737-1.951872-2.933935$

C $-6.633309-1.202110-1.293683$

C $-5.434368-1.775183-2.086510$

O $-6.8816350 .139247-1.715577$

C -1.9347762 .5658312 .134291$

$\begin{array}{llll}\text { O } & -1.665300 & 3.748170 & 2.296806\end{array}$

C -1.096181 1.4894782 .710381

C $-1.129461 \quad 0.215246 \quad 2.288399$

C $-0.298420-0.8824962 .791877$

C $0.682874-0.5688123 .891336$

C $-4.082008-1.125406-1.758027$

C $-2.931418-1.867740-2.418169$

C $-0.450174-2.072409-2.333804$

O $-3.095264-2.737328-3.270188$

C $-0.136433-3.141172-1.333644$

C $0.988044-3.177425-0.603355$

C $1.285525-4.2404440 .414043$

C $1.486241-3.6783421 .804775$

C $2.720328-3.5406092 .318182$

C $0.261197-3.3807232 .640988$

C $-0.442572-2.1104892 .249389$

N $-1.697500-1.436021-1.950196$

H $-1.9410071 .538409-0.744642$

H -3.634304 4.034973-1.261082

H $-3.808706 \quad 3.9666241 .182457$

H $-5.188178 \quad 1.3752740 .292039$

H $-5.8241382 .947786-0.127570$

H $-6.699561 \quad 1.6885273 .722613$

H -7.081890 3.8850893 .212798

H -5.7239834 .6221992 .360071$

H -7.207518 4.195308 1.479349

H $-4.869201-0.0278701 .924336$

H $-5.178293-0.1895243 .634055$

H -6.415136 -1.919467 2.594242

H -7.609322 $-0.735123 \quad 3.135504$

H -7.6000510 .2119860 .748185$

H $-9.378563-1.4008291 .496048$
H -8.325057 -2.747013 1.111825

H -10.739555 -2.656840 -0.126534

H -7.628945 -3.117096 -1.400116

H -10.696222 -0.316907-0.337383

H -10.236364 -0.507300 - 2.014062

H $-9.2135070 .444460-0.920125$

H $-9.052781-2.516604-3.063567$

H $-5.349326-2.848383-1.869088$

H $-5.616834-1.674891-3.163976$

H $-6.0361160 .572187-1.911447$

H $-0.402247 \quad 1.8203603 .476442$

H - $-1.820264-0.0583061 .492643$

H $0.159318-0.2071604 .783208$

H 1.3942360 .1979253 .564113

H $1.278669-1.4295544 .201814$

H $-4.057013-0.089644-2.110877$

H $-3.911643-1.126884-0.675552$

H $0.318126-1.294699-2.369580$

H $-0.550313-2.513102-3.330645$

H $-0.879516-3.924234-1.199662$

H $1.743958-2.410093-0.747274$

H $2.189679-4.7703350 .086439$

H $0.494705-5.0004650 .442680$

H $2.877128-3.1753603 .327323$

H $3.603440-3.7925771 .741136$

H $0.513400-3.3675553 .707803$

H $-0.448286-4.2132342 .545269$

H - $1.172186-2.2428381 .450213$

H $-1.666558-0.766333-1.187171$

C -0.192416 $2.931739-2.540144$

O $0.4691041 .847604-1.853201$

C $1.2264022 .401523-0.755957$

C $1.5116053 .834496-1.167379$

C $0.1885364 .220408-1.801774$

H 0.5614352 .3767230 .116301

C $2.4556701 .526513-0.505093$

H $1.7798364 .474823-0.322138$

H $2.3208463 .874497-1.906931$

C $0.2763115 .437604-2.706426$

H $-0.5261564 .437315-0.998570$

C $3.427147 \quad 2.0920090 .543884$

$\begin{array}{lllll}\text { O } & 1.994545 & 0.250241 & -0.049052\end{array}$

H $2.9754541 .353858-1.453617$

H 3.8593643 .0333320 .184451

$\begin{array}{lllll}\text { O } & 2.674653 & 2.413279 & 1.721671\end{array}$

C 4.5556531 .1242170 .955175

C $5.4193120 .671039-0.229483$

H $\quad 4.1221840 .2695821 .486045$

O 5.3824551 .8210601 .893258

H $4.8033920 .195643-1.000833$

H $5.8589871 .556120-0.710002$

H $0.5959256 .317126-2.138013$

H $0.9946035 .283504-3.518690$

H $-0.6979485 .658538-3.154059$

C $-1.6987722 .649965-2.628501$

H $1.326356-0.060037-0.683114$

$\begin{array}{llll}H & 3.300230 & 2.767676 & 2.376657\end{array}$

H 5.9412971 .1629632 .339788

H $-1.856278 \quad 1.742866-3.225151$

H $-2.200423 \quad 3.472511-3.150977$

H $0.2181772 .941756-3.557465$

C $8.463024-1.341277-0.974684$

O $7.282427-0.535526-1.088814$

C $6.576852-0.2802240 .134382$

C $6.110934-1.6035790 .762093$

C $7.266563-2.5961440 .920635$

C $8.131037-2.703382-0.339849$

H $8.780626-1.524756-2.009502$

C $9.604430-0.600079-0.260626$

H 7.2493310 .2432760 .820513

O $5.078641-2.197266-0.022188$

H $5.680553-1.4014841 .749255$

H $6.857822-3.5828461 .171154$

O $8.071995-2.1670452 .016628$

H $9.062554-3.232652-0.109789$

O $7.444930-3.512450-1.299684$

C $10.0766230 .612887-1.035655$

H $9.307683-0.2562450 .733432$ 
H $\quad 10.463235-1.273589-0.157130$

H $5.331756-2.135207-0.957457$

H $8.773486-2.8287252 .141726$

H 6.872575 -2.937774 -1.834081

O $11.0834601 .217482-0.336842$

O $9.628587 \quad 0.985523-2.110892$

C $11.6368732 .388965-0.944634$

H $10.8668143 .158566-1.054167$

H $12.4246132 .771641-0.290333$

H $12.0765602 .140514-1.915315$

SCF Energy (B3LYP/6-31G**//MMFF) $=-3245.90836201$

08_00635

MMFF Geometry

C 2.1070635 .2610380 .465746

C 2.8926604 .2388760 .836027

C 4.0651193 .7642230 .015353

O $3.8285602 .385790-0.357766$

C 5.3553603 .8216280 .847523

C $6.6014503 .677327-0.002876$

C $7.3327792 .551767-0.114284$

C $7.0396674 .932432-0.715157$

C 7.0702041 .2119930 .515216

C $7.2050480 .081770-0.515551$

C $7.102407-1.3007830 .142718$

C $7.418877-2.406081-0.866010$

C $7.262520-3.802026-0.245313$

O $5.771586-1.4666270 .643034$

O $7.264396-4.762638-1.312297$

C $5.884102-3.889000 \quad 0.439922$

C $8.442984-4.1614970 .664625$

O $5.748527-5.1115591 .161340$

C $5.569334-2.6890381 .365743$

C $4.089669-2.7572431 .807128$

O $6.400215-2.7567812 .526385$

C $2.9926362 .174321-1.411035$

O $2.4653353 .023616-2.112797$

C $2.7853610 .715943-1.543747$

C $1.6993110 .290108-2.205342$

C $1.291651-1.098557-2.420697$

C $2.269844-2.181412-2.056993$

C $3.564322-1.4819572 .477868$

C $2.112800-1.6629802 .877977$

C $-0.177523-1.8450661 .909828$

O $1.782862-1.8368734 .048601$

C $-0.631732-2.740740 \quad 0.800335$

C -1.563730 -2.398685 -0.101884

C $-2.060650-3.317140-1.181335$

C $-1.974076-2.708777-2.562836$

C $-3.077733-2.301422-3.212299$

C $-0.613331-2.635114-3.223866$

C $0.069434-1.324660-2.944714$

N $1.253564-1.6482251 .790935$

H $2.3303435 .795341-0.454519$

H 2.6709383 .6952451 .751191

H $4.1789904 .371547-0.891432$

H 5.3318173 .0541101 .629838

H 5.4201704 .7805981 .379593

H $8.2358952 .593274-0.722467$

H $7.9684664 .789773-1.277690$

H $6.2746695 .261497-1.424923$

H 7.2137435 .7371780 .006720

H 7.8067101 .0652801 .314182

H $6.079497 \quad 1.1610660 .975268$

H $\quad 6.4163730 .194655-1.270954$

H $8.1706670 .166722-1.029991$

H $7.811503-1.3365490 .978312$

H $8.428031-2.281941-1.278375$

H $6.732953-2.326688-1.721257$

H $8.101807-4.669198-1.798056$

H $5.127768-3.915593-0.357532$

H $9.382671-4.1370500 .099892$

H $8.350218-5.184357 \quad 1.046057$

H $8.551297-3.4848461 .514718$

H $\quad 5.939097 \quad-5.836508 \quad 0.541597$

H $3.465762-2.9587750 .928868$

H 3.945633 -3.600769 2.494951

H $5.963028-2.2906163 .256215$
H $3.4973650 .048093-1.073411$

H $1.0166091 .037213-2.610397$

H $3.230894-2.015249-2.556981$

H $1.941434-3.181056-2.348345$

H $2.439661-2.199846-0.978436$

H $4.136882-1.2386623 .378861$

H $3.637329-0.6190161 .805976$

H $-0.646178-0.8579381 .868804$

H $-0.415474-2.2985802 .877110$

H $-0.188328-3.7328710 .756263$

H $-2.022237-1.416247-0.040526$

H $-3.103350-3.567548-0.945842$

H -1.518141 -4.270697 -1.174971

H -3.022216 -1.884558 -4.212591

H -4.058564 -2.362590 -2.754585

H $-0.727166-2.725491-4.312203$

H $-0.021623-3.502662-2.920730$

H $-0.539143-0.459274-3.211622$

H $1.612158-1.3378760 .893087$

C -0.4311695 .2923790 .641469$

$\begin{array}{lllll}\text { O } & -0.590454 & 3.866409 & 0.767927\end{array}$

C - $-1.3028683 .375367-0.391507$

C $-1.7677134 .608902-1.156285$

C $-0.6725565 .607040-0.833134$

H $-0.5633462 .815012-0.976945$

C -2.4351332 .4512770 .075185$

H -1.874321 $4.420824-2.228915$

H $-2.7335364 .964354-0.775619$

C -1.058539 $7.050322-1.101417$

H $0.2042015 .351189-1.440069$

C $-3.3371071 .943845-1.066854$

$\begin{array}{llll}\text { O } & -1.818665 & 1.338252 & 0.728125\end{array}$

H -3.0238322 .9797180 .833013$

H -3.840101 $2.790992-1.547015$

O $-2.5056441 .354950-2.075928$

C $-4.3975430 .896623-0.665363$

C $-5.349048 \quad 1.3853030 .441280$

H $-3.908256-0.036819-0.368933$

O $\quad-5.1554530 .603989-1.844706$

H $-4.773767 \quad 1.774210 \quad 1.286514$

H -5.9568052 .2012450 .030259$

H -1.290637 $7.195782-2.161501$

H -1.939447 $7.345266-0.521355$

H $-0.2392437 .726834-0.838710$

C 0.9091155 .7287201 .241374

H $-2.5102560 .858063 \quad 1.213625$

H -2.022718 $0.618993-1.662285$

H $-5.771938-0.116047-1.628231$

H $0.973278 \quad 5.3543042 .271035$

H $0.947716 \quad 6.822727 \quad 1.300810$

H -1.2297975 .7372151 .250580$

C -8.094985 -1.163244 0.248004

O $-7.185691-0.098810-0.064056$

C -6.2805820 .2777120 .977190$

C -7.0556920 .7369472 .225289$

C $-8.064848-0.3246792 .672897$

C $-8.917293-0.8286761 .504906$

H $-8.788890-1.198300-0.601955$

C $-7.385600-2.5244270 .334918$

H -5.654563 -0.5818661 .240109$

O -7.7663001 .9452791 .961496$

H -6.3482660 .9387393 .038799$

H -8.7257310 .0984553 .439591$

O $-7.374547-1.428863 \quad 3.250115$

H -9.491048 -1.7102831 .812429$

O $-9.8764120 .175722 \quad 1.161136$

C $-6.918381-3.014681-1.019952$

H $-6.510098-2.5018750 .988188$

H $-8.093901-3.2677220 .720370$

H -7.1165862 .6657351 .903142$

H $-6.874500-1.0955034 .014566$

H -9.3905520 .9496850 .828160$

O $-6.613803-4.343449-0.926905$

O $-6.816296-2.324518-2.024311$

C $-6.175296-4.967980-2.137574$

H $-5.180596-4.602916-2.405809$

H -6.117584 -6.045527 -1.961889

H $-6.887055-4.784561-2.948283$ 
H $-8.137800-5.0812990 .389515$

H -7.456682 -4.091487 1.656791

H -5.650439 -7.7696020 .797203$

H -7.096998 -7.183036 -0.200910

H -4.784773 -6.404929 2.549116

H -5.646112 -4.9680102 .997076$

H -3.391616 -5.262455 0.895158

H $-4.024997-1.360595-0.968885$

C $5.049057-3.385679-1.507278$

O $5.161741-2.668753-0.261442$

C $5.874368-1.442768-0.507834$

C $6.632848-1.673038-1.806192$

C $5.595220-2.458074-2.593904$

H $5.120599-0.669424-0.693121$

C $6.706787-1.0555160 .716475$

H $6.924777-0.737838-2.292807$

H $7.532606-2.278948-1.648196$

C $6.162014-3.188198-3.798058$

H $4.818294-1.762721-2.936187$

C $5.877824-0.9855832 .020041$

O $7.735178-2.0344230 .899079$

H $7.216996-0.1032730 .531120$

H $5.591368-2.0008272 .319262$

O $6.727375-0.4948663 .062742$

C $4.619614-0.1016171 .933912$

C $3.811129-0.0853823 .245811$

H $3.962170-0.4695741 .139443$

O $4.994417 \quad 1.236053 \quad 1.609334$

H $3.696684-1.1111533 .615069$

H 4.3556420 .4994403 .997261

H $6.589420-2.477115-4.512544$

H $6.952144-3.888846-3.508175$

H $5.379190-3.755393-4.311504$

C $3.611362-3.881185-1.686253$

H $8.239860-1.7802261 .690679$

H $\quad 6.9400810 .4313632 .855375$

H $5.362531 \quad 1.2352510 .710601$

H $3.372655-4.535261-0.837767$

H $3.545494-4.488014-2.596909$

H $5.700319-4.263845-1.406943$

C 1.3799352 .6272392 .404759

O $2.577411 \quad 1.9218962 .750796$

C 2.4123380 .5300263 .032228

C 1.4947300 .3534634 .248645

C 0.1499031 .0581984 .029680

C 0.3243832 .4937333 .516970

H 1.6838193 .6804192 .350246

C 0.8679882 .2228851 .017386

H 1.9577830 .0331372 .167914

O 2.1231120 .8684875 .420307

H $1.327064-0.7167714 .416400$

H -0.4014891 .0718254 .978052$

O $-0.611854 \quad 0.299694 \quad 3.094101$

H -0.6356132 .8930473 .169681$

O 0.7358353 .2988304 .626669

C $1.9193572 .476103-0.039329$

H $\quad 0.569220 \quad 1.171958 \quad 0.977445$

H $-0.0135872 .811196 \quad 0.744974$

H 2.3325201 .8043465 .258237

H -1.4787530 .7306123 .003744$

H $\quad 0.775376 \quad 4.2214014 .321937$

O $2.202378 \quad 1.320299-0.705638$

O $2.4270743 .570247-0.249632$

C $3.1849601 .429878-1.740322$

H $4.1491681 .724334-1.316320$

H $3.2930150 .450111-2.212350$

H $2.8639052 .149622-2.498802$

SCF Energy (B3LYP/6-31G**//MMFF) = -3245.91533099

\section{7}

MM̄FF Geometry

C $2.583750-2.720234-2.633701$

C $1.450027-2.350366-2.019953$

C $0.381590-3.323892-1.598623$

O $-0.892690-2.726662-1.946700$

C $0.394706-3.567883-0.080360$

C $1.503324-4.4795320 .396947$ 
C $2.584373-4.0771921 .092357$

C $1.303814-5.943156 \quad 0.096523$

C $2.961282-2.6750641 .477040$

C $2.776192-2.4083892 .979984$

C $1.873345-1.1950673 .253444$

C $1.767556-0.9236314 .756166$

C $\quad 0.7739550 .204846 \quad 5.073567$

O $0.579783-1.4856302 .717026$

O $\quad 0.520943 \quad 0.153199 \quad 6.485955$

C $-0.555676-0.0805754 .340326$

C $1.381721 \quad 1.5846224 .798430$

O $-1.4673001 .003798 \quad 4.499738$

C $-0.370452-0.4222972 .846301$

C -1.686503 -0.876875 2.174604

O $0.072576 \quad 0.749004 \quad 2.153811$

C - $1.931902-3.568325-2.185783$

O $-1.887906-4.791360-2.206468$

C $-3.193962-2.834365-2.435114$

C -3.360128 -1.526065 -2.186713

C $-4.595749-0.758664-2.377565$

C $-5.796084-1.490848-2.917418$

C $-2.278284-2.1700982 .736854$

C $-3.421386-2.6644121 .873308$

C $-5.809957-2.2189611 .333934$

O $-3.295584-3.6381721 .134614$

C $-6.497700-1.0109580 .771850$

C -6.1007790 .2702840 .834959$

C $-6.8587631 .401500 \quad 0.198703$

C $-6.0200692 .183659-0.788972$

C $-5.6135143 .433869-0.511092$

C $-5.7216601 .545061-2.130344$

C $-4.5953530 .550754-2.050692$

N -4.567141 -1.896809 2.008200

H $2.761470-3.775500-2.827877$

H $1.280003-1.298562-1.805628$

H $0.497185-4.262296-2.154506$

H $-0.561323-4.009660 \quad 0.230552$

H $0.411571-2.6037120 .436077$

H $3.300385-4.8335861 .411712$

H $2.090515-6.5684190 .532218$

H $0.347923-6.2878620 .504538$

H $1.305763-6.119536-0.983264$

H $4.023576-2.554808 \quad 1.229783$

H $2.439631-1.9309150 .867798$

H $2.350322-3.2916793 .473764$

H $3.760363-2.2313113 .431292$

H $2.294429-0.3249252 .736110$

H $2.753797-0.6992205 .181390$

H $1.425238-1.8360995 .264989$

H $-0.163507 \quad 0.814585 \quad 6.687044$

H -1.012955 -0.9369734 .850452$

H $2.288127 \quad 1.7285145 .398825$

H 0.6973742 .3867635 .095684

H 1.6504831 .7325463 .751346

H -1.185922 1.7173743 .901915

H $-2.426523-0.0675742 .218303$

H -1.497315 -1.029720 1.102341

H $-0.1391380 .647583 \quad 1.211203$

H -4.006458 -3.452091 -2.804380

H -2.521363 -0.957909-1.788859

H $-5.580017-1.900854-3.910277$

H -6.681736 -0.862030 -3.025034

H -6.077290 -2.314136 -2.251491

H -1.524727 -2.964688 2.776319

H -2.650040 -2.027295 3.757191

H $-6.459637-2.7021512 .070840$

H -5.619569 -2.935809 0.528522

H -7.419689 -1.2326470 .236395$ H -5.1892950 .5378591 .362501$ H -7.2120892 .0588701 .003532$ H $-7.762127 \quad 1.041747-0.309586$ H -5.036341 4.012381-1.224526 H -5.8450363 .9065010 .437604$ H -5.422448 $2.315978-2.852828$ $\mathrm{H}-6.6462221 .121170-2.530259$ H $-3.6684860 .981891-1.674378$ H -4.602139 -1.211050 2.757178

C $4.610405-1.363811-1.986146$
O $4.044663-0.259306-1.243127$

C $5.0656640 .740470-1.049074$

C $6.3841460 .055851-1.382102$

C $5.958182-0.863791-2.507233$

H $4.8849971 .529321-1.788189$

C $5.001058 \quad 1.3152070 .368698$

H $7.1601510 .768401-1.677673$

H $6.762483-0.527279-0.534085$

C $6.957843-1.970790-2.795149$

H $5.811735-0.265323-3.416679$

C 3.6838372 .0479660 .698145

$\begin{array}{llll}\text { O } & 5.159880 & 0.245926 & 1.308781\end{array}$

H $5.850226 \quad 1.987512 \quad 0.535777$

H $2.837698 \quad 1.3550380 .633041$

O 3.7598022 .4720452 .066106

C $3.4255793 .295203-0.172803$

C 2.2046304 .1268150 .266166

H $3.3078933 .005554-1.221491$

O $4.5720084 .150445-0.111640$

H 2.2332974 .2875391 .350444

H $2.2965115 .123652-0.186863$

H $7.920407-1.548841-3.102483$

H $7.131306-2.594479-1.911755$

H $6.601449-2.620360-3.600602$

C $3.648706-1.769413-3.103613$

H $4.419231-0.3678381 .172597$

H 3.9379431 .6821312 .605036

H 4.6995354 .4025910 .819219

H $4.191442-2.271549-3.913175$

H $3.180534-0.880801-3.541749$

H $4.758081-2.186462-1.274540$

C $-0.4490413 .168578-2.170745$

O $0.7466673 .676445-1.567320$

C $0.8628143 .503501-0.149645$

C -0.3178204 .1640610 .579831$

C -1.6604453 .6776120 .027571$

C -1.703065 $3.754270-1.499913$

H $-0.4193453 .542454-3.202491$

C $-0.4469021 .635816-2.229772$

H 0.8783282 .4355330 .080523

O $-\begin{array}{llll}0.228820 & 5.581459 & 0.444741\end{array}$

H -0.2440143 .9301801 .649066$

H $-2.464494 \quad 4.2940580 .448175$

O -1.8722792 .3355420 .452219$

H -2.596901 $3.247150-1.879106$

O $-1.8220355 .130699-1.869725$

C $0.4849831 .153782-3.318801$

H $-0.1356281 .154390-1.299423$

H -1.454785 $1.276926-2.461588$

H $-0.9700875 .971000 \quad 0.938854$

H -2.7640782 .0750830 .165811$

H -1.908742 $5.169542-2.837372$

O $-0.209051 \quad 0.404835-4.223023$

O $1.6803921 .413739-3.361687$

C $0.539221-0.084542-5.340195$

H $\quad \begin{array}{llll}\text { H } & 138124 & 0.712560 & -5.791475\end{array}$

H $1.175841-0.914273-5.023909$

H $-0.169343-0.451724-6.087323$

SCF Energy (B3LYP/6-31G**//MMFF) $=-3245.91047519$

0800638

MM̄FF Geometry

C 1.6917094 .3340660 .439832

C 2.1626643 .1166820 .132053

C $2.4354502 .658272-1.274705$

O $1.6139191 .489169-1.506719$

C $3.9056732 .240920-1.431167$

C $4.3010092 .026513-2.877875$

C $4.3542020 .827703-3.490224$

C $4.6902393 .275614-3.625534$

C $3.981838-0.515834-2.927404$

C $5.186218-1.456523-2.789720$

C $6.147998-1.064022-1.656353$

C $7.334051-2.030559-1.607020$

C $8.258189-1.747954-0.414406$

O $5.420164-1.121033-0.426059$

O $9.136750-2.876240-0.288871$

C $7.402974-1.6647750 .867811$ 
C $9.154865-0.532190-0.675132$

O $8.204487-1.2840851 .984240$

C $6.171337-0.7385920 .733795$

C $5.247742-0.9122191 .962251$

O $6.617021 \quad 0.6156440 .658048$

C $0.3955911 .666896-2.076090$

O $-0.0810572 .724470-2.465372$

C $-0.3428070 .387958-2.200226$

C $0.033595-0.758192-1.610731$

C $-0.666565-2.043882-1.695016$

C $-1.940569-2.097268-2.497267$

C $3.971073-0.0647731 .910729$

C $2.959603-0.5523252 .926316$

C $1.148674-2.2165593 .243753$

O $2.862403-0.0463504 .041553$

C $0.744390-3.5081612 .611017$

C $-0.494840-3.7563122 .162674$

C $-0.901386-5.0416971 .503426$

C - $-1.551333-4.8284170 .153104$

C $-2.875995-4.993559-0.002028$

C $-0.663598-4.524955-1.034039$

C $-0.146915-3.110898-1.051424$

N $2.216000-1.6255312 .460662$

H $1.5216765 .056474-0.353977$

H 2.3179672 .3919220 .927420

H $2.1919863 .445753-1.998588$

H $4.0997181 .332073-0.853950$

H $4.5663463 .007315-1.003810$

H $4.6761860 .798828-4.530658$

H $4.9868763 .067224-4.658996$

H $3.8532013 .979838-3.659396$

H $5.5367163 .764587-3.132170$

H $3.453950-0.436653-1.973271$

H $3.265314-0.971737-3.622724$

H $4.792743-2.461641-2.588430$

H $5.729884-1.507384-3.741282$

H $6.499507-0.039703-1.822344$

H $7.899359-2.002265-2.547070$

H $6.959530-3.060085-1.516077$

H $9.676554-2.7417680 .509170$

H $7.050648-2.6826971 .083736$

H $9.774534-0.700352-1.564223$

H $9.857092-0.3717730 .150255$

H $8.591858 \quad 0.390017-0.833313$

H $8.360204-0.326046 \quad 1.923657$

H $4.966622-1.9702872 .044999$

H $5.788114-0.6505982 .880739$

H $5.877946 \quad 1.2048870 .875009$

H -1.247310 $0.450518-2.796928$

H $\quad 0.938220-0.766783-1.005231$

H -2.427335 -3.074551 -2.476250

H -1.743272 -1.861435 -3.548731

H -2.671105 -1.380844 -2.107014

H 4.1869920 .9874282 .126074

H $3.507687-0.0991490 .918875$

H $0.327152-1.4932633 .286707$

H $1.508210-2.3840644 .264390$

H $1.510988-4.2729902 .512017$

H -1.260665 -2.994066 2.276581

H -1.596538 -5.5552612 .180315$

H $-0.046171-5.7187221 .385639$

H -3.351324 -4.879676 -0.970472

H $-3.517534-5.2409870 .837147$

H -1.177937 -4.757215 -1.974041

H $\quad 0.195287-5.208731-1.021685$

H $\quad 0.778196-2.977676-0.490019$

H $2.270363-1.8572721 .472424$

C $-0.156917 \quad 4.6837792 .148190$

O -0.6060143 .3172472 .027385$

C $-1.743417 \quad 3.286117 \quad 1.135861$

C -2.3284994 .6858481 .215861$

C -1.068658 5.5303081 .255674

H -1.3224183 .0973230 .142373$

C -2.670024 2.1383381 .549046

H $-2.9710904 .933990 \quad 0.366858$

H -2.9042804 .8151222 .140617$

C $-1.292657 \quad 6.939511 \quad 1.776569$

H $\quad-0.672504 \quad 5.597614 \quad 0.235778$
C $-4.002104 \quad 1.975860 \quad 0.782610$

O

H -2.902093 2.264547 2.614176

H -4.5117931 .1148501 .229683$

O $-4.8424993 .106017 \quad 0.996345$

C $-3.8838201 .704146-0.731767$

C $-5.2340991 .501808-1.449480$

H $-3.2541090 .822989-0.892491$

O $-3.2365182 .805378-1.369432$

H $-5.8235852 .426588-1.407119$

H $-5.0074841 .347638-2.513030$

H -1.9879987 .4831401 .128668$

H -1.7125036 .9323182 .787965$

H -0.3503247 .4960611 .805676$

C $1.3438284 .760271 \quad 1.840242$

H -1.573542 0.8425410 .558359

H -4.9512983 .2143061 .956451$

H $-3.0871172 .563259-2.299281$

H 1.8806074 .1221322 .554099

H 1.7068555 .7826441 .996451

H -0.3039144 .9544853 .201469$

C $-5.850584-2.045772-0.464117$

O $-5.257498-0.850442-0.986538$

C $-6.0703770 .323449-0.927088$

C $-7.3574770 .117692-1.741585$

C $-8.108218-1.139690-1.287625$

C -7.177993 -2.354104-1.177555

H $-5.141446-2.843692-0.718114$

C $-5.977197-2.0031211 .066463$

H $-6.340576 \quad 0.5295740 .112787$

O $\quad-7.057737 \quad 0.007871-3.132113$

H -7.997969 $0.998985-1.621052$

H $-8.908813-1.363252-2.003347$

O $-8.713035-0.906695-0.019118$

H -7.683208 -3.181622 -0.665846

O $-6.854342-2.810079-2.494786$

C $-4.633291-1.8841901 .754343$

H $-6.584633-1.1570101 .397773$

H $-6.444587-2.9301571 .418011$

H -6.426164 -0.723263 -3.243085

H $-9.321801-0.154836-0.117474$

H $-7.686786-3.060891-2.930546$

O $-4.837342-1.7941063 .102313$

O $-3.548299-1.8706261 .190563$

C $-3.658878-1.6642163 .903809$

H $-3.967345-1.4135254 .922185$

H $-3.120388-2.6155993 .924345$

H $-3.016339-0.8623933 .527939$

SCF Energy (B3LYP/6-31G**//MMFF) $=-3245.91316963$

0800639

MM̄FF Geometry

C $-2.698212-4.0756610 .230883$

C -1.484664 -3.509149 0.313289

C $-0.713692-3.4052621 .603620$

O $-0.034965-2.1292541 .654880$

C $0.359904-4.5083821 .664127$

C $1.174565-4.4952672 .944820$

C $2.512734-4.3437822 .996318$

C $0.397116-4.6906034 .221524$

C $3.469941-4.2095281 .845475$

C $3.809124-2.7463121 .563246$

C $4.723051-2.5562570 .343194$

C $6.117869-3.1485170 .557998$

C $7.052902-2.852843-0.623937$

O $4.828937-1.1438260 .130329$

O $8.396732-3.145368-0.209801$

C $7.002591-1.344083-0.943731$

C $6.759292-3.773673-1.814649$

O $7.723311-1.052471-2.138663$

C $5.569407-0.769907-1.040127$

C $5.6175050 .772539-1.079597$

O $4.911655-1.188149-2.237704$

C $-0.738034-1.0584412 .110772$

O $-1.926432-1.0210852 .385890$

C 0.2139190 .0690002 .246401

C -0.2302801 .2691652 .648120$

C 0.5832972 .4697072 .863155 
C 2.0667552 .3639282 .625454

C $4.2274321 .416668-1.136053$

C $4.2962982 .933662-1.168370$

C $2.7857184 .919428-1.312875$

O $5.3481143 .565139-1.127420$

C 2.5968795 .4575810 .070795

C 1.4471865 .9939610 .508055

C 1.2883906 .6547841 .846262

C 0.1951826 .0413722 .689015

C -1.049098 6.5503692 .678902

C 0.5783004 .9248863 .633908

C -0.0345463 .5988343 .268398$

N $3.0301473 .492785-1.251439$

H $-3.139971-4.4910471 .132309$

H -1.008254 -3.127697 -0.585364

H -1.389918 -3.4941182 .463079$

H $1.012333-4.4007740 .791075$

H $-0.114674-5.4944471 .573160$

H $2.991311-4.3341743 .975032$

H $1.051581-4.8469395 .085894$

H $-0.224127-3.8154914 .434855$

H $-0.249821-5.5702864 .141298$

H $4.377051-4.7647152 .110720$

H $3.083775-4.6917820 .942137$

H $2.883964-2.1793521 .392608$

H $4.271555-2.2849672 .446310$

H $4.234655-3.008968-0.527791$

H $6.071500-4.2297890 .730963$

H $6.559923-2.7173251 .467099$

H $8.427553-4.0744200 .076315$

H $7.525663-0.821385-0.130316$

H $6.926101-4.821983-1.538224$

H $7.440383-3.573049-2.648906$

H $5.731906-3.691518-2.176077$

H $8.623251-1.404374-2.027226$

H $6.136094 \quad 1.146318-0.187118$

H $6.1892271 .105816-1.955136$

H $5.542724-1.682065-2.784947$

H $1.257591-0.1243492 .025370$

H $-1.294768 \quad 1.3867692 .848233$

H 2.5202651 .6646713 .336278

H 2.5937483 .3149592 .725775

H 2.2709522 .0110731 .609391

H $3.6933851 .083904-2.033793$

H $3.6395051 .123534-0.259142$

H $1.9005605 .070807-1.939108$

H $3.6358255 .417256-1.790444$

H 3.4669375 .4559150 .723048

H $0.5859886 .017797-0.156246$

H 1.0619267 .7142991 .665032

H 2.2315806 .6570602 .406633

H -1.829295 $6.147173 \quad 3.316044$

H -1.314888 7.3791452 .031051

H 0.2286665 .1851884 .642660

H $1.6656644 .867783 \quad 3.733421$

H -1.1164613 .5705243 .406806$

H $2.2222322 .879230-1.245632$

C $-4.903688-3.721478-1.016452$

O $-4.933177-2.302565-0.781106$

C $-6.119656-1.993053-0.019801$

C $-6.927685-3.2864250 .073777$

C $-5.834147-4.335938 \quad 0.027222$

H $-5.781036-1.7194900 .986258$

C $-6.875183-0.820345-0.654946$

H -7.528563 -3.3315390 .987353$

H $-7.607511-3.407673-0.777311$

C $-6.333809-5.724986-0.327479$

H $-5.357232-4.3673831 .013518$

C $-6.0169710 .441362-0.874963$

O $-7.361367-1.237513-1.938451$

H -7.760583 $-0.583340-0.053108$

H $-5.2769270 .231848-1.655627$

O $-6.8942011 .445086-1.401878$

C $-5.3306390 .975366 \quad 0.396622$

C -4.6058742 .3231910 .218188$

H -4.6152960 .2317860 .764716$

O -6.3226641 .1416801 .413472$

H $-5.3278683 .105129-0.047307$
H -4.2091222 .6119531 .201105$

H $-7.059725-6.0735660 .414373$

H $-6.822390-5.738456-1.307566$

H -5.506772 -6.441441-0.352661

C $-3.462092-4.236580-1.053602$

H $-7.845144-0.485880-2.322058$

H $-6.3433572 .146825-1.787273$

H $\quad-5.865458 \quad 1.4080582 .229295$

H -2.930985 -3.713296-1.859099

H -3.468461 -5.301512 -1.313899

H $-5.331684-3.868949-2.017777$

C $-1.3650531 .132766-1.177039$

O $-2.4829241 .358689-0.311043$

C $-3.4608002 .279046-0.803733$

C $-2.8292023 .662305-1.020470$

C $-1.6173523 .572793-1.954424$

C $-0.6601772 .452923-1.538056$

H $-0.6707200 .542297-0.569103$

C -1.770822 $0.266250-2.373630$

H $-3.8480091 .916753-1.761086$

O $-2.431823 \quad 4.2314660 .225400$

H -3.581887 4.331975 -1.452259

H -1.077493 $4.527921-1.942931$

O $-2.0540883 .334903-3.288651$

H $0.0860572 .283625-2.322017$

O $0.0440392 .910059-0.381087$

C $-0.571327-0.318749-3.079665$

H $-2.414635-0.561387-2.049887$

H $-2.3629830 .825256-3.104174$

H -1.800399 3.6217820 .644143

H $-2.6283254 .075974-3.546397$

H $0.6462082 .199903-0.102761$

O $0.082074-1.187494-2.256574$

O $-0.254835-0.036748-4.228131$

C $1.267581-1.781295-2.794314$

H $1.014874-2.420816-3.644932$

H $1.981082-1.006375-3.090436$

H $1.723426-2.396292-2.013604$

SCF Energy $\left(B 3 L Y P / 6-31 G^{* *} / / M M F F\right)=-3245.90755939$

0800640

MMFF Geometry

C $-3.2186852 .876497-1.502520$

C $-4.1104803 .587253-0.796775$

C $-4.538303 \quad 3.2829880 .621691$

O $-3.6269692 .340396 \quad 1.236966$

C -5.9523412 .6771330 .632206$

C -6.4911442 .4050282 .025908$

C -6.8406451 .1868192 .485755$

C -6.6529613 .6122492 .913650$

C $-6.807002-0.1137821 .735416$

C $-5.552344-0.9265432 .072319$

C $-5.385385-2.1202641 .122342$

C $-4.225052-3.0093321 .567967$

C $-3.961200-4.148610 \quad 0.573347$

O $-5.119079-1.592152-0.180159$

O $-2.687779-4.7130040 .922566$

C $-3.847168-3.555889-0.850500$

C $-4.988867-5.2749710 .731442$

O $-3.775188-4.585635-1.832153$

C $-4.988740-2.578031-1.212621$

C $-4.750568-1.815378-2.537910$

O $-6.225899-3.273333-1.414891$

C -2.6116752 .8528031 .980758$

O -2.4123654 .0334682 .233723$

C -1.7403041 .7889932 .524970$

C -1.530399 0.6122291 .913847

C $-0.649576-0.4572562 .399610$

C $-0.035671-0.2806803 .765518$

C $-3.492788-0.940437-2.582006$

C $-2.267610-1.664880-3.111980$

C $0.218831-1.595005-3.035417$

O $-2.334503-2.674818-3.808052$

C $0.573318-2.564333-1.951510$

C $1.612241-2.407481-1.118736$

C $1.948057-3.368789-0.018683$

C $1.761772-2.7641451 .354799$

C $2.817610-2.3326992 .064199$ 
C $0.371888-2.768178 \quad 1.951088$ C $-0.432482-1.5382761 .619445$ N -1.087474 -1.033918 -2.742464 H $-2.7848971 .981226-1.065216$ H $-4.5536034 .472279-1.248083$ H $-4.5397194 .227143 \quad 1.179260$ H -5.9331181 .7593390 .036024$ H $-6.651002 \quad 3.3548130 .123614$ H -7.205732 1.1051203 .508582 H -7.1912503 .3778533 .838541$ H -5.6772674 .0188643 .196422$ H -7.221597 4.3913222 .395477 H $-7.698095-0.6929062 .009814$ H -6.894271 0.0489320 .657516 H -4.669796 -0.2791491 .993089$ H -5.613703 -1.275147 3.110645 H $-6.322655-2.6883421 .100742$ H -4.404125 -3.410708 2.573291 H -3.314743 -2.401198 1.648326 H $-2.518424-5.4656640 .331127$ H -2.893474 -3.015677 -0.884730 H $-4.909986-5.7245761 .728740$ H $-4.800557-6.0873400 .021009$ H $-6.019301-4.9362720 .604274$ H -2.972245 -5.104468 -1.657538 H $-4.784257-2.512411-3.384195$ H -5.609951-1.144621 -2.682430 H $-6.053028-4.228113-1.395361$ H -1.223006 2.0592053 .439873 H -2.0314790 .4140730 .967730$ H $-0.815478-0.153356 \quad 4.524773$ H $\quad 0.6194430 .5975973 .780795$ H $\quad 0.575688-1.1264364 .085374$ H -3.670893 -0.107244 -3.272844 H -3.281148 -0.510346 -1.597387 H $0.927533-0.763834-3.101666$ H $0.190233-2.109843-4.000960$ H $-0.066840-3.436917-1.841499$ H $2.263328-1.544589-1.231147$ H $2.990348-3.684716-0.158051$ H $1.354573-4.288431-0.095385$ H $2.703586-1.9345483 .066503$ H $3.821238-2.3672561 .655511$ H $0.427422-2.9249653 .033988$ H $-0.176758-3.6464071 .588391$ H -0.909417 -1.574710 0.639781 H -1.125872 -0.241391-2.107571 C - $-1.2349913 .522159-2.930054$ O $-0.5002252 .431542-2.334043$ C $0.3312622 .969744-1.283691$ C $0.573574 \quad 4.411798-1.689363$ C $-0.8032584 .797725-2.197797$ H $-0.2697752 .928224-0.365859$ C $1.5763322 .095404-1.135542$ H $0.9097155 .038568-0.858491$ H $1.3146754 .471227-2.495990$ C $-0.8041506 .035531-3.078250$ H -1.450332 $4.987742-1.332329$ C $2.6514562 .664390-0.195840$ O $1.1424190 .827233-0.635750$ H $2.0002091 .907513-2.127756$ H $3.0772553 .576602-0.630440$ O 2.0112153 .0566351 .026698 C $3.781347 \quad 1.680470 \quad 0.169714$ C $4.4874031 .062930-1.050893$ H 3.3869260 .8942640 .824743 O 4.7287312 .4251120 .943219 H $3.7492220 .623448-1.728388$ H $5.0137111 .865297-1.582715$ H $-0.4456246 .905779-2.518847$ H $-0.1559815 .907692-3.951747$ H -1.814908 $6.255467-3.436530$ C $-2.739063 \quad 3.222458-2.884180$ H $\quad 0.8403020 .9601350 .278709$ H 2.6991263 .4236231 .608284 H 5.4246871 .8099141 .229166 H -2.944443 $2.353626-3.521471$ H -3.300404 $4.066380-3.300939$
H $-0.921310 \quad 3.563427-3.980545$

C $7.591736-0.3335370 .514026$

O 6.5876880 .5641610 .023160

C $5.494009-0.042424-0.669427$

C $6.002019-0.812365-1.900478$

C $7.083957-1.823797-1.511513$

C $8.164953-1.192988-0.624734$

H 8.3941720 .3206790 .879076

C $7.089911-1.1614631 .701993$

H $4.982957-0.7396950 .002958$

O $6.5491650 .082627-2.866299$

H $5.162361-1.338461-2.371228$

H $7.543473-2.221372-2.424732$

O $6.465093-2.910752-0.829381$

H $8.818143-1.966020-0.203263$

O $8.989671-0.342798-1.426824$

C $6.753074-0.2705802 .873988$

H $\quad 6.213642-1.7685271 .460591$

H $7.861760-1.8624722 .041327$

H $5.8131100 .577839-3.263259$

H $7.160103-3.555805-0.614358$

H $9.382628-0.890941-2.127314$

O $5.404260-0.0870562 .947038$

O 7.5905880 .2198813 .620100

C 4.9493390 .7525814 .012517

H $\quad 5.3936841 .748874 \quad 3.928774$

H 3.8631120 .8456343 .930312

H 5.1924110 .3001184 .978451

SCF Energy $\left(B 3 L Y P / 6-31 G^{* *} / / M M F F\right)=-3245.90962110$

0800641

MMFF Geometry

C $-0.443212-3.619527-0.816484$

C $-1.280461-2.572923-0.757124$

C $-1.832403-1.898315-1.987337$

O $-1.669192-0.469140-1.840884$

C $-3.342627-2.158007-2.107583$

C $-3.847971-2.028326-3.529918$

C $-4.403486-0.920740-4.057929$

C $-3.733219-3.282910-4.358175$

C $-4.5663470 .433912-3.426576$

C $-6.0254630 .754495-3.076009$

C $-6.4640190 .138393-1.739194$

C $-7.9531460 .383176-1.493379$

C $-8.400428-0.168475-0.130974$

O $-5.6980850 .768400-0.707178$

$\begin{array}{lllll}\text { O } & -9.693728 & 0.387179 & 0.163568\end{array}$

C -7.4373010 .3473690 .961698$

C $-8.596355-1.688170-0.174832$

O $-7.705090-0.2656842 .220356$

C $\begin{array}{llll}-5.931308 & 0.253722 & 0.607537\end{array}$

C -5.1172501 .1333321 .584751$

O $-5.420020-1.0704550 .755994$

C $-0.4415470 .070804-2.045782$

O $0.582334-0.510585-2.363327$

C $-0.5708891 .519539-1.776579$

C $0.5294772 .262761-1.599534$

C $0.5601153 .690150-1.277888$

C $-0.7530134 .428507-1.246749$

C $-3.612704 \quad 1.1901451 .275747$

C -2.8636122 .0148442 .310912$

C -0.5600032 .6885862 .974655$

O $-3.4243262 .590025 \quad 3.240945$

C -0.2000264 .0365852 .434349$

C 1.0651844 .4714442 .322785

C 1.4406045 .8498671 .856504

C 2.4999005 .8412510 .776232

C 3.7950326 .0387231 .075293

C $2.0459585 .690121-0.657760$

C $1.7530794 .261338-1.012596$

N -1.4946002 .0288352 .081324$

H -0.173147 -4.001560 -1.799864

H -1.589533 -2.166370 0.201191

H -1.306890 -2.228842 -2.892253

H -3.890479-1.472175-1.452636

H $-3.591999-3.167844-1.755014$

H $-4.757745-0.970269-5.087246$

H $-4.104601-3.144626-5.379055$ 
H $-2.687993-3.600245-4.428530$ H -4.312714 -4.092608 -3.902544 H $-3.9224200 .562435-2.552923$ H -4.216802 $1.171394-4.160662$ H $-6.115648 \quad 1.846515-3.005555$ H $-6.6896360 .425124-3.884452$ H $-6.251722-0.935877-1.750044$ H $-8.558238-0.050810-2.299076$ H -8.154650 $1.463441-1.514465$ H -10.294409 $0.153765-0.564756$ H $-7.678693 \quad 1.411017 \quad 1.097359$ H -9.390702 -1.949740 -0.884919 H $-8.927683-2.0747660 .794934$ H -7.701258 -2.231859-0.481296 H -7.327003 -1.160804 2.205993 H -5.5048672 .1604671 .560875$ H -5.2482540 .7592032 .608592$ H $-6.119152-1.7084680 .556920$ H -1.572393 $1.927115-1.695479$ H $1.5001421 .776445-1.676480$ H $-0.6581865 .478987-0.965130$ H -1.435951 $3.976418-0.519197$ H -1.228166 $4.404233-2.233876$ H $-3.1869890 .180324 \quad 1.270838$ H $-3.443625 \quad 1.6425860 .292249$ H 0.3115282 .0368663 .068541 H -1.015525 2.8000843 .963925 H -1.017094 4.700601 2.162863 H 1.8782963 .8177002 .630291 H 1.8093756 .4047122 .729417 H $\quad 0.5633706 .4057921 .502899$ H 4.5567396 .0606690 .302929 H 4.1229866 .1754982 .100457 H $2.8316776 .040020-1.340914$ H $1.2022426 .360287-0.844206$ H $2.6466923 .637427-1.052584$ H -1.1205361 .5586551 .262142$ C $0.083779-3.7298431 .723989$ O $0.830264-2.4941951 .751948$ C $1.790608-2.5593752 .819742$ C $1.238670-3.5966343 .785136$ C $0.692803-4.6232552 .812182$ H $2.718908-2.9486382 .384191$ C $2.061202-1.1717093 .408777$ H $2.006470-4.0000514 .451735$ H $0.423358-3.1956114 .397155$ C $-0.298741-5.5897623 .437466$ H $1.534168-5.1980652 .401817$ C $2.432263-0.0987922 .364817$ O $0.890037-0.6930214 .080981$ H $2.849877-1.2402994 .168183$ H $1.5343550 .197672 \quad 1.808885$ O 2.8532911 .0704503 .081334 C $3.534048-0.4962581 .366666$ C 3.9365140 .6851210 .462248 H $3.176627-1.3257240 .746700$ O $4.668974-0.9540742 .099248$ H 3.0328131 .1983750 .117085 H 4.5319951 .3928091 .052712 H $0.178163-6.1663244 .236806$ H - $-1.155813-5.0623113 .869285$ H $-0.678000-6.2948702 .691052$ C $0.192466-4.3592170 .330514$ H $0.827417-1.1580264 .932120$ H 3.6892000 .8543623 .529337 H $5.342958-1.2300991 .455253$ H $-0.234797-5.3688830 .348752$ H $1.256151-4.4633640 .079295$ H $-0.957114-3.4870061 .968254$ C $6.873895-0.798222-1.318618$ O $6.002453-0.281756-0.304897$ C $4.755176 \quad 0.243039-0.766653$ C $4.9926801 .405209-1.743745$ C $5.9132150 .984884-2.893415$ C $7.1699580 .268293-2.385679$ H $7.812799-1.015790-0.792778$ C $6.349476-2.115270-1.901580$ H $4.194688-0.548872-1.275641$
O $5.5695262 .506103-1.044633$

H $4.0256391 .735579-2.140684$

H $6.202684 \quad 1.875477-3.464399$

O $5.1838800 .129291-3.768353$

H $7.715560-0.192137-3.217531$

O $8.0466051 .231560-1.793883$

C $6.306648-3.186295-0.837022$

H $5.354395-2.019854-2.345055$

H $7.007816-2.479112-2.699265$

H $5.6627423 .238913-1.676825$

H $5.772801-0.109024-4.504386$

H $8.2729861 .882625-2.479761$

O $5.018438-3.410772-0.450684$

O $7.302191-3.739307-0.387597$

C $4.844242-4.3995450 .568714$

H $5.297274-5.3485620 .266025$

H $5.276555-4.0464241 .509473$

H $3.772034-4.5559080 .710606$

SCF Energy (B3LYP/6-31G**//MMFF) $=-3245.89627258$

\section{2}

MM̄FF Geometry

C 4.3104530 .8903672 .541259

C $5.437448 \quad 0.831337 \quad 1.814876$

C $5.7782701 .717154 \quad 0.635572$

O 4.7089852 .6760350 .445316

C $5.9158810 .907066-0.670204$

C $7.040925-0.107515-0.681524$

C $6.873202-1.434843-0.511847$

C $8.412056 \quad 0.459812-0.937598$

C $5.587292-2.150688-0.206871$

C $5.128095-3.034480-1.373447$

C $3.683818-3.512552-1.164697$

C $3.278293-4.538646-2.223388$

C $1.796032-4.931556-2.101356$

O $2.844231-2.356941-1.258769$

O $1.427513-5.637629-3.296241$

C $0.942111-3.645516-2.055637$

C $1.552079-5.896354-0.935408$

O $-0.427855-3.942885-1.799365$

C $1.452299-2.608292-1.033946$

C $0.730783-1.245972-1.142388$

$\begin{array}{lllll}\text { O } & 1.229052 & -3.099953 & 0.291807\end{array}$

C $4.9852623 .873915-0.121958$

O $6.074804 \quad 4.255287-0.525918$

C $3.7702974 .724299-0.200887$

C $2.555387 \quad 4.333896 \quad 0.219636$

C 1.3207025 .1252650 .182523

C $1.3988126 .508211-0.409083$

C $0.759607-0.616679-2.537561$

C $0.4544920 .871111-2.516466$

C $-0.2726612 .717771-4.032745$

O $0.6838441 .586364-1.544722$

C $-1.4197393 .241806-3.227443$

C $-1.2937194 .261943-2.365744$

C $-2.4021114 .784515-1.501691$

C $-2.221498 \quad 4.422204-0.043131$

C $-3.012212 \quad 3.5062410 .541088$

C $-1.188568 \quad 5.1834690 .761570$

$\begin{array}{llll}C & 0.186887 & 4.575188 & 0.669155\end{array}$

N $-0.0562941 .313525-3.731040$

H $3.5652681 .650614 \quad 2.323494$

H 6.1716810 .0699822 .068940

H 6.7078412 .2507290 .868308

H $6.0708141 .600284-1.508539$

H $4.9533660 .438641-0.911929$

H $7.749769-2.078906-0.562010$

H $9.185073-0.315590-0.963282$

H $8.4342320 .979592-1.900999$

H $8.6846691 .171141-0.151708$

H $5.745043-2.7779300 .679678$

H $4.797517-1.4477840 .070705$

H $5.187495-2.461244-2.308046$

H $5.803313-3.893638-1.466609$

H $3.598260-3.944159-0.160352$

H $3.917286-5.428590-2.167734$

H $3.442288-4.114357-3.223941$

H $2.009251-6.412812-3.377624$ 
H $\quad 0.982195-3.208268-3.061150$

H $2.130948-6.817514-1.073705$

H $0.502073-6.206196-0.892713$

H $1.828308-5.4775910 .034427$

H $-0.712147-4.595111-2.462339$

H $-0.309597-1.345205-0.811939$

H $1.200049-0.560443-0.423374$

H $1.192504-2.3413970 .896867$

H $3.9372865 .708489-0.627006$

H $2.439616 \quad 3.3361120 .639247$

H 2.0676217 .1419220 .183743

H $0.4354217 .019270-0.456921$

H $1.7747196 .465762-1.437620$

H $1.751006-0.725178-2.992303$

H $0.033769-1.125849-3.181349$

H $-0.4984002 .797579-5.100530$

H $0.6589223 .257202-3.830090$

H - $2.3814652 .750102-3.344883$

H $-0.3280974 .751100-2.273194$

H -3.368401 $4.413166-1.868034$

H -2.452276 $5.875429-1.604541$

H -2.910114 3.2587521 .592540

H -3.776983 $2.980980-0.020294$

H -1.4712315 .1769741 .823164$

H $-1.215440 \quad 6.238157 \quad 0.475601$

H 0.2389413 .5642061 .077027

H $-0.1248970 .656761-4.502791$

C $2.652214-0.796093 \quad 3.292217$

$\begin{array}{lllll}\text { O } & 1.562275 & 0.144231 & 3.329936\end{array}$

C $0.377938-0.5249333 .815886$

C $0.763513-1.9861154 .006715$

C $2.255266-1.8947034 .271356$

H $0.148927-0.0590884 .781843$

C $-0.752562-0.2737392 .810653$

H $0.213540-2.4594784 .825563$

H $0.569924-2.5653613 .096532$

C $2.987195-3.208344 \quad 4.057617$

H $2.410385-1.5532955 .303442$

C $-2.049229-1.0618183 .063541$

O -1.0442651 .1286992 .822209$

H $-0.373666-0.4960801 .807426$

H $-1.866081-2.1390132 .971782$

O $-2.490392-0.8319734 .407430$

C $-3.224918-0.6704882 .142876$

C -2.876572 -0.7170260 .645157$

H -3.594246 0.3282702 .406069

O $-4.298442-1.5817012 .395568$

$\begin{array}{llll}\text { H }-2.044932 & -0.033508 & 0.446848\end{array}$

H $-2.558349-1.736406 \quad 0.392837$

H $2.601151-3.9777734 .734355$

H $2.867775-3.5722793 .031473$

H $4.058347-3.0955294 .251566$

C $3.956419-0.0756053 .638745$

H -1.422436 1.3429293 .692291

H -1.858896-1.264583 5.005980

H $-4.510115-1.5254023 .343184$

H $4.763564-0.8010783 .788164$

H 3.8398090 .4941134 .568217

H $2.700097-1.1942542 .269536$

C -6.201339-1.143101-1.026789

O $-5.004883-1.382359-0.273585$

C $-4.053577-0.314592-0.264894$

C $-3.580476-0.018434-1.696753$

C $-4.7664240 .275660-2.620741$

C -5.868286 $-0.780506-2.485267$

H $-6.725677-2.107429-1.036114$

C $-7.114188-0.118199-0.335833$

H -4.5162880 .5827020 .158602$

O $-2.850923-1.137141-2.197048$

H -2.896407 $0.837400-1.672380$

H $-4.4235530 .302461-3.662332$

O $-5.3115101 .554198-2.309074$

H $-6.778068-0.442813-2.994441$

O $-5.446436-1.971584-3.157574$

C $-7.591065-0.6002371 .018733$

H $-6.6003480 .834854-0.184294$

H $-8.0045230 .059666-0.949562$

H $-2.529832-0.903758-3.084629$
H $-4.6051512 .211965-2.425572$

H $-4.681627-2.329968-2.675109$

O $-8.144490 \quad 0.450594 \quad 1.693995$

O $-7.509343-1.7500681 .428510$

C -8.6423720 .1472003 .000942$

H -7.832218 -0.2173693 .639872$

H $-9.043526 \quad 1.0674093 .434065$

H -9.447410 -0.590976 2.937170

SCF Energy (B3LYP/6-31G**//MMFF) $=-3245.90219205$

0800643

MMFF Geometry

C - $-1.772522 \quad 2.8909342 .169919$

C -1.8386824 .0078341 .427806$

C $-2.408108 \quad 4.113922 \quad 0.031260$

O $-3.1549162 .931122-0.327871$

C $-1.231553 \quad 4.262849-0.957257$

C $-1.6274854 .254304-2.420824$

C $-1.2416153 .308081-3.301947$

C $-2.4929435 .401658-2.869257$

C $-0.3617352 .116658-3.028154$

C $-1.1863330 .927595-2.541033$

C $-0.348657-0.232966-1.991396$

C $0.448552-0.953926-3.079112$

C $1.137222-2.222799-2.547440$

O $-1.286388-1.133667-1.391139$

O $1.559340-3.002307-3.677293$

C $0.106496-3.073928-1.772510$

C $2.406663-1.890885-1.753924$

O $0.735143-4.186062-1.139594$

C $-0.699142-2.262447-0.735687$

C $-1.851697-3.112133-0.149882$

$\begin{array}{llllll}\text { O } & 0.171895 & -1.871387 & 0.325771\end{array}$

C $-4.473096 \quad 2.890366 \quad 0.005527$

$\begin{array}{lllll}\text { O } & -5.100197 & 3.733252 & 0.626742\end{array}$

C $-5.0316971 .629965-0.537962$

C $-6.3414651 .373593-0.401319$

C $-7.0608750 .209123-0.926310$

C $-6.259000-0.842741-1.645833$

C $-2.848779-2.2947030 .686782$

C $-3.980130-3.1459891 .233659$

C $-6.171458-2.9267472 .405002$

O $-3.979893-4.3731951 .192377$

C $-7.222350-3.1163671 .357011$

C -8.449136 -2.577940 1.427590

C $-9.528268-2.8374820 .416792$

C $-10.121339-1.570984-0.154843$

C -11.323885 $-1.126696 \quad 0.248342$

C $-9.368020-0.877088-1.266849$

C $-8.3988920 .152266-0.757674$

N $-4.979725-2.3645821 .799668$

H -2.194827 1.9636001 .794278

H $-1.3864634 .918278 \quad 1.816656$

H -3.057302 $4.995961-0.028116$

H $-0.5132573 .460317-0.758623$

H $-0.6922405 .197100-0.749848$

H $-1.590777 \quad 3.384741-4.330601$

H -2.660221 $5.396831-3.951596$

H $-3.4731905 .357870-2.384582$

H -2.019375 $6.355621-2.615302$

H $0.1631561 .856067-3.954920$

H $0.4222762 .368811-2.306815$

H $-1.8665741 .249471-1.744650$

H $-1.8418300 .567186-3.345478$

H $\quad 0.3162290 .154532-1.210597$

H $1.187575-0.284459-3.535151$

H $-0.230768-1.247331-3.891663$

H $2.150008-2.450448-4.218139$

H $-0.595781-3.490913-2.507855$

H $3.130378-1.366977-2.389761$

H $2.910131-2.802684-1.415388$

H $2.215761-1.260155-0.883804$

H $1.220781-4.673810-1.826768$

H $-2.412518-3.579227-0.970058$

H $-1.441975-3.9206500 .468402$

H $-0.339248-1.769198 \quad 1.142982$

H $-4.3561550 .961676-1.059584$

H $-6.958302 \quad 2.0999520 .126814$ 
H $-5.822532-0.432520-2.563076$ H $-6.843733-1.719706-1.930523$ H -5.449579 -1.212790 -1.008956 H -2.349321 -1.8280241 .542478$ H $-3.285975-1.4988700 .073896$ H $-6.491820-2.2401843 .194895$ H -5.925472 -3.8921062 .859290$ H $-6.973610-3.7622570 .518066$ H -8.709429 -1.955817 2.280869 H -10.310958 -3.4254390 .914313$ H -9.164802 -3.468889-0.403748 H -11.770146 $-0.236742-0.183255$ H -11.885785 -1.639046 1.022146 H -10.080913 -0.354928 -1.919914 H -8.901281 -1.624614 -1.913723 H $-8.8855970 .971710-0.226106$ H -4.924273 -1.3532451 .718531$ C 0.3340522 .1869403 .388673 O 1.1522152 .9684702 .489388 C 1.7122732 .0826561 .498626 C 1.7167050 .7169832 .163664 C 0.3818780 .7408852 .883206 H 1.0151312 .0908980 .650893 C 3.0844252 .6019501 .055806 H $1.803587-0.1045421 .449206$ H 2.5385630 .6375302 .884395 C $0.262900-0.2968643 .985645$ H -0.4069210 .5540042 .145138$ C 3.7930691 .6634370 .056718 O 2.8933313 .8961630 .478403 H 3.7200442 .7516741 .935403 H 3.8740390 .6771120 .522085 O $2.977721 \quad 1.502567-1.104537$ C $5.2069842 .117291-0.359874$ C $5.8796961 .194823-1.396495$ H 5.8338632 .1936550 .536265 O $5.1384143 .422586-0.937408$ H $5.302697 \quad 1.207901-2.329987$ H $6.8521381 .641572-1.643994$ H $0.354968-1.3075213 .574670$ H $1.044246-0.1704974 .742608$ H $-0.708091-0.2213454 .485487$ C -1.0618802 .8169313 .493827$ H $2.2838103 .802298-0.273030$ H $3.0042542 .338338-1.600672$ H $6.0490683 .715105-1.113186$ H -0.9587893 .8290233 .905855$ H -1.680638 2.2495324 .198238 H 0.8167982 .2575534 .371657

C $7.210985-1.4936700 .832369$ O $6.939423-0.2242180 .221543$ C $6.086692-0.254272-0.927616$ C $6.694067-1.132478-2.032878$ C $7.008740-2.535760-1.504859$

C $7.793985-2.483010-0.191747$ H $7.996059-1.2850511 .570964$ C $5.986700-2.0372581 .583582$ H $5.116090-0.673191-0.650366$ O $7.887815-0.527479-2.524948$ H $5.984807-1.190648-2.867335$

H $7.594956-3.087617-2.249692$

O $5.795426-3.248884-1.285230$

H $7.846923-3.4825090 .254322$

O $9.142625-2.096981-0.473930$

C $5.838415-1.3699402 .931778$

H $5.042473-1.9190911 .045882$

H $6.110696-3.1088951 .781546$

H $8.229720-1.091454-3.239507$

H $5.328874-3.303216-2.136549$

H $9.127813-1.181243-0.801275$

O $5.496068-0.0595722 .778466$

O $6.004355-1.9508453 .996981$

C $5.3587610 .684588 \quad 3.992899$

H 5.1331531 .7219103 .733099

H $4.533798 \quad 0.283848 \quad 4.589471$

H 6.2935650 .6623944 .561160

SCF Energy (B3LYP/6-31G**//MMFF)= -3245.90057107
0800644

MM̄FF Geometry

C $0.4394193 .554083-0.459893$

C 0.0053214 .1013040 .686586

C $-0.6328003 .346872 \quad 1.834474$

O

C -1.8338644 .1503932 .366826$

C -2.4955883 .5372873 .583725$

C -3.6559882 .8520713 .555677$

C -1.7893203 .7823184 .891311$

C -4.4705952 .4936782 .342752$

C -4.8988501 .0206312 .380459$

C -5.5732660 .5869081 .071187$

C $-6.121919-0.8349931 .203350$

C $-6.717893-1.342840-0.116113$

$\begin{array}{lllll}\text { O } & -4.592646 & 0.638741 & 0.028096\end{array}$

O $-6.915213-2.7597510 .004968$

C $-5.686798-1.124599-1.240344$

C $-8.096496-0.733541-0.395383$

O $-6.244351-1.451689-2.511640$

C $-5.0905470 .304462-1.273381$

C $-3.9068370 .335068-2.267835$

O $-6.0929301 .224257-1.705772$

C -0.8707710 .9455812 .055836$

O $-0.318160 \quad 0.849647 \quad 3.137913$

C $-1.395380-0.1771731 .248406$

C -1.233616 -1.451707 1.629892

C $-1.696906-2.6026970 .853737$

C $-2.289382-3.7013781 .689846$

C $-3.0996611 .641257-2.294484$

C $-1.9329261 .479417-3.252013$

C $0.1943130 .193075-3.471553$

O $-1.991798 \quad 1.868514-4.415884$

C $-0.229838-1.209716-3.786634$

C $0.313579-2.302442-3.228653$

C $-0.219542-3.691004-3.441224$

C $-0.718518-4.336299-2.161250$

C $-0.165745-5.472173-1.703253$

C -1.916604 -3.725085 -1.459610

C $-1.528367-2.646512-0.483673$

N $-0.8699880 .792769-2.684902$

H $0.3843182 .478141-0.599812$

H 0.1025695 .1780540 .811282

H 0.1217633 .2119122 .618243

H -2.5680574 .2890691 .564167$

H -1.5122025 .1665272 .633971$

H -4.0646682 .4982544 .501273$

H -2.3239343 .3451185 .741316$

H -0.7865683 .3445114 .873796$

H -1.6967174 .8573055 .077408$

H $-5.364416 \quad 3.1282792 .321342$

H -3.9182972 .6835611 .418869$

H -4.0177870 .3927632 .561288$

H -5.5871030 .8667013 .221103$

H $-6.381288 \quad 1.2921990 .843684$

H $-6.866260-0.8926232 .007392$

H $-5.308805-1.5131381 .499400$

H -7.508375 -2.916205 0.759707

H -4.866256 -1.836224 -1.070750

H $-8.795388-0.9821070 .412265$

H $-8.534870-1.150421-1.308947$

H $-8.0740330 .354152-0.488465$

H $-6.590856-2.358364-2.451376$

H -3.218868 -0.482403 -2.018260

H $-4.2736470 .140682-3.284225$

H $-5.6617752 .016928-2.061690$

H $-1.906307 \quad 0.072357 \quad 0.325153$

H $-0.736539-1.6839662 .568610$

H -1.583694 -4.020316 2.465268

H $-2.548884-4.5924231 .114379$

H $-3.203978-3.3511412 .181177$

H -3.705967 $2.485169-2.638510$

H -2.718976 $1.899046-1.300459$

H $1.1080310 .226485-2.870126$

H $\quad 0.351370 \quad 0.760447 \quad-4.393647$

H - $1.071484-1.323592-4.466320$

H $1.147836-2.192349-2540882$

H $\quad 0.582064-4.291543-3.889629$ 
H -1.039409-3.689712 -4.170678 H $-0.536905-5.957528-0.806530$ H $0.672052-5.939405-2.209793$ H -2.504560 -4.512671-0.977776 H -2.591198 -3.291862 -2.209254 H $-1.064887-1.782347-0.953845$ H $-0.9551660 .481799-1.722474$

C $2.4807894 .283927-1.806689$

O $2.9485382 .921274-1.854742$

C $4.1986852 .851050-1.139707$

C $4.6415904 .292151-0.912170$

C $3.3039284 .979449-0.723416$

H $3.9764622 .408115-0.163708$

C $5.2133811 .988203-1.892763$

H $5.3033174 .391579-0.046304$

H $5.1623384 .699191-1.787072$

C $3.3693026 .489644-0.864726$

H 2.9238984 .7289930 .274931

C $4.7749250 .523373-2.095318$

O $5.4291132 .570708-3.184355$

H $6.1801772 .022526-1.376160$

H $3.9359850 .484805-2.800925$

O $5.873762-0.142836-2.731099$

C $4.405099-0.220629-0.794873$

C $4.141987-1.722361-1.017670$

H $3.5068890 .229654-0.357813$

O $5.468191-0.092690 \quad 0.149930$

H $3.499457-1.856195-1.895179$

H $5.101057-2.218344-1.212128$

H $4.0374336 .917565-0.110068$

H $3.7434216 .785432-1.850631$

H $2.3794476 .937156-0.731117$

C $0.9636514 .338616-1.629035$

H $6.0765842 .012499-3.648391$

H $5.543784-0.985299-3.086555$

H $5.478907 \quad 0.8242850 .470032$

H $\quad 0.4912773 .916098-2.525542$

H $0.6341005 .382698-1.572782$

H $2.7203384 .721028-2.785485$

C $3.861593-2.8022112 .560012$

O $4.355790-2.2836231 .319260$

C $3.473660-2.3971110 .199881$

C $3.151189-3.875592-0.068761$

C $2.620694-4.5762831 .187791$

C $3.489432-4.2873612 .416889$

H $4.713346-2.7339693 .249232$

C $2.729437-1.9401243 .129397$

H $2.545336-1.8555190 .413077$

O $4.313293-4.561965-0.531540$

H $2.407662-3.933451-0.870810$

H $2.589251-5.6588251 .014339$

O $1.293460-4.1415721 .460044$

H $2.989413-4.6270083 .331610$

O $4.710467-5.0243272 .306048$

C $3.219957-0.5454263 .438943$

H $1.871806-1.8664682 .455668$

H $2.348214-2.3646804 .065504$

H $5.009458-4.4528110 .138971$

H $\quad 0.749520-4.3520880 .683111$

H $4.480330-5.9685312 .270382$

$\begin{array}{llll}\text { O } & 2.743109 & 0.333696 & 2.512325\end{array}$

O $3.952455-0.2744924 .381785$

C 3.1268881 .6986772 .701715

H 2.8130962 .0509473 .688991

H 4.2084871 .8056872 .577095

H 2.6234962 .3009861 .941288

SCF Energy (B3LYP/6-31G**//MMFF)= -3245.90041375

\section{5}

MM̄FF Geometry

C $-2.9603703 .859738-0.453659$

C $-3.2167794 .285963 \quad 0.792971$

C $-3.9080763 .469691 \quad 1.861027$

O -3.5774032 .0653491 .734333$

C -5.4324013 .6227391 .744701$

C -6.1858112 .9140152 .852049$

C -6.8007791 .7227312 .711480$

C -6.2597303 .6586994 .159191$
C -6.8126310 .8441121 .489631$

C $-6.655477-0.6375651 .862359$

C $-6.636453-1.5340810 .615778$

C -6.748295-3.007964 1.007323

C $-6.657243-3.928451-0.218309$

O $-5.402522-1.309266-0.075440$

O $-6.461800-5.2708090 .251305$

C $-5.405665-3.546906-1.033293$

C $-7.958473-3.936657-1.028943$

O $-5.355394-4.273532-2.259480$

C $-5.277586-2.029091-1.310306$

C $-3.879427-1.735752-1.902562$

O $-6.274074-1.630595-2.252323$

C -2.3404791 .6776512 .150736$

O -1.4620532 .3865162 .616005$

C -2.2212310 .2244791 .908758$

C $-1.002743-0.3001651 .710288$

C $-0.709394-1.6991471 .399985$

C -1.799246-2.712205 1.634241

C $-3.554863-0.246227-2.068685$

C $-2.059030-0.042760-2.199902$

C $-0.118099-0.568130-3.658337$

O $-1.3992740 .483111-1.307071$

C $0.420078-1.870258-3.151940$

C $1.458848-1.978263-2.309491$

C $1.996906-3.299259-1.838570$

C $2.213913-3.366337-0.343223$

C $3.451637-3.4883620 .164854$

C $1.001536-3.3972980 .565247$

C $0.522765-2.0217720 .953153$

N $-1.543006-0.522861-3.390869$

H -3.311964 $2.879242-0.764782$

H -2.8752275 .2728111 .096502$

H -3.571790 3.8182282 .845882

H -5.7698093 .2563800 .767508$

H $-5.7050514 .686613 \quad 1.762533$

H $-7.357121 \quad 1.3325113 .562823$

H -6.8471303 .1215624 .911460$

H -5.2573973 .8101134 .571903$

H -6.7279844 .6375564 .012966$

H -7.7706910 .9846210 .975150$

H $-6.021186 \quad 1.1204750 .787039$

H $-5.723510-0.7699212 .427235$

H -7.485308 -0.9329822 .516711$

H -7.473464 -1.247934 -0.032008

H $-7.678136-3.1963241 .558469$

H $-5.932376-3.2653301 .697453$

H $-7.212070-5.4991410 .826722$

H $-4.529825-3.865180-0.450329$

H -8.796291 -4.269578 -0.404639

H -7.902147 -4.649822 -1.858740

H $-8.217890-2.956320-1.434043$

H -5.421887 -5.218480 -2.039021

H -3.115749 -2.178507-1.252120

H -3.784069-2.223236 -2.880996

H $-5.992322-0.803117-2.673947$

H -3.133399 -0.3574841 .849956$

H $-0.1396730 .364632 \quad 1.729834$

H -2.171647 -2.642104 2.662754

H -1.463062 -3.743615 1.498308

H $-2.637945-2.5500110 .951906$

H -4.050542 $0.172260-2.951336$

H $-3.8889990 .335165-1.202649$

H $\quad 0.3695760 .291507-3.187629$

H $0.015604-0.502425-4.742247$

H $-0.065168-2.774779-3.512361$

H $1.966820-1.078825-1.969442$

H $2.944922-3.472494-2.364536$

H $1.335308-4.123568-2.133680$

H $3.615275-3.5763501 .233625$

H $4.328494-3.501867-0.473855$

H $1.249586-3.9424891 .485368$

H $\quad 0.209538-3.967320 \quad 0.066218$

H $1.262286-1.2259360 .858586$

H $-2.159239-1.003970-4.039123$

C $-0.9368183 .810666-1.887088$

O $-0.1022533 .553076-0.741454$

C $1.2811603 .630412-1.143054$ 
C $1.2701873 .733836-2.660995$

C $-0.0262804 .486026-2.906225$

H $1.6651464 .560139-0.704114$

C $2.0230362 .423671-0.556454$

H $2.1487194 .254211-3.053434$

H $1.2287292 .738373-3.120063$

C $-0.5210894 .390604-4.338353$

H $\quad 0.1202845 .541487-2.640998$

C $3.4723062 .273491-1.059116$

O 2.0136202 .5729380 .866508

H $1.4710151 .500300-0.771086$

H $3.4390312 .041023-2.129764$

O $4.1600233 .519154-0.931579$

C $4.2798431 .171280-0.340981$

C $5.6389760 .933569-1.028796$

H $3.6984770 .241712-0.343987$

O 4.4907861 .5570221 .014816

H $5.4673250 .752242-2.096694$

H $6.2464241 .841818-0.926926$

H $0.2098934 .826181-5.027370$

H $-0.6863993 .349942-4.637681$

H - $1.4650814 .930638-4.461529$

C $-2.1514524 .633646-1.458915$

H 1.0841702 .6438111 .145137

H 4.2049143 .7282330 .017426

H $4.959908 \quad 0.831767 \quad 1.459934$

H -2.783393 $4.859173-2.324508$

H $-1.8230195 .586674-1.026918$

H -1.265084 2.838881-2.273211

C $7.593145-0.8842211 .601442$

O $6.867563 \quad 0.1152220 .873925$

C $6.421521-0.256567-0.434732$

C $7.618584-0.640951-1.319541$

C $8.474584-1.724147-0.657329$

C $8.808156-1.3745500 .796893$

H $7.969372-0.3627042 .491183$

C $6.683256-2.0220892 .074485$

H $5.738204-1.108706-0.356110$

O $8.4175890 .514900-1.566092$

H $7.243043-0.996054-2.286817$

H $9.404668-1.845549-1.225813$

O $7.765032-2.958238-0.712464$

H $9.254411-2.239474 \quad 1.300422$

O $9.801746-0.3452060 .808375$

C $5.720941-1.536453 \quad 3.132224$

H $6.110882-2.4823581 .265723$

H $7.273047-2.8275552 .528492$

H $9.1419120 .249786-2.158070$

H $8.330425-3.639878-0.311137$

H 9.4023940 .4582510 .432457

O $4.539409-1.1711052 .559275$

O $5.996673-1.4719754 .323429$

C $3.540915-0.6804203 .459593$

H $3.209144-1.4836894 .123900$

H $3.926944 \quad 0.1656134 .036397$

H $2.688609-0.3371672 .867646$

SCF Energy (B3LYP/6-31G**//MMFF) $=-3245.90671401$

0800646

MM̄FF Geometry

C $-1.685468-3.976788-1.126274$

C $-2.806116-3.599246-0.493692$

C $-4.180184-3.611696-1.111694$

O $-4.637504-2.240337-1.145581$

C $-5.142135-4.432082-0.234118$

C $-6.566521-4.463999-0.756819$

C -7.608964 -3.819858 -0.193209

C $-6.781393-5.309935-1.985466$

C -7.593211 -2.941522 1.026950

C $-7.772064-1.4653130 .650154$

C $-7.287915-0.5261351 .764706$

C $-7.5848310 .931061 \quad 1.406374$

C $-6.984867 \quad 1.9140982 .421822$

O $-5.875657-0.721703 \quad 1.899573$

O $\quad-7.0463973 .2192721 .825904$

C $-5.496917 \quad 1.5621712 .641480$

C -7.8305611 .9852933 .698226$

O -4.9352512 .3680453 .674371$
C $-5.253590 \quad 0.065130 \quad 2.922727$

C $-3.751166-0.3107802 .945180$

O $-5.786859-0.2562764 .213169$

C $-4.404706-1.537990-2.288113$

O $-3.936997-1.963133-3.332436$

C $-4.777224-0.128986-2.034025$

C $-4.3754740 .822946-2.889151$

C $-4.6336772 .259649-2.770940$

C $-5.4159992 .733378-1.574828$

C $-2.995259-0.0827211 .631614$

C $-2.2696561 .249221 \quad 1.547386$

C $-0.9959802 .656974-0.071103$

O $-2.096961 \quad 1.9860422 .514091$

C -1.884858 $3.745924-0.583351$

C $-1.6771004 .380799-1.747282$

C $-2.5191465 .523041-2.240381$

C $-3.0192745 .318868-3.653016$

C $-2.3704455 .856014-4.700057$

C $-4.3202994 .572870-3.841308$

C -4.153222 $3.081839-3.726998$

N $-1.793877 \quad 1.4933900 .265567$

H -1.739152 -4.333449 -2.151373

H -2.736343 -3.235522 0.529440

H $-4.153758-4.036919-2.122257$

H $-5.113300-4.0430000 .788163$

H $-4.779599-5.466313-0.157591$

H -8.588839 -3.920063 -0.658375

H -7.837101 -5.367276 -2.271604

H $-6.232326-4.898732-2.837939$

H -6.434886 -6.333264 -1.807265

H $-8.413752-3.2472451 .687915$

H -6.682887 -3.0836171 .612736$

H -7.204487 -1.252601 -0.264067

H -8.829118 -1.2746360 .427248$

H -7.784646 -0.8033872 .701980$

H -8.6649931 .0942581 .304072$

H $-7.158347 \quad 1.1529380 .419325$

H -6.5983763 .8388392 .427364$

H -4.9736951 .8421991 .720285$

H -8.8497852 .3134803 .461106$

H $-7.4340492 .729843 \quad 4.397029$

H -7.903533 1.0275914 .217789

H -5.2307582 .0058544 .526932$

H -3.2520440 .1766443 .791245$

H -3.691724 -1.388425 3.156103

H $-5.356658-1.0672204 .531450$

H $-5.3458690 .089928-1.138578$

H $-3.7933120 .525390-3.760678$

H $-6.4421152 .353037-1.615707$

H $-5.4775213 .820413-1.493601$

H $-4.9475742 .388510-0.647105$

H -2.216945 -0.851890 1.545979

H $-3.659309-0.1945410 .770186$

H $-0.2582082 .340285-0.815113$

H -0.4642993 .0044190 .820628$

H -2.7149484 .0512670 .049399$

H -0.826474 $4.094617-2.362150$

H -1.904257 $6.431677-2.194390$

H -3.370470 $5.710665-1.574292$

H $-2.7392045 .740590-5.713921$

H $-1.4523306 .418760-4.566554$

H $-4.7276334 .774751-4.841431$

H $-5.0707574 .974690-3.154944$

H $-3.5880602 .644401-4.551329$

H $-2.0347660 .852097-0.484477$

C $0.659237-2.982905-1.168314$

O $1.916175-3.091302-0.461723$

C $2.292075-1.7875820 .022663$

C $1.597382-0.814449-0.908874$

C $0.263304-1.501323-1.131195$

H $1.886929-1.7105061 .039944$

C $3.821115-1.6824920 .044255$

H $1.4916060 .185463-0.477802$

H $2.146227-0.726341-1.854841$

C $-0.431307-1.010004-2.391853$

H $-0.381940-1.304585-0.265481$

C $4.344467-0.2756970 .381457$

O $4.312642-2.5988941 .027733$ 
H $4.219935-2.012647-0.921404$ H $4.0564380 .426941-0.408970$ O $3.696813 \quad 0.186768 \quad 1.574845$ C $5.866129-0.1757450 .613540$ C $6.704464-0.699271-0.565483$ H $6.145877-0.7004831 .534672$ O $\quad 6.178544 \quad 1.2063250 .816481$ H $\quad 6.415076-1.729155-0.797309$ H $6.492216-0.074706-1.442373$ H $-0.6224870 .066210-2.333864$ H $0.179252-1.192992-3.282591$ H -1.389244 -1.512939-2.536029 C $-0.330626-3.934259-0.485809$ H $3.971841-3.4805010 .798020$ H $3.906555-0.4442342 .284767$ H 5.6402601 .5188191 .563843 H $-0.405701-3.6749100 .578787$ H $0.088848-4.948688-0.497653$ H $0.849218-3.315689-2.196211$ C $10.039807 \quad 0.877698 \quad 0.108426$ O $8.6547270 .679768-0.203043$ C $8.223150-0.680707-0.286042$ C $9.007860-1.419131-1.383215$

C $10.519160-1.294719-1.166639$ C $\quad 10.9403660 .156400-0.907342$ H $\quad 10.199274 \quad 1.957406-0.009753$ C $\quad 10.355968 \quad 0.515734 \quad 1.563933$ H $8.392672-1.1748680 .676864$ O $8.660565-0.882602-2.658417$ H $8.713332-2.475632-1.377718$

H $11.040725-1.676177-2.052760$

O $10.888480-2.115094-0.062070$

H $11.9813890 .202026-0.566984$

O $10.8708210 .887532-2.135033$

C 9.6295471 .4405512 .511578

H $10.099143-0.5169581 .816453$

H 11.4278680 .6233541 .768290

H $\quad 9.145650-1.388779-3.331953$

H $11.851739-2.0423590 .048427$

H $\quad 11.475675 \quad 0.460655-2.765347$

O $8.531230 \quad 0.815864 \quad 3.023489$

O 9.9850502 .5876572 .749132

C 7.7378711 .5998543 .919665

H 6.8699141 .0041374 .214383

H 8.3156321 .8483274 .814934

H 7.3855632 .5084773 .421894

SCF Energy (B3LYP/6-31G**//MMFF) $=-3245.90453756$

0800647

MM̄FF Geometry

C $0.3704174 .528456-0.247828$

C $-0.3326183 .436558-0.581763$

C $-1.3496933 .404725-1.692387$

O $-2.5622422 .886426-1.100551$

C $-0.8744292 .458254-2.806800$

C $-1.6808242 .568130-4.086775$

C $-2.5878941 .656516-4.489863$

C $-1.3645523 .759026-4.952672$

C $-3.0229500 .436704-3.728885$

C $-2.192994-0.794015-4.111761$

C $-2.389191-1.993454-3.171879$

C $-3.809881-2.557859-3.221444$

C $-3.996625-3.713203-2.226192$

O $-2.065412-1.567318-1.844510$

O $-5.404084-3.971953-2.109444$

C $-3.505491-3.252707-0.838486$

C $-3.356400-5.013308-2.725506$

O $-3.531843-4.3304240 .095258$

C $-2.100908-2.604520-0.858682$

C -1.808774 -1.960105 0.516135

O $-1.124106-3.612082-1.122993$

C $-3.7523893 .422269-1.468559$

O $-3.9368704 .282122-2.318757$

C $-4.8659982 .869553-0.659327$

C $-4.719847 \quad 1.8592390 .212421$

C -5.7488751 .3278091 .109752$

C -7.120419 1.9461091 .054056

C $-0.568097-1.0571890 .548930$
C $-0.349981-0.5421031 .957639$

C -1.3460091 .0138373 .631713$

O $0.458121-1.0667762 .719969$

C -2.5031280 .2926564 .252421$

C -3.6387860 .8906894 .643313$

C -4.8235810 .1427655 .188090$

C -6.0898360 .3880594 .391418$

C $-7.084397 \quad 1.1356234 .899152$

C $-6.241325-0.2914083 .044706$

C $-5.4024650 .351278 \quad 1.974198$

N -1.1876910 .5152722 .277056$

H $0.2151595 .453771-0.797291$

H $-0.1683712 .511119-0.034335$

H -1.512835 $4.419239-2.075374$

H $-0.8827811 .425638-2.437200$

H $0.1744872 .670162-3.056701$

H -3.086154 $1.802072-5.446620$

H -1.596422 $4.690306-4.427306$

H $-0.302353 \quad 3.764227-5.218212$

H -1.937315 $3.757269-5.886161$

H $-2.9740950 .623315-2.651721$

H $-4.0820800 .259175-3.949836$

H -2.417637 -1.089464 -5.144066

H $-1.129171-0.522469-4.083385$

H -1.669992 -2.762198 -3.478988

H $-4.065574-2.879025-4.238628$

H $-4.533928-1.774184-2.962615$

H $-5.742725-4.190759-2.994541$

H $-4.224642-2.507120-0.470242$

H -3.799321 -5.314832 -3.682290

H $-3.549627-5.839826-2.032620$

H -2.277600 -4.932208 -2.873352

H $-4.431386-4.7001170 .085188$

H -2.670492 -1.3493690.812162

H -1.695776 -2.743642 1.276561

H $-0.286610-3.363143-0.701882$

H $-5.8195563 .368052-0.801868$

H $-3.743952 \quad 1.387680 \quad 0.309577$

H -7.0710613 .0158971 .285259$

H -7.8244491 .5044001 .762672$

H -7.5570421 .8211260 .057020$

H $\quad 0.333037-1.594625 \quad 0.241289$

H $-0.680372-0.204529-0.130467$

H -1.5152162 .0929883 .566178$

H -0.4343450 .8307994 .208462$

H $-2.409944-0.7863294 .355539$

H -3.7341751 .9697694 .547694$

H $-4.9632520 .455722 \quad 6.230815$

H -4.631639 -0.9369005 .224551$

H $-8.006998 \quad 1.297046 \quad 4.351848$

H -6.9977801 .6066225 .872633$

H -7.293674 -0.329001 2.741153

H -5.945755 -1.344303 3.138381

H $-4.384082-0.0329731 .936972$

H -1.8598040 .8322541 .584938$

C 2.8038424 .6663850 .299823

O $3.1772853 .399166-0.281392$

C 4.5312723 .0939100 .101820

C 5.0976484 .3730650 .705170

C 3.8630874 .9593191 .361466

H 4.4727782 .3382690 .892974

C $5.3155672 .541759-1.092509$

H 5.9087844 .1749071 .412168

H $5.4787715 .052587-0.066107$

C 4.0023016 .4310871 .709274

H 3.6459284 .3911502 .276194

C $4.6872191 .282185-1.727007$

O $5.3774703 .561326-2.097138$

H $6.3499092 .340263-0.788722$

H $3.7418261 .536573-2.221908$

O $5.5718950 .819054-2.755073$

C $4.4514450 .124206-0.737200$

C $3.979968-1.163083-1.444022$

H $3.6970350 .425562-0.002088$

O $5.669622-0.123100-0.036334$

H $3.153527-0.918527-2.121886$

H $4.811675-1.559859-2.039388$

H 4.8214966 .5820832 .419856 
H 4.2128007 .0354070 .820482

H 3.0842776 .8133242 .166499

C 1.3856244 .5655740 .860273

H $6.0416853 .287432-2.751654$

H $5.5466851 .468519-3.477672$

H $5.496976-0.8174450 .621294$

H 1.1599275 .4307561 .493754

H $1.2940703 .669177 \quad 1.486498$

H $2.8521445 .413316-0.503796$

C $4.381847-3.656327 \quad 1.320224$

O $4.646012-2.6816280 .303388$

C $3.513472-2.254020-0.457797$

C $2.884064-3.452737-1.185744$

C $2.554673-4.593139-0.216444$

C $3.729816-4.9109710 .716596$

H $5.372958-3.9396531 .697543$

C $3.589613-3.0627462 .489958$

H $2.775062-1.8059450 .215096$

O $3.765713-3.935882-2.198320$

H $1.969549-3.120062-1.690366$

H $2.291849-5.486360-0.796332$

O $1.413688-4.2242990 .551597$

H $3.413851-5.5813191 .524342$

O $4.739096-5.598818-0.028279$

C $4.354465-1.9345003 .141091$

H $2.607767-2.6913852 .185819$

H $3.405420-3.8197013 .261156$

H $4.610270-4.163568-1.773074$

H $1.197666-4.9716381 .135019$

H $4.343661-6.419197-0.369402$

O $3.757762-0.7417032 .856736$

O $5.370240-2.0936043 .805587$

C 4.3899140 .4149393 .413032

H 3.7793071 .2874043 .165507

H 4.4527430 .3296984 .501995

H 5.3846090 .5459122 .976839

SCF Energy (B3LYP/6-31G**//MMFF) $=-3245.91403542$

08_00648

MM̄FF Geometry

C $-0.069345-4.1035250 .548858$

C $-1.277673-3.5561180 .349750$

C -2.039876 -3.609556-0.948259

O $-2.129118-2.240623-1.408550$

C $-3.453385-4.168710-0.689814$

C $-4.318940-4.315936-1.928022$

C $-5.454685-3.622725-2.151973$

C $-3.868308-5.358428-2.918876$

C $-6.108543-2.631952-1.229775$

C $-5.721161-1.187169-1.563078$

C $-6.012811-0.237289-0.392557$

C $-5.721310 \quad 1.210847-0.783344$

C $-5.881510 \quad 2.169406 \quad 0.405721$

O $-5.158636-0.625708 \quad 0.686789$

O -5.2810663 .4236780 .043452$

C -5.0833971 .6226401 .613152$

C -7.358853 2.4678020 .688094

O $\begin{array}{llll}-5.355831 & 2.409279 & 2.769823\end{array}$

C $-5.334070 \quad 0.122606 \quad 1.897293$

C $-4.366323-0.4909742 .936319$

O $-6.638797-0.0919922 .449413$

C -1.869255 -1.985664 -2.715597

O $-1.644420-2.809627-3.592079$

C - $1.873989-0.534846-3.011031$

C -1.872612 $0.429263-2.077082$

C $-1.8435201 .873102-2.335664$

C -1.788535 2.323621 -3.772800

C $-2.878163-0.4148002 .578791$

C $-2.1705790 .798028 \quad 3.158216$

C -0.0655472 .1120852 .924441$

O $\begin{array}{llll}-2.633916 & 1.475799 & 4.071548\end{array}$

C -0.4949833 .3387492 .185183$

C 0.2230373 .9039591 .204268

C -0.2091685 .1282120 .453800$

C $-0.5256274 .836175-0.995583$

C $0.3267965 .184361-1.973915$

C -1.873338 4.227476 -1.315000

C $-1.8587852 .722934-1.286830$
N -0.9373641 .0118742 .558682$

H $\quad 0.426093-4.632634-0.260773$

H -1.749233 -3.015636 1.167697

H -1.499843 -4.234189-1.669571

H -3.944838 -3.524494 0.046573

H $-3.375708-5.156577-0.215465$

H -5.997905 -3.802280 -3.078576

H $-4.568876-5.467634-3.753717$

H -2.893001 -5.107237 -3.342496

H -3.790134 -6.334325 -2.428206

H $-7.196960-2.741288-1.319882$

H $-5.890075-2.874539-0.185725$

H -4.651395 -1.142497 -1.793921

H -6.262851-0.863191-2.460063

H -7.059985 -0.356973 -0.091298

H $-6.3584041 .524280-1.619836$

H $-4.6902331 .287859-1.150192$

H $-5.7112343 .739456-0.769684$

H $-4.0239021 .772627 \quad 1.371071$

H -7.822155 2.958615 -0.176442

H -7.4699123 .1679641 .523295$

H -7.9430231 .5708180 .904536$

H -4.7331892 .1416403 .466301$

H -4.564746 -0.075973 3.931776

H -4.619069-1.558449 3.017666

H -7.0439760 .7700572 .635349$

H -1.839366 $-0.298896-4.070114$

H $-1.8878140 .146180-1.026147$

H -2.689914 $2.006801-4.308972$

H $-0.9116831 .900969-4.276064$

H $-1.7131103 .406356-3.890297$

H -2.372278 -1.288693 3.008789

H $-2.729858-0.4524081 .494229$

H $\quad 0.958873 \quad 1.8162872 .677020$

H -0.1275272 .2856334 .003619$

H -1.446365 3.7809062 .471582

H 1.1746903 .4641020 .920551

H 0.5982295 .8681820 .527962

H -1.079760 5.5998850 .926728

H $0.0914105 .007345-3.017866$

H $1.2818095 .649249-1.752431$

H -2.241207 $4.608642-2.273451$

H $-2.6154524 .580347-0.587350$

H $-1.8980172 .301356-0.282857$

H $-0.6662950 .440702 \quad 1.763486$

C $1.806816-3.0046631 .786406$

O $2.917619-3.5750761 .065820$

C $4.150162-3.0957501 .657516$

C $3.738918-2.0972872 .732368$

C $2.379135-2.6200983 .146702$

H $4.624703-3.9739052 .112077$

C $5.033094-2.5177430 .543914$

H $4.452342-2.0537743 .560600$

H $3.643346-1.0874632 .313027$

C $1.552648-1.5980093 .907427$

H $2.515162-3.5162523 .766563$

C $6.232746-1.6828881 .028513$

O $5.502682-3.602166-0.263457$

H $4.398527-1.918098-0.112159$

H $5.901342-0.8502881 .658473$

O $7.076169-2.4995611 .850202$

C $7.115014-1.125735-0.104495$

C $6.380639-0.341999-1.201721$

H $7.666259-1.946871-0.579355$

O $8.112619-0.2736460 .466146$

H $7.1203170 .119694-1.869701$

H $5.837176-1.055957-1.835452$

H $2.056179-1.3118754 .836615$

H $1.400858-0.6901183 .315846$

H $\quad 0.568693-1.9994594 .167667$

C $0.659733-4.0111061 .861650$

H $6.113404-4.1293710 .279704$

H $6.578049-2.7301882 .652005$

H $8.582989-0.7905901 .142513$

H -0.043154 -3.724912 2.652661

H $1.039637-5.0088242 .112176$

H $1.494356-2.1041861 .239515$

C $3.6572212 .120870-1.758121$ 
O $4.744377 \quad 1.206943-1.947184$

C $5.3805180 .740814-0.747482$

C $5.992631 \quad 1.9270530 .006743$

C 4.9314042 .9970880 .294665

C $4.1083933 .349424-0.951241$

H $3.4003712 .458785-2.770655$

C 2.421126 1.419963-1.182094

H $4.6218000 .269976-0.118027$

O $7.0479362 .488833-0.768364$

H $6.419071 \quad 1.5772340 .952914$

H $5.428507 \quad 3.8982190 .674194$

O 4.0718202 .5134851 .323608

H $3.2315513 .948287-0.680117$

O $4.9117334 .155061-1.819366$

C $\quad 1.995090 \quad 0.280293-2.077897$

H $2.5848861 .034275-0.171410$

H $1.5767222 .114201-1.113552$

H $7.4357903 .216186-0.252904$

H 3.4501443 .2275421 .544109

H $5.1541124 .960435-1.331520$

O $2.299103-0.910891-1.489780$

O $1.4855490 .433556-3.180192$

C $2.030314-2.081336-2.267228$

H $2.414180-1.973470-3.286191$

H $2.536384-2.927184-1.794679$

H $0.956554-2.277079-2.277037$

SCF Energy (B3LYP/6-31G**//MMFF) $=-3245.90182273$

0800649

MM̄FF Geometry

C $-2.658615-3.548769-0.360060$

C $-1.893648-2.462145-0.535409$

C $-0.909867-2.284634-1.659234$

O $0.366871-1.929968-1.072930$

C -1.334882 -1.121931-2.573735

C $-0.878563-1.326651-4.003589$

C $0.274363-0.866728-4.525667$

C $-1.838265-2.107126-4.865909$

C $1.367631-0.096286-3.839742$

C $2.647252-0.938422-3.766899$

C $3.862671-0.135299-3.282871$

C $5.088178-1.047861-3.175786$

C $6.312726-0.305225-2.620841$

O $3.565214 \quad 0.437445-2.006117$

O $7.308218-1.281638-2.279010$

C $5.9028790 .442675-1.334108$

C $6.9651700 .574609-3.692502$

O $6.9738101 .253215-0.854932$

C $4.6066641 .275838-1.485416$

C $4.1626671 .797001-0.100730$

O $4.8720862 .388961-2.340806$

C $1.160149-2.939630-0.626238$

O $0.952773-4.138066-0.726839$

C $2.326770-2.3242640 .045120$

C $3.258878-3.1113460 .600652$

C $4.440454-2.6389881 .315422$

C $5.693267-3.3885560 .954836$

C $2.7963792 .493446-0.088499$

C 2.4782572 .9691831 .314895

C 1.8926852 .0909113 .572174

O 2.6589774 .1346951 .658960

C 3.1783851 .6325194 .184195

C 3.3284880 .4605454 .819254

C $4.650397-0.0326675 .333453$

C $5.137076-1.2598544 .592268$

C $5.336771-2.4242905 .232378$

C $5.458023-1.1200283 .116730$

C $4.356378-1.6647652 .243863$

N $2.024397 \quad 1.9487762 .134213$

H -2.601548 -4.369670 -1.069835

H -1.979418 -1.6483790 .175566$

H -0.805612 -3.211901-2.236999

H $-0.954505-0.169385-2.187773$

H -2.428508 -1.017168 -2.585888

H $\quad 0.474300-1.075097-5.576510$

H -1.462194 -2.251720 -5.884303

H -2.017710 -3.098311 -4.437284

H $-2.795904-1.581352-4.938670$
H $1.555410 \quad 0.816161-4.418474$

H $1.0761030 .225448-2.836019$

H $2.469120-1.781528-3.088362$

H $2.873910-1.364385-4.752975$

H $4.0449320 .670615-4.003701$

H $5.322852-1.503237-4.146048$

H $4.855482-1.885065-2.502598$

H $\quad 6.933938-1.876092-1.607205$

H $5.733843-0.317317-0.560013$

H $7.280917-0.036783-4.546595$

H $7.8775831 .048857-3.314441$

H $6.3016711 .354258-4.072187$

H $7.0151612 .048774-1.412376$

H $4.1183140 .953176 \quad 0.598842$

H $4.9120092 .497350 \quad 0.290349$

H $4.2161523 .083185-2.174271$

H $2.386069-1.2418390 .085511$

H $3.175269-4.1938960 .525088$

H $5.802394-3.449597-0.134311$

H $5.655624-4.4085801 .352646$

H $6.604115-2.9161621 .328260$

H $2.7765123 .366566-0.748193$

H $2.0058911 .815728-0.428364$

H 1.0413241 .4822273 .889770

H 1.6970053 .1349113 .834944

H 4.0403862 .2833244 .055747

H $2.471597-0.1976994 .940392$

H $4.538626-0.2422586 .404633$

H 5.4183860 .7475945 .258141

H $5.708192-3.2981434 .706939$

H $5.132434-2.5304706 .292455$

H $6.420297-1.6030992 .921937$

H $5.612615-0.0631812 .866558$

H $3.383019-1.2059002 .415506$

H 1.9706301 .0070121 .756255

C $-5.032504-3.3403050 .412090$

O $-5.129809-1.9092290 .256451$

C $-6.400949-1.4694960 .773758$

C $-7.182419-2.732423 \quad 1.122724$

C $-6.067482-3.6965981 .476180$

H $-6.194106-0.9188541 .698304$

C -7.096254 -0.559886 -0.243271

H $-7.885815-2.5682281 .944907$

H -7.753923 -3.1066630 .265285$

C $-6.499658-5.1524291 .461010$

H $-5.684778-3.4422282 .473615$

C $-6.2913290 .704523-0.611723$

O $-7.305430-1.313946-1.445293$

H $-8.091484-0.2850480 .125611$

H $\quad-5.3587750 .414650-1.107486$

O $-7.0529521 .417278-1.597476$

C $-5.9980201 .649799 \quad 0.573724$

C -5.3510522 .9833270 .148400$

H $\quad-5.3607591 .1468291 .308832$

O $\quad-7.2390431 .9477691 .220015$

H $-5.8959493 .396999-0.708242$

H -5.4820793 .7098830 .961954$

H -7.296973 -5.3242842 .191463$

H $-6.876360-5.4490670 .476345$

H $-5.663661-5.8118151 .714049$

C $-3.602407-3.7097480 .799705$

H -7.738661 -0.723564 -2.085380

H $-7.8689041 .727842-1.168393$

H -7.0379462 .4740392 .012581$

H -3.547912 -4.751645 1.134757

H $-3.269642-3.0851861 .638571$

H -5.301859-3.789835 -0.553122

C -1.7275842 .7458901 .013212$

$\begin{array}{llll}\text { O } & -3.156917 & 2.849747 & 1.078764\end{array}$

C $-3.8537552 .849019-0.175113$

C $-3.3638843 .999520-1.066429$

C $-1.8447903 .951444-1.245773$

C -1.1239083 .8248430 .096325$

H -1.386059 2.9548112 .035512

C -1.2896171 .3202990 .654005$

H $-3.6747671 .899556-0.687833$

O $-3.7456525 .257613-0.513964$

H $-3.8532923 .919760-2.044289$ 
H -1.506924 $4.864939-1.750533$ O $-1.4900772 .838503-2.061963$ H $-0.0609943 .623129-0.071994$ O -1.2050515 .0874770 .763571$ C $-1.3701000 .414162 \quad 1.864851$ H $-1.899807 \quad 0.865818-0.129029$ H -0.2510381 .3312160 .307851$ H $-3.375190 \quad 5.3136780 .383353$ H -1.935382 $2.949024-2.919201$ H $-0.6690385 .026911 \quad 1.572634$ O $-\begin{array}{llll}0.265882 & -0.387146 & 1.913638\end{array}$ $\begin{array}{lllll}\text { O } & -2.306636 & 0.394125 & 2.652835\end{array}$ C $-0.225066-1.3201732 .997736$ H $-0.301605-0.7977323 .955990$ H -1.029801 -2.053499 2.891666 H $\quad 0.734359-1.8426802 .960876$ SCF Energy (B3LYP/6-31G**//MMFF) $=-3245.90664618$

0800650

MM̄FF Geometry

C $-2.4987312 .989483-0.438472$

C $-3.5316193 .385850 \quad 0.320956$

C $-4.2202552 .592151 \quad 1.405773$

O -3.4975341 .3877081 .735850$

C -5.6059382 .1427200 .909680$

C -6.5816581 .8487222 .030193$

C -6.8342610 .6278212 .540624$

C -7.3395183 .0491952 .537808$

C $-6.156453-0.6766442 .227164$

C $-7.022220-1.6244421 .385528$

C $-6.865514-1.387797-0.124428$

C $-7.807839-2.293451-0.918515$

C $-7.586296-2.158003-2.432812$

O $-5.505539-1.683890-0.464174$

O $-8.269905-3.243529-3.077664$

C $-6.082576-2.328023-2.734090$

C -8.199722 $-0.867228-2.988070$

O $-5.805154-2.069867-4.108815$

C $-5.166861-1.461797-1.837499$

C $-3.688988-1.880888-2.039723$

O $-5.318428-0.089019-2.203139$

C $-2.496527 \quad 1.4507142 .649888$

O -2.0573532 .4430203 .205239$

C -2.0264320 .0617862 .846143$

C $-0.872051-0.1869133 .479509$

C $-0.297085-1.5153553 .707658$

C -1.100388 -2.7097573 .257685$

C $-2.710604-1.274304-1.022355$

C $-1.272153-1.686671-1.295960$

C $1.047989-1.320838-0.450017$

O $-0.953060-2.446228-2.206989$

C $1.423578-2.4237180 .489186$

C $2.383033-2.3007901 .419544$

C $2.811155-3.4116882 .336290$

C $2.957624-2.9697053 .777333$

C $4.171721-2.7750824 .319200$

C $1.705076-2.8411774 .615705$

C $0.917783-1.6027964 .288459$

N $-0.386237-1.102097-0.400464$

H -2.070338 $2.001016-0.304536$

H $-3.945853 \quad 4.3782180 .149727$

H -4.3113263 .2221272 .299341$

H $-5.495128 \quad 1.2586260 .272326$

H -6.0575182 .9119520 .268541$

H $-7.593841 \quad 0.549576 \quad 3.318171$

H $-8.0341702 .793895 \quad 3.345115$

H -6.646251 3.8019282 .926577

H -7.924164 3.4994361 .728933

H $-5.179108-0.5278501 .762412$

H -5.950202 -1.162635 3.189794

H $-6.700426-2.6497991 .610686$

H -8.075864 -1.543255 1.678778

H $-7.079044-0.336727-0.344570$

H $-8.854529-2.085926-0.663128$

H -7.632682 -3.341015 -0.635761

H $-9.210831-3.190301-2.836893$

H -5.833776 -3.384595 -2.561538

H $-9.279554-0.844084-2.797869$
H $-8.086574-0.812735-4.076348$

H $-7.7690780 .035744-2.550539$

H $-6.389452-2.642378-4.634726$

H -3.609649 -2.973052-1.957839

H -3.364322 -1.606216 -3.051481

H $-4.5100450 .394573-1.972558$

H -2.645895 -0.7297602 .439150$

H -0.2874850 .6543043 .849045$

H -2.051534 -2.756580 3.799816

H $-0.596847-3.6646933 .417990$

H $-1.312031-2.6537682 .184283$

H -2.757099 -0.182050 -1.050266

H -2.967592 -1.604894 -0.009712

H $1.528125-0.376237-0.180343$

H $1.343393-1.586706-1.470026$

H $\quad 0.907213-3.3739670 .377845$

H $2.914808-1.3561381 .504898$

H $3.772279-3.7917941 .965366$

H $2.115066-4.2582132 .288703$

H $4.288248-2.4857975 .358411$

H $5.077704-2.8958723 .734723$

H $1.966616-2.7865155 .681111$

H $1.119154-3.7594254 .522951$

H $1.424272-0.6795254 .573627$

H $-0.749859-0.5208750 .348534$

C $-0.3923964 .072435-1.379169$

O $0.3272902 .847563-1.607585$

C $1.5319512 .868891-0.805704$

C $1.5783904 .240288-0.139173$

C $0.1069464 .592526-0.034973$

H $1.4005182 .083486-0.051783$

C $2.7308002 .550811-1.712068$

H 2.0830424 .2154900 .831499

H $2.1004584 .966704-0.774651$

C -0.1554416 .0695680 .195057$

H -0.3228484 .0157530 .793554$

C $4.0890412 .645363-0.989891$

O $2.5447331 .224541-2.211066$

H $2.7213433 .219664-2.580894$

H $4.1565493 .649650-0.553200$

O 4.1109601 .7304370 .100030

C $5.3391932 .473905-1.879816$

C $5.4698251 .193555-2.725351$

H $6.2258902 .554256-1.238599$

O $5.3731913 .592803-2.774628$

H $4.6487671 .135539-3.448427$

H $6.3733291 .315302-3.339792$

H $0.297436 \quad 6.3985721 .136049$

H $0.2620996 .682264-0.610999$

H -1.230176 6.2699960 .249116

C $-1.8979943 .843713-1.519822$

H $1.6796601 .198753-2.654838$

H 4.9392081 .8789930 .586569

H $6.2153123 .556754-3.259693$

H -2.090992 $3.339987-2.475259$

H $-2.4029734 .816197-1.562282$

H $-0.0830844 .756032-2.181904$

C $7.089904-1.176244-0.335713$

O $6.799486-0.044972-1.167549$

C $5.590502-0.116213-1.930563$

C $5.607103-1.342344-2.858216$

C $5.874505-2.627747-2.068942$

C $7.090505-2.482799-1.148255$

H $8.114623-1.0078310 .020002$

C $6.167695-1.2276180 .890178$

H $4.736499-0.205142-1.255206$

O $6.611612-1.177170-3.856935$

H $4.640119-1.407097-3.371540$

H $6.048397-3.456334-2.766047$

O $4.734216-2.957602-1.281455$

H $7.156823-3.336418-0.463397$

O $8.280892-2.491475-1.941678$

C $6.382633-0.054547 \quad 1.823189$

H $5.118528-1.2171130 .589331$

H $6.361620-2.1390691 .466363$

H $6.574747-1.953106-4.441684$

H $3.977181-3.054968-1.883933$

H $8.308883-3.338051-2.419060$ 
O $5.363506-0.0219822 .732186$

O $7.321645 \quad 0.727578 \quad 1.773092$

C 5.4200651 .0409183 .688192

H $5.387763 \quad 2.0097053 .180784$

H 4.5466280 .9574424 .340452

H 6.3240670 .9529264 .298186

SCF Energy (B3LYP/6-31G*//MMFF) $=-3245.90472636$

08_00651

MM̄FF Geometry

C $1.781199 \quad 4.6164901 .067522$

C 2.8398423 .8018531 .183212

C 3.9135533 .6836090 .132826

O $3.753877 \quad 2.385251-0.488039$

C $5.304473 \quad 3.759851 \quad 0.779280$

C $6.4293063 .682499-0.233992$

C $7.1665722 .580166-0.473981$

C $6.7248994 .965680-0.966466$

C 7.0090581 .2233540 .153363

C $6.9407840 .115975-0.907716$

C $6.702488-1.254810-0.260055$

C $6.829928-2.378558-1.287544$

C $6.563345-3.752838-0.652751$

O $5.378761-1.2581540 .282752$

O $6.366893-4.696859-1.719691$

C $5.237517-3.6958290 .138813$

C $7.778136-4.256248 \quad 0.135254$

O $5.029407-4.8875760 .892032$

C $5.053526-2.437821 \quad 1.026547$

C $3.570767-2.3189201 .440338$

O $5.793409-2.5112142 .244661$

C $2.8564372 .310581-1.510503$

O $2.2655733 .239255-2.039901$

C $2.6534120 .883219-1.838196$

C $1.5226890 .532228-2.469621$

C $1.101610-0.825683-2.816058$

C $2.098397-1.936833-2.628225$

C $3.235587-1.0400322 .217532$

C $1.745017-0.9849272 .487933$

C $-0.433135-0.6402711 .319864$

O $1.273546-1.3104343 .574884$

C $-0.824583-1.7931630 .447503$

C $-1.748238-1.705062-0.521373$

C $-2.126086-2.846594-1.422785$

C $-2.162368-2.454584-2.885429$

C $-3.331544-2.311750-3.532701$

C $-0.843550-2.301713-3.617074$

C $-0.153837-1.008156-3.276758$

N $1.015710-0.584447 \quad 1.378580$

H 1.6897235 .2582830 .194870

H 2.9035373 .1323082 .038175

H $3.8195114 .475538-0.620879$

H 5.4111962 .9701181 .531417

H 5.4037854 .7006401 .337827

H $7.9755202 .652984-1.199843$

H $7.5742124 .867914-1.650989$

H $5.8599175 .278420-1.559333$

H $6.9661855 .762110-0.254928$

H 7.8722751 .0452050 .805950

H 6.1181851 .1681150 .783845

H $6.1267610 .339148-1.609503$

H $7.8774930 .100822-1.478276$

H $7.429803-1.3889310 .549338$

H $7.817823-2.363348-1.764472$

H $6.102045-2.219449-2.095561$

H $7.157861-4.680553-2.285253$

H $4.439311-3.685211-0.616828$

H $8.640122-4.381264-0.531650$

H $7.590666-5.2462040 .564817$

H $8.085810-3.5804110 .934679$

H $5.591721-4.8462021 .683418$

H $2.942886-2.3519270 .543722$

H $3.285571-3.1808262 .057903$

H $6.593763-3.0357822 .102114$

H $3.3967390 .168263-1.506447$

H $0.8086191 .314693-2.726011$

H $3.057594-1.682198-3.093324$

H $1.787490-2.880213-3.082158$
H $2.263870-2.122990-1.564496$

H $3.763899-1.0113853 .176795$

H $3.526684-0.1420961 .660585$

H -0.7775040 .3144870 .912668$

H $-0.843962-0.7705692 .324953$

H $-0.322222-2.742846 \quad 0.616648$

H -2.269636 $-0.762402-0.666650$

H -3.112603 -3.209509-1.109311

H $-1.441817-3.694962-1.297575$

H $-3.366916-2.053691-4.585766$

H $-4.280233-2.443312-3.022892$

H $-1.009837-2.312033-4.702056$

H $-0.231320-3.181767-3.402637$

H $-0.788245-0.129728-3.407316$

H $1.509822-0.3581650 .520858$

C - 0.4403203 .6391721 .638705

$\begin{array}{llll}\text { O } & -0.996718 & 4.003417 & 0.362139\end{array}$

C $-2.404158 \quad 3.677526 \quad 0.354130$

C -2.689662 2.9923351 .685708

C -1.629505 3.5883192 .590596

H $-2.9314594 .637798 \quad 0.297206$

C $-2.7077812 .824924-0.886910$

H -3.708461 3.1771652 .039797

H $-2.553968 \quad 1.9069671 .604876$

C $-1.384503 \quad 2.7740603 .849172$

H -1.9345534 .6061472 .867714$

C $-4.1586342 .300981-0.927743$

O $-2.4346103 .643662-2.026810$

H $-2.0043861 .984044-0.926475$

H $-4.3385121 .750518-0.000413$

O $-5.0496143 .415076-0.939303$

C $-4.4516701 .364667-2.118017$

C $-5.8769230 .777704-2.143697$

H $-3.7253220 .545007-2.101982$

O $-4.252358 \quad 2.068270-3.345256$

H $-6.6047601 .571450-2.353623$

H $-5.9260730 .101677-3.007766$

H -2.299583 2.7048604 .446370

H $-1.060704 \quad 1.7550553 .611551$

H $-0.611134 \quad 3.2367164 .469932$

C $0.6428304 .638786 \quad 2.047432$

H $-2.5467253 .092180-2.819204$

H $-5.9491993 .079586-0.790304$

H $-4.3704831 .432762-4.071846$

H $1.009174 \quad 4.4133603 .055604$

H 0.2365505 .6571902 .070697

H $-0.0028012 .638561 \quad 1.522015$

C $-5.481813-1.7274050 .593472$

O $-5.308950-1.003822-0.632224$

C $-6.2829590 .016621-0.870767$

C $-7.681384-0.609259-0.987009$

C $-8.019520-1.427217 \quad 0.264302$

C $-6.877477-2.3725100 .659957$

H $-4.747495-2.5403880 .540497$

C $-5.116922-0.8445541 .790774$

H $-6.2839830 .721265-0.032614$

O $-7.731361-1.453642-2.135297$

H $-8.4184660 .190433-1.128268$

H $-8.928087-2.0122900 .076193$

O $-8.299340-0.5251241 .330530$

H $-7.050741-2.7767281 .662869$

O $-6.889021-3.492783-0.232581$

C $-4.729029-1.6684632 .992261$

H $-4.274915-0.1858061 .543061$

H -5.927502 -0.1709392 .083352$

H $-8.635859-1.804346-2.202024$

H $-8.538375-1.0562352 .109337$

H -6.671437 -3.165785 -1.122564

O $-3.427379-2.059442 \quad 2.885344$

O $-5.494881-1.9431893 .906551$

C $-2.930784-2.857963 \quad 3.963479$

H -2.986409-2.301533 4.903992

H $-3.494697-3.792984 \quad 4.032708$

H $-1.883511-3.0937873 .756859$

SCF Energy (B3LYP/6-31G*//MMFF) $=-3245.89548107$

0800652

MM̄FF Geometry 
C $0.194591-2.960525-2.144364$ C $-0.682678-2.213206-2.832220$ C $-0.587596-0.731988-3.114123$ O $0.592252-0.151959-2.517153$ C -1.805466 -0.024979-2.499248 C $-1.8721881 .449771-2.833384$ C $-1.5790272 .437252-1.965273$ C $-2.3617701 .777610-4.219555$ C -1.079502 $2.291640-0.553291$ C $-0.0790993 .403727-0.209240$ C 0.4307603 .2945201 .234805 C 1.1967914 .5624371 .619131 C $1.7967294 .463373 \quad 3.027251$ O 1.2928172 .1538381 .322891 O $2.736868 \quad 5.537107 \quad 3.183675$ C 2.5912303 .1479543 .130482 C 0.7365754 .6506524 .118762 O $3.0544962 .938176 \quad 4.463147$ C 1.8090661 .9052492 .638970 C 2.7763090 .7014002 .560035 O 0.7638341 .6109183 .566510 C $1.696380-0.014640-3.297316$ O $1.828580-0.363105-4.459204$ C $2.7398340 .629687-2.468975$ C $4.0094360 .639123-2.900604$ C $5.1613041 .192566-2.185504$ C $4.8908111 .982091-0.932502$ C $2.181917-0.5643151 .935901$ C $3.273749-1.6049051 .785154$ C $5.011171-2.4180740 .192776$ O $3.596864-2.3364892 .718728$ C $6.248086-1.5771360 .263983$ C $7.175391-1.535933-0.705229$ C $8.412859-0.685593-0.637385$ C $8.6260190 .150742-1.881900$ C $9.626895-0.124553-2.735280$ C $7.7269701 .351193-2.099543$ C $6.3948200 .958739-2.680118$ N $3.867737-1.5911140 .533737$ H $1.098452-2.507415-1.749898$ H -1.572817 -2.699719 -3.228298 H $-0.581775-0.584049-4.200783$ H - $1.795058-0.166070-1.412820$ H -2.733777 - $0.498211-2.846138$ H -1.716048 $3.464013-2.301616$ H $-2.4511102 .856684-4.384783$ H -1.672303 $1.386170-4.973811$ H $-3.3500301 .336978-4.387514$ H -1.938797 2.3579270 .124434 H $-0.6029411 .319958-0.392055$ H $\quad 0.7687313 .350909-0.904940$ H $-0.5645874 .377670-0.352289$ H -0.4285403 .1513231 .900530$ H 0.5517625 .4466651 .540723 H $2.015243 \quad 4.7245610 .903371$ H $2.260406 \quad 6.3744443 .049526$ H 3.4887103 .2596692 .505399 H 0.2568975 .6321204 .021907 H 1.1899774 .6365275 .116042 H -0.0507893 .8948164 .086885$ H 3.5498653 .7327884 .726117 H $3.6563600 .988457 \quad 1.972124$ H 3.1366630 .4496393 .565980 H 0.5479760 .6668643 .514743 H $2.4399131 .049961-1.515496$ H $4.2361210 .168325-3.856509$ H $4.4871761 .332158-0.151916$ H $4.1688272 .783149-1.128668$ H $5.7777502 .467778-0.521094$ H $1.396541-0.9934592 .566289$ H $1.731653-0.3564930 .958961$ H $4.837428-2.814065-0.812568$ H $\quad 5.087230-3.2539440 .895092$ H $6.390353-0.9756011 .159095$ H $7.048403-2.158112-1.588448$ H $9.267352-1.357528-0.484055$ H $8.391669-0.0222620 .236212$ H $9.8014950 .483991-3.616547$
H $10.291494-0.967156-2.575426$

H $8.1982472 .051917-2.801318$

H $7.6434151 .896746-1.156239$

H $6.4774010 .395310-3.611184$

H $3.560415-0.910019-0.153350$

C $-0.085658-4.775446-0.381959$

O $\quad-1.114615-3.996780 \quad 0.267944$

C $-0.576085-3.4821371 .501129$

C $0.631233-4.3572961 .812945$

C $1.199044-4.5595530 .421924$

H $-0.220380-2.4677101 .285991$

C -1.642682 -3.426725 2.598201

H $1.328880-3.8773182 .503527$

H $0.339154-5.3234252 .239118$

C $2.191708-5.7042220 .325592$

H $1.700358-3.6351190 .115317$

C $-2.891036-2.5927762 .234978$

O $-2.105088-4.7541202 .881014$

H -1.203433 -3.0452263 .528452$

H -3.466653 -3.141626 1.485404

O $-3.700502-2.5513653 .417943$

C $-2.594320-1.1530011 .762483$

C $-3.802632-0.2026901 .677289$

H -2.084602 -1.1827760 .793810$

O $-1.672196-0.5740222 .697199$

H $-4.451971-0.3072722 .555133$

H -3.435696 0.8306111 .773143

H $3.055941-5.5179800 .971688$

H $1.741431-6.6542430 .632113$

H $2.555458-5.818163-0.700388$

C $-0.009970-4.426704-1.872152$

H -1.427297 -5.1942063 .420754$

H -4.614400 -2.369293 3.140442

H -1.376204 0.2755712 .327696

H -0.942908 -4.757144 -2.346895

H $\quad 0.801930-4.990732-2.345108$

H $-0.401291-5.823612-0.295081$

C $-6.4967990 .996432-0.581762$

$\begin{array}{lllll}0 & -5.633565 & 0.772038 & 0.539445\end{array}$

C $-4.636425-0.250610 \quad 0.384492$

C $-5.303922-1.5947920 .075851$

C $-6.266153-1.495877-1.112797$

C $-7.214618-0.300441-0.985413$

H $-7.2580711 .696582-0.213171$

C $-5.7633151 .675897-1.747923$

H $-3.9780950 .030398-0.442043$

O $-6.012219-2.0665821 .219184$

H $-4.532612-2.331985-0.165942$

H $-6.848375-2.423414-1.175117$

O $-5.500118-1.382896-2.308824$

H $-7.762625-0.138576-1.920787$

O $-8.186891-0.584106 \quad 0.024951$

C $-5.3298313 .086981-1.407990$

H $-4.869077 \quad 1.126893-2.049157$

H $-6.4430151 .740133-2.605813$

H $-6.655994-1.3833501 .473543$

H -6.123078 -1.351184 -3.054667

H $-8.673712-1.379211-0.251761$

O $\quad-4.9291203 .714265-2.553731$

O $-5.326653 \quad 3.575757-0.286803$

C $-4.4849605 .065221-2.393459$

H -3.672296 $5.119383-1.662614$

H $-4.1105595 .416228-3.358708$

H -5.321943 $5.699933-2.087786$

SCF Energy $(B 3 L Y P / 6-31 G * * / / M M F F)=-3245.90633885$

0800653

MM̄FF Geometry

C -1.716997 -0.226616 -2.855729

C $-0.447417-0.656440-2.865276$

C $0.028618-1.865073-2.103085$

O $0.985883-1.362445-1.144121$

C $0.718152-2.856099-3.052021$

C $1.116081-4.153700-2.377762$

C $2.366736-4.455185-1.975850$

C $0.002461-5.153997-2.204551$

C $3.582925-3.573641-2.043510$

C $4.283651-3.493340-0.680372$ 
C $5.401888-2.441787-0.677556$

C $6.165176-2.4740220 .646672$

C $7.239263-1.3791530 .705966$

O $4.794839-1.158267-0.849427$

O $7.683763-1.2683872 .067255$

C $6.597662-0.0253010 .333470$

C $8.474396-1.755520-0.120773$

O $7.588151 \quad 0.9919970 .206644$

C $5.717749-0.066512-0.940897$

C $4.8884981 .230815-1.041558$

O $6.503071-0.139416-2.131453$

C $0.854429-1.7342890 .156496$

O $0.104648-2.5778390 .618484$

C $1.754676-0.8631930 .940862$

C $1.594943-0.7434872 .266417$

C $2.3582990 .169363 \quad 3.116652$

C $2.680777-0.3939094 .472285$

C $3.918478 \quad 1.262201-2.230673$

C $3.1710462 .581013-2.236413$

C $1.4597653 .833849-0.927794$

O $3.5298323 .523494-2.938517$

C 2.1705684 .3439710 .289020

C 1.6128144 .4145441 .507031

C 2.3646964 .8132322 .744660

C 2.4563263 .6881293 .756740

C 1.8042223 .7563944 .929498

C 3.3656182 .5154553 .445474

C 2.6496921 .4202722 .704853

N $2.1185012 .604127-1.333579$

H -2.465081 -0.783478 -2.296520

H $0.301550-0.089705-3.414263$

H $-0.817436-2.344463-1.596225$

H $\quad 1.590889-2.382447-3.515917$

H $\quad 0.047122-3.097354-3.887877$

H $2.541666-5.437028-1.537969$

H $0.347784-6.085450-1.743455$

H $-0.786317-4.744552-1.565713$

H $-0.433487-5.409113-3.175979$

H $4.276974-3.991391-2.782344$

H $3.330450-2.565464-2.380571$

H $3.545347-3.2399750 .090706$

H $4.696796-4.477611-0.426663$

H $\quad 6.074223-2.641684-1.520101$

H $6.613553-3.4610970 .815459$

H $5.462975-2.3169141 .477883$

H $8.014093-2.1393392 .347031$

H $5.9577380 .269602 \quad 1.177525$

H $8.934056-2.6701890 .273008$

H $9.246554-0.981325-0.055239$

H $8.247245-1.932301-1.174260$

H 8.0976841 .0051811 .035106

H $4.3119771 .364821-0.117819$

H $5.5604672 .095134-1.125308$

H $7.442088-0.146050-1.888344$

H $2.495874-0.287580 \quad 0.399321$

H $0.837077-1.3319372 .779147$

H $3.322247-1.2766774 .372209$

H $1.764083-0.6922984 .993469$

H $3.200870 \quad 0.310940 \quad 5.124158$

H $4.4531291 .167317-3.182012$

H $3.1975160 .438621-2.180799$

H $0.4111443 .594155-0.726717$

H $1.5095024 .572495-1.733389$

H 3.2148404 .6229930 .166745

H 0.5697754 .1370601 .632611

H 1.8560755 .6827463 .180019

H 3.3790685 .1533432 .500855

H 1.8869992 .9642695 .666618

H 1.1699814 .6010415 .176244

H 3.8266492 .1504194 .368583

H 4.2074302 .8584612 .830490

H 2.3348171 .7078441 .703578

H $1.9463351 .779950-0.765658$

C $-2.4666932 .152773-2.571864$

O $-3.5163361 .767413-1.662259$

C $-4.5700822 .740076-1.724872$

C $-3.9076134 .005997-2.247883$

C $-2.9483043 .428107-3.271439$
H $-5.2847982 .375670-2.473802$

C $-5.2847442 .871451-0.374570$

H -4.626942 $4.707508-2.680391$

H -3.346886 $4.526933-1.463391$

C $-1.8229704 .373702-3.655814$

H $-3.5167503 .170270-4.175248$

C $-5.658274 \quad 1.5302860 .293490$

O $-4.432080 \quad 3.574198 \quad 0.538213$

H $-6.1740303 .500387-0.501614$

H -4.7571291 .0934610 .734145$

O -6.5258721 .8210031 .396327$

C $-6.3344440 .502304-0.625601$

C $-6.848008-0.7648050 .081598$

H $-5.6358600 .202430-1.414244$

O $\quad-7.4480991 .127809-1.269465$

H $-7.738348-0.5273970 .678522$

H -7.206562 -1.434003 -0.711496

H $-2.2245655 .283709-4.113434$

H -1.231593 $4.669606-2.782911$

H $-1.1471803 .901789-4.376061$

C $-2.1885411 .013732-3.558997$

H -4.9113293 .6530241 .380863$

H -7.3615182 .1582211 .030096$

H -7.828044 $0.483228-1.890685$

H -1.449961 $1.338158-4.301668$

H $-3.1042280 .754297-4.104745$

H -1.578112 2.352264 -1.959921

C $-3.525874-2.2893540 .939980$

O $-4.607493-1.6697650 .226018$

C $-5.821246-1.5001600 .963831$

C $-6.359822-2.8600231 .447092$

C $-5.292130-3.6677512 .191828$

C $-3.947607-3.676551 \quad 1.456897$

H -2.742520 -2.439277 0.186650

C $-2.952446-1.3911782 .050239$

H $-5.621995-0.8685211 .835187$

O $-6.846673-3.6356710 .353100$

H -7.216481 -2.681341 2.107457

H $-5.636500-4.7015412 .318701$

O $-5.098431-3.111863 \quad 3.490104$

H $-3.167197-4.0700922 .118516$

O $-4.028619-4.5851130 .354952$

C $-2.265413-0.1578941 .508535$

H -3.731105 -1.053748 2.738746

H -2.199605 -1.948849 2.619542

H $-6.182922-3.610443-0.355302$

H -5.947845 -3.161351 3.960650

H -4.282504 -4.086336 -0.439013

O

O -2.0329650 .0604390 .328985$

C -1.2859481 .8829752 .188175$

H -0.4317711 .6899081 .532475$

H -1.9984292 .5528721 .699163$

$\mathrm{H}-0.9237172 .3591903 .102985$

SCF Energy $(B 3 L Y P / 6-31 G * * / / M M F F)=-3245.90711459$

08_00654

MM̄FF Geometry

C $1.082611-0.873847-0.764517$

C $-0.156421-0.563480-0.357149$

C -0.4478690 .1113550 .956580$

O $-1.493276-0.6616731 .587346$

C $-0.964537 \quad 1.538813 \quad 0.714917$

C -0.9045052 .4143531 .949233$

C -1.9657892 .7435312 .710984$

C 0.4583862 .9722802 .271884

C -3.3899402 .2844782 .564092$

C $-4.330703 \quad 3.4144842 .122625$

C -4.1434153 .8272940 .653888$

C -5.0592835 .0005020 .302424$

C $-4.9749355 .364924-1.187437$

O $-4.4713472 .698546-0.160713$

O $-6.0735796 .239904-1.487542$

C $-5.1698304 .085840-2.030173$

C $-3.6964376 .147598-1.511625$

O $-4.953810 \quad 4.379821-3.408925$

C -4.286402 $2.900555-1.568112$

C $-4.7165621 .596805-2.284603$ 
O $-2.9230683 .183443-1.888681$ C $-1.421609-0.8917082 .921566$ O $-0.540118-0.5197883 .683551$ C $-2.566893-1.7096073 .387584$ C $-3.651762-1.9596142 .637342$ C $-4.804571-2.777653 \quad 3.021757$ C $-4.764197-3.4617154 .362826$ C $-4.071300 \quad 0.324968-1.714734$ C $-4.584848-0.932382-2.395178$ C $-4.519157-3.416573-2.173806$ O $-5.311222-0.913438-3.385131$ C $-5.781646-3.783087-1.458543$ C $-5.879933-4.792643-0.580309$ C -7.149792 $-5.143310 \quad 0.141977$ C $-7.009018-5.0640841 .647133$ C $-6.895096-6.1802102 .386602$ C -7.089111-3.702075 2.303420 C $-5.824763-2.9030342 .147167$ N -4.121069-2.081147 -1.767031 H $1.925390-0.625009-0.124356$ H -1.002075 $-0.794070-0.999500$ H $\quad 0.456440 \quad 0.1144491 .576684$ H -1.9870641 .4966340 .326575$ H $-0.3733672 .030707-0.068627$ H -1.799706 3.4061073 .559760 H 0.4441513 .6222153 .153272 H 1.1698842 .1652642 .471854 H $0.8331693 .566111 \quad 1.431819$ H -3.4832901 .4251181 .893881$ H -3.7184631 .9253713 .547770$ H -5.3595983 .0581072 .263175$ H -4.1975354 .2853132 .776666$ H -3.0961884 .1033360 .489516$ H -4.8294355 .8741340 .925148$ H $-6.100054 \quad 4.7333520 .534086$ H $-5.998907 \quad 7.022314-0.914816$ H $-6.2263583 .798632-1.932704$ H $-3.6553667 .073388-0.924942$ H $-3.6793456 .458913-2.561925$ H -2.784089 $5.585060-1.302733$ H $-5.2816633 .627735-3.929392$ H $-5.8053501 .483305-2.199381$ H -4.476485 $1.660416-3.353016$ H -2.439432 2.349916 -1.996601 H -2.469983 -2.090645 4.399164 H $-3.709943-1.5312641 .638034$ H -3.880099 -4.104146 4.441640 H -5.628454 -4.102446 4.550458 H -4.734761 -2.721621 5.170126 H -2.986028 $0.352879-1.850599$ H $-4.2839670 .239660-0.642955$ H -3.693023 -4.092635 -1.931748 H -4.684451 -3.437412 -3.255653 H -6.661808 -3.182266 -1.676303 H -5.005136 -5.402950 -0.368385 H -7.432924 -6.160328 -0.158782 H -7.981089 -4.501222 -0.175067 H $-6.824716-6.1373993 .468370$ H $-6.865586-7.1623861 .926510$ H -7.364462 -3.795403 3.359947 H $-7.916788-3.1353281 .857422$ H $-5.762340-2.3667001 .200304$ H $-3.585629-1.991703-0.908218$ C $2.281542-2.754470-1.903745$ O $3.573249-2.352933-1.406146$ C $4.590563-3.145936-2.045867$ C $3.857451-4.211944-2.853775$ C $2.566978-3.498063-3.206220$ H $5.120835-2.481088-2.737246$ C $5.560141-3.695100-0.994214$ H $4.427565-4.528656-3.732499$ H $3.646321-5.105091-2.254302$ C $1.455033-4.436130-3.642523$ H $2.771829-2.780286-4.011885$ C $6.206625-2.615454-0.100277$ O $4.850687-4.595586-0.131065$ H $6.336481-4.296102$-1.480906 H $5.439823-2.1447420 .524675$ $\begin{array}{llll}\text { O } & 7.102206 & -3.282076 & 0.800308\end{array}$

C $7.000354-1.537464-0.865987$

C $7.771468-0.5693180 .054211$

H $6.327583-0.968051-1.516241$

O $7.948785-2.195631-1.710751$

H $8.356694-1.1430810 .782796$

H $8.506603-0.027495-0.556467$

H $1.751478-4.993400-4.537038$

H $1.212127-5.161996-2.859350$

H $\quad 0.543413-3.878886-3.879461$

C $1.398221-1.520432-2.084680$

H $4.117395-4.099186 \quad 0.271445$

H $6.588106-3.9601601 .271322$

H $8.390991-1.512890-2.243556$

H $0.474019-1.792230-2.607149$

H $1.921691-0.780038-2.701209$

H $1.845224-3.432500-1.158128$

C 5.6314702 .4829540 .259934

O $6.488687 \quad 1.429865-0.202326$

C $6.8608810 .443448 \quad 0.768654$

C 7.5720601 .1014771 .960380

C 6.7149792 .2156712 .566965

C 6.2158893 .1877051 .496003

H $5.6273643 .214742-0.559183$

C $4.191644 \quad 1.989366 \quad 0.453194$

H $5.962300-0.064761 \quad 1.128930$

$\begin{array}{lllll}\text { O } & 8.835537 & 1.628396 & 1.561417\end{array}$

H $7.7714580 .335076 \quad 2.718499$

H 7.3044622 .7664823 .309844

O $5.5885691 .649887 \quad 3.230936$

H 5.4680163 .8714821 .914344

O $7.3116903 .992887 \quad 1.050611$

C $3.5142351 .857753-0.893045$

H 4.1185421 .0182340 .949387

H 3.6241652 .7064561 .057862

H 8.6814412 .2487430 .828359

H 5.9241041 .0557693 .923758

H 7.6507374 .4786491 .821869

O $2.4634482 .723075-0.973483$

O $3.8862251 .091726-1.772667$

C $1.7684662 .741484-2.224251$

H $2.4499193 .023826-3.032446$

H $1.3166741 .766160-2.423539$

H $0.9726813 .488332-2.159070$

SCF Energy (B3LYP/6-31G*//MMFF)= -3245.90554737

0800655

MM̄FF Geometry

C $-0.162443-3.3301460 .987912$

C $0.789749-2.387507 \quad 0.928074$

C $1.283173-1.5811952 .098765$

O $0.982569-0.2006811 .796185$

C $2.810945-1.7445482 .212714$

C $3.403475-1.1760753 .483865$

$\begin{array}{llll}\text { C } & 4.010290 & 0.023514 & 3.577609\end{array}$

C $3.316586-2.0780284 .687391$

C 4.1421721 .0781432 .513758

C 5.5916641 .3293752 .082188

C $6.1776290 .220536 \quad 1.192964$

$\begin{array}{llll}\text { C } 7.622106 & 0.561734 & 0.814983\end{array}$

C $8.214018-0.441477-0.184580$

$\begin{array}{lllll}\text { O } & 5.371127 & 0.127625 & 0.013690\end{array}$

O $9.4158040 .129363-0.724638$

C $7.229496-0.613596-1.359877$

C $8.624904-1.7509380 .498706$

O $7.672596-1.640479-2.244874$

C $5.776381-0.891431-0.910863$

C $4.825876-0.822463-2.131623$

O $5.712460-2.193288-0.328030$

C 0.5822320 .6123352 .805152

$\begin{array}{lllll}\mathrm{O} & 0.394313 & 0.293474 & 3.971118\end{array}$

C 0.3799522 .0035242 .334534

C $0.6861572 .427201 \quad 1.098206$

C $0.491446 \quad 3.780922 \quad 0.571414$

C $-0.220498 \quad 4.779483 \quad 1.445340$

C $3.351497-1.056369-1.789593$

C $2.461483-0.779458-2.983795$

C $1.1408161 .001485-4.085012$ 
O $2.147378-1.666110-3.774760$

C $1.1360642 .495134-4.121398$

C $0.0658013 .243802-3.813619$

C $0.0681154 .745498-3.831141$

C $-0.2440515 .347904-2.478016$

C $-1.4299285 .932982-2.239464$

C $0.848916 \quad 5.354112-1.430794$

C $0.9341914 .055036-0.673680$

N $2.086745 \quad 0.551607-3.080687$

H $-0.656250-3.5459811 .930417$

H $1.261225-2.188927-0.030784$

H $0.778281-1.9017933 .017912$

H $3.295150-1.2856651 .344006$

H $3.078387-2.8091292 .162685$

H 4.4246250 .3128614 .542698

H $3.772910-1.6309645 .576855$

H $2.271142-2.2952184 .927820$

H $3.832740-3.0236684 .491497$

H $3.519594 \quad 0.868147 \quad 1.640039$

H 3.7468932 .0102312 .938175

H 5.6014342 .2723901 .519453

H $6.225446 \quad 1.4757692 .965657$

H $6.138092-0.7314721 .734364$

H $8.2519310 .629527 \quad 1.710847$

H $7.650888 \quad 1.5599100 .355097$

H $10.018567 \quad 0.314841 \quad 0.015784$

H $7.2397420 .320575-1.938878$

H $9.387652-1.5603901 .263335$

H $9.084496-2.442697-0.215693$

H $7.791541-2.2602750 .986894$

H $8.582429-1.421608-2.509921$

H $4.9214070 .164135-2.603729$

H $5.121071-1.568503-2.880189$

H $4.785265-2.470645-0.272166$

H $-0.0376352 .673798 \quad 3.079146$

H $1.123543 \quad 1.7214670 .393695$

H 0.3703974 .9945892 .342265

H $-1.199097 \quad 4.3957041 .752784$

H -0.4106165 .7340920 .950326$

H $3.177593-2.094815-1.489271$

H $3.033538-0.423155-0.954092$

H $\quad 0.1605050 .585857-3.827401$

H $1.4346150 .594918-5.058362$

H $2.0635492 .983715-4.410734$

H $-0.8641812 .755194-3.531683$

H -0.673344 5.070106-4.572636

H $1.0295225 .138838-4.184450$

H -1.650501 6.392821-1.282026

H -2.209619 5.959115-2.993536

H $0.7220296 .204769-0.752586$

H $1.8159755 .538663-1.916040$

H $1.4416263 .262700-1.222775$

H $2.2699351 .161374-2.288415$

C $-1.773286-3.619676-0.938386$

O $-2.975608-3.903833-0.187954$

C $-3.910330-4.558674-1.059818$

C $-3.458003-4.239281-2.477303$

C $-1.953871-4.279820-2.310456$

H -3.770544 $-5.637051-0.897138$

C $-5.370141-4.222994-0.731815$

H $-3.829260-4.959803-3.211900$

H -3.767478 -3.236502 -2.789498

C $-1.198200-3.581968-3.429547$

H -1.634782 -5.330192 -2.267748

C $-5.864532-2.806220-1.092944$

O $-6.175546-5.163216-1.457637$

H $-5.550644-4.4111310 .333648$

H $-5.839591-2.673126-2.179841$

O $-7.253641-2.729319-0.734777$

C $-5.118778-1.653023-0.402981$

C $-5.665167-0.278219-0.837527$

H $-4.055544-1.695445-0.653621$

O $-5.247719-1.8196111 .005957$

H $-5.627186-0.222081-1.932131$

H -6.713016 $-0.192776-0.523584$

H $-1.408977-4.059303-4.391998$

H -1.481309 -2.526923 -3.508235

H $-0.118111-3.628254-3.258675$
C $-0.547002-4.163041-0.202511$

H $-7.104976-4.975941-1.240754$

H $-7.308361-2.784507 \quad 0.234914$

H $-4.753362-1.1020221 .435707$

H $0.305424-4.219048-0.891047$

H $-0.736369-5.184890 \quad 0.149049$

H $-1.712724-2.528766-1.041018$

C $-4.6072572 .060547 \quad 1.883830$

O $-5.255207 \quad 1.023236 \quad 1.137463$

C $-4.8702250 .899152-0.236537$

C $-5.1420212 .208669-0.992926$

C $-4.4627993 .395652-0.303120$

C $-4.769868 \quad 3.427184 \quad 1.196439$

H $-5.152307 \quad 2.1004942 .835927$

C -3.1490301 .7103902 .189937$

H $-3.8021150 .662901-0.295249$

O $-6.5485592 .433705-1.064037$

H $-4.7719622 .106186-2.020099$

H $-4.805763 \quad 4.332811-0.757939$

O $-3.052332 \quad 3.311459-0.483933$

H -4.1308154 .1652691 .694493$

$\begin{array}{llll}\text { O } & -6.117660 & 3.868844 & 1.384306\end{array}$

C $-3.0668080 .590250 \quad 3.198608$

H $-2.576444 \quad 1.4277501 .302365$

H -2.6336242 .5684032 .635728$

H $-6.6894793 .250574-1.572395$

H -2.876498 $3.320121-1.440162$

H $-6.707798 \quad 3.188601 \quad 1.016677$

O $-2.854705-0.5975882 .565143$

O $-3.195510 \quad 0.753263 \quad 4.405263$

C $-2.796195-1.7474283 .415307$

H $-1.860637-1.7426673 .981584$

H $-3.657281-1.7775294 .090255$

H -2.824457-2.638278 2.783009

SCF Energy (B3LYP/6-31G**//MMFF)= -3245.91482097

0800656

MM̄FF Geometry

C -0.979262 -2.885203 2.567744

C $-0.435496-1.7657123 .072164$

C $-1.121022-0.4201233 .184190$

O $-2.433669-0.5160032 .582160$

C -0.2920090 .6788612 .480786$

C $0.427244 \quad 1.593328 \quad 3.454448$

C $0.1462802 .897766 \quad 3.646478$

C 1.5604930 .9570614 .219480

C $-0.907918 \quad 3.738514 \quad 2.980727$

C -0.3204014 .9425242 .230021$

C 0.6228234 .5555991 .081146

C 1.1539105 .8038630 .373339

C $2.0466395 .441545-0.823057$

$\begin{array}{lllll}\text { O } & -0.103334 & 3.743561 & 0.152446\end{array}$

O $2.2317076 .627041-1.611463$

C $1.3049284 .419415-1.709255$

C $3.443410 \quad 4.994746-0.378134$

O $2.1524603 .934280-2.748257$

C $0.697174 \quad 3.232800-0.921974$

C $-0.2131312 .404527-1.859598$

$\begin{array}{lllll}\text { O } & 1.751162 & 2.399580 & -0.437611\end{array}$

C $-3.3570020 .429902 \quad 2.882328$

\begin{tabular}{llll}
\hline & -3.206224 & 1.438157 & 3.551193
\end{tabular}

C -4.6238170 .0112342 .254126$

$\begin{array}{llll}\text { C } & -5.378682 & 0.925506 & 1.630362\end{array}$

$\begin{array}{llll}C & -6.663327 & 0.658892 & 0.985798\end{array}$

C $-7.404930-0.5847621 .400876$

C $-1.069696 \quad 1.350125-1.147075$

C $-1.9268370 .565989-2.124195$

C $-3.844400-1.018477-2.195277$

O $-1.7648290 .607744-3.341149$

C $-5.030083-0.177022-2.555157$

C $-6.299864-0.575802-2.387875$

C $-7.492020 \quad 0.252177-2.778804$

C $-8.5273730 .338342-1.679588$

C $-9.554649-0.528091-1.642054$

C $-8.434432 \quad 1.470475-0.679429$

C $-7.139013 \quad 1.5407610 .082714$

N -2.880872 $-0.207021-1.474679$

H $-2.011593-2.8750542 .231689$ 
H $0.589137-1.807333 \quad 3.434472$ H -1.255786 -0.2020614 .251539$ H $-0.935563 \quad 1.2554581 .812911$ H $\quad 0.461308 \quad 0.235343 \quad 1.814912$ H $\quad 0.743086 \quad 3.4427814 .377684$ H 2.1283331 .6863934 .806928 H 1.1808550 .2008674 .913252 H 2.2624960 .4782253 .529828 H -1.538205 3.1628312 .299224 H -1.578566 $4.113973 \quad 3.763740$ H -1.162266 $5.521217 \quad 1.828097$ H 0.2087365 .5937662 .937102 H 1.4576393 .9776321 .492964 H 1.6961836 .4495641 .075221 H $\quad 0.308644 \quad 6.4027320 .006049$ H $2.6336667 .305607-1.042317$ H $0.4848574 .956731-2.206262$ H 3.9357285 .7893710 .195213 H $4.091174 \quad 4.804025-1.240646$ H 3.4250894 .1005840 .246876 H $2.5002854 .708103-3.223623$ H $-0.9006123 .080710-2.385134$ H $\quad 0.399904 \quad 1.910439-2.623454$ H $1.4114381 .502210-0.297491$ H -4.883594 -1.039961 2.304737 H -5.0161001 .9505041 .566704$ H -6.933145 -1.475657 0.972873 H $-8.452336-0.5784291 .089596$ H -7.417552 -0.6846012 .492347$ H $-0.4401630 .628990-0.617049$ H $-1.732021 \quad 1.836443-0.422246$ H -4.112535 -1.860088 -1.549129 H -3.375704 -1.404834 -3.106242 H $-4.8375890 .797644-2.997948$ H -6.494235 -1.564295 -1.977496 H $-7.938776-0.208362-3.669947$ H -7.198205 $1.263887-3.084981$ H -10.324182 -0.461589-0.880069 H -9.647035 -1.326119-2.371477 H -9.2653391 .4432130 .037059$ H -8.559885 2.414200 -1.226666 H -6.540881 $2.422416-0.148970$ H -2.988286 $-0.123088-0.467865$ C $-0.134923-4.7178201 .012308$ O $0.664081-3.8266450 .209169$ C $0.143830-3.836250-1.135355$ C $-0.914448-4.937412-1.183063$ C $-1.436866-4.9219180 .239250$ H - $0.351877-2.869987-1.285302$ C $1.269835-4.016308-2.159194$ H -1.689200 -4.732374 -1.928335 H $-0.476518-5.914818-1.417086$ C $-2.197593-6.1773090 .626441$ H $-2.099931-4.0550280 .341720$

C $2.378872-2.945846-2.105933$

O $1.888718-5.290277-1.933545$ H $0.837116-4.060698-3.166156$ H $2.979958-3.089191-1.201715$

O $3.262815-3.198690-3.207332$

C $1.886120-1.490039-2.181620$

C $2.990084-0.447354-2.441463$

H $1.356091-1.239250-1.255903$

O $0.939361-1.384982-3.249904$

H $3.381146-0.566392-3.460318$

H $2.5041270 .536329-2.433612$

H -3.073521 -6.309831 -0.017152

H -1.572715 -7.071563 0.531139

H -2.545753 $-6.117267 \quad 1.662351$

C $-0.256301-4.1970322 .448415$

H $2.594172-5.390789-2.595499$

H $2.766648-3.027799-4.026450$

H $0.597288-0.474846-3.252437$

H $\quad 0.751010-4.0991732 .870647$ H $-0.785953-4.9365873 .060299$

H $\quad 0.419470-5.6655141 .050369$

C $4.557898-0.3277140 .949962$

O $3.608559-0.291295-0.124448$

C $4.151444-0.461119-1.435900$
C $5.1839230 .636801-1.744499$

C $6.2645950 .709053-0.660443$

C 5.6598650 .7275640 .746595

H $3.983173-0.0464621 .840781$

C $5.118257-1.7402471 .180436$

H $4.644545-1.435899-1.498573$

O $4.5283071 .898791-1.842464$

H $5.6382290 .427173-2.720303$

H $6.8662041 .615553-0.800918$

O $7.136911-0.411016-0.776311$

H 6.4493540 .5894351 .494740

O 5.0976042 .0232760 .968033

C $4.055928-2.7186491 .631388$

H $5.568990-2.1490730 .272619$

H $5.880621-1.7063941 .967398$

H $5.2005802 .564333-2.066685$

H $7.532235-0.384775-1.664338$

H 4.7570002 .0460661 .878438

O $4.612032-3.9645431 .705846$

O $2.895531-2.4334811 .889582$

C $3.730161-5.0149612 .112364$

H $4.322287-5.9248942 .240798$

H $3.253459-4.7714163 .066678$

H $2.980634-5.1913021 .336349$

SCF Energy $\left(B 3 L Y P / 6-31 G^{* *} / / M M F F\right)=-3245.89078485$

0800657

MM̄FF Geometry

C $2.614078-0.8069084 .253904$

C $2.805078-0.9713702 .936295$

C $4.134582-0.7587132 .257355$

$\begin{array}{lllll}\text { O } & 4.002169 & 0.326189 & 1.314103\end{array}$

C $4.494086-2.0121801 .442998$

C $5.957771-2.0968771 .065712$

C $6.475034-1.721830-0.120570$

C $6.847139-2.7118162 .116013$

C $5.784633-1.037176-1.267220$

C $5.484360-1.971247-2.448107$

C $4.141376-2.703619-2.303683$

C $3.913290-3.664575-3.471549$

C $2.528453-4.325658-3.402275$

O $3.111409-1.707921-2.284176$

O $2.304178-4.961028-4.669705$

C $1.461015-3.225006-3.224696$

C $2.487488-5.438989-2.349376$

O $0.168494-3.797089-3.033800$

C $1.786555-2.215205-2.098126$

C $0.802702-1.023781-2.169774$

O $1.639091-2.868320-0.835920$

C 4.1720471 .5963981 .760983

O 4.3956571 .9579222 .903890

C 4.0232262 .4815550 .585110

C 3.8604493 .8011940 .753019

C $3.6631604 .782123-0.316792$

C $3.7163844 .284830-1.738805$

C $1.1682450 .170807-1.275252$

C $\quad 0.1203891 .270722-1.359797$

C $-0.5085963 .450881-0.324270$

O $\quad-0.827117 \quad 1.233631-2.141023$

C $0.0301464 .590689-1.130376$

C $0.1622365 .836628-0.649035$

C $0.6634236 .999014-1.459206$

C $1.7667907 .768741-0.765384$

C $1.5083978 .924296-0.129991$

C $3.1802177 .241842-0.880126$

C $3.426415 \quad 6.0661340 .024011$

N $0.3658102 .299108-0.460500$

H $3.455578-0.463928 \quad 4.853677$

H $1.985584-1.2939452 .300695$

H $4.926014-0.5311112 .982242$

H $3.877334-2.0426010 .539448$

H $4.239853-2.9210332 .005381$

H $7.540889-1.879794-0.283197$

H $7.894218-2.7619171 .799220$

H $6.810671-2.1243513 .039010$

H $6.520632-3.7326592 .340282$

H $4.881511-0.513508-0.943842$

H $6.464953-0.249730-1.617188$ 
H $5.444267-1.351062-3.353539$ H $6.300079-2.691446-2.584731$ H $4.137348-3.256494-1.358865$ H $4.701856-4.426511-3.509823$ H $3.986219-3.111267-4.418530$ H $1.399102-5.317609-4.664472$ H $1.409275-2.678610-4.176492$ H $3.235692-6.207318-2.578752$ H $1.520803-5.954580-2.354316$ H $2.680727-5.078999-1.336691$ H $0.116581-4.109724-2.114493$ H $0.758724-0.655523-3.203348$ H $-0.203947-1.368988-1.904743$ H $1.414089-2.210094-0.159749$ H $4.0253382 .013984-0.393194$ H 3.8493884 .1993011 .766689 H $3.5193175 .059159-2.482412$ H $2.9671593 .503080-1.905083$ H $4.7067683 .873147-1.962404$ H $1.254846-0.139938-0.229162$ H $2.1276810 .597407-1.588386$ H -0.5657023 .6897140 .742327$ H -1.510915 $3.193321-0.680570$ H $0.2981794 .389195-2.164783$ H -0.1379996 .0425430 .376141$ H $-0.1903247 .662990-1.648560$ H $1.0133896 .676864-2.447827$ H 2.2975189 .4964380 .346629 H $0.5005979 .321343-0.067395$ H $3.8991218 .023235-0.598906$ H $3.3990727 .029925-1.930126$ H 3.4025606 .3247861 .083742 H 1.1976972 .2638470 .120321 C $0.283795-1.9315904 .365504$ O $-0.398356-1.1786543 .343766$ C $-1.797300-1.5244773 .364232$ C -1.941353 -2.677691 4.351638 C $-0.815899-2.3888435 .324136$ H -2.327446 -0.657026 3.770509 C -2.295485 -1.841313 1.949807 H $-2.927183-2.6935164 .826612$ H -1.788252 -3.6498903 .869119$ C $-0.441399-3.5861526 .181150$ H -1.116782 -1.556923 5.975014 C -2.095553 -0.7123720 .919548$ O $-1.573479-2.9880631 .473751$ H -3.348944 -2.140551 1.987996 H -1.017573 -0.5640460 .785193$ O $-2.587689-1.190132-0.336590$ C $-2.759003 \quad 0.642410 \quad 1.231865$ C $-4.213071 \quad 0.600102 \quad 1.741277$ H $-2.730863 \quad 1.259796 \quad 0.326494$ O $-1.958046 \quad 1.3166022 .205211$ H -4.2637260 .0478992 .686530$ H $-4.489256 \quad 1.632740 \quad 1.995718$ H -1.298214 -3.912971 6.779368 H $-0.117325-4.4332825 .567302$ H $0.372888-3.3382426 .868719$ C $1.352161-1.0630695 .037998$ H -1.898438 -3.1823900 .577769$ H -2.374499 -0.519228 -1.007555 H -2.326196 2.2086752 .323696 H $1.662907-1.5501295 .970060$ H $0.926129-0.0889945 .307932$ H $\quad 0.744009-2.8015553 .878484$ C $-6.1339610 .498878-1.465443$ $\begin{array}{lllll}\text { O } & -5.241207 & 0.877582 & -0.408659\end{array}$ C $\begin{array}{llll}-5.243183 & 0.036148 & 0.748567\end{array}$ C $-6.648784-0.0304631 .370030$ C $-7.690944-0.4595250 .334097$ C $-7.5784590 .362703-0.953777$ H $-6.1130471 .343922-2.165890$ C $-5.636058-0.747483-2.214473$ H $-4.941257-0.9750040 .463853$ O $\quad-7.000502 \quad 1.244727 \quad 1.902896$ H $-6.630682-0.7429632 .203688$ H -8.693324 -0.3378980 .762106$ O $\quad \begin{array}{llll}-7.506750 & -1.842840 & 0.048242\end{array}$
H $-8.204329-0.069389-1.743092$

O $-8.0722571 .683025-0.709206$

C $-4.369157-0.476273-2.999732$

H $-5.425694-1.572950-1.529637$

H $-6.395845-1.069861-2.935744$

H $-7.877301 \quad 1.1593742 .314365$

H -8.187671 -2.102769 -0.595455

H $-8.9995911 .602056-0.428381$

O $-3.937561-1.646349-3.558518$

O $-3.827404 \quad 0.614117-3.116044$

C $-2.738755-1.552887-4.334519$

H $-2.456192-2.561407-4.647431$

H $-2.916373-0.943624-5.225512$

H $-1.926328-1.132389-3.735631$

SCF Energy $(B 3 L Y P / 6-31 G * * / / M M F F)=-3245.91208062$

08 00658

MM̄FF Geometry

C $1.248377-3.6889950 .696732$

C $-0.047307-3.4537590 .442930$

C $-0.656150-3.516768-0.934493$

O $-1.407241-2.296187-1.143344$

C $-1.624816-4.708087-1.026703$

C $-2.243740-4.881555-2.402010$

C $-3.534770-4.636665-2.705313$

C $-1.309101-5.396178-3.467409$

C $-4.615929-4.140040-1.787726$

C $-5.006322-2.694858-2.121310$

C $-5.813248-2.048185-0.986485$

C $-6.287771-0.651553-1.388328$

C $-6.9815970 .081033-0.229157$

O $-4.949561-1.9624420 .151759$

O $-7.0891801 .467418-0.589168$

C -6.0792080 .0072891 .022754$

C $-8.413891-0.423133-0.017625$

O $-6.743260 \quad 0.5524492 .160245$

C $-5.557416-1.4126671 .326627$

C $-4.491872-1.4419402 .450393$

O $-6.653373-2.2250201 .764992$

C $-0.700882-1.184342-1.490395$

O $0.501385-1.110562-1.689666$

C -1.650224 -0.050075 -1.558658

C $-1.1834931 .192890-1.748441$

C $-1.9797062 .419619-1.827395$

C $-3.4696142 .298463-1.650982$

C $-3.210888-0.6496812 .163851$

C $-3.215231 \quad 0.7628122 .723689$

C -1.8742142 .8632742 .695481$

O -4.0628871 .1735493 .511259$

C -2.4557303 .8162621 .699806$

C -1.7552214 .8151241 .141486$

C $-2.336627 \quad 5.8155770 .184950$

C $-1.5615135 .905193-1.110161$

C $-0.6235676 .850331-1.291187$

C -1.939835 $4.957983-2.225235$

C $-1.3427153 .588636-2.048895$

N -2.129468 1.5002802 .269481

H $1.908471-3.980064-0.116630$

H $-0.707180-3.1659601 .258435$

H $\quad 0.119207-3.604565-1.705545$

H $-2.398247-4.598712-0.260468$

H -1.095121 -5.636570 -0.773201

H -3.863067 -4.804773 -3.730376

H -1.825909 $-5.597621-4.411989$

H $-0.520073-4.667189-3.674928$

H $-0.843476-6.332823-3.143841$

H -5.494284 -4.787395 -1.901976

H -4.324922 -4.226625 -0.738991

H $-4.097121-2.105580-2.293744$

H $-5.585816-2.680827-3.052652$

H $-6.667213-2.693133-0.748450$

H -6.949076 -0.702404 -2.262238

H $-5.423344-0.053645-1.705247$

H $-7.593373 \quad 1.523701-1.418854$

H $\quad-5.2255170 .6658770 .826053$

H $-9.005704-0.288817-0.931237$

H $-8.9273580 .157356 \quad 0.756794$

H $-8.460535-1.4802090 .251928$ 
H -7.380700 -0.109704 2.477099 H -4.943571 -1.152774 3.406888 H -4.188139 -2.490949 2.579491 H -6.298094 -2.978874 2.264480 H -2.704753 $-0.265764-1.436240$ H $-0.1098621 .330025-1.862058$ H -3.907099 $1.735056-2.481900$ H $-3.9847843 .260055-1.604245$ H -3.705485 $1.785263-0.712890$ H -2.370964 -1.163770 2.649037 H $-2.997664-0.6186081 .091689$ H -0.7905192 .9747492 .791086$ H -2.3297603 .0319593 .676608$ H -3.507728 3.6987661 .450939 H -0.7117264 .9490601 .417264$ H $-2.336174 \quad 6.7929600 .685275$ H $-3.3897855 .598127-0.032315$ H $-0.0843816 .939693-2.228480$ H $-0.3701247 .551863-0.503250$ H -1.574907 5.346891-3.185703 H $-3.0279234 .940831-2.333550$ H $-0.2572713 .575626-2.151915$ H -1.5059331 .0953841 .577008$ C $3.031207-2.5505722 .013386$ O $4.192561-3.2091991 .458585$ C $5.342219-2.8314622 .227040$ C $4.908637-1.6665793 .108527$ C $3.464192-2.0364353 .385218$ H $5.559382-3.6802392 .890696$ C $6.586608-2.5931811 .363839$ H $5.509952-1.5799314 .018454$ H $4.961493-0.7134832 .571408$ C $2.629856-0.8776553 .904533$

H $3.441606-2.8544414 .117761$ C $6.491833-1.5019680 .273074$ O $7.649613-2.2469422 .262049$ H $6.874714-3.5410940 .893279$ H $6.283665-0.5367840 .743229$ O $7.797955-1.372340-0.311914$ C $5.485195-1.779488-0.860487$ C $5.538913-0.762604-2.019226$ H $4.468197-1.804816-0.463578$ O $5.760462-3.075054-1.400654$ H $6.552514-0.714688-2.434712$ H $4.903608-1.153826-2.825065$ H $3.036043-0.5049674 .850420$ H $2.613040-0.0442753 .194911$ H $1.595362-1.1883384 .080626$ C $1.863927-3.5372892 .060697$ H $8.448820-2.1088061 .725265$ H $7.996649-2.207149-0.770334$ H $5.065294-3.276024-2.050204$ H $\quad 1.107557-3.1937602 .775615$ H $2.213043-4.5226822 .391171$ H $2.796610-1.7040921 .353736$ C $3.0695151 .713152-0.757248$ O $3.6707620 .517814-1.269398$ C $5.0532980 .636853-1.620992$

C $5.2211981 .668842-2.747296$

C $4.6218193 .019774-2.342357$

C $3.2095312 .874059-1.757836$

H $2.0047851 .462974-0.676551$

C 3.5909952 .0065900 .656430

H $5.6218290 .972546-0.747808$

O $4.5747481 .228985-3.939338$

H $6.2878031 .792968-2.971210$

H $4.5885183 .670709-3.224731$

O $5.4855323 .632212-1.389703$

H $2.8890523 .809089-1.285003$

O $2.2985302 .615853-2.831428$

C 2.6292152 .8337571 .479548

H 3.7396101 .0562461 .183016

H 4.5545192 .5219660 .630753

H $5.0966170 .495934-4.306596$

H $5.108414 \quad 4.500686-1.167685$

H $2.3368453 .374753-3.438033$

O 3.1602213 .0158052 .725097

O 1.5522253 .2625661 .090061
C 2.3592093 .7781573 .633370

H 2.0892734 .7437293 .194851

H 1.4637613 .2116283 .904290

H 2.9478893 .9573524 .536936

SCF Energy (B3LYP/6-31G**//MMFF) $=-3245.90272098$

08_00659

MM̄FF Geometry

C -2.468425 5.3301830 .074178

C $-3.2440044 .281276-0.237845$

C -4.2129093 .6510600 .727446$

$\begin{array}{llll}\text { O } & -3.898659 & 2.246100 & 0.861470\end{array}$

C -5.6421103 .7636340 .172468$

C -6.6907813 .2207891 .122548$

C -7.3026862 .0291480 .974702$

C -7.0723394 .1364542 .255955$

C $-7.031621 \quad 1.001322-0.090155$

C $-7.172558-0.4272530 .454887$

C $-6.889848-1.472458-0.633443$

C $-7.324689-2.864044-0.172082$

C $-6.993397-3.936275-1.219360$

O $-5.482895-1.467160-0.904316$

O $-7.146728-5.221735-0.598854$

C $-5.510503-3.797853-1.615019$

C $-7.967016-3.906150-2.403232$

O $-5.186121-4.674613-2.692105$

C $-5.085554-2.349420-1.963338$

C $-3.545558-2.294386-2.095082$

O $-5.673538-1.970562-3.208642$

C $-2.9407411 .903396 \quad 1.764143$

O -2.2399142 .6607102 .416390$

C -2.9089880 .4249041 .840434$

C $-1.980405-0.1766912 .598481$

C -1.818884 -1.619798 2.791573

C $-2.882666-2.5262252 .229704$

C $-2.951856-0.882493-2.190334$

C $-1.436066-0.955005-2.244788$

C $0.540204-1.572552-0.846396$

O $-0.812368-0.715081-3.275637$

C $0.711842-2.7754690 .025928$

C $1.427318-2.7805931 .160929$

C $1.560780-3.9852702 .049212$

C $1.139770-3.7151253 .478611$

C $2.033039-3.7538524 .481924$

C $-0.331476-3.4773453 .760170$

C $-0.742283-2.0581353 .477685$

N -0.879718 -1.349281-1.039165

H $-2.5267145 .757640 \quad 1.072119$

H -3.186396 $3.852366-1.235529$

H -4.1617294 .1335051 .711780$

H $-5.7069113 .249999-0.794574$

H -5.879584 $4.814593-0.041525$

H -8.0820321 .7635781 .687605$

H -7.870955 3.7178942 .877808

H -6.2125754 .3218182 .907060$

H -7.4276585 .0958581 .866239$

H -7.755755 $1.147970-0.900497$

H $-6.0309111 .120594-0.515818$

H $-6.475854-0.5650251 .292147$

H -8.190133 -0.5634420 .842265$

H -7.435237 -1.186775 -1.540774

H -8.395906 -2.8805330 .064709$

H $-6.806064-3.1173790 .763381$

H $-8.063186-5.293674-0.281238$

H $-4.914531-4.133027-0.754115$

H $-8.995517-4.063455-2.056860$

H -7.760071 -4.721600 -3.105211

H -7.944286 -2.963514 -2.953838

H $-5.452993-5.571240-2.426407$

H -3.101985 -2.792883 -1.224740

H $-3.227899-2.862970-2.978931$

H -5.136217 -1.270194-3.610966

H -3.651593 -0.1269901 .275243$

H -1.255496 0.4459623 .121760

H $-3.876062-2.2118912 .569989$

H $-2.774245-3.5674202 .540894$

H -2.869555 -2.510094 1.136701

H $-3.300052-0.365313-3.090262$ 
H $-3.240018-0.267191-1.331109$ H $0.947913-0.670830-0.383787$ H $1.029908-1.727814-1.812387$ H $\quad 0.211427-3.688329-0.289635$ H $\quad 1.944447-1.875053 \quad 1.470229$ H $2.608801-4.3092232 .014417$ H $0.973296-4.8283241 .663973$ H $1.733568-3.5857805 .511145$ H $3.083308-3.9525304 .298618$ H -0.546672 -3.680672 4.817191 H - $0.910253-4.2088683 .189526$ H $-0.055732-1.3143173 .885508$ H $-1.473115-1.365335-0.215727$ C $-0.0541835 .602500-0.689006$ O $0.1387124 .213950-1.019001$ C $1.1094113 .654331-0.106376$ C 1.7071194 .8345500 .648383 C $0.528766 \quad 5.787163 \quad 0.709911$ H $0.538786 \quad 3.0119700 .576300$ C $2.1159342 .820770-0.907161$ H 2.0835574 .5540591 .636651 H 2.5329525 .2829540 .082006 C 0.9133257 .2194201 .034068 H $-0.163153 \quad 5.419525 \quad 1.477414$ C $3.2806212 .274213-0.058588$ O $1.4004351 .734936-1.500152$ H $2.518743 \quad 3.415788-1.735816$ H 3.8390343 .1240240 .350327 O 2.7629841 .5508361 .058535 C $4.2720601 .384802-0.839570$ C 5.4760930 .9899770 .027534 H $4.6182801 .933323-1.722269$ O $3.6413610 .187523-1.286397$ H $5.978887 \quad 1.8892360 .405737$ H 5.1247810 .4543000 .920278 H 1.3912037 .2760592 .017547 H 1.6142997 .6243370 .296409 H 0.0295617 .8649021 .049753 C -1.520928 $5.987586-0.888774$ H $0.6720232 .114834-2.021145$ H 2.2957010 .7731090 .709586 H $2.9170760 .440438-1.883246$ H $-1.8144715 .733395-1.915499$ H -1.634331 $7.073603-0.790901$ H $0.5429646 .168305-1.416853$ C $8.521383-1.141127-0.100155$ O $7.504978-0.2158610 .306585$ C $6.4976960 .073009-0.672632$

C $7.1406290 .689060-1.923706$

C $8.266591-0.197090-2.470402$

C $9.233087-0.645436-1.368985$

H $9.250994-1.1297190 .720297$

C $7.980331-2.570760-0.218960$

H $5.989767-0.859332-0.940129$

O $7.6580691 .984275-1.625370$

H $6.3769740 .818989-2.698066$

H $8.8245860 .355849-3.235966$

O $7.709195-1.352999-3.089466$

H $9.904327-1.428910-1.739767$

O $10.0518260 .465537-0.991368$

C $7.516169-3.0767191 .126129$

H $7.152138-2.669237-0.926476$

H $8.765417-3.251384-0.569876$

H $8.2928511 .889586-0.894592$

H $7.125032-1.050423-3.805541$

H $10.5473260 .745270-1.780029$

O $6.216495-2.7193721 .332418$

O $8.230694-3.6915421 .906913$

C $5.664514-3.0873772 .599446$

H $6.298301-2.7299583 .416795$

H $4.683059-2.6145682 .689260$

H $5.541129-4.1728592 .651242$

SCF Energy (B3LYP/6-31G*//MMFF) $=-3245.92558254$

08_00660

MM̄FF Geometry

C $-4.102118-1.662646-2.530666$

C $-2.969890-1.450454-3.218362$
C $-1.589659-1.759889-2.710206$

O $-0.846584-0.526818-2.876206$

C $-0.901144-2.872591-3.522645$

C $-1.420014-4.263821-3.220345$

C $-0.923715-5.068543-2.259645$

C $-2.537901-4.755617-4.103587$

C $0.228951-4.795627-1.335966$

C $-0.238691-4.403928 \quad 0.069892$

C $0.936097-4.2907901 .053558$

C $0.457597-3.780757 \quad 2.414030$

C $1.622035-3.5472693 .389544$

O $1.898019-3.385446 \quad 0.505352$

O $1.131537-2.7596234 .485108$

C $2.711970-2.7101972 .683804$

C $2.125200-4.8625153 .996143$

O $3.876566-2.5966853 .497803$

C $3.090651-3.2520461 .288318$

C $4.031264-2.2664650 .555456$

O $3.770926-4.5015661 .442224$

C $0.208038-0.283768-2.055924$

O $0.665413-1.020317-1.198463$

C $0.698642 \quad 1.074623-2.384158$

C $1.8211551 .546674-1.822780$

C $2.3771382 .888993-2.017525$

C $1.642853 \quad 3.824311-2.943134$

C $4.197385-2.588576-0.936968$

C $5.504408-2.056300-1.485210$

C $6.6824010 .019207-2.127408$

O $6.407327-2.811791-1.839667$

C $6.7030331 .433866-1.650581$

C $6.5844362 .491894-2.466421$

C $6.6162263 .916799-1.995318$

C $5.3408864 .661848-2.319599$

C $5.2615685 .450204-3.404834$

C $4.1937214 .568353-1.339698$

C $3.5013493 .232424-1.354685$

N $5.556499-0.672475-1.529068$

H $-4.053317-2.122093-1.547853$

H $-3.030792-1.003111-4.208710$

H -1.625107 -2.012916-1.643889

H $0.181254-2.829776-3.354608$

H $-0.995680-2.667777-4.598245$

H $-1.376984-6.049979-2.125833$

H -2.804199 $-5.797444-3.894295$

H $-3.441040-4.156264-3.967746$

H $-2.239755-4.702172-5.155964$

H $0.837087-5.707331-1.280880$

H $0.888282-4.022971-1.741778$

H $-0.757756-3.4395150 .009317$

H $-0.961658-5.1367660 .449516$

H $1.392268-5.2830061 .153295$

H $-0.280990-4.4649022 .849995$

H -0.065936 -2.826612 2.274078

H $0.383129-3.2348334 .885122$

H $2.318559-1.6917282 .561859$

H $1.321834-5.3581974 .554598$

H $2.926225-4.6827444 .721512$

H $2.488096-5.5694853 .247314$

H $3.593679-2.2483874 .360713$

H $3.628845-1.2476530 .629384$

H $5.013299-2.2642921 .045465$

H $4.413826-4.6113590 .724224$

H $\quad 0.1015341 .663779-3.071700$

H $2.3733790 .901147-1.141411$

H $1.5529203 .384876-3.942927$

H $2.1431184 .785900-3.076421$

H $0.6389754 .035949-2.558714$

H $4.185091-3.668309-1.122347$

H $3.369546-2.163723-1.516860$

H $6.573934-0.055034-3.214793$

H $7.610599-0.488738-1.845123$

H $6.8307921 .594317-0.582699$

H $6.4699202 .329295-3.535784$

H $7.4721304 .408616-2.475869$

H $6.8113703 .979324-0.917423$

H $4.3635506 .015525-3.629665$

H $6.0930755 .544840-4.095388$

H $3.4729685 .378193-1.502634$ 
H $4.5745174 .748903-0.325795$

H $3.9700412 .490760-0.707708$

H $4.692184-0.154661-1.395280$

C $-6.271959-0.395114-2.124804$

O $-5.4736240 .702000-1.633630$

C $-5.7837310 .894896-0.239057$

C -7.061555 0.1060490 .020675

C -6.854707 -1.086631 -0.892504

H $-4.976487 \quad 0.427036 \quad 0.334464$

C -5.8803132 .3849660 .095880$

H -7.172473 -0.1715621 .073245$

H -7.952203 $0.670803-0.279510$

C -8.126732 -1.871090 -1.163297

H $-6.113703-1.754146-0.435137$

C $-4.5510993 .153701-0.072445$

O $-6.8618092 .972752-0.765231$

H -6.2542752 .5052721 .120130$

H $-4.2589753 .194460-1.128737$

O $\quad-4.776444 \quad 4.507614 \quad 0.340804$

C -3.3857132 .5817140 .763588$

C $-2.153675 \quad 3.5111110 .789975$

H $-3.0933031 .608550 \quad 0.353970$

O -3.8575012 .3698512 .094967$

H -1.899158 $3.807317-0.234513$

H $-2.4158984 .413856 \quad 1.355589$

H -8.534542 -2.275085 -0.230942

H -8.897099 -1.242112 -1.621735

H -7.931608 -2.710534 -1.837813

C $-5.455578-1.283936-3.066430$

H -7.065732 $3.856641-0.416032$

H $-5.3512154 .924163-0.323060$

H $-3.148773 \quad 1.9302382 .593611$

H -5.327946 -0.740992 -4.012108

H $-6.016378-2.195183-3.302416$

H $-7.0869240 .056063-2.707019$

C -0.2280491 .8939983 .563933$

O -1.2420912 .5638992 .807910$

C -0.9147922 .8630081 .448595$

C $0.3181393 .780793 \quad 1.393848$

C 1.4934883 .1946112 .186376

C 1.0669342 .7198013 .580512

H -0.6205561 .8609194 .588632$

C $-0.0249450 .449518 \quad 3.095362$

H -0.7014861 .9308230 .913721$

O $-0.0179945 .060786 \quad 1.927396$

H $0.601632 \quad 3.9267700 .345468$

H 2.2766843 .9566732 .280379

O 2.0410422 .1022021 .457160

H 1.8647712 .1333924 .051101

O $0.8383893 .860142 \quad 4.412552$

C -1.298855 $-0.349132 \quad 3.237905$

H $\quad 0.3131420 .3821662 .057412$

H $0.742042-0.0471923 .699804$

H 0.7647785 .6308101 .838503

H 2.8060111 .7682691 .955642

H 1.6701904 .3617644 .457758

O $-1.826258-0.6061452 .006419$

O $-1.773504-0.6918434 .312816$

C $-3.034869-1.3713431 .998565$

H -3.835566 -0.8159522 .495819$

H $-3.319929-1.5439230 .957497$

H -2.875753 -2.339650 2.482650

SCF Energy (B3LYP/6-31G**//MMFF) $=-3245.89212042$

$08 \_00661$

MM̄MF Geometry

C -2.857807 5.4219370 .455867

C -3.2937364 .1989830 .115103$

C -4.4492893 .5135330 .799880$

O $-4.0929792 .130168 \quad 1.039061$

C $-5.6858803 .527232-0.111980$

C -6.9423093 .0686200 .601046$

C -7.5059361 .8532530 .460027$

C $-7.5964114 .105378 \quad 1.478966$

C -7.010735 $0.698040-0.365609$

C $-7.084705-0.6190430 .420841$

C $-6.701690-1.821761-0.452479$

C $-6.972370-3.1344650 .283931$
C $-6.539610-4.350365-0.548345$

O $-5.309617-1.723815-0.771880$

O $-6.531780-5.4965410 .316044$

C $-5.090120-4.133829-1.026811$

C $-7.534657-4.660959-1.672673$

O $-4.691167-5.163840-1.928849$

C $-4.841815-2.744801-1.664532$

C $-3.324501-2.546341-1.881196$

O $-5.501765-2.678309-2.929702$

C -3.2686811 .8642882 .087604$

O -2.7896402 .6690732 .870202$

C -3.0292990 .4034752 .105115$

C $-2.037879-0.0918062 .861205$

C -1.654809-1.5016112.973081

C $-2.555000-2.5206382 .326963$

C $-2.912397-1.119992-2.264222$

C $-1.400030-1.016423-2.288257$

C $0.570346-0.911978-0.757010$

O $-0.764526-1.089950-3.337037$

C $0.881154-2.1450590 .032497$

C $1.591489-2.1335891 .170441$

C $1.888738-3.3611681 .982534$

C $1.558355-3.1874723 .450317$

C $2.532965-3.1217874 .373150$

C $0.098411-3.1634703 .858047$

C $-0.528355-1.8107713 .649257$

N $-0.855708-0.883528-1.020562$

H -3.3882735 .9543921 .243500$

H $-2.8067673 .643732-0.680972$

H -4.6785943 .9952591 .758747$

H $-5.5019472 .911730-1.000349$

H -5.863607 $4.542393-0.492485$

H $-8.438278 \quad 1.6624800 .990662$

H -8.526709 3.7430861 .929247

H -6.9290674 .3964502 .295790$

H -7.8408834 .9982030 .894241$

H -7.644822 $0.623506-1.257186$

H $-5.9838870 .851213-0.709239$

H $-6.410525-0.5562111 .284958$

H $-8.103222-0.7589510 .804685$

H $-7.290285-1.777148-1.376534$

H -8.031142 -3.2172910 .559593$

H $-6.413684-3.1437891 .230516$

H -7.427133 -5.6028660 .680720$

H -4.441072 -4.227662 -0.144545

H -8.530482 -4.859342 -1.258535

H -7.249051 -5.570662 -2.212514

H -7.633662 -3.848112 -2.394977

H $-4.848416-6.014748-1.484800$

H $-2.795174-2.819280-0.961363$

H $-2.966908-3.230652-2.661728$

H -5.064293 -2.008072 -3.478054

H -3.658213 -0.2191891 .478819$

H -1.429081 0.6029893 .438629

H -3.588655 -2.395492 2.669500

H $-2.283247-3.5529392 .555689$

H -2.532982 -2.413444 1.239958

H $-3.290852-0.845736-3.254354$

H $-3.303713-0.382828-1.553817$

H $0.811147-0.001973-0.201336$

H $1.130632-0.915641-1.696755$

H $\quad 0.502613-3.090540-0.349139$

H $1.989200-1.1910341 .537844$

H $2.955227-3.5894331 .860051$

H $1.346995-4.2338881 .597174$

H $2.305051-3.0207835 .429122$

H 3.580642 -3.161979 4.093894

H $0.003759-3.4097314 .923777$

H $-0.420627-3.9639663 .324294$

H $0.034180-0.9966484 .109359$

H - $1.478701-0.814749-0.221442$

C $-0.6426725 .328400-0.872120$

O -0.0371564 .4013600 .050536$

C $1.3920654 .400503-0.149846$

C $1.6234425 .123666-1.466275$

C $0.5110286 .154058-1.441794$

H 1.8120994 .9777870 .683582

C $1.8865132 .952716-0.097105$ 
H $2.6200335 .570022-1.532674$

H $1.4947314 .440555-2.315181$

C $0.2209726 .767625-2.800304$

H $0.7867906 .951723-0.738978$

C $3.3768862 .782616-0.449125$

O $1.647862 \quad 2.4662961 .227142$

H $1.2834902 .331771-0.770399$

H $3.5283883 .113905-1.482813$

$\begin{array}{llllll}\text { O } & 4.169123 & 3.634489 & 0.377486\end{array}$

C $3.8803961 .328838-0.328824$

$\begin{array}{llll}C & 5.305537 & 1.167874 & -0.874493\end{array}$

H $3.2018390 .677509-0.888522$

O 3.8812210 .9013821 .030973

H $5.3492341 .504319-1.918237$

H $5.9877851 .830026-0.324227$

H $1.103207 \quad 7.292303-3.181329$

H $-0.0587906 .004344-3.534197$

H $-0.5993227 .489321-2.735473$

C $-1.6840706 .167844-0.126733$

H 0.6983752 .5738241 .409490

H $4.0511693 .338171 \quad 1.296414$

H $2.972104 \quad 0.9778791 .365422$

H $-2.0735086 .955685-0.781441$

H $-1.184908 \quad 6.6656080 .715224$

H -1.109851 $4.738470-1.670191$

C $7.949224-1.429379-1.244762$

O $7.194896-0.211781-1.309771$

C $5.871006-0.262682-0.761061$

C $5.032258-1.323539-1.490597$

C $5.729316-2.688093-1.497405$

C $7.194282-2.580424-1.930734$

H $8.854202-1.229752-1.833445$

C $8.387832-1.7572760 .191156$

H $5.942009-0.5158020 .301810$

O $4.776838-0.913729-2.832665$

H $4.060711-1.421708-0.995352$

H $5.200065-3.362960-2.181245$

O $5.674821-3.260681-0.193970$

H $7.721346-3.523791-1.745382$

O $7.244225-2.341987-3.340642$

C $9.353106-0.7300750 .746703$

H $7.536916-1.8053490 .875465$

H $8.903246-2.724820 \quad 0.200191$

H $5.634545-0.761306-3.265167$

H $4.737515-3.3497630 .048702$

H $6.815803-3.096070-3.780608$

O $9.692248-1.0887302 .021151$

$\begin{array}{lllll}\text { O } & 9.760557 & 0.256285 & 0.149165\end{array}$

C $10.603295-0.2078832 .686094$

H $10.165093 \quad 0.7904142 .779131$

H $10.789233-0.6040473 .687876$

H $11.552508-0.1649352 .143687$

SCF Energy (B3LYP/6-31G**//MMFF) $=-3245.92274027$

08_00662

MM̄FF Geometry

C $-0.8063094 .294790-0.927528$

C $-0.308926 \quad 3.437788-0.024107$

C $0.559473 \quad 3.8444551 .137809$

O 1.7249992 .9867691 .132976

C $-0.199843 \quad 3.6281672 .458396$

C $0.579648 \quad 4.0644913 .686344$

C $1.087648 \quad 3.2295394 .615744$

C 0.7477695 .5541063 .847400

C 1.0053731 .7296284 .642268

C 2.3482771 .0921604 .263992

C $2.182124-0.3738443 .840015$

C $3.542510-1.0181363 .574980$

C $3.409786-2.4389743 .006196$

O $1.398836-0.3783332 .642673$

O $4.693888-2.8257412 .491113$

C $2.430668-2.4179391 .811114$

C $3.057128-3.4564164 .098014$

O $2.180433-3.7507091 .370320$

C $1.109597-1.6791852 .115783$

C $0.219093-1.4654740 .868102$

O $0.347020-2.453193 \quad 3.049602$

C $2.727575 \quad 3.3093090 .269978$
O $2.788366 \quad 4.291704-0.452211$

C $3.731526 \quad 2.223293 \quad 0.322462$

C $4.7238682 .196742-0.579557$

C $5.7714751 .178824-0.686406$

$\begin{array}{llll}\text { C } & 5.724050 & 0.023790 & 0.278207\end{array}$

C $0.840830-0.631392-0.256656$

C $1.518007-1.454935-1.338585$

C $2.956239-1.195092-3.357873$

O $1.385498-2.671431-1.441354$

C $4.367501-1.522462-2.981999$

C $5.432979-1.102011-3.681433$

C $6.850922-1.476835-3.355163$

C $7.770474-0.279145-3.266156$

C $8.5207370 .094998-4.316484$

C $7.887495 \quad 0.425965-1.934104$

C $6.7097021 .317503-1.646431$

N $2.255897-0.659602-2.205608$

H $-0.6061825 .358404-0.829596$

H $-0.5215912 .376250-0.127958$

H $0.871415 \quad 4.8923701 .051000$

H -0.4884332 .5751552 .531714$

H $-1.144375 \quad 4.1888862 .439032$

H 1.6298783 .6616975 .455734

H 1.2178695 .8184904 .800819

H 1.3749495 .9614593 .048799

H $-0.226816 \quad 6.0520553 .815181$

H $\quad 0.728517 \quad 1.413498 \quad 5.655698$

H 0.2029831 .3621953 .998790

H $2.796215 \quad 1.6530203 .434283$

H 3.0366531 .1648605 .115027

H $1.645189-0.9121234 .629845$

H $4.153824-1.0234714 .486086$

H $4.096752-0.4078382 .850054$

H $5.336673-2.7753543 .219288$

H $2.951376-1.9079150 .992071$

H $3.832330-3.4694694 .873670$

H $3.022040-4.4742573 .694148$

H $2.104776-3.2455074 .588940$

H $1.697313-3.7011710 .528690$

H $-0.147450-2.4281890 .499780$

H -0.677007 -0.9240111 .201874$

H $-0.588254-2.2022392 .970291$

H 3.6113541 .4612751 .083290

H $4.7738942 .994287-1.319970$

H $4.750249-0.4748220 .232113$

H $5.8940490 .374077 \quad 1.301859$

H $6.467247-0.749447 \quad 0.074316$

H $0.037259-0.078755-0.760236$

H 1.5445370 .1052280 .141296

H $2.902471-0.441259-4.149702$

H $2.447639-2.101212-3.702951$

H $4.518160-2.163633-2.116633$

H $5.276791-0.488268-4.565873$

H $7.203975-2.155224-4.143176$

H $6.909347-2.052870-2.423284$

H $9.2055010 .934170-4.252341$

H $8.462533-0.426801-5.265991$

H $8.7829991 .062245-1.922144$

H $8.066453-0.314248-1.149234$

H $6.6534382 .185505-2.304982$

H $2.3535800 .332617-2.010636$

C $-3.115255 \quad 4.129733-1.904864$

O $-3.6119573 .267145-0.858331$

C $-4.6711352 .459587-1.392925$

C $-5.220838 \quad 3.262171-2.563337$

C $-3.9381713 .809299-3.158296$

H $-4.2104301 .547683-1.786610$

C $-5.6898452 .101988-0.308976$

H $-5.7907962 .644884-3.264009$

H $-5.8611424 .086508-2.229128$

C $-4.1493605 .004754-4.071338$

H $-3.4523383 .009633-3.734180$

C $-5.121948 \quad 1.505606 \quad 0.997544$

$\begin{array}{lllll}\text { O } & -6.408751 & 3.290158 & 0.059176\end{array}$

H $-6.4429241 .425422-0.728910$

H -4.5806432 .2878441 .544900$

$\begin{array}{llll}\text { O } & -6.242297 & 1.141518 & 1.820308\end{array}$

$\begin{array}{lll}\text { C }-4.224896 & 0.257640 & 0.903403\end{array}$ 
C $-4.755378-0.835716-0.034681$

H $-4.150762-0.1635021 .912540$

O $-2.8994920 .633602 \quad 0.539676$

H -5.812803 -1.0397810 .171525$

H $-4.723915-0.479628-1.071168$

H $-4.776194 \quad 4.730258-4.926053$

H -4.641035 5.829849-3.545091

H -3.193036 $5.372741-4.456564$

C -1.624065 $3.858550-2.109719$

H -5.7580403 .9396970 .377002$

H $-6.7973561 .933944 \quad 1.920885$

H -2.858935 $0.748065-0.423096$

H -1.257782 4.392964 -2.993966

H $-1.4642502 .787910-2.292948$

H -3.283078 $5.162087-1.573975$

C $-3.651829-4.136415-1.337642$

O $-4.361760-2.911269-1.122007$

C $-3.943445-2.1458520 .016322$

C $-4.154395-2.9592321 .303885$

C $-3.475410-4.332129 \quad 1.220334$

C $-3.771220-5.047598-0.104771$

H $-4.177149-4.620248-2.171244$

C $-2.204535-3.895712-1.779618$

H $-2.883167-1.899805-0.097618$

O $-5.552212-3.1407901 .524380$

H -3.753612 -2.3989362 .155941$

H -3.823573 -4.9548722 .053631$

O $-2.071874-4.1517051 .376718$

H -3.109089 $-5.912396-0.225123$

O $-5.105959-5.561648-0.057097$

C $-2.147920-3.027029-3.014039$

H -1.600236 -3.427580 -0.999645

H $-1.707558-4.843720-2.017080$

H -5.657406 -3.613471 2.367628

H $-1.657180-5.0308141 .352961$

H $-5.714573-4.804173-0.012752$

O $-1.765772-1.762630-2.673013$

O $-2.431802-3.418377-4.138401$

C $-1.694045-0.822494-3.748673$

H $-0.946846-1.142585-4.480992$

H $-2.675340-0.709102-4.219052$

H $-1.3897590 .142950-3.335331$

SCF Energy (B3LYP/6-31G**//MMFF) $=-3245.92046333$

08_00663

MMFF Geometry

C -2.495458 $5.537853-0.504034$

C $-3.2230024 .412203-0.553119$

C -3.993909 3.8798490 .626135

$\begin{array}{lllll}\text { O } & -3.445555 & 2.587331 & 0.980267\end{array}$

$\begin{array}{llll}\text { C } & -5.474995 & 3.680268 & 0.264521\end{array}$

C -6.2948113 .1880781 .443107$

C -6.7161741 .9166841 .596908$

C -6.6309354 .2283452 .479933$

C -6.4807150 .7775290 .642599$

C -7.471192 -0.3722250 .875993$

C $-7.381049-1.440245-0.222979$

C $-8.391586-2.5606550 .031986$

C $-8.281033-3.679663-1.013947$

O $-6.052235-1.974227-0.240747$

O $-9.009476-4.815837-0.524340$

C $-6.803529-4.107128-1.133025$

C $-8.935340-3.285710-2.343608$

O $-6.626883-5.035926-2.200548$

C $-5.824000-2.920143-1.294758$

C $-4.366703-3.430025-1.195640$

O $-6.015636-2.330113-2.583738$

C -2.3231822 .5807931 .748982$

O $-1.696175 \quad 3.5515612 .143081$

C $-1.985081 \quad 1.1702872 .041176$

C -0.7979080 .8904782 .598739$

C $-0.308929-0.4311462 .995978$

C -1.256587-1.594203 2.872805

C $-3.313960-2.322083-1.045410$

C $-1.901108-2.860322-1.206861$

C $0.480421-2.136811-1.107199$

O $-1.656164-4.014609-1.548677$

C $1.031337-2.773240 \quad 0.130122$
C $2.120996-2.3253260 .771502$

C $2.723377-2.999731 \quad 1.969047$

C $2.897323-2.0655033 .143816$

C $4.108453-1.5913053 .480806$

C $1.676609-1.7433763 .975642$

C $0.950885-0.5277013 .469429$

N $-0.941181-1.890581-0.950352$

H -2.481142 6.1224290 .412637

H -3.224982 $3.815317-1.462054$

H $-3.913816 \quad 4.5562501 .486573$

H $-5.5494122 .979683-0.574385$

H $-5.9074774 .622998-0.095790$

H -7.272109 1.6691062 .499993

H -7.279867 3.8334263 .268988

H -5.7226614 .6051042 .959670$

H -7.1554045 .0700122 .015892$

H $-6.5878851 .120585-0.391738$

H -5.4575490 .4080350 .776098$

H $-7.255016-0.8265541 .851819$

H -8.495566 0.0194250 .915428

H $-7.586485-0.954537-1.184444$

H $-9.414249-2.164356 \quad 0.062877$

H -8.209213 -2.9959051 .024729$

H $-9.929916-4.539782-0.374223$

H $-6.543709-4.643977-0.209701$

H $-9.999061-3.063682-2.195444$

H $-8.900025-4.112487-3.061613$

H -8.477270 -2.408198 -2.804229

H $-7.246820-5.770190-2.051244$

H $-4.266001-4.090392-0.324217$

H $-4.137739-4.031124-2.085012$

H $-5.186680-1.919094-2.874599$

H -2.720335 $0.410672 \quad 1.802413$

H $-0.110176 \quad 1.7139562 .789983$

H -2.127715 -1.4468323 .520692$

H $-0.811663-2.5522663 .148425$

H $-1.606158-1.7034301 .842035$

H -3.454393 -1.542098 -1.800862

H $-3.401625-1.858550-0.056023$

H $0.953419-1.175462-1.324885$

H $0.632704-2.804339-1.961740$

H $\quad 0.534400-3.669614 \quad 0.494046$

H $2.639329-1.4493620 .392513$

H $3.696945-3.4030971 .662508$

H $2.129055-3.8671852 .281989$

H $4.241185-0.9406734 .338877$

H $4.991105-1.8336152 .900059$

H $1.980716-1.5337885 .010192$

H $1.039772-2.6288144 .048474$

H $1.5492770 .383183 \quad 3.527482$

H $-1.231892-0.977878-0.611654$

C $-0.1640725 .666316-1.501106$

O $-0.0025974 .241158-1.646233$

C $0.9764493 .784014-0.687401$

C $1.6618925 .038257-0.163240$

C $0.5254186 .041701-0.189616$

H $\quad 0.406703 \quad 3.2939770 .111487$

C $1.9108852 .777848-1.368303$

H $2.085787 \quad 4.899438 \quad 0.835917$

H $2.4678955 .353609-0.837598$

C $0.9813947 .488479-0.127576$

H -0.1219495 .8367770 .671206$

C $3.0379632 .267206-0.449310$

O $1.1158701 .669559-1.799555$

H $2.3442993 .225490-2.270810$

H $3.6055483 .125326-0.072432$

O 2.4645361 .6231560 .688793

C $4.0379141 .317024-1.144544$

C $5.1612190 .874876-0.193682$

H $4.4696281 .841971-2.003715$

$\begin{array}{llll}\text { O } & 3.388562 & 0.149703 & -1.635955\end{array}$

H $5.6821401 .755186 \quad 0.204340$

H 4.7276050 .3721110 .681479

H $1.524075 \quad 7.6811980 .803640$

H $1.6468617 .736268-0.961373$

H $0.1239288 .167725-0.166638$

C -1.644902 $6.039061-1.636134$

H $0.4077712 .022985-2.365264$ 
$\begin{array}{llll}\text { H } & 1.941246 & 0.870528 & 0.363725\end{array}$

H $2.7244290 .431943-2.286998$

H $-2.0272215 .636539-2.582873$

H -1.750946 7.128787-1.690049

H $0.374767 \quad 6.114152-2.346634$

C $8.188235-1.301716-0.117443$

$\begin{array}{lllll}0 & 7.148737 & -0.371562 & 0.216867\end{array}$

C $6.182333-0.103667-0.810243$

C $6.8722650 .437047-2.072168$

C $8.001604-0.486511-2.538464$

C $8.936185-0.866222-1.388258$

H $8.900224-1.2368750 .715511$

C $7.663442-2.745281-0.183091$

H $5.657839-1.035181-1.047335$

O $7.394227 \quad 1.741999-1.829295$

H $6.1343570 .531718-2.876040$

H $8.5809260 .014889-3.323431$

O $7.451243-1.676637-3.095408$

H $9.623794-1.662156-1.697380$

O $9.7376640 .268254-1.045058$

C $7.301993-3.2873731 .184872$

H $6.774034-2.823838-0.813338$

H $8.442446-3.402728-0.586293$

H $8.0010121 .683216-1.071464$

H $6.884412-1.417404-3.841645$

H $10.252624 \quad 0.512024-1.833064$

O $6.701314-4.5054541 .033022$

O $7.534604-2.7334282 .250241$

C $6.334723-5.1685752 .247195$

H $5.677720-4.5346502 .849775$

H $5.794753-6.0823001 .985648$

H $7.232424-5.4388602 .811035$

SCF Energy (B3LYP/6-31G**//MMFF) $=-3245.93005002$

08_00664

MM̄FF Geometry

C $-0.635312-3.8309030 .433831$

C $-1.357226-2.7359550 .148002$

C $-1.835566-2.398917-1.241080$

O $-1.538091-1.006701-1.501697$

C $-3.362230-2.546417-1.334259$

C $-3.839559-2.779983-2.753001$

C $-4.275985-1.821493-3.592626$

C $-3.842706-4.223216-3.189150$

C $-4.311270-0.334128-3.375800$

C $-5.7372050 .202189-3.192888$

C $-6.2439650 .050182-1.751200$

C $-7.717445 \quad 0.446754-1.651009$

C -8.227802 $0.360277-0.206132$

O $-5.4549420 .916131-0.927672$

O $-9.4932171 .041531-0.169933$

C -7.2566591 .1298720 .715701$

C $-8.523729-1.0886560 .196976$

O \begin{tabular}{lllll}
\hline & -7.595931 & 0.947252 & 2.087934
\end{tabular}

C $-5.752977 \quad 0.844895 \quad 0.470339$

C $-4.908395 \quad 1.936453 \quad 1.167194$

O $-5.335802-0.3968181 .036669$

C $-0.252802-0.662885-1.765600$

O $0.708914-1.407753-1.844566$

C $-0.2229390 .807352-1.915889$

C $0.9409921 .460594-1.793645$

C $1.1336112 .906465-1.917574$

C $-0.0688013 .745554-2.263951$

C $-3.392907 \quad 1.8072420 .945903$

C -2.6333252 .8844831 .705868$

C -0.3254963 .5978222 .308100$

O -3.1945693 .7815672 .331114$

C $0.2239604 .618663 \quad 1.362744$

C $1.533163 \quad 4.898246 \quad 1.269020$

C 2.1076835 .9577720 .373568

C $3.2167045 .435840-0.512271$

C $4.5067925 .574667-0.162317$

C $2.8306084 .833645-1.842874$

C $2.3698953 .408583-1.719376$

N -1.2572382 .7292221 .610472$

H $-0.415072-4.525913-0.374566$

H $-1.620150-2.0294340 .929311$

H $-1.335581-3.020059-1.995204$
H $-3.848195-1.657451-0.917227$

H $-3.708777-3.389532-0.721443$

H $-4.623086-2.128959-4.578745$

H $-4.188979-4.346874-4.220783$

H -2.833699 $-4.643153-3.128269$

H $-4.504161-4.812494-2.545693$

H $-3.672098-0.021585-2.546217$

H $-3.8790590 .128789-4.272253$

H $-5.7259351 .269425-3.450732$

H $-6.420042-0.294488-3.893056$

H $-6.107820-0.986994-1.428219$

H $-8.335301-0.173119-2.312718$

H $-7.8421251 .480028-2.004620$

H $-9.794138 \quad 1.0548820 .755163$

H -7.4296962 .1958890 .510990$

H $-9.327772-1.498070-0.427142$

H $-8.888886-1.1495031 .227820$

H -7.663488 -1.7520250 .092749$

H -7.2720530 .0735872 .363992$

H -5.2195052 .9241810 .801916$

H -5.1021851 .9129432 .247600$

H $-6.069224-1.0267201 .002349$

H -1.163656 $1.311048-2.106670$

H $1.839750 \quad 0.883765-1.580009$

H $0.1353714 .818153-2.273480$

H $-0.8700873 .595588-1.531824$

H $-0.4477853 .480129-3.256947$

H $-3.0400750 .829091 \quad 1.291604$

H $-3.152631 \quad 1.907195-0.118444$

H $\quad 0.4595152 .9631182 .727618$

H $-0.8396874 .101347 \quad 3.132987$

H -0.4855505 .1753540 .755541$

H 2.2344354 .3642001 .905941

H $2.4940456 .761062 \quad 1.014874$

H $1.3315036 .423924-0.245830$

H $5.3077825 .227025-0.806355$

H 4.7896556 .0324860 .779817

H $3.6958254 .835161-2.520028$

H $2.0977825 .478366-2.336311$

H $3.1718142 .718031-1.457878$

H -0.8763171 .9836061 .035139$

C $0.149361-3.0877192 .774465$

O $1.212203-2.2373862 .296860$

C $1.981082-1.7778933 .426419$

C $1.252826-2.2786514 .670061$

C $0.606270-3.5501164 .155465$

H $2.958019-2.2700203 .372848$

C $2.134227-0.2553383 .364263$

H $1.935984-2.4513475 .507428$

H $0.489040-1.5656125 .003493$

C $-0.513295-4.0615395 .044941$

H $1.377662-4.3258724 .058402$

C $2.997377 \quad 0.2487162 .184586$

$\begin{array}{llll}\text { O } & 0.829452 & 0.327680 & 3.253415\end{array}$

H 2.5483830 .1155064 .308693

H $2.534899-0.0389871 .234426$

$\begin{array}{lllll}\text { O } & 2.974109 & 1.681267 & 2.216707\end{array}$

C $4.467401-0.2246132 .229440$

C 5.3999500 .4932851 .233231

H $4.513284-1.3067402 .069123$

$\begin{array}{lllll}\text { O } & 4.977077 & 0.026445 & 3.544343\end{array}$

H 5.3111821 .5794591 .356991

H $6.434306 \quad 0.249274 \quad 1.512778$

H $-0.133106-4.2958716 .044616$

H $-1.310444-3.3185465 .154385$

H $-0.956727-4.9724784 .631358$

C $-0.076046-4.2260581 .777385$

H 0.4342380 .0052572 .425167

H 3.3926451 .9634613 .048059

H $5.867618-0.3610343 .592908$

H $-0.740010-4.9852202 .205618$

H $0.889767-4.7123861 .586875$

H $-0.748906-2.4669522 .878075$

C $5.465392-1.831391-1.682121$

O $5.597670-1.260097-0.374051$

C $5.1716140 .100257-0.233761$

C $5.9582941 .011927-1.190546$

C $5.8646380 .513918-2.635252$ 
C $6.186605-0.980541-2.739525$

H $5.985419-2.796189-1.617935$

C $4.000686-2.110837-2.026161$

H $4.1040180 .172775-0.460432$

O $7.3264411 .060924-0.789344$

H $5.5581282 .029929-1.115498$

H $6.5643381 .085144-3.257241$

O $4.5496590 .764981-3.120906$

H $5.942064-1.359361-3.738809$

O $7.592433-1.170147-2.555831$

C $3.446650-3.186814-1.123993$

H $3.360543-1.232926-1.913875$

H $3.895304-2.439955-3.066780$

H $7.7867591 .671425-1.389980$

H $4.5132610 .459570-4.043276$

H $8.049617-0.666488-3.250581$

O $3.599389-4.409925-1.706216$

O $2.962954-2.966772-0.021584$

C $3.133012-5.520442-0.933739$

H $3.631250-5.5462240 .040216$

H $2.047681-5.462654-0.811690$

H $3.376872-6.437854-1.475933$

SCF Energy (B3LYP/6-31G*//MMFF) $=-3245.90025213$

08_00665

MM̄FF Geometry

C -2.781871 $3.405735-1.399143$

C -3.929393 $3.970274-0.990080$

C $-4.9834523 .317675-0.120146$

O $-4.523052 \quad 2.045951 \quad 0.395332$

C $-6.2738703 .084885-0.923742$

C $-7.4564252 .703312-0.050752$

C -7.9892971 .4684930 .039269$

C $-8.066952 \quad 3.838144 \quad 0.733458$

C -7.548303 $0.219710-0.668452$

C $-6.935204-0.7865990 .313066$

C $-6.322302-1.989487-0.416809$

C $-5.752661-2.9994170 .580034$

C $-5.024143-4.155590-0.123017$

O $-5.277418-1.496870-1.260876$

O $-4.251188-4.8549340 .865121$

C $-4.024624-3.574911-1.147650$

C $-6.007960-5.176711-0.706212$

O $-3.446303-4.611354-1.936654$

C $-4.640278-2.498011-2.064689$

C $-3.594475-1.773973-2.947468$

O $-5.579533-3.118376-2.951341$

C -3.7063602 .0897021 .486059$

$\begin{array}{llll}\text { O } & -3.388736 & 3.081229 & 2.124656\end{array}$

C $-3.208816 \quad 0.7196881 .742217$

C $-2.154616 \quad 0.5583842 .555850$

C -1.523711 -0.7072502 .933791$

C $-2.105134-1.9763322 .372365$

C $-2.490488-1.029676-2.187246$

C -1.207353-1.827737-2.029335

C $1.045941-1.682249-0.980715$

O $-1.016517-2.915477-2.565894$

C $1.055897-2.3838650 .339498$

C $1.853167-2.0289321 .358060$

C $1.951818-2.7879352 .648324$

C $1.705557-1.9231823 .861195$

C $2.725938-1.3427114 .515368$

C $0.288979-1.821108 \quad 4.374314$

C $-0.466912-0.6700373 .772206$

N $-0.280551-1.159237-1.241144$

H $-2.5705092 .367386-1.159061$

H -4.116092 $5.011812-1.244519$

H $-5.189536 \quad 3.9928180 .719438$

H $-6.0886802 .326259-1.690491$

H $-6.5440563 .995972-1.474961$

H -8.8454341 .3259830 .697851$

H -8.966304 3.5312411 .278323

H $-7.356607 \quad 4.228108 \quad 1.468423$

H $-8.355407 \quad 4.6524370 .060595$

H $-8.423548-0.231068-1.152347$

H $-6.8440620 .441065-1.473133$

H $-6.156331-0.2830290 .898510$

H $-7.702719-1.1293221 .018112$
H $-7.100030-2.453882-1.034522$

H $-6.541803-3.3848501 .237548$

H $-5.036668-2.4920401 .240311$

H $-4.859489-5.1627701 .558776$

H $-3.206127-3.126276-0.572428$

H $-6.632708-5.6030180 .088095$

H -5.478626 -6.023690 -1.156270

H $-6.676488-4.748487-1.455838$

H $-3.049835-5.254647-1.324334$

H -3.180692 -2.468297 -3.688716

H $-4.136306-1.017013-3.533124$

H $-5.702396-2.542177-3.723813$

H $-3.706057-0.1058341 .247880$

H -1.7045641 .4462692 .999447$

H -3.117431 -2.136080 2.758344

H $-1.520851-2.8669602 .613810$

H -2.148054 -1.929624 1.279304

H $-2.222248-0.132698-2.760518$

H -2.837072 -0.696765 -1.203449

H $1.740318-0.838391-1.009520$

H $1.330523-2.383804-1.771550$

H $0.412238-3.2541680 .443008$

H $2.517822-1.1773291 .238233$

H $2.960356-3.2178362 .704910$

H $1.270459-3.6474582 .660985$

H $2.559263-0.7485305 .407656$

H $3.748934-1.4427464 .167331$

H $0.303593-1.6628695 .461562$

H $-0.223831-2.7794274 .249872$

H $-0.113390 \quad 0.303356 \quad 4.116075$

H $-0.541942-0.283128-0.797786$

C $-0.4174894 .308806-1.327149$

O $0.1411603 .020037-0.991438$

C 0.4733083 .0240110 .408699

C 0.5648804 .4885850 .810693

C $-0.5687705 .078091-0.009032$

H -0.3890792 .5885870 .931573$

C 1.6845132 .1267680 .688030

H 0.4428914 .6400121 .887070

H 1.5083944 .9450320 .499657

C $-0.4924476 .586574-0.168425$

H $-1.5122014 .827196 \quad 0.489611$

C 3.0010232 .5620760 .008654

O 1.8459792 .0303982 .101074

H $1.427526 \quad 1.128640 \quad 0.316199$

H $2.7886662 .868434-1.022222$

O 3.5475953 .6969250 .679680

C $4.0696871 .447683-0.041280$

C $5.3744521 .946128-0.692769$

H $3.6675000 .601485-0.609393$

O $4.340371 \quad 0.992882 \quad 1.282327$

H $5.1331102 .532665-1.587341$

H 5.8888932 .6015130 .021296

H -0.5451317 .0795820 .807891$

H $0.4424686 .893306-0.649373$

H $-1.3230846 .954737-0.778875$

C -1.702705 $4.136266-2.148566$

H 2.5536691 .3863132 .272836

H 3.7303343 .4318211 .597437

H 4.9733900 .2575591 .217913

H - $1.477973 \quad 3.552151-3.049396$

H -2.057806 $5.118580-2.481485$

H $0.3298304 .800258-1.963320$

C $7.602840-1.015062-0.119921$

O $\quad 6.7351130 .108710 \quad 0.080716$

C $6.3266830 .802662-1.100421$

C $7.5500521 .337252-1.863982$

C $8.5602530 .221895-2.150135$

C $8.866163-0.600355-0.894454$

H $7.914517-1.3123840 .890060$

C $6.869369-2.207098-0.755090$

H $5.7668150 .116874-1.745513$

O $8.1794182 .363800-1.098943$

H $7.2090211 .789719-2.802866$

H $9.4922090 .658673-2.529151$

O $8.047604-0.644642-3.157716$

H $9.444776-1.494740-1.153598$

O $9.6759910 .178098-0.009046$ 
C $5.868761-2.8366490 .191727$

H $6.323379-1.933427-1.661056$

H $7.606874-2.978002-1.009575$

H $8.9259742 .703691-1.620954$

H $7.887814-0.108616-3.953022$

H $10.4950220 .403018-0.482297$

O $5.573868-4.094240-0.253250$

O $5.388989-2.291701 \quad 1.175891$

C $4.639409-4.836787 \quad 0.535982$

H $4.680776-5.881548 \quad 0.216503$

H $4.895401-4.7884201 .598818$

H $3.629490-4.456845 \quad 0.362377$

SCF Energy (B3LYP/6-31G**//MMFF) $=-3245.91677233$

08_00666

MMFF Geometry

C $-2.3517612 .544770-1.317056$

C $-3.383033 \quad 3.146659-0.703230$

C $-4.0283712 .740566 \quad 0.602555$

O -3.3825331 .5900251 .190541$

C $-5.502590 \quad 2.357450 \quad 0.386027$

C -6.3666122 .6776591 .589471$

C $-6.6882701 .809622 \quad 2.567830$

C $-6.911276 \quad 4.083214 \quad 1.619734$

C -6.2228710 .3915932 .744771$

C $-7.344326-0.6308542 .516200$

C -7.637333 -0.8731491 .028104$

C $-8.824045-1.8230730 .856810$

C $-9.065366-2.174560-0.619039$

O $-6.462966-1.4519960 .449568$

O $-9.966924-3.290931-0.665313$

C $-7.734441-2.642250-1.243895$

C $-9.751220-1.030683-1.375046$

O $-7.872723-2.842972-2.648808$

C $-6.547702-1.691769-0.959366$

C $-5.224497-2.356979-1.407430$

O $-6.733024-0.479673-1.693011$

C -2.2405301 .7798771 .900817$

O $-1.6689262 .833532 \quad 2.124460$

C $-1.7767850 .439558 \quad 2.314935$

C -0.4695690 .2288052 .522069$

C $0.144295-1.0517432 .877967$

C $-0.774991-2.219197 \quad 3.130785$

C $-3.954391-1.634191-0.930898$

C $-2.697497-2.311366-1.452776$

C $-0.217538-2.084755-1.446084$

O $-2.721999-3.308341-2.170169$

C $0.404073-2.930945-0.379466$

C $1.675676-2.7793090 .021443$

C $2.347985-3.6433601 .050191$

C $3.106094-2.8424472 .087352$

C $4.434758-2.6678551 .988871$

C $2.339402-2.3200083 .280993$

C $1.490050-1.1249122 .942774$

$\mathrm{N}-1.543514-1.664134-1.032641$

H $-1.9195671 .638159-0.904470$

H -3.778709 $4.060624-1.143470$

H -3.9511493 .5883901 .295503$

H $-5.579967 \quad \begin{array}{lll}1.292495 & 0.141519\end{array}$

H $-5.9246842 .889112-0.477232$

H -7.3429382 .1602503 .365137$

H -7.524305 4.274617 2.506877

H -6.0930374 .8102841 .621947$

H -7.5369594 .2663920 .740091$

H -5.3577850 .1589312 .118557$

H -5.8722810 .2952033 .780291$

H -7.024480 -1.577412 2.971534

H $-8.256340-0.3123273 .035988$

H -7.8481190 .0861420 .542871$

H $-9.731220-1.3974991 .303765$

H $-8.628340-2.7545651 .406393$

H -10.786206 -3.033494 -0.208745

H -7.500483 -3.625798 -0.812460

H -10.720406 -0.796955 -0.918099

H $-9.969787-1.316378-2.410017$

H $-9.162903-0.110797-1.387528$

H -8.617683 -3.453894 -2.782076

H $-5.183721-3.380880-1.012731$
H $-5.203834-2.430160-2.502493$

H $-5.867435-0.090932-1.893383$

H -2.520706 $-0.345640 \quad 2.387857$

H $\quad 0.218752 \quad 1.0634122 .393855$

H $-0.250857-3.1503063 .353701$

H -1.396137 -2.421333 2.251082

H -1.431729 -2.011108 3.982685

H $-3.945257-0.595768-1.277883$

H $-3.908100-1.6322830 .164122$

H $0.360129-1.176959-1.639762$

H $-0.289500-2.658725-2.375465$

H $-0.202363-3.7204560 .057468$

H $2.283661-2.011762-0.448954$

H $3.041537-4.3095790 .520340$

H $1.628451-4.3017581 .552158$

H $4.991989-2.1179192 .740063$

H $4.994910-3.0648121 .148575$

H $3.035282-2.0066774 .070967$

H $1.768431-3.1386233 .727518$

H $2.070490-0.2257352 .730478$

H $-1.617131-0.873876-0.398827$

C $-0.2989763 .663590-2.305887$

O $0.6106282 .628284-1.883175$

C $1.4192053 .135631-0.806496$

C $1.2341734 .649880-0.822856$

C $-0.2133264 .770260-1.252472$

H 0.9936102 .7262540 .119065

C $2.8855132 .689530-0.919134$

H 1.4327775 .1030050 .153109

H $1.8883975 .130216-1.559689$

C $-0.5886346 .146044-1.773914$

H $-0.8391904 .539853-0.382620$

C $3.0868521 .161687-0.940445$

O $3.4759153 .251634-2.091710$

H $3.4142993 .116990-0.061594$

H $2.5944840 .703014-0.074199$

O $2.4683190 .611000-2.109420$

C $4.5644720 .717501-0.982035$

C $5.3904251 .223172 \quad 0.214822$

H $5.0326481 .042600-1.918749$

O $4.592378-0.712012-0.988467$

H $5.407198 \quad 2.3177150 .206925$

H $4.8993010 .891713 \quad 1.138758$

H $-0.4437806 .904386-0.997550$

H $0.0223886 .428939-2.637677$

H $-1.6392136 .171996-2.080113$

C $-1.6963393 .083528-2.558900$

H $2.9944332 .894935-2.858052$

H $1.5209330 .824248-2.069731$

H $4.088771-1.008278-1.765999$

H -1.620262 $2.255890-3.274887$

H $-2.3274943 .850558-3.023486$

H $0.0933324 .029630-3.263975$

C $8.103831-1.3403540 .534511$

O $6.832932-0.678004 \quad 0.547520$

$\begin{array}{llll}\text { C } & 6.846180 & 0.711451 & 0.209677\end{array}$

C 7.7210351 .4807551 .211952

C $9.130673 \quad 0.8854101 .295870$

C $9.099609-0.6364931 .472211$

H $7.905182-2.3365150 .950885$

C $8.632528-1.527403-0.895665$

H $7.2482090 .840965-0.800907$

$\begin{array}{lllll}\text { O } & 7.120673 & 1.465677 & 2.506207\end{array}$

H 7.7800992 .5296870 .899400

H 9.6654601 .3353402 .141173

O $9.854651 \quad 1.1955210 .108784$

H $10.099386-1.0628061 .328767$

O $8.703584-0.9378092 .814115$

C $7.722587-2.408012-1.727568$

H $8.728176-0.568999-1.412772$

H $9.616502-2.008955-0.869575$

H 6.9879790 .5364422 .761274

H 9.8913702 .1642700 .031750

H $9.362973-0.5426973 .409645$

O $8.058539-2.275363-3.045054$

O $6.844234-3.134800-1.283741$

C $7.278156-3.052247-3.958956$

H $6.222855-2.770801-3.890985$ 
H $7.632376-2.842829-4.971862$

H $7.408111-4.119377-3.754950$

SCF Energy (B3LYP/6-31G**//MMFF) $=-3245.92161424$

$08 \_00667$

MM̄FF Geometry

C $3.474178 \quad 1.0384930 .263112$

C 3.7011491 .8542971 .302676

C 2.7097322 .2228922 .380466

O $1.3533791 .902267 \quad 1.996337$

C 2.7544423 .7453972 .639913

C 1.7352714 .2129313 .662266

C 0.7394295 .0859253 .410704

C 1.8920493 .6388555 .047769

C 0.4463755 .8332942 .137801

C -0.6473905 .1684141 .292361$

C -0.1101514 .4592550 .038909$

C $0.3396385 .453242-1.037448$

C $0.7901764 .744129-2.325233$

O $-1.169373 \quad 3.638518-0.474295$

O $0.8808845 .733272-3.362695$

C $-0.3054343 .742170-2.749088$

C $2.1926444 .141865-2.180102$

O $\quad 0.1206792 .960765-3.863100$

C $-0.7868072 .831477-1.597356$

C $-1.9954111 .957828-1.999603$

O $0.2747961 .934375-1.249741$

C 0.7851830 .7666192 .481265

O $1.342824-0.1538213 .054856$

C -0.6734710 .8881492 .248500$

C -1.520709 -0.0484692 .700483$

C -2.9861170 .0280992 .655388$

C -3.5994381 .2673162 .058550$

C $-3.2711772 .724754-2.349888$

C $-4.3958961 .767837-2.697170$

C $-5.9424830 .107472-1.703052$

O $-4.7393641 .568695-3.860275$

C $-6.480536-0.229180-0.349357$

C $-6.306317-1.4192670 .245877$

C $-6.903377-1.7871601 .572291$

C $-5.866017-2.1736232 .601888$

C $-5.634347-3.4656022 .892167$

C $-5.202523-1.0592473 .380868$

C $-3.708020-0.9807143 .187978$

N $-4.9536161 .160498-1.583504$

H 2.5018540 .5691300 .145235

H $4.6953372 .285527 \quad 1.407070$

H 2.9863251 .6901623 .298673

H 2.6095134 .2680341 .688666

H 3.7546534 .0324692 .990613

H $0.055391 \quad 5.3250134 .224702$

H 1.2243354 .1160815 .773245

H 1.6687872 .5677855 .052085

H 2.9168553 .7850455 .404431

H $0.094817 \quad 6.8307462 .433672$

H 1.3599196 .0148921 .564913

H -1.227791 4.4570671 .894811

H -1.372944 5.9305070 .977641

H $\quad 0.7189563 .8115620 .333981$

H $1.1385166 .108220-0.671670$

H $-0.4984956 .118962-1.287631$

H $1.5143286 .412731-3.074876$

H -1.149838 4.342872 -3.107489

H $2.9216924 .923632-1.934991$

H $2.5342223 .701497-3.123246$

H $2.2533793 .381535-1.399904$

H $0.7375522 .285583-3.532414$

H -1.718811 $1.295337-2.830386$

H -2.219186 $1.282649-1.163802$

H $-0.098667 \quad 1.202407-0.732075$

H -1.0191351 .7947531 .764913$

H -1.109571 -0.9310513 .188493$

H -3.391909 2.1389692 .688845

H -4.6822281 .2022841 .934601$

H $-3.193131 \quad 1.4568741 .060643$

H $-3.5925163 .360024-1.516587$

H -3.118695 $3.377875-3.214822$

H $-5.463335-0.749843-2.188160$
H $-6.7479060 .453913-2.359356$

H $-7.065807 \quad 0.5377920 .152073$

H $-5.738617-2.192222-0.266663$

H -7.593101 -2.624573 1.402459

H -7.526705 -0.976437 1.969832

H $-4.930046-3.7533393 .665584$

H $-6.143087-4.2640812 .362222$

H $-5.387742-1.2293254 .450713$

H $-5.697571-0.1072073 .170421$

H -3.167997 -1.832010 3.604789

H $-4.5004531 .292532-0.685156$

C $5.148247-0.651681-0.644599$

O $4.153013-1.677840-0.451436$

C $4.568863-2.5078020 .649004$

C $6.063807-2.2646360 .801011$

C $6.135553-0.7766000 .518580$

H $4.064733-2.1252211 .544680$

C $4.173321-3.9669500 .412473$

H $6.432170-2.5325181 .795689$

H $6.643264-2.8230490 .056512$

C $7.535123-0.2834350 .194938$

H $5.767205-0.2389901 .400774$

C $2.660228-4.192490 \quad 0.205111$

O $4.853170-4.458330-0.749106$

H $4.529117-4.5800821 .248746$

H $2.365802-3.807184-0.777108$

O $2.436559-5.6072420 .154985$

C $1.763720-3.5779361 .295647$

C $0.287941-4.0102831 .243168$

H $1.820766-2.4852301 .236332$

O $2.275965-3.9565262 .577290$

H $\quad 0.200403-5.0752301 .493630$

H $-0.223971-3.4770212 .055608$

H $8.208815-0.4572291 .040491$

H $7.951401-0.798803-0.677044$

H $7.5299350 .790127-0.018060$

C $4.4891810 .721841-0.800051$

H $4.601231-3.891146-1.498087$

H $2.650800-5.969317 \quad 1.031995$

H $1.752256-3.4903793 .251226$

H $3.9596270 .753064-1.760605$

H $5.2580631 .501775-0.851201$

H $5.656832-0.894690-1.586646$

C $-0.861967-1.886761-1.610523$

O $-0.282319-2.331787-0.377166$

C $-0.423753-3.726221-0.091518$

C $-1.907582-4.129394-0.039724$

C $-2.650362-3.698419-1.308409$

C $-2.356149-2.238998-1.665466$

H $-0.785193-0.794693-1.576258$

C $-0.072618-2.376326-2.833985$

H $\quad 0.072976-4.306793-0.874902$

O $-2.527459-3.5244031 .093192$

H $-1.973577-5.2160900 .090853$

H $-3.730907-3.817181-1.161757$

O $-2.265115-4.530200-2.397742$

H -2.752356 -2.006331-2.660285

O $-3.042457-1.389534-0.743199$

C $1.301697-1.747579-2.913467$

H $\quad 0.064057-3.460553-2.819757$

H $-0.605132-2.098864-3.750859$

H -3.453577 -3.819279 1.111489

H -2.489087 -5.446507 -2.161562

H $-2.662491-1.5379780 .139497$

O $2.004446-2.344011-3.921347$

O $1.705605-0.843102-2.196858$

C $3.331503-1.849686-4.127078$

H $3.972461-2.151795-3.294487$

H $3.719963-2.293016-5.047873$

H $3.328337-0.760976-4.238485$

SCF Energy $\left(B 3 L Y P / 6-31 G^{* *} / / M M F F\right)=-3245.91504935$

08_00668

MMFF Geometry

C -1.1084623 .1331911 .512245$

C -0.4812961 .9888941 .204474$

C $-0.096032 \quad 0.9553122 .233039$

O 1.2607650 .5289151 .969670 
C $-1.020749-0.2661512 .104491$ C $-0.788600-1.3283293 .162401$ C $-0.246108-2.5413982 .933603$ C -1.253858 -0.9659534 .549763$ C $0.256318-3.0935281 .629560$ C $1.783860-3.2293281 .639727$ C $2.345714-3.4510690 .228240$ C $3.856147-3.6834580 .280523$ C $4.480634-3.767971-1.120800$ O $2.056466-2.273926-0.532683$ O $5.904782-3.651374-0.973356$ C $4.011179-2.553919-1.952723$ C $4.223525-5.131003-1.773584$ O $4.455211-2.661331-3.303037$ C $2.486925-2.319465-1.898114$ C $2.052626-0.988645-2.560047$

O $1.830129-3.376576-2.608080$ C 2.2630021 .3263092 .431290 O 2.1445292 .3705623 .052264 C 3.5481020 .7075772 .035085 C 4.6979911 .3598872 .261656 C 6.0435510 .8831091 .932450 C $6.163337-0.4465151 .236331$ C $2.6124920 .282876-1.912070$ C $3.880560 \quad 0.808872-2.563385$ C $5.6432852 .544598-2.247011$ O $4.307797 \quad 0.397212-3.638211$ C $6.8567411 .911571-1.642764$ C $7.7791772 .598023-0.950961$ C $9.0323821 .987831-0.391632$ C 9.2231942 .2826261 .079230 C 10.0215753 .2843221 .485421 C $8.563451 \quad 1.3583212 .076743$ C 7.1008581 .6537662 .263115 N $4.4563161 .829512-1.817465$ H $-1.359537 \quad 3.3388292 .550295$ H $-0.237719 \quad 1.7740080 .166930$ H $-0.159409 \quad 1.3671203 .247833$ H $-0.908206-0.6857921 .101335$ H -2.0694880 .0537762 .173148$ H $-0.142371-3.224913 \quad 3.775528$ H -1.161606 -1.802182 5.251114 H $-0.665003-0.1354864 .950548$ H -2.307943 -0.670338 4.531811 H $-0.195093-4.080776 \quad 1.471509$ H $-0.065623-2.4850770 .782757$ H $2.223326-2.3155732 .059236$ H $2.071417-4.0606592 .295173$ H $1.837456-4.310908-0.223915$ H $4.090620-4.5842120 .861480$ H $4.330501-2.8524510 .818649$ H $6.205704-4.368551-0.389358$ H $4.521084-1.678332-1.534918$ H $4.633331-5.936391-1.151990$ H $4.736206-5.210789-2.738422$ H $3.162645-5.340316-1.925140$ H $3.879068-3.301203-3.754690$ H $2.252523-1.018278-3.637765$ H $0.958922-0.928817-2.470417$ H $0.944136-3.071969-2.865069$ H $3.512922-0.2672271 .563395$ H 4.6548002 .3376732 .739823 H $7.178278-0.6792110 .907707$ H $5.544127-0.4666180 .333204$ H $5.843457-1.2547551 .902250$ H $1.866144 \quad 1.081013-2.017458$ H $2.7832610 .137509-0.841609$ H $5.523053 \quad 3.587697-1.937758$ H $5.7153312 .510661-3.338811$ H $6.9998470 .846699-1.810771$ H $7.6484053 .668922-0.811744$ H $9.8799382 .389227-0.962714$ H $9.0568990 .902918-0.552846$ H $10.188158 \quad 3.4830972 .538866$ H 10.5220553 .9315760 .772663 H $9.041148 \quad 1.465996 \quad 3.060173$ H $8.756974 \quad 0.320331 \quad 1.791985$ H 6.9129002 .6073482 .758731
H $4.0614792 .068797-0.912535$

C $-3.0152114 .187845 \quad 0.288719$

O $-3.3731003 .107643-0.601683$

C $-4.3440213 .593290-1.544406$

C $-4.8017314 .954149-1.036676$

C $-3.5328915 .461479-0.379309$

H $-3.8009863 .764620-2.484501$

C $-5.4404032 .554434-1.831861$

H $-5.1518825 .605693-1.843408$

H $-5.6087714 .863729-0.302146$

C $-3.770596 \quad 6.6130980 .582427$

H $-2.8338795 .784187-1.162714$

C $-5.858376 \quad 1.676979-0.628934$

O $-6.5490203 .225148-2.423250$

H $-5.0422671 .870585-2.592928$

H $-4.960511 \quad 1.166579-0.266332$

$\begin{array}{lllll}\text { O } & -6.353079 & 2.477159 & 0.440597\end{array}$

C $-6.9199960 .612242-0.978659$

C -7.219072 -0.3719420 .172002$

H $-6.5909950 .055713-1.863921$

O $\quad-8.1445291 .263406-1.325444$

H -7.5648080 .1786391 .055595$

H -8.066314 $-0.995972-0.141990$

H -4.202462 7.4705310 .055811

H $-4.460030 \quad 6.3297091 .384819$

H -2.8328616 .9381241 .043647$

C -1.5020384 .1813140 .509476$

H $-7.1971642 .552089-2.690628$

H -5.5958172 .9418850 .833531$

H $-8.7728260 .577555-1.608671$

H -1.1642375 .1491290 .896778$

H $-0.9831074 .010523-0.441813$

H $-3.5471514 .014028 \quad 1.233405$

C $-4.508957-2.838440-0.519806$

O $-5.673356-2.012113-0.634380$

C $-6.028430-1.2726830 .538071$

C $-6.344351-2.2344641 .691799$

C $-5.163105-3.1798261 .947403$

C $-4.652249-3.824700 \quad 0.653149$

H $-4.485418-3.424173-1.447991$

C $-3.224697-1.998569-0.464570$

H $-5.192975-0.6352470 .838998$

O $-7.507112-2.9960971 .374746$

H $-6.565847-1.6487712 .591918$

H -5.469255 -3.971015 2.642889

O $-4.094613-2.4578312 .552586$

H -3.691140 -4.319845 0.832914

O $-5.570077-4.8514530 .262556$

C $-3.029972-1.160383-1.711831$

H $-3.238521-1.3157850 .387405$

H $-2.355185-2.658674-0.373298$

H -7.695996 -3.572641 2.134668

H $-4.426087-2.0853243 .387406$

H $-6.413350-4.4243020 .033259$

O $-2.008578-0.278154-1.499870$

O $-3.677039-1.269352-2.744252$

C $-1.7050860 .587063-2.598530$

H $-1.3412720 .001772-3.448183$

H $-2.5859251 .170182-2.881472$

$\mathrm{H}-0.9167191 .275351-2.282930$

SCF Energy (B3LYP/6-31G**//MMFF) $=-3245.90275285$

08_00669

MM̄MF Geometry

C $3.277386 \quad 1.1362892 .919054$

C 2.3606440 .1597302 .998717

C $\quad 0.8680350 .3085322 .843667$

O $0.556759 \quad 1.6198142 .313639$

C $0.0950240 .161778 \quad 4.165748$

C $0.165753-1.2039474 .810447$

C $-0.641101-2.2360414 .495828$

C $1.171772-1.3459055 .924013$

C $-1.720684-2.2821153 .447828$

C - $-1.552347-3.5132122 .546217$

C $-2.747468-3.7237181 .604586$

C $-2.562830-5.0093070 .794707$

C -3.705855 -5.224779 -0.205864

O $-2.844699-2.6033140 .720520$ 
O $-3.286624-6.264209-1.102638$

C -3.895946 -3.933411-1.027400

C $-4.971549-5.7406090 .488419$

O $-5.034406-4.042306-1.880015$

C $-3.974729-2.649443-0.166136$

C $-3.909996-1.408098-1.089063$

O $-5.209065-2.6467040 .550120$

C -0.3460381 .6970321 .300918$

O $\quad-0.902043 \quad 0.7683220 .738759$

C $-0.562543 \quad 3.1312391 .003177$

C -1.5104603 .4865440 .123083$

C -1.876207 $4.852294-0.257495$

C $-1.0955225 .984596 \quad 0.356067$

C -3.878440 -0.062312 -0.353939

C $-5.2401160 .578143-0.144352$

C -6.2560882 .6428950 .829610$

O $-6.2853380 .107930-0.583023$

C $-6.2062233 .751330-0.174581$

C -5.9855945 .0355870 .144634$

C $-5.9147426 .146879-0.863787$

C $-4.5900686 .878708-0.828346$

C $-4.4596018 .028617-0.145347$

C $-3.4444856 .329545-1.650804$

C $-2.8997575 .031494-1.118844$

N $-5.134676 \quad 1.7558660 .584082$

H 2.9647712 .1659812 .769096

H $2.708713-0.8580523 .162823$

H $0.541362-0.4647602 .139492$

H $-0.957048 \quad 0.4278593 .994674$

H $0.4372040 .925748 \quad 4.877986$

H $-0.529161-3.1582865 .065180$

H $1.154382-2.3435026 .375876$

H $2.187168-1.1713765 .557906$

H $0.960874-0.6222486 .718423$

H -2.687632 -2.336619 3.962193

H -1.733442 -1.3768632 .833490$

H -0.635209 -3.3923171 .957901$

H - $-1.425318-4.4105103 .165447$

H -3.657074 -3.782481 2.213895

H -2.464911 $-5.878521 \quad 1.457071$

H -1.618049 -4.9525020 .235187$

H -3.970218 -6.354793-1.788811

H -3.028788 -3.851290 -1.696233

H $-4.765072-6.6879091 .001215$

H -5.760922 $-5.962408-0.238240$

H $-5.369728-5.0424191 .227641$

H -5.826976 -3.904946 -1.333638

H $-2.981218-1.471861-1.669523$

H $-4.739502-1.423737-1.805227$

H $-5.383446-1.7464650 .866442$

H 0.0477943 .8544741 .532036

H -2.088171 $2.701675-0.361974$

H -1.182456 5.9650341 .448238

H -1.4371006 .9712240 .035116$

H -0.0361665 .9116990 .085865$

H $-3.377917-0.1515860 .615307$

H -3.301609 $0.642381-0.965920$

H $-6.1623473 .009893 \quad 1.856477$

H -7.1988312 .0948840 .737894$

H $-6.3528963 .478827-1.217223$

H $-5.8493805 .308021 \quad 1.188817$

H $-6.7347586 .844909-0.649985$

H $-6.1001845 .779436-1.880756$

H -3.524846 $8.579219-0.136567$

H $-5.284730 \quad 8.443483 \quad 0.424103$

H $-2.6423837 .069883-1.752811$

H -3.793316 $6.163495-2.678592$

H -3.413942 4.151524 -1.506450

H -4.2126152 .0826480 .858812$

C 5.5194391 .5292391 .864130

O 5.1043120 .9603010 .607998

C $6.2207561 .001034-0.305474$

C 7.4025681 .5811110 .471936

C 7.0249251 .2905661 .911217

H $6.447038-0.035576-0.576951$

C $5.8490301 .809577-1.555703$

H 8.3504421 .1185370 .178801

H 7.5054442 .6606990 .311378
C 7.7662062 .1540582 .916566

H 7.2307910 .2319322 .119007

C $4.6289921 .269989-2.330079$

O $5.5482763 .157426-1.163897$

H $6.7148431 .879089-2.224099$

H $3.7335381 .337665-1.702973$

O $4.4053702 .146323-3.444068$

C $4.794154-0.165289-2.872163$

C $3.638008-0.611473-3.792126$

H $4.897387-0.869927-2.040518$

O $6.007188-0.223420-3.627760$

H $3.4615760 .155156-4.555973$

H $3.956944-1.508442-4.340240$

H 8.8469631 .9987962 .833601

H 7.5658493 .2186792 .755630

H 7.4690171 .9063933 .940204

C 4.7575020 .8888393 .025095

H $4.7933623 .123339-0.551611$

H $4.3087873 .047001-3.089948$

H $\quad 6.137681-1.146917-3.902871$

H 5.1073641 .3089573 .974651

H $4.958784-0.1884633 .048755$

H 5.3010762 .6050671 .821295

C $1.409667-2.688711-1.642234$

O $2.503251-2.218945-2.441360$

C $2.344676-0.917410-3.021076$

C $1.109792-0.884445-3.931386$

C $-0.140994-1.311518-3.157274$

C $0.079529-2.629615-2.412161$

H $1.633909-3.748044-1.459608$

C $1.369133-1.975014-0.287350$

H $2.214742-0.179222-2.225286$

O $1.313264-1.749229-5.045812$

H $0.9841040 .133353-4.319743$

H -0.983556 -1.427709 -3.849775

O $-0.483524-0.302450-2.211638$

H $-0.748827-2.814449-1.719972$

O $0.058115-3.702984-3.358150$

C $2.414678-2.5593920 .633111$

H $1.528444-0.894972-0.345964$

H $\quad 0.399269-2.1252340 .199617$

H $\quad 0.524665-1.688738-5.611510$

H $\quad-0.6577260 .516863-2.705345$

H $\quad 0.832257-3.603548-3.938468$

O $3.645119-2.060590 \quad 0.323596$

O $2.167717-3.3813361 .506506$

C $4.733770-2.5995711 .079589$

H $5.638218-2.0435300 .820314$

H $4.552096-2.4917872 .152899$

H $4.881596-3.6517390 .818961$

SCF Energy (B3LYP/6-31G**//MMFF $)=-3245.92003783$

08_00670

MM̄FF Geometry

C $-2.698776-2.033796-3.439983$

C $-3.832581-2.717097-3.219035$

C $-4.966192-2.311135-2.301092$

O $-4.579771-1.218410-1.436484$

C $-5.344680-3.504806-1.401794$

C $-6.476364-3.209062-0.435501$

C $-6.329058-3.0398710 .894310$

C $-7.848417-3.147179-1.055734$

C $-5.050895-3.0555351 .686818$

C $-4.878446-1.7614032 .494483$

C $-3.467611-1.6455573 .091685$

C $-3.353958-0.3990803 .971269$

C -1.920028 -0.171989 4.473755

O $-2.544724-1.5641482 .000558$

O -1.8429891 .1632124 .997181$

C $-0.952124-0.240873 \quad 3.273162$

C $-1.565240-1.1122475 .631395$

O $0.404059-0.1858783 .709142$

C $-1.165939-1.4823002 .383816$

C $-0.325353-1.4439781 .083178$

O $-0.767574-2.6515523 .108627$

C $-4.8827190 .047671-1.832764$

O $\quad-5.410894 \quad 0.386485-2.879246$

C $-4.4498190 .963795-0.753047$ 
C $-4.505365 \quad 2.291860-0.931864$

C $-4.1508503 .312302 \quad 0.059331$

C -3.651696 $2.841678 \quad 1.400225$

C $-0.668177-0.3094590 .113214$

C 0.1872620 .9360250 .265325

C $0.3899853 .252021-0.643841$

$\begin{array}{lllll}\text { O } & 1.170299 & 0.998672 & 0.997431\end{array}$

C $-0.344124 \quad 4.232327 \quad 0.214241$

C $-0.8910595 .362600-0.258238$

C $-1.576975 \quad 6.3825690 .603088$

C $-2.974694 \quad 6.707807 \quad 0.128501$

C $-3.2155467 .801338-0.614633$

C $-4.104930 \quad 5.8238120 .602361$

C $-4.306213 \quad 4.612682-0.267522$

N $-0.2642691 .961168-0.556138$

H -2.546341 -1.069552 -2.963718

H $-3.967310-3.665008-3.737702$

H -5.831289-2.030490 -2.914129

H $-4.455650-3.849220-0.863046$

H -5.643052 -4.358279-2.026405

H $-7.223780-2.8629031 .490084$

H -8.636318 $-3.000121-0.309231$

H $-7.911529-2.318090-1.767156$

H $-8.068489-4.079620-1.585761$

H -5.076879 -3.9089542 .374955$

H $-4.180803-3.2018101 .043722$

H -5.064083 -0.9022691 .838102$

H -5.627803 -1.728495 3.295114

H -3.258692 -2.549479 3.675955

H $-4.053145-0.4543084 .815031$

H -3.6577890 .4824773 .391486$

H -2.502656 1.2455765 .706978

H - 1.1266140 .6609272 .673578

H $-2.241929-0.9481466 .478822$

H $-0.557398-0.9100326 .010476$

H $-1.628031-2.1684005 .361500$

H $\quad 0.5035250 .618514 \quad 4.246558$

H $0.744433-1.4642221 .321050$

H $-0.519680-2.3828250 .544325$

H $-0.576989-3.3602182 .472010$

H -4.1067710 .5165860 .172080$

H $-4.8615002 .672409-1.888381$

H -4.448546 2.3194331 .940366

H -3.298592 3.6504932 .043341

H -2.802405 $2.160551 \quad 1.281831$

H $-0.497403-0.669760-0.908115$

H $-1.723559-0.0334430 .185943$

H $0.3894373 .544921-1.698421$

H $1.4282473 .167635-0.307954$

H $-0.4017294 .014867 \quad 1.278219$

H $-0.8085205 .589035-1.319003$

H -0.9607907 .2913300 .594583$

H -1.612200 6.0631591 .652071

H -4.221040 8.059673 -0.930021

H -2.411767 $8.458857-0.929008$

H $-5.046846 \quad 6.389614 \quad 0.586770$

H $-3.957730 \quad 5.574421 \quad 1.656921$

H $-4.6710514 .854626-1.266840$

H -1.111322 $1.824357-1.100233$

C $-0.229664-2.298013-3.625116$

O $0.114091-0.900920-3.739470$

C $1.536185-0.786749-3.975733$

C $2.101694-2.184069-3.775175$

C $0.945191-3.047910-4.244212$

H $1.642864-0.483233-5.025020$

C $2.0819530 .304742-3.051208$

H $3.021397-2.351655-4.343223$

H $2.313406-2.372666-2.715035$

C $1.058094-4.497220-3.805009$

H $0.887121-3.002479-5.339934$

C $3.6155440 .354375-2.937523$

O $1.5902441 .557839-3.533775$

H $1.6659150 .175172-2.046862$

H $3.942099-0.581802-2.476353$

O $4.2042010 .396573-4.236326$

C $4.1251151 .544165-2.095695$

C $5.6145991 .427297-1.718255$

H $3.5180211 .632652-1.187618$
O $3.9503762 .753029-2.838382$

H $6.2098111 .201427-2.611382$

H $5.9519142 .411880-1.368058$

H $\quad 0.197019-5.078586-4.148603$

H $1.961680-4.955238-4.220409$

H $1.108892-4.583530-2.714398$

C $-1.576030-2.535435-4.310584$

H $0.6192341 .502562-3.548911$

H $3.920701 \quad 1.227435-4.655184$

H $4.1874263 .493305-2.253955$

H $-1.712142-3.601955-4.520653$

H $-1.612059-1.992040-5.262170$

H $-0.297130-2.527503-2.552975$

C $5.225415-0.0916031 .660996$

$\begin{array}{lllll}\text { O } & 5.187481 & 0.822152 & 0.557254\end{array}$

C $5.8730600 .381640-0.620632$

C $7.367707 \quad 0.209143-0.318013$

C $7.577585-0.7635430 .848801$

C $6.677390-0.4319552 .046589$

H 4.7797580 .4720872 .490809

C $4.324339-1.3034791 .379341$

H $5.460775-0.580581-0.939448$

O 7.9397741 .4749220 .003620

H $7.870849-0.162848-1.218449$

H $8.626326-0.7304601 .168206$

O $7.303124-2.0935900 .417870$

H $6.683835-1.2559572 .768916$

O 7.2133650 .7146332 .716219

C $3.849927-1.9928492 .639965$

H $3.433834-0.9651170 .834952$

H $4.830809-2.0423020 .752497$

H $8.888003 \quad 1.335960 \quad 0.167095$

H $7.906253-2.294817-0.317864$

H 8.1063380 .4836923 .023845

O $3.022104-3.0235532 .294409$

O $4.161105-1.6774183 .779984$

C $2.489332-3.7759893 .388829$

H $1.792560-4.5155322 .985504$

H $3.295399-4.2998143 .911035$

H $1.946048-3.1204294 .076111$

SCF Energy (B3LYP/6-31G**//MMFF) $=-3245.90235779$

08_00671

MMFF Geometry

C $-4.632922 \quad 2.3094890 .633441$

C $-4.4754443 .397949-0.135597$

C $-3.3075113 .702798-1.043344$

O $-2.3054782 .662803-1.012996$

C $-2.617818 \quad 5.021472-0.648249$

C $-1.6892265 .538512-1.733909$

C $-0.3450485 .593579-1.657036$

C $-2.3766606 .059597-2.973631$

C $0.5586595 .205453-0.521705$

C $1.4157073 .984731-0.872336$

C 2.5498453 .7444190 .137406

C $3.7065594 .731512-0.062334$

C 4.9004884 .4304170 .858749

O $3.0216002 .402003-0.058356$

$\begin{array}{llllll}\text { O } & 6.031634 & 5.167147 & 0.366972\end{array}$

C 5.2534242 .9316300 .743335

$\begin{array}{llll}\text { C } 4.651812 & 4.928424 & 2.287440\end{array}$

O 6.2681712 .5714441 .677316

C 4.0297212 .0028390 .882705

C 4.3688830 .5179500 .634631

O 3.5405482 .0839252 .228463

C $-2.4467751 .629533-1.887940$

O $-3.3852221 .421257-2.639653$

C $-1.2197230 .809310-1.797778$

C $-0.991877-0.148170-2.708160$

C $0.218387-0.963888-2.807870$

C $1.301848-0.746381-1.783689$

C $4.9870020 .178555-0.722838$

C $5.179853-1.322961-0.877205$

C $6.093230-3.024773-2.444762$

O $4.811857-2.137034-0.033514$

C $4.986504-3.525933-3.316495$

C $3.994978-4.319070-2.881705$

C $2.883054-4.821720-3.758674$ 
C $1.585894-4.073903-3.521182$

C $0.593051-4.647451-2.820203$

C $1.520864-2.697321-4.153845$

C $0.320660-1.857636-3.813671$

N $5.813769-1.651289-2.067306$

H -3.843802 1.5659850 .697506

H $-5.2949814 .113052-0.187465$

H $-3.7101263 .789906-2.059849$

H -2.078592 4.8762220 .293296

H $-3.3674875 .798915-0.449748$

H $\quad 0.1928475 .994938-2.516300$

H -1.682162 $6.565711-3.653072$

H $-2.8433665 .243776-3.533113$

H -3.149824 $6.786077-2.702577$

H $1.1946016 .071784-0.308663$

H 0.0058305 .0046450 .400022

H $0.7716993 .097497-0.907831$

H $1.8385894 .077349-1.881394$

H 2.1347253 .8268441 .148828

H 3.3774665 .7671610 .079535

H $4.0549394 .671260-1.103229$

H 5.7955556 .1106370 .360307

H $5.6946742 .794021-0.251427$

H 4.4781026 .0113772 .290020

H 5.5305824 .7674072 .921269

H 3.7887294 .4579702 .762613

H 5.8531352 .5029932 .554026

H 5.0208650 .1493351 .438077

H $3.439431-0.0579210 .729492$

H $2.983213 \quad 1.3062752 .397136$

H $-0.5113861 .050714-1.014779$

H - $1.742298-0.327535-3.476818$

H $1.7201570 .261733-1.872950$

H $2.130416-1.453396-1.872106$

H $\quad 0.901938-0.873580-0.772944$

H $4.3415910 .528348-1.535582$

H $5.9686870 .652662-0.824442$

H $\quad 6.211557-3.638184-1.545092$

H $7.039607-3.037645-2.993833$

H $5.005930-3.218677-4.359298$

H $3.969716-4.618489-1.836437$

H $2.757056-5.894843-3.565650$

H $3.152937-4.746891-4.819838$

H $-0.354681-4.153211-2.639685$

H $0.705654-5.643620-2.404458$

H $\quad 1.538897-2.820494-5.244967$

H $2.430947-2.135015-3.916950$

H $-0.514683-1.974532-4.504218$

H $6.019673-0.918873-2.740524$

C $-6.653760 \quad 0.783548 \quad 0.826544$

O $-5.855141-0.4054360 .996114$

C $-5.716790-1.045571-0.284430$

C $-6.933230-0.595378-1.081106$

C $-7.0314210 .858982-0.659564$

H -4.810683 -0.632669-0.746173

C $-5.550027-2.560543-0.131266$

H $-6.798058-0.722007-2.159313$

H -7.839581 -1.133221 -0.781053

C $-8.3949361 .480550-0.908856$

H $-6.2871631 .424491-1.231452$

C $-4.524750-2.9955990 .934018$

O $-6.805471-3.149400 \quad 0.232486$

H -5.290565 -2.995509-1.103449

H $-4.950504-2.8558971 .935769$

O $-4.310441-4.4048690 .785386$

C $-3.162787-2.2821050 .869224$

C $-2.186938-2.8252791 .921977$

H $-3.318125-1.2133281 .033310$

O $-2.589094-2.429972-0.427004$

H -2.686440 -2.897807 2.896750

H - $-1.898661-3.8534511 .662478$

H -8.634900 $1.466355-1.977042$

H $-9.186440 \quad 0.939167-0.379810$

H -8.413093 $2.521672-0.570741$

C $-5.9017932 .002312 \quad 1.383802$

H -7.098552 -2.7293751 .059660$

H -5.179038 -4.8381120 .848506$

H $-2.490662-3.382225-0.599258$
H $-5.626818 \quad 1.8105432 .428113$

H -6.5717402 .8703411 .380818$

H $-7.5513450 .627501 \quad 1.437967$

C $1.187138-2.1829583 .333756$

O $-0.107387-2.7218183 .046608$

C $-0.888563-2.0104962 .075669$

C -1.117039-0.559035 2.532121

C 0.1959300 .1405502 .898630

C $1.077339-0.7234493 .804154$

H $1.556343-2.7830514 .175493$

C $2.160855-2.3754422 .167824$

H $-0.358601-2.0214871 .117637$

O $-1.995363-0.5265173 .656064$

H -1.606785 0.0006781 .728035

H $-0.0251331 .084076 \quad 3.413157$

O $0.915444 \quad 0.451770 \quad 1.711400$

H $2.077455-0.2831283 .889718$

O $0.501141-0.7115625 .113751$

C $2.449220-3.8397711 .942275$

H $1.805586-1.9471171 .227560$

H $3.119779-1.8911902 .387047$

H -1.591691 -1.057778 4.363709

H $\quad 0.372413 \quad 1.068341 \quad 1.191659$

H $1.098955-1.2030725 .702476$

O $1.554996-4.3604971 .054389$

O $3.348491-4.4501562 .505664$

C $1.700656-5.7571560 .780996$

H $2.619627-5.9314950 .213990$

H $1.703452-6.3351971 .710282$

H $\quad 0.846815-6.0761350 .177790$

SCF Energy (B3LYP/6-31G**//MMFF $)=-3245.92530234$

08_00672

MMFF Geometry

C $-3.315311-2.495926-2.462836$

C $-3.436522-1.369778-3.182416$

C $-4.435753-0.255058-2.978820$

O $\quad-3.698110 \quad 0.946482-2.641455$

C $-5.476622-0.472268-1.864537$

C $-6.6412180 .497007-1.944668$

C $-6.8257821 .552924-1.129122$

C $-7.6751770 .172573-2.993925$

C $-5.9266672 .053648-0.034008$

C -6.4992311 .7741951 .363733$

C -6.2969600 .3193311 .815632$

C $-6.9833860 .063543 \quad 3.157846$

C $-6.689614-1.3466393 .692845$

O $-4.888802 \quad 0.1096901 .945629$

O $-7.122831-1.3645915 .061297$

C $-5.163682-1.5838753 .665804$

C $-7.512664-2.4117242 .958548$

O $-4.845221-2.9313534 .006506$

C $-4.507328-1.2206982 .312891$

C $-2.966128-1.2352322 .453838$

O $-4.899689-2.176781 \quad 1.327860$

C $-3.4474751 .842693-3.628136$

O $-3.7138401 .714023-4.815749$

C $-2.8193203 .078726-3.105001$

C $-2.4232073 .254590-1.834171$

C $-1.8338504 .478226-1.279944$

C $-1.6986835 .667221-2.196282$

C $-2.216686-0.688378 \quad 1.228417$

C $-0.747397-0.451607 \quad 1.532542$

C 1.2000440 .8836650 .740639

O $-0.175739-0.9439682 .501951$

C 1.1202252 .2049941 .436511

C 1.5340223 .3567040 .888470

C 1.4231234 .6874861 .572517

C 0.6487205 .6954930 .752097

C 1.2902046 .6627160 .074568

C -0.8632365 .6418860 .790732$

C -1.4460424 .4908940 .013197$

N $-0.147737 \quad 0.3675130 .585386$

H -3.958018 -2.670861-1.606106

H -2.772061 -1.235216 -4.034652

H $-4.961194-0.123708-3.932565$

H $-4.985310-0.398345-0.889080$

H $-5.895619-1.485115-1.920117$ 
H $-7.7241402 .153739-1.268480$ H -8.530473 $0.856004-2.961178$ H $-7.2428270 .237745-3.996862$ H -8.061189 -0.841383 -2.845896 H $-4.9082861 .664593-0.124317$ H $-5.8378183 .140178-0.161620$ H -5.9827832 .4379502 .069647$ H -7.564369 2.0354901 .392623 H $-6.698432-0.3551401 .051845$ H $-8.065530 \quad 0.226927 \quad 3.079637$ H -6.6236440 .7951553 .895416$ H -6.998444 -2.264808 5.406146 H $-4.721015-0.9510094 .447678$ H -8.584673 -2.224072 3.093142 H -7.328865 -3.409392 3.372329 H -7.312997 -2.443701 1.885331 H -5.174473 -3.094165 4.906217 H -2.687334 -0.6192013 .319184$ H -2.614733 -2.255487 2.653007 H -4.210135 -2.2319860 .648597$ H -2.704891 $3.863084-3.847059$ H -2.544616 2.437896-1.124873 H -1.047306 $5.427167-3.044008$ H -1.266534 $6.544325-1.710523$ H -2.679067 $5.971762-2.579244$ H $-2.277109-1.3910880 .391864$ $\begin{array}{llll}\text { H } & -2.655157 & 0.263238 & 0.907907\end{array}$ H $1.6353570 .972945-0.258498$ H $1.803336 \quad 0.1853241 .328731$ H 0.6921862 .2137482 .436192 H $1.9751013 .345463-0.105721$ H 2.4409525 .0531461 .759714 H 0.9573394 .5935142 .561377 H $0.7484997 .414098-0.489838$ H $2.373555 \quad 6.723843 \quad 0.067125$ H $-1.297070 \quad 6.5897240 .450579$ H -1.1902275 .5560681 .835393$ H -1.5773673 .5846050 .604745$ H $-0.7203900 .770259-0.150902$ C $-1.221496-3.688977-1.755590$ O $-0.297546-2.602984-1.976066$ C $1.042962-3.135712-2.039981$ C $0.960382-4.510201-1.400535$ C $-0.400283-4.973777-1.878985$ H $1.282788-3.222783-3.107720$ C $1.997623-2.150891-1.361327$ H $1.771881-5.173202-1.714407$ H $0.977773-4.433664-0.306246$ C -0.959639-6.139311-1.081482 H $-0.319838-5.269065-2.933990$ C $3.437680-2.675166-1.211811$ O $2.004012-0.956231-2.147596$ H $1.608574-1.879299-0.373685$ H $3.414486-3.587813-0.605567$ O $3.948559-3.044396-2.492715$ C $4.403759-1.678859-0.534826$ C $5.791123-2.309518-0.302403$ H $3.983746-1.3691370 .429639$ O $4.565111-0.511519-1.335840$ H $5.665008-3.2798980 .192561$ H $6.268945-2.477946-1.275740$ H -1.950371 -6.424134-1.449552 H $-0.303976-7.012206-1.164942$ H -1.053731 -5.890340 -0.019012 C $-2.347924-3.601683-2.784983$ H $1.083882-0.647638-2.214464$ H $3.991542-2.235366-3.031197$ H $3.699046-0.075061-1.399296$ H -2.915006 -4.539651 -2.807551 H -1.926751 -3.453391 -3.787216 H - $-1.611039-3.576798-0.735806$ C 7.8624370 .7109630 .476623 O $7.057432-0.261762-0.201065$ C $6.712028-1.4222230 .559808$ C $7.986703-2.1675620 .985749$ C $8.951321-1.2428241 .734808$ C $9.1669890 .082126 \quad 0.993239$ H $8.128117 \quad 1.439459-0.300187$
C 7.0697051 .4501671 .560709

H $6.152226-1.1200781 .451810$

O $8.644679-2.716905-0.154636$

H $7.707018-3.0086081 .630956$

H $9.912180-1.7572701 .858434$

O $8.422835-0.9916943 .033591$

H $9.6912610 .803527 \quad 1.630712$

O $10.002979-0.156384-0.143547$

C 5.8892042 .1818980 .967673

H 6.6771520 .7791112 .330115

H 7.7002922 .1805512 .081487

H $8.826588-1.988001-0.772340$

H $9.059985-0.4290303 .505832$

H $10.849330-0.5065020 .183354$

O $6.311282 \quad 3.307180 \quad 0.323896$

O $4.7322671 .792805 \quad 1.061315$

C $5.2830654 .082246-0.299987$

H 4.6359824 .5248640 .462322

H $5.7600904 .886931-0.865879$

H $4.7014953 .465464-0.991977$

SCF Energy (B3LYP/6-31G**//MMFF) $=-3245.91188267$

08_00673

MMFF Geometry

C $-4.930538 \quad 1.3505382 .564843$

C -4.8946082 .3889651 .713628$

C -5.2558642 .3351440 .244551$

O $-5.2769990 .948226-0.174168$

C $-4.2286763 .062936-0.651466$

C $-4.0088164 .528421-0.337406$

C -2.8320235 .0698810 .036974$

C $-5.2234995 .409192-0.488577$

C -1.5025924 .3840220 .197384$

C $-0.3913695 .163158-0.524071$

C $0.9227684 .370379-0.568768$

C $2.0672235 .242487-1.086850$

C $3.3675614 .441161-1.251815$

O $0.7230713 .262652-1.452573$

O $4.2798545 .235623-2.025166$

C $3.0711013 .167514-2.071017$

C 4.0540714 .1800360 .093774

O $4.2133192 .314657-2.119029$

C $1.8428502 .376705-1.563880$

C $1.474071 \quad 1.272247-2.583159$

O $2.1605351 .774386-0.310864$

C $-6.0568900 .580041-1.217453$

O $-6.8067711 .302031-1.858861$

C $-5.889105-0.862420-1.525983$

C $-5.053202-1.672876-0.855796$

C $-4.819499-3.096541-1.116022$

C $-5.571756-3.727889-2.257751$

C $0.1649610 .535176-2.269693$

C $-0.254384-0.396560-3.394099$

C $-2.151717-1.870840-4.072434$

O $0.453795-0.638724-4.367408$

C $-2.112212-3.284391-3.571223$

C -1.457769 -3.757131-2.498597

C $-1.512229-5.192696-2.058149$

C $-2.024906-5.349608-0.642369$

C $-1.194886-5.6850490 .359801$

C $-3.513205-5.196972-0.403847$

C $-3.937739-3.755211-0.333396$

N -1.513009 $-0.935978-3.166084$

H $-5.277495 \quad 0.383173 \quad 2.214742$

H -4.5823603 .3549872 .103168$

H -6.2545062 .7717620 .121879$

H -4.556310 $2.989588-1.697505$

H -3.294873 2.492021 -0.630567

H -2.8124996 .1371430 .256600$

H $-4.9841606 .471461-0.370324$

H $-5.6616285 .282647-1.483984$

H -5.9798885 .1575120 .260961$

H $-1.2656844 .329249 \quad 1.266686$

H $-1.5248443 .357046-0.172847$

H $-0.7146295 .385430-1.549811$

H $-0.2339346 .120641-0.011717$

H $1.146453 \quad 3.9996460 .438622$

H $2.2286766 .104809-0.428092$ 
H $1.7924815 .660992-2.065427$ H $4.4194876 .075090-1.554264$ H $2.8757823 .482910-3.105723$ H 4.2820025 .1272060 .597428 H $5.0175243 .678991-0.048263$ H 3.4487503 .5808110 .776973 H $4.9563612 .843998-2.456132$ H $\quad 1.3717761 .728702-3.576642$ H $2.2835580 .534190-2.645218$ H $1.4798611 .115022-0.105815$ H -6.492643 -1.221105 -2.353477 H $-4.467864-1.262527-0.034530$ H $-6.650584-3.694224-2.069767$ H $-5.312864-4.774369-2.428070$ H -5.363378 -3.201461 -3.195839 H $0.277489-0.073020-1.367566$ H $-0.6470881 .252374-2.105431$ H - -1.682625 -1.816199 -5.059803 H -3.194647 -1.551564 -4.168173 H -2.707369 -3.983969-4.155728 H $-0.845360-3.091705-1.897299$ H $-0.498383-5.604743-2.143459$ H -2.134944 -5.794602 -2.731784 H $-1.558708-5.8237001 .372430$ H $-0.131480-5.8201550 .192058$ H $-3.788016-5.6620920 .552398$ H -4.052585 -5.770203-1.162072 H -3.453083 -3.203077 0.473839 H $-2.001413-0.706575-2.305099$ C $-3.2975790 .517625 \quad 4.318571$ O $\begin{array}{llll}-2.215276 & 0.805387 & 3.408117\end{array}$ C -1.823086 -0.4268802 .769644$ C $-2.232645-1.5170493 .744603$ C $-3.570164-0.9884424 .226263$ H -2.417098 -0.4944121 .848432$ C $-0.329700-0.3726012 .434922$ H -2.310294 -2.501843 3.274741 H - $1.521048-1.5824914 .576923$ C $-4.033030-1.6046435 .535701$ H $-4.321924-1.2068763 .459217$ C $0.234881-1.6953991 .888912$ O -0.1391890 .6638851 .466407$ H $0.226031-0.0644913 .327062$ H $\quad 0.193555-2.4632622 .670290$

O $-0.628615-2.1429050 .834851$

C $1.660973-1.6174431 .310238$

C $2.703895-1.0126892 .265724$

H $1.643508-1.0562560 .371185$

O $2.052060-2.9505460 .959806$

H $2.347060-0.0537492 .654072$

H $2.838640-1.6980163 .111644$

H -4.168147 -2.685585 5.425644

H $-3.306380-1.4373576 .337861$

H -4.988855 -1.173599 5.850290

C $-4.492618 \quad 1.4274334 .003085$ H $-0.664457 \quad 0.4323660 .681516$ H $-0.253052-2.9691700 .485851$ H $2.125086-3.4659661 .781247$ H -4.210006 2.4608164 .240861 H -5.3389301 .1708994 .650174$ H $-2.927586 \quad 0.770705 \quad 5.320131$

C $5.867890-1.9660200 .492085$

O $4.627362-2.0277841 .208565$

C $4.066372-0.7674301 .582324$

C 5.034574-0.006485 2.501726

C 6.4170440 .1360681 .855249

C $6.927750-1.1974851 .299311$

H $6.196657-3.0107530 .415738$

C $5.681964-1.430385-0.932856$

H $3.883314-0.1727790 .681388$

O $5.155441-0.6999253 .742236$

H 4.6135490 .9831432 .716493

H 7.1317330 .5133422 .596918

O $\quad 6.354743 \quad 1.084444 \quad 0.794433$

H $7.815802-1.0395830 .675993$

O $7.324411-2.0351062 .389112$

C $4.849287-2.385171-1.754337$

H $5.215021-0.442084-0.970360$
H $\quad 6.649579-1.330378-1.438851$

H $5.751578-0.1833964 .310730$

H $6.062728 \quad 1.929975 \quad 1.175192$

H $8.032109-1.5738502 .870744$

O $3.541936-2.000658-1.732441$

O $5.304825-3.368355-2.324200$

C $2.633832-2.853176-2.436128$

H $2.806030-2.772875-3.513198$

H $2.737835-3.890110-2.102294$

H $1.618082-2.518815-2.213964$

SCF Energy $(B 3 L Y P / 6-31 G * * / / M M F F)=-3245.90737137$ 